PNNL-18823

WTP-RPT-190, Rev 0

U.S. DEPARTMENT OF ENERGY

Prepared for the U.S. Department of Energy

under Contract DE-AC05-76RL01830

\title{
PEP Run Report for Simulant Shakedown/Functional Testing
}

\author{
GB Josephson \\ EC Golovich \\ JGH Geeting \\ OP Bredt \\ CE Guzman-Leong \\ CA Burns \\ DE Kurath \\ GJ Sevigny
}

December 2009

\section{Pacific Northwest}

NATIONAL LABORATORY

Proudly Operated by Battelle Since 1965 
PNNL-18823

WTP-RPT-190, Rev 0

\section{PEP Run Report for Simulant Shakedown/Functional Testing}

GB Josephson
JGH Geeting
OP Bredt
CA Burns

\author{
EC Golovich \\ CE Guzman-Leong \\ DE Kurath \\ GJ Sevigny
}

December 2009

Test Specification: 24590-PTF-TSP-RT-07-001 Rev 2

Work Authorization: WA\#2007-024

Test Plan: TP-RPP-WTP-506, Rev. 0.4; TP-WTP-PEP-044, Rev 0.2

Test Exceptions: 24590-PTF-TEF-RT-08-00002

$$
\begin{aligned}
& \text { 24590-WTP-TEF-RT-09-00003 } \\
& \text { 24590-PTF-TEF-RT-09-00001 } \\
& \text { 24590-WTP-TEF-RT-09-00002 Rev 0 } \\
& \text { 24590-WTP-TEF-RT-09-00001 Rev } 1
\end{aligned}
$$

R\&T focus area: Pretreatment

Test Scoping Statement(s): NA

Prepared for

the U.S. Department of Energy

under Contract DE-AC05-76RL01830

Pacific Northwest National Laboratory

Richland, Washington 99352 
WTP-RPT-190, Rev 0

Completeness of Testing

This report describes the results of work and testing specified by Test Specification 24590-PTF-TSP-RT-07-001 Rev 2 "Pretreatment Engineering Platform (PEP) Testing (Phase 1)" and Test Plans TP-RPP-WTP-506 Rev 0.4 "Pretreatment Engineering Platform (PEP) Testing (Phase 1) and TP-WTP-PEP-044 Rev 0.2 "Test Plan for the PEP Parallel Laboratory Testing." The work and any associated testing followed the quality assurance requirements outlined in the Test Specification/Plan. The descriptions provided in this test report are an accurate account of both the conduct of the work and the data collected. Test plan results are reported. Also reported are any unusual or anomalous occurrences that are different from expected results. The test results and this report have been reviewed and verified.

Approved:

Mellemathor

Gordon H. Burman

Gordon H. Beeman, Manager WTP R\&T Support Project

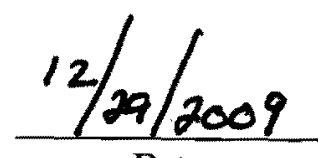

Date 


\section{Contents}

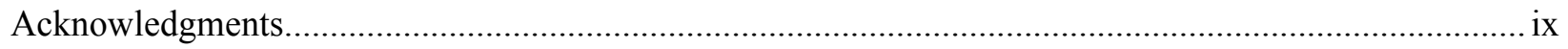

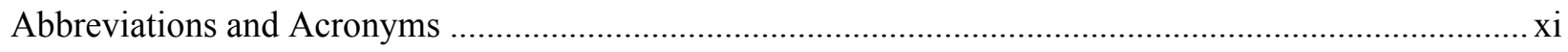

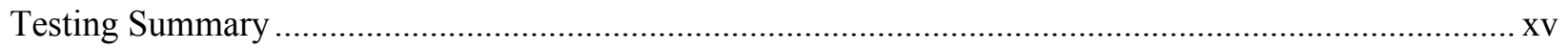

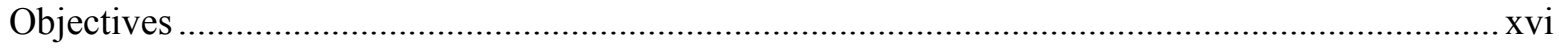

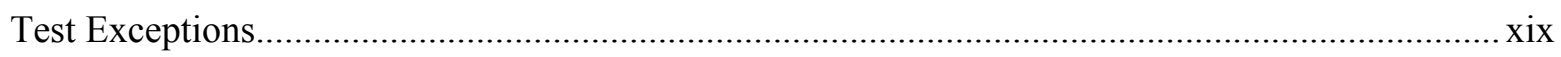

Results and Performance Against Success Criteria .....................................................................xii

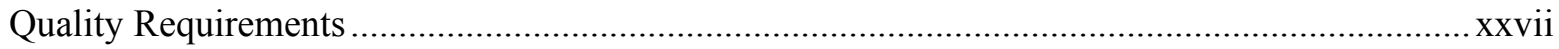

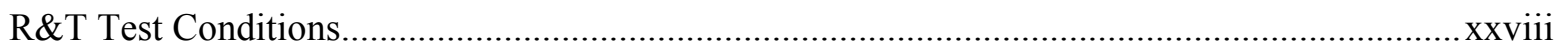

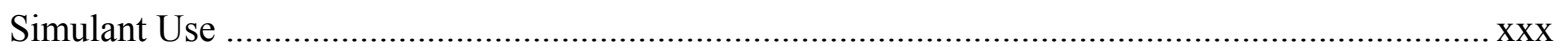

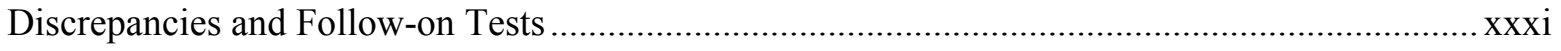

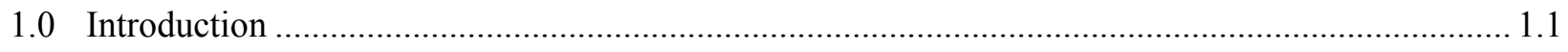

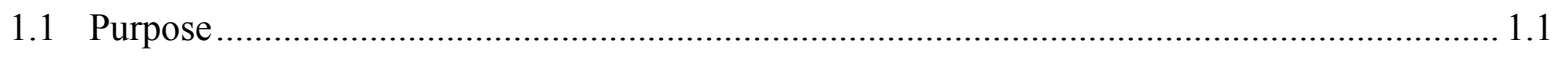

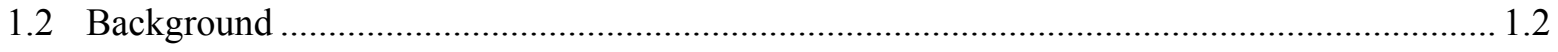

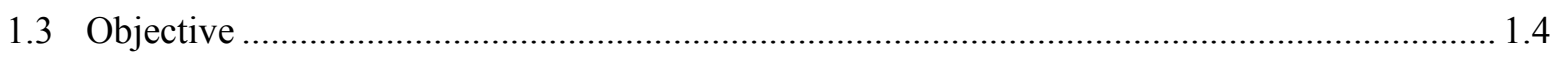

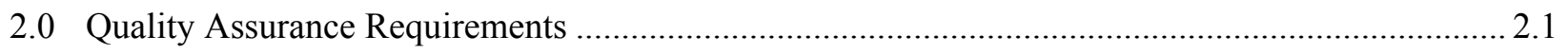

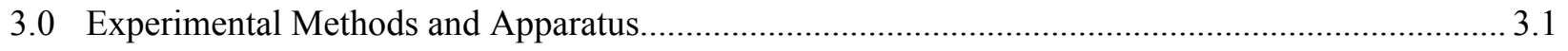

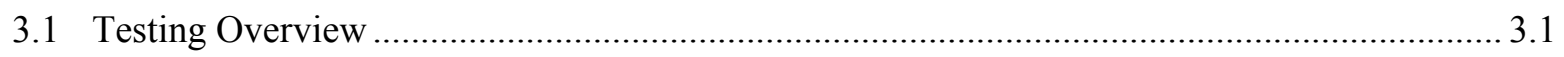

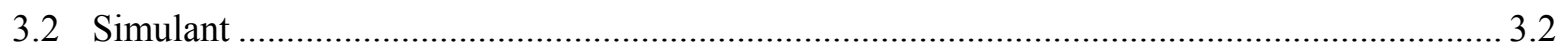

3.3 Pretreatment Engineering Platform Overview …........................................................... 3.3

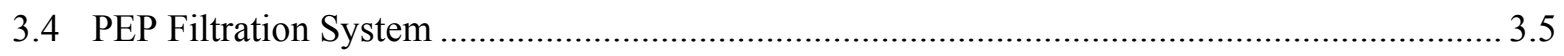

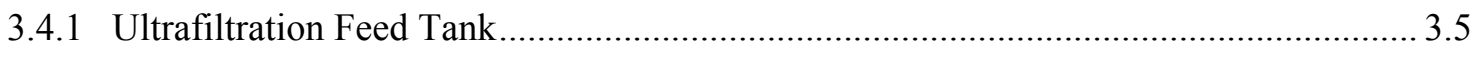

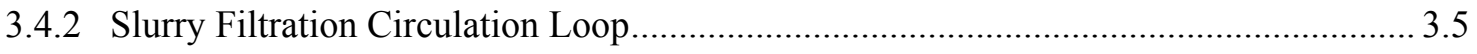

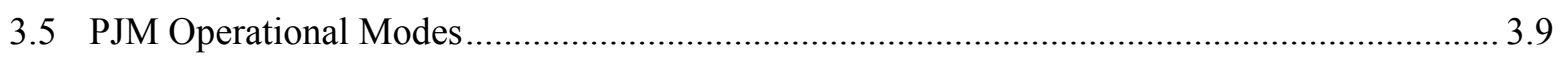

3.5.1 Standard Mode (Regular) Description ................................................................ 3.9

3.5.2 Standard Mode (Short-Cycle) …...................................................................... 3.10

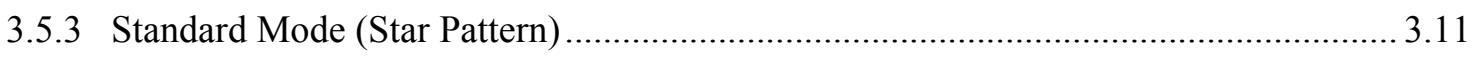

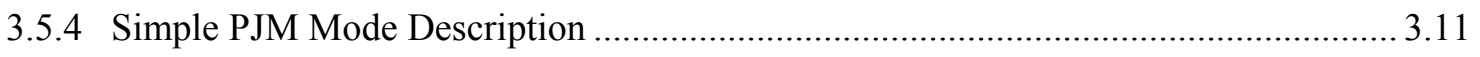

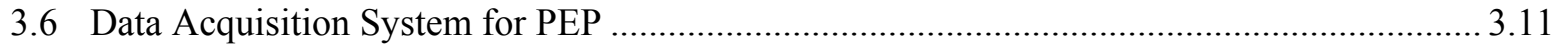

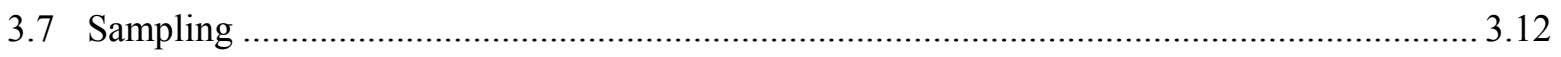

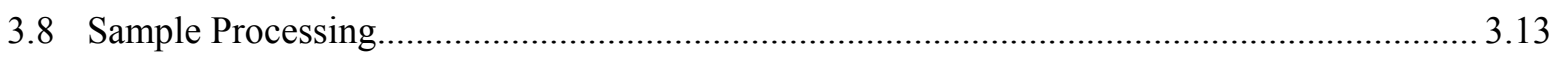

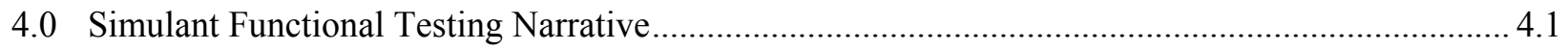

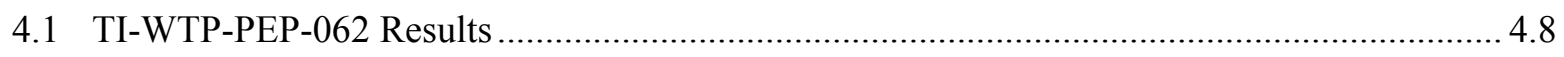

4.1.1 Wash Ultrafilters with Oxalic Acid.................................................................. 4.10

4.1.2 Prepare, Stage and Characterize Simulant in HLP-VSL-T22 and Reagents ................ 4.10

4.1.3 Transfer from UFP-VSL-T01A and -T01B........................................................... 4.11

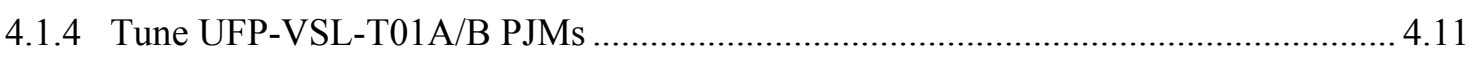




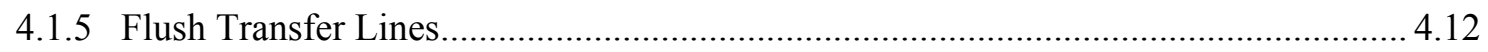

4.1.6 Simulant Transfer from UFP-VSL-T01A to UFP-VSL-T02A …................................ 4.12

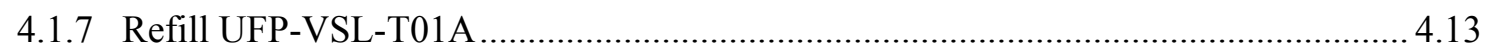

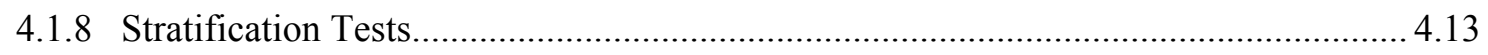

4.1.9 Low-Solids Filter Test (filter conditioning) ............................................................ 4.14

4.1.10 Flush Filter-Loop - (preparation for down time) ...................................................... 4.17

4.1.11 Concentrate Solids to 20-wt\% UDS in UFP-VSL-T02A with Filters \#2 to \#5 ........... 4.17

4.1.12 Add Caustic in UFP-VSL-T02A …............................................................................ 4.19

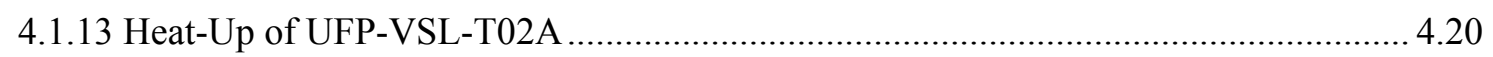

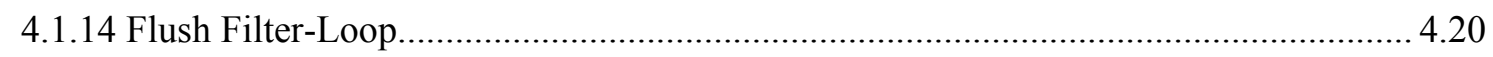

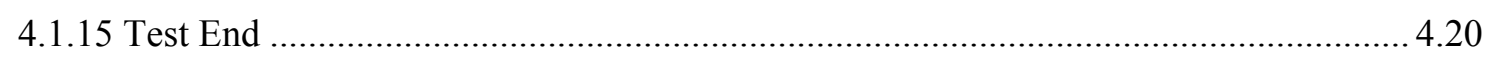

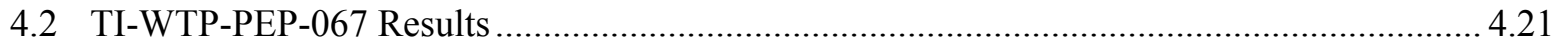

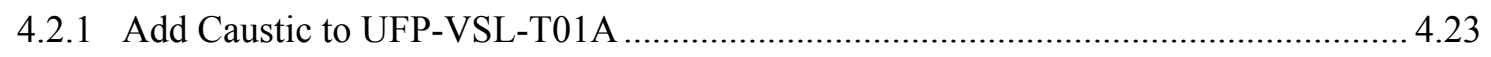

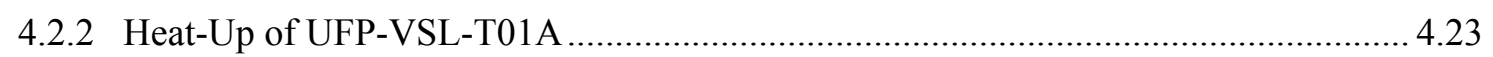

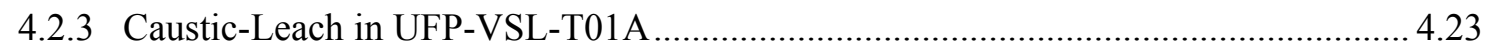

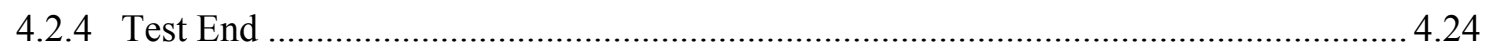

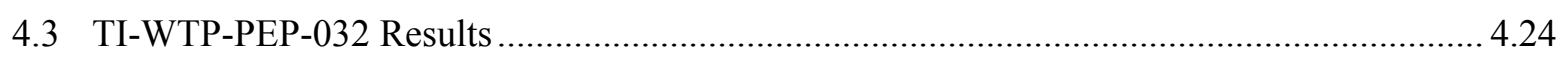

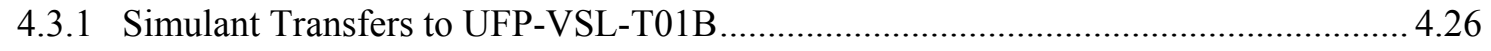

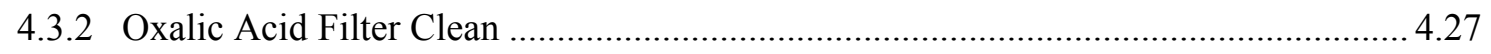

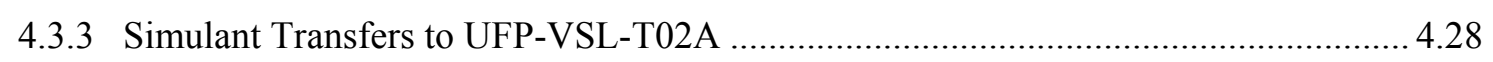

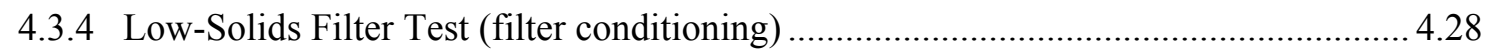

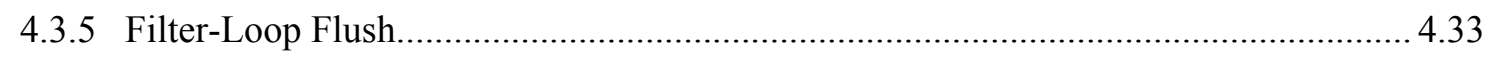

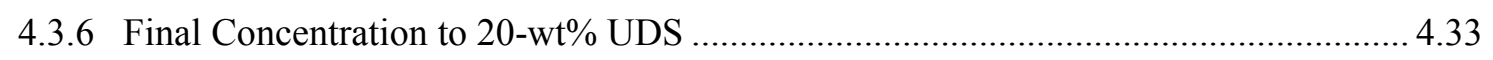

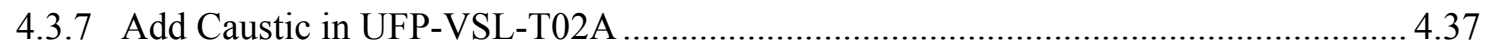

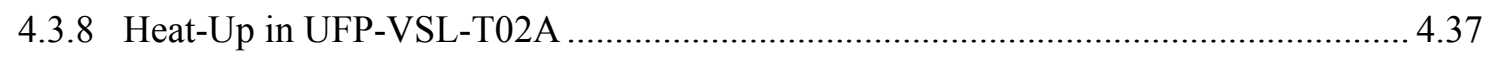

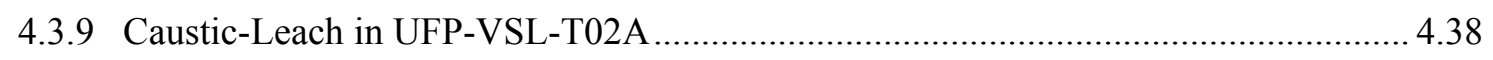

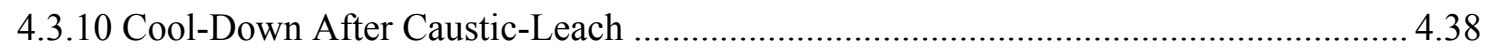

4.3.11 Parallel Processing (upfront leaching) Conducted in T01A........................................ 4.39

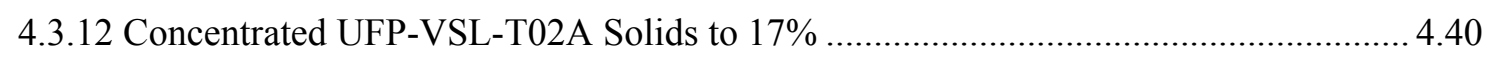

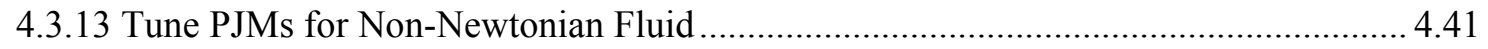

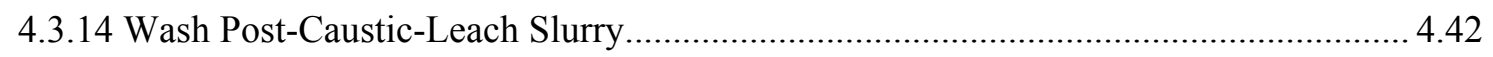

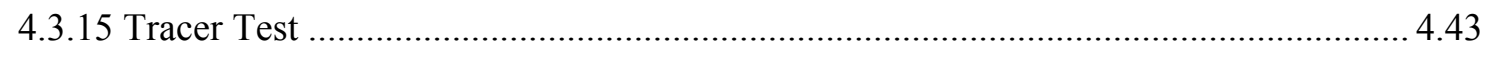

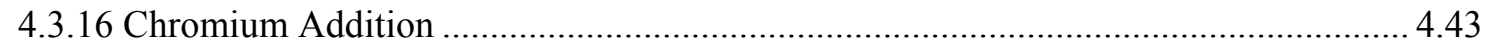

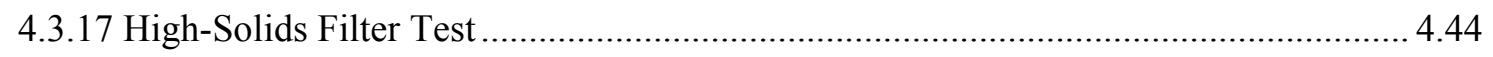

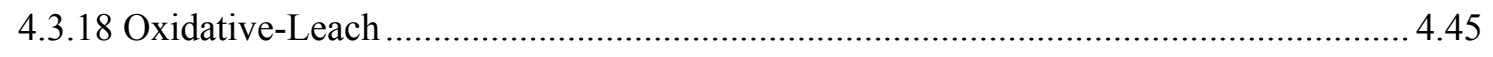

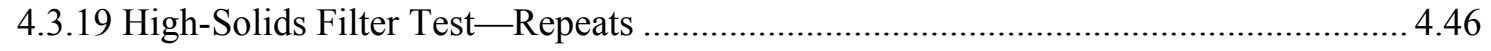

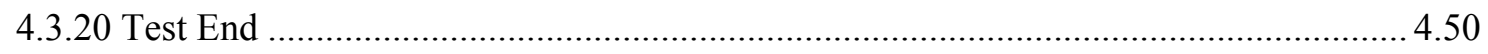

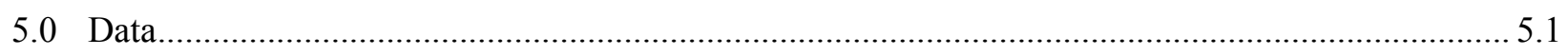

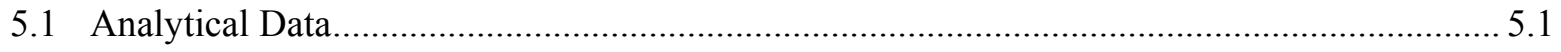




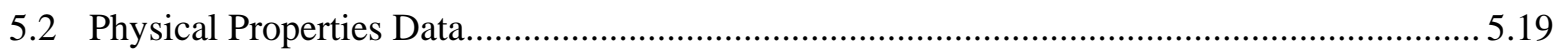

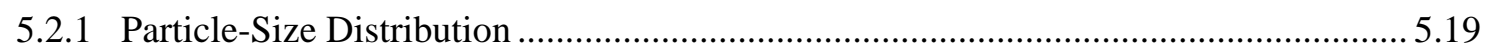

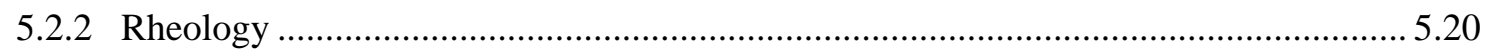

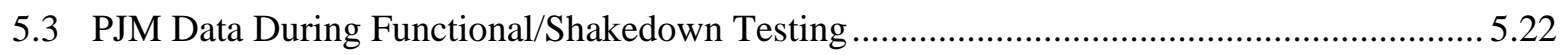

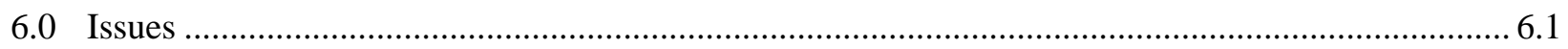

6.1 Maintaining Targeted Flow Rate in Filter-Loop ................................................................ 6.1

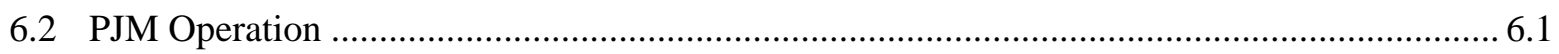

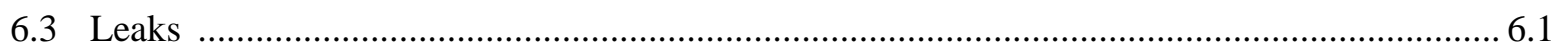

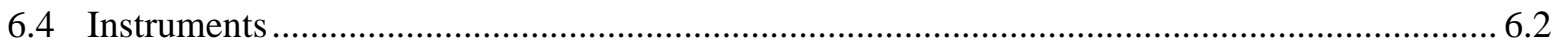

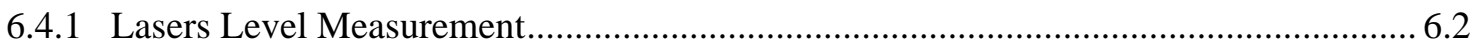

6.4.2 Bubblers Level/Density Measurement ...................................................................... 6.2

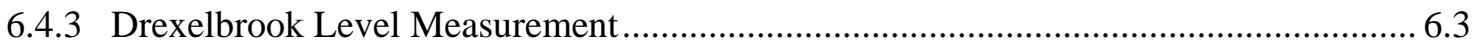

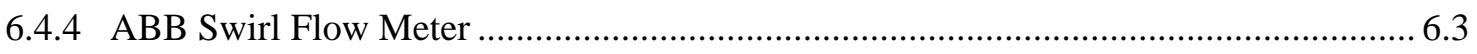

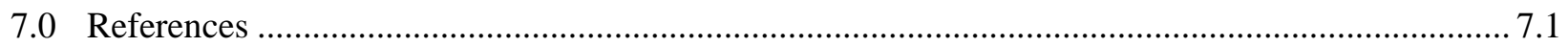

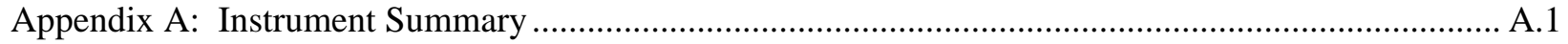

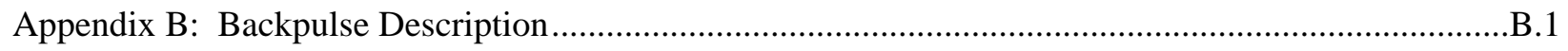

Appendix C: Sample List and Naming Convention............................................................................. 1

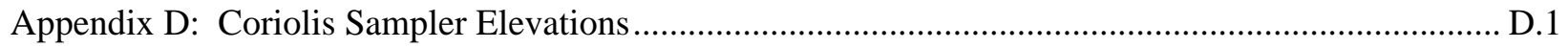

Appendix E: Sample Processing and Methods ...................................................................................

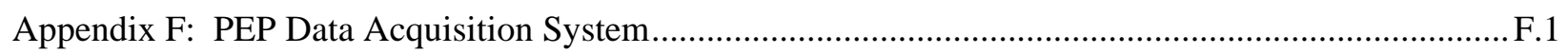

Appendix G: Evaluation of PJM Peak Average Velocity ......................................................................... G.1

Appendix H: Oxalic Acid Cleaning Instructions for the PEP Filters................................................... H.1

Appendix I: T02A Systems Operations Guidance for Simulant Shakedown/Functional Testing ...............1

Appendix J: Shakedown/Functional Data Plots........................................................................................

Appendix K: Shakedown/Functional Summary ................................................................................ K.1

Appendix L: Physical Properties...........................................................................................................

Appendix M: Operational Process Sheets............................................................................................. 


\section{Figures}

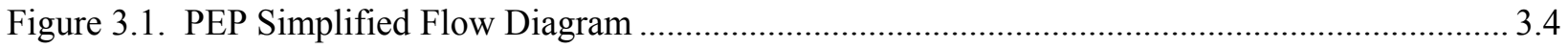

Figure 3.2. Schematic of UFP-VSL-T02A Loop and Filtration ...................................................... 3.6

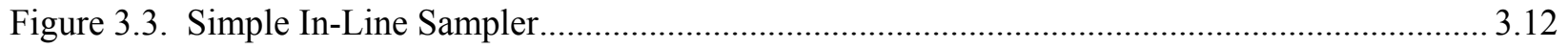

Figure 3.4. In-Tank Sampling Showing the Three Radial Positions at Three Heights and

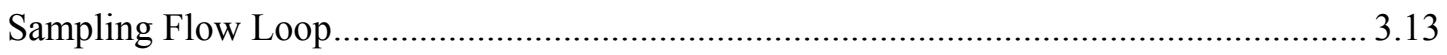

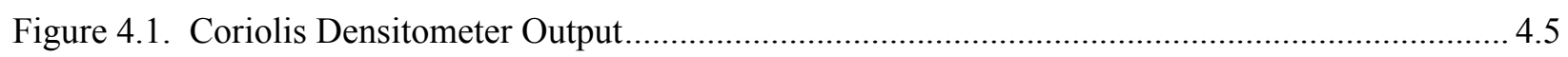

Figure 4.2. Functional Test Filter Conditioning .......................................................................... 4.6

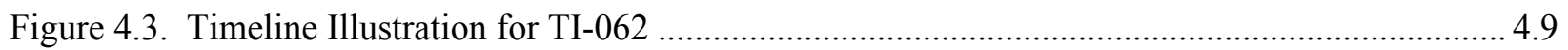

Figure 4.4. Permeate Production Rate During the Initial Functional Test (TI-062) Pre-Leach Concentration of Solids on Filter 1

Figure 4.5. TMP and Temperature Corrected Filter Flux for the Initial Functional Test (TI-062) Pre-Leach Concentration of Solids on Filter 1

Figure 4.6. Permeate Production Rate During the Final Functional Test (TI-062)

Pre-Leach Concentration of Solids on Filters 2-5 ......................................................... 4.18

Figure 4.7. TMP and Temperature Corrected Filter Flux for the Final Functional Test (TI-062)

Pre-Leach Concentration of Solids on Filters 2-5 ....................................................... 4.19

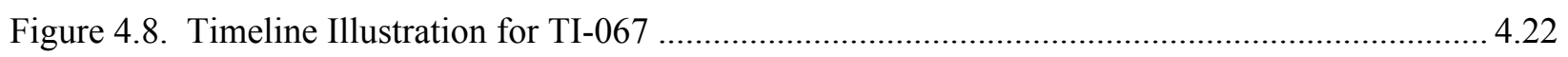

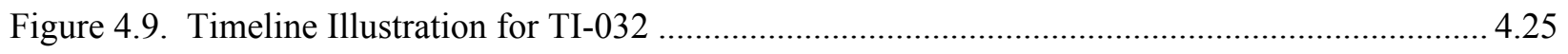

Figure 4.10. Measured and Estimated UDS Concentration Evolution for the Initial Pre-Leach Concentration Operations in Tank T02A (one filter).

Figure 4.11. Permeate Production Rate During the Initial Functional Test (TI-032) Pre-Leach Concentration of Solids on Filter 1

Figure 4.12. TMP and Temperature Corrected Filter Flux for the Initial Functional Test (TI-032)

Pre-Leach Concentration of Solids on Filter 1.

Figure 4.13. Dewatering Curve for the Initial Functional Test (TI-032) Pre-Leach Concentration of Solids on Filter 1

Figure 4.14. Measured and Estimated UDS Concentration Evolution for the Final Pre-Leach Concentration Operations in Tank T02A (filters 2-5).

Figure 4.15. Permeate Production Rate During the Final Functional Test (TI-032) Pre-Leach Concentration of Solids on Filters 2-5

Figure 4.16. TMP and Temperature Corrected Filter Flux for the Final Functional Test (TI-032) Pre-Leach Concentration of Solids on Filters 2-5

Figure 4.17. Dewatering Curve for the Final Functional Test (TI-032) Pre-Leach Concentration of Solids on Filters 2-5.

Figure 4.18. Comparison of Dewatering Curves for Initial and Final Pre-Leach Concentration Solids During TI-032. 
Figure 4.19. Permeate Production Rate During the Functional Test (TI-032) Post-Caustic-Leach Concentration of Solids on Filters 2-5

Figure 4.20. Permeate Production Rate During Functional Test (TI-032) Post-Caustic-Leach Slurry Washing on Filters 2-5

Figure 4.21. Permeate Production Rate During Functional Test (TI-032) Dewatering and Washing Associated with Chromium Addition 4.44

Figure 4.22. Frame from Oxidative-Leach Video-01:40 01/09/2009

Figure 4.23. Permeate Production Rate Evaluation for the Second High-Solids Dewatering Attempt.. 4.47

Figure 4.24. Measured and Estimated UDS Concentration Evolution for High-Solids Dewatering (third attempt) for TI-032

Figure 4.25. Permeate Production Rate During the Third Attempt of the Functional Test (TI-032) High-Solids Dewatering Operation on Filter 1 4.48

Figure 4.26 Dewatering Curve for the Third Attempt of the Functional Test (TI-032) High-Solids Dewatering Operation on Filter 1

Figure 5.1. Simulant Line Flush 5.5 


\section{Tables}

Table S.1. Summary of Shakedown/Functional Test Objectives and Results ........................................xvi

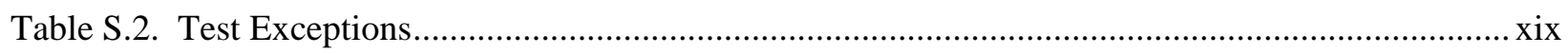

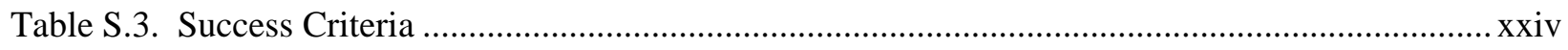

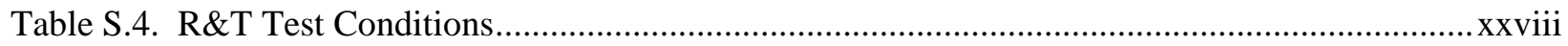

Table 2.1. Description of NCRs Active During Shakedown/Functional Test ...................................... 2.2

Table 3.1. Specifications of the Five PEP Cross-Flow Filtration Bundles …........................................... 3.8

Table 3.2. Permeate Metering and Pulse-Pot Configurations for PEP..................................................... 3.8

Table 4.1. Stratification Data (wt\% UDS) from UFP-VSL-T01A......................................................... 4.14

Table 4.2. Stratification Data (wt\% UDS) from UFP-VSL-T01B .........................................................24

Table 4.3. Functional Testing Detailed Description ............................................................................. 4.51

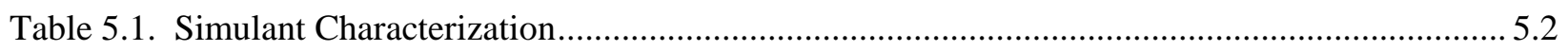

Table 5.2. UDS and Density Analysis of Samples Collected at Multiple Elevations and

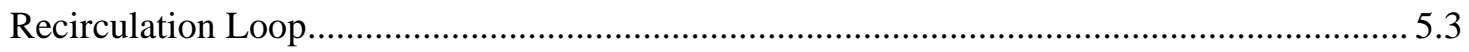

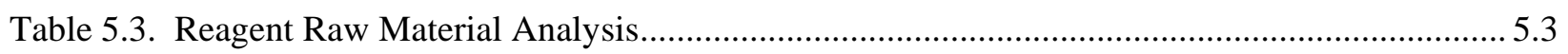

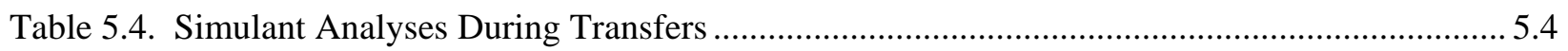

Table 5.5. PEP Flush Line Between HLP-VSL-T22 and Tank T01B .................................................. 5.4

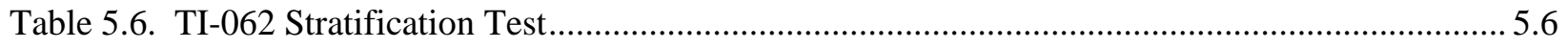

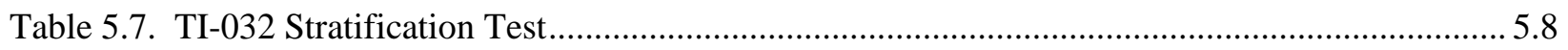

Table 5.8. Low-Solids Filter Test—Establish Baseline Sampling Variation........................................ 5.10

Table 5.9. TI-062 Low-Solids Filter Test, 36-Hr Conditioning and Concentration to 20-wt\%.............. 5.11

Table 5.10. TI-032 Low-Solids Filter Test, 36-Hr Conditioning and Concentration to 20-wt\%............ 5.12

Table 5.11. Pre-Caustic-Leach Simulant Characterization .................................................................. 5.13

Table 5.12. TI-032 Caustic-Leach Analytical Data for Tank T02A ..................................................... 5.15

Table 5.13. TI-032 Analytical Data for Caustic-Leach in Tank T01A ................................................ 5.16

Table 5.14. TI-067 Analytical Data for Caustic-Leach in Tank T01A ................................................. 5.17

Table 5.15. Post-Caustic-Leach Washing Analytical Data for TI-032 ................................................ 5.18

Table 5.16. Reconcentration After Chrome Addition and High-Solids Filter TI-032

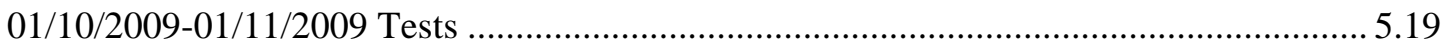

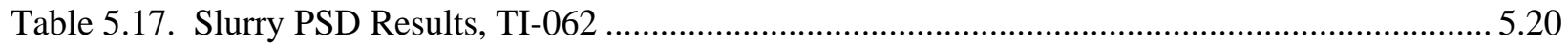

Table 5.18. Slurry Particle-Size Distribution Results, TI-032 ............................................................ 5.20

Table 5.19. Shakedown/Functional Rheology Data Summary, TI-062 ............................................... 5.21

Table 5.20. Shakedown/Functional Rheology Data Summary, TI-032 ............................................... 5.21

Table 5.21 PJM Parameters for Functional Test from TI-WTP-PEP-062 ............................................ 5.23

Table 5.22 PJM Parameters for Functional Test from TI-WTP-PEP-032 ........................................... 5.24 


\section{Acknowledgments}

This report was supported by many dedicated staff involved in laboratory experiments, sampling, data acquisition, and technical reviews. Individuals on the operations and sampling crew are especially recognized for supporting the Pretreatment Engineering Platform Project by working unpredictable, long, and off-hour shifts for the 10 months of PEP operations. The tests and reports could not have gotten this far without their extraordinary effort. The authors gratefully acknowledge the help and support of the following staff:

$\begin{array}{lll}\text { JM Alzheimer } & \text { MS Fountain } & \text { JH Sachs } \\ \text { EJ Antonio } & \text { K Groves } & \text { R Shannon } \\ \text { EBK Baer } & \text { AD Guzman } & \text { SV Shoemaker } \\ \text { R Bang } & \text { SD Halstead } & \text { DS Sklarew } \\ \text { NN Bauman } & \text { GL Harvey } & \text { C Smith } \\ \text { JD Bohlke } & \text { RE Hohimer } & \text { DM Smith } \\ \text { JR Bontha } & \text { DE Hurley } & \text { SA Smith } \\ \text { CF Brown } & \text { S Lehrman } & \text { JJ Soelberg } \\ \text { WC Buchmiller } & \text { ML Kimura } & \text { B Stiver } \\ \text { KJ Cantrell } & \text { SN Kundu } & \text { Y Su } \\ \text { CR Chapman } & \text { JB Lang } & \text { JC Tucker } \\ \text { J Chun } & \text { JS Loveland } & \text { PLJ Valdez } \\ \text { DA Clark } & \text { JR Markillie } & \text { D Vonghalath } \\ \text { E Cordova } & \text { ES Mast } & \text { BE Wells } \\ \text { BP Crume } & \text { K Miller } & \text { A Woodstock } \\ \text { JM Cuta } & \text { DP Mendoza } & \text { S Wright } \\ \text { RL Daubert } & \text { KE Parker } & \text { ST Yokuda } \\ \text { C Dreyer } & \text { RP Pires } & \text { JK Young } \\ \text { EP Dresel } & \text { K Quigley } & \text { L Zhong } \\ \text { BM Dunn } & \text { BJ Riley } & \text { MR Zumhoff } \\ \text { A Edmondson } & \text { CP Rodriguez } & \end{array}$

The authors also thank Wayne Cosby for valuable editorial support, Dave MacPherson and Kirsten Meier for Quality Assurance support throughout the project, Chrissy Charron, Andrea Boehler, and Mona Champion for their administrative support through the months of PEP testing, and Wesley Lawrence, Dean Kurath, James Huckaby, Steve Barnes, John Truax, Donald Bachand, Robert Burk, and Gordon Beeman for their leadership and technical guidance during PEP operations. We also thank Brady Hanson and Michael Minette for the management and coordination of data analysis activities. 



\section{Abbreviations and Acronyms}

\begin{tabular}{|c|c|}
\hline AFA & antifoam agent \\
\hline APEL & Applied Processing Engineering Laboratory \\
\hline ASO & Analytical Support Operations \\
\hline ASR & Analytical Services Request \\
\hline $\mathrm{AV}$ & axial velocity \\
\hline BET & Brunauer, Emmett, and Teller (a method for measuring surface areas) \\
\hline $\mathrm{BNI}$ & Bechtel National Inc. \\
\hline $\mathrm{CD}$ & Coriolis densitometer \\
\hline CFR & Code of Federal Regulations \\
\hline CUF & Cell Unit Filter - cross-flow ultrafiltration testing system \\
\hline DACS & data acquisition collection system \\
\hline DAS & data acquisition system \\
\hline DIW & deionized water \\
\hline DOE & U.S. Department of Energy \\
\hline EDS & Energy Dispersive Spectroscopy \\
\hline EFRT & External Flowsheet Review Team \\
\hline EQL & estimated quantitation limit \\
\hline FEP & Feed Evaporator Product \\
\hline fps & feet per second \\
\hline $\mathrm{ft}$ & feet \\
\hline GEA & gamma energy analysis \\
\hline GPC & Gel permeation chromatography \\
\hline gpm & gallons per minute \\
\hline HDPE & high-density polyethylene \\
\hline HLW & high-level waste \\
\hline IC & ion chromatography \\
\hline ICP & inductively coupled plasma \\
\hline IW & inhibited water \\
\hline JTG & Joint Test Group \\
\hline LAW & low-activity waste \\
\hline LRB & laboratory record book \\
\hline LTE & lead test engineer \\
\hline $\mathrm{m}$ & meter \\
\hline MDL & minimum detection limit \\
\hline
\end{tabular}




\begin{tabular}{|c|c|}
\hline M\&TE & measuring and test equipment \\
\hline $\mathrm{NaOH}$ & sodium hydroxide \\
\hline NMR & nuclear magnetic resonance \\
\hline NPSH & net positive suction head \\
\hline OES & optical emission spectroscopy \\
\hline ORP & Office of River Protection \\
\hline PDMS & polydimethylsiloxane \\
\hline PDL-W & Process Development Laboratory-West \\
\hline PEP & Pretreatment Engineering Platform \\
\hline PJM & pulse jet mixer \\
\hline PNNL & Pacific Northwest National Laboratory \\
\hline PNWD & Battelle_-Pacific Northwest Division \\
\hline PPG & polypropyleneglycol \\
\hline PSD & particle-size distribution \\
\hline psid & pounds per square inch differential \\
\hline psig & pounds per square inch gauge \\
\hline PTF & Pretreatment Facility \\
\hline QA & quality assurance \\
\hline QAM & Quality Assurance Manual \\
\hline QARD & Quality Assurance Requirements and Descriptions \\
\hline RPD & relative percent difference \\
\hline RPL & Radiochemical Processing Laboratory \\
\hline RPP-WTP & River Protection Project-Waste Treatment Plant \\
\hline RSD & relative standard deviation \\
\hline RTD & resistance temperature detector \\
\hline R\&T & Research and Technology \\
\hline SAL & Shielded Analytical Laboratory \\
\hline SBMS & Standards-Based Management System \\
\hline SEM & scanning electron microscopy \\
\hline SwRI & Southwest Research Institute \\
\hline $\mathrm{TC}$ & thermocouple \\
\hline TDS & total dissolved solids \\
\hline TEM & transmission electron microscopy \\
\hline TI & test instruction \\
\hline TIC & total inorganic carbon \\
\hline TMP & transmembrane pressure \\
\hline
\end{tabular}




$\begin{array}{ll}\text { TRU } & \text { transuranic } \\ \text { UDS } & \text { undissolved solids } \\ \text { UFP } & \text { ultrafiltration process } \\ \text { WTP } & \text { Waste Treatment and Immobilization Plant } \\ \text { XPS } & \text { X-ray photo-electron spectroscopy } \\ \text { XRD } & \text { X-ray diffraction }\end{array}$





\section{Testing Summary}

Pacific Northwest National Laboratory (PNNL) has been tasked by Bechtel National Inc. (BNI) on the River Protection Project-Hanford Tank Waste Treatment and Immobilization Plant (RPP-WTP) project to perform research and development activities to resolve technical issues identified for the Pretreatment Facility (PTF). The Pretreatment Engineering Platform (PEP) was designed, constructed, and operated as part of a plan to respond to issue M12, "Undemonstrated Leaching Processes." The PEP is a $1 / 4.5$-scale test platform designed to simulate the WTP pretreatment caustic leaching, oxidative leaching, ultrafiltration solids concentration, and slurry washing processes. ${ }^{\text {(a) }}$ The PEP replicates the WTP leaching processes using prototypic equipment and control strategies. The PEP also includes nonprototypic ancillary equipment to support the core processing.

Two process flowsheets are currently being evaluated for the ultrafiltration process (UFP) and leaching operations. The baseline flowsheet has caustic leaching conducted in the UFP-1 ultrafiltration feed preparation vessels (i.e., vessels UFP-VSL-T01A and B in the PEP; vessels UFP-VSL-00001A and $\mathrm{B}$ [UFP-1] in the WTP PTF). The alternative scenario has caustic leaching performed in the UFP-2 ultrafiltration feed vessels (i.e., vessel UFP-VSL-T02 ${ }^{\text {(b) }}$ in the PEP and vessels UFP-VSL-00002A and B [UFP-2] in the WTP PTF).

With both flowsheets, 19-M sodium hydroxide solution ( $\mathrm{NaOH}$, caustic) is added to the waste slurry to leach solid aluminum compounds (e.g., gibbsite, boehmite). Caustic addition is followed by a heating step that uses direct injection of steam to accelerate the leaching process. Following the caustic-leach, the vessel contents are cooled using vessel cooling jackets and/or external heat exchangers. The main difference between the two scenarios is that for leaching in UFP-1, the $19-\mathrm{M} \mathrm{NaOH}$ is added to unconcentrated waste slurry (3- to 8-wt\% solids), while for leaching in UFP-2, the slurry is concentrated to nominally 20 -wt $\%$ solids using cross-flow ultrafiltration before adding caustic. After cooling, the leached slurry is concentrated and washed with an aqueous solution of $0.01-\mathrm{M} \mathrm{NaOH}$ (referred to as inhibited water) to remove soluble salts. If the resulting waste solids remain high in chromium, sodium permanganate reagent is added, and the slurry is circulated to oxidize and dissolve the chromium solids. Following the oxidative leaching of chromium-containing solids, the slurry is washed to remove the dissolved chromium and concentrated.

The PEP testing program was conducted using a waste simulant, which was developed in response to Task 5 from the M-12 External Flowsheet Review Team (EFRT) issue response plan. ${ }^{\left({ }^{c}\right)}$ The testing included the following tests with simulated Hanford tank waste:

(a) The scale of $1 / 4.5$ was chosen because this scale enables the ultrafiltration loop to be configured to meet two important criteria: 1) using one filter bundle, the ratio of solids in the feed tank to filter surface area will be the same as in the plant, and 2) using five filter bundles, the type and extent of mixing in the feed vessel will be approximately prototypic during the solids washing processes.

(b) In this report, the UFP vessels are generally denoted as Tank T01A/B and Tank T02A. In some cases, alternative designations are used to maintain continuity with previous documentation (e.g., Test Specification/Plan).

(c) SM Barnes, and R Voke. 2006. "Issue Response Plan for Implementation of External Flowsheet Review Team (EFRT) Recommendations - M12: Undemonstrated Leaching Process.” 24590-WTP-PL-ENG-06-0024 Rev. 0, Bechtel National, Inc., Richland, Washington. 
- Shakedown/Functional testing: Tested process operations (e.g., slurry transfers, steam heating of the vessels and the accumulation of condensate, filter backpulsing and flushing), process controls (e.g., transmembrane pressure [TMP] and axial flow velocity in the filter-loop), and certain test functions (e.g., in-line slurry sampling accuracy and precision).

- Integrated Test A: Demonstrated integrated processing when caustic leaching $\left(98^{\circ} \mathrm{C}\right)$ is performed in UFP-VSL-00001A/B with the Cr simulant component added after the post-caustic-leach washing step.

- Integrated Test B: Demonstrated integrated processing when the caustic leaching $\left(98^{\circ} \mathrm{C}\right)$ is performed in UFP-VSL-00002A with the Cr simulant component added after the post-caustic-leach washing step.

- Integrated Test D: Demonstrated integrated processing when the caustic leaching is performed at a lower temperature $\left(85^{\circ} \mathrm{C}\right)$ in UFP-VSL-00002A and with the $\mathrm{Cr}$ simulant component added to the initial batch of simulant.

- Integrated Test $\mathrm{C}$ was deleted from the scope of Phase I testing (ICN-TP-RPP-WTP-506_R0.2).

The work described in this report includes a narrative of the Shakedown/Functional Test and the data obtained during the test.

\section{Objectives}

Table S.1 summarizes the objectives and results of the Shakedown/Functional testing along with a discussion of how the objectives were met. Only objectives relevant to the Shakedown/Functional testing are presented.

Table S.1. Summary of Shakedown/Functional Test Objectives and Results

\begin{tabular}{|c|c|c|}
\hline Test Objective & $\begin{array}{c}\text { Objective } \\
\text { Met? }\end{array}$ & Discussion \\
\hline $\begin{array}{l}\text { Demonstrate that the PEP steam vessel } \\
\text { heating control strategy heats the caustic } \\
\text { leaching vessels to the required setpoint in } \\
\text { the specified heat-up time, controls the } \\
\text { vessel at the leaching temperature for the } \\
\text { duration of caustic leaching, and confirms } \\
\text { that the resulting accumulation of } \\
\text { condensate is approximately prototypic. } \\
\text { Due to scaling issues associated with heat } \\
\text { loss to the surroundings and the evaporation } \\
\text { of water (i.e., into the vessel headspace, air } \\
\text { sparge mixing bubbles, and steam ring air } \\
\text { purge), the PEP vessels will not } \\
\text { automatically mimic the thermal behavior } \\
\text { of the PTF. Prototypic thermal behavior } \\
\text { will be achieved in the PEP with } \\
\text { nonprototypic controls that need to be } \\
\text { tested before integrated process testing }\end{array}$ & $\mathrm{Y}$ & $\begin{array}{l}\text { Results of thermal modeling indicated a target amount of } \\
\text { condensate accumulation (viz. dilution) that WTP will } \\
\text { accumulate during the leaching time. With a higher } \\
\text { surface area-to-volume ratio, PEP had greater relative } \\
\text { heat losses than the plant and would have accumulated } \\
\text { more condensate, resulting in more dilution. } \\
\text { Through preheating without adding direct steam, the } \\
\text { Functional testing showed that caustic leaching could be } \\
\text { conducted in PEP and could obtain a total condensate } \\
\text { accumulation that was volumetrically proportional to the } \\
\text { WTP model. That is, both the PEP test processes had } \\
\text { approximately the same total dilution as that expected in } \\
\text { the WTP. } \\
\text { Analysis of condensate accumulation is presented in } \\
\text { WTP-RPT-186 and WTP-RPT-197. }\end{array}$ \\
\hline
\end{tabular}


Table S.1. Summary of Shakedown/Functional Test Objectives and Results

\begin{tabular}{|c|c|c|}
\hline Test Objective & $\begin{array}{l}\text { Objective } \\
\text { Met? }\end{array}$ & Discussion \\
\hline $\begin{array}{l}\text { Demonstrate that the PEP vessel cool-down } \\
\text { control strategy achieves calculated } \\
\text { prototypic vessel temperatures when caustic } \\
\text { leaching is conducted in the PEP } \\
\text { UFP-VSL-T02A vessel. As discussed } \\
\text { above, prototypic thermal behavior in the } \\
\text { PEP will require nonprototypic controls, } \\
\text { and the controls to cool down } \\
\text { UFP-VSL-T02A need to be tested before } \\
\text { integrated process testing. }\end{array}$ & $\mathrm{Y}$ & $\begin{array}{l}\text { Early testing showed that ambient cooling of PEP without } \\
\text { any cooling water was sufficient to meet the initial } \\
\text { cooling curve predicted for WTP with their maximum } \\
\text { cooling water flow. After the vessel cooled somewhat, } \\
\text { then a small amount of cooling water was needed, but the } \\
\text { thermal mass of the system made system response very } \\
\text { slow. Rather than try to tune the system PID controller to } \\
\text { control the unresponsive system, it was decided to use } \\
\text { manual control with operators making } 1 \text { to } 5 \% \text { changes in } \\
\text { the manual valve position based upon periodic ( } \sim 15-m i n) \\
\text { readings of the system temperature. } \\
\text { Cooling curves are presented in WTP-RPT-186 and } \\
\text { WTP-RPT-197. }\end{array}$ \\
\hline $\begin{array}{l}\text { Demonstrate that the PEP slurry cool-down } \\
\text { strategy achieves prototypic thermal } \\
\text { quenching of the caustic-leach when caustic } \\
\text { leaching is conducted in the PEP } \\
\text { UFP-VSL-T01A/B. As discussed above, } \\
\text { prototypic thermal behavior in the PEP will } \\
\text { require nonprototypic controls. In addition } \\
\text { to general thermal scaling difficulties, the } \\
\text { PEP UFP-VSL-T01A/B vessels do not have } \\
\text { water jackets like the plant, so an additional } \\
\text { (nonprototypic) process step has been } \\
\text { introduced to achieve prototypic cooling of } \\
\text { the vessel contents using an external heat } \\
\text { exchanger. The strategy and controls used } \\
\text { to cool down UFP-VSL-T01A need to be } \\
\text { tested before integrated process testing. }\end{array}$ & $\mathrm{Y}$ & $\begin{array}{l}\text { UFP-VSL-T01A/B had external cooling heat exchangers. } \\
\text { When the leach was complete, flow was initiated through } \\
\text { the heat exchangers, and the cooling water flow was } \\
\text { controlled manually in the same manner that the cooling } \\
\text { water flow was controlled through the cooling jacket on } \\
\text { UFP-VSL-T02A, described above. } \\
\text { Cooling curves are presented in WTP-RPT-186 and } \\
\text { WTP-RPT-197. }\end{array}$ \\
\hline $\begin{array}{l}\text { Condition the five filter bundles using PEP } \\
\text { Phase I simulant and collect filter } \\
\text { conditioning data for comparison to } \\
\text { analogous Cell Unit Filter (CUF) } \\
\text { conditioning (low-solids filter testing). }\end{array}$ & $\mathrm{Y}$ & $\begin{array}{l}\text { Filter conditioning was conducted twice during } \\
\text { Functional process testing. Each conditioning consisted } \\
\text { of } 36 \text { hours of filtration with total permeate recycle. } \\
\text { Between hours } 12 \text { and } 24 \text {, each of the five filters was } \\
\text { backpulsed every } 30 \text { minutes. The backpulsing of five } \\
\text { filters demonstrated a relationship between filter position } \\
\text { and filter flux that could not be observed in the CUF. In } \\
\text { short, extended backpulsing had little or no impact on the } \\
\text { long-term flux for filter \#1. However, the flux from } \\
\text { filters \#2 to \#5 was progressively worse after } 12 \text { hours of } \\
\text { backpulsing. } \\
\text { Analyses of the low-solids filter conditioning tests are } \\
\text { presented in WTP-RPT-185 and WTP-RPT-197. }\end{array}$ \\
\hline $\begin{array}{l}\text { Collect data for the comparison of CUF and } \\
\text { PEP filter performance under conditions }\end{array}$ & $\mathrm{N}$ & $\begin{array}{l}\text { During the Functional testing, the high-solids filter test } \\
\text { could not be completed to the solids target desired. }\end{array}$ \\
\hline
\end{tabular}


Table S.1. Summary of Shakedown/Functional Test Objectives and Results

\begin{tabular}{|c|c|c|}
\hline Test Objective & $\begin{array}{l}\text { Objective } \\
\text { Met? }\end{array}$ & Discussion \\
\hline $\begin{array}{l}\text { that produce filter cake on the cross-flow } \\
\text { filters (high-solids filter testing). }\end{array}$ & & $\begin{array}{l}\text { Conditions for the high-solids filter test were adjusted, } \\
\text { and the test was completed during integrated process } \\
\text { testing. Results of the high-solids filter testing are } \\
\text { presented in WTP-RPT-185 and WTP-RPT-197. }\end{array}$ \\
\hline $\begin{array}{l}\text { Demonstrate the PEP filter-loop flush } \\
\text { strategy. The PEP was not designed to } \\
\text { have equipment to prototypically mimic the } \\
\text { filter-loop flush because this operation was } \\
\text { tested with a full-scale test platform. } \\
\text { However, during Integrated testing, it is } \\
\text { important to flush the filter-loop to avoid } \\
\text { plugging the ultrafilter tubes when not } \\
\text { circulating and to recover the slurry } \\
\text { contents without introducing excessive } \\
\text { nonprototypic flush water to } \\
\text { UFP-VSL-T02A. This will be achieved by } \\
\text { a nonprototypic filter-loop flush in the PEP, } \\
\text { and the adequacy of this step needs to be } \\
\text { understood before Integrated testing. }\end{array}$ & $\mathrm{Y}$ & $\begin{array}{l}\text { It was determined that solids recovery was not important } \\
\text { for the scaled tests in UFP-VSL-T02A. Therefore, } \\
\text { Integrated testing was conducted using a minimal flush so } \\
\text { as not to introduce extra water. Then the loop was } \\
\text { cleaned out in a nonprototypic manner. No evidence of } \\
\text { tube plugging was observed using only a minimal flush. }\end{array}$ \\
\hline $\begin{array}{l}\text { Demonstrate that the PEP equipment, } \\
\text { controls, and procedures are adequate to } \\
\text { conduct Integrated process testing. This } \\
\text { objective addresses the need to verify that } \\
\text { all system components are adequate to } \\
\text { complete the Integrated process testing with } \\
\text { the Phase I PEP simulant. }\end{array}$ & $\mathrm{Y}$ & $\begin{array}{l}\text { Recognizing the importance of PEP testing, PNNL } \\
\text { management conducted a management review to } \\
\text { determine whether PEP equipment, controls, and } \\
\text { procedures were adequate to safely accomplish the } \\
\text { defined testing. The results of the review were published } \\
\text { as PNNL-18046 "Pretreatment Engineering Platform } \\
\text { Startup Management Assessment" and led to PNNL } \\
\text { management authorization that testing could proceed. } \\
\text { Proceeding to Integrated testing also required Joint Test } \\
\text { Group authorization. } \\
\text { During simulant Shakedown/Functional testing, several } \\
\text { changes were made to equipment, instrumentation, and } \\
\text { operating procedures to prepare for Integrated testing, } \\
\text { including: } \\
\text { - Installation of liquid drains on PJM vent lines to } \\
\text { mitigate condensate accumulation above } 65^{\circ} \mathrm{C} \text {. } \\
\text { - Control of reagent additions and vessel cooling using } \\
\text { manual adjustment of the control valve rather than } \\
\text { "automatic" control. } \\
\text { - Modification of laser level output signal filtering to the } \\
\text { - Rata acquisition system (DAS) and control system. } \\
\text { - Redesign of the laser mounting system, air cooling. } \\
\text { probes. }\end{array}$ \\
\hline
\end{tabular}


Table S.1. Summary of Shakedown/Functional Test Objectives and Results

\begin{tabular}{|c|c|c|}
\hline Test Objective & $\begin{array}{c}\text { Objective } \\
\text { Met? }\end{array}$ & Discussion \\
\hline & & $\begin{array}{l}\text { tank density monitoring. } \\
\text { - Issue of multiple operating procedures to execute } \\
\text { testing steps. }\end{array}$ \\
\hline $\begin{array}{l}\text { Evaluate stratification of solids in } \\
\text { UFP-VSL-T01A/B and UFP-VSL-T02A } \\
\text { under prototypic mixing conditions. If } \\
\text { mixing in these vessels does not result in an } \\
\text { approximately homogeneous distribution of } \\
\text { solids (i.e., solids settling is negligible), } \\
\text { then sample collection and analyses during } \\
\text { Integrated process testing may need to be } \\
\text { increased to properly characterize the vessel } \\
\text { contents. }\end{array}$ & $\mathrm{Y}$ & $\begin{array}{l}\text { Samples were taken in quadruplicate at three evenly } \\
\text { spaced times during a 36-hr stratification test. Results of } \\
\text { solids measurements (\% undissolved solids [UDS]) } \\
\text { indicated there was no statistical difference in mean wt\% } \\
\text { UDS between locations at any one time, nor is there a } \\
\text { difference between locations over time. } \\
\text { The results of the stratification tests indicated that solids } \\
\text { settling was negligible, and no changes in sampling } \\
\text { strategy were necessary. Data and statistics are presented } \\
\text { in WTP-RPT-197. }\end{array}$ \\
\hline $\begin{array}{l}\text { Evaluate the precision of filter-loop in-line } \\
\text { slurry samples and their agreement with } \\
\text { in-tank samples. In-line slurry sampling } \\
\text { precision and accuracy are needed to } \\
\text { calculate test result uncertainties, and the } \\
\text { number of samples collected may need to } \\
\text { be increased if sample precision is poor. }\end{array}$ & $\mathrm{Y}$ & $\begin{array}{l}\text { During the low-solids filter test, } 30 \text { samples were taken } \\
\text { simultaneously from the in-line sampling location and } \\
\text { from the UFP-VSL-T02A middle/middle location. } \\
\text { Variance between the samples led to the conclusion that } \\
\text { the in-tank sampling location was less variable and hence } \\
\text { a better location to sample for fluid characteristics during } \\
\text { subsequent tests. Sample analyses are in the sample } \\
\text { analytical table, and statistics are presented in } \\
\text { WTP-RPT-197. }\end{array}$ \\
\hline $\begin{array}{l}\text { Estimate variance of the combined sample } \\
\text { acquisition, handling, and analysis for } \\
\text { critical data, including wt } \% \text { UDS and } \\
\text { chemical analyses. }\end{array}$ & $\mathrm{Y}$ & $\begin{array}{l}\text { Variance for sample collection and } \mathrm{wt} \% \text { UDS analysis are } \\
\text { derived from the sampling described immediately above. } \\
\text { Estimates of the variance for each chemical analysis } \\
\text { method are presented in WTP-RPT-197. }\end{array}$ \\
\hline
\end{tabular}

\section{Test Exceptions}

A summary description of the Test Exceptions applied to these tests is shown in Table S.2.

Table S.2. Test Exceptions

\begin{tabular}{||l|l||}
\hline \multicolumn{1}{|c|}{ Test Exceptions } & \multicolumn{1}{c|}{ Description of Test Exceptions } \\
\hline 1) 24590-PTF-TEF-RT-08- \\
ICN1 to Test Plan \\
TP-RPP-WTP-506.
\end{tabular}$\quad \begin{aligned} & \text { This Test Exception: } \\
& \text { 1. } \begin{array}{l}\text { Added a stage during the filter conditioning section of the } \\
\text { Shakedown/Functional Test where the simulant slurry is concentrated from } \\
\text { approximately 5-wt\% solids to 20-wt\% solids in one operation. This is in } \\
\text { addition to the previously specified low-solids filter and high-solids filter } \\
\text { testing. }\end{array}\end{aligned}$


Table S.2. Test Exceptions

\begin{tabular}{|c|c|}
\hline Test Exceptions & Description of Test Exceptions \\
\hline & $\begin{array}{l}\text { 4. The sampling specified in the low-solids filtration test over-specifies the } \\
\text { sample collection timing required. The technical requirement is to get } 30 \\
\text { unique samples. The sampling schedule specified is not required to achieve } \\
\text { this test objective. }\end{array}$ \\
\hline $\begin{array}{l}\text { 2) } 24590-\text { PTF-TEF-RT-09- } \\
\text { 00001 incorporated into } \\
\text { ICN-2 and ICN-3 to Test } \\
\text { Plan TP-RPP-WTP-506. }\end{array}$ & 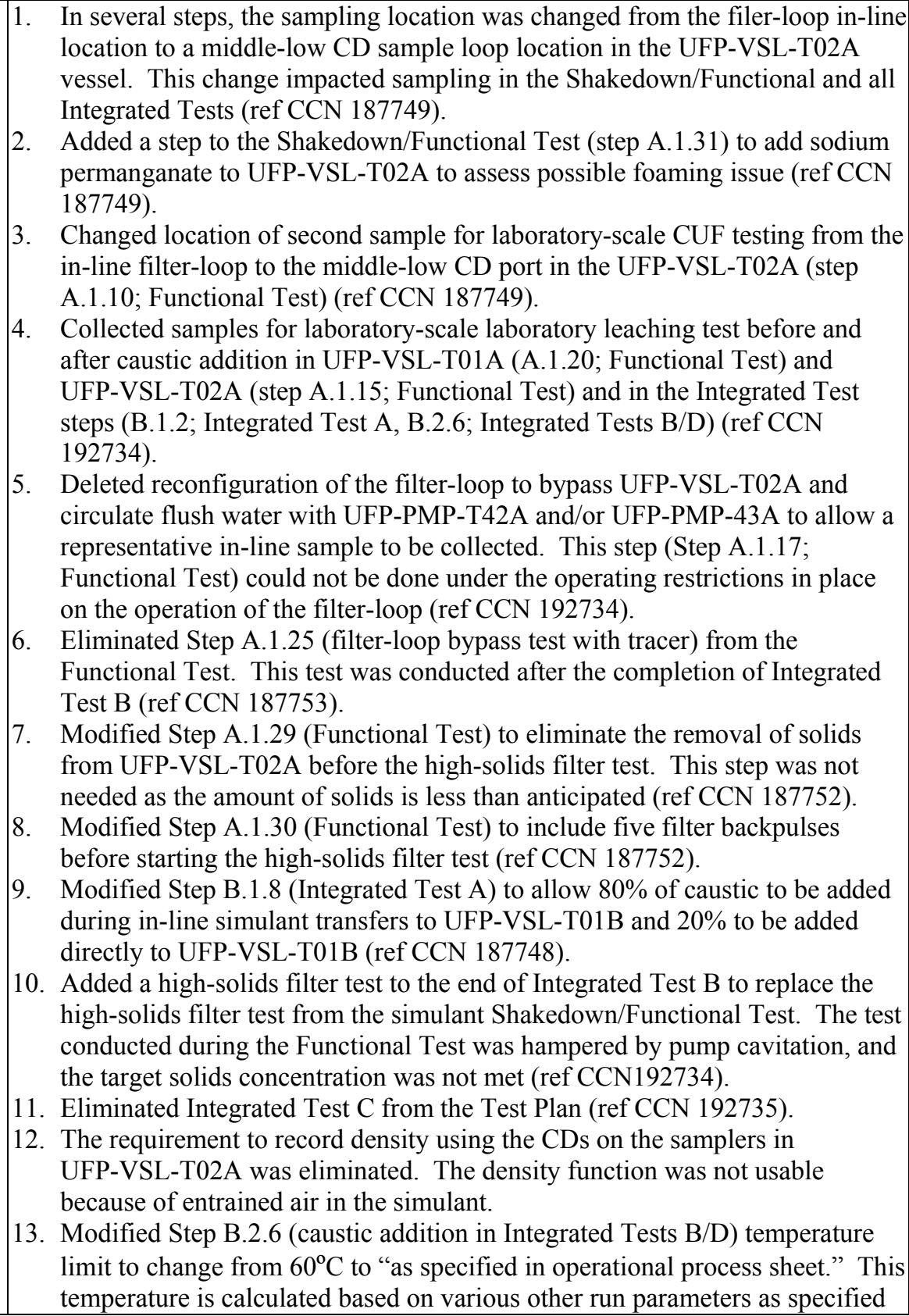 \\
\hline
\end{tabular}


Table S.2. Test Exceptions

\begin{tabular}{|c|c|}
\hline Test Exceptions & Description of Test Exceptions \\
\hline & $\begin{array}{l}\text { in the operational process sheet. } \\
\text { 14. Eliminated the monitoring of Integrated Test D permeate samples for } 30 \text { days } \\
\text { to look for precipitation. This scope was deleted, and a revised scope was } \\
\text { incorporated into the laboratory testing plan. (a) } \\
\text { 15. Step B.2.20 (Integrated Tests B and D) sampling of the heel in } \\
\text { UFP-VSL-T01A was deleted. This sample was not needed since the heels } \\
\text { were removed before follow-on testing. } \\
\text { 16. Step B.1.26 (Integrated Test A) sampling of heel in UFP-VSL-T01B was } \\
\text { deleted. This sample was not needed since the heels were removed before } \\
\text { follow-on testing. } \\
\text { 17. Steps B.1.25 (Integrated Test A) and B.2.19 (Integrated Tests B/D) were } \\
\text { modified from the following: "transfer slurry from UFP-VSL-T02A to } \\
\text { HLP-VSL-T27" to "transfer slurry from UFP-VSL-T02A to } \\
\text { UFP-VSL-62A/B or to totes for storage as directed by the WTP test director." } \\
\text { The HLP-VSL-T27 vessel was no longer available for use since it served as } \\
\text { the receipt vessel for the filter-loop pressure safety valves. } \\
\text { 18. Added a second batch of leaching to Integrated Tests B/D in } \\
\text { UFP-VSL-T02A. This additional leaching batch was needed to provide a } \\
\text { sufficient quantity of solids to operate the UFP-VSL-T02A at prototypic } \\
\text { levels for the steps following caustic leaching. } \\
\text { 19. Added a filter bypass tracer test following the post-caustic-leach dewatering } \\
\text { step in Integrated Test B. This test replaced the filter bypass tracer test that } \\
\text { could not be conducted during the simulant Shakedown/Functional testing. } \\
\text { 20. Deleted instructions to route permeate to a specific tank } \\
\text { (i.e., UFP-VSL-T62A/B). There was no need to segregate various permeate } \\
\text { streams. } \\
\text { 21. Minor changes were made to make the Test Plan consistent with the approved } \\
\text { operational process sheets. }\end{array}$ \\
\hline $\begin{array}{l}\text { 24) } \\
00003 \text { incorporated into } \\
\text { ICN-1 to Test Plan } \\
\text { TP-WTP-PEP-044. }\end{array}$ & $\begin{array}{l}\text { This Test Exception specified activities to be performed with permeate samples } \\
\text { obtained from Integrated Test D. The Integrated Test D permeate samples were } \\
\text { originally stored in a temperature-controlled environment and then moved to a } \\
\text { location with a reduced temperature where precipitation was likely to occur. The } \\
\text { Test Exception requested that the approximate size distribution of the solids be } \\
\text { measured in several ( } 3 \text { or } 4 \text { ) selected PEP samples from Integrated Test D using } \\
\text { polarized light microscopy (PLM). Size-calibrated photographs should be } \\
\text { provided along with the analysis. If possible, record the mineral identification of } \\
\text { the solids phase(s) along with the particle-size distribution. WTP personnel will } \\
\text { select the samples in consultation with the subcontractor, and this will be based in } \\
\text { part on observing which samples contain the most solids or appear to contain } \\
\text { different types of solids. Repeat the size-distribution analysis approximately } \\
1 \text { week after the initial measurements to determine whether there was a significant } \\
\text { change in crystal size, habit, or composition. } \\
\text { Perform each size-distribution analysis by measuring the diameter (or length and } \\
\text { width for elongated crystals) of approximately } 100 \text { individual particles in each } \\
\text { sample. The size may be measured either on the microscope slide, using a } \\
\text { calibrated ocular scale, or on the size-calibrated photographs. The program }\end{array}$ \\
\hline
\end{tabular}

(a) RL Russell. 2008. Test Plan for the PEP Parallel Laboratory Testing. TP-WTP-PEP-044, Pacific Northwest National Laboratory, Richland, Washington. 
Table S.2. Test Exceptions

\begin{tabular}{|c|c|c|}
\hline \multirow{2}{*}{\multicolumn{2}{|c|}{ Test Exceptions }} & Description of Test Exceptions \\
\hline & & $\begin{array}{l}\text { recognizes the limitations of the statistical significance of a size-distribution } \\
\text { measurement based on such a small population. This Test Exception did not } \\
\text { affect any of the existing test plan objectives. }\end{array}$ \\
\hline & $\begin{array}{l}\text { 24590-WTP-TEF-RT-09- } \\
00002 \text { Rev 0, incorporated } \\
\text { into ICN-4 to Test Plan } \\
\text { TP-RPP-WTP-506 }\end{array}$ & $\begin{array}{l}\text { This Test Exception: } \\
\text { 1. Requests a report summarizing the lessons learned during scale-up, } \\
\text { manufacture, and transport of the PEP simulant. } \\
\text { 2. Specifies the sampling and analysis scope to be performed to complete the } \\
\text { prototypic nitric acid PEP filter cleaning process. } \\
\text { 3. Deletes the Engineering Ties report scope. } \\
\text { 4. Specifies additional experimental and analytical work required to estimate the } \\
\text { amount of excess caustic in caustic-leachate samples and post-caustic-leach } \\
\text { wash solutions containing } \approx 3.5-\mathrm{M} \mathrm{Na.}\end{array}$ \\
\hline & $\begin{array}{l}\text { 24590-WTP-TEF-RT-09- } \\
00001 \text { Rev } 1 \text { incorporated } \\
\text { into ICN-2 to Test Plan } \\
\text { TP-WTP-PEP-044 }\end{array}$ & $\begin{array}{l}\text { This Test Exception specifies additional work to be conducted with caustic-leach } \\
\text { solutions and post-caustic-leach washing permeate samples obtained from PEP } \\
\text { Integrated Tests A, B, and D. It contains the following tasks: } \\
\text { 1. Determination of precipitate mineralogy, precipitate phase compositions, and } \\
\text { solution saturation composition. } \\
\text { 2. Determination of rate of approach to saturation concentrations. } \\
\text { 3. Identification and characterization of precipitates formed in } \\
\text { post-caustic-leach filtrate. } \\
\text { 4. Determination of the dilution required to redissolve the precipitate. } \\
\text { 5. Determination of super-saturation in post-caustic-leach filtrates from } \\
\text { Integrated Test B in the PEP. } \\
\text { 6. Determine the effects of blending during the post-caustic-leach dewatering } \\
\text { and wash cycle. }\end{array}$ \\
\hline
\end{tabular}

\section{Results and Performance Against Success Criteria}

The PEP system tests were designed to generate the data necessary to:

- Provide engineering-scale system performance data. This information is used to support the WTP computer process models' projections of the waste processing campaign.

Assessment of this success criterion requires data analysis and will be addressed in report WTP-RPT-197.

- Confirm the operability and functionality of UFP system components.

The operability and functionality of the pretreatment systems, including PJM mixing, steam heating, temperature control, ultrafiltration, backpulsing, loop flushing, two-pumps-in-series operation, and spiral heat exchanger operation, were confirmed. Two operability issues were most evident: level control measurement and air-entrainment at low tank levels in UFP-VSL-T02A. The PEP had three methods of level control: bubbler, laser, and emittance (capacitance) sensors. None of these methods were satisfactory under all process conditions. 
The Research and Technology (R\&T) success criteria for achieving the objectives are further discussed in Table S.3. The success criteria for the entire PEP testing program are provided with discussion limited to the success criteria covered by the scope of this report. The success criteria are shaded in gray if the Shakedown/Functional testing made no contribution to meeting them. 
Table S.3. Success Criteria

\begin{tabular}{|c|c|}
\hline Success Criteria & How Testing Did or Did Not Meet Success Criteria \\
\hline \multicolumn{2}{|l|}{ UFP System Process Performance } \\
\hline $\begin{array}{l}\text { Measure the aluminum leaching } \\
\text { performance of the PEP and laboratory } \\
\text { systems as a function of time under WTP } \\
\text { UFP-1 and UFP-2 projected leaching } \\
\text { conditions at bounding high and low } \\
\text { process temperatures (nominally } 100^{\circ} \mathrm{C} \\
\text { and } 80^{\circ} \mathrm{C} \text { ). }\end{array}$ & $\begin{array}{l}\text { Aluminum leaching was conducted under UFP-1 and UFP-2 projected } \\
\text { leaching conditions and is analyzed and presented in WTP-RPT- } 197 . \\
\text { Partial results for Integrated Tests A and B are presented in } \\
\text { WTP-RPT-186. }\end{array}$ \\
\hline $\begin{array}{l}\text { Compare aluminum leach performance in } \\
\text { UFP-1 where all of the } \mathrm{NaOH} \text { is added } \\
\text { in-line to the case where a fraction of the } \\
\text { total } \mathrm{NaOH} \text { is added directly to the tank. }\end{array}$ & $\begin{array}{l}\text { Results to meet this success criterion are discussed in report } \\
\text { WTP-RPT-197. }\end{array}$ \\
\hline $\begin{array}{l}\text { Measure chromium leaching performance } \\
\text { in the PEP and laboratory systems as a } \\
\text { function of time at the WTP projected } \\
\text { conditions in UFP-2 for both the UFP-1 } \\
\text { and UFP-2 aluminum leaching } \\
\text { flowsheets. }\end{array}$ & $\begin{array}{l}\text { Chromium leaching results are presented in reports WTP-RPT-188 and } \\
\text { WTP-RPT-197. Data from the Shakedown/Functional testing were not } \\
\text { used to assess chromium leaching. }\end{array}$ \\
\hline $\begin{array}{l}\text { Evaluate the process control strategy for } \\
\text { specifying required reagent additions, } \\
\text { including } \mathrm{NaOH}, \mathrm{NaMnO}_{4} \text {, and wash } \\
\text { solutions provided in the PEP Phase I } \\
\text { Testing Process Description. }\end{array}$ & $\begin{array}{l}\text { The process control strategy for specifying the amount of reagent was } \\
\text { to analyze a sample of the simulant feed for aluminum and chrome } \\
\text { content and specify the amount of } \mathrm{NaOH} \text { and } \mathrm{NaMnO}_{4} \text { based on the } \\
\text { result. The amount of reagent added was correct within the analytical } \\
\text { error of the analysis method. A comparison of targeted and delivered } \\
\text { reagent additions is provided in report WTP-RPT- } 188 \text { for Integrated } \\
\text { Tests A and B. Additional discussion and results for Integrated Test D } \\
\text { are provided in report WTP-RPT-197. }\end{array}$ \\
\hline $\begin{array}{l}\text { Measure the filter system performance at } \\
\text { the nominal flow velocity and TMPs for } \\
\text { the solids concentration and washing } \\
\text { stages for the UFP-1 and UFP-2 } \\
\text { aluminum leaching flowsheets. }\end{array}$ & $\begin{array}{l}\text { Filter conditioning was performed for } 36 \text { hours, including } 24 \\
\text { backpulses conducted at } 30 \text {-min intervals between hours } 12 \text { to } 24 \text {. } \\
\text { These low solids filter test data were used to determine a relationship } \\
\text { between the laboratory-scale CUF filter system and PEP } \\
\text { (WTP-RPT-185). Additional filter performance data were collected } \\
\text { during dewatering and washing process steps in integrated process } \\
\text { testing. }\end{array}$ \\
\hline $\begin{array}{l}\text { Evaluate the control strategy for make-up } \\
\text { additions from UFP-VSL-00001A/B to } \\
\text { UFP-VSL-00002A/B during the initial } \\
\text { dewatering process. }\end{array}$ & $\begin{array}{l}\text { The control strategy for make-up additions between UFP-VSL-T01A } \\
\text { and UFP-VSL-T02A during the initial dewatering process was } \\
\text { demonstrated during Shakedown/Functional testing and was } \\
\text { determined satisfactory to implement for integrated process testing. } \\
\text { Results are presented in report WTP-RPT-197. }\end{array}$ \\
\hline $\begin{array}{l}\text { Measure the wash-water volumes } \\
\text { required to remove or reduce: } \\
\text { 1) the free hydroxide following the } \\
\text { aluminum leaching stage, and } \\
\text { 2) dissolved chromium after the } \\
\text { oxidative leaching process to the } \\
\text { specified concentrations. }\end{array}$ & $\begin{array}{l}\text { Data from the Shakedown/Functional testing were not used for } \\
\text { assessment of washing. Solids washing results are analyzed and } \\
\text { presented in WTP-RPT-185 and WTP-RPT-197. }\end{array}$ \\
\hline
\end{tabular}


Table S.3. Success Criteria

\begin{tabular}{|c|c|}
\hline Success Criteria & How Testing Did or Did Not Meet Success Criteria \\
\hline $\begin{array}{l}\text { Perform mass balances for selected } \\
\text { constituents, including aluminum, } \\
\text { chromium, manganese, sodium, } \\
\text { hydroxide, oxalate, phosphate, sulfate, } \\
\text { and water to evaluate leaching and } \\
\text { washing process performance. }\end{array}$ & $\begin{array}{l}\text { Mass-balance results for selected constituents are in report } \\
\text { WTP-RPT-197. }\end{array}$ \\
\hline $\begin{array}{l}\text { Measure solids distribution under scaled } \\
\text { mixing conditions before and after caustic } \\
\text { leaching evolutions. }\end{array}$ & $\begin{array}{l}\text { Data from the Shakedown/Functional testing were used to assess the } \\
\text { homogeneity of tank contents before and after caustic leaching. Data } \\
\text { are analyzed in report WTP-RPT-197. }\end{array}$ \\
\hline $\begin{array}{l}\text { Measure the rheology of the slurry } \\
\text { simulant and the shear strength of the } \\
\text { settled solids before and after each } \\
\text { leaching and washing unit operation and } \\
\text { following the final concentration. }\end{array}$ & $\begin{array}{l}\text { The rheology of the slurry simulant was measured for } \\
\text { Shakedown/Functional testing and is provided in this report. }\end{array}$ \\
\hline $\begin{array}{l}\text { Estimate the quantity of excess hydroxide } \\
\text { added in the process that may not be } \\
\text { needed to keep aluminate in solution } \\
\text { following filtration. }\end{array}$ & $\begin{array}{l}\text { Results to meet this success criterion are discussed in report } \\
\text { WTP-RPT-197. }\end{array}$ \\
\hline $\begin{array}{l}\text { Collect and retain permeate samples for } \\
\text { extended precipitation studies (including } \\
\text { permeate/simulated supernatant blended } \\
\text { cases) from each concentration cycle. }\end{array}$ & $\begin{array}{l}\text { Collection of permeate samples was not part of the } \\
\text { Shakedown/Functional Test scope. This success criterion was } \\
\text { addressed during Integrated testing. The results of the precipitation } \\
\text { studies are discussed in WTP-RPT-197, WTP-RPT-200, and } \\
\text { WTP-RPT-205. }\end{array}$ \\
\hline \multicolumn{2}{|c|}{ UFP System Operability and Functionality } \\
\hline $\begin{array}{l}\text { Verify that the dual, in-series pump } \\
\text { configuration is controllable and } \\
\text { maintains the required slurry velocity and } \\
\text { pressures for ultrafilter operation. }\end{array}$ & $\begin{array}{l}\text { The data required to meet this success criterion were provided on } \\
\text { compact disks transmitted in the following reference: Letter from } \\
\text { GH Beeman to H Hazen, "Subcontract No. } \\
\text { 24590-QL-HC9-WA49-00001, Project No. } 53569 \text { (WA-024) } \\
\text { Engineering Ties Data Transmittal: The Electronic File Enclosed with } \\
\text { this Letter Has Been Reviewed for Technical Accuracy Per the Quality } \\
\text { Assurance (QA) Program," WTP/RPP-MOA-PNNL-00392, dated } \\
\text { 4/10/2009. } \\
\text { During Shakedown/Functional testing, the desired flow rate (109 gpm) } \\
\text { could not be maintained in the filter-loop when the tank level was low } \\
\text { (e.g., }<15 \text { to } 18 \text { inches). Air entrainment appeared as a problem at low } \\
\text { tank levels when the filter-loop return nozzle was above the slurry } \\
\text { surface or when PJM overblows occurred. }\end{array}$ \\
\hline $\begin{array}{l}\text { Measure the operating characteristics for } \\
\text { the cooling heat exchanger for the filter } \\
\text { recirculation loop (UFP-HX-T02A) } \\
\text { (temperature changes as a function of } \\
\text { flow to determine how to achieve the } \\
\text { desired performance in the PTF analog). }\end{array}$ & $\begin{array}{l}\text { The data required to meet this success criterion were provided on } \\
\text { compact disks transmitted in the following reference: Letter from } \\
\text { GH Beeman to H Hazen, "Subcontract No. } \\
\text { 24590-QL-HC9-WA49-00001, Project No. } 53569 \text { (WA-024) } \\
\text { Engineering Ties Data Transmittal: The Electronic File Enclosed with } \\
\text { this Letter Has Been Reviewed for Technical Accuracy Per the QA } \\
\text { Program," WTP/RPP-MOA-PNNL-00392, dated 4/10/2009. }\end{array}$ \\
\hline
\end{tabular}


Table S.3. Success Criteria

\begin{tabular}{|c|c|}
\hline Success Criteria & How Testing Did or Did Not Meet Success Criteria \\
\hline $\begin{array}{l}\text { Confirm whether the WTP process } \\
\text { control strategies for ultrafilter system } \\
\text { filling, operating, backpulsing, draining, } \\
\text { flushing, and cleaning are adequate for } \\
\text { stable operation. Provide to WTP data to } \\
\text { determine whether backpulsing is a } \\
\text { required and effective means of restoring } \\
\text { the filter permeate rates to confirm that } \\
\text { production throughput is maintained and } \\
\text { whether operation of the backpulse } \\
\text { system induces any process or equipment } \\
\text { operations issues. }\end{array}$ & $\begin{array}{l}\text { Operating experience for the UF system filling and backpulsing are } \\
\text { included in the Shakedown/Functional testing narrative in this report. } \\
\text { Quantitative results to meet this success criterion are discussed in } \\
\text { report WTP-RPT-197. }\end{array}$ \\
\hline $\begin{array}{l}\text { Use only the process information and data } \\
\text { available to the WTP PTF operating staff } \\
\text { during WTP operations (e.g., caustic and } \\
\text { permanganate addition volumes, } \\
\text { permeate mass balances for solids } \\
\text { concentration) to operate the PEP. }\end{array}$ & $\begin{array}{l}\text { This success criterion was met by developing an operational process } \\
\text { sheet of all the operating parameters (e.g., transfer volumes, reagent } \\
\text { addition volumes, control levels) based on prototypic characterization } \\
\text { data before the start of the Integrated tests. Changes to the operational } \\
\text { process sheet made during the test itself were based only on data that } \\
\text { would be available to the plant, and were not, for example, based on } \\
\text { information from nonprototypic samples. Results to meet this success } \\
\text { criterion are discussed in report WTP-RPT-197. }\end{array}$ \\
\hline $\begin{array}{l}\text { Confirm whether the elevated } \\
\text { temperature PJM operating strategy is } \\
\text { adequate for stable PEP and WTP } \\
\text { operation. }\end{array}$ & $\begin{array}{l}\text { Results to meet this success criterion are discussed in report } \\
\text { WTP-RPT-197. }\end{array}$ \\
\hline $\begin{array}{l}\text { Measure the heat-up rate and } \\
\text { controllability of the PEP } \\
\text { UFP-VSL-00001 and UFP-VSL- } 00002 \\
\text { vessels and the cooling performance for } \\
\text { UFP vessels. }\end{array}$ & $\begin{array}{l}\text { The heat-up, thermal control at } 98^{\circ} \mathrm{C} \text {, and cool-down performance of } \\
\text { UFP-VSL-T02A was measured during Shakedown/Functional testing } \\
\text { to make certain that Integrated testing was successfully executed. } \\
\text { Quantitative measures to fulfill this criterion are not part of } \\
\text { Shakedown/Functional testing. }\end{array}$ \\
\hline $\begin{array}{l}\text { Measure the performance of the in-line } \\
\text { addition of process chemicals into the } \\
\text { simulated wastes and determine the } \\
\text { extent of blending in the process vessels. }\end{array}$ & $\begin{array}{l}\text { The blending of process chemicals added in-line was determined in } \\
\text { Shakedown/Functional testing. Analysis of the data is presented in } \\
\text { WTP-RPT-197. }\end{array}$ \\
\hline $\begin{array}{l}\text { Monitor ultrafilter performance (to } \\
\text { include visual inspection of the filter } \\
\text { tubes, tube sheets, and heads from an } \\
\text { ultrafilter for any evidence of flow } \\
\text { mal-distribution and/or solids buildup at } \\
\text { least once during Phase I). }\end{array}$ & $\begin{array}{l}\text { Ultrafilter visual inspection results are presented in reports } \\
\text { WTP-RPT-193 and WTP-RPT-197. }\end{array}$ \\
\hline $\begin{array}{l}\text { Measure, record, and control } \\
\text { ultrafiltration temperature, TMP, and } \\
\text { slurry flow during filter-loop operations. }\end{array}$ & $\begin{array}{l}\text { Slurry flow rate, temperature, and TMP were recorded and controlled } \\
\text { during Shakedown/Functional testing. Results are provided in this } \\
\text { report. Data analyses are included in WTP-RPT-197. }\end{array}$ \\
\hline $\begin{array}{l}\text { Record any solids accumulations } \\
\text { observed during any operating stage or } \\
\text { maintenance evolution. }\end{array}$ & $\begin{array}{l}\text { A solids plugging event occurred because of a valve misalignment. No } \\
\text { significant solids accumulations occurred during proper operating or } \\
\text { maintenance evolutions. }\end{array}$ \\
\hline $\begin{array}{l}\text { Monitor the permeate production rate of } \\
\text { each ultrafilter assembly in operation. }\end{array}$ & $\begin{array}{l}\text { The permeate production rate of each ultrafilter was recorded during } \\
\text { Shakedown/Functional testing. Results are provided in this report. }\end{array}$ \\
\hline
\end{tabular}


Table S.3. Success Criteria

\begin{tabular}{||l|l||}
\hline \multicolumn{1}{|c|}{ Success Criteria } & \multicolumn{1}{c|}{ How Testing Did or Did Not Meet Success Criteria } \\
\hline $\begin{array}{l}\text { Record operating time of each ultrafilter } \\
\text { assembly. }\end{array}$ & $\begin{array}{l}\text { The operating time of each ultrafilter assembly was recorded during the } \\
\text { Shakedown/Functional testing. Results are provided in this report. }\end{array}$ \\
\hline $\begin{array}{l}\text { Record each ultrafilter assembly cleaning } \\
\text { event (backpulse, flush, chemical } \\
\text { cleaning, etc.). }\end{array}$ & $\begin{array}{l}\text { Each ultrafilter cleaning event was recorded in Shakedown/Functional } \\
\text { testing. A summary of the results is provided in the narrative section of } \\
\text { this report, with more details recorded in the Operations and Testing } \\
\text { Laboratory Record Books. }\end{array}$ \\
\hline $\begin{array}{l}\text { Evaluate the pulse-pot operation and } \\
\text { backpulse operation strategies contained } \\
\text { in PEP Phase I Testing Process } \\
\text { Description. }\end{array}$ & $\begin{array}{l}\text { Backpulsing was conducted as a part of the filter conditioning in } \\
\text { Shakedown/Functional testing. Backpulse operations are evaluated in } \\
\text { report WTP-RPT-197. }\end{array}$ \\
\hline $\begin{array}{l}\text { Evaluate permeate and permeate blends } \\
\text { for precipitation of solids, particularly } \\
\text { aluminum and oxalate solids. }\end{array}$ & $\begin{array}{l}\text { Results to meet this success criterion are discussed in reports } \\
\text { WTP-RPT-197, WTP-RPT-200, and WTP-RPT-205. }\end{array}$ \\
\hline
\end{tabular}

\section{Quality Requirements}

The PNNL quality assurance (QA) program is based upon the requirements as defined in DOE Order 414.1C, Quality Assurance and 10 CFR 830, Energy/Nuclear Safety Management, Subpart A--Quality Assurance Requirements (a.k.a. the Quality Rule). PNNL has chosen to implement the following consensus standards in a graded approach:

- ASME NQA-1-2000, Quality Assurance Requirements for Nuclear Facility Applications, Part 1, Requirements for Quality Assurance Programs for Nuclear Facilities.

- ASME NQA-1-2000, Part II, Subpart 2.7, Quality Assurance Requirements for Computer Software for Nuclear Facility Applications.

- ASME NQA-1-2000, Part IV, Subpart 4.2, Graded Approach Application of Quality Assurance Requirements for Research and Development.

The procedures necessary to implement the requirements are documented through PNNL's "How Do I...?" (HDI). ${ }^{\text {(a) }}$ PNNL implements the RPP-WTP quality requirements by performing work in accordance with the River Protection Project-Waste Treatment Plant Support Program (RPP-WTP) Quality Assurance Plan (RPP-WTP-QA-001, QAP). Work was performed to the quality requirements of NQA-1-1989, Part I, Basic and Supplementary Requirements, NQA-2a-1990, Part 2.7, and DOE/RW-0333P, Rev 13, Quality Assurance Requirements and Descriptions (QARD) as applicable. These quality requirements are implemented through the River Protection Project-Waste Treatment Plant Support Program (RPP-WTP) Quality Assurance Manual (RPP-WTP-QA-003, QAM). The requirements of DOE/RW-0333P, Rev 13, Quality Assurance Requirements and Descriptions (QARD) and 10 CFR 830 Subpart A were not required for this work.

RPP-WTP addresses internal verification and validation activities by conducting an Independent Technical Review of the final data report in accordance with RPP-WTP's procedure QA-RPP-WTP-604.

(a) PNNL's system for managing the delivery of laboratory-level policies, requirements, and procedures. 
This review procedure is part of PNNL's RPP-WTP Quality Assurance Manual (RPP-WTP-QA-003). Following this procedure, a technical review verified that the reported results are traceable, that inferences and conclusions are soundly based, and the reported work satisfies the objectives.

\section{R\&T Test Conditions}

The research and technology (R\&T) test conditions as defined in the Test Specification are summarized in Table S.4. The R\&T test conditions for the entire PEP testing program are provided with discussion limited to the R\&T test conditions implemented during Functional process testing. The test conditions are shaded in gray if the Shakedown/Functional testing made no contribution to meeting them.

Table S.4. R\&T Test Conditions

\begin{tabular}{|c|c|}
\hline List R\&T Test Conditions & Were Test Conditions Followed? \\
\hline \multicolumn{2}{|l|}{ General Requirements } \\
\hline $\begin{array}{l}\text { Perform mass balances for selected constituents, } \\
\text { including aluminum, chromium, manganese, sodium, } \\
\text { hydroxide, oxalate, phosphate, sulfate, and water, to } \\
\text { evaluate leaching and washing process performance. }\end{array}$ & $\begin{array}{l}\text { Yes. This R\&T test condition is discussed for Cr in the } \\
\text { oxidative-leach process in Integrated Tests A and B in } \\
\text { WTP-RPT-188 and is fully discussed in report } \\
\text { WTP-RPT-197. }\end{array}$ \\
\hline $\begin{array}{l}\text { Evaluate ultrafilter performance (to include visual } \\
\text { inspection of the filter tubes, tube sheets, and heads from } \\
\text { an ultrafilter for any evidence of flow mal-distribution } \\
\text { and/or solids buildup or evidence of potential failure). }\end{array}$ & $\begin{array}{l}\text { Data to evaluate filter flux were collected. Visual } \\
\text { inspection was completed after Integrated Test D and is } \\
\text { discussed in WTP-RPT-193 and WTP-RTP-197. }\end{array}$ \\
\hline $\begin{array}{l}\text { Assess the blending achieved during in-line additions of } \\
\text { leaching and washing solutions. }\end{array}$ & $\begin{array}{l}\text { In-line blending of } \mathrm{NaOH} \text { for UFP-VSL-T02A leaching } \\
\text { and in-line blending of wash solutions was conducted. } \\
\text { In-line blending of NaOH for up-front leaching } \\
\text { (UFP-VSL-T01A/B) was not conducted. In-line } \\
\text { addition of wash water during Integrated Tests D and B } \\
\text { is discussed in WTP-RPT-187 and is fully discussed in } \\
\text { report WTP-RPT-197. }\end{array}$ \\
\hline $\begin{array}{l}\text { Record any solids accumulations observed during any } \\
\text { operating stage or maintenance evolution (e.g., } \\
\text { photography, particle-size distribution). }\end{array}$ & $\begin{array}{l}\text { Not conducted during Shakedown/Functional testing. } \\
\text { This R\&T test condition is discussed in report } \\
\text { WTP-RPT-197. }\end{array}$ \\
\hline \multicolumn{2}{|l|}{ Leaching Operations } \\
\hline $\begin{array}{l}\text { Maintain caustic leaching temperature at the required } \\
\text { setpoint and record steam usage to remain in the } \\
\text { temperature range. }\end{array}$ & $\begin{array}{l}\text { Yes. This R\&T test condition was met for the leaching } \\
\text { tests discussed in this report. The conditions for the } \\
\text { remaining tests are discussed in WTP-RPT- } 197 \text {. }\end{array}$ \\
\hline $\begin{array}{l}\text { Maintain oxidative leaching temperature at the required } \\
\text { setpoint. }\end{array}$ & $\begin{array}{l}\text { Oxidative leaching was not conducted during the } \\
\text { Shakedown/Functional testing. This R\&T test } \\
\text { condition is discussed in reports WTP-RPT-188 and } \\
\text { WTP-RPT-197. }\end{array}$ \\
\hline $\begin{array}{l}\text { Obtain periodic samples during the leaching operations to } \\
\text { monitor the amount of aluminum or chromium that has } \\
\text { dissolved and concentrations of the reactants and } \\
\text { products in the liquid fraction in the vessel. }\end{array}$ & $\begin{array}{l}\text { Yes. This R\&T condition was met for the caustic } \\
\text { leaching tests discussed in this report. Additional } \\
\text { discussion of this R\&T condition is provided in } \\
\text { WTP-RPT-186, WTP-RPT-188, and WTP-RPT-197. }\end{array}$ \\
\hline
\end{tabular}


Table S.4. R\&T Test Conditions

\begin{tabular}{|c|c|}
\hline List R\&T Test Conditions & Were Test Conditions Followed? \\
\hline $\begin{array}{l}\text { Provide data to demonstrate the WTP process control } \\
\text { strategy for the caustic and permanganate addition. }\end{array}$ & $\begin{array}{l}\text { The strategy for in-line addition of chemicals was } \\
\text { implemented. The strategy to determine permanganate } \\
\text { addition based upon one Cr analysis after } \\
\text { laboratory-scale caustic addition was not part of the } \\
\text { Shakedown/Functional Test scope. This R\&T test } \\
\text { condition is discussed in report WTP-RPT-197. }\end{array}$ \\
\hline $\begin{array}{l}\text { Measure the rheology of the slurry simulant and shear } \\
\text { strength of the settled solids before and following each } \\
\text { leaching unit operation. }\end{array}$ & $\begin{array}{l}\text { Yes. Rheology measurements were obtained and are } \\
\text { included in this report. This R\&T test condition is } \\
\text { discussed in report WTP-RPT-197. }\end{array}$ \\
\hline \multicolumn{2}{|l|}{ Concentration Operations } \\
\hline $\begin{array}{l}\text { Monitor the permeate production rate of each ultrafilter } \\
\text { assembly in operation. }\end{array}$ & $\begin{array}{l}\text { The permeate production rates were monitored during } \\
\text { the Shakedown/Functional testing. This R\&T test } \\
\text { condition is discussed in report WTP-RPT-185, the run } \\
\text { reports for the individual tests (WTP-RPT-191, } \\
\text { WTP-RPT-192, WTP-RPT-193), and WTP-RPT-197. }\end{array}$ \\
\hline Record operating time of each ultrafilter assembly. & $\begin{array}{l}\text { Yes. The operating time for each ultrafilter assembly is } \\
\text { included in this report for the Shakedown/Functional } \\
\text { Test. This R\&T test condition is discussed in report } \\
\text { WTP-RPT-197. }\end{array}$ \\
\hline $\begin{array}{l}\text { Record each ultrafilter assembly "cleaning' event" } \\
\text { (backpulse, flush, chemical cleaning, etc.). }\end{array}$ & $\begin{array}{l}\text { Yes. Ultrafilter cleaning events during } \\
\text { Shakedown/Functional testing are included in this } \\
\text { report. This R\&T test condition is discussed in report } \\
\text { WTP-RPT-197. }\end{array}$ \\
\hline $\begin{array}{l}\text { Confirm pulse-pot operation and backpulse operation } \\
\text { strategies. }\end{array}$ & $\begin{array}{l}\text { Yes. Pulse-pot and backpulse operation strategies were } \\
\text { confirmed during the Shakedown/Functional testing. } \\
\text { This R\&T test condition is discussed in report } \\
\text { WTP-RPT-197. }\end{array}$ \\
\hline $\begin{array}{l}\text { Control ultrafiltration temperature, TMP, and slurry flow } \\
\text { as specified in test-specific operational process sheets. }\end{array}$ & $\begin{array}{l}\text { Yes. This R\&T test condition is discussed in report } \\
\text { WTP-RPT-185, the run reports for the individual tests } \\
\text { (WTP-RPT-191, WTP-RPT-192, WTP-RPT-193), and } \\
\text { WTP-RPT-197. }\end{array}$ \\
\hline $\begin{array}{l}\text { Collect and retain permeate samples for extended } \\
\text { precipitation studies (including permeate/simulated } \\
\text { supernatant blended cases) from each concentration } \\
\text { cycle. }\end{array}$ & $\begin{array}{l}\text { Collection of permeate samples was not part of the } \\
\text { Shakedown/Functional Test scope. The results of the } \\
\text { precipitation studies are discussed in WTP-RPT-197, } \\
\text { WTP-RPT-200, and WTP-RPT-205. }\end{array}$ \\
\hline $\begin{array}{l}\text { Demonstrate WTP ultrafiltration system control scheme } \\
\text { in normal operating modes (e.g., fill and startup, } \\
\text { operation, backpulsing, flush and drain, cleaning and } \\
\text { return to service). }\end{array}$ & $\begin{array}{l}\text { Yes. This R\&T test condition is discussed in report } \\
\text { WTP-RPT-197. }\end{array}$ \\
\hline \multicolumn{2}{|l|}{ Washing Operations } \\
\hline $\begin{array}{l}\text { Wash slurries using a washing protocol to be specified in } \\
\text { test-specific operational process sheets. }\end{array}$ & $\begin{array}{l}\text { Yes. Post-caustic-leach washing was conducted during } \\
\text { the Shakedown/Functional testing, but the results are } \\
\text { not used to calculate washing efficiencies. This R\&T } \\
\text { test condition is discussed in reports WTP-RPT-187 } \\
\text { and WTP-RPT-197. }\end{array}$ \\
\hline Sample permeate immediately before each wash solution & Yes. Per the revised Test Plan, washing performance \\
\hline
\end{tabular}


Table S.4. R\&T Test Conditions

\begin{tabular}{|l|l||}
\hline List R\&T Test Conditions & Were Test Conditions Followed? \\
\hline addition to monitor washing performance/efficiency. & $\begin{array}{l}\text { was monitored by sampling and analyzing the washed } \\
\text { slurry, not the permeate. Nineteen samples were taken } \\
\text { during 34 wash additions rather than every batch. The } \\
\text { results obtained during the Shakedown/Functional Test } \\
\text { are not used to determine washing efficiencies. This } \\
\text { R\&T test condition for the Integrated tests is discussed } \\
\text { in reports WTP-RPT-187 and WTP-RPT-197. }\end{array}$ \\
\hline Measure rheology of the washed solids. & $\begin{array}{l}\text { Yes. The rheology of the washed solids was measured } \\
\text { after the solids were washed. This R\&T test condition } \\
\text { is discussed in reports WTP-RPT-187 and } \\
\text { WTP-RPT-197. }\end{array}$ \\
\hline
\end{tabular}

\section{Simulant Use}

PEP process testing was performed with a nonradioactive aqueous slurry of simulant waste chemicals and solids. The simulant composition and make-up recipe were provided by WTP as documented in Simulant Recommendation for Phase 1 Testing in the Pretreatment Engineering Platform. ${ }^{\text {(a) }}$ Aqueous chemical concentrations were within the ranges expected for waste feeds to the PTF. The hydroxide concentration was marginally one standard deviation lower than the average concentration expected in the feeds to the plant. The oxalate and phosphate components were at the lower end of the expected ranges, but the oxalate component was at the solubility limit, and the phosphate component was at or near the solubility limit. The solids components and blend were selected to obtain targeted solids mass loss (aluminum and chromium leaching and oxalate washing) and treatment time. The simulant was not selected to represent any particular Hanford tank waste type.

The simulant was blended from the components listed below. The basis for selecting the individual components and the comparison to actual waste behavior are provided where applicable in the indicated references.

- Boehmite (for Al) (Russell et al. 2009a)

- Gibbsite (for Al) (Russell et al. 2009b)

- Chromium oxyhydroxide (CrOOH) slurry (Rapko et al. 2007)

- Sodium oxalate

- Filtration simulant (Russell et al. 2009c)

- Supernate.

A separate chromium solids slurry simulant was prepared and added to the PEP process after post-caustic-leach washing (a nonprototypic addition) during the Shakedown/Functional Tests and Integrated Tests A and B. This approach was taken because laboratory-scale tests had shown that the

(a) P Sundar. 2008. Simulant Recommendation for Phase 1 Testing in the Pretreatment Engineering Platform, 24590-PTF-RPT-RT-08-006 Rev 0, Bechtel National, Inc., Richland, WA. 
high-temperature caustic leaching step dissolved significant amounts of the $\mathrm{CrOOH}$ solids (Russell et al. 2009a). In Integrated Test D, the chromium solids component of the simulant was added during the simulant make-up process to demonstrate the PTF permanganate addition strategy. Simulant was procured from NOAH Technologies Corporation (San Antonio, TX). Samples of each simulant batch were characterized to make certain that chemical and physical property requirements were met. Batches of the simulant were procured as follows:

- A 15-gallon trial batch of the blended simulant for laboratory testing to demonstrate the efficacy of the simulant fabrication procedure.

- A 250-gallon scale-up batch of the blended simulant to demonstrate scale-up of the simulant fabrication procedure to an intermediate scale.

- Batches 0, 1, and 2, each nominally 3500 gallons, of blended simulant for the Shakedown/Functional Tests and Integrated Tests A and B. These batches did not contain the $\mathrm{CrOOH}$ component.

- Batch 3, nominally 1200 gallons, for Integrated Test D. This batch contained the CrOOH solids component.

- The CrOOH solids slurry for the Shakedown/Functional Test and Integrated Tests A and B was obtained in two separate batches containing nominally 18 - and $36-\mathrm{kg}$ of $\mathrm{Cr}$ as $\mathrm{CrOOH}$.

\section{Discrepancies and Follow-on Tests}

No discrepancies or follow-on tests were identified. 



\subsection{Introduction}

This is one in a series of reports that summarize Phase I testing results from the Pretreatment Engineering Platform (PEP) located in the Process Development Laboratory-West (PDL-W) in Richland, Washington. Separate run reports have been prepared for each Phase I test (i.e., Shakedown/Functional testing (Josephson et al. 2009), Integrated Test A (Guzman-Leong et al. 2009), Integrated Test B (Geeting et al. 2009), and Integrated Test D (Sevigny et al. 2009). In addition, reports have been written that specifically address:

1. Filtration scale-up (Daniel et al. 2009)

2. Caustic Leaching scale-up (Mahoney et al. 2009)

3. Solids Washing (Baldwin, et al. 2009)

4. Oxidative Leaching scale-up (Rapko et al. 2009).

This report focuses on Shakedown/Functional testing, summarizes test conduct and results, and includes only very limited data analysis. This includes a test narrative, sample analyses, summaries of PEP monitoring data, evaluations or summaries of problems encountered, and deviations from the test protocols. Data files have been supplied on transportable hard drives because of the large amount of data. The testing reported here was conducted according to TI-WTP-PEP-062, TI-WTP-PEP-067, and TI-WTP-PEP-032, which were written in accordance with Test Plan TP-RPP-WTP-506, Rev. 0.4, ${ }^{\text {(a) }}$ which was written in response to Test Specification 24590-PTF-TSP-RT-07-001, Rev 2. ${ }^{\text {(b) }}$

\subsection{Purpose}

The purpose of this testing program is to provide WTP with data to "...confirm the ultrafiltration process (UFP) system design and sludge treatment process flowsheet." ${ }^{\text {(c) }}$ The system design and treatment process flowsheet will be confirmed by evaluating the following operations on an engineering-scale integrated platform (the PEP):

- Solids concentration

- Aluminum leaching

- Washing

- Chromium leaching.

Tests were used to collect data needed for design input and to improve prediction of full-scale plant performance:

(a) GB Josephson, OP Bredt, JK Young, and DE Kurath. 2009. Test Plan for Pretreatment Engineering Platform (PEP) Testing (Phase I). TP-RPP-WTP-506, Rev. 0.4, Pacific Northwest National Laboratory, Richland, Washington.

(b) JL Huckaby and JR Markillie. 2008. Pretreatment Engineering Platform (PEP) Testing (Phase I). 24590-PTF-TSP-RT-07-001, Rev 2, Bechtel National, Inc., Richland, Washington.

(c) S Barnes and R Voke. 2006. Issue Response Plan for Implementation of External Flowsheet Review Team (EFRT) Recommendations - M12, Undemonstrated Leaching Processes. 24590-WTP-PL-ENG-06-0024, Rev. 0, Bechtel National, Inc., Richland, Washington. 
- Demonstration of component operation and determination of operating characteristics

- Integrated simulations of leaching scenarios.

The PEP data will be used by WTP along with laboratory-scale data and appropriate application of scaling to improve the WTP plant models used to predict plant operating performance.

\subsection{Background}

An EFRT was assembled in October 2005 to challenge and provide a critical review of the design of the Hanford Tank Waste Treatment and Immobilization Plant (WTP). The review ${ }^{(a)}$ identified a number $^{2}$ of issues and potential issues that if not addressed could impact operation of the plant. One of the issues that must be addressed in the Pretreatment Facility (PTF) is "Undemonstrated Leaching Processes." The EFRT reported that neither the caustic leaching nor the oxidative leaching process has been demonstrated at greater than bench-scale. Without a scale-up study, the EFRT indicated that the ability to predict the effectiveness of these processes is limited.

The WTP project under BNI and the U.S. Department of Energy (DOE) developed the "Issue Response Plan for Implementation of External Flowsheet Review Team (EFRT) Recommendations--M12, Undemonstrated Leaching Processes" to resolve the "Undemonstrated Leaching Processes" issue. (a) The plan addresses related topics that are not specifically in response to EFRT concerns. These include caustic addition and leaching concerns that were better understood after the EFRT report was issued, information to support revision of the contract design basis for the PTF, including system capacities, and earlier initiatives on enhancing plant throughput capacity. The solution for closing this issue includes conducting engineering-scale testing of all leaching (caustic and oxidative), washing processes and filtration scenarios. ${ }^{(b)}$

The flowsheet and equipment design demonstration will be performed in the following two phases:

- Phase 1 - Confirm the UFP system design and sludge treatment process flowsheet ${ }^{(a)}$

- Phase 2-Confirm the UFP system performance over a range of anticipated plant process conditions, including the principal types of WTP feeds.

The data provided by the Phase I engineering-scale testing will be used to confirm the performance of the selected process flowsheet design and equipment based on post-Phase I test modeling.

A technical review meeting to discuss the WTP project ultrafiltration leaching process was held 10/23/06 to 10/24/06 to review the Issue Response Plan for M12. ${ }^{\text {(b) }}$ Participants included members of DOE, EFRT, the WTP project, and Pacific Northwest National Laboratory (PNNL). At the meeting, PNNL made recommendations on the methodology and scale for the leaching processes.

(a) JP Henschel. 2006. Contract No. DE-AC27-01RV14136 - Report of External Flowsheet Review Team for the Hanford Tank Waste Treatment and Immobilization Plant: "Comprehensive Review of the Waste Treatment Plant Flowsheet and Throughput.” Letter to RJ Schepens. CCN: 132846, Bechtel National, Inc., Richland, Washington.

(b) S Barnes and R Voke. 2006. Issue Response Plan for Implementation of External Flowsheet Review Team (EFRT) Recommendations - M12, Undemonstrated Leaching Processes. 24590-WTP-PL-ENG-06-0024 Rev. 0, Bechtel National, Inc., Richland, Washington. 
Members of the EFRT also presented their assessment (Integrated Test Platform, M12 - Test Review) with specific recommendations. ${ }^{\text {(a) }}$ The general system requirements recommended by EFRT and PNNL staff members included the following:

- Principal processing equipment elements of the UFP system need to be included in the demonstration (including UFP-VSL-00001A/B, UFP-VSL-00002, the ultrafiltration loop recirculation pumps, and the ultrafilters with supporting equipment.).

- The demonstration system needs to be flexible to accommodate testing of the baseline processes and process options for a range of operating conditions and processing times.

- Geometric similarity (prototypic) is needed in the key pieces of processing equipment (including UFP-VSL-00001A/B and UFP-VSL-00002).

- Ultrafilter elements must be prototypic length and diameter to obtain expected filter performance data. The test equipment should be scaled down by using fewer filter elements in each assembly.

The following system parameters were developed and approved by the EFRT consultants:

- The engineering-scale test facility should be a factor of 4.5 smaller than the full-scale Pretreatment Facility.

- Each ultrafilter assembly should contain 12 full-scale elements.

- Five ultrafilter assemblies should be provided (based on M-13 recommendations).

Additional bases for the engineering-scale test system factor of 4.5 are provided in Scaling Relationships for the Pretreatment Engineering Platform (Kuhn et al. 2008). Some of the scaling factors are listed below.

- Selected processes within the PEP will be operated at both scale-time (4.5 times shorter than plant time) and plant-time in separate test runs to address scaling issues.

- Prototypic tanks will be dimensionally scaled with a radius $1 / 4.5$ times their full-scale radius and operating height $1 / 4.5$ that of full-scale. The tank volume will be $1 /(4.5)^{3}$ or $\sim 1 / 90^{\text {th }}$ of the plant.

- The filtration design is scaled by filter area. The total filtration area is $1 /(4.5)^{2}$ or $\sim 1 / 20^{\text {th }}$ that of the plant.

- Fluid velocities in pipes are kept approximately the same (using commercially-available pipe diameters) to mimic solids settling that may occur in plant piping.

- Chemical reaction times are not scaled.

- The pulse jet mixer (PJM) nozzle velocity in the PEP will be scaled to provide the same mixing power per volume of tank contents. The plant targets a nozzle discharge velocity of $8-\mathrm{m} / \mathrm{s}$ in UFP-VSL-00001A/B and 12-m/s in UFP-VSL-00002A/B. For non-Newtonian fluids, the nozzle velocity will be the same in the PEP and WTP. For Newtonian fluids, the velocity is scaled by $1 /(4.5)^{0.333}$. The total PJM cycle time will be scaled to maintain the fraction of the cycle in active drive mode. It is recognized that there is not a sharp distinction from Newtonian to non-Newtonian behavior. For the purpose of Phase I testing, the PJM velocities were scaled to maintain equal power per volume for the initial concentration and caustic-leach. After the slurry is concentrated following the caustic-leach, the PJMs will be adjusted to have the same PJM nozzle velocities as the plant (non-Newtonian scaling) and kept in that regime for the remainder of the process (post-caustic-leach wash, slurry wash, oxidative leaching, final slurry washing, and final concentration). 
Section 6.4 of Pretreatment Engineering Platform (PEP) Testing (Phase I) Test Specification ${ }^{(a)}$ identifies the requirements for engineering-scale tests. The PEP data will be used by WTP along with laboratory-scale data and appropriate application of scaling to improve the WTP plant models used to predict plant operating performance.

\subsection{Objective}

The objective of this report is to present the results of the Shakedown/Functional Test with only very limited data analysis. This includes a test narrative, sample analyses, summaries of PEP monitoring data, evaluations or summaries of problems encountered, and deviations from the test protocols. Some specific test objectives and summary results are provided in the summary section of this report.

(a) JL Huckaby and JR Markillie. 2008. Pretreatment Engineering Platform (PEP) Testing (Phase I). 24590-PTF-TSP-RT-07-001, Rev 2, Bechtel National, Inc., Richland, Washington. 


\subsection{Quality Assurance Requirements}

The PNNL quality assurance (QA) program is based upon the requirements as defined in DOE Order 414.1C, Quality Assurance and 10 CFR 830, Energy/Nuclear Safety Management, Subpart A--Quality Assurance Requirements (a.k.a. the Quality Rule). PNNL has chosen to implement the following consensus standards in a graded approach:

- ASME NQA-1-2000, Quality Assurance Requirements for Nuclear Facility Applications, Part 1, Requirements for Quality Assurance Programs for Nuclear Facilities.

- ASME NQA-1-2000, Part II, Subpart 2.7, Quality Assurance Requirements for Computer Software for Nuclear Facility Applications.

- ASME NQA-1-2000, Part IV, Subpart 4.2, Graded Approach Application of Quality Assurance Requirements for Research and Development.

The procedures necessary to implement the requirements are documented through PNNL's "How Do I...?" (HDI). ${ }^{\text {(a) }}$ PNNL implements the RPP-WTP quality requirements by performing work in accordance with the River Protection Project-Waste Treatment Plant Support Program (RPP-WTP) Quality Assurance Plan (RPP-WTP-QA-001, QAP). Work was performed to the quality requirements of NQA-1-1989, Part I, Basic and Supplementary Requirements, NQA-2a-1990, Part 2.7, and DOE/RW-0333P, Rev 13, Quality Assurance Requirements and Descriptions (QARD) as applicable. These quality requirements are implemented through the River Protection Project-Waste Treatment Plant Support Program (RPP-WTP) Quality Assurance Manual (RPP-WTP-QA-003, QAM). The requirements of DOE/RW-0333P, Rev 13, Quality Assurance Requirements and Descriptions (QARD) and 10 CFR 830 Subpart A were not required for this work.

RPP-WTP addresses internal verification and validation activities by conducting an Independent Technical Review of the final data report in accordance with RPP-WTP's procedure QA-RPP-WTP-604. This review procedure is part of PNNL's RPP-WTP Quality Assurance Manual (RPP-WTP-QA-003). Following this procedure, a technical review would verify that the reported results are traceable, that inferences and conclusions are soundly based, and the reported work satisfies the objectives.

Table 2.1 shows a list of NCRs that impact simulant Shakedown/Functional testing data.

(a) PNNL's system for managing the delivery of laboratory-level policies, requirements, and procedures. 
Table 2.1. Description of NCRs Active During Shakedown/Functional Test

\begin{tabular}{|c|c|}
\hline $\begin{array}{l}\text { Nonconformance } \\
\text { Report Number }\end{array}$ & Description \\
\hline \multirow[t]{4}{*}{ NCR 38764.1} & $\begin{array}{l}\text { Measurement: 1) Inhibited Water (IW) supply to HLP-VSL-T22; 2) sodium } \\
\text { permanganate supply to UFP-VSL-T02A; 3) nitric acid supply to } \\
\text { UFP-VSL-T02A; 4) cooling water line for UFP-VSL-T02A; and 5) flush water } \\
\text { line for the PJMs. }\end{array}$ \\
\hline & $\begin{array}{l}\text { Issue: Due to the proximity of equipment vibration created by the operations } \\
\text { process, the vibration creates irregularities in the data collected, which are } \\
\text { recorded in the DAS. }\end{array}$ \\
\hline & $\begin{array}{l}\text { Affected instruments follow: FT-0135, FT-0651, FT-0659, FT-066, FT-0677, } \\
\text { FT-1621, and FT-1721. }\end{array}$ \\
\hline & What it means: Data from these instruments are For Information Only. \\
\hline \multirow[t]{4}{*}{ NCR 38767.1} & Measurement: Air flow rate to spargers in Tank UFP-VSL-T02A. \\
\hline & $\begin{array}{l}\text { Issue: Micro-Motion identifies flow rates, below which the uncertainty is } \\
\text { greater than } 0.5 \% \text {. In the case of the PEP, air flow rates below } 0.090-\mathrm{kg} / \mathrm{min} \\
\text { have uncertainties greater than } 0.5 \% \text {. For the lowest flow rate reported } \\
(0.012-\mathrm{kg} / \mathrm{min} \text { on FT-1977), the estimated uncertainty is } \sim 4 \% \text {. }\end{array}$ \\
\hline & $\begin{array}{l}\text { Affected instruments follow: FT-1901, FT-1973, FT-1977, FT-1981, and } \\
\text { FT-1995. }\end{array}$ \\
\hline & $\begin{array}{l}\text { What it means: Recognize the greater uncertainty that accompanies data with air } \\
\text { flow rates }<0.090-\mathrm{kg} / \mathrm{min} \text {. }\end{array}$ \\
\hline \multirow[t]{4}{*}{ NCR 41090.1} & $\begin{array}{l}\text { Measurement: Flow and temperature measurement for bottom air sparger to } \\
\text { UFP-VSL-T02A. }\end{array}$ \\
\hline & $\begin{array}{l}\text { Issue: The two analog outputs were cross-wired. The coriolis temperature } \\
\text { output designated as TT-1976 was wired to channel } 177 \text { of DAS2 instead of } \\
\text { channel } 174 \text {. The flow rate output from FT-1977 was wired to channel } 174 \\
\text { instead of } 177 \text {. Wiring was corrected on } 1 / 05 / 09 \text { at } 11: 30 \text {. }\end{array}$ \\
\hline & Affected instruments follow: FT-1977 and TT-1976. \\
\hline & $\begin{array}{l}\text { What it means: Affected data taken before } 1 / 05 / 09 \text { need to be corrected as } \\
\text { follows: }\left[\mathrm{TT}-1976\left({ }^{\circ} \mathrm{C}\right)\right]^{*}(0.73 / 110)=[\mathrm{FT}-1977(\mathrm{~kg} / \mathrm{min})] \text {. }\end{array}$ \\
\hline \multirow[t]{3}{*}{ NCR 43398.1} & Measurement: Temperature measurement in Tank UFP-VSL-T01B. \\
\hline & $\begin{array}{l}\text { Issue: TTK- } 0427 \text { failed at installation. All data from TTK- } 0427 \text { are invalid and } \\
\text { unusable. }\end{array}$ \\
\hline & $\begin{array}{l}\text { What it means: Do not use data from TTK-0427. (There are } 19 \text { other } \\
\text { temperature measurements available from this tank, with TTK- } 0425 \text { the } \\
\text { prototypic measurement.) }\end{array}$ \\
\hline
\end{tabular}


Table 2.1. Description of NCRs Active During Shakedown/Functional Test

\begin{tabular}{ll}
$\begin{array}{l}\text { Nonconformance } \\
\text { Report Number }\end{array}$ & \multicolumn{1}{c}{ Description } \\
\hline NCR 42402.1 & Measurement: Temperature measurement of the slurry in the filter-loop. \\
& $\begin{array}{l}\text { Issue: The following RTD thermowells in the filter-loop do not extend into the } \\
\text { process stream: }\end{array}$ \\
& TT-0513: HX-T02A (cooling) slurry outlet temperature. No data are to be used \\
& for quality-affecting work. Data may be used for qualitative purposes only. \\
& $\begin{array}{l}\text { TT-0515: HX-T03A (heating) slurry outlet temperature. No data are to be used } \\
\text { for quality-affecting work. Data may be used for qualitative purposes only. }\end{array}$ \\
& $\begin{array}{l}\text { TT-0537: Filter \#5 outlet temperature (HX02A inlet). No data are to be used for } \\
\text { quality-affecting work. Data may be used for qualitative purposes only. }\end{array}$ \\
& $\begin{array}{l}\text { TT-0791: Filter \#1 inlet temperature. Data from this should not be used at all. } \\
\text { Tank temperature data will be used for quality-affecting filter processing data. }\end{array}$ \\
& $\begin{array}{l}\text { Measurement: On-line density measurement in UFP-VSL-T01B coriolis sample } \\
\text { station. }\end{array}$ \\
& Issue: DT-2101 failed. \\
& What it means: All readings after 12/23/2008 are not valid. \\
\hline
\end{tabular}





\subsection{Experimental Methods and Apparatus}

\subsection{Testing Overview}

The operations log during Shakedown/Functional testing is recorded in OpLog2 pg. 157-168 and OpsLog 3 pg. 1-94. The Lead Test Engineer (LTE) observations were recorded in laboratory record book (LRB) BNW-59944 and 60108. The filters were cleaned with oxalic acid immediately before Shakedown/Functional testing and again before the start of Test Instruction (TI) TI-032. This was after initial partial testing under TI-062 and TI-067. The instruction for acid cleaning is provided in Appendix $\mathrm{H}$, and observations during the cleaning are recorded in LRB BNW-59944.

The following is a summary level overview of testing reported herein. Refer to the test narrative (Section 4), Test Instructions TI-WTP-PEP-062, TI-WTP-PEP-067, and TI-WTP-PEP-032, and the LRBs listed above for additional details.

1. Acid clean UFP-FILT-T01A through -T05A with 0.5-M oxalic acid.

2. Rinse filters and filter-loop to achieve targeted $\mathrm{pH}$ of $\sim 3$.

3. Prepare, stage, and characterize simulant in HLP-VSL-T22 and reagents to be used.

4. Transfer simulant from HLP-VSL-T22 to UFP-VSL-T01A (T01A) and conduct a performance check on level instruments.

5. Transfer simulant from HLP-VSL-T22 to UFP-VSL-T01B (T01B) and conduct a performance check on level instruments.

6. Conduct a line flush on the transfer line between HLP-VSL-T22 to T01B and sample transfer contents for five line volumes of transfer.

7. Transfer simulant from T01A to UFP-VSL-T02A (T02A) and conduct a performance check on level instruments.

8. Conduct a solids stratification test on T01A at prototypic PJM conditions.

9. Condition ultrafilters (low-solids filter flux test) for 36 hours, including 24 backpulses at 30 -min. intervals during hours 12 to 24 .

10. Concentrate solids in Tank T02A to $20-\mathrm{wt} \%$ with only the first filter bundle (UFP-FILT-T01A).

11. Concentrate a larger volume in Tank T02A to $20-w t \%$ with four filter bundles, UFP-FILT-T02A through -T05A.

12. Caustic-leach concentrated simulant in Tank T02A at $98^{\circ} \mathrm{C}$ for 16 hours.

13. Cool leached simulant to $25^{\circ} \mathrm{C}$ at controlled rate.

14. Caustic-leach unconcentrated simulant in $\mathrm{T} 01 \mathrm{~A} / \mathrm{B}$ at $98^{\circ} \mathrm{C}$ for 16 hours.

15. Cool leached simulant to $25^{\circ} \mathrm{C}$ at controlled rate.

16. Dewater leached material to $17-\mathrm{wt} \%$ using four filters, UFP-FILT-T02A through -T05A at constant level control feeding from T01A.

17. Wash leached slurry and remove permeate using four filters, UFP-FILT-T02A through -T05A.

18. Add chromium slurry simulant. 
19. Reconcentrate slurry and wash using four filters, UFP-FILT-T02A through -T05A.

20. Conduct high-solids filter test removing permeate through one filter, UFP-FILT-T01A.

21. Conduct oxidative-leach on slurry for 6 hours at $25^{\circ} \mathrm{C}$. View level of foaming during oxidative-leach.

22. Clean out system and prepare for integrated process testing.

\subsection{Simulant}

PEP process testing was performed with a nonradioactive aqueous slurry of simulant waste solids and liquids. The simulant composition and make-up recipe were provided by WTP as documented in Simulant Recommendation for Phase I Testing in the Pretreatment Engineering Platform. ${ }^{\text {(a) Aqueous }}$ chemical concentrations were within the ranges expected for waste feeds to the PTF. The hydroxide concentration was marginally one standard deviation lower than the average concentration expected in the feeds to the plant. The oxalate and phosphate components were at the lower end of the expected ranges, but the oxalate component was at the solubility limit, and the phosphate component was at or near the solubility limit. The solids components and blend were selected to obtain targeted solids mass loss (aluminum and chromium leaching and oxalate washing) and treatment time. The simulant was not selected to represent any particular Hanford tank waste type.

The simulant was blended from the components listed below. The basis for selecting the individual components and the comparison to actual waste behavior is provided where applicable in the indicated references.

- Boehmite (for Al) (Russell et al. 2009a)

- Gibbsite (for Al) (Russell et al. 2009b)

- Chromium oxyhydroxide (CrOOH) slurry (Rapko et al. 2007)

- Sodium oxalate

- Filtration simulant (Russell et al. 2009c)

- Supernate.

A separate chromium solids slurry simulant was prepared and added to the PEP process after post-caustic-leach washing (a nonprototypic addition) during the Shakedown/Functional Tests and Integrated Tests A and B. This approach was taken because laboratory-scale tests had shown that the high-temperature caustic leaching step dissolved significant amounts of the $\mathrm{CrOOH}$ solids (Russell et al. 2009a). In Integrated Test $D$, the chromium solids component of the simulant was added during the simulant make-up process to demonstrate the PTF permanganate addition strategy. Simulant was procured from NOAH Technologies Corporation (San Antonio, TX). Samples of each simulant batch were characterized to make certain that chemical and physical property requirements were met. Batches of the simulant were procured as follows:

- A 15-gallon trial batch of the blended simulant for laboratory testing to demonstrate the efficacy of the simulant fabrication procedure.

(a) PS Sundar. 2008. Simulant Recommendation for Phase I Testing in the Pretreatment Engineering Platform. 24590-PTF-RPT-RT-08-006, Rev. 0, Bechtel National, Inc., Richland Washington. 
- A 250-gallon scale-up batch of the blended simulant to demonstrate scale-up of the simulant fabrication procedure to an intermediate scale.

- Batches 0, 1, and 2, each nominally 3500 gallons, of blended simulant for the Shakedown/Functional Test and Integrated Tests A and B. These batches did not contain the $\mathrm{CrOOH}$ component.

- Batch 3, nominally 1200 gallons, for Integrated Test D. This batch contained the CrOOH solids component.

- The CrOOH solids slurry for the Shakedown/Functional Test and Integrated Tests A and B was obtained in two separate batches containing nominally 18- and 36-kg of $\mathrm{Cr}$ as $\mathrm{CrOOH}$.

Characterization data for the simulant are detailed and discussed further as part of analytical data in Section 5-Data.

\subsection{Pretreatment Engineering Platform Overview}

The following section provides an overview of the PEP to orient the reader. This section does not provide details on PEP equipment or instrumentation. The interested reader is referred to the following documents for more details on the PEP:

- P\&IDs and mechanical data books kept with the PEP (currently available at the Process Development Laboratory-West [PDL-W]).

- Pretreatment Engineering Platform (PEP) Phase I Testing Process Description, 24590-WTP-RPT-PET-07-002, Rev 1.

- Functional Requirements for Engineering-Scale Pretreatment System, 24590-PTF-3YD-UFP-00002, Rev 1.

The PEP test system is designed to perform engineering-scale demonstrations of most WTP pretreatment processes. These include (but are not limited to) vessel-to-vessel transfers, waste pumping, cross-flow filtration, filter cleaning, waste solids washing, chemical reagent addition and mixing, waste slurry heating and cooling, and waste chemical leaching. Figure 3.1 presents a highly simplified process diagram showing the vessels, pumps, heat exchangers, and filter systems associated with the PEP. Equipment that has been considered critical for evaluating the integrated system performance has been scaled to be prototypic. Specifically, slurry-handling vessels have been scaled to be geometrically similar to WTP with a $1 / 4.5$ scale; the working height and diameter are scaled to $1 / 4.5$. Pipe sizes are scaled to have approximately $1 / 4.5$ the diameter, but the fluid velocity is to be approximately the same as the full-scale plant.

Waste simulant feed can be received from three primary sources: High Level Waste (HLW) Feed Receipt Vessel (HLP-VSL-T22), Low-Activity Waste (LAW) Waste Feed Receipt Product (FRP) Vessels (FRP-VSL-T01), or Feed Evaporator Product (FEP) Vessel (FEP-VSL-T01). Feed is received into the Ultrafiltration Feed Preparation Vessels, T01A/B. In Shakedown/Functional testing, feed from T01A/B was used to provide simulant feed to Tank T02A. Simulant concentration, caustic leaching, oxidative leaching, and washing were conducted in Tank T02A and the associated filter-loop. The separated solids were transferred to HLW storage (HLP-VSL-T27A) while the liquid fraction (permeate) was stored in a permeate collection vessel (UFP-VSL-T62A/B). Wash and leach solutions were sent to UFPVSL-T62A/B. 


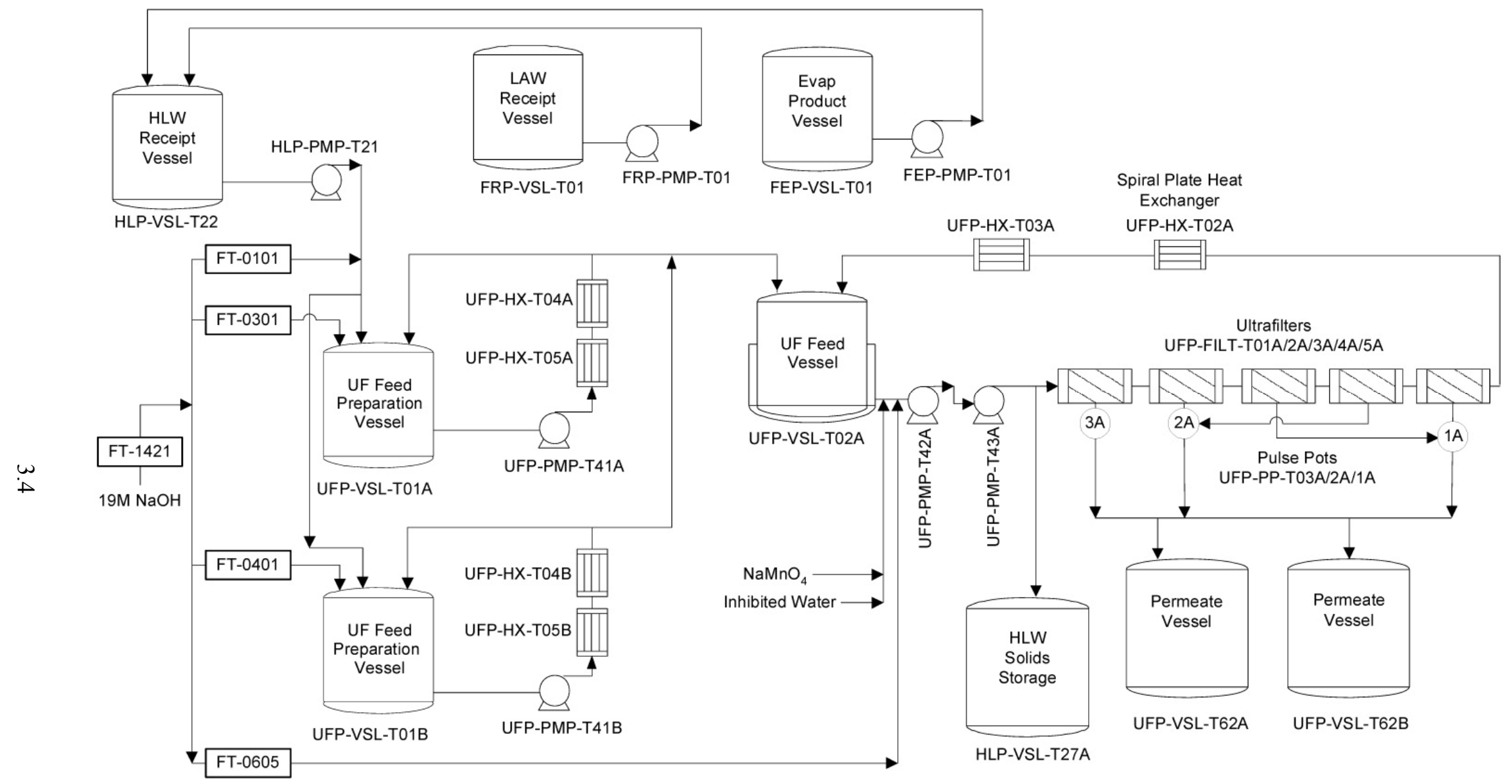

Figure 3.1. PEP Simplified Flow Diagram 


\subsection{PEP Filtration System}

The PEP filtration system is composed of an ultrafiltration feed tank (Tank T02A), a slurry circulation and filter-loop, a permeate metering and collection system, and a filter backpulse and cleaning system. A simplified schematic of the filtration system is shown in Figure 3.2. The PEP filtration system is configured to measure the feed flow rate, temperatures, and axial and transmembrane pressure (TMP) drop across each filter bundle. In addition, the system is configurable such that filter bundles 1 through 5 may be connected in-series to the slurry circulation loop or bypassed such that flow is directly through filter bundle 1 or through filter bundles 2 through 5. A summary of process instrumentation is provided in Appendix A. In the following paragraphs, key process equipment for slurry filtration operations are identified and discussed. Interested readers are referred to the documents listed in Section 3.1 if more information is needed.

\subsubsection{Ultrafiltration Feed Tank}

Tank T02A serves as a primary supply and mixing reservoir for slurry being circulated through the filter-loop. The contents of this tank are mixed using an array of six PJMs. Ancillary systems for Tank T02A include air spargers to limit flammable gas hold-up in the actual waste treatment system, bubblers to measure slurry density and level, laser level sensors, and an array of resistance temperature detectors (RTDs) to measure the tank temperature profile. (Specific locations of the RTD arrays for Tanks T02A and T01A are available in LRB 59944, pages 12-17.) Tank T02A is equipped with a water jacket supplied with chilled water to cool the contained slurry.

\subsubsection{Slurry Filtration Circulation Loop}

The filter-loop contains process equipment key to slurry dewatering and washing operations. It is composed of two slurry pumps, a series of five filter bundles, and two heat exchangers.

Two centrifugal slurry pumps, UFP-PMP-T42A and UFP-PMP-T43A (hereafter referred to as T42A and $\mathrm{T} 43 \mathrm{~A}$, respectively), are operated in-series to provide the required slurry flow rate and pressure for the cross-flow filter bundles. The suction to T42A is fed by Tank T02A. In addition, the feed to pump $\mathrm{T} 42 \mathrm{~A}$ is connected to process $\mathrm{IW}^{(\mathrm{a})}$ supplies used for slurry washing and dilution operations. The discharge from pump T42A feeds pump T43A. Slurry discharge from pump T43A can be fed through, or bypassed around, the cross-flow filter banks. Pumps T42A and T43A provide a combined filter-loop flow rate and pressure of up to $150 \mathrm{gpm}$ and $243 \mathrm{psig}$. (Note: Only pump T42A was used during Shakedown/Functional testing under TI-032. T43A was locked out because of pressure/safety concerns.)

(a) IW typically refers to a $0.01-\mathrm{M}$ solution of $\mathrm{NaOH}$. 


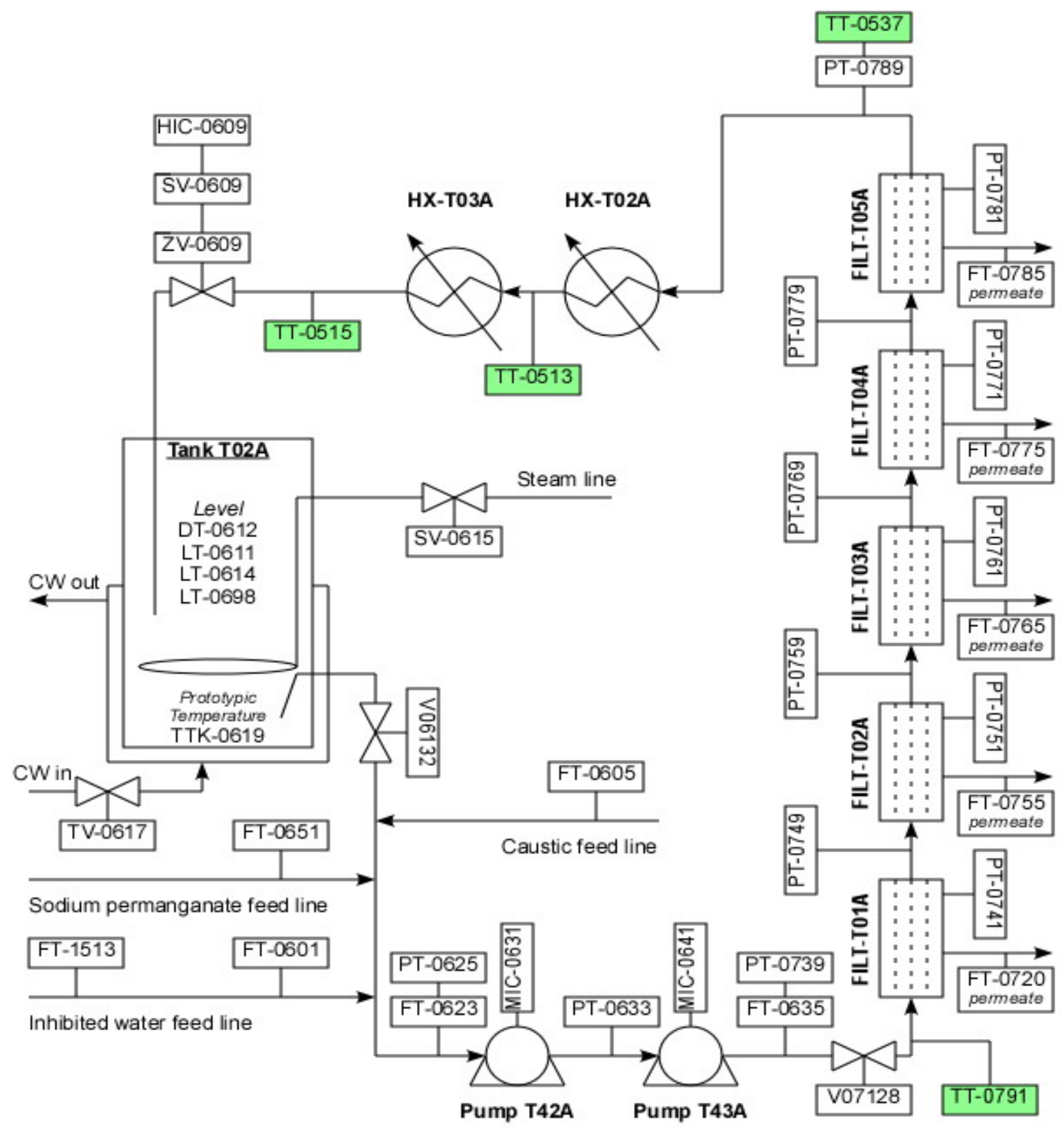

Figure 3.2. Schematic of UFP-VSL-T02A Loop and Filtration

(Note: During TI-062, both HX-T02A and HX-T03A were aligned. It was demonstrated that HX-T03A would not be needed. Thereafter, HX-T03A was bypassed during TI-032 and subsequent Integrated testing.) (Legend: DT — density transmitter, HX — heat exchanger, LT - level transmitter, FT - flow transmitter, PT - pressure transmitter, TT - temperature transmitter (including matching TE (temperature element)), $\mathrm{MIC}=$ Motor Indicating Controller, $\mathrm{V}=$ valve, $\mathrm{SV}=$ solenoid valve, $\mathrm{ZV}=$ positioning valve, and $\mathrm{HIC}=$ human interface control. Instruments with Nonconformance Reports issued during testing are shaded.) 
The cross-flow filter system is the core of slurry liquid-solid separations. It is composed of five filter bundles operated in-series. These filter bundles are designated as UFP-FILT-T01A to -T05A (hereafter referred to as filters 1 through 5). The filter-loop is equipped with slurry bypass valves to allow slurry flow through filter bundle 1 and/or filters 2 through 5 .

The filters purchased for the PEP were obtained from the Mott Corporation (Farmington, CT) using the same specifications for the filters being purchased for the WTP PTF. The filters are constructed of porous sintered 316 stainless steel with an effective filtration rating of $0.1-\mu \mathrm{m}$. The PEP test system employs a combination of 8-ft-long and 10-ft-long filter elements (which were formed by welding either four or five 2-ft filter elements together). As such, the PEP elements have the same radial dimensions and filtration ratings as the 2 - $\mathrm{ft}$ elements, but have a longer filtration length of either 96 or 120 inches. A summary of the geometries of the five filter bundles is provided in Table 3.1.

Tubeside slurry flow rate and pressure are monitored by a series of flow meters and pressure transducers. Slurry flow to pump T42A is measured by magnetic flow meter FT-0623. Slurry discharge flow from pump T43A is measured by a second magnetic flow meter (FT-0635). Circulation loop pressure is monitored by a series of pressure transducers located at the entrance to each slurry pump, filter bundle, and heat exchanger.

The temperature in both Tank T02A and the slurry filter-loop is controlled with a combination of two in-line heat exchangers. The first heat exchanger, UFP-HX-T02A, is a spiral-plate heat exchanger that uses chilled water to cool the circulating slurry. The second heat exchanger, UFP-HX-T03A, is a steam exchanger intended to heat the circulating slurry if needed. Both heat exchangers are equipped with a bypass loop so that they can be isolated from slurry flow. RTDs installed in thermowells monitor and control the performance of the heat exchanger. ${ }^{\text {(a) }}$ For the current testing, UFP-HX-T03A was not used and was bypassed. The final process element in the slurry circulation loop is a pressure control valve (SV-0609) that can be adjusted in combination with the slurry pumps to provide adequate backpressure for permeate production. After passing through SV-0609, the dewatered circulating slurry is recycled back into Tank T02A.

(a) The RTD measuring the slurry outlet temperature (TT-0513) did not extend into the process stream, and data were to be used for qualitative purposes only. See discussion in Section 2 for a description of this nonconformance (NCR 42402.1). The impact for testing was that during Shakedown/Functional testing, the temperature control for the cooling heat exchanger in the filter-loop, UFP-HX-T02A, was a hybrid method of control. The cooling water control valve (TV-0513) was in automatic, controlling the outlet slurry temperature,TT-0513. However, the temperature setpoint was manually changed to try to control the temperature in Tank $\mathrm{T} 02 \mathrm{~A}$ at $25^{\circ} \mathrm{C}$. This control scheme resulted in more temperature variation than under automatic control. 
Table 3.1. Specifications of the Five PEP Cross-Flow Filtration Bundles

\begin{tabular}{cccccc}
\hline Filter \# & Filter ID & $\begin{array}{c}\text { Number of } \\
\text { Elements in } \\
\text { Bundle }\end{array}$ & $\begin{array}{c}\text { Element } \\
\text { Inside } \\
\text { Diameter } \\
\text { (inches) }\end{array}$ & $\begin{array}{c}\text { Element } \\
\text { Length }(\mathrm{ft})\end{array}$ & $\begin{array}{c}\text { Burface Area } \\
\left(\mathrm{ft}^{2}\right)\end{array}$ \\
\hline 1 & UFP-FILT-T01A & 12 & 0.5 & 10 & 15.7 \\
2 & UFP-FILT-T02A & 12 & 0.5 & 10 & 15.7 \\
3 & UFP-FILT-T03A & 12 & 0.5 & 10 & 15.7 \\
4 & UFP-FILT-T04A & 12 & 0.5 & 8 & 12.6 \\
5 & UFP-FILT-T05A & 12 & 0.5 & 8 & 12.6 \\
Total & -- & -- & -- & -- & 72.3 \\
\hline
\end{tabular}

\subsubsection{Permeate Metering and Collection Systems/Filtration Backpulse Systems}

The permeate metering and collection systems consist of Coriolis mass flow meters for monitoring permeate production rates, permeate collection tanks, and three pulse-pots connected to high-pressure air supplies for backpulsing the filter bundles.

Permeate (shellside) mass production rates from filters 1 through 5 are monitored by Coriolis flow meters. Permeate flow from each of the filter bundles is directed to three pulse-pots (designated as UFP-PP-T01A to UFP-PP-T03A). Pulse-pot UFP-PP-T03A serves filter bundle 1, pulse-pot UFP-PP-T02A serves filter bundles 2 and 4, and pulse-pot UFP-PP-T01A serves filter bundles 3 and 5 . The pulse-pots are filled with a sufficient volume of collected permeate to backpulse the filter bundles. Overflow from the pulse-pots may be directed to 1) permeate or process slurry collection tanks (UFP-VSL-T62A and -T62B) during slurry dewatering operations, or 2) a return line to Tank T02A during continuous recycle filtration operations. Table 3.2 summarizes the permeate metering and pulse-pot systems.

Table 3.2. Permeate Metering and Pulse-Pot Configurations for PEP

\begin{tabular}{lcc}
\hline Filter Bundle No. / ID & Permeate Coriolis Meter & Associated Pulse-Pot \\
\hline 1 - UFP-FILT-T01A & FT-0720 & UFP-PP-T03A \\
2- UFP-FILT-T02A & FT-0755 & UFP-PP-T02A \\
3 - UFP-FILT-T03A & FT-0765 & UFP-PP-T01A \\
4- UFP-FILT-T04A & FT-0775 & UFP-PP-T02A \\
5- UFP-FILT-T05A & FT-0785 & UFP-PP-T01A \\
\hline
\end{tabular}

In addition, the pulse-pots also facilitate backflushing of the filter bundles (i.e., backpulsing operations). During backpulsing, one of the pulse-pots is isolated and charged with high-pressure air until the pulse-pot pressure exceeds the tubeside pressure of the filter bundle to be backpulsed by a given amount (typically 40 psid). After the target pulse-pot pressure is reached, the valve isolating the pulse-pot 
from the filter is opened, and the permeate collected in the pulse-pot flows back through the filter element. The back-flow of permeate loosens any particles that are weakly entrained in the filter pores or that have caked on the filter surface. A description of the backpulsing process, as it was implemented in the PEP, is provided in Appendix B.

\subsection{PJM Operational Modes}

The following modes of PJM operation were used during the testing: standard mode (regular), standard mode (star pattern-Tank T02A only), short-cycle mode, and simple mode. Testing started with TI-062 using the standard mode, but the remaining Shakedown/Functional testing was conducted using the simple mode after the Drexelbrook level probes failure in Tank T02A during the caustic-leach. The short-cycle function would automatically activate when the tank level dropped to 12 inches and would remain active until the tank level rose above 20 inches.

All PJM operating modes have the same basic cycle: Drive Phase, Vent Phase, and Vacuum Phase, followed by a Hold (or Vent) Phase. PJM mixing technology involves a pulse tube coupled with a jet nozzle. One end of the tube is immersed in the tank while periodic pressure, vacuum, and venting are supplied to the opposite end. Changing the applied pressure creates four operating modes for the pulse tube: 1) the drive mode, when pressure is applied to discharge the contents of the PJM tube through the nozzle, 2) the vent mode, when the pressure is vented to the atmosphere, 3) the refill mode, when vacuum is applied to refill the pulse tube (at temperatures above $60^{\circ} \mathrm{C}$, the vacuum is disabled, and the pulse tubes refill by gravity), and 4) the Hold mode, when all valves to the PJMs are closed, causing the fluid level in each PJM to remain constant until the next drive phase. The PJM system uses these operating modes to produce a sequence of drive cycles that provide mixing in the vessel.

A timer set to the overall cycle time starts at the beginning of each Drive Phase. A new Drive Phase begins when either the cycle timer runs out or the cycle enters the final Hold/Vent Phase, whichever is later. This condition exists to make sure the Vacuum Phase has completed before moving on to the next cycle.

PJM operations at PEP consisted of one of several control modes: standard mode (regular), standard mode (star pattern-Tank T02A only), standard mode (short-cycle), and simple mode. However, PJM controls at PEP were not prototypic. During Integrated Test B, the standard mode (regular) was used whenever possible, and the standard mode (star pattern) was used when the level was low, typically $<23$ inches. If standard mode was nonfunctional (e.g., during caustic leaching, the PJM Drexelbrook level probes ceased functioning), the PJMs were operated in simple mode. For all tanks, if the PJMs were operating in standard mode, the short-cycle function would automatically activate when the tank level dropped to 12 inches and would remain active until the tank level rose above 22 for Tank T01 A and Tank T01B. The short-cycle activation occurred automatically in Tank T02A when the level dropped to 11.5 inches and remained until the level returned to 16 inches.

\subsubsection{Standard Mode (Regular) Description}

Standard mode operations control each PJM individually based on that PJM level instrument and one overall cycle time. Each cycle is controlled by five main variables: cycle time, drive time set point, vent time set point, L1 level, and L2 level. (L1 and L2 levels are user-definable upper and lower level set 
points used by the control software to stop the vacuum in the PJM [PJM full], or identify overblows, respectively.) Additionally, each PJM could be enabled or disabled individually.

The specified cycle time entered controlled the peak-to-peak distance between cycles. If a very short-cycle time is entered, the PJM cycles will run as quickly as possible while still completing the first three phases (Drive, Vent, and Vacuum), but may be longer than the set point.

The Drive Phase is controlled by the time entered as the drive time set point (corrected for temperature if enabled). Each PJM begins the Drive Phase at the same instant, but can have different lengths of time specified for driving each PJM. If any PJM does not finish within 30 seconds of the PJM with the shortest drive time, that PJM was disabled, and the cycle continued to the next phase.

The Vent Phase is controlled by the vent time set point, which has no correction factors. Each PJM begins the Vent Phase at the same instant, but can have different lengths of time specified for each PJM. If any PJM does not finish within 30 seconds of the PJM with the shortest vent time, that PJM will be disabled, and the cycle will continue to the next phase. Generally, each vent time set point was set to a small number such as 500 milliseconds to smooth out the transition between the Drive and Vacuum phases.

The Vacuum Phase is controlled by the L1 Level, which is set at the top of the desired PJM stroke. All PJMs begin the Vacuum Phase at the same instant, but each PJM L1 Level is set separately. If any PJM does not reach the L1 Level within 30 seconds of the first PJM to reach its L1 Level, that PJM is disabled, and the cycle continues to the next phase. When tank temperatures are greater than $60^{\circ} \mathrm{C}$, the Vacuum Phase is disabled, and each PJM vents to atmosphere until it reaches the L1 Level set point. This is commonly referred to as gravity refill. The final phase in the standard mode is a Hold Phase. All valves to the PJM are closed, causing the fluid level in each PJM to remain constant at the L1 Level.

The standard mode operation also contains a temperature linearization variable that reduces the drive time to prevent overblows because of the difference in fluid viscosity as the temperature increases. The variable is expressed as a slope, the percent of drive time decrease per degree Celsius increase from $0^{\circ} \mathrm{C}$. This function was largely untested. A modest $0.25 \%$ linearization factor was used in early testing and was adopted for the remainder of testing simply because it seemed to work. Further optimization was not conducted.

\subsubsection{Standard Mode (Short-Cycle)}

A short-cycle consists of a Drive Phase in which each enabled PJM drives for $20 \%$ of the drive time set point followed by a 10-second Vent Phase. The short-cycle function would automatically activate when the tank level dropped to 12 inches and would remain active until the tank level rose above 22 inches for Tank T01A and Tank T01B. The short-cycle activation occurred automatically in Tank T02A when the level dropped to 11.5 inches and remained until the level returned to 16 inches. Additionally, the short-cycle would activate during high temperature operations $\left(>60^{\circ} \mathrm{C}\right)$ if the tank level fell below 44 inches (T01A/B) or 46 inches (T02A) to confirm that there was enough fluid to gravity fill above the L1 Level for each PJM due to the disabling of the vacuum at high temperatures. 


\subsubsection{Standard Mode (Star Pattern)}

Tank T02A additionally can operate in standard mode (star pattern). The star pattern option follows the same rules as the regular standard PJM mode. The difference is that only two PJMs are enabled at a given time. When the operator sets the star pattern button on the human machine interface (HMI), the center PJM and one other PJM will operate through a set number of cycles, typically five. Then the outer PJM will be disabled, and a different outer PJM will be enabled for the same number of cycles. The pattern of enabling and disabling the five outer PJMs forms a five point star. The star mode is used to reduce the level fluctuations in Tank T02A because fewer PJMs are filling and discharging in this mode. The star mode was developed during Integrated process testing. This mode did not apply during simulant Shakedown/Functional process testing.

\subsubsection{Simple PJM Mode Description}

As mentioned above, the simple mode operates substantially the same as the standard mode. Each cycle consists of Drive, Vent, Vacuum, and Hold/Vent phases. There are two main differences. The first difference is that all PJMs operate together as one unit. There are no individual levels used or individual enabling or disabling of PJMs. The second difference is that each phase is controlled by a time (instead of level). An operator sets the cycle time, drive time set point (uncorrected), vent time set point, and vacuum time set point (uncorrected). Additionally, the operator can choose whether the last phase holds the PJM level or vents (returning the PJM level to the same as the tank level).

During Shakedown/Functional testing, the simple mode was used when the standard mode was not working. The standard mode did not work during leaching operations because the PJM level probe ceased to function at elevated temperatures in high-caustic solutions.

The drive time had additional correction factors for temperature (same as in standard mode) and tank level. The tank level correction (if enabled) reduced the drive time according to the user-entered slope (\% drive time/inch) for every inch below the top of the PJM head (46 inches by default). For example, if the drive time set point was 10,000 milliseconds, the drive slope was $1 \% /$ inch, and the tank level was at 36 inches, then the corrected drive time would be $(10,000$ milliseconds $) *\{1.00-[0.01 /$ inch $*$ (46 to 36 inches) $]\}=9000$ milliseconds. The operator could select which the tank level measurement was used for the slope compensation, either the tank bubbler, laser, or Drexelbrook probe.

The vacuum time also contained a correction factor for tank level, but not for temperature. Although the vacuum correction has its own set point, the calculation is the same as for the drive time, except that the vacuum time increases when the tank level is below 46 inches, whereas the drive time decreases.

The operator-selectable hold phase was created to give the option to more closely resemble standard PJM operation. If the hold phase is disabled, each PJM just vents to the tank level during the remainder of the PJM cycle.

\subsection{Data Acquisition System for PEP}

The DAS for PEP is described in Appendix F. 


\subsection{Sampling}

A list of samples taken and their purpose is provided in Appendix C. Actual sample times were recorded in the Test Instruction.

In general, slurry samples were collected using either an in-tank sampler or an in-line sampler. In-line samples were obtained from the slurry recirculation loop or transfer piping by drawing a side stream from the process flow as shown in Figure 3.3. To obtain a sample, the second valve was fully opened, and then the first valve was opened sufficiently to allow samples to be safely obtained. The sample line and valves were purged with at least three line volumes before each sampling event.

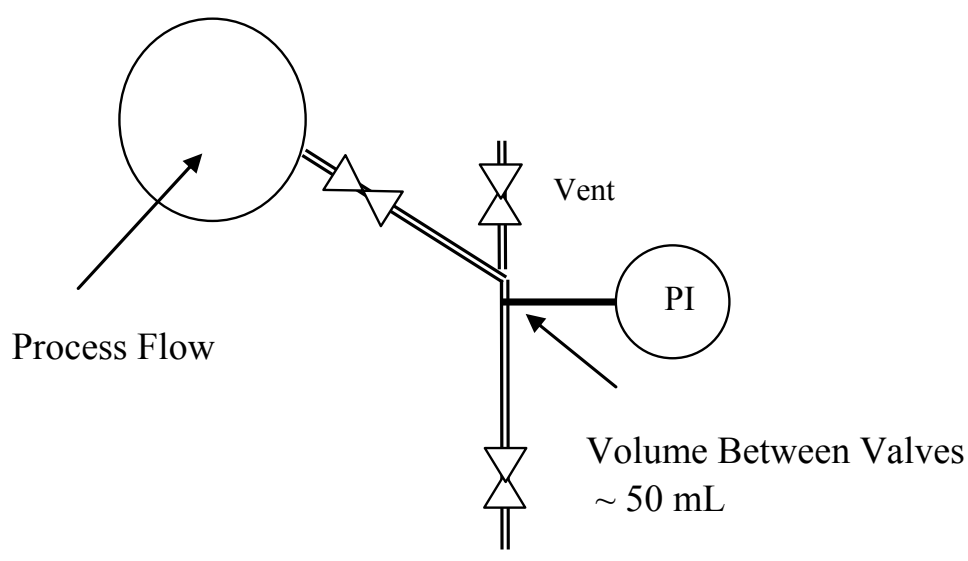

Figure 3.3. Simple In-Line Sampler

Figure 3.4 is a schematic of the in-tank sampling system for Tank T01A/B and Tank T02A. Samples were obtained with the sample loop in recirculation mode with slurry returned to the vessel. To obtain a sample, a valve was used to divert the entire flow to the sample bottle. The sampling valve and line were purged before each sample to minimize cross contamination with previous sampling events. Sample heights are provided in Appendix D. 


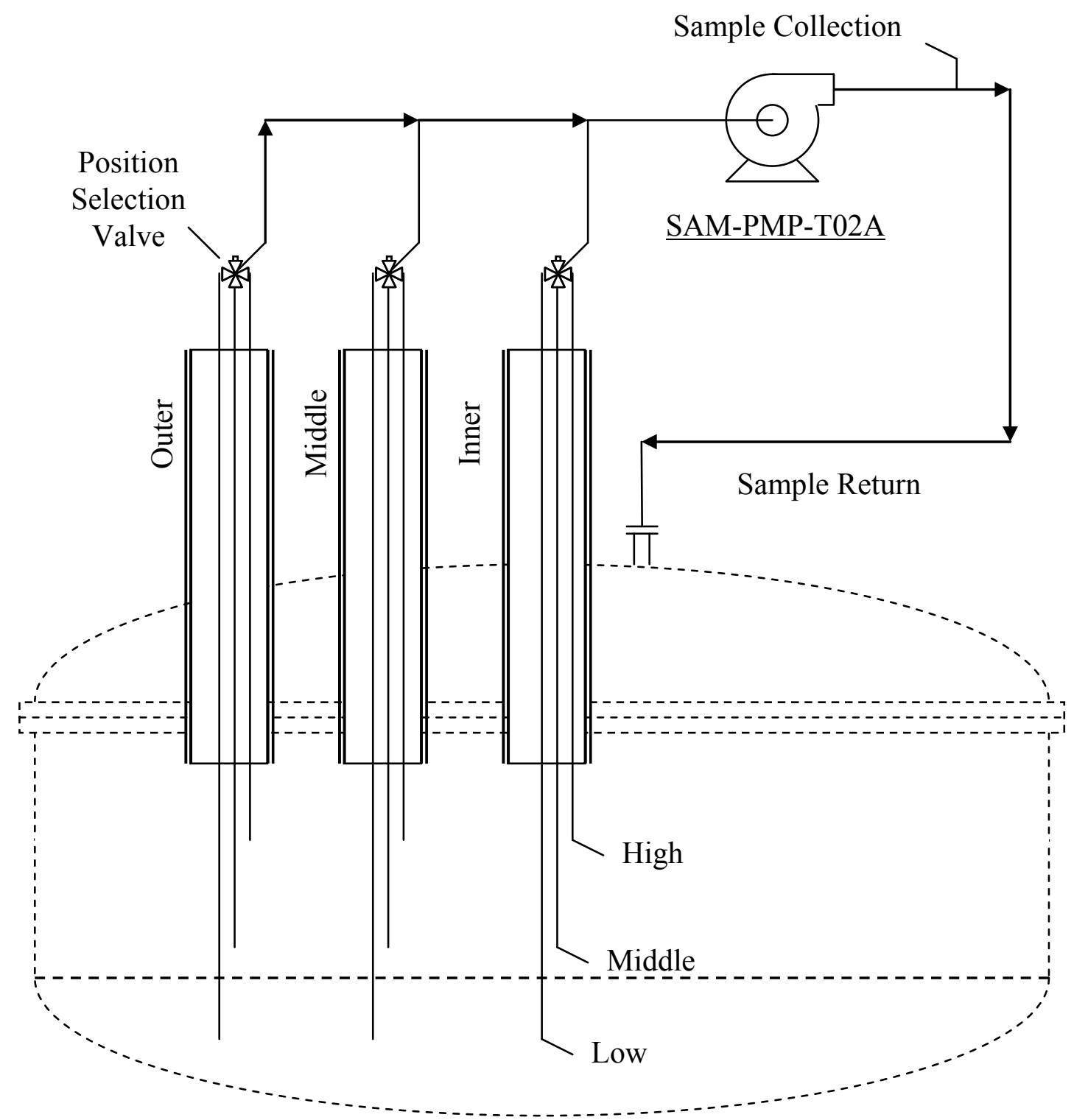

Figure 3.4. In-Tank Sampling Showing the Three Radial Positions at Three Heights and Sampling Flow Loop

Permeate (liquid) samples were taken from the permeate piping between the filter and the pulse-pot. The sample line and valves were purged with at least three line volumes before each sampling event.

\subsection{Sample Processing}

The samples were collected in pre-labeled sample containers that were prepared and staged within PDL-W based upon the Sample Collection and Analysis table in the governing Test Instruction. The required analysis determined the sample volume. All samples for chemical analyses approximately 40-mL of sample was taken in a 50-mL plastic centrifuge cones with a screw top lid. If centrifugation 
was needed it was done in the original sample container. If centrifuging wasn't needed the cone was handled as a regular sample bottle. Samples for yield stress analyses and samples for parallel lab testing were taken in larger containers, but all other samples were $50-\mathrm{mL}$ sample cones. For analyses requiring larger volumes (e.g. shear stress analyses) multiple 50-mL cones were filled and then combined at the analytical lab. Sample handling flow diagrams and method descriptions are detailed in Appendix E. 


\subsection{Simulant Functional Testing Narrative}

PEP simulant Shakedown and Functional testing evaluated the performance of the individual components of the PEP and finalized the operational characteristics (thermal distribution, solids distribution, etc.) before the Integrated process tests. This included finalizing the strategies for managing condensate, tuning PJMs using simulant, and operating spargers at elevated temperatures. The Functional Test performed transfer, mixing, heating, cooling, and filtration unit operations with simulant to 1) confirm testing procedures as well as equipment and instrument functions and performance, and 2) determine limited baseline process/equipment performance needed to perform Integrated process testing. The success criterion of the Functional testing was to obtain the qualified data (i.e., in accordance with the RPP-WTP QAP) needed to perform final planning for the Integrated process tests.

The data reported in this narrative section were generally taken from the Test Instruction (TI) and are considered For Information Only. They are not qualified per the RPP-WTP QAP because data recorded in the TI were usually obtained from the HMI (rather than the DAS or field transmitters). Qualified data can be obtained from tables in Section 5 of this report, the DAS files provided separately on transportable hard drives, and the figures in Appendix J, which were plotted using DAS data and meet project quality requirements.

Simulant Functional testing was conducted under three Test Instructions, TI-062, TI-067, and TI-032, to perform testing identified in the PEP testing (Phase I) Test Plan, TP-RPP-WTP-506. ${ }^{\text {(a) }}$ Three separate TIs were issued because some TI steps had to be repeated. Testing began with TI-062 on $11 / 22 / 2008$, and was suspended for the Thanksgiving holiday. Shortly after testing resumed on 11/30/2008, the ultrafilter housing gaskets developed leaks, and the PJM level probes in Tank T02A failed. While repairs were underway, leaching in Tank T01A was tested under TI-067. After repairs were completed, simulant Functional testing was fully executed under TI-032. The partial testing conducted under TI-062 and TI-067 was repeated, and test steps not previously executed because of equipment problems were executed completely.

Findings from simulant Functional testing included:

- Transfer Simulant from HLP-VSL-T22 to Tank T01A/B. The intent was to demonstrate steady-state solids distribution in Phase I simulant before and during transfer to UFP vessels. The four samples collected during transfer in TI-062 showed adequate mixing during transfers. ${ }^{\text {(b) }}$

- Evaluate Stratification of Solids in Tanks T01A/B and T02A. The intent was to demonstrate steady-state solids distribution in Tank T01A with Phase I simulant at 5-wt\% UDS after transfer. The data from Test Instructions TI-032 and TI-062 demonstrated that the objective of negligible solids settling was met satisfactorily both during stratification tests in Tanks T01A/B and during the caustic-leach processes in Tank T01A, Tank T01B, and Tank T02A. ${ }^{(\mathrm{c})}$

(a) GB Josephson, OP Bredt, JK Young, and DE Kurath. 2009. Test Plan for Pretreatment Engineering Platform (PEP) Testing (Phase I). TP-RPP-WTP-506, Rev. 0.4, Pacific Northwest National Laboratory, Richland, Washington.

(b) Mixing is considered adequate when there is no statistically significant (95\%) difference between the samples and/or no pattern to the observed variance.

(c) BD Hanson, FunTest Closure-Stratification r1, 2/03/09. Unpublished—presented to Test Director. 
- Evaluate Laser and Bubbler Level Probe Operation. The PEP test platform was instrumented to provide two independent means to measure the level in the tanks. The primary method used three bubbler tubes and measured the pressure difference in the air supply between two tubes. Two tubes had a fixed elevation difference between the ends of the tubes ( $\sim 6$ inches), and a third tube terminated in the headspace above the liquid. Comparing the pressure difference between the headspace and the lower tube with the headspace and the upper tube allowed one to calculate the density of the fluid. The level could then be determined by measuring the pressure difference between the headspace and the lower tube and compensating for density. This is the same approach planned for the WTP, although the specific design was not the same. PEP was also equipped with a laser to measure the level to provide another independent data source.

Simulant Functional testing demonstrated that both level measurement methods had their problems. Eventually, the laser method emerged as the preferred method, but still needed to be applied with caution. The laser could not distinguish between foam and liquid level, so if foam was present, the laser would read too high. Initially, the laser was sensitive to tank temperature and particulate in the headspace. With modifications to equipment and operating strategies, these issues were resolved and were not problematic during Integrated testing. Process control based on level was switched between laser readings and bubbler readings, depending on operator decisions on the reliability of the instrument during a given processing step.

The bubbler level measurements were shown to be sensitive to fluid flow across the face of the tube. This occurred during every PJM discharge and when the filter-loop pumps were operating in Tank T02A. Additionally, it was discovered during early testing that the simulant would tend to "plug" the bubbler tubes, giving an erroneous high pressure in the tube. Blowing the tubes with pressurized air or washing the tubes with water could remove the "plugs."

To obtain the most reliable level measurement, PJMs, pumps, and sparger air were temporarily turned off to get a "stable level" measurement. If the bubbler-measured density gave an accurate value, then the bubbler level was considered accurate. However, if the density measured by the bubbler system was unrealistic, the level measurement was also unreliable.

Between the laser level, bubbler level, and "expected" volumes from transfers, sufficient information could be deduced to operate the plant and execute the Test Instruction, although other methods would also be employed to accurately determine level during data analysis.

- Verify PJM Operation at Target Velocities and Cycle Times. The level of mixing by the PJMs in the prototypic vessels was controlled by three parameters: nozzle velocity, stroke length, and cycle time. The values for each parameter were selected so that mixing in the PEP would be appropriately scaled to mixing in the full-scale plant. For Newtonian fluids, the PEP nozzle velocity was selected to provide the same mixing power per unit tank volume as the plant. In Tank T01A/B, the plant velocities were to be $8-\mathrm{m} / \mathrm{s}$. The scaled PEP velocity was $8^{*}\left({ }^{1} / 4.5\right)^{1 / 3}$, or $4.8-\mathrm{m} / \mathrm{s}$. In Tank T02A, the plant velocity was to be $12-\mathrm{m} / \mathrm{s}$. The scaled PEP velocity was $12 *(1 / 4.5)^{1 / 3}$, or $7.3-\mathrm{m} / \mathrm{s}$. After the post-caustic leaching concentration step, it was expected that the slurry would demonstrate non-Newtonian behavior, and the target nozzle velocity in PEP was the same as the plant, $12-\mathrm{m} / \mathrm{s}$. Cycle times were selected to provide the same energy/tank volume in PEP as the plant. The cycle time scaled as $\left({ }^{1} / 4.5\right)^{2 / 3}$ for Newtonian fluids and $1 / 4.5$ for non-Newtonian fluids. Cycle times in Tank T01A/B were to be 95 seconds in the plant. The scaled PEP cycle times were to be $95 *(1 / 4.5)^{2 / 3}$, or 35 seconds. Cycle times in Tank T02A were to be 90 seconds in the plant. The scaled PEP cycle 
times were to be $90 *(1 / 4.5)^{2 / 3}$, or 33 seconds, for Newtonian fluids; and $90 *(1 / 4.5)$, or 20 seconds, for non-Newtonian fluids.

The programmable logic controller (PLC) controlled the cycle time, and control was quite precise. The nozzle velocity and the stroke length were determined by controlling the pressure and duration of the driving air. The proper settings for the drive pressure and drive time were established by monitoring the drive pressure during the course of the PJM cycle and calculating the nozzle velocity and stroke length using the Bernoulli equation:

$$
\begin{aligned}
& P_{t}+\rho g\left(L_{i n i}-x-H\right)-P_{H S P}=\frac{1}{2} \rho\left[\left(1+k_{f}\right)\left(\frac{R}{R_{n}}\right)^{4}-1\right] \dot{x}^{2} \\
& \text { where } \quad \dot{x}=\text { liquid velocity in the PJM tube } \\
& X=\text { length of the displaced liquid inside the PJM tube } \\
& P_{t}=\text { averaged PJM tube pressure } \\
& P_{H S P}=\text { head space pressure of the PJM tank } \\
& L_{\text {ini }}=\text { initial liquid level height inside the PJM tube } \\
& H=\text { liquid level height inside the tank } \\
& \rho=\text { liquid density } \\
& g=\text { gravitational acceleration } 9.81\left(\mathrm{~m} / \mathrm{s}^{2}\right) \\
& k_{f}=\text { empirically determined loss coefficient } \\
& R=\text { PJM tube radius } \\
& R_{n}=\text { nozzle radius. }
\end{aligned}
$$

The PEP design included level sensors (Drexelbrook capacitance probes) in each PJM body. The original intent was that the level probe would continuously measure the level, and the measured level would be used to control when the PJM cycles would be executed. The "standard" control strategy was to:

1) Fill the PJM by applying vacuum until the level measurement indicated that the PJM body was full.

2) Open the air valve to drive the level down for a prescribed time to achieve the target stroke length.

3) Vent the pressure from the PJM.

4) Refill the PJM to target level and wait for cycle time to complete.

The control strategy was abandoned when the Drexelbrook level probes failed during the first caustic-leach. The strategy could not be executed without a valid level measurement. A "simple" control strategy was established and used for most of the Integrated testing. In the "simple" mode, a vacuum was applied for a prescribed time to refill the PJM body. It was recognized that refilling the PJM required a longer time when the tank level was low. Another parameter, level compensation, was added to the control scheme.

During the course of testing, the stroke length could be roughly estimated by measuring the overall level change in the tank with the laser level probes and making adjustments to compensate for overblows. If PJM control had been the objective of the testing, additional modifications to the sensors would have been made. However, since the major objective of PEP was to confirm the performance of each unit 
operation in the WTP flowsheet at an engineering scale with the prototypic mixing as provided by the PJMs, it was decided to proceed with testing using nothing more complicated for control than planned for the full-scale plant.

- Demonstrate In-Line Addition of Process Chemicals. Caustic was added to the suction of the T42A pump at a prototypic rate $(10.3-\mathrm{kg} / \mathrm{min})$ to a total target mass of $531-\mathrm{kg}$ during TI-062. It was discovered that the caustic addition rate varied wildly when on "auto" control because the control valve was much larger than needed for the addition rate implemented, and the controller settings were not optimized for the low flow. It was determined that steady control could be achieved by operating the control valve manually and making minor adjustments during the addition. This control method was applied during TI-032 and the subsequent Integrated testing.

- Demonstrate Simulant Heating and Cooling. Heating and temperature control of Tank T01A and Tank T02A vessels using steam injection was demonstrated. Data from TI-032 for caustic leaching in Tanks T01A and T02A showed that direct steam injection prototypically ramped the vessel temperature during heat up, and the control system maintained it during the caustic-leach operation.

Cool-down after leaching was achieved with a combination of ambient cooling and manually controlling water flow through the vessel heat exchangers and cooling jacket. The PEP was designed to have automatic control of the cooling heat exchangers including the capability for single pass cooling. This caused the system to have a very slow response time due to the large heat sink, and tuning the controller for slow cooling rates was not possible. Therefore operating with the heat exchanger cooling water off for the initial cooling and in manual later on was used.

- Test PJM, Air Sparge Mixer, and Steam Ring Air Purge Operations at $98^{\circ} \mathrm{C}$. Systems operated successfully at $98^{\circ} \mathrm{C}$.

- Calculate Caustic-Leach Factors. The data from caustic-leach tests TI-067 and TI-032 permitted aluminum leach factors to be calculated for tests conducted in Tanks T01A and T02A.

- Steam Condensate Accumulation Estimation and Measurement: Prototypic heating of Tank T01A and Tank T02A was conducted to determine the steam condensate accumulation through the course of heat-up, and the temperature was maintained throughout the caustic-leach step. This was done to demonstrate that the volume of condensate accumulated at the end of the heating could be controlled to match the prototypic, scaled WTP PTF model value. Information from testing was used to adjust PEP operations to obtain prototypic vessel temperatures and prototypic steam condensate volumes during the PEP caustic leaching tests.

Early operation of PEP with water at elevated temperatures revealed significant condensation in the PJM vapor lines, which drained back into the vessel (Tank T01A/B or Tank T02A) and caused more condensate accumulation than expected for WTP. Based on this observation, the PJM vapor lines were plumbed to drain condensate away from the system rather than drain back to the tank. Opening the condensate drain valves was established as part of normal operation during the Functional testing.

While leaching in Tank T01A under TI-032, the volume of condensate accumulated during the $\sim 16-\mathrm{hr}$ $98^{\circ} \mathrm{C}$ hold period was less than the scaled WTP model estimate by $\sim 13$ gallons. The volume of condensate accumulated during the $98^{\circ} \mathrm{C}$ hold period in Tank T02A was greater than expected by $\sim 8$ gallons. The final accumulation including heat-up was within 1 gallon of the scaled WTP model estimate. Analysis of the level and volume addition data supported this conclusion so that Integrated testing could begin, and this is presented by Kurath et al. (2009). 
- Real-Time Density Measurement Using CD. The intent had been to demonstrate monitoring the density continuously using the CD. However, the circulation loop was sensitive to air in-leakage and entrained air that created incorrect specific gravity readings. Also, the circulating pumps were prone to failure if they lost prime. Therefore, continuous circulation was abandoned, and during Integrated process testing, the pumps were only turned on to take samples. The CD density measured by the micro-motion flow meter was also abandoned as a data source for process analysis. Figure 4.1 shows the output from the T01B while it was filling. At times, the density measurement was moderately stable, but most of the time, the measurement had too much scatter to be useful. The plot of the flow rate shows that the density scatter coincides with periods when the flow was unstable, which is attributed to air entrainment.

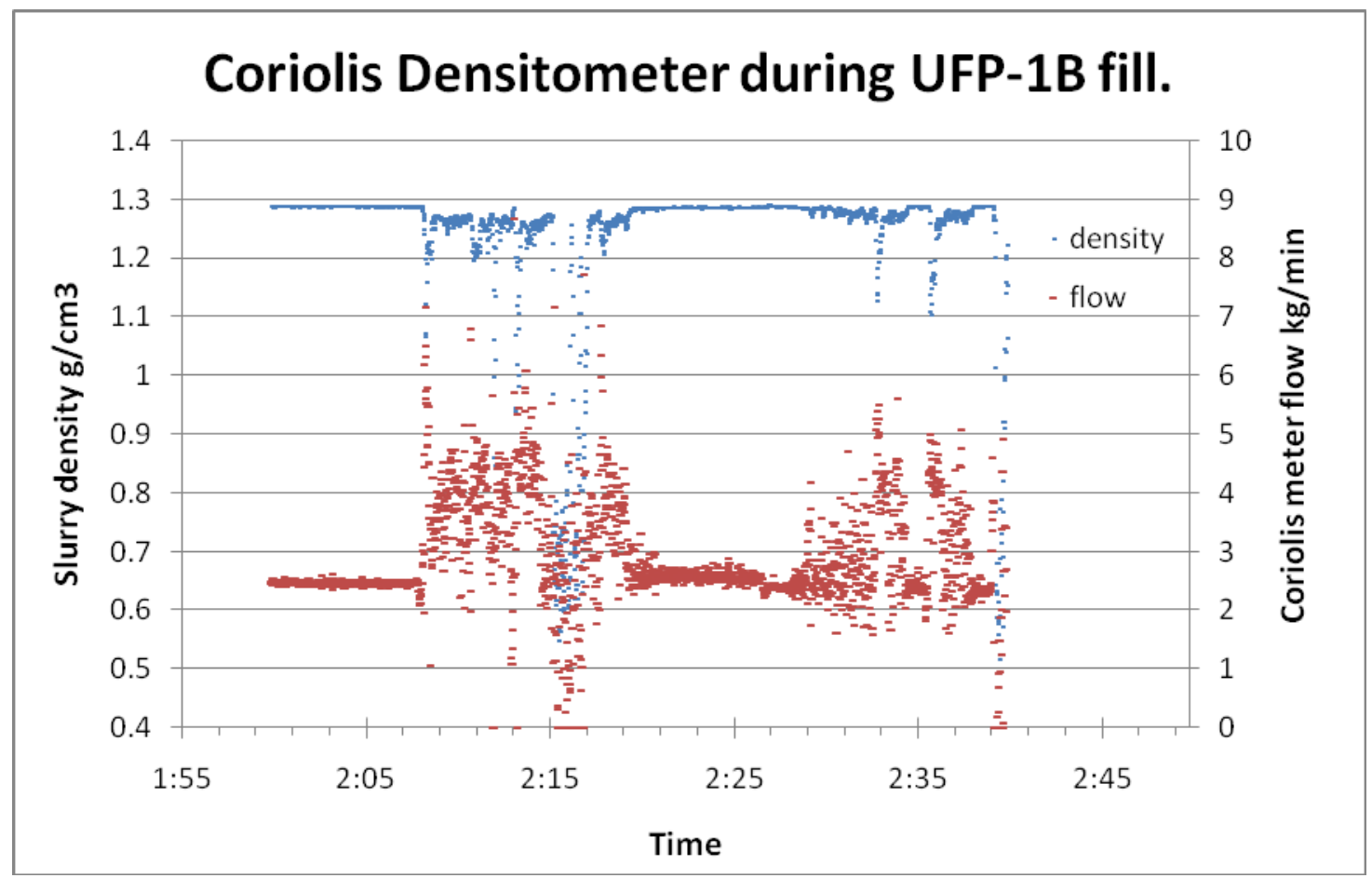

Figure 4.1. Coriolis Densitometer Output

- Filter Conditioning. Filters were conditioned in TI-062 and TI-032. The intent was to monitor the reduction in filter flux due to progressive fouling of the filter by small particles that enter a filter pore, lodge in the filter media, and plug the path to permeate collection (a.k.a., "depth-fouling"). Filter conditioning included backpulsing to dislodge these particles and restore filter flux to satisfactory levels. Backpulsing had five steps, which were automatically controlled:

1. Isolate filters connected to the pulse-pot (close outlet valves).

2. Empty the pulse-pot to a prescribed level.

3. Pressurize the pulse-pot to a prescribed target pressure (40 psi) above the inlet pressure of the filter tubes.

4. Open a fast-acting valve to pressurize the filter shell and cause permeate to flow backwards through the filter until the pulse-pot pressure reached a prescribed target ( 5 psi above inlet pressure on the tubeside of the filter). 
5. Open the pulse-pot valves to the permeate system and restart TMP control on the filter shell.

Backpulsing was conducted at 30-min intervals during hours 12 to 24 of the 36 -hr filter conditioning. Executing backpulsing was operationally easy, being entirely automatic after the operator "initiated" a backpulse. On occasion, the control system would mistakenly conduct two backpulses on the same filter. The error was irregular, and the cause was attributed to glitches in the PLC code. The effectiveness of the backpulsing during Functional testing is discussed in the final report (Kurath et al. 2009). Figure 4.2 illustrates an interesting behavior of the backpulsing, which will be further analyzed in the final report (Kurath et al. 2009).

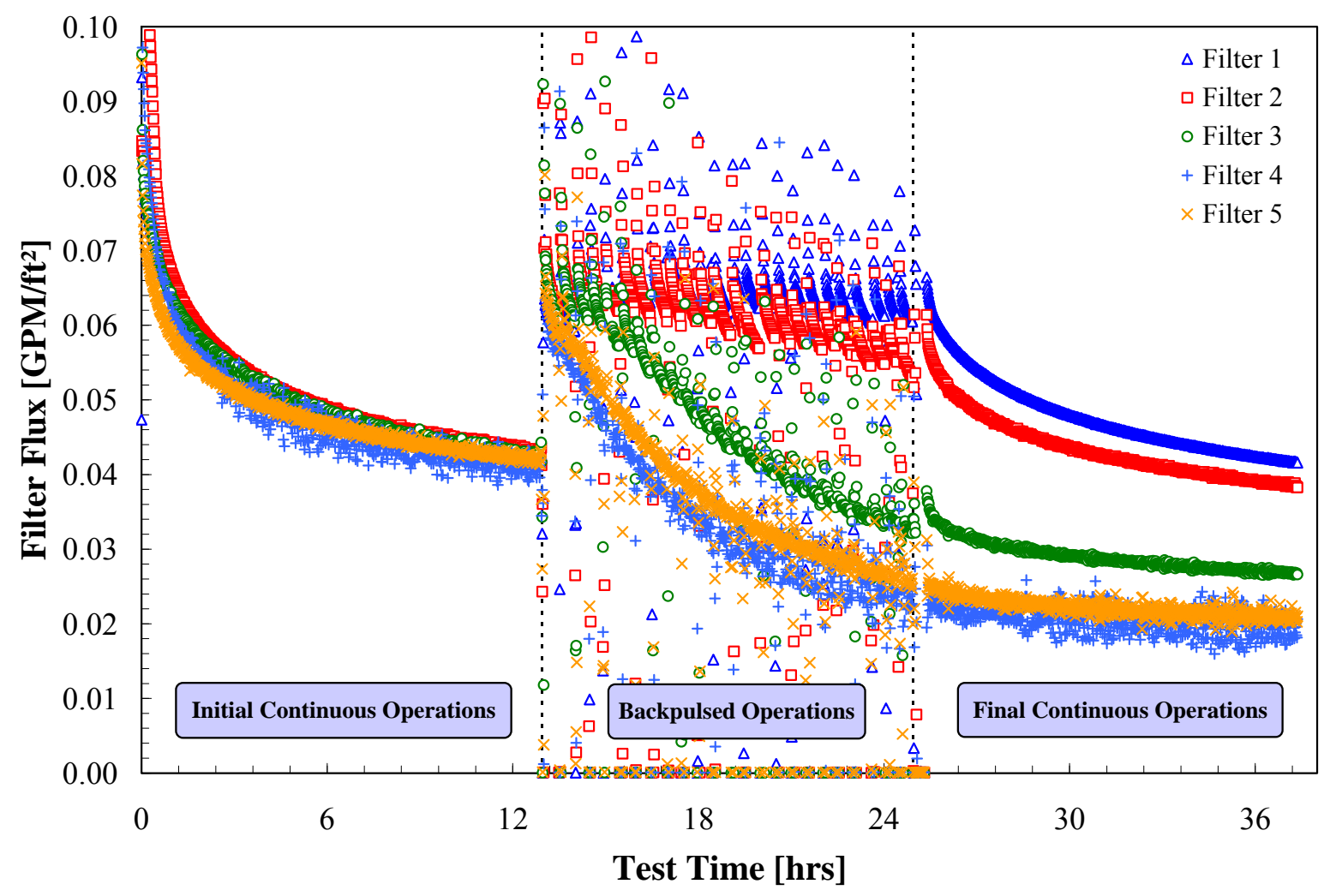

Figure 4.2. Functional Test Filter Conditioning

All five filters demonstrated approximately the same flux performance during the first 12 hours of filter conditioning. Each filter began with a very high flux that dropped nearly exponentially and then leveled off at a gradual rate of decline. Backpulsing began at the 12-hr mark (shown by random fluxes as data occurred at different points in the backpulse cycle). The 12 hours of backpulsing ended at hour 24, and each filter demonstrated very different behavior than at the beginning of the backpulsing. After backpulsing, filter 1 had a noticeably higher flux than filter 2 , which is higher than filter 3 , etc. The fundamental cause is presumed to be related to a redistribution of fines that cause depth-fouling, with more fines accumulating in filters \#4 and \#5. It should be noted that during TI-062, the backpulse sequence usually (but not always) was $\# 2, \# 3, \# 1, \# 4, \# 5$. The first three filters would be backpulsed rapidly, almost simultaneously. After the backpulse was completed on filters \#2 and \#3 and the permeate level was raised in the pulse-pot, filters \#4 and \#5 would be rapidly backpulsed. 
During TI-032, the sequence was usually \#1, \#2, \#3,\#4, \#5. Both backpulsing conditioning tests gave similar results to Figure 4.2. Filters \#4 and \#5 had greater reduction in filter flux due to backpulsing. Additional analysis of the data will be presented in the final report (Kurath et al. 2009).

- Filter-Loop Flush Strategy. Flushing of the filter-loop is primarily conducted to prevent plugging of the filters during extended periods with no fluid flow through the tubes. Solids left in the filter-loop also represent "wasted" production, so WTP operations will, with experience, optimize the amount of solids that are recovered through additional flushing vs. the extra dilution water that enters the system.

The PEP was not designed to prototypically mimic the filter-loop flush, and no attempt was made to optimize the amount of dilution water vs. solids recovery; solids recovery to optimize production efficiency was not part of Phase I testing. The initial strategy was to flush approximately half the filter-loop to Tank T02A. During Functional testing, samples from Tank T02A were taken before and after the flush to determine the extent of dilution caused by flushing. The results were inconclusive. ${ }^{\text {(a) }}$ There were no problems with plugging of the filter tubes during the long down periods, so it was decided that for later testing, it would be better to avoid uncertainties about dilution in Tank T02A and add about one-half the flush water to avoid tube plugging and minimizing the chance of dilution. This strategy was carried forward to Integrated testing, and no plugging problems occurred (due to a lack of flushing).

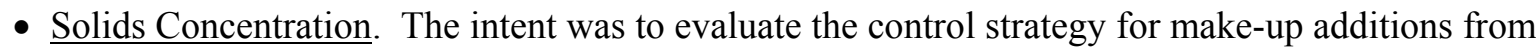
Tank T01A/B to Tank T02A during dewatering. The control strategy was to monitor the level in Tank T02A as permeate was removed. When the level reached a pre-determined target, a prototypic batch volume of simulant would be transferred from Tank T01A to make up for the permeate volume removed. This system worked well, subject only to reproducible level measurement to serve as a trigger.

- Solids Washing. During TI-032, solids washing was conducted after the caustic-leach and after the chrome addition preparatory for oxidative-leach. The intent was to evaluate whether the wash-water volumes sufficiently reduced the free hydroxide concentration to the specified concentrations. Both washing events were successful and operated as planned so washing volumes could be established for Integrated process testing.

- Dual Pump Operation. Functional testing for TI-062 and TI-032 demonstrated that the dual, in-series, pump configuration was controllable and maintained the required slurry velocity and pressures for ultrafilter operation when the tank level was sufficient to provide adequate suction head to the pumps. During Integrated testing, problems emerged with entrained air in the pumps when the tank levels were low, and the solids content was such as to retain trained air. (It should be noted that the PEP dual pumps were sized to provide higher-than-prototypic pressure. The impact of tank level and entrained air issues observed in PEP could have been worse had the pumps been designed to deliver only prototypic pressures.)

- High-Solids Filter Test. Previous laboratory testing had demonstrated a generally linear relationship between the filter flux rate and the natural logarithm of the solids concentration (wt $\%$ UDS). The linear relationship showed a shift in slope at a solids range known as the "knee." The high-solids filter test was intended to dewater a tank of simulant in Tank T02A through a concentration range

(a) LE Mahoney, FunTest Closure-Demonstrate PEP Filter Loop Flush Strategy. 1/25/09. Unpublished data presented to Test Director. 
high enough to identify the "knee" for the Phase I simulant. The test was repeated three times during TI-032. The first time, the test was terminated when flow could not be maintained in the filter-loop. During the last part of the test, a failure of the DAS caused a data loss. The second attempt was aborted because of plugging in the \#1 filter, and the third time, the test could not be finished because the slurry pumps could not maintain the target $109 \mathrm{gpm}$. At the time, it was considered that the failure was caused by insufficient net positive suction head (NPSH) for the pumps as the tank level decreased. Entrained air, which was determined to be an issue during Integrated testing, could also have been a factor. The high-solids filter test was successfully conducted after Integrated Test B.

A narrative follows that describes the detailed TI activities and the starting and stopping points for each TI. Shorthand versions of names, such as Tank T01A and HX-04A, are used. The times given are local time, PST, and a 24-hr clock.

\subsection{TI-WTP-PEP-062 Results}

The purpose of TI-062 was to complete the entire Functional process testing as a shakedown to establish readiness for actual Functional process testing. To the extent that processing was completed successfully, the data could be used to satisfy Functional process testing data requirements. As a shakedown, the TI-062 would identify equipment, procedural, or processing problems that would be addressed before testing to complete Test Plan requirements.

HLP-VSL-T22 was filled with 2590 gallons of simulant from FEP on 11/21/2008. Simulant Shakedown testing formally commenced on 11/22/2008 when simulant was transferred from HLP-VSL-T22 to fill Tank T01A. Figure 4.3 provides a timeline for TI-062 and a list of test activities and completion dates. 
PEP Functional Test TI-062

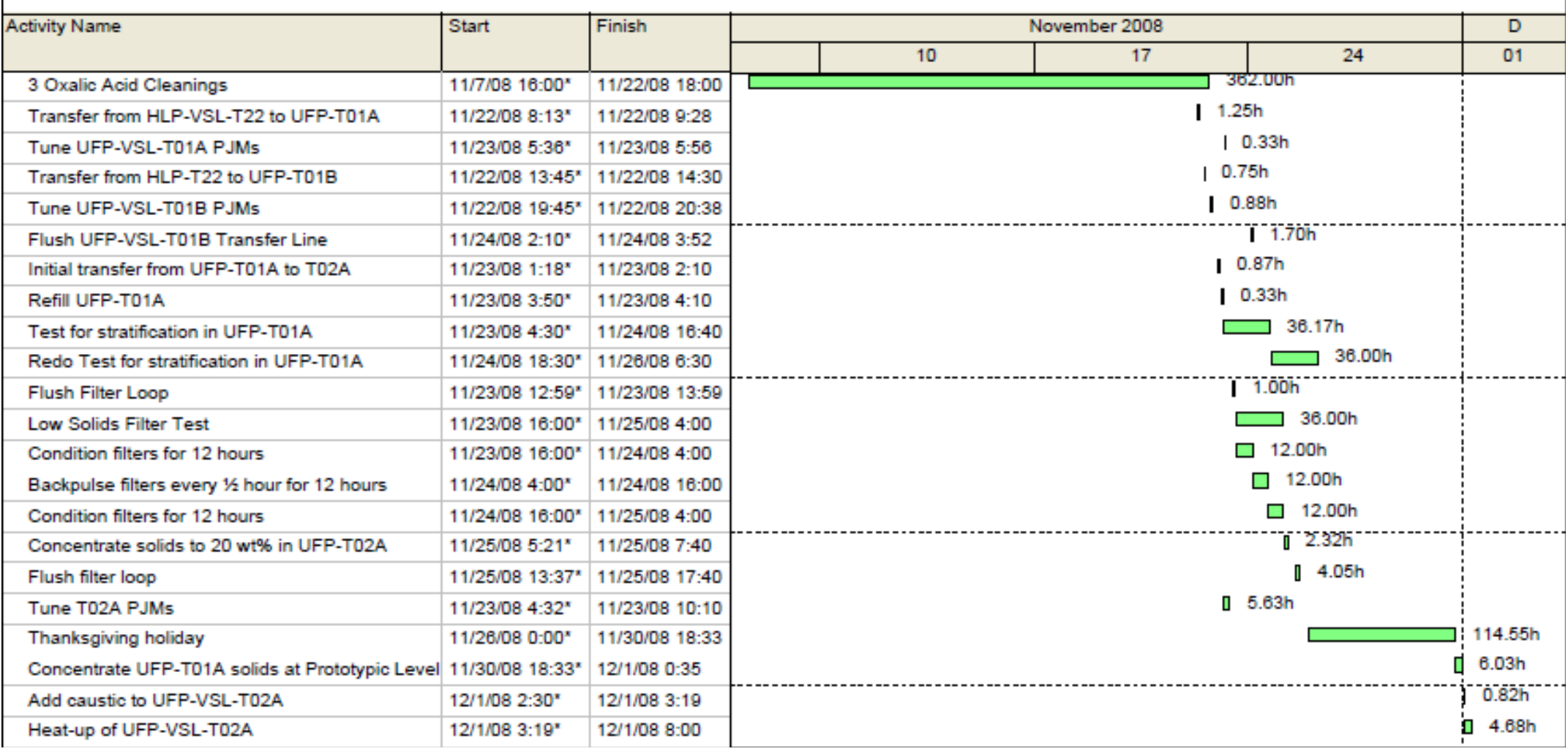

Figure 4.3. Timeline Illustration for TI-062. (For information only. Do not use times from this illustration; detailed times and processes are listed in the text.) 


\subsubsection{Wash Ultrafilters with Oxalic Acid}

Before simulant Shakedown/Functional testing began, the filters were cleaned with oxalic acid to achieve as close to a "pristine" condition as possible. During water testing, the filter flux degraded to $<2-\mathrm{kg} / \mathrm{min}\left(0.044 \mathrm{gpm} / \mathrm{ft}^{2}\right)$ at $40 \mathrm{psig}$, and the JTG decided to clean the filters. The first cleaning (1A) was performed on $11 / 7 / 2008$. The instructions for the cleaning were based on laboratory-scale tests using oxalic acid, soap (Alconox), and subsequent rinses (Billing et al. 2009). The initial oxalic wash used $0.5-\mathrm{M}$ acid, and at the conclusion, the rate of $\sim 0.65 \mathrm{gpm} / \mathrm{ft}^{2}$ was considered inadequate for proceeding. A second acid wash (1B) was completed followed by a soap wash and IW rinse. During the second wash, 30 backpulses of the filters were performed, and Tank T02A was drained along with the loop and the deadlegs. The tank was refilled with DIW and rinsed a couple of times. The cleaning on 11/11/2008 at 22:00 resulted in a permeate flux of $\sim 1.6 \mathrm{gpm} / \mathrm{ft}^{2}$. The tank was filled with IW after the oxalic flush/rinse, and the permeate rates decreased to $0.5 \mathrm{gpm} / \mathrm{ft}^{2}$. Samples were taken and visually inspected. The inspection showed some solids in the solution that were not seen in the acidic rinse solutions and were believed to be iron hydroxide. The loop was drained and refilled with IW. On 11/13/2008 and $11 / 14 / 2008$, additional permeate rates were measured using IW, and the permeate flux rates decreased again. The filtration rates were low, and a third cleaning (2A) process was prepared.

The third filter cleaning was started on 11/15/2008 at 23:00. Oxalic acid was added to Tank T02A and recirculated through the loop. The filtration rates improved immediately and increased to 7 - to $10-\mathrm{kg} / \mathrm{min}$ with low TMP or $\sim 2 \mathrm{gpm} / \mathrm{ft}^{2}$ at $40 \mathrm{psig}$. The acid wash was followed by four to five complete rinses using DIW that passed through the empty nitric acid tank and into Tank T02A. The T02A vessel was drained before the rinse water was added, but a small heel could not be removed by normal drain legs. The $\mathrm{pH}$ of the solution in the tank and the loop rose to $\sim 3.5$ after several rinses. The cleaning was completed on 11/18/2008, and the loop was left filled with IW (pH 12). The filter flux rates at the end of the IW rinse had dropped to $0.25 \mathrm{gpm} / \mathrm{ft}^{2}$.

A fourth cleaning was initiated on $11 / 21 / 2008$ with 0.5 -M oxalic acid with the intent of leaving the final solution at a low $\mathrm{pH}$ to avoid iron hydroxide precipitation. The cleaning was completed on 11/22/2008 at 13:00 hrs. The oxalic acid was flushed through the loop for over an hour with 15 backpulses. The flux rates were not all above the desired $8-\mathrm{kg} / \mathrm{min}$, so additional backpulses were performed. After initial backpulsing, all the filters were above $8-\mathrm{kg} / \mathrm{min}$ except filter \#2, which only had $7.7-\mathrm{kg} / \mathrm{min}$. It was decided to move ahead, and the acid wash was followed by two rinses of DIW that passed through the empty nitric acid tank. The last rinse left the $\mathrm{pH}$ in Tank T02A between 3 and 4 and avoided the precipitation of iron hydroxide. The loop and tank were left with the low $\mathrm{pH}$ water. The tank and loop were drained on 11/23/2008 before simulant was added to Tank T02A as part of Shakedown/Functional testing.

\subsubsection{Prepare, Stage and Characterize Simulant in HLP-VSL-T22 and Reagents}

HLP-VSL-T22 samples were taken on 11/26/2009 from three elevations and analyzed for w\% UDS to determine homogeneity of mixing. Samples for full simulant characterization had been taken earlier from the tank recirculation line while the tank was being circulated and mixed at maximum agitator speed. The only reagent characterized for simulant Functional testing was the 19-M caustic to be used for 
the caustic-leach. On 11/20/2008, fresh 19-M caustic was transferred from the totes to the 19-M tank. After the transfer, the crew noted a large mass of white crystals inside the caustic tote. A "quick-turnaround" titration of the caustic indicated that the concentration was 17.9-M instead of the nominal 19-M $(50 \% \mathrm{NaOH})$. The new lower caustic value was used to recalculate the amount of $\mathrm{NaOH}$ to add for the leaching and was included in a revised operational process sheet for TI-062. Subsequent, "official" analysis of the caustic was 18.7-M (closer to target) as shown in Table 5.3.

\subsubsection{Transfer from UFP-VSL-T01A and -T01B}

On 11/21/2008, simulant was transferred from HLP-VSL-T22 to Tank T01A. Approximately 593 gallons were transferred at a nominal rate of $15 \mathrm{gpm}$. The transfer was completed between 08:13 and 09:28 during which time data were collected to conduct a performance check on the level measuring instruments in UFP-VSL-1A. Four samples from the transfer line were collected during the transfer and analyzed for wt\% UDS as another measure of homogeneity. During the transfer, the PJMs were not operated. After the transfer, 1003-g of antifoam agent (AFA) ${ }^{(a)}$ were added to Tank T01A on 11/22/2008 at 09:41 to achieve a target $350 \mathrm{ppm}$ concentration in the simulant, and PJMs were started.

Immediately after Tank T01A was filled and before the PJMs were completely adjusted, simulant was transferred from HLP-VSL-T22 to Tank T01B. Approximately 593 gallons were transferred at a nominal rate of $15 \mathrm{gpm}$. The transfer was completed between 13:45 and 14:30 on 11/22/2008. During the transfer, data were collected to conduct a performance check on the level measuring instruments in Tank T01B; PJMs were not operated. AFA was NOT added to the vessel because the simulant was returned back to HLP-VSL-T22 after the PJM tuning was complete.

\subsubsection{Tune UFP-VSL-T01A/B PJMs}

The Tank T01A PJMs were initially adjusted by operating staff around $05: 00$ on 11/23/2008 to achieve a peak average nozzle velocity of $4.8-\mathrm{m} / \mathrm{s}$ with an $80 \%$ stroke length and $35-\mathrm{s}$ cycle time. The nozzle velocity was calculated based upon the rate of level change in the PJM tube measured by the Drexelbrook level probes, $\mathrm{dL} / \mathrm{dt}$, according to:

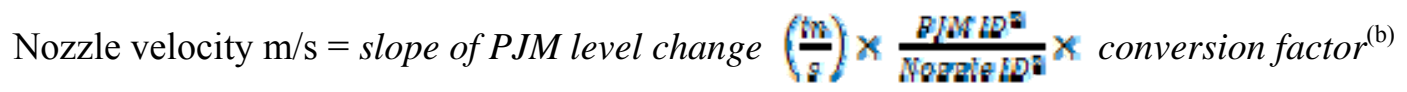

$$
\text { Nozzle velocity } \mathrm{m} / \mathrm{s}=\frac{\Delta \boldsymbol{L} \backslash \mathrm{m} / \mathrm{m}}{\Delta \boldsymbol{r}(\Omega)} \times 2,02(\text { for Tank T01A/B) }
$$

Adjusting PJMs in Tank T01B was completed at 19:45 on 11/22/2008 (LRB 59944, page 168, and continued on LRB 60108, page 1). Tuning in Tank T01A was completed at 00:58 on 11/23/2008. Both vessels were tuned to the same peak average nozzle velocity, stroke length, and cycle time. It was observed that PJM nozzle velocities changed in Tank T01B, depending on whether Tank T01A PJMs were on or off. This is likely due to the common air header supplying all the PJMs. Turning off a set of

(a) Note: The AFA was very viscous, so it was always diluted with three parts water for each part AFA to facilitate addition.

(b) The conversion factor converts the change in level measured in inches/second to meters/second (desired units for nozzle velocity) and is $1 / 39.39(.0254)$. 
PJMs reduces the total air demand, and this likely slightly increases the supply pressure. PJMs turned off during tuning problems with the Tank T01B laser level probe, so level control was switched from the laser to the bubbler at 21:56. Note: The PJM "tunings" or "adjustments" were directed by PEP LTEs and operating staff. When the nozzle velocities were checked during the day on 11/24/20 using the "official" methods (i.e., using data from the DAS and a methodology qualified to the RPP-WTP QAP), it was determined that the velocities were significantly too high $(7-\mathrm{m} / \mathrm{s}$ vs. $4.8-\mathrm{m} / \mathrm{s})$. The PJMs in both Tank T01A and Tank T01B were then officially "tuned."

\subsubsection{Flush Transfer Lines}

After the PJM tuning was complete and at a convenient time during test execution, the simulant was pumped back to HLP-VSL-T22, and the transfer line to Tank T01B was flushed with approximately five line volumes of IW to test the line flushing efficiency. This test was conducted twice. During the first attempt, the IW flow rate was set incorrectly to be $28-\mathrm{kg} / \mathrm{min}$ rather than a target $28 \mathrm{gal} / \mathrm{min}$. The error was quickly discovered, and the test was repeated after another small simulant transfer ( 20 gallons) to refill the line. During the second attempt, the maximum IW rate was $\sim 20 \mathrm{gpm}$. Flush samples were taken at approximately 20 -s intervals, which should have been approximately every 6 gallons (1 line volume) transferred. The samples were analyzed for $\mathrm{wt} \%$ UDS. The second line flush was completed between 03:51 and 03:53 on 11/24/2008.

\subsubsection{Simulant Transfer from UFP-VSL-T01A to UFP-VSL-T02A}

The filters were cleaned with oxalic acid immediately before the Functional process testing using simulant. The transition to alkaline conditions was to be accomplished by quickly quenching the remaining acid in the final oxalic acid rinse with highly alkaline simulant with entrained solids to prevent plugging. This is believed to provide nucleation sites so that precipitating iron hydroxide could grow on existing particles rather than nucleate rapidly into very small particles that could foul the filter media. This was done by isolating Tank T02A from the filter-loop and draining as much acid rinse as possible. Then Tank T02A was filled from Tank T01A, and the filter-loop pumps were turned on to rapidly displace the last acid rinse with alkaline simulant.

Approximately 324 gallons of simulant were transferred from Tank T01A at prototypic rates ( 6.9 gpm). During the transfer, a performance check was conducted on the level measuring devices in Tank T02A, so the PJMs were not operated, keeping level disturbances to a minimum. The transfer commenced at 01:14 and was completed at $02: 10$ on 11/23/2008. The transfer was terminated a few gallons short of the target volume of 329 gallons because Tank T02A hit a high-high level alarm.

PJM tuning could not be completed because of the high-high level alarm, so the filter-loop was opened, and the pumps were turned "ON" to fill the loop, lowering the tank level from 72.8 inches to 58 inches. Both heat exchangers were in-line, and the filter bundles were bypassed. The filter-loop filling commenced at 03:06 on 11/23/2008. After the loop was filled, the level was adequate to tune the PJMs. The pumps were turned "OFF," and PJM tuning was conducted.

PJM tuning was conducted to achieve PJM target parameters: $7.3-\mathrm{m} / \mathrm{s}$ nozzle velocity, $80 \%$ stroke length, and 33-s cycle time. PJM tuning was completed at 09:02 on 11/23/2008. 


\subsubsection{Refill UFP-VSL-T01A}

A second batch of simulant was transferred from HLP-VSL-T22 to Tank T01A to refill the vessel while the PJMs were being tuned in Tank T02A. On 11/23/2008, Tank T01A was refilled with simulant from 03:49 to 04:10 at a target rate of $30 \mathrm{gpm}$. During the transfer, two samples were collected, the first at approximately 30 seconds into the transfer and the second when about 200 gallons were transferred. After the transfer, additional AFA was added to Tank T01A (target 550-mL) to maintain a target $350 \mathrm{ppm}$.

\subsubsection{Stratification Tests}

The stratification test was conducted to determine whether solids stratified or "settled" over an extended time (i.e., 36 hours) in Tank T01A/B. Prototypic mixing was maintained with the PJMs tuned by the operators to be near the operating targets (i.e., $4.8-\mathrm{m} / \mathrm{s}$ nozzle velocity, $80 \%$ stroke length, $35-\mathrm{s}$ cycle time), and the air purge on the steam ring at the target flow rate $(0.13-\mathrm{kg} / \mathrm{min})$.

The stratification test was first initiated at 04:20 on 11/23/2008. An initial sample set was taken at 04:30 and then was repeated every 12 hours. Samples were taken at 16:50 on 11/23/2008, which was 12 hours from the original starting time. The test was originally planned to extend 36 hours. However, after several hours, the PJM velocity was checked using data from the DAS and following the official methodology, and it was determined that the velocities were actually too high. The PJMs were retuned on $11 / 24 / 2008$ to lower drive pressures. It was decided that the test time should restart after the PJM retune. The stratification test was restarted at 12:00 on 11/24/2008. The 12-hr samples based on the restart time were retaken at 00:01 to 00:10 on 11/25/2008 and then repeated at 12-hr intervals. The final samples were taken at 00:07 to 00:16 on 11/26/2008, completing the 36-hr stratification. Data from the stratification test are shown in Table 4.1. 
Table 4.1. Stratification Data (wt $\%$ UDS) from UFP-VSL-T01A ${ }^{(a)}$

\begin{tabular}{|c|c|c|c|c|}
\hline Location & $\mathrm{t}=12$ hour & $\mathrm{t}=12$ hour repeat & $\mathrm{t}=24$ hour & $\mathrm{t}=36$ hour \\
\hline Inner-Low & 5.44 & 5.39 & 5.39 & 5.42 \\
\hline “ & 5.35 & 5.43 & 5.40 & 5.41 \\
\hline “" & 5.37 & 5.45 & 5.44 & 5.43 \\
\hline “ & 5.33 & 5.40 & 5.38 & 5.39 \\
\hline Mean & 5.37 & 5.42 & 5.40 & 5.41 \\
\hline Inner-Middle & 5.46 & 5.42 & 5.36 & 5.50 \\
\hline “ & 5.38 & 5.40 & 5.39 & 5.48 \\
\hline " & 5.38 & 5.39 & 5.38 & 5.41 \\
\hline “ & 5.39 & 5.39 & 5.35 & 5.40 \\
\hline Mean & 5.40 & 5.40 & 5.37 & 5.45 \\
\hline Inner-High & 5.08 & 5.43 & 5.33 & 5.44 \\
\hline “ & 5.39 & 5.43 & 5.36 & 5.40 \\
\hline “" & 5.41 & 5.44 & 5.39 & 5.38 \\
\hline “ & 5.37 & 5.40 & 5.38 & 5.40 \\
\hline Mean & 5.31 & 5.43 & 5.37 & 5.41 \\
\hline
\end{tabular}

\subsubsection{Low-Solids Filter Test (filter conditioning)}

The prime objective was to determine a rate of decline in the low-solids filter flux as filtration progressed. All filter conditioning steps were to be performed at a feed flow rate corresponding to an axial velocity of $15 \pm 1-\mathrm{ft} / \mathrm{sec}$, a TMP pressure of $40 \pm 4 \mathrm{psid}$, and with the feed temperature maintained at $25 \pm 2{ }^{\circ} \mathrm{C}$.

Filter conditioning consisted of three 12 -hr periods performed in the following manner: 1) before starting, drain the tubeside and shellside of the filter-loop and the pump, 2) align all five filters for filtering, 3) fill the tubeside with simulant and circulate for $\sim 10$ minutes, 4) open permeate valves, fill the shellside, and return all permeate to Tank T02A, 5) collect a feed sample for parallel CUF filtration, 6) condition the filters for a 12-hr period, 7) backpulse each filter every 30 minutes for a period of 12 hours, and 8) condition the filters for a third 12-hr period without backpulsing. For the filter conditioning, the loop alignment included all five filters and both heat exchangers, UFP-HX-T02A and UFP-HX-T03A. During filter conditioning, permeate was returned to Tank T02A, so the solids concentration did not change.

Before starting the recirculation pumps, the shellside of each filter was drained, the permeate control valves manually opened, and the pulse-pots drained. DAS data indicate that the pulse-pots were drained at 12:24 on 11/23/2008. The procedure to drain the shells was completed at 13:00. The tubeside of the filters had been previously drained when the loop was filled to remove extra volume from Tank T02A so the PJMs could be tuned. The filter-loop flow was set to $109 \mathrm{gpm}$, and the loop recirculated for several

(a) FunTest Closure stratification r1.doc - presented to WTP Test Director to close stratification issue prior to advancing to integrated process testing. 
minutes. The backpressure control valve, ZV-0609, was adjusted to 53\%, establishing a backpressure on the end for UFP-FILT-T05A of approximately $55 \mathrm{psig}$, and the permeate valves were opened. After all of the filter shells had filled with permeate and a 40 psi TMP was established, the pumps were turned off, and a stable level was taken in Tank T02A at 14:50 on 11/23/2008. The level drop in Tank T02A between this measurement and the previous was 24.8 inches, indicating that the volume of the filter shells, pulse-pots, and associated piping back to Tank T02A was about 116 gallons.

After the stable level measurement, recirculation and filtration were continued for 30 minutes. Then permeate flow was stopped to take a slurry sample for parallel laboratory testing in the CUF and samples from the filter-loop to establish initial slurry conditions. When this sampling was complete, permeate flow was restarted at 16:00 on 11/23/2008, beginning the first 12-hr filter conditioning period. During the first 5 hours of the period, a set of six samples was removed from the filter-loop, and simultaneously, six samples were removed from the CD sampling station. After the second set of samples were taken, it was difficult to sample from that location. The final three sets of samples were taken from the middle-low location. A total of 30 samples ( 5 sets of 6 samples) were taken from each location and analyzed for wt $\%$ UDS. Statistical analysis of the 60 samples (30 samples each from two locations) was used to determine the variability of sampling systems and wt\% UDS analytical method.

Samples of permeate were also taken from each filter and visually examined for breakthrough of solids. If a filter tube was broken or the seals between the shell and tubes leak, solids would be in the permeate. (Note: No solids were ever observed in the permeate when examined immediately. It was noted that clear/white needle-like solids did appear in many permeate samples after they were allowed to sit for several days. These solids are attributed to post-processing crystallization of super-saturated components [e.g., oxalate] and not solids breakthrough from the tubeside.)

The second conditioning period was completed between $04: 16$ and 16:16 on 11/24/2008 during which time DAS data were collected at $10 \mathrm{~Hz}$ instead of the normal $1 \mathrm{~Hz}$. During the second 12-hr period of conditioning, the filters were backpulsed every 30 minutes. The backpulse was characterized by three control parameters.

- Initial pulse-pot overpressure-This established the driving force to push permeate backwards through the filter pores. During filter conditioning, the target overpressure was 40 psi.

- Initial pulse-pot level-This established the headspace volume of the pressurized gas in the pulse-pot at the beginning of the pulse. This parameter controls the pressure vs. time (volume) relationship during the backpulse. During filter conditioning, the target level was 9 inches.

- Deadband-This controls the final pressure in the pulse-pot, ending the backpulse. The "deadband" is the pressure in the pulse-pot above the filter tubeside pressure. During filter conditioning, the deadband was 5 psi.

After the second conditioning period, a second sample was collected for parallel laboratory testing in the CUF.

During the third 12-hr period of conditioning, the filters were operated identical to the first period; there was no backpulse. The third 12-hr conditioning period was completed between 16:40 11/24/2008 and $04: 42,11 / 25 / 2008$. 


\subsubsection{Concentrate Solids in UFP-VSL-T02A with One Filter}

After the filter conditioning was complete, the filter-loop was reconfigured to flow only through filters UFP-FILT-T01A and UFP-HX-T02A, bypassing the other four filters and the steam heat exchanger, UFP-HX-T03A. After the circulation was restarted, permeate was removed, and the tank level was allowed to drop. At the beginning of the concentration step, PJMs were operated with the same drive pressures, drive time, and cycle time as during filter conditioning. When the level dropped to $\sim 27$ inches in Tank T02A, the PJMs were turned off (06:00 on 11/25/2008). The bottom air spargers were operated at full rate, $\sim 0.4-\mathrm{kg} / \mathrm{min}$, the upper air sparger was at idle rate, $\sim 0.01-\mathrm{kg} / \mathrm{min}$, and the steam ring air purge was on at $0.1-\mathrm{kg} / \mathrm{min}$.

The concentration began at $05: 21$ on 11/25/2008 with the tank level at 43.7 inches (by laser, 35.4 inches by bubbler). The concentration step was terminated when a target permeate removal had been accomplished, 422-kg, at 07:40. The tank level was then 22.5 inches by laser (13.7 inches by bubbler). Note: At 07:55 on 11/25/2008, the laser measurement was "recalibrated" to match the bubbler measurement.

The initial pre-leach concentration operation performed during TI-062 achieved an average axial velocity of 14.7-ft/s (as measured by the discharge flow from Pump T43A) and TMP of 39.6 psid. Figure 4.4 and Figure 4.5 show the permeate production rate and TMP/temperature corrected filter flux measured during pre-leach concentration for filter bundle 1. Filter flux shows a slight decline from 0.05 $\mathrm{gpm} / \mathrm{ft}^{2}$ (initial) to $0.04 \mathrm{gpm} / \mathrm{ft}^{2}$ (final) during dewatering operations. Analytical samples taken during the dewatering operations were not analyzed, and as such, insufficient information exists to perform a mass balance and analyze the UDS functionality of filter flux.

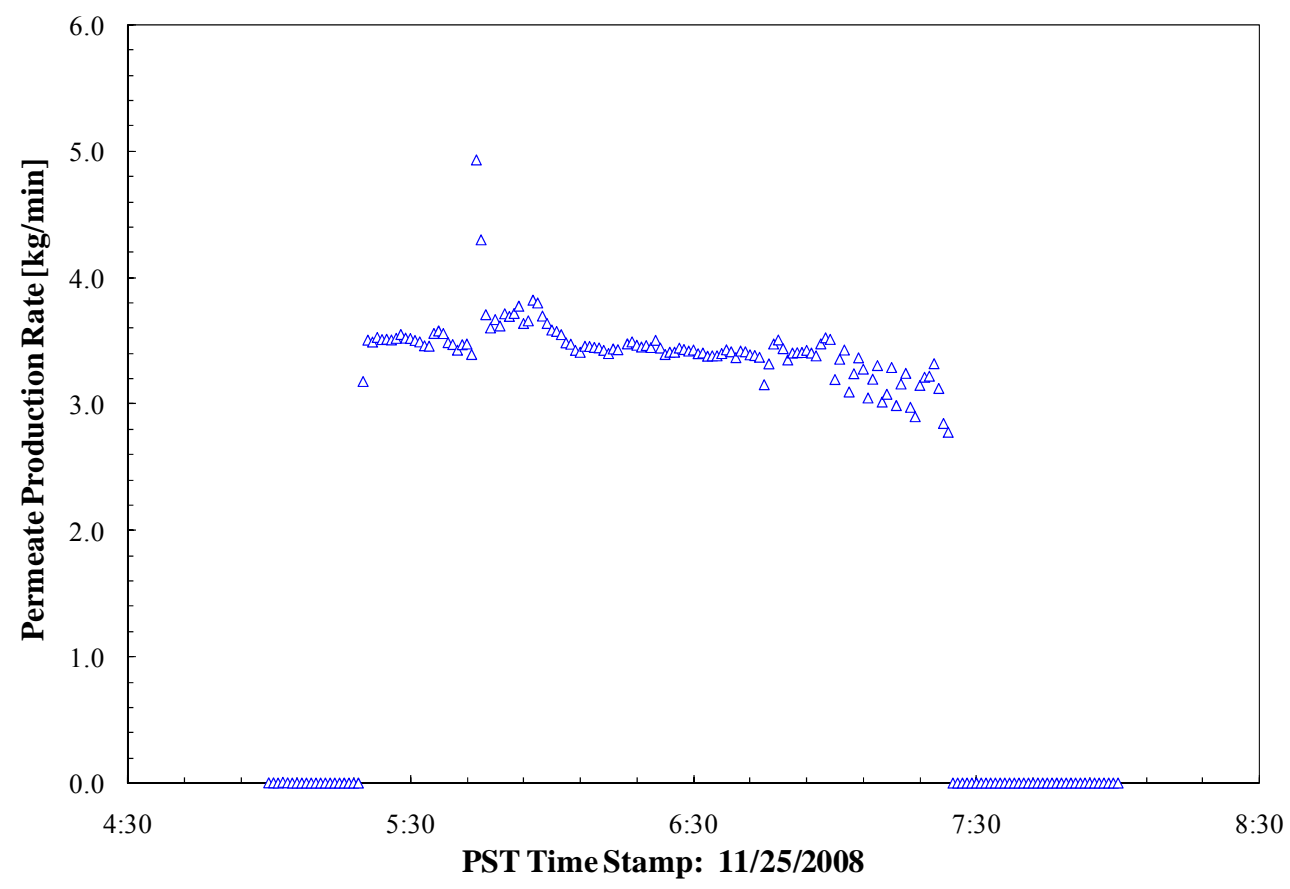

Figure 4.4. Permeate Production Rate During the Initial Functional Test (TI-062) Pre-Leach Concentration of Solids on Filter 1 


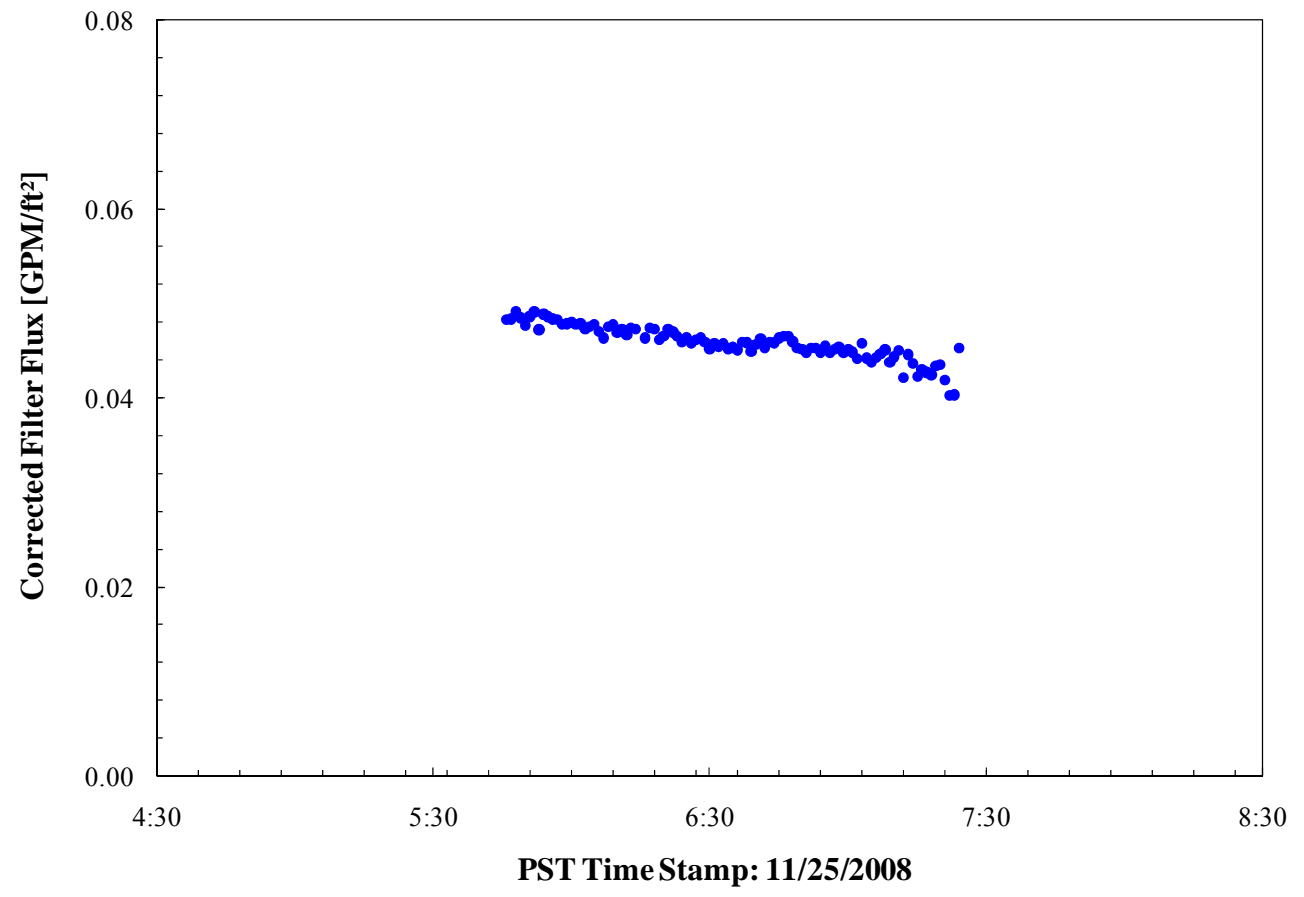

Figure 4.5. TMP and Temperature Corrected Filter Flux for the Initial Functional Test (TI-062) Pre-Leach Concentration of Solids on Filter 1

\subsubsection{Flush Filter-Loop-(preparation for down time)}

After concentration, the filter-loop was flushed with 201-kg of IW from the suction of the pump to Tank T02A. Then the loop was recirculated with Tank T02A isolated. The next step was to flush approximately two line volumes through the filter-loop to T62A. However, because T62A was full, the flush could not be done. The pumps were left in recirculation mode from 04:00 to 17:46 on 11/25/2008 and then shut down. The pumps were re-started at 12:24 the next day and recirculated for about 10 minutes, and then the flush to T62A was completed.

The entire PEP system was secured from 11/26 to 11/29 for the Thanksgiving holiday. During the off time, the PJM operation and air purge to the steam rings were continued in the UFP vessels. Operations resumed on $11 / 30 / 2008$.

\subsubsection{Concentrate Solids to 20-wt\% UDS in UFP-VSL-T02A with Filters \#2 to \#5}

When the concentration was terminated before the holiday, the solids concentration was not yet up to $20 \%$. The concentration was completed by transferring simulant from Tank T01A to Tank T02A while continuing to remove permeate. The transfer was controlled by a batch-wise level control strategy in Tank T02A. When the measured level in Tank T02A dropped below a target, a batch (11 gallons) of thin simulant was transferred from Tank T01A. By calculation, the final wt\% UDS target would be reached when 22 batches of simulant had been transferred, and the level in Tank T02A was at a prototypic level (19 inches). 
The filters were reconfigured for this concentration step to bypass UFP-FILT-T01A and flow through UFP-FILT-T02A to -T05A. The concentration process restarted at 21:47 on 11/30/2008 when 55 gallons of thin simulant were transferred from Tank T01A to Tank T02A. The extra volume was added to Tank T02A before the filter recirculation pumps were started to make sure that there was sufficient volume in Tank T02A to refill the filter-loop, which had been filled with IW over the holiday break and then drained. Circulation through the filter-loop started at 22:30 at $109 \mathrm{gpm}$. The lower spargers were operated at "idle" rate $(0.037-\mathrm{kg} / \mathrm{min})$, and the upper spargers were off. The steam air purge was on at $0.1-\mathrm{kg} / \mathrm{min}$. Permeate flow was initiated from UFP-FILT-T02A through T05A at 23:00, and the initial samples were collected from the filter-loop. Additional slurry samples were collected after each five batches (55 gallons) were transferred from Tank T01A and after the last batch. After 22 batches had been transferred and the Tank T02A level was reduced to the target 19-inch level, the concentration was completed at $01: 55$ on 12/01/2008. Approximately $868-\mathrm{kg}$ of permeate had been removed from the 22 batches, and the subsequent level adjustment was down to the target.

The final pre-leach concentration operation for TI-062 achieved an average axial velocity of 14.8-ft/s (as measured by the discharge flow from Pump T43A) and TMP of $40.0 \pm 0.5$ psid on filters 2 through 5. Tank T02A had an average temperature of $25.4^{\circ} \mathrm{C}$. Figure 4.6 shows the permeate production rates for filters 2-5 during this dewatering operation. Figure 4.7 shows the TMP/temperature corrected (areaaveraged) filter flux.

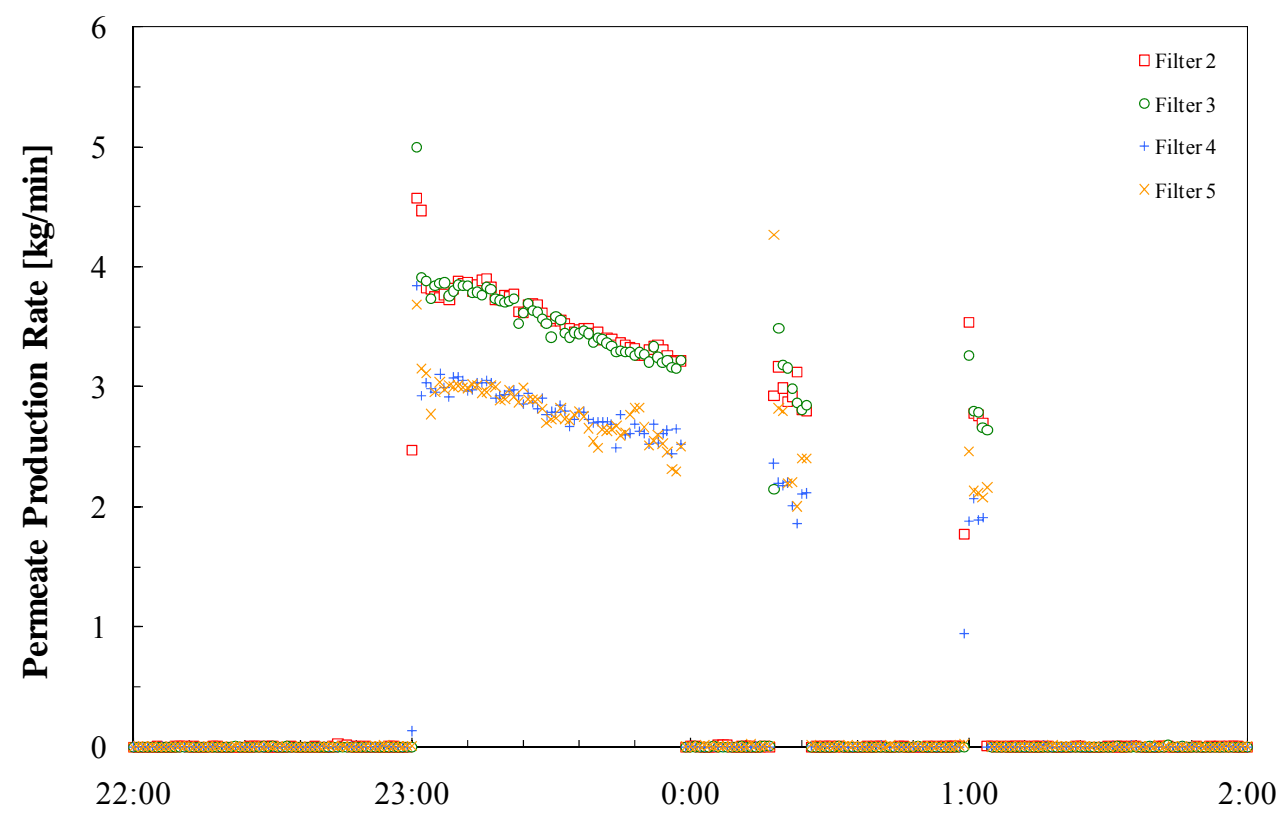

PST Time Stamp: 11/30/2008-12/1/2008

Figure 4.6. Permeate Production Rate During the Final Functional Test (TI-062) Pre-Leach Concentration of Solids on Filters 2-5 


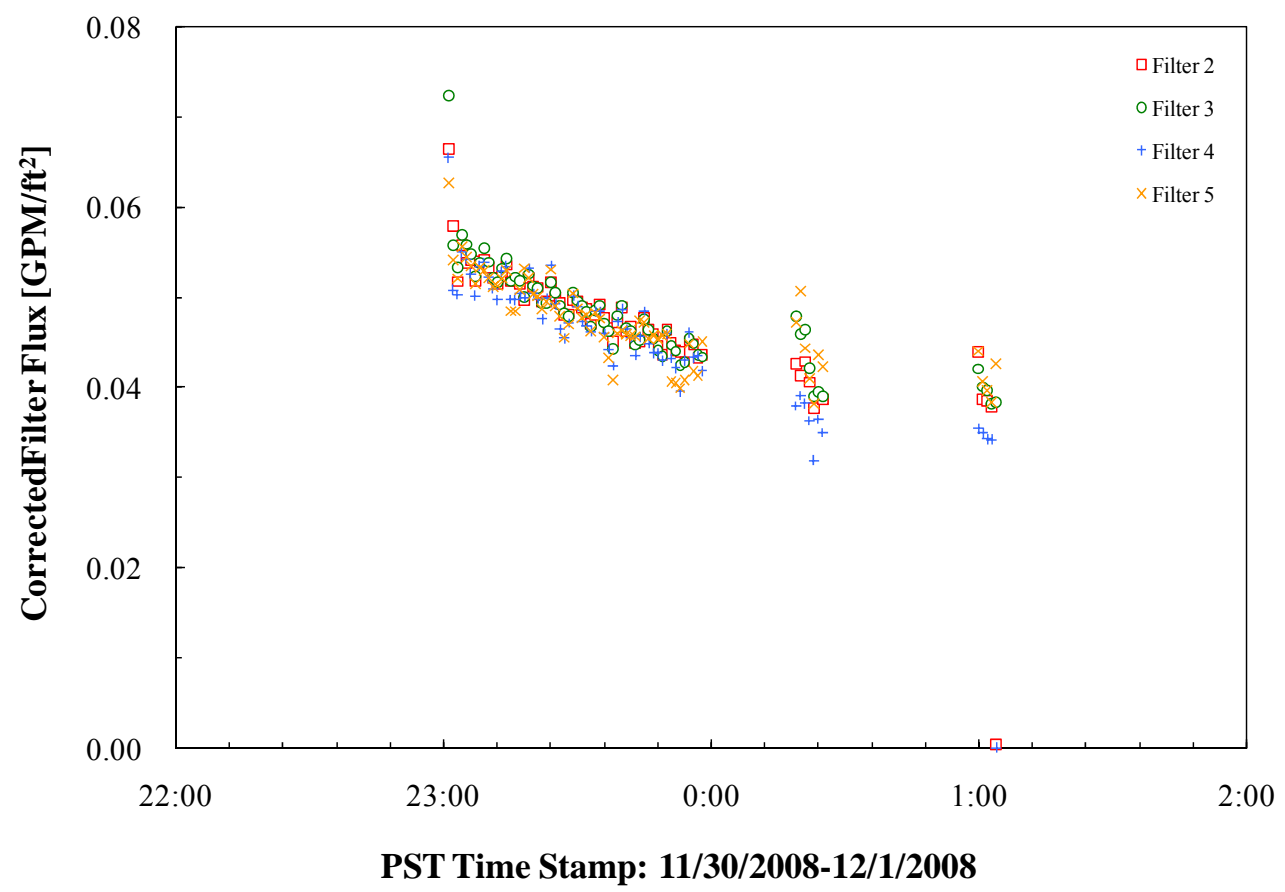

Figure 4.7. TMP and Temperature Corrected Filter Flux for the Final Functional Test (TI-062) Pre-Leach Concentration of Solids on Filters 2-5

Filter flux shows a decline from $\sim 0.06 \mathrm{gpm} / \mathrm{ft}^{2}$ (initial) to $0.04 \mathrm{gpm} / \mathrm{ft}^{2}$ (final) during dewatering operations. Relative to the pre-leach dewatering results in Figure 4.5, the rate of flux decline shown in Figure 4.7 is greater, likely because of faster filtering with four filters instead of one. A material balance of the final pre-leaching dewatering operation could not be performed because there were insufficient analytical results.

\subsubsection{Add Caustic in UFP-VSL-T02A}

To prepare for the caustic addition, the filter-loop was reconfigured to align the steam heat exchanger in case additional heating was needed to reach the initial target temperature before engaging direct steam injection. Also, AFA was added on 12/1/2008 at 02:30 to keep the antifoam concentration up to $350 \mathrm{ppm}$ when the caustic was added. When the UFP-HX-T03A exchanger was aligned and the pumps were started, the level in Tank T02A dropped 9 inches. Even with both pumps operating at $100 \%$, a circulation rate of only about 90 gpm could be maintained. After caustic addition started, the level in the tank rose, and the pumps could again maintain the $109 \mathrm{gpm}$ circulation rate.

Caustic was added to the suction of T42A pump at a prototypic rate $(10.3-\mathrm{kg} / \mathrm{min})$ to a total target mass of 531-kg. It was discovered during the addition that the caustic addition rate varied wildly when on "auto" control. During the addition, the flow rate control was switched to "manual," and the valve was adjusted to maintain a steadier rate. The caustic addition was completed in 49 minutes at 03:19 on $12 / 01 / 2008$. 


\subsubsection{Heat-Up of UFP-VSL-T02A}

The temperature was $72^{\circ} \mathrm{C}$ after the caustic addition was complete, and samples were taken, which met the target temperature for the pre-heating so that additional heating with the steam heat exchanger was not needed.

At 03:23, the operators observed that the PJMs were operating in short stroke mode rather than full stroke. Further investigation identified that the PJM level probes were not accurately reporting the level. Diagnostic testing later identified that the probes were covered with a Kynar material, which was not recommended for high-caustic service. Eventually, all the probes in the three UFP vessels were replaced with fluorinated ethylene propylene (FEP) coated probes. ${ }^{\text {(a) }}$

While the operators were investigating issues with the PJMs, the slurry with caustic added was continuing to circulate through the filter-loop, maintaining the temperature by heating with the pumps. Leaks were observed from the end flange on the $\# 5$ filter at 06:00,12/01/2008. The leaks were reported to maintenance, which eventually led to terminating the test.

\subsubsection{Flush Filter-Loop}

After the test was terminated, the filter-loop was flushed with IW to remove hazardous material from the loop and prepare the system for maintenance. PEP testing was resumed after the EDPM filter flange gaskets were replaced on all five filter bundles with Teflon gaskets.

\subsubsection{Test End}

After the test ended, a "simple" mode PJM control option was implemented for the Tank T02A PJMs. The new "simple" mode did not require level input from the Drexelbrook level sensors to control the PJM refill. Rather, the "simple" mode used timers for the vent stage and vacuum refill stage to the PJM tube after each discharge. Because the refill was "blind," it was impossible to select appropriate conditions (time and vacuum) to completely fill the PJM tube without going up into the air piping. The tuning approach was "trial and error" and was crude based upon using the tank level increase to estimate the stroke length. If the stroke length could not be obtained without overblows, the length of the vacuum refill stage was increased. The Tank T02A PJMs were tuned in simple mode on 12/3/2009 to a cycle time of 33 seconds, a nozzle velocity of $\sim 7-\mathrm{m} / \mathrm{s}$, a drive pressure rising by 6 psi over $8 \mathrm{sec}$, and a vent time of 1 second. The vacuum ratio was set to $100 \%$ (the vacuum time was set as a ratio of the drive time; at $100 \%$, the vacuum time and drive time would be equal). On 12/4/2008, the laser continued to malfunction in Tank T02A jumping from 52 inches to 112 inches when the tank level was 42 inches. On $12 / 6 / 2008$, the problems with the Tank T02A PJM level probes were again observed. Drexelbrook fill levels inside Tank T02A PJMs returned to the levels seen before caustic addition. A $10 \mathrm{~Hz}$ PJM file was collected at 23:54 to document this condition (LRB 60108, pg 20). Diagnostic tests were used to troubleshoot the PJM Level probes (LRB 60108, page 22). It was thought that pulling the simulant onto the Kynar that was coating the probes resulted in false high-level readings inside the PJM tubes. A memo

(a) During the conduct of testing, the probe coating was commonly referred to as "Teflon." The FEP coating is similarly a fluorinated polymer with very high chemical resistance, but is not exactly the same product that carries the trade name Teflon ${ }^{\circledR}$. 
on page 25 of the LRB describes the outcome of diagnostic testing. The conclusion was to replace the Kynar-coated level probes with FEP-coated probes.

Some of the Tank T02A level probes were replaced on 12/11/2009. Four Drexelbrook level probes were removed from Tank T02A and replaced on 12/12/2008 with FEP-coated probes. On 12/12/2008, transfers of water (deionized water [DIW] mixed with dilute oxalic acid) from the nitric acid (NAR) vessel to Tank T02A were used to calibrate the span of the new PJM level probes. Transfers from the NAR vessel to Tank T02A began at 22:40 with the PJMs vented, bubblers on, and spargers/steam rings off. Instrument technicians set the zero and span for the new level probes during the transfer. The zero level for the new probes was recorded at 11 inches, and the $100 \%(20-\mathrm{mA})$ level was set at 47.0 to 47.5 inches (LRB 60108, page 27). Verification of the spans was confirmed during subsequent transfer from Tank T02A to T62A (LRB 60108, page 29).

\subsection{TI-WTP-PEP-067 Results}

The testing associated with TI-WTP-PEP-067 was started on 12/09/2008.

TI-067 was conducted to opportunistically conduct the prototypic up-front caustic-leach to obtain data if possible, but also to identify additional equipment problems that could arise in the T01 vessels similar to those that caused the early termination of TI-062. Figure 4.8 provides a timeline for test activities. Other steps were completed in TI-032. [Reference: LRB 60108]

After data were analyzed, it was discovered that during the 8 days that PEP testing was suspended between the end of TI-062 and the start of TI-067, the volume in Tank T01A had gradually increased by approximately 70 gallons. It was concluded at the time that the steam control valve did not shut off completely, allowing steam and/or condensate to leak into the vessel. The tank temperature did not increase noticeably, but it was concluded that a small leak of condensate would not cause a measurable temperature increase. As a precaution, during subsequent testing the hand valves on the steam supply were closed to assure there was no leakage into the tank. 
PEP Functional Test TI-067

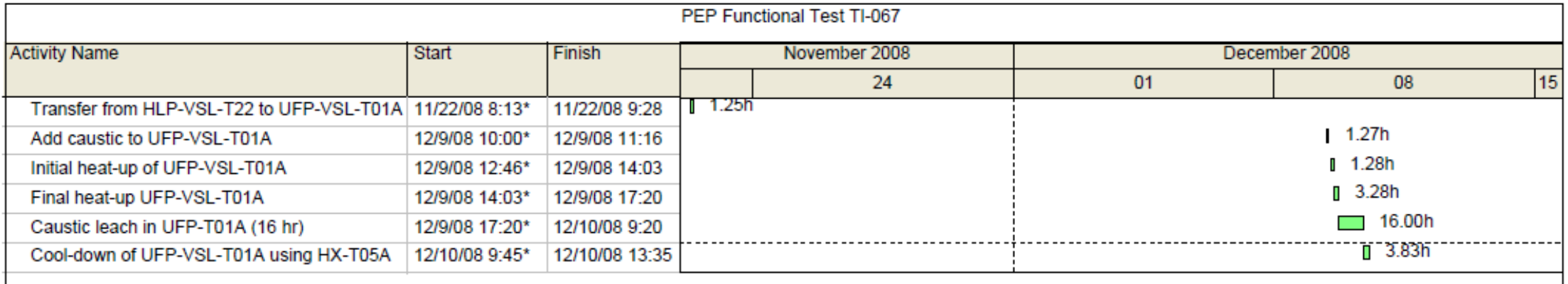

Figure 4.8. Timeline Illustration for TI-067. (For information only. Do not use times from this illustration; detailed times and processes are listed in the text.) 


\subsubsection{Add Caustic to UFP-VSL-T01A}

Section 7.4 of TI-067, leaching in Tank T01A, was performed on 12/9/2008 and 12/10/2008. The main functions performed in this test were adding 19-M NaOH (Section 7.4.1), heating at a controlled rate, holding at the specified leaching temperature, and cooling at a controlled rate (Section 7.4.2).

Simulant was already in Tank T01A from the sections of TI-062 that had been performed previously. AFA (400-mL) was added to Tank T01A at 09:04 on the morning of Tuesday, 12/9/2008. A stable level in Tank T01A (Section 7.4.1.6) of about 49 inches (bubbler, 48.8, laser, 50.4) was recorded. PJMs were started and run, controlled with the "simplified control program," to make sure of adequate mixing for subsequent $\mathrm{NaOH}$ addition.

Adding 554-kg of 19-M NaOH to the top of Tank T01A (TI Section 7.4.1.9) was started at 10:00. Manual control of the flow was used to achieve the target flow rate $(10-\mathrm{kg} / \mathrm{min})$ because automatic control was unstable. Samples were taken using the CD sampling station both before and during caustic addition. Density and temperature measurements were taken using the CD sampling station on Tank-T01A during transfer and after mixing.

\subsubsection{Heat-Up of UFP-VSL-T01A}

Caustic-leach operations in Tank T01A (7.4.2.2 T01A Heating) started on 12/9/2008 at 12:46. Initial heating was performed with a heat exchanger; final heating used steam injection.

The temperature in Tank T01A after adding caustic was $45^{\circ} \mathrm{C}$. Initial heating with HX-T04A began at 12:24 and used the tank temperature (TEK-0325) as the controlling parameter. The steam to the heat exchanger was controlled manually to maintain an outlet temperature of the recirculating simulant and caustic at $<80^{\circ} \mathrm{C}$. The target temperature of $63^{\circ} \mathrm{C}$ for initial heat-up was reached at 14:03. Verification was made that PJMs switched from vacuum to vent fill when the temperature reached $60^{\circ} \mathrm{C}$.

Steam injection (Section 7.4.2.6) was used for heating Tank T01A from $63^{\circ} \mathrm{C}$ to $98^{\circ} \mathrm{C}$, starting at 14:11 and continuing for about 3 hours. When the tank temperature rose above $65^{\circ} \mathrm{C}$, the PJM piping was switched to drain any accumulated condensate to a collection carboy so condensate did not drain back into the tank. Samples were taken at the CD sampling station when Tank T01A contents reached $88^{\circ} \mathrm{C}$ at 16:33.

\subsubsection{Caustic-Leach in UFP-VSL-T01A}

Caustic leaching in Tank T01A started at $17: 15$ when the target temperature of $98^{\circ} \mathrm{C}$ was reached. This began 16 hours of leaching. Stable level readings in Tank T01A (Step 7.4.2.6.1) at $98^{\circ} \mathrm{C}$ were 67.0 inches (bubbler) and 67.2 inches (laser). At 18:04, 56 gallons of leached simulant was transferred from Tank T01A to Tank T02A to reduce the level in Tank T01A to approximately 62 inches. After the transfer, levels in Tank T01A were recorded as 60.8 inches (bubbler) and 61.2 inches (laser). Samples were collected via the Tank T01A sample station at the beginning of leaching, at 1 hour, 2 hours, and 4 hours into leaching, and thereafter every 4 hours. PJM readings were recorded every half-hour from 2.5 hours into the leach through the remaining leach duration. 
The leach was completed at 09:15 on 12/10/2008, the automatic temperature control using direct steam was stopped, and sampling was begun. Sampling was complete at 09:45. Stable level readings at the end of leaching were 64.2 inches (bubbler) and 65.4 inches (laser).

The leached slurry in Tank T01A was cooled (7.4.2.9) to $60^{\circ} \mathrm{C}$ by recirculating slurry through HX-T04A (heater, with steam off) while aligning HX-T05A as needed to achieve the target temperature in 4 hours. The temperature of the Tank T01A was logged every 15 minutes to monitor and adjust the cooling rate.

When the tank temperature reached $68^{\circ} \mathrm{C}, \mathrm{HX}-\mathrm{T} 05 \mathrm{~A}$ was left aligned, and control was placed in auto mode. When the temperature in Tank T01A went below $60^{\circ} \mathrm{C}$, it was confirmed that PJMs had switched to vacuum fill.

\subsubsection{Test End}

TI-067 (Section 7.4) was completed on 12/10/2008 at 13:35. On 12/17/2008, from 19:25 to 22:55, one of the Drexelbrook level probes (LI-1609) was replaced inside a Tank T01A PJM. The level probe quit working on 12/20/2008 at 22:16 just after caustic addition was complete (LRB 60108, page 31), but the other probes were apparently not damaged.

\subsection{TI-WTP-PEP-032 Results}

The overall objective of Shakedown/Functional testing was to confirm the performance of all the components of the PEP and determine their important operational characteristics for final preparations before initiating Integrated process testing (ref. Table A.1 of the PEP Test Plan, TP-RPP-WTP-506). ${ }^{\text {(a) }}$ The first attempt to complete the entire testing (TI-062) had been terminated because of equipment failures. After the requisite repairs were completed, the entire Functional process testing was again undertaken under TI-032. This included some duplication of steps previously executed under previous TIs. It was considered important that all the process steps be executed in appropriate succession to demonstrate that the PEP was ready to commence Integrated process testing.

The necessary system alignments for HLP-VSL-T22, Tank T01A/B, Tank T02A, ultrafilters, and heat exchangers were completed before the test. The individual steps of the tests are described below. Figure 4.9 provides a timeline of test activities.

(a) GB Josephson, OP Bredt, JK Young, and DE Kurath. 2009. Test Plan for Pretreatment Engineering Platform (PEP) Testing (Phase I). TP-RPP-WTP-506, Rev. 0.4, Pacific Northwest National Laboratory, Richland, Washington. 


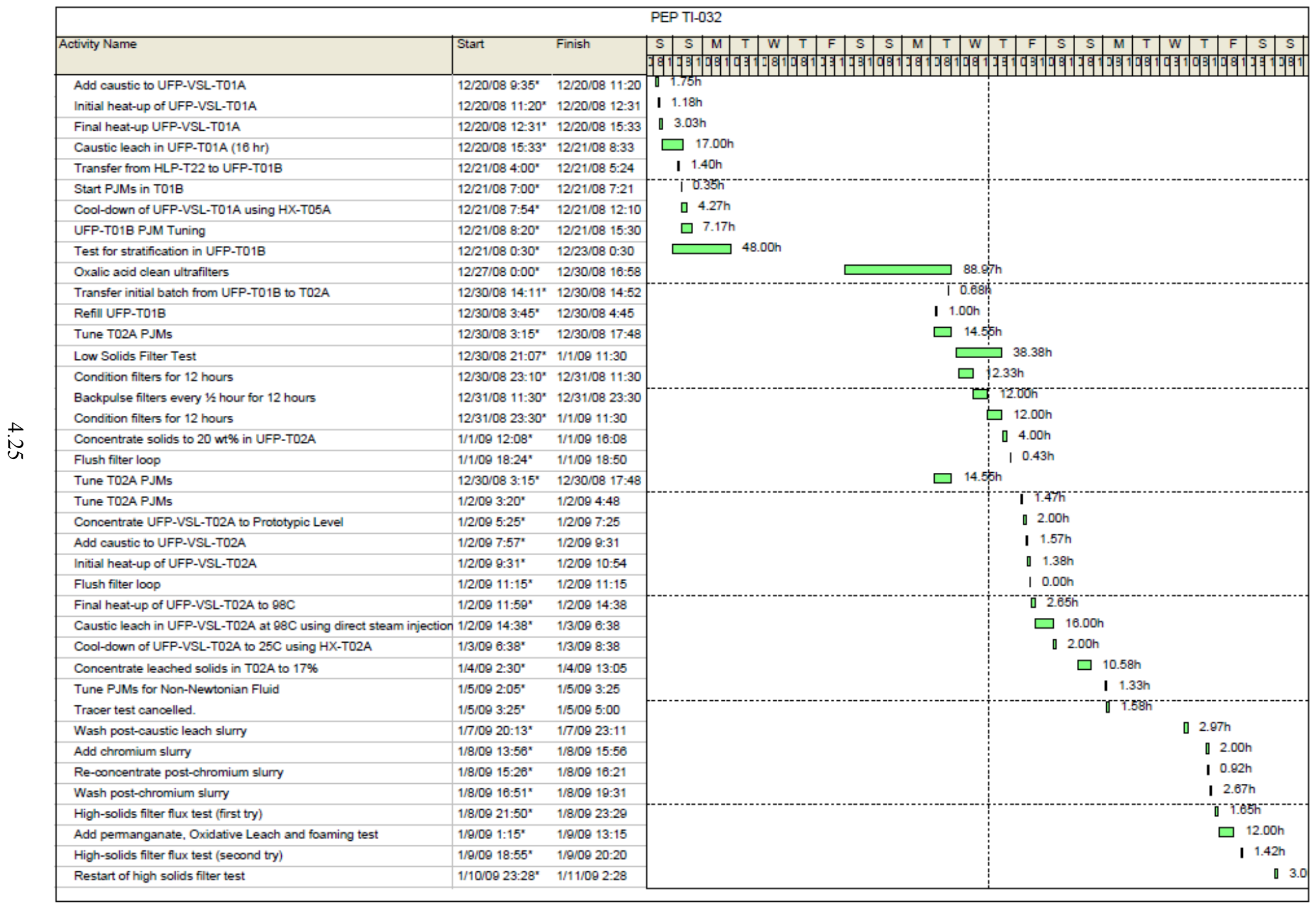

Figure 4.9. Timeline Illustration for TI-032. (For information only. Do not use times from this illustration; detailed times and processes are listed in the text.) 


\subsubsection{Simulant Transfers to UFP-VSL-T01B}

On 12/21/2008, Tank T01B was filled to 66.3 inches when 593 gallons was transferred from HLP-VSL-T22. The PJMs were off during the transfer. A performance check on Tank T01B level measurements was included in this activity. Flow totalizer FQI-0119 readings for volume, bubbler LI-0423 and laser LI-0420 levels, and capacitance probes LI-1701 and LI-1709 levels (in PJMs) were recorded during the transfer. Diluted AFA was added to the top of the tank after the transfer. The PJMs were tuned to $4.8-\mathrm{m} / \mathrm{s}$ with an $80 \%$ stroke length and 35-s cycle time in preparation for the stratification test. PJMs were operated in simple mode for the rest of the test. Tank mixing was checked by monitoring the CD densities at all elevations on the inner tank radial locations. All density readings were observed to be $1.285 \pm 0.004$.

\subsubsection{Stratification Test}

The Tank T01B stratification test was initiated on 12/21/2008 at 12:20. Quadruplicate UDS samples were to be taken from each of three elevations at 12, 24, and 36 hours. An initial sample set was taken at 12:30. The last sampling was completed on 12/23/2008 at 00:25 after 36 hours. The Tank T01B PJM nozzle velocity after the transfer was $4.8-\mathrm{m} / \mathrm{s}$ with a stroke length of $76 \%$. The steam ring air purge rate was set to $0.13-\mathrm{kg} / \mathrm{m}$. Sampling to determine Tank T01B stratification was conducted for 36 hours, which is described below.

Analysis of the wt\% UDS for the 12, 24, and 36-hr samples showed essentially the same results as the stratification test performed in Tank T01A during TI-062. There was no statistical difference in mean wt\% UDS between locations at any one time, nor was there a difference for each location over time. The results are shown in Table 4.2. 
Table 4.2. Stratification Data (wt $\%$ UDS) from UFP-VSL-T01B ${ }^{(a)}$

\begin{tabular}{|c|c|c|c|c|}
\hline \multicolumn{2}{|l|}{ Location } & $\mathrm{t}=12$ hour & $\mathrm{t}=24$ hour & $\mathrm{t}=36$ hour \\
\hline \multicolumn{2}{|l|}{ Inner-Low } & 5.30 & 5.33 & 5.37 \\
\hline “ & & 5.30 & 5.34 & 5.37 \\
\hline \multicolumn{2}{|l|}{ “ } & 5.33 & 5.33 & 5.34 \\
\hline \multirow[t]{2}{*}{ “" } & & 5.32 & 5.32 & 5.36 \\
\hline & Mean & 5.31 & 5.33 & 5.36 \\
\hline \multicolumn{2}{|l|}{ Inner-Middle } & 5.32 & 5.31 & 5.36 \\
\hline \multicolumn{2}{|l|}{ “ } & 5.37 & 5.27 & 5.36 \\
\hline \multicolumn{2}{|l|}{ “ } & 5.34 & 5.26 & 5.33 \\
\hline \multicolumn{2}{|l|}{ “ } & 5.36 & 5.30 & 5.35 \\
\hline & Mean & 5.35 & 5.29 & 5.35 \\
\hline \multicolumn{2}{|l|}{ Inner-High } & 5.33 & 5.28 & 5.42 \\
\hline \multicolumn{2}{|l|}{ “ } & 5.30 & 5.30 & 5.36 \\
\hline \multicolumn{2}{|l|}{ “ } & 5.25 & 5.28 & 5.29 \\
\hline \multicolumn{2}{|l|}{ “" } & 5.28 & 5.32 & 5.35 \\
\hline & Mean & 5.29 & 5.30 & 5.36 \\
\hline
\end{tabular}

For the most part, there was no statistical difference in the wt $\%$ UDS at different vessel locations at the same time. Therefore, it was decided by the JTG that Integrated process testing could proceed with sampling as described in the Test Plan, which was based upon the tank being essentially homogeneous.

\subsubsection{Oxalic Acid Filter Clean}

The filter-loop was filled with IW before filter conditioning, and the clean water filter flux was checked on $12 / 27 / 2008$ after 15 backpulses. The flux ranged from $3.8-\mathrm{kg} / \mathrm{min}$ on filter T05A to 9.9- $\mathrm{kg} / \mathrm{min}$ on filter T03A. The filter flux criterion was provided by BNI, and filters 1,2 , and 3 failed the clean water filter flux criterion $\left(1.24 \mathrm{gal} / \mathrm{min} / \mathrm{ft}^{2}\right.$ at $40 \mathrm{psi}$.).

On 12/27/2008, the ultrafilters were cleaned and backpulsed with oxalic acid. Oxalic acid cleaning was completed on 12/28/2009 at 06:40. After cleaning, the oxalic acid was drained and the filter-loop refilled with DIW. Filters 1, 2, and 3 failed the clean water filter flux criterion (1.24 gal $/ \mathrm{min} / \mathrm{ft}^{2}$ at $\left.40 \mathrm{psi}\right)$, and filters 4 and 5 passed (LRB 60108 pg 38 -39). On 12/28, it was determined that the filters should be cleaned again. The filter-loop was drained on 12/28/2008 at 12:26, and the oxalic acid cleaning procedure was performed again. Flow through the filters started at 14:08, and backpulsing was finished at 16:24. The first DIW rinse was performed by filling the acid tank, NAR-VSL-T01, (now empty of oxalic acid) with DIW on 12/29 11:20, and recirculation started at 12:05.

For the second rinse, 219 gallons of DIW were transferred from the acid tank with a pH below 3.5 at 22:25. The second rinse was completed at 23:47, and the flux rates were considered acceptable. The

(a) FunTest Closure stratification r1.doc-presented to WTP Test Director to close the stratification issue before advancing to Integrated process testing. 
Tank T02A, loop, and filters were drained, and Shakedown testing proceeded. It was observed that the drain solution samples from the loop had significant amounts of magnetic particles, and solution drained from the filter shells had tiny specks of magnetic material. Note: After the second acidic rinse, the system was NOT rinsed with IW. Intentionally, highly alkaline simulant was loaded into Tank T02A, and circulation was initiated so that any precipitation caused by the switch from acidic to alkaline conditions would have ample particle surfaces to grow crystals rather than nucleate into very small particles that would plug the filter. Whether the mechanism was correct or not was not determined, but testing was resumed.

\subsubsection{Simulant Transfers to UFP-VSL-T02A}

To prepare for simulant transfer from Tank T01B to Tank T02A, recirculation of Tank T01B was started with the variable frequency drive (VFD) at $70 \%$ and $7.3 \mathrm{gpm}$ with the heat exchangers bypassed. An initial batch was transferred from Tank T01B to Tank T02A on 12/30/2008 from 02:11 to 02:52. The initial batch neutralized the remaining acidity from the oxalic acid wash, provided slurry to fill the filter-loop, and provided volume to conduct the filter conditioning. The total transfer was 283 gallons (VFD at 65\% and $6.8 \mathrm{gpm}$ ) to achieve a Tank T02A level of 63 inches. The flow batch controller (FQIC-0431) on the transfer line was used to totalize the amount of the transfer. Overblows were heard in Tank T01B during the transfer as the Tank T01B level decreased, which identified the need for level compensation strategy.

A performance check on Tank T02A level instruments was performed during the initial transfer from Tank T01B to Tank T02A. After 46 gallons were transferred, the LI-0611 (bubbler) read 14 inches, and the LI-0623 (laser) read 27.6 inches. By the end of the transfer, the LI-0611 (bubbler) read 72 inches, and the LI-0623 (laser) read 77.6 inches. The total transfer volume based on the change in Tank T02A level was closer to 324 gallons rather than the recorded 283 gallons. Air spargers were turned on (upper $0.1-\mathrm{kg} / \mathrm{m}$ and lower $0.4-\mathrm{kg} / \mathrm{min}$ ), and PJMs were tuned in Tank T02A after the initial transfer from Tank T01B.

On 12/31/2008, HLP-VSL-T22 was used to refill Tank T01B after the initial transfer of 283 gallons from Tank T01B to Tank T02A. Tank T01B was refilled to 65 inches with a transfer of 390 gallons. The Tank T01B bubbler was clogged, so the laser was used for the stable level after the transfer. Additional diluted AFA was added to Tank T01B, and recirculation of Tank T01B was stopped.

\subsubsection{UFP-VSL-T02A PJM Tuning}

PJMs were difficult to tune in Tank T02A: the procedure extended from 03:15 to 17:48 on 12/30/2008. Parameters for the tuning were from TI-062. The PJM nozzle velocities were 7.4-m/s, and the stroke length was at $80 \%$ per laser after tuning.

\subsubsection{Low-Solids Filter Test (filter conditioning)}

Filters were conditioned by recirculating the contents of Tank T02A through the filter-loop for 36 hours. Filter conditioning consisted of three parts, each 12 hours long. All five filters and heat 
exchanger UFP-HX-T02A (cooling) were aligned. The steam heat exchanger, UFP-HX-T03A, was bypassed. ${ }^{(a)}$ This test is a duplicate of the first filter conditioning test performed under TI-062.

Filtration was initiated to fill the filter-loop on 12/30/2008 at 21:07. The filter-loop flow rate was set to $109 \mathrm{gpm}$ (ZV-0609 at $49 \%$ and T42A VFD at 91\%). The permeate was returned to Tank T02A. The Tank T02A level dropped to 35.7 inches (bubbler) and 43.1 inches (laser) when the pulse-pots were filled at 22:32 on 12/30/2008. An additional volume (89.6 gallons) was transferred from Tank T01B to reach the Tank T02A target level of 62.5 inches.

The first 12-hr period started at $23: 10$ on $12 / 30 / 2008$ and ended at 11:11 on 12/31/2008. Permeate flow was stopped after 10 minutes to take a slurry sample for parallel laboratory testing in the CUF. Permeate samples were taken at 60 minutes, 6 hours, and 12 hours. Tiny bits of magnetic particulates were observed in the final permeate samples. CD samples were taken at 60 minutes and 6, 9, and 12 hours.

The second 12-hr conditioning was from 11:30 to 23:30 on 12/31/2008. Filters were backpulsed every $1 / 2$ hour for 12 hours. CD samples were taken at the end of backpulsing. Each backpulse was controlled by the PLC and consisted of seven steps:

1. The permeate TMP control valve was closed and the pulse-pot isolated.

2. The pulse-pot was pressurized to approximately $50 \mathrm{psi}$ using high-pressure air.

3. Liquid was pushed out of the pulse-pot into the permeate piping until the pulse-pot liquid level reached a target (e.g., 9 to 10 inches), and then the permeate discharge valve was closed. The purge level was established during the water testing to be just above where the purge outlet would be uncovered, and the air blow traveled through the purge outlet into the permeate line.

4. The pulse-pot was pressurized to a target pressure above (overpressure) the filter inlet pressure. The standard overpressure was $40 \mathrm{psi}$, but the overpressure often went up to 45 to 50 psi because of the time to close the air valve and open the backpulse valves.

5. The "fast-acting" valve between the pulse-pot and the filter shell was open, allowing the air pressure to push permeate backwards through the filters.

6. The "fast-acting" valve remained open until the pressure in the pulse-pot reached a target overpressure above the filter inlet and then closed. The target overpressure was 5 psi.

7. After the fast acting valve closed, the pulse-pot was vented, and the TMP control valve again activated.

The backpulse generally worked well, and the three control parameters were not changed during the backpulsing: purge level, backpulse pressure, and final overpressure. During the third 12-hr period of conditioning, the filters were operated identical to the first period; there was no backpulse.

The third 12-hr conditioning period was from $23: 30$ on $12 / 31 / 2008$ to $11: 28$ on $1 / 1 / 2009$. At the end of filter conditioning, the filter-loop was reconfigured to align to one filter with permeate valves for filters 2 to 5 closed.

(a) Operating procedures required that both heat exchangers be initially aligned. Then the steam heat exchanger was isolated by a separation procedure before flow was started. 


\subsubsection{Concentrate Solids in UFP-VSL-T02A with One Filter}

Solids were concentrated to 20 -wt $\%$ UDS in Tank T02A using filter one, reducing the tank level without adding any new feed from Tank T01B. The concentration began on 1/1/2009 at 12:08. The PJMs were operated with the same drive pressures, drive time, and cycle time as during filter conditioning. The upper spargers were set at $0.01-\mathrm{kg} / \mathrm{min}$, and the lower spargers were set at $0.037-\mathrm{kg} / \mathrm{min}$. Tank T02A used the bubbler for level control as the laser level was reading high because of foam. The filter flow rate was $109 \mathrm{gpm}$ and was obtained using only the T42A pump. (Note: The T43A pump was locked out during execution of TI-032 because of a pressure relief design flaw. With only one pump, the system could not exceed the system design limits if the pump was deadheaded. After modifications to the pressure relief system, Integrated testing with two pumps was resumed.)

Overblows were observed on PJMs when the level in Tank T02A dropped below 27.4 inches, and PJMs were turned off. The concentration continued until the level reached the target 10 inches. No problems in maintaining the loop flow rate at $109 \mathrm{gpm}$ were noticed at this time. The total permeate removed (as recorded on HMI, for information only) was about $673-\mathrm{kg}$ (which was low because filters 2 to 5 permeate values recorded negative readings on flow meters when actually they were off.)

The initial pre-leach solids concentration operation in Tank T02A employed an average axial velocity of 14.5-ft/s (Pump T43A discharge) and an average TMP of 39.8 psid. The average Tank T02A temperature during this initial dewater operation was $25.3^{\circ} \mathrm{C}$. Analytical samples corresponding to the slurry at the beginning and end of the initial filtration period were taken, and as such, sufficient information exists to perform a material balance on the filtration operation. The result of this analysis is shown in Figure 4.10, which indicates the time dependency of UDS increase for the initial pre-leach dewatering concentration. This information provides the basis for estimating the dewatering curve for this operation. 


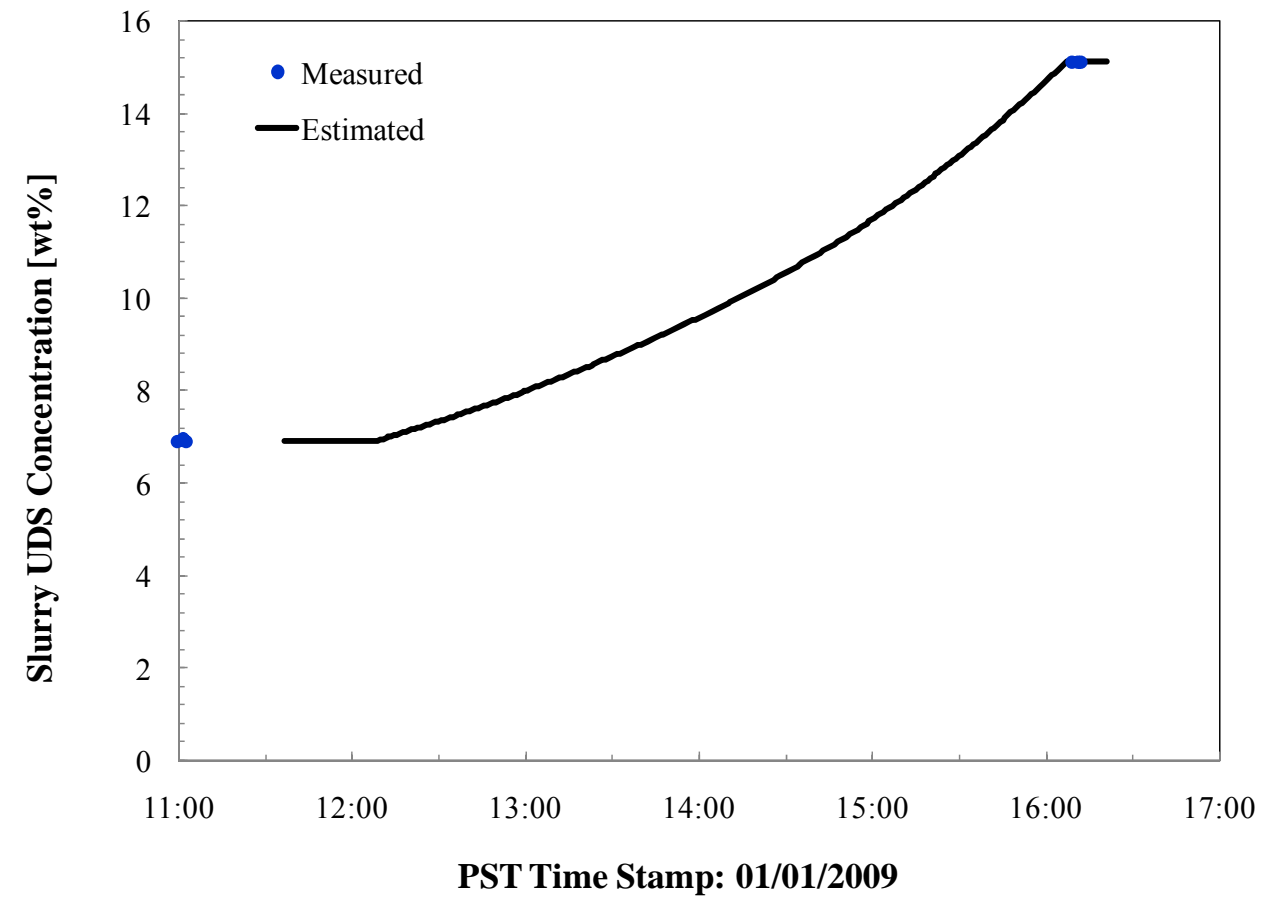

Figure 4.10. Measured and Estimated UDS Concentration Evolution for the Initial Pre-Leach Concentration Operations in Tank T02A (one filter)

Figure 4.11 and Figure 4.12 show the evolution of permeate production rate and TMP/temperature corrected filter flux during the initial pre-leach solids concentration under TI-032. Figure 4.13 shows the corresponding dewatering curve for this operation. Filter flux declines steadily with time, and the dewatering curve does not evidence a dewatering knee (that would suggest a transition to a cake-resistance-limited filtration region). Given the low starting UDS of $\sim 5$-wt $\%$, it can be tentatively concluded that dewater operations occur in the membrane-resistance limited regime. 


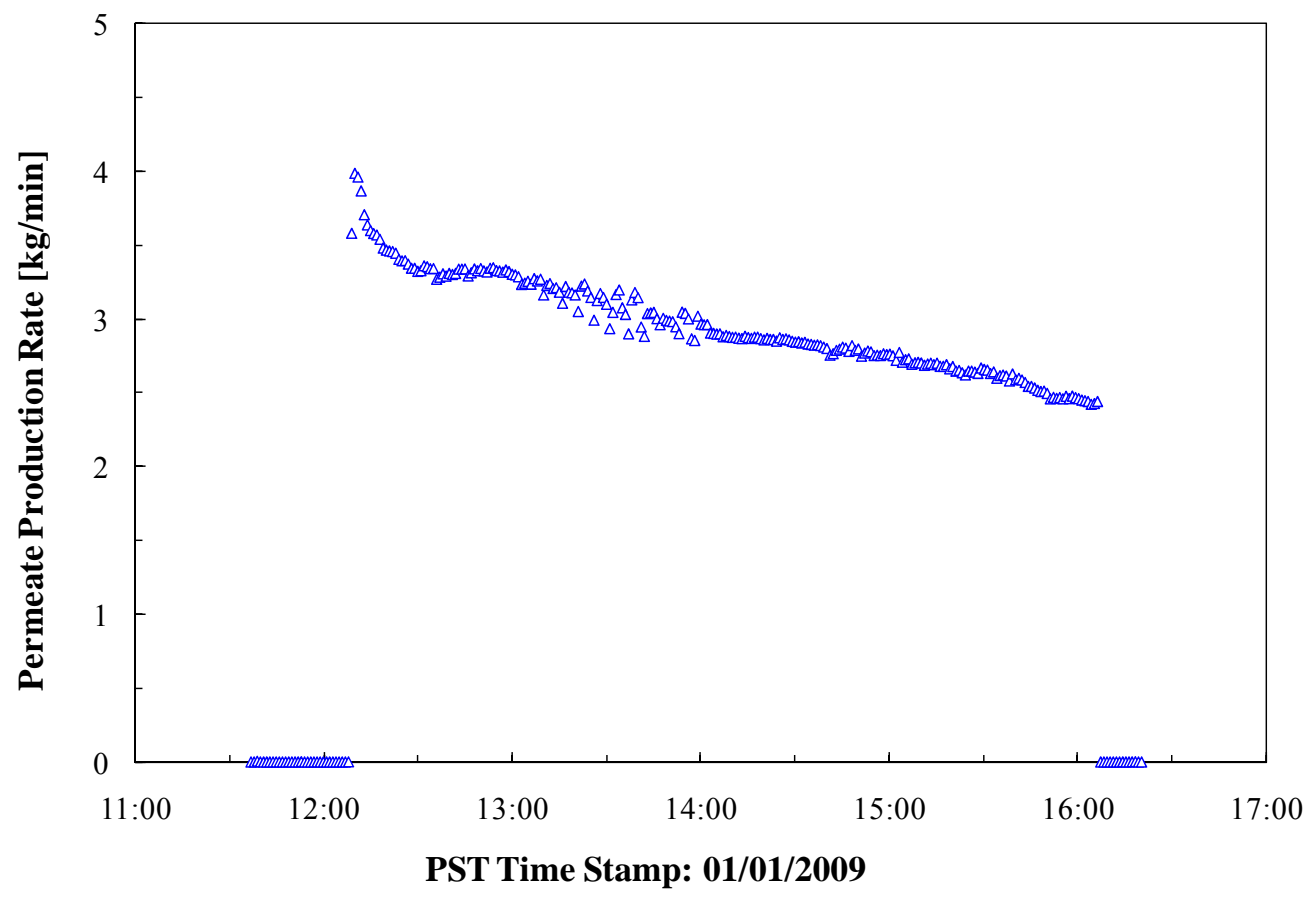

Figure 4.11. Permeate Production Rate During the Initial Functional Test (TI-032) Pre-Leach Concentration of Solids on Filter 1

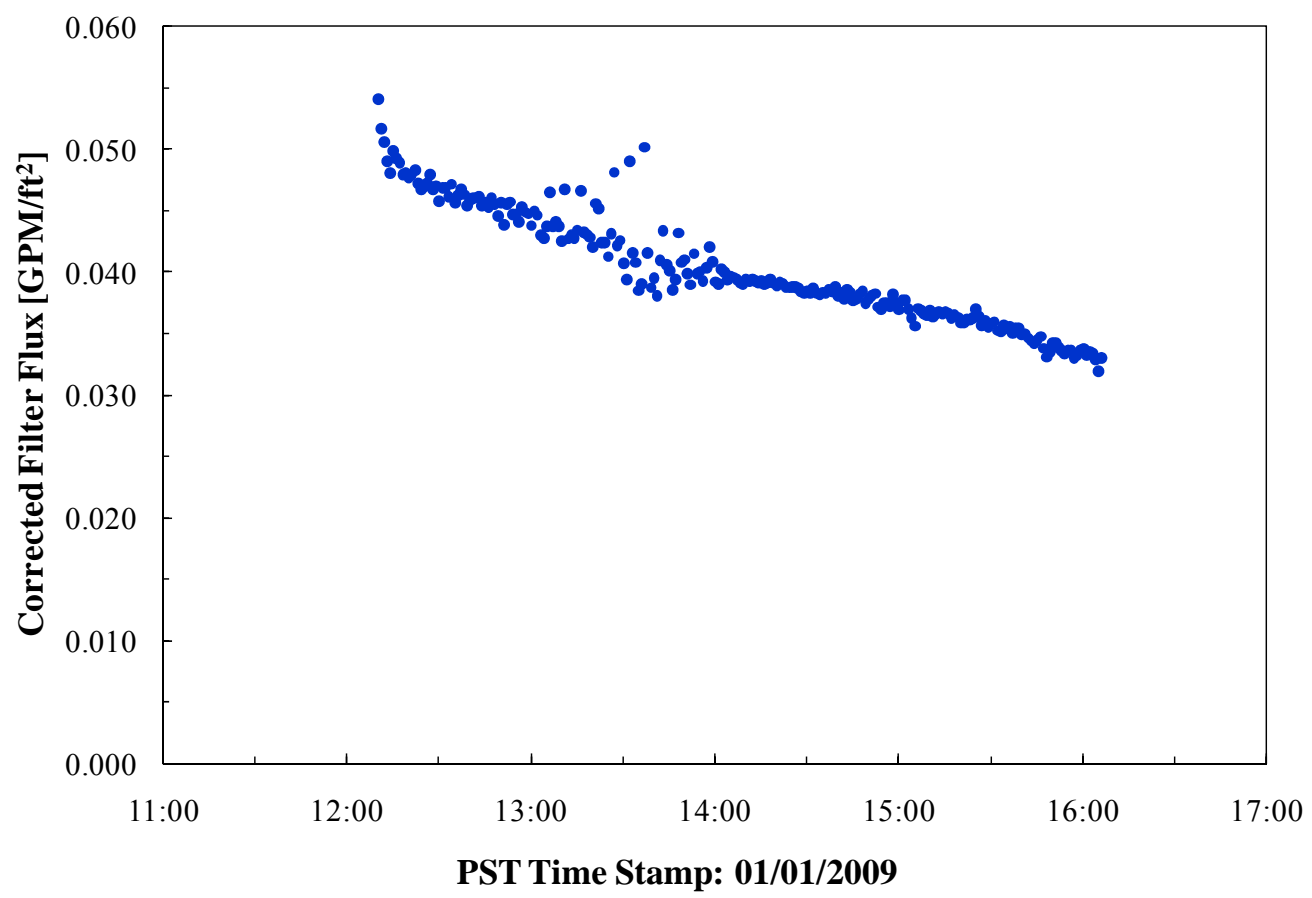

Figure 4.12. TMP and Temperature Corrected Filter Flux for the Initial Functional Test (TI-032) Pre-Leach Concentration of Solids on Filter 1 


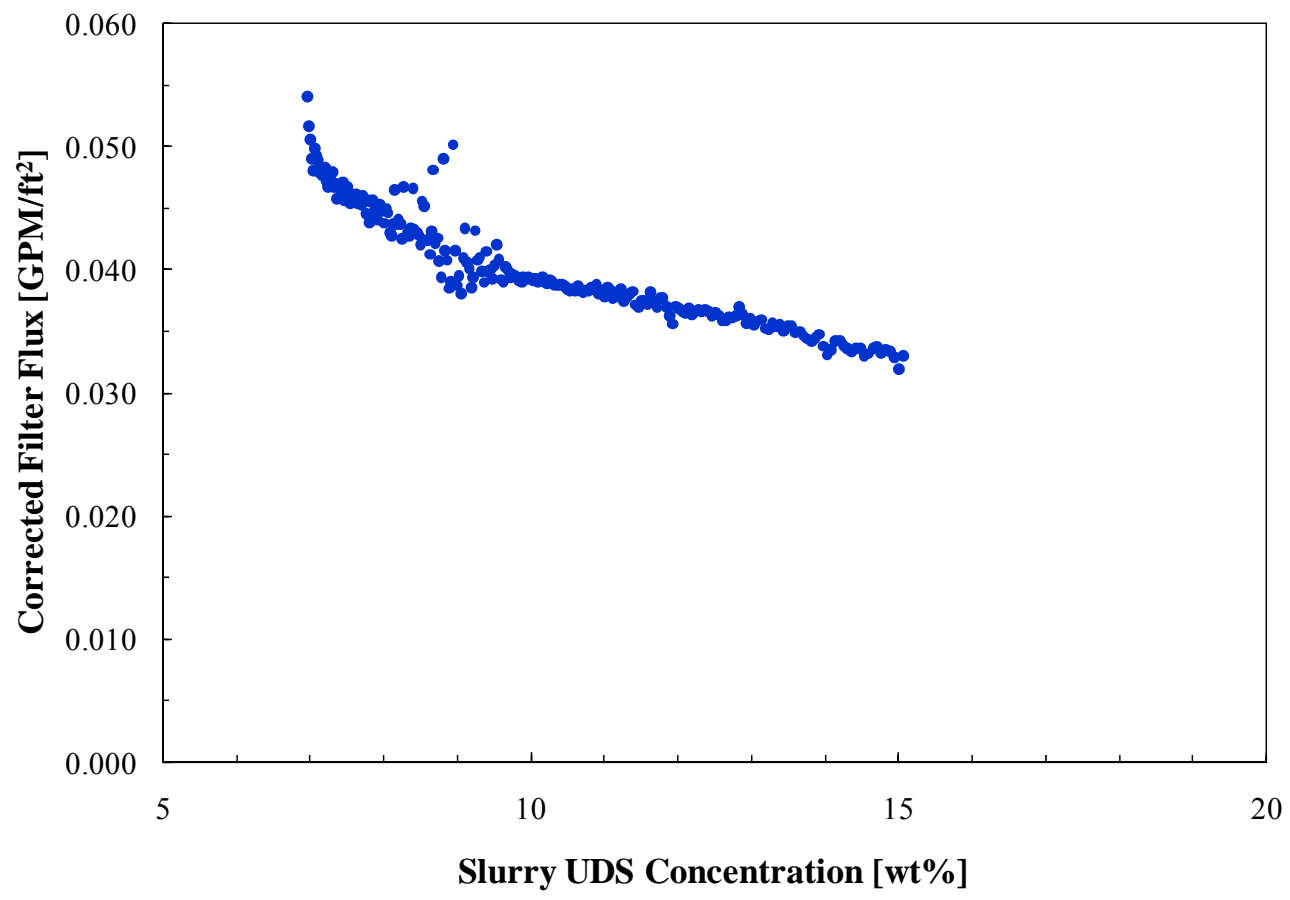

Figure 4.13. Dewatering Curve for the Initial Functional Test (TI-032) Pre-Leach Concentration of Solids on Filter 1

\subsubsection{Filter-Loop Flush}

After the low-solids filter test was complete, the filter-loop was flushed with approximately 43 gallons of water, and the filter-loop was reconfigured to bypass filter \#1 and filter through filters 2 to 5. When the pumps were restarted, they could not maintain the $109 \mathrm{gpm}$ flow rate. An additional 30 gallons of IW was added to Tank T02A, and a higher flow rate could be established, but it was very unsteady, fluctuating between $60 \mathrm{gpm}$ and $109 \mathrm{gpm}$. After many trials and starts and stops, the system was successfully restarted with the air spargers turned off and PJMs off. When PJMs were turned back on, the flow rate deteriorated. With PJMs off and bottom air spargers on, a 109 gpm flow rate could be maintained. The culprit for causing air entrainment was considered to be the PJMs, so more adjustments were made to the PJM vacuum refill parameter drive times to make sure that all the PJM tubes filled sufficiently to prevent overblows and obtain a 6-in. level change in the tank. Conditions were established finally at $04: 40$ on $01 / 02 / 2009$ to proceed with the final concentration to 20 -wt $\%$ solids.

\subsubsection{Final Concentration to $20-w t \%$ UDS}

On 1/2/2009 at 05:25, operators began concentrating Tank T02A solids using filters 2 to 5 with Tank T01B in autofill. After 18 batches had been transferred, the Tank T01B level changed from 66 to 46 inches. Operators continued concentration until the Tank T02A level was reduced from 40 inches to the target 18-in. level. The concentration was completed at 07:25 on 1/02/2009. Approximately 857-kg of permeate had been removed from the 18 batches that had been transferred.,

The final concentration of the pre-leach slurry on filters 2-5 employed an average axial velocity of 14.3-ft/s (Pump T43A discharge) and a TMP of $40 \pm 1$ psid. A significant deviation from target 
temperature of $25^{\circ} \mathrm{C}$ occurred during the test. During the first hour of the two hour dewatering operation, Tank T02A temperature was approximately 45 to $50^{\circ} \mathrm{C}$. During the final hour, temperature control was applied, but $\sim 25^{\circ} \mathrm{C}$ was not attained in Tank T02A until the last 30 minutes of testing. The filter TMP also shows occasional deviations of up to $10 \mathrm{psid}$ in certain cases. Both temperature and TMP deviations impact the quality of filter flux corrections for TMP and temperature, which are typically only applied to small deviations from the target test conditions. For the current operations, the quality of correction is uncertain because the deviations in temperature and TMP being corrected are large for certain test periods.

Analytical samples for UDS determination were taken at the start and end of this final pre-leach concentration operation. These samples provided sufficient information to perform a material balance on the filtration operation. This result of this analysis is shown in Figure 4.14. This material balance provides the required information to calculate the dewatering curve for this operation.

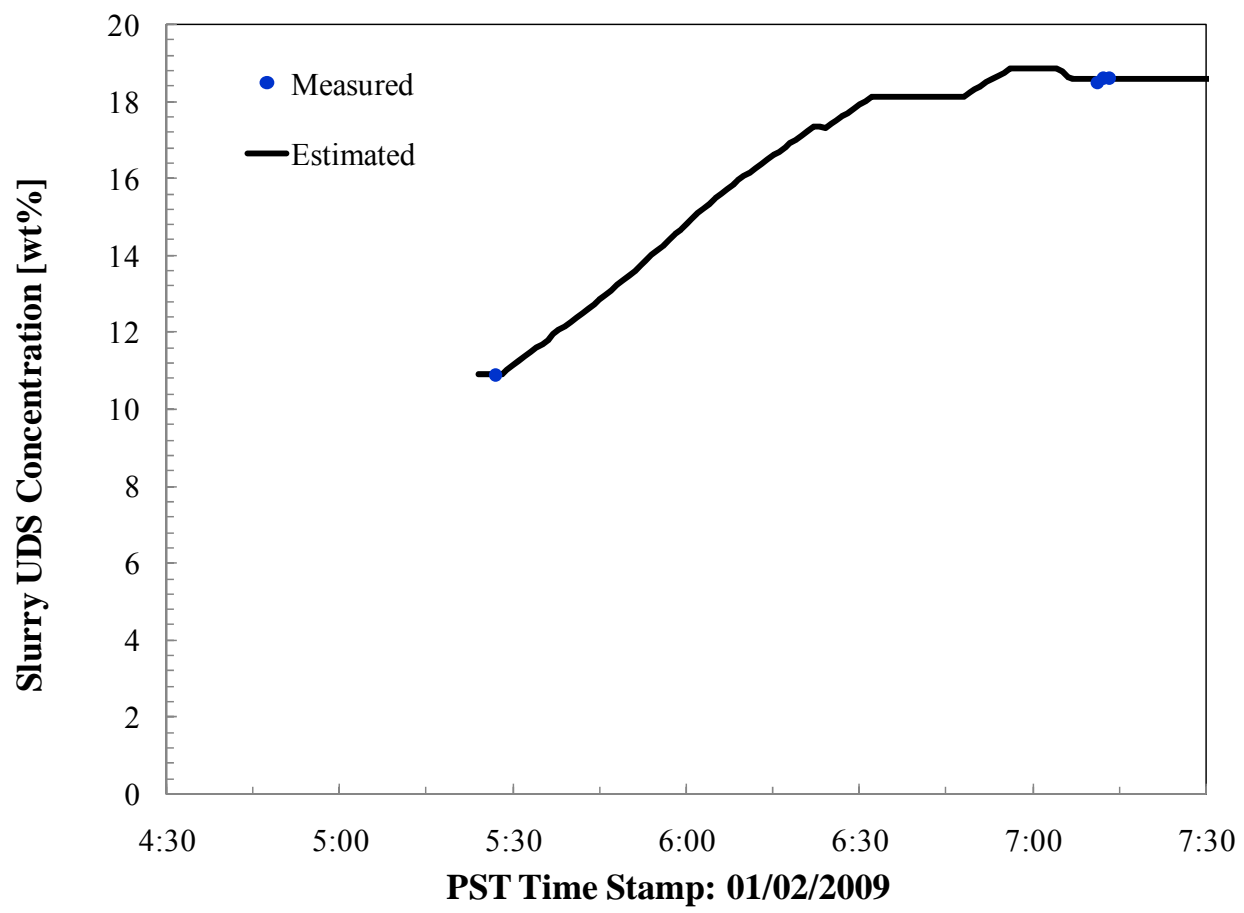

Figure 4.14. Measured and Estimated UDS Concentration Evolution for the Final Pre-Leach Concentration Operations in Tank T02A (filters 2-5)

Figure 4.15 and Figure 4.16 show the evolution of permeate production rate and TMP/temperature corrected flux during final pre-leach dewatering operations. The impact of significant temperature deviation is observed in Figure 4.15. Here, a significant (apparent) drop-off in permeate production occurs around $0600 \mathrm{hrs}$ as a result of cooling the contents of Tank T02A from $\sim 50^{\circ} \mathrm{C}$ to $25^{\circ} \mathrm{C}$. As indicated by the results in Figure 4.16, application of temperature correction formula appears to eliminate most of the variability in permeate production associated with the temperature excursion. The filter flux data are still noisy and exhibit $\sim 0.005 \mathrm{gpm} / \mathrm{ft}^{2}$ jumps associated with TMP spikes previously discussed. Overall, the flux data suggest a decline in flux but do not evidence a dewatering knee. The final pre-leach dewatering operation is a continuation of the initial operation discussed in the previous section. Figure 4.17 shows the dewatering curve for the second dewatering operation. Given the starting UDS of 
$10-\mathrm{wt} \%$, it can be tentatively concluded that final dewatering operations also fall in the membrane resistance limited regime.

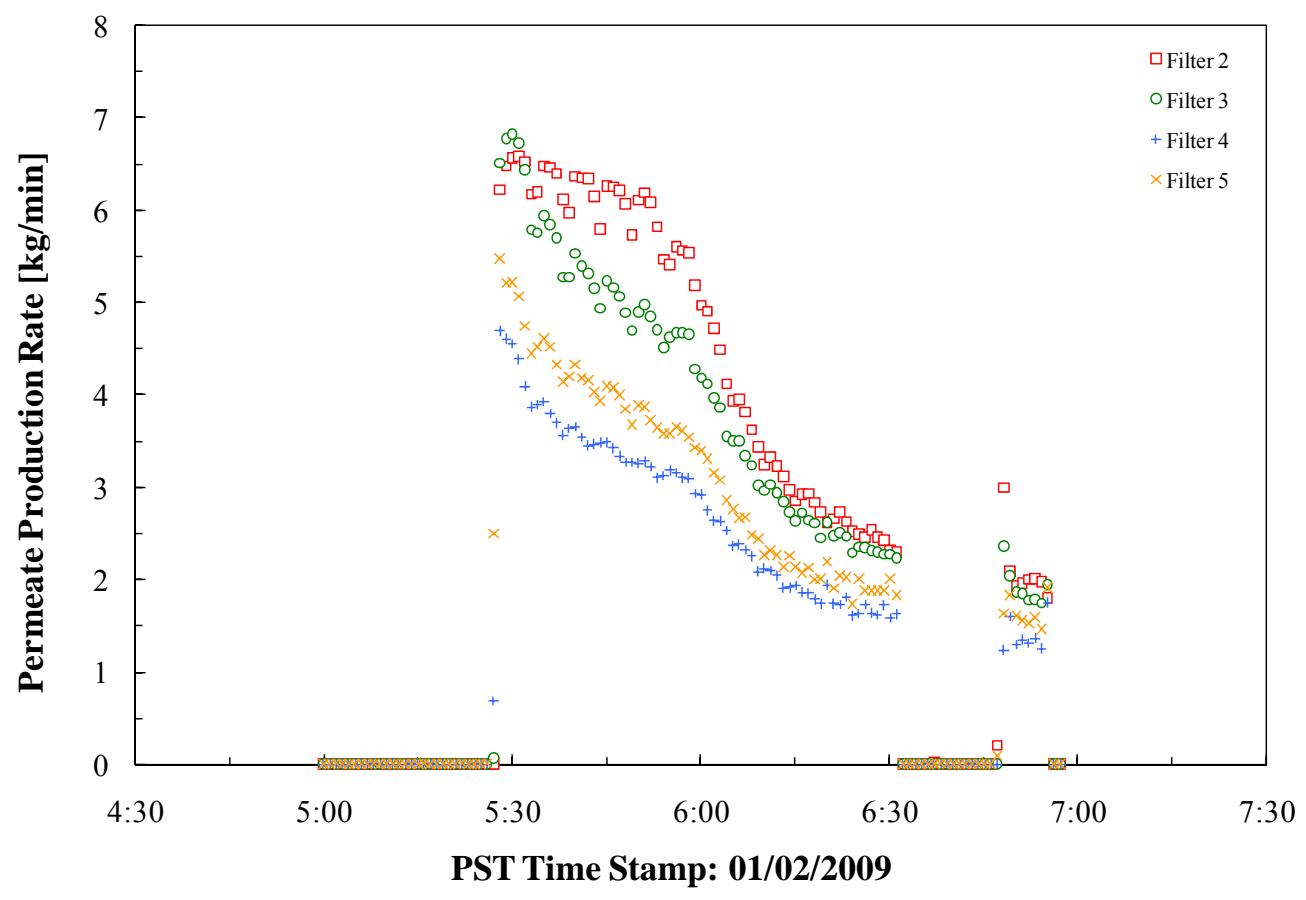

Figure 4.15. Permeate Production Rate During the Final Functional Test (TI-032) Pre-Leach Concentration of Solids on Filters 2-5 


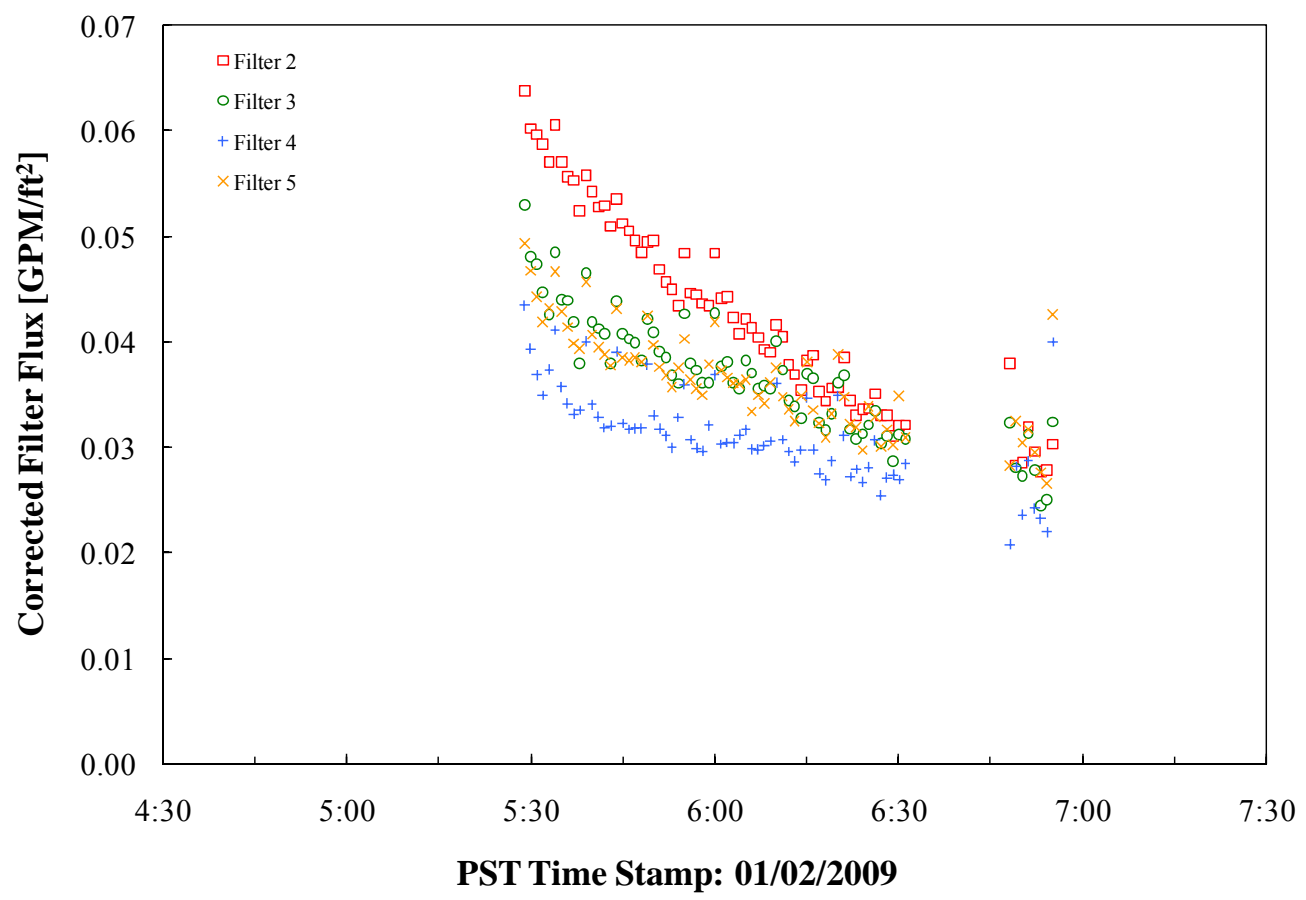

Figure 4.16. TMP and Temperature Corrected Filter Flux for the Final Functional Test (TI-032) Pre-Leach Concentration of Solids on Filters 2-5

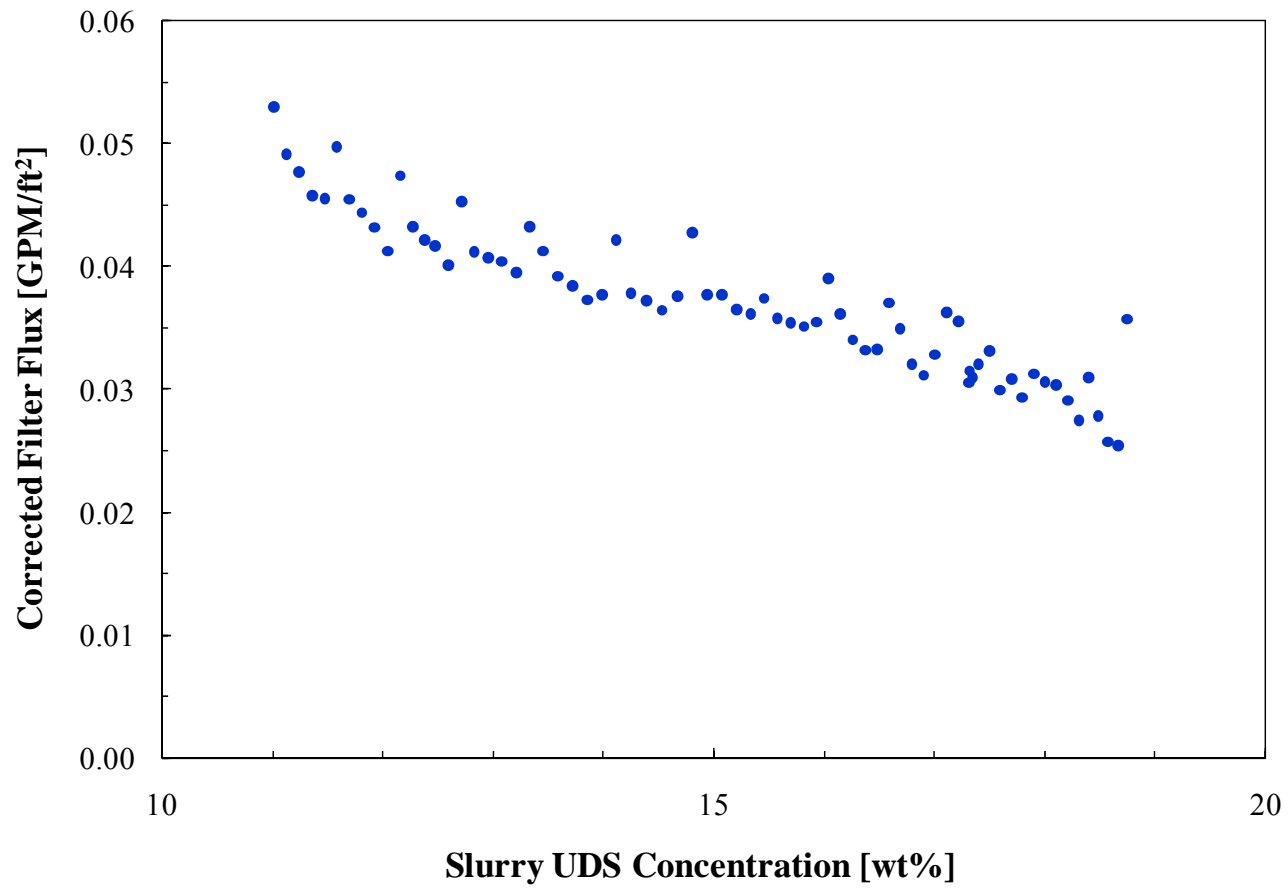

Figure 4.17. Dewatering Curve for the Final Functional Test (TI-032) Pre-Leach Concentration of Solids on Filters 2-5 
Figure 4.18 compares the initial and final dewatering curves. Filter flux for both dewatering operation is comparable. For similar UDS ranges, the final dewatering operation shows slightly higher filter flux. This difference can be attributed to fouling or potentially because of imperfect correction of the significant $\left(>25^{\circ} \mathrm{C}\right)$ temperature deviation. Overall, the filter flux behavior during final dewatering operation on filters 2-5 appears to be a reasonable continuation of that during the initial dewatering operation on filter 1.

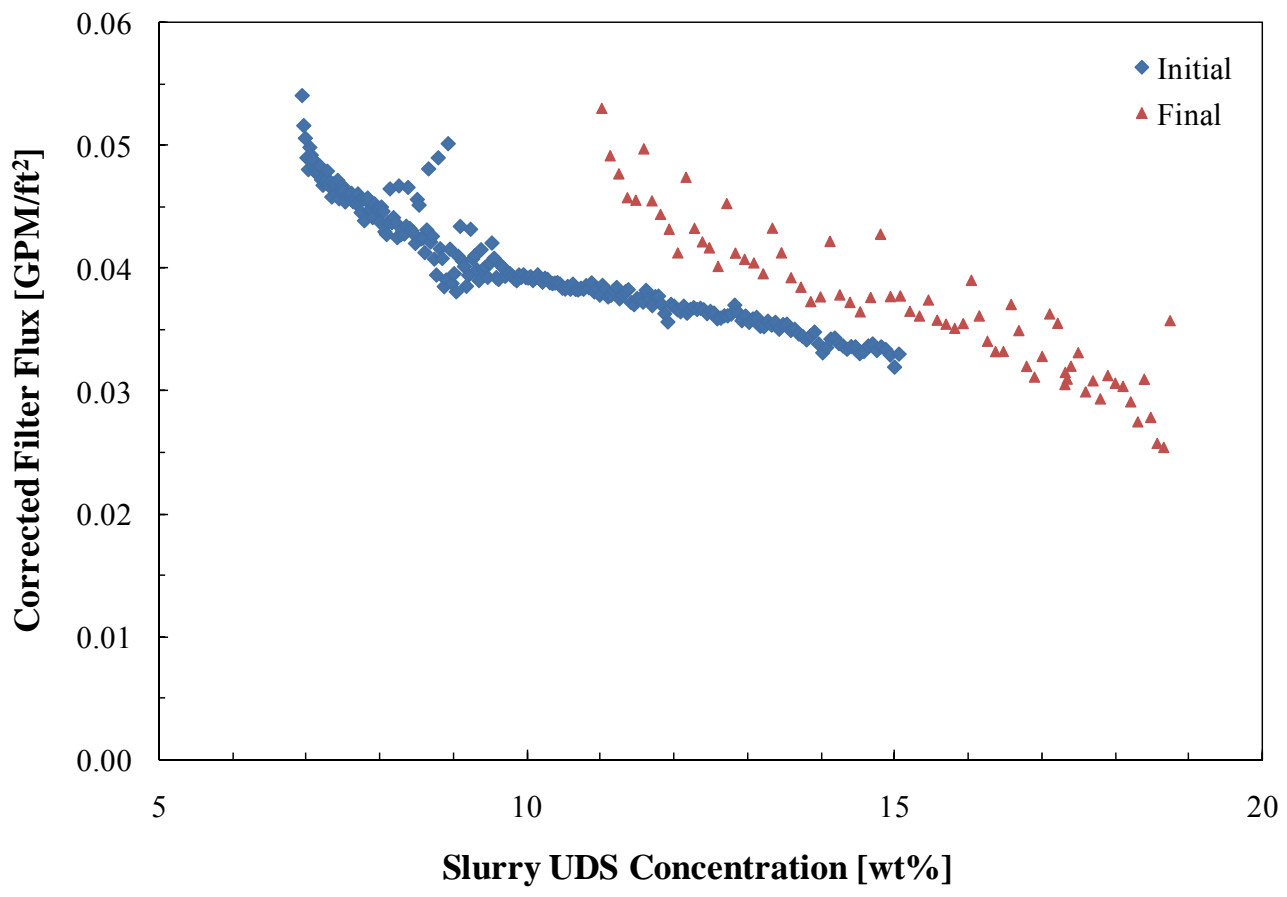

Figure 4.18. Comparison of Dewatering Curves for Initial and Final Pre-Leach Concentration Solids During TI-032

\subsubsection{Add Caustic in UFP-VSL-T02A}

Caustic was added to Tank T02A from 07:57 to 09:18 on 1/2/2009. After the concentrated caustic addition was completed, a batch temperature of $\sim 45^{\circ} \mathrm{C}$ was achieved as a result of caustic addition and pump heat associated with recirculation flow through the filter-loop and (inactive) heat exchangers. DAS data indicate that chilled water flow through the vessel cooling jacket was initiated at $45^{\circ} \mathrm{C}$ because the water jacket control valve was set to automatic control (inadvertently) with a temperature set point of $45^{\circ} \mathrm{C}$. During a brief period of mixing after caustic addition, the temperature dropped to $\sim 39.3^{\circ} \mathrm{C}$ as a result of the chilled water in the cooling jacket. Without the jacket cooling, the temperature increase from the combination of pump heat and $\mathrm{NaOH}$ dilution heat would be considerably greater than the $\sim 11^{\circ} \mathrm{C}$ observed in this test.

\subsubsection{Heat-Up in UFP-VSL-T02A}

Caustic addition was complete at $09: 18$, and heating was continued by recirculating through the filter-loop. The slurry temperature increased to $\sim 56.1^{\circ} \mathrm{C}$ before the water flow was stopped at 09:48 by 
closing one of the manual jacket valves (V06117). Tank T02A was heated to $60^{\circ} \mathrm{C}$ by $10: 54$ in spite of the jacket cooling. After stopping the water jacket cooling, recirculation through the filter-loop continued, and pump heat brought the temperature up to $72.8^{\circ} \mathrm{C}$ by $11: 10$. After recirculation ceased, the slurry batch temperature dropped to $65.9^{\circ} \mathrm{C}$ before direct steam heating in Tank T02A started at 11:59.

The delay from the end of pre-heating to the start of direct steam injection was due to several processing steps, including an initial single-volume loop flush into Tank T02A, further loop flushing into waste tank T62A, slurry sampling, and preparation of steam system components. The efficiency of these steps was improved during Integrated testing. The final heat-up of Tank T02A to $98^{\circ} \mathrm{C}$ was completed on 1/2/2009 at 11:59 using direct steam injection. Approximately $0.9-\mathrm{L}$ of condensate were drained and collected from the PJM lines during the heat-up.

\subsubsection{Caustic-Leach in UFP-VSL-T02A}

Shortly after reaching the $98^{\circ} \mathrm{C}$ set point and while in automatic control mode, hand-written notes on the TI (pg. 99) and in Operations Logbook \#3 (pg. 38) indicate that a high-temperature alarm for a temperature exceeding $100^{\circ} \mathrm{C}$ was reported by the PLC/HMI control system (e.g., $100.2^{\circ} \mathrm{C}$ noted), and the steam valve was closed, resulting in a low-temperature excursion and unplanned shutdown of the PJMs. Several other incidents during the $98^{\circ} \mathrm{C}$ hold period caused the steam control valve to switch from auto to manual mode (before being reset to auto control).

In Tank T02A, the volume of condensate accumulated during the $98^{\circ} \mathrm{C}$ hold period was greater than expected by $\sim 8$ gallons. Misoperation of the water cooling jacket and other unexpected heat sinks in Tank T02A during high-temperature caustic leaching were the primary causes for the excess condensate to be accumulated.

At $15: 40$ on $01 / 02 / 2009$ ( 1 hour after reaching $98^{\circ} \mathrm{C}$ ), the PJM nozzle velocity was checked and found to be $8.3-\mathrm{m} / \mathrm{s}$ with a 6.2 -in. stroke length and re-adjusted to $7.3-\mathrm{m} / \mathrm{s}$. CD samples were collected at $1,2,4,8,12$, and 16 hours. The Tank T02A bubbler read 57 inches at the end of the leach (after cleaning bubblers). The target level was 62.5 inches $(+5 /-9)$.

\subsubsection{Cool-Down After Caustic-Leach}

After 16 hours, the caustic-leach was completed. Tank T02A was cooled to $25^{\circ} \mathrm{C}$ using HX-T02A. The cooling water valve was operated manually to achieve the prototypic cool-down curve. The tank temperature was checked every 30 minutes and compared to the target temperature. If the temperature was above target, the cooling water valve would be opened $5 \%$. If the temperature was below target, the valve would be closed 5\%. Most of the time, the valve was open to less than $20 \%$ (minimal flow). The manual water jacket valve that had to be closed in the middle of the pre-heating process was inadvertently left closed after $\sim 2.5$ hours into the cooling period. The manual closure valve(s) should have been opened at the start of the cooling period to allow jacket cooling to be turned on via the control valve for the water jacket flow. The oversight meant that ambient cooling alone was used for an extended period, and the slurry temperatures remained higher than the target profiles until after cooling water flow was initiated. The cooling water swirl meter indicated flow when none existed, which made the problem less obvious. Simultaneous to processing occurring in Tank T02A, parallel processing was being done in Tank T01A to produce a leached slurry that could supplement the leached material in Tank T02A for the subsequent 
concentration to target $17-\mathrm{wt} \%$ UDS. Section 4.3.11 describes the parallel processing. The serial processing re-commences with Section 4.3.12.

\subsubsection{Parallel Processing (upfront leaching) Conducted in T01A}

\subsubsection{Add Caustic to T01A}

On 12/17/2008 (before test start), Tank T01A was already filled to 45 inches, and PJM tuning in Tank T01A was conducted using the new LI-1609 Drexelbrook probe. Results were recorded in LRB 60108, pp. 30-31. The PJMs were tuned to achieve a peak average nozzle velocity of $4.8-\mathrm{m} / \mathrm{s}, 80 \%$ stroke length, and 35-s cycle time. PJMs were operated in simple mode for the test. Tank T01A was placed into recirculation with the recirculation loop aligned with the steam heat exchanger in case additional heating was needed to reach the initial target temperature before engaging direct steam injection. Also, AFA was added to keep the antifoam concentration up to $350 \mathrm{ppm}$ when the caustic was added.

AFA was added to Tank T01A on 12/20/2008 at 07:52. Caustic (571-kg) was added to the top of Tank T01A at a rate of approximately $17.7-\mathrm{kg} / \mathrm{min}$ on $12 / 20 / 2008$ at $09: 35$. The caustic addition was completed in about 30 minutes at 10:06. The temperature after the caustic addition was $44^{\circ} \mathrm{C}$.

\subsubsection{Heat-Up of T01A}

Tank T01A was heated to $58^{\circ} \mathrm{C}$ over a period of 1 hour using HX-T04A and then to a temp of $98^{\circ} \mathrm{C}$ using direct steam injection over a 3-hr period. The 16-hr caustic-leach commenced when the tank temperature hit $98^{\circ} \mathrm{C}$ at $15: 33$ on $12 / 20 / 2008$.

\subsubsection{Caustic-Leach in UFP-VSL-T01A}

The Tank T01A caustic-leach started at $15: 33$ on 12/20/2008 and ended at 07:33 on 12/21/2008. Problems were encountered with the PJMs stopping, frequent Tank T01A high-density alarms sounding, and the bubblers plugging during the caustic-leach. Problems were noted with reading the temperature on Tank T01A CD sampling loops and with operating the Tank T01A sampling pump. The last CD sample was taken at 07:47 on 12/21/2008. Tank T01A collected 19-L of steam condensate from the PJM lines during heat-up and caustic-leach. On two occasions, the Tank T01A temperature controller (TIC 0325) switched from automatic to manual on its own and had to be reset. At one point, Tank T01A reached $100.2^{\circ} \mathrm{C}$ in auto control mode, which was outside the $\pm 2{ }^{\circ} \mathrm{C}$ allowance. The laser data measuring tank level, LI-0320, were very noisy unless the steam valve was on; see LRB 60108, pages 32-37.

\subsubsection{Cool-Down After Caustic-Leach in UFP-VSL-T01A}

After the caustic-leach (07:54 on 12/21/2008), Tank T01A was cooled to $25^{\circ} \mathrm{C}$ using HX-T05A. The slurry was recirculated through HX-T05A with the cooling valve operated in manual mode. During the cooling, it was observed that cooling water did not appear to flow until the control valve was opened to $>25 \%$ (no indication on flow meter and an elevated inlet cooling water temperature to the heat

exchanger). The recirculation rate for PMP-T41A was $8.9 \mathrm{gpm}$ during cool-down. The vacuum ratio for 
the PJMs was set to $100 \%$ when the PJMs switched to vacuum fill at $60^{\circ} \mathrm{C}$. Cool-down was completed at $12: 10$ on $12 / 21 / 2008$.

Tests were conducted during the cool-down to verify the elevation of each of the CD sampler legs in Tank T01A. This was done by bubbler air through each of the CD legs and measuring the static pressure of the air, which is the same principle employed by the bubbler level gauges (LRB 60108, pages 35-38). Similar tests were also conducted on Tanks T01B and T02A. The test identified that two of the Tank T01B samples legs were reversed (NCR 38770.1).

\section{This is the last step of the Parallel Processing in UFP-VSL-T01A.}

\subsubsection{Concentrated UFP-VSL-T02A Solids to $17 \%$}

Leached solids in Tank T02A were concentrated to 17\% using filters 2 through 5 on 1/04/2009 starting at 02:30. The concentration was initially done at "constant" level using "Autofill" from Tank T01A. Forty-two 11-gallon batches were transferred, which drained Tank T01A from 57.9 inches (55.9 on bubbler) to the target of 10 inches. Problems with bubblers in Tank T02A triggered multiple autobatches. Autofill from Tank T01A was temporarily disabled (refer to LRB 60108, pg 55) while the bubbler problem was investigated. The slurry in Tank T02A was also accidently heated to $35^{\circ} \mathrm{C}$ during the test because the automatic temperature control using HX-T02A was not initially engaged.

After the Tank T01A level reached its target, the "Autofill" feature was turned off at 13:05 on 1/04/2009. Dewatering was continued for another 5 minutes to reach a target 37 -in. level in Tank T02A. The total permeate production was $2429-\mathrm{kg}$.

Concentration of the caustic-leached solids to $17-\mathrm{wt} \%$ UDS employed an average axial velocity of $14.3-\mathrm{ft} / \mathrm{s}$ and a TMP of $\sim 40 \pm 1 \mathrm{psid}$ over most of the test period. There was difficulty in maintaining both axial velocity and TMP targets toward the end of testing. An average Tank T02A temperature of $\sim 27^{\circ} \mathrm{C}$ (including the temperature excursions) was observed during testing. Insufficient analytical data were taken during this operation to perform a material balance of the filtration operation. Because no supernate density determinations were done, the filter flux during this operation was not calculated. As such, only permeate production rate evolution is reported for dewatering of the caustic-leached solids. This is shown in Figure 4.19. 


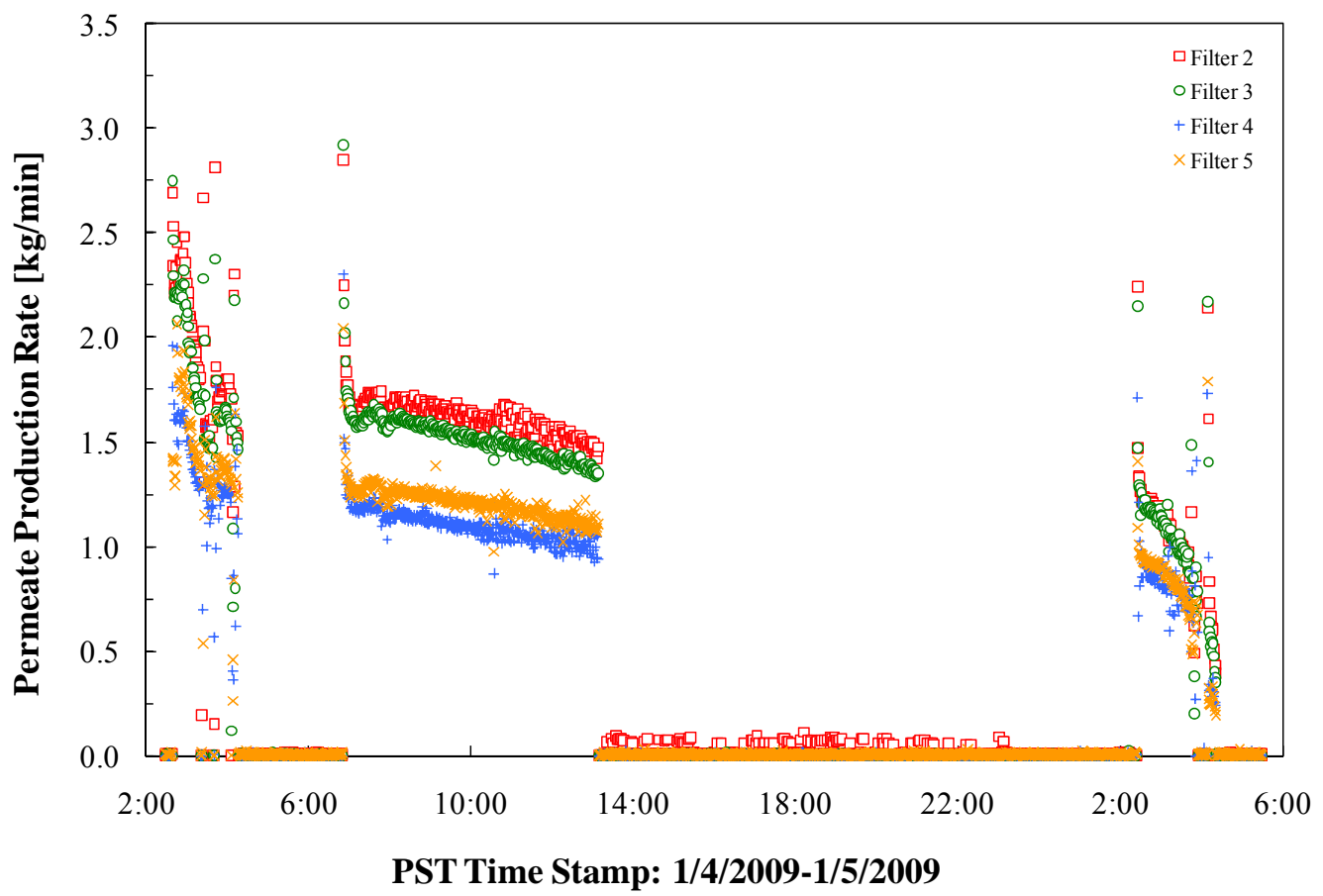

Figure 4.19. Permeate Production Rate During the Functional Test (TI-032) Post-Caustic-Leach Concentration of Solids on Filters 2-5

\subsubsection{Tune PJMs for Non-Newtonian Fluid}

On 1/5/2009 at 02:05, PJMs in Tank T02A were tuned for a nozzle velocity of $12.3-\mathrm{m} / \mathrm{s}$ to achieve proper mixing with non-Newtonian fluid produced during solids washing. The test used a method for calculating nozzle velocities based upon the slope of the linear section on the tank level vs. the time curve during the PJM drive cycle (ref. LRB 60108, pg. 58). The vacuum ratio was set at 400\%, and settings for auto level compensation and temperature linearization were enabled.

After the PJMs were adjusted to $\sim 12-\mathrm{m} / \mathrm{s}$, dewatering was restarted at 03:25 on 1/05/2009 to reach an 18-in. level in Tank T02A. However, the pump could not maintain the target flow rate of $109 \mathrm{gpm}$, and operators reported hearing "cavitation," so the pump was shut down. Ad hoc "studies" were conducted on the pumping system until dewatering recommenced at 23:55 on 1/06/2009. Among the ad hoc "studies," it was determined that doubling the air purge rate on the steam ring did not impact the loop flow. Many combinations of pump speed and backpressure control valve position were tried, but no effective combination was found that would produce the target $109 \mathrm{gpm}$ flow. A camera was lowered into the vessel, and a video of the tank liquid and return line was recorded. From this, it was discovered that the liquid returning to the tank impinged directly upon the steam ring. In the full-scale design, the liquid return leg is not supposed to hit the steam ring.

A washer was accidentally dropped into Tank T02A when the system was ready to restart at 23:55 on $1 / 06 / 2009$. The pumps were stopped as soon as the incident was discovered, and testing was terminated. Attempts were made to retrieve the washer using magnets in the vessel. After a day of searching with no success, it was decided to proceed with the water wash step of the test with a control level of 18 inches 
(raised from 13 inches to avoid pump/air problems). Note: When the system was later disassembled, the washer was found in the suction of the T43A pump, which had not been running. Evidently, the washer had been sucked into the pump suction before the T42A pump could be turned off and passed through the pump without causing damage.

\subsubsection{Wash Post-Caustic-Leach Slurry}

The washing of the post-caustic-leach slurry commenced at 20:13 on 1/7/09; the target was a free hydroxide concentration of $0.25-\mathrm{M}$ or less. Washing also removed oxalate in preparation for the high-solids filter flux test. The IW tank level was 114 inches before the wash and 92.6 inches after the wash. The T62A level increased from 7 inches to 17.5 inches. After the wash, the Tank T02A level was at a target level of 18.4 inches using the laser (23.1 per bubbler with $1.06-\mathrm{spG}$ ). The total wash mass was $1416-\mathrm{kg}$. During the wash, the IW was transferred to Tank T02A in $1 / 4$ to $1 / 3$ the expected time. To decrease the autobatch transfer time, the IW flow rate controller, FQIC-1523, was subsequently controlled in manual at $20 \%$ open (flow rate of $19.3-\mathrm{kg} / \mathrm{m}$ instead of the $16.3-\mathrm{kg} / \mathrm{m}$ target) to avoid the wide fluctuations in the flow rate from operating the FQIC 1523 control valve in automatic mode during the batch transfers.

Washing of the post-caustic-leach slurry employed filter bundles 2 to 5 , an average axial velocity of 14.9-ft/s, an average TMP $\sim 40 \pm 1 \mathrm{psid}$. Tank T02A showed deviations from the target temperature of $25^{\circ} \mathrm{C}$ of up to $3^{\circ} \mathrm{C}$. An average Tank T02A temperature of 25.6 was achieved during testing. Insufficient analytical data were taken during this operation to perform a material balance of the washing operation. Lack of analytical sample for a material balance prevents estimation of dissolved solids evolution, which in turn prevents determination of the filter flux (which requires knowledge of permeate density). Thus, for post-caustic-leach slurry washing, only permeate production rate evolution is reported. This is shown in Figure 4.20. 


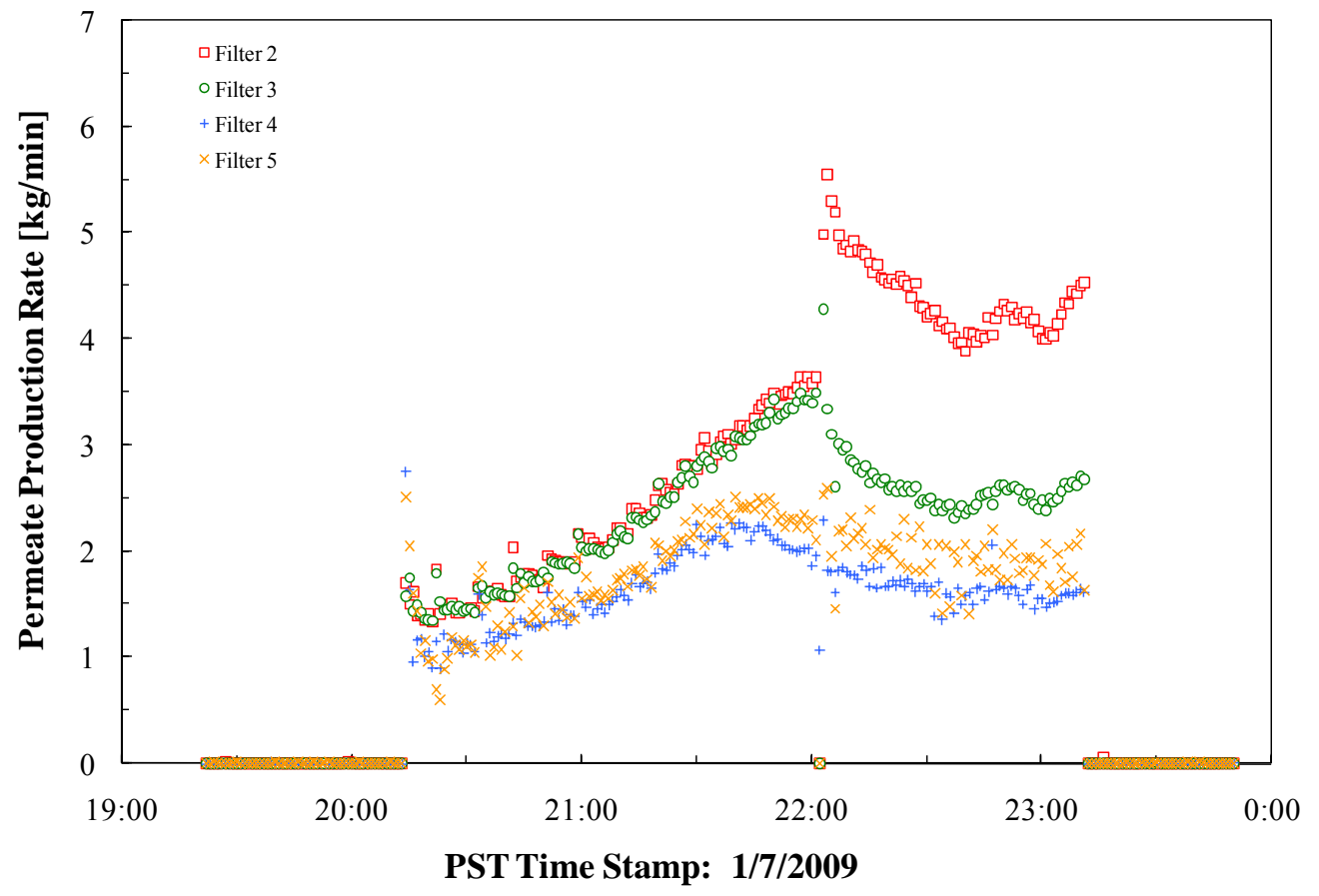

Figure 4.20. Permeate Production Rate During Functional Test (TI-032) Post-Caustic-Leach Slurry Washing on Filters 2-5

\subsubsection{Tracer Test}

A tracer test was to be completed before the post-caustic-leach water wash, but it was deleted and conducted during the Integrated tests.

\subsubsection{Chromium Addition}

Before the chromium addition, it was noted that the 55-gallon drums containing $\mathrm{CrOOH}$ had painted internals that were peeling. The test was put on hold until another drum of chromium could be obtained. On 1/8/2009 beginning at 15:29, $631 \mathrm{lbs}$ of chromium slurry was added to Tank T02A. The level in Tank T02A after chromium addition was 40 inches.

On 1/8/2009 from 15:36 to 15:59, filters T02A through T05A were aligned to re-concentrate the post-chromium slurry and remove the water added with the chromium. Tank T02A levels were 19.5 inches on the bubbler and 22.2 inches on the laser after the first dewatering.

On 1/8/2009 from $16: 00$ to $16: 51$, the post-chromium slurry was washed by adding $833-\mathrm{kg}$ of IW in 11-gal batches and using filters UFP-FILT-T02A through -T05A to remove the wash-water. Tank T02A levels were now 22.9 inches on the bubbler and 35.6 inches on the laser after the wash.

Washing and dewatering operations associated with chromium addition employed filters 2-5, an average axial velocity of $14.4-\mathrm{ft} / \mathrm{s}$, and an average TMP of $40 \pm 1 \mathrm{psid}$ on active filters. Temperature deviations from the target Tank T02A temperature of $25^{\circ} \mathrm{C}$ were typically less than $2^{\circ} \mathrm{C}$. The average 
Tank T02A temperature during filtration and washing was $\sim 26^{\circ} \mathrm{C}$. No analytical samples were available to support material balance estimations. As a result, only permeate production rates are reported for operations associated with TI-032 chromium addition. Permeate production rates for these steps are shown in Figure 4.21.

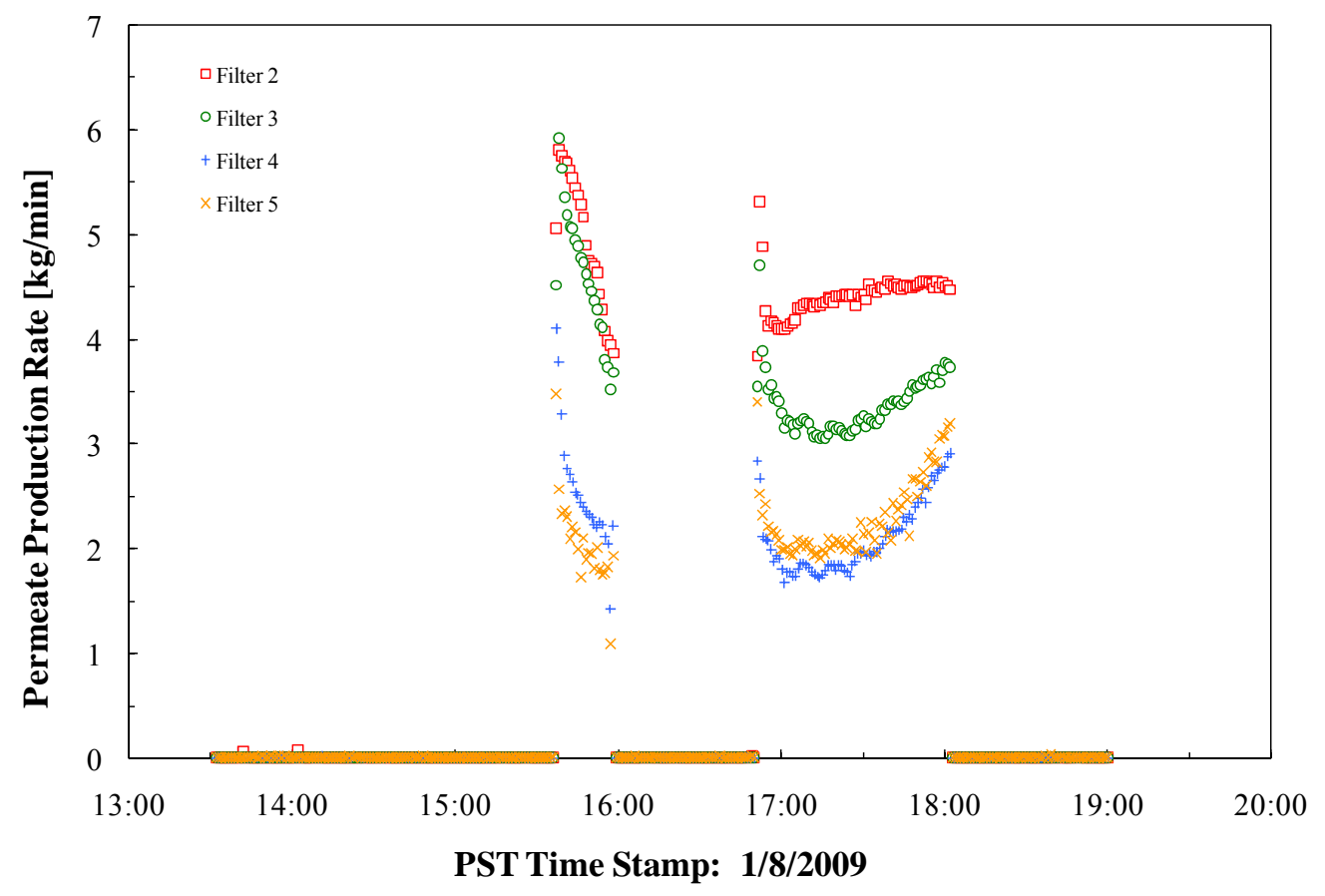

Figure 4.21. Permeate Production Rate During Functional Test (TI-032) Dewatering and Washing Associated with Chromium Addition (dewatering and washing on filter 2-5)

\subsubsection{High-Solids Filter Test}

IW was added in-line to the suction of UFP-PMP-T42A until the slurry level in Tank T02A was at the "High Mixing Volume" level ( $\geq 62.5$ inches) on $1 / 8 / 2009$ at 19:31. The Tank T02A level was now 63.5 inches on the laser (64.5 inches on the bubbler). Air spargers were set at $0.1-\mathrm{kg} / \mathrm{m}$ (upper) and $0.04-\mathrm{kg} / \mathrm{m}$ (lower). A PJM nozzle velocity of $12-\mathrm{m} / \mathrm{s}$ was verified.

The filters were configured for flow through all five filters, the T02A was mixed for 5 minutes, and initial samples were taken. Slurry in Tank T02A was aligned for recirculation through five filters, but permeate valves were opened at 21:02 for only the first filter bundle. Permeate valves on filters 2 through 5 remained closed. After the permeate valve was opened, operators backpulsed filter 1 five times. The high-solids filter test was started on 1/8/2009 at 21:55, and one 40-mL sample was collected every 15 minutes.

Several difficulties were encountered as soon as the test started, especially with pump operations, because of entrained air in the waste.

1) The DAS failed about 35 minutes before the end of the test, so critical data were not recorded. 
2) The testing crew was unable to operate the pump down to the target level of 10 inches in the vessel. A stable level measurement indicated the level was about 15 inches.

3) Preliminary plots of the data indicate that limited samples and data were obtained after the knee in the curve.

The concentration ended after 2 hours because of pumping problems. After consultation between Steve Barnes, Jim Huckaby, and Reid Peterson, it was decided to redo the high-solids filter test after permanganate addition.

\subsubsection{Oxidative-Leach}

Permanganate was added to Tank T02A at the inlet of pump T42A beginning at 01:15 on 1/09/2009. Foaming during the 6 -hr oxidative leach at $25^{\circ} \mathrm{C}$ was recorded at $02: 35$ on 1/09/2009 on a 2-min video tape labeled “Oxidative Leaching for TI-032.” Foaming was not excessive. The foam level dropped as the test progressed (see LRB 60108, pg 66). Figure 4.22 shows a frame from the videotape.

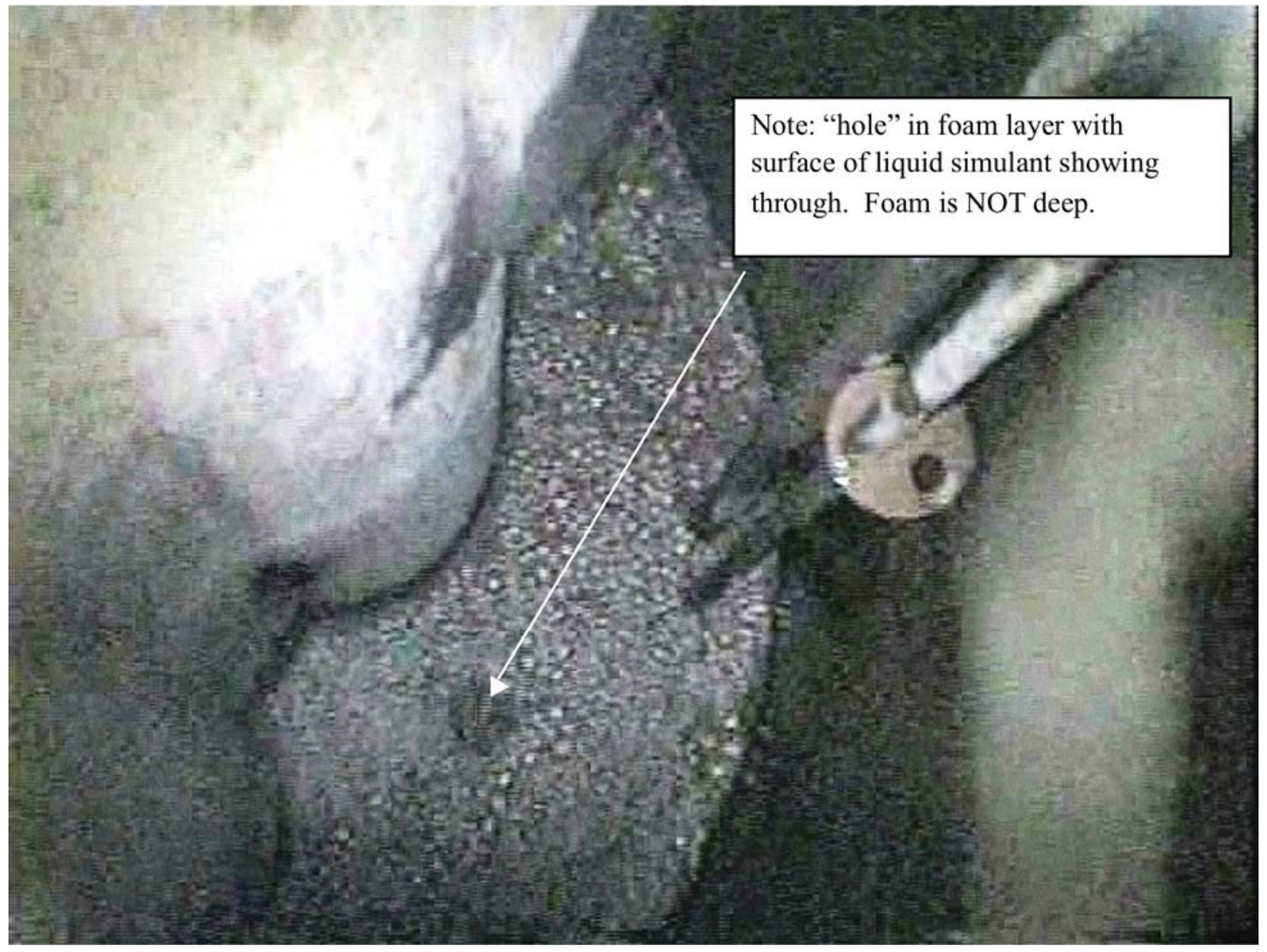

Figure 4.22. Frame from Oxidative-Leach Video—01:40 01/09/2009

The resolution of the frame is not good enough to estimate the depth of the foam, but it is good enough to qualitatively determine that the foam is not very deep. The initial conclusion would be that the 
oxidative-leach does not cause excessive foaming. However, subsequent analyses of "ad-hoc" samples taken raised doubts whether all of the $\mathrm{NaMnO}_{4}$ may have gotten into the tank. Without the prescribed amount of $\mathrm{NaMnO}_{4}$ in the vessel, one could not conclude confidently based on this test alone that foaming will not occur during oxidative leaching. Fortunately, subsequent Integrated testing did not encounter any excessive foaming problems during oxidative-leach, so the conclusion may still be correct.

\subsubsection{High-Solids Filter Test-Repeats}

The high-solids filter test was repeated three times to obtain data to represent the change in filtering character as the solids increase, and the solids cake on the inside surface of the filter tube dominates the filter resistance. A plot of filter flux vs. logarithm of \% solids demonstrates a characteristic "knee" commonly attributed to this change in overall filter resistance. The high-solids filter test was to determine where the "knee" would be for this simulant with prototypic flow conditions (109 gpm, 40 psi TMP).

For the test, simulant previously concentrated to $\sim 17$ to $20 \%$ solids was diluted with IW and then re-concentrated to the original volume (and original \% solids). One filter bundle, UFP-FILT-T01A, was used to conduct the filtration.

The first high-solids filter test is reported above in Section 4.3.18. A second attempt at the high-solids filter test was conducted after the oxidative-leach step commenced at 19:00 on 1/09/2009. The test was secured at 20:20 when the flow rate in filter 1 dropped to zero. It was discovered that filter 1 was plugged as a result of improper valve alignment. The bypass around filter 1 had been left open, so the flow through the filter tubes was insufficient to keep solids from accumulating.

Filter bundle 1 was plugged with solids during the second attempt at the high-solids test. Figure 4.23 shows the evolution of permeate production rate during this attempt at the high-solids dewatering operation. The initial permeate rate variation from $\sim 1830 \mathrm{hrs}$ to $1900 \mathrm{hrs}$ is caused by backpulsing of filter 1 before the start of dewatering. The decline in permeate rate from $1900 \mathrm{hrs}$ to $2000 \mathrm{hrs}$ is associated with deposition of solids in the filter elements on bundle 1. The plugging in filter \#1 was cleared by filling the pulse-pot with IW and backpulsing. After 2 to 3 pulse-pots of water were backpulsed through filter 1 , flow through the tubes was restored, and a filter flux rate of $6.5-\mathrm{kg} / \mathrm{min}$ at a TMP of 40 psi was achieved. 


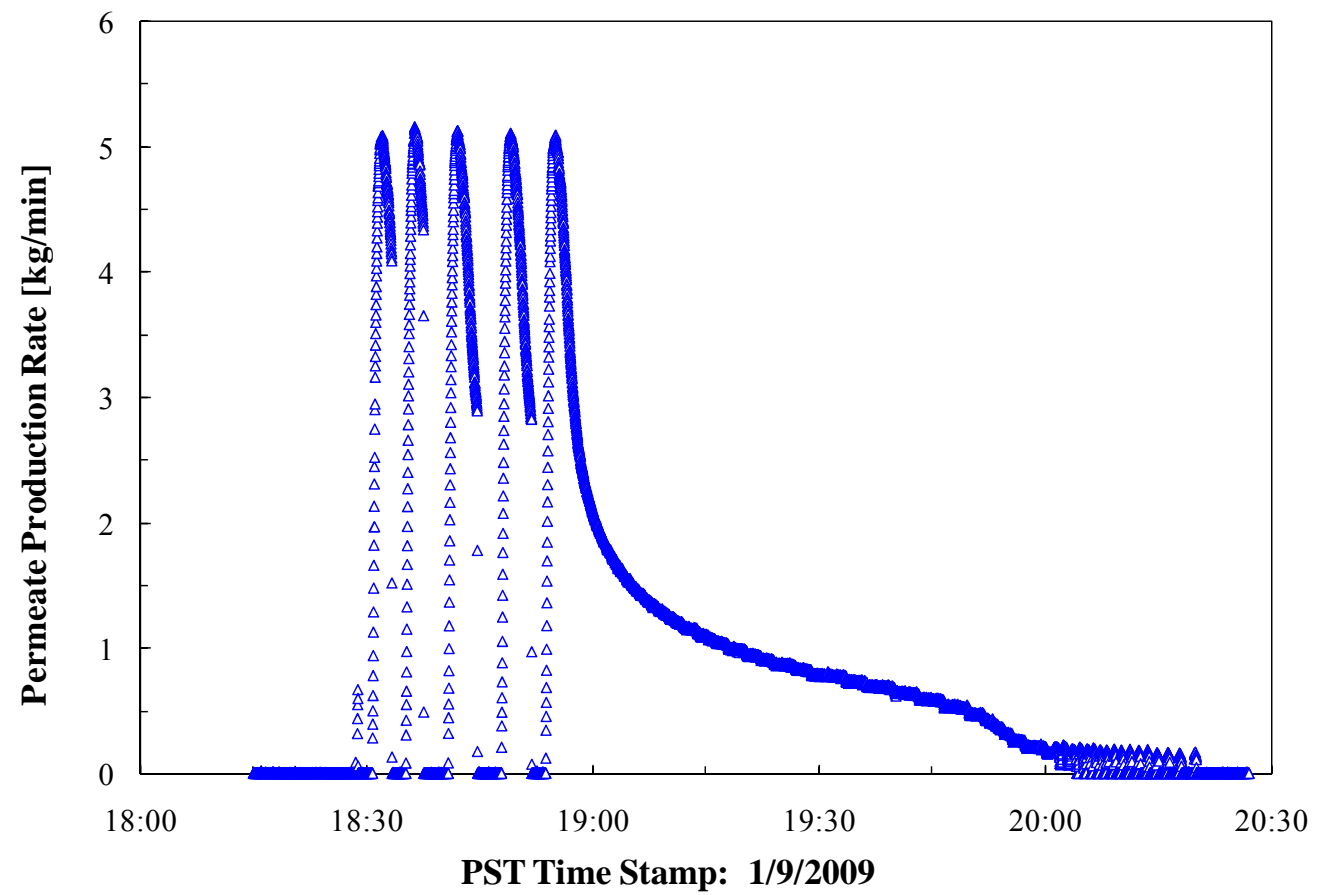

Figure 4.23. Permeate Production Rate Evaluation for the Second High-Solids Dewatering Attempt

A third attempt at the high-solids filter test commenced with five backpulses at 20:35 on 1/10/2009, and permeate removal began at 23:28. (Note: The interval between backpulses and the start of permeate removal was spent investigating the high pressure in the \#1 filter permeate line. It was determined that the pressure was due entirely to the relatively high permeate rate flow through a small control valve.) As the test progressed, the level in Tank T02A dropped below 52 inches, which called for a reduction in the sparger air flow rates from full rate $(0.4-\mathrm{kg} / \mathrm{min}$ lower, $0.1-\mathrm{kg} / \mathrm{min}$ upper $)$ to idle rate $(0.04-\mathrm{kg} / \mathrm{min}$ lower, $0.01-\mathrm{kg} / \mathrm{min}$ upper). Within 10 minutes of the sparger rate being reduced, the tank level measured by the laser dropped from 52 to 40 inches. The excessive drop was attributed to either a foam layer or air holdup in the slurry.

The test progressed without difficulty until the pump could not maintain the target $109 \mathrm{gpm}$ rate around 02:21. The test was terminated at 02:29 on 1/11/2009. The final tank level was about 16 inches (15.9 inches by bubbler; 16.7 inches by laser). The final target had been 10 inches.

The third attempt at the high-solids dewatering operation achieved an average axial velocity of 14.2-ft/s and an average TMP of $39.5 \mathrm{psid}$ (during actual dewatering). Tank T02A temperature was approximately $25^{\circ} \mathrm{C}$ during this time. Sufficient analytical data were taken to perform a material balance on the dewatering operation. The result of the material balance is the estimated UDS evolution shown in Figure 4.24. Knowledge of the UDS content allows determination of the dewatering curve. 


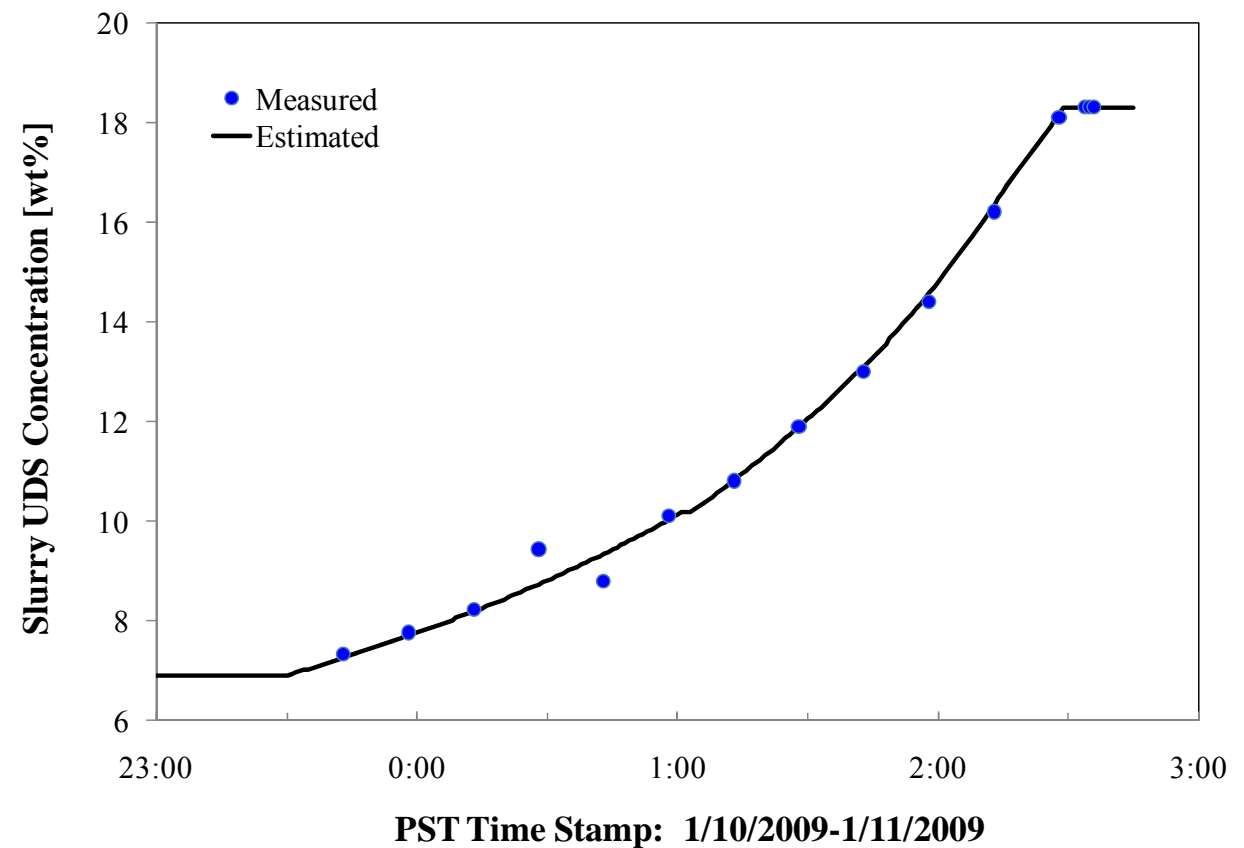

Figure 4.24. Measured and Estimated UDS Concentration Evolution for High-Solids Dewatering (third attempt) for TI-032

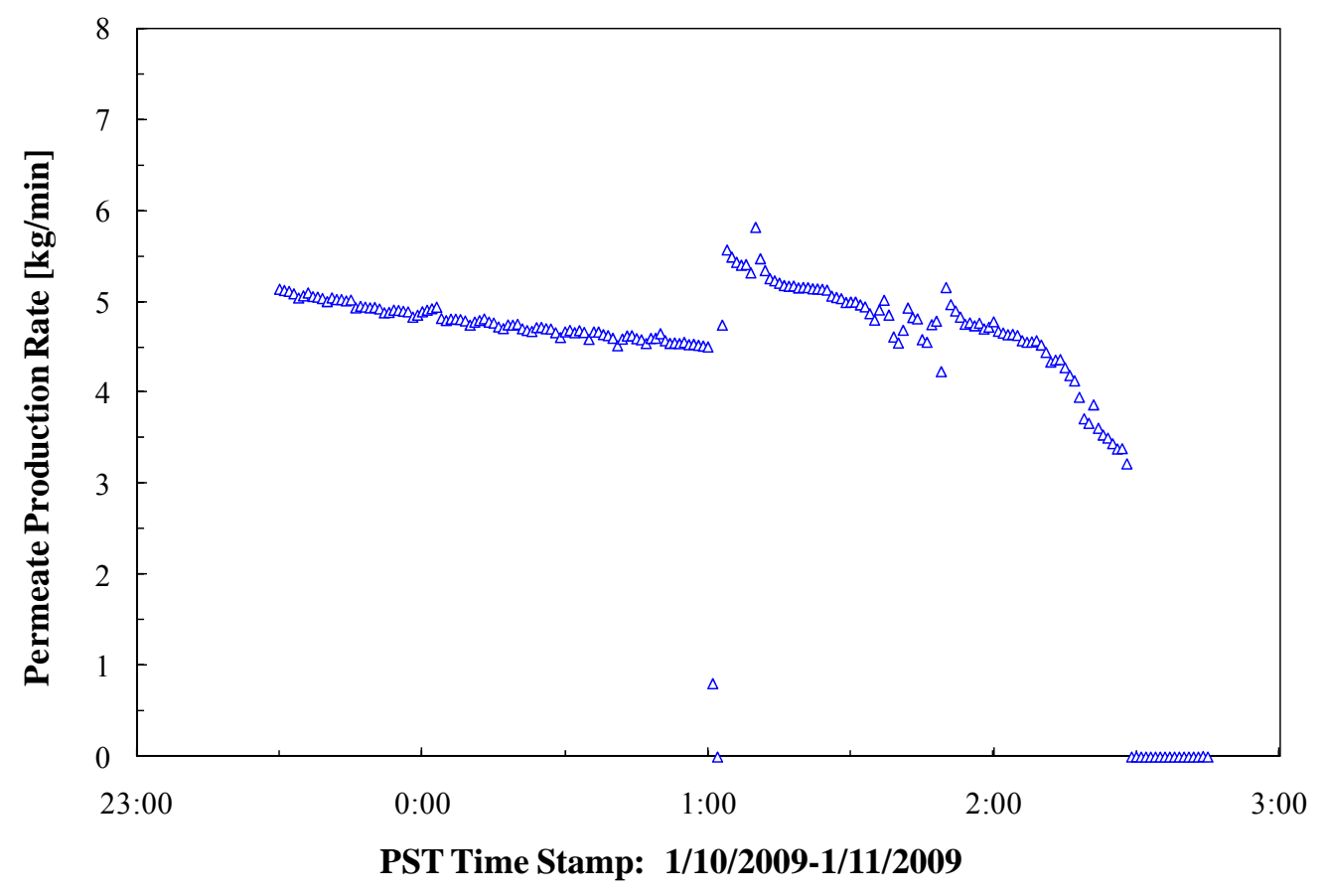

Figure 4.25. Permeate Production Rate During the Third Attempt of the Functional Test (TI-032) High-Solids Dewatering Operation on Filter 1 
Figure 4.25 and Figure 4.26 show the permeate production rate and TMP/temperature corrected filter flux evolution during the third attempt at the high-solids dewatering operation. The most notable feature in Figure 4.25 is a sudden increase in flux around 0100 hrs. This increase is likely associated with the brief process stoppage at this time. During this time, permeate production was stopped and axial velocity reduced. During this time, shellside pressure was likely relieved by a back-flow of permeate through the filter element. This back-flow would have similar effects to a backpulse. As such, the jump in permeate production at 0100 hrs is likely a result of cake disruption.

Neglecting the jump in permeate production associated with the filtration stoppage, permeate rates show a steady decline over the course of the filtration process until $0210 \mathrm{hrs}$, where permeate production begins to decline more rapidly until the end of filtration at 0230 hrs. This decline is accompanied by a gradual decline in axial velocity from 14.8- to $12.6-\mathrm{ft} / \mathrm{s}$ over the same time period. It is unknown if the decline in permeate production is associated solely by the decline in axial velocity or if it is also caused by a transition from membrane-resistance-limited filtration to cake-resistance-limited filtration.

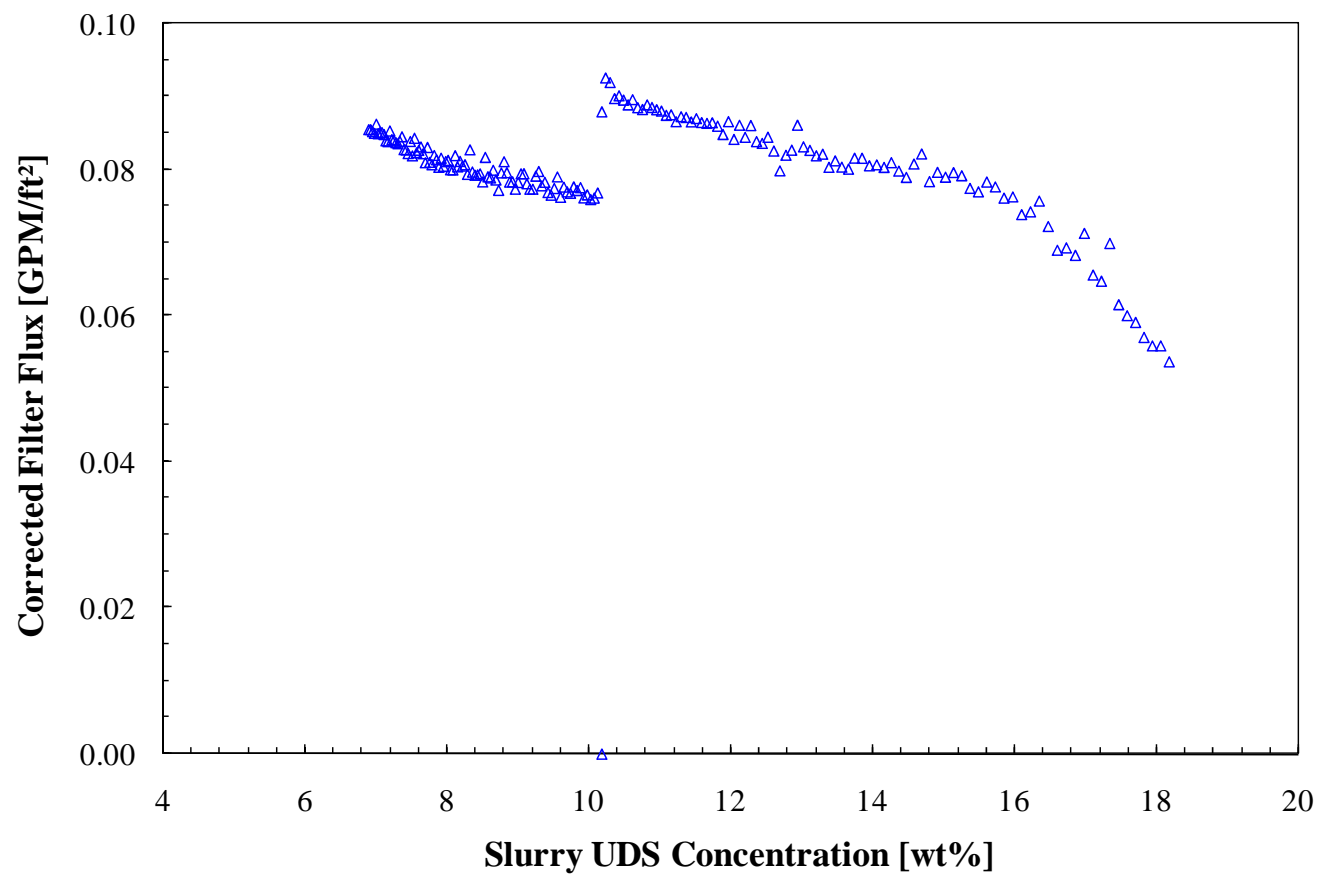

Figure 4.26 Dewatering Curve for the Third Attempt of the Functional Test (TI-032) High-Solids Dewatering Operation on Filter 1

Figure 4.26 shows the dewatering curve for the third attempt at high-solids test. Filtration below $16-w t \%$ is likely membrane-resistance limited. At 16-wt\% UDS and above, there is increased decline in flux with increasing wt\% UDS. This could indicate a transition to cake-resistance-limited filtration at 16-wt\% UDS; however, the decline is also accompanied by reduced axial velocity (which can also lower filter flux). As such, no definitive conclusion regarding the existence of a dewatering knee can be made for data presented in Figure 4.26. Because of the difficulties in maintaining loop flow when the tank level was low, the high-solids filter test was re-designed to include more initial solids. The test was then repeated at the end of Integrated Test B using combined leached and washed solids from Integrated Tests A and B. Analysis of the data from the high-solids filter tests is presented in the EFRT filtration report 
A and B. Analysis of the data from the high-solids filter tests is presented in the EFRT filtration report (Daniel et al. 2009) and the filtration section of the final report (Kurath et al. 2009). Data from the Functional testing high-solids filter test attempts were never fully analyzed.

\subsubsection{Test End}

Filter-loop flush and transfer from Tank T02A to HLP-T22 was conducted on 1/14/2009 as part of operations.

Table 4.3 is derived from Table A.1 from the Test Plan, TP-RPP-WTP-506. ${ }^{\text {(a) }}$ It has been modified to describe how each of the steps identified in the Test Plan were accomplished through the three Test Instructions executed as part of Shakedown/Functional testing. Like Table A.1 in the Test Plan, the table includes sampling and analyses requirements at each step of processing. Objectives and samples that were used as the basis for planning further PEP operations are colored in blue in the table. Objectives and samples that were used for EFRT reporting are colored in red. Other samples, whose analysis required less urgency, are black.

(a) GB Josephson, OP Bredt, JK Young, and DE Kurath. 2009. Test Plan for Pretreatment Engineering Platform (PEP) Testing (Phase I). TP-RPP-WTP-506, Rev. 0.4, Pacific Northwest National Laboratory, Richland, Washington. 
Table 4.3. Functional Testing Detailed Description

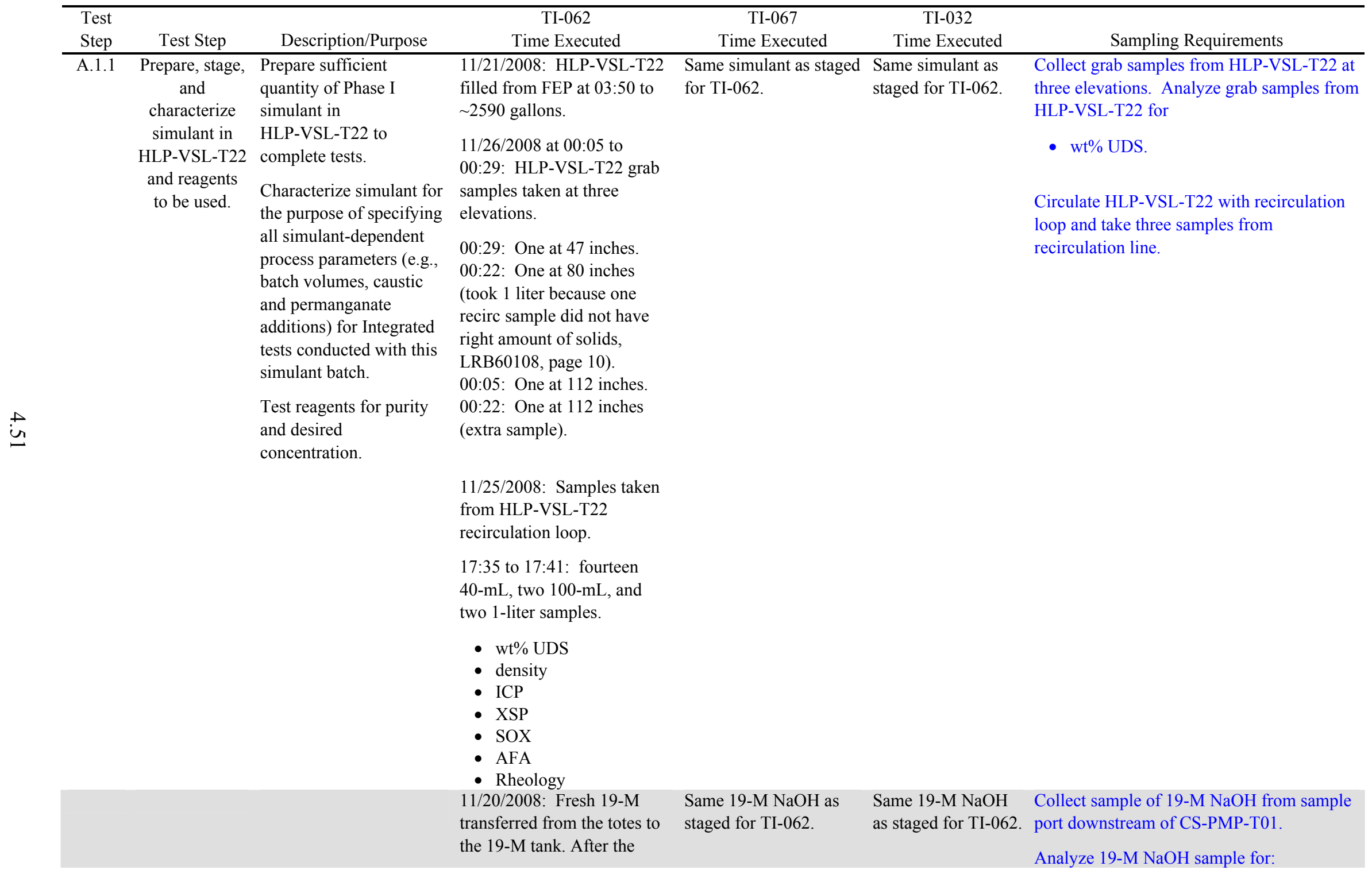


Table 4.3. Functional Testing Detailed Description

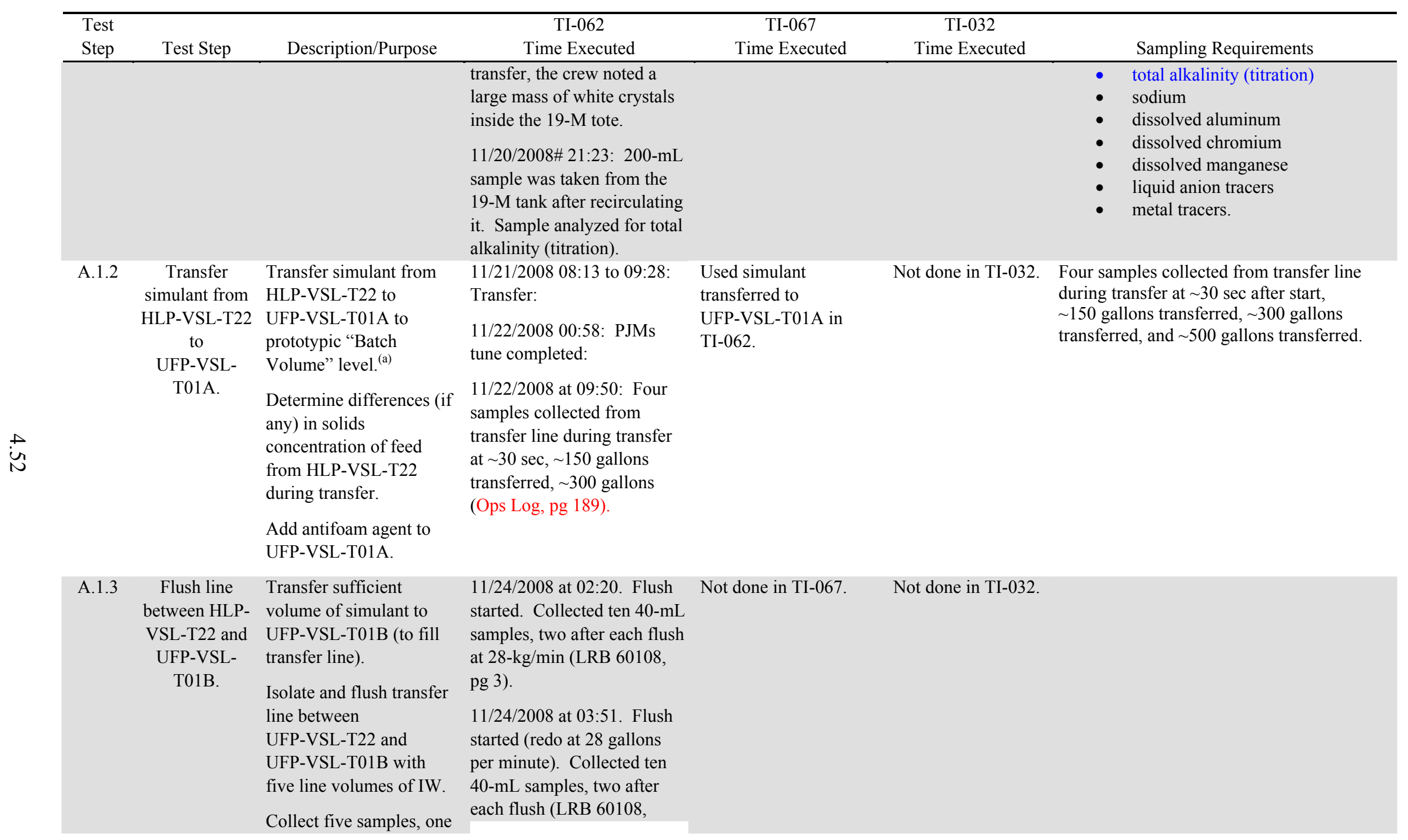

(a) Named vessel levels indicated with single quotation marks (e.g., "Batch Volume") refer to levels specified in PEP Process Description (Lehrman 2008). 
Table 4.3. Functional Testing Detailed Description

\begin{tabular}{|c|c|c|c|c|c|c|}
\hline $\begin{array}{l}\text { Test } \\
\text { Step }\end{array}$ & Test Step & Description/Purpose & $\begin{array}{c}\text { TI-062 } \\
\text { Time Executed }\end{array}$ & $\begin{array}{c}\text { TI-067 } \\
\text { Time Executed }\end{array}$ & $\begin{array}{c}\text { TI-032 } \\
\text { Time Executed }\end{array}$ & Sampling Requirements \\
\hline & & $\begin{array}{l}\text { sample representing each } \\
\text { line volume of flush } \\
\text { water added. }\end{array}$ & $\begin{array}{l}\text { pg } 3) \\
\text { Five sets of } 2 \times 40-\mathrm{mL} \\
\text { samples taken: } \\
03: 51: 21 \\
03: 51: 41 \\
03: 52: 02 \\
03: 52: 23 \\
03: 52: 44\end{array}$ & & & \\
\hline A. 1.4 & $\begin{array}{l}\text { Transfer } \\
\text { simulant from } \\
\text { UFP-VSL- } \\
\text { T01A/B to } \\
\text { UFP-VSL- } \\
\text { T02A. }\end{array}$ & $\begin{array}{l}\text { Transfer simulant from } \\
\text { UFP-VSL-T01A to } \\
\text { UFP-VSL-T02A to fill } \\
\text { UFP-VSL-T02A to the } \\
\text { maximum batch level. } \\
\text { Monitor UFP-VSL-T01A } \\
\text { for solids settling that } \\
\text { may impact transfer of } \\
\text { solids. } \\
\text { Determine differences (if } \\
\text { any) in solids } \\
\text { concentration of feed } \\
\text { from UFP-VSL-T01A } \\
\text { during transfer. }\end{array}$ & $\begin{array}{l}\text { Completed at 3:52:44. } \\
\text { 11/23/2008 at 01:14 } \\
\text { Transfer started. } \\
\text { 11/23/2008 at } 02: 10 \\
\text { Completed. } \\
\text { Four samples collected } \\
\text { during transfer: } \\
01: 13 \\
01: 24 \\
01: 34 \\
01: 50 \text {. }\end{array}$ & Not done in TI-067. & $\begin{array}{l}\text { Transferred from } \\
\text { UFP-VSL-T01B to } \\
\text { UFP-VSL-T02A. } \\
\text { 02:11 12/30/2008 } \\
\text { Transfer started. } \\
\text { 02:52 12/30/2008 } \\
\text { Completed. }\end{array}$ & \\
\hline
\end{tabular}


Table 4.3. Functional Testing Detailed Description

\begin{tabular}{|c|c|c|c|c|c|c|}
\hline $\begin{array}{l}\text { Test } \\
\text { Step }\end{array}$ & Test Step & Description/Purpose & $\begin{array}{c}\text { TI-062 } \\
\text { Time Executed }\end{array}$ & $\begin{array}{c}\text { TI-067 } \\
\text { Time Executed }\end{array}$ & $\begin{array}{c}\text { TI-032 } \\
\text { Time Executed }\end{array}$ & Sampling Requirements \\
\hline A.1.5 & $\begin{array}{c}\text { Transfer } \\
\text { simulant from } \\
\text { HLP-VSL-T22 } \\
\text { to } \\
\text { UFP-VSL- } \\
\text { T01A/B } \\
\text { (to refill). }\end{array}$ & $\begin{array}{l}\text { Transfer simulant from } \\
\text { HLP-VSL-T22 to } \\
\text { UFP-VSL-T01A to } \\
\text { prototypic "Batch } \\
\text { Volume" level. } \\
\text { Determine differences (if } \\
\text { any) in solids } \\
\text { concentration of feed } \\
\text { from HLP-VSL-T22 } \\
\text { during transfer and/or } \\
\text { between transfers in steps } \\
\text { A.1.2 and A.1.3. } \\
\text { Add antifoam agent. }\end{array}$ & $\begin{array}{l}\text { Transfer started: } 03: 49 \\
\text { 11/23/2008. } \\
\text { Completed: } 04: 00 \\
\text { 11/23/2008. }\end{array}$ & Not done in TI-067. & $\begin{array}{l}\text { Transfer started: } \\
\text { 05:24 12/21/2008. } \\
\text { Completed: } 06: 06 \\
\text { 12/21/2008. } \\
\text { No record of } \\
\text { samples collected } \\
\text { during transfer. }\end{array}$ & \\
\hline
\end{tabular}


Table 4.3. Functional Testing Detailed Description

\begin{tabular}{|c|c|c|c|c|c|c|}
\hline $\begin{array}{l}\text { Test } \\
\text { Step }\end{array}$ & Test Step & Description/Purpose & $\begin{array}{c}\text { TI-062 } \\
\text { Time Executed }\end{array}$ & $\begin{array}{c}\text { TI-067 } \\
\text { Time Executed }\end{array}$ & $\begin{array}{c}\text { TI-032 } \\
\text { Time Executed }\end{array}$ & Sampling Requirements \\
\hline A.1.6 & $\begin{array}{c}\text { Test for } \\
\text { stratification of } \\
\text { solids in UFP- } \\
\text { VSL-T01A/B. }\end{array}$ & $\begin{array}{l}\text { Determine steady state } \\
\text { solids distribution in } \\
\text { UFP-VSL-T01A/B with } \\
\text { Phase I simulant, 5-wt\% } \\
\text { solids. }\end{array}$ & $\begin{array}{l}\text { 11/23/2008 at 04:20: } \\
\text { UFP-VSL-T01A } \\
\text { stratification test start. } \\
\text { 11/24/2008 morning PJMs } \\
\text { re-tuned. } \\
\text { 11/24/2008 at } 12 \text { noon: } \\
\text { Restart the stratification test } \\
\text { after the PJM re-tune. } \\
\text { Four inner CD samples taken } \\
\text { every } 12 \text { hours. } \\
11 / 25 / 2008 \text { at } 00: 00 \text { to } 00: 09 \\
\text { (12 hours). } \\
11 / 25 / 2008 \text { at } 12: 00 \text { to } 12: 17 \\
\text { ( } 24 \text { hours). } \\
11 / 26 / 2008 \text { at } 00: 07 \text { to } 00: 16 \\
\text { ( } 36 \text { hours). }\end{array}$ & Not done in TI-067. & $\begin{array}{l}\text { 12/21/2008 at 12:30: } \\
\text { UFP-VSL-T01B } \\
\text { stratification Test } \\
\text { start. } \\
\text { 12/23/2008 00:30: } \\
\text { UFP-VSL-T01B } \\
\text { stratification test } \\
\text { end. } \\
\text { Four Inner CD } \\
\text { samples taken from } \\
\text { each port every } \\
\text { 12 hours (twelve } \\
40 \text {-mL samples). } \\
\text { 12/22/2008 at } 00: 36 \\
\text { to 00:55 (12 hours). } \\
\text { 12/22/2008 at } 12: 30 \\
\text { to } 12: 39 \text { ( } 24 \text { hours). } \\
\text { 12/23/2008 at } 00: 25 \\
\text { to 00:39 (36 hours). } \\
\text { 1/1/2009 at } 22: 54 \text { to } \\
23: 03: \text { four } 40-m L \\
\text { samples taken from } \\
\text { CD middle-low } \\
1 \text { hour before } \\
\text { transfer to } \\
\text { UFP-VSL-T02A. }\end{array}$ & $\begin{array}{l}\text { Record UFP-VSL-T01A CD densities at all } \\
\text { submerged locations every } 2 \text { hours. Record } \\
\text { density at inner CD port between recordings. } \\
\text { Collect four samples from UFP-VSL-T01A } \\
\text { from the each of the three submerged CD } \\
\text { sample ports on the inner CD sample tree } \\
\text { (low, middle, high) after } 12 \text { and } 24 \text { hours } \\
\text { and about } 1 \text { hour before slurry is transferred } \\
\text { out. The number of replicate samples was } \\
\text { specified by the JTG with input from the } \\
\text { data analysis team. } \\
\text { Analyze all CD port samples for } \\
\text { - wt } \% \text { UDS. } \\
\text { Analyze } 12 \text {-hr and final CD port samples } \\
\text { from top and bottom CD ports for } \\
\text { - total aluminum solids } \\
\text { - PSD (not in replicate). }\end{array}$ \\
\hline A. 1.7 & $\begin{array}{l}\text { Drain filter- } \\
\text { loop } \\
\text { and permeate } \\
\text { lines. }\end{array}$ & $\begin{array}{l}\text { Filter-loop and permeate } \\
\text { system initially filled } \\
\text { with IW. } \\
\text { Drain IW from } \\
\text { filter-loop. }\end{array}$ & $\begin{array}{l}\sim 13: 00 \text { 11/23/2008. } \\
\text { No samples taken. }\end{array}$ & Not done in TI-067. & $\begin{array}{l}11 / 23 / 2008 \text { at } 15: 30 \\
\text { Start Drain. } \\
11 / 23 / 2008 \text { at } 16: 58 \\
\text { End Drain. } \\
\text { No samples taken. }\end{array}$ & None. \\
\hline
\end{tabular}


Table 4.3. Functional Testing Detailed Description

\begin{tabular}{|c|c|c|c|c|c|c|}
\hline $\begin{array}{l}\text { Test } \\
\text { Step }\end{array}$ & Test Step & Description/Purpose & $\begin{array}{c}\text { TI-062 } \\
\text { Time Executed }\end{array}$ & $\begin{array}{c}\text { TI-067 } \\
\text { Time Executed }\end{array}$ & $\begin{array}{c}\text { TI-032 } \\
\text { Time Executed }\end{array}$ & Sampling Requirements \\
\hline & & $\begin{array}{l}\text { Drain IW from shellside } \\
\text { of each filter bundle, } \\
\text { pulse-pots and permeate } \\
\text { lines. } \\
\text { Fill shellside of filters, } \\
\text { pulse-pots and permeate } \\
\text { lines with supernate. }\end{array}$ & & & & \\
\hline A. 1.8 & $\begin{array}{l}\text { Conduct low- } \\
\text { solids filter } \\
\text { flux test (initial } \\
\text { loading). }\end{array}$ & $\begin{array}{l}\text { Record permeate flows } \\
\text { from each filter bundle as } \\
\text { functions of time for } 12 \\
\text { hours. } \\
\text { Collect in-line slurry } \\
\text { samples to establish } \\
\text { baseline sampling } \\
\text { variation for in-line } \\
\text { sampling. } \\
\text { Filter-loop, pulse-pots } \\
\text { and shellside of filters } \\
\text { initially drained. } \\
\text { Filter-loop flow through } \\
\text { all } 5 \text { filter bundles and } \\
\text { UFP-HX-T02A. } \\
\text { - } 25^{\circ} \mathrm{C} \text { filtration temp } \\
\text { - TMP } 40 \text { psid } \\
\text { - Filter-loop flow } \\
109 \text { gpm (15-ft/sec) } \\
\text { - UFP-HX-T03A } \\
\text { bypassed } \\
\text { - Permeate return to } \\
\text { UFP-VSL-T02A. } \\
\text { UFP-VSL-T02A PJM } \\
\text { velocities and cycle times }\end{array}$ & $\begin{array}{l}\text { 11/23/2008 at 15:40: Start. } \\
\text { 11/24/2008 at 03:40: End. } \\
\text { 11/23/2008 Samples from } \\
\text { the middle-low CD port, one } \\
\text { for parallel CUF and three } \\
\text { for physical properties, about } \\
\text { 10 minutes after the } \\
\text { permeate system is known to } \\
\text { be flowing back into } \\
\text { UFP-VSL-T02A. Collect a } \\
\text { total of } 30 \text { samples from the } \\
\text { middle-middle CD port at } \\
\sim-1 \text {-hr intervals over a } \\
\text { period of } 5 \text { hours. } \\
\text { 11/23/2008: Collected } \\
\text { filter-loop in-line samples at } \\
\text { 10 minutes and } \\
\text { - } 1 \text { hour for } 5 \text { hours. } \\
\text { 15:50 to 16:01 (sever } 40-\mathrm{mL} \text {, } \\
\text { two } 100 \text { mL). } \\
\text { 17:27 to 17:31 (six } 40-\mathrm{mL} \text { ) } \\
\text { 18:03 to } 18: 09 \text { (six } 40-\mathrm{mL} \text { ) } \\
\text { 19:14 to 19:22 (six } 40-\mathrm{mL} \text { ) } \\
\text { 20:01 to } 20: 08 \text { (six } 40-\mathrm{mL} \text { ) } \\
\text { 21:03 to } 21: 10 \text { (six } 40-\mathrm{mL} \text { ) }\end{array}$ & Not done in TI-067. & $\begin{array}{l}\text { Start/end times are } \\
\text { for the entire } \\
\text { low-solids filter flux } \\
\text { test: } \\
\text { 12/30/2008 at 22:28: } \\
\text { Start. } \\
\text { 01/01/2009 at } \\
\text { 11:28:End. } \\
\text { 12/30/2008 at } 23: 23 \\
\text { to 23:31: Took } \\
\text { eight } 40-\text { mL } \\
\text { samples from the } \\
\text { middle-low CD port } \\
\text { for physical } \\
\text { properties, about } \\
\text { 10 minutes after } \\
\text { permeate was } \\
\text { flowing back into } \\
\text { UFP-VSL-T02A. } \\
\text { 12/30/2008 at } 08: 09 \\
\text { to 08:12: Took five } \\
\text { filter-loop in-line } \\
\text { samples; see LRB } \\
60108-43 \text {. Observed } \\
\text { tiny amounts of } \\
\text { metal particulates. }\end{array}$ & $\begin{array}{l}\text { Collect samples from the middle-low CD } \\
\text { port, one for parallel CUF and three (3) for } \\
\text { physical properties, about } 10 \text { minutes after } \\
\text { permeate system is known to be flowing } \\
\text { back into UFP-VSL-T02A (with permeate } \\
\text { valves closed). } \\
\text { Transport CUF sample to APEL. } \\
\text { Analyze physical properties filter-loop } \\
\text { in-line sample for } \\
\text { - wt\% UDS } \\
\text { - slurry density } \\
\text { - shear stress vs. shear rate (not in } \\
\text { replicate) } \\
\text { - liquid viscosity (not in replicate) } \\
\text { - PSD (not in replicate). } \\
\text { Intervals over a period of } 5 \text { hours for in-line } \\
\text { sampling standard deviation estimate. } \\
\text { Samples may be collected in batches of six. } \\
\text { Samples should be collected in parallel with } \\
\text { the CD samples. } \\
\text { Analyze filter-loop in-line samples for } \\
\text { - wt\% UDS. } \\
\text { Collect a total of } 30 \text { samples from the } \\
\text { middle-middle CD port at } ~-1 \text {-hr intervals } \\
\text { over a period of } 5 \text { hours for CD port }\end{array}$ \\
\hline
\end{tabular}


Table 4.3. Functional Testing Detailed Description

\begin{tabular}{|c|c|c|c|c|c|c|}
\hline $\begin{array}{l}\text { Test } \\
\text { Step }\end{array}$ & Test Step & Description/Purpose & $\begin{array}{c}\text { TI-062 } \\
\text { Time Executed }\end{array}$ & $\begin{array}{c}\text { TI-067 } \\
\text { Time Executed }\end{array}$ & $\begin{array}{c}\text { TI-032 } \\
\text { Time Executed }\end{array}$ & Sampling Requirements \\
\hline & & match PTF PJM. & $\begin{array}{l}\text { Collect permeate samples } \\
\text { between each filter bundle. } \\
\text { 11/23/2008: } 17: 48 \text { to } 17: 52 \\
\text { 11/23/2008: } 22: 01 \text { to } 22: 07 \\
\text { 11/24/2008: } 04: 02 \text { to } 04: 06 \text {. }\end{array}$ & & $\begin{array}{l}\text { Collected permeate } \\
\text { samples between } \\
\text { each filter bundle } \\
\text { and the pulse-pots at } \\
3 \text { evenly spaced } \\
\text { times and inspect } \\
\text { for particle } \\
\text { breakthrough. } \\
\text { 12/31/2008 at } 00: 30 \text { : } \\
\text { (five } 40 \text {-mL without } \\
\text { IDs; inspected by } \\
\text { GJ and found clear). } \\
12 / 30 / 2008 \text { at } 05: 30 \\
\text { to } 05: 40 \text { (fifteen } \\
40-\mathrm{mL} \text {, five } \\
100 \text {-mL). } \\
12 / 30 / 2008 \text { at } 11: 10 \\
\text { to } 11: 14 \text { (fifteen } \\
40-\mathrm{mL} \text {; five } \\
100 \text {-mL). } \\
12 / 31 / 2008 \text { at } 10: 45 \\
\text { to } 11: 02 \text { : Took four } \\
4.2-\mathrm{L} \text { samples from } \\
\text { the middle-low CD } \\
\text { port at client } \\
\text { request. }\end{array}$ & $\begin{array}{l}\text { sampling standard deviation estimate. } \\
\text { Samples may be collected in batches of six. } \\
\text { Samples should be collected in parallel with } \\
\text { the filter-loop in-line samples. } \\
\text { Analyze middle-middle CD port samples for } \\
\text { - wt\% UDS. } \\
\text { Collect permeate samples between each } \\
\text { filter bundle and the pulse-pots at three } \\
\text { evenly spaced times and inspect for particle } \\
\text { breakthrough. }\end{array}$ \\
\hline A. 1.9 & $\begin{array}{l}\text { Backpulse } \\
\text { filters. }\end{array}$ & $\begin{array}{l}\text { Backpulse filters } 24 \text { times } \\
\text { at approximately } 30 \text {-min } \\
\text { intervals and record } \\
\text { permeate flows from each } \\
\text { filter bundle. No changes } \\
\text { from Step A.1.8. }\end{array}$ & $\begin{array}{l}\text { 11/24/2008 04:16: Start. } \\
\text { 11/24/2008 16:16: End. } \\
\text { 11/24/2008 at 16:25 to } \\
\text { 16:28: Collect filter-loop } \\
\text { sample at } 25^{\text {th }} \text { backpulse } \\
\text { (four } 40-\mathrm{mL} \text { ). }\end{array}$ & Not done in TI-067. & $\begin{array}{l}\text { 12/31/2008 at } 11: 30: \\
\text { Start. } \\
\text { 12/31/2008 at } 23: 30: \\
\text { End. } \\
12 / 31 / 2008 \text { at } 23: 40:\end{array}$ & $\begin{array}{l}\text { Collect a sample from the middle-low CD } \\
\text { port for characterization near the end of this } \\
\text { step. Analyze filter-loop in-line } \\
\text { characterization sample for } \\
\text { - wt } \% \text { UDS } \\
\text { - PSD. }\end{array}$ \\
\hline
\end{tabular}


Table 4.3. Functional Testing Detailed Description

\begin{tabular}{|c|c|c|c|c|c|c|}
\hline $\begin{array}{l}\text { Test } \\
\text { Step }\end{array}$ & Test Step & Description/Purpose & $\begin{array}{c}\text { TI-062 } \\
\text { Time Executed }\end{array}$ & $\begin{array}{c}\text { TI-067 } \\
\text { Time Executed }\end{array}$ & $\begin{array}{c}\text { TI-032 } \\
\text { Time Executed }\end{array}$ & Sampling Requirements \\
\hline & & & & & $\begin{array}{l}\text { Collect sample (two } \\
40 \text {-mL) from the } \\
\text { middle-low CD port } \\
\text { for characterization } \\
\text { near the end of this } \\
\text { step. }\end{array}$ & \\
\hline A. 1.10 & $\begin{array}{l}\text { Conduct low- } \\
\text { solids filter } \\
\text { flux test } \\
\text { (conditioned } \\
\text { filter flux and } \\
\text { its change with } \\
\text { time). }\end{array}$ & $\begin{array}{l}\text { Record permeate flows } \\
\text { from each filter bundle as } \\
\text { functions of time for } \\
12 \text { hours. No changes } \\
\text { from Step A.1.9. }\end{array}$ & $\begin{array}{l}\text { 11/24/2008 16:00: Start. } \\
\text { 1/25/2008 04:00: End. } \\
\text { 11/25/2008 at 04:22 to } \\
\text { 04:24: Collect filter-loop } \\
\text { sample at end (four 40-mL). }\end{array}$ & Not done in TI-067. & $\begin{array}{l}\text { 12/31/2008 at } 23: 30 \text { : } \\
\text { Start. } \\
\text { 1/1/2009 at } 11: 30 \text { : } \\
\text { End. } \\
\text { 1/1/2009 at } 11: 00- \\
11: 14 \text { : Collect } \\
\text { sample (five } 40-\mathrm{mL} \\
\text { and one } 14.2-\mathrm{L} \text { ) } \\
\text { from the middle-low } \\
\text { CD port for } \\
\text { characterization near } \\
\text { the end of this step. }\end{array}$ & $\begin{array}{l}\text { Collect three samples from the middle-low } \\
\text { CD sample port near the end of this step for } \\
\text { characterization and also collect a sample } \\
\text { for parallel CUF testing. } \\
\text { Transport the CUF sample to APEL. } \\
\text { Analyze the three characterization samples } \\
\text { for } \\
\text { - wt } \% \text { UDS } \\
\text { - PSD (not in replicate). }\end{array}$ \\
\hline A.1.11 & $\begin{array}{l}\text { Concentrate } \\
\text { solids in } \\
\text { UFP-VSL- } \\
\text { T02A. }\end{array}$ & $\begin{array}{l}\text { Reconfigure filter-loop to } \\
\text { bypass filters } \\
\text { UFP-FILT-T02A through } \\
\text { UFP-FILT-T05A. } \\
\text { Concentrate slurry in } \\
\text { UFP-VSL-T02A to the } \\
\text { lesser of } 20 \text {-wt } \% \text { UDS or } \\
\text { the maximum achievable. } \\
\text { Record permeate flows } \\
\text { from UFP-FILT-T01A. } \\
\text { Filter-loop flow through } \\
\text { first filter bundle and } \\
\text { UFP-HX-T02A. } \\
\text { - } 25^{\circ} \mathrm{C} \text { filtration temp } \\
\text { - TMP } 40 \text { psid }\end{array}$ & $\begin{array}{l}11 / 25 / 2008 \text { at } 08: 14 \text { to } \\
08: 17 \text { : Collect in-line } \\
\text { samples when concentration } \\
\text { is complete (three } 40-\mathrm{mL} \text {, } \\
\text { one } 100-\mathrm{mL} \text { ). }\end{array}$ & Not done in TI-067. & $\begin{array}{l}\text { 1/1/2009 at } 16: 09- \\
16: 13 \text { : Collect three } \\
\text { (three } 40-\mathrm{mL} \text { ) } \\
\text { samples from the } \\
\text { middle-low CD port } \\
\text { when slurry } \\
\text { concentration } \\
\text { complete. }\end{array}$ & $\begin{array}{l}\text { Collect three samples from the middle-low } \\
\text { CD port when slurry concentration is } \\
\text { complete. } \\
\text { Analyze filter-loop in-line sample for } \\
\text { - wt } \% \text { UDS } \\
\text { - shear stress vs. shear rate (not } \\
\text { replicate). }\end{array}$ \\
\hline
\end{tabular}


Table 4.3. Functional Testing Detailed Description

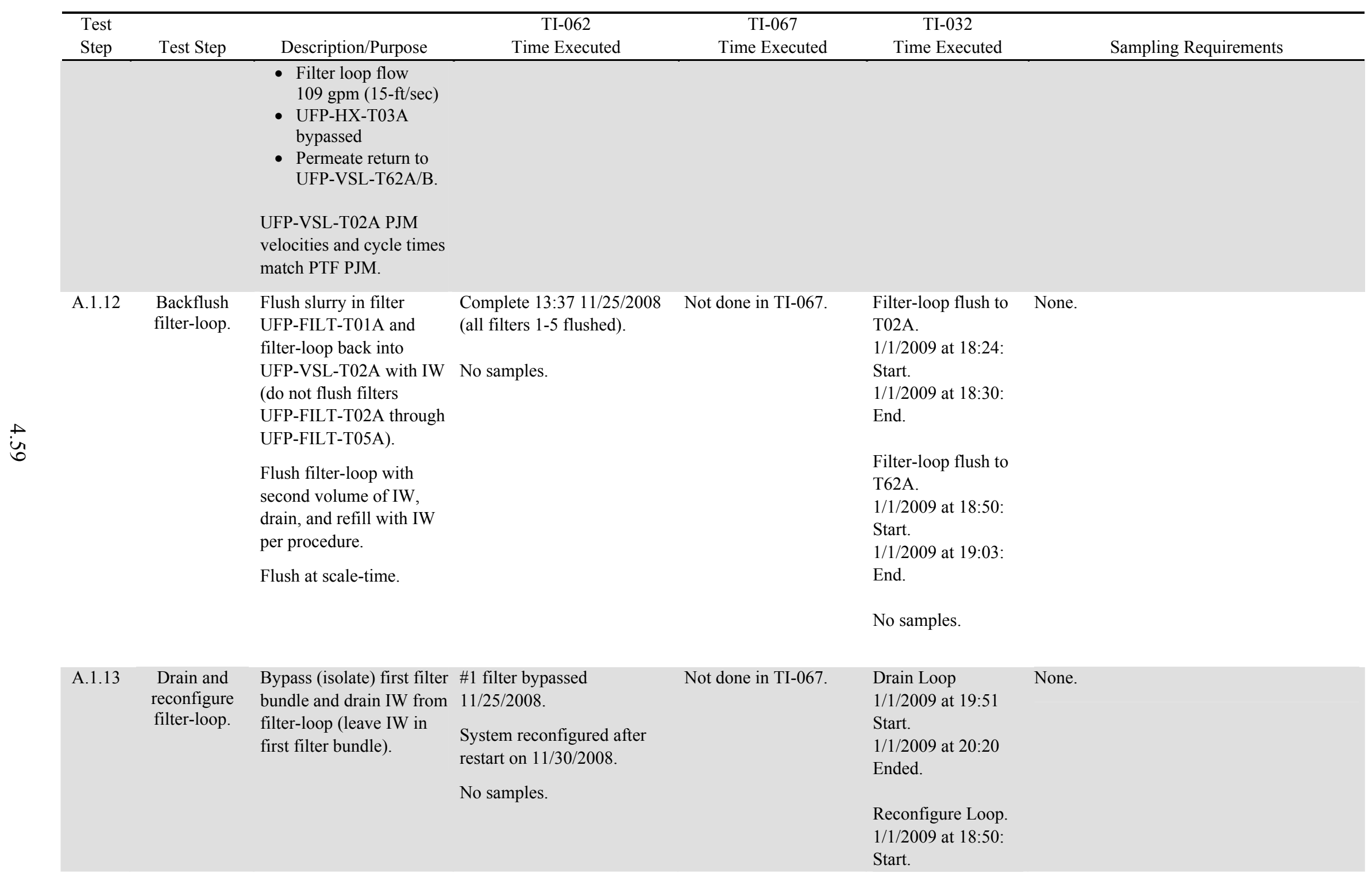


Table 4.3. Functional Testing Detailed Description

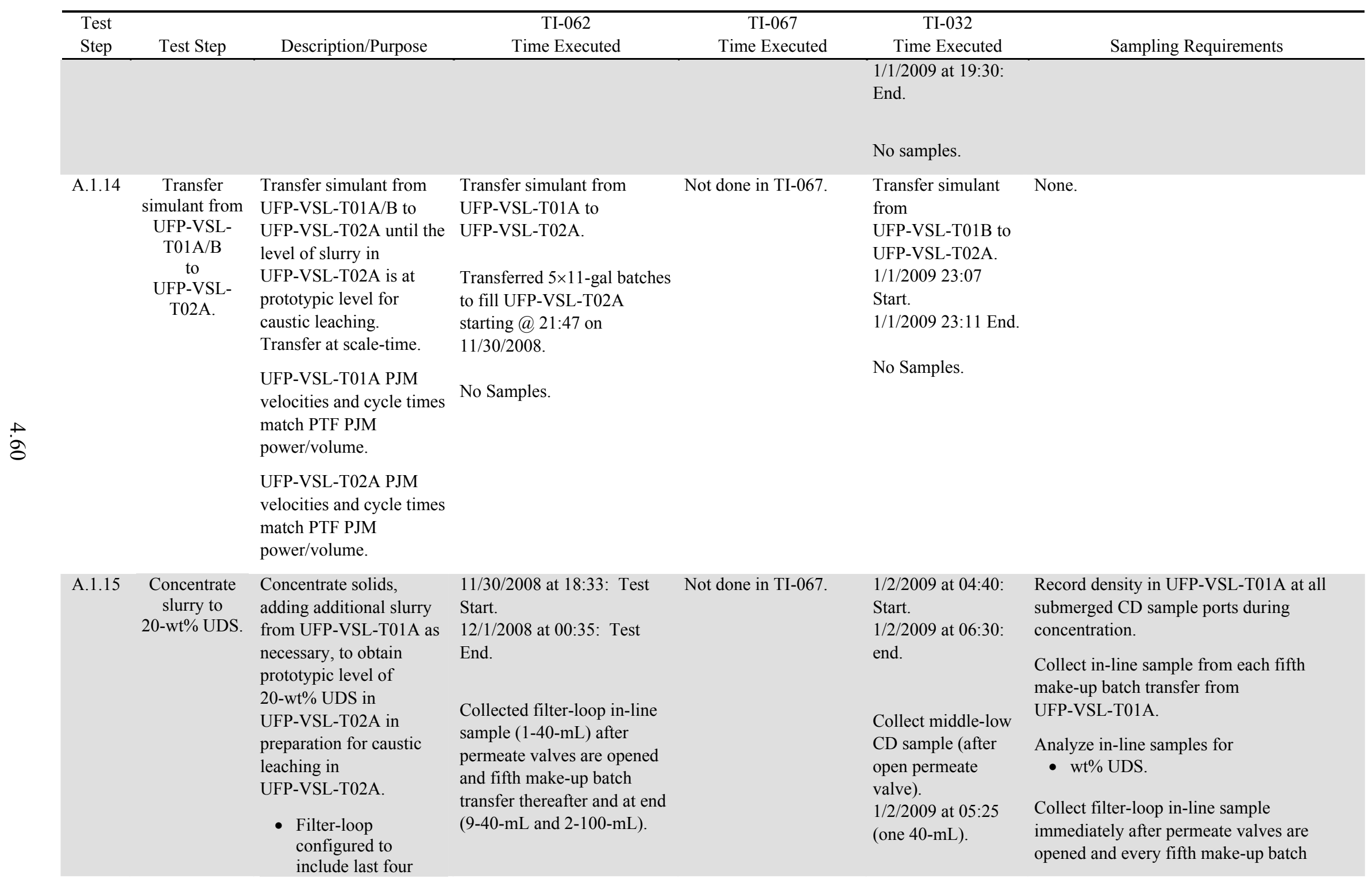


Table 4.3. Functional Testing Detailed Description

\begin{tabular}{|c|c|c|c|c|c|c|}
\hline $\begin{array}{l}\text { Test } \\
\text { Step }\end{array}$ & Test Step & Description/Purpose & $\begin{array}{c}\text { TI-062 } \\
\text { Time Executed }\end{array}$ & $\begin{array}{c}\text { TI-067 } \\
\text { Time Executed }\end{array}$ & $\begin{array}{c}\text { TI-032 } \\
\text { Time Executed }\end{array}$ & Sampling Requirements \\
\hline & & $\begin{array}{l}\text { filter bundles and } \\
\text { UFP-HX-T02A. } \\
\text { - Route permeate to } \\
\text { UFP-VSL-T62B. } \\
\text { - UFP-VSL- } \\
\text { T01A/T02A PJM } \\
\text { velocities and cycle } \\
\text { times match PTF } \\
\text { PJM power/volume. } \\
\text { Test control strategy for } \\
\text { make-up transfers from } \\
\text { UFP-VSL-T01A/B to } \\
\text { UFP-VSL-T02A during } \\
\text { concentration of solids. } \\
\text { Monitor UFP-VSL-T01A } \\
\text { for solids settling that } \\
\text { may impact the transfer } \\
\text { of solids. } \\
\text { Determine differences (if } \\
\text { any) in solids } \\
\text { concentration of feed } \\
\text { from UFP-VSL-T01A } \\
\text { during transfer and/or } \\
\text { between transfers in Steps } \\
\text { A.1.4 and A.1.11. } \\
\text { Test capability of control } \\
\text { scheme to achieve } \\
\text { 20-wt\% UDS } \\
\text { ultrafiltration endpoint. } \\
\text { Examine solid particles } \\
\text { from initial and final } \\
\text { slurry samples for } \\
\text { differences due to } \\
\text { processing. }\end{array}$ & $\begin{array}{l}11 / 30 / 2008 \text { at } 23: 02 \\
\text { (permeate valves open). } \\
11 / 30 / 2008 \text { at } 22: 26\left(5^{\text {th }}\right. \\
\text { batch). } \\
11 / 30 / 2008 \text { at } 23: 08\left(10^{\text {th }}\right. \\
\text { batch). } \\
11 / 30 / 2008 \text { at } 23: 26\left(15^{\text {th }}\right. \\
\text { batch). } \\
11 / 30 / 2008 \text { at } 23: 46\left(20^{\text {th }}\right. \\
\text { batch). } \\
11 / 30 / 2008 \text { at } 23: 55\left(22^{\text {nd }}\right. \\
\text { batch). } \\
12 / 1 / 2009 \text { at } 01: 48 \text { to } 01: 53 \\
\text { (at end). } \\
\text { Collect in-line sample from } \\
\text { fifth make-up batch transfer } \\
\text { from UFP-VSL-T01A } \\
\text { (40-mL). } \\
11 / 30 / 2008 \text { at } 22: 00\left(5^{\text {th }}\right. \\
\text { batch). } \\
11 / 30 / 2008 \text { at } 23: 07\left(10^{\text {th }}\right. \\
\text { batch). } \\
11 / 30 / 2008 \text { at } 23: 26\left(15^{\text {th }}\right. \\
\text { batch). } \\
11 / 30 / 2008 \text { at } 23: 46\left(20^{\text {th }}\right. \\
\text { batch). }\end{array}$ & & $\begin{array}{l}\text { After concentration } \\
\text { complete collect } \\
\text { middle-low CD } \\
\text { samples. } \\
1 / 2 / 2009 \text { at } 07: 11 \text { to } \\
07: 19 \text { (nine } 40-\mathrm{mL} \text {, } \\
\text { one } 100-\mathrm{mL}, 1-\mathrm{L} \text { for } \\
\text { parallel laboratory } \\
\text { tests.) }\end{array}$ & $\begin{array}{l}\text { transfer thereafter. } \\
\text { Analyze all filter-loop in-line samples for } \\
\text { - wt\% UDS. } \\
\text { Collect three final filter-loop in-line samples } \\
\text { after solids concentration is completed. } \\
\text { Collect a sample from the middle-low CD } \\
\text { sample port for parallel laboratory leach } \\
\text { testing after solids concentration is } \\
\text { complete. Transport sample to APEL. } \\
\text { Analyze initial and final filter-loop in-line } \\
\text { samples for shear stress vs. shear rate (not in } \\
\text { replicate). } \\
\text { Analyze the three final filter-loop in-line } \\
\text { samples for } \\
\text { - wt\% UDS } \\
\text { - slurry density } \\
\text { - liquid viscosity (not in replicate) } \\
\text { - liquid density } \\
\text { - dissolved aluminum } \\
\text { - liquid anion tracers } \\
\text { - total aluminum solids } \\
\text { - solid metal tracers. }\end{array}$ \\
\hline
\end{tabular}


Table 4.3. Functional Testing Detailed Description

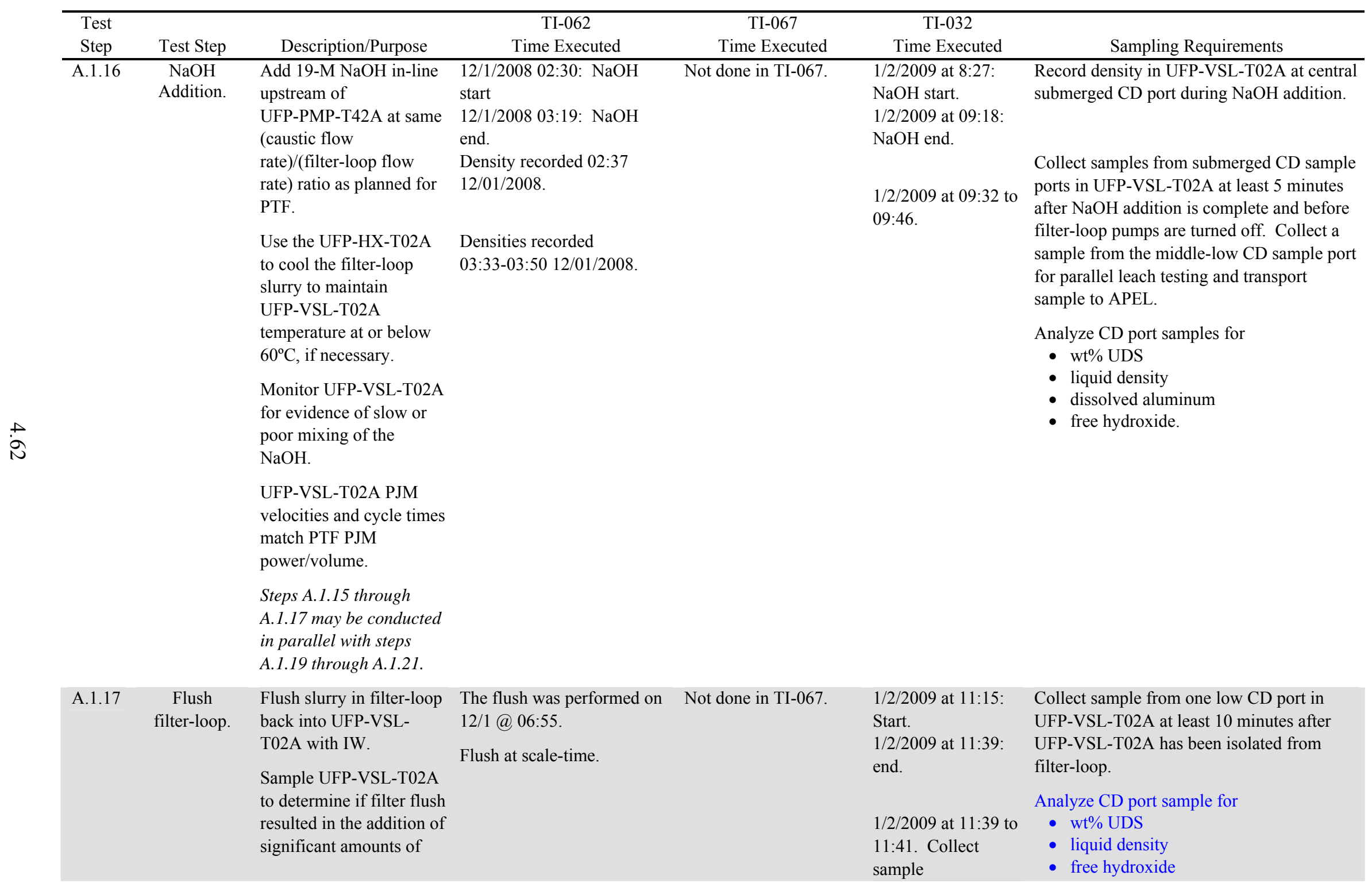


Table 4.3. Functional Testing Detailed Description

\begin{tabular}{|c|c|c|c|c|c|c|}
\hline $\begin{array}{l}\text { Test } \\
\text { Step }\end{array}$ & Test Step & Description/Purpose & $\begin{array}{c}\text { TI-062 } \\
\text { Time Executed }\end{array}$ & $\begin{array}{c}\text { TI-067 } \\
\text { Time Executed }\end{array}$ & $\begin{array}{c}\text { TI-032 } \\
\text { Time Executed }\end{array}$ & Sampling Requirements \\
\hline & & $\begin{array}{l}\text { flush water. } \\
\text { Flush filter-loop with } \\
\text { second volume of IW, } \\
\text { drain, and refill with IW } \\
\text { per procedure. }\end{array}$ & (1) & (1) & $\begin{array}{l}\text { UFP-VSL-T02A } \\
\text { low CD port in } \\
\text { UFP-VSL-T02A } \\
\text { after flush. }\end{array}$ & liquid anion tracers. \\
\hline A. 1.18 & $\begin{array}{c}\text { Heat } \\
\text { UFP-VSL- } \\
\text { T02A } \\
\text { to } 98^{\circ} \mathrm{C} \text { and } \\
\text { caustic-leach } \\
\text { for } \\
16 \text { hours. }\end{array}$ & $\begin{array}{l}\text { Heat, leach, and mix at } \\
\text { plant-time; } 98^{\circ} \mathrm{C} \\
\text { temperature, } 16 \text {-hr } \\
\text { caustic-leach. } \\
\text { UFP-VSL-T02A PJM } \\
\text { velocities and cycle times } \\
\text { match PTF PJM } \\
\text { power/volume. } \\
\text { UFP-VSL-T02A to } 98^{\circ} \mathrm{C} \text {. } \\
\text { Test steam injection } \\
\text { heating equipment and } \\
\text { controls. Determine if } \\
\text { condensate accumulation } \\
\text { is within target range. } \\
\text { Test PJM, air sparge } \\
\text { mixer and steam ring air } \\
\text { purge operations at } 98^{\circ} \mathrm{C} \text {. } \\
\text { Examine solids for } \\
\text { chemical and physical } \\
\text { changes during caustic } \\
\text { leaching. } \\
\text { Test for stratification of } \\
\text { solids in UFP-VSL-T02A } \\
\text { during and at the end of } \\
\text { caustic leaching. }\end{array}$ & $\begin{array}{l}\text { Not done in TI-062. } \\
\text { All testing was terminated } \\
\text { because of leaks in the } \\
\text { filter-loop. }\end{array}$ & Not done in TI-067. & $\begin{array}{l}\text { 1/2/2009 at } 09: 31 \text { : } \\
\text { Start. } \\
\text { 1/2/2009 at } 14: 58 \text { : } \\
\text { End. } \\
\text { Collect sample from } \\
\text { middle-middle CD } \\
\text { Port. } \\
\text { 1/2/2009 at } 13: 25- \\
\left.\text { 13:29 ( } 88^{\circ} \mathrm{C}\right) \text { nine } \\
40-\mathrm{mL} \text {. } \\
\text { 1/2/2009 at } 14: 39- \\
\left.\text { 14:42 ( } 98^{\circ} \mathrm{C}\right) \text { six } \\
40-\mathrm{mL} \text {. } \\
\text { 1/2/2009 at } 15: 37 \text { to } \\
\text { 15:40 ( } 1 \text { hour) six } \\
40-\mathrm{mL} \text {. } \\
\text { 1/2/2009 at } 16: 37 \text { to } \\
\text { 16:41 (2 hour) six } \\
40-\mathrm{mL} \text {. } \\
\text { 1/2/2009 at } 18: 36 \text { to } \\
\text { 18:38 ( } 4 \text { hours) six } \\
40-\mathrm{mL} \text {. } \\
1 / 2 / 2009 \text { at } 22: 36 \text { to } \\
22: 38 \text { ( } 8 \text { hours) six } \\
40-\mathrm{mL} \text {. } \\
\text { 1/3/2009 at } 02: 36 \text { to } \\
02: 38 \text { ( } 12 \text { hours) six }\end{array}$ & \\
\hline
\end{tabular}


Table 4.3. Functional Testing Detailed Description

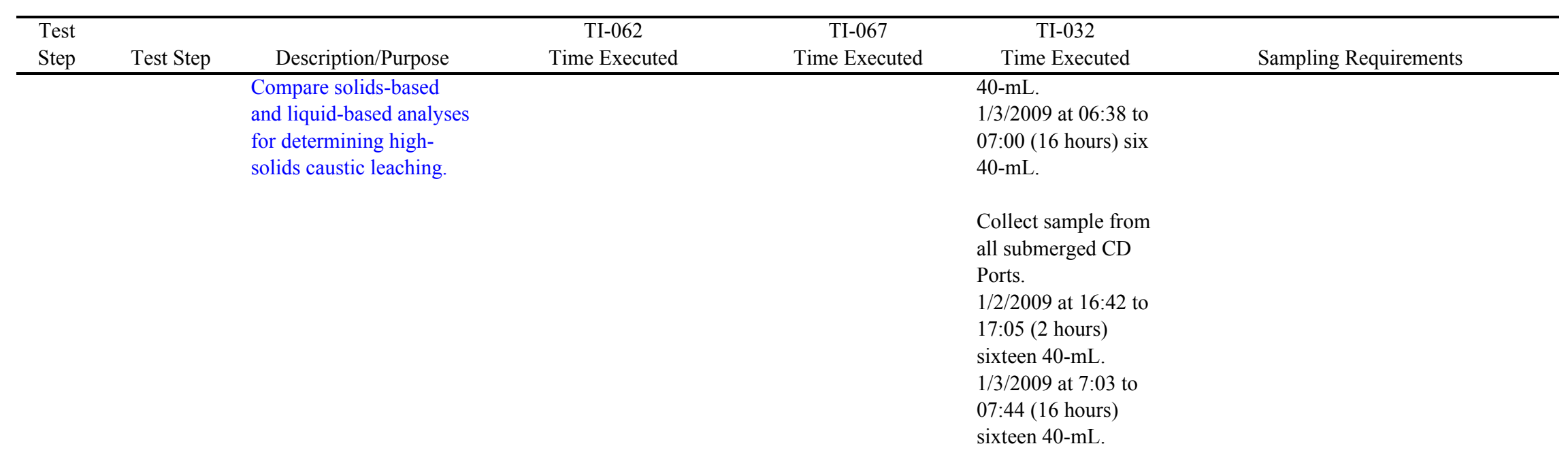

\begin{tabular}{|c|c|c|c|c|c|}
\hline A. 1.19 & $\begin{array}{l}\text { Cool } \\
\text { UFP-VSL- } \\
\text { T02A. }\end{array}$ & $\begin{array}{l}\text { Cool UFP-VSL-T02A } \\
\text { contents to the filtration } \\
\text { temperature in plant time } \\
\text { using the cooling jacket } \\
\text { or HX-T02A if necessary. } \\
\text { UFP-VSL-T02A PJM } \\
\text { velocities and cycle times } \\
\text { match PTF PJM } \\
\text { power/volume. } \\
\text { Test cooling strategy and } \\
\text { controls provide } \\
\text { prototypic cool-down } \\
\text { curve. }\end{array}$ & Not done in TI-062. & Not done in TI-067. & $\begin{array}{l}\text { Cool to } 60 \text { degrees } \\
\text { C. } \\
\text { 1/3/2009 at } 06: 30 \text { : } \\
\text { Start. } \\
\text { 1/3/2009 at 10:00: } \\
\text { End. } \\
\text { Cool to } 25 \text { degrees } \\
\text { C. } \\
\text { 1/3/2009 10:00: } \\
\text { Start. } \\
\text { 1/3/2009 22:15: } \\
\text { End. } \\
\text { No samples. }\end{array}$ \\
\hline A. 1.20 & $\begin{array}{l}\text { Prepare } \\
\text { UFP-VSL- } \\
\text { T01A } \\
\text { for caustic } \\
\text { leaching. }\end{array}$ & $\begin{array}{l}\text { Adjust slurry volume in } \\
\text { UFP-VSL-T01A to } \\
\text { achieve prototypic caustic } \\
\text { leaching batch size. } \\
\text { Add prototypic quantity }\end{array}$ & Not done in TI-062. & $\begin{array}{l}12 / 9 / 2008 \text { at } 10: 04 \\
\text { Caustic Add Start. } \\
12 / 9 / 2008 \text { at } 11: 11 \\
\text { Caustic Add End. }\end{array}$ & $\begin{array}{l}12 / 20 / 2008 \text { at } 09: 30 \\
\text { Caustic Add Start. } \\
\text { 12/20/2008 at } \\
\text { 10:05-Caustic Add } \\
\text { End (Per DAS data } \\
\text { FT-1421). }\end{array}$ \\
\hline
\end{tabular}


Table 4.3. Functional Testing Detailed Description

\begin{tabular}{|c|c|c|c|c|c|c|}
\hline $\begin{array}{l}\text { Test } \\
\text { Step }\end{array}$ & Test Step & Description/Purpose & $\begin{array}{c}\text { TI-062 } \\
\text { Time Executed }\end{array}$ & $\begin{array}{c}\text { TI-067 } \\
\text { Time Executed }\end{array}$ & $\begin{array}{c}\text { TI-032 } \\
\text { Time Executed }\end{array}$ & Sampling Requirements \\
\hline & & $\begin{array}{l}\text { of 19-M NaOH directly } \\
\text { to UFP-VSL-T01A for } \\
\text { caustic leaching. } \\
\text { HLP-VSL-T22 agitator } \\
\text { speed set so vessel is well } \\
\text { mixed. } \\
\text { Set transfer rate to make } \\
\text { certain of turbulent flow } \\
\text { in transfer pipes. } \\
\text { UFP-VSL-T01A PJM } \\
\text { velocities and cycle times } \\
\text { match PTF PJM. }\end{array}$ & & $\begin{array}{l}\text { Collect samples from } \\
\text { the inner-middle CD } \\
\text { port. } \\
\text { 12/9/2008 at } 09: 37 \text { to } \\
09: 45: \text { (prior to) nine } \\
\text { 40-mL. } \\
\text { 12/9/2008 at } 11: 34 \text { to } \\
11: 40 \text { (after) six } 40-\mathrm{mL} \text {. } \\
\text { 12/9/2009-Record } \\
\text { density in } \\
\text { UFP-VSL-T01A at all } \\
\text { submerged CD ports } \\
\text { (during). } \\
\text { Tune PJMs. }\end{array}$ & $\begin{array}{l}\text { Collect samples } \\
\text { from the } \\
\text { inner-middle CD } \\
\text { port. } \\
12 / 20 / 2008 \text { at } 09: 17 \\
\text { to } 09: 23 \text { :(prior to) } \\
9-40-\mathrm{mL} \text { and } 1-\mathrm{L} \\
\text { for CUF. } \\
12 / 5 / 2008 \text { at } 10: 31 \\
\text { to } 10: 38 \text { (after) } \\
6-40-\mathrm{mL} \& 1-\mathrm{L} \text { for } \\
\text { CUF. }\end{array}$ & \\
\hline A. 1.21 & $\begin{array}{c}\text { Heat } \\
\text { UFP-VSL- } \\
\text { T01A } \\
\text { to } 98^{\circ} \mathrm{C} \text { and } \\
\text { caustic-leach } \\
\text { for } \\
16 \text { hours. }\end{array}$ & $\begin{array}{l}\text { Heat slurry in } \\
\text { UFP-VSL-T01A at } \\
\text { plant-time to } 98^{\circ} \mathrm{C} \text { for } \\
\text { 16-hr caustic-leach. } \\
\text { UFP-VSL-T01A PJM } \\
\text { velocities and cycle times } \\
\text { match PTF PJM } \\
\text { power/volume. } \\
\text { Test steam injection } \\
\text { heating equipment and } \\
\text { controls. } \\
\text { Determine if condensate } \\
\text { accumulation is within } \\
\text { target range. } \\
\text { Test PJM and steam ring } \\
\text { air purge operations at } \\
98^{\circ} \mathrm{C} \text {. } \\
\text { Test for stratification of }\end{array}$ & Not done in TI-062. & $\begin{array}{l}12 / 9 / 2008 \text { at } 17: 20 \\
\text { Caustic-Leach Start. } \\
\text { 12/10/2008 at } 09: 20 \\
\text { Caustic-Leach End. } \\
\text { Collect samples from } \\
\text { the inner-middle CD } \\
\text { port. } \\
\text { 12/9/2008 at } 16: 33 \text { to } \\
\left.\text { 16:35 ( } 88^{\circ} \mathrm{C}\right) \text { three } \\
40-\mathrm{mL} \text {. } \\
\text { 12/9/2008 at } 17: 17 \text { to } \\
\left.\text { 17:18 ( } 98^{\circ} \mathrm{C}\right) \text { two } \\
40-\mathrm{mL} \text {. } \\
12 / 9 / 2008 \text { at } 18: 17 \text { to } \\
18: 18(1 \text { hour) two }\end{array}$ & $\begin{array}{l}\text { 12/20/2008 15:33 } \\
\text { Caustic-Leach Start. } \\
\text { 12/21/2008 08:33 } \\
\text { Caustic-Leach End. } \\
\text { Collect samples } \\
\text { from the } \\
\text { inner-middle CD } \\
\text { port. } \\
\text { 12/20/2008 at } 14: 49 \\
\text { to } 14: 53\left(88^{\circ} \mathrm{C}\right) \text { nine } \\
40-\mathrm{mL} \text {. } \\
12 / 20 / 2008 \text { at } 15: 33 \\
\text { to } 15: 36\left(98^{\circ} \mathrm{C}\right) \text { six } \\
40-\mathrm{mL} \text {. } \\
12 / 20 / 2008 \text { at } 16: 34\end{array}$ & \\
\hline
\end{tabular}


Table 4.3. Functional Testing Detailed Description

\begin{tabular}{|c|c|c|c|c|c|c|}
\hline $\begin{array}{l}\text { Test } \\
\text { Step }\end{array}$ & Test Step & Description/Purpose & $\begin{array}{c}\text { TI-062 } \\
\text { Time Executed }\end{array}$ & $\begin{array}{c}\text { TI-067 } \\
\text { Time Executed }\end{array}$ & $\begin{array}{c}\text { TI-032 } \\
\text { Time Executed }\end{array}$ & Sampling Requirements \\
\hline & & solids in UFP-VSL-T01A & & 40-mL. & to $16: 36$ ( 1 hour) six & \\
\hline & & during and at the end of & & $12 / 9 / 2008$ at $19: 15$ to & 40-mL. & \\
\hline & & caustic leaching. & & $19: 16$ (2 hours) two & $12 / 20 / 200817: 35$ to & \\
\hline & & & & 40-mL. & 17:38 (2 hour). & \\
\hline & & Compare solids-based & & $12 / 9 / 2008$ at $21: 16$ to & $12 / 20 / 2008$ at $19: 24$ & \\
\hline & & for determining low- & & 21:17 (4 hours) two & to $19: 28$ (4 hours) & \\
\hline & & olids constic loubing & & 40-mL. & six 40-mL. & \\
\hline & & & & $12 / 10 / 2008$ at $01: 15$ to & $12 / 20 / 2008$ at $23: 23$ & \\
\hline & & & & 01:16 (8 hours) two & to $23: 26$ ( 8 hours) & \\
\hline & & & & 40-mL. & six 40-mL. & \\
\hline & & & & $12 / 10 / 2008$ at $05: 14:$ & $12 / 21 / 2008$ at $03: 39$ & \\
\hline & & & & 05:15 (12 hours) six & to $03: 431$ ( 12 hours) & \\
\hline & & & & 40-mL. & six 40-mL. & \\
\hline & & & & & $12 / 21 / 2008$ at $7: 31$ & \\
\hline & & & & Collect samples from all & to $7: 33$ ( 16 hours). & \\
\hline & & & & submerged CD ports. & Collect samples & \\
\hline & & & & $12 / 9 / 2008$ at $19: 20$ to & from all submerged & \\
\hline & & & & 20:03 (2 hours) eighteen & CD ports. & \\
\hline & & & & $40-\mathrm{mL}$ & $12 / 20 / 2008$ at $17: 40$ & \\
\hline & & & & $12 / 10 / 2008$ at $09: 21$ to & to $17: 46$ (2 hours) & \\
\hline & & & & 09:42 (16 hours) sixteen & eighteen $40-\mathrm{mL}$. & \\
\hline & & & & $40-\mathrm{mL}$ & $12 / 21 / 2008$ at $07: 34:$ & \\
\hline & & & & Record density in & 07:40 (16 hours) & \\
\hline & & & & UFP-VSL-T01A at & eighteen 40-mL. & \\
\hline & & & & submerged CD ports. & & \\
\hline & & & & $\begin{array}{l}\text { (2 hours). } \\
\text { (2/9008 at }\end{array}$ & Record density in & \\
\hline & & & & $12 / 10 / 2008$ at $10: 13$ & UFP-VSL-T01A at & \\
\hline & & & & (16 hours). & submerged CD & \\
\hline & & & & & ports. & \\
\hline & & & & & (2 hours). & \\
\hline & & & & & (16 hours). & \\
\hline \multirow[t]{3}{*}{ A.1.22 } & \multirow{3}{*}{$\begin{array}{l}\text { Partial } \\
\text { cool-down of } \\
\text { UFP-VSL- }\end{array}$} & \multirow{3}{*}{$\begin{array}{l}\text { Cool-down of } \\
\text { UFP-VSL-T01A contents } \\
\text { to quench the caustic }\end{array}$} & \multirow[t]{3}{*}{ Not done in TI-062. } & $12 / 10 / 2008$ at $09: 45:$ & $12 / 21 / 2008$ at $07: 54:$ & \\
\hline & & & & Start. & Start. & \\
\hline & & & & $12 / 10 / 2008$ at 13.35. & $12 / 21 / 2008$ at $12: 10:$ & \\
\hline
\end{tabular}


Table 4.3. Functional Testing Detailed Description

\begin{tabular}{|c|c|c|c|c|c|c|}
\hline $\begin{array}{l}\text { Test } \\
\text { Step }\end{array}$ & Test Step & Description/Purpose & $\begin{array}{c}\text { TI-062 } \\
\text { Time Executed }\end{array}$ & $\begin{array}{c}\text { TI-067 } \\
\text { Time Executed }\end{array}$ & $\begin{array}{c}\text { TI-032 } \\
\text { Time Executed }\end{array}$ & Sampling Requirements \\
\hline & & leaching process. & & End. & End. & \\
\hline & & $\begin{array}{l}\text { Recirculate slurry in } \\
\text { UFP-VSL-T01A through } \\
\text { external chiller } \\
\text { (UFP-HX-T05A) to } \\
\text { reduce vessel contents to } \\
60^{\circ} \mathrm{C} \text {. }\end{array}$ & & $\begin{array}{l}\text { 12/10/2008 at 11:59: } \\
\text { Record density in } \\
\text { UFP-VSL-T01A at } \\
\text { inner CD port during } \\
\text { cool-down. }\end{array}$ & $\begin{array}{l}12 / 21 / 2008 \text { Record } \\
\text { density in } \\
\text { UFP-VSL-T01A at } \\
\text { inner CD port } \\
\text { during cool-down. }\end{array}$ & \\
\hline & & $\begin{array}{l}\text { UFP-VSL-T01A PJM } \\
\text { velocities and cycle times } \\
\text { match PTF PJM } \\
\text { power/volume. }\end{array}$ & & & & \\
\hline \multirow[t]{2}{*}{ A. 1.23} & $\begin{array}{c}\text { Drain } \\
\text { filter-loop. }\end{array}$ & $\begin{array}{l}\text { Bypass first filter bundle } \\
\text { and drain IW from } \\
\text { filter-loop (leave IW in } \\
\text { first filter bundle). }\end{array}$ & Not done in TI-062. & Not done in TI-067. & $\begin{array}{l}\text { 1/1/2009: Start. } \\
\text { 1/1/2009: End. } \\
\text { No Samples. }\end{array}$ & None. \\
\hline & & $\begin{array}{l}\text { Filter-loop configured to } \\
\text { include last four filter } \\
\text { bundles and } \\
\text { UFP-HX-T02A. }\end{array}$ & & & & \\
\hline \multirow[t]{3}{*}{ A. 1.24} & $\begin{array}{l}\text { Concentrate } \\
\text { solids in } \\
\text { UFP-VSL- } \\
\text { T02A. }\end{array}$ & $\begin{array}{l}\text { Ultrafilters } \\
\text { preconditioned and } \\
\text { flushed. } \\
\text { UFP-VSL-T01A PJM } \\
\text { velocities and cycle times } \\
\text { match PTF PJM } \\
\text { power/volume. }\end{array}$ & Not done in TI-062. & Not done in TI-067. & $\begin{array}{l}\text { Start: 1/4/2009@ } \\
\text { 01:30. } \\
\text { End:1/7/2009@ } \\
\text { 14:31. } \\
\text { No Samples. }\end{array}$ & $\begin{array}{l}\text { Record density in UFP-VSL-T02A at } \\
\text { middle-middle CD port location during } \\
\text { concentration. }\end{array}$ \\
\hline & & $\begin{array}{l}\text { UFP-VSL-T02A PJM } \\
\text { velocities and cycle times } \\
\text { match PTF PJM } \\
\text { power/volume. }\end{array}$ & & & $\begin{array}{l}\text { Record density in } \\
\text { UFP-VSL-T02A at } \\
\text { middle-middle CD } \\
\text { port location during } \\
\text { concentration. }\end{array}$ & \\
\hline & & $\begin{array}{l}\text { Route permeate to } \\
\text { UFP-VSL-T62B. }\end{array}$ & & & & \\
\hline
\end{tabular}


Table 4.3. Functional Testing Detailed Description

\begin{tabular}{|c|c|c|c|c|c|c|}
\hline $\begin{array}{l}\text { Test } \\
\text { Step }\end{array}$ & Test Step & Description/Purpose & $\begin{array}{c}\text { TI-062 } \\
\text { Time Executed }\end{array}$ & $\begin{array}{c}\text { TI-067 } \\
\text { Time Executed }\end{array}$ & $\begin{array}{c}\text { TI-032 } \\
\text { Time Executed }\end{array}$ & Sampling Requirements \\
\hline & & $\begin{array}{l}\text { Concentrate solids in } \\
\text { UFP-VSL-T02A until } \\
\text { "Leaching in } \\
\text { UFP-VSL-T01A/B } \\
\text { Concentration Setpoint" } \\
\text { level is reached, four } \\
\text { filter bundles, } \\
\text { nonprototypic } \\
\text { concentration. }\end{array}$ & & & & \\
\hline & & $\begin{array}{l}\text { Transfer 11-gal batches } \\
\text { of slurry from } \\
\text { UFP-VSL-T01A to } \\
\text { UFP-VSL-T02A as } \\
\text { needed to maintain the } \\
\text { "setpoint" level in } \\
\text { UFP-VSL-T02A. }\end{array}$ & & & & \\
\hline & & $\begin{array}{l}\text { Cool transfers from } \\
\text { UFP-VSL-T01A with } \\
\text { UFP-HX-T05A. }\end{array}$ & & & & \\
\hline & & $\begin{array}{l}\text { When UFP-VSL-T01A } \\
\text { has been emptied to its } \\
\text { heel, continue to dewater } \\
\text { UFP-VSL-T02A to } \\
\text { achieve } 17 \text {-wt } \% \text { UDS. }\end{array}$ & & & & \\
\hline & & $\begin{array}{l}\text { Use autocontrol strategy } \\
\text { for make-up additions } \\
\text { from the UFP-VSL- } \\
\text { T01A/B to } \\
\text { UFP-VSL-T02A during } \\
\text { solids concentration. }\end{array}$ & & & & \\
\hline A. 1.26 & $\begin{array}{l}\text { Post-caustic- } \\
\text { leach slurry } \\
\text { wash. }\end{array}$ & $\begin{array}{l}\text { Wash the } \\
\text { post-caustic-leach slurry } \\
\text { to below } 0.25-\mathrm{M} \text { free }\end{array}$ & Not done in TI-062. & Not done in TI-067. & $\begin{array}{l}\text { 1/7/2009 at } 20: 13: \\
\text { Start. } \\
1 / 7 / 2009 \text { at } 23: 10: \\
\text { End. }\end{array}$ & $\begin{array}{l}\text { Analyze all even numbered samples for: } \\
\text { Analyze all inner CD port samples for } \\
\text { - liquid density }\end{array}$ \\
\hline
\end{tabular}


Table 4.3. Functional Testing Detailed Description

\begin{tabular}{|c|c|c|c|c|c|c|}
\hline $\begin{array}{l}\text { Test } \\
\text { Step }\end{array}$ & Test Step & Description/Purpose & $\begin{array}{c}\text { TI-062 } \\
\text { Time Executed }\end{array}$ & $\begin{array}{c}\text { TI-067 } \\
\text { Time Executed }\end{array}$ & $\begin{array}{c}\text { TI-032 } \\
\text { Time Executed }\end{array}$ & Sampling Requirements \\
\hline & & $\begin{array}{l}\text { hydroxide. } \\
\text { Remove oxalate in } \\
\text { preparation for high- } \\
\text { solids filter flux test. } \\
\text { Nonprototypic wash. } \\
\text { Four ultrafilter bundles. } \\
\text { UFP-VSL-T02A PJM } \\
\text { velocities match PTF } \\
\text { PJM velocities, and cycle } \\
\text { time is } 1 / 4.5 \text { that of PTF. }\end{array}$ & & & $\begin{array}{l}\text { Collect } 19 \text { samples } \\
\text { from the middle-low } \\
\text { CD loop. Samples } \\
\text { collected } 1 \text { minute } \\
\text { after wash-water } \\
\text { addition and after } \\
\text { every } 2^{\text {nd }} \text { batch. }\end{array}$ & $\begin{array}{l}\text { - dissolved aluminum } \\
\text { - free hydroxide } \\
\text { - liquid anion tracers. } \\
\text { Archive all odd numbered samples. }\end{array}$ \\
\hline A. 1.27 & $\begin{array}{l}\text { Add chromium } \\
\text { solids slurry. }\end{array}$ & $\begin{array}{l}\text { Test nonprototypic } \\
\text { addition of chromium } \\
\text { solids slurry to suction } \\
\text { side of UFP-PMP-T42A. }\end{array}$ & Not done in TI-062. & See TI-032. & $\begin{array}{l}\text { 1/8/2009 at } 13: 56: \\
\text { Start. } \\
\text { 1/8/2009 at } 15: 36: \\
\text { End. } \\
\text { No Samples. }\end{array}$ & None. \\
\hline \multirow[t]{4}{*}{ A. 1.28} & \multirow[t]{4}{*}{$\begin{array}{l}\text { Reconcentrate } \\
\text { solids and } \\
\text { wash slurry. }\end{array}$} & $\begin{array}{l}\text { Filter to remove liquid } \\
\text { volume introduced with } \\
\text { chromium slurry and } \\
\text { concentrate to } 20 \text {-wt } \% \\
\text { solids. }\end{array}$ & Not done in TI-062. & Not done in TI-067. & $\begin{array}{l}\text { 1/8/2009 at } 15: 26: \\
\text { Start. } \\
1 / 8 / 2009 \text { at } 16: 51: \\
\text { End. }\end{array}$ & $\begin{array}{l}\text { Collect filter-loop in-line sample after final } \\
\text { wash. } \\
\text { Analyze filter-loop in-line sample for } \\
\text { - wt } \% \text { UDS. }\end{array}$ \\
\hline & & $\begin{array}{l}\text { Wash to reduce } \\
\text { hydroxide concentration } \\
\text { to approximately } 0.25-\mathrm{M} \text {. }\end{array}$ & & & $\begin{array}{l}\text { Collect one } 40-\mathrm{mL} \\
\text { CD sample from } \\
\text { middle-low port. }\end{array}$ & \\
\hline & & $\begin{array}{l}\text { Nonprototypic solids } \\
\text { concentration and wash. } \\
\text { Four ultrafilter bundle. }\end{array}$ & & & $\begin{array}{l}1 / 8 / 2009 \text { at } 18: 38 \\
\text { after wash (one } \\
40-\mathrm{mL} \text { ). }\end{array}$ & \\
\hline & & $\begin{array}{l}\text { UFP-VSL-T02A PJM } \\
\text { velocities match PTF } \\
\text { PJM velocities, and cycle } \\
\text { time is } 1 / 4.5 \text { that of PTF. }\end{array}$ & & & $\begin{array}{l}1 / 8 / 2009 \text { Collect } \\
\text { filter-loop in-line } \\
\text { sample after final } \\
\text { wash. }\end{array}$ & \\
\hline A.1.29 & $\begin{array}{l}\text { Prepare for } \\
\text { high-solids }\end{array}$ & $\begin{array}{l}\text { Add IW in-line to suction } \\
\text { of UFP-PMP-T42A until }\end{array}$ & Not done in TI-062. & Not done in TI-067. & $\begin{array}{l}\text { Collect CD samples } \\
\text { from middle-low }\end{array}$ & $\begin{array}{l}\text { Collect three samples from the middle-low } \\
\text { CD sample port for parallel CUF test and }\end{array}$ \\
\hline
\end{tabular}


Table 4.3. Functional Testing Detailed Description

\begin{tabular}{|c|c|c|c|c|c|c|}
\hline $\begin{array}{l}\text { Test } \\
\text { Step }\end{array}$ & Test Step & Description/Purpose & $\begin{array}{c}\text { TI-062 } \\
\text { Time Executed }\end{array}$ & $\begin{array}{c}\text { TI-067 } \\
\text { Time Executed }\end{array}$ & $\begin{array}{c}\text { TI-032 } \\
\text { Time Executed }\end{array}$ & Sampling Requirements \\
\hline & filter test. & $\begin{array}{l}\text { slurry level in } \\
\text { UFP-VSL-T02A is at the } \\
\text { "High Mixing Volume" } \\
\text { level. } \\
\text { Mix UFP-VSL-T02A } \\
\text { with filter-loop jet for } \\
10 \text { minutes. } \\
\text { UFP-VSL-T02A PJM } \\
\text { velocities match PTF } \\
\text { PJM velocities, and cycle } \\
\text { time is } 1 / 4.5 \text { that of PTF. }\end{array}$ & & & $\begin{array}{l}\text { port, two } 40-\mathrm{mL}, \\
\text { one } 100-\mathrm{mL}, \text { one } \\
22.9-\mathrm{L} \text {. } \\
\text { 1/8/2009 at } 21: 21 \text { to } \\
21: 38 \text {. } \\
1 / 9 / 2009 \text { at } 17: 49 \text { to } \\
17: 50 \text {. } \\
1 / 10 / 2009 \text { at } \\
20: 07-\text { Collect CD } \\
\text { samples from } \\
\text { middle-low port, } \\
3-40-\mathrm{mL} \text { before } \\
\text { open permeate } \\
\text { valves. } \\
1 / 8 / 2009 \text { at } 20: 35 \text { to } \\
20: 36 \text {. } \\
1 / 9 / 2009 \text { at } 18: 55 \text {. } \\
1 / 10 / 2009 \text { at } 21: 07 \\
\text { to } 21: 09 \text {. }\end{array}$ & $\begin{array}{l}\text { characterization at end of 10-min mixing } \\
\text { period. } \\
\text { Analyze characterization filter-loop in-line } \\
\text { sample for } \\
\text { - wt\% UDS } \\
\text { - liquid viscosity } \\
\text { - PSD. }\end{array}$ \\
\hline A. 1.30 & $\begin{array}{l}\text { High-solids } \\
\text { filter test. }\end{array}$ & $\begin{array}{l}\text { Configure filters to flow } \\
\text { slurry through all five } \\
\text { filters. Concentrate } \\
\text { slurry in UFP-VSL-T02A } \\
\text { to } 20 \text {-wt } \% \text { solids using } \\
\text { only the first filter } \\
\text { bundle. After the } \\
\text { permeate valves are } \\
\text { opened, backpulse filter } 1 \\
\text { five times as rapidly as } \\
\text { possible. } \\
\text { Record permeate flow } \\
\text { from first filter bundle as } \\
\text { a function of time. } \\
\text { Collect in-line slurry }\end{array}$ & Not done in TI-062. & Not done in TI-067. & $\begin{array}{l}\text { 1/8/2009 at } 21: 50 \text { : } \\
\text { Start. } \\
\text { 1/8/2009 at } 23: 39 \text { : } \\
\text { End. } \\
\text { Collect } 3-40-\mathrm{mL} \mathrm{CD} \\
\text { samples: } \\
1 / 8 / 2009 \text { at } 22: 07 \\
(15 \text { minutes). } \\
1 / 8 / 2009 \text { at } 22: 15 \text {. } \\
1 / 8 / 2009 \text { at } 22: 30 \text {. } \\
1 / 8 / 2009 \text { at } 22: 45 \text {. } \\
1 / 8 / 2009 \text { at } 23: 00 \text {. } \\
1 / 8 / 2009 \text { at } 23: 15 \text {. } \\
1 / 8 / 2009 \text { at } 23: 29\end{array}$ & $\begin{array}{l}\text { Collect three samples from the middle-low } \\
\text { CD sample port after backpulsing is } \\
\text { complete. After the permeate flow has been } \\
\text { routed to T- } 62 \mathrm{~A} / \mathrm{B} \text {, begin taking samples } \\
\text { from the middle-low CD sample port and at } \\
15 \text {-min intervals thereafter. The final } \\
\text { samples are to be taken and analyzed in } \\
\text { triplicate. } \\
\text { Analyze CD sample port samples for } \\
\text { - wt\% UDS. }\end{array}$ \\
\hline
\end{tabular}


Table 4.3. Functional Testing Detailed Description

\begin{tabular}{|c|c|c|c|c|c|c|}
\hline $\begin{array}{l}\text { Test } \\
\text { Step }\end{array}$ & Test Step & Description/Purpose & $\begin{array}{c}\text { TI-062 } \\
\text { Time Executed }\end{array}$ & $\begin{array}{c}\text { TI-067 } \\
\text { Time Executed }\end{array}$ & $\begin{array}{c}\text { TI-032 } \\
\text { Time Executed }\end{array}$ & Sampling Requirements \\
\hline & & $\begin{array}{l}\text { samples to establish wt\% } \\
\text { UDS periodically during } \\
\text { solids concentration } \\
\text { process. }\end{array}$ & & & $\begin{array}{l}\text { (ended due to pump } \\
\text { problems). } \\
1 / 8 / 2009 \text { at } 23: 32 \\
\text { (final sample). }\end{array}$ & \\
\hline & & $\begin{array}{l}\text { Filter-loop flow through } \\
\text { all filter bundles. } \\
\text { Withdraw permeate from } \\
\text { first filter bundle only. }\end{array}$ & & & $\begin{array}{l}\text { 1/9/2009 at } 18: 55: \\
\text { Start. } \\
\text { 1/9/2009 at } 20: 20: \\
\text { End. }\end{array}$ & \\
\hline & & TMP 40 psid. & & & Collect 3-40-mL CD & \\
\hline & & $\begin{array}{l}\text { Filter-loop flow } 109 \mathrm{gpm} \\
\text { (15-ft/sec). }\end{array}$ & & & $\begin{array}{l}\text { samples: } \\
\text { 1/9/2009 at 19:15 }\end{array}$ & \\
\hline & & $\begin{array}{l}\text { Permeate flow routed to } \\
\text { UFP-VSL-T62A or B. }\end{array}$ & & & $\begin{array}{l}\text { (15 minutes). } \\
1 / 9 / 2009 \text { at } 19: 30 . \\
1 / 9 / 2009 \text { at } 19: 45 .\end{array}$ & \\
\hline & & $\begin{array}{l}\text { UFP-VSL-T02A PJM } \\
\text { velocities match PTF } \\
\text { PJM velocities, and cycle } \\
\text { time is } 1 / 4.5 \text { that of PTF. }\end{array}$ & & & $\begin{array}{l}\text { 1/9/2009 at 20:00. } \\
\text { 1/9/2009 at 20:16. } \\
\text { (Secured at 20:20 } \\
\text { due to zero } \\
\text { permeate flow.) } \\
\text { 1/9/2009 at 20:22 } \\
\text { (final sample). }\end{array}$ & \\
\hline & & & & & $\begin{array}{l}1 / 10 / 2009 \text { at } 23: 28: \\
\text { Start. } \\
1 / 11 / 2009 \text { at } 02: 28: \\
\text { End. }\end{array}$ & \\
\hline & & & & & $\begin{array}{l}\text { Collect } 3-40-\mathrm{mL} \\
\text { CD samples: } \\
1 / 10 / 2009 \text { at } 23: 43 \\
\text { (15 minutes). } \\
1 / 10 / 2009 \text { at } 23: 58 . \\
1 / 11 / 2009 \text { at } 00: 13 .\end{array}$ & \\
\hline
\end{tabular}


Table 4.3. Functional Testing Detailed Description

\begin{tabular}{|c|c|c|c|c|c|c|}
\hline $\begin{array}{l}\text { Test } \\
\text { Step }\end{array}$ & Test Step & Description/Purpose & $\begin{array}{c}\text { TI-062 } \\
\text { Time Executed }\end{array}$ & $\begin{array}{c}\text { TI-067 } \\
\text { Time Executed }\end{array}$ & $\begin{array}{c}\text { TI-032 } \\
\text { Time Executed }\end{array}$ & Sampling Requirements \\
\hline & & & & & $\begin{array}{l}1 / 11 / 2009 \text { at } 00: 28 \text {. } \\
1 / 11 / 2009 \text { at } 00: 43 \text {. } \\
1 / 11 / 2009 \text { at } 00: 58 \text {. } \\
1 / 11 / 2009 \text { at } 01: 13 \text {. } \\
1 / 11 / 2009 \text { at } 01: 28 \text {. } \\
1 / 11 / 2009 \text { at } 01: 43 \text {. } \\
1 / 11 / 2009 \text { at } 01: 58 \text {. } \\
1 / 11 / 2009 \text { at } 02: 13 \text {. } \\
1 / 11 / 2009 \text { at } 02: 28 \text {. } \\
\text { Stopped at } 02: 29 \\
\text { because could not } \\
\text { keep target flow. } \\
1 / 11 / 2009 \text { at } 02: 34 \\
\text { (final sample } 1 \text { extra } \\
\text { set for client). }\end{array}$ & \\
\hline A. 1.31 & $\begin{array}{l}\text { Oxidative- } \\
\text { leach }\end{array}$ & $\begin{array}{l}\text { Add sodium } \\
\text { permanganate to } \\
\text { UFP-VSL-T02A to assess } \\
\text { potential for foaming. } \\
\text { UFP-VSL-T02A PJM } \\
\text { velocities match PTF } \\
\text { PJM velocities, and cycle } \\
\text { time is } 1 / 4.5 \text { that of PTF. }\end{array}$ & Not done in TI-062. & Not done in TI-067. & $\begin{array}{l}\text { 1/9/2009 at 01:15. } \\
\text { 1/9/2009 at 08:00. } \\
\text { No samples. } \\
\text { The degree of } \\
\text { foaming assessed } \\
\text { visually with a } \\
\text { borescope inserted } \\
\text { into the vessel. }\end{array}$ & \\
\hline A. 1.32 & $\begin{array}{c}\text { Flush } \\
\text { filter-loop. }\end{array}$ & $\begin{array}{l}\text { Flush slurry in filter-loop } \\
\text { back into } \\
\text { UFP-VSL-T02A at } \\
\text { scale-time. } \\
\text { UFP-VSL-T01A PJM } \\
\text { velocities and cycle times } \\
\text { match PTF PJM } \\
\text { power/volume. }\end{array}$ & Not done in TI-062. & Not done in TI-067. & Per procedure. & None. \\
\hline A.1.33 & $\begin{array}{l}\text { Transfer slurry } \\
\text { from } \\
\text { UFP-VSL- } \\
\text { T02A }\end{array}$ & $\begin{array}{l}\text { Transfer contents of } \\
\text { UFP-VSL-T02A to } \\
\text { HLP-VSL-T27 per }\end{array}$ & Not done in TI-062. & Not done in TI-067. & Per procedure. & None. \\
\hline
\end{tabular}


Table 4.3. Functional Testing Detailed Description

\begin{tabular}{|c|c|c|c|c|c|c|}
\hline $\begin{array}{l}\text { Test } \\
\text { Step }\end{array}$ & Test Step & Description/Purpose & $\begin{array}{c}\text { TI-062 } \\
\text { Time Executed }\end{array}$ & $\begin{array}{c}\text { TI-067 } \\
\text { Time Executed }\end{array}$ & $\begin{array}{c}\text { TI-032 } \\
\text { Time Executed }\end{array}$ & Sampling Requirements \\
\hline & $\begin{array}{c}\text { to } \\
\text { HLP-VSL- } \\
\text { T27. }\end{array}$ & procedure. & & & & \\
\hline A. 1.34 & $\begin{array}{c}\text { Prepare } \\
\text { UFP-VSL- } \\
\text { T01A and } \\
\text { UFP-VSL- } \\
\text { T02A } \\
\text { for Integrated } \\
\text { process testing. }\end{array}$ & $\begin{array}{l}\text { Remove slurry heel from } \\
\text { UFP-VSL-T01A, rinse } \\
\text { and drain. } \\
\text { Remove slurry heel from } \\
\text { UFP-VSL-T02A, rinse } \\
\text { and drain. }\end{array}$ & Not done in TI-062. & Not done in TI-067. & Per procedure. & None. \\
\hline
\end{tabular}





\subsection{Data}

Selected data obtained from the DAS during Shakedown/Functional process testing have been plotted in 12-hr intervals, and this is provided in Appendix J. Tables summarizing the results of sample measurements taken during testing are provided in Section 5.1 (analytical results) and Section 5.2 (physical-property results). The analysis of the analytical data is done in Sections 9, 10, 11, and 12 of the PEP Phase 1 Final Test Report (Kurath et al. 2009). PJM peak average velocity and stroke length, tuned iteratively to target values during testing, are provided in Section 5.3.

\subsection{Analytical Data}

This section provides analytical summary tables for key processes. Reporting limits and uncertainties and special analytical notes are in the final analytical reports submitted by each analytical laboratory. These reports are identified for each sample in Appendix C, which provides a list of all samples collected, the disposition of each sample, and references to final electronic analytical reports.

In the case of the initial simulant characterization, total species concentrations in the original samples slurry have been calculated. In other cases, data are presented in a more raw form: the species concentration in the supernatant, the concentration in the wet centrifuged solids, and the masses of tare sample vial, vial plus total slurry, and vial plus wet centrifuged solids. The latter three masses can be used to calculate the mass fraction of wet centrifuged solids in the slurry, which in turn can be used in calculating the total species concentration in the original sample slurries. (See Mahoney et al. 2009, Section A.5 of Appendix A for more information on calculating the species concentration in the slurry.)

Table 5.1 shows the analytical characterization of the simulant used for all Shakedown/Functional testing. The concentration of key metal analytes, anions, TIC, wt \% UDS, and density are provided. The concentrations of the metals and TIC in the slurry were calculated from samples phase-separated at PDL-W. The liquid analysis results are supernatant analytical results obtained from samples phase-separated in PDL-W. Physical properties (i.e., rheology and PSD) of the initial simulant are included in Section 5.2. Qualitative physical properties (i.e., SEM micrographs and XRD analyses) are included in Appendices L.3 and L.4, respectively. 
Table 5.1. Simulant Characterization

\begin{tabular}{|c|c|c|}
\hline \multirow[b]{2}{*}{ Analyte } & \multicolumn{2}{|c|}{$\begin{array}{l}\text { PEP Shakedown Feed Composition } \\
\text { TI-062 Samples Taken 11/25/2009 }\end{array}$} \\
\hline & $\begin{array}{c}\text { Slurry } \\
\mu \mathrm{g} / \mathrm{g}\end{array}$ & $\begin{array}{l}\text { Liquid Phase } \\
\mu \mathrm{g} / \mathrm{g}\end{array}$ \\
\hline $\mathrm{Al}$ & $20023 \pm 344$ & $2977 \pm 52$ \\
\hline $\mathrm{Ca}$ & $154.5 \pm 4.0$ & $\mathrm{n} / \mathrm{d}$ \\
\hline $\mathrm{Ce}$ & $53.0 \pm 1.1$ & $0.0097 \pm 0.007$ \\
\hline $\mathrm{Cr}$ & $\mathrm{n} / \mathrm{d}$ & $1.32 \pm 0.03$ \\
\hline $\mathrm{Fe}$ & $4736 \pm 94$ & $\mathrm{n} / \mathrm{d}$ \\
\hline $\mathrm{K}$ & $1029 \pm 17$ & $1090 \pm 20$ \\
\hline $\mathrm{La}$ & $40.0 \pm 0.8$ & $0.010 \pm 0.001$ \\
\hline $\mathrm{Mg}$ & $105.1 \pm 2.9$ & $\mathrm{n} / \mathrm{d}$ \\
\hline $\mathrm{Mn}$ & $1027 \pm 20$ & $\mathrm{n} / \mathrm{d}$ \\
\hline $\mathrm{Na}$ & $88920 \pm 1372$ & $91600 \pm 1587$ \\
\hline $\mathrm{Nd}$ & $108.5 \pm 2.1$ & $0.026 \pm 0.001$ \\
\hline $\mathrm{Ni}$ & $142.8 \pm 2.8$ & $\mathrm{n} / \mathrm{d}$ \\
\hline $\mathrm{P}$ & $1784 \pm 28$ & $1873 \pm 32$ \\
\hline $\mathrm{Si}^{(b, \mathrm{~d})}$ & $48.72 \pm 5.7$ & $2.80 \pm 0.36$ \\
\hline $\mathrm{Sr}$ & $43.8 \pm 1.3$ & $\mathrm{n} / \mathrm{d}$ \\
\hline $\mathrm{Zr}$ & $135.7 \pm 2.6$ & $0.737 \pm 0.022$ \\
\hline $\mathrm{Cl}$ & - & $1137 \pm 20$ \\
\hline $\mathrm{NO}_{2}$ & - & $18267 \pm 329$ \\
\hline $\mathrm{NO}_{3}$ & - & $79333 \pm 1380$ \\
\hline $\mathrm{PO}_{4}$ & - & $5547 \pm 96$ \\
\hline $\mathrm{SO}_{4}$ & - & $14167 \pm 247$ \\
\hline $\mathrm{C}_{2} \mathrm{O}_{4}$ & - & $625 \pm 11$ \\
\hline Free $\mathrm{OH}^{-}$ & - & $15239 \pm 660$ \\
\hline $\mathrm{TIC}^{(\mathrm{a})}$ & $1510 \pm 253$ & $6905 \pm 133$ \\
\hline PDMS (mg/g) wet solids & 0.046 & - \\
\hline PDMS (mg/g) liquid & - & $\mathrm{n} / \mathrm{d}^{(\mathrm{c})}$ \\
\hline PPG (mg/g) wet solids & trace & - \\
\hline PPG (mg/g) liquid & - & $\mathrm{n} / \mathrm{d}^{(\mathrm{c})}$ \\
\hline $\mathrm{wt} \%$ UDS & $5.52 \pm 0.03$ & - \\
\hline Slurry Density (g/mL) & $1.278 \pm 0.006$ & - \\
\hline Liquid Density (g/mL) & - & $1.239 \pm 0.001$ \\
\hline $\mathrm{Wt} \% \mathrm{H}_{2} \mathrm{O}$ in liquid & - & $72.7 \pm 0.1$ \\
\hline \multicolumn{3}{|c|}{$\begin{array}{l}\text { (a) TIC = total inorganic carbon. } \\
\text { (b) The AFA slurry sample was phase separated at SwRI with the following information reported } \\
\text { wet centrifuged solids had } 590-\mu \mathrm{g} / \mathrm{g} \mathrm{Si} \text {; percent total solids in the wet centrifuged solids was } \\
56.73 \% \text {; liquid fraction of slurry sample had } 3.07-\mu \mathrm{g} / \mathrm{g} \text { Si. } \\
\text { (c) Single sample non-detect with analysis results below method reporting limit. } \\
\text { (d) Si results from sample named "ICP" rather than sample named "AFA." } \\
\text { - Analysis not required by Test Plan. } \\
\text { trace-A peak in the spectrum was detected, but was below the quantitation limit, which is not } \\
\text { specified in the Dow Corning reports. }\end{array}$} \\
\hline \multicolumn{3}{|c|}{$\begin{array}{l}\text { Bold numbers are calculated values. } \\
\mathrm{n} / \mathrm{d} \text { - Triplicate samples with analysis results below method reporting limit. } \\
\text { Uncertainty values reported are } 1 \sigma \text {, instead of the } 2-\sigma \text { values reported by SwRI. }\end{array}$} \\
\hline
\end{tabular}


Immediately before transferring simulant from the storage tank (HLP-VSL-T22), the tank was mixed, and samples were collected at different elevations. They were analyzed for $\%$ UDS to determine the homogeneity of the feed to Tanks T01A and T01B. The analyses are shown in Table 5.2.

Table 5.2. UDS and Density Analysis of Samples Collected at Multiple Elevations and Recirculation Loop

\begin{tabular}{ccc}
\hline & \multicolumn{2}{c}{$\begin{array}{c}\text { HLP-VSL-T22 Simulant } \\
\text { (Functional Tests) }\end{array}$} \\
\cline { 2 - 3 } Depth & $\begin{array}{c}\text { Slurry } \\
\text { wt\% UDS }\end{array}$ & $\begin{array}{c}\text { Liquid Density } \\
\mathrm{g} / \mathrm{mL}\end{array}$ \\
\hline 47 inches & 5.29 & 1.198 \\
80 inches & 5.30 & 1.207 \\
112 inches & 5.27 & 1.191 \\
\hline & 5.65 & 1.195 \\
Recirculation Loop & 5.47 & 1.198 \\
& 5.43 & 1.202 \\
\hline
\end{tabular}

The reagents used for Shakedown/Functional testing were sampled and analyzed. The analyses are shown in Table 5.3. Not shown in the table are the impromptu 19-M NaOH samples taken throughout testing. The real time results for those "quick result" samples were molarities: 19.27, 19.22, 18.8, 18.6, 19.12, and 18.95 .

Table 5.3. Reagent Raw Material Analysis

\begin{tabular}{cc|cc}
\hline & & \multicolumn{2}{|c}{ Reagents } \\
\hline Measurement & $\mathrm{Units}$ & $19-\mathrm{M} \mathrm{NaOH}$ & $\mathrm{NaMnO}_{4}$ \\
\hline Anion & $\mathrm{M}$ & 18.7 & 0.92 \\
Density & $\mathrm{g} / \mathrm{mL}$ & 1.522 & 1.085 \\
$\mathrm{C}_{2} \mathrm{O}_{4}$ & $\mu \mathrm{g} / \mathrm{g}$ & $<19.5$ & $<19.4$ \\
$\mathrm{NO}_{3}$ & $\mu \mathrm{g} / \mathrm{g}$ & $<86.4$ & $<85.8$ \\
$\mathrm{NO}_{2}$ & $\mu \mathrm{g} / \mathrm{g}$ & $<64.2$ & $<63.7$ \\
$\mathrm{SO}_{4}$ & $\mu \mathrm{g} / \mathrm{g}$ & $<19.5$ & 841 \\
$\mathrm{PO}_{4}$ & $\mu \mathrm{g} / \mathrm{g}$ & $<59.7$ & $<59.3$ \\
$\mathrm{Al}$ & $\mu \mathrm{g} / \mathrm{g}$ & $<9.60$ & $<4.60$ \\
$\mathrm{Ca}$ & $\mu \mathrm{g} / \mathrm{g}$ & $<4.70$ & $<4.60$ \\
$\mathrm{Cr}$ & $\mu \mathrm{g} / \mathrm{g}$ & $<0.960$ & 1.17 \\
$\mathrm{Fe}$ & $\mu \mathrm{g} / \mathrm{g}$ & $<9.60$ & $<4.60$ \\
$\mathrm{Mg}$ & $\mu \mathrm{g} / \mathrm{g}$ & $<4.70$ & $<4.60$ \\
$\mathrm{Mn}$ & $\mu \mathrm{g} / \mathrm{g}$ & $<0.960$ & 46800 \\
$\mathrm{Na}$ & $\mu \mathrm{g} / \mathrm{g}$ & 285000 & 19900 \\
$\mathrm{Nd}$ & $\mu \mathrm{g} / \mathrm{g}$ & $<0.0188$ & $<0.0460$ \\
$\mathrm{Sr}$ & $\mu \mathrm{g} / \mathrm{g}$ & $<0.960$ & $<0.460$ \\
$\mathrm{Cl}$ & $\mu \mathrm{g} / \mathrm{g}$ & $<19.5$ & 21.2 \\
\hline $\mathrm{M} \mathrm{Molarity.}$ & & & \\
\hline
\end{tabular}

Table 5.4 shows data that contributes to the understanding of homogeneity during the transfer from samples taken at different points during the transfers. A transfer of approximately 593 gallons from HLP-VSL-T22 to Tank T01B was also conducted, but samples were not taken. 
Table 5.4. Simulant Analyses During Transfers

\begin{tabular}{|c|c|c|c|}
\hline TI Step & Process Condition & Slurry UDS (Wt $\%)$ & Liquid Density $(\mathrm{g} / \mathrm{mL})$ \\
\hline \multirow{2}{*}{ 7.2.1.2 Transfer:HLP-VSL-T22 to Tank T01A } & $30 \mathrm{sec}$ & 5.35 & 1.223 \\
\hline & $150 \mathrm{gal}$ & 5.42 & 1.228 \\
\hline \multirow{2}{*}{ Total $=593$ gallons $^{(\mathrm{a})}$} & $300 \mathrm{gal}$ & 5.38 & 1.215 \\
\hline & $500 \mathrm{gal}$ & 5.57 & 1.224 \\
\hline \multirow{2}{*}{ 7.2.4.4 Transfer: Tank T01A to Tank T02A } & $30 \mathrm{sec}$. & 5.33 & 1.220 \\
\hline & $75 \mathrm{gal}$ & 5.37 & 1.218 \\
\hline \multirow{2}{*}{ Total $=324$ gallons $^{(\mathrm{a})}$} & $150 \mathrm{gal}$ & 5.37 & 1.211 \\
\hline & $250 \mathrm{gal}$ & 5.33 & 1.219 \\
\hline 7.5.2 Transfer:HLP-VSL-T22 to Tank T01A & $30 \mathrm{sec}$ & 6.14 & 1.225 \\
\hline Total $=326$ gallons ${ }^{(\mathrm{a})}$ & $200 \mathrm{gal}$ & 5.44 & 1.226 \\
\hline
\end{tabular}

(a) Total transfer volume from TI-062 Operational process sheet.

The plan for WTP operations currently includes a line flush after extended operating periods for $\mathrm{H}_{2}$ control. Table 5.5 shows the results of flushing a line after transferring simulant to Tank T01B. The flush was conducted at the maximum achievable rate in PEP, $20 \mathrm{gpm}$, which was about $70 \%$ of the scaled prototypic rate of 28 gpm planned for WTP.

Table 5.5. PEP Flush Line Between HLP-VSL-T22 and Tank T01B

\begin{tabular}{|c|c|c|c|c|c|c|c|c|}
\hline \multicolumn{2}{|c|}{ Slurry } & \multicolumn{7}{|c|}{ Liquid Analyses } \\
\hline \multirow{3}{*}{ Flush } & \multirow[b]{2}{*}{ UDS } & \multirow[b]{2}{*}{ Liquid Density } & \multirow{2}{*}{$\begin{array}{l}\text { Free } \\
\mathrm{OH}^{-(\mathrm{a})}\end{array}$} & \multicolumn{3}{|c|}{ (tracer) } & \multirow{3}{*}{$\begin{array}{c}\mathrm{PO}_{4}^{3-(\mathrm{b})} \\
\mu \mathrm{g} / \mathrm{g} \\
{[\mathrm{M}]}\end{array}$} & \multirow{3}{*}{$\begin{array}{c}\mathrm{SO}_{4}{ }^{2-(b)} \\
\mu \mathrm{g} / \mathrm{g} \\
{[\mathrm{M}]}\end{array}$} \\
\hline & & & & $\mathrm{NO}_{3}^{-(b)}$ & $\mathrm{Cl}-$ & $\mathrm{NO}_{2}^{-(\mathrm{b})}$ & & \\
\hline & $\mathrm{Wt} \%$ & $\mathrm{~g} / \mathrm{mL}$ & $\mathrm{M}$ & $\begin{array}{c}\mu \mathrm{g} / \mathrm{g} \\
{[\mathrm{M}]}\end{array}$ & $\mu \mathrm{g} / \mathrm{g}$ & $\begin{array}{c}\mu \mathrm{g} / \mathrm{g} \\
{[\mathrm{M}]}\end{array}$ & & \\
\hline$\# 1$ & 5.45 & 1.204 & $<0.04$ & $\begin{array}{c}1850 \\
{[0.039]}\end{array}$ & 35.5 & $\begin{array}{c}457 \\
{[0.034]^{(\mathrm{c})}}\end{array}$ & $\begin{array}{c}127 \\
{[<0.01]}\end{array}$ & $\begin{array}{c}356 \\
{[0.004]^{(\mathrm{c})}}\end{array}$ \\
\hline$\# 2$ & 0.075 & 0.966 & $<0.04$ & $\begin{array}{c}773 \\
{[0.024]}\end{array}$ & $<19.9$ & $\begin{array}{c}210 \\
{[0.047]^{(c)}}\end{array}$ & $\begin{array}{c}63 \\
{[<0.01]}\end{array}$ & $\begin{array}{c}146 \\
{[0.002]^{(\mathrm{c})}}\end{array}$ \\
\hline \#3 & 0.052 & 0.974 & $<0.04$ & $\begin{array}{c}582 \\
{[0.019]^{(\mathrm{c})}}\end{array}$ & $<19.9$ & $\begin{array}{c}165 \\
{[<0.03]}\end{array}$ & $\begin{array}{l}<61.0 \\
{[<0.01]}\end{array}$ & $\begin{array}{c}106 \\
{[0.002]^{(\mathrm{c})}}\end{array}$ \\
\hline \#4 & 0.019 & 0.970 & $<0.04$ & $\begin{array}{c}379 \\
{[0.017]^{(\mathrm{c})}}\end{array}$ & $<19.0$ & $\begin{array}{c}112 \\
{[0.043]^{(\mathrm{c})}}\end{array}$ & $\begin{array}{l}<58.2 \\
{[<0.01]}\end{array}$ & $\begin{array}{c}64.3 \\
{[0.002]^{(\mathrm{c})}}\end{array}$ \\
\hline$\# 5$ & 0.008 & 0.987 & $<0.04$ & $\begin{array}{c}265 \\
{[0.015]^{(\mathrm{c})}}\end{array}$ & $<19.0$ & $\begin{array}{c}87.4 \\
{[0.038]^{(\mathrm{c})}}\end{array}$ & $\begin{array}{c}<61.1 \\
{[<0.01]}\end{array}$ & $\begin{array}{c}41.4 \\
{[<0.001]}\end{array}$ \\
\hline \multicolumn{9}{|c|}{ (a) Raman. } \\
\hline \multicolumn{9}{|c|}{ (b) IC and Raman. Raman shown in brackets [ ]. } \\
\hline \multicolumn{9}{|c|}{ (c) Detected result is qualitative. Result $>$ IDL but $<$ EQL (estimated quantitation limit). } \\
\hline \multicolumn{9}{|l|}{ M } \\
\hline
\end{tabular}

Figure 5.1 shows the nitrate, free hydroxide, \%UDS, and density concentrations plotted as a function of line volumes of flush water. ${ }^{\text {(a) }}$

(a) One line volume is the volume contained between the HLP-T22 pump and the Tank T01B vessel. In this case, one line volume is approximately 6 gallons. 


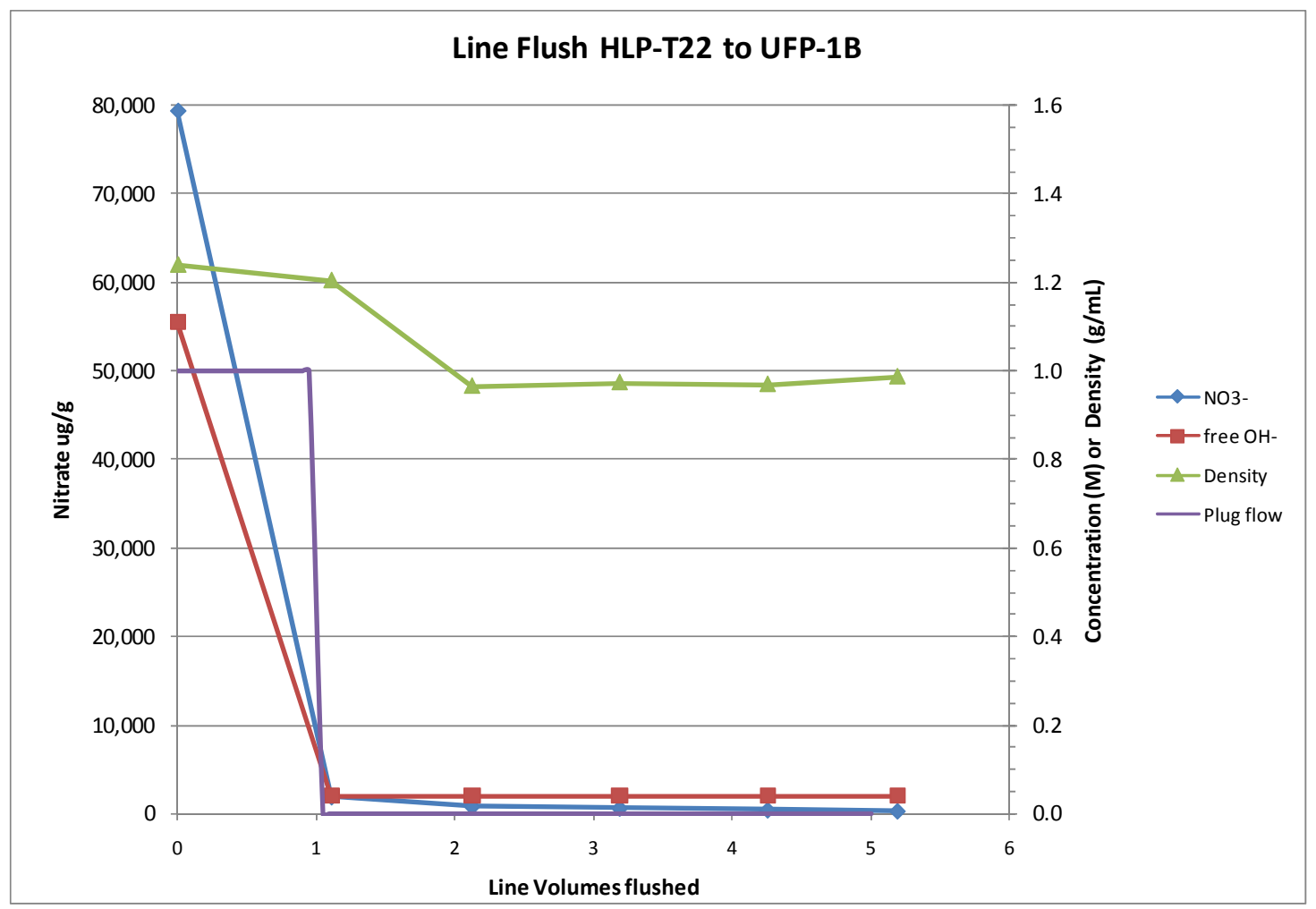

Figure 5.1. Simulant Line Flush

Table 5.6 and Table 5.7 show UDS results and aluminum concentration in the wet centrifuged solids for samples collected during the stratification tests in TI-062 (Tank T01A) and TI-032 (Tank T01B), respectively. PSD data from the stratification tests are in Table 5.17 and Table 5.18 in Section 5.2, Physical Properties Data. The wt $\%$ of wet centrifuged solids in the slurry can be used in calculating the total species concentration in the original slurry samples. See Section A.5 in Appendix A of Mahoney et al. (2009) for more information on calculating the species concentration in the slurry. An aliquot of the wet centrifuged solids was dried at $105^{\circ} \mathrm{C}$ to determine the percent solids that are reported with the reported analytes in the wet centrifuged solids. 
Table 5.6. TI-062 Stratification Test

\begin{tabular}{|c|c|c|c|}
\hline \multirow[b]{2}{*}{ Process Conditions } & UDS & Wet Centrifuged & Solids Analyses \\
\hline & $\mathrm{Wt} \%$ & \multicolumn{2}{|c|}{$\mathrm{Al}$} \\
\hline \multicolumn{4}{|c|}{$\begin{array}{l}12 \mathrm{hr} \text { after initial slurry transfer. PJMs incorrect velocity } \\
\text { (TI-062 7.2.5.8 } 11 / 23 / 2008)\end{array}$} \\
\hline \multirow{5}{*}{ Low CD sampling location } & 5.44 & - & - \\
\hline & 5.35 & - & - \\
\hline & 5.37 & - & - \\
\hline & 5.33 & - & - \\
\hline & & 121000 & $\mu \mathrm{g} / \mathrm{g}$ \\
\hline $\mathrm{Wt} \%$ centrifuged solids & - & 13.8 & $\mathrm{wt} \%$ \\
\hline$\%$ solids in centrifuged sol ids & - & 56.26 & $\mathrm{wt} \%$ \\
\hline \multirow{4}{*}{ Middle CD sampling location } & 5.46 & - & - \\
\hline & 5.38 & - & - \\
\hline & 5.38 & - & - \\
\hline & 5.39 & - & - \\
\hline \multirow{5}{*}{ High CD sampling location } & 5.08 & - & - \\
\hline & 5.39 & - & - \\
\hline & 5.41 & - & - \\
\hline & 5.37 & - & - \\
\hline & - & 121000 & $\mu \mathrm{g} / \mathrm{g}$ \\
\hline \multirow{2}{*}{$\begin{array}{l}\mathrm{Wt} \% \text { centrifuged solids } \\
\% \text { solids in centrifuged solids }\end{array}$} & - & 13.9 & $\mathrm{wt} \%$ \\
\hline & - & 56.67 & $\mathrm{wt} \%$ \\
\hline \multicolumn{4}{|c|}{$\begin{array}{l}2 \mathrm{hr} \text { after PJMs retuned, restarted Stratification test 7.2.5.8 } \\
\text { (restart } 11 / 24 / 2008-11 / 26 / 2008)\end{array}$} \\
\hline \multirow{5}{*}{ Low CD sampling location } & 5.39 & - & - \\
\hline & 5.43 & - & - \\
\hline & 5.45 & - & - \\
\hline & 5.40 & - & - \\
\hline & - & 122000 & $\mu \mathrm{g} / \mathrm{g}$ \\
\hline $\mathrm{Wt} \%$ centrifuged solids & - & 13.9 & $\mathrm{wt} \%$ \\
\hline$\%$ solids in centrifuged solids & - & 55.99 & $\mathrm{wt} \%$ \\
\hline \multirow{4}{*}{ Middle CD sampling location } & 5.42 & - & - \\
\hline & 5.40 & - & - \\
\hline & 5.39 & - & - \\
\hline & 5.39 & - & - \\
\hline \multirow{5}{*}{ High CD sampling location } & 5.43 & - & - \\
\hline & 5.43 & - & - \\
\hline & 5.44 & - & - \\
\hline & 5.40 & - & - \\
\hline & - & 119000 & $\mu \mathrm{g} / \mathrm{g}$ \\
\hline \multirow{2}{*}{$\begin{array}{l}\mathrm{Wt} \% \text { centrifuged solids } \\
\% \text { solids in centrifuged solids }\end{array}$} & - & 14.1 & $\mathrm{wt} \%$ \\
\hline & - & 56.32 & $\mathrm{wt} \%$ \\
\hline \multicolumn{4}{|c|}{$24 \mathrm{hr}$ after restart (7.2.5. } \\
\hline \multirow[t]{2}{*}{ Low CD sampling location } & 5.38 & - & - \\
\hline & 5.44 & - & - \\
\hline
\end{tabular}


Table 5.6. TI-062 Stratification Test

\begin{tabular}{|c|c|c|c|}
\hline \multirow{4}{*}{ Process Conditions } & \multirow{3}{*}{$\begin{array}{c}\text { UDS } \\
\mathrm{Wt} \% \\
5.40\end{array}$} & \multicolumn{2}{|c|}{$\begin{array}{l}\text { Wet Centrifuged } \\
\text { Solids Analyses }\end{array}$} \\
\hline & & \multicolumn{2}{|c|}{$\mathrm{Al}$} \\
\hline & & - & - \\
\hline & 5.39 & - & - \\
\hline \multirow{4}{*}{ Middle CD sampling location } & 5.36 & - & - \\
\hline & 5.39 & - & - \\
\hline & 5.38 & - & - \\
\hline & 5.35 & - & - \\
\hline \multirow{4}{*}{ High CD sampling location } & 5.33 & - & - \\
\hline & 5.36 & - & - \\
\hline & 5.39 & - & - \\
\hline & 5.38 & - & - \\
\hline \multicolumn{4}{|c|}{$36 \mathrm{hr}$ after restart (7.2.5.8) } \\
\hline \multirow{4}{*}{ Low CD sampling location } & 5.42 & - & - \\
\hline & 5.41 & - & - \\
\hline & 5.43 & - & - \\
\hline & 5.39 & - & - \\
\hline \multirow{4}{*}{ Middle CD sampling location } & 5.50 & - & - \\
\hline & 5.48 & - & - \\
\hline & 5.41 & - & - \\
\hline & 5.40 & - & - \\
\hline \multirow{4}{*}{ High CD sampling location } & 5.44 & - & - \\
\hline & 5.40 & - & - \\
\hline & 5.38 & - & - \\
\hline & 5.40 & - & - \\
\hline \multicolumn{4}{|c|}{$\sim 1 \mathrm{hr}$ before transfer from Tank T01A to Tank T02A (7.2.11.6.1) } \\
\hline Low CD sampling location & - & 124000 & $\mu \mathrm{g} / \mathrm{g}$ \\
\hline $\mathrm{Wt} \%$ centrifuged solids & - & 14.1 & $\mathrm{wt} \%$ \\
\hline$\%$ solids in centrifuged solids & - & 56.86 & $\mathrm{wt} \%$ \\
\hline High CD sampling location & - & 123000 & $\mu \mathrm{g} / \mathrm{g}$ \\
\hline $\mathrm{Wt} \%$ centrifuged solids & - & 14.3 & $\mathrm{wt} \%$ \\
\hline$\%$ solids in centrifuged solids & - & 56.91 & $\mathrm{wt} \%$ \\
\hline
\end{tabular}


Table 5.7. TI-032 Stratification Test

\begin{tabular}{|c|c|c|c|}
\hline Process Conditions & $\begin{array}{l}\text { UDS } \\
\mathrm{Wt} \%\end{array}$ & \multicolumn{2}{|c|}{$\begin{array}{l}\text { Wet Centrifuged } \\
\text { Solids Analyses }\end{array}$} \\
\hline \multicolumn{4}{|c|}{$12 \mathrm{hr}$ after slurry transferred (7.2.4.8) } \\
\hline \multirow{5}{*}{ Low CD sampling location } & 5.30 & - & - \\
\hline & 5.30 & - & - \\
\hline & 5.33 & - & - \\
\hline & 5.32 & - & - \\
\hline & - & 115000 & $\mu \mathrm{g} / \mathrm{g}$ \\
\hline \multirow{2}{*}{$\begin{array}{l}\mathrm{Wt} \% \text { centrifuged solids } \\
\% \text { solids in centrifuged solids }\end{array}$} & - & 14.2 & $w t \%$ \\
\hline & - & 55.60 & $\mathrm{wt} \%$ \\
\hline \multirow{4}{*}{ Middle CD sampling location } & 5.32 & - & - \\
\hline & 5.37 & - & - \\
\hline & 5.34 & - & - \\
\hline & 5.36 & - & - \\
\hline \multirow{5}{*}{ High CD sampling location } & 5.33 & - & - \\
\hline & 5.30 & - & - \\
\hline & 5.25 & - & - \\
\hline & 5.28 & - & - \\
\hline & - & 118000 & $\mu \mathrm{g} / \mathrm{g}$ \\
\hline $\mathrm{Wt} \%$ centrifuged solids & - & 14.2 & $\mathrm{wt} \%$ \\
\hline$\%$ solids in centrifuged solids & - & 55.09 & $\mathrm{wt} \%$ \\
\hline \multicolumn{4}{|c|}{$24 \mathrm{hr}$ after slurry transferred } \\
\hline \multirow{4}{*}{ Low CD sampling location } & 5.33 & - & - \\
\hline & 5.34 & - & - \\
\hline & 5.33 & - & - \\
\hline & 5.32 & - & - \\
\hline \multirow{4}{*}{ Medium CD sampling location } & 5.31 & - & - \\
\hline & 5.27 & - & - \\
\hline & 5.26 & - & - \\
\hline & 5.30 & - & - \\
\hline \multirow{4}{*}{ High CD sampling location } & 5.28 & - & - \\
\hline & 5.30 & - & - \\
\hline & 5.28 & - & - \\
\hline & 5.32 & - & - \\
\hline \multicolumn{4}{|c|}{$36 \mathrm{hr}$ after slurry transferred } \\
\hline \multirow{4}{*}{ Low CD sampling location } & 5.37 & - & - \\
\hline & 5.37 & - & - \\
\hline & 5.34 & - & - \\
\hline & 5.36 & - & - \\
\hline \multirow{4}{*}{ Medium CD sampling location } & 5.36 & - & - \\
\hline & 5.36 & - & - \\
\hline & 5.33 & - & - \\
\hline & 5.35 & - & - \\
\hline \multirow[t]{2}{*}{ High CD sampling location } & 5.42 & - & - \\
\hline & 5.36 & - & - \\
\hline
\end{tabular}


Table 5.7. TI-032 Stratification Test

\begin{tabular}{|c|c|c|c|}
\hline \multirow[t]{3}{*}{ Process Conditions } & \multirow{2}{*}{$\begin{array}{l}\text { UDS } \\
\mathrm{Wt} \% \\
5.29\end{array}$} & \multicolumn{2}{|c|}{$\begin{array}{c}\text { Wet Centrifuged } \\
\text { Solids Analyses } \\
\text { Al }\end{array}$} \\
\hline & & - & - \\
\hline & 5.35 & - & - \\
\hline \multicolumn{4}{|c|}{1 hour before transfer to Tank T02A $(7.2 .10 .6 .101 / 01 / 2009)$} \\
\hline Low CD sampling location & - & 113000 & $\mu \mathrm{g} / \mathrm{g}$ \\
\hline $\mathrm{Wt} \%$ centrifuged solids & - & 14.5 & $\mathrm{wt} \%$ \\
\hline$\%$ solids in centrifuged solids & - & 55.94 & $\mathrm{wt} \%$ \\
\hline High CD sampling location & - & 113000 & $\mu \mathrm{g} / \mathrm{g}$ \\
\hline $\mathrm{Wt} \%$ centrifuged solids & - & 14.4 & $\mathrm{wt} \%$ \\
\hline$\%$ solids in centrifuged solids & - & 55.94 & $\mathrm{wt} \%$ \\
\hline $\begin{array}{l}\text { - Analysis not required by Test Plan. } \\
\text { Bold numbers are calculated values. }\end{array}$ & & & \\
\hline
\end{tabular}

During TI-062, multiple (30) samples were collected from the in-line and the middle-low CD sampling port in Tank T02A to monitor low-solids filtration during the initial low-solids filter flux loading. Data from these samples are presented in Table 5.8. These data were used to determine the baseline sampling variance and the preferred sampling location (from tank or from filter-loop) to represent process conditions during ultrafiltration processing. 
Table 5.8. Low-Solids Filter Test—Establish Baseline Sampling Variation

\begin{tabular}{|c|c|c|c|c|c|}
\hline \multirow[b]{2}{*}{ Elapsed Process Time: } & \multicolumn{2}{|c|}{$\begin{array}{c}\text { In-Tank Samples Middle- } \\
\text { Middle CD Port }\end{array}$} & \multicolumn{3}{|c|}{ Filter-Loop Samples } \\
\hline & $\begin{array}{l}\mathrm{UDS} \\
\mathrm{Wt} \%\end{array}$ & $\begin{array}{c}\text { Slurry } \\
\text { Density } \\
\text { g/mL }\end{array}$ & $\begin{array}{l}\text { UDS } \\
\mathrm{Wt} \%\end{array}$ & $\begin{array}{c}\text { Slurry } \\
\text { Density } \\
\mathrm{g} / \mathrm{mL}\end{array}$ & $\begin{array}{c}\text { Liquid } \\
\text { Density } \\
\mathrm{g} / \mathrm{mL}\end{array}$ \\
\hline \multirow{6}{*}{$0 \mathrm{hr}$} & - & - & 7.25 & - & 1.174 \\
\hline & - & - & 7.18 & - & 1.171 \\
\hline & - & - & 7.32 & - & 1.157 \\
\hline & - & - & - & 1.269 & - \\
\hline & - & - & - & 1.275 & - \\
\hline & - & - & - & 1.267 & - \\
\hline \multirow{6}{*}{$1 \mathrm{hr}$} & 6.83 & 1.228 & 7.08 & 1.249 & 1.154 \\
\hline & 6.80 & 1.234 & 6.99 & 1.247 & 1.184 \\
\hline & 6.81 & 1.244 & 6.98 & 1.243 & 1.187 \\
\hline & 6.85 & 1.252 & 6.89 & 1.237 & 1.185 \\
\hline & 6.80 & 1.259 & 6.95 & 1.247 & 1.192 \\
\hline & 6.80 & 1.257 & 6.86 & 1.250 & 1.195 \\
\hline \multirow{6}{*}{$2 \mathrm{hr}$} & 6.85 & 1.260 & 7.16 & 1.258 & 1.186 \\
\hline & 6.87 & 1.248 & 7.12 & 1.255 & 1.194 \\
\hline & 6.84 & 1.257 & 6.96 & 1.252 & 1.190 \\
\hline & 6.83 & 1.237 & 6.82 & 1.251 & 1.194 \\
\hline & 6.82 & 1.241 & 6.76 & 1.248 & 1.193 \\
\hline & 6.79 & 1.246 & 6.79 & 1.256 & 1.196 \\
\hline \multirow{6}{*}{$3 \mathrm{hr}^{(\mathrm{a})}$} & 6.79 & 1.241 & 7.07 & 1.247 & 1.188 \\
\hline & 6.78 & 1.245 & 6.90 & 1.233 & 1.163 \\
\hline & 6.81 & 1.250 & 6.90 & 1.243 & 1.170 \\
\hline & 6.81 & 1.259 & 6.93 & 1.230 & 1.155 \\
\hline & 6.80 & 1.266 & 6.81 & 1.230 & 1.154 \\
\hline & 6.78 & 1.267 & 6.61 & 1.230 & 1.161 \\
\hline \multirow{6}{*}{$4 \mathrm{hr}^{(\mathrm{a})}$} & 6.80 & 1.249 & 6.45 & 1.245 & 1.152 \\
\hline & 6.78 & 1.260 & 6.85 & 1.238 & 1.156 \\
\hline & 6.80 & 1.259 & 7.04 & 1.247 & 1.160 \\
\hline & 7.13 & 1.250 & 7.00 & 1.240 & 1.155 \\
\hline & 6.80 & 1.251 & 7.02 & 1.235 & 1.159 \\
\hline & 6.80 & 1.251 & 7.01 & 1.248 & 1.173 \\
\hline \multirow{6}{*}{$5 \mathrm{hr}^{(\mathrm{a})}$} & 6.84 & 1.256 & 6.91 & 1.240 & 1.168 \\
\hline & 6.80 & 1.258 & 6.79 & 1.250 & 1.182 \\
\hline & 6.79 & 1.261 & 6.91 & 1.236 & 1.159 \\
\hline & 6.96 & 1.257 & 7.13 & 1.252 & 1.176 \\
\hline & 6.79 & 1.250 & 7.03 & 1.228 & 1.153 \\
\hline & 6.77 & 1.253 & 6.98 & 1.231 & 1.161 \\
\hline
\end{tabular}

- Analysis not required by Test Plan.

(a) Tank sampled from middle-low port. 
Based on the variance of the sampling, it was determined immediately after execution of TI-062 that the in-tank location demonstrated less sample variance and was the preferred location to collect representative process samples. The sampling variance test was not repeated during TI-032.

After the filters were conditioned during Shakedown/Functional testing, the filter-loop was reconfigured to remove permeate using only filter \#1, and the level in Tank 02A was reduced to achieve approximately 20 -wt $\%$ UDS. Then the filters were reconfigured again to filter through filters \#2 to \#5, and filtration was continued adding simulant from Tank T01A while on constant level control. This filtration was continued to achieve a target 20 -wt\% UDS with the higher level. (Refer to narrative Section 4 of this report). Table 5.9 and show the solids analyses for samples taken during backpulsing and the two subsequent concentration steps for TI-062 and TI-032, respectively.

Rheology data and PSD data are in Section 5.2. Table 5.9 shows that the second concentration step did not reach the $\sim 20$-wt $\%$ UDS target. The samples for the first concentration step (\#1 filter only) were not analyzed for TI-062.

Table 5.9. TI-062 Low-Solids Filter Test, $36-\mathrm{Hr}$ Conditioning and Concentration to 20 -wt $\%$

\begin{tabular}{|c|c|c|c|}
\hline \multirow[b]{2}{*}{ Process Condition: } & \multicolumn{3}{|c|}{ In-Line Filter-Loop Samples } \\
\hline & $\begin{array}{l}\text { UDS } \\
\mathrm{Wt} \%\end{array}$ & $\begin{array}{l}\text { Slurry } \\
\text { Density } \\
\mathrm{g} / \mathrm{mL}\end{array}$ & $\begin{array}{c}\text { Liquid } \\
\text { Density } \\
\mathrm{g} / \mathrm{mL}\end{array}$ \\
\hline After $24^{\text {th }}$ backpulse & 6.57 & 1.268 & 1.210 \\
\hline \multirow{3}{*}{$\begin{array}{l}\text { End of low-solids filter } \\
\text { flux test }\end{array}$} & 6.96 & 1.262 & 1.214 \\
\hline & 6.85 & 1.261 & 1.210 \\
\hline & 6.86 & 1.271 & 1.209 \\
\hline \multicolumn{4}{|l|}{$\begin{array}{l}\text { First concentration } \\
\text { complete }^{(a)}\end{array}$} \\
\hline \multirow{3}{*}{$\begin{array}{l}\text { Second solids conc. } \\
\text { (dewater) to } 20 \text {-wt } \% \\
\text { UDS }\end{array}$} & 16.81 & 1.372 & 1.209 \\
\hline & 16.72 & 1.372 & 1.220 \\
\hline & 16.79 & 1.371 & 1.221 \\
\hline
\end{tabular}

Table 5.10 includes the analytical data from the middle-low location in Tank 02A during 36 hours of filter condition and the subsequent dewatering step to prepare for caustic addition. Chemical composition at the end of the filter conditioning from the same location is included in Table 5.11, and physical properties data from the filter conditioning is in Section 5.2. 
Table 5.10. TI-032 Low-Solids Filter Test, $36-\mathrm{Hr}$ Conditioning and Concentration to $20-\mathrm{wt} \%$

\begin{tabular}{|c|c|c|c|}
\hline Process Conditions & $\begin{array}{l}\mathrm{UDS} \\
\mathrm{Wt} \%\end{array}$ & $\begin{array}{c}\text { Slurry } \\
\text { Density } \\
\mathrm{g} / \mathrm{mL}\end{array}$ & $\begin{array}{c}\text { Liquid } \\
\text { Density } \\
\mathrm{g} / \mathrm{mL}\end{array}$ \\
\hline \multirow{3}{*}{$\begin{array}{l}1^{\text {st }} 12 \text {-hr filter conditioning. } \\
\text { No backpulsing }\end{array}$} & 6.82 & 1.287 & 1.192 \\
\hline & 6.82 & 1.283 & 1.176 \\
\hline & 6.81 & 1.283 & 1.195 \\
\hline $\begin{array}{l}\text { Near completion of } 2^{\text {nd }} \\
\text { 12-hr filter conditioning } \\
\text { (backpulsing) }\end{array}$ & 6.92 & 1.287 & 1.222 \\
\hline \multirow{3}{*}{$\begin{array}{l}\text { Near completion of } 3^{\text {rd }} \\
\text { 12-hr filter conditioning }\end{array}$} & 6.92 & 1.299 & 1.245 \\
\hline & 6.96 & 1.289 & 1.221 \\
\hline & 6.92 & 1.97 & 1.241 \\
\hline \multirow{3}{*}{$\begin{array}{l}\text { Concentrate solids in Tank } \\
\text { T02A }\left(1^{\text {st }} \text { conc. complete }\right)\end{array}$} & 15.10 & 1.353 & 1.216 \\
\hline & 15.10 & 1.333 & 1.226 \\
\hline & 15.10 & 1.336 & 1.237 \\
\hline $\begin{array}{l}\text { Concentrate slurry to } \\
20 \text {-wt } \% \text { UDS: Start of } 2^{\text {nd }} \\
\text { concentration }\end{array}$ & 10.90 & 1.318 & 1.235 \\
\hline \multirow{3}{*}{$\begin{array}{l}\text { Concentrate slurry to } \\
20 \text {-wt } \% \text { UDS: end of } 2^{\text {nd }} \\
\text { concentration }\end{array}$} & 18.50 & 1.371 & 1.234 \\
\hline & 18.60 & 1.379 & 1.130 \\
\hline & 18.60 & 1.375 & 1.231 \\
\hline
\end{tabular}

Table 5.11 shows the analytical characterization of the simulant before adding $\mathrm{NaOH}$ for the caustic-leach. The UDS and density analyses were performed on the intact slurry. The wt $\%$ of wet centrifuged solids in the slurry can be used in calculating the total species concentration in the original samples slurry. See Section A.5 in Appendix A of Mahoney et al. (2009) for more information on calculating the species concentration in the slurry. An aliquot of the wet centrifuged solids was dried at $105^{\circ} \mathrm{C}$ to determine the percent solids that are reported with the reported analytes in the wet centrifuged solids. 
Table 5.11. Pre-Caustic-Leach Simulant Characterization

\begin{tabular}{|c|c|c|c|c|c|c|}
\hline \multirow[b]{2}{*}{ Analyte } & \multicolumn{2}{|c|}{$\begin{array}{c}\text { Tank T01A Caustic-Leach } \\
\text { Pre-NaOH Addition } \\
\text { TI-032 12/20/2008 - } \\
12 / 21 / 2008\end{array}$} & \multicolumn{2}{|c|}{$\begin{array}{c}\text { Tank T02A Caustic-Leach } \\
\text { Pre-NaOH Addition } \\
\\
\text { TI-032 01/02/2009-01/03/2009 }\end{array}$} & \multicolumn{2}{|c|}{$\begin{array}{l}\text { Tank T01A Caustic-Leach } \\
\text { Pre-NaOH Addition } \\
\text { TI-067 12/2009/2008 - 12/10/2008 }\end{array}$} \\
\hline & $\begin{array}{c}\text { Wet } \\
\text { Centrifuged } \\
\text { Solids } \\
\mu \mathrm{g} / \mathrm{g}\end{array}$ & $\begin{array}{c}\text { Liquid Phase } \\
\text { (Raman) } \\
\mu \mathrm{g} / \mathrm{g}\end{array}$ & $\begin{array}{l}\text { Wet Centrifuged } \\
\text { Solids } \\
\mu \mathrm{g} / \mathrm{g}\end{array}$ & $\begin{array}{l}\text { Liquid Phase } \\
\text { (Raman) } \\
\mu \mathrm{g} / \mathrm{g}\end{array}$ & $\begin{array}{c}\text { Wet } \\
\text { Centrifuged } \\
\text { Solids } \\
\mu \mathrm{g} / \mathrm{g}\end{array}$ & $\begin{array}{l}\text { Liquid Phase } \\
\text { (Raman) } \\
\mu \mathrm{g} / \mathrm{g}\end{array}$ \\
\hline $\mathrm{Al}$ & 115000 & 3735 & 136000 & 3924 & 124000 & 2920 \\
\hline $\mathrm{Ca}$ & 1023 & - & 1260 & - & 1010 & - \\
\hline $\mathrm{Ce}$ & 381 & - & 504 & - & 422 & - \\
\hline $\mathrm{Cr}$ & 45 & - & 63 & - & $<47.9$ & - \\
\hline $\mathrm{Fe}$ & 32933 & - & 42633 & - & 34500 & - \\
\hline $\mathrm{K}$ & 660 & - & 604 & - & 525 & - \\
\hline $\mathrm{La}$ & 289 & - & 381 & - & 318 & - \\
\hline $\mathrm{Mg}$ & 700 & - & 864 & - & 695 & - \\
\hline $\mathrm{Mn}$ & 7143 & - & 8873 & - & 7390 & - \\
\hline $\mathrm{Na}$ & 71567 & - & 68467 & - & 63100 & - \\
\hline $\mathrm{Nd}$ & 776 & - & 998 & - & 855 & - \\
\hline $\mathrm{Ni}$ & 956 & - & 1317 & - & 1030 & - \\
\hline $\mathrm{P}$ & 1350 & - & 1313 & - & 1070 & - \\
\hline $\mathrm{Si}$ & 410 & - & 793 & - & $<384$ & - \\
\hline $\mathrm{Sr}$ & 284 & - & 371 & - & 322 & - \\
\hline $\mathrm{Zr}$ & 921 & - & 1247 & - & 958 & - \\
\hline $\mathrm{Cl}$ & - & - & - & - & - & - \\
\hline $\mathrm{NO}_{2}$ & - & 18322 & - & 19265 & - & 18323 \\
\hline $\mathrm{NO}_{3}$ & - & 84716 & - & 84480 & - & 71859 \\
\hline $\mathrm{PO}_{4}$ & - & (a) & - & (a) & - & (a) \\
\hline $\mathrm{SO}_{4}$ & - & 14863 & - & 15071 & - & 12767 \\
\hline $\mathrm{C}_{2} \mathrm{O}_{4}$ & - & $\mathrm{n} / \mathrm{d}$ & - & $\mathrm{n} / \mathrm{d}$ & - & $\mathrm{n} / \mathrm{d}$ \\
\hline Free $\mathrm{OH}^{-}$ & - & $0.98^{(\mathrm{c})}$ & - & $1.05^{(\mathrm{c})}$ & - & $0.92^{(\mathrm{c})}$ \\
\hline $\mathrm{TIC}^{(\mathrm{b})}$ & - & - & - & - & - & - \\
\hline Liquid Density (g/mL) & $1.232 \pm 0.007$ & - & $1.198 \pm 0.008$ & - & $1.180 \pm 0.011$ & - \\
\hline $\mathrm{Wt} \% \mathrm{H}_{2} \mathrm{O}$ in liquid & $72.4 \pm 0.1$ & - & $72.6 \pm 0.1$ & - & $75.8 \pm 0.2$ & - \\
\hline Slurry UDS (wt\%) & 5.37 & - & 18.6 & - & 4.67 & - \\
\hline Slurry Density (g/mL) & $1.284 \pm 0.006$ & - & $1.375 \pm 0.006$ & - & $1.242 \pm 0.010$ & - \\
\hline $\mathrm{Wt} \%$ centrifuged solids ${ }^{(\mathrm{d})}$ & 14.4 & $\%$ & 39.7 & $\%$ & 11.8 & $\%$ \\
\hline$\%$ solids in cent. solids ${ }^{(\mathrm{d})}$ & 55.91 & $\%$ & 62.40 & $\%$ & 55.98 & $\%$ \\
\hline $\begin{array}{l}\text { - Analysis not required by Te } \\
\text { Bold numbers are calculated v } \\
\text { (a) Additional information, in } \\
\text { (b) } \mathrm{TIC}=\text { total inorganic carbc } \\
\text { (c) Free } \mathrm{OH}^{-} \text {is expressed in } \mathrm{M} \\
\text { (d) Values of first sample coll }\end{array}$ & $\begin{array}{l}\text { Plan. } \\
\text { ues. } \\
\text { uding phosphate } \\
\text { larity. } \\
\text { ted from a set of }\end{array}$ & $\begin{array}{l}\text { larities, reporte } \\
\text { plicate samples }\end{array}$ & $\begin{array}{l}\mathrm{d} \text { in individual caustic } \\
\text { collected. }\end{array}$ & c-leach tables. & & \\
\hline
\end{tabular}

Table 5.12 shows the analytical data associated with the baseline caustic-leach in Tank T02A for TI-032. Liquid analyses data are available, but are not shown for the start of the baseline caustic-leach for TI-062 because this activity was terminated.

Two caustic-leaches were conducted in Tank T01A, which is typical of upfront leaching. Analytical data for the first upfront leaching (TI-067) are shown in Table 5.13, and analytical data for the second upfront leaching (TI-032) are shown in Table 5.14. The slurry density results were obtained from intact 
slurry samples submitted to the analytical laboratory. The wt\% UDS, aluminum, iron, and strontium slurry concentrations were calculated from samples phase-separated at PDL-W. (See Mahoney et al. [2009] Section A.5 of Appendix A for more information on calculating the species concentration in the slurry.)

The liquid analysis results are analytical results obtained from the phase-separated supernatants.

Table 5.15 shows the analytical data associated with the post-caustic-leach washing. Two separate slurry samples were collected for the wt $\%$ UDS and slurry density. The liquid analysis results are supernatant analytical results obtained from samples phase-separated in PDL-W, and the liquid density results were calculated from data collected in PDL-W. IC results are provided as part of the liquid analyses and presented in $\mu \mathrm{g} / \mathrm{g}$. 
Table 5.12. TI-032 Caustic-Leach Analytical Data for Tank T02A (middle-middle location)

\begin{tabular}{|c|c|c|c|c|c|c|c|c|c|c|c|c|c|c|}
\hline \multirow[b]{2}{*}{$\begin{array}{c}\text { Process } \\
\text { Description }\end{array}$} & & \multicolumn{8}{|c|}{ Liquid Analyses $^{(\mathrm{a}, \mathrm{b}, \mathrm{c}, \mathrm{e})}$} \\
\hline & $\begin{array}{l}\text { UDS } \\
\mathrm{Wt} \% \\
\end{array}$ & $\begin{array}{l}\text { Slurry } \\
\text { Density } \\
\mathrm{g} / \mathrm{mL}\end{array}$ & $\begin{array}{c}\text { Wt } \% \\
\text { Centrifuged } \\
\text { Solids } \\
\mathrm{Wt} \% \\
\end{array}$ & $\begin{array}{c}\mathrm{Al} \\
\mu \mathrm{g} / \mathrm{g}\end{array}$ & $\begin{array}{c}\mathrm{Fe} \\
\mu \mathrm{g} / \mathrm{g} \\
\end{array}$ & $\begin{array}{c}\mathrm{Sr} \\
\text { (tracer) } \\
\mu \mathrm{g} / \mathrm{g}\end{array}$ & $\begin{array}{c}\text { Supernate } \\
\text { Density } \\
\mathrm{g} / \mathrm{mL}\end{array}$ & $\begin{array}{c}\mathrm{Al}(\mathrm{OH})_{4}^{-} \\
\mathrm{M}\end{array}$ & $\begin{array}{l}\mathrm{OH}^{-} \\
\mathrm{M} \\
\end{array}$ & $\begin{array}{c}\mathrm{NO}_{3}^{-} \\
\text {(tracer) } \\
\mathrm{M} \\
\end{array}$ & $\begin{array}{c}\mathrm{NO}_{2}^{-} \\
\text {(tracer) } \\
\mathrm{M} \\
\end{array}$ & $\begin{array}{c}\mathrm{C}_{2} \mathrm{O}_{4}{ }^{2-} \\
\mathrm{M} \\
\end{array}$ & $\begin{array}{c}\mathrm{PO}_{4}{ }^{3-} \\
\mathrm{M} \\
\end{array}$ & $\begin{array}{c}\mathrm{SO}_{4}{ }^{2-} \\
\mathrm{M}\end{array}$ \\
\hline $\begin{array}{c}\text { Pre-NaOH } \\
\text { addition }\end{array}$ & 18.6 & 1.375 & 45.4 & 56444 & 16953 & 148 & 1.198 & 0.174 & 1.053 & 1.632 & 0.502 & $\mathrm{n} / \mathrm{d}$ & 0.078 & 0.188 \\
\hline \multirow{4}{*}{$\begin{array}{l}\text { Post-NaOH } \\
\text { addition }\end{array}$} & 7.18 & - & 26.1 & - & - & - & 1.380 & 0.563 & 8.617 & 0.957 & 0.315 & $<0.01$ & $0.028^{(\mathrm{f})}$ & 0.030 \\
\hline & 8.34 & - & 26.1 & - & - & - & 1.387 & 0.568 & 8.402 & 0.961 & 0.311 & $<0.01$ & $0.029^{(\mathrm{f})}$ & 0.030 \\
\hline & 7.15 & - & 25.4 & - & - & - & 1.382 & 0.581 & 8.425 & 0.961 & 0.314 & $<0.01$ & $0.041^{(f)}$ & 0.030 \\
\hline & $12.97^{(\mathrm{d})}$ & - & 25.4 & - & - & - & 1.384 & 0.590 & 8.433 & 0.969 & 0.313 & $<0.01$ & $0.031^{(\mathrm{f})}$ & 0.031 \\
\hline \multirow{4}{*}{$\begin{array}{l}\text { Post-loop flush } \\
88^{\circ} \mathrm{C} \\
98^{\circ} \mathrm{C} \text { - hour } 0 \\
98^{\circ} \mathrm{C} \text { - hour } 1\end{array}$} & 6.41 & 1.434 & 29.9 & - & - & - & 1.362 & 0.622 & 7.680 & 1.032 & 0.326 & $<0.01$ & $0.022^{(\mathrm{f})}$ & 0.063 \\
\hline & 6.08 & 1.420 & 19.3 & 27735 & 8663 & 76 & 1.365 & 0.595 & 7.072 & 0.965 & 0.298 & $\mathrm{n} / \mathrm{d}$ & $\mathbf{0 . 0 2 1}^{(\mathrm{f})}$ & 0.067 \\
\hline & 5.49 & - & 17.0 & 26723 & 8381 & 74 & 1.353 & 0.572 & 6.550 & 0.883 & 0.264 & $\mathrm{n} / \mathrm{d}$ & $0.032^{(\mathrm{f})}$ & 0.098 \\
\hline & 5.39 & - & 17.6 & 26565 & 8320 & 73 & 1.352 & 0.582 & 6.522 & 0.876 & 0.269 & $\mathrm{n} / \mathrm{d}$ & $0.026^{(f)}$ & 0.098 \\
\hline \multirow{8}{*}{$98^{\circ} \mathrm{C}-$ hour 2} & 5.27 & - & 16.9 & 26502 & 8283 & 72 & 1.351 & 0.606 & 6.392 & 0.885 & 0.267 & $\mathrm{n} / \mathrm{d}$ & $0.032^{(\mathrm{f})}$ & 0.099 \\
\hline & 5.37 & 1.395 & 16.7 & - & - & - & 1.357 & - & - & - & - & - & - & - \\
\hline & 5.46 & 1.396 & 16.7 & - & - & - & 1.351 & - & - & - & - & - & - & - \\
\hline & 5.32 & 1.399 & 16.6 & - & - & - & 1.352 & - & - & - & - & - & - & - \\
\hline & (d) & 1.391 & 16.6 & - & - & - & 1.355 & - & - & - & - & - & - & - \\
\hline & 5.28 & 1.395 & 16.8 & - & - & - & 1.348 & - & - & - & - & - & - & - \\
\hline & 5.35 & 1.394 & 16.5 & - & - & - & 1.350 & - & - & - & - & - & - & - \\
\hline & 5.23 & 1.399 & 16.8 & - & - & - & 1.352 & - & - & - & - & - & - & - \\
\hline $98^{\circ} \mathrm{C}$ - hour 4 & 5.15 & - & 15.8 & 26005 & 8203 & 72 & 1.342 & 0.617 & 6.262 & 0.850 & 0.258 & $\mathrm{n} / \mathrm{d}$ & $0.033^{(\bar{f})}$ & 0.095 \\
\hline $98^{\circ} \mathrm{C}-$ hour 8 & 4.94 & - & 14.9 & 25079 & 7863 & 69 & 1.335 & 0.661 & 5.957 & 0.828 & 0.252 & $\mathrm{n} / \mathrm{d}$ & $0.018^{(f)}$ & 0.093 \\
\hline $98^{\circ} \mathrm{C}$ - hour 12 & 4.60 & - & 13.9 & 24190 & 7559 & 66 & 1.329 & 0.683 & 5.645 & 0.795 & 0.241 & $\mathrm{n} / \mathrm{d}$ & $\mathbf{0 . 0 2 0}^{(\mathrm{f})}$ & 0.090 \\
\hline \multirow{9}{*}{$98^{\circ} \mathrm{C}-$ hour 16} & 4.52 & - & 13.7 & 23460 & 7461 & 65 & 1.321 & 0.697 & 5.510 & 0.768 & 0.238 & $\mathrm{n} / \mathrm{d}$ & $0.017^{(\mathrm{f})}$ & 0.088 \\
\hline & 4.54 & 1.353 & 14.2 & - & - & - & 1.322 & - & - & - & - & - & - & - \\
\hline & 4.50 & 1.356 & 14.6 & - & - & - & 1.322 & - & - & - & - & - & - & - \\
\hline & 4.29 & 1.356 & 13.8 & - & - & - & 1.321 & - & - & - & - & - & - & - \\
\hline & 4.34 & 1.357 & 13.5 & - & - & - & 1.327 & - & - & - & - & - & - & - \\
\hline & 4.73 & 1.360 & 14.4 & - & - & - & 1.333 & - & - & - & - & - & - & - \\
\hline & 4.36 & 1.359 & 13.8 & - & - & - & 1.315 & - & - & - & - & - & - & - \\
\hline & 4.50 & 1.355 & 13.9 & - & - & - & 1.326 & - & - & - & - & - & - & - \\
\hline & 4.37 & 1.353 & 14.2 & - & - & - & 1.327 & - & - & - & - & - & - & - \\
\hline
\end{tabular}

- Analysis not required by Test Plan.

Bold numbers are calculated values.

(a) Supernate concentration of chromate is $<0.001$ based on Raman analysis.

(b) Phosphate and sulfate not reliable tracers. Not used in caustic leaching analysis.

(c) Supernate analyzed by Raman method.

(d) Error in analysis.

(e) $\mathrm{Ca}, \mathrm{Nd}, \mathrm{Mg}$ are not provided in this table because they were not used in the caustic-leach data analysis.

(f) Detected result is qualitative. Result $>$ IDL but $<$ EQL.

$\mathrm{n} / \mathrm{d}$ Triplicate samples with analysis results below method reporting limit.

M Molarity. 
Table 5.13. TI-032 Analytical Data for Caustic-Leach in Tank T01A (upfront leaching, inner-middle location)

\begin{tabular}{|c|c|c|c|c|c|c|c|c|c|c|c|c|c|c|}
\hline \multirow[b]{3}{*}{ Process Description } & \multicolumn{6}{|c|}{ Slurry Analyses } & \multicolumn{8}{|c|}{ Liquid Analyses $^{(\mathrm{a}, \mathrm{b}, \mathrm{c}, \mathrm{d})}$} \\
\hline & UDS & Slurry Density & $\begin{array}{c}\mathrm{Wt} \% \text { Centrifuged } \\
\text { Solids }\end{array}$ & $\mathrm{Al}$ & $\mathrm{Fe}$ & Sr (tracer) & Supernate Density & $\mathrm{Al}(\mathrm{OH})_{4}^{-}$ & $\mathrm{OH}^{-}$ & $\mathrm{NO}_{3}{ }^{-}$(tracer) & $\mathrm{NO}_{2}^{-}$(tracer) & $\mathrm{C}_{2} \mathrm{O}_{4}^{2-}$ & $\mathrm{PO}_{4}^{3-}$ & $\mathrm{SO}_{4}^{2-}$ \\
\hline & $\mathrm{Wt} \%$ & $\mathrm{~g} / \mathrm{mL}$ & $\mathrm{Wt} \%$ & $\mu \mathrm{g} / \mathrm{g}$ & $\mu \mathrm{g} / \mathrm{g}$ & $\mu \mathrm{g} / \mathrm{g}$ & $\mathrm{g} / \mathrm{mL}$ & M & M & M & M & M & M & M \\
\hline \multirow[t]{2}{*}{ Prior } & 5.37 & 1.284 & 16.5 & 19751 & 4741 & 41 & 1.240 & 0.171 & 0.980 & 1.683 & 0.491 & $\mathrm{n} / \mathrm{d}$ & 0.075 & 0.191 \\
\hline & 3.09 & 1.349 & 13.3 & 14956 & 3630 & 31 & 1.321 & 0.197 & 4.975 & 1.379 & 0.423 & $<0.01$ & $0.019^{(\mathrm{e})}$ & 0.153 \\
\hline \multirow[t]{2}{*}{ Post-NaOH addition } & 3.64 & 1.350 & 13.3 & - & - & - & 1.322 & 0.189 & 4.925 & 1.358 & 0.510 & $<0.01$ & $0.012^{(\mathrm{e})}$ & 0.148 \\
\hline & 3.17 & 1.348 & 13.2 & - & - & $\overline{--1}$ & 1.324 & 0.194 & 4.963 & 1.365 & 0.422 & $<0.01$ & $0.012^{(\mathrm{e})}$ & 0.052 \\
\hline $88^{\circ} \mathrm{C}$ & 2.46 & 1.329 & 9.4 & 13523 & 3319 & 30 & 1.309 & 0.332 & $4.202^{(\mathrm{f})}$ & 1.202 & 0.352 & $\mathrm{n} / \mathrm{d}$ & $\mathrm{n} / \mathrm{d}$ & 0.133 \\
\hline hour 0 & 2.57 & - & 9.8 & 13333 & 3374 & 29 & 1.304 & 0.334 & 4.222 & 1.184 & 0.356 & $\mathrm{n} / \mathrm{d}$ & $0.017^{(\mathrm{e})}$ & 0.132 \\
\hline \multirow[t]{4}{*}{ hour 1 . } & 2.51 & - & 9.6 & 13516 & 3372 & 29 & 1.306 & 0.350 & 4.297 & 1.218 & 0.338 & $\mathrm{n} / \mathrm{d}$ & $\mathbf{0 . 0 1 6}^{(\mathrm{e})}$ & 0.136 \\
\hline & 2.43 & - & 9.6 & 13234 & 3333 & 29 & 1.299 & 0.295 & $\mathbf{3 . 6 6 4} 4^{(t)}$ & 1.143 & $0.206^{(\mathrm{g})}$ & $\mathrm{n} / \mathrm{d}$ & $0.102^{(\mathrm{g})}$ & 0.118 \\
\hline & 2.54 & 1.322 & 9.0 & - & - & - & 1.308 & - & - & - & - & - & - & - \\
\hline & 2.36 & 1.328 & 9.1 & - & - & - & 1.302 & - & - & - & - & - & - & - \\
\hline \multirow[t]{4}{*}{ hour 2} & 2.32 & 1.324 & 9.1 & - & - & - & 1.294 & - & - & - & - & - & - & - \\
\hline & 2.39 & 1.325 & 9.1 & - & - & - & 1.297 & - & - & - & - & - & - & - \\
\hline & 2.42 & 1.319 & 9.0 & - & - & - & 1.304 & - & - & - & - & - & - & - \\
\hline & 2.35 & 1.317 & 9.3 & - & - & $\overline{-}$ & 1.300 & $-\overline{-}$ & - & - & - & - & - & - \\
\hline \multirow{3}{*}{$\begin{array}{l}\text { hour } 4 \\
\text { hour } 8 \\
\text { hour } 12\end{array}$} & 2.31 & - & 8.8 & 13422 & 3348 & 30 & 1.296 & 0.345 & $\mathbf{4 . 0 6 8} 8^{(t)}$ & 1.185 & 0.345 & $\mathrm{n} / \mathrm{d}$ & $\mathrm{n} / \mathrm{d}$ & 0.130 \\
\hline & 2.43 & - & 7.8 & 13058 & 3204 & 29 & 1.292 & 0.370 & 4.002 & 1.179 & 0.345 & $\mathrm{n} / \mathrm{d}$ & $0.027^{(\mathrm{e})}$ & 0.131 \\
\hline & 2.35 & - & 7.8 & 12939 & 3231 & 29 & 1.290 & 0.379 & 3.903 & 1.143 & 0.338 & $\mathrm{n} / \mathrm{d}$ & $0.013^{(\mathrm{e})}$ & 0.128 \\
\hline \multirow{7}{*}{ hour 16} & 2.17 & - & 7.3 & 12432 & 3202 & 28 & 1.287 & 0.387 & $3.819^{(t)}$ & 1.134 & 0.346 & $\mathrm{n} / \mathrm{d}$ & $0.012^{(\bar{e})}$ & 0.125 \\
\hline & 2.28 & 1.311 & 7.4 & - & - & - & 1.289 & - & - & - & - & - & - & - \\
\hline & 2.46 & 1.308 & 7.8 & - & - & - & 1.288 & - & - & - & - & - & - & - \\
\hline & 2.11 & 1.304 & 7.3 & - & - & - & 1.292 & - & - & - & - & - & - & - \\
\hline & 2.12 & 1.309 & 7.4 & - & - & - & 1.284 & - & - & - & - & - & - & - \\
\hline & 2.16 & 1.302 & 7.3 & - & - & - & 1.290 & - & - & - & - & - & - & - \\
\hline & $\begin{array}{l}2.09 \\
\end{array}$ & 1.312 & 7.3 & - & - & - & 1.295 & - & - & - & - & - & - & - \\
\hline
\end{tabular}

- Analysis not required by Test Plan.

Bold numbers are calculated values.

(a) Supernate concentration chromate is $<0.001$ based on Raman analysis.

(b) Phosphate and sulfate not reliable tracers. Not used in caustic leaching analysis.

(c) Supernate analyzed by Raman method.

(d) $\mathrm{Ca}, \mathrm{Nd}$, and $\mathrm{Mg}$ not provided in this table. Not used in caustic-leach data analysis.

(e) Detected result is qualitative. Result $>$ IDL but $<$ EQL.

(f) Results are estimates. Results are potentially biased low because the continuing calibration verification is less than $90 \%$, but greater than $80 \%$.

(g) One of the values is not consistent with the other two measurements.

$\mathrm{n} / \mathrm{d}$ Triplicate samples with analysis results below method reporting limit.

M Molarity. 
Table 5.14. TI-067 Analytical Data for Caustic-Leach in Tank T01A (upfront leaching, inner-middle location)

\begin{tabular}{|c|c|c|c|c|c|c|c|c|c|c|c|c|c|c|}
\hline \multirow[b]{2}{*}{$\begin{array}{c}\text { Process } \\
\text { Description }\end{array}$} & \multicolumn{6}{|c|}{ Slurry Analyses } & \multicolumn{8}{|c|}{ Liquid Analyses $^{(a, b, c, d)}$} \\
\hline & $\begin{array}{l}\text { UDS } \\
\mathrm{Wt} \%\end{array}$ & $\begin{array}{l}\text { Slurry } \\
\text { Density } \\
\mathrm{g} / \mathrm{mL}\end{array}$ & $\begin{array}{c}\mathrm{Wt} \% \\
\text { Centrifuged } \\
\text { Solids } \\
\mathrm{Wt} \%\end{array}$ & $\begin{array}{c}\mathrm{Al} \\
\mu \mathrm{g} / \mathrm{g}\end{array}$ & $\begin{array}{c}\mathrm{Fe} \\
\mu \mathrm{g} / \mathrm{g}\end{array}$ & $\begin{array}{c}\mathrm{Sr} \text { (tracer) } \\
\mu \mathrm{g} / \mathrm{g}\end{array}$ & $\begin{array}{c}\text { Supernate } \\
\text { Density } \\
\mathrm{g} / \mathrm{mL}\end{array}$ & $\begin{array}{c}\mathrm{Al}(\mathrm{OH})_{4}^{-} \\
\mathrm{M}\end{array}$ & $\begin{array}{c}\mathrm{OH}^{-} \\
\mathrm{M}\end{array}$ & $\begin{array}{c}\mathrm{NO}_{3}^{-} \text {(tracer) } \\
M\end{array}$ & $\begin{array}{c}\mathrm{NO}_{2}^{-} \\
\text {(tracer) } \\
\mathrm{M}\end{array}$ & $\begin{array}{c}\mathrm{C}_{2} \mathrm{O}_{4}{ }^{2-} \\
\mathrm{M}\end{array}$ & $\begin{array}{c}\mathrm{PO}_{4}{ }^{3-} \\
\mathrm{M}\end{array}$ & $\begin{array}{c}\mathrm{SO}_{4}{ }^{2-} \\
\mathrm{M}\end{array}$ \\
\hline Prior & 4.67 & 1.242 & 13.7 & 17222 & 4075 & 38 & 1.181 & 0.128 & 0.920 & 1.368 & 0.470 & $<0.01^{(\mathrm{f})}$ & 0.067 & 0.157 \\
\hline \multirow{2}{*}{$\begin{array}{l}\text { Post-NaOH } \\
\text { addition }\end{array}$} & 3.11 & 1.307 & 12.1 & 13276 & 3230 & 30 & 1.265 & 0.162 & 4.470 & 1.186 & 0.382 & $<0.01^{(\mathrm{f})}$ & $0.026^{(\mathrm{e})}$ & 0.134 \\
\hline & 3.03 & 1.317 & 12.4 & - & - & - & 1.256 & 0.154 & 4.309 & 1.116 & 0.395 & $<0.01^{(\mathrm{f})}$ & $0.033^{(\mathrm{e})}$ & 0.125 \\
\hline \multirow{3}{*}{$\begin{array}{l}88^{\circ} \mathrm{C} \\
\text { hour } 0 \\
\text { hour } 1\end{array}$} & 2.25 & 1.281 & 10.5 & 12568 & 2970 & 29 & 1.232 & 0.306 & 3.839 & 1.093 & 0.354 & $<0.01^{(f)}$ & $0.027^{(\mathrm{e})}$ & 0.124 \\
\hline & 2.24 & 1.271 & 9.56 & 12287 & 2938 & 28 & 1.233 & 0.295 & 3.718 & 1.043 & 0.331 & $<0.01^{(\mathrm{f})}$ & $0.025^{(\mathrm{e})}$ & 0.117 \\
\hline & 2.13 & 1.267 & 10.2 & 12284 & 2952 & 28 & 1.221 & 0.289 & 3.631 & 1.018 & 0.327 & $<0.01^{(\mathrm{f})}$ & $0.031^{(\mathrm{e})}$ & 0.115 \\
\hline \multirow{9}{*}{ hour 2} & 2.20 & 1.264 & 9.69 & 12016 & 2931 & 28 & 1.225 & 0.292 & 3.642 & 1.021 & 0.322 & $<0.01^{(f)}$ & $0.026^{(\mathrm{e})}$ & 0.115 \\
\hline & 2.10 & 1.276 & 9.4 & - & - & - & 1.251 & - & - & - & - & - & - & - \\
\hline & 2.04 & 1.278 & 9.3 & - & - & - & 1.222 & - & - & - & - & - & - & - \\
\hline & 2.19 & 1.277 & 10.3 & - & - & - & 1.212 & - & - & - & - & - & - & - \\
\hline & 2.15 & 1.278 & 9.64 & - & - & - & 1.216 & - & - & - & - & - & - & - \\
\hline & 2.18 & 1.283 & 10.2 & - & - & - & 1.226 & - & - & - & - & - & - & - \\
\hline & 2.09 & 1.276 & 10.1 & - & - & - & 1.216 & - & - & - & - & - & - & - \\
\hline & 2.26 & 1.284 & 9.62 & - & - & - & 1.246 & - & - & - & - & - & - & - \\
\hline & 2.19 & 1.290 & 9.16 & $-\overline{-}$ & $-\overline{-}$ & - & 1.251 & - & - & $-\overline{-}$ & - & - & - & - \\
\hline \multirow{3}{*}{$\begin{array}{l}\text { hour } 4 \\
\text { hour } 8 \\
\text { hour } 12\end{array}$} & 2.15 & 1.262 & 9.90 & 12229 & 2902 & 28 & 1.221 & 0.306 & 3.620 & 1.046 & 0.343 & $<0.01^{(\mathrm{f})}$ & $0.031^{(\mathrm{e})}$ & 0.118 \\
\hline & 2.10 & 1.267 & 10.0 & 11968 & 2850 & 27 & 1.223 & 0.321 & 3.589 & 1.029 & 0.344 & $<0.01^{(\mathrm{f})}$ & $0.032^{(\mathrm{e})}$ & 0.117 \\
\hline & 1.96 & 1.254 & 9.41 & 11826 & 2794 & 27 & 1.219 & 0.332 & 3.493 & 1.013 & 0.328 & $<0.01^{(\mathrm{f})}$ & $0.031^{(\mathrm{e})}$ & 0.115 \\
\hline \multirow{8}{*}{ hour 16} & 1.80 & 1.255 & 9.09 & 11715 & 2776 & 27 & 1.219 & 0.334 & 3.432 & 0.993 & 0.327 & $<0.01^{(\mathrm{f})}$ & $0.028^{(\mathrm{e})}$ & 0.112 \\
\hline & 1.68 & 1.271 & 9.27 & - & - & - & 1.220 & - & - & - & - & - & - & - \\
\hline & 1.72 & 1.271 & 8.04 & - & - & - & 1.214 & - & - & - & - & - & - & - \\
\hline & 1.71 & 1.274 & 7.78 & - & - & - & 1.220 & - & - & - & - & - & - & - \\
\hline & 1.80 & 1.271 & 8.29 & - & - & - & 1.219 & - & - & - & - & - & - & - \\
\hline & 1.67 & 1.271 & 7.87 & - & - & - & 1.238 & - & - & - & - & - & - & - \\
\hline & 1.82 & 1.274 & 8.65 & - & - & - & 1.226 & - & - & - & - & - & - & - \\
\hline & 1.78 & 1.272 & 8.28 & - & - & - & 1.223 & - & - & - & - & - & - & - \\
\hline
\end{tabular}

\section{Analysis not required by Test Tan.}

(a) Supernate concentration chromate is $<0.001$ based on Raman analysis.

(b) Phosphate and sulfate not reliable tracers. Not used in caustic leaching analysis.

(c) Supernate analyzed by Raman method.

(d) $\mathrm{Ca}, \mathrm{Nd}, \mathrm{Mg}$ not provided in this table. Not used in caustic-leach data analysis.

(e) Detected result is qualitative. Result $>$ IDL but $<$ EQL.

(f) Results are estimates. Results are potentially biased low because the continuing calibration verification is less than $90 \%$, but greater than $80 \%$.

$\mathrm{n} / \mathrm{d}$ Triplicate samples with analysis results below method reporting limit.

M Molarity. 
Table 5.15. Post-Caustic-Leach Washing Analytical Data for TI-032 (middle-low location)

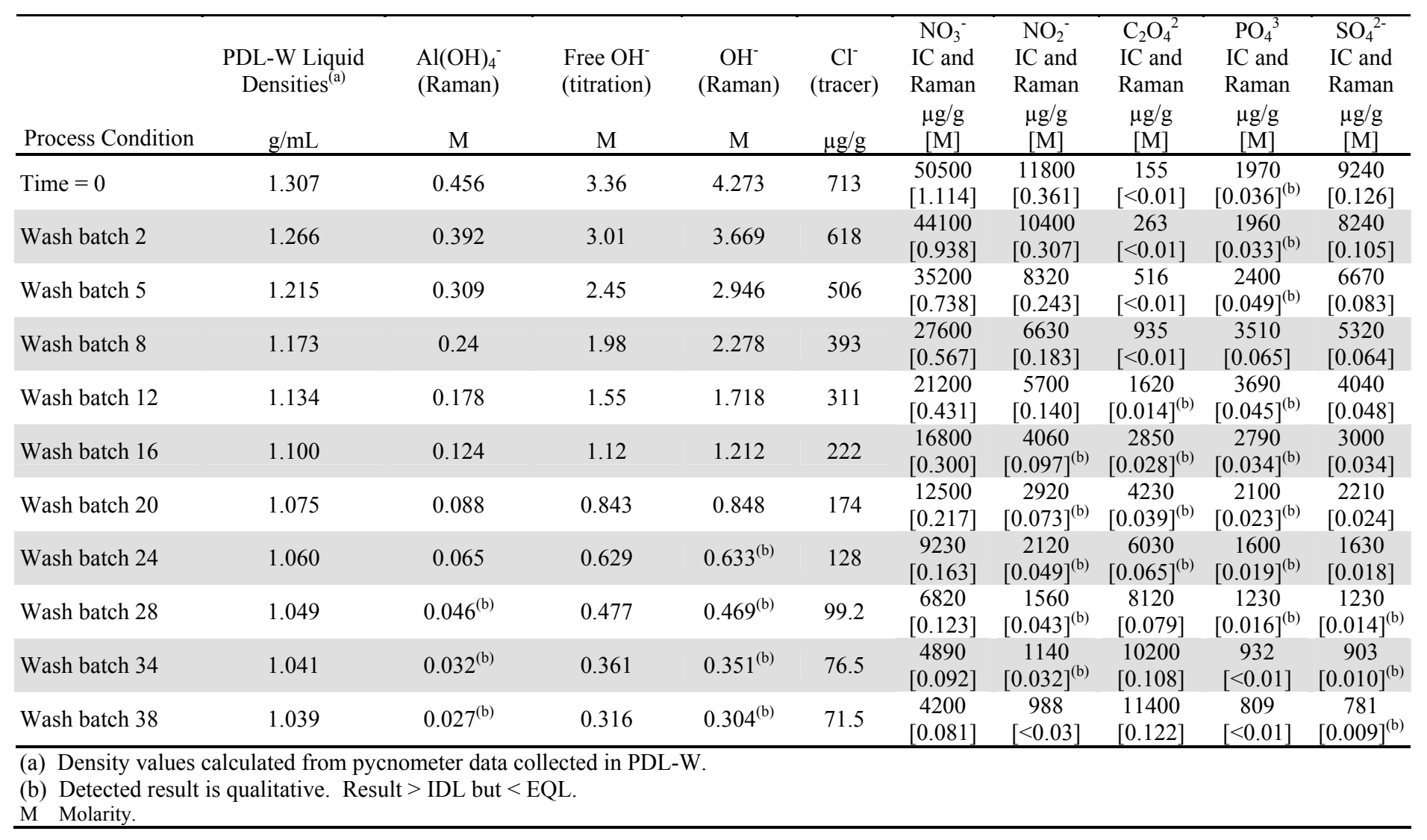


Table 5.16 shows the analytical data associated with the third high-solids filter test attempt conducted during Shakedown/Functional testing, TI-032. Rheological data are recorded in Section 5.2. All of the high-solids filter test attempts were terminated early because of problems with maintaining the filter-loop flow rate. Further attempts to produce a high enough solids concentration to allow observation of the "knee" in the flux vs. log concentration curve (refer to Section 4.3.19) were conducted during Integrated process testing.

Table 5.16. Reconcentration After Chrome Addition and High-Solids Filter TI-032 01/10/2009-01/11/2009 Tests (middle-low location)

\begin{tabular}{|c|c|c|c|c|}
\hline Process Description & $\begin{array}{l}\text { Step Time / } \\
\text { Condition }\end{array}$ & $\begin{array}{l}\text { UDS } \\
\mathrm{Wt} \%\end{array}$ & $\begin{array}{l}\text { Slurry } \\
\text { Density } \\
\mathrm{g} / \mathrm{mL}\end{array}$ & $\begin{array}{c}\text { Liquid } \\
\text { Density } \\
\mathrm{g} / \mathrm{mL}\end{array}$ \\
\hline $\begin{array}{l}\text { Reconcentrate solids and } \\
\text { wash slurry after chrome } \\
\text { introduction }\end{array}$ & & 14.3 & 1.113 & 1.014 \\
\hline $\begin{array}{l}\text { Prepare for high-solids } \\
\text { test at end of } 10 \mathrm{~min} \\
\text { mixing }\end{array}$ & & 6.88 & 1.042 & 0.988 \\
\hline $\begin{array}{l}\text { Immediately before } \\
\text { opening permeate valves }\end{array}$ & $0 \mathrm{~min}$ & 6.97 & 1.046 & 0.992 \\
\hline \multirow{14}{*}{ High-solids filter test } & $15 \mathrm{~min}$ & 7.33 & 1.051 & 0.991 \\
\hline & $30 \mathrm{~min}$ & 7.76 & 1.047 & 0.983 \\
\hline & $45 \mathrm{~min}$ & 8.22 & 1.059 & 0.996 \\
\hline & $60 \mathrm{~min}$ & 9.43 & 1.066 & 1.003 \\
\hline & $1 \mathrm{hr} 15 \mathrm{~min}$ & 8.79 & 1.051 & 0.985 \\
\hline & $1 \mathrm{hr} 30 \mathrm{~min}$ & 10.1 & 1.064 & 1.002 \\
\hline & $1 \mathrm{hr} 45 \mathrm{~min}$ & 10.8 & 1.069 & 0.996 \\
\hline & $2 \mathrm{hr}$ & 11.9 & 1.111 & 1.040 \\
\hline & $2 \mathrm{hr} 15 \mathrm{~min}$ & 13.0 & 1.120 & 1.014 \\
\hline & $2 \mathrm{hr} 30 \mathrm{~min}$ & 14.4 & 1.153 & 1.036 \\
\hline & $2 \mathrm{hr} 45 \mathrm{~min}$ & 16.2 & 1.108 & 0.989 \\
\hline & $3 \mathrm{hr}$ & 18.1 & 1.132 & 0.976 \\
\hline & Final sample & 18.3 & 1.136 & 0.978 \\
\hline & Final sample & 18.3 & 1.138 & 1.026 \\
\hline
\end{tabular}

\subsection{Physical Properties Data}

This section summarizes the PSD and rheology results.

\subsubsection{Particle-Size Distribution}

Table 5.17 and Table 5.18 provide the PSD data at key process steps, before and after sonication. The average particle size, i.e., the average equivalent diameter, is defined as the diameter where 50 mass \% (of the particles) have a larger equivalent diameter, and the other 50 mass $\%$ have a smaller equivalent diameter. Hence, the average particle size is denoted as equivalent $\mathrm{d}(0.500)$. The definition of $\mathrm{d}(0.100)$ is the equivalent diameter where 10 mass \% (of the particles) has a smaller diameter (and hence the remaining $90 \%$ is coarser). The definition of $\mathrm{d}(0.900)$ can be derived similarly. 
Table 5.17. Slurry PSD Results, TI-062

\begin{tabular}{|c|c|c|c|c|c|c|}
\hline \multirow[b]{2}{*}{ Process Step Description } & \multicolumn{3}{|c|}{$\begin{array}{l}\text { Pre-Sonication } \\
\text { (microns) }\end{array}$} & \multicolumn{3}{|c|}{$\begin{array}{l}\text { Sonicated } \\
\text { (microns) }\end{array}$} \\
\hline & $\mathrm{d}(0.100)$ & $\mathrm{d}(0.500)$ & $\mathrm{d}(0.900)$ & $\mathrm{d}(0.100)$ & $\mathrm{d}(0.500)$ & $\mathrm{d}(0.900)$ \\
\hline $\begin{array}{l}\text { Prepare, stage and characterize simulant } \\
\text { (HLP-VSL-T22) and characterize reagents }\end{array}$ & 1.65 & 7.014 & 22.321 & 1.646 & 6.718 & 19.168 \\
\hline Test for stratification of solids in Tank T01A @ & 1.479 & 6.125 & 20.79 & 1.472 & 5.804 & 17.504 \\
\hline $\begin{array}{l}\text { plant PJM frequency,-12 hours after transfer } \\
(11 / 23 / 2008)\end{array}$ & 1.417 & 5.969 & 18.552 & 1.469 & 5.932 & 17.485 \\
\hline Test for stratification of solids in Tank T01A @ & 1.566 & 6.356 & 19.554 & 1.566 & 6.24 & 18.233 \\
\hline $\begin{array}{l}\text { plant PJM frequency,-1 hour before transferring } \\
\text { to Tank T02A }\end{array}$ & 1.555 & 6.465 & 20.175 & 1.569 & 6.354 & 18.764 \\
\hline Repeat of Test for stratification of solids in Tank & 1.491 & 6.328 & 21.588 & 1.5 & 5.914 & 17.696 \\
\hline $\begin{array}{l}\text { T01A @ plant PJM frequency,-12 hours after } \\
\text { transfer (11/24/2008) }\end{array}$ & 1.419 & 5.853 & 17.926 & 1.52 & 5.849 & 16.918 \\
\hline Conduct low-solids filter flux test & 1.316 & 5.891 & 18.383 & 1.399 & 5.676 & 16.152 \\
\hline $\begin{array}{l}\text { Backpulse filters- in-line sample at conclusion of } \\
\text { 24th backpulse }\end{array}$ & 1.213 & 4.8 & 14.386 & 1.181 & 4.641 & 12.568 \\
\hline $\begin{array}{l}\text { Conduct low-solids filter flux test near end of } \\
\text { filtering ( } \sim 12 \mathrm{hrs})\end{array}$ & 1.001 & 4.656 & 15.698 & 1.053 & 4.618 & 14.553 \\
\hline
\end{tabular}

Table 5.18. Slurry Particle-Size Distribution Results, TI-032

\begin{tabular}{|c|c|c|c|c|c|c|}
\hline \multirow[b]{2}{*}{ Process Step Description } & \multicolumn{3}{|c|}{$\begin{array}{c}\begin{array}{c}\text { Pre-Sonication } \\
\text { (microns) }\end{array} \\
\end{array}$} & \multicolumn{3}{|c|}{$\begin{array}{l}\text { Sonicated } \\
\text { (microns) }\end{array}$} \\
\hline & $\mathrm{d}(0.100)$ & $\mathrm{d}(0.500)$ & $\mathrm{d}(0.900)$ & $\mathrm{d}(0.100)$ & $\mathrm{d}(0.500)$ & $\mathrm{d}(0.900)$ \\
\hline \multirow{2}{*}{$\begin{array}{l}\text { Test for stratification of solids in Tank T01B @ } \\
\text { plant PJM frequency-12 hours after slurry } \\
\text { transferred to Tank T01B. }\end{array}$} & 0.106 & 0.492 & 17.873 & 0.108 & 0.406 & 15.066 \\
\hline & 1.732 & 7.355 & 24.351 & 1.702 & 6.791 & 19.275 \\
\hline \multirow{2}{*}{$\begin{array}{l}\text { Test for stratification of solids in Tank T01B @ } \\
\text { plant PJM frequency -1 hour before transferring } \\
\text { to Tank T02A. }\end{array}$} & 1.53 & 6.394 & 19.803 & 1.616 & 6.417 & 18.129 \\
\hline & 1.663 & 6.926 & 21.646 & 1.659 & 6.664 & 19.232 \\
\hline $\begin{array}{l}\text { Conduct low-solids filter flux test- }-10 \text { minutes } \\
\text { after permeate system is known to be flowing } \\
\text { back to Tank T02A. }\end{array}$ & 1.237 & 5.936 & 17.789 & 1.312 & 5.952 & 17.336 \\
\hline $\begin{array}{l}\text { Conduct low-solids filter flux test, sampled near } \\
\text { the completion of the second 12-hr filtering } \\
\text { period. }\end{array}$ & 1.008 & 4.615 & 15.147 & 1.109 & 4.715 & 14.928 \\
\hline $\begin{array}{l}\text { Conduct low-solids filter flux, sampled near the } \\
\text { completion of the last } 12 \text {-hr filtering period. }\end{array}$ & 0.966 & 4.279 & 14.39 & 1.05 & 4.357 & 14.104 \\
\hline Prepare for high-solids filter test sample. & 0.506 & 1.115 & 3.434 & 0.494 & 1.083 & 3.378 \\
\hline $\begin{array}{l}\text { Prepare for high-solids filter test sample } \\
\text { (repeated 01/09/2009). }\end{array}$ & 0.494 & 1.079 & 3.175 & 0.492 & 1.083 & 3.681 \\
\hline
\end{tabular}

\subsubsection{Rheology}

Table 5.19 and Table 5.20 provide a summary of rheology measurements performed at key points throughout Shakedown/Functional testing. For simplicity, only Bingham yield stress and consistency fits for the down ramps have been reported here. A more extensive analysis of the rheology measurements taken are provided in Appendix L with flow curve analysis using both the Bingham and Casson models for both the up and down ramps. 
Table 5.19. Shakedown/Functional Rheology Data Summary, TI-062

\begin{tabular}{|c|c|c|c|c|c|}
\hline Process Test Step & Form & $\begin{array}{l}\text { Shear } \\
\text { Strength } \\
{[\mathrm{Pa}]^{(\mathrm{b})}}\end{array}$ & $\begin{array}{l}\text { Bingham } \\
\text { Yield } \\
{[\mathrm{Pa}]^{(\mathrm{a})}}\end{array}$ & $\begin{array}{l}\text { Bingham } \\
\text { Consistency } \\
{[\mathrm{mPa} \cdot \mathrm{s}]^{(\mathrm{a})}}\end{array}$ & $\begin{array}{l}\text { Supernate } \\
\text { Viscosity } \\
{[\mathrm{mPa} \cdot \mathrm{s}]^{(\mathrm{a})}}\end{array}$ \\
\hline \multirow{5}{*}{$\begin{array}{l}\text { Prepare, stage, and characterize simulant } \\
\text { (HLP-VSL-T22) and characterize reagents. }\end{array}$} & slurry & - & 0.009 & 0.004 & - \\
\hline & slurry & (c) & - & - & - \\
\hline & slurry & (c) & - & - & - \\
\hline & slurry & - & 0.002 & 0.005 & - \\
\hline & supernate & - & - & - & 0.003 \\
\hline \multirow{3}{*}{$\begin{array}{l}\text { Low-solids filter flux test } 10 \text { minutes after } \\
\text { permeate has filled the lines. }\end{array}$} & slurry & - & 0.051 & 0.004 & - \\
\hline & slurry & - & 0.052 & 0.004 & - \\
\hline & supernate & - & - & - & 0.003 \\
\hline \multirow{2}{*}{ Concentration of solids in Tank T02A. } & slurry & - & 0.294 & 0.006 & - \\
\hline & supernate & - & - & - & 0.003 \\
\hline $\begin{array}{l}\text { Beginning of solids concentrations (dewater) to } \\
20 \% \text { UDS, immediately after permeate valves } \\
\text { are opened. }\end{array}$ & slurry & - & 0.072 & 0.005 & - \\
\hline End of solids concentrations (dewater) to $20 \%$ & slurry & - & 0.780 & 0.008 & - \\
\hline UDS. & supernate & - & - & - & 0.003 \\
\hline \multicolumn{6}{|c|}{$\begin{array}{l}\text { - Analysis not required by Test Plan. } \\
\text { (a) All results derived from flow-curve analysis correspond to the down-ramp. } \\
\text { (b) Shear strength measurements correspond to a } 72-\mathrm{hr} \text { gel time. } \\
\text { (c) Found to have no measureable shear strength. }\end{array}$} \\
\hline
\end{tabular}

Table 5.20. Shakedown/Functional Rheology Data Summary, TI-032

\begin{tabular}{lcccc}
\hline \multicolumn{1}{c}{ Process Test Step } & Form & $\begin{array}{c}\text { Bingham } \\
\text { Yield } \\
{[\mathrm{Pa}]^{(\mathrm{a})}}\end{array}$ & $\begin{array}{c}\text { Bingham } \\
\text { Consistency } \\
{[\mathrm{mPa} \cdot \mathrm{s}]^{(\mathrm{a})}}\end{array}$ & $\begin{array}{c}\text { Supernate } \\
\text { Viscosity } \\
{[\mathrm{mPa} \cdot \mathrm{s}]^{(\mathrm{a})}}\end{array}$ \\
\hline Conduct low-solids filter flux test & $\begin{array}{c}\text { slurry } \\
\text { slurry } \\
\text { Concentrate solids in Tank T01A }\end{array}$ & 0.065 & 0.005 & - \\
Concentrated slurry to 20-wt\% UDS & supernate & - & 0.005 & - \\
Evaluate bypass of filter-loop jet within Tank T02A. & slurry & 0.572 & 0.008 & - \\
1 min after tracer injected. & slurry & 1.241 & 0.010 & - \\
Prepare for high-solids test & slurry & 2.027 & 0.018 & - \\
Prepare for high-solids test (repeat 01/10/2009) & slurry & 0.179 & 0.003 & - \\
\hline
\end{tabular}

- Analysis not required by Test Plan.

(a) All results derived from flow-curve analysis correspond to the down-ramp. 


\subsection{PJM Data During Functional/Shakedown Testing}

Tables 5.21 and 5.20 contain PJM nozzle velocities and stroke lengths obtained during tuning steps in TI-062 and TI-032. 
Table 5.21 PJM Parameters for Functional Test from TI-WTP-PEP-062

\begin{tabular}{|c|c|c|c|c|c|c|c|c|c|c|}
\hline $\begin{array}{c}\text { Data Set } \\
\text { Date:Time } \\
(\mathrm{mm} / \mathrm{dd} / \mathrm{yr}) \\
(\mathrm{hr} ; \mathrm{min})\end{array}$ & $\begin{array}{l}\text { TI Step \# } \\
\text { (TI p\#) }\end{array}$ & Vessel & $\begin{array}{c}\text { PJM } \\
\text { Operation } \\
\text { Mode }\end{array}$ & $\begin{array}{c}\text { Process Step } \\
\text { Description }\end{array}$ & $\begin{array}{c}\text { Target } \\
\text { PJM } \\
\text { Nozzle } \\
\text { Velocity } \\
(\mathrm{m} / \mathrm{s})\end{array}$ & $\begin{array}{c}\text { Actual } \\
\text { PJM } \\
\text { Nozzle } \\
\text { Velocity } \\
(\mathrm{m} / \mathrm{s})\end{array}$ & $\begin{array}{l}\text { Target } \\
\text { Stroke } \\
\text { Length } \\
\text { (in.) } \\
(\%)\end{array}$ & $\begin{array}{l}\text { Actual } \\
\text { Stroke } \\
\text { Length } \\
\text { (in.) } \\
(\%)\end{array}$ & $\begin{array}{l}\text { Target } \\
\text { PJM } \\
\text { Cycle } \\
\text { Time } \\
\text { (s) }\end{array}$ & $\begin{array}{l}\text { Actual } \\
\text { PJM } \\
\text { Cycle } \\
\text { Time } \\
\text { (s) }\end{array}$ \\
\hline $\begin{array}{c}11 / 22 / 08 \\
20: 13-20: 38\end{array}$ & $\begin{array}{l}7.2 .2 .9 \\
(\mathrm{p} 42)\end{array}$ & $\begin{array}{l}\text { UFP- } \\
\text { VSL- } \\
\text { T01B }\end{array}$ & Standard & $\begin{array}{l}\text { After transfer } \\
\text { from HLP- } \\
\text { VSL-T22 to } \\
\text { UFP-VSL- } \\
\text { T01B }\end{array}$ & $4.8 \pm 0.3$ & 4.2 & $\begin{array}{c}28.1 \pm 1.7 " \\
80 \pm 5 \%\end{array}$ & $\begin{array}{l}22.9 " \\
65 \%\end{array}$ & $35 \pm 1$ & 35.0 \\
\hline $\begin{array}{c}11 / 23 / 08 \\
09: 51\end{array}$ & N/A & $\begin{array}{l}\text { UFP- } \\
\text { VSL- } \\
\text { T01A }\end{array}$ & Standard & $\begin{array}{l}\text { After initial } \\
\text { transfer from } \\
\text { UFP-VSL- } \\
\text { T01A to UFP- } \\
\text { VSL-T02A }\end{array}$ & $4.8 \pm 0.3$ & 4.9 & $\begin{array}{c}28.1 \pm 1.7 " \\
80 \pm 5 \%\end{array}$ & $\begin{array}{l}27.5 \% \\
78 \%\end{array}$ & $35 \pm 1$ & 35.2 \\
\hline $\begin{array}{c}11 / 23 / 08 \\
09: 04-10: 10\end{array}$ & $\begin{array}{l}7.2 .4 .6 \\
(p 47)\end{array}$ & $\begin{array}{l}\text { UFP- } \\
\text { VSL- } \\
\text { T02A }\end{array}$ & Standard & $\begin{array}{l}\text { After initial } \\
\text { transfer from } \\
\text { UFP-VSL- } \\
\text { T01A to UFP- } \\
\text { VSL-T02A }\end{array}$ & $7.3 \pm 0.4$ & 5.9 & $\begin{array}{c}30.3 \pm 1.8 \% \\
80 \pm 5 \%\end{array}$ & $\begin{array}{l}31 \% \\
82 \%\end{array}$ & $33 \pm 1$ & 33.2 \\
\hline $\begin{array}{c}11 / 24 / 08 \\
04: 16\end{array}$ & $\begin{array}{l}7.2 .8 .2 \\
(p \text { 65) }\end{array}$ & $\begin{array}{l}\text { UFP- } \\
\text { VSL- } \\
\text { T02A }\end{array}$ & Standard & $\begin{array}{l}\text { During filtration } \\
\text { with } \\
\text { backpulsing }\end{array}$ & $7.3 \pm 0.4$ & 6.3 & $\begin{array}{c}30.3 \pm 1.8 " \\
80 \pm 5 \%\end{array}$ & $\begin{array}{l}29.2 \% \\
77 \%\end{array}$ & $33 \pm 1$ & 33.2 \\
\hline
\end{tabular}


Table 5.22 PJM Parameters for Functional Test from TI-WTP-PEP-032

\begin{tabular}{|c|c|c|c|c|c|c|c|c|c|c|}
\hline $\begin{array}{c}\text { Data Set } \\
\text { Date:Time } \\
(\mathrm{mm} / \mathrm{dd} / \mathrm{yr}) \\
(\mathrm{hr} ; \mathrm{min})\end{array}$ & $\begin{array}{l}\text { TI Step \# } \\
\text { (TI p\#) }\end{array}$ & Vessel & $\begin{array}{c}\text { PJM } \\
\text { Operation } \\
\text { Mode }\end{array}$ & $\begin{array}{c}\text { Process Step } \\
\text { Description }\end{array}$ & $\begin{array}{c}\text { Target } \\
\text { PJM } \\
\text { Nozzle } \\
\text { Velocity } \\
(\mathrm{m} / \mathrm{s})\end{array}$ & $\begin{array}{c}\text { Actual } \\
\text { PJM } \\
\text { Nozzle } \\
\text { Velocity } \\
(\mathrm{m} / \mathrm{s})\end{array}$ & $\begin{array}{c}\text { Target } \\
\text { Stroke } \\
\text { Length } \\
\text { (in.) } \\
(\%)\end{array}$ & $\begin{array}{c}\text { Actual } \\
\text { Stroke } \\
\text { Length } \\
\text { (in.) } \\
(\%)\end{array}$ & $\begin{array}{l}\text { Target } \\
\text { PJM } \\
\text { Cycle } \\
\text { Time } \\
\text { (s) }\end{array}$ & $\begin{array}{c}\text { Actual } \\
\text { PJM } \\
\text { Cycle } \\
\text { Time } \\
\text { (s) }\end{array}$ \\
\hline $\begin{array}{c}12 / 17 / 08 \\
19: 24\end{array}$ & $\begin{array}{c}\text { LRB } \\
60108, p \\
30-31 \text { (in } \\
\text { place of } \\
7.2 .1 .7 \text { ) }\end{array}$ & $\begin{array}{l}\text { UFP- } \\
\text { VSL- } \\
\text { T01A }\end{array}$ & Simple & $\begin{array}{l}\text { After transfer } \\
\text { from HLP-VSL- } \\
\text { T22 to UFP- } \\
\text { VSL-T01A }\end{array}$ & $4.8 \pm 0.3$ & 4.9 & $\begin{array}{c}28.1 \pm 1.7 " \\
80 \pm 5 \%\end{array}$ & $\begin{array}{c}31.8 " \\
90.6 \%\end{array}$ & $35 \pm 1$ & 35.3 \\
\hline $\begin{array}{c}12 / 21 / 08 \\
08: 15\end{array}$ & $\begin{array}{c}7.2 .2 .7 .1 \\
(\mathrm{p} \mathrm{39})\end{array}$ & $\begin{array}{l}\text { UFP- } \\
\text { VSL- } \\
\text { T01B }\end{array}$ & Simple & $\begin{array}{l}\text { After transfer } \\
\text { from HLP-VSL- } \\
\text { T22 to UFP- } \\
\text { VSL-T01B }\end{array}$ & $4.8 \pm 0.3$ & 4.8 & $\begin{array}{c}28.1 \pm 1.7 " \\
80 \pm 5 \%\end{array}$ & $\begin{array}{l}32.3^{\prime \prime} \\
92 \%\end{array}$ & $35 \pm 1$ & 35.1 \\
\hline $\begin{array}{c}12 / 30 / 08 \\
03: 55-13: 42\end{array}$ & $\begin{array}{l}7.2 .5 .2 \\
(p 51)\end{array}$ & $\begin{array}{l}\text { UFP- } \\
\text { VSL- } \\
\text { T02A }\end{array}$ & Simple & $\begin{array}{l}\text { Before draining } \\
\text { filters }\end{array}$ & $7.3 \pm 0.4$ & 7.2 & $\begin{array}{c}30.3 \pm 1.8 \% \\
80 \pm 5 \%\end{array}$ & $\begin{array}{l}28.9 " \\
76 \%\end{array}$ & $33 \pm 1$ & 33.2 \\
\hline $\begin{array}{c}12 / 31 / 08 \\
11: 24-23: 44\end{array}$ & $\begin{array}{l}7.2 .7 .1 \\
(p 58)\end{array}$ & $\begin{array}{l}\text { UFP- } \\
\text { VSL- } \\
\text { T02A }\end{array}$ & Simple & $\begin{array}{l}\text { During filtration } \\
\text { with backpulsing }\end{array}$ & $7.3 \pm 0.4$ & 7.2 & $\begin{array}{c}30.3 \pm 1.8 \% \\
80 \pm 5 \%\end{array}$ & $\begin{array}{l}26 " \\
69 \%\end{array}$ & $33 \pm 1$ & 33.2 \\
\hline $\begin{array}{c}01 / 02 / 09 \\
18: 44-19: 40\end{array}$ & $\begin{array}{c}7.3 .1 .15 \\
(\mathrm{p} 81)\end{array}$ & $\begin{array}{l}\text { UFP- } \\
\text { VSL- } \\
\text { T02A }\end{array}$ & Simple & $\begin{array}{l}\text { During caustic } \\
\text { leaching }\end{array}$ & $7.3 \pm 0.4$ & 7.1 & $\begin{array}{c}30.3 \pm 1.8 \% \\
80 \pm 5 \%\end{array}$ & $\begin{array}{l}26.4 " \\
70 \%\end{array}$ & $33 \pm 1$ & 33.2 \\
\hline $\begin{array}{c}01 / 04 / 09 \\
13: 43 \\
- \\
01 / 05 / 09 \\
02: 19\end{array}$ & $\begin{array}{l}7.5 .2 .9 \\
(\mathrm{p} 116)\end{array}$ & $\begin{array}{l}\text { UFP- } \\
\text { VSL- } \\
\text { T02A }\end{array}$ & Simple & $\begin{array}{l}\text { Between dewater } \\
\text { operations }\end{array}$ & $12 \pm 0.6$ & 11.6 & $\begin{array}{c}30.3 \pm 1.8 \% \\
80 \pm 5 \%\end{array}$ & $\begin{array}{l}29.3 \\
77 \%\end{array}$ & $20 \pm 1$ & 21.2 \\
\hline
\end{tabular}




\subsection{Issues}

\subsection{Maintaining Targeted Flow Rate in Filter-Loop}

The flow rate in the filter-loop was unable to meet the targeted rate of 109 gpm when the level in the tank was below $\sim 22$ inches. During Shakedown/Functional testing, the reason for low flow rates was thought to be due to insufficient NPSH for the pumps, but was more likely due to air entrainment. Air entrainment was not identified as an issue during Shakedown/Functional testing, but later, during Integrated testing, it was determined that air entrainment was an issue and could be caused by PJM overblows, high sparging rates or a low tank level.

\subsection{PJM Operation}

Standard mode-The Drexelbrook level instruments in the PJMs (which determined how long pressure and vacuum to the PJMs would be applied) did not work at elevated temperatures in caustic solution and did not work after simulant was drawn above the PJM tube into the PJM piping. As a result, PJMs were operated in simple mode for Shakedown/Functional testing after the first Drexelbrook failure at the beginning of the caustic-leach step during the first test (TI-062).

Simple mode - In simple mode, pressure and vacuum are applied for predetermined time intervals. These time intervals are modified by tank level and temperature. These corrections generally worked, but operators would have to closely monitor the PJMs as these correction factors did not work perfectly. The PJMs would overblow (that is, pressurized air would be on too long, causing the air to come out the bottom of the PJM) and overdraw (that is, vacuum would be applied too long and draw slurry up into the piping). Both issues caused operational problems.

In Tank T02A, air from PJM overblows was entrained into the pump suction causing severe pump vibration. Because the pump flow rate in Tank T01A/B was much lower, PJM overblows in these tanks did not present an immediate operational issue.

Overdraws during the refill stage of the PJM cycle when vacuum pulled slurry into the PJM did not cause immediate problems, but could eventually be observed if there were any leaks in the air delivery piping. Also, it is expected that over extended operating times, this would cause plugging.

\subsection{Leaks}

The pilot plant was subject to periodic leaks. The main leaks reported during Shakedown/Functional testing were:

1. Gaskets between the ultrafiltration filter bundles and the housing began leaking at the beginning of the caustic-leach in TI-062. All of the gaskets were replaced with Teflon gaskets.

2. The RTD inlet nozzle on Tank T02A leaked when the simulant level was too high. Several RTD leads penetrated the Tank T02A wall through a $1 / 2$-in. pipe nozzle. The nozzle was then "sealed" with a polymer sealant to fill the gaps around the bundle of RTD leads, but the sealant leaked when the tank level came up to the nozzle. The issue was resolved by keeping the tank level below the nozzle level. 
3. The pressure relief system vent piping leaked through plugs on the low-pressure side of the pressure relief valves. Investigation of the leaks led to the discovery that the pressure relief system was not designed properly. The system was redesigned and repiped while extra precautions to prevent generating high pressures were instituted (i.e., only one slurry pump could be used). Shakedown/Functional testing proceeded while the retro-fit was being planned.

\subsection{Instruments}

\subsubsection{Lasers Level Measurement}

The laser signal is inherently noisy as the laser light gets reflected off many surfaces and aerosols in the vapor phase. The laser instruments were fitted with an automatic program to decipher the "quality" of the laser signal and determine what output to send to the signal transmitters. The lasers were programmed to output a maximum output if the signal was deemed to be poor quality, which caused high-level alarms and interlocks to be engaged. To avoid spurious alarms, the control system was programmed to use time-averaging for several seconds before alarms were engaged. Maximum output values were still recorded on the DAS, but the alarm system avoided unwanted interlock actions. During Shakedown/Functional testing, the signals were generally very noisy and could not be used for process control. It was also determined that the lasers were susceptible to overheating during the $98^{\circ} \mathrm{C}$ leach. These issues were gradually mitigated in later testing.

\subsubsection{Bubblers Level/Density Measurement}

Bubblers were susceptible to plugging on a regular basis. Blowing high pressure air through the bubbler piping left bubblers operational-sometimes. When high-pressure air was not successful by itself, the bubblers could be cleaned by injecting water, soaking, and then blowing with air. Sometimes multiple water addition/air blowing cycles were needed to clear the bubbler. When bubbler cleaning was successful, the duration of functionality was unpredictable.

Another issue with the bubbler is that the readings in Tank T02A were affected when the filter-loop pumps (UFP-PMP-T42A and/or UFP-PMP-T43A) were running. The flow from these pumps, which was returned to the vessel through the filter-loop return nozzle, would cause pressure changes at the bubbler dip legs. This was a hydrodynamic effect caused by the flowing fluid impinging on or flowing past the dip legs. Consequently, the bubbler level and density readings were wrong when the filter-loop pumps were on. The bubblers in Tanks T01A/B were not influenced by the recirculation pumps in these tanks because of lower flow or better placement within the tank.

The bubblers in all UFP vessels were also affected by the PJM cycles. When the PJMs were in the Drive Phase, the level readings by the bubblers were incorrect. This was a hydrodynamic effect caused by the flowing fluid impinging on or flowing past the dip legs.

The lesson learned on the bubblers is that they need to be equipped with an effective cleaning capability, and the placement of the dip legs should be chosen so as not to be influenced by moving liquid within the tank. 


\subsubsection{Drexelbrook Level Measurement}

The Drexelbrook level indicators did not function properly at elevated temperatures and elevated caustic solutions. During the temperature ramp from ambient to $98^{\circ} \mathrm{C}$ in both caustic leaching batches, the PJMs quit functioning in standard mode (which relied on the Drexelbrook for vacuum and drive level set points) and had to be switched to simple mode (which did not rely on the Drexelbrook level). Residual simulant in the piping above the PJMs also impacted the Drexelbrooks to make the level appear higher. It was concluded that conductive simulant could coat the Drexelbrook probe and cause an erroneous "admittance" between the probe and the wall of the piping above the PJM that was sensed by the probe electronics. Regardless of the actual mechanism, the Drexelbrook probes would only work around ambient temperatures and if there was no simulant in the PJM piping above the PJM tube. Since it is not known when simulant in the piping was affecting the signal, the Drexelbrook level readings should only be used for qualitative purposes.

The Drexelbrook probes were all replaced before starting the Integrated tests. These level probes had an FEP coating with a larger diameter coating around the probe in the air line above the PJM. The intent was to prevent the erroneous "admittance" readings, but results obtained during the Integrated tests indicate that the replacement probes did not function as intended.

\subsubsection{ABB Swirl Flow Meter}

Swirl flow meters require a minimum Reynolds number to provide an accurate flow rate. Below the minimum Reynolds number, the flow meters are programmed to output zero flow rate. At issue in the PEP was that the ABB swirl flow meters were susceptible to ambient vibration (from pumps, people walking in close proximity to the flow meter, etc.) causing some of the PEP swirl flow meters to read flow with none in the pipe. The flow meters were used for totalizing inputs and could have some error. Steps were taken to reduce the vibration by isolating the swirl flow meters. Such steps reduced, but did not eliminate, errant readings. Swirl flow meter DAS data should be compared against the Test Instruction to validate whether flow indicated is actual or due to vibration. 



\subsection{References}

Baldwin DL, PP Schonewill, JJ Toth, JL Huckaby, PW Eslinger, BD Hanson, DE Kurath, and MJ Minette. 2009. EFRT M12 Issue Resolution: Solids Washing. WTP-RPT-187, PNNL-18499, Pacific Northwest National Laboratory, Richland, Washington.

Billing JM, RC Daniel, DE Kurath, and RA Peterson. 2009. Bench-Scale Filtration Testing I Support of the Pretreatment Engineering Platform. WTP-RPT-203, PNNL-18673, Pacific Northwest National Laboratory, Richland Washington.

Daniel RC, JM Billing, JR Bontha, CF Brown, PW Eslinger, BD Hanson, JL Huckaby, NK Karri, ML Kimura, DE Kurath, and MJ Minette. 2009. EFRT M12 Issue Resolution: Comparison of Filter Performance at PEP and CUF Scale. WTP-RPT-185, PNNL-18498, Pacific Northwest National Laboratory, Richland, Washington.

Geeting JGH, OP Bredt, CA Burns, EC Golovich, CE Guzman-Leong, GB Josephson, DE Kurath, GJ Sevigny, and RL Aaberg. 2009. Pretreatment Engineering Platform (PEP) Integrated Test B Run Report-Caustic and Oxidative Leaching in UFP-VSL-T02A. WTP-RPT-192, PNNL-18723, Pacific Northwest National Laboratory, Richland, Washington.

Guzman-Leong CE, OP Bredt, CA Burns, RC Daniel, YF Su, JGH Geeting, EC Golovich, GB Josephson, DE Kurath, GJ Sevigny, DM Smith, PLJ Valdez, ST Yokuda, and JK Young. 2009. PEP Run Report for Integrated Test A; Caustic Leaching in UFP-VSL-T01A, Oxidative Leaching in UFP-VSL-T02A. WTP-RPT-191, PNNL-18755, Pacific Northwest National Laboratory, Richland, Washington.

Kuhn WL, ST Arm, JL Huckaby, DE Kurath, and SD Rassat. 2008. Scaling Relationships for the Pretreatment Engineering Platform. WTP-RPT-160, PNNL-16948, Pacific Northwest National Laboratory, Richland, Washington.

Kurath DE, BD Hanson, MJ Minette, DL Baldwin, BM Rapko, LA Mahoney, PP Schonewill, RC Daniel, PW Eslinger, JL Huckaby, JM Billing, PS Sundar, GJ Josephson, JJ Toth, ST Yokuda, EBK Baer, SM-Barnes, EC Golovich, SD Rassat, CF Brown, JGH Geeting, GJ Sevigny, JR Bontha, RL Aaberg, PM Aker, CE Guzman-Leong, ML Kimura, SK Sundaram, RP Pires, BE Wells, and OP Bredt. 2009. Pretreatment Engineering Platform Phase I Final Test Report. WTP-RPT-197, PNNL-18894, Pacific Northwest National Laboratory, Richland, Washington.

Mahoney LA, SD Rassat, PW Eslinger, RL Aaberg, PM Aker, EC Golovich, BD Hanson, TS Hausmann, JL Huckaby, DE Kurath, MJ Minette, SK Sundaram, and ST Yokuda. 2009. EFRT Issue Resolution: Caustic Leach Rate Constants from PEP and Lab-Scale Tests. WTP-RPT-186, PNNL-18502, Pacific Northwest National Laboratory, Richland, Washington.

Rapko BM, GJ Lumetta, JR Deschane, and RA Peterson. 2007. Process Development for Permanganate Addition During Oxidative Leaching of Hanford Tank Sludge Simulants. WTP-RPT-164, PNNL-16794, Pacific Northwest National Laboratory, Richland, Washington. 
Rapko BM, CF Brown, PW Eslinger, MS Fountain, TS Hausmann, JL Huckaby, BD Hanson, DE Kurath, and MJ Minette. 2009. EFRT M12 Issue Resolution: Comparison of PEP and Bench-Scale Oxidative Leaching Results. WTP-RPT-188, PNNL-18500, Pacific Northwest National Laboratory, Richland, Washington.

Russell RL, RA Peterson, HD Smith, DE Rinehart, PM Aker, and EC Buck. 2009a. Development and Characterization of Boehmite Component Simulant. WTP-RPT-184, PNNL-18176, Pacific Northwest National Laboratory, Richland, Washington.

Russell RL, Peterson RA, DE Rinehart, and HD Smith. 2009b. Development and Characterization of Gibbsite Component Simulant. WTP-RPT-176, PNNL-18013, Pacific Northwest National Laboratory, Richland Washington.

Russell RL, JM Billing, RA Peterson, DE Rinehart, and HD Smith. 2009c. Development and Demonstration of Ultrafiltration Simulants. WTP-RPT-183, PNNL-18090, Pacific Northwest National Laboratory, Richland, Washington.

Sevigny GJ, OP Bredt, CA Burns, DE Kurath, JGH Geeting, EC Golovich, CE Guzman-Leong, and GB Josephson. 2009. PEP Integrated Test D Run Report-Caustic and Oxidative Leaching in UFP-VSL-T02A. WTP-RPT-193, PNNL-18741, Pacific Northwest National Laboratory, Richland, Washington 
Appendix A

Instrument Summary 



\section{Appendix A: Instrument Summary}

This table provides a partial list of instruments used in the PEP. The interested reader is referred to the P\&IDs and the Measurement and Test Equipment (M\&TE) list for a complete list of instruments.

\begin{tabular}{|c|c|c|c|c|c|c|}
\hline \multirow[b]{2}{*}{ Component } & \multicolumn{3}{|l|}{ Instrument } & \multicolumn{3}{|c|}{ Tolerance } \\
\hline & ID & Min & Max & Units & $( \pm)$ & Notes \\
\hline \multicolumn{7}{|l|}{ Vessel T02A } \\
\hline T02A level (bubbler) & LT-0611 & 0.00 & 200.00 & in $\mathrm{H}_{2} \mathrm{O}$ & 0.15 & \\
\hline T02A density (bubbler) & DT-0612 & 0.00 & 200.00 & in $\mathrm{H}_{2} \mathrm{O}$ & 0.15 & \\
\hline T02A level (laser) & LT-0614 & -3.95 & 96.00 & in & 0.25 & \\
\hline T02A prototypic temperature & TTK-0619 & 10.00 & 110.00 & $\mathrm{C}$ & 2 & \\
\hline \multicolumn{7}{|l|}{ T02A Temperature Array } \\
\hline $\mathrm{x}=\mathrm{A}, \mathrm{B}, \mathrm{D}, \mathrm{E}, \mathrm{F}, \mathrm{G}, \mathrm{H}, \mathrm{J}, \mathrm{K}, \mathrm{L}$ & TTx-0619 & 10.00 & 110.00 & $\mathrm{C}$ & 2 & \\
\hline $\mathrm{x}=\mathrm{A}, \mathrm{B}, \mathrm{D}, \mathrm{E}, \mathrm{F}, \mathrm{G}, \mathrm{H}, \mathrm{J}, \mathrm{K}, \mathrm{L}$ & TTx-0621 & 10.00 & 110.00 & $\mathrm{C}$ & 2 & \\
\hline $\mathrm{x}=1-7$ & N39-x & 10.00 & 110.00 & $\mathrm{C}$ & 2 & \\
\hline$x=1-7$ & N41-x & 10.00 & 110.00 & $\mathrm{C}$ & 2 & \\
\hline$x=1-7$ & N42-x & 10.00 & 110.00 & $\mathrm{C}$ & 2 & \\
\hline$x=1-7$ & N52-X & 10.00 & 110.00 & $\mathrm{C}$ & 2 & \\
\hline \multicolumn{7}{|l|}{ HX-T02A } \\
\hline HX-T02A cooling water flow & FT-0541 & 0.00 & 70.00 & gpm & 0.88 & \\
\hline $\begin{array}{l}\mathrm{HX}-\mathrm{T} 02 \mathrm{~A} \text { cooling water inlet } \\
\text { temperature }\end{array}$ & TT-0539 & 0.00 & 110.00 & $\mathrm{C}$ & 1.8 & \\
\hline $\begin{array}{l}\text { HX-T02A cooling water outlet } \\
\text { temperature }\end{array}$ & TT-0540 & 0.00 & 110.00 & $\mathrm{C}$ & 1.8 & \\
\hline $\begin{array}{l}\text { HX-T02A process fluid inlet } \\
\text { temperature }\end{array}$ & TT-0537 & 0.00 & 110.00 & $\mathrm{C}$ & 1.8 & $\begin{array}{l}\text { For Information Only } \\
\text { re: NCR } 42402.1\end{array}$ \\
\hline $\begin{array}{l}\text { HX-T02A process fluid outlet } \\
\text { temperature }\end{array}$ & TT-0513 & 0.00 & 110.00 & $\mathrm{C}$ & 1.8 & $\begin{array}{l}\text { For Information Only } \\
\text { re: NCR 42402.1 }\end{array}$ \\
\hline \multicolumn{7}{|l|}{ Pumps T42A/T43A } \\
\hline $\begin{array}{l}\text { T42A pump discharge pressure } \\
\text { (T43A suction) }\end{array}$ & PT-0633 & 0.00 & 348.00 & psig & 0.26 & \\
\hline T42A pump inlet flow & FT-0623 & 0.00 & 220.00 & gpm & 1.6 & \\
\hline T42A pump speed & ST-0627 & 0.00 & 1785.00 & RPM & 4 & \\
\hline T42A pump suction pressure & PT-0625 & -260.00 & 260.00 & in $\mathrm{H}_{2} \mathrm{O}$ & 0.4 & \\
\hline T42A pump motor hp & JT-0629 & 0.00 & 100.00 & $\mathrm{hp}$ & 1.5 & For Information Only \\
\hline T43A outlet temperature & TT-0791 & 0.00 & 110.00 & $\mathrm{C}$ & 1.8 & $\begin{array}{l}\text { Should not be used as } \\
\text { an indicator of } \\
\text { temperature re: NCR } \\
42402.1\end{array}$ \\
\hline T43A pump discharge pressure & PT-0739 & 0.00 & 348.00 & psig & 0.26 & \\
\hline T43A pump outlet flow & FT-0635 & 0.00 & 220.00 & gpm & 1.6 & \\
\hline T43A pump speed & ST-0639 & 0.00 & 1785.00 & RPM & 4 & \\
\hline T43A pump motor hp & JT-0637 & 0.00 & 100.00 & $\mathrm{hp}$ & 1.5 & For Information Only \\
\hline \multicolumn{7}{|l|}{$\begin{array}{c}\text { Pumps Air Entrainment Trouble } \\
\text { Shoot }\end{array}$} \\
\hline $\begin{array}{l}\text { Air purge flow to steam sparger } \\
\text { FT-1995 }\end{array}$ & FT-1995 & 0.00 & 0.73 & $\mathrm{~kg} / \mathrm{min}$ & 0.02 & $\begin{array}{l}\text { Accuracy revised at } \\
\text { flowrates below } \\
0.090-\mathrm{kg} / \mathrm{min} \text { re: } \mathrm{NCR} \\
38767.1 \\
(0.00045-\mathrm{kg} / \mathrm{min} / \mathrm{flow} \\
\text { rate in } \mathrm{kg} / \mathrm{min} \times 100 \%)\end{array}$ \\
\hline Upper air sparger flow FT-1901 & FT-1901 & 0.00 & 0.73 & $\mathrm{~kg} / \mathrm{min}$ & 0.01 & Accuracy revised at \\
\hline
\end{tabular}




\begin{tabular}{|c|c|c|c|c|c|c|}
\hline Component & $\begin{array}{l}\text { Instrument } \\
\text { ID }\end{array}$ & Min & $\operatorname{Max}$ & Units & $\begin{array}{c}\text { oleran } \\
( \pm)\end{array}$ & Notes \\
\hline & & & & & & $\begin{array}{l}\text { flow rates below } \\
0.090-\mathrm{kg} / \mathrm{min} \text { re: } \mathrm{NCR} \\
38767.1 \\
(0.00045-\mathrm{kg} / \mathrm{min} / \mathrm{flow} \\
\text { rate in } \mathrm{kg} / \mathrm{min} \times 100 \%)\end{array}$ \\
\hline Lower air sparger flow FT-1977 & FT-1977 & 0.00 & 0.73 & $\mathrm{~kg} / \mathrm{min}$ & 0.01 & $\begin{array}{l}\text { Accuracy revised at } \\
\text { flow rates below } \\
0.090-\mathrm{kg} / \mathrm{min} \text { re: NCR } \\
38767.1 \\
(0.00045-\mathrm{kg} / \mathrm{min} / \mathrm{flow} \\
\text { rate in } \mathrm{kg} / \mathrm{min} \times 100 \%)\end{array}$ \\
\hline High-pressure steam flow & FT-0645 & 0.00 & 33.43 & $\mathrm{cfm}$ & 0.41 & \\
\hline High-pressure steam pressure & PT-0643 & 0.00 & 150.00 & psig & 0.11 & \\
\hline Inhibited water process water & FT-0601 & 0.00 & 189.27 & $\mathrm{~kg} / \mathrm{min}$ & 0.24 & \\
\hline 19-M sodium hydroxide & FT-0605 & 0.00 & 75.71 & $\mathrm{~kg} / \mathrm{min}$ & 0.95 & \\
\hline 1-M sodium permanganate & FT-0651 & 0.00 & 15.00 & gpm & 0.11 & \\
\hline 2-M caustic flow & FT-0655 & 0.00 & 57.10 & $\mathrm{~kg} / \mathrm{min}$ & 0.71 & \\
\hline 2-M nitric acid flow & FT-0659 & 0.00 & 15.00 & gpm & 0.11 & \\
\hline \multicolumn{7}{|l|}{ Filter UFP-FILT-T01A } \\
\hline Tubeside inlet temp. & TT-0791 & 0.00 & 110.00 & $\mathrm{C}$ & 1.8 & $\begin{array}{l}\text { Should not be used as } \\
\text { an indicator of } \\
\text { temperature re: NCR } \\
42402.1\end{array}$ \\
\hline Tubeside inlet press. & PT-0739 & 0.00 & 348.00 & psig & 0.26 & \\
\hline Tubeside flow & FT-0635 & 0.00 & 220.00 & gpm & 1.6 & \\
\hline Shellside inlet press. & PT-0741 & 0.00 & 700.00 & psig & 0.53 & \\
\hline Shellside outlet flow & FT-0720 & 0.00 & 35.85 & $\mathrm{~kg} / \mathrm{min}$ & 0.45 & \\
\hline \multicolumn{7}{|l|}{ Filter UFP-FILT-T02A } \\
\hline Tubeside inlet temp. & TT-0791 & 0.00 & 110.00 & $\mathrm{C}$ & 1.8 & $\begin{array}{l}\text { Should not be used as } \\
\text { an indicator of } \\
\text { temperature re: NCR } \\
42402.1\end{array}$ \\
\hline Tubeside inlet press. & PT-0749 & 0.00 & 348.00 & psig & 0.26 & \\
\hline Shellside inlet press. & PT-0751 & 0.00 & 700.00 & psig & 0.53 & \\
\hline Shellside flow & FT-0755 & 0.00 & 37.85 & $\mathrm{~kg} / \mathrm{min}$ & 0.05 & \\
\hline \multicolumn{7}{|l|}{ Filter UFP-FILT-T03A } \\
\hline Tubeside inlet temp. & TT-0791 & 0.00 & 110.00 & $\mathrm{C}$ & 1.8 & $\begin{array}{l}\text { Should not be used as } \\
\text { an indicator of } \\
\text { temperature re: NCR } \\
42402.1\end{array}$ \\
\hline Tubeside inlet press. & PT-0759 & 0.00 & 348.00 & psig & 0.26 & \\
\hline Shellside inlet press. & PT-0761 & 0.00 & 700.00 & psig & 0.53 & \\
\hline Shellside flow & FT-0765 & 0.00 & 37.85 & $\mathrm{~kg} / \mathrm{min}$ & 0.05 & \\
\hline \multicolumn{7}{|l|}{ Filter UFP-FILT-T04A } \\
\hline Tubeside inlet temp. & TT-0791 & 0.00 & 110.00 & $\mathrm{C}$ & 1.8 & $\begin{array}{l}\text { Should not be used as } \\
\text { an indicator of } \\
\text { temperature re: NCR } \\
42402.1\end{array}$ \\
\hline Tubeside inlet press. & PT-0769 & 0.00 & 348.00 & psig & 0.26 & \\
\hline Shellside inlet press. & PT-0771 & 0.00 & 700.00 & psig & 0.53 & \\
\hline Shellside flow & FT-0775 & 0.00 & 37.85 & $\mathrm{~kg} / \mathrm{min}$ & 0.47 & \\
\hline \multicolumn{7}{|l|}{ Filter UFP-FILT-T05A } \\
\hline Tubeside inlet temp. & TT-0791 & 0.00 & 110.00 & $\mathrm{C}$ & 1.8 & $\begin{array}{l}\text { Should not be used as } \\
\text { an indicator of }\end{array}$ \\
\hline
\end{tabular}




\begin{tabular}{ccccccc}
\hline Component & $\begin{array}{c}\text { Instrument } \\
\text { ID }\end{array}$ & Min & Max & Units & $\begin{array}{c}\text { Tolerance } \\
( \pm)\end{array}$ & Notes \\
\hline & & & & & & temperature re: NCR \\
Tubeside inlet press. & PT-0779 & 0.00 & 348.00 & $\mathrm{psig}$ & 0.26 & 42402.1 \\
Tubeside outlet press. & PT-0789 & 0.00 & 348.00 & $\mathrm{psig}$ & 0.26 & \\
Shellside inlet press. & PT-0781 & 0.00 & 700.00 & $\mathrm{psig}$ & 0.53 & \\
Shellside flow & FT-0785 & 0.00 & 37.85 & $\mathrm{~kg} / \mathrm{min}$ & 0.24 & \\
Pulse-pot UFP-PP-T03A & & & & & & \\
Level & LT-0733 & 0.00 & 44.00 & $\mathrm{In}$. & 0.1 & For Information Only \\
Permeate flow from FILT-T01A & FT-0720 & 0.00 & 35.85 & $\mathrm{~kg} / \mathrm{min}$ & 0.45 & \\
Inlet pressure & PT-0731 & 0.00 & 700.00 & $\mathrm{psig}$ & 0.53 & \\
Outlet pressure & PT-0741 & 0.00 & 700.00 & $\mathrm{psig}$ & 0.53 & \\
Pulse-pot UFP-PP-T02A & & & & & & \\
Level & LT-0725 & 0.00 & 44.00 & $\mathrm{In}$. & 0.1 & For Information Only \\
Permeate flow from FILT-T02A & FT-0755 & 0.00 & 37.85 & $\mathrm{~kg} / \mathrm{min}$ & 0.05 & \\
Permeate flow from FILT-T04A & FT-0775 & 0.00 & 37.85 & $\mathrm{~kg} / \mathrm{min}$ & 0.47 & \\
Inlet pressure & PT-0723 & 0.00 & 700.00 & $\mathrm{psig}$ & 0.53 & \\
Outlet pressure to FILT-T02A & PT-0751 & 0.00 & 700.00 & $\mathrm{psig}$ & 0.53 & \\
Outlet pressure to FILT-T04A & PT-0771 & 0.00 & 700.00 & $\mathrm{psig}$ & 0.53 & \\
Pulse-pot UFP-PP-T01A & & & & & & \\
Level & LT-0717 & 0.00 & 44.00 & $\mathrm{In}$. & 0.1 & For Information Only \\
\hline Permeate flow from FILT-T03A & FT-0765 & 0.00 & 37.85 & $\mathrm{~kg} / \mathrm{min}$ & 0.05 & \\
\hline Permeate flow from FILT-T05A & FT-0785 & 0.00 & 37.85 & $\mathrm{~kg} / \mathrm{min}$ & 0.24 & \\
Inlet pressure & PT-0715 & 0.00 & 700.00 & $\mathrm{psig}$ & 0.53 & \\
Outlet pressure to FILT-T03A & PT-0761 & 0.00 & 700.00 & $\mathrm{psig}$ & 0.53 & \\
Outlet pressure to FILT-T05A & PT-0781 & 0.00 & 700.00 & $\mathrm{psig}$ & 0.53 & \\
\hline & & & & & & \\
\hline & & & & & \\
\hline
\end{tabular}



Appendix B

\section{Backpulse Description}





\section{Appendix B: Backpulse Description}

Backpulse Description:

The backpulsing function of the filter-loop can be operated only when actively filtering T02A contents. There are three variables that can be set by the operator: level drain set point, backpulse pressure set point, and pressure deadband for completion.

The level drain set point is the height of fluid in the pulse-pot used for the backpulse.

The backpulse pressure set point is the amount above the filter inlet pressure to which the pulse-pot should be charged (i.e., if the inlet pressure is 100 psig and the backpulse pressure set point is set to $40 \mathrm{psig}$, the control system will charge the pulse-pot to $140 \mathrm{psig}$ ).

The pressure deadband for completion is the amount above the filter inlet pressure that will cause the backpulse to be marked as finished (i.e., if the inlet pressure is $100 \mathrm{psig}$ and the deadband is set to $10 \mathrm{psig}$, the backpulse will complete when the pulse-pot pressure indicator gets down to $110 \mathrm{psig}$ ).

During filtering, the operator presses the "Start Backpulse" button in the HMI, which initiates the backpulse cycle. The first step is to close all valves entering and leaving the pulse-pot. Next, the high-pressure air line is opened and the pulse-pot is pressurized to $50 \mathrm{psig}$. The high-pressure air valve is closed and the drain valve to $\mathrm{T} 62 \mathrm{~A} / \mathrm{B}$ is opened. The pulse-pot fluid level falls until reaching the level drain set point when the drain valve is closed. The high-pressure air valve is opened again and pressurizes the pulse-pot to the sum of the filter inlet pressure plus the backpulse pressure set point $(100 \mathrm{psig}+40 \mathrm{psig}=140 \mathrm{psig}$ in the above example). The air valve is closed and the backpulse cycle pauses for 15 seconds. The fast-acting valve then opens and the pressure in the pulse-pot pushes fluid back through the filter until the pressure in the pulse-pot is equal to the filter inlet pressure plus the deadband (100 psig $+10 \mathrm{psig}=110 \mathrm{psig}$ in the above example). The final step is to return to filtering conditions. The fast-acting valve closes and the filter outlet valve and pulse-pot outlet valve to T62A/B (not the drain valve) are opened. 

Appendix C

Sample List and Naming Convention 



\section{Appendix C: Sample List and Naming Convention}

\subsection{Sample Naming Convention}

Every sample was given a unique sample name. The sample names were a composite of either six or seven separate descriptors. The descriptors are defined in Table C.1 and sample naming examples are provided following the table. The acronyms for each descriptor are defined in Table C.2. The spacings between descriptors were separated by either an underscore symbol ( _ ) or a space ( ). 
Table C.1. Sample Naming Nomenclature Definitions

\begin{tabular}{|c|c|c|}
\hline Descriptor & Identification & Explanation \\
\hline Descriptor 1 & Test Name & $\begin{array}{l}\text { Refers to the Shakedown Test, Functional Test, Integrated Test A, Integrated Test B, or } \\
\text { Integrated Test D. }\end{array}$ \\
\hline Descriptor 2 & Location & $\begin{array}{l}\text { Identifies the location from which the sample is being collected, based on Table } 3 \text {. For } \\
\text { example, a sample collected from a tank will include the acronym of the tank followed } \\
\text { by the location within the tank. }\end{array}$ \\
\hline Descriptor 3 & Test Process Step & $\begin{array}{l}\text { Refers to the process step as identified in the sample collection and analysis table in the } \\
\text { governing Test Instruction. The test process step includes an identifier for processes } \\
\text { that are repeated during testing. }\end{array}$ \\
\hline Descriptor 4 & Sequential Number & $\begin{array}{l}\text { Unique number identifier that increments by } 1 \text { each time a sample was collected. All } \\
\text { sub-samples and separated samples generated from the original sample had the same } \\
\text { unique number identifier. }\end{array}$ \\
\hline Descriptor 5 & Sample Routing & Refers to the sample destination, type of analysis, storage, or archive. \\
\hline Descriptor 6 & Store Code & Final destination code. \\
\hline Descriptor 7 & Separated-Sample Identifier & $\begin{array}{l}\text { Refers to the type of sub-sample; e.g., decantate, solids, rinse solution, etc. For a } \\
\text { separated sample, a descriptor of the matrix was added after the sample routing } \\
\text { identifier. }\end{array}$ \\
\hline
\end{tabular}


Table C.2. Sample Naming Acronyms

\begin{tabular}{|c|c|}
\hline Acronym & Definition \\
\hline \multicolumn{2}{|c|}{ Test Name (1 character) Descriptor 1} \\
\hline $\mathrm{S}$ & Shakedown testing \\
\hline $\mathrm{F}$ & Functional testing \\
\hline A & First Integrated test to be performed \\
\hline B & Second Integrated test to be performed \\
\hline $\mathrm{C}$ & Third Integrated test to be performed \\
\hline $\mathrm{D}$ & Fourth Integrated test to be performed \\
\hline \multicolumn{2}{|c|}{$\begin{array}{l}\text { Location (maximum of } 5 \text { characters) Descriptor } 2 \\
\text { Vessels Name ( } 3 \text { characters) }\end{array}$} \\
\hline $\mathrm{T} 22$ & HLP-VSL-T22 \\
\hline FRP & FRP-VSL-T01 \\
\hline FEP & FEP-VSL-T01 \\
\hline $01 \mathrm{~A}$ & UFP-VSL-T01A \\
\hline 01B & UFP-VSL-T01B \\
\hline $02 \mathrm{~A}$ & UFP-VSL-T02A \\
\hline $\mathrm{T} 27$ & HLP-VSL-T27A \\
\hline $62 \mathrm{~A}$ & UFP-VSL-T62A \\
\hline $62 \mathrm{~B}$ & UFP-VSL-T62B \\
\hline \multicolumn{2}{|c|}{ Vessel Sampling Location (2 characters) } \\
\hline $\mathrm{IH}$ & Inner High \\
\hline IM & Inner Middle \\
\hline IL & Inner Low \\
\hline $\mathrm{MH}$ & Middle High \\
\hline MM & Middle Middle \\
\hline ML & Middle Low \\
\hline $\mathrm{OH}$ & Outer High \\
\hline $\mathrm{OM}$ & Outer Middle \\
\hline OL & Outer Low \\
\hline RL & Recirculation Line \\
\hline GT & Grab sample at top of a tank \\
\hline GM & Grab sample at middle of a tank \\
\hline GB & Grab sample at bottom of a tank \\
\hline \multicolumn{2}{|c|}{ In-Line/Transfer Sampling Locations (5 characters) } \\
\hline $\mathrm{T} 221 \mathrm{~A}$ & In-line transfer from HLP-T22 to Tank T01A \\
\hline 01A2A & In-line transfer from Tank T01A to Tank T02A \\
\hline 01B2A & In-line transfer from Tank T01B to Tank T02A \\
\hline $\mathrm{T} 221 \mathrm{~B}$ & In-line transfer from HLP-T22 to Tank T01B \\
\hline 000FL & Filter-loop in-line sample \\
\hline 00PF1 & Permeate filter number 1 \\
\hline 00PF2 & Permeate filter number 2 \\
\hline 00PF3 & Permeate filter number 3 \\
\hline
\end{tabular}


Table C.2. Sample Naming Acronyms

\begin{tabular}{ll}
\hline Acronym & Definition \\
\hline $00 \mathrm{PF} 4$ & Permeate filter number 4 \\
$00 \mathrm{PF} 5$ & Permeate filter number 5 \\
$00 \mathrm{C} 01$ & Downstream of CS-PMP-T01 \\
$00 \mathrm{C} 02$ & Downstream of CS-PMP-T02 \\
$00 \mathrm{C} 03$ & Downstream of CS-PMP-T03
\end{tabular}

Test Process Step ( 3 characters ${ }^{(a)}$ plus 2 repetitive process characters ${ }^{(b)}$ ) Descriptor 3

(a) 001 through 032 (initial 3 characters) As described in the guiding document such as

(b) A two-character field to identify any

Appendix A or B in TP-RPP-WTP-506 Rev.0 or repetitive process. the governing Test Instruction.

Repetitive Process (2 characters)

\begin{tabular}{|c|c|}
\hline $\mathrm{XX}$ & No repeating process \\
\hline BT & $10^{\circ} \mathrm{C}$ below set point temperature \\
\hline 00 & $\begin{array}{l}\text { Time when set point temperature is reached (in } \\
\mathrm{hr} \text { ) }\end{array}$ \\
\hline 01 through 16 & $\begin{array}{l}\text { Time after set point temperature reached (in } \mathrm{hr} \text { ) or } \\
\text { cycle number, depending on the process step. }\end{array}$ \\
\hline
\end{tabular}

Sequence Number (4 characters) Descriptor 4

0001 through 9999

Sequential number incremented by 1 for replicate samples

Sample Routing ( 3 characters) Descriptor 5

\begin{tabular}{ll} 
ARC & Archive \\
XSP & Undissolved solids \\
AFA & XRD, SEM, and/or PSD \\
SOX & Antifoaming agent analysis \\
HTC & Solid oxalate analysis \\
DEN & Heat capacity \\
ICP & Density \\
& Inductively coupled plasma (ICP) optical \\
RAM & emission spectrometry analysis \\
RHE & Raman analysis \\
TDS & Rheology (shear strength/shear stress) \\
CUF & Total dissolved solids \\
TFI & Cross-flow ultrafilter (CUF)/parallel testing \\
& Total inorganic carbon (TIC) analysis/free \\
& hydroxide (FOH) analysis/ion chromatography \\
ALK & (IC) analysis \\
\hline ADT & Total alkalinity \\
OST & Acidity titration \\
\hline OTR & Oxidation state titration \\
\hline
\end{tabular}


Table C.2. Sample Naming Acronyms

\begin{tabular}{ll}
\hline Acronym & Definition \\
\hline SUP & Supernate, used when no Raman required \\
Separated-samples (1 character) Descriptor 6 & \\
\hline 0 & RTL-520 store \\
1 & RTL-520 archive \\
2 & SwRI \\
3 & RPL \\
4 & APEL \\
5 & Dow Corning \\
6 & Other \\
Separated-samples (1 character) Descriptor 7 & \\
\hdashline D & \\
B & Decanted supernatant (decantate) \\
R & Bulk solids not rinsed \\
S & Rinsate composite \\
P & Rinsed solids \\
F & Process, samples that were filtered/processed \\
\hline
\end{tabular}

The sample names are constructed in the following manner:

Descriptor1_Descriptor2_Descriptor3a_Descriptor3b_Descriptor4_Descriptor5_Descriptor6_ Descriptor7

The following description is an example of sample naming for samples collected from HLP-VSL-T22 during Functional testing.

- The sample name for the first Functional test grab slurry sample collected from the top of HLP-VSL-T22 during process step 1 that was to be archived would have been:

F_T22GT_001_XX_0001_ARC_1.

- For the same sample location and type described above, analyzed for PSD on a bulk solid that was not rinsed and ICP on the decantate, the original sample vial would have been labeled:

F_T22GM_001_XX_0009_XSP_4_B for the solid.

- Since the solid phase always stayed in the original sample vial, and the liquid was decanted off into a new vial, then the new vial would have been labeled: F_T22GM_001_XX_0009_DEN_0_D for the decantate.

- The decantate from the above sample container could have been sub-sampled for ICP and Raman; the ICP container would have been labeled as follows:

F_T22GM_001_XX_0009_ICP_2_D.

- The Raman container would have been labeled as follows:

F_T22GM_001_XX_0009_RAM_3_D. 
TI-WTP-PEP-062 (testing dates 11/19/2008 through 12/01/2008)

\begin{tabular}{|c|c|c|c|c|c|c|}
\hline TI \# & $\begin{array}{c}\text { TI Step } \\
\#\end{array}$ & TI Step Description & Sample ID & Analysis & Folder & Final Electronic Report File Name \\
\hline TI-062 & 1.1 .4 & $\begin{array}{c}\text { Prepare, stage, and characterize } \\
\text { simulant (HLP-T22) and characterize } \\
\text { reagents }\end{array}$ & S T22GB 001 XX 0152 UDS 2 & UDS & 081203-6 & \\
\hline TI-062 & 1.1 .4 & $\begin{array}{c}\text { Prepare, stage, and characterize } \\
\text { simulant (HLP-T22) and characterize } \\
\text { reagents }\end{array}$ & S T22GM 001 XX 0151 UDS 2 & UDS & 081203-6 & \\
\hline TI-062 & 1.1 .4 & $\begin{array}{c}\text { Prepare, stage, and characterize } \\
\text { simulant (HLP-T22) and characterize } \\
\text { reagents }\end{array}$ & S T22GT 001 XX 0150 UDS 2 & UDS & 081203-6 & \\
\hline TI-062 & 1.1 .5 & $\begin{array}{c}\text { Prepare, stage, and characterize } \\
\text { simulant (HLP-T22) and characterize } \\
\text { reagents }\end{array}$ & S T22GM 001 XX 0703 RHE 4 & Rheology & TDP-WTP-355 & $\begin{array}{c}\text { TDP-WTP-355 Shakedown RHE Data on PEP } \\
\text { Slurry }\end{array}$ \\
\hline TI-062 & 1.1 .5 & $\begin{array}{c}\text { Prepare, stage, and characterize } \\
\text { simulant (HLP-T22) and characterize } \\
\text { reagents }\end{array}$ & S T22RL 001 XX 0153 UDS 2 & UDS & 081203-6 & \\
\hline TI-062 & 1.1 .5 & $\begin{array}{c}\text { Prepare, stage, and characterize } \\
\text { simulant (HLP-T22) and characterize } \\
\text { reagents }\end{array}$ & S T22RL 001 XX 0154 UDS 2 & UDS & 081203-6 & \\
\hline TI-062 & 1.1 .5 & $\begin{array}{c}\text { Prepare, stage, and characterize } \\
\text { simulant (HLP-T22) and characterize } \\
\text { reagents }\end{array}$ & S T22RL 001 XX 0155 UDS 2 & UDS & 081203-6 & \\
\hline TI-062 & 1.1 .5 & $\begin{array}{c}\text { Prepare, stage, and characterize } \\
\text { simulant (HLP-T22) and characterize } \\
\text { reagents }\end{array}$ & S T22RL 001 XX 0156 DEN 2 & Density Slurry & 081203-11 & 081203-11DEN \\
\hline TI-062 & 1.1 .5 & $\begin{array}{c}\text { Prepare, stage, and characterize } \\
\text { simulant (HLP-T22) and characterize } \\
\text { reagents }\end{array}$ & S T22RL 001 XX 0157 DEN 2 & Density Slurry & 081203-11 & 081203-11DEN \\
\hline TI-062 & 1.1 .5 & $\begin{array}{c}\text { Prepare, stage, and characterize } \\
\text { simulant (HLP-T22) and characterize } \\
\text { reagents }\end{array}$ & S T22RL 001 XX 0158 DEN 2 & Density Slurry & 081203-11 & 081203-11DEN \\
\hline TI-062 & 1.1 .5 & $\begin{array}{c}\text { Prepare, stage, and characterize } \\
\text { simulant (HLP-T22) and characterize } \\
\text { reagents }\end{array}$ & S T22RL 001 XX 0159 RHE 4 & Rheology & TDP-WTP-355 & $\begin{array}{l}\text { TDP-WTP-355 Shakedown RHE Data on PEP } \\
\text { Slurry }\end{array}$ \\
\hline TI-062 & 1.1 .5 & $\begin{array}{c}\text { Prepare, stage, and characterize } \\
\text { simulant (HLP-T22) and characterize } \\
\text { reagents }\end{array}$ & S T22RL 001 XX 0160 RHE 4 & Rheology & TDP-WTP-355 & $\begin{array}{c}\text { TDP-WTP-355 Shakedown RHE Data on PEP } \\
\text { Slurry }\end{array}$ \\
\hline TI-062 & 1.1 .5 & $\begin{array}{c}\text { Prepare, stage, and characterize } \\
\text { simulant (HLP-T22) and characterize } \\
\text { reagents }\end{array}$ & S T22RL 001 XX 0161 RHE 4 & Rheology & TDP-WTP-355 & $\begin{array}{l}\text { TDP-WTP-355 Shakedown RHE Data on PEP } \\
\text { Slurry }\end{array}$ \\
\hline
\end{tabular}


TI-WTP-PEP-062 (testing dates 11/19/2008 through 12/01/2008)

\begin{tabular}{|c|c|c|c|c|c|c|}
\hline TI \# & $\begin{array}{c}\text { TI Step } \\
\# \\
\end{array}$ & TI Step Description & Sample ID & Analysis & Folder & Final Electronic Report File Name \\
\hline TI-062 & 1.1 .5 & $\begin{array}{c}\text { Prepare, stage, and characterize } \\
\text { simulant (HLP-T22) and characterize } \\
\text { reagents }\end{array}$ & S T22RL 001 XX 0162 RHE 4 & Rheology & TDP-WTP-355 & $\begin{array}{c}\text { TDP-WTP-355 Shakedown RHE Data on PEP } \\
\text { Slurry }\end{array}$ \\
\hline TI-062 & 1.1 .5 & $\begin{array}{c}\text { Prepare, stage, and characterize } \\
\text { simulant (HLP-T22) and characterize } \\
\text { reagents }\end{array}$ & S T22RL 001 XX 0163 ICP 2 B & ICP Wet Solids & 081203-2 & 081125-13 and 081203-2 Solid ICP ICPMS \\
\hline TI-062 & 1.1 .5 & $\begin{array}{c}\text { Prepare, stage, and characterize } \\
\text { simulant (HLP-T22) and characterize } \\
\text { reagents }\end{array}$ & S T22RL 001 XX 0163 ICP 2 D & ICP supernatant & 081203-3 & 081203-1 and 081203-3 Liquid ICP ICPMS \\
\hline TI-062 & 1.1 .5 & $\begin{array}{c}\text { Prepare, stage, and characterize } \\
\text { simulant (HLP-T22) and characterize } \\
\text { reagents }\end{array}$ & S T22RL 001 XX 0163 TFI 2 D & IC & $081125-12$ & 081125-12 and 081203-13 IC TIC TOC \\
\hline TI-062 & 1.1 .5 & $\begin{array}{c}\text { Prepare, stage, and characterize } \\
\text { simulant (HLP-T22) and characterize } \\
\text { reagents }\end{array}$ & S T22RL 001 XX 0163 TFI 2 D & TIC/TOC & $081203-13$ & 081125-12 and 081203-13 IC TIC TOC \\
\hline TI-062 & 1.1 .5 & $\begin{array}{c}\text { Prepare, stage, and characterize } \\
\text { simulant (HLP-T22) and characterize } \\
\text { reagents }\end{array}$ & S T22RL 001 XX 0163 TFI 2 D & Titration & 081203-12 & \\
\hline TI-062 & 1.1 .5 & $\begin{array}{c}\text { Prepare, stage, and characterize } \\
\text { simulant (HLP-T22) and characterize } \\
\text { reagents }\end{array}$ & S T22RL 001 XX 0164 ICP 2 B & ICP Wet Solids & 081203-2 & 081125-13 and 081203-2 Solid ICP ICPMS \\
\hline TI-062 & 1.1 .5 & $\begin{array}{c}\text { Prepare, stage, and characterize } \\
\text { simulant (HLP-T22) and characterize } \\
\text { reagents }\end{array}$ & S T22RL 001 XX 0164 ICP 2 D & $\begin{array}{c}\text { ICP } \\
\text { Supernatant }\end{array}$ & 081203-3 & 081203-1 and 081203-3 Liquid ICP ICPMS \\
\hline TI-062 & 1.1 .5 & $\begin{array}{c}\text { Prepare, stage, and characterize } \\
\text { simulant (HLP-T22) and characterize } \\
\text { reagents }\end{array}$ & S T22RL 001 XX 0164 TFI 2 D & IC & 081125-12 & 081125-12 and 081203-13 IC TIC TOC \\
\hline TI-062 & 1.1 .5 & $\begin{array}{c}\text { Prepare, stage, and characterize } \\
\text { simulant (HLP-T22) and characterize } \\
\text { reagents }\end{array}$ & S T22RL 001 XX 0164 TFI 2 D & TIC/TOC & $081203-13$ & 081125-12 and 081203-13 IC TIC TOC \\
\hline TI-062 & 1.1 .5 & $\begin{array}{c}\text { Prepare, stage, and characterize } \\
\text { simulant (HLP-T22) and characterize } \\
\text { reagents }\end{array}$ & S T22RL 001 XX 0164 TFI 2 D & Titration & 081203-12 & \\
\hline TI-062 & 1.1 .5 & $\begin{array}{c}\text { Prepare, stage, and characterize } \\
\text { simulant (HLP-T22) and characterize } \\
\text { reagents }\end{array}$ & S T22RL 001 XX 0165 ICP 2 B & ICP Wet Solids & 081203-2 & 081125-13 and 081203-2 Solid ICP ICPMS \\
\hline TI-062 & 1.1 .5 & $\begin{array}{c}\text { Prepare, stage, and characterize } \\
\text { simulant (HLP-T22) and characterize } \\
\text { reagents }\end{array}$ & S T22RL 001 XX 0165 ICP 2 D & $\begin{array}{c}\text { ICP } \\
\text { Supernatant }\end{array}$ & 081203-3 & 081203-1 and 081203-3 Liquid ICP ICPMS \\
\hline
\end{tabular}


TI-WTP-PEP-062 (testing dates 11/19/2008 through 12/01/2008)

\begin{tabular}{|c|c|c|c|c|c|c|}
\hline TI \# & $\begin{array}{c}\text { TI Step } \\
\#\end{array}$ & TI Step Description & Sample ID & Analysis & Folder & Final Electronic Report File Name \\
\hline TI-062 & 1.1 .5 & $\begin{array}{c}\text { Prepare, stage, and characterize } \\
\text { simulant (HLP-T22) and characterize } \\
\text { reagents }\end{array}$ & S T22RL 001 XX 0165 TFI 2 D & IC & 081125-12 & 081125-12 and 081203-13 IC TIC TOC \\
\hline TI-062 & 1.1 .5 & $\begin{array}{c}\text { Prepare, stage, and characterize } \\
\text { simulant (HLP-T22) and characterize } \\
\text { reagents }\end{array}$ & S T22RL 001 XX 0165 TFI 2 D & TIC/TOC & 081203-13 & 081125-12 and 081203-13 IC TIC TOC \\
\hline TI-062 & 1.1 .5 & $\begin{array}{c}\text { Prepare, stage, and characterize } \\
\text { simulant (HLP-T22) and characterize } \\
\text { reagents }\end{array}$ & S T22RL 001 XX 0165 TFI 2 D & Titration & 081203-12 & \\
\hline TI-062 & 1.1 .5 & $\begin{array}{c}\text { Prepare, stage, and characterize } \\
\text { simulant (HLP-T22) and characterize } \\
\text { reagents }\end{array}$ & S T22RL 001 XX 0166 XSP 4 B & SEM & TDP-WTP 352 & $\begin{array}{c}\text { TDP WTP } 352 \text { Shakedown Test SEM Data } \\
\text { Analysis }\end{array}$ \\
\hline TI-062 & 1.1 .5 & $\begin{array}{c}\text { Prepare, stage, and characterize } \\
\text { simulant (HLP-T22) and characterize } \\
\text { reagents }\end{array}$ & S T22RL 001 XX 0166 XSP 4 B & PSD & TDP-WTP-354 & $\begin{array}{c}\text { TDP WTP } 354 \text { PEP Shakedown Test PSD Data } \\
\text { Analysis }\end{array}$ \\
\hline TI-062 & 1.1 .5 & $\begin{array}{c}\text { Prepare, stage, and characterize } \\
\text { simulant (HLP-T22) and characterize } \\
\text { reagents }\end{array}$ & S T22RL 001 XX 0166 XSP 4 B & XRD & TDP-WTP-353 & $\begin{array}{c}\text { TDP WTP } 353 \text { Shakedown Test XRD Data } \\
\text { Analysis }\end{array}$ \\
\hline TI-062 & 1.1 .5 & $\begin{array}{c}\text { Prepare, stage, and characterize } \\
\text { simulant (HLP-T22) and characterize } \\
\text { reagents }\end{array}$ & S T22RL 001 XX 0167 SOX 2 & TIC/TOC & 081203-14 & 081203-14 TIC TOC \\
\hline TI-062 & 1.1 .5 & $\begin{array}{c}\text { Prepare, stage, and characterize } \\
\text { simulant (HLP-T22) and characterize } \\
\text { reagents }\end{array}$ & S T22RL 001 XX 0169 AFA 5 B & $\begin{array}{l}\text { Toluene Wet } \\
\text { Solids }\end{array}$ & 47516 & PNNL 47516 Report.pdf \\
\hline TI-062 & 1.1 .5 & $\begin{array}{c}\text { Prepare, stage, and characterize } \\
\text { simulant (HLP-T22) and characterize } \\
\text { reagents }\end{array}$ & S T22RL 001 XX 0169 AFA 5 D & $\begin{array}{l}\text { Toluene } \\
\text { Supernate }\end{array}$ & 47516 & PNNL 47516 Report.pdf \\
\hline TI-062 & 1.1 .5 & $\begin{array}{c}\text { Prepare, stage, and characterize } \\
\text { simulant (HLP-T22) and characterize } \\
\text { reagents }\end{array}$ & S T22RL 001 XX 0170 AFA 5 B & $\begin{array}{l}\text { THF Wet } \\
\text { Solids }\end{array}$ & 47516 & PNNL 47516 Report.pdf \\
\hline TI-062 & 1.1 .5 & $\begin{array}{c}\text { Prepare, stage, and characterize } \\
\text { simulant (HLP-T22) and characterize } \\
\text { reagents }\end{array}$ & S T22RL 001 XX 0170 AFA 5 D & THF Supernate & 47516 & PNNL 47516 Report.pdf \\
\hline TI-062 & 1.1 .5 & $\begin{array}{c}\text { Prepare, stage, and characterize } \\
\text { simulant (HLP-T22) and characterize } \\
\text { reagents }\end{array}$ & S T22RL 001 XX 0168 AFA 2 & ICP Wet Solids & $081203-4$ & 081203-4 ICP ICPMS \\
\hline TI-062 & 1.1 .5 & $\begin{array}{c}\text { Prepare, stage, and characterize } \\
\text { simulant (HLP-T22) and characterize } \\
\text { reagents }\end{array}$ & S T22RL 001 XX 0168 AFA 2 & $\begin{array}{c}\text { ICP } \\
\text { Supernatant }\end{array}$ & 081203-4 & 081203-4 ICP ICPMS \\
\hline
\end{tabular}


TI-WTP-PEP-062 (testing dates 11/19/2008 through 12/01/2008)

\begin{tabular}{|c|c|c|c|c|c|c|}
\hline TI \# & $\begin{array}{c}\text { TI Step } \\
\#\end{array}$ & TI Step Description & Sample ID & Analysis & Folder & Final Electronic Report File Name \\
\hline TI-062 & $\mathrm{n} / \mathrm{a}$ & $\begin{array}{c}\text { Prepare, stage, and characterize } \\
\text { simulant (HLP-T22) and characterize } \\
\text { reagents }\end{array}$ & S T22RL 001 XX 0705 AFA 5 D & $\begin{array}{l}\text { Toluene } \\
\text { Supernate }\end{array}$ & 47516 & PNNL 47516 Report.pdf \\
\hline TI-062 & $\mathrm{n} / \mathrm{a}$ & $\begin{array}{c}\text { Prepare, stage, and characterize } \\
\text { simulant (HLP-T22) and characterize } \\
\text { reagents }\end{array}$ & S T22RL 001 XX 0706 AFA 5 D & $\begin{array}{l}\text { Toluene } \\
\text { Supernate }\end{array}$ & 47516 & PNNL 47516 Report.pdf \\
\hline TI-062 & 1.1 .5 & $\begin{array}{l}\text { Prepare, stage, and characterize } \\
\text { simulant }\end{array}$ & S T22RL 001 XX 163 RAM 3 D & Raman & ASR 8303 & ASR 8303; ASR 8303 rev. 1 \\
\hline TI-062 & 1.1 .5 & $\begin{array}{l}\text { Prepare, stage, and characterize } \\
\text { simulant }\end{array}$ & S T22RL 001 XX 164 RAM 3 D & Raman & ASR 8303 & ASR 8303; ASR 8303 rev. 1 \\
\hline TI-062 & 1.1 .5 & $\begin{array}{l}\text { Prepare, stage, and characterize } \\
\text { simulant }\end{array}$ & S T22RL 001 XX 165 RAM 3 D & Raman & ASR 8303 & ASR 8303; ASR 8303 rev. 1 \\
\hline TI-062 & 2.1 .2 & $\begin{array}{c}\text { Transfer from HLP-VSL-T22 to } \\
\text { UFP-VSL-01A }\end{array}$ & S T221A 002 XX 0177 UDS 2 & UDS & $081202-1$ & \\
\hline TI-062 & 2.1 .2 & $\begin{array}{c}\text { Transfer from HLP-VSL-T22 to } \\
\text { UFP-VSL-01A }\end{array}$ & S T221A 002 XX 0178 UDS 2 & UDS & $081125-10$ & \\
\hline TI-062 & 2.1 .2 & $\begin{array}{c}\text { Transfer from HLP-VSL-T22 to } \\
\text { UFP-VSL-01A }\end{array}$ & S T221A 002 XX 0179 UDS 2 & UDS & $081125-10$ & \\
\hline TI-062 & 2.1 .2 & $\begin{array}{c}\text { Transfer from HLP-VSL-T22 to } \\
\text { UFP-VSL-01A }\end{array}$ & S T221A 002 XX 0180 UDS 2 & UDS & $081125-10$ & \\
\hline TI-062 & 2.3 .1 & $\begin{array}{c}\text { Flush line between HLP-VSL-T22 and } \\
\text { UFP-VSL-T01B }\end{array}$ & S T221B 003 XX 0186 TFI 2 D & IC & $\begin{array}{l}081125-12 \\
081203-13\end{array}$ & 081125-12 and 081203-12 IC TIC TOC \\
\hline TI-062 & 2.3 .1 & $\begin{array}{c}\text { Flush line between HLP-VSL-T22 and } \\
\text { UFP-VSL-T01B }\end{array}$ & S T221B 003 XX 0187 TFI 2 D & $\mathrm{IC}$ & $\begin{array}{l}081125-12 \\
081203-13\end{array}$ & 081125-12 and 081203-12 IC TIC TOC \\
\hline TI-062 & 2.3 .1 & $\begin{array}{c}\text { Flush line between HLP-VSL-T22 and } \\
\text { UFP-VSL-T01B }\end{array}$ & S T221B 003 XX 0188 TFI 2 D & $\mathrm{IC}$ & $\begin{array}{c}081125-12 \\
081203-13\end{array}$ & 081125-12 and 081203-12 IC TIC TOC \\
\hline TI-062 & 2.3 .1 & $\begin{array}{c}\text { Flush line between HLP-VSL-T22 and } \\
\text { UFP-VSL-T01B }\end{array}$ & S T221B 003 XX 0189 TFI 2 D & $\mathrm{IC}$ & $\begin{array}{l}081125-12 \\
081203-13\end{array}$ & 081125-12 and 081203-12 IC TIC TOC \\
\hline TI-062 & 2.3 .1 & $\begin{array}{c}\text { Flush line between HLP-VSL-T22 and } \\
\text { UFP-VSL-T01B }\end{array}$ & S T221B 003 XX 0190 TFI 2 D & $\mathrm{IC}$ & $\begin{array}{l}081125-12 \\
081203-13\end{array}$ & 081125-12 and 081203-12 IC TIC TOC \\
\hline TI-062 & 2.3 .1 & $\begin{array}{c}\text { Flush line between UFP-VSL-T22 and } \\
\text { UFP-VSL-T01B }\end{array}$ & S T221B 003 XX 186 RAM 3 D & Raman & ASR 8300 & ASR 8300 RAM rev. 1 \\
\hline TI-062 & 2.3 .1 & $\begin{array}{l}\text { Flush line between UFP-VSL-T22 and } \\
\text { UFP-VSL-T01B }\end{array}$ & S T221B 003 XX 187 RAM 3 D & Raman & ASR 8300 & ASR 8300 RAM rev. 1 \\
\hline TI-062 & 2.3 .1 & $\begin{array}{c}\text { Flush line between UFP-VSL-T22 and } \\
\text { UFP-VSL-T01B }\end{array}$ & S T221B 003 XX 188 RAM 3 D & Raman & ASR 8300 & ASR 8300 RAM rev. 1 \\
\hline TI-062 & 2.3 .1 & $\begin{array}{l}\text { Flush line between UFP-VSL-T22 and } \\
\text { UFP-VSL-T01B }\end{array}$ & S T221B 003 XX 189 RAM 3 D & Raman & ASR 8300 & ASR 8300 RAM rev. 1 \\
\hline TI-062 & 2.3 .1 & $\begin{array}{c}\text { Flush line between UFP-VSL-T22 and } \\
\text { UFP-VSL-T01B }\end{array}$ & S T221B 003 XX 190 RAM 3 D & Raman & ASR 8300 & ASR 8300 RAM rev. 1 \\
\hline
\end{tabular}


TI-WTP-PEP-062 (testing dates 11/19/2008 through 12/01/2008)

\begin{tabular}{|c|c|c|c|c|c|c|}
\hline TI \# & $\begin{array}{c}\text { TI Step } \\
\#\end{array}$ & TI Step Description & Sample ID & Analysis & Folder & Final Electronic Report File Name \\
\hline TI-062 & 2.3 .1 & $\begin{array}{c}\text { Flush line between HLP-VSL-T22 and } \\
\text { UFP-VSL-T01B }\end{array}$ & $\begin{array}{l}\text { SPARE-02 S T221B } 003 \text { XX } \\
\text { 0181 UDS } 2\end{array}$ & UDS & $081125-10$ & \\
\hline TI-062 & 2.3 .1 & $\begin{array}{l}\text { Flush line between HLP-VSL-T22 and } \\
\text { UFP-VSL-T01B }\end{array}$ & $\begin{array}{l}\text { SPARE-03 S T221B } 003 \mathrm{XX} \\
0182 \text { UDS } 2\end{array}$ & UDS & $081125-10$ & \\
\hline TI-062 & 2.3 .1 & $\begin{array}{l}\text { Flush line between HLP-VSL-T22 and } \\
\text { UFP-VSL-T01B }\end{array}$ & $\begin{array}{l}\text { SPARE-04 S T221B } 003 \text { XX } \\
0183 \text { UDS } 2\end{array}$ & UDS & $081125-10$ & \\
\hline TI-062 & 2.3 .1 & $\begin{array}{c}\text { Flush line between HLP-VSL-T22 and } \\
\text { UFP-VSL-T01B }\end{array}$ & $\begin{array}{l}\text { SPARE-05 S T221B } 003 \text { XX } \\
0184 \text { UDS } 2\end{array}$ & UDS & $081125-10$ & \\
\hline TI-062 & 2.3 .1 & $\begin{array}{c}\text { Flush line between HLP-VSL-T22 and } \\
\text { UFP-VSL-T01B }\end{array}$ & $\begin{array}{l}\text { SPARE-06 S T221B } 003 \mathrm{XX} \\
0185 \text { UDS } 2\end{array}$ & UDS & $081125-10$ & \\
\hline TI-062 & 2.4 .4 & $\begin{array}{c}\text { Transfer from UFP-VSL-T01A to } \\
\text { UFP-VSL-T02A }\end{array}$ & S 01A2A 004 XX 0191 UDS 2 & UDS & $081202-1$ & \\
\hline TI-062 & 2.4 .4 & $\begin{array}{c}\text { Transfer from UFP-VSL-T01A to } \\
\text { UFP-VSL-T02A }\end{array}$ & S 01A2A 004 XX 0192 UDS 2 & UDS & $081202-1$ & \\
\hline TI-062 & 2.4 .4 & $\begin{array}{c}\text { Transfer from UFP-VSL-T01A to } \\
\text { UFP-VSL-T02A }\end{array}$ & S 01A2A 004 XX 0193 UDS 2 & UDS & $081202-1$ & \\
\hline TI-062 & 2.4 .4 & $\begin{array}{c}\text { Transfer from UFP-VSL-T01A to } \\
\text { UFP-VSL-T02A }\end{array}$ & S 01A2A 004 XX 0194 UDS 2 & UDS & $081202-1$ & \\
\hline TI-062 & 2.5 .2 & $\begin{array}{c}\text { Transfer from HLP-VSL-T22 to } \\
\text { UFP-VSL-T01A }\end{array}$ & S T221A 005 XX 0195 UDS 2 & UDS & $081125-10$ & \\
\hline TI-062 & 2.5 .2 & $\begin{array}{c}\text { Transfer from HLP-VSL-T22 to } \\
\text { UFP-VSL-T01A }\end{array}$ & S T221A 005 XX 0196 UDS 2 & UDS & $081125-10$ & \\
\hline TI-062 & 2.5.8 & $\begin{array}{l}\text { Test for stratification of solids in } \\
\text { UFP-T01A @ plant PJM frequency }\end{array}$ & S 01AIH 006120197 UDS 2 & UDS & $081202-1$ & \\
\hline TI-062 & 2.5 .8 & $\begin{array}{l}\text { Test for stratification of solids in } \\
\text { UFP-T01A @ plant PJM frequency }\end{array}$ & S 01AIH 006120198 UDS 2 & UDS & $081202-1$ & \\
\hline TI-062 & 2.5 .8 & $\begin{array}{l}\text { Test for stratification of solids in } \\
\text { UFP-T01A@plant PJM frequency }\end{array}$ & S 01AIH 006120199 UDS 2 & UDS & $081202-1$ & \\
\hline TI-062 & 2.5 .8 & $\begin{array}{l}\text { Test for stratification of solids in } \\
\text { UFP-T01A @ plant PJM frequency }\end{array}$ & S 01AIH 006120200 UDS 2 & UDS & $081202-1$ & \\
\hline TI-062 & 2.5 .8 & $\begin{array}{l}\text { Test for stratification of solids in } \\
\text { UFP-T01A@plant PJM frequency }\end{array}$ & S 01AIH 006120209 ICP 2 B & ICP Wet Solids & $081125-13$ & 081125-13 and 081203-2 ICP ICPMS \\
\hline TI-062 & 2.5 .8 & $\begin{array}{l}\text { Test for stratification of solids in } \\
\text { UFP-T01A @ plant PJM frequency }\end{array}$ & S 01AIH 006120210 XSP 4 B & PSD & TDP-WTP-354 & $\begin{array}{c}\text { TDP WTP } 354 \text { PEP Shakedown Test PSD Data } \\
\text { Analysis }\end{array}$ \\
\hline TI-062 & 2.5.8 & $\begin{array}{l}\text { Test for stratification of solids in } \\
\text { UFP-T01A @ plant PJM frequency }\end{array}$ & S 01AIH 006120702 XSP 4 B & PSD & TDP-WTP-354 & $\begin{array}{c}\text { TDP WTP } 354 \text { PEP Shakedown Test PSD Data } \\
\text { Analysis }\end{array}$ \\
\hline TI-062 & 2.5 .8 & $\begin{array}{l}\text { Test for stratification of solids in } \\
\text { UFP-T01A @ plant PJM frequency }\end{array}$ & S 01AIM 006120201 UDS 2 & UDS & $\begin{array}{l}081202-1 \\
081202-1 \\
081202-1 \\
081202-1\end{array}$ & $\begin{array}{l}081202-1 \\
081202-1 \\
081202-1 \\
081202-1\end{array}$ \\
\hline
\end{tabular}


TI-WTP-PEP-062 (testing dates 11/19/2008 through 12/01/2008)

\begin{tabular}{|c|c|c|c|c|c|c|}
\hline TI \# & $\begin{array}{c}\text { TI Step } \\
\#\end{array}$ & TI Step Description & Sample ID & Analysis & Folder & Final Electronic Report File Name \\
\hline TI-062 & 2.5 .8 & $\begin{array}{l}\text { Test for stratification of solids in } \\
\text { UFP-T01A@plant PJM frequency }\end{array}$ & S 01AIM 006120202 UDS 2 & UDS & 081202-1 & \\
\hline TI-062 & 2.5 .8 & $\begin{array}{l}\text { Test for stratification of solids in } \\
\text { UFP-T01A @ plant PJM frequency }\end{array}$ & S 01AIM 006120203 UDS 2 & UDS & $081202-1$ & \\
\hline TI-062 & 2.5 .8 & $\begin{array}{l}\text { Test for stratification of solids in } \\
\text { UFP-T01A@plant PJM frequency }\end{array}$ & S 01AIM 006120204 UDS 2 & UDS & 081202-1 & \\
\hline TI-062 & 2.5 .8 & $\begin{array}{l}\text { Test for stratification of solids in } \\
\text { UFP-T01A @ plant PJM frequency }\end{array}$ & S 01AIL 006120205 UDS 2 & UDS & $081202-1$ & \\
\hline TI-062 & 2.5 .8 & $\begin{array}{l}\text { Test for stratification of solids in } \\
\text { UFP-T01A@plant PJM frequency }\end{array}$ & S 01AIL 006120206 UDS 2 & UDS & $081202-1$ & \\
\hline TI-062 & 2.5 .8 & $\begin{array}{l}\text { Test for stratification of solids in } \\
\text { UFP-T01A@plant PJM frequency }\end{array}$ & S 01AIL 006120207 UDS 2 & UDS & $081202-1$ & \\
\hline TI-062 & 2.5 .8 & $\begin{array}{l}\text { Test for stratification of solids in } \\
\text { UFP-T01A@plant PJM frequency }\end{array}$ & S 01AIL 006120208 UDS 2 & UDS & 081202-1 & \\
\hline TI-062 & 2.5 .8 & $\begin{array}{l}\text { Test for stratification of solids in } \\
\text { UFP-T01A@plant PJM frequency }\end{array}$ & S 01AIL 006120211 ICP 2 B & ICP Wet Solids & 081202-1 & 081125-13 and 081203-2 ICP ICPMS \\
\hline TI-062 & 2.5 .8 & $\begin{array}{l}\text { Test for stratification of solids in } \\
\text { UFP-T01A @ plant PJM frequency }\end{array}$ & S 01AIL 006120212 XSP 4 B & PSD & TDP-WTP-354 & $\begin{array}{c}\text { TDP WTP } 354 \text { PEP Shakedown Test PSD Data } \\
\text { Analysis }\end{array}$ \\
\hline TI-062 & 2.5 .8 & $\begin{array}{l}\text { Test for stratification of solids in } \\
\text { UFP-T01A@ plant PJM frequency }\end{array}$ & S 01AIL 006120700 XSP 4 B & PSD & TDP-WTP-354 & $\begin{array}{c}\text { TDP WTP } 354 \text { PEP Shakedown Test PSD Data } \\
\text { Analysis }\end{array}$ \\
\hline TI-062 & 2.5 .8 & $\begin{array}{l}\text { Test for stratification of solids in } \\
\text { UFP-T01A@ plant PJM frequency }\end{array}$ & $\begin{array}{c}\text { SPARE-12 S 01AIL } 006120687 \\
\text { UDS } 2\end{array}$ & UDS & 081203-5 & \\
\hline TI-062 & 2.5 .8 & $\begin{array}{l}\text { Test for stratification of solids in } \\
\text { UFP-T01A @ plant PJM frequency }\end{array}$ & $\begin{array}{l}\text { SPARE-13 S 01AIL } 006120688 \\
\text { UDS } 2\end{array}$ & UDS & $081203-5$ & \\
\hline TI-062 & 2.5 .8 & $\begin{array}{l}\text { Test for stratification of solids in } \\
\text { UFP-T01A@plant PJM frequency }\end{array}$ & $\begin{array}{c}\text { SPARE-14 S 01AIL } 006120689 \\
\text { UDS } 2\end{array}$ & UDS & 081203-5 & \\
\hline TI-062 & 2.5 .8 & $\begin{array}{l}\text { Test for stratification of solids in } \\
\text { UFP-T01A@plant PJM frequency }\end{array}$ & $\begin{array}{l}\text { SPARE-15 S 01AIL } 006120690 \\
\text { UDS } 2\end{array}$ & UDS & $081203-5$ & \\
\hline TI-062 & 2.5 .8 & $\begin{array}{l}\text { Test for stratification of solids in } \\
\text { UFP-T01A @ plant PJM frequency }\end{array}$ & $\begin{array}{c}\text { SPARE-16 S 01AIM } 00612 \\
0691 \text { UDS } 2\end{array}$ & UDS & 081203-5 & \\
\hline TI-062 & 2.5 .8 & $\begin{array}{l}\text { Test for stratification of solids in } \\
\text { UFP-T01A @ plant PJM frequency }\end{array}$ & $\begin{array}{c}\text { SPARE-17 S 01AIM } 00612 \\
0692 \text { UDS } 2\end{array}$ & UDS & $081203-5$ & \\
\hline TI-062 & 2.5 .8 & $\begin{array}{l}\text { Test for stratification of solids in } \\
\text { UFP-T01A @ plant PJM frequency }\end{array}$ & $\begin{array}{c}\text { SPARE-18 S 01AIM } 00612 \\
0693 \text { UDS } 2\end{array}$ & UDS & $081203-5$ & \\
\hline TI-062 & 2.5 .8 & $\begin{array}{l}\text { Test for stratification of solids in } \\
\text { UFP-T01A@ plant PJM frequency }\end{array}$ & $\begin{array}{l}\text { SPARE-19 S 01AIM } 00612 \\
0694 \text { UDS } 2\end{array}$ & UDS & $081203-5$ & \\
\hline TI-062 & 2.5 .8 & $\begin{array}{l}\text { Test for stratification of solids in } \\
\text { UFP-T01A @ plant PJM frequency }\end{array}$ & $\begin{array}{c}\text { SPARE-20 S 01AIH } 006120695 \\
\text { UDS } 2\end{array}$ & UDS & $081203-5$ & \\
\hline TI-062 & 2.5 .8 & $\begin{array}{l}\text { Test for stratification of solids in } \\
\text { UFP-T01A @ plant PJM frequency }\end{array}$ & $\begin{array}{c}\text { SPARE-21 S 01AIH } 006120696 \\
\text { UDS } 2\end{array}$ & UDS & $081203-5$ & \\
\hline
\end{tabular}


TI-WTP-PEP-062 (testing dates 11/19/2008 through 12/01/2008)

\begin{tabular}{|c|c|c|c|c|c|c|}
\hline TI \# & $\begin{array}{c}\text { TI Step } \\
\#\end{array}$ & TI Step Description & Sample ID & Analysis & Folder & Final Electronic Report File Name \\
\hline TI-062 & 2.5.8 & $\begin{array}{l}\text { Test for stratification of solids in } \\
\text { UFP-T01A @ plant PJM frequency }\end{array}$ & $\begin{array}{c}\text { SPARE-22 S 01AIH } 006120697 \\
\text { UDS } 2\end{array}$ & UDS & $081203-5$ & \\
\hline TI-062 & 2.5 .8 & $\begin{array}{l}\text { Test for stratification of solids in } \\
\text { UFP-T01A @ plant PJM frequency }\end{array}$ & $\begin{array}{c}\text { SPARE-23 S 01AIH } 006120698 \\
\text { UDS } 2\end{array}$ & UDS & 081203-5 & \\
\hline TI-062 & 2.5 .8 & $\begin{array}{l}\text { Test for stratification of solids in } \\
\text { UFP-T01A @ plant PJM frequency }\end{array}$ & $\begin{array}{l}\text { SPARE-24 S 01AIL } 006120699 \\
\text { ICP } 2 \text { B }\end{array}$ & ICP Wet Solids & $081125-13$ & 081125-13 and 081203-2 ICP ICPMS \\
\hline TI-062 & 2.5 .8 & $\begin{array}{l}\text { Test for stratification of solids in } \\
\text { UFP-T01A @ plant PJM frequency }\end{array}$ & $\begin{array}{l}\text { SPARE-26 S 01AIH } 006120701 \\
\text { ICP } 2 \text { B }\end{array}$ & ICP Wet Solids & $081125-13$ & 081125-13 and 081203-2 ICP ICPMS \\
\hline TI-062 & 2.5 .8 & $\begin{array}{l}\text { Test for stratification of solids in } \\
\text { UFP-T01A @ plant PJM frequency }\end{array}$ & $\begin{array}{l}\text { SPARE-28 S 01AIL } 006120699 \\
\text { ICP } 2 \text { D }\end{array}$ & $\begin{array}{c}\text { ICP } \\
\text { Supernatant }\end{array}$ & 081203-1 & 081203-1 and 081203-3 Liquid ICP ICPMS \\
\hline TI-062 & 2.5.8 & $\begin{array}{l}\text { Test for stratification of solids in } \\
\text { UFP-T01A @ plant PJM frequency }\end{array}$ & $\begin{array}{c}\text { SPARE-30 S 01AIH } 006120701 \\
\text { ICP } 2 \text { D }\end{array}$ & $\begin{array}{c}\text { ICP } \\
\text { Supernatant }\end{array}$ & 081203-1 & 081203-1 and 081203-3 Liquid ICP ICPMS \\
\hline TI-062 & 2.5 .8 & $\begin{array}{l}\text { Test for stratification of solids in } \\
\text { UFP-T01A@plant PJM frequency }\end{array}$ & S 01AIH 006240213 UDS 2 & UDS & 0081203-7 & \\
\hline TI-062 & 2.5 .8 & $\begin{array}{l}\text { Test for stratification of solids in } \\
\text { UFP-T01A @ plant PJM frequency }\end{array}$ & S 01AIH 006240214 UDS 2 & UDS & 0081203-7 & \\
\hline TI-062 & 2.5 .8 & $\begin{array}{l}\text { Test for stratification of solids in } \\
\text { UFP-T01A @ plant PJM frequency }\end{array}$ & S 01AIH 006240215 UDS 2 & UDS & $0081203-7$ & \\
\hline TI-062 & 2.5 .8 & $\begin{array}{l}\text { Test for stratification of solids in } \\
\text { UFP-T01A @ plant PJM frequency }\end{array}$ & S 01AIH 006240216 UDS 2 & UDS & 0081203-7 & \\
\hline TI-062 & 2.5 .8 & $\begin{array}{l}\text { Test for stratification of solids in } \\
\text { UFP-T01A@ plant PJM frequency }\end{array}$ & S 01AIM 006240217 UDS 2 & UDS & $081203-10$ & \\
\hline TI-062 & 2.5 .8 & $\begin{array}{l}\text { Test for stratification of solids in } \\
\text { UFP-T01A @ plant PJM frequency }\end{array}$ & S 01AIM 006240218 UDS 2 & UDS & $081203-10$ & \\
\hline TI-062 & 2.5 .8 & $\begin{array}{l}\text { Test for stratification of solids in } \\
\text { UFP-T01A @ plant PJM frequency }\end{array}$ & S 01AIM 006240219 UDS 2 & UDS & $081203-10$ & \\
\hline TI-062 & 2.5 .8 & $\begin{array}{l}\text { Test for stratification of solids in } \\
\text { UFP-T01A @ plant PJM frequency }\end{array}$ & S 01AIM 006240220 UDS 2 & UDS & $081203-10$ & \\
\hline TI-062 & 2.5 .8 & $\begin{array}{l}\text { Test for stratification of solids in } \\
\text { UFP-T01A @ plant PJM frequency }\end{array}$ & S 01AIL 006240221 UDS 2 & UDS & 0081203-7 & \\
\hline TI-062 & 2.5 .8 & $\begin{array}{l}\text { Test for stratification of solids in } \\
\text { UFP-T01A @ plant PJM frequency }\end{array}$ & S 01AIL 006240222 UDS 2 & UDS & $081203-10$ & \\
\hline TI-062 & 2.5 .8 & $\begin{array}{l}\text { Test for stratification of solids in } \\
\text { UFP-T01A @ plant PJM frequency }\end{array}$ & S 01AIL 006240223 UDS 2 & UDS & $081203-10$ & \\
\hline TI-062 & 2.5 .8 & $\begin{array}{l}\text { Test for stratification of solids in } \\
\text { UFP-T01A @ plant PJM frequency }\end{array}$ & S 01AIL 006240224 UDS 2 & UDS & 081203-10 & \\
\hline TI-062 & 2.5 .8 & $\begin{array}{l}\text { Test for stratification of solids in } \\
\text { UFP-T01A@ plant PJM frequency }\end{array}$ & S 01AIH 006360225 UDS 2 & UDS & 0081203-7 & \\
\hline TI-062 & 2.5 .8 & $\begin{array}{l}\text { Test for stratification of solids in } \\
\text { UFP-T01A @ plant PJM frequency }\end{array}$ & S 01AIH 006360226 UDS 2 & UDS & 0081203-7 & \\
\hline
\end{tabular}


TI-WTP-PEP-062 (testing dates 11/19/2008 through 12/01/2008)

\begin{tabular}{|c|c|c|c|c|c|c|}
\hline TI \# & $\begin{array}{c}\text { TI Step } \\
\#\end{array}$ & TI Step Description & Sample ID & Analysis & Folder & Final Electronic Report File Name \\
\hline TI-062 & 2.5 .8 & $\begin{array}{l}\text { Test for stratification of solids in } \\
\text { UFP-T01A@plant PJM frequency }\end{array}$ & S 01AIH 006360227 UDS 2 & UDS & 0081203-7 & \\
\hline TI-062 & 2.5 .8 & $\begin{array}{l}\text { Test for stratification of solids in } \\
\text { UFP-T01A @ plant PJM frequency }\end{array}$ & S 01AIH 006360228 UDS 2 & UDS & $0081203-7$ & \\
\hline TI-062 & 2.5 .8 & $\begin{array}{l}\text { Test for stratification of solids in } \\
\text { UFP-T01A@plant PJM frequency }\end{array}$ & S 01AIL 006360233 UDS 2 & UDS & $081203-10$ & \\
\hline TI-062 & 2.5 .8 & $\begin{array}{l}\text { Test for stratification of solids in } \\
\text { UFP-T01A @ plant PJM frequency }\end{array}$ & S 01AIL 006360234 UDS 2 & UDS & $081203-10$ & \\
\hline TI-062 & 2.5 .8 & $\begin{array}{l}\text { Test for stratification of solids in } \\
\text { UFP-T01A@plant PJM frequency }\end{array}$ & S 01AIL 006360235 UDS 2 & UDS & $081203-10$ & \\
\hline TI-062 & 2.5 .8 & $\begin{array}{l}\text { Test for stratification of solids in } \\
\text { UFP-T01A@plant PJM frequency }\end{array}$ & S 01AIL 006360236 UDS 2 & UDS & $081203-10$ & \\
\hline TI-062 & 2.5 .8 & $\begin{array}{l}\text { Test for stratification of solids in } \\
\text { UFP-T01A@plant PJM frequency }\end{array}$ & S 01AIM 006360229 UDS 2 & UDS & $081203-10$ & \\
\hline TI-062 & 2.5 .8 & $\begin{array}{l}\text { Test for stratification of solids in } \\
\text { UFP-T01A@plant PJM frequency }\end{array}$ & S 01AIM 006360230 UDS 2 & UDS & $081203-10$ & \\
\hline TI-062 & 2.5 .8 & $\begin{array}{l}\text { Test for stratification of solids in } \\
\text { UFP-T01A@plant PJM frequency }\end{array}$ & S 01AIM 006360231 UDS 2 & UDS & $081203-10$ & \\
\hline TI-062 & 2.5 .8 & $\begin{array}{l}\text { Test for stratification of solids in } \\
\text { UFP-T01A@plant PJM frequency }\end{array}$ & S01AIM 006360232 UDS 2 & UDS & $081203-10$ & \\
\hline TI-062 & 2.11.6.1 & $\begin{array}{l}\text { Test for stratification of solids in } \\
\text { UFP-T01A@plant PJM frequency }\end{array}$ & S 01AIH 006360237 ICP 2 B & ICP Wet Solids & $081125-13$ & 081125-13 and 081203-2 ICP ICPMS \\
\hline TI-062 & 2.11.6.1 & $\begin{array}{l}\text { Test for stratification of solids in } \\
\text { UFP-T01A @ plant PJM frequency }\end{array}$ & S 01AIH 006360238 XSP 4 B & PSD & TDP-WTP-354 & $\begin{array}{c}\text { TDP WTP } 354 \text { PEP Shakedown Test PSD Data } \\
\text { Analysis }\end{array}$ \\
\hline TI-062 & 2.11.6.1 & $\begin{array}{l}\text { Test for stratification of solids in } \\
\text { UFP-T01A@plant PJM frequency }\end{array}$ & S 01AIL 006360239 ICP 2 B & ICP Wet Solids & $081125-13$ & 081125-13 and 081203-2 ICP ICPMS \\
\hline TI-062 & 2.11.6.1 & $\begin{array}{l}\text { Test for stratification of solids in } \\
\text { UFP-T01A@plant PJM frequency }\end{array}$ & S 01AIL 006360240 XSP 4 B & PSD & TDP-WTP-354 & $\begin{array}{c}\text { TDP WTP } 354 \text { PEP Shakedown Test PSD Data } \\
\text { Analysis }\end{array}$ \\
\hline TI-062 & 2.7 .6 & Conduct low-solids filter flux test & S 000FL 008 XX 0241 UDS 2 & UDS & $081125-6$ & \\
\hline TI-062 & 2.7 .6 & Conduct low-solids filter flux test & S 000FL 008 XX 0242 UDS 2 & UDS & $081125-6$ & \\
\hline TI-062 & 2.7 .6 & Conduct low-solids filter flux test & S 000FL 008 XX 0243 UDS 2 & UDS & $081125-6$ & \\
\hline TI-062 & 2.7 .6 & Conduct low-solids filter flux test & S 000FL 008 XX 0244 DEN 2 & Slurry Density & $081125-11$ & 081125-11 Den \\
\hline TI-062 & 2.7 .6 & Conduct low-solids filter flux test & S 000FL 008 XX 0245 DEN 2 & Slurry Density & $081125-11$ & 081125-11 Den \\
\hline TI-062 & 2.7 .6 & Conduct low-solids filter flux test & S 000FL 008 XX 0246 DEN 2 & Slurry Density & 081125-11 & 081125-11 Den \\
\hline TI-062 & 2.7 .6 & Conduct low-solids filter flux test & S 000FL 008 XX 0247 RHE 4 & Rheology & TDP-WTP-355 & Shakedown Rheology Data on PEP Slurry \\
\hline TI-062 & 2.7 .6 & Conduct low-solids filter flux test & S 000FL 008 XX 0248 RHE 4 & Rheology & TDP-WTP-355 & Shakedown Rheology Data on PEP Slurry \\
\hline TI-062 & 2.7 .6 & Conduct low-solids filter flux test & S 000FL 008 XX 0249 XSP 4 B & PSD & TDP-WTP-354 & $\begin{array}{c}\text { TDP WTP } 354 \text { PEP Shakedown Test PSD Data } \\
\text { Analysis }\end{array}$ \\
\hline TI-062 & 2.7 .6 & Conduct low-solids filter flux test & S 000FL 008 XX 0250 CUF 4 & CUF & & \\
\hline TI-062 & 2.7 .7 & Conduct low-solids filter flux test & S 000FL 008 XX 0266 UDS 2 & UDS & $081125-6$ & \\
\hline TI-062 & 2.7 .7 & Conduct low-solids filter flux test & S 000FL 008 XX 0267 UDS 2 & UDS & $081125-6$ & \\
\hline
\end{tabular}


TI-WTP-PEP-062 (testing dates 11/19/2008 through 12/01/2008)

\begin{tabular}{|c|c|c|c|c|c|c|}
\hline TI \# & $\begin{array}{c}\text { TI Step } \\
\#\end{array}$ & TI Step Description & Sample ID & Analysis & Folder & Final Electronic Report File Name \\
\hline TI-062 & 2.7 .7 & Conduct low-solids filter flux test & S 000FL 008 XX 0268 UDS 2 & UDS & $081125-6$ & \\
\hline TI-062 & 2.7 .7 & Conduct low-solids filter flux test & S 000FL 008 XX 0269 UDS 2 & UDS & $081125-6$ & \\
\hline TI-062 & 2.7 .7 & Conduct low-solids filter flux test & S 000FL 008 XX 0270 UDS 2 & UDS & $081125-6$ & \\
\hline TI-062 & 2.7 .7 & Conduct low-solids filter flux test & S 000FL 008 XX 0271 UDS 2 & UDS & $081125-6$ & \\
\hline TI-062 & 2.7 .7 & Conduct low-solids filter flux test & S 000FL 008 XX 0272 UDS 2 & UDS & $081125-6$ & \\
\hline TI-062 & 2.7 .7 & Conduct low-solids filter flux test & S 000FL 008 XX 0273 UDS 2 & UDS & $081125-6$ & \\
\hline TI-062 & 2.7 .7 & Conduct low-solids filter flux test & S 000FL 008 XX 0274 UDS 2 & UDS & $081125-6$ & \\
\hline TI-062 & 2.7 .7 & Conduct low-solids filter flux test & S 000FL 008 XX 0275 UDS 2 & UDS & $081125-6$ & \\
\hline TI-062 & 2.7 .7 & Conduct low-solids filter flux test & S 000FL 008 XX 0276 UDS 2 & UDS & $081125-6$ & \\
\hline TI-062 & 2.7 .7 & Conduct low-solids filter flux test & S 000FL 008 XX 0277 UDS 2 & UDS & $081125-6$ & \\
\hline TI-062 & 2.7 .7 & Conduct low-solids filter flux test & S 000FL 008 XX 0278 UDS 2 & UDS & $081125-6$ & \\
\hline TI-062 & 2.7 .7 & Conduct low-solids filter flux test & S 000FL 008 XX 0279 UDS 2 & UDS & $081125-6$ & \\
\hline TI-062 & 2.7 .7 & Conduct low-solids filter flux test & S 000FL 008 XX 0280 UDS 2 & UDS & $081125-6$ & \\
\hline TI-062 & 2.7 .7 & Conduct low-solids filter flux test & S 000FL 008 XX 0281 UDS 2 & UDS & $081125-6$ & \\
\hline TI-062 & 2.7.7 & Conduct low-solids filter flux test & S 000FL 008 XX 0282 UDS 2 & UDS & $081125-6$ & \\
\hline TI-062 & 2.7 .7 & Conduct low-solids filter flux test & S 000FL 008 XX 0283 UDS 2 & UDS & $081125-7$ & \\
\hline TI-062 & 2.7 .7 & Conduct low-solids filter flux test & S 000FL 008 XX 0284 UDS 2 & UDS & $081125-7$ & \\
\hline TI-062 & 2.7 .7 & Conduct low-solids filter flux test & S 000FL 008 XX 0285 UDS 2 & UDS & $081125-7$ & \\
\hline TI-062 & 2.7 .7 & Conduct low-solids filter flux test & S 000FL 008 XX 0286 UDS 2 & UDS & $081125-7$ & \\
\hline TI-062 & 2.7 .7 & Conduct low-solids filter flux test & S 000FL 008 XX 0287 UDS 2 & UDS & $081125-7$ & \\
\hline TI-062 & 2.7 .7 & Conduct low-solids filter flux test & S 000FL 008 XX 0288 UDS 2 & UDS & $081125-7$ & \\
\hline TI-062 & 2.7 .7 & Conduct low-solids filter flux test & S 000FL 008 XX 0289 UDS 2 & UDS & $081125-7$ & \\
\hline TI-062 & 2.7 .7 & Conduct low-solids filter flux test & S 000FL 008 XX 0290 UDS 2 & UDS & $081125-7$ & \\
\hline TI-062 & 2.7 .7 & Conduct low-solids filter flux test & S 000FL 008 XX 0291 UDS 2 & UDS & $081125-7$ & \\
\hline TI-062 & 2.7 .7 & Conduct low-solids filter flux test & S 000FL 008 XX 0292 UDS 2 & UDS & $081125-7$ & \\
\hline TI-062 & 2.7 .7 & Conduct low-solids filter flux test & S 000FL 008 XX 0293 UDS 2 & UDS & $081125-7$ & \\
\hline TI-062 & 2.7 .7 & Conduct low-solids filter flux test & S 000FL 008 XX 0294 UDS 2 & UDS & $081125-7$ & \\
\hline TI-062 & 2.7 .7 & Conduct low-solids filter flux test & S 000FL 008 XX 0295 UDS 2 & UDS & 081125-7 & \\
\hline TI-062 & 2.7 .7 & Conduct low-solids filter flux test & S 02AMM 008 XX 0296 UDS 2 & UDS & $081125-8$ & \\
\hline TI-062 & 2.7 .7 & Conduct low-solids filter flux test & S 02AMM 008 XX 0297 UDS 2 & UDS & $081125-8$ & \\
\hline TI-062 & 2.7 .7 & Conduct low-solids filter flux test & S 02AMM 008 XX 0298 UDS 2 & UDS & $081125-8$ & \\
\hline TI-062 & 2.7 .7 & Conduct low-solids filter flux test & S 02AMM 008 XX 0299 UDS 2 & UDS & $081125-8$ & \\
\hline TI-062 & 2.7 .7 & Conduct low-solids filter flux test & S 02AMM 008 XX 0300 UDS 2 & UDS & $081125-8$ & \\
\hline TI-062 & 2.7 .7 & Conduct low-solids filter flux test & S 02AMM 008 XX 0301 UDS 2 & UDS & $081125-8$ & \\
\hline TI-062 & 2.7 .7 & Conduct low-solids filter flux test & S 02AMM 008 XX 0302 UDS 2 & UDS & $081125-8$ & \\
\hline TI-062 & 2.7.7 & Conduct low-solids filter flux test & S 02AMM 008 XX 0303 UDS 2 & UDS & $081125-8$ & \\
\hline TI-062 & 2.7 .7 & Conduct low-solids filter flux test & S 02AMM 008 XX 0304 UDS 2 & UDS & $081125-8$ & \\
\hline TI-062 & 2.7.7 & Conduct low-solids filter flux test & S 02AMM 008 XX 0305 UDS 2 & UDS & $081125-8$ & \\
\hline TI-062 & 2.7 .7 & Conduct low-solids filter flux test & S 02AMM 008 XX 0306 UDS 2 & UDS & $081125-8$ & \\
\hline TI-062 & 2.7.7 & Conduct low-solids filter flux test & S 02AMM 008 XX 0307 UDS 2 & UDS & $081125-9$ & \\
\hline TI-062 & 2.7 .7 & Conduct low-solids filter flux test & S 02AMM 008 XX 0308 UDS 2 & UDS & $081125-9$ & \\
\hline
\end{tabular}


TI-WTP-PEP-062 (testing dates 11/19/2008 through 12/01/2008)

\begin{tabular}{|c|c|c|c|c|c|c|}
\hline TI \# & $\begin{array}{c}\text { TI Step } \\
\#\end{array}$ & TI Step Description & Sample ID & Analysis & Folder & Final Electronic Report File Name \\
\hline TI-062 & 2.7 .7 & Conduct low-solids filter flux test & S 02AMM 008 XX 0309 UDS 2 & UDS & $081125-9$ & \\
\hline TI-062 & 2.7 .7 & Conduct low-solids filter flux test & S 02AMM 008 XX 0310 UDS 2 & UDS & $081125-9$ & \\
\hline TI-062 & 2.7.7 & Conduct low-solids filter flux test & S 02AMM 008 XX 0311 UDS 2 & UDS & $081125-9$ & \\
\hline TI-062 & 2.7.7 & Conduct low-solids filter flux test & S 02AMM 008 XX 0312 UDS 2 & UDS & $081125-9$ & \\
\hline TI-062 & 2.7.7 & Conduct low-solids filter flux test & S 02AMM 008 XX 0313 UDS 2 & UDS & $081125-9$ & \\
\hline TI-062 & 2.7 .7 & Conduct low-solids filter flux test & S 02AMM 008 XX 0314 UDS 2 & UDS & $081125-9$ & \\
\hline TI-062 & 2.7 .7 & Conduct low-solids filter flux test & S 02AMM 008 XX 0315 UDS 2 & UDS & $081125-9$ & \\
\hline TI-062 & 2.7 .7 & Conduct low-solids filter flux test & S 02AMM 008 XX 0316 UDS 2 & UDS & $081125-9$ & \\
\hline TI-062 & 2.7 .7 & Conduct low-solids filter flux test & S 02AMM 008 XX 0317 UDS 2 & UDS & $081125-9$ & \\
\hline TI-062 & 2.7 .7 & Conduct low-solids filter flux test & S 02AMM 008 XX 0318 UDS 2 & UDS & $081125-9$ & \\
\hline TI-062 & 2.7 .7 & Conduct low-solids filter flux test & S 02AMM 008 XX 0319 UDS 2 & UDS & $081125-9$ & \\
\hline TI-062 & 2.7 .7 & Conduct low-solids filter flux test & S 02AMM 008 XX 0320 UDS 2 & UDS & $081125-9$ & \\
\hline TI-062 & 2.7 .7 & Conduct low-solids filter flux test & S 02AMM 008 XX 0321 UDS 2 & UDS & 081125-9 & \\
\hline TI-062 & 2.7 .7 & Conduct low-solids filter flux test & S 02AMM 008 XX 0322 UDS 2 & UDS & $081125-9$ & \\
\hline TI-062 & 2.7 .7 & Conduct low-solids filter flux test & S 02AMM 008 XX 0323 UDS 2 & UDS & $081125-9$ & \\
\hline TI-062 & 2.7 .7 & Conduct low-solids filter flux test & S 02AMM 008 XX 0324 UDS 2 & UDS & $081125-9$ & \\
\hline TI-062 & 2.7 .7 & Conduct low-solids filter flux test & S 02AMM 008 XX 0325 UDS 2 & UDS & 081125-9 & \\
\hline TI-062 & 2.7 .7 & Conduct low-solids filter flux test & S 00PF4 008 XX 0260 ARC 1 & \multirow{2}{*}{\multicolumn{2}{|c|}{$\begin{array}{l}\text { Archive } \\
\text { Archive }\end{array}$}} & \\
\hline TI-062 & 2.7 .7 & Conduct low-solids filter flux test & S 00PF3 008 XX 0257 ARC 1 & & & \\
\hline TI-062 & 2.7 .7 & Conduct low-solids filter flux test & S 00PF2 008 XX 0254 ARC 1 & \multicolumn{2}{|c|}{$\begin{array}{l}\text { Archive } \\
\text { Archive }\end{array}$} & \\
\hline TI-062 & 2.7 .7 & Conduct low-solids filter flux test & S 00PF1 008 XX 0251 ARC 1 & \multicolumn{2}{|c|}{ Archive } & \\
\hline TI-062 & 2.7 .7 & Conduct low-solids filter flux test & S 00PF5 008 XX 0263 ARC 1 & \multicolumn{2}{|c|}{ Archive } & \\
\hline TI-062 & 2.7 .7 & Conduct low-solids filter flux test & S 00PF1 008 XX 0252 ARC 1 & \multicolumn{2}{|c|}{ Archive } & \\
\hline TI-062 & 2.7 .7 & Conduct low-solids filter flux test & S 00PF2 008 XX 0255 ARC 1 & \multicolumn{2}{|c|}{ Archive } & \\
\hline TI-062 & 2.7 .7 & Conduct low-solids filter flux test & S 00PF3 008 XX 0258 ARC 1 & \multicolumn{2}{|c|}{ Archive } & \\
\hline TI-062 & 2.7 .7 & Conduct low-solids filter flux test & S 00PF5 008 XX 0264 ARC 1 & \multicolumn{2}{|c|}{ Archive } & \\
\hline TI-062 & 2.7 .7 & Conduct low-solids filter flux test & S 00PF4 008 XX 0261 ARC 1 & \multicolumn{2}{|c|}{ Archive } & \\
\hline TI-062 & 2.7 .7 & Conduct low-solids filter flux test & S 00PF5 008 XX 0265 ARC 1 & \multicolumn{2}{|c|}{ Archive } & \\
\hline TI-062 & 2.7.7 & Conduct low-solids filter flux test & S 00PF4 008 XX 0262 ARC 1 & \multicolumn{2}{|c|}{ Archive } & \\
\hline TI-062 & 2.7.7 & Conduct low-solids filter flux test & S 00PF3 008 XX 0259 ARC 1 & \multicolumn{2}{|c|}{ Archive } & \\
\hline TI-062 & 2.7 .7 & Conduct low-solids filter flux test & S 00PF2 008 XX 0256 ARC 1 & \multicolumn{2}{|c|}{ Archive } & \\
\hline TI-062 & 2.7 .7 & Conduct low-solids filter flux test & S 00PF1 008 XX 0253 ARC 1 & \multicolumn{2}{|c|}{ Archive } & \\
\hline TI-062 & 2.8.3.1 & Backpulse filters & S 000FL 009 XX 0326 UDS 2 & UDS & 081203-15 & \\
\hline TI-062 & 2.8.3.1 & Backpulse filters & S 000FL 009 XX 0327 XSP 4 B & PSD & TDP-WTP-354 & $\begin{array}{c}\text { TDP WTP } 354 \text { PEP Shakedown Test PSD Data } \\
\text { Analysis }\end{array}$ \\
\hline TI-062 & 2.8.3.1 & Backpulse filters & S 000FL 009 XX 0328 CUF 4 & CUF & & \\
\hline TI-062 & 2.9 .2 & Conduct low-solids filter flux test & S 000FL 010 XX 0329 UDS 2 & UDS & $081203-15$ & \\
\hline TI-062 & 2.9 .2 & Conduct low-solids filter flux test & S 000FL 010 XX 0330 UDS 2 & UDS & $081203-15$ & \\
\hline TI-062 & 2.9 .2 & Conduct low-solids filter flux test & S 000FL 010 XX 0331 UDS 2 & UDS & 081203-15 & \\
\hline TI-062 & 2.9.2 & Conduct low-solids filter flux test & S 000FL 010 XX 0332 XSP 4 B & PSD & TDP-WTP-354 & $\begin{array}{c}\text { TDP WTP } 354 \text { PEP Shakedown Test PSD Data } \\
\text { Analysis }\end{array}$ \\
\hline
\end{tabular}


TI-WTP-PEP-062 (testing dates 11/19/2008 through 12/01/2008)

\begin{tabular}{|c|c|c|c|c|c|c|}
\hline TI \# & $\begin{array}{c}\text { TI Step } \\
\#\end{array}$ & TI Step Description & Sample ID & Analysis & Folder & Final Electronic Report File Name \\
\hline TI-062 & 2.10 .10 & Concentrate solids in UFP-VSL-T02A & S 000FL 011 XX 0333 UDS 2 & (1) & & \\
\hline TI-062 & 2.10 .10 & Concentrate solids in UFP-VSL-T02A & S 000FL 011 XX 0334 UDS 2 & (1) & & \\
\hline TI-062 & 2.10 .10 & Concentrate solids in UFP-VSL-T02A & S 000FL 011 XX 0335 UDS 2 & (1) & & \\
\hline TI-062 & 2.10 .10 & Concentrate solids in UFP-VSL-T02A & S 000FL 011 XX 0336 RHE 4 & Rheology & TDP-WTP-355 & Shakedown Rheology Data on PEP Slurry \\
\hline TI-062 & 2.12 .8 & $\begin{array}{c}\text { Initial solids concentration (dewater) } \\
\text { to } 20-\mathrm{wt} \% \text { UDS }\end{array}$ & S 000FL 015 XX 0338 UDS 2 & (1) & & \\
\hline TI-062 & 2.12 .8 & $\begin{array}{c}\text { Initial solids concentration (dewater) } \\
\text { to } 20-\mathrm{wt} \% \text { UDS }\end{array}$ & S 000FL 015 XX 0340 UDS 2 & (1) & & \\
\hline TI-062 & 2.12 .8 & $\begin{array}{c}\text { Initial solids concentration (dewater) } \\
\text { to } 20-\mathrm{wt} \% \text { UDS }\end{array}$ & S 000FL 015 XX 0342 UDS 2 & (1) & & \\
\hline TI-062 & 2.12 .8 & $\begin{array}{l}\text { Initial solids concentration (dewater) } \\
\text { to } 20-\mathrm{wt} \% \text { UDS }\end{array}$ & S 000FL 015 XX 0344 UDS 2 & (1) & & \\
\hline TI-062 & 2.12 .8 & $\begin{array}{l}\text { Initial solids concentration (dewater) } \\
\text { to } 20-\mathrm{wt} \% \text { UDS }\end{array}$ & S 000FL 015 XX 0348 UDS 2 & (1) & & \\
\hline TI-062 & 2.12 .8 & $\begin{array}{l}\text { Initial solids concentration (dewater) } \\
\text { to } 20-\mathrm{wt} \% \text { UDS }\end{array}$ & S 000FL 015 XX 0349 UDS 2 & (1) & & \\
\hline TI-062 & 2.12 .8 & $\begin{array}{c}\text { Initial solids concentrations (dewater) } \\
\text { to } 20 \text {-wt } \% \text { UDS }\end{array}$ & S 000FL 015 XX 0350 RHE 4 & Rheology & TDP-WTP-355 & $\begin{array}{l}\text { TDP-WTP-355 Shakedown RHE Data on PEP } \\
\text { Slurry }\end{array}$ \\
\hline TI-062 & 2.12 .8 & $\begin{array}{l}\text { Initial solids concentration (dewater) } \\
\text { to } 20-\mathrm{wt} \% \text { UDS }\end{array}$ & S 01A2A 015 XX 0337 UDS 2 & (1) & & \\
\hline TI-062 & 2.12 .8 & $\begin{array}{l}\text { Initial solids concentration (dewater) } \\
\text { to } 20-\mathrm{wt} \% \text { UDS }\end{array}$ & S 01A2A 015 XX 0339 UDS 2 & (1) & & \\
\hline TI-062 & 2.12 .8 & $\begin{array}{c}\text { Initial solids concentration (dewater) } \\
\text { to } 20-\mathrm{wt} \% \text { UDS }\end{array}$ & S 01A2A 015 XX 0341 UDS 2 & (1) & & \\
\hline TI-062 & 2.12 .8 & $\begin{array}{c}\text { Initial solids concentration (dewater) } \\
\text { to } 20-w t \% \text { UDS }\end{array}$ & S 01A2A 015 XX 0343 UDS 2 & (1) & & \\
\hline TI-062 & 2.12 .8 & $\begin{array}{l}\text { Initial solids concentration (dewater) } \\
\text { to } 20-\mathrm{wt} \% \text { UDS }\end{array}$ & S 01A2A 015 XX 0345 UDS 2 & (1) & & \\
\hline TI-062 & 2.12 .9 & $\begin{array}{c}\text { Initial solids concentration (dewater) } \\
\text { to } 20-\mathrm{wt} \% \text { UDS }\end{array}$ & S 000FL 015 XX 0354 DEN 2 & (1) & & \\
\hline TI-062 & 2.12 .9 & $\begin{array}{c}\text { Initial solids concentration (dewater) } \\
\text { to } 20-\mathrm{wt} \% \text { UDS }\end{array}$ & S 000FL 015 XX 0355 DEN 2 & (1) & & \\
\hline TI-062 & 2.12 .9 & $\begin{array}{l}\text { Initial solids concentration (dewater) } \\
\text { to } 20-w t \% \text { UDS }\end{array}$ & S 000FL 015 XX 0356 DEN 2 & (1) & & \\
\hline TI-062 & 2.12 .9 & $\begin{array}{c}\text { Initial solids concentrations (dewater) } \\
\text { to } 20-w t \% \text { UDS }\end{array}$ & S 000FL 015 XX 0357 RHE 4 & Rheology & TDP-WTP-355 & $\begin{array}{l}\text { TDP-WTP-355 Shakedown RHE Data on PEP } \\
\text { Slurry }\end{array}$ \\
\hline TI-062 & 2.12 .9 & $\begin{array}{c}\text { Initial solids concentrations (dewater) } \\
\text { to } 20-\mathrm{wt} \% \text { UDS }\end{array}$ & S 000FL 015 XX 0358 RHE 4 & Rheology & TDP-WTP-355 & $\begin{array}{c}\text { TDP-WTP-355 Shakedown RHE Data on PEP } \\
\text { Slurry }\end{array}$ \\
\hline TI-062 & 2.12 .9 & $\begin{array}{l}\text { Initial solids concentration (dewater) } \\
\text { to } 20-w t \% \text { UDS }\end{array}$ & S 000FL 015 XX 0359 ICP 2 B & $(1),(2)$ & 081203-2 & 081125-13 and 081203-2 Solid ICP ICPMS \\
\hline
\end{tabular}


TI-WTP-PEP-062 (testing dates 11/19/2008 through 12/01/2008)

\begin{tabular}{|c|c|c|c|c|c|c|}
\hline TI\# & $\begin{array}{c}\text { TI Step } \\
\text { \# }\end{array}$ & TI Step Description & Sample ID & Analysis & Folder & Final Electronic Report File Name \\
\hline TI-062 & 2.12 .9 & $\begin{array}{l}\text { Initial solids concentration (dewater) } \\
\text { to } 20 \text {-wt } \% \text { UDS }\end{array}$ & S 000FL 015 XX 0359 TFI 2 D & (1) & & \\
\hline TI-062 & 2.12 .9 & $\begin{array}{l}\text { Initial solids concentration (dewater) } \\
\text { to } 20 \text {-wt } \% \text { UDS }\end{array}$ & S 000FL 015 XX 0359 TFI 2 D & (1) & & \\
\hline TI-062 & 2.12 .9 & Initial solids concentration & S 000FL 015 XX 0359 RAM 3 D & Raman & ASR 8303 & ASR 8303; ASR 8303 rev. 1 \\
\hline TI-062 & 2.12 .9 & $\begin{array}{c}\text { Initial solids concentration (dewater) } \\
\text { to } 20 \text {-wt } \% \text { UDS }\end{array}$ & S 000FL 015 XX 0360 ICP 2 B & $(1),(2)$ & 081203-2 & 081125-13 and 081203-2 Solid ICP ICPMS \\
\hline TI-062 & 2.12 .9 & $\begin{array}{l}\text { Initial solids concentration (dewater) } \\
\text { to } 20 \text {-wt } \% \text { UDS }\end{array}$ & S 000FL 015 XX 0360 TFI 2 D & (1) & & \\
\hline TI-062 & 2.12 .9 & $\begin{array}{c}\text { Initial solids concentration (dewater) } \\
\text { to } 20 \text { - } \mathrm{wt} \% \text { UDS }\end{array}$ & S 000FL 015 XX 0360 TFI 2 D & (1) & & \\
\hline TI-062 & 2.12 .9 & Initial solids concentration & S 000FL 015 XX 0360 RAM 3 D & Raman & ASR 8303 & ASR 8303; ASR 8303 rev. 1 \\
\hline TI-062 & 2.12 .9 & $\begin{array}{l}\text { Initial solids concentration (dewater) } \\
\text { to } 20 \text {-wt } \% \text { UDS }\end{array}$ & S 000FL 015 XX 0361 ICP 2 B & $(1),(2)$ & 081203-2 & 081125-13 and 081203-2 Solid ICP ICPMS \\
\hline TI-062 & 2.12 .9 & $\begin{array}{l}\text { Initial solids concentration (dewater) } \\
\text { to } 20 \text {-wt } \% \text { UDS }\end{array}$ & S 000FL 015 XX 0361 TFI 2 D & (1) & & \\
\hline TI-062 & 2.12 .9 & $\begin{array}{l}\text { Initial solids concentration (dewater) } \\
\text { to } 20 \text {-wt } \% \text { UDS }\end{array}$ & S 000FL 015 XX 0361 TFI 2 D & (1) & & \\
\hline TI-062 & 2.12 .9 & $\begin{array}{l}\text { Initial solids concentration (dewater) } \\
\text { to } 20 \text {-wt } \% \text { UDS }\end{array}$ & S 000FL 015 XX 0361 RAM 3 D & Raman & ASR 8303 & ASR 8303; ASR 8303 rev. 1 \\
\hline TI-062 & 2.12 .9 & $\begin{array}{l}\text { Initial solids concentration (dewater) } \\
\text { to } 20 \text {-wt } \% \text { UDS }\end{array}$ & $\begin{array}{l}\text { SPARE } 32 \mathrm{~S} \text { 000FL } 015 \mathrm{XX} \\
0351 \text { UDS } 2\end{array}$ & (1) & & \\
\hline TI-062 & 2.12 .9 & $\begin{array}{c}\text { Initial solids concentration (dewater) } \\
\text { to } 20 \text {-wt } \% \text { UDS }\end{array}$ & $\begin{array}{l}\text { SPARE } 33 \text { S 000FL } 015 \text { XX } \\
0352 \text { UDS } 2\end{array}$ & (1) & & \\
\hline TI-062 & 2.12 .9 & $\begin{array}{c}\text { Initial solids concentration (dewater) } \\
\text { to } 20 \text {-wt } \% \text { UDS }\end{array}$ & $\begin{array}{l}\text { SPARE } 34 \text { S 000FL } 015 \text { XX } \\
0353 \text { UDS } 2\end{array}$ & (1) & & \\
\hline TI-062 & 3.1 .6 & $\mathrm{NaOH}$ addition & S 02AML 016 XX 0364 UDS 2 & (1) & & \\
\hline TI-062 & 3.1 .6 & $\mathrm{NaOH}$ addition & S 02AML 016 XX 365 RAM 3 D & Raman & ASR 8303 & ASR 8303; ASR 8303 rev. 1 \\
\hline TI-062 & 3.1 .6 & $\mathrm{NaOH}$ addition & S 02AMM 016 XX 0362 UDS 2 & (1) & & \\
\hline TI-062 & 3.1 .6 & $\mathrm{NaOH}$ addition & $\begin{array}{c}\text { S 02AMM } 016 \text { XX } 363 \text { RAM } 3 \\
\text { D }\end{array}$ & Raman & ASR 8303 & ASR 8303; ASR 8303 rev. 1 \\
\hline TI-062 & 3.1 .6 & $\mathrm{NaOH}$ addition & S 02AOL 016 XX 0368 UDS 2 & (1) & & \\
\hline TI-062 & 3.1 .6 & $\mathrm{NaOH}$ addition & S 02AOL 016 XX 369 RAM 3 D & Raman & ASR 8303 & ASR 8303; ASR 8303 rev. 1 \\
\hline TI-062 & 3.1 .6 & $\mathrm{NaOH}$ addition & S 02AOM 016 XX 0366 UDS 2 & (1) & & \\
\hline TI-062 & 3.1 .6 & $\mathrm{NaOH}$ addition & $\begin{array}{c}\text { S 02AOM } 016 \text { XX } 367 \text { RAM } 3 \\
\text { D }\end{array}$ & Raman & ASR 8303 & ASR 8303; ASR 8303 rev. 1 \\
\hline TI-062 & $\mathrm{n} / \mathrm{a}$ & no description listed & S 02AOL 016 XX 0703 RHE 4 & Rheology & TDP-WTP-355 & $\begin{array}{l}\text { TDP-WTP-355 Shakedown RHE Data on PEP } \\
\text { Slurry }\end{array}$ \\
\hline TI-062 & $\mathrm{n} / \mathrm{a}$ & no description listed & S 02AOL 016 XX 0704 RHE 4 & Rheology & TDP-WTP-355 & $\begin{array}{c}\text { TDP-WTP-355 Shakedown RHE Data on PEP } \\
\text { Slurry }\end{array}$ \\
\hline
\end{tabular}


TI-WTP-PEP-062 (testing dates 11/19/2008 through 12/01/2008)

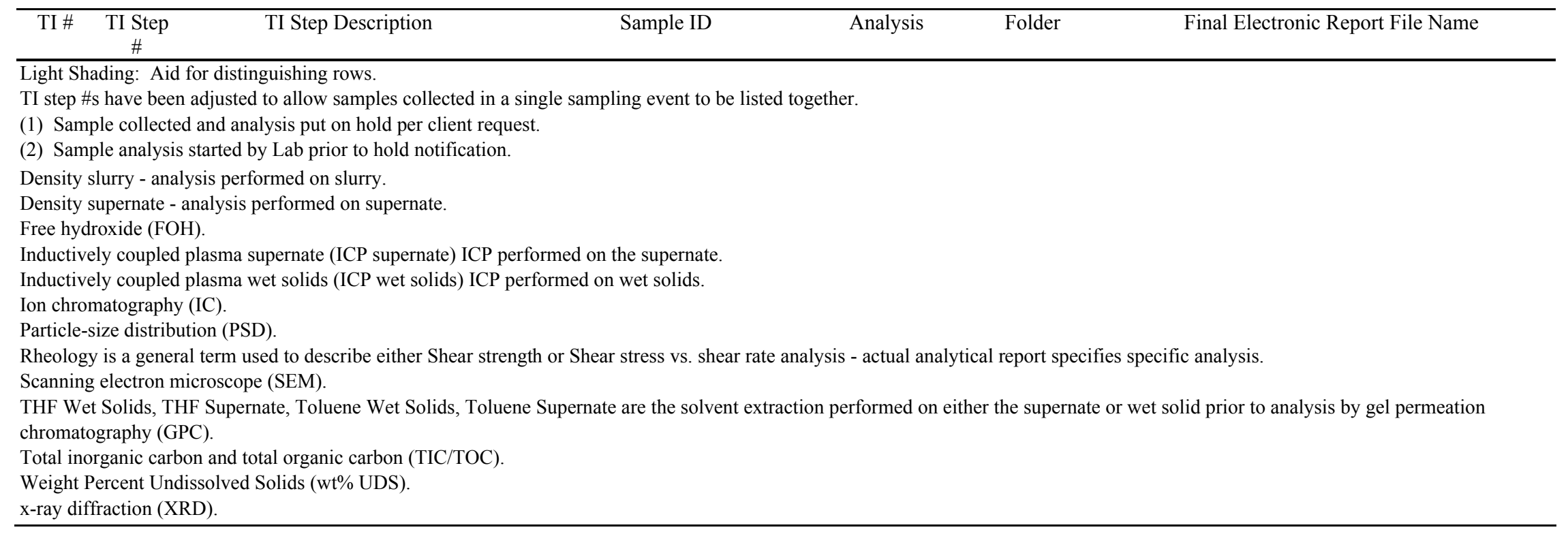


TI-WTP-PEP-067 (testing dates 12/03/08 through 12/10/08)

\begin{tabular}{|c|c|c|c|c|c|c|}
\hline TI \# & $\begin{array}{l}\text { TI Step } \\
\#\end{array}$ & TI Step Description & Sample ID & Analysis & Folder & Final Electronic Report File Name \\
\hline TI-067 & 4.1 .9 & Prepare UFP-VSL-T01A for caustic leaching & S 01AIM 020 XX 0457 UDS 2 & UDS & $081212-9$ & \\
\hline TI-067 & 4.1 .9 & Prepare UFP-VSL-T01A for caustic leaching & S 01AIM 020 XX 0458 UDS 2 & $(1)$ & & \\
\hline TI-067 & 4.1 .9 & Prepare UFP-VSL-T01A for caustic leaching & S 01AIM 020 XX 0459 UDS 2 & (1) & & \\
\hline TI-067 & 4.1 .9 & Prepare UFP-VSL-T01A for caustic leaching & S 01AIM 020 XX 0460 DEN 2 & Density Slurry & 081212-8 & 081212-8DEN \\
\hline TI-067 & 4.1.9 & Prepare UFP-VSL-T01A for caustic leaching & S 01AIM 020 XX 0461 DEN 2 & (1) & & \\
\hline TI-067 & 4.1 .9 & Prepare UFP-VSL-T01A for caustic leaching & S 01AIM 020 XX 0462 DEN 2 & (1) & & \\
\hline TI-067 & 4.1.9 & Prepare UFP-VSL-T01A for caustic leaching & S 01AIM 020 XX 0463 ICP 2 B & ICP Wet Solids & $081212-7$ & 081212-7 081215-2 ICP ICPMS \\
\hline TI-067 & 4.1 .9 & Prepare UFP-VSL-T01A for caustic leaching & S 01AIM 020 XX 0463 RAM 3 D & Raman & ASR 8309 & ASR 8309 RAM rev 1 \\
\hline TI-067 & 4.1 .9 & Prepare UFP-VSL-T01A for caustic leaching & S 01AIM 020 XX 0464 ICP 2 B & (1) & & \\
\hline TI-067 & 4.1 .9 & Prepare UFP-VSL-T01A for caustic leaching & S 01AIM 020 XX 0464 RAM 3 D & (1) & & \\
\hline TI-067 & 4.1 .9 & Prepare UFP-VSL-T01A for caustic leaching & S 01AIM 020 XX 0465 ICP 2 B & (1) & & \\
\hline TI-067 & 4.1 .9 & Prepare UFP-VSL-T01A for caustic leaching & S 01AIM 020 XX 0465 RAM 3 D & (1) & & \\
\hline TI-067 & 4.1.9.4 & Prepare UFP-VSL-T01A for caustic leaching & S 01AIL 020 XX 0472 UDS 2 & UDS & 081216-6 & \\
\hline TI-067 & 4.1.9.4 & Prepare UFP-VSL-T01A for caustic leaching & S 01AIL 020 XX 0473 DEN 2 & Density Slurry & $081216-8$ & 081216-8DEN \\
\hline TI-067 & 4.1.9.4 & Prepare UFP-VSL-T01A for caustic leaching & S 01AIL 020 XX 0474 ICP 2 B & ICP Wet Solids & $081215-2$ & 081212-7 081215-2 ICP ICPMS \\
\hline TI-067 & 4.1.9.4 & Prepare UFP-VSL-T01A for caustic leaching & S 01AIL 020 XX 0474 RAM 3 D & Raman & ASR 8309 & ASR 8309 RAM rev 1 \\
\hline TI-067 & 4.1.9.4 & Prepare UFP-VSL-T01A for caustic leaching & S 01AIM 020 XX 0469 UDS 2 & UDS & 081216-6 & \\
\hline TI-067 & 4.1.9.4 & Prepare UFP-VSL-T01A for caustic leaching & S 01AIM 020 XX 0470 DEN 2 & Density Slurry & 081216-8 & 081216-8DEN \\
\hline TI-067 & 4.1.9.4 & Prepare UFP-VSL-T01A for caustic leaching & S 01AIM 020 XX 0471 ICP 2 B & ICP Wet Solids & $081215-2$ & 081212-7 081215-2 ICP ICPMS \\
\hline TI-067 & 4.1.9.4 & Prepare UFP-VSL-T01A for caustic leaching & S 01AIM 020 XX 0471 RAM 3 D & Raman & ASR 8309 & ASR 8309 RAM rev 1 \\
\hline TI-067 & 4.2.5.3 & $\begin{array}{l}\text { Heat UFP-VSL-T01A to } 88^{\circ} \mathrm{C} \text { and } \\
\text { caustic-leach for } 16 \mathrm{hr}\end{array}$ & S 01AIM 021 BT 0475 UDS 2 & UDS & $081216-6$ & \\
\hline TI-067 & 4.2.5.3 & $\begin{array}{l}\text { Heat UFP-VSL-T01A to } 88^{\circ} \mathrm{C} \text { and } \\
\text { caustic-leach for } 16 \mathrm{hr}\end{array}$ & S 01AIM 021 BT 0478 DEN 2 & Density Slurry & 081216-8 & 081216-8DEN \\
\hline TI-067 & 4.2.5.3 & $\begin{array}{l}\text { Heat UFP-VSL-T01A to } 88^{\circ} \mathrm{C} \text { and } \\
\text { caustic-leach for } 16 \mathrm{hr}\end{array}$ & S 01AIM 021 BT 0481 ICP 2 B & ICP Wet Solids & 081215-2 & 081212-7 081215-2 ICP ICPMS \\
\hline TI-067 & 4.2.5.3 & $\begin{array}{l}\text { Heat UFP-VSL-T01A to } 88^{\circ} \mathrm{C} \text { and } \\
\text { caustic-leach for } 16 \mathrm{hr}\end{array}$ & S 01AIM 021 BT 0481 RAM 3 D & Raman & ASR 8309 & ASR 8309 RAM rev 1 \\
\hline TI-067 & 4.2.6 & $\begin{array}{l}\text { Heat UFP-VSL-T01A to } 98^{\circ} \mathrm{C} \text { and } \\
\text { caustic-leach for } 16 \mathrm{hr}\end{array}$ & S 01AIM 021000484 UDS 2 & UDS & 081216-6 & \\
\hline TI-067 & 4.2 .6 & $\begin{array}{l}\text { Heat UFP-VSL-T01A to } 98^{\circ} \mathrm{C} \text { and } \\
\text { caustic-leach for } 16 \mathrm{hr}\end{array}$ & S 01AIM 021000487 ICP 2 B & ICP Wet Solids & $081215-2$ & 081212-7 081215-2 ICP ICPMS \\
\hline TI-067 & 4.2 .6 & $\begin{array}{l}\text { Heat UFP-VSL-T01A to } 98^{\circ} \mathrm{C} \text { and } \\
\text { caustic-leach for } 16 \mathrm{hr}\end{array}$ & S 01AIM 021000487 RAM 3 D & Raman & ASR 8309 & ASR 8309 RAM rev 1 \\
\hline TI-067 & 4.2 .7 & $\begin{array}{l}\text { Heat UFP-VSL-T01A to } 98^{\circ} \mathrm{C} \text { and } \\
\text { caustic-leach for } 16 \mathrm{hr}\end{array}$ & S 01AIM 021010490 UDS 2 & UDS & 081216-6 & \\
\hline TI-067 & 4.2 .7 & $\begin{array}{l}\text { Heat UFP-VSL-T01A to } 98^{\circ} \mathrm{C} \text { and } \\
\text { caustic-leach for } 16 \mathrm{hr}\end{array}$ & S 01AIM 021010493 ICP 2 B & ICP Wet Solids & $081215-2$ & 081212-7 081215-2 ICP ICPMS \\
\hline
\end{tabular}


TI-WTP-PEP-067 (testing dates 12/03/08 through 12/10/08)

\begin{tabular}{|c|c|c|c|c|c|c|}
\hline TI \# & $\begin{array}{l}\text { TI Step } \\
\#\end{array}$ & TI Step Description & Sample ID & Analysis & Folder & Final Electronic Report File Name \\
\hline TI-067 & 4.2 .7 & $\begin{array}{l}\text { Heat UFP-VSL-T01A to } 98^{\circ} \mathrm{C} \text { and } \\
\text { caustic-leach for } 16 \mathrm{hr}\end{array}$ & S 01AIM 021010493 RAM 3 D & Raman & ASR 8309 & ASR 8309 RAM rev 1 \\
\hline TI-067 & 4.2 .7 & $\begin{array}{l}\text { Heat UFP-VSL-T01A to } 98^{\circ} \mathrm{C} \text { and } \\
\text { caustic-leach for } 16 \mathrm{hr}\end{array}$ & S 01AIM 021020496 UDS 2 & UDS & 081216-6 & \\
\hline TI-067 & 4.2 .7 & $\begin{array}{l}\text { Heat UFP-VSL-T01A to } 98^{\circ} \mathrm{C} \text { and } \\
\text { caustic-leach for } 16 \mathrm{hr}\end{array}$ & S 01AIM 021020499 ICP 2 B & ICP Wet Solids & $081215-2$ & 081212-7 081215-2 ICP ICPMS \\
\hline TI-067 & 4.2 .7 & $\begin{array}{l}\text { Heat UFP-VSL-T01A to } 98^{\circ} \mathrm{C} \text { and } \\
\text { caustic-leach for } 16 \mathrm{hr}\end{array}$ & S 01AIM 021020499 RAM 3 D & Raman & ASR 8309 & ASR 8309 RAM rev 1 \\
\hline TI-067 & 4.2 .7 & $\begin{array}{l}\text { Heat UFP-VSL-T01A to } 98^{\circ} \mathrm{C} \text { and } \\
\text { caustic-leach for } 16 \mathrm{hrs}\end{array}$ & S 01AIH 021020502 UDS 2 & UDS & 081216-6 & \\
\hline TI-067 & 4.2 .7 & $\begin{array}{l}\text { Heat UFP-VSL-T01A to } 98^{\circ} \mathrm{C} \text { and } \\
\text { caustic-leach for } 16 \mathrm{hr}\end{array}$ & S 01AIH 021020503 DEN 2 & Density Slurry & 081216-8 & 081216-8DEN \\
\hline TI-067 & 4.2 .7 & $\begin{array}{l}\text { Heat UFP-VSL-T01A to } 98^{\circ} \mathrm{C} \text { and } \\
\text { caustic-leach for } 16 \mathrm{hr}\end{array}$ & S 01AML 021020504 UDS 2 & UDS & $081216-7$ & \\
\hline TI-067 & 4.2 .7 & $\begin{array}{l}\text { Heat UFP-VSL-T01A to } 98^{\circ} \mathrm{C} \text { and } \\
\text { caustic-leach for } 16 \mathrm{hr}\end{array}$ & S 01AML 021020505 DEN 2 & Density Slurry & $081216-8$ & 081216-8DEN \\
\hline TI-067 & 4.2 .7 & $\begin{array}{l}\text { Heat UFP-VSL-T01A to } 98^{\circ} \mathrm{C} \text { and } \\
\text { caustic-leach for } 16 \mathrm{hr}\end{array}$ & S 01AMM 021020506 UDS 2 & UDS & $081216-7$ & \\
\hline TI-067 & 4.2 .7 & $\begin{array}{l}\text { Heat UFP-VSL-T01A to } 98^{\circ} \mathrm{C} \text { and } \\
\text { caustic-leach for } 16 \mathrm{hr}\end{array}$ & S 01AMM 021020507 DEN 2 & Density Slurry & $081216-8$ & 081216-8DEN \\
\hline TI-067 & 4.2 .7 & $\begin{array}{l}\text { Heat UFP-VSL-T01A to } 98^{\circ} \mathrm{C} \text { and } \\
\text { caustic-leach for } 16 \mathrm{hr}\end{array}$ & S 01AMH 021020508 UDS 2 & UDS & 081216-6 & \\
\hline TI-067 & 4.2 .7 & $\begin{array}{l}\text { Heat UFP-VSL-T01A to } 98^{\circ} \mathrm{C} \text { and } \\
\text { caustic-leach for } 16 \mathrm{hr}\end{array}$ & S 01AMH 021020509 DEN 2 & Density Slurry & $081216-8$ & 081216-8DEN \\
\hline TI-067 & 4.2 .7 & $\begin{array}{l}\text { Heat UFP-VSL-T01A to } 98^{\circ} \mathrm{C} \text { and } \\
\text { caustic-leach for } 16 \mathrm{hr}\end{array}$ & S 01AOL 021020510 UDS 2 & UDS & $081216-7$ & \\
\hline TI-067 & 4.2 .7 & $\begin{array}{l}\text { Heat UFP-VSL-T01A to } 98^{\circ} \mathrm{C} \text { and } \\
\text { caustic-leach for } 16 \mathrm{hr}\end{array}$ & S 01AOL 021020511 DEN 2 & Density Slurry & 081216-8 & 081216-8DEN \\
\hline TI-067 & 4.2 .7 & $\begin{array}{l}\text { Heat UFP-VSL-T01A to } 98^{\circ} \mathrm{C} \text { and } \\
\text { caustic-leach for } 16 \mathrm{hr}\end{array}$ & S 01AOM 021020512 UDS 2 & UDS & 081216-7 & \\
\hline TI-067 & 4.2 .7 & $\begin{array}{l}\text { Heat UFP-VSL-T01A to } 98^{\circ} \mathrm{C} \text { and } \\
\text { caustic-leach for } 16 \mathrm{hr}\end{array}$ & S 01AOM 021020513 DEN 2 & Density Slurry & 081216-8 & 081216-8DEN \\
\hline TI-067 & 4.2 .7 & $\begin{array}{l}\text { Heat UFP-VSL-T01A to } 98^{\circ} \mathrm{C} \text { and } \\
\text { caustic-leach for } 16 \mathrm{hr}\end{array}$ & S 01AOH 021020514 UDS 2 & UDS & $081216-7$ & \\
\hline TI-067 & 4.2 .7 & $\begin{array}{l}\text { Heat UFP-VSL-T01A to } 98^{\circ} \mathrm{C} \text { and } \\
\text { caustic-leach for } 16 \mathrm{hr}\end{array}$ & S 01AOH 021020515 DEN 2 & Density Slurry & 081216-8 & 081216-8DEN \\
\hline TI-067 & 4.2 .7 & $\begin{array}{l}\text { Heat UFP-VSL-T01A to } 98^{\circ} \mathrm{C} \text { and } \\
\text { caustic-leach for } 16 \mathrm{hr}\end{array}$ & S 01AIL 021020516 UDS 2 & UDS & 081216-6 & \\
\hline TI-067 & 4.2 .7 & $\begin{array}{l}\text { Heat UFP-VSL-T01A to } 98^{\circ} \mathrm{C} \text { and } \\
\text { caustic-leach for } 16 \mathrm{hr}\end{array}$ & S 01AIL 021020517 DEN 2 & Density Slurry & $081216-8$ & 081216-8DEN \\
\hline
\end{tabular}


TI-WTP-PEP-067 (testing dates 12/03/08 through 12/10/08)

\begin{tabular}{|c|c|c|c|c|c|c|}
\hline TI \# & $\begin{array}{l}\text { TI Step } \\
\#\end{array}$ & TI Step Description & Sample ID & Analysis & Folder & Final Electronic Report File Name \\
\hline TI-067 & 4.2 .7 & $\begin{array}{l}\text { Heat UFP-VSL-T01A to } 98^{\circ} \mathrm{C} \text { and } \\
\text { caustic-leach for } 16 \mathrm{hr}\end{array}$ & S 01AIM 021040518 UDS 2 & UDS & 081216-6 & \\
\hline TI-067 & 4.2 .7 & $\begin{array}{c}\text { Heat UFP-VSL-T01A to } 98^{\circ} \mathrm{C} \text { and } \\
\text { caustic-leach for } 16 \mathrm{hr}\end{array}$ & S 01AIM 021040521 ICP 2 B & ICP Wet Solids & $081215-2$ & 081212-7 081215-2 ICP ICPMS \\
\hline TI-067 & 4.2 .7 & $\begin{array}{l}\text { Heat UFP-VSL-T01A to } 98^{\circ} \mathrm{C} \text { and } \\
\text { caustic-leach for } 16 \mathrm{hr}\end{array}$ & S 01AIM 021040521 RAM 3 D & Raman & ASR 8309 & ASR 8309 RAM rev 1 \\
\hline TI-067 & 4.2 .7 & $\begin{array}{l}\text { Heat UFP-VSL-T01A to } 98^{\circ} \mathrm{C} \text { and } \\
\text { caustic-leach for } 16 \mathrm{hr}\end{array}$ & S 01AIM 021080524 UDS 2 & UDS & 081216-6 & \\
\hline TI-067 & 4.2 .7 & $\begin{array}{l}\text { Heat UFP-VSL-T01A to } 98^{\circ} \mathrm{C} \text { and } \\
\text { caustic-leach for } 16 \mathrm{hr}\end{array}$ & S 01AIM 021080527 ICP 2 B & ICP Wet Solids & $081215-2$ & 081212-7 081215-2 ICP ICPMS \\
\hline TI-067 & 4.2 .7 & $\begin{array}{l}\text { Heat UFP-VSL-T01A to } 98^{\circ} \mathrm{C} \text { and } \\
\text { caustic-leach for } 16 \mathrm{hr}\end{array}$ & S 01AIM 021080527 RAM 3 D & Raman & ASR 8309 & ASR 8309 RAM rev 1 \\
\hline TI-067 & 4.2 .7 & $\begin{array}{l}\text { Heat UFP-VSL-T01A to } 98^{\circ} \mathrm{C} \text { and } \\
\text { caustic-leach for } 16 \mathrm{hr}\end{array}$ & S 01AIM 021120530 UDS 2 & UDS & 081216-6 & \\
\hline TI-067 & 4.2 .7 & $\begin{array}{l}\text { Heat UFP-VSL-T01A to } 98^{\circ} \mathrm{C} \text { and } \\
\text { caustic-leach for } 16 \mathrm{hr}\end{array}$ & S 01AIM 021120533 ICP 2 B & ICP Wet Solids & $081215-2$ & 081212-7 081215-2 ICP ICPMS \\
\hline TI-067 & 4.2 .7 & $\begin{array}{l}\text { Heat UFP-VSL-T01A to } 98^{\circ} \mathrm{C} \text { and } \\
\text { caustic-leach for } 16 \mathrm{hr}\end{array}$ & S 01AIM 021120533 RAM 3 D & Raman & ASR 8309 & ASR 8309 RAM rev 1 \\
\hline TI-067 & 4.2 .7 & $\begin{array}{l}\text { Heat UFP-VSL-T01A to } 98^{\circ} \mathrm{C} \text { and } \\
\text { caustic-leach for } 16 \mathrm{hr}\end{array}$ & S 01AIM 021160536 UDS 2 & UDS & $081216-7$ & \\
\hline TI-067 & 4.2 .7 & $\begin{array}{l}\text { Heat UFP-VSL-T01A to } 98^{\circ} \mathrm{C} \text { and } \\
\text { caustic-leach for } 16 \mathrm{hr}\end{array}$ & S 01AIM 021160539 ICP 2 B & ICP Wet Solids & $081215-2$ & 081212-7 081215-2 ICP ICPMS \\
\hline TI-067 & 4.2 .7 & $\begin{array}{l}\text { Heat UFP-VSL-T01A to } 98^{\circ} \mathrm{C} \text { and } \\
\text { caustic-leach for } 16 \mathrm{hr}\end{array}$ & S 01AIM 021160539 RAM 3 D & Raman & ASR 8309 & ASR 8309 RAM rev 1 \\
\hline TI-067 & 4.2 .7 & $\begin{array}{l}\text { Heat UFP-VSL-T01A to } 98^{\circ} \mathrm{C} \text { and } \\
\text { caustic-leach for } 16 \mathrm{hr}\end{array}$ & S 01AIL 021160556 UDS 2 & UDS & 081216-7 & \\
\hline TI-067 & 4.2 .7 & $\begin{array}{l}\text { Heat UFP-VSL-T01A to } 98^{\circ} \mathrm{C} \text { and } \\
\text { caustic-leach for } 16 \mathrm{hr}\end{array}$ & S 01AIL 021160557 DEN 2 & Density Slurry & 081216-8 & 081216-8DEN \\
\hline TI-067 & 4.2 .7 & $\begin{array}{l}\text { Heat UFP-VSL-T01A to } 98^{\circ} \mathrm{C} \text { and } \\
\text { caustic-leach for } 16 \mathrm{hr}\end{array}$ & S 01AMH 021160548 UDS 2 & UDS & 081216-7 & \\
\hline TI-067 & 4.2 .7 & $\begin{array}{l}\text { Heat UFP-VSL-T01A to } 98^{\circ} \mathrm{C} \text { and } \\
\text { caustic-leach for } 16 \mathrm{hr}\end{array}$ & S 01AMH 021160549 DEN 2 & Density Slurry & 081216-8 & 081216-8DEN \\
\hline TI-067 & 4.2 .7 & $\begin{array}{l}\text { Heat UFP-VSL-T01A to } 98^{\circ} \mathrm{C} \text { and } \\
\text { caustic-leach for } 16 \mathrm{hr}\end{array}$ & S 01AML 021160544 UDS 2 & UDS & $081216-7$ & \\
\hline TI-067 & 4.2 .7 & $\begin{array}{l}\text { Heat UFP-VSL-T01A to } 98^{\circ} \mathrm{C} \text { and } \\
\text { caustic-leach for } 16 \mathrm{hr}\end{array}$ & S 01AML 021160545 DEN 2 & Density Slurry & 081216-8 & 081216-8DEN \\
\hline TI-067 & 4.2 .7 & $\begin{array}{l}\text { Heat UFP-VSL-T01A to } 98^{\circ} \mathrm{C} \text { and } \\
\text { caustic-leach for } 16 \mathrm{hr}\end{array}$ & S 01AMM 021160546 UDS 2 & UDS & 081216-7 & \\
\hline TI-067 & 4.2 .7 & $\begin{array}{l}\text { Heat UFP-VSL-T01A to } 98^{\circ} \mathrm{C} \text { and } \\
\text { caustic-leach for } 16 \mathrm{hr}\end{array}$ & S 01AMM 021160547 DEN 2 & Density Slurry & $081216-8$ & 081216-8DEN \\
\hline
\end{tabular}


TI-WTP-PEP-067 (testing dates 12/03/08 through 12/10/08)

\begin{tabular}{|c|c|c|c|c|c|c|}
\hline TI \# & $\begin{array}{l}\text { TI Step } \\
\#\end{array}$ & TI Step Description & Sample ID & Analysis & Folder & Final Electronic Report File Name \\
\hline TI-067 & 4.2 .7 & $\begin{array}{l}\text { Heat UFP-VSL-T01A to } 98^{\circ} \mathrm{C} \text { and } \\
\text { caustic-leach for } 16 \mathrm{hr}\end{array}$ & S 01AOH 021160554 UDS 2 & UDS & 081216-7 & \\
\hline TI-067 & 4.2 .7 & $\begin{array}{l}\text { Heat UFP-VSL-T01A to } 98^{\circ} \mathrm{C} \text { and } \\
\text { caustic-leach for } 16 \mathrm{hr}\end{array}$ & S 01AOH 021160555 DEN 2 & Density Slurry & $081216-8$ & 081216-8DEN \\
\hline TI-067 & 4.2 .7 & $\begin{array}{l}\text { Heat UFP-VSL-T01A to } 98^{\circ} \mathrm{C} \text { and } \\
\text { caustic-leach for } 16 \mathrm{hr}\end{array}$ & S 01AOL 021160550 UDS 2 & UDS & 081216-7 & \\
\hline TI-067 & 4.2 .7 & $\begin{array}{l}\text { Heat UFP-VSL-T01A to } 98^{\circ} \mathrm{C} \text { and } \\
\text { caustic-leach for } 16 \mathrm{hr}\end{array}$ & S 01AOL 021160551 DEN 2 & Density Slurry & 081216-8 & 081216-8DEN \\
\hline TI-067 & 4.2 .7 & $\begin{array}{l}\text { Heat UFP-VSL-T01A to } 98^{\circ} \mathrm{C} \text { and } \\
\text { caustic-leach for } 16 \mathrm{hr}\end{array}$ & S 01AOM 021160552 UDS 2 & UDS & $081216-7$ & \\
\hline TI-067 & 4.2 .7 & $\begin{array}{l}\text { Heat UFP-VSL-T01A to } 98^{\circ} \mathrm{C} \text { and } \\
\text { caustic-leach for } 16 \mathrm{hr}\end{array}$ & S 01AOM 021160553 DEN 2 & Density Slurry & $081216-8$ & 081216-8DEN \\
\hline
\end{tabular}

Dark Shading: Samples were sent to storage/archive in RTL or sent out of PDL-W for additional studies.

Light Shading: Aid for distinguishing rows.

TI step \# have been adjusted to allow samples collected in a single sampling event to be listed together.

(1) Sample collected and analysis put on hold per client request.

(2) Sample Analysis started by Lab prior to hold notification.

$\Omega$ Density slurry - analysis performed on slurry.

N Density supernate - analysis performed on supernate.

Free hydroxide $(\mathrm{FOH})$.

Inductively coupled plasma supernate (ICP supernate) ICP performed on the supernate.

Inductively coupled plasma wet solids (ICP wet solids) ICP performed on wet solids.

Ion chromatography (IC).

Particle-size distribution (PSD).

Rheology is a general term used to describe either Shear strength or Shear stress vs. shear rate analysis - actual analytical report specifies specific analysis.

Scanning electron microscope (SEM.)

THF Wet Solids, THF Supernate, Toluene Wet Solids, Toluene Supernate are the solvent extraction performed on either the supernate or wet solid prior to analysis by gel permeation chromatography (GPC).

Total inorganic carbon and total organic carbon (TIC/TOC).

Weight Percent Undissolved Solids (wt\% UDS).

$\mathrm{x}$-ray diffraction (XRD). 
TI-WTP-PEP-032 (testing dates 12/19/2008 through 01/11/2009)

\begin{tabular}{|c|c|c|c|c|c|c|}
\hline TI \# & $\begin{array}{c}\text { TI Step } \\
\#\end{array}$ & TI Step Description & Sample ID & Analysis & Folder & Final Electronic Report File Name \\
\hline TI-032 & 2.4 .8 & $\begin{array}{l}\text { Test for stratification of solids in UFP-T01B @ } \\
\text { plant PJM frequency }\end{array}$ & S 01BIH 006120814 UDS 2 & UDS & 090102-10 & \\
\hline TI-032 & 2.4 .8 & $\begin{array}{c}\text { Test for stratification of solids in UFP-T01B @ } \\
\text { plant PJM frequency }\end{array}$ & S 01BIH 006120815 UDS 2 & UDS & 090102-10 & \\
\hline TI-032 & 2.4 .8 & $\begin{array}{c}\text { Test for stratification of solids in UFP-T01B @ } \\
\text { plant PJM frequency }\end{array}$ & S 01BIH 006120816 UDS 2 & UDS & 090102-10 & \\
\hline TI-032 & 2.4 .8 & $\begin{array}{c}\text { Test for stratification of solids in UFP-T01B @ } \\
\text { plant PJM frequency }\end{array}$ & S 01BIH 006120817 UDS 2 & UDS & 090102-10 & \\
\hline TI-032 & 2.4 .8 & $\begin{array}{c}\text { Test for stratification of solids in UFP-T01B @ } \\
\text { plant PJM frequency }\end{array}$ & S 01BIM 006120818 UDS 2 & UDS & $090102-11$ & \\
\hline TI-032 & 2.4 .8 & $\begin{array}{l}\text { Test for stratification of solids in UFP-T01B @ } \\
\text { plant PJM frequency }\end{array}$ & S 01BIM 006120819 UDS 2 & UDS & 090102-11 & \\
\hline TI-032 & 2.4 .8 & $\begin{array}{c}\text { Test for stratification of solids in UFP-T01B @ } \\
\text { plant PJM frequency }\end{array}$ & S 01BIM 006120820 UDS 2 & UDS & 090102-11 & \\
\hline TI-032 & 2.4 .8 & $\begin{array}{c}\text { Test for stratification of solids in UFP-T01B @ } \\
\text { plant PJM frequency }\end{array}$ & S 01BIM 006120821 UDS 2 & UDS & 090102-11 & \\
\hline TI-032 & 2.4 .8 & $\begin{array}{c}\text { Test for stratification of solids in UFP-T01B @ } \\
\text { plant PJM frequency }\end{array}$ & S 01BIL 006120822 UDS 2 & UDS & 090102-10 & \\
\hline TI-032 & 2.4 .8 & $\begin{array}{c}\text { Test for stratification of solids in UFP-T01B @ } \\
\text { plant PJM frequency }\end{array}$ & S 01BIL 006120823 UDS 2 & UDS & 090102-10 & \\
\hline TI-032 & 2.4 .8 & $\begin{array}{c}\text { Test for stratification of solids in UFP-T01B @ } \\
\text { plant PJM frequency }\end{array}$ & S 01BIL 006120824 UDS 2 & UDS & 090102-10 & \\
\hline TI-032 & 2.4 .8 & $\begin{array}{c}\text { Test for stratification of solids in UFP-T01B @ } \\
\text { plant PJM frequency }\end{array}$ & S 01BIL 006120825 UDS 2 & UDS & 090102-10 & \\
\hline TI-032 & 2.4 .8 & $\begin{array}{c}\text { Test for stratification of solids in UFP-T01B @ } \\
\text { plant PJM frequency }\end{array}$ & S 01BIH 006120826 ICP 2 B & $\begin{array}{l}\text { ICP Wet } \\
\text { Solids }\end{array}$ & $090102-4$ & 090102-4 ICP and ICPMS \\
\hline TI-032 & 2.4 .8 & $\begin{array}{c}\text { Test for stratification of solids in UFP-T01B @ } \\
\text { plant PJM frequency }\end{array}$ & S 01BIH 006120827 XSP 4 B & PSD & TDP-WTP-354 & $\begin{array}{c}\text { TDP WTP } 354 \text { PEP Shakedown Test PSD } \\
\text { Data Analysis }\end{array}$ \\
\hline TI-032 & 2.4 .8 & $\begin{array}{c}\text { Test for stratification of solids in UFP-T01B @ } \\
\text { plant PJM frequency }\end{array}$ & S 01BIL 006120828 ICP 2 B & $\begin{array}{l}\text { ICP Wet } \\
\text { Solids }\end{array}$ & $090102-4$ & 090102-4 ICP and ICPMS \\
\hline TI-032 & 2.4 .8 & $\begin{array}{c}\text { Test for stratification of solids in UFP-T01B @ } \\
\text { plant PJM frequency }\end{array}$ & S 01BIL 006120829 XSP 4 B & PSD & TDP-WTP-354 & $\begin{array}{c}\text { TDP WTP } 354 \text { PEP Shakedown Test PSD } \\
\text { Data Analysis }\end{array}$ \\
\hline TI-032 & 2.4 .8 & $\begin{array}{c}\text { Test for stratification of solids in UFP-T01B @ } \\
\text { plant PJM frequency }\end{array}$ & S 01BIH 006240830 UDS 2 & UDS & 090102-10 & \\
\hline TI-032 & 2.4 .8 & $\begin{array}{c}\text { Test for stratification of solids in UFP-T01B @ } \\
\text { plant PJM frequency }\end{array}$ & S 01BIH 006240831 UDS 2 & UDS & 090102-10 & \\
\hline TI-032 & 2.4 .8 & $\begin{array}{c}\text { Test for stratification of solids in UFP-T01B @ } \\
\text { plant PJM frequency }\end{array}$ & S 01BIH 006240832 UDS 2 & UDS & 090102-10 & \\
\hline
\end{tabular}


TI-WTP-PEP-032 (testing dates 12/19/2008 through 01/11/2009)

\begin{tabular}{|c|c|c|c|c|c|}
\hline TI \# & $\begin{array}{c}\text { TI Step } \\
\#\end{array}$ & TI Step Description & Sample ID & Analysis & Final Electronic Report File Name \\
\hline TI-032 & 2.4 .8 & $\begin{array}{c}\text { Test for stratification of solids in UFP-T01B @ } \\
\text { plant PJM frequency }\end{array}$ & S 01BIH 006240833 UDS 2 & UDS & 090102-10 \\
\hline TI-032 & 2.4 .8 & $\begin{array}{c}\text { Test for stratification of solids in UFP-T01B @ } \\
\text { plant PJM frequency }\end{array}$ & S 01BIM 006240834 UDS 2 & UDS & 090102-12 \\
\hline TI-032 & 2.4 .8 & $\begin{array}{c}\text { Test for stratification of solids in UFP-T01B @ } \\
\text { plant PJM frequency }\end{array}$ & S 01BIM 006240835 UDS 2 & UDS & $090102-12$ \\
\hline TI-032 & 2.4 .8 & $\begin{array}{c}\text { Test for stratification of solids in UFP-T01B @ } \\
\text { plant PJM frequency }\end{array}$ & S 01BIM 006240836 UDS 2 & UDS & 090102-12 \\
\hline TI-032 & 2.4 .8 & $\begin{array}{c}\text { Test for stratification of solids in UFP-T01B @ } \\
\text { plant PJM frequency }\end{array}$ & S 01BIM 006240837 UDS 2 & UDS & 090102-12 \\
\hline TI-032 & 2.4 .8 & $\begin{array}{c}\text { Test for stratification of solids in UFP-T01B @ } \\
\text { plant PJM frequency }\end{array}$ & S 01BIL 006240838 UDS 2 & UDS & $090102-11$ \\
\hline TI-032 & 2.4 .8 & $\begin{array}{c}\text { Test for stratification of solids in UFP-T01B @ } \\
\text { plant PJM frequency }\end{array}$ & S 01BIL 006240839 UDS 2 & UDS & $090102-11$ \\
\hline TI-032 & 2.4 .8 & $\begin{array}{c}\text { Test for stratification of solids in UFP-T01B @ } \\
\text { plant PJM frequency }\end{array}$ & S 01BIL 006240840 UDS 2 & UDS & $090102-11$ \\
\hline TI-032 & 2.4 .8 & $\begin{array}{l}\text { Test for stratification of solids in UFP-T01B @ } \\
\text { plant PJM frequency }\end{array}$ & S 01BIL 006240841 UDS 2 & UDS & $090102-11$ \\
\hline TI-032 & 2.4 .8 & $\begin{array}{c}\text { Test for stratification of solids in UFP-T01B @ } \\
\text { plant PJM frequency }\end{array}$ & S 01BIH 006360842 UDS 2 & UDS & 090102-10 \\
\hline TI-032 & 2.4 .8 & $\begin{array}{l}\text { Test for stratification of solids in UFP-T01B @ } \\
\text { plant PJM frequency }\end{array}$ & S 01BIH 006360843 UDS 2 & UDS & 090102-10 \\
\hline TI-032 & 2.4 .8 & $\begin{array}{c}\text { Test for stratification of solids in UFP-T01B @ } \\
\text { plant PJM frequency }\end{array}$ & S 01BIH 006360844 UDS 2 & UDS & 090102-10 \\
\hline TI-032 & 2.4 .8 & $\begin{array}{c}\text { Test for stratification of solids in UFP-T01B @ } \\
\text { plant PJM frequency }\end{array}$ & S 01BIH 006360845 UDS 2 & UDS & 090102-10 \\
\hline TI-032 & 2.4 .8 & $\begin{array}{c}\text { Test for stratification of solids in UFP-T01B @ } \\
\text { plant PJM frequency }\end{array}$ & S 01BIL 006360850 UDS 2 & UDS & $090102-11$ \\
\hline TI-032 & 2.4 .8 & $\begin{array}{c}\text { Test for stratification of solids in UFP-T01B @ } \\
\text { plant PJM frequency }\end{array}$ & S 01BIL 006360851 UDS 2 & UDS & $090102-11$ \\
\hline TI-032 & 2.4 .8 & $\begin{array}{c}\text { Test for stratification of solids in UFP-T01B @ } \\
\text { plant PJM frequency }\end{array}$ & S 01BIL 006360852 UDS 2 & UDS & $090102-11$ \\
\hline TI-032 & 2.4 .8 & $\begin{array}{l}\text { Test for stratification of solids in UFP-T01B @ } \\
\text { plant PJM frequency }\end{array}$ & S 01BIL 006360853 UDS 2 & UDS & $090102-11$ \\
\hline TI-032 & 2.4 .8 & $\begin{array}{l}\text { Test for stratification of solids in UFP-T01B @ } \\
\text { plant PJM frequency }\end{array}$ & S 01BIM 006360846 UDS 2 & UDS & $090102-11$ \\
\hline TI-032 & 2.4 .8 & $\begin{array}{c}\text { Test for stratification of solids in UFP-T01B @ } \\
\text { plant PJM frequency }\end{array}$ & S 01BIM 006360847 UDS 2 & UDS & $090102-11$ \\
\hline TI-032 & 2.4 .8 & $\begin{array}{c}\text { Test for stratification of solids in UFP-T01B @ } \\
\text { plant PJM frequency }\end{array}$ & S 01BIM 006360848 UDS 2 & UDS & $090102-11$ \\
\hline
\end{tabular}


TI-WTP-PEP-032 (testing dates 12/19/2008 through 01/11/2009)

\begin{tabular}{|c|c|c|c|c|c|c|}
\hline TI \# & $\begin{array}{c}\text { TI Step } \\
\text { \# }\end{array}$ & TI Step Description & Sample ID & Analysis & Folder & Final Electronic Report File Name \\
\hline TI-032 & 2.4 .8 & $\begin{array}{l}\text { Test for stratification of solids in UFP-T01B @ } \\
\text { plant PJM frequency }\end{array}$ & S 01BIM 006360849 UDS 2 & UDS & 090102-11 & \\
\hline TI-032 & 2.10 .6 .1 & $\begin{array}{c}\text { Test for stratification of solids in UFP-T01B @ } \\
\text { plant PJM frequency }\end{array}$ & S 01BIH 006360854 ICP 2 B & $\begin{array}{l}\text { ICP Wet } \\
\text { Solids }\end{array}$ & 090103-6 & 090103-3 and 090103-6 ICP and ICPMS \\
\hline TI-032 & 2.10.6.1 & $\begin{array}{c}\text { Test for stratification of solids in UFP-T01B @ } \\
\text { plant PJM frequency }\end{array}$ & S 01BIH 006360855 XSP 4 B & PSD & TDP-WTP-354 & $\begin{array}{c}\text { TDP WTP } 354 \text { PEP Shakedown Test PSD } \\
\text { Data Analysis }\end{array}$ \\
\hline TI-032 & 2.10 .6 .1 & $\begin{array}{c}\text { Test for stratification of solids in UFP-T01B @ } \\
\text { plant PJM frequency }\end{array}$ & S 01BIL 006360856 ICP 2 B & $\begin{array}{l}\text { ICP Wet } \\
\text { Solids }\end{array}$ & 090103-6 & 090103-3 and 090103-6 ICP and ICPMS \\
\hline TI-032 & 2.10.6.1 & $\begin{array}{c}\text { Test for stratification of solids in UFP-T01B @ } \\
\text { plant PJM frequency }\end{array}$ & S 01BIL 006360857 XSP 4 B & PSD & TDP-WTP-354 & $\begin{array}{c}\text { TDP WTP } 354 \text { PEP Shakedown Test PSD } \\
\text { Data Analysis }\end{array}$ \\
\hline TI-032 & 2.6 .5 & Conduct low-solids filter flux test & S 02AML 008 XX 0858 UDS 2 & UDS & 090102-14 & \\
\hline TI-032 & 2.6 .5 & Conduct low-solids filter flux test & S 02AML 008 XX 0859 UDS 2 & UDS & 090102-14 & \\
\hline TI-032 & 2.6 .5 & Conduct low-solids filter flux test & S 02AML 008 XX 0860 UDS 2 & UDS & 090102-14 & \\
\hline TI-032 & 2.6 .5 & Conduct low-solids filter flux test & S 02AML 008 XX 0861 DEN 2 & Density Slurry & 090102-15 & 090102-15DEN \\
\hline TI-032 & 2.6 .5 & Conduct low-solids filter flux test & S 02AML 008 XX 0862 DEN 2 & Density Slurry & $090102-15$ & 090102-15DEN \\
\hline TI-032 & 2.6 .5 & Conduct low-solids filter flux test & S 02AML 008 XX 0863 DEN 2 & Density Slurry & 090102-15 & 090102-15DEN \\
\hline TI-032 & 2.6 .5 & Conduct low-solids filter flux test & S 02AML 008 XX 0865 RHE 4 & Rheology & TDP-WTP-355 & $\begin{array}{c}\text { TDP-WTP-355 Shakedown RHE Data on } \\
\text { PEP Slurry }\end{array}$ \\
\hline TI-032 & 2.6 .5 & Conduct low-solids filter flux test & S 02AML 008 XX 0866 RHE 4 & Rheology & TDP-WTP-355 & $\begin{array}{c}\text { TDP-WTP-355 Shakedown RHE Data on } \\
\text { PEP Slurry }\end{array}$ \\
\hline TI-032 & 2.6 .5 & Conduct low-solids filter flux test & S 02AML 008 XX 0868 CUF 4 & CUF & & \\
\hline TI-032 & 2.6 .5 & Conduct low-solids filter flux test & $\begin{array}{c}\text { S 02AML } 008 \text { XX } 0867 \text { XSP } 4 \\
\text { B }\end{array}$ & PSD & TDP-WTP-354 & $\begin{array}{c}\text { TDP WTP } 354 \text { PEP Shakedown Test PSD } \\
\text { Data Analysis }\end{array}$ \\
\hline TI-032 & 2.6 .5 & Conduct low-solids filter flux test & $\begin{array}{c}\text { S 02AML } 008 \text { XX } 0864 \text { RAM } 3 \\
\text { D }\end{array}$ & Raman & ASR 8319 & ASR 8319 RAM rev 1 \\
\hline TI-032 & 2.6 .5 & Conduct low-solids filter flux test & S 00PF1 008 XX 0869 ARC 1 & Archive & & \\
\hline TI-032 & 2.6 .5 & Conduct low-solids filter flux test & S 00PF1 008 XX 0874 ARC 1 & Archive & & \\
\hline TI-032 & 2.6 .5 & Conduct low-solids filter flux test & S 00PF2 008 XX 0875 ARC 1 & Archive & & \\
\hline TI-032 & 2.6 .5 & Conduct low-solids filter flux test & S 00PF3 008 XX 0876 ARC 1 & Archive & & \\
\hline TI-032 & 2.6 .5 & Conduct low-solids filter flux test & S 00PF4 008 XX 0877 ARC 1 & Archive & & \\
\hline TI-032 & 2.6 .5 & Conduct low-solids filter flux test & S 00PF5 008 XX 0878 ARC 1 & Archive & & \\
\hline TI-032 & 2.6 .5 & Conduct low-solids filter flux test & S 00PF1 008 XX 0985 ARC 1 & Archive & & \\
\hline TI-032 & 2.6 .5 & Conduct low-solids filter flux test & S 00PF2 008 XX 0986 ARC 1 & Archive & & \\
\hline TI-032 & 2.6 .5 & Conduct low-solids filter flux test & S 00PF3 008 XX 0987 ARC 1 & Archive & & \\
\hline TI-032 & 2.6 .5 & Conduct low-solids filter flux test & S 00PF4 008 XX 0988 ARC 1 & Archive & & \\
\hline TI-032 & 2.6 .5 & Conduct low-solids filter flux test & S 00PF5 008 XX 0989 ARC 1 & Archive & & \\
\hline TI-032 & 2.6 .5 & Conduct low-solids filter flux test & S 00PF1 008 XX 0879 ARC 1 & Archive & & \\
\hline TI-032 & 2.6 .5 & Conduct low-solids filter flux test & S 00PF2 008 XX 0880 ARC 1 & Archive & & \\
\hline TI-032 & 2.6 .5 & Conduct low-solids filter flux test & S 00PF3 008 XX 0881 ARC 1 & Archive & & \\
\hline TI-032 & 2.6 .5 & Conduct low-solids filter flux test & S 00PF4 008 XX 0882 ARC 1 & Archive & & \\
\hline
\end{tabular}


TI-WTP-PEP-032 (testing dates 12/19/2008 through 01/11/2009)

\begin{tabular}{|c|c|c|c|c|c|c|}
\hline TI \# & $\begin{array}{c}\text { TI Step } \\
\#\end{array}$ & TI Step Description & Sample ID & Analysis & Folder & Final Electronic Report File Name \\
\hline TI-032 & 2.6 .5 & Conduct low-solids filter flux test & S 00PF5 008 XX 0883 ARC 1 & Archive & & \\
\hline TI-032 & 2.7 .3 & Conduct low-solids filter flux test & S 02AML 009 XX 0884 UDS 2 & UDS & 090103-1 & \\
\hline TI-032 & 2.7 .3 & Conduct low-solids filter flux test & $\begin{array}{c}\text { S 02AML } 009 \text { XX } 0885 \text { XSP } 4 \\
\text { B, }\end{array}$ & PSD & TDP-WTP-354 & $\begin{array}{c}\text { TDP WTP } 354 \text { PEP Shakedown Test PSD } \\
\text { Data Analysis }\end{array}$ \\
\hline TI-032 & 2.8 .2 & Conduct low-solids filter flux test & $\begin{array}{c}\text { S 02AML } 010 \text { XX } 0889 \text { RAM } 3 \\
\text { D }\end{array}$ & Raman & ASR 8319 & ASR 8319 RAM rev 1 \\
\hline TI-032 & 2.8 .2 & Conduct low-solids filter flux test & S 02AML 010 XX 0886 UDS 2 & UDS & 090103-1 & \\
\hline TI-032 & 2.8 .2 & Conduct low-solids filter flux test & S 02AML 010 XX 0887 UDS 2 & UDS & 090103-1 & \\
\hline TI-032 & 2.8 .2 & Conduct low-solids filter flux test & S 02AML 010 XX 0888 UDS 2 & UDS & 090103-1 & \\
\hline TI-032 & 2.8 .2 & Conduct low-solids filter flux test & $\begin{array}{c}\text { S 02AML } 010 \text { XX } 0890 \text { XSP } 4 \\
\text { B }\end{array}$ & PSD & TDP-WTP-354 & $\begin{array}{c}\text { TDP WTP } 354 \text { PEP Shakedown Test PSD } \\
\text { Data Analysis }\end{array}$ \\
\hline TI-032 & 2.8 .2 & Conduct low-solids filter flux test & S 02AML 010 XX 0891 CUF 4 & CUF & & \\
\hline TI-032 & 2.9 .9 & Concentrate solids in UFP-VSL-T02A & S 02AML 011 XX 0892 UDS 2 & UDS & 090103-1 & \\
\hline TI-032 & 2.9 .9 & Concentrate solids in UFP-VSL-T02A & S 02AML 011 XX 0893 UDS 2 & UDS & 090103-1 & \\
\hline TI-032 & 2.9 .9 & Concentrate solids in UFP-VSL-T02A & S 02AML 011 XX 0894 UDS 2 & UDS & 090103-1 & \\
\hline TI-032 & 2.9 .9 & Concentrate solids in UFP-VSL-T01A & S 02AML 011 XX 0895 RHE 4 & Rheology & TDP-WTP-355 & $\begin{array}{c}\text { TDP-WTP-355 Shakedown RHE Data on } \\
\text { PEP Slurry }\end{array}$ \\
\hline TI-032 & 2.11 .9 & Concentrate slurry to 20 -wt $\%$ UDS & S 02AML 015 XX 0896 UDS 2 & UDS & 090103-5 & \\
\hline TI-032 & 2.11 .14 & Concentrate slurry to 20 -wt $\%$ UDS & S 02AML 015 XX 0897 UDS 2 & UDS & 090103-1 & \\
\hline TI-032 & 2.11 .14 & Concentrate slurry to 20 -wt $\%$ UDS & S 02AML 015 XX 0898 UDS 2 & UDS & 090103-1 & \\
\hline TI-032 & 2.11 .14 & Concentrate slurry to $20-\mathrm{wt} \%$ UDS & S 02AML 015 XX 0899 UDS 2 & UDS & 090103-1 & \\
\hline TI-032 & 2.11 .14 & Concentrate slurry to 20 -wt $\%$ UDS & S 02AML 015 XX 0900 DEN 2 & Density Slurry & 090103-2 & 090103-2DEN \\
\hline TI-032 & 2.11 .14 & Concentrate slurry to $20-\mathrm{wt} \%$ UDS & S 02AML 015 XX 0901 DEN 2 & Density Slurry & 090103-2 & 090103-2DEN \\
\hline TI-032 & 2.11 .14 & Concentrate slurry to 20 -wt $\%$ UDS & S 02AML 015 XX 0902 DEN 2 & Density Slurry & 090103-2 & 090103-2DEN \\
\hline TI-032 & 2.11 .14 & Concentrate slurry to $20-\mathrm{wt} \%$ UDS & S 02AML 015 XX 0903 RHE 4 & Rheology & TDP-WTP-355 & $\begin{array}{c}\text { TDP-WTP-355 Shakedown RHE Data on } \\
\text { PEP Slurry }\end{array}$ \\
\hline TI-032 & 2.11 .14 & Concentrate slurry to $20-\mathrm{wt} \%$ UDS & $\begin{array}{c}\text { S 02AML } 015 \text { XX } 0904 \text { ICP } 2 \\
\text { B }\end{array}$ & $\begin{array}{l}\text { ICP Wet } \\
\text { Solids }\end{array}$ & 090103-3 & 090103-3 and 090103-6 ICP and ICPMS \\
\hline TI-032 & 2.11 .14 & Concentrate slurry to $20-\mathrm{wt} \%$ UDS & $\begin{array}{c}\text { S 02AML } 015 \text { XX } 0904 \text { RAM } 3 \\
\text { D }\end{array}$ & Raman & ASR 8319 & ASR 8319 RAM rev 1 \\
\hline TI-032 & 2.11 .14 & Concentrate slurry to 20 -wt $\%$ UDS & $\begin{array}{c}\text { S 02AML } 015 \text { XX } 0904 \text { TFI } 2 \\
\text { D }\end{array}$ & IC & 090103-4 & 090103-4IC \\
\hline TI-032 & 2.11 .14 & Concentrate slurry to $20-\mathrm{wt} \%$ UDS & $\begin{array}{c}\text { S 02AML } 015 \text { XX } 0905 \text { ICP } 2 \\
\text { B }\end{array}$ & $\begin{array}{l}\text { ICP Wet } \\
\text { Solids }\end{array}$ & 090103-3 & 090103-3 and 090103-6 ICP and ICPMS \\
\hline TI-032 & 2.11 .14 & Concentrate slurry to 20 -wt $\%$ UDS & $\begin{array}{c}\text { S 02AML } 015 \text { XX } 0905 \text { RAM } 3 \\
\text { D }\end{array}$ & Raman & ASR 8319 & ASR 8319 RAM rev 1 \\
\hline TI-032 & 2.11 .14 & Concentrate slurry to $20-\mathrm{wt} \%$ UDS & $\begin{array}{c}\text { S 02AML } 015 \text { XX } 0905 \text { TFI } 2 \\
\text { D }\end{array}$ & IC & 090103-4 & 090103-4IC \\
\hline TI-032 & 2.11 .14 & Concentrate slurry to $20-\mathrm{wt} \%$ UDS & $\begin{array}{c}\text { S 02AML } 015 \text { XX } 0906 \text { ICP } 2 \\
\text { B }\end{array}$ & $\begin{array}{l}\text { ICP Wet } \\
\text { Solids }\end{array}$ & 090103-3 & 090103-3 and 090103-6 ICP and ICPMS \\
\hline
\end{tabular}


TI-WTP-PEP-032 (testing dates 12/19/2008 through 01/11/2009)

\begin{tabular}{|c|c|c|c|c|c|c|}
\hline TI \# & $\begin{array}{c}\text { TI Step } \\
\#\end{array}$ & TI Step Description & Sample ID & Analysis & Folder & Final Electronic Report File Name \\
\hline TI-032 & 2.11 .14 & Concentrate slurry to $20-\mathrm{wt} \%$ UDS & $\begin{array}{c}\text { S 02AML } 015 \text { XX } 0906 \text { RAM } 3 \\
\text { D }\end{array}$ & Raman & ASR 8319 & ASR 8319 RAM rev 1 \\
\hline TI-032 & 2.11 .14 & Concentrate slurry to $20-w t \%$ UDS & $\begin{array}{c}\text { S 02AML } 015 \text { XX } 0906 \text { TFI } 2 \\
\text { D }\end{array}$ & IC & 090103-4 & 090103-4IC \\
\hline TI-032 & 2.11 .14 & Concentrate slurry to $20-\mathrm{wt} \%$ UDS & S 02AML 015 XX 0976 CUF 4 & $\begin{array}{c}\text { Parallel Bench } \\
\text { Test }\end{array}$ & & \\
\hline TI-032 & 3.1 .7 & $\mathrm{NaOH}$ addition & $\begin{array}{c}\text { S 02AML } 016 \text { XX } 0738 \text { UDS } 2 \\
\text { B }\end{array}$ & UDS & 090106-3 & \\
\hline TI-032 & 3.1 .7 & $\mathrm{NaOH}$ addition & $\begin{array}{c}\text { S 02AML } 016 \text { XX } 0738 \text { UDS } 2 \\
\text { D }\end{array}$ & UDS & 090106-3 & \\
\hline TI-032 & 3.1 .7 & $\mathrm{NaOH}$ addition & $\begin{array}{c}\text { S 02AML } 016 \text { XX } 0739 \text { RAM } 3 \\
\text { D }\end{array}$ & Raman & ASR 8319 & ASR 8319 RAM rev 1 \\
\hline TI-032 & 3.1 .7 & $\mathrm{NaOH}$ addition & $\begin{array}{c}\text { S 02AMM } 016 \text { XX } 0736 \text { UDS } 2 \\
\text { B }\end{array}$ & UDS & 090106-3 & \\
\hline TI-032 & 3.1 .7 & $\mathrm{NaOH}$ addition & $\begin{array}{c}\text { S 02AMM } 016 \text { XX } 0736 \text { UDS } 2 \\
\text { D }\end{array}$ & UDS & 090106-3 & \\
\hline TI-032 & 3.1 .7 & $\mathrm{NaOH}$ addition & $\begin{array}{c}\text { S 02AMM } 016 \text { XX } 0737 \text { RAM } \\
3 \text { D }\end{array}$ & Raman & ASR 8319 & ASR 8319 RAM rev 1 \\
\hline TI-032 & 3.1 .7 & $\mathrm{NaOH}$ addition & $\begin{array}{c}\text { S 02AOL } 016 \text { XX } 0742 \text { UDS } 2 \\
\text { B }\end{array}$ & UDS & 090106-4 & \\
\hline TI-032 & 3.1 .7 & $\mathrm{NaOH}$ addition & $\begin{array}{c}\text { S 02AOL } 016 \text { XX } 0742 \text { UDS } 2 \\
\text { D }\end{array}$ & UDS & 090106-4 & \\
\hline TI-032 & 3.1 .7 & $\mathrm{NaOH}$ addition & $\begin{array}{c}\text { S 02AOL } 016 \text { XX } 0743 \text { RAM } 3 \\
\text { D }\end{array}$ & Raman & ASR 8319 & ASR 8319 RAM rev 1 \\
\hline TI-032 & 3.1 .7 & $\mathrm{NaOH}$ addition & $\begin{array}{c}\text { S } 02 \mathrm{AOM} 016 \mathrm{XX} 0740 \text { UDS } 2 \\
\text { B }\end{array}$ & UDS & $090106-5$ & \\
\hline TI-032 & 3.1 .7 & $\mathrm{NaOH}$ addition & $\begin{array}{c}\text { S 02AOM } 016 \text { XX } 0740 \text { UDS } 2 \\
\text { D }\end{array}$ & UDS & 090106-5 & \\
\hline TI-032 & 3.1 .7 & $\mathrm{NaOH}$ addition & $\begin{array}{c}\text { S 02AOM } 016 \text { XX } 0741 \text { RAM } \\
3 \text { D }\end{array}$ & Raman & ASR 8319 & ASR 8319 RAM rev 1 \\
\hline TI-032 & 3.1.9.7 & Filter-loop flush & S 02AML 017 XX 0744 UDS 2 & UDS & 090106-6 & \\
\hline TI-032 & 3.1.9.7 & Filter-loop flush & $\begin{array}{c}\text { S 02AML } 017 \text { XX } 0745 \text { RAM } 3 \\
\text { D }\end{array}$ & Raman & ASR 8319 & ASR 8319 RAM rev 1 \\
\hline TI-032 & 3.1.9.7 & Filter-loop flush & S 02AML 017 XX 0977 CUF 4 & $\begin{array}{c}\text { Parallel Bench } \\
\text { Test }\end{array}$ & & \\
\hline TI-032 & 3.1 .13 .1 & Heat UFP-VSL-T02A to $88^{\circ} \mathrm{C}$ & $\begin{array}{c}\text { S 02AMM } 018 \text { BT } 0374 \text { UDS } 2 \\
\text { B }\end{array}$ & UDS & 090106-4 & \\
\hline TI-032 & 3.1.13.1 & Heat UFP-VSL-T02A to $88^{\circ} \mathrm{C}$ & $\begin{array}{c}\text { S 02AMM } 018 \text { BT } 0374 \text { UDS } 2 \\
\text { D }\end{array}$ & UDS & 090106-4 & \\
\hline TI-032 & 3.1 .13 .1 & Heat UFP-VSL-T02A to $88^{\circ} \mathrm{C}$ & S 02AMM 018 BT 0375 UDS 2 & UDS & 090106-4 & \\
\hline
\end{tabular}


TI-WTP-PEP-032 (testing dates 12/19/2008 through 01/11/2009)

\begin{tabular}{|c|c|c|c|c|c|c|}
\hline TI \# & $\begin{array}{c}\text { TI Step } \\
\#\end{array}$ & TI Step Description & Sample ID & Analysis & Folder & Final Electronic Report File Name \\
\hline & & & B & & & \\
\hline TI-032 & 3.1 .13 .1 & Heat UFP-VSL-T02A to $88^{\circ} \mathrm{C}$ & $\begin{array}{c}\text { S 02AMM } 018 \text { BT } 0375 \text { UDS } 2 \\
\text { D }\end{array}$ & UDS & 090106-4 & \\
\hline TI-032 & 3.1 .13 .1 & Heat UFP-VSL-T02A to $88^{\circ} \mathrm{C}$ & $\begin{array}{c}\text { S 02AMM } 018 \text { BT } 0376 \text { UDS } 2 \\
\text { B }\end{array}$ & UDS & 090106-4 & \\
\hline TI-032 & 3.1 .13 .1 & Heat UFP-VSL-T02A to $88^{\circ} \mathrm{C}$ & $\begin{array}{c}\text { S 02AMM } 018 \text { BT } 0376 \text { UDS } 2 \\
\text { D }\end{array}$ & UDS & 090106-4 & \\
\hline TI-032 & 3.1 .13 .1 & Heat UFP-VSL-T02A to $88^{\circ} \mathrm{C}$ & S 02AMM 018 BT 0377 DEN 2 & Density Slurry & 090106-1 & 090106-1DEN \\
\hline TI-032 & 3.1 .13 .1 & Heat UFP-VSL-T02A to $88^{\circ} \mathrm{C}$ & S 02AMM 018 BT 0378 DEN 2 & Density Slurry & 090106-1 & 090106-1DEN \\
\hline TI-032 & 3.1 .13 .1 & Heat UFP-VSL-T02A to $88^{\circ} \mathrm{C}$ & S 02AMM 018 BT 0379 DEN 2 & Density Slurry & 090106-1 & 090106-1DEN \\
\hline TI-032 & 3.1 .13 .1 & Heat UFP-VSL-T02A to $88^{\circ} \mathrm{C}$ & $\begin{array}{c}\text { S 02AMM } 018 \text { BT } 0380 \text { ICP } 2 \\
\text { B }\end{array}$ & $\begin{array}{l}\text { ICP Wet } \\
\text { Solids }\end{array}$ & 090106-2 & 090106-2 ICP ICPMS \\
\hline TI-032 & 3.1 .13 .1 & Heat UFP-VSL-T02A to $88^{\circ} \mathrm{C}$ & $\begin{array}{c}\text { S 02AMM } 018 \text { BT } 0380 \text { RAM } \\
\text { 3 D }\end{array}$ & Raman & ASR 8319 & ASR 8319 RAM rev 1 \\
\hline TI-032 & 3.1.13.1 & Heat UFP-VSL-T02A to $88^{\circ} \mathrm{C}$ & $\begin{array}{c}\text { S 02AMM } 018 \text { BT } 0381 \text { ICP } 2 \\
\text { B }\end{array}$ & $\begin{array}{l}\text { ICP Wet } \\
\text { Solids }\end{array}$ & 090106-2 & 090106-2 ICP ICPMS \\
\hline TI-032 & 3.1.13.1 & Heat UFP-VSL-T02A to $88^{\circ} \mathrm{C}$ & $\begin{array}{c}\text { S 02AMM } 018 \text { BT } 0381 \text { RAM } \\
\text { 3 D }\end{array}$ & Raman & ASR 8319 & ASR 8319 RAM rev 1 \\
\hline TI-032 & 3.1.13.1 & Heat UFP-VSL-T02A to $88^{\circ} \mathrm{C}$ & $\begin{array}{c}\text { S 02AMM } 018 \text { BT } 0382 \text { ICP } 2 \\
\text { B }\end{array}$ & $\begin{array}{l}\text { ICP Wet } \\
\text { Solids }\end{array}$ & 090106-2 & 090106-2 ICP ICPMS \\
\hline TI-032 & 3.1 .13 .1 & Heat UFP-VSL-T02A to $88^{\circ} \mathrm{C}$ & $\begin{array}{c}\text { S 02AMM } 018 \text { BT } 0382 \text { RAM } \\
\text { 3 D }\end{array}$ & Raman & ASR 8319 & ASR 8319 RAM rev 1 \\
\hline TI-032 & 3.1 .14 & UFP-VSL-T02A Caustic-leach $98^{\circ} \mathrm{C}$ & $\begin{array}{c}\text { S 02AMM } 018000383 \text { UDS } 2 \\
\text { B }\end{array}$ & UDS & 090106-3 & \\
\hline TI-032 & 3.1 .14 & UFP-VSL-T02A Caustic-leach $98^{\circ} \mathrm{C}$ & $\begin{array}{c}\text { S 02AMM } 018000383 \text { UDS } 2 \\
\text { D }\end{array}$ & UDS & 090106-3 & \\
\hline TI-032 & 3.1 .14 & UFP-VSL-T02A Caustic-leach $98^{\circ} \mathrm{C}$ & $\begin{array}{c}\text { S 02AMM } 018000384 \text { UDS } 2 \\
\text { B }\end{array}$ & UDS & 090106-3 & \\
\hline TI-032 & 3.1 .14 & UFP-VSL-T02A Caustic-leach $98^{\circ} \mathrm{C}$ & $\begin{array}{c}\text { S 02AMM } 018000384 \text { UDS } 2 \\
\text { D }\end{array}$ & UDS & 090106-3 & \\
\hline TI-032 & 3.1 .14 & UFP-VSL-T02A Caustic-leach $98^{\circ} \mathrm{C}$ & $\begin{array}{c}\text { S 02AMM } 018000385 \text { UDS } 2 \\
\text { B }\end{array}$ & UDS & 090106-3 & \\
\hline TI-032 & 3.1 .14 & UFP-VSL-T02A Caustic-leach $98^{\circ} \mathrm{C}$ & $\begin{array}{c}\text { S 02AMM } 018000385 \text { UDS } 2 \\
\text { D }\end{array}$ & UDS & 090106-3 & \\
\hline TI-032 & 3.1 .14 & UFP-VSL-T02A Caustic-leach $98^{\circ} \mathrm{C}$ & S 02AMM 018000386 ICP 2 B & $\begin{array}{l}\text { ICP Wet } \\
\text { Solids }\end{array}$ & 090106-2 & 090106-2 ICP ICPMS \\
\hline TI-032 & 3.1 .14 & UFP-VSL-T02A Caustic-leach $98^{\circ} \mathrm{C}$ & $\begin{array}{c}\text { S 02AMM } 018000386 \text { RAM } 3 \\
\text { D }\end{array}$ & Raman & ASR 8319 & ASR 8319 RAM rev 1 \\
\hline TI-032 & 3.1 .14 & UFP-VSL-T02A Caustic-leach $98^{\circ} \mathrm{C}$ & S 02AMM 018000387 ICP 2 B & $\begin{array}{l}\text { ICP Wet } \\
\text { Solids }\end{array}$ & 090106-2 & 090106-2 ICP ICPMS \\
\hline
\end{tabular}


TI-WTP-PEP-032 (testing dates 12/19/2008 through 01/11/2009)

\begin{tabular}{|c|c|c|c|c|c|c|}
\hline TI \# & $\begin{array}{c}\text { TI Step } \\
\#\end{array}$ & TI Step Description & Sample ID & Analysis & Folder & Final Electronic Report File Name \\
\hline TI-032 & 3.1 .14 & UFP-VSL-T02A Caustic-leach $98^{\circ} \mathrm{C}$ & $\begin{array}{c}\text { S 02AMM } 018000387 \text { RAM } 3 \\
\text { D }\end{array}$ & Raman & ASR 8319 & ASR 8319 RAM rev 1 \\
\hline TI-032 & 3.1 .14 & UFP-VSL-T02A Caustic-leach $98^{\circ} \mathrm{C}$ & S 02AMM 018000388 ICP 2 B & $\begin{array}{l}\text { ICP Wet } \\
\text { Solids }\end{array}$ & 090106-2 & 090106-2 ICP ICPMS \\
\hline TI-032 & 3.1 .14 & UFP-VSL-T02A Caustic-leach $98^{\circ} \mathrm{C}$ & $\begin{array}{c}\text { S 02AMM } 018000388 \text { RAM } 3 \\
\text { D }\end{array}$ & Raman & ASR 8319 & ASR 8319 RAM rev 1 \\
\hline TI-032 & 3.1 .15 & UFP-VSL-T02A Caustic-leach $98^{\circ} \mathrm{C}$ & $\begin{array}{c}\text { S 02AMM } 018010389 \text { UDS } 2 \\
\text { B }\end{array}$ & UDS & 090106-3 & \\
\hline TI-032 & 3.1 .15 & UFP-VSL-T02A Caustic-leach $98^{\circ} \mathrm{C}$ & $\begin{array}{c}\text { S 02AMM } 018010389 \text { UDS } 2 \\
\text { D }\end{array}$ & UDS & 090106-3 & \\
\hline TI-032 & 3.1 .15 & UFP-VSL-T02A Caustic-leach $98^{\circ} \mathrm{C}$ & $\begin{array}{c}\text { S 02AMM } 018010390 \text { UDS } 2 \\
\text { B }\end{array}$ & UDS & 090106-3 & \\
\hline TI-032 & 3.1 .15 & UFP-VSL-T02A Caustic-leach $98^{\circ} \mathrm{C}$ & $\begin{array}{c}\text { S 02AMM } 018010390 \text { UDS } 2 \\
\text { D }\end{array}$ & UDS & 090106-3 & \\
\hline TI-032 & 3.1 .15 & UFP-VSL-T02A Caustic-leach $98^{\circ} \mathrm{C}$ & $\begin{array}{c}\text { S 02AMM } 018010391 \text { UDS } 2 \\
\text { B }\end{array}$ & UDS & 090106-3 & \\
\hline TI-032 & 3.1 .15 & UFP-VSL-T02A Caustic-leach $98^{\circ} \mathrm{C}$ & $\begin{array}{c}\text { S 02AMM } 018010391 \text { UDS } 2 \\
\text { D }\end{array}$ & UDS & 090106-3 & \\
\hline TI-032 & 3.1 .15 & UFP-VSL-T02A Caustic-leach $98^{\circ} \mathrm{C}$ & S 02AMM 018010392 ICP 2 B & $\begin{array}{l}\text { ICP Wet } \\
\text { Solids }\end{array}$ & 090106-2 & 090106-2 ICP ICPMS \\
\hline TI-032 & 3.1 .15 & UFP-VSL-T02A Caustic-leach $98^{\circ} \mathrm{C}$ & $\begin{array}{c}\text { S 02AMM } 018010392 \text { RAM } 3 \\
\text { D }\end{array}$ & Raman & ASR 8319 & ASR 8319 RAM rev 1 \\
\hline TI-032 & 3.1 .15 & UFP-VSL-T02A Caustic-leach $98^{\circ} \mathrm{C}$ & S 02AMM 018010393 ICP 2 B & $\begin{array}{l}\text { ICP Wet } \\
\text { Solids }\end{array}$ & 090106-2 & 090106-2 ICP ICPMS \\
\hline TI-032 & 3.1 .15 & UFP-VSL-T02A Caustic-leach $98^{\circ} \mathrm{C}$ & $\begin{array}{c}\text { S 02AMM } 018010393 \text { RAM } 3 \\
\text { D }\end{array}$ & Raman & ASR 8319 & ASR 8319 RAM rev 1 \\
\hline TI-032 & 3.1 .15 & UFP-VSL-T02A Caustic-leach $98^{\circ} \mathrm{C}$ & S 02AMM 018010394 ICP 2 B & $\begin{array}{l}\text { ICP Wet } \\
\text { Solids }\end{array}$ & 090106-2 & 090106-2 ICP ICPMS \\
\hline TI-032 & 3.1 .15 & UFP-VSL-T02A Caustic-leach $98^{\circ} \mathrm{C}$ & $\begin{array}{c}\text { S 02AMM } 018010394 \text { RAM } 3 \\
\text { D }\end{array}$ & Raman & ASR 8319 & ASR 8319 RAM rev 1 \\
\hline TI-032 & 3.1 .15 & UFP-VSL-T02A Caustic-leach $98^{\circ} \mathrm{C}$ & $\begin{array}{c}\text { S 02AMM } 018020395 \text { UDS } 2 \\
\text { B }\end{array}$ & UDS & 090106-3 & \\
\hline TI-032 & 3.1 .15 & UFP-VSL-T02A Caustic-leach $98^{\circ} \mathrm{C}$ & $\begin{array}{c}\text { S 02AMM } 018020395 \text { UDS } 2 \\
\text { D }\end{array}$ & UDS & 090106-3 & \\
\hline TI-032 & 3.1 .15 & UFP-VSL-T02A Caustic-leach $98^{\circ} \mathrm{C}$ & $\begin{array}{c}\text { S 02AMM } 018020396 \text { UDS } 2 \\
\text { B }\end{array}$ & UDS & 090106-3 & \\
\hline TI-032 & 3.1 .15 & UFP-VSL-T02A Caustic-leach $98^{\circ} \mathrm{C}$ & $\begin{array}{c}\text { S 02AMM } 018020396 \text { UDS } 2 \\
\text { D }\end{array}$ & UDS & 090106-3 & \\
\hline TI-032 & 3.1 .15 & UFP-VSL-T02A Caustic-leach $98^{\circ} \mathrm{C}$ & $\begin{array}{c}\text { S 02AMM } 018020397 \text { UDS } 2 \\
\text { B }\end{array}$ & UDS & 090106-3 & \\
\hline
\end{tabular}


TI-WTP-PEP-032 (testing dates 12/19/2008 through 01/11/2009)

\begin{tabular}{|c|c|c|c|c|c|c|}
\hline TI \# & $\begin{array}{c}\text { TI Step } \\
\#\end{array}$ & TI Step Description & Sample ID & Analysis & Folder & Final Electronic Report File Name \\
\hline TI-032 & 3.1 .15 & UFP-VSL-T02A Caustic-leach $98^{\circ} \mathrm{C}$ & $\begin{array}{c}\text { S 02AMM } 018020397 \text { UDS } 2 \\
\text { D }\end{array}$ & UDS & 090106-3 & \\
\hline TI-032 & 3.1 .15 & UFP-VSL-T02A Caustic-leach $98^{\circ} \mathrm{C}$ & S 02AMM 018020398 ICP 2 B & $\begin{array}{l}\text { ICP Wet } \\
\text { Solids }\end{array}$ & 090106-2 & 090106-2 ICP ICPMS \\
\hline TI-032 & 3.1 .15 & UFP-VSL-T02A Caustic-leach $98^{\circ} \mathrm{C}$ & $\begin{array}{c}\text { S 02AMM } 018020398 \text { RAM } 3 \\
\text { D }\end{array}$ & Raman & ASR 8319 & ASR 8319 RAM rev 1 \\
\hline TI-032 & 3.1 .15 & UFP-VSL-T02A Caustic-leach $98^{\circ} \mathrm{C}$ & S 02AMM 018020399 ICP 2 B & $\begin{array}{l}\text { ICP Wet } \\
\text { Solids }\end{array}$ & 090106-2 & 090106-2 ICP ICPMS \\
\hline TI-032 & 3.1 .15 & UFP-VSL-T02A Caustic-leach $98^{\circ} \mathrm{C}$ & $\begin{array}{c}\text { S 02AMM } 018020399 \text { RAM } 3 \\
\text { D }\end{array}$ & Raman & ASR 8319 & ASR 8319 RAM rev 1 \\
\hline TI-032 & 3.1 .15 & UFP-VSL-T02A Caustic-leach $98^{\circ} \mathrm{C}$ & $\begin{array}{c}\text { S 02AMM } 018020400 \text { RAM } 3 \\
\text { D }\end{array}$ & Raman & ASR 8319 & ASR 8319 RAM rev 1 \\
\hline TI-032 & 3.1 .15 & UFP-VSL-T02A Caustic-leach $98^{\circ} \mathrm{C}$ & S 02AMM 018020400 ICP 2 B & $\begin{array}{l}\text { ICP Wet } \\
\text { Solids }\end{array}$ & 090106-2 & 090106-2 ICP ICPMS \\
\hline TI-032 & 3.1 .15 & UFP-VSL-T02A Caustic-leach $98^{\circ} \mathrm{C}$ & $\begin{array}{c}\text { S 02AMH } 018020401 \text { UDS } 2 \\
\text { B }\end{array}$ & UDS & 090106-3 & \\
\hline TI-032 & 3.1 .15 & UFP-VSL-T02A Caustic-leach $98^{\circ} \mathrm{C}$ & $\begin{array}{c}\text { S 02AMH } 018020401 \text { UDS } 2 \\
\text { D }\end{array}$ & UDS & 090106-3 & \\
\hline TI-032 & 3.1 .15 & UFP-VSL-T02A Caustic-leach $98^{\circ} \mathrm{C}$ & S 02AMH 018020402 DEN 2 & Density Slurry & 090106-1 & 090106-1DEN \\
\hline TI-032 & 3.1 .15 & UFP-VSL-T02A Caustic-leach $98^{\circ} \mathrm{C}$ & S 02AOL 018020403 UDS 2 B & UDS & 090106-4 & \\
\hline TI-032 & 3.1 .15 & UFP-VSL-T02A Caustic-leach $98^{\circ} \mathrm{C}$ & S 02AOL 018020403 UDS 2 D & UDS & 090106-4 & \\
\hline TI-032 & 3.1 .15 & UFP-VSL-T02A Caustic-leach $98^{\circ} \mathrm{C}$ & S 02AOL 018020404 DEN 2 & Density Slurry & 090106-1 & 090106-1DEN \\
\hline TI-032 & 3.1 .15 & UFP-VSL-T02A Caustic-leach $98^{\circ} \mathrm{C}$ & $\begin{array}{c}\text { S 02AOM } 018020405 \text { UDS } 2 \\
\text { B }\end{array}$ & UDS & $090106-5$ & \\
\hline TI-032 & 3.1 .15 & UFP-VSL-T02A Caustic-leach $98^{\circ} \mathrm{C}$ & $\begin{array}{c}\text { S 02AOM } 018020405 \text { UDS } 2 \\
\text { D }\end{array}$ & UDS & 090106-5 & \\
\hline TI-032 & 3.1 .15 & UFP-VSL-T02A Caustic-leach $98^{\circ} \mathrm{C}$ & S 02AOM 018020406 DEN 2 & Density Slurry & 090106-1 & 090106-1DEN \\
\hline TI-032 & 3.1 .15 & UFP-VSL-T02A Caustic-leach $98^{\circ} \mathrm{C}$ & $\begin{array}{c}\text { S 02AOH } 018020407 \text { UDS } 2 \\
\text { B }\end{array}$ & UDS & 090106-4 & \\
\hline TI-032 & 3.1 .15 & UFP-VSL-T02A Caustic-leach $98^{\circ} \mathrm{C}$ & $\begin{array}{c}\text { S 02AOH } 018020407 \text { UDS } 2 \\
\text { D }\end{array}$ & UDS & 090106-4 & \\
\hline TI-032 & 3.1 .15 & UFP-VSL-T02A Caustic-leach $98^{\circ} \mathrm{C}$ & $\mathrm{S} 02 \mathrm{AOH} 018020408$ DEN 2 & Density Slurry & 090106-1 & 090106-1DEN \\
\hline TI-032 & 3.1 .15 & UFP-VSL-T02A Caustic-leach $98^{\circ} \mathrm{C}$ & S 02AIL 018020409 UDS 2 B & UDS & 090106-3 & \\
\hline TI-032 & 3.1 .15 & UFP-VSL-T02A Caustic-leach $98^{\circ} \mathrm{C}$ & S 02AIL 018020409 UDS 2 D & UDS & 090106-3 & \\
\hline TI-032 & 3.1 .15 & UFP-VSL-T02A Caustic-leach $98^{\circ} \mathrm{C}$ & S 02AIL 018020410 DEN 2 & Density Slurry & 090106-1 & 090106-1DEN \\
\hline TI-032 & 3.1 .15 & UFP-VSL-T02A Caustic-leach $98^{\circ} \mathrm{C}$ & S 02AIM 018020411 UDS 2 B & UDS & 090106-3 & \\
\hline TI-032 & 3.1 .15 & UFP-VSL-T02A Caustic-leach $98^{\circ} \mathrm{C}$ & S 02AIM 018020411 UDS 2 D & UDS & 090106-3 & \\
\hline TI-032 & 3.1 .15 & UFP-VSL-T02A Caustic-leach $98^{\circ} \mathrm{C}$ & S 02AIM 018020412 DEN 2 & Density Slurry & 090106-1 & 090106-1DEN \\
\hline TI-032 & 3.1 .15 & UFP-VSL-T02A Caustic-leach $98^{\circ} \mathrm{C}$ & $\begin{array}{c}\text { S 02AML } 018020415 \text { UDS } 2 \\
\text { B }\end{array}$ & UDS & 090106-3 & \\
\hline
\end{tabular}


TI-WTP-PEP-032 (testing dates 12/19/2008 through 01/11/2009)

\begin{tabular}{|c|c|c|c|c|c|c|}
\hline TI \# & $\begin{array}{c}\text { TI Step } \\
\#\end{array}$ & TI Step Description & Sample ID & Analysis & Folder & Final Electronic Report File Name \\
\hline TI-032 & 3.1 .15 & UFP-VSL-T02A Caustic-leach $98^{\circ} \mathrm{C}$ & $\begin{array}{c}\text { S 02AML } 018020415 \text { UDS } 2 \\
\text { D }\end{array}$ & UDS & 090106-3 & \\
\hline TI-032 & 3.1 .15 & UFP-VSL-T02A Caustic-leach $98^{\circ} \mathrm{C}$ & S 02AML 018020416 DEN 2 & Density Slurry & 090106-1 & 090106-1DEN \\
\hline TI-032 & 3.1 .15 & UFP-VSL-T02A Caustic-leach $98^{\circ} \mathrm{C}$ & $\begin{array}{c}\text { S 02AMM } 018040417 \text { UDS } 2 \\
\text { B }\end{array}$ & UDS & 090106-4 & \\
\hline TI-032 & 3.1 .15 & UFP-VSL-T02A Caustic-leach $98^{\circ} \mathrm{C}$ & $\begin{array}{c}\text { S 02AMM } 018040417 \text { UDS } 2 \\
\text { D }\end{array}$ & UDS & 090106-4 & \\
\hline TI-032 & 3.1 .15 & UFP-VSL-T02A Caustic-leach $98^{\circ} \mathrm{C}$ & $\begin{array}{c}\text { S 02AMM } 018040418 \text { UDS } 2 \\
\text { B }\end{array}$ & UDS & 090106-4 & \\
\hline TI-032 & 3.1 .15 & UFP-VSL-T02A Caustic-leach $98^{\circ} \mathrm{C}$ & $\begin{array}{c}\text { S 02AMM } 018040418 \text { UDS } 2 \\
\text { D }\end{array}$ & UDS & 090106-4 & \\
\hline TI-032 & 3.1 .15 & UFP-VSL-T02A Caustic-leach $98^{\circ} \mathrm{C}$ & $\begin{array}{c}\text { S 02AMM } 018040419 \text { UDS } 2 \\
\text { B }\end{array}$ & UDS & 090106-4 & \\
\hline TI-032 & 3.1 .15 & UFP-VSL-T02A Caustic-leach $98^{\circ} \mathrm{C}$ & $\begin{array}{c}\text { S 02AMM } 018040419 \text { UDS } 2 \\
\text { D }\end{array}$ & UDS & 090106-4 & \\
\hline TI-032 & 3.1 .15 & UFP-VSL-T02A Caustic-leach $98^{\circ} \mathrm{C}$ & S 02AMM 018040420 ICP 2 B & $\begin{array}{l}\text { ICP Wet } \\
\text { Solids }\end{array}$ & 090106-2 & 090106-2 ICP ICPMS \\
\hline TI-032 & 3.1 .15 & UFP-VSL-T02A Caustic-leach $98^{\circ} \mathrm{C}$ & $\begin{array}{c}\text { S 02AMM } 018040420 \text { RAM } 3 \\
\text { D }\end{array}$ & Raman & ASR 8319 & ASR 8319 RAM rev 1 \\
\hline TI-032 & 3.1 .15 & UFP-VSL-T02A Caustic-leach $98^{\circ} \mathrm{C}$ & S 02AMM 018040421 ICP 2 B & $\begin{array}{l}\text { ICP Wet } \\
\text { Solids }\end{array}$ & 090106-2 & 090106-2 ICP ICPMS \\
\hline TI-032 & 3.1 .15 & UFP-VSL-T02A Caustic-leach $98^{\circ} \mathrm{C}$ & $\begin{array}{c}\text { S 02AMM } 018040421 \text { RAM } 3 \\
\text { D }\end{array}$ & Raman & ASR 8319 & ASR 8319 RAM rev 1 \\
\hline TI-032 & 3.1 .15 & UFP-VSL-T02A Caustic-leach $98^{\circ} \mathrm{C}$ & S 02AMM 018040422 ICP 2 B & $\begin{array}{l}\text { ICP Wet } \\
\text { Solids }\end{array}$ & 090106-2 & 090106-2 ICP ICPMS \\
\hline TI-032 & 3.1 .15 & UFP-VSL-T02A Caustic-leach $98^{\circ} \mathrm{C}$ & $\begin{array}{c}\text { S 02AMM } 018040422 \text { RAM } 3 \\
\text { D }\end{array}$ & Raman & ASR 8319 & ASR 8319 RAM rev 1 \\
\hline TI-032 & 3.1 .15 & UFP-VSL-T02A Caustic-leach $98^{\circ} \mathrm{C}$ & $\begin{array}{c}\text { S 02AMM } 018080423 \text { UDS } 2 \\
\text { B }\end{array}$ & UDS & 090106-4 & \\
\hline TI-032 & 3.1 .15 & UFP-VSL-T02A Caustic-leach $98^{\circ} \mathrm{C}$ & $\begin{array}{c}\text { S 02AMM } 018080423 \text { UDS } 2 \\
\text { D }\end{array}$ & UDS & 090106-4 & \\
\hline TI-032 & 3.1 .15 & UFP-VSL-T02A Caustic-leach $98^{\circ} \mathrm{C}$ & $\begin{array}{c}\text { S 02AMM } 018080424 \text { UDS } 2 \\
\text { B }\end{array}$ & UDS & 090106-4 & \\
\hline TI-032 & 3.1 .15 & UFP-VSL-T02A Caustic-leach $98^{\circ} \mathrm{C}$ & $\begin{array}{c}\text { S 02AMM } 018080424 \text { UDS } 2 \\
\text { D }\end{array}$ & UDS & 090106-4 & \\
\hline TI-032 & 3.1 .15 & UFP-VSL-T02A Caustic-leach $98^{\circ} \mathrm{C}$ & $\begin{array}{c}\text { S 02AMM } 018080425 \text { UDS } 2 \\
\text { B }\end{array}$ & UDS & 090106-4 & \\
\hline TI-032 & 3.1 .15 & UFP-VSL-T02A Caustic-leach $98^{\circ} \mathrm{C}$ & $\begin{array}{c}\text { S 02AMM } 018080425 \text { UDS } 2 \\
\text { D }\end{array}$ & UDS & 090106-4 & \\
\hline TI-032 & 3.1 .15 & UFP-VSL-T02A Caustic-leach $98^{\circ} \mathrm{C}$ & S 02AMM 018080426 ICP 2 B & ICP Wet & 090106-2 & 090106-2 ICP ICPMS \\
\hline
\end{tabular}


TI-WTP-PEP-032 (testing dates 12/19/2008 through 01/11/2009)

\begin{tabular}{|c|c|c|c|c|c|c|}
\hline TI \# & $\begin{array}{c}\text { TI Step } \\
\#\end{array}$ & TI Step Description & Sample ID & Analysis & Folder & Final Electronic Report File Name \\
\hline & & & & Solids & & \\
\hline TI-032 & 3.1 .15 & UFP-VSL-T02A Caustic-leach $98^{\circ} \mathrm{C}$ & $\begin{array}{c}\text { S 02AMM } 018080426 \text { RAM } 3 \\
\text { D }\end{array}$ & Raman & ASR 8319 & ASR 8319 RAM rev 1 \\
\hline TI-032 & 3.1 .15 & UFP-VSL-T02A Caustic-leach $98^{\circ} \mathrm{C}$ & S 02AMM 018080427 ICP 2 B & $\begin{array}{l}\text { ICP Wet } \\
\text { Solids }\end{array}$ & 090106-2 & 090106-2 ICP ICPMS \\
\hline TI-032 & 3.1 .15 & UFP-VSL-T02A Caustic-leach $98^{\circ} \mathrm{C}$ & $\begin{array}{c}\text { S 02AMM } 018080427 \text { RAM } 3 \\
\text { D }\end{array}$ & Raman & ASR 8319 & ASR 8319 RAM rev 1 \\
\hline TI-032 & 3.1 .15 & UFP-VSL-T02A Caustic-leach $98^{\circ} \mathrm{C}$ & S 02AMM 018080428 ICP 2 B & $\begin{array}{l}\text { ICP Wet } \\
\text { Solids }\end{array}$ & 090106-2 & 090106-2 ICP ICPMS \\
\hline TI-032 & 3.1 .15 & UFP-VSL-T02A Caustic-leach $98^{\circ} \mathrm{C}$ & $\begin{array}{c}\text { S 02AMM } 018080428 \text { RAM } 3 \\
\text { D }\end{array}$ & Raman & ASR 8319 & ASR 8319 RAM rev 1 \\
\hline TI-032 & 3.1 .15 & UFP-VSL-T02A Caustic-leach $98^{\circ} \mathrm{C}$ & $\begin{array}{c}\text { S 02AMM } 018120429 \text { UDS } 2 \\
\text { B }\end{array}$ & UDS & 090106-4 & \\
\hline TI-032 & 3.1 .15 & UFP-VSL-T02A Caustic-leach $98^{\circ} \mathrm{C}$ & $\begin{array}{c}\text { S 02AMM } 018120429 \text { UDS } 2 \\
\text { D }\end{array}$ & UDS & 090106-4 & \\
\hline TI-032 & 3.1 .15 & UFP-VSL-T02A Caustic-leach $98^{\circ} \mathrm{C}$ & $\begin{array}{c}\text { S 02AMM } 018120430 \text { UDS } 2 \\
\text { B }\end{array}$ & UDS & 090106-4 & \\
\hline TI-032 & 3.1 .15 & UFP-VSL-T02A Caustic-leach $98^{\circ} \mathrm{C}$ & $\begin{array}{c}\text { S 02AMM } 018120430 \text { UDS } 2 \\
\text { D }\end{array}$ & UDS & 090106-4 & \\
\hline TI-032 & 3.1 .15 & UFP-VSL-T02A Caustic-leach $98^{\circ} \mathrm{C}$ & $\begin{array}{c}\text { S 02AMM } 018120431 \text { UDS } 2 \\
\text { B }\end{array}$ & UDS & 090106-4 & \\
\hline TI-032 & 3.1 .15 & UFP-VSL-T02A Caustic-leach $98^{\circ} \mathrm{C}$ & $\begin{array}{c}\text { S 02AMM } 018120431 \text { UDS } 2 \\
\text { D }\end{array}$ & UDS & 090106-4 & \\
\hline TI-032 & 3.1 .15 & UFP-VSL-T02A Caustic-leach $98^{\circ} \mathrm{C}$ & S 02AMM 018120432 ICP 2 B & $\begin{array}{l}\text { ICP Wet } \\
\text { Solids }\end{array}$ & 090106-2 & 090106-2 ICP ICPMS \\
\hline TI-032 & 3.1 .15 & UFP-VSL-T02A Caustic-leach $98^{\circ} \mathrm{C}$ & $\begin{array}{c}\text { S 02AMM } 018120432 \text { RAM } 3 \\
\text { D }\end{array}$ & Raman & ASR 8319 & ASR 8319 RAM rev 1 \\
\hline TI-032 & 3.1 .15 & UFP-VSL-T02A Caustic-leach $98^{\circ} \mathrm{C}$ & S 02AMM 018120433 ICP 2 B & $\begin{array}{l}\text { ICP Wet } \\
\text { Solids }\end{array}$ & 090106-2 & 090106-2 ICP ICPMS \\
\hline TI-032 & 3.1 .15 & UFP-VSL-T02A Caustic-leach $98^{\circ} \mathrm{C}$ & $\begin{array}{c}\text { S 02AMM } 018120433 \text { RAM } 3 \\
\text { D }\end{array}$ & Raman & ASR 8319 & ASR 8319 RAM rev 1 \\
\hline TI-032 & 3.1 .15 & UFP-VSL-T02A Caustic-leach $98^{\circ} \mathrm{C}$ & S 02AMM 018120434 ICP 2 B & $\begin{array}{l}\text { ICP Wet } \\
\text { Solids }\end{array}$ & 090106-2 & 090106-2 ICP ICPMS \\
\hline TI-032 & 3.1 .15 & UFP-VSL-T02A Caustic-leach $98^{\circ} \mathrm{C}$ & $\begin{array}{c}\text { S 02AMM } 018120434 \text { RAM } 3 \\
\text { D }\end{array}$ & Raman & ASR 8319 & ASR 8319 RAM rev 1 \\
\hline TI-032 & 3.1 .15 & UFP-VSL-T02A Caustic-leach $98^{\circ} \mathrm{C}$ & S 02AIL 018160449 UDS 2 B & UDS & 090106-3 & \\
\hline TI-032 & 3.1 .15 & UFP-VSL-T02A Caustic-leach $98^{\circ} \mathrm{C}$ & S 02AIL 018160449 UDS 2 D & UDS & 090106-3 & \\
\hline TI-032 & 3.1 .15 & UFP-VSL-T02A Caustic-leach $98^{\circ} \mathrm{C}$ & S 02AIL 018160450 DEN 2 & Density Slurry & 090106-1 & 090106-1DEN \\
\hline TI-032 & 3.1 .15 & UFP-VSL-T02A Caustic-leach $98^{\circ} \mathrm{C}$ & S 02AIM 018160451 UDS 2 B & UDS & 090106-3 & \\
\hline TI-032 & 3.1 .15 & UFP-VSL-T02A Caustic-leach $98^{\circ} \mathrm{C}$ & S 02AIM 018160451 UDS 2 D & UDS & 090106-3 & \\
\hline
\end{tabular}


TI-WTP-PEP-032 (testing dates 12/19/2008 through 01/11/2009)

\begin{tabular}{|c|c|c|c|c|c|c|}
\hline TI \# & $\begin{array}{c}\text { TI Step } \\
\#\end{array}$ & TI Step Description & Sample ID & Analysis & Folder & Final Electronic Report File Name \\
\hline TI-032 & 3.1 .15 & UFP-VSL-T02A Caustic-leach $98^{\circ} \mathrm{C}$ & S 02AIM 018160452 DEN 2 & Density Slurry & 090106-1 & 090106-1DEN \\
\hline TI-032 & 3.1 .15 & UFP-VSL-T02A Caustic-leach $98^{\circ} \mathrm{C}$ & $\begin{array}{c}\text { S 02AMH } 018160441 \text { UDS } 2 \\
\text { B }\end{array}$ & UDS & 090106-3 & \\
\hline TI-032 & 3.1 .15 & UFP-VSL-T02A Caustic-leach $98^{\circ} \mathrm{C}$ & $\begin{array}{c}\text { S 02AMH } 018160441 \text { UDS } 2 \\
\text { D }\end{array}$ & UDS & 090106-3 & \\
\hline TI-032 & 3.1 .15 & UFP-VSL-T02A Caustic-leach $98^{\circ} \mathrm{C}$ & S 02AMH 018160442 DEN 2 & Density Slurry & 090106-1 & 090106-1DEN \\
\hline TI-032 & 3.1 .15 & UFP-VSL-T02A Caustic-leach $98^{\circ} \mathrm{C}$ & $\begin{array}{c}\text { S 02AML } 018160455 \text { UDS } 2 \\
\text { B }\end{array}$ & UDS & 090106-3 & \\
\hline TI-032 & 3.1 .15 & UFP-VSL-T02A Caustic-leach $98^{\circ} \mathrm{C}$ & $\begin{array}{c}\text { S 02AML } 018160455 \text { UDS } 2 \\
\text { D }\end{array}$ & UDS & 090106-3 & \\
\hline TI-032 & 3.1 .15 & UFP-VSL-T02A Caustic-leach $98^{\circ} \mathrm{C}$ & S 02AML 018160456 DEN 2 & Density Slurry & 090106-1 & 090106-1DEN \\
\hline TI-032 & 3.1 .15 & UFP-VSL-T02A Caustic-leach $98^{\circ} \mathrm{C}$ & S 02AIH 018160453 UDS 2 B & UDS & 090106-3 & \\
\hline TI-032 & 3.1 .15 & UFP-VSL-T02A Caustic-leach $98^{\circ} \mathrm{C}$ & S 02AIH 018160453 UDS 2 D & UDS & 090106-3 & \\
\hline TI-032 & 3.1 .15 & UFP-VSL-T02A Caustic-leach $98^{\circ} \mathrm{C}$ & S 02AIH 018160454 DEN 2 & Density Slurry & 090106-1 & 090106-1DEN \\
\hline TI-032 & 3.1 .15 & UFP-VSL-T02A Caustic-leach $98^{\circ} \mathrm{C}$ & $\begin{array}{c}\text { S 02AMM } 018160435 \text { UDS } 2 \\
\text { B }\end{array}$ & UDS & 090106-4 & \\
\hline TI-032 & 3.1 .15 & UFP-VSL-T02A Caustic-leach $98^{\circ} \mathrm{C}$ & $\begin{array}{c}\text { S 02AMM } 018160435 \text { UDS } 2 \\
\text { D }\end{array}$ & UDS & 090106-4 & \\
\hline TI-032 & 3.1 .15 & UFP-VSL-T02A Caustic-leach $98^{\circ} \mathrm{C}$ & $\begin{array}{c}\text { S 02AMM } 018160436 \text { UDS } 2 \\
\text { B }\end{array}$ & UDS & 090106-4 & \\
\hline TI-032 & 3.1 .15 & UFP-VSL-T02A Caustic-leach $98^{\circ} \mathrm{C}$ & $\begin{array}{c}\text { S 02AMM } 018160436 \text { UDS } 2 \\
\text { D }\end{array}$ & UDS & 090106-4 & \\
\hline TI-032 & 3.1 .15 & UFP-VSL-T02A Caustic-leach $98^{\circ} \mathrm{C}$ & $\begin{array}{c}\text { S 02AMM } 018160437 \text { UDS } 2 \\
\text { B }\end{array}$ & UDS & 090106-4 & \\
\hline TI-032 & 3.1 .15 & UFP-VSL-T02A Caustic-leach $98^{\circ} \mathrm{C}$ & $\begin{array}{c}\text { S 02AMM } 018160437 \text { UDS } 2 \\
\text { D }\end{array}$ & UDS & 090106-4 & \\
\hline TI-032 & 3.1 .15 & UFP-VSL-T02A Caustic-leach $98^{\circ} \mathrm{C}$ & S 02AMM 018160438 ICP 2 B & $\begin{array}{l}\text { ICP Wet } \\
\text { Solids }\end{array}$ & 090106-2 & 090106-2 ICP ICPMS \\
\hline TI-032 & 3.1 .15 & UFP-VSL-T02A Caustic-leach $98^{\circ} \mathrm{C}$ & $\begin{array}{c}\text { S 02AMM } 018160438 \text { RAM } 3 \\
\text { D }\end{array}$ & Raman & ASR 8319 & ASR 8319 RAM rev 1 \\
\hline TI-032 & 3.1 .15 & UFP-VSL-T02A Caustic-leach $98^{\circ} \mathrm{C}$ & S 02AMM 018160439 ICP 2 B & $\begin{array}{l}\text { ICP Wet } \\
\text { Solids }\end{array}$ & 090106-2 & 090106-2 ICP ICPMS \\
\hline TI-032 & 3.1 .15 & UFP-VSL-T02A Caustic-leach $98^{\circ} \mathrm{C}$ & $\begin{array}{c}\text { S 02AMM } 018160439 \text { RAM } 3 \\
\text { D }\end{array}$ & Raman & ASR 8319 & ASR 8319 RAM rev 1 \\
\hline TI-032 & 3.1 .15 & UFP-VSL-T02A Caustic-leach $98^{\circ} \mathrm{C}$ & S 02AMM 018160440 ICP 2 B & $\begin{array}{l}\text { ICP Wet } \\
\text { Solids }\end{array}$ & 090106-2 & 090106-2 ICP ICPMS \\
\hline TI-032 & 3.1 .15 & UFP-VSL-T02A Caustic-leach $98^{\circ} \mathrm{C}$ & $\begin{array}{c}\text { S 02AMM } 018160440 \text { RAM } 3 \\
\text { D }\end{array}$ & Raman & ASR 8319 & ASR 8319 RAM rev 1 \\
\hline TI-032 & 3.1 .15 & UFP-VSL-T02A Caustic-leach $98^{\circ} \mathrm{C}$ & $\begin{array}{c}\text { S 02AOH } 018160447 \text { UDS } 2 \\
\text { B }\end{array}$ & UDS & 090106-4 & \\
\hline
\end{tabular}


TI-WTP-PEP-032 (testing dates 12/19/2008 through 01/11/2009)

\begin{tabular}{|c|c|c|c|c|c|c|}
\hline TI \# & $\begin{array}{c}\text { TI Step } \\
\#\end{array}$ & TI Step Description & Sample ID & Analysis & Folder & Final Electronic Report File Name \\
\hline TI-032 & 3.1 .15 & UFP-VSL-T02A Caustic-leach $98^{\circ} \mathrm{C}$ & $\begin{array}{c}\text { S 02AOH } 018160447 \text { UDS } 2 \\
\text { D }\end{array}$ & UDS & 090106-4 & \\
\hline TI-032 & 3.1 .15 & UFP-VSL-T02A Caustic-leach $98^{\circ} \mathrm{C}$ & $\mathrm{S} 02 \mathrm{AOH} 018160448 \mathrm{DEN} 2$ & Density Slurry & 090106-1 & 090106-1DEN \\
\hline TI-032 & 3.1 .15 & UFP-VSL-T02A Caustic-leach $98^{\circ} \mathrm{C}$ & S 02AOL 018160443 UDS 2 B & UDS & 090106-4 & \\
\hline TI-032 & 3.1 .15 & UFP-VSL-T02A Caustic-leach $98^{\circ} \mathrm{C}$ & S 02AOL 018160443 UDS 2 D & UDS & 090106-4 & \\
\hline TI-032 & 3.1 .15 & UFP-VSL-T02A Caustic-leach $98^{\circ} \mathrm{C}$ & S 02AOL 018160444 DEN 2 & Density Slurry & 090106-1 & 090106-1DEN \\
\hline TI-032 & 3.1 .15 & UFP-VSL-T02A Caustic-leach $98^{\circ} \mathrm{C}$ & $\begin{array}{c}\text { S 02AOM } 018160445 \text { UDS } 2 \\
\text { B }\end{array}$ & UDS & 090106-5 & \\
\hline TI-032 & 3.1 .15 & UFP-VSL-T02A Caustic-leach $98^{\circ} \mathrm{C}$ & $\begin{array}{c}\text { S 02AOM } 018160445 \text { UDS } 2 \\
\text { D }\end{array}$ & UDS & 090106-5 & \\
\hline TI-032 & 3.1 .15 & UFP-VSL-T02A Caustic-leach $98^{\circ} \mathrm{C}$ & S 02AOM 018160446 DEN 2 & Density Slurry & 090106-1 & 090106-1DEN \\
\hline TI-032 & 4.1 .9 & Prepare UFP-VSL-T01A for caustic leaching & S 01AIM 020 XX 0748 CUF 4 & $\begin{array}{l}\text { Parallel Bench } \\
\text { Test }\end{array}$ & & \\
\hline TI-032 & 4.1 .9 & Prepare UFP-VSL-T01A for caustic leaching & S 01AIM 020 XX 0750 UDS 2 & UDS & 090102-9 & \\
\hline TI-032 & 4.1 .9 & Prepare UFP-VSL-T01A for caustic leaching & S 01AIM 020 XX 0751 UDS 2 & UDS & 090102-9 & \\
\hline TI-032 & 4.1 .9 & Prepare UFP-VSL-T01A for caustic leaching & S 01AIM 020 XX 0752 UDS 2 & UDS & 090102-9 & \\
\hline TI-032 & 4.1 .9 & Prepare UFP-VSL-T01A for caustic leaching & S 01AIM 020 XX 0753 DEN 2 & Density Slurry & 090102-7 & 090102-7 DEN \\
\hline TI-032 & 4.1 .9 & Prepare UFP-VSL-T01A for caustic leaching & S 01AIM 020 XX 0754 DEN 2 & Density Slurry & 090102-7 & 090102-7 DEN \\
\hline TI-032 & 4.1 .9 & Prepare UFP-VSL-T01A for caustic leaching & S 01AIM 020 XX 0755 DEN 2 & Density Slurry & 090102-7 & 090102-7 DEN \\
\hline TI-032 & 4.1 .9 & Prepare UFP-VSL-T01A for caustic leaching & S 01AIM 020 XX 0756 ICP 2 B & $\begin{array}{l}\text { ICP Wet } \\
\text { Solids }\end{array}$ & 090102-4 & 090102-4 ICP and ICPMS \\
\hline TI-032 & 4.1.9 & Prepare UFP-VSL-T01A for caustic leaching & $\begin{array}{c}\text { S 01AIM } 020 \text { XX } 0756 \text { RAM } 3 \\
\text { D }\end{array}$ & Raman & ASR 8316 & ASR 8316 RAM rev 1 \\
\hline TI-032 & 4.1 .9 & Prepare UFP-VSL-T01A for caustic leaching & S 01AIM 020 XX 0757 ICP 2 B & $\begin{array}{l}\text { ICP Wet } \\
\text { Solids }\end{array}$ & 090102-4 & 090102-4 ICP and ICPMS \\
\hline TI-032 & 4.1 .9 & Prepare UFP-VSL-T01A for caustic leaching & $\begin{array}{c}\text { S 01AIM } 020 \text { XX } 0757 \text { RAM } 3 \\
\text { D }\end{array}$ & Raman & ASR 8316 & ASR 8316 RAM rev 1 \\
\hline TI-032 & 4.1 .9 & Prepare UFP-VSL-T01A for caustic leaching & $\begin{array}{c}\text { S 01AIM } 020 \text { XX } 0757 \text { RAM } 3 \\
\text { D }\end{array}$ & Raman & ASR 8316 & ASR 8316 RAM rev 1 \\
\hline TI-032 & 4.1 .9 & Prepare UFP-VSL-T01A for caustic leaching & S 01AIM 020 XX 0758 ICP 2 B & $\begin{array}{l}\text { ICP Wet } \\
\text { Solids }\end{array}$ & $090102-4$ & 090102-4 ICP and ICPMS \\
\hline TI-032 & 4.1 .9 & Prepare UFP-VSL-T01A for caustic leaching & $\begin{array}{c}\text { S 01AIM } 020 \text { XX } 0758 \text { RAM } 3 \\
\text { D }\end{array}$ & Raman & ASR 8316 & ASR 8316 RAM rev 1 \\
\hline TI-032 & 4.1.9.5 & Prepare UFP-VSL-T01A for caustic leaching & $\begin{array}{c}\text { S 01AIH } 020 \text { XX } 0466 \text { UDS } 2 \\
\text { B }\end{array}$ & UDS & $090102-5$ & \\
\hline TI-032 & 4.1.9.5 & Prepare UFP-VSL-T01A for caustic leaching & $\begin{array}{c}\text { S } 01 \text { AIH } 020 \text { XX } 0466 \text { UDS } 2 \\
\text { D }\end{array}$ & UDS & 090102-5 & \\
\hline TI-032 & 4.1.9.5 & Prepare UFP-VSL-T01A for caustic leaching & S 01AIH 020 XX 0467 DEN 2 & Density Slurry & $090102-7$ & 090102-7 DEN \\
\hline TI-032 & 4.1.9.5 & Prepare UFP-VSL-T01A for caustic leaching & S 01AIH 020 XX 0468 ICP 2 B & $\begin{array}{l}\text { ICP Wet } \\
\text { Solids }\end{array}$ & 090102-4 & 090102-4 ICP and ICPMS \\
\hline
\end{tabular}


TI-WTP-PEP-032 (testing dates 12/19/2008 through 01/11/2009)

\begin{tabular}{|c|c|c|c|c|c|c|}
\hline TI \# & $\begin{array}{c}\text { TI Step } \\
\#\end{array}$ & TI Step Description & Sample ID & Analysis & Folder & Final Electronic Report File Name \\
\hline TI-032 & 4.1.9.5 & Prepare UFP-VSL-T01A for caustic leaching & $\begin{array}{c}\text { S 01AIH } 020 \text { XX } 0468 \text { RAM } 3 \\
\text { D }\end{array}$ & Raman & ASR 8316 & ASR 8316 RAM rev 1 \\
\hline TI-032 & 4.1.9.5 & Prepare UFP-VSL-T01A for caustic leaching & S 01AIL 020 XX 0759 UDS 2 B & UDS & $090102-5$ & \\
\hline TI-032 & 4.1.9.5 & Prepare UFP-VSL-T01A for caustic leaching & $\begin{array}{c}\text { S 01AIL } 020 \text { XX } 0759 \text { UDS } 2 \\
\text { D }\end{array}$ & UDS & $090102-5$ & \\
\hline TI-032 & 4.1.9.5 & Prepare UFP-VSL-T01A for caustic leaching & S 01AIL 020 XX 0760 DEN 2 & Density Slurry & 090102-7 & 090102-7 DEN \\
\hline TI-032 & 4.1.9.5 & Prepare UFP-VSL-T01A for caustic leaching & S 01AIL 020 XX 0761 ICP 2 B & $\begin{array}{l}\text { ICP Wet } \\
\text { Solids }\end{array}$ & $090102-4$ & 090102-4 ICP and ICPMS \\
\hline TI-032 & 4.1.9.5 & Prepare UFP-VSL-T01A for caustic leaching & $\begin{array}{c}\text { S 01AIL } 020 \text { XX } 0761 \text { RAM } 3 \\
\text { D }\end{array}$ & Raman & ASR 8316 & ASR 8316 RAM rev 1 \\
\hline TI-032 & 4.1.9.5 & Prepare UFP-VSL-T01A for caustic leaching & S 01AIM 020 XX 0749 CUF 4 & $\begin{array}{l}\text { Parallel Bench } \\
\text { Test }\end{array}$ & & \\
\hline TI-032 & 4.1.9.5 & Prepare UFP-VSL-T01A for caustic leaching & $\begin{array}{c}\text { S 01AIM } 020 \text { XX } 0762 \text { UDS } 2 \\
\text { B }\end{array}$ & UDS & $090102-5$ & \\
\hline TI-032 & 4.1.9.5 & Prepare UFP-VSL-T01A for caustic leaching & $\begin{array}{c}\text { S 01AIM } 020 \text { XX } 0762 \text { UDS } 2 \\
\text { D }\end{array}$ & UDS & $090102-5$ & \\
\hline TI-032 & 4.1.9.5 & Prepare UFP-VSL-T01A for caustic leaching & S 01AIM 020 XX 0763 DEN 2 & Density Slurry & 090102-7 & 090102-7 DEN \\
\hline TI-032 & 4.1.9.5 & Prepare UFP-VSL-T01A for caustic leaching & S 01AIM 020 XX 0764 ICP 2 B & $\begin{array}{l}\text { ICP Wet } \\
\text { Solids }\end{array}$ & $090102-4$ & 090102-4 ICP and ICPMS \\
\hline TI-032 & 4.1.9.5 & Prepare UFP-VSL-T01A for caustic leaching & $\begin{array}{c}\text { S 01AIM } 020 \text { XX } 0764 \text { RAM } 3 \\
\text { D }\end{array}$ & Raman & ASR 8316 & ASR 8316 RAM rev 1 \\
\hline TI-032 & 4.2.6.3 & Heat UFP-VSL-T01A to $88^{\circ} \mathrm{C}$ & $\begin{array}{c}\text { S 01AIM } 021 \text { BT } 0476 \text { UDS } 2 \\
\text { B }\end{array}$ & UDS & 090102-6 & \\
\hline TI-032 & 4.2.6.3 & Heat UFP-VSL-T01A to $88^{\circ} \mathrm{C}$ & $\begin{array}{c}\text { S 01AIM } 021 \text { BT } 0476 \text { UDS } 2 \\
\text { D }\end{array}$ & UDS & $090102-6$ & \\
\hline TI-032 & 4.2.6.3 & Heat UFP-VSL-T01A to $88^{\circ} \mathrm{C}$ & $\begin{array}{c}\text { S 01AIM } 021 \text { BT } 0477 \text { UDS } 2 \\
\text { B }\end{array}$ & UDS & 090102-6 & \\
\hline TI-032 & 4.2.6.3 & Heat UFP-VSL-T01A to $88^{\circ} \mathrm{C}$ & $\begin{array}{c}\text { S 01AIM } 021 \text { BT } 0477 \text { UDS } 2 \\
\text { D }\end{array}$ & UDS & 090102-6 & \\
\hline TI-032 & 4.2.6.3 & Heat UFP-VSL-T01A to $88^{\circ} \mathrm{C}$ & S 01AIM 021 BT 0479 DEN 2 & Density Slurry & 090102-7 & 090102-7 DEN \\
\hline TI-032 & 4.2.6.3 & Heat UFP-VSL-T01A to $88^{\circ} \mathrm{C}$ & S 01AIM 021 BT 0480 DEN 2 & Density Slurry & $090102-7$ & 090102-7 DEN \\
\hline TI-032 & 4.2.6.3 & Heat UFP-VSL-T01A to $88^{\circ} \mathrm{C}$ & S 01AIM 021 BT 0482 ICP 2 B & $\begin{array}{l}\text { ICP Wet } \\
\text { Solids }\end{array}$ & 090102-4 & 090102-4 ICP and ICPMS \\
\hline TI-032 & 4.2.6.3 & Heat UFP-VSL-T01A to $88^{\circ} \mathrm{C}$ & $\begin{array}{c}\text { S 01AIM } 021 \text { BT } 0482 \text { RAM } 3 \\
\text { D }\end{array}$ & Raman & ASR 8316 & ASR 8316 RAM rev 1 \\
\hline TI-032 & 4.2.6.3 & Heat UFP-VSL-T01A to $88^{\circ} \mathrm{C}$ & S 01AIM 021 BT 0483 ICP 2 B & $\begin{array}{l}\text { ICP Wet } \\
\text { Solids }\end{array}$ & 090102-4 & 090102-4 ICP and ICPMS \\
\hline TI-032 & 4.2.6.3 & Heat UFP-VSL-T01A to $88^{\circ} \mathrm{C}$ & $\begin{array}{c}\text { S 01AIM } 021 \text { BT } 0483 \text { RAM } 3 \\
\text { D }\end{array}$ & Raman & ASR 8316 & ASR 8316 RAM rev 1 \\
\hline TI-032 & 4.2.6.3 & Heat UFP-VSL-T01A to $88^{\circ} \mathrm{C}$ & S 01AIM 021 BT 0765 UDS 2 & UDS & $090102-6$ & \\
\hline
\end{tabular}


TI-WTP-PEP-032 (testing dates 12/19/2008 through 01/11/2009)

\begin{tabular}{|c|c|c|c|c|c|c|}
\hline TI \# & $\begin{array}{c}\text { TI Step } \\
\#\end{array}$ & TI Step Description & Sample ID & Analysis & Folder & Final Electronic Report File Name \\
\hline & & & B & & & \\
\hline TI-032 & 4.2 .6 .3 & Heat UFP-VSL-T01A to $88^{\circ} \mathrm{C}$ & $\begin{array}{c}\text { S 01AIM } 021 \text { BT } 0765 \text { UDS } 2 \\
\text { D }\end{array}$ & UDS & $090102-6$ & \\
\hline TI-032 & 4.2.6.3 & Heat UFP-VSL-T01A to $88^{\circ} \mathrm{C}$ & S 01AIM 021 BT 0766 DEN 2 & Density Slurry & 090102-7 & 090102-7 DEN \\
\hline TI-032 & 4.2.6.3 & Heat UFP-VSL-T01A to $88^{\circ} \mathrm{C}$ & S 01AIM 021 BT 0767 ICP 2 B & $\begin{array}{l}\text { ICP Wet } \\
\text { Solids }\end{array}$ & 090102-4 & 090102-4 ICP and ICPMS \\
\hline TI-032 & 4.2.6.3 & Heat UFP-VSL-T01A to $88^{\circ} \mathrm{C}$ & $\begin{array}{c}\text { S 01AIM } 021 \text { BT } 0767 \text { RAM } 3 \\
\text { D }\end{array}$ & Raman & ASR 8316 & ASR 8316 RAM rev 1 \\
\hline TI-032 & 4.2 .7 & $\begin{array}{l}\text { Heat UFP-VSL-T01A to } 98^{\circ} \mathrm{C} \text { and } \\
\text { caustic-leach for } 16 \mathrm{hr}\end{array}$ & S 01AIM 021000485 UDS 2 B & UDS & $090102-5$ & \\
\hline TI-032 & 4.2 .7 & $\begin{array}{l}\text { Heat UFP-VSL-T01A to } 98^{\circ} \mathrm{C} \text { and } \\
\text { caustic-leach for } 16 \mathrm{hr}\end{array}$ & S 01AIM 021000485 UDS 2 D & UDS & $090102-5$ & \\
\hline TI-032 & 4.2 .7 & $\begin{array}{l}\text { Heat UFP-VSL-T01A to } 98^{\circ} \mathrm{C} \text { and } \\
\text { caustic-leach for } 16 \mathrm{hr}\end{array}$ & S 01AIM 021000486 UDS 2 B & UDS & $090102-5$ & \\
\hline TI-032 & 4.2 .7 & $\begin{array}{l}\text { Heat UFP-VSL-T01A to } 98^{\circ} \mathrm{C} \text { and } \\
\text { caustic-leach for } 16 \mathrm{hr}\end{array}$ & S 01AIM 021000486 UDS 2 D & UDS & $090102-5$ & \\
\hline TI-032 & 4.2 .7 & $\begin{array}{l}\text { Heat UFP-VSL-T01A to } 98^{\circ} \mathrm{C} \text { and } \\
\text { caustic-leach for } 16 \mathrm{hr}\end{array}$ & S 01AIM 021000488 ICP 2 B & $\begin{array}{l}\text { ICP Wet } \\
\text { Solids }\end{array}$ & 090102-4 & 090102-4 ICP and ICPMS \\
\hline TI-032 & 4.2 .7 & $\begin{array}{l}\text { Heat UFP-VSL-T01A to } 98^{\circ} \mathrm{C} \text { and } \\
\text { caustic-leach for } 16 \mathrm{hr}\end{array}$ & $\begin{array}{c}\text { S 01AIM } 021000488 \text { RAM } 3 \\
\text { D }\end{array}$ & Raman & ASR 8316 & ASR 8316 RAM rev 1 \\
\hline TI-032 & 4.2 .7 & $\begin{array}{l}\text { Heat UFP-VSL-T01A to } 98^{\circ} \mathrm{C} \text { and } \\
\text { caustic-leach for } 16 \mathrm{hr}\end{array}$ & S 01AIM 021000489 ICP 2 B & $\begin{array}{l}\text { ICP Wet } \\
\text { Solids }\end{array}$ & $090102-4$ & 090102-4 ICP and ICPMS \\
\hline TI-032 & 4.2 .7 & $\begin{array}{c}\text { Heat UFP-VSL-T01A to } 98^{\circ} \mathrm{C} \text { and } \\
\text { caustic-leach for } 16 \mathrm{hr}\end{array}$ & $\begin{array}{c}\text { S 01AIM } 021000489 \text { RAM } 3 \\
\text { D }\end{array}$ & Raman & ASR 8316 & ASR 8316 RAM rev 1 \\
\hline TI-032 & 4.2 .7 & $\begin{array}{l}\text { Heat UFP-VSL-T01A to } 98^{\circ} \mathrm{C} \text { and } \\
\text { caustic-leach for } 16 \mathrm{hr}\end{array}$ & S 01AIM 021000768 UDS 2 B & UDS & $090102-5$ & \\
\hline TI-032 & 4.2 .7 & $\begin{array}{l}\text { Heat UFP-VSL-T01A to } 98^{\circ} \mathrm{C} \text { and } \\
\text { caustic-leach for } 16 \mathrm{hr}\end{array}$ & S 01AIM 021000768 UDS 2 D & UDS & $090102-5$ & \\
\hline TI-032 & 4.2 .7 & $\begin{array}{l}\text { Heat UFP-VSL-T01A to } 98^{\circ} \mathrm{C} \text { and } \\
\text { caustic-leach for } 16 \mathrm{hr}\end{array}$ & S 01AIM 021000769 ICP 2 B & $\begin{array}{l}\text { ICP Wet } \\
\text { Solids }\end{array}$ & $090102-4$ & 090102-4 ICP and ICPMS \\
\hline TI-032 & 4.2 .7 & $\begin{array}{l}\text { Heat UFP-VSL-T01A to } 98^{\circ} \mathrm{C} \text { and } \\
\text { caustic-leach for } 16 \mathrm{hr}\end{array}$ & $\begin{array}{c}\text { S 01AIM } 021000769 \text { RAM } 3 \\
\text { D }\end{array}$ & Raman & ASR 8316 & ASR 8316 RAM rev 1 \\
\hline TI-032 & 4.2 .8 & $\begin{array}{l}\text { Heat UFP-VSL-T01A to } 98^{\circ} \mathrm{C} \text { and } \\
\text { caustic-leach for } 16 \mathrm{hr}\end{array}$ & S 01AIM 021010491 UDS 2 B & UDS & $090102-5$ & \\
\hline TI-032 & 4.2 .8 & $\begin{array}{l}\text { Heat UFP-VSL-T01A to } 98^{\circ} \mathrm{C} \text { and } \\
\text { caustic-leach for } 16 \mathrm{hr}\end{array}$ & S 01AIM 021010491 UDS 2 D & UDS & $090102-5$ & \\
\hline TI-032 & 4.2 .8 & $\begin{array}{l}\text { Heat UFP-VSL-T01A to } 98^{\circ} \mathrm{C} \text { and } \\
\text { caustic-leach for } 16 \mathrm{hr}\end{array}$ & S 01AIM 021010492 UDS 2 B & UDS & $090102-5$ & \\
\hline TI-032 & 4.2 .8 & $\begin{array}{l}\text { Heat UFP-VSL-T01A to } 98^{\circ} \mathrm{C} \text { and } \\
\text { caustic-leach for } 16 \mathrm{hr}\end{array}$ & S 01AIM 021010492 UDS 2 D & UDS & $090102-5$ & \\
\hline
\end{tabular}


TI-WTP-PEP-032 (testing dates 12/19/2008 through 01/11/2009)

\begin{tabular}{|c|c|c|c|c|c|c|}
\hline TI \# & $\begin{array}{c}\text { TI Step } \\
\#\end{array}$ & TI Step Description & Sample ID & Analysis & Folder & Final Electronic Report File Name \\
\hline TI-032 & 4.2 .8 & $\begin{array}{l}\text { Heat UFP-VSL-T01A to } 98^{\circ} \mathrm{C} \text { and } \\
\text { caustic-leach for } 16 \mathrm{hr}\end{array}$ & S 01AIM 021010494 ICP 2 B & $\begin{array}{l}\text { ICP Wet } \\
\text { Solids }\end{array}$ & 090102-4 & 090102-4 ICP and ICPMS \\
\hline TI-032 & 4.2 .8 & $\begin{array}{l}\text { Heat UFP-VSL-T01A to } 98^{\circ} \mathrm{C} \text { and } \\
\text { caustic-leach for } 16 \mathrm{hr}\end{array}$ & $\begin{array}{c}\text { S 01AIM } 021010494 \text { RAM } 3 \\
\text { D }\end{array}$ & Raman & ASR 8316 & ASR 8316 RAM rev 1 \\
\hline TI-032 & 4.2 .8 & $\begin{array}{l}\text { Heat UFP-VSL-T01A to } 98^{\circ} \mathrm{C} \text { and } \\
\text { caustic-leach for } 16 \mathrm{hr}\end{array}$ & S 01AIM 021010495 ICP 2 B & $\begin{array}{l}\text { ICP Wet } \\
\text { Solids }\end{array}$ & 090102-4 & 090102-4 ICP and ICPMS \\
\hline TI-032 & 4.2 .8 & $\begin{array}{l}\text { Heat UFP-VSL-T01A to } 98^{\circ} \mathrm{C} \text { and } \\
\text { caustic-leach for } 16 \mathrm{hr}\end{array}$ & $\begin{array}{c}\text { S 01AIM } 021010495 \text { RAM } 3 \\
\text { D }\end{array}$ & Raman & ASR 8316 & ASR 8316 RAM rev 1 \\
\hline TI-032 & 4.2 .8 & $\begin{array}{l}\text { Heat UFP-VSL-T01A to } 98^{\circ} \mathrm{C} \text { and } \\
\text { caustic-leach for } 16 \mathrm{hr}\end{array}$ & S 01AIM 021010770 UDS 2 B & UDS & $090102-5$ & \\
\hline TI-032 & 4.2 .8 & $\begin{array}{l}\text { Heat UFP-VSL-T01A to } 98^{\circ} \mathrm{C} \text { and } \\
\text { caustic-leach for } 16 \mathrm{hr}\end{array}$ & S 01AIM 021010770 UDS 2 D & UDS & $090102-5$ & \\
\hline TI-032 & 4.2 .8 & $\begin{array}{l}\text { Heat UFP-VSL-T01A to } 98^{\circ} \mathrm{C} \text { and } \\
\text { caustic-leach for } 16 \mathrm{hr}\end{array}$ & S 01AIM 021010771 ICP 2 B & $\begin{array}{l}\text { ICP Wet } \\
\text { Solids }\end{array}$ & $090102-4$ & 090102-4 ICP and ICPMS \\
\hline TI-032 & 4.2 .8 & $\begin{array}{l}\text { Heat UFP-VSL-T01A to } 98^{\circ} \mathrm{C} \text { and } \\
\text { caustic-leach for } 16 \mathrm{hr}\end{array}$ & $\begin{array}{c}\text { S 01AIM } 021010771 \text { RAM } 3 \\
\text { D }\end{array}$ & Raman & ASR 8316 & ASR 8316 RAM rev 1 \\
\hline TI-032 & 4.2 .8 & $\begin{array}{l}\text { Heat UFP-VSL-T01A to } 98^{\circ} \mathrm{C} \text { and } \\
\text { caustic-leach for } 16 \mathrm{hr}\end{array}$ & S 01AIM 021020497 UDS 2 B & UDS & $090102-5$ & \\
\hline TI-032 & 4.2 .8 & $\begin{array}{l}\text { Heat UFP-VSL-T01A to } 98^{\circ} \mathrm{C} \text { and } \\
\text { caustic-leach for } 16 \mathrm{hr}\end{array}$ & S 01AIM 021020497 UDS 2 D & UDS & $090102-5$ & \\
\hline TI-032 & 4.2 .8 & $\begin{array}{l}\text { Heat UFP-VSL-T01A to } 98^{\circ} \mathrm{C} \text { and } \\
\text { caustic-leach for } 16 \mathrm{hr}\end{array}$ & S 01AIM 021020498 UDS 2 B & UDS & $090102-5$ & \\
\hline TI-032 & 4.2 .8 & $\begin{array}{l}\text { Heat UFP-VSL-T01A to } 98^{\circ} \mathrm{C} \text { and } \\
\text { caustic-leach for } 16 \mathrm{hr}\end{array}$ & S 01AIM 021020498 UDS 2 D & UDS & $090102-5$ & \\
\hline TI-032 & 4.2 .8 & $\begin{array}{l}\text { Heat UFP-VSL-T01A to } 98^{\circ} \mathrm{C} \text { and } \\
\text { caustic-leach for } 16 \mathrm{hr}\end{array}$ & S 01AIM 021020500 ICP 2 B & $\begin{array}{l}\text { ICP Wet } \\
\text { Solids }\end{array}$ & 090102-4 & 090102-4 ICP and ICPMS \\
\hline TI-032 & 4.2 .8 & $\begin{array}{l}\text { Heat UFP-VSL-T01A to } 98^{\circ} \mathrm{C} \text { and } \\
\text { caustic-leach for } 16 \mathrm{hr}\end{array}$ & $\begin{array}{c}\text { S 01AIM } 021020500 \text { RAM } 3 \\
\text { D }\end{array}$ & Raman & ASR 8316 & ASR 8316 RAM rev 1 \\
\hline TI-032 & 4.2 .8 & $\begin{array}{l}\text { Heat UFP-VSL-T01A to } 98^{\circ} \mathrm{C} \text { and } \\
\text { caustic-leach for } 16 \mathrm{hr}\end{array}$ & S 01AIM 021020501 ICP 2 B & $\begin{array}{l}\text { ICP Wet } \\
\text { Solids }\end{array}$ & 090102-4 & 090102-4 ICP and ICPMS \\
\hline TI-032 & 4.2 .8 & $\begin{array}{l}\text { Heat UFP-VSL-T01A to } 98^{\circ} \mathrm{C} \text { and } \\
\text { caustic-leach for } 16 \mathrm{hr}\end{array}$ & $\begin{array}{c}\text { S 01AIM } 021020501 \text { RAM } 3 \\
\text { D }\end{array}$ & Raman & ASR 8316 & ASR 8316 RAM rev 1 \\
\hline TI-032 & 4.2 .8 & $\begin{array}{l}\text { Heat UFP-VSL-T01A to } 98^{\circ} \mathrm{C} \text { and } \\
\text { caustic-leach for } 16 \mathrm{hr}\end{array}$ & S 01AIM 021020772 UDS 2 B & UDS & $090102-5$ & \\
\hline TI-032 & 4.2 .8 & $\begin{array}{l}\text { Heat UFP-VSL-T01A to } 98^{\circ} \mathrm{C} \text { and } \\
\text { caustic-leach for } 16 \mathrm{hr}\end{array}$ & S 01AIM 021020772 UDS 2 D & UDS & $090102-5$ & \\
\hline TI-032 & 4.2 .8 & $\begin{array}{l}\text { Heat UFP-VSL-T01A to } 98^{\circ} \mathrm{C} \text { and } \\
\text { caustic-leach for } 16 \mathrm{hr}\end{array}$ & S 01AIM 021020773 ICP 2 B & $\begin{array}{l}\text { ICP Wet } \\
\text { Solids }\end{array}$ & 090102-4 & 090102-4 ICP and ICPMS \\
\hline TI-032 & 4.2 .8 & $\begin{array}{l}\text { Heat UFP-VSL-T01A to } 98^{\circ} \mathrm{C} \text { and } \\
\text { caustic-leach for } 16 \mathrm{hr}\end{array}$ & $\begin{array}{c}\text { S 01AIM } 021020773 \text { RAM } 3 \\
\text { D }\end{array}$ & Raman & ASR 8316 & ASR 8316 RAM rev 1 \\
\hline
\end{tabular}


TI-WTP-PEP-032 (testing dates 12/19/2008 through 01/11/2009)

\begin{tabular}{|c|c|c|c|c|c|c|}
\hline TI \# & $\begin{array}{c}\text { TI Step } \\
\#\end{array}$ & TI Step Description & Sample ID & Analysis & Folder & Final Electronic Report File Name \\
\hline TI-032 & 4.2 .8 & $\begin{array}{l}\text { Heat UFP-VSL-T01A to } 98^{\circ} \mathrm{C} \text { and } \\
\text { caustic-leach for } 16 \mathrm{hr}\end{array}$ & $\begin{array}{c}\text { S 01AML } 021020776 \text { UDS } 2 \\
\text { B }\end{array}$ & UDS & $090102-6$ & \\
\hline TI-032 & 4.2 .8 & $\begin{array}{l}\text { Heat UFP-VSL-T01A to } 98^{\circ} \mathrm{C} \text { and } \\
\text { caustic-leach for } 16 \mathrm{hr}\end{array}$ & $\begin{array}{c}\text { S 01AML } 021020776 \text { UDS } 2 \\
\text { D }\end{array}$ & UDS & 090102-6 & \\
\hline TI-032 & 4.2 .8 & $\begin{array}{l}\text { Heat UFP-VSL-T01A to } 98^{\circ} \mathrm{C} \text { and } \\
\text { caustic-leach for } 16 \mathrm{hr}\end{array}$ & S 01AML 021020777 DEN 2 & Density Slurry & 090102-7 & 090102-7 DEN \\
\hline TI-032 & 4.2 .8 & $\begin{array}{l}\text { Heat UFP-VSL-T01A to } 98^{\circ} \mathrm{C} \text { and } \\
\text { caustic-leach for } 16 \mathrm{hr}\end{array}$ & $\begin{array}{c}\text { S 01AMM } 021020778 \text { UDS } 2 \\
\text { B }\end{array}$ & UDS & 090102-6 & \\
\hline TI-032 & 4.2 .8 & $\begin{array}{l}\text { Heat UFP-VSL-T01A to } 98^{\circ} \mathrm{C} \text { and } \\
\text { caustic-leach for } 16 \mathrm{hr}\end{array}$ & $\begin{array}{c}\text { S 01AMM } 021020778 \text { UDS } 2 \\
\text { D }\end{array}$ & UDS & 090102-6 & \\
\hline TI-032 & 4.2 .8 & $\begin{array}{l}\text { Heat UFP-VSL-T01A to } 98^{\circ} \mathrm{C} \text { and } \\
\text { caustic-leach for } 16 \mathrm{hr}\end{array}$ & S 01AIMM 021020779 DEN 2 & Density Slurry & 090102-8 & 090102-8 DEN \\
\hline TI-032 & 4.2 .8 & $\begin{array}{l}\text { Heat UFP-VSL-T01A to } 98^{\circ} \mathrm{C} \text { and } \\
\text { caustic-leach for } 16 \mathrm{hr}\end{array}$ & S 01AOL 021020782 UDS 2 D & UDS & $090102-6$ & \\
\hline TI-032 & 4.2 .8 & $\begin{array}{l}\text { Heat UFP-VSL-T01A to } 98^{\circ} \mathrm{C} \text { and } \\
\text { caustic-leach for } 16 \mathrm{hr}\end{array}$ & S 01AOL 021020783 DEN 2 & Density Slurry & $090102-7$ & 090102-7 DEN \\
\hline TI-032 & 4.2 .8 & $\begin{array}{l}\text { Heat UFP-VSL-T01A to } 98^{\circ} \mathrm{C} \text { and } \\
\text { caustic-leach for } 16 \mathrm{hr}\end{array}$ & $\begin{array}{c}\text { S 01AOM } 021020784 \text { UDS } 2 \\
\text { B }\end{array}$ & UDS & 090102-6 & \\
\hline TI-032 & 4.2 .8 & $\begin{array}{l}\text { Heat UFP-VSL-T01A to } 98^{\circ} \mathrm{C} \text { and } \\
\text { caustic-leach for } 16 \mathrm{hr}\end{array}$ & $\begin{array}{c}\text { S 01AOM } 021020784 \text { UDS } 2 \\
\text { D }\end{array}$ & UDS & $090102-6$ & \\
\hline TI-032 & 4.2 .8 & $\begin{array}{l}\text { Heat UFP-VSL-T01A to } 98^{\circ} \mathrm{C} \text { and } \\
\text { caustic-leach for } 16 \mathrm{hr}\end{array}$ & $\begin{array}{c}\mathrm{S} 01 \mathrm{AOH} 021020786 \text { UDS } 2 \\
\mathrm{~B}\end{array}$ & UDS & 090102-6 & \\
\hline TI-032 & 4.2 .8 & $\begin{array}{c}\text { Heat UFP-VSL-T01A to } 98^{\circ} \mathrm{C} \text { and } \\
\text { caustic-leach for } 16 \mathrm{hr}\end{array}$ & $\begin{array}{c}\text { S 01AOH } 021020786 \text { UDS } 2 \\
\text { D }\end{array}$ & UDS & $090102-6$ & \\
\hline TI-032 & 4.2 .8 & $\begin{array}{l}\text { Heat UFP-VSL-T01A to } 98^{\circ} \mathrm{C} \text { and } \\
\text { caustic-leach for } 16 \mathrm{hr}\end{array}$ & S 01AOH 021020787 DEN 2 & Density Slurry & 090102-7 & 090102-7 DEN \\
\hline TI-032 & 4.2 .8 & $\begin{array}{l}\text { Heat UFP-VSL-T01A to } 98^{\circ} \mathrm{C} \text { and } \\
\text { caustic-leach for } 16 \mathrm{hr}\end{array}$ & S 01AIL 021020788 UDS 2 B & UDS & $090102-5$ & \\
\hline TI-032 & 4.2.8 & $\begin{array}{l}\text { Heat UFP-VSL-T01A to } 98^{\circ} \mathrm{C} \text { and } \\
\text { caustic-leach for } 16 \mathrm{hr}\end{array}$ & S 01AIL 021020788 UDS 2 D & UDS & $090102-5$ & \\
\hline TI-032 & 4.2 .8 & $\begin{array}{l}\text { Heat UFP-VSL-T01A to } 98^{\circ} \mathrm{C} \text { and } \\
\text { caustic-leach for } 16 \mathrm{hr}\end{array}$ & S 01AIL 021020789 DEN 2 & Density Slurry & $090102-7$ & 090102-7 DEN \\
\hline TI-032 & 4.2 .8 & $\begin{array}{l}\text { Heat UFP-VSL-T01A to } 98^{\circ} \mathrm{C} \text { and } \\
\text { caustic-leach for } 16 \mathrm{hr}\end{array}$ & S 01AIM 021040519 UDS 2 B & UDS & $090102-5$ & \\
\hline TI-032 & 4.2 .8 & $\begin{array}{l}\text { Heat UFP-VSL-T01A to } 98^{\circ} \mathrm{C} \text { and } \\
\text { caustic-leach for } 16 \mathrm{hr}\end{array}$ & S 01AIM 021040519 UDS 2 D & UDS & $090102-5$ & \\
\hline TI-032 & 4.2 .8 & $\begin{array}{l}\text { Heat UFP-VSL-T01A to } 98^{\circ} \mathrm{C} \text { and } \\
\text { caustic-leach for } 16 \mathrm{hr}\end{array}$ & S 01AIM 021040520 UDS 2 B & UDS & 090102-5 & \\
\hline TI-032 & 4.2 .8 & $\begin{array}{l}\text { Heat UFP-VSL-T01A to } 98^{\circ} \mathrm{C} \text { and } \\
\text { caustic-leach for } 16 \mathrm{hr}\end{array}$ & S 01AIM 021040520 UDS 2 D & UDS & $090102-5$ & \\
\hline
\end{tabular}


TI-WTP-PEP-032 (testing dates 12/19/2008 through 01/11/2009)

\begin{tabular}{|c|c|c|c|c|c|c|}
\hline TI \# & $\begin{array}{c}\text { TI Step } \\
\#\end{array}$ & TI Step Description & Sample ID & Analysis & Folder & Final Electronic Report File Name \\
\hline TI-032 & 4.2 .8 & $\begin{array}{l}\text { Heat UFP-VSL-T01A to } 98^{\circ} \mathrm{C} \text { and } \\
\text { caustic-leach for } 16 \mathrm{hr}\end{array}$ & S 01AIM 021040522 ICP 2 B & $\begin{array}{l}\text { ICP Wet } \\
\text { Solids }\end{array}$ & 090102-4 & 090102-4 ICP and ICPMS \\
\hline TI-032 & 4.2 .8 & $\begin{array}{l}\text { Heat UFP-VSL-T01A to } 98^{\circ} \mathrm{C} \text { and } \\
\text { caustic-leach for } 16 \mathrm{hr}\end{array}$ & $\begin{array}{c}\text { S 01AIM } 021040522 \text { RAM } 3 \\
\text { D }\end{array}$ & Raman & ASR 8316 & ASR 8316 RAM rev 1 \\
\hline TI-032 & 4.2 .8 & $\begin{array}{l}\text { Heat UFP-VSL-T01A to } 98^{\circ} \mathrm{C} \text { and } \\
\text { caustic-leach for } 16 \mathrm{hr}\end{array}$ & S 01AIM 021040523 ICP 2 B & $\begin{array}{l}\text { ICP Wet } \\
\text { Solids }\end{array}$ & 090102-4 & 090102-4 ICP and ICPMS \\
\hline TI-032 & 4.2 .8 & $\begin{array}{l}\text { Heat UFP-VSL-T01A to } 98^{\circ} \mathrm{C} \text { and } \\
\text { caustic-leach for } 16 \mathrm{hr}\end{array}$ & $\begin{array}{c}\text { S 01AIM } 021040523 \text { RAM } 3 \\
\text { D }\end{array}$ & Raman & ASR 8316 & ASR 8316 RAM rev 1 \\
\hline TI-032 & 4.2 .8 & $\begin{array}{l}\text { Heat UFP-VSL-T01A to } 98^{\circ} \mathrm{C} \text { and } \\
\text { caustic-leach for } 16 \mathrm{hr}\end{array}$ & S 01AIM 021040790 UDS 2 B & UDS & $090102-5$ & \\
\hline TI-032 & 4.2 .8 & $\begin{array}{l}\text { Heat UFP-VSL-T01A to } 98^{\circ} \mathrm{C} \text { and } \\
\text { caustic-leach for } 16 \mathrm{hr}\end{array}$ & S 01AIM 021040790 UDS 2 D & UDS & $090102-5$ & \\
\hline TI-032 & 4.2 .8 & $\begin{array}{l}\text { Heat UFP-VSL-T01A to } 98^{\circ} \mathrm{C} \text { and } \\
\text { caustic-leach for } 16 \mathrm{hr}\end{array}$ & S 01AIM 021040791 ICP 2 B & $\begin{array}{l}\text { ICP Wet } \\
\text { Solids }\end{array}$ & $090102-4$ & 090102-4 ICP and ICPMS \\
\hline TI-032 & 4.2 .8 & $\begin{array}{l}\text { Heat UFP-VSL-T01A to } 98^{\circ} \mathrm{C} \text { and } \\
\text { caustic-leach for } 16 \mathrm{hr}\end{array}$ & $\begin{array}{c}\text { S 01AIM } 021040791 \text { RAM } 3 \\
\text { D }\end{array}$ & Raman & ASR 8316 & ASR 8316 RAM rev 1 \\
\hline TI-032 & 4.2 .8 & $\begin{array}{l}\text { Heat UFP-VSL-T01A to } 98^{\circ} \mathrm{C} \text { and } \\
\text { caustic-leach for } 16 \mathrm{hr}\end{array}$ & S 01AIM 021080525 UDS 2 B & UDS & $090102-5$ & \\
\hline TI-032 & 4.2 .8 & $\begin{array}{l}\text { Heat UFP-VSL-T01A to } 98^{\circ} \mathrm{C} \text { and } \\
\text { caustic-leach for } 16 \mathrm{hr}\end{array}$ & S 01AIM 021080525 UDS 2 D & UDS & $090102-5$ & \\
\hline TI-032 & 4.2 .8 & $\begin{array}{l}\text { Heat UFP-VSL-T01A to } 98^{\circ} \mathrm{C} \text { and } \\
\text { caustic-leach for } 16 \mathrm{hr}\end{array}$ & S 01AIM 021080526 UDS 2 B & UDS & $090102-5$ & \\
\hline TI-032 & 4.2 .8 & $\begin{array}{l}\text { Heat UFP-VSL-T01A to } 98^{\circ} \mathrm{C} \text { and } \\
\text { caustic-leach for } 16 \mathrm{hr}\end{array}$ & S 01AIM 021080526 UDS 2 D & UDS & $090102-5$ & \\
\hline TI-032 & 4.2 .8 & $\begin{array}{l}\text { Heat UFP-VSL-T01A to } 98^{\circ} \mathrm{C} \text { and } \\
\text { caustic-leach for } 16 \mathrm{hr}\end{array}$ & S 01AIM 021080528 ICP 2 B & $\begin{array}{l}\text { ICP Wet } \\
\text { Solids }\end{array}$ & 090102-4 & 090102-4 ICP and ICPMS \\
\hline TI-032 & 4.2 .8 & $\begin{array}{l}\text { Heat UFP-VSL-T01A to } 98^{\circ} \mathrm{C} \text { and } \\
\text { caustic-leach for } 16 \mathrm{hr}\end{array}$ & $\begin{array}{c}\text { S 01AIM } 021080528 \text { RAM } 3 \\
\text { D }\end{array}$ & Raman & ASR 8316 & ASR 8316 RAM rev 1 \\
\hline TI-032 & 4.2 .8 & $\begin{array}{l}\text { Heat UFP-VSL-T01A to } 98^{\circ} \mathrm{C} \text { and } \\
\text { caustic-leach for } 16 \mathrm{hr}\end{array}$ & S 01AIM 021080529 ICP 2 B & $\begin{array}{l}\text { ICP Wet } \\
\text { Solids }\end{array}$ & 090102-4 & 090102-4 ICP and ICPMS \\
\hline TI-032 & 4.2 .8 & $\begin{array}{l}\text { Heat UFP-VSL-T01A to } 98^{\circ} \mathrm{C} \text { and } \\
\text { caustic-leach for } 16 \mathrm{hr}\end{array}$ & $\begin{array}{c}\text { S 01AIM } 021080529 \text { RAM } 3 \\
\text { D }\end{array}$ & Raman & ASR 8316 & ASR 8316 RAM rev 1 \\
\hline TI-032 & 4.2 .8 & $\begin{array}{l}\text { Heat UFP-VSL-T01A to } 98^{\circ} \mathrm{C} \text { and } \\
\text { caustic-leach for } 16 \mathrm{hr}\end{array}$ & S 01AIM 021080792 UDS 2 B & UDS & $090102-5$ & \\
\hline TI-032 & 4.2 .8 & $\begin{array}{l}\text { Heat UFP-VSL-T01A to } 98^{\circ} \mathrm{C} \text { and } \\
\text { caustic-leach for } 16 \mathrm{hr}\end{array}$ & S 01AIM 021080792 UDS 2 D & UDS & $090102-5$ & \\
\hline TI-032 & 4.2 .8 & $\begin{array}{l}\text { Heat UFP-VSL-T01A to } 98^{\circ} \mathrm{C} \text { and } \\
\text { caustic-leach for } 16 \mathrm{hr}\end{array}$ & S 01AIM 021080793 ICP 2 B & $\begin{array}{l}\text { ICP Wet } \\
\text { Solids }\end{array}$ & 090102-4 & 090102-4 ICP and ICPMS \\
\hline TI-032 & 4.2 .8 & $\begin{array}{l}\text { Heat UFP-VSL-T01A to } 98^{\circ} \mathrm{C} \text { and } \\
\text { caustic-leach for } 16 \mathrm{hr}\end{array}$ & $\begin{array}{c}\text { S 01AIM } 021080793 \text { RAM } 3 \\
\text { D }\end{array}$ & Raman & ASR 8316 & ASR 8316 RAM rev 1 \\
\hline
\end{tabular}


TI-WTP-PEP-032 (testing dates 12/19/2008 through 01/11/2009)

\begin{tabular}{|c|c|c|c|c|c|c|}
\hline TI \# & $\begin{array}{c}\text { TI Step } \\
\#\end{array}$ & TI Step Description & Sample ID & Analysis & Folder & Final Electronic Report File Name \\
\hline TI-032 & 4.2 .8 & $\begin{array}{l}\text { Heat UFP-VSL-T01A to } 98^{\circ} \mathrm{C} \text { and } \\
\text { caustic-leach for } 16 \mathrm{hr}\end{array}$ & S 01AIM 021120531 UDS 2 B & UDS & $090102-5$ & \\
\hline TI-032 & 4.2 .8 & $\begin{array}{l}\text { Heat UFP-VSL-T01A to } 98^{\circ} \mathrm{C} \text { and } \\
\text { caustic-leach for } 16 \mathrm{hr}\end{array}$ & S 01AIM 021120531 UDS 2 D & UDS & $090102-5$ & \\
\hline TI-032 & 4.2 .8 & $\begin{array}{l}\text { Heat UFP-VSL-T01A to } 98^{\circ} \mathrm{C} \text { and } \\
\text { caustic-leach for } 16 \mathrm{hr}\end{array}$ & S 01AIM 021120532 UDS 2 B & UDS & $090102-6$ & \\
\hline TI-032 & 4.2 .8 & $\begin{array}{l}\text { Heat UFP-VSL-T01A to } 98^{\circ} \mathrm{C} \text { and } \\
\text { caustic-leach for } 16 \mathrm{hr}\end{array}$ & S 01AIM 021120532 UDS 2 D & UDS & 090102-6 & \\
\hline TI-032 & 4.2 .8 & $\begin{array}{l}\text { Heat UFP-VSL-T01A to } 98^{\circ} \mathrm{C} \text { and } \\
\text { caustic-leach for } 16 \mathrm{hr}\end{array}$ & S 01AIM 021120534 ICP 2 B & $\begin{array}{l}\text { ICP Wet } \\
\text { Solids }\end{array}$ & $090102-4$ & 090102-4 ICP and ICPMS \\
\hline TI-032 & 4.2 .8 & $\begin{array}{l}\text { Heat UFP-VSL-T01A to } 98^{\circ} \mathrm{C} \text { and } \\
\text { caustic-leach for } 16 \mathrm{hr}\end{array}$ & $\begin{array}{c}\text { S 01AIM } 021120534 \text { RAM } 3 \\
\text { D }\end{array}$ & Raman & ASR 8316 & ASR 8316 RAM rev 1 \\
\hline TI-032 & 4.2 .8 & $\begin{array}{l}\text { Heat UFP-VSL-T01A to } 98^{\circ} \mathrm{C} \text { and } \\
\text { caustic-leach for } 16 \mathrm{hr}\end{array}$ & S 01AIM 021120535 ICP 2 B & $\begin{array}{l}\text { ICP Wet } \\
\text { Solids }\end{array}$ & 090102-4 & 090102-4 ICP and ICPMS \\
\hline TI-032 & 4.2 .8 & $\begin{array}{l}\text { Heat UFP-VSL-T01A to } 98^{\circ} \mathrm{C} \text { and } \\
\text { caustic-leach for } 16 \mathrm{hr}\end{array}$ & $\begin{array}{c}\text { S 01AIM } 021120535 \text { RAM } 3 \\
\text { D }\end{array}$ & Raman & ASR 8316 & ASR 8316 RAM rev 1 \\
\hline TI-032 & 4.2 .8 & $\begin{array}{l}\text { Heat UFP-VSL-T01A to } 98^{\circ} \mathrm{C} \text { and } \\
\text { caustic-leach for } 16 \mathrm{hr}\end{array}$ & S 01AIM 021120794 UDS 2 B & UDS & $090102-6$ & \\
\hline TI-032 & 4.2 .8 & $\begin{array}{l}\text { Heat UFP-VSL-T01A to } 98^{\circ} \mathrm{C} \text { and } \\
\text { caustic-leach for } 16 \mathrm{hr}\end{array}$ & S 01AIM 021120794 UDS 2 D & UDS & $090102-6$ & \\
\hline TI-032 & 4.2 .8 & $\begin{array}{l}\text { Heat UFP-VSL-T01A to } 98^{\circ} \mathrm{C} \text { and } \\
\text { caustic-leach for } 16 \text { ho }\end{array}$ & S 01AIM 021120795 ICP 2 B & $\begin{array}{l}\text { ICP Wet } \\
\text { Solids }\end{array}$ & $090102-4$ & 090102-4 ICP and ICPMS \\
\hline TI-032 & 4.2 .8 & $\begin{array}{l}\text { Heat UFP-VSL-T01A to } 98^{\circ} \mathrm{C} \text { and } \\
\text { caustic-leach for } 16 \mathrm{hr}\end{array}$ & $\begin{array}{c}\text { S 01AIM } 021120795 \text { RAM } 3 \\
\text { D }\end{array}$ & Raman & ASR 8316 & ASR 8316 RAM rev 1 \\
\hline TI-032 & 4.2 .8 & $\begin{array}{l}\text { Heat UFP-VSL-T01A to } 98^{\circ} \mathrm{C} \text { and } \\
\text { caustic-leach for } 16 \mathrm{hr}\end{array}$ & S 01AIM 021160537 UDS 2 B & UDS & $090102-6$ & \\
\hline TI-032 & 4.2 .8 & $\begin{array}{l}\text { Heat UFP-VSL-T01A to } 98^{\circ} \mathrm{C} \text { and } \\
\text { caustic-leach for } 16 \text { us }\end{array}$ & S 01AIM 021160537 UDS 2 D & UDS & $090102-6$ & \\
\hline TI-032 & 4.2 .8 & $\begin{array}{l}\text { Heat UFP-VSL-T01A to } 98^{\circ} \mathrm{C} \text { and } \\
\text { caustic-leach for } 16 \mathrm{hr}\end{array}$ & S 01AIM 021160538 UDS 2 B & UDS & 090102-6 & \\
\hline TI-032 & 4.2 .8 & $\begin{array}{l}\text { Heat UFP-VSL-T01A to } 98^{\circ} \mathrm{C} \text { and } \\
\text { caustic-leach for } 16 \mathrm{hr}\end{array}$ & S 01AIM 021160538 UDS 2 D & UDS & 090102-6 & \\
\hline TI-032 & 4.2 .8 & $\begin{array}{l}\text { Heat UFP-VSL-T01A to } 98^{\circ} \mathrm{C} \text { and } \\
\text { caustic-leach for } 16 \mathrm{hr}\end{array}$ & S 01AIM 021160540 ICP 2 B & $\begin{array}{l}\text { ICP Wet } \\
\text { Solids }\end{array}$ & $090102-4$ & 090102-4 ICP and ICPMS \\
\hline TI-032 & 4.2 .8 & $\begin{array}{l}\text { Heat UFP-VSL-T01A to } 98^{\circ} \mathrm{C} \text { and } \\
\text { caustic-leach for } 16 \mathrm{hr}\end{array}$ & $\begin{array}{c}\text { S 01AIM } 021160540 \text { RAM } 3 \\
\text { D }\end{array}$ & Raman & ASR 8316 & ASR 8316 RAM rev 1 \\
\hline TI-032 & 4.2 .8 & $\begin{array}{l}\text { Heat UFP-VSL-T01A to } 98^{\circ} \mathrm{C} \text { and } \\
\text { caustic-leach for } 16 \mathrm{hr}\end{array}$ & S 01AIM 021160541 ICP 2 B & $\begin{array}{l}\text { ICP Wet } \\
\text { Solids }\end{array}$ & $090102-4$ & 090102-4 ICP and ICPMS \\
\hline TI-032 & 4.2 .8 & $\begin{array}{l}\text { Heat UFP-VSL-T01A to } 98^{\circ} \mathrm{C} \text { and } \\
\text { caustic-leach for } 16 \mathrm{hr}\end{array}$ & $\begin{array}{c}\text { S 01AIM } 021160541 \text { RAM } 3 \\
\text { D }\end{array}$ & Raman & ASR 8316 & ASR 8316 RAM rev 1 \\
\hline
\end{tabular}


TI-WTP-PEP-032 (testing dates 12/19/2008 through 01/11/2009)

\begin{tabular}{|c|c|c|c|c|c|c|}
\hline TI \# & $\begin{array}{c}\text { TI Step } \\
\#\end{array}$ & TI Step Description & Sample ID & Analysis & Folder & Final Electronic Report File Name \\
\hline TI-032 & 4.2 .8 & $\begin{array}{l}\text { Heat UFP-VSL-T01A to } 98^{\circ} \mathrm{C} \text { and } \\
\text { caustic-leach for } 16 \mathrm{hr}\end{array}$ & S 01AIM 021160796 UDS 2 B & UDS & $090102-6$ & \\
\hline TI-032 & 4.2 .8 & $\begin{array}{l}\text { Heat UFP-VSL-T01A to } 98^{\circ} \mathrm{C} \text { and } \\
\text { caustic-leach for } 16 \mathrm{hr}\end{array}$ & S 01AIM 021160796 UDS 2 D & UDS & $090102-6$ & \\
\hline TI-032 & 4.2 .8 & $\begin{array}{l}\text { Heat UFP-VSL-T01A to } 98^{\circ} \mathrm{C} \text { and } \\
\text { caustic-leach for } 16 \mathrm{hr}\end{array}$ & S 01AIM 021160797 ICP 2 B & $\begin{array}{l}\text { ICP Wet } \\
\text { Solids }\end{array}$ & 090102-4 & 090102-4 ICP and ICPMS \\
\hline TI-032 & 4.2 .8 & $\begin{array}{l}\text { Heat UFP-VSL-T01A to } 98^{\circ} \mathrm{C} \text { and } \\
\text { caustic-leach for } 16 \mathrm{hr}\end{array}$ & $\begin{array}{c}\text { S 01AIM } 021160797 \text { RAM } 3 \\
\text { D }\end{array}$ & Raman & ASR 8316 & ASR 8316 RAM rev 1 \\
\hline TI-032 & 4.2 .8 & $\begin{array}{l}\text { Heat UFP-VSL-T01A to } 98^{\circ} \mathrm{C} \text { and } \\
\text { caustic-leach for } 16 \mathrm{hr}\end{array}$ & $\begin{array}{c}\text { S 01AML } 021160800 \text { UDS } 2 \\
\text { B }\end{array}$ & UDS & $090102-6$ & \\
\hline TI-032 & 4.2 .8 & $\begin{array}{l}\text { Heat UFP-VSL-T01A to } 98^{\circ} \mathrm{C} \text { and } \\
\text { caustic-leach for } 16 \mathrm{hr}\end{array}$ & $\begin{array}{c}\text { S 01AML } 021160800 \text { UDS } 2 \\
\text { D }\end{array}$ & UDS & $090102-6$ & \\
\hline TI-032 & 4.2 .8 & $\begin{array}{l}\text { Heat UFP-VSL-T01A to } 98^{\circ} \mathrm{C} \text { and } \\
\text { caustic-leach for } 16 \mathrm{hr}\end{array}$ & S 01AML 021160801 DEN 2 & Density Slurry & $090102-7$ & 090102-7 DEN \\
\hline TI-032 & 4.2 .8 & $\begin{array}{l}\text { Heat UFP-VSL-T01A to } 98^{\circ} \mathrm{C} \text { and } \\
\text { caustic-leach for } 16 \mathrm{hr}\end{array}$ & $\begin{array}{c}\text { S 01AMM } 021160802 \text { UDS } 2 \\
\text { B }\end{array}$ & UDS & $090102-6$ & \\
\hline TI-032 & 4.2 .8 & $\begin{array}{l}\text { Heat UFP-VSL-T01A to } 98^{\circ} \mathrm{C} \text { and } \\
\text { caustic-leach for } 16 \mathrm{hr}\end{array}$ & $\begin{array}{c}\text { S 01AMM } 021160802 \text { UDS } 2 \\
\text { D }\end{array}$ & UDS & $090102-6$ & \\
\hline TI-032 & 4.2 .8 & $\begin{array}{l}\text { Heat UFP-VSL-T01A to } 98^{\circ} \mathrm{C} \text { and } \\
\text { caustic-leach for } 16 \mathrm{hr}\end{array}$ & S 01AMM 021160803 DEN 2 & Density Slurry & $090102-7$ & 090102-7 DEN \\
\hline TI-032 & 4.2 .8 & $\begin{array}{l}\text { Heat UFP-VSL-T01A to } 98^{\circ} \mathrm{C} \text { and } \\
\text { caustic-leach for } 16 \mathrm{hr}\end{array}$ & S 01AIL 021160812 UDS 2 B & UDS & $090102-6$ & \\
\hline TI-032 & 4.2 .8 & $\begin{array}{l}\text { Heat UFP-VSL-T01A to } 98^{\circ} \mathrm{C} \text { and } \\
\text { caustic-leach for } 16 \mathrm{hr}\end{array}$ & S 01AIL 021160812 UDS 2 D & UDS & 090102-6 & \\
\hline TI-032 & 4.2 .8 & $\begin{array}{l}\text { Heat UFP-VSL-T01A to } 98^{\circ} \mathrm{C} \text { and } \\
\text { caustic-leach for } 16 \mathrm{hr}\end{array}$ & S 01AIL 021160813 DEN 2 & Density Slurry & $090102-7$ & 090102-7 DEN \\
\hline TI-032 & 4.2 .8 & $\begin{array}{l}\text { Heat UFP-VSL-T01A to } 98^{\circ} \mathrm{C} \text { and } \\
\text { caustic-leach for } 16 \mathrm{hr}\end{array}$ & $\begin{array}{c}\text { S 01AOH } 021160810 \text { UDS } 2 \\
\text { B }\end{array}$ & UDS & $090102-6$ & \\
\hline TI-032 & 4.2 .8 & $\begin{array}{l}\text { Heat UFP-VSL-T01A to } 98^{\circ} \mathrm{C} \text { and } \\
\text { caustic-leach for } 16 \mathrm{hr}\end{array}$ & $\begin{array}{c}\text { S 01AOH } 021160810 \text { UDS } 2 \\
\text { D }\end{array}$ & UDS & 090102-6 & \\
\hline TI-032 & 4.2 .8 & $\begin{array}{l}\text { Heat UFP-VSL-T01A to } 98^{\circ} \mathrm{C} \text { and } \\
\text { caustic-leach for } 16 \mathrm{hr}\end{array}$ & S 01AOH 021160811 DEN 2 & Density Slurry & $090102-7$ & 090102-7 DEN \\
\hline TI-032 & 4.2 .8 & $\begin{array}{l}\text { Heat UFP-VSL-T01A to } 98^{\circ} \mathrm{C} \text { and } \\
\text { caustic-leach for } 16 \mathrm{hr}\end{array}$ & S 01AOL 021020782 UDS 2 B & UDS & $090102-6$ & \\
\hline TI-032 & 4.2 .8 & $\begin{array}{l}\text { Heat UFP-VSL-T01A to } 98^{\circ} \mathrm{C} \text { and } \\
\text { caustic-leach for } 16 \mathrm{hr}\end{array}$ & S 01AOL 021160806 UDS 2 B & UDS & $090102-6$ & \\
\hline TI-032 & 4.2 .8 & $\begin{array}{l}\text { Heat UFP-VSL-T01A to } 98^{\circ} \mathrm{C} \text { and } \\
\text { caustic-leach for } 16 \mathrm{hr}\end{array}$ & S 01AOL 021160806 UDS 2 D & UDS & 090102-6 & \\
\hline TI-032 & 4.2 .8 & $\begin{array}{l}\text { Heat UFP-VSL-T01A to } 98^{\circ} \mathrm{C} \text { and } \\
\text { caustic-leach for } 16 \mathrm{hr}\end{array}$ & S 01AOL 021160807 DEN 2 & Density Slurry & $090102-7$ & 090102-7 DEN \\
\hline
\end{tabular}


TI-WTP-PEP-032 (testing dates 12/19/2008 through 01/11/2009)

\begin{tabular}{|c|c|c|c|c|c|c|}
\hline TI \# & $\begin{array}{c}\text { TI Step } \\
\#\end{array}$ & TI Step Description & Sample ID & Analysis & Folder & Final Electronic Report File Name \\
\hline TI-032 & 4.2 .8 & $\begin{array}{l}\text { Heat UFP-VSL-T01A to } 98^{\circ} \mathrm{C} \text { and } \\
\text { caustic-leach for } 16 \mathrm{hr}\end{array}$ & S 01AOM 021020785 DEN 2 & Density Slurry & $090102-7$ & 090102-7 DEN \\
\hline TI-032 & 4.2 .8 & $\begin{array}{l}\text { Heat UFP-VSL-T01A to } 98^{\circ} \mathrm{C} \text { and } \\
\text { caustic-leach for } 16 \mathrm{hr}\end{array}$ & $\begin{array}{c}\text { S 01AOM } 021160808 \text { UDS } 2 \\
\text { B }\end{array}$ & UDS & $090102-6$ & \\
\hline TI-032 & 4.2 .8 & $\begin{array}{l}\text { Heat UFP-VSL-T01A to } 98^{\circ} \mathrm{C} \text { and } \\
\text { caustic-leach for } 16 \mathrm{hr}\end{array}$ & $\begin{array}{c}\text { S } 01 \text { AOM } 021160808 \text { UDS } 2 \\
\text { D }\end{array}$ & UDS & $090102-6$ & \\
\hline TI-032 & 4.2 .8 & $\begin{array}{l}\text { Heat UFP-VSL-T01A to } 98^{\circ} \mathrm{C} \text { and } \\
\text { caustic-leach for } 16 \mathrm{hr}\end{array}$ & S 01AOM 021160809 DEN 2 & Density Slurry & $020102-7$ & 090102-7 DEN \\
\hline TI-032 & 7.1.8 & Post-caustic-leach slurry wash & $\begin{array}{c}\text { S 02AML } 026 \text { XX } 0907 \text { RAM } 3 \\
\text { D }\end{array}$ & Raman & ASR 8321 & ASR 8321 RAM rev 1 \\
\hline TI-032 & 7.1.8 & Post-caustic-leach slurry wash & $\begin{array}{c}\text { S 02AML } 026 \text { XX } 0907 \text { TFI } 2 \\
\text { D }\end{array}$ & IC & 090109-15 & 090109-15 IC and OH \\
\hline TI-032 & 7.1.8 & Post-caustic-leach slurry wash & $\begin{array}{c}\text { S 02AML 026 XX } 0907 \text { TFI } 2 \\
\text { D }\end{array}$ & OH Titration & 090109-15 & 090109-15 IC and $\mathrm{OH}$ \\
\hline TI-032 & 7.1.8 & Post-caustic-leach slurry wash & $\begin{array}{c}\text { S 02AML } 026 \text { XX } 0909 \text { RAM } 3 \\
\text { D }\end{array}$ & Raman & ASR 8321 & ASR 8321 RAM rev 1 \\
\hline TI-032 & 7.1.8 & Post-caustic-leach slurry wash & $\begin{array}{c}\text { S 02AML } 026 \text { XX } 0909 \text { TFI } 2 \\
\text { D }\end{array}$ & IC & 090109-15 & 090109-15 IC and $\mathrm{OH}$ \\
\hline TI-032 & 7.1.8 & Post-caustic-leach slurry wash & $\begin{array}{c}\text { S 02AML } 026 \text { XX } 0909 \text { TFI } 2 \\
\text { D }\end{array}$ & OH Titration & 090109-15 & 090109-15 IC and OH \\
\hline TI-032 & 7.1.8 & Post-caustic-leach slurry wash & $\begin{array}{c}\text { S 02AML } 026 \text { XX } 0911 \text { RAM } 3 \\
\text { D }\end{array}$ & Raman & ASR 8321 & ASR 8321 RAM rev 1 \\
\hline TI-032 & 7.1.8 & Post-caustic-leach slurry wash & $\begin{array}{c}\text { S 02AML } 026 \text { XX } 0911 \text { TFI } 2 \\
\text { D }\end{array}$ & IC & 090109-15 & 090109-15 IC and OH \\
\hline TI-032 & 7.1.8 & Post-caustic-leach slurry wash & $\begin{array}{c}\text { S 02AML } 026 \text { XX } 0911 \text { TFI } 2 \\
\text { D }\end{array}$ & OH Titration & 090109-15 & 090109-15 IC and $\mathrm{OH}$ \\
\hline TI-032 & 7.1.8 & Post-caustic-leach slurry wash & $\begin{array}{c}\text { S 02AML } 026 \text { XX } 0913 \text { RAM } 3 \\
\text { D }\end{array}$ & Raman & ASR 8321 & ASR 8321 RAM rev 1 \\
\hline TI-032 & 7.1.8 & Post-caustic-leach slurry wash & $\begin{array}{c}\text { S 02AML } 026 \text { XX } 0913 \text { TFI } 2 \\
\text { D }\end{array}$ & IC & 090109-15 & 090109-15 IC and $\mathrm{OH}$ \\
\hline TI-032 & 7.1.8 & Post-caustic-leach slurry wash & $\begin{array}{c}\text { S 02AML } 026 \text { XX } 0913 \text { TFI } 2 \\
\text { D }\end{array}$ & OH Titration & 090109-15 & 090109-15 IC and $\mathrm{OH}$ \\
\hline TI-032 & 7.1.8 & Post-caustic-leach slurry wash & $\begin{array}{c}\text { S 02AML } 026 \text { XX } 0915 \text { RAM } 3 \\
\text { D }\end{array}$ & Raman & ASR 8321 & ASR 8321 RAM rev 1 \\
\hline TI-032 & 7.1 .8 & Post-caustic-leach slurry wash & $\begin{array}{c}\text { S 02AML } 026 \text { XX } 0915 \text { TFI } 2 \\
\text { D }\end{array}$ & IC & 090109-15 & 090109-15 IC and OH \\
\hline TI-032 & 7.1.8 & Post-caustic-leach slurry wash & $\begin{array}{c}\text { S 02AML } 026 \text { XX } 0915 \text { TFI } 2 \\
\text { D }\end{array}$ & OH Titration & 090109-15 & 090109-15 IC and OH \\
\hline TI-032 & 7.1.8 & Post-caustic-leach slurry wash & $\begin{array}{c}\text { S 02AML } 026 \text { XX } 0917 \text { RAM } 3 \\
\text { D }\end{array}$ & Raman & ASR 8321 & ASR 8321 RAM rev 1 \\
\hline
\end{tabular}


TI-WTP-PEP-032 (testing dates 12/19/2008 through 01/11/2009)

\begin{tabular}{|c|c|c|c|c|c|c|}
\hline TI \# & $\begin{array}{c}\text { TI Step } \\
\#\end{array}$ & TI Step Description & Sample ID & Analysis & Folder & Final Electronic Report File Name \\
\hline TI-032 & 7.1.8 & Post-caustic-leach slurry wash & $\begin{array}{c}\text { S 02AML } 026 \text { XX } 0917 \text { TFI } 2 \\
\text { D }\end{array}$ & IC & 090109-15 & 090109-15 IC and $\mathrm{OH}$ \\
\hline TI-032 & 7.1.8 & Post-caustic-leach slurry wash & $\begin{array}{c}\text { S 02AML } 026 \text { XX } 0917 \text { TFI } 2 \\
\text { D }\end{array}$ & OH Titration & 090109-15 & 090109-15 IC and OH \\
\hline TI-032 & 7.1.8 & Post-caustic-leach slurry wash & $\begin{array}{c}\text { S 02AML } 026 \text { XX } 0919 \text { RAM } 3 \\
\text { D }\end{array}$ & Raman & ASR 8321 & ASR 8321 RAM rev 1 \\
\hline TI-032 & 7.1.8 & Post-caustic-leach slurry wash & $\begin{array}{c}\text { S 02AML } 026 \text { XX } 0919 \text { TFI } 2 \\
\text { D }\end{array}$ & IC & 090109-15 & 090109-15 IC and OH \\
\hline TI-032 & 7.1.8 & Post-caustic-leach slurry wash & $\begin{array}{c}\text { S 02AML } 026 \text { XX } 0919 \text { TFI } 2 \\
\text { D }\end{array}$ & OH Titration & 090109-15 & 090109-15 IC and $\mathrm{OH}$ \\
\hline TI-032 & 7.1.8 & Post-caustic-leach slurry wash & $\begin{array}{c}\text { S 02AML } 026 \text { XX } 0921 \text { RAM } 3 \\
\text { D }\end{array}$ & Raman & ASR 8321 & ASR 8321 RAM rev 1 \\
\hline TI-032 & 7.1.8 & Post-caustic-leach slurry wash & $\begin{array}{c}\text { S 02AML } 026 \text { XX } 0921 \text { TFI } 2 \\
\text { D }\end{array}$ & IC & 090109-15 & 090109-15 IC and $\mathrm{OH}$ \\
\hline TI-032 & 7.1.8 & Post-caustic-leach slurry wash & $\begin{array}{c}\text { S 02AML } 026 \text { XX } 0921 \text { TFI } 2 \\
\text { D }\end{array}$ & OH Titration & 090109-15 & 090109-15 IC and $\mathrm{OH}$ \\
\hline TI-032 & 7.1.8 & Post-caustic-leach slurry wash & $\begin{array}{c}\text { S 02AML } 026 \text { XX } 0923 \text { RAM } 3 \\
\text { D }\end{array}$ & Raman & ASR 8321 & ASR 8321 RAM rev 1 \\
\hline TI-032 & 7.1.8 & Post-caustic-leach slurry wash & $\begin{array}{c}\text { S 02AML } 026 \text { XX } 0923 \text { TFI } 2 \\
\text { D }\end{array}$ & IC & $090109-15$ & 090109-15 IC and $\mathrm{OH}$ \\
\hline TI-032 & 7.1.8 & Post-caustic-leach slurry wash & $\begin{array}{c}\text { S 02AML } 026 \text { XX } 0923 \text { TFI } 2 \\
\text { D }\end{array}$ & OH Titration & 090109-15 & 090109-15 IC and $\mathrm{OH}$ \\
\hline TI-032 & 7.1.8 & Post-caustic-leach slurry wash & $\begin{array}{c}\text { S 02AML } 026 \text { XX } 0925 \text { RAM } 3 \\
\text { D }\end{array}$ & Raman & ASR 8321 & ASR 8321 RAM rev 1 \\
\hline TI-032 & 7.1.8 & Post-caustic-leach slurry wash & $\begin{array}{c}\text { S 02AML } 026 \text { XX } 0925 \text { TFI } 2 \\
\text { D }\end{array}$ & IC & 090109-15 & 090109-15 IC and OH \\
\hline TI-032 & 7.1.8 & Post-caustic-leach slurry wash & $\begin{array}{c}\text { S 02AML } 026 \text { XX } 0925 \text { TFI } 2 \\
\text { D }\end{array}$ & OH Titration & 090109-15 & 090109-15 IC and $\mathrm{OH}$ \\
\hline TI-032 & 7.1.8 & Post-caustic-leach slurry wash & $\begin{array}{c}\text { S 02AML } 026 \text { XX } 0992 \text { RAM } 3 \\
\text { D }\end{array}$ & Raman & ASR 8321 & ASR 8321 RAM rev 1 \\
\hline TI-032 & 7.1.8 & Post-caustic-leach slurry wash & $\begin{array}{c}\text { S 02AML } 026 \text { XX } 0992 \text { TFI } 2 \\
\text { D }\end{array}$ & IC & 090109-15 & 090109-15 IC and $\mathrm{OH}$ \\
\hline TI-032 & 7.1.8 & Post-caustic-leach slurry wash & $\begin{array}{c}\text { S 02AML } 026 \text { XX } 0992 \text { TFI } 2 \\
\text { D }\end{array}$ & OH Titration & 090109-15 & 090109-15 IC and $\mathrm{OH}$ \\
\hline TI-032 & 7.1.8 & Post-caustic-leach slurry wash & S 02AML 026 XX 0908 ARC 1 & Archive & & \\
\hline TI-032 & 7.1.8 & Post-caustic-leach slurry wash & S 02AML 026 XX 0910 ARC 1 & Archive & & \\
\hline TI-032 & 7.1.8 & Post-caustic-leach slurry wash & S 02AML 026 XX 0912 ARC 1 & Archive & & \\
\hline TI-032 & 7.1.8 & Post-caustic-leach slurry wash & S 02AML 026 XX 0914 ARC 1 & Archive & & \\
\hline TI-032 & 7.1.8 & Post-caustic-leach slurry wash & S 02AML 026 XX 0916 ARC 1 & Archive & & \\
\hline TI-032 & 7.1.8 & Post-caustic-leach slurry wash & S 02AML 026 XX 0918 ARC 1 & Archive & & \\
\hline
\end{tabular}


TI-WTP-PEP-032 (testing dates 12/19/2008 through 01/11/2009)

\begin{tabular}{|c|c|c|c|c|c|c|}
\hline TI \# & $\begin{array}{c}\text { TI Step } \\
\quad \#\end{array}$ & TI Step Description & Sample ID & Analysis & Folder & Final Electronic Report File Name \\
\hline TI-032 & 7.1 .8 & Post-caustic-leach slurry wash & S 02AML 026 XX 0920 ARC 1 & Archive & & \\
\hline TI-032 & 7.1.8 & Post-caustic-leach slurry wash & S 02AML 026 XX 0922 ARC 1 & Archive & & \\
\hline TI-032 & 7.1 .8 & Post-caustic-leach slurry wash & S 02AML 026 XX 0924 ARC 1 & Archive & & \\
\hline TI-032 & 8.2.2.4 & Reconcentrate solids and wash slurry & S 02AML 028 XX 0926 UDS 2 & UDS & 0901113-6 & \\
\hline TI-032 & $\begin{array}{l}9.1 .7- \\
9.1 .8\end{array}$ & Prepare for high-solids test & S 02AML 029 XX 0927 UDS 1 & (1) & & \\
\hline TI-032 & $\begin{array}{l}9.1 .7- \\
9.1 .8\end{array}$ & Prepare for high-solids test & S 02AML 029 XX 0928 RHE 4 & Rheology & TDP-WTP-355 & $\begin{array}{c}\text { TDP-WTP-355 Shakedown RHE Data on } \\
\text { PEP Slurry }\end{array}$ \\
\hline TI-032 & $\begin{array}{l}9.1 .7- \\
9.1 .8\end{array}$ & Prepare for high-solids test & $\begin{array}{c}\text { S 02AML } 029 \text { XX } 0929 \text { XSP } 4 \\
\text { B }\end{array}$ & PSD & TDP-WTP-354 & $\begin{array}{c}\text { TDP WTP } 354 \text { PEP Shakedown Test PSD } \\
\text { Data Analysis }\end{array}$ \\
\hline TI-032 & $\begin{array}{l}9.1 .7- \\
9.1 .8\end{array}$ & Prepare for high-solids test & S 02AML 029 XX 0997 UDS 2 & UDS & 0901113-6 & \\
\hline TI-032 & $\begin{array}{l}9.1 .7- \\
9.1 .8\end{array}$ & Prepare for high-solids test & S 02AML 029 XX 0998 RHE 4 & Rheology & TDP-WTP-355 & $\begin{array}{c}\text { TDP-WTP-355 Shakedown RHE Data on } \\
\text { PEP Slurry }\end{array}$ \\
\hline TI-032 & $\begin{array}{l}9.1 .7- \\
9.1 .8\end{array}$ & Prepare for high-solids test & $\begin{array}{c}\text { S 02AML } 029 \text { XX } 0999 \text { XSP } 4 \\
\text { B }\end{array}$ & PSD & TDP-WTP-354 & $\begin{array}{c}\text { TDP WTP } 354 \text { PEP Shakedown Test PSD } \\
\text { Data Analysis }\end{array}$ \\
\hline TI-032 & $\begin{array}{l}9.1 .7- \\
9.1 .8\end{array}$ & Prepare for high-solids test & S 02AML 029 XX 1000 CUF 4 & CUF & & \\
\hline TI-032 & $\begin{array}{l}9.1 .7- \\
9.1 .8\end{array}$ & Prepare for high-solids test & S 02AML 029 XX 0978 CUF 4 & CUF & & \\
\hline TI-032 & 9.2 .3 & High-solids filter test & S 02AML 030 XX 0930 UDS 2 & (1) & & \\
\hline TI-032 & 9.2 .3 & High-solids filter test & S 02AML 030 XX 0931 UDS 2 & (1) & & \\
\hline TI-032 & 9.2 .3 & High-solids filter test & S 02AML 030 XX 0932 UDS 2 & (1) & & \\
\hline TI-032 & 9.2 .3 & High-solids filter test & S 02AML 030 XX 0933 UDS 2 & (1) & & \\
\hline TI-032 & 9.2 .3 & High-solids filter test & S 02AML 030 XX 0934 UDS 2 & (1) & & \\
\hline TI-032 & 9.2 .3 & High-solids filter test & S 02AML 030 XX 0935 UDS 2 & (1) & & \\
\hline TI-032 & 9.2 .3 & High-solids filter test & S 02AML 030 XX 0936 UDS 2 & (1) & & \\
\hline TI-032 & 9.2 .3 & High-solids filter test & S 02AML 030 XX 0937 UDS 2 & (1) & & \\
\hline TI-032 & 9.2 .3 & High-solids filter test & S 02AML 030 XX 0938 UDS 2 & (1) & & \\
\hline TI-032 & 9.2 .3 & High-solids filter test & S 02AML 030 XX 0939 UDS 2 & (1) & & \\
\hline TI-032 & 9.2 .3 & High-solids filter test & S 02AML 030 XX 1001 UDS 2 & UDS & 090113-4 & \\
\hline TI-032 & 9.2 .3 & High-solids filter test & S 02AML 030 XX 1002 UDS 2 & UDS & 090113-4 & \\
\hline TI-032 & 9.2 .3 & High-solids filter test & S 02AML 030 XX 1003 UDS 2 & UDS & 090113-4 & \\
\hline TI-032 & 9.2 .3 & High-solids filter test & S 02AML 030 XX 1004 UDS 2 & UDS & 090113-4 & \\
\hline TI-032 & 9.2 .3 & High-solids filter test & S 02AML 030 XX 1005 UDS 2 & UDS & 090113-4 & \\
\hline TI-032 & 9.2 .3 & High-solids filter test & S 02AML 030 XX 1006 UDS 2 & UDS & 090113-4 & \\
\hline TI-032 & 9.2 .3 & High-solids filter test & S 02AML 030 XX 1007 UDS 2 & UDS & 090113-4 & \\
\hline TI-032 & 9.2 .3 & High-solids filter test & S 02AML 030 XX 1008 UDS 2 & UDS & 090113-4 & \\
\hline TI-032 & 9.2 .3 & High-solids filter test & S 02AML 030 XX 1009 UDS 2 & UDS & 090113-4 & \\
\hline TI-032 & 9.2 .3 & High-solids filter test & S 02AML 030 XX 1010 UDS 2 & UDS & 090113-4 & \\
\hline
\end{tabular}


TI-WTP-PEP-032 (testing dates 12/19/2008 through 01/11/2009)

\begin{tabular}{|c|c|c|c|c|c|c|}
\hline TI \# & $\begin{array}{c}\text { TI Step } \\
\#\end{array}$ & TI Step Description & Sample ID & Analysis & Folder & Final Electronic Report File Name \\
\hline TI-032 & 9.2 .3 & High-solids filter test & S 02AML 030 XX 1011 UDS 2 & UDS & $090113-4$ & \\
\hline TI-032 & 9.2 .3 & High-solids filter test & S 02AML 030 XX 1012 UDS 2 & UDS & 090113-4 & \\
\hline TI-032 & 9.2 .3 & High-solids filter test & S 02AML 030 XX 1013 UDS 2 & UDS & 090113-4 & \\
\hline TI-032 & 9.2 .3 & High-solids filter test & S 02AML 030 XX 1014 UDS 2 & UDS & 090113-4 & \\
\hline TI-032 & 9.2 .3 & High-solids filter test & S 02AML 030 XX 1015 UDS 2 & UDS & 090113-4 & \\
\hline TI-032 & 9.2 .3 & High-solids filter test & S 02AML 030 XX 1021 UDS 2 & & & \\
\hline TI-032 & 9.2 .3 & High-solids filter test & S 02AML 030 XX 1021 UDS 2 & UDS & $\begin{array}{l}\text { CCP-WTPSP- } \\
754\end{array}$ & $\begin{array}{c}\text { CCP WTPSP } 754 \text { Shakedown UDS Data } \\
\text { Analysis }\end{array}$ \\
\hline TI-032 & 9.2 .3 & High-solids filter test & S 02AML 030 XX 1021 UDS 2 & UDS & $\begin{array}{l}\text { CCP-WTPSP- } \\
755\end{array}$ & $\begin{array}{c}\text { CCP WTPSP } 755 \text { Integrated Test A UDS } \\
\text { Data Analysis }\end{array}$ \\
\hline TI-032 & 9.2 .3 & High-solids filter test & S 02AML 030 XX 1022 UDS 2 & UDS & 090113-4 & \\
\hline TI-032 & 9.2 .3 & High-solids filter test & S 02AML 030 XX 1023 UDS 2 & UDS & 090113-5 & \\
\hline TI-032 & 9.2 .3 & High-solids filter test & S 02AML 030 XX 1024 UDS 2 & UDS & 090113-5 & \\
\hline TI-032 & 9.2 .6 & High-solids filter test & S 02AML 030 XX 0973 UDS 2 & $(1)$ & & \\
\hline TI-032 & 9.2 .6 & High-solids filter test & S 02AML 030 XX 0974 UDS 2 & (1) & & \\
\hline TI-032 & 9.2 .6 & High-solids filter test & S 02AML 030 XX 0975 UDS 2 & (1) & & \\
\hline TI-032 & 12.1 .4 & $\begin{array}{l}\text { Evaluate bypass of filter-loop jet within } \\
\text { UFP-VSL-TO2A. 1-min after tracer injected. }\end{array}$ & S 000FL 025 XX 0991 RHE 4 & Rheology & TDP-WTP-355 & $\begin{array}{c}\text { TDP-WTP-355 Shakedown RHE Data on } \\
\text { PEP Slurry }\end{array}$ \\
\hline TI-032 & $\mathrm{n} / \mathrm{a}$ & $\mathrm{n} / \mathrm{a}$ & S 000FL XXX XX 0979 CUF 4 & & & \\
\hline TI-032 & $\mathrm{n} / \mathrm{a}$ & $\mathrm{n} / \mathrm{a}$ & S 000FL XXX XX 0980 CUF 4 & & & \\
\hline TI-032 & $\mathrm{n} / \mathrm{a}$ & $\mathrm{n} / \mathrm{a}$ & S 02AML 008 XX 0981 CUF 4 & CUF & & \\
\hline TI-032 & $\mathrm{n} / \mathrm{a}$ & $\mathrm{n} / \mathrm{a}$ & S 02AML 008 XX 0982 CUF 4 & CUF & & \\
\hline TI-032 & $\mathrm{n} / \mathrm{a}$ & $\mathrm{n} / \mathrm{a}$ & S 02AML 008 XX 0983 CUF 4 & CUF & & \\
\hline TI-032 & $\mathrm{n} / \mathrm{a}$ & $\mathrm{n} / \mathrm{a}$ & S 02AML 008 XX 0984 CUF 4 & CUF & & \\
\hline TI-032 & $\mathrm{n} / \mathrm{a}$ & $\mathrm{n} / \mathrm{a}$ & S 02AML 029 XX 978A UDS 4 & UDS & $\begin{array}{l}\text { CCP-WTPSP- } \\
754\end{array}$ & $\begin{array}{c}\text { CCP WTPSP } 754 \text { Shakedown UDS Data } \\
\text { Analysis }\end{array}$ \\
\hline TI-032 & $\mathrm{n} / \mathrm{a}$ & $\mathrm{n} / \mathrm{a}$ & S 02AML 029 XX 978B UDS 4 & UDS & $\begin{array}{l}\text { CCP-WTPSP- } \\
754\end{array}$ & $\begin{array}{c}\text { CCP WTPSP } 754 \text { Shakedown UDS Data } \\
\text { Analysis }\end{array}$ \\
\hline TI-032 & $\mathrm{n} / \mathrm{a}$ & $\mathrm{n} / \mathrm{a}$ & S 02AML 029 XX 978C UDS 4 & UDS & $\begin{array}{l}\text { CCP-WTPSP- } \\
754\end{array}$ & $\begin{array}{c}\text { CCP WTPSP } 754 \text { Shakedown UDS Data } \\
\text { Analysis }\end{array}$ \\
\hline TI-032 & $\mathrm{n} / \mathrm{a}$ & $\mathrm{n} / \mathrm{a}$ & S 00CUF 029 XX 1000 UDS 4 & UDS & $\begin{array}{l}\text { CCP-WTPSP- } \\
754\end{array}$ & $\begin{array}{c}\text { CCP WTPSP } 754 \text { Shakedown UDS Data } \\
\text { Analysis }\end{array}$ \\
\hline TI-032 & $\mathrm{n} / \mathrm{a}$ & $\mathrm{n} / \mathrm{a}$ & S 00CUF 029 XX 1000 UDS 4 & UDS & $\begin{array}{l}\text { CCP-WTPSP- } \\
755\end{array}$ & $\begin{array}{c}\text { CCP WTPSP } 755 \text { Integrated Test A UDS } \\
\text { Data Analysis }\end{array}$ \\
\hline TI-032 & $\mathrm{n} / \mathrm{a}$ & $\mathrm{n} / \mathrm{a}$ & $\begin{array}{l}\text { S 02AML XXX XX } 0994 \text { UDS } \\
2 \text { B }\end{array}$ & UDS & $090113-5$ & \\
\hline TI-032 & $\mathrm{n} / \mathrm{a}$ & $\mathrm{n} / \mathrm{a}$ & $\begin{array}{l}\text { S 02AML XXX XX } 0994 \text { UDS } \\
2 \text { D }\end{array}$ & UDS & 090113-5 & \\
\hline TI-032 & $\mathrm{n} / \mathrm{a}$ & $\mathrm{n} / \mathrm{a}$ & S 02AML XXX XX 0995 DEN & Density Slurry & 090113-2 & 090113-2DEN \\
\hline
\end{tabular}


TI-WTP-PEP-032 (testing dates 12/19/2008 through 01/11/2009)

\begin{tabular}{cccccc}
\hline TI \# & $\begin{array}{c}\text { TI Step } \\
\#\end{array}$ & TI Step Description & Sample ID & Analysis & Folder \\
\hline TI-032 & n/a & n/a & S 02AML XXX XX 0996 ICP 2 & $\begin{array}{c}\text { ICP Wet } \\
\text { Solids }\end{array}$ & $090113-3$ \\
TI-032 & n/a & n/a & S 02AML XXX XX 0996 RAM & Raman & ASR 8324 \\
TI-032 & n/a & n/a & S 02AML XXX XX 0997 ARC & 090113-3 ICP ICPMS & ASR 8324 RAM rev 1 \\
\hline
\end{tabular}

Dark Shading: Samples were sent to storage/archive in RTL or sent out of PDL-W for additional studies.

Light Shading: Aid for distinguishing rows.

TI step \# have been adjusted to allow samples collected in a single sampling event to be listed together.

(1) Sample collected and analysis put on hold per client request.

(2) Sample Analysis started by lab prior to hold notification.

Density slurry - analysis performed on slurry.

Density supernate - analysis performed on supernate.

Free hydroxide $(\mathrm{FOH})$

Inductively coupled plasma supernate (ICP supernate) ICP performed on the supernate.

Inductively coupled plasma wet solids (ICP wet solids) ICP performed on wet solids.

$\Omega$ Ion chromatography (IC).

is Particle -size distribution (PSD).

Rheology is a general term used to describe either Shear strength or Shear stress vs. shear rate analysis - actual analytical report specifies specific analysis.

Scanning electron microscope (SEM)

THF Wet Solids, THF Supernate, Toluene Wet Solids, Toluene Supernate are the solvent extraction performed on either the supernate or wet solid prior to analysis by gel permeation chromatography (GPC).

Total inorganic carbon and total organic carbon (TIC/TOC).

Weight Percent Undissolved Solids (wt\% UDS).

$\mathrm{x}$-ray diffraction (XRD). 
Appendix D

\section{Coriolis Sampler Elevations}





\section{Appendix D: Coriolis Sampler Elevations (Kurath 2008)}

\begin{tabular}{llll} 
Date: & $\mathbf{1 2 / 3 1 / 2 0 0 8}$ & Project No.: & $<53032>$ \\
To: & Dean Kurath & Internal & File/LB \\
From: & Matt Fountain & Distribution: & \\
& Gary Josephson & & \\
Subject: & Coriolis Sampler Elevations & & \\
\hline
\end{tabular}

'As installed' elevations of the Coriolis samplers were estimated by bubbling air through each sampler leg and measuring the bubbler pressure on the tank's lower bubbler leg calibrated pressure transmitter.

Method: After recording the pressure of the lowest bubbler leg the bubbler tubing was disconnected from the air supply and the supply attached to the Coriolis sampler stand via the low point drain and connecting Teflon tubing with Swagelock fittings. Air was bubbled through the tubing to each sampler leg and the pressure recorded. In UFP-1A and UFP-1B the PJMs were operating and the "lowest" pressure was recorded, when the PJMs were filled. Levels in UFP-1A, UFP-1B, and UFP-2A were measured on LT-0323, LT-0423, and LT-0611, respectively.

The measured pressures were compared with the measured differences in tubing lengths between the bottom-middle and middle-upper to make the final estimate sample elevations. Measured tubing differences were considered absolute and measured elevations were adjusted as much as an in. so that the offsets matched measured lengths.

Below are listed the "expected" sampler elevations based upon initial design and the results of this study: 


\begin{tabular}{|c|c|c|c|c|c|c|}
\hline \multirow{2}{*}{$\begin{array}{l}\text { Vessel } \\
\text { UFP-1A }\end{array}$} & \multicolumn{3}{|c|}{ Initial Design Elevation } & \multicolumn{3}{|c|}{ "As Installed" Sample Tube Elevation } \\
\hline & Low & Middle & High & Low & Middle & High \\
\hline Inner & 2 & 33 & 64 & 11 & 33 & 64 \\
\hline Middle & 11 & 33 & 55 & 9 & 31 & 53 \\
\hline Outer & 11 & 33 & 55 & 9 & 31 & 53 \\
\hline \multicolumn{7}{|l|}{ UFP-1B } \\
\hline Inner & 2 & 33 & 64 & 10 & 32 & 63 \\
\hline Middle & 11 & 33 & 55 & 6 & 28 & 51 \\
\hline Outer & 11 & 33 & 55 & 6 & 51 & 29 \\
\hline \multicolumn{7}{|l|}{ UFP-2A } \\
\hline Inner & 50 & 57 & 64 & 51 & 58 & 65 \\
\hline Middle & $\begin{array}{l}2 \text { in. off } \\
\text { bottom }\end{array}$ & 33 & 55 & 6 & 34 & 56 \\
\hline Outer & 11 & 33 & 55 & 11 & 33 & 56 \\
\hline
\end{tabular}

Conclusions: The 'as installed' sampler levels agree quite well with the design with the following exceptions:

- The UFP-1B outer-middle and outer-high sampling tubing has been switched. (An NCR is being written and the tubing needs to be corrected and the correction date recorded. Samples taken until then are incorrect.)

- The inner low sampler tubes were cut shorter than originally anticipated. Instead of sampling very close (2-in.) from the bottom of the vessel the sample is 10-in.-11-in. off the bottom.

- The middle and outer samplers in UFP-1B were installed about 4-in.-5-in. lower than initially intended.

The design of the UFP-2A middle-low position was designed to be 2-in. off the vessel bottom at the middle radial location. With a $2: 1$ elliptical head the elevation rise for the middle sampling location is estimated to be $\sim 3.5$-in. so the measured 6 -in. elevation from the center tank bottom is actually very close. 


\section{Appendix E}

Sample Processing and Methods 



\section{Appendix E: Sample Processing and Methods}

\subsection{Sample Processing}

The samples were collected in pre-labeled sample containers that were prepared and staged within PDL-W based upon the Sample Collection and Analysis table in the governing Test Instruction. The required analysis determined the sample volume. All samples for chemical analyses approximately 40-mL of sample was taken in a 50-mL plastic centrifuge cones with a screw top lid. If centrifugation was needed it was done in the original sample container. If centrifuging wasn't needed the cone was handled as a regular sample bottle. Samples for yield stress analyses and samples for parallel lab testing were taken in larger containers, but all other samples were $50-\mathrm{mL}$ sample cones. For analyses requiring larger volumes (e.g., shear stress analyses) multiple $50-\mathrm{mL}$ cones were filled and then combined at the analytical lab. Sample handling flow diagrams and method descriptions are detailed in Appendix E. in Figure E.1 through Figure E.3 for samples collected and processed in PDL-W. The letters "D," "B," "R," and "S," which are shown in the figures, were included in the sample names for phase-separated processed samples. The letter "D" was included in the decanted supernatant sample names. The letter "B" was included in the wet centrifuged solids sample names. The letter " $R$ " was included in the sample name of the combined rinse solution. The letter " $\mathrm{S}$ " was included in the sample names of the wet rinsed centrifuged solids. These figures represent two basic sample processing methods. One approach was used during non-leaching test steps, and another was used during leaching.

\subsubsection{Non-Leaching Samples}

Non-leaching samples were not always processed immediately after collection. Because sample collection could occur at any time during testing and testing was being performed $24 \mathrm{hr}$ per day, non-leached samples collected after $0600 \mathrm{hr}$ were typically processed and prepared for shipment by $0600 \mathrm{hr}$ the morning following sample collection. Non-leached samples used for the analyses listed below were shipped as collected in the container size specified and with the exception of the archive samples, tare and full sample weights were not required.

- weight percent undissolved solids (wt\% UDS) $(50-\mathrm{mL})$

- density $(50-\mathrm{mL})$

- heat Capacity $(50-\mathrm{mL})$

- shear strength $(2 \times 1-\mathrm{L})$

- shear stress vs. shear rate on original intact slurry $(100-\mathrm{mL})$

- shear stress vs. shear rate on supernatant (100-mL)

- total organic carbon on the slurry $(50-\mathrm{mL})$

- ICP-Silicon (50-mL)

- $\operatorname{archive}(50-\mathrm{mL})$.

During a sampling event, if an intact slurry sample was collected for density analysis and another intact slurry sample was collected for wt $\%$ UDS, then density measurements were performed on the supernatant in addition to the intact slurry submitted for density analysis. These density measurements 
were higher accuracy than density measurements obtained following the $\mathrm{wt} \%$ UDS method described in 24590-WTP-GPG-RTD-001 Rev 0. ${ }^{\text {(a) }}$

(a) GL Smith and K Prindiville. 2002. Guidelines for Performing Chemical, Physical, and Rheological Properties Measurements. 24590-WTP-GPG-RTD-001 Rev. 0, Bechtel National, Inc., Richland, Washington. 


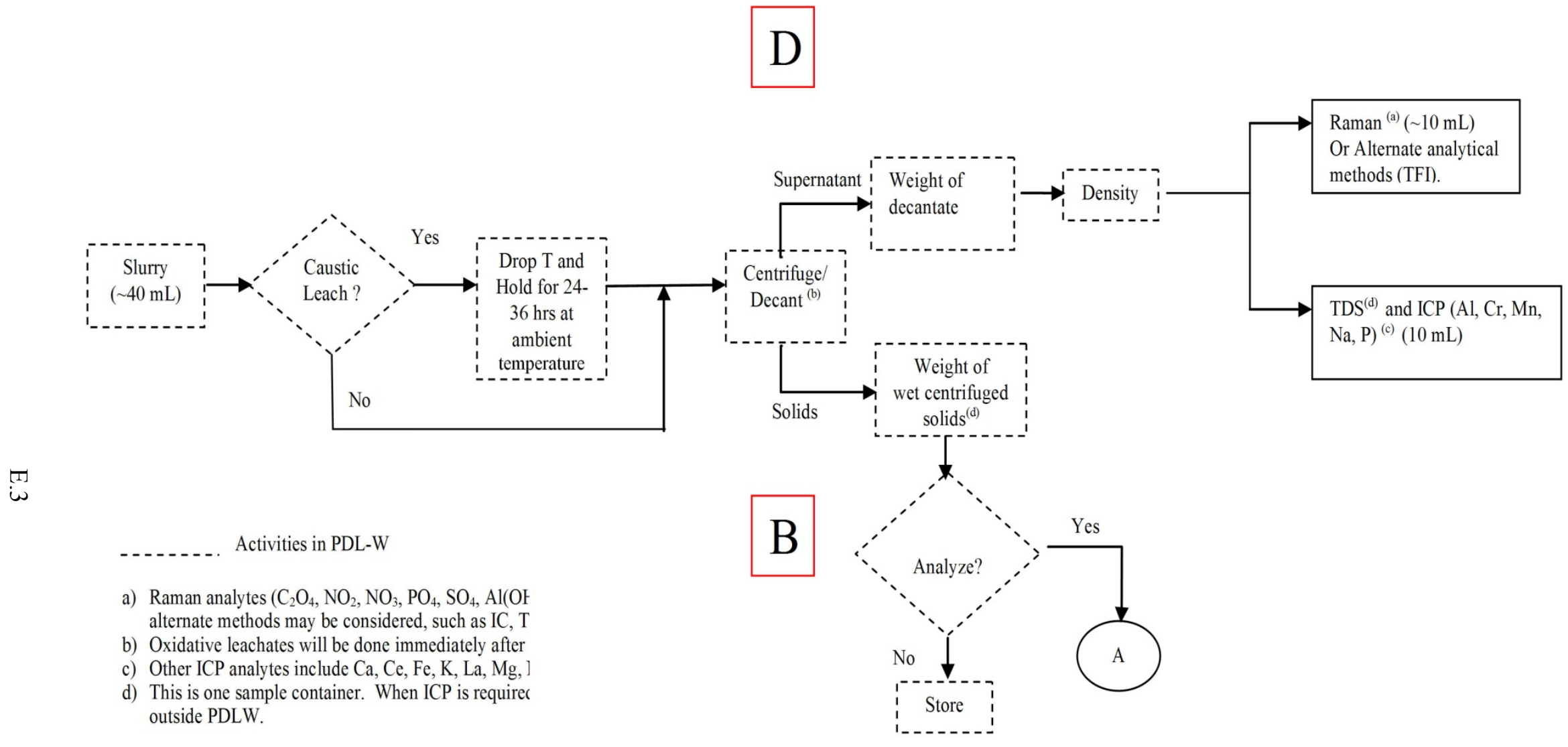

Figure E.1. Sample Processing for Decantate Handling 


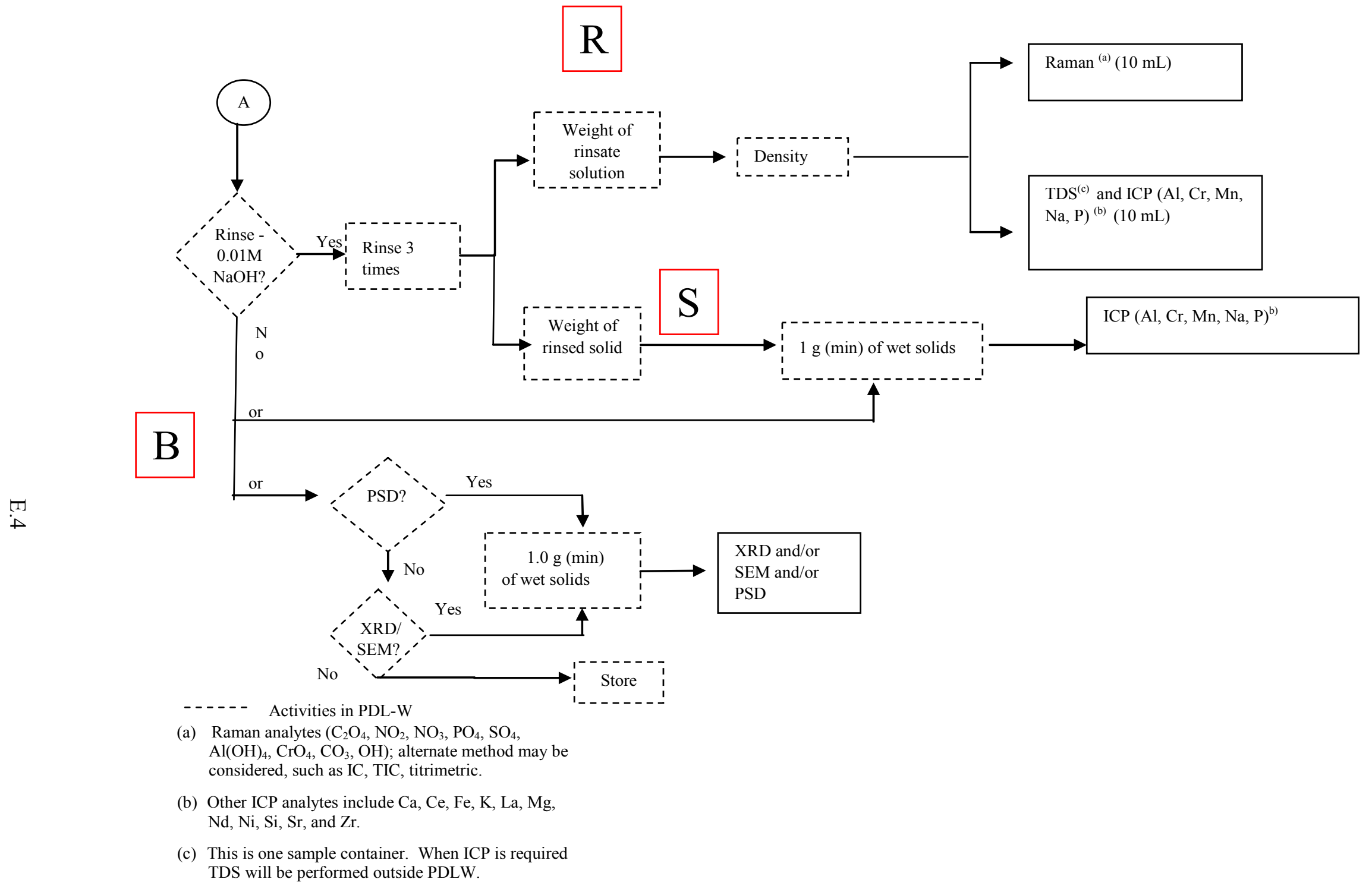

Figure E.2. Sample Processing for Solids 


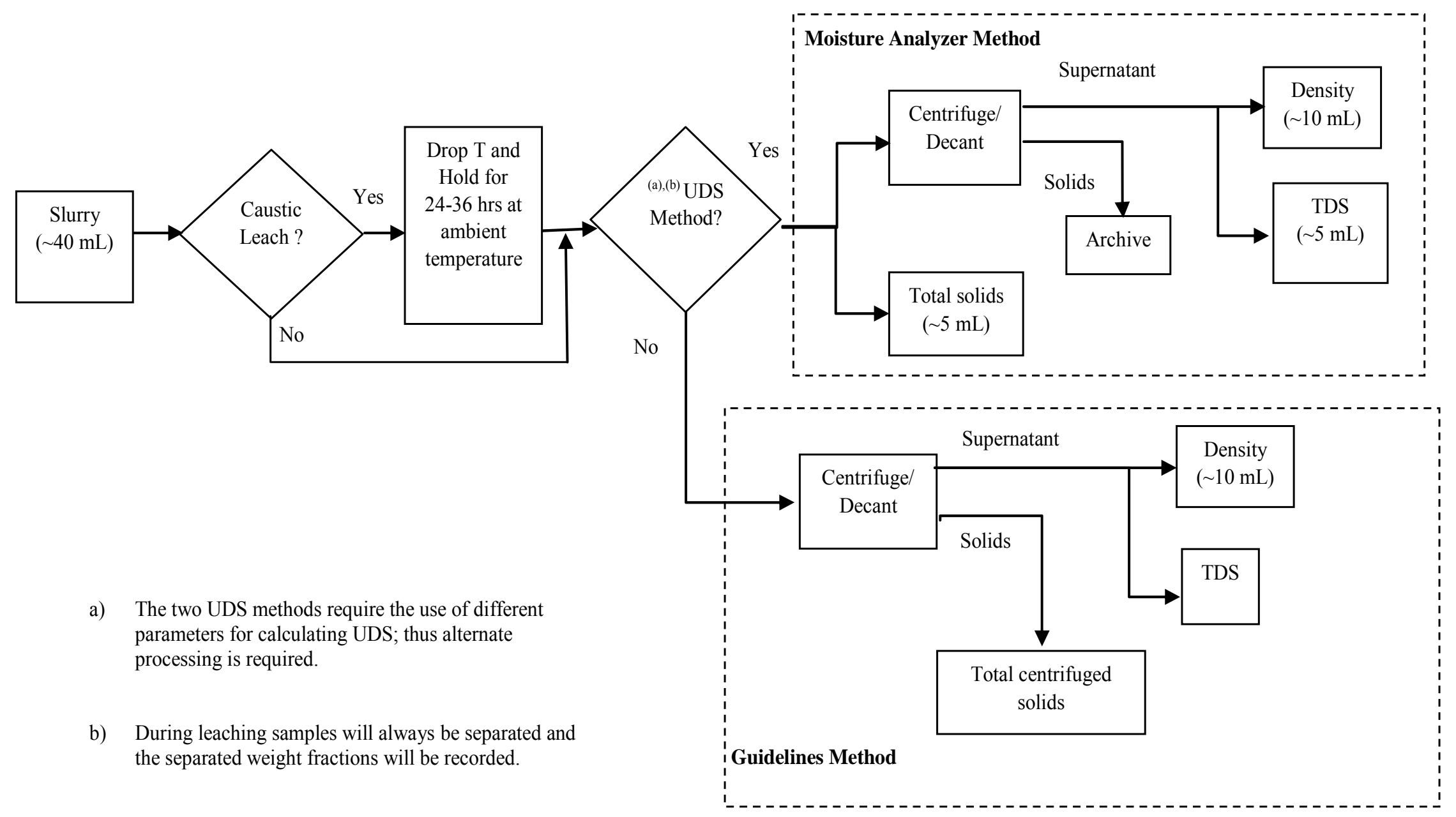

Figure E.3. Sample Processing for UDS 
A single 50-mL sample was centrifuged and the supernatant decanted to allow multiple laboratories to perform several analyses simultaneously on the supernatant. To perform this phase separation, the original sample was centrifuged at $\sim 4,500 \mathrm{G}$ with a swinging bucket rotor in PDL-W. The centrifuging time was initially set for 10-min. This centrifuging time was sufficient to cause phase separation during Shakedown, Integrated Test A, and oxidative leaching. However, during some of the Integrated Test B and Test D process steps, phase separation was not achieved after 10-min., so the samples were centrifuged for an hr. The actual centrifuging time is recorded on the sample benchsheets. After centrifuging, the supernatant was decanted and submitted for some or all the analyses listed below:

- ICP

- total dissolved solids

- density

- IC

- $\mathrm{TOC}$

- Raman

- free hydroxide

- density.

Not all of these analyses were necessarily performed on every decanted supernatant. The specific analysis was identified in the Sample Collection and Analysis table in the governing Test Instruction. The density was determined on the decanted supernatant at PDL-W for all samples being analyzed by Raman spectroscopy before the samples were transported for analysis. In addition, at times, density was determined by Southwest Research Institute (SwRI) before ICP analysis. The wet centrifuged solids from this same container were either submitted for ICP analysis, if it was required, or stored in the original sample collection container as excess. A tare weight (before filling) and gross weight (after filling) of the sample container were documented on sample benchsheets.

For DOW Corning to quantify the antifoam agent (AFA) compounds by gel permeation chromatography (GPC) in the solid and supernatant fractions of the slurry, two 50-mL slurry samples were collected and centrifuged at 4,500 $\mathrm{G}$ for 10-min., and the supernatant was decanted into two additional properly labeled $50-\mathrm{mL}$ containers before shipping. Weighing empty and filled containers was not required.

For SwRI to quantify silicon by ICP in the AFA, a 50-mL slurry sample was collected and shipped as collected during non-leaching process steps. Once at SwRI, the slurry was centrifuged at 2,200 G for an $\mathrm{hr}$, the supernatant was separated from the wet centrifuged solids, and then each phase was analyzed separately. During leaching, the phase separation occurred in PDL-W following the standard practice of centrifuging at $4,500 \mathrm{G}$ for 10 -min.

A single 50-mL container was filled for particle-size distribution (PSD), $\mathrm{x}$-ray diffraction (XRD), and scanning electron microscopy (SEM). Initially, these samples were centrifuged. The supernatant was decanted and stored while the wet solids were submitted for analysis. Intact slurry samples were submitted after Shakedown and Integrated Test A and initial Integrated Test B simulant characterization. If a phase-separated sample was submitted, then descriptor 7 was included in the sample name. If 
descriptor 7 was not in the name, then an intact sample was submitted for analysis. Weighing empty and filled containers was not required.

For parallel Cell Unit Filter (CUF) and bench-scale testing, varying amounts of either permeate or slurry samples were collected. A tare weight (before filling) and gross weight (after filling) of the sample container was recorded on the sample container. These samples were delivered to the Applied Processing Engineering Laboratory (APEL) by the next business day after sample collection.

\subsubsection{Leaching Samples}

The core critical analyses for leached samples were wt\% UDS, slurry density, ICP, IC, Raman and free hydroxide. Two 50-mL samples were collected for the UDS and density analyses. Another 50-mL sample was collected for the remaining core analyses.

- During caustic leaching, the original samples were immediately placed in a thermostatically controlled water bath at $20^{\circ} \pm 2^{\circ} \mathrm{C}$ to decrease the sample temperature to $25 \pm 5^{\circ} \mathrm{C}$ after collection. Once the desired temperature was reached, which typically took less than $10-\mathrm{min}$. for $50-\mathrm{mL}$ containers, the samples were allowed to sit for $24 \mathrm{hr}$. in PDL-W. The density sample was shipped as collected. The two other samples to be used for analysis were centrifuged following the sample process described for non-leached samples.

- During oxidative leaching, sample processing began immediately after sample collection. The oxidative-leach density sample was shipped as collected while the other two samples were phase-separated immediately after collection by centrifuging and decanting the supernatant as described above for non-leached samples. To allow for consistency in comparing analytical results, the samples for ICP analysis collected immediately before adding $\mathrm{NaMnO}_{4}$ were phase-separated immediately after collection. The oxidative-leached centrifuged solids from samples collected immediately before adding $\mathrm{NaMnO}_{4}$ and at the end of oxidative leaching for analysis were weighed and rinsed three times with $0.01-\mathrm{M} \mathrm{NaOH}$ solution. The actual quantity of rinse is recorded on sample benchsheets. The wet solids were vortexed three times to suspend and rinse the solids after each addition of $\mathrm{NaOH}$ solution. The rinsate was collected and weighed. The density of the rinsate was measured at PDL-W, and then it was split into sub-samples for ICP and Raman analysis.

During leaching, additional 50-mL samples were collected for archiving. Caustic-leached archived samples were also cooled and phase-separated as described above before storage. The oxidatively leached archived samples were also immediately phase-separated after collection as described above. The centrifuged oxidatively leached solids that went to storage were not rinsed.

\subsubsection{Undissolved Solids}

The UDS was measured at PNNL only when results were needed the same day the sample was collected. A halogen moisture analyzer (HG63, Mettler Toledo) was used to determine the wt $\%$ UDS under procedure TPR-RPP-WTP-648. If results for multiple samples were required, then a modified version of the oven method outlined in Guidelines for Performing Chemical, Physical, and Rheological Properties Measurements ${ }^{(\mathrm{a})}$ was used.

(a) Smith GL and K Prindiville. 2002. Guidelines for Performing Chemical, Physical, and Rheological Properties Measurements. 24590-WTP-GPG-RTD-001 Rev. 0, Bechtel National, Inc., Richland, Washington. 


\subsubsection{Supernatant Density}

Density measurements were performed at PDL-W on samples that were analyzed by Raman. Density was measured in accordance with TPR-WTP-PEP-054, Determination of Density using Pycnometer or Graduated Cylinder. All other density values were analyzed externally by SwRI.

\subsection{Analytical Methods}

This section describes the analytical methods used to determine the chemical composition of the PEP simulant samples collected during testing. Analytical results are provided in Section 5.

\subsubsection{Preparation for ICP or ICP-MS}

For the solutions, aliquots were diluted with hydrochloric acid and then analyzed.

For solids, two preparative techniques were used. The first preparative technique was lithium metaborate/tetraborate fusion, referred to as prep method "80/20 Fusion." Aluminum, barium, cerium, chromium, iron, lanthanum, manganese, neodymium, silicon, and strontium were reported from the fusion. The second preparative method used concentrated nitric, perchloric, hydrofluoric, and hydrochloric acids in an open vessel and was referred to as the "Teflon" preparative method. If residue remained from the "Teflon" method, then the residue was separated, dried, and fused using the lithium metaborate/tetraborate fusion technique. Both the "Teflon" and residue preparations were analyzed. The remaining metals were reported from either the "Teflon" digestion only or the combination of the two. If an analyte was detected in both the Teflon digestate and the residue fusions, then the reported result was the sum of the results obtained from the two preparations. These results were identified as "combined" on Sample Analysis Data Sheets while analytes reported from only the fusion were identified as " $80 / 20$ Fusion," and analytes reported solely from the acid digestions were identified as "Teflon" on the Sample Analysis Data Sheets. The preparative QC samples included a sample duplicate, preparation blank (PB), solid laboratory control samples (LCSs) consisting of obsidian rock and basalt rock, and an aqueous LCS and a matrix spike (MS), for "Teflon." The results are reported on an "as received" or wet-weight basis. The percent solids were determined at $105^{\circ} \mathrm{C}$ and reported on the Sample Analysis Data Sheets for all solids.

\subsubsection{Metals Analysis by ICP-AES}

All metals were determined by ICP-AES using SwRI procedure TAP01-0406-130 with the exception of cerium, cesium, lanthanum, and neodymium. The preparative QC samples (duplicate, PB, LCS, and MS) were processed along with analytical workstation QC (initial and continuing calibration verifications, initial and continuing blanks, interference check samples, and post-digestion spikes).

\subsubsection{Metals Analysis by ICP-MS}

Cerium, cesium, lanthanum, and neodymium were determined by ICP-MS in accordance with SwRI procedure TAP01-0406-046. The preparative QC samples (duplicate, PB, LCS, MS) were processed along with analytical workstation QC (initial and continuing calibration verifications, initial and continuing blanks, interference check samples and post-digestion spikes). 


\subsubsection{Anions (IC)}

Decanted supernatant and rinsates were analyzed by IC for chloride, nitrate, nitrite phosphate, sulfate, and oxalate at SwRI in accordance with procedure SwRI TAP 01-0406-042. Approximately 0.25-g of the sample was diluted to 50-mL using deionized water (DIW). Since the dilution was performed by weight, the sample results are reported on a weight basis. The standard reporting by the laboratory is nitrate as $\mathrm{N}$, nitrite as N, and phosphate as P. Shortly after PEP testing began, PNNL requested that results be reported as nitrate, nitrite, and phosphate and not as nitrogen or phosphorus. The IC analytical report narrative identifies conversion factors used to report as anions. QC samples generated at the analytical workstation included a sample replicate determination, process blank, LCS, and MS.

\subsubsection{Total Inorganic Carbon/Total Organic Carbon (TIC/TOC)}

The samples were analyzed for total carbon (TC) using a Dohrman DC-80 Carbon Analyzer in accordance with procedure SwRI TAP 01-0406-001. The liquids were directly injected, and the slurry was analyzed using the soil/sludge sampler. Another aliquot of the sample was acidified with sulfuric acid and sparged to remove inorganic carbon and then analyzed for TOC. The TIC was calculated from the difference in the TC and TOC results. All samples were analyzed in duplicate and average results reported when the relative percent differences (RPDs) were less than 20\%. If the RPD was greater than $20 \%$, then the sample was analyzed twice more, and the average of the quadruplicate analysis was reported. The liquids were corrected for density, and all sample results were reported on a weight basis. QC samples were generated at the analytical workstation and included a sample replicate determination, process blank, LCS, and MS.

\subsubsection{Hydroxide}

The free-hydroxide concentration was determined by potentiometric titration with standardized $\mathrm{HCl}$ according to procedure RPG-CMC-228, "Determination of Hydroxyl (OH-), and Alkalinity of Aqueous Solutions, Leachates, and Supernates and Operation of Brinkman 636 Auto-Titrator." The free hydroxide was defined as the first inflection point on the titration curve. QC samples were generated at the analytical workstation and included a sample replicate determination, process blank, and blank spike (BS).

\subsubsection{Raman}

Raman was used to quantify aluminate, carbonate, chromate, hydroxide, nitrate, nitrite, oxalate, phosphate, and sulfate following procedure RPG-CMC-240. If precipitate formed in the solution samples submitted for Raman before the analysis, then the samples were centrifuged, and aliquots of the liquid were pipetted and analyzed. Two sets of Raman results were reported for Shakedown, Integrated Test A, and some of the Integrated Test B samples. The first set of results was generated using calibrations that were periodically adjusted to optimize the performance of QC check samples. The second set of results was recalculated based on the original calibration parameters. The generation of these two sets of results and the discovery of the calibration adjustments are documented as RPP-WTP CAR, number 42708.1. Only Raman results from the recalculation are provided. As a result of using the original calibration parameters, an occasional QC sample falls outside established performance limits. QC samples were 
generated at the analytical workstation and included a sample replicate determination, process blank, BS, and MS.

\subsubsection{Preparation for Gel Permeation Chromatography (AFA components)}

Duplicate samples were collected to support this analysis. Both samples were centrifuged and phase-separated at PDL-W before shipping. Once at the analytical laboratory, one of the two liquid fractions was mixed with toluene while the other was mixed with tetrahydrofuran (THF). Both were shaken for an hr and allowed to settle overnight. The upper organic layer was removed and allowed to concentrate. The toluene extract was dried at room temperature to completeness and brought to $3.0-\mathrm{mL}$ toluene. The THF extracts were concentrated to approximately $2-\mathrm{mL}$ at room temperature and then placed in an $80^{\circ} \mathrm{C}$ oven to dry to completeness and brought up to 3.0-mL THF. One of the two solids fractions was extracted with $10-\mathrm{mL}$ of toluene while the other was extracted with $10.0-\mathrm{mL}$ of THF. In both cases, the solvent was added directly into the receipt vessel, and the initial sample and solvent were shaken for $2 \mathrm{hr}$. All solutions were filtered through 0.45- $\mu \mathrm{m}$ PTFE syringe filters. Calibration verification was performed before sample analysis and after every 15 samples.

\subsubsection{Gel Permeation Chromatography (AFA components)}

The toluene extract allowed polydimethylsiloxane (PDMS) to be analyzed by GPC while the THF extract allowed polypropylene glycol (PPG) to be analyzed by GPC. The toluene extract was analyzed by a Waters 717 autosampler and a Waters 2410 differential refractometer. Two columns, a PLgel 5- $\mu \mathrm{m}$ guard column and a PLgel 5- $\mu \mathrm{m}$ Mixed-C column, were used for separation, and an HPLC-grade toluene at a flowrate of $1.0-\mathrm{mL} / \mathrm{min}$ was used as the eluent. Both the columns and detector were heated to $45^{\circ} \mathrm{C}$. The THF extract was analyzed by a Waters 2695 Separation Module equipped with a vacuum degasser and a Waters 2410 differential refractometer. Two columns, a PLgel 5- $\mu \mathrm{m}$ guard column and a PLgel $5-\mu \mathrm{m}$ Mixed-C column, were used for separation, and a certified-grade THF at a flowrate of $1.0-\mathrm{mL} / \mathrm{min}$ was used as the eluent. Both the columns and detector were heated to $35^{\circ} \mathrm{C}$. The quantity of PDMS and PPG was used to determine the amount of AFA, Q2-3183A Antifoam, in the samples. Since the AFA is a proprietary composition belonging to Dow Corning, they performed the analytical work. Calibration verification was performed before sample analysis and after every 15 samples.

\subsection{Physical Properties}

This section describes the methods used to determine the physical properties of the PEP simulant samples, the crystal form and habit of the solids and the density, wt\% UDS, rheology, particle-size attributes, and heat capacity. A more detailed outline of the methods used in this section is given, where applicable, in the appropriate Test Data Package supplied with the sample results for each characterization.

\subsubsection{Percent UDS and Density}

Weight percent UDS, weight percent dissolved solids, bulk slurry density, and supernatant density were determined following the Guidelines for Performing Chemical, Physical and Rheological Properties 
Measurements. ${ }^{(a)}$ Some steps in the Guidelines were not performed because the results generated from these steps were not needed, or steps were slightly modified to reduce analysis time. These modifications were not believed to impact the final results.

Slurry sample UDS analysis was performed with the following modifications:

- Steps 4, 6, and 7 were omitted because the settling data were not required.

- Steps 8 and 9. Centrifuge the cones at $\sim 1,000 \mathrm{G}$ for $1 \mathrm{hr}$. Record the volume of the total sample and the volume of centrifuged solids on the physical properties data sheet. After this, the WTP procedures required that the supernatant be decanted into a pre-weighed graduated cylinder to obtain the supernatant mass and volume. Rather than use a graduated cylinder, the volume of supernatant was calculated as the difference between the volume of the total sample and the volume of centrifuged solids.

- Step 11 requires decanting the centrifuged supernatant liquid to a pre-weighed graduated cylinder. Because of the high concentration of $\mathrm{NaOH}$ in some of the samples, some of the liquid was left in the cylinder. Rather than decant, the centrifuged supernatant liquid was pipetted to a pre-weighed glass Petri dish or vial, and the mass of the supernatant liquid was recorded.

- Step 12 was omitted because air-drying was not necessary.

Separated slurry samples UDS analysis was performed with the following modifications:

- Step 8 requires centrifuging at $\sim 1,000 \mathrm{G}$ for $1 \mathrm{hr}$. Samples were centrifuged at $\sim 4,500 \mathrm{G}$ for either 10 -min. or $1 \mathrm{hr}$. The centrifuge time is documented on the sample handling benchsheets in TDP-WTP-349.

- Wet solids (designated as "B") and decanted supernatants (designated as "D") were sent to SwRI along with empty vial and separated sample weights. These weights are also documented on the sample handing benchsheets in TDP-WTP-349.

- The wet solids were transferred to a tared container using DIW to completely remove the solids. The solids and DIW rinses were then oven dried to constant weight.

- The total weight of the supernatant was calculated from the sample-handling benchsheets. Since it was not feasible for the entire volume of supernatant to be oven dried, approximately $5-\mathrm{mL}$ was transferred to a Petri dish for drying.

- The density was determined on the supernatant liquid using a 5-mL volumetric flask.

Two sets of UDS reports were issued for Shakedown, Integrated Test A, Integrated Test B, and some of the Integrated Test D samples. The uncertainty associated with the first set of UDS results used a Taylor series expansion. This first estimate of uncertainty provided reasonable results until the quantity of total dissolved solids approached the detection limit of the method. The quantity of dissolved solids was used to calculate the UDS so the uncertainty for the UDS would increase as the quantity of total dissolved solids decreased. The estimate of uncertainty provided in the revised reports was calculated more rigorously. Only UDS reports with the more rigorous uncertainty calculation were used in data analysis.

(') GL Smith and K Prindiville. 2002. Guidelines for Performing Chemical, Physical, and Rheological Properties Measurements. 24590-WTP-GPG-RTD-001 Rev. 0, Bechtel National, Inc., Richland, Washington. 


\subsubsection{Density}

Three pre-weighed 5-mL Class A volumetric flasks were filled to the specified line with the liquid sample and reweighed. The density was calculated as the mass of the sample divided by the certified volume of the volumetric flask.

Slurry samples requiring a higher accuracy supernatant density than provided by the UDS analysis were sub-sampled and centrifuged for $1 \mathrm{hr}$. at 1,000 G, and the phase-separated supernatant was analyzed as described in the paragraph above.

\subsubsection{Rheology Measurements}

Rheological testing was conducted on the solids in contact with the supernatant generated as part of the homogenization process. Testing was conducted according to RPL-COLLIOD-02, Measurement of Physical and Rheological Properties of Solutions, Slurries and Sludges. For the current study, two regions of tank waste flow behavior are considered: 1) incipient motion in settled tank waste solids (shear strength), and 2) non-elastic flow of tank waste slurries and supernates (flow curve).

\subsubsection{Shear-Strength Testing}

For tank waste slurries, a finite stress must be applied before the material will begin to flow. The stress required to transition the material from elastic deformation to viscous flow is referred to as the shear strength, and its origin can be attributed to static and kinetic friction between individual particles and/or aggregates, the strength of the matrix supporting the coarse fraction (i.e., the interstitial fluid), and sludge cohesion arising from interparticle adhesive forces such as van der Waals forces.

The shear strength was measured with the vane method. For the vane technique, the stress required to begin motion is determined by slowly rotating a vane immersed in the test sample's settled solids while continuously monitoring the resisting torque as a function of time. A material's static shear strength is then associated with the maximum torque measured during the transition from initial to steady-state vane rotation.

The maximum torque required for incipient motion is dependent on vane geometry. To account for vane-geometry effects, the shear strength is expressed in terms of the uniform and isotropic stress acting over the surface area of the cylinder of rotation swept out by the vane. The shear strength is related to the maximal torque during incipient motion according to Equation 3.1 (Barnes and Dzuy 2001):

$$
\tau_{S S}=\frac{M_{\max }}{4 \pi R^{3}\left(\frac{H}{2 R}+\frac{1}{3}\right)}
$$

Here, $\tau_{s s}$ is the shear strength $\left(\mathrm{N} / \mathrm{m}^{2}\right), M_{\max }$ is the maximum torque $(\mathrm{N} \cdot \mathrm{m})$, and $R$ and $H$ are the radius and height of the cylinder of rotation swept out by the vane $(\mathrm{m})$. Because the shear band observed upon slow rotation of the vane does not extend appreciably beyond the vane paddles, $R$ and $H$ are taken to be the dimensions of the vane itself. 
The proximity of the vane to the sample container inner surfaces as well as the free surface of the settled solids can impact shear-strength results. As such, certain geometric constraints must be satisfied for the test to be considered independent of container geometry. Table E.1 identifies the geometric constraints.

Table E.1. Vane Immersion Depth and Container Geometry Constraints for Shear-Strength Tests Using the Vane Technique

\begin{tabular}{lll}
\hline Constraint & Criterion & For $8 \times 16-\mathrm{mm}(\mathrm{R} \times \mathrm{H})$ Vane \\
\hline Vane height to radius & $\mathrm{H}<7 \mathrm{R}$ & $\mathrm{H}<56-\mathrm{mm}$ (Satisfied) \\
Container radius to vane radius & $\mathrm{R}_{\text {cont }}>2 \mathrm{R}$ & $\mathrm{R}_{\text {cont }}>16-\mathrm{mm}$ \\
Immersion depth to vane height & $\mathrm{h}>\mathrm{H}$ & $\mathrm{h}>16-\mathrm{mm}$ \\
$\begin{array}{l}\text { Separation between bottom of vane and } \\
\text { container floor }\left(\mathrm{h}_{\mathrm{floor}}\right)\end{array}$ & $\mathrm{h}_{\text {floor }}>0.5 \mathrm{H}$ & $\mathrm{h}_{\text {floor }}>8-\mathrm{mm}$ \\
\hline $\mathrm{R}=$ radius & & \\
$\mathrm{H}=$ height & & \\
\hline
\end{tabular}

\subsubsection{Flow-Curve Testing}

The non-elastic flow of tank waste slurries and supernates is characterized with rotational viscometry. The typical result of such testing is a set of flow-curve data, which shows the stress response of a material to a range of applied rates-of-deformation. Specifically, flow-curve testing allows characterization of a material's shear stress, $\tau$, which is the response as a function of applied shear rate, $\dot{\gamma}$. Once measured, the flow-curve data can be interpreted with several constitutive equations for the viscous stress/rate-of-strain relationship. Such analysis allows the flow behavior over a broad range of conditions to be described with just a few rheological descriptors, such as viscosity, yield stress, consistency, and flow index.

A concentric cylinder rotational viscometer operated in controlled-rate mode was used for flow-curve testing of tank waste slurries and supernates. Rotational viscometers operate by placing a given volume of test sample into a measurement cup of known geometry. A cylindrical rotor attached to a torque sensor is then lowered into the sample until the slurry is even with, but does not cover, the top of the rotor. A single-point determination of a fluid's flow properties is made by spinning a rotor at a known rotational speed, $\Omega$, and measuring the resisting torque, $M$, acting on the rotor. The torque acting on the rotor can be directly related to the shear stress at the rotor using the equation,

$$
\tau=\frac{M}{2 \pi H R_{I}^{2}}
$$

The shear stress has units of force per area $\left(\mathrm{N} / \mathrm{m}^{2}\right)$. The rotational rate is related to the shear rate. However, calculating the fluid shear rate at the rotor is complicated by the fact that the shear rate depends on both the measurement-system geometry and the fluid rheological properties. For the simplest fluids (i.e., Newtonian fluids), the shear rate of the fluid at the rotor can be calculated given the geometry of the cup rotor shear by using the equation, 


$$
\dot{\gamma}=\left(\frac{2 R_{O}^{2}}{R_{O}^{2}-R_{I}^{2}}\right) \Omega
$$

Here, the shear rate has units of inverse seconds $\left(\mathrm{s}^{-1}\right)$. Calculating the shear rate for materials showing more complex shear-stress versus shear-rate behavior (i.e., non-Newtonian fluids) requires estimates of yield stress and a degree of shear-thinning or shear-thickening. As the goal of rheological testing is to determine and quantify such behavior, these values are typically not known. This requirement can be circumvented by using a cup-and-rotor system with a small gap ( $\sim 1-\mathrm{mm})$ for fluid shear. For fluid flow in small-gap cup and rotor systems, shear-rate effects introduced by fluid properties are minimized such that Equation 3.3 provides an accurate determination of shear rate for non-Newtonian materials.

The resistance of a fluid to flow is often described in terms of the fluid's apparent viscosity, $\eta_{a p p}$, which is defined as the ratio of the shear stress to the shear rate:

$$
\eta_{\text {app }}=\frac{\tau}{\dot{\gamma}}
$$

For Newtonian fluids, the apparent viscosity is independent of the shear rate. For non-Newtonian fluids, the apparent viscosity will vary as a function of the shear rate. The unit of apparent viscosity is $\mathrm{Pa} \cdot \mathrm{s}$, although it is typically reported in the unit of centipoise (cP; where $1 \mathrm{cP}=1-\mathrm{mPa} \cdot \mathrm{s})$.

Flow-curve data are usually combined plots of $\tau$ and $\eta_{\text {app }}$ as a function of $\dot{\gamma}$. As stated above, flow-curve data can be interpreted with several constitutive equations (i.e., flow curves), allowing characterization of those data with just a few rheological descriptors. The behavior of tank waste sludges, slurries, and supernates can be described by four common flow-curve equations:

Newtonian-Newtonian fluids flow as a result of any applied stress and show constant viscosity over all shear conditions. The flow curve for Newtonian fluids is

$$
\tau=\eta \dot{\gamma}
$$

where $\eta$ is the Newtonian viscosity.

Ostwald (Power Law) - Power-law fluids flow as a result of any applied stress and have viscosities that either increase or decrease with increasing shear rate. They are described by,

$$
\tau=m \dot{\gamma}^{n}
$$

where $m$ is the power-law consistency index, and $n$ is the power-law index. Power-law fluids with $n<1$ are referred to as pseudoplastic (shear-thinning), whereas power-law fluids with $n>1$ are referred to as dilatant (shear-thickening). 
Bingham Plastic - Bingham plastics are fluids that show finite yield points. A finite stress (i.e., the yield stress), must be exceeded before these types of materials flow. Once flow is initiated, the stress response of the material is Newtonian over the rest of the shear-rate range. Bingham plastics are described by

$$
\tau=\tau_{O}^{B}+k_{B} \dot{\gamma}
$$

where $\tau_{O}^{B}$ is the Bingham yield index, and $k_{B}$ is the Bingham consistency index.

Casson-Fluids that behave in accordance with a Casson model show a finite yield followed by pseudoplastic behavior. They are described by,

$$
(\tau)^{0.5}=\left(\tau_{o}^{C}\right)^{0.5}+\left(k_{C} \dot{\gamma}\right)^{0.5}
$$

where $\tau_{o}^{C}$ is the Casson yield index, and $k_{C}$ is the Casson consistency index. Although more limited in the types of flow behavior that it can describe relative to the Herschel-Bulkley equation, the Casson model is popular because it is capable of accurately describing many shear-thinning fluids and because units on the parameters are more physically meaningful (e.g., the consistency is in Pa.s versus Pa. ${ }^{\mathrm{n}}$ for the Herschel-Bulkley model).

Power-law fluids, Bingham plastics, and Casson fluids are referred to as non-Newtonian fluids. Generally, liquids without internal and/or interconnected structures (such as tank waste supernatants) are Newtonian. Sludges and slurries are typically non-Newtonian, but their exact behavior depends on the concentration of solids and suspending phase chemistry. Sufficiently dilute slurries may show Newtonian behavior.

\subsubsection{Rheology Instrumentation}

Rheological characterization was accomplished with an Anton Parr Rheometer (MCR 301) for shear-rate measurements, and the shear strength was measured with a Rotovisco RV20 Measuring System M equipped with an M5 measuring head and RC20 controller sold by HAAKE Mess-Technik GmbH u Co. (now the Thermo Electron Corporation). The M5 measuring head is a "Searle" type viscometer capable of producing rotational speeds up to $500 \mathrm{rpm}$ and measuring torques up to $0.049 \mathrm{~N} \cdot \mathrm{m}$. The minimum rotational speed and torque resolution achievable by this measuring head are $0.05 \mathrm{rpm}$ and $0.49-\mathrm{mN} \cdot \mathrm{m}$, respectively.

Calibration and analysis were performed according to TPR-WTP-PEP-049.

Specific measurement tools, such as cup-and-rotor assemblies and shear vanes, are attached to measure selected rheological properties. Shear-strength measurements employ an 8-mm $\times 16-\mathrm{mm}$ $(\mathrm{R} \times \mathrm{H})$ shear vane tool. Flow-curve measurements employed an MV1 stainless steel measuring cup and rotor. The dimensions of the MV1 and vane measuring systems are listed in Table E.2. 
Table E.2. Vane and Cup and Rotor Measuring System Dimensions

\begin{tabular}{lcccc}
\hline Measuring System & $\begin{array}{c}\text { Vane/Rotor Radius } \\
(\mathrm{mm})\end{array}$ & $\begin{array}{c}\text { Vane/Rotor Height } \\
(\mathrm{mm})\end{array}$ & $\begin{array}{c}\text { Container Radius } \\
(\mathrm{mm})\end{array}$ & $\begin{array}{c}\text { Gap Width } \\
(\mathrm{mm})\end{array}$ \\
\hline Vane Tool & 8 & 16 & $>16$ & $>8$ \\
MV1 & 20.04 & 60 & 21 & 0.96 \\
\hline
\end{tabular}

The temperature is controlled with a combination of the standard measuring system temperature jacket and a temperature-controlled recirculator. The jacket temperature is monitored with a Type-K thermocouple calibrated over $0^{\circ}$ to $100^{\circ} \mathrm{C}$ and connected to a calibrated multichannel temperature display. The temperature control is employed only for flow-curve measurements. Shear-strength measurements are carried out at ambient cell temperature.

The rheometer was controlled, and data were acquired with a remote computer connection using the RheoWin Pro Job Manager Software, Version 2.96. During measurement, the software automatically collects and converts rotor torque readings into shear stresses based on Equation 5.1 (for vane testing) or Equation 5.2 (for flow-curve testing). Likewise, the software also automatically converts the rotational rate readings into shear rates based on Equation 3.2.

\subsubsection{Rheology Materials and Methods}

No sample treatment was performed before analysis with the exception of the mechanical agitation required to mix and sub-sample selected sample containers.

\subsubsection{Shear-Strength Testing}

Before testing, the simulant slurries that were provided for shear-strength testing were mixed thoroughly and subsequently allowed to settle for at least 48 to $72 \mathrm{hr}$. When possible, the shear strength was measured by immersing the $16-\times 16-\mathrm{mm}$ vane tool to a depth of $15-\mathrm{mm}$ into the settled solids. The vane was slowly rotated at $0.3 \mathrm{rpm}$ for 180 seconds. For the entire duration of rotation, the time, rotational rate, and vane torque were continuously monitored and recorded. At the end of the measurement, shear stress versus time data were parsed, and the maximum measured shear stress (i.e., the material's shear strength) was determined.

\subsubsection{Flow-Curve Testing}

Each flow curve was measured over an 11-min period and split into three intervals. Over the first 5-min., the shear rate was smoothly increased from zero to $1,000-\mathrm{s}^{-1}$. For the next min, the shear rate was held constant at 1,000-s ${ }^{-1}$. For the final 5-min., the shear rate was smoothly reduced back to zero. During this time, the resisting torque and rotational rate were continuously monitored and recorded. 
Results are provided in the Test Data Packages listed below, with summary results provided in Appendix L.

- Shakedown/Functional testing TDP-WTP-353.

\subsubsection{Particle-Size Distribution}

Particle sizes were characterized according to procedure RPL-COLLOID-01, Rev. 1, Particle Size Analysis Using Malvern MS2000. This procedure uses a Mastersizer 2000 (Malvern Instruments, Inc., Southborough, MA 01772 USA) with a Hydro S wet dispersion accessory. Malvern lists the Mastersizer particle-size measurement range as nominally 0.02 to $2000-\mu \mathrm{m}$. The actual PSD measurement range is dependent on the accessory used as well as the properties of the solids being analyzed. The Malvern 2000 uses laser diffraction technology to define PSD.

The Hydro S wet-dispersion accessory consisted of a 150-mL dispersion unit coupled with a sample flow cell with a continuous variable and independent pump and stirrer and ultrasound. The flow, stirring rate, and sonication can be controlled and altered during measurement. PSD measurements can be made before, during, and after sonication, allowing the influence of each on the sample PSD to be determined. The primary measurement functions of the Malvern analyzer were controlled through Mastersizer 2000 software (Malvern Instruments, Ltd. Copyright 1998-2002). The properties applied to the test samples are summarized in Table E.3.

The PSD measurements were conducted in either DIW or in a $0.01-\mathrm{M} \mathrm{NaOH}$ dispersion solution matrix, depending on the sample being analyzed. The sample dispersion was added drop-wise to the dispersion unit (while the pump and stirrer were active) until an obscuration in the range of 10 to $20 \%$ was reached.

Table E.3. Properties Applied to Group 8 Test Materials

\begin{tabular}{cc}
\hline \multicolumn{2}{c}{ Property } \\
\hline $\begin{array}{c}\text { Material selected for optical } \\
\text { properties }\end{array}$ & Ferric Oxide Hydroxide \\
Refractive index (RI) & 2.94 \\
Absorption & 0 \\
Analysis mode & General purpose \\
Sensitivity & normal \\
Suspending phase & Water/0.01-M NaOH \\
\hline
\end{tabular}

The size distributions of particles were measured under varying flow conditions before and after sonication. For each condition, multiple measurements of PSD were taken. The analyzer software then generated an average of these measurements. Both the individual measurement and average were saved to the analyzer data file.

\subsubsection{X-Ray Diffraction}

The sample mounts for XRD examination were prepared by first cleaning the solids. This procedure included centrifuging the solids into a pellet and decanting the solute. Fresh washing solution was added to the pellet, and it was resuspended. The pellets in solution were vortexed to reconstitute them into the 
solution, and the centrifuging procedure was repeated three times. Following the final centrifuging and decant, the remaining pellet was left to dry in a $105^{\circ} \mathrm{C}$ oven overnight. The pellet was then pulverized to a powder with a tungsten carbide milling chamber for 1-min. in the Angstrom shaker mill, mixed with an internal standard (rutile, $\mathrm{TiO}_{2}$, or alumina, $\mathrm{Al}_{2} \mathrm{O}_{3}$ ), milled for another 2-min. to make sure that the two powders were a homogenous mixture, and then mounted into an off-axis, zero background, quartz sample holder. The XRD examination was conducted according to procedure APEL-PAD-V, Operation of Scintag Pad-V X-Ray Diffractometer. The XRD instrument used for these samples was the PNNL Scintag PAD V XRD (property number WD33356), located in Laboratory 102 in the APEL building. The data range for the sample was $5^{\circ}$ to $80^{\circ} 2 \theta$, with a step size of $0.02^{\circ} 2 \theta$ and count time of 2.0 seconds per step. Copper K $\alpha$ X-rays were used. The x-ray tube operating conditions were $45 \mathrm{kV}$ and 40-mA. Phase identification was done with the JADE search match routines (Version 6.0, Materials Data Inc.) with comparison to the International Centre for Diffraction Data (ICDD) database PDF-2, release 1999, which includes the Inorganic Crystal Structure Database (ICSD) maintained by Fachinformationszentrum (FIZ), Karlsruhe, Germany. The chemistry provided for Group 7, in order of decreasing concentration, was $\mathrm{Fe}, \mathrm{Na}, \mathrm{U}, \mathrm{P}, \mathrm{Ca}, \mathrm{Al}, \mathrm{Si}, \mathrm{Bi}, \mathrm{Sr}$, and $\mathrm{Mg}$. Phase identifications were first done without chemistry restrictions. Searches were restricted to the PDF and ICSD inorganic sections.

The pattern was also examined with RIQAS (release 4.0.0.26, 6/10/2002, Materials Data Inc.) Rietveld analysis software. The phases identified above were input into the analysis along with a polynomial background and an amorphous hump at $\sim 35^{\circ} 2 \theta$.

Details of the XRD analysis and results are provided in Test Data Package, TDP-WTP-353, with summary results provided in Appendix L.

\subsubsection{Scanning Electron Microscopy}

Within the same sample processing as above for the XRD sample preparation, just before the supernatant was decanted, the specimen was vortexed and a small volume of slurry was drawn up using a pipette and placed on an aluminum stub. The slurry was placed in an oven at $105^{\circ} \mathrm{C}$ to dry overnight. The sample was then coated with gold-palladium using a Polaron Range plasma sputter coater and analyzed with a JEOL SEM (property number WD30596) according to APEL-102-SEM, Scanning Electron Microscope Examination. Selected sample areas were evaluated by x-ray energy dispersive spectroscopy (EDS) for qualitative elemental composition.

Results are provided in the Test Data Package, TDP-WTP-352, provided in Appendix L.

\subsubsection{Heat Capacity}

Approximately eight samples at key process steps were collected and analyzed for heat capacity during Integrated Tests A and B. Heat capacity analyses were NOT conducted on samples taken during Shakedown/Functional testing. 
Appendix F PEP Data Acquisition System 



\section{Appendix F: PEP Data Acquisition System}

The data acquisition system (DAS) for PEP is composed of four data acquisition servers and one graphical user interface (GUI) PC. They are networked together, along with the rest of the PEP equipment as depicted below.

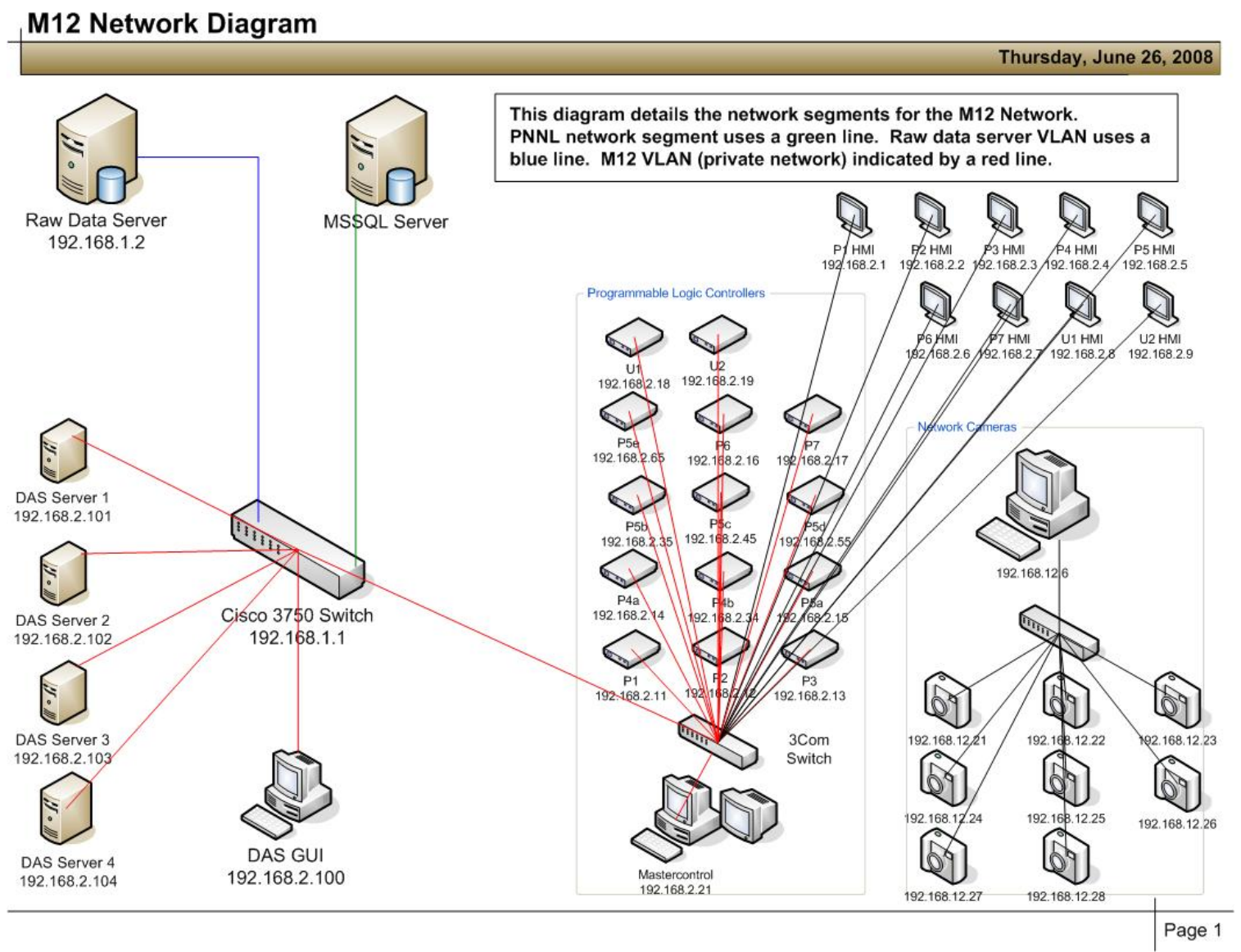

The GUI PC resides in the control room and is used to start and stop the data acquisition servers and set the recording rate. Each server that is located out in the high bay is independent of the others while collecting data. A global positioning system (GPS) receiver is used to provide a time stamp to each DAS server.

\subsection{Background}

The PEP data acquisition and control system was designed to be very robust and flexible in operation. While the same sensors are used for controlling the PEP and recording data, the sensor outputs are split into two loops to make certain that the DAS is completely separate from the control system. This requirement was put into the system to allow changes to the control system of the PEP to be performed without the rigorous Verification and Validation process, which is required of any quality-affecting software program. This allowed many changes to the control system to be made during operation of the 
PEP system without affecting the quality of the data. The DAS and all related software did undergo PNNL software control procedures to make certain that the data meet quality standards.

From each signal splitter, one loop went to the control system and the other went to the DAS. 4-20 ma outputs from all sensors were selected to confirm the reliability of the system and prevent long cable runs from introducing error in the signals. The data acquisition servers are manufactured by Microstar Laboratories, located in Bellevue, Washington. Each server is capable of storing a large number of channels at a high sampling rate. For PEP, they have been set to a maximum sampling rate of $100 \mathrm{~Hz}$. Each server stores its information in a file local to that server. After data was collected, it was transferred to the raw data server and to the database server for analysis.

\subsection{Data Retrieval}

During operations of the PEP during Shakedown and Phase 1 testing, over a terabyte of data was recorded by the four data acquisition servers. The data are stored in binary files that are not human readable. Software was developed to provide analysts with an easy tool to retrieve the data they required by specifying the date/time, instruments, and sampling rate. This tool is composed of three main components: the data ingest server, the database itself, and the data retrieval tool. The data ingest server watches a specified directory on the database server and when new files are put there it uploads the data from that location into the database and transfers those data files into a directory where processed files are stored. The database itself utilizes Microsoft's SQL server as a basis for the large database that stores all data and sensor information for the PEP. Finally, the data retrieval tool is a web-based application that allows staff to recover the data they need by specifying the time/date, instruments, and sampling rate necessary. The software produces a text file that can easily be imported into Excel or any other analysis package. This method provides a data file that meets quality standards for the PEP project.

Another method for retrieving data was commonly referred to the DAS widget. This tool is a simple application that allows a user with detailed knowledge of the system to retrieve data if they do not have access to the web-based tool, or if they need access to the data before the data has been imported into the database. This tool did not go through the software control procedure and does not provide data that meets quality standards for PEP. It will read a file from one server and produce a file with all the raw (ma) values from every instrument on that server. With that data, and the information in the M\&TE list, staff can retrieve the data from all sensors in engineering units. It is more time-consuming to retrieve data in this manner, but it allows data to be read in near real time. This method was commonly used to provide near real-time data for PJM tuning.

\subsection{Data Storage}

All data recorded during operation of the PEP was first copied to the raw data server in PDL-W and then copied to the database server in LSB. This procedure is detailed in the data management plan and provides redundant storage of all data to make certain that data are not lost. 
Appendix G

\section{Evaluation of PJM Peak Average Velocity}





\section{Appendix G: Evaluation of PJM Peak Average Velocity}

\subsection{Introduction}

A number of Microsoft Excel macros were developed in the Visual Basic language to evaluate the peak average velocity and average PJM stroke length. The definitions of the peak average velocity and stroke length and the background theory of evaluating these quantities are given in Section G.2. In Section G.3, the Excel macros developed to evaluate the peak average velocity and stroke length, and their implementation procedures are described. Finally, the loss coefficients used for the nozzle velocity evaluation from the PJM tube pressures are presented in Section G.4.

\subsection{Background Theory}

This section defines the peak average velocity and stroke length, and describes the methods used to evaluate these quantities.

\subsubsection{Definitions of Peak Average Velocity and Stroke Length}

In the PJM operation, steady periodic variation in PJM tube pressure drives flow through the PJM nozzle, which results in cyclic vertical motion of the liquid level in the PJM tube and the PJM tank. Typical nozzle velocity and PJM tube level profiles as a function of time for a complete operating cycle are shown in Figure G.1 and Figure G.2, respectively.

From Figure G.1, the peak average time $t_{\text {peak }}$ and the discharge time $t_{\text {disch arge }}$ are defined as:

$$
\begin{gathered}
t_{\text {peak }}=t_{\text {end }}-t_{\max } \\
t_{\text {disch arge }}=t_{\text {end }}-t_{o}
\end{gathered}
$$

where $t_{o}$ is the start of pressurization (start of drive cycle), $t_{\text {end }}$ is the end of pressurization (nozzle velocity begins to decrease rapidly), and $t_{\max }$ is the time at maximum discharge.

The peak average velocity $\bar{U}_{\text {peak }}$ for the discharge process is defined as:

$$
\bar{U}_{\text {peak }}=\frac{1}{t_{\text {peak }}} \int_{t_{\max }}^{t_{\text {end }}} U(t) d t
$$

where $U(t)$ is the nozzle velocity. 


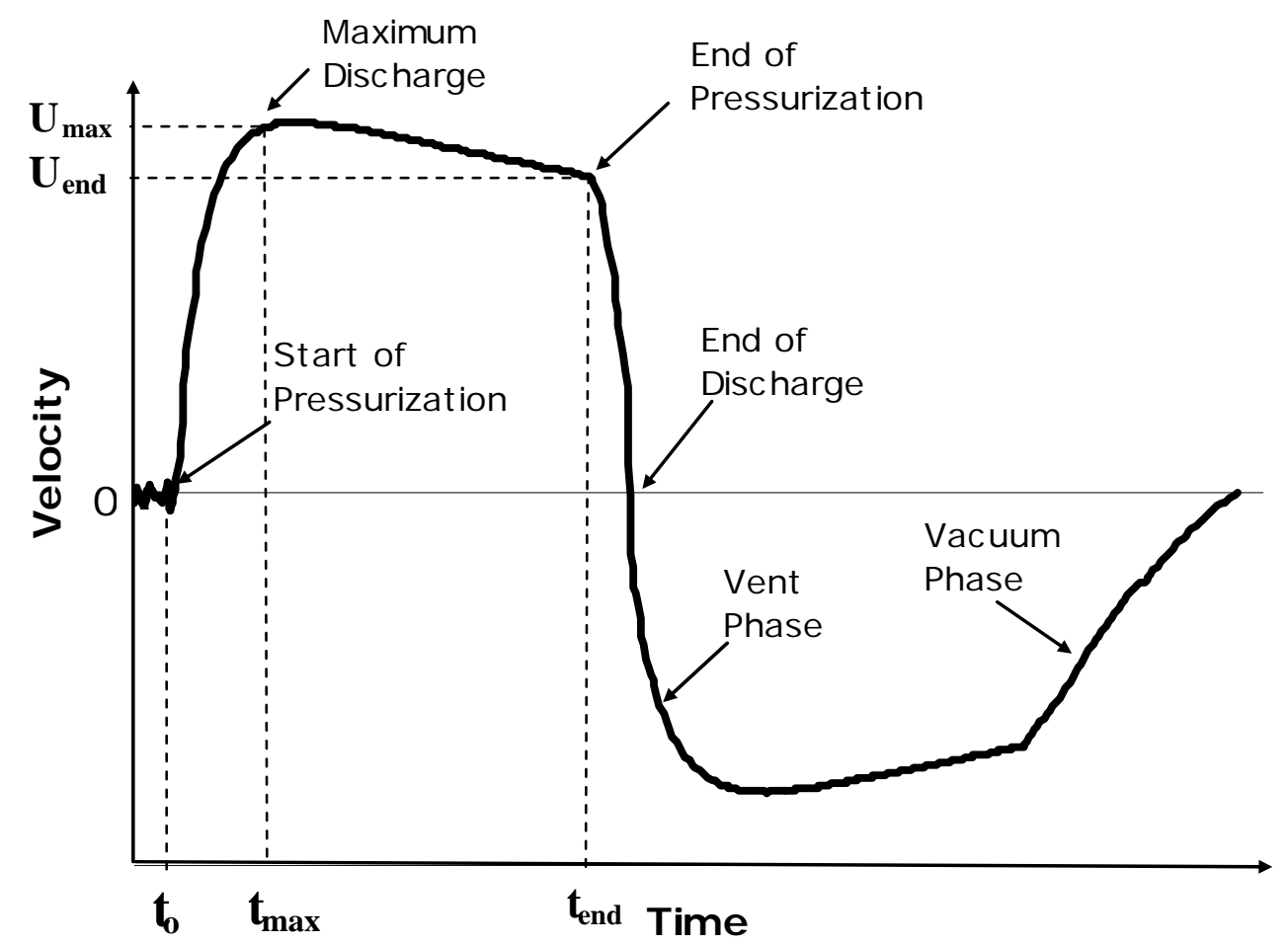

Figure G.1. Typical Nozzle Velocity Time Distribution

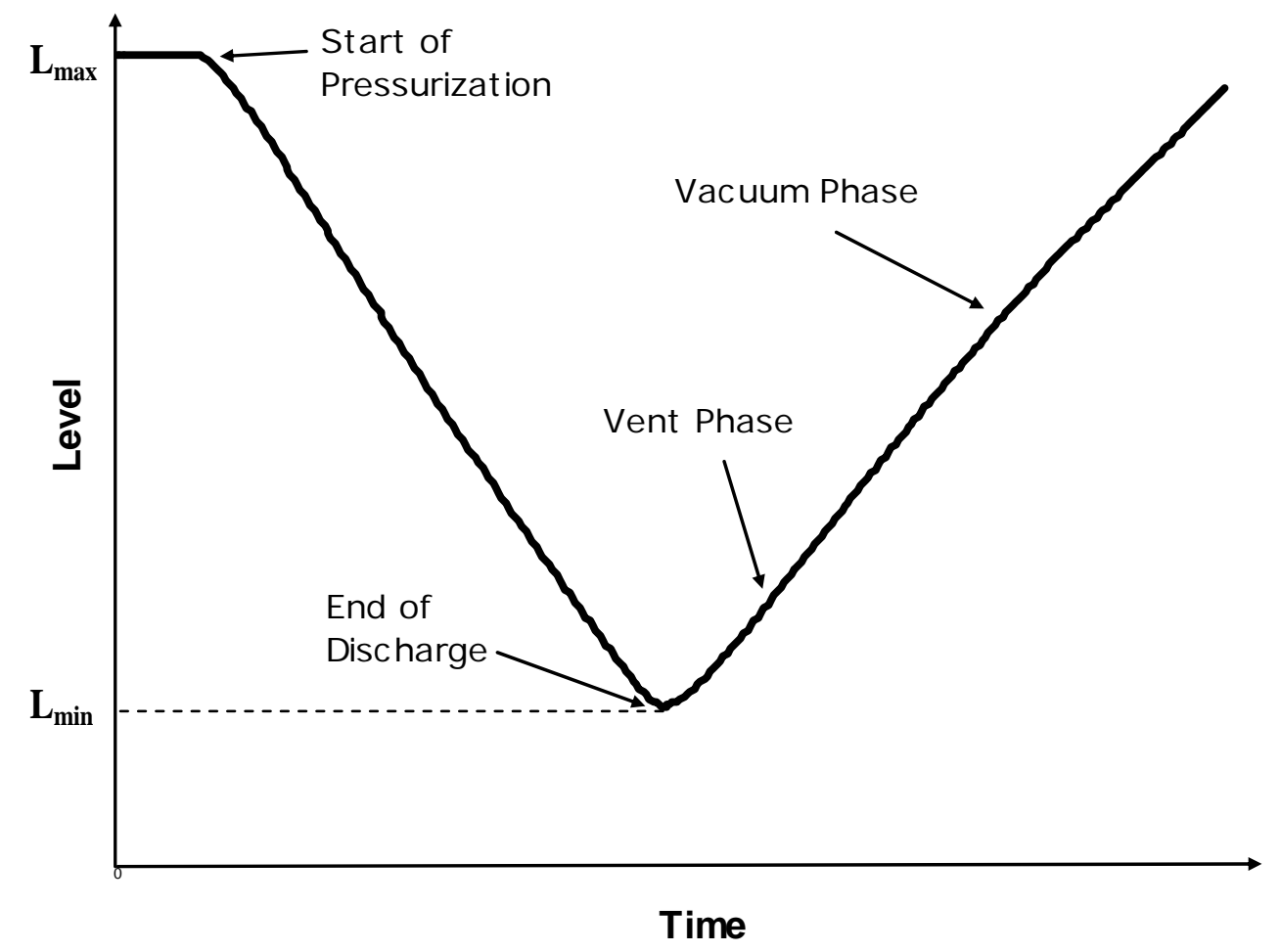

Figure G.2. Typical PJM Tube Level Time Distribution 
The nozzle velocity $U(t)$ is evaluated from the measured PJM tube liquid level and the PJM tank level, and the PJM tube pressure. Calculation of the nozzle velocity $U(t)$ is described in section G.3 in detail. The Trapezoidal rule is used for the numerical integration of Equation (G.3).

From Figure G.2, the PJM tube stroke length $L_{S T R}$ is defined as:

$$
L_{S T R}=L_{\max }-L_{\min }
$$

where $L_{\max }$ is the PJM tube level at start of pressurization, and $L_{\min }$ is the PJM tube level at the end of discharge.

Along with Equation (G.4), the PJM tube stroke length percentage $L_{S T R P}$ is defined as:

$$
L_{\text {STRP }}=\frac{L_{\text {STR }} \cdot A_{\text {tube }}}{V}
$$

where $A_{\text {tube }}$ is the PJM tube cross-sectional area, and $V$ is the PJM tube volume. Namely, the PJM tube stroke length percentage $L_{\text {STRP }}$ is defined as the ratio of the volume of the PJM liquid displacement to the entire PJM tube volume.

\subsection{Methods}

The methods used to evaluate the nozzle velocity $U(t)$ from the level and pressure data are described in this section.

\subsubsection{Nozzle Velocity Evaluation from PJM Tube and Tank Liquid Level Data}

Significantly large fluctuations were contained in the level data measured for the PJM tubes and tanks, due to the hydrodynamics of the mixing operation. To smooth out these fluctuations and capture the effective level motion in an operating cycle, the level measurements were averaged. The measured level data was averaged over multiple sequential sampling cycles (typically 10 cycles) to obtain the average cyclic level distribution for each tube, or for the PJM tank. In addition, the averages for each PJM tube were combined to obtain the average cyclic level distribution for all of the PJM tubes. Prior to the data averaging, the PJM tank level data were filtered to remove the outliers.

For the case in which averaging was insufficient to remove fluctuations, the averaged liquid level distribution was further smoothed by applying the $6^{\text {th }}$ power binomial coefficients $(1,6,15,20,15,6$, 1)/64 for smoothing of the interior data points, the $3^{\text {rd }}$ power binomial coefficients $(1,4,6,4,1) / 16$ for smoothing at the $3^{\text {rd }}$ exterior data point, the $2^{\text {nd }}$ power binomial coefficients $(1,2,1) / 4$ for smoothing at the $2^{\text {nd }}$ exterior data point.

The averaged liquid surface levels with or without smoothing were used to evaluate the nozzle velocity $U$ as: 


$$
U=\left(\frac{A}{A_{n}}\right) \frac{d L}{d t}
$$

where

$$
\begin{aligned}
A & =\text { averaged liquid level } \\
A / A_{n} & =\text { area ratio } \\
A & =\text { area of the PJM tube or the PJM tank liquid surface } \\
A_{n} & =\text { area of the PJM nozzle. }
\end{aligned}
$$

The finite central difference method is used to evaluate $\frac{d L}{d t}$.

\subsubsection{Nozzle Velocity Evaluation from Tube Pressure Data}

The average of the PJM tube pressure data was taken prior to the nozzle velocity evaluation, using the same approach as described above for averaging of the level measurements. The averaged cyclic distribution in pressure was used to evaluate the nozzle velocity by applying the Bernoulli equation with the pressure loss effect taken into account.

The Bernoulli's equation taking account of the pressure loss coefficient is given as:

$$
P_{t}+\rho g\left(L_{i n i}-x-H\right)-P_{H S P}=\frac{1}{2} \rho\left[\left(1+k_{f}\right)\left(\frac{R}{R_{n}}\right)^{4}-1\right] \dot{x}^{2}
$$

where

$$
\begin{aligned}
\dot{x} & =\text { liquid velocity in the PJM tube } \\
x & =\text { length of the displaced liquid inside the PJM tube } \\
P_{t} & =\text { averaged PJM tube pressure } \\
P_{H S P} & =\text { headspace pressure of the PJM tank } \\
L_{i n i} & =\text { initial liquid level height inside the PJM tube } \\
H & =\text { liquid level height inside the tank } \\
\rho & =\text { liquid density } \\
g & =\text { gravitational acceleration } 9.81\left(\mathrm{~m} / \mathrm{s}^{2}\right) \\
k_{f} & =\text { empirically determined loss coefficient } \\
R & =\text { PJM tube radius } \\
R_{n} & =\text { nozzle radius. }
\end{aligned}
$$

The coefficient $\left(\frac{R}{R_{n}}\right)^{2}$ in Equation (G.7) is exactly equivalent to the area ratio defined in Equation (G.6) for the ratio of the pulse tube area to the pulse tube nozzle area. The parameters $x, P_{t}$, $P_{H S P}, L_{i n i}$, and $H$ used for Equation (G.7) are illustrated in the schematic diagram of Figure G.3. 
Along with Equation (G.7), the nozzle velocity $U$, the length of the displaced liquid $x$, the liquid level height of the tank $H$ are evaluated as:

$$
\begin{gathered}
U=\left(\frac{R}{R_{n}}\right)^{2} \dot{x} \\
x=x_{\text {old }}+\Delta t \dot{x} \\
H=H_{\text {ini }}+\left(\frac{A_{P L}}{A_{T L}}\right) x
\end{gathered}
$$

where $\Delta t=$ sampling time

$X_{\text {old }}=$ length of the displaced liquid at the previous pressure measurement time

$H_{\text {ini }}=$ initial tank liquid level height

$A_{P L}=$ total liquid surface area of all of the PJM tubes

$A_{T L} \quad$ tank liquid surface area.

To solve Equation (G.7) numerically, the initial liquid level height inside the PJM tube $L_{\text {ini }}$ is needed as a starting point. This can be determined by imposing the zero initial velocity condition for the given initial tank liquid level height $H_{i n i}$ as:

$$
L_{i n i}=\left(\frac{P_{H S P}-P_{t i}}{\rho g}\right)+H_{i n i}
$$

where $P_{t i}$ is the averaged PJM tube pressure at the pressurization start point of the PJM pressure cycle process (see Figure G.1). 


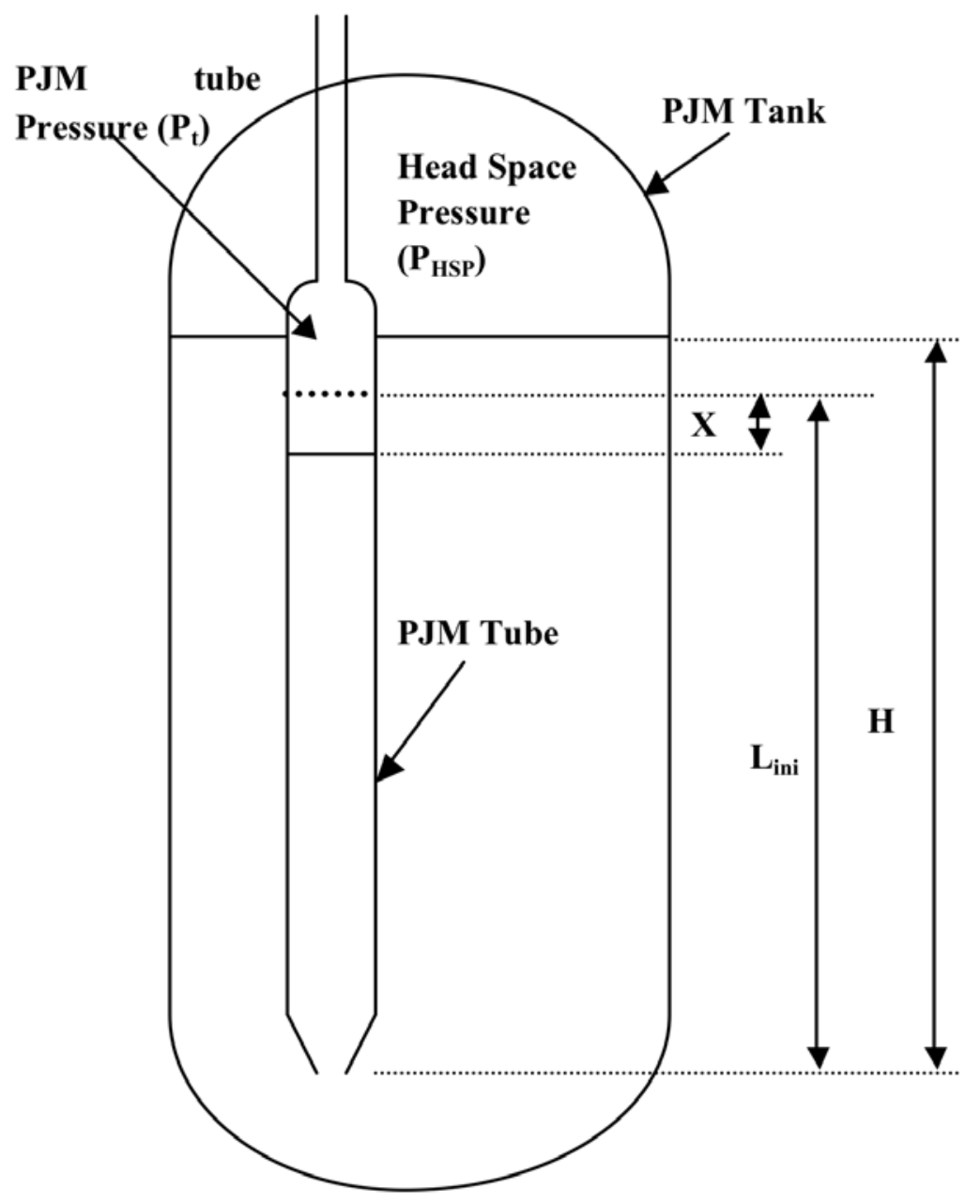

Figure G.3. Schematic of PJM Tank and Tube

The loss coefficients were obtained by iterating to a "best fit" of the nozzle velocity distribution evaluated by applying Equation (G.7) through Equation (G.11), compared to the distribution determined from the tank level data measured by a laser anemometer.

\subsection{Loss Coefficient Evaluations}

To evaluate the nozzle velocity time distribution from the PJM pressure data with Equation (G. 7) through Equation (G.11), the empirically determined loss coefficients were needed. The loss coefficients were obtained by iterating to a "best fit" of the nozzle velocity distribution evaluated from the pressure data, compared to the distribution determined from the tank level data measured by a laser anemometer.

This section presents the loss coefficients evaluated in the RPP-WTP PEP testing in Table G.1. The plots used for the evaluations of the loss coefficients are given in Figure G.4 through Figure G.7. 
Table G.1. Loss Coefficients Evaluated

\begin{tabular}{cccc}
\hline Tank & $\begin{array}{c}\text { Loss Coefficient for } \\
\text { Forward Flow }\end{array}$ & $\begin{array}{c}\text { Loss Coefficient for } \\
\text { Backward Flow }\end{array}$ & $\begin{array}{c}\text { Target Velocity } \\
(\mathrm{m} / \mathrm{s})\end{array}$ \\
\hline UFP-VSL-T01A & 0.25 & 1.2 & 4.8 \\
UFP-VSL-T01B & 0.38 & 1.3 & 4.8 \\
UFP-VSL-T02A & 0.34 & 0.1 & 7.3 \\
UFP-VSL-T02A & 0.1 & 0.1 & 12 \\
\hline
\end{tabular}

T01A: $K_{-} f=0.25 \& K_{-} b=1.2$ for $4.9(\mathrm{~m} / \mathrm{s})$

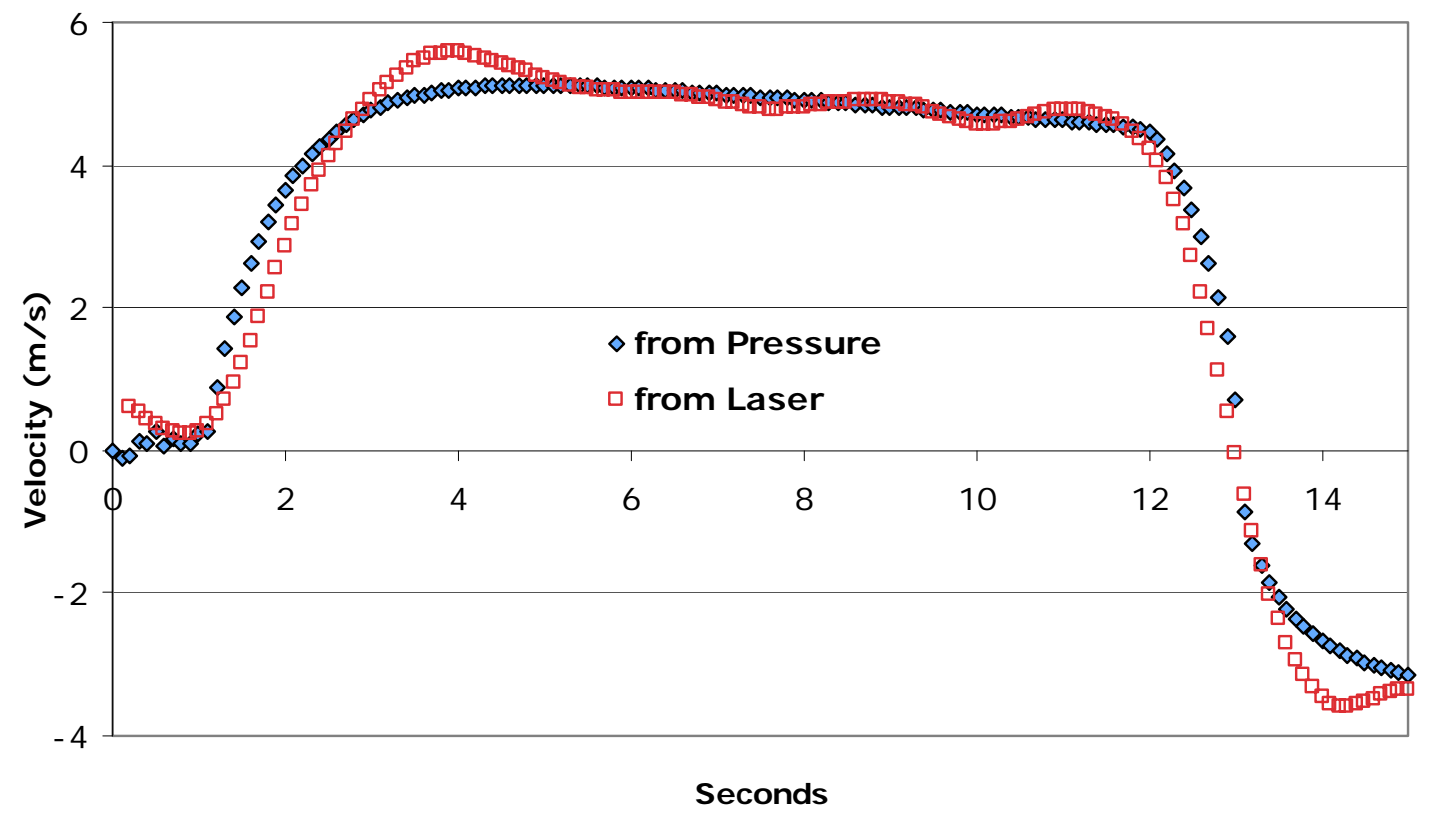

Figure G.4. Nozzle Velocity Distributions Used to Evaluate the Loss Coefficients for UFP-VSL-T01A Tank at the Peak Average Velocity of $4.9(\mathrm{~m} / \mathrm{s}) ; \mathrm{k}_{-} \mathrm{f}=0.25$ is the loss coefficient of the forward flow, and $\mathrm{k}_{-} \mathrm{b}=1.2$ is the loss coefficient of the backward flow. 


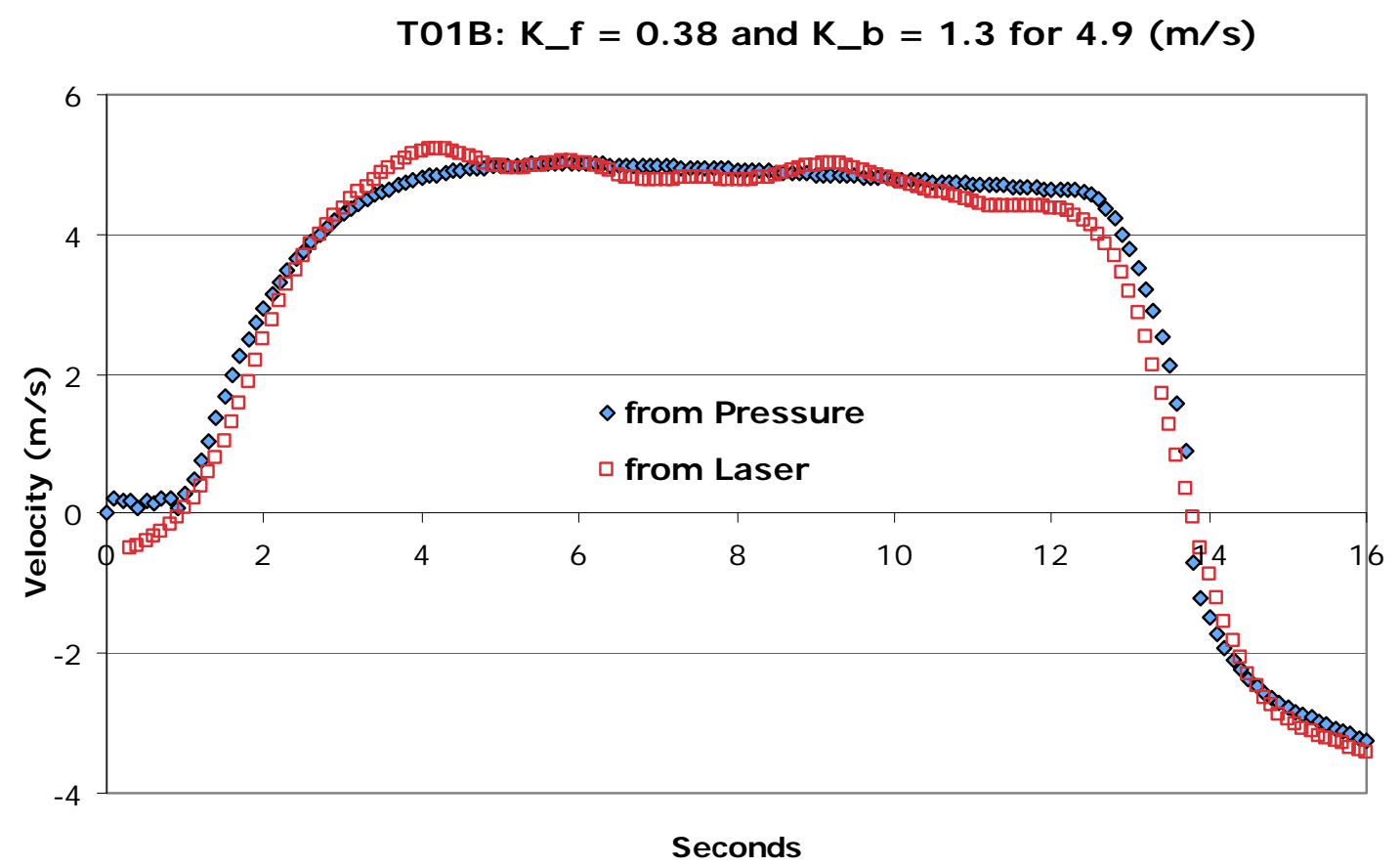

Figure G.5. Nozzle Velocity Distributions Used to Evaluate the Loss Coefficients for UFP-VSL-T01B Tank at the Peak Average Velocity of $4.9(\mathrm{~m} / \mathrm{s}) ; \mathrm{k}_{-} \mathrm{f}=0.38$ is the loss coefficient of the forward flow, and $\mathrm{k} \_\mathrm{b}=1.3$ is the loss coefficient of the backward flow. 


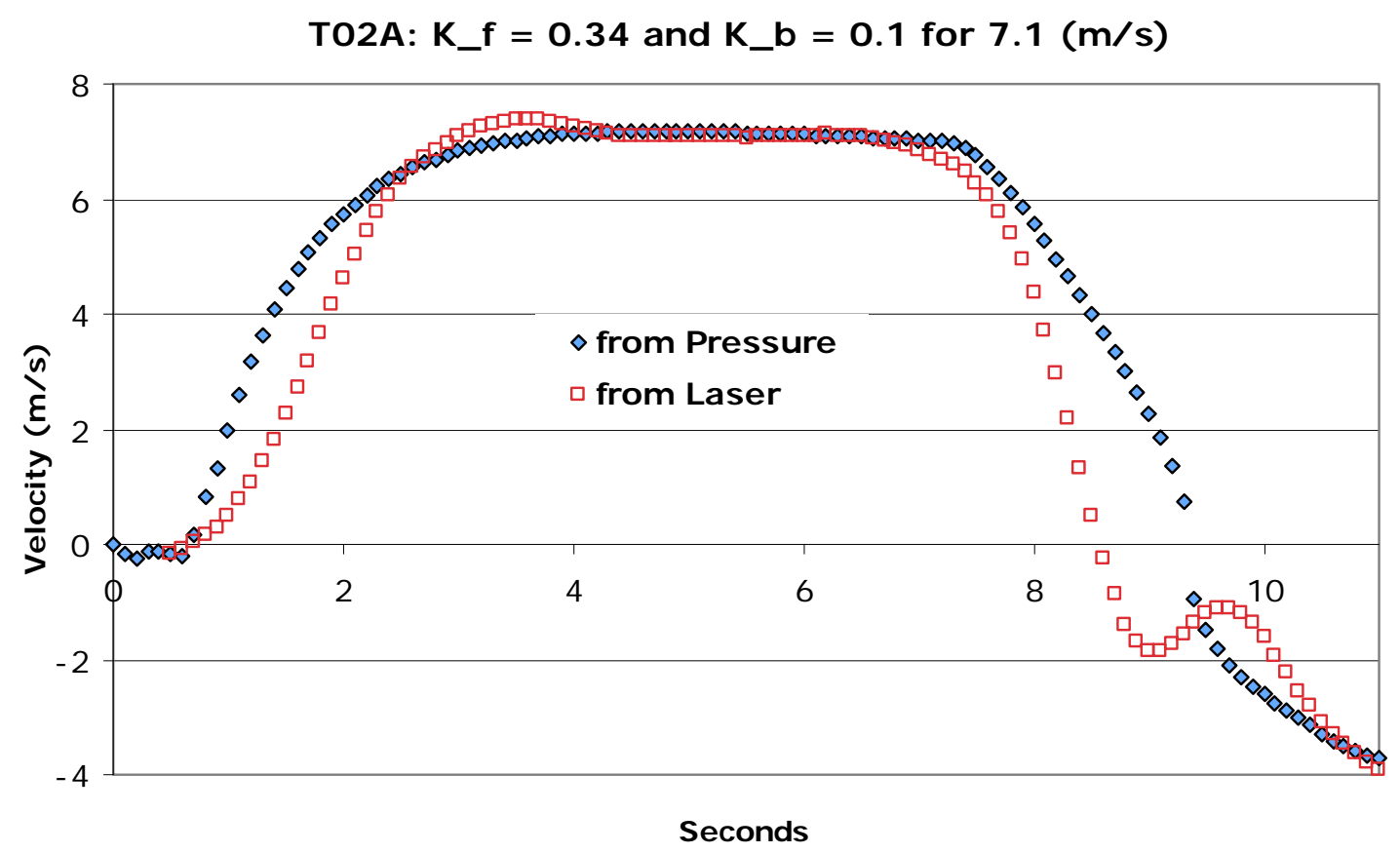

Figure G.6. Nozzle Velocity Distributions Used to Evaluate the Loss Coefficients for UFP-VSL-T02A Tank at the Peak Average Velocity of $7.1(\mathrm{~m} / \mathrm{s}) ; \mathrm{k}_{-} \mathrm{f}=0.34$ is the loss coefficient of the forward flow, and $\mathrm{k} \_\mathrm{b}=0.1$ is the loss coefficient of the backward flow.

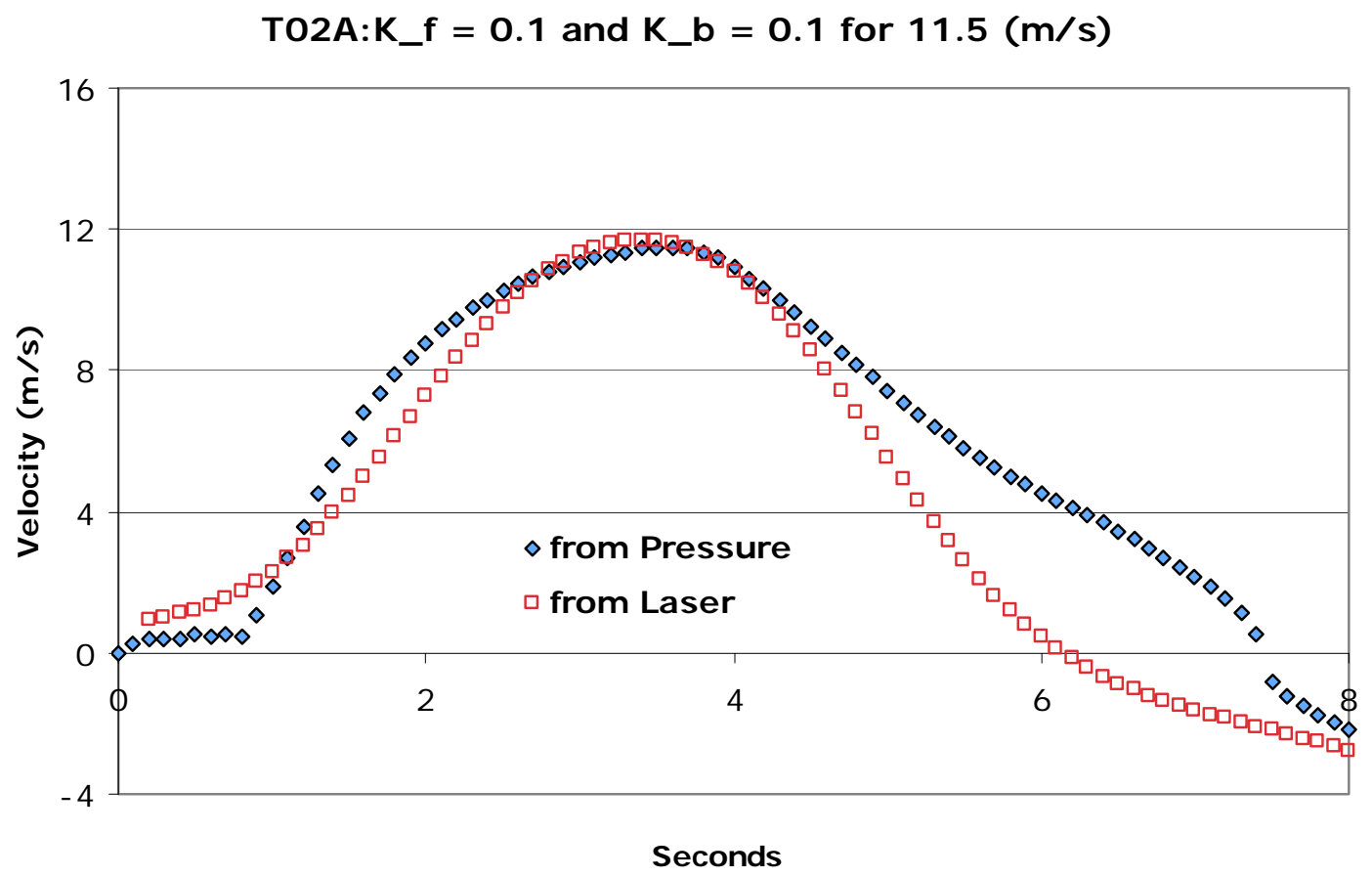

Figure G.7. Nozzle Velocity Distributions Used to Evaluate the Loss Coefficients for UFP-VSL-T02A Tank at the Peak Average Velocity of $11.5(\mathrm{~m} / \mathrm{s}) ; \mathrm{k}_{-} \mathrm{f}=0.1$ is the loss coefficient of the forward flow, and $\mathrm{k} \_\mathrm{b}=0.1$ is the loss coefficient of the backward flow. 



\section{Appendix $\mathrm{H}$}

\section{Oxalic Acid Cleaning Instructions for the PEP Filters}





\section{Appendix H: Oxalic Acid Cleaning Instructions for the PEP Filters}

\subsection{Filter Cleaning Before the Functional Test}

During water testing for the PEP the filter flux degraded to $<2-\mathrm{kg} / \mathrm{min}\left(0.044 \mathrm{gpm} / \mathrm{ft}^{2}\right)$ at $40 \mathrm{psig}$ and the JTG decided to clean the filters before Shakedown and Functional testing with the simulant. The first cleaning (1A) was performed on 11/07/2008. The instructions for the cleaning were based on laboratory-scale tests with the use of oxalic acid, detergent (Alconox), and subsequent rinses. The initial oxalic flush obtained a flux rate of $\sim 0.65 \mathrm{gpm} / \mathrm{ft}^{2}$ and was considered inadequate for proceeding. The acid clean was repeated followed by a detergent wash and inhibited water rinse. During the acid cleaning 30 backpulses of the filters were performed, after which UFP-T02A was drained along with the loop and the deadlegs. The tank was refilled with DIW and rinsed a couple of times. The results of the cleaning on $11 / 11 / 2008$ at $2200 \mathrm{hr}$ resulted in a permeate flux of $\sim 1.6 \mathrm{gpm} / \mathrm{ft}^{2}$. The tank was refilled with inhibited water after the DIW rinse and the permeate rates decreased to $0.5 \mathrm{gpm} / \mathrm{ft}^{2}$. Samples were taken and visually inspected. The inspection showed some solids in the solution that were not seen in the acidic rinse solutions and were believed to be iron hydroxide. The loop was drained and refilled with inhibited water. On 11/13/2008 and 11/14/2008, additional permeate rates were measured using inhibited water and the permeate flux rates decreased again. The filtration rates were low and a second cleaning process was prepared.

The next filter cleaning was started on 11/15/2008 at $2300 \mathrm{hr}$. Oxalic acid was added to T02A and recirculated through the loop. The filtration rates improved immediately and increased to $7-10-\mathrm{kg} / \mathrm{min}$ with low TMP ( $\sim 2 \mathrm{gpm} / \mathrm{ft}^{2}$ at $\left.40 \mathrm{psig}\right)$. The acid wash was followed by $4-5$ complete rinses using DIW that passed through the empty nitric acid tank and into T02A. The T02A vessel was drained before the rinse water was added, but a small heel remained. The $\mathrm{pH}$ of the solution in the tank and the loop rose to $\sim 3.5$ after several rinses. The cleaning was completed on 11/18/2008 and the loop was left with inhibited water. The filter flux rate at the end of the IW wash had dropped to $0.25 \mathrm{gpm} / \mathrm{ft}^{2}$.

Another oxalic acid cleaning was performed on 11/21/2008 with the intent of leaving the final solution at a low $\mathrm{pH}$ to avoid iron hydroxide precipitation. Instructions for the oxalic cleaning conducted immediately before Shakedown/Functional testing are included below.

\section{Third Cleaning Sequence of the UFP System}

\section{Cleaning the Ultrafilters:}

1. Drain the recirculation loop (UFP-VSL-T02A and both sides of the filters, heat exchangers, piping, and deadlegs) to UFP-VSL-62B or as directed by the LTE.

2. Ensure the 19-M NaOH line to the filter-loop has been charged and flushed. 
3. Prepare $\sim 200$ gal of $\sim 0.5-\mathrm{M}$ oxalic acid solution in NAR-VSL-T01. Add $\sim 200$ gal of oxalic acid from the tote to NAR-VSL-T01. Measure oxalic quantity by tank level change.

4. Start PJMs.

5. Align recirculation loop for flow through all five filters and both heat exchangers. Align system with permeate flow back to UFP-VSL-T02A.

6. Start filtration according to procedures. Operate filter with $0.5-\mathrm{M}$ oxalic acid solution with recirculation back to UFP-VSL-T02A for at least 10-min. and then backpulse the filter at 30 times.

7. Run the filter-loop with the oxalic acid solution for $1 \mathrm{hr}$ after the 30 backpulses to obtain a stable filter flux. Record values every 20-min.

8. Drain UFP-VSL-T02A and entire loop to UFP-VSL-62B or as directed by LTE. Also drain the filters (both sides), piping, and the deadlegs of the oxalic acid solution.

9. Fill UFP-VSL-T02A with 180 gal of fresh RO water. Circulate the water through the filters for 15-min. and then backpulse filters 15 times. Obtain sample from in-line sampler immediately after last backpulse and check pH. pH should be about 2. Recirculate for another 15-min. and record filtration flux. Drain system, including tank, completely.

10. Fill UFP-VSL-T02A with 180 gal of $\mathrm{RO}$ water adjusted to have a $\mathrm{pH}$ of 3-3.5. Check to ensure correct $\mathrm{pH}$ in acid tank before transferring to UFP-VSL-T02A. Circulate the water through the filters for 30-min. to obtain a stable filter flux. Record stable filter flux rate.

11. Stop circulation and perform one more backpulse of all filters, or as directed by LTE.

12. Isolate filters and drain UFP-VSL-T02A and filter piping and heat exchangers.

The cleaning was completed on 11/22/2008 at $1300 \mathrm{hr}$. The T02A tank was filled with simulant on $11 / 22 / 2008$ at $0023 \mathrm{hr}$ as part of TI-062 and circulation through the loop started to neutralize. The filter flux did not drop off as drastically as previously, so the cleaning was considered successful and the TI continued.

After an extended break in testing (due to the repairs to the filters, replacement of Drexelbrook level probes, and Christmas holiday) another oxalic acid cleaning was conducted before the start of TI- 032 . The results of the first cleaning were not acceptable for all five filters so the oxalic acid cleaning was repeated and TI-032 filter conditioning proceeded. A time table of all the acid cleanings is shown below. 


\subsection{Timeline for Filter Cleaning}

\begin{tabular}{|c|c|c|c|}
\hline & Start Date & Finish Date & \\
\hline 1st cleaning 0.5-M oxalic & $11 / 07 / 20081600$ & 11/07/2008 2200 & No rinses. Permeate flow not adequate. \\
\hline \multirow[t]{6}{*}{ 1st cleaning repeated } & 11/11/2009 2200 & 11/15/2008 1345 & $\begin{array}{l}\text { Transferred } 72 \text { gal 2-M oxalic acid to } \\
\text { NAR-T01. }\end{array}$ \\
\hline & $11 / 11 / 20086100$ & & $\begin{array}{l}\text { Oxalic acid recirculation in T02A. Started } \\
\text { permeate flow. }\end{array}$ \\
\hline & $11 / 11 / 20082305$ & & Completed 30 backpulses. \\
\hline & $11 / 12 / 20082014$ & & Filtering and draining. \\
\hline & $11 / 15 / 20081100$ & $11 / 15 / 20081345$ & Acid wash complete. \\
\hline & $11 / 15 / 20081404$ & $11 / 15 / 20081630$ & $\begin{array}{l}\text { Transfer DIW from NAR to T02A } \\
\text { recirculating drain. }\end{array}$ \\
\hline \multirow[t]{5}{*}{ 2nd cleaning 0.5-M oxalic } & 11/15/2008 2259 & 11/18/2008 1001 & $\begin{array}{l}\text { Stopped 2nd cleaning sequence @ step } 8 \\
\text { after backpulse - no draining. }\end{array}$ \\
\hline & $11 / 15 / 20082259$ & $11 / 16 / 20081137$ & $\begin{array}{l}\text { Filled T02A, } 268 \text { gal oxalic \& rinsed with } \\
\text { DIW. }\end{array}$ \\
\hline & $11 / 16 / 20086000$ & & Drained T02A. 11/16 1345. \\
\hline & $11 / 16 / 20082000$ & & Drained T02A, refilled 250 gal. \\
\hline & $11 / 17 / 20082200$ & & $\begin{array}{l}\text { Added } 18 \text { gal of } 0.17-\mathrm{M} \mathrm{NaOH} \text { from } 19-\mathrm{M} \\
\text { tank to T02A. }\end{array}$ \\
\hline \multirow[t]{6}{*}{ 3rd cleaning Oxalic } & $11 / 21 / 20082200$ & $11 / 22 / 20081800$ & 1 oxalic acid wash and 1 DIW rinse. \\
\hline & $11 / 21 / 20082243$ & & $\begin{array}{l}\text { Transferred oxalic acid to NAR, Drain } \\
\text { T02A and the loop. }\end{array}$ \\
\hline & $11 / 22 / 20080150$ & & Filled T02A with oxalic acid. \\
\hline & $11 / 22 / 20082240$ & & Restarted filter-loop flow. \\
\hline & $11 / 22 / 20081223$ & & $\begin{array}{l}\text { Transferred } 200 \text { gal DIW from NAR to } \\
\text { T02A. }\end{array}$ \\
\hline & $11 / 22 / 20081800$ & & Drain T02A and the loop. \\
\hline \multirow[t]{2}{*}{$\begin{array}{l}\text { TI-62 started (Functional } \\
\text { Test) }\end{array}$} & $11 / 23 / 2008114$ & & Simulant to T02A transfer commenced. \\
\hline & $11 / 23 / 20080600$ & & Condition filters. \\
\hline \multirow[t]{2}{*}{ 4th cleaning oxalic acid } & $12 / 27 / 2008000$ & $12 / 28 / 20080640$ & \\
\hline & $12 / 28 / 20081226$ & & Drained loop. \\
\hline \multirow[t]{4}{*}{ 4th cleaning oxalic acid } & $12 / 28 / 20081226$ & $12 / 29 / 20092347$ & $\begin{array}{l}\text { Filter cleaning procedures repeated; } \\
\text { flux rate did not meet specified criteria. }\end{array}$ \\
\hline & $12 / 28 / 20081408$ & $12 / 28 / 20081624$ & Filter-loop flow started. \\
\hline & $12 / 29 / 20091120$ & & Started 1 st rinse. \\
\hline & $12 / 29 / 20092225$ & $12 / 29 / 20092347$ & Second rinse -219 gal $\mathrm{pH}<3$ \\
\hline Functional Testing cont. & $12 / 30 / 20082107$ & & $\begin{array}{l}\text { Filtration was initiated to fill the } \\
\text { filter-loop. }\end{array}$ \\
\hline
\end{tabular}





\section{Appendix I}

T02A Systems Operations Guidance for Simulant Shakedown/Functional Testing 



\section{Appendix I: T02A Systems Operations Guidance for Simulant Shakedown/Functional Testing}

During simulant Shakedown/Functional testing it was first observed that flow through the ultrafiltration loop could not be maintained when the level in the T02A vessel got below about 22 in. and also PJM overblows could cause a loss of target flow, $109 \mathrm{gpm}$. During subsequent Integrated process testing it was further elaborated that the root cause to losing flow at low tank levels was due to air entrainment, either from air sources such as PJM overblows or sparger air or perhaps from air entrained by the return line into the tank. For later, Integrated process testing guidance was developed to remove air from the system after air entrainment compromised the ability to maintain flow targets. Guidance to operate the flow loop after flow was lost due to air entrainment and guidance to remove entrained air are reported in WTP-RPT-192 and WTP-RPT-193. 

Appendix $\mathrm{J}$

Shakedown/Functional Data Plots 



\section{Appendix J: Shakedown/Functional Data Plots 11/21/2008 00:00-11/21/2008 12:00}

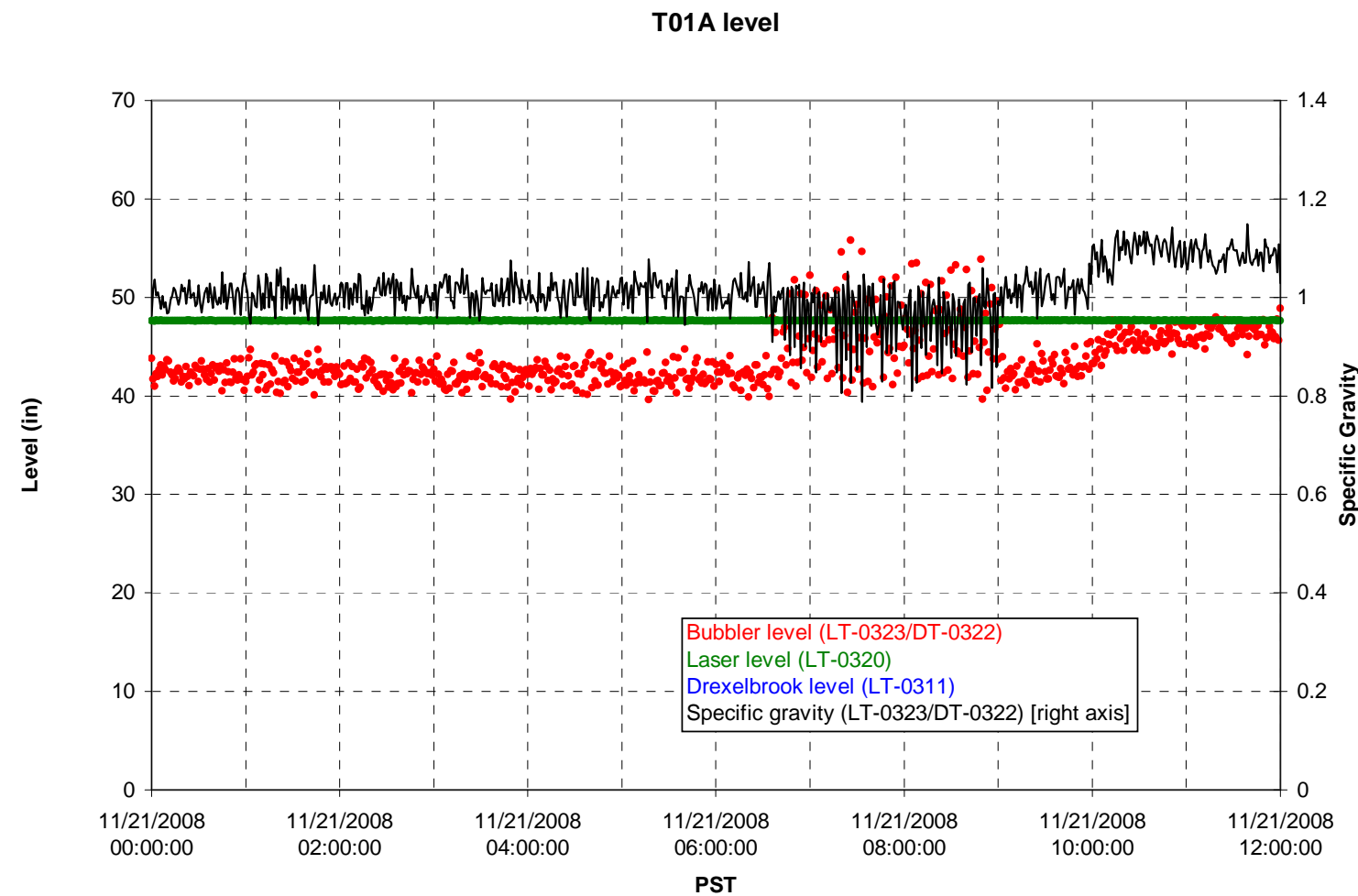

T01A temperatures

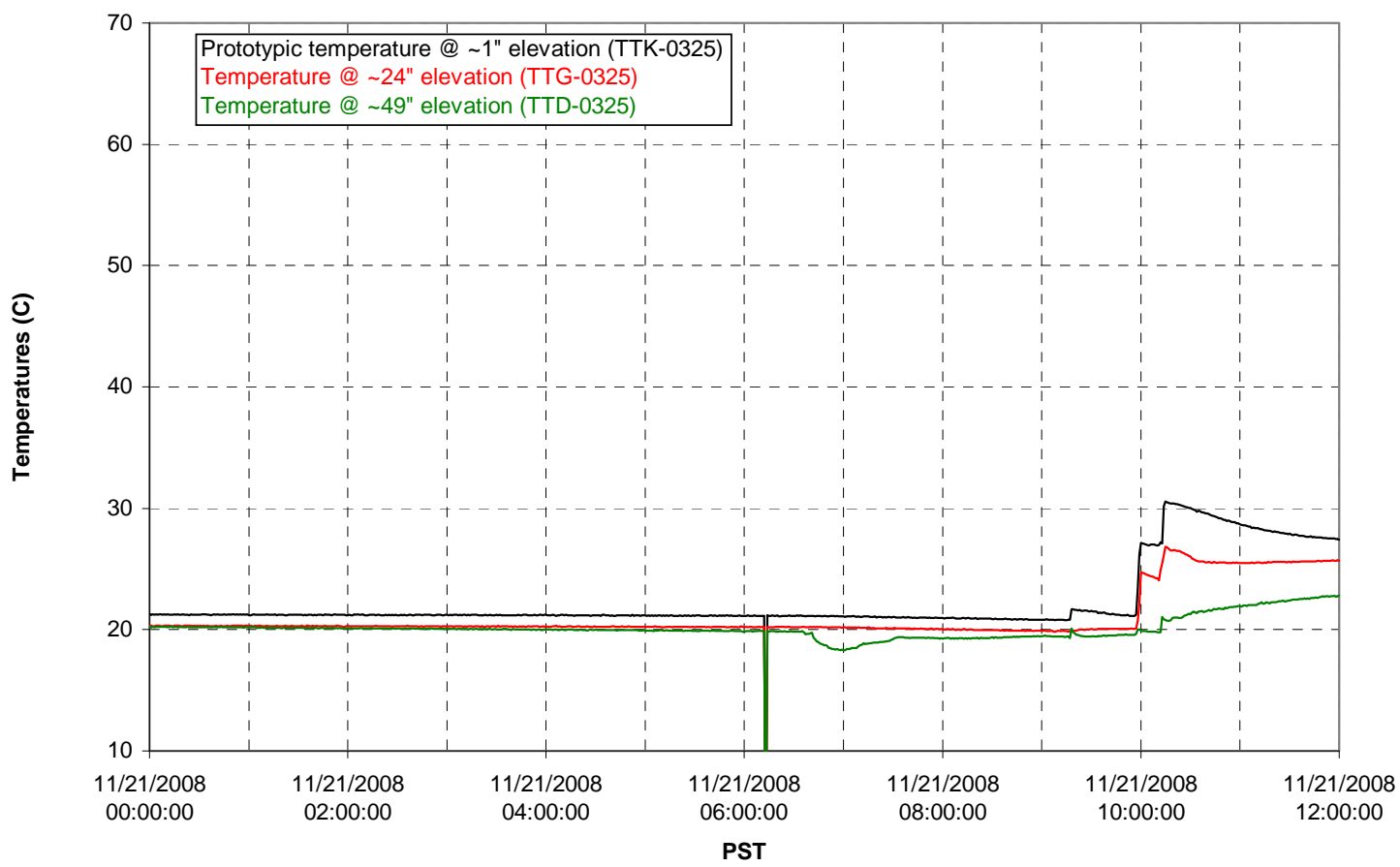


T01B level

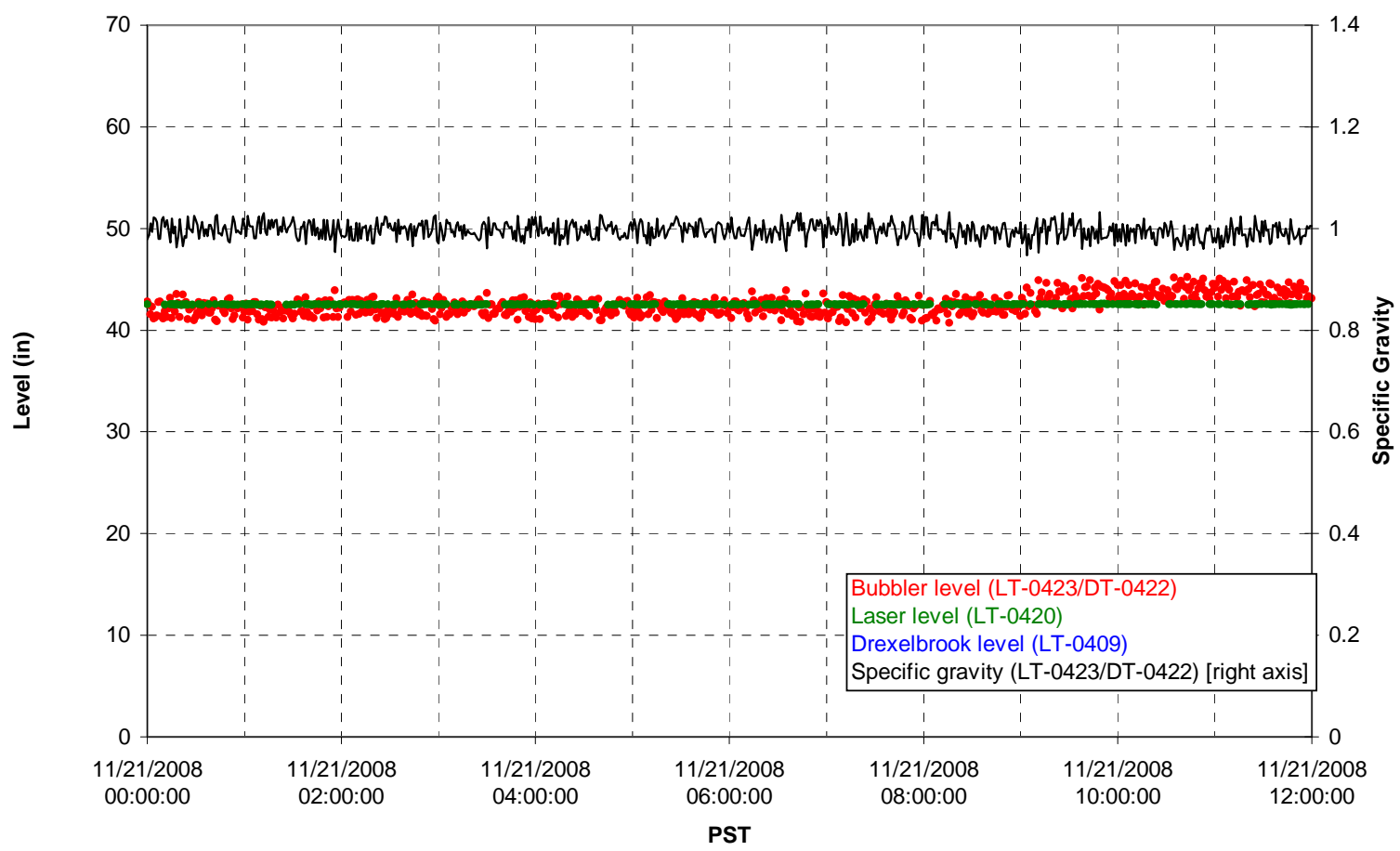

T01B temperatures

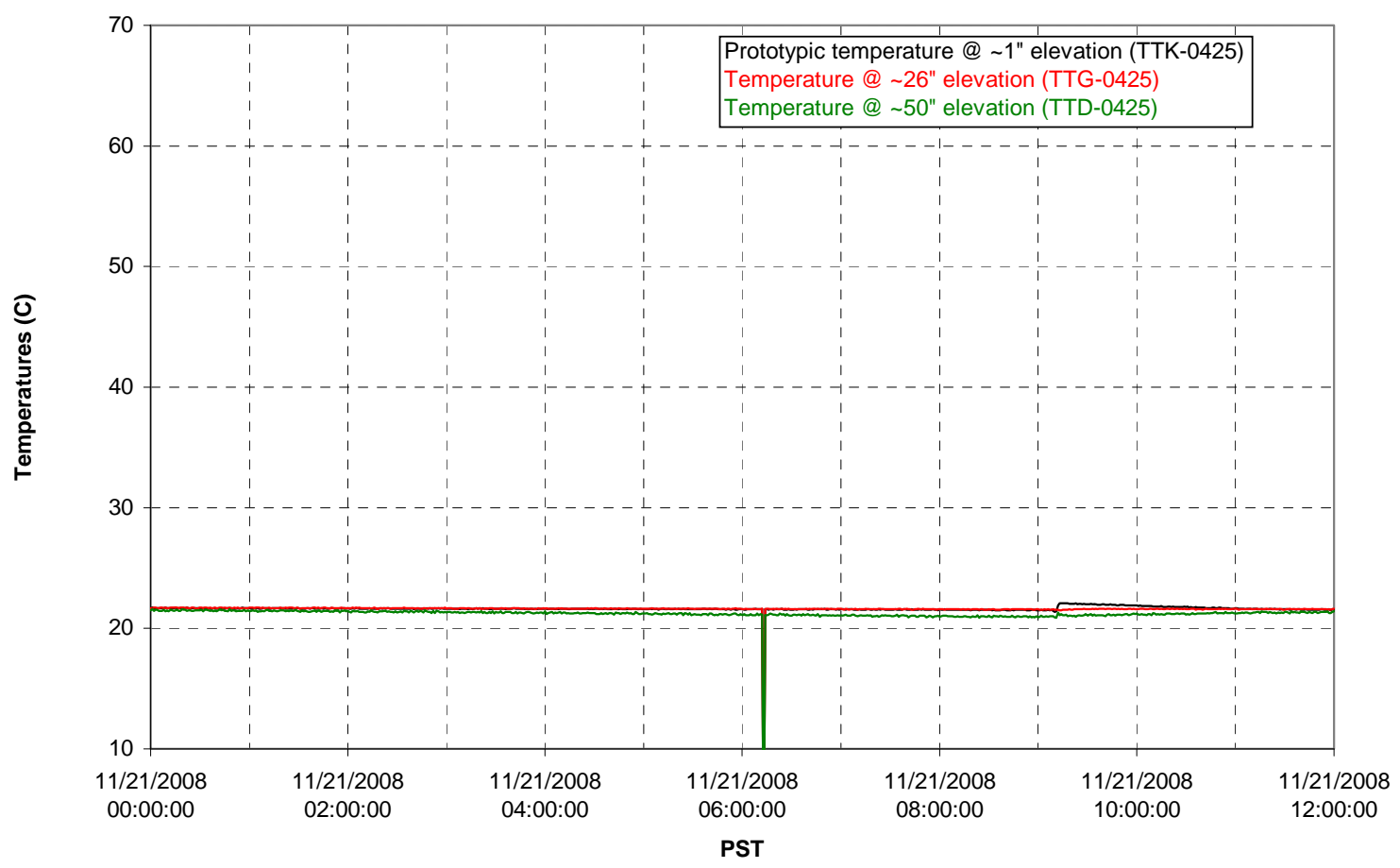


T02A level

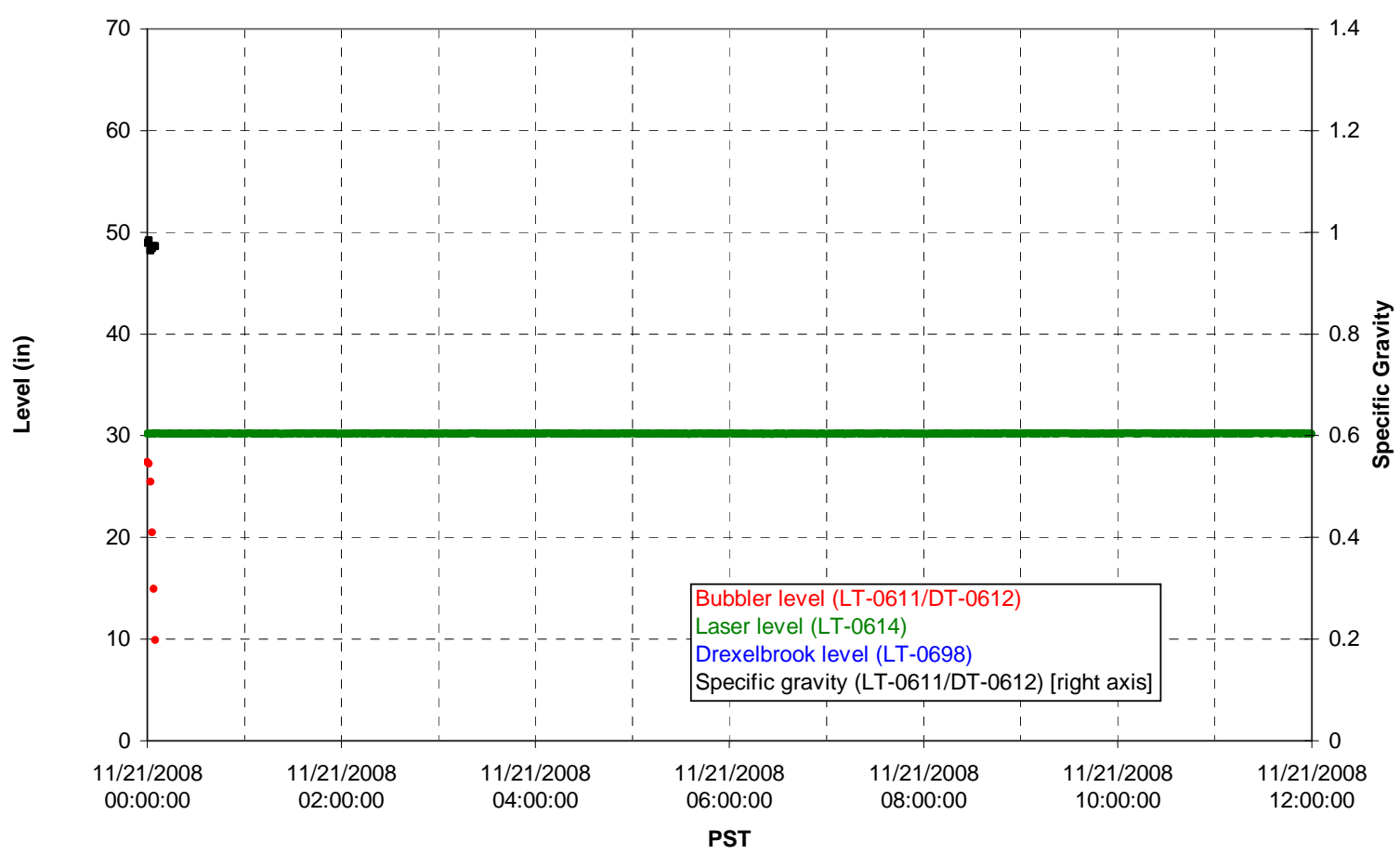

T02A temperatures

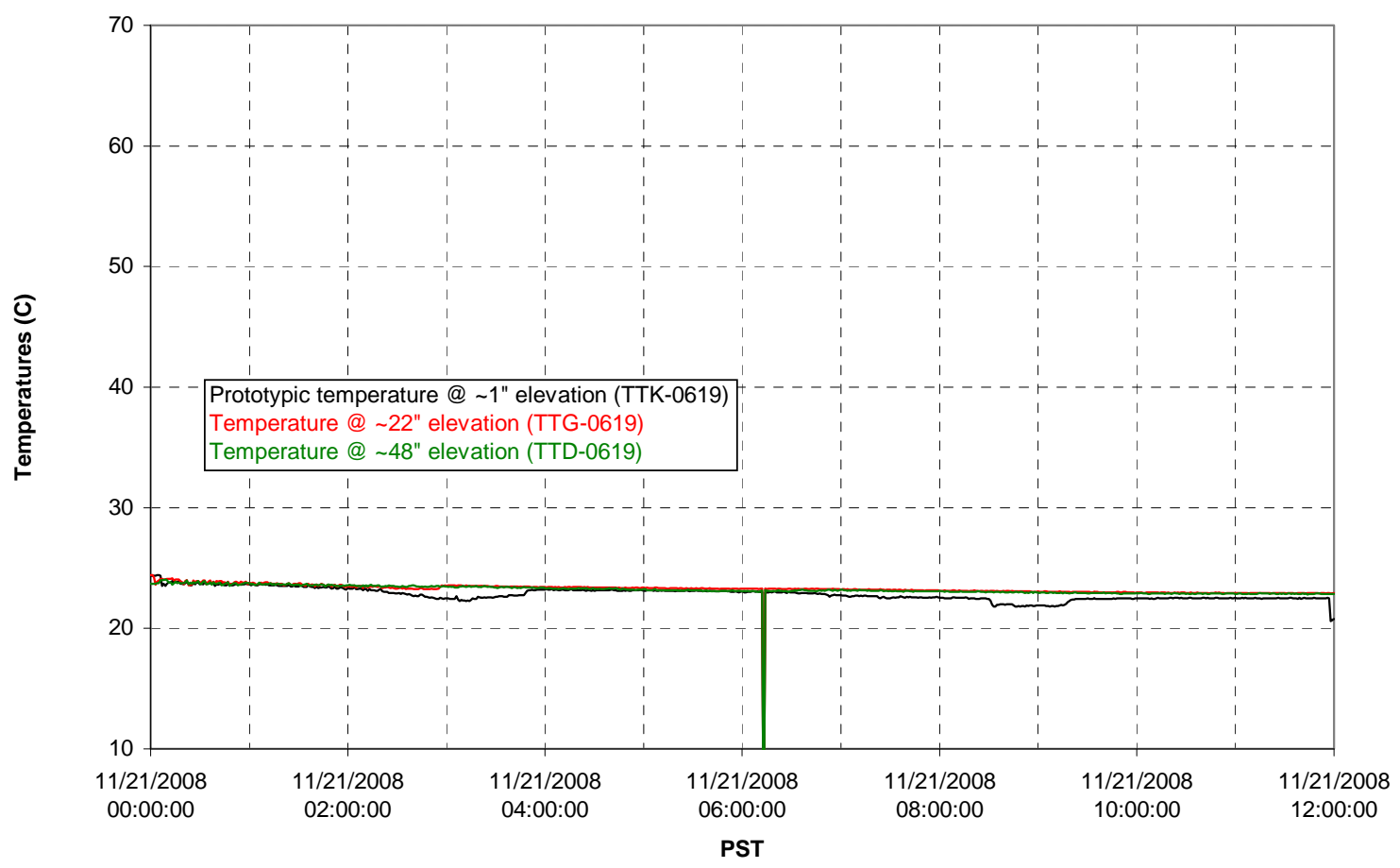


T02A and filter loop temperatures

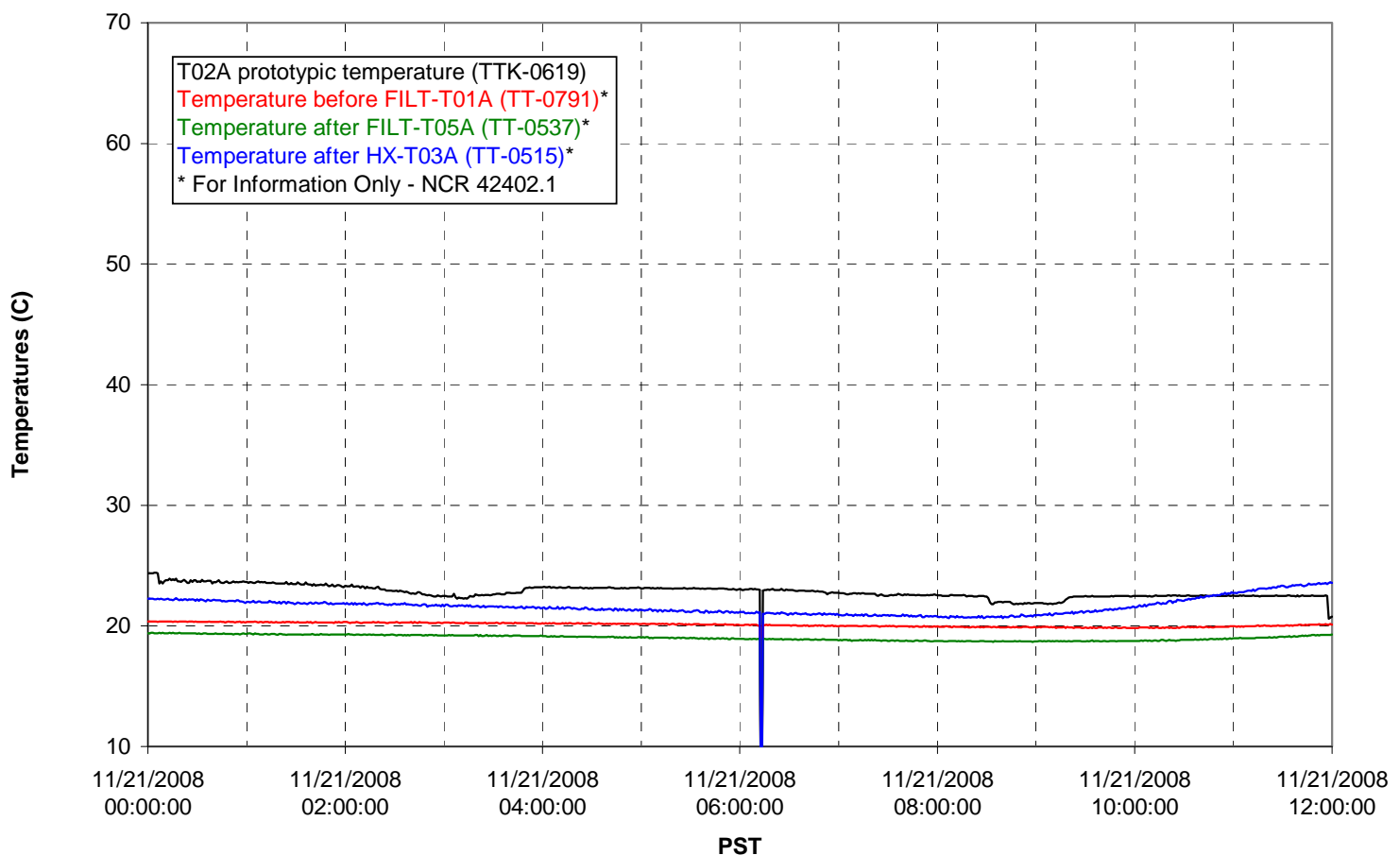

Pump Pressures and Flow

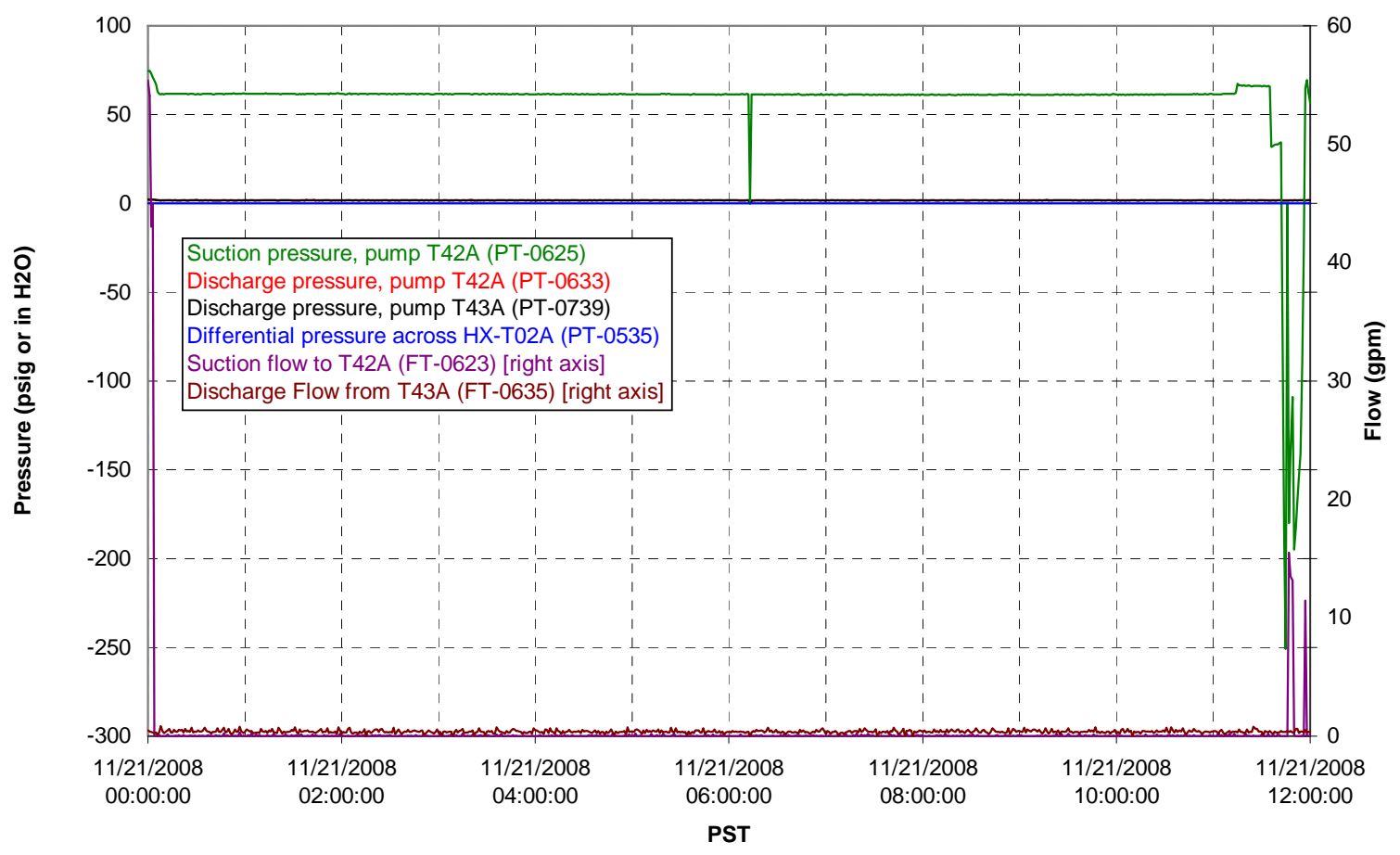


Axial pressure drop

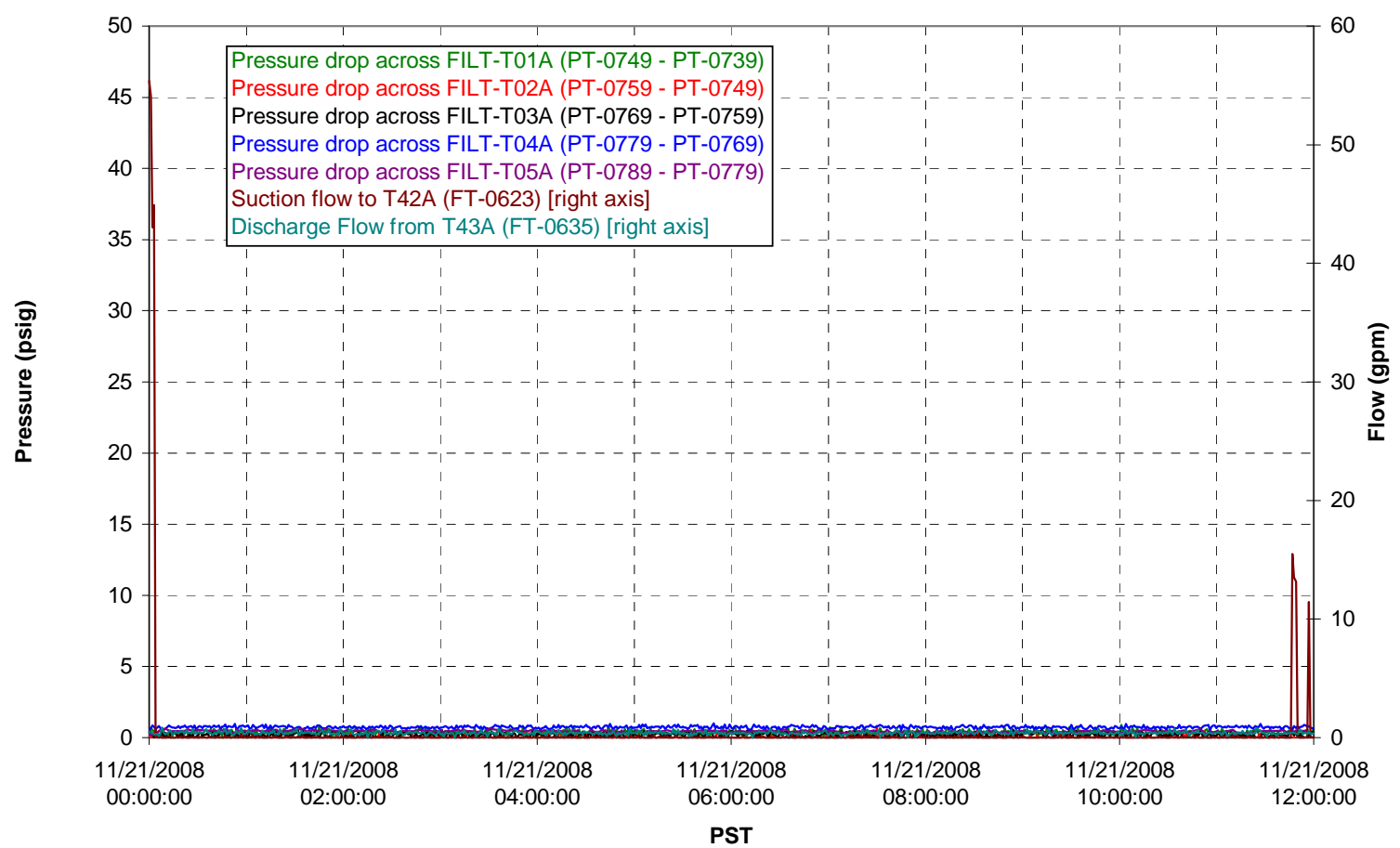

Permeate flow rates

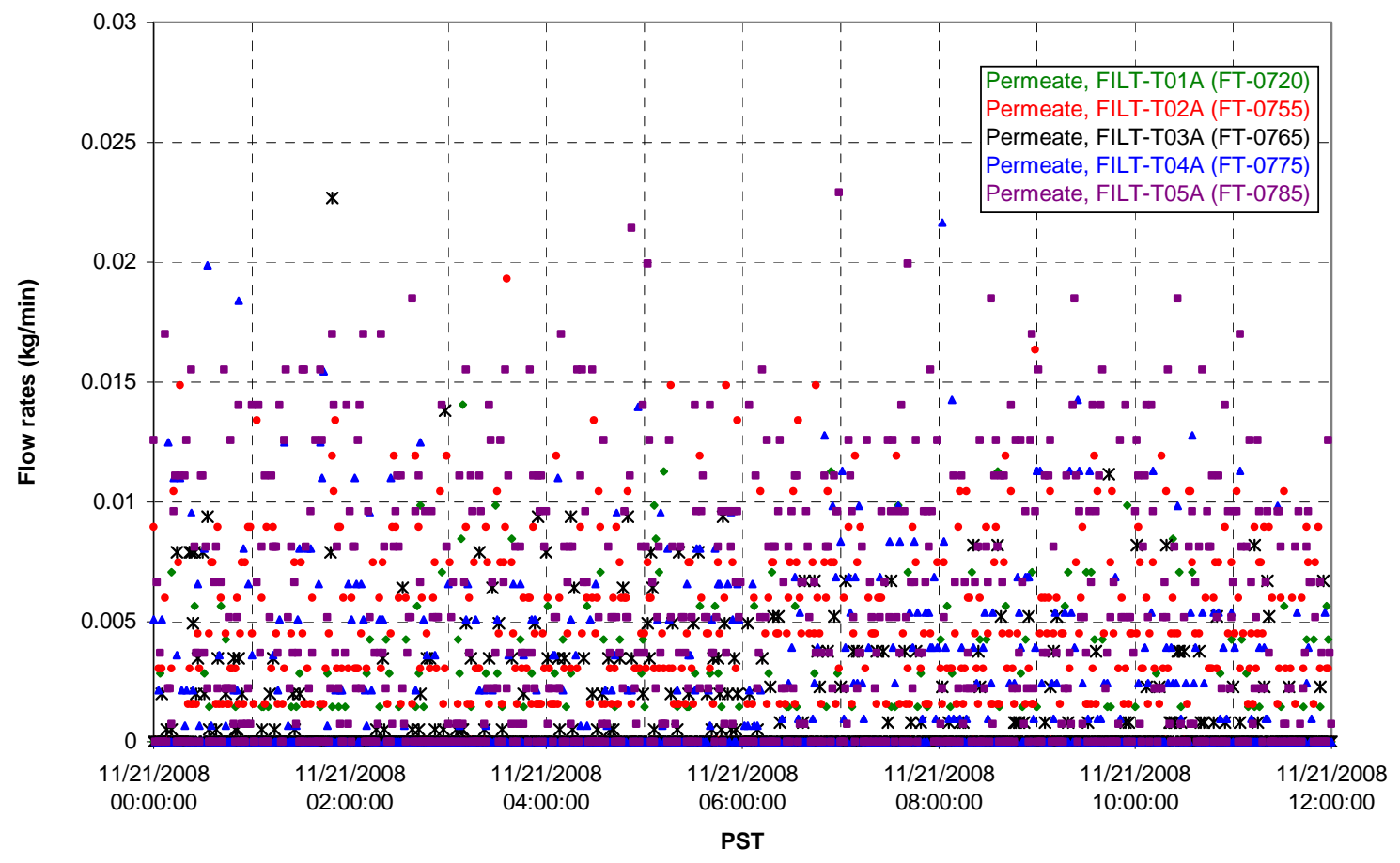


T02A Inner Temperature Tree

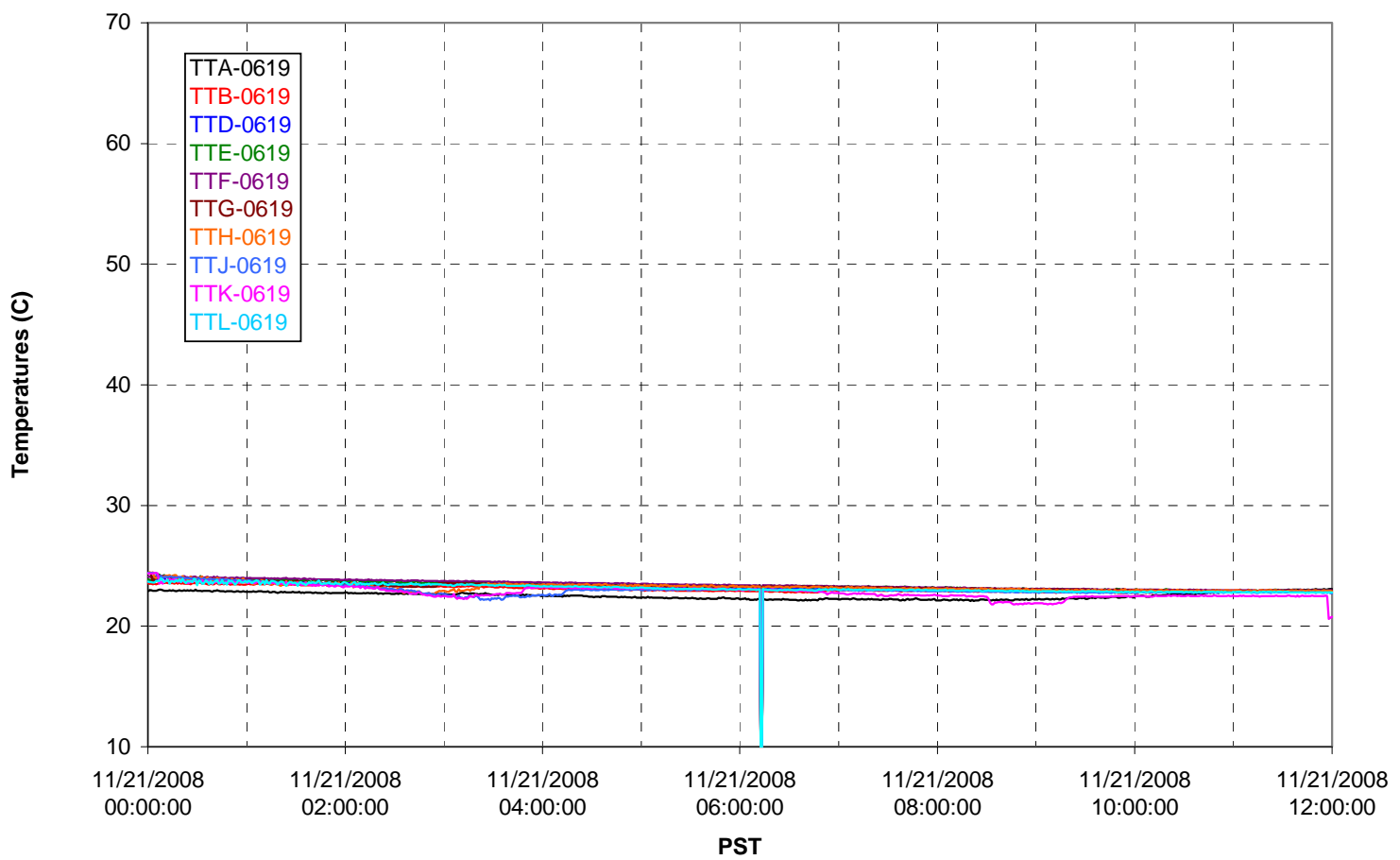

T02A Outer Temperature Tree

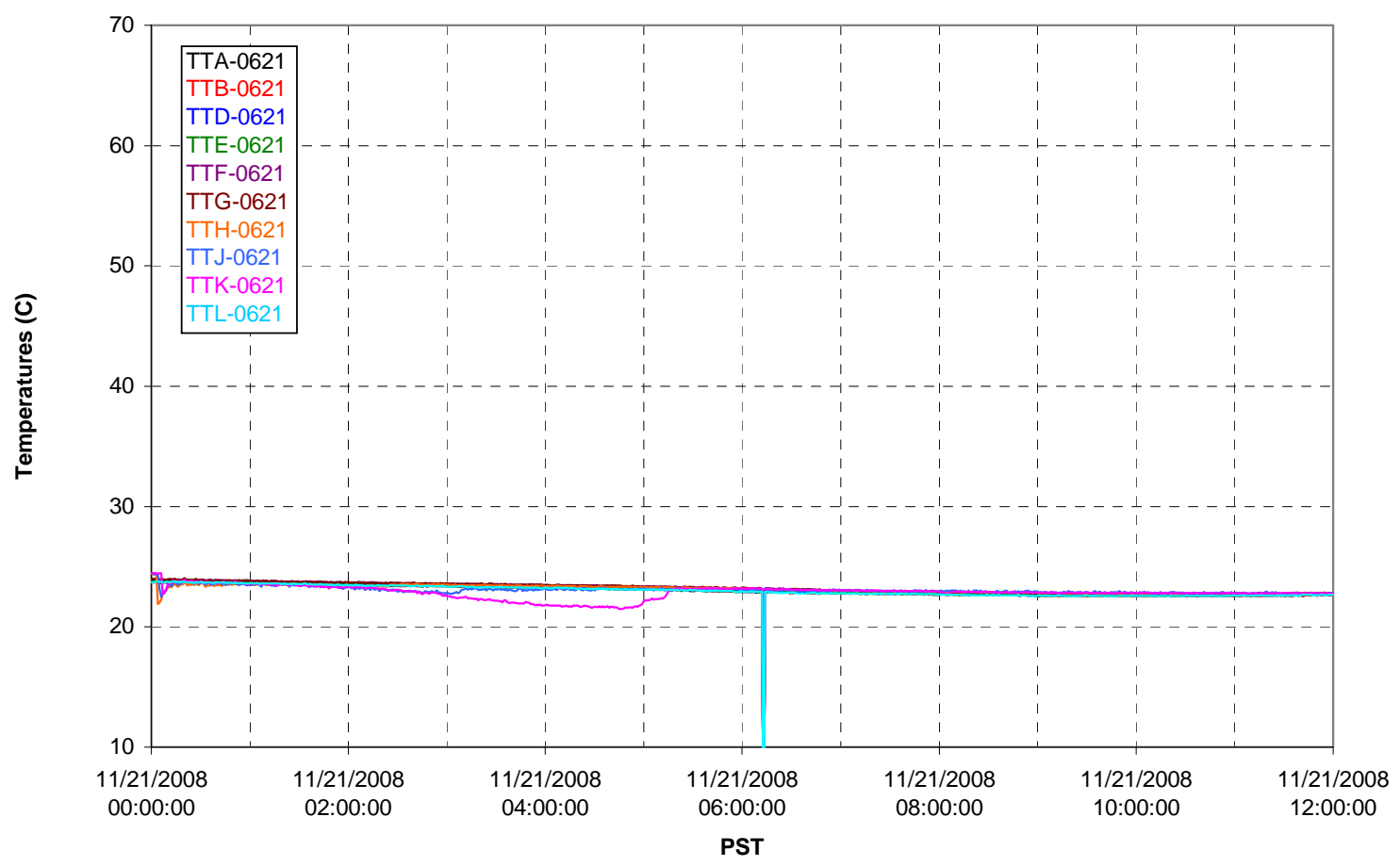


T02A temperatures

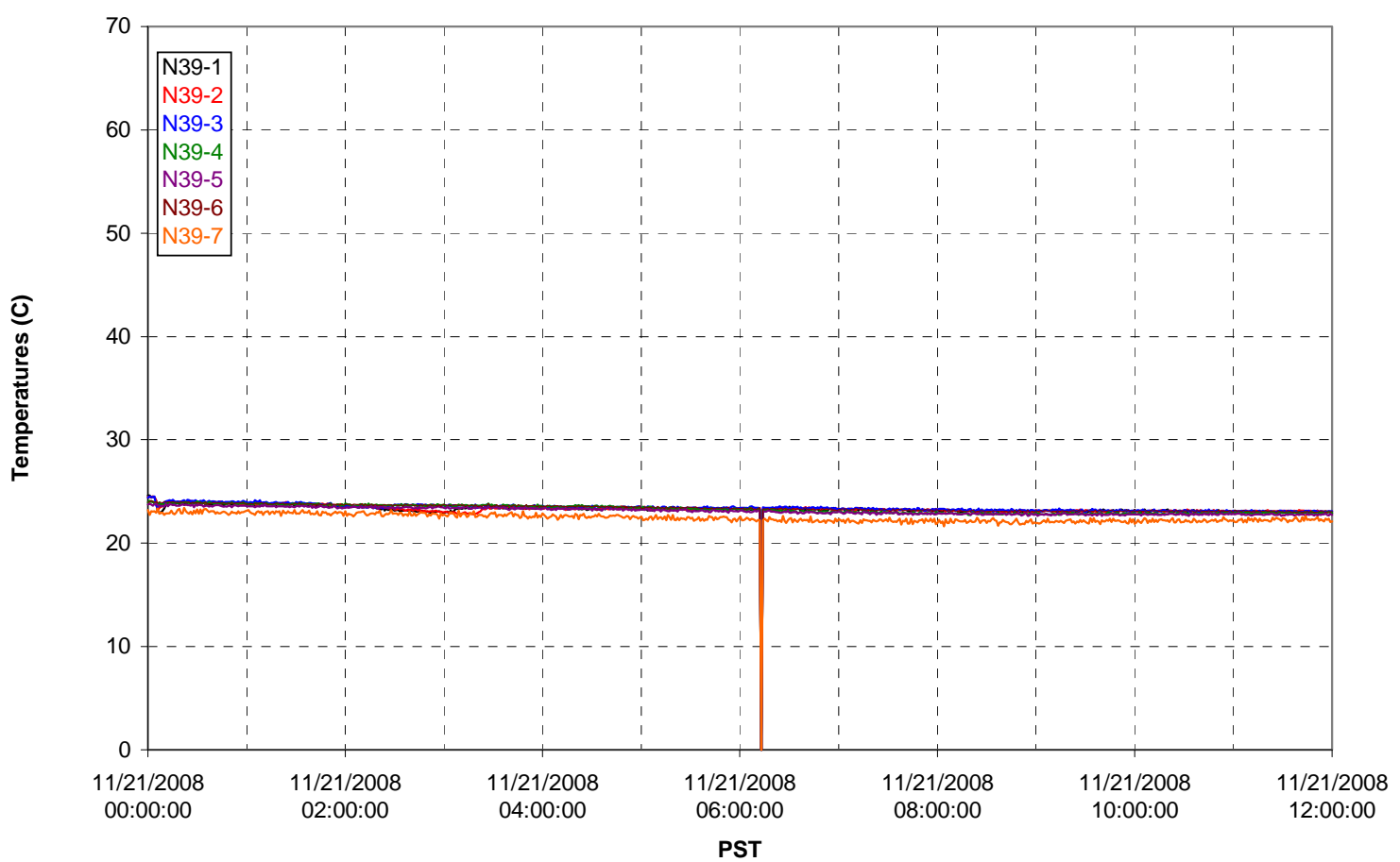

T02A temperatures

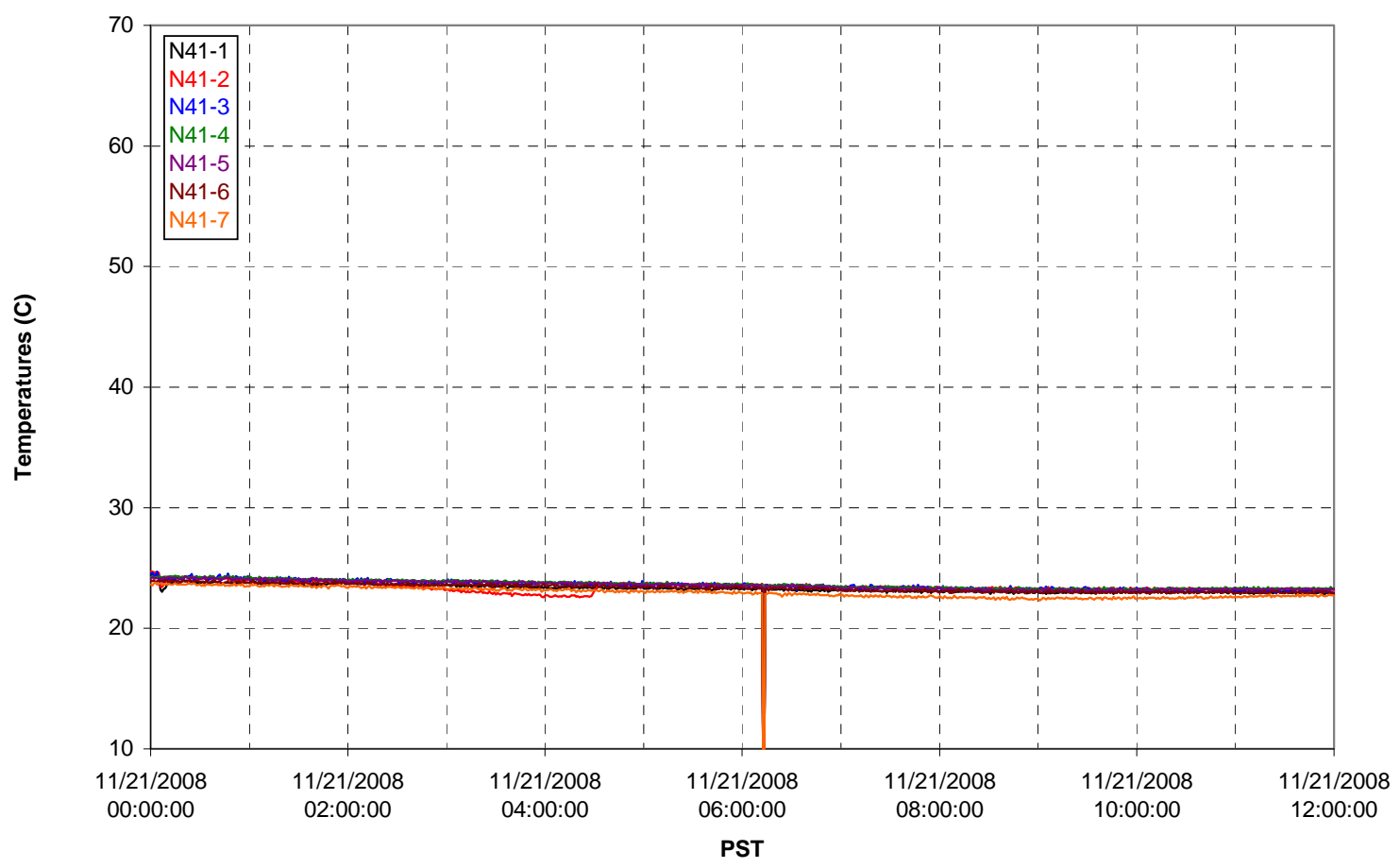


T02A temperatures

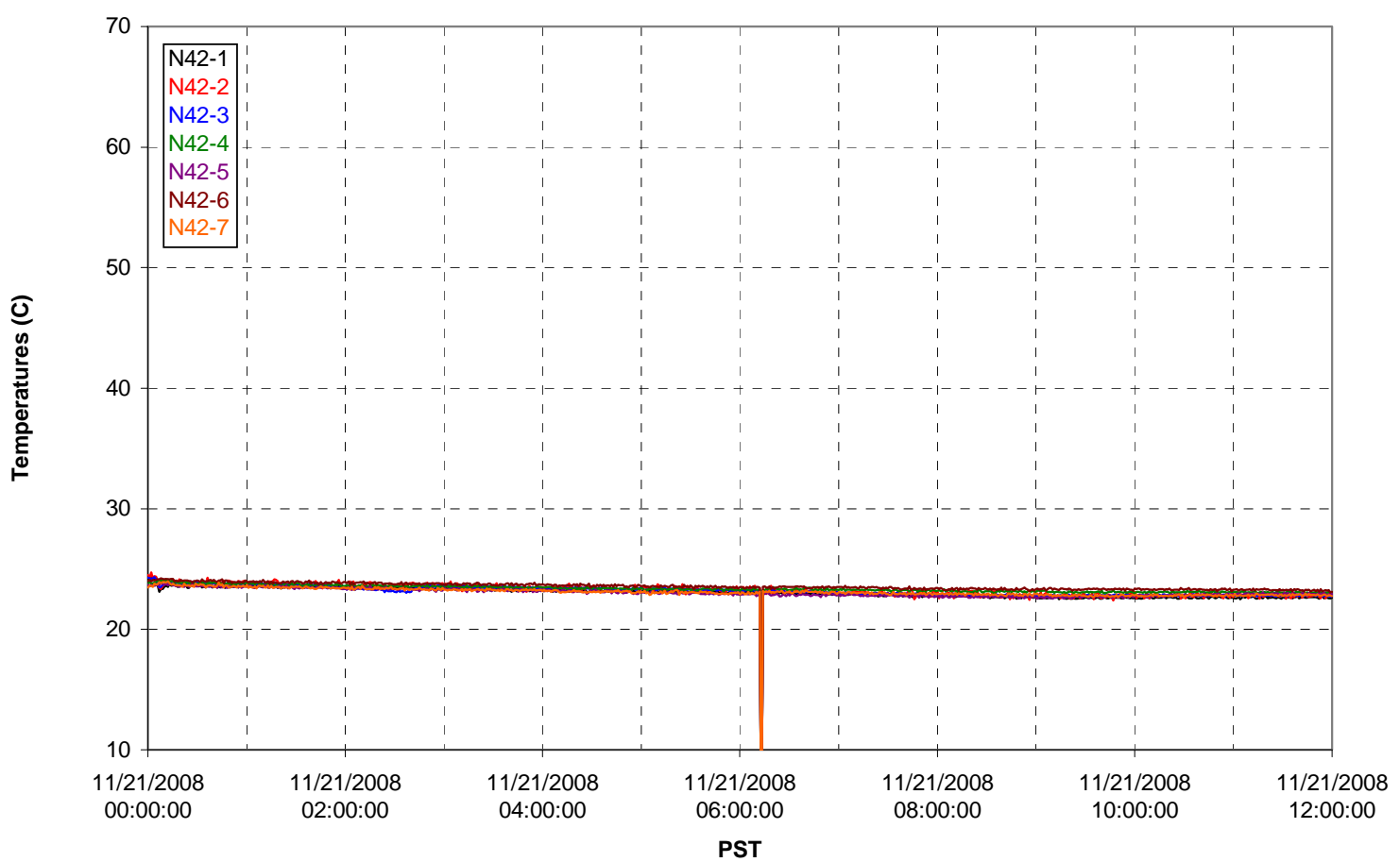

T02A temperatures

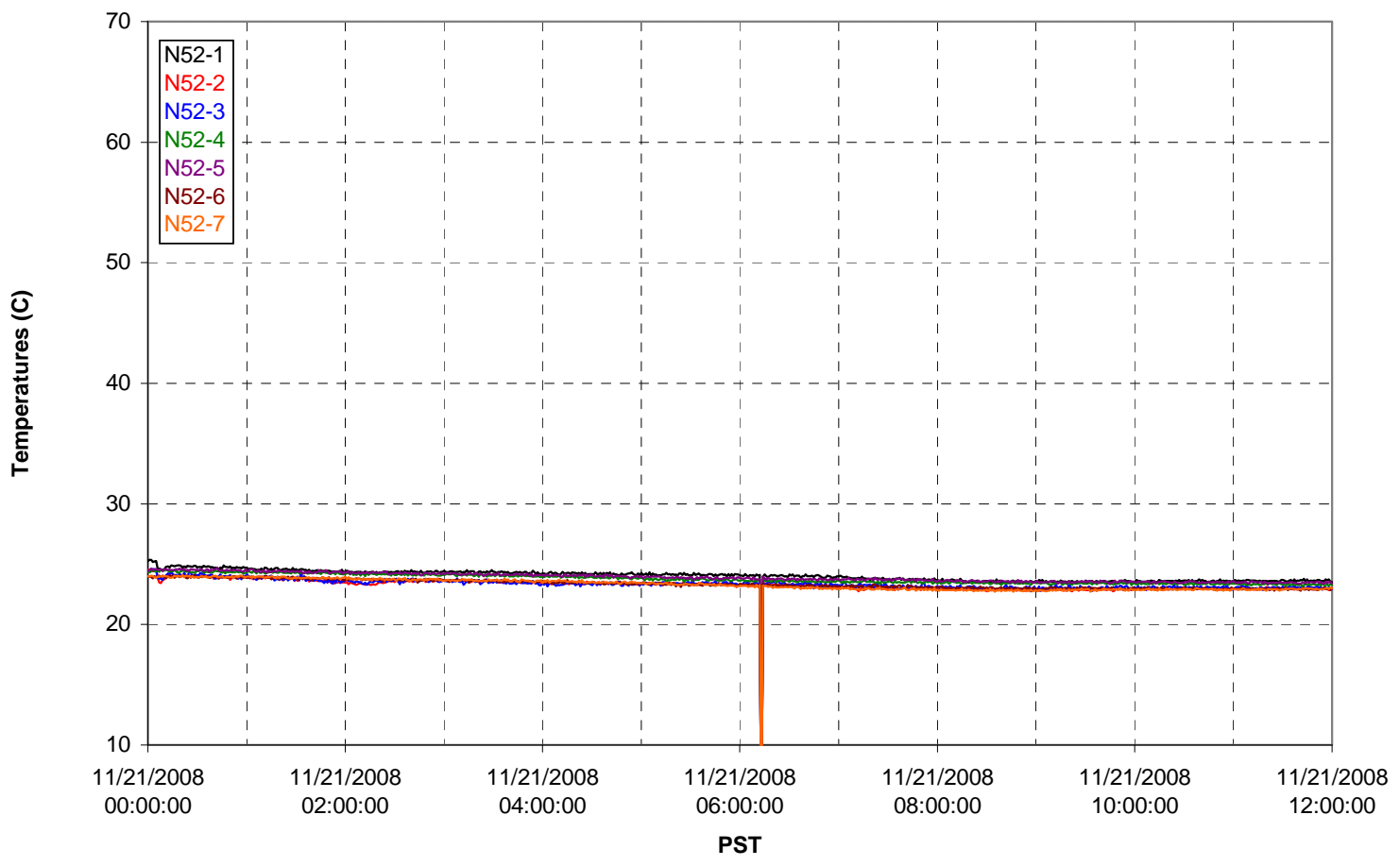


T02A Heating and Cooling

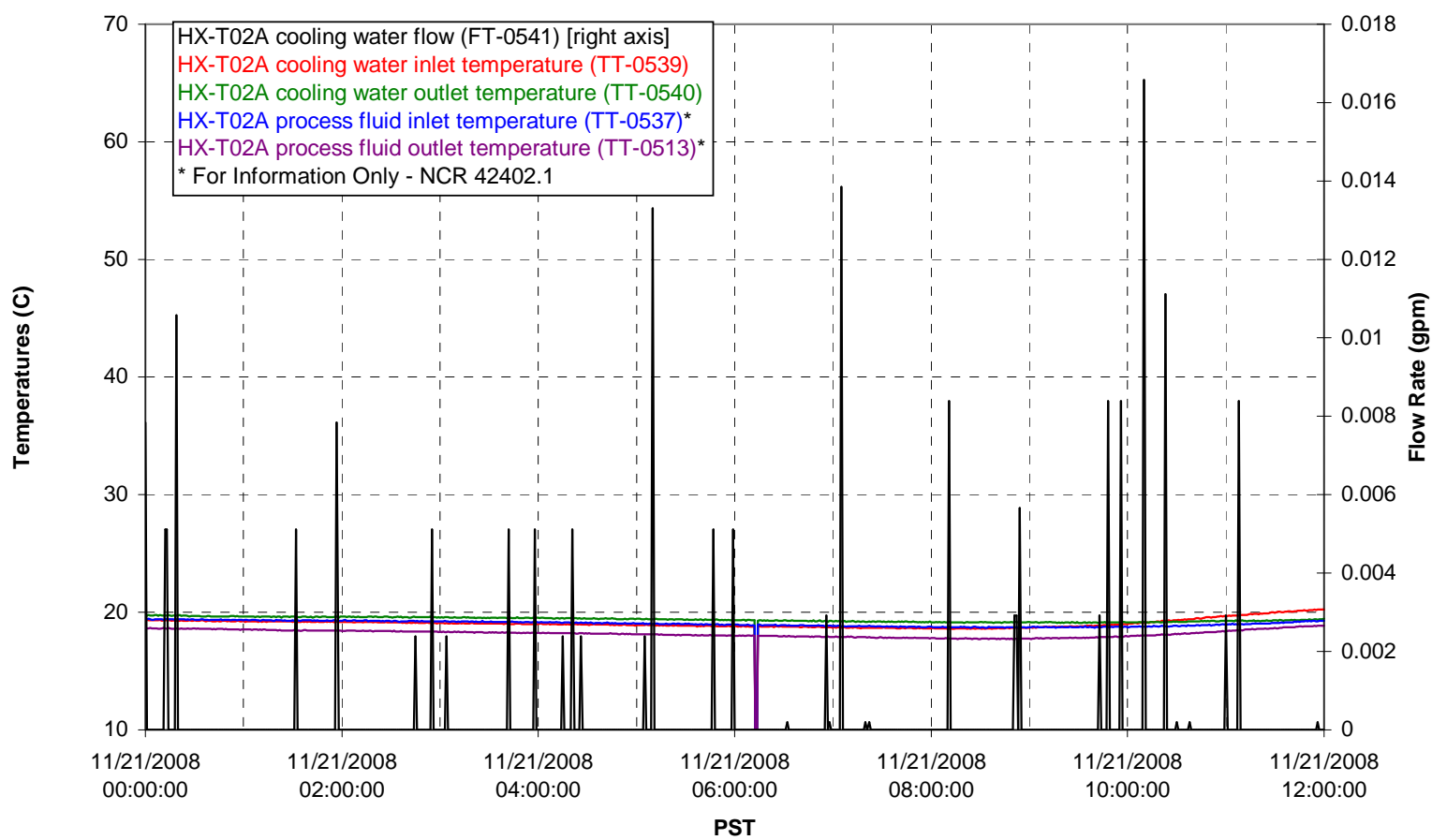

Pump Operation

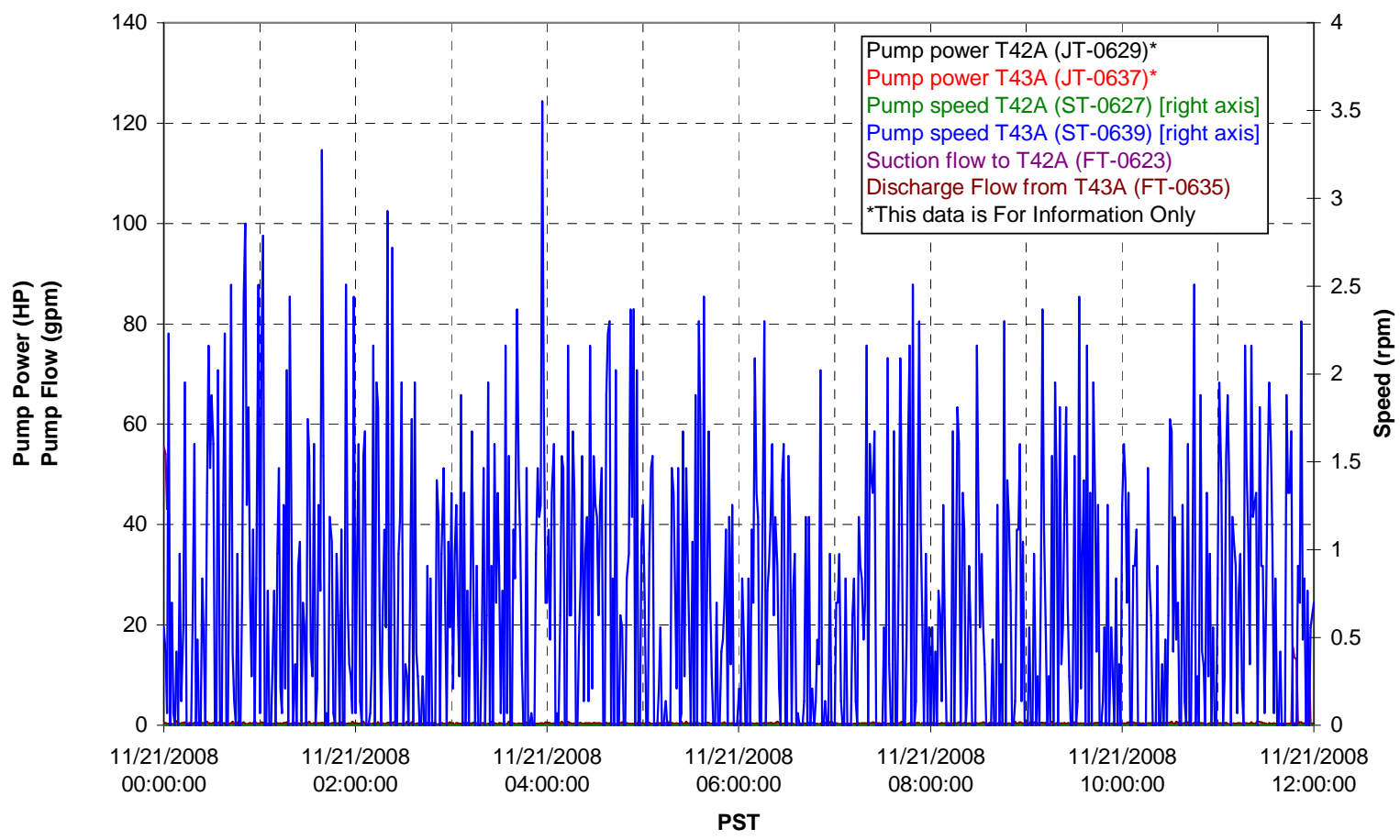


Pulsepot UFP-PP-T01A

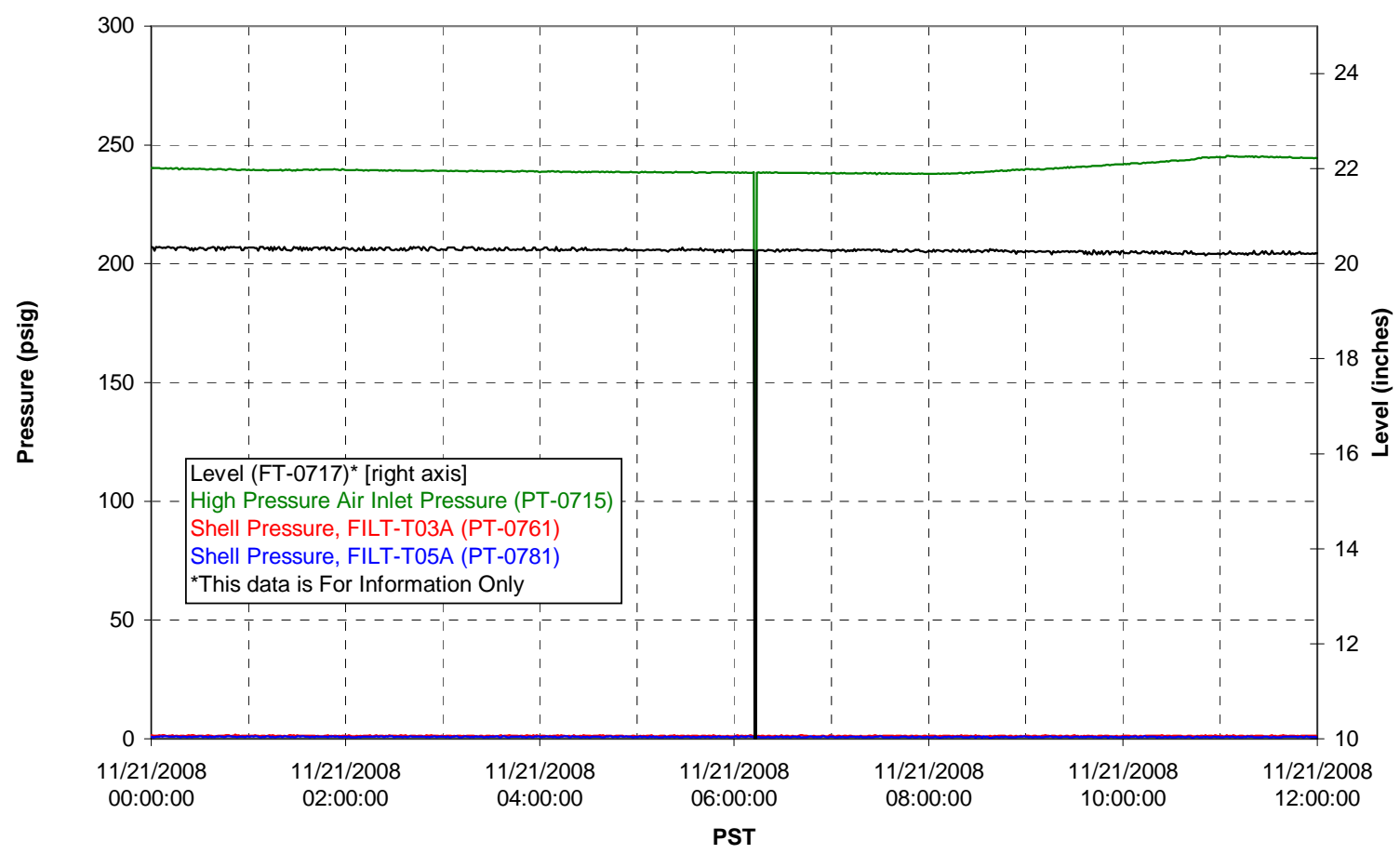

Pulsepot UFP-PP-T02A

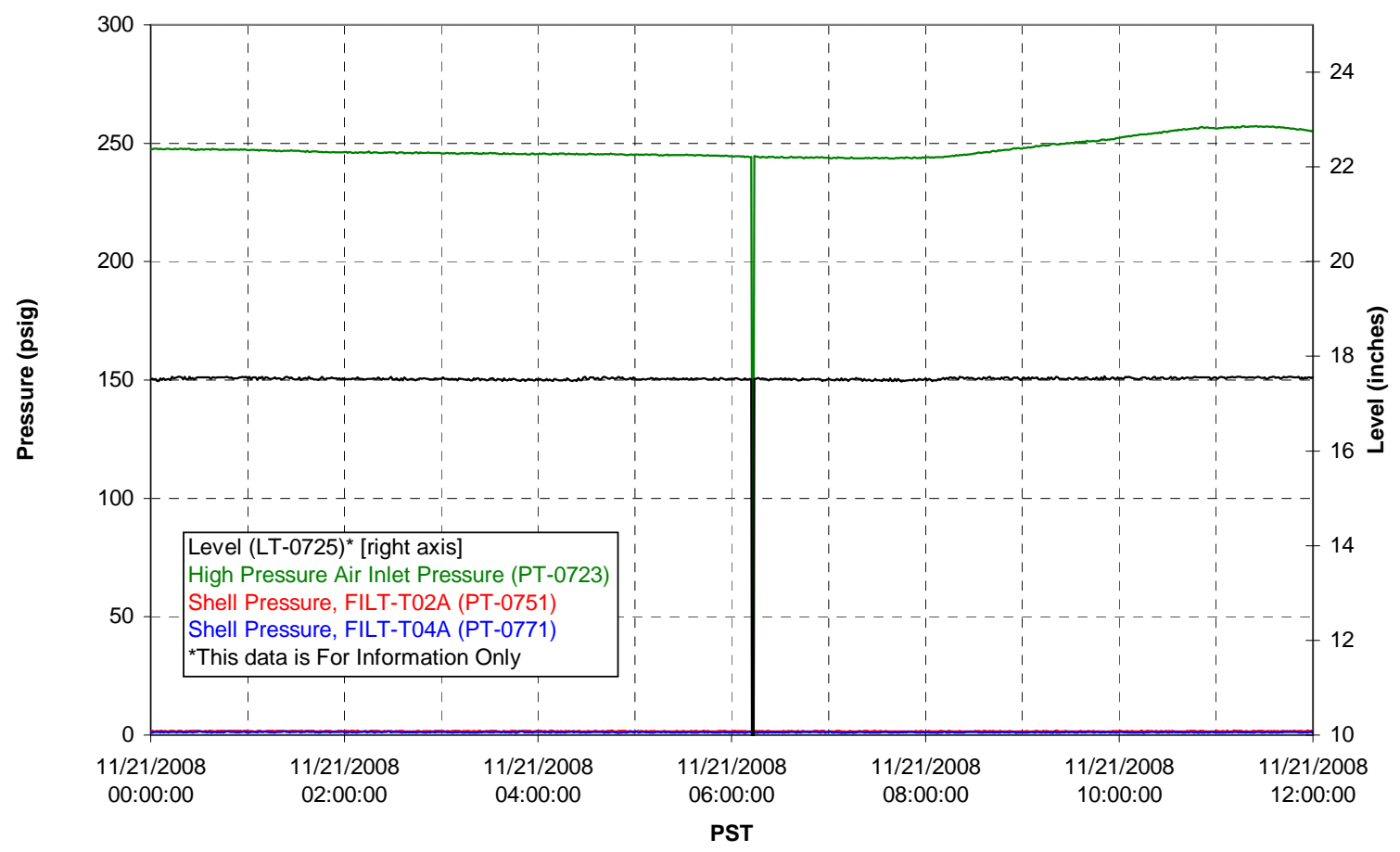


Pulsepot UFP-PP-T03A

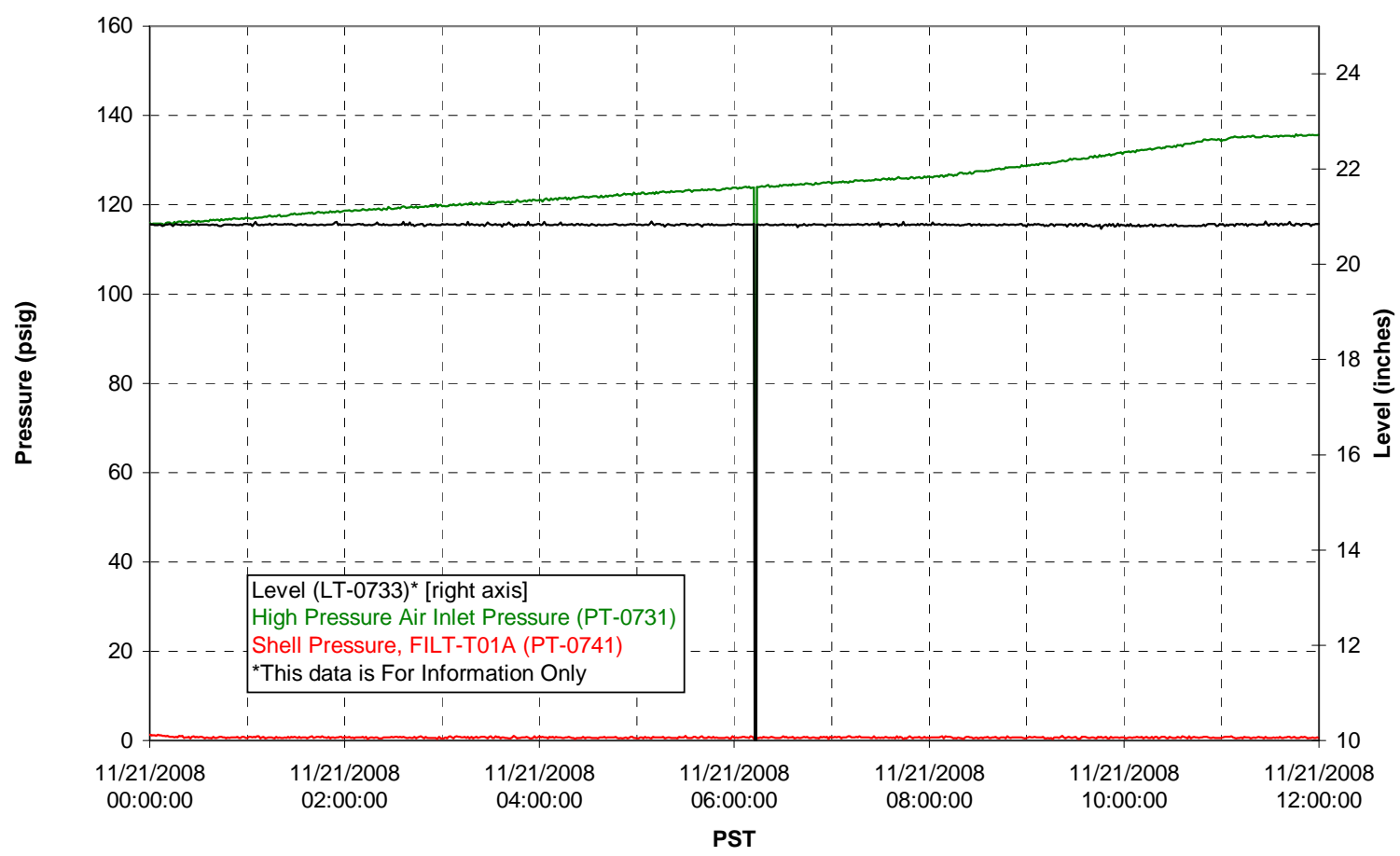

Pulsepot Levels

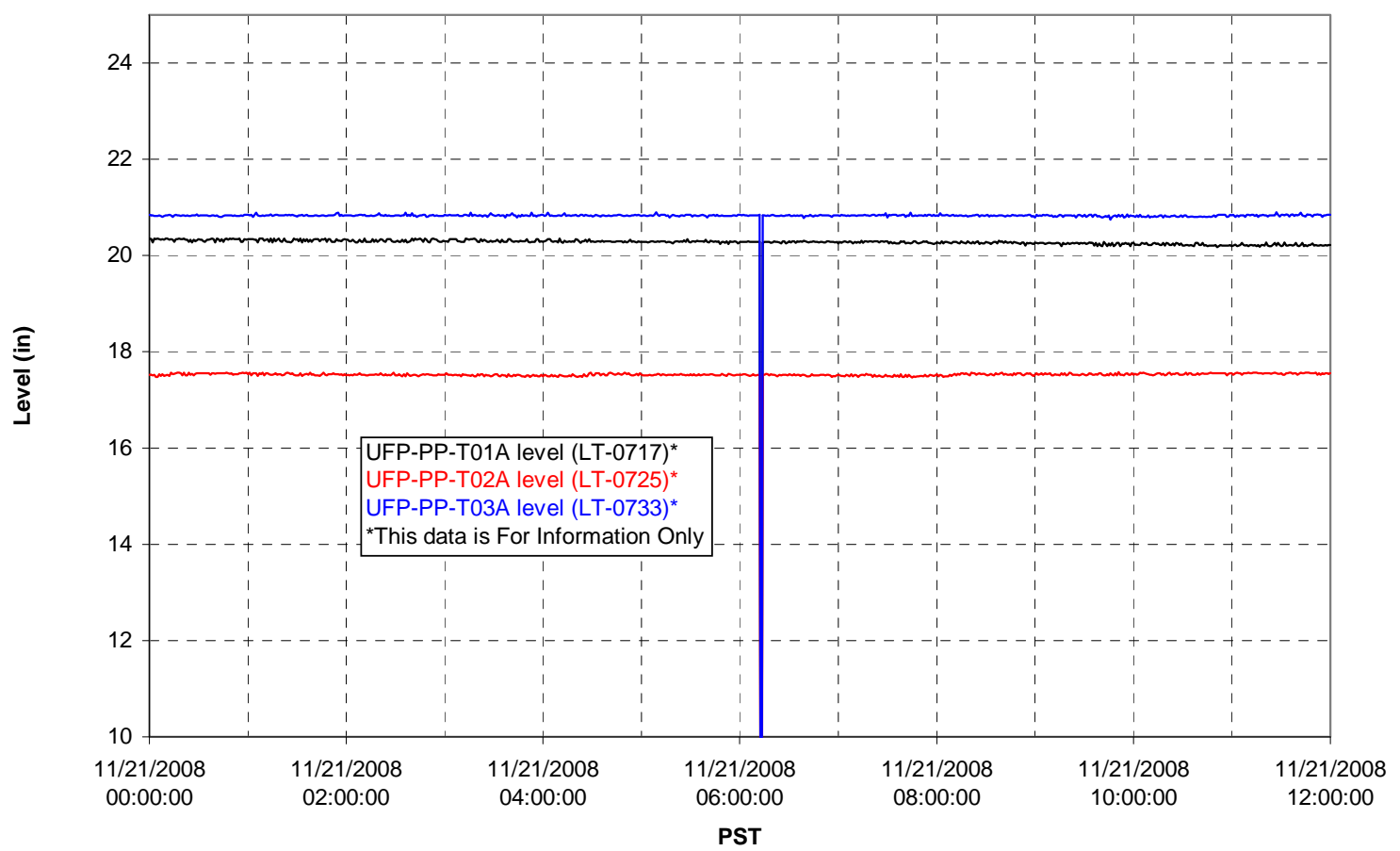


Filter UFP-FILT-T01A

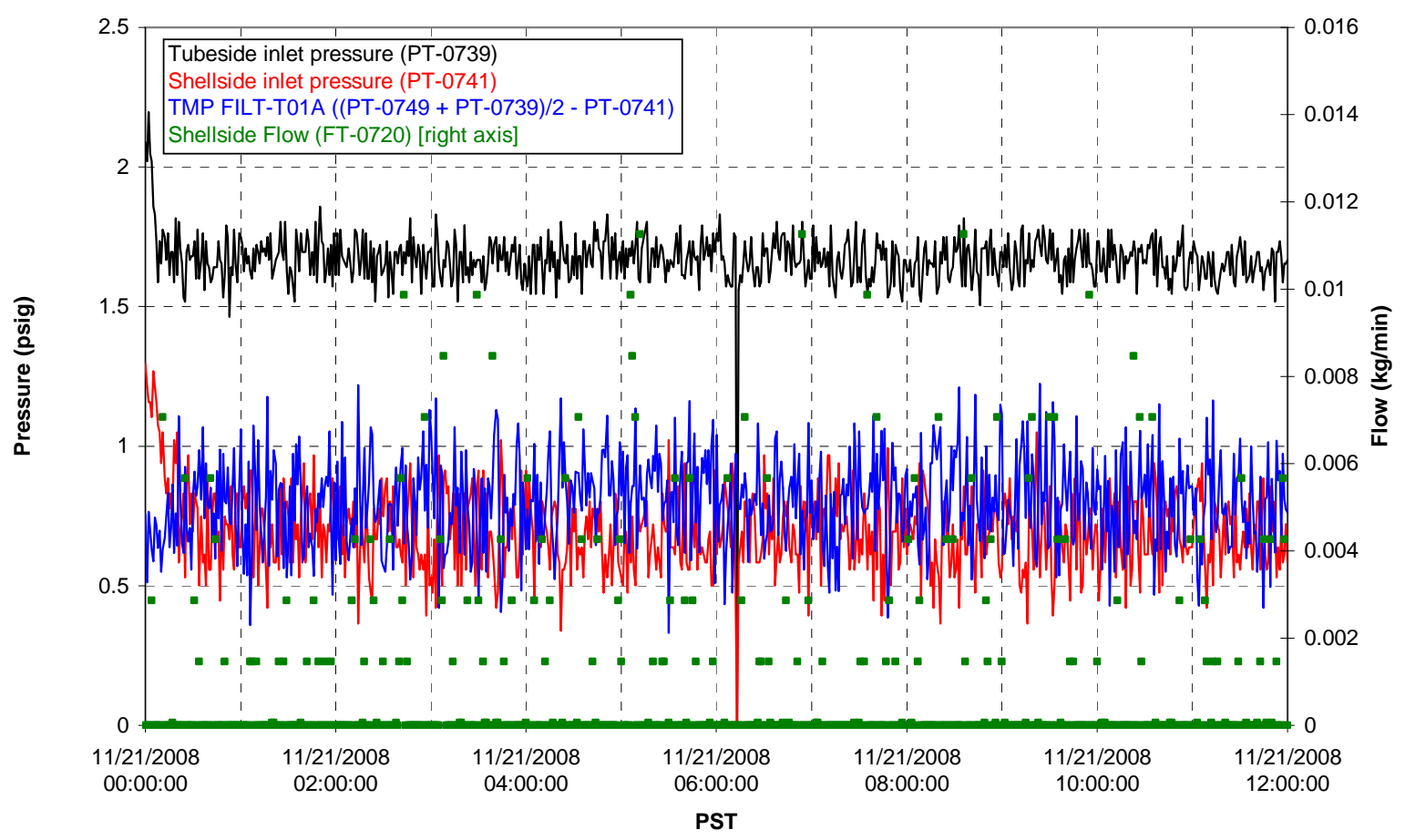

Filter UFP-FILT-T02A

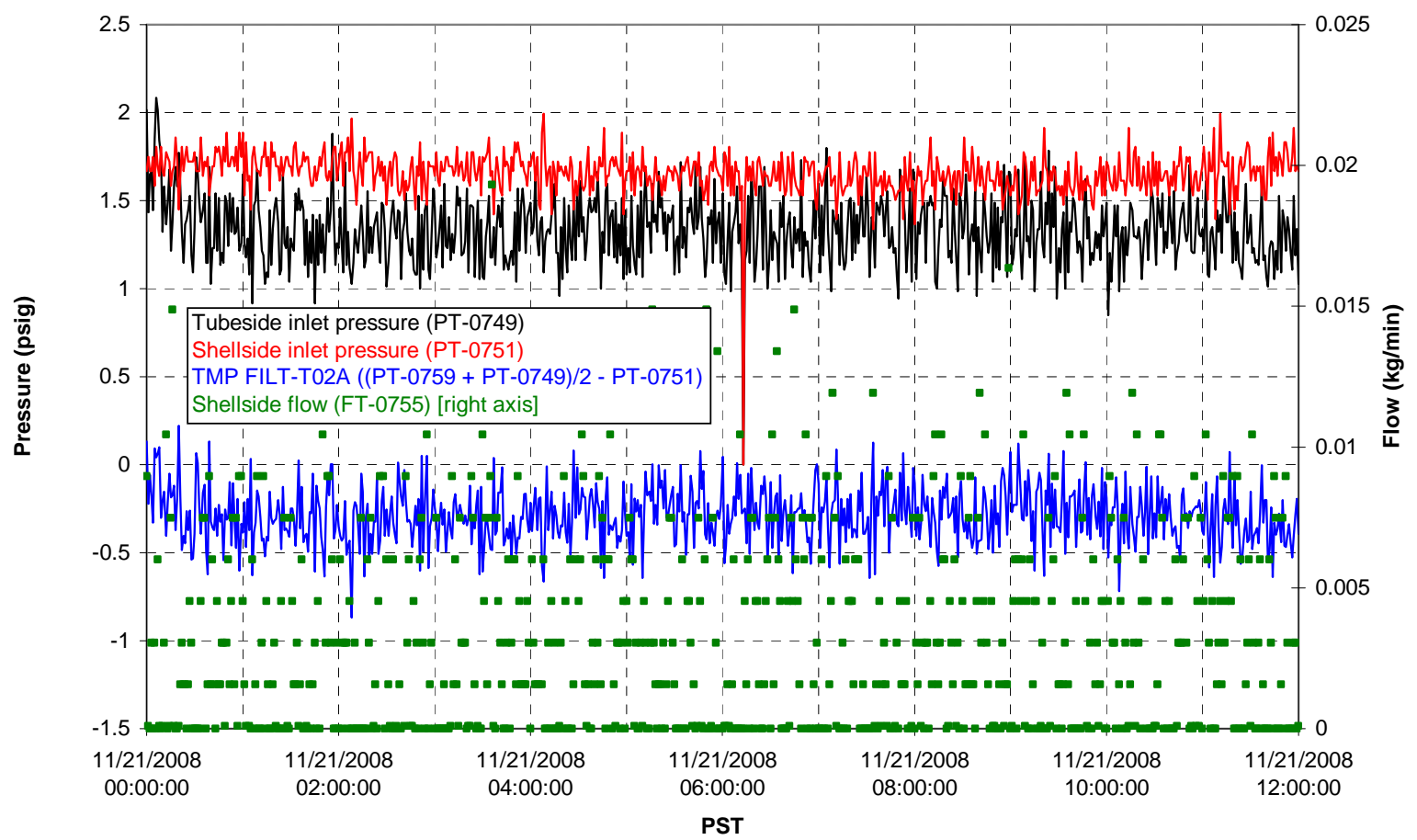


Filter UFP-FILT-T03A

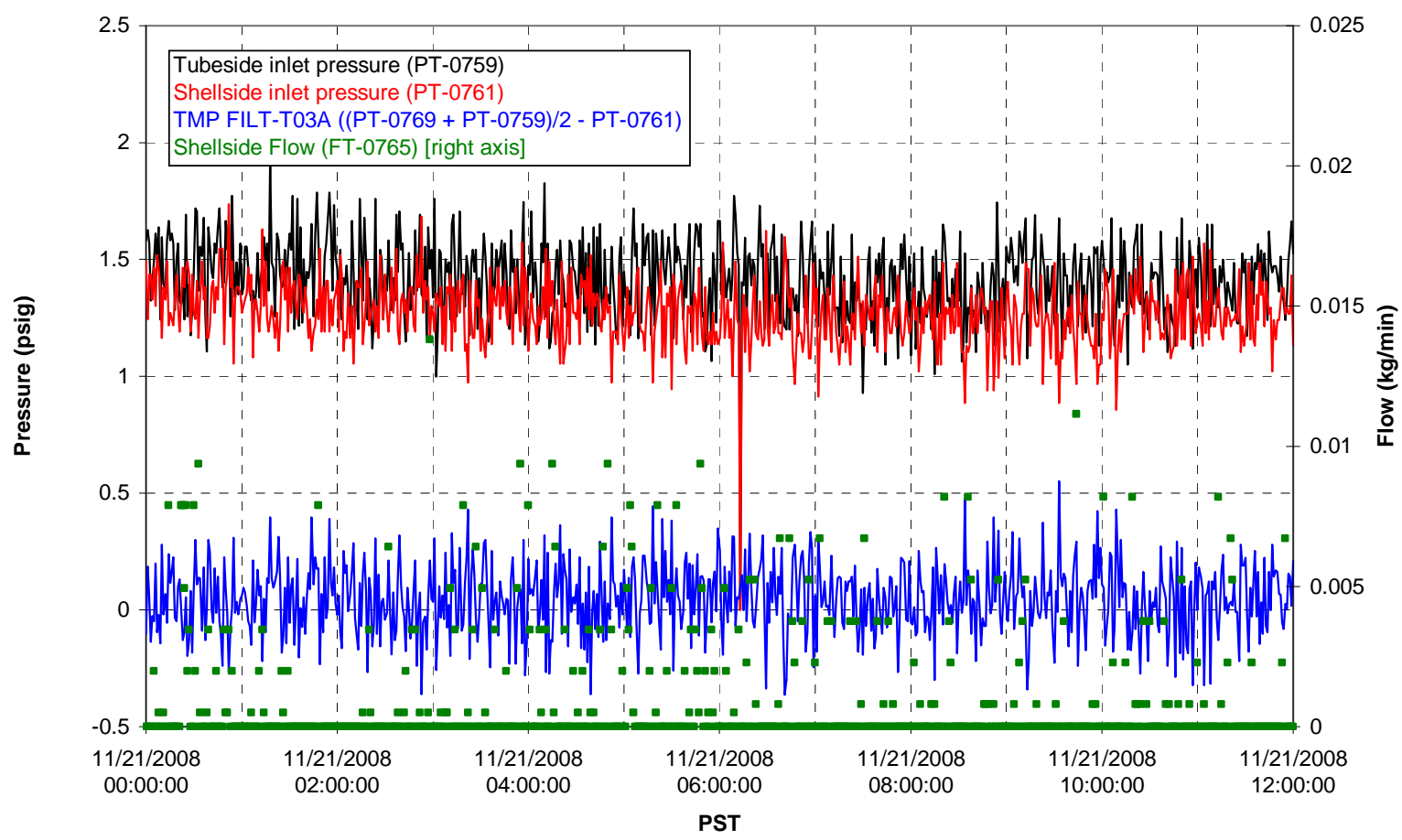

Filter UFP-FILT-T04A

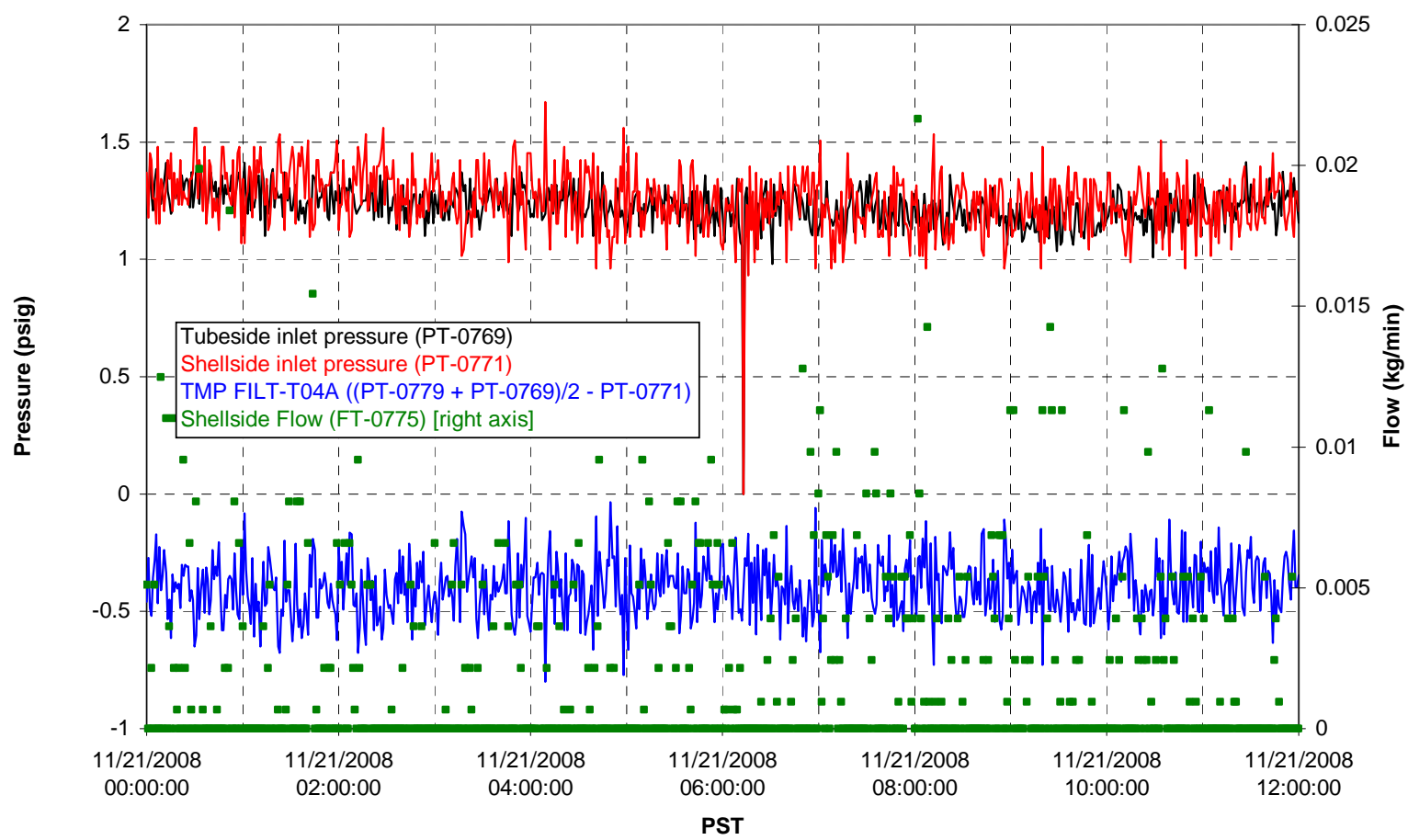


Filter UFP-FILT-T05A

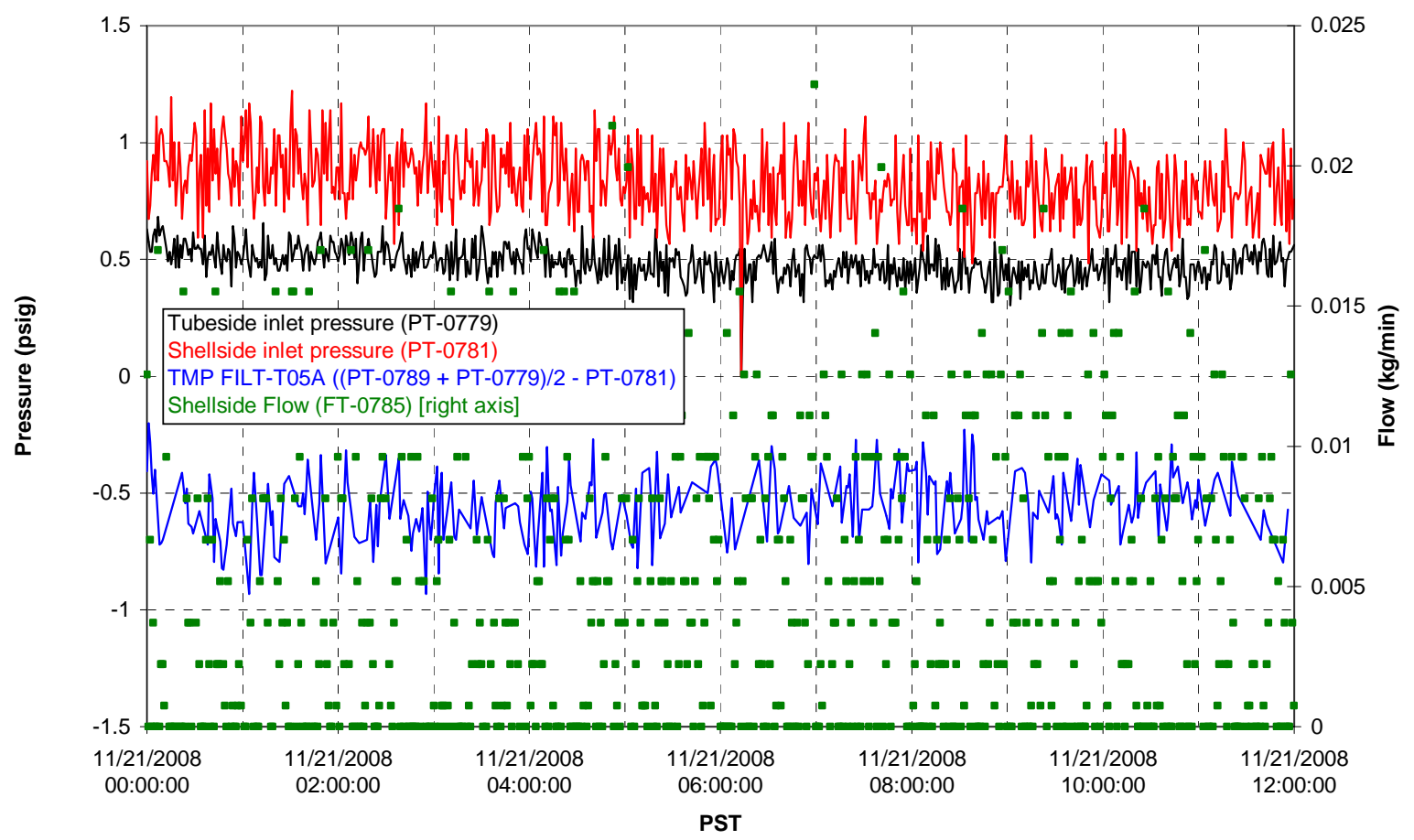

Chemical Flow

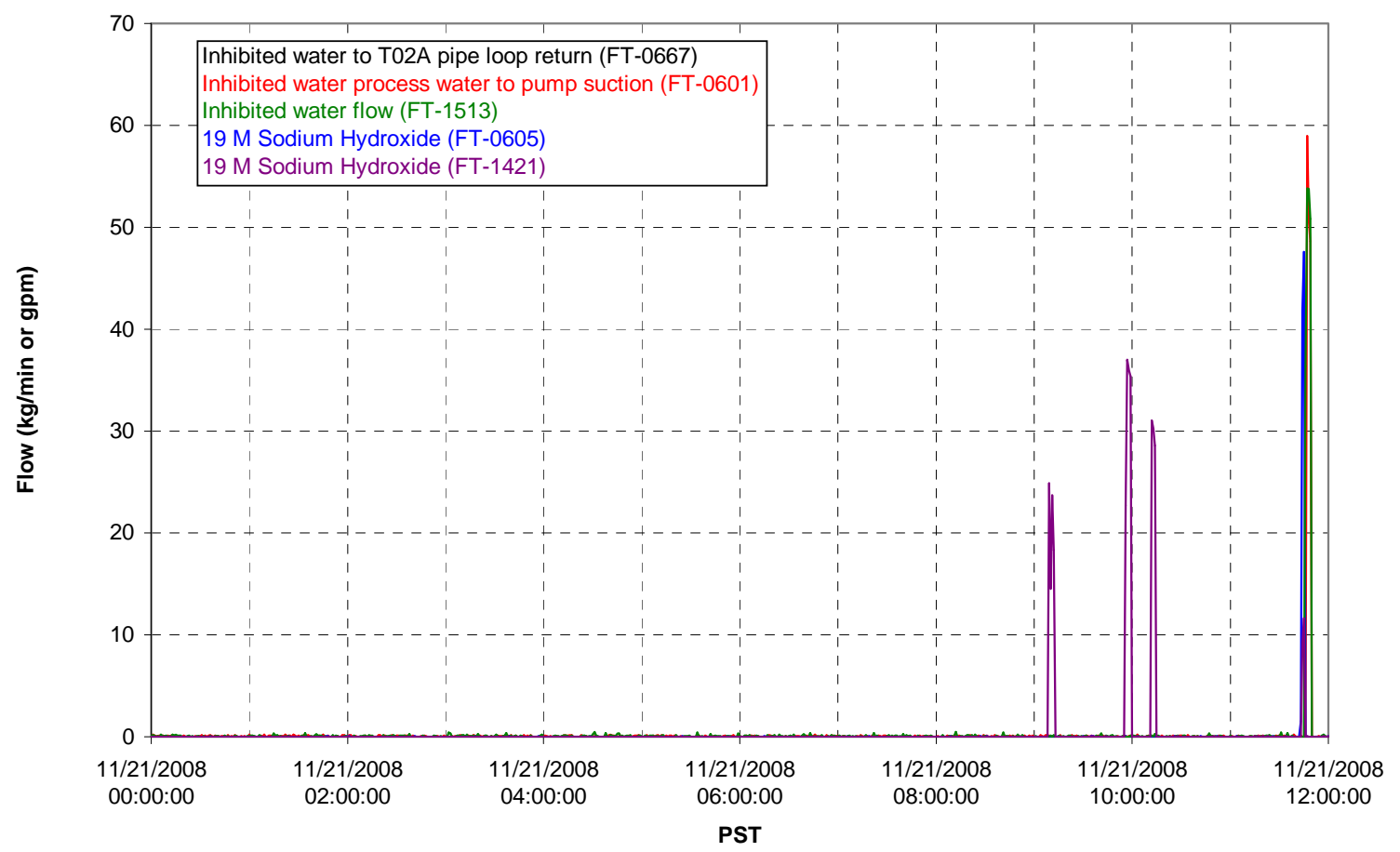




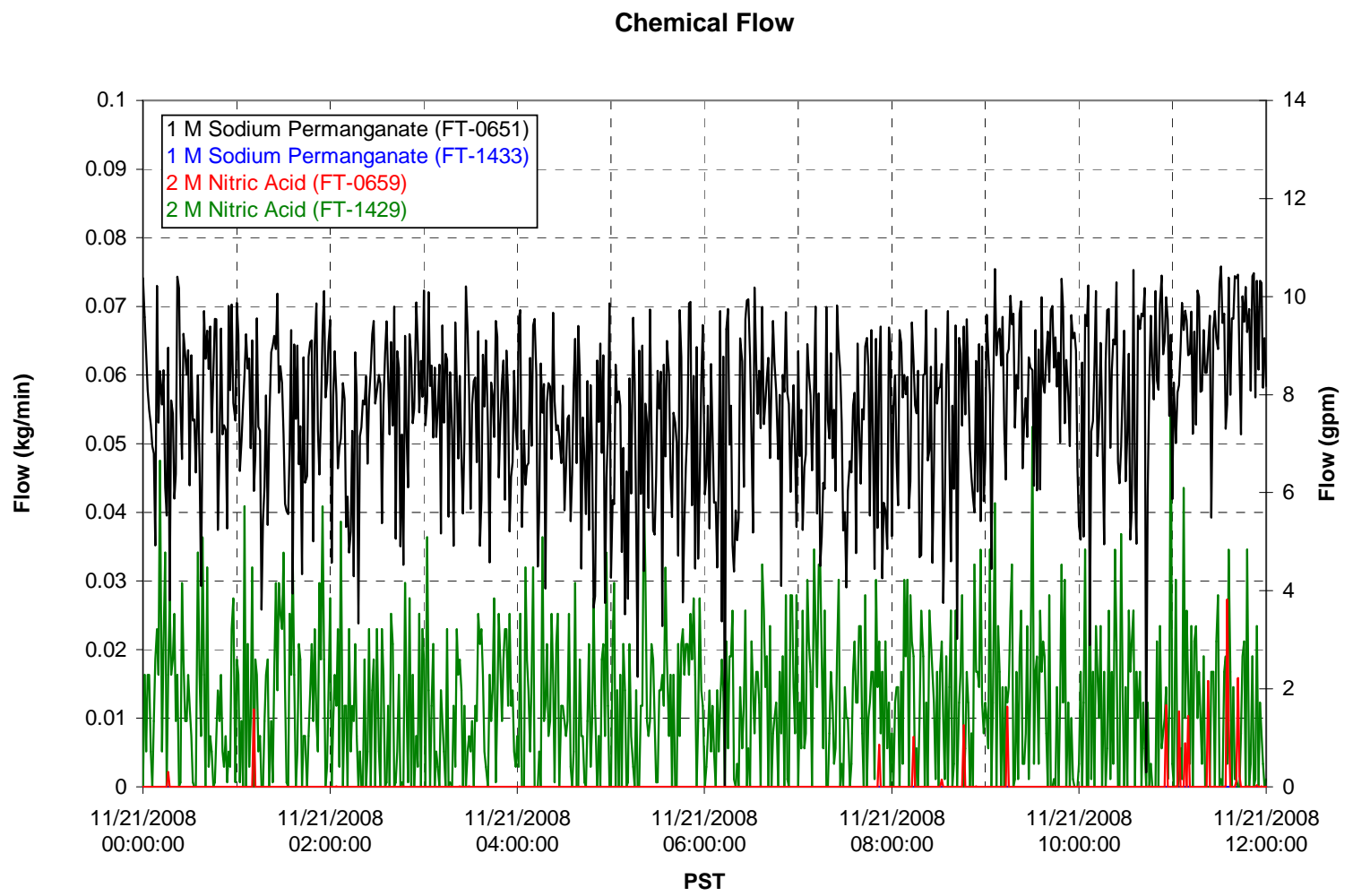

Air Flows

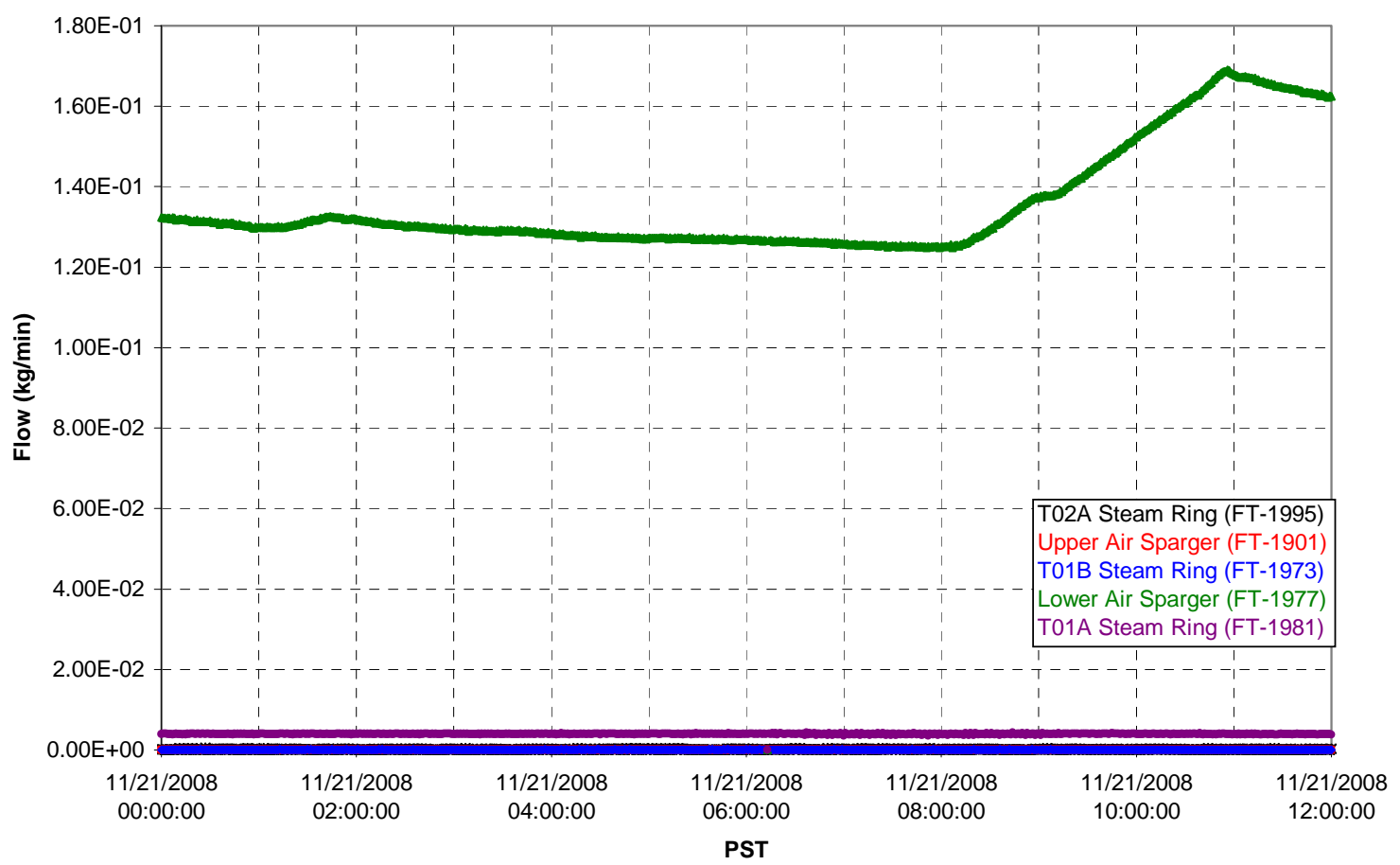


T02A Steam

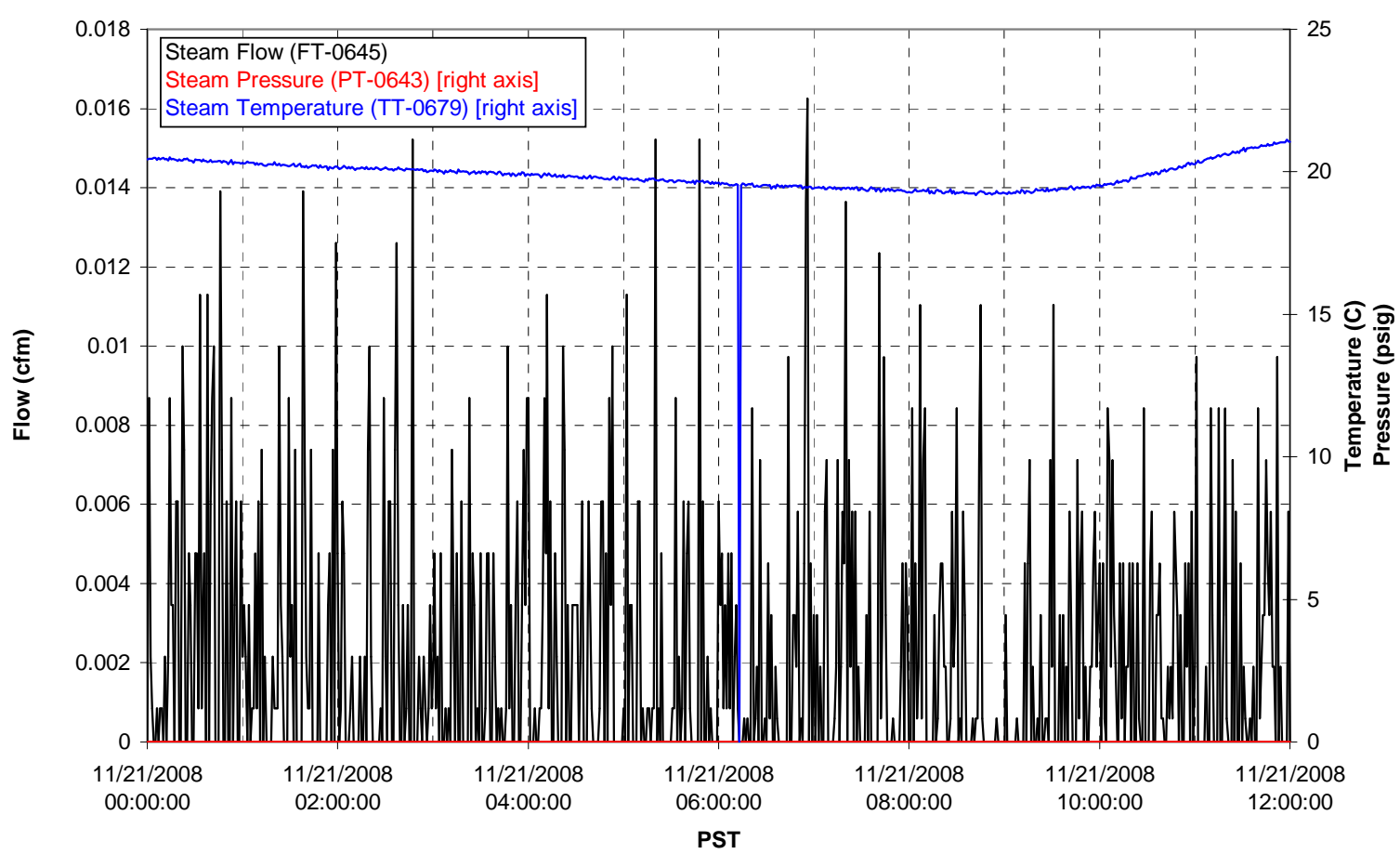

T01A Steam

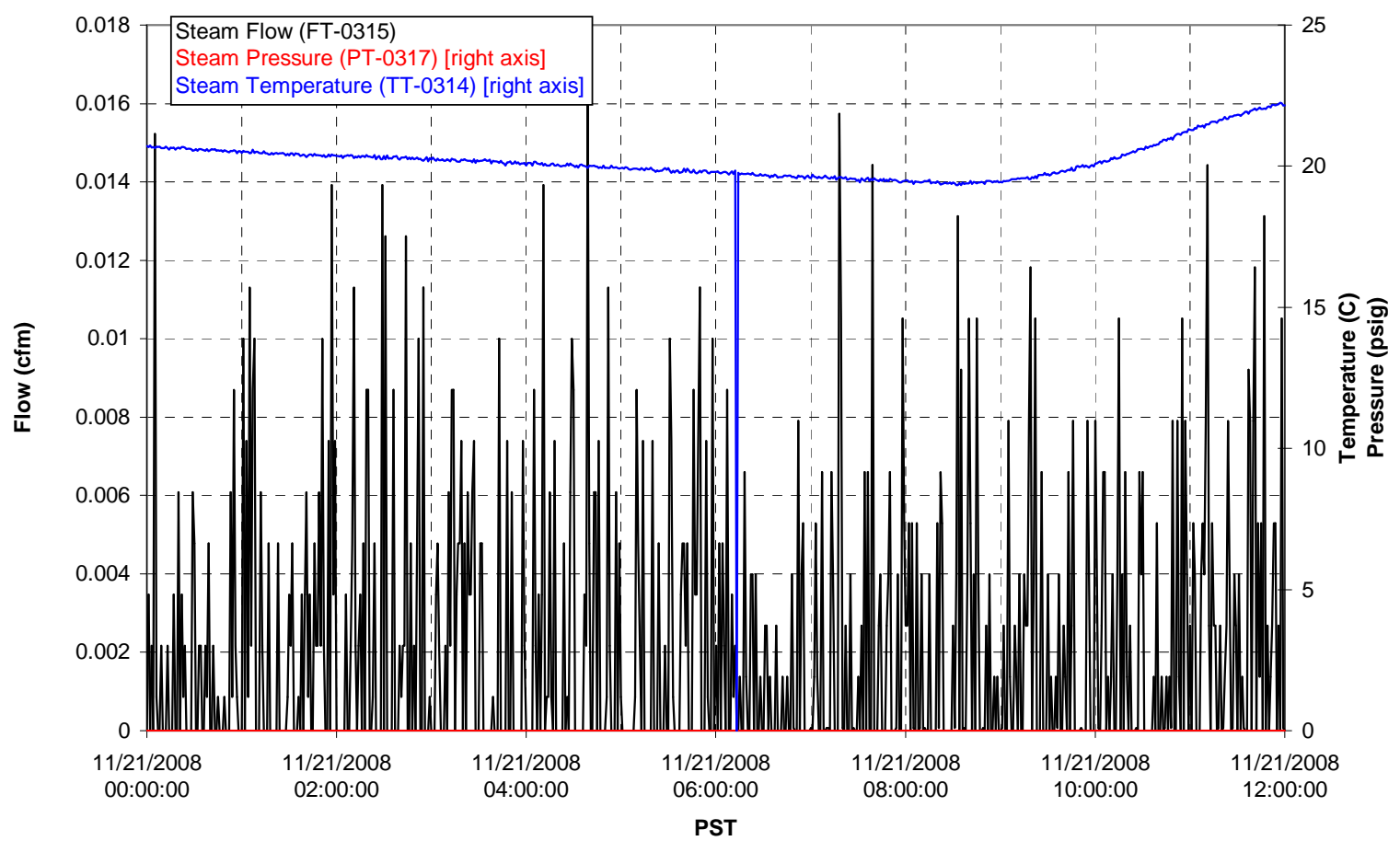


T01B Steam

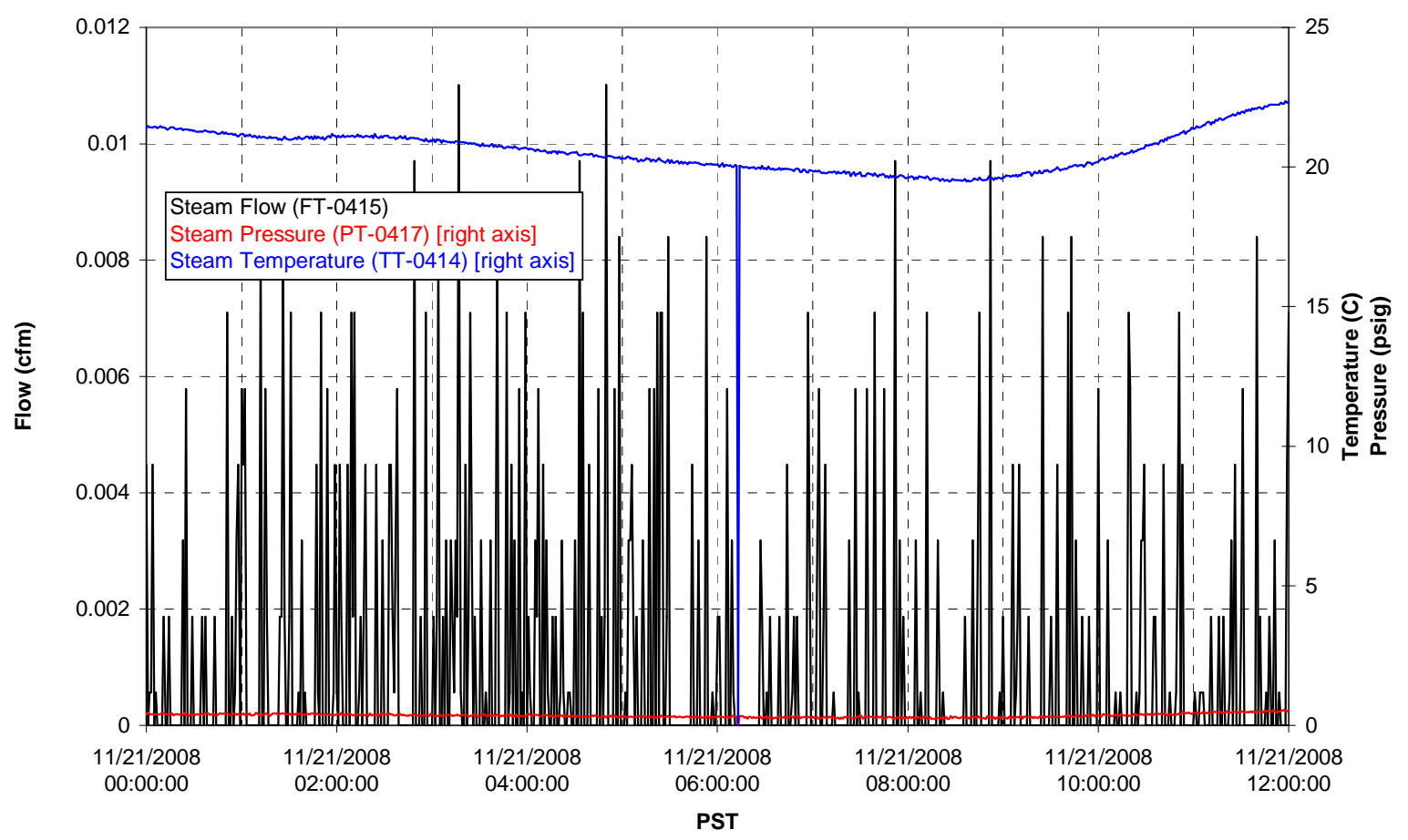


Functional Test Data Plots

11/21/2008 12:00 - 11/22/2008 00:00 
T01A level

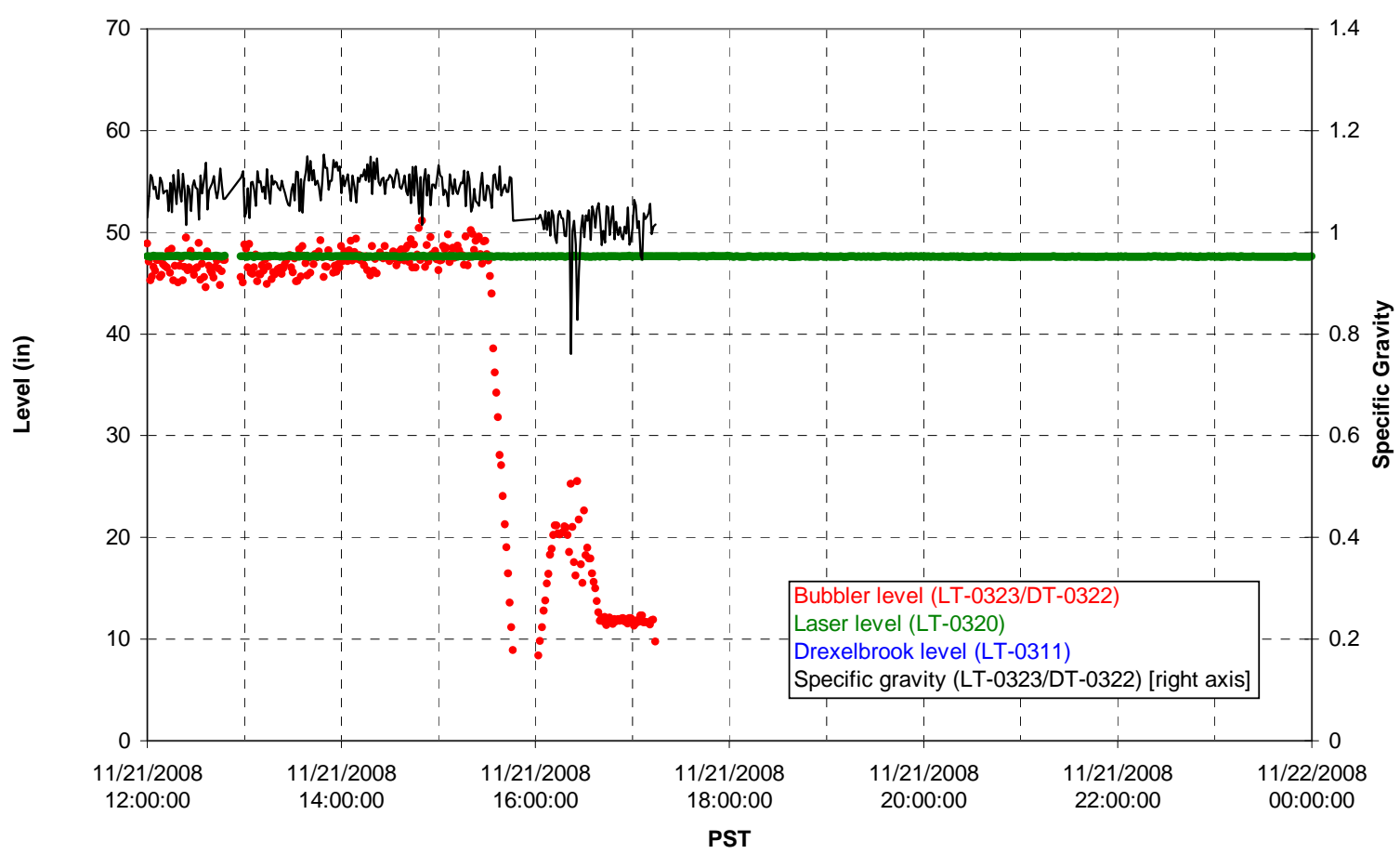

T01A temperatures

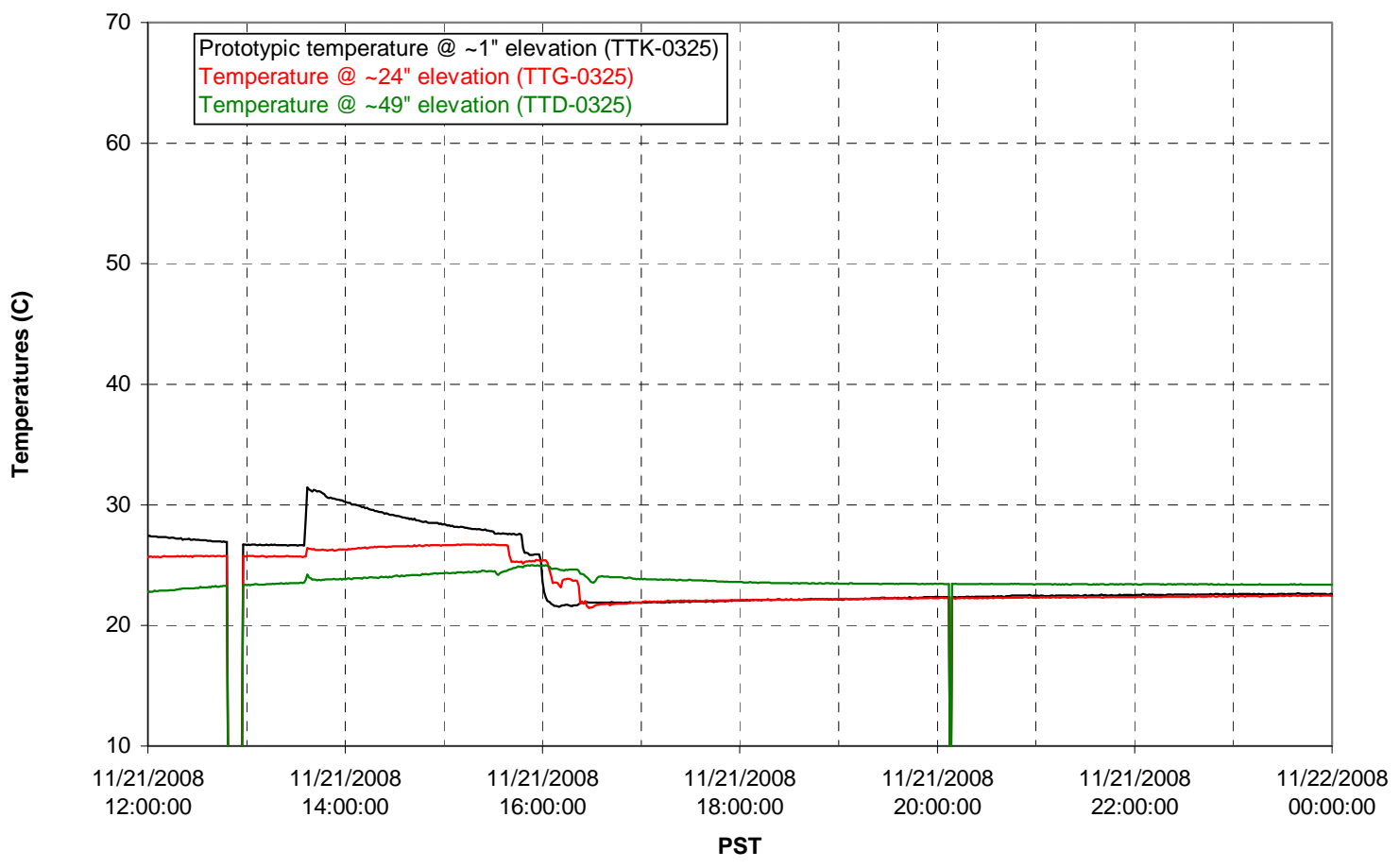


T01B level

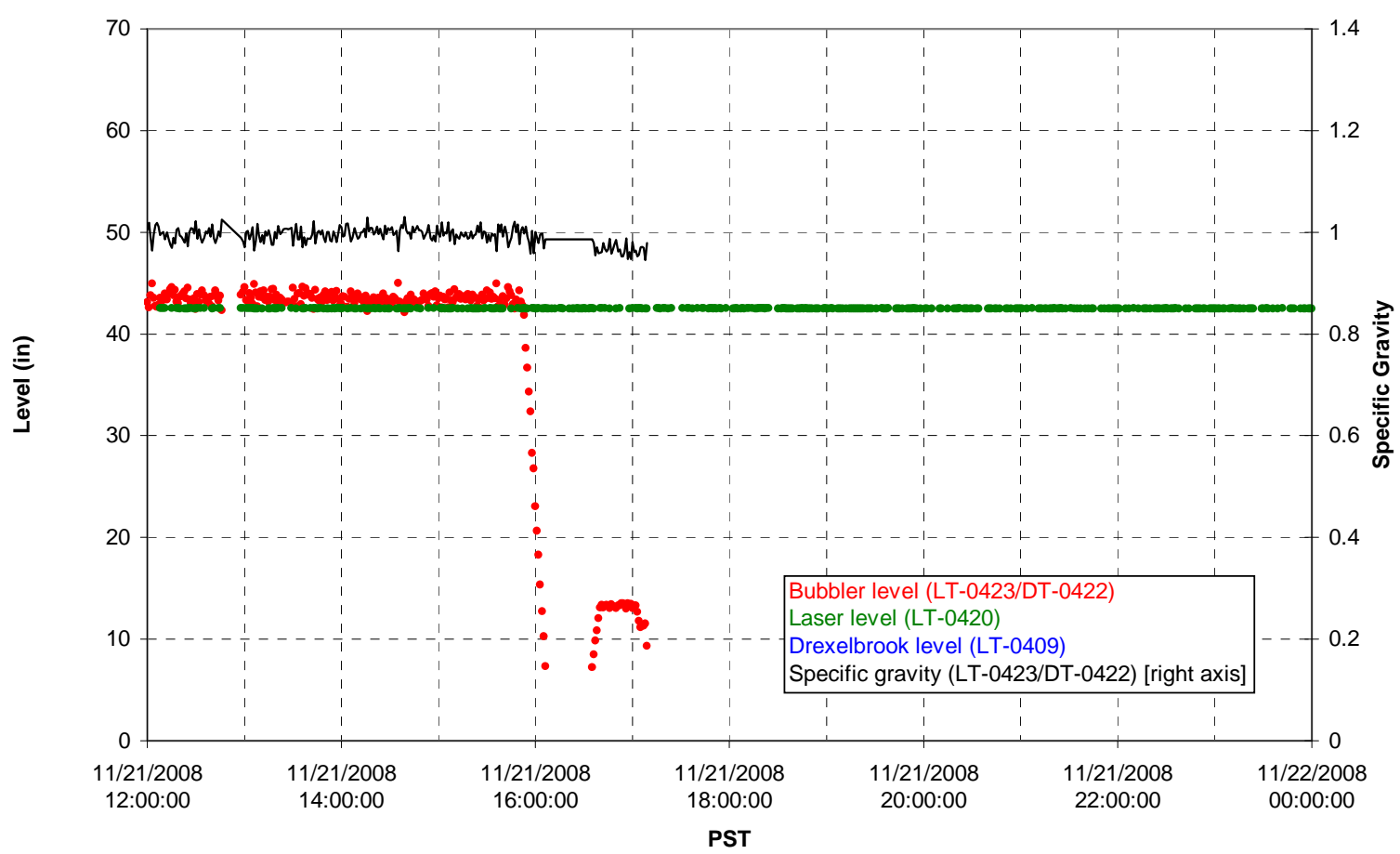

T01B temperatures

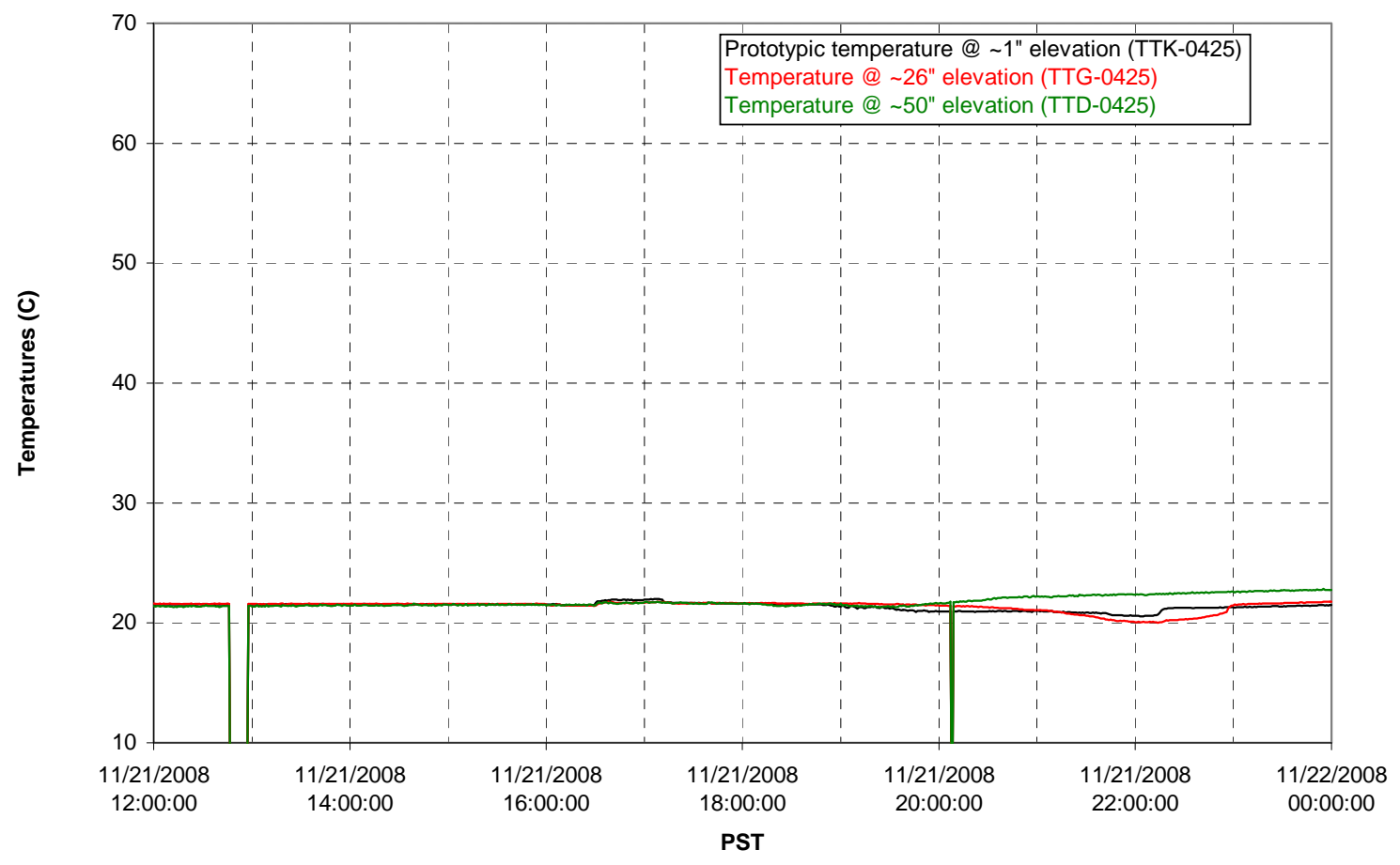


T02A level

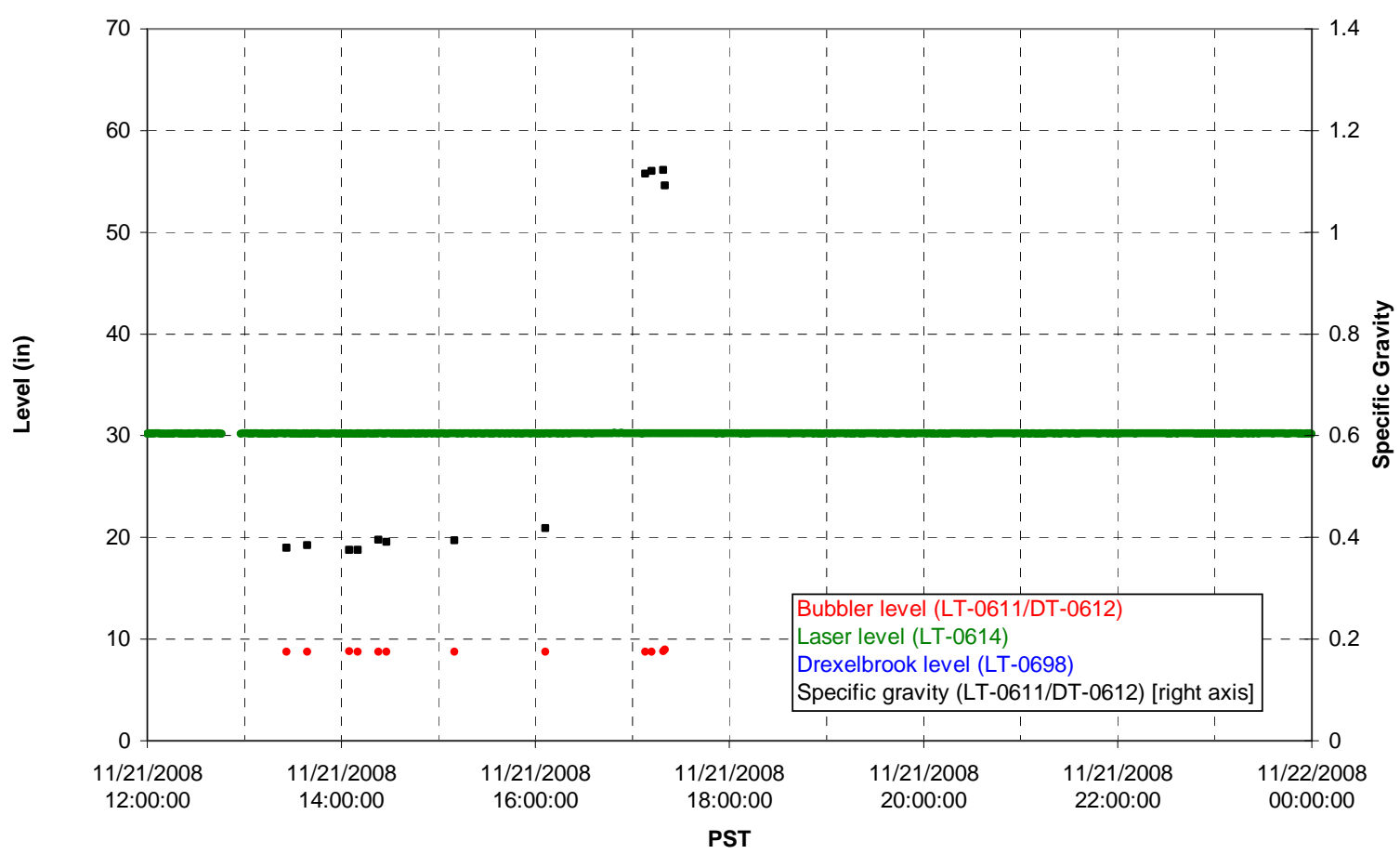

T02A temperatures

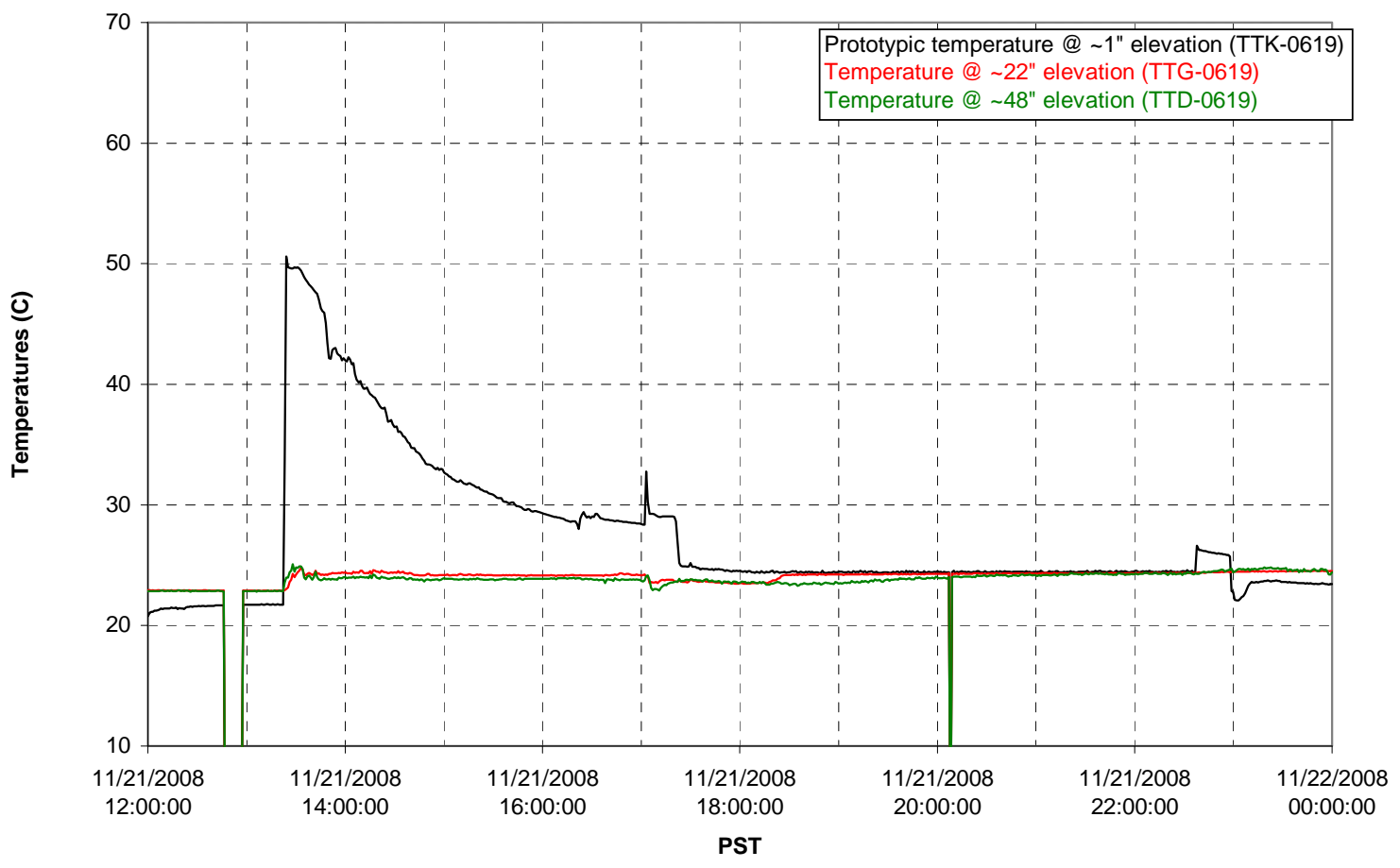


T02A and filter loop temperatures

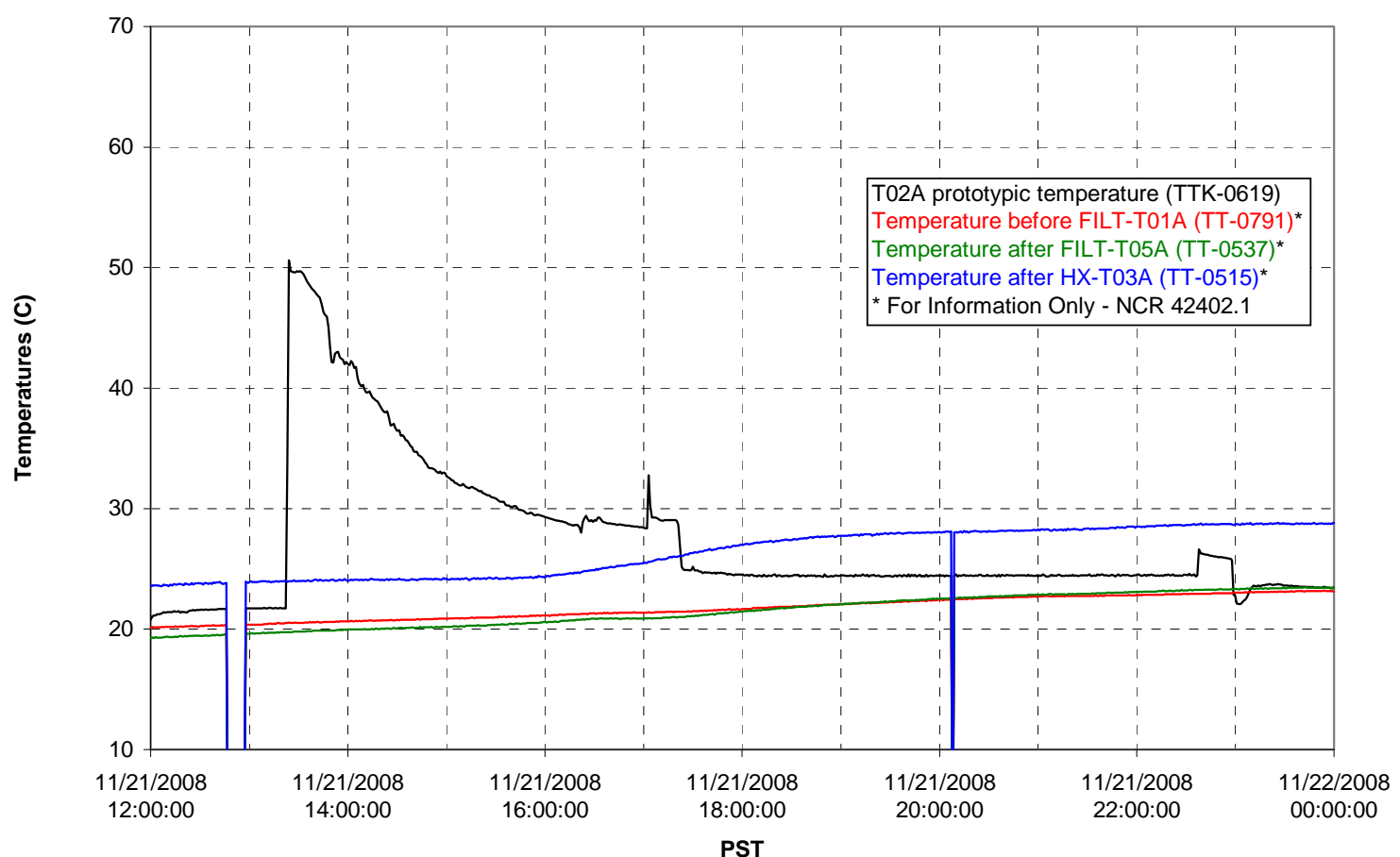

Pump Pressures and Flow

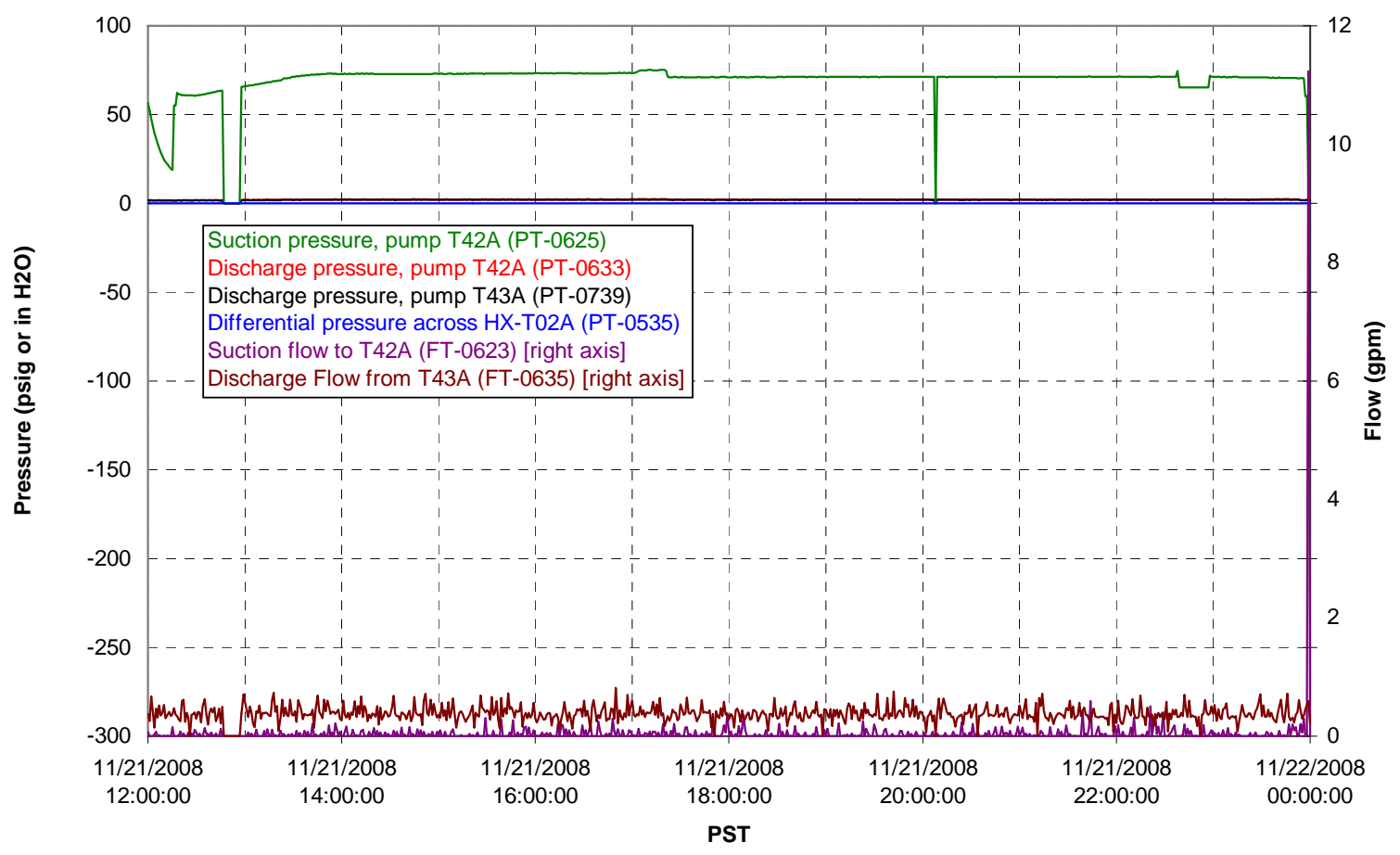


Axial pressure drop

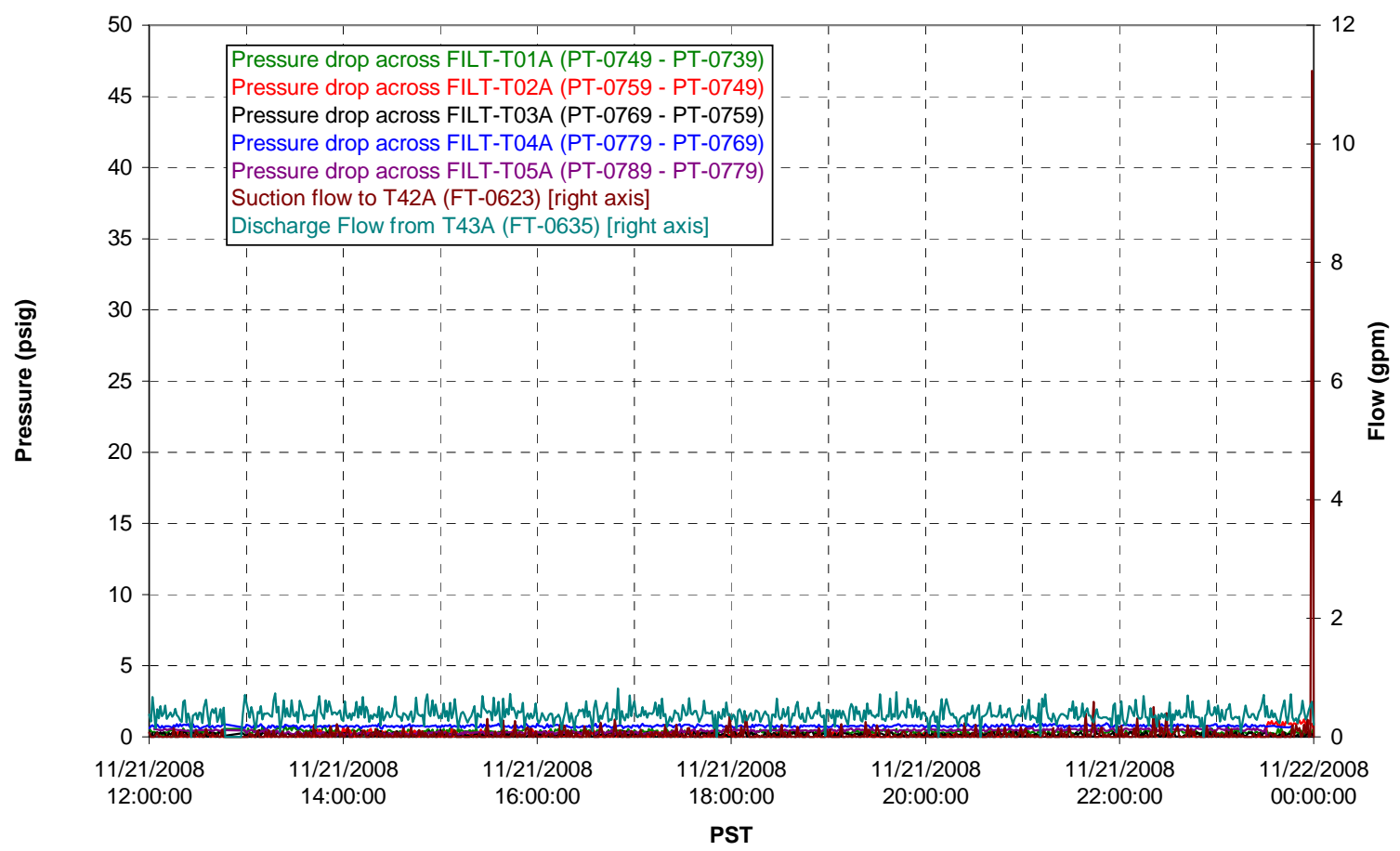

Permeate flow rates

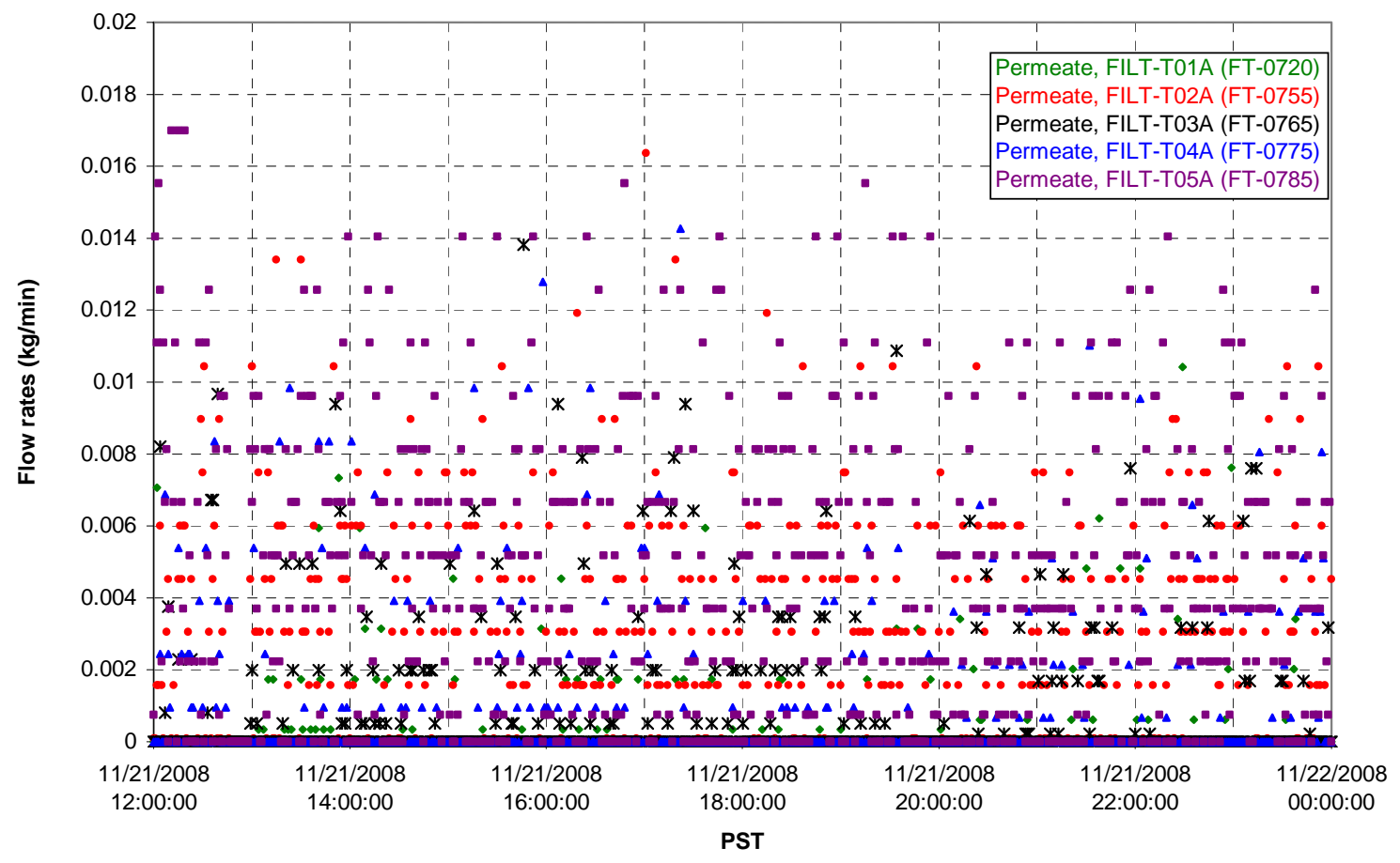


T02A Inner Temperature Tree

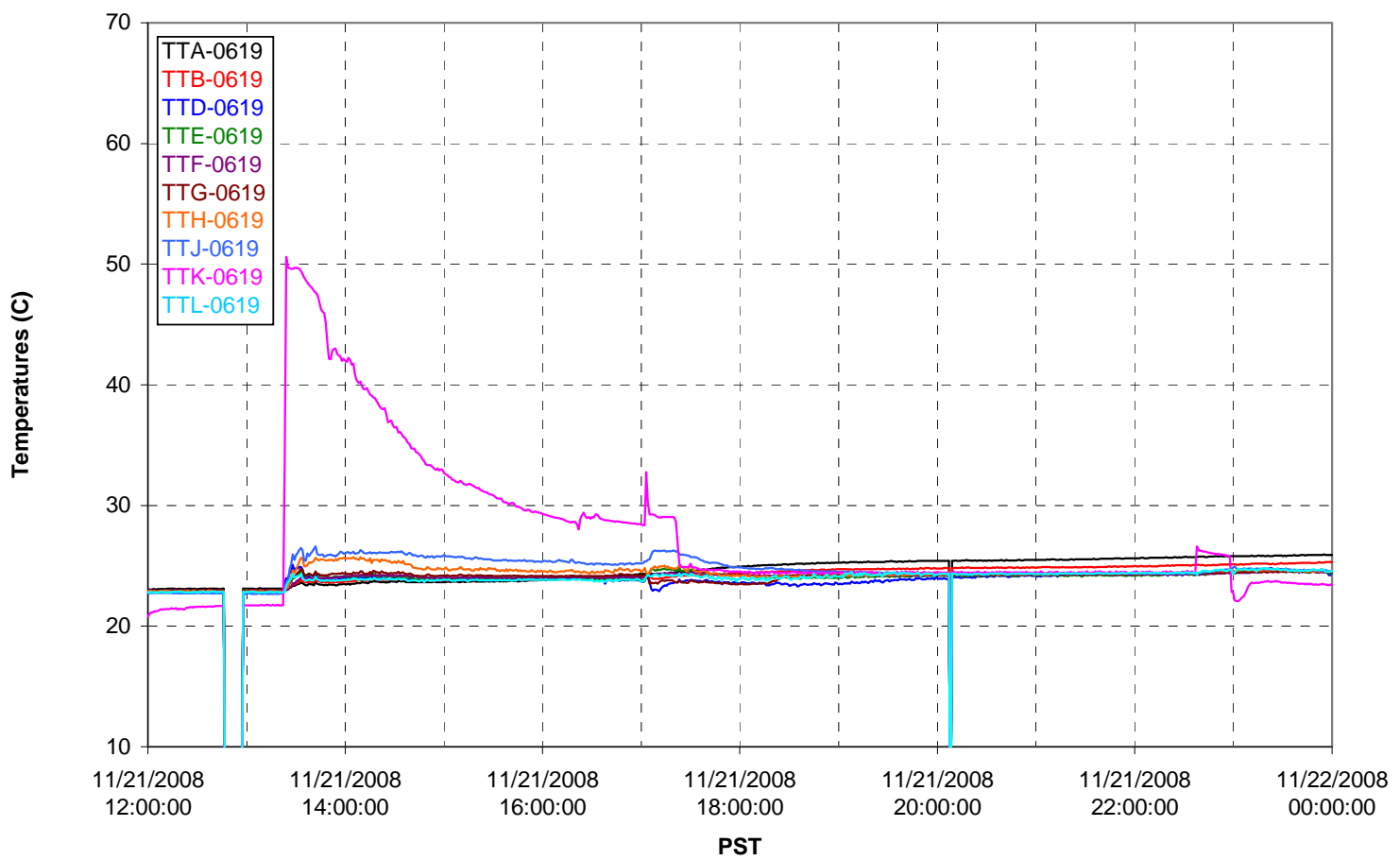

T02A Outer Temperature Tree

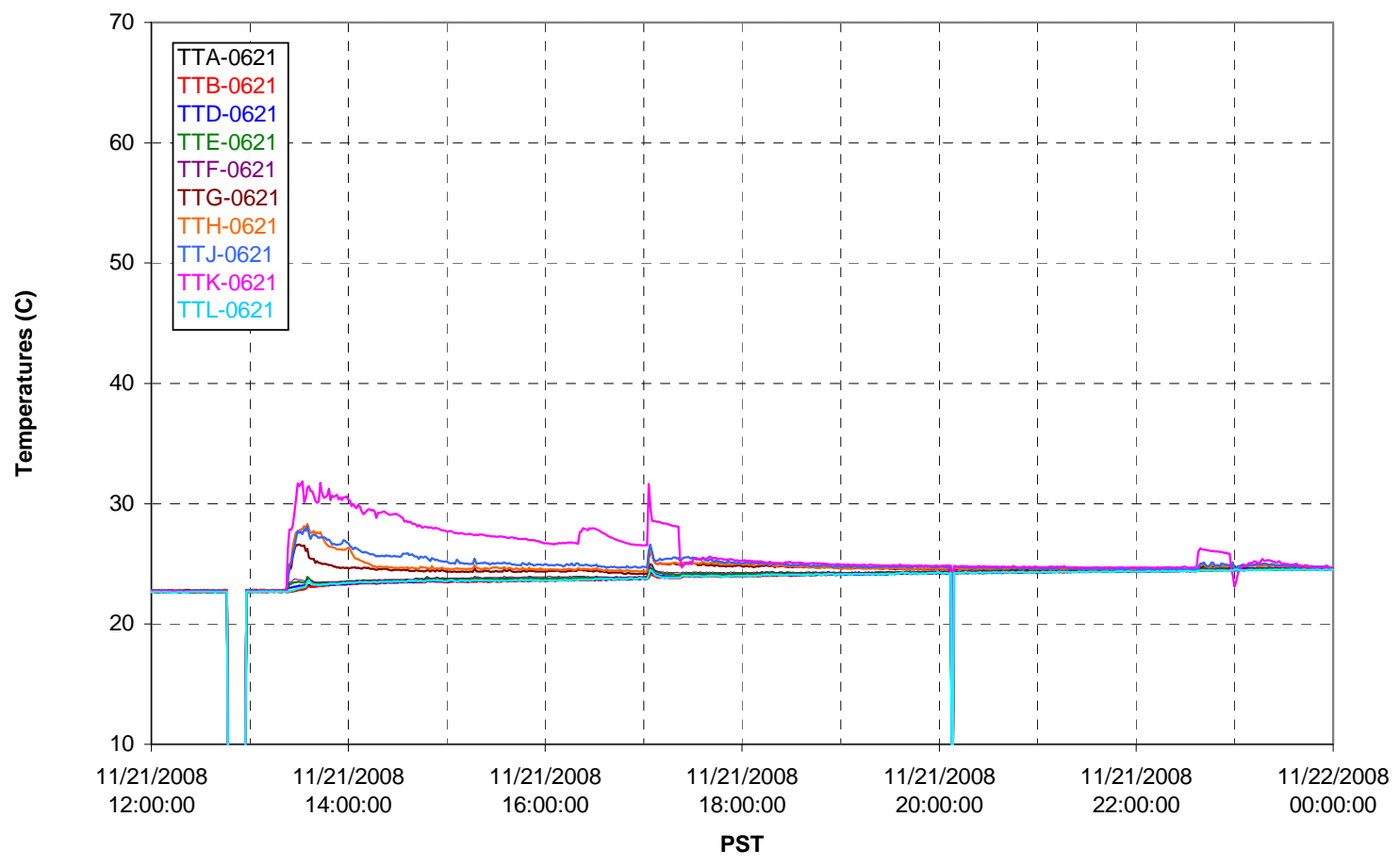


T02A temperatures

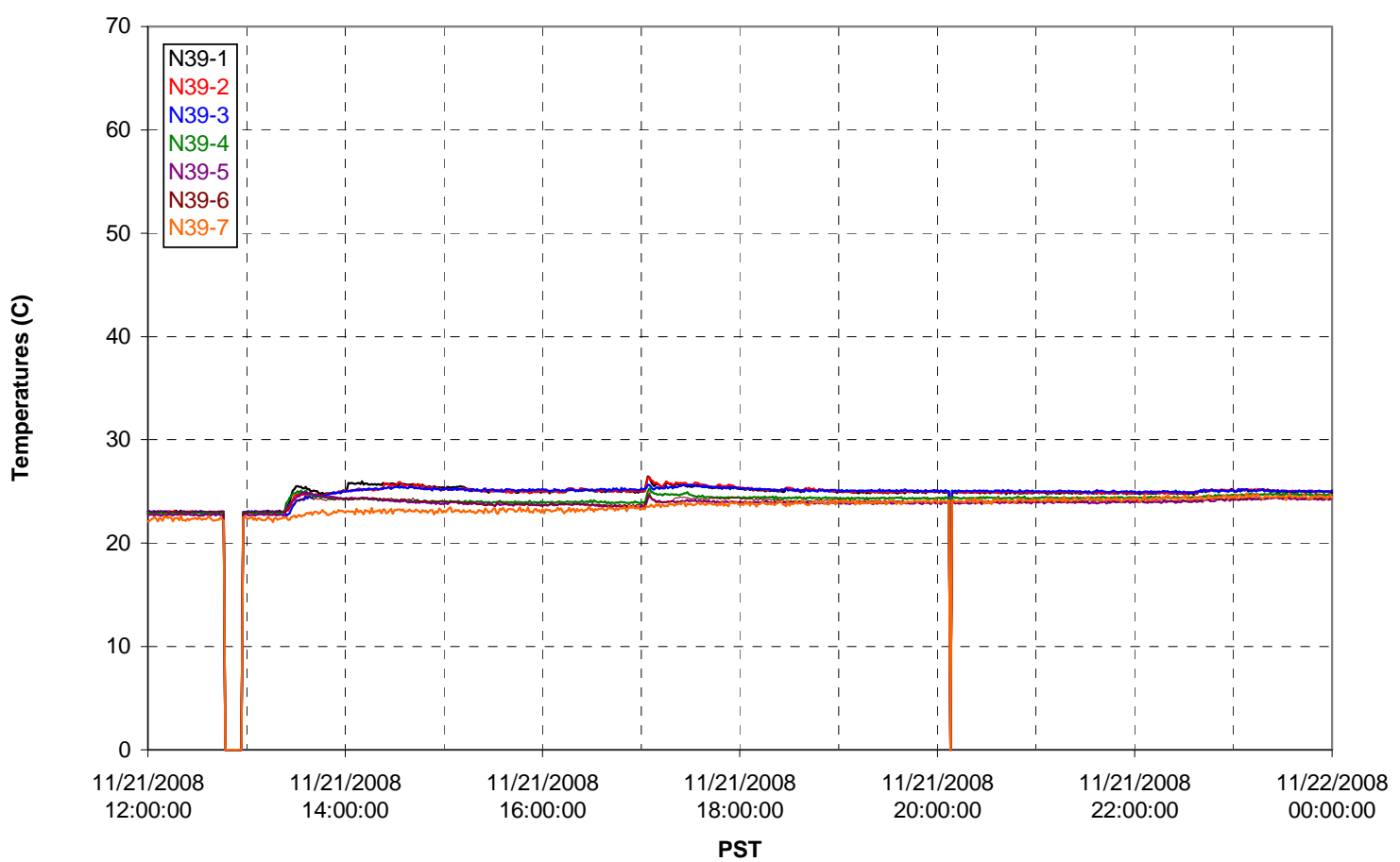

T02A temperatures

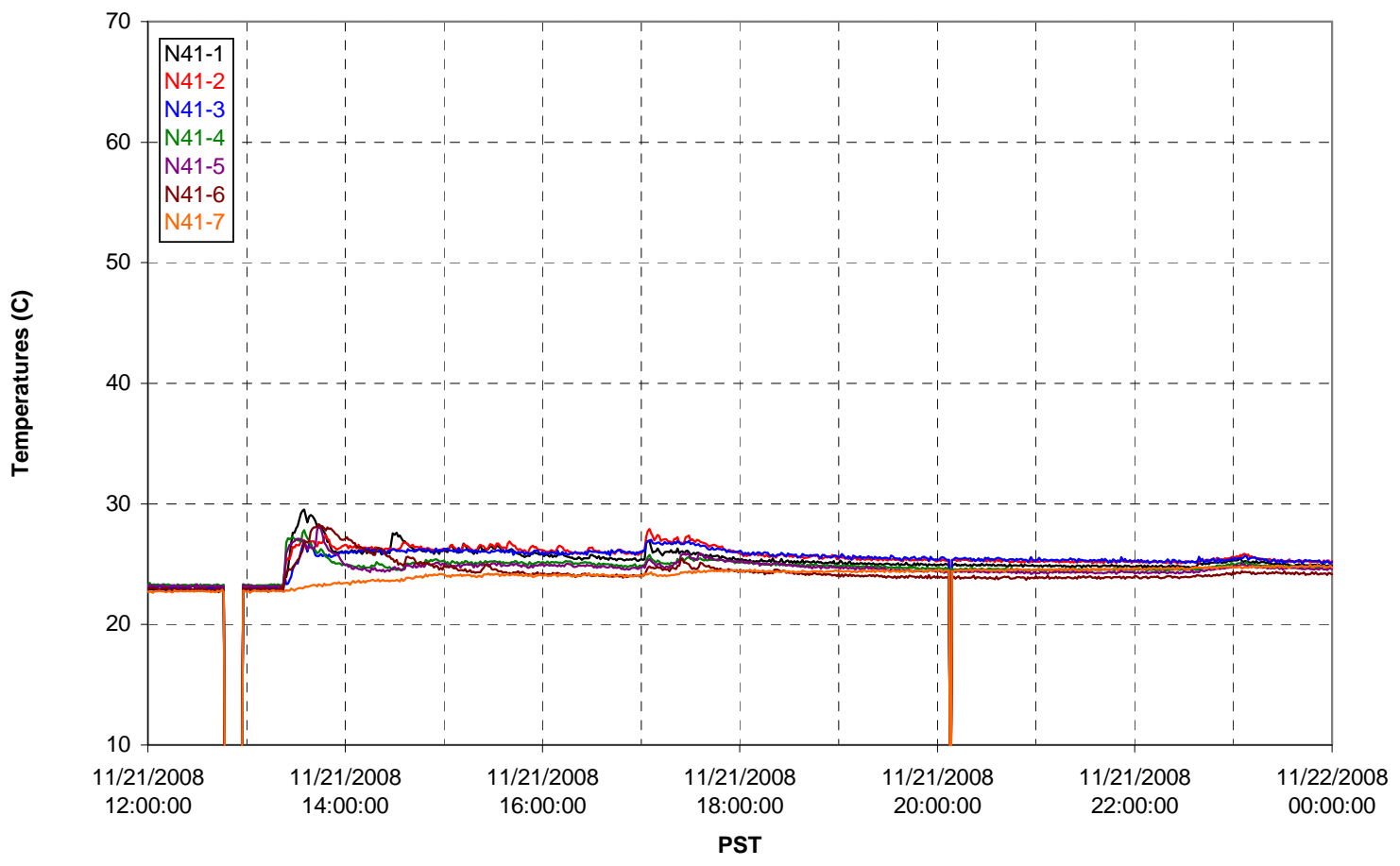


T02A temperatures

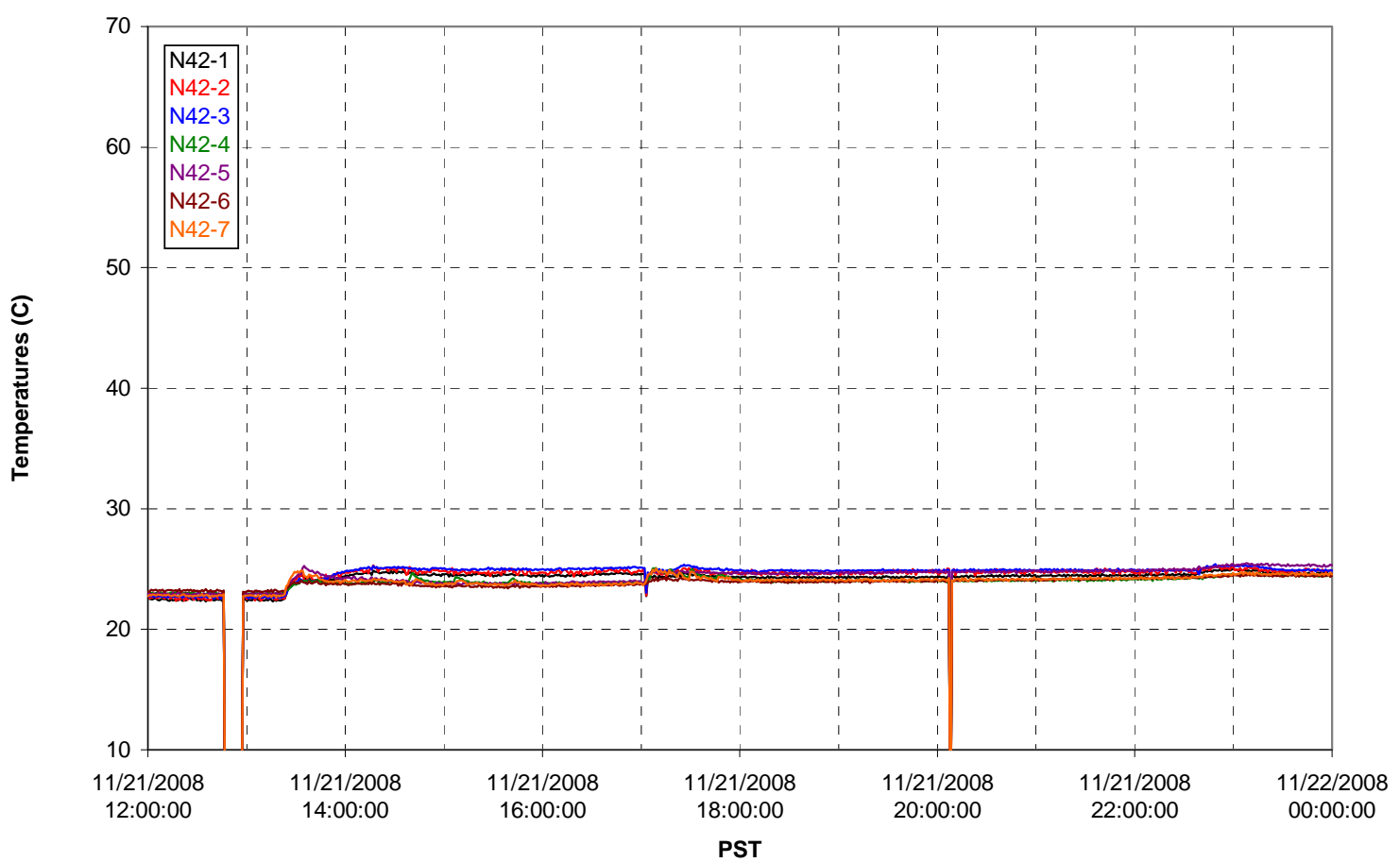

T02A temperatures

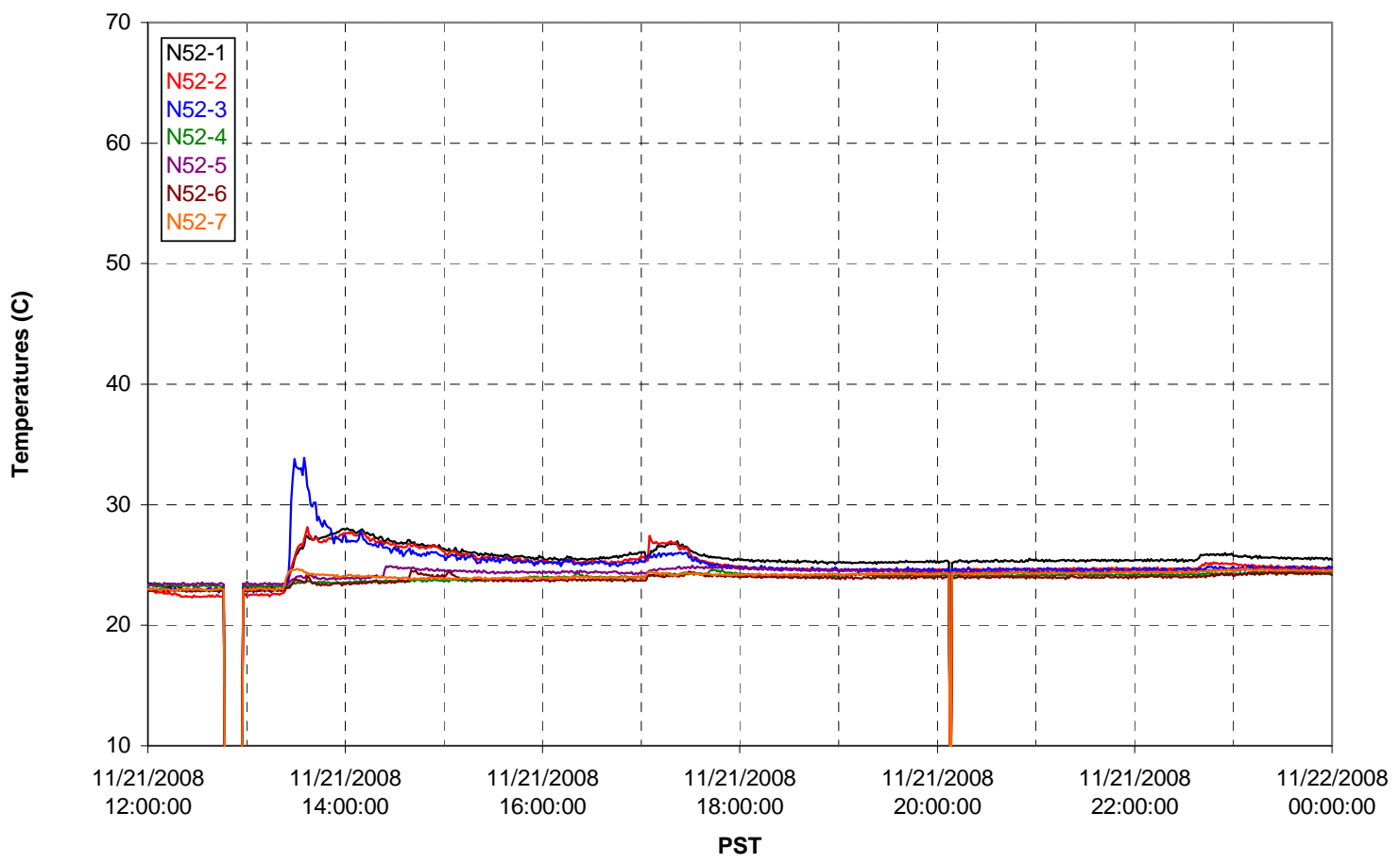


T02A Heating and Cooling

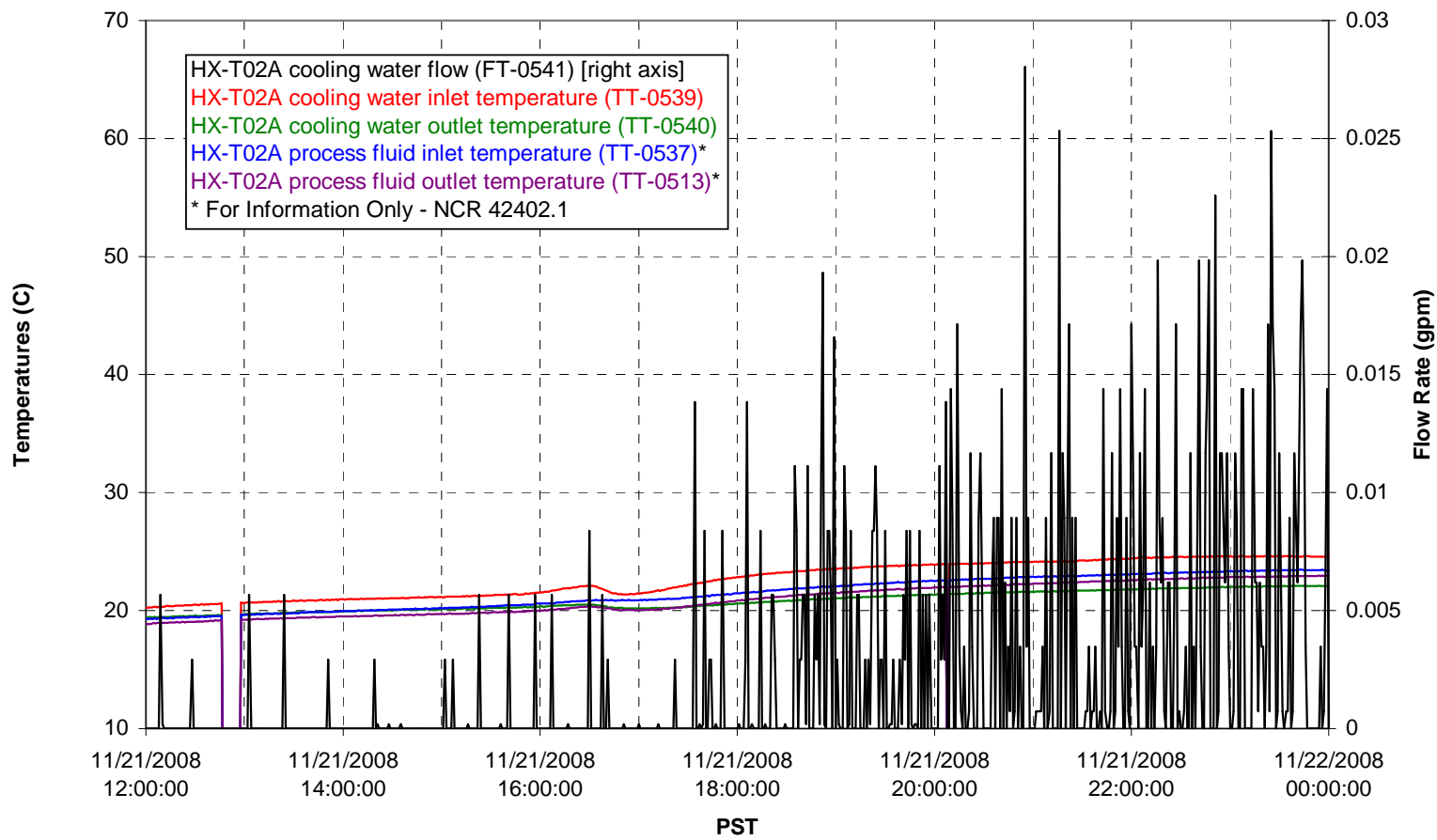

Pump Operation

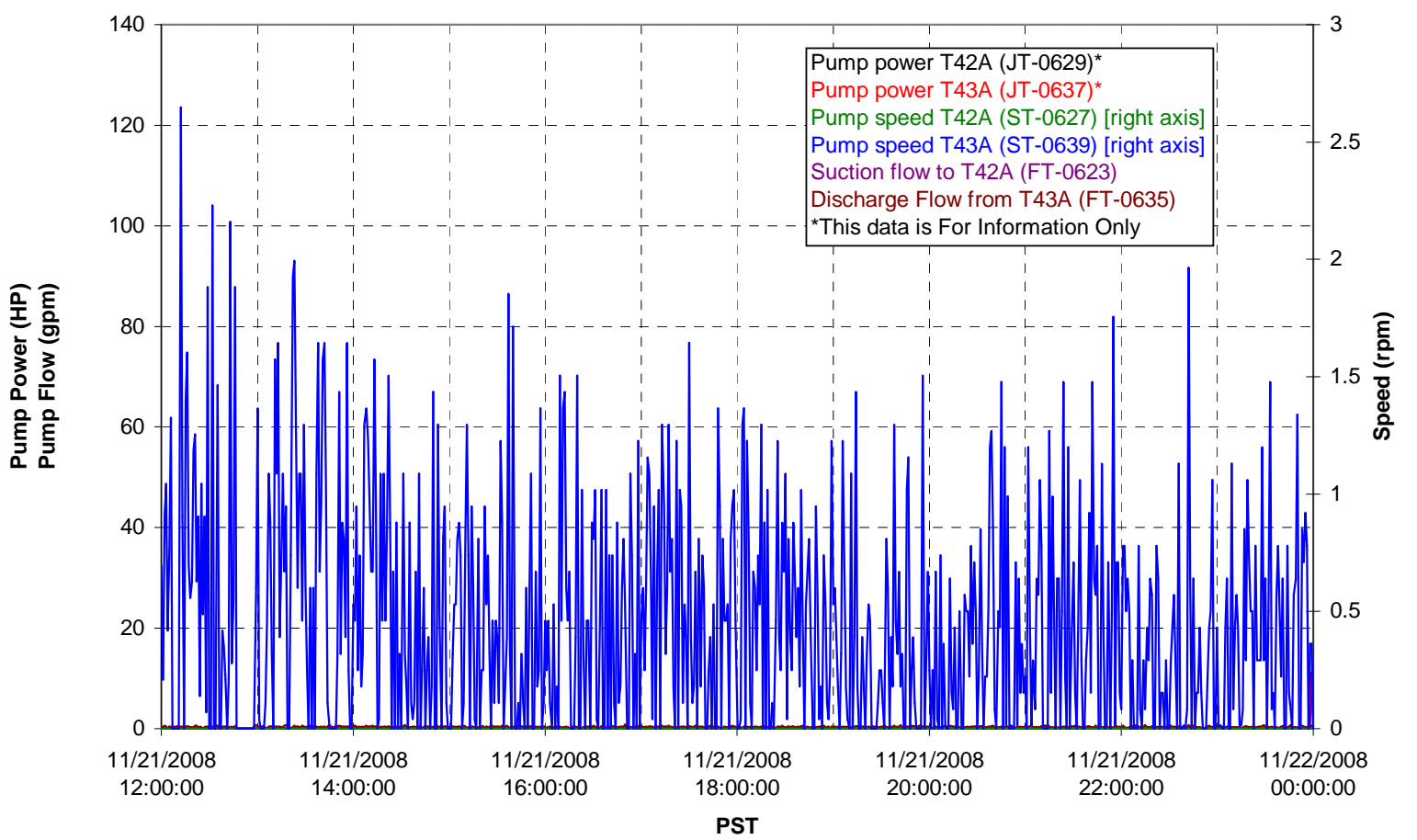


Pulsepot UFP-PP-T01A

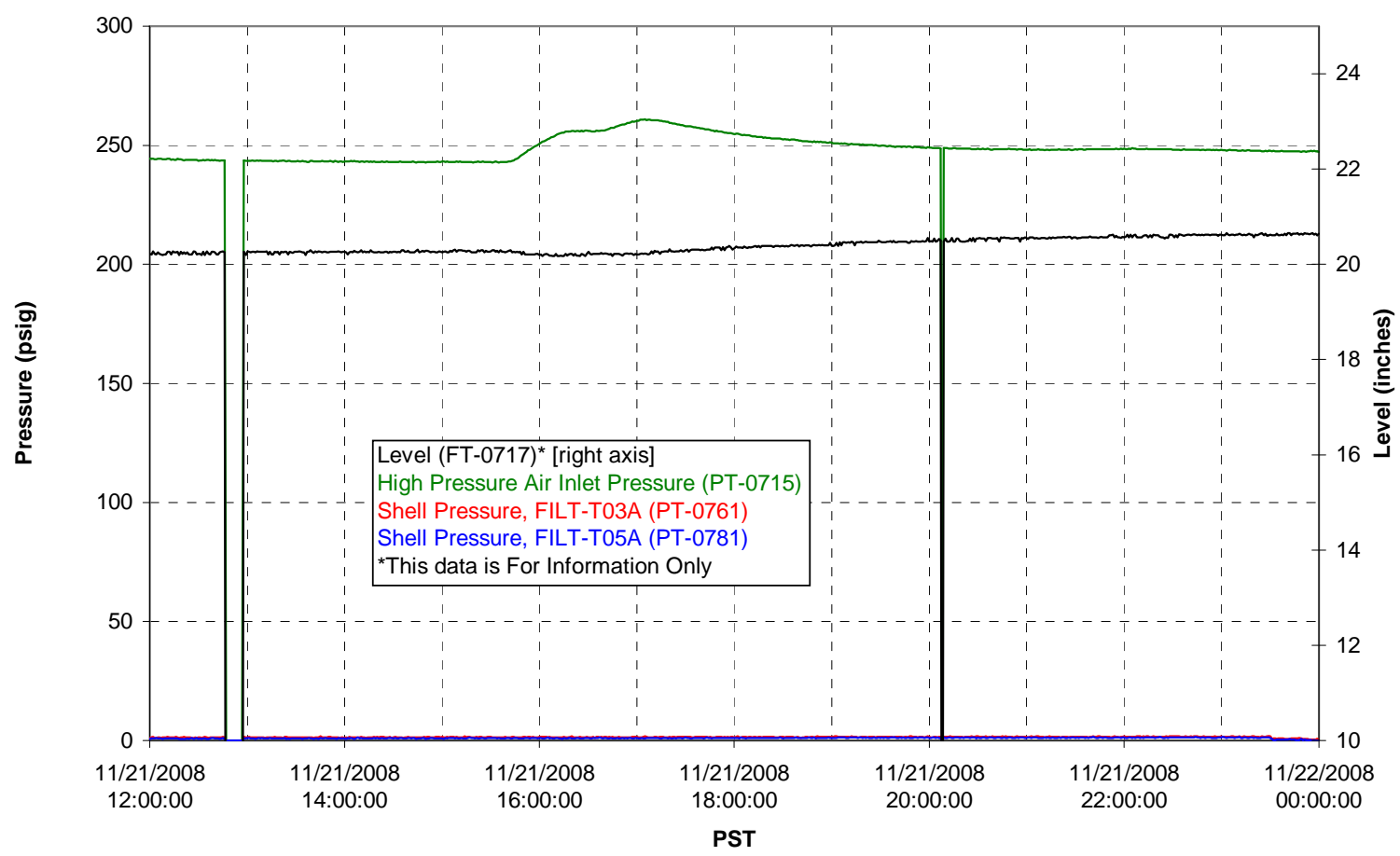

Pulsepot UFP-PP-T02A

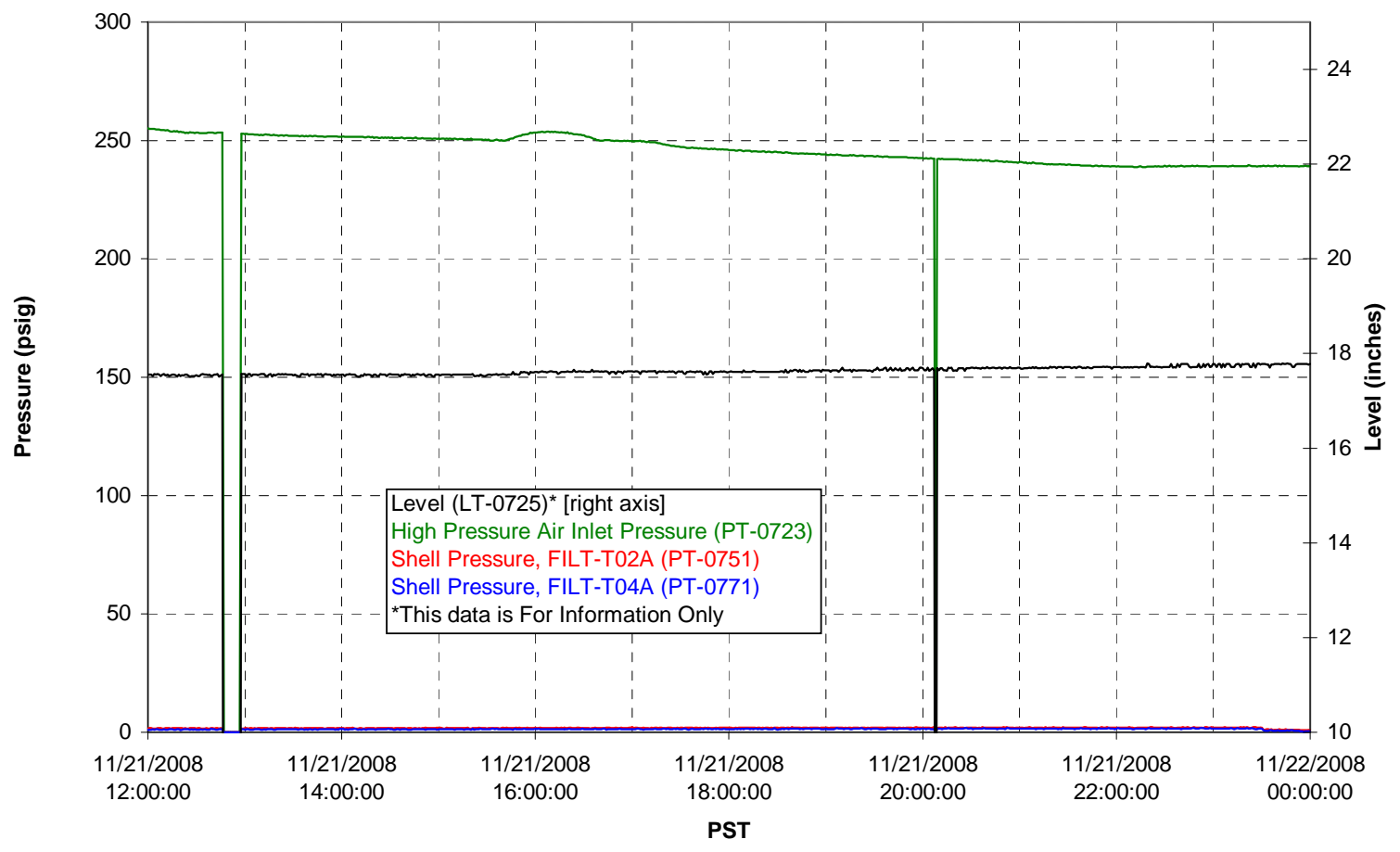


Pulsepot UFP-PP-T03A

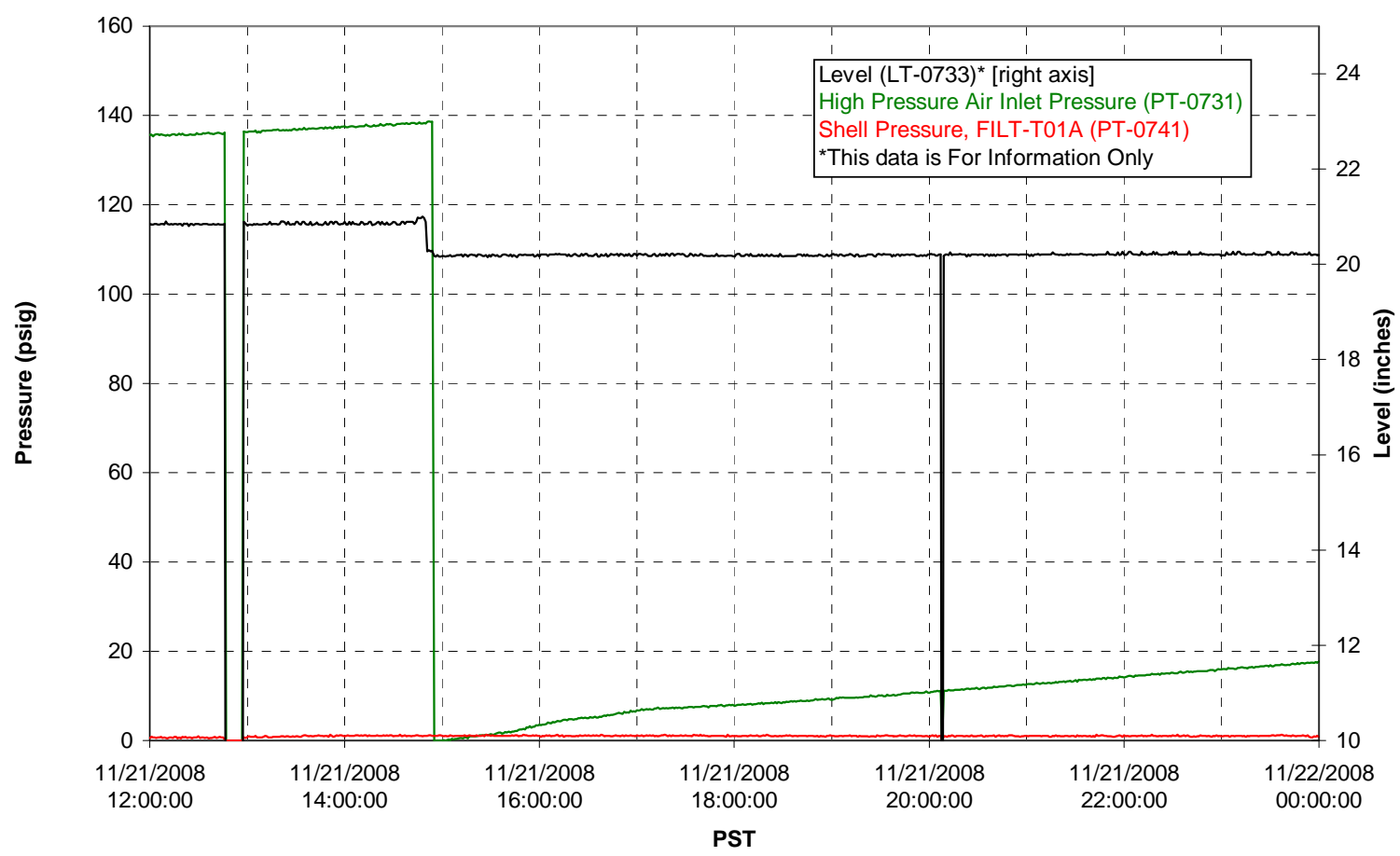

Pulsepot Levels

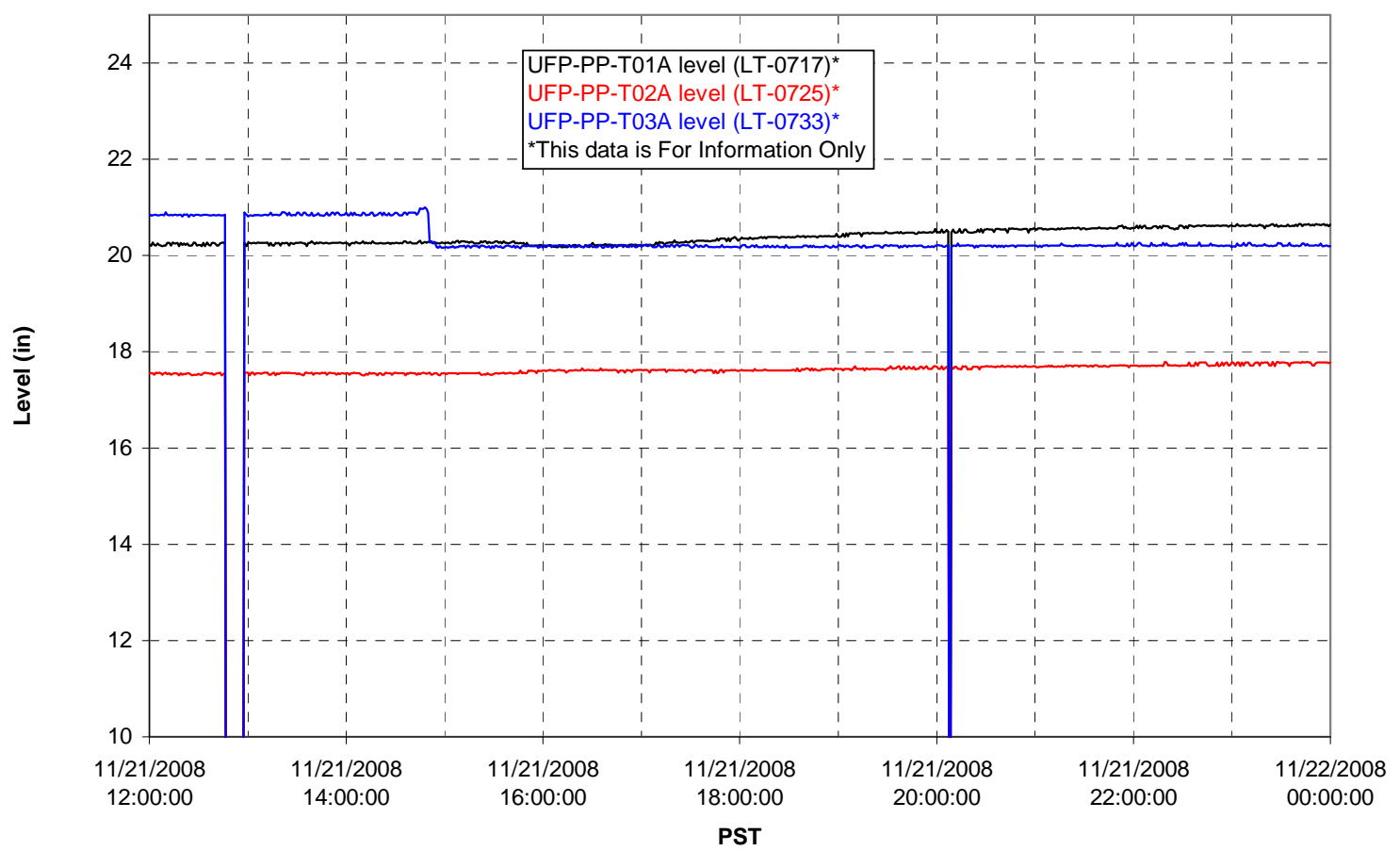


Filter UFP-FILT-T01A

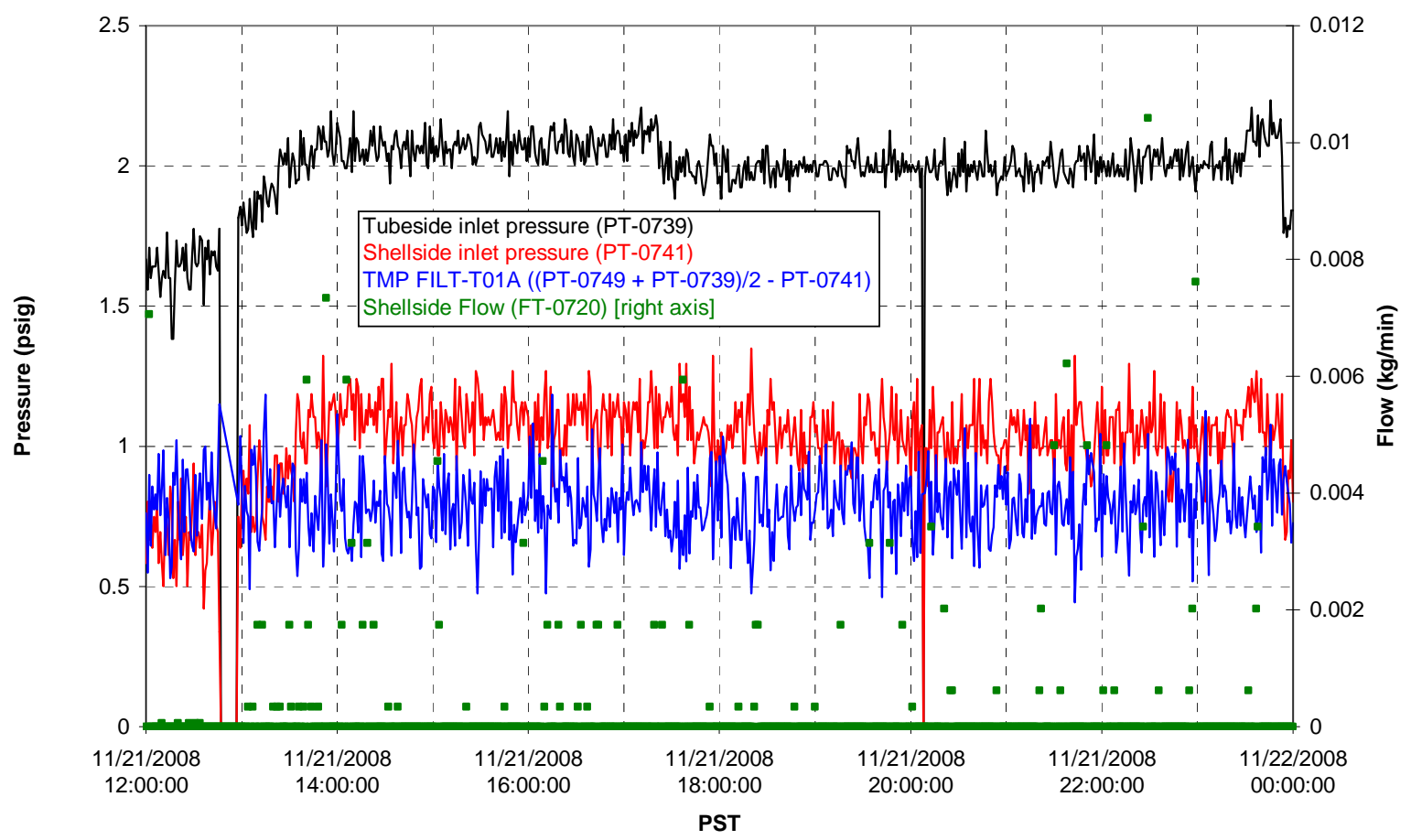

Filter UFP-FILT-T02A

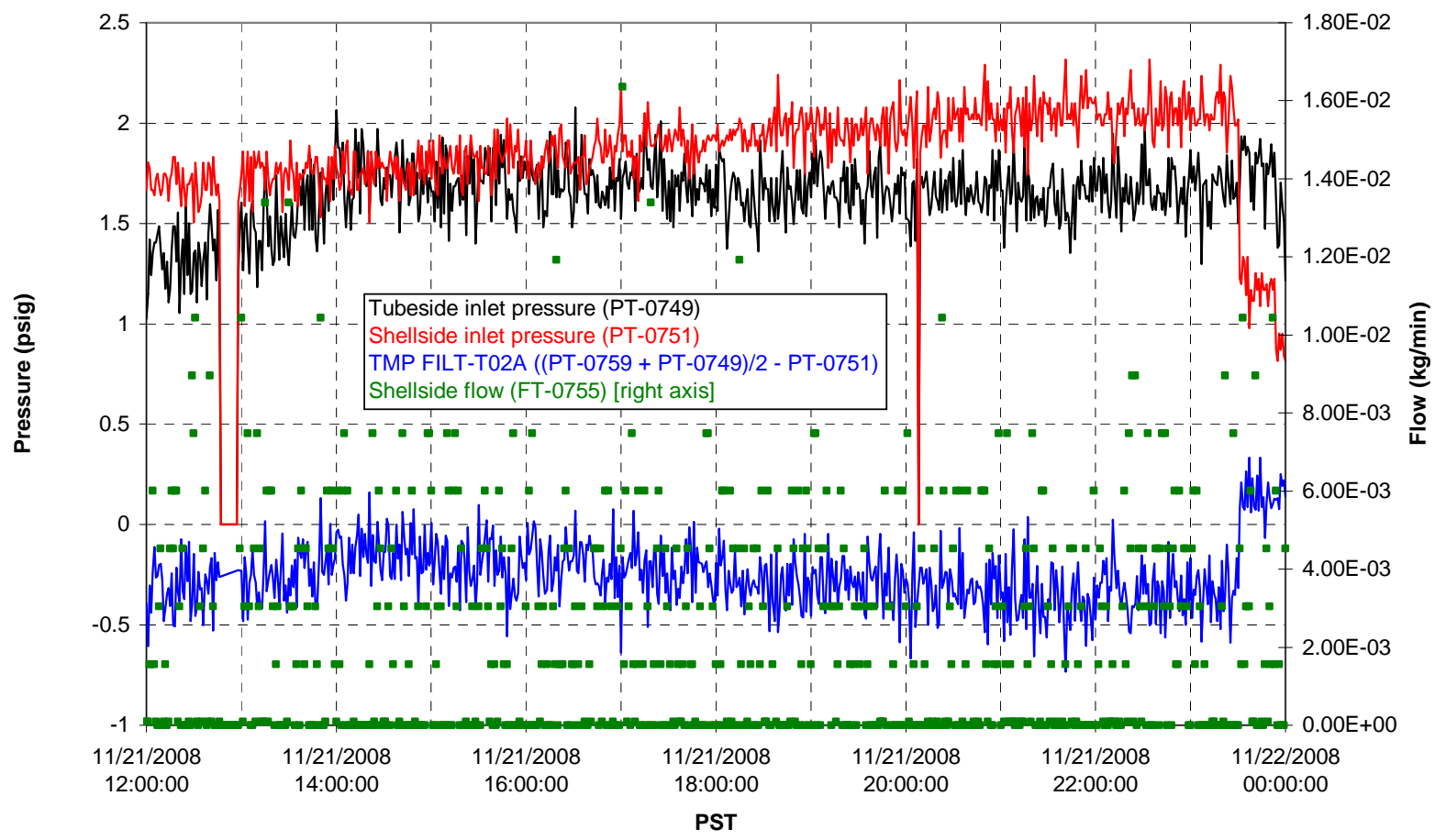


Filter UFP-FILT-T03A

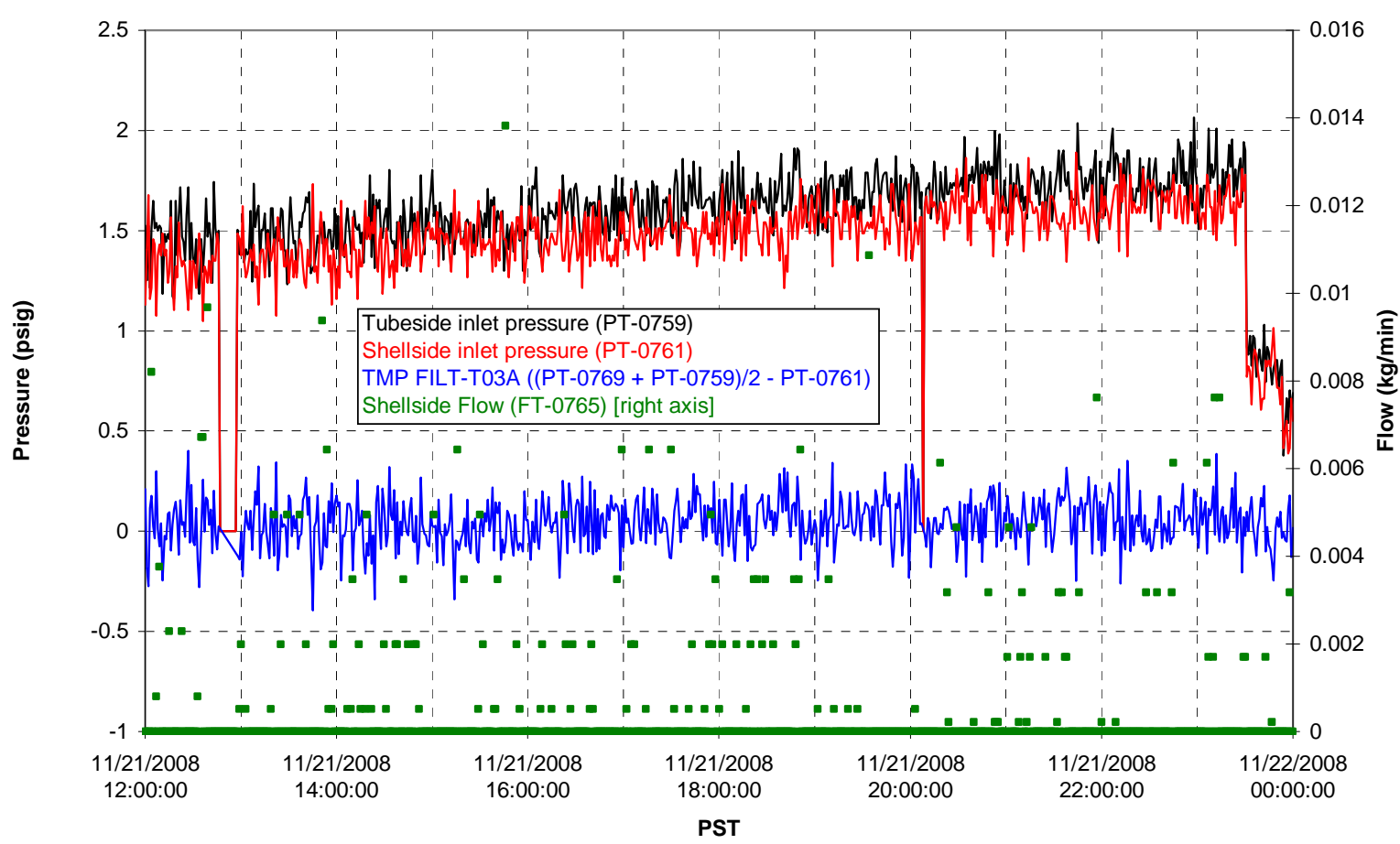

Filter UFP-FILT-T04A

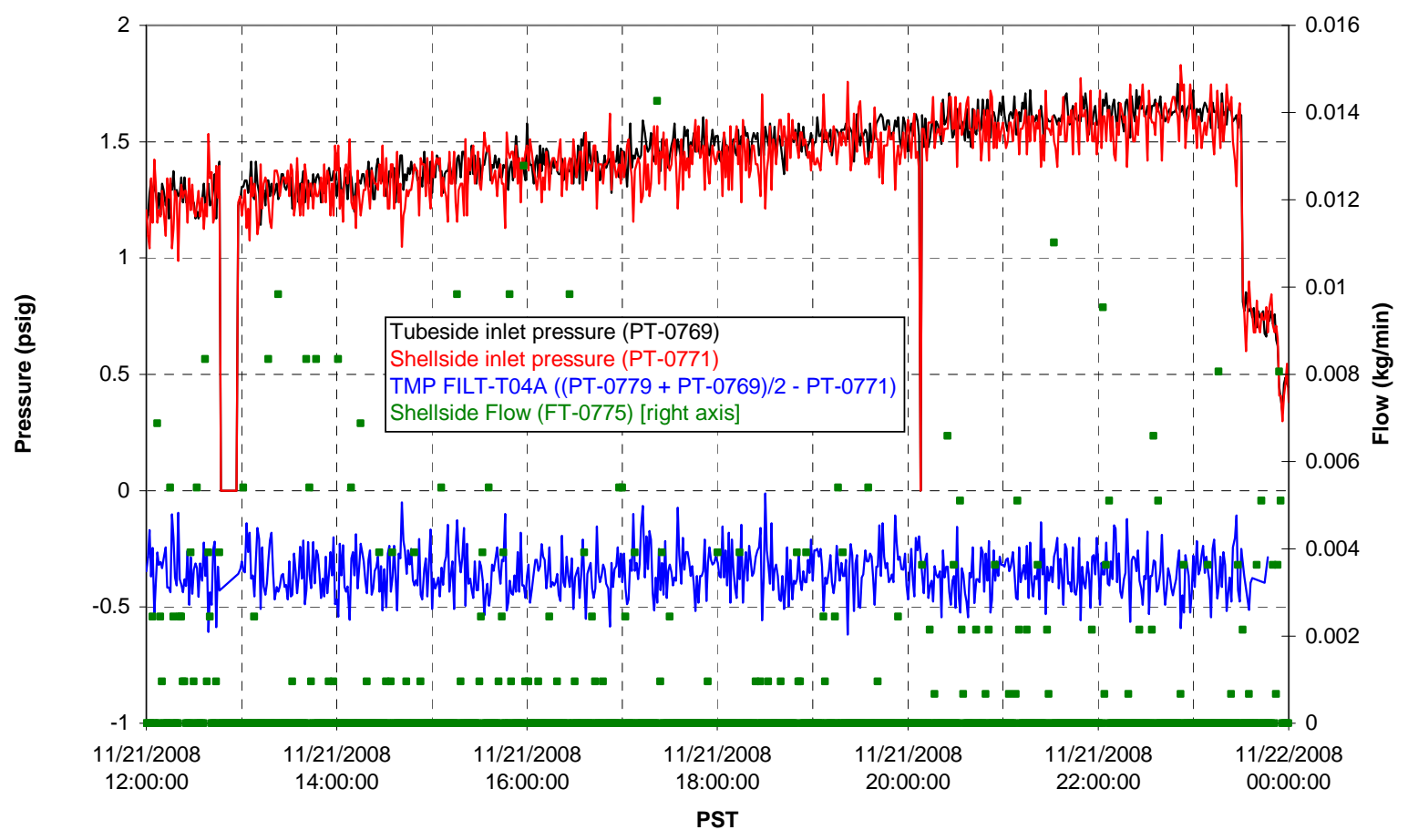


Filter UFP-FILT-T05A

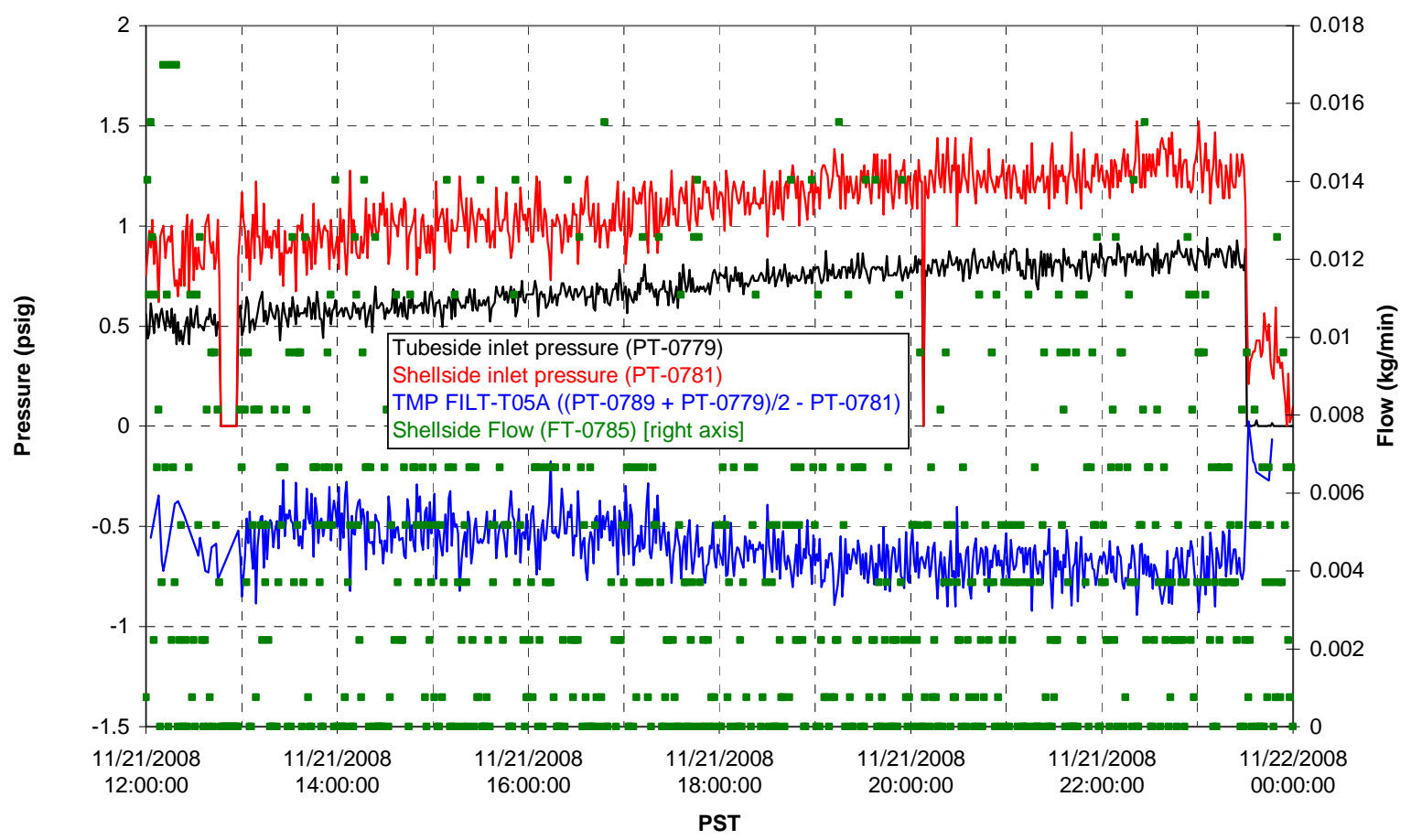

Chemical Flow

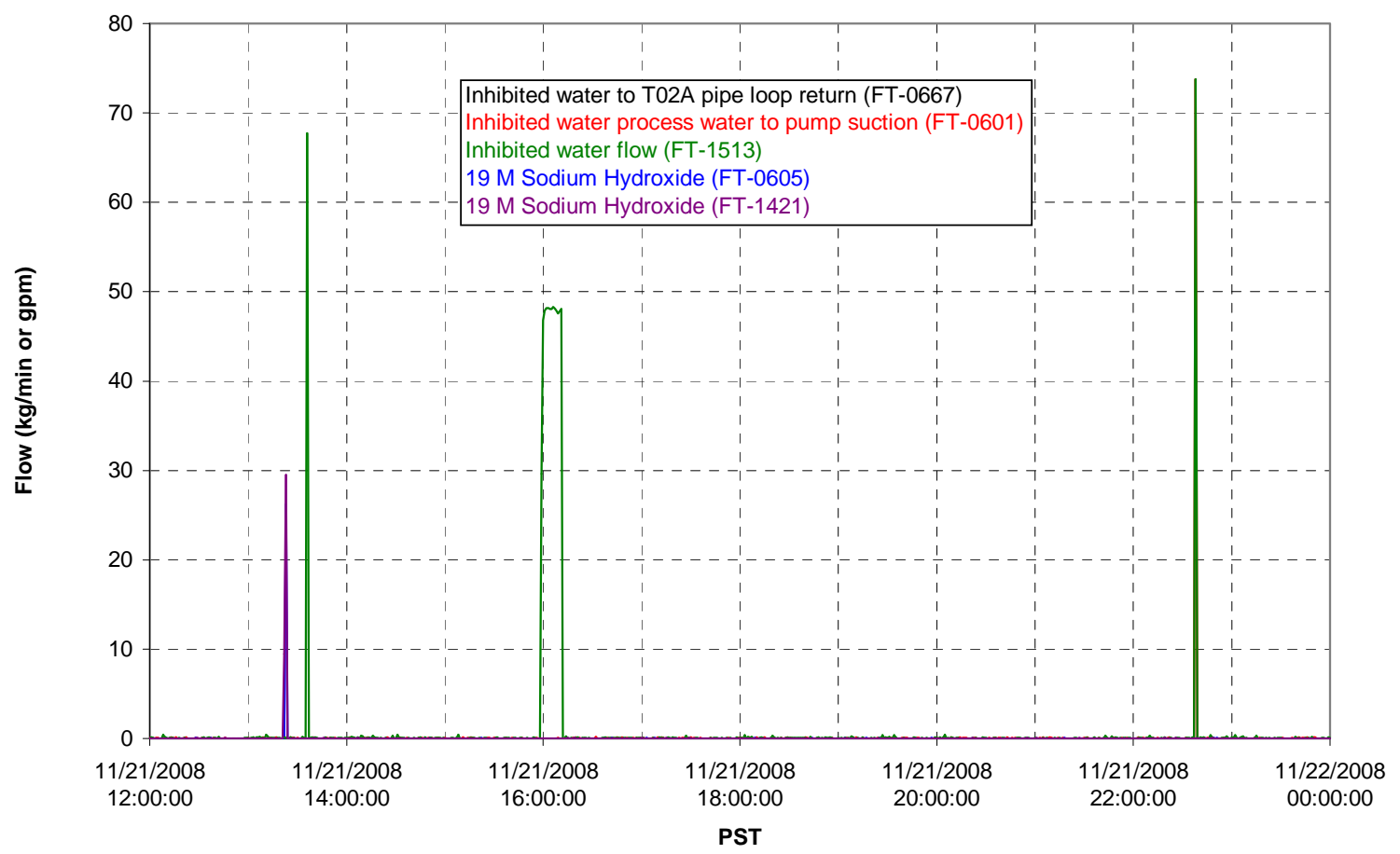




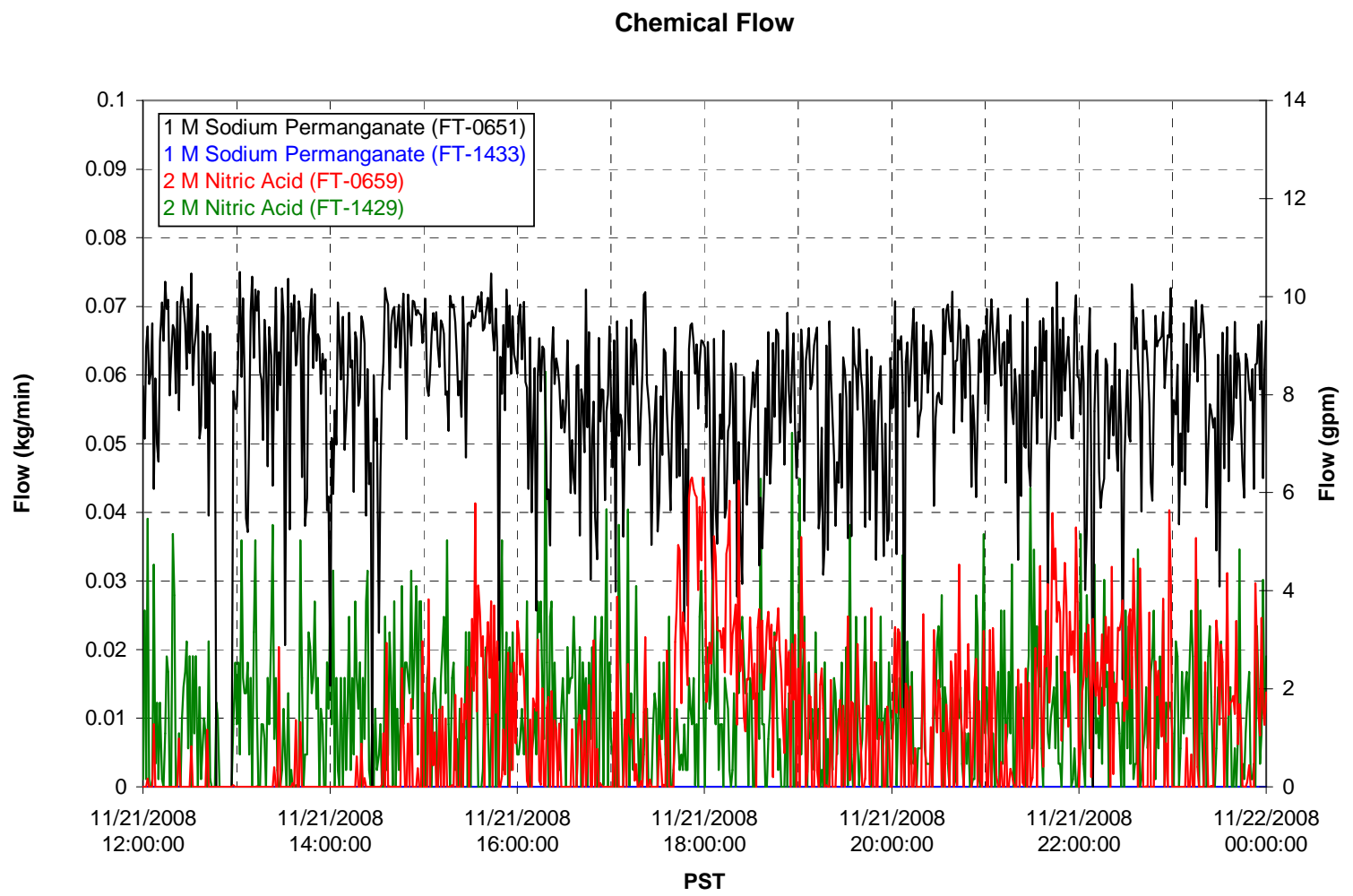

Air Flows

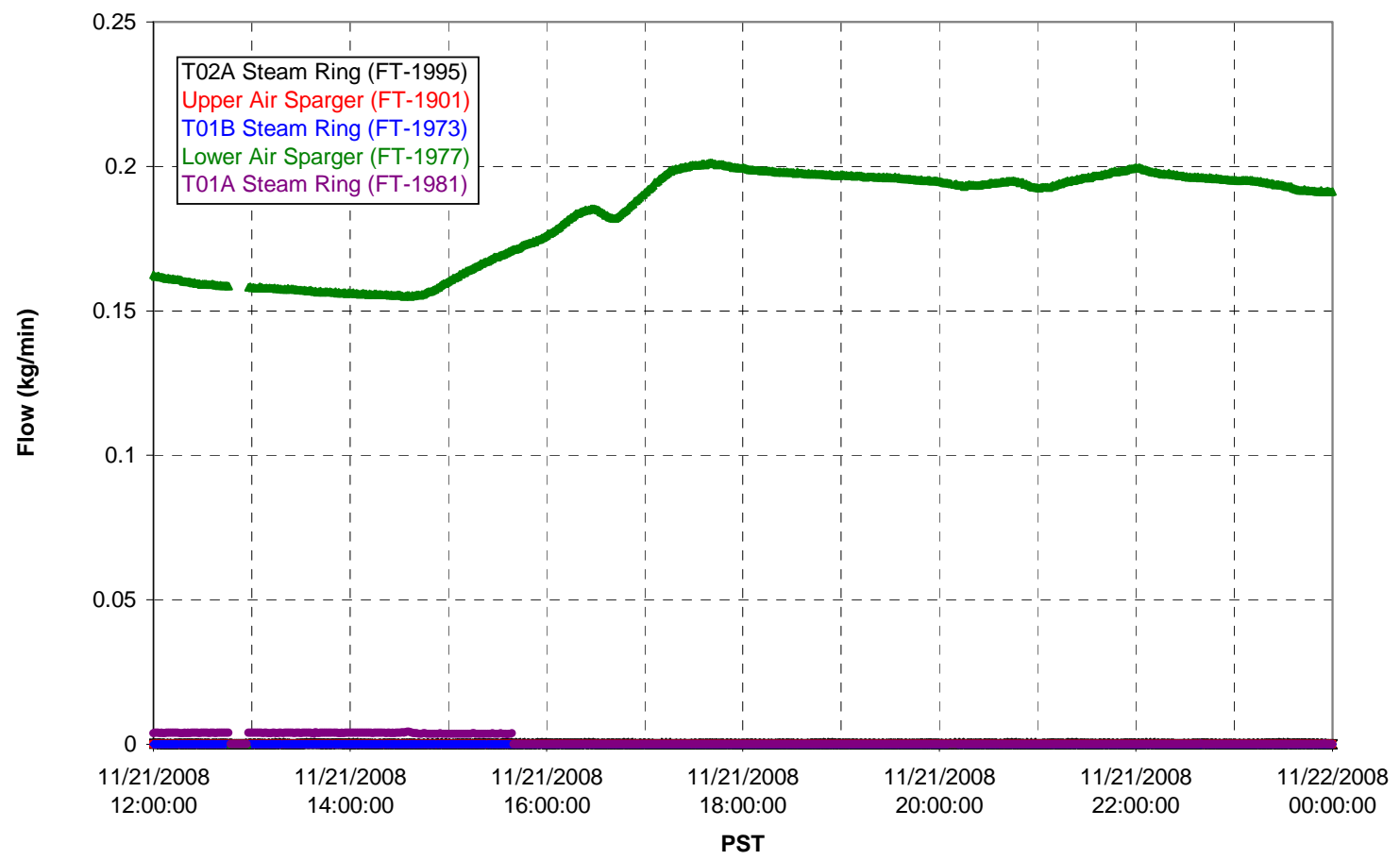


T02A Steam

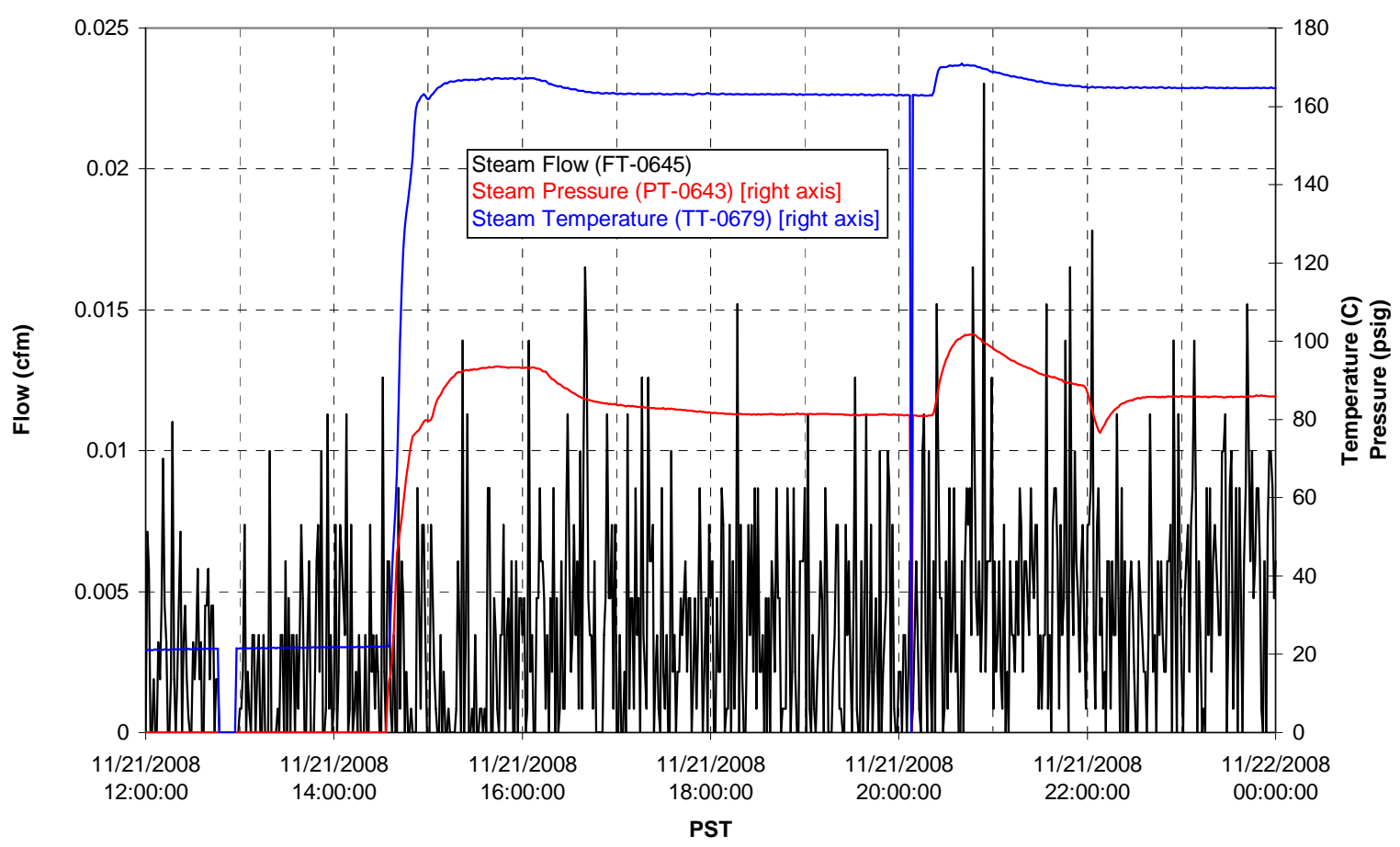

T01A Steam

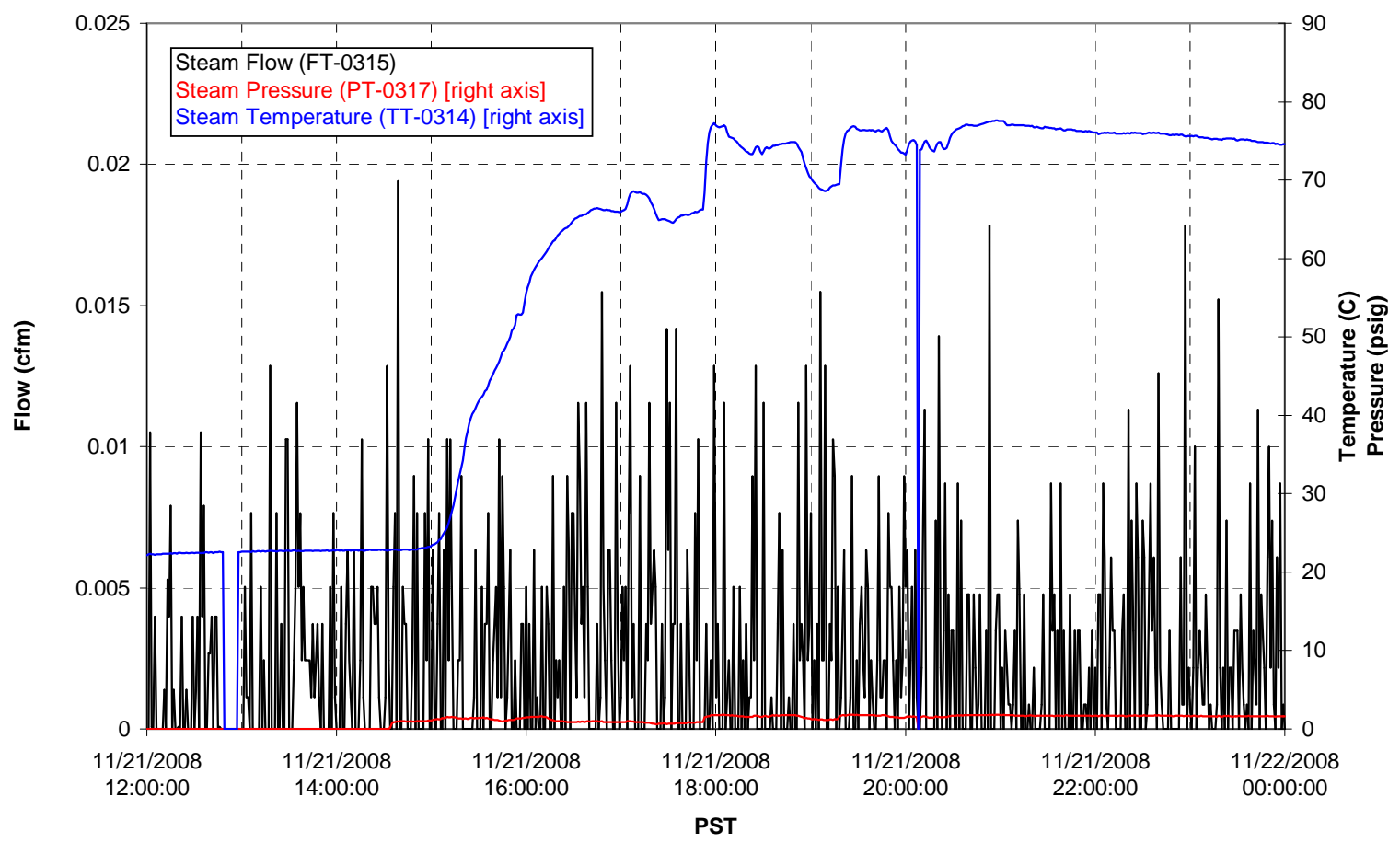


T01B Steam

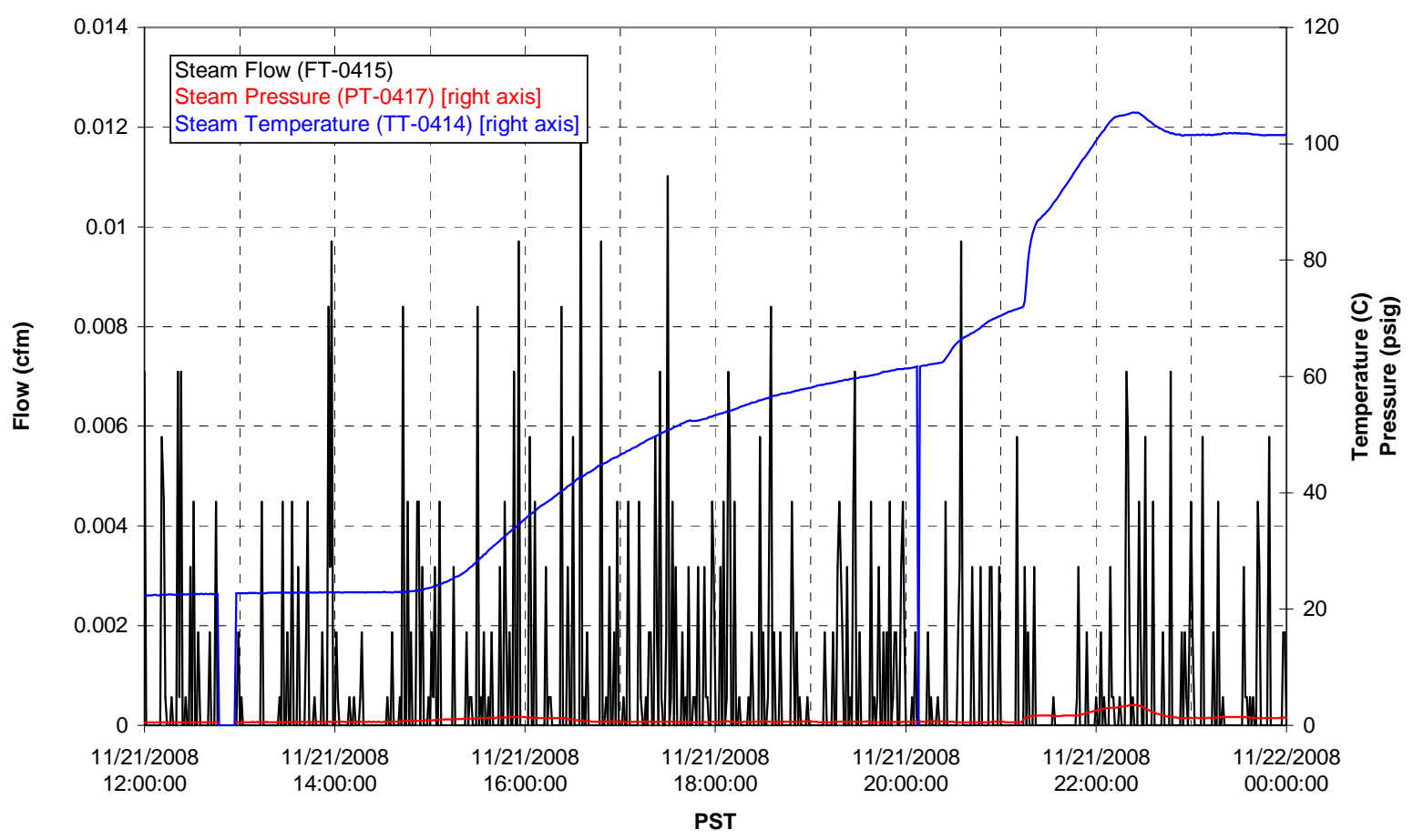


Functional Test Data Plots

11/22/2008 00:00 - 11/22/2008 12:00 
T01A level

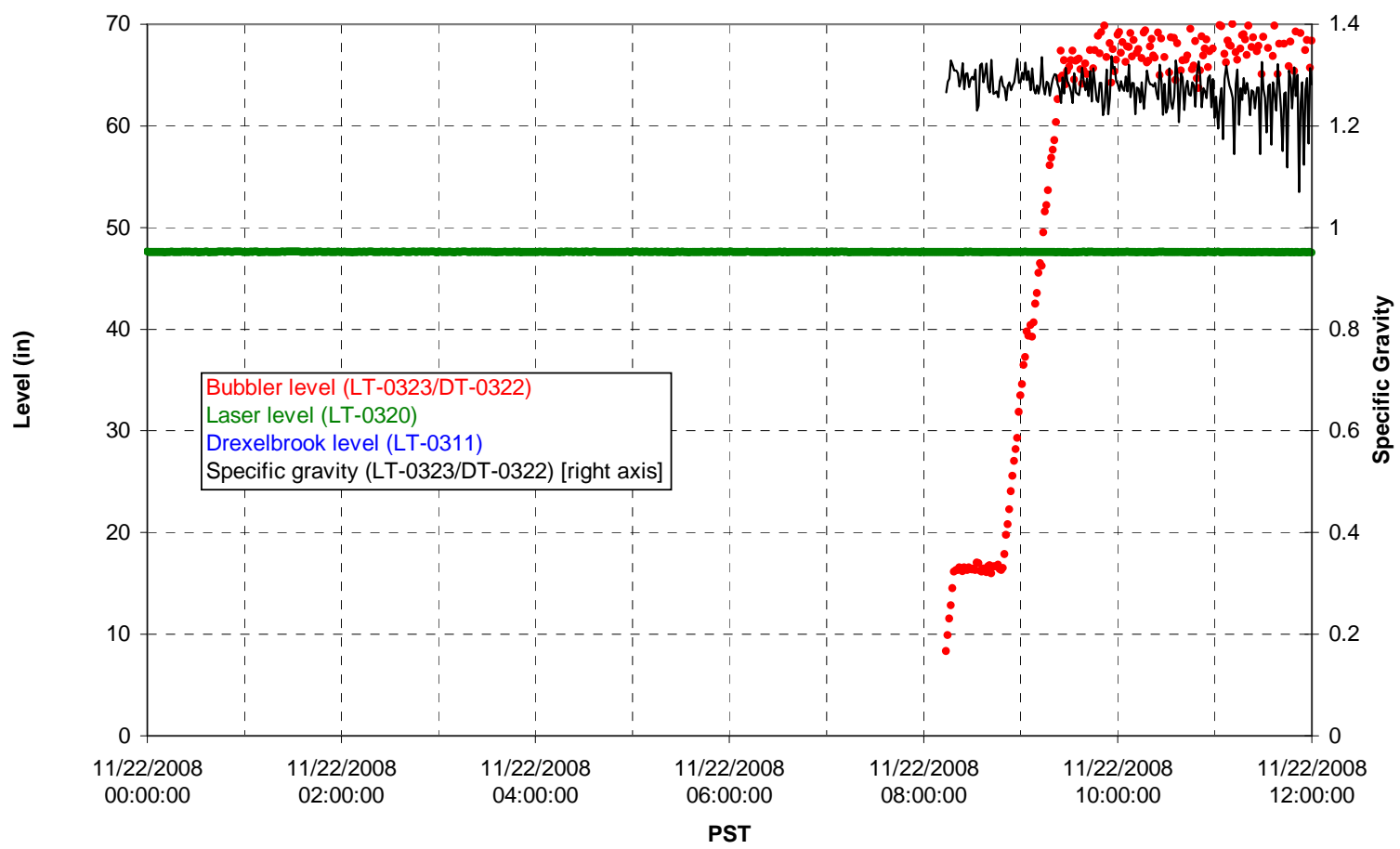

T01A temperatures

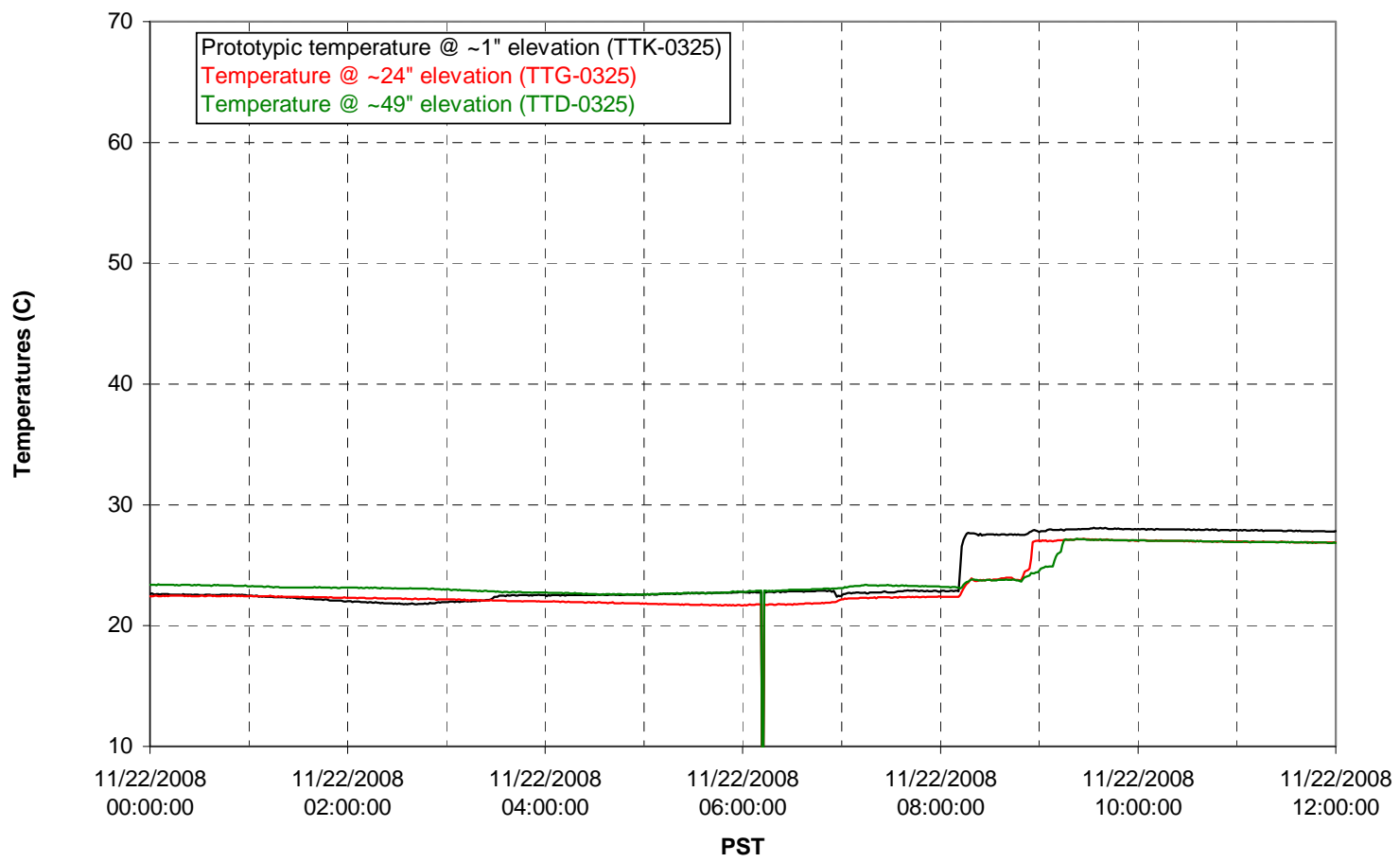


T01B level

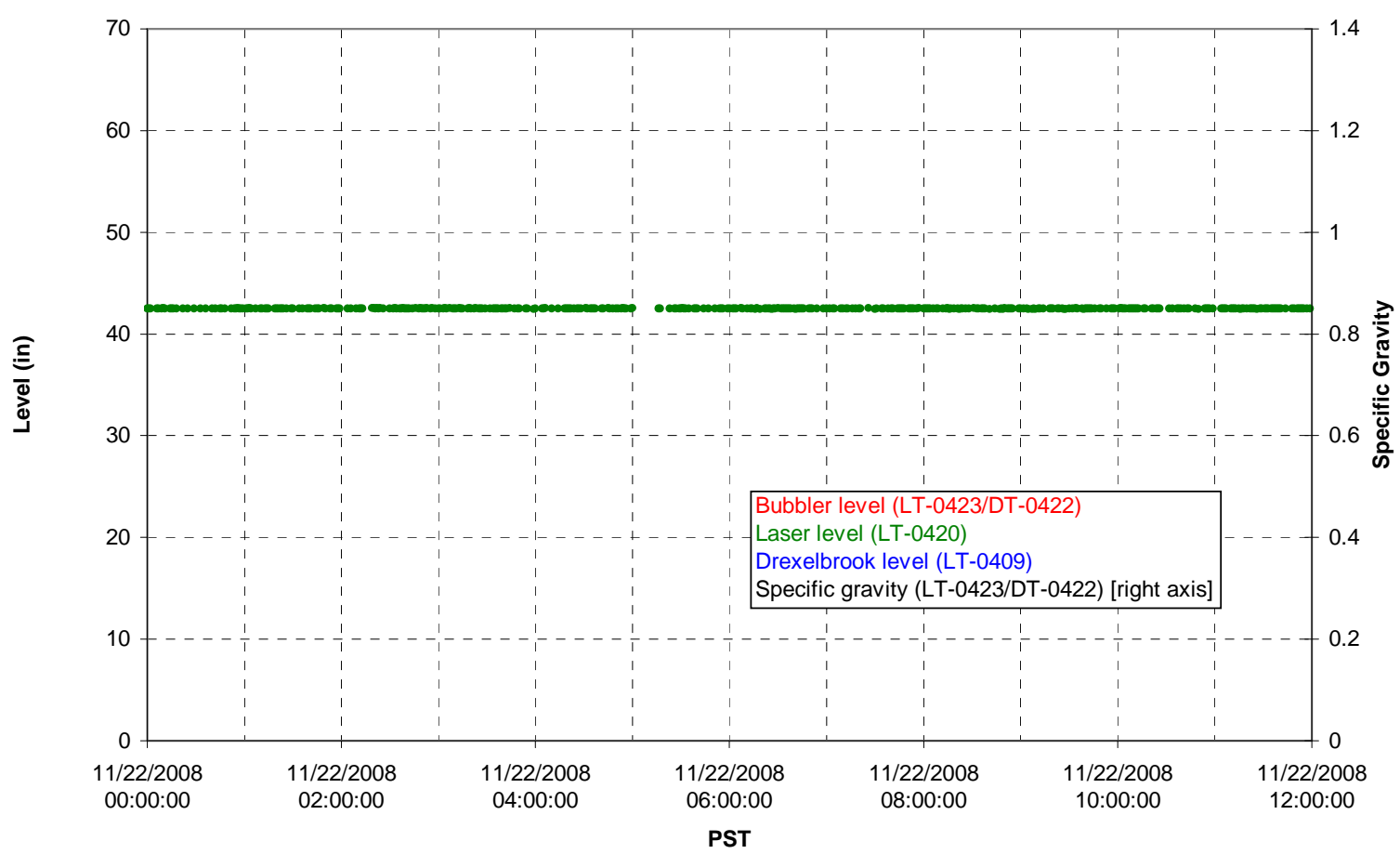

T01B temperatures

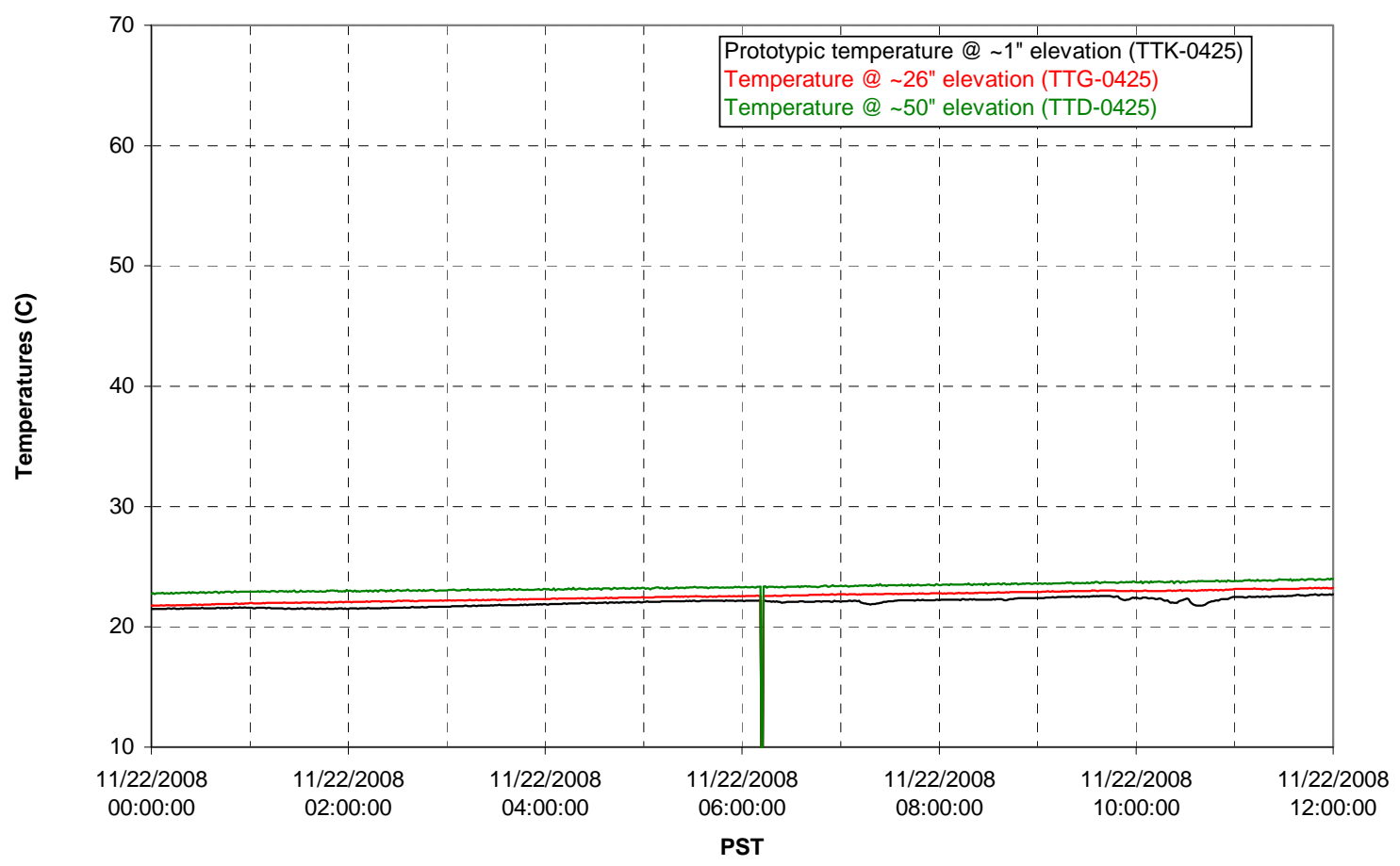


T02A level

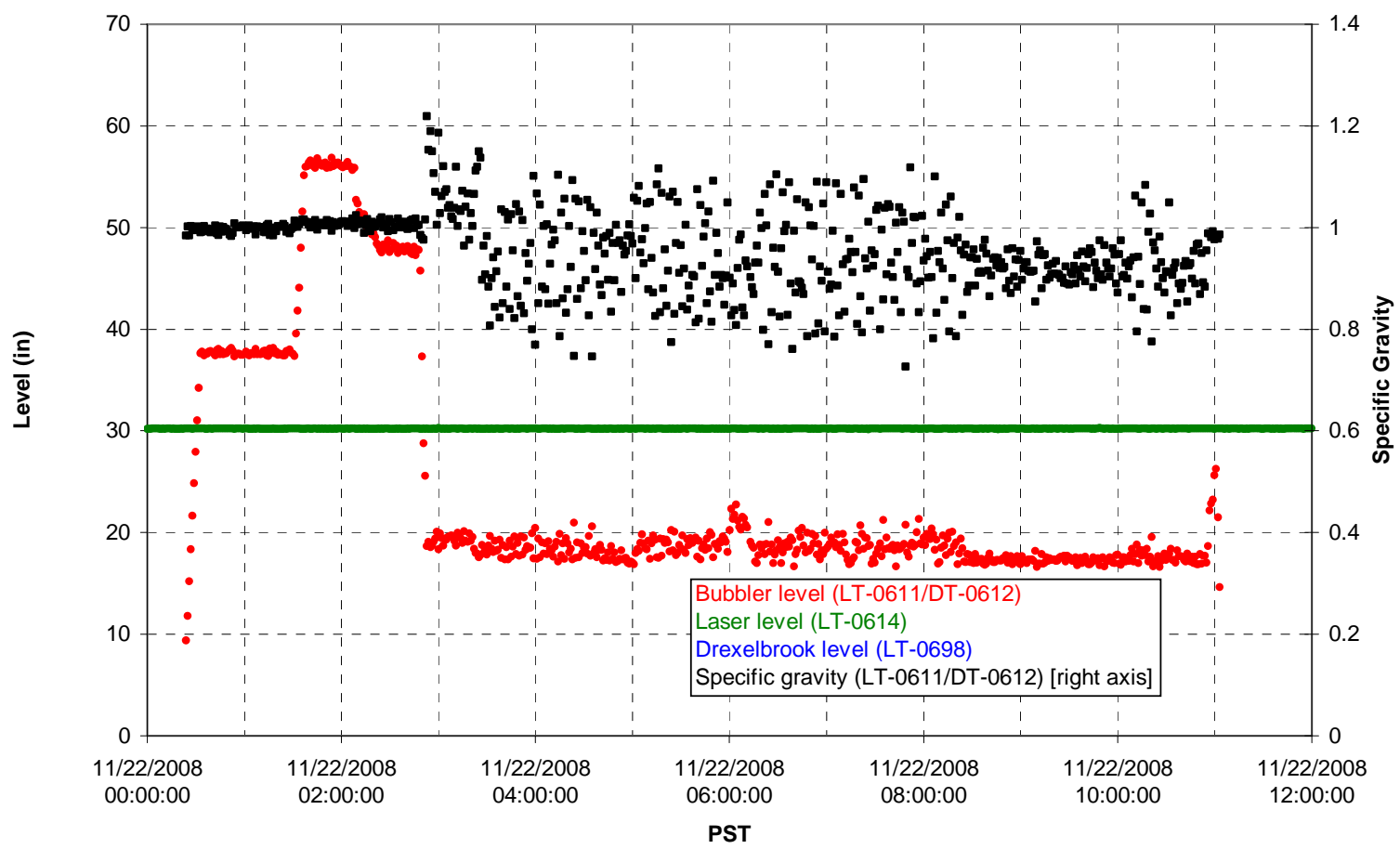

T02A temperatures

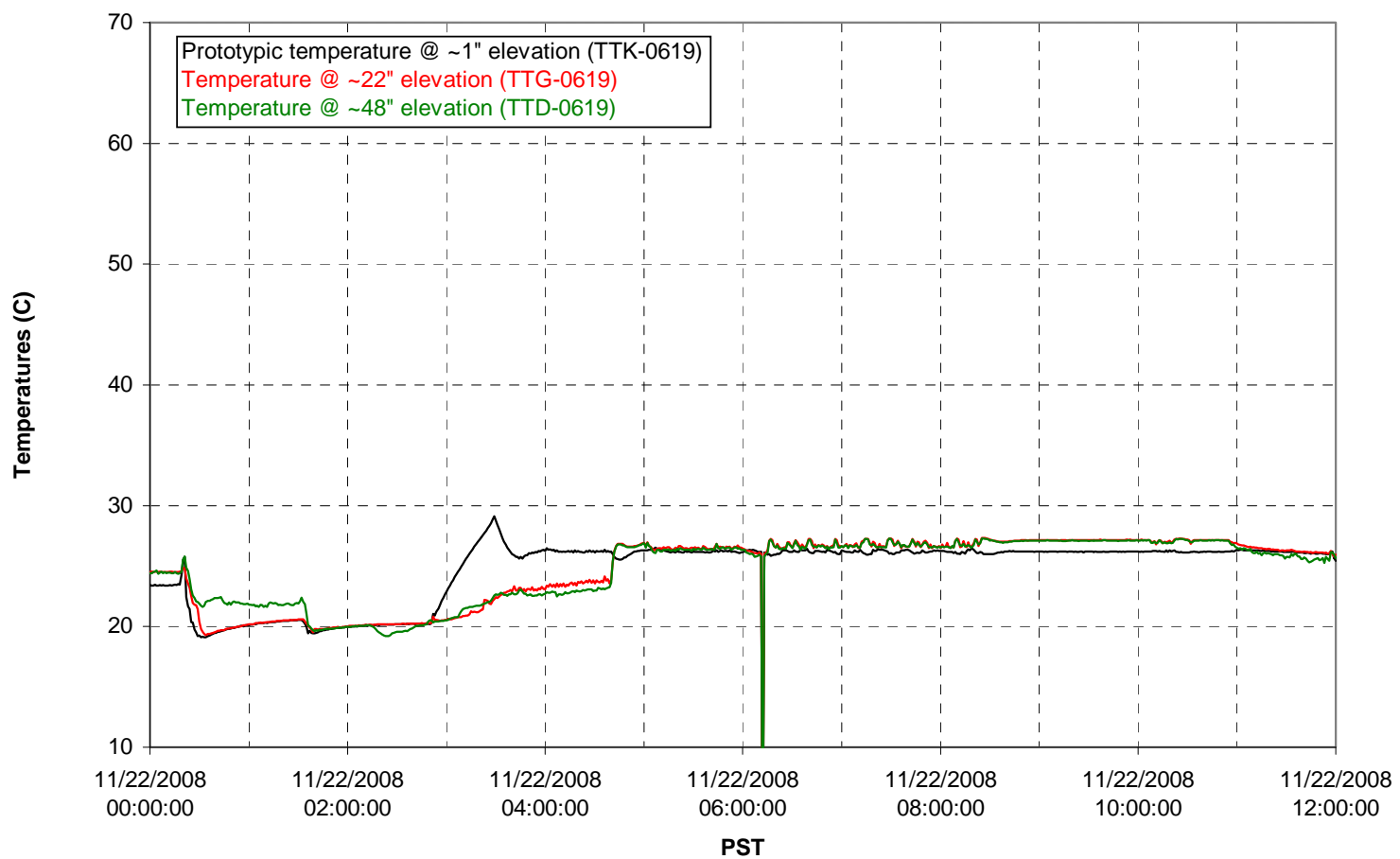


T02A and filter loop temperatures

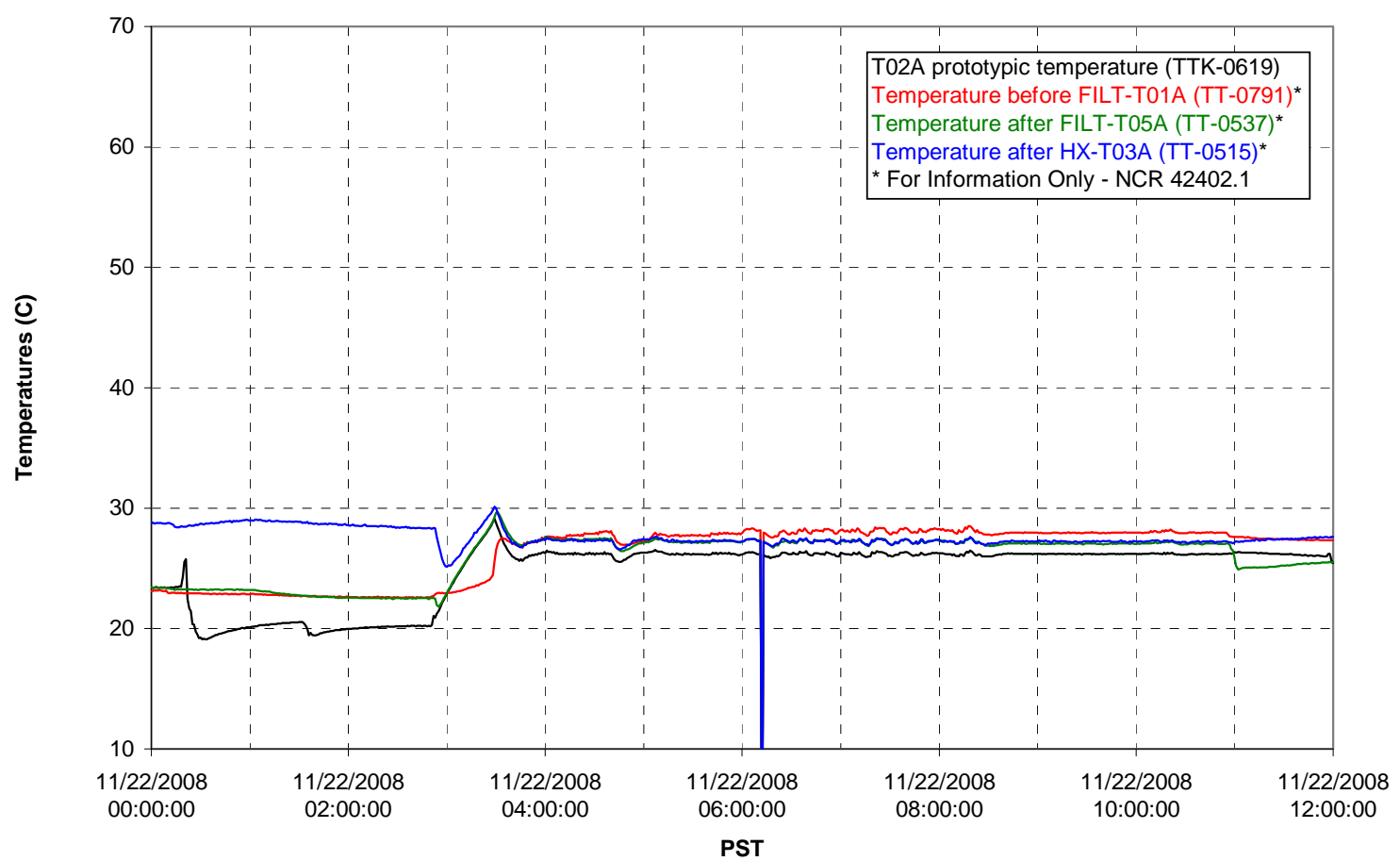

Pump Pressures and Flow

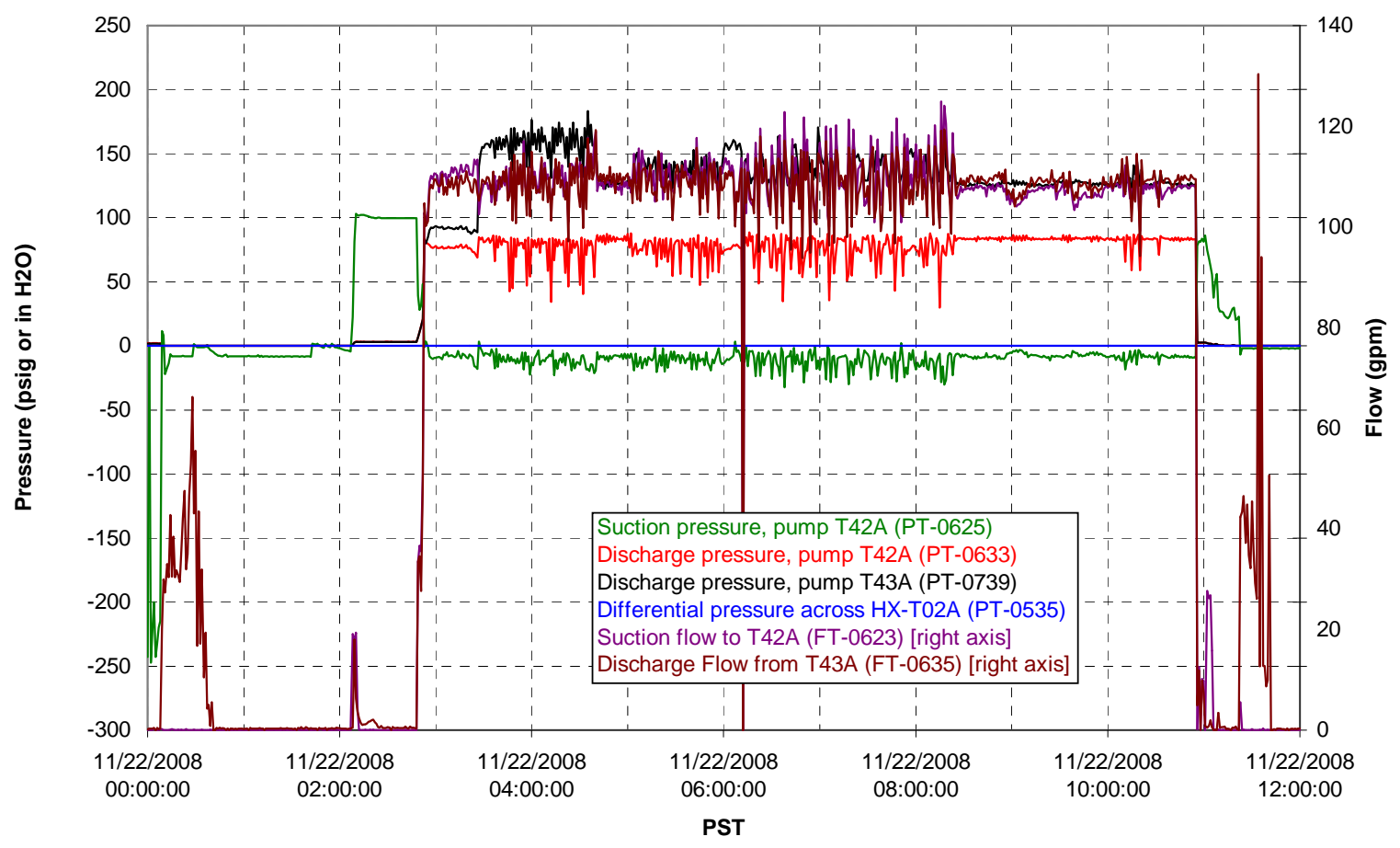




\section{Axial pressure drop}

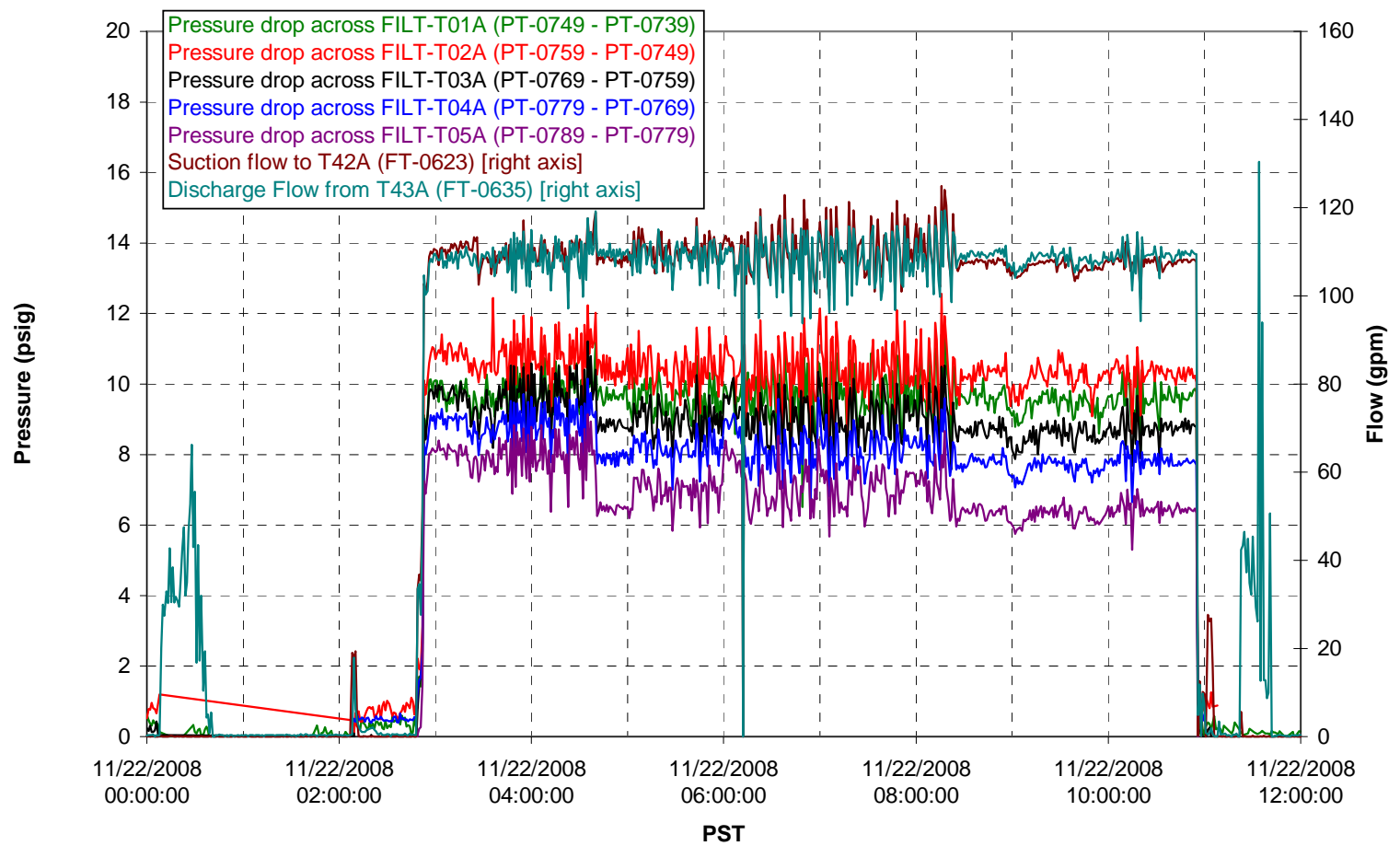

Permeate flow rates

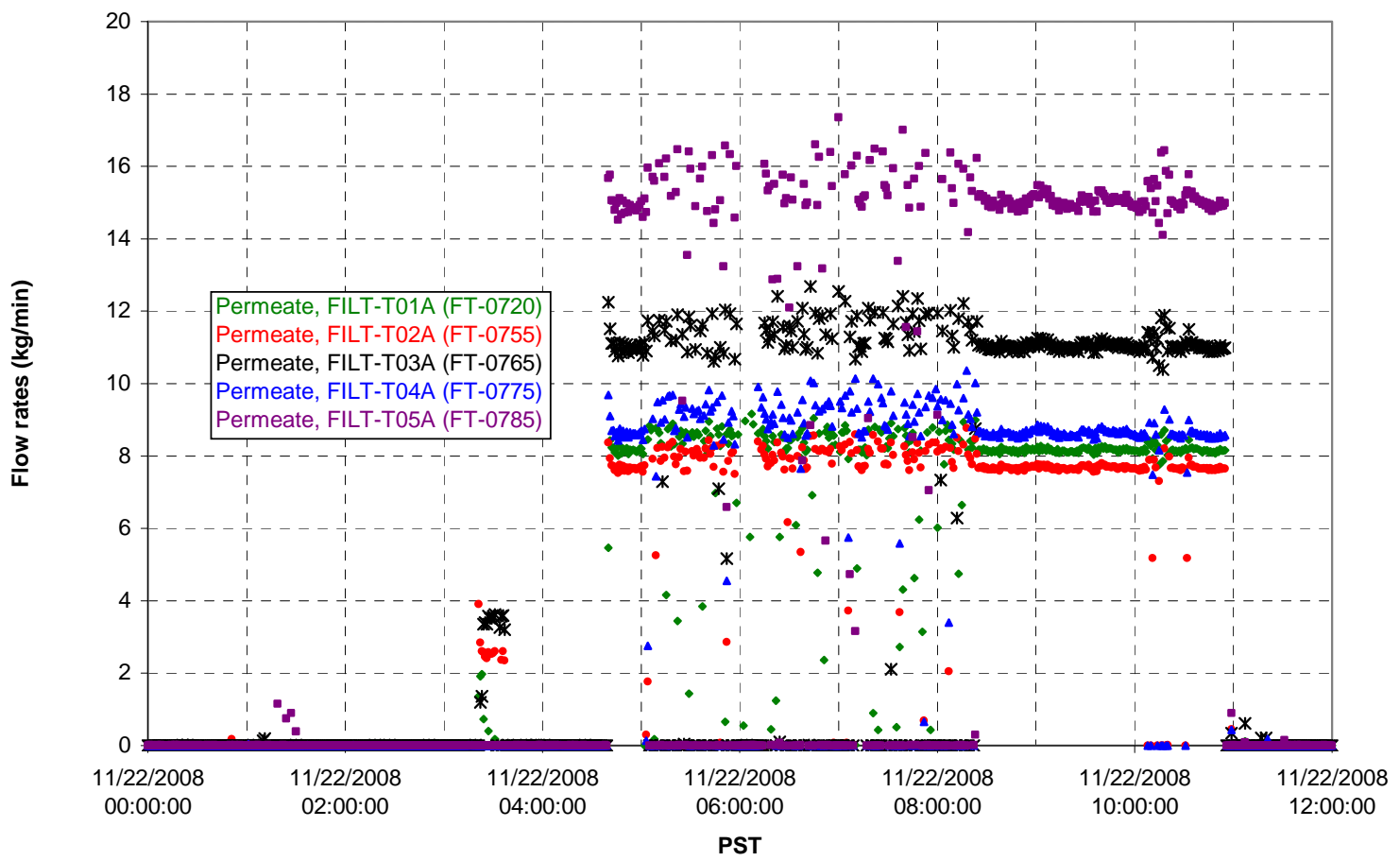


T02A Inner Temperature Tree

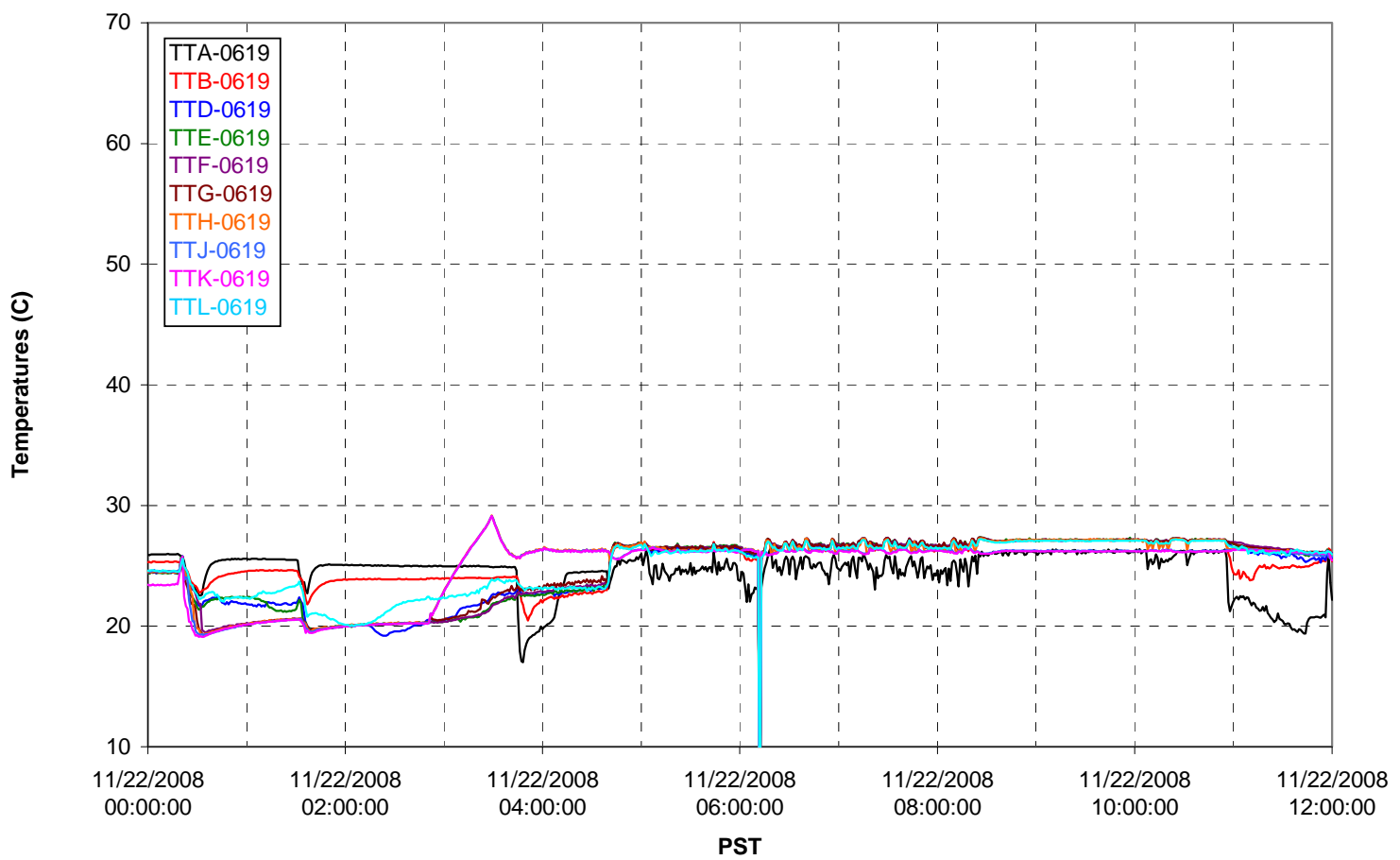

T02A Outer Temperature Tree

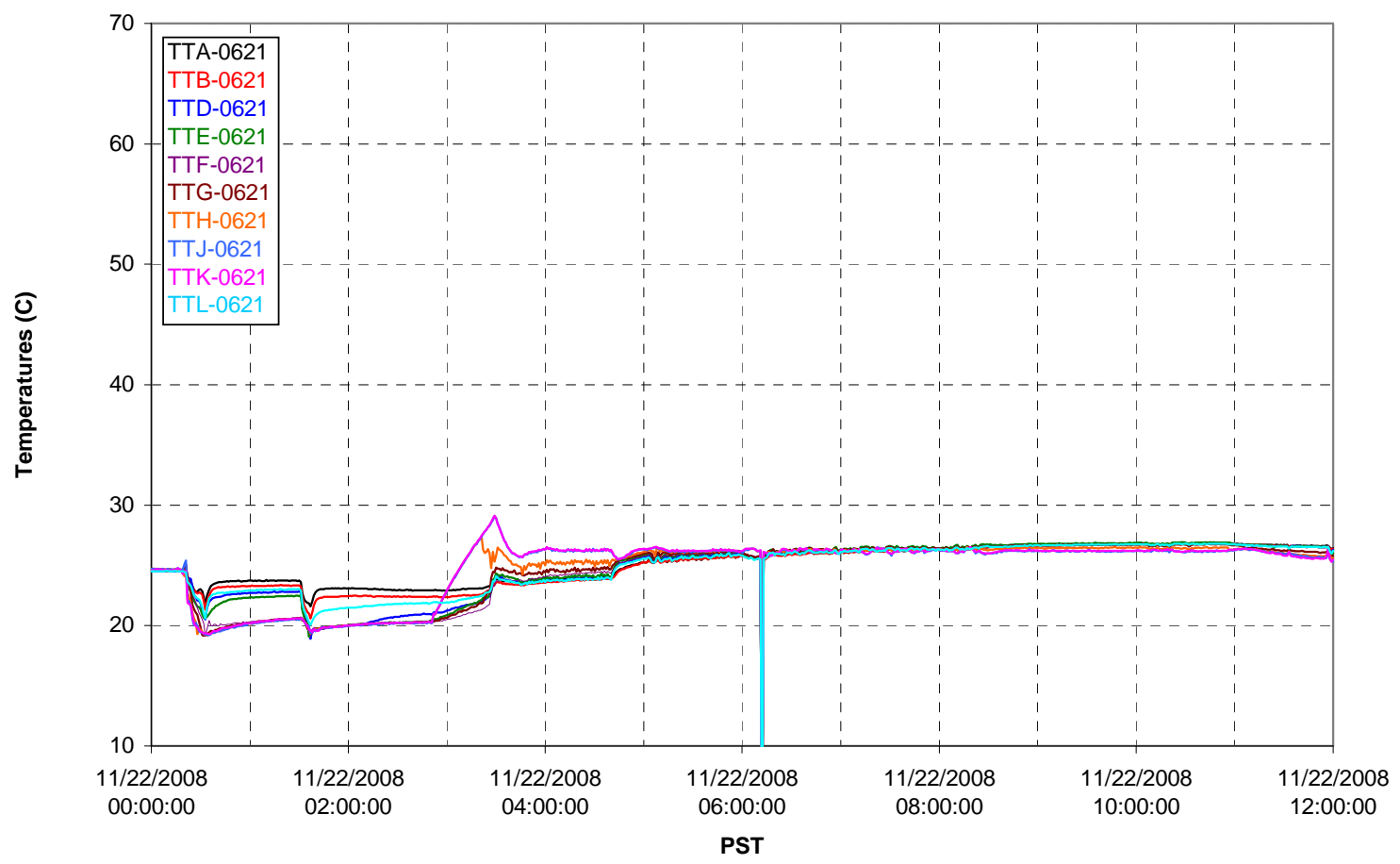


T02A temperatures

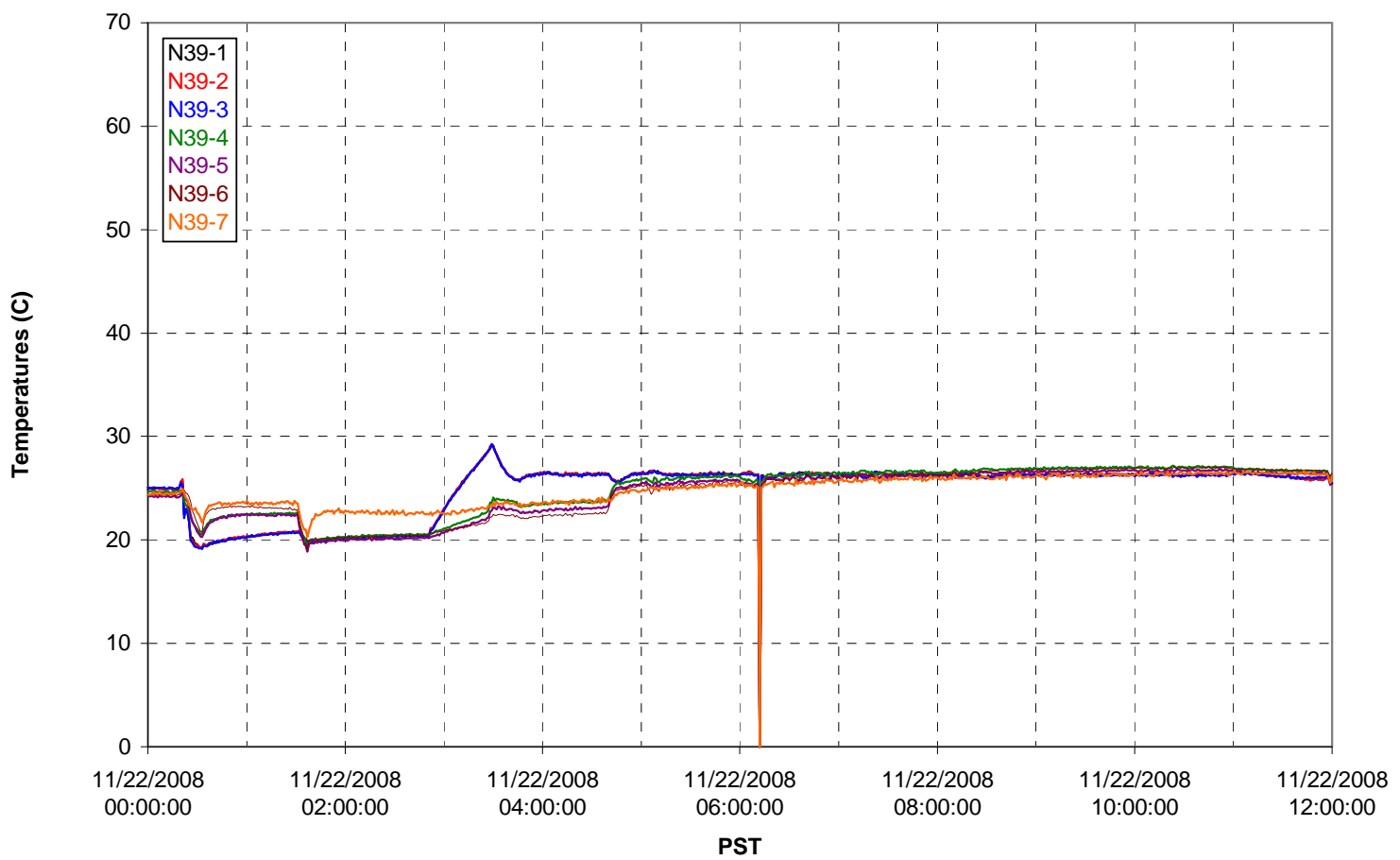

T02A temperatures

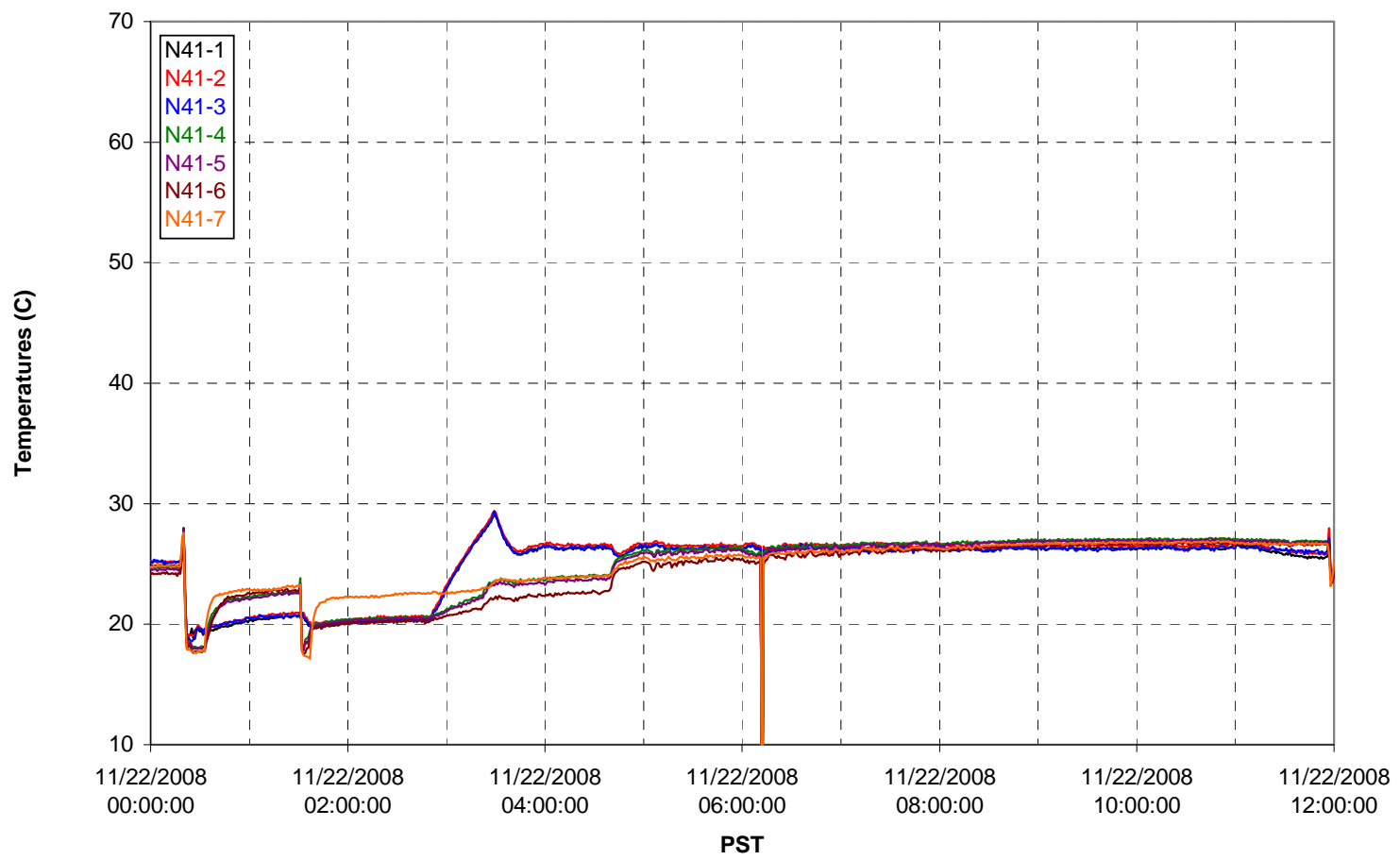


T02A temperatures

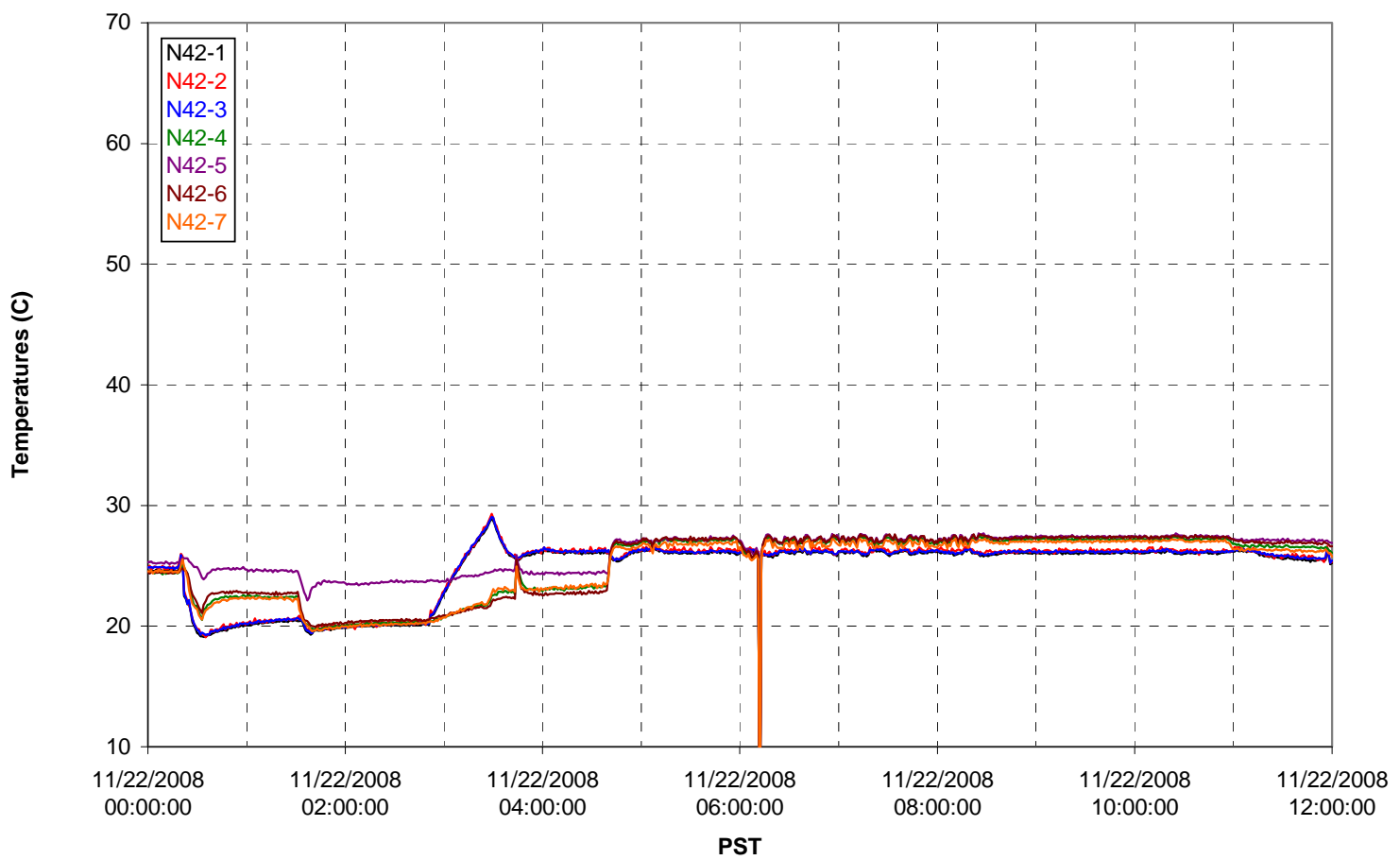

T02A temperatures

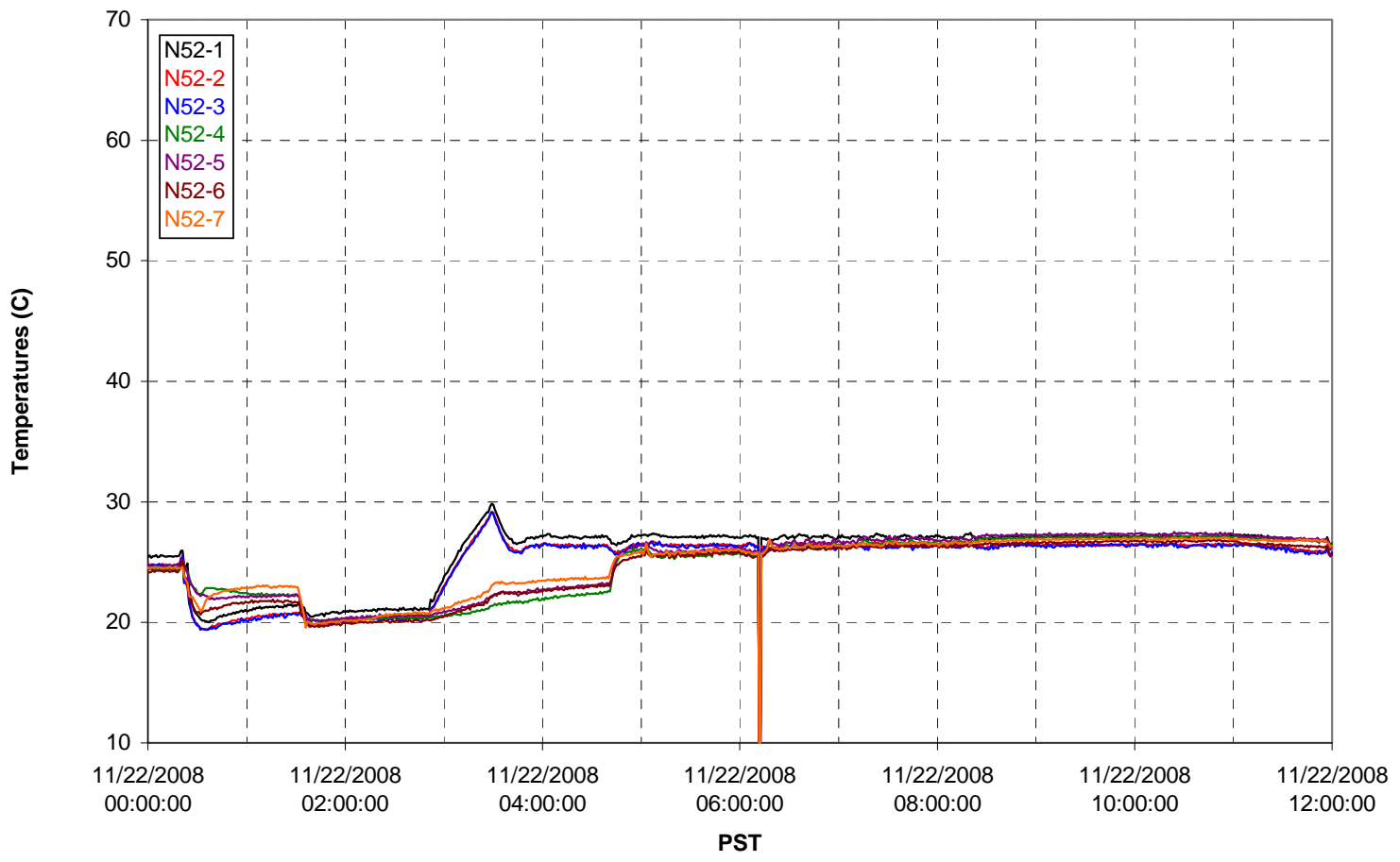


T02A Heating and Cooling
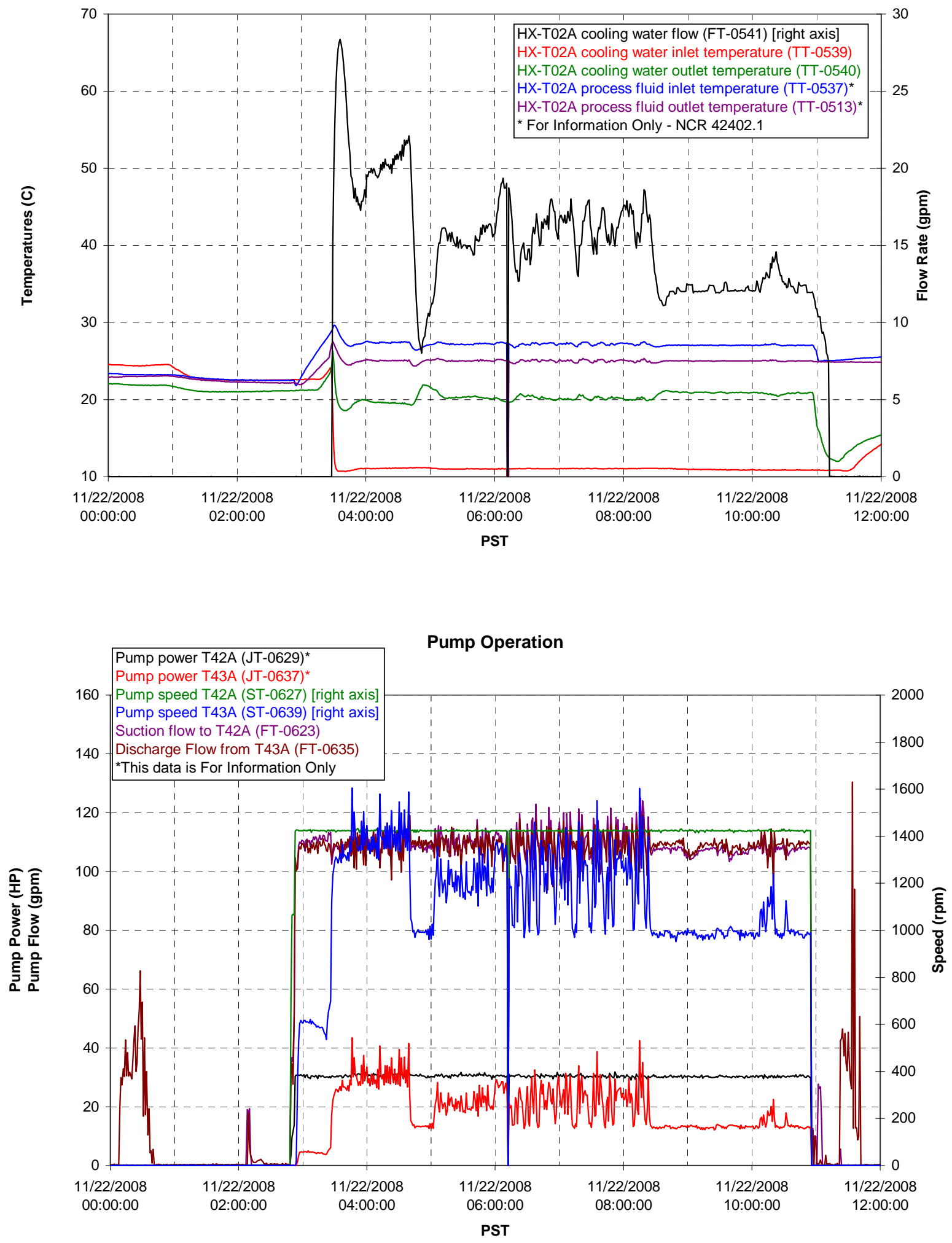
Pulsepot UFP-PP-T01A

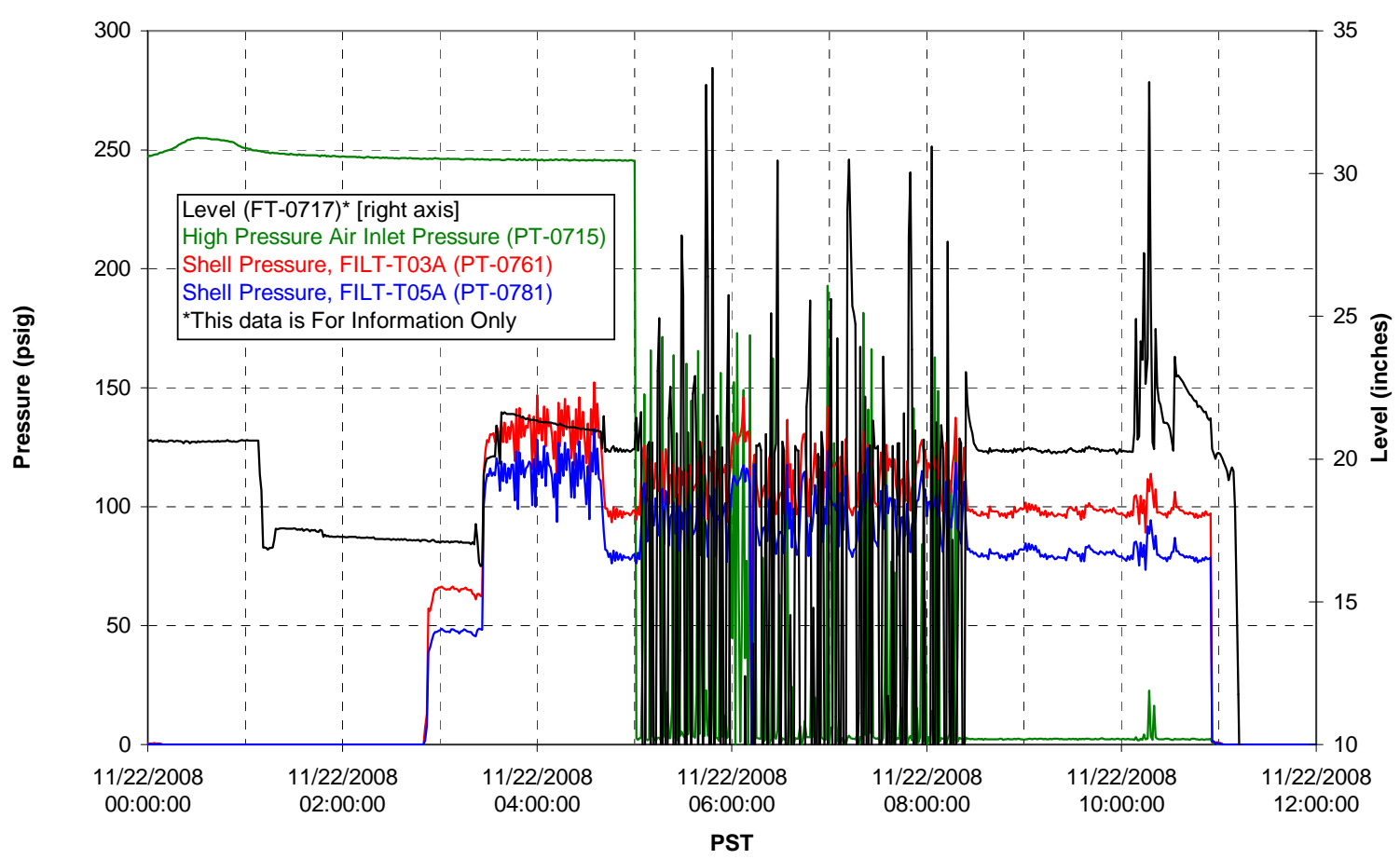

Pulsepot UFP-PP-T02A

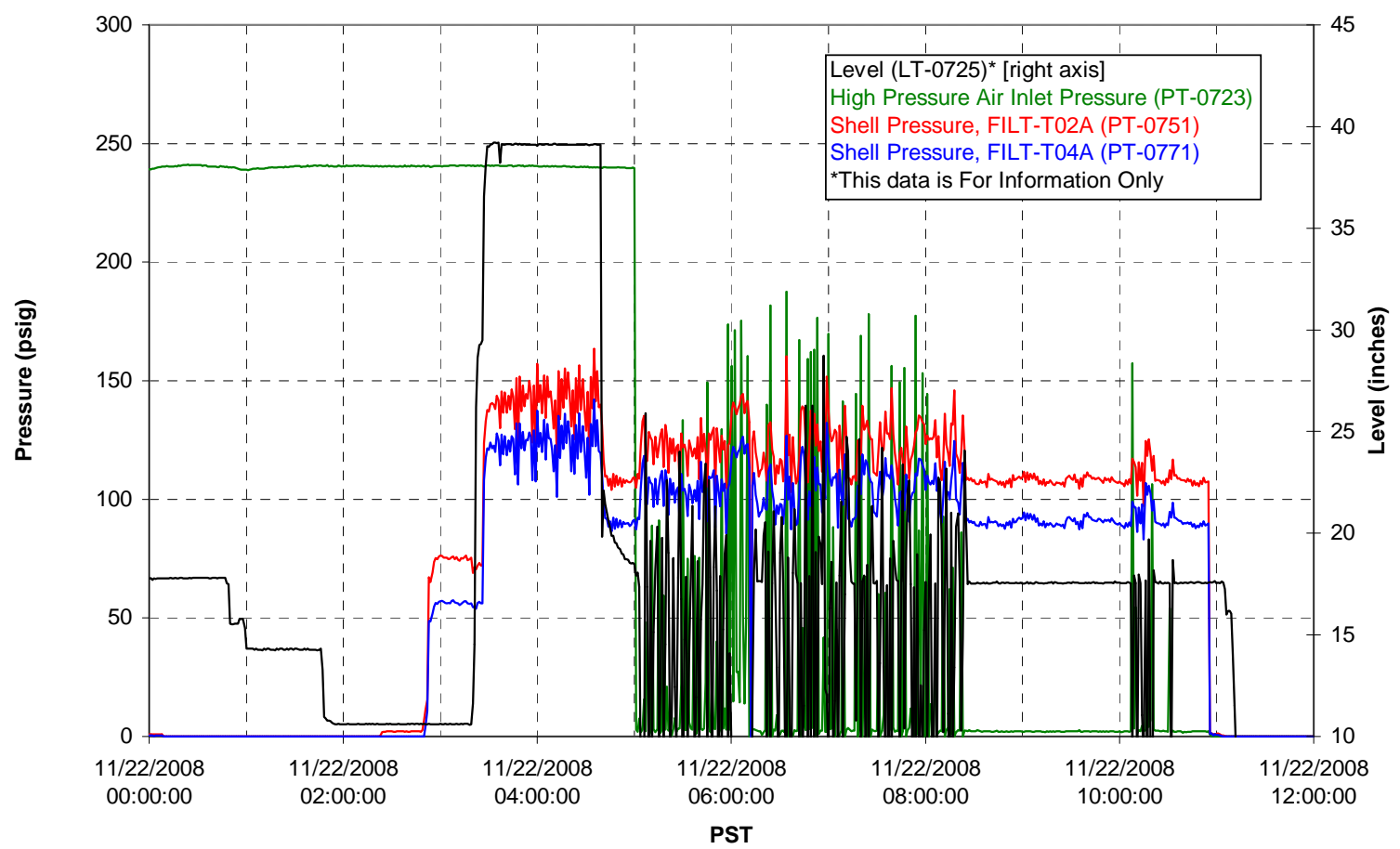


Pulsepot UFP-PP-T03A

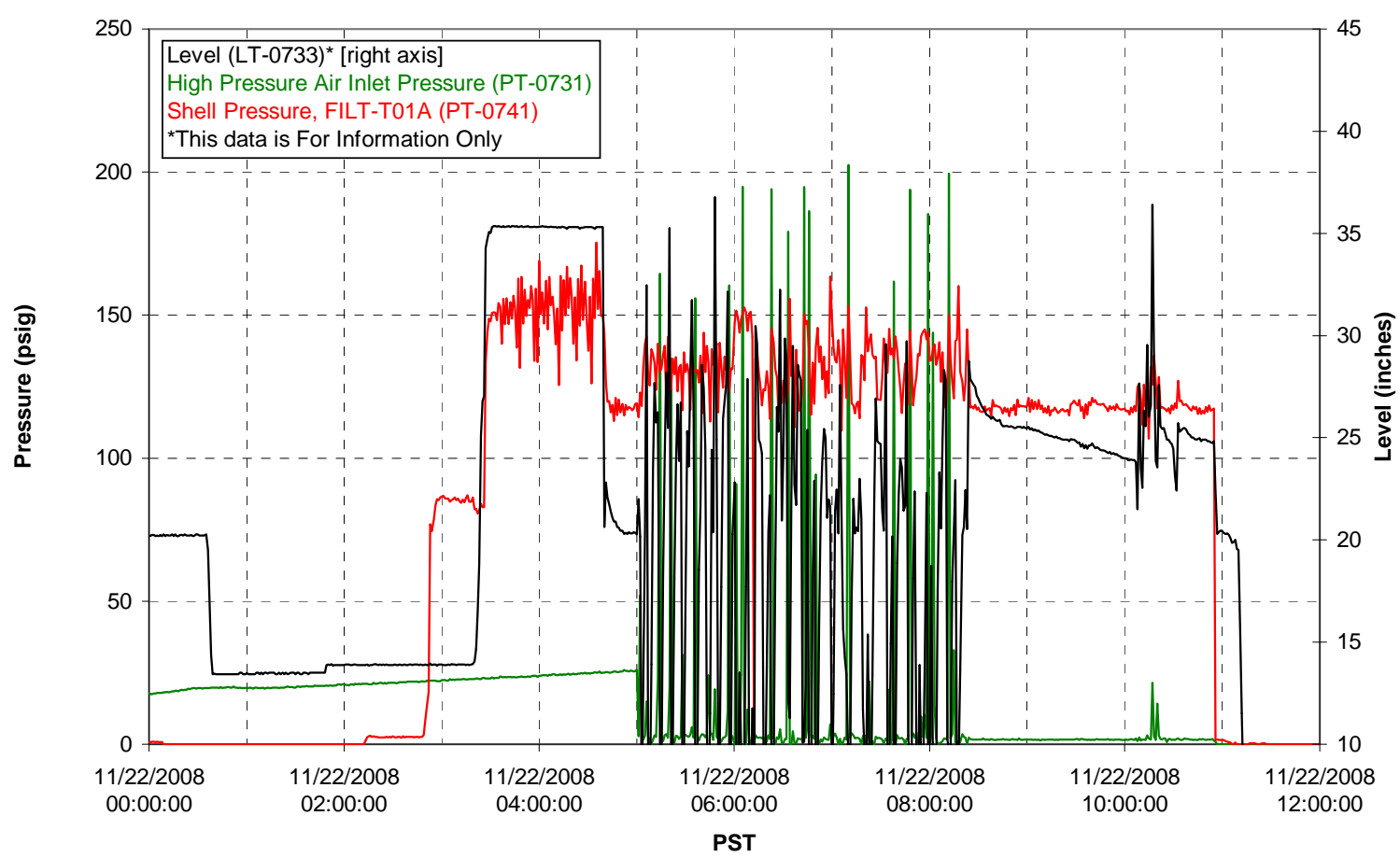

Pulsepot Levels

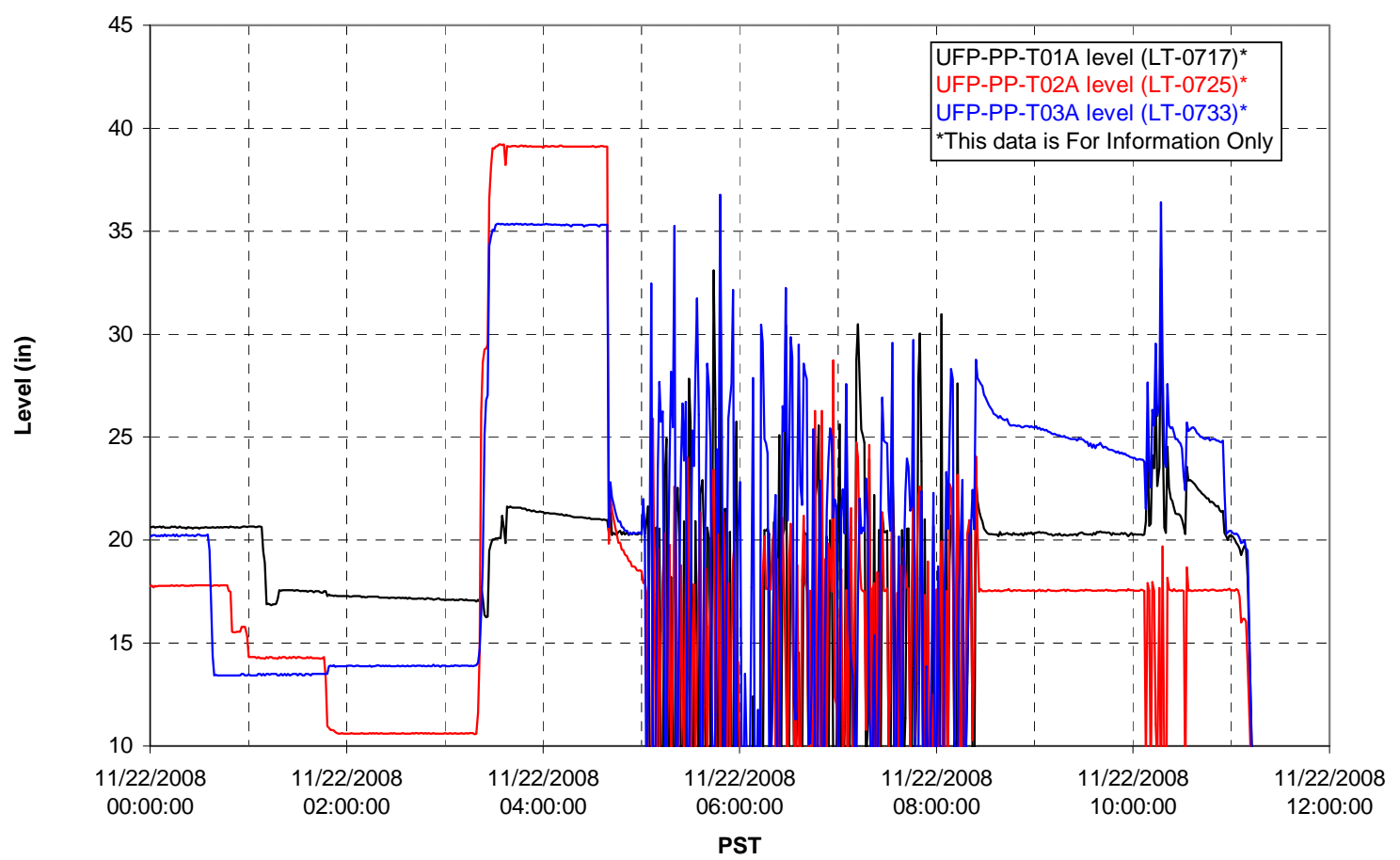


Filter UFP-FILT-T01A

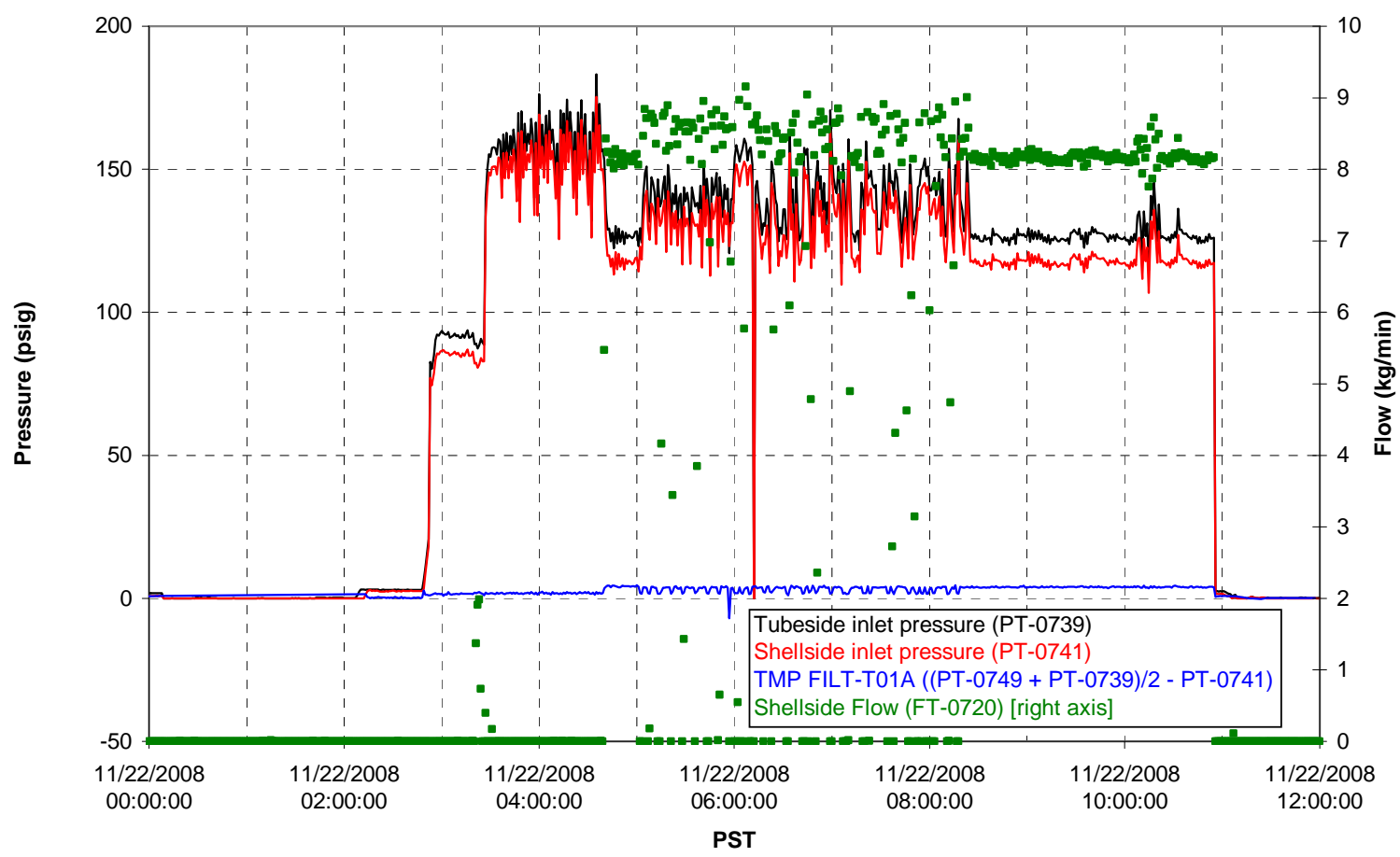

Filter UFP-FILT-T02A

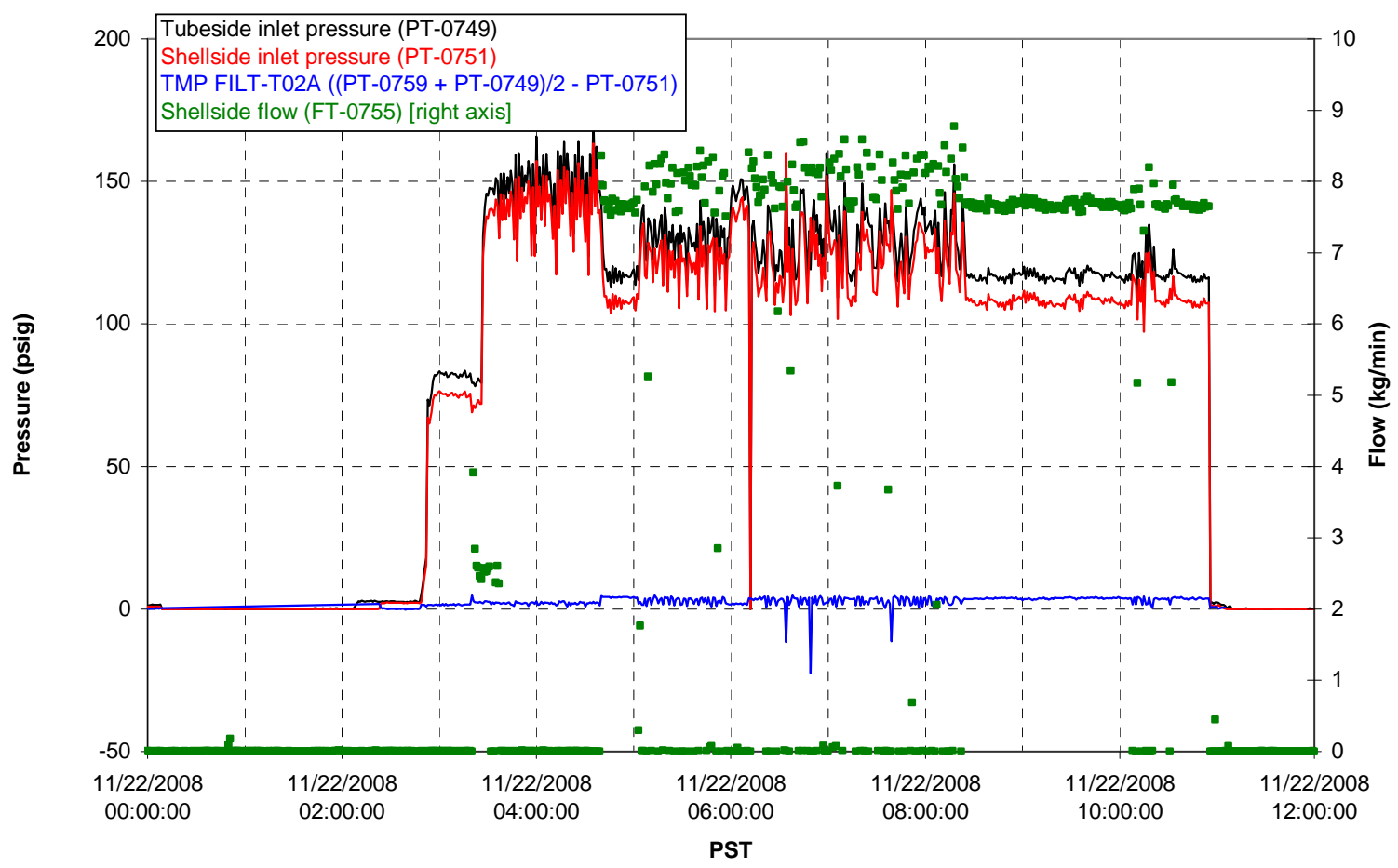


Filter UFP-FILT-T03A

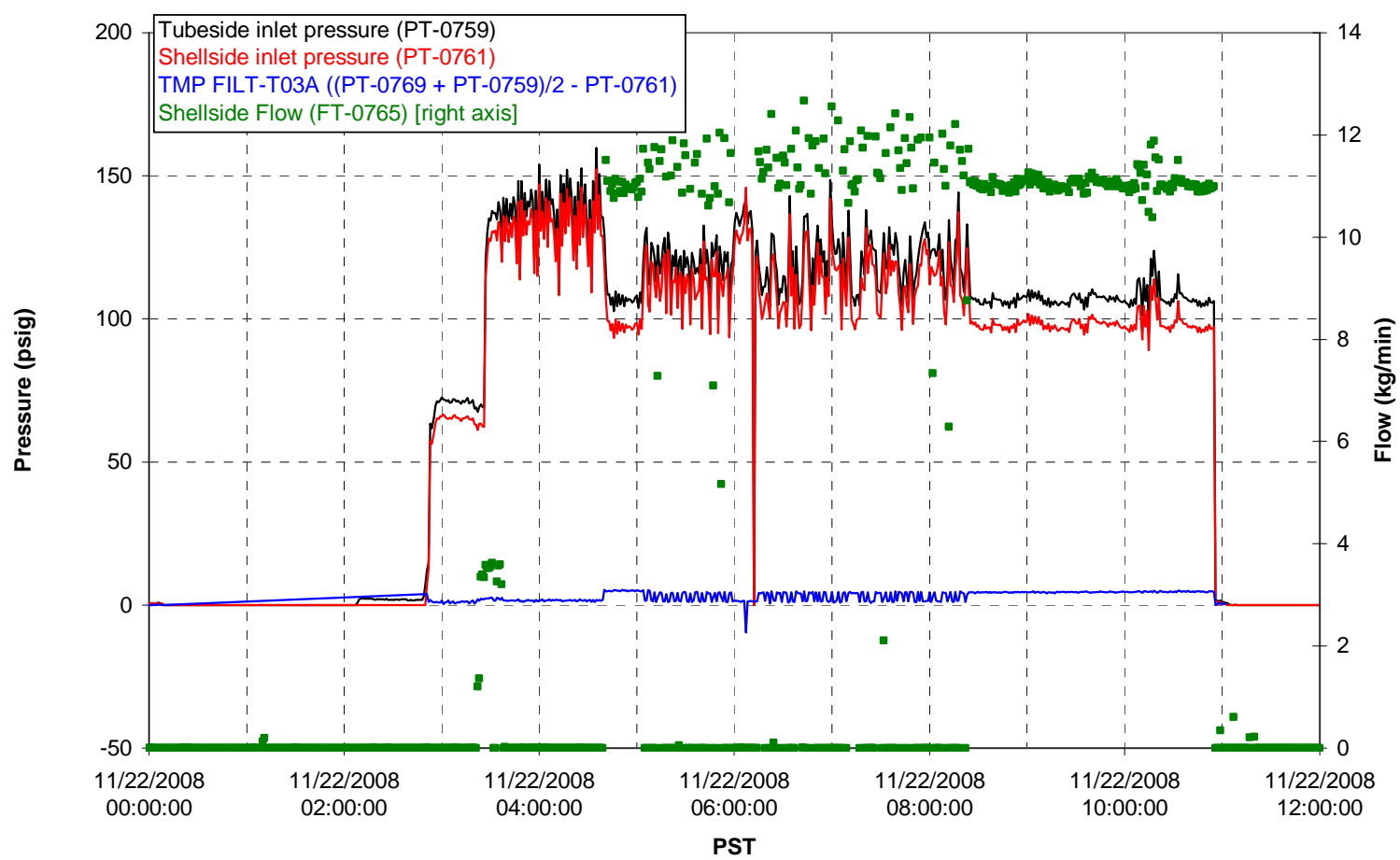

Filter UFP-FILT-T04A

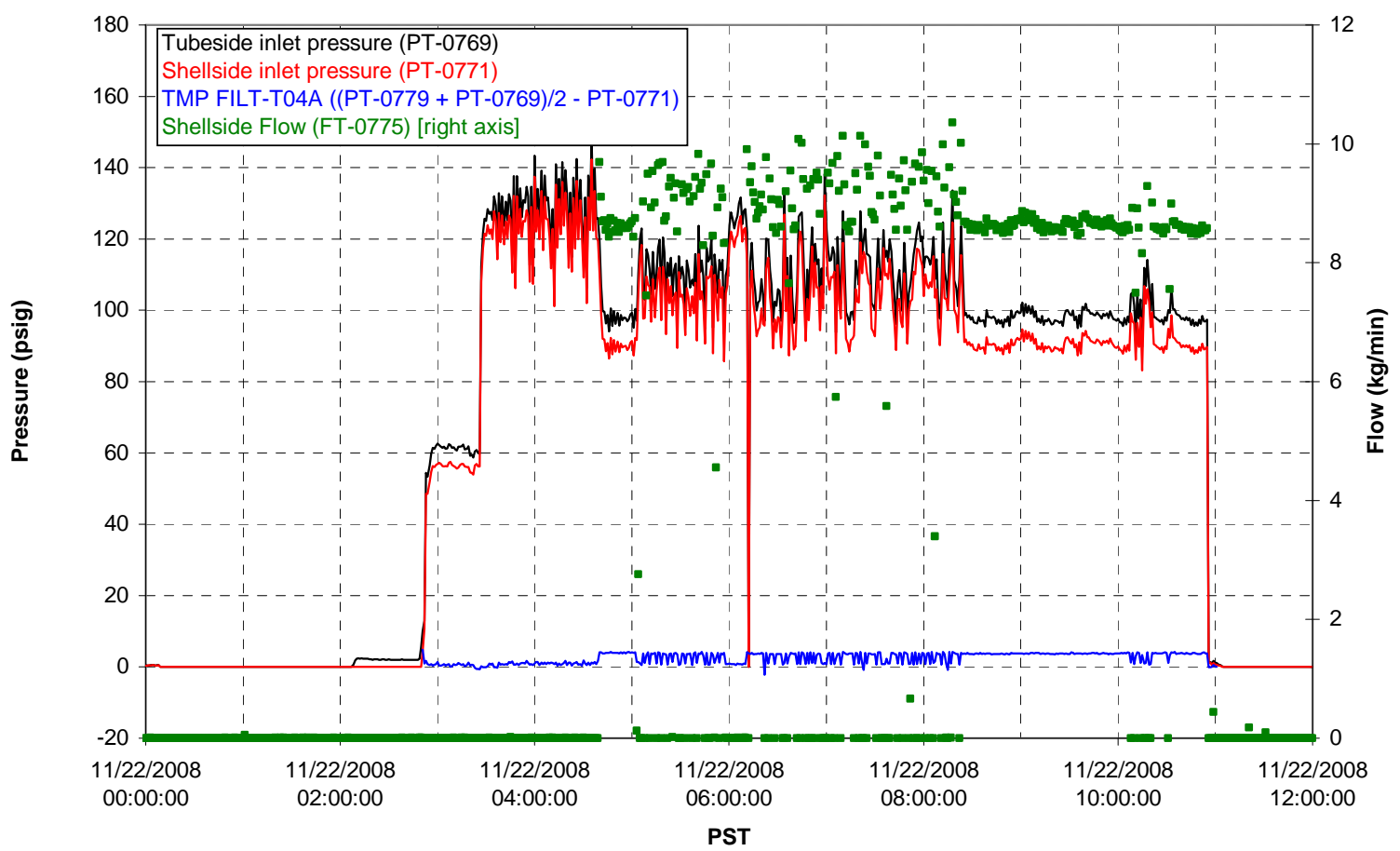


Filter UFP-FILT-T05A

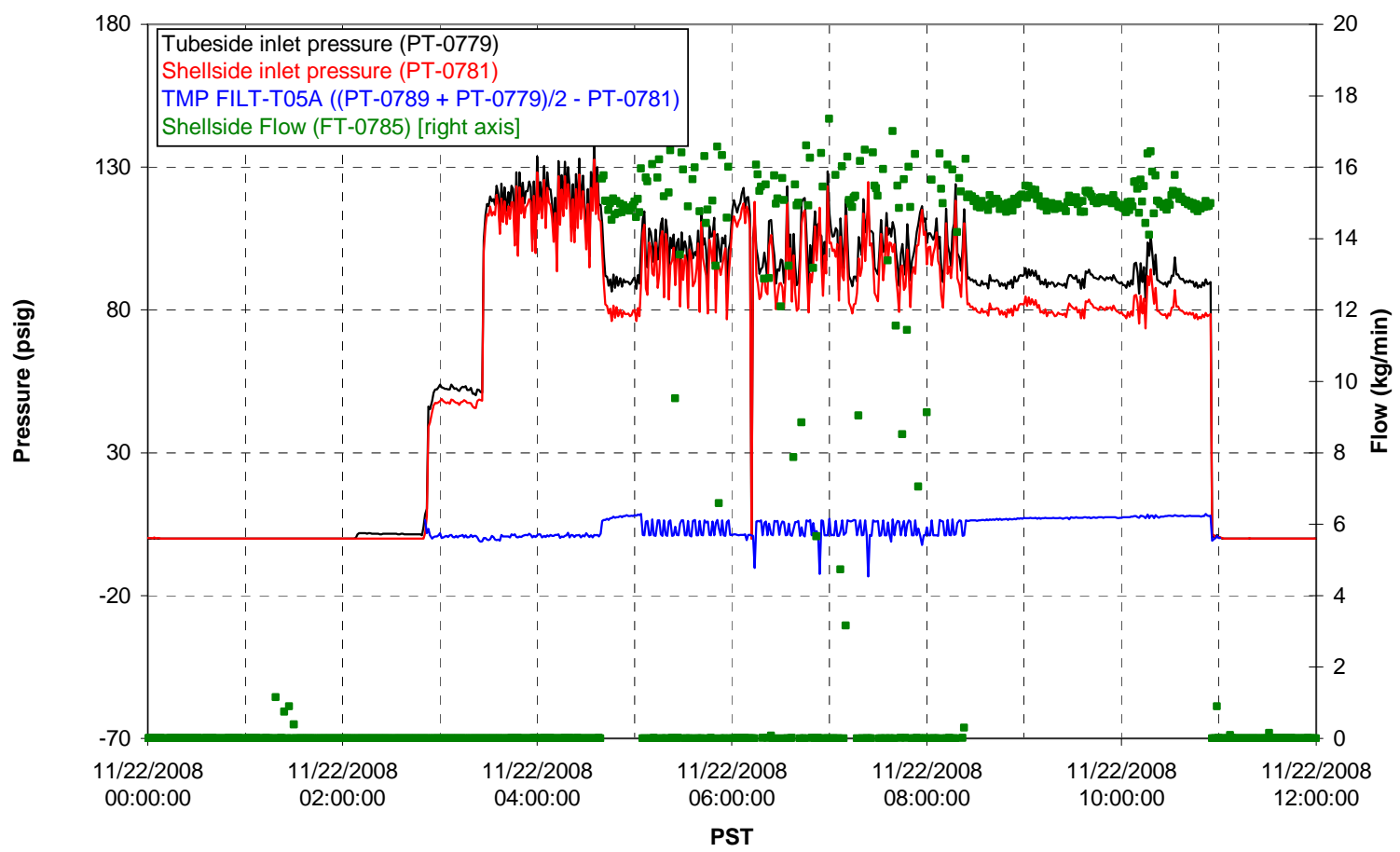

Chemical Flow

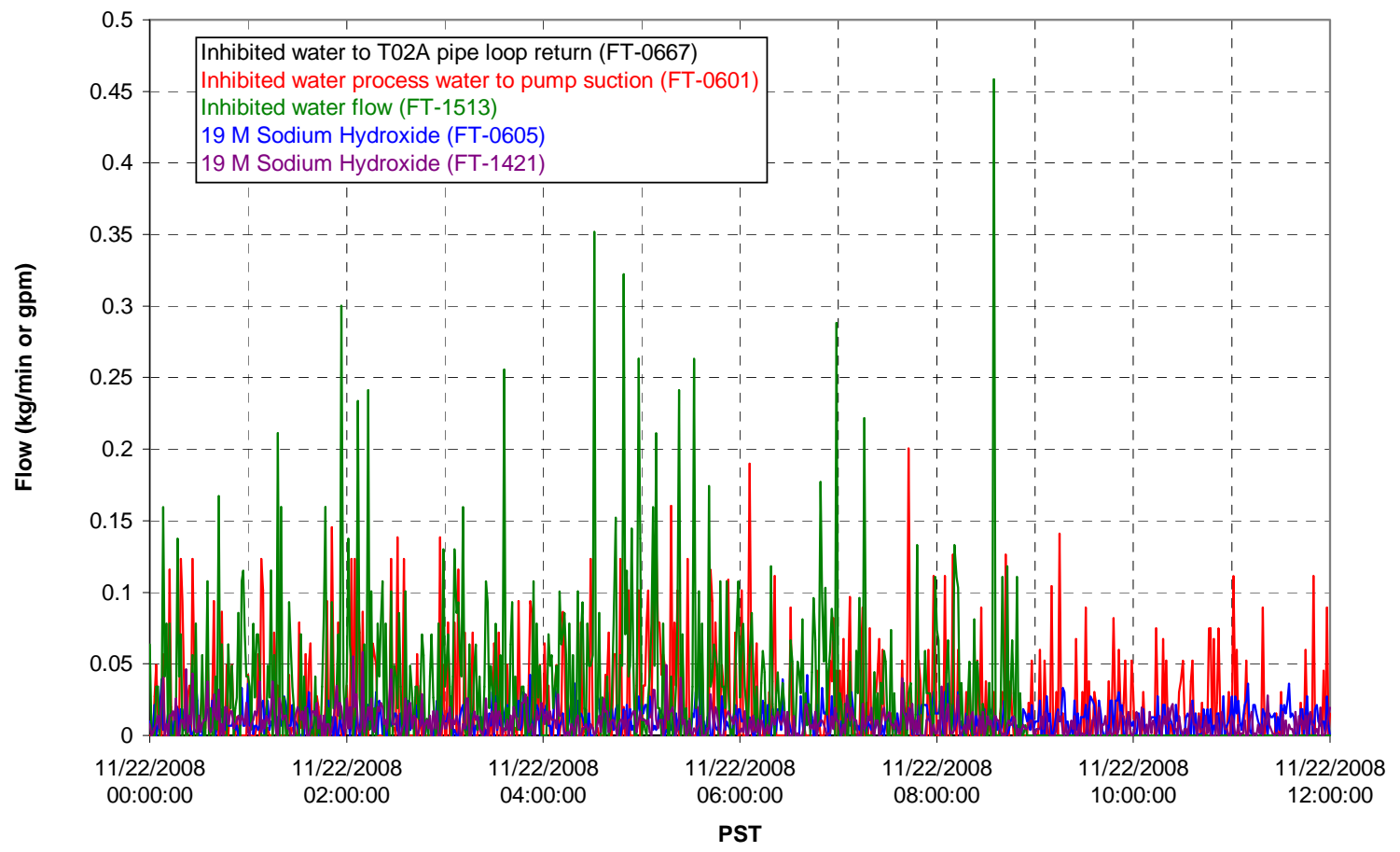


Chemical Flow

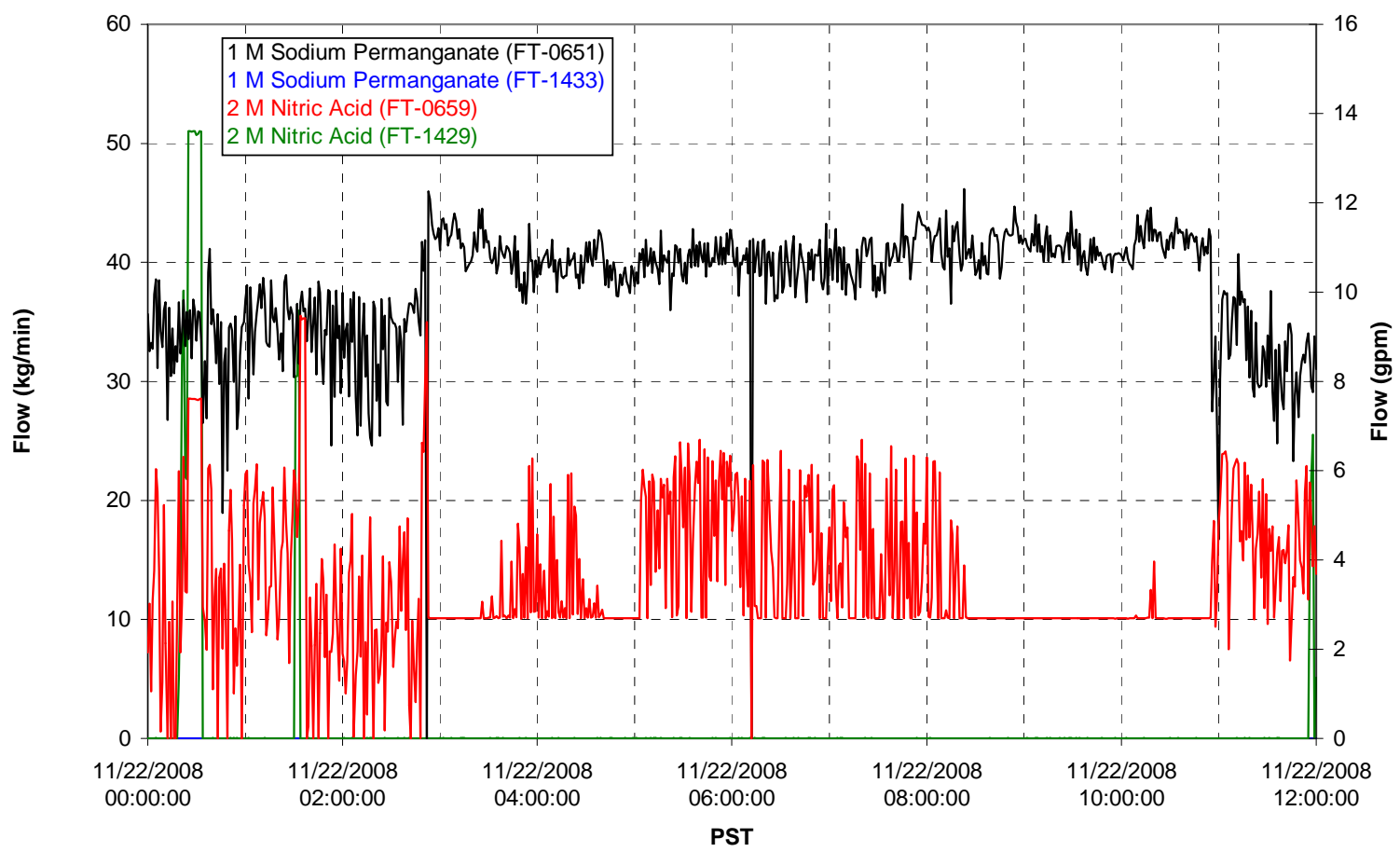

Air Flows

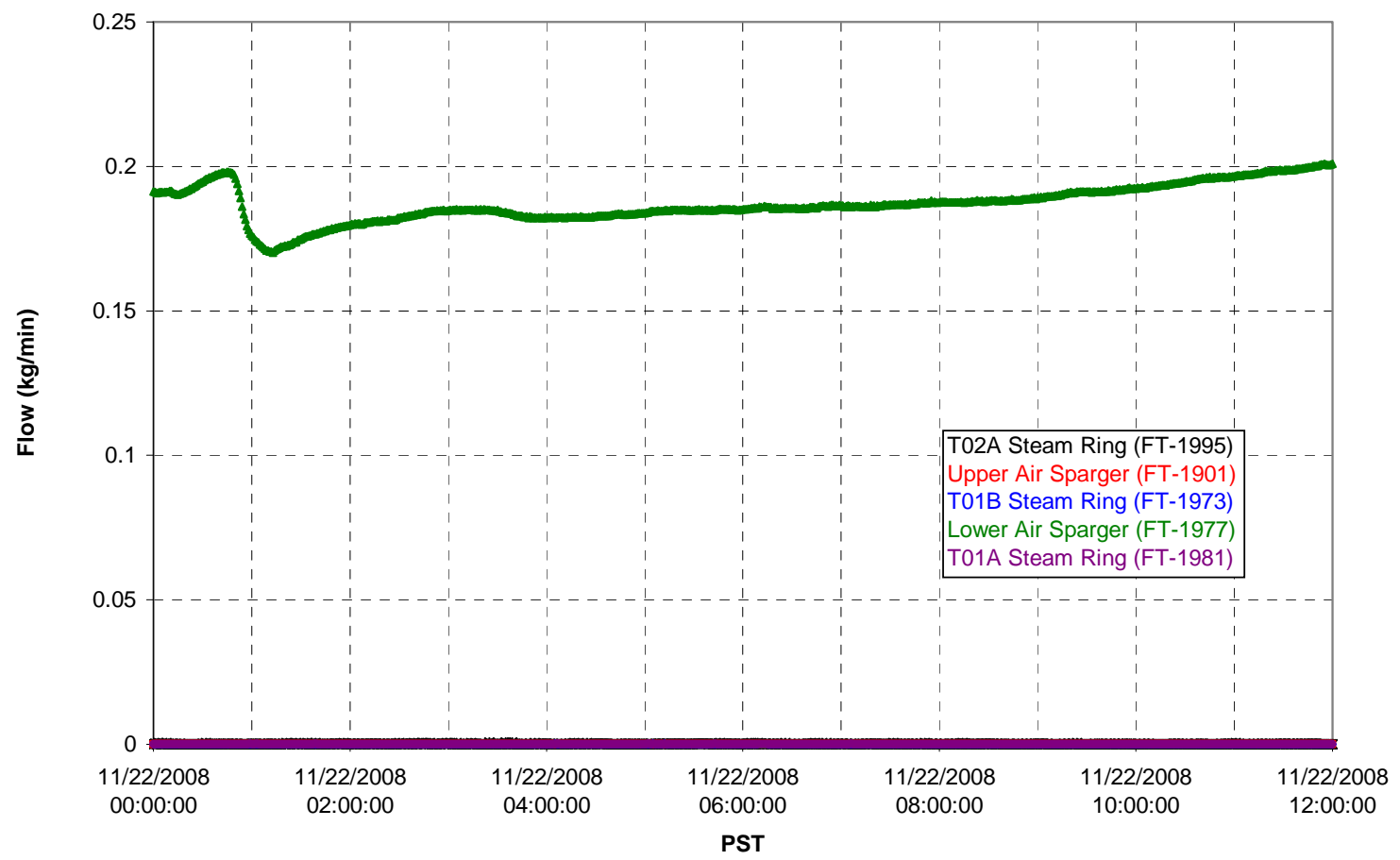


T02A Steam

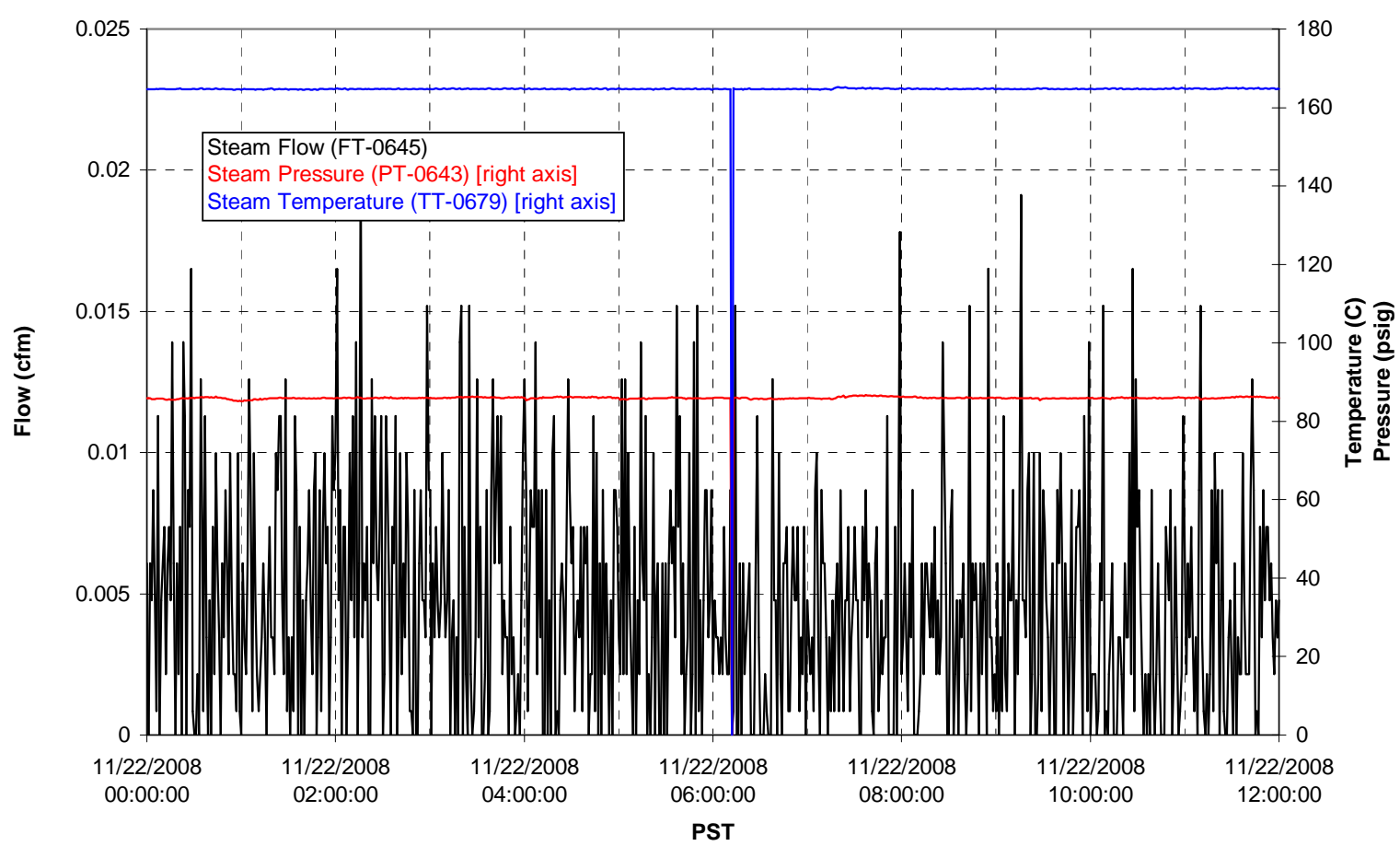

T01A Steam

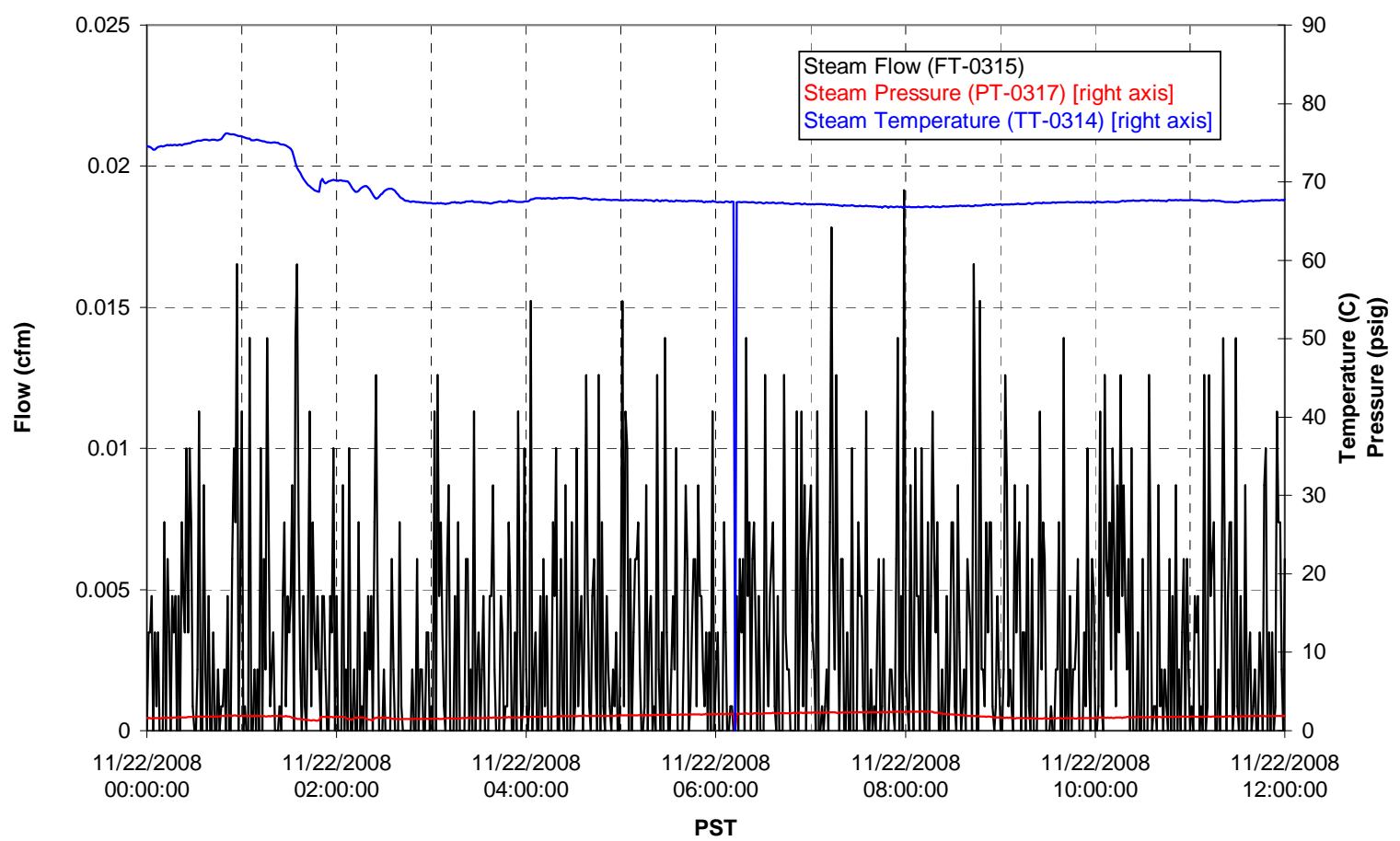


T01B Steam

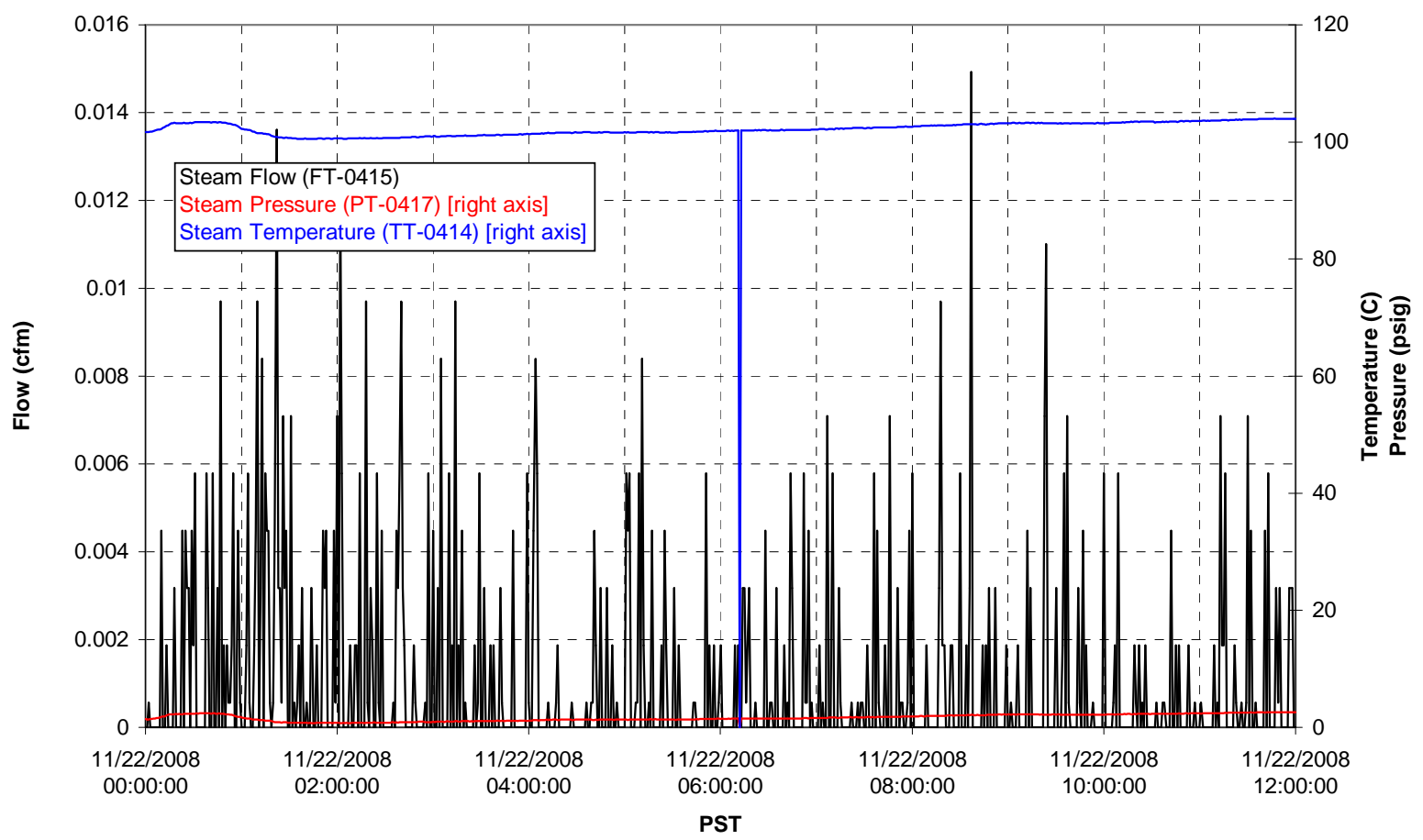


Functional Test Data Plots

11/22/2008 12:00 - 11/23/2008 00:00 
T01A level

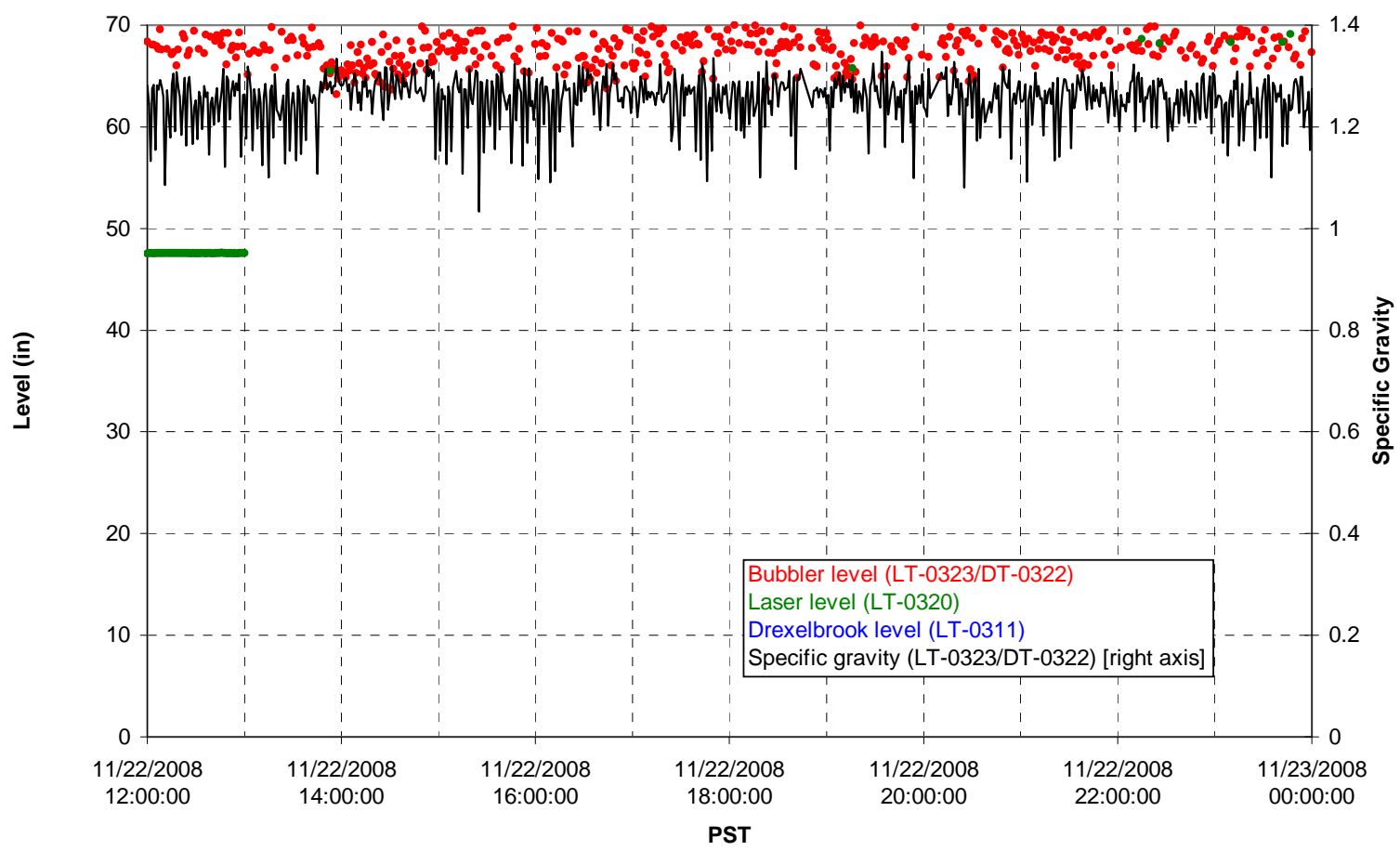

T01A temperatures

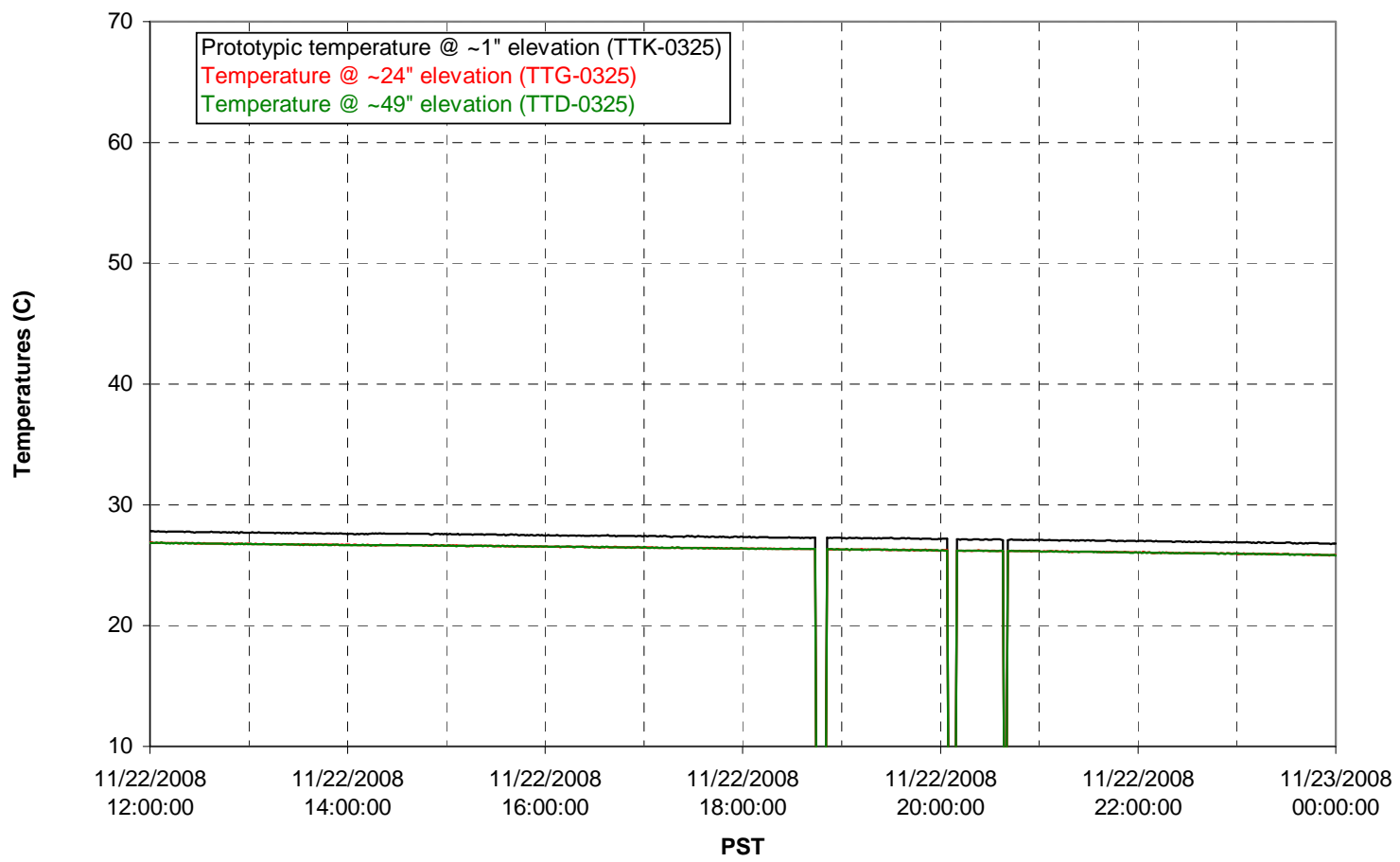


T01B level

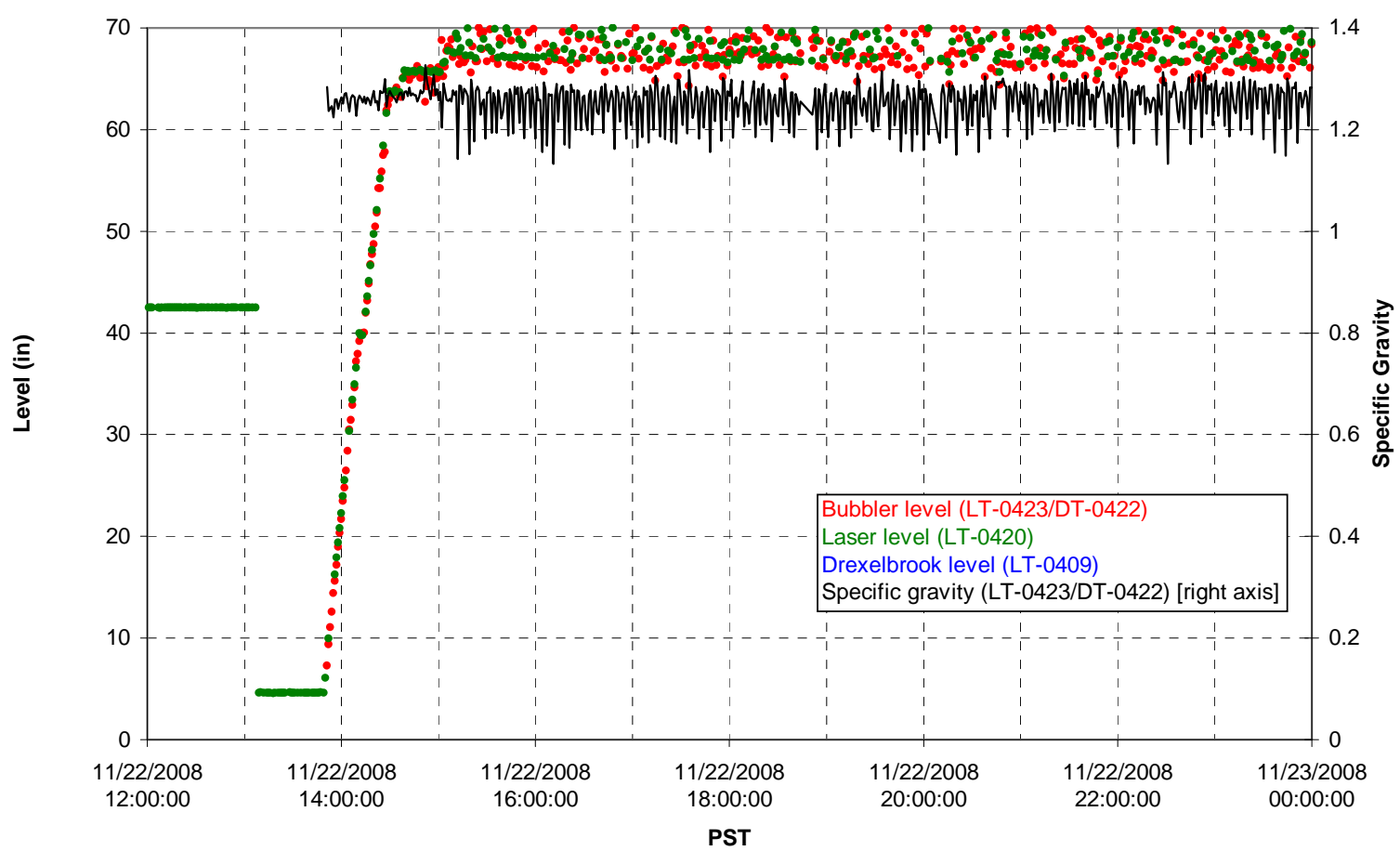

T01B temperatures

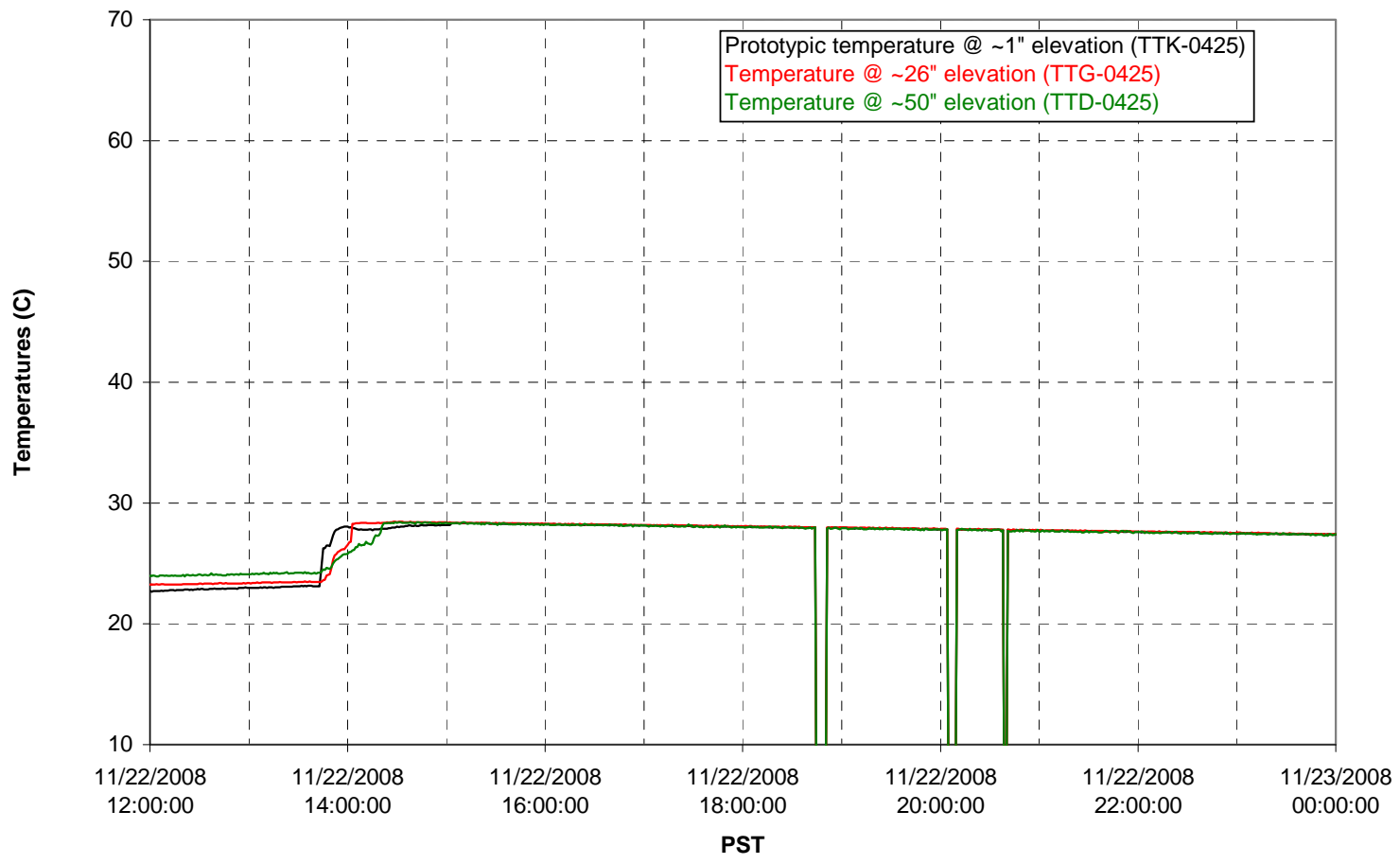


T02A level

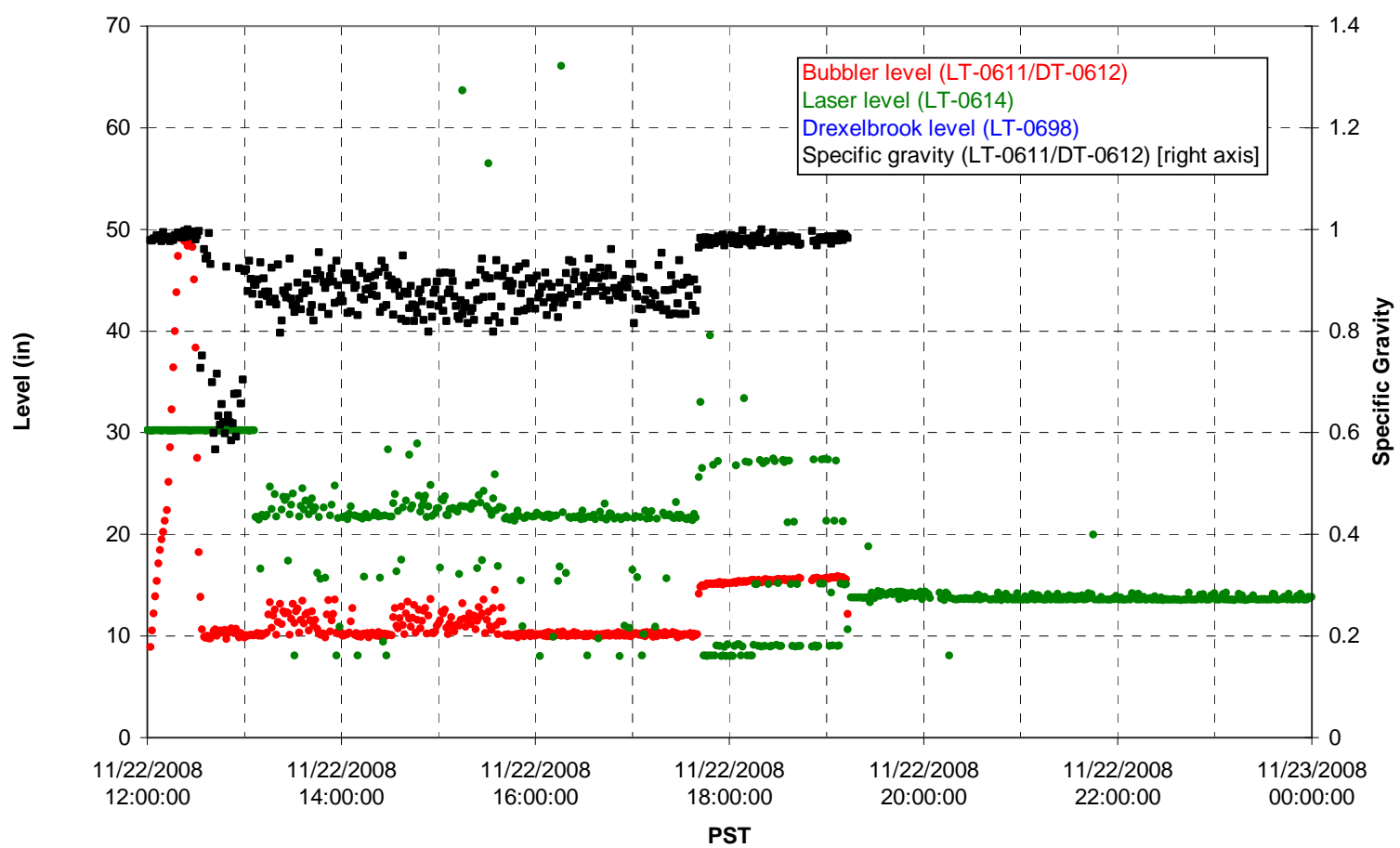

T02A temperatures

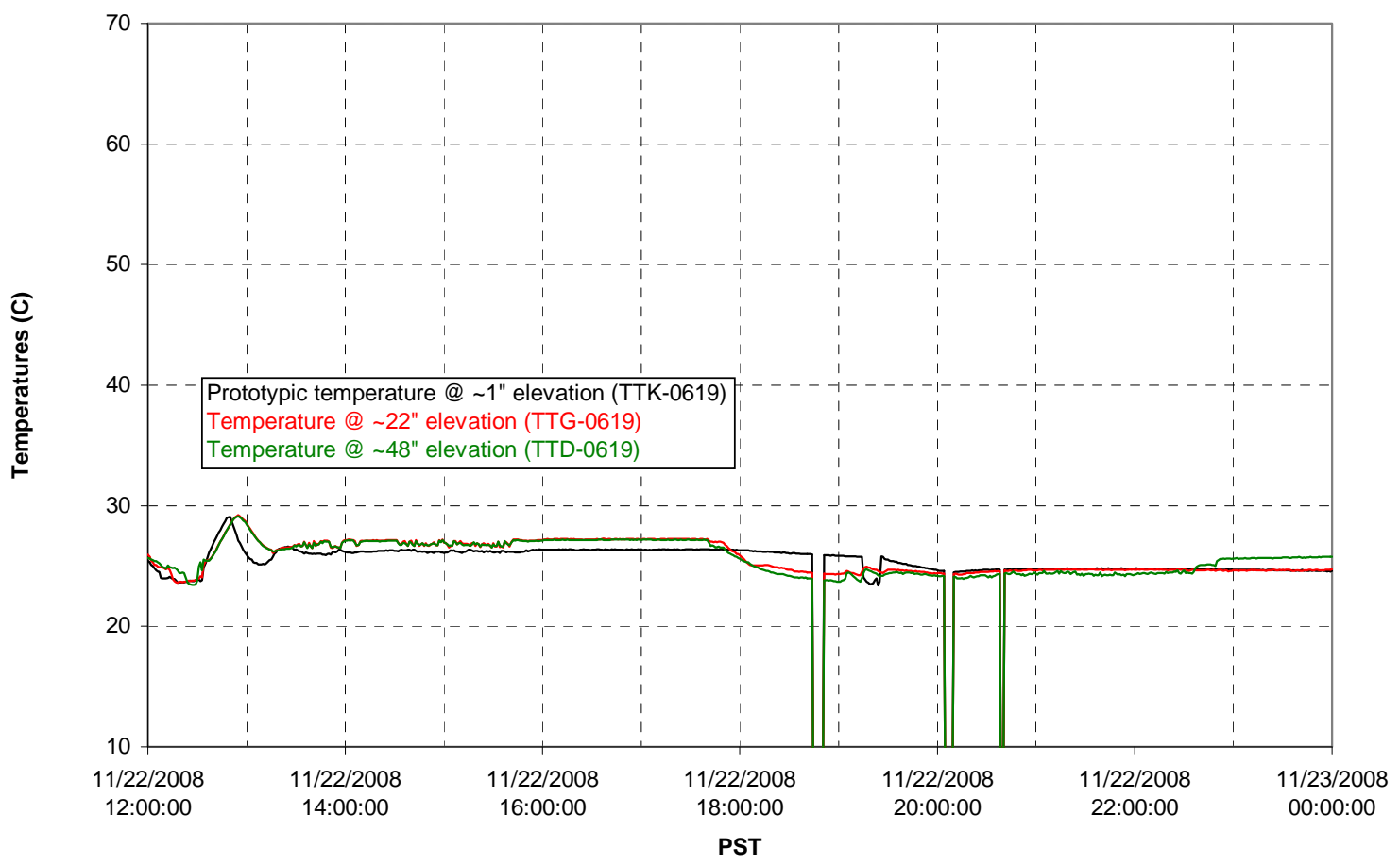


T02A and filter loop temperatures
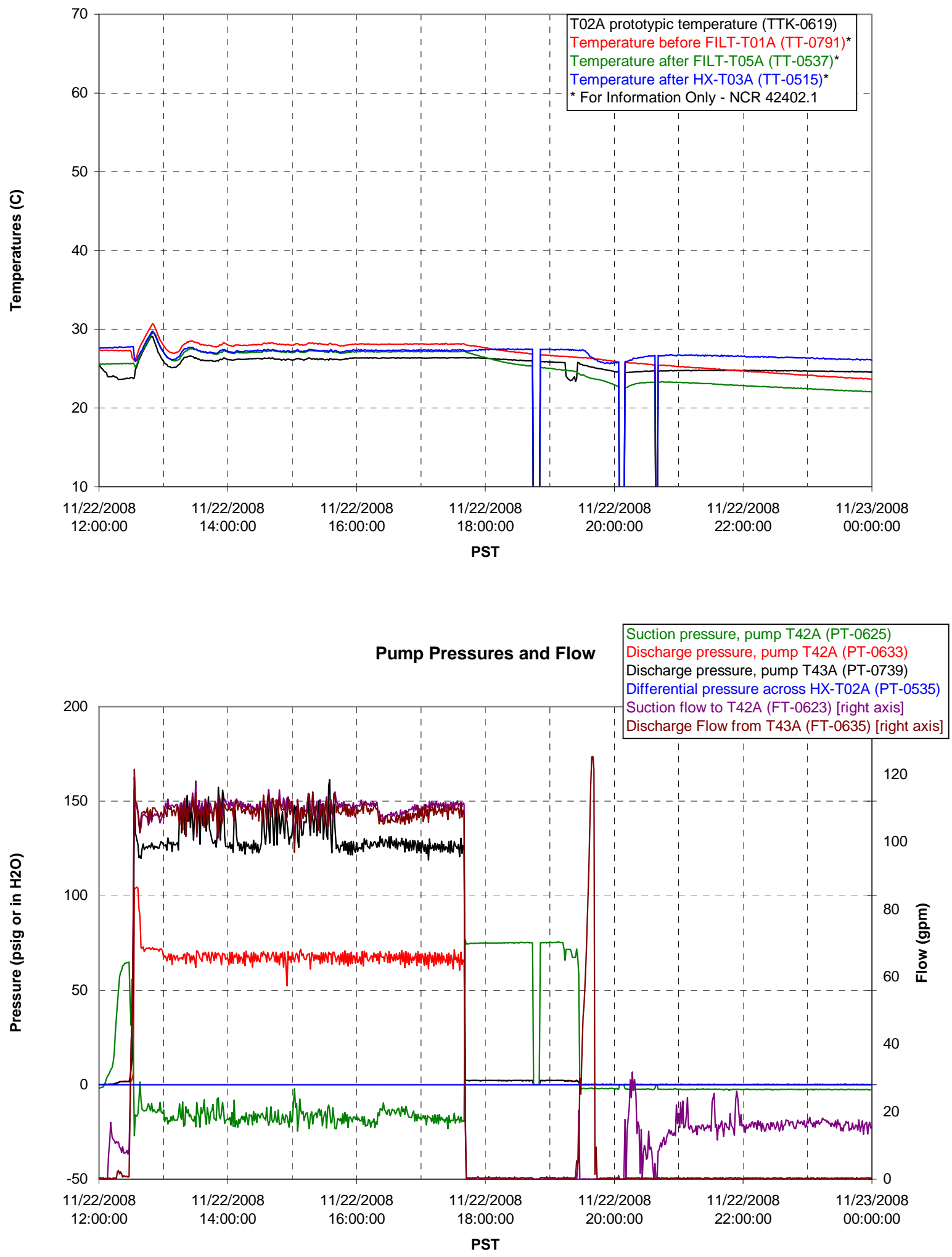


\section{Axial pressure drop}

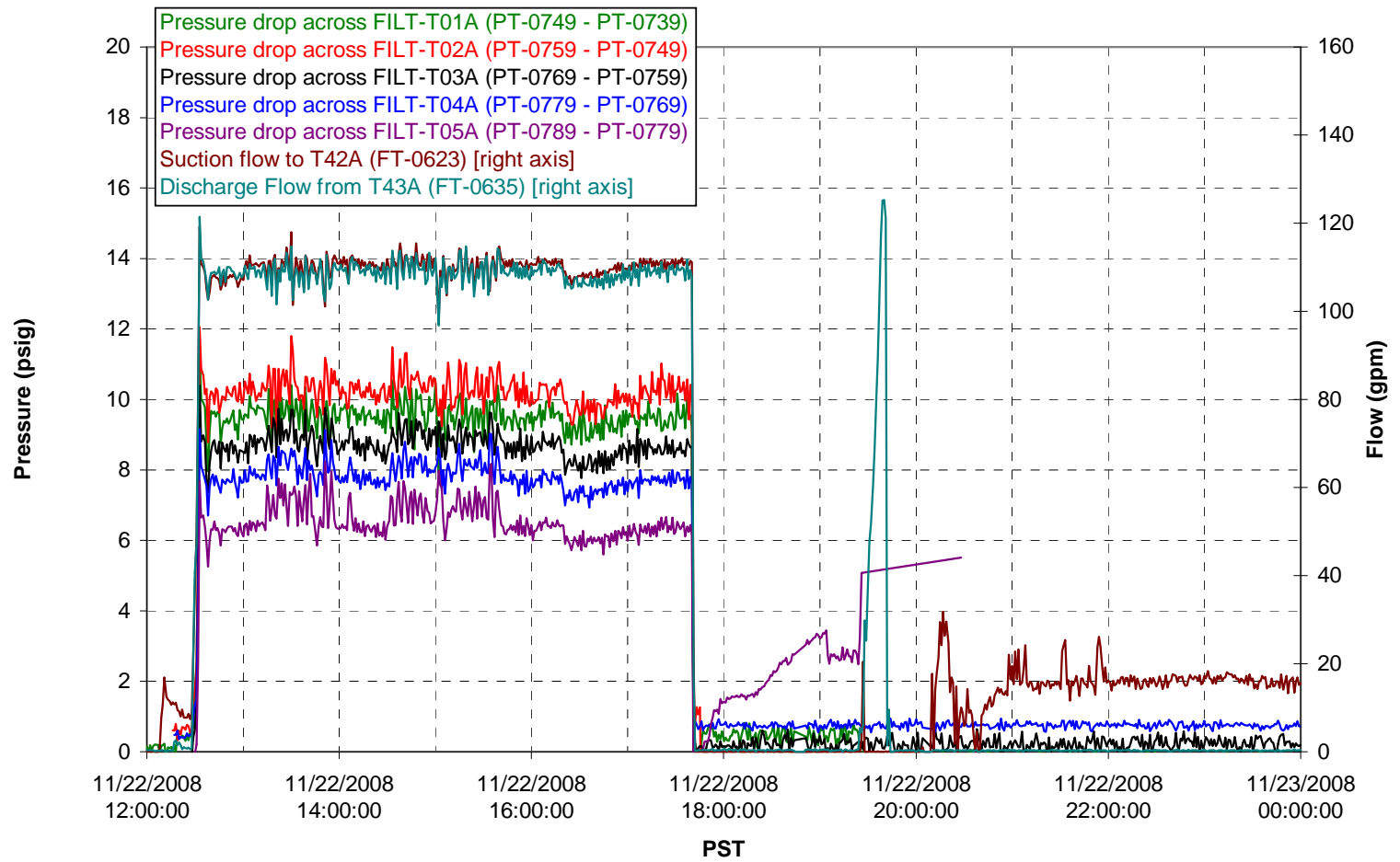

Permeate flow rates

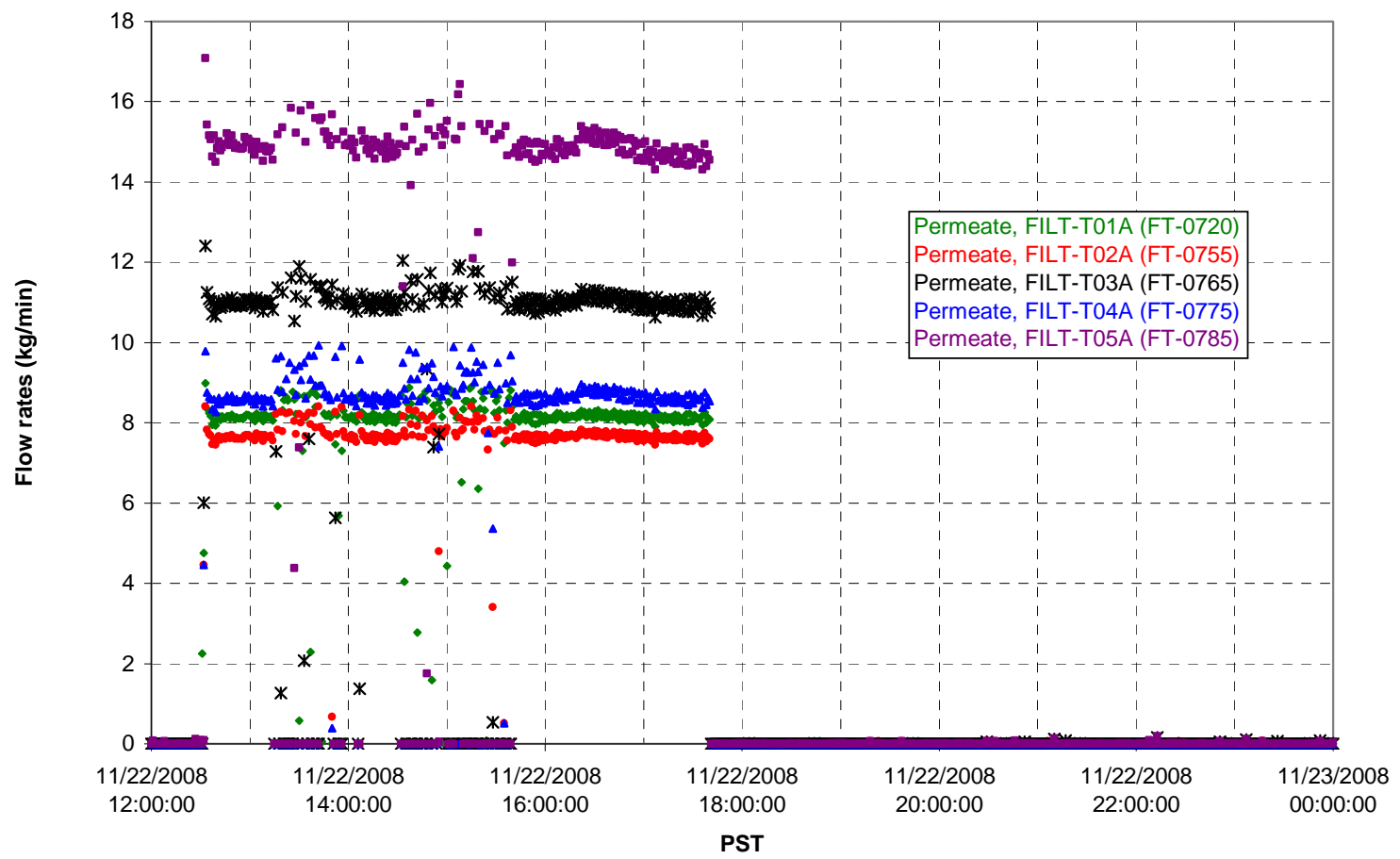


T02A Inner Temperature Tree

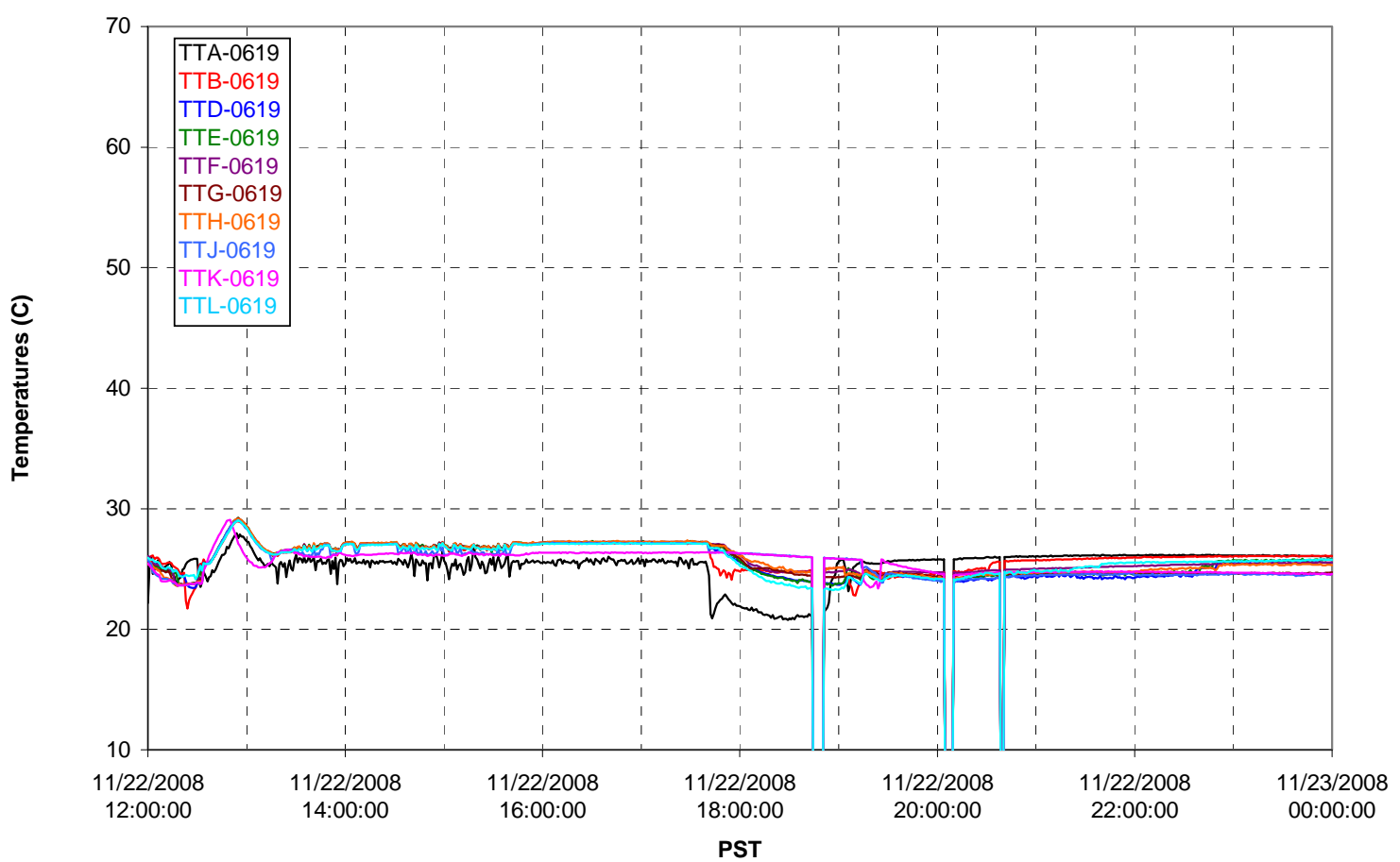

T02A Outer Temperature Tree

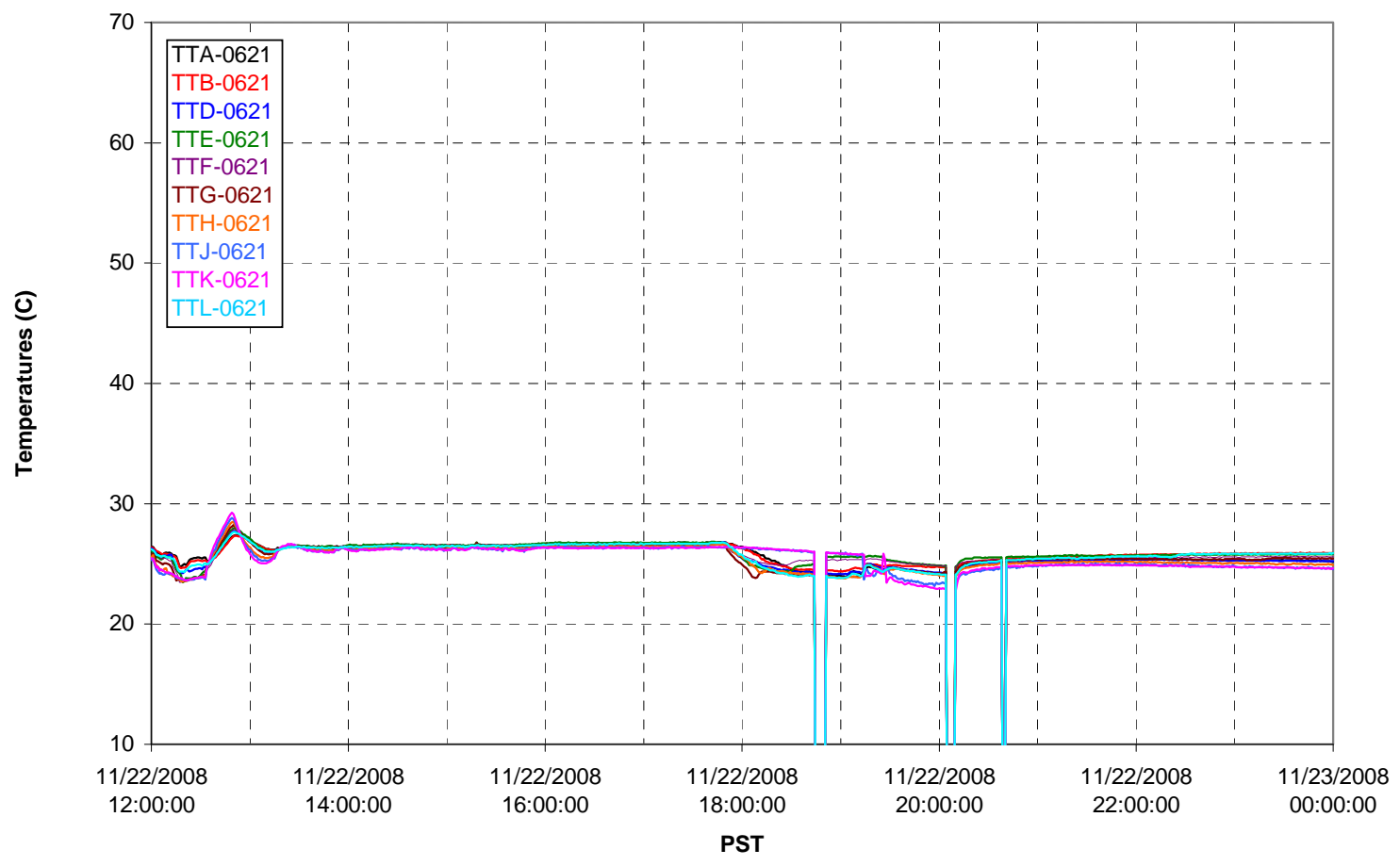


T02A temperatures

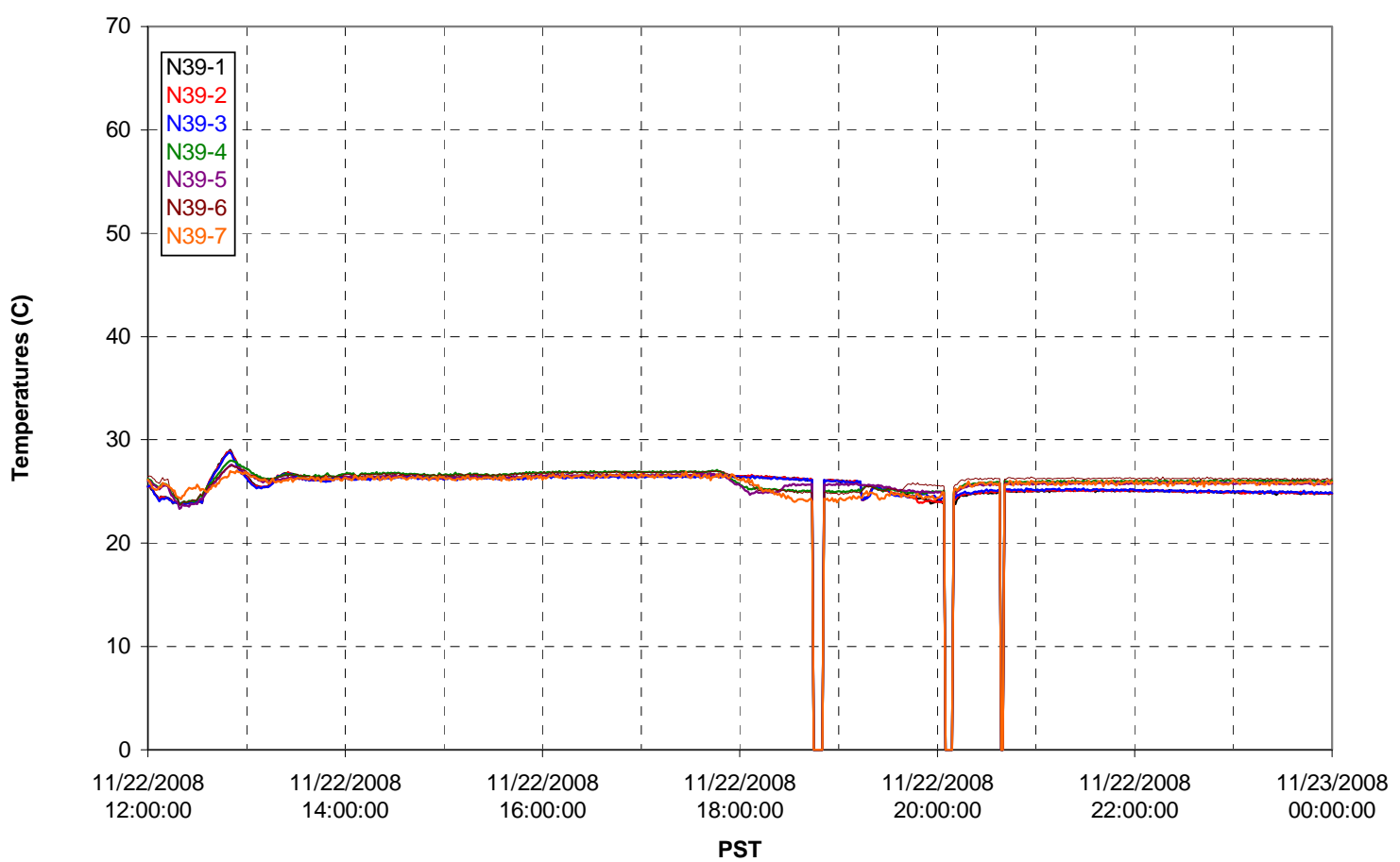

T02A temperatures

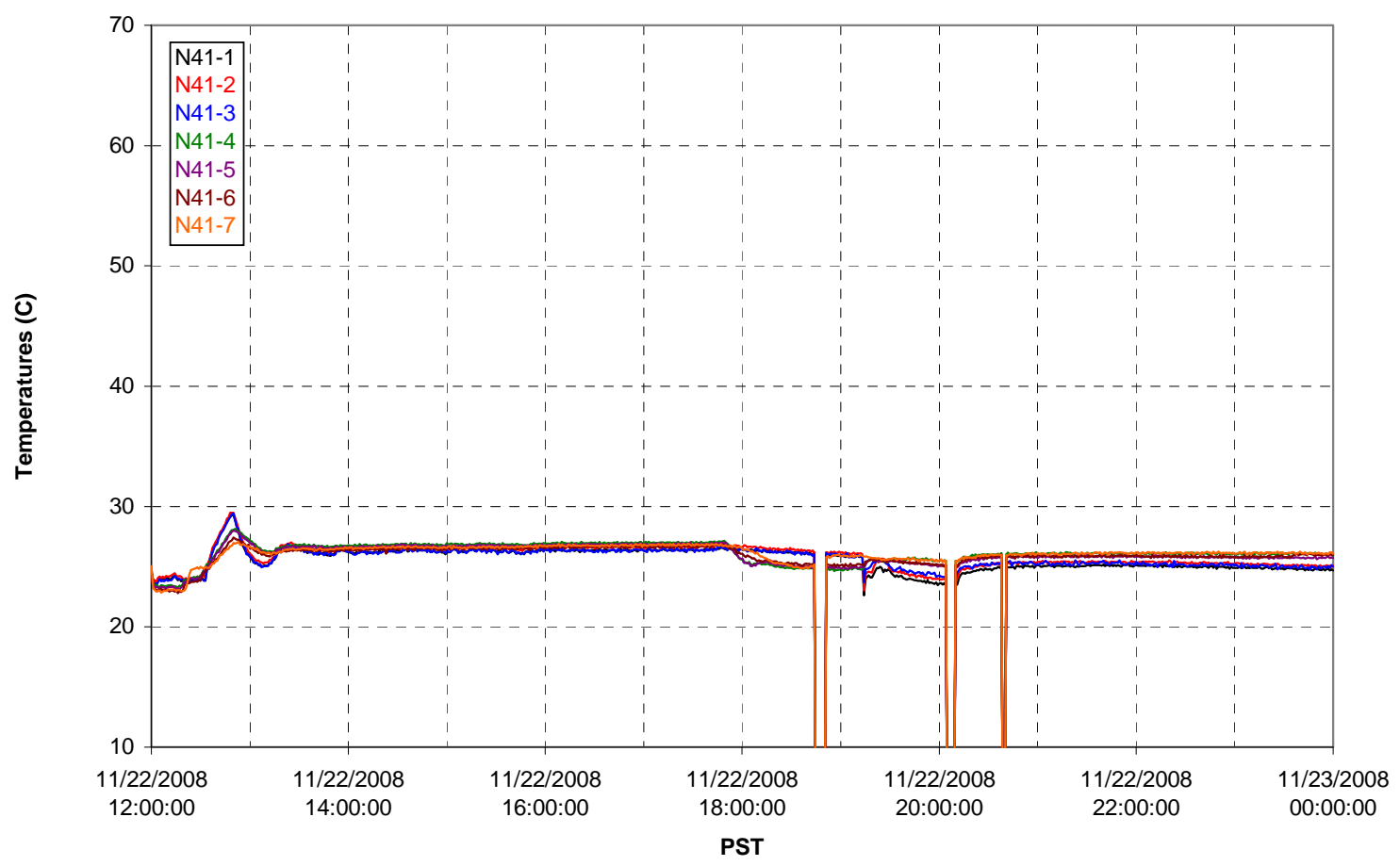


T02A temperatures

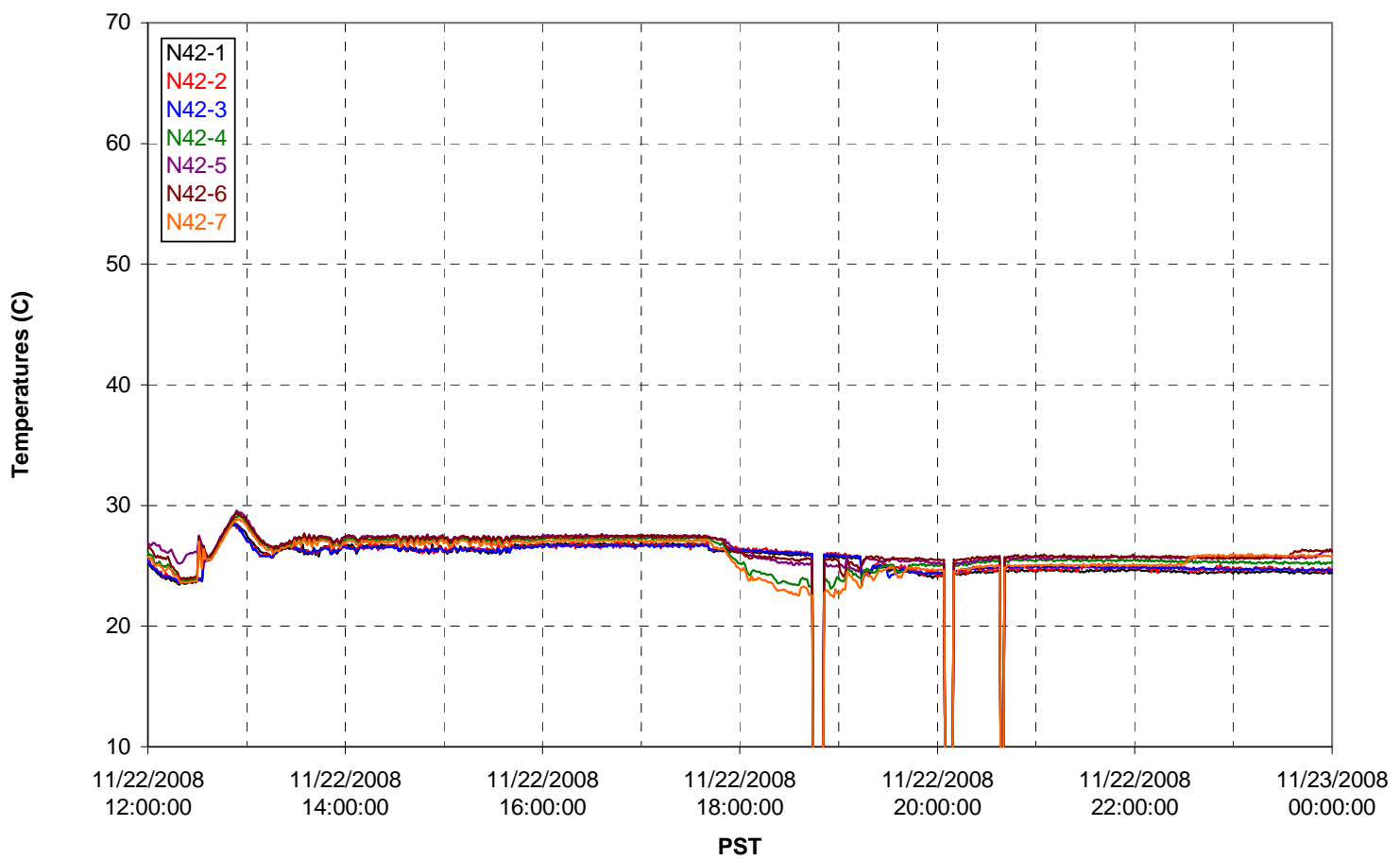

T02A temperatures

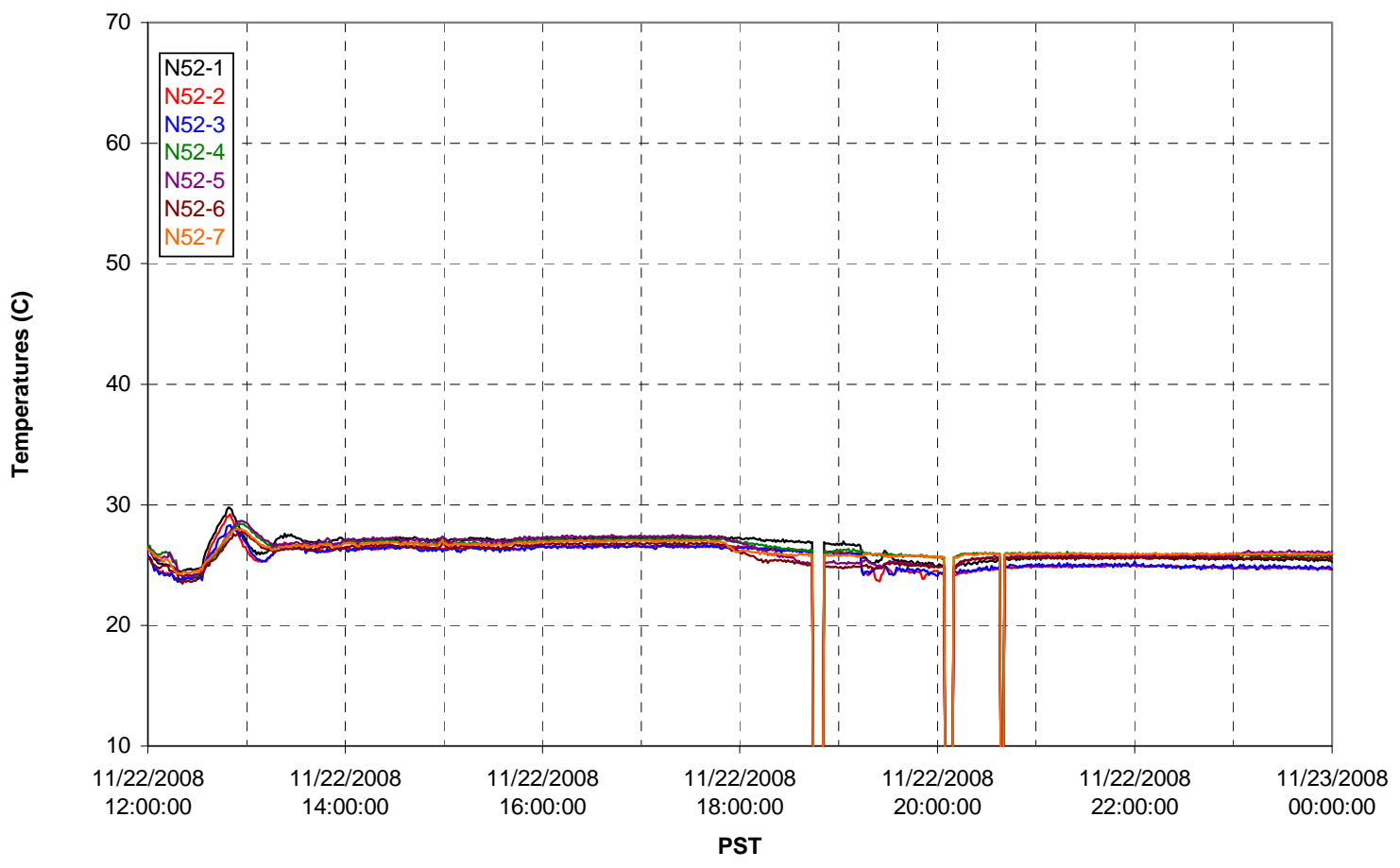


T02A Heating and Cooling

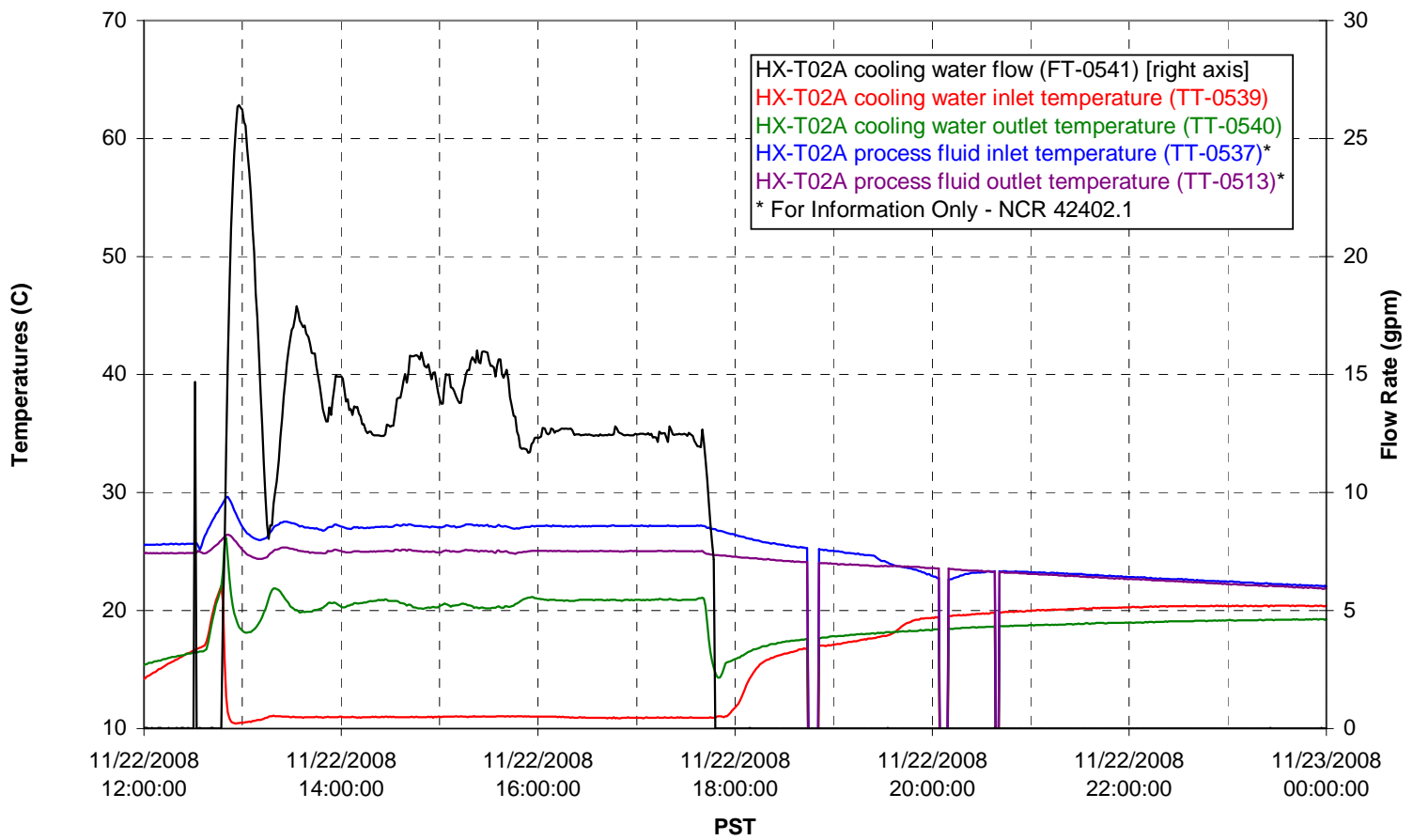

Pump Operation

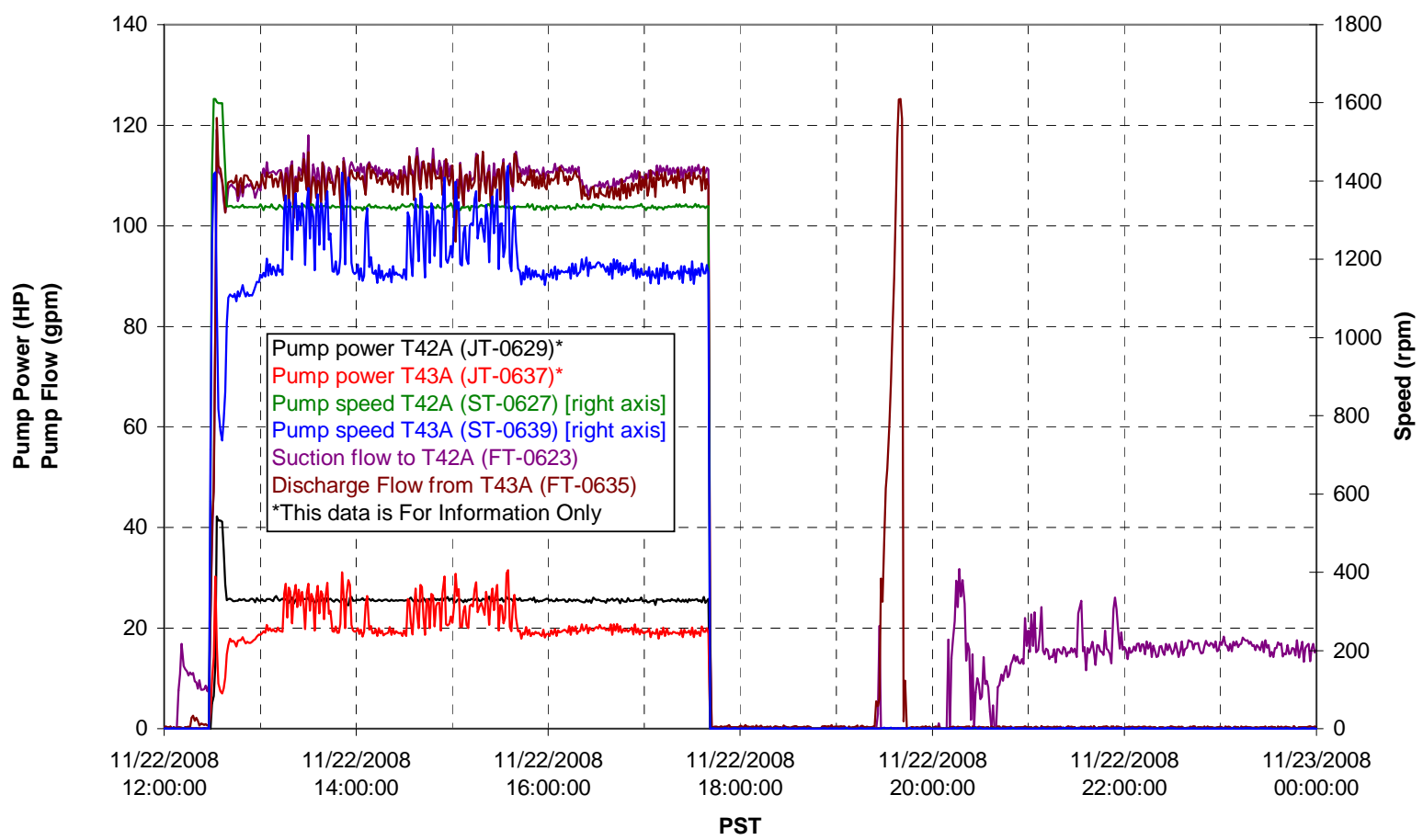


Pulsepot UFP-PP-T01A

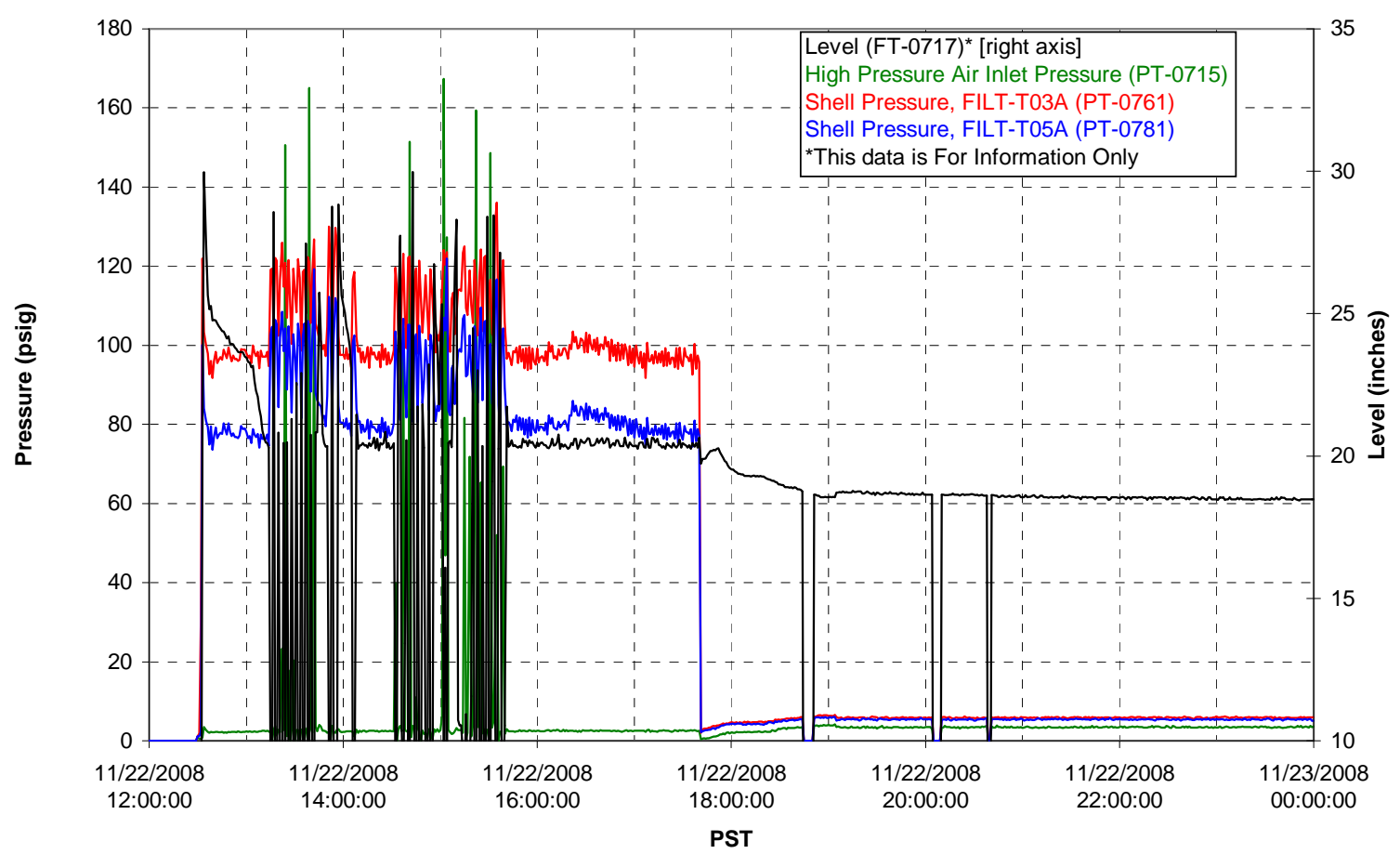

Pulsepot UFP-PP-T02A

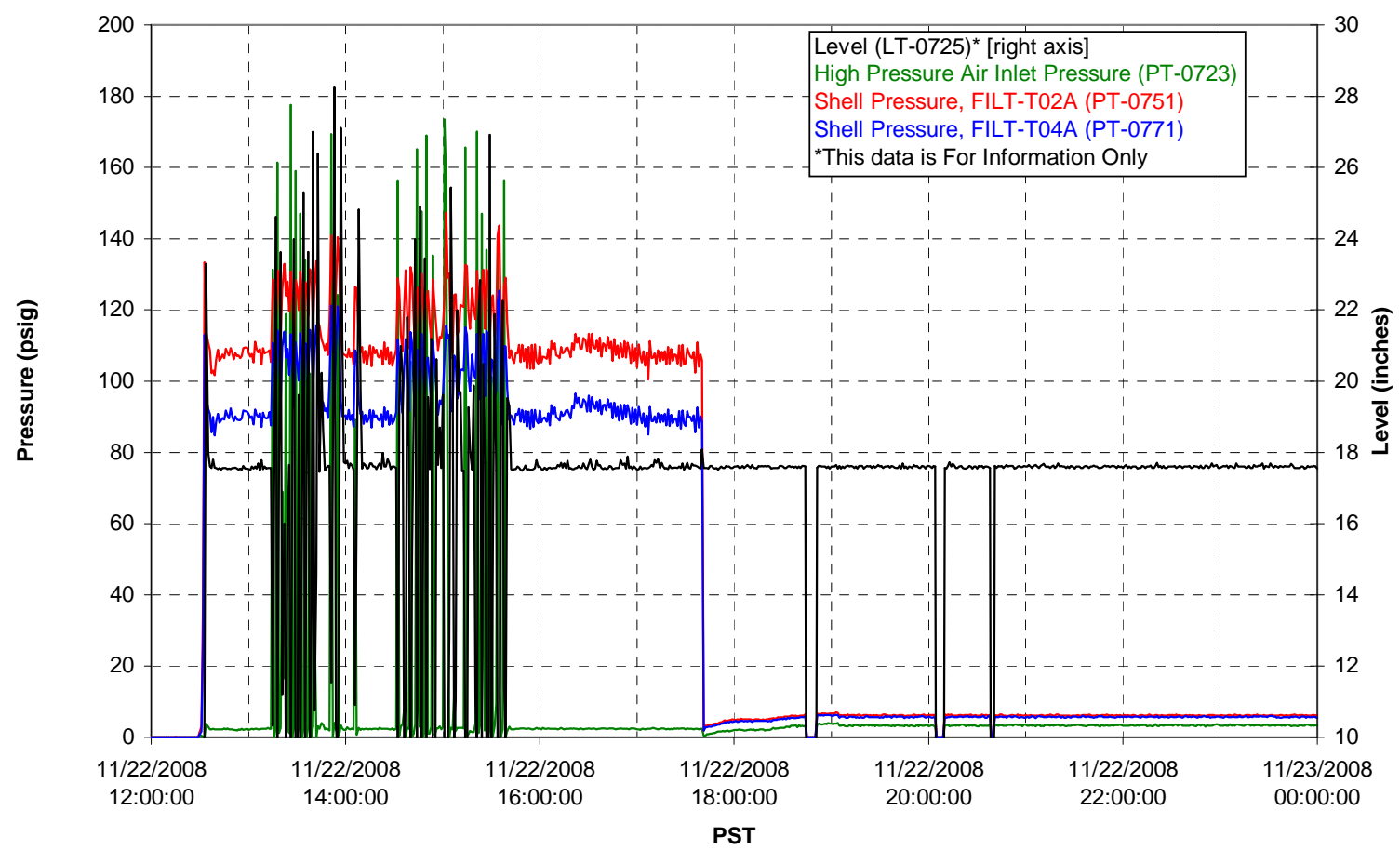


Pulsepot UFP-PP-T03A

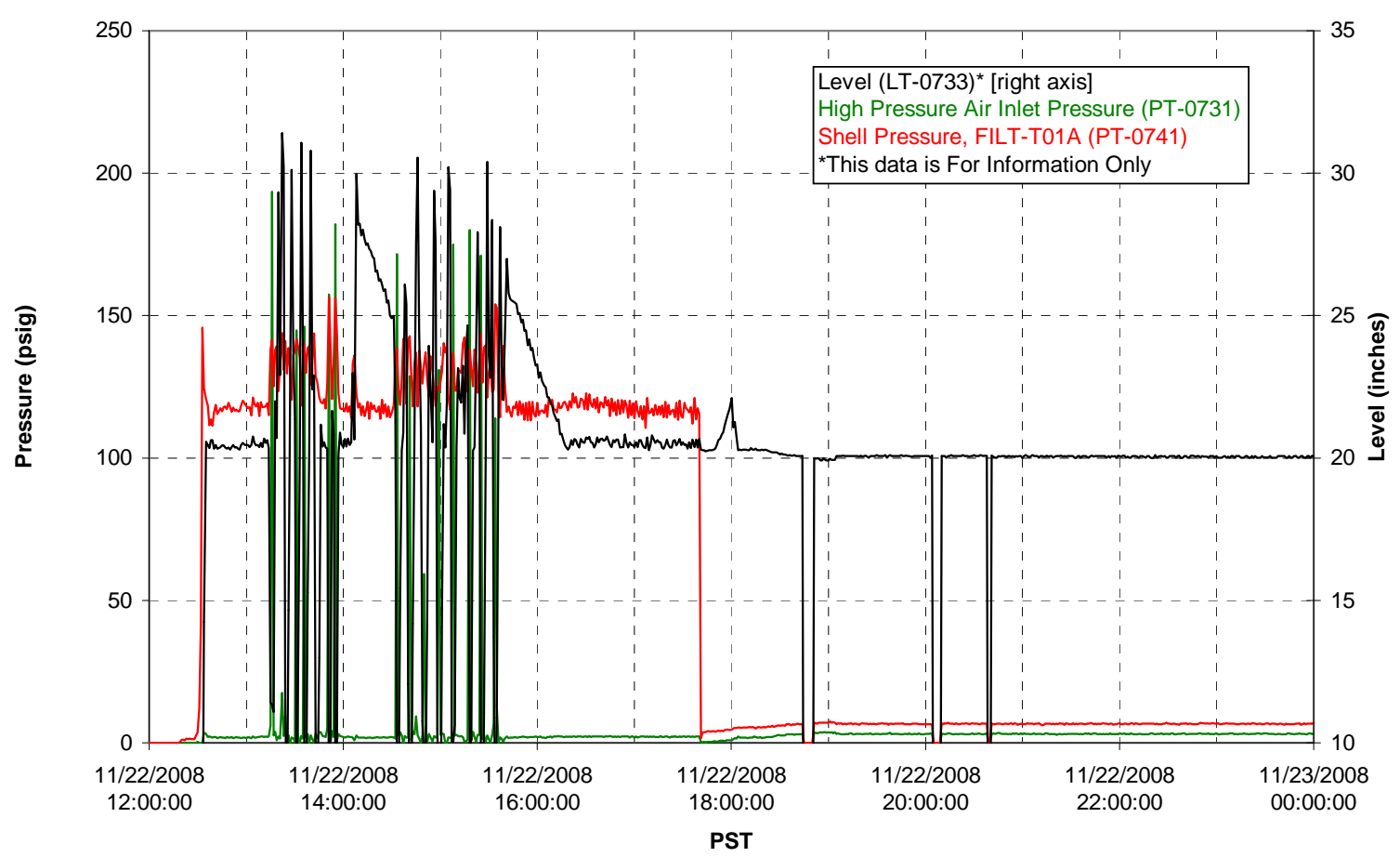

Pulsepot Levels

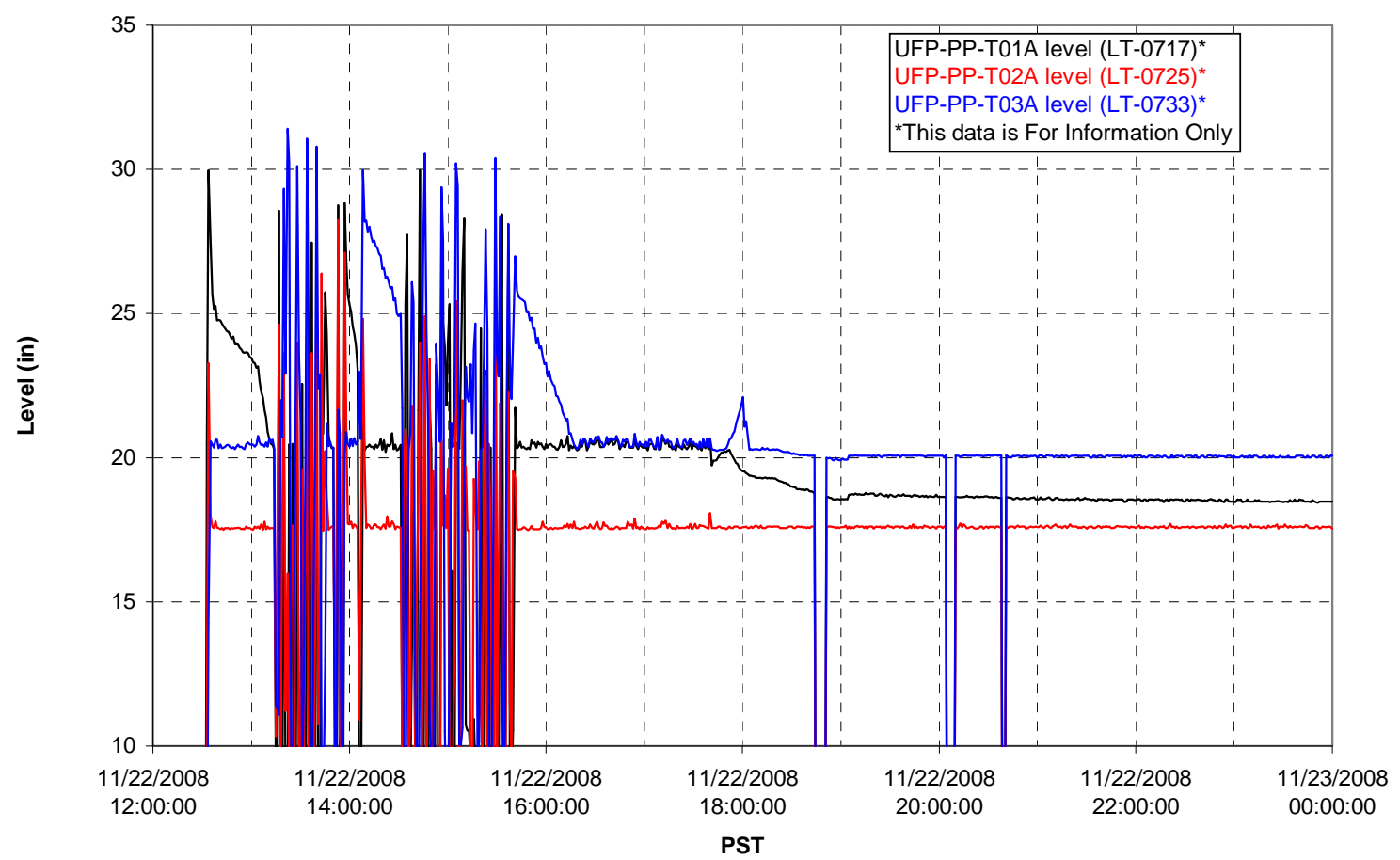


Filter UFP-FILT-T01A

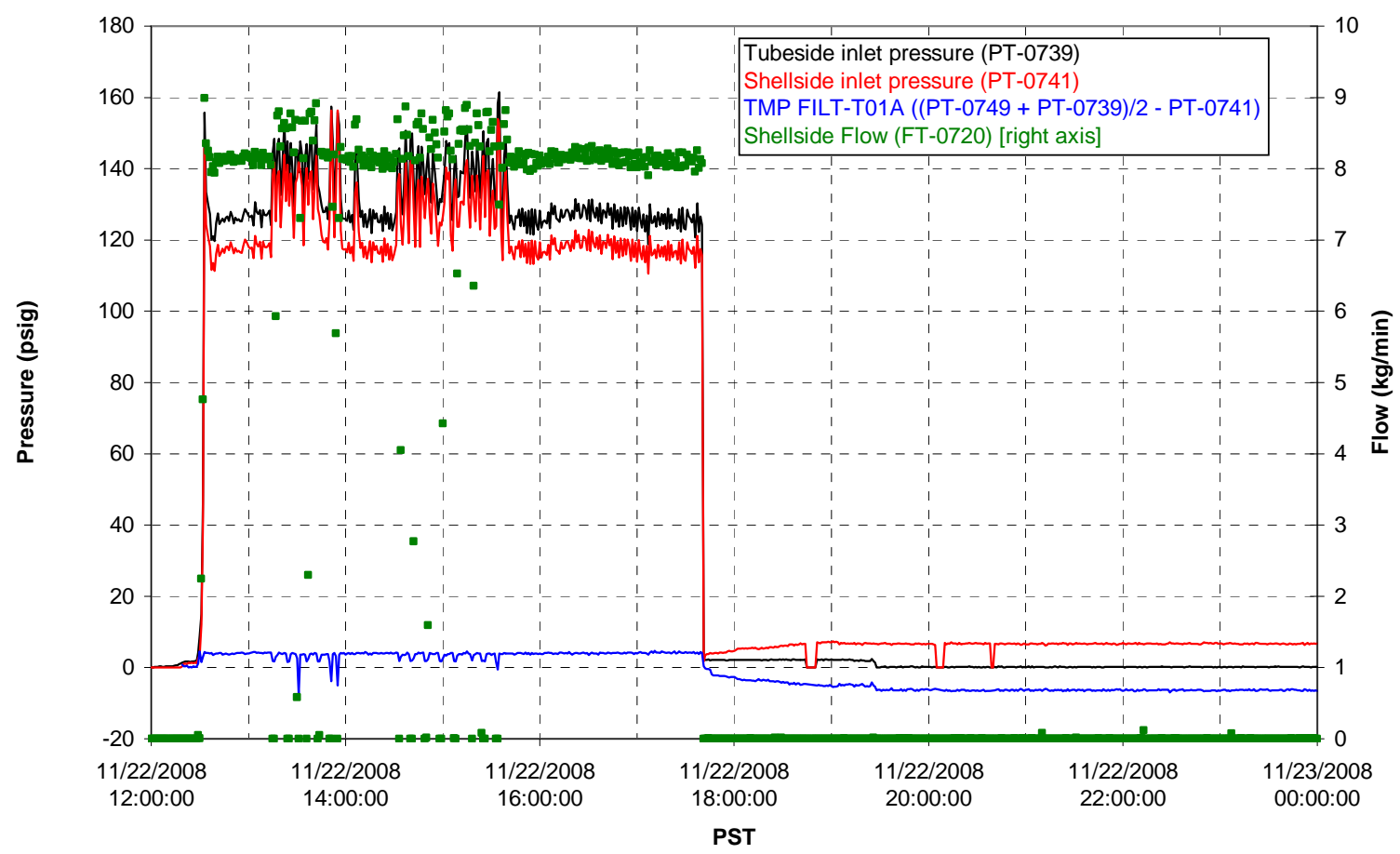

Filter UFP-FILT-T02A

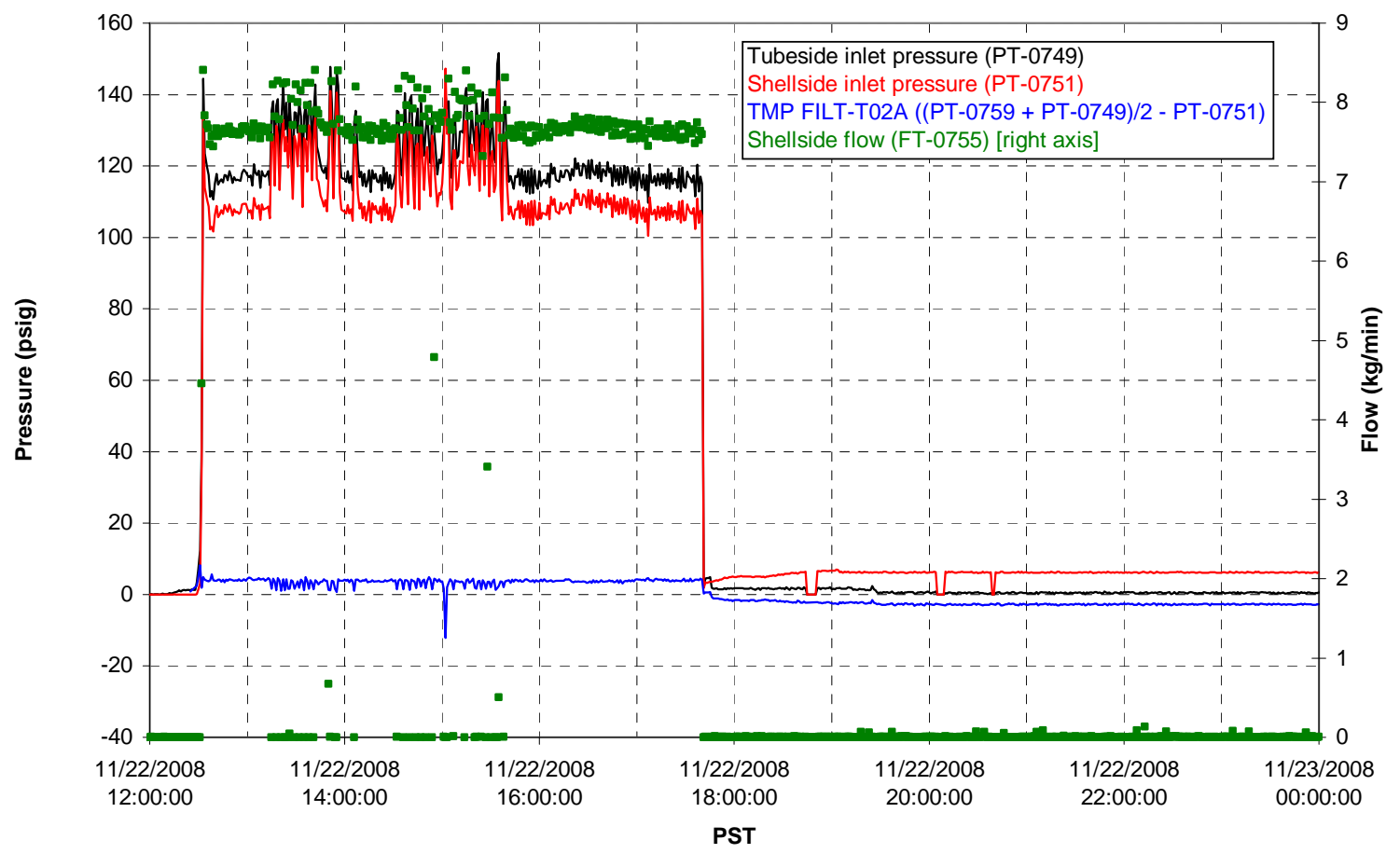


Filter UFP-FILT-T03A

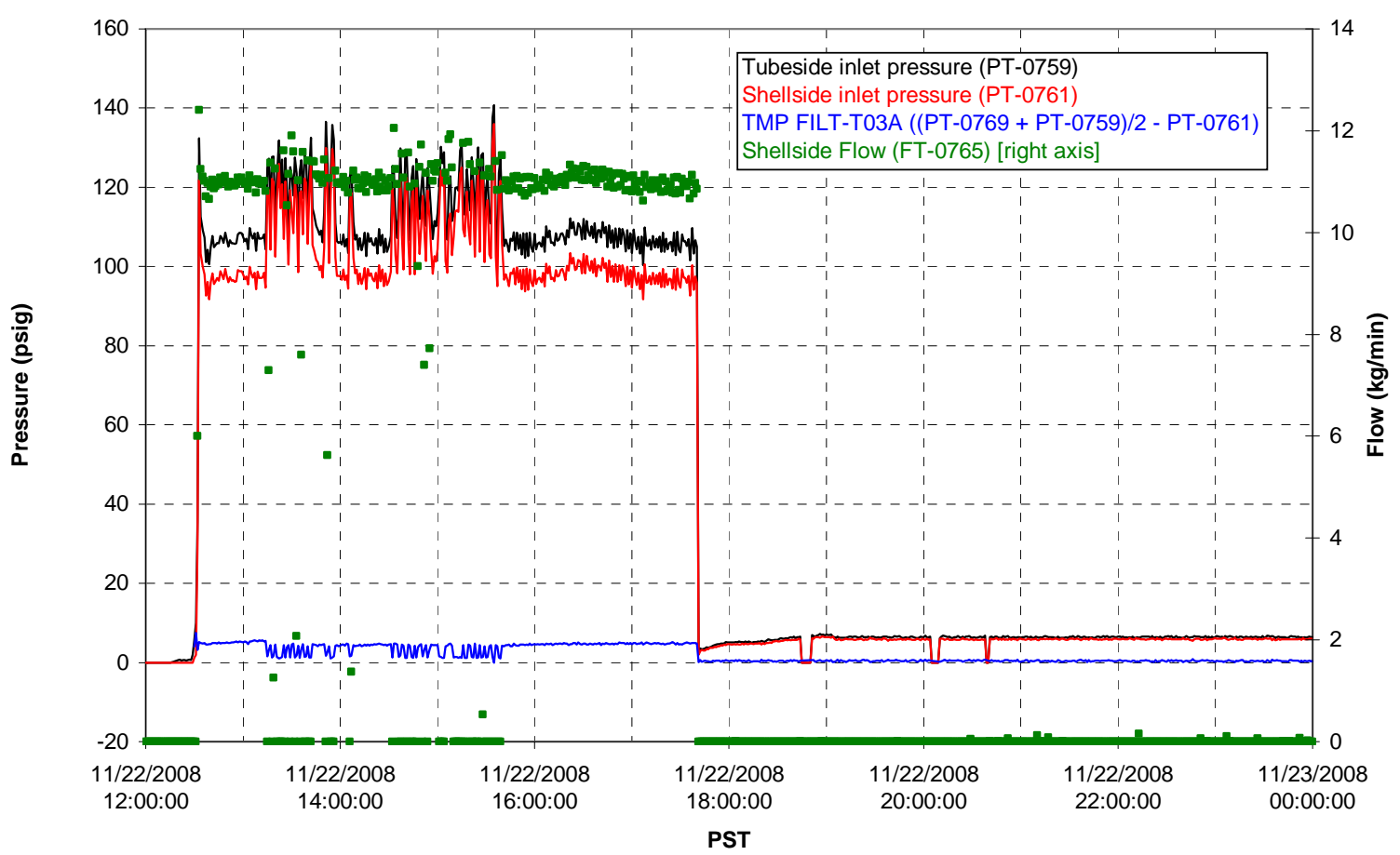

Filter UFP-FILT-T04A

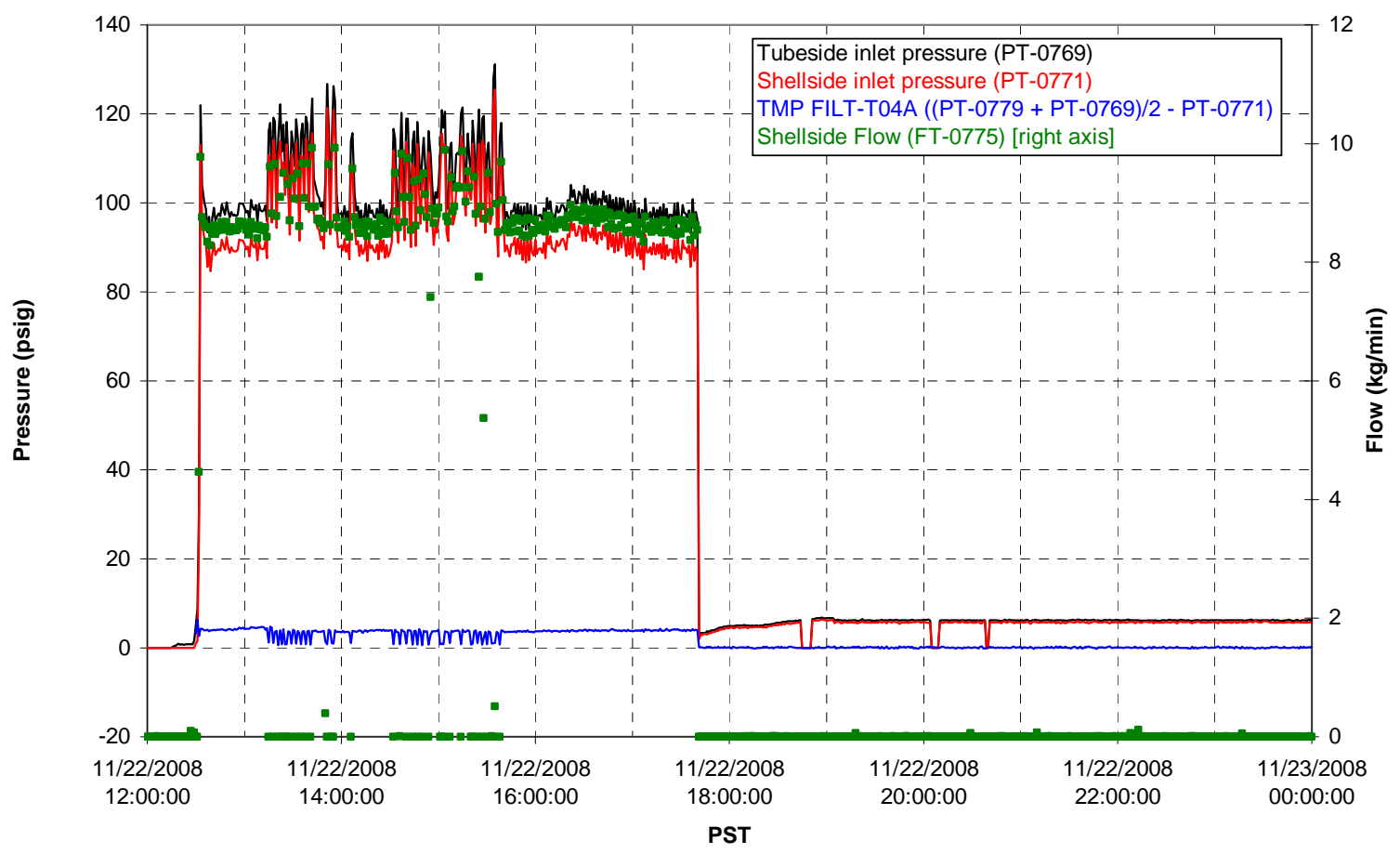


Filter UFP-FILT-T05A

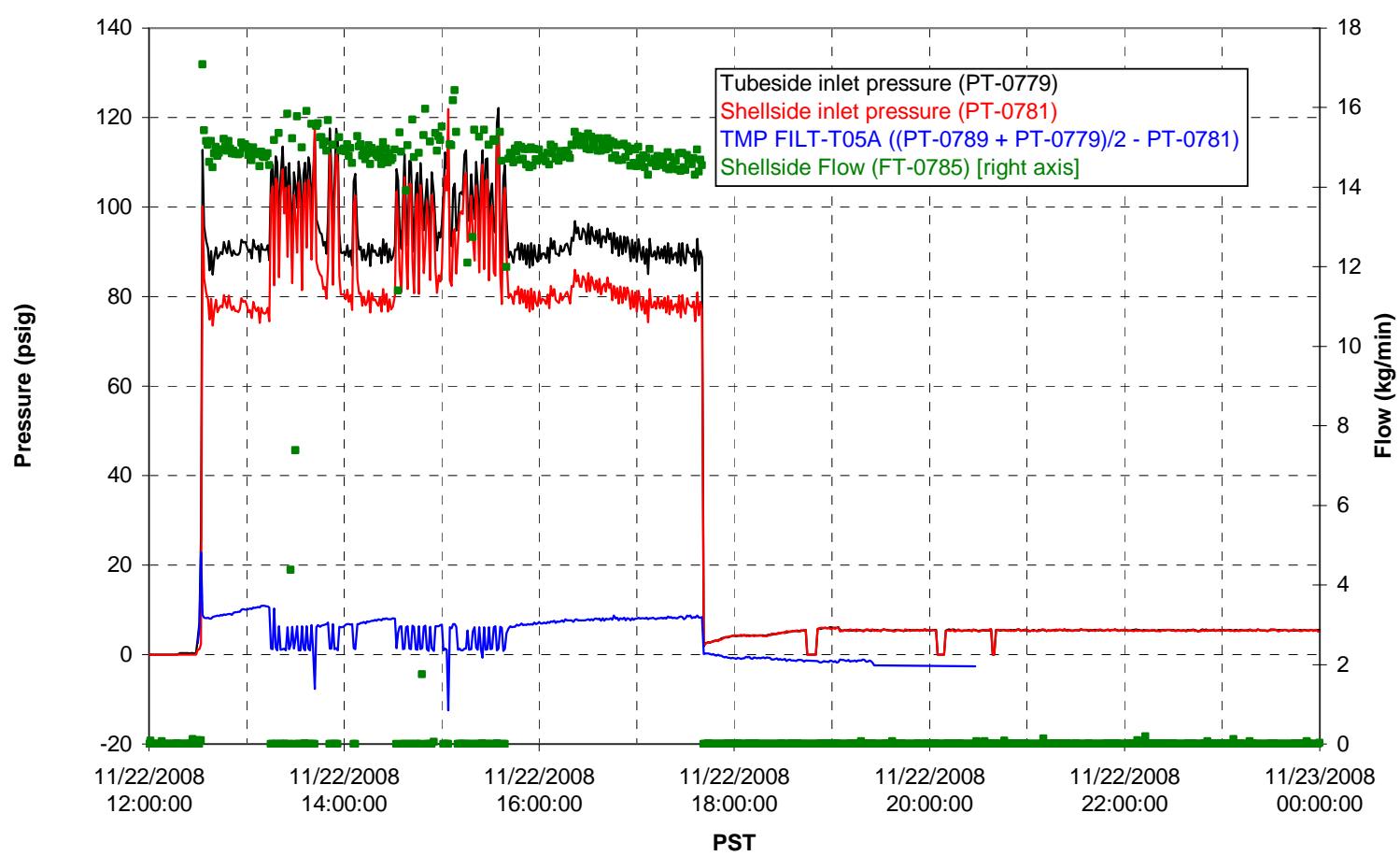

Chemical Flow

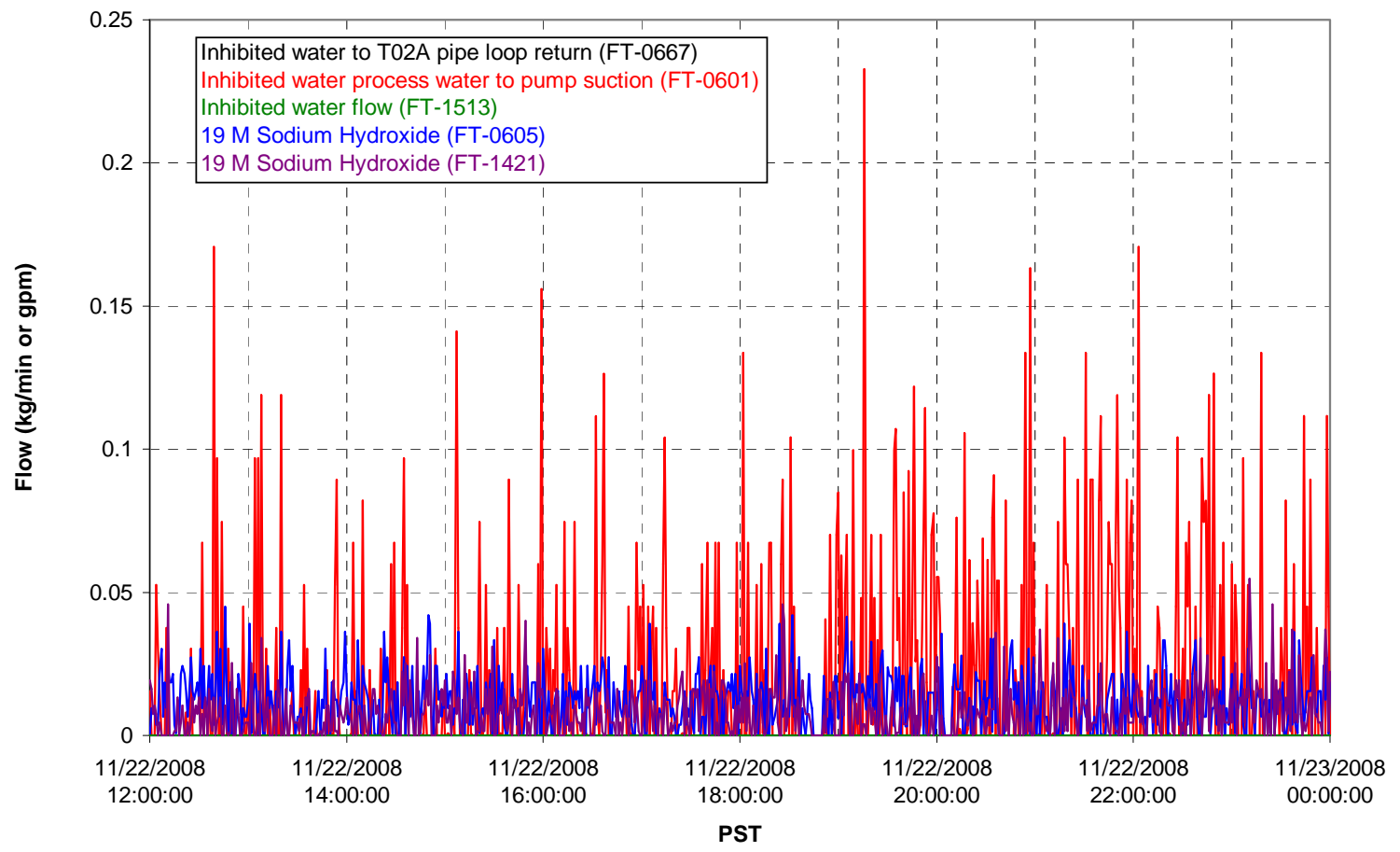




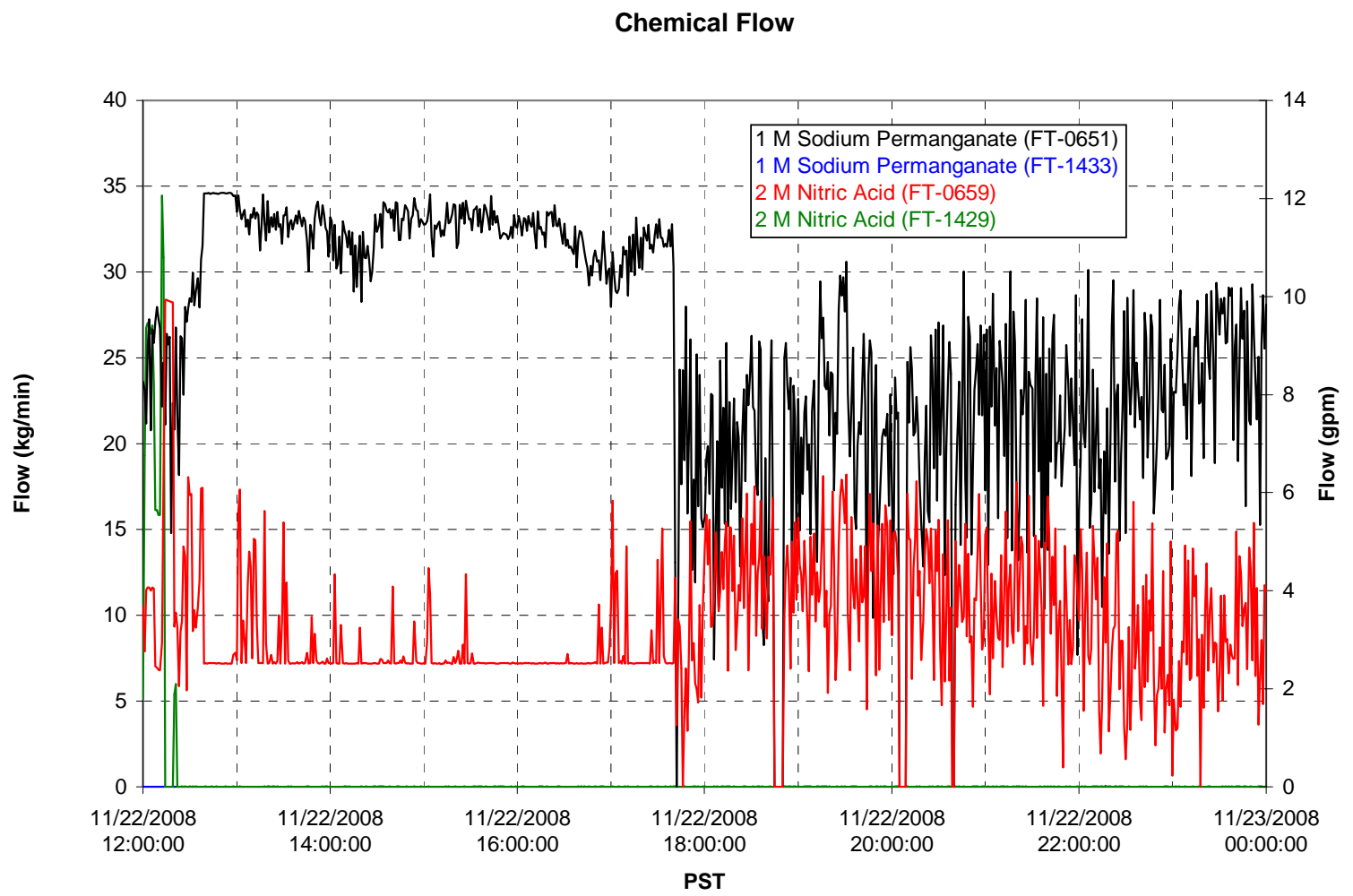

Air Flows

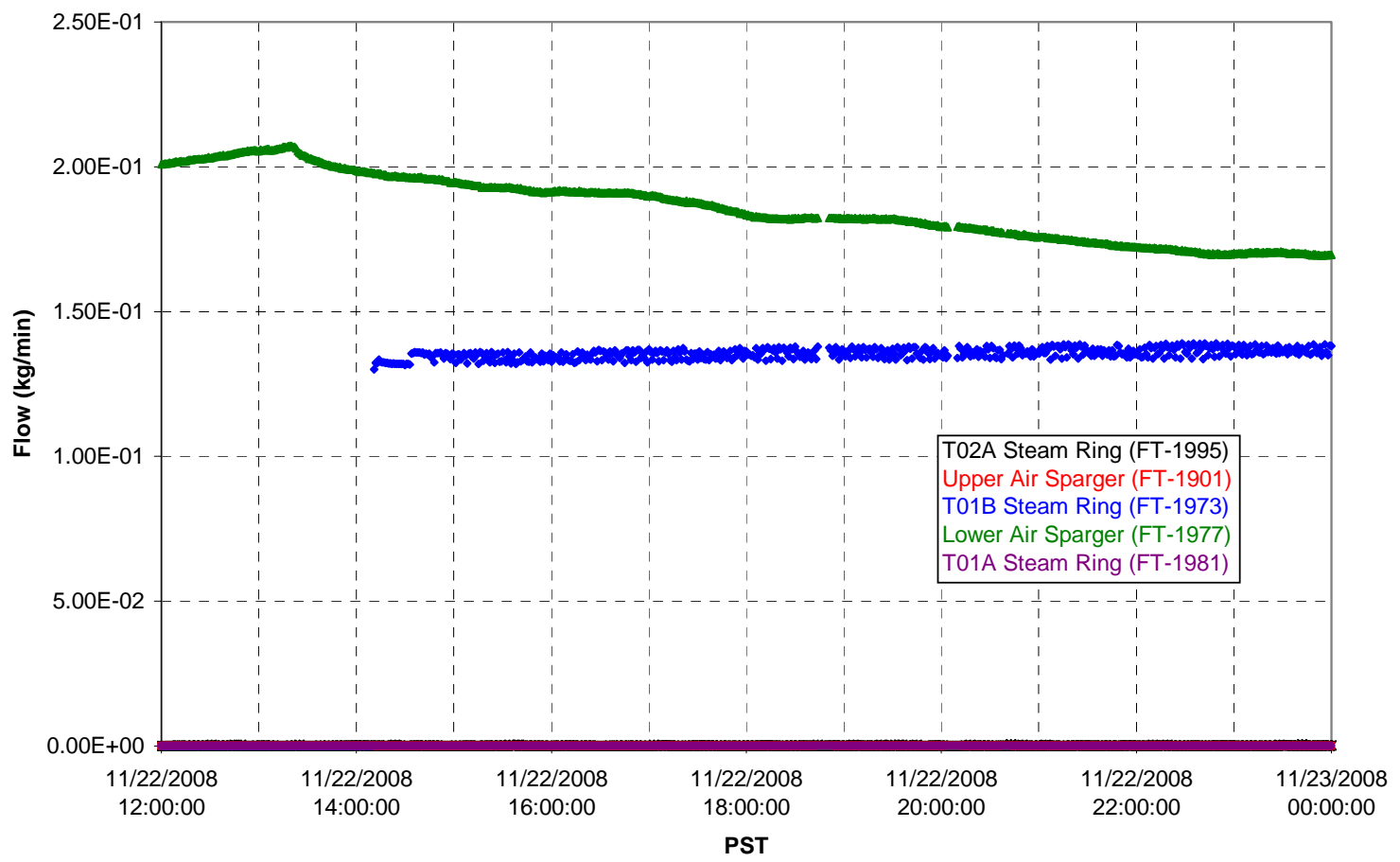


T02A Steam

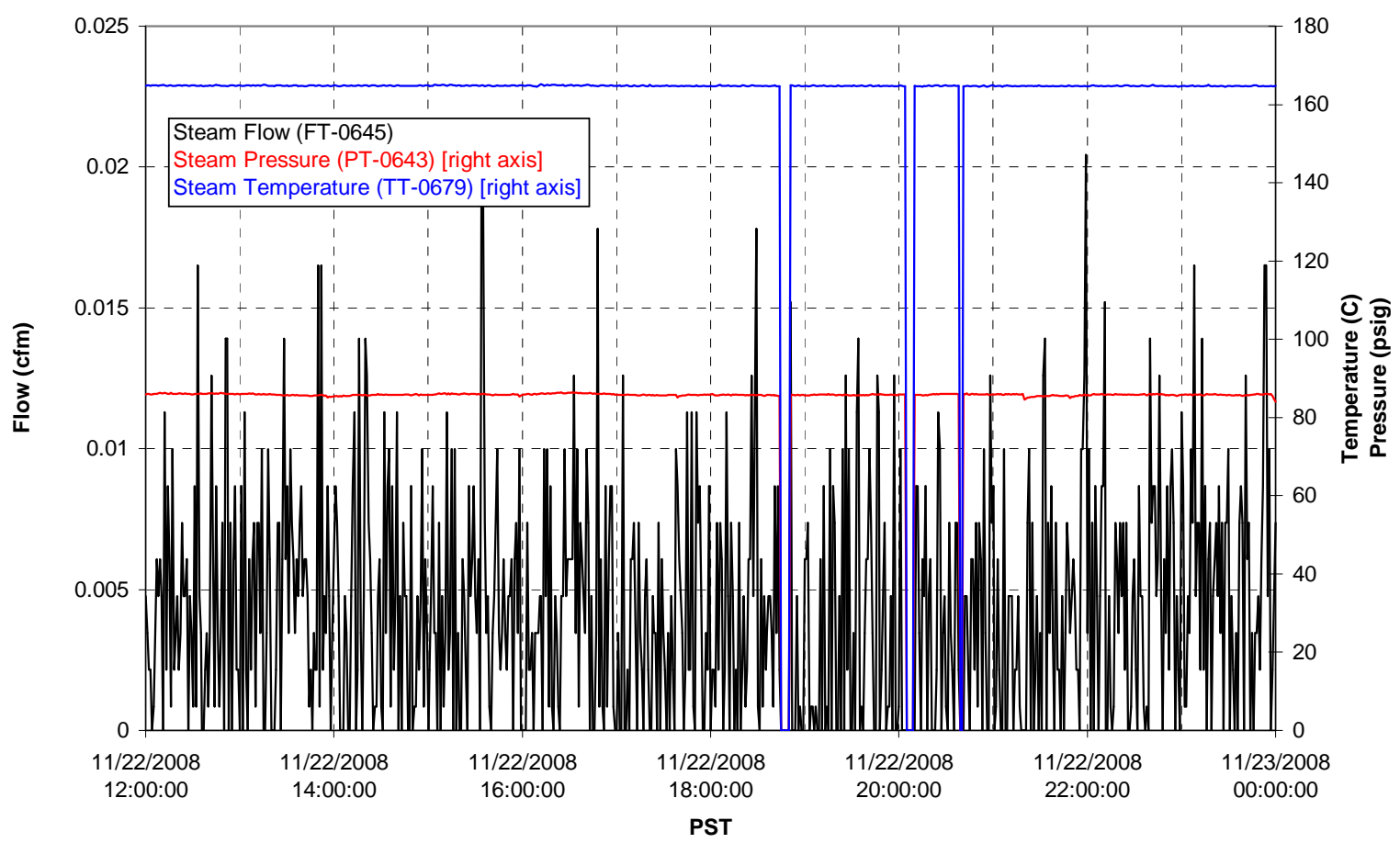

T01A Steam

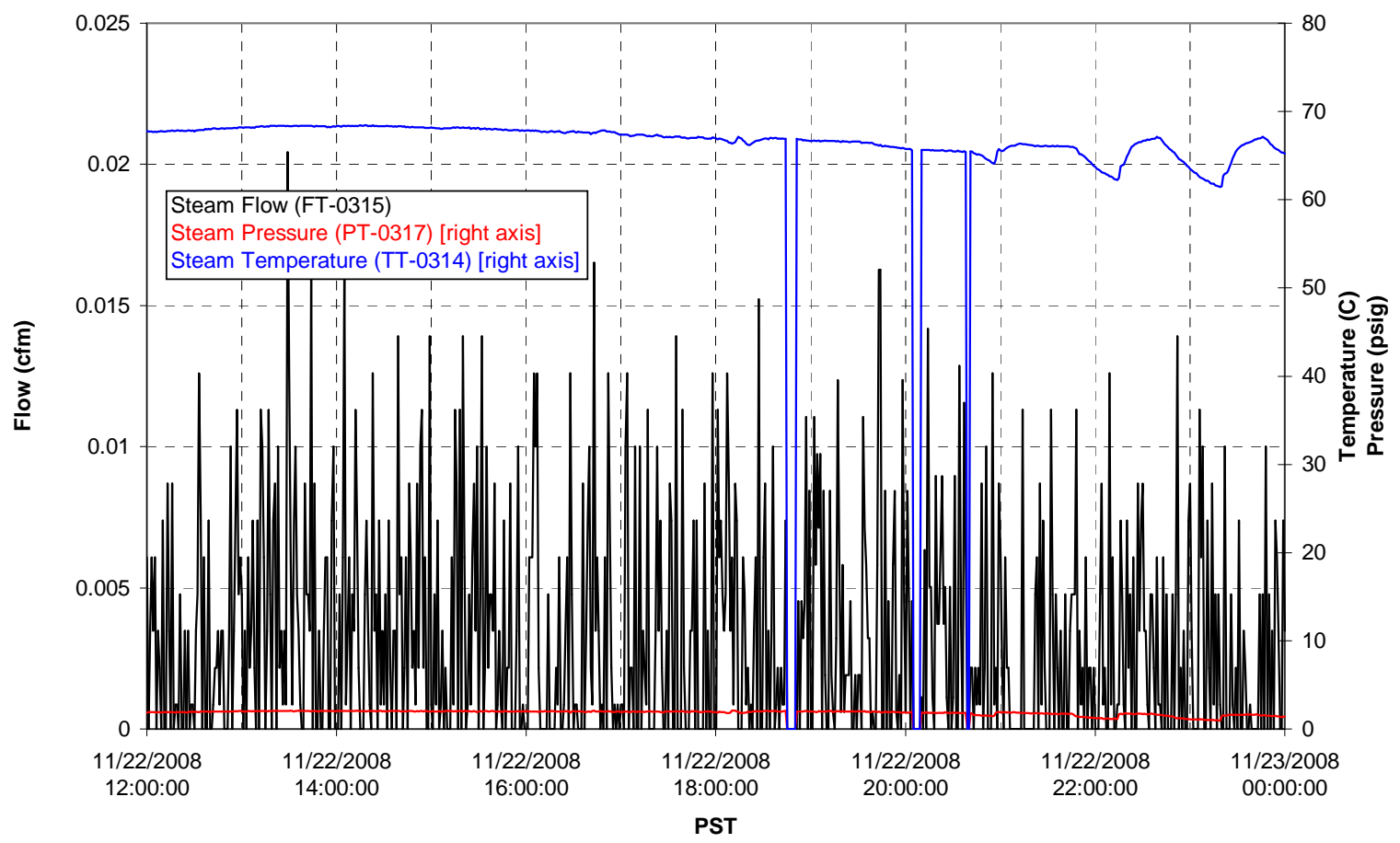


T01B Steam

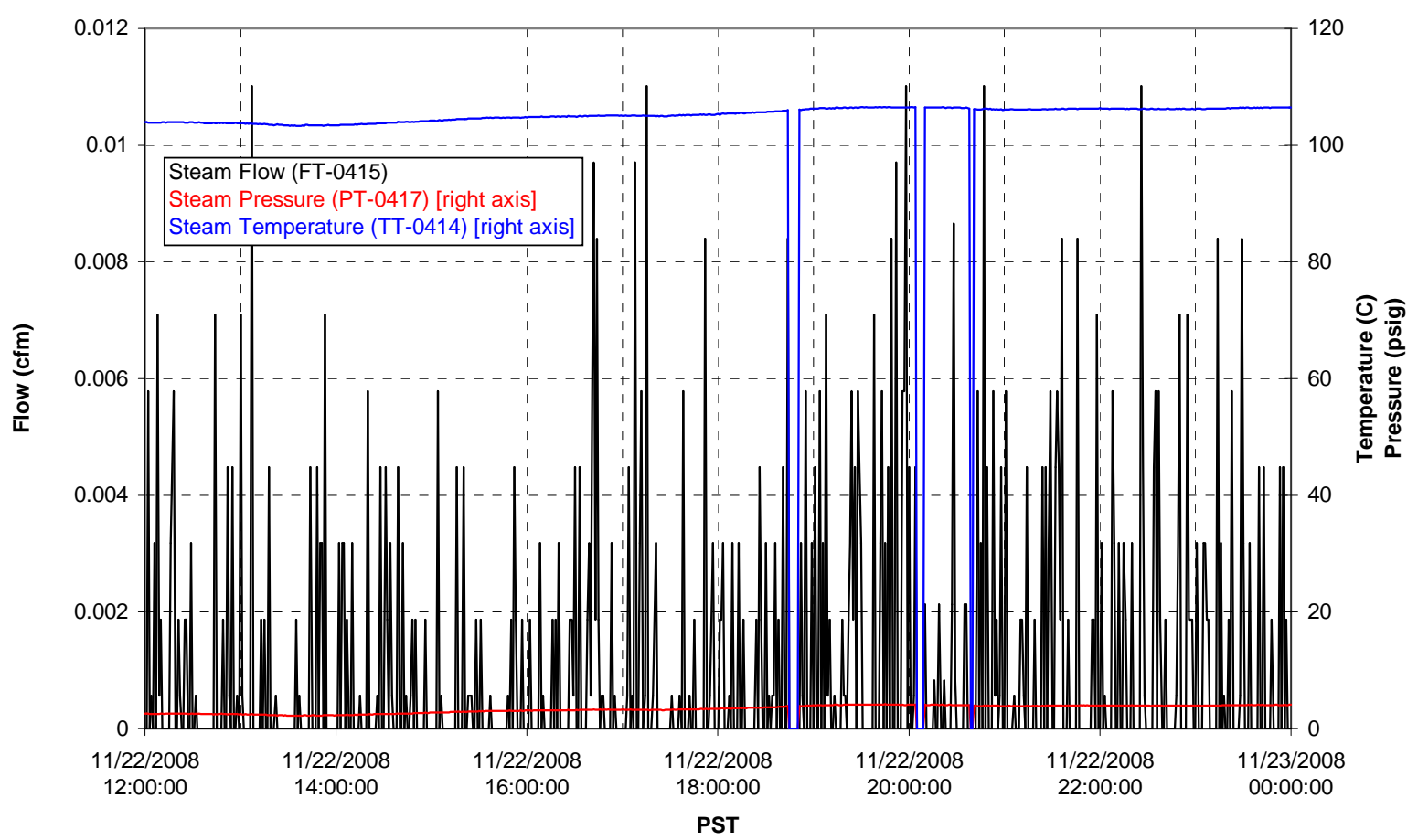


Functional Test Data Plots

11/23/2008 00:00 - 11/23/2008 12:00 
T01A level

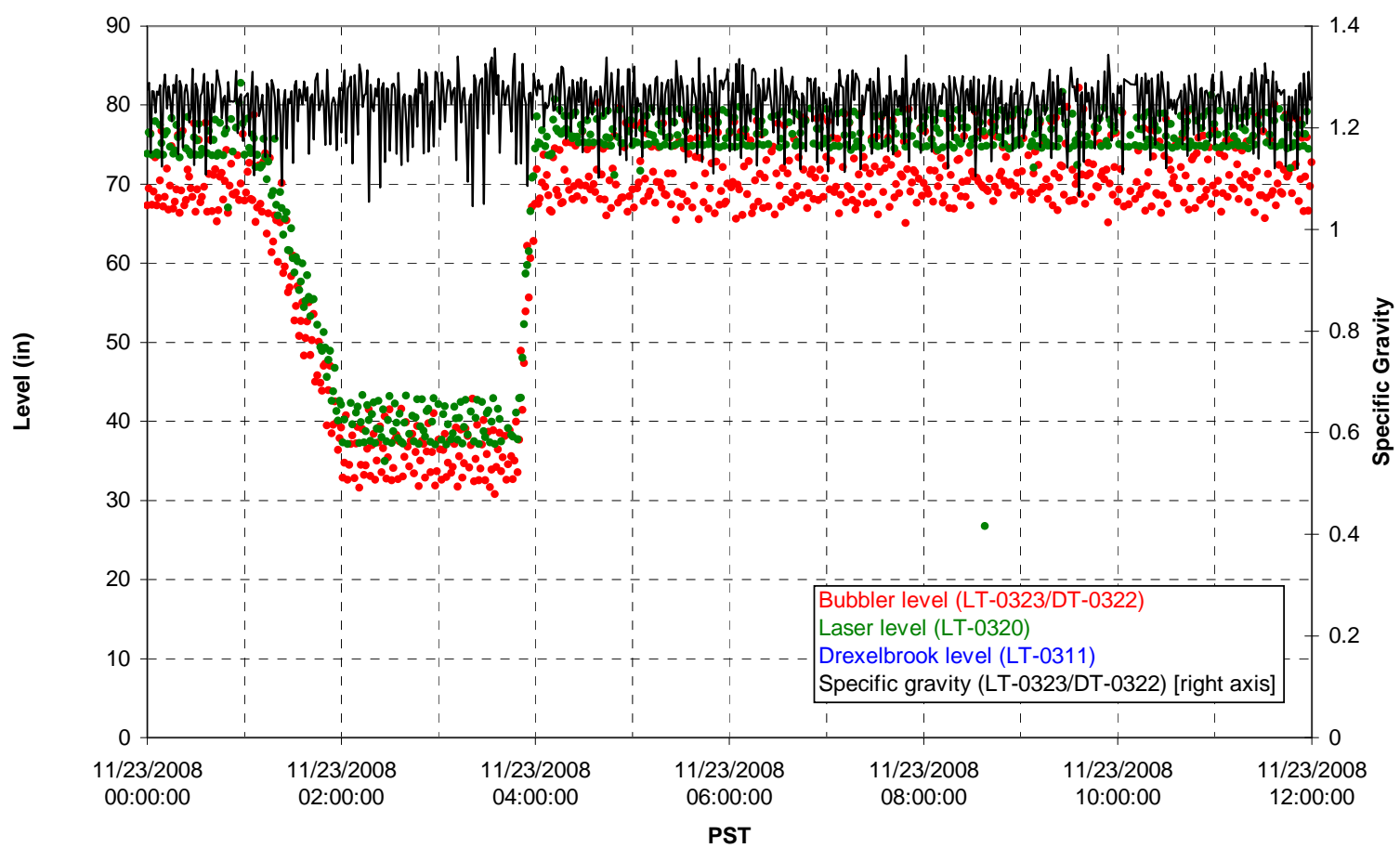

T01A temperatures

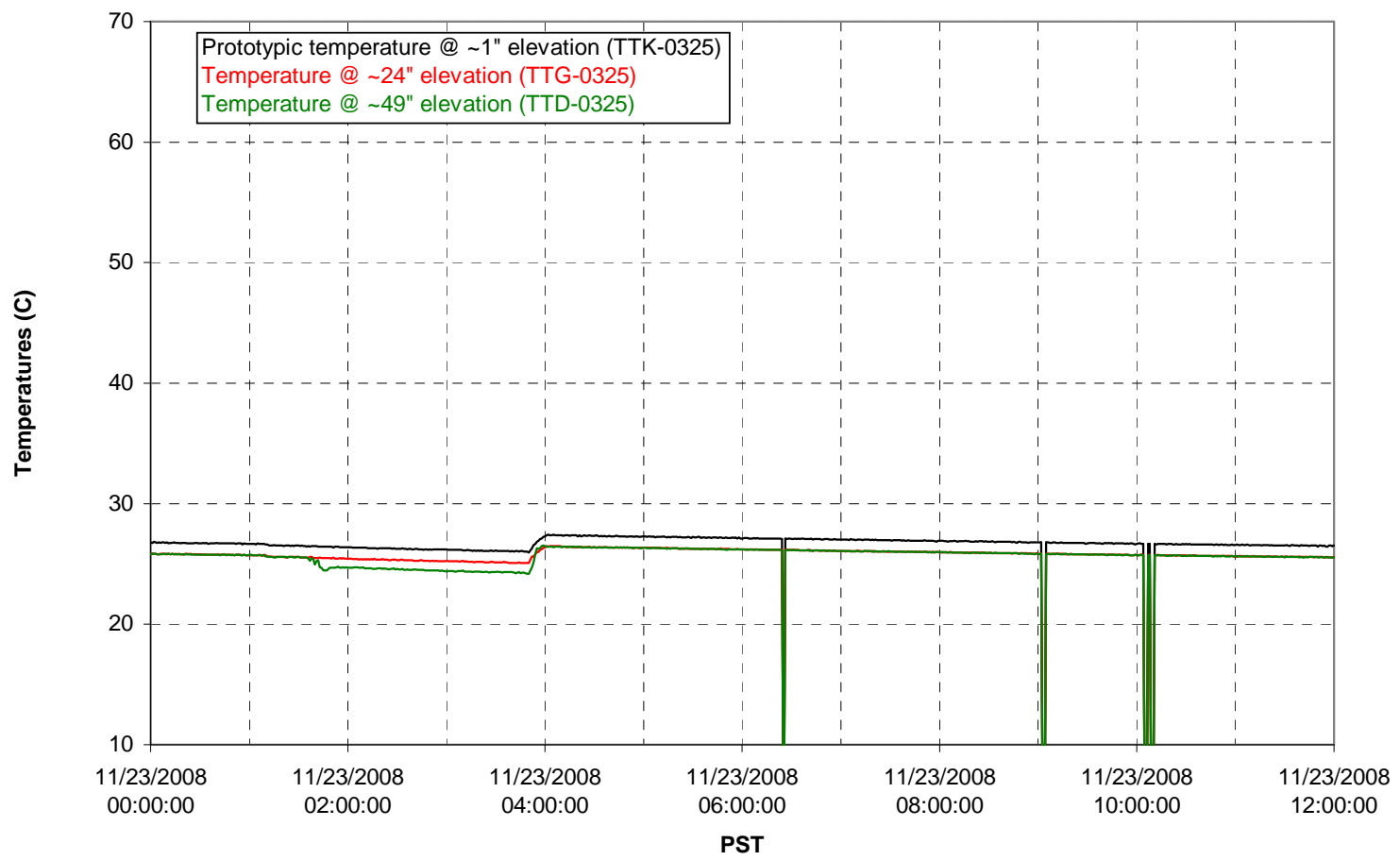


T01B level

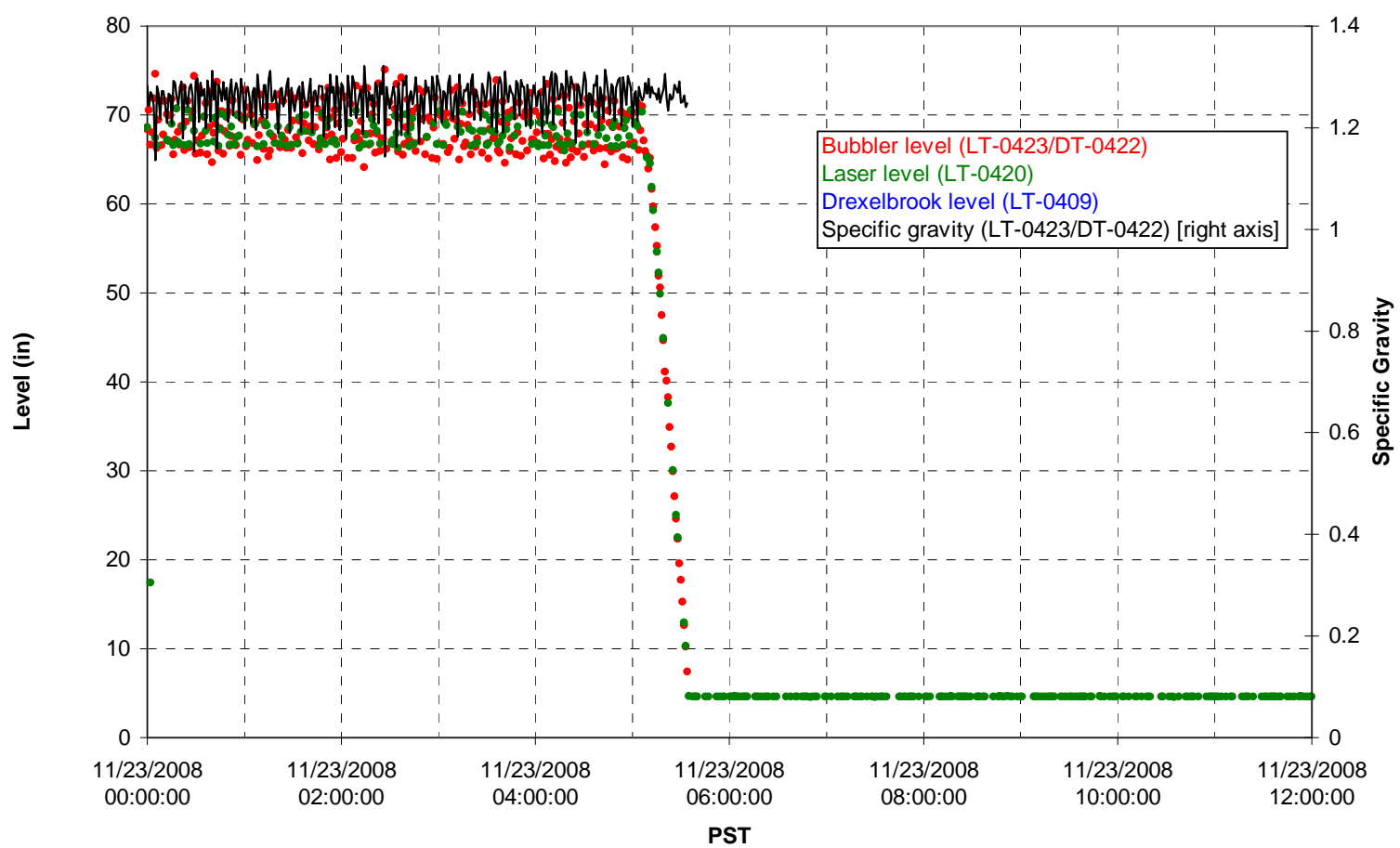

T01B temperatures

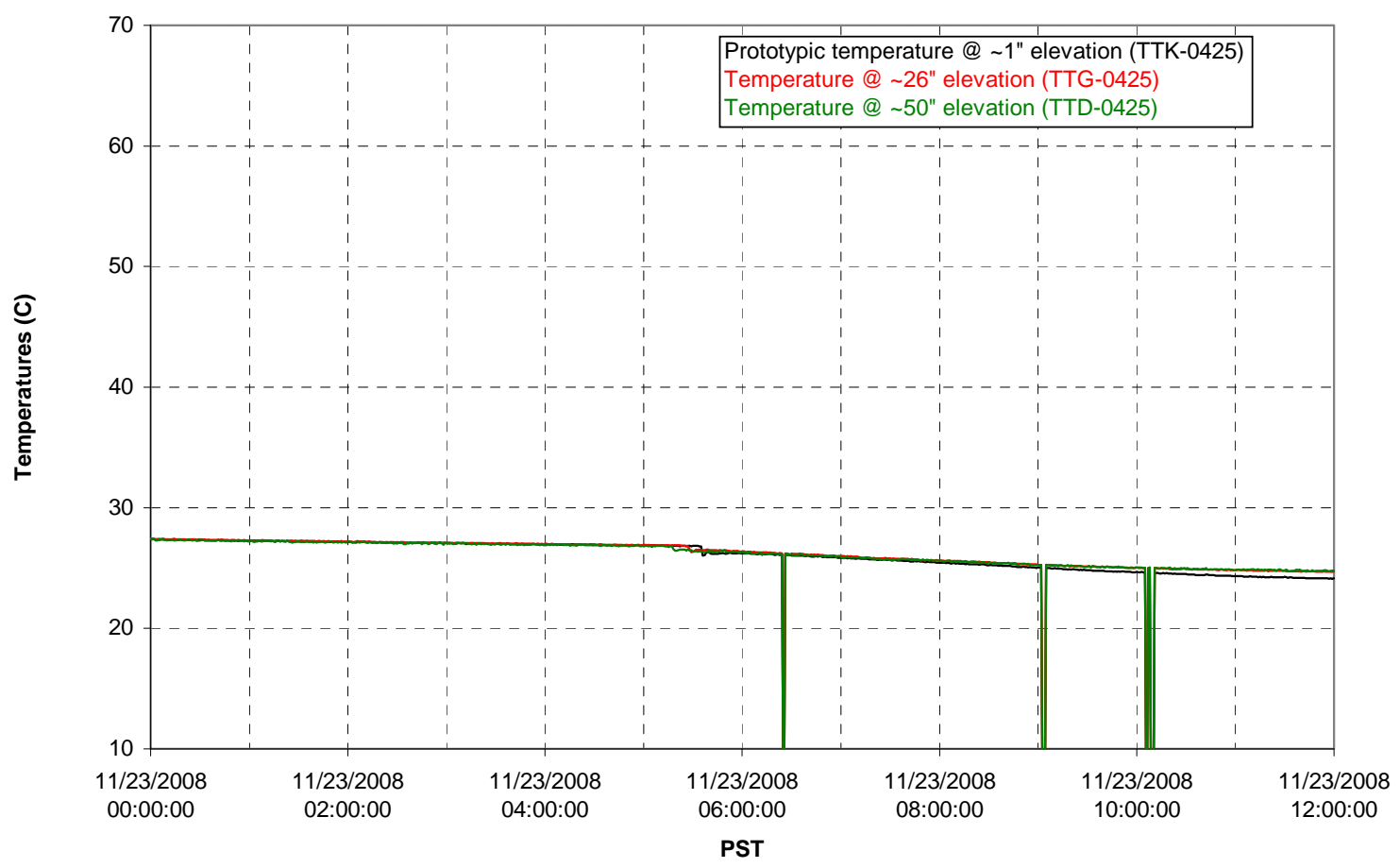


T02A level

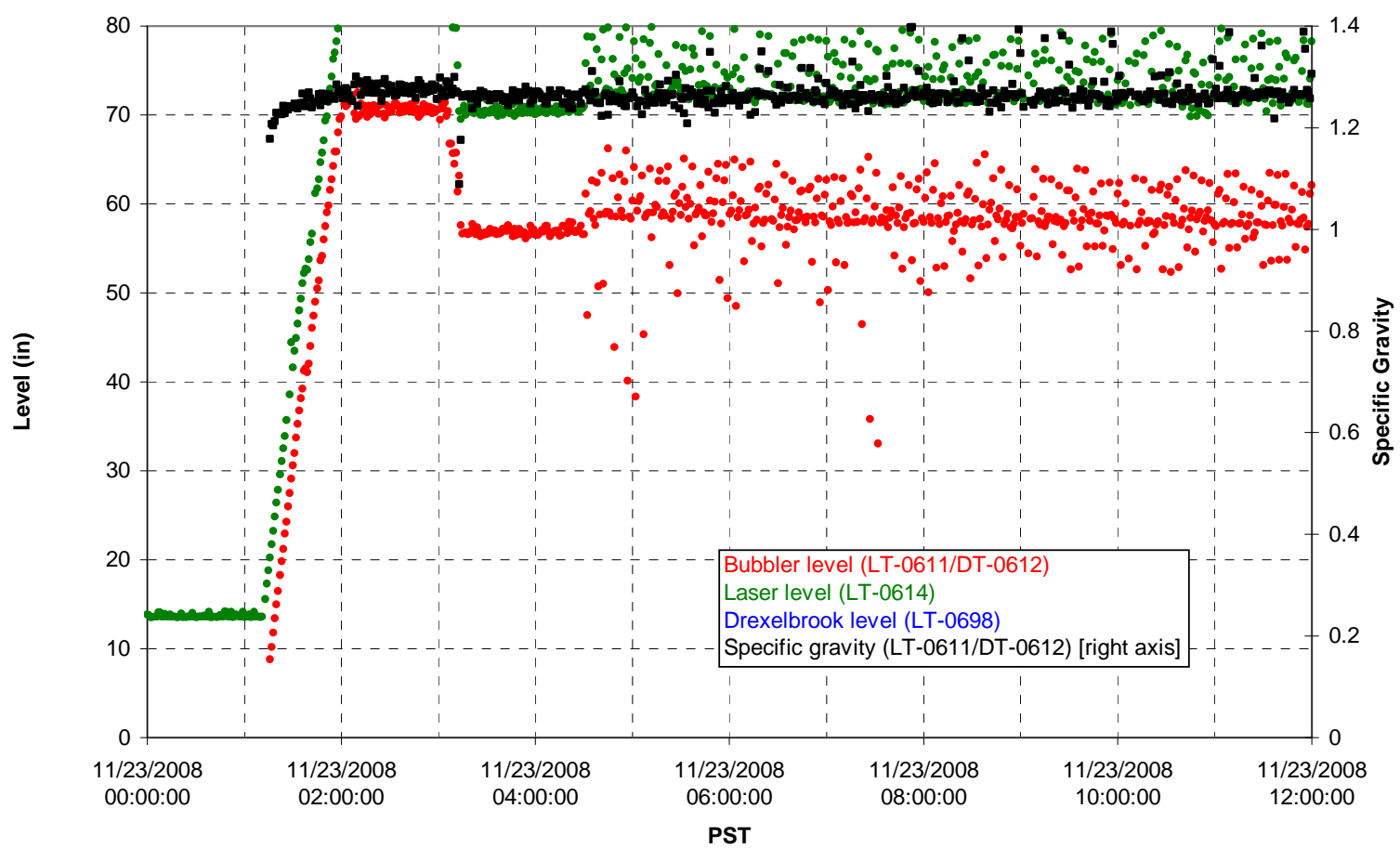

T02A temperatures

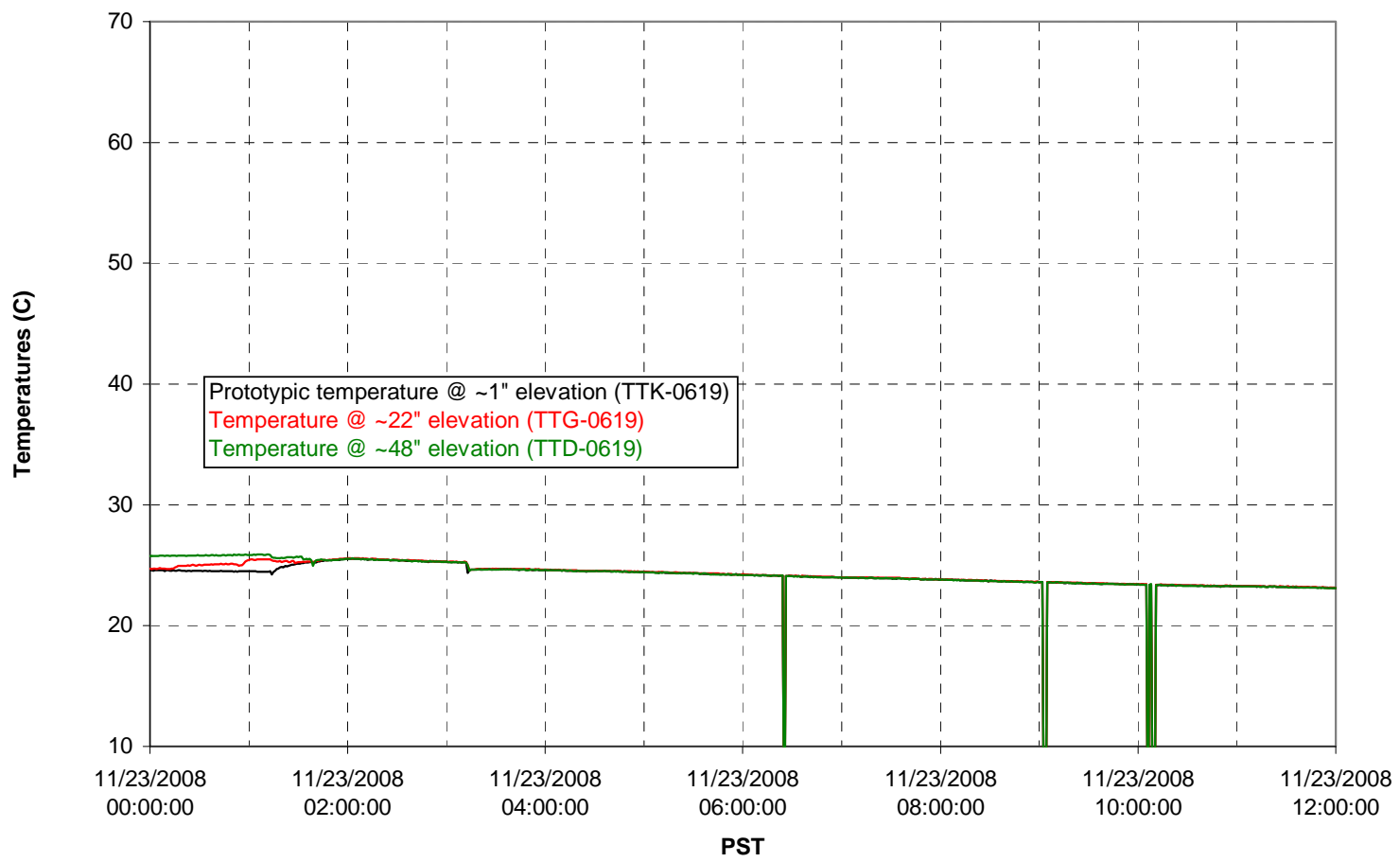


T02A and filter loop temperatures

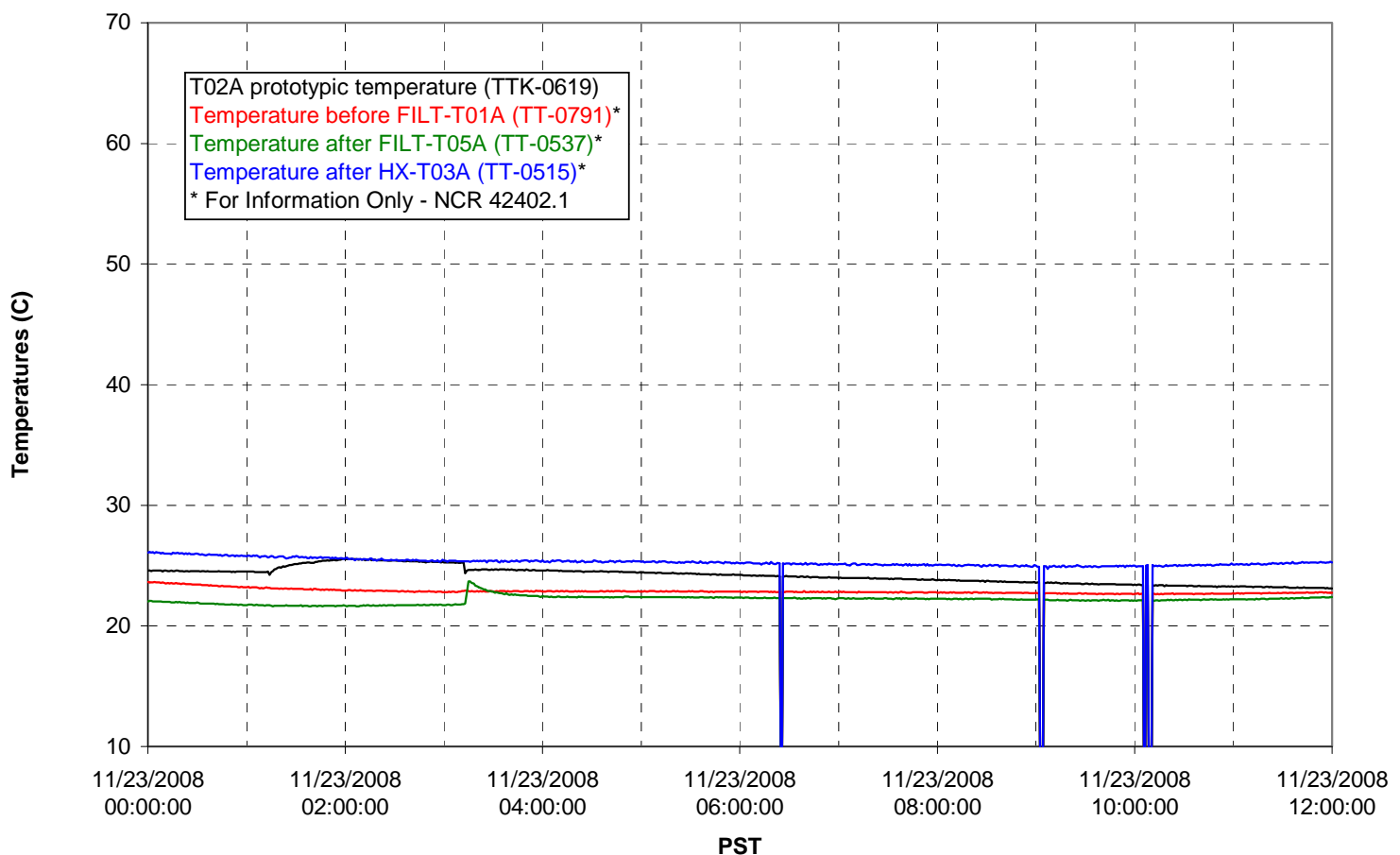

Pump Pressures and Flow

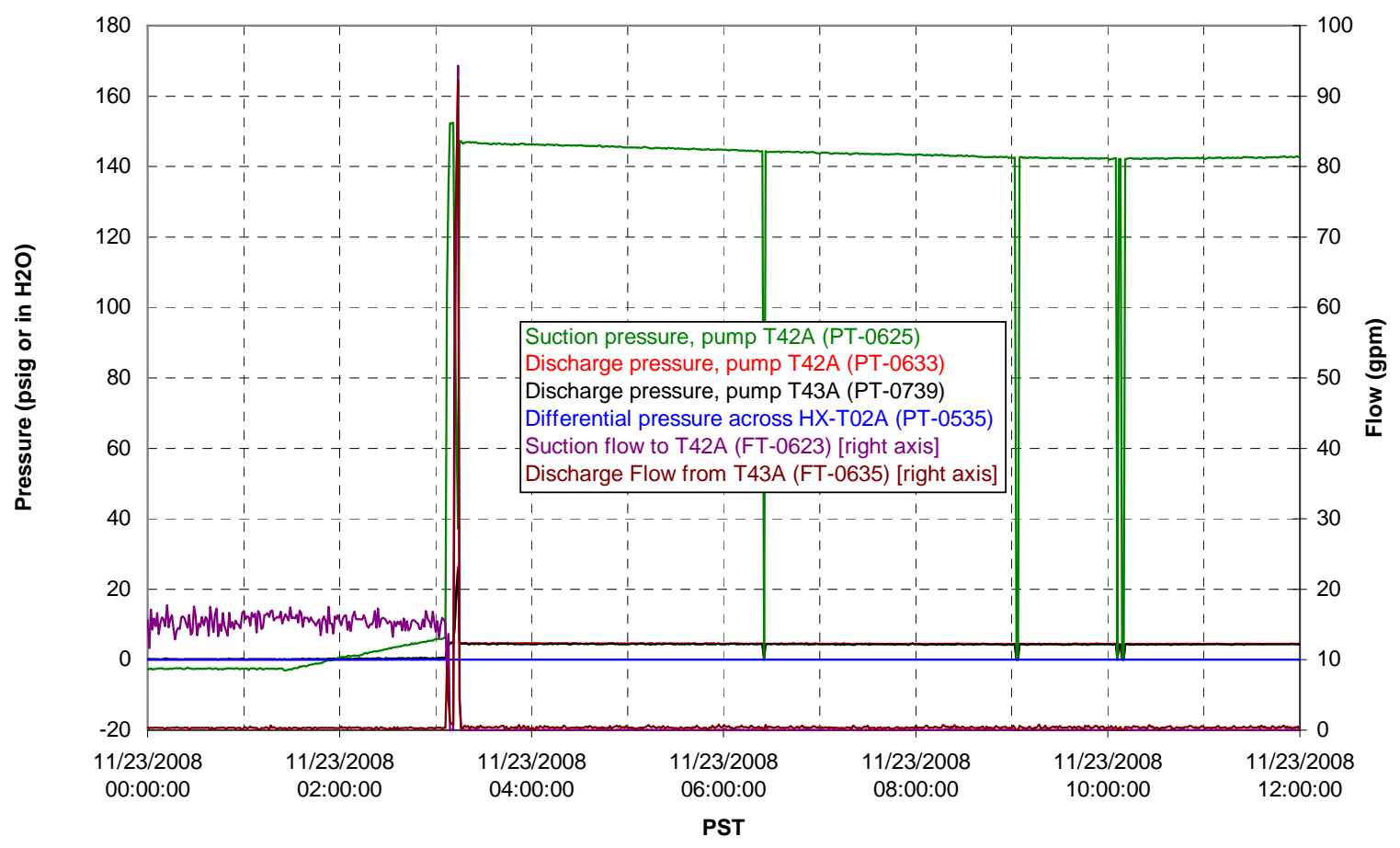


Axial pressure drop

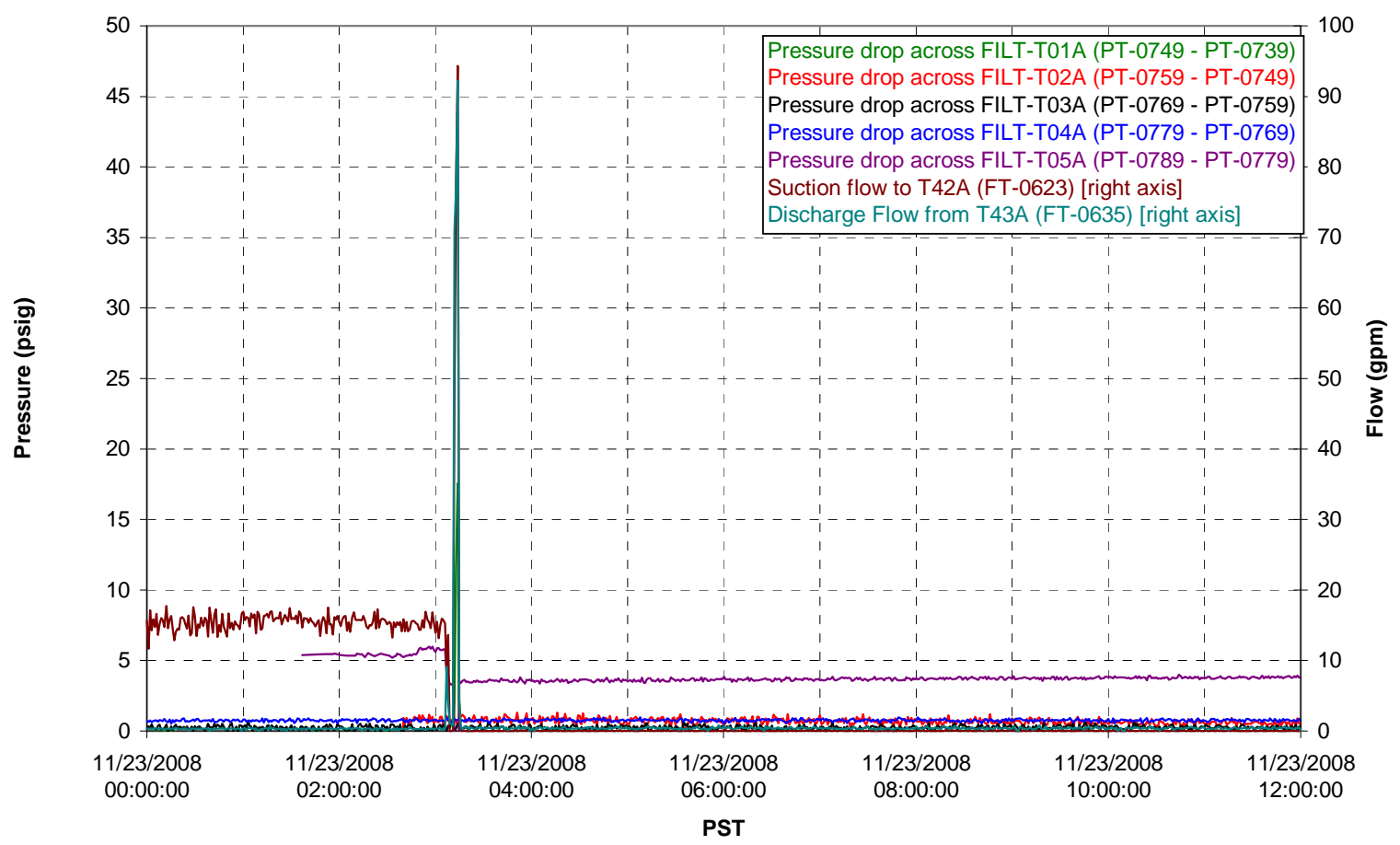

Permeate flow rates

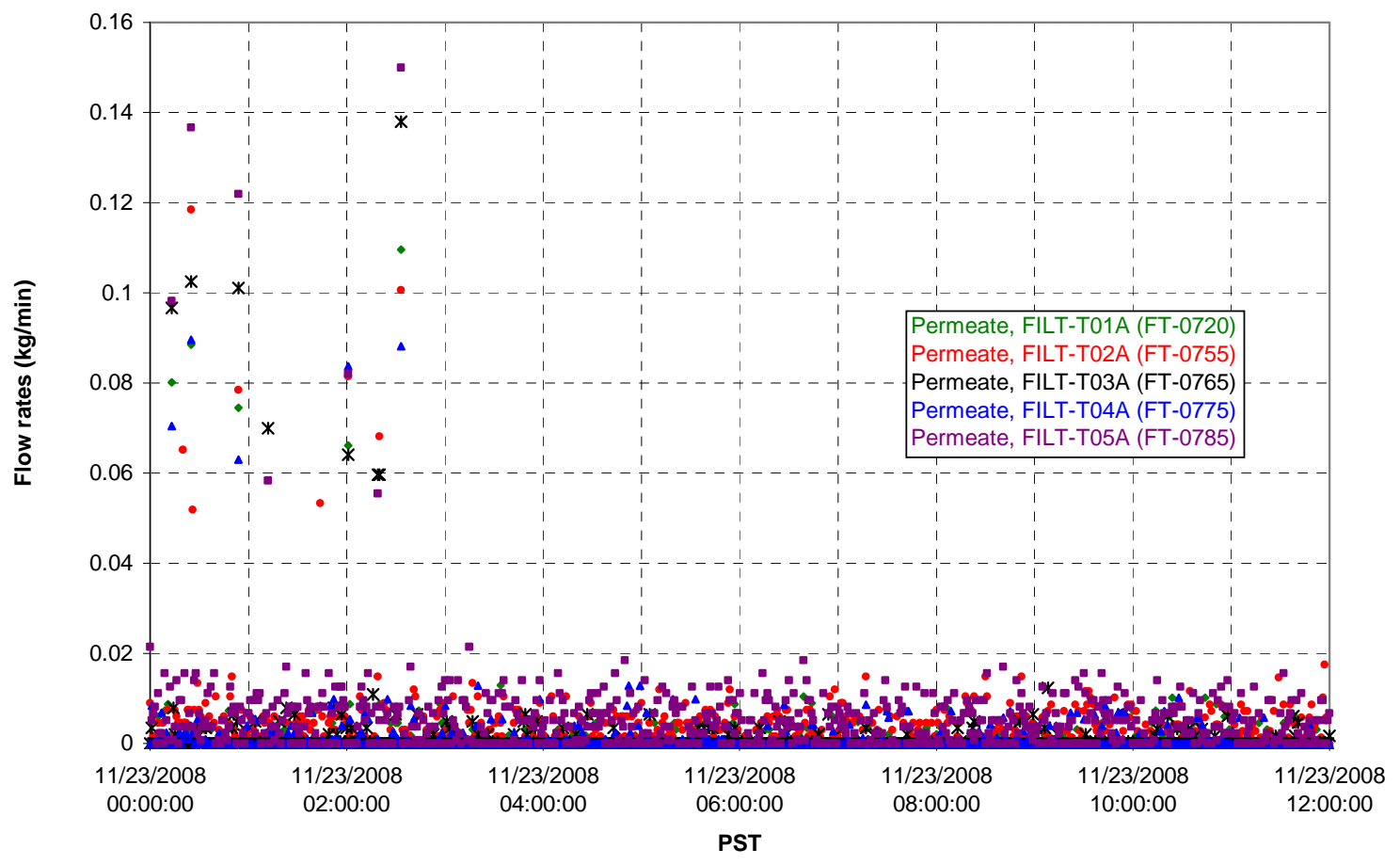


T02A Inner Temperature Tree

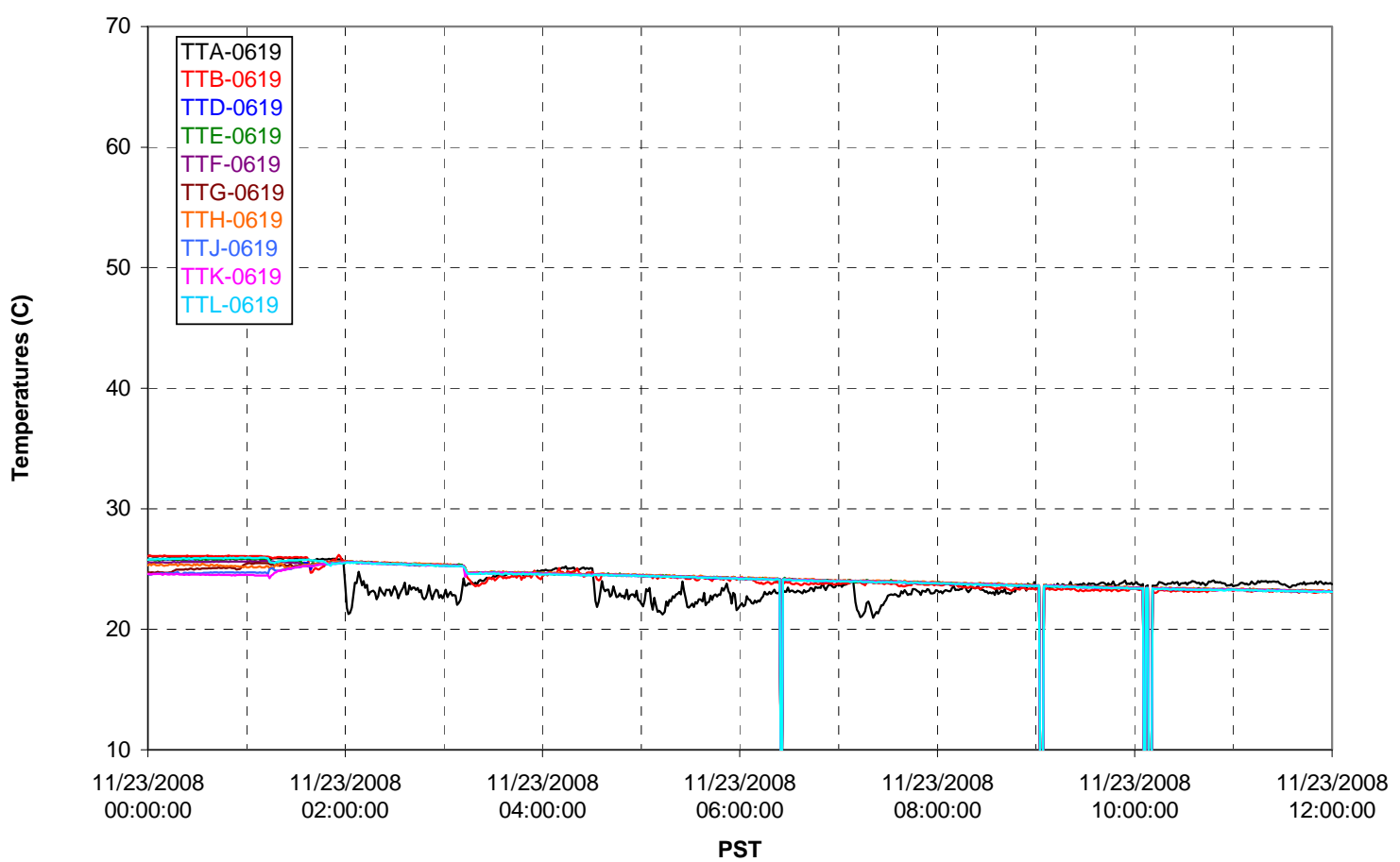

T02A Outer Temperature Tree

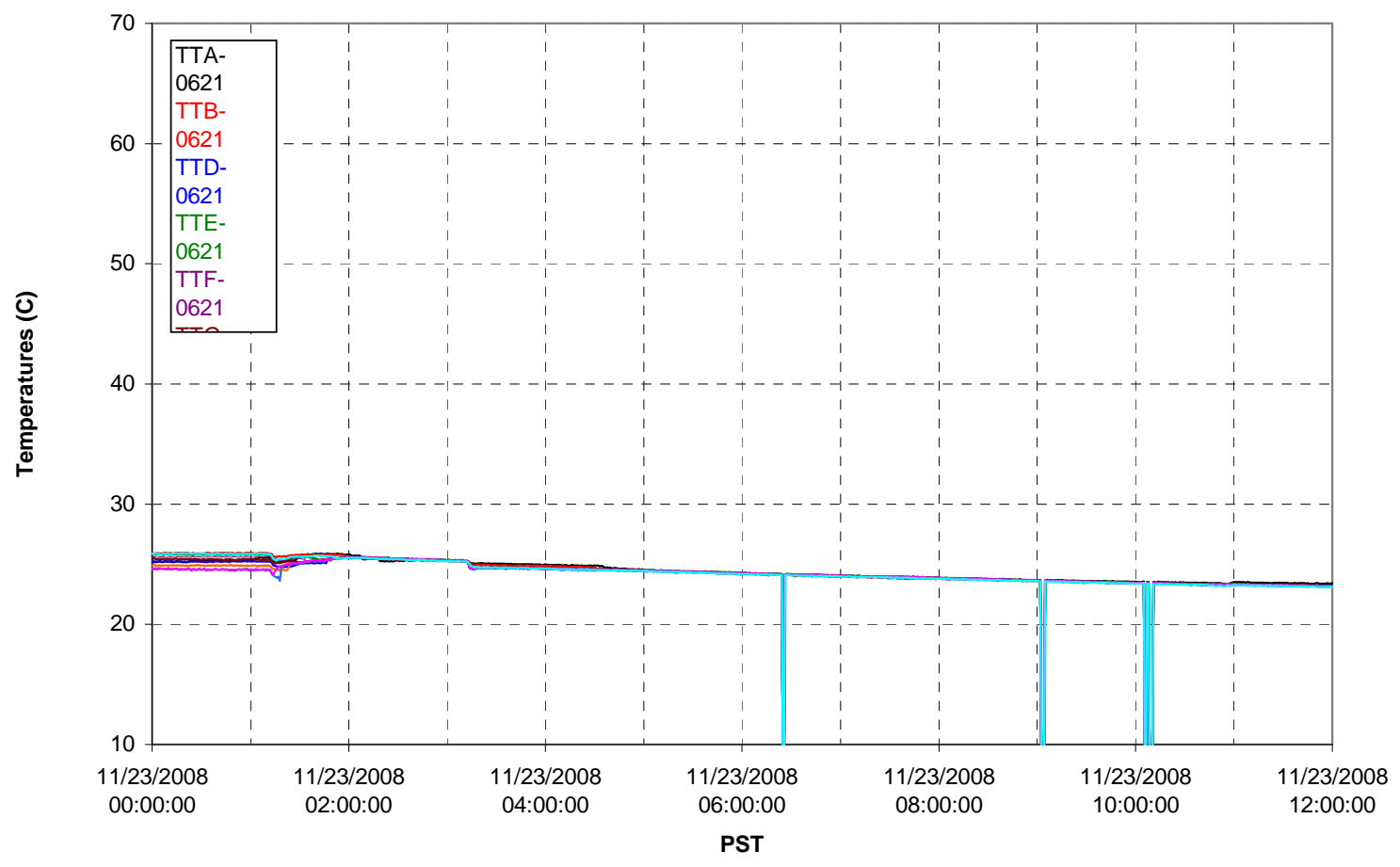


T02A temperatures

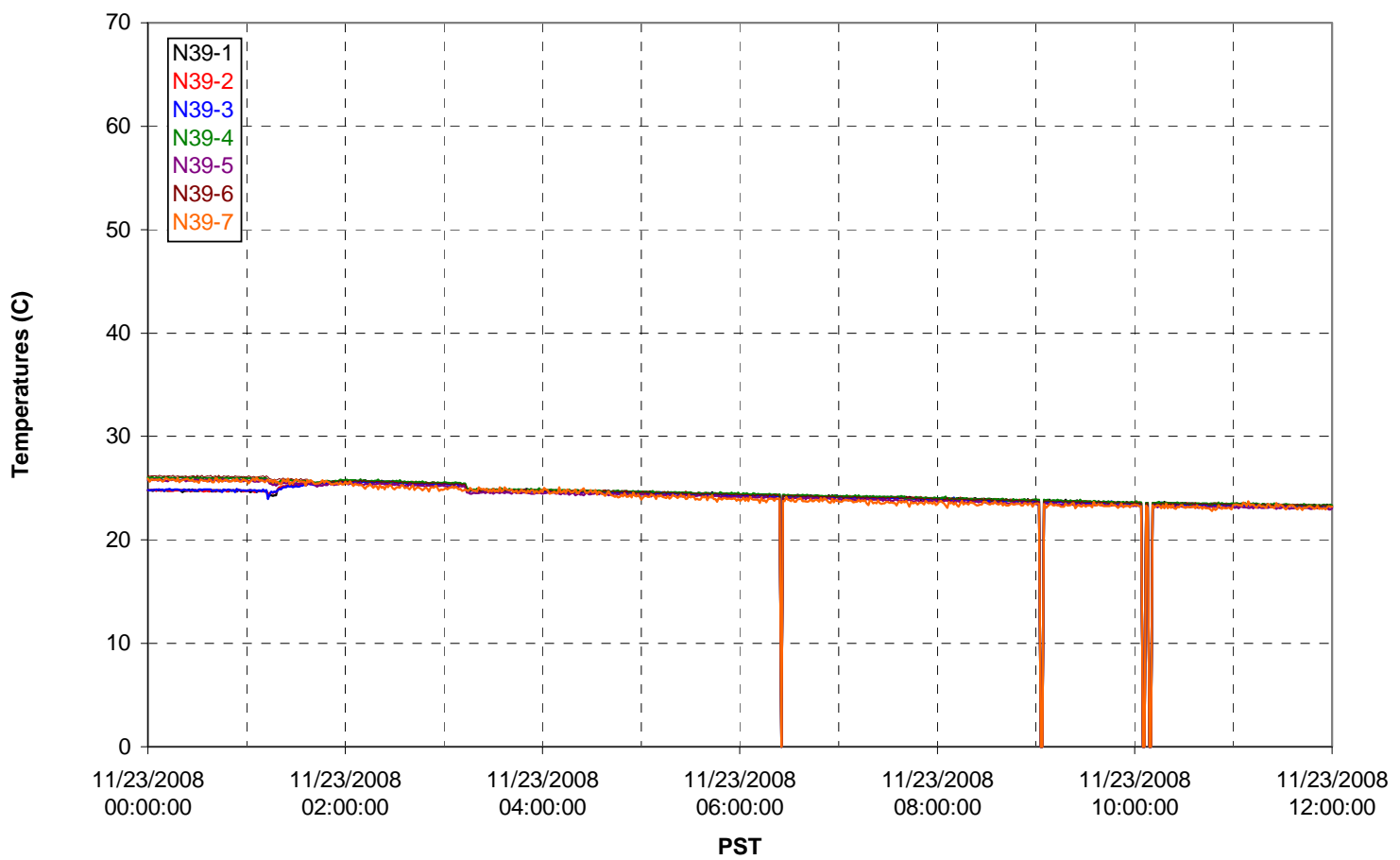

T02A temperatures

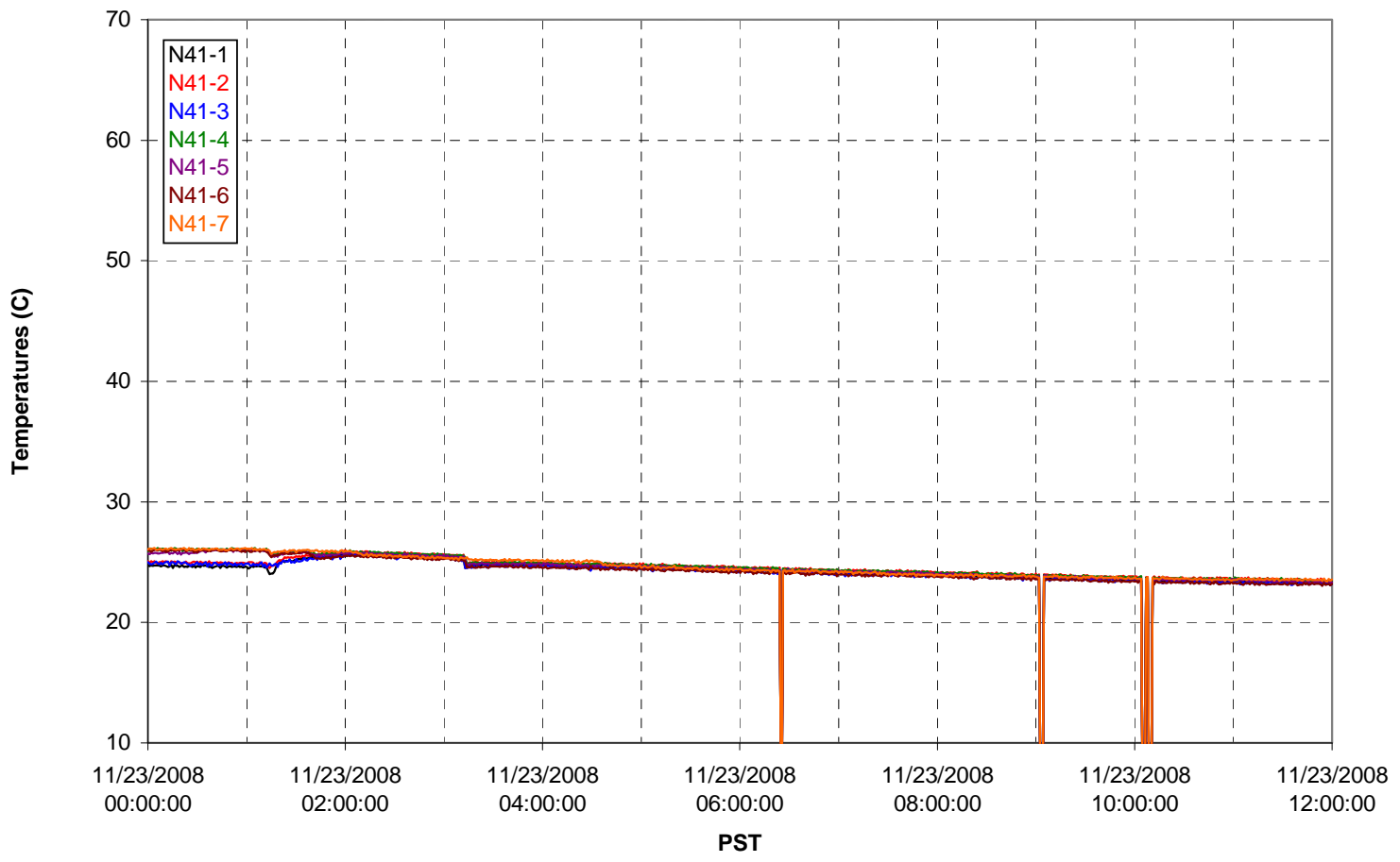


T02A temperatures

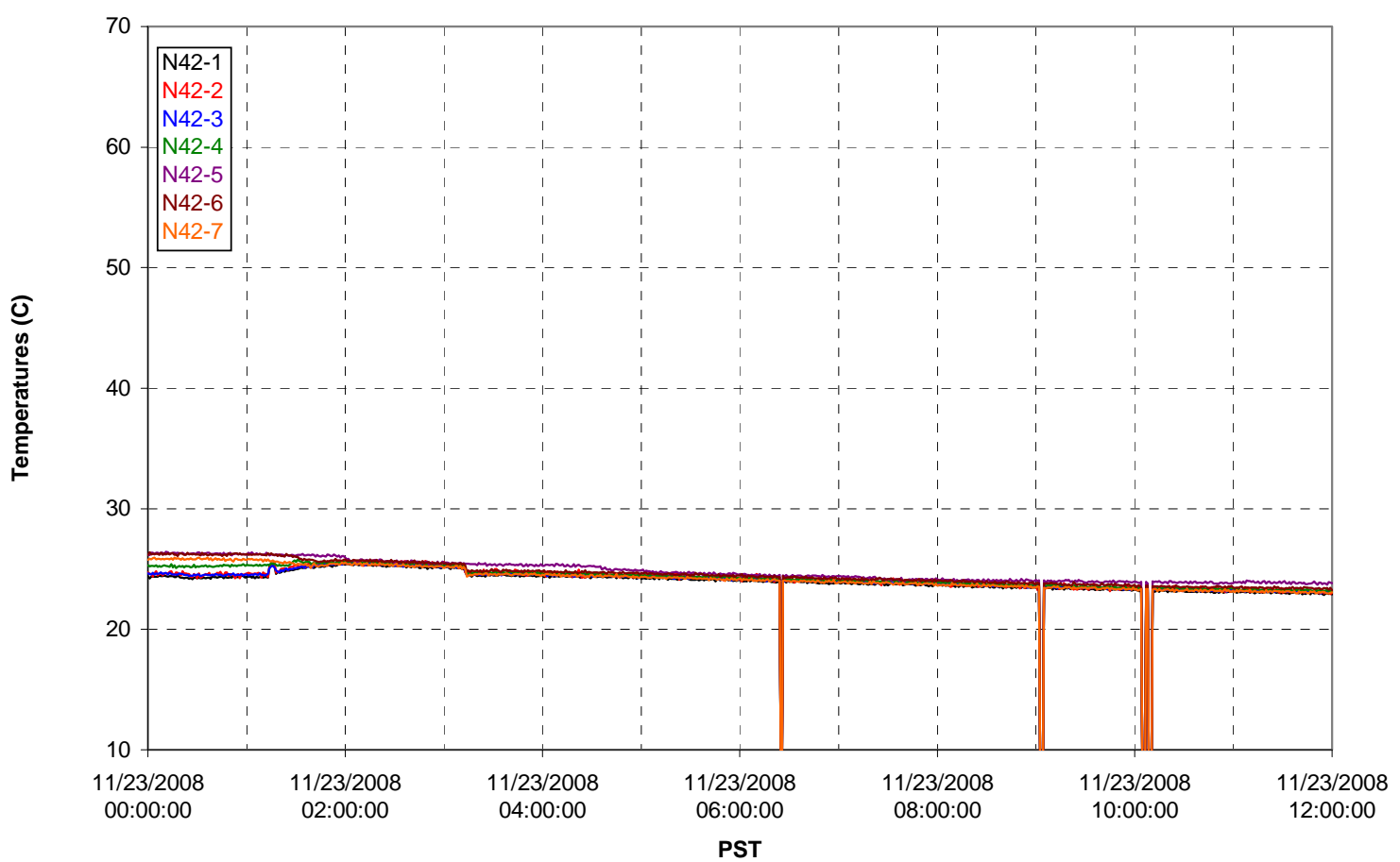

T02A temperatures

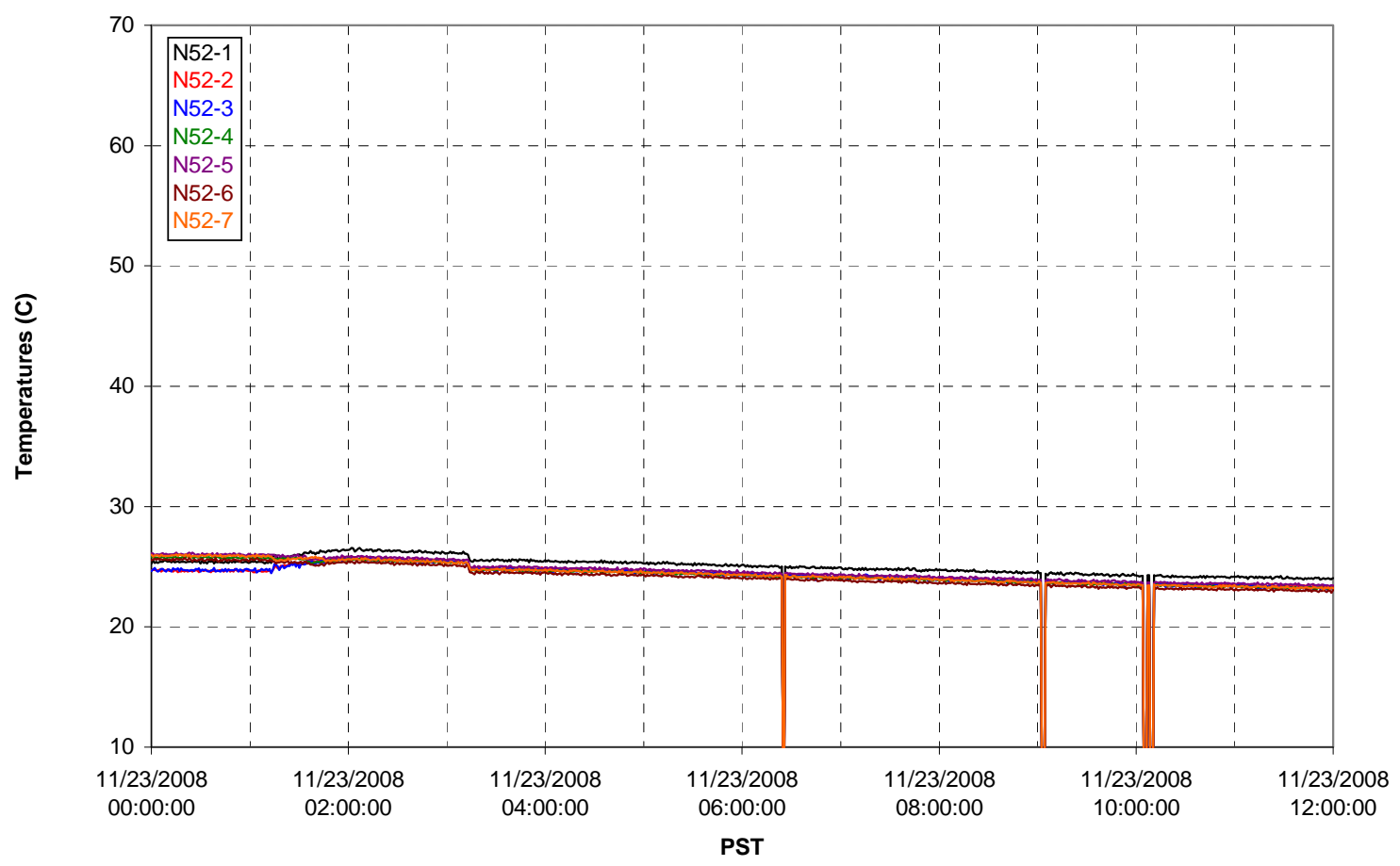


T02A Heating and Cooling

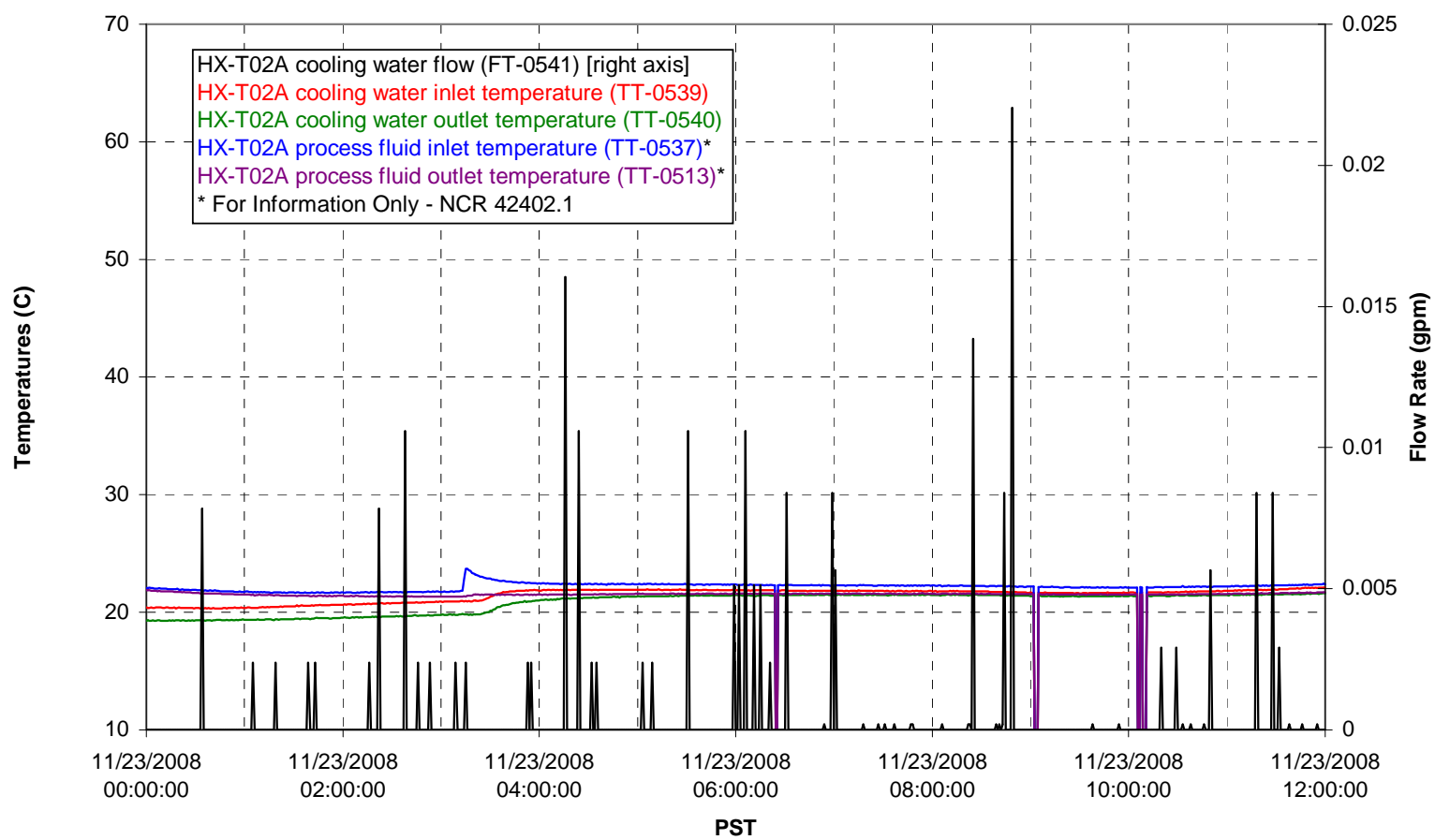

Pump Operation

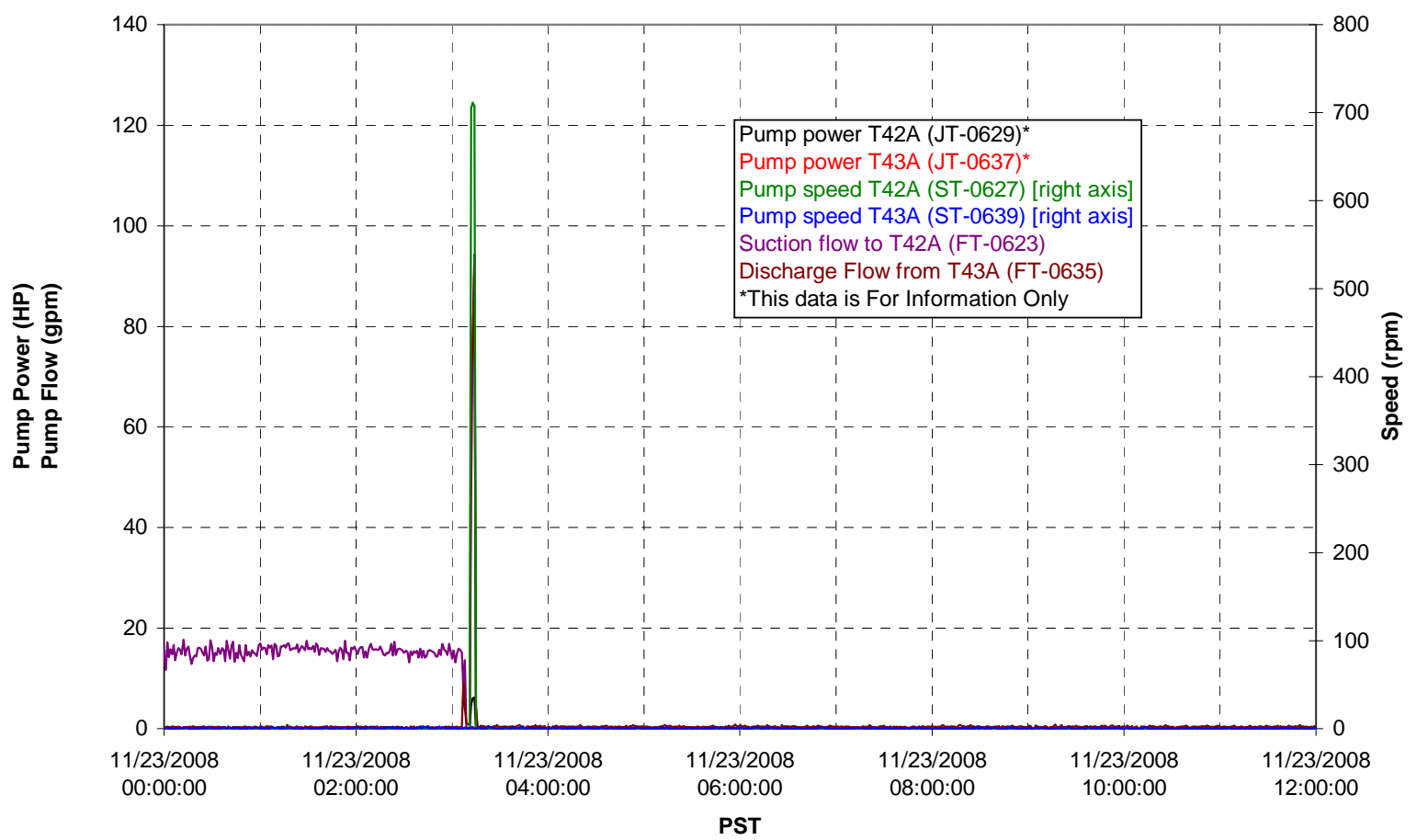


Pulsepot UFP-PP-T01A

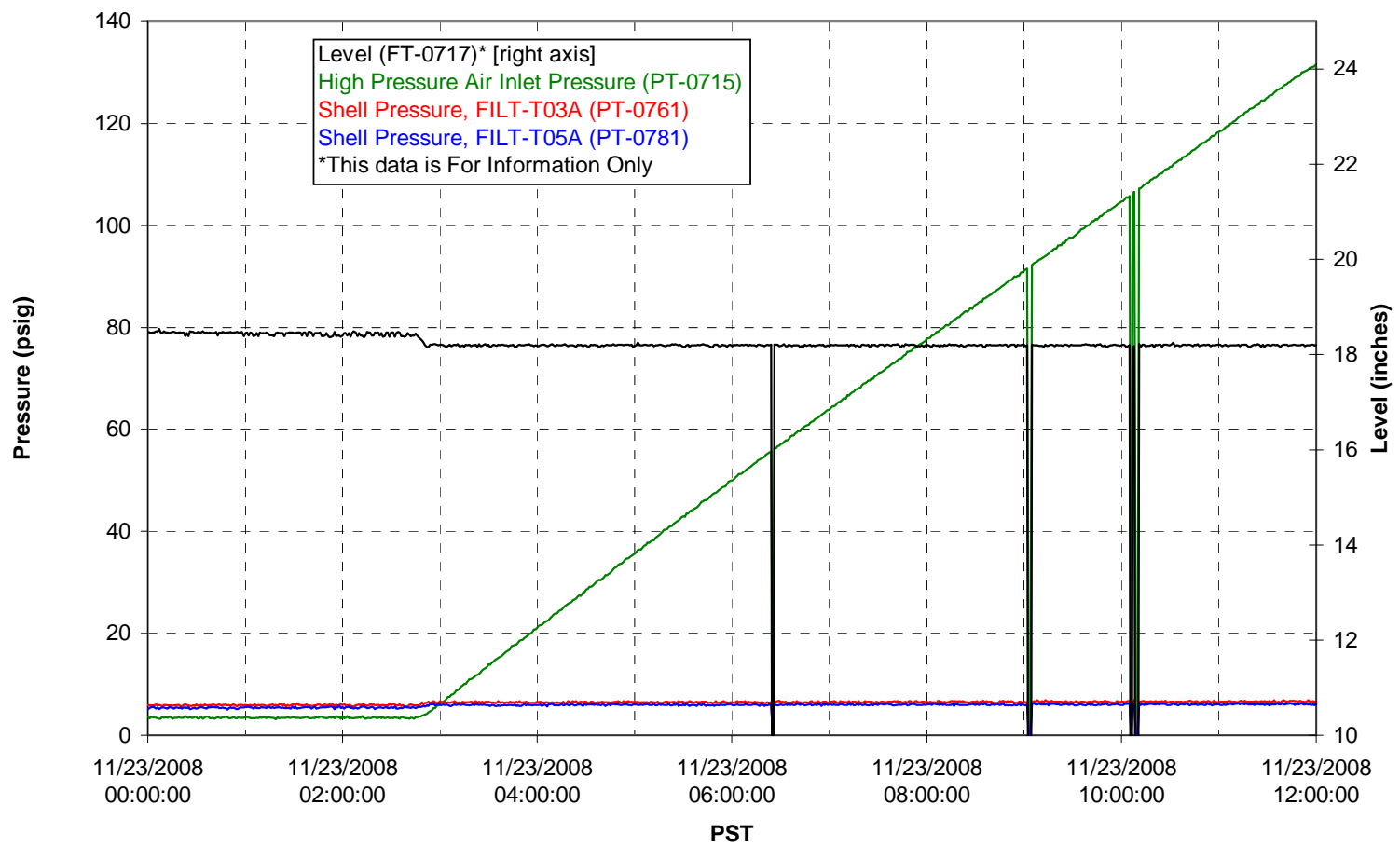

Pulsepot UFP-PP-T02A

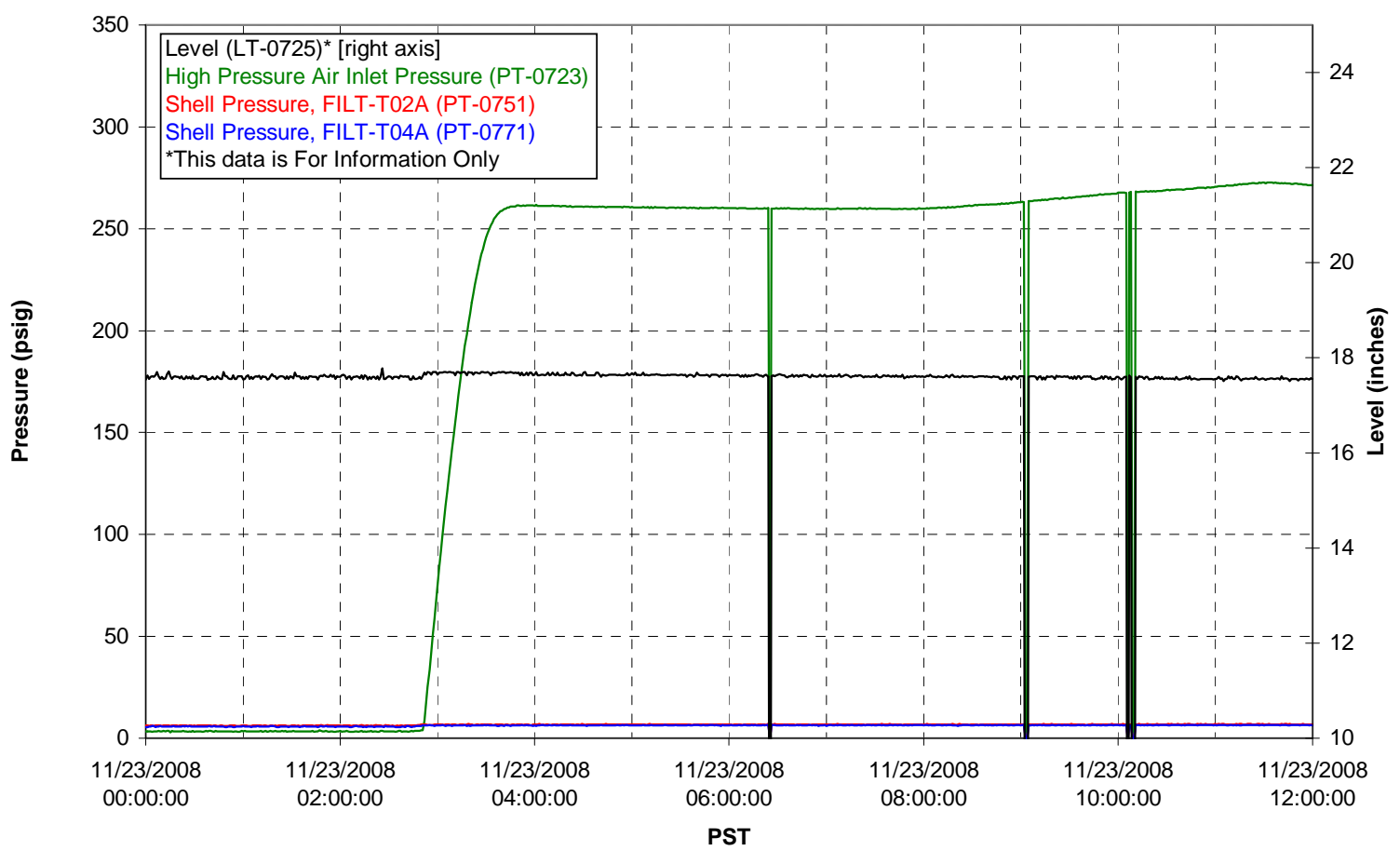


Pulsepot UFP-PP-T03A

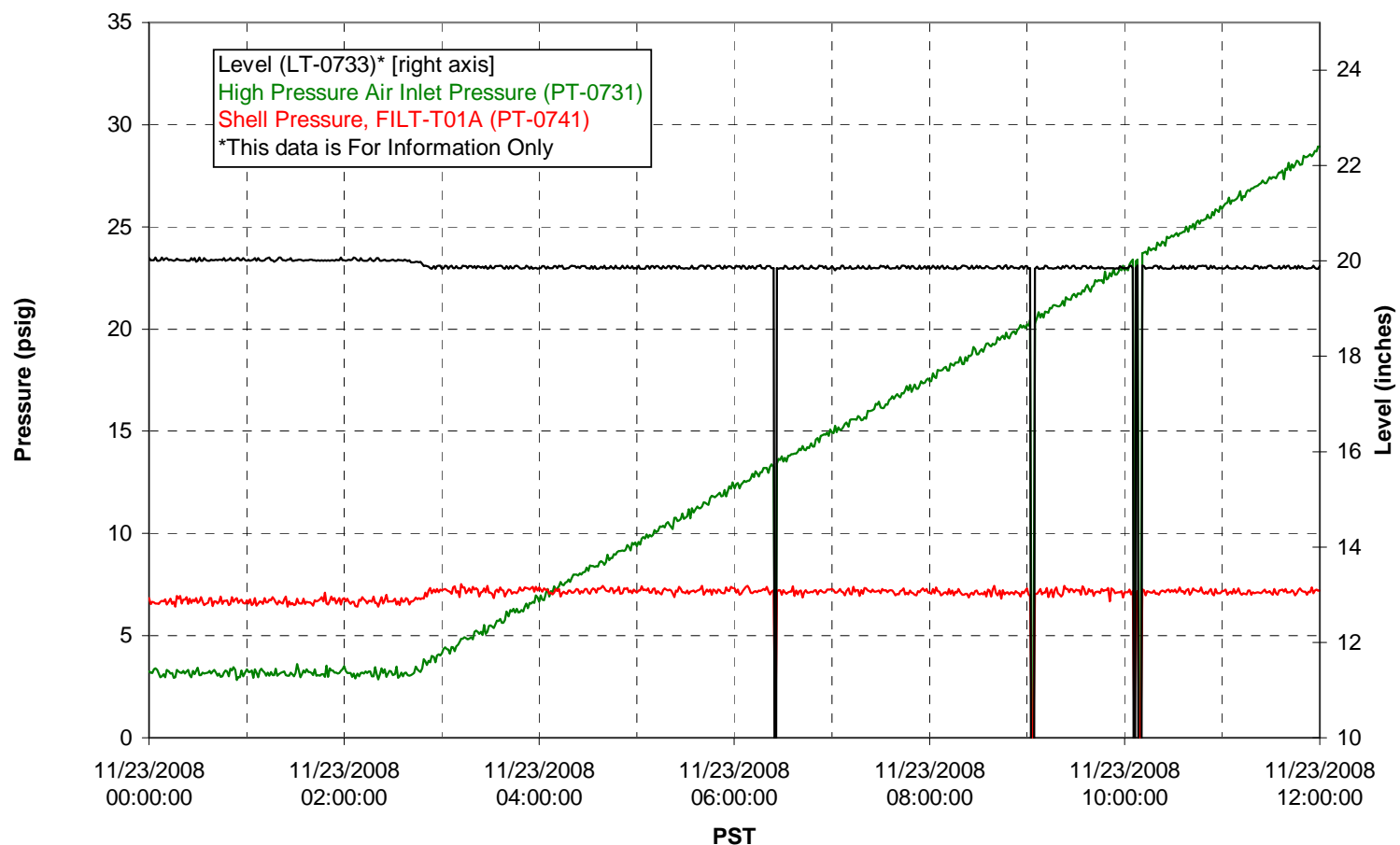

Pulsepot Levels

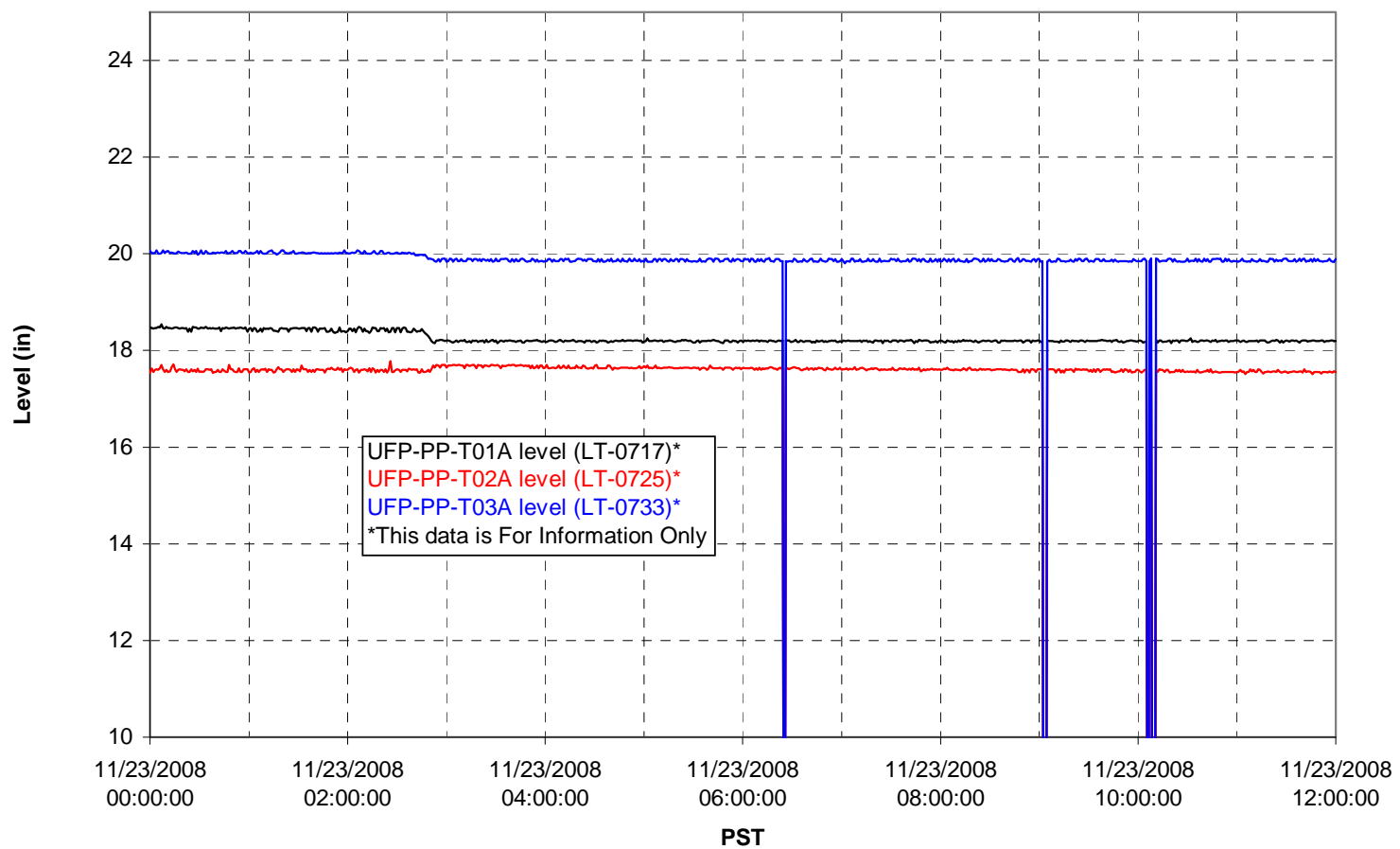


Filter UFP-FILT-T01A

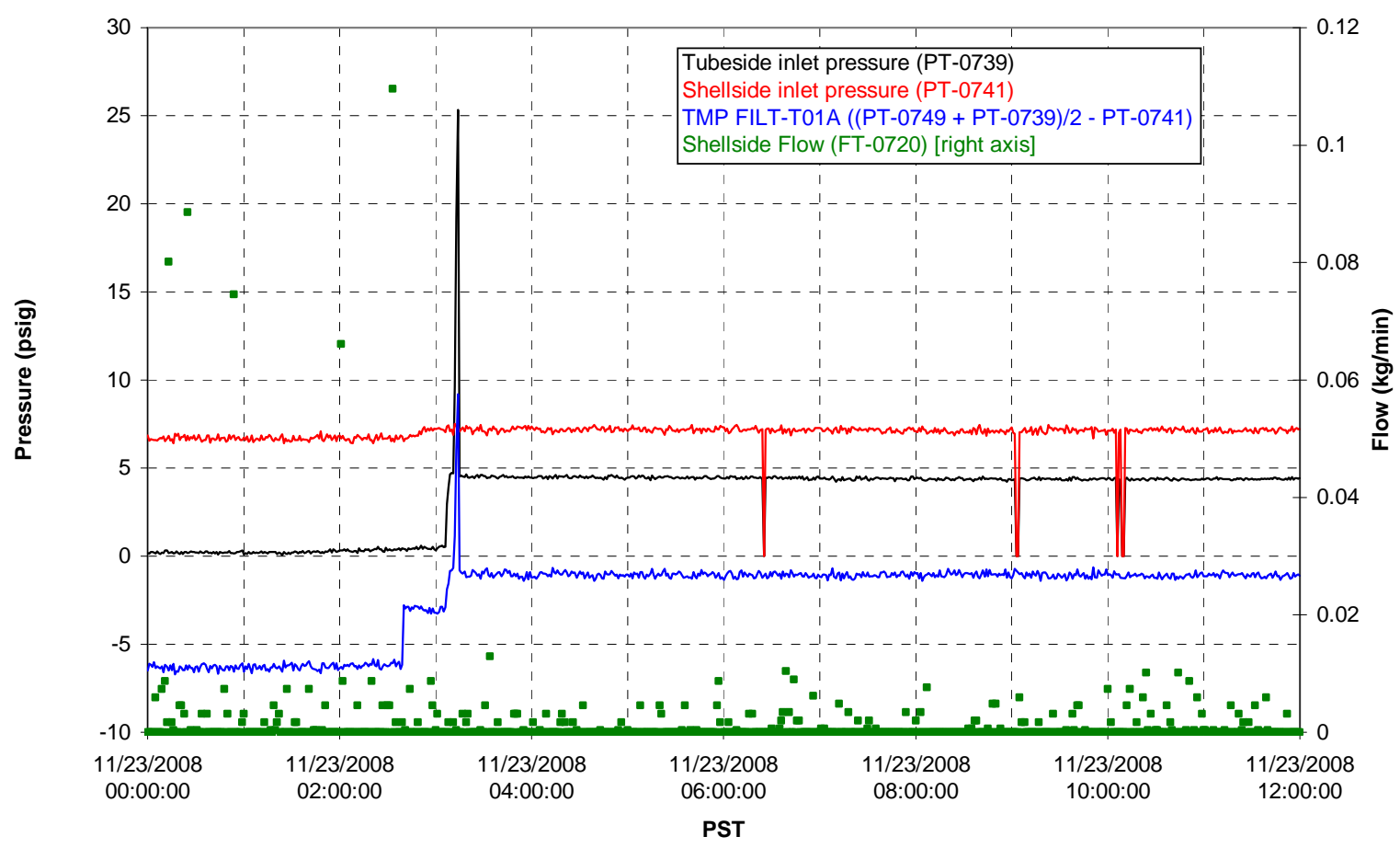

Filter UFP-FILT-T02A

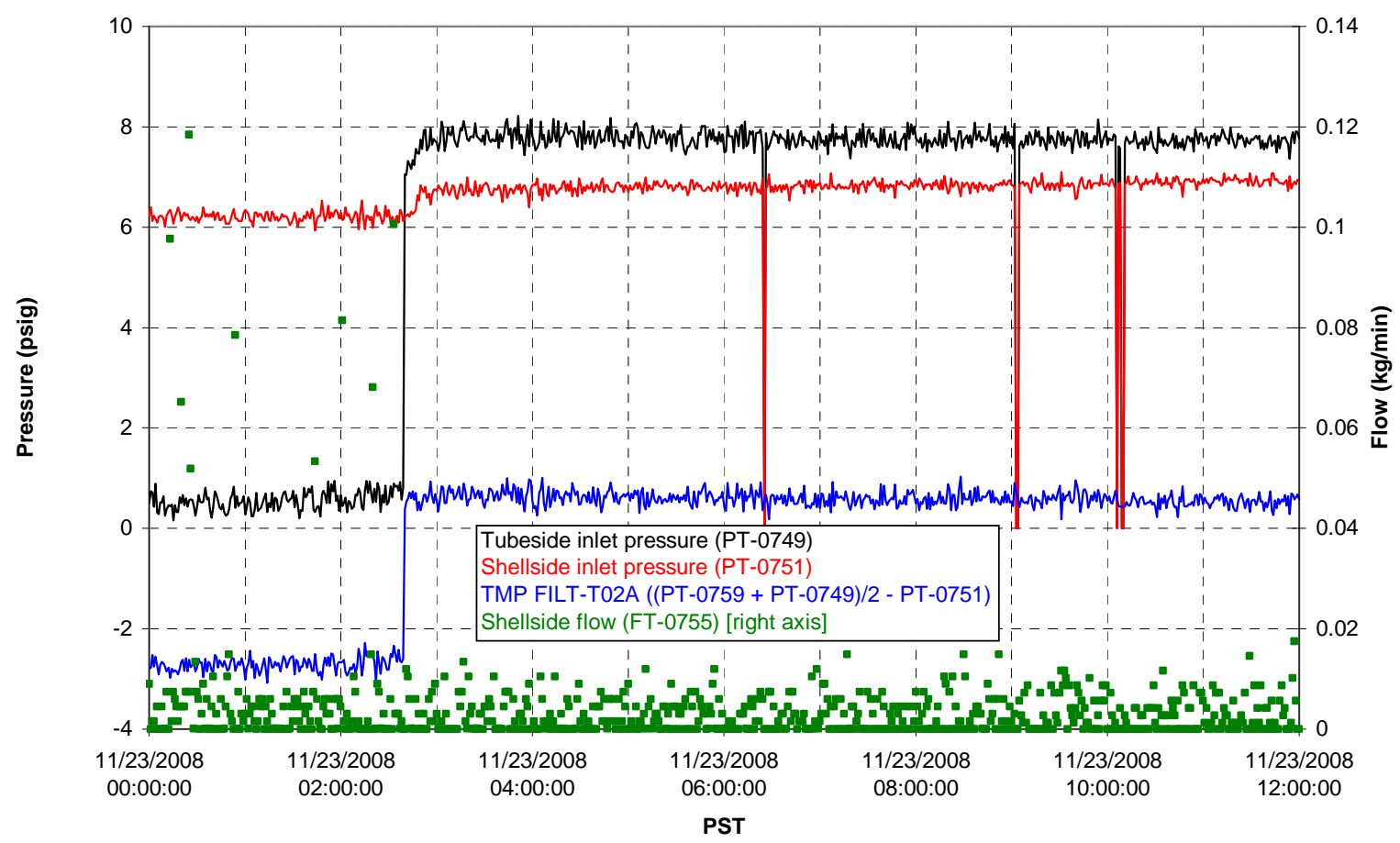


Filter UFP-FILT-T03A

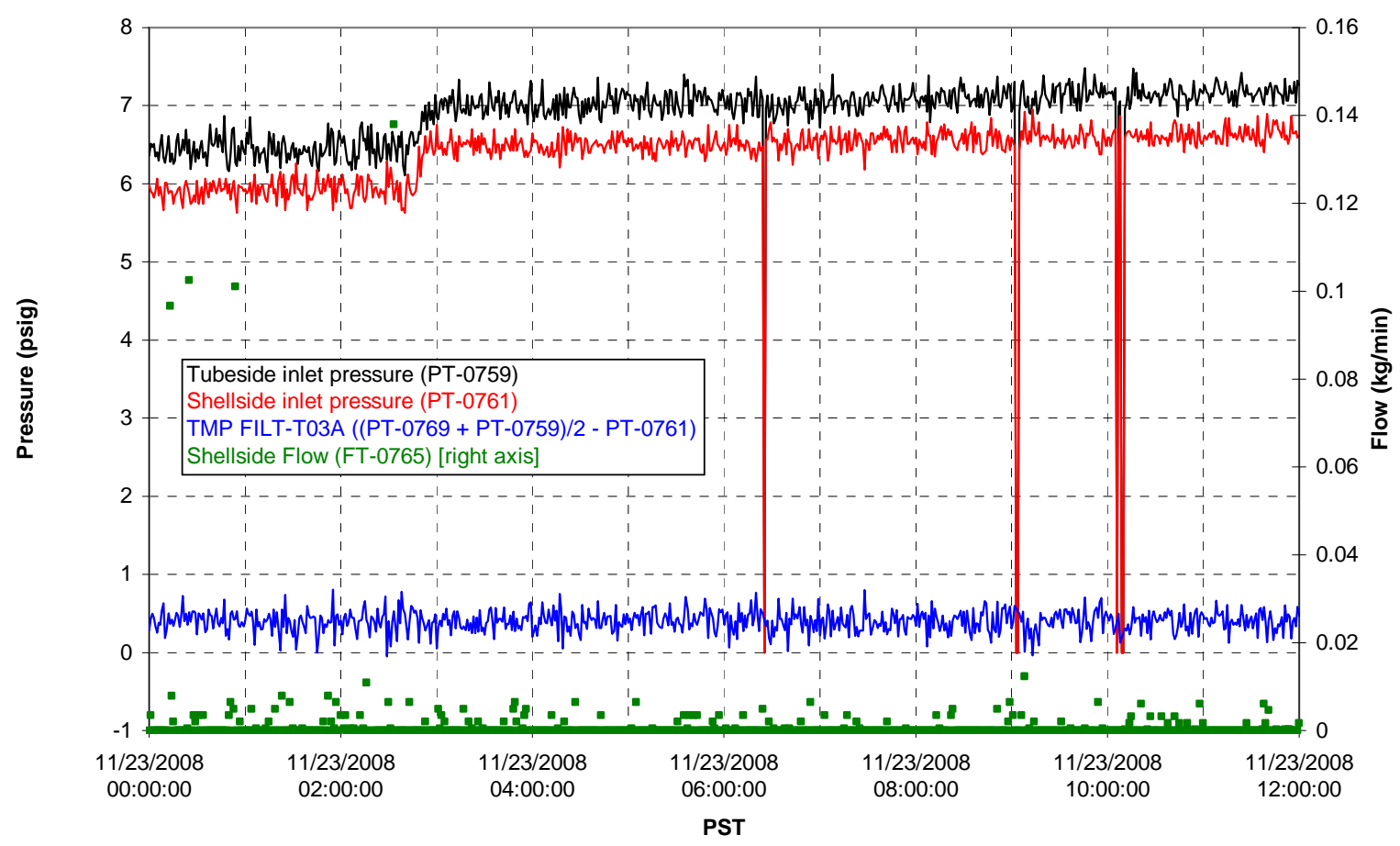

Filter UFP-FILT-T04A

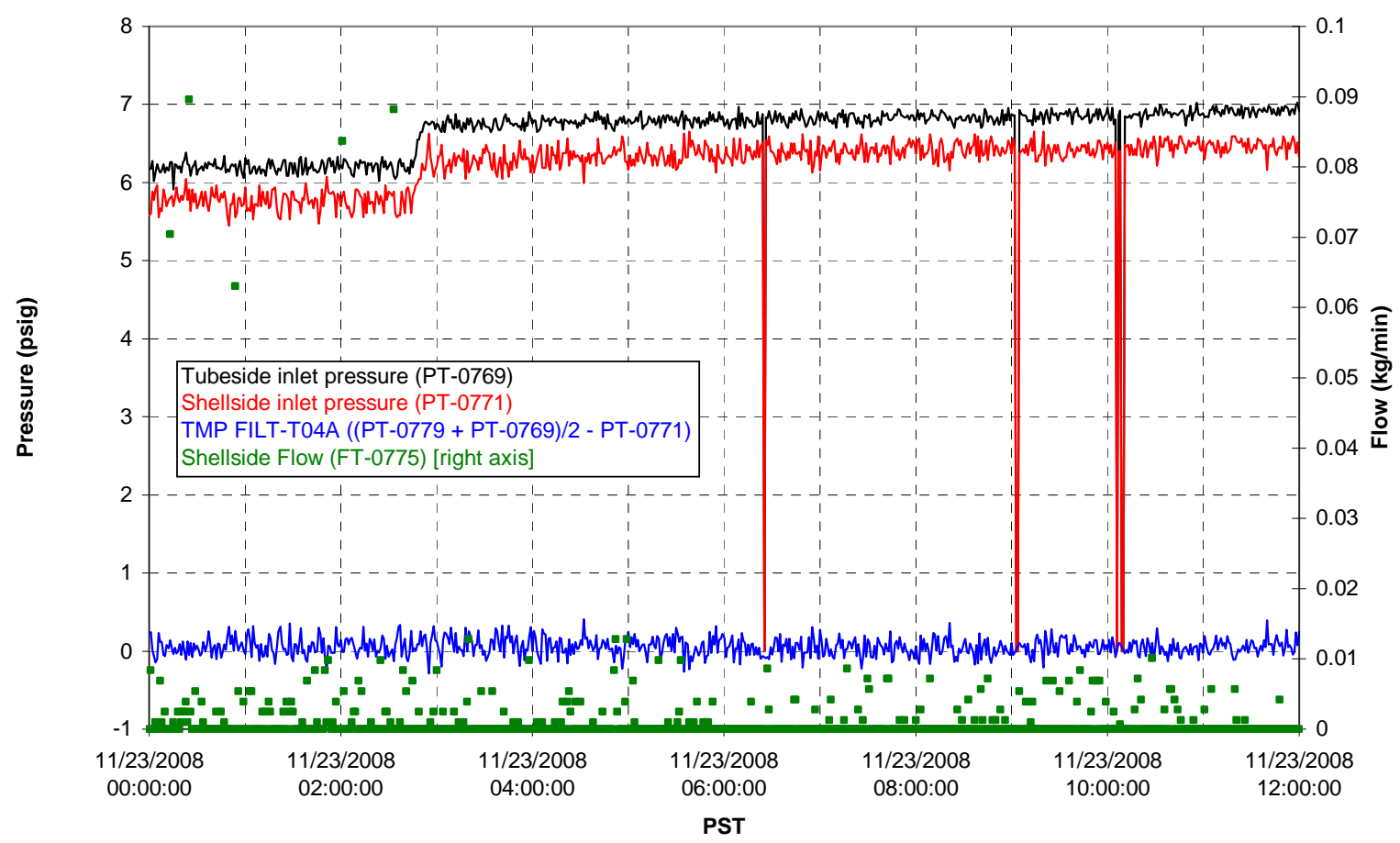


Filter UFP-FILT-T05A

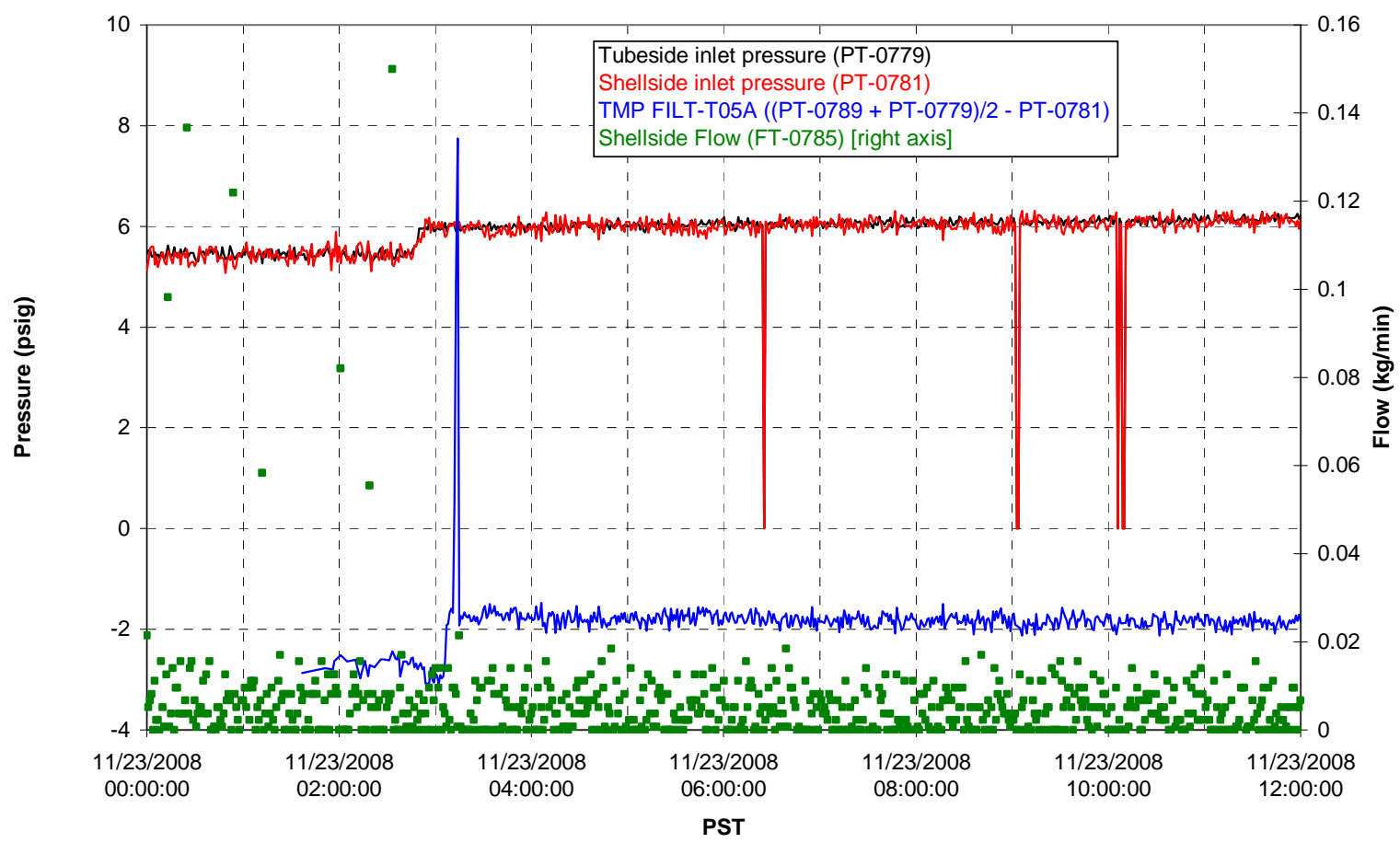

Chemical Flow

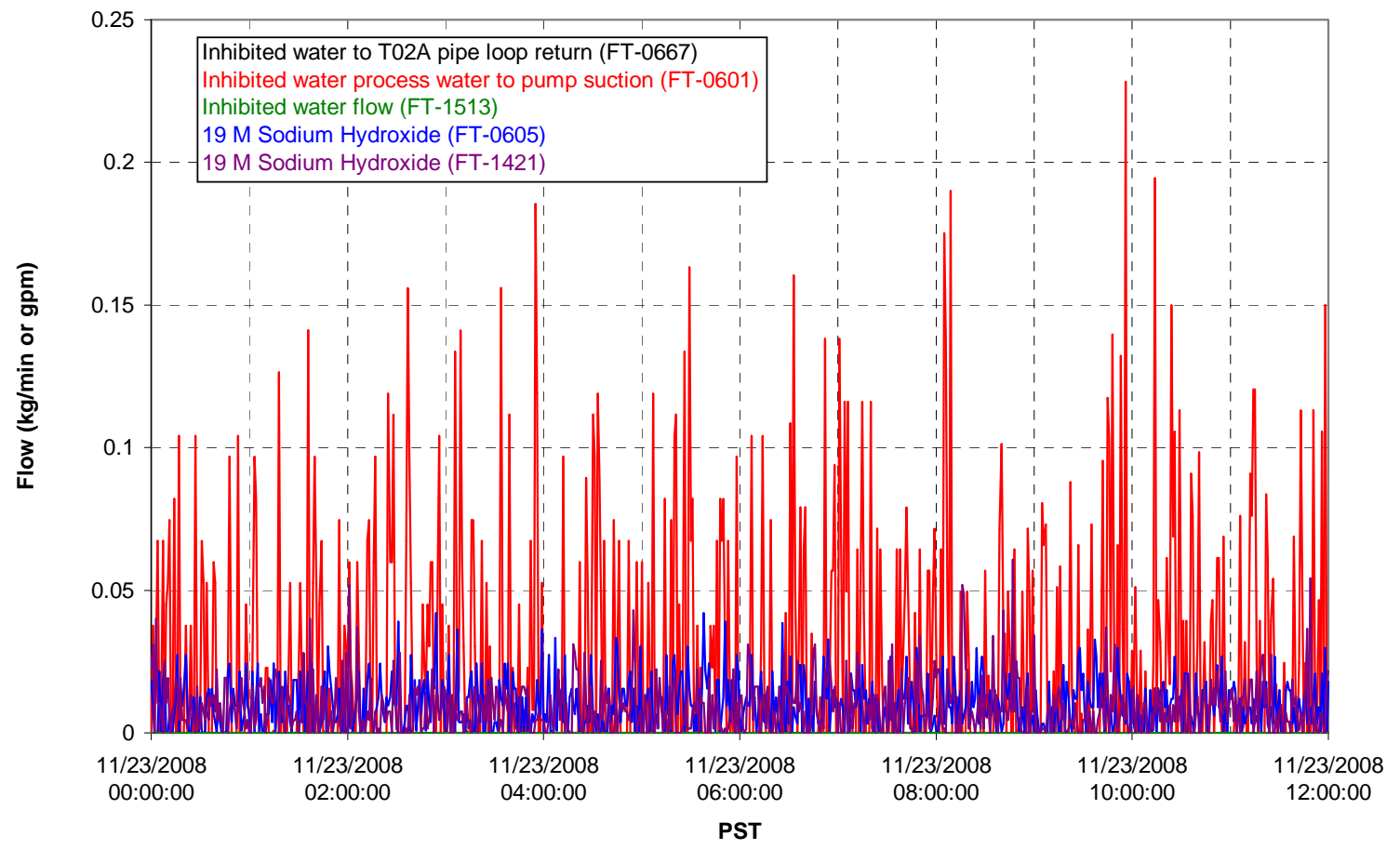




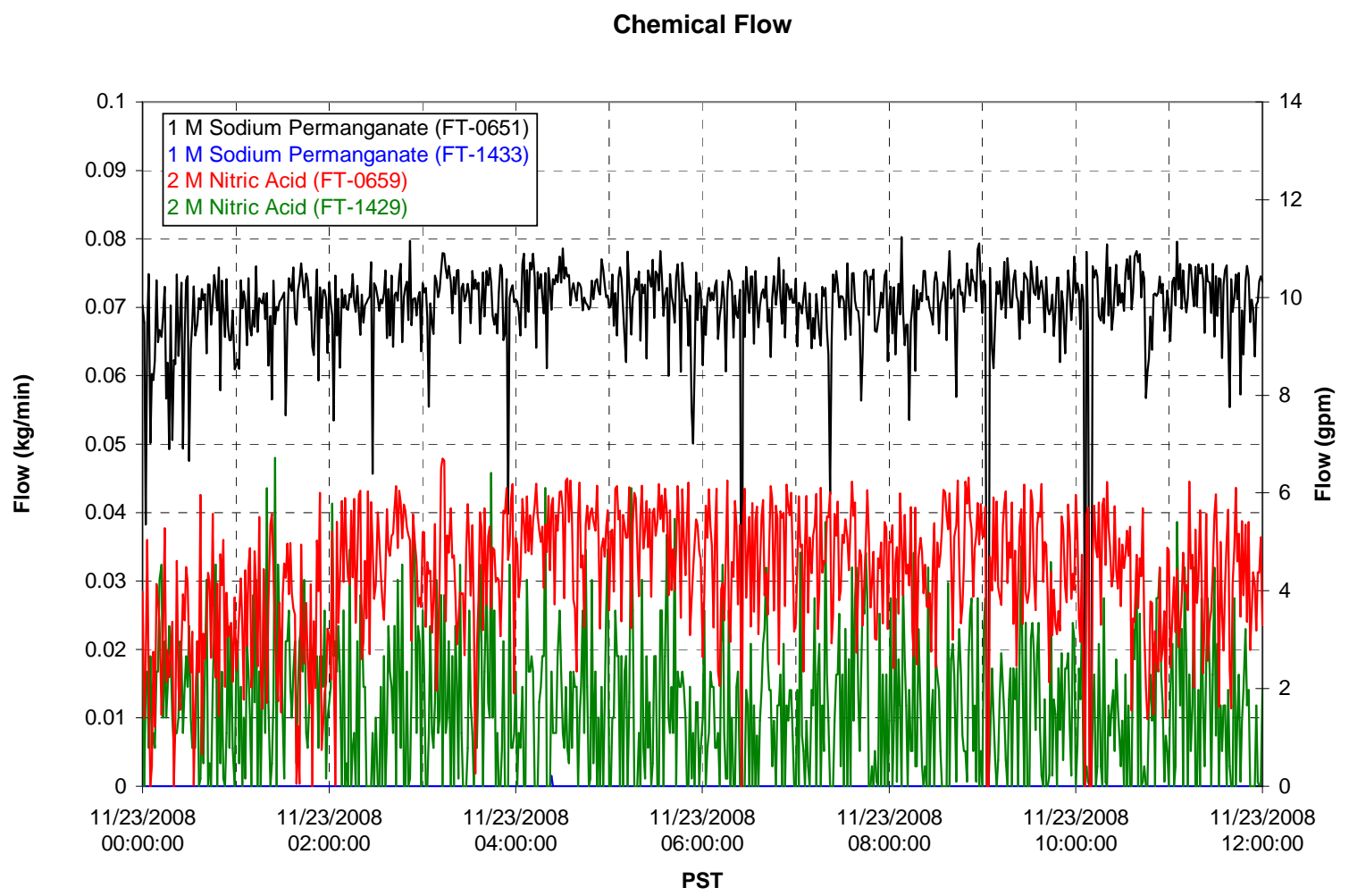

Air Flows

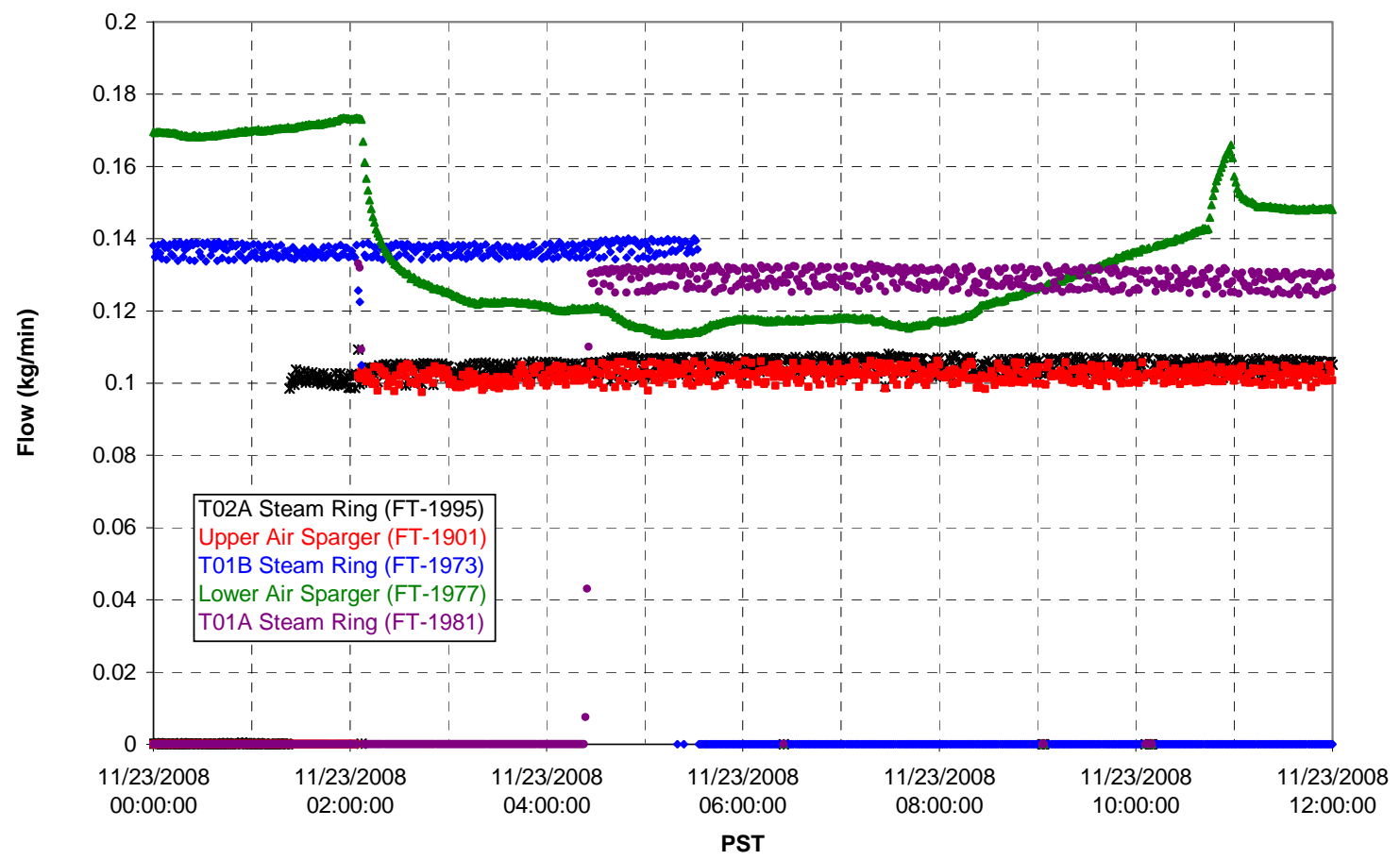


T02A Steam

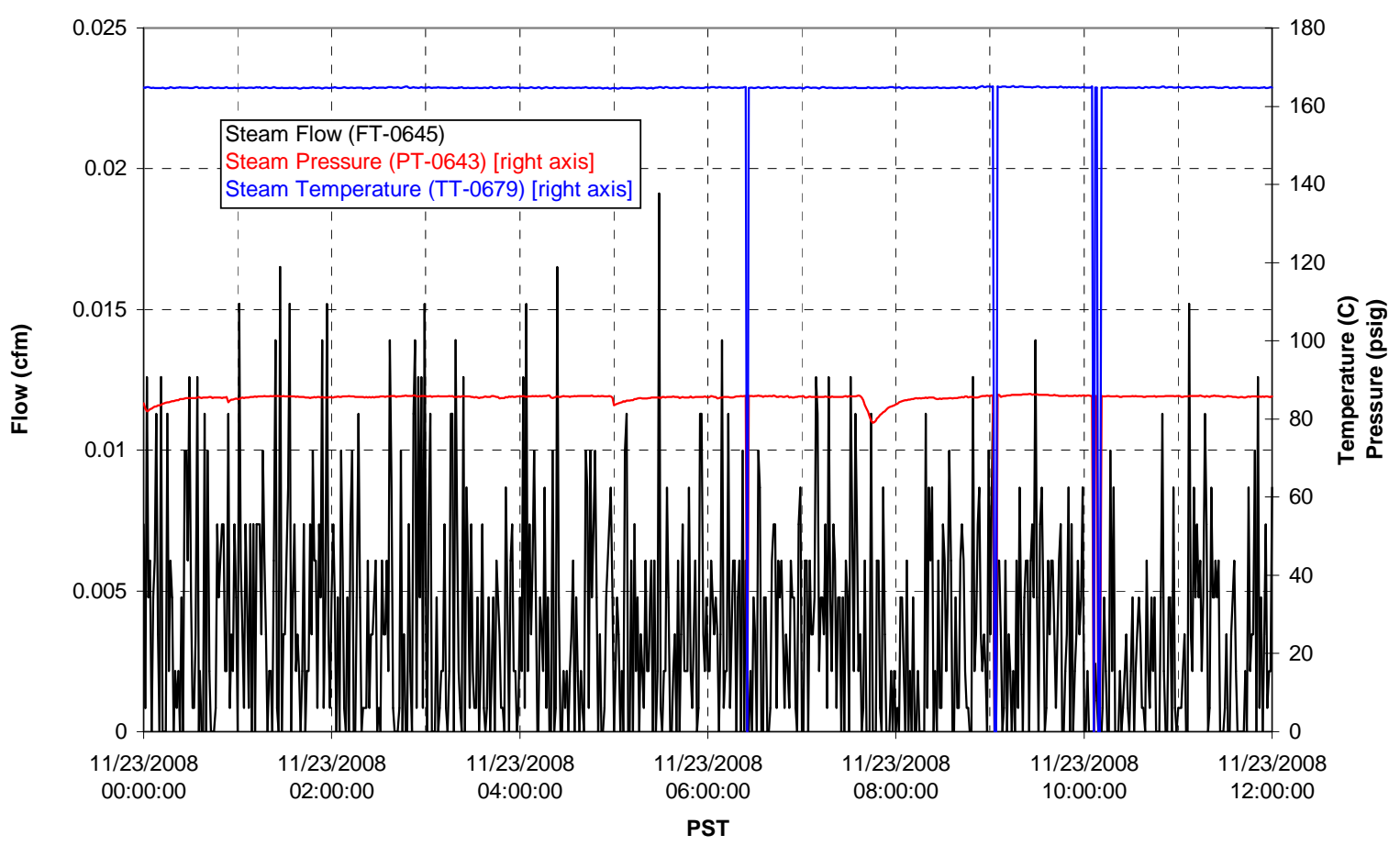

T01A Steam

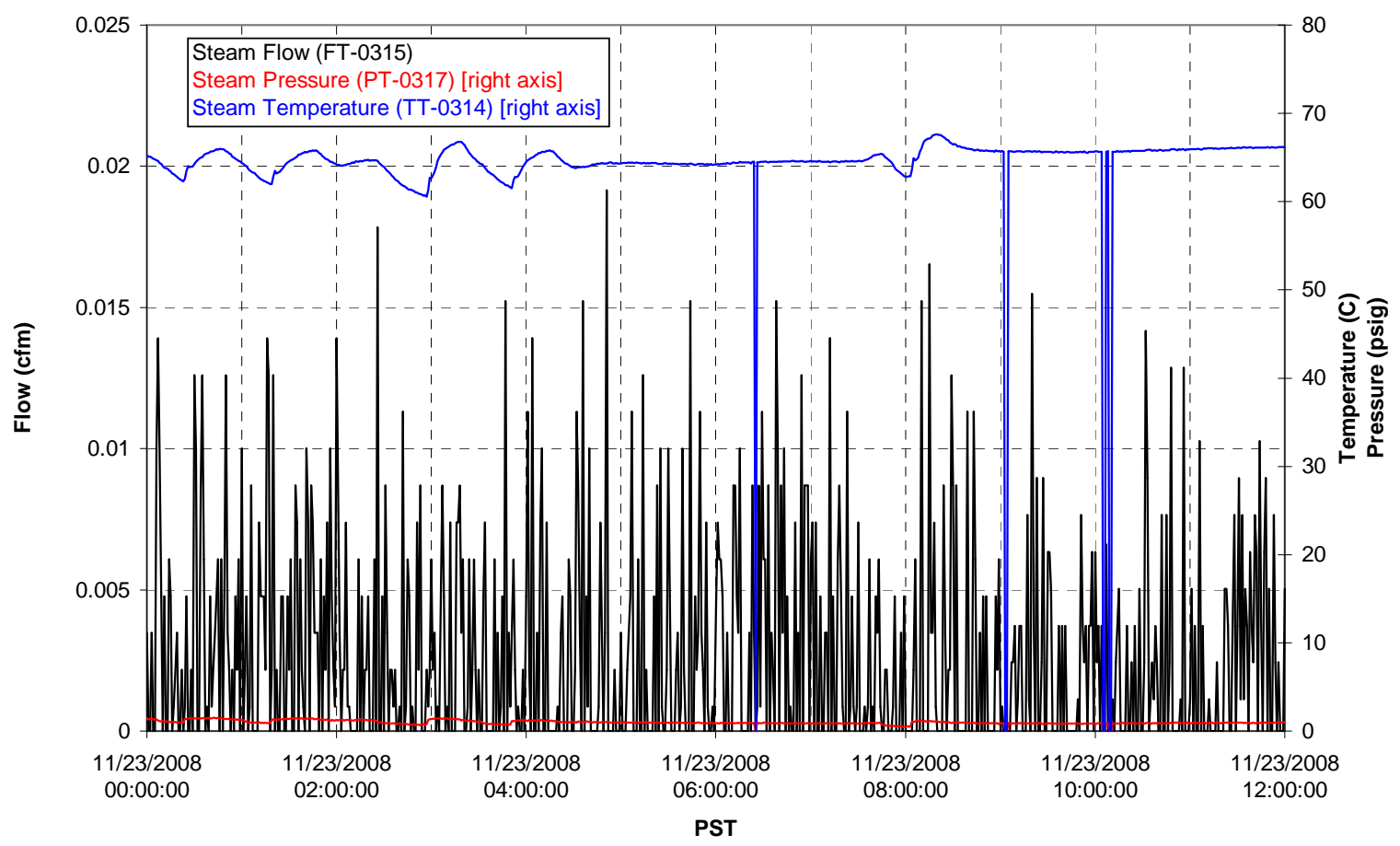


T01B Steam

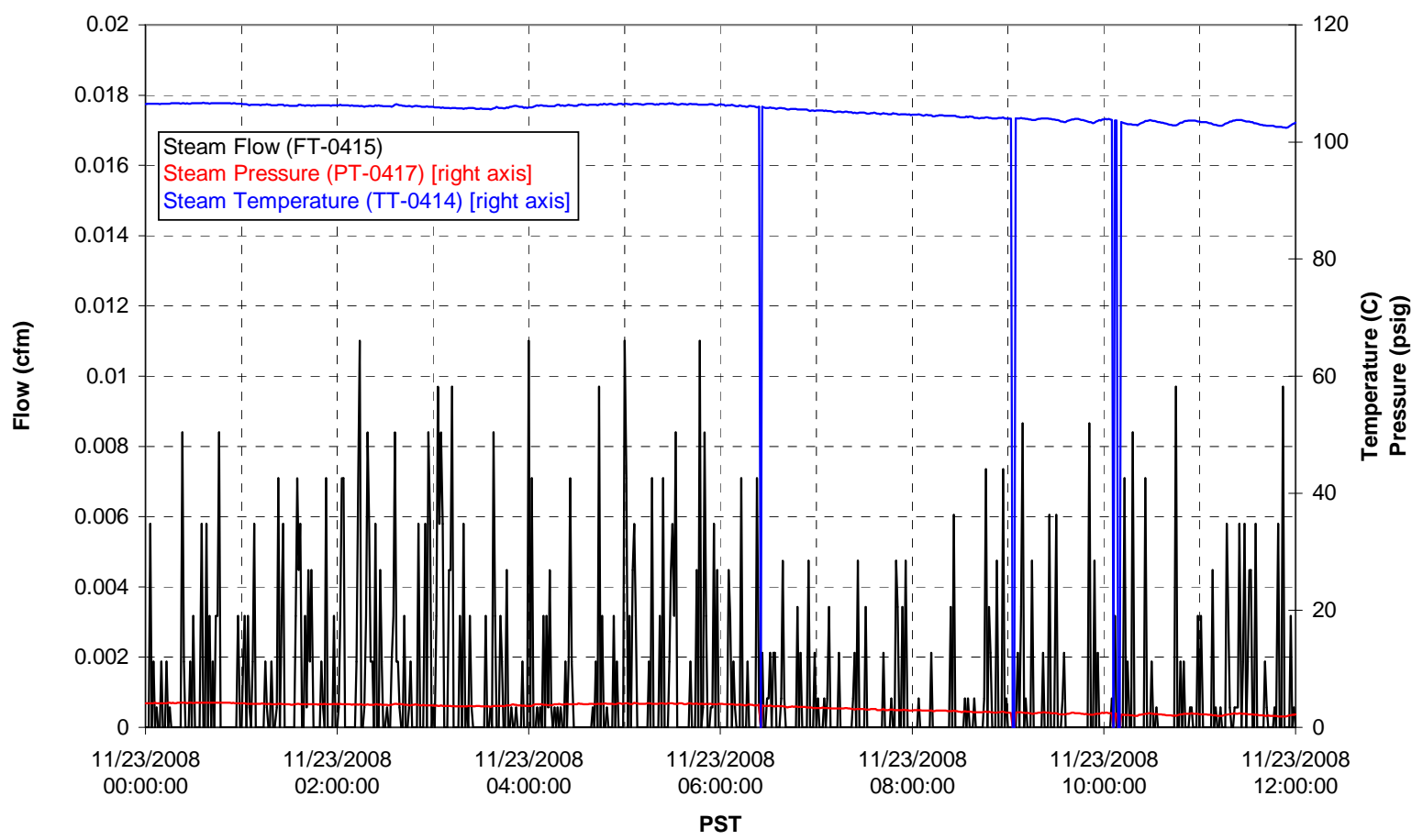


Functional Test Data Plots

11/23/2008 12:00 - 11/24/2008 00:00 
T01A level

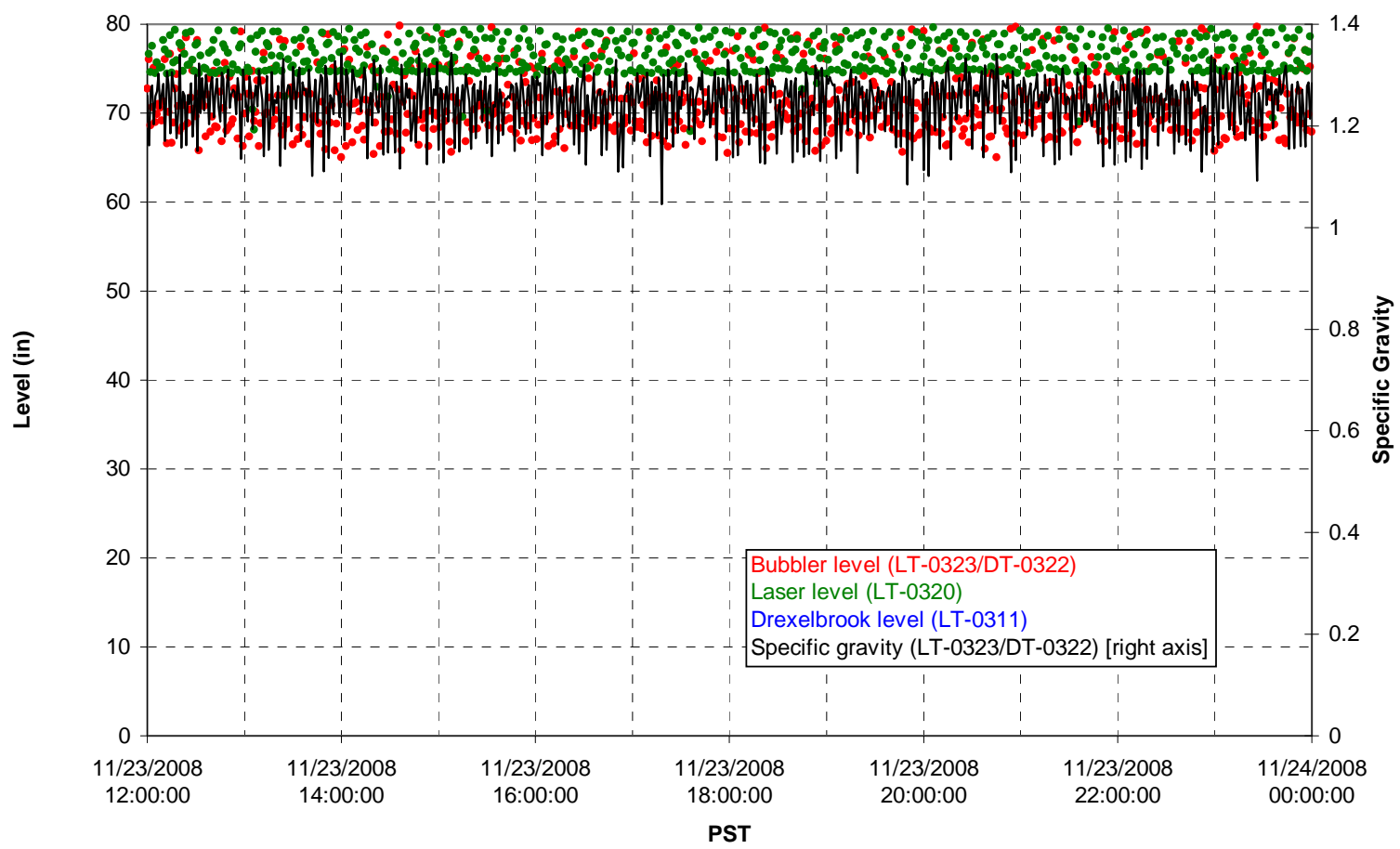

T01A temperatures

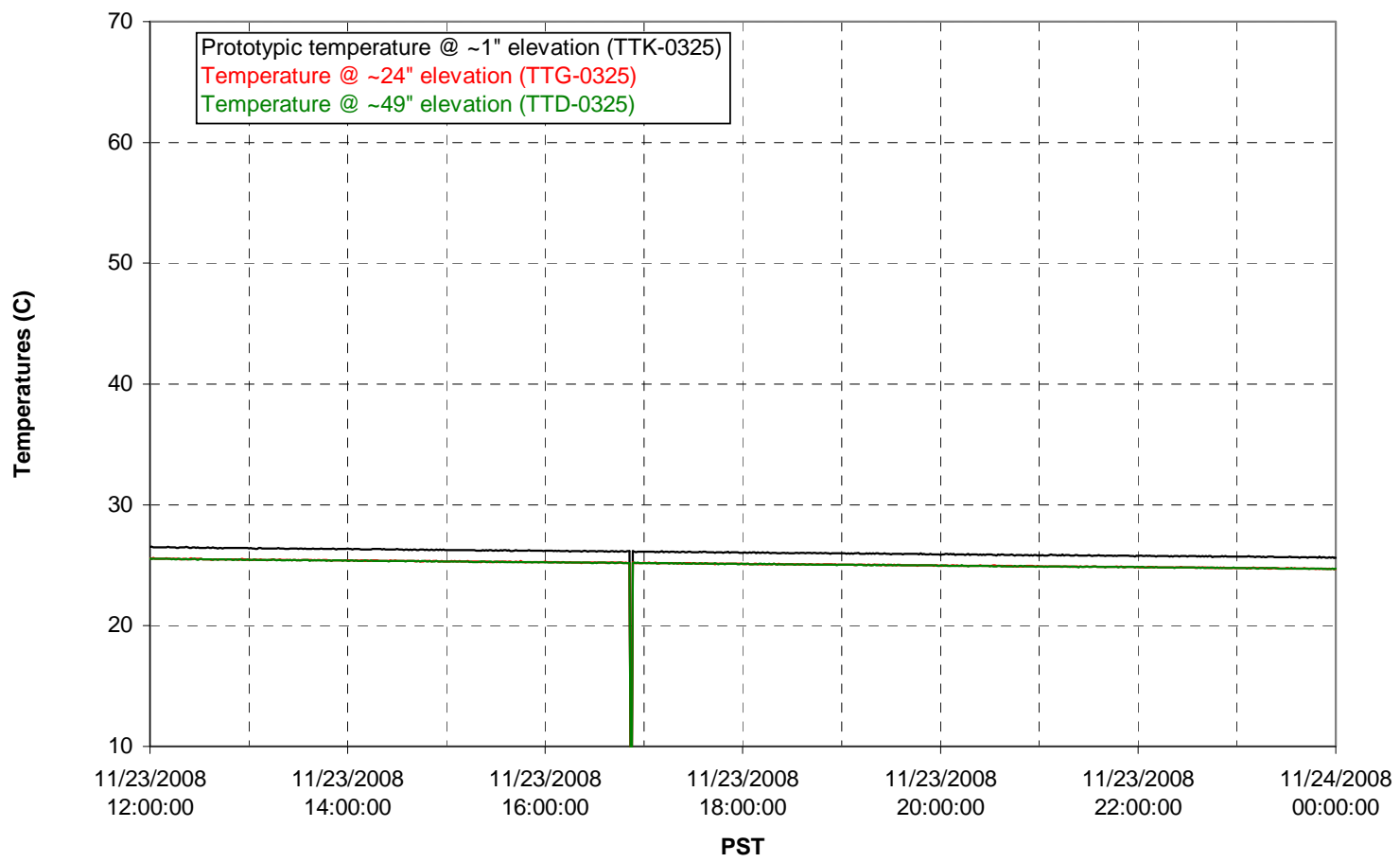


T01B level

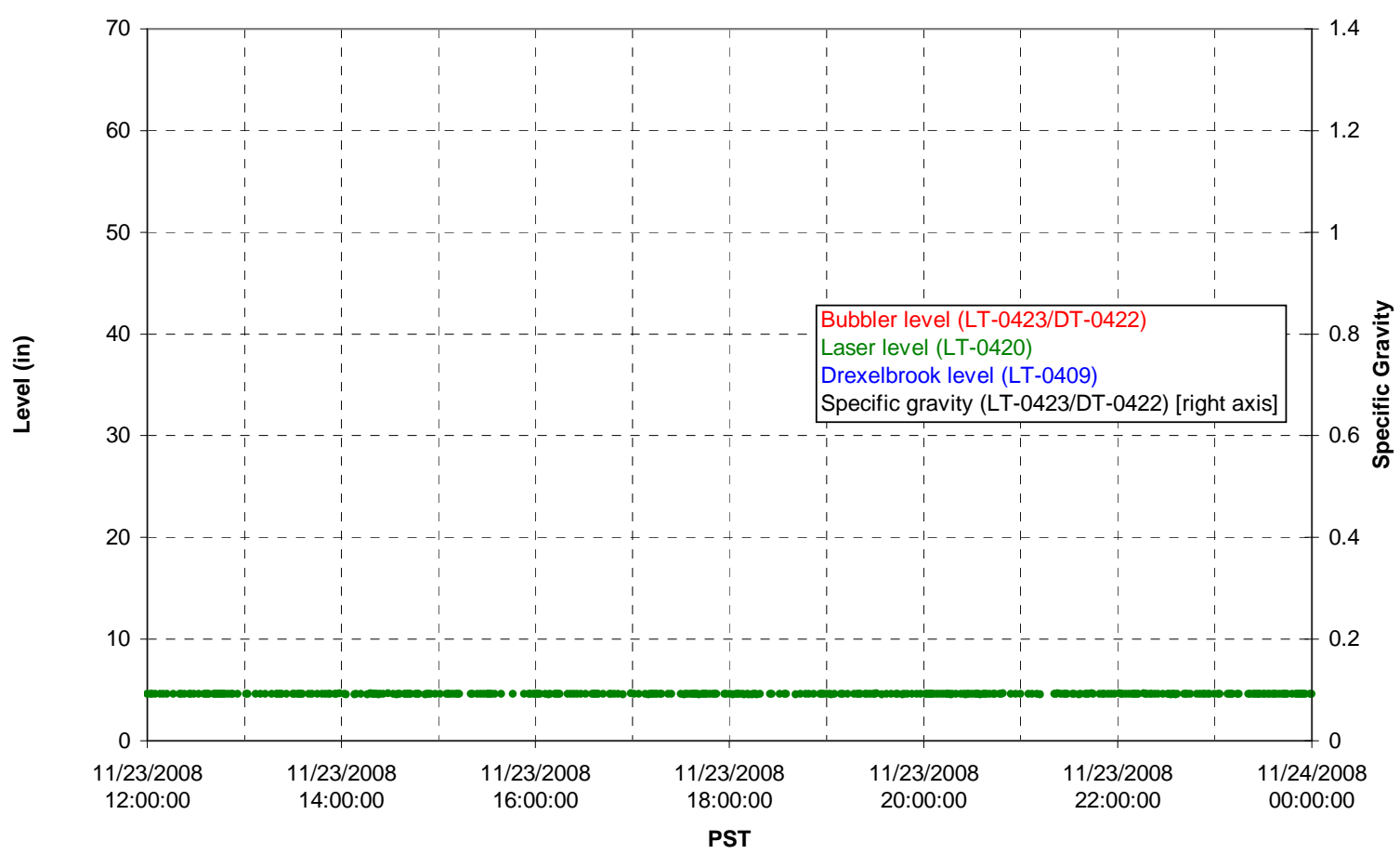

T01B temperatures

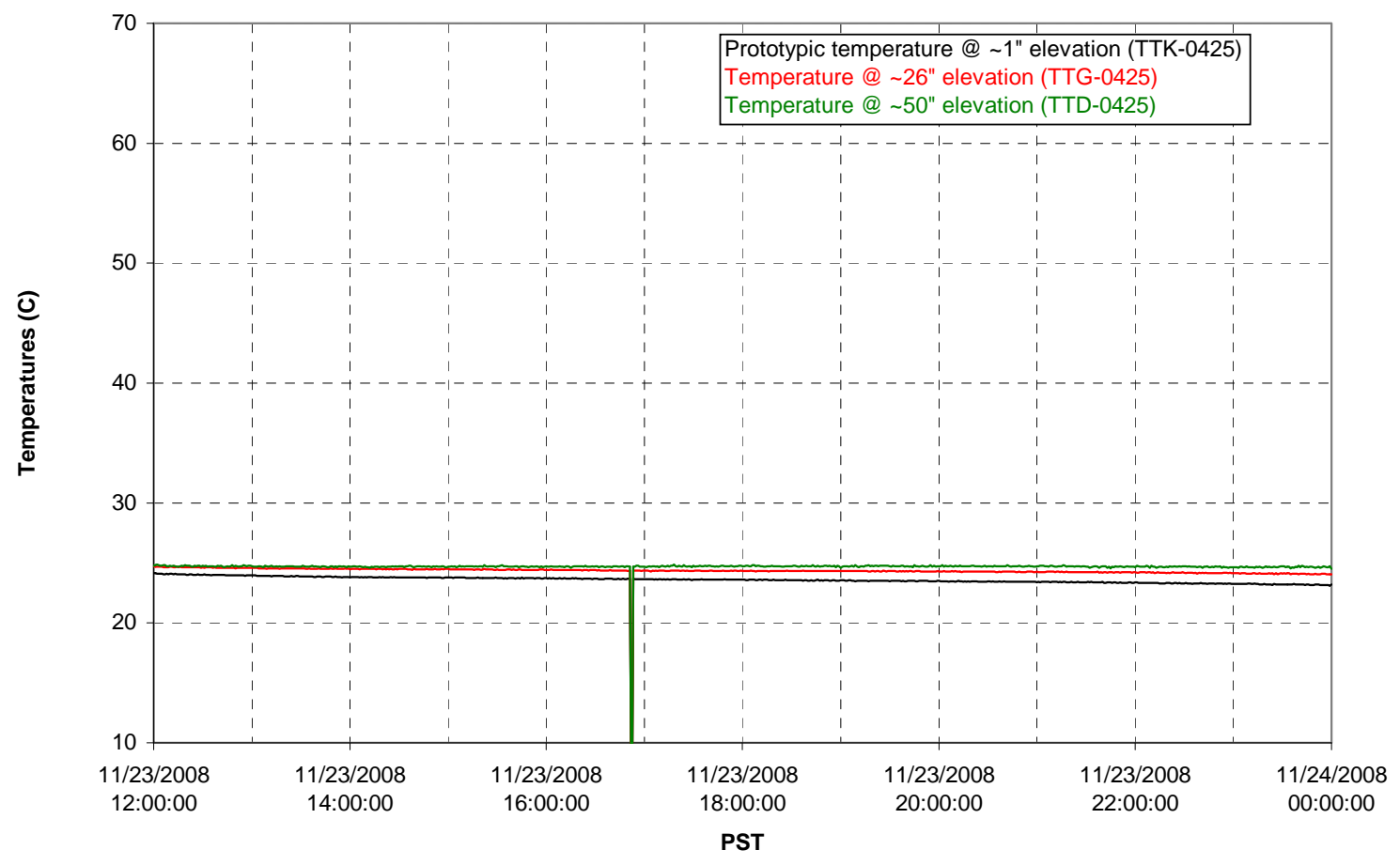


T02A level

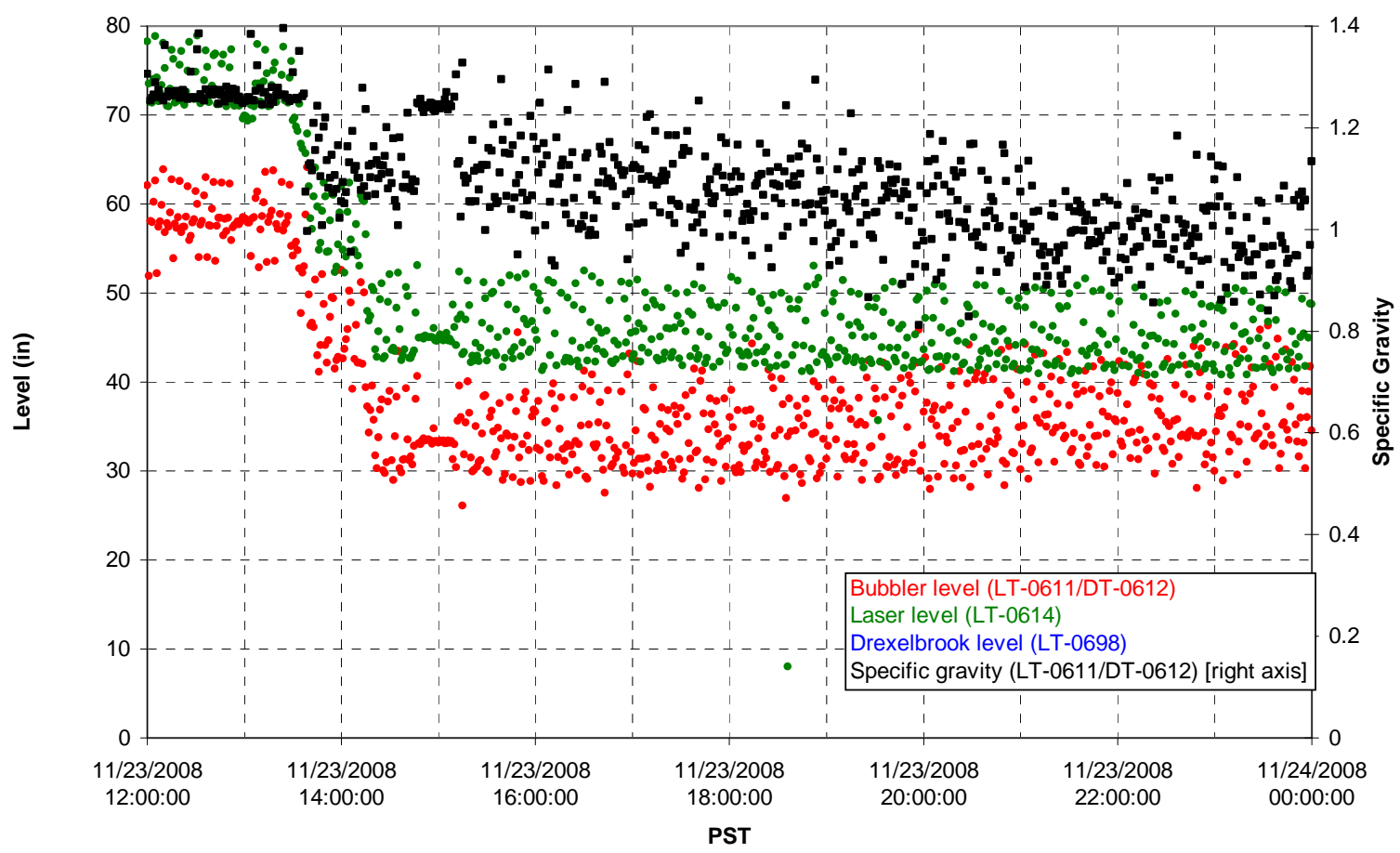

T02A temperatures

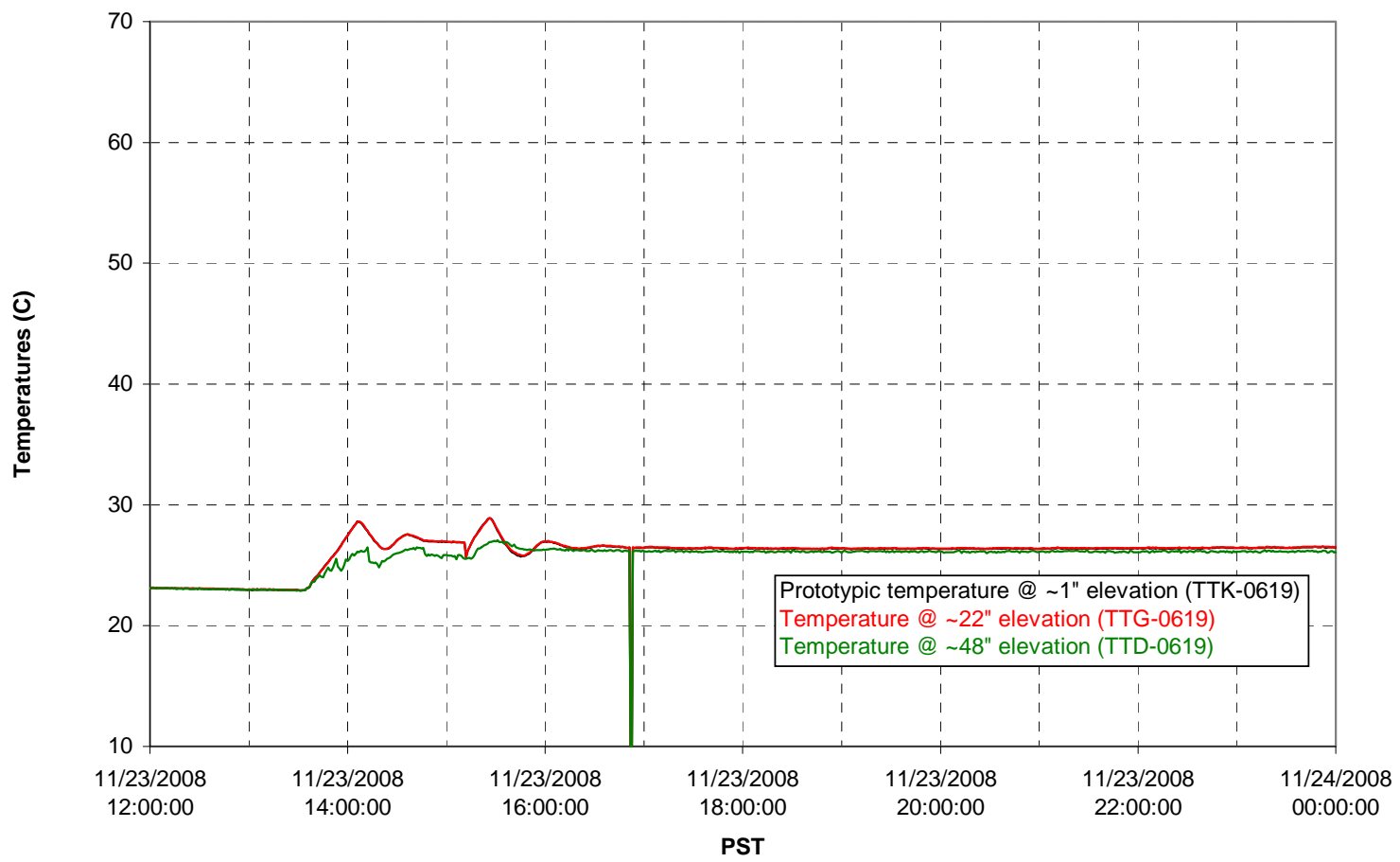


T02A and filter loop temperatures

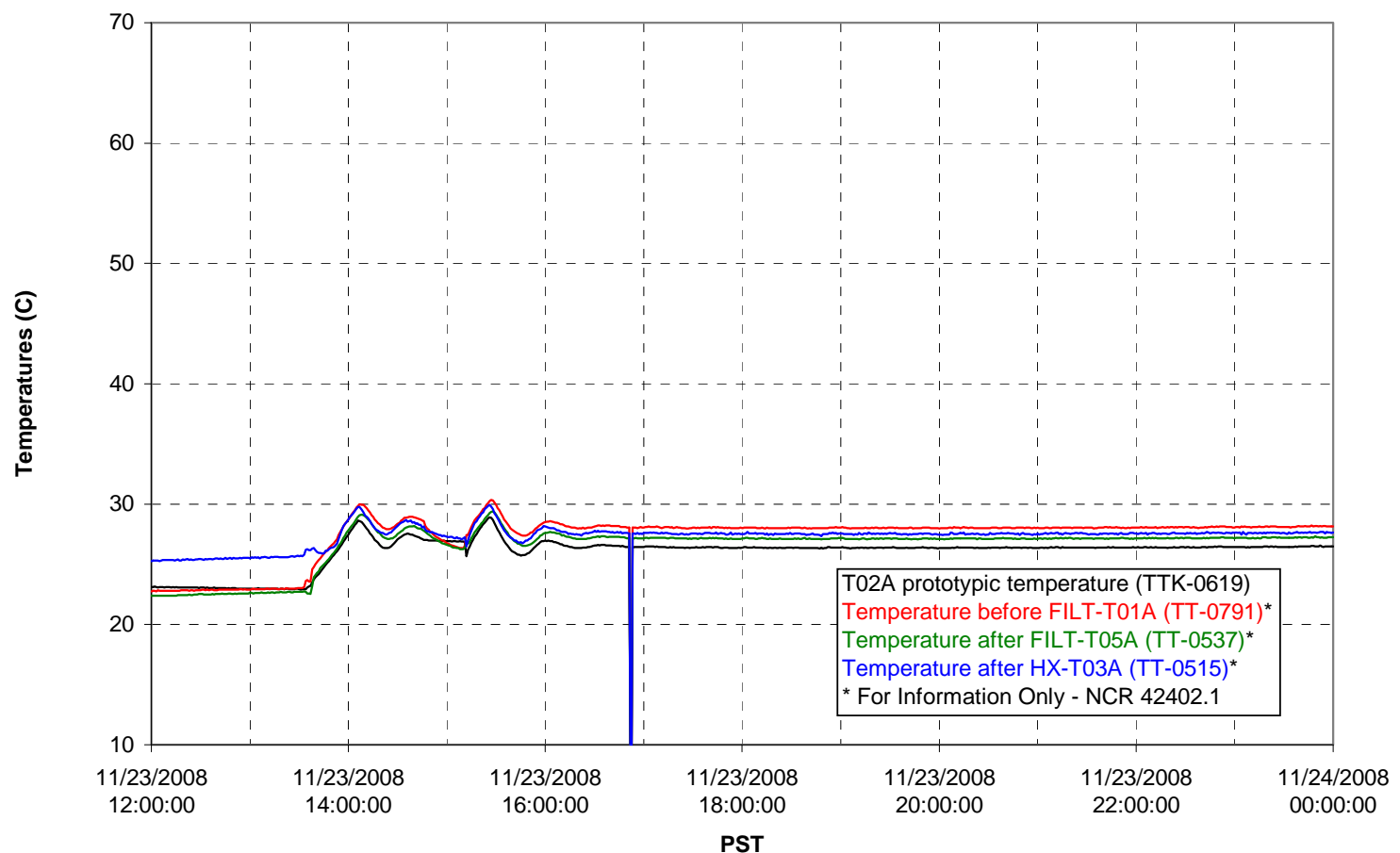

Pump Pressures and Flow

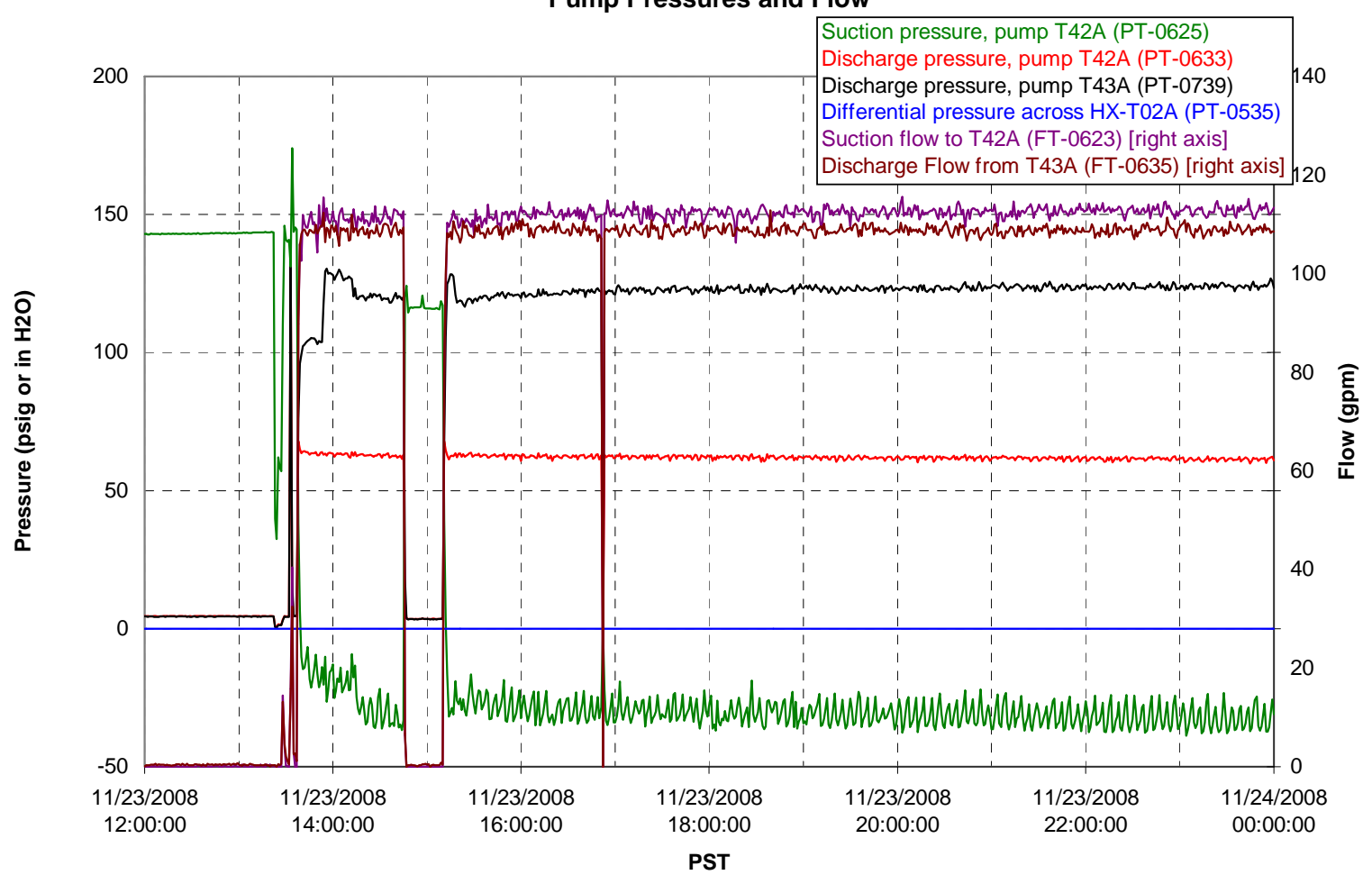


Axial pressure drop

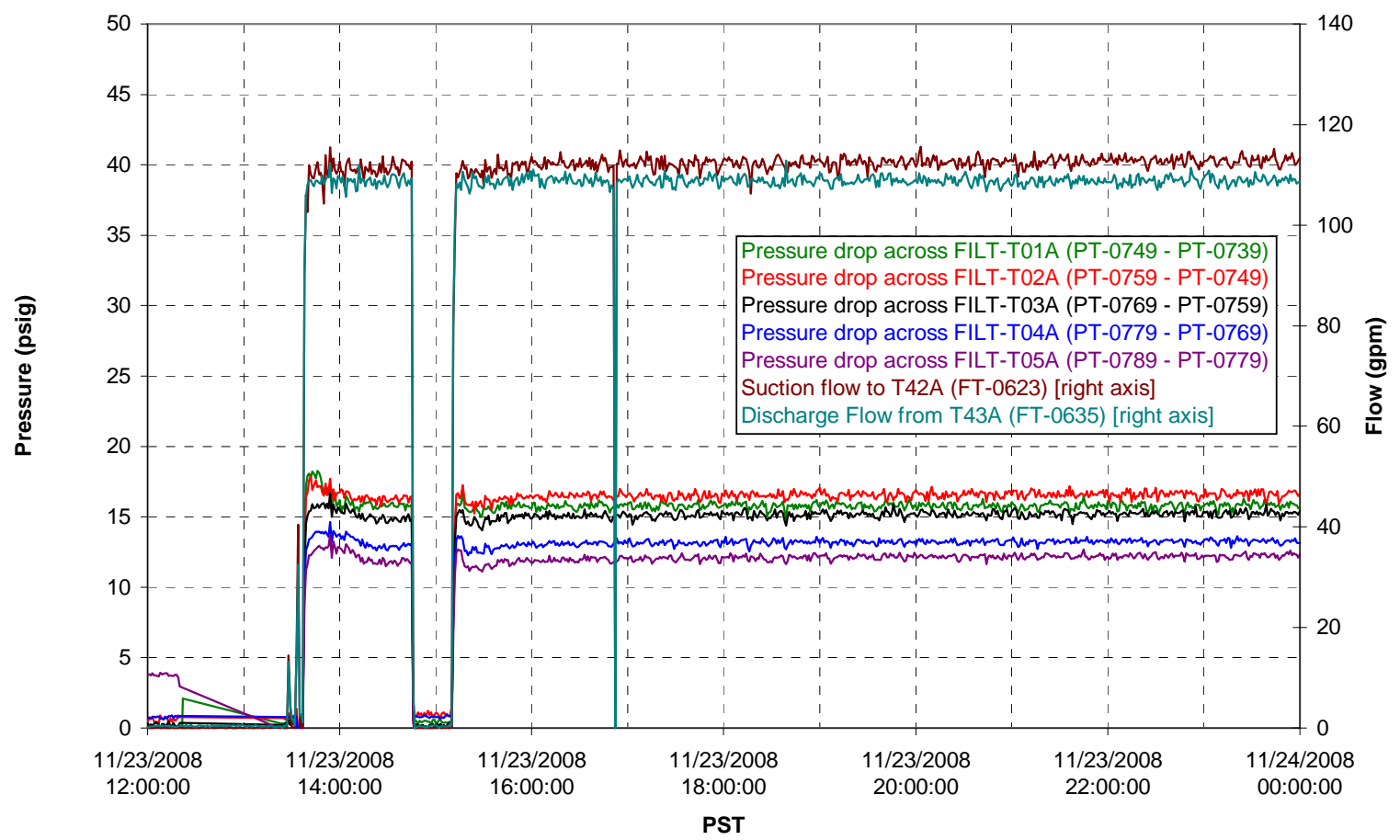

Permeate flow rates

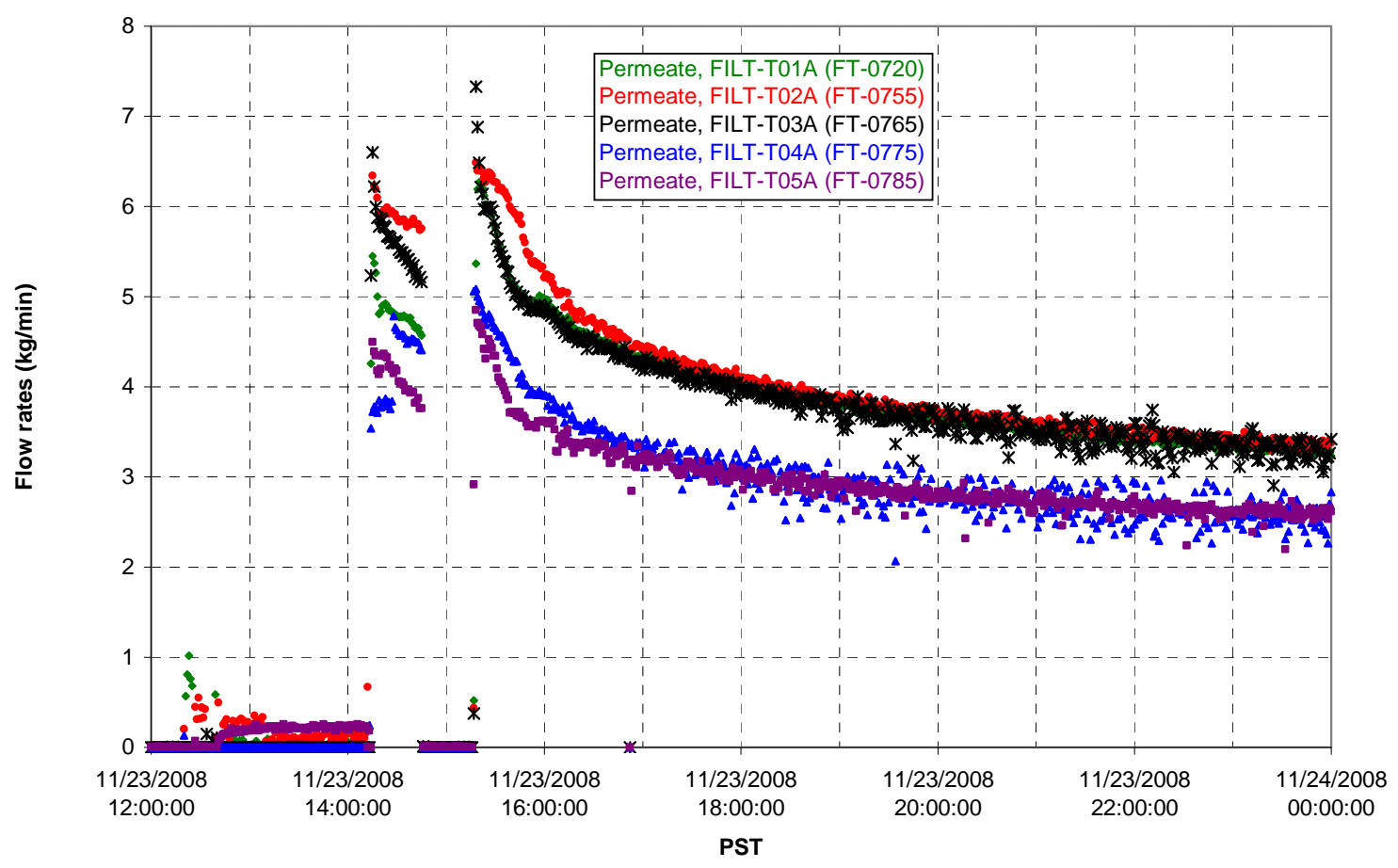


T02A Inner Temperature Tree

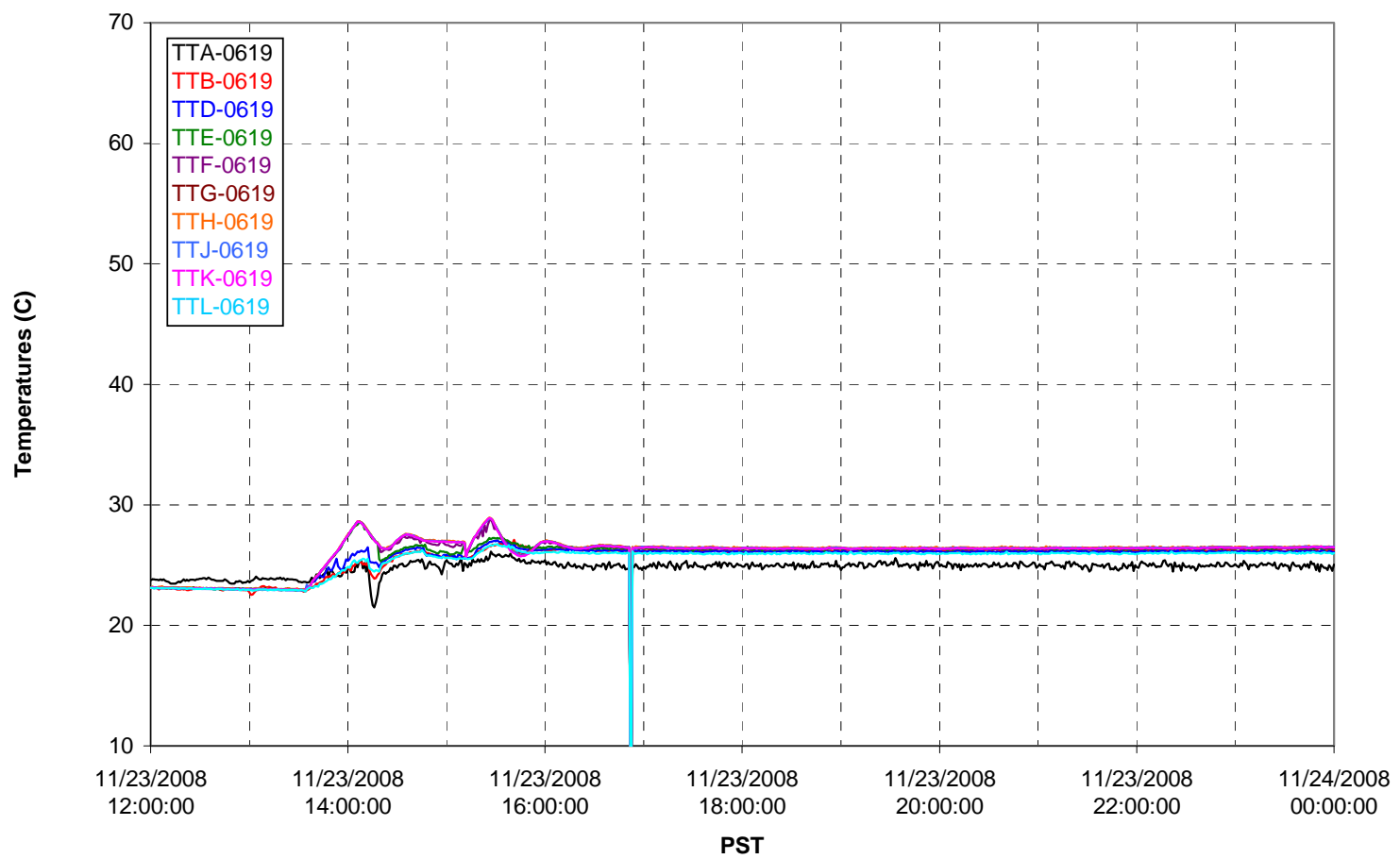

T02A Outer Temperature Tree

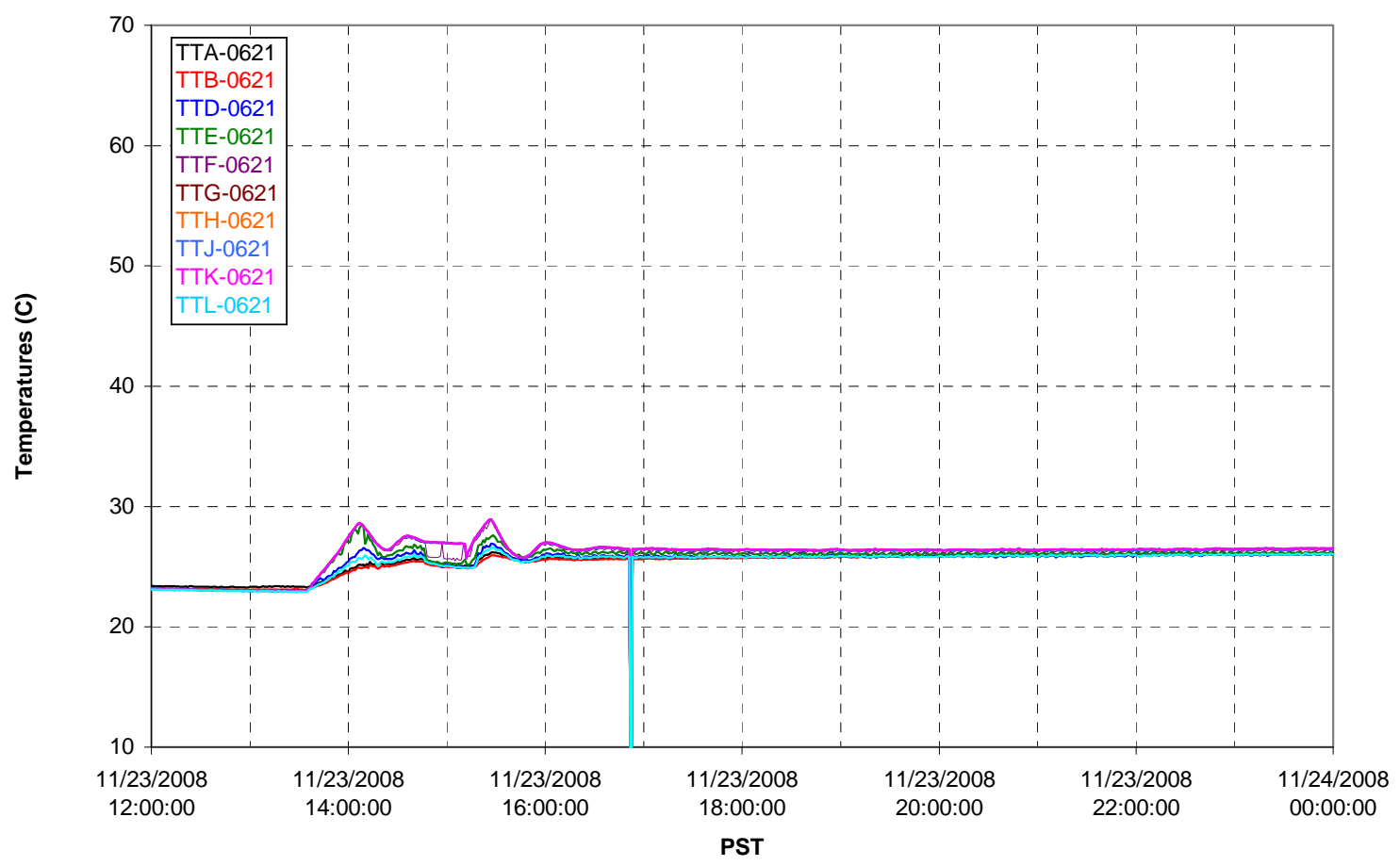


T02A temperatures

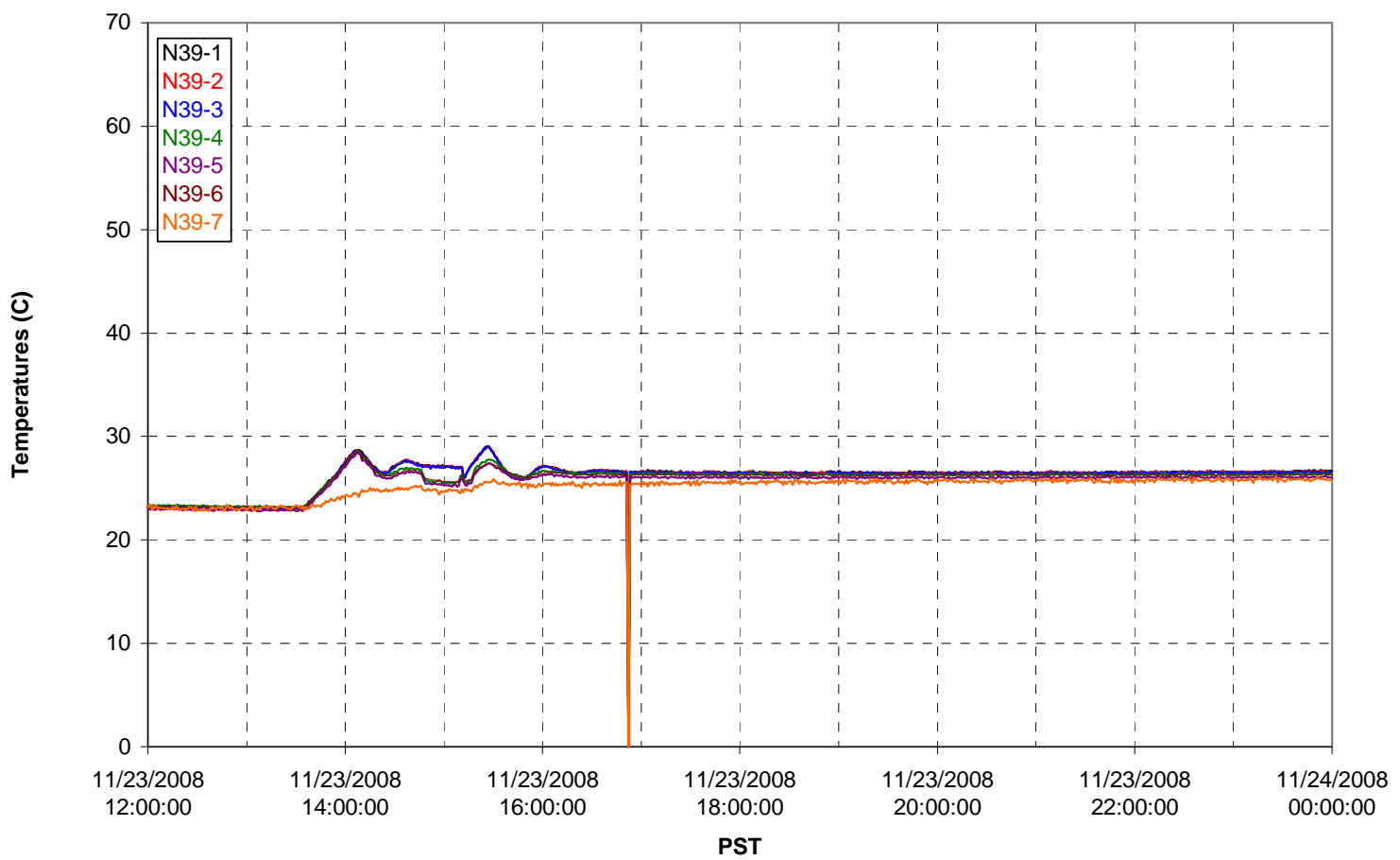

T02A temperatures

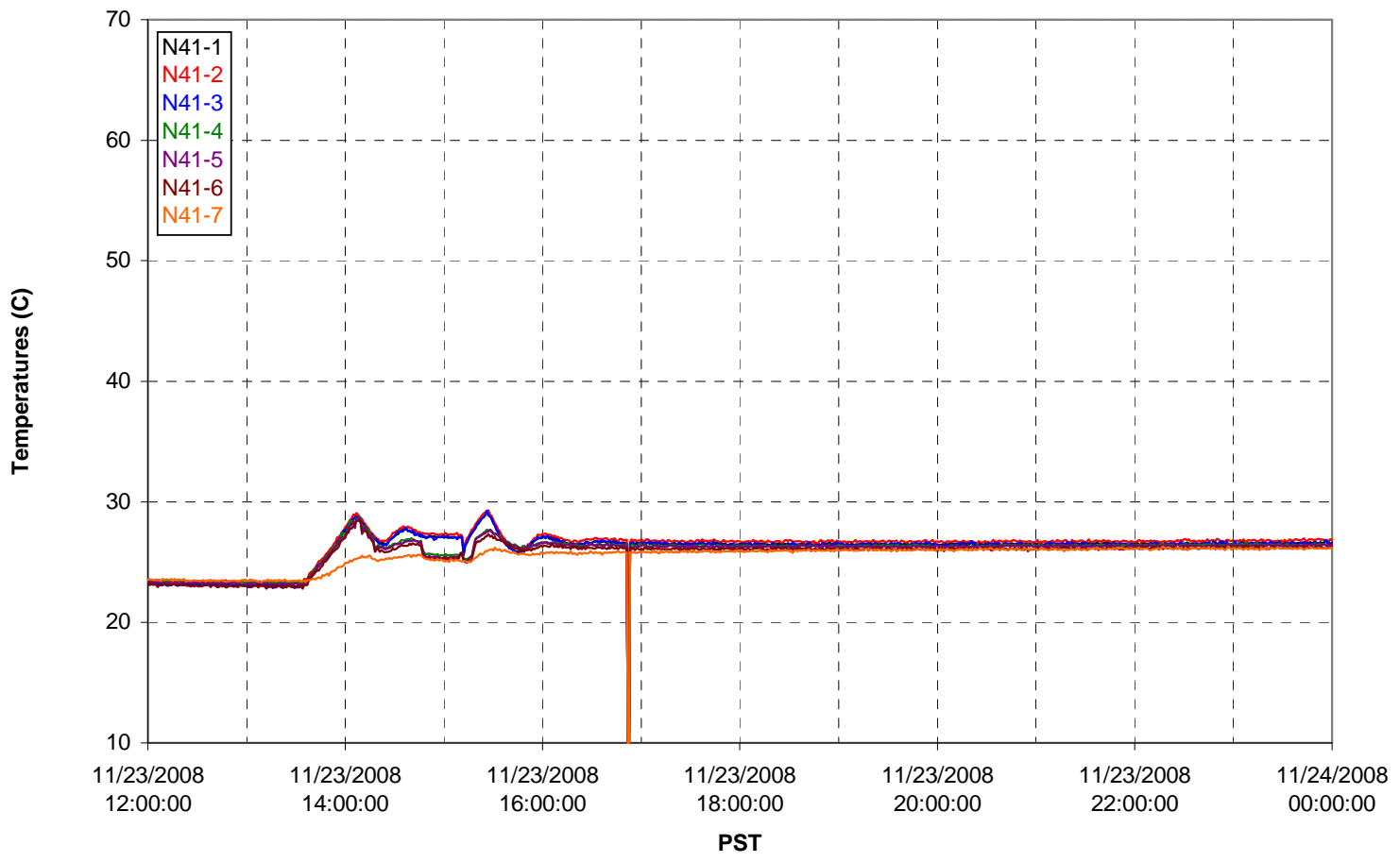


T02A temperatures

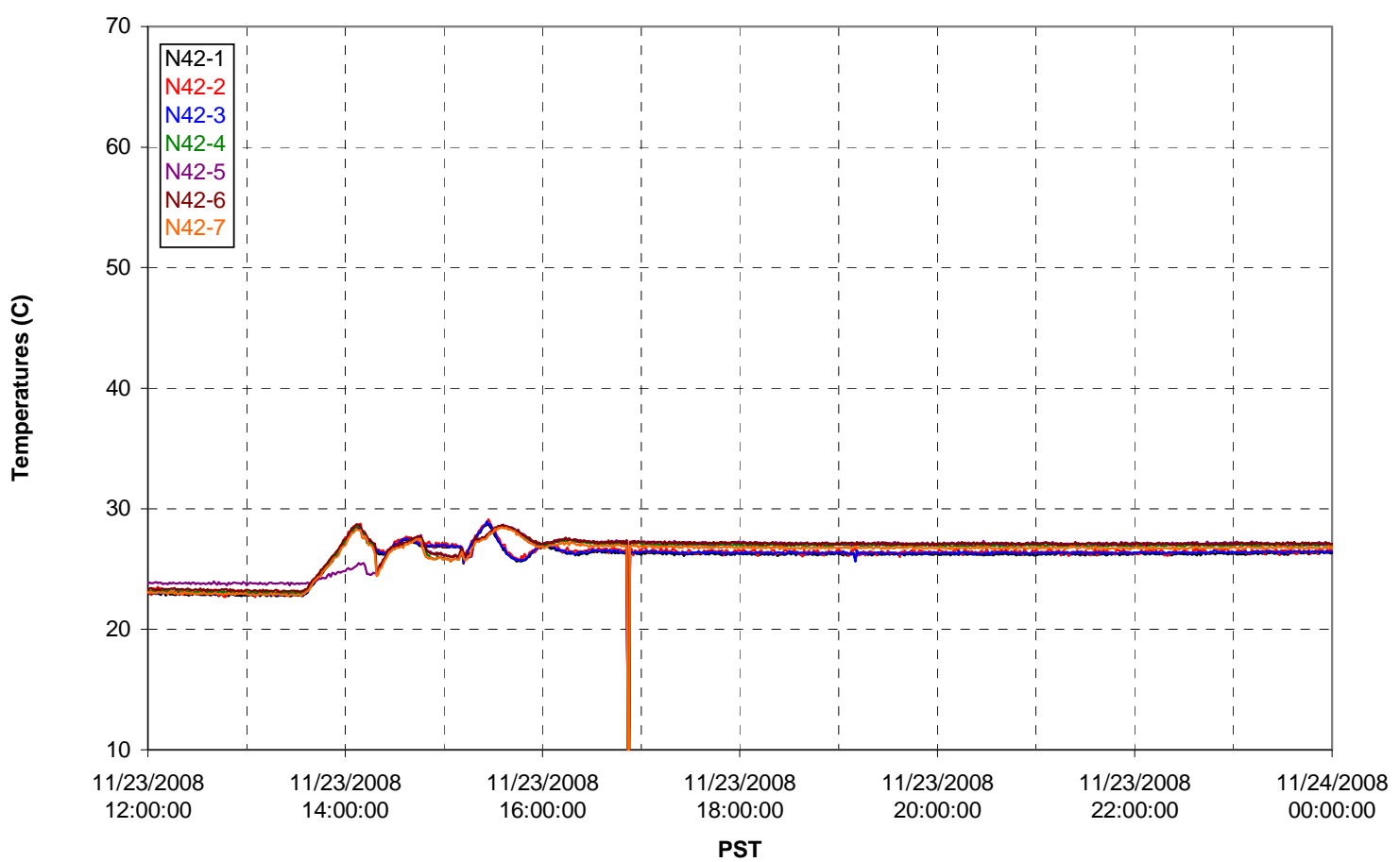

T02A temperatures

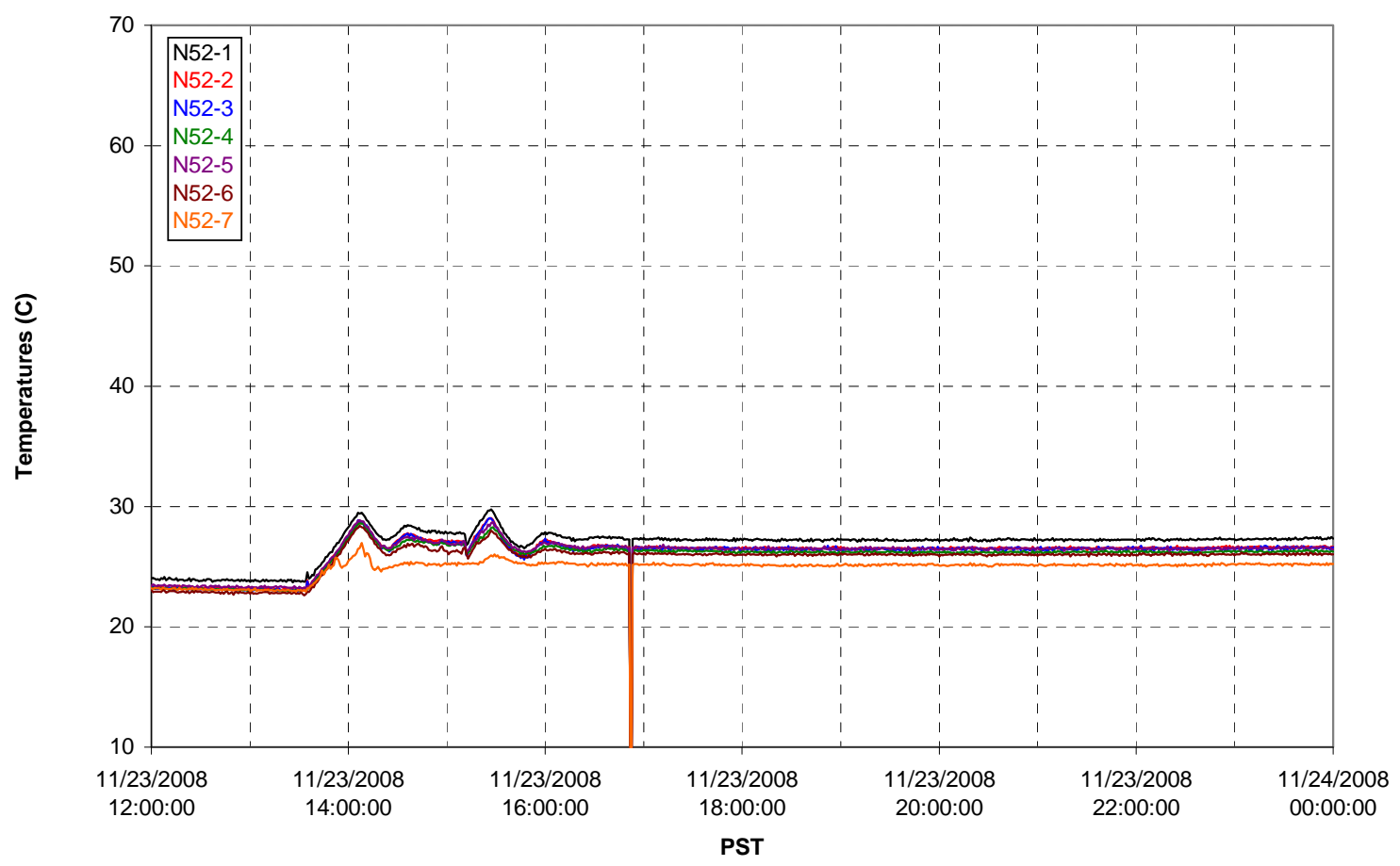


T02A Heating and Cooling

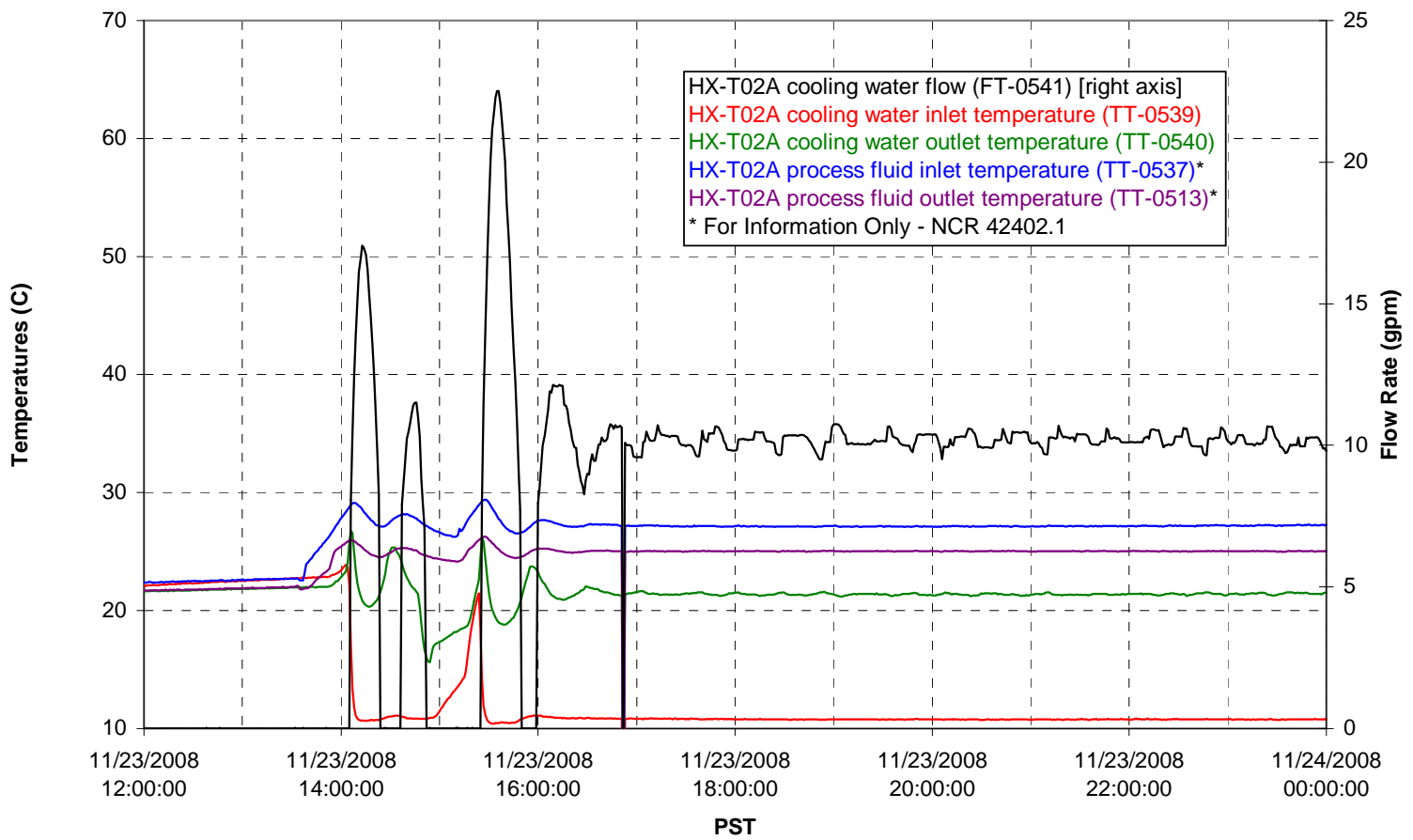

Pump Operation

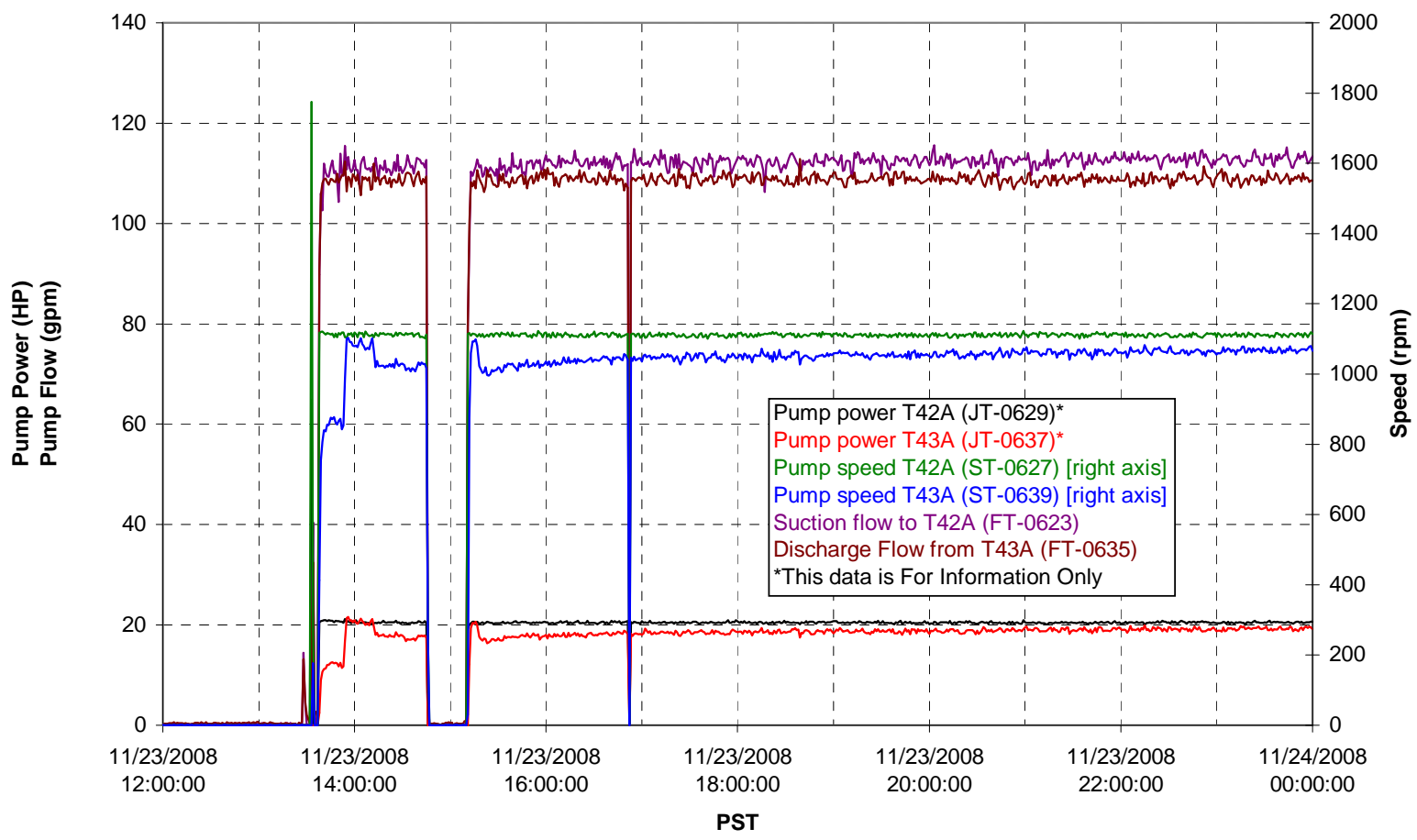


Pulsepot UFP-PP-T01A

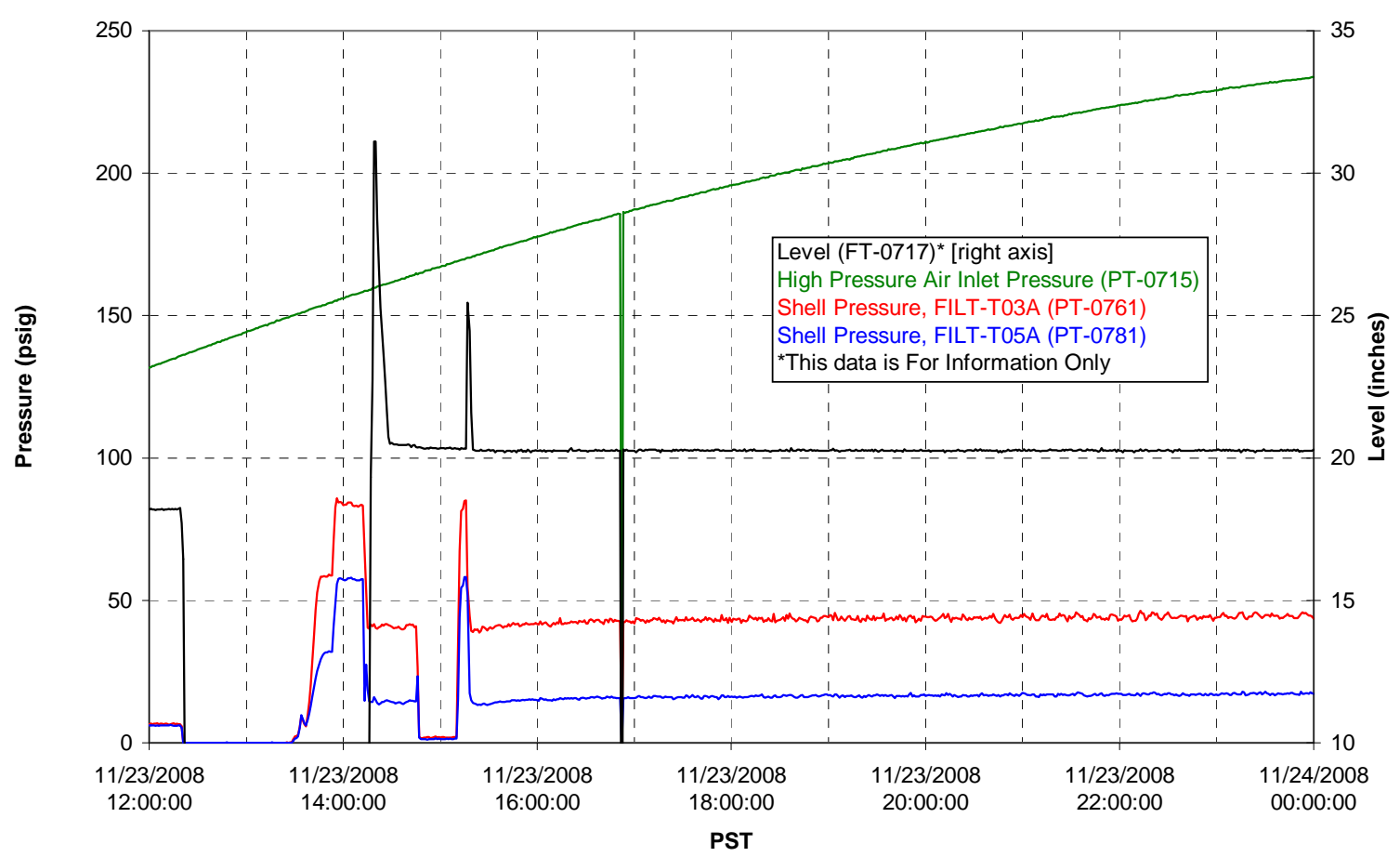

Pulsepot UFP-PP-T02A

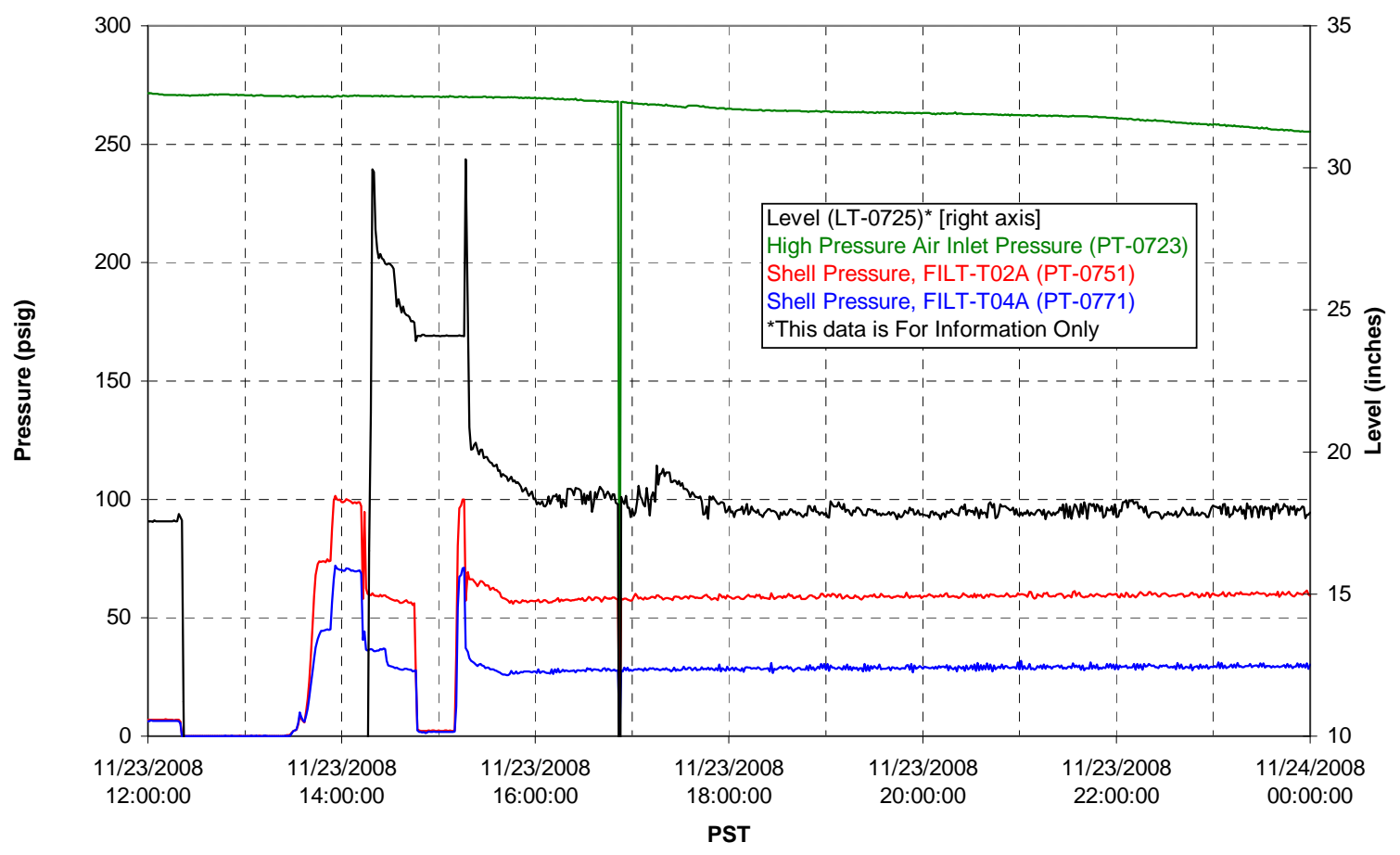


Pulsepot UFP-PP-T03A

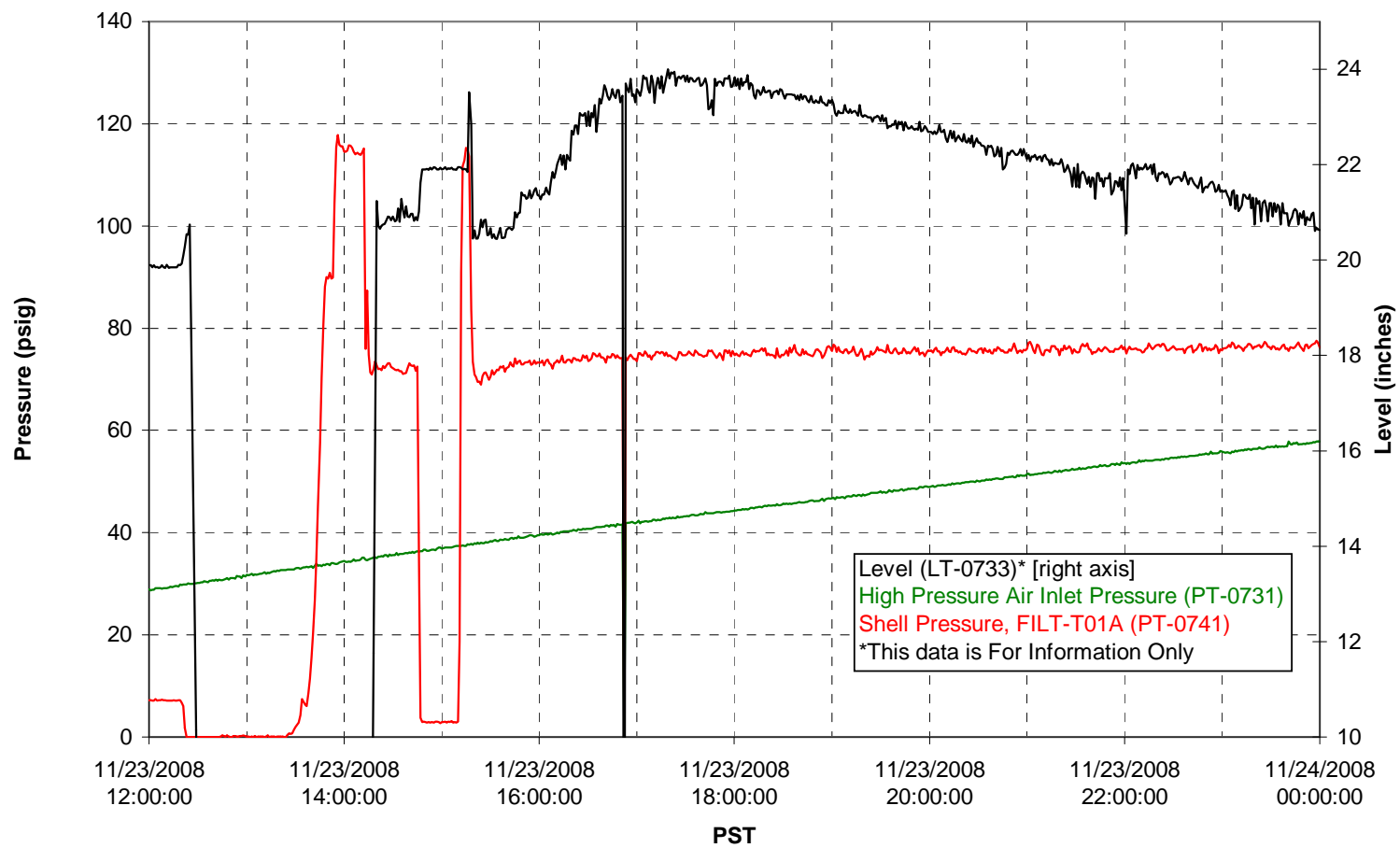

Pulsepot Levels

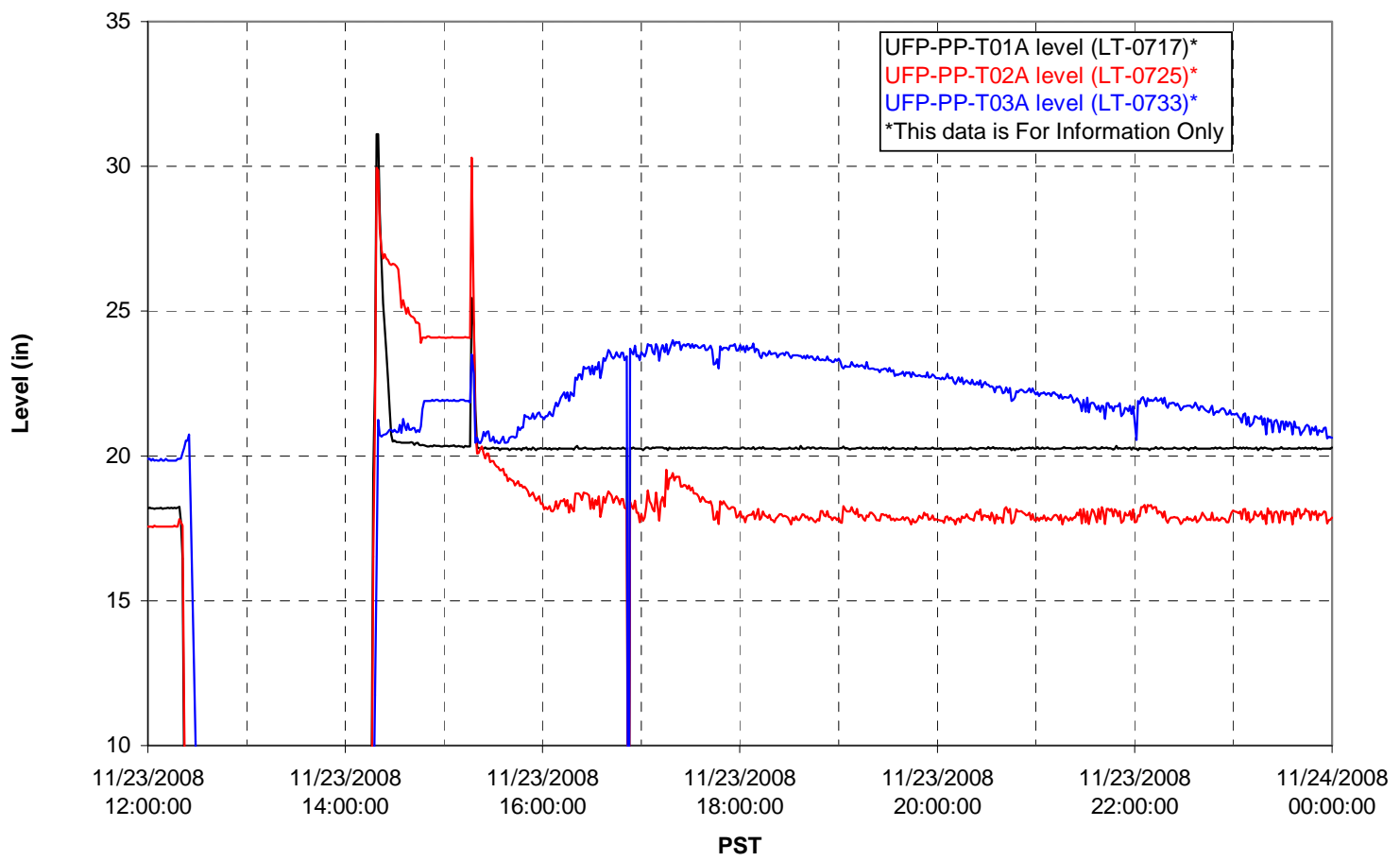


Filter UFP-FILT-T01A

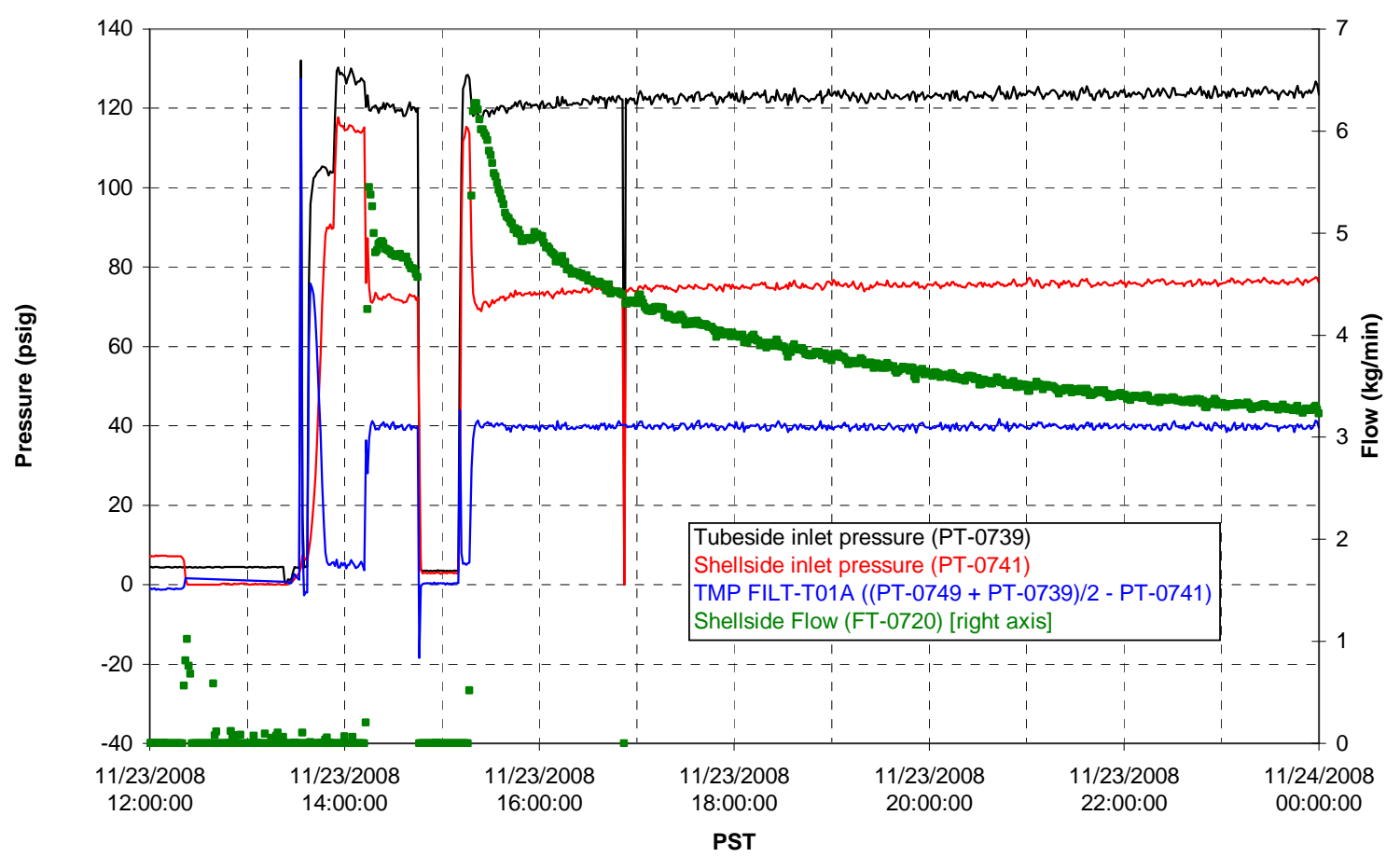

Filter UFP-FILT-T02A

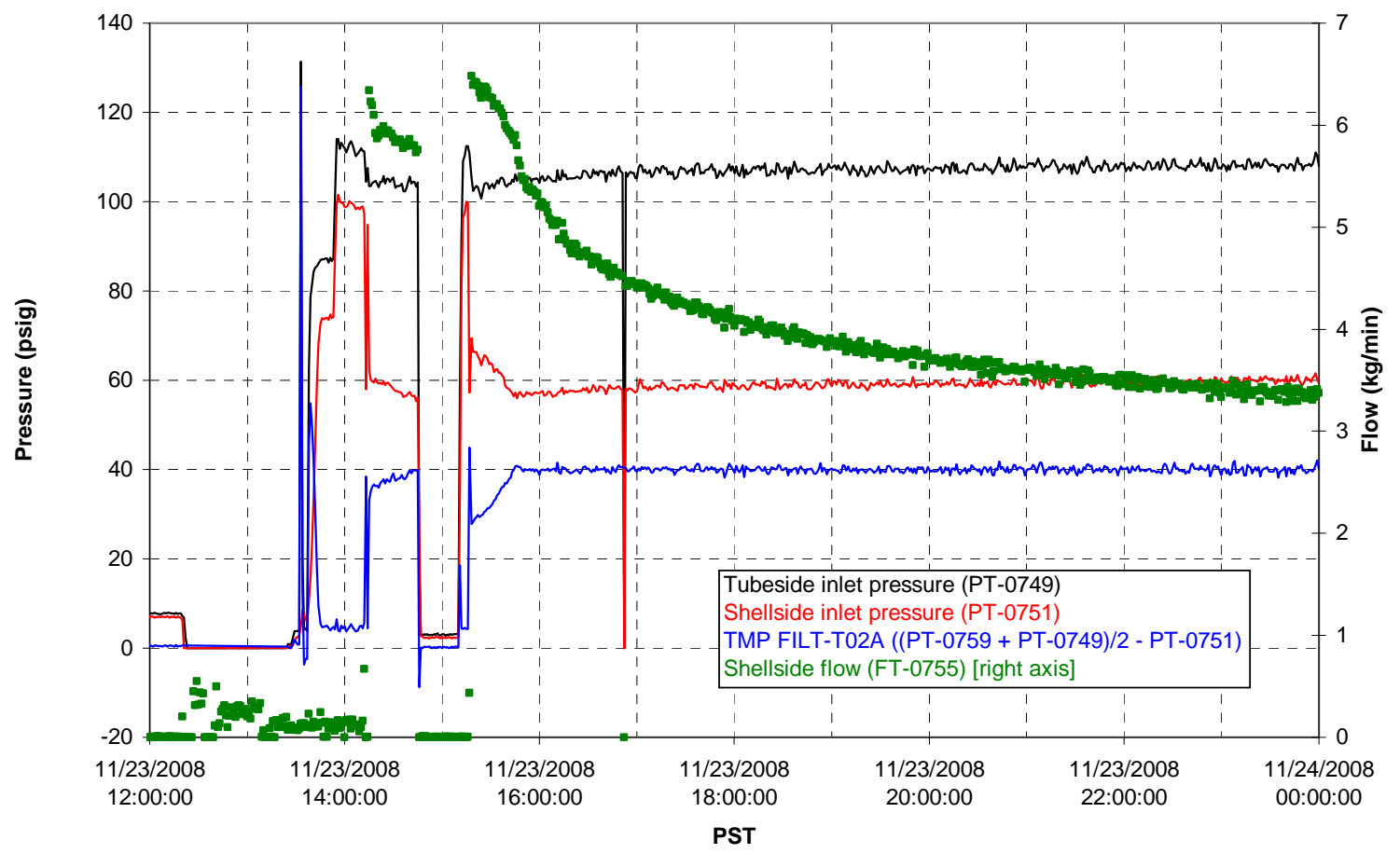


Filter UFP-FILT-T03A

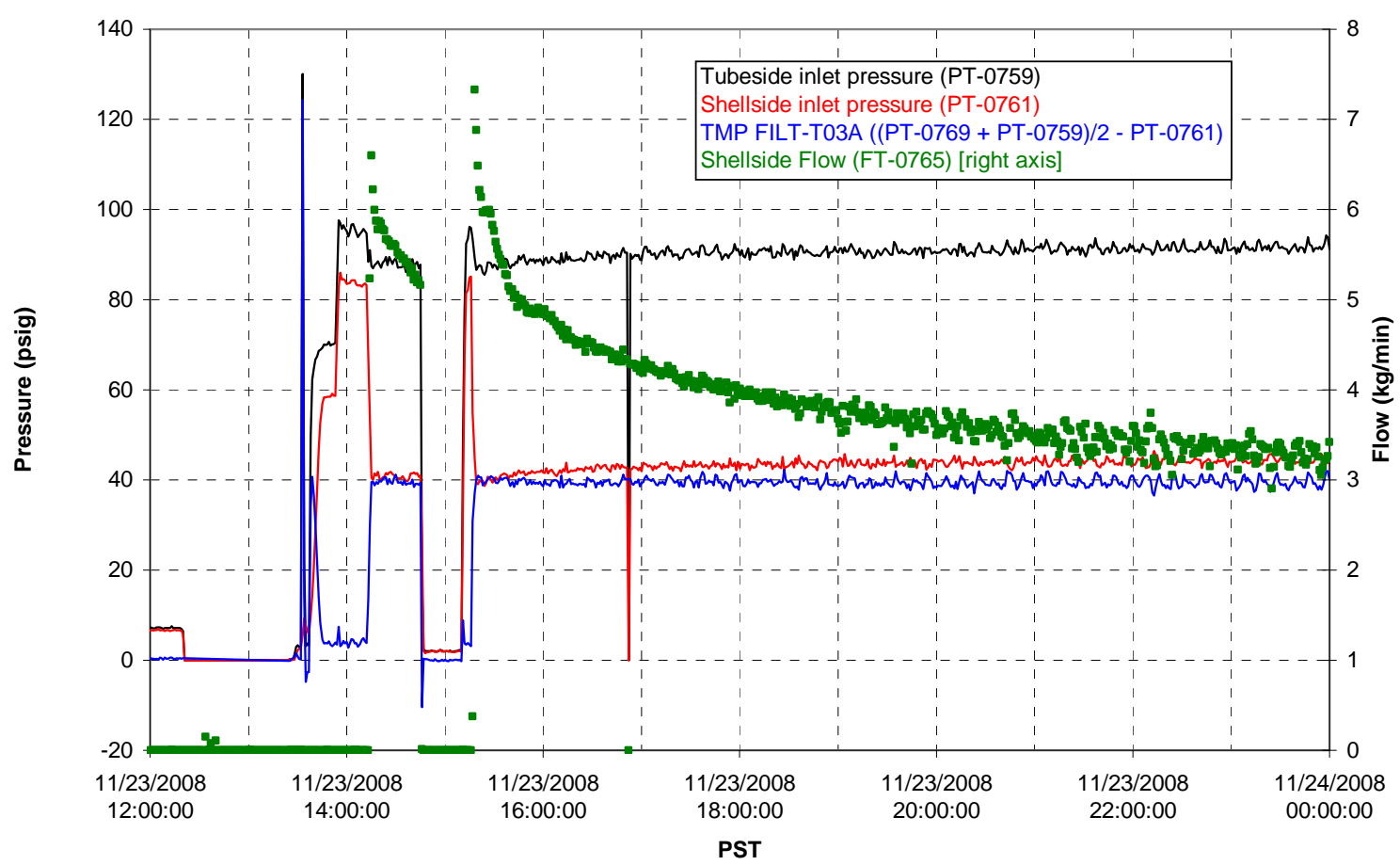

Filter UFP-FILT-T04A

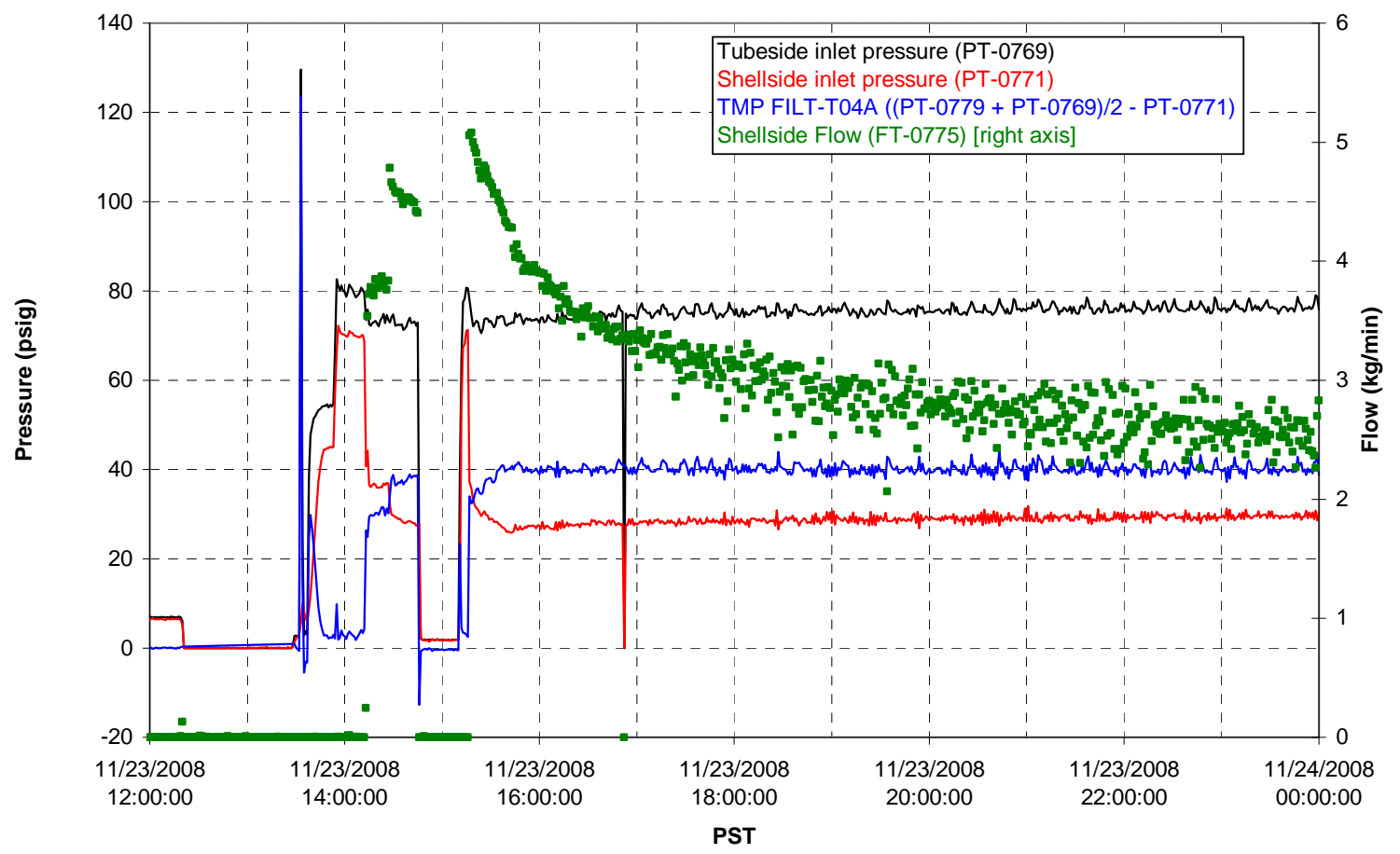


Filter UFP-FILT-T05A

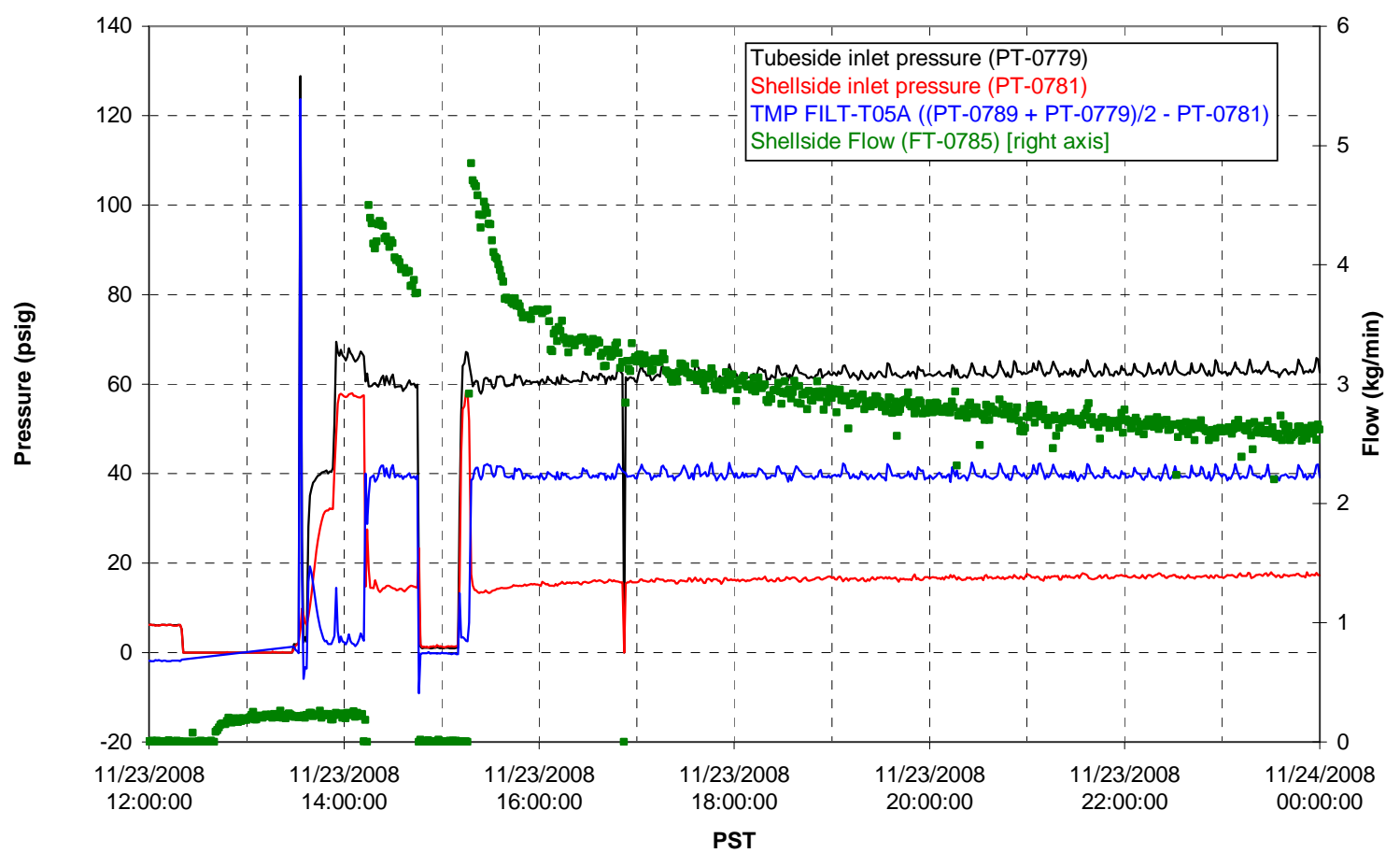

Chemical Flow

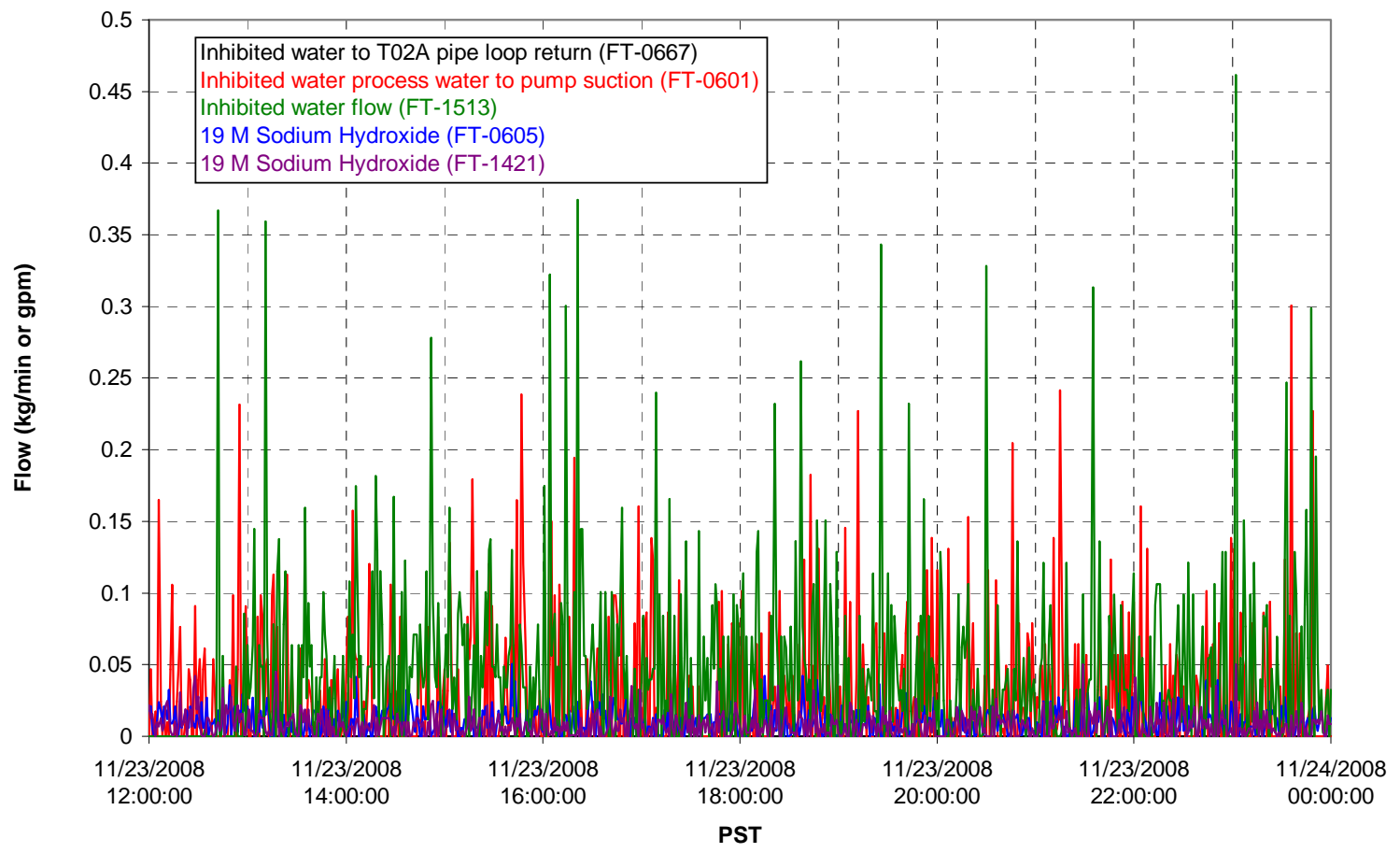


Chemical Flow

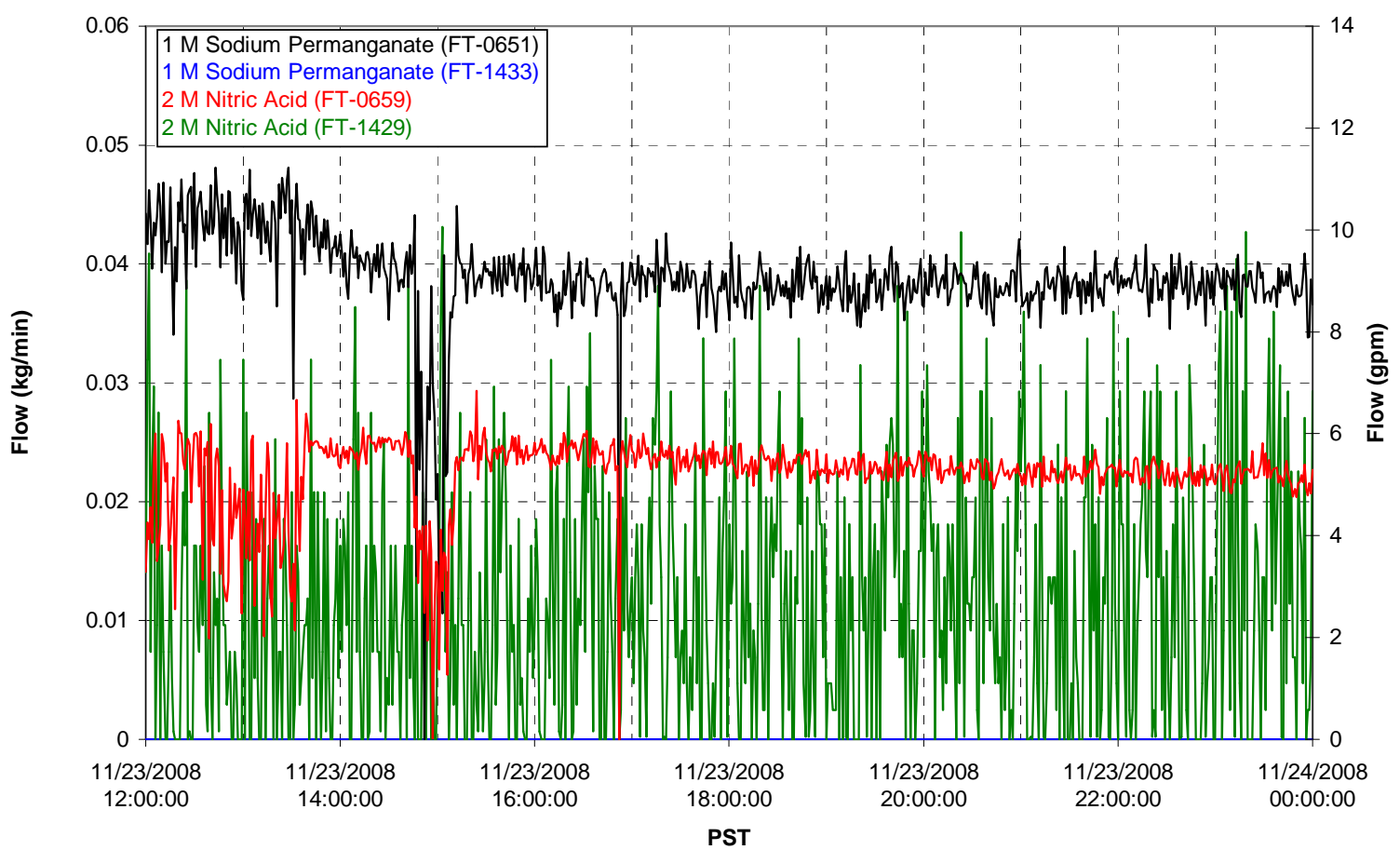

Air Flows

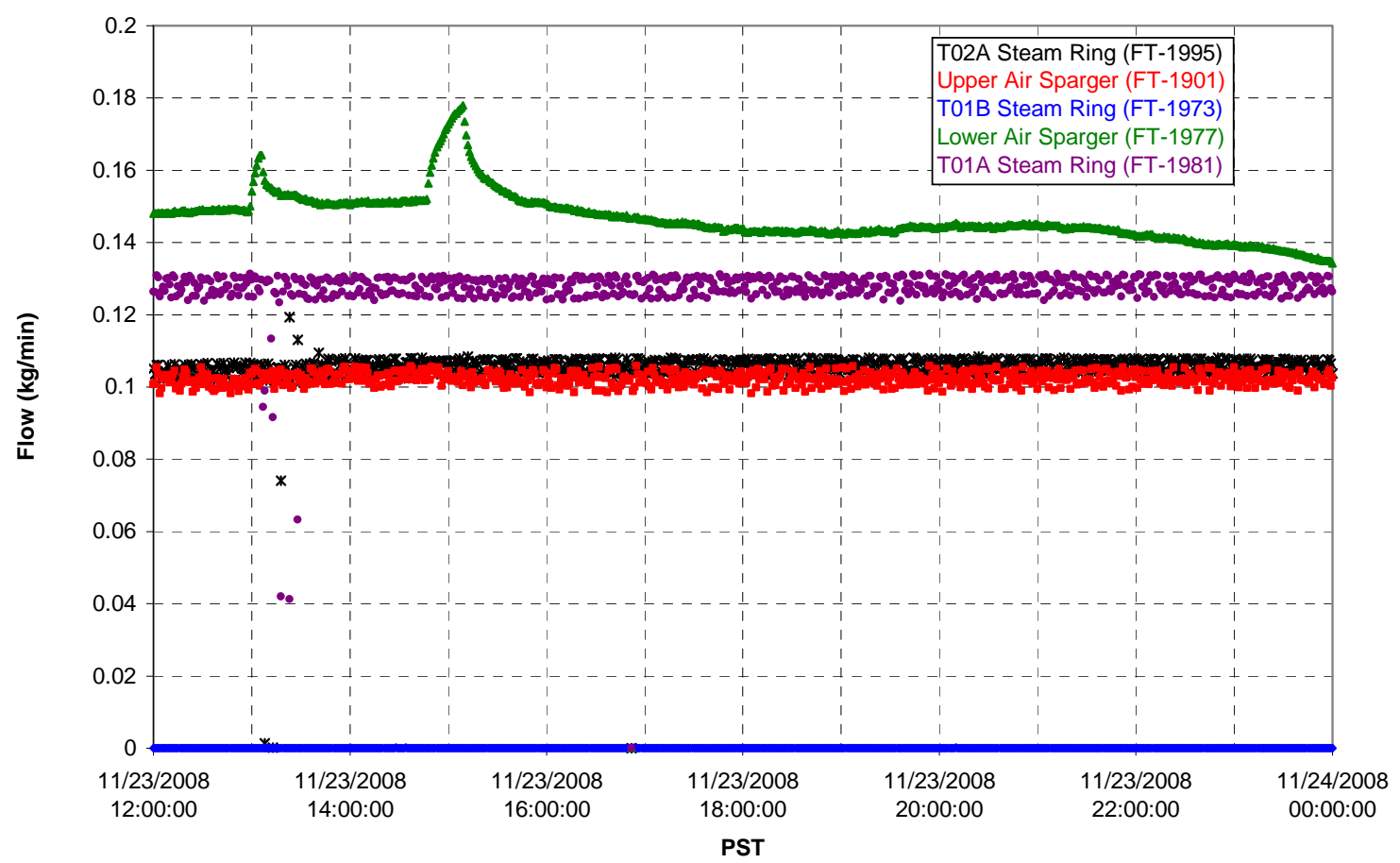


T02A Steam

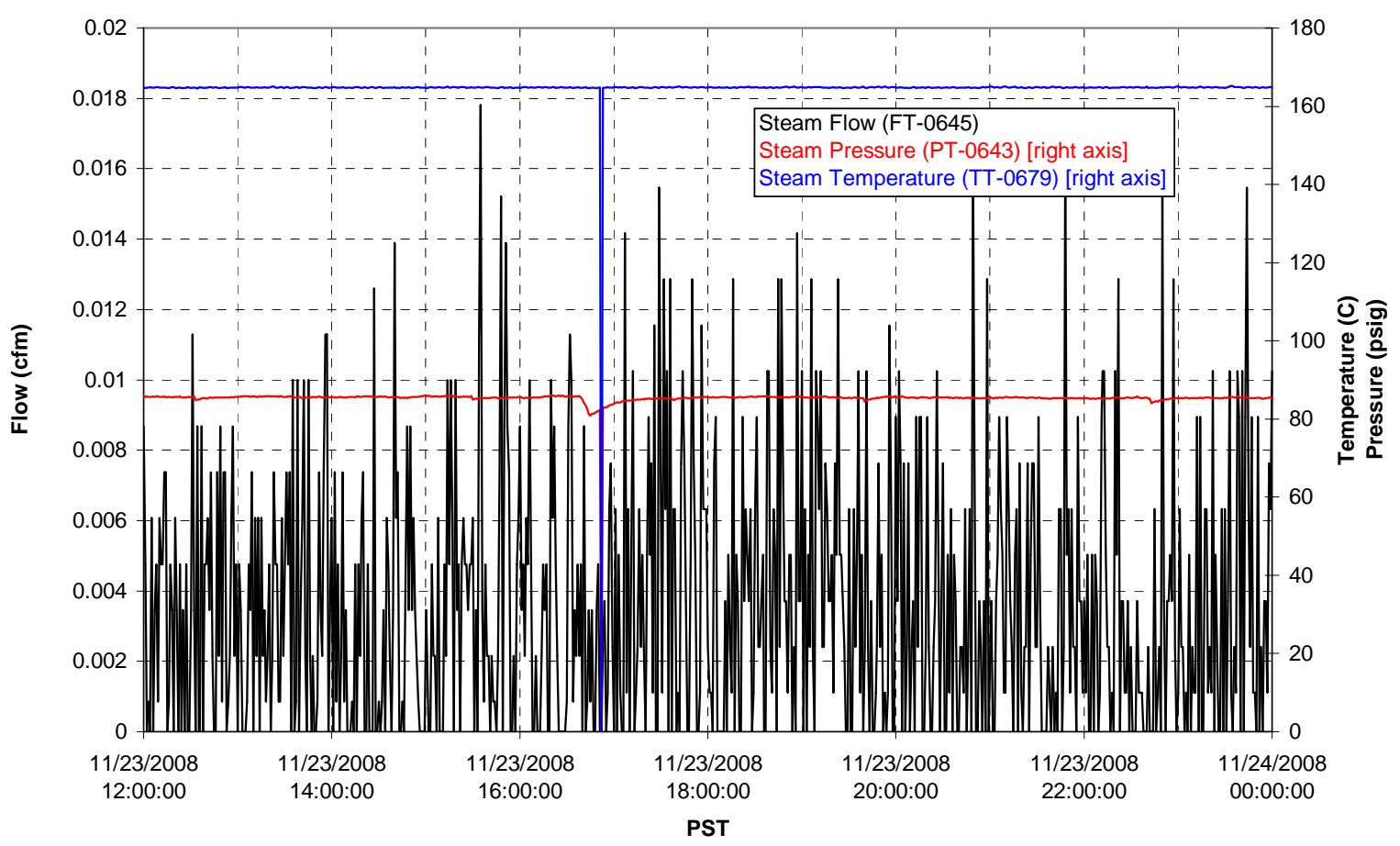

T01A Steam

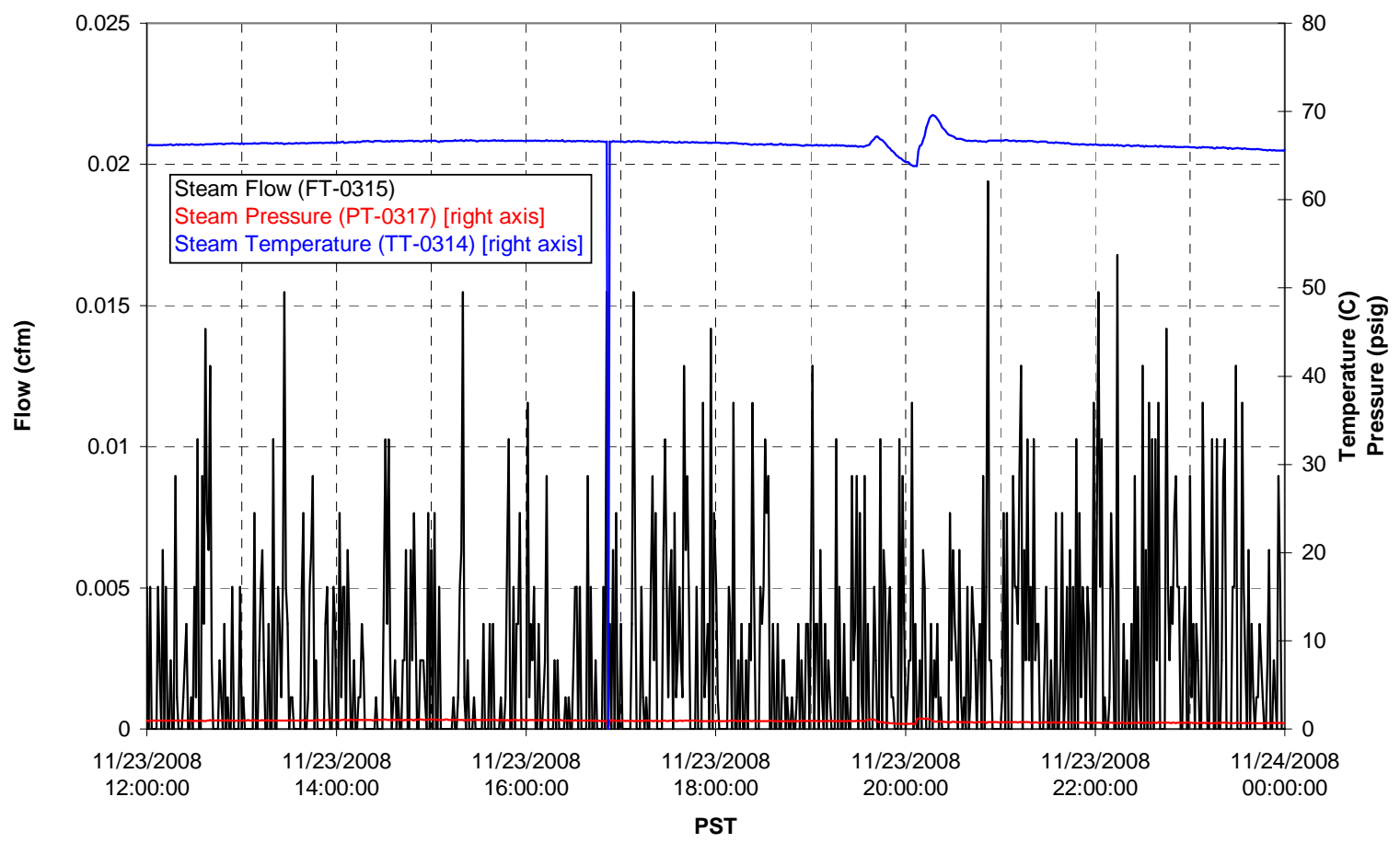


T01B Steam

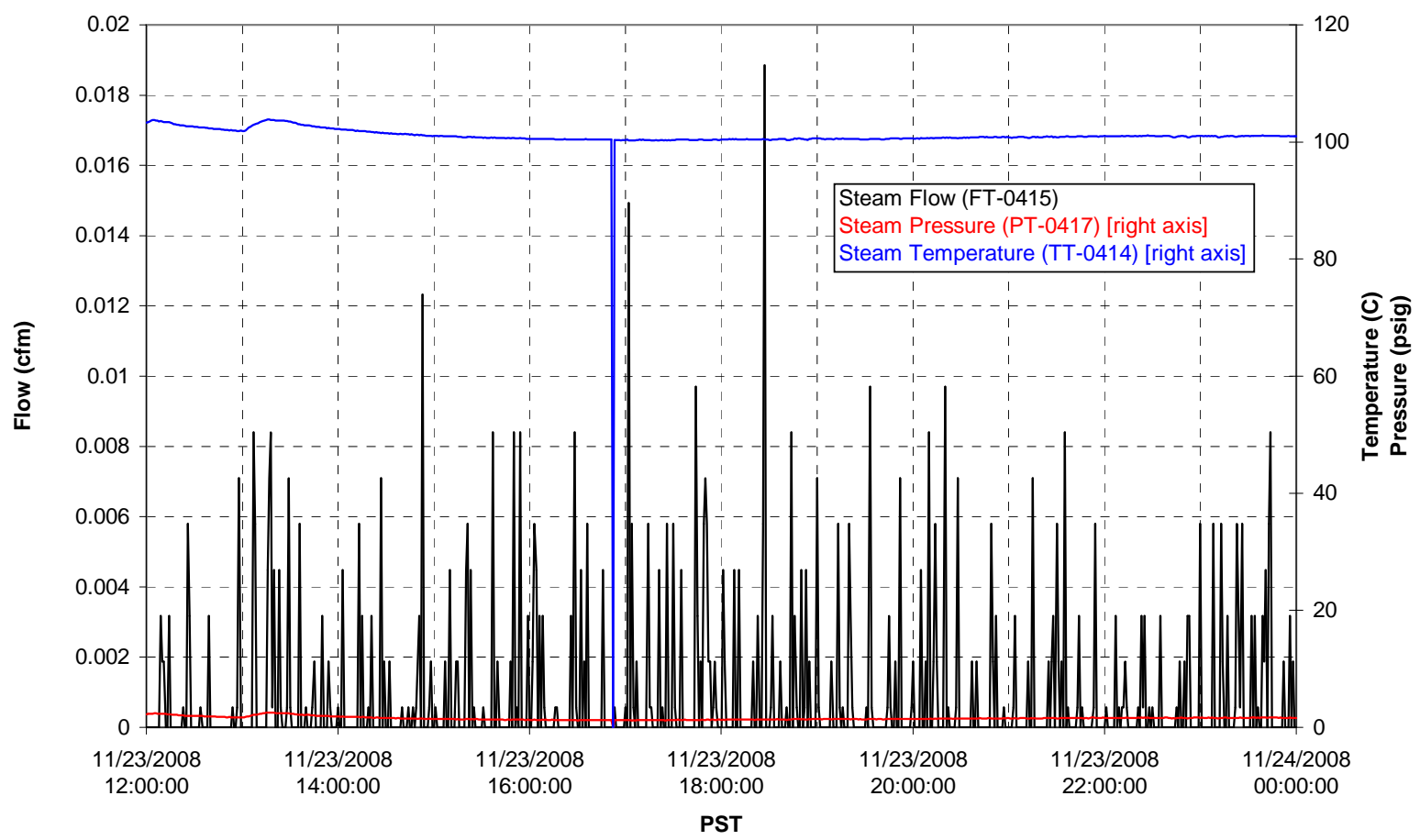


Functional Test Data Plots

11/24/2008 00:00 - 11/24/2008 12:00 
T01A level

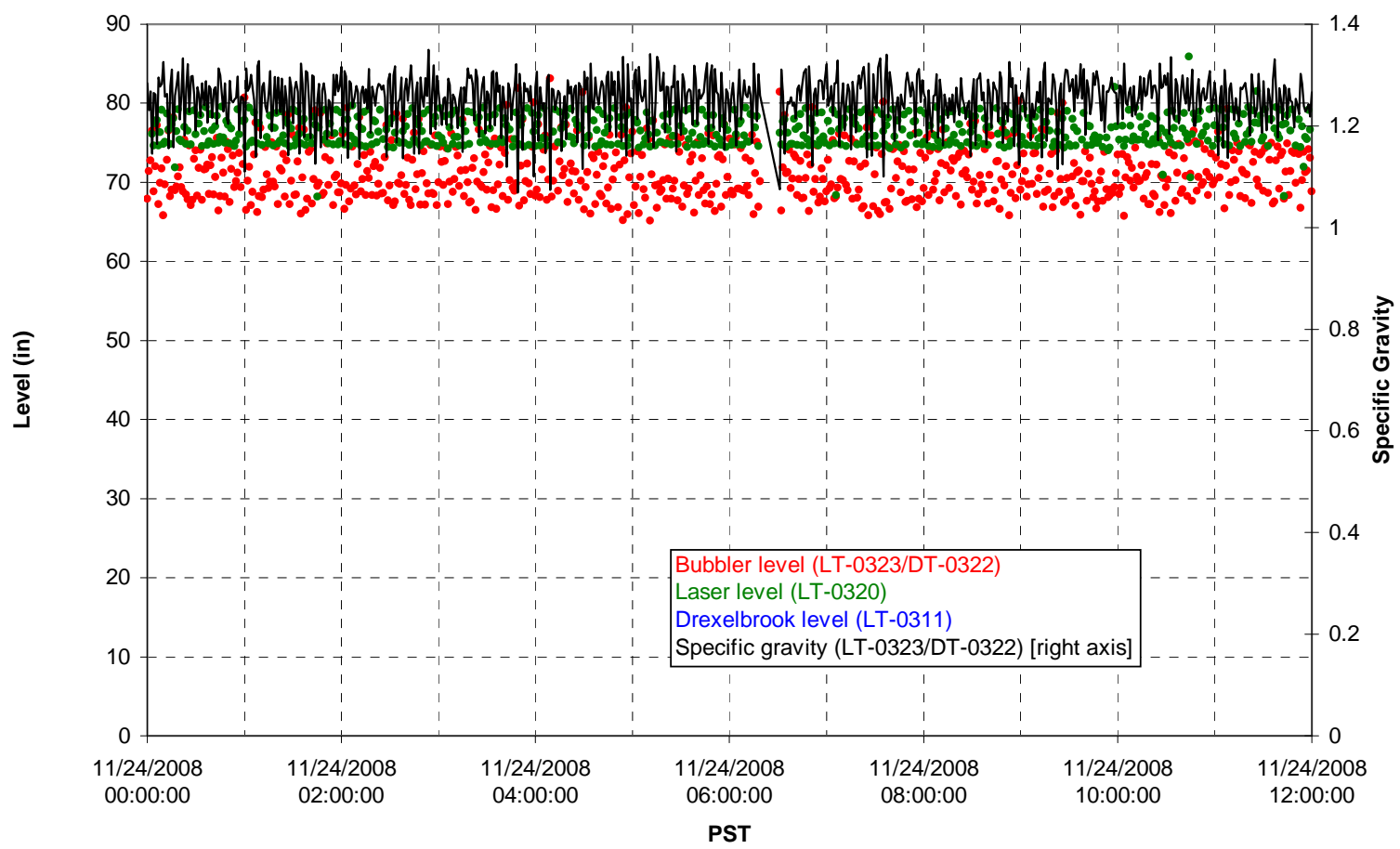

T01A temperatures

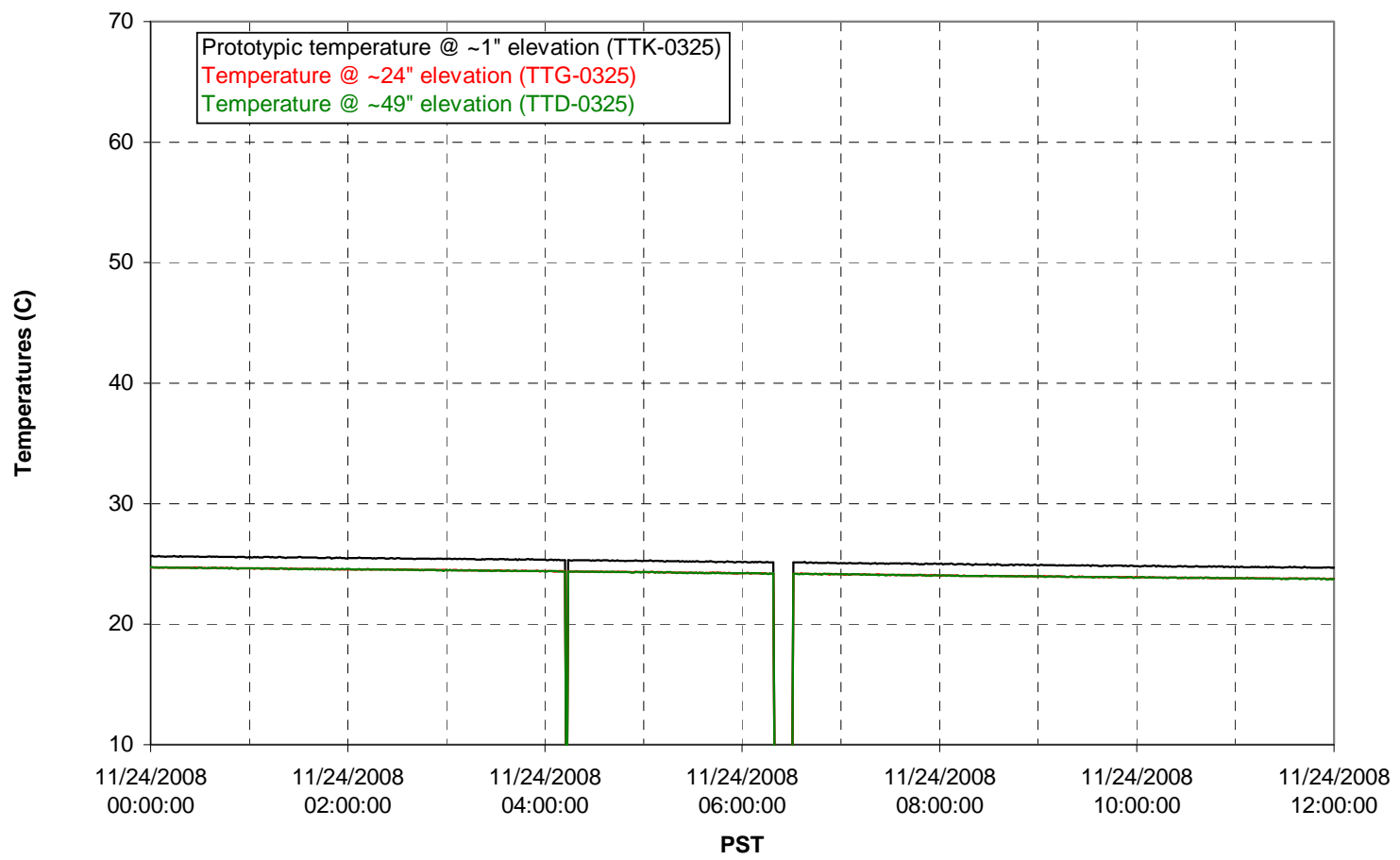


T01B level

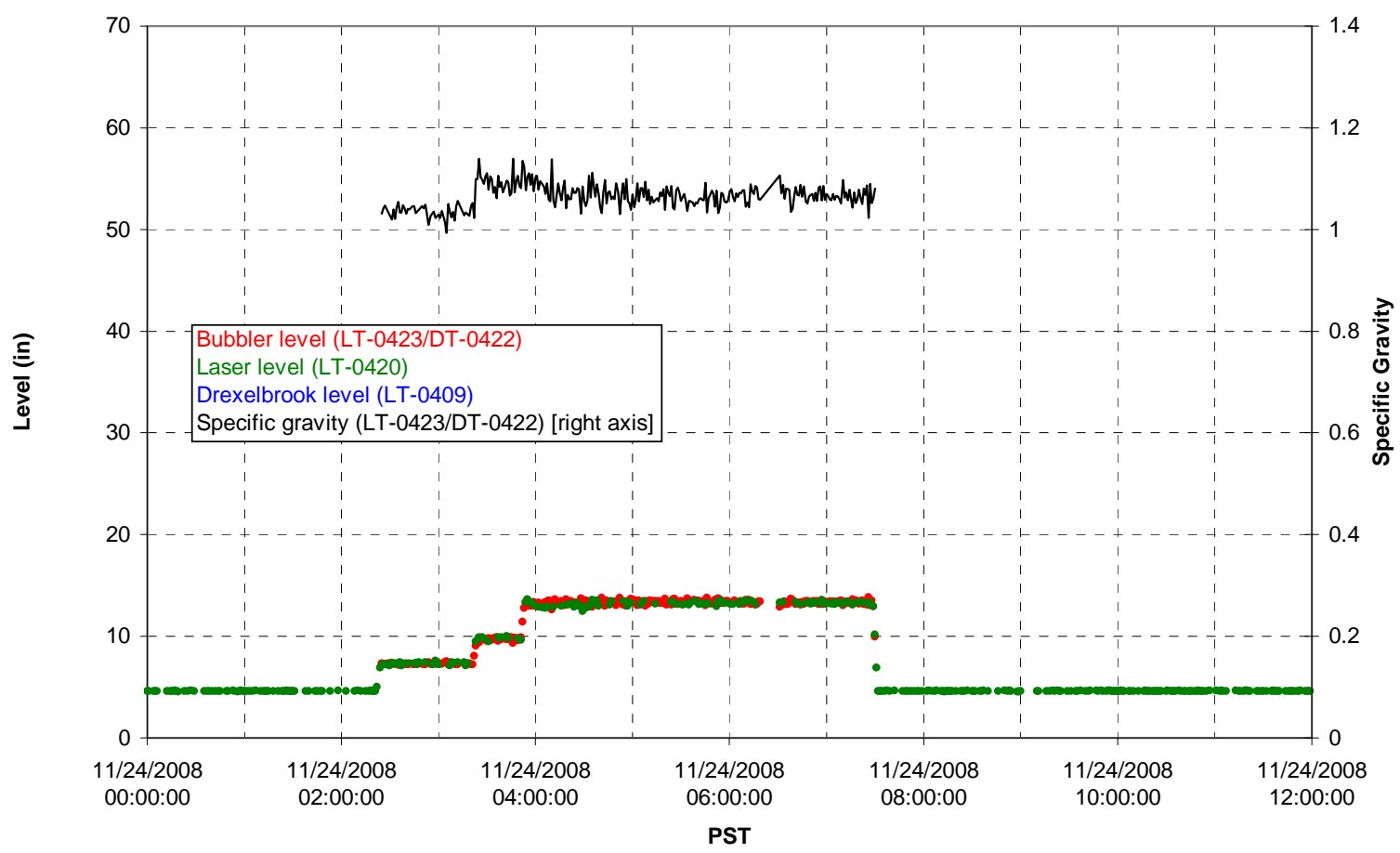

T01B temperatures

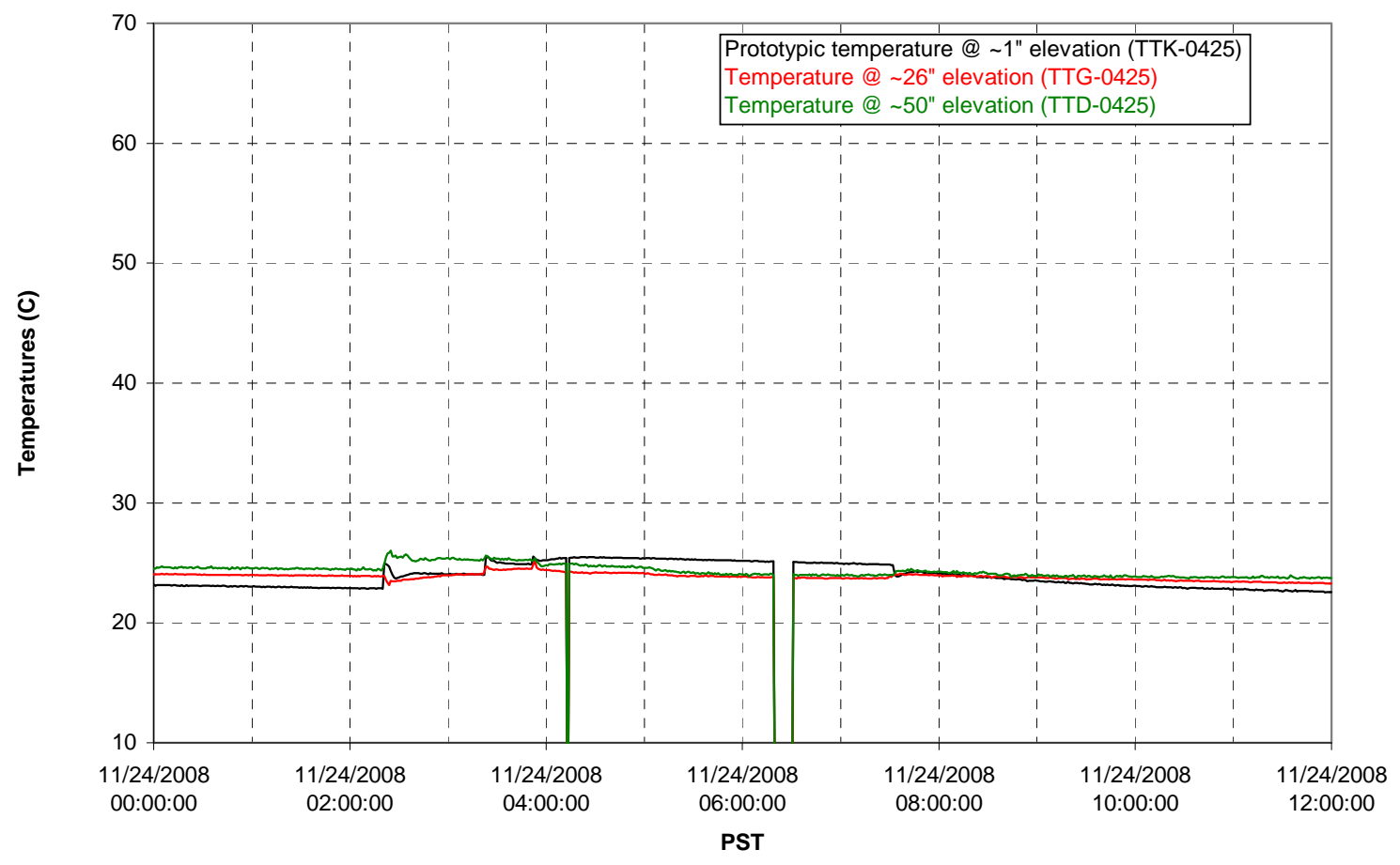


T02A level

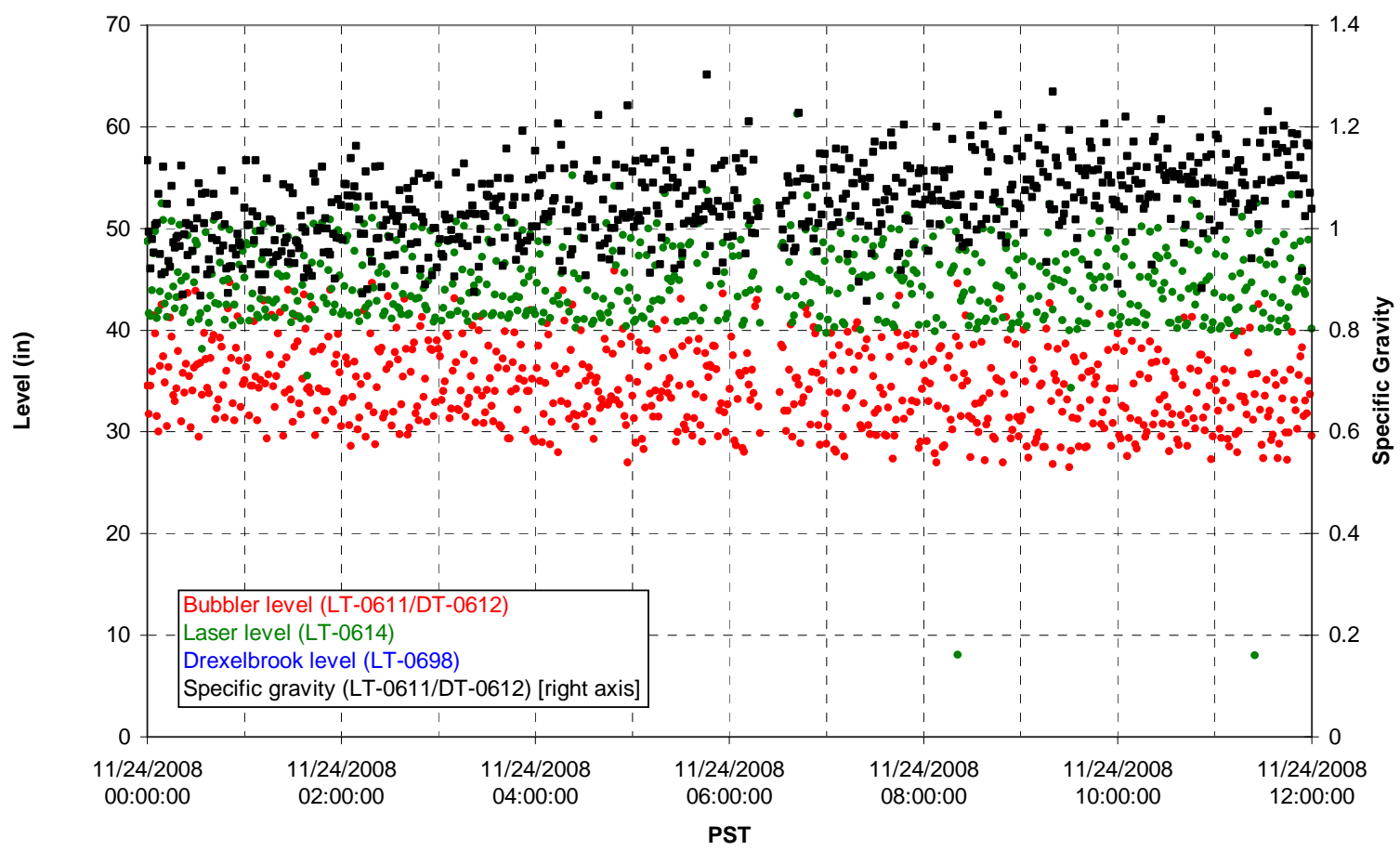

T02A temperatures

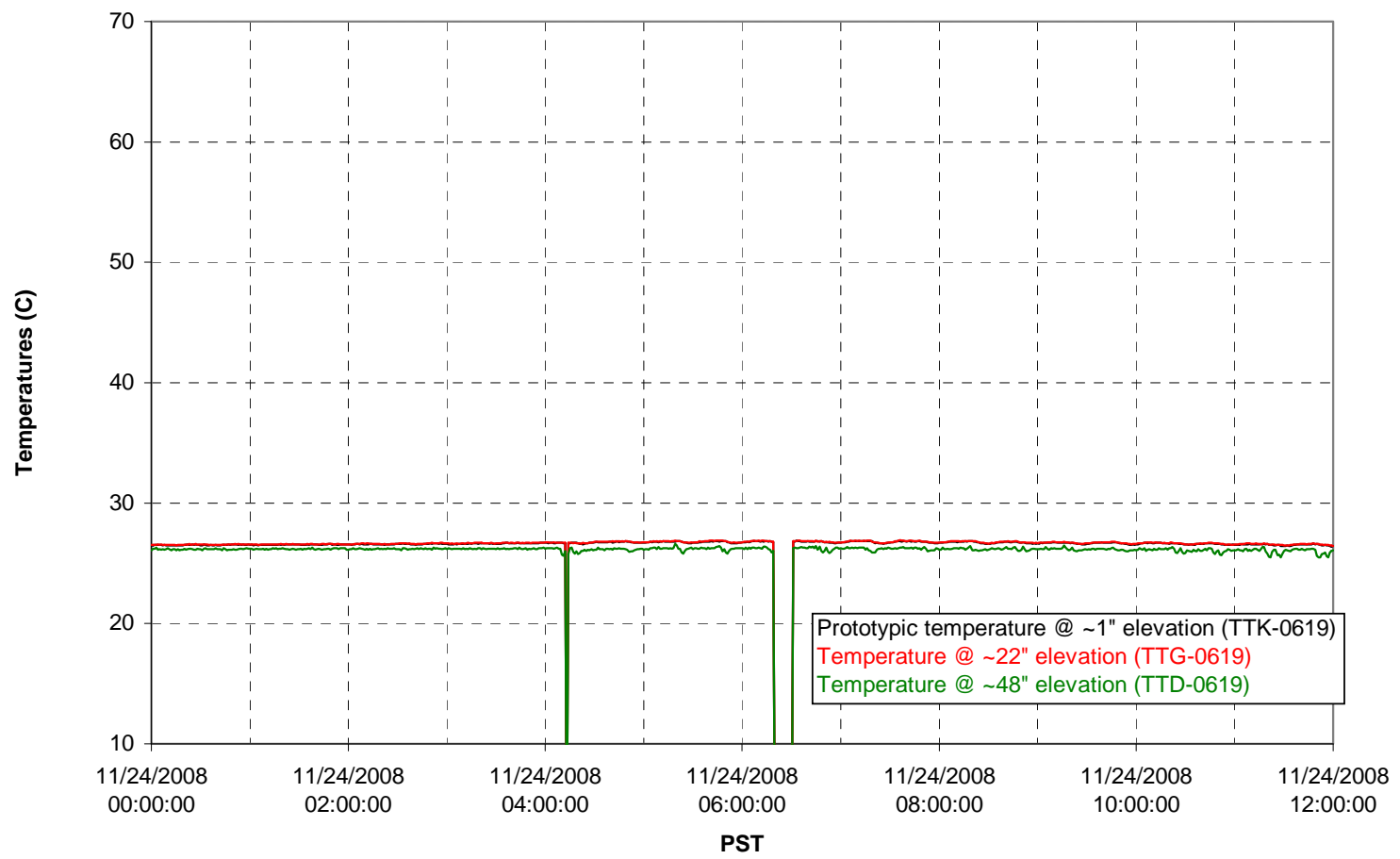


T02A and filter loop temperatures

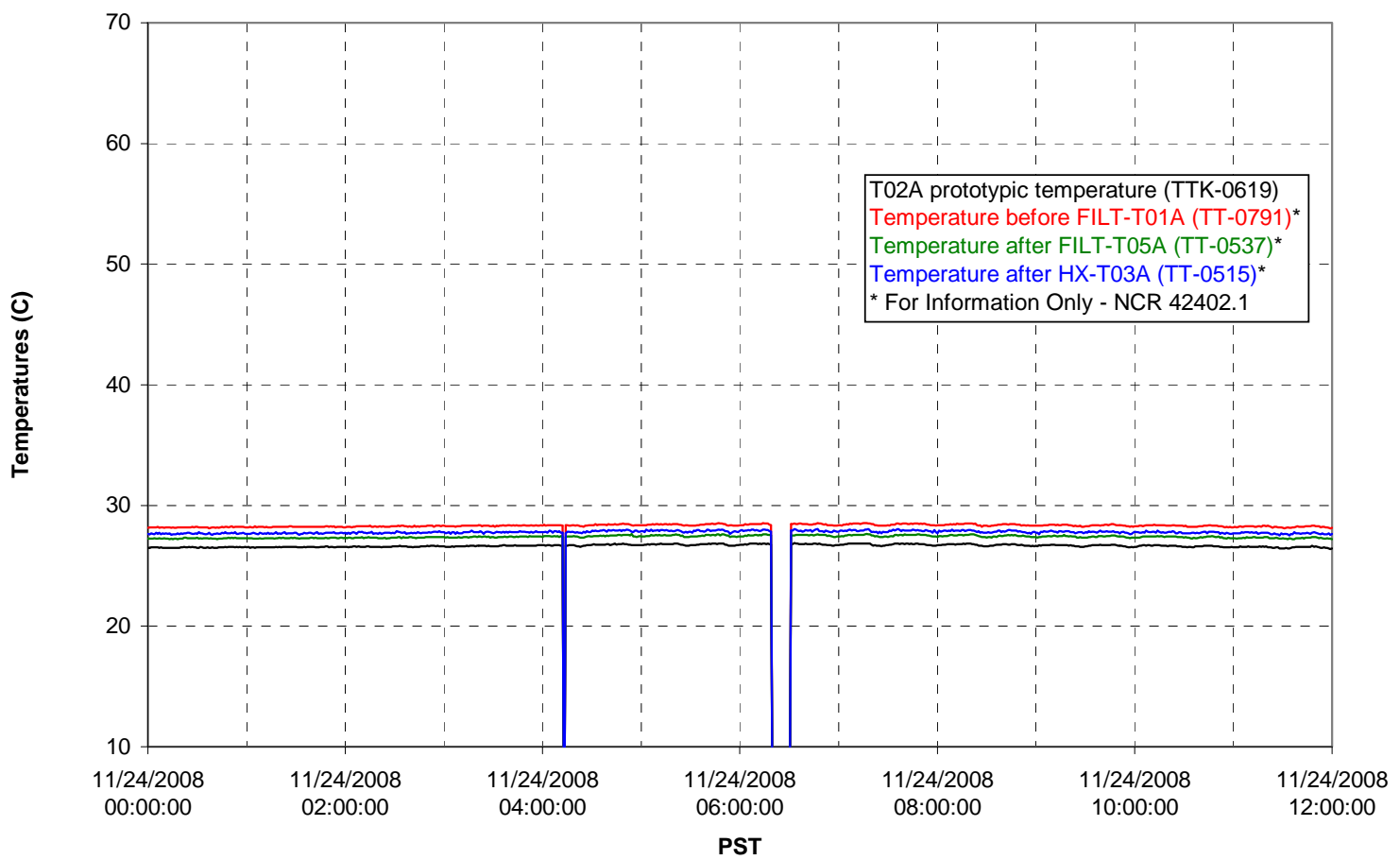

Pump Pressures and Flow

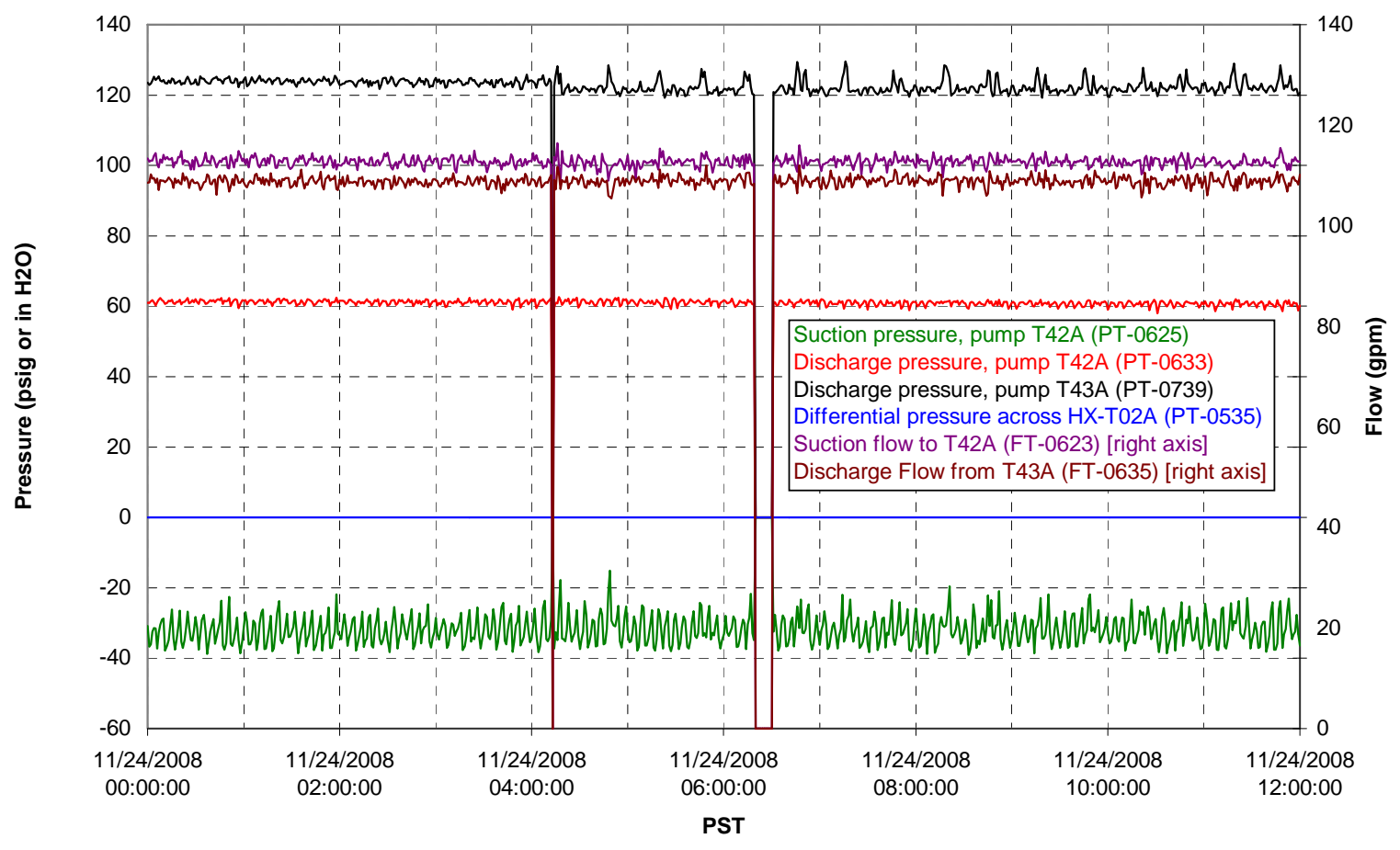


Axial pressure drop

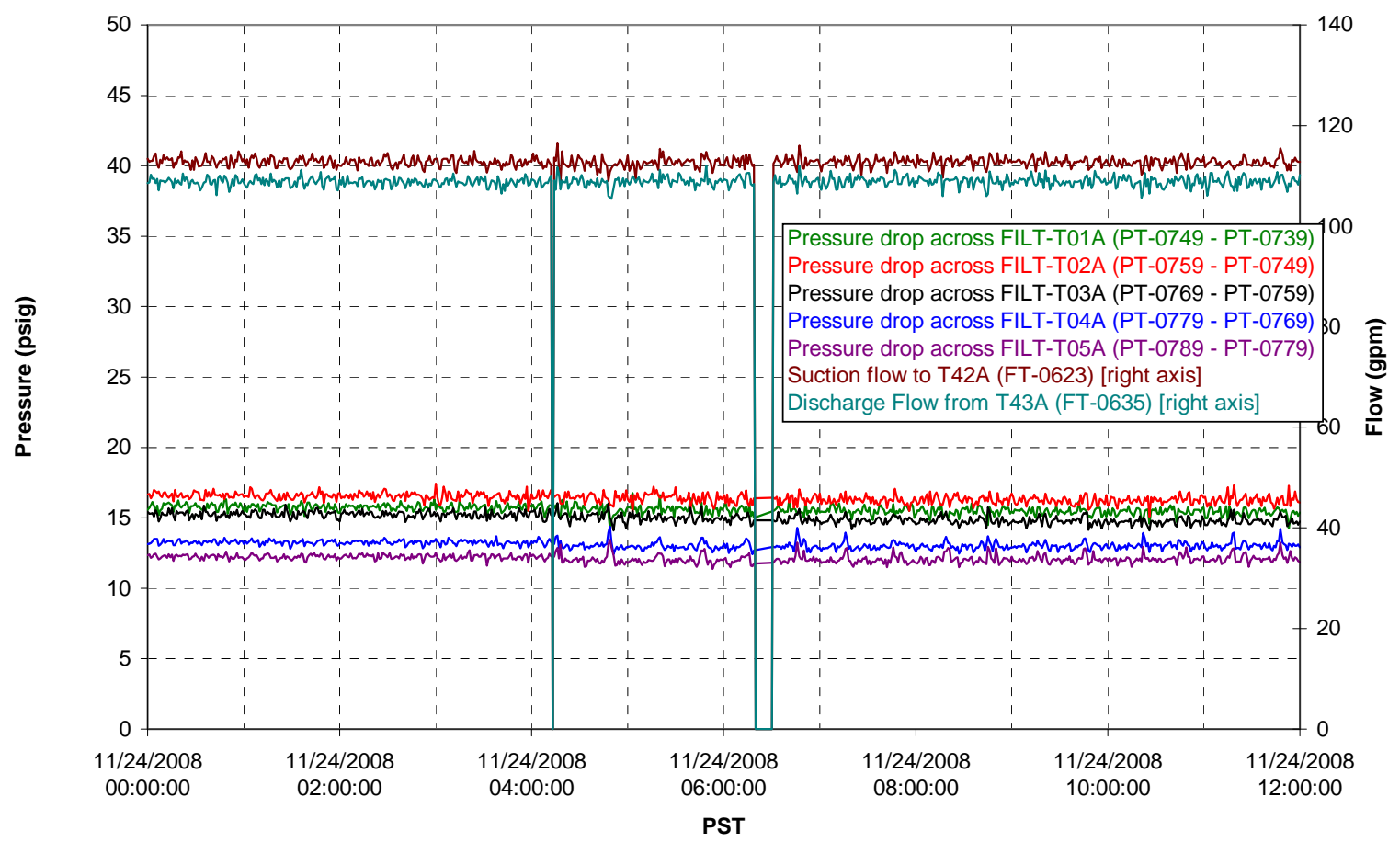

Permeate flow rates

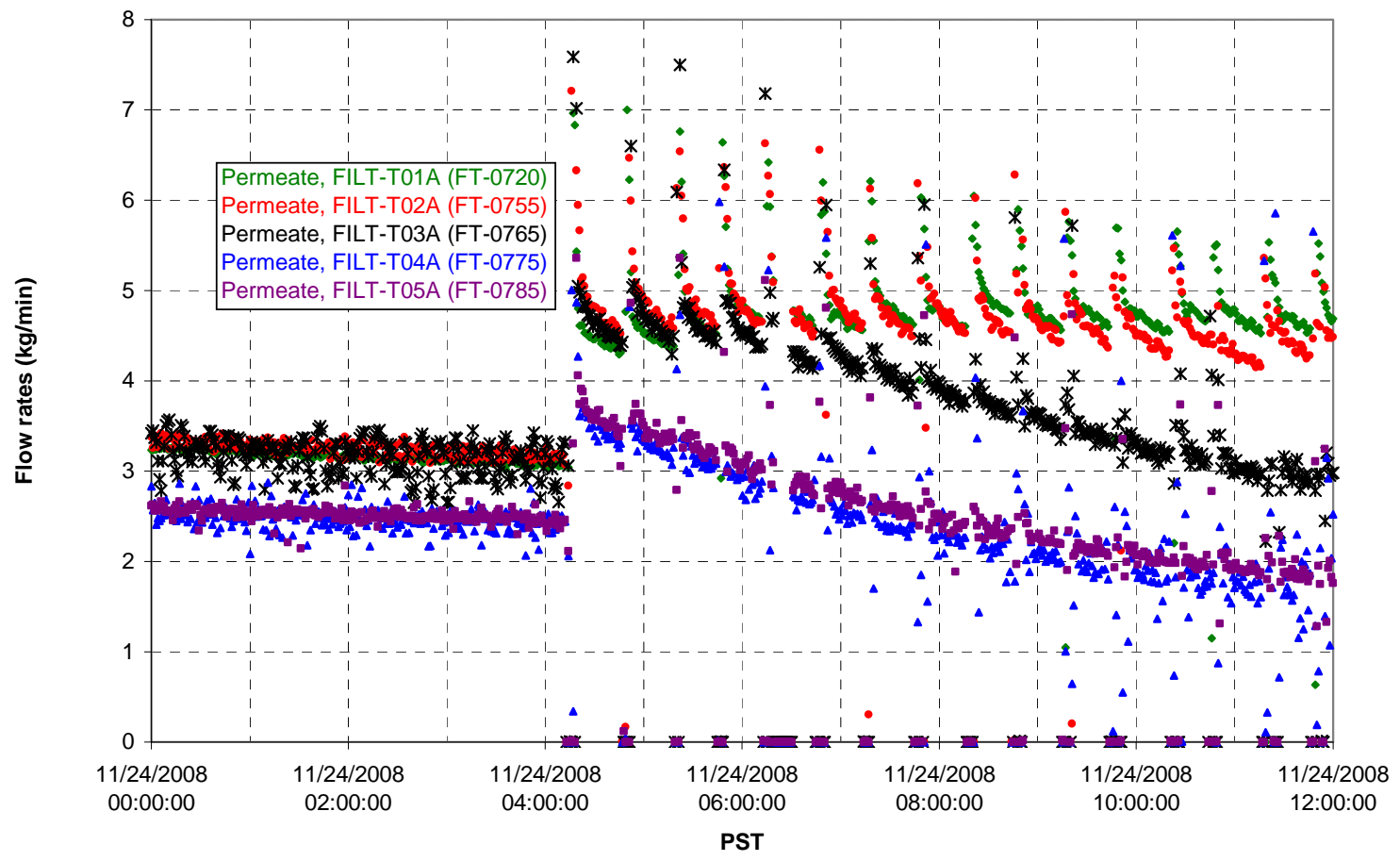


T02A Inner Temperature Tree

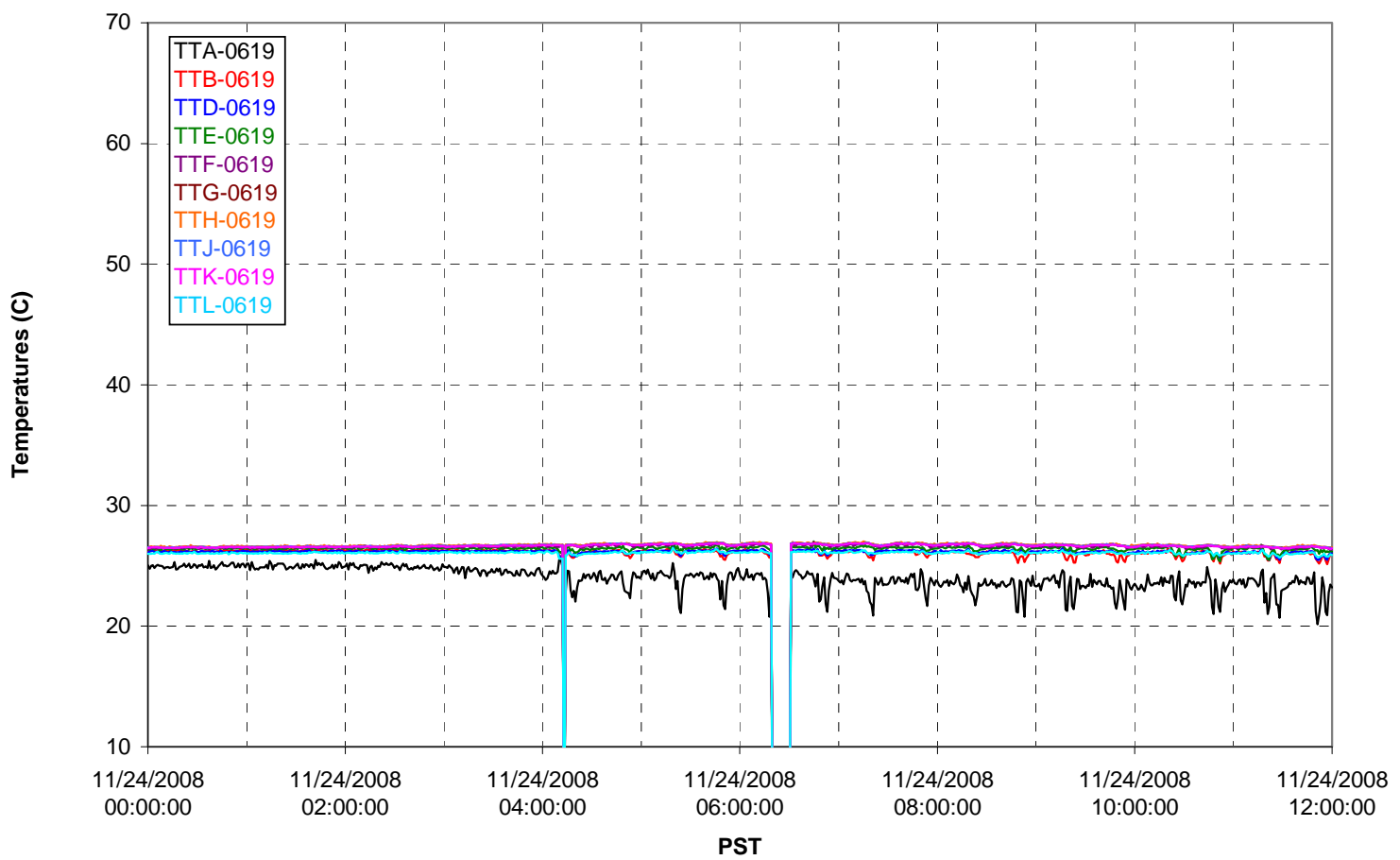

T02A Outer Temperature Tree

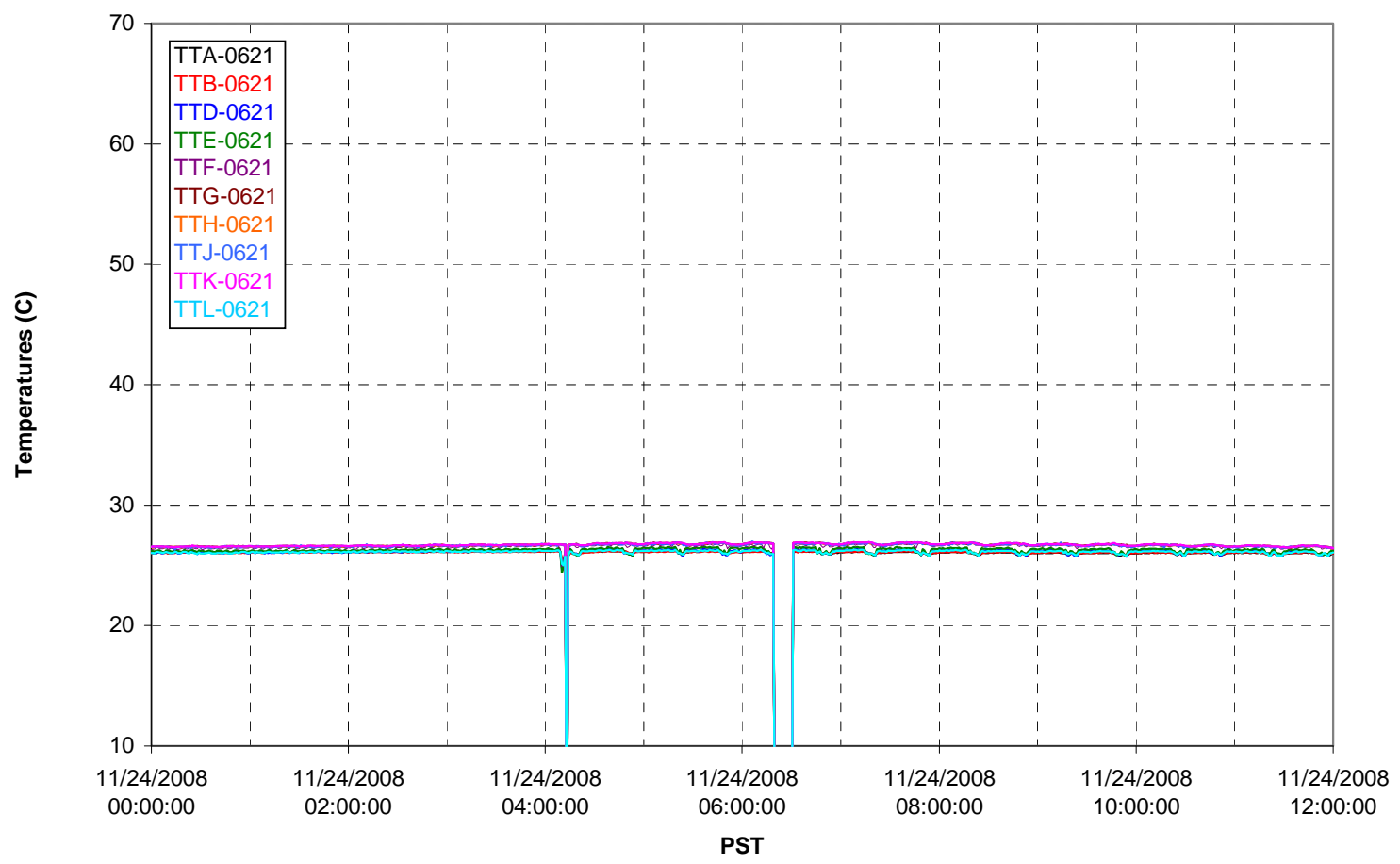


T02A temperatures

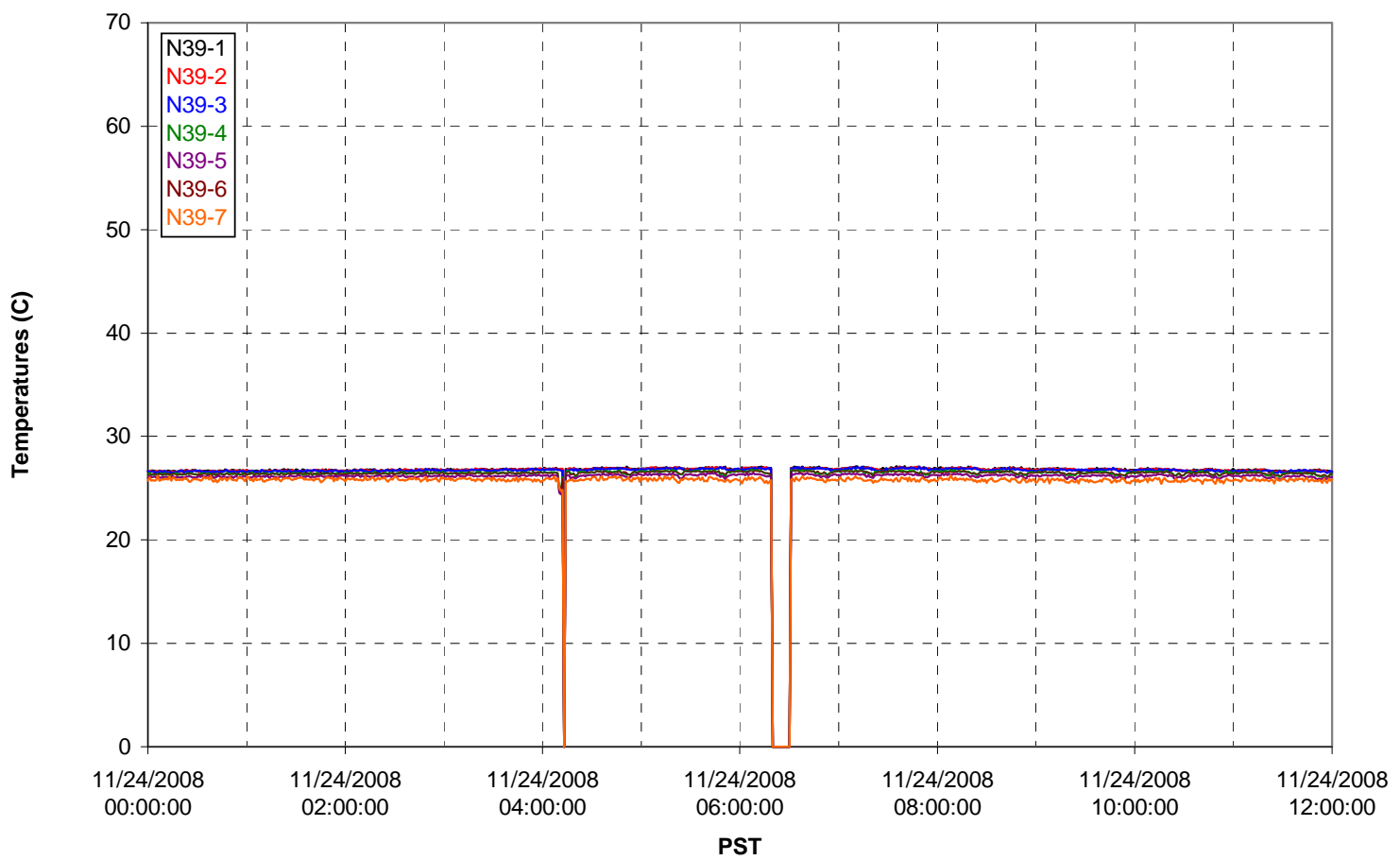

T02A temperatures

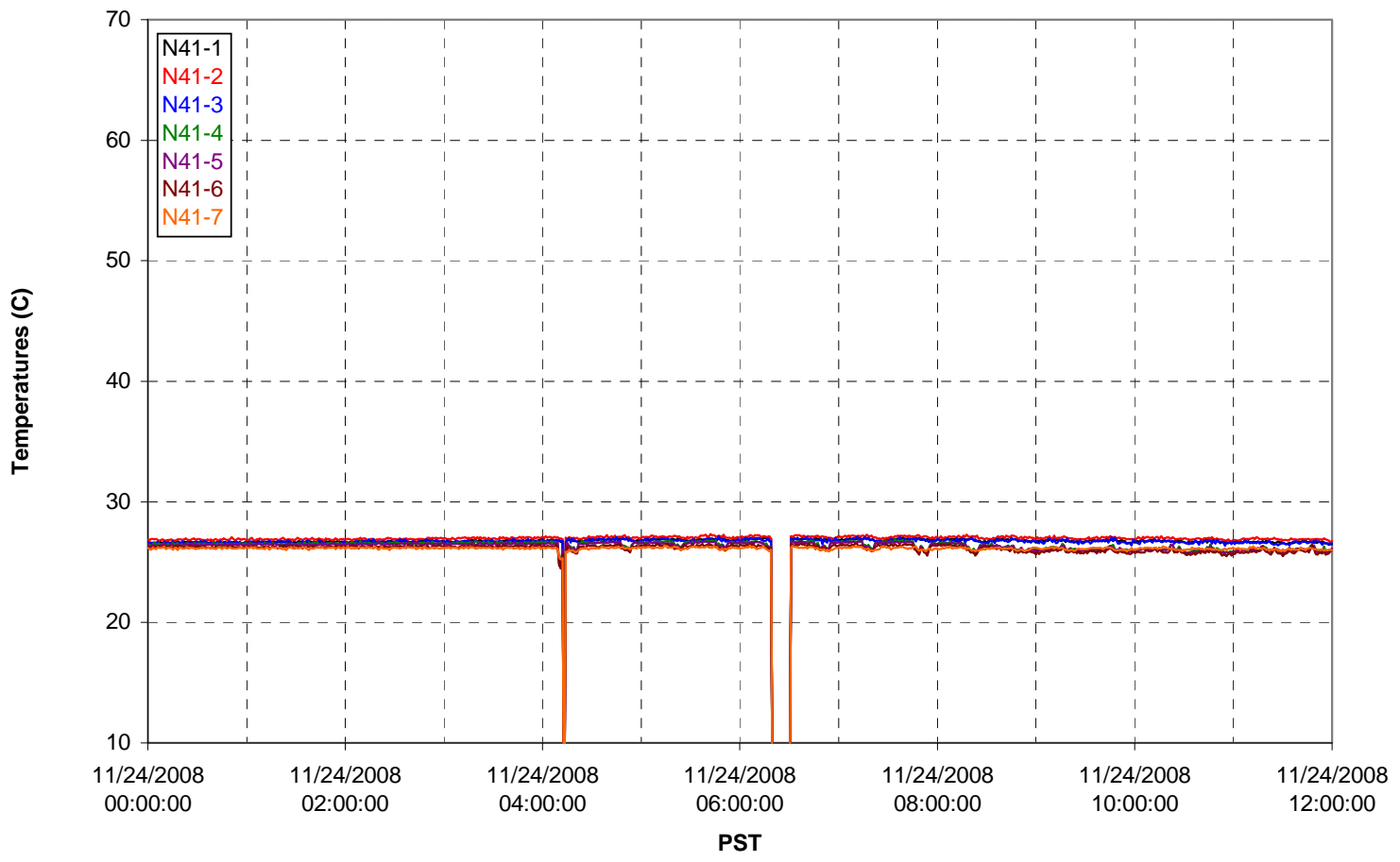


T02A temperatures

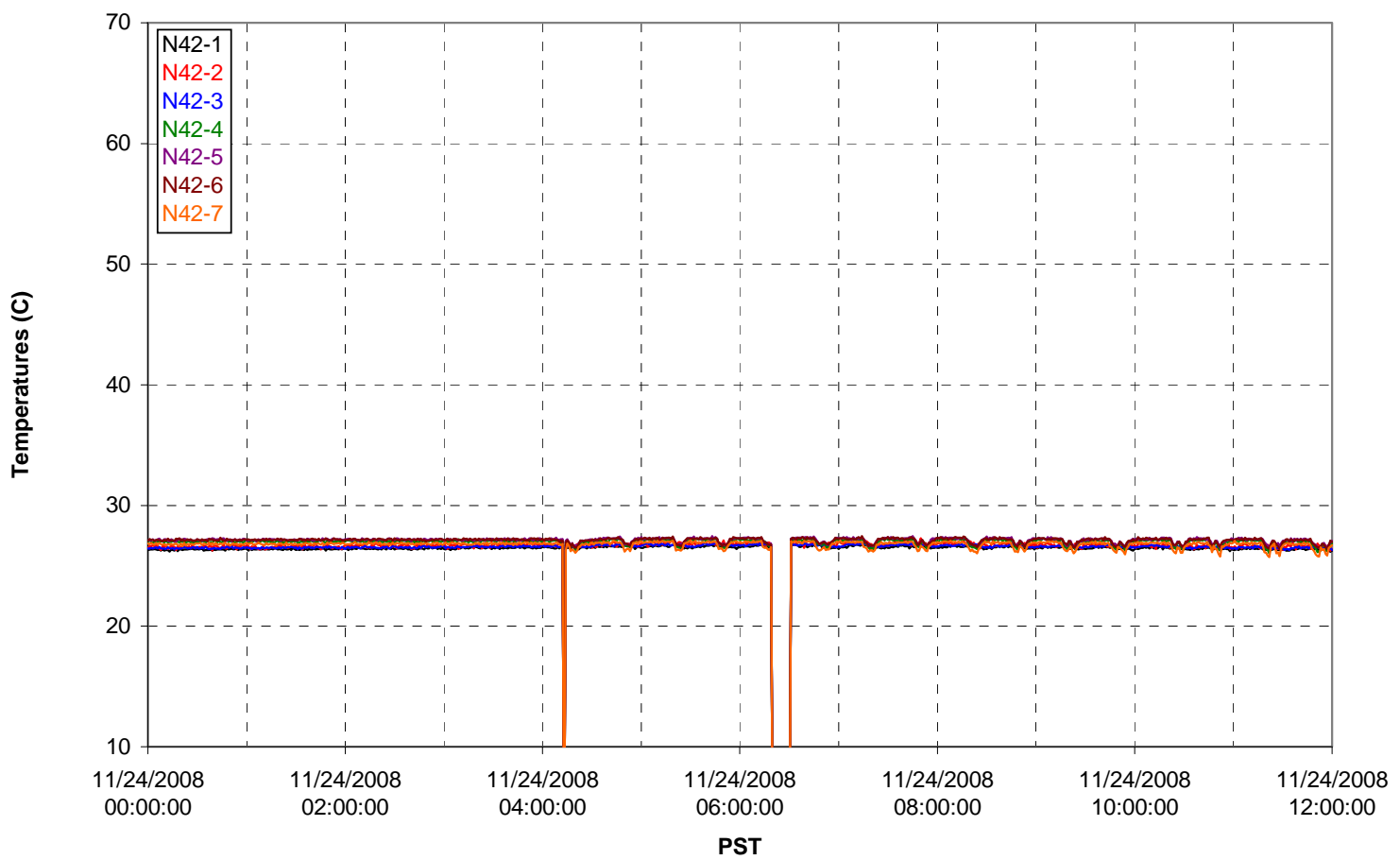

T02A temperatures

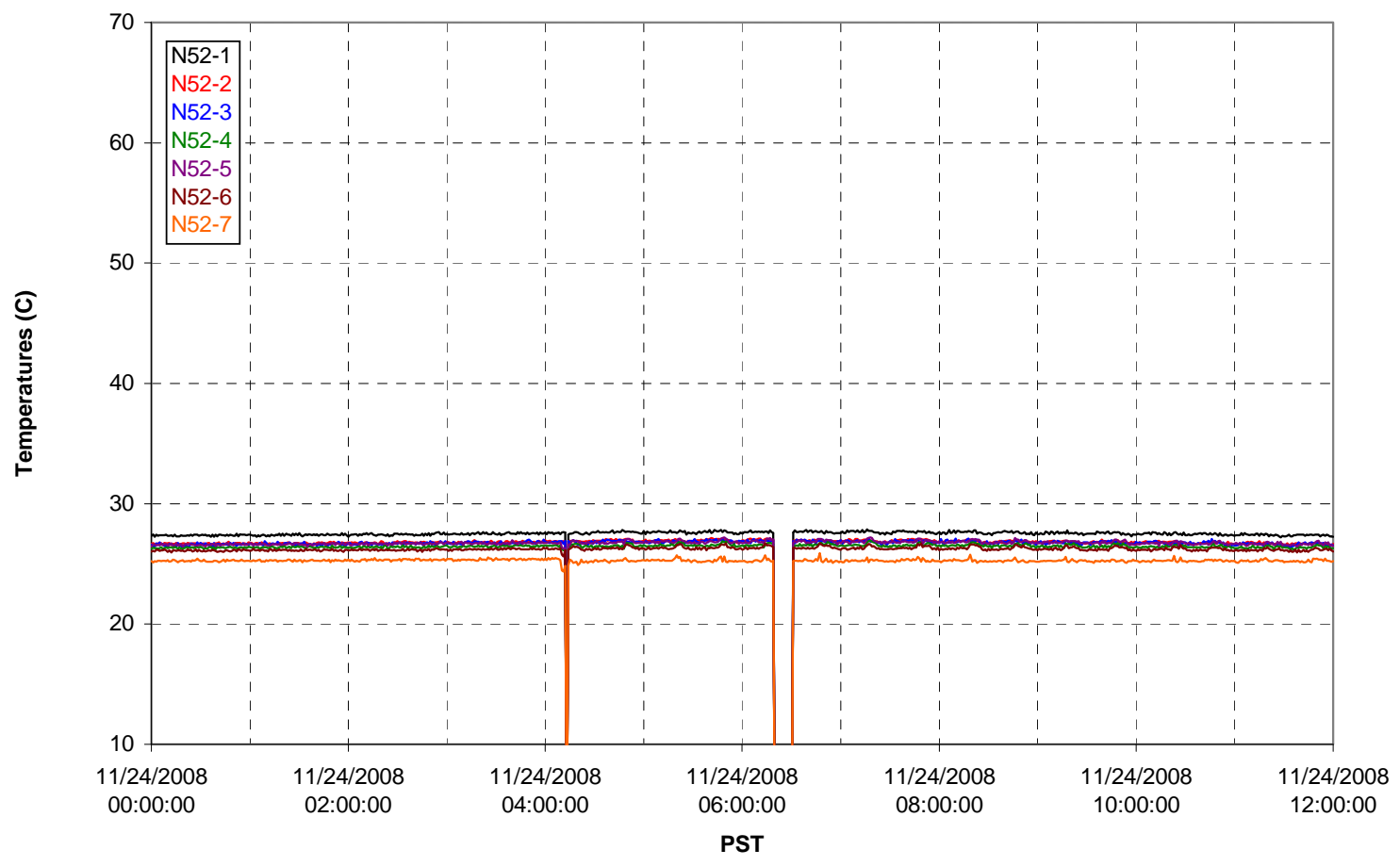


T02A Heating and Cooling

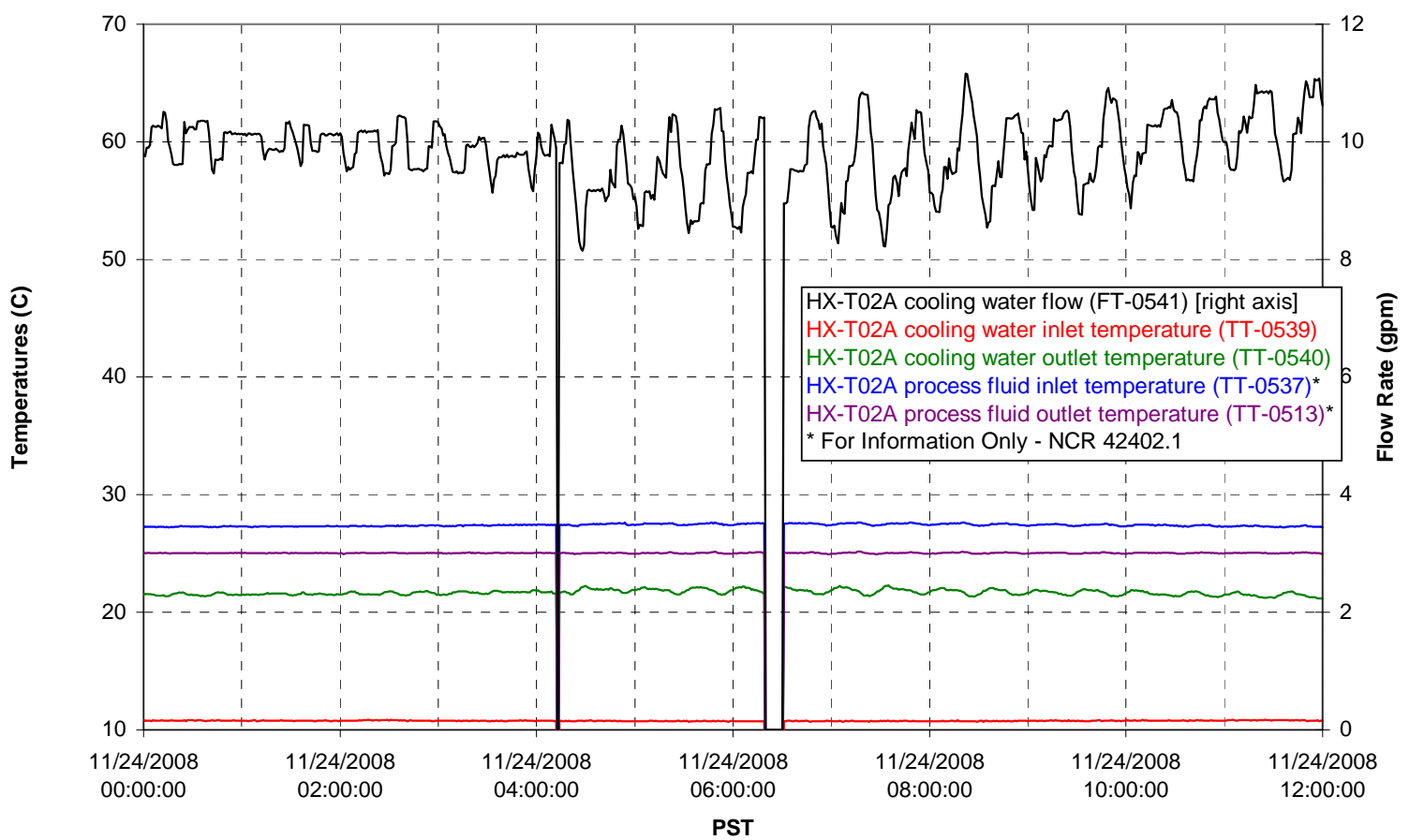

Pump Operation

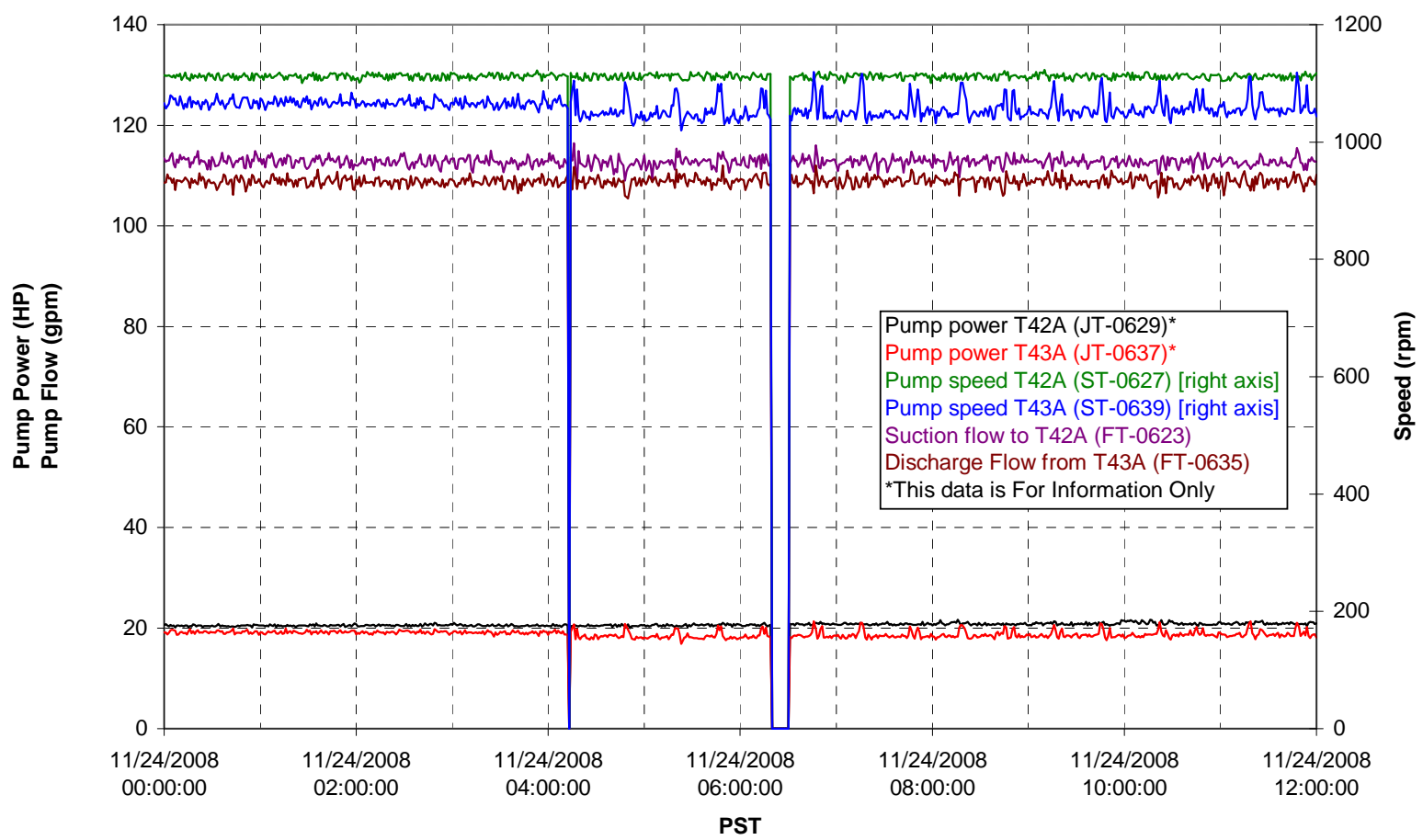


Pulsepot UFP-PP-T01A

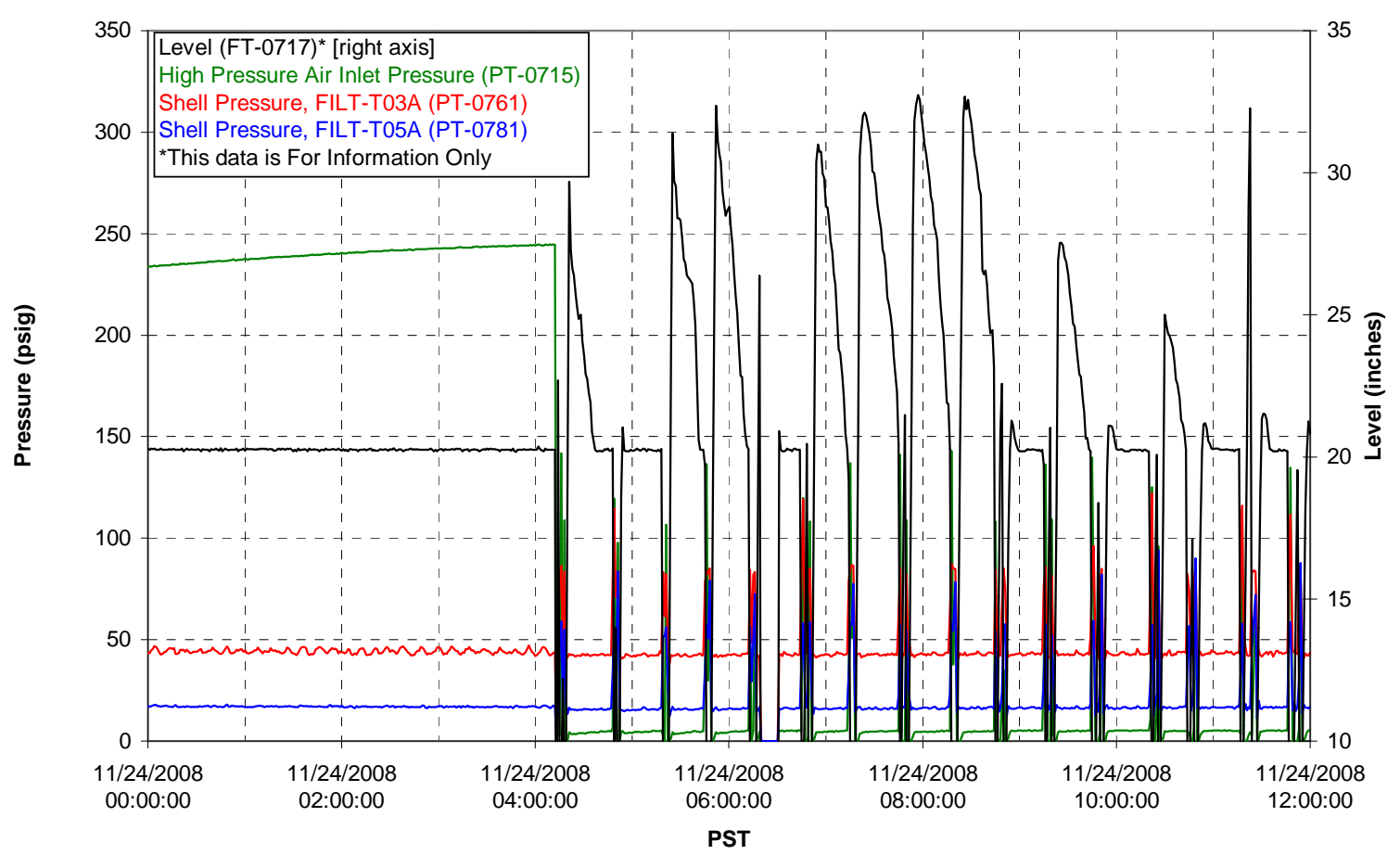

Pulsepot UFP-PP-T02A

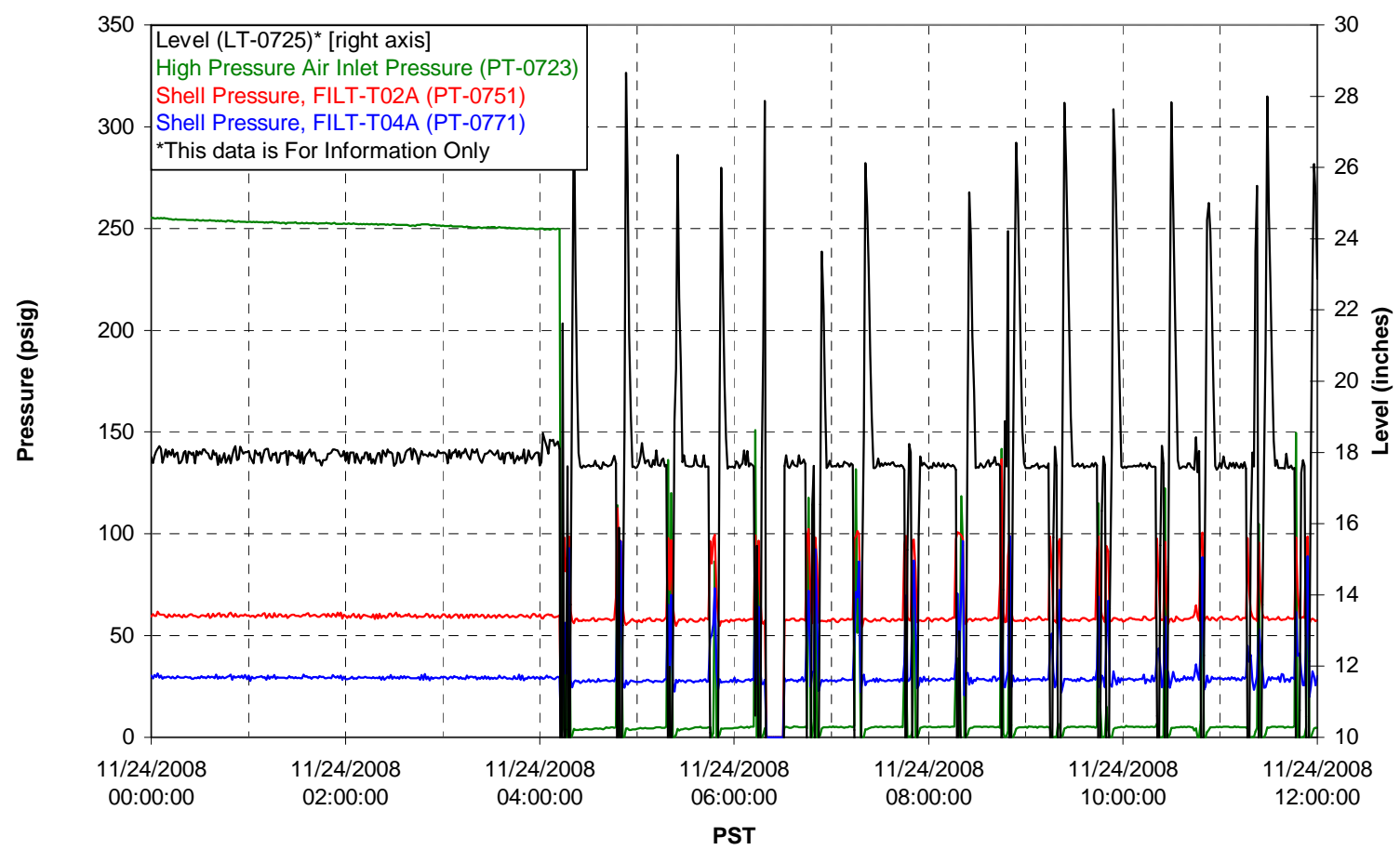


Pulsepot UFP-PP-T03A

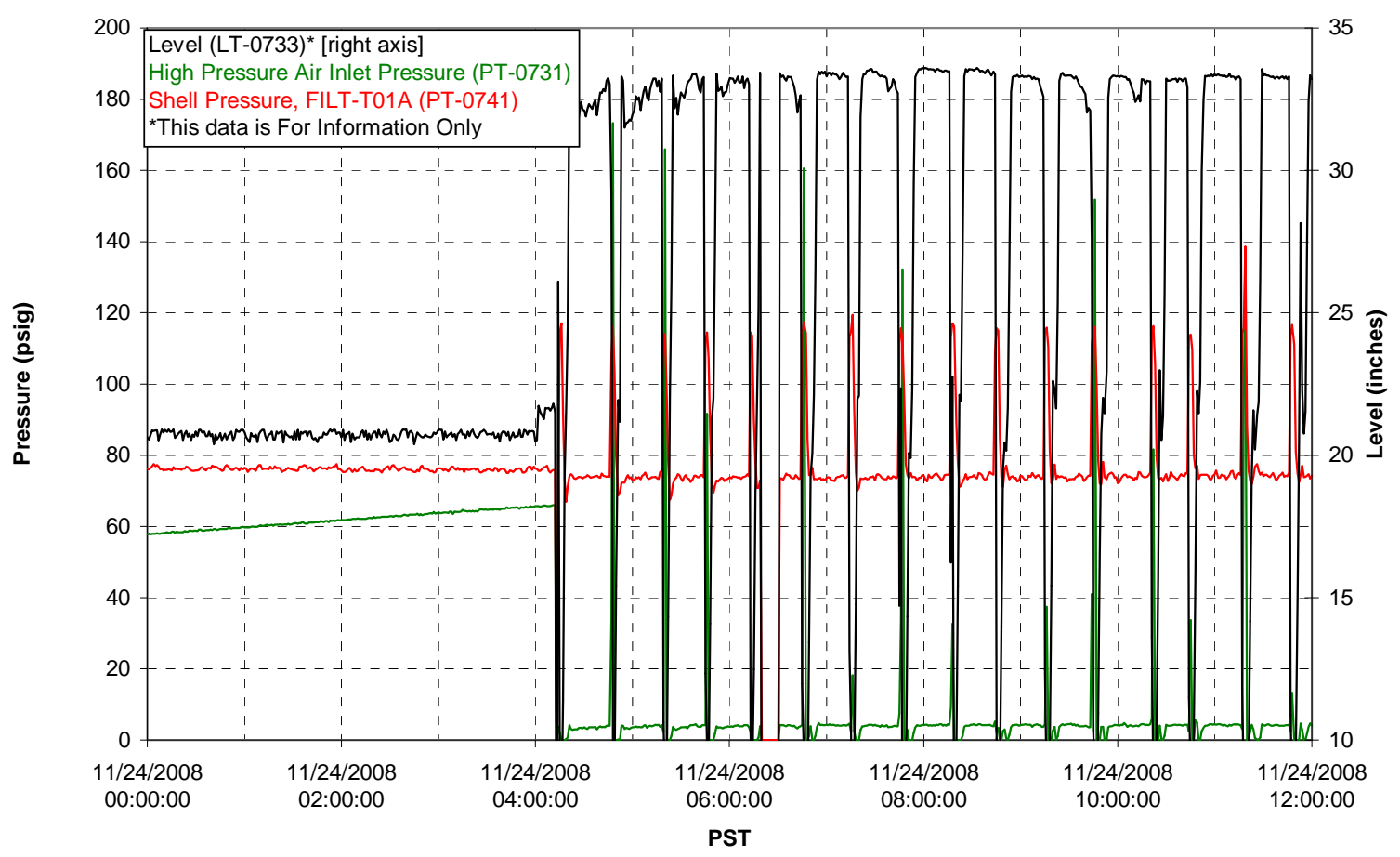

Pulsepot Levels

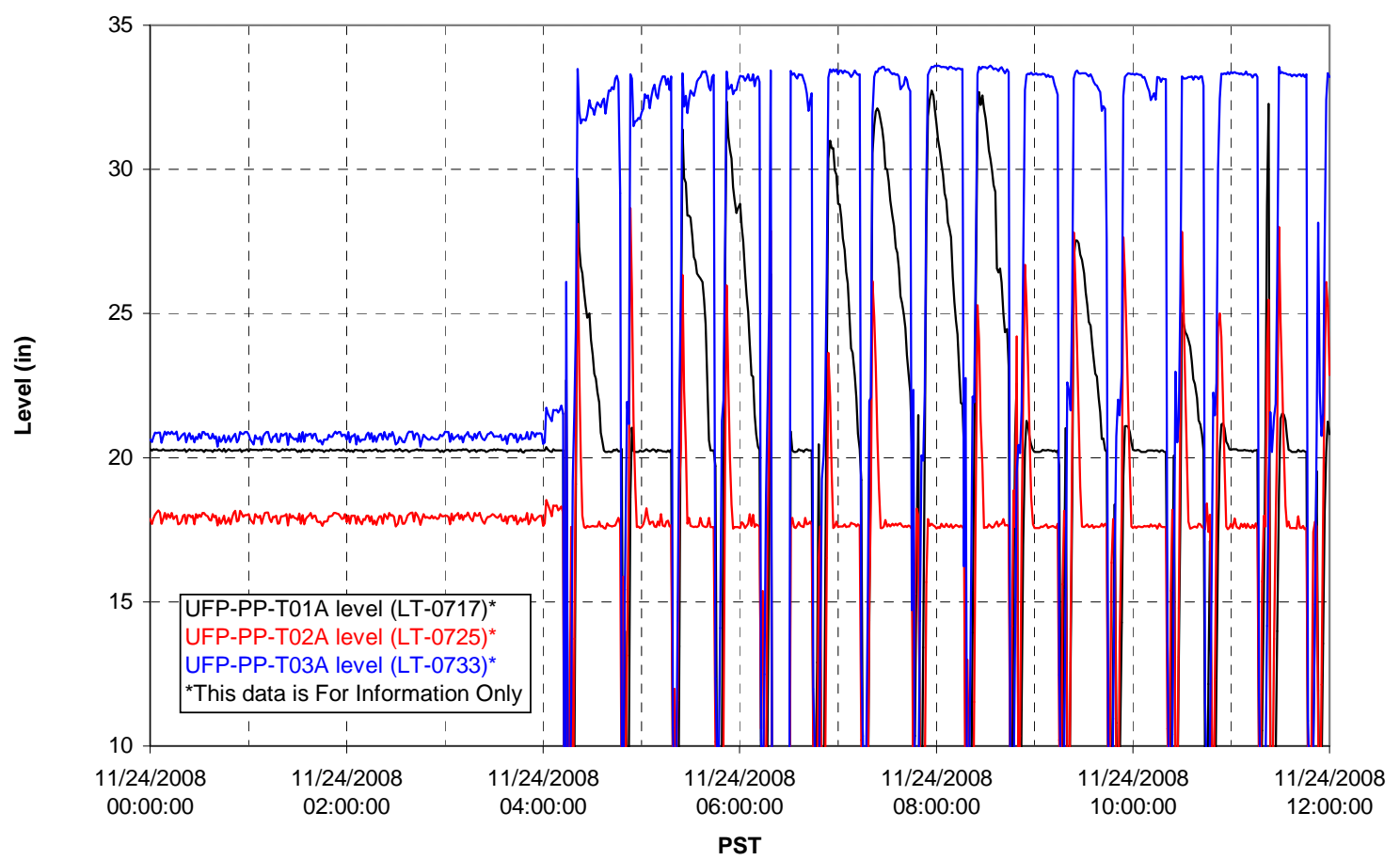


Filter UFP-FILT-T01A

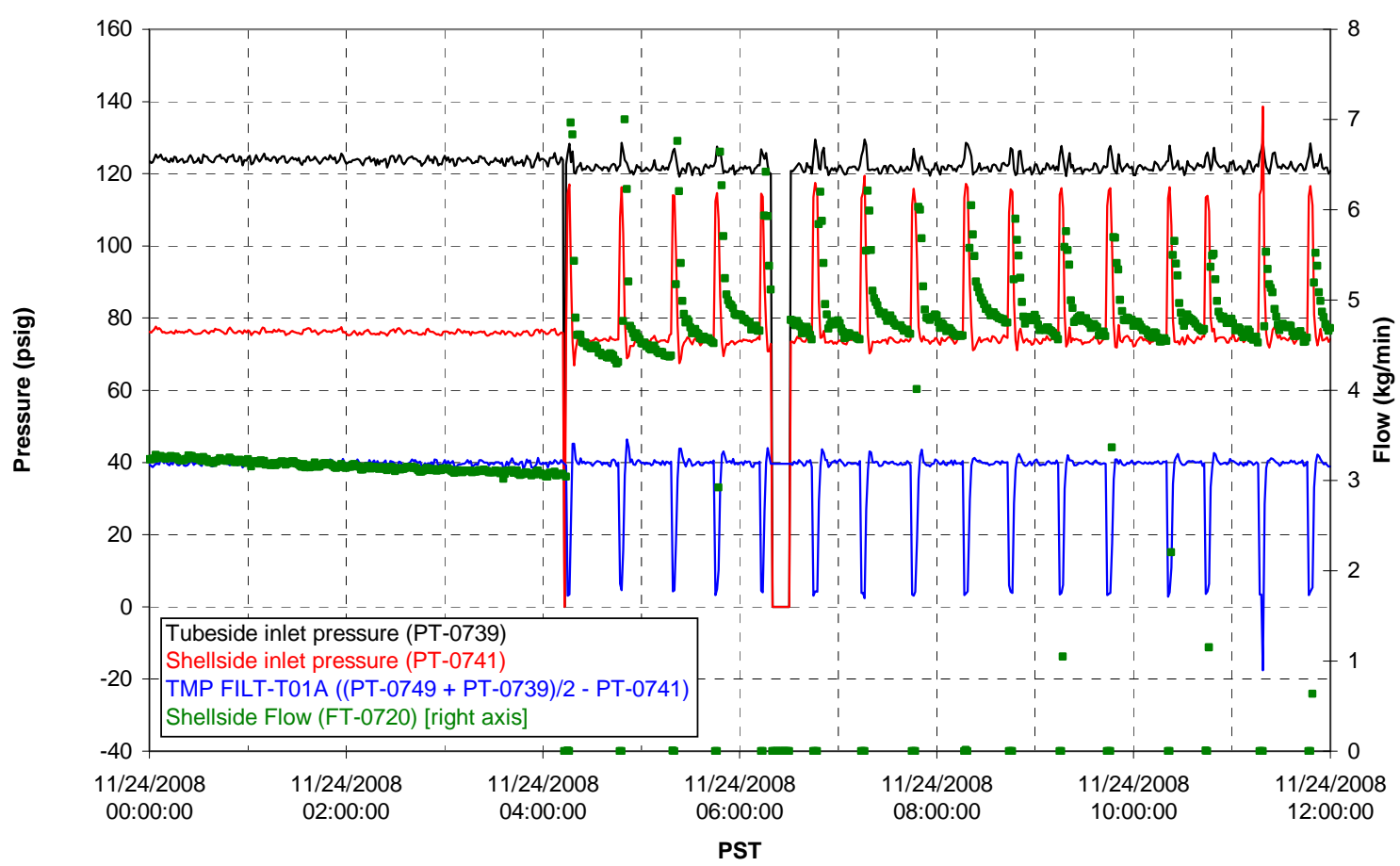

Filter UFP-FILT-T02A

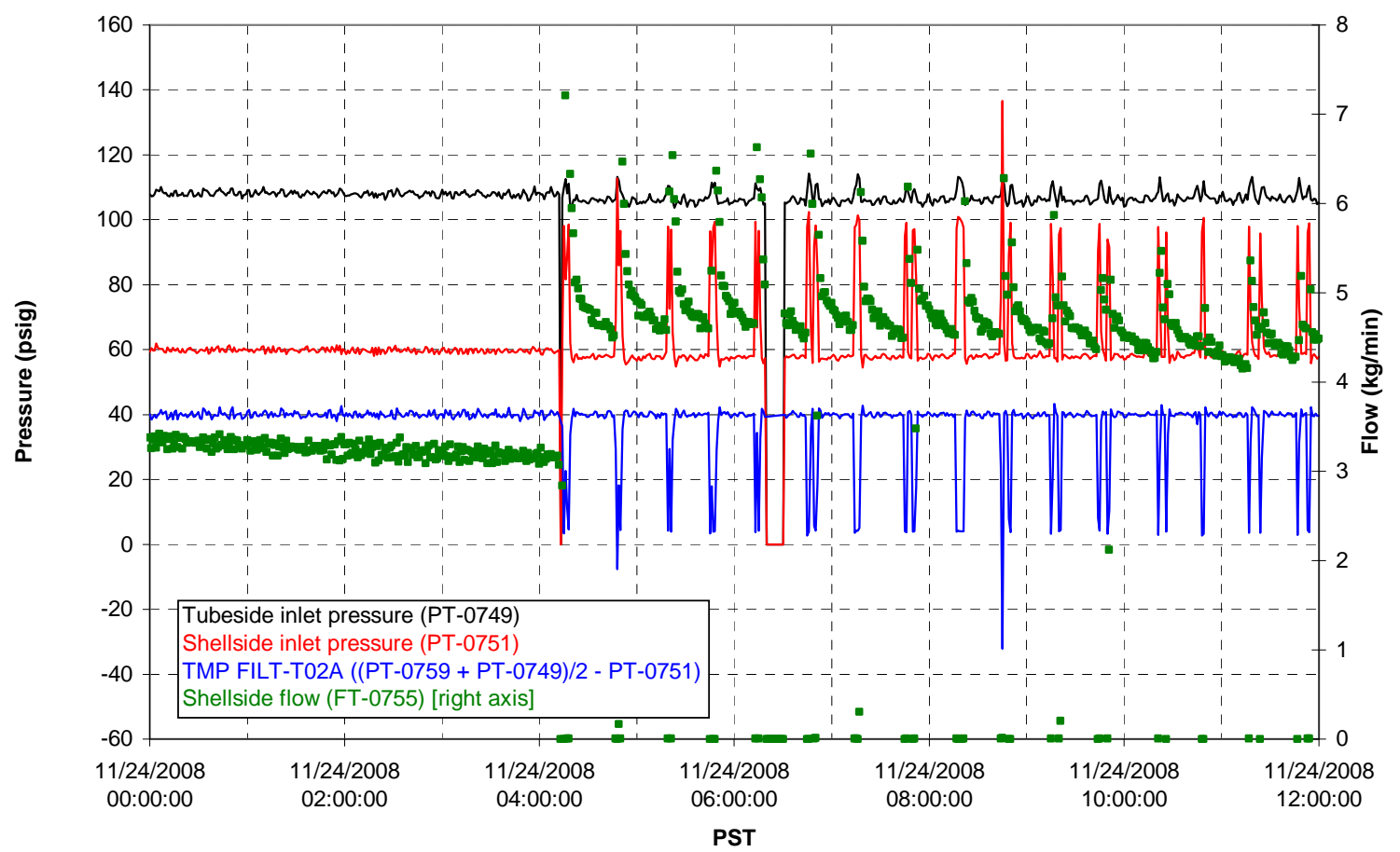


Filter UFP-FILT-T03A

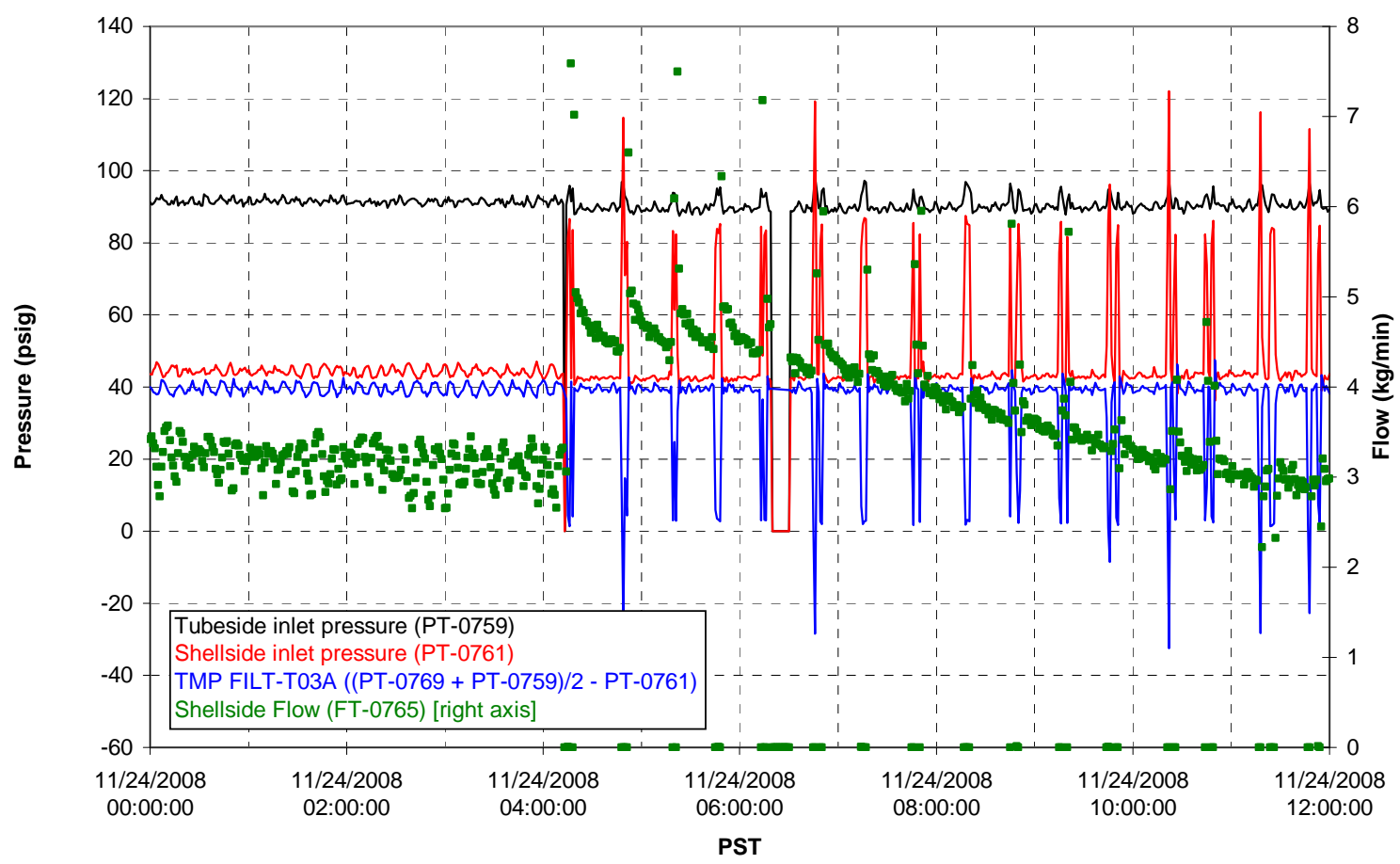

Filter UFP-FILT-T04A

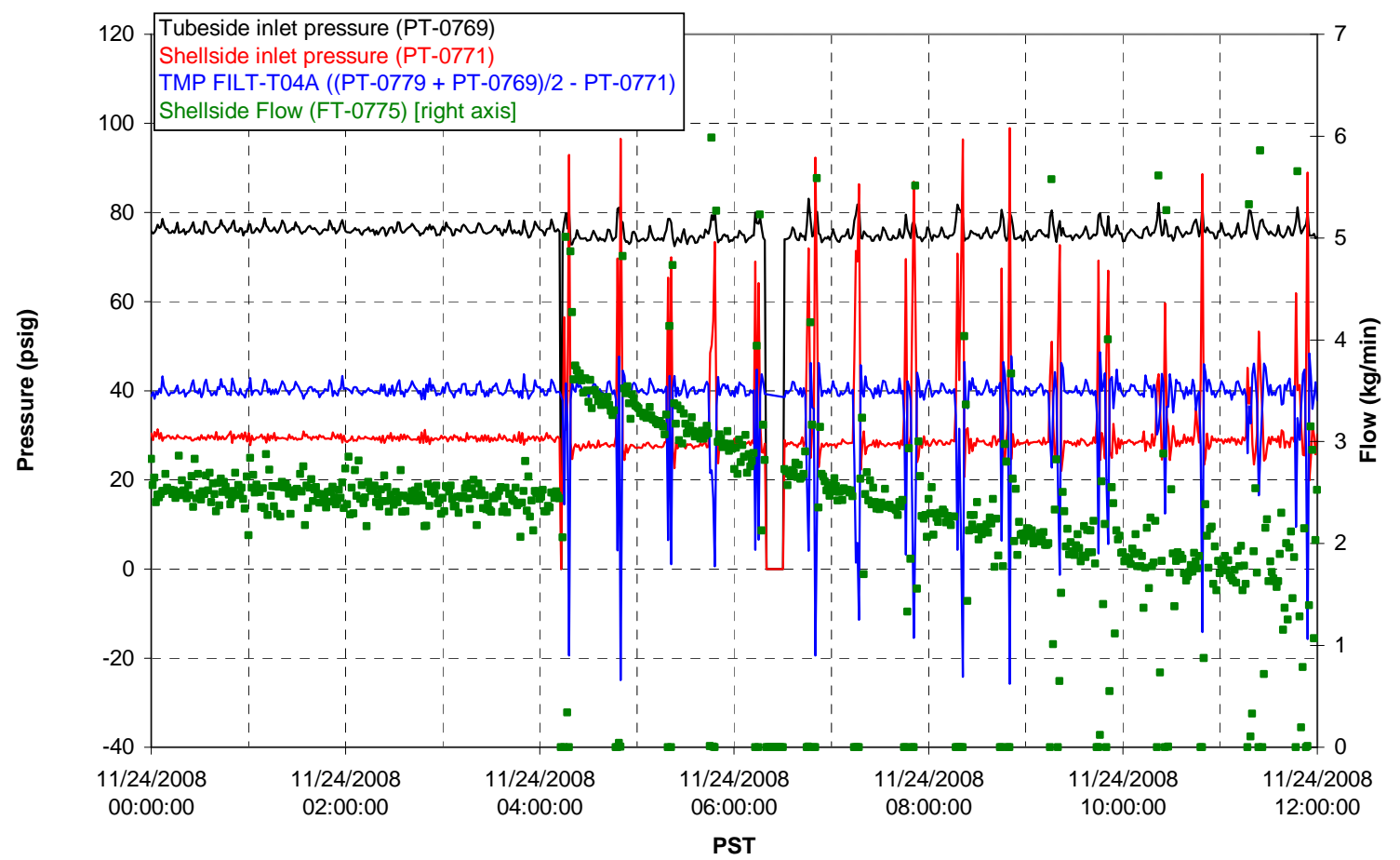


Filter UFP-FILT-T05A

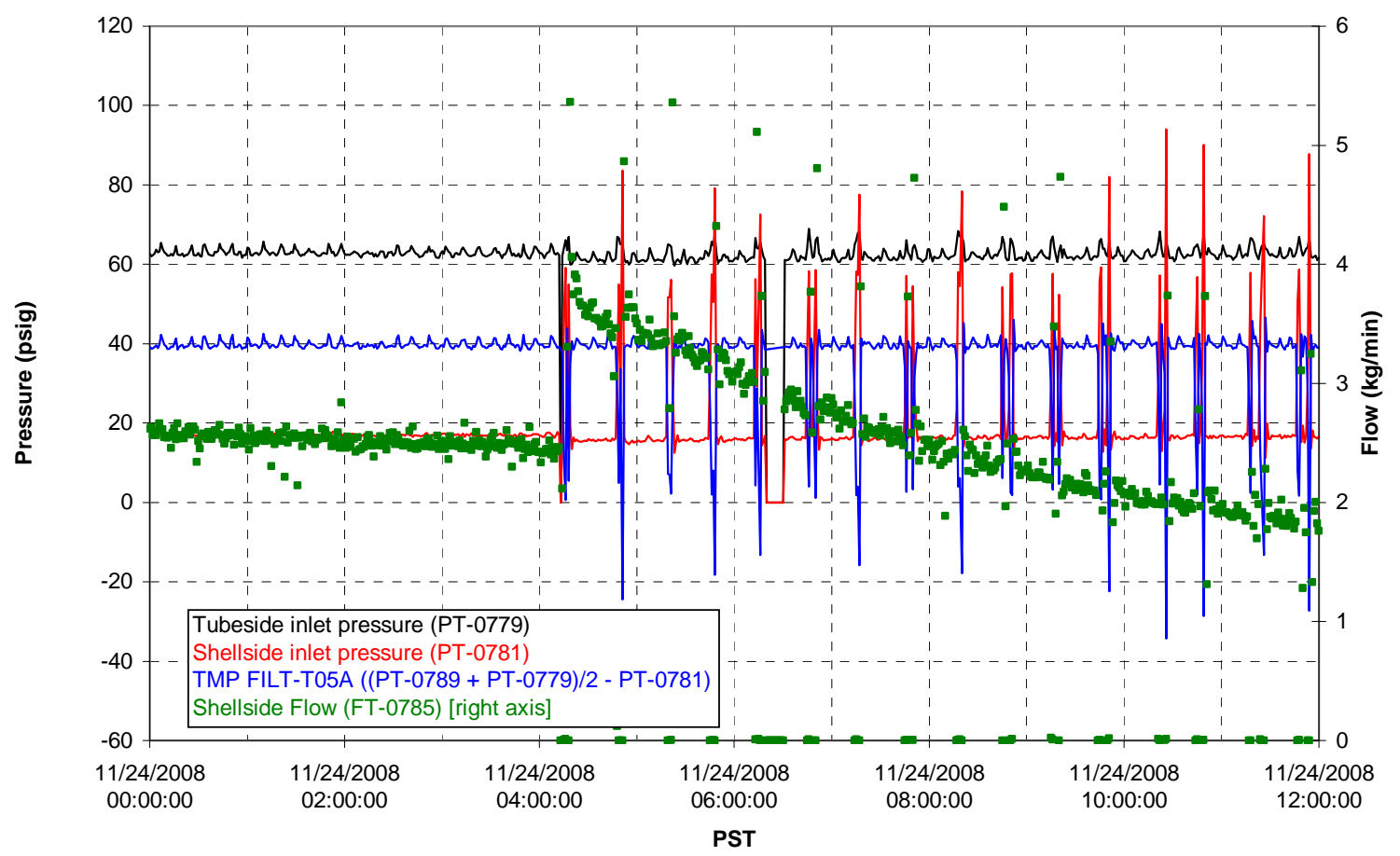

Chemical Flow

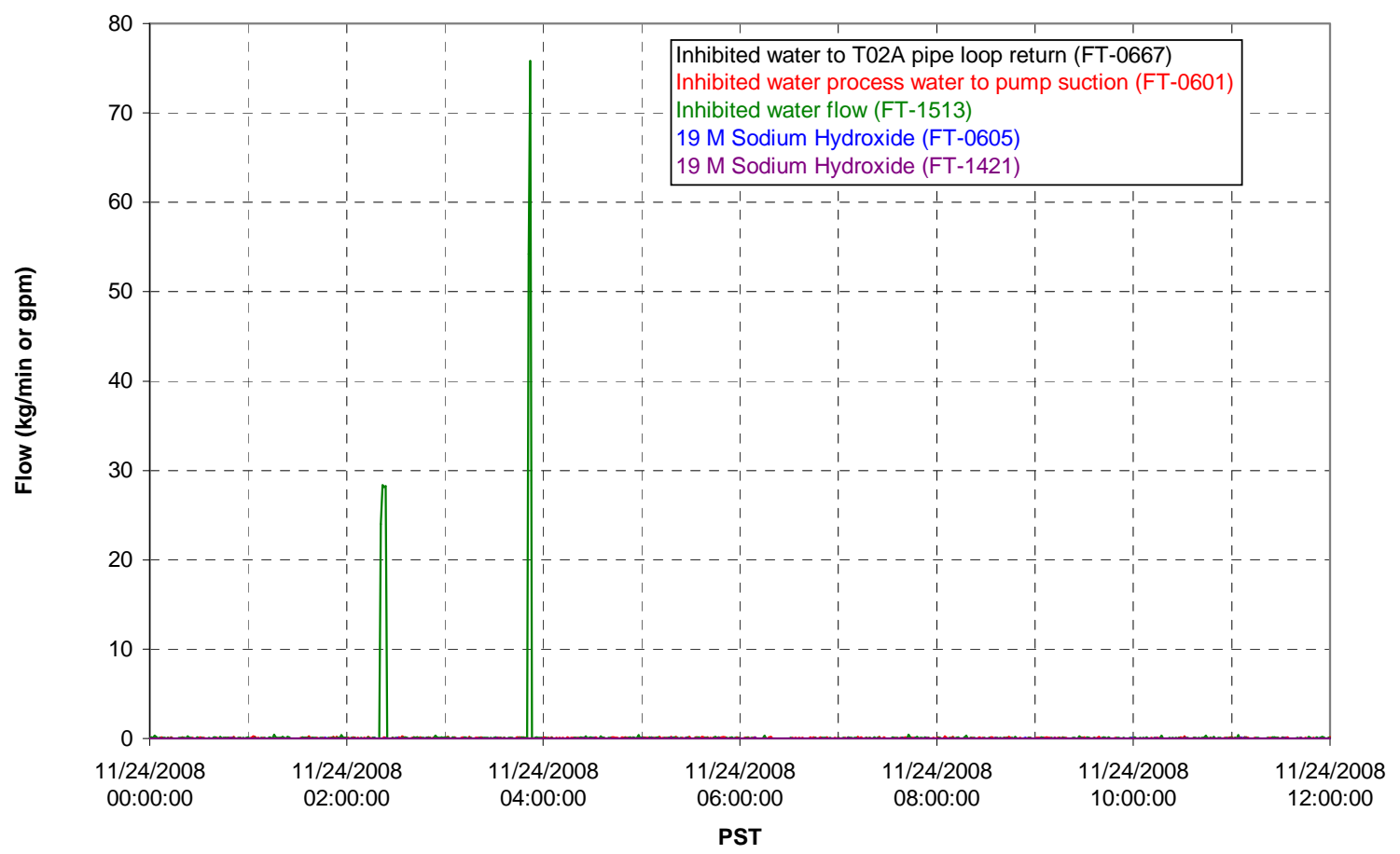


Chemical Flow

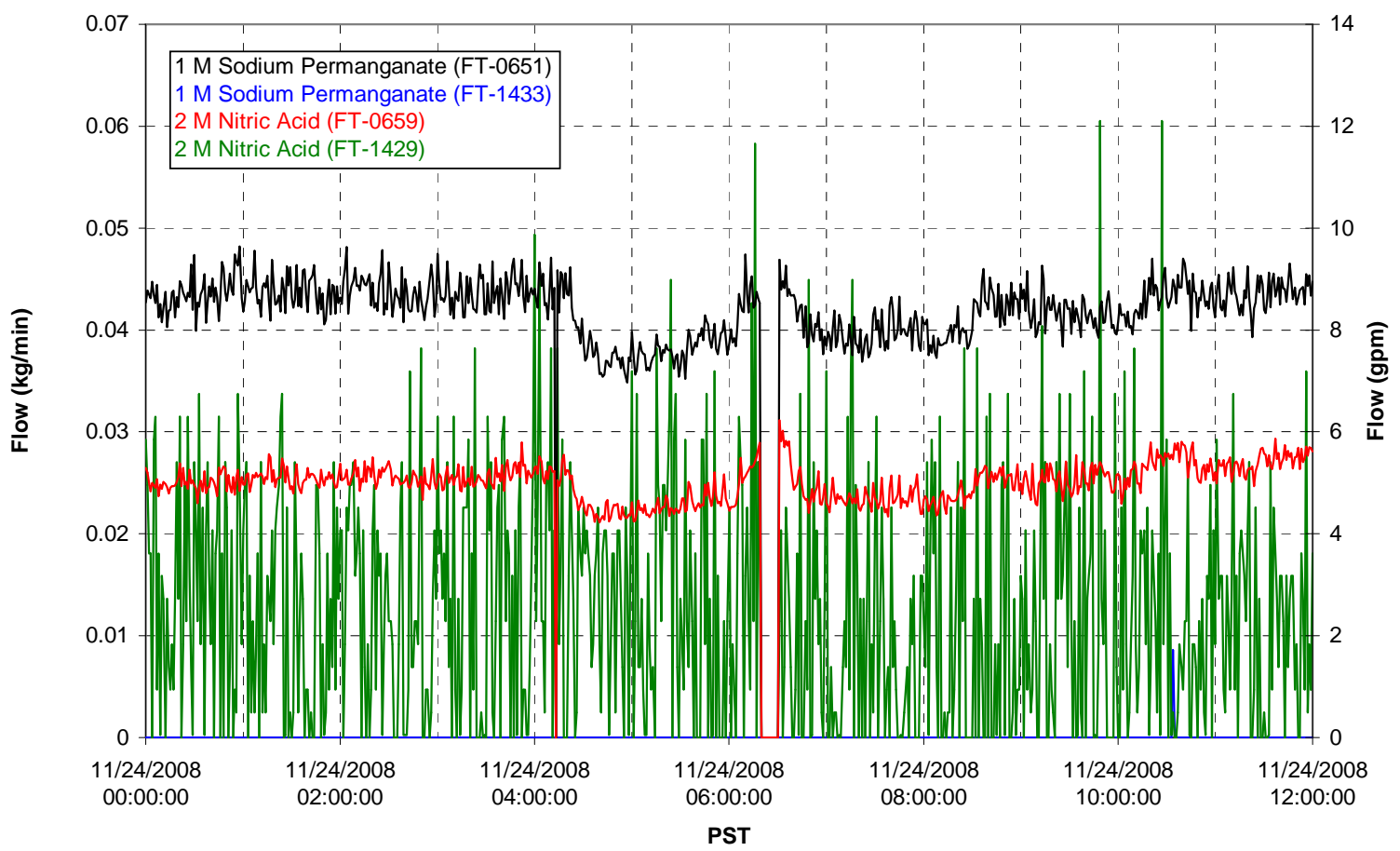

Air Flows

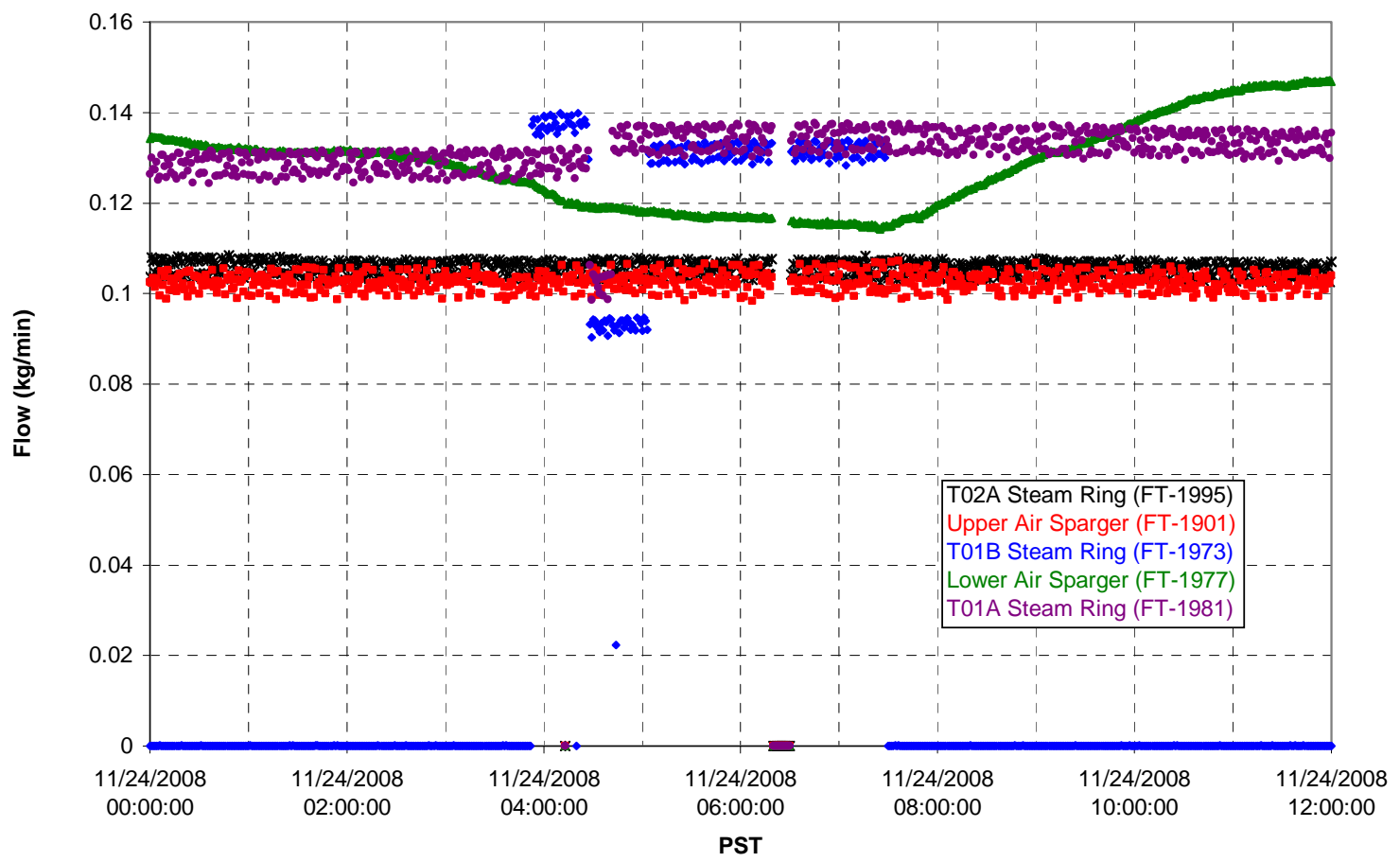


T02A Steam

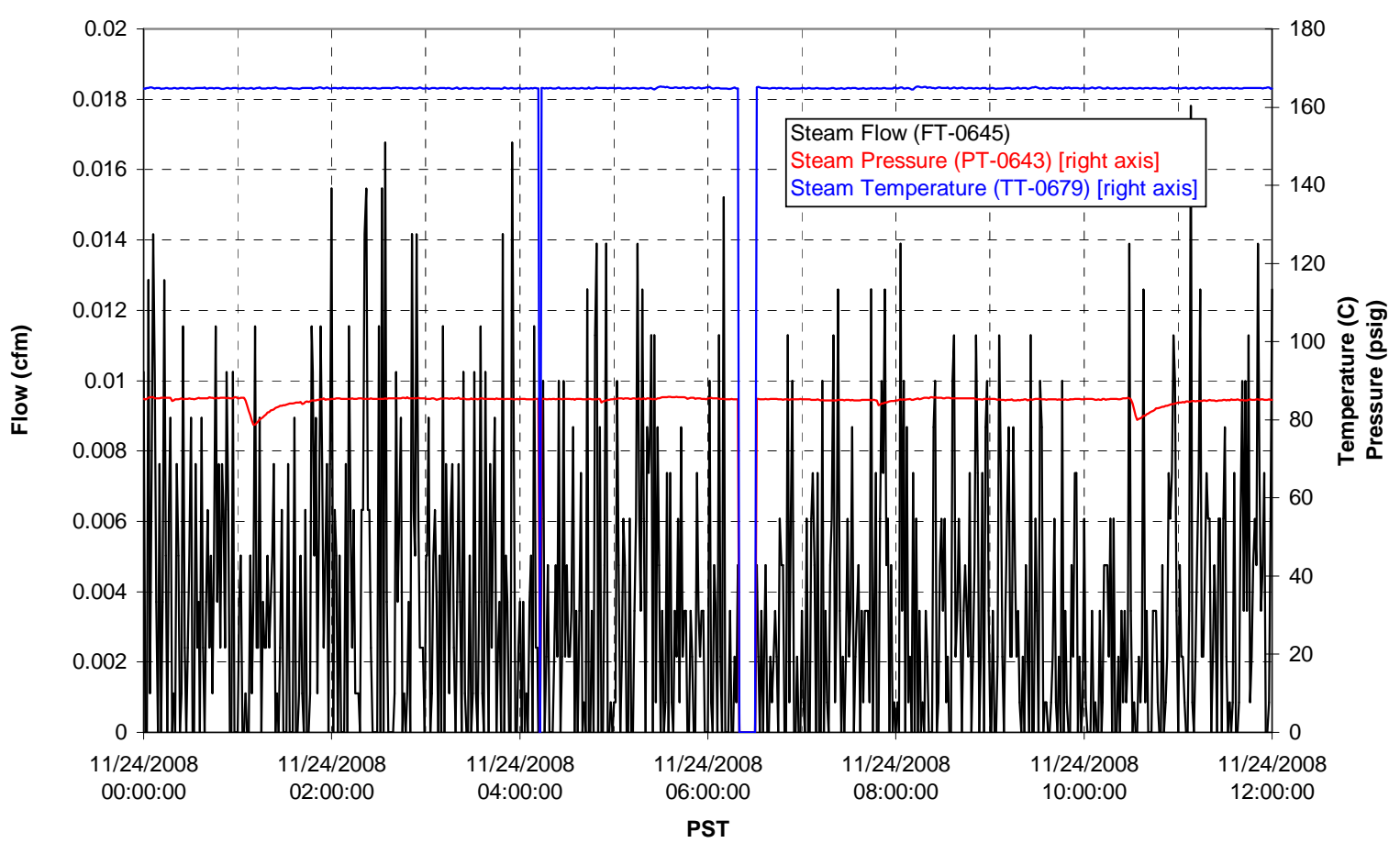

T01A Steam

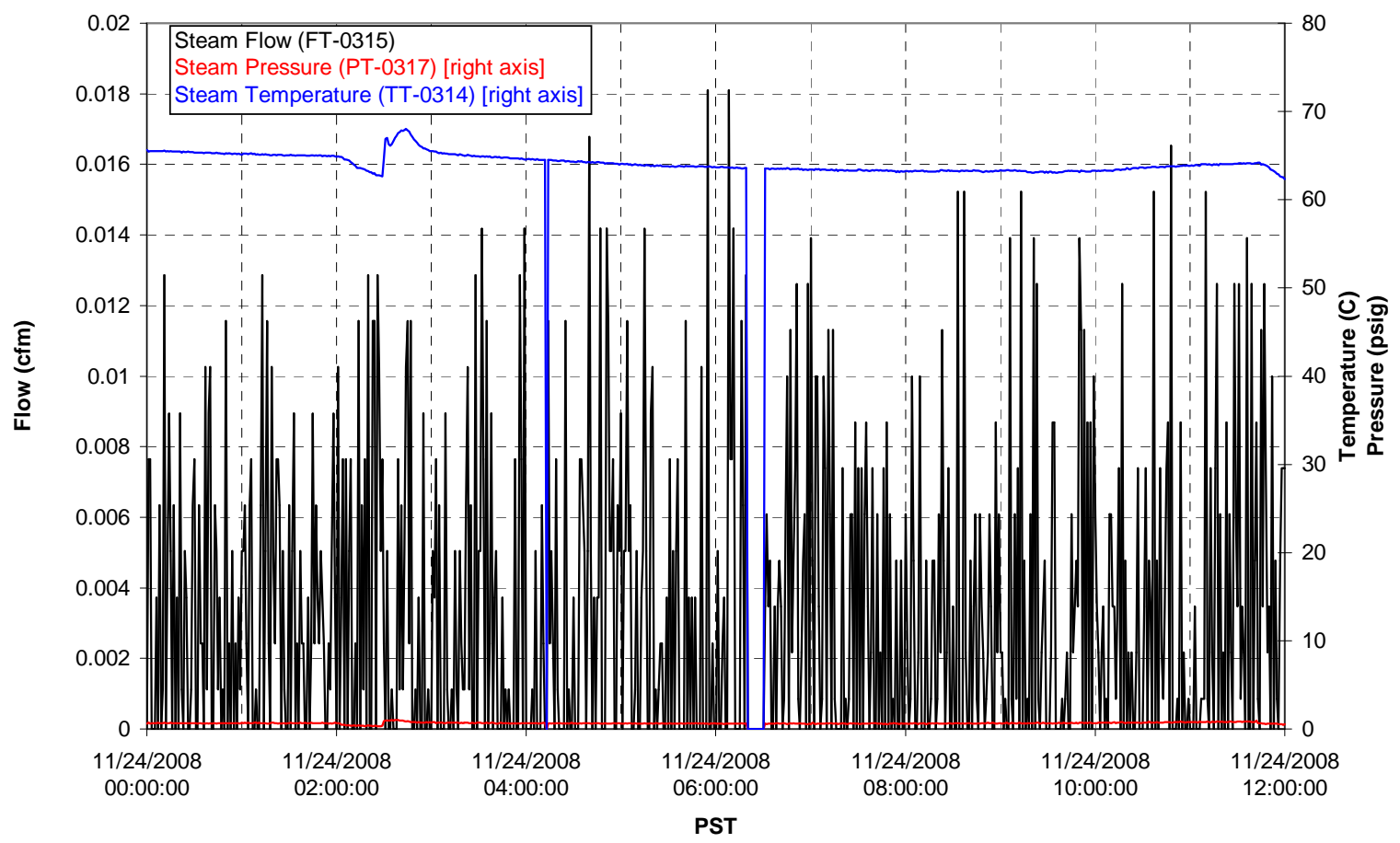


T01B Steam

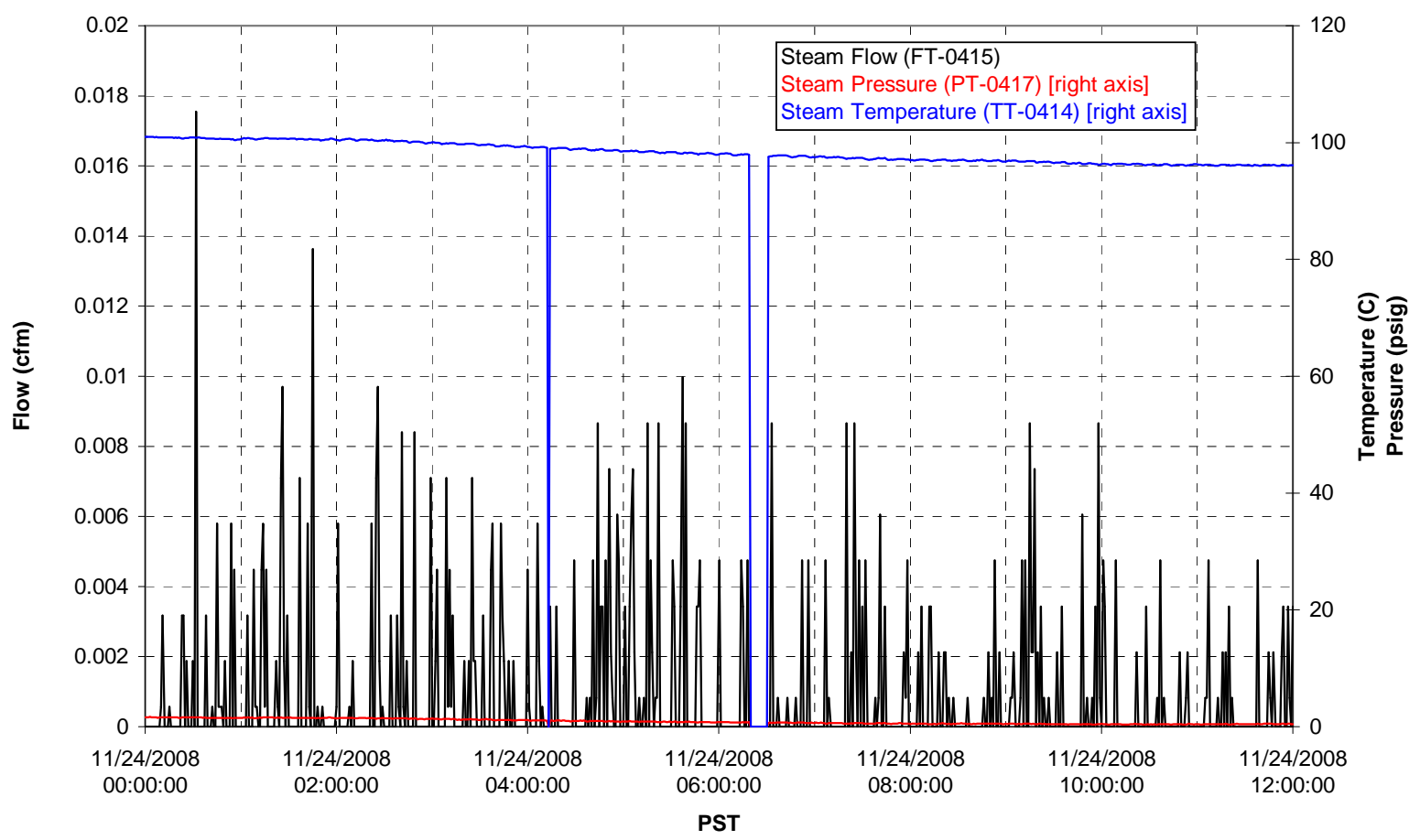


Functional Test Data Plots

11/24/2008 12:00 - 11/25/2008 00:00 
T01A level

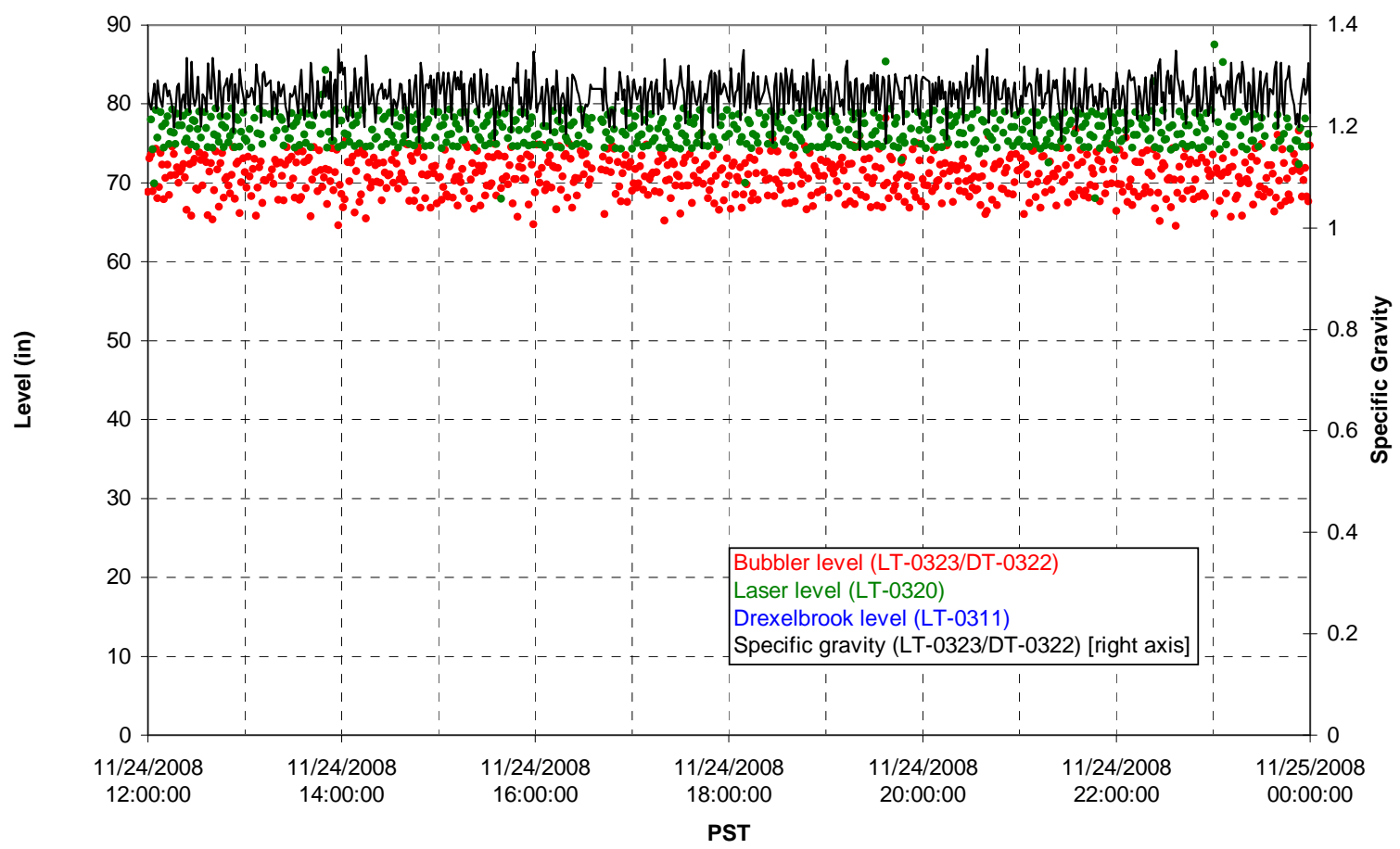

T01A temperatures

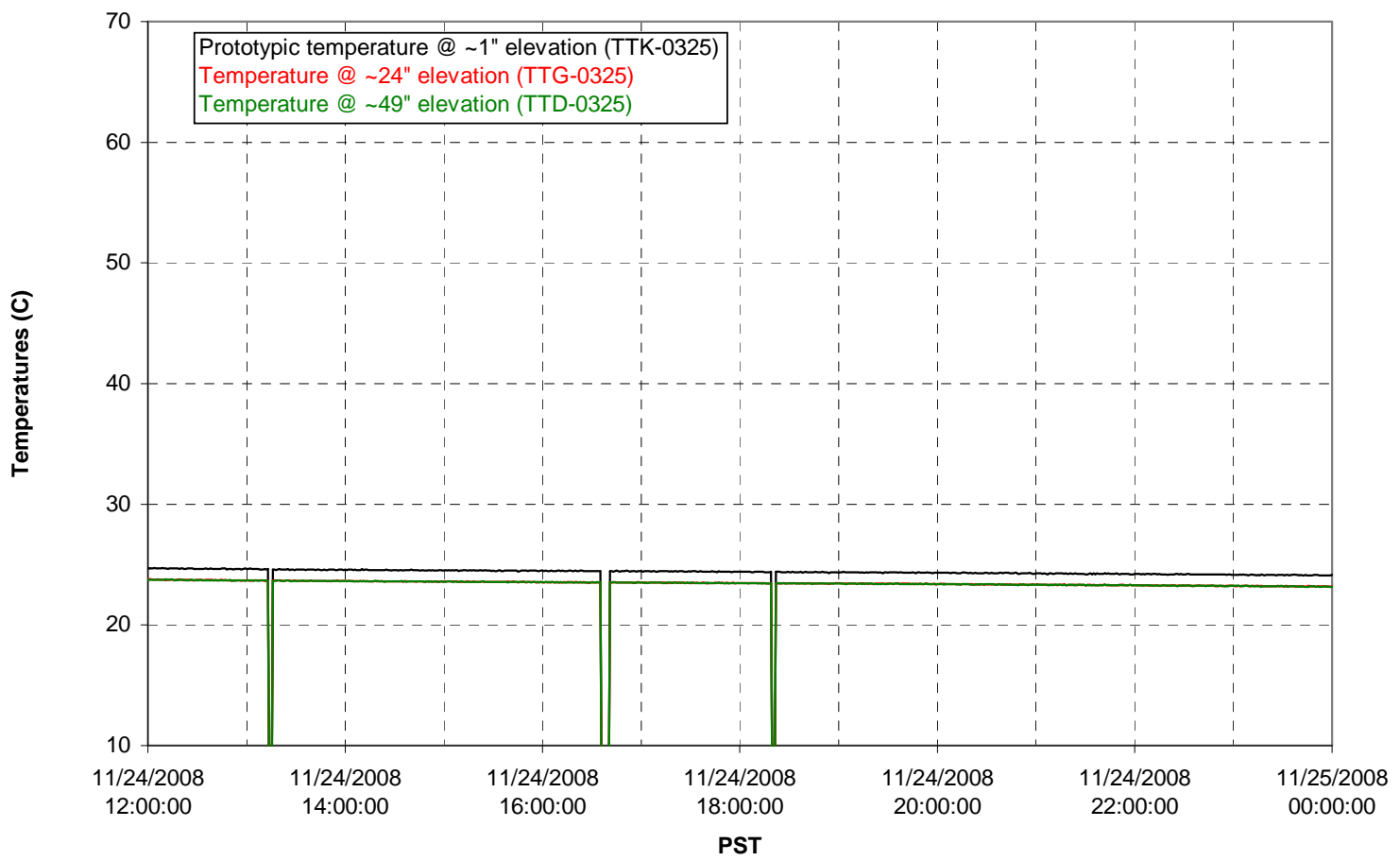


T01B level

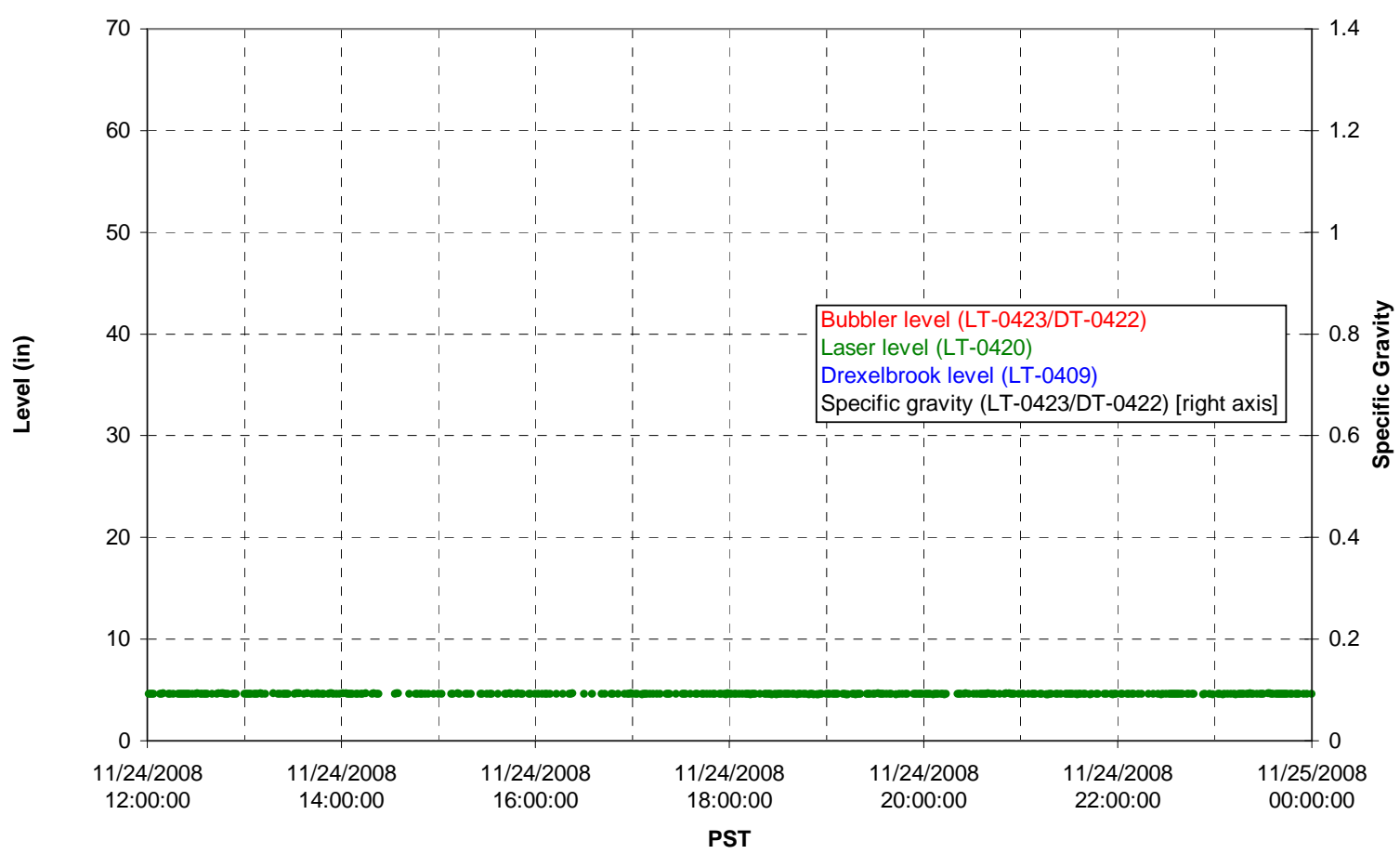

T01B temperatures

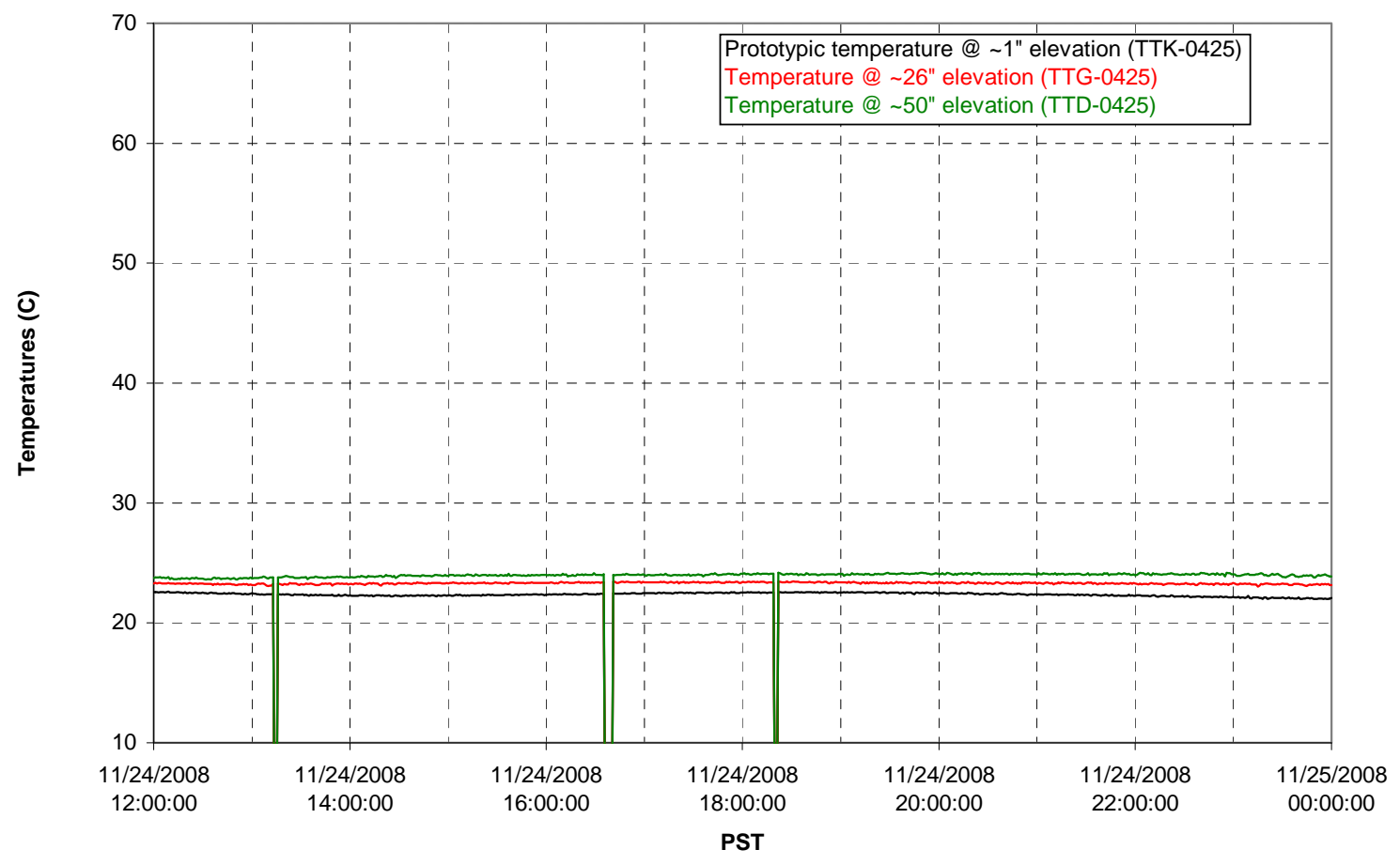


T02A level

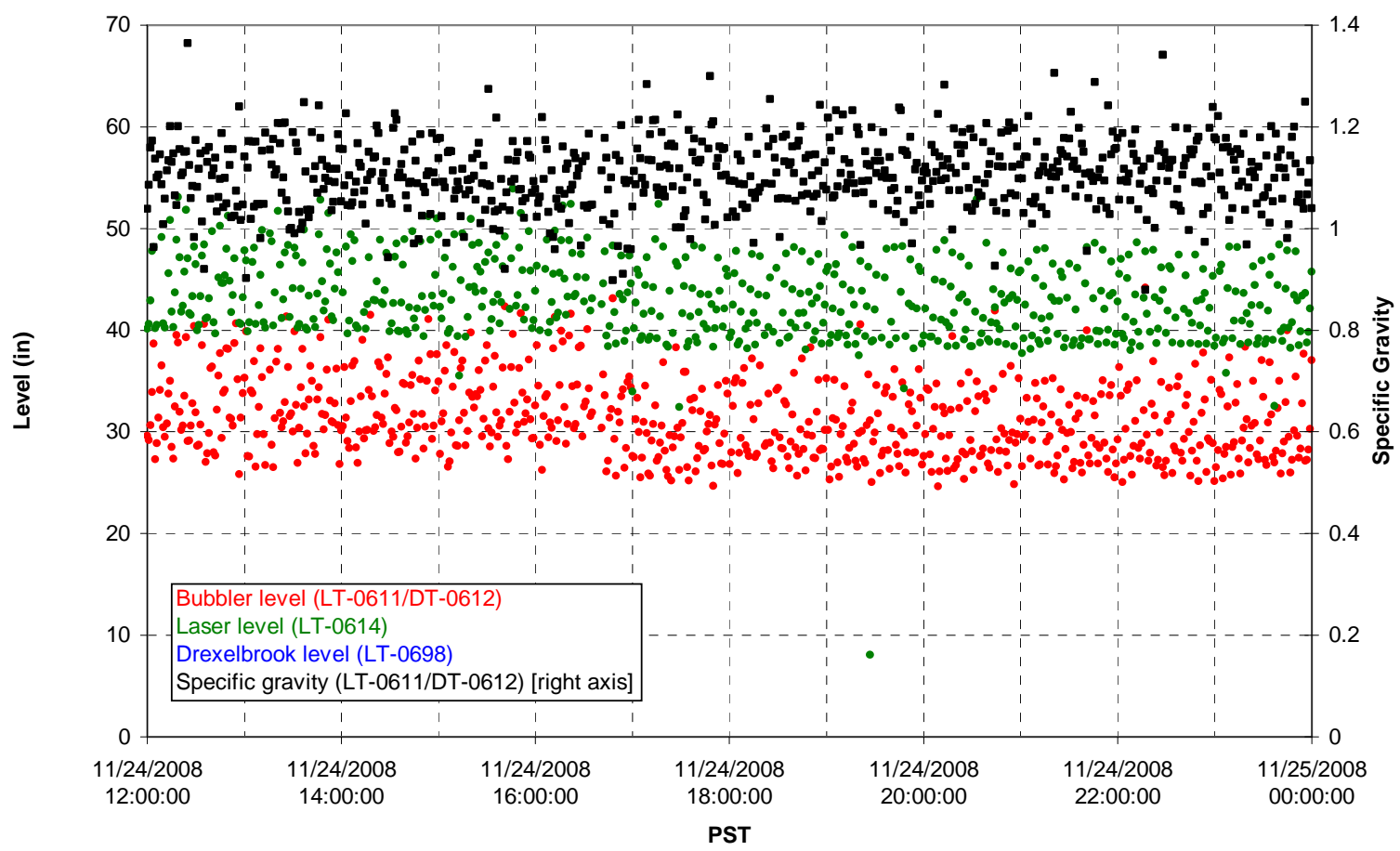

T02A temperatures

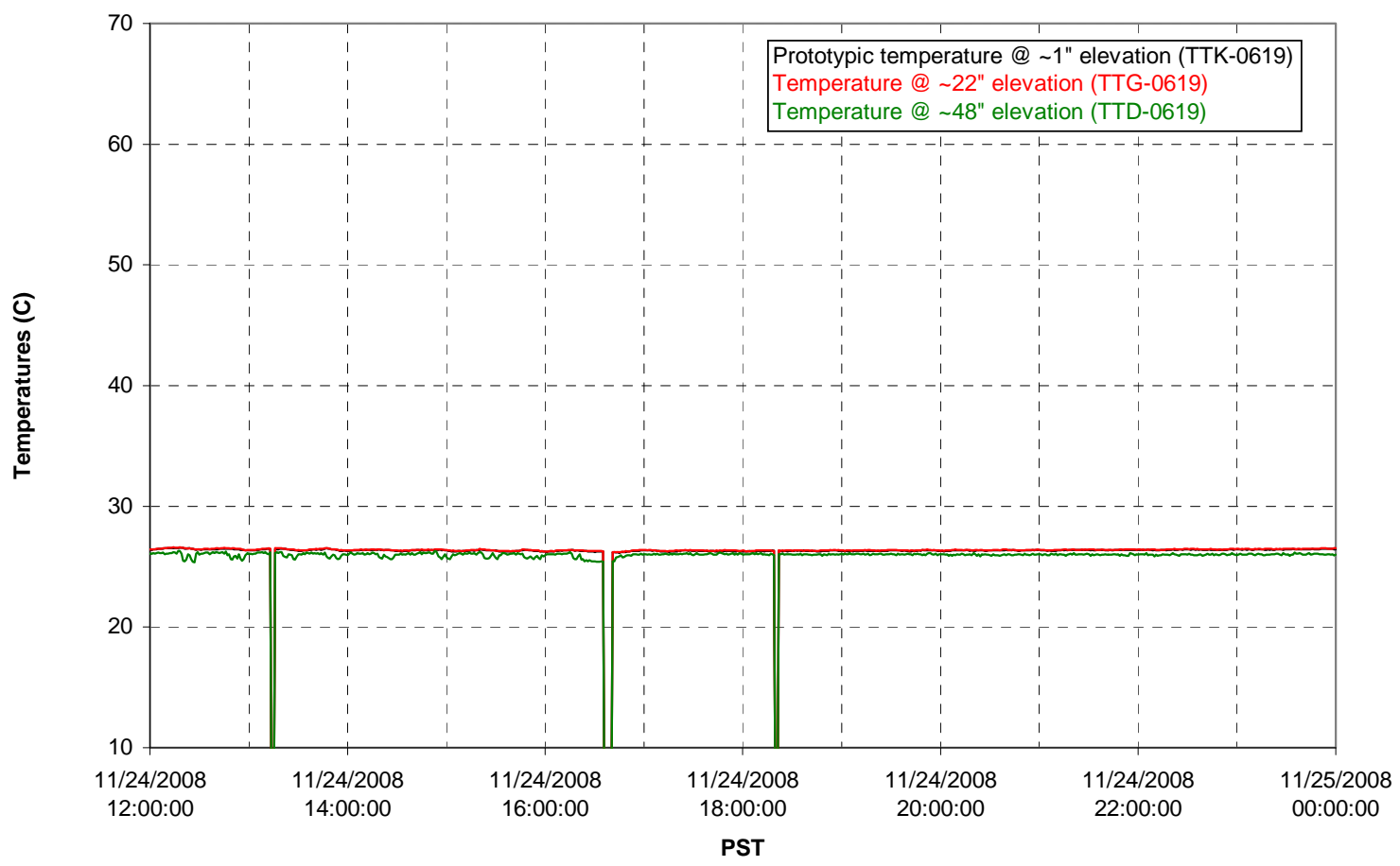


T02A and filter loop temperatures

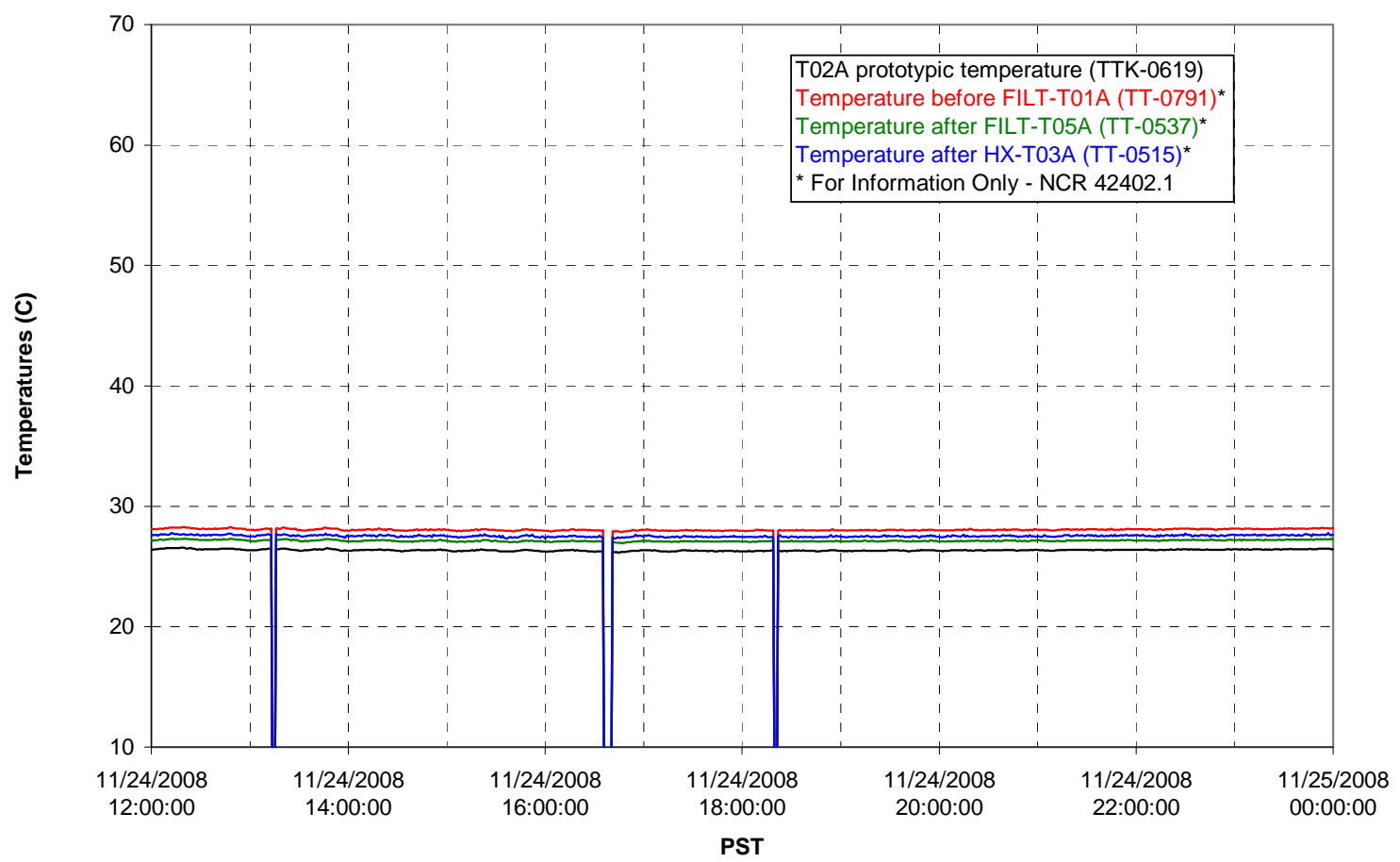

Pump Pressures and Flow

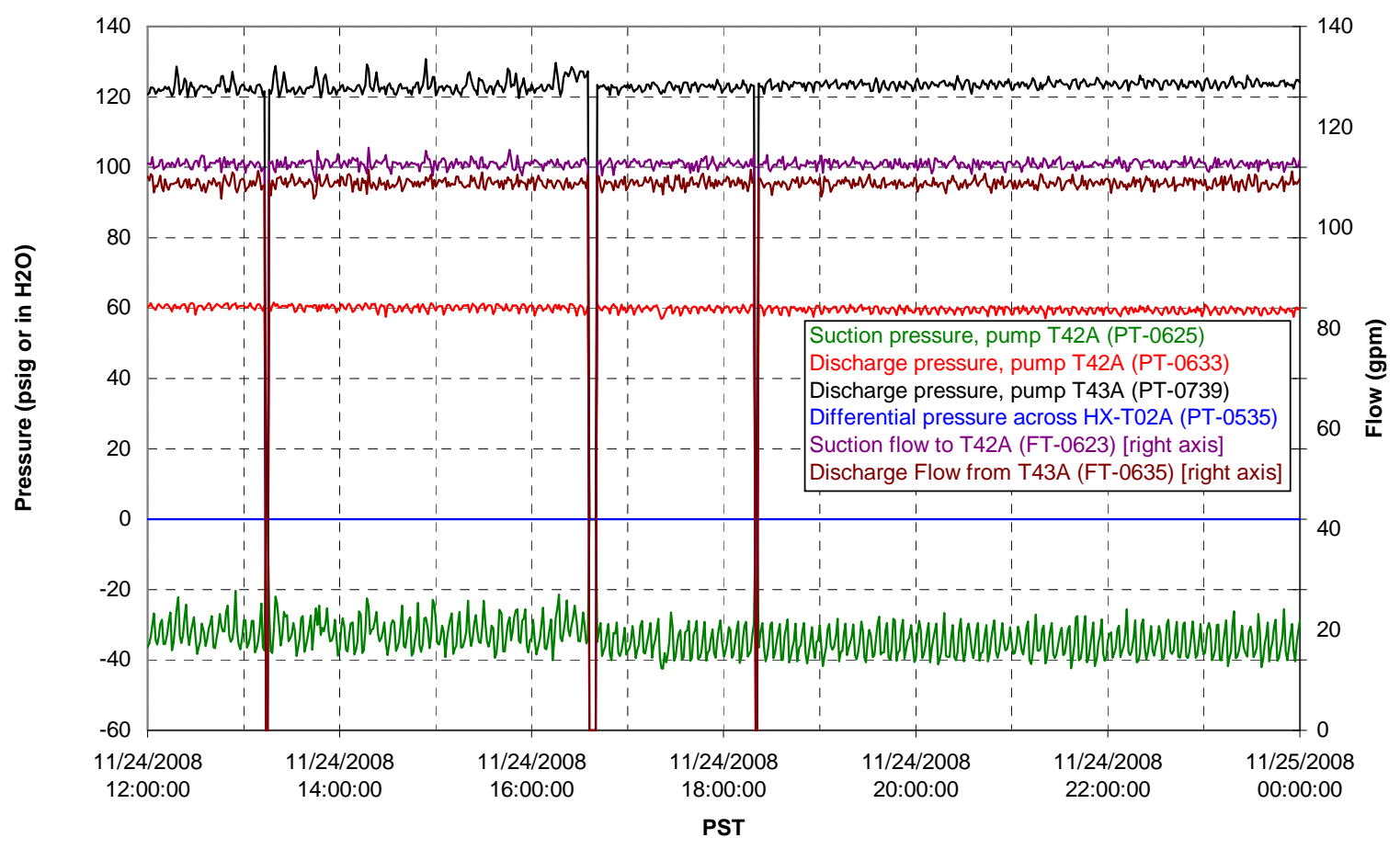


Axial pressure drop

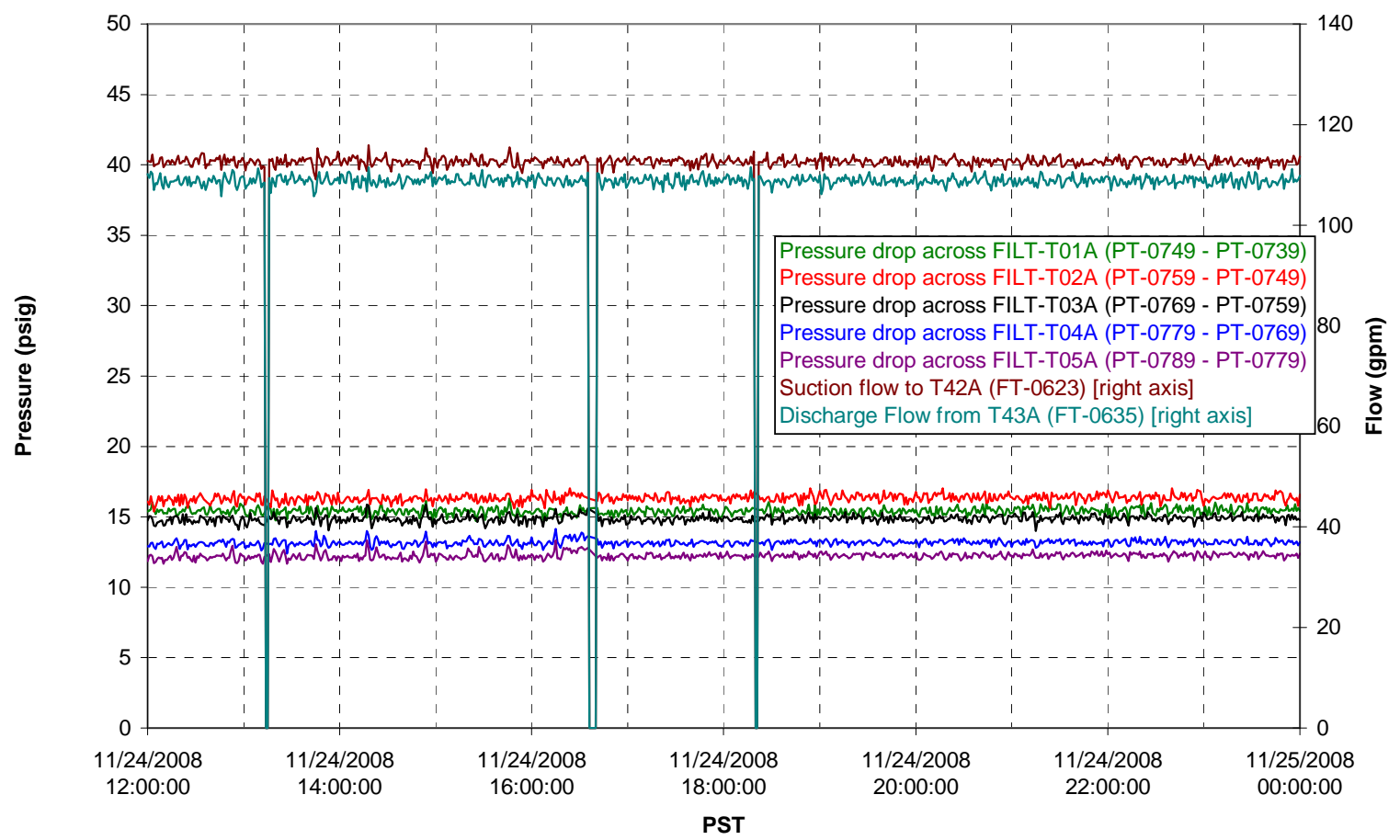

Permeate flow rates

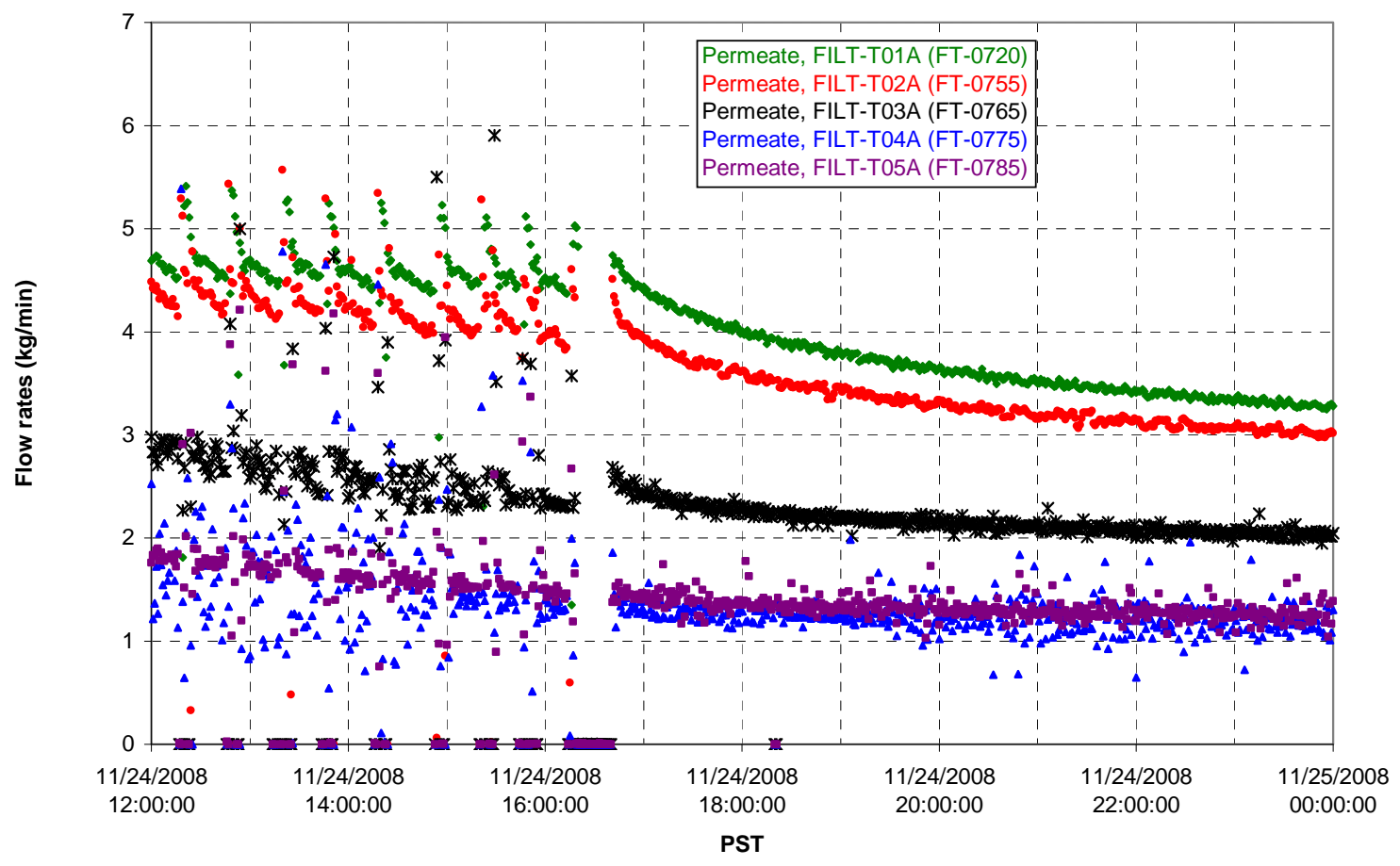


T02A Inner Temperature Tree

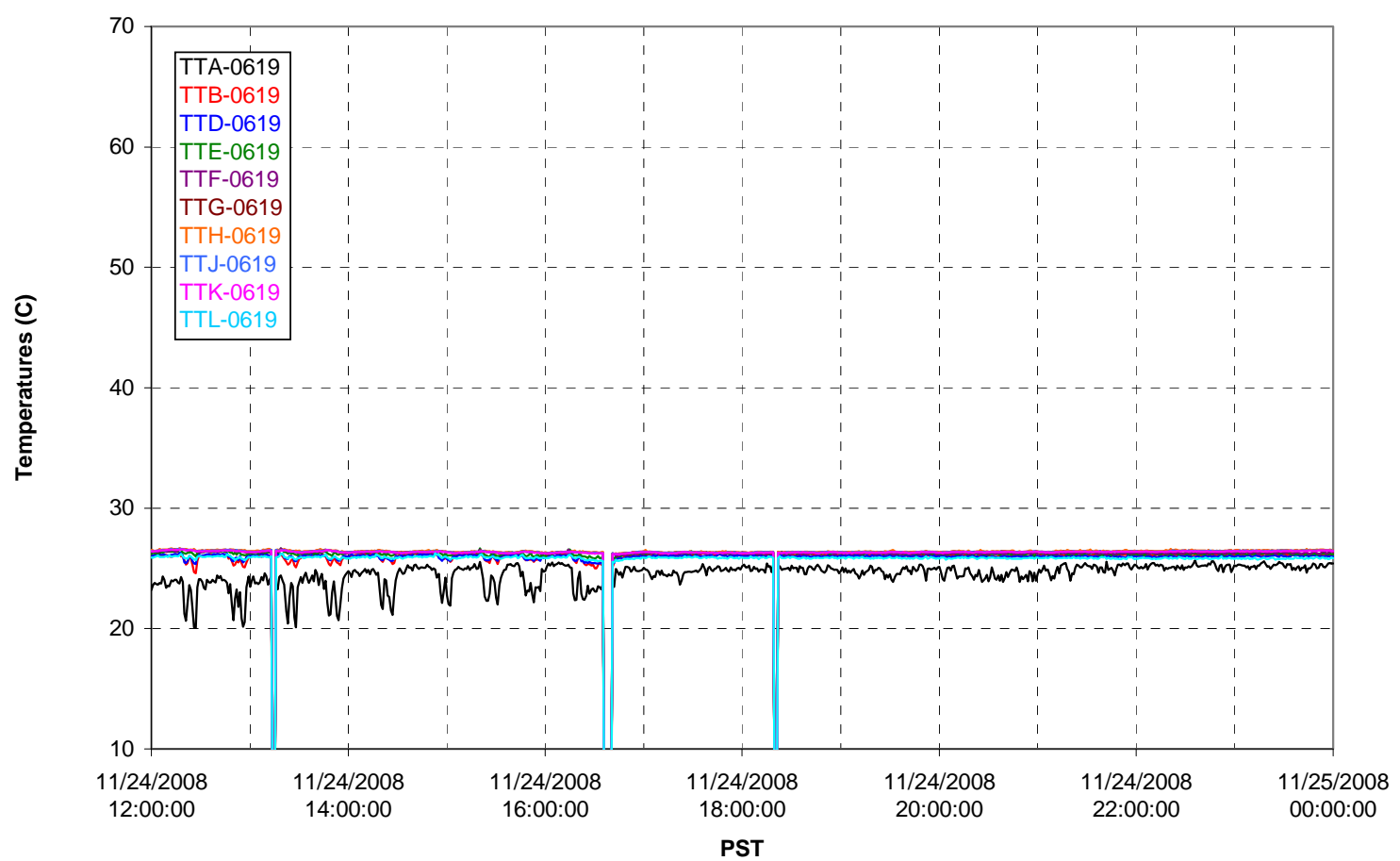

T02A Outer Temperature Tree

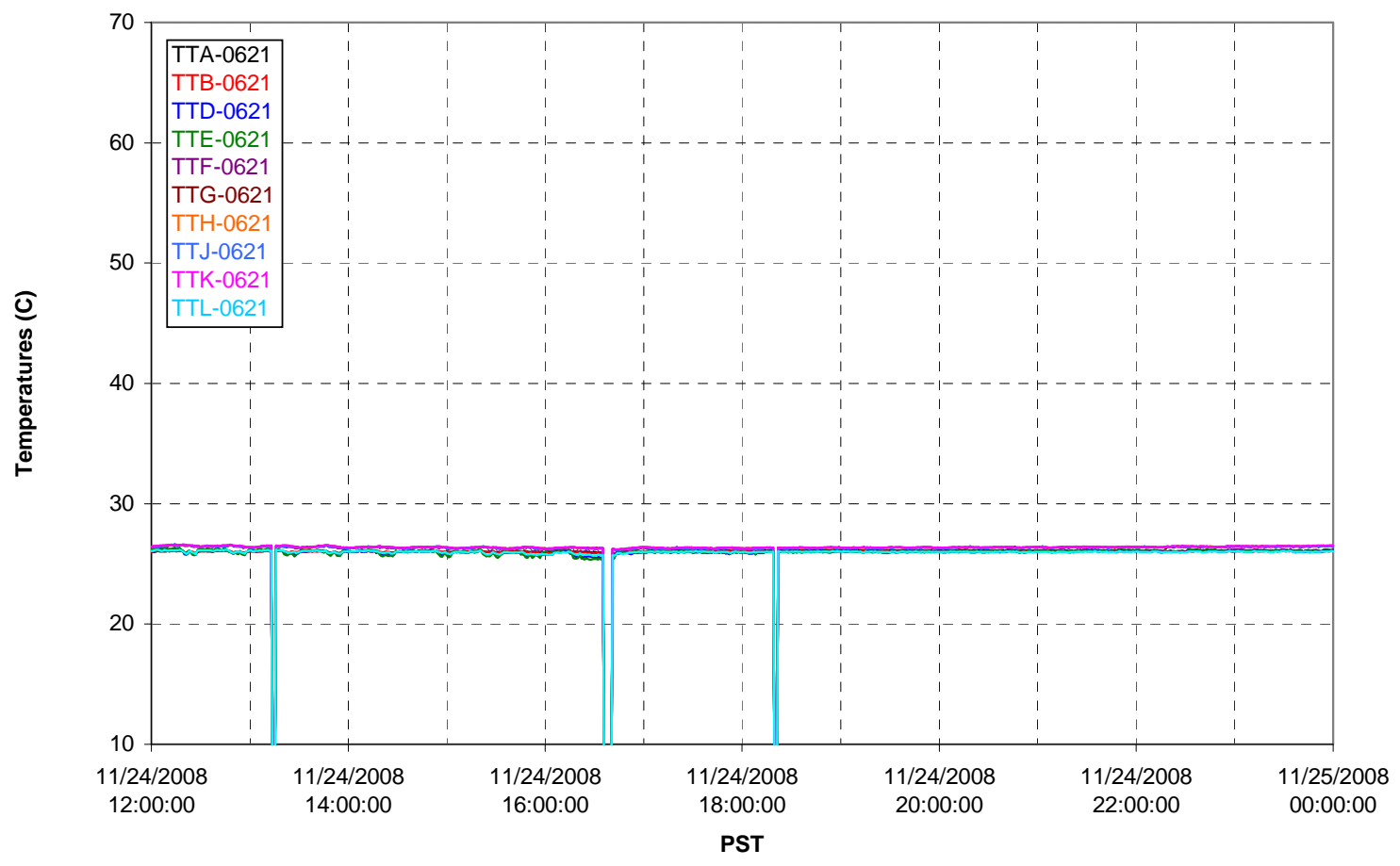


T02A temperatures

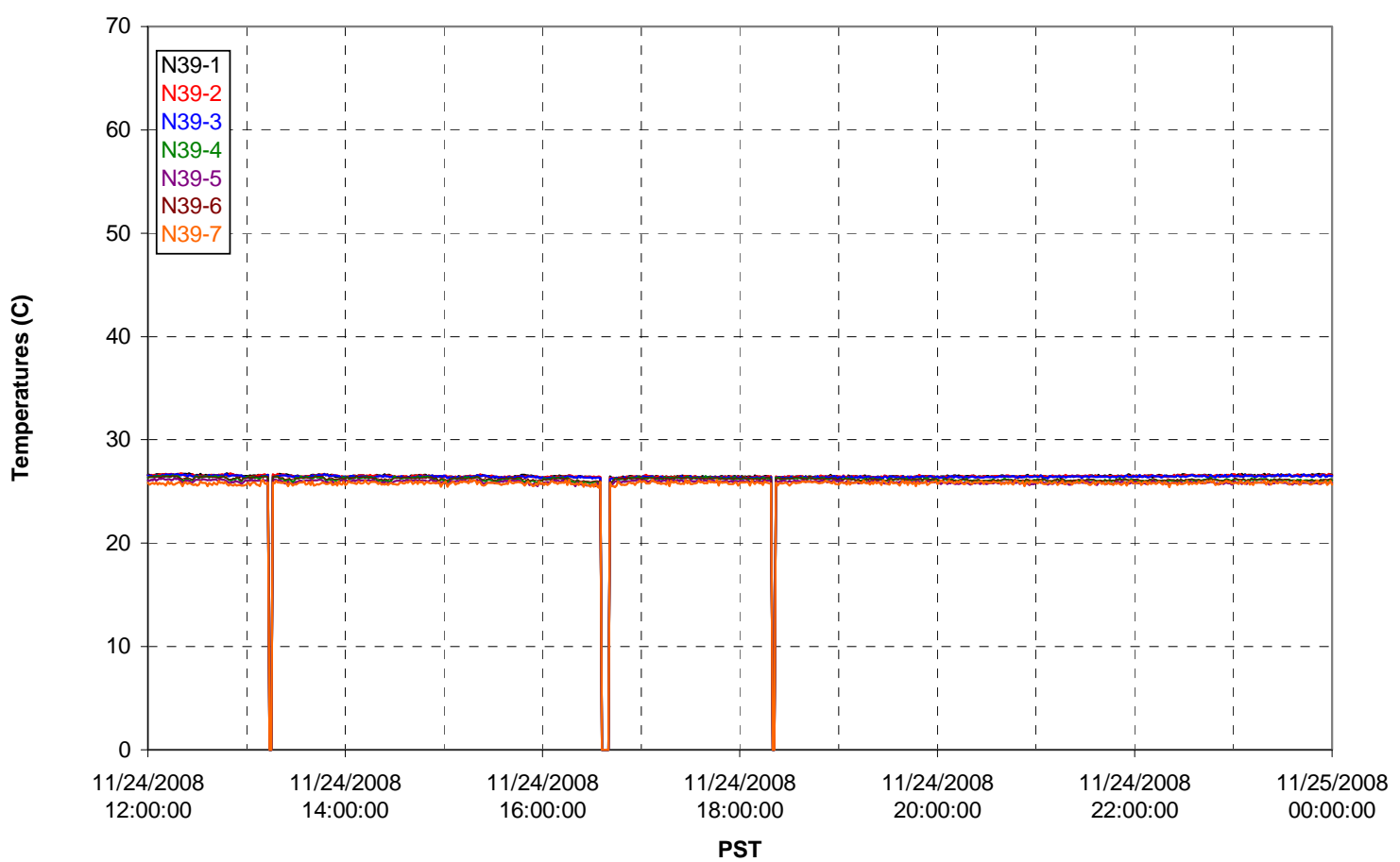

T02A temperatures

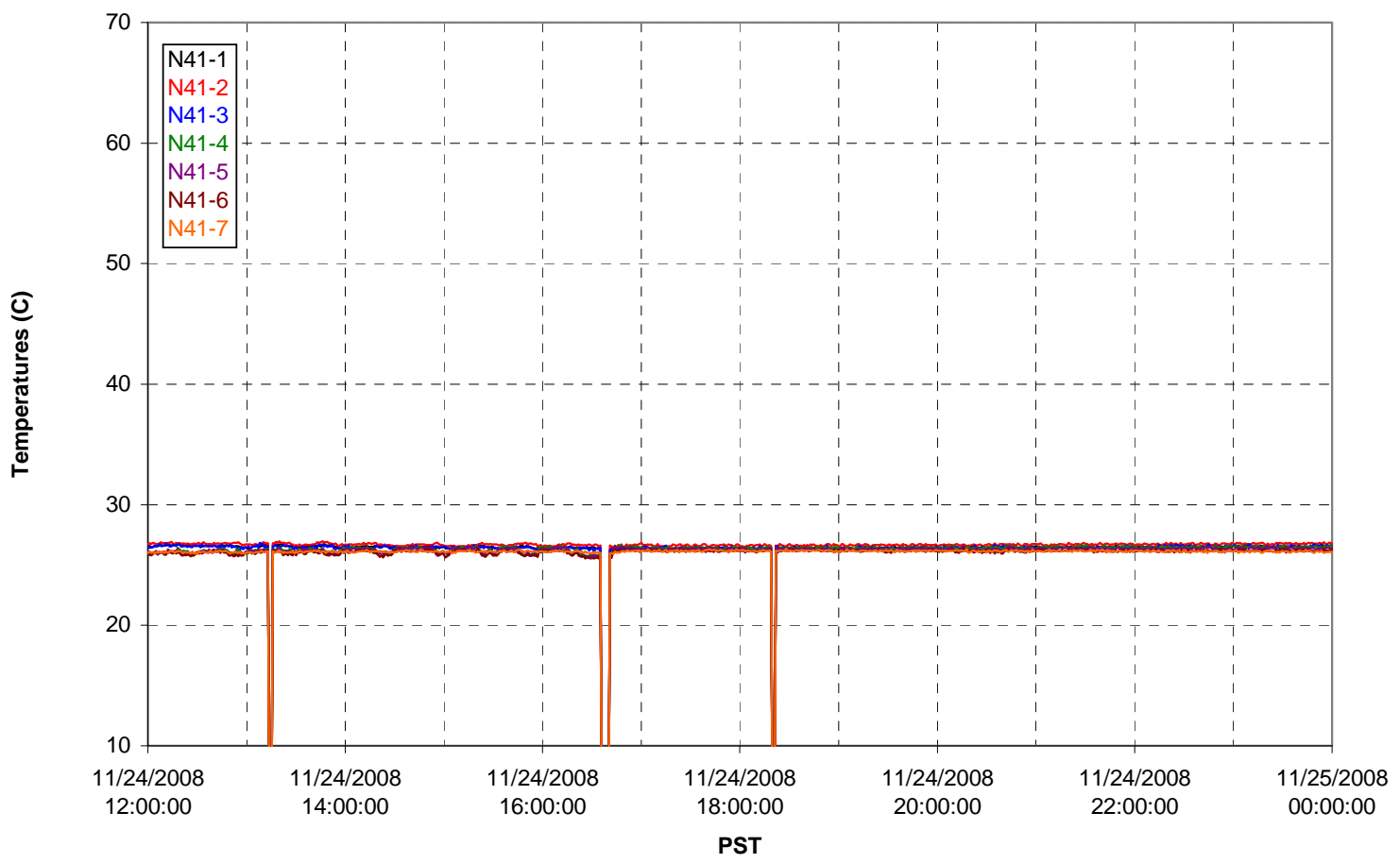


T02A temperatures

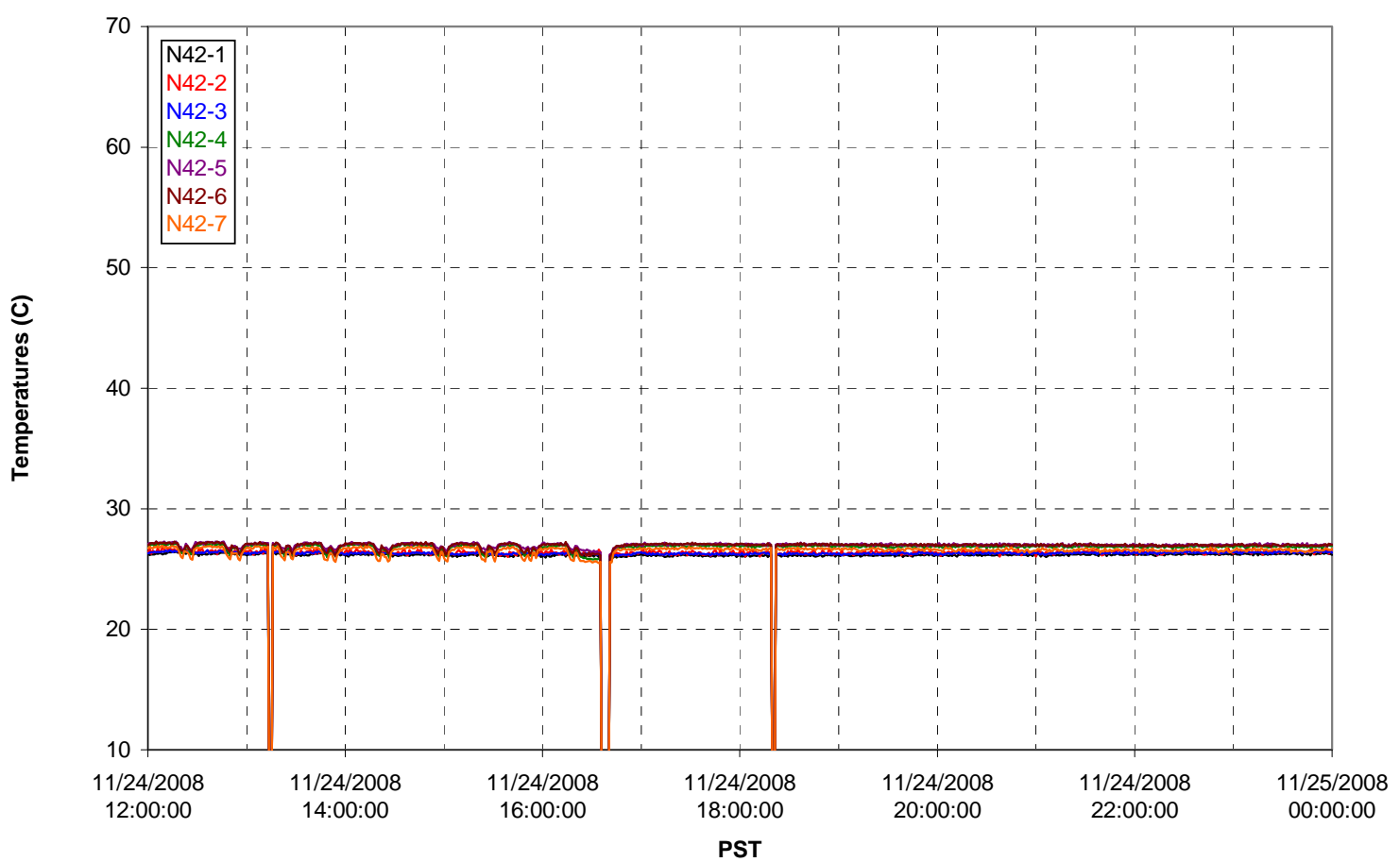

T02A temperatures

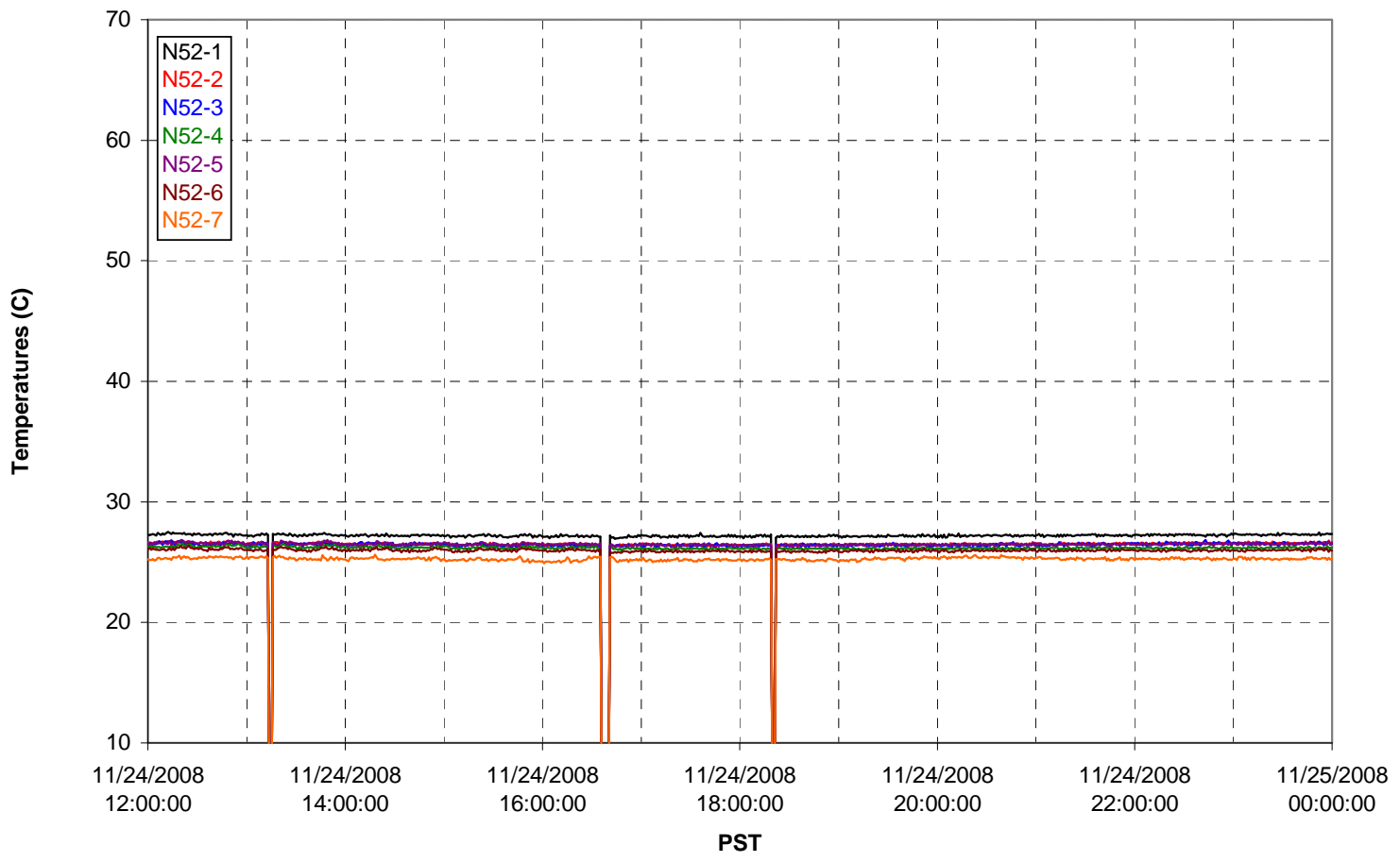


T02A Heating and Cooling

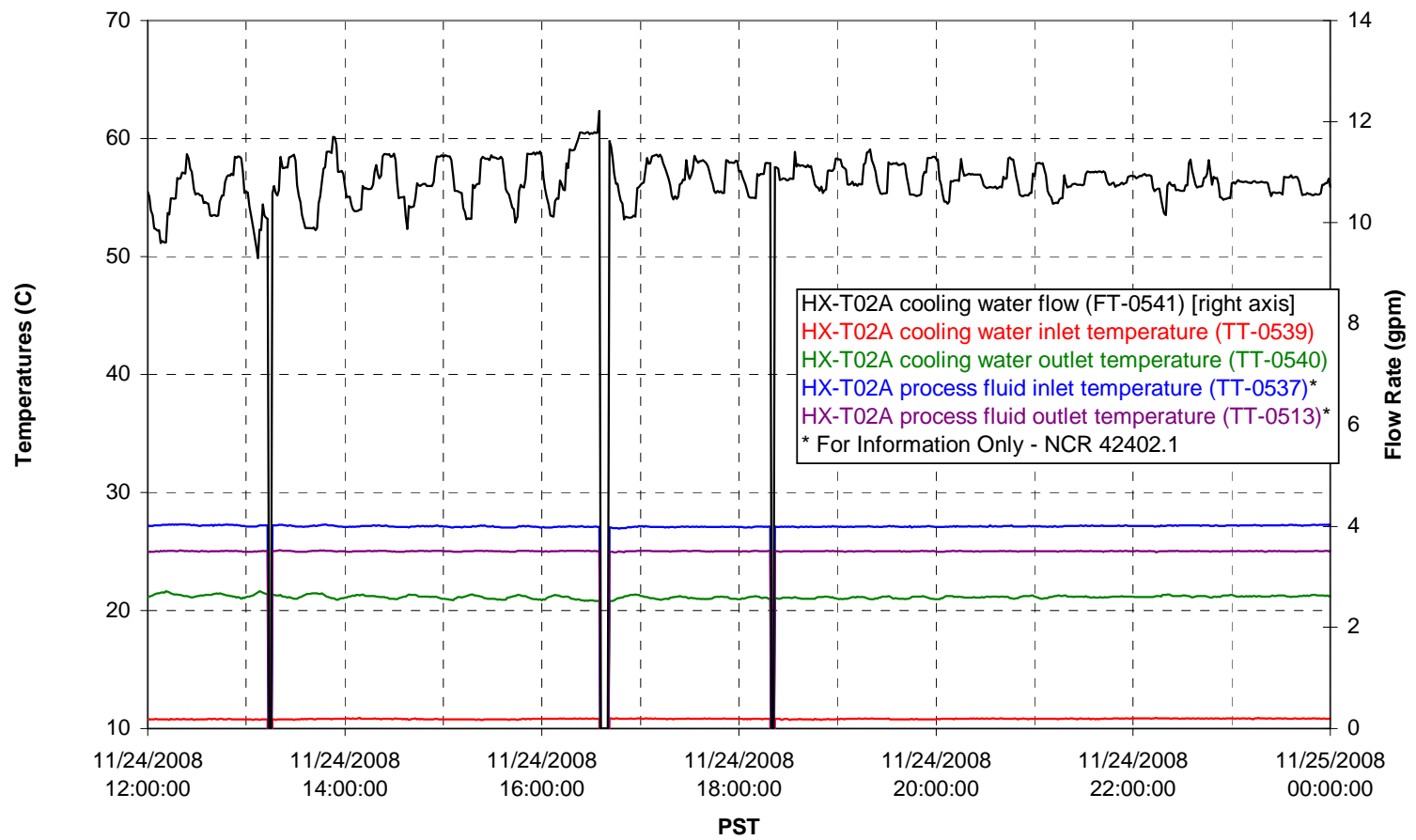

Pump Operation

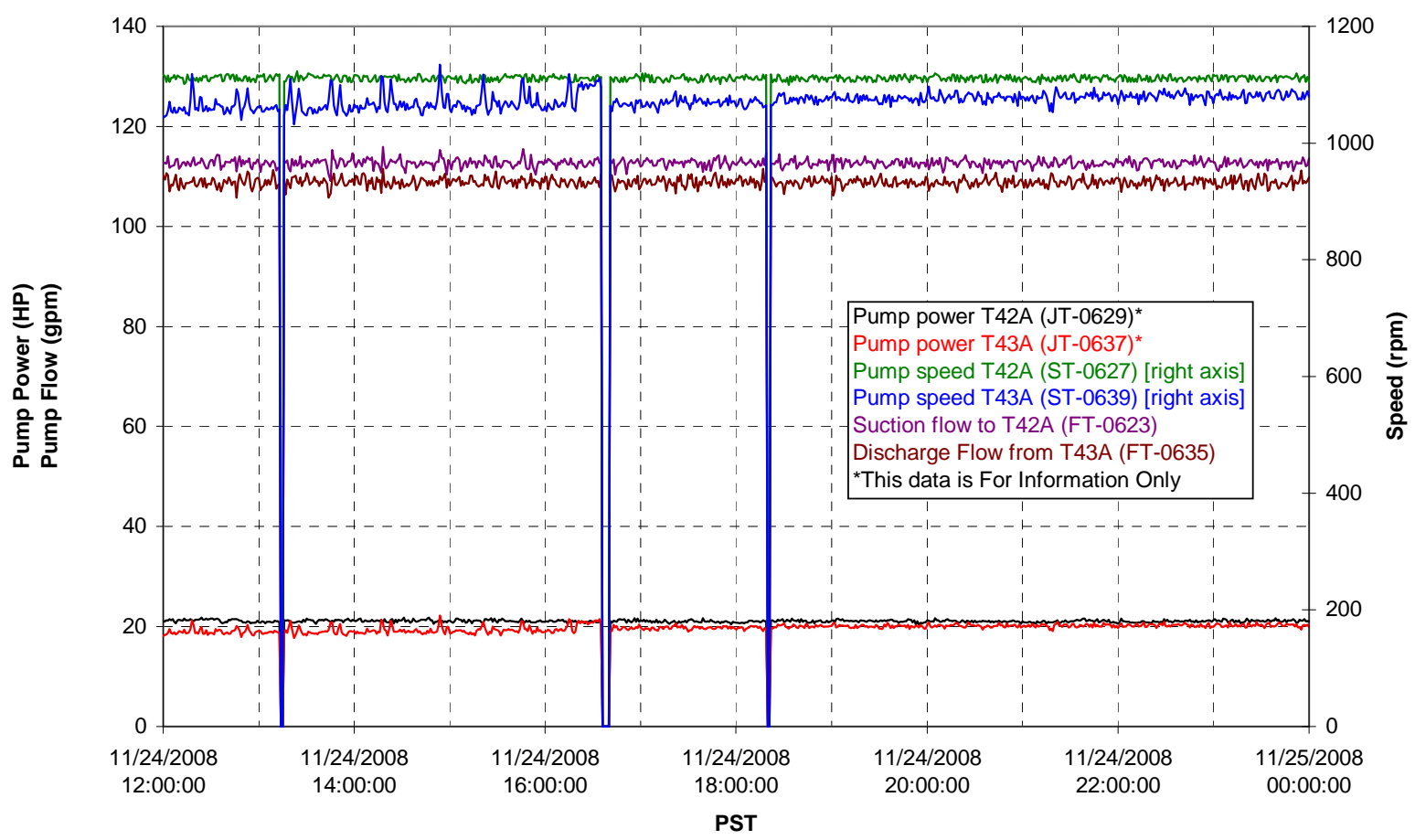


Pulsepot UFP-PP-T01A

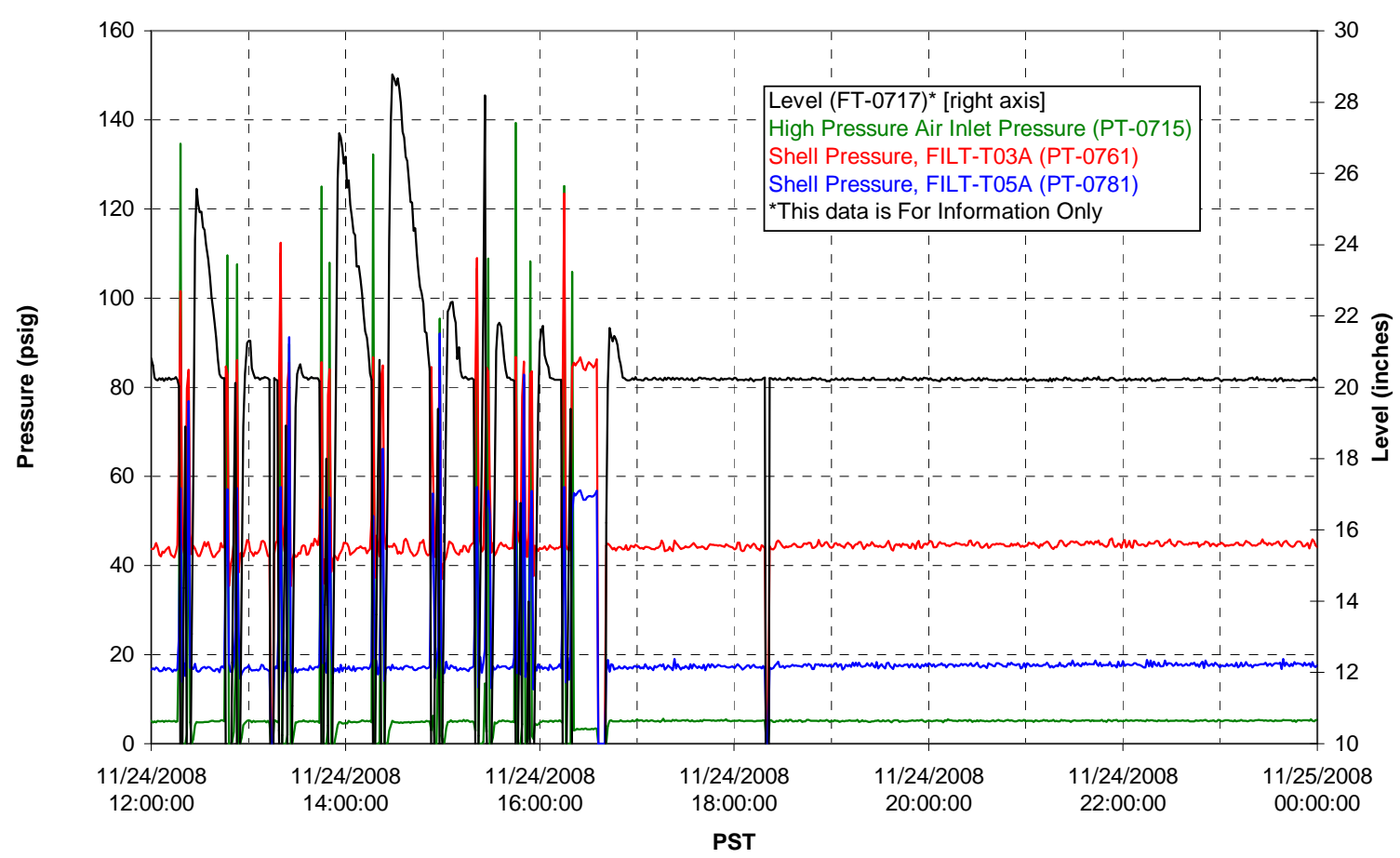

Pulsepot UFP-PP-T02A

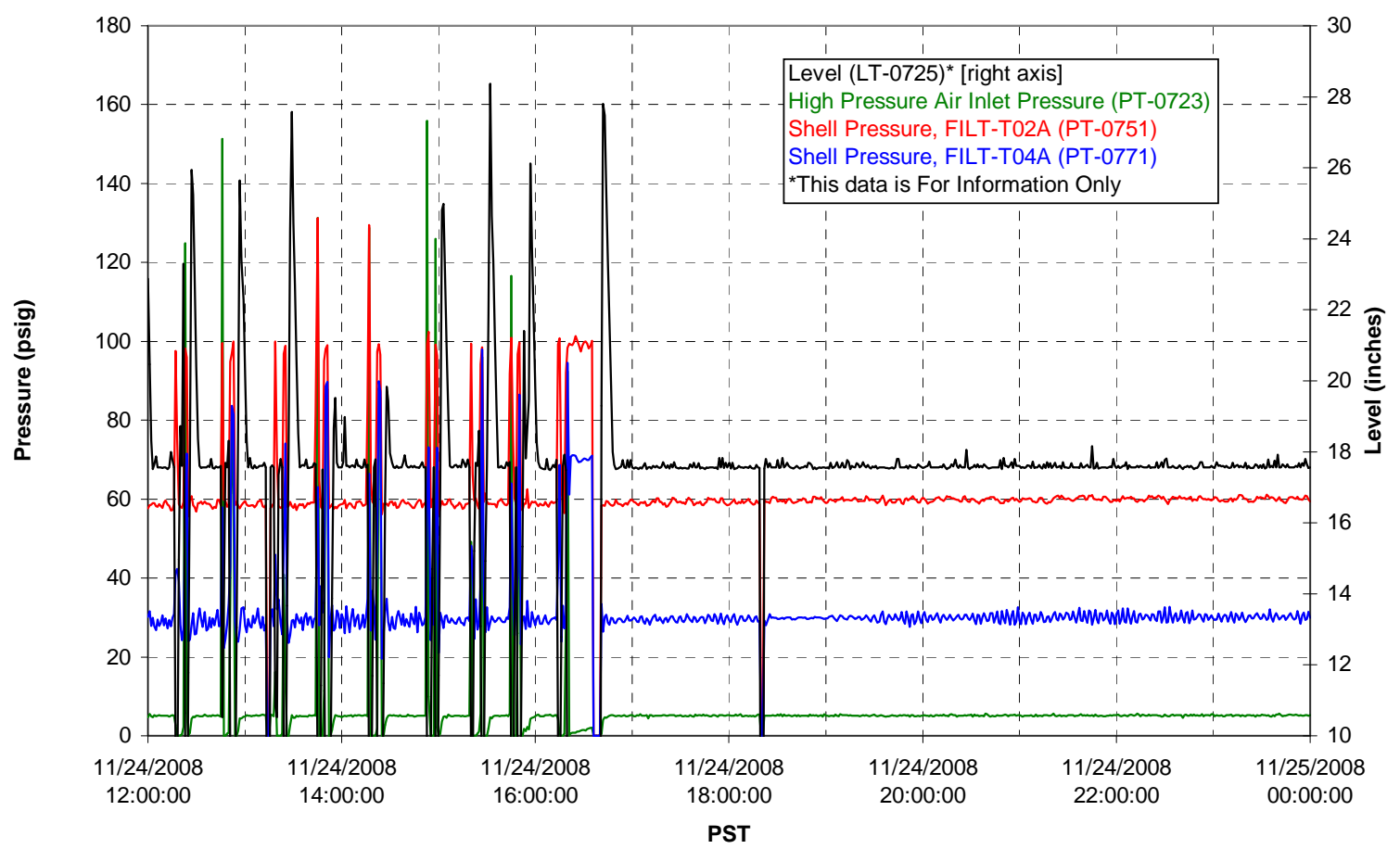


Pulsepot UFP-PP-T03A

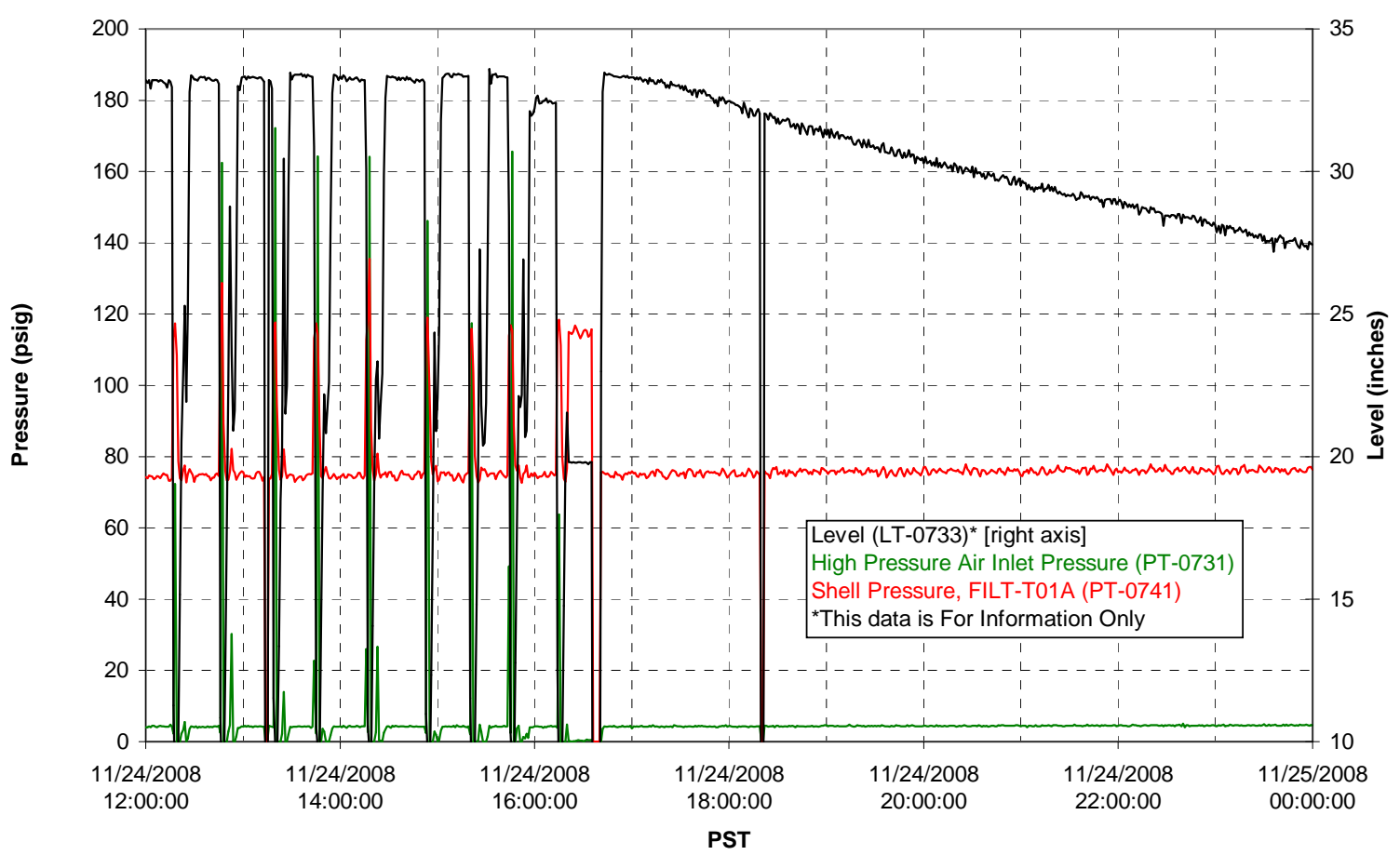

Pulsepot Levels

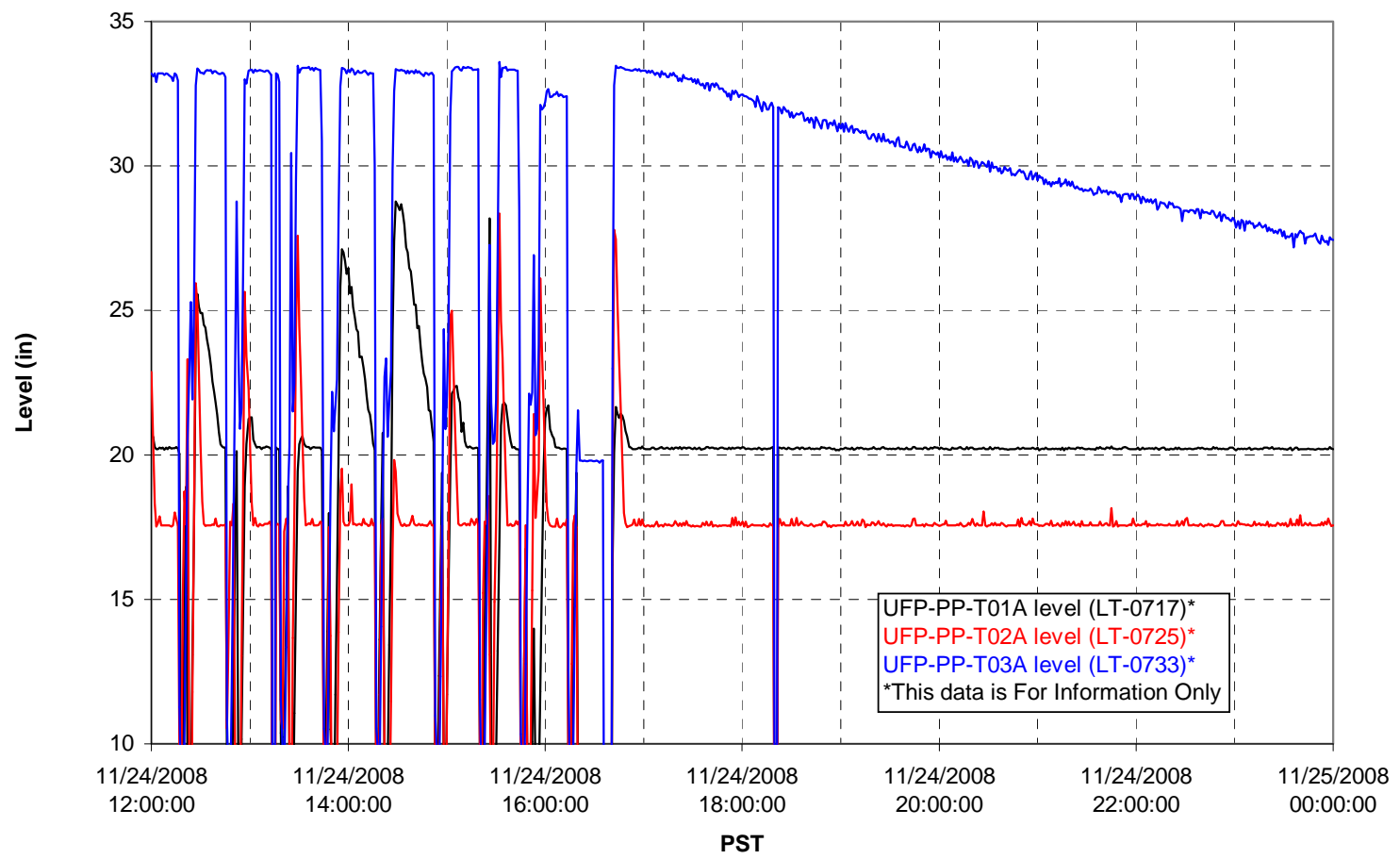


Filter UFP-FILT-T01A

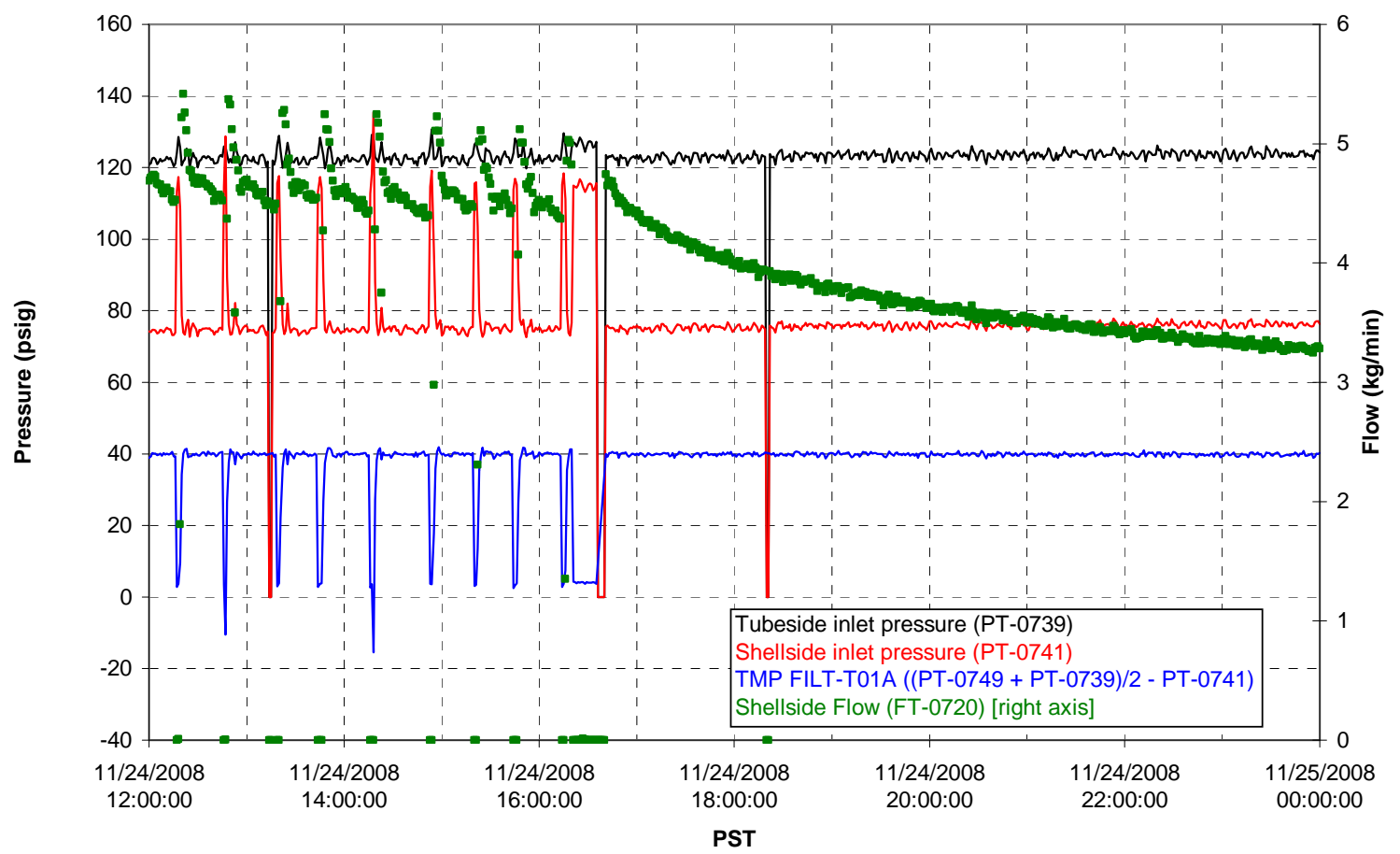

Filter UFP-FILT-T02A

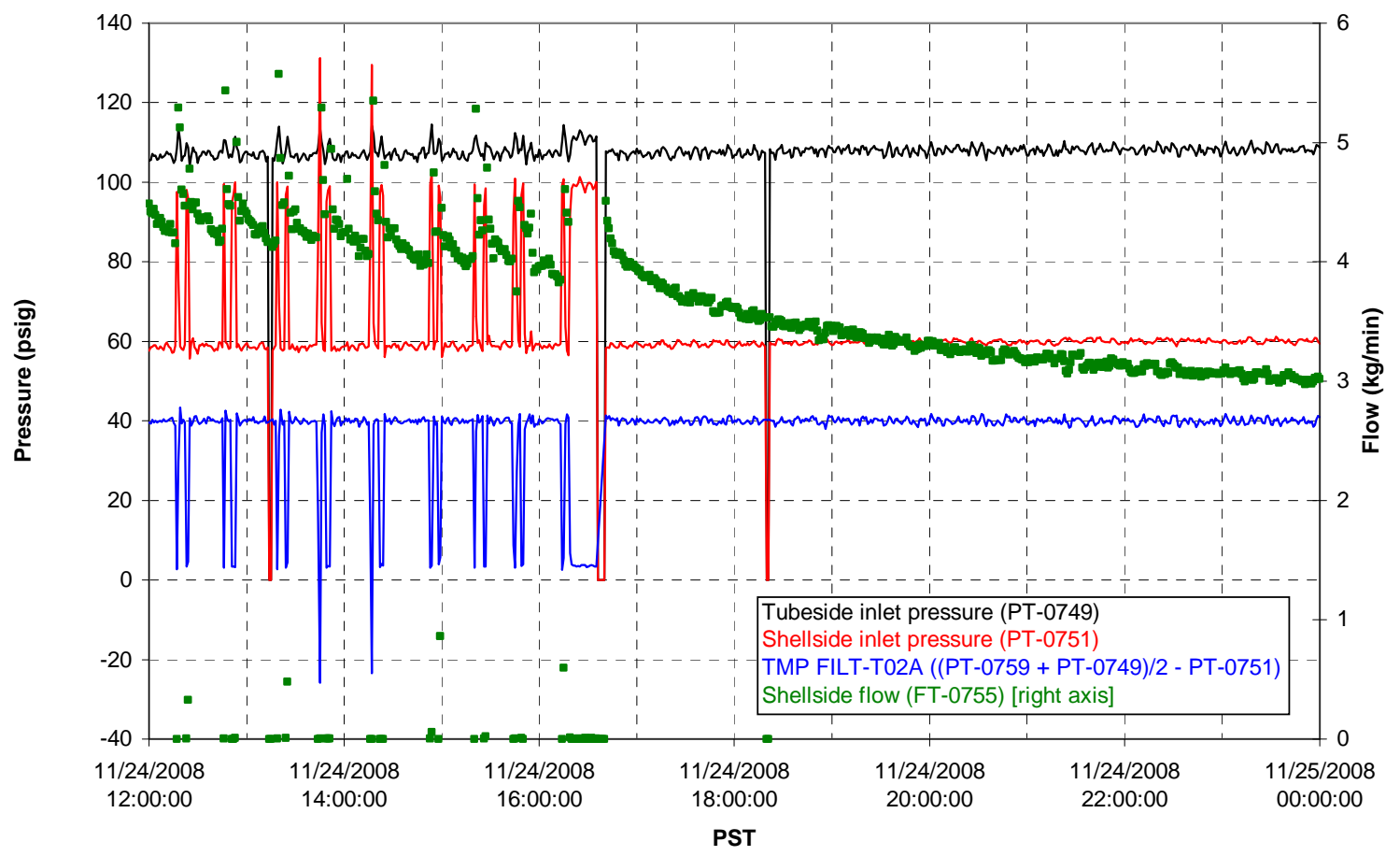


Filter UFP-FILT-T03A

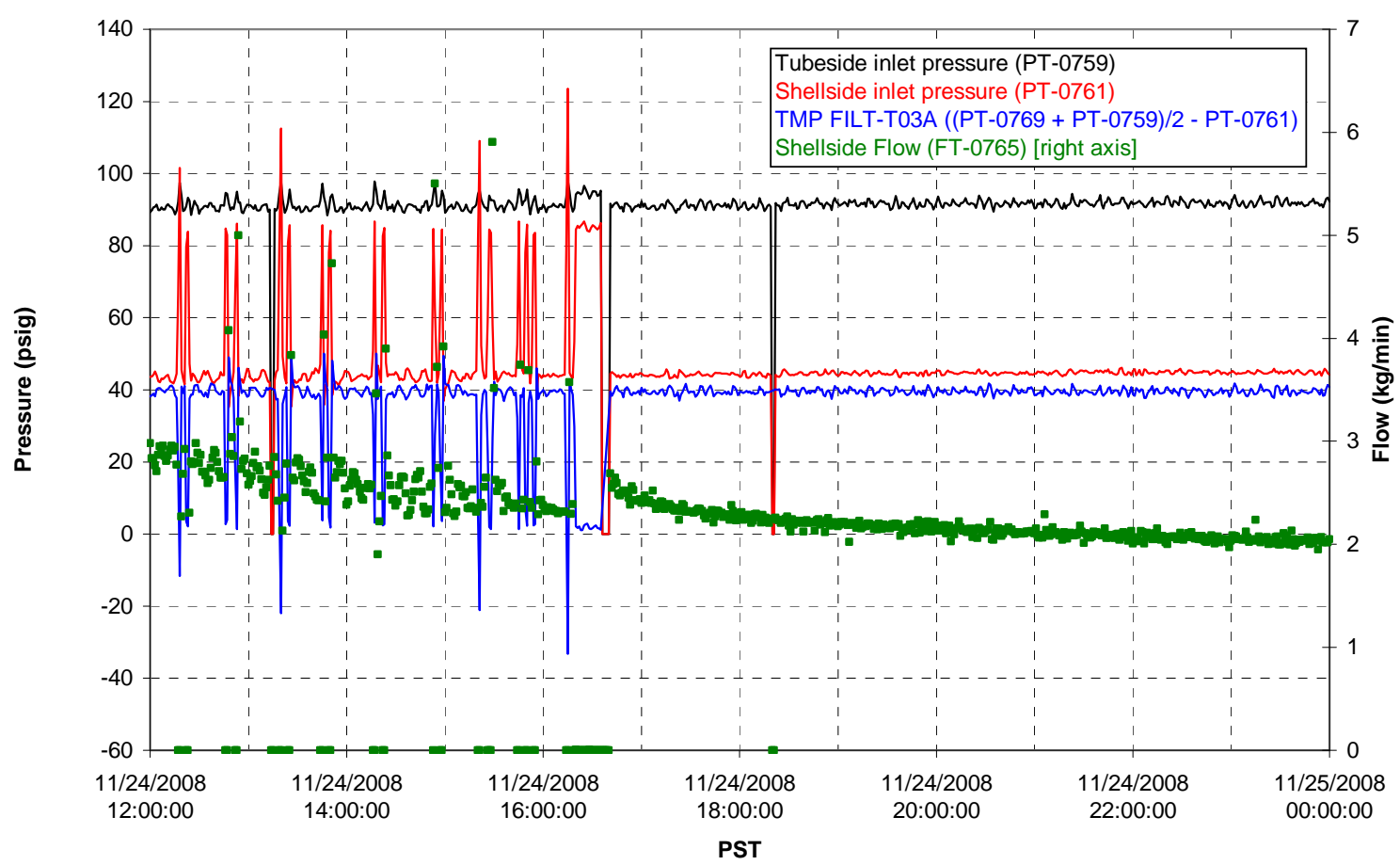

Filter UFP-FILT-T04A

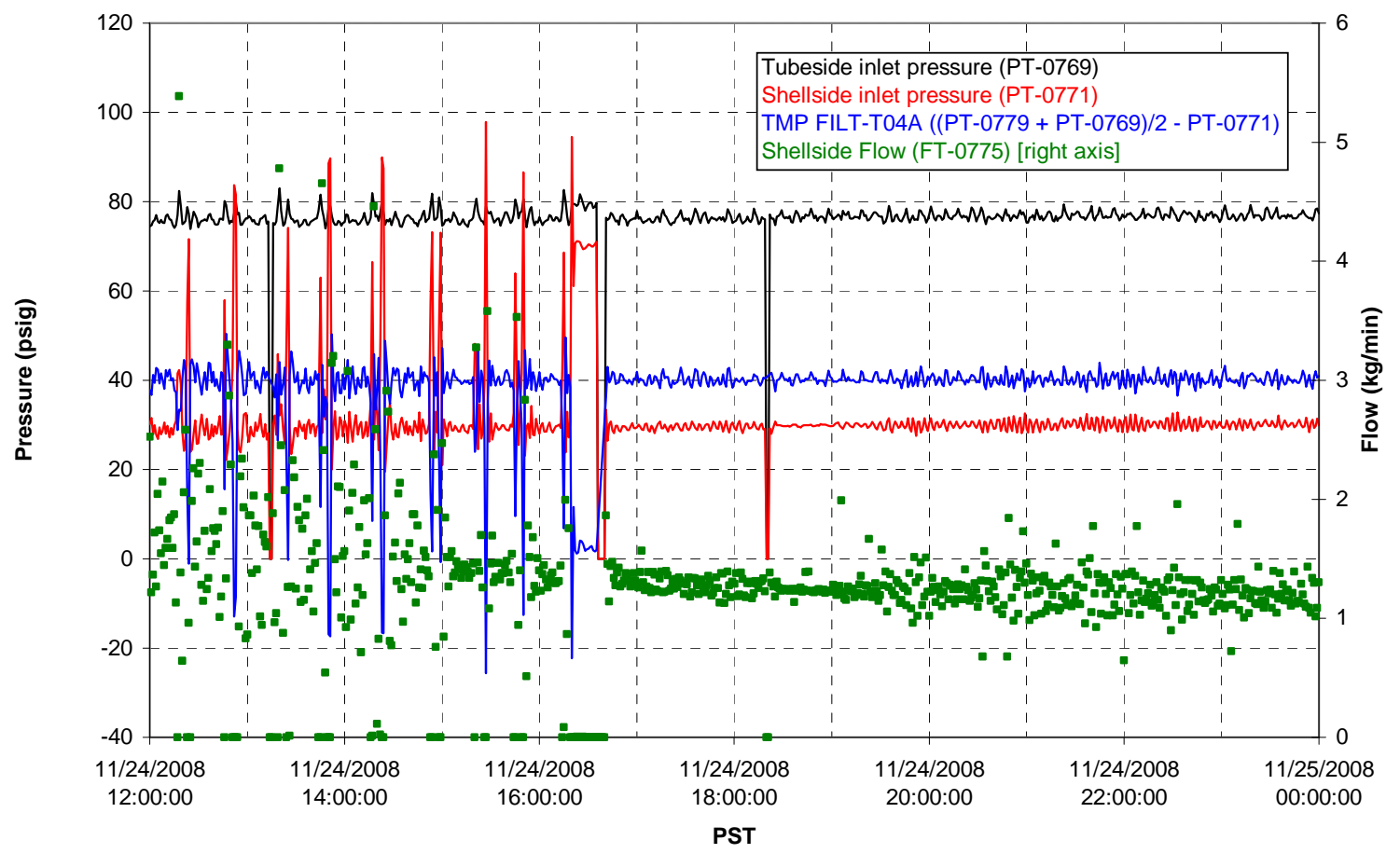


Filter UFP-FILT-T05A

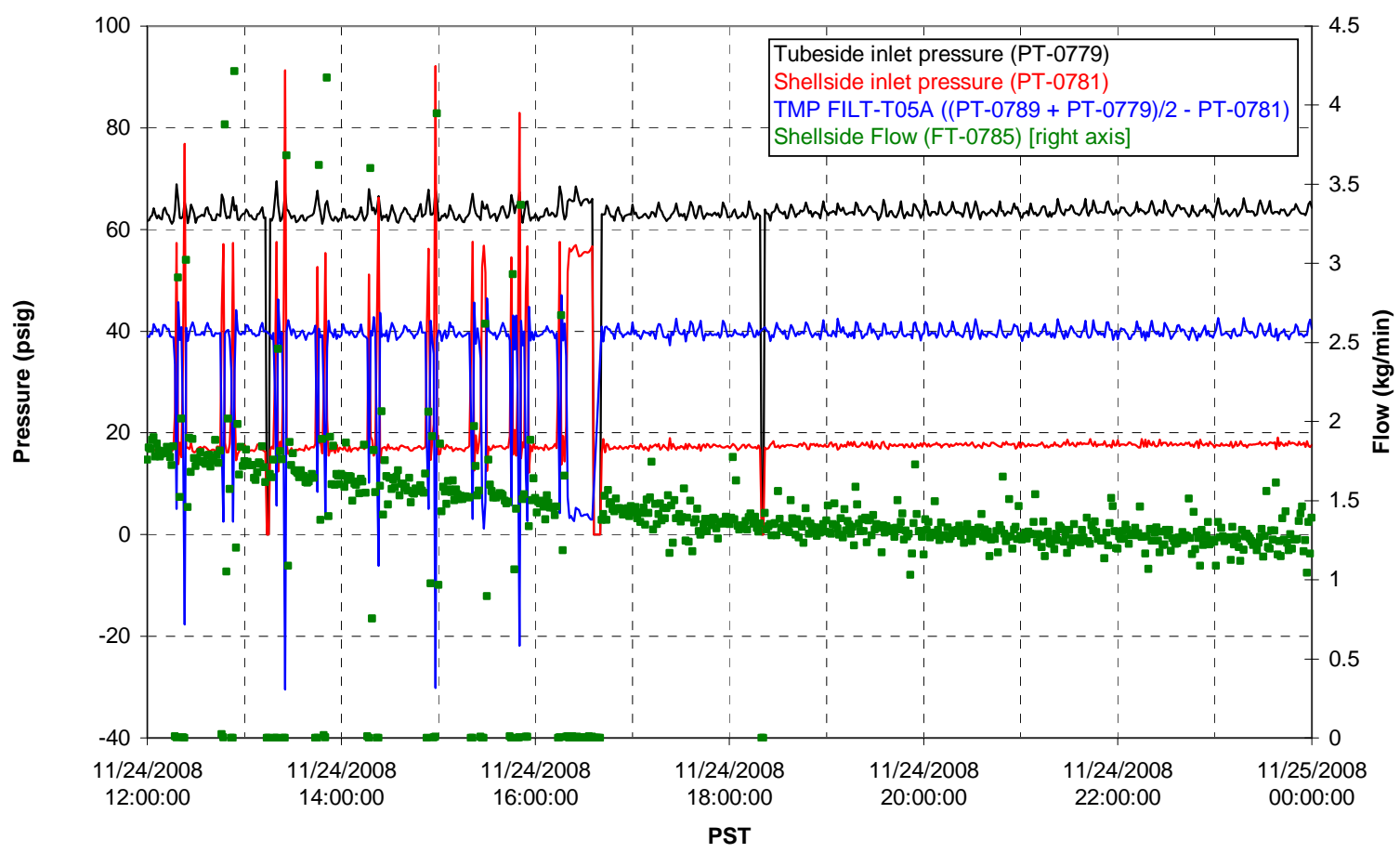

Chemical Flow

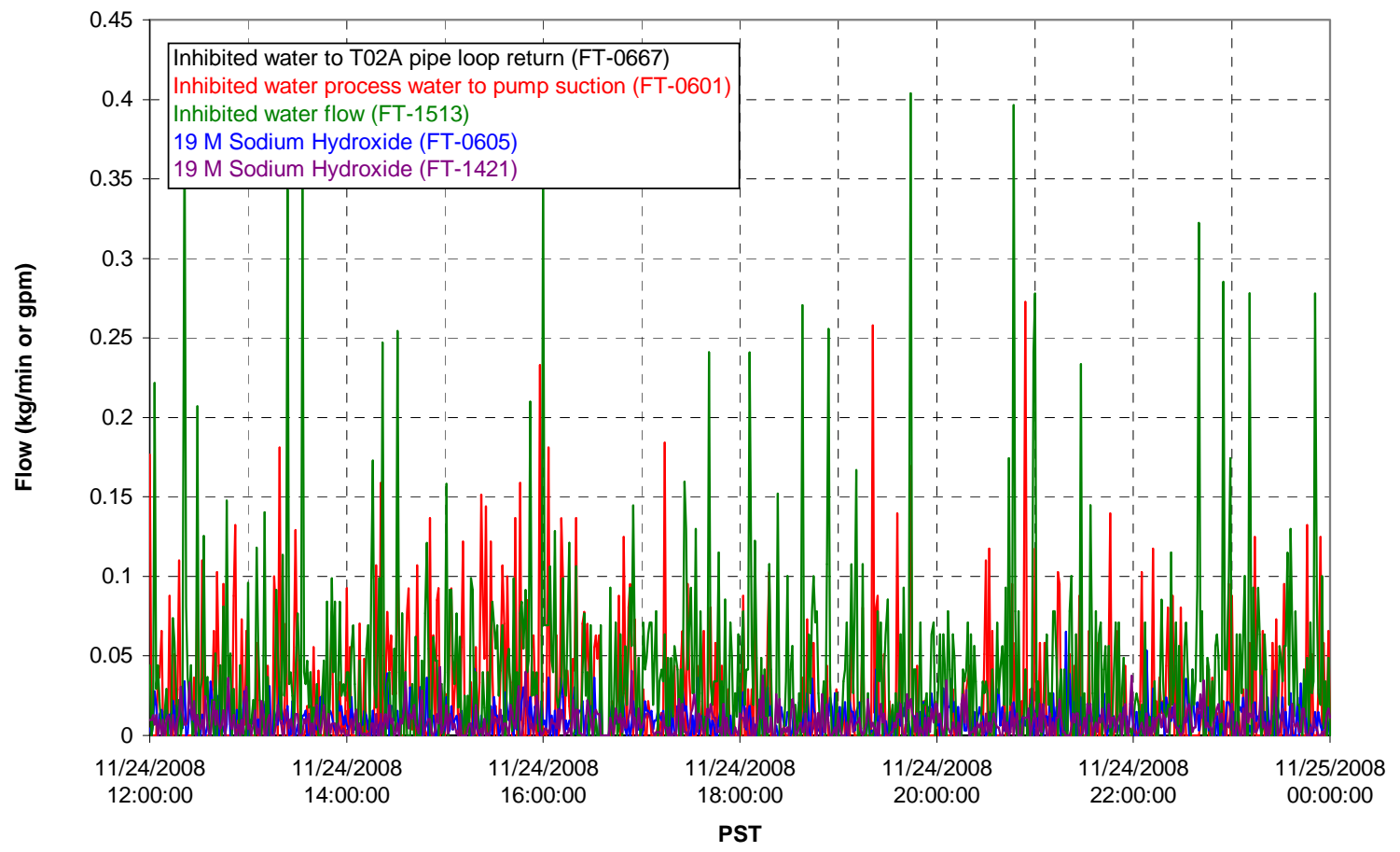




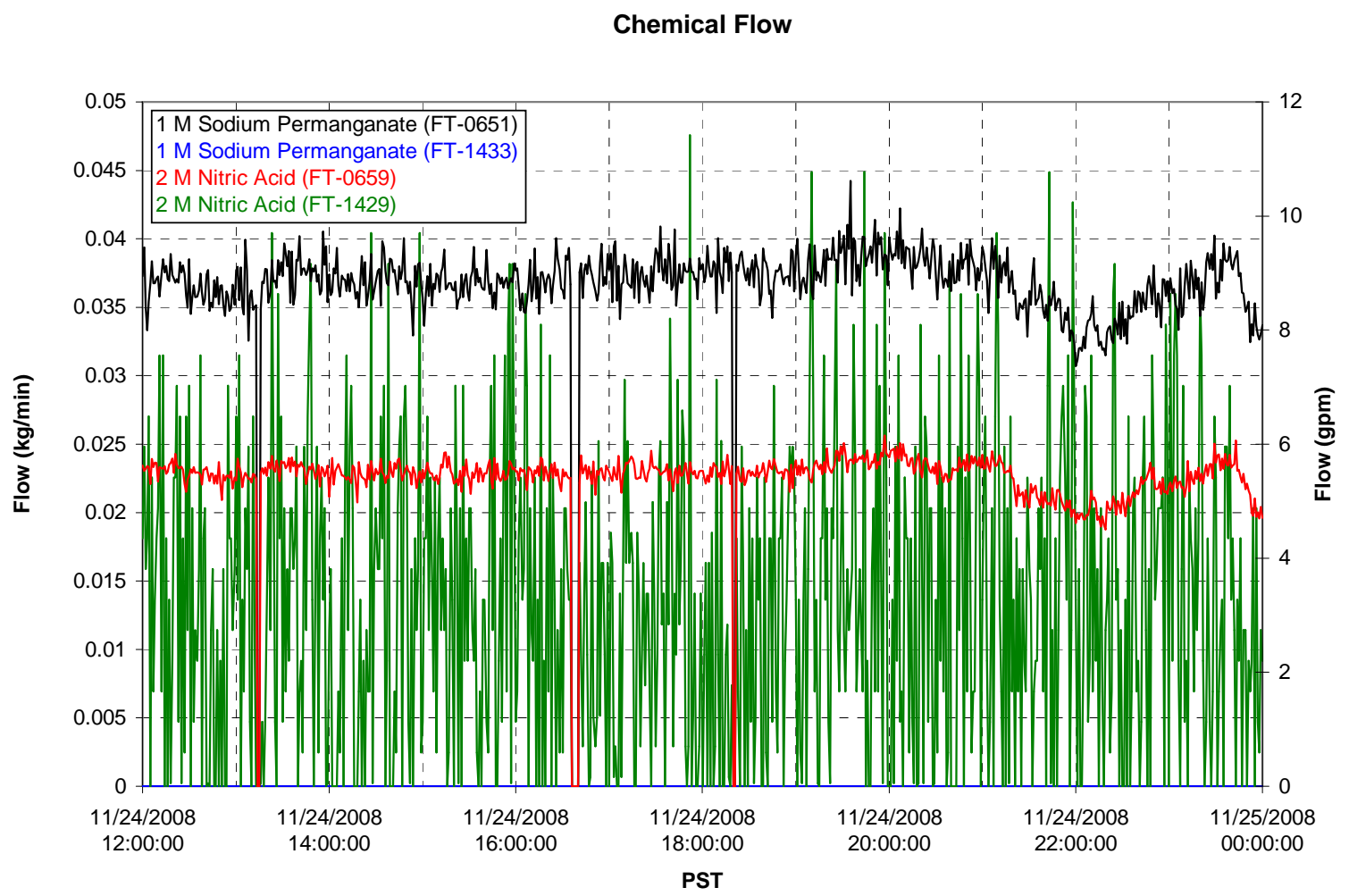

Air Flows

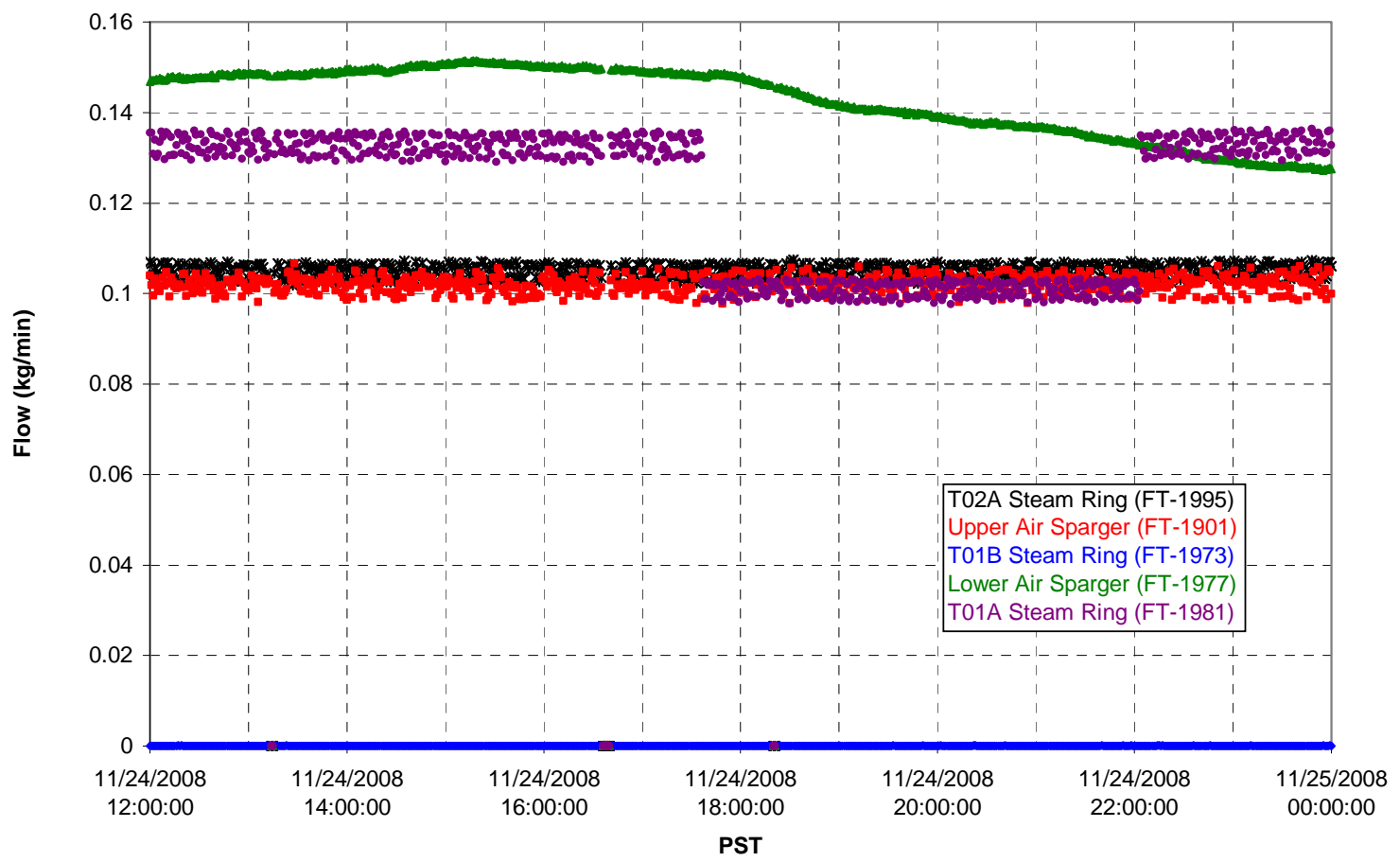


T02A Steam

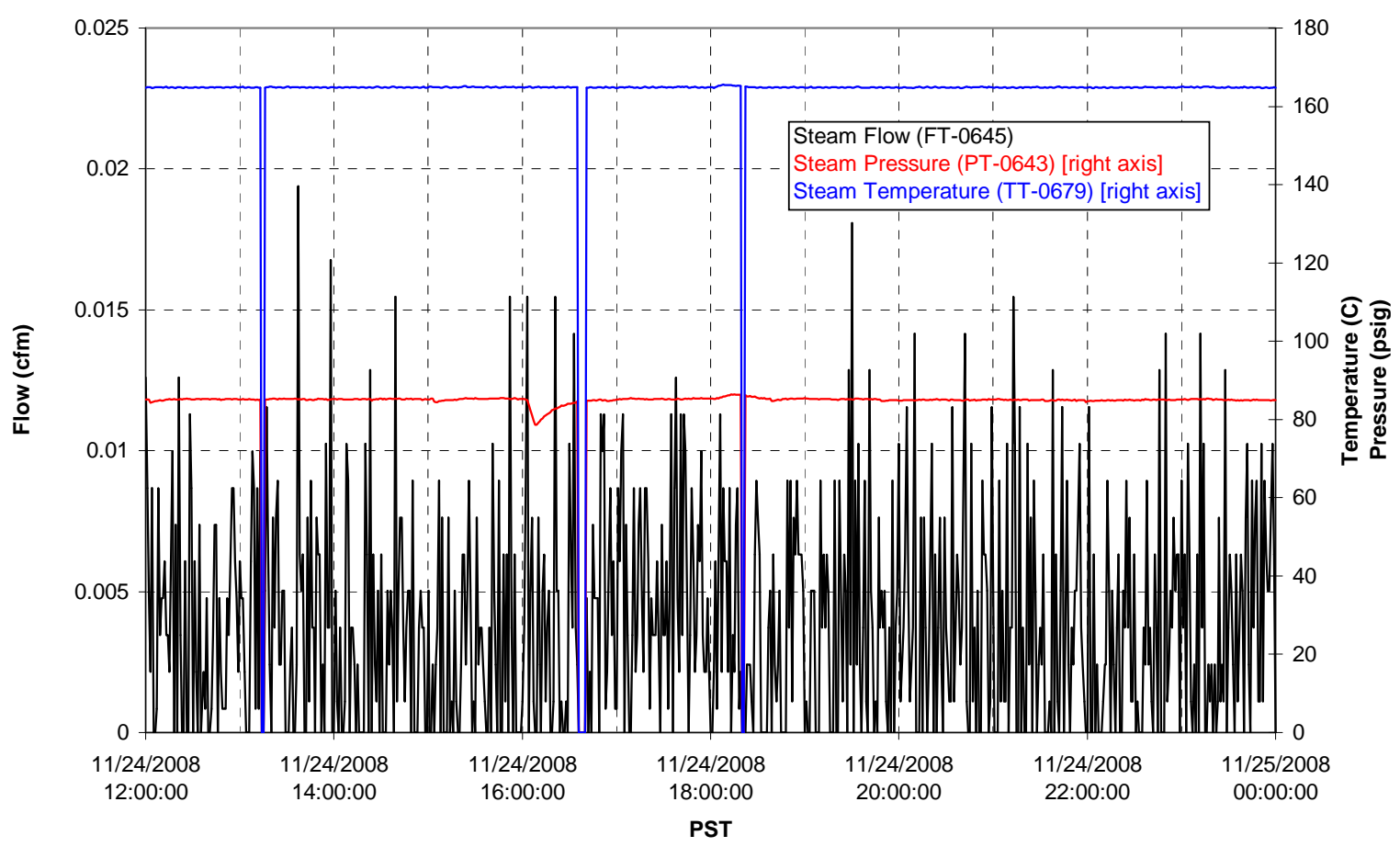

T01A Steam

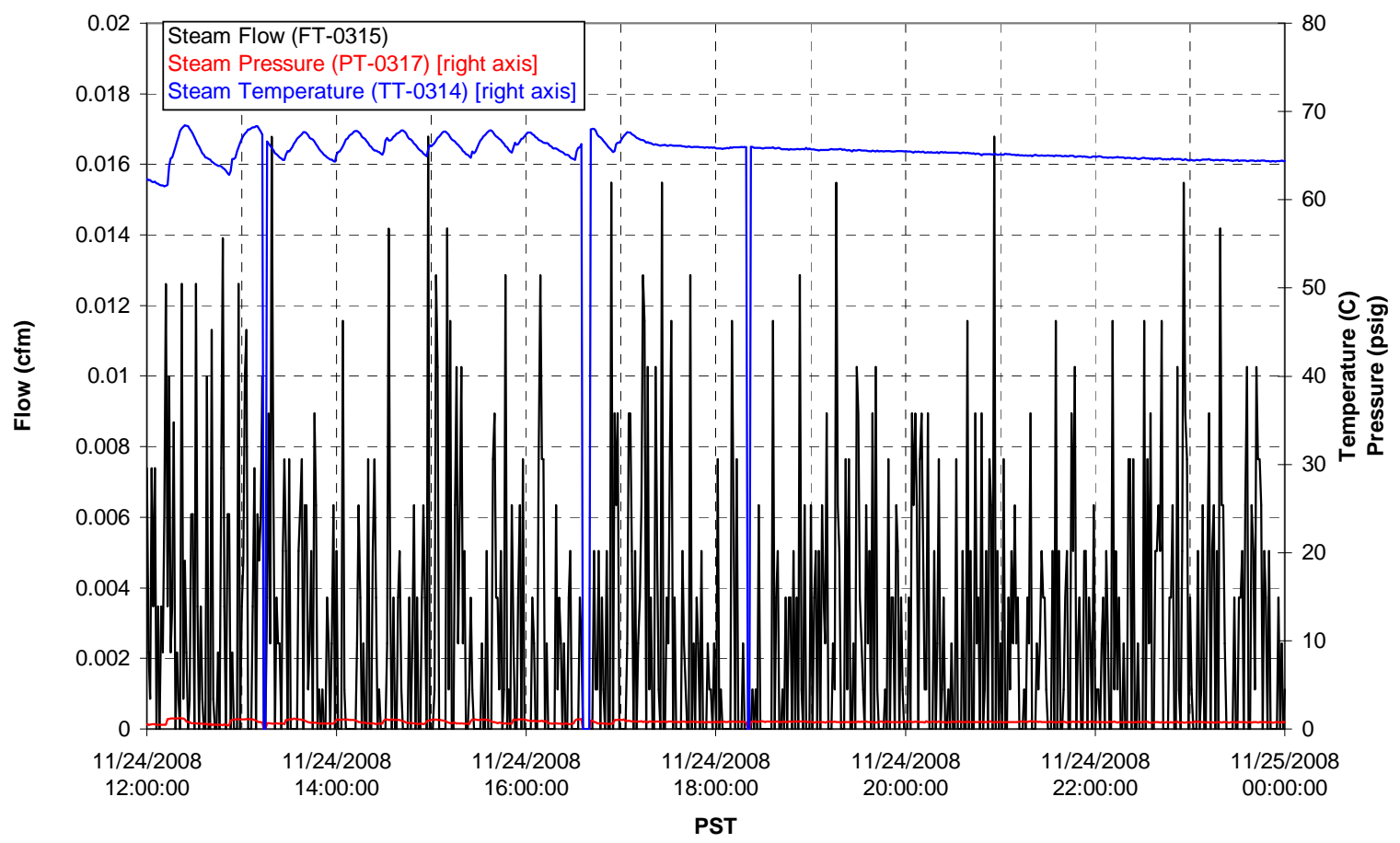


T01B Steam

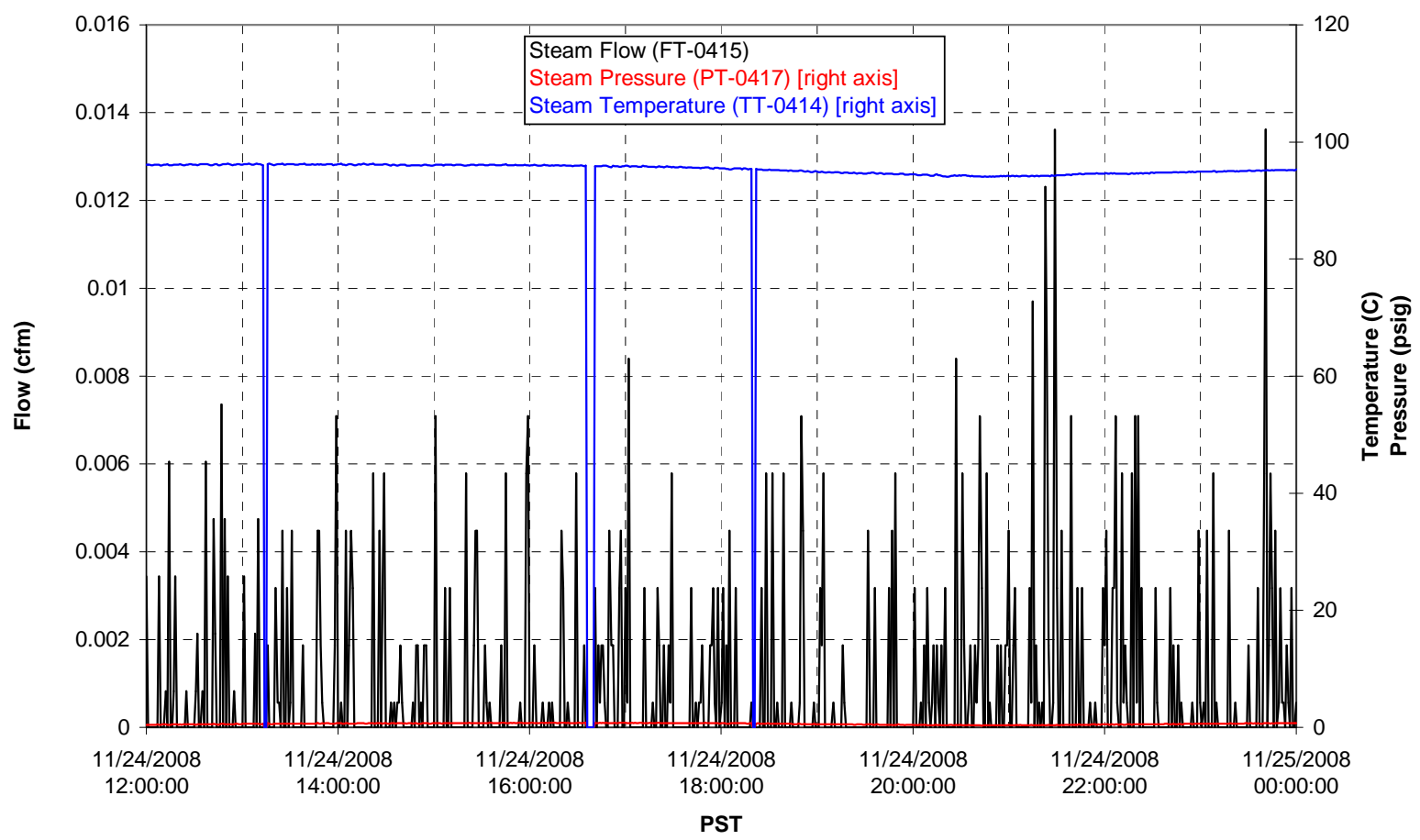


Functional Test Data Plots

11/25/2008 00:00 - 11/25/2008 12:00 
T01A level

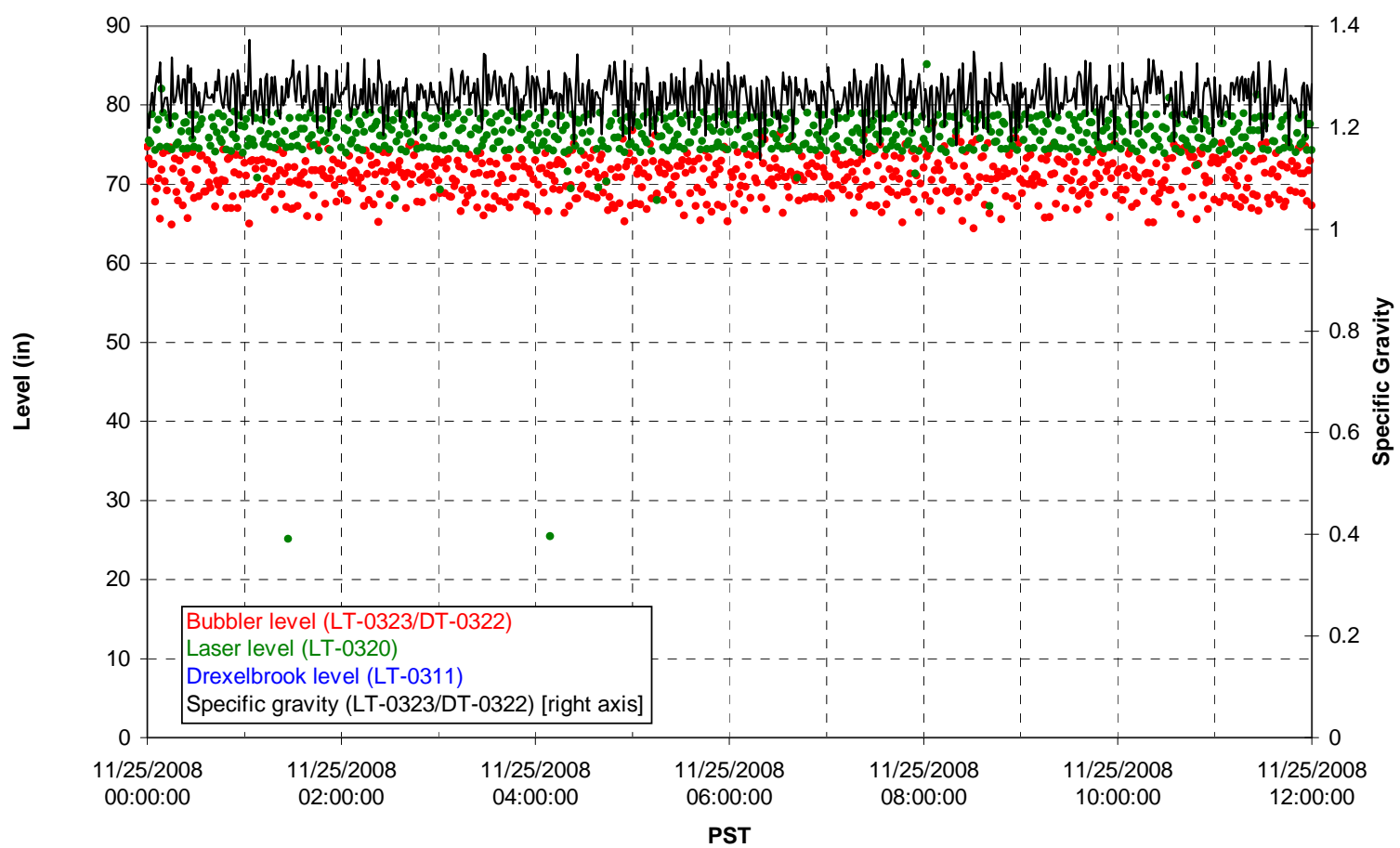

T01A temperatures

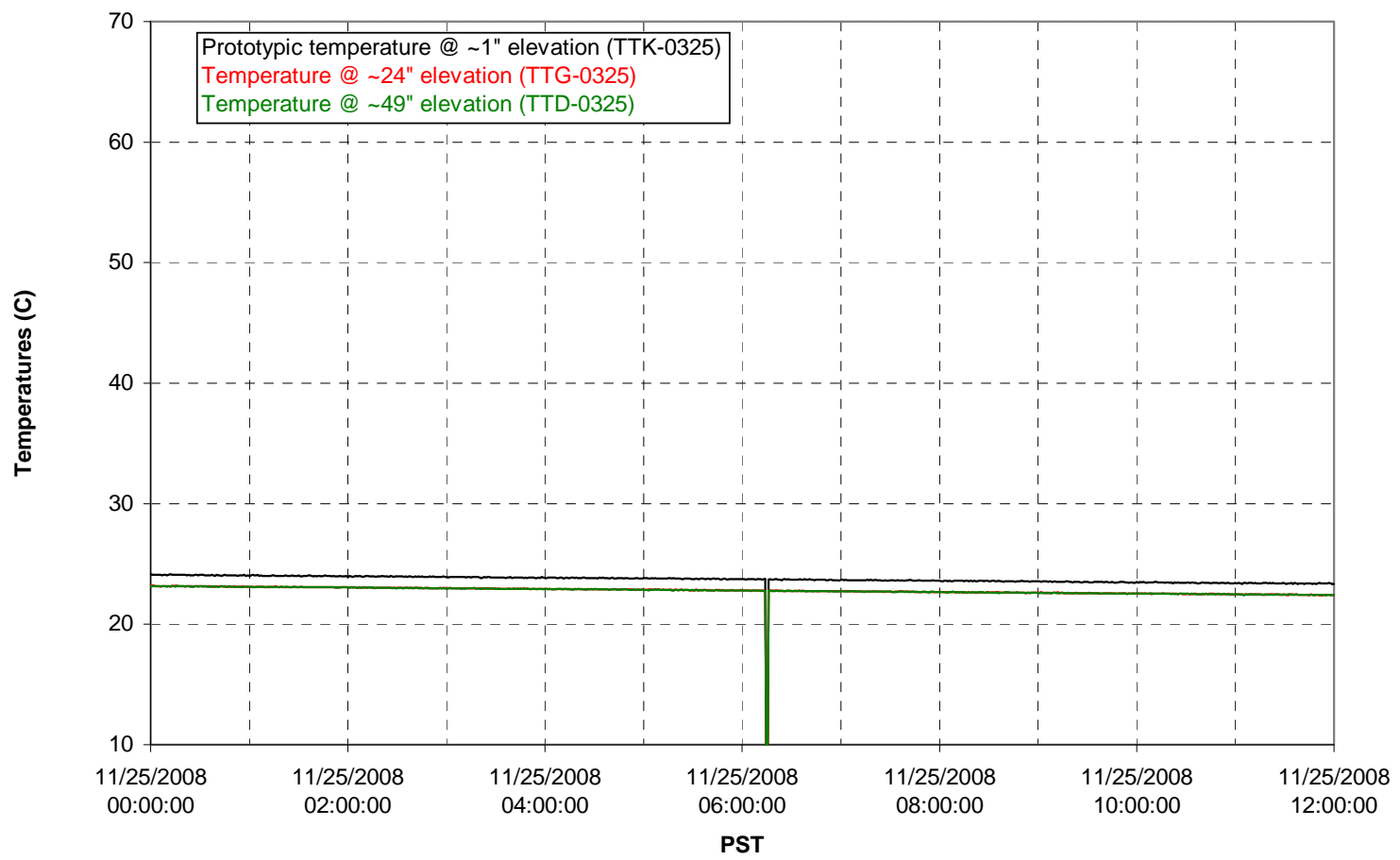


T01B level

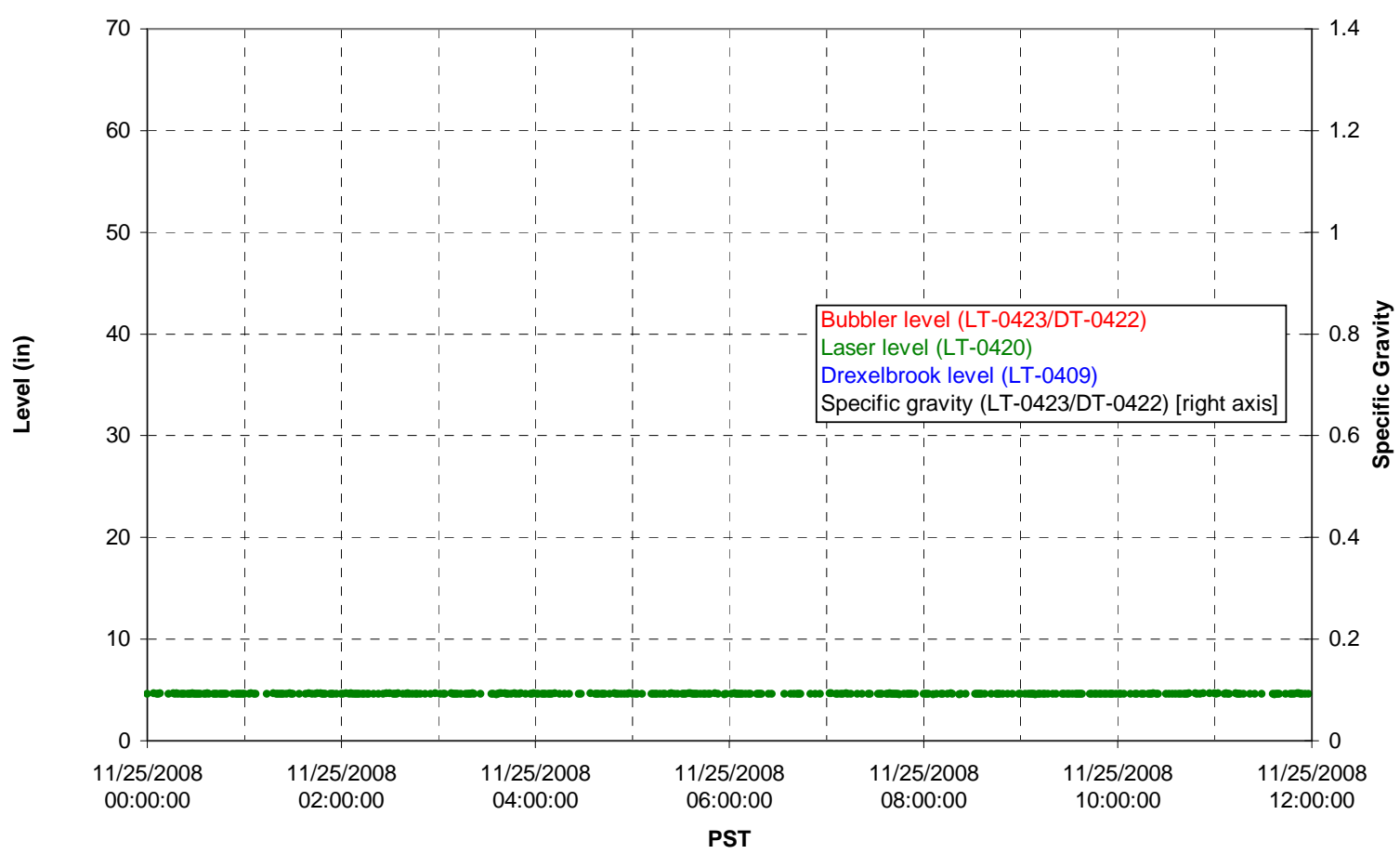

T01B temperatures

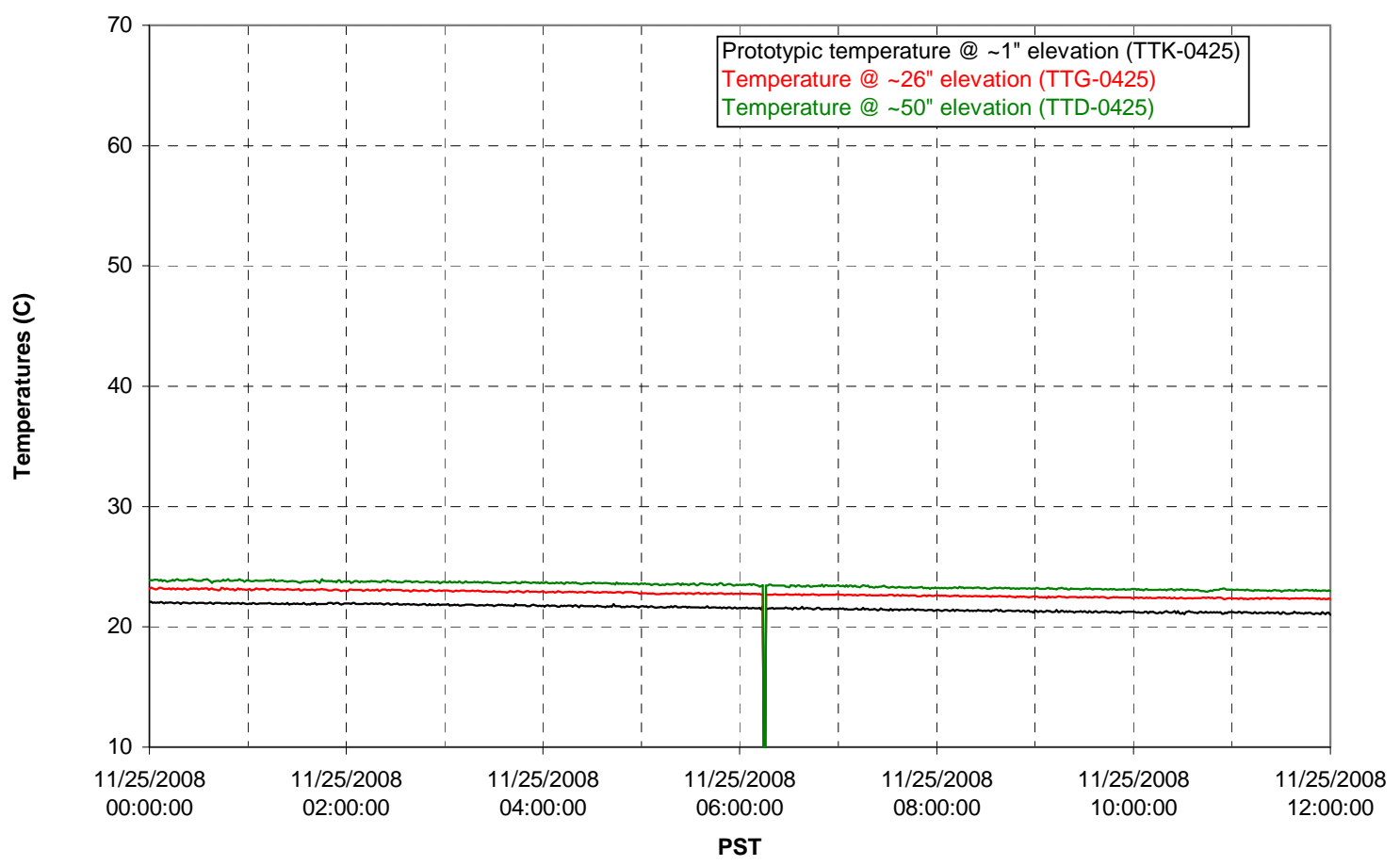


T02A level

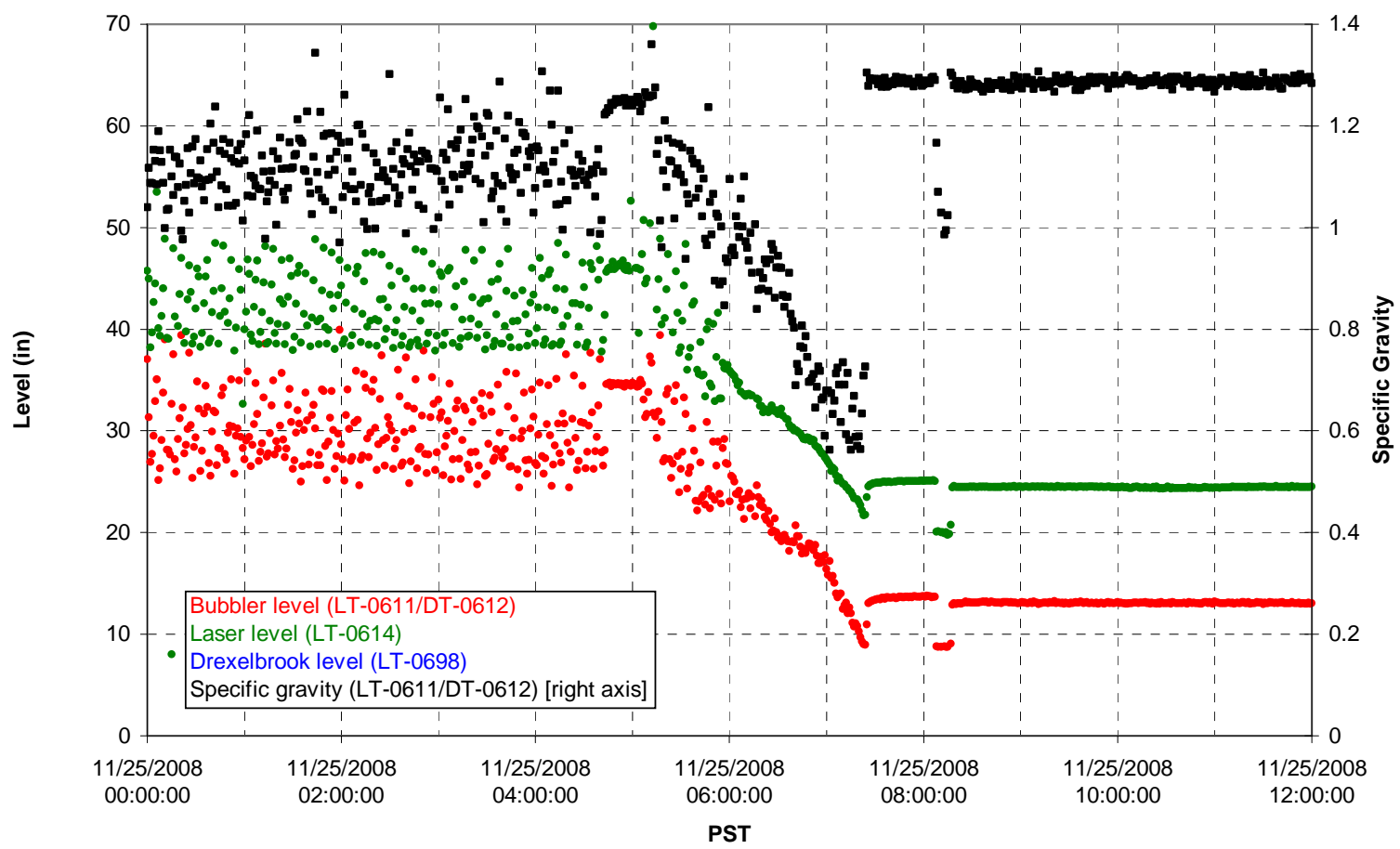

T02A temperatures

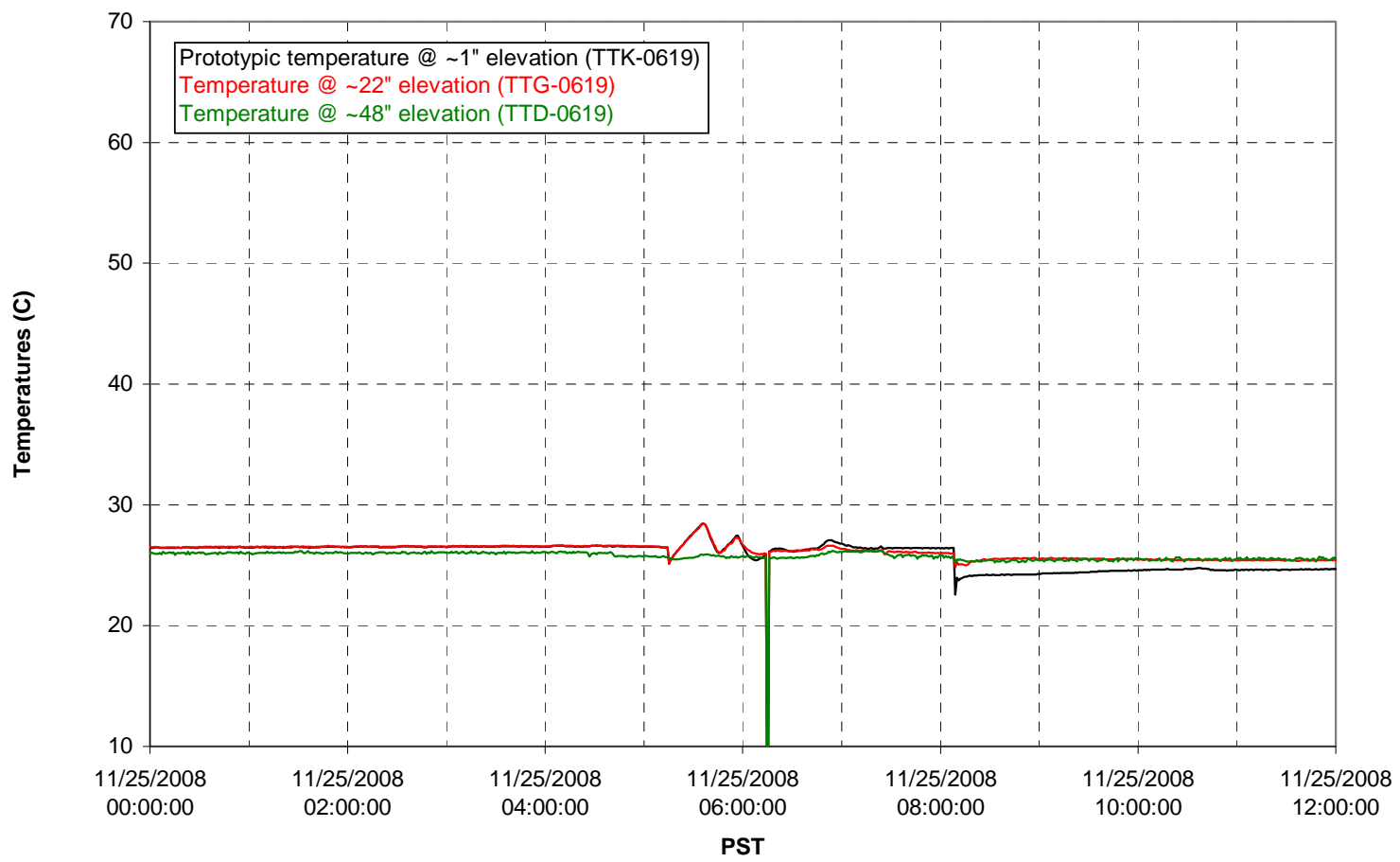


T02A and filter loop temperatures
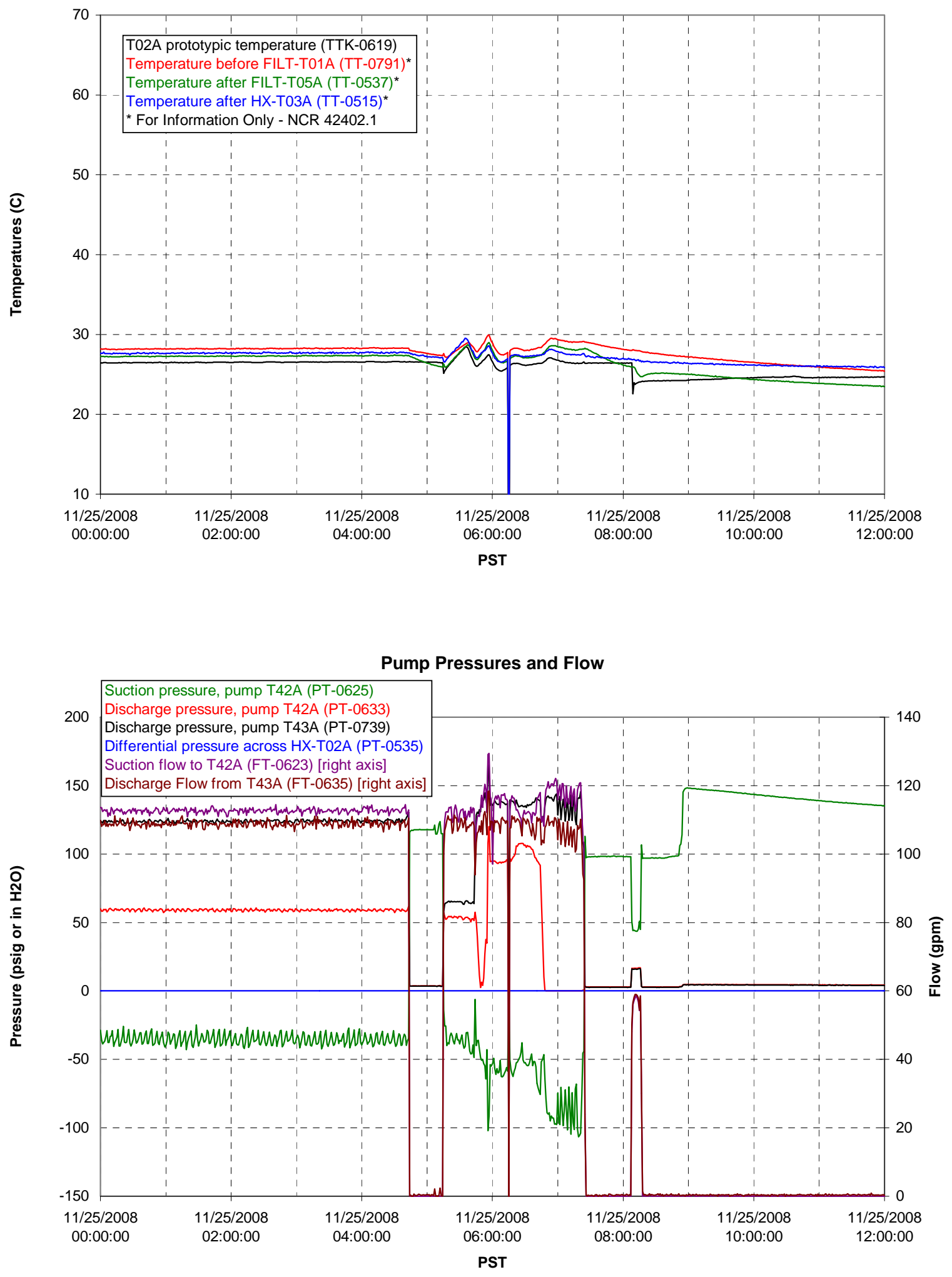


\section{Axial pressure drop}

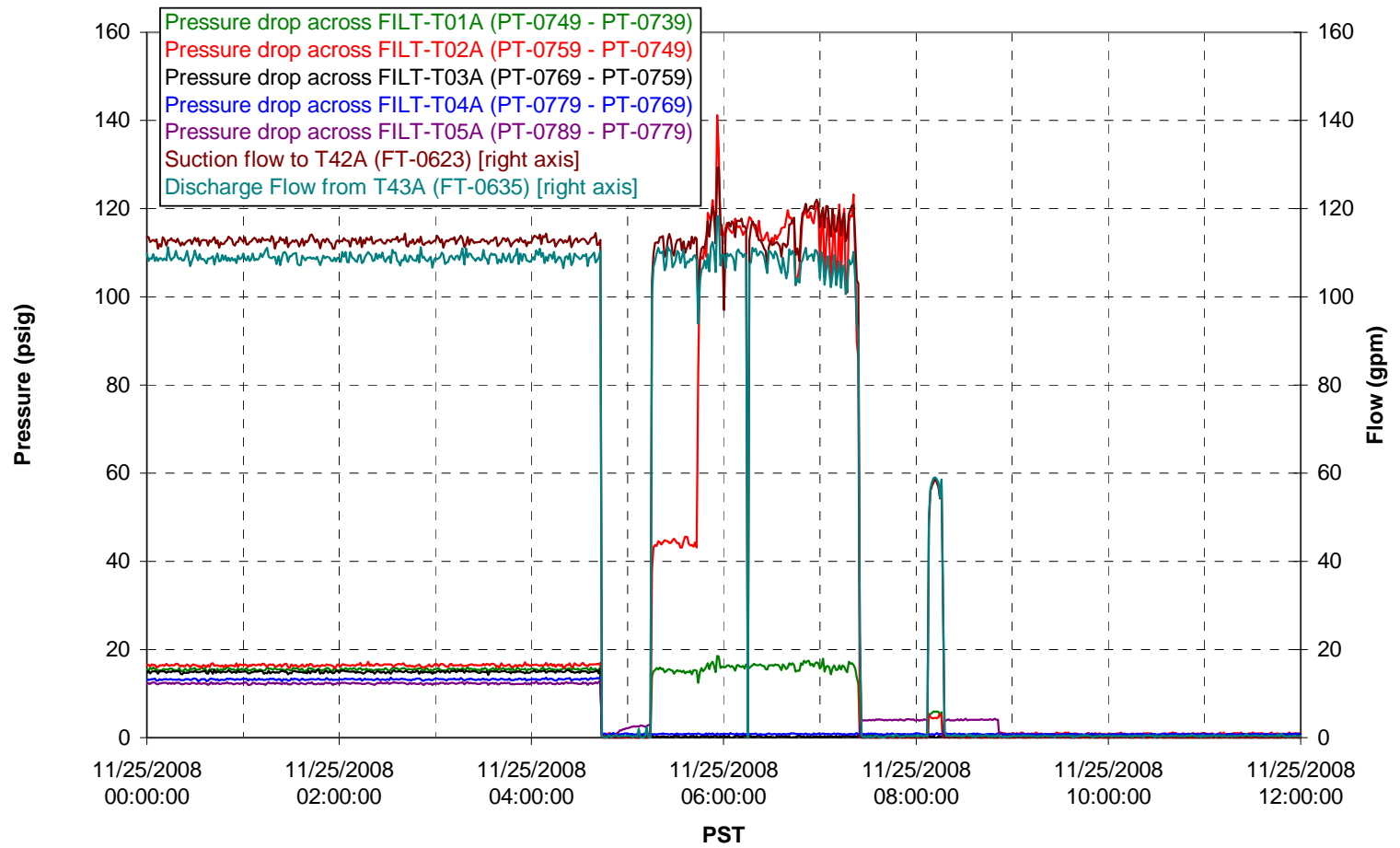

Permeate flow rates

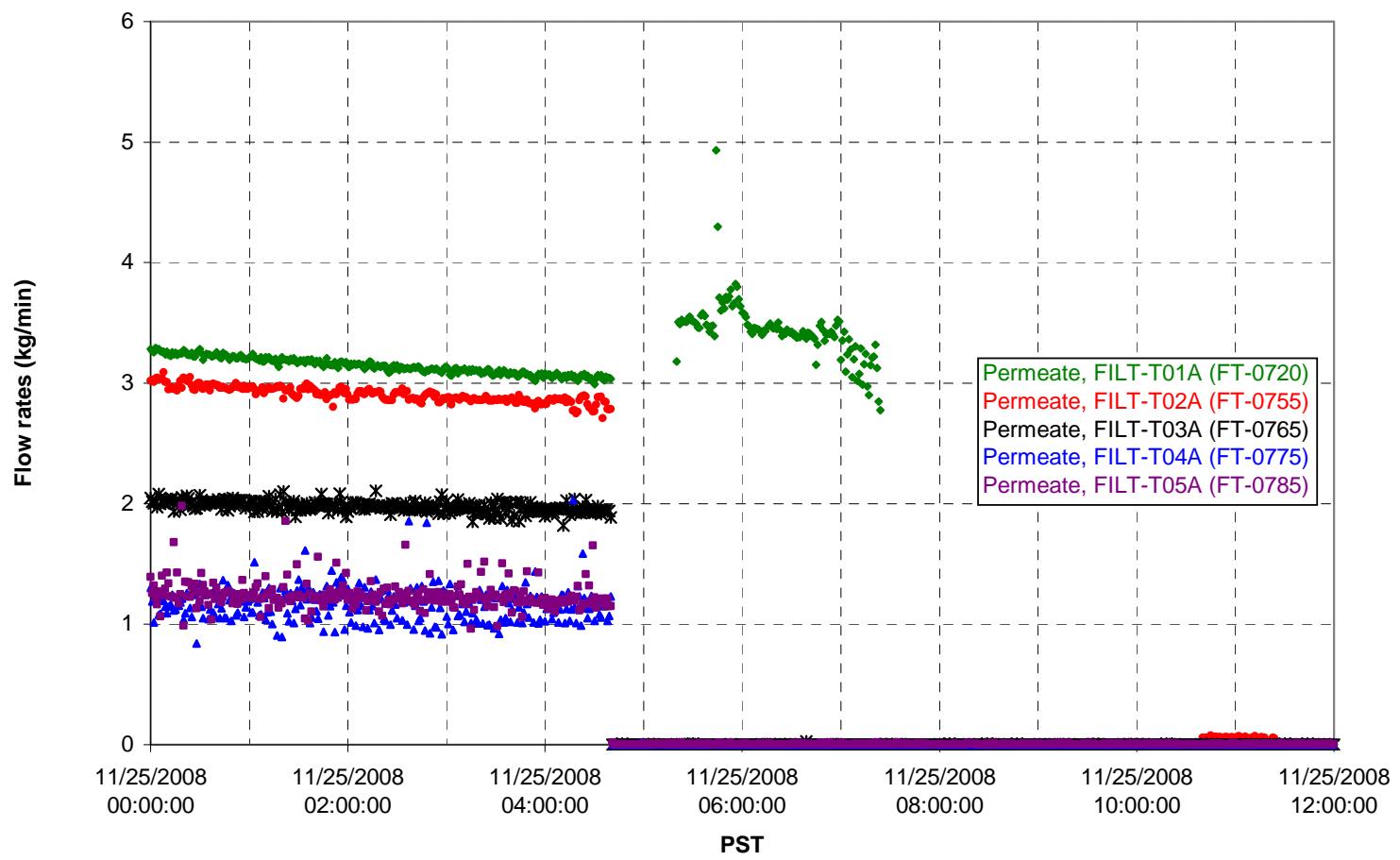


T02A Inner Temperature Tree

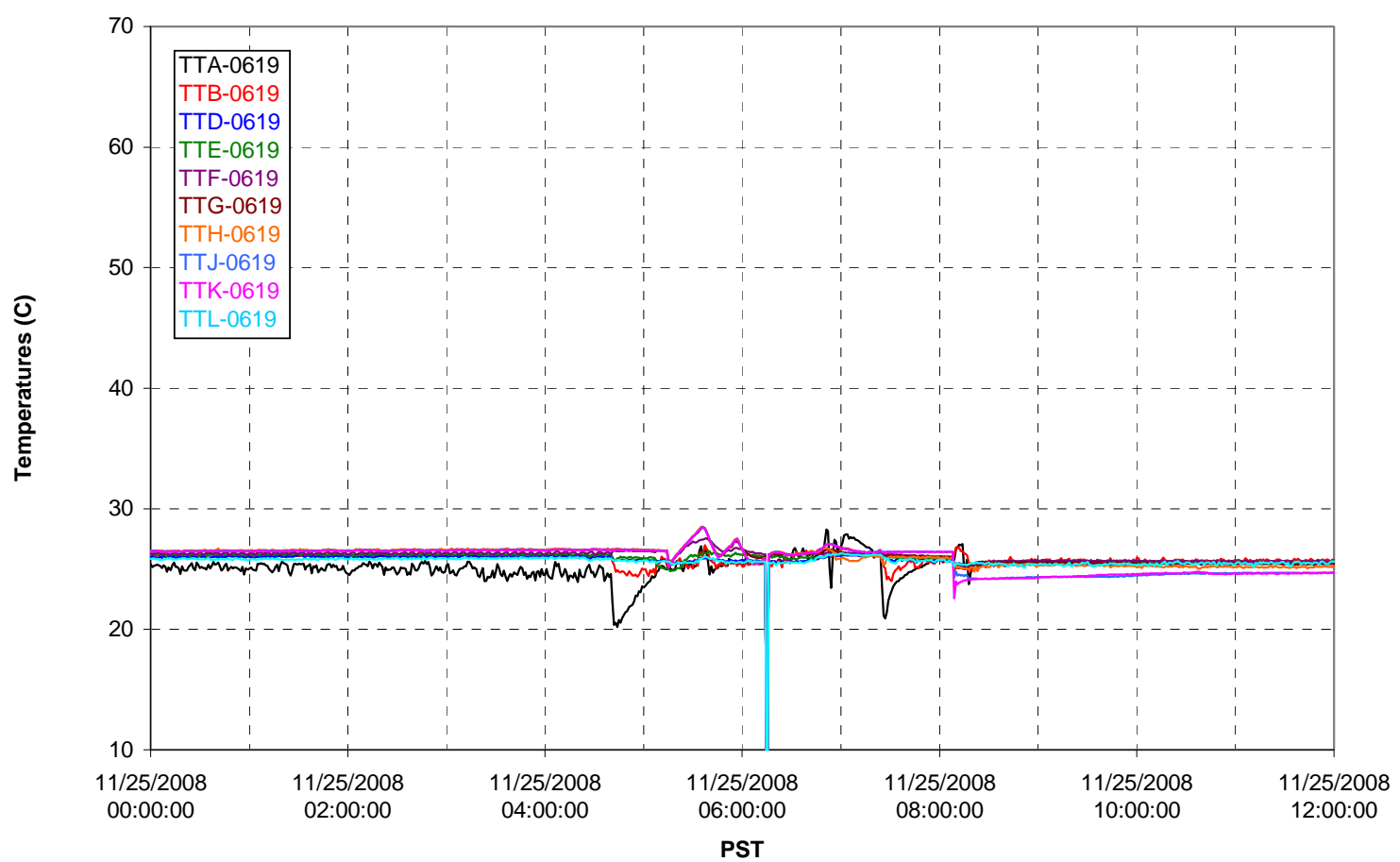

T02A Outer Temperature Tree

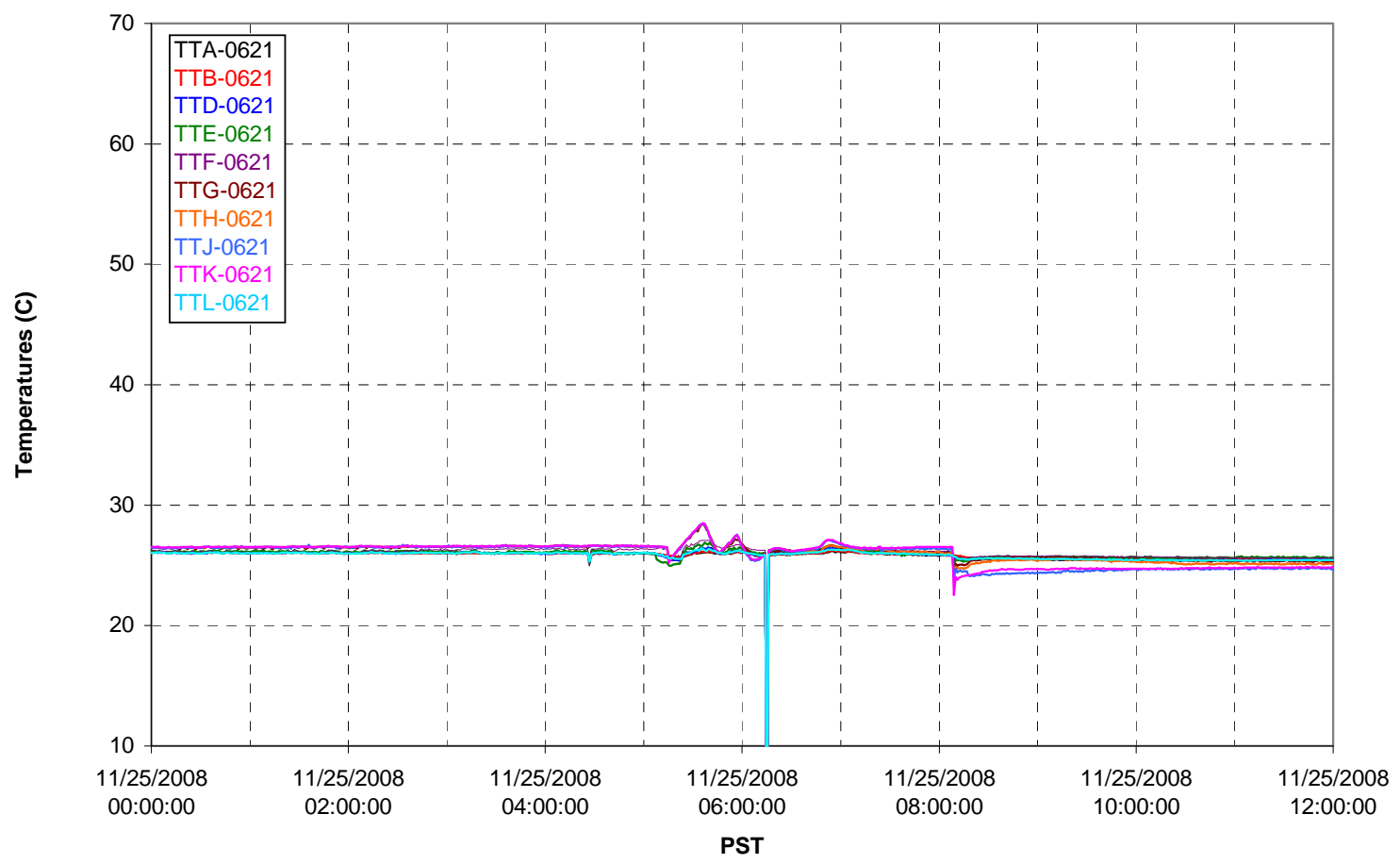


T02A temperatures

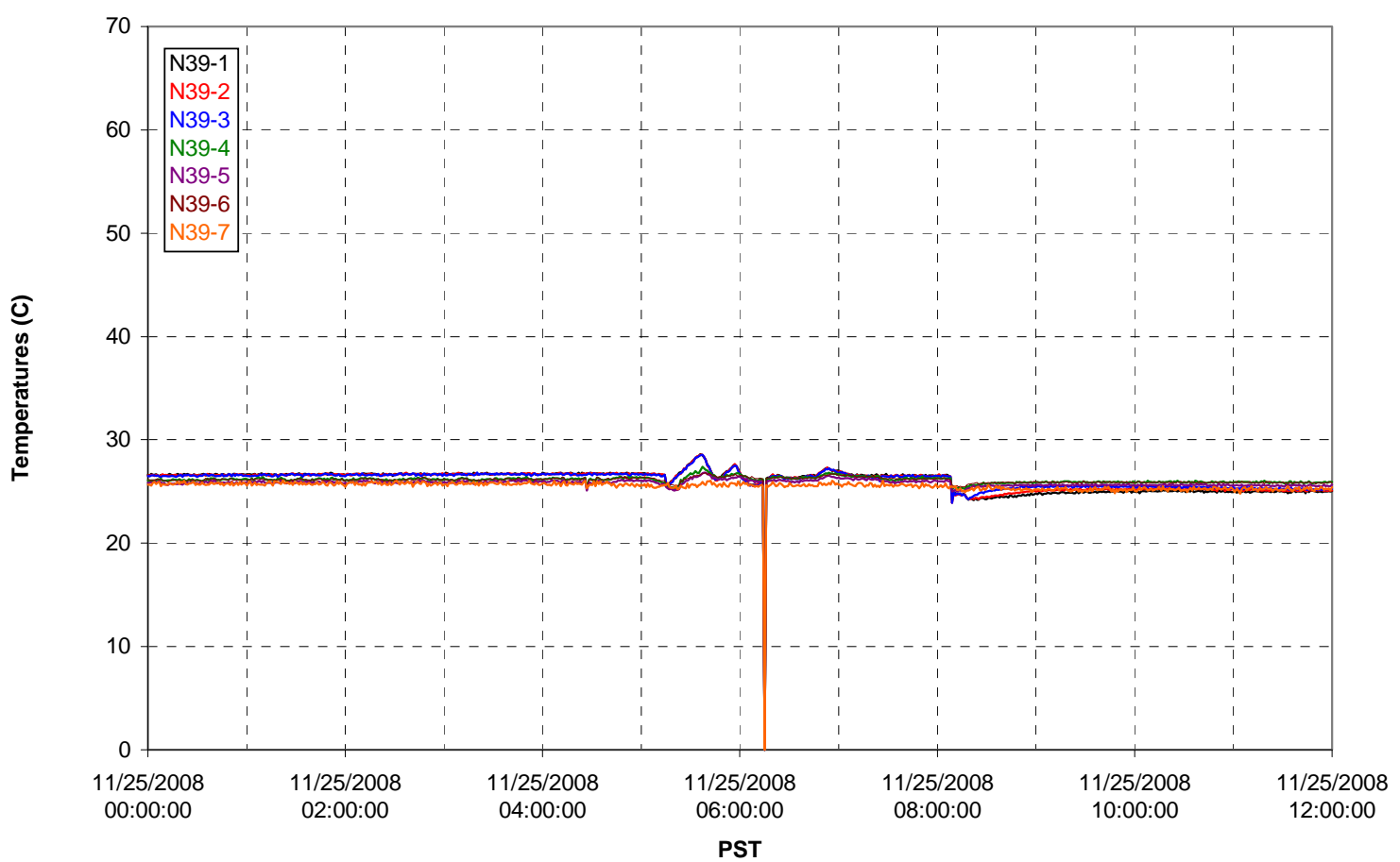

T02A temperatures

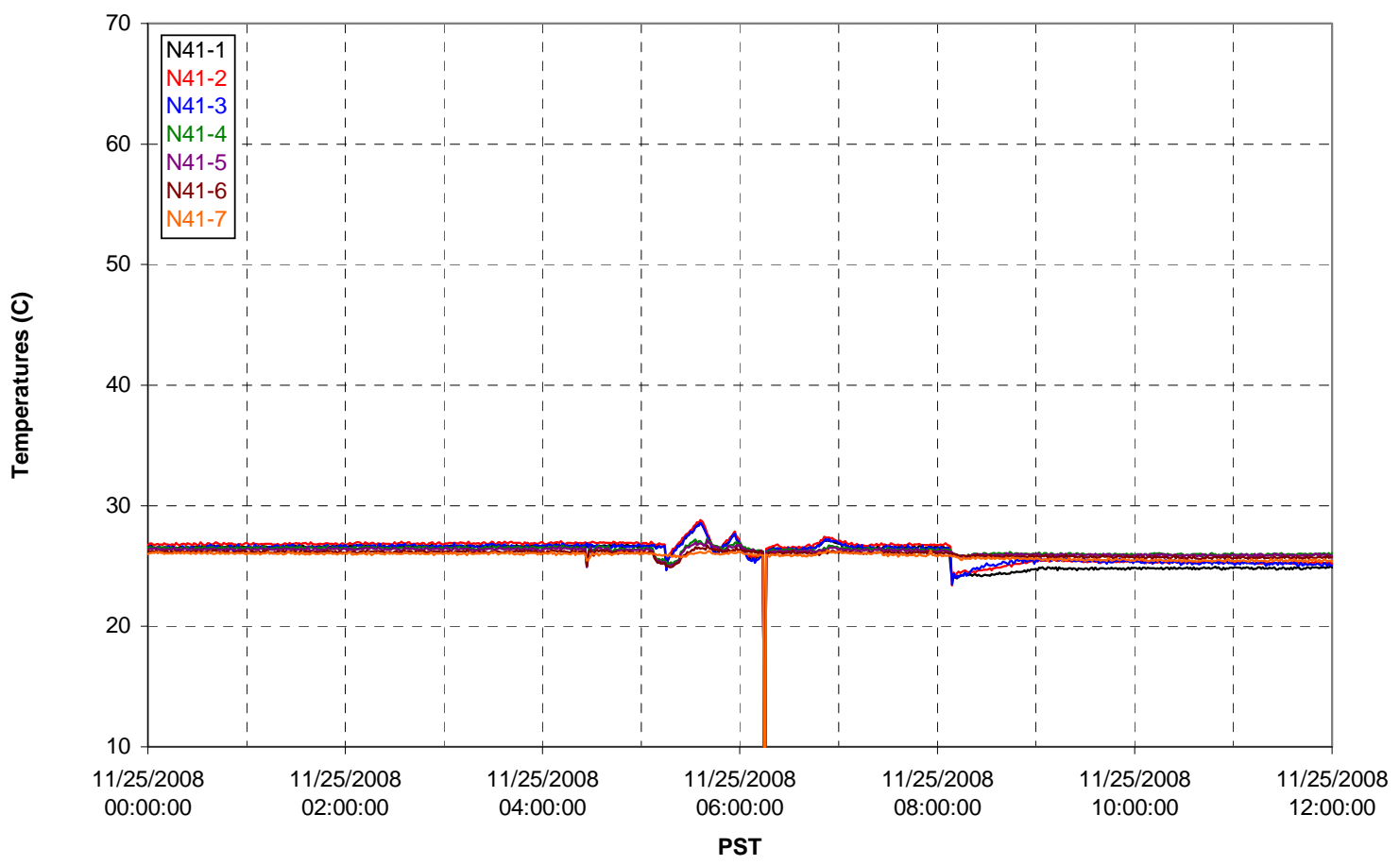


T02A temperatures

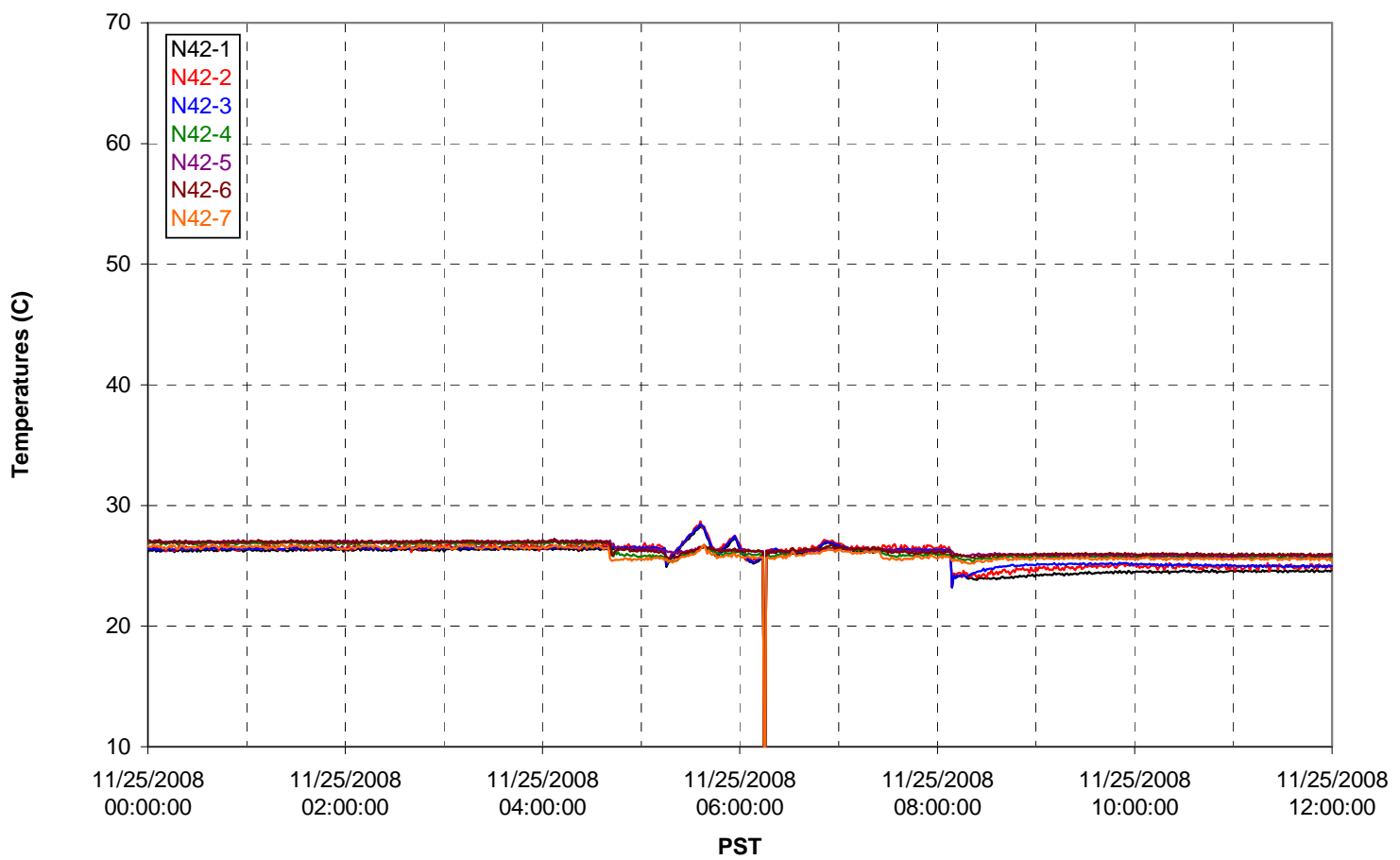

T02A temperatures

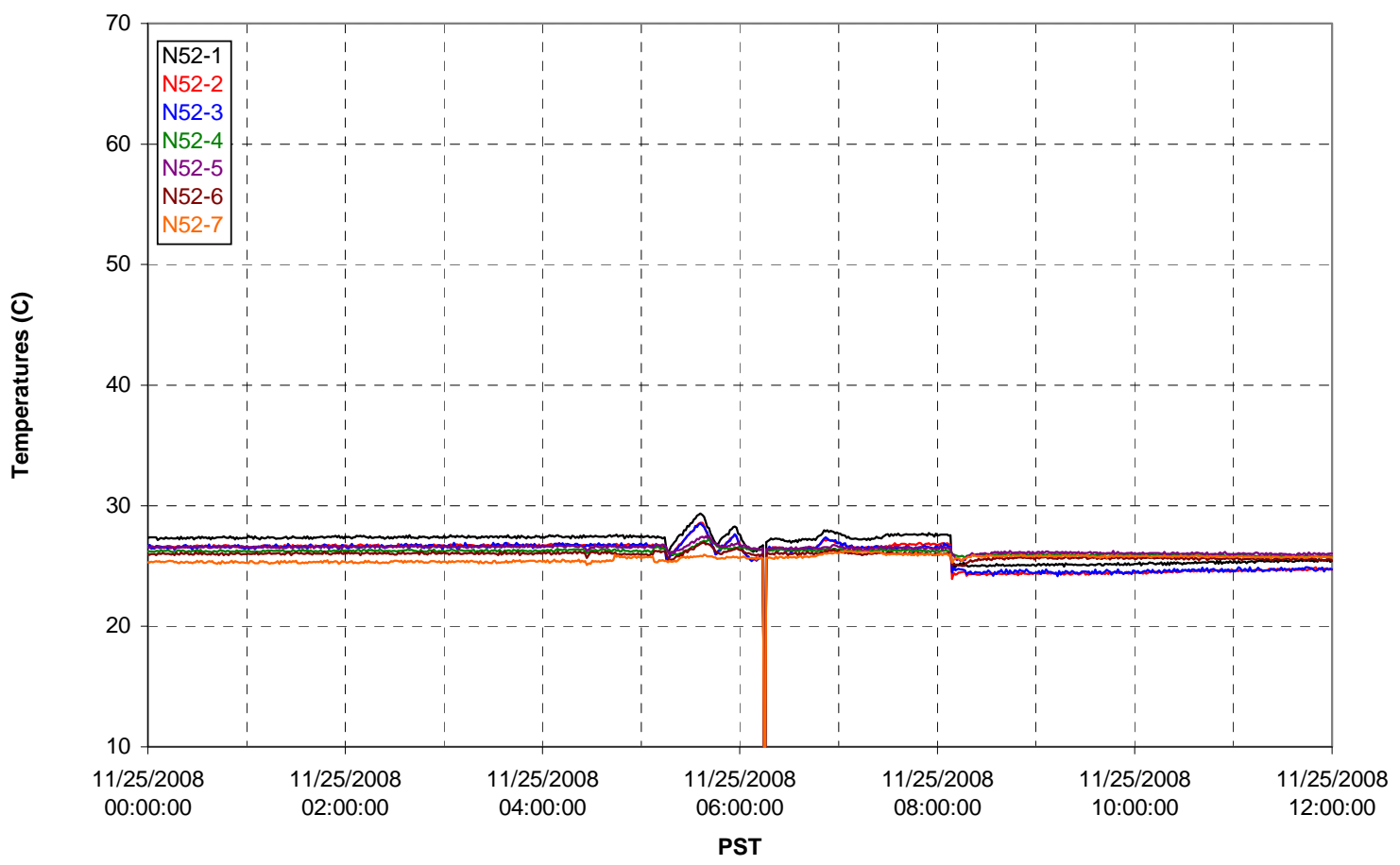


T02A Heating and Cooling

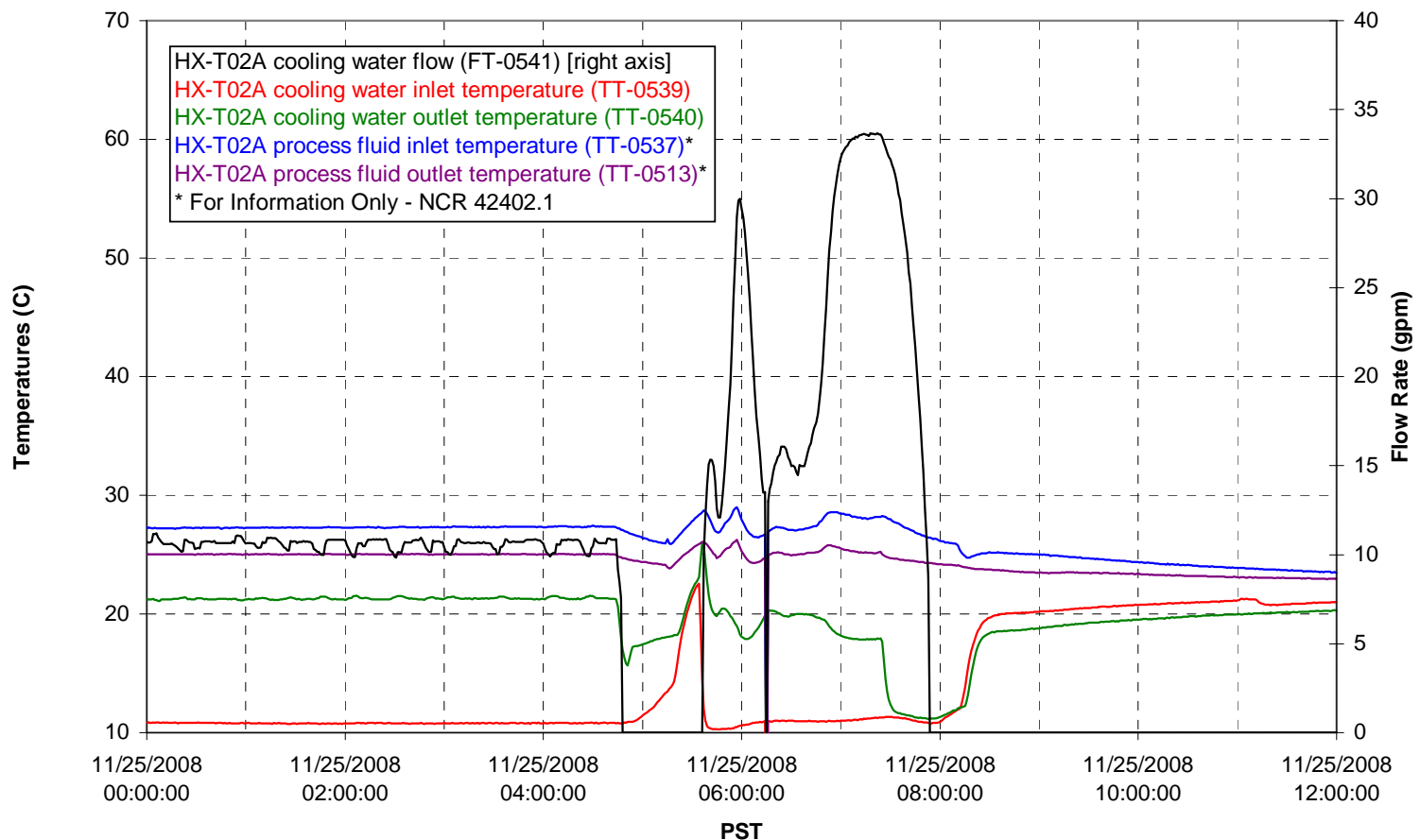

Pump Operation

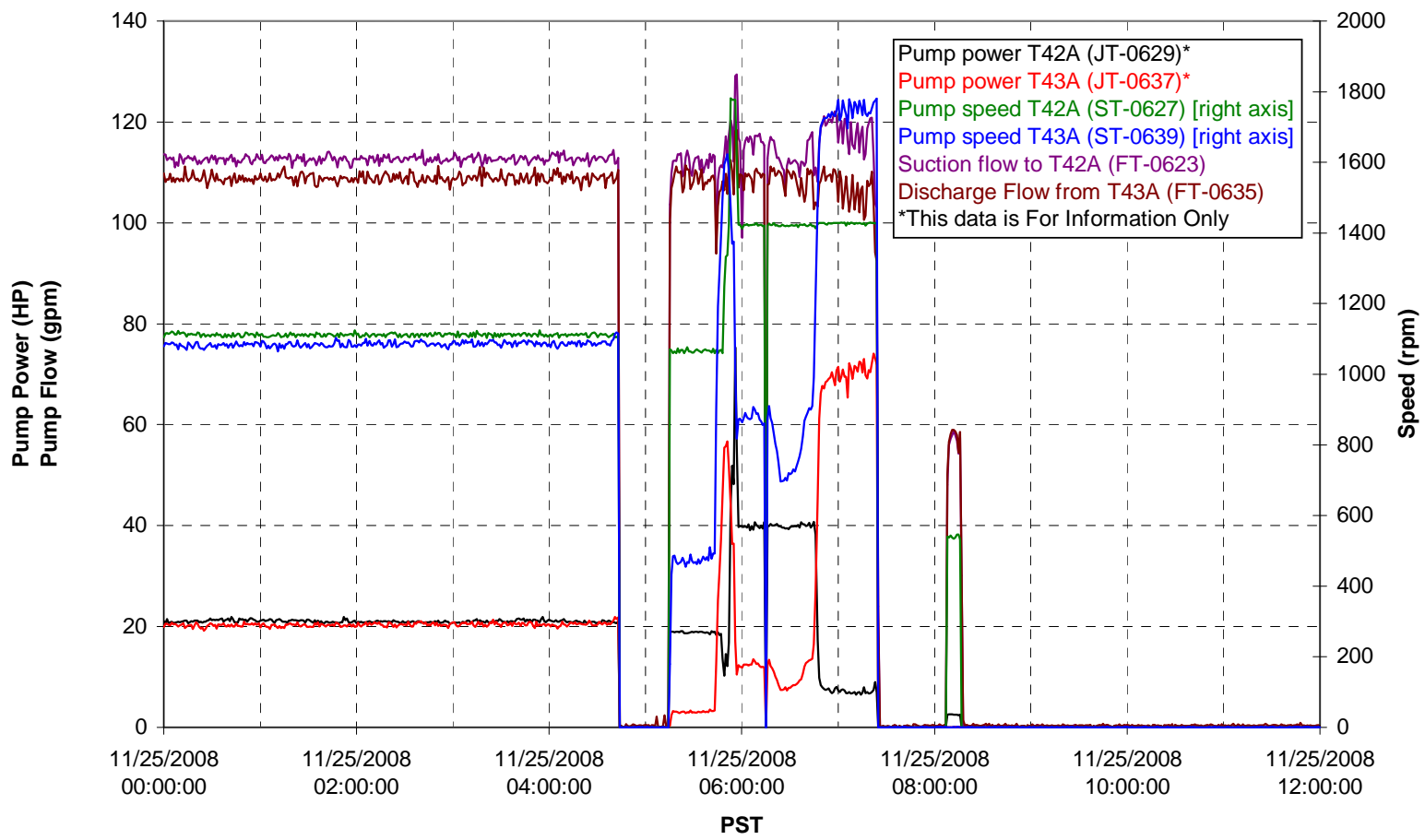


Pulsepot UFP-PP-T01A

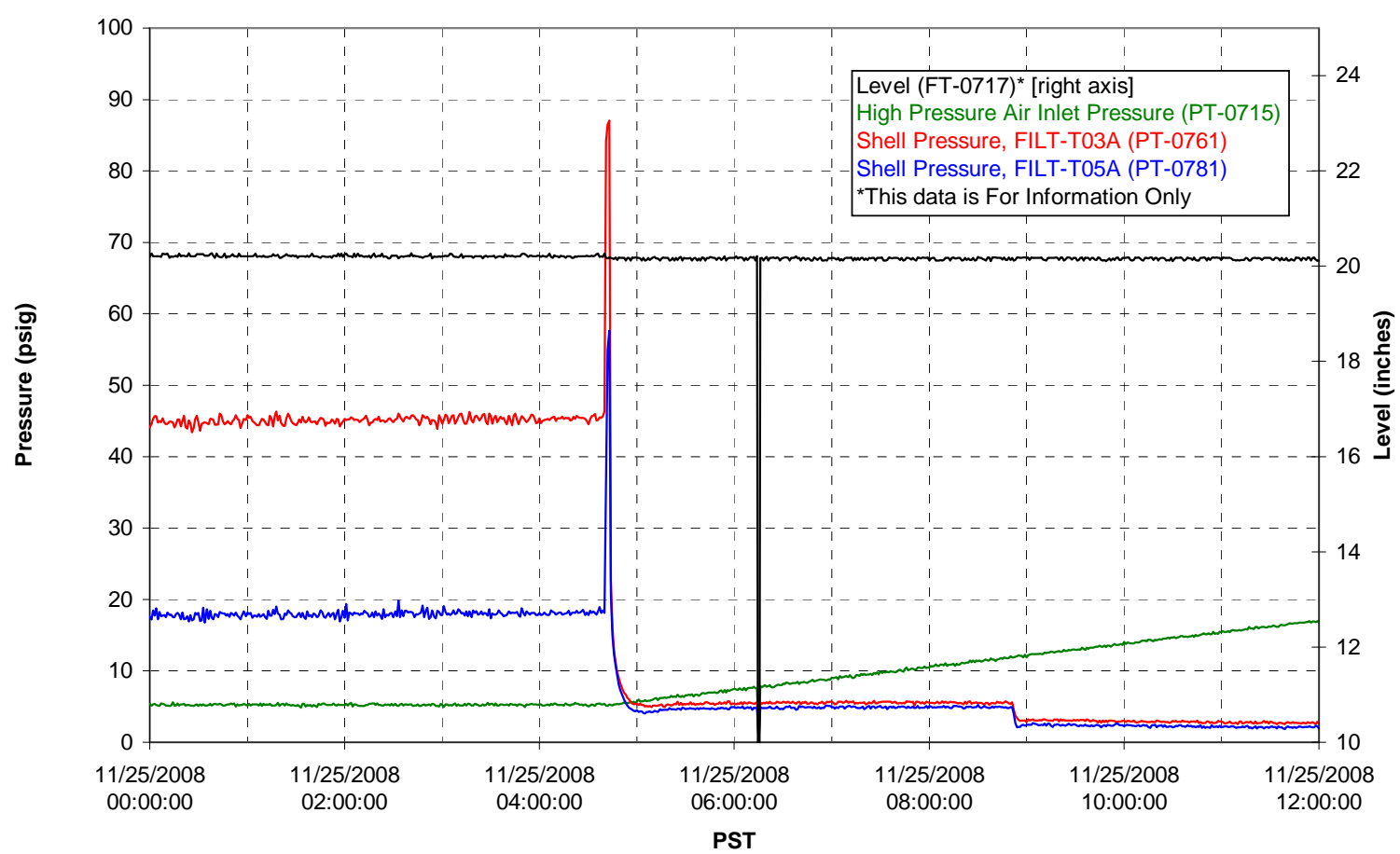

Pulsepot UFP-PP-T02A

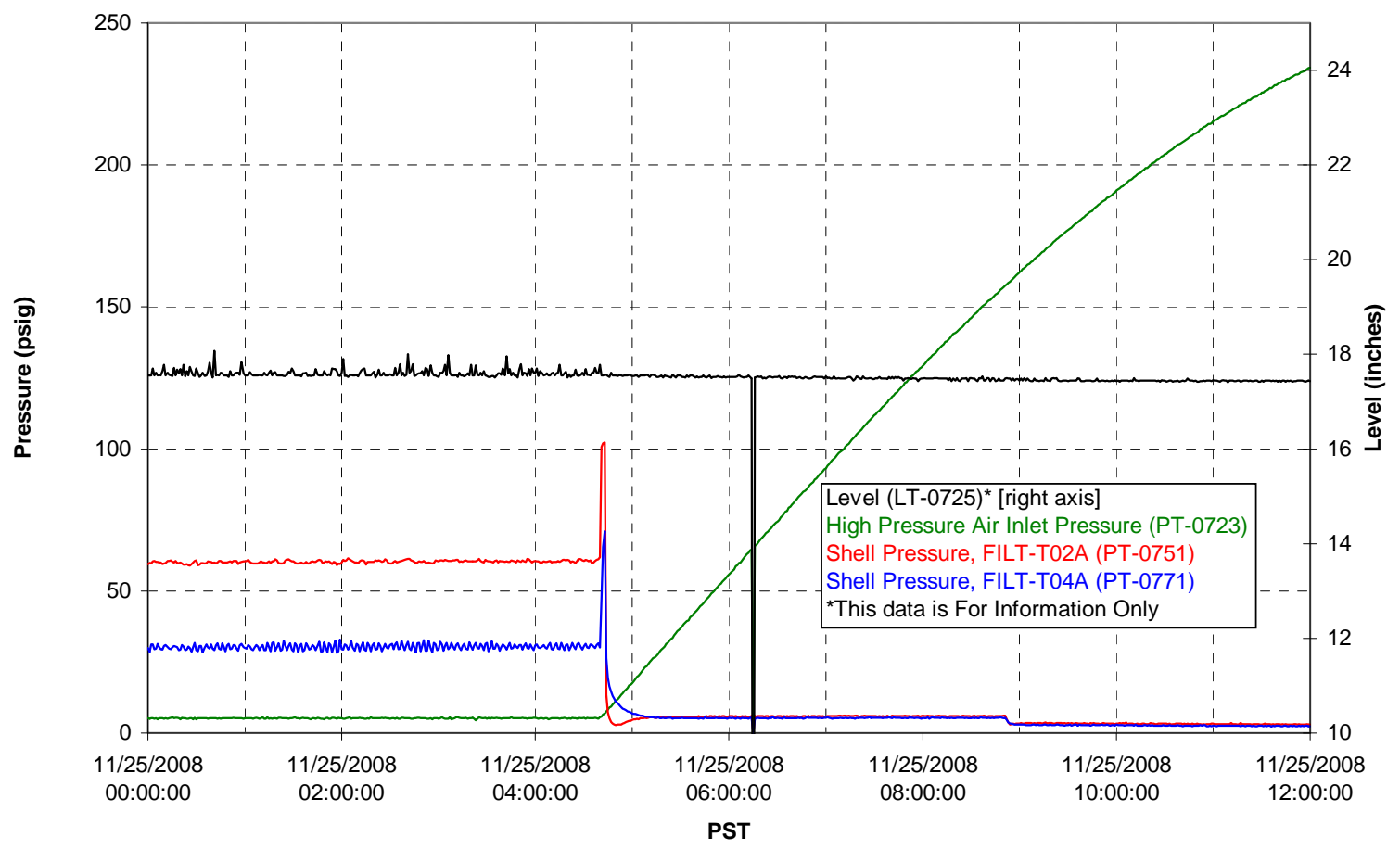


Pulsepot UFP-PP-T03A

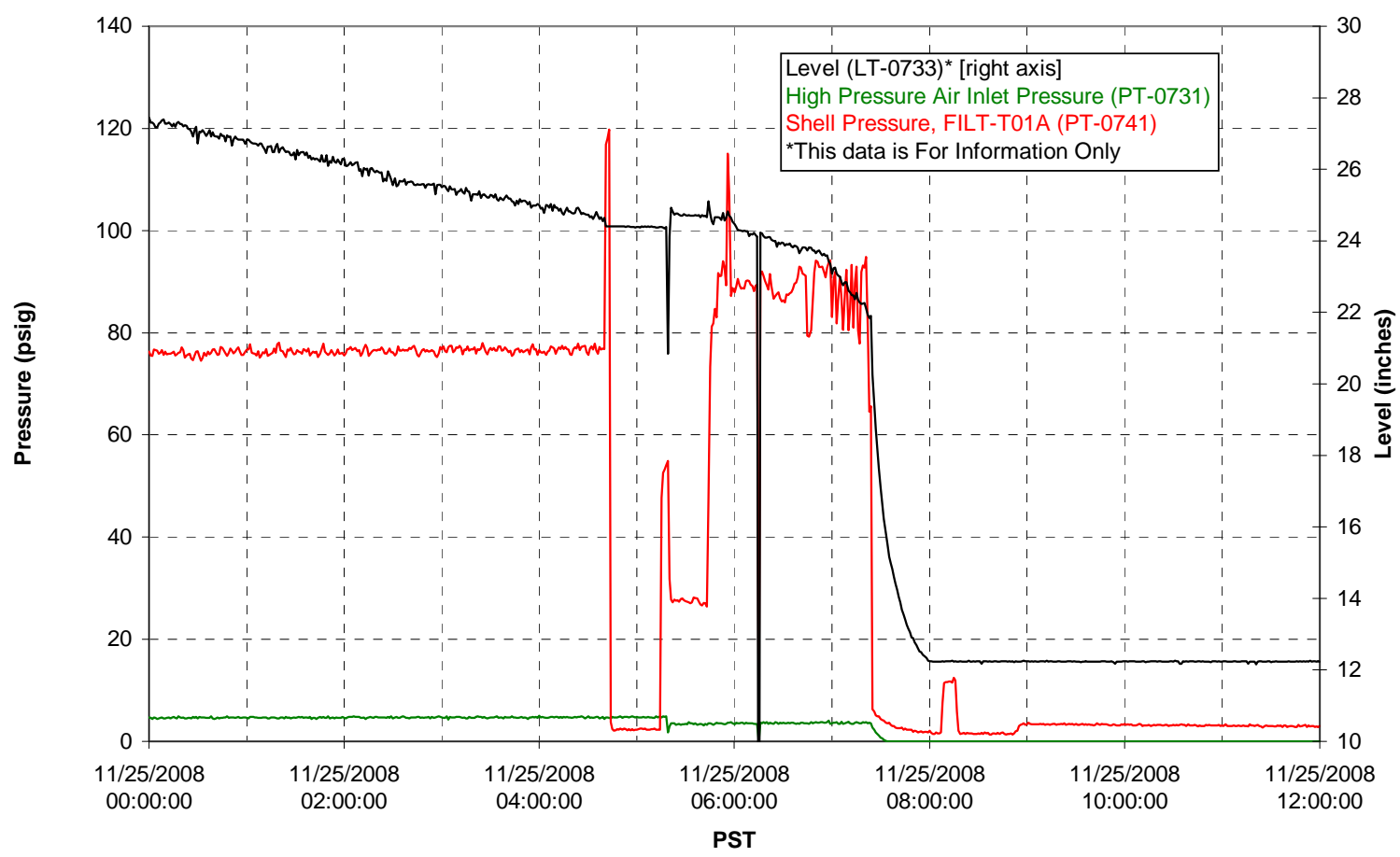

Pulsepot Levels

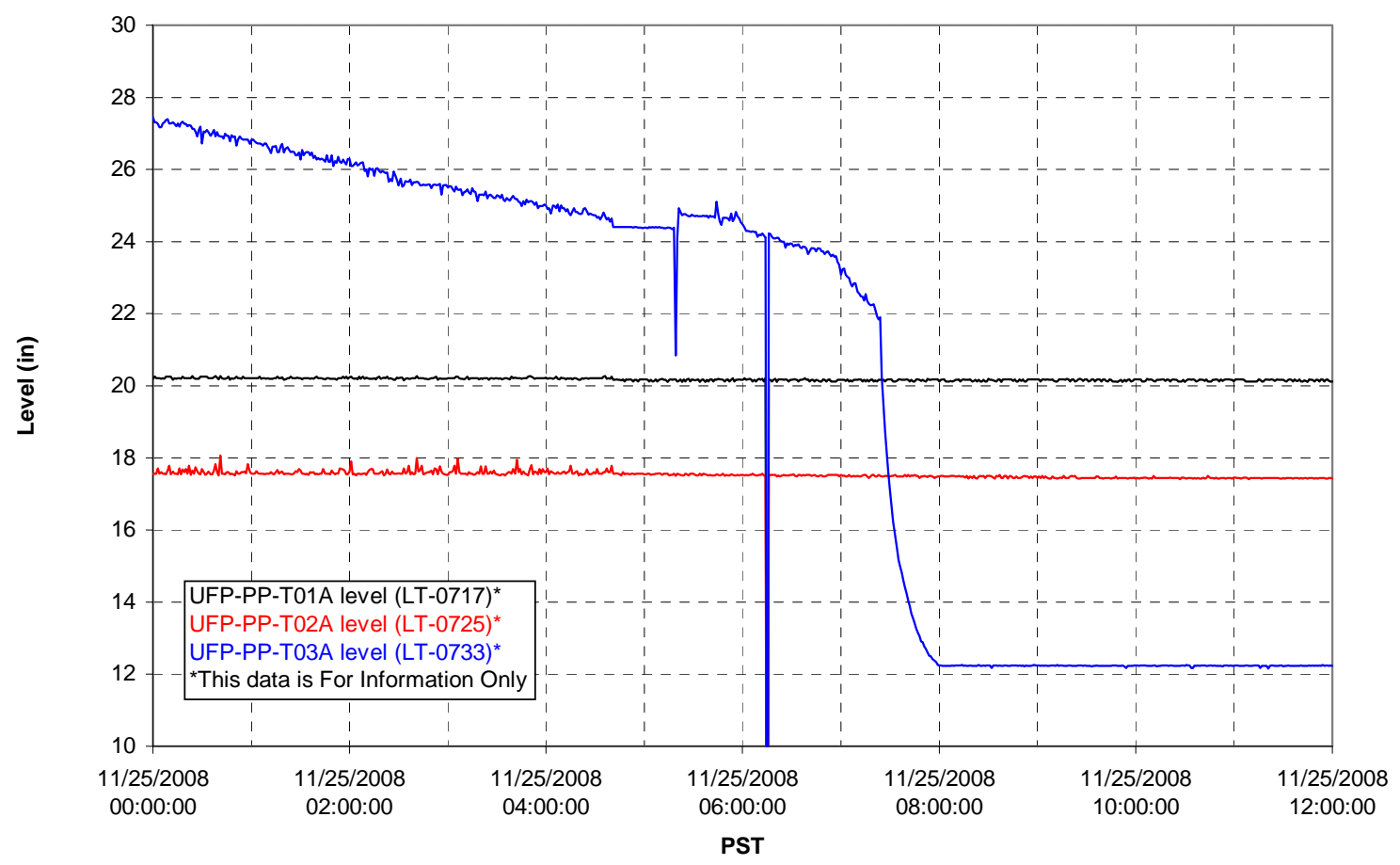


Filter UFP-FILT-T01A

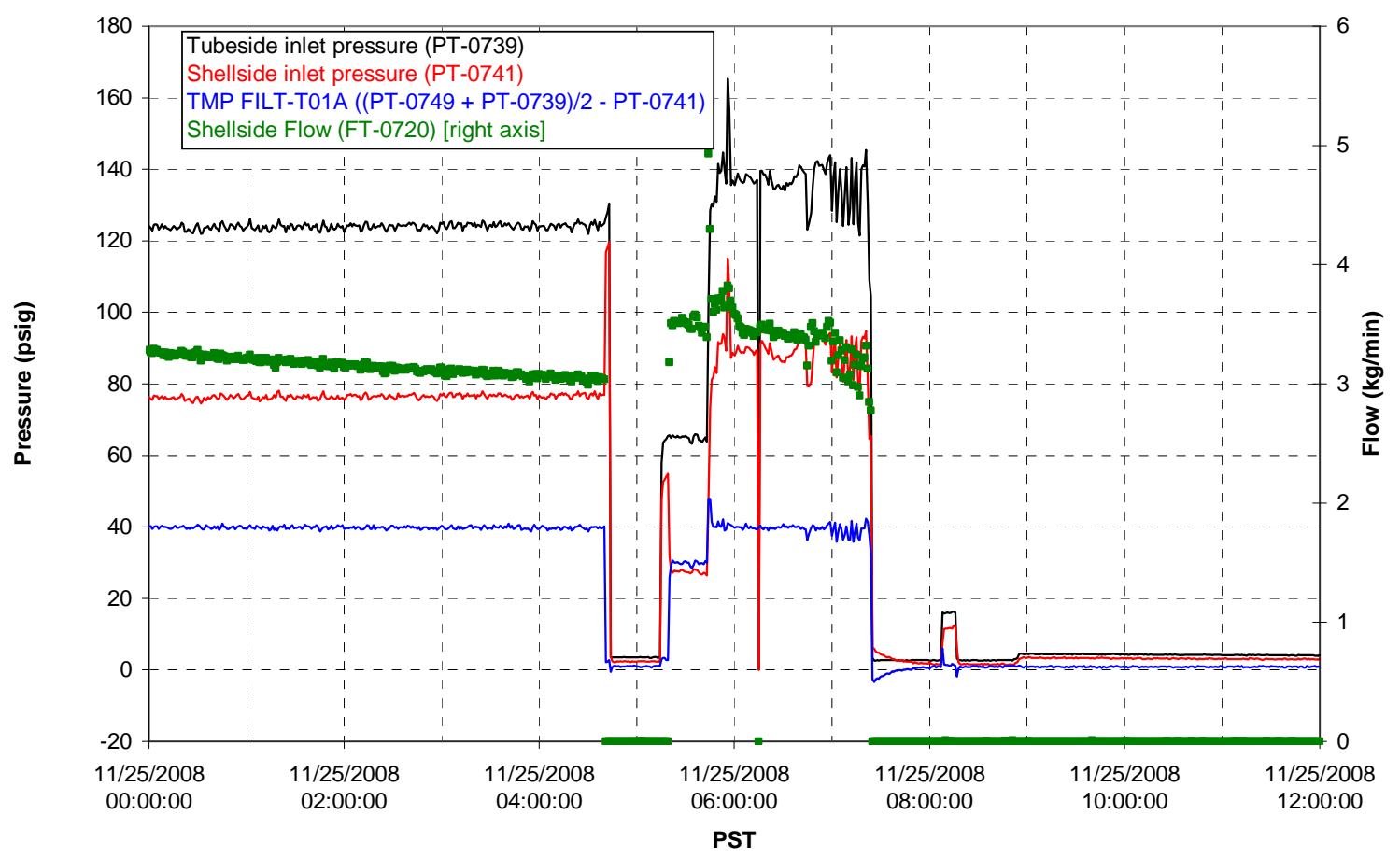

Filter UFP-FILT-T02A

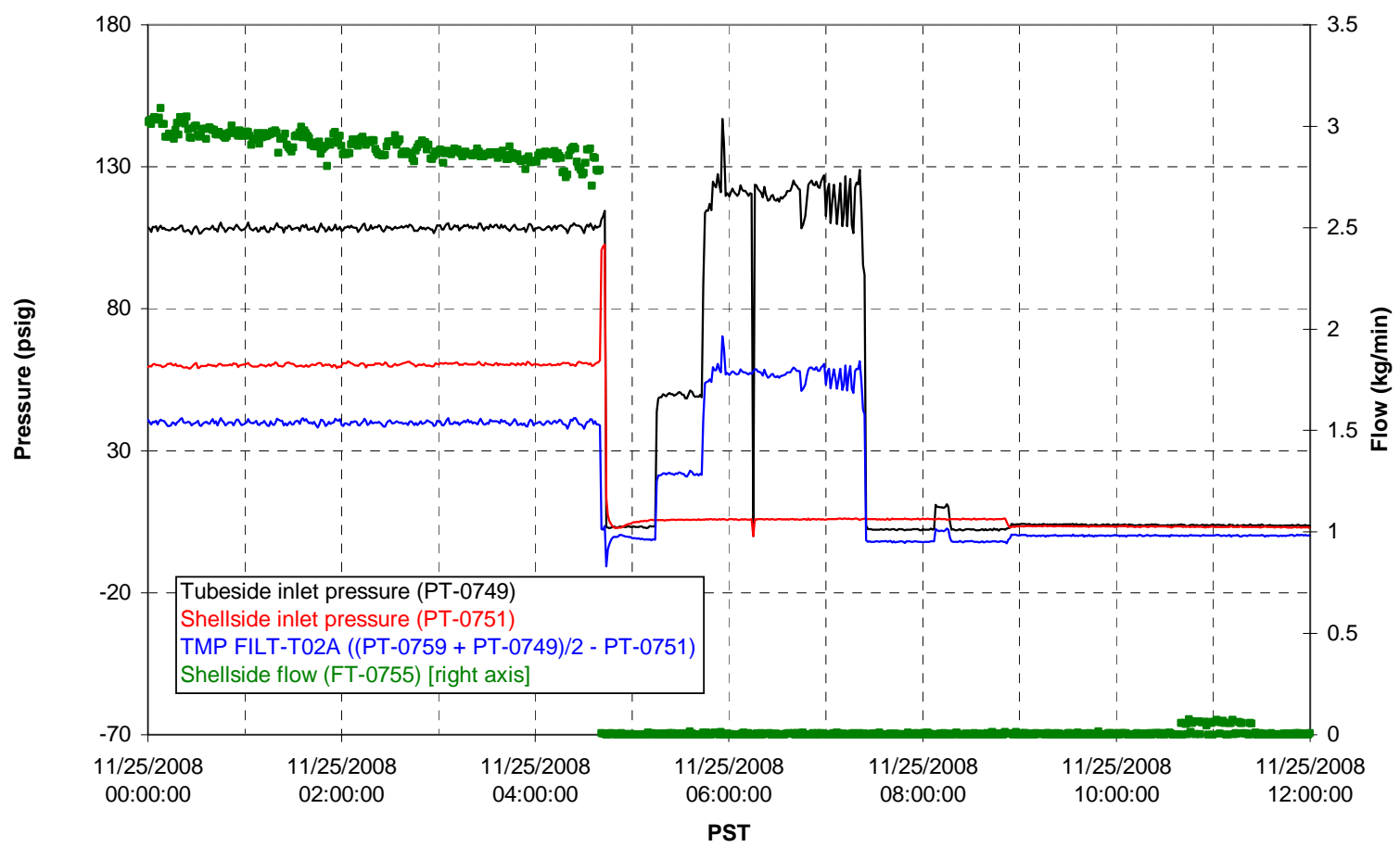


Filter UFP-FILT-T03A

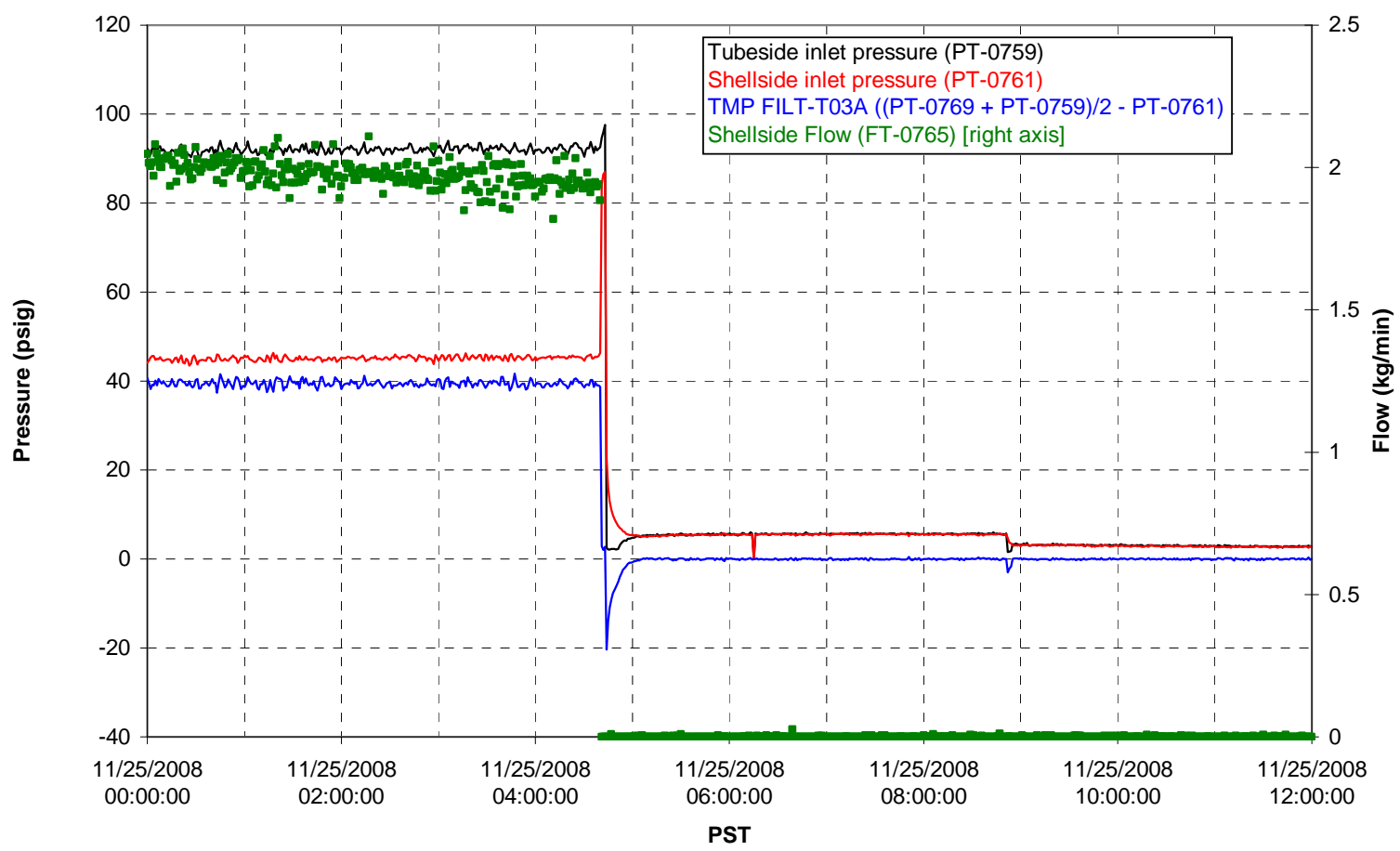

Filter UFP-FILT-T04A

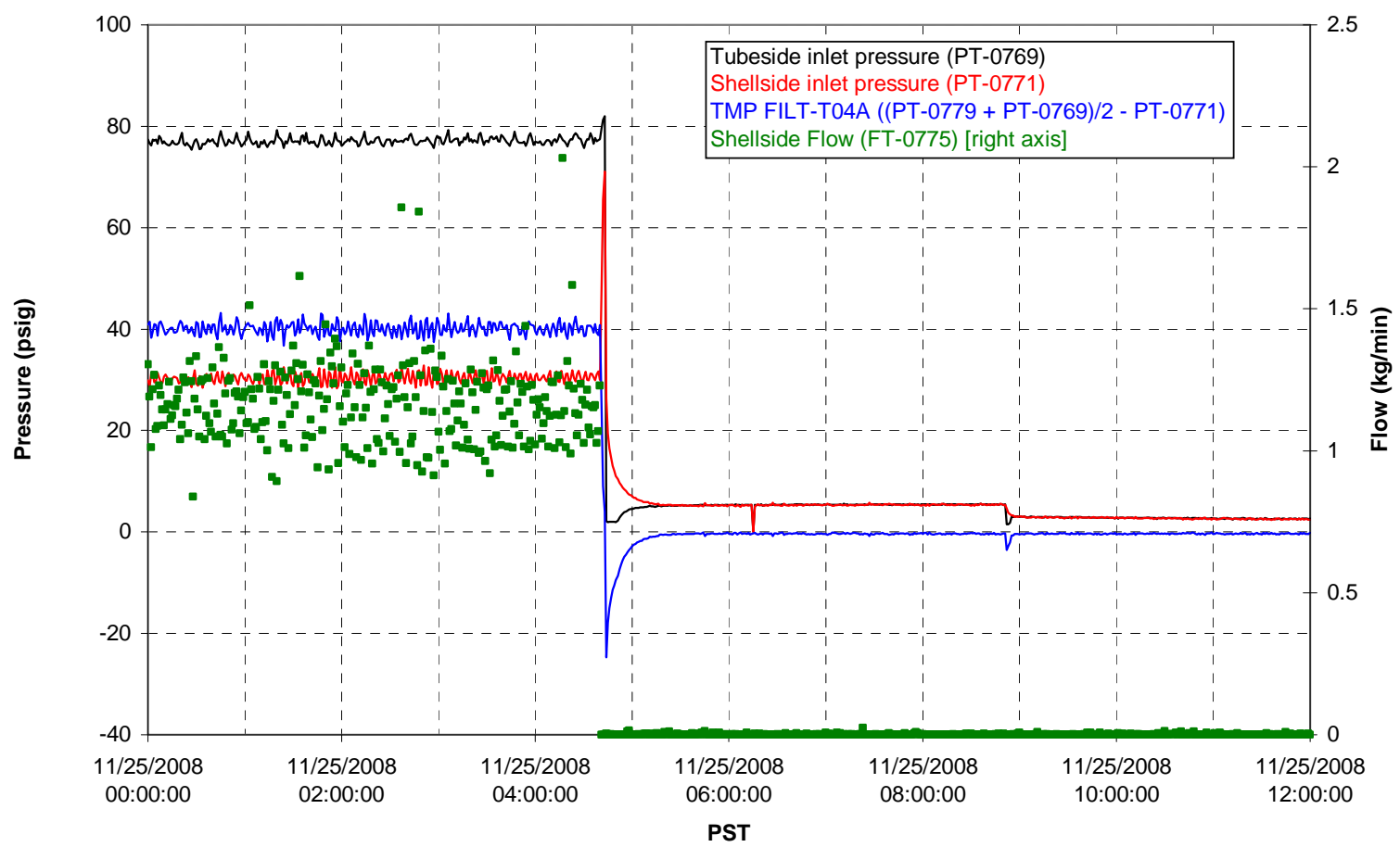


Filter UFP-FILT-T05A

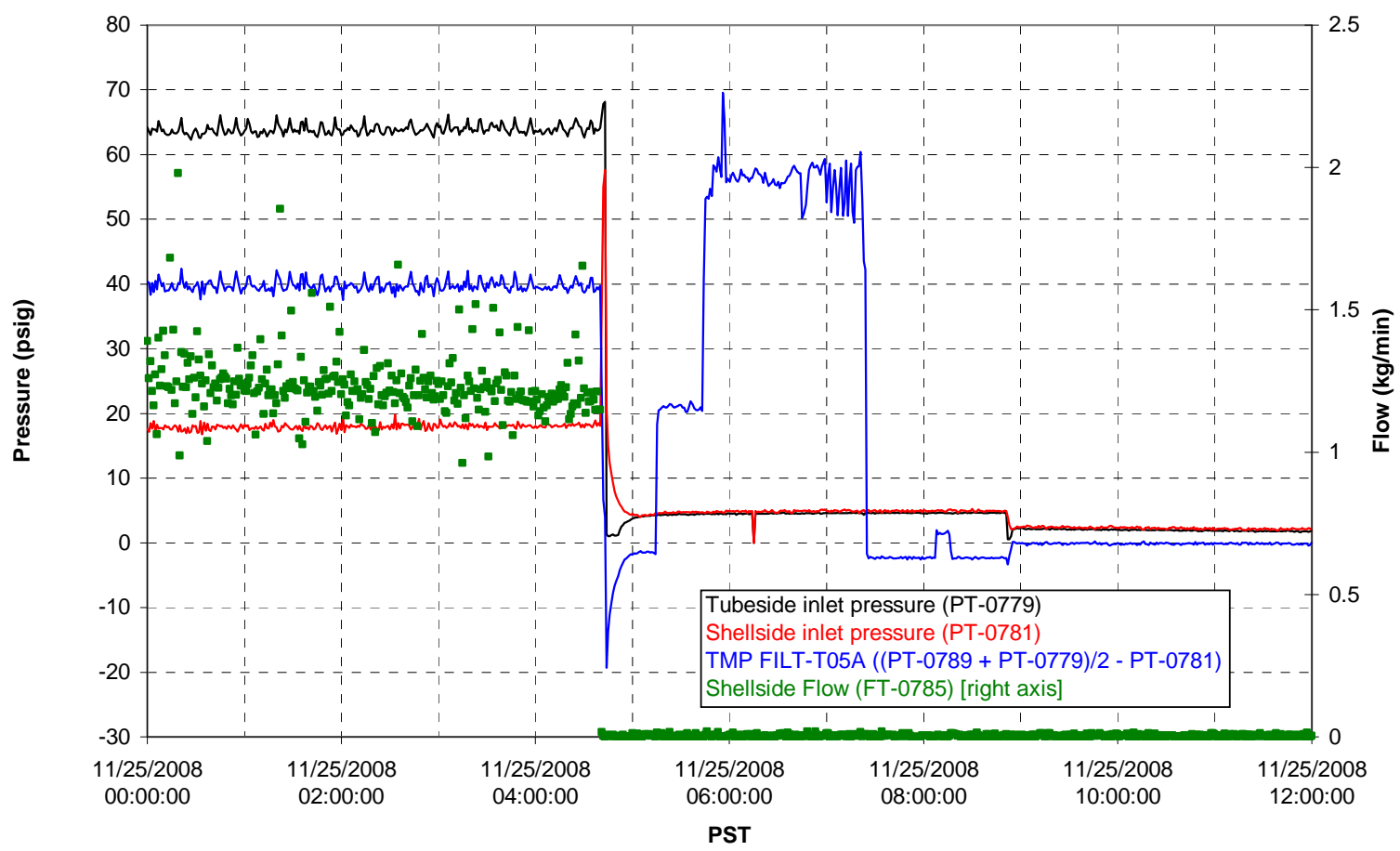

Chemical Flow

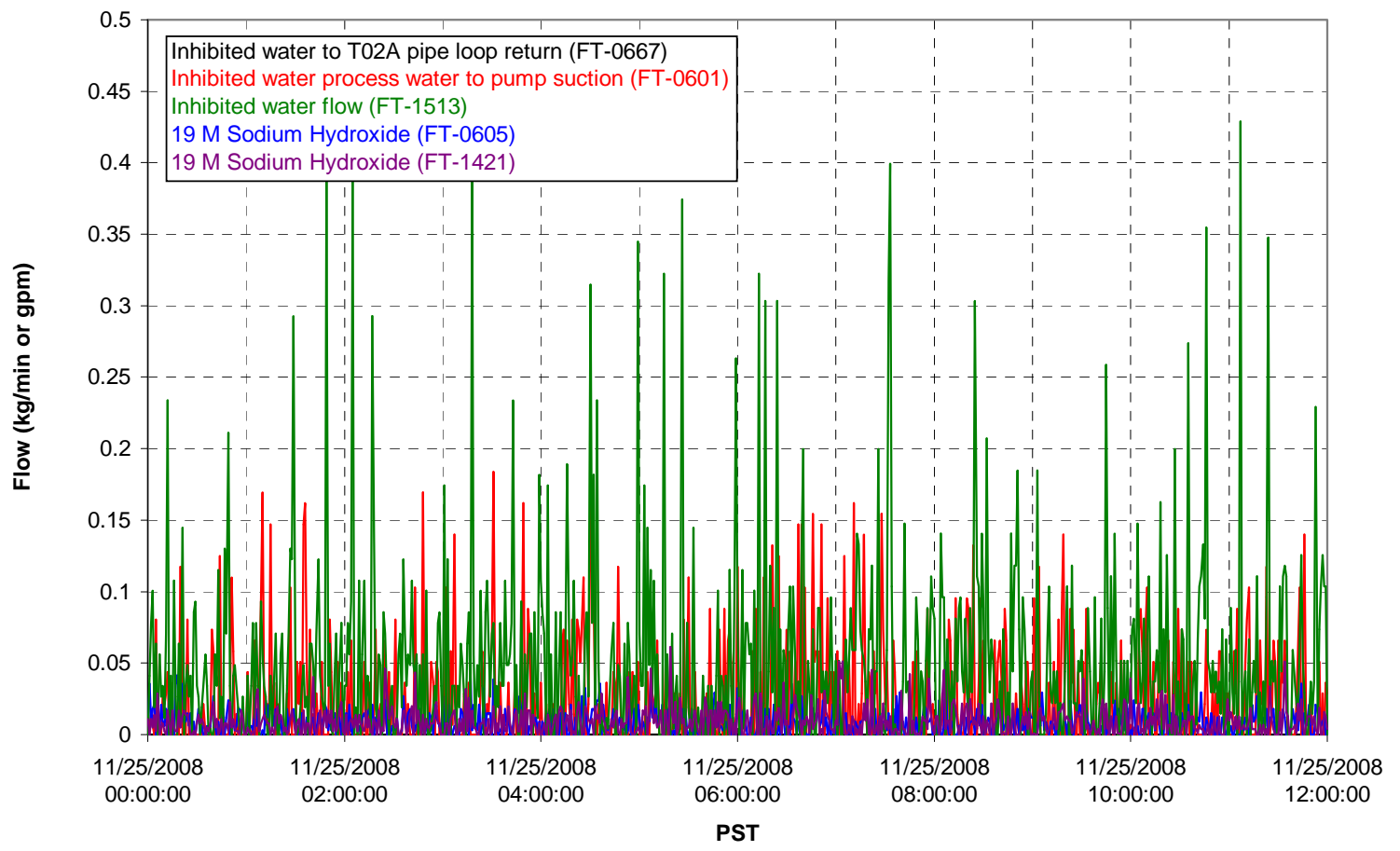




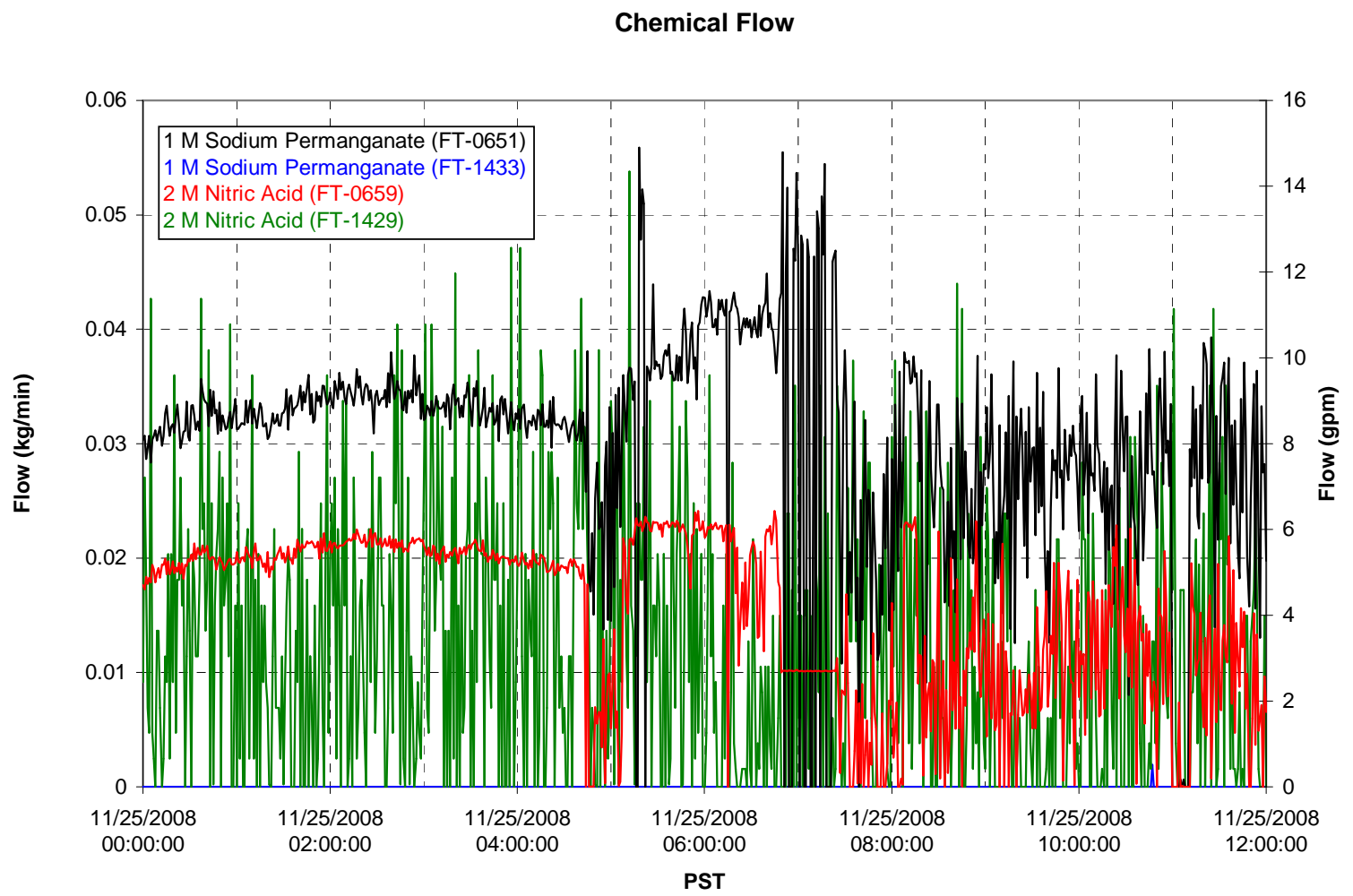

Air Flows

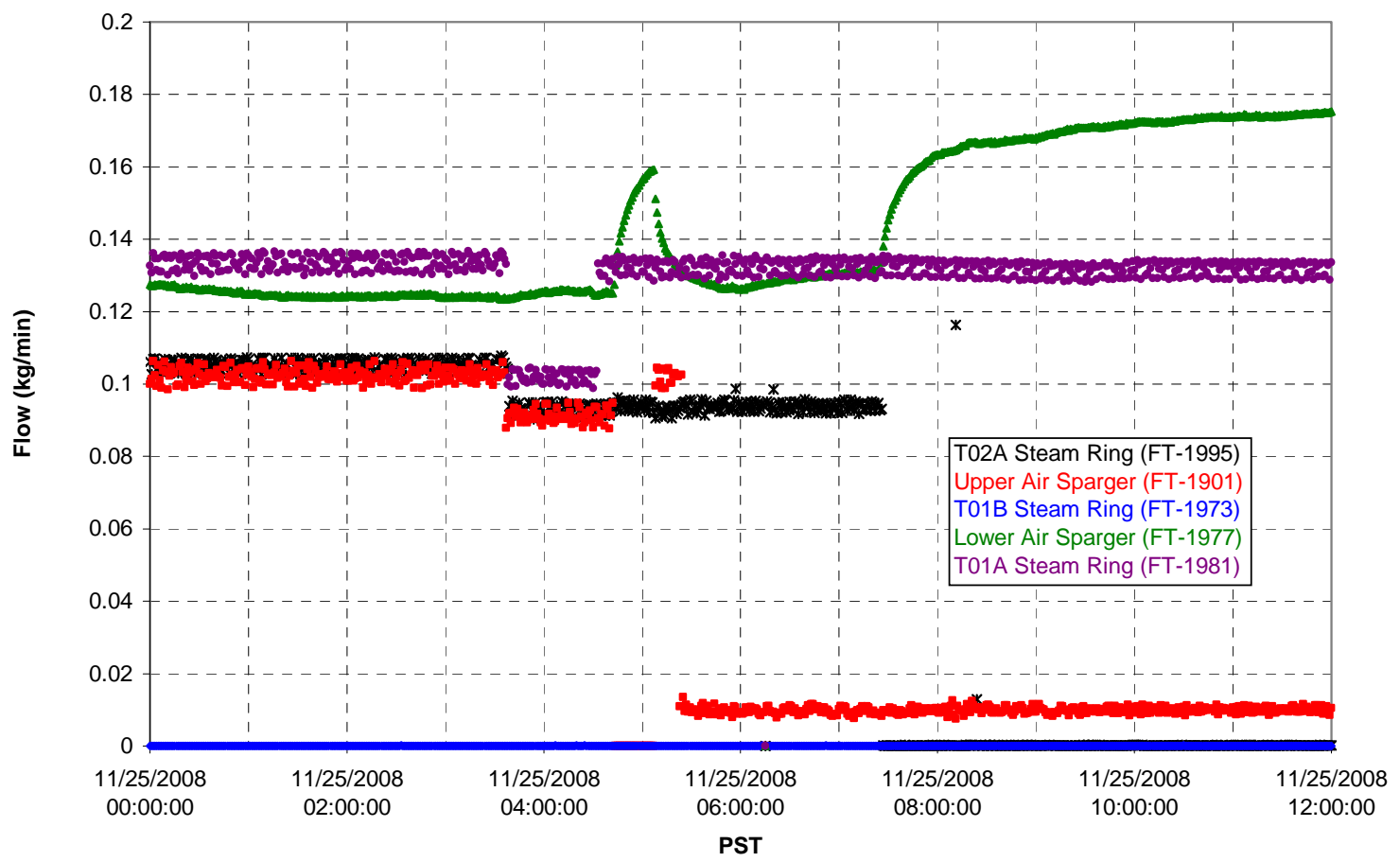


T02A Steam

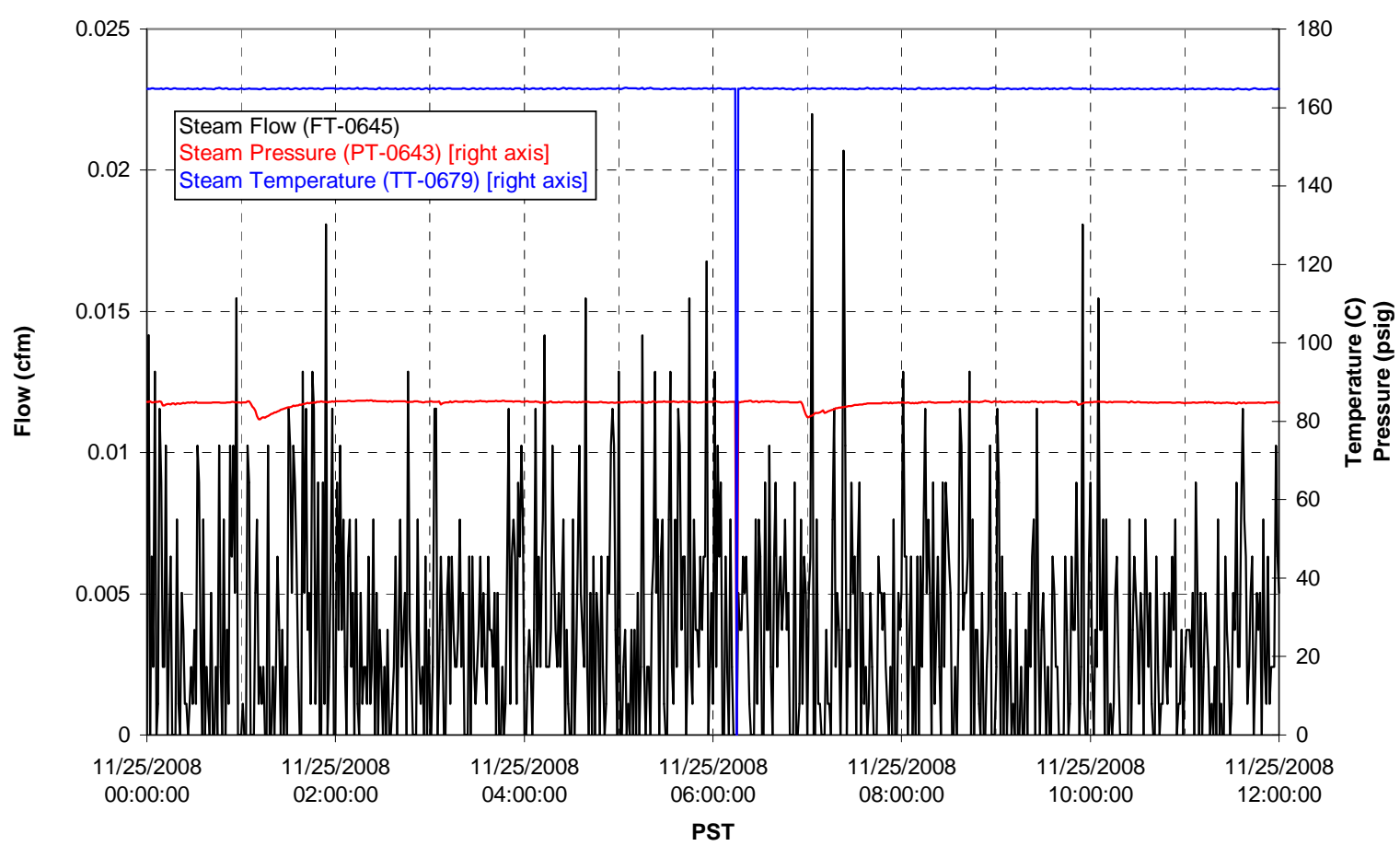

T01A Steam

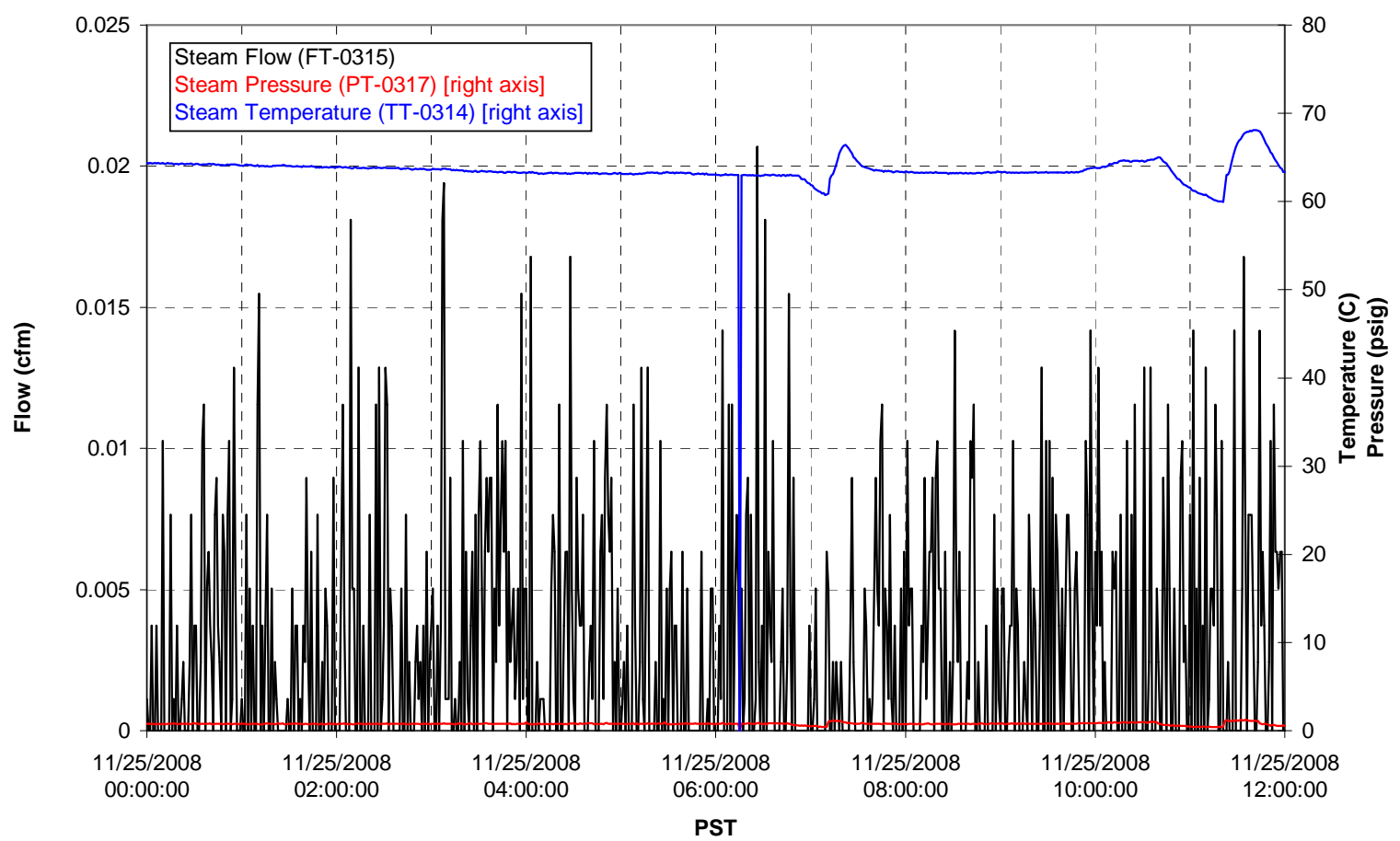


T01B Steam

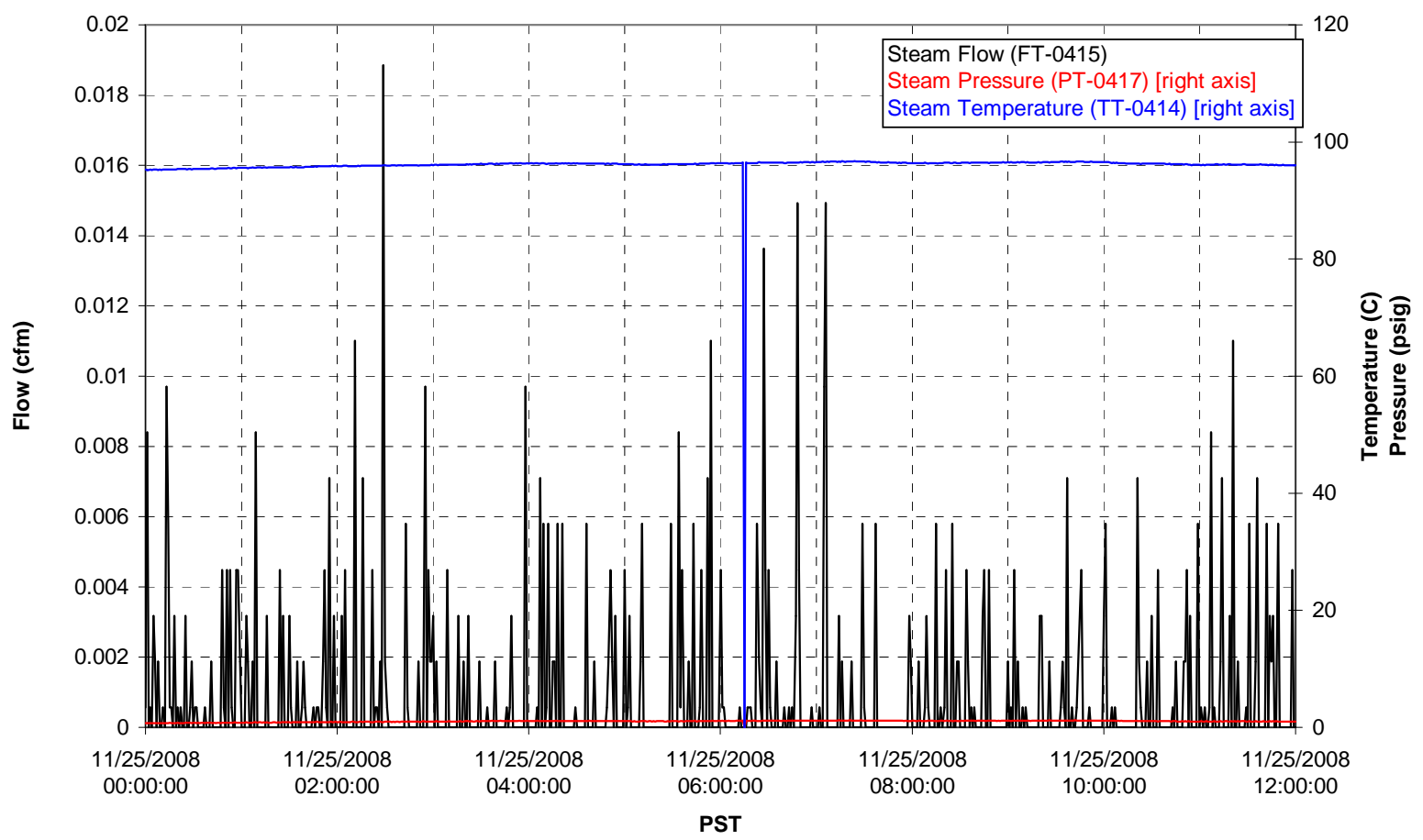


Functional Test Data Plots

11/25/2008 12:00 - 11/26/2008 00:00 
T01A level

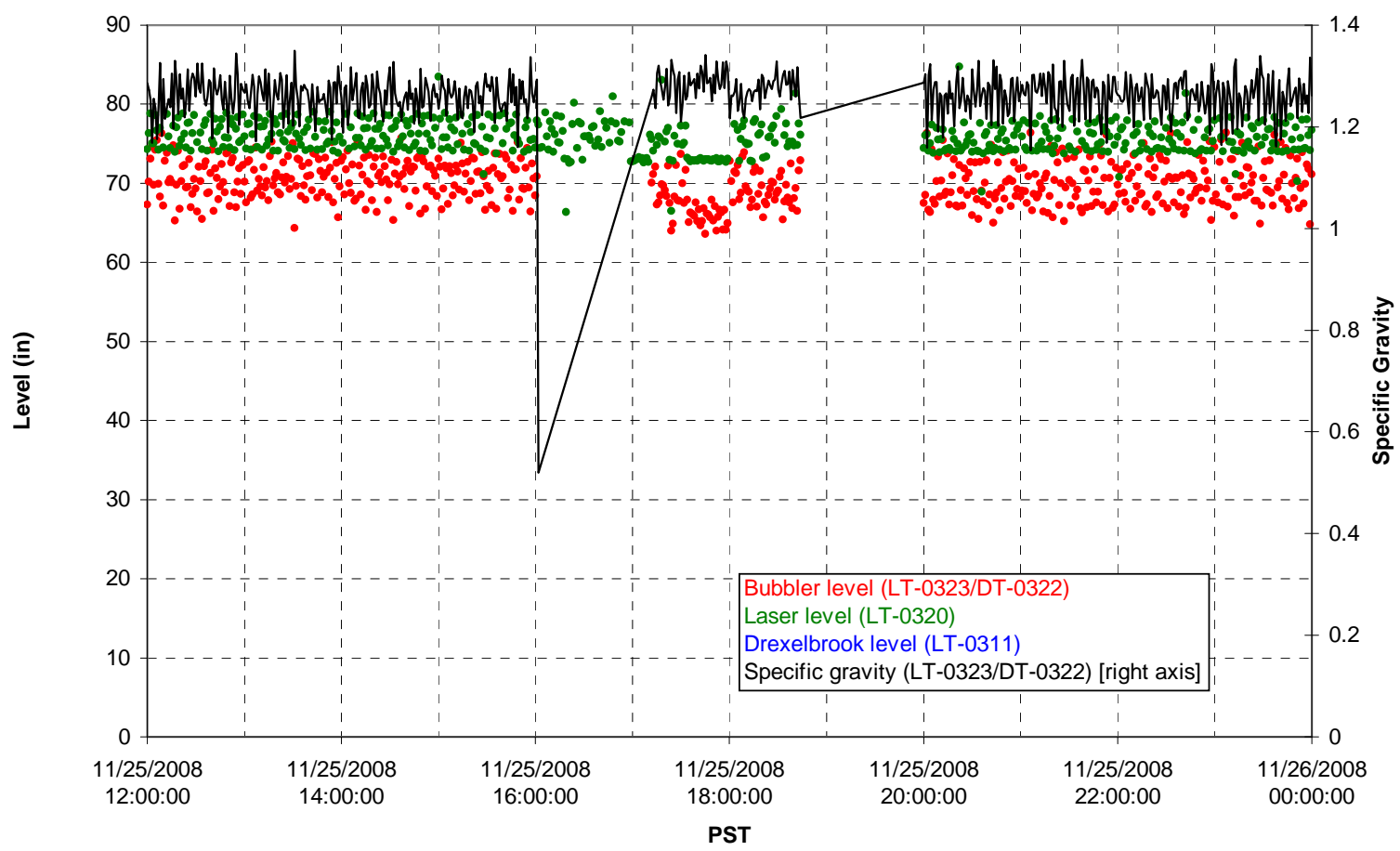

T01A temperatures

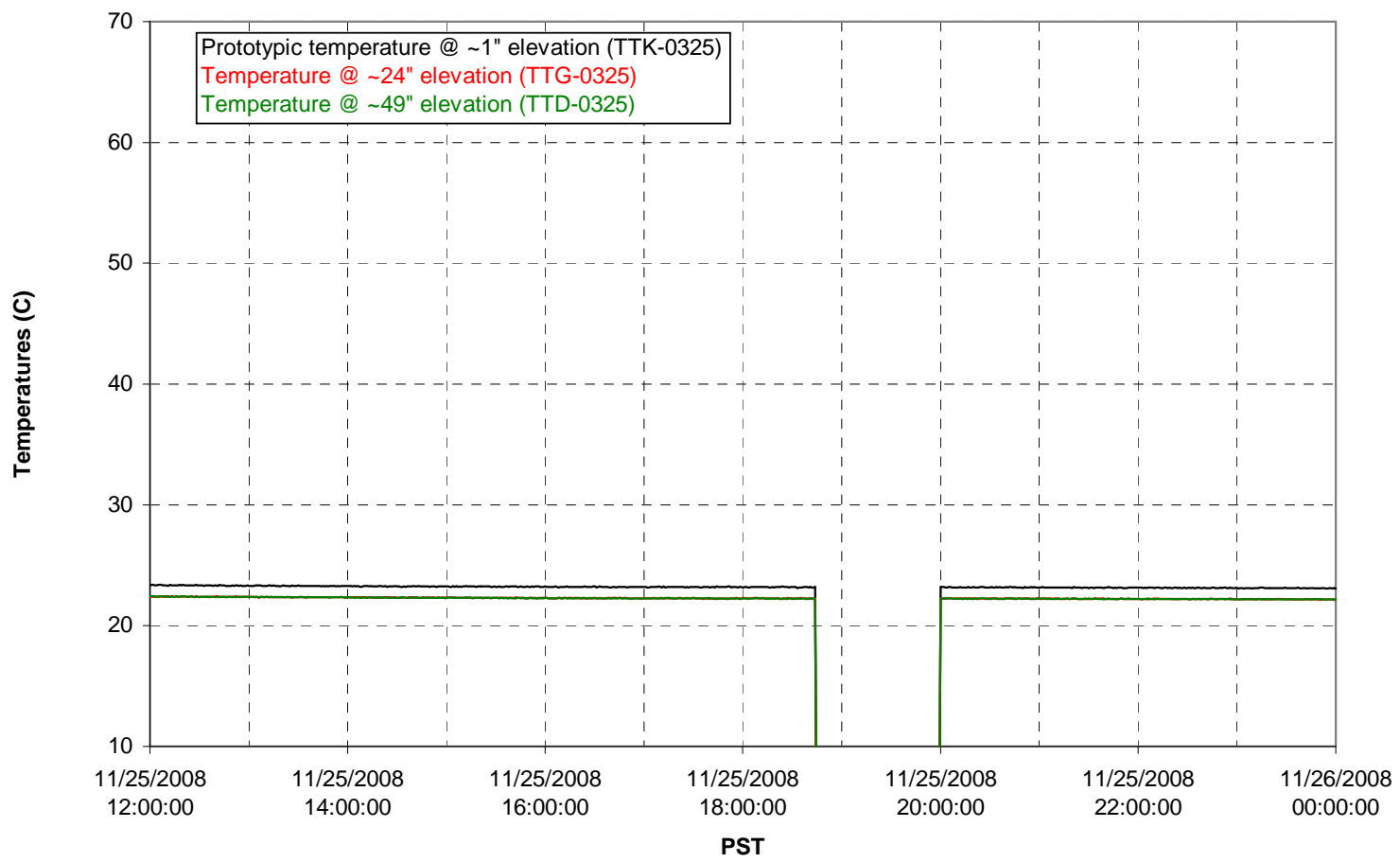


T01B level

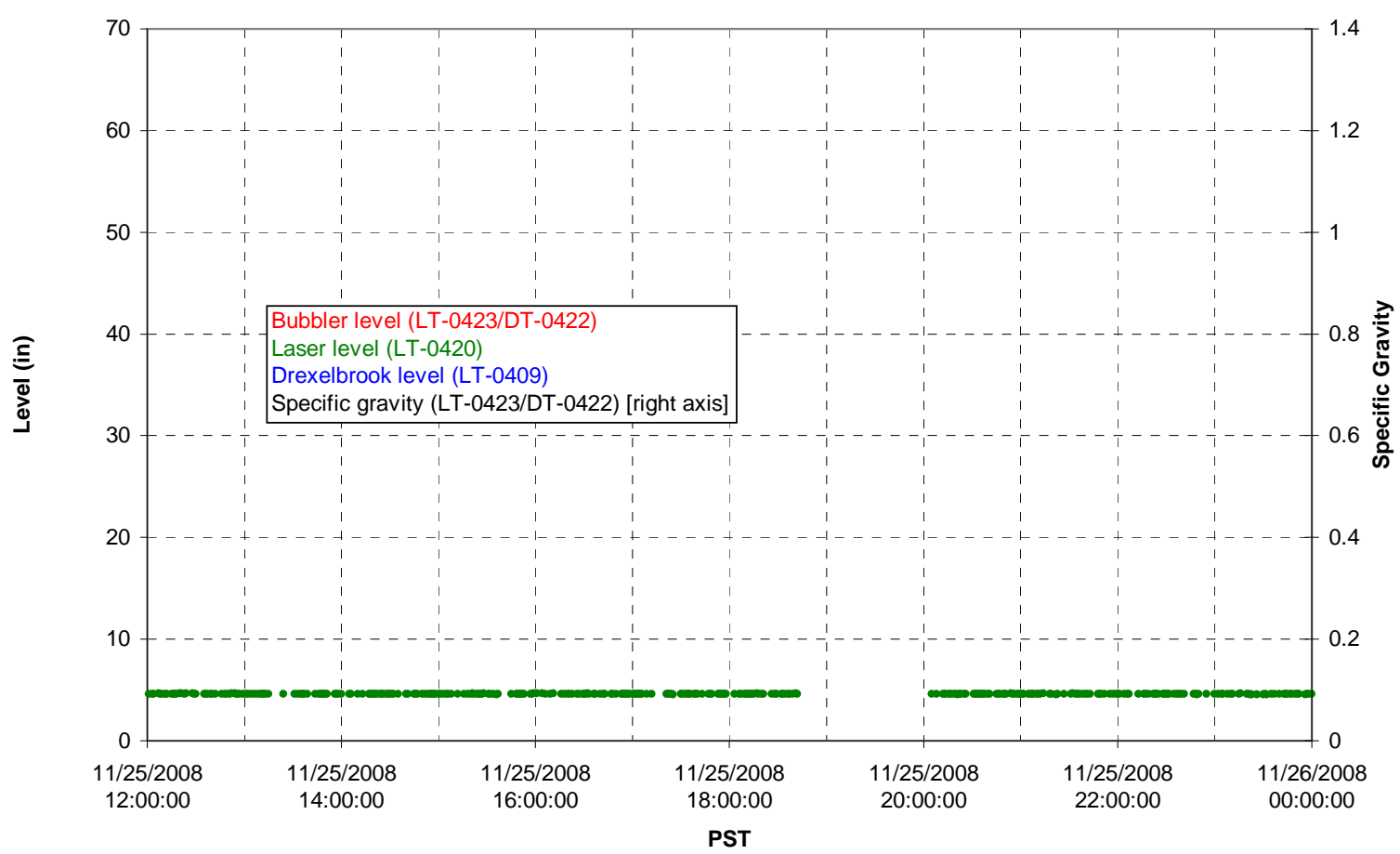

T01B temperatures

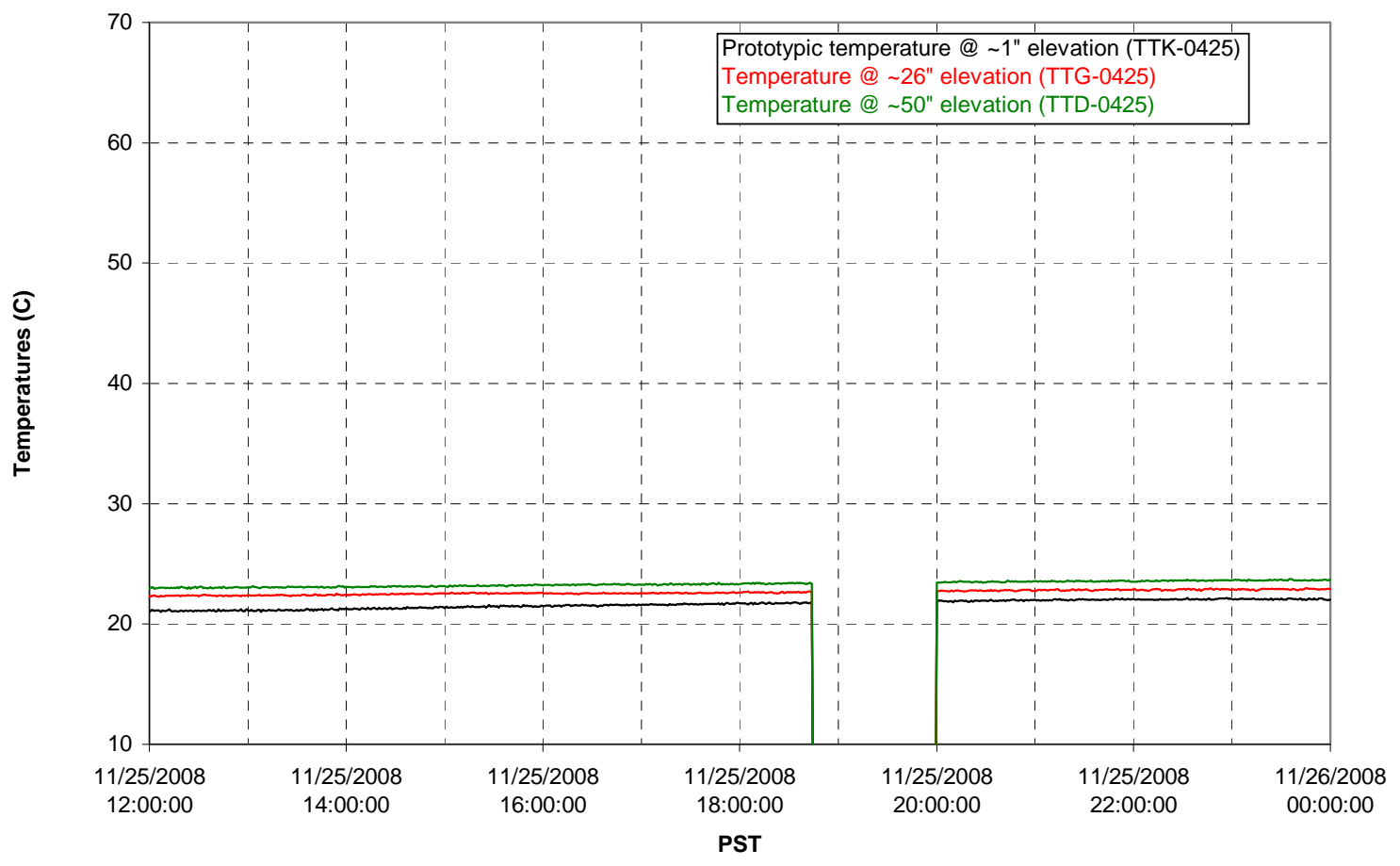


T02A level

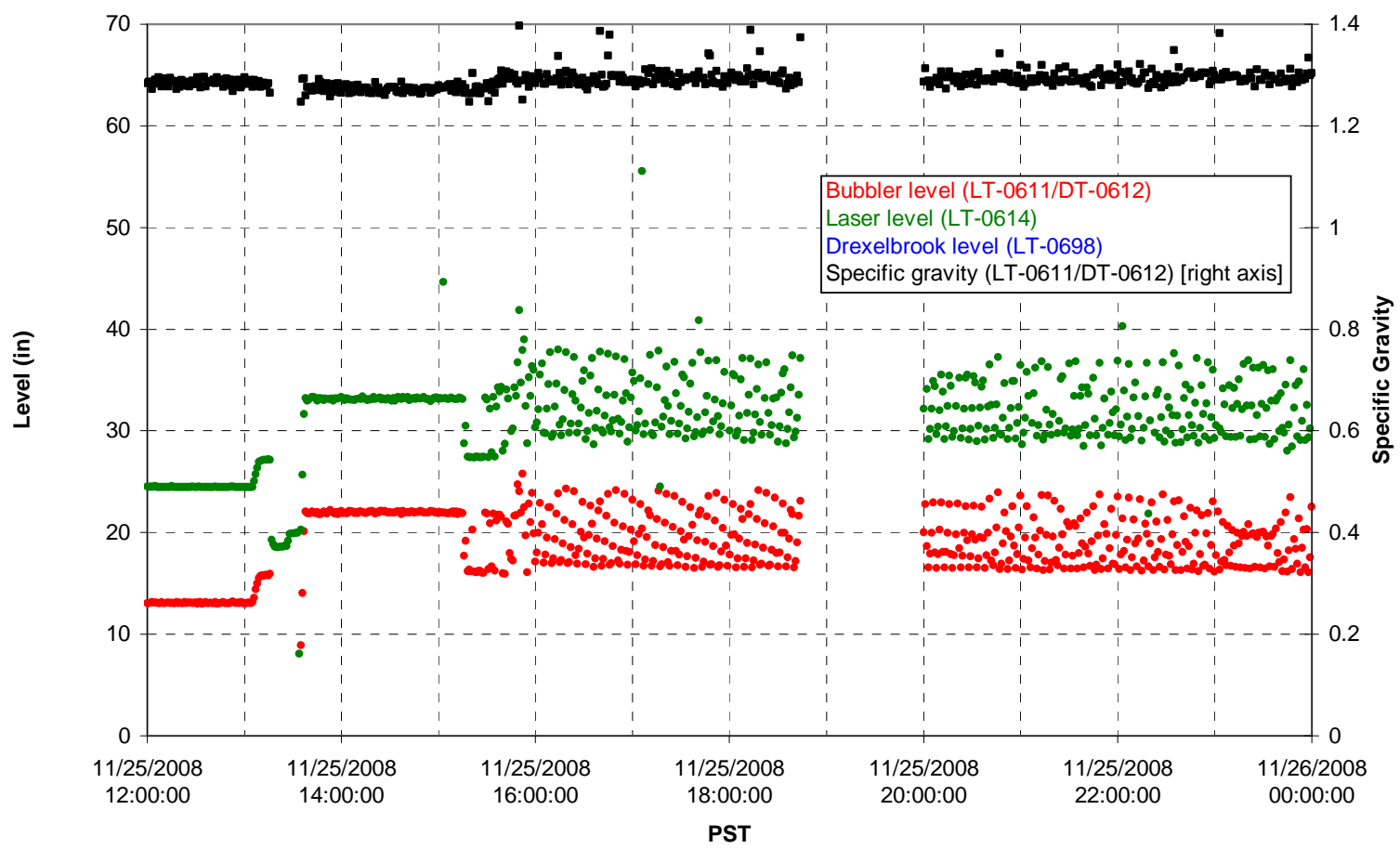

T02A temperatures

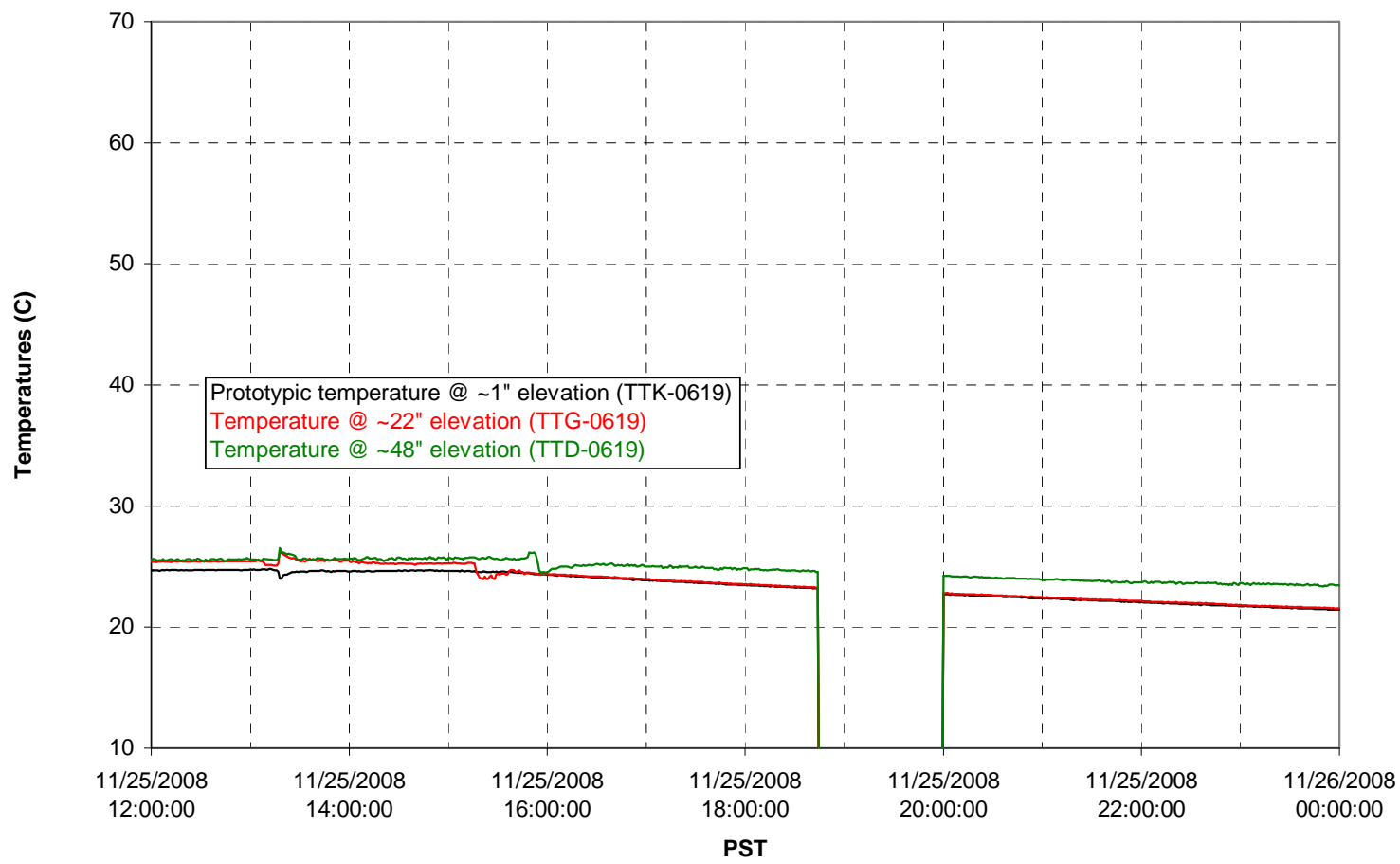


T02A and filter loop temperatures

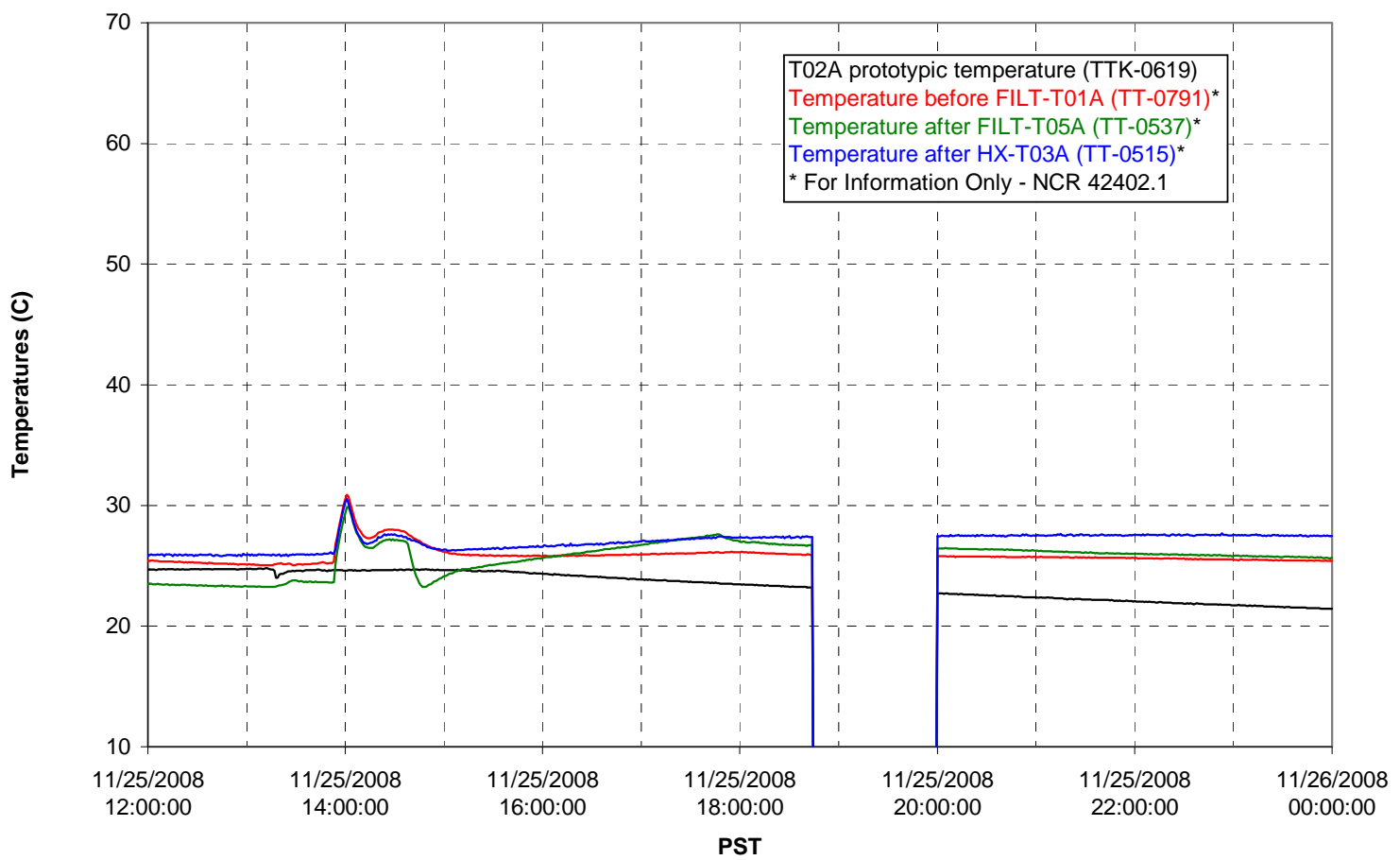

Pump Pressures and Flow

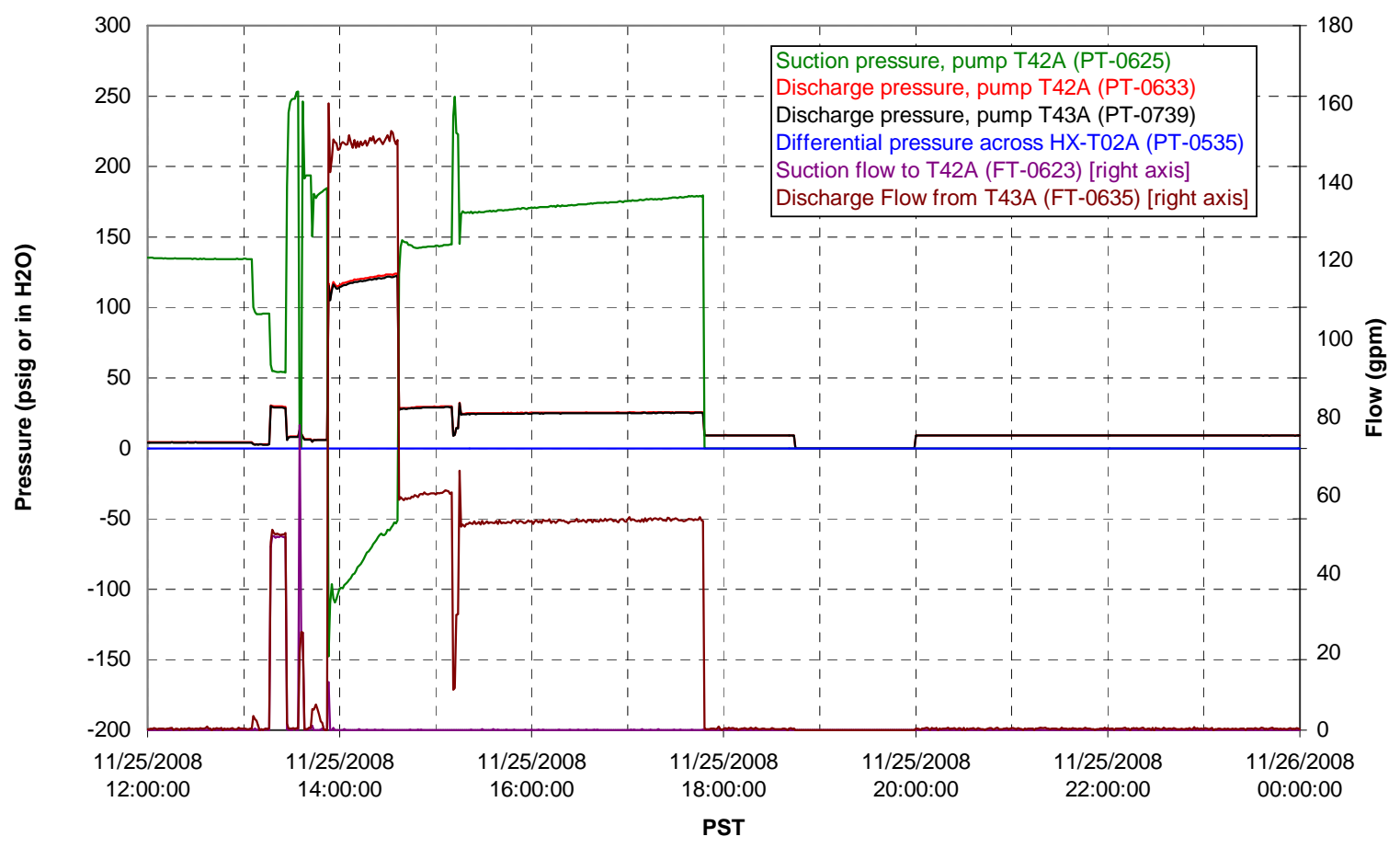


Axial pressure drop

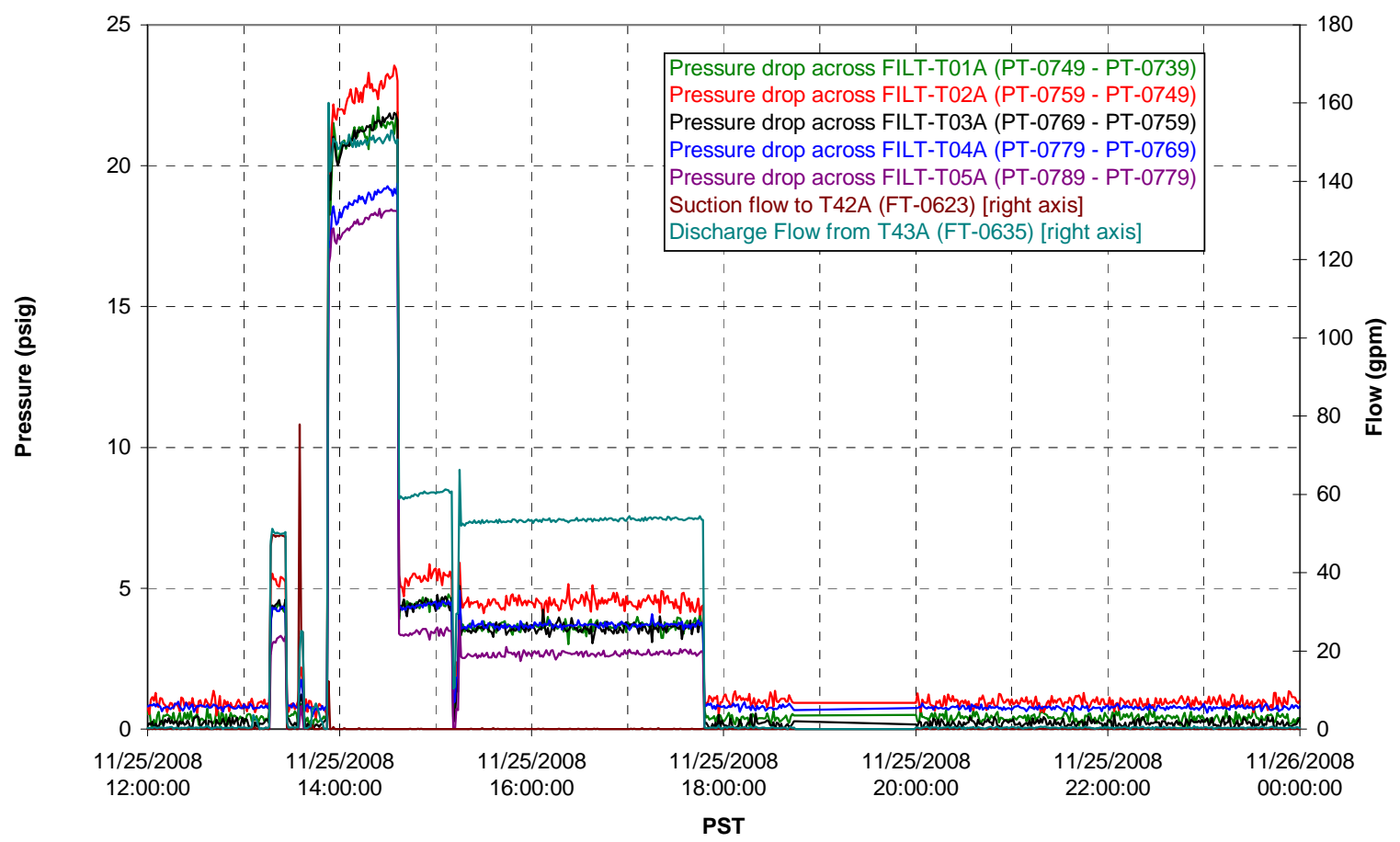

Permeate flow rates

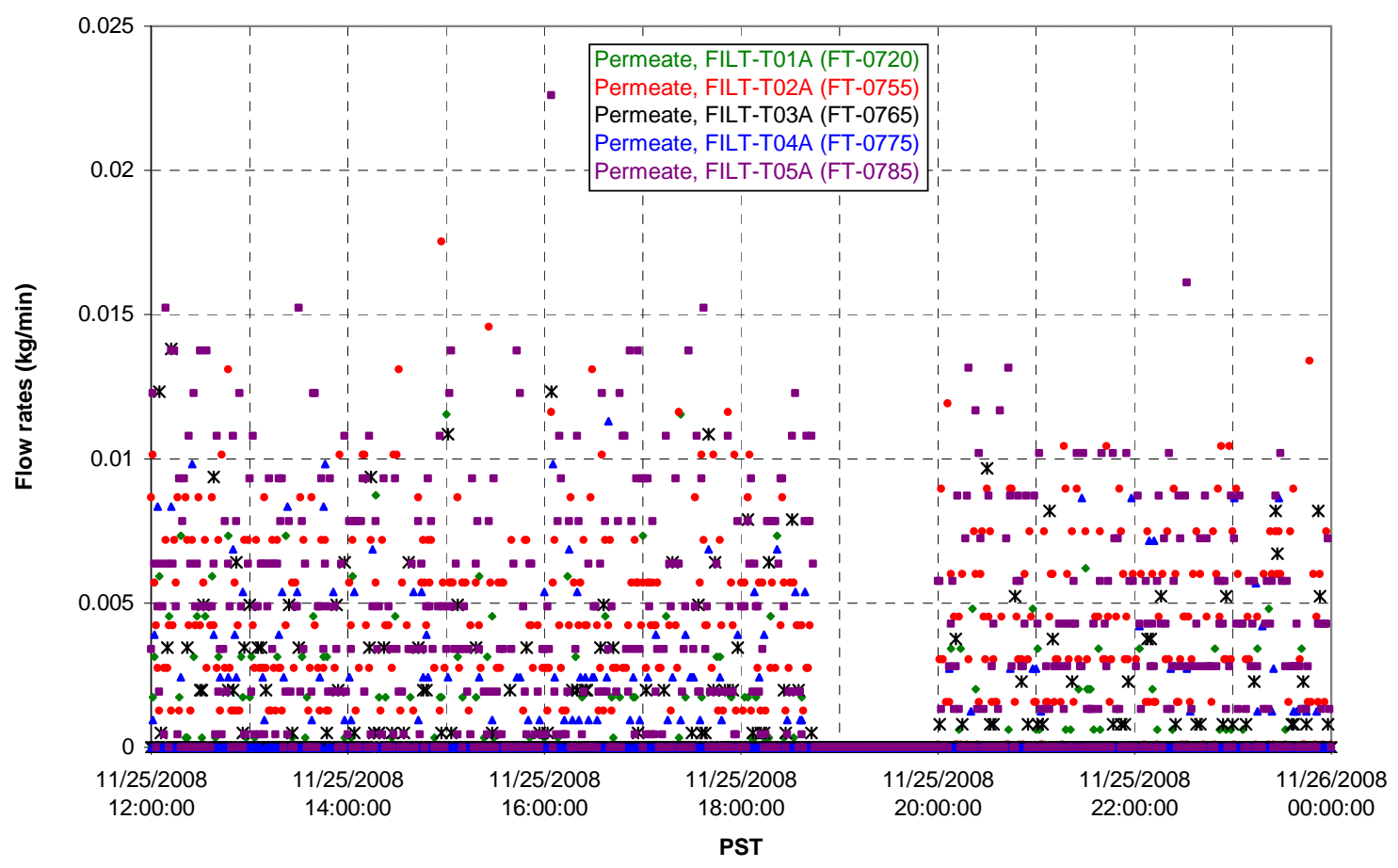


T02A Inner Temperature Tree

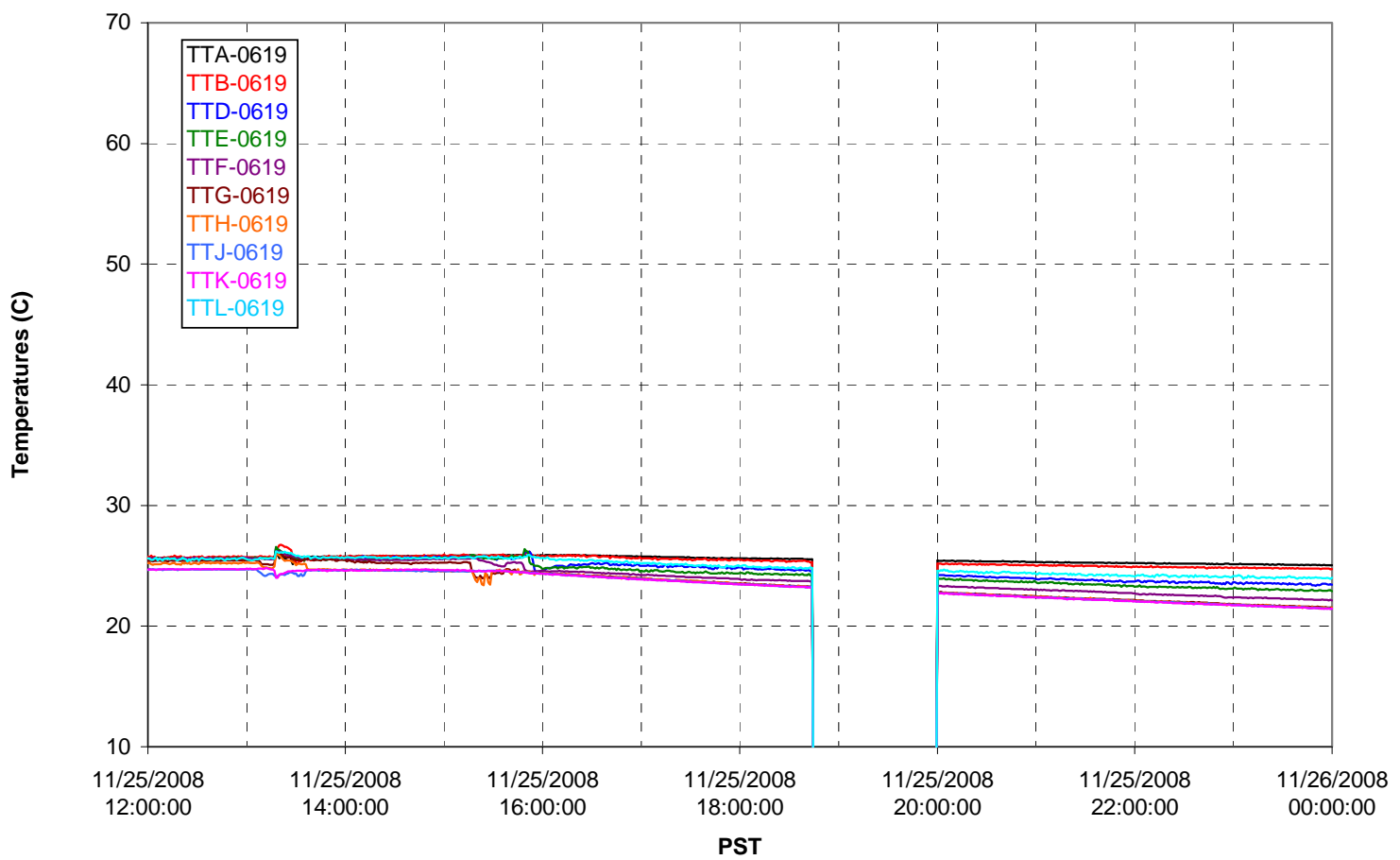

T02A Outer Temperature Tree

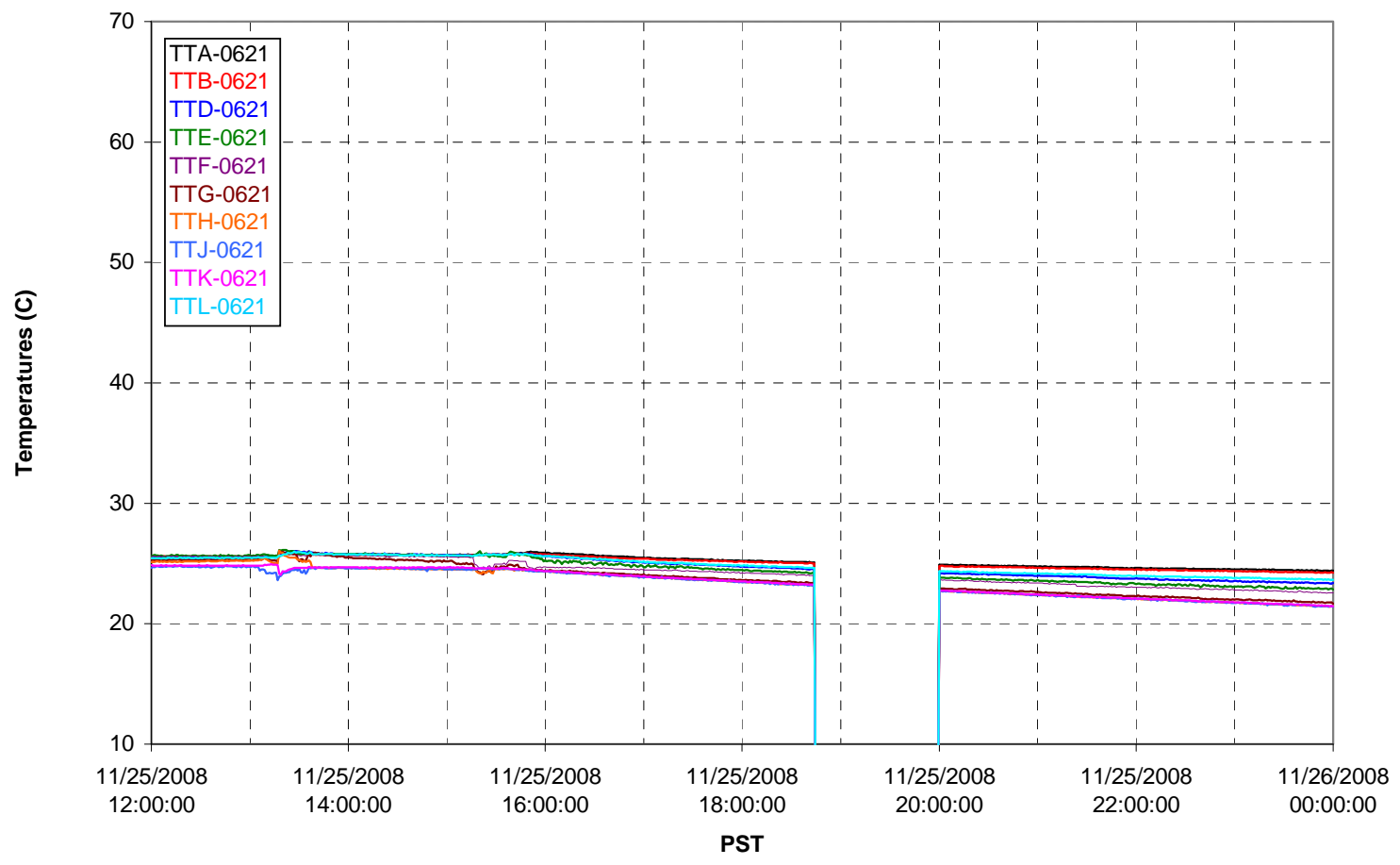


T02A temperatures

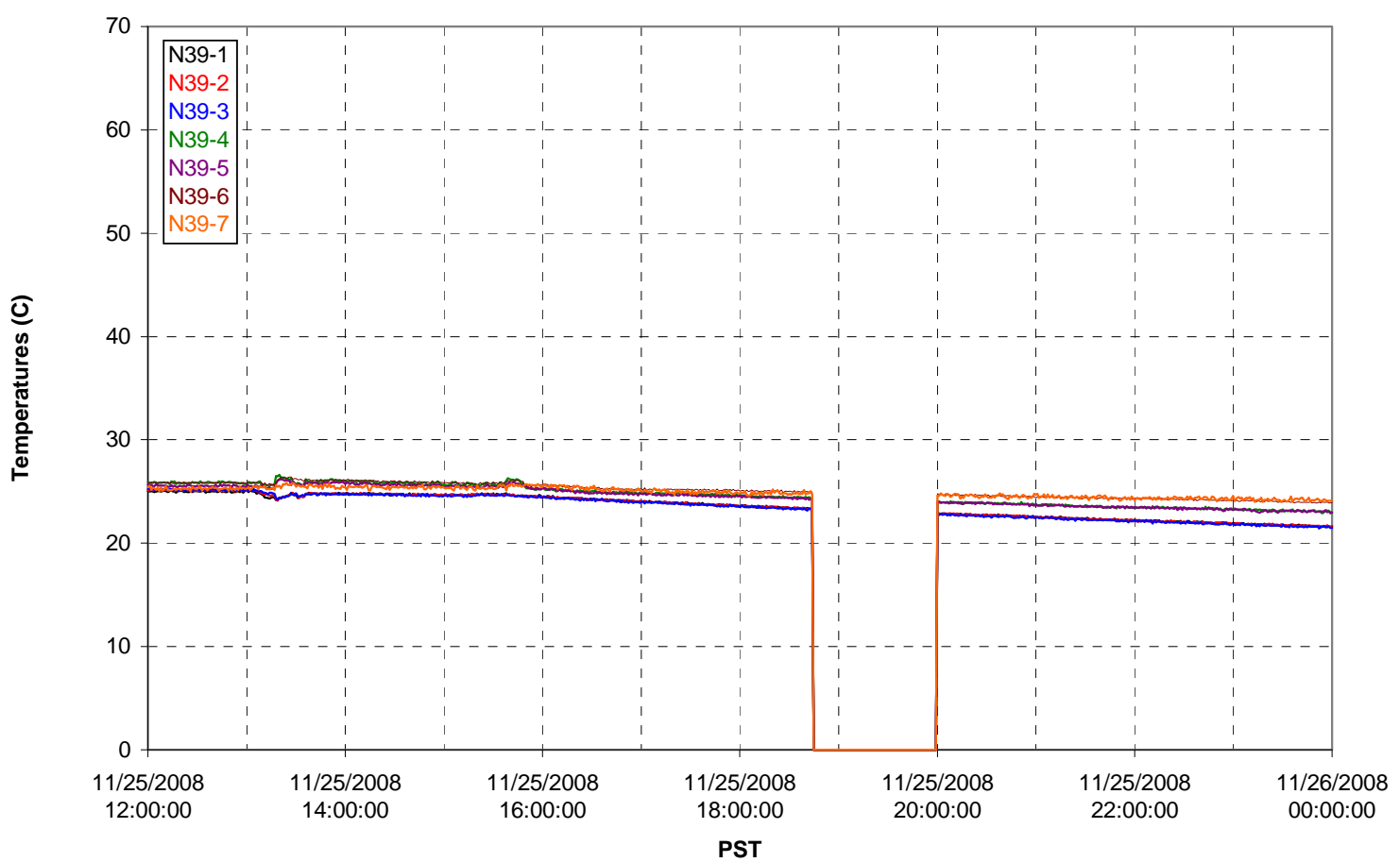

T02A temperatures

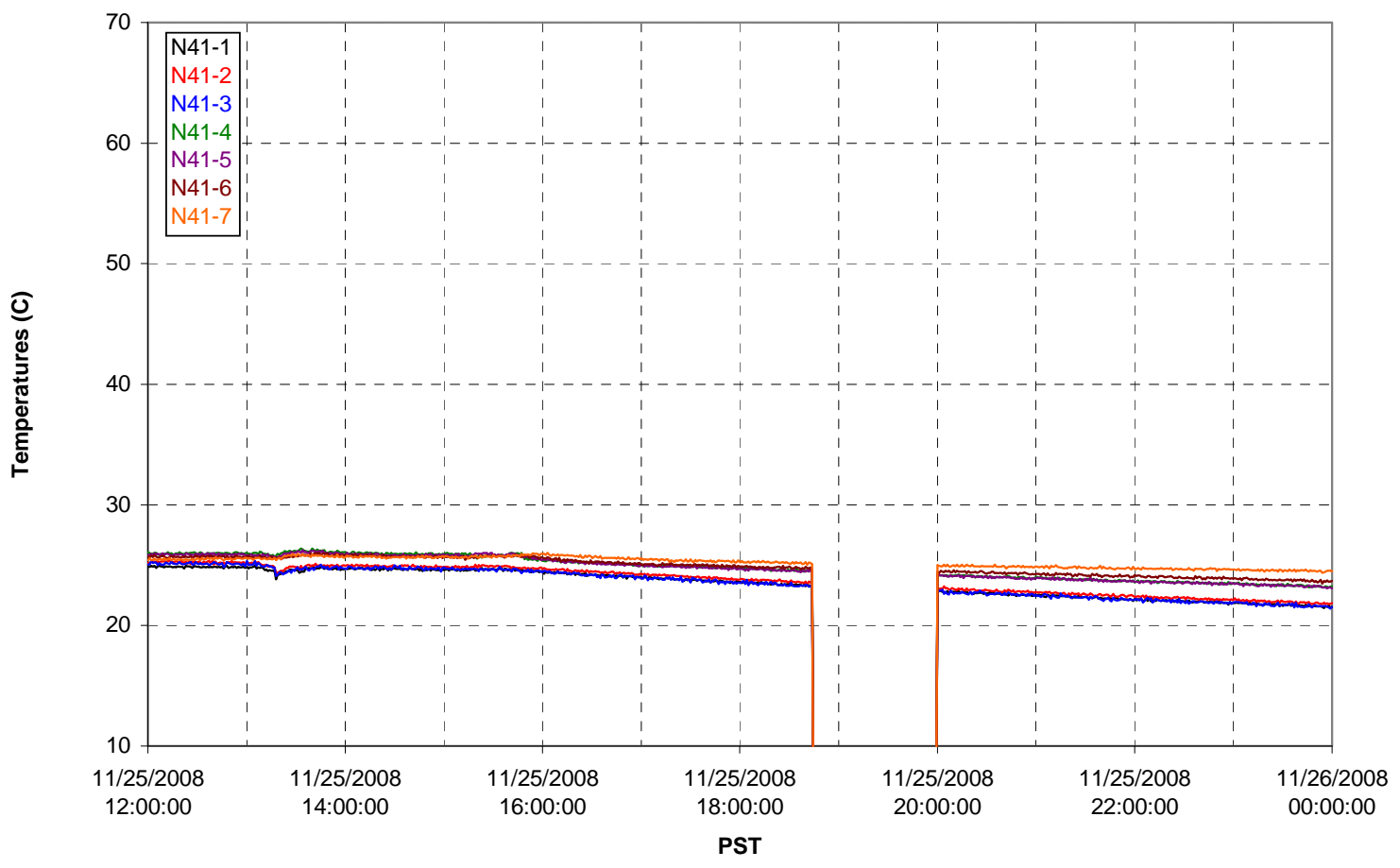


T02A temperatures

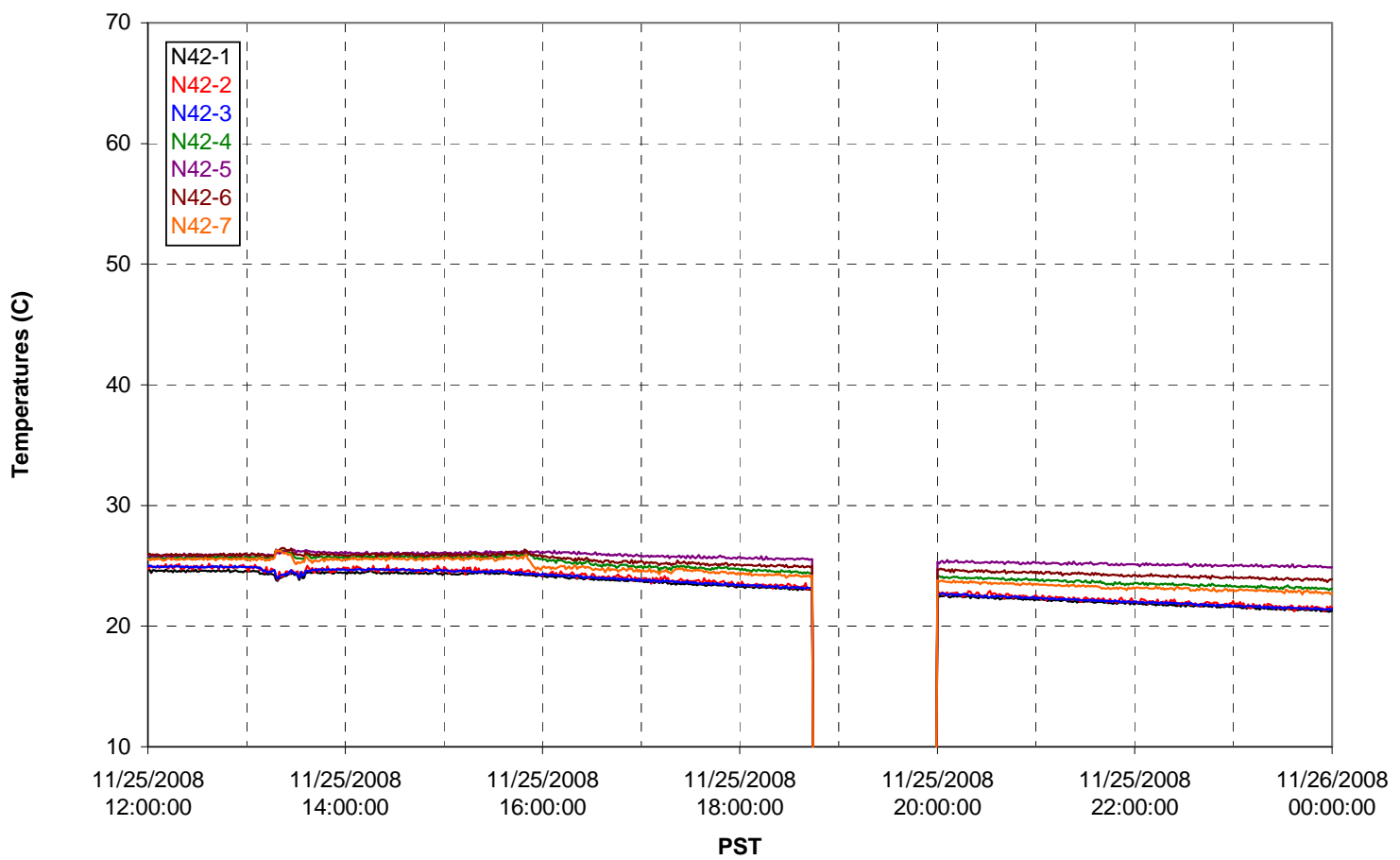

T02A temperatures

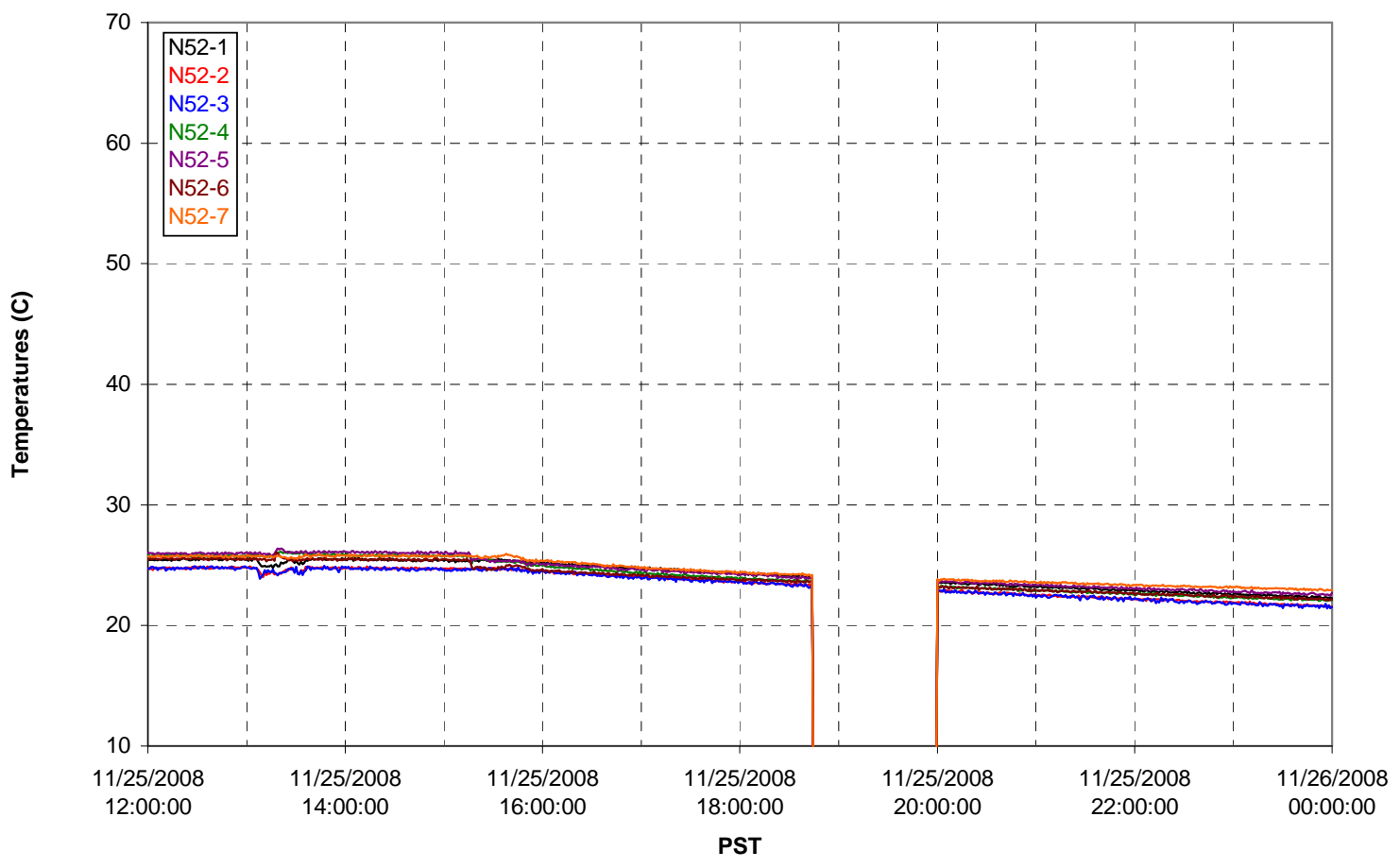


T02A Heating and Cooling

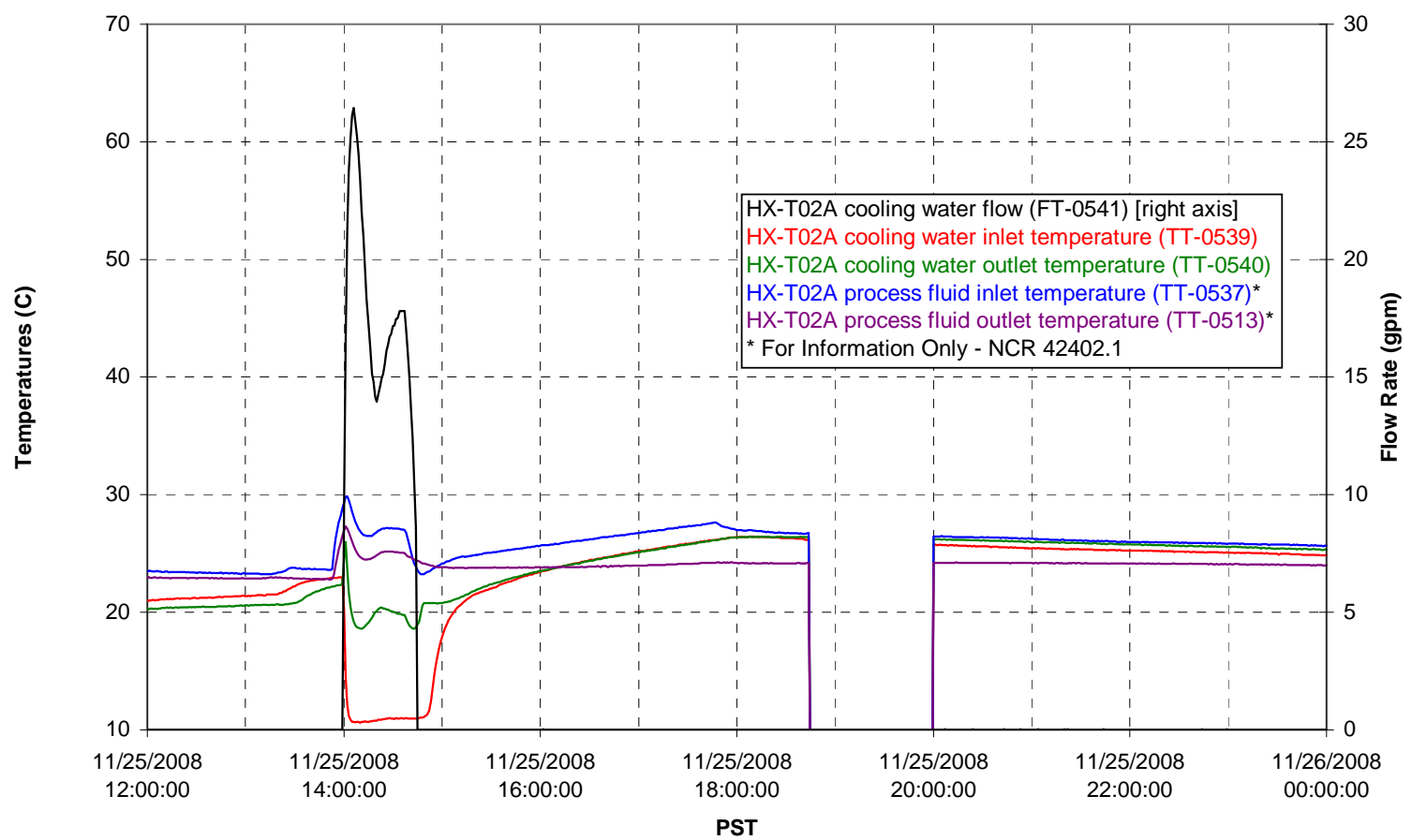

Pump Operation

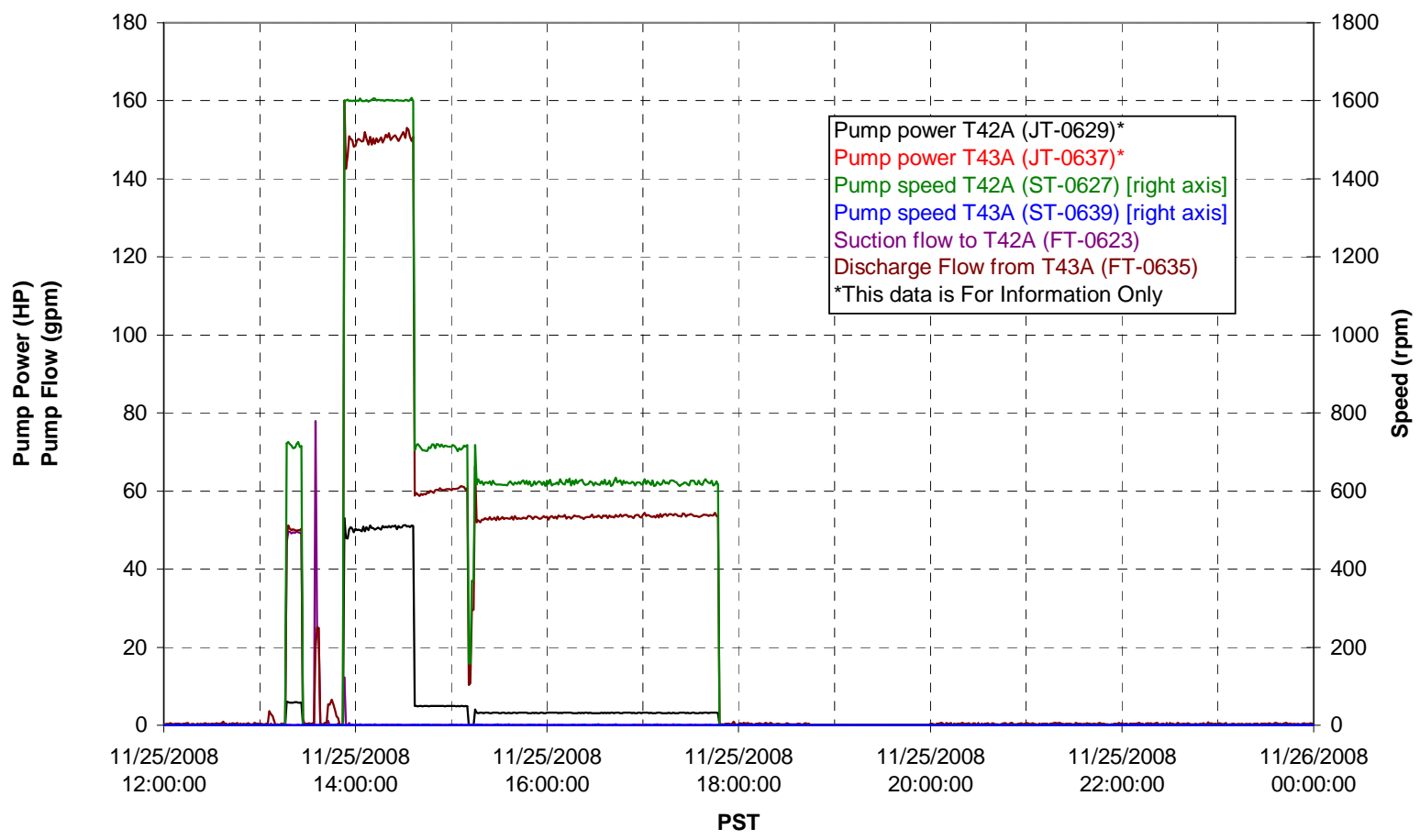


Pulsepot UFP-PP-T01A

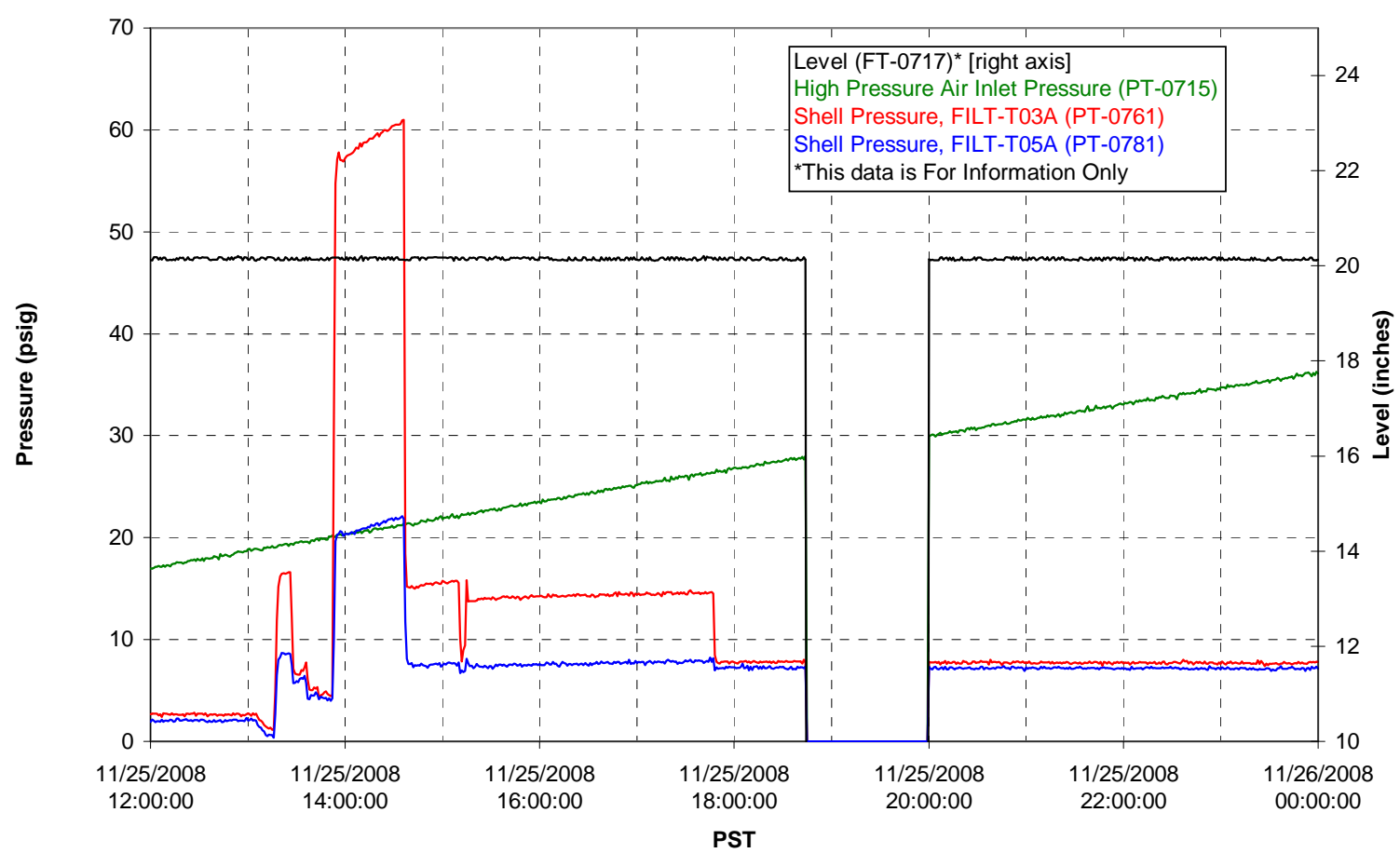

Pulsepot UFP-PP-T02A

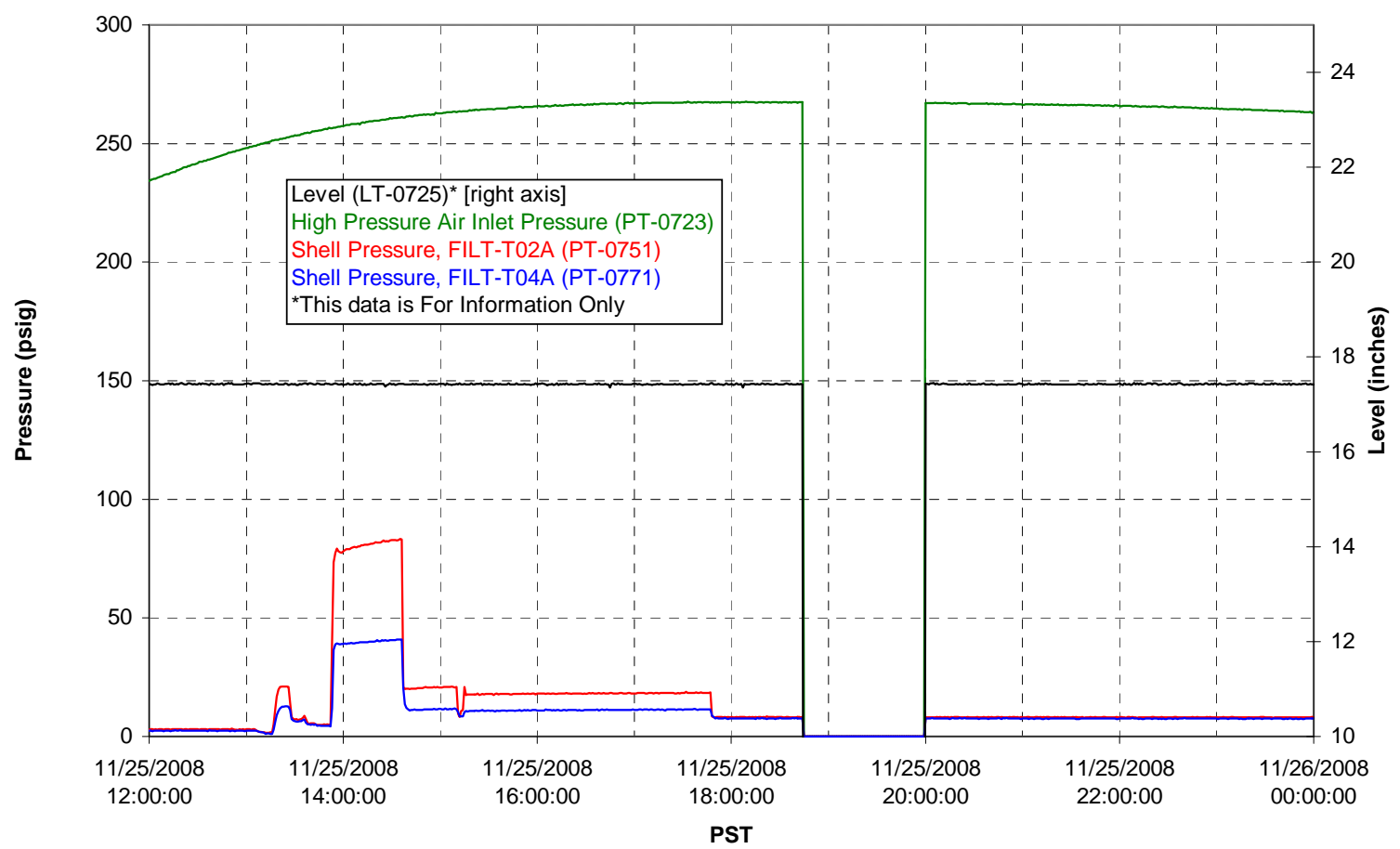


Pulsepot UFP-PP-T03A

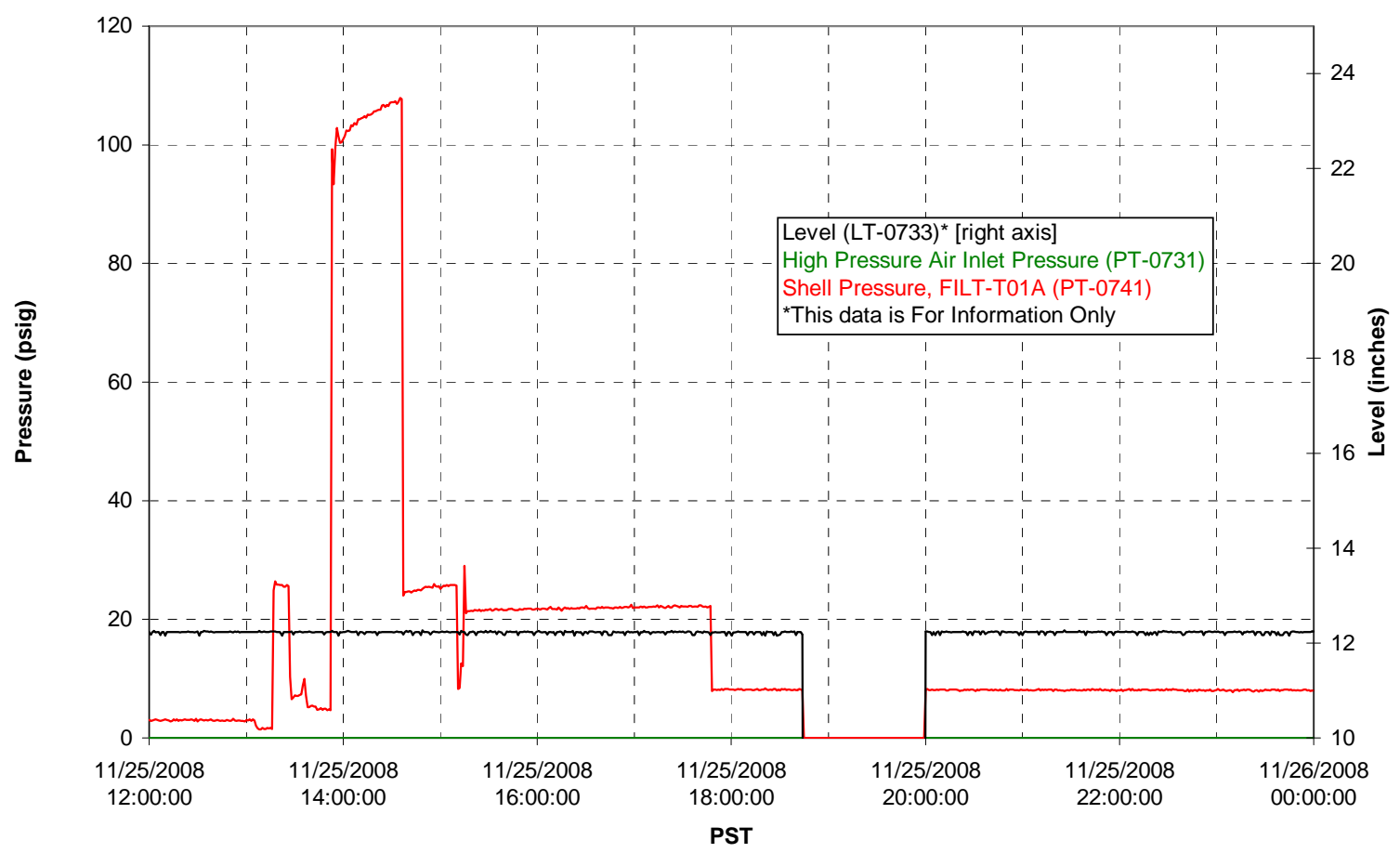

Pulsepot Levels

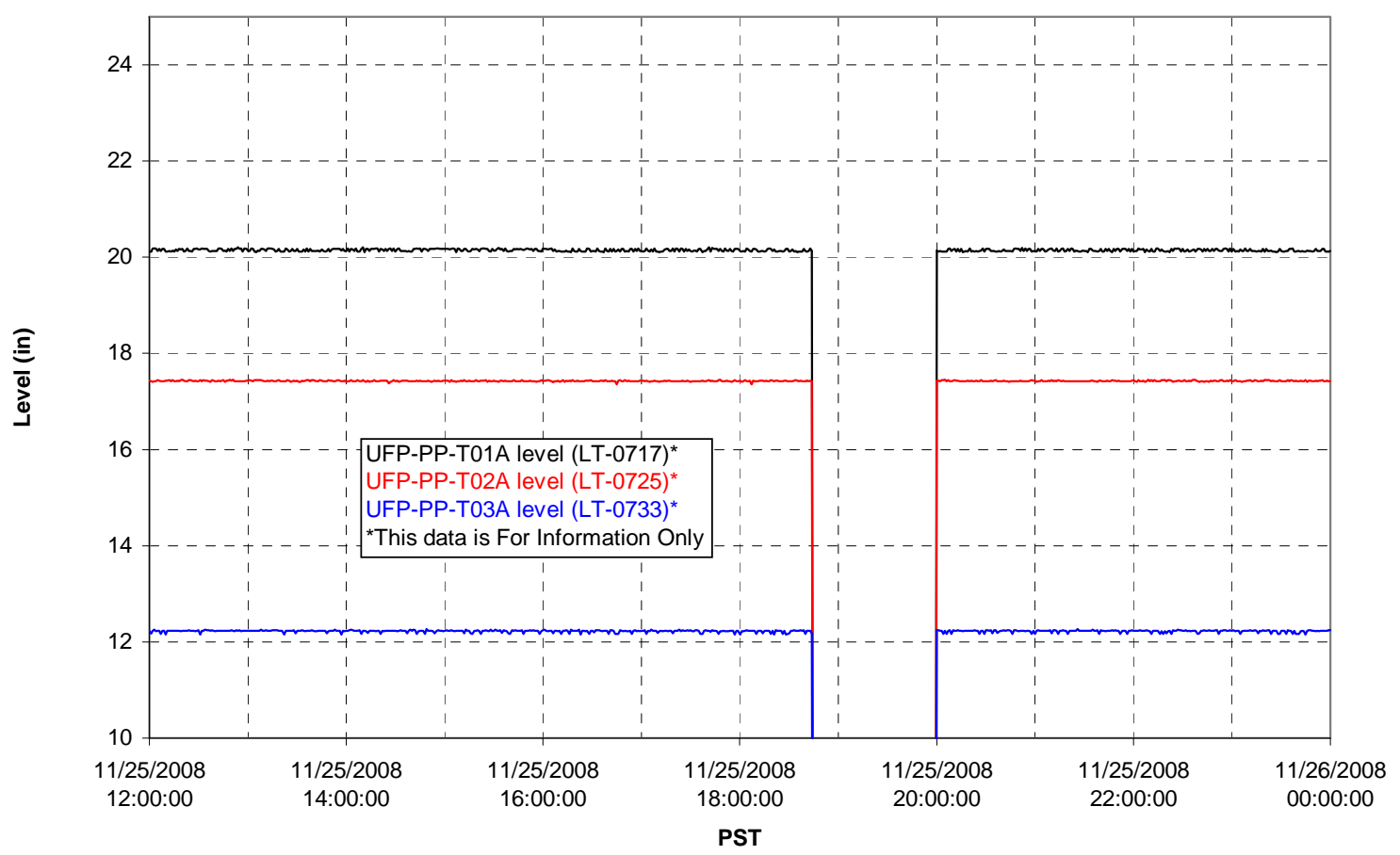


Filter UFP-FILT-T01A

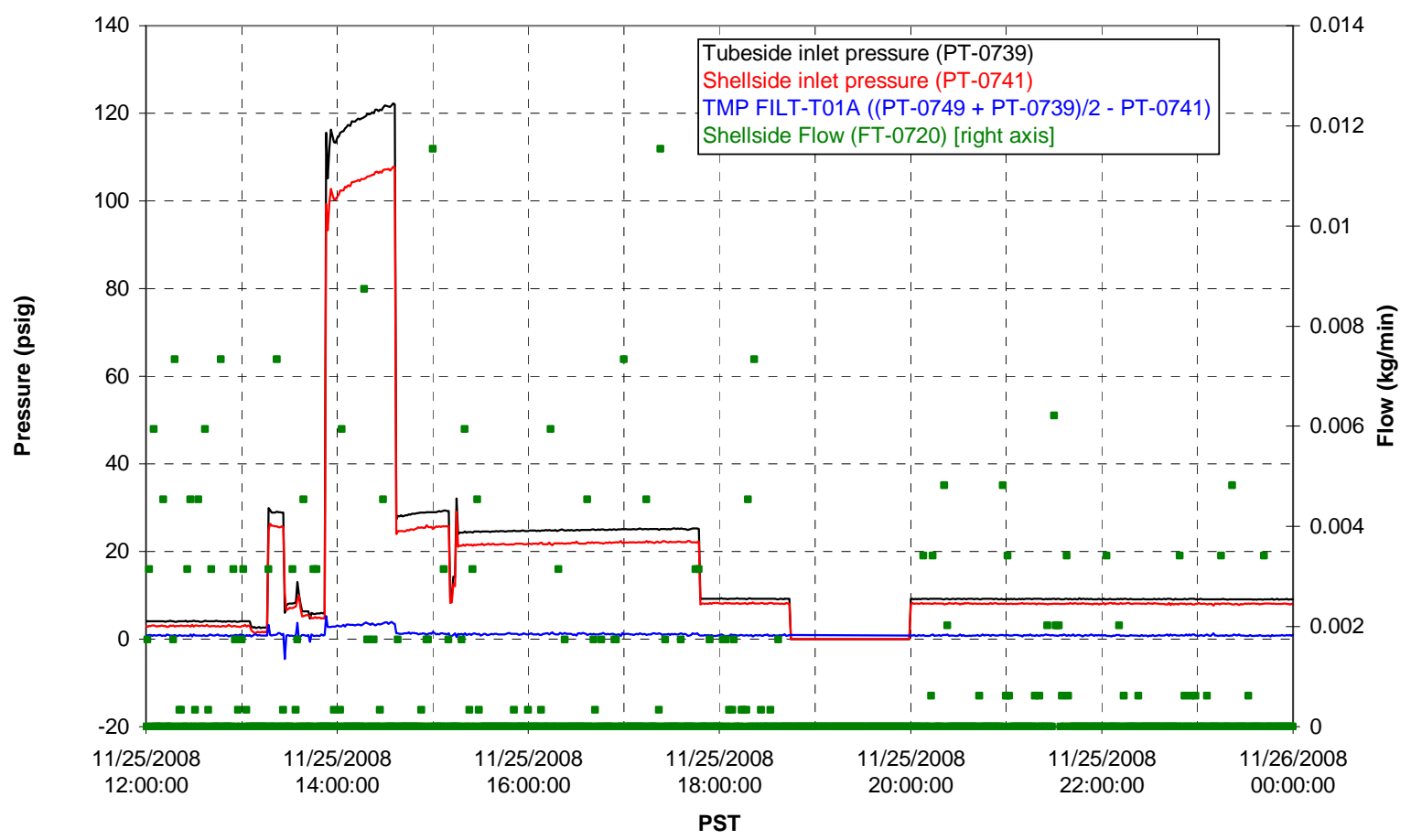

Filter UFP-FILT-T02A

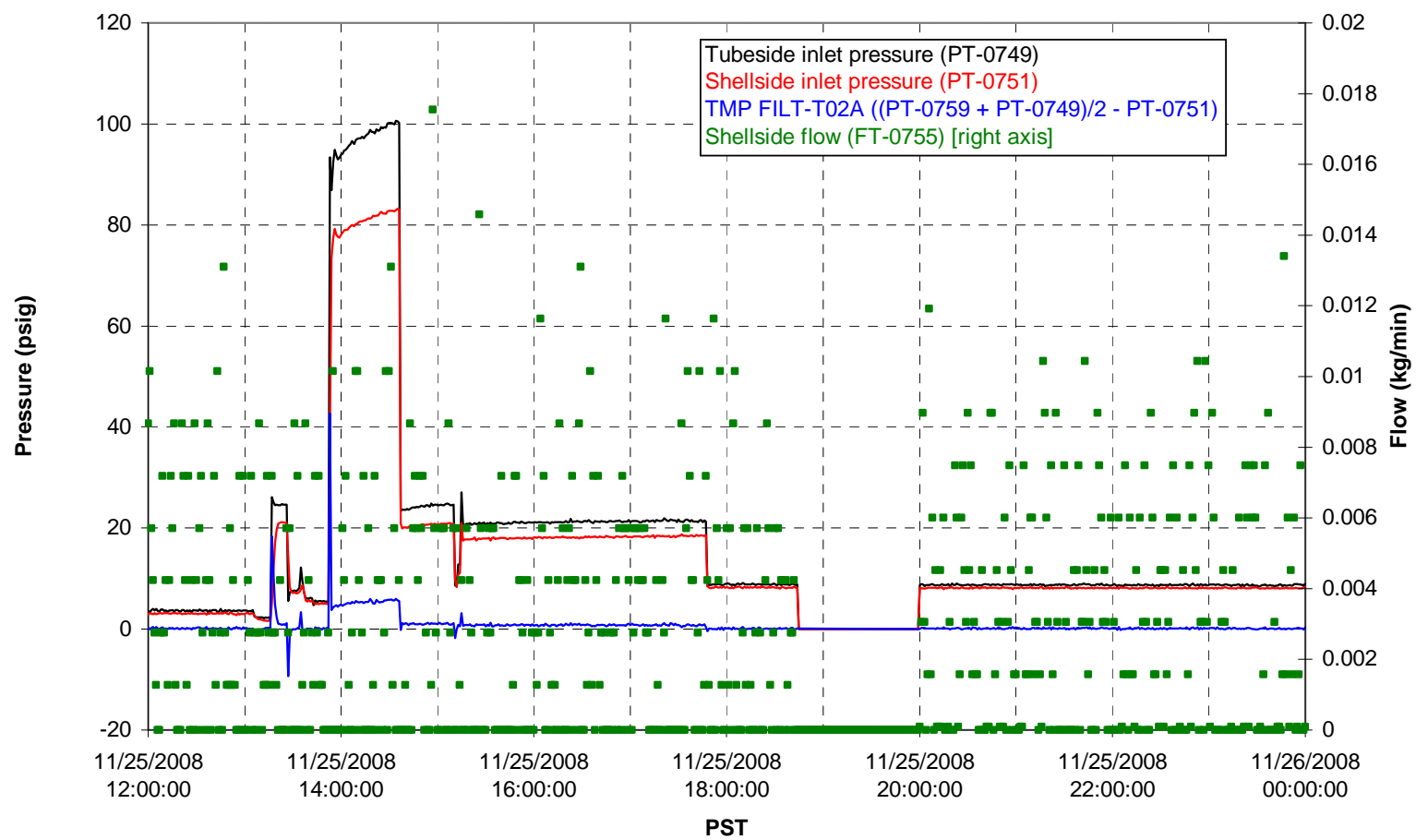


Filter UFP-FILT-T03A

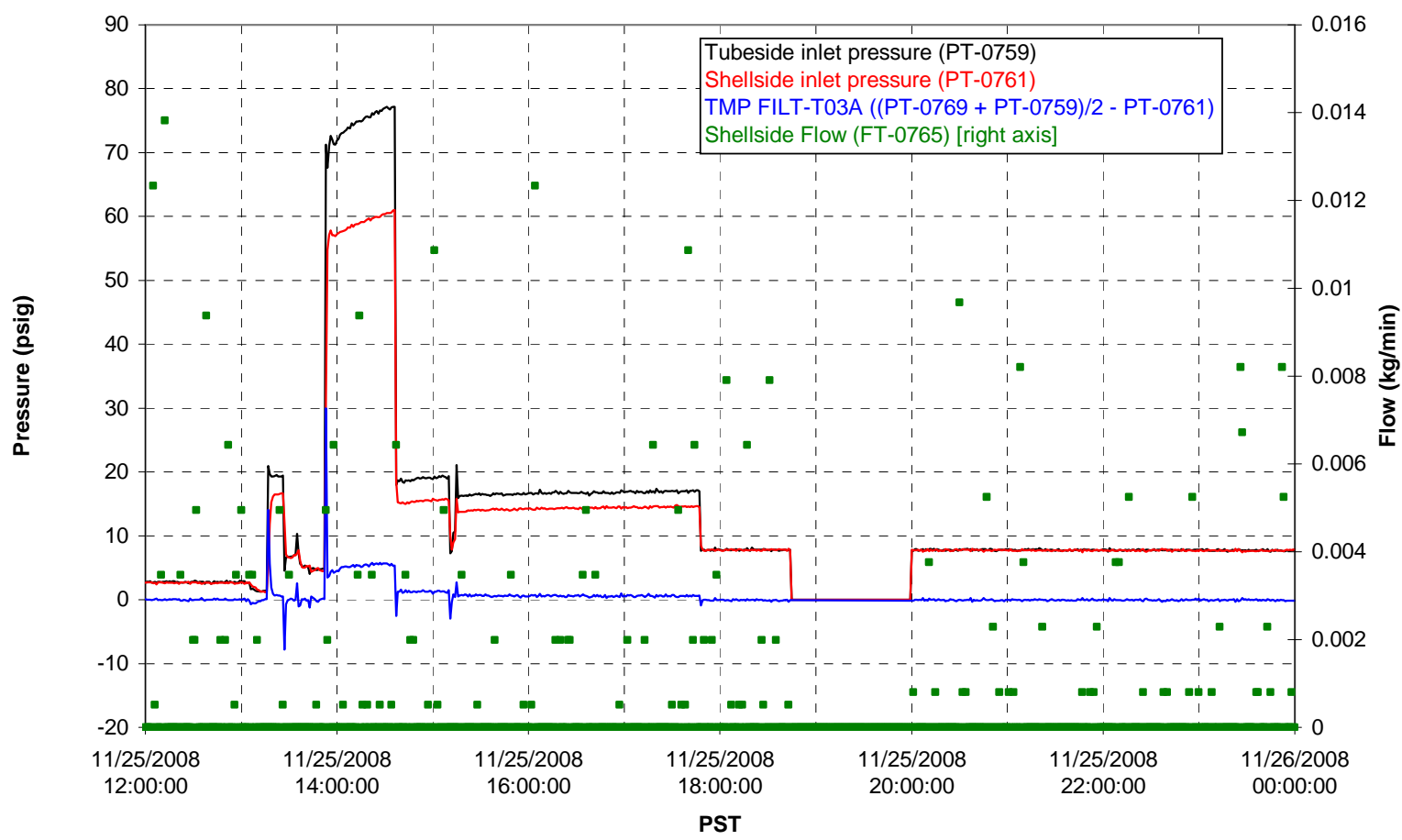

Filter UFP-FILT-T04A

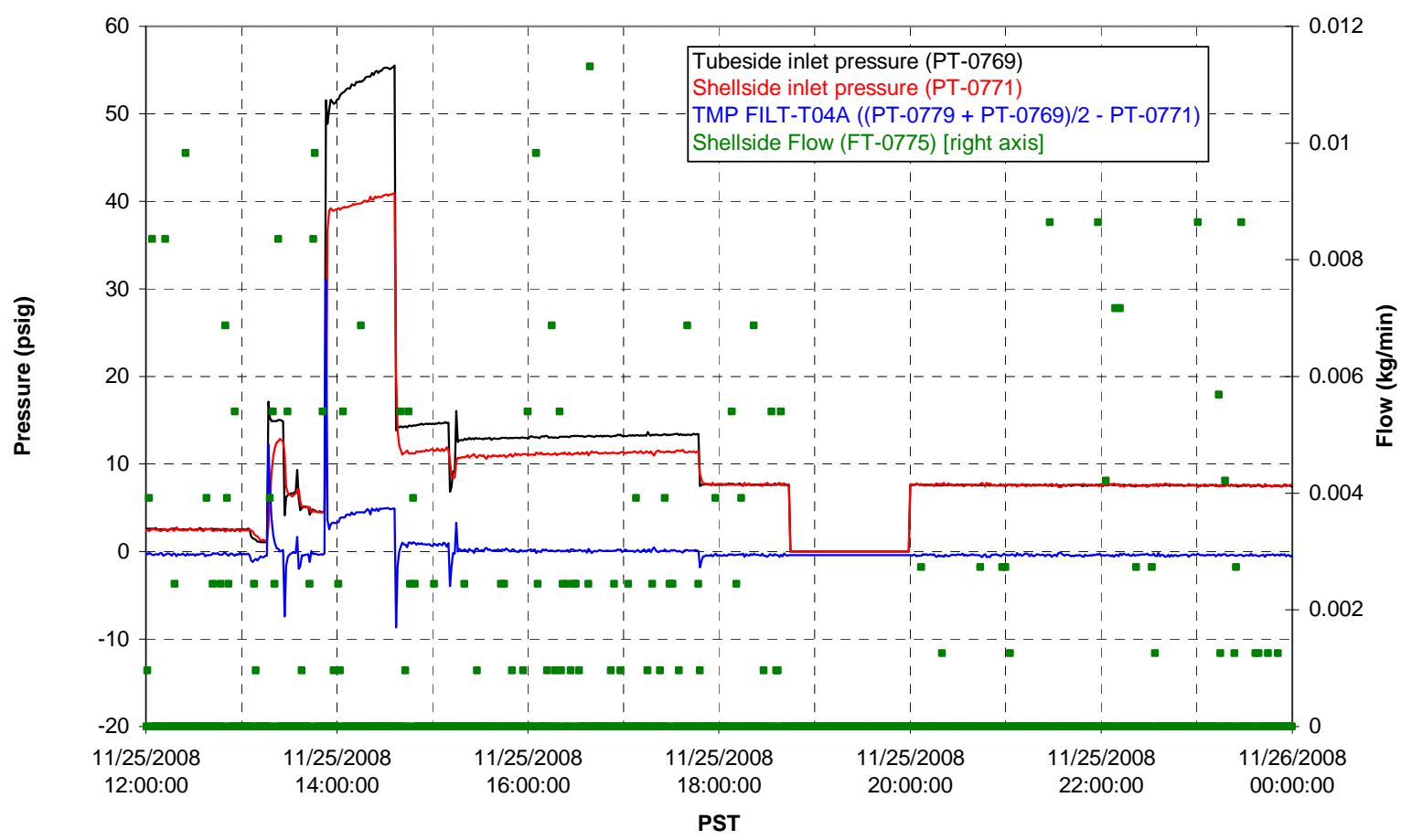


Filter UFP-FILT-T05A

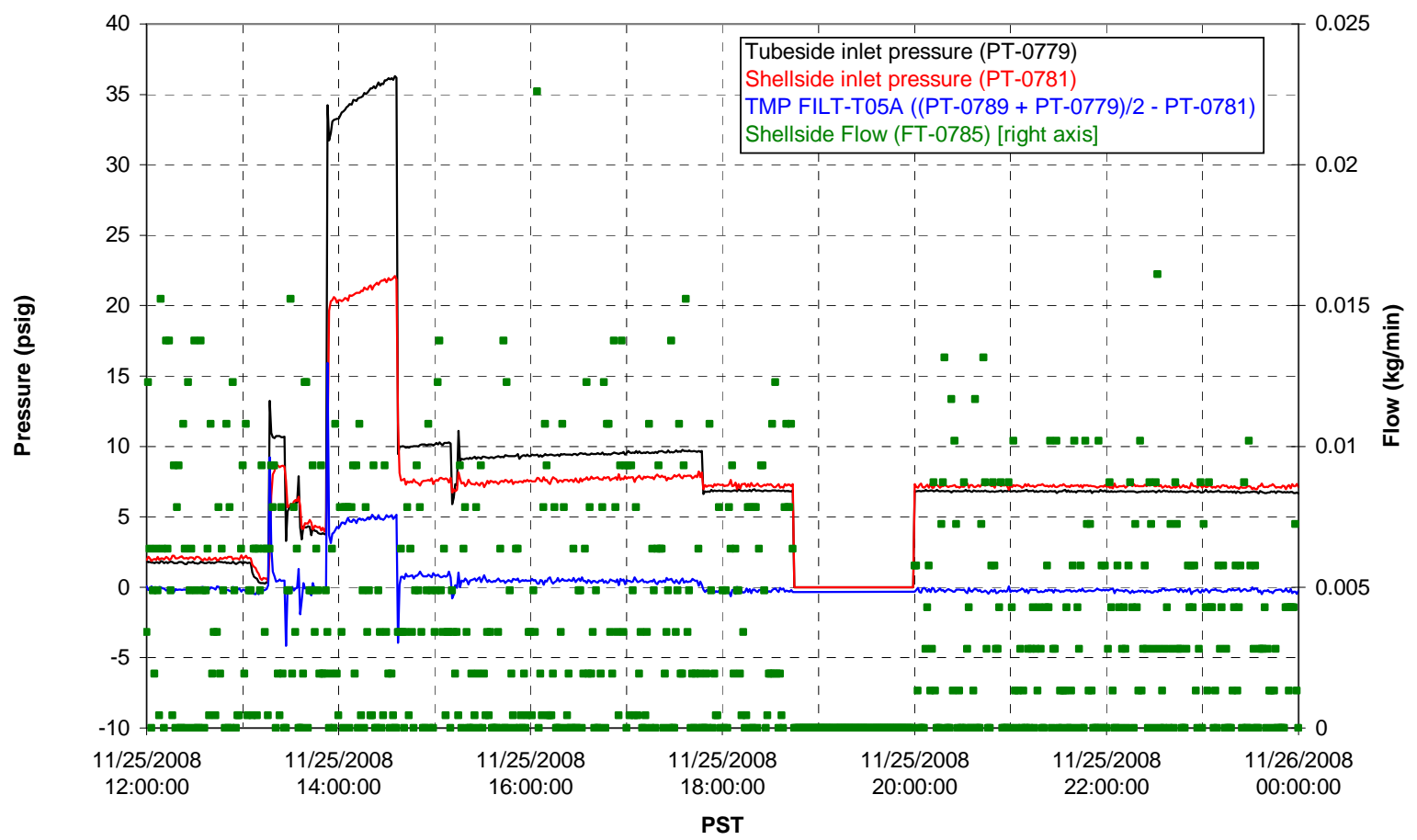

Chemical Flow

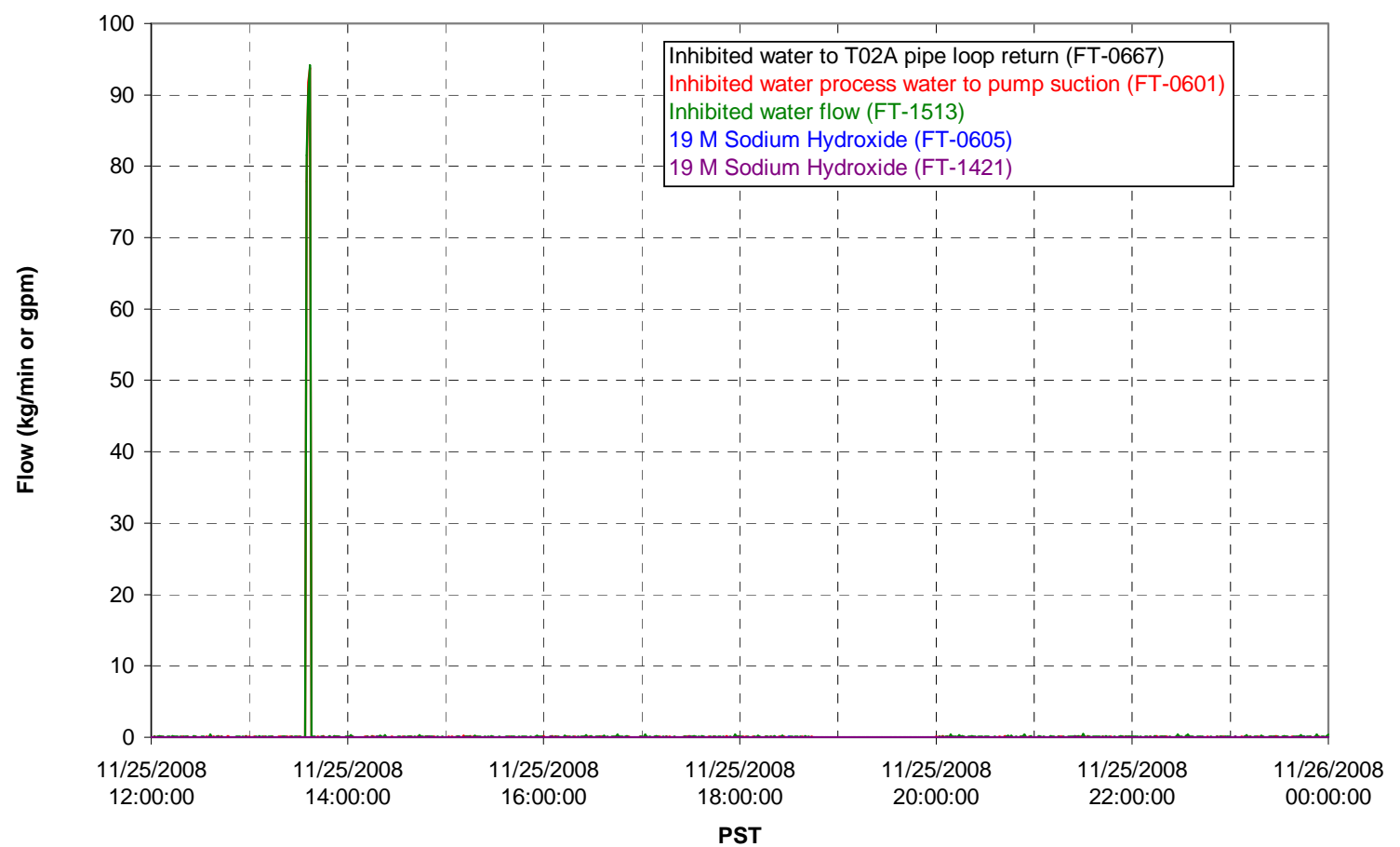


Chemical Flow

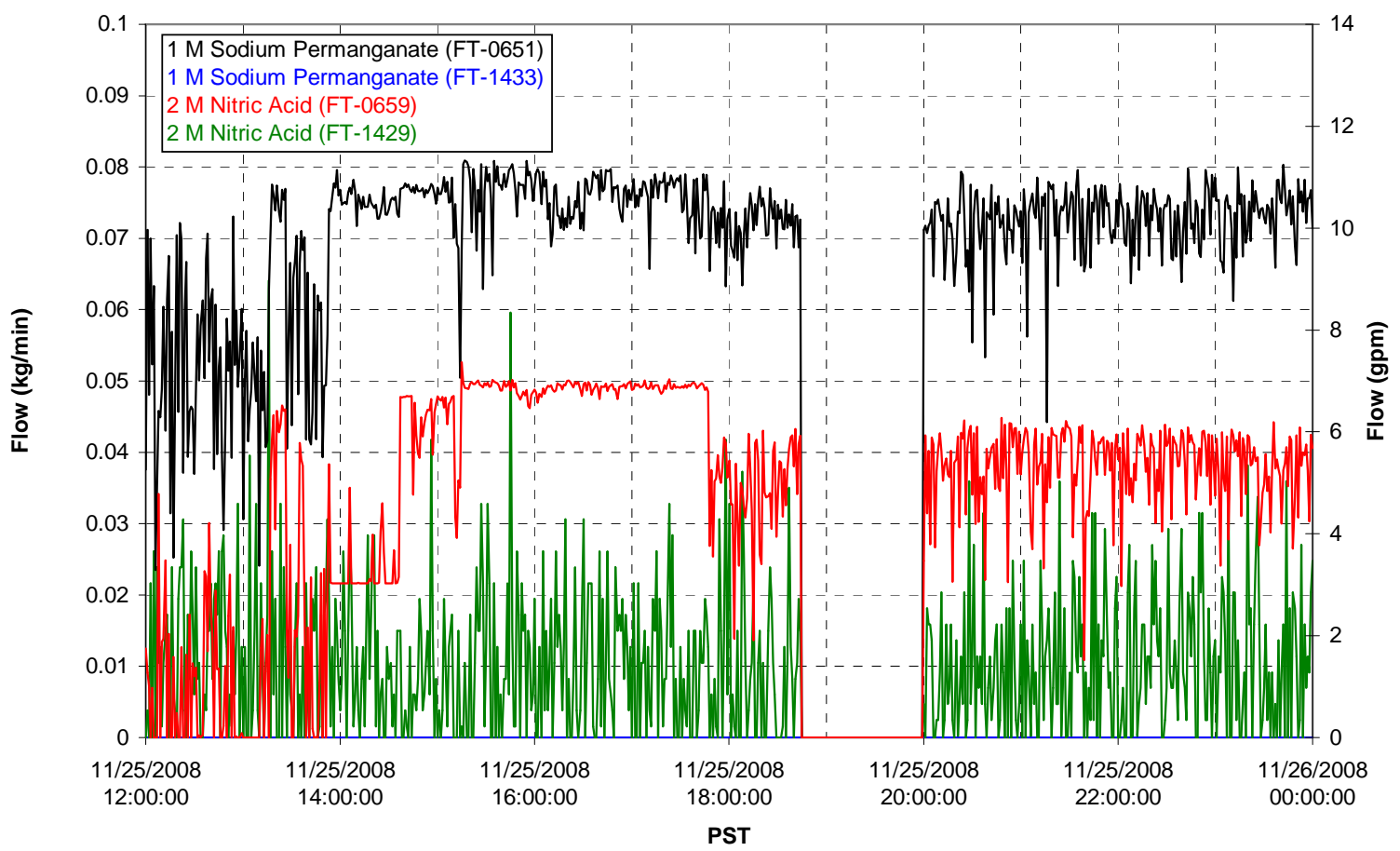

Air Flows

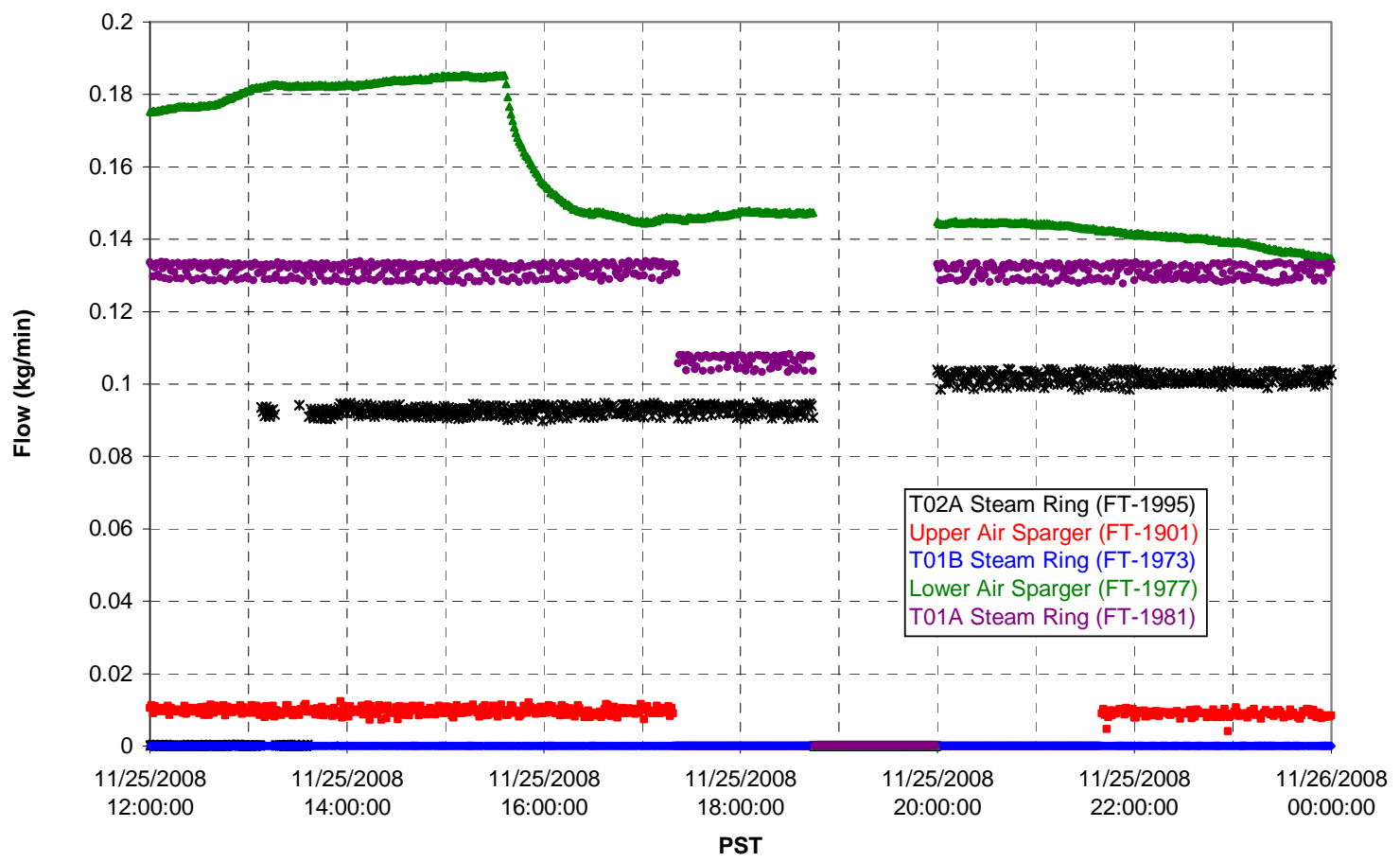


T02A Steam

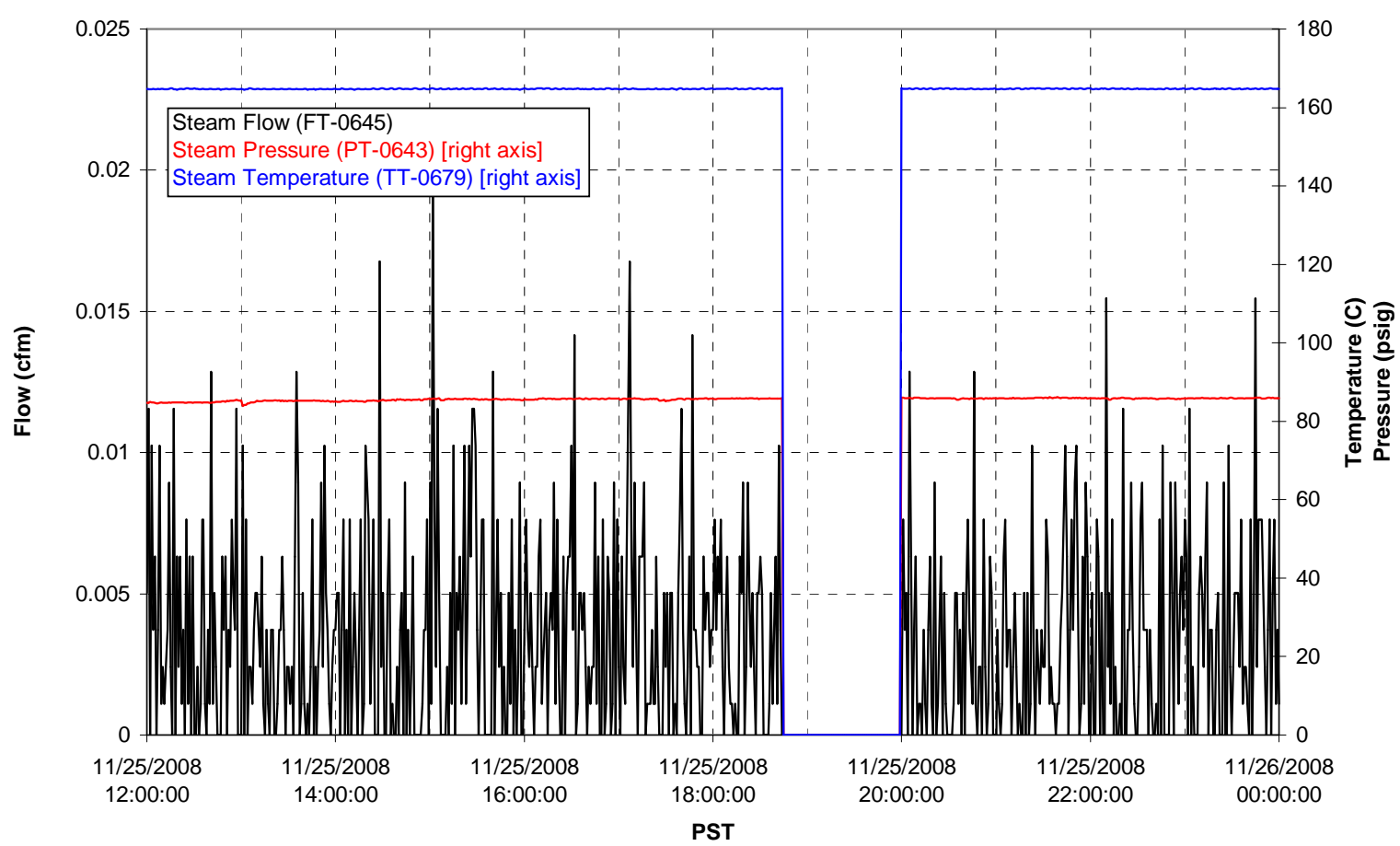

T01A Steam

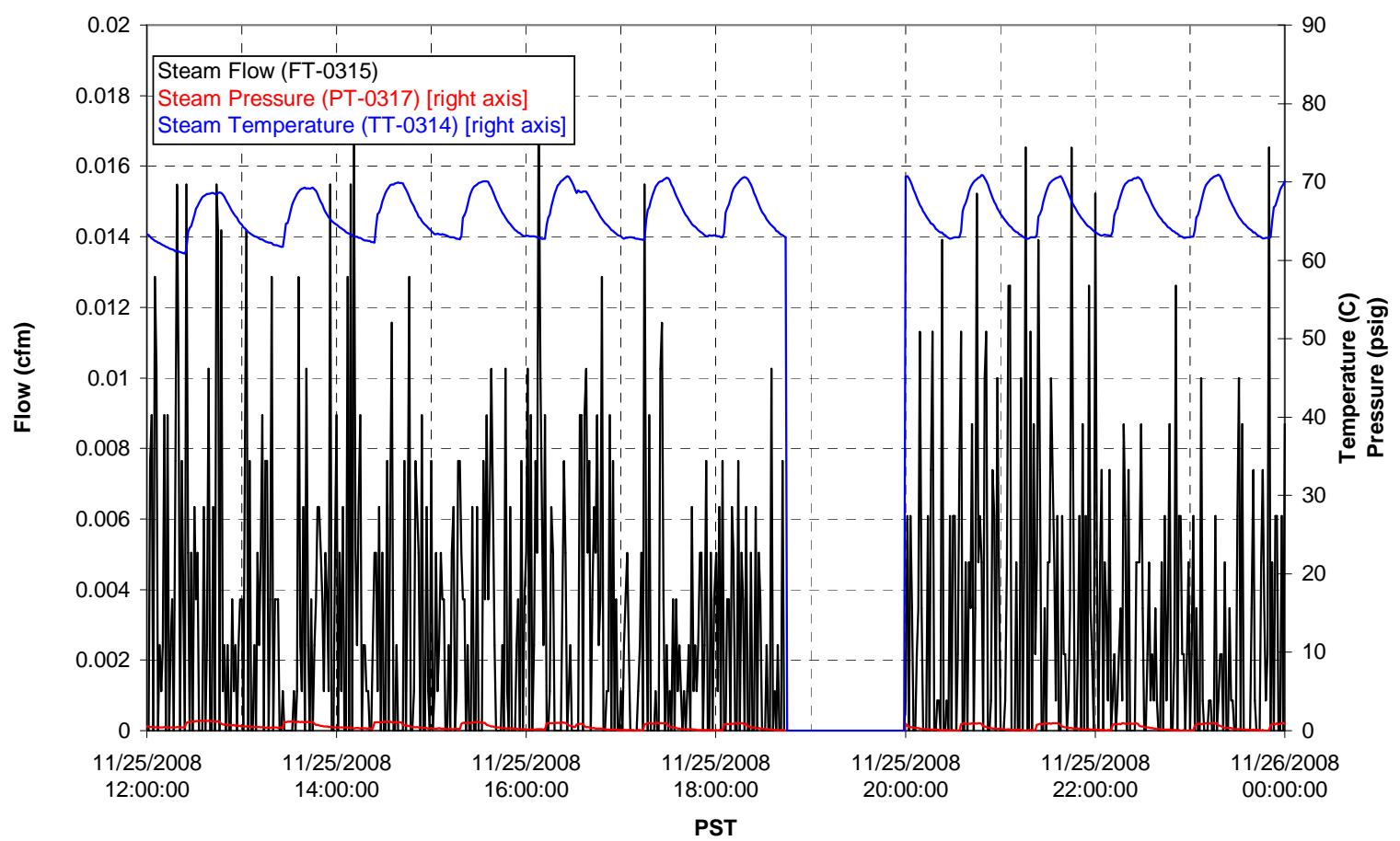


T01B Steam

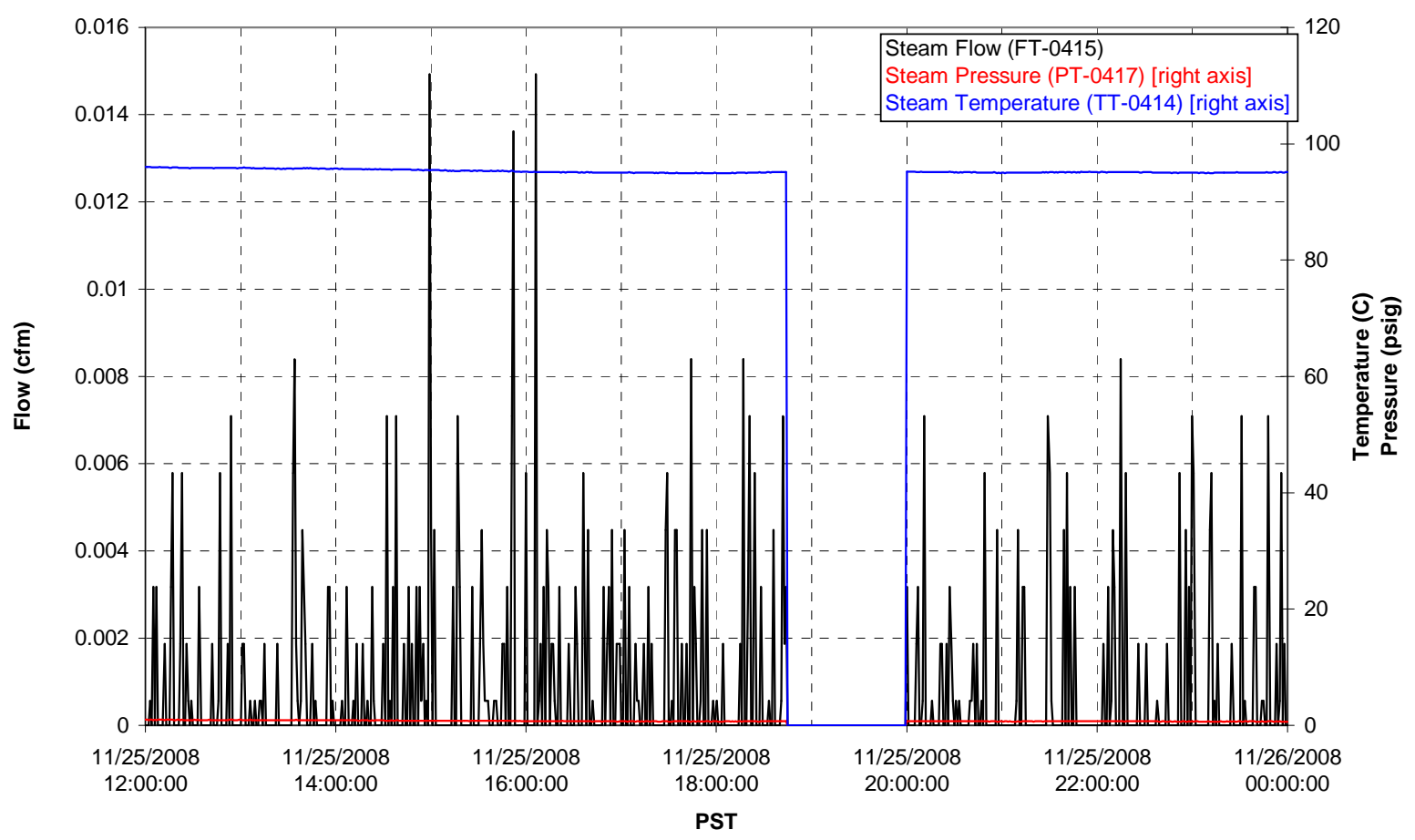


Functional Test Data Plots

11/30/2008 12:00 - 12/01/2008 00:00 
T01A level

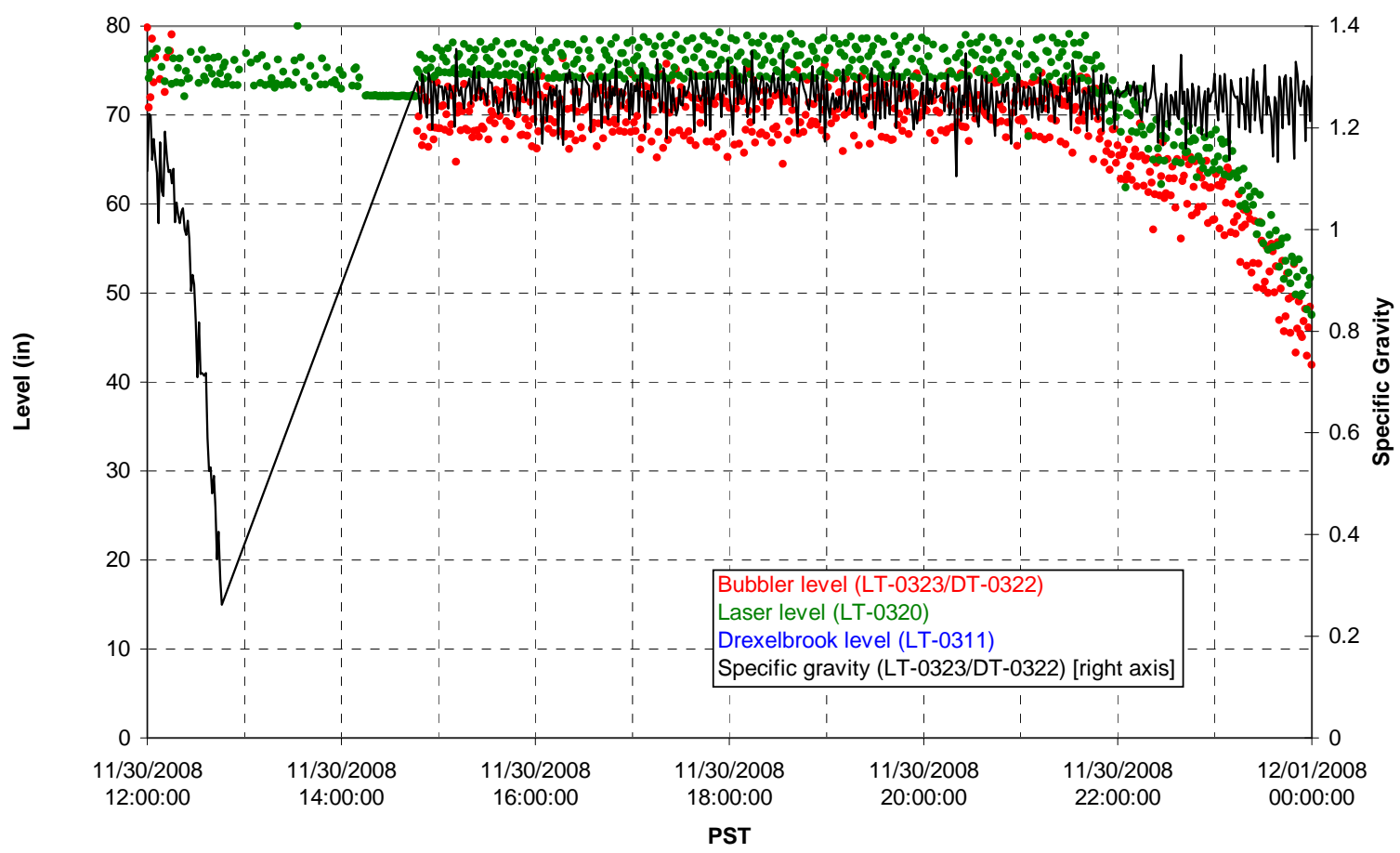

T01A temperatures

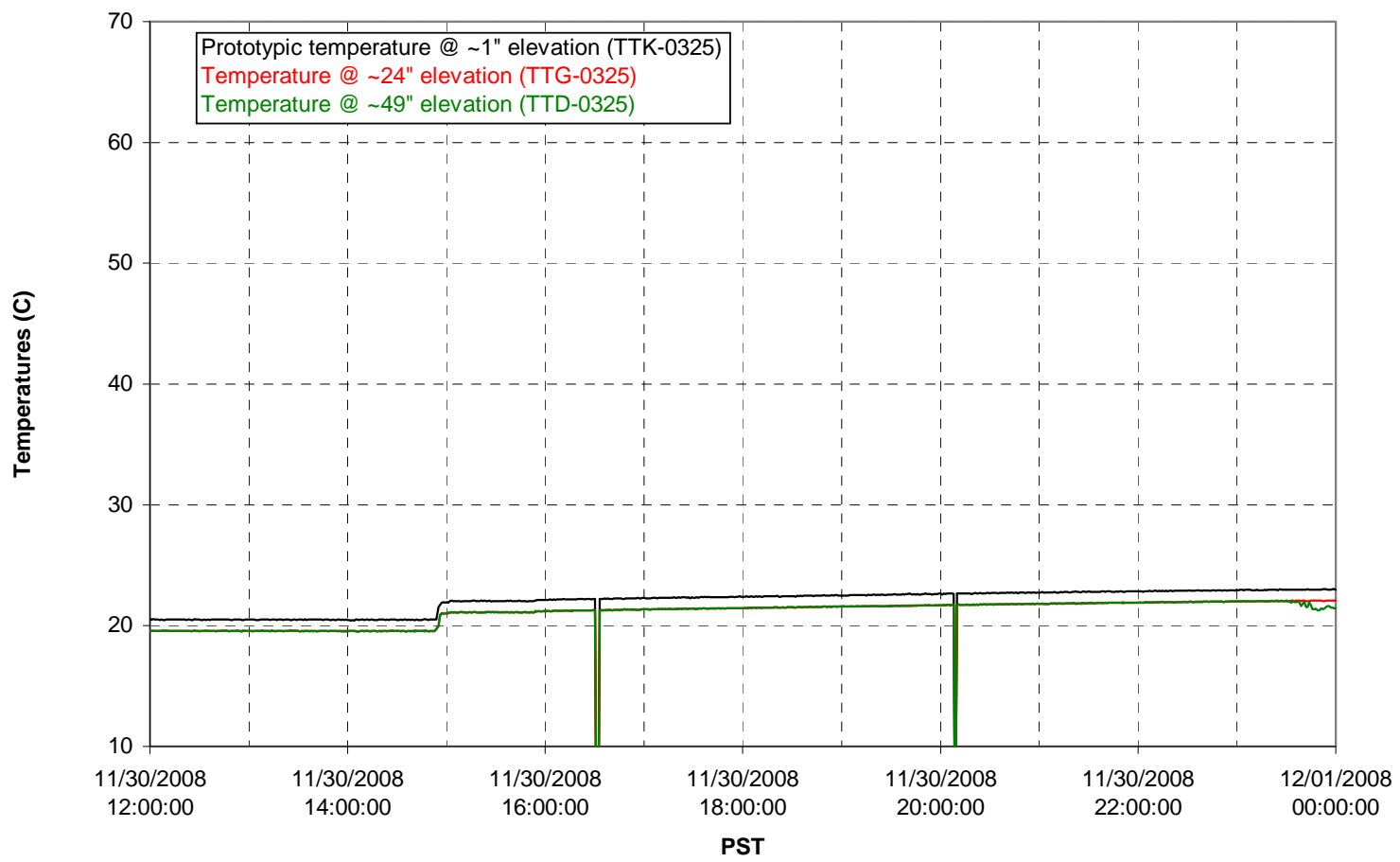


T01B level

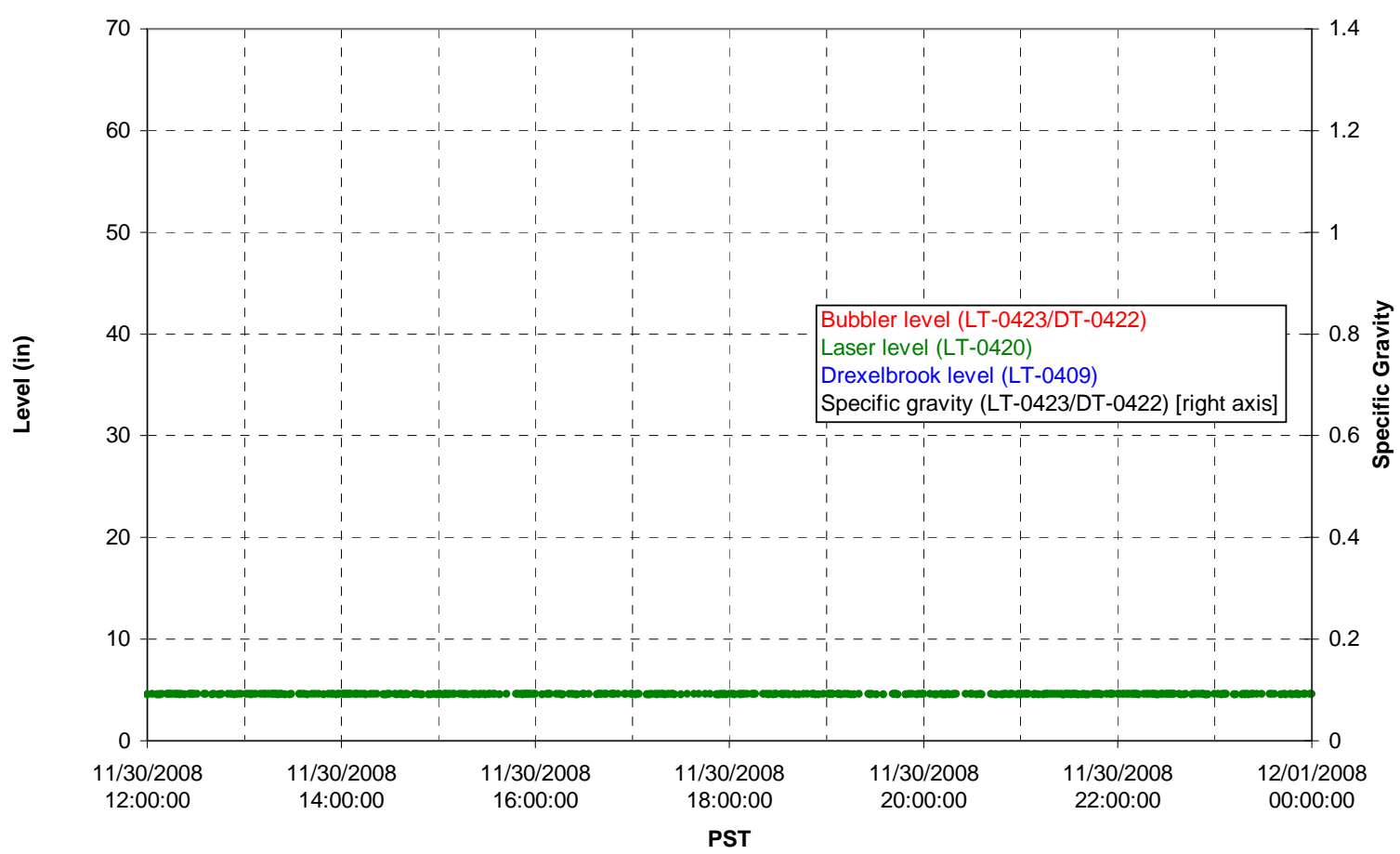

T01B temperatures

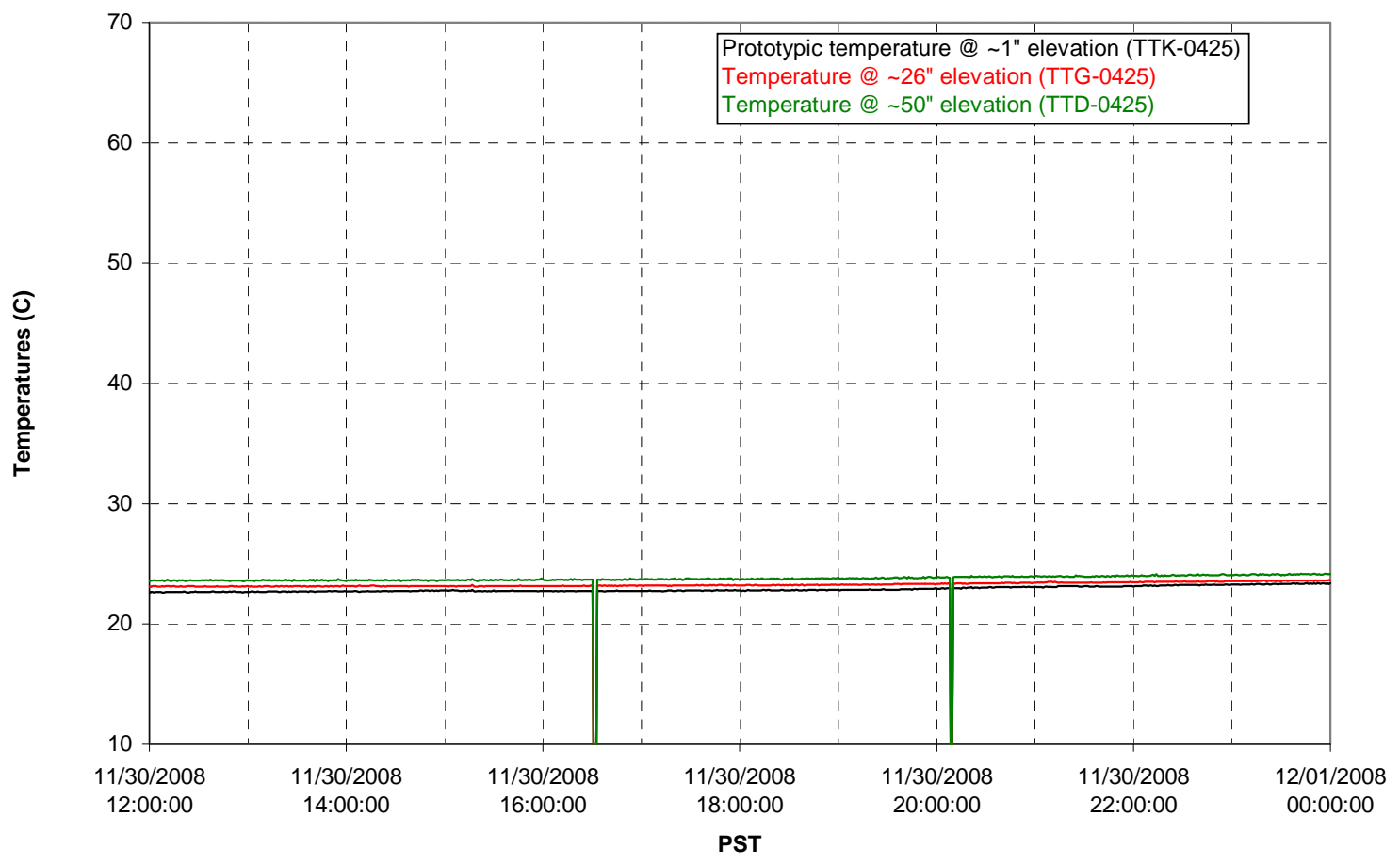


T02A level

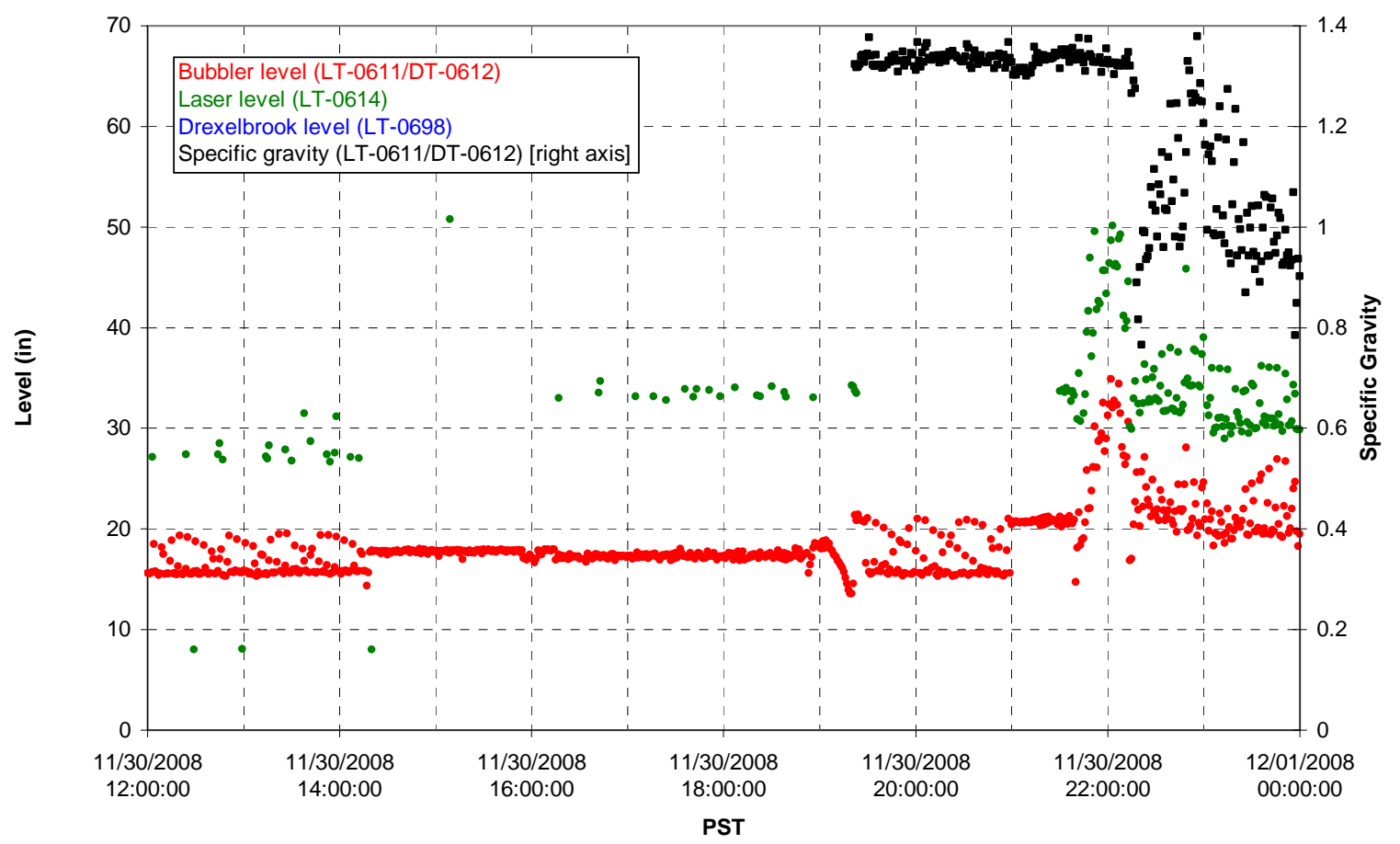

T02A temperatures

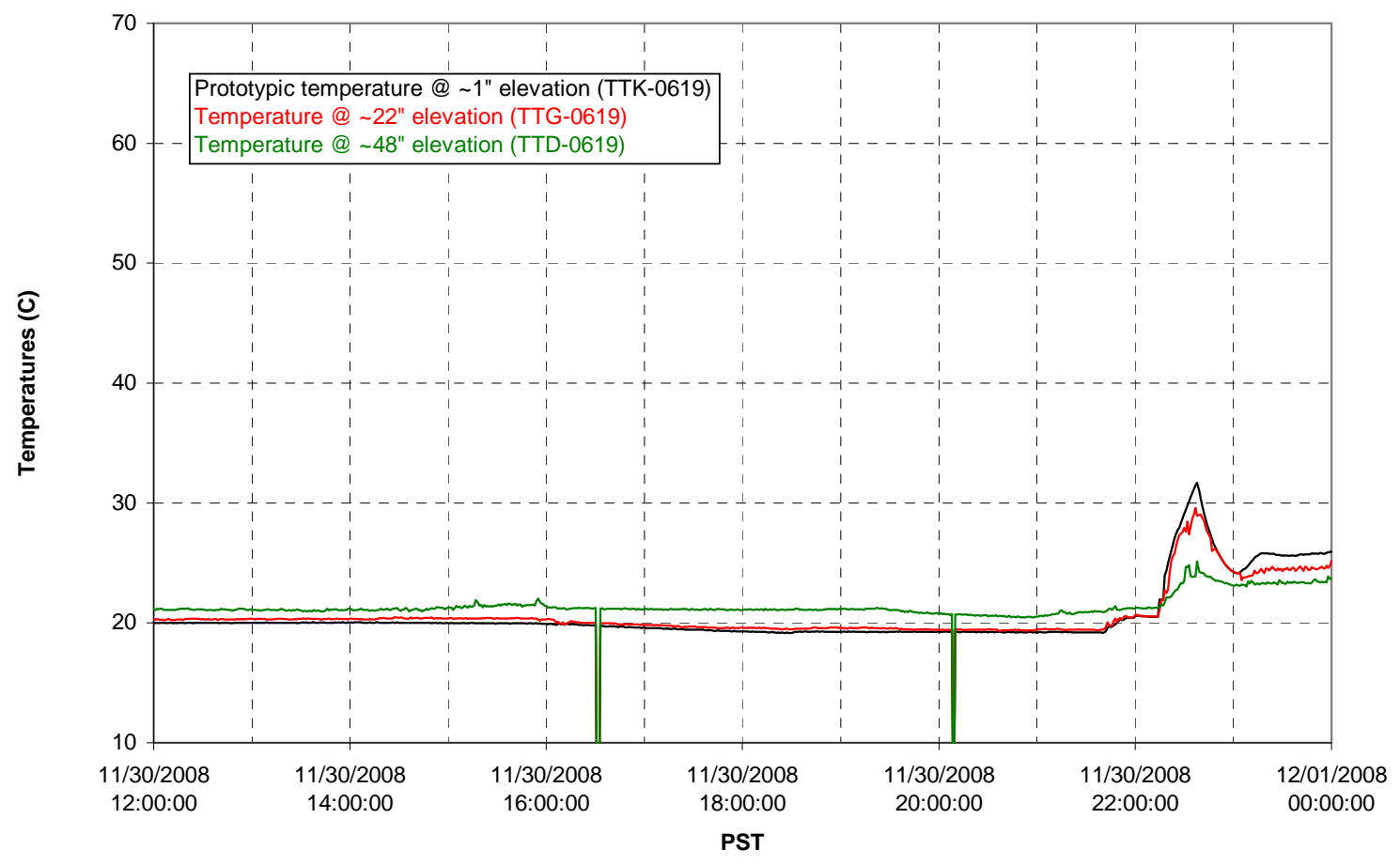


T02A and filter loop temperatures

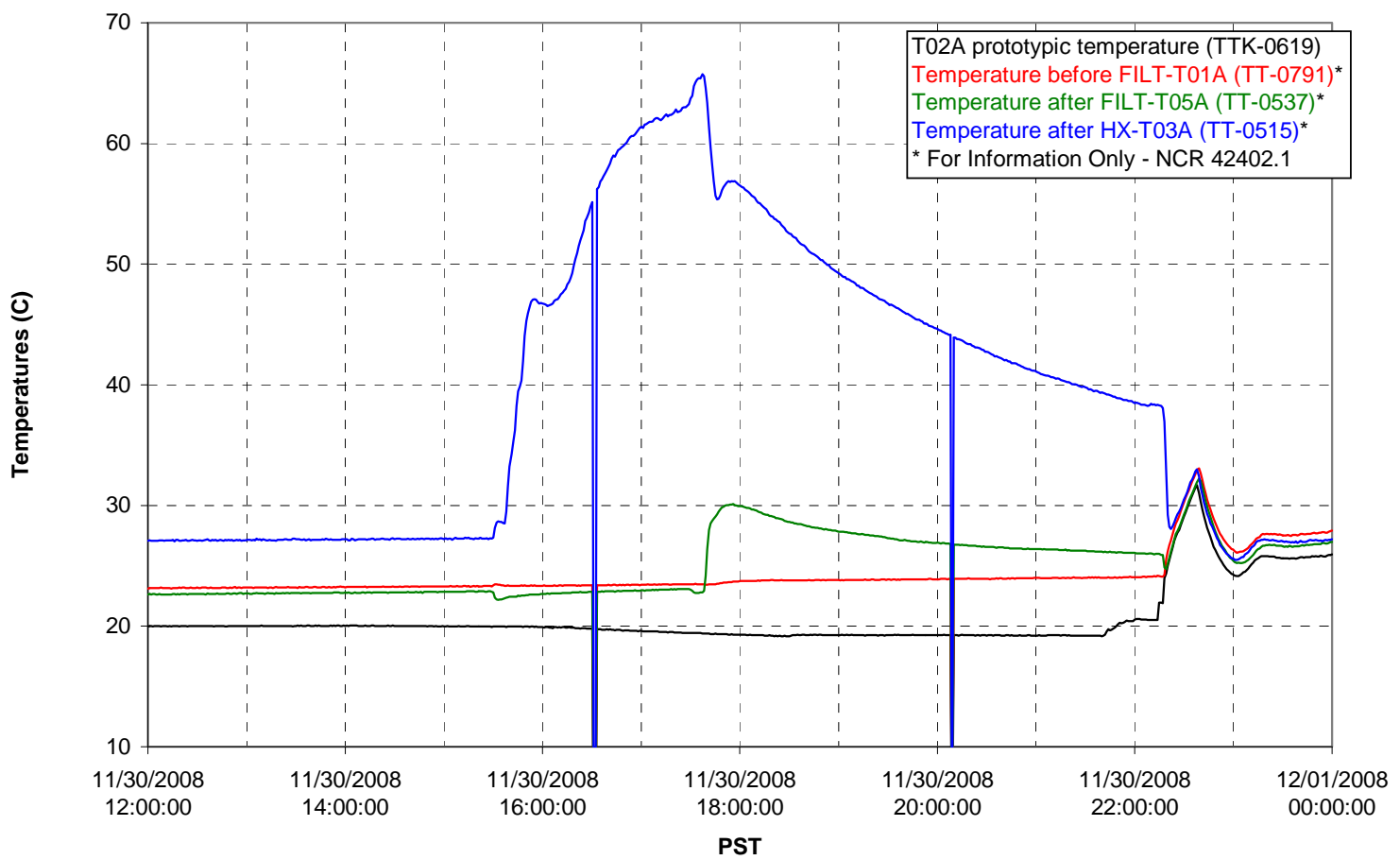

Pump Pressures and Flow

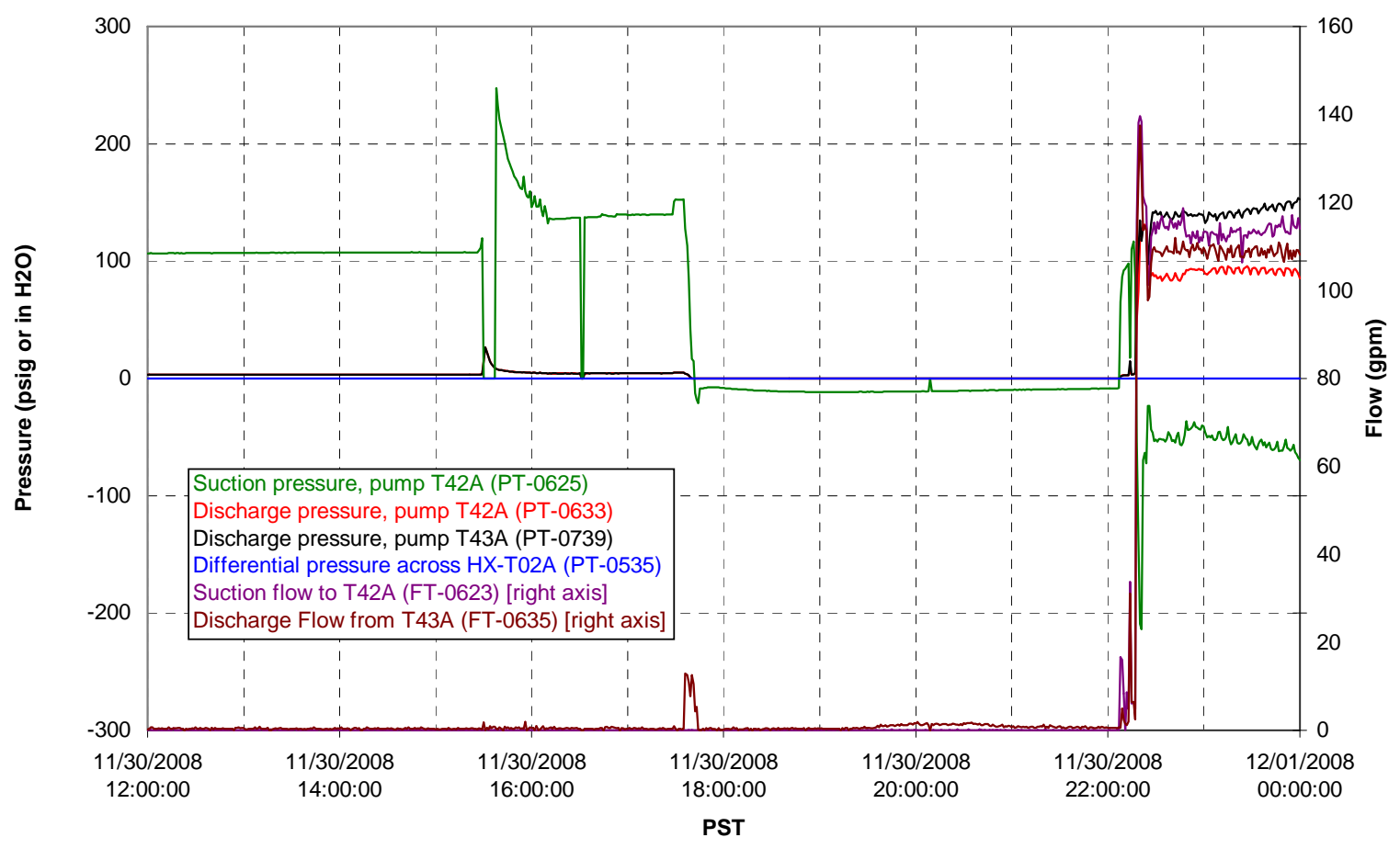




\section{Axial pressure drop}

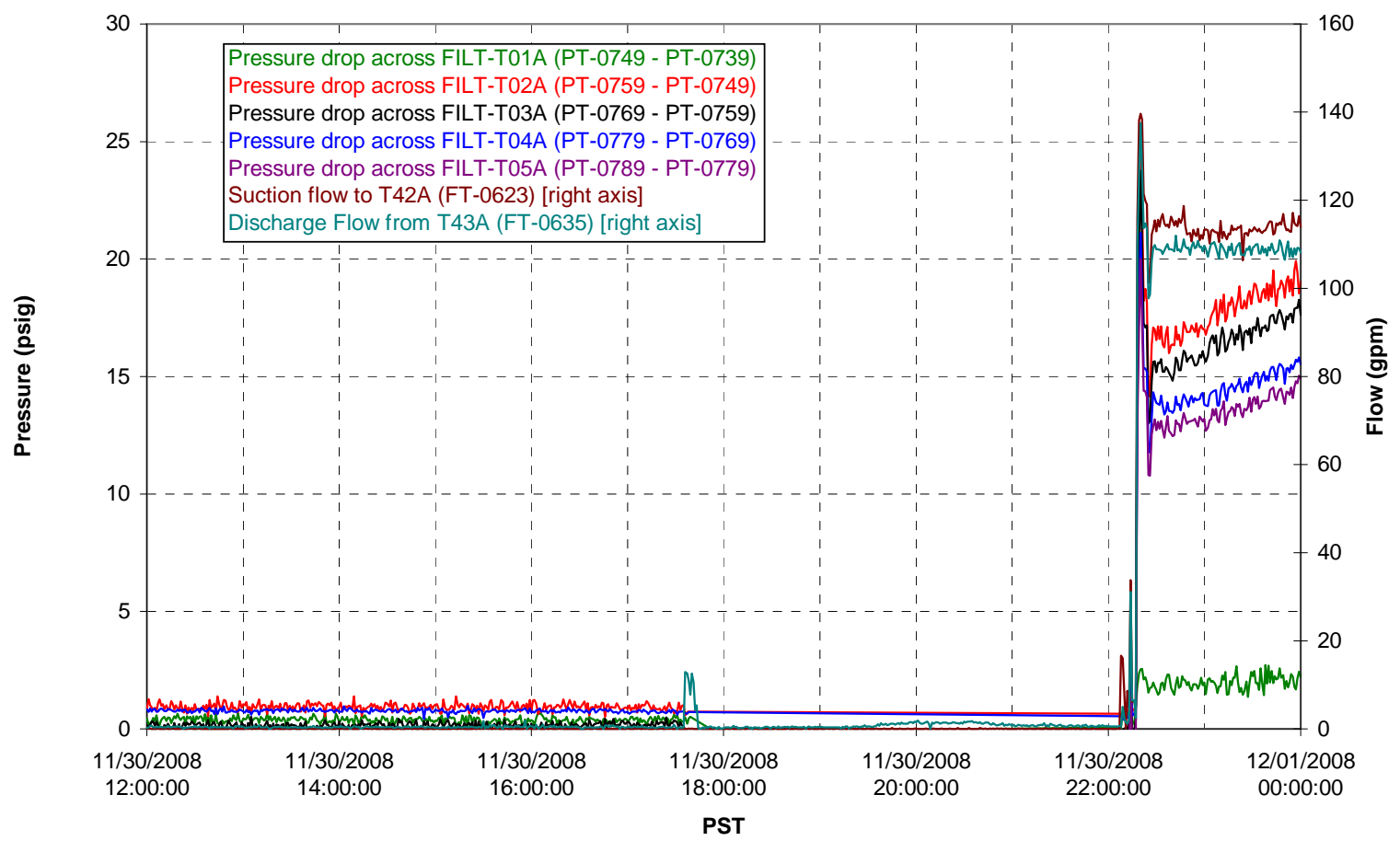

Permeate flow rates

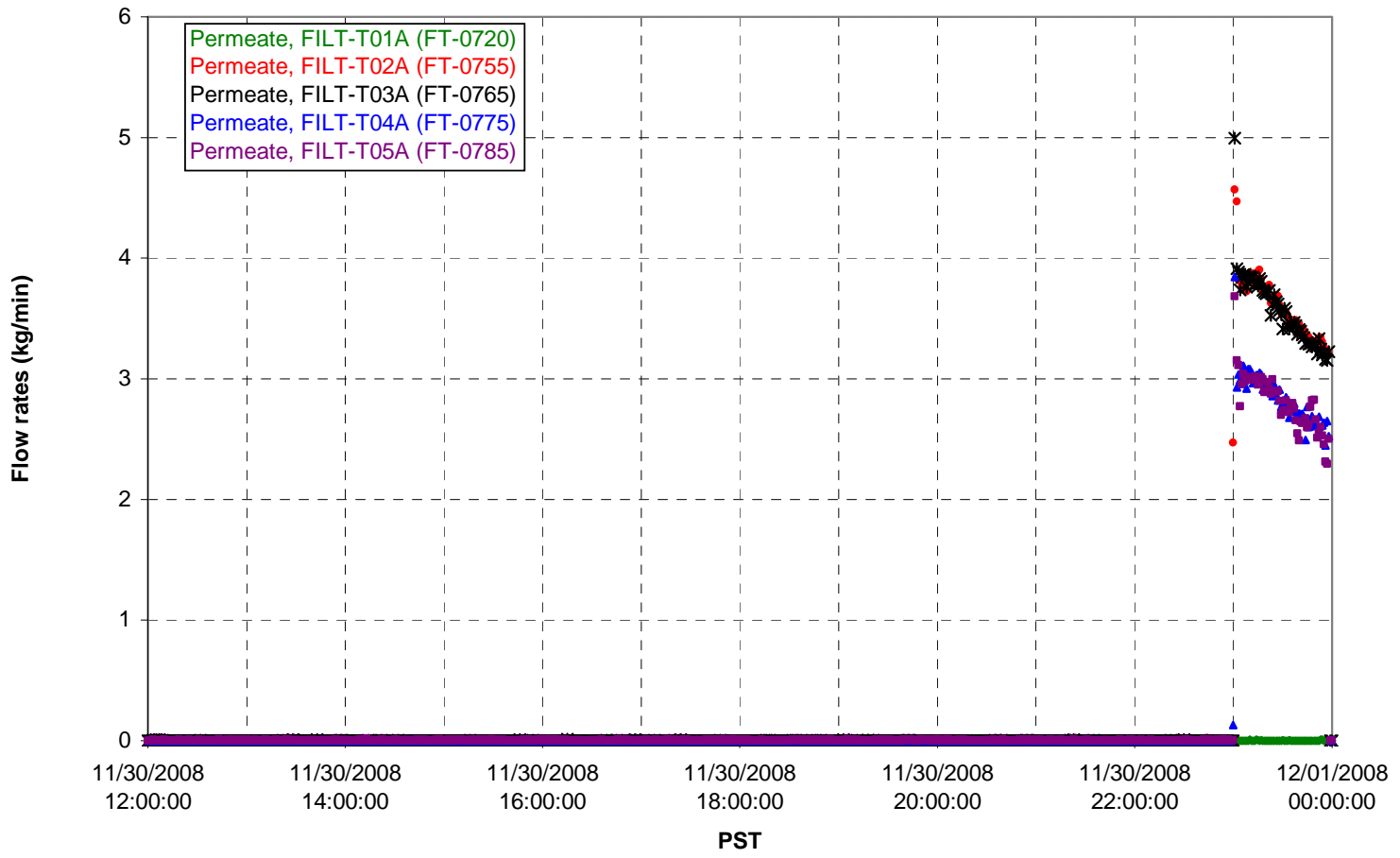


T02A Inner Temperature Tree

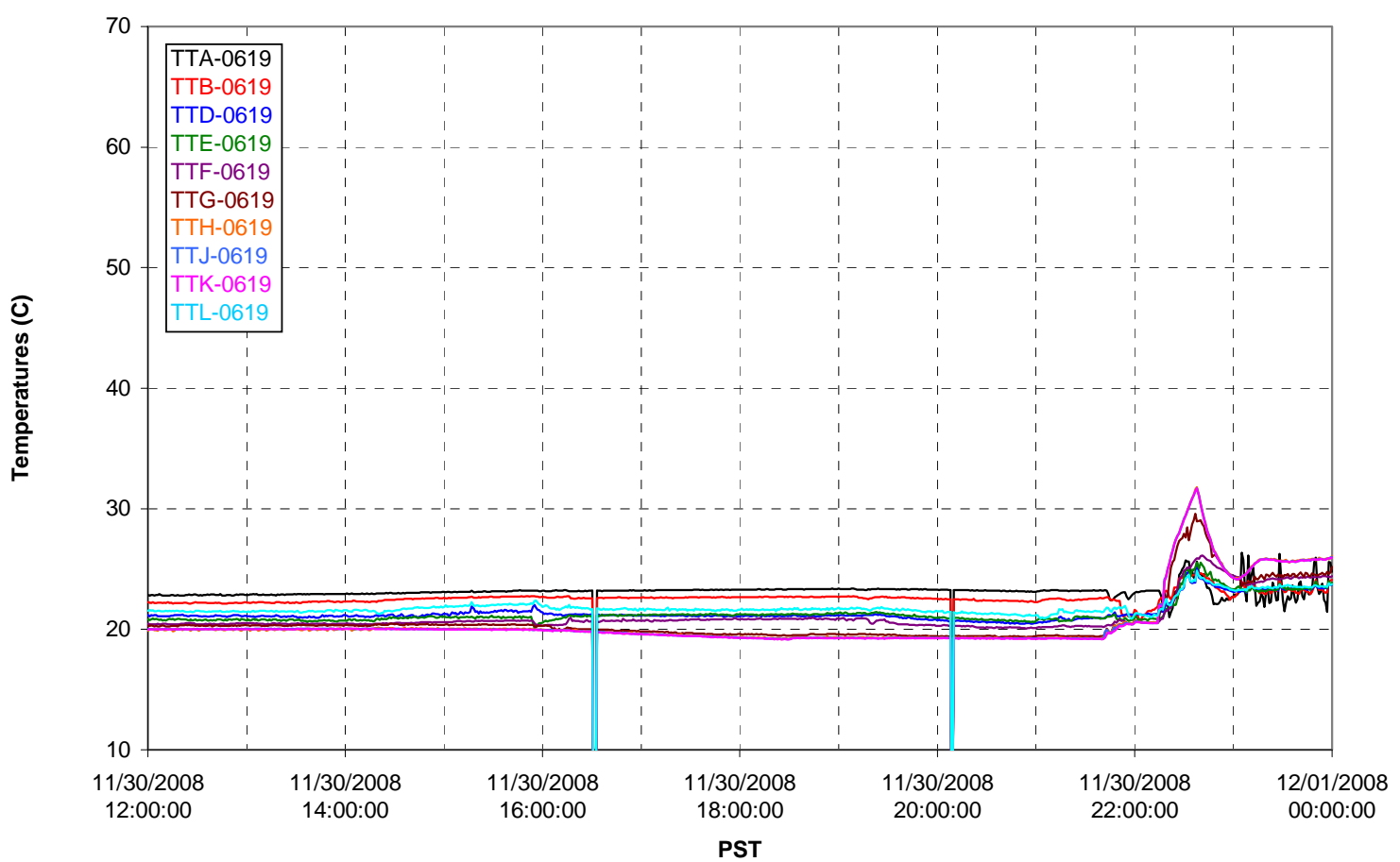

T02A Outer Temperature Tree

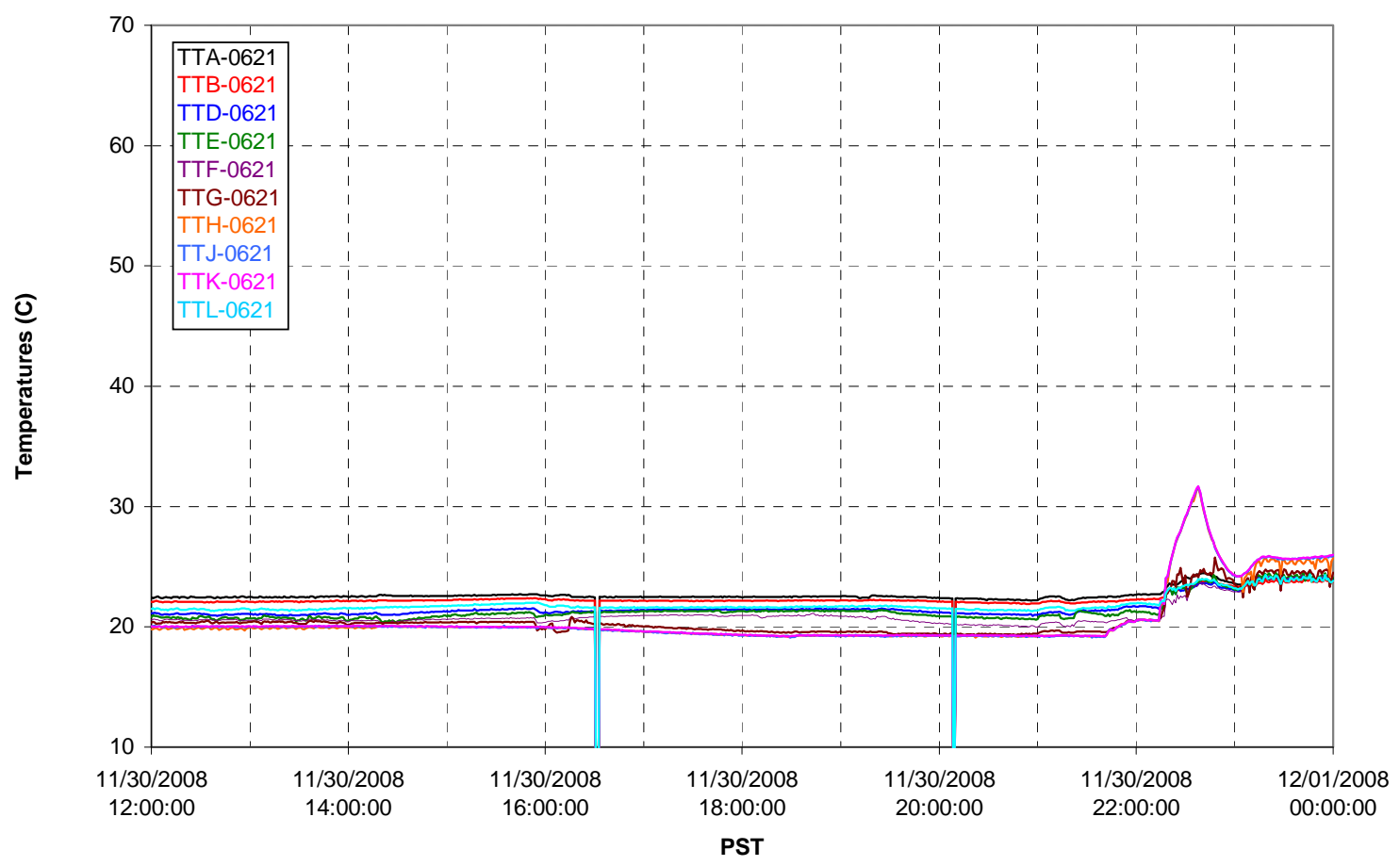


T02A temperatures

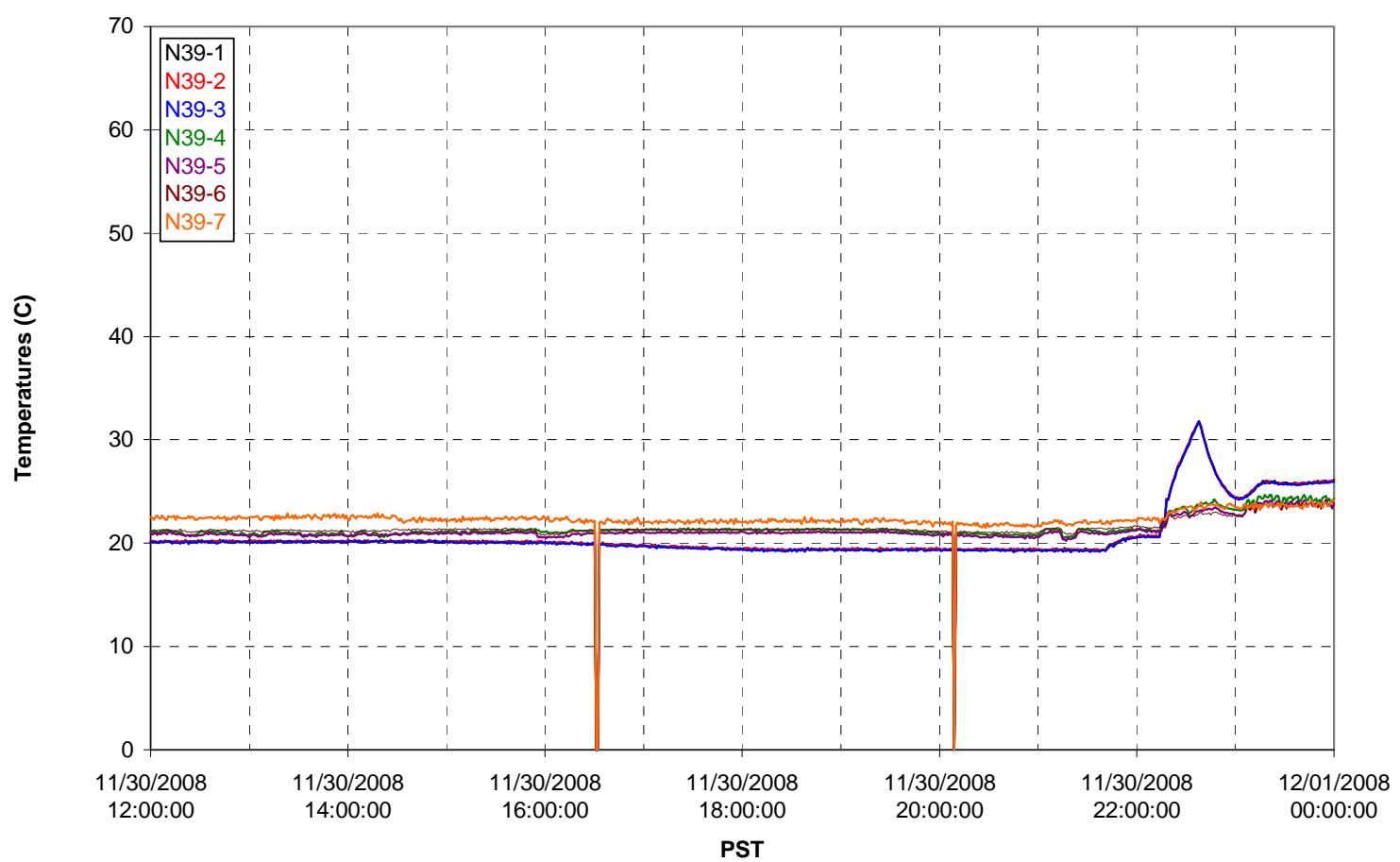

T02A temperatures

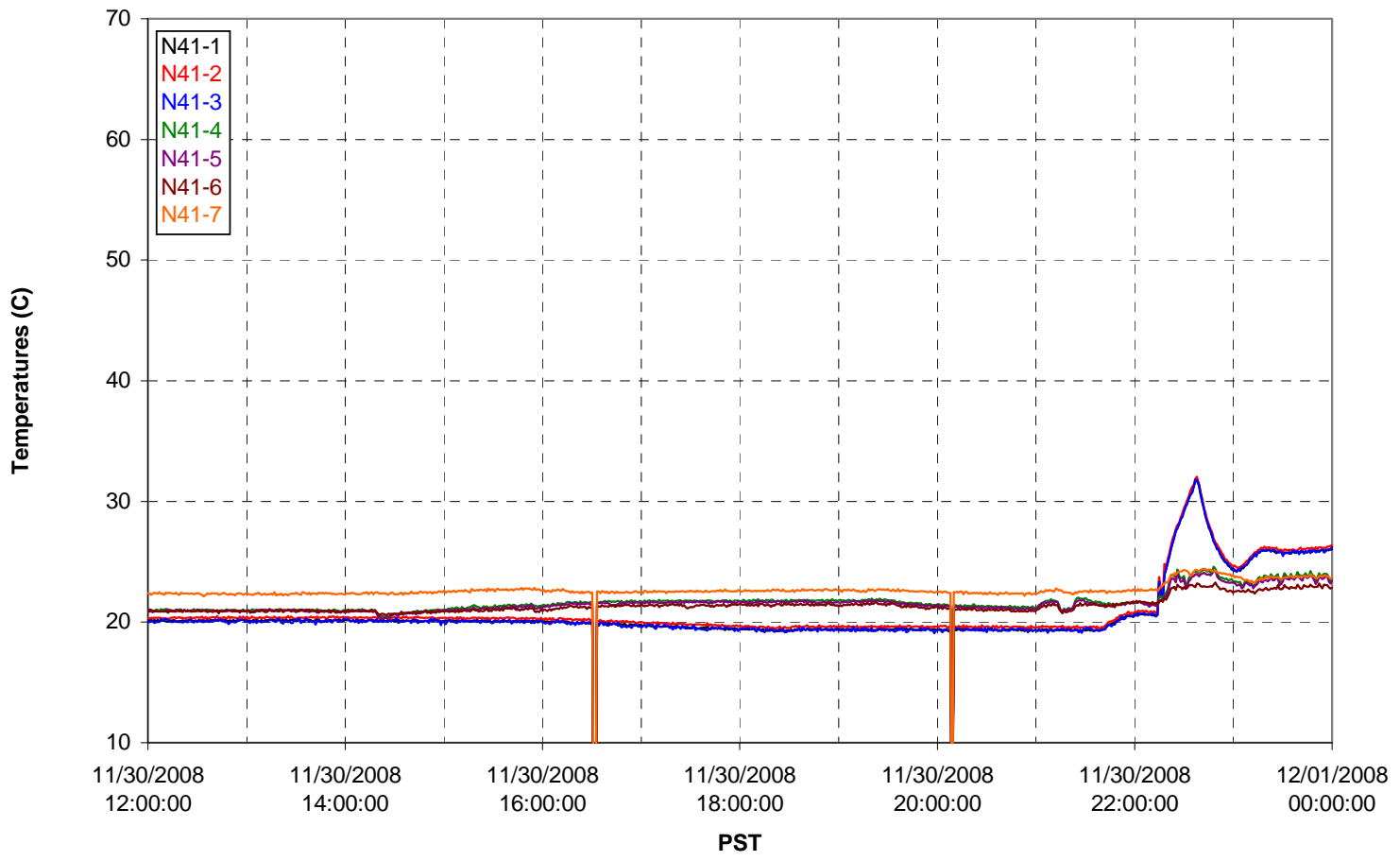


T02A temperatures

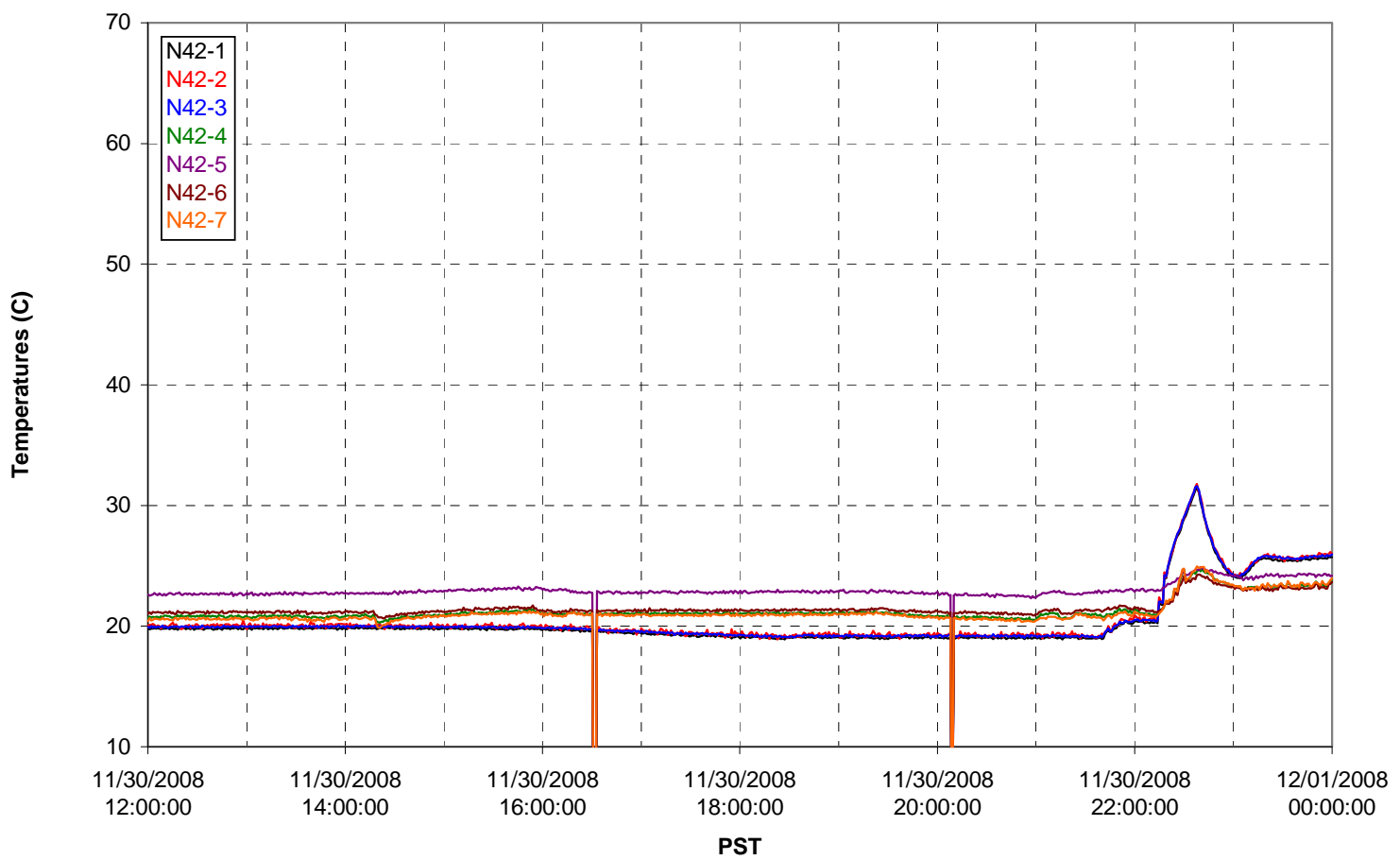

T02A temperatures

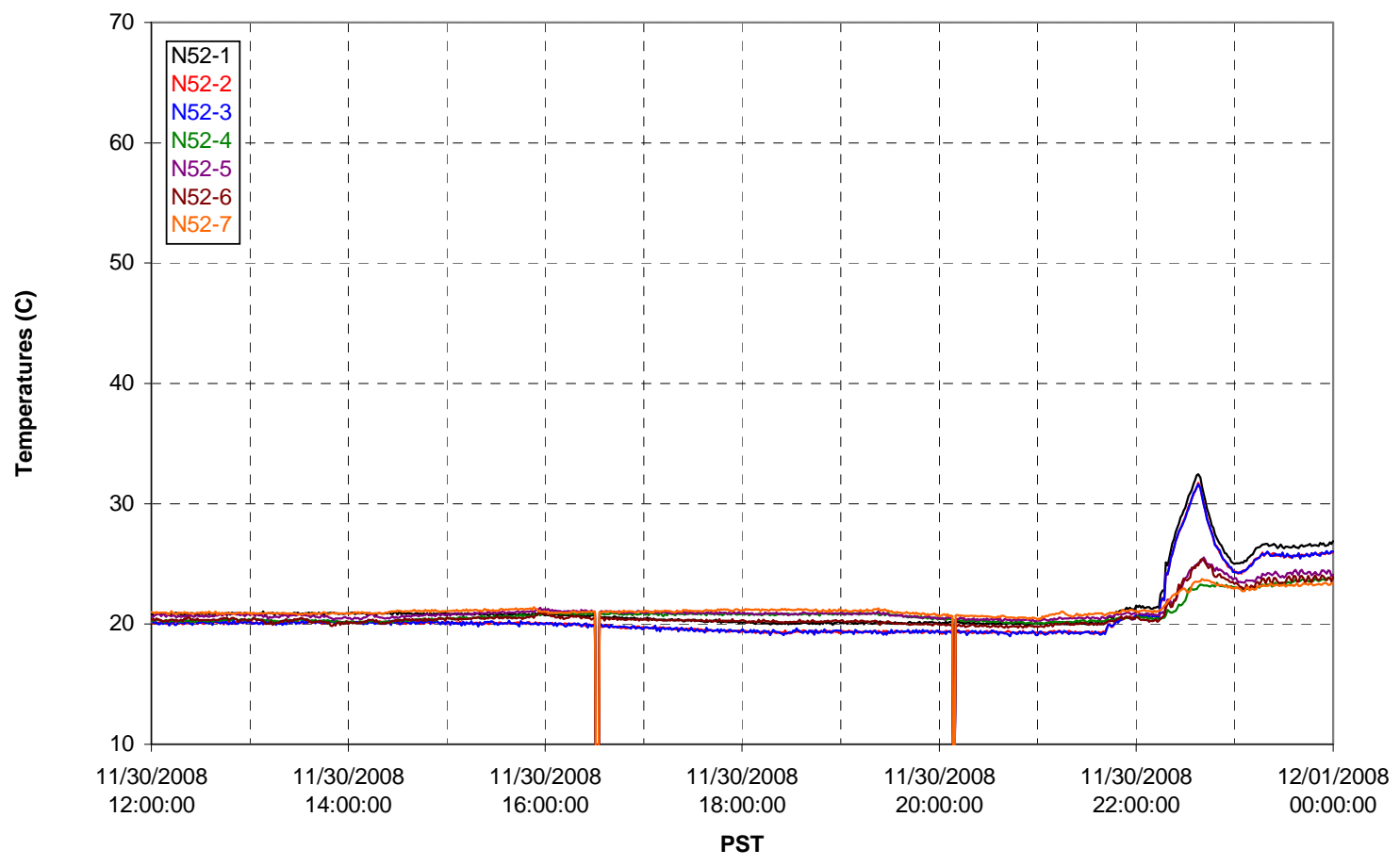


T02A Heating and Cooling

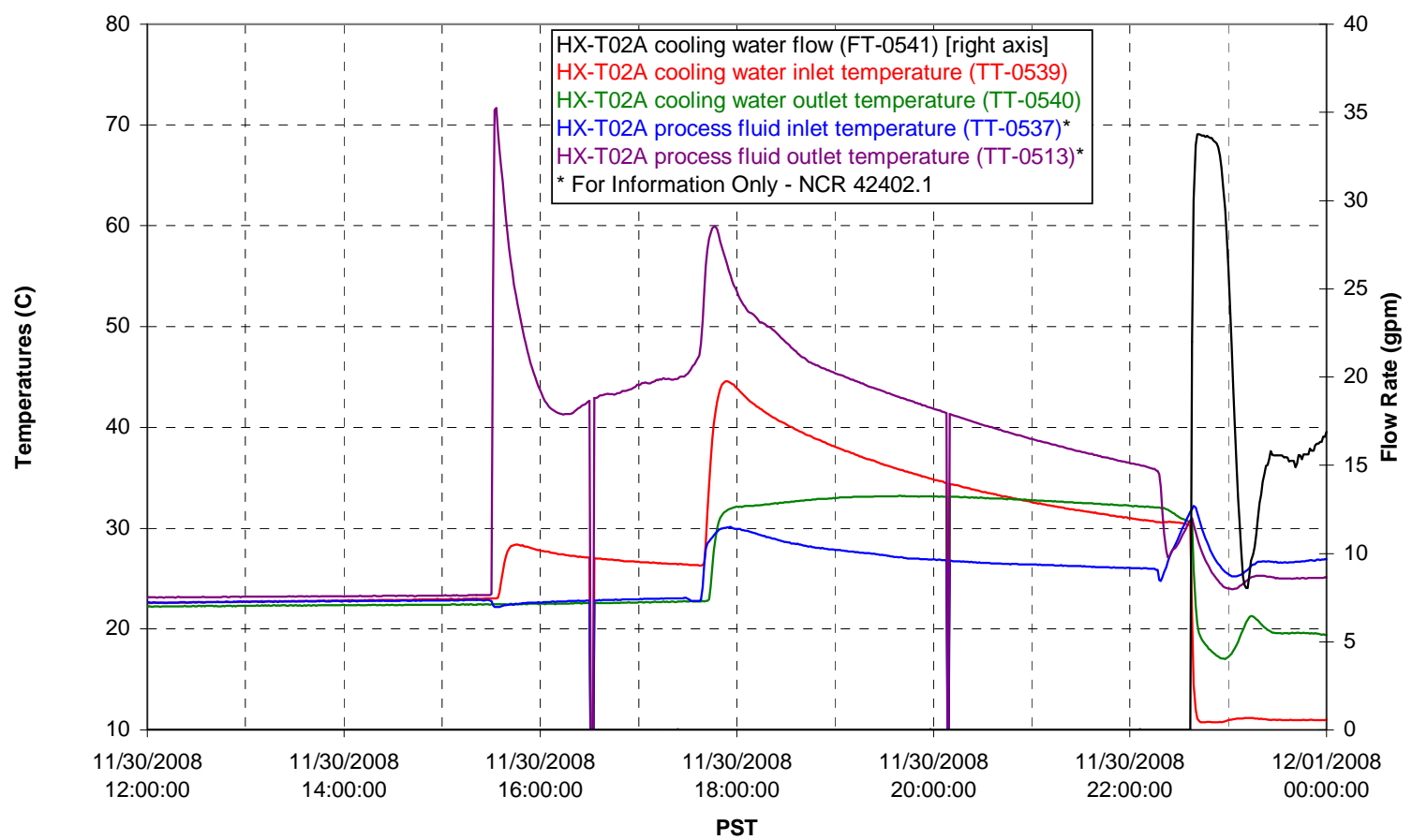

Pump Operation

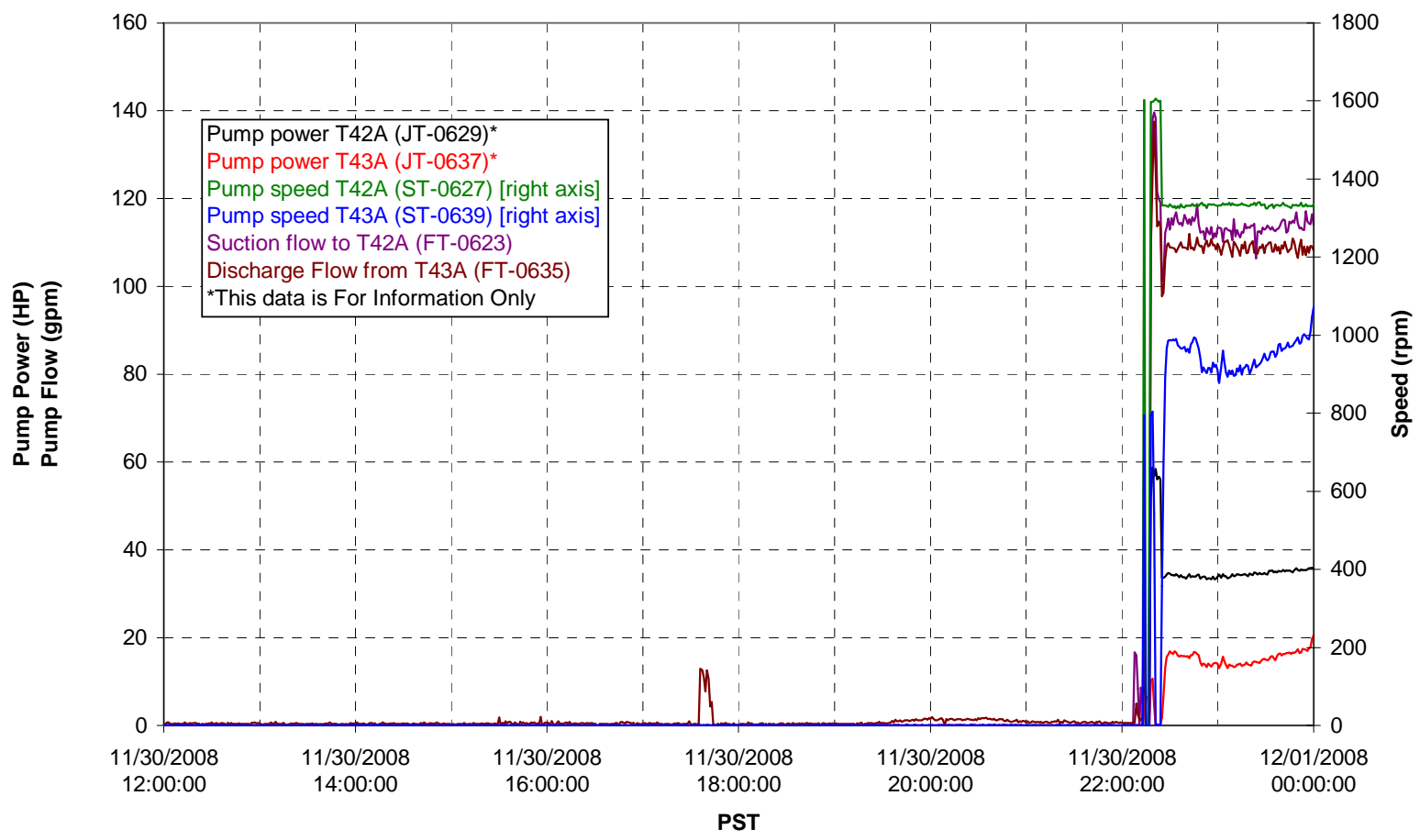


Pulsepot UFP-PP-T01A

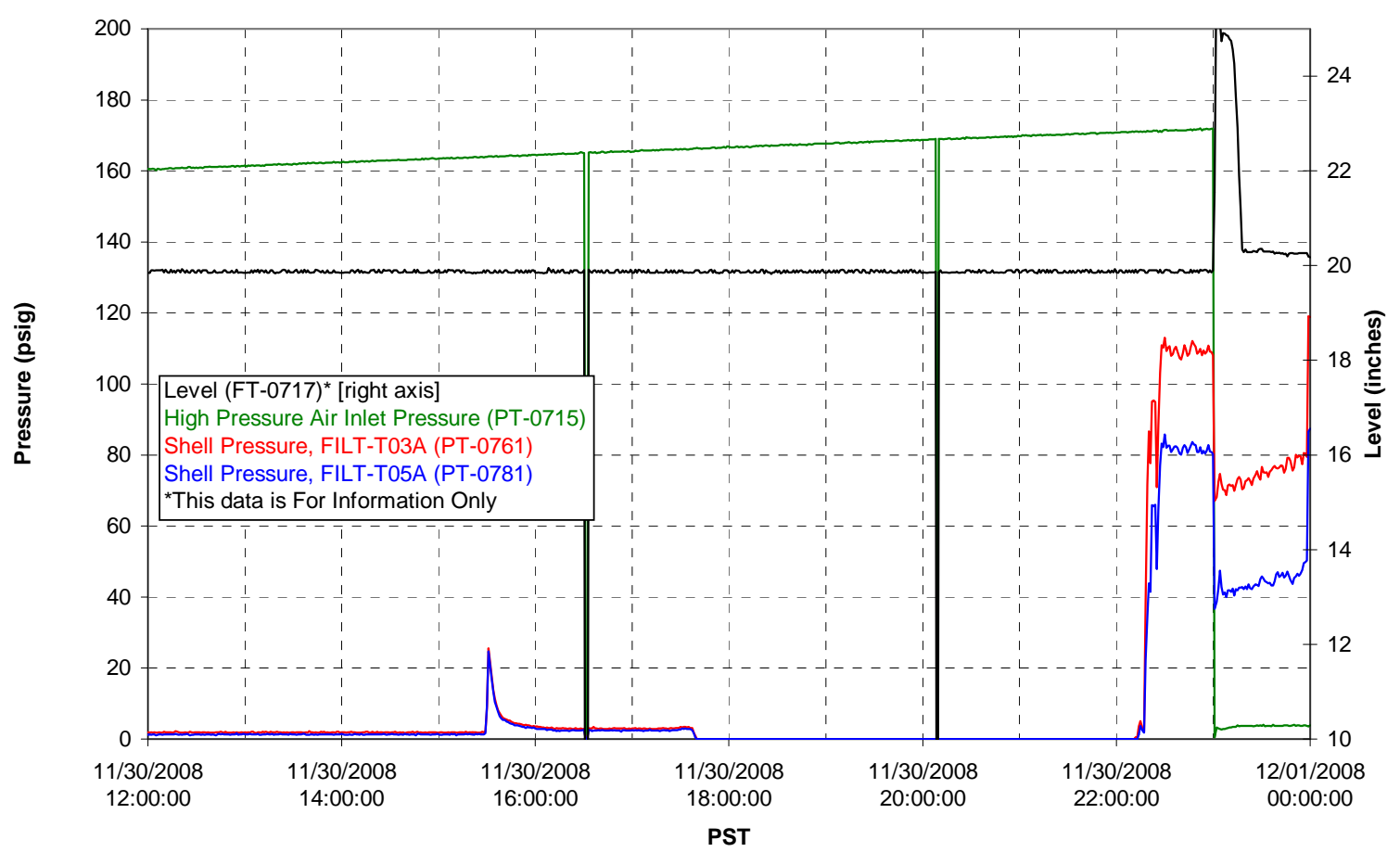

Pulsepot UFP-PP-T02A

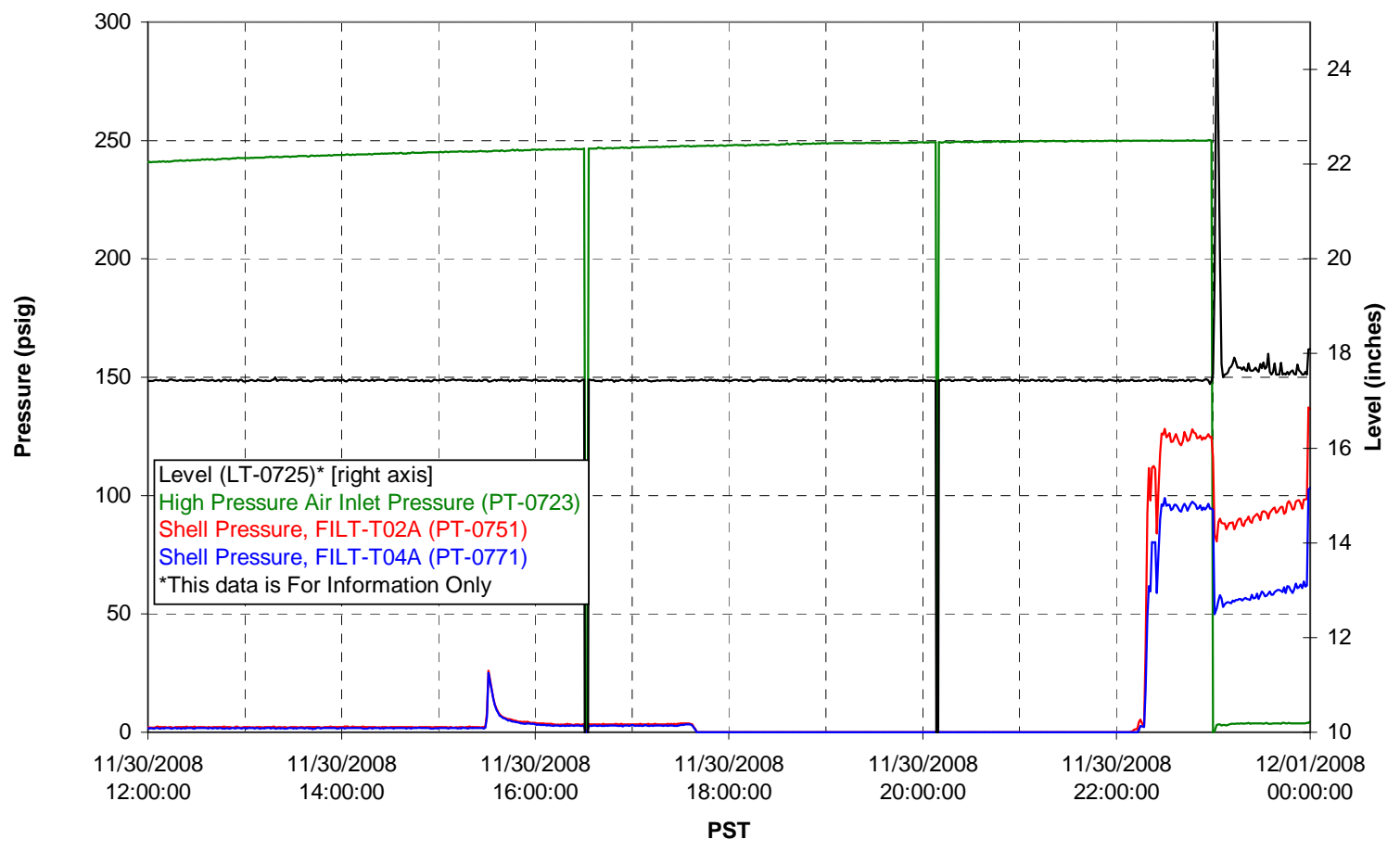


Pulsepot UFP-PP-T03A

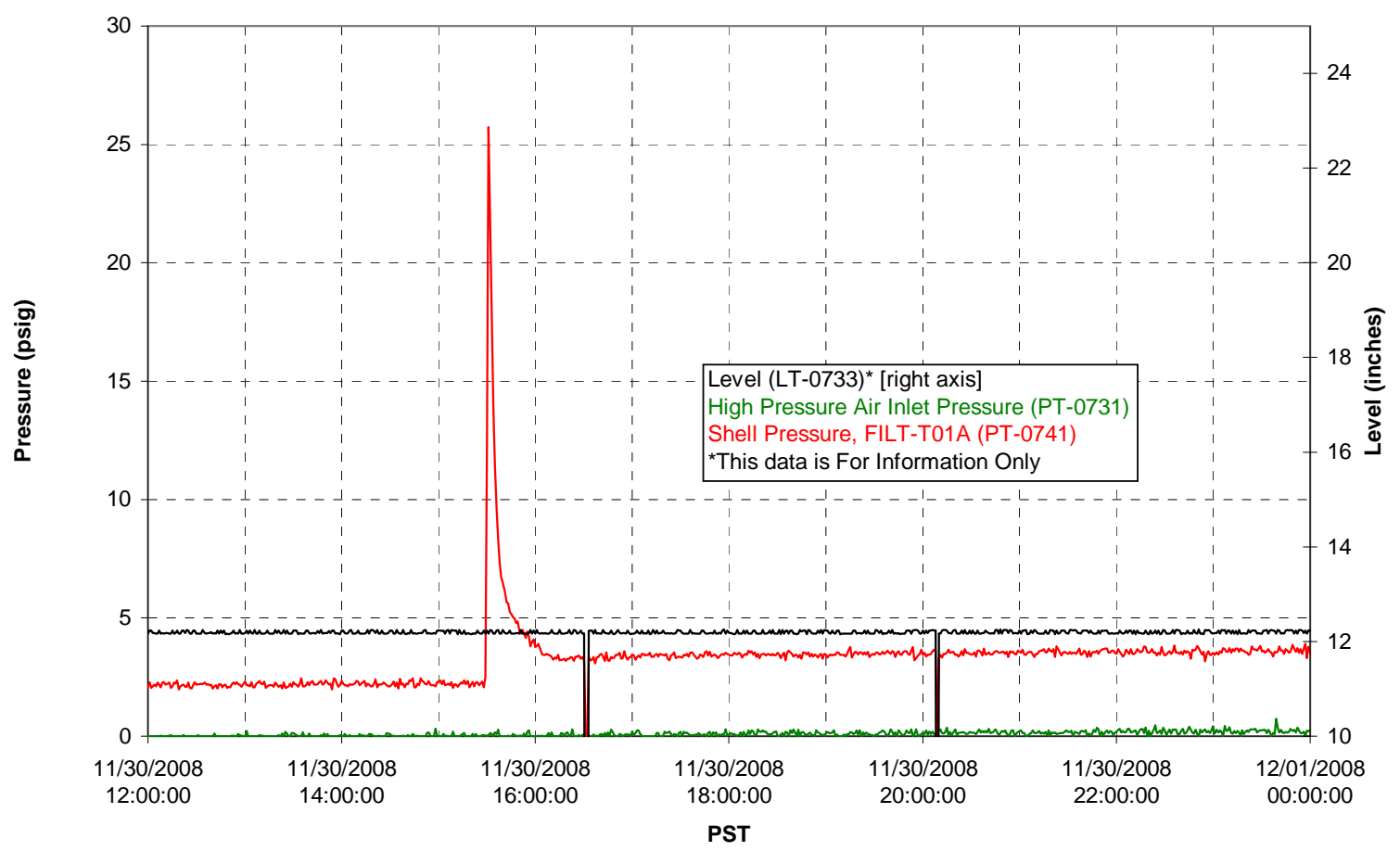

Pulsepot Levels

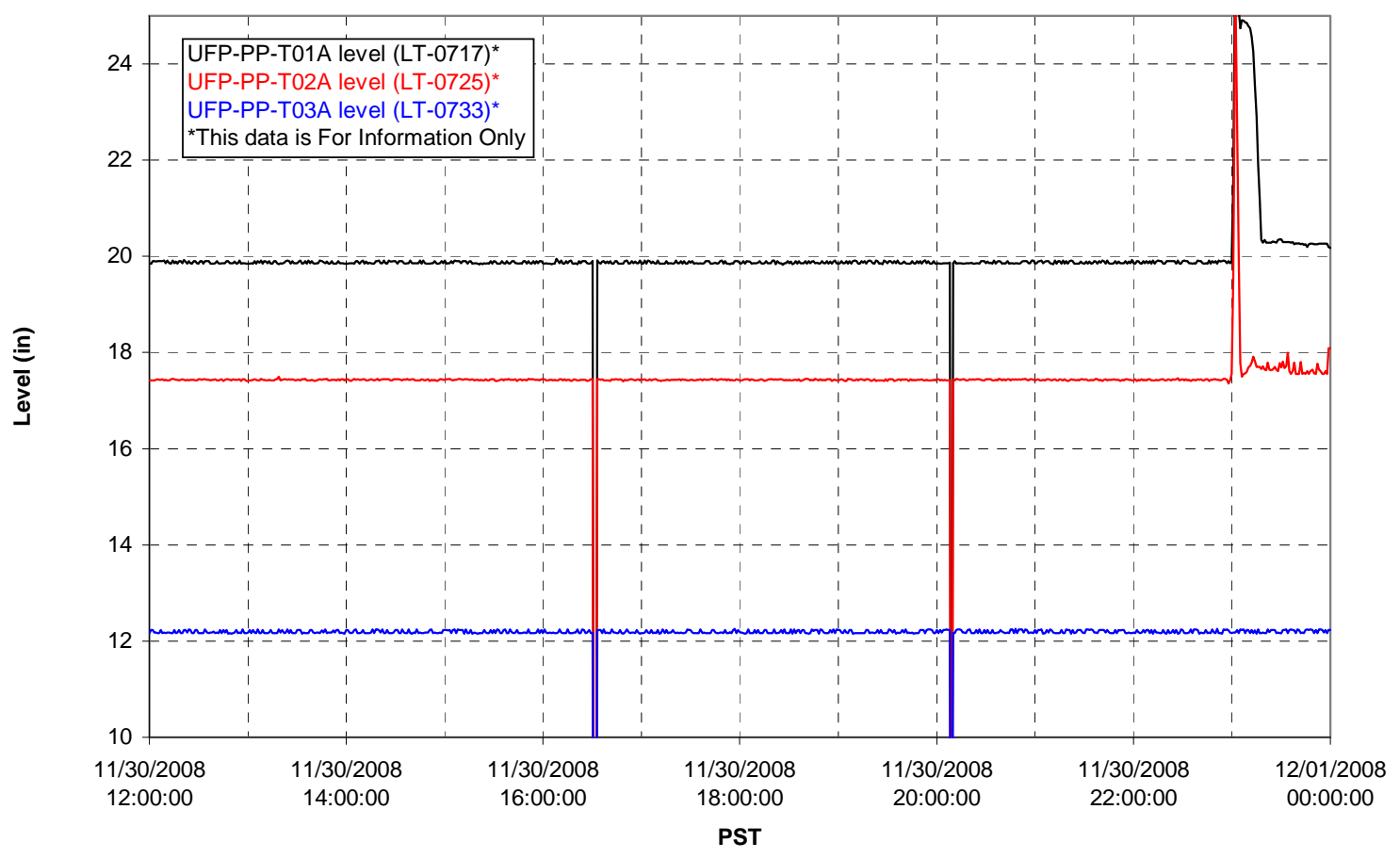


Filter UFP-FILT-T01A

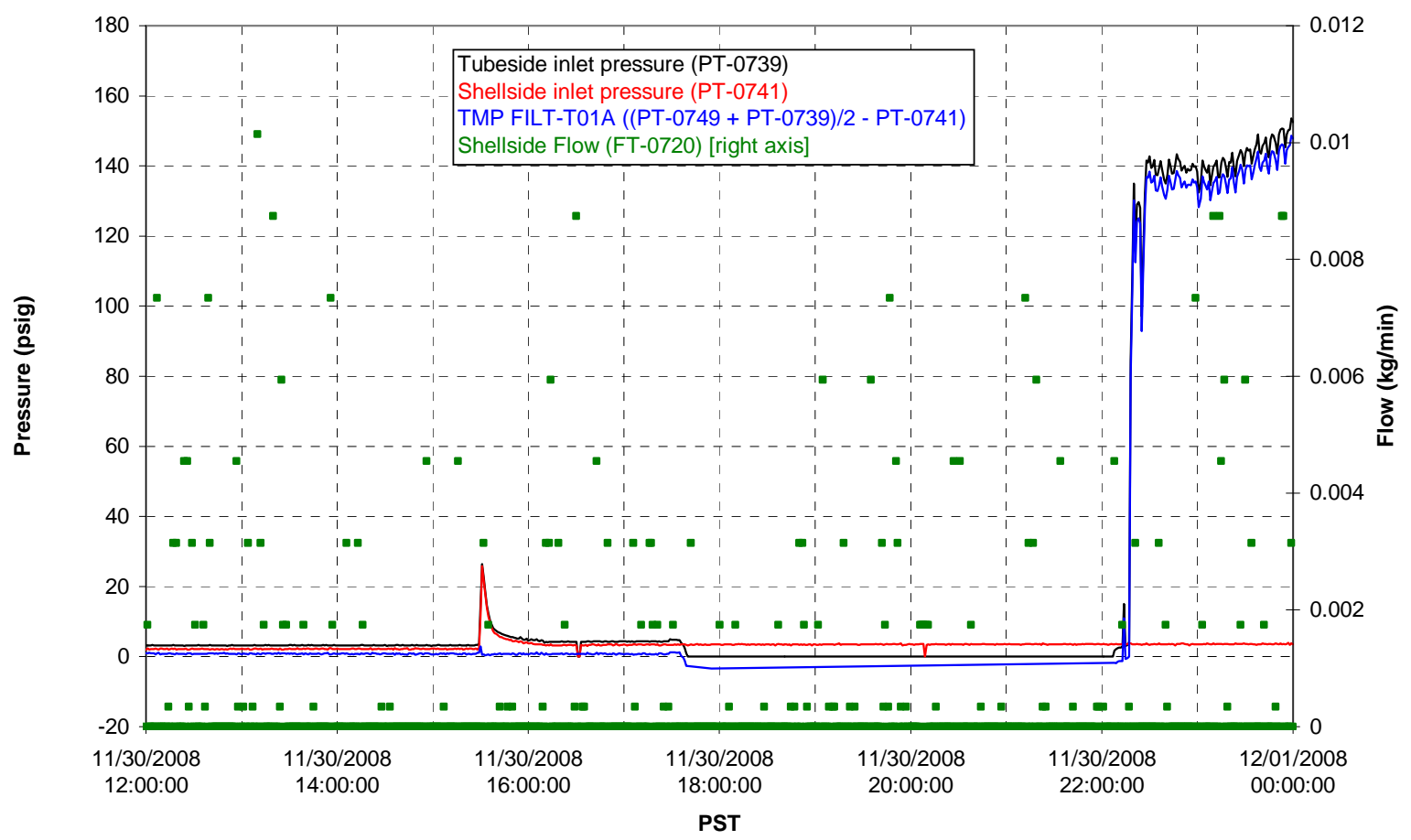

Filter UFP-FILT-T02A

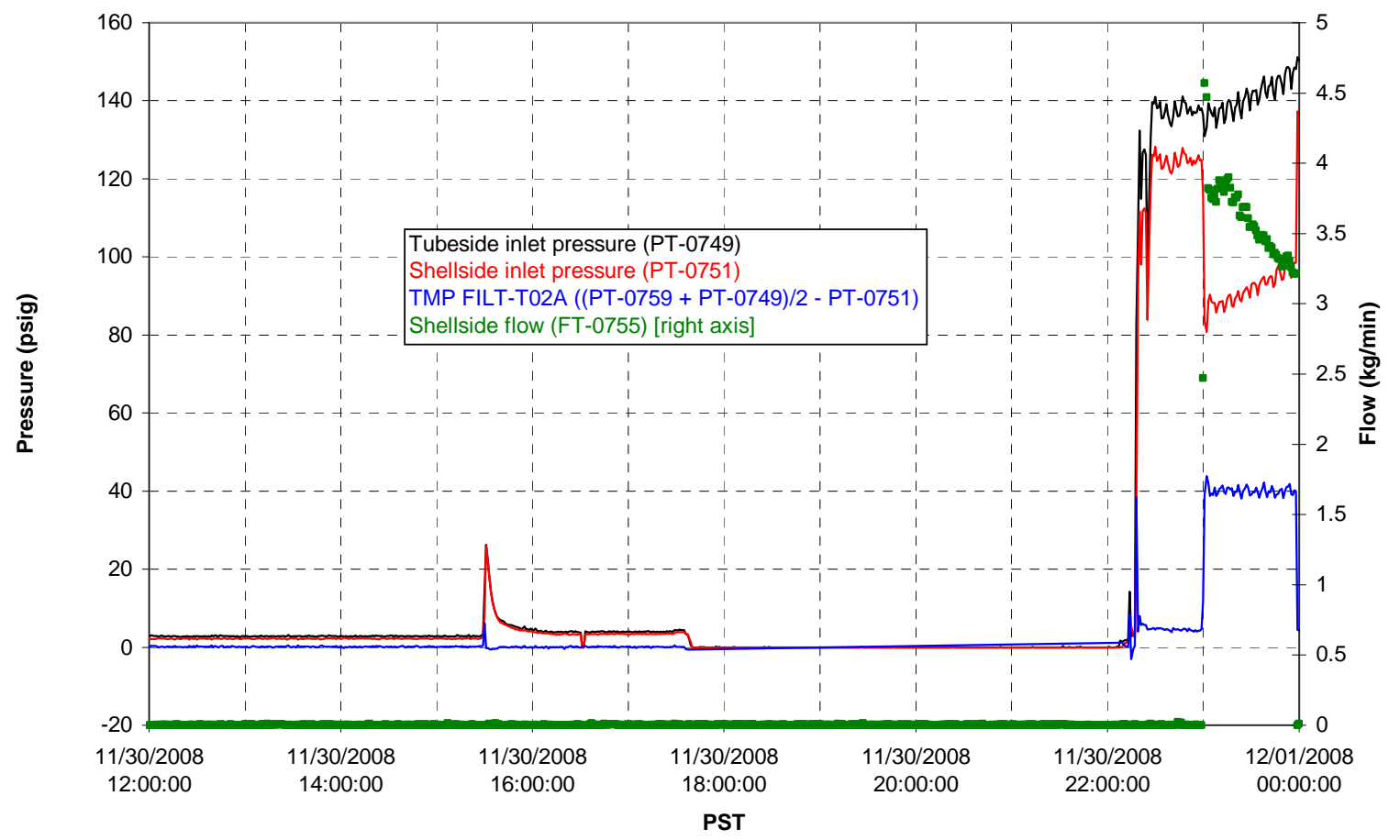


Filter UFP-FILT-T03A

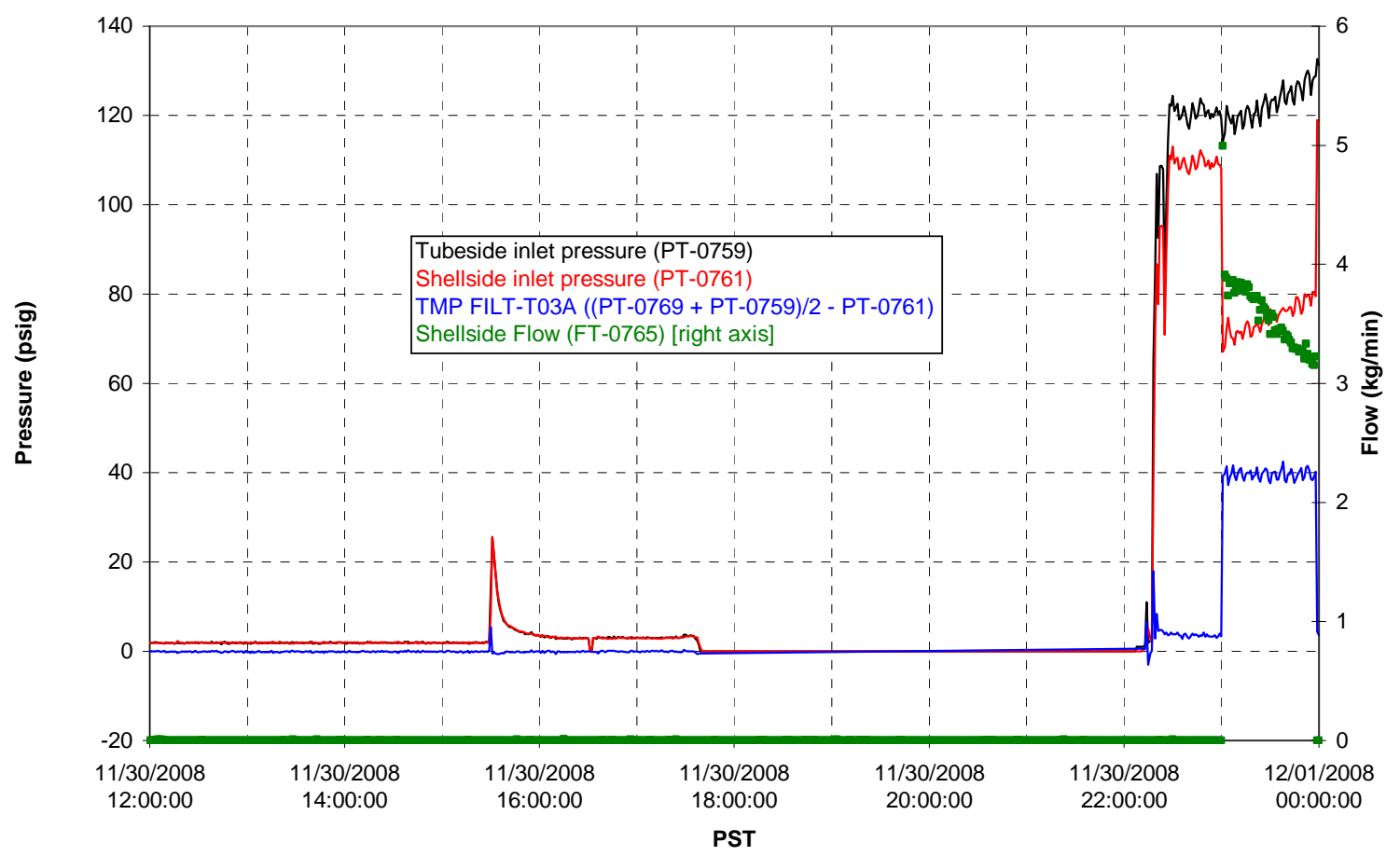

Filter UFP-FILT-T04A

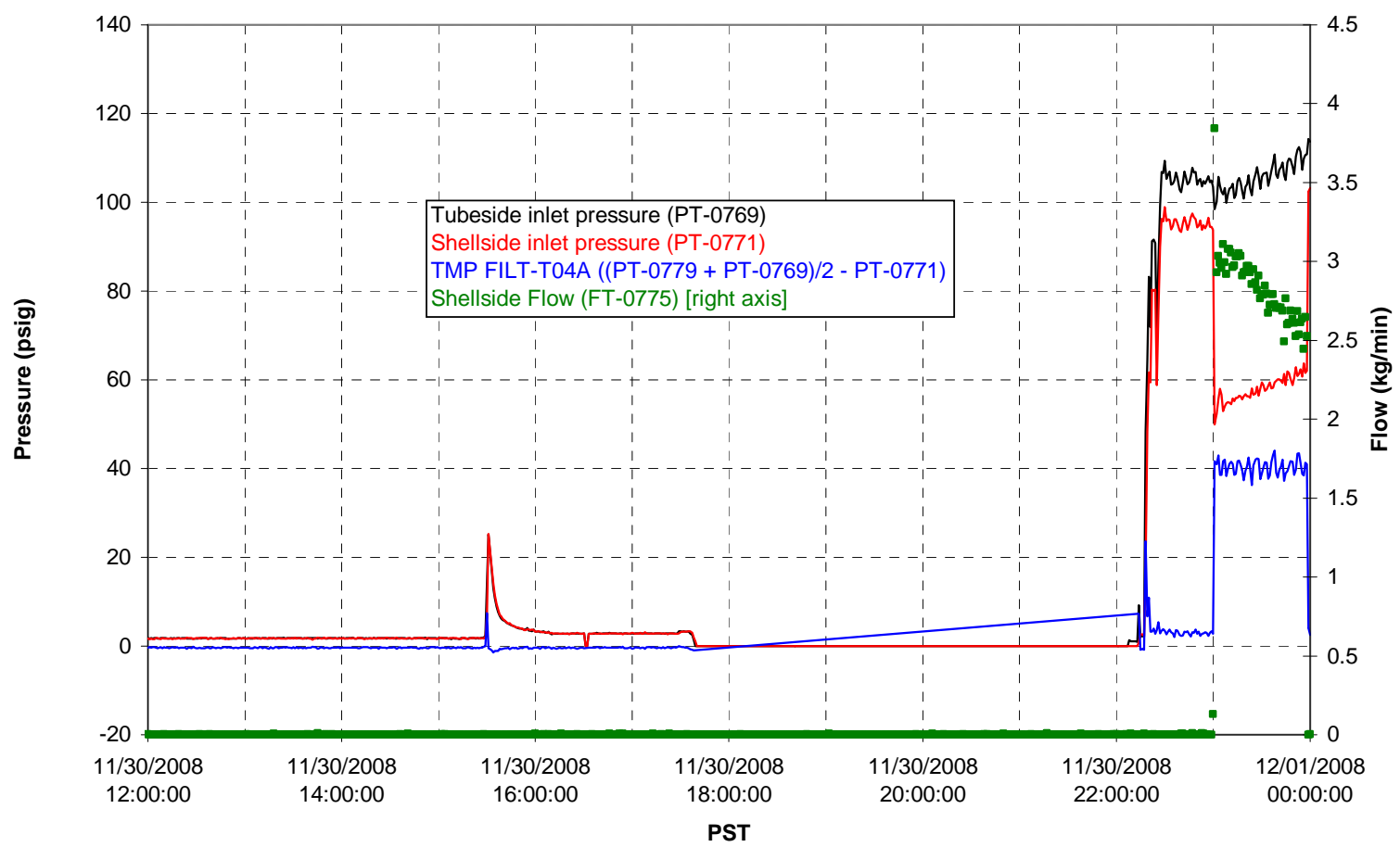


Filter UFP-FILT-T05A

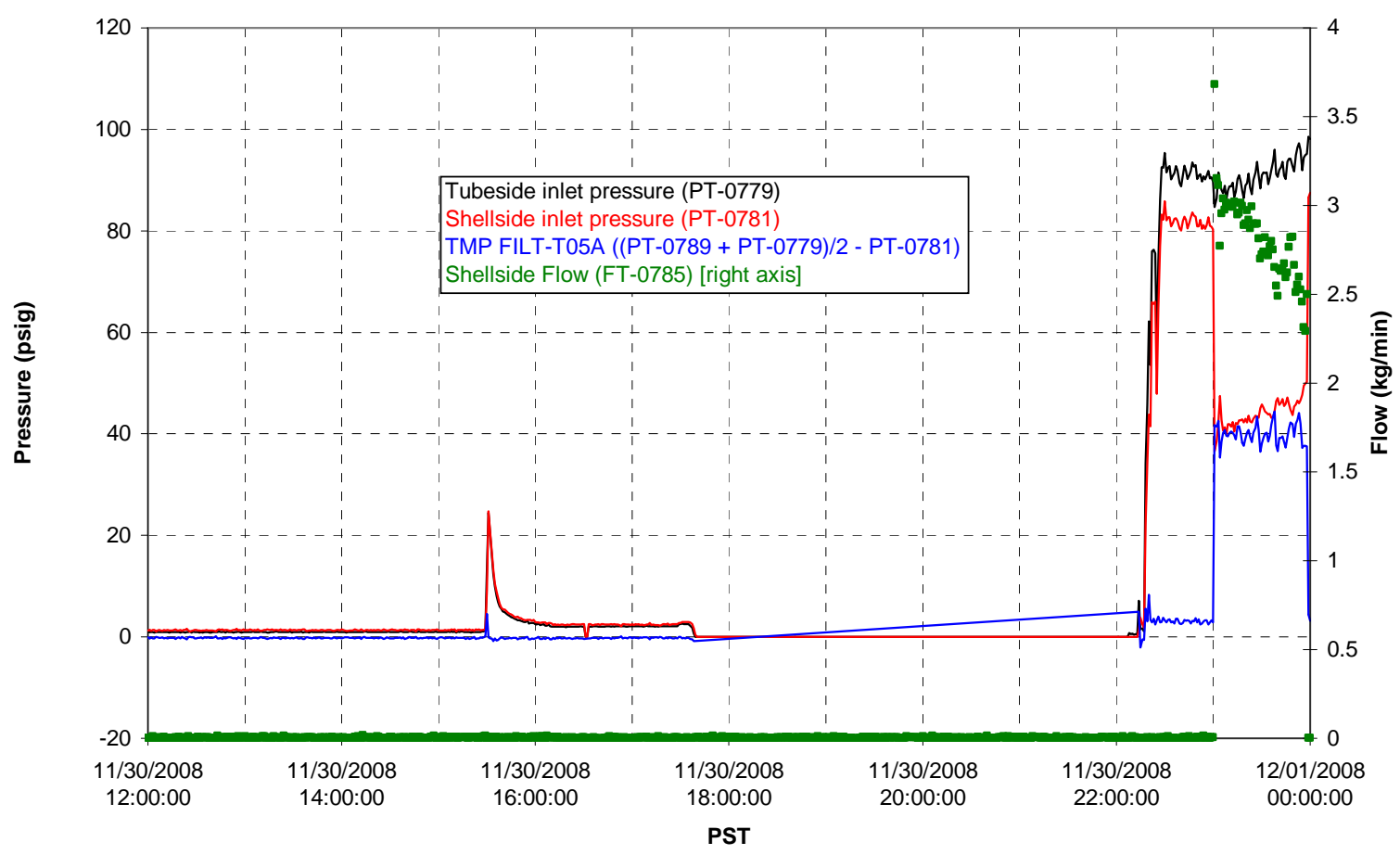

Chemical Flow

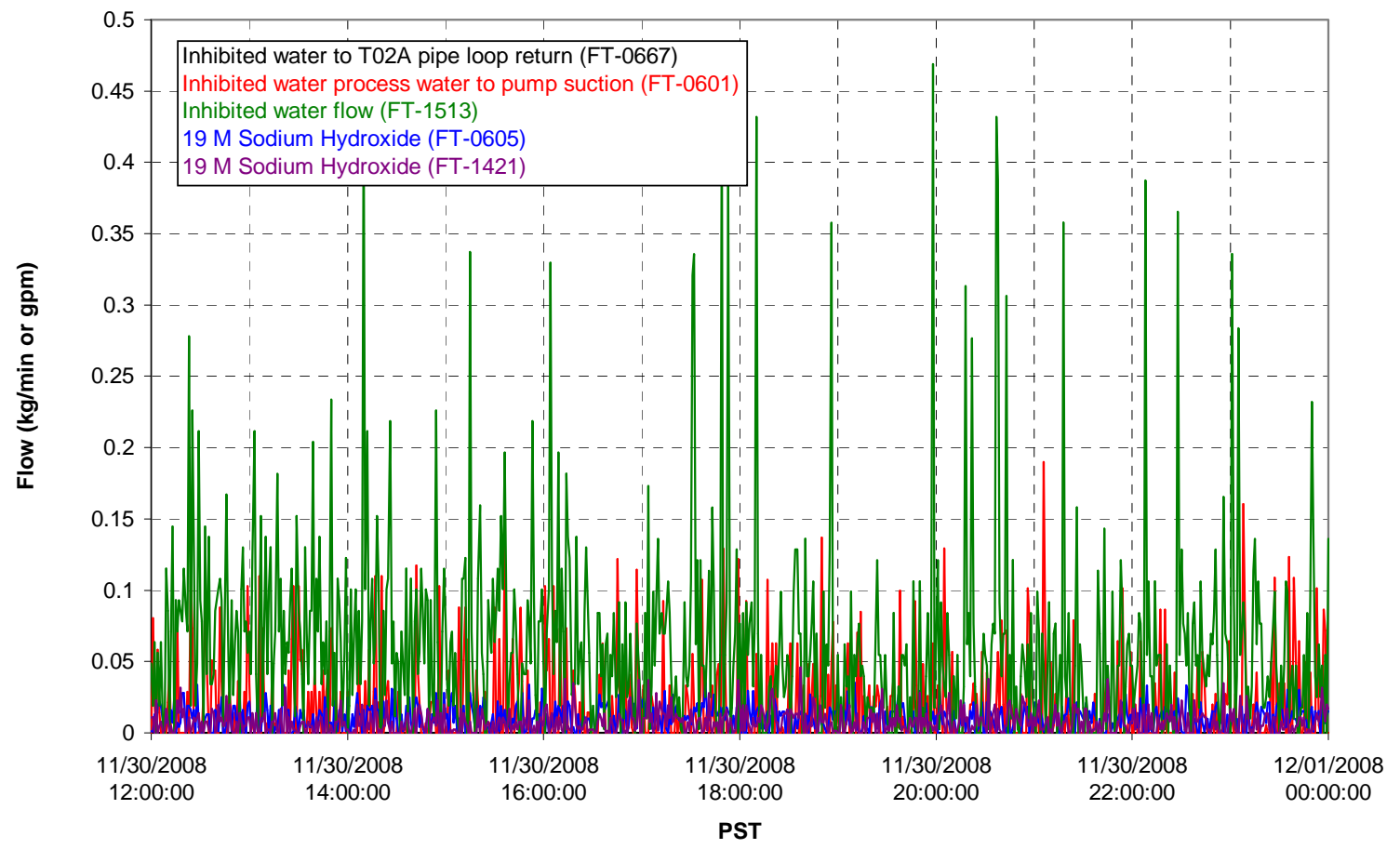


Chemical Flow

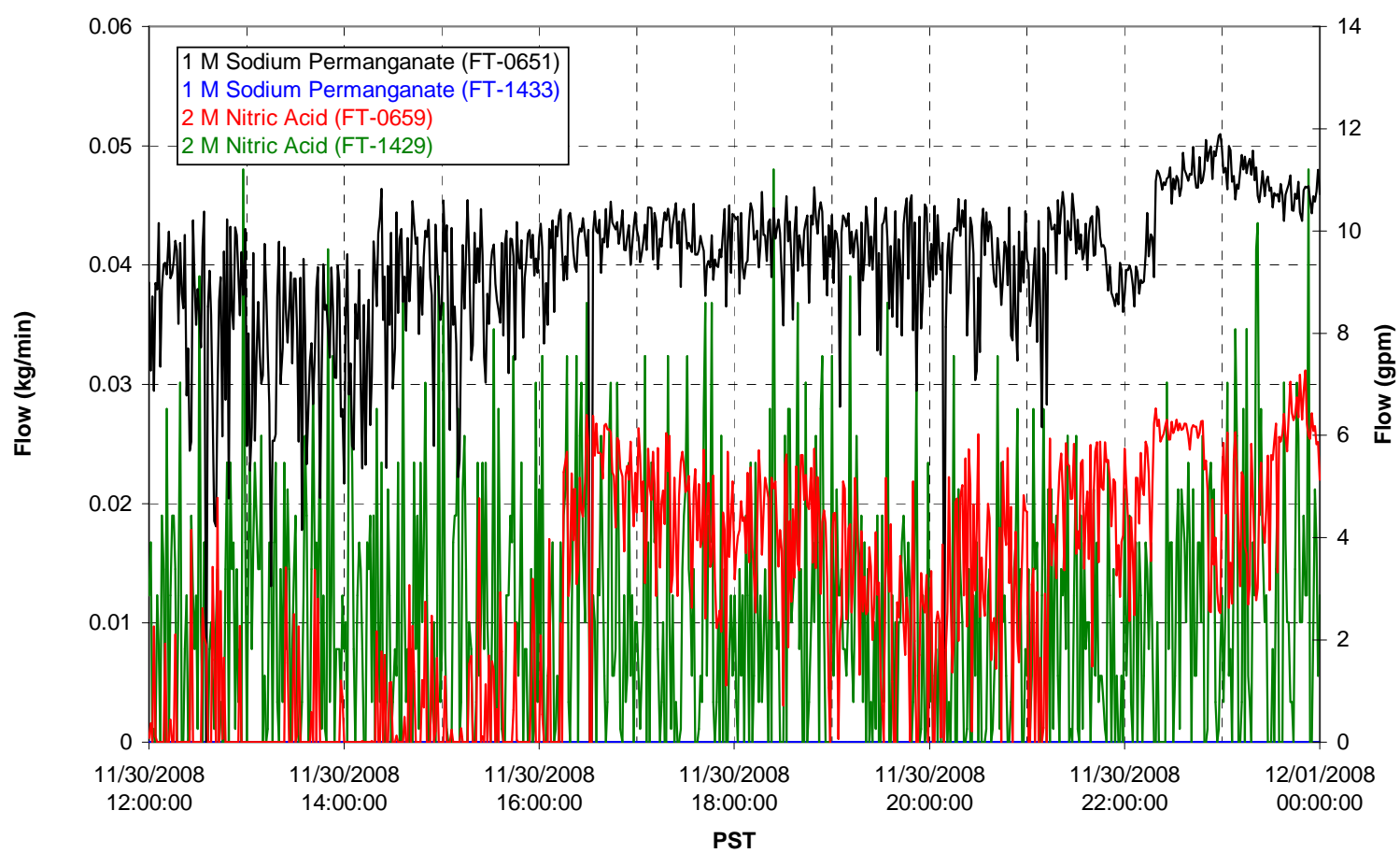

Air Flows

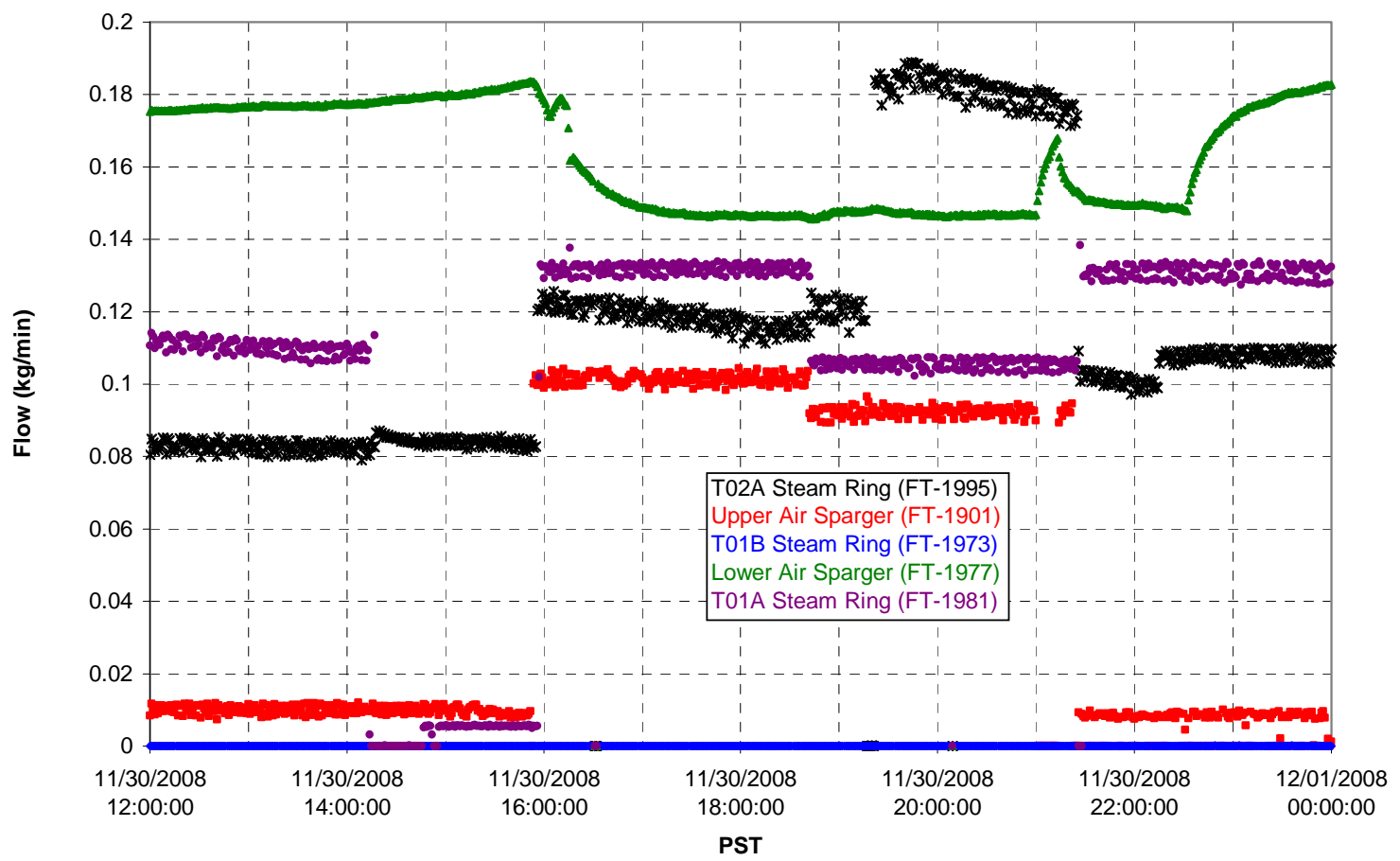


T02A Steam

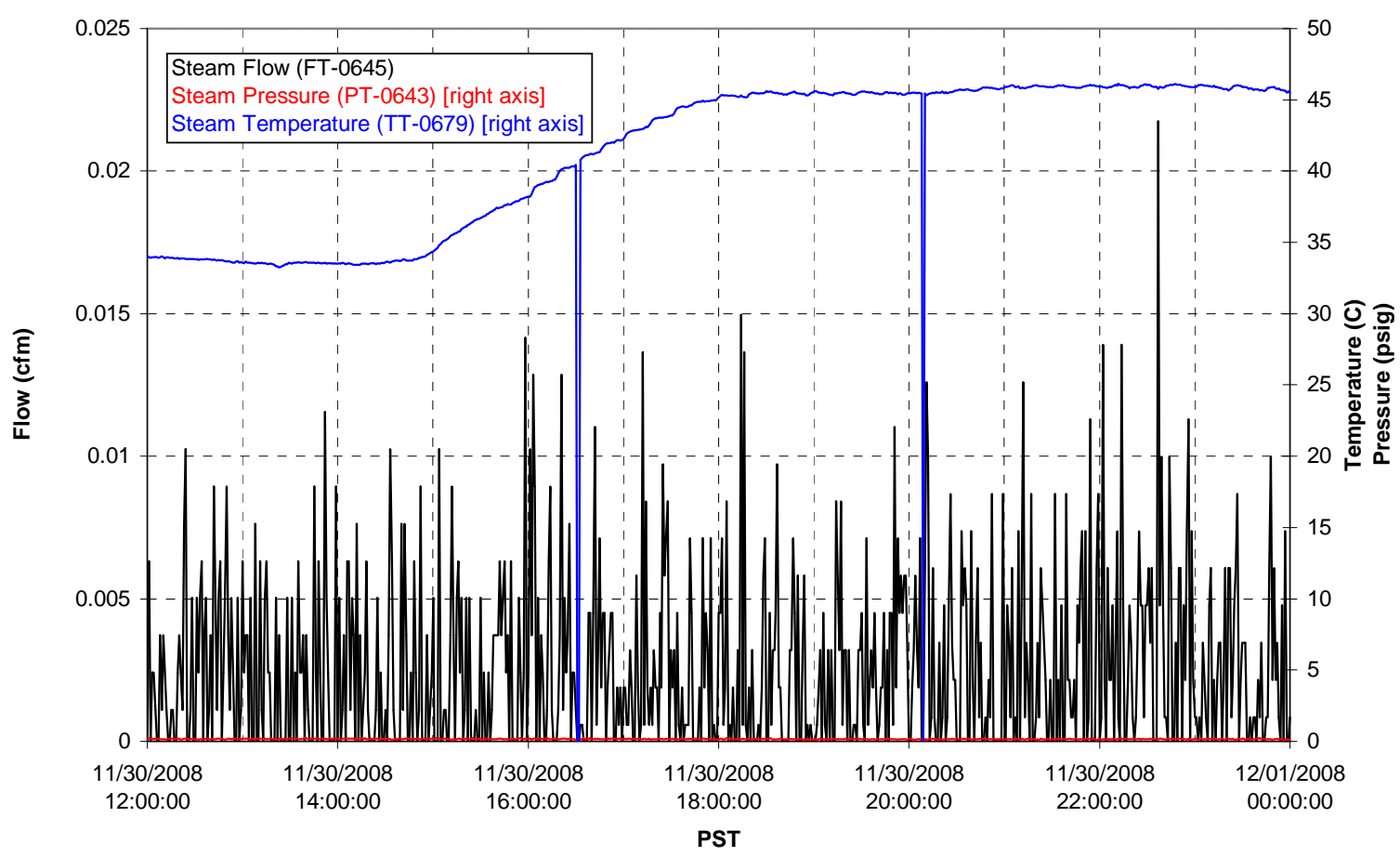

T01A Steam

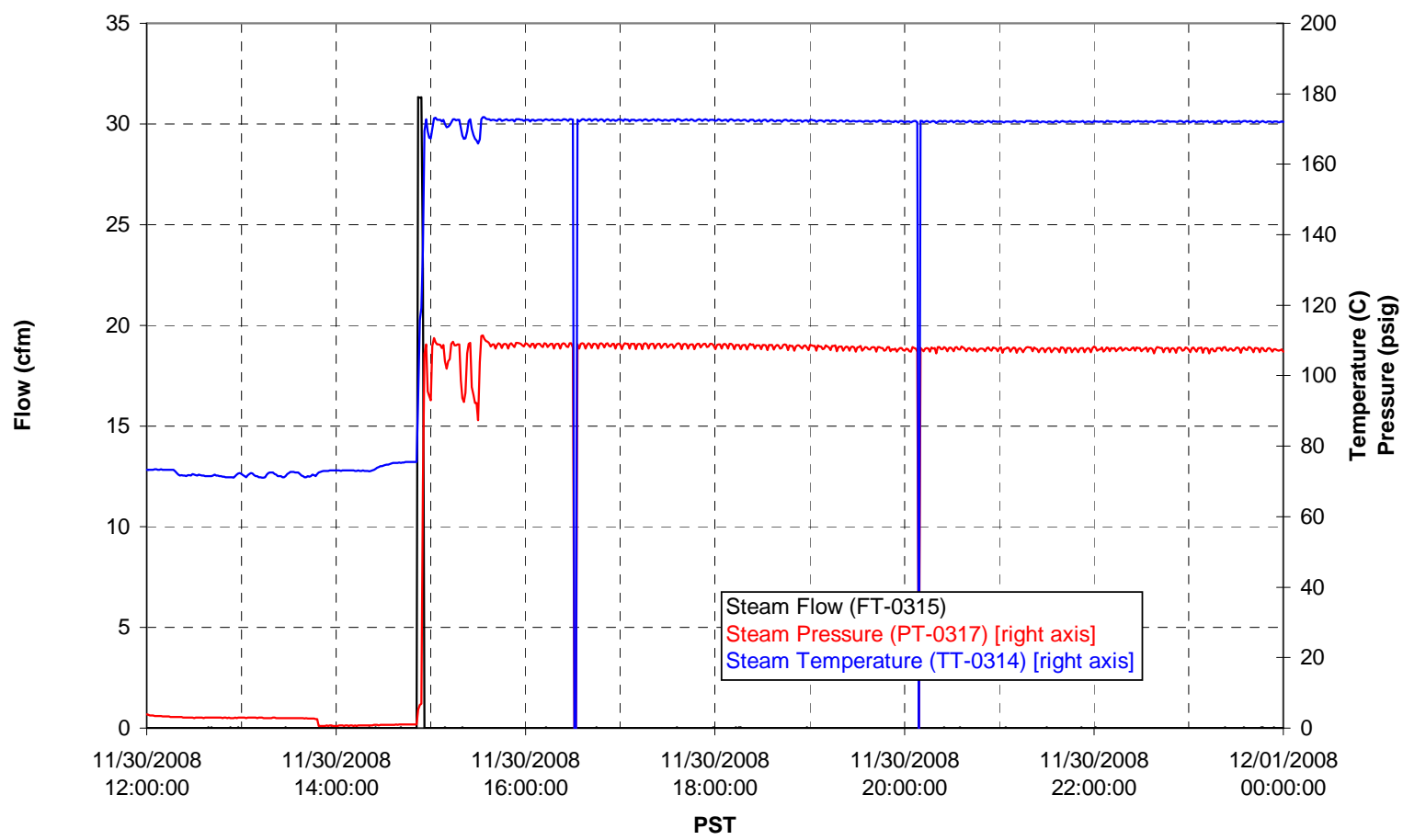


T01B Steam

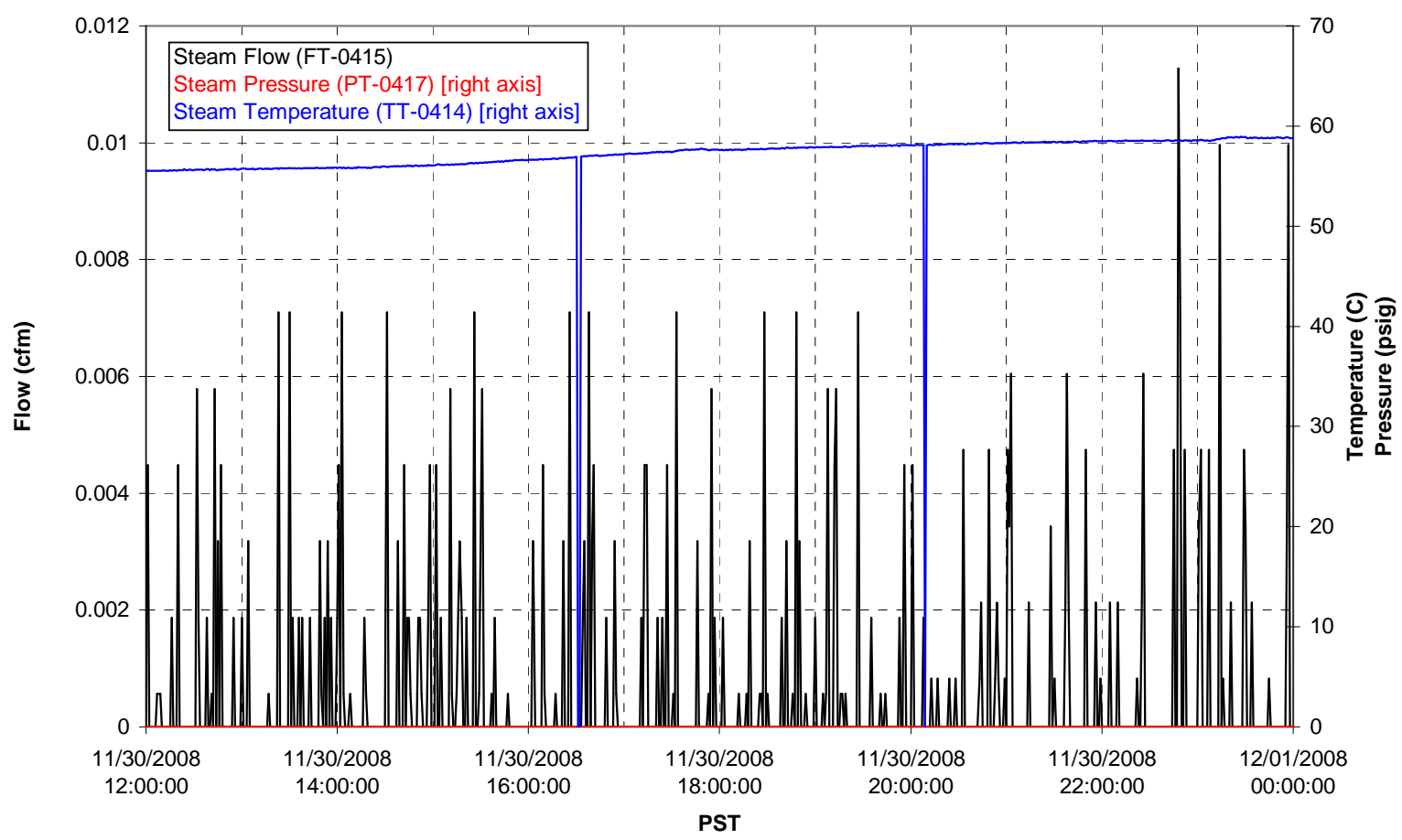


Functional Test Data Plots

12/01/08 00:00 - 12/01/08 12:00 
T01A level

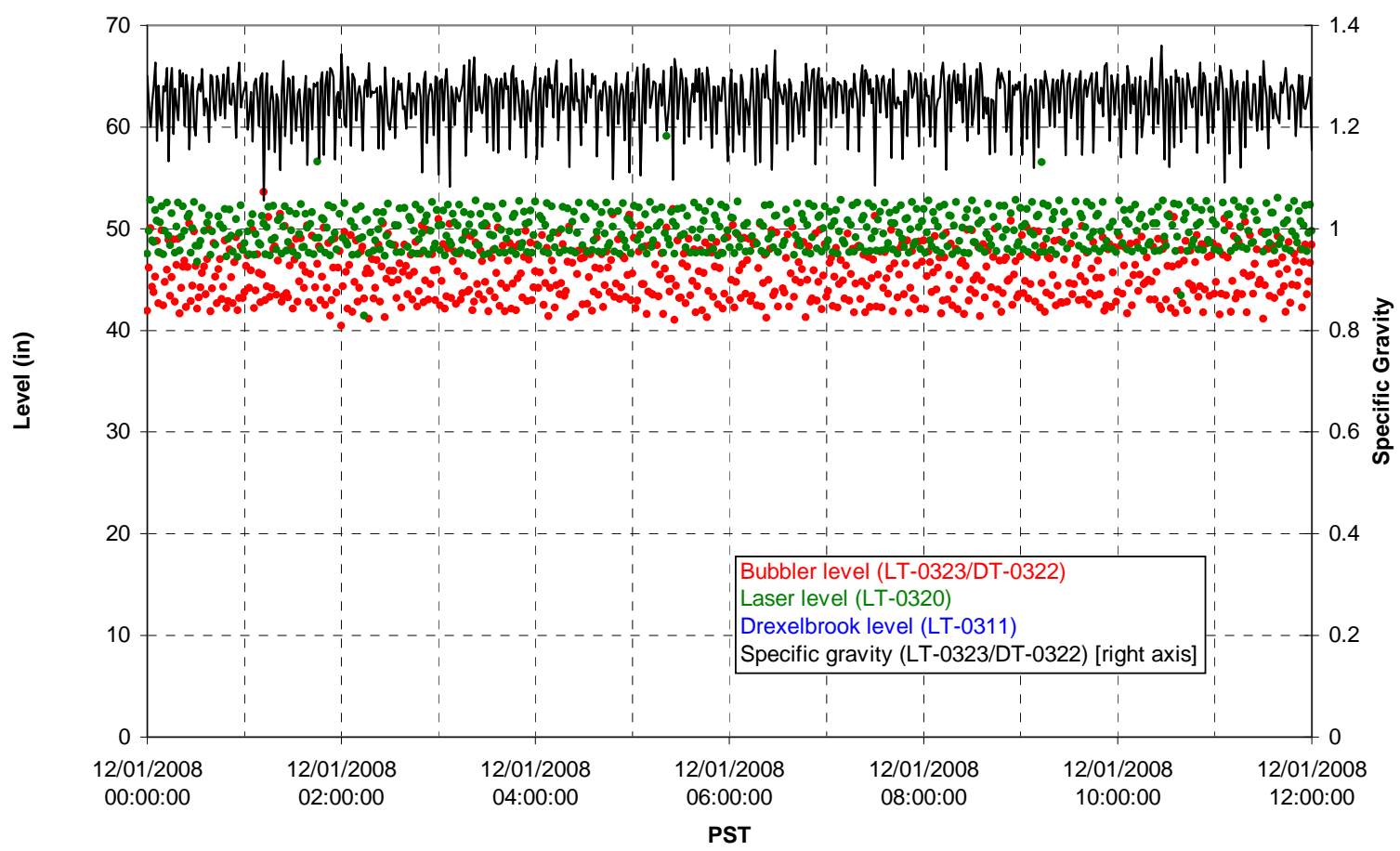

T01A temperatures

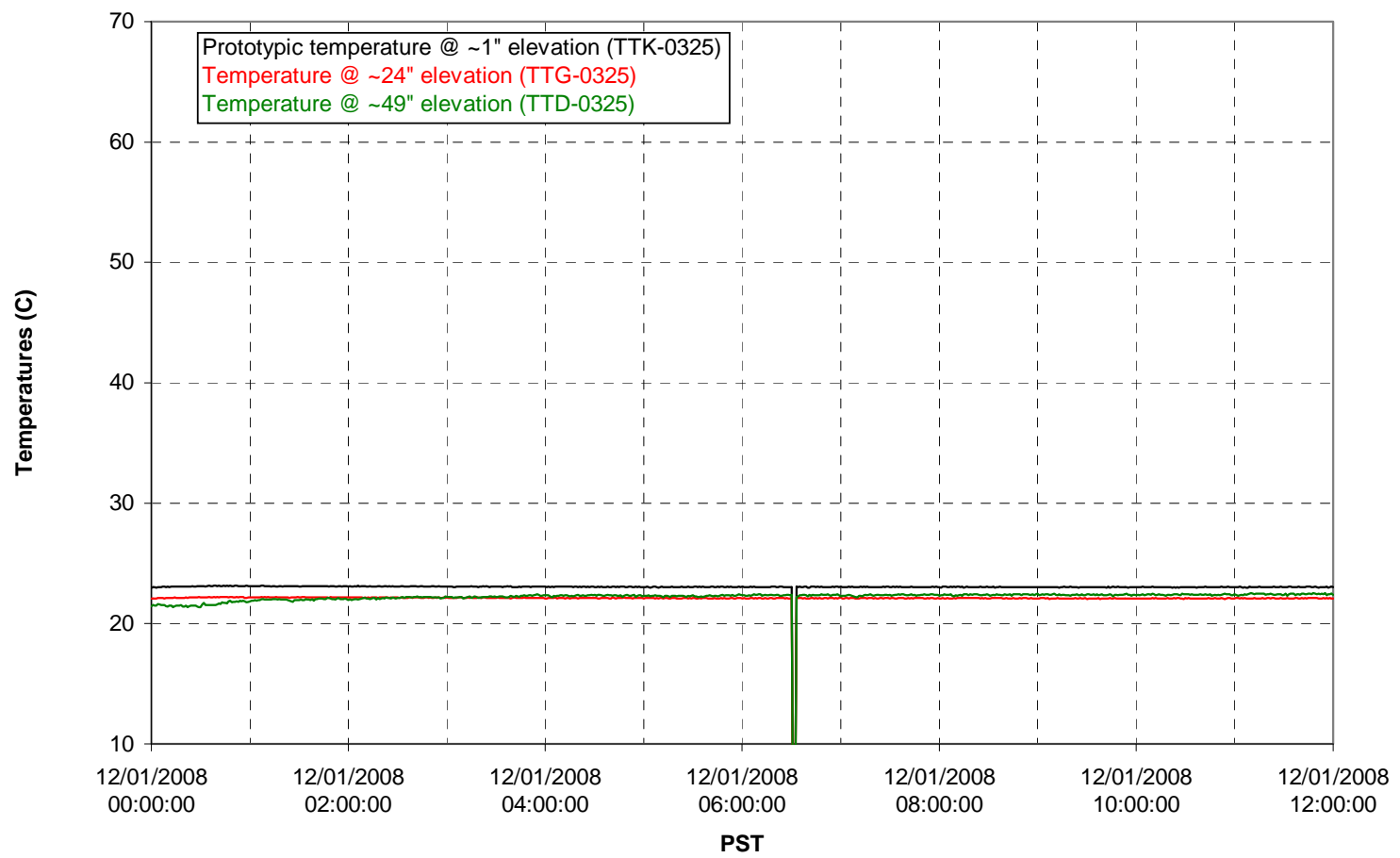


T01B level

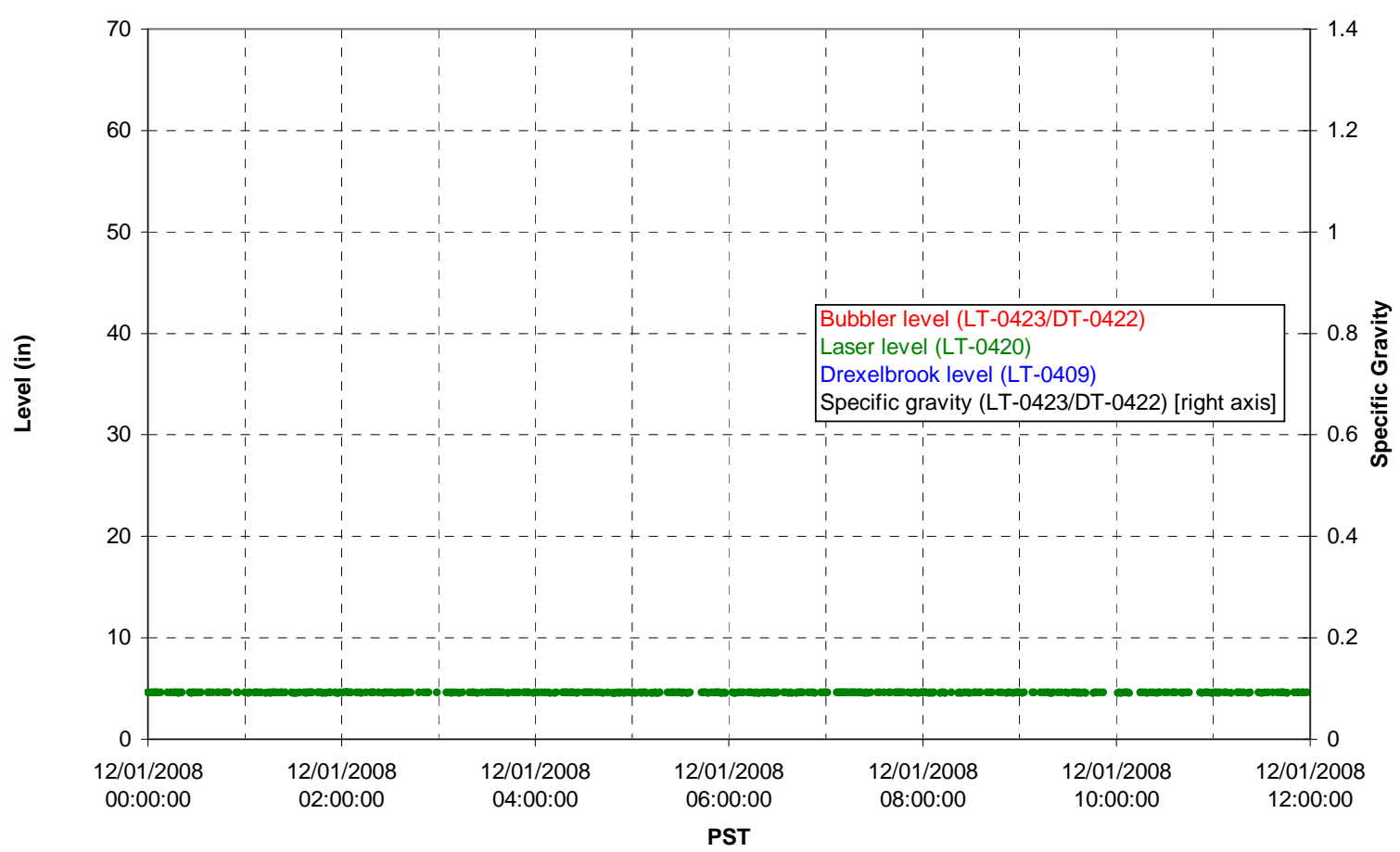

T01B temperatures

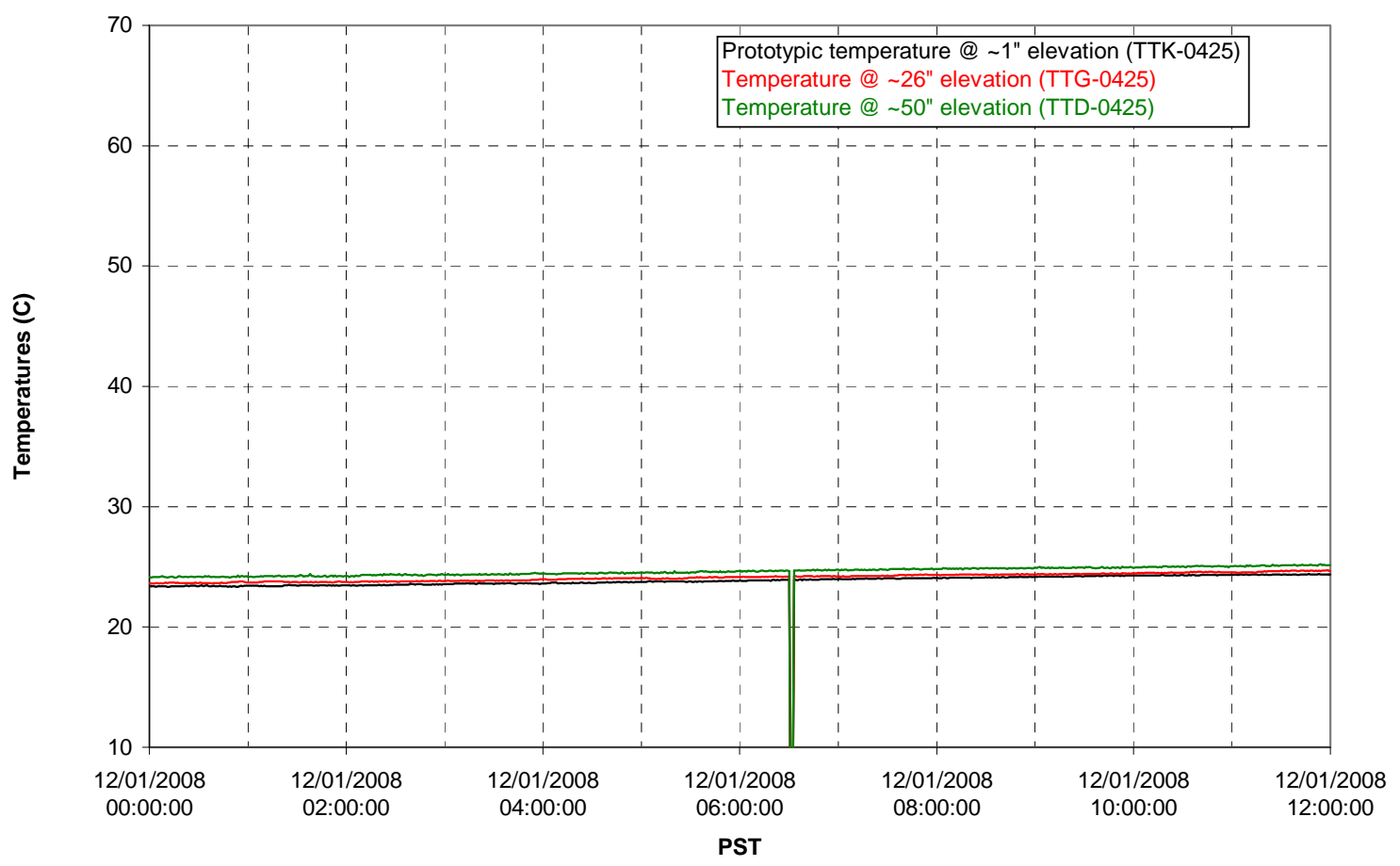


T02A level

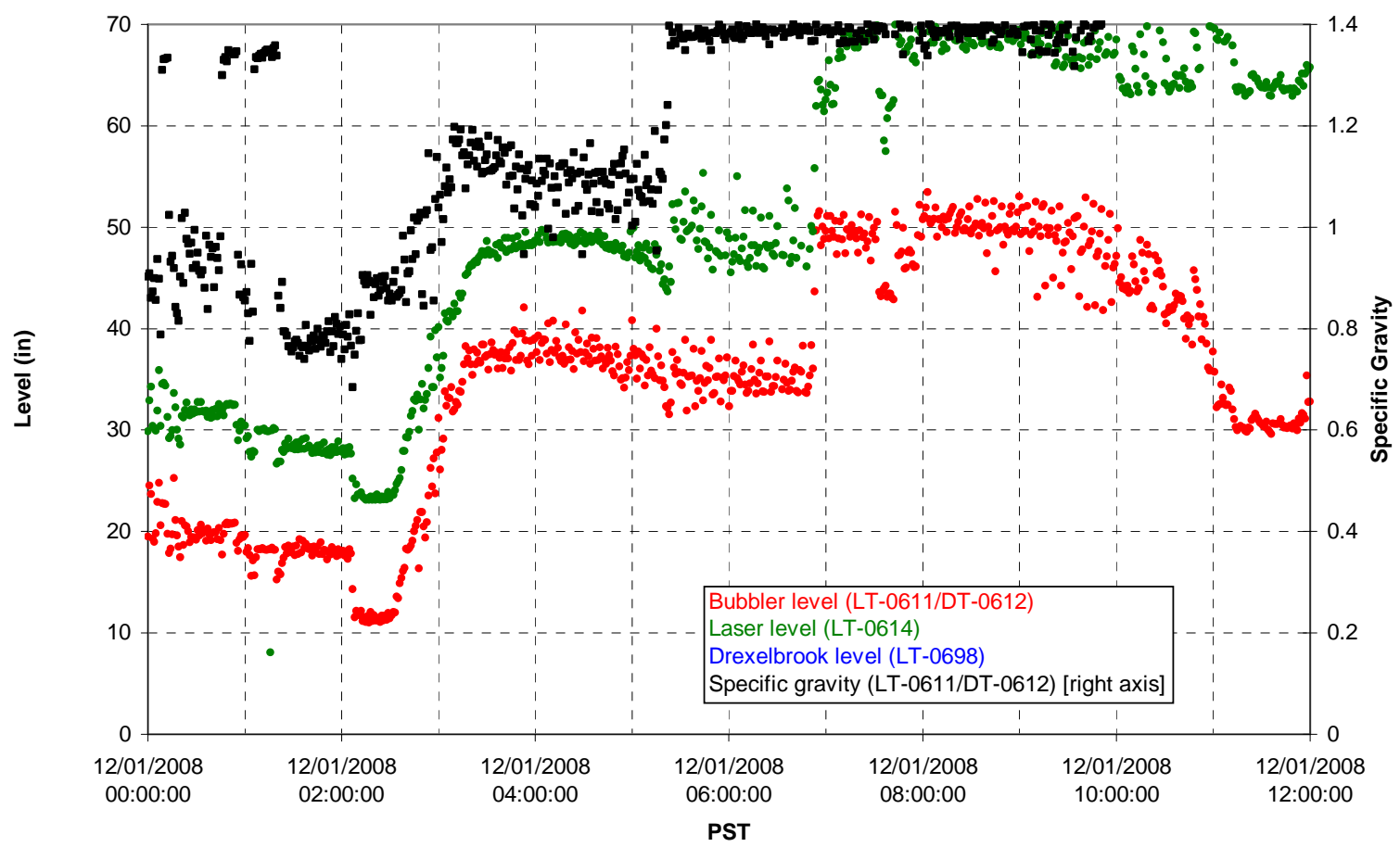

T02A temperatures

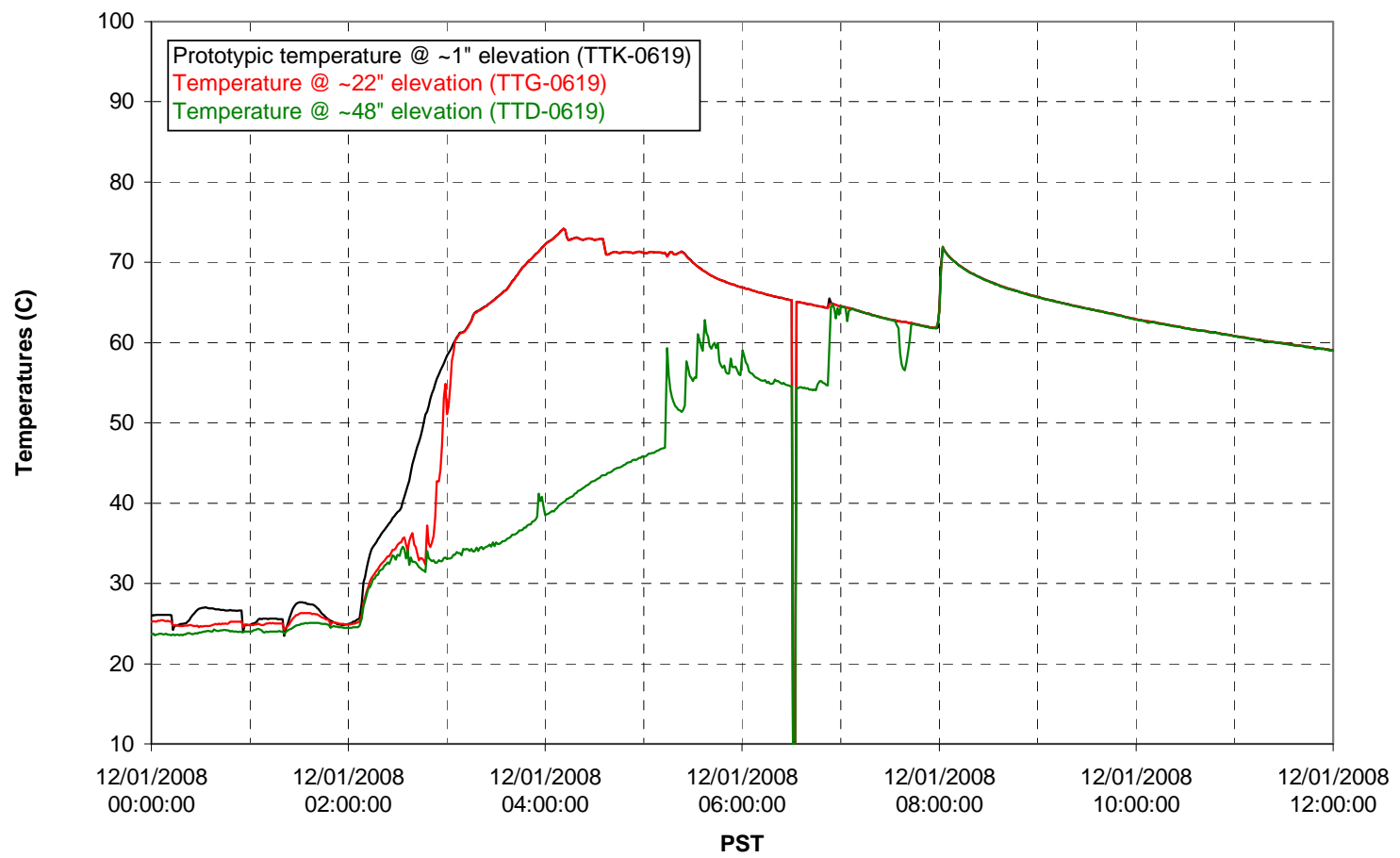


T02A and filter loop temperatures

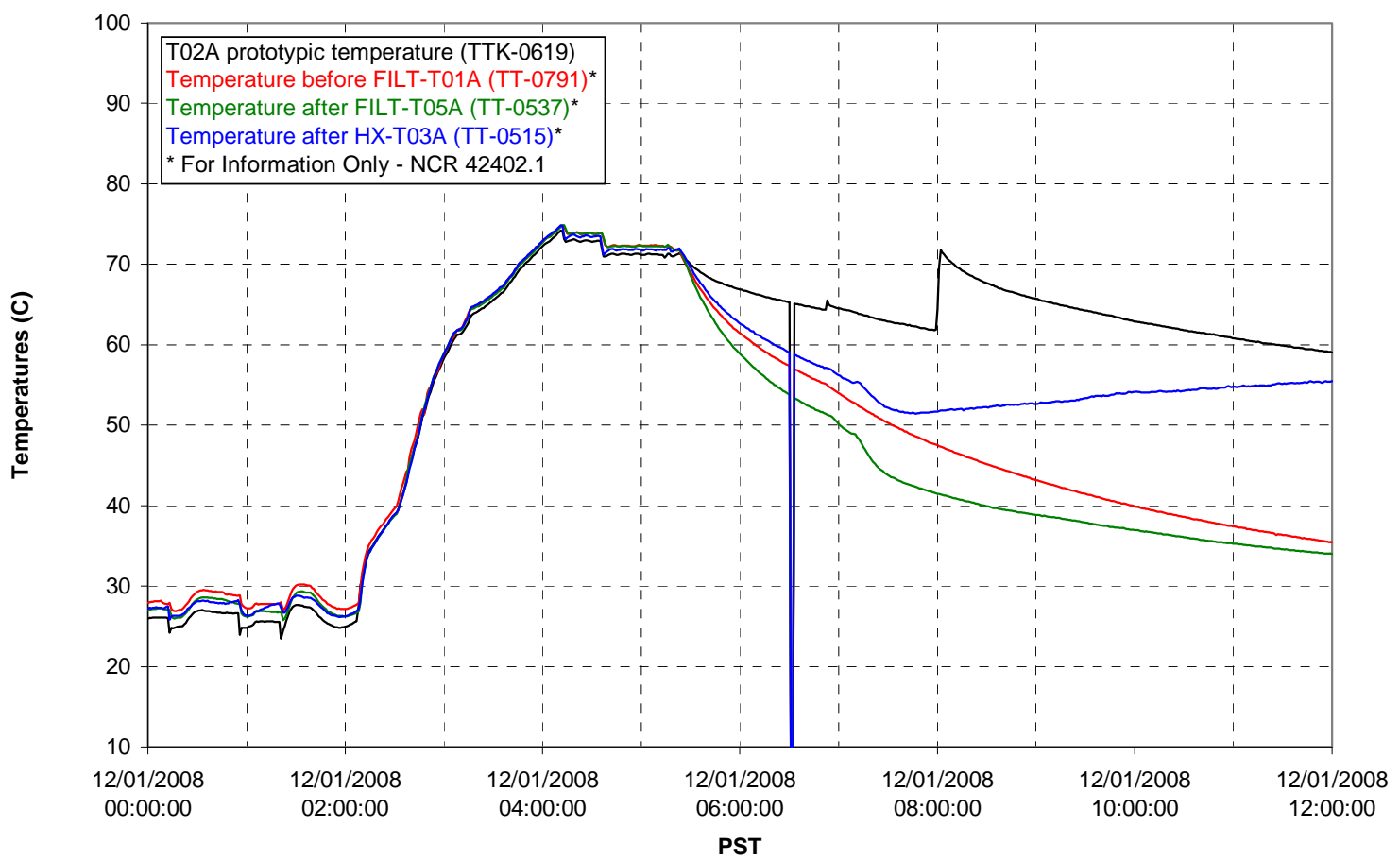

Pump Pressures and Flow

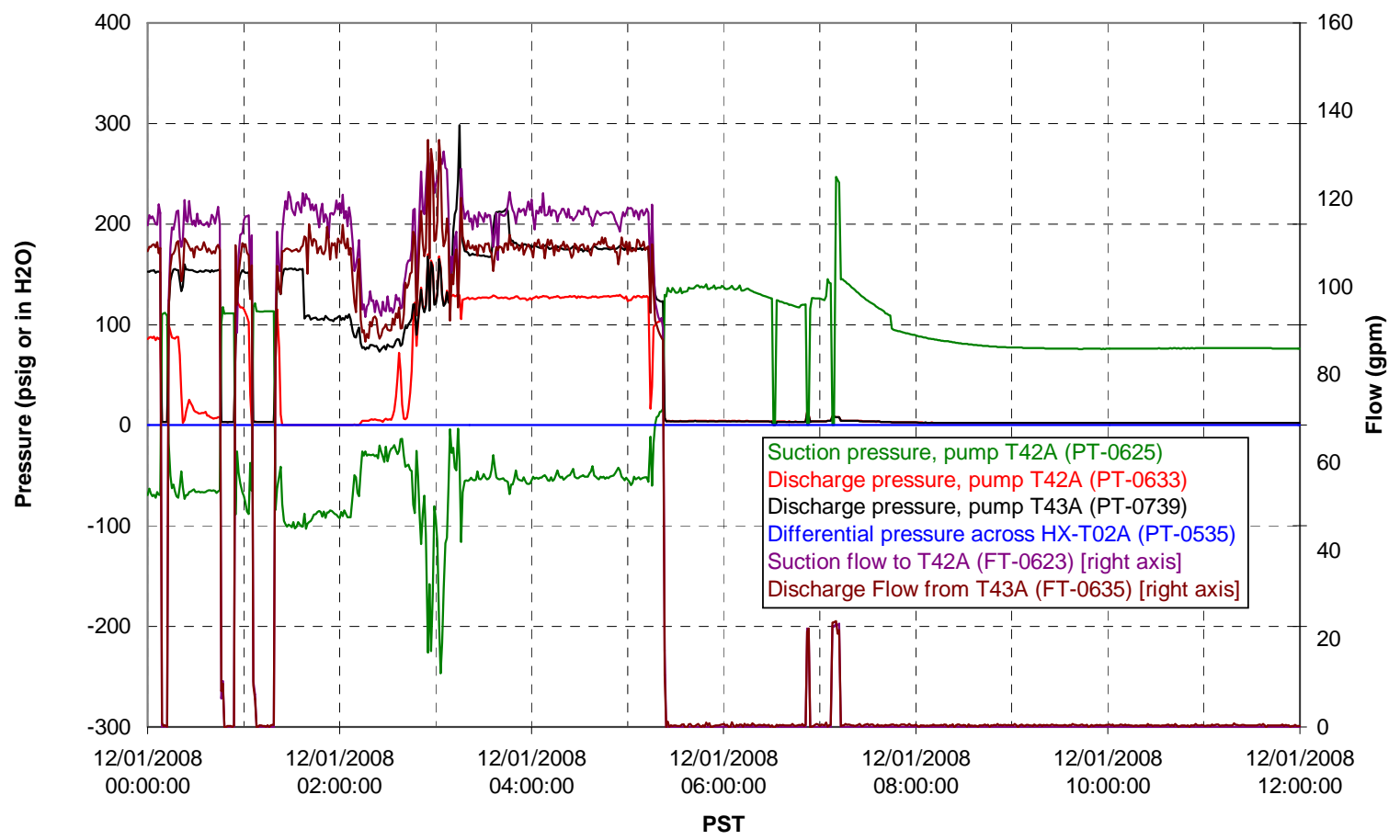




\section{Axial pressure drop}

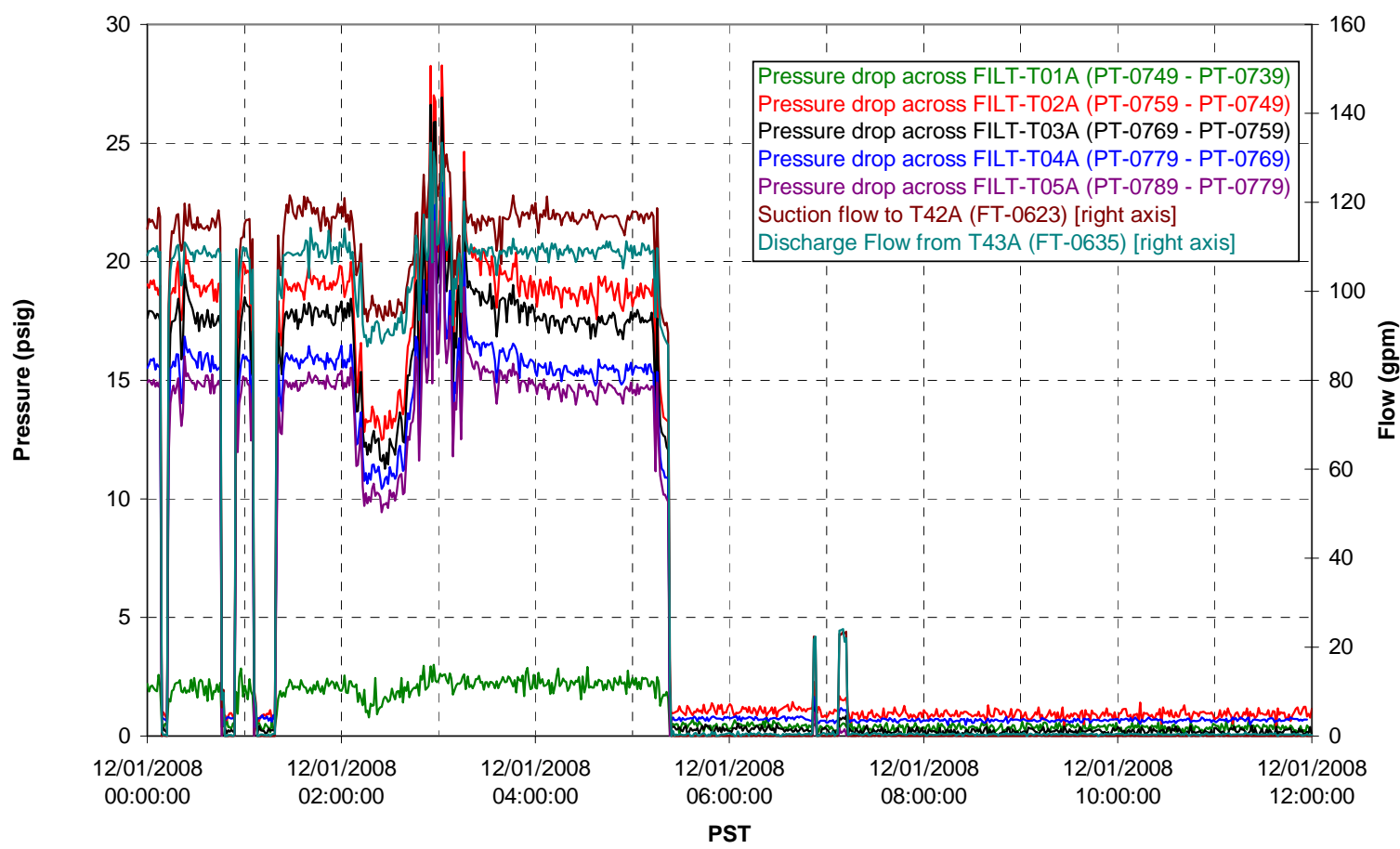

Permeate flow rates

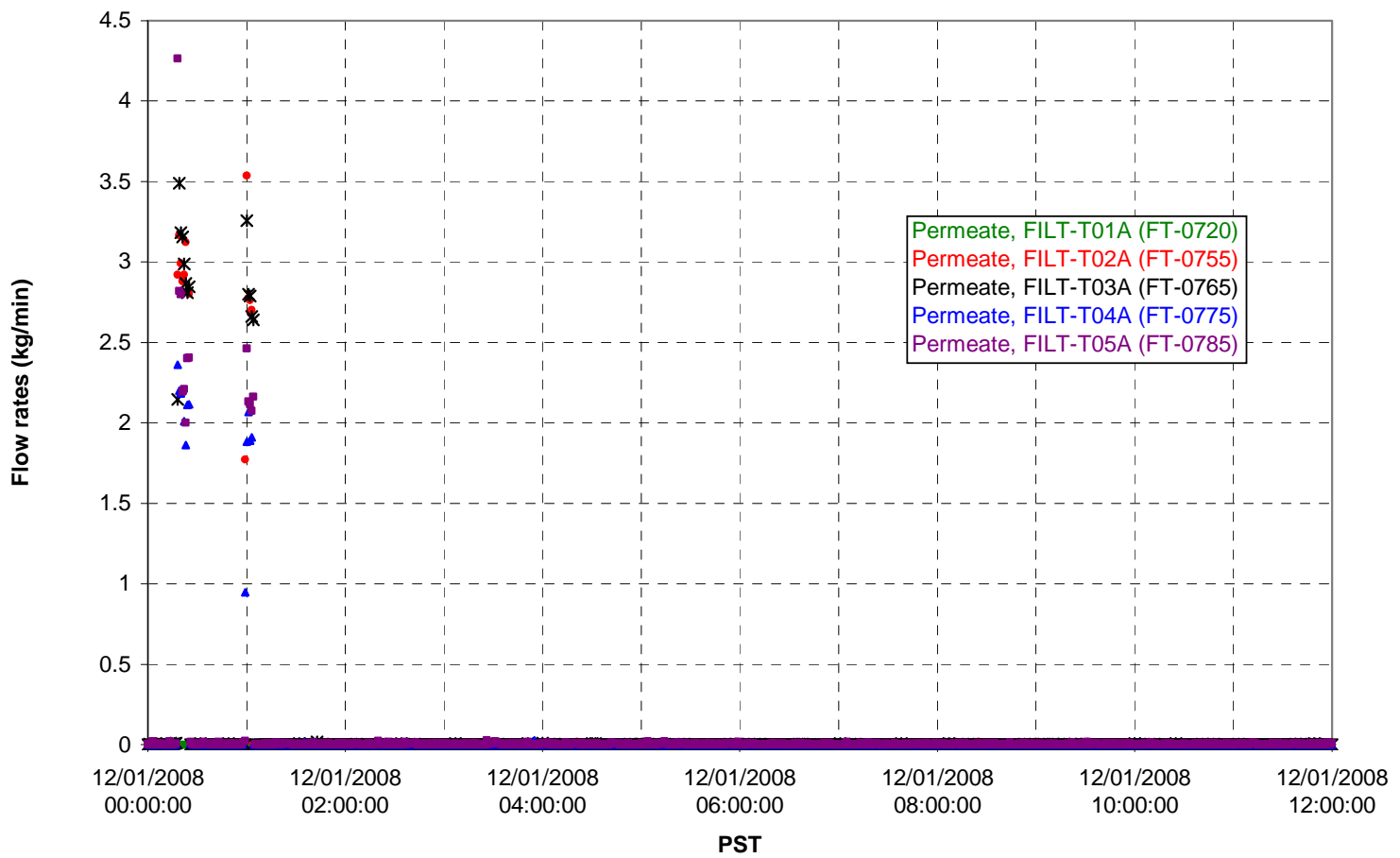


T02A Inner Temperature Tree

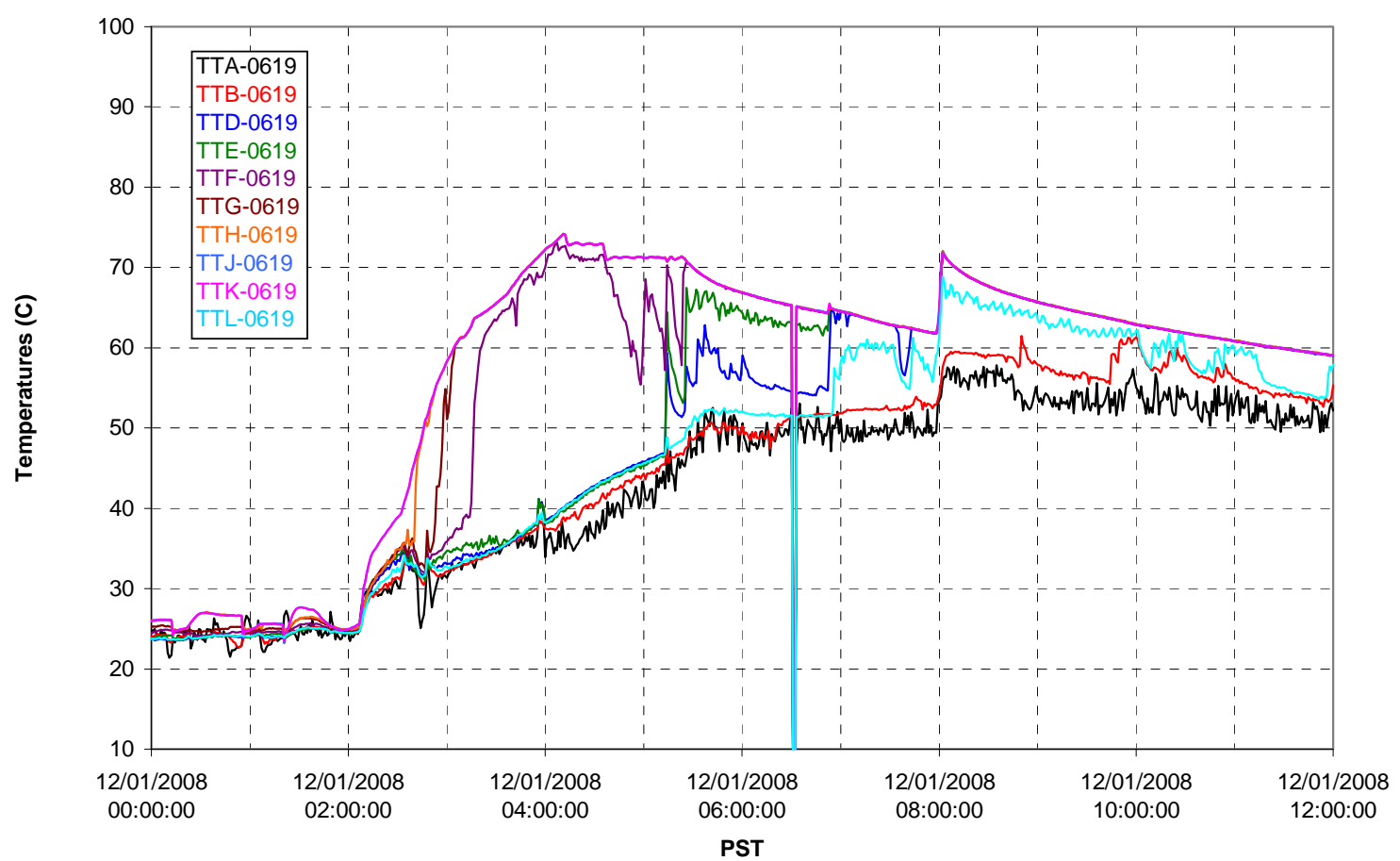

T02A Outer Temperature Tree

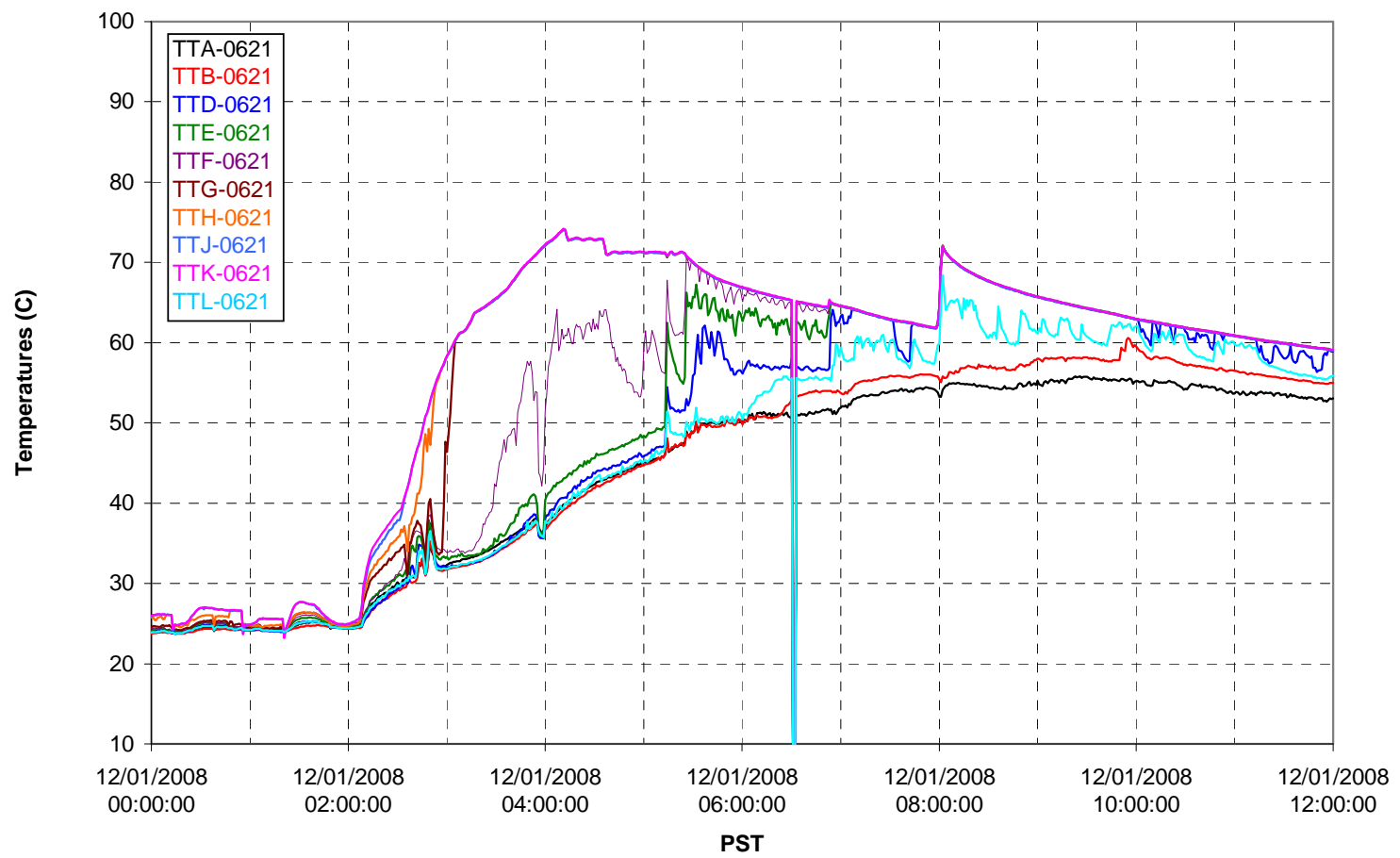


T02A temperatures

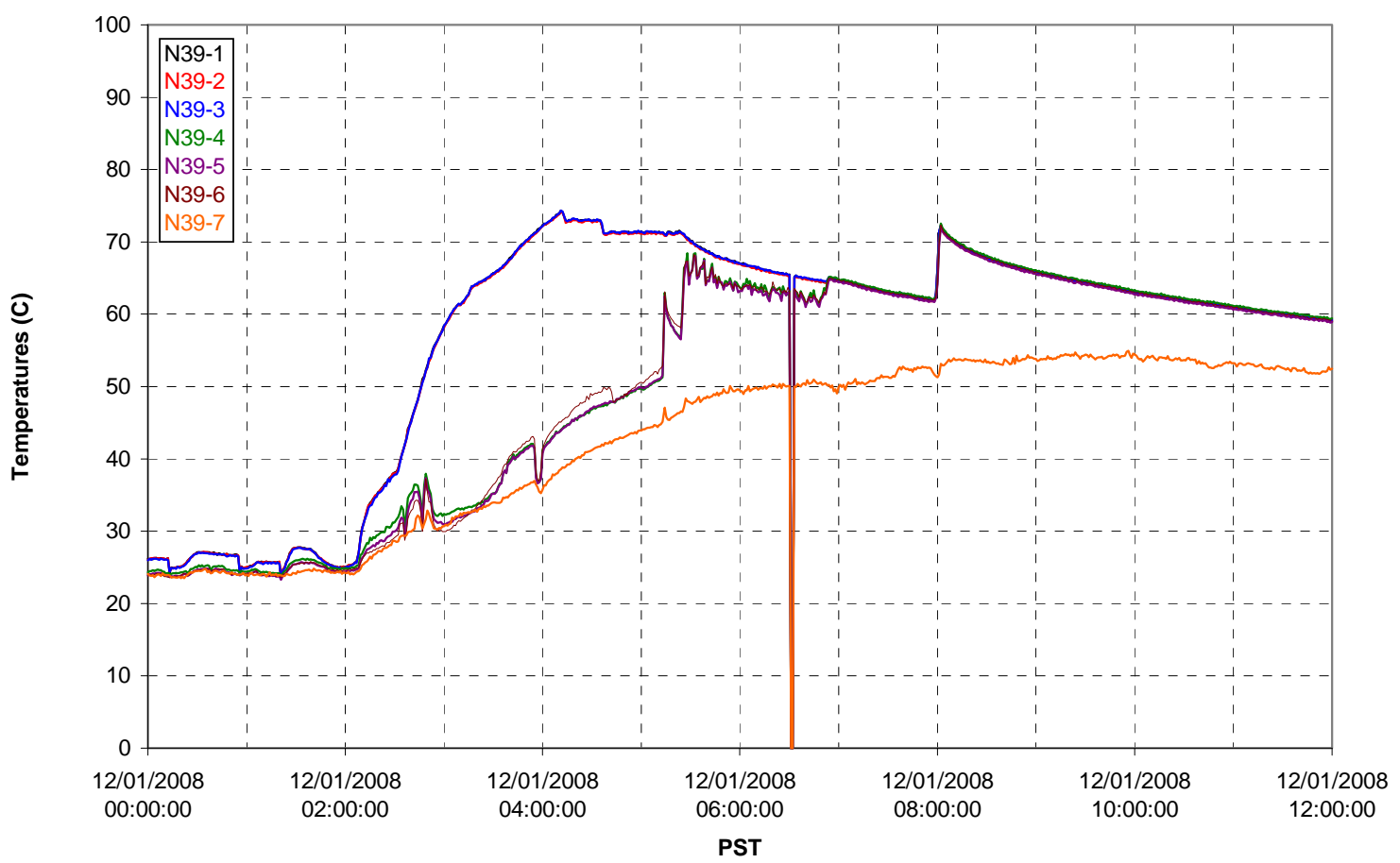

T02A temperatures

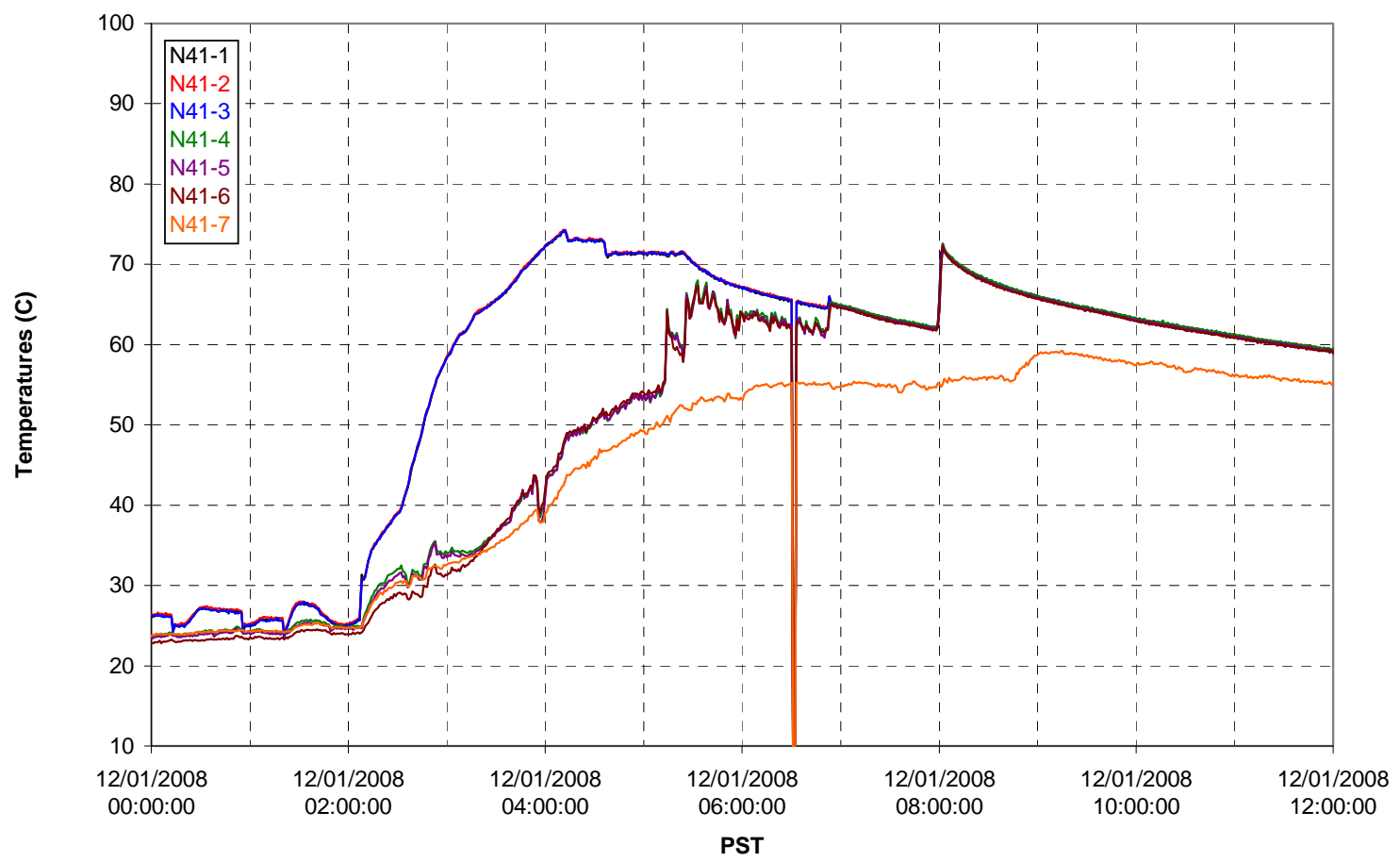


T02A temperatures

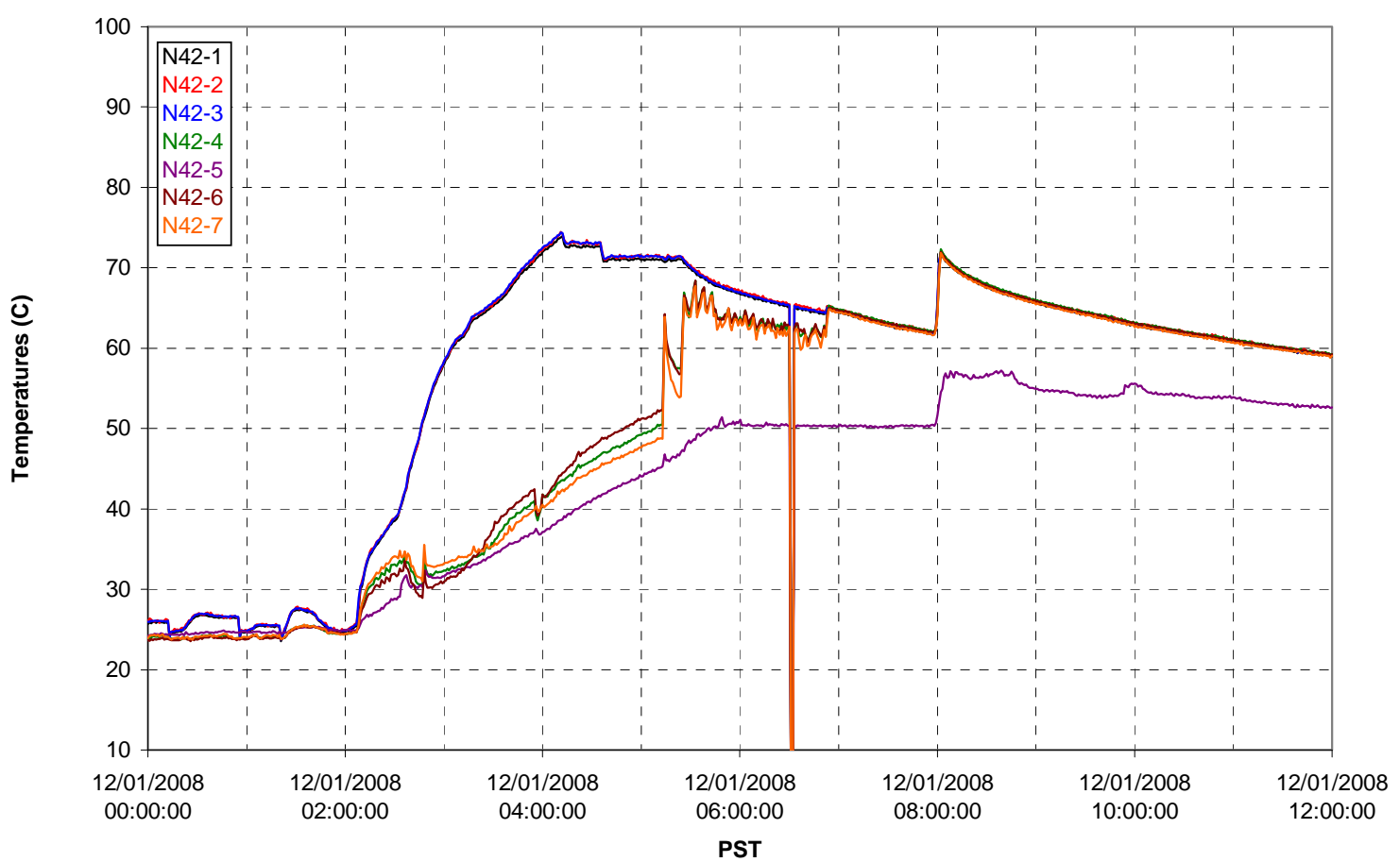

T02A temperatures

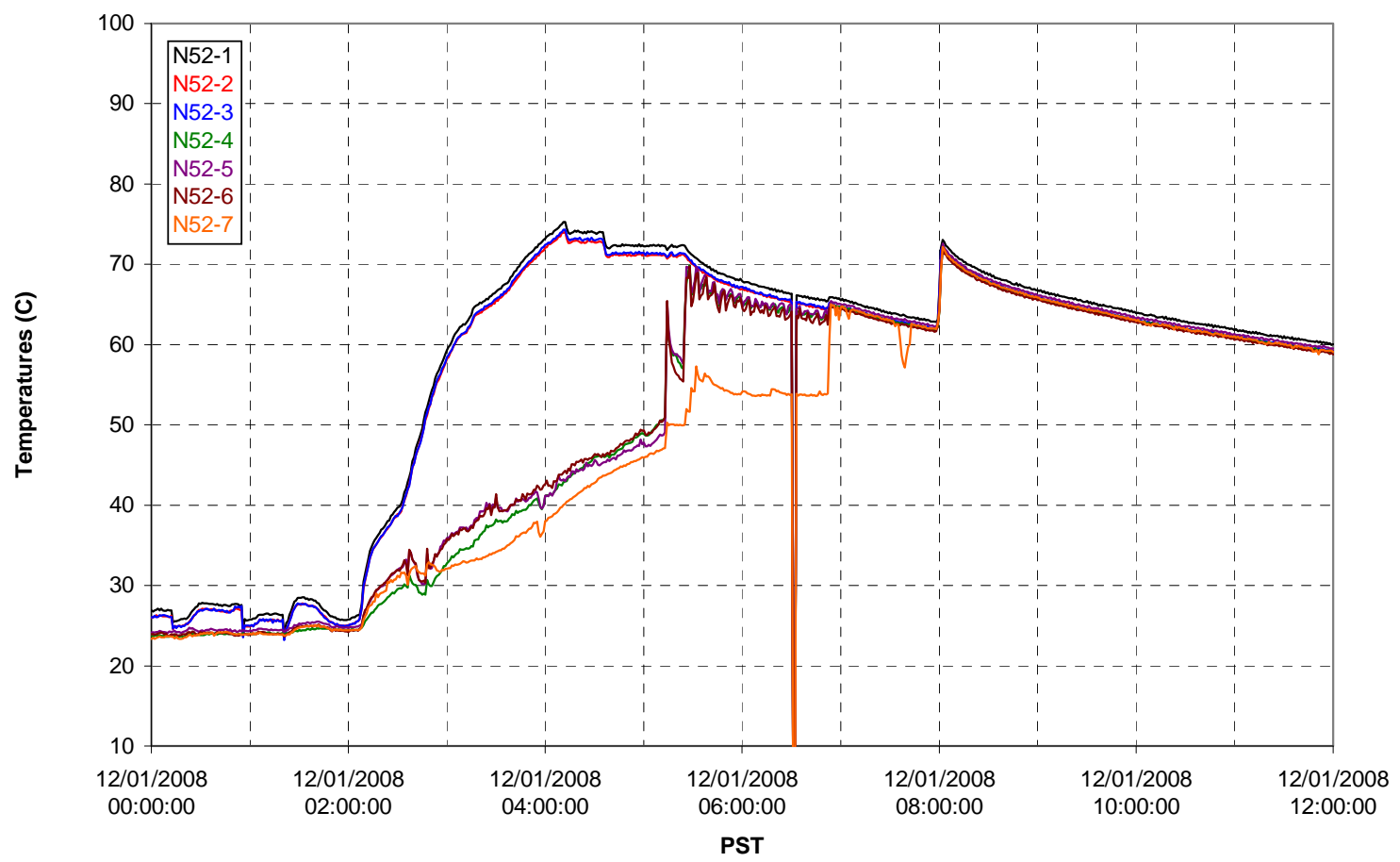


T02A Heating and Cooling

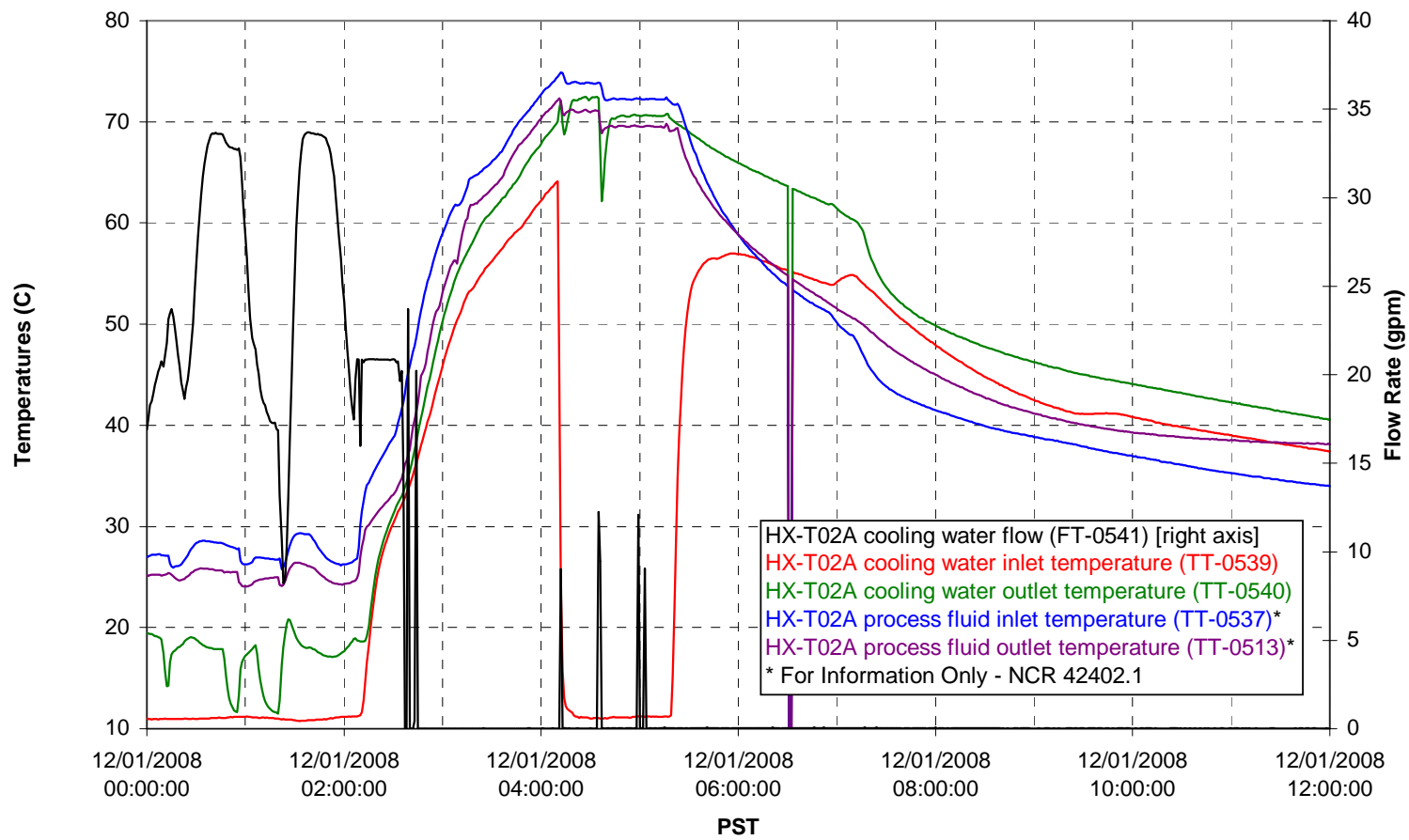

Pump Operation

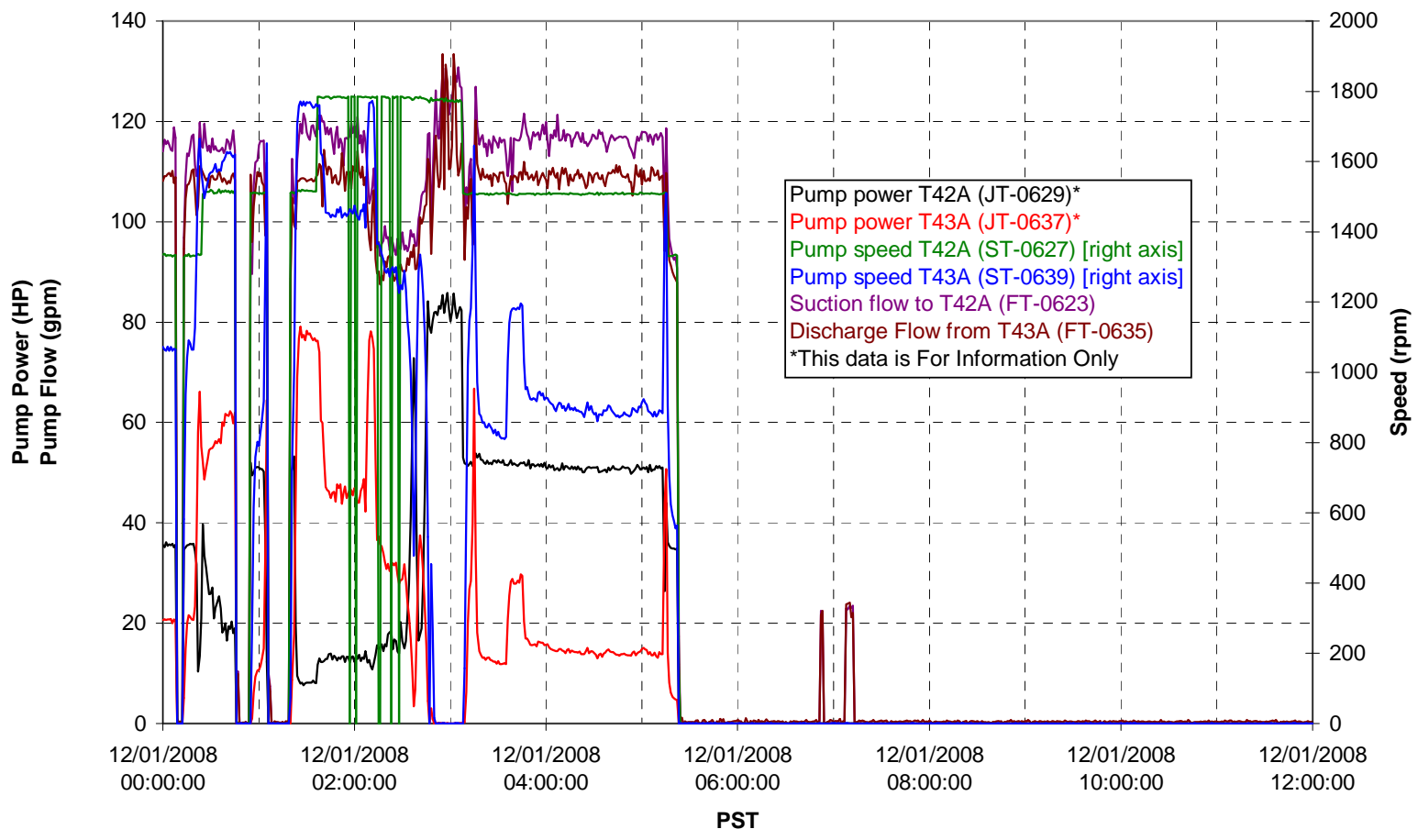


Pulsepot UFP-PP-T01A

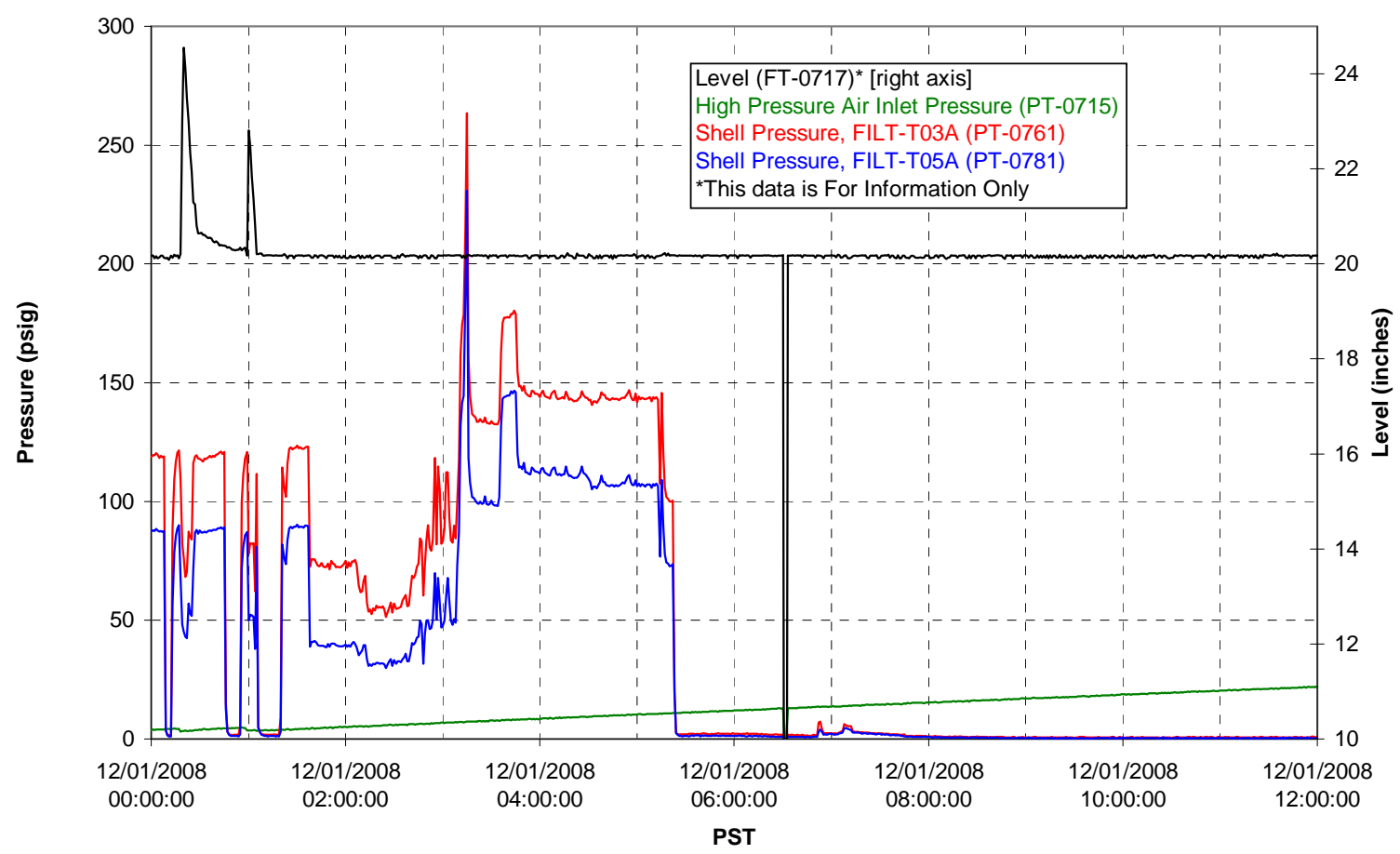

Pulsepot UFP-PP-T02A

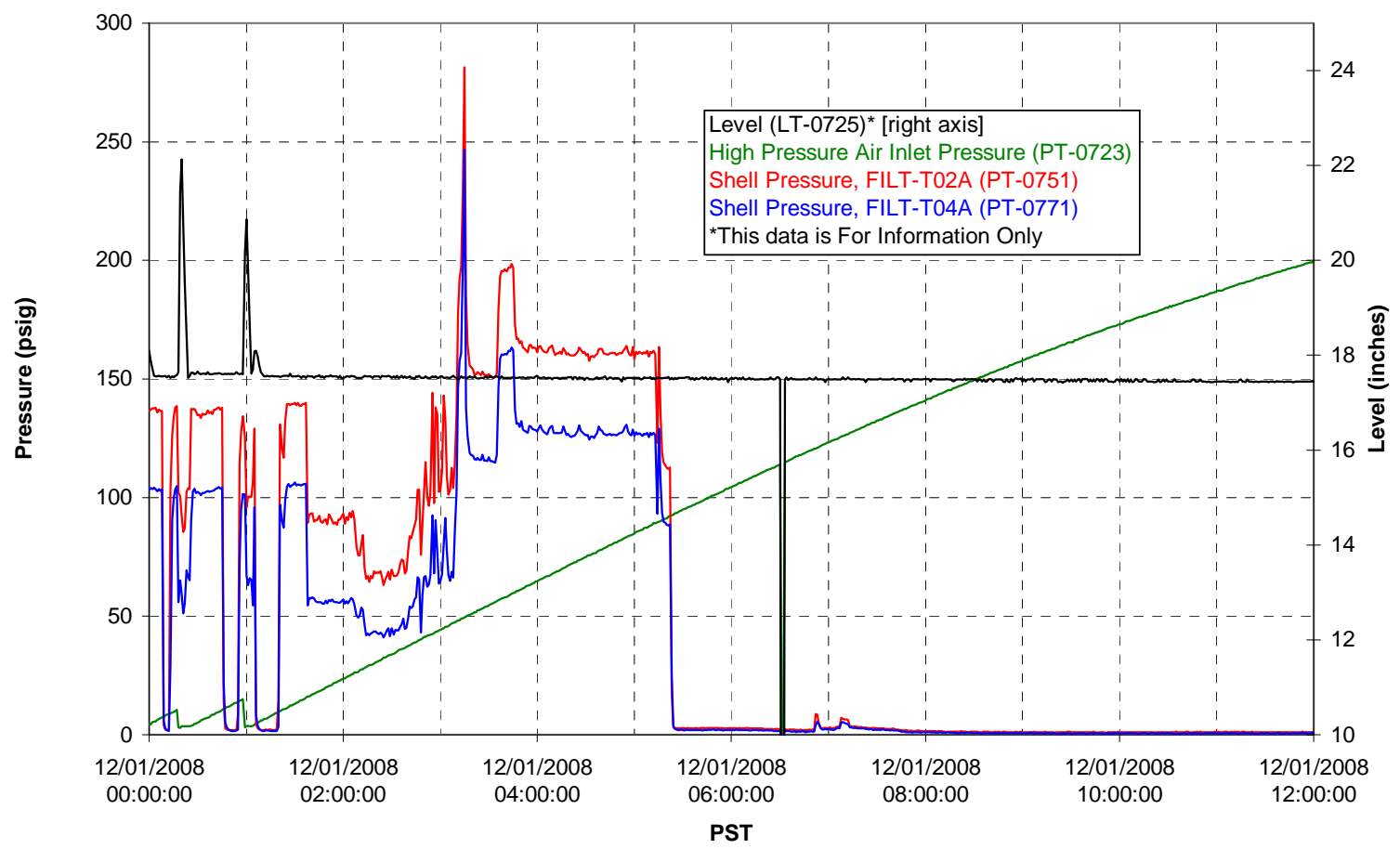


Pulsepot UFP-PP-T03A

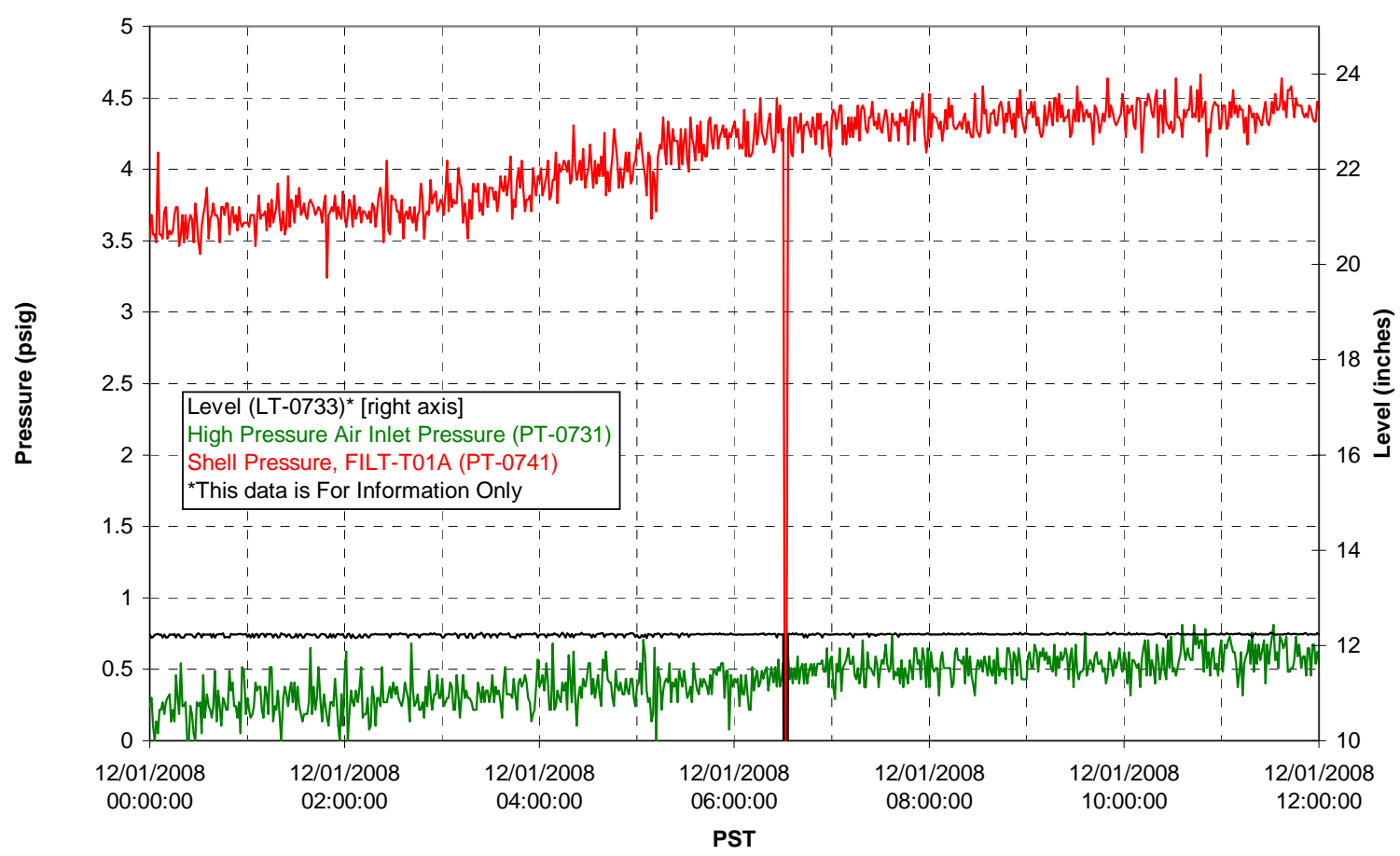

Pulsepot Levels

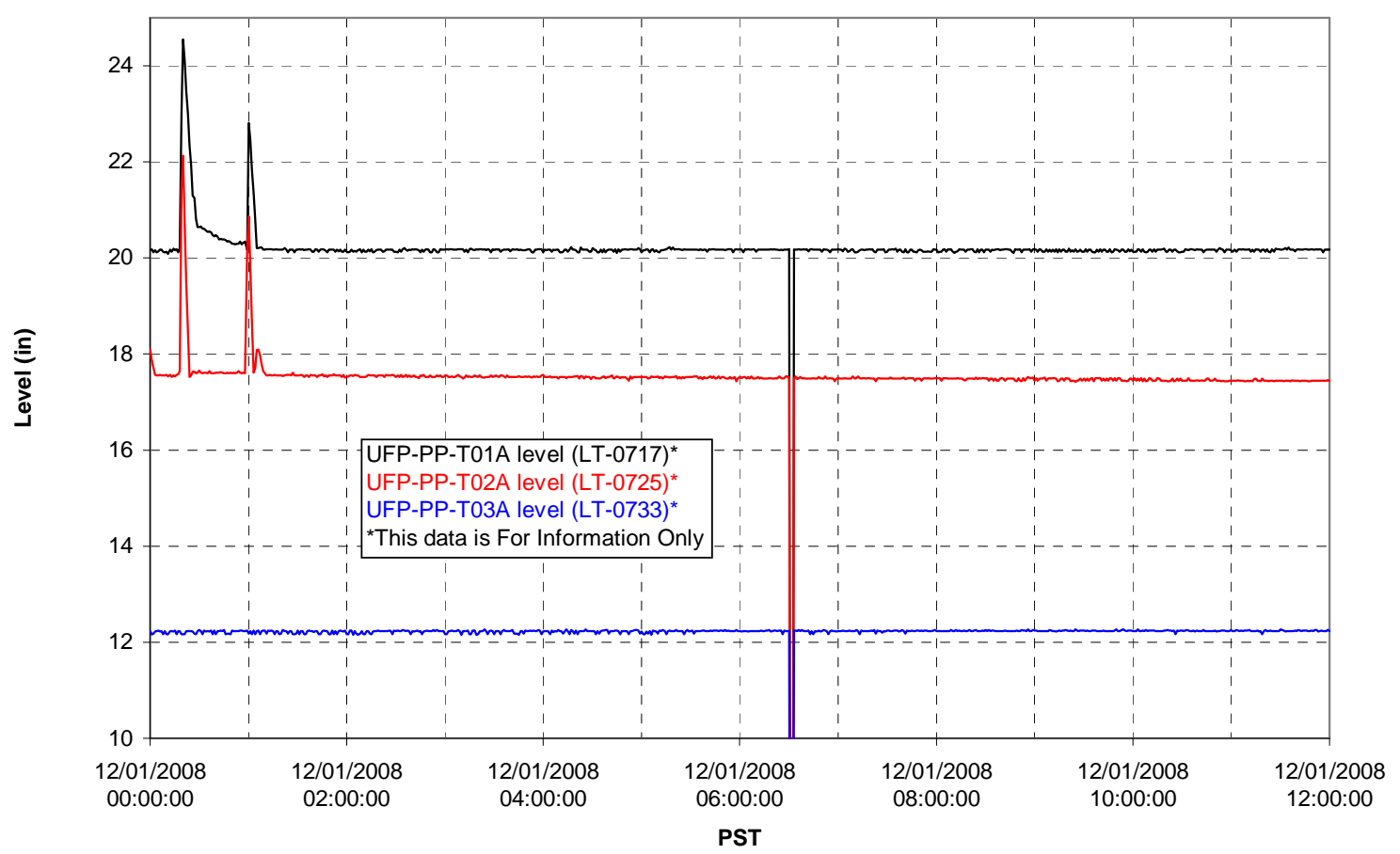


Filter UFP-FILT-T01A

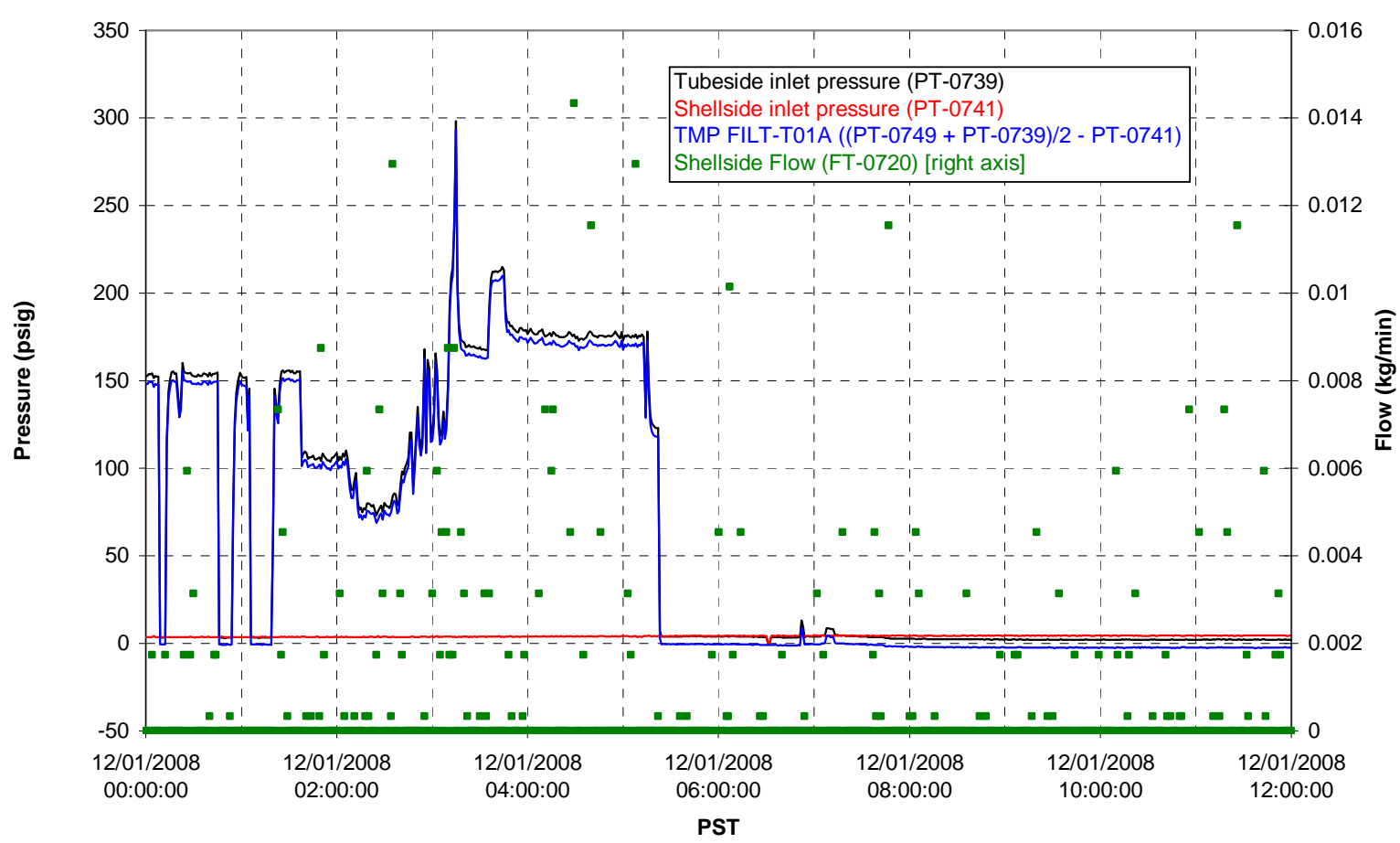

Filter UFP-FILT-T02A

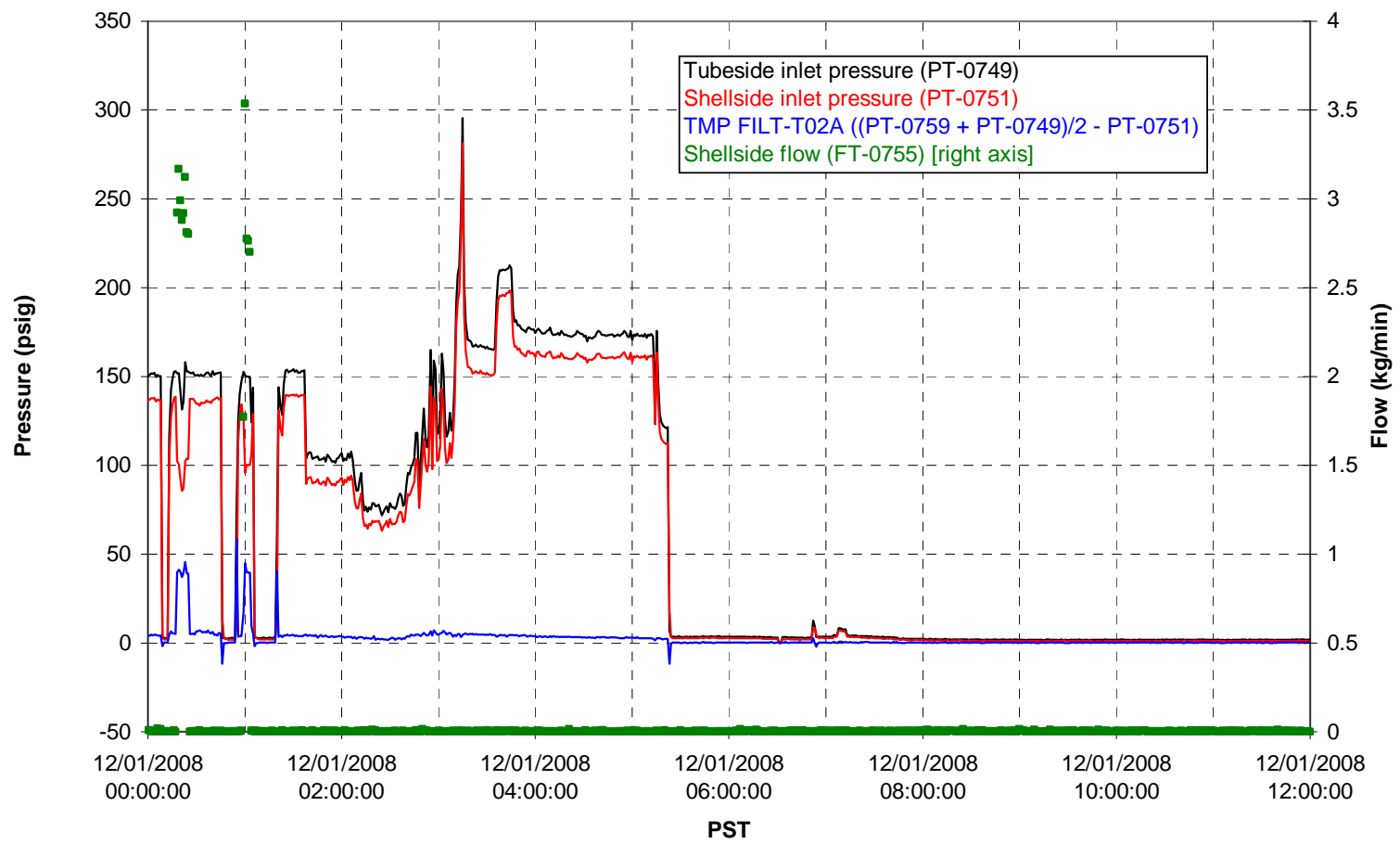


Filter UFP-FILT-T03A

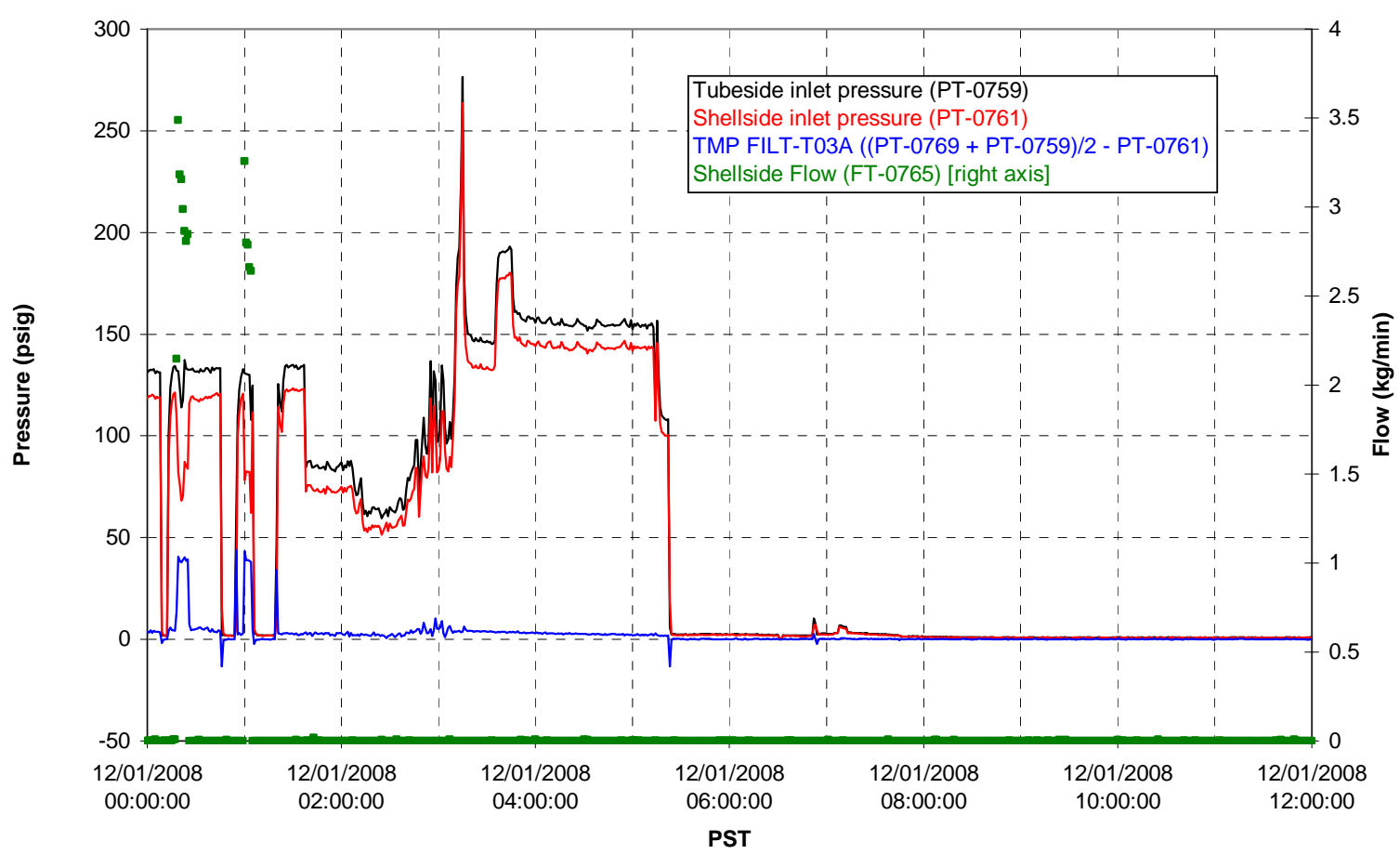

Filter UFP-FILT-T04A

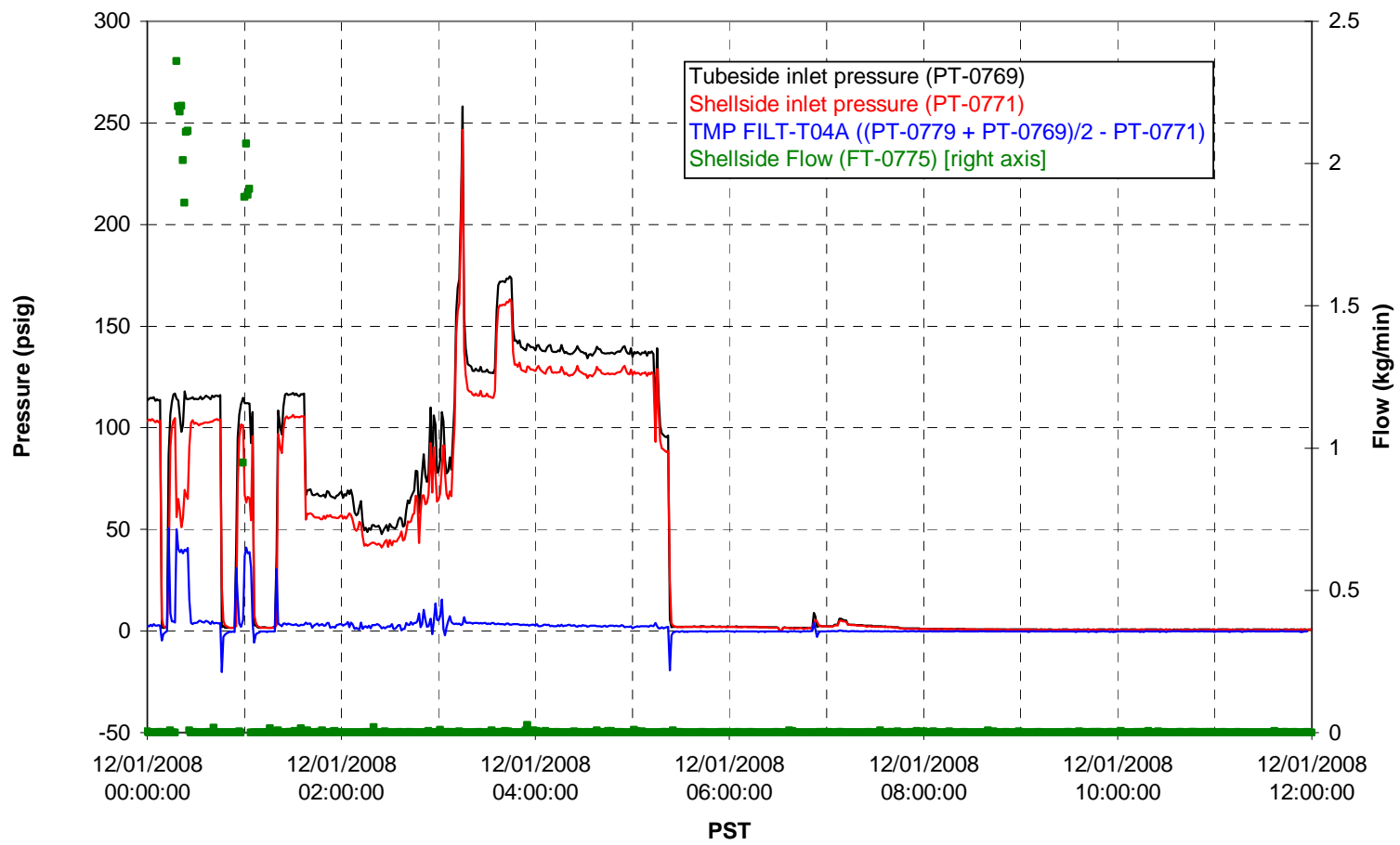


Filter UFP-FILT-T05A

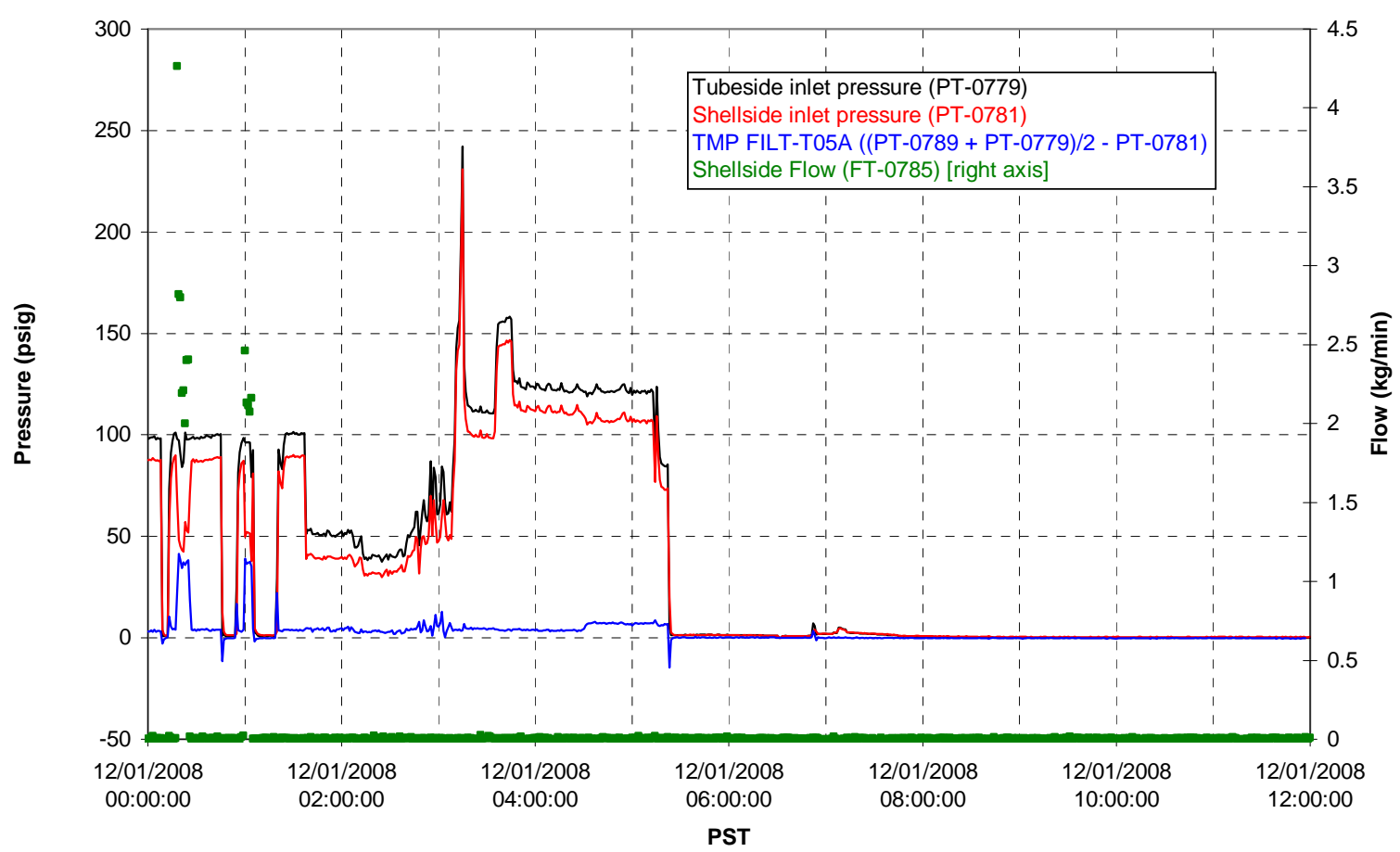

Chemical Flow

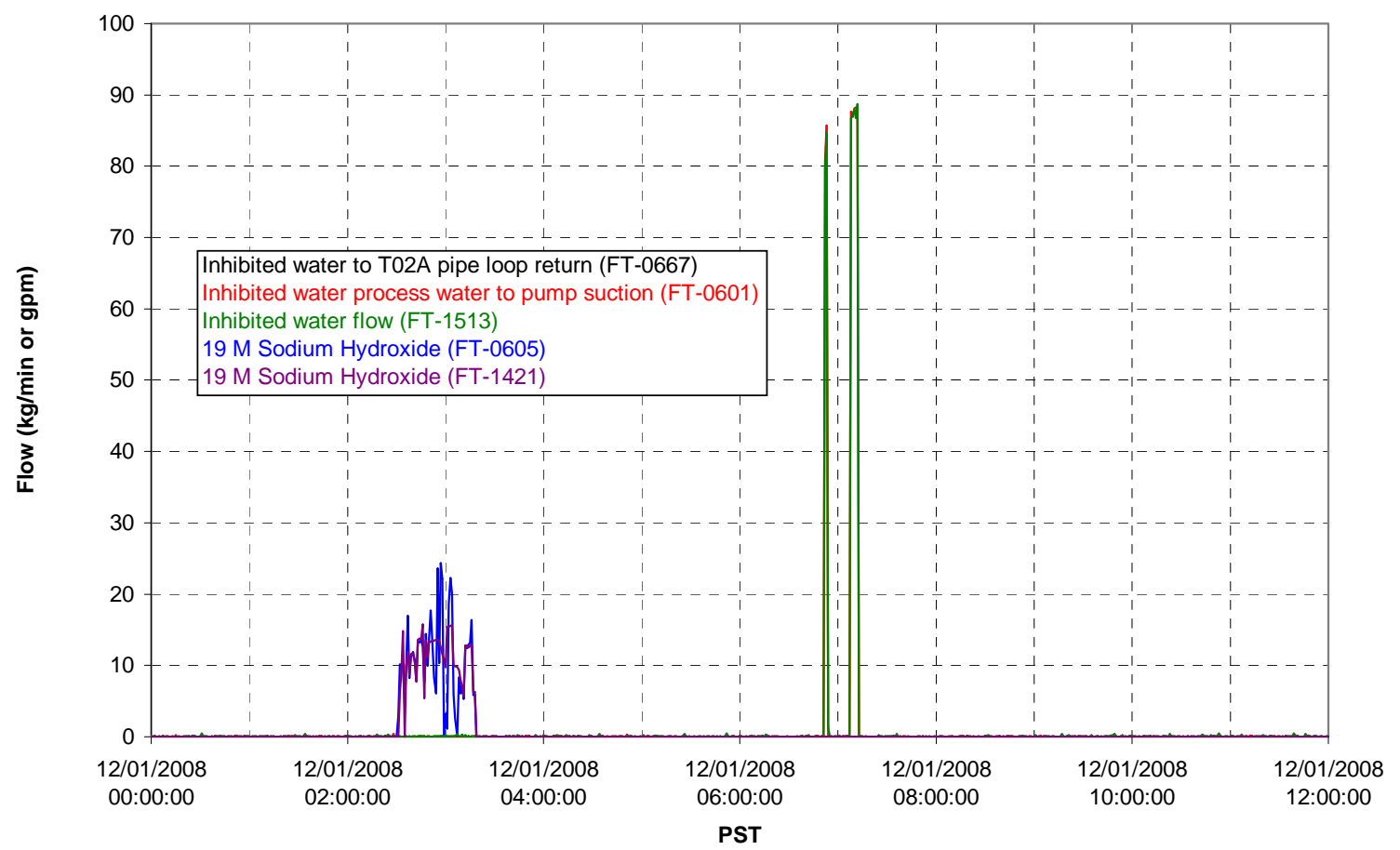


Chemical Flow

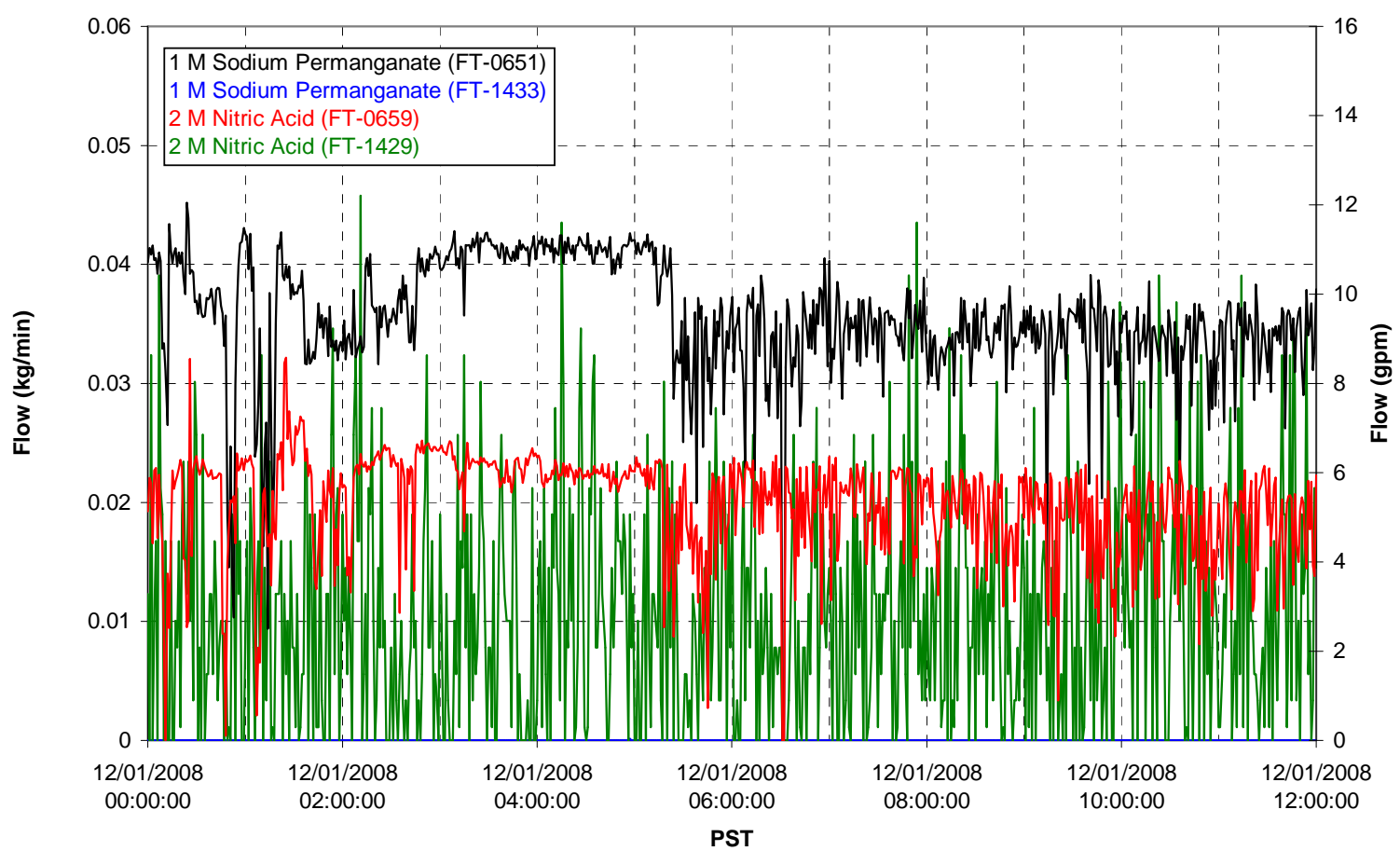

Air Flows

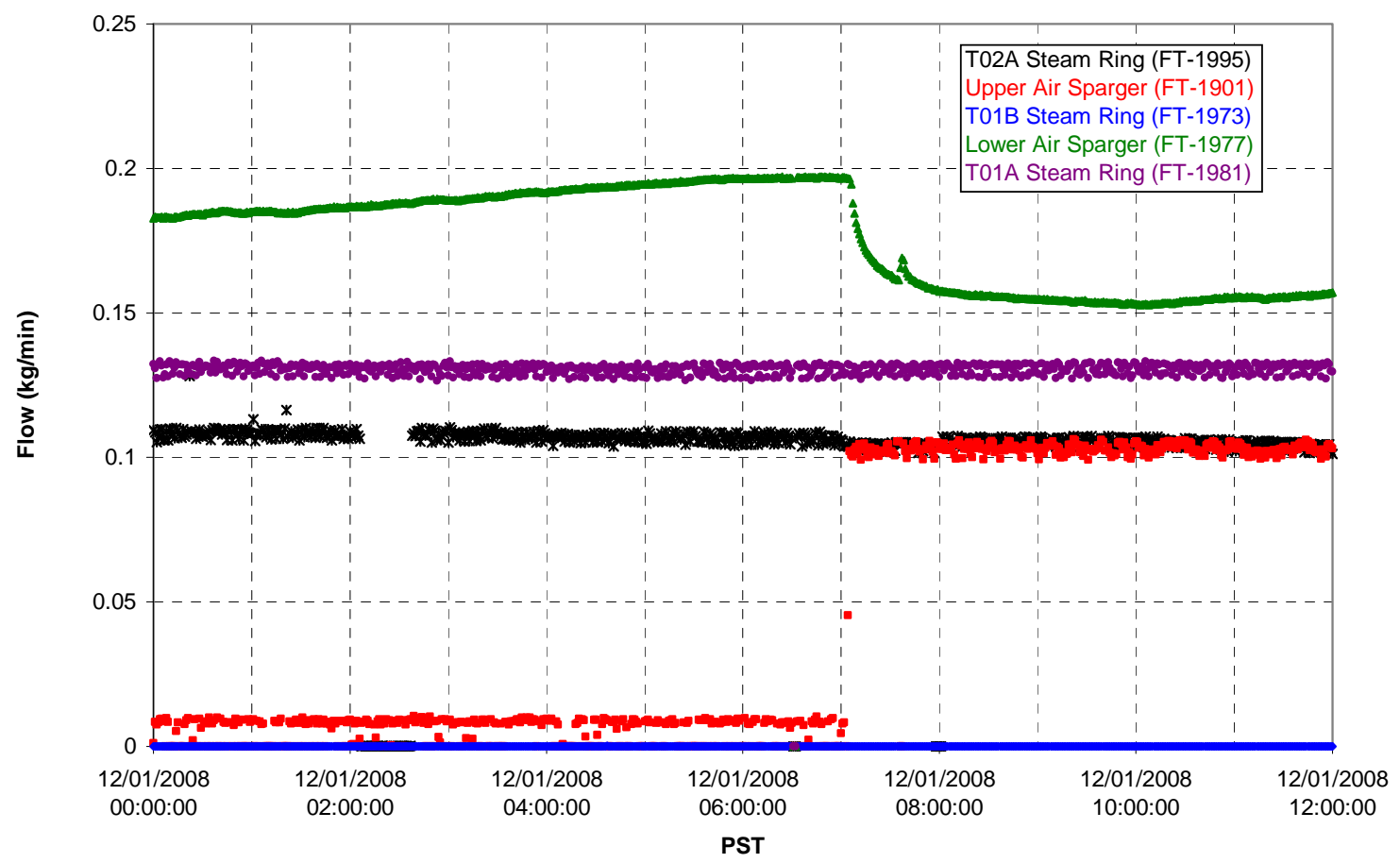


T02A Steam

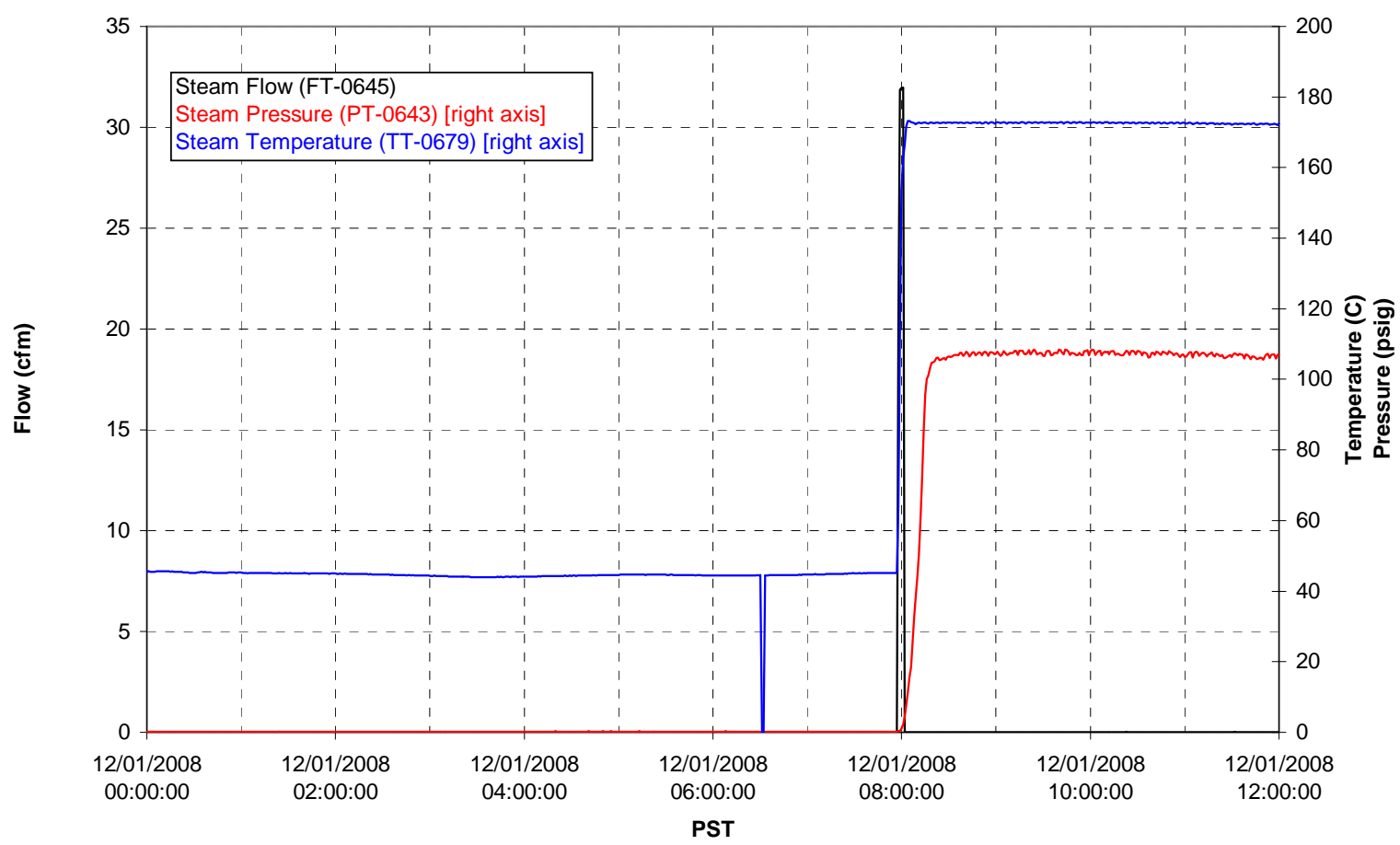

T01A Steam

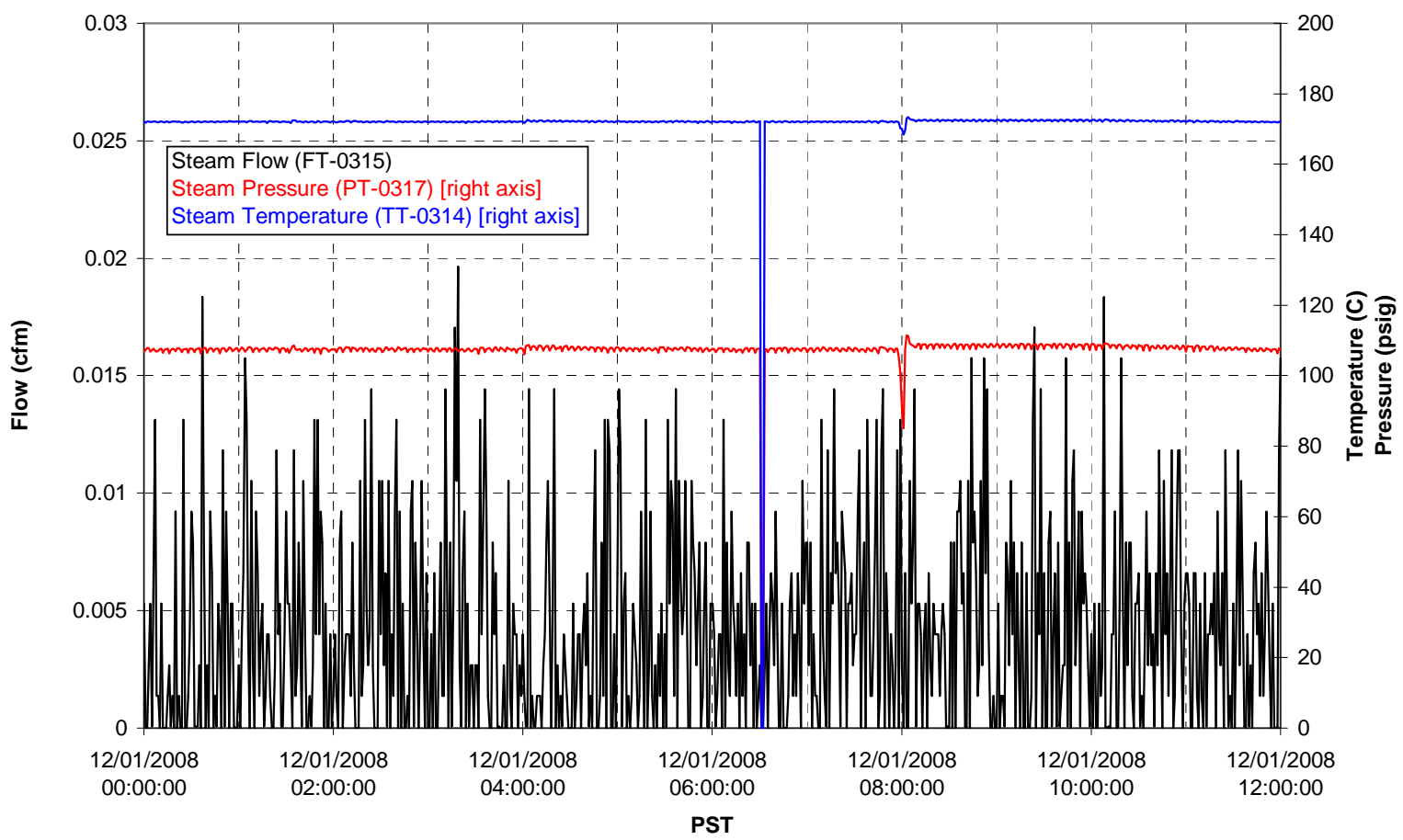


T01B Steam

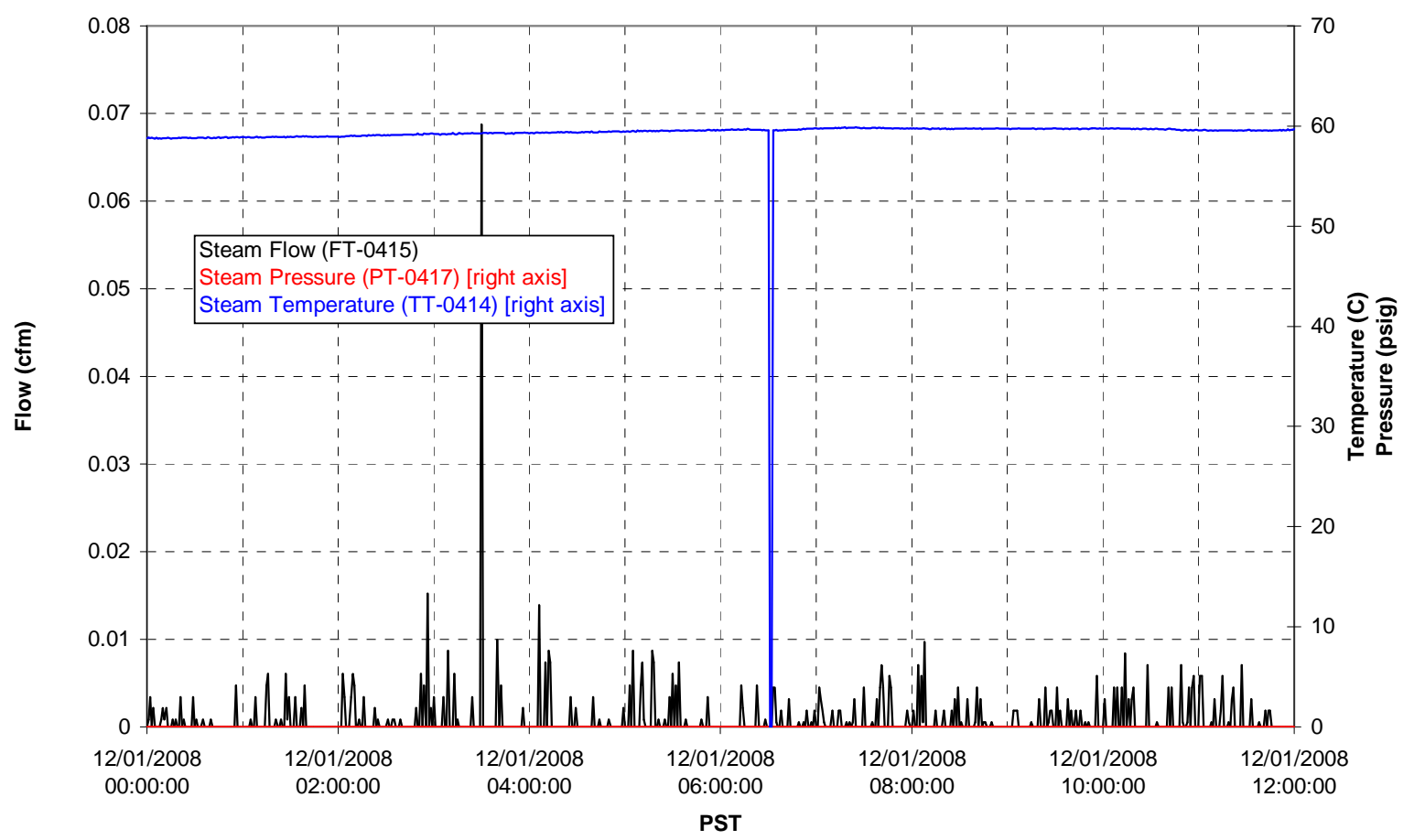


Functional Test Data Plots

12/09/2008 00:00 - 12/09/2008 12:00 
T01A level

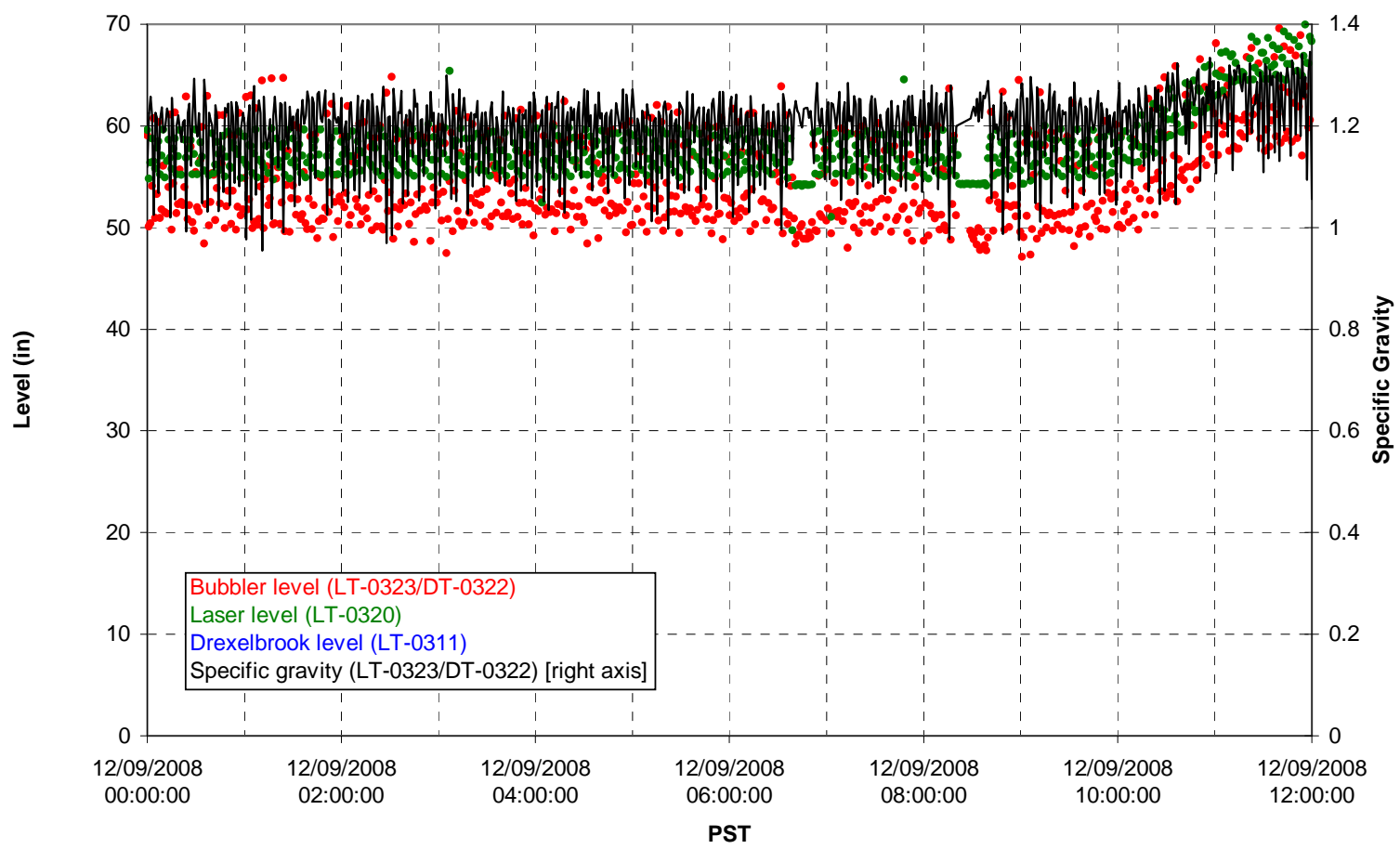

T01A temperatures

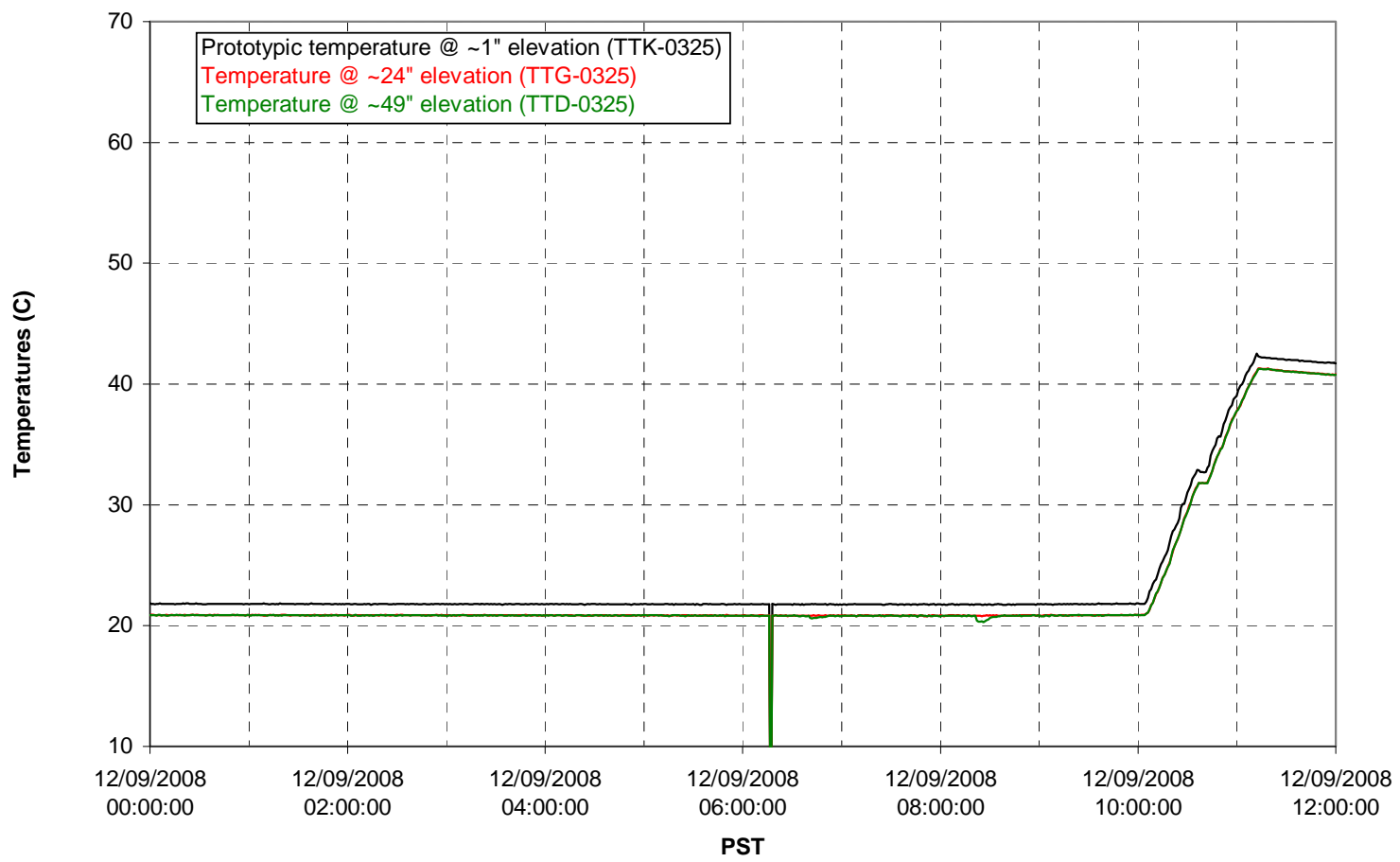


T01B level

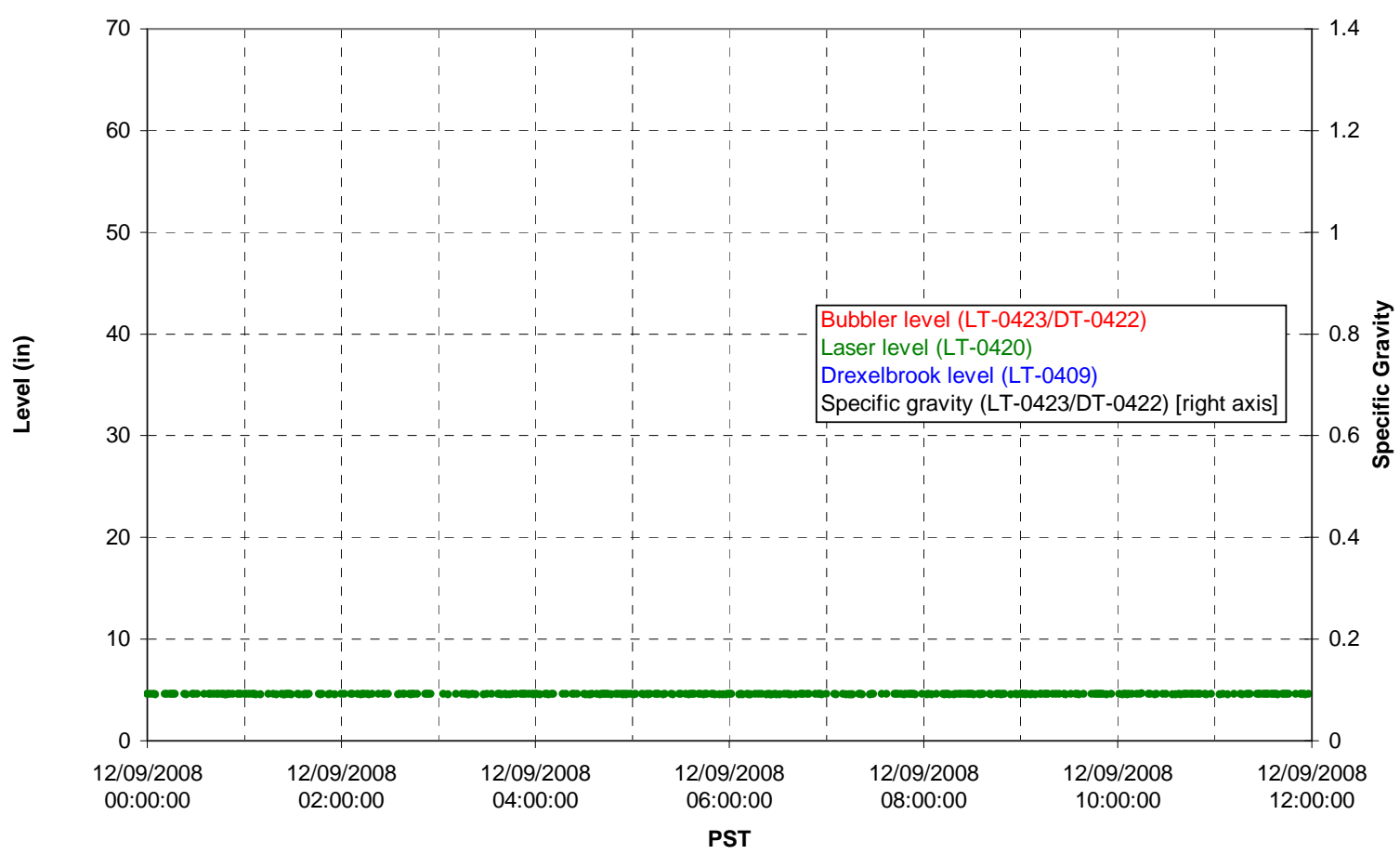

T01B temperatures

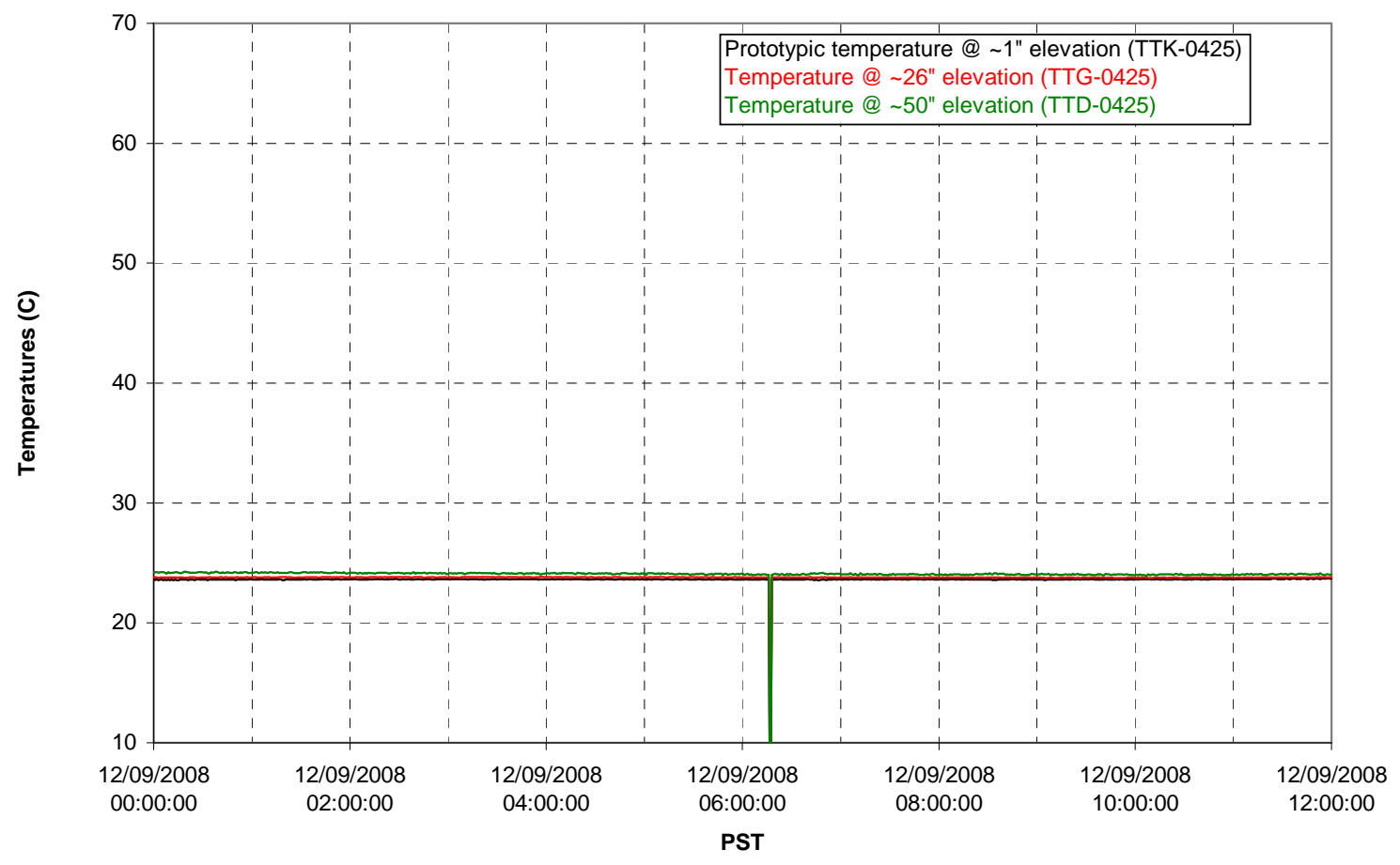




\section{T02A level}

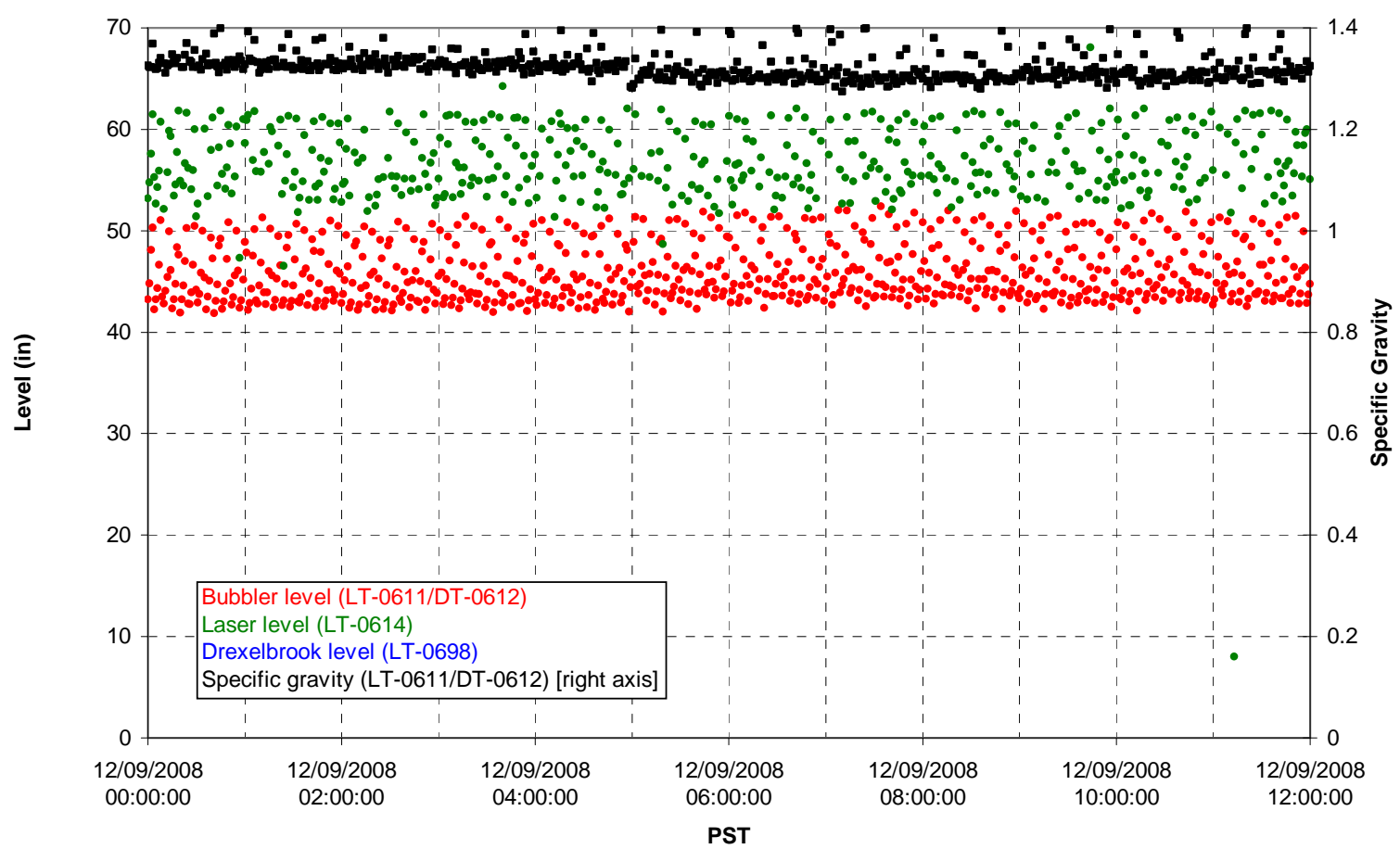

T02A temperatures

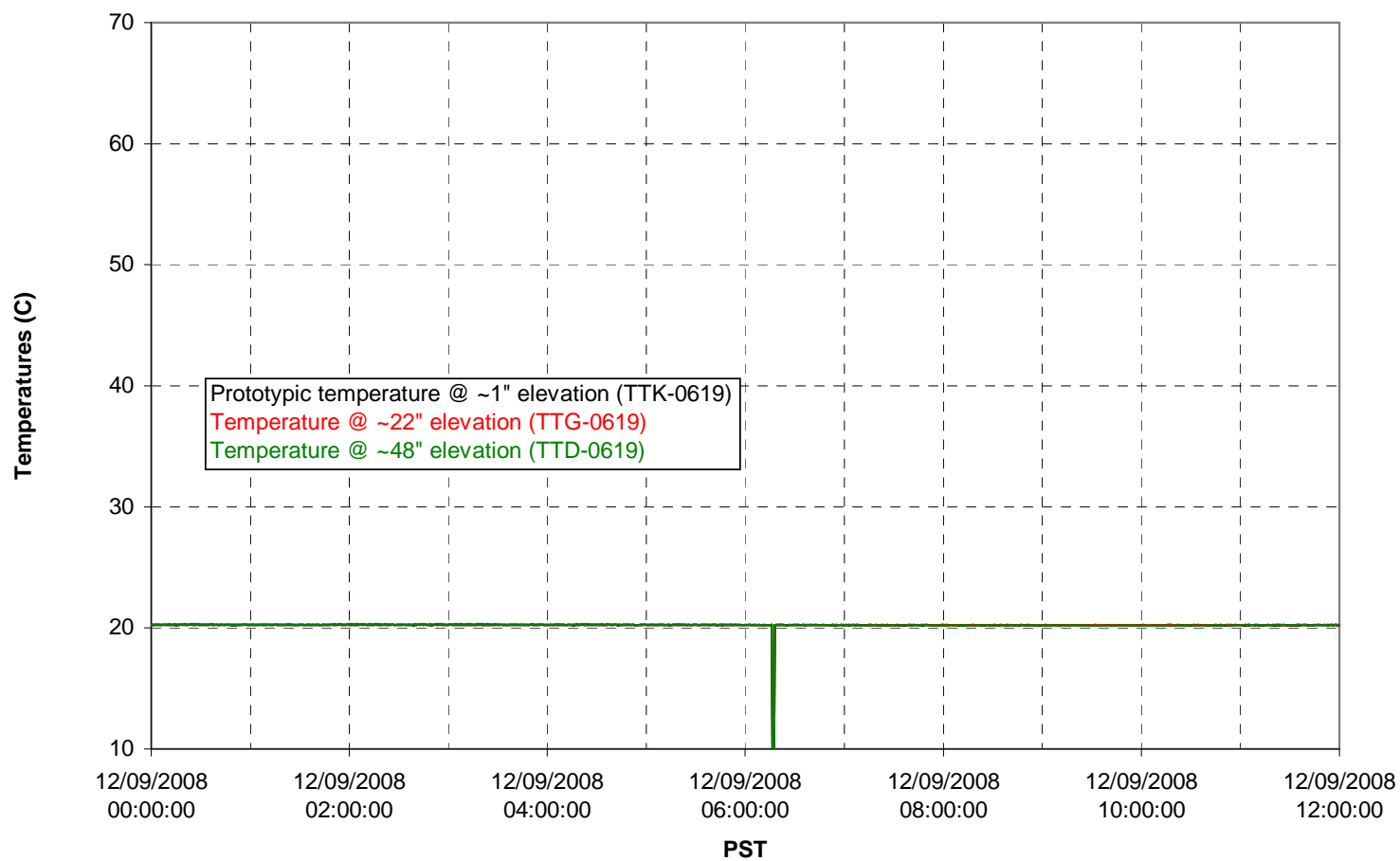


T02A and filter loop temperatures

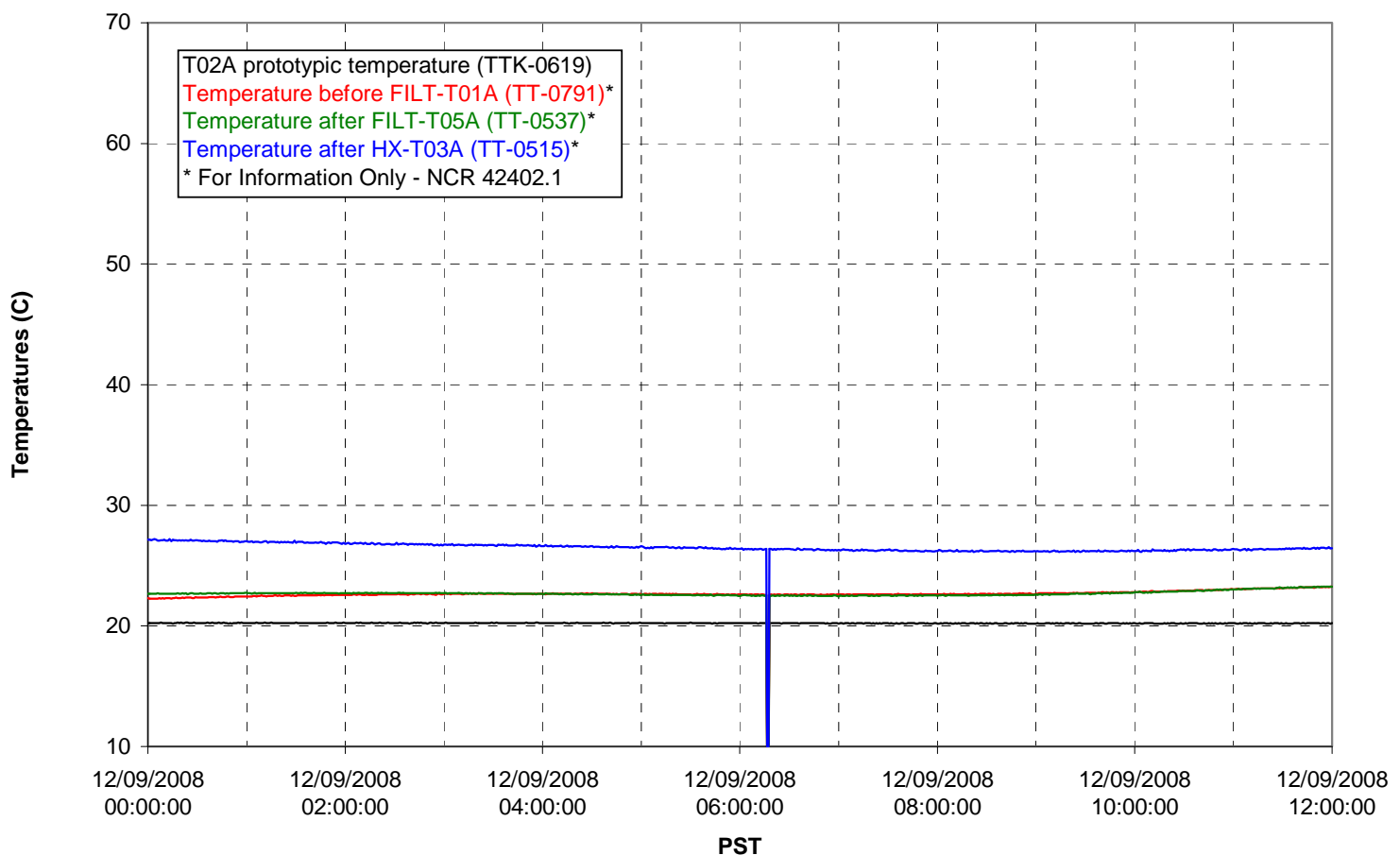

Pump Pressures and Flow

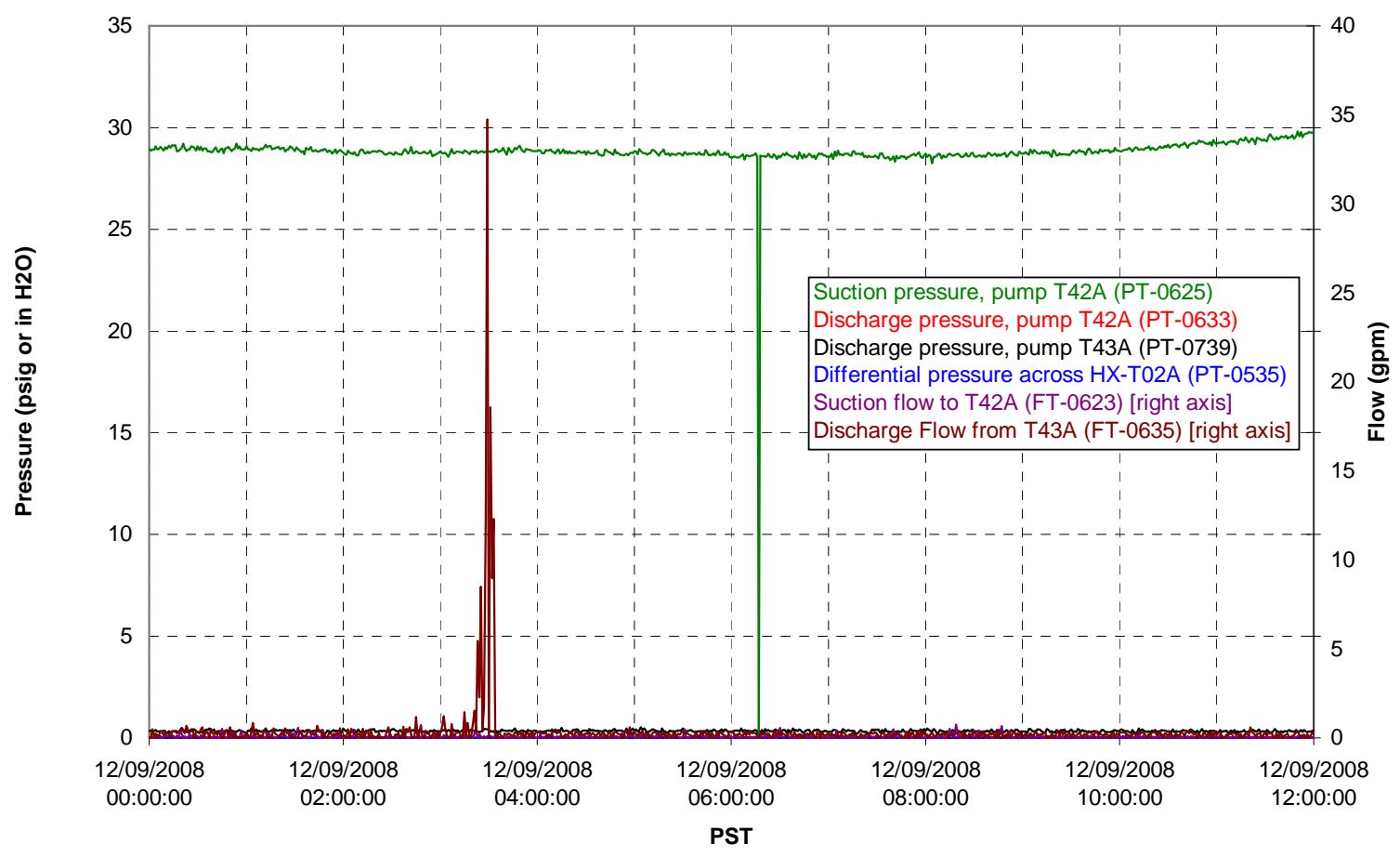


Axial pressure drop

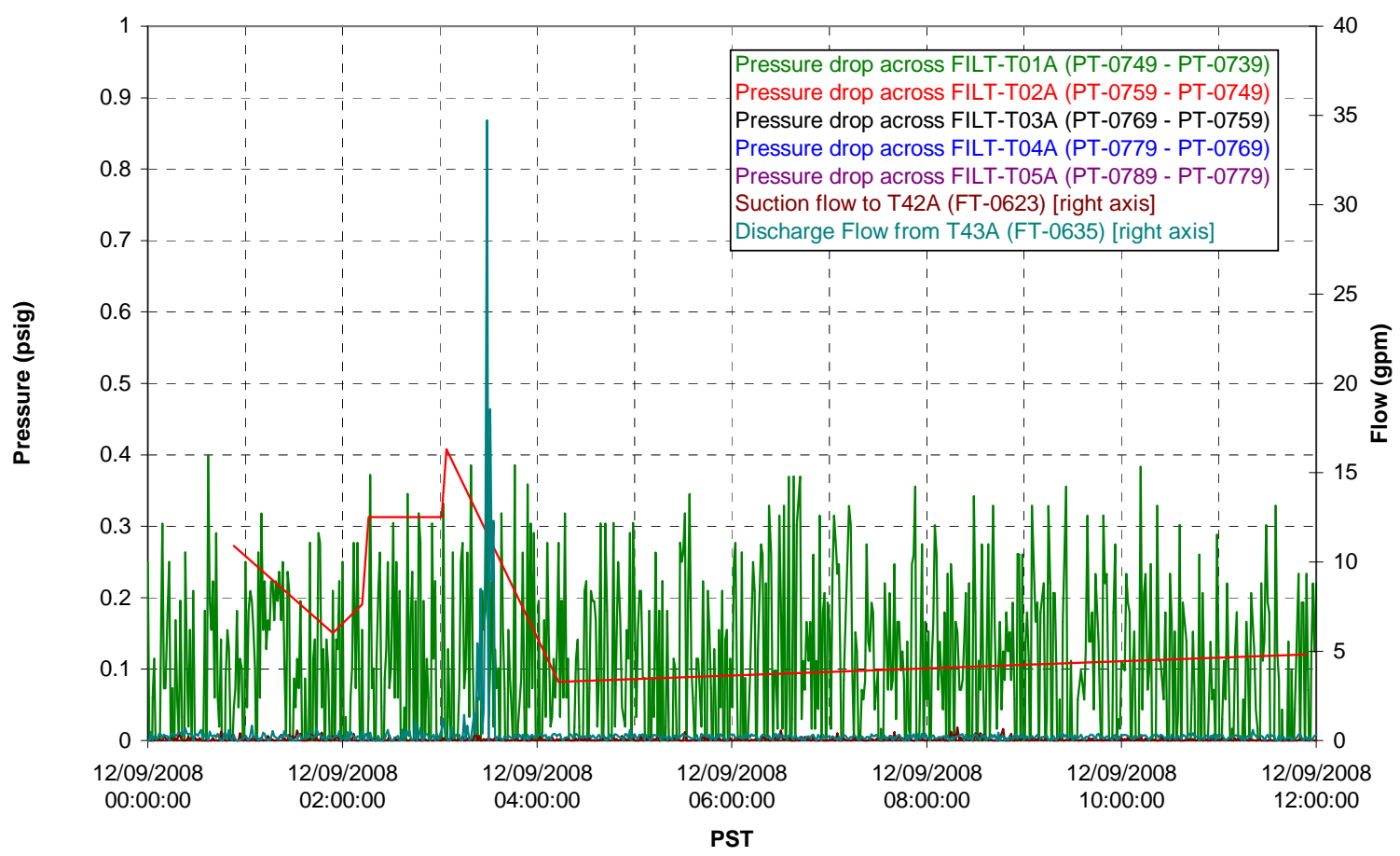

Permeate flow rates

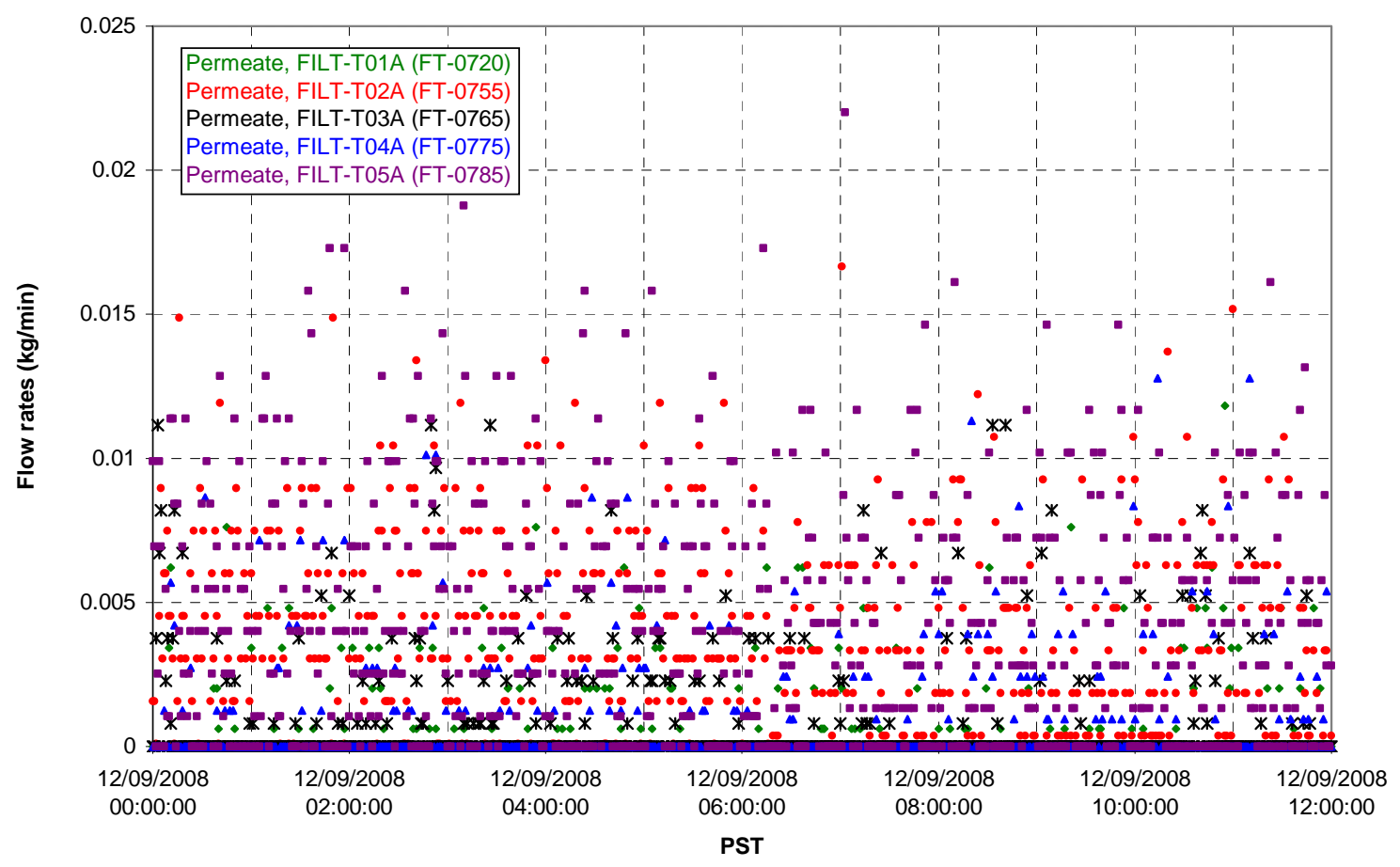


T02A Inner Temperature Tree

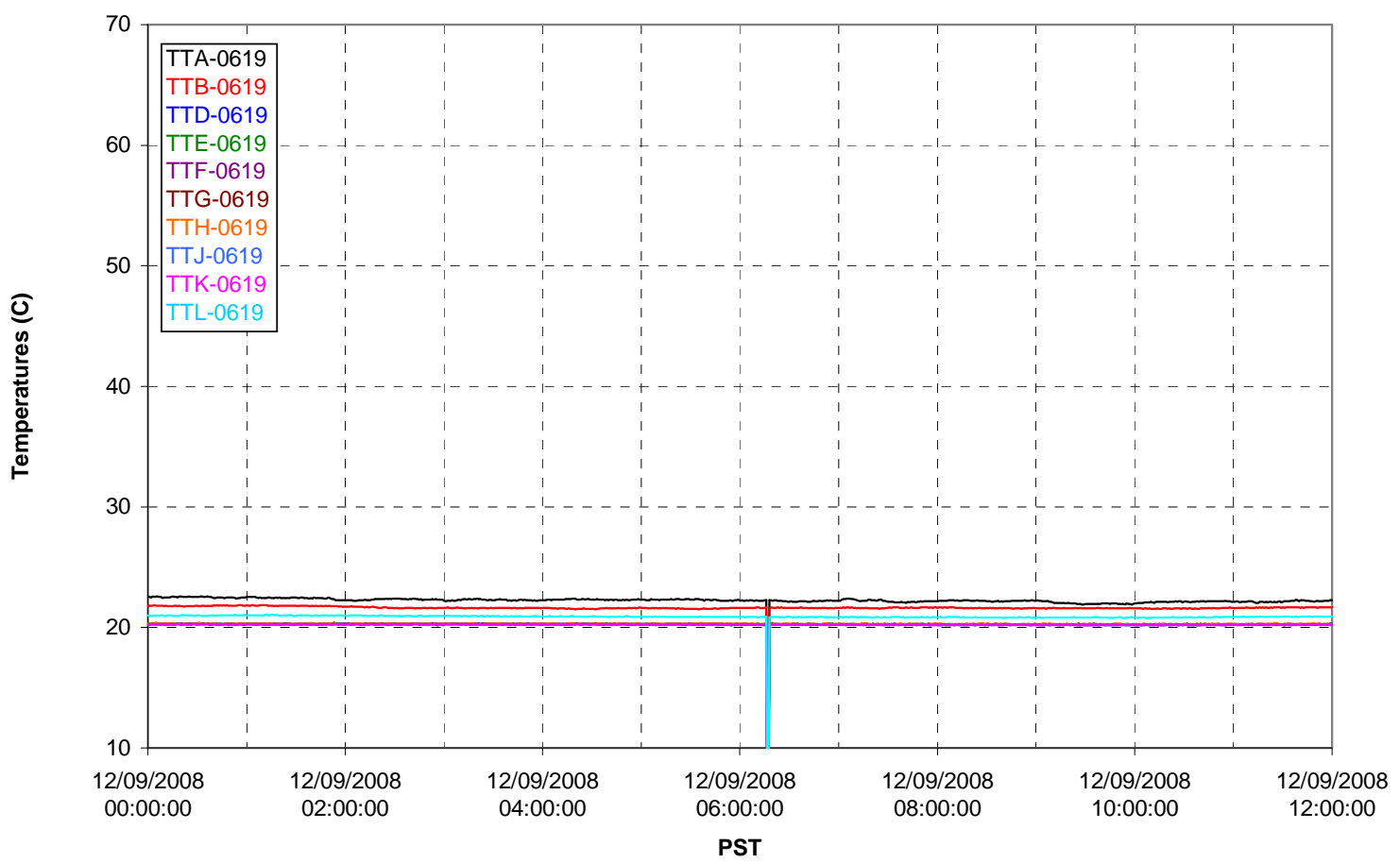

T02A Outer Temperature Tree

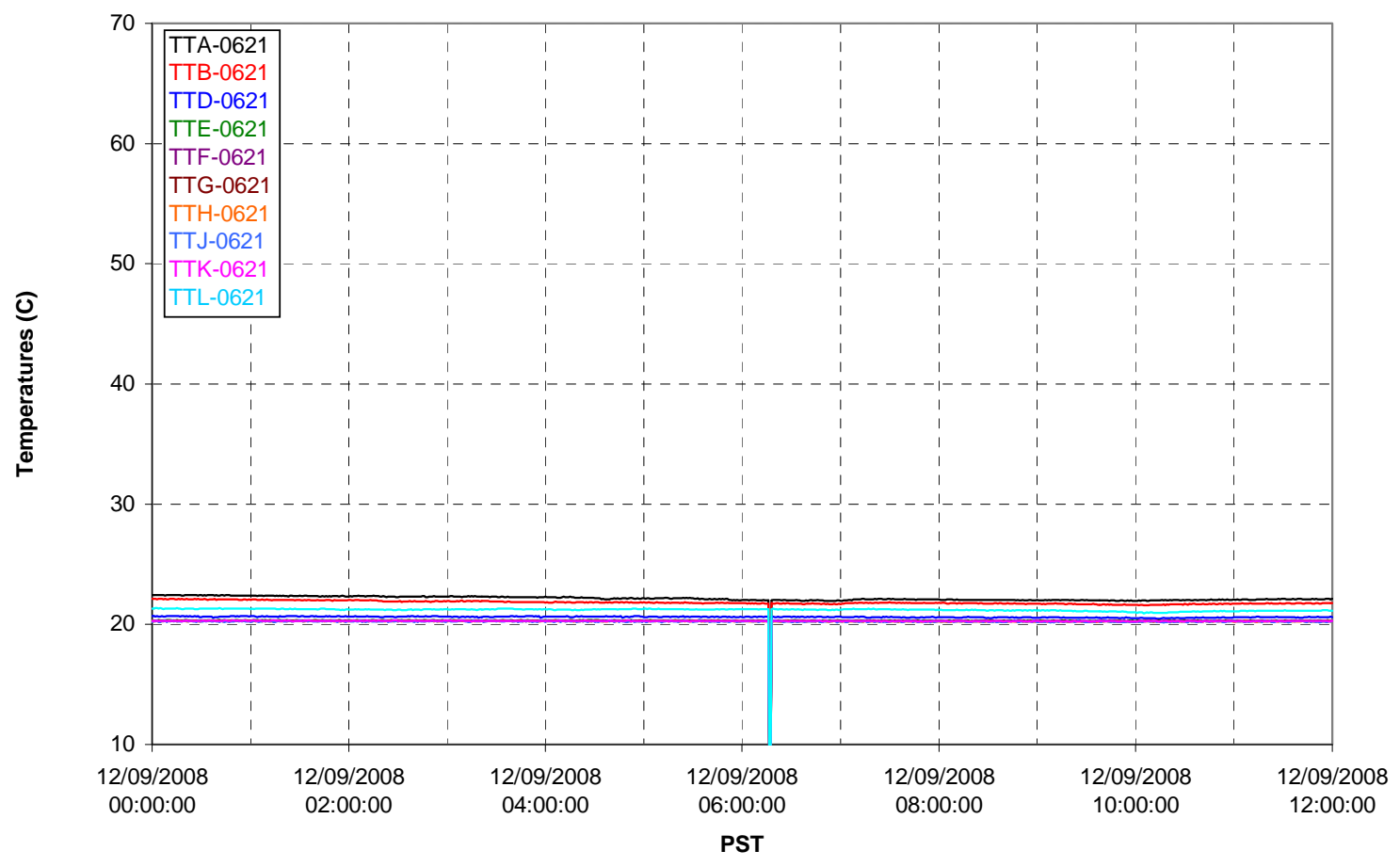


T02A temperatures

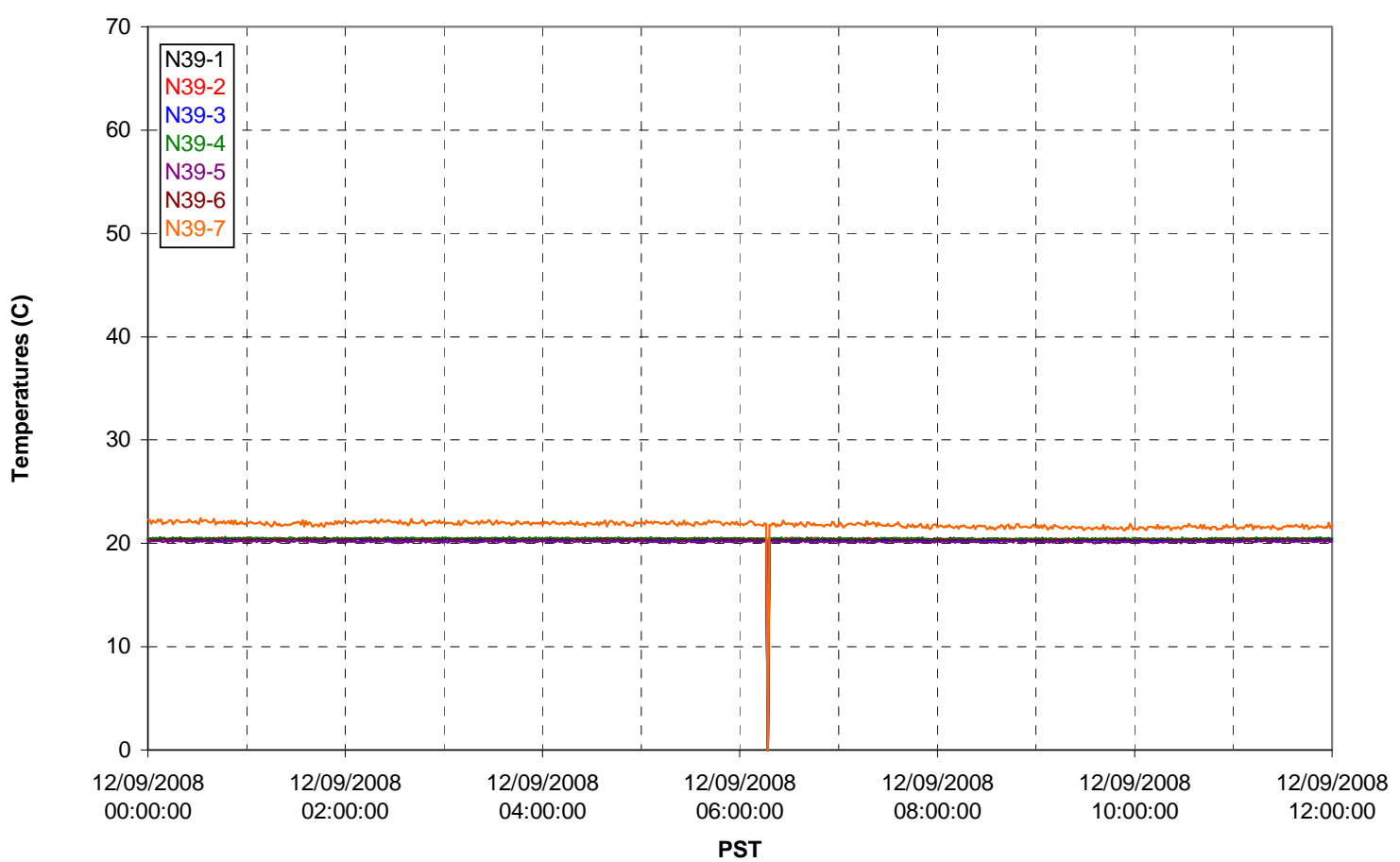

T02A temperatures

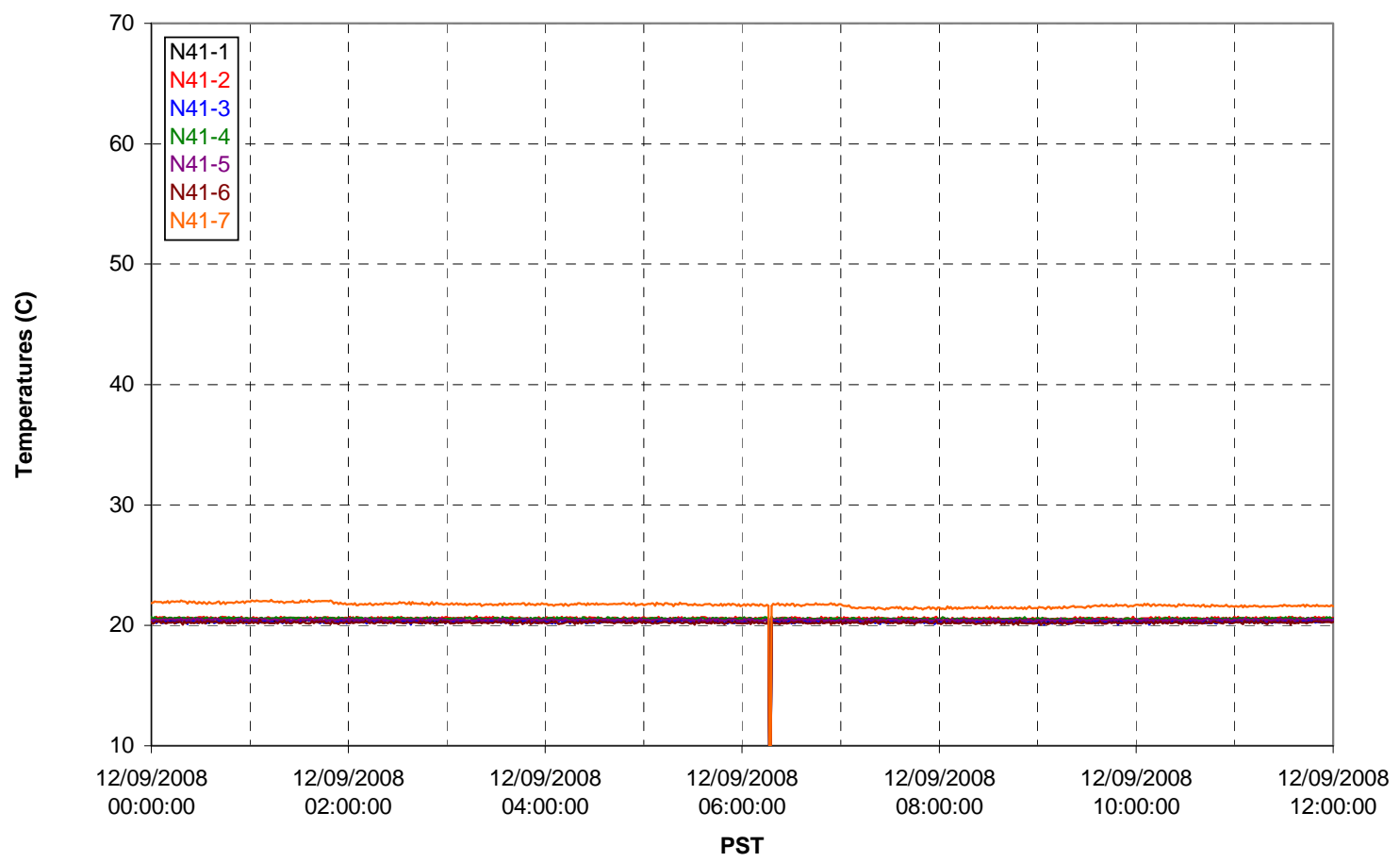


T02A temperatures

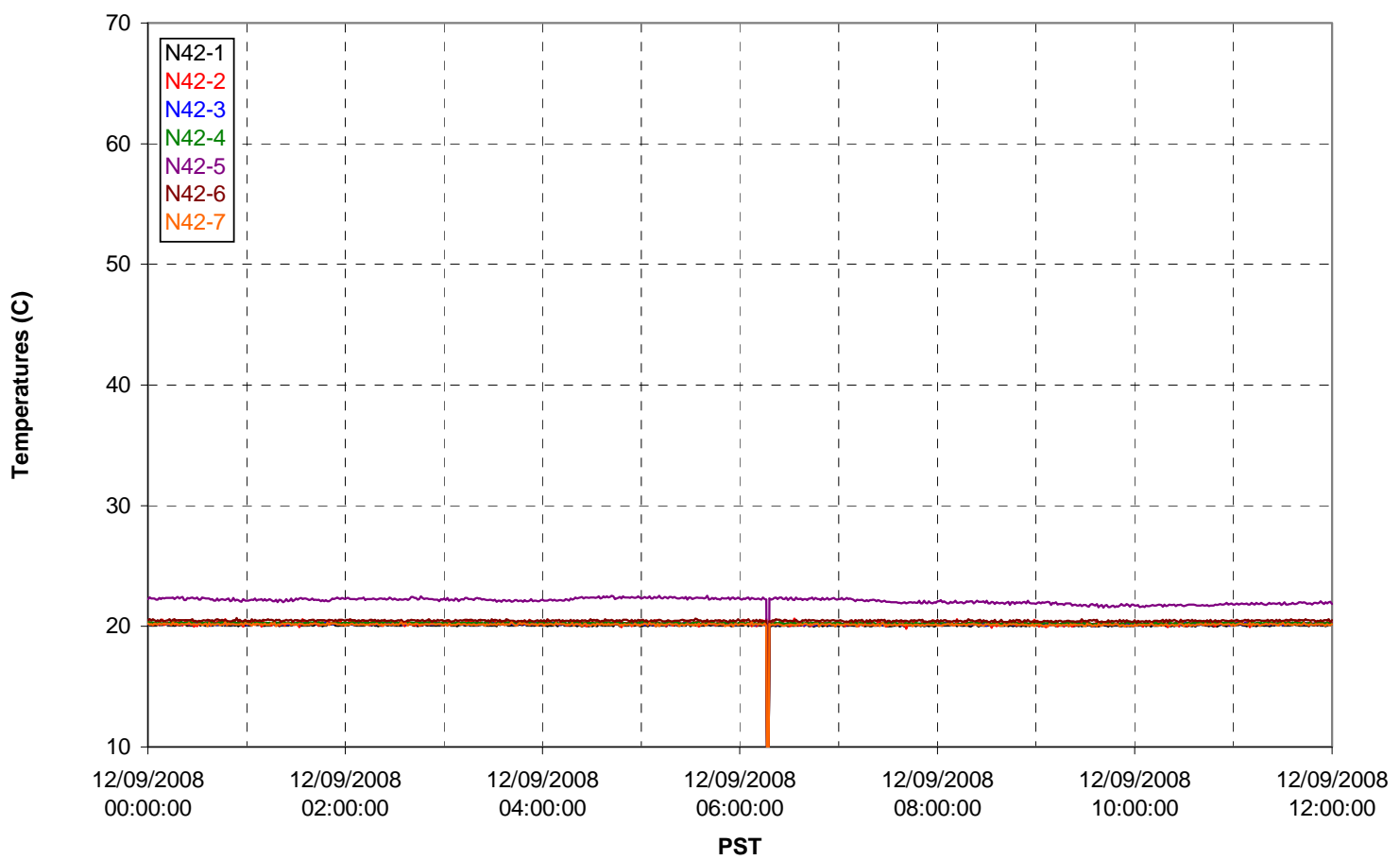

T02A temperatures

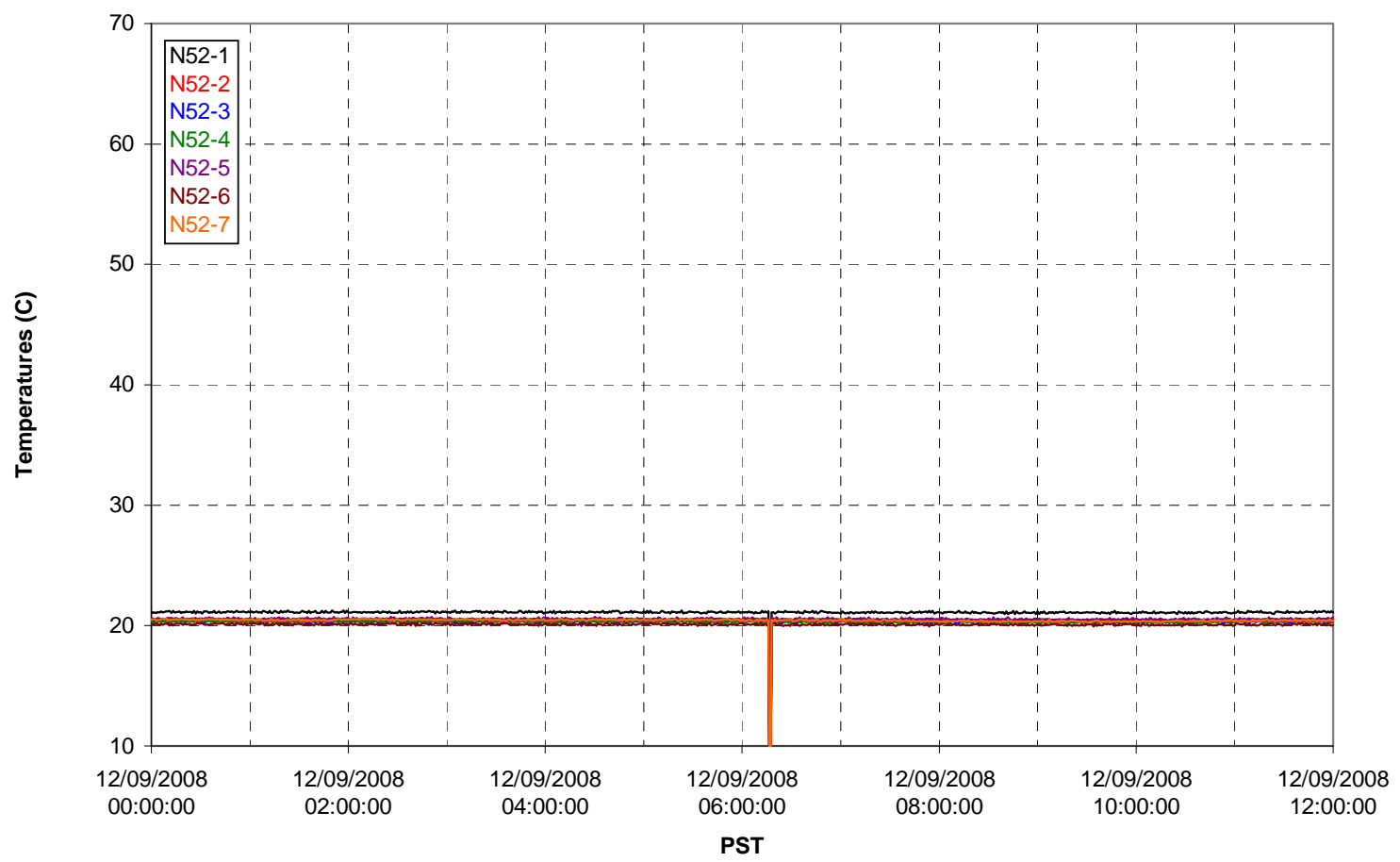


T02A Heating and Cooling

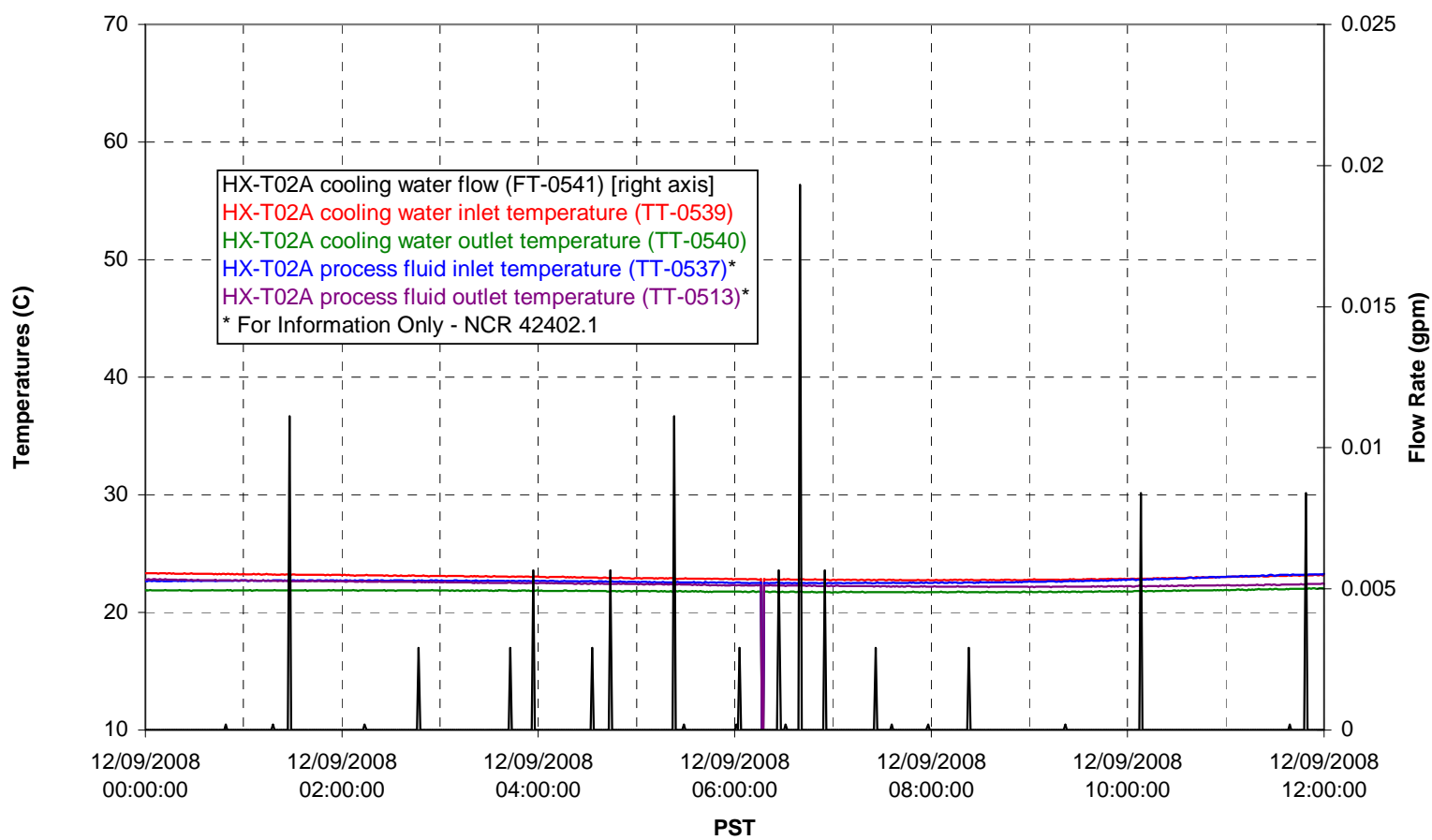

Pump Operation

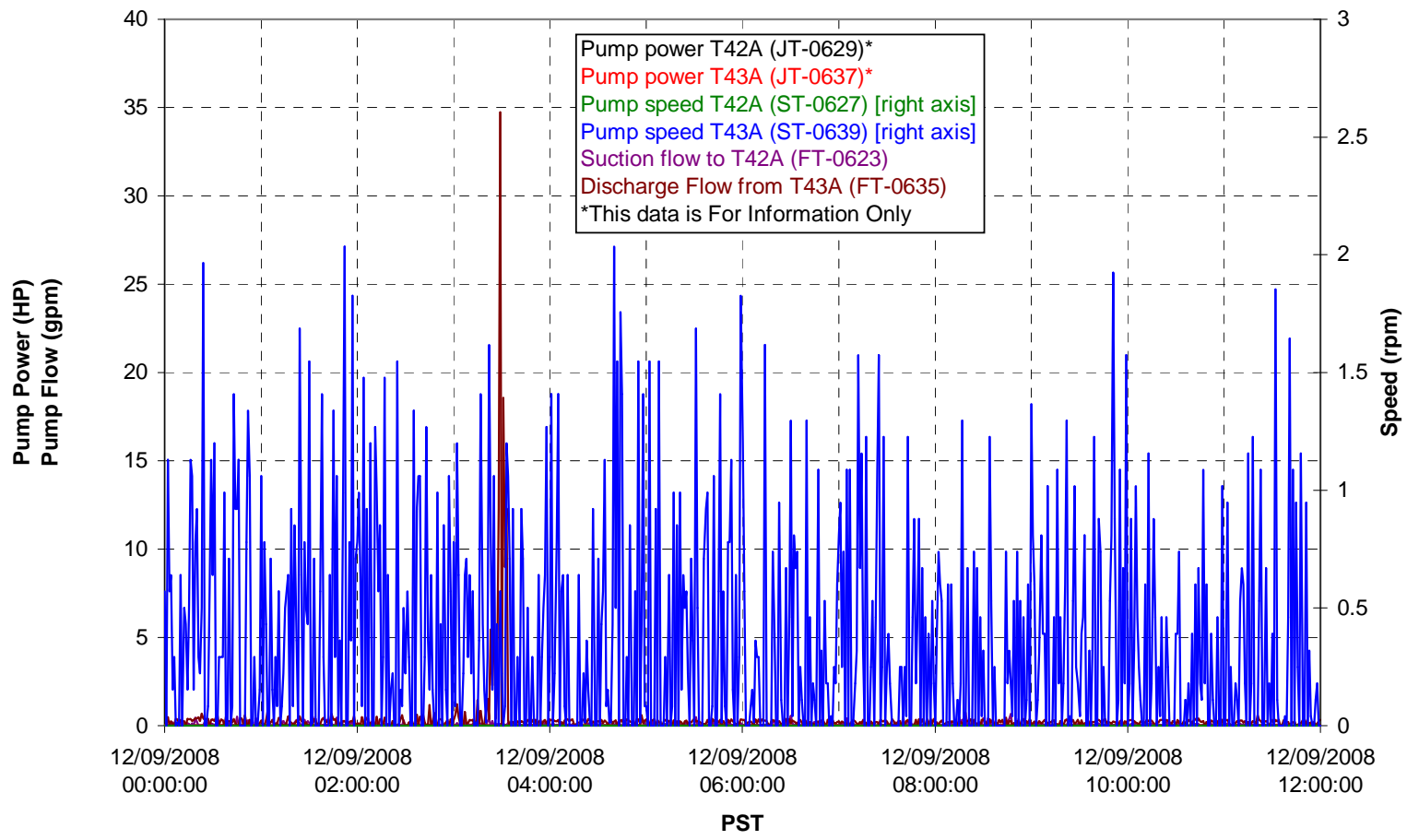


Pulsepot UFP-PP-T01A

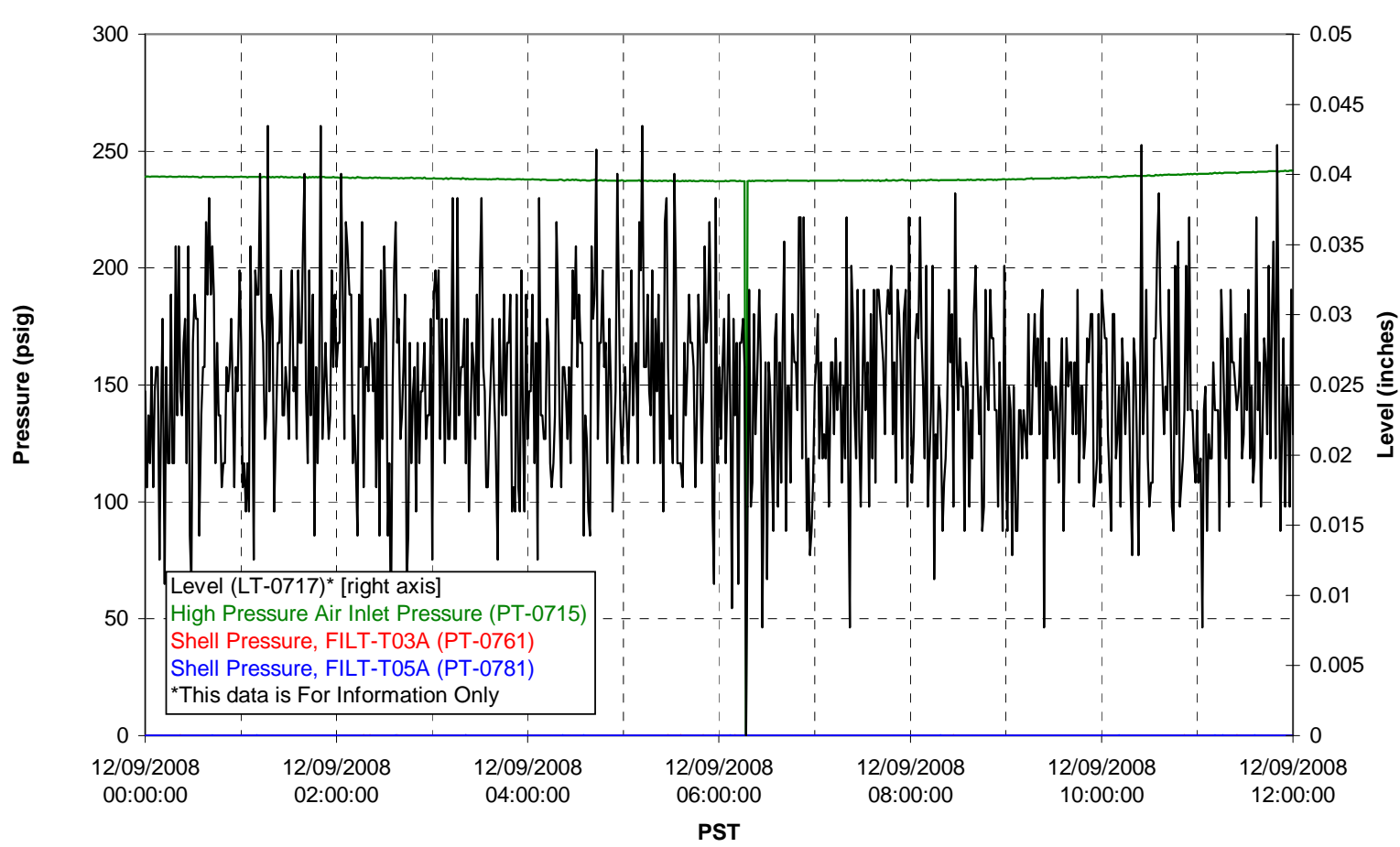

Pulsepot UFP-PP-T02A

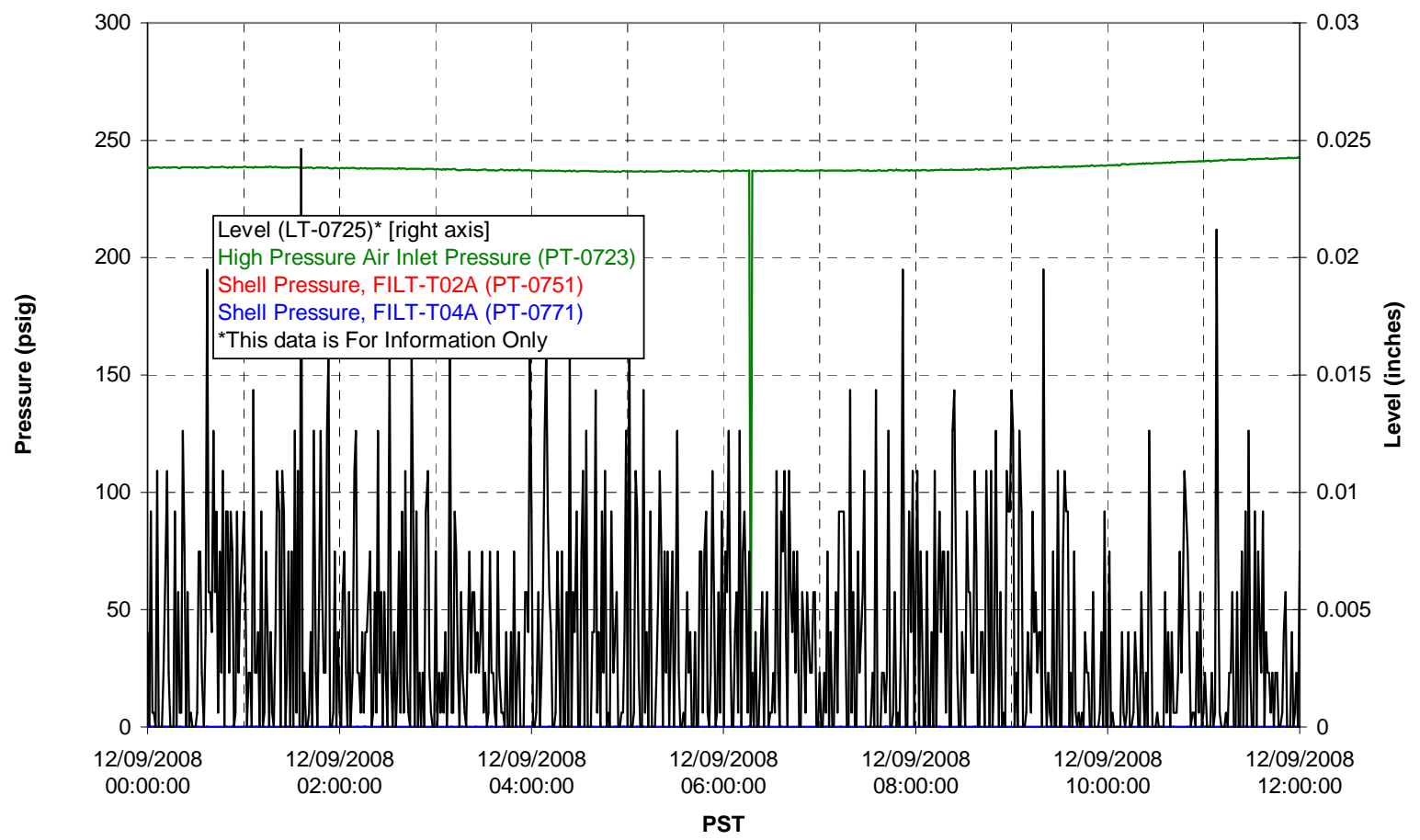


Pulsepot UFP-PP-T03A

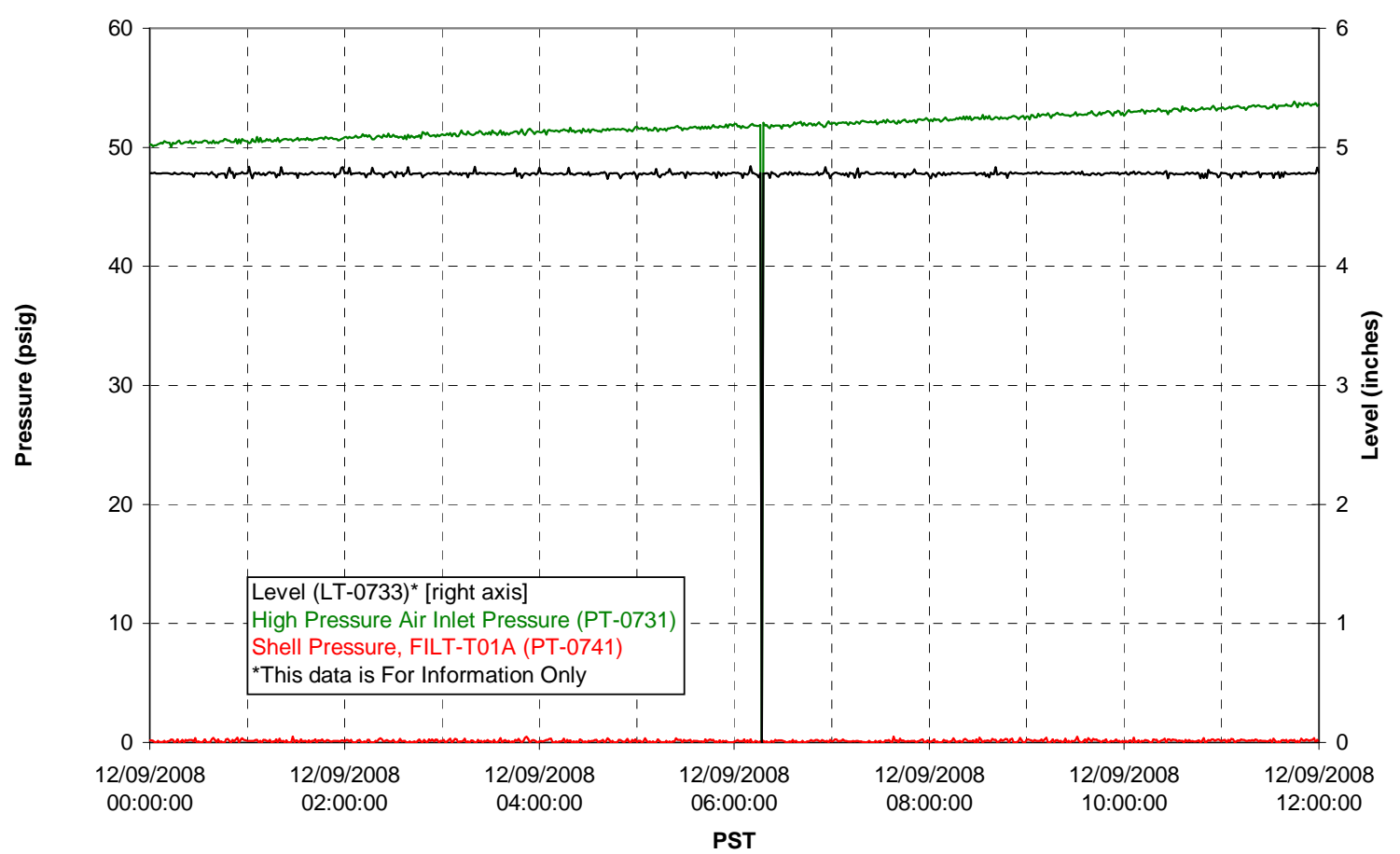

Pulsepot Levels

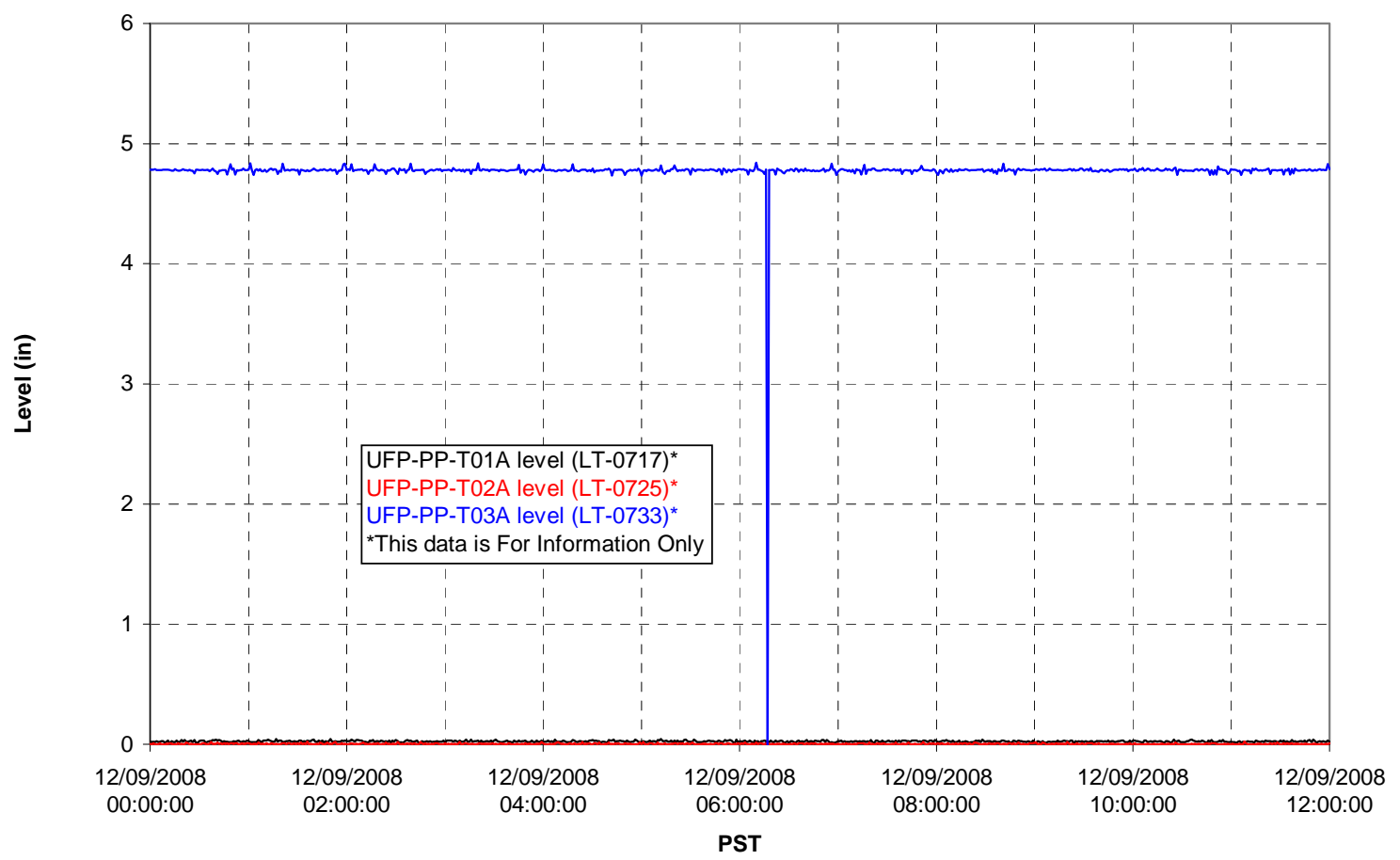


Filter UFP-FILT-T01A

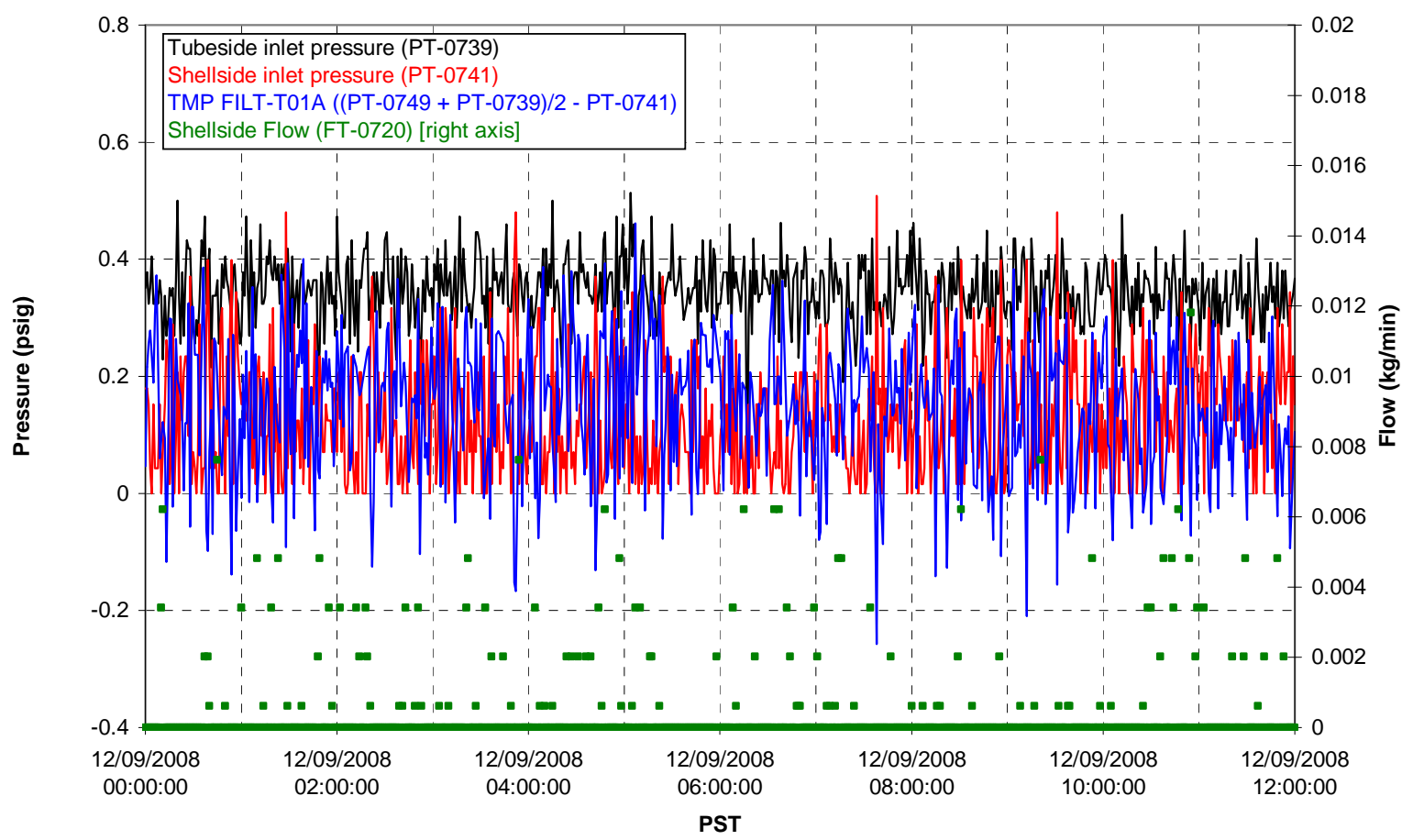

Filter UFP-FILT-T02A

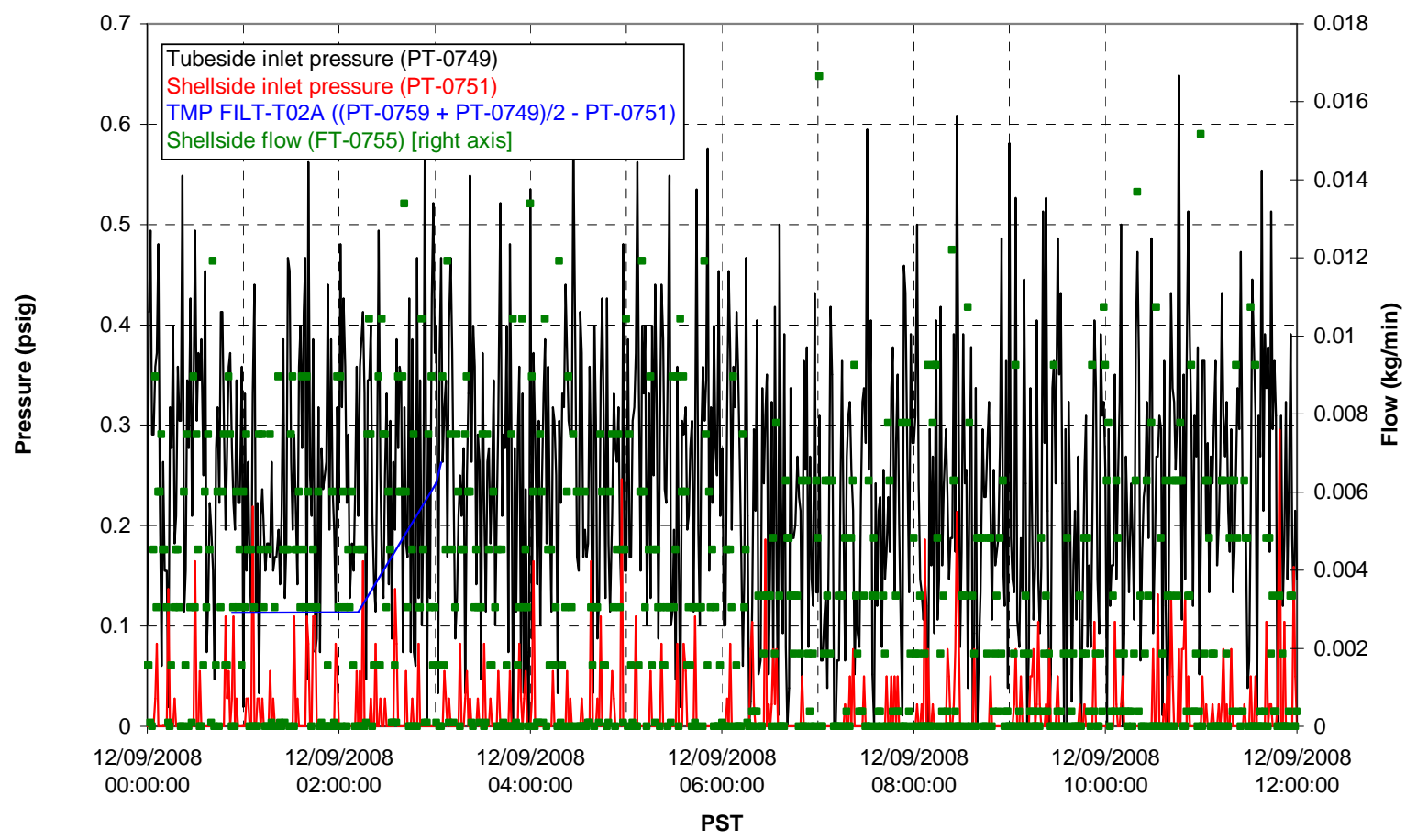


Filter UFP-FILT-T03A

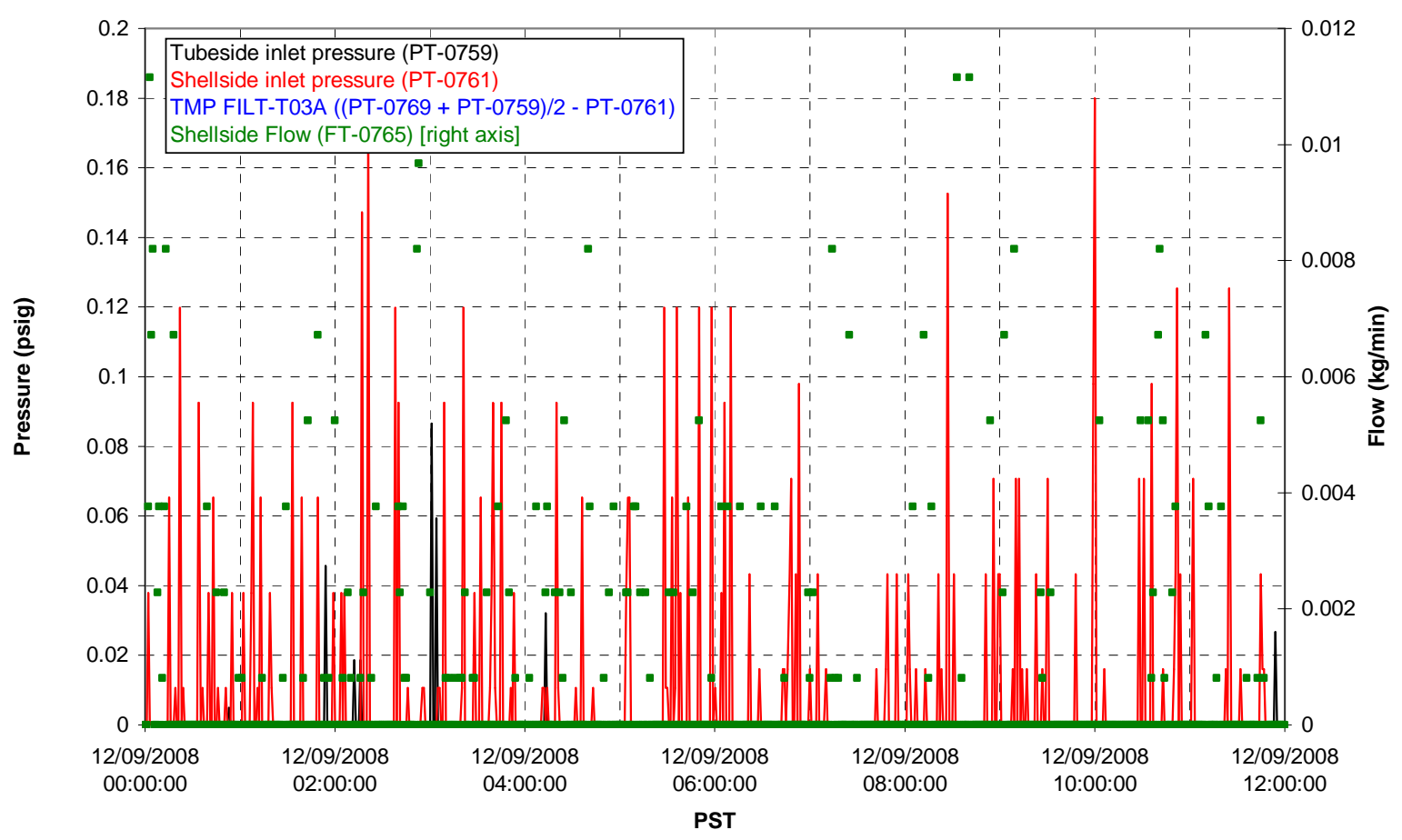

Filter UFP-FILT-T04A

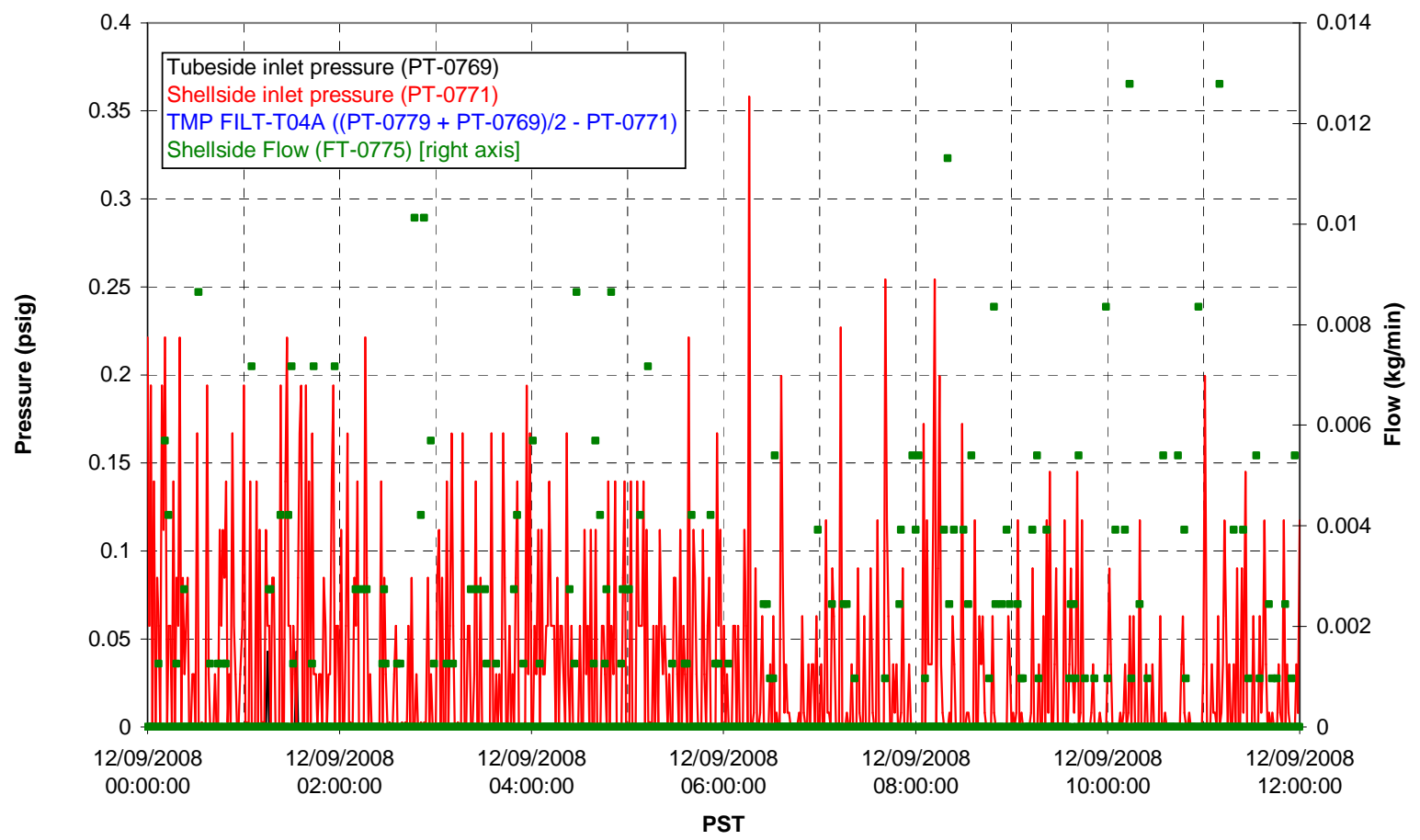


Filter UFP-FILT-T05A

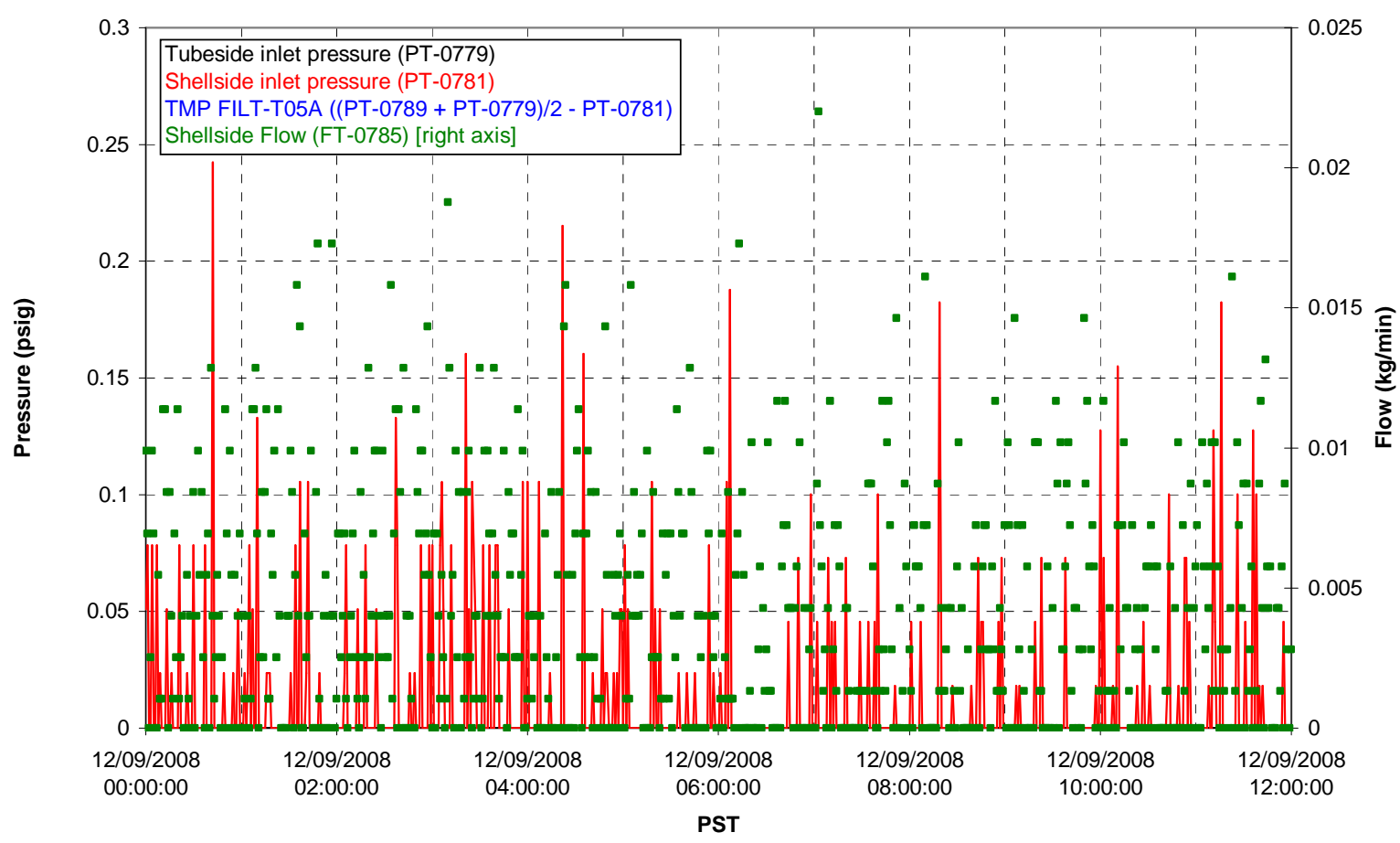

Chemical Flow

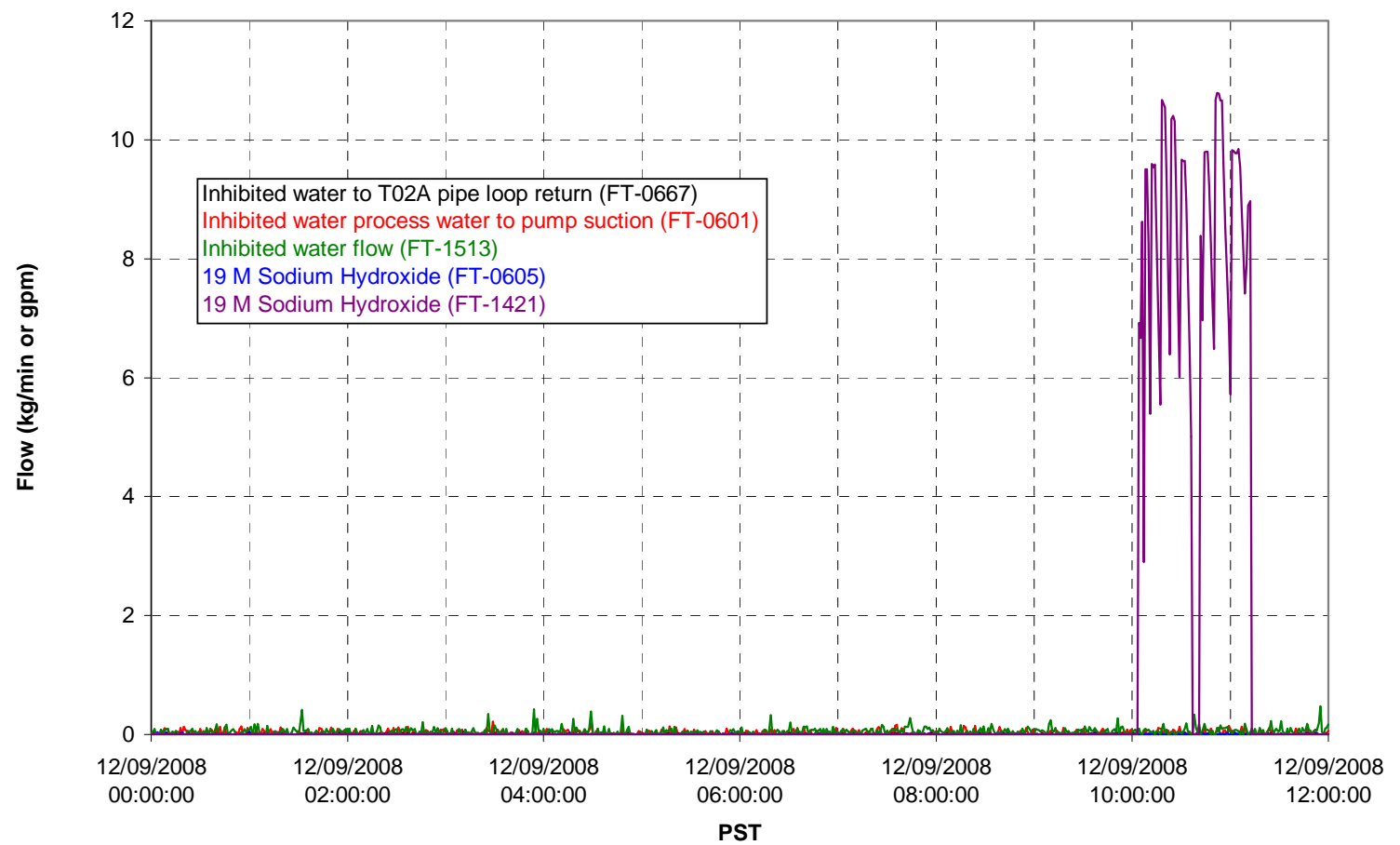


Chemical Flow

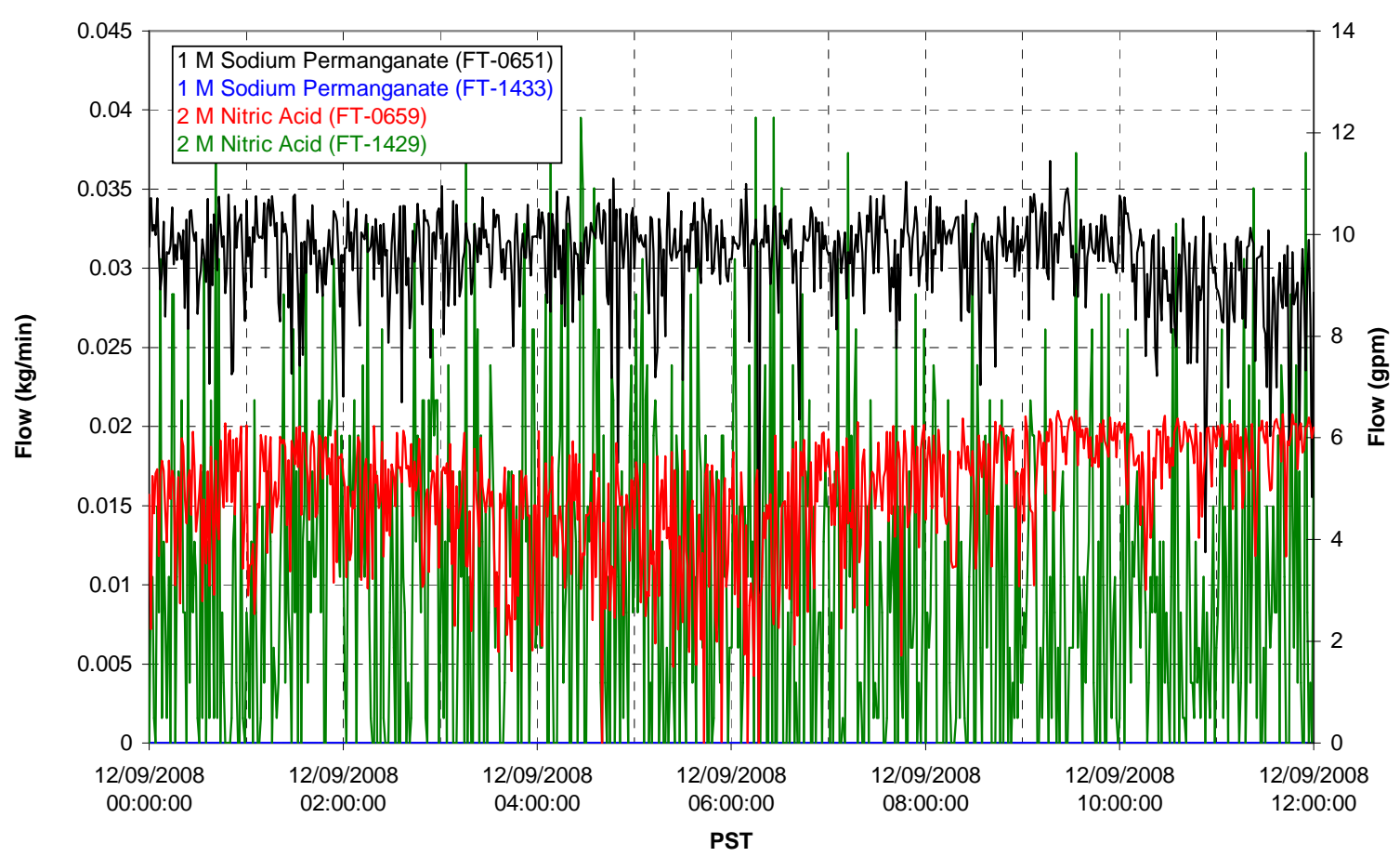

Air Flows

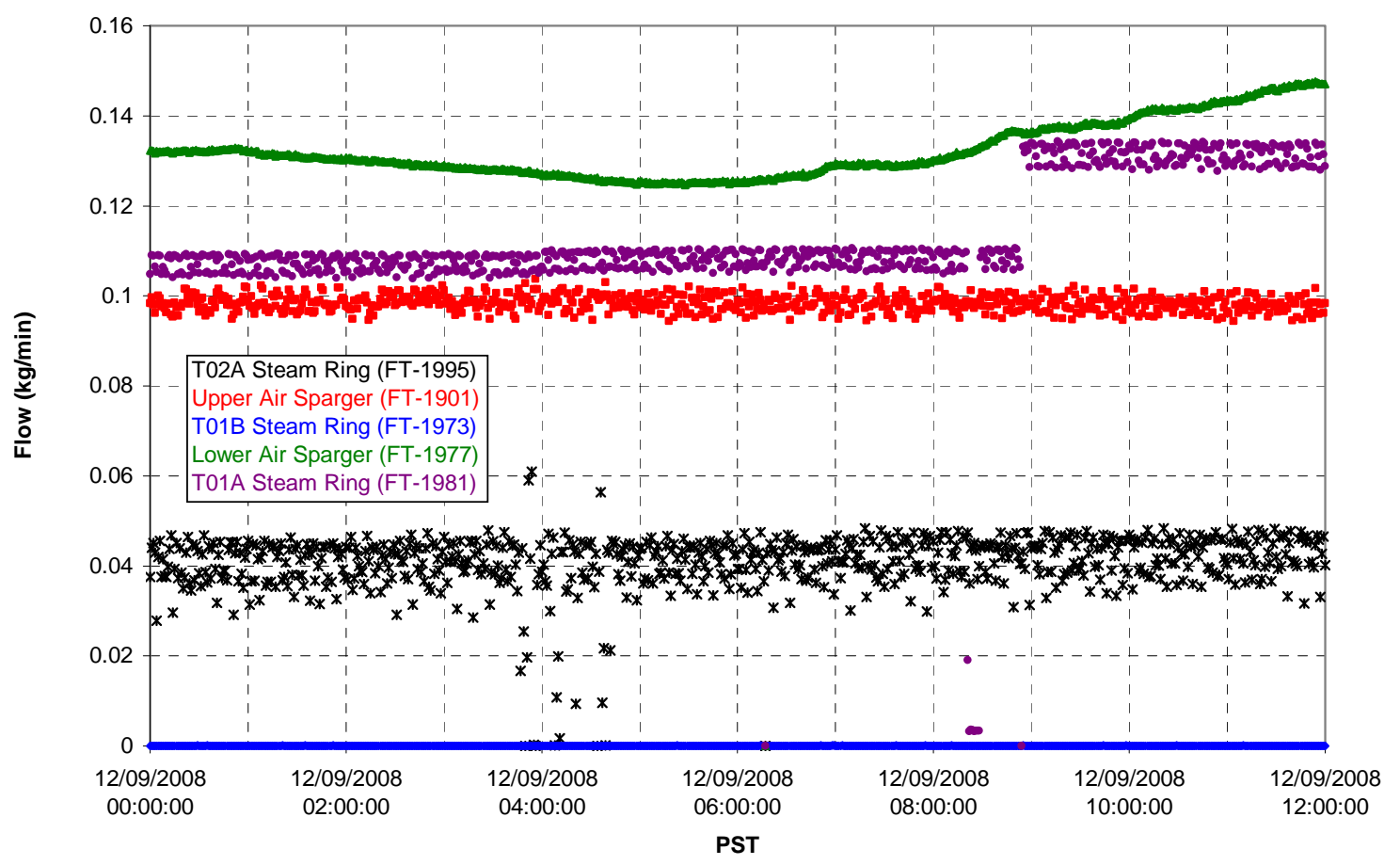


T02A Steam

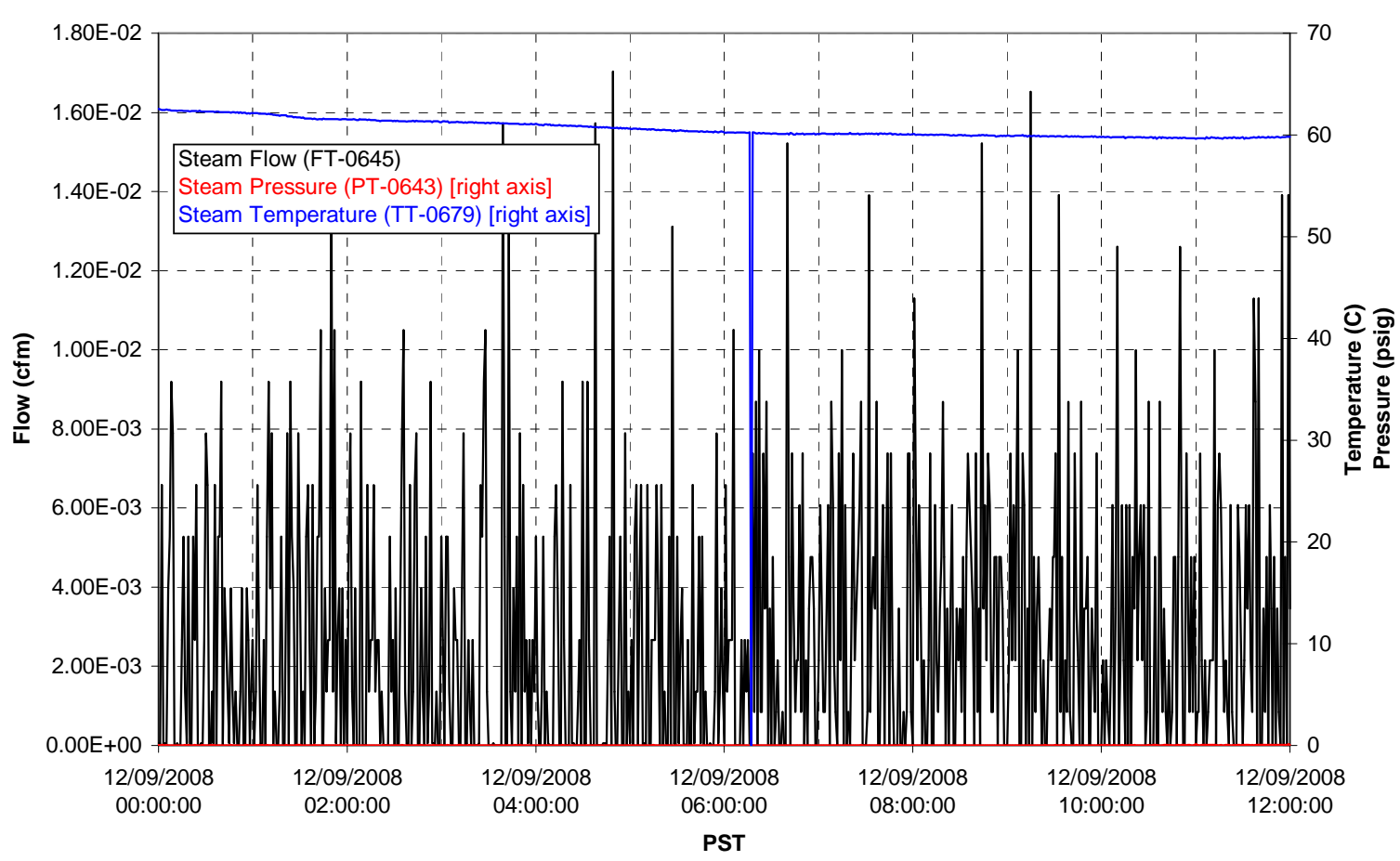

T01A Steam

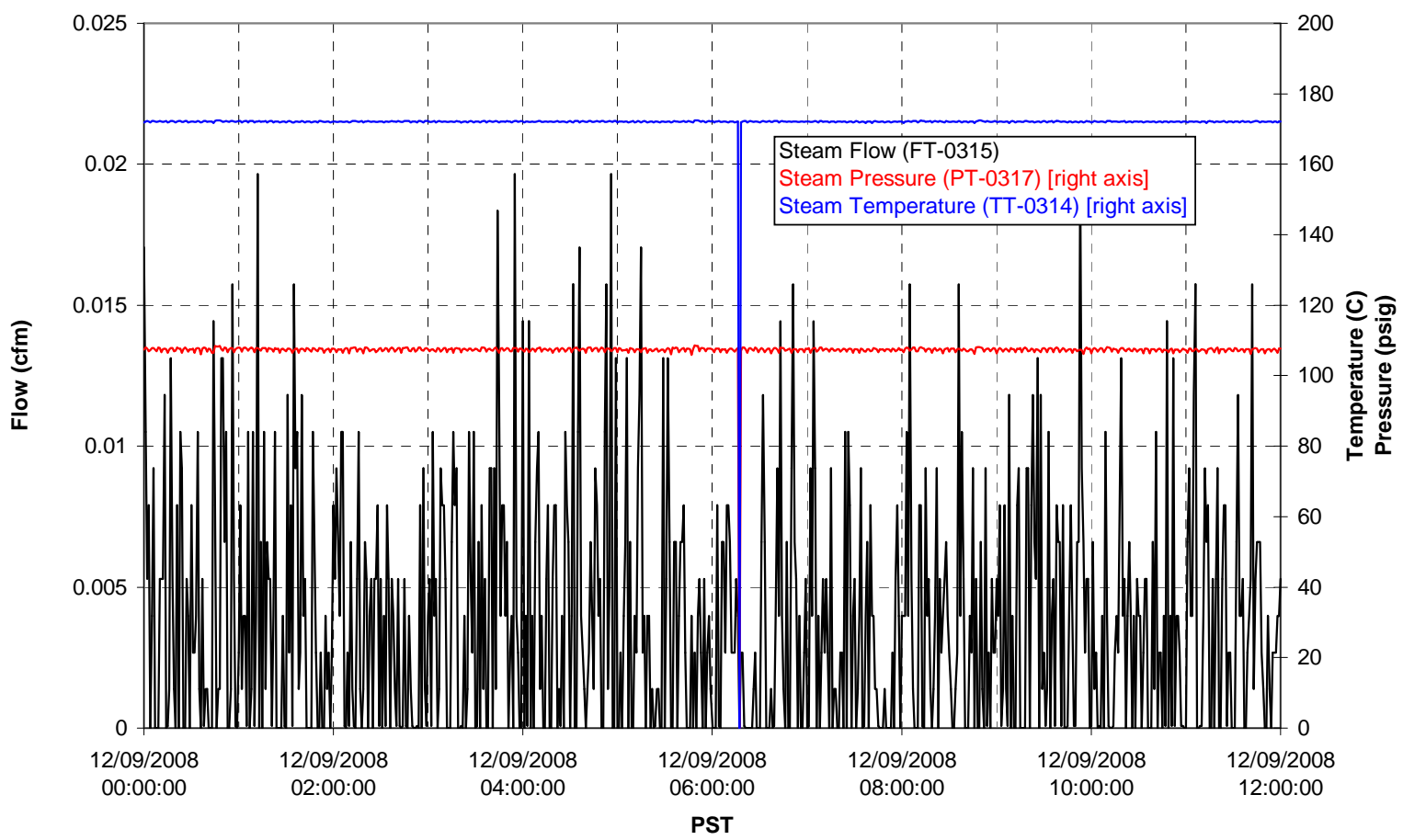


T01B Steam

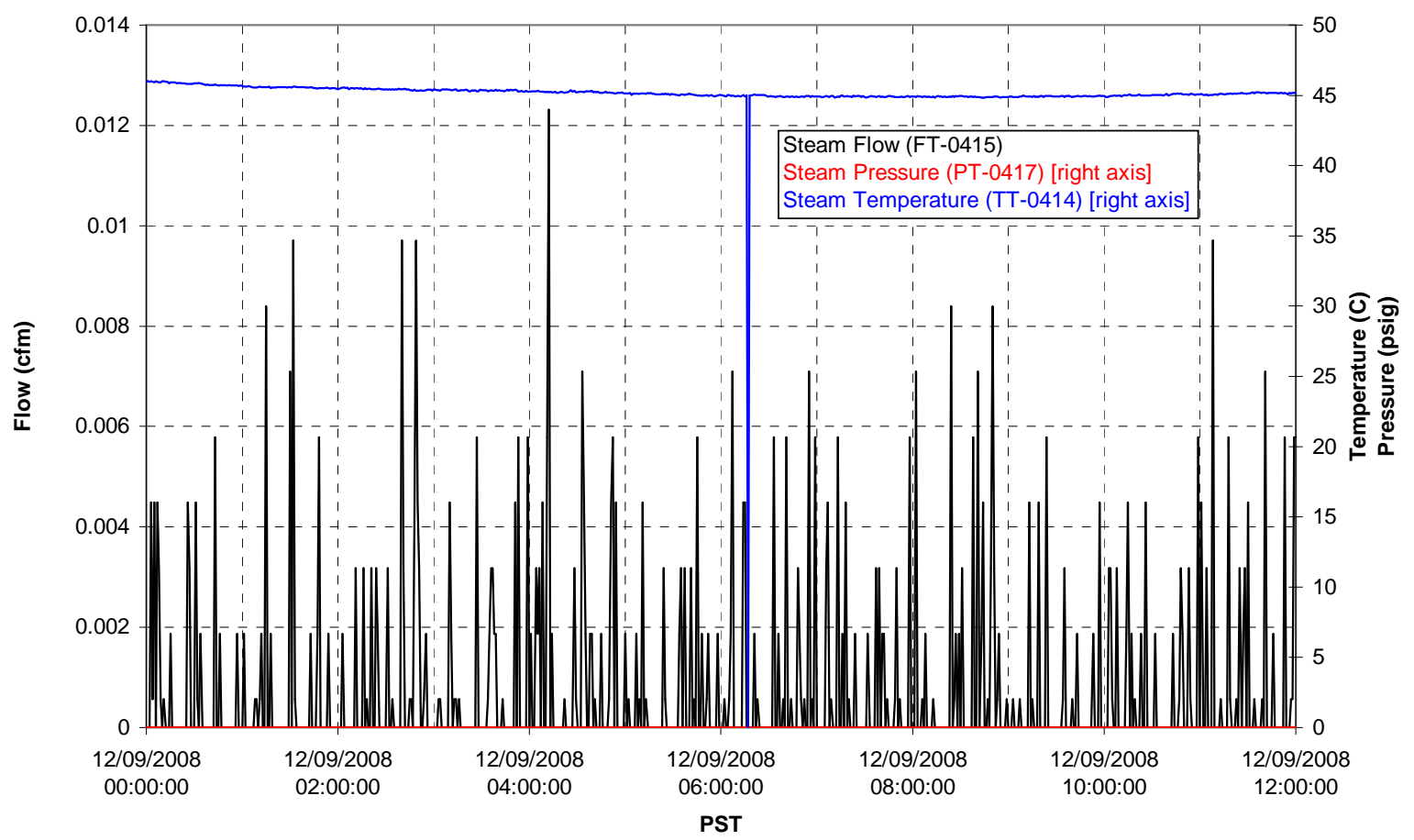


Functional Test Data Plots

12/09/2008 12:00 - 12/10/2008 00:00 
T01A level

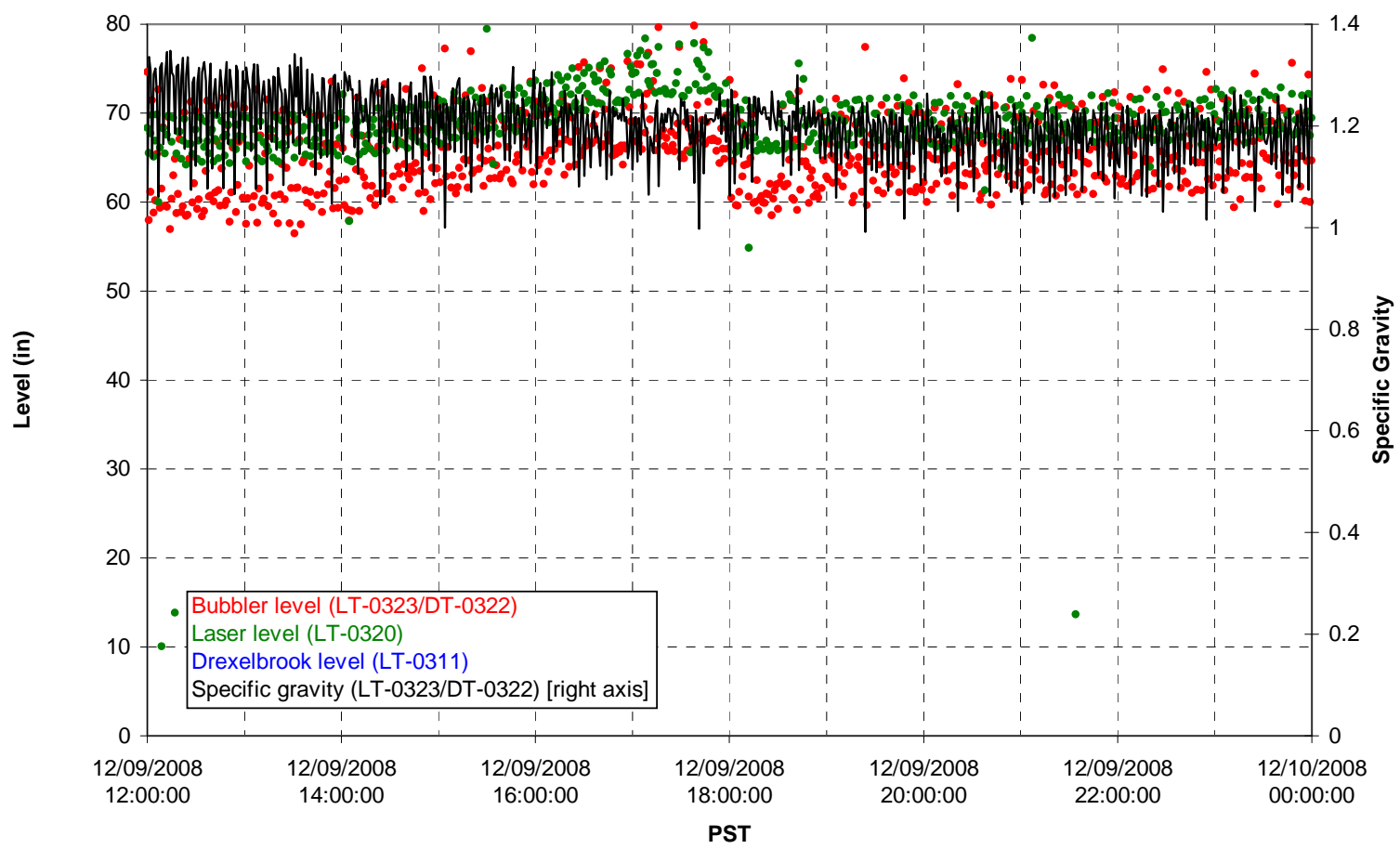

T01A temperatures

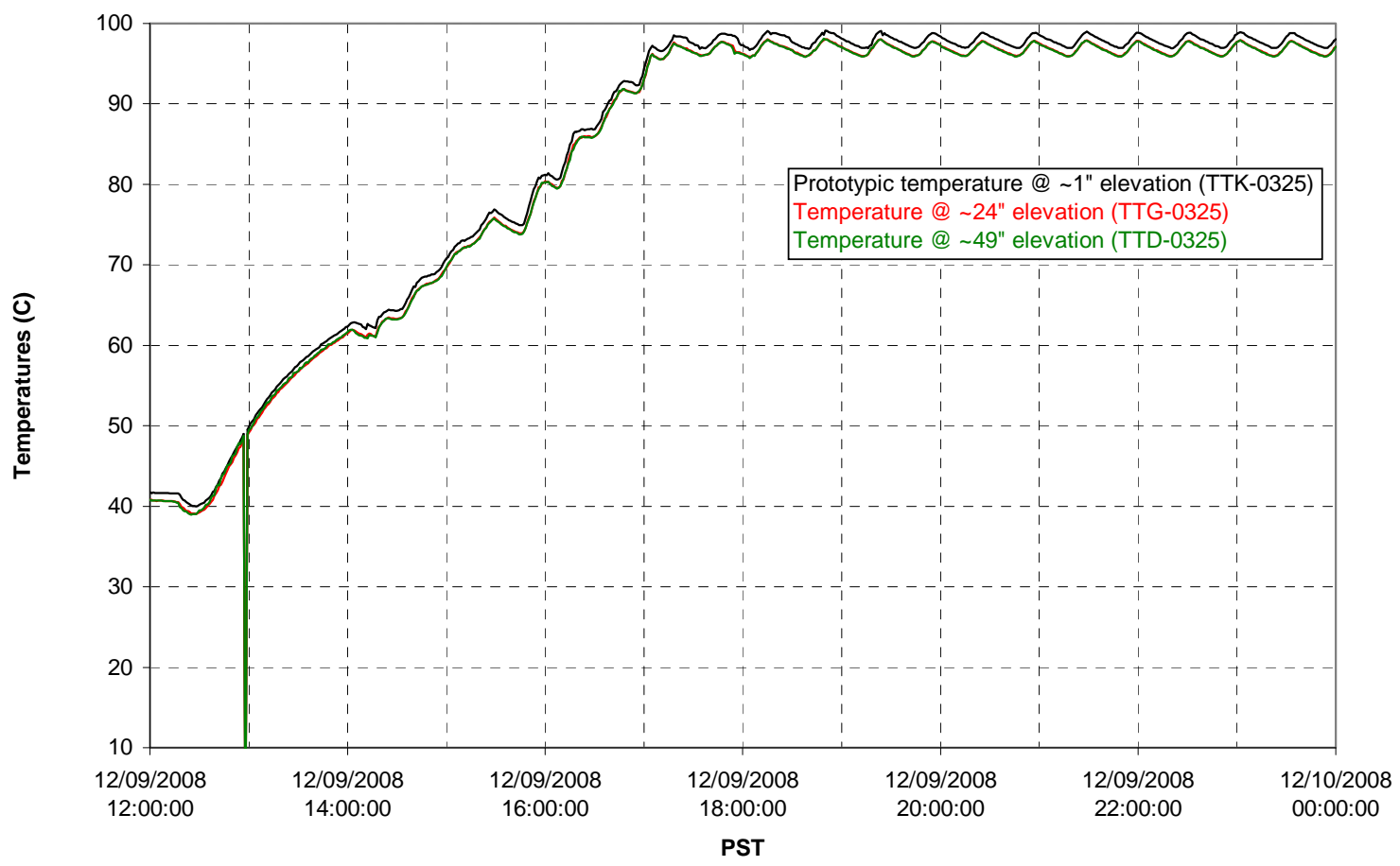


T01B level

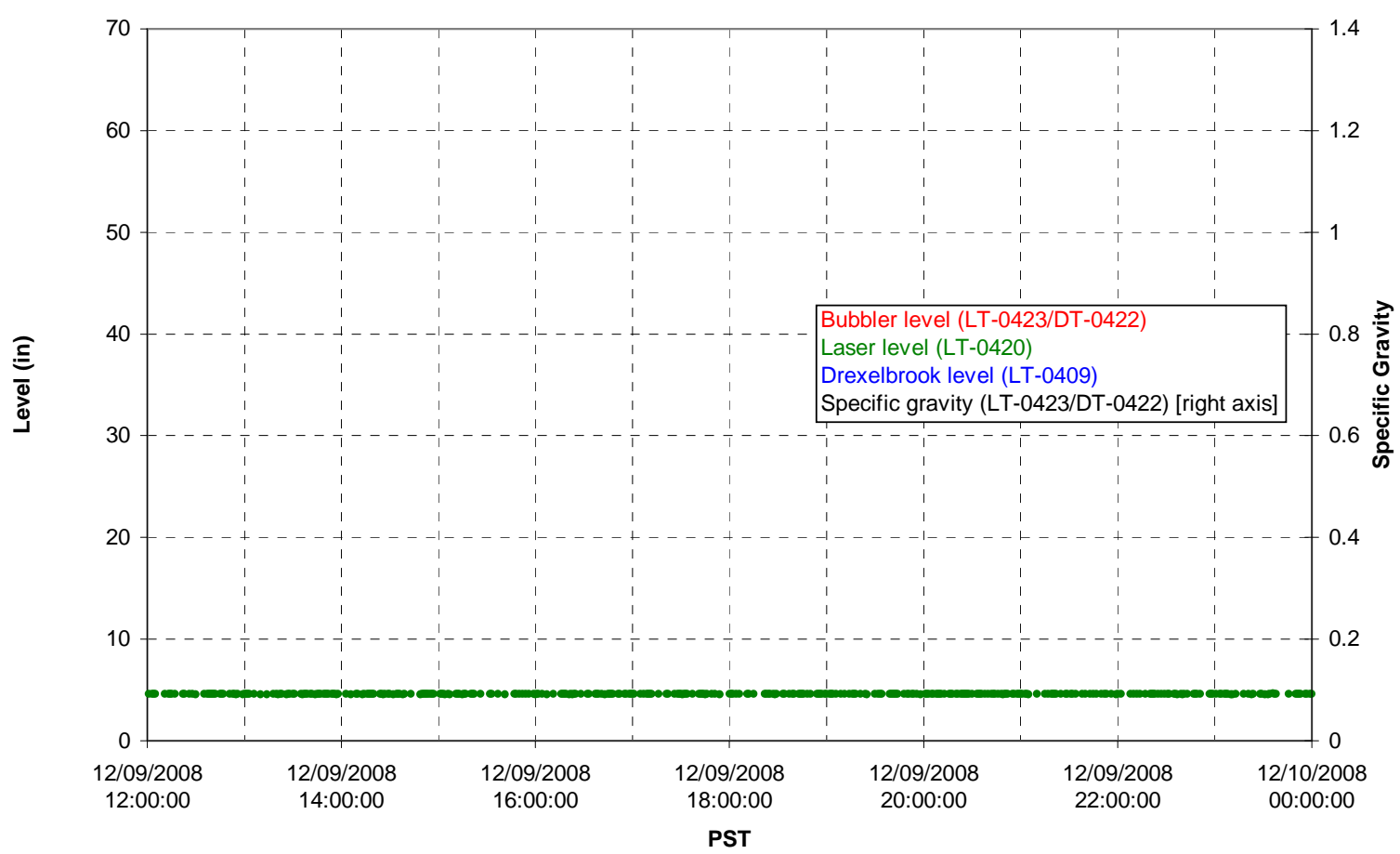

T01B temperatures

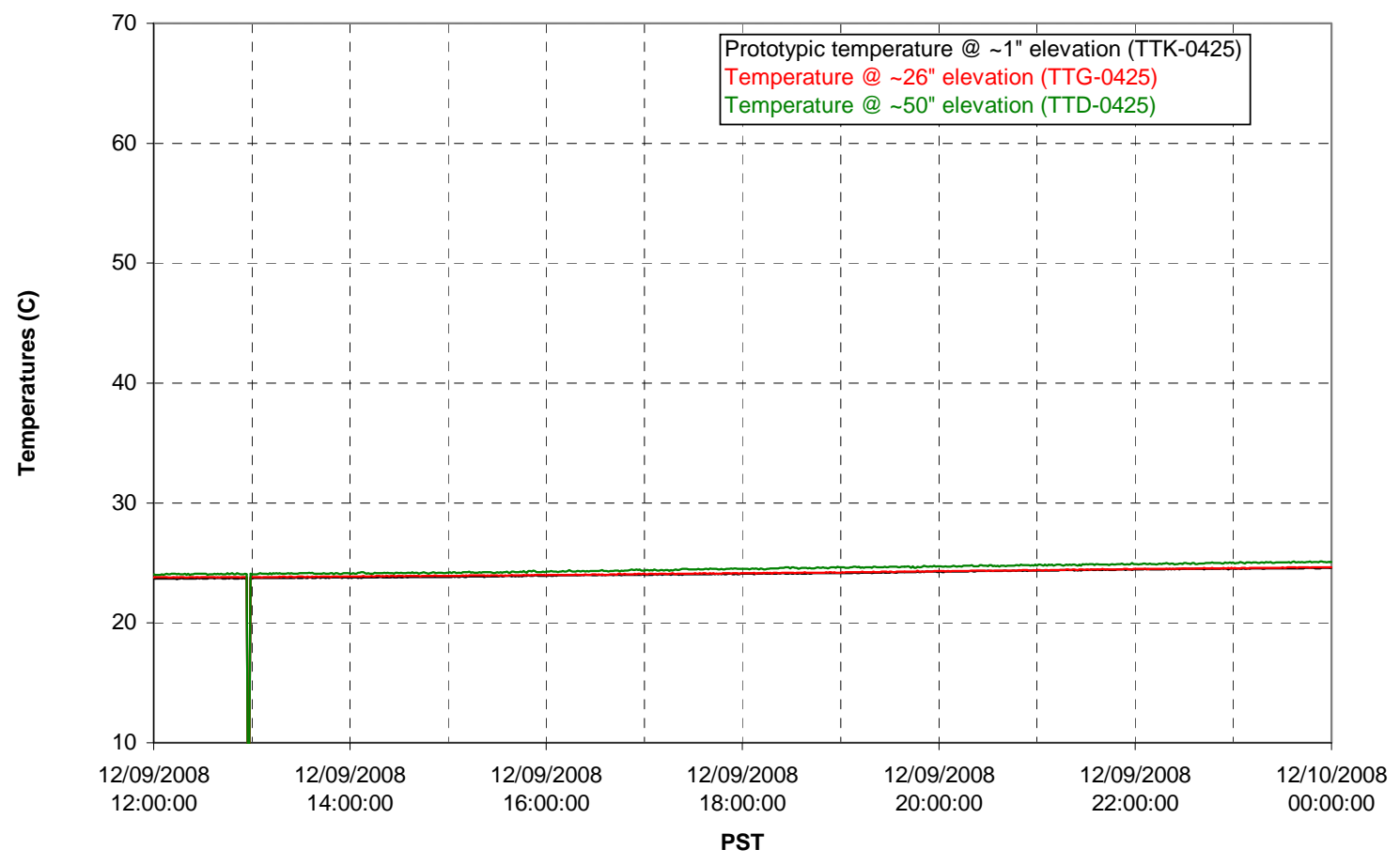


T02A level

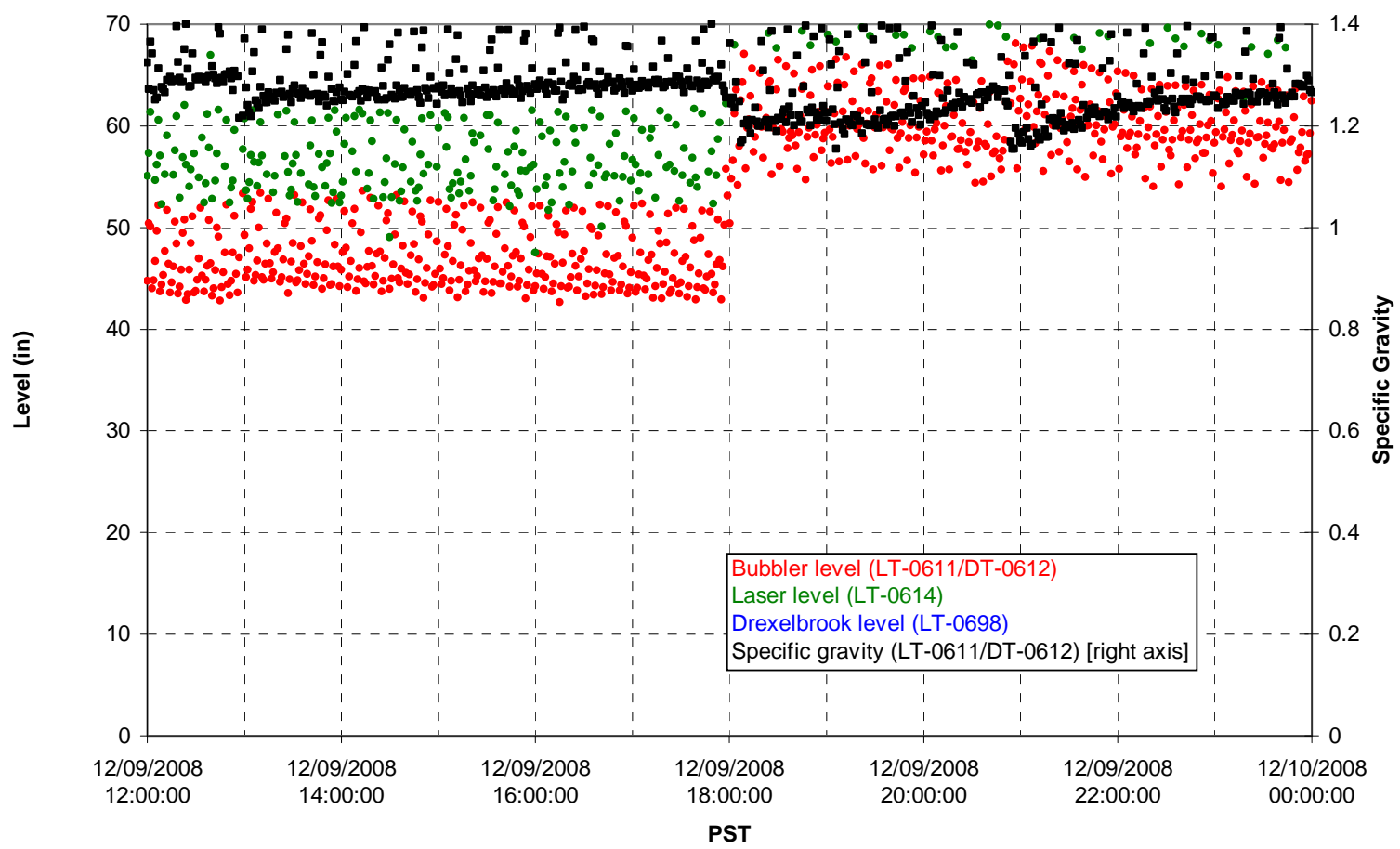

T02A temperatures

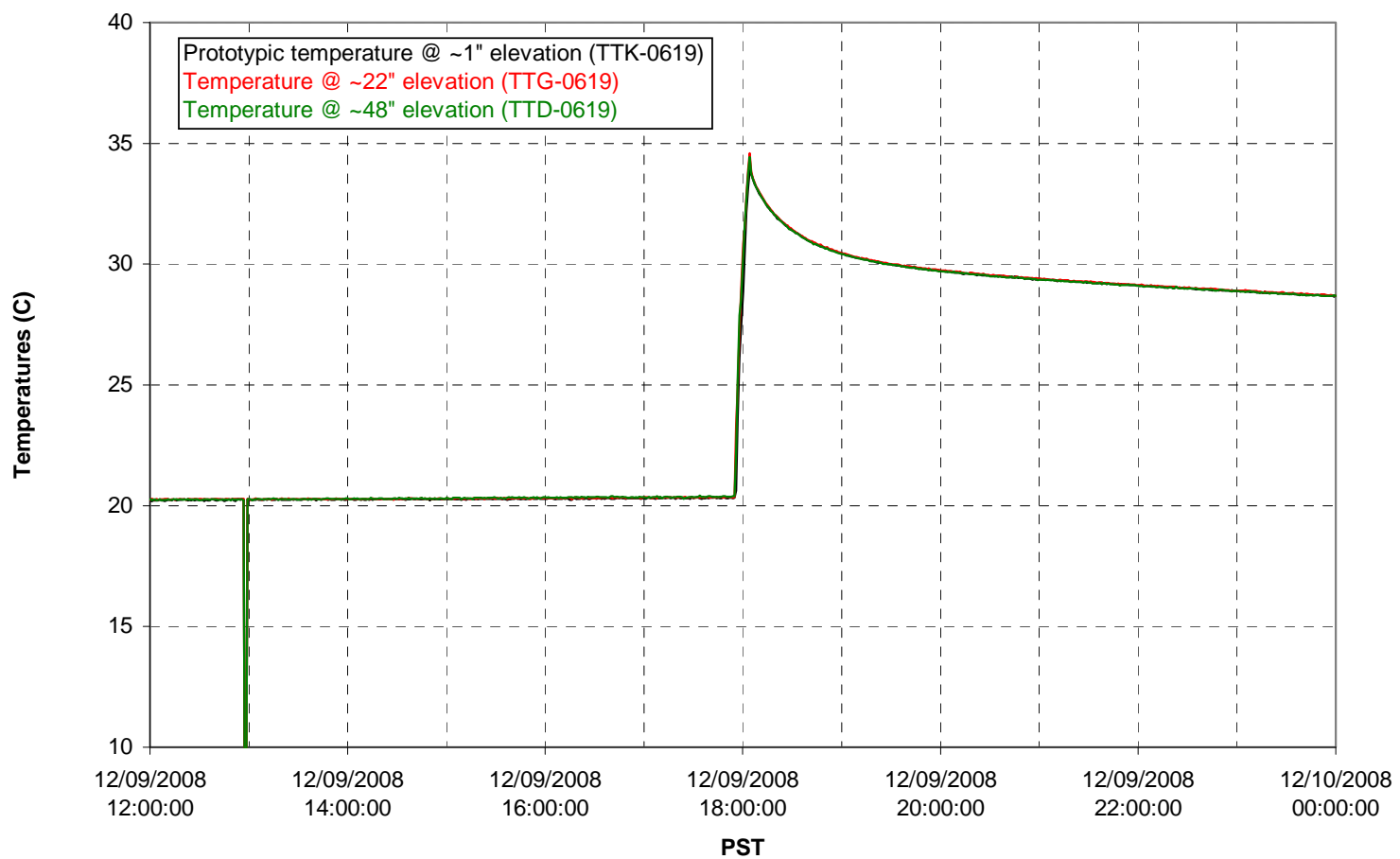


T02A and filter loop temperatures

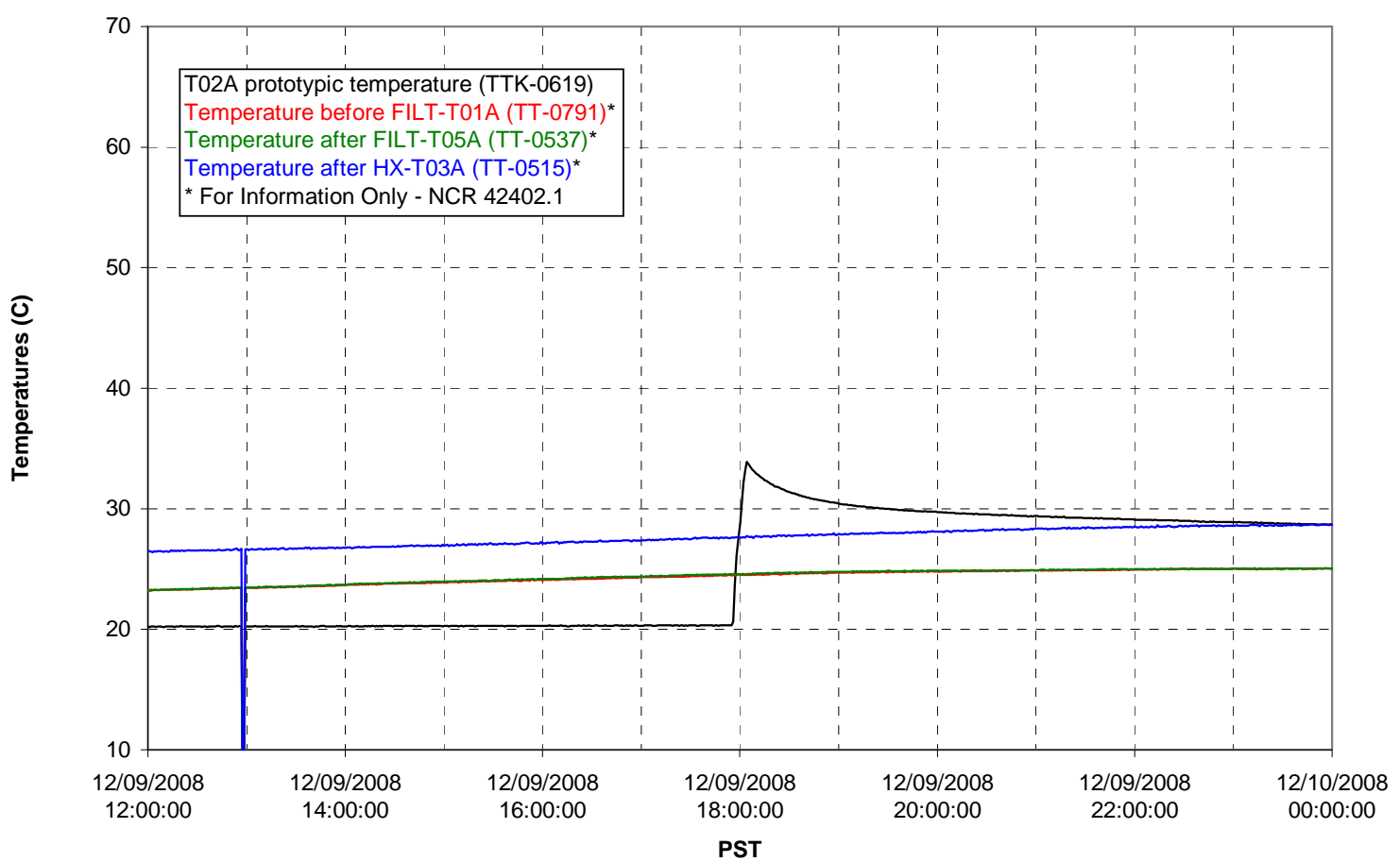

Pump Pressures and Flow

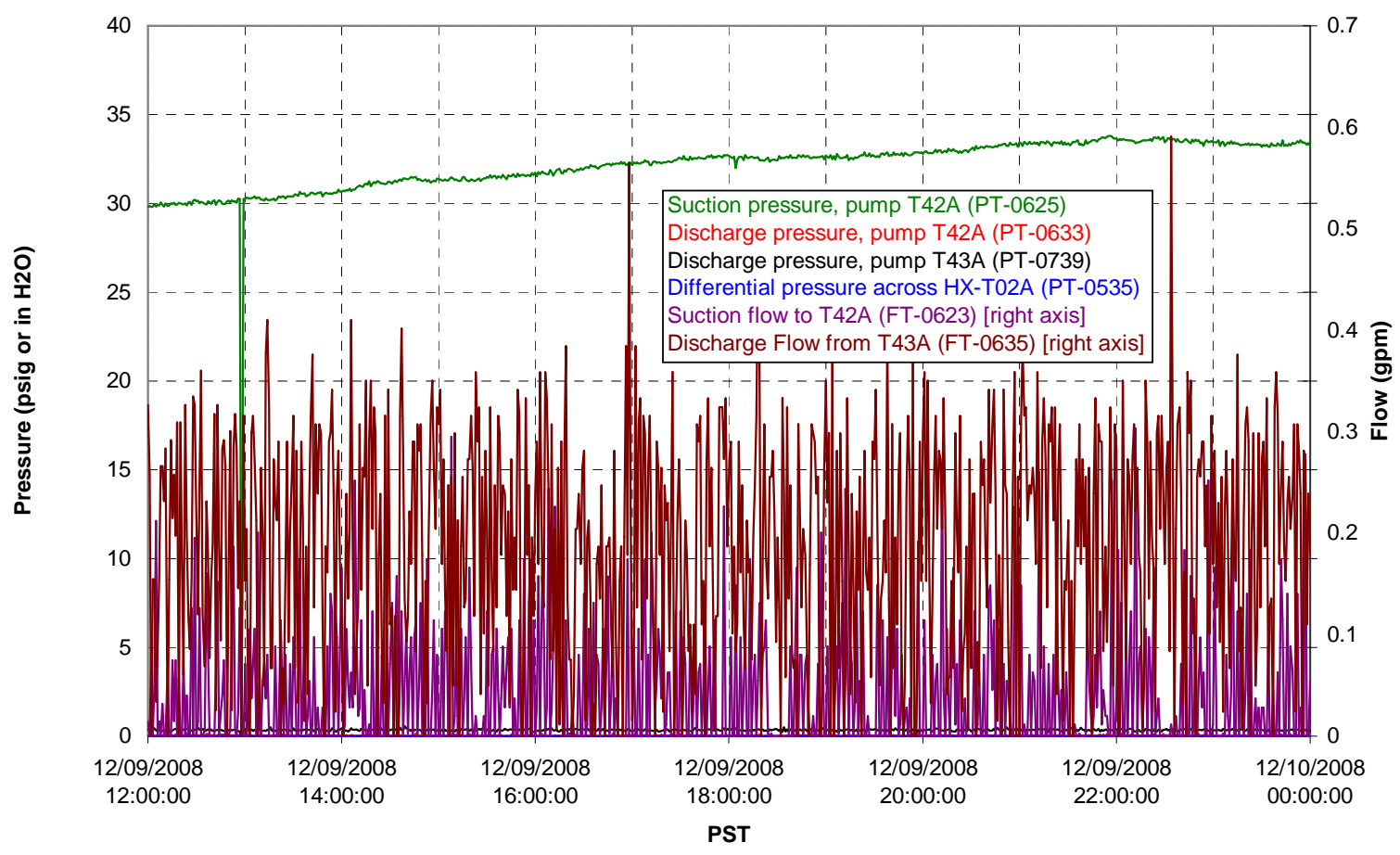


Axial pressure drop

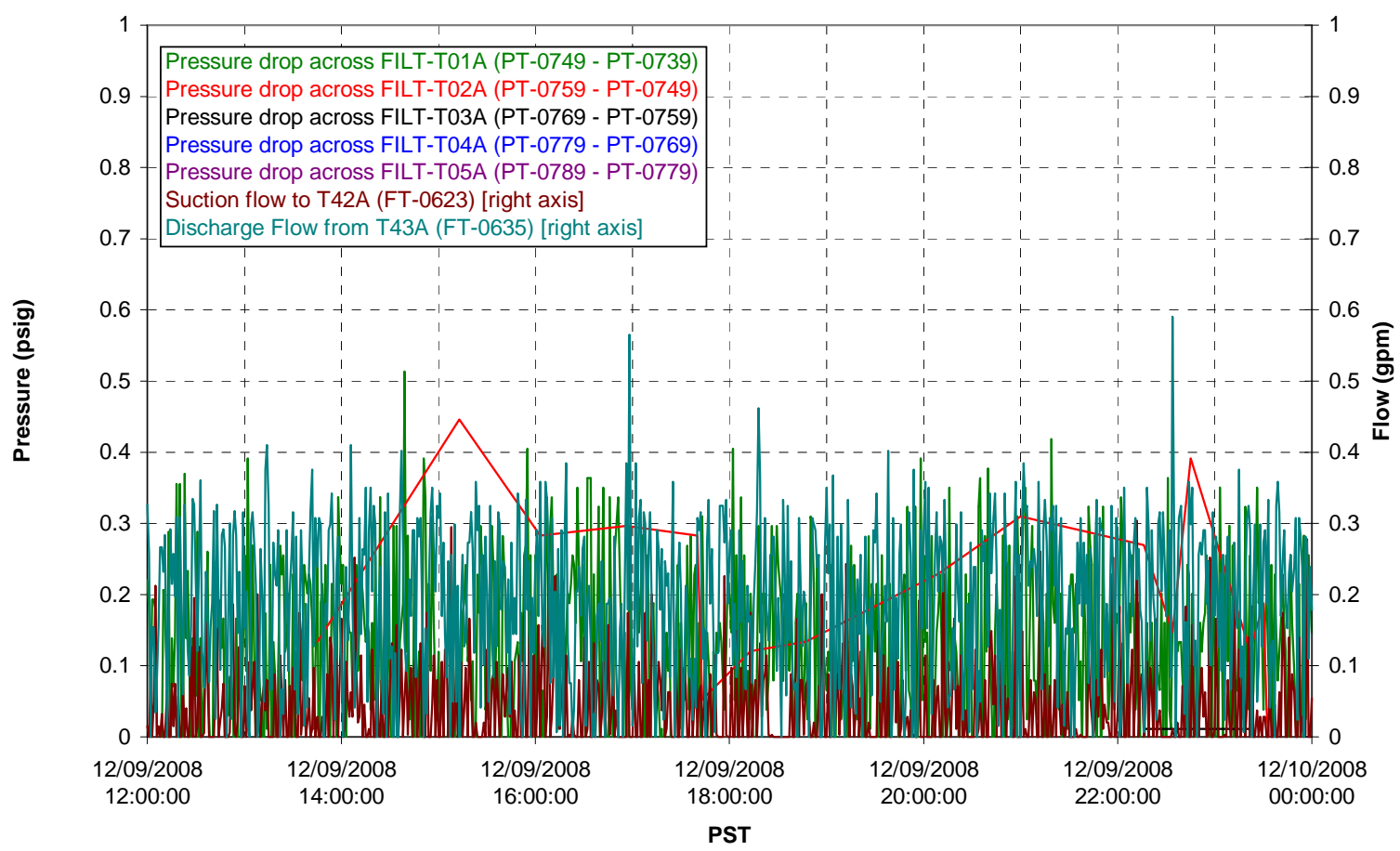

Permeate flow rates

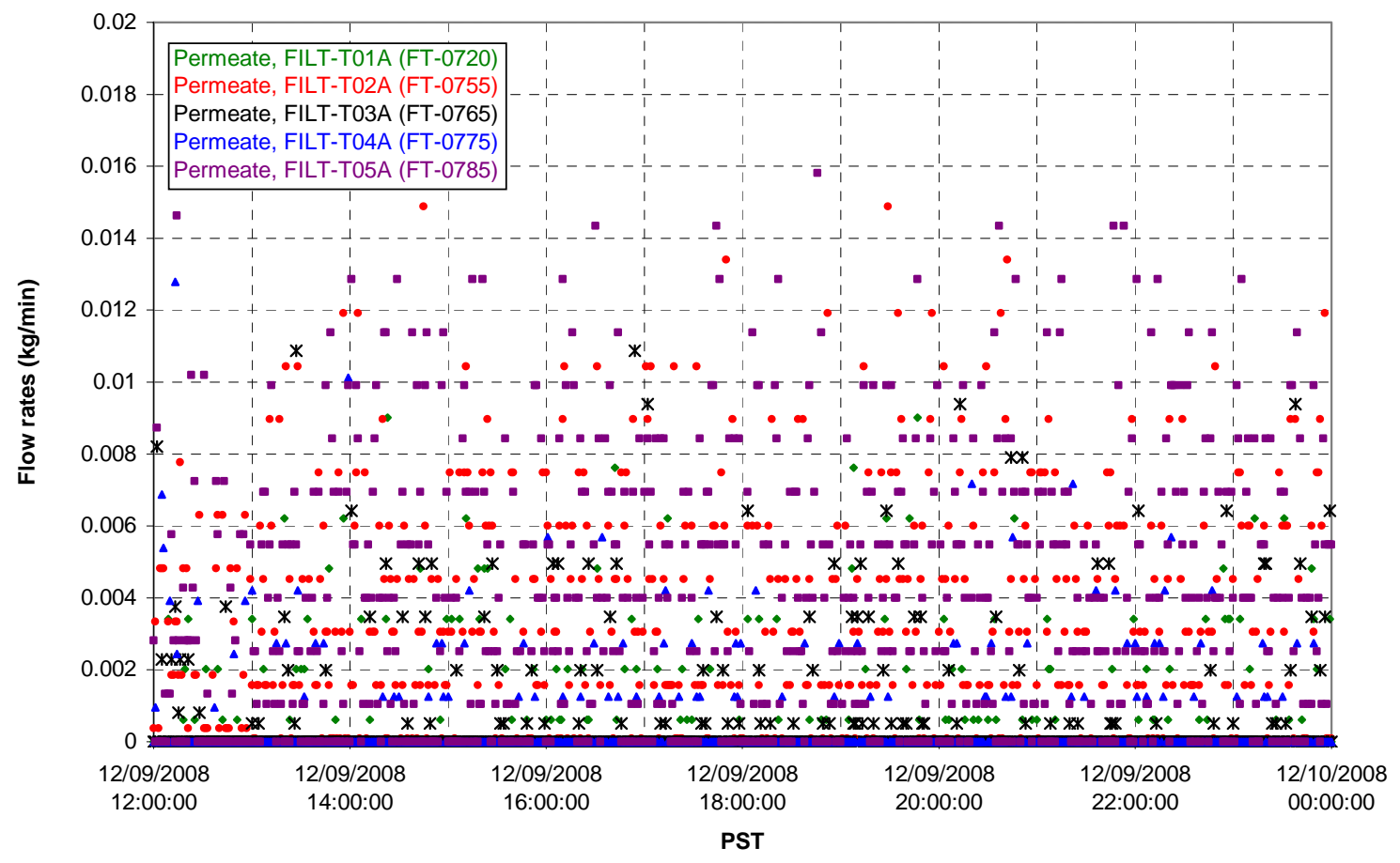


T02A Inner Temperature Tree

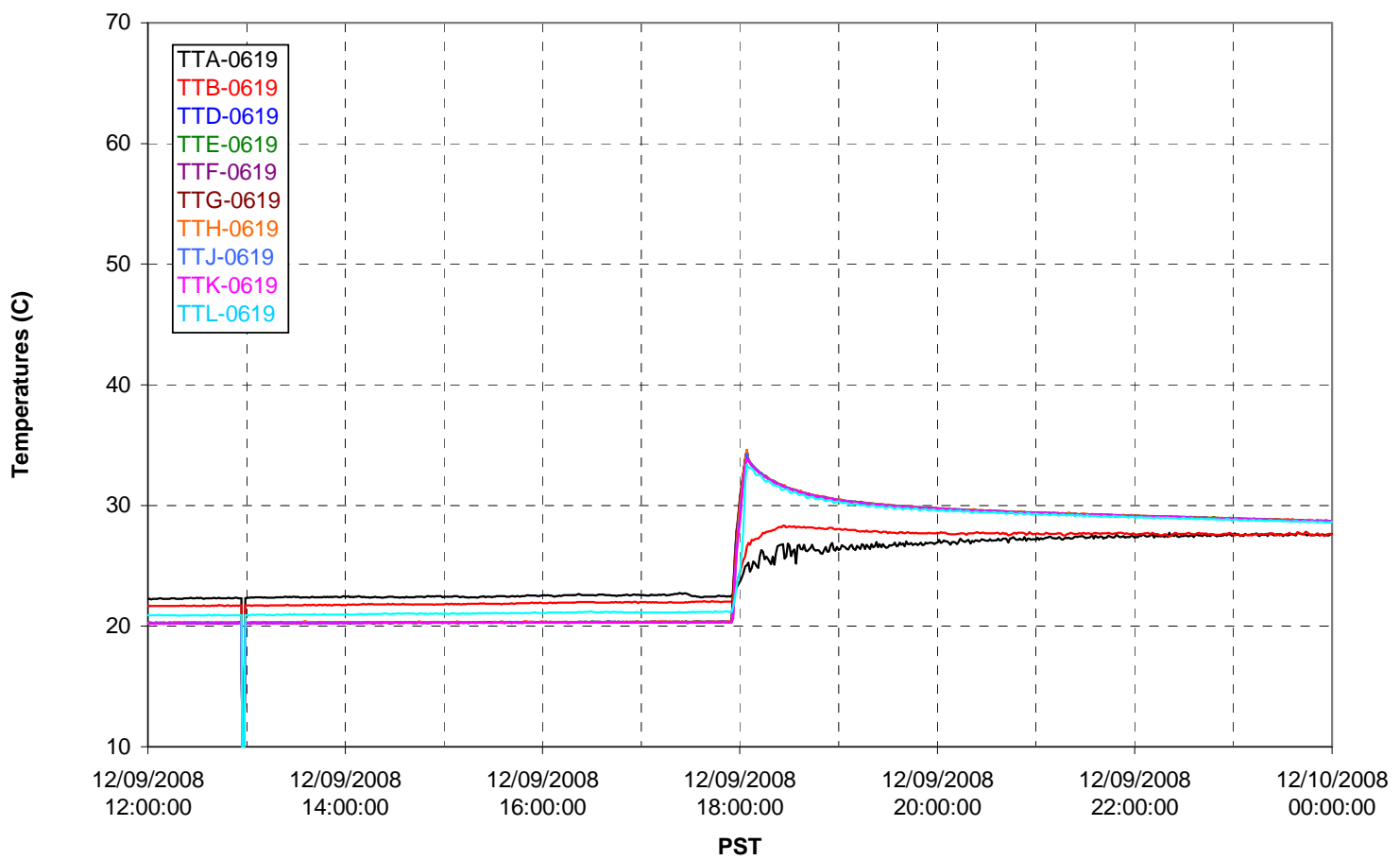

T02A Outer Temperature Tree

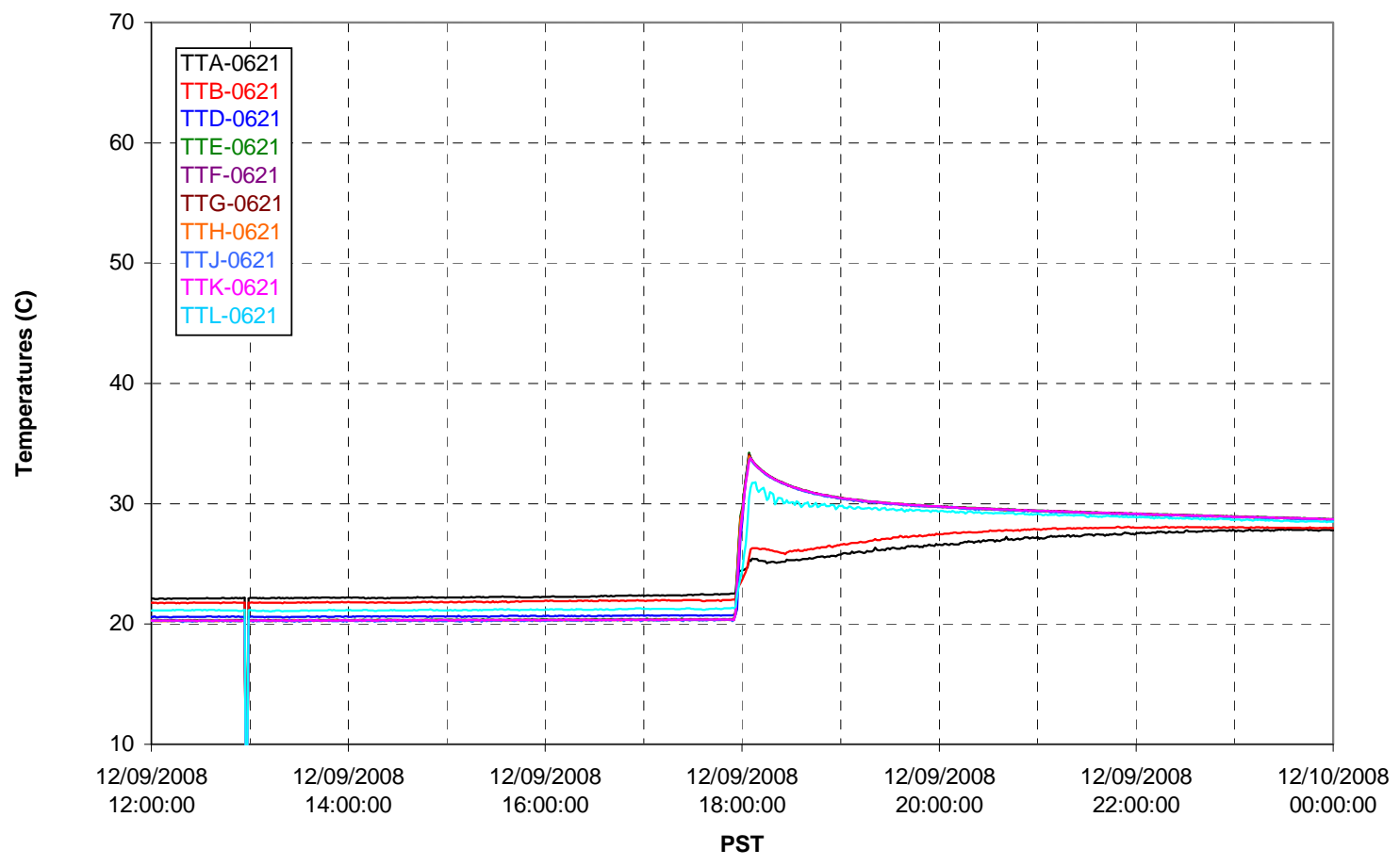


T02A temperatures

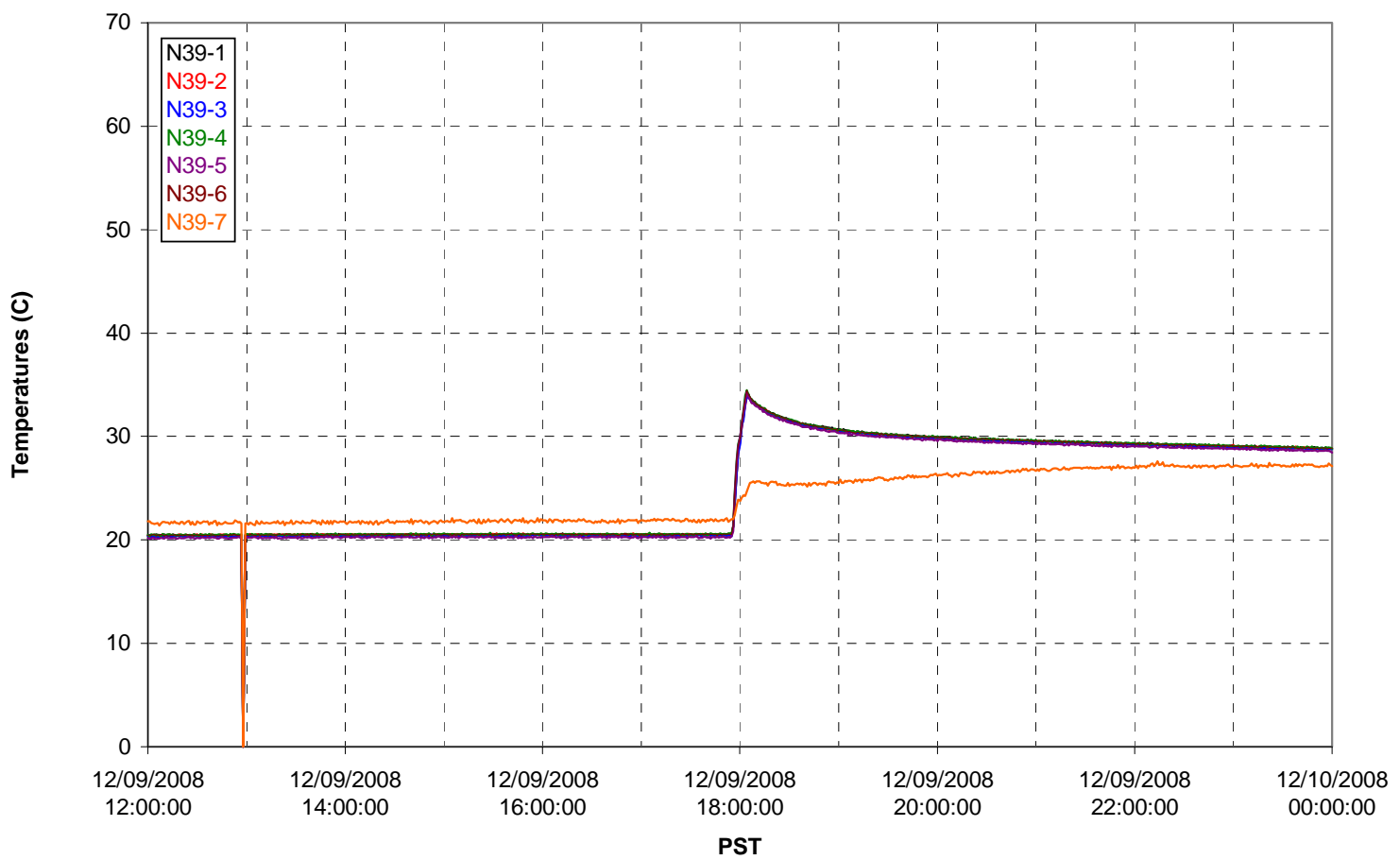

T02A temperatures

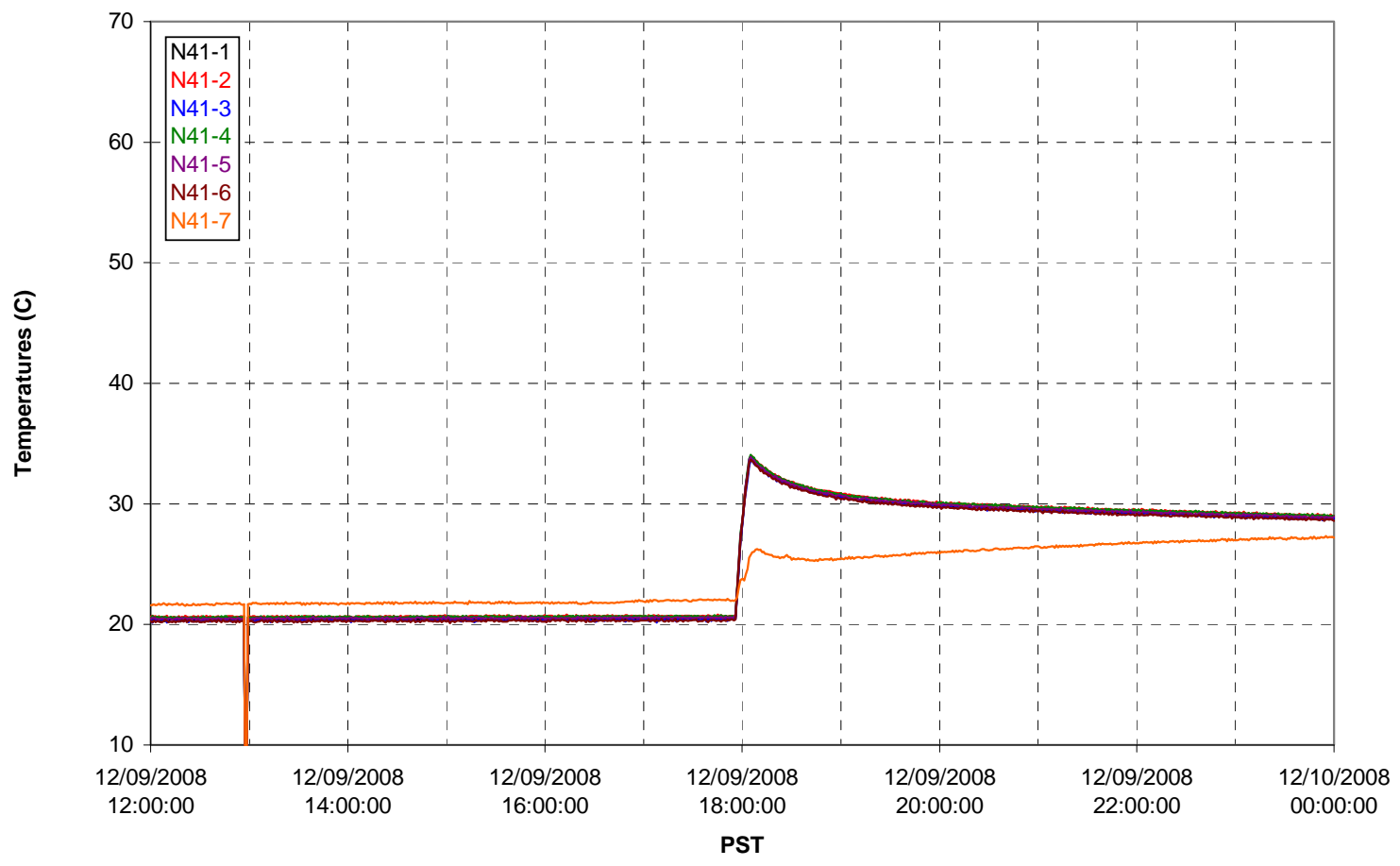


T02A temperatures

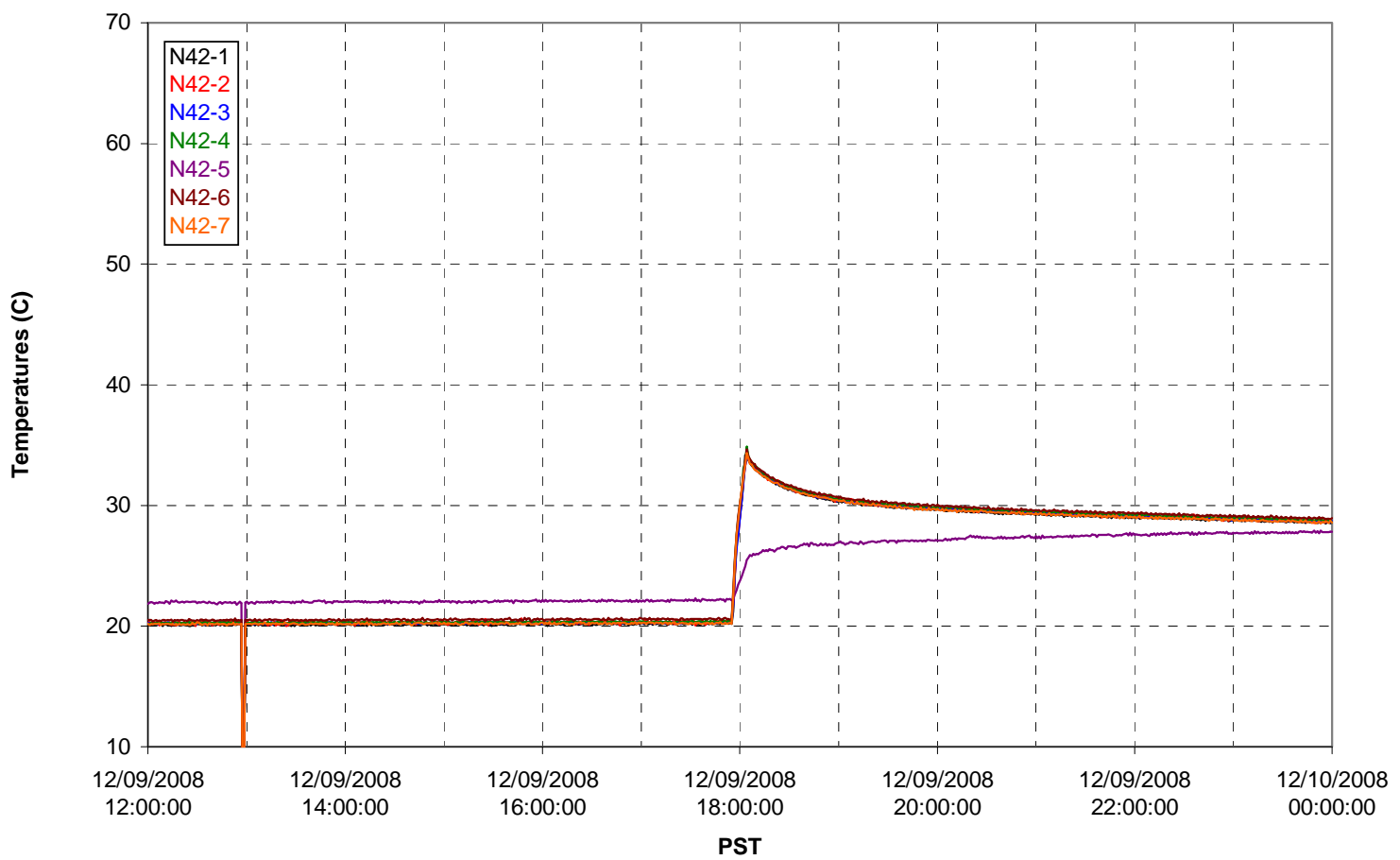

T02A temperatures

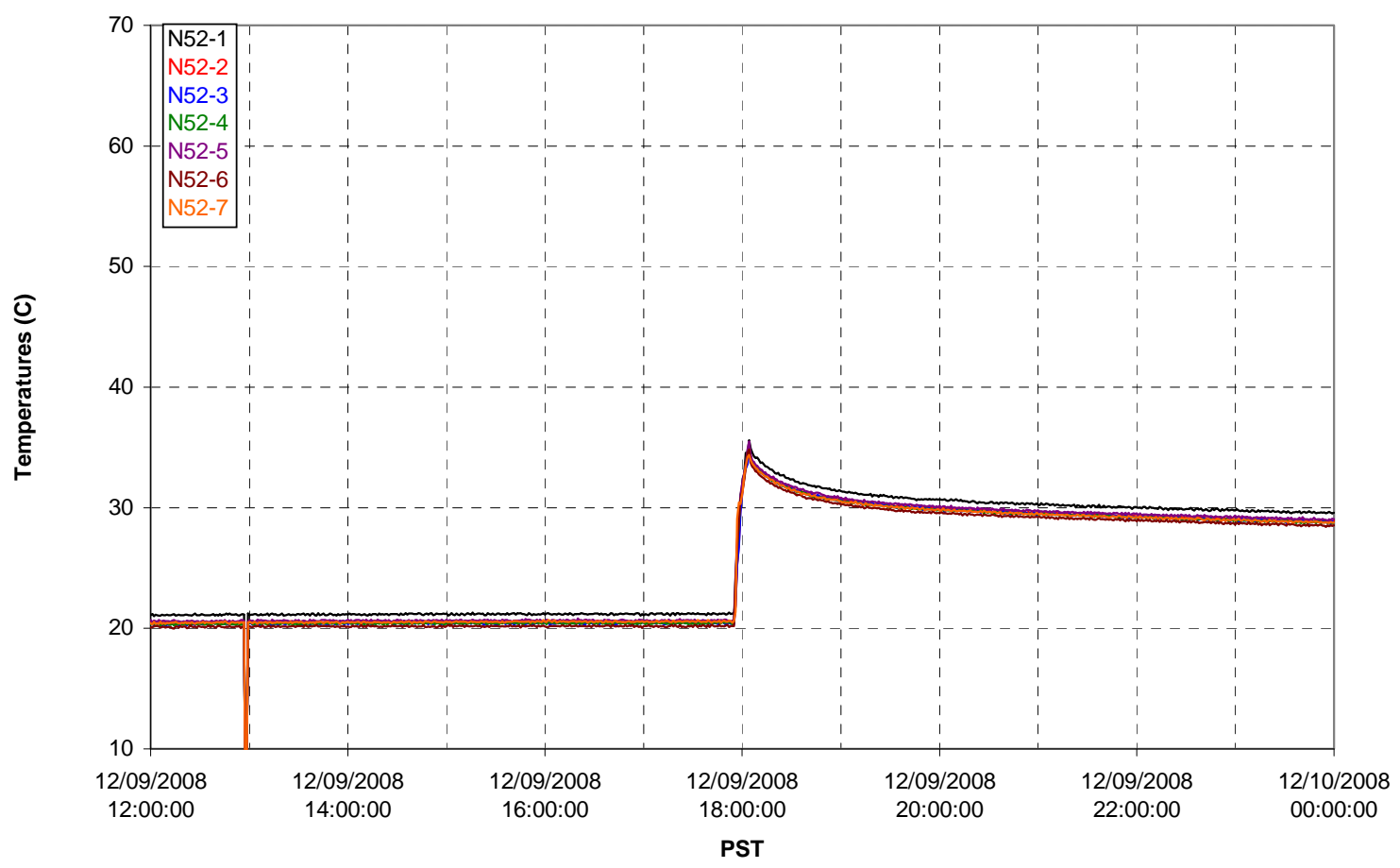


T02A Heating and Cooling

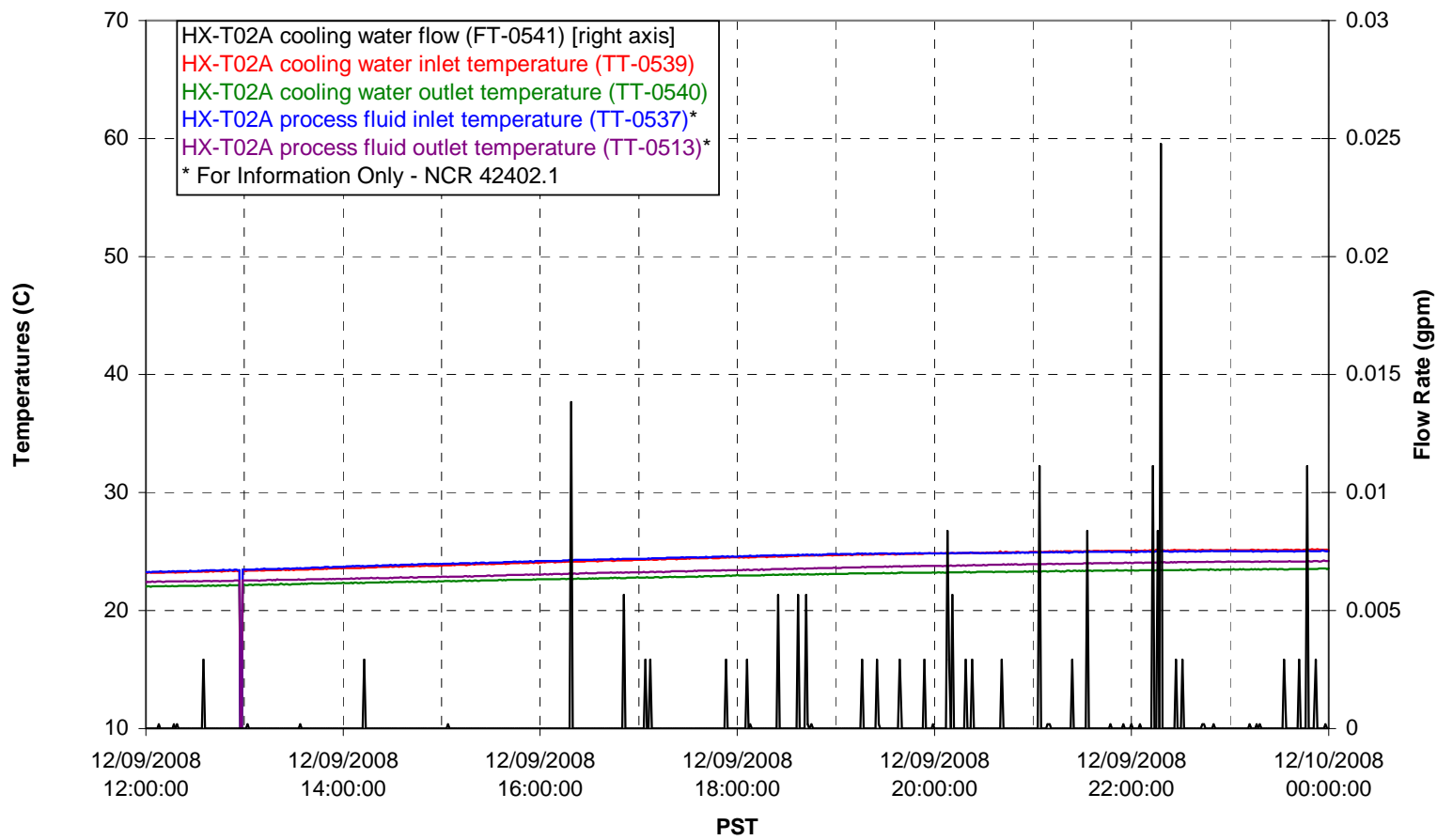

Pump Operation

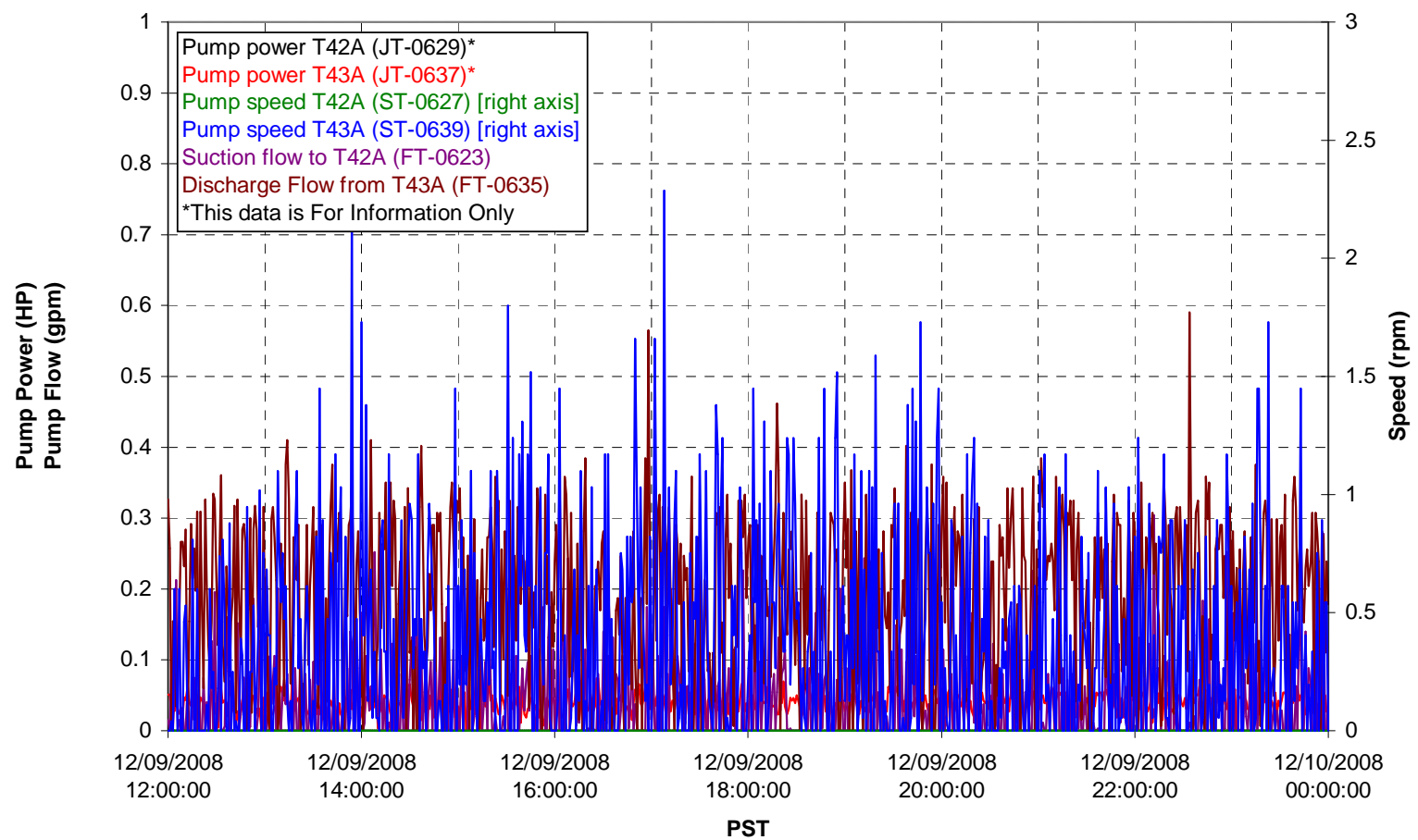


Pulsepot UFP-PP-T01A

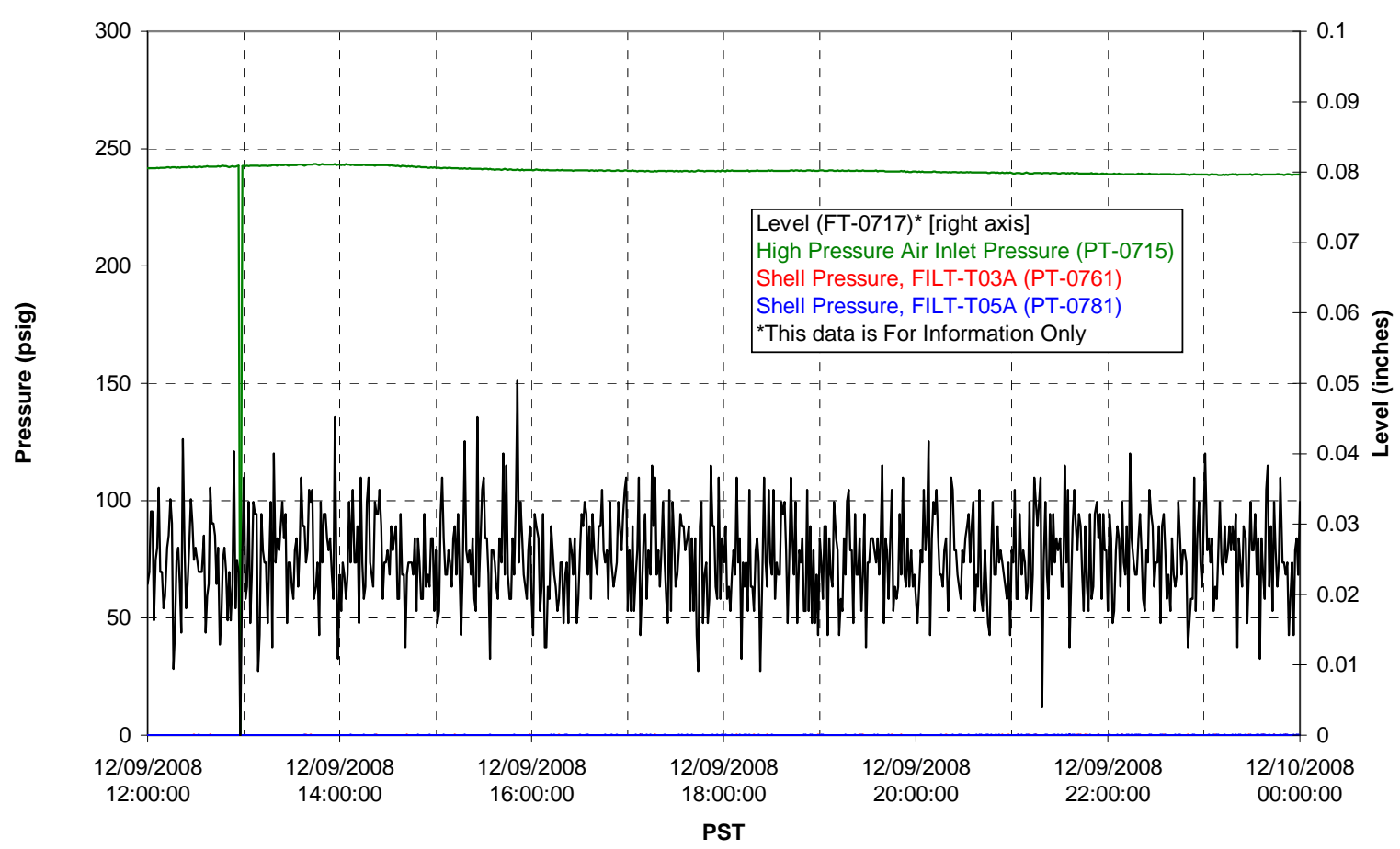

Pulsepot UFP-PP-T02A

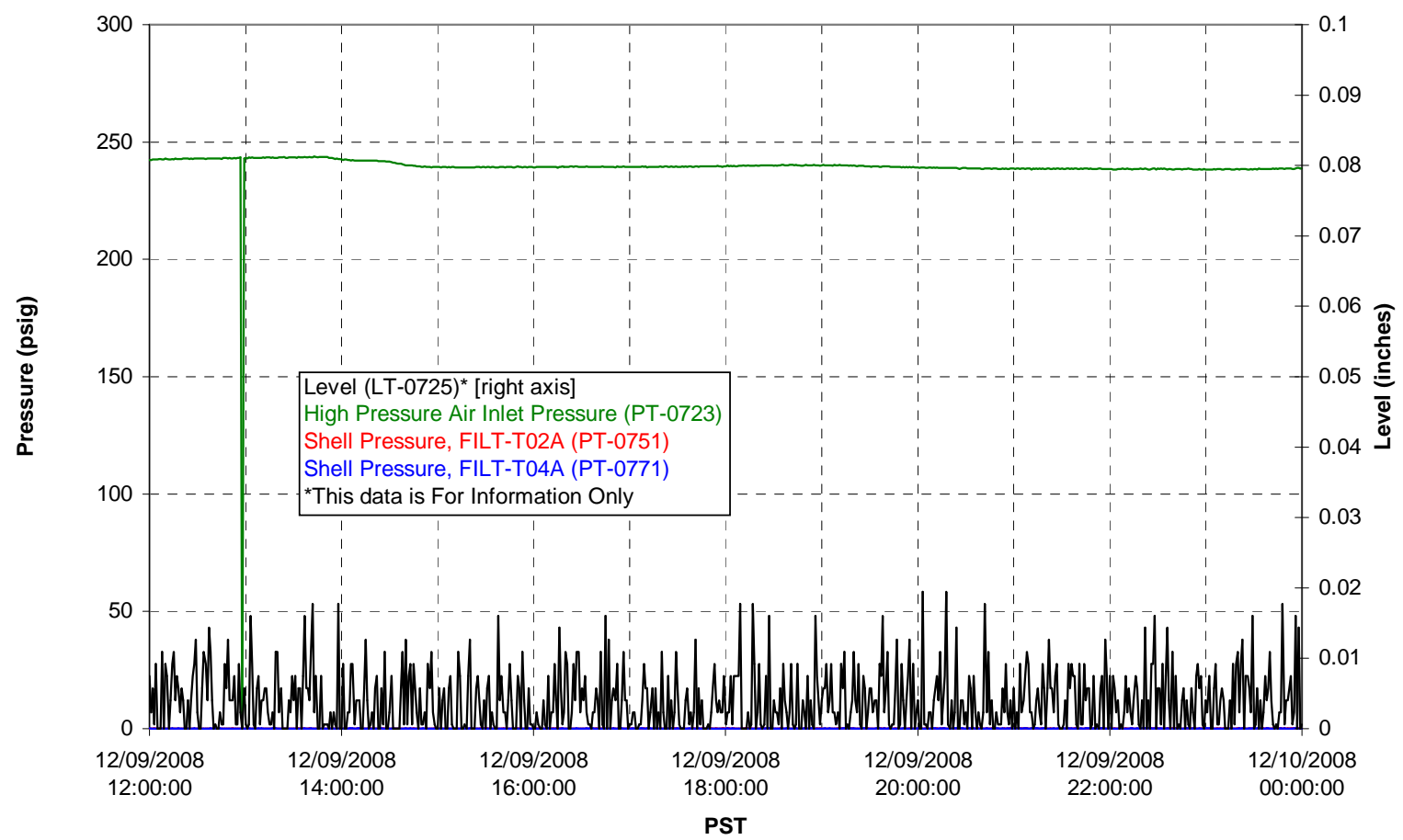


Pulsepot UFP-PP-T03A

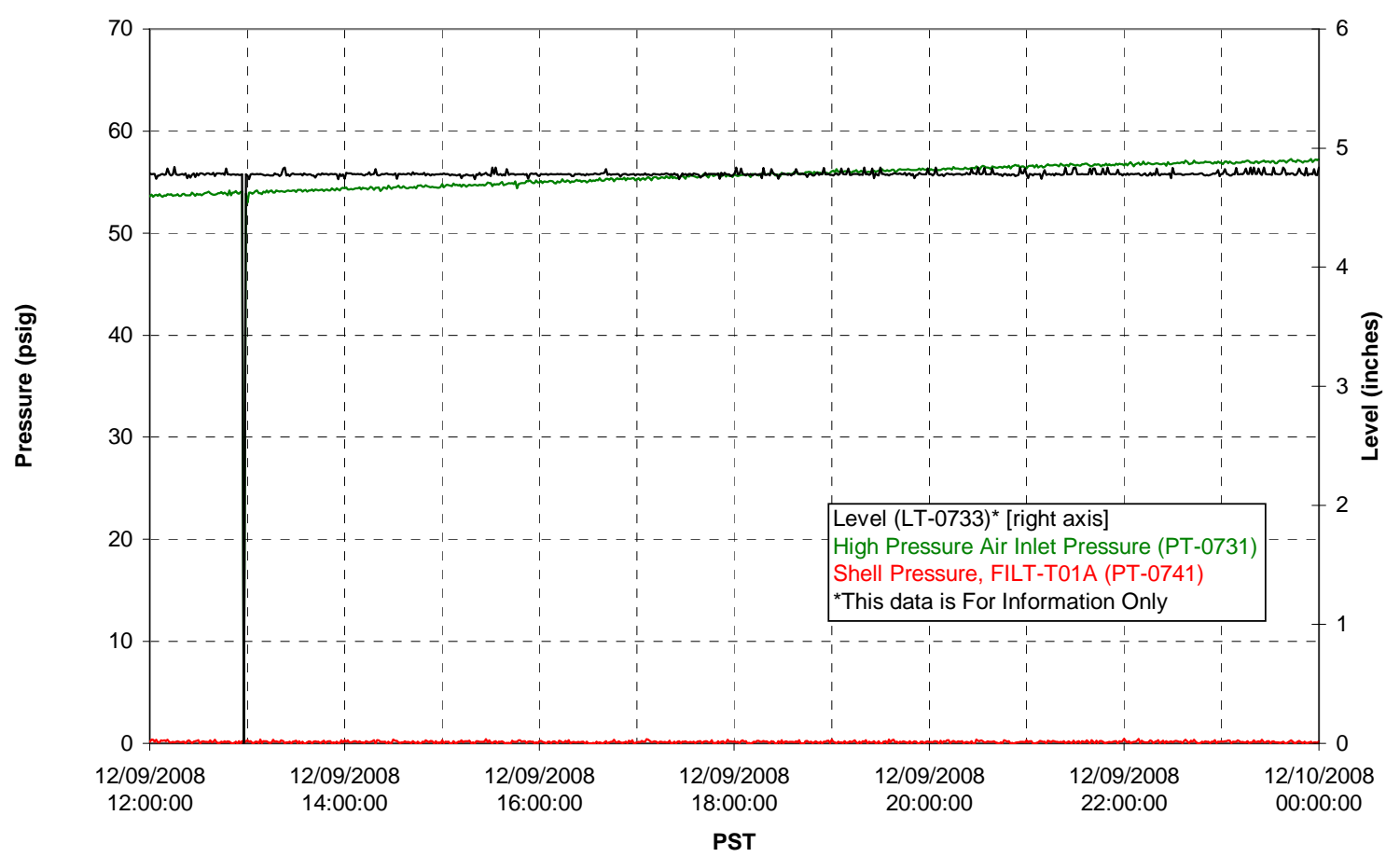

Pulsepot Levels

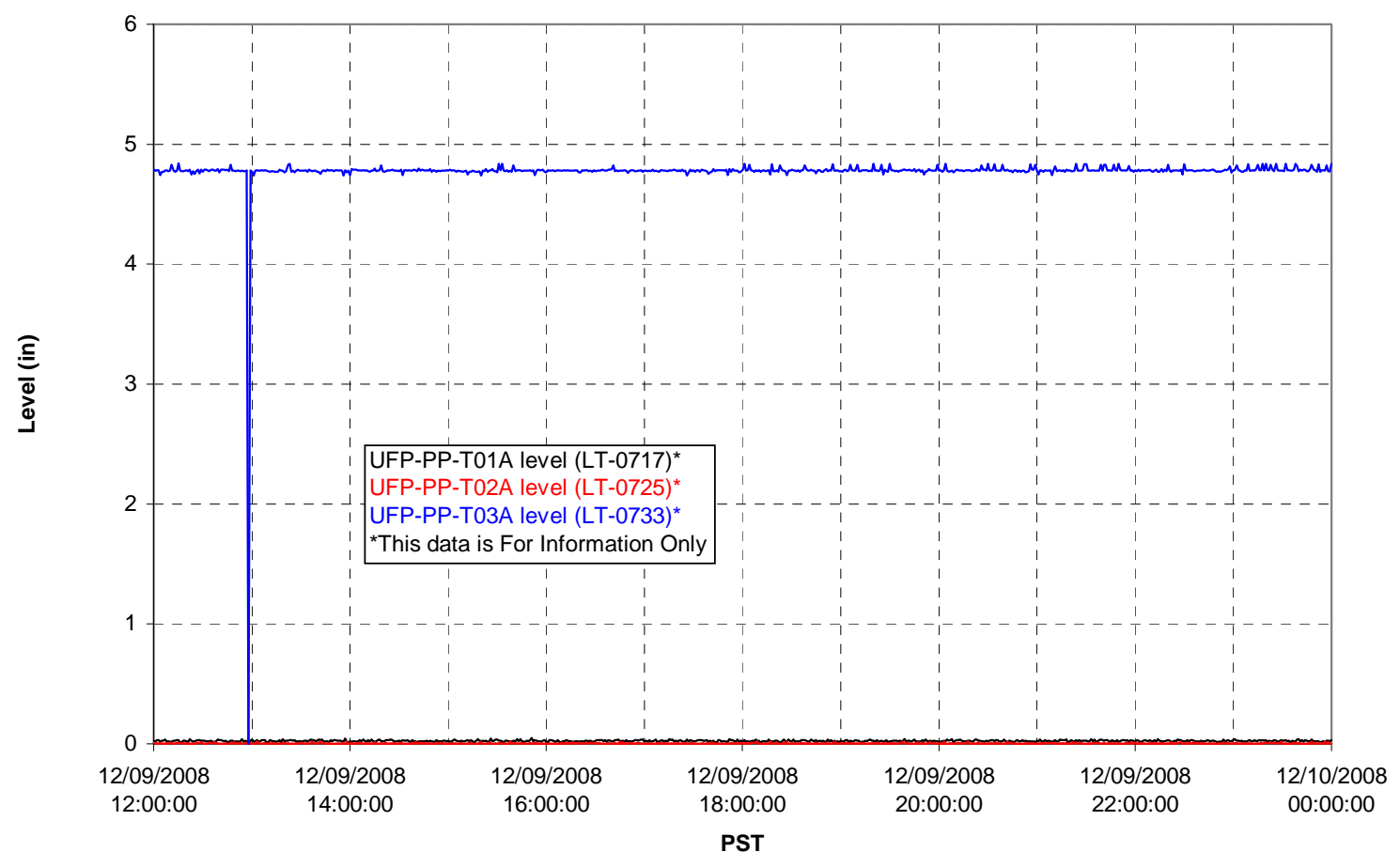


Filter UFP-FILT-T01A

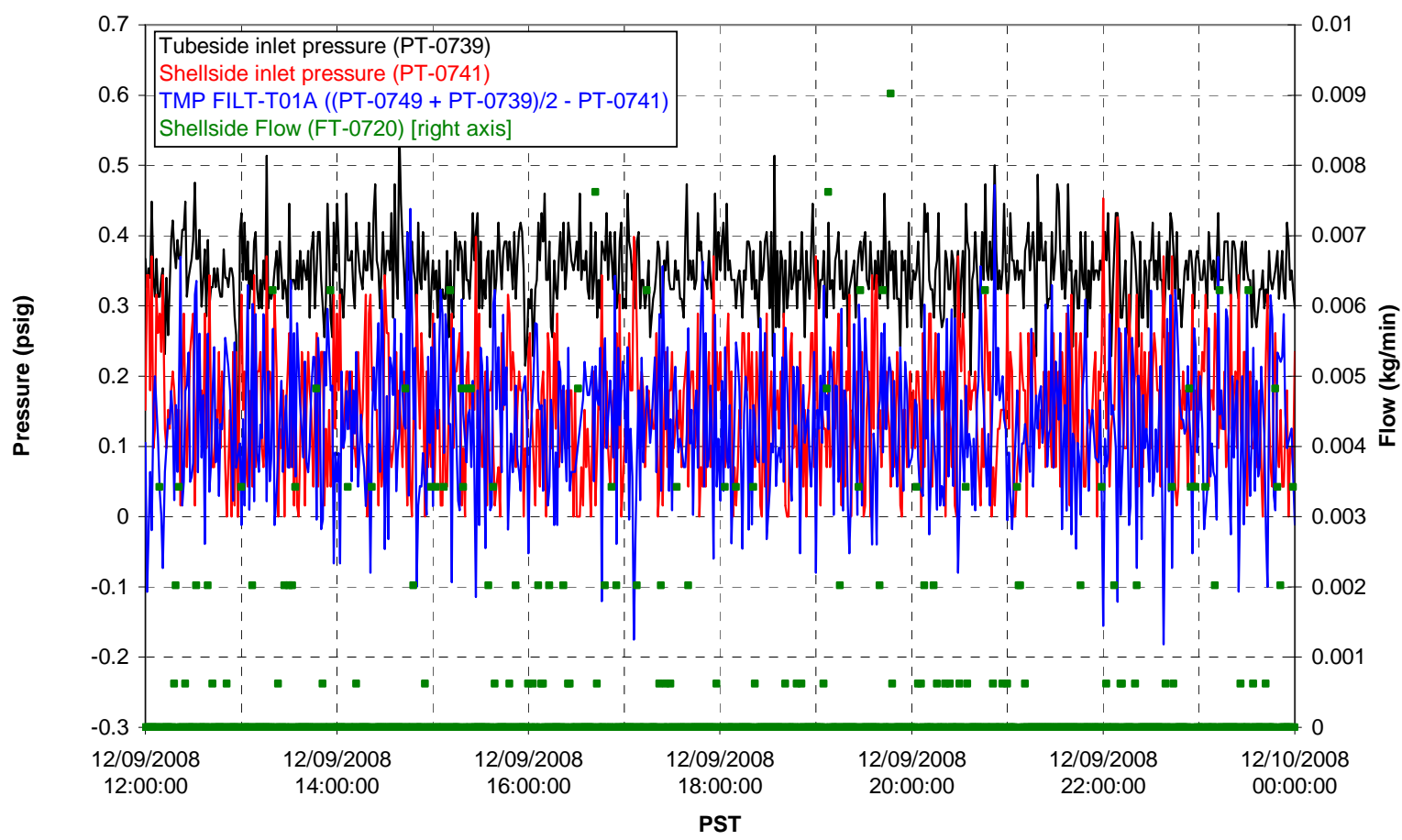

Filter UFP-FILT-T02A

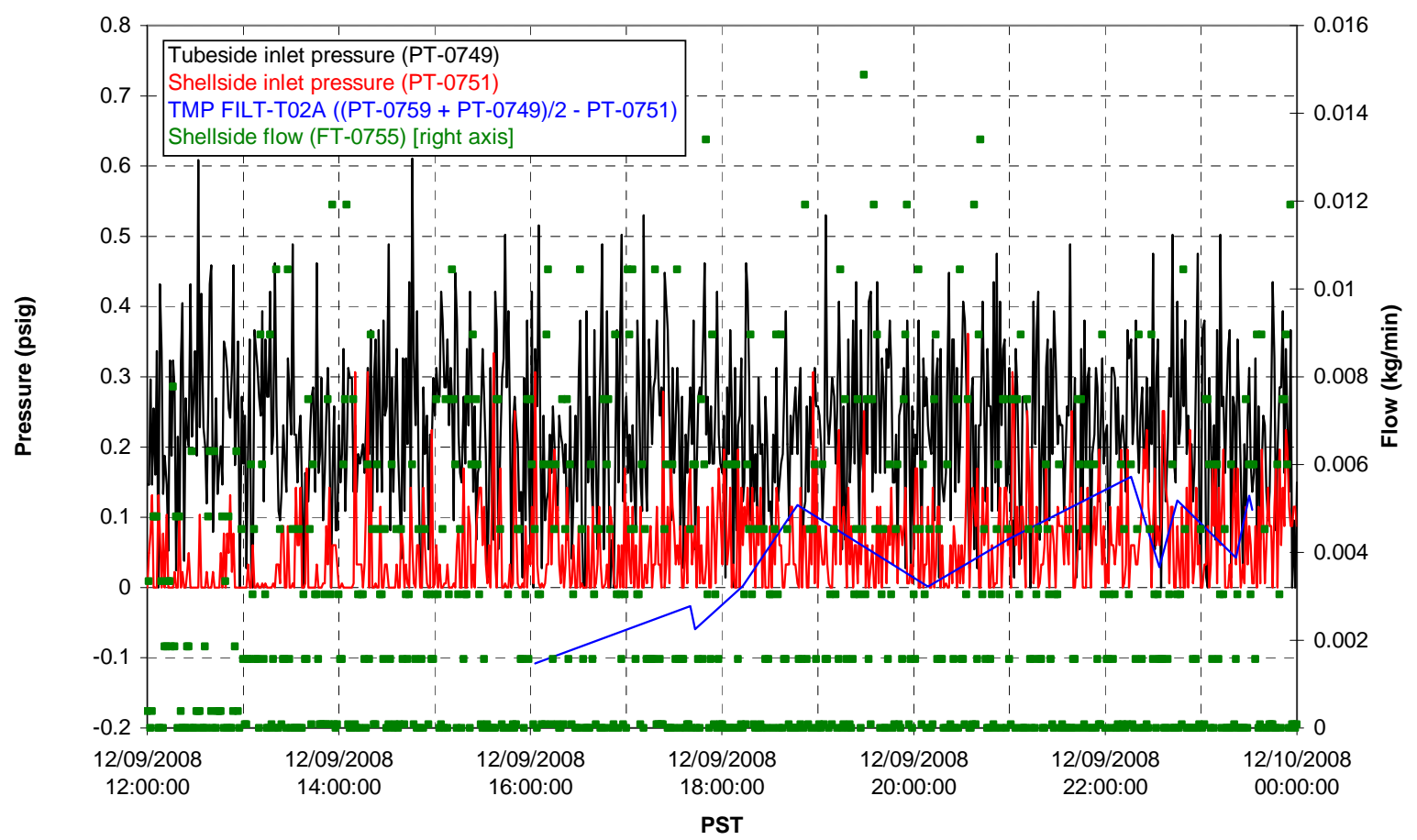


Filter UFP-FILT-T03A

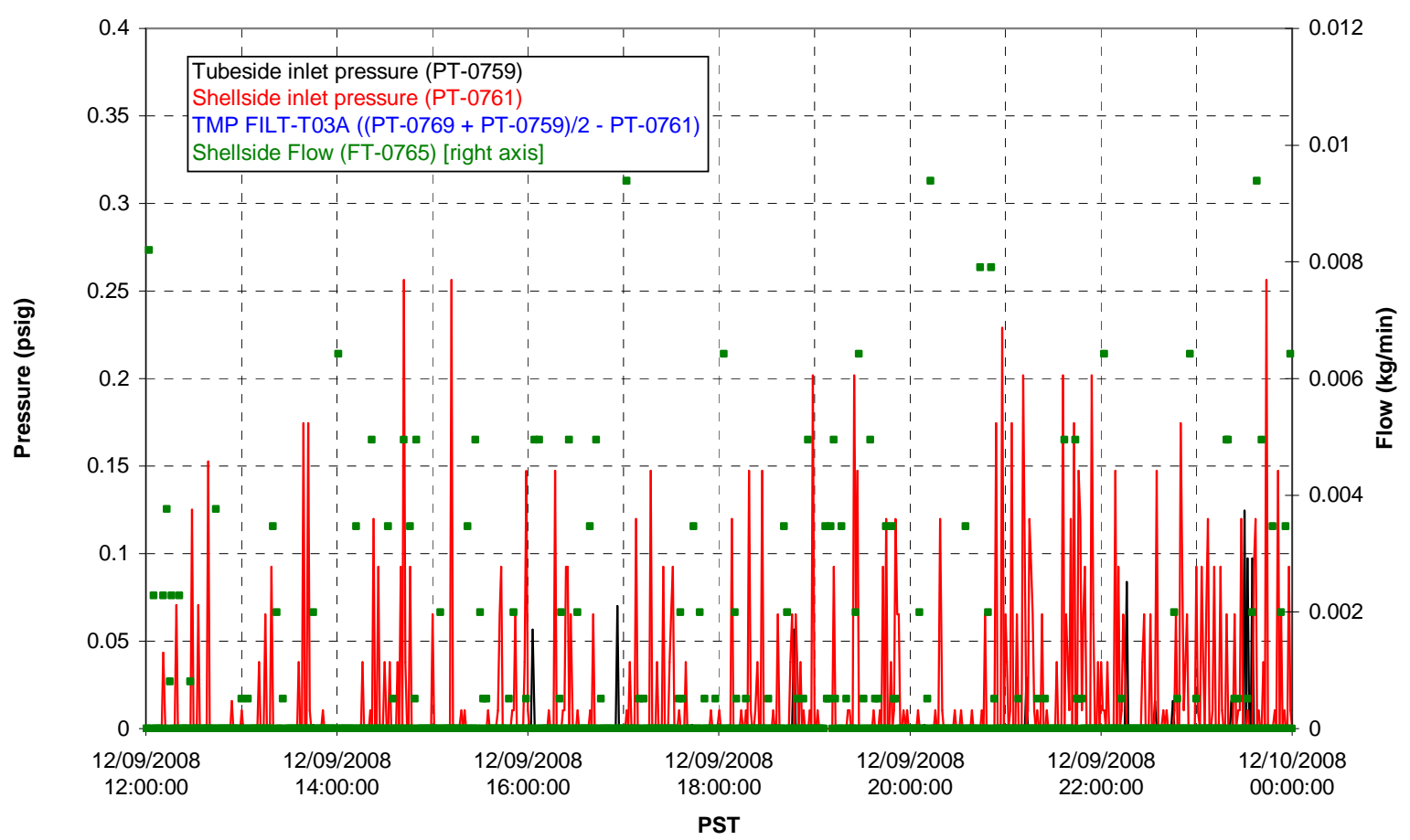

Filter UFP-FILT-T04A

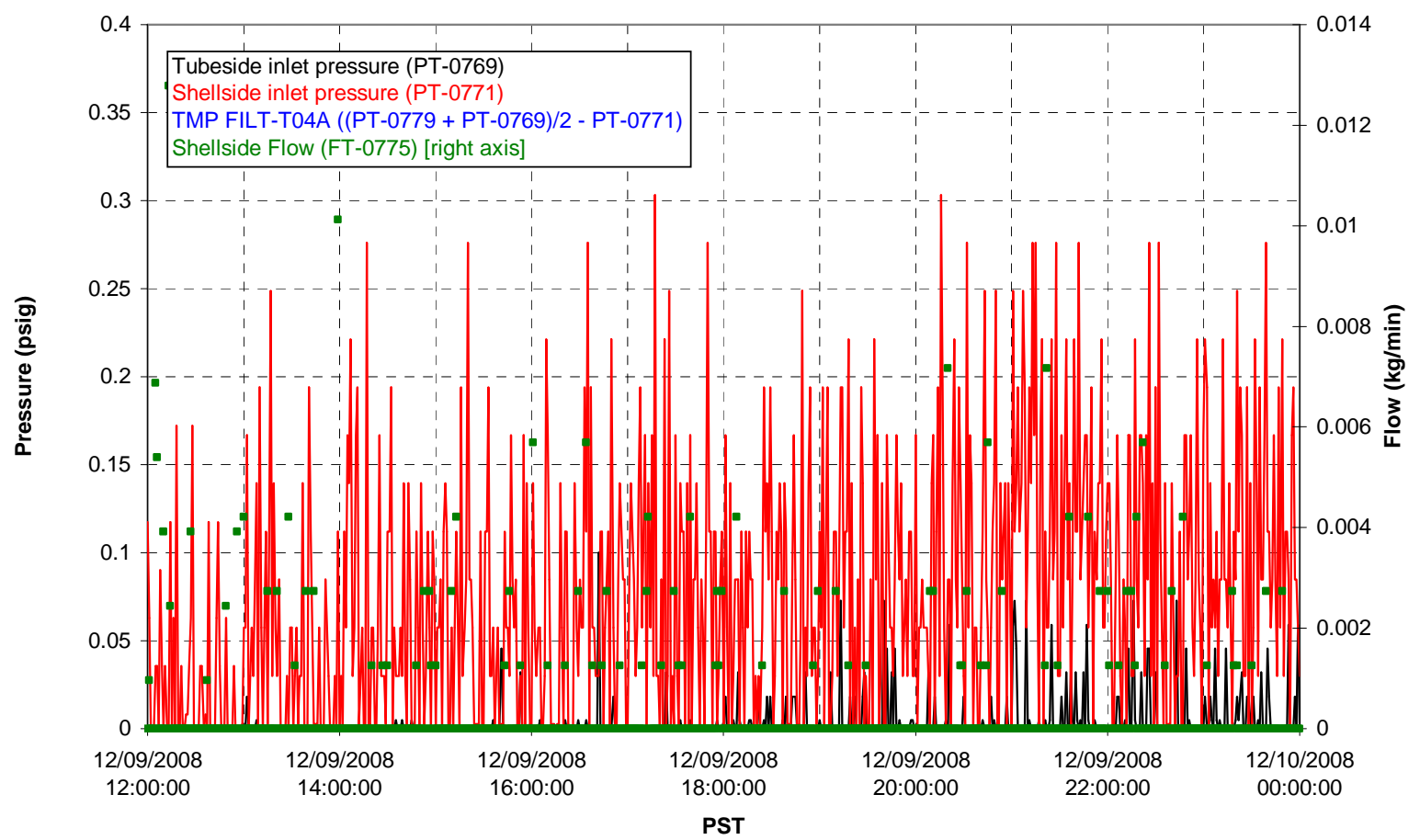


Filter UFP-FILT-T05A

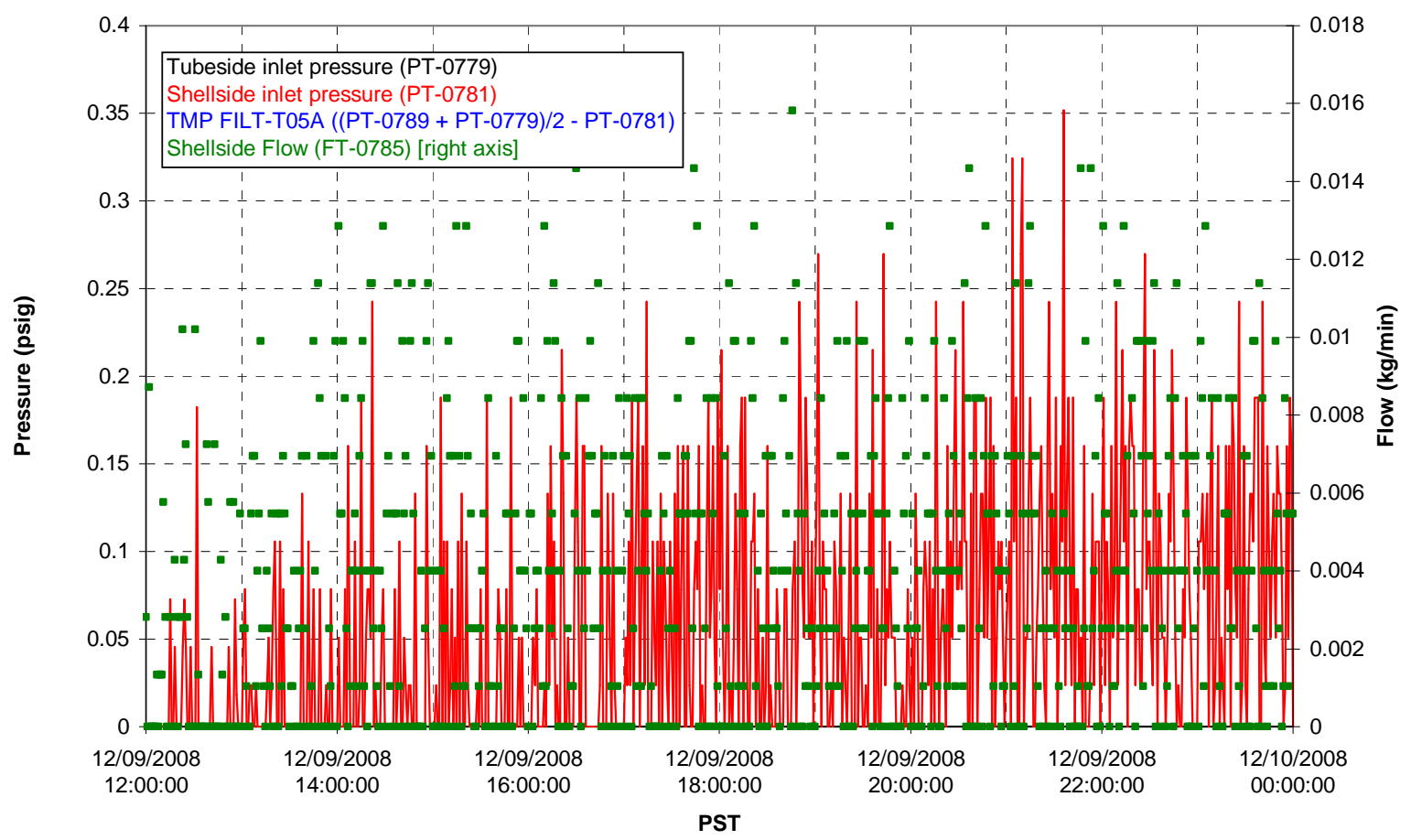

Chemical Flow

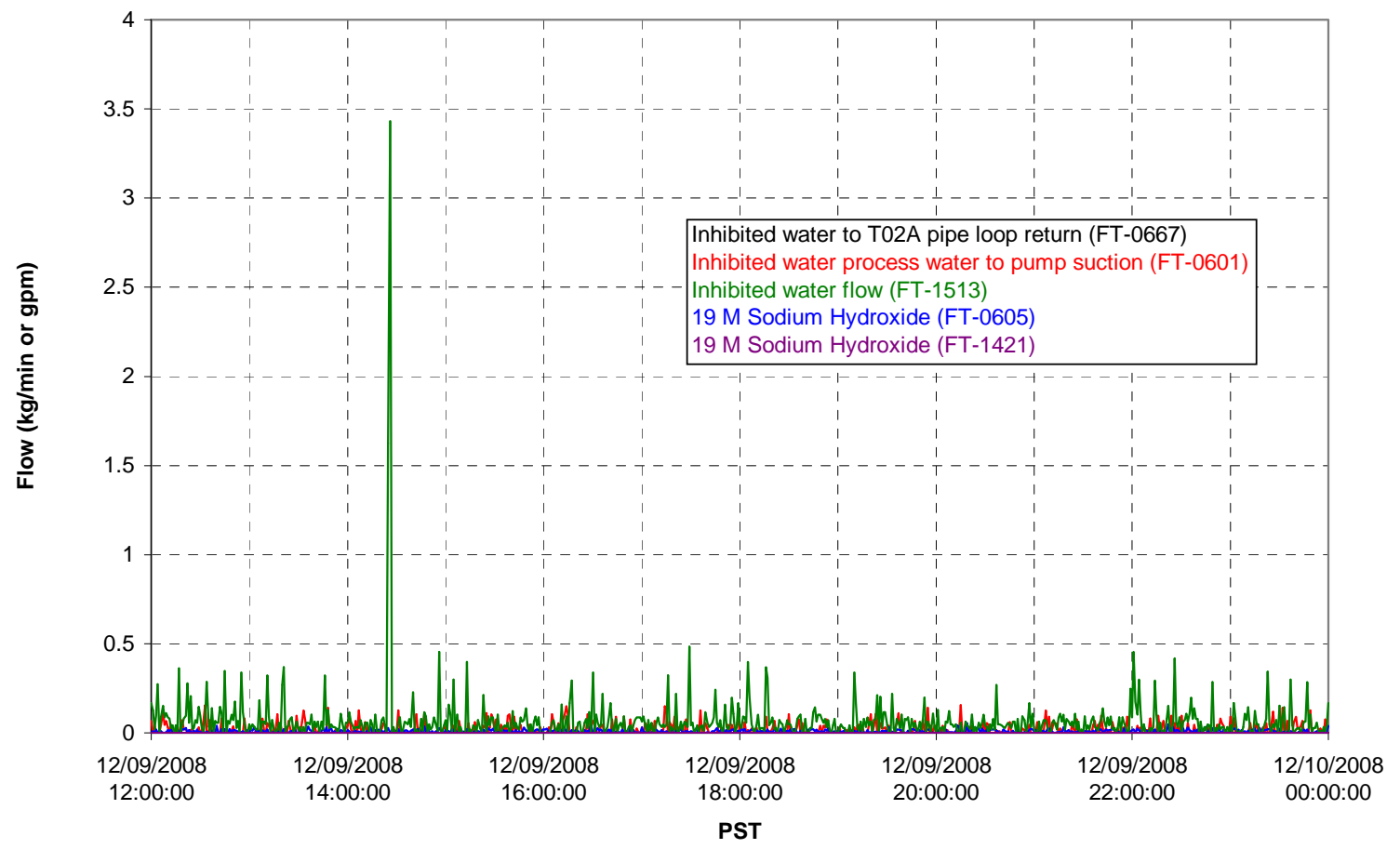




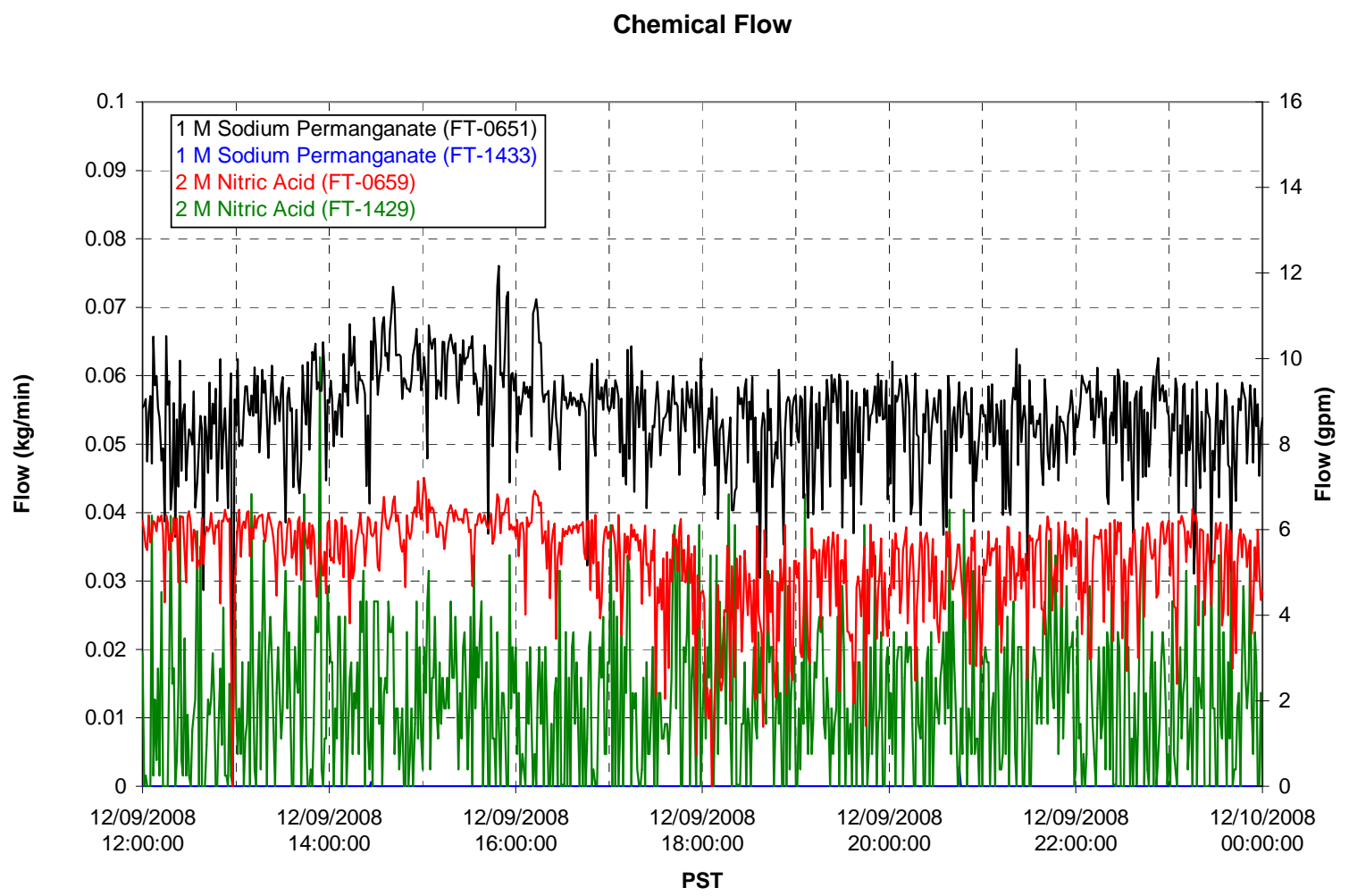

Air Flows

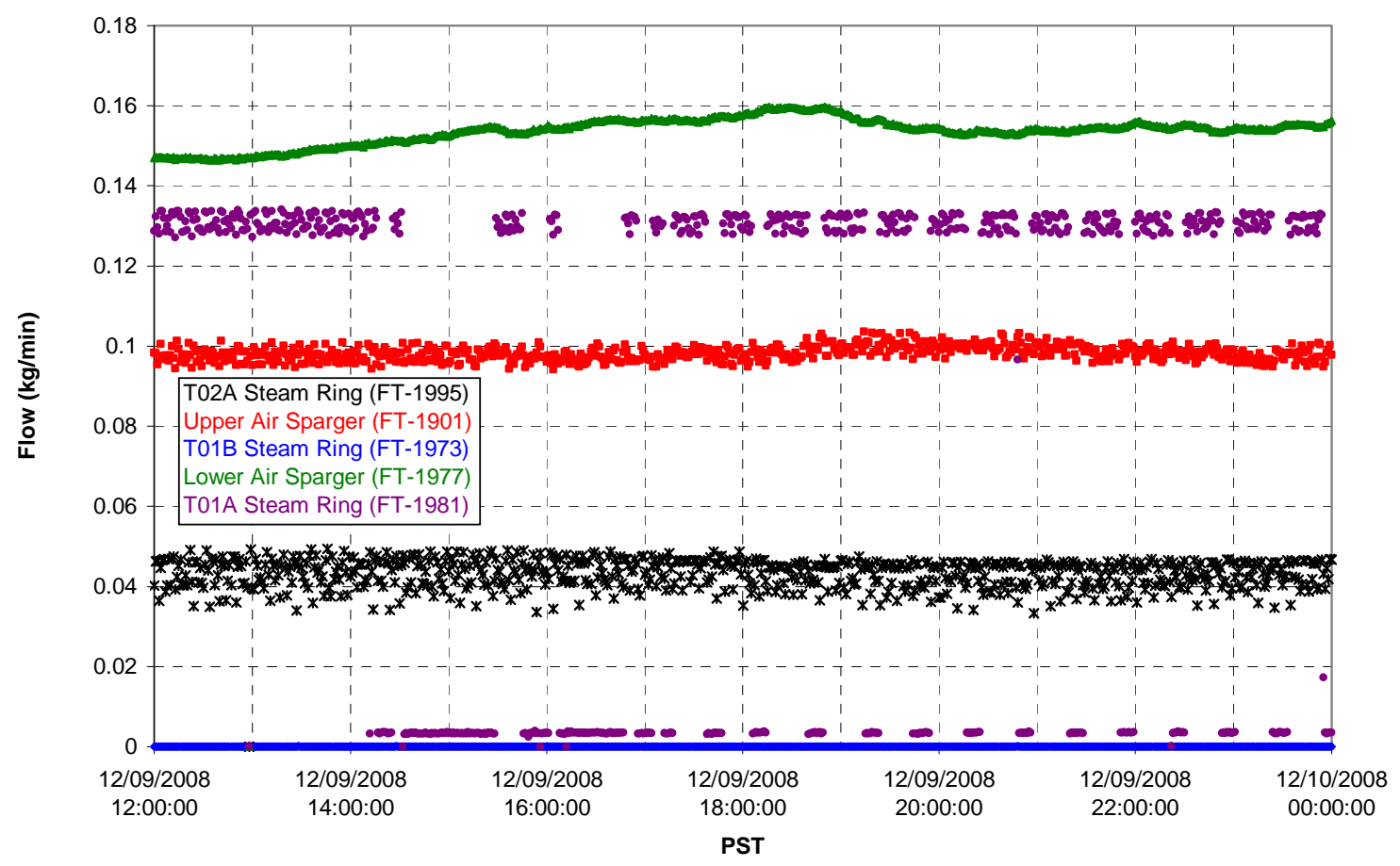


T02A Steam

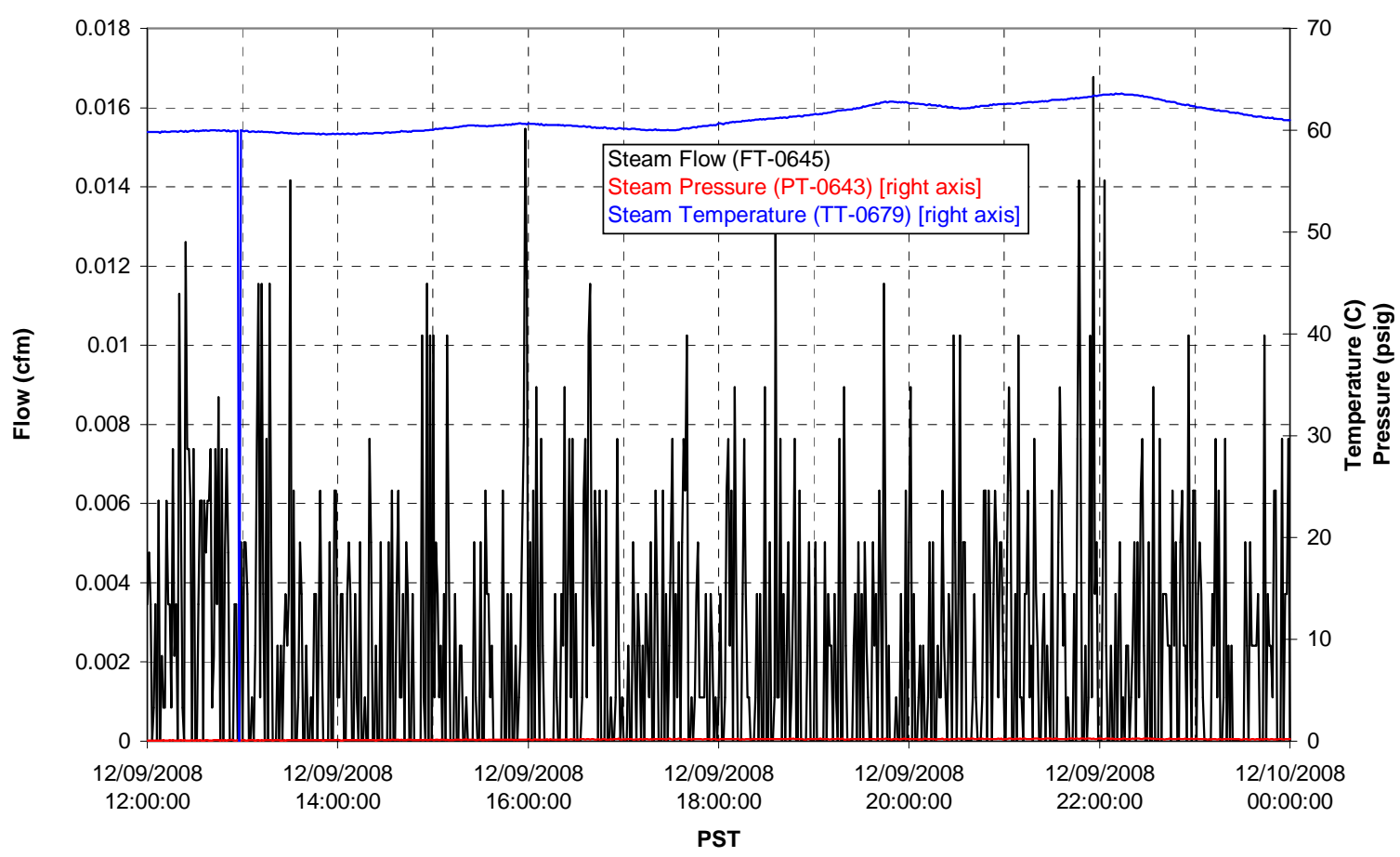

T01A Steam

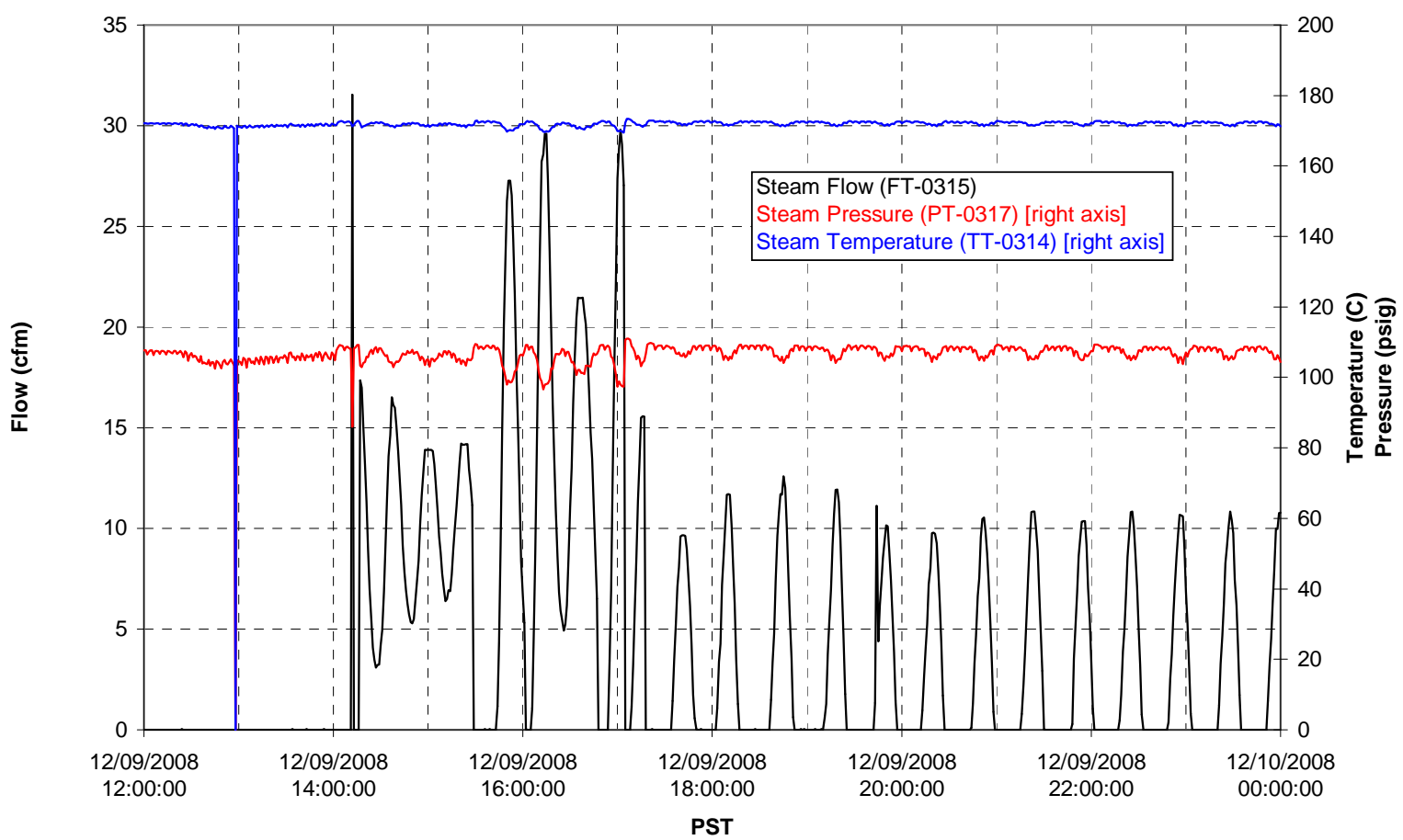


T01B Steam

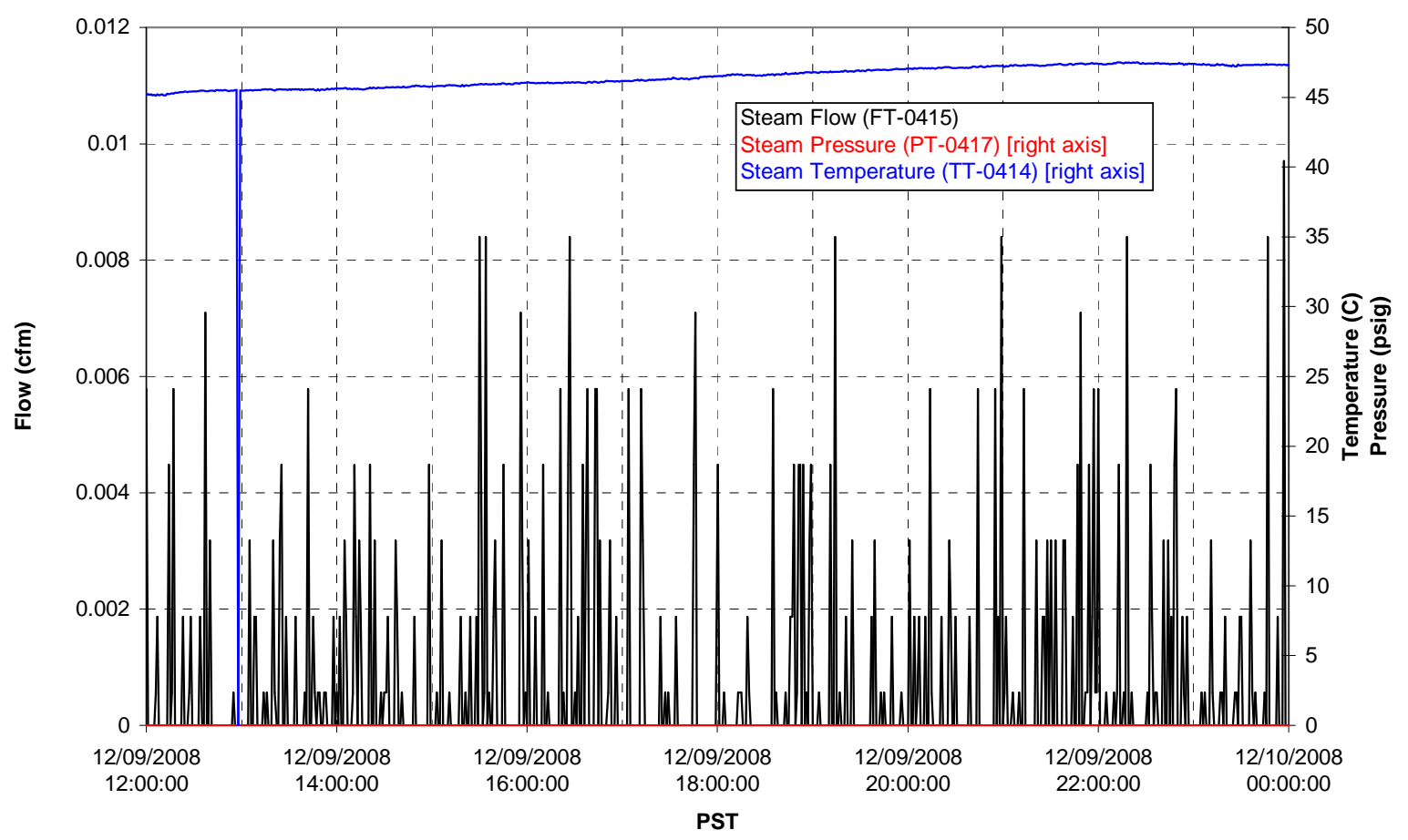


Functional Test Data Plots

12/10/08 00:00 - 12/10/08 12:00 
T01A level

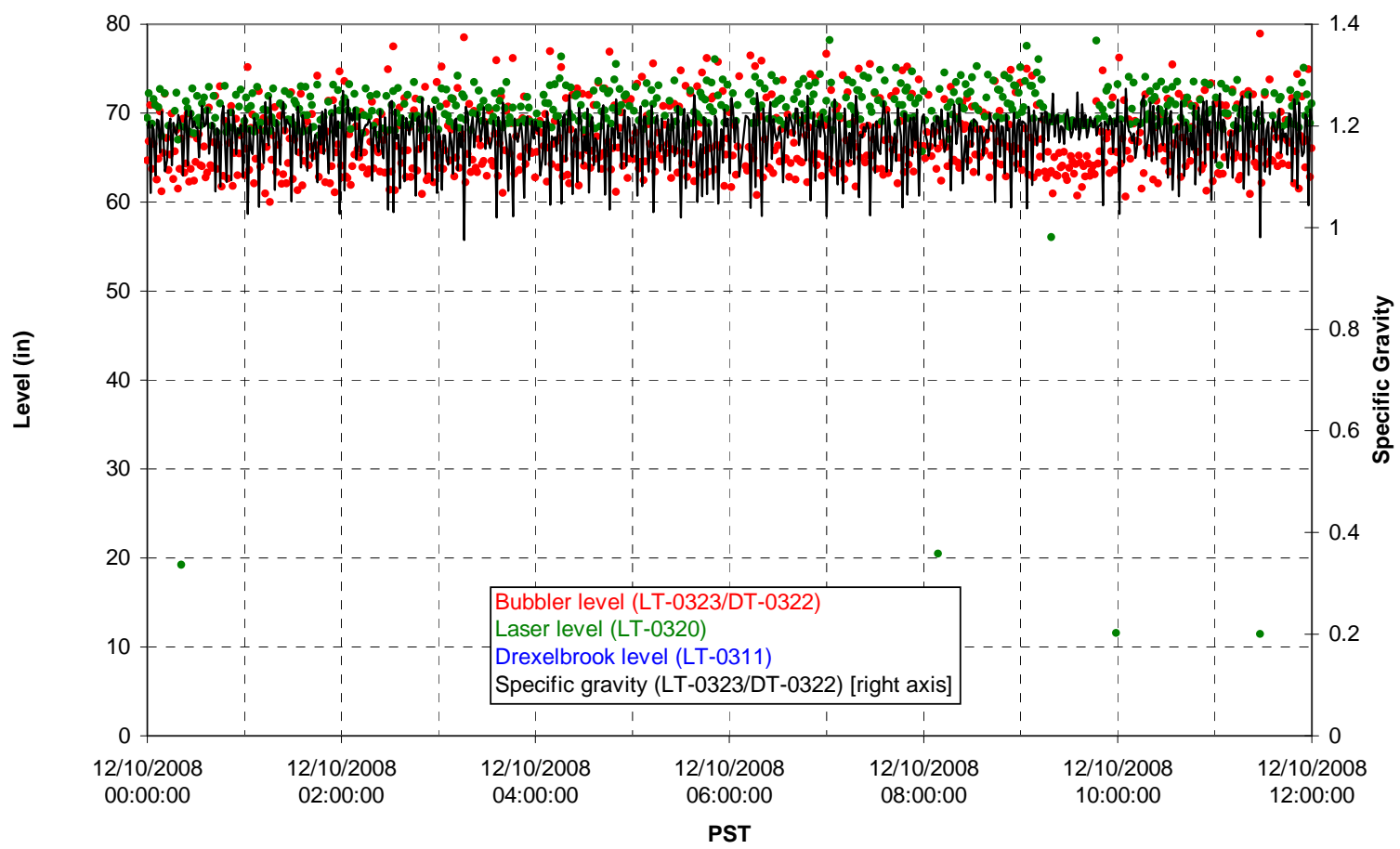

T01A temperatures

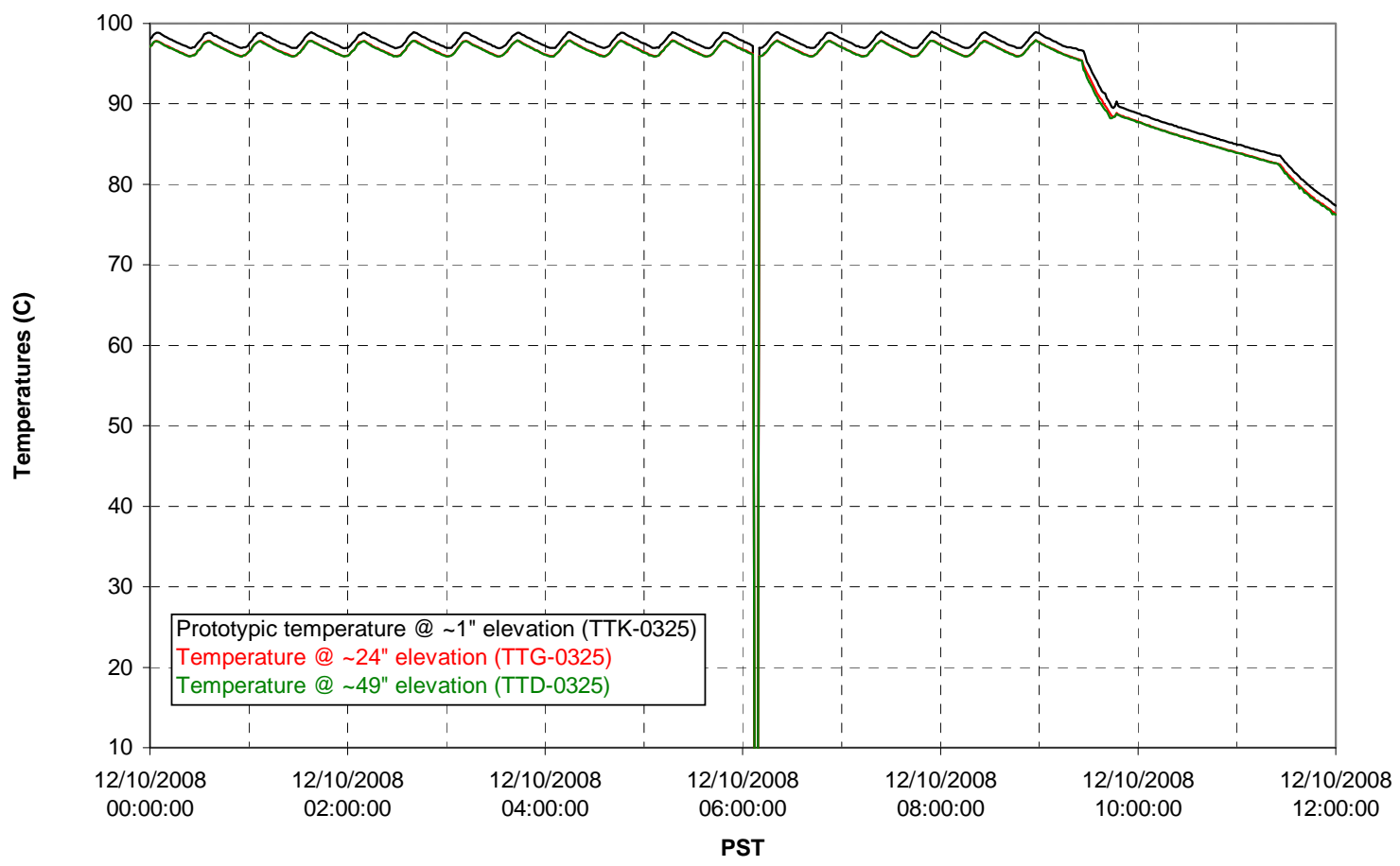


T01B level

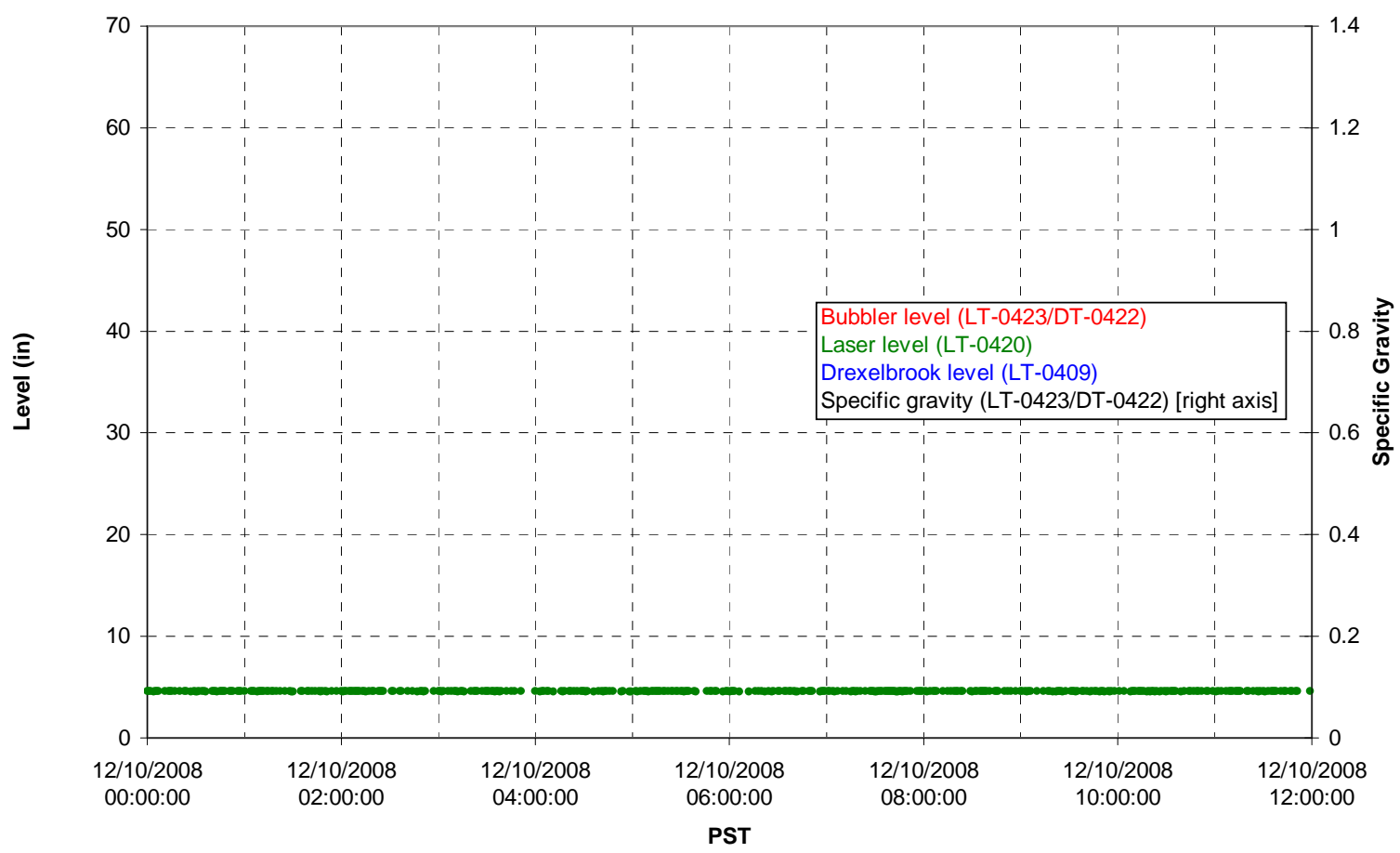

T01B temperatures

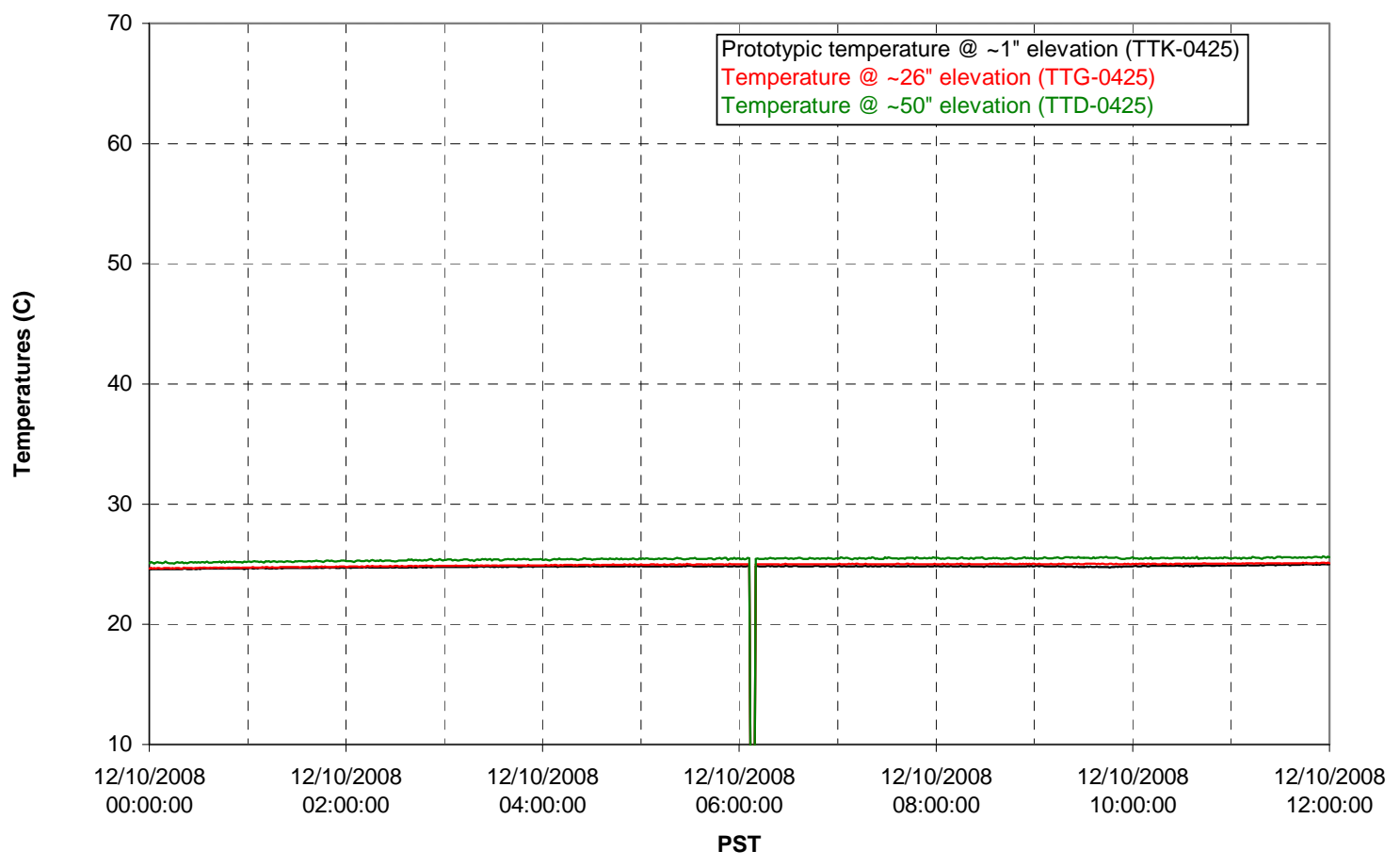


T02A level

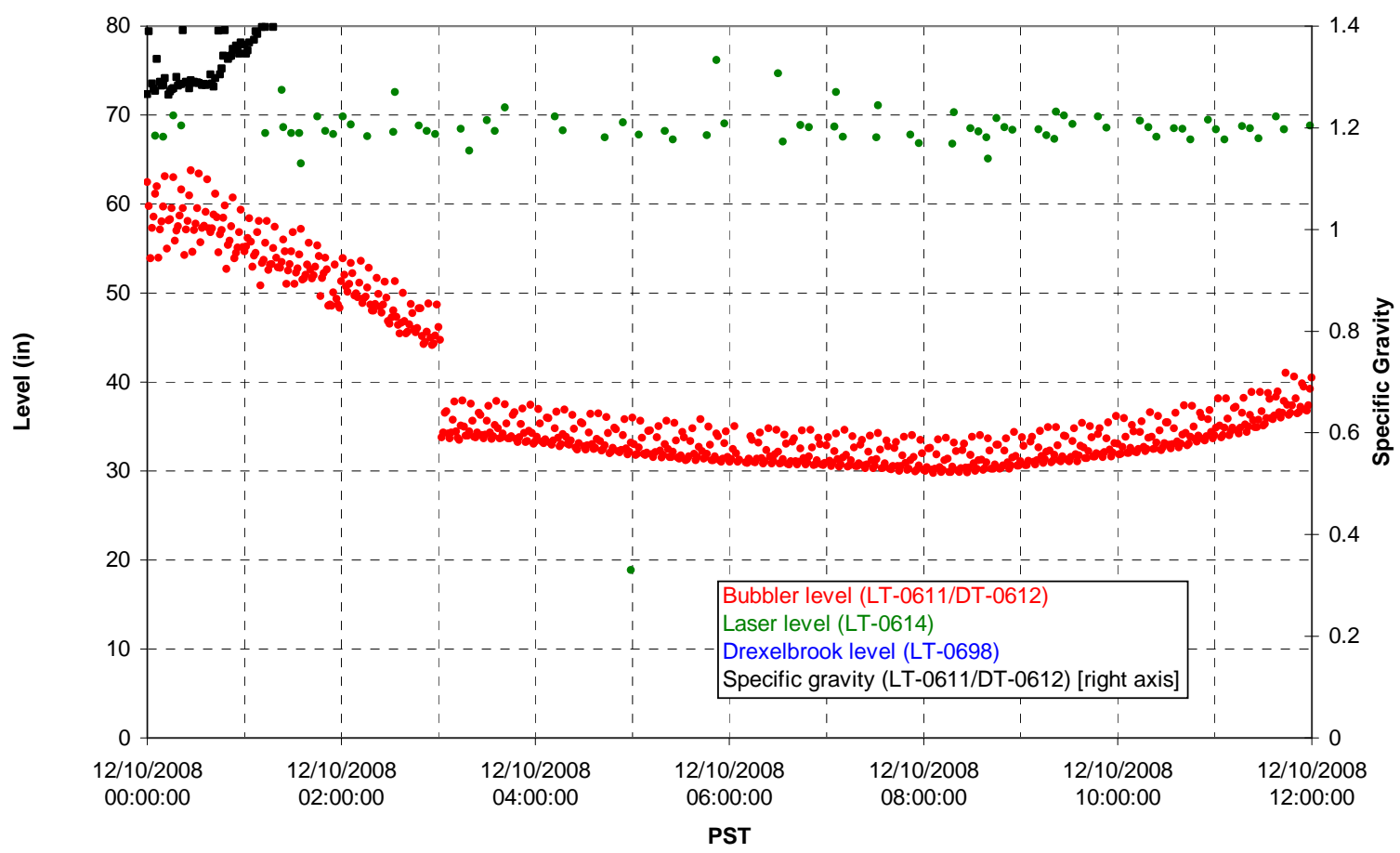

T02A temperatures

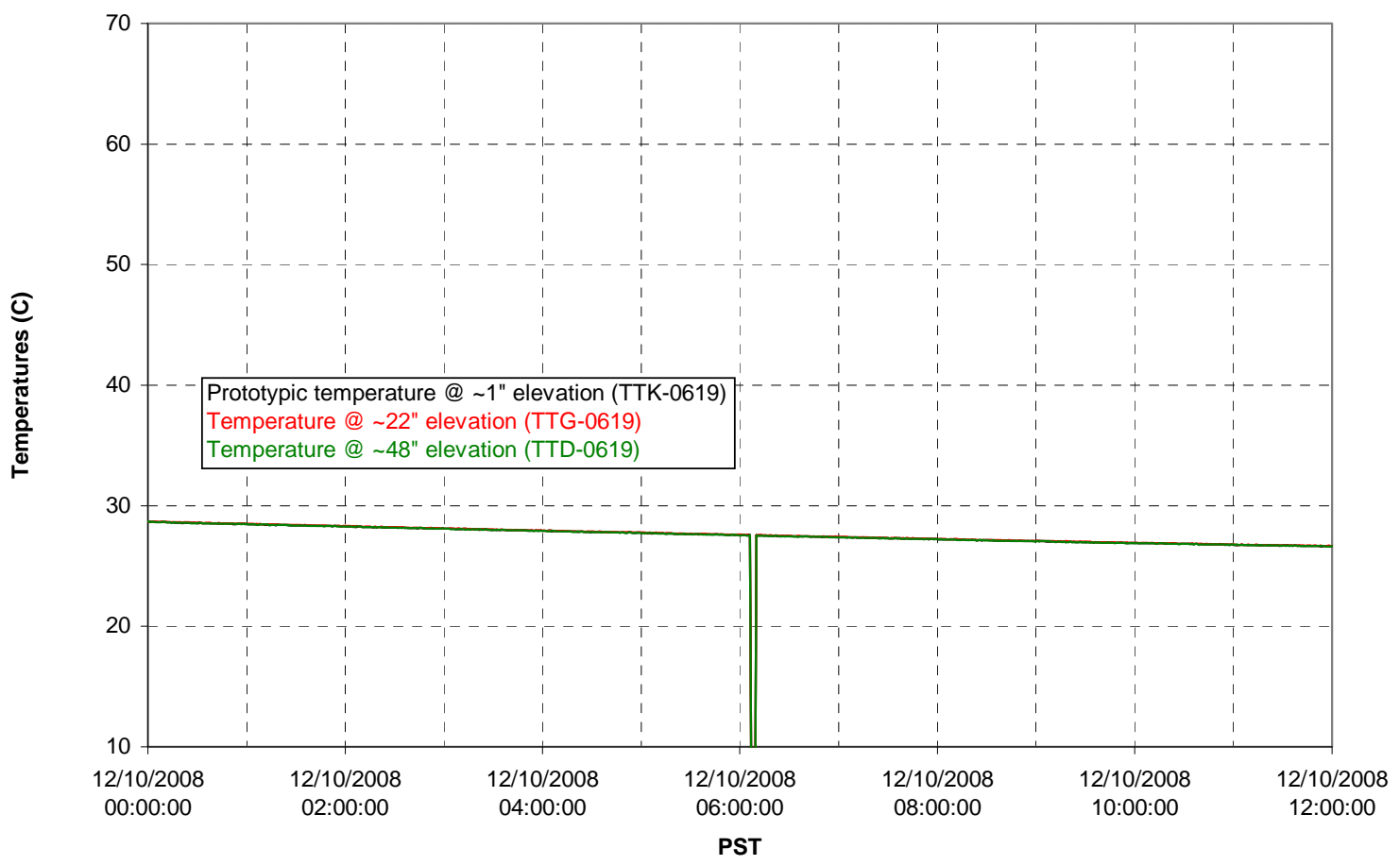


T02A and filter loop temperatures

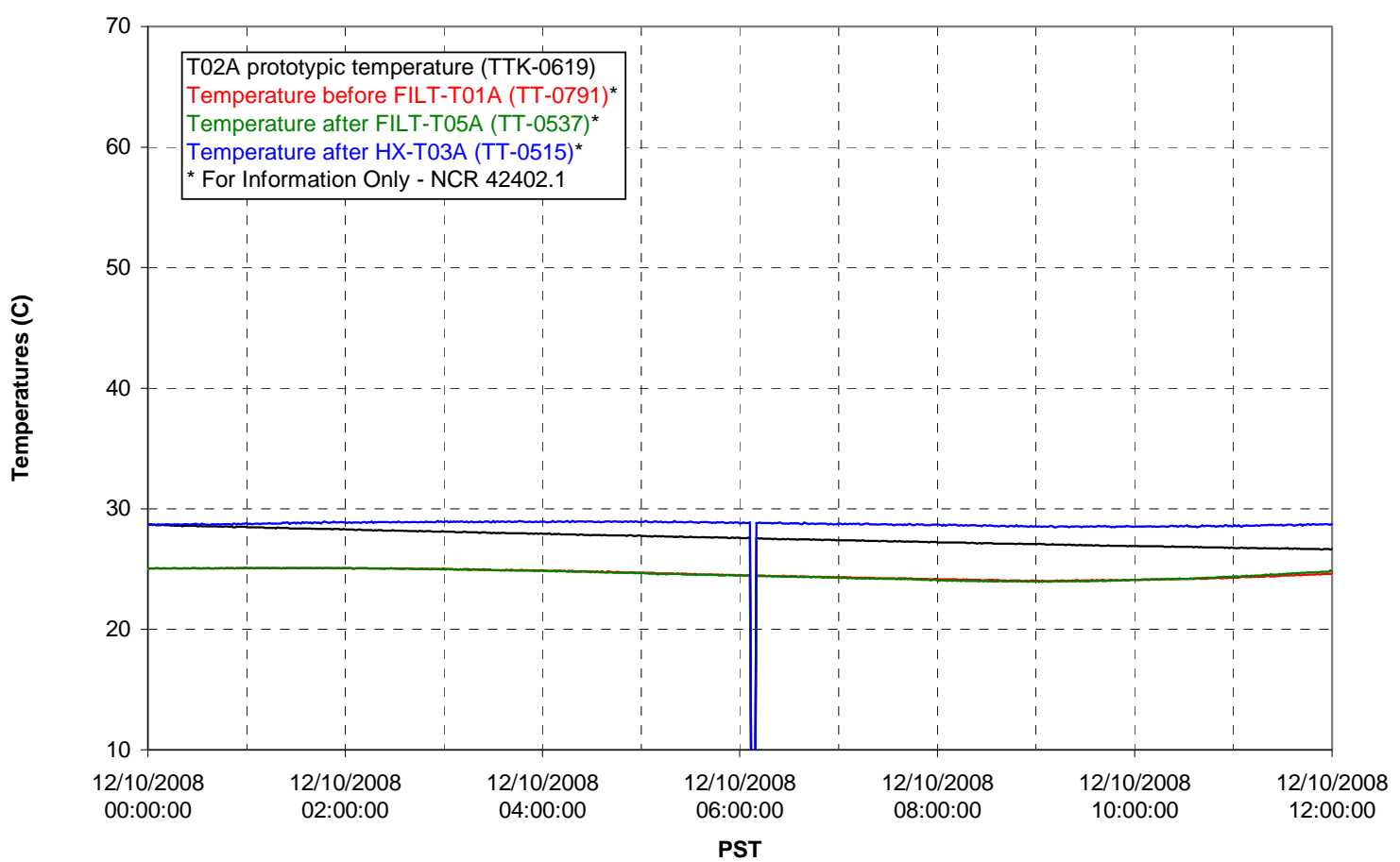

Pump Pressures and Flow

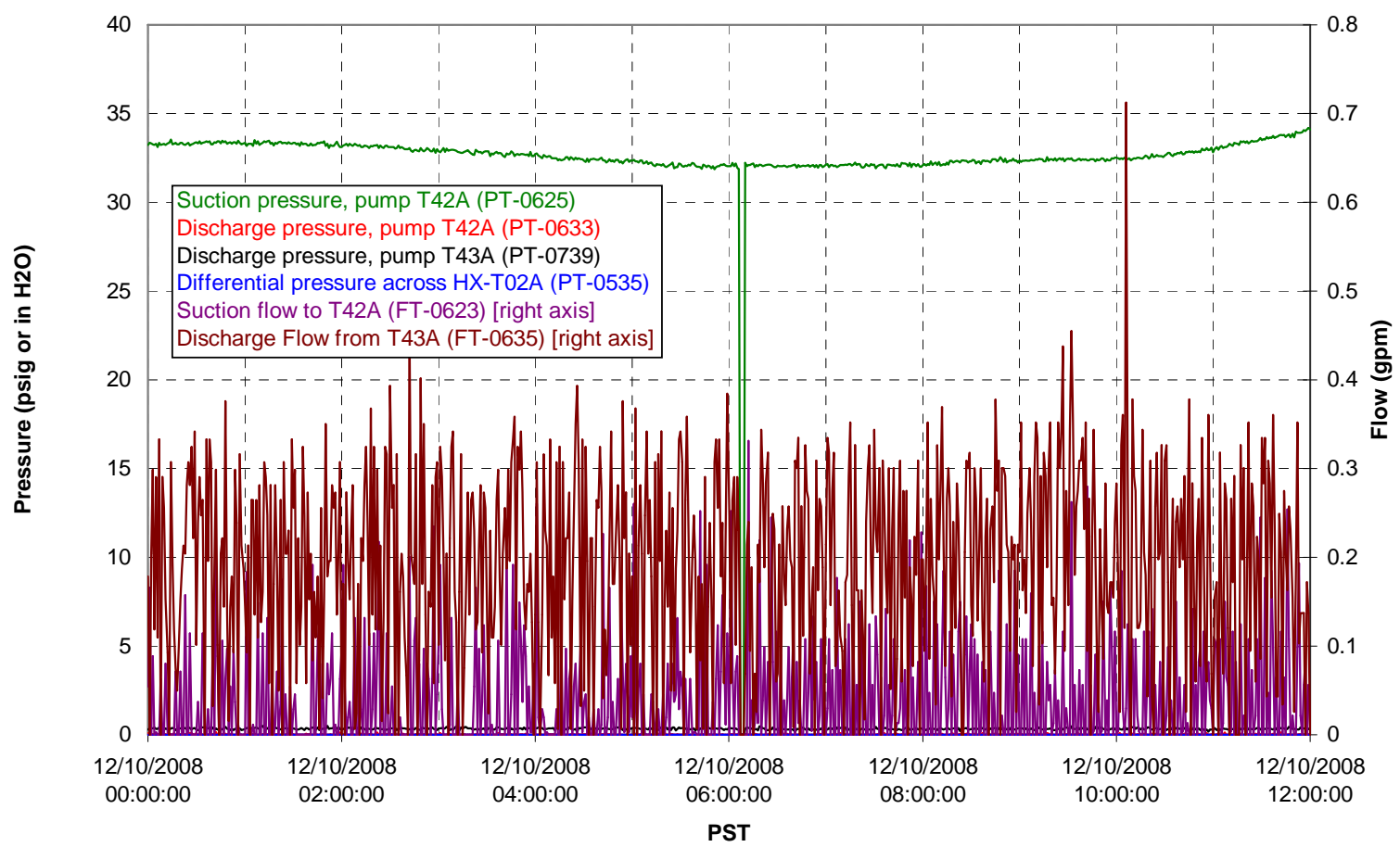


Axial pressure drop

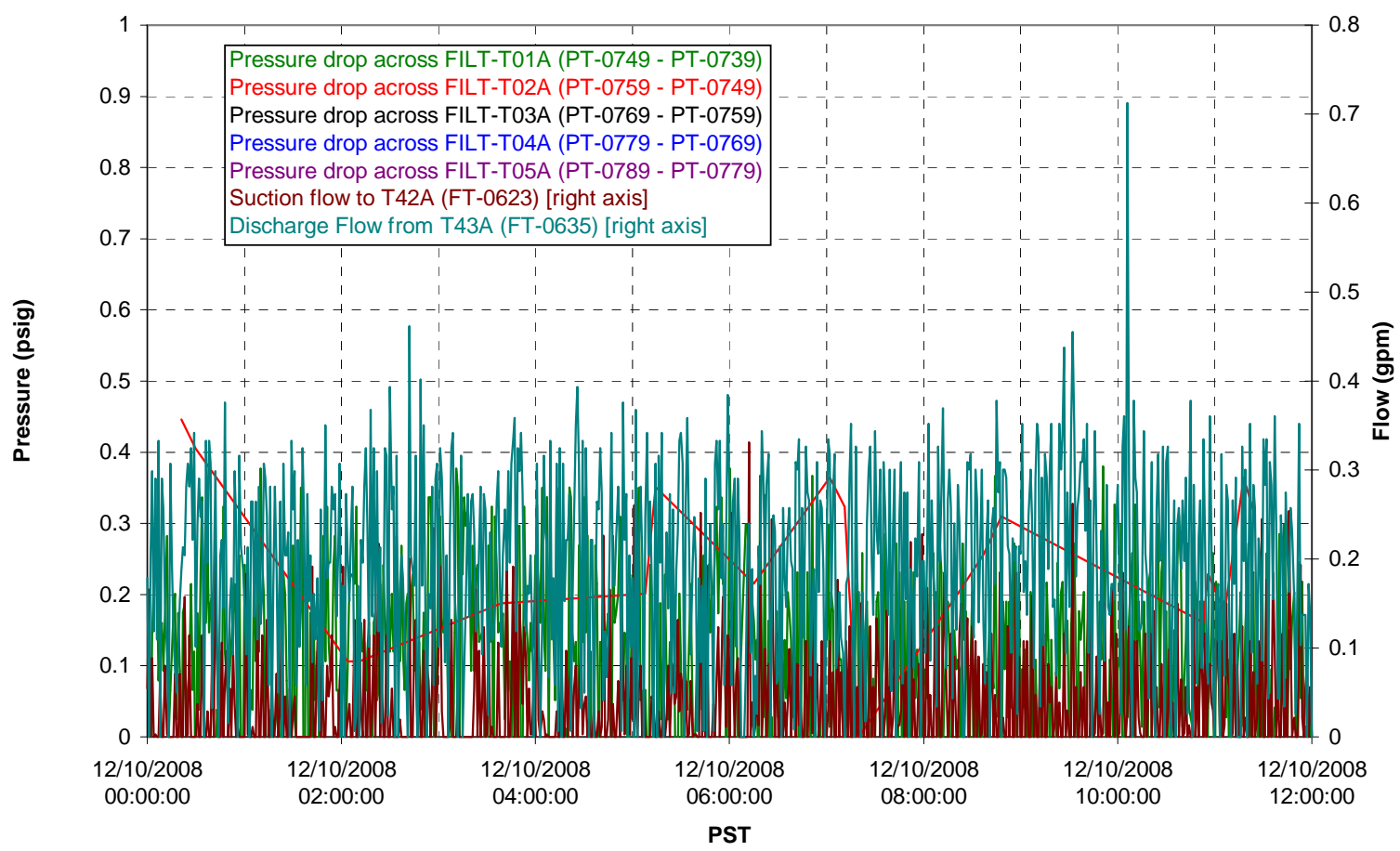

Permeate flow rates

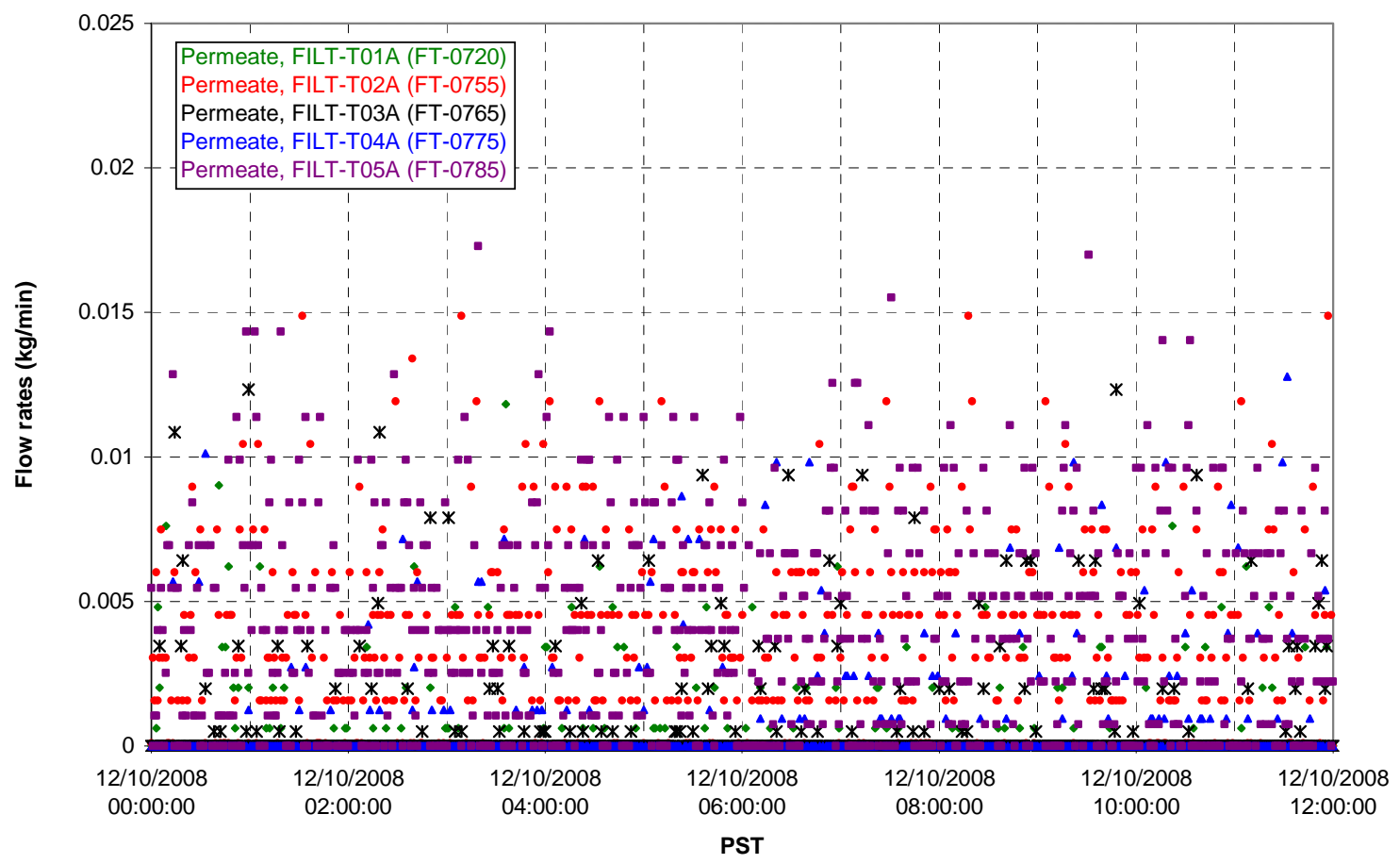


T02A Inner Temperature Tree

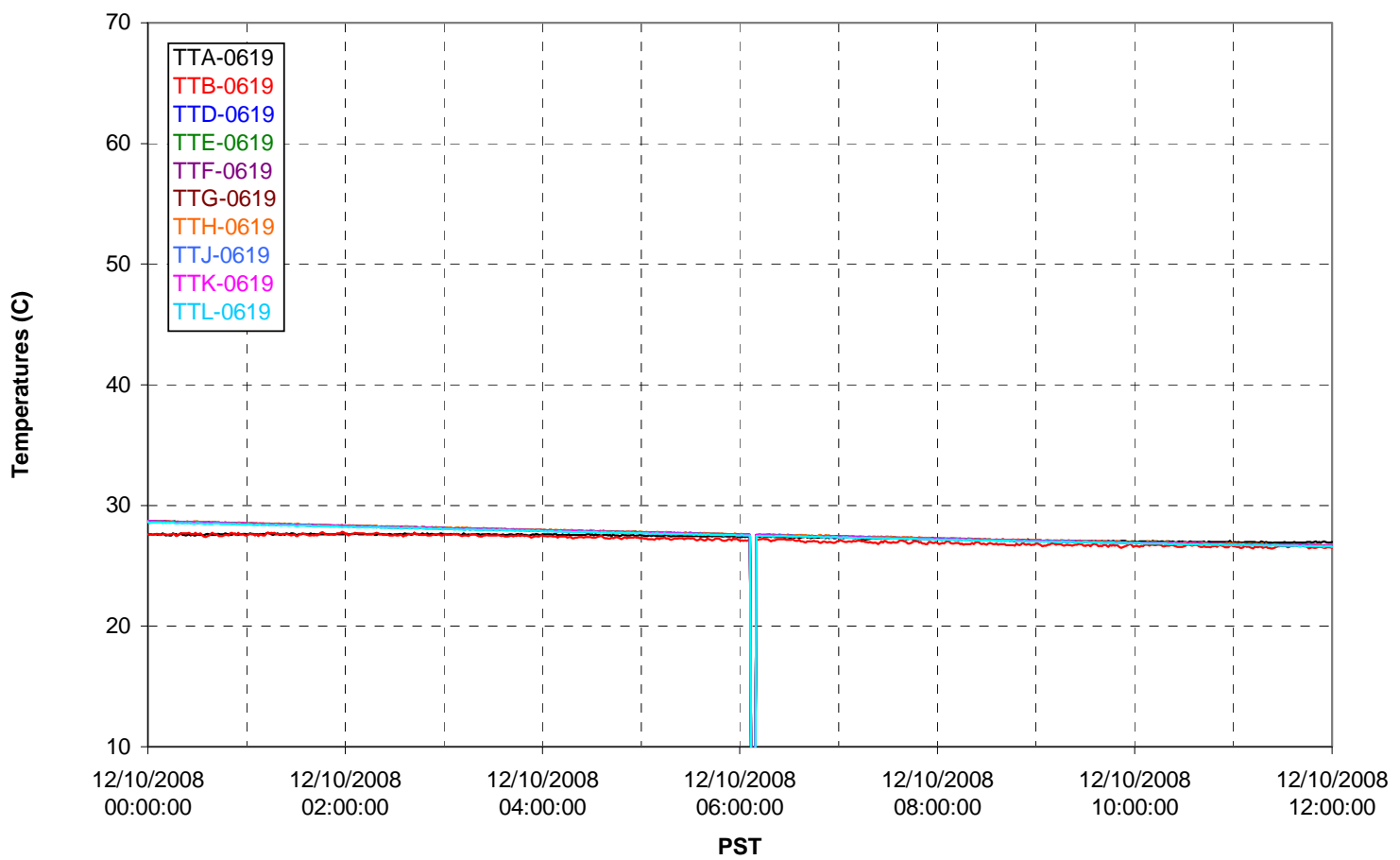

T02A Outer Temperature Tree

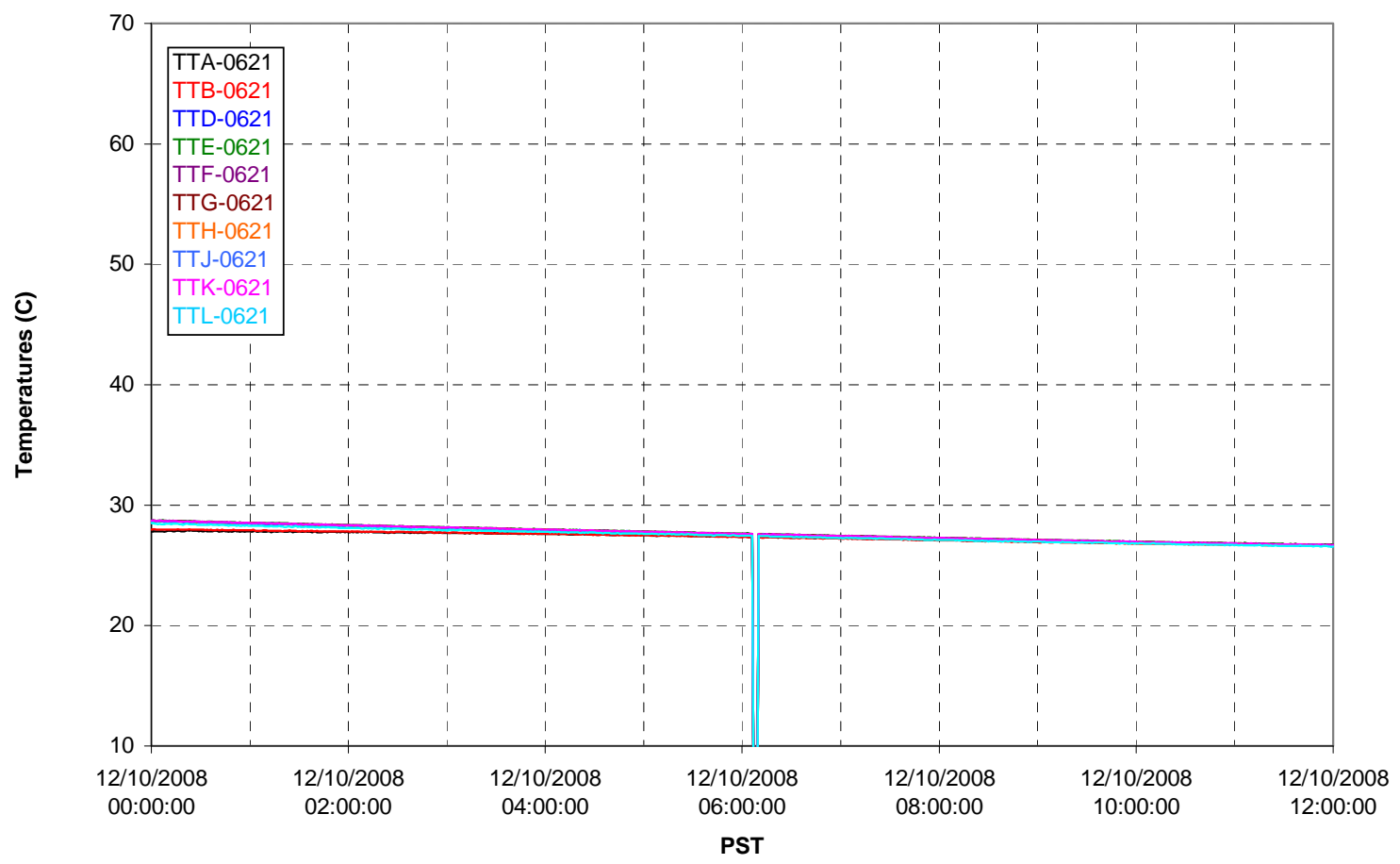


T02A temperatures

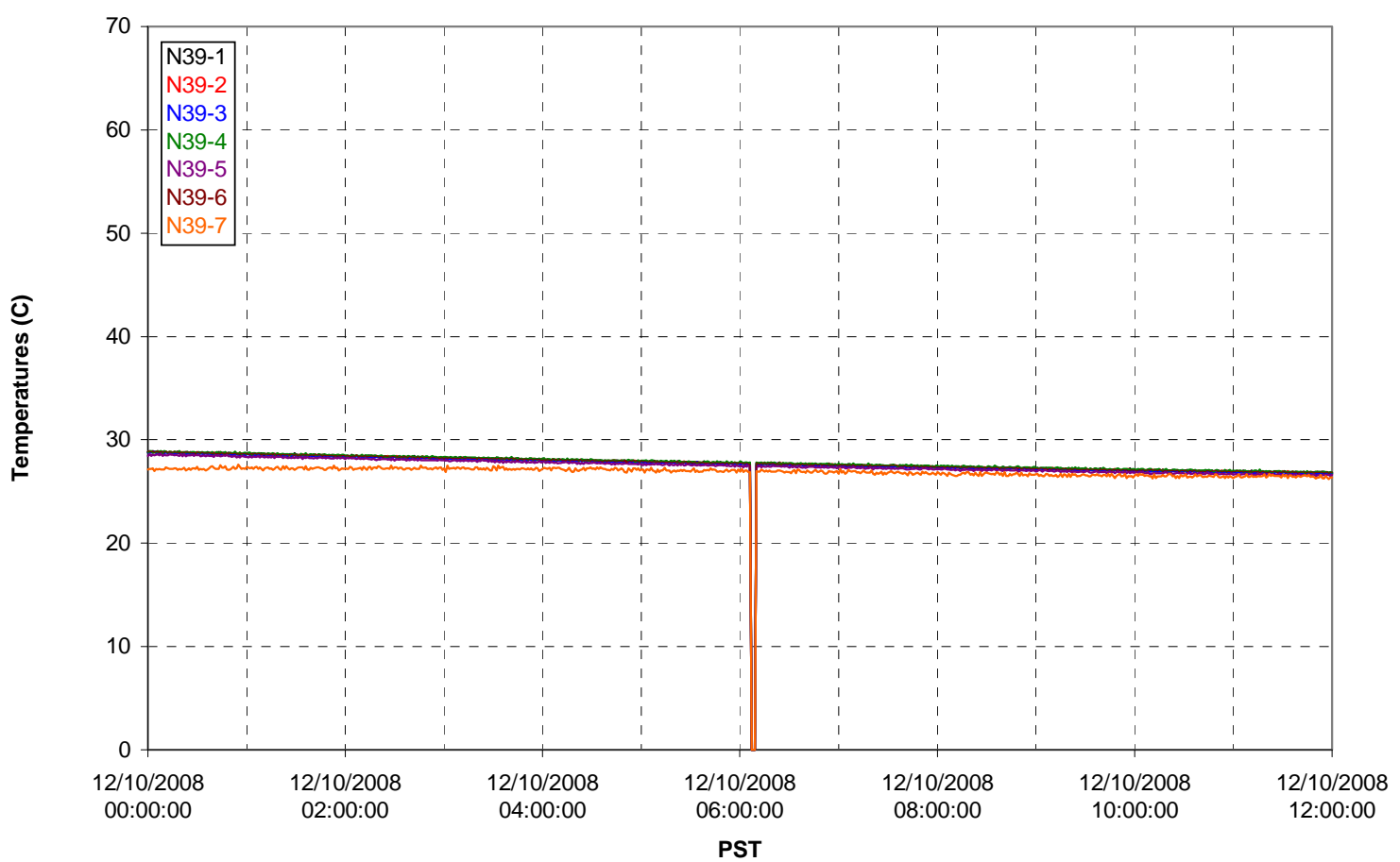

T02A temperatures

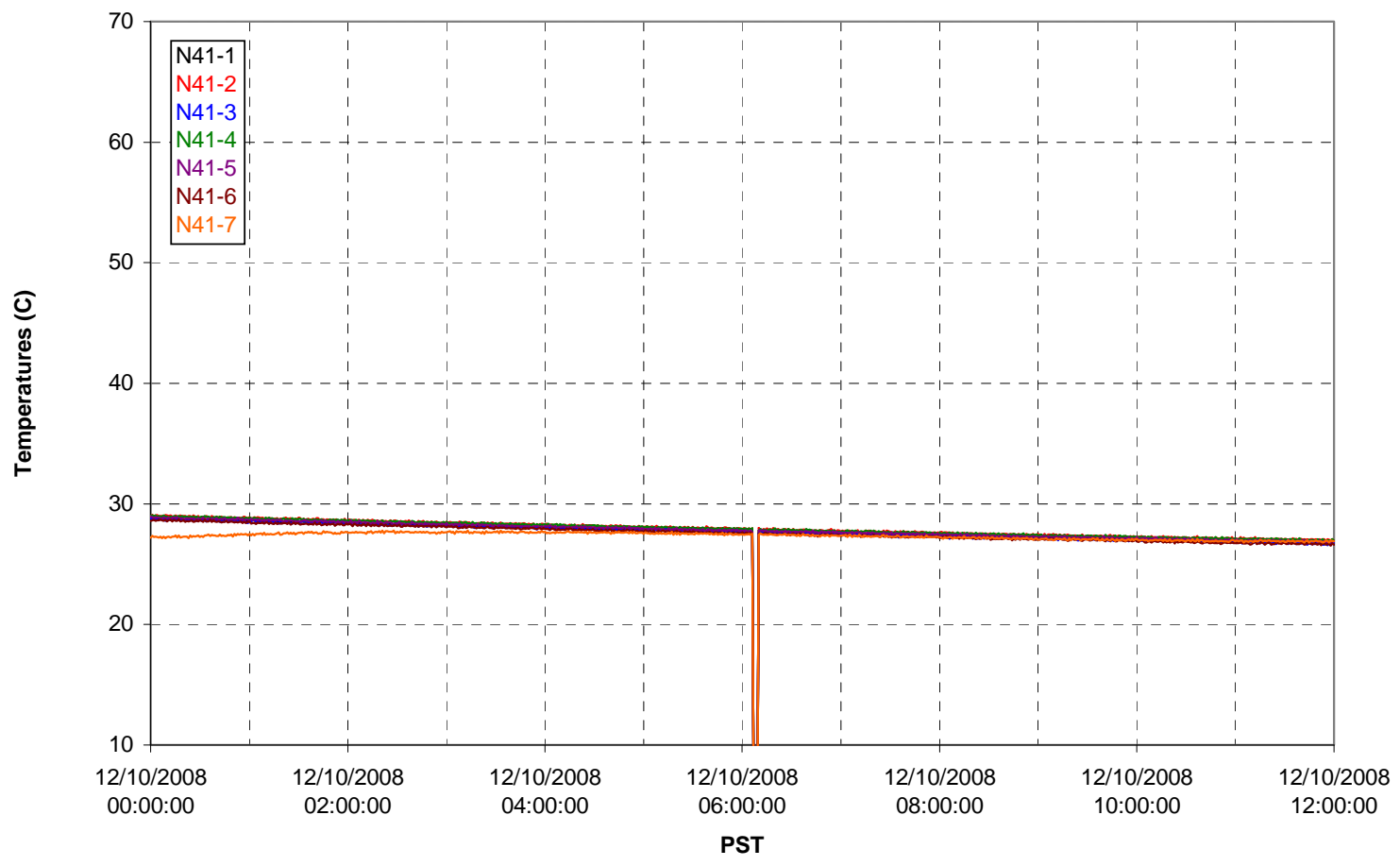


T02A temperatures

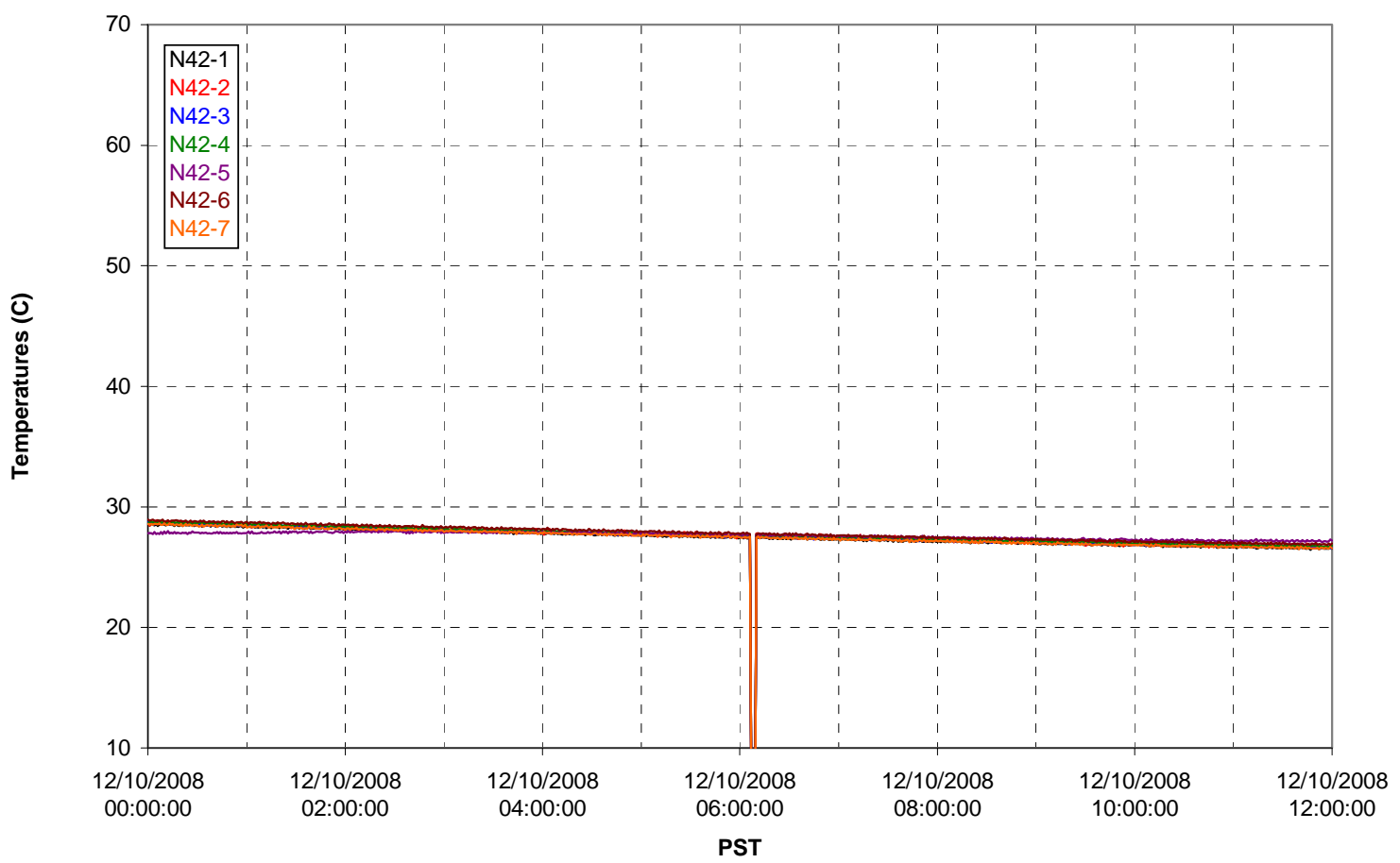

T02A temperatures

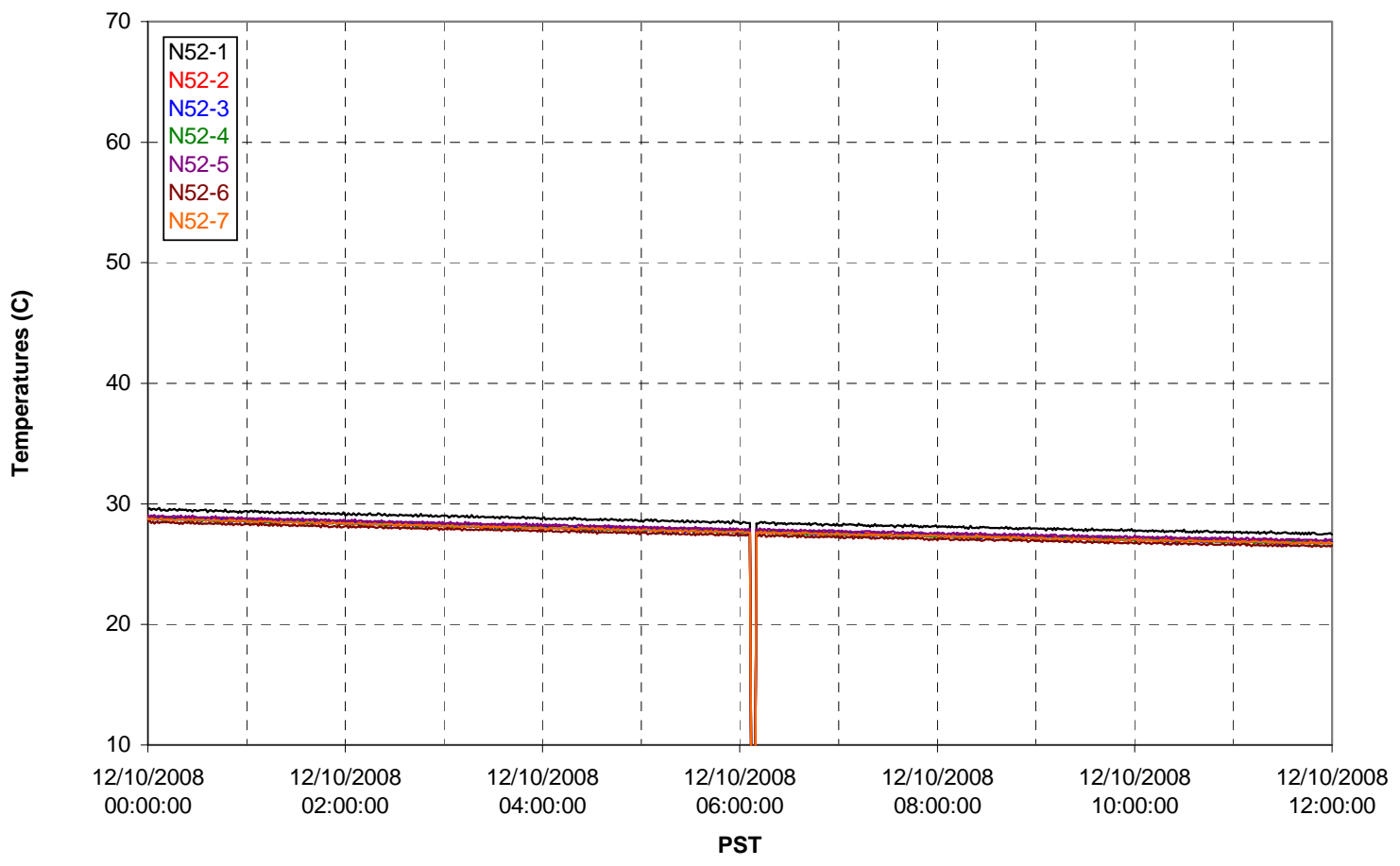


T02A Heating and Cooling

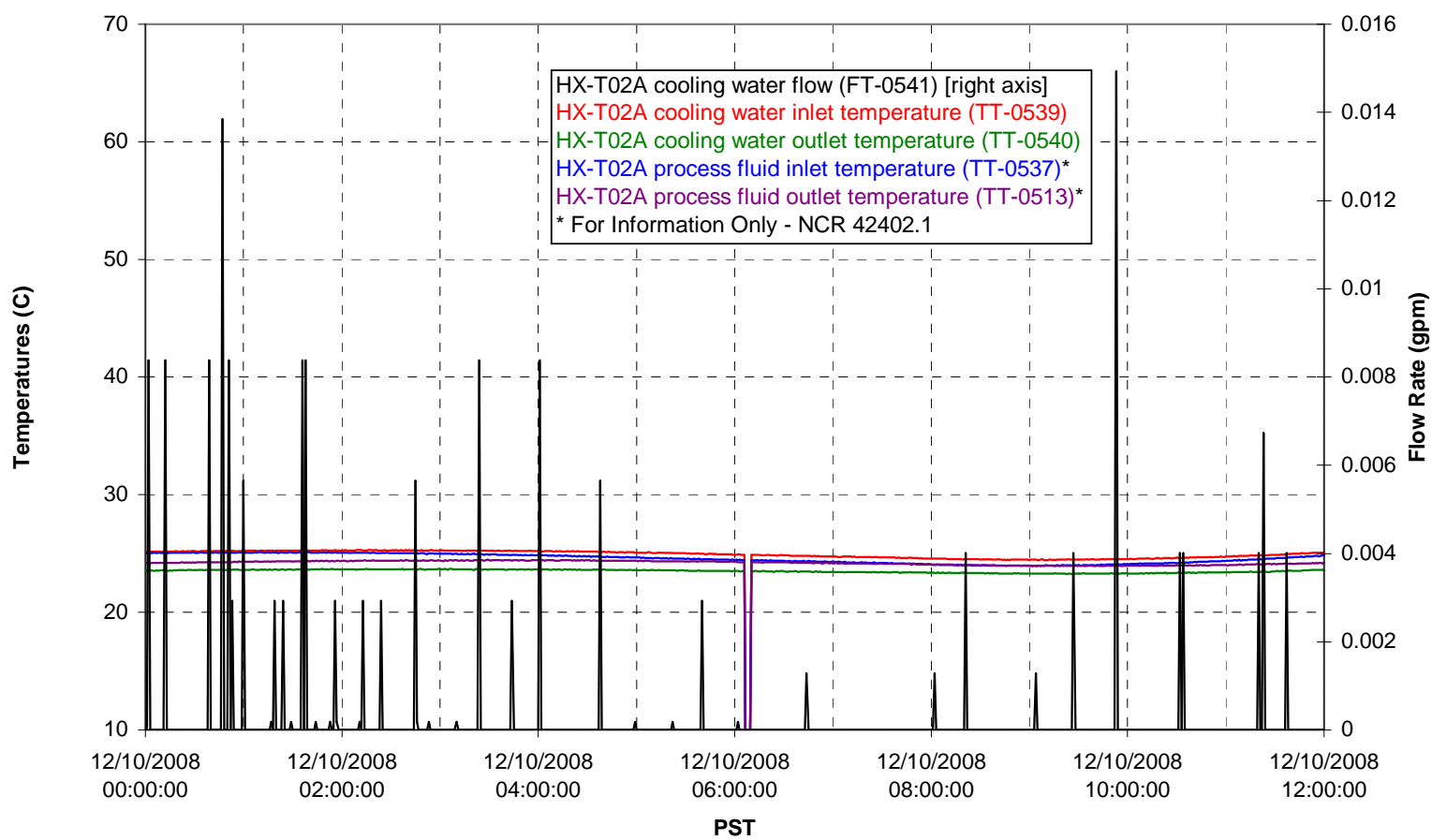

Pump Operation

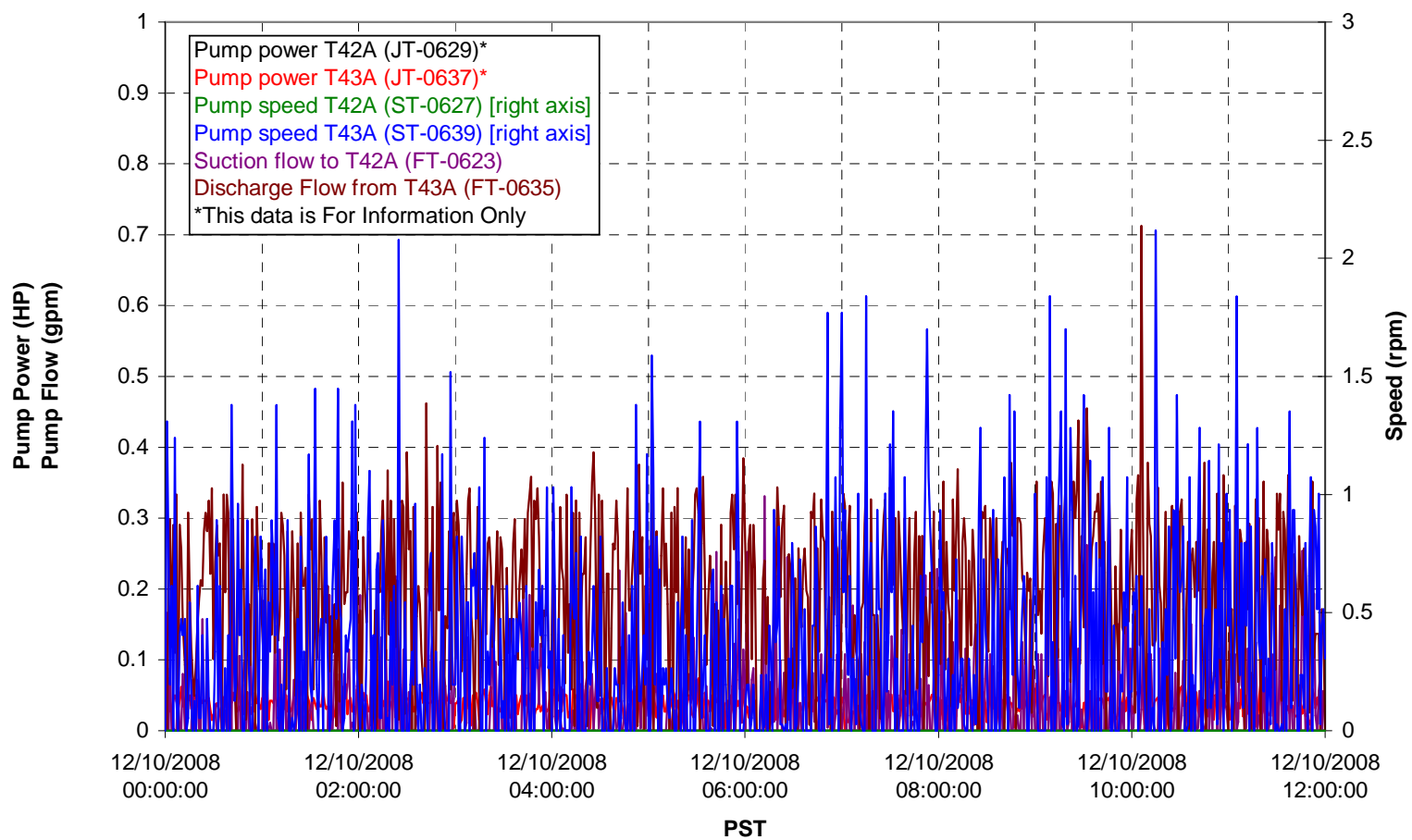


Pulsepot UFP-PP-T01A

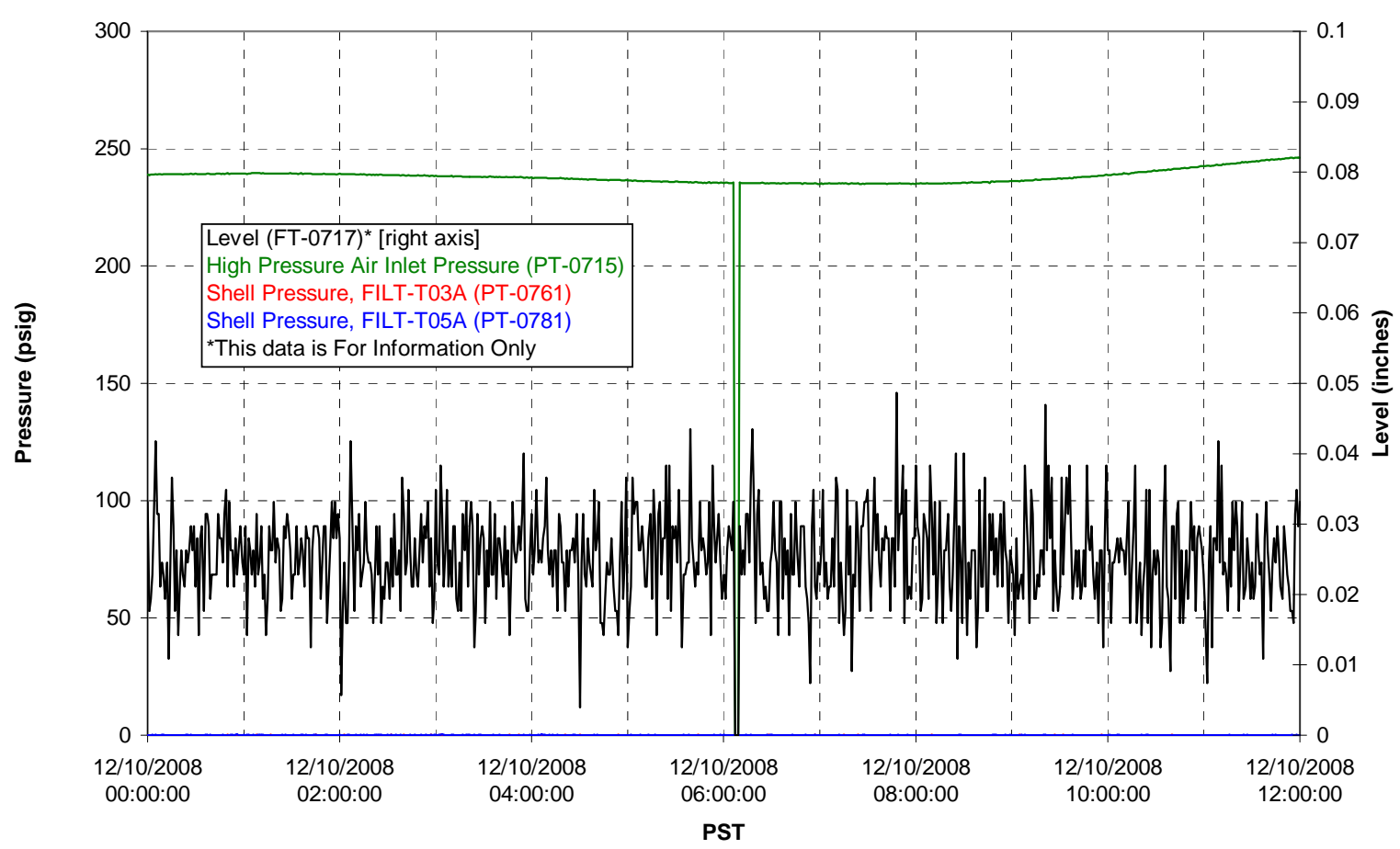

Pulsepot UFP-PP-T02A

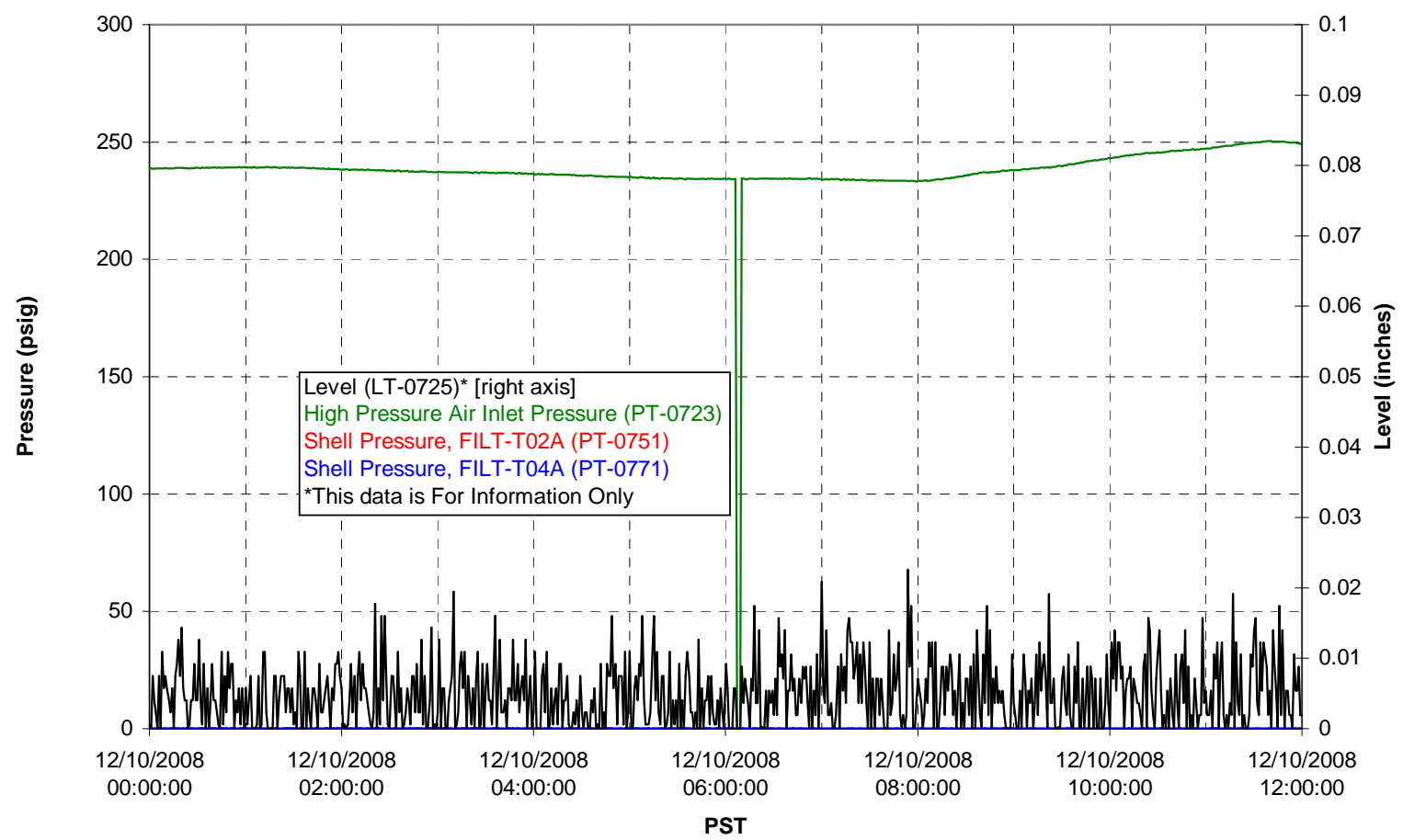


Pulsepot UFP-PP-T03A

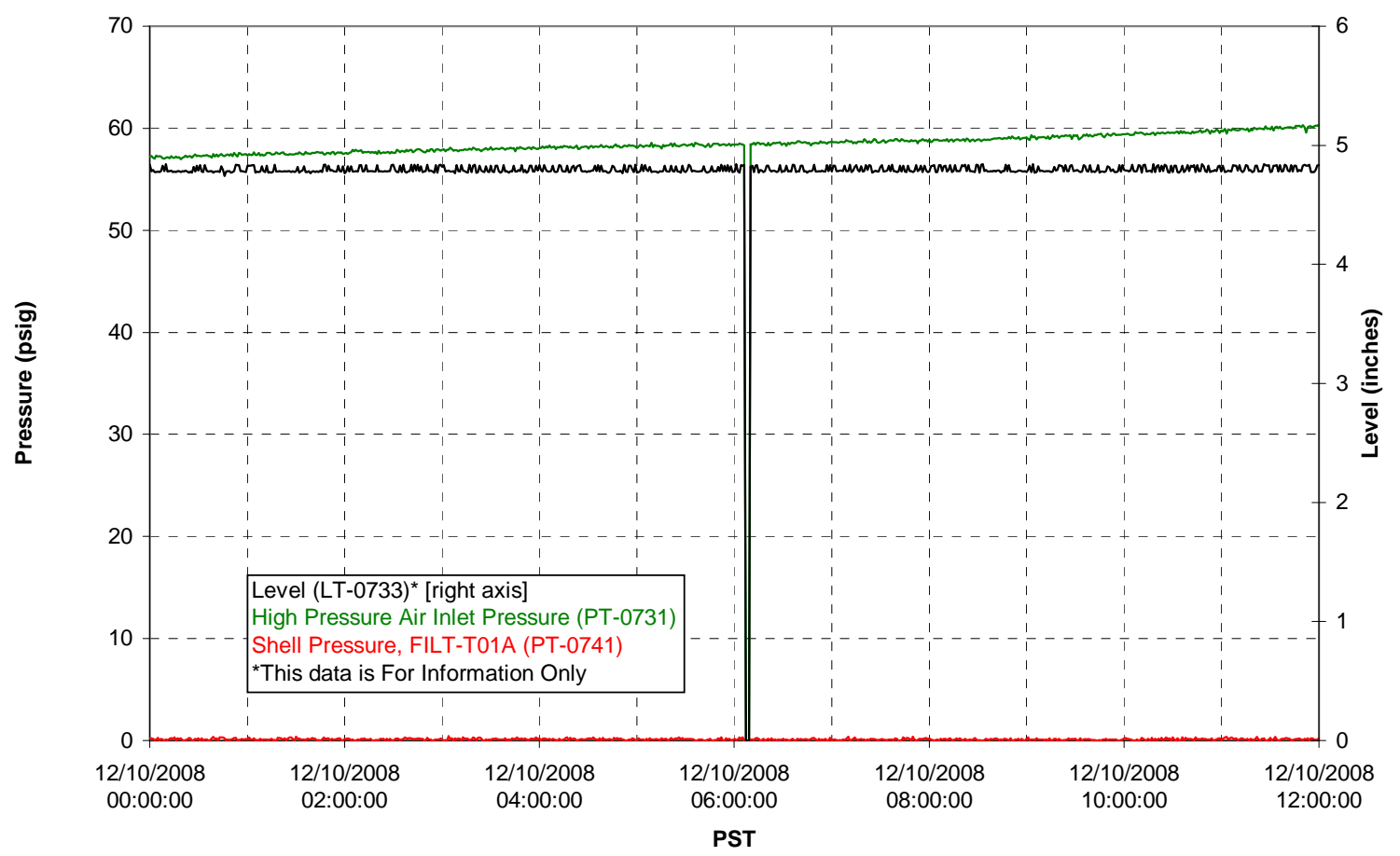

Pulsepot Levels

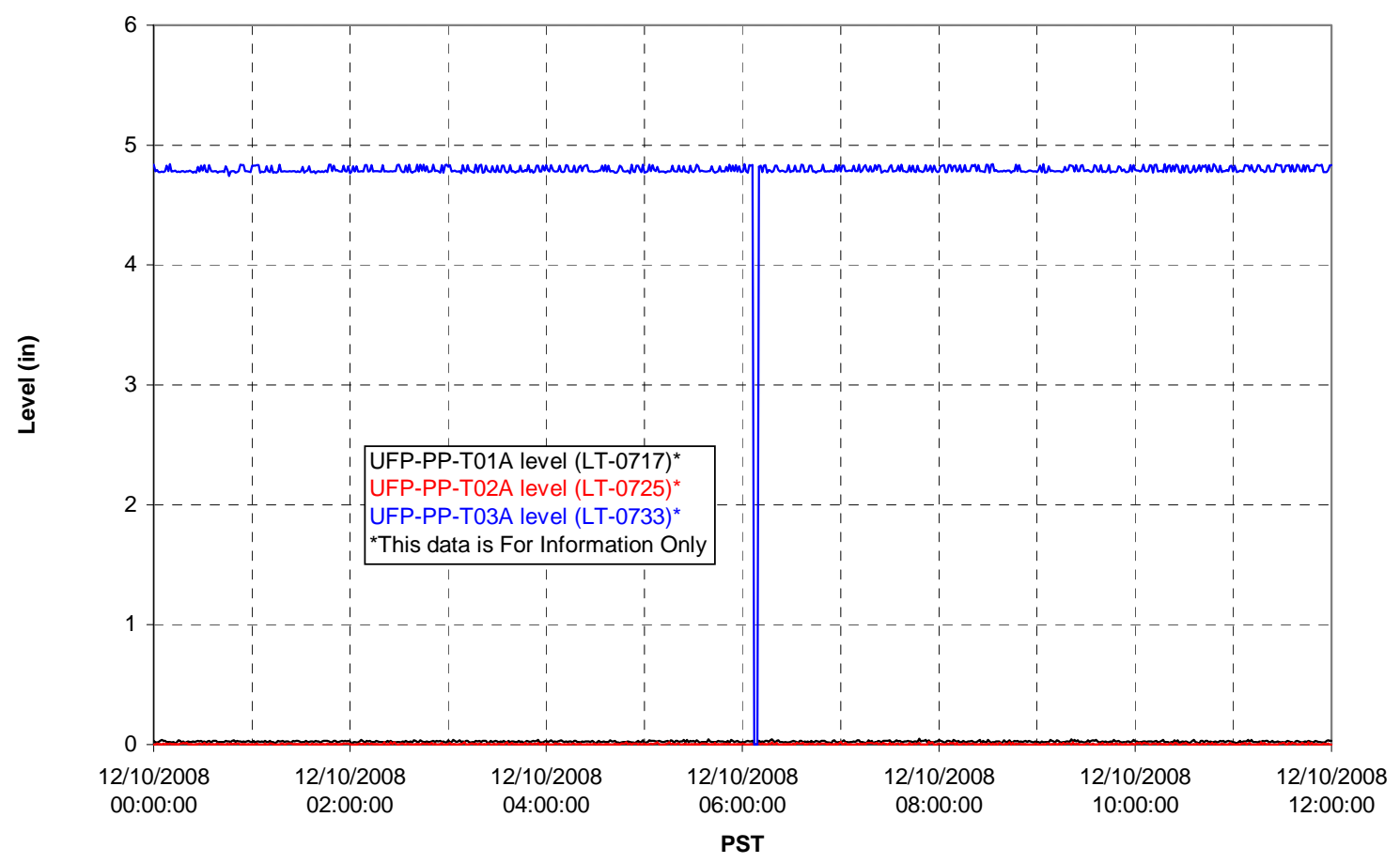


Filter UFP-FILT-T01A

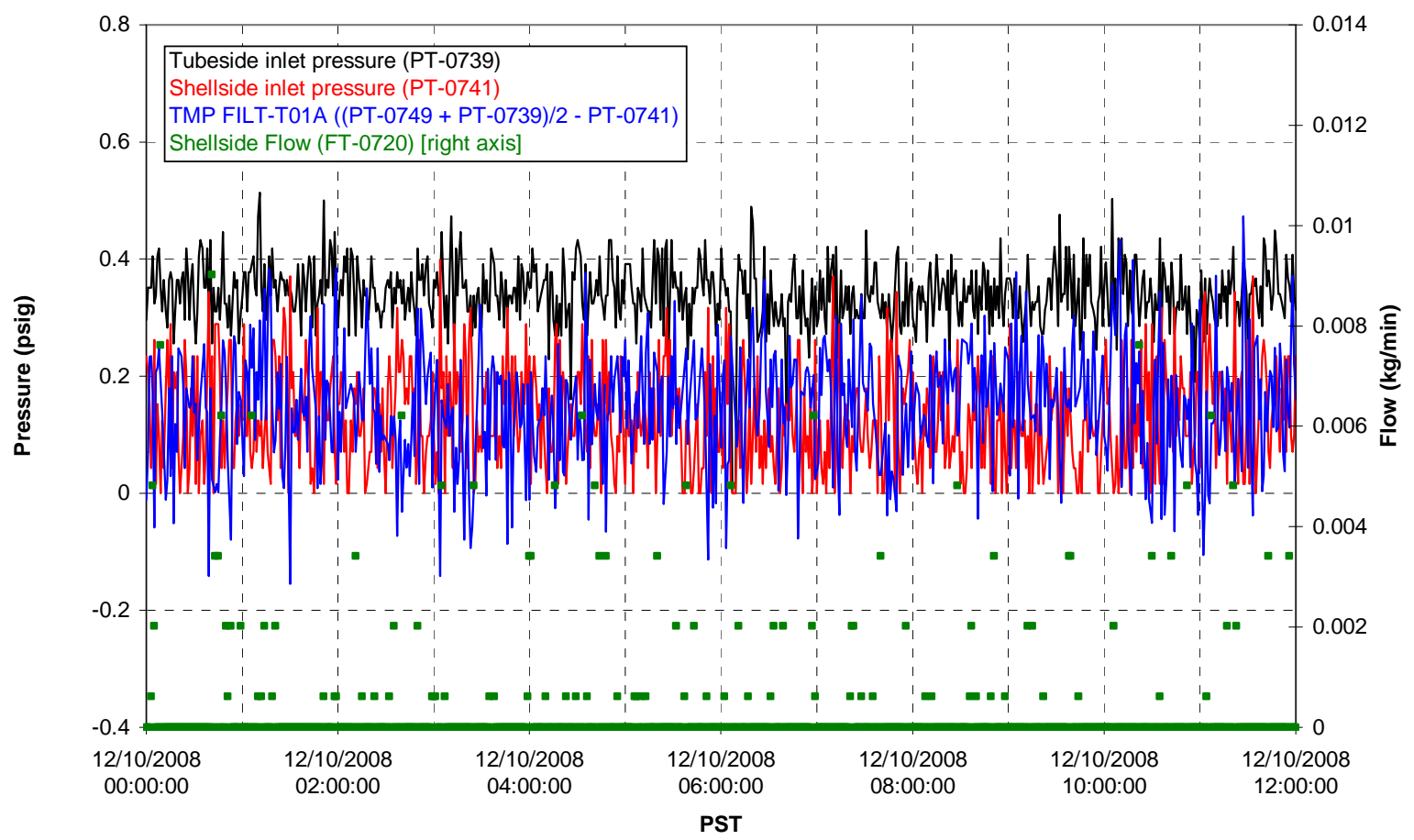

Filter UFP-FILT-T02A

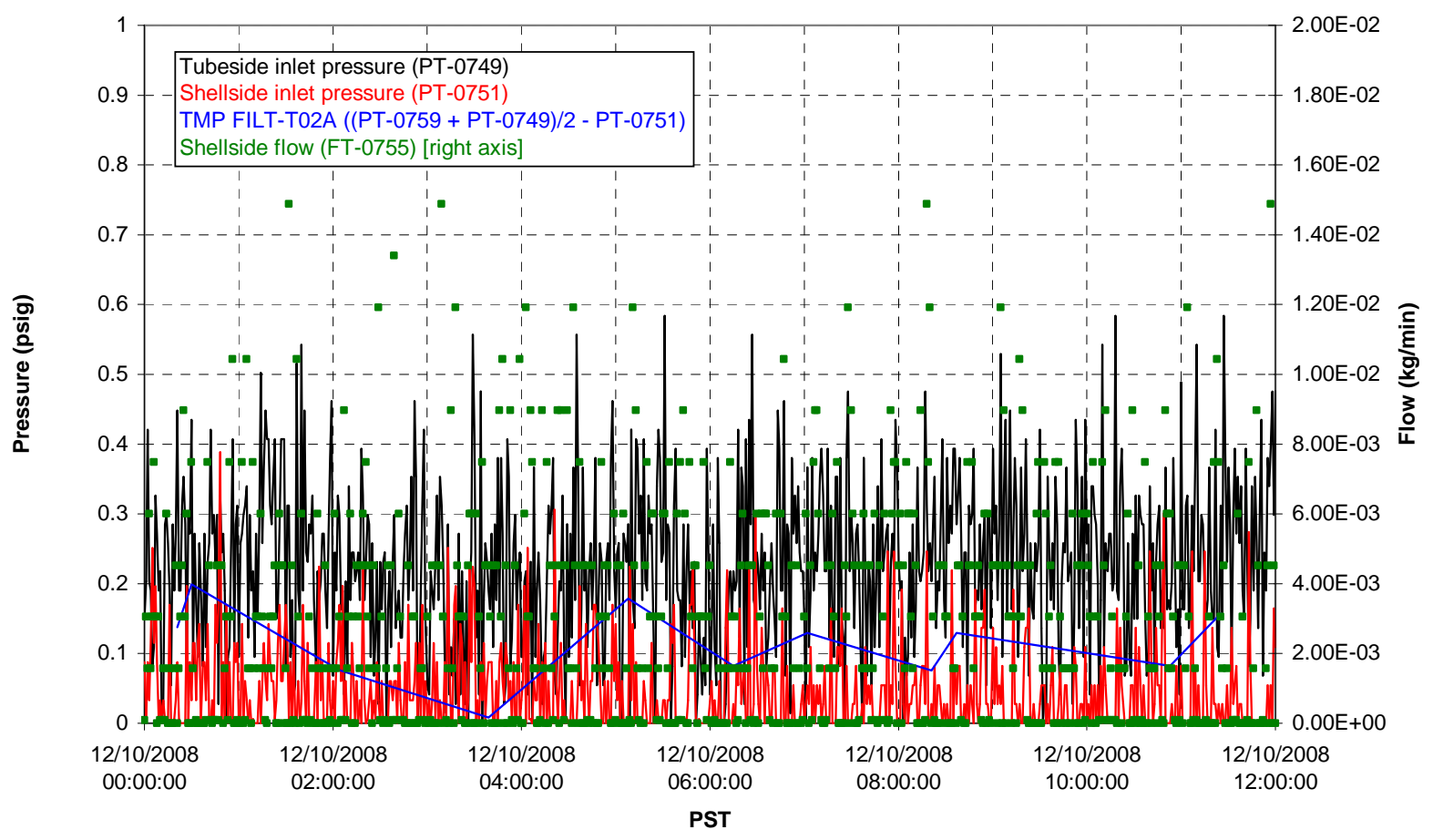


Filter UFP-FILT-T03A

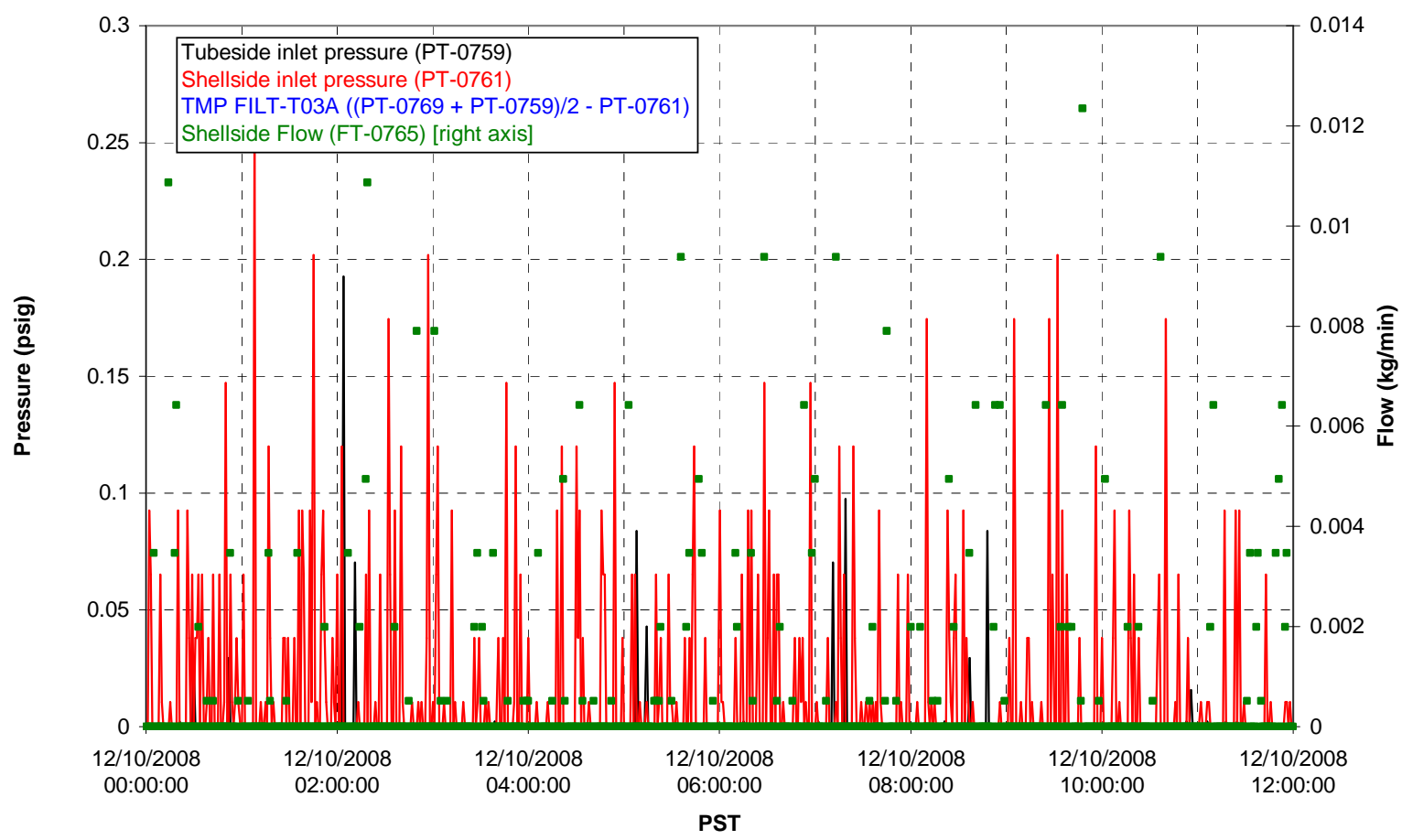

Filter UFP-FILT-T04A

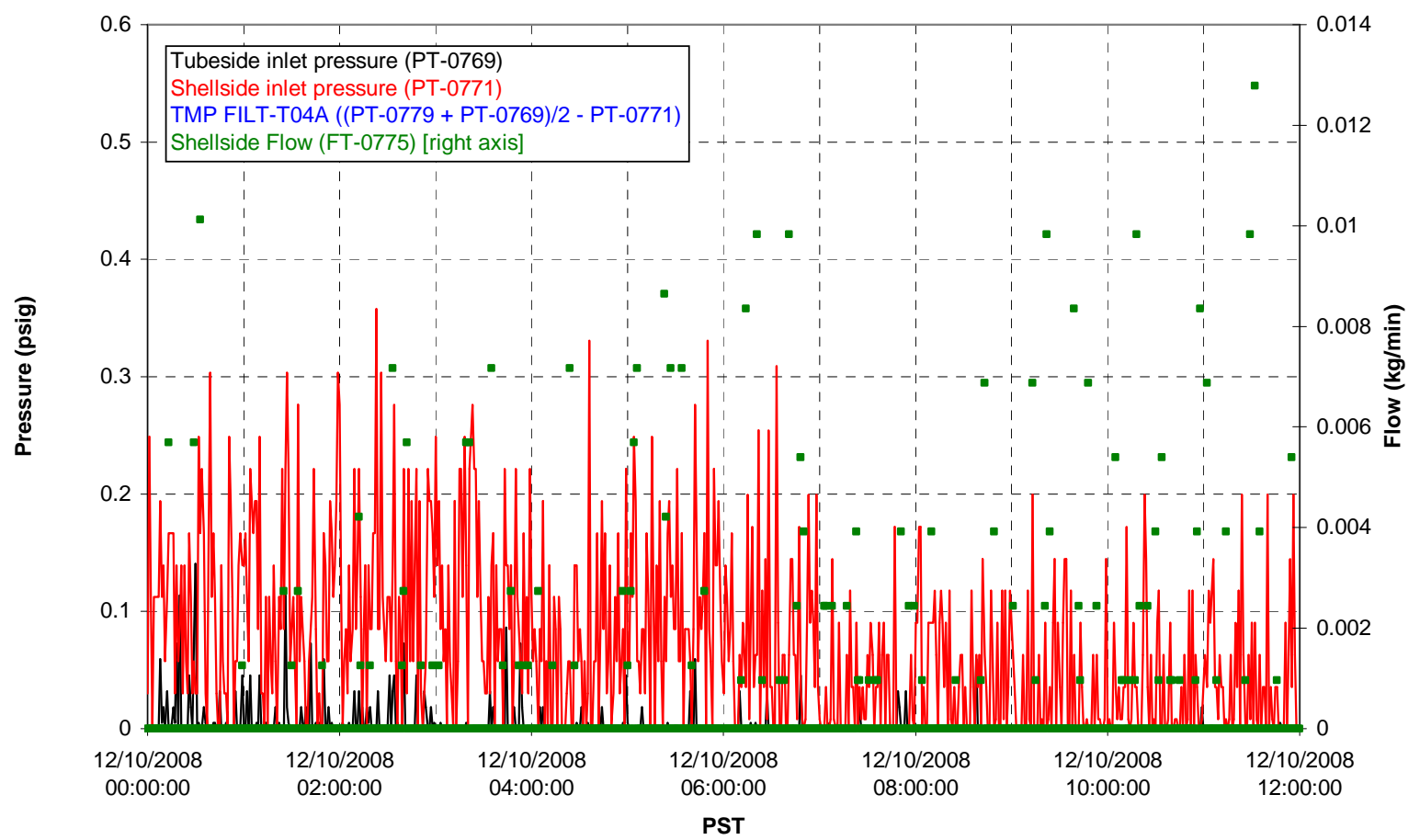


Filter UFP-FILT-T05A

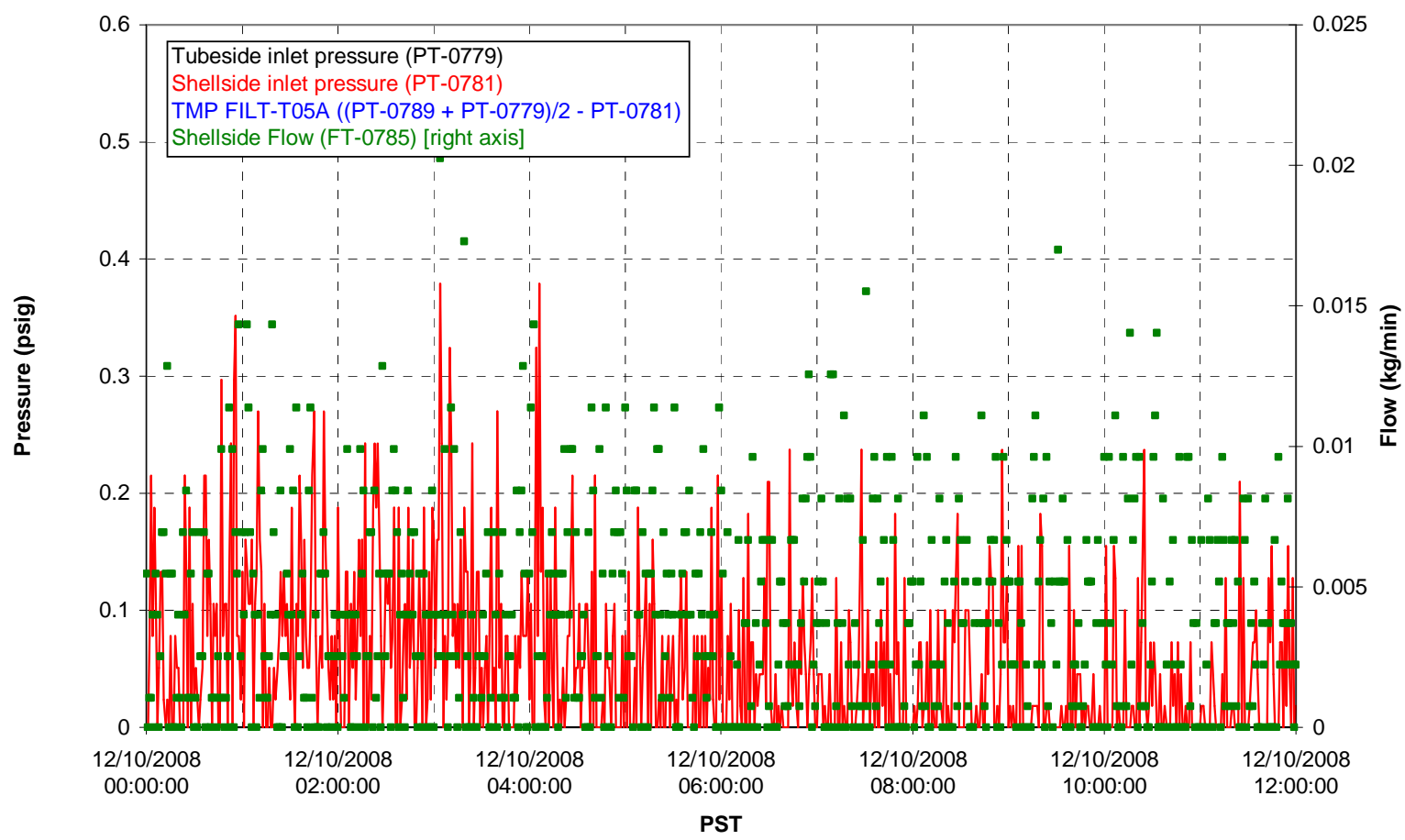

Chemical Flow

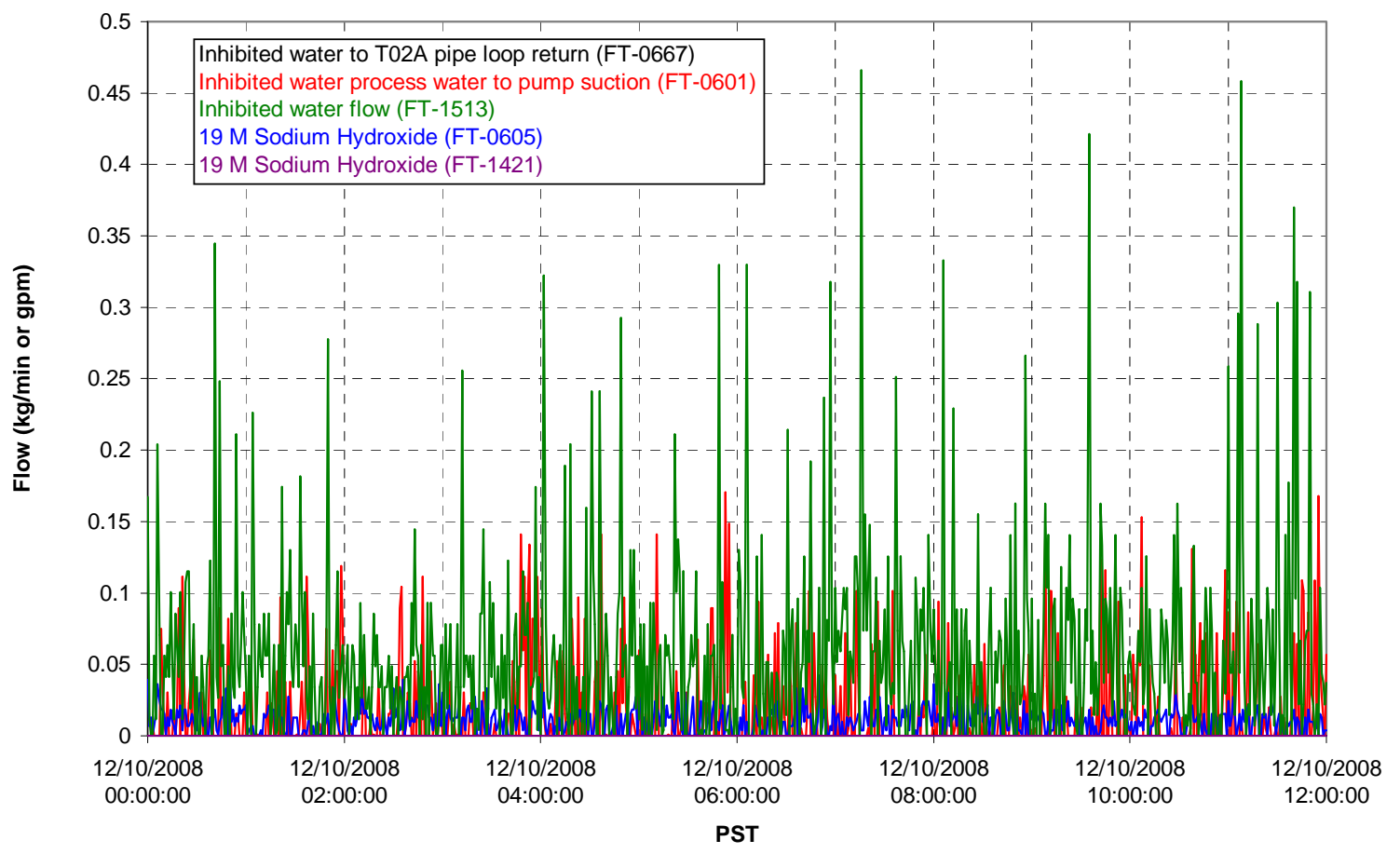




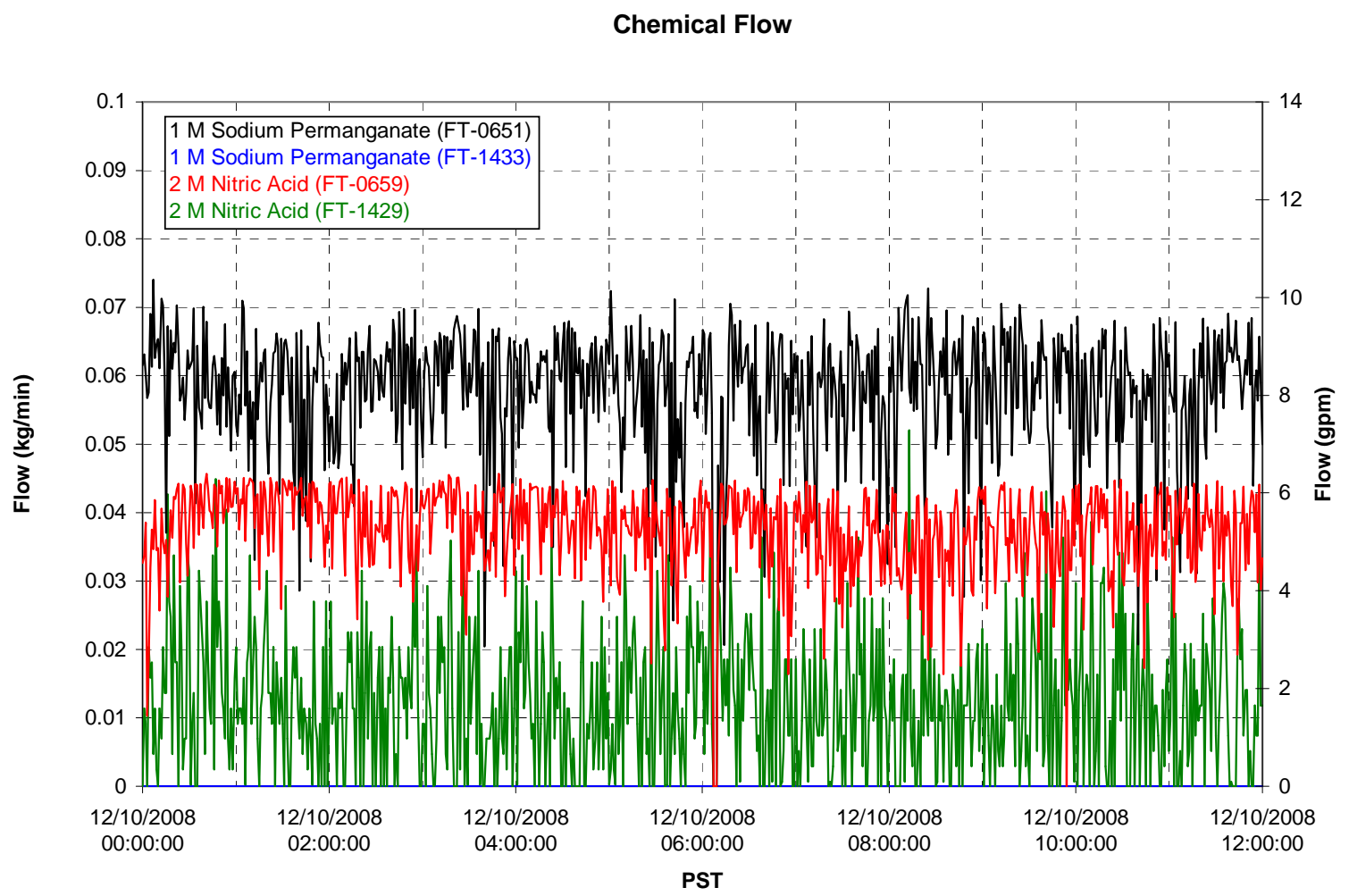

Air Flows

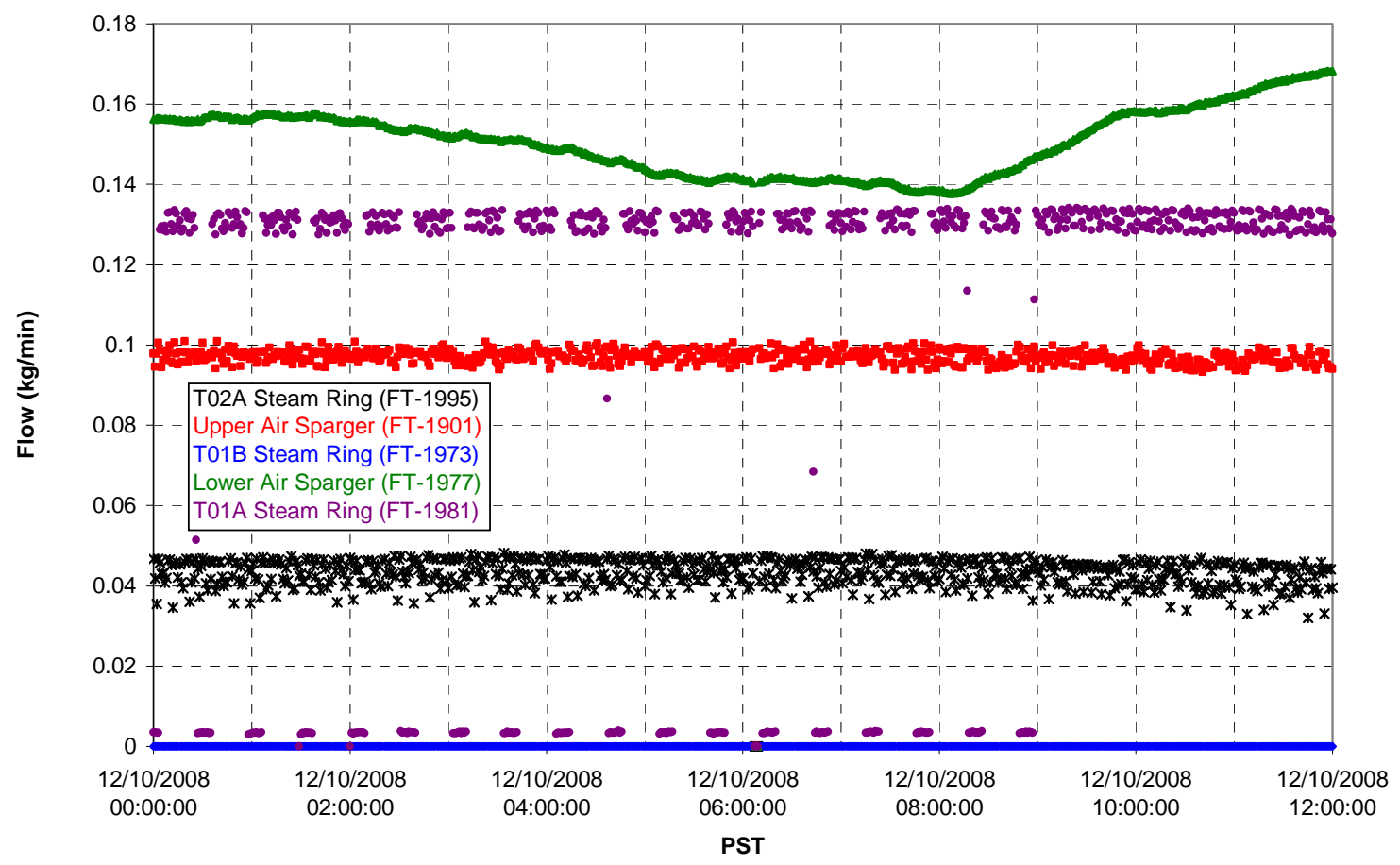


T02A Steam

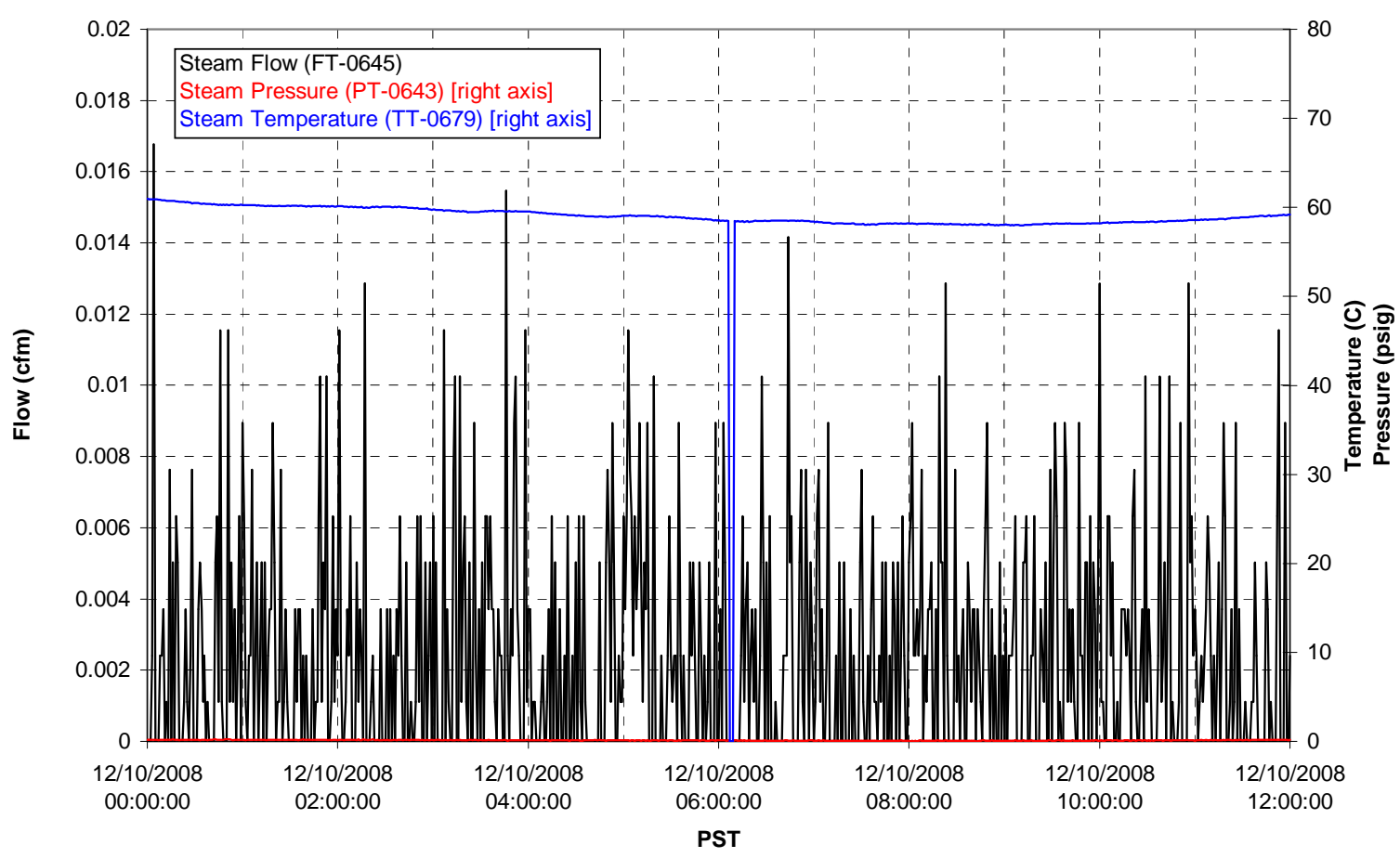

T01A Steam

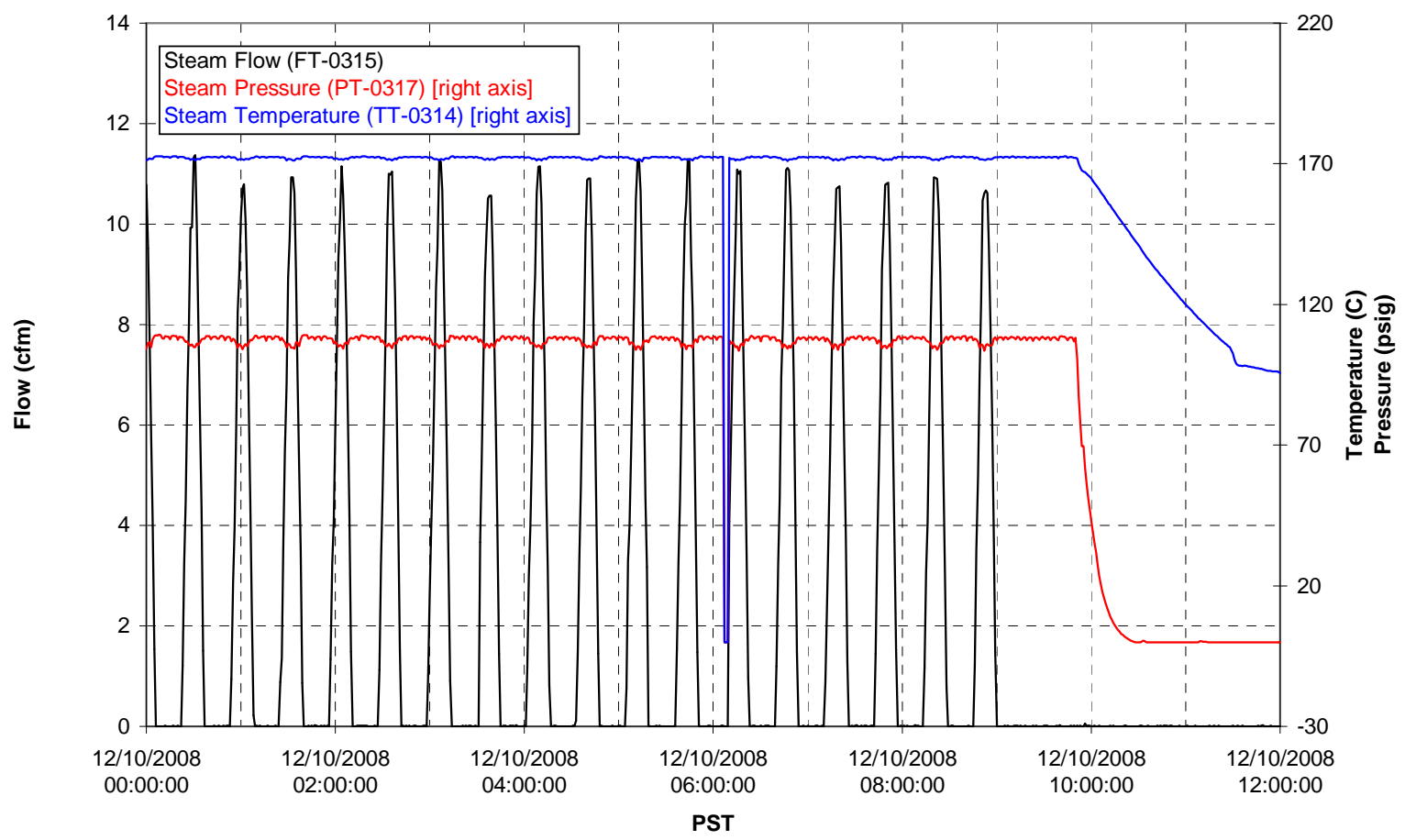


T01B Steam

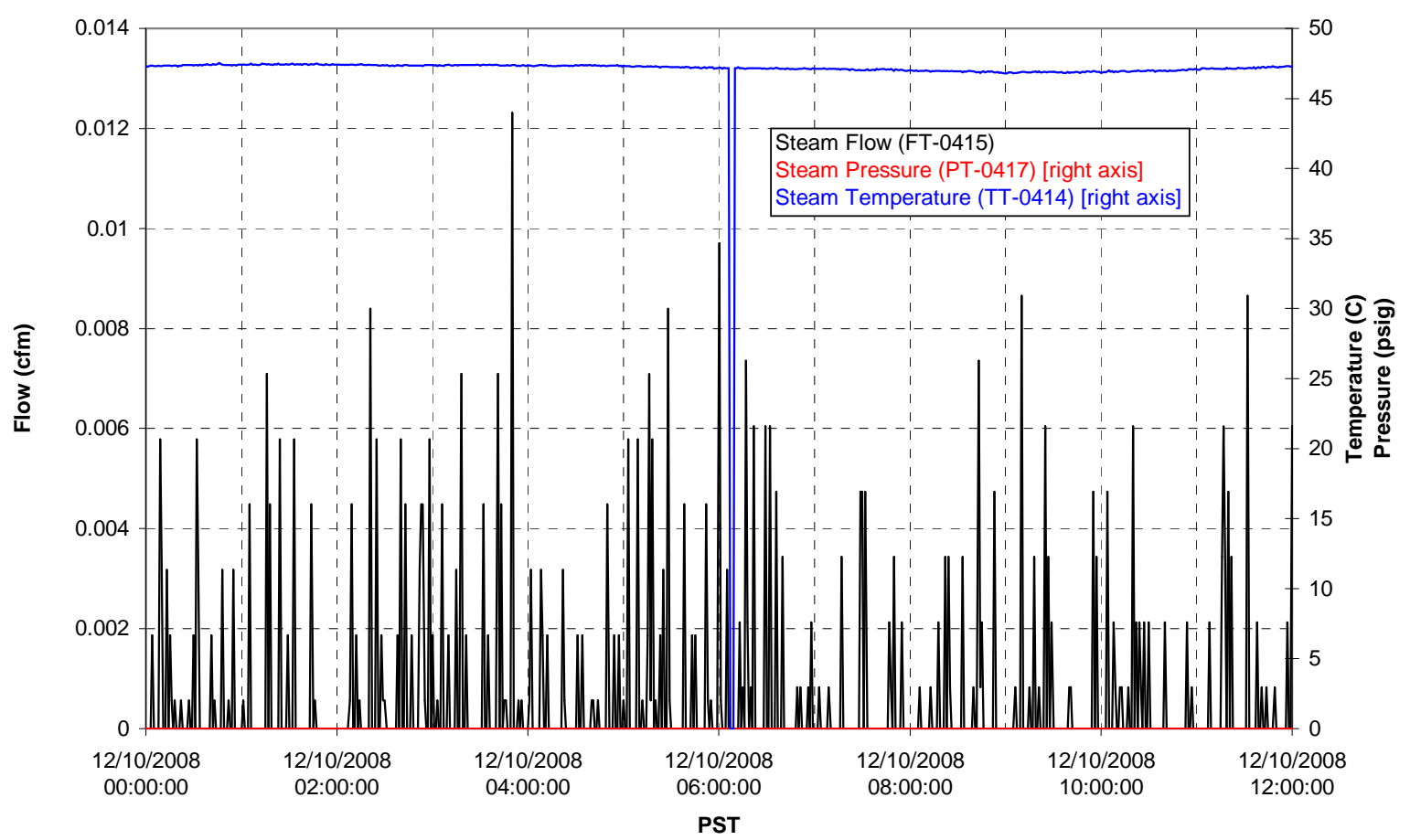


Functional Test Data Plots

12/10/2008 12:00 - 12/11/2008 00:00 
T01A level

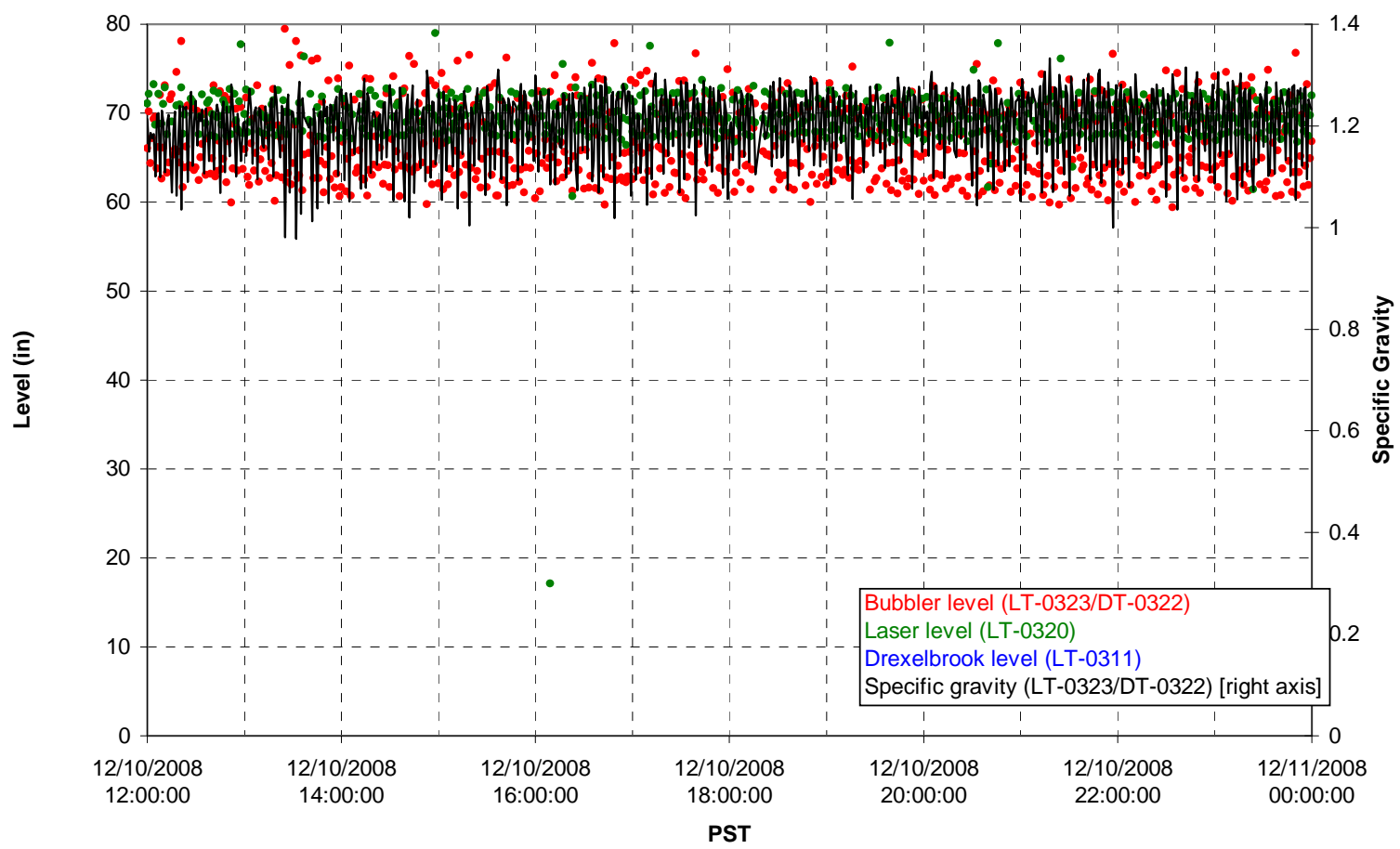

T01A temperatures

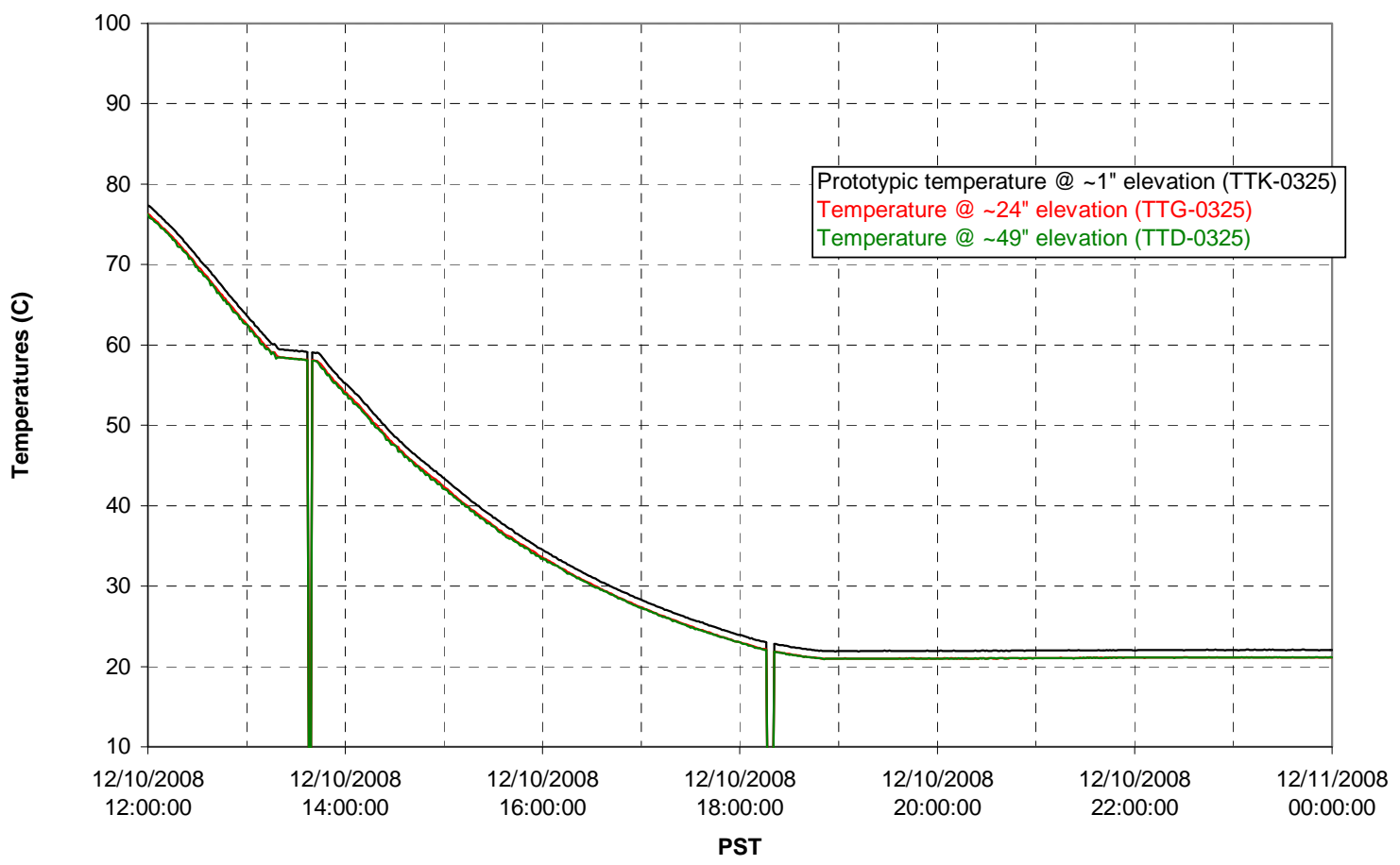


T01B level

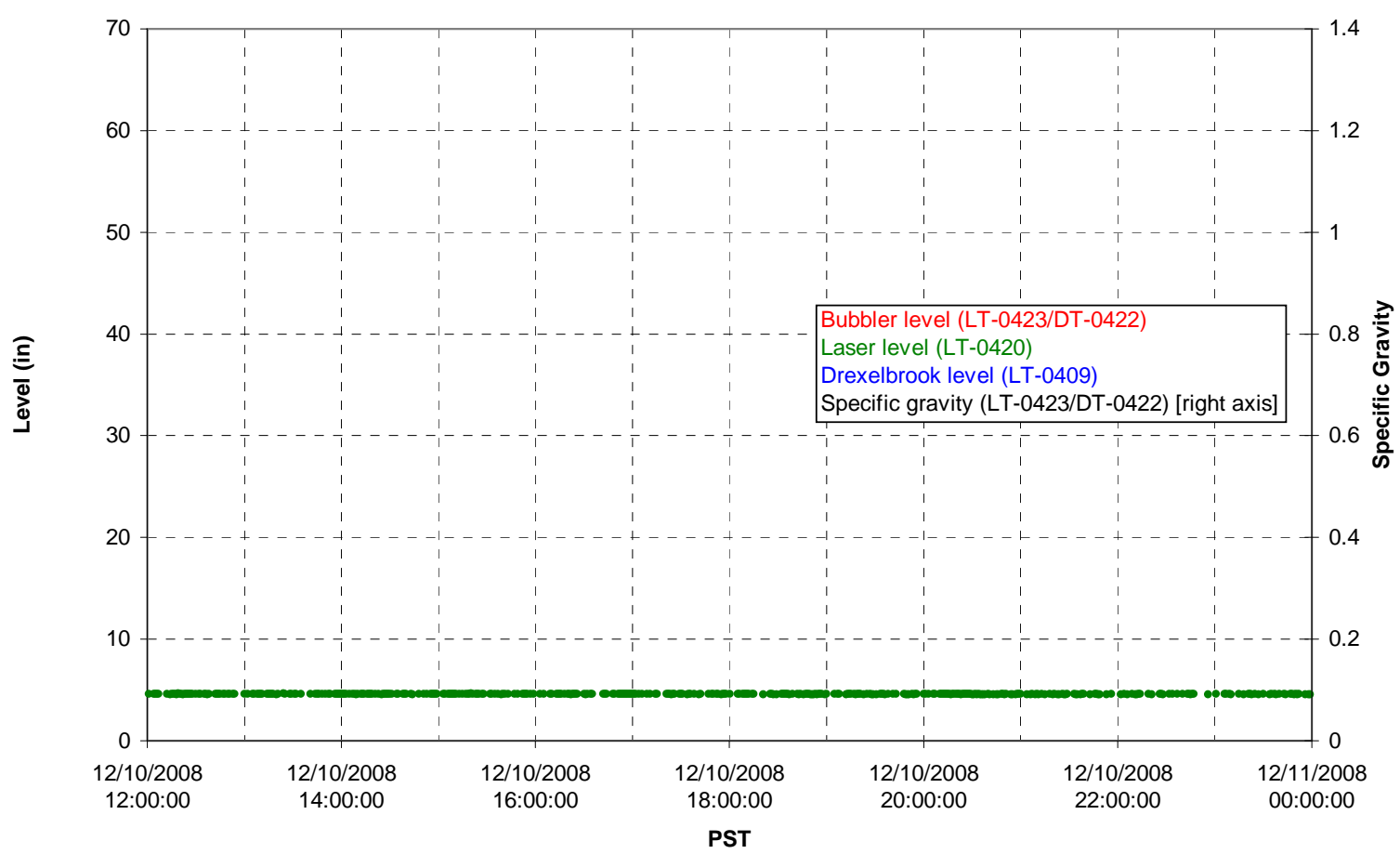

T01B temperatures

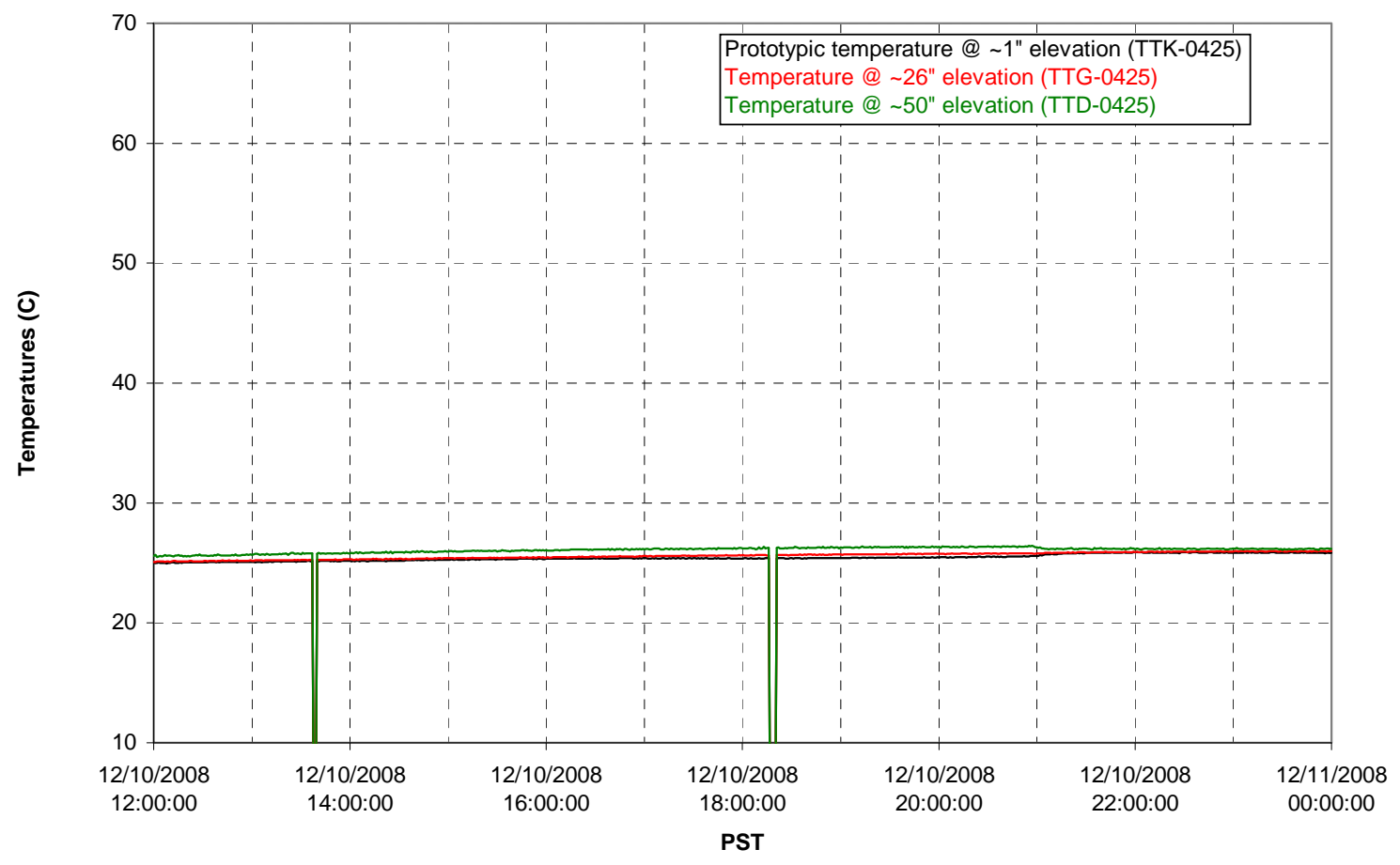


T02A level

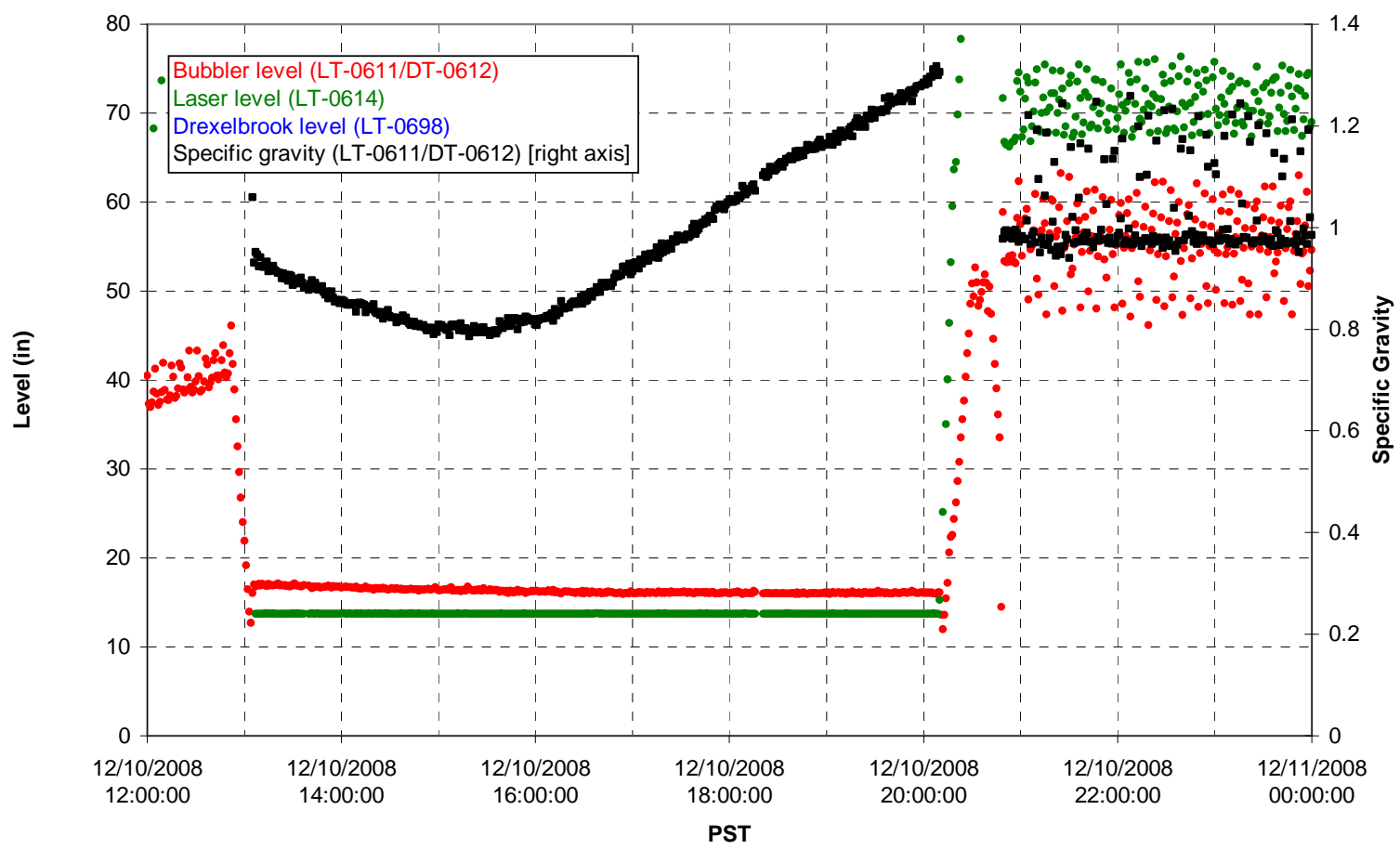

T02A temperatures

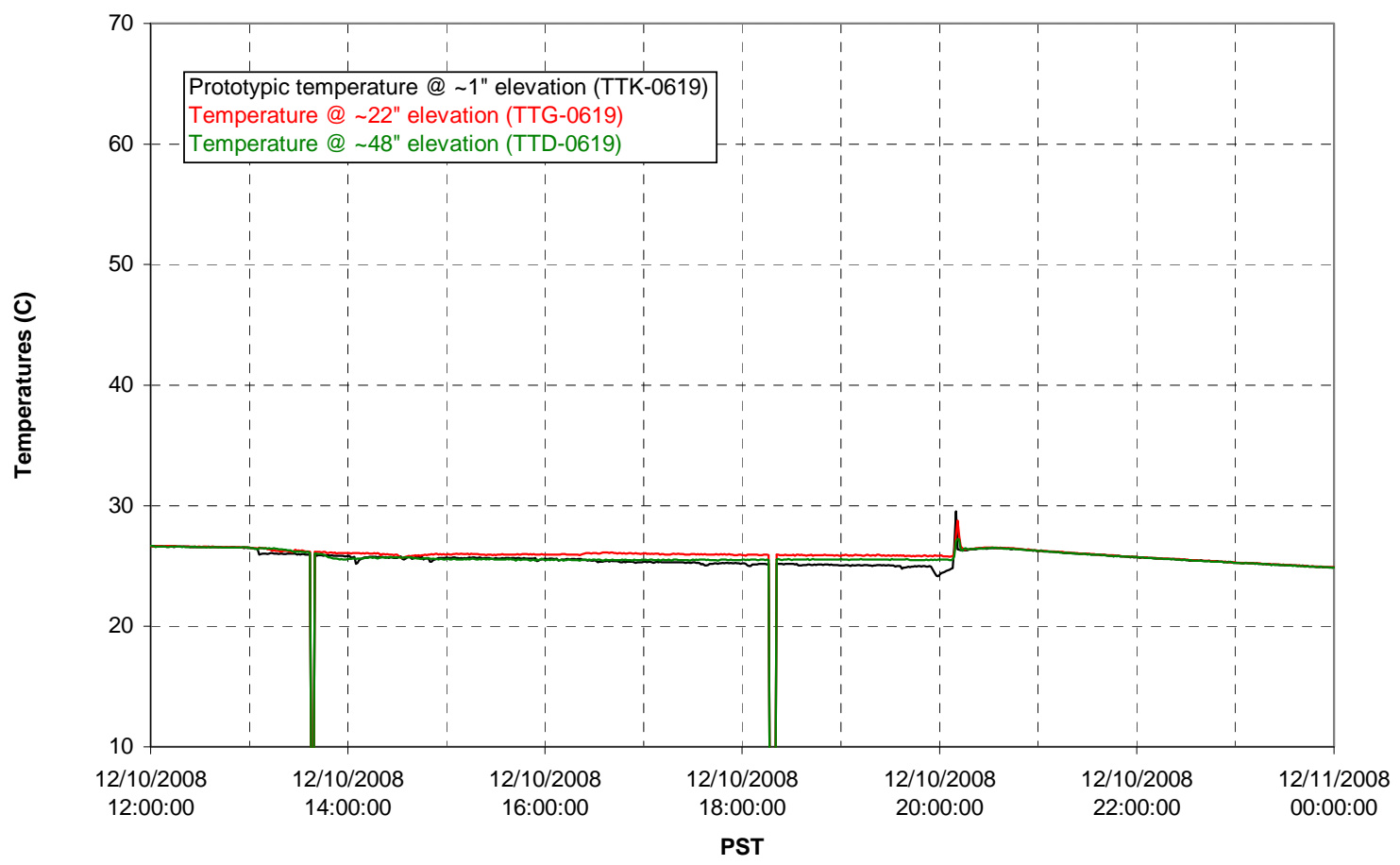


T02A and filter loop temperatures

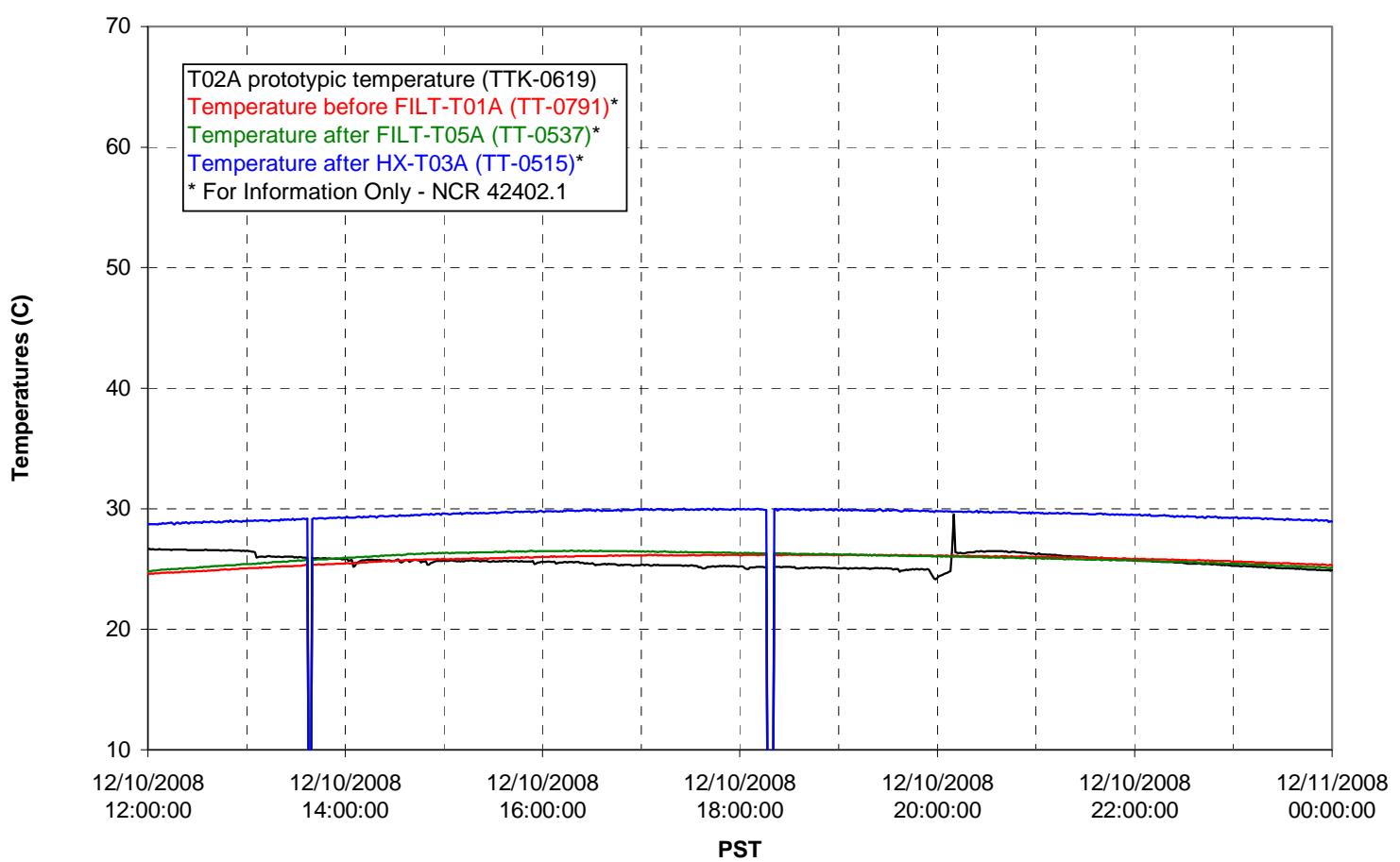

Pump Pressures and Flow

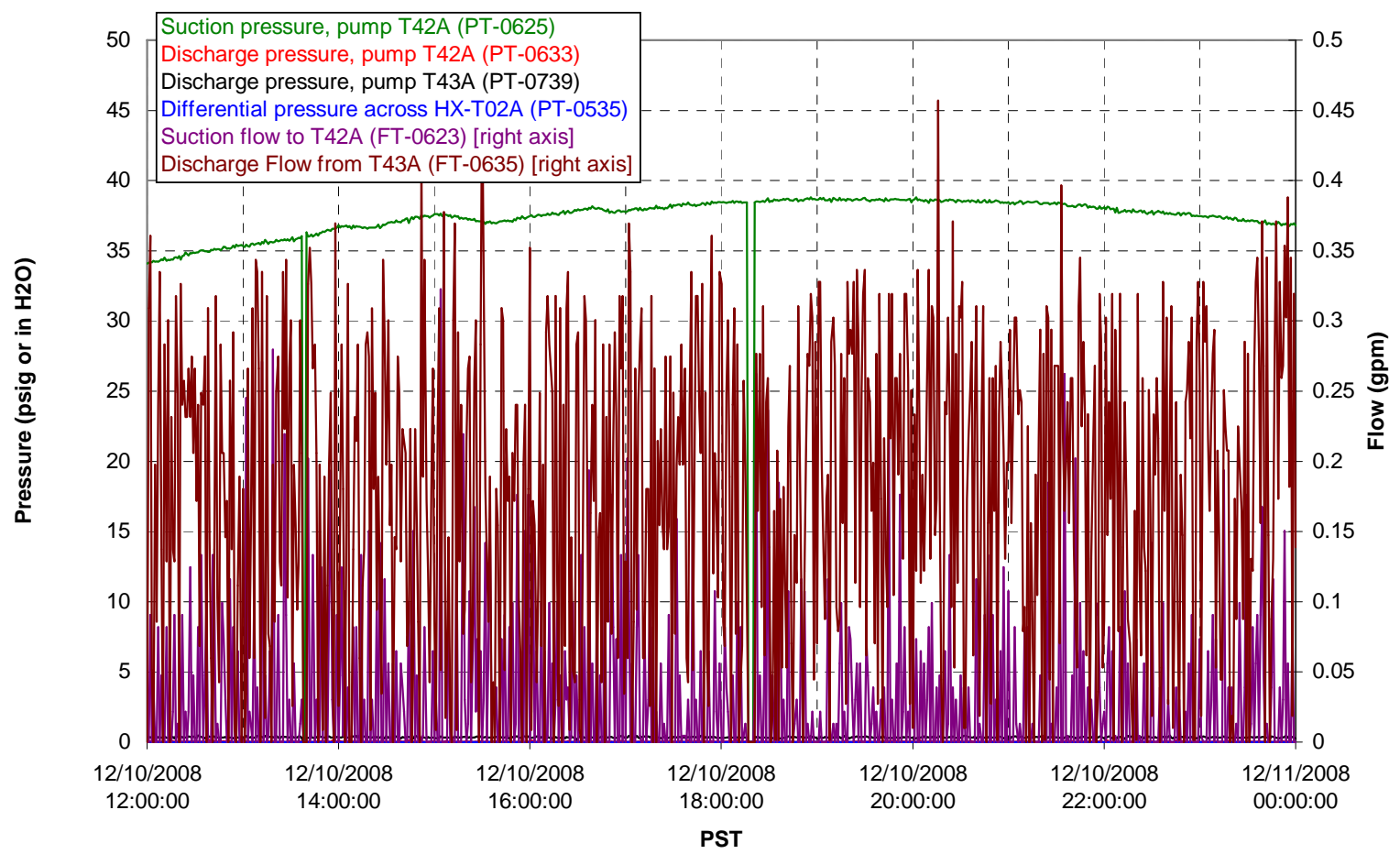


Axial pressure drop

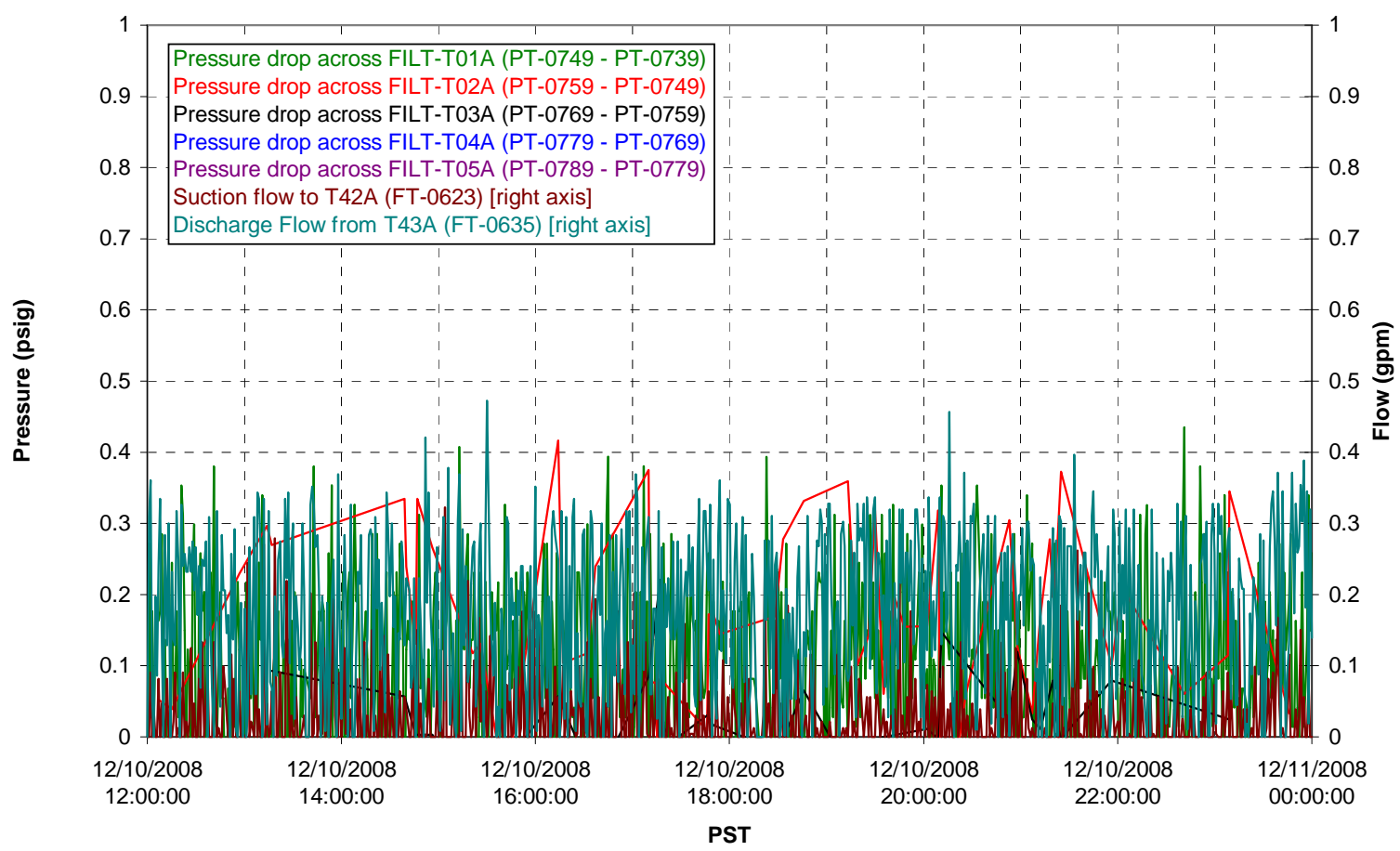

Permeate flow rates

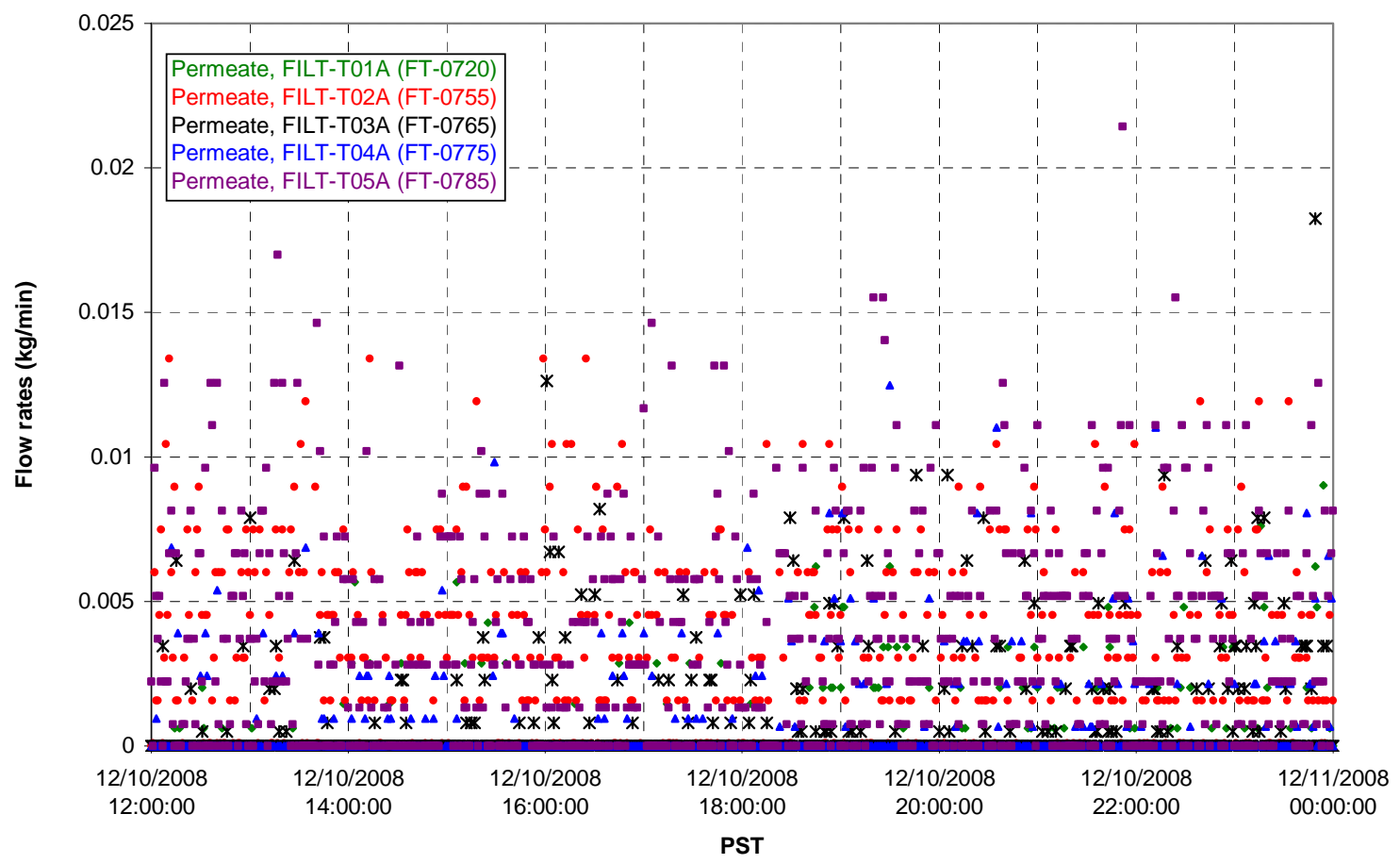


T02A Inner Temperature Tree

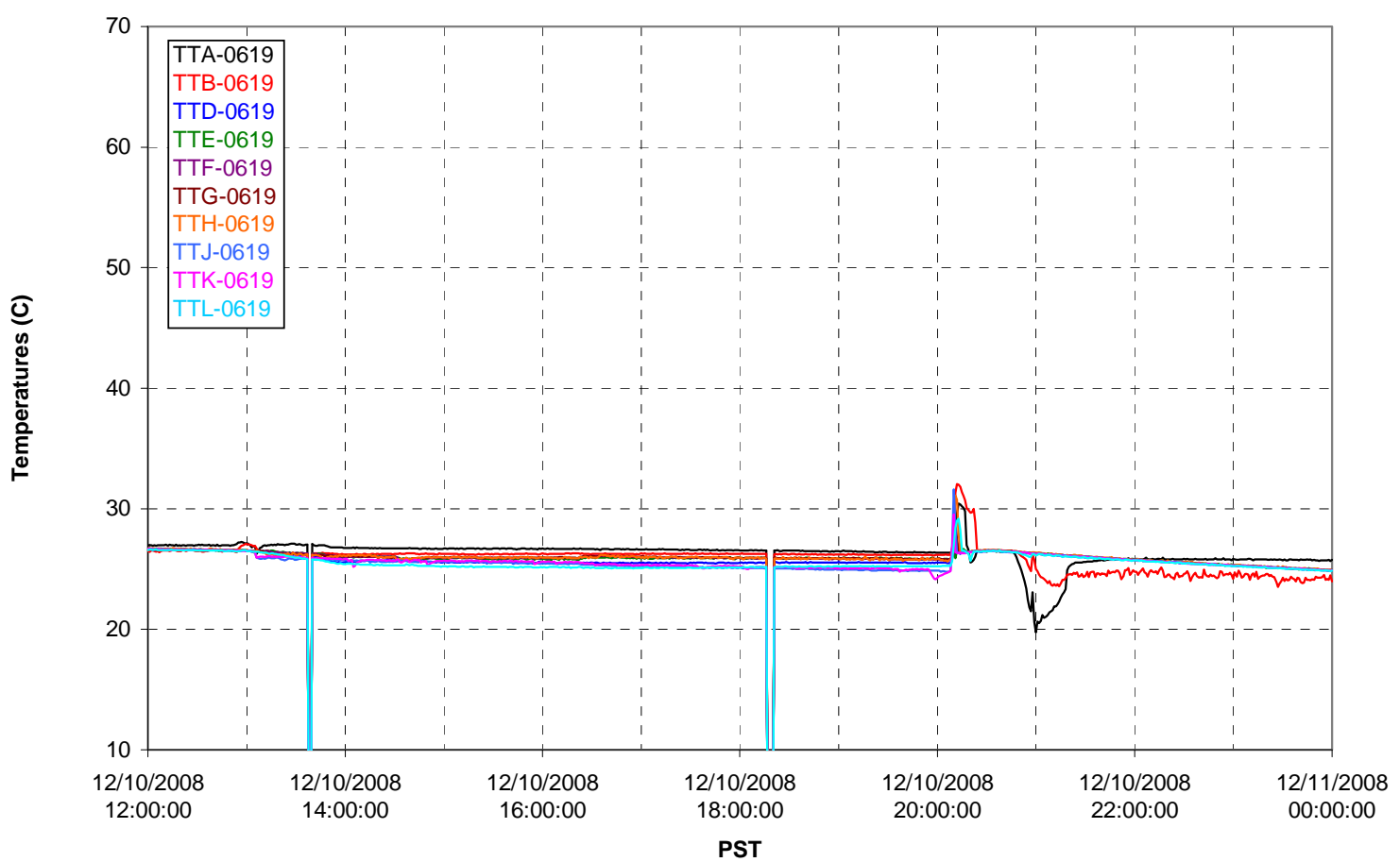

T02A Outer Temperature Tree

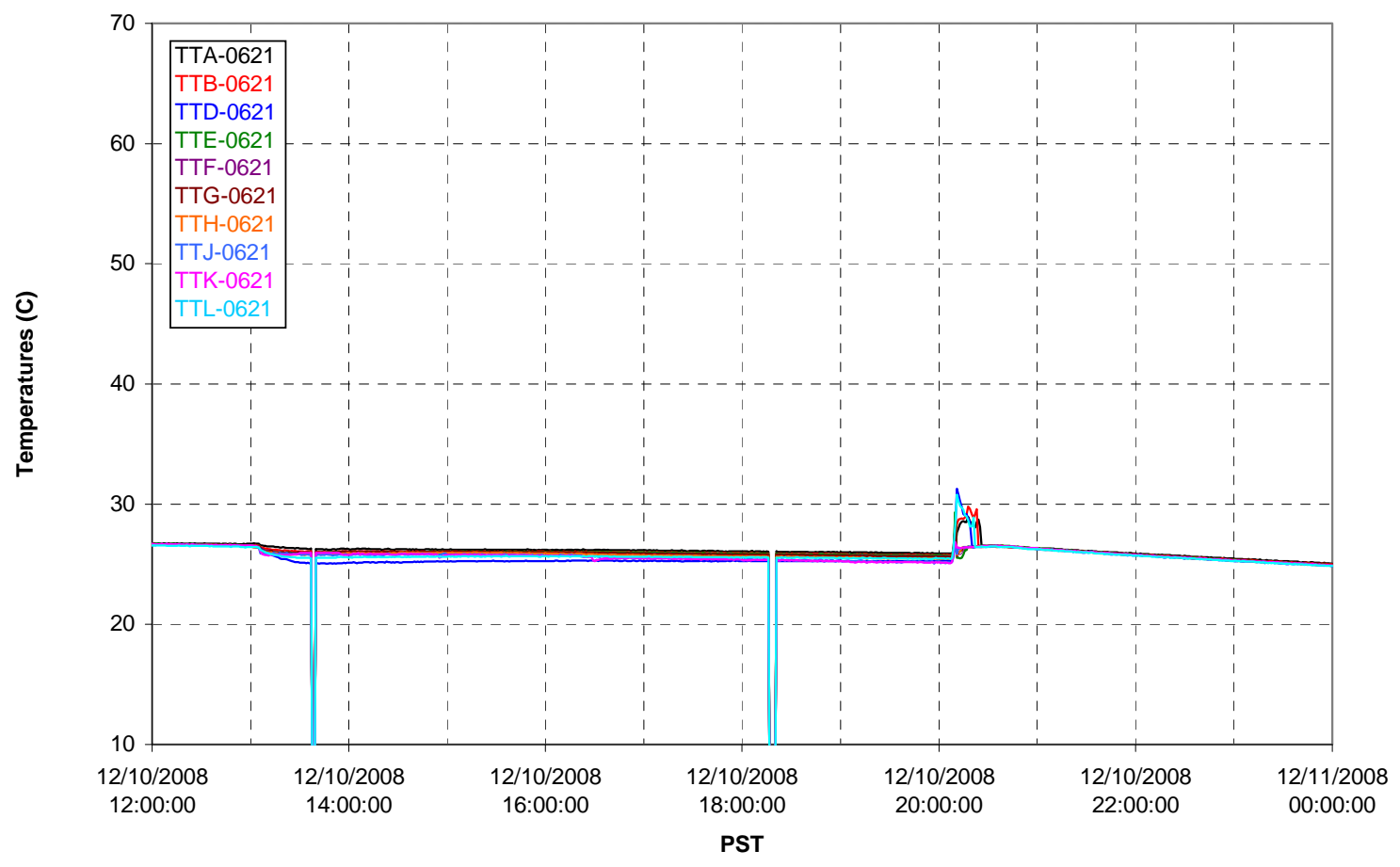


T02A temperatures

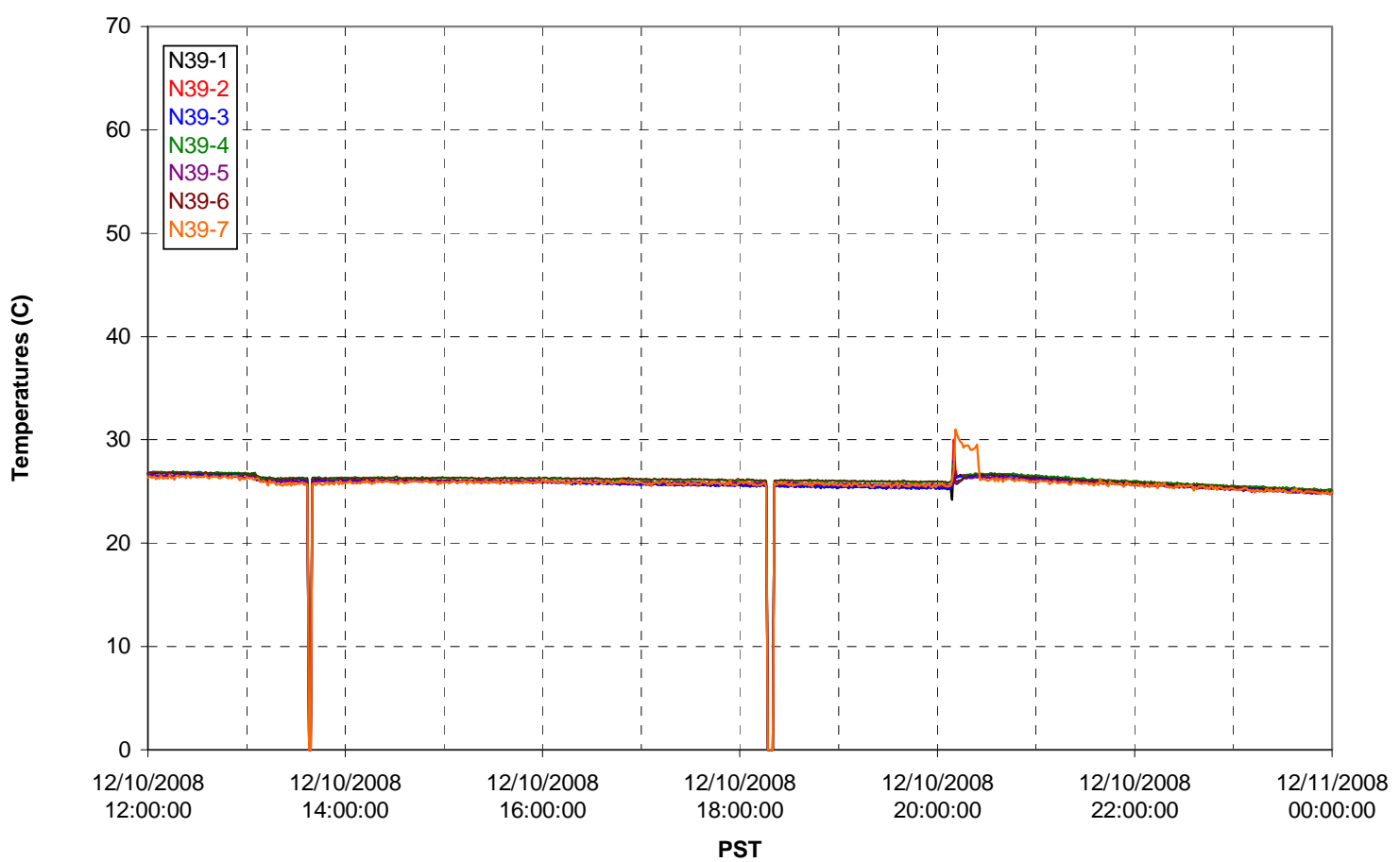

T02A temperatures

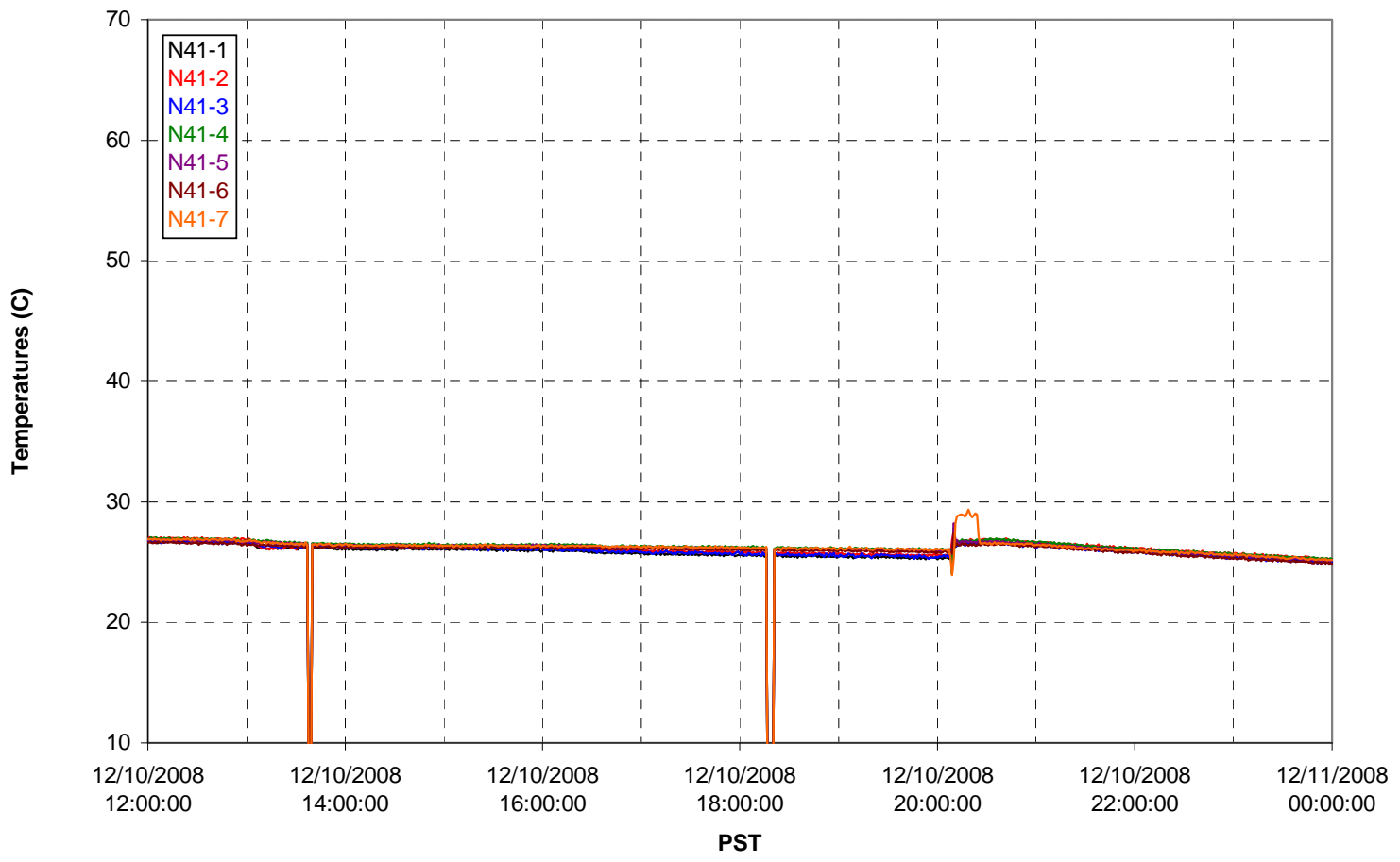


T02A temperatures

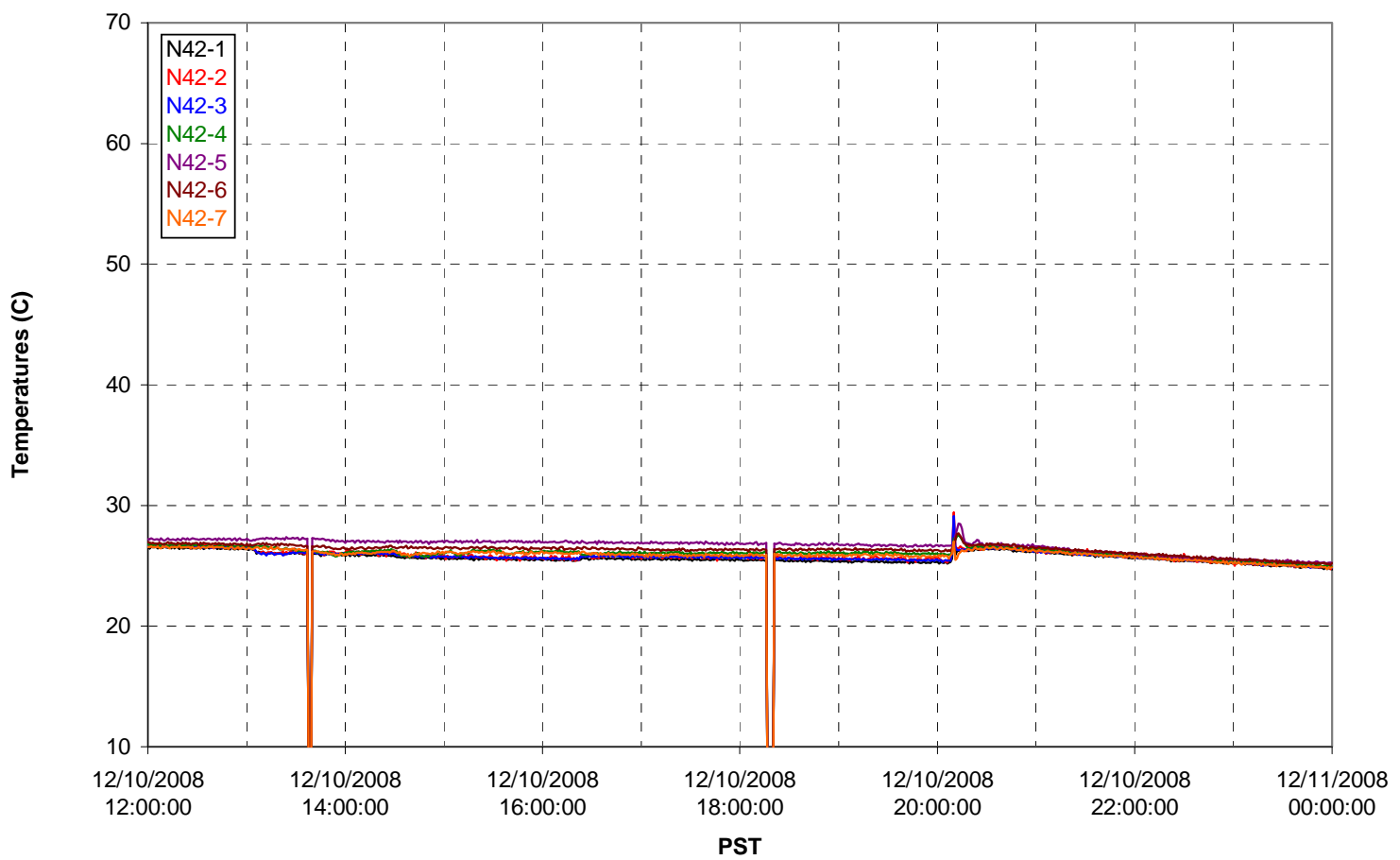

T02A temperatures

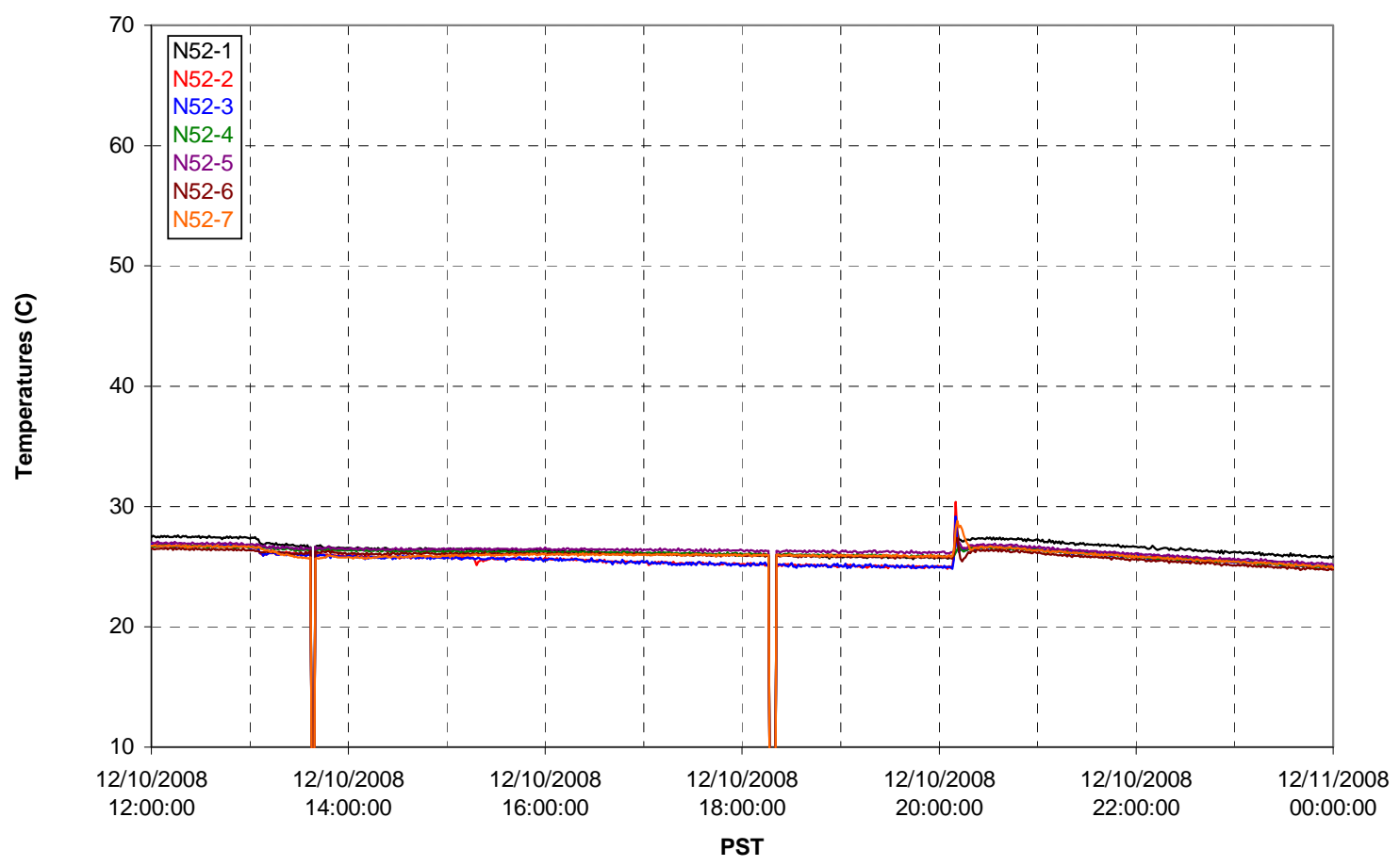


T02A Heating and Cooling

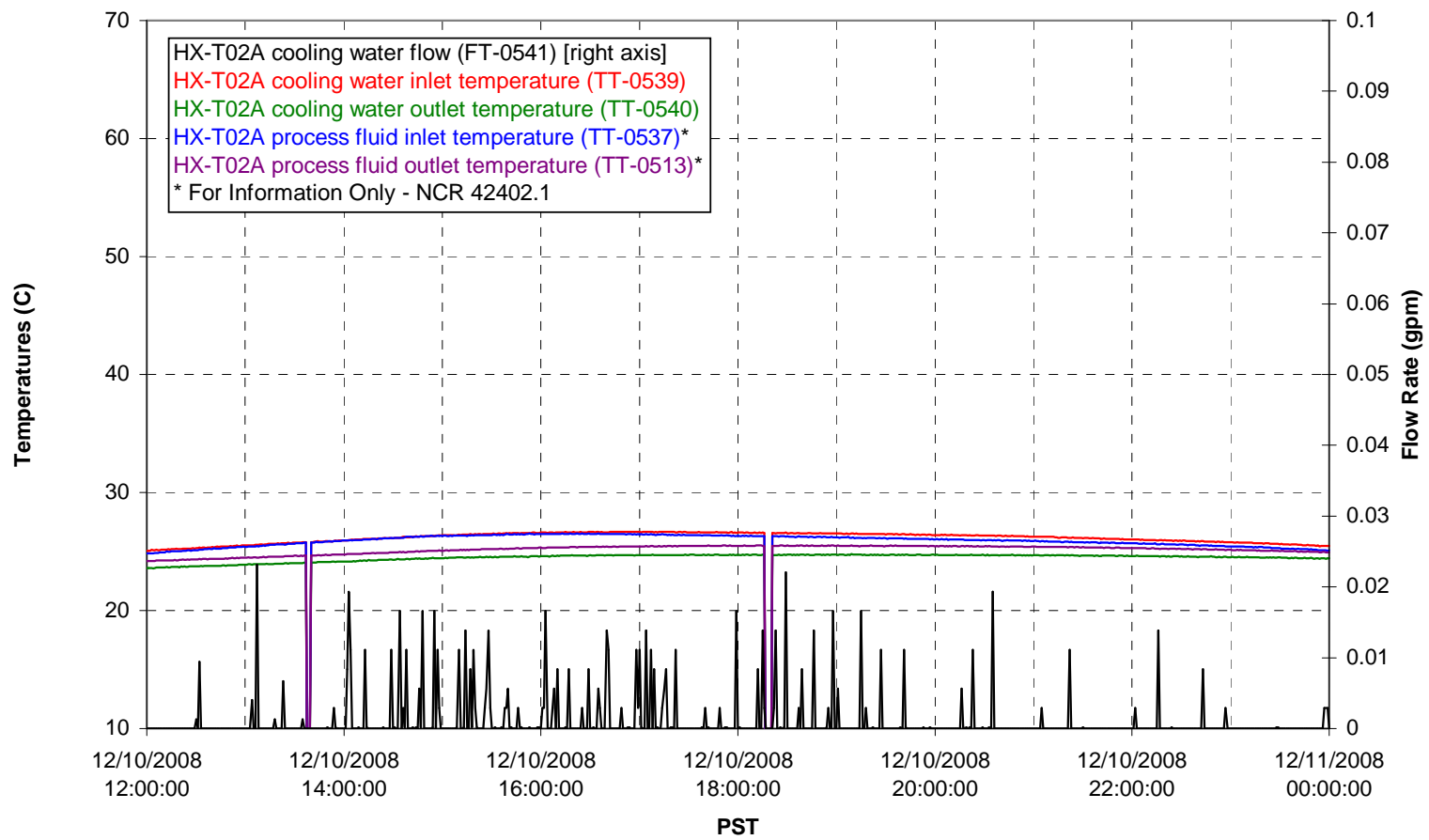

Pump Operation

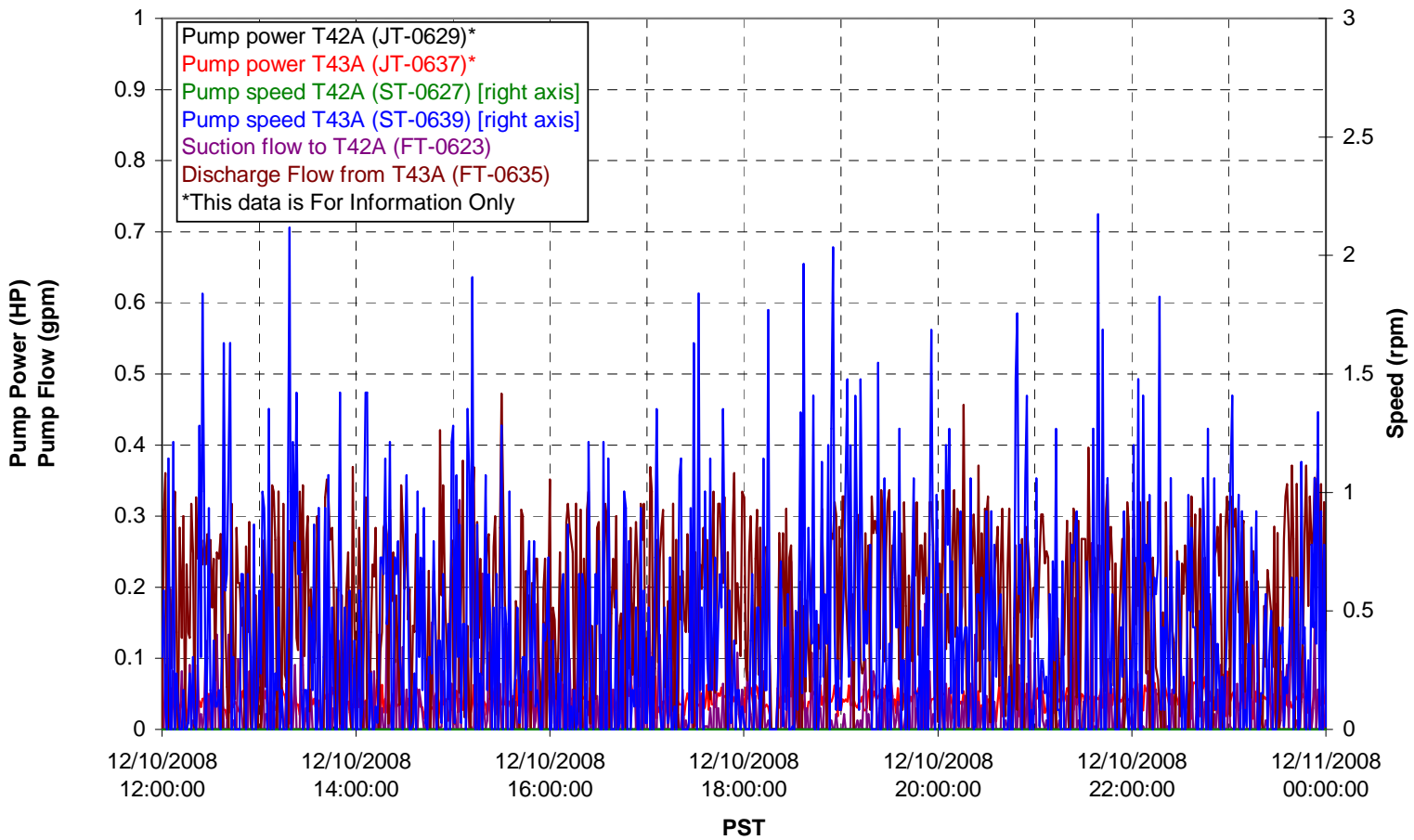


Pulsepot UFP-PP-T01A

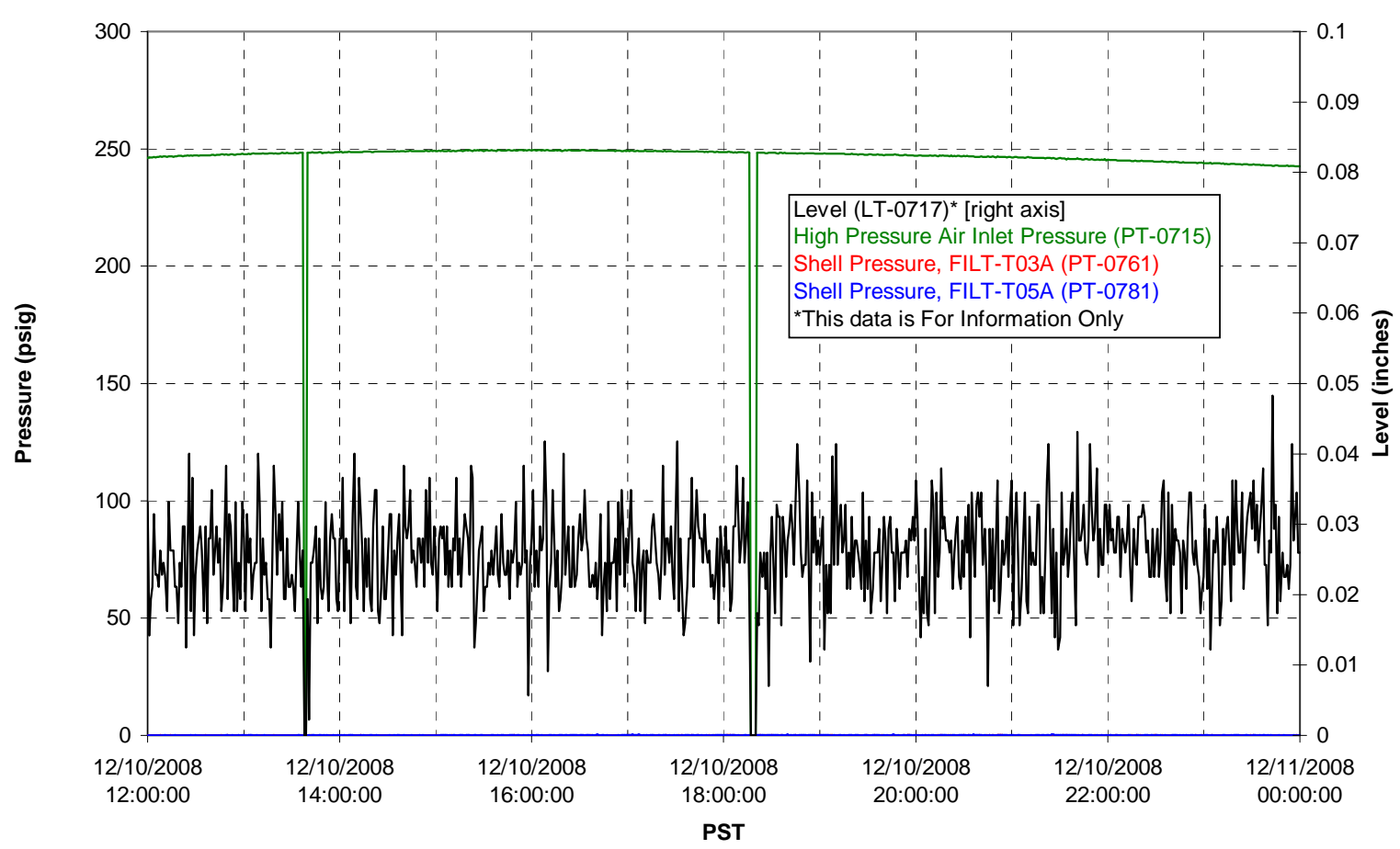

Pulsepot UFP-PP-T02A

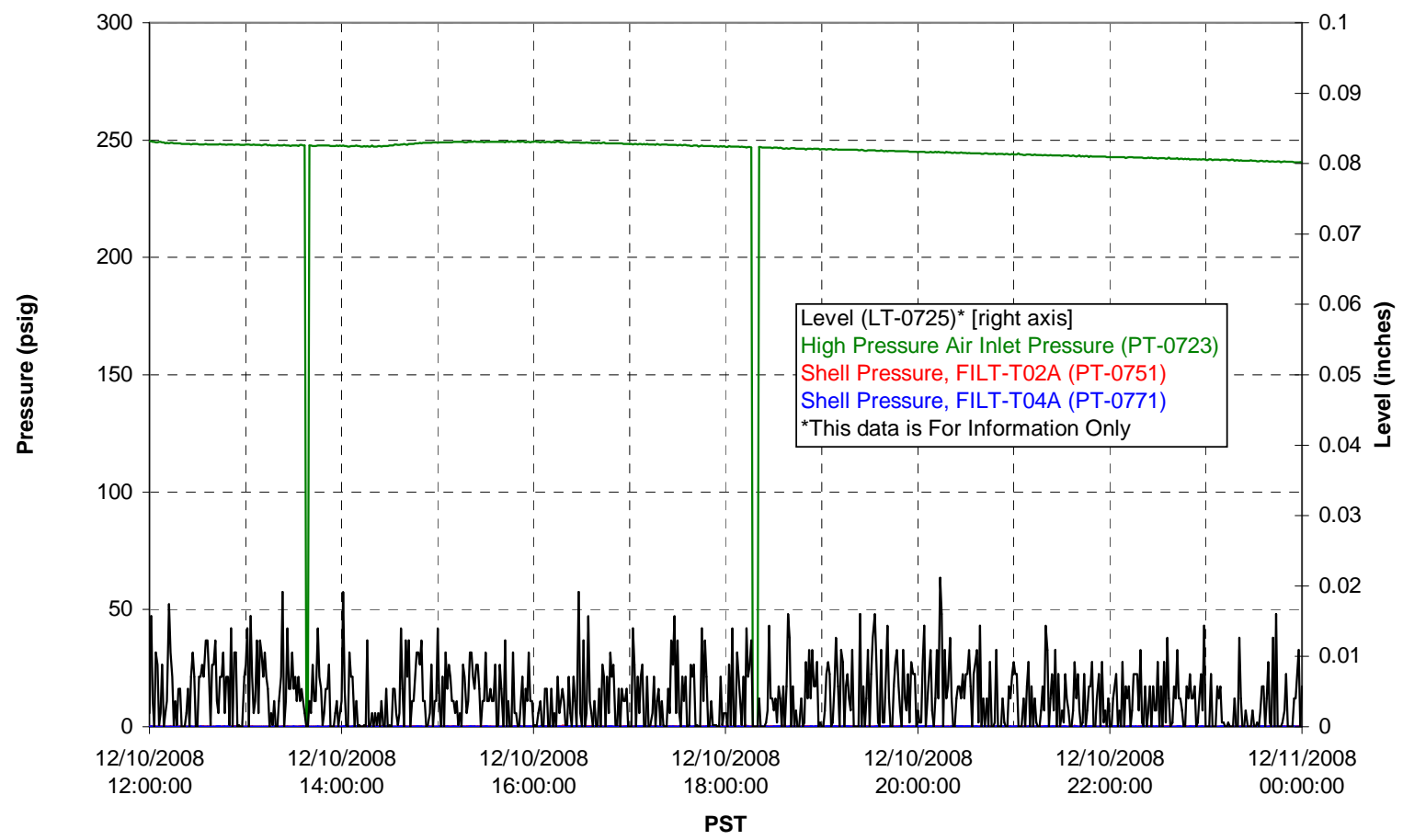


Pulsepot UFP-PP-T03A

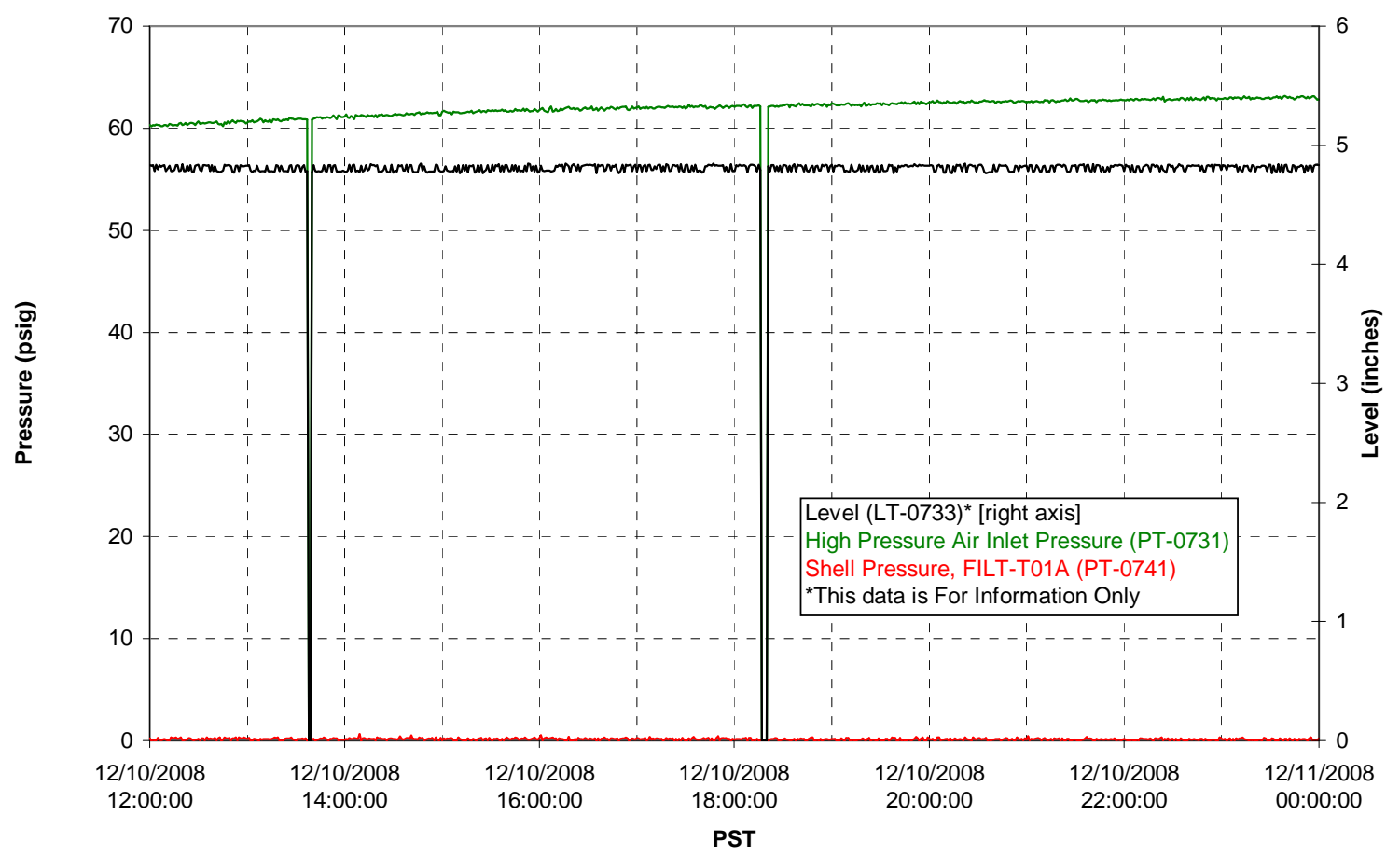

Pulsepot Levels

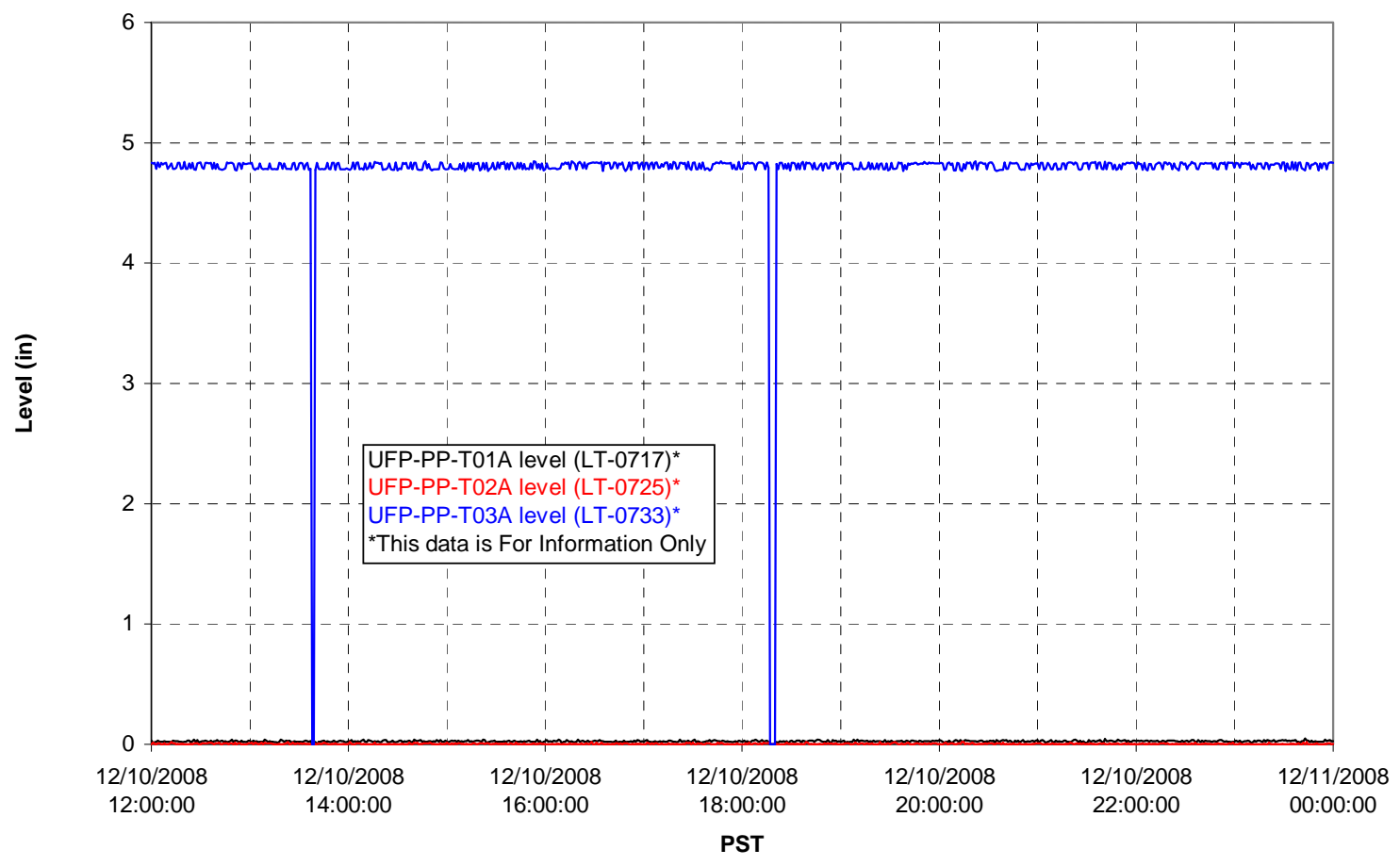


Filter UFP-FILT-T01A

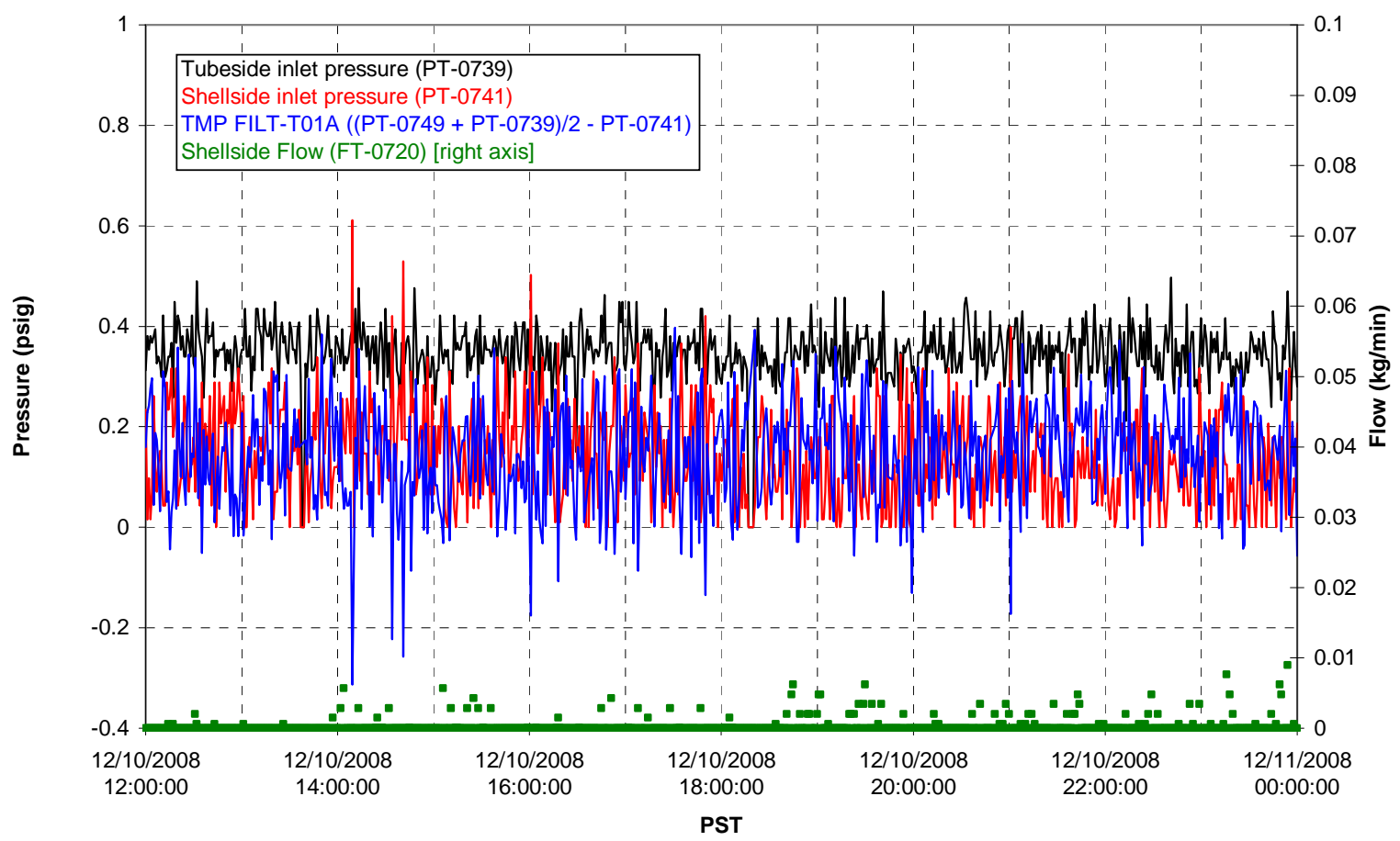

Filter UFP-FILT-T02A

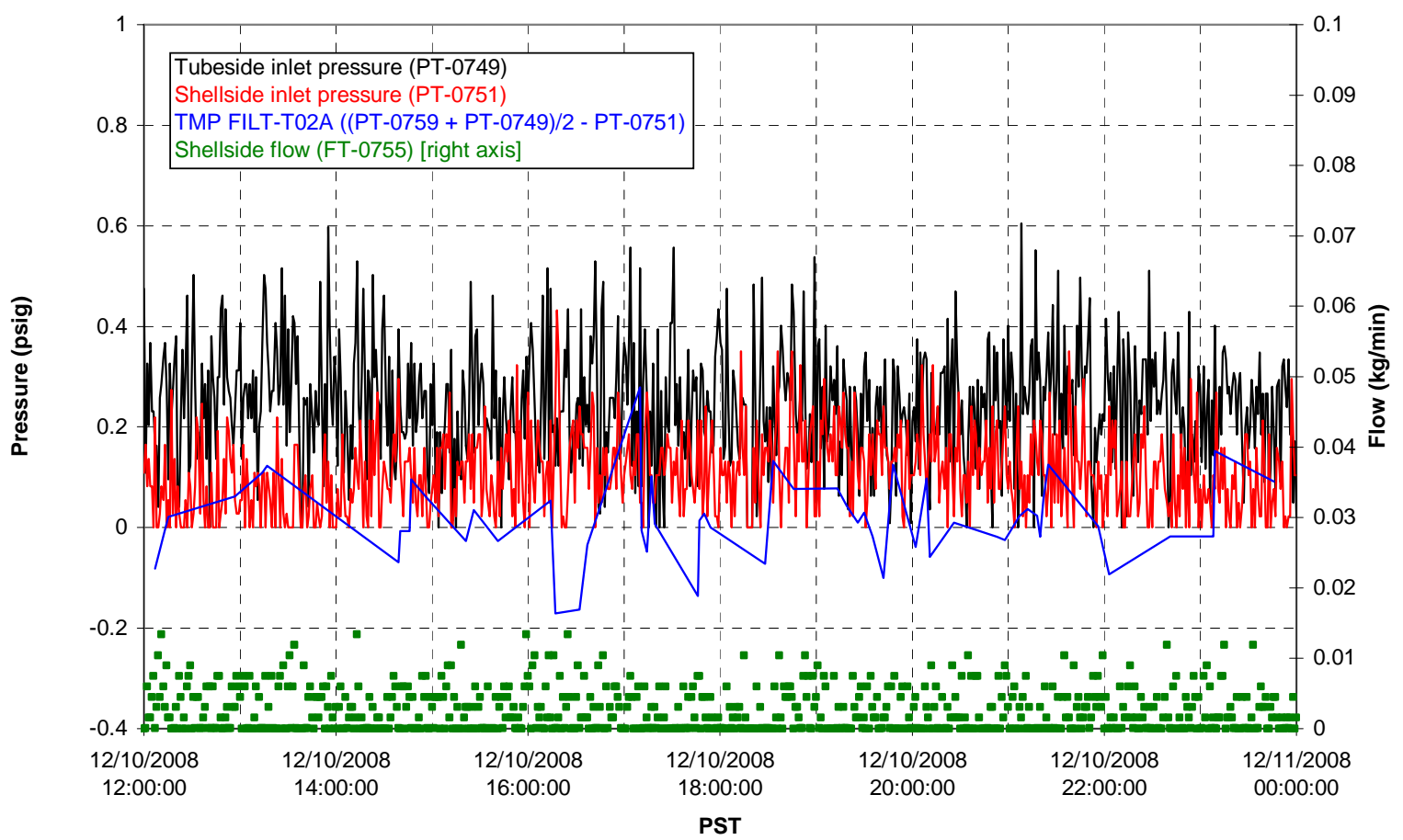


Filter UFP-FILT-T03A

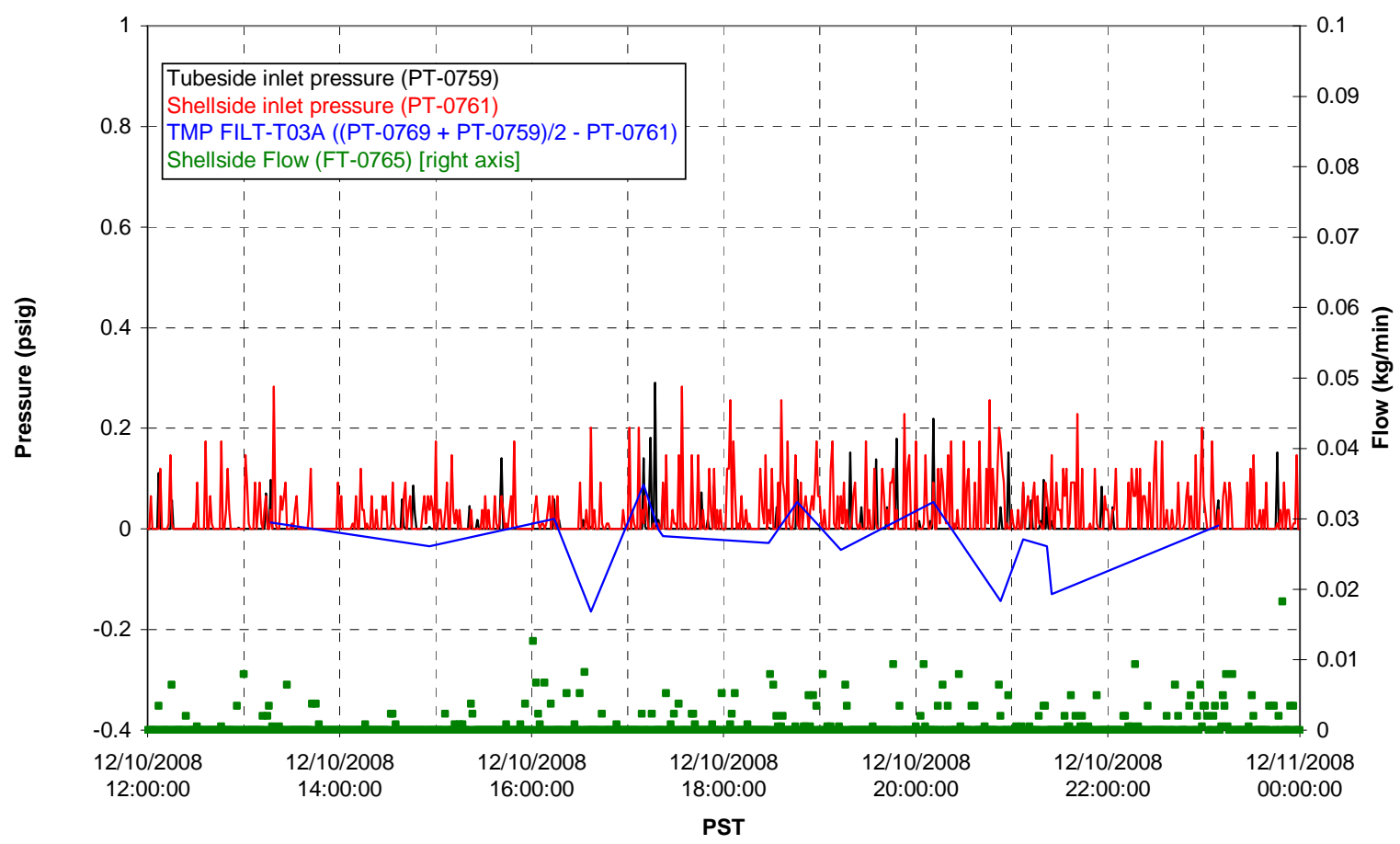

Filter UFP-FILT-T04A

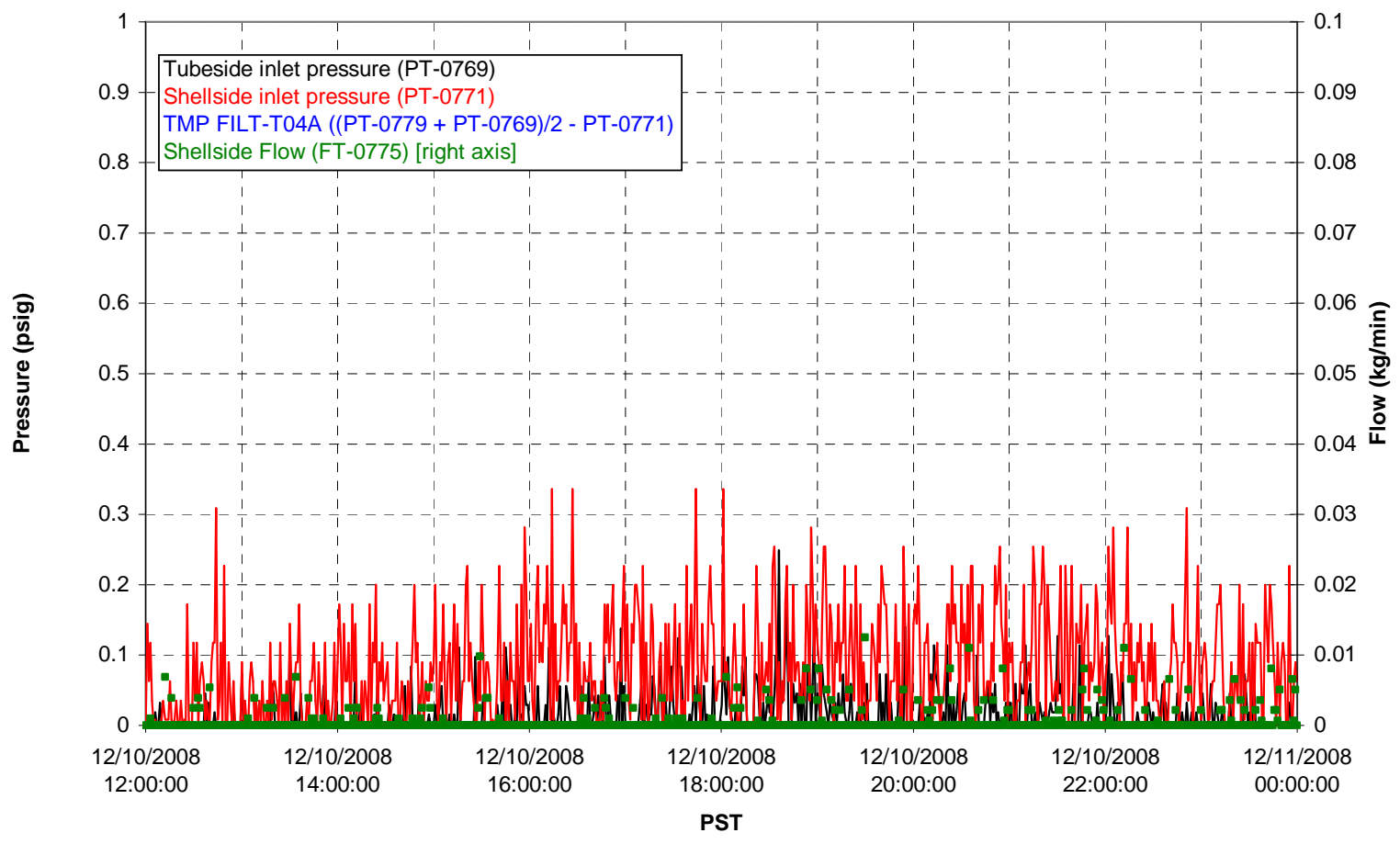


Filter UFP-FILT-T05A

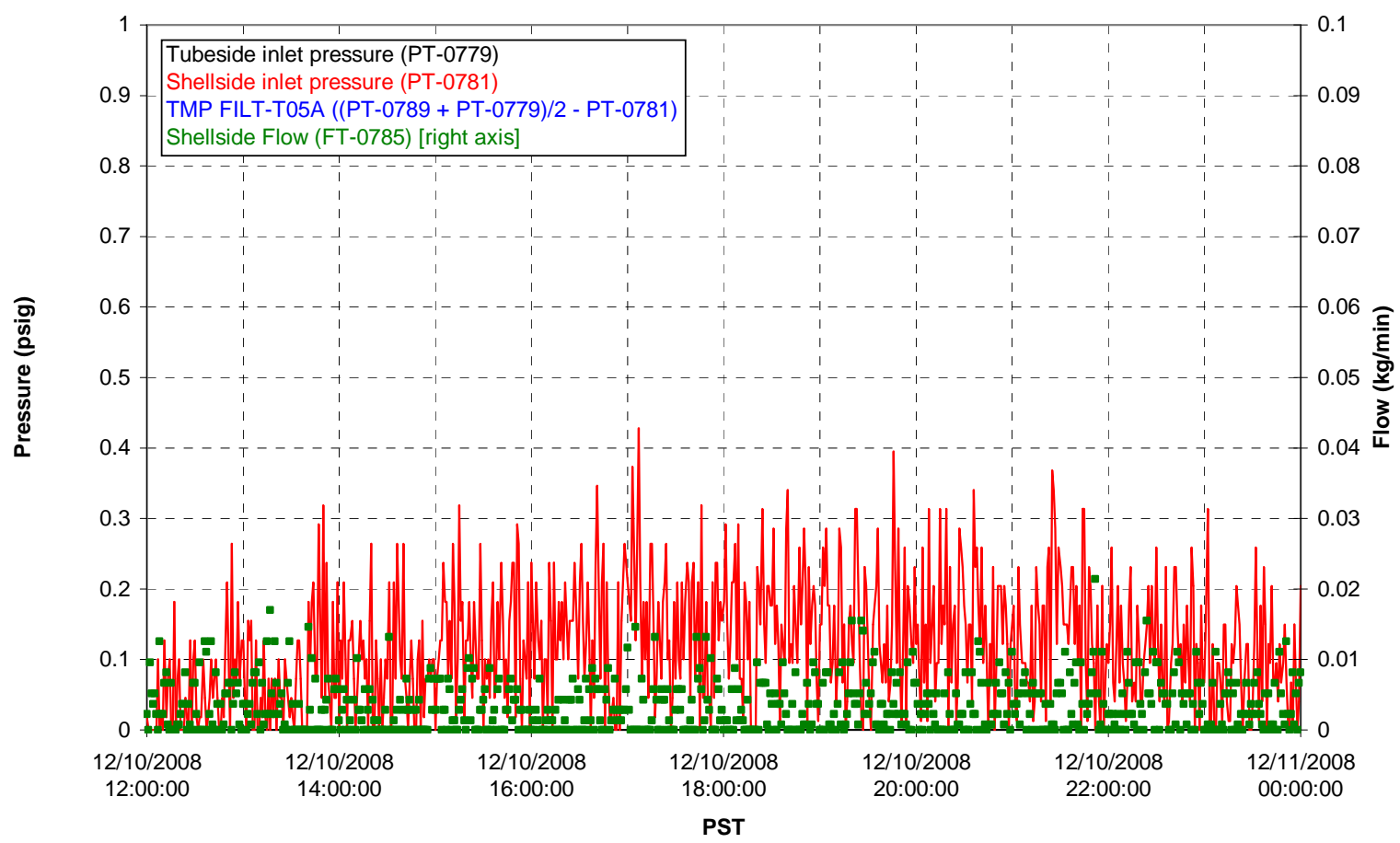

Chemical Flow

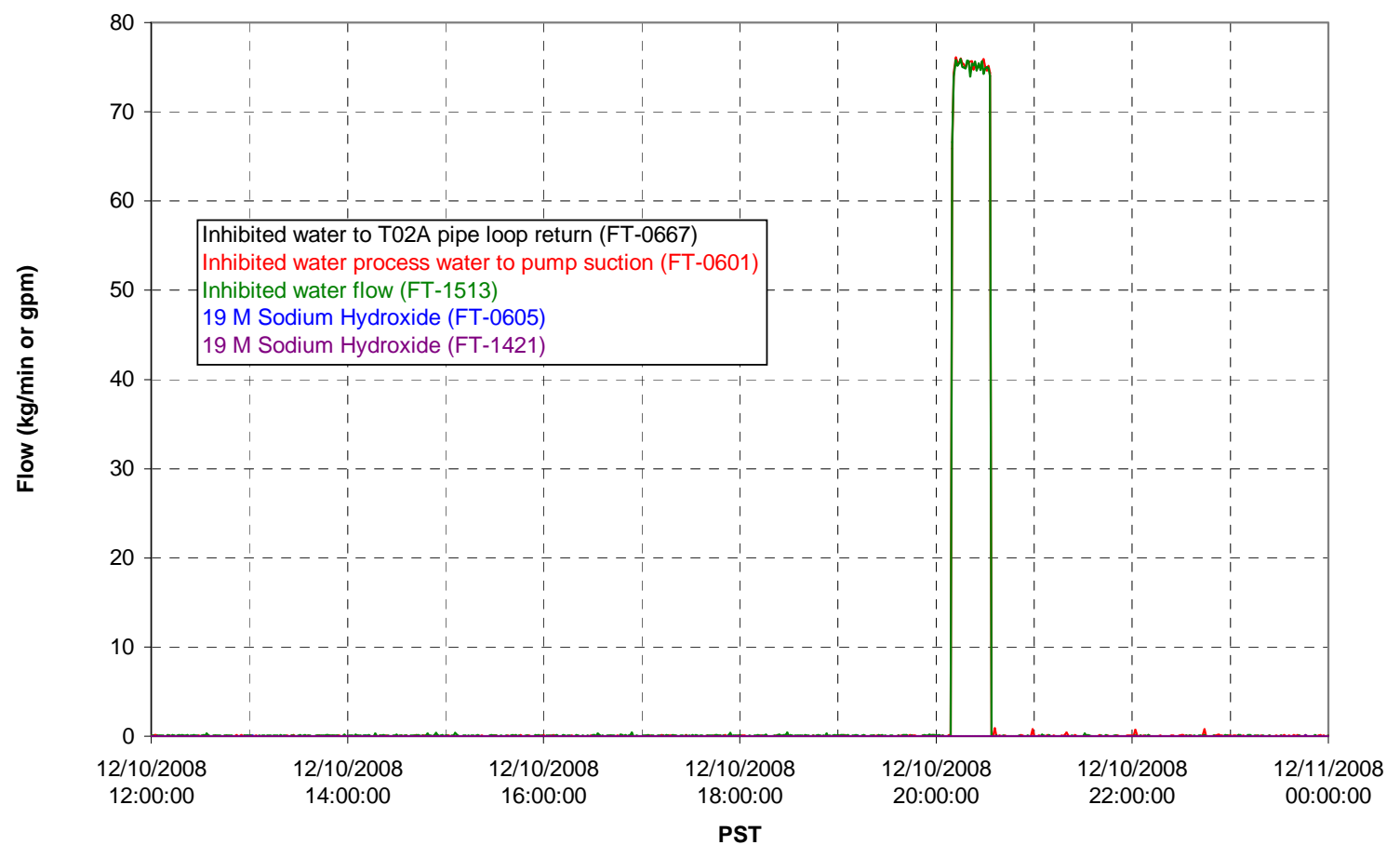




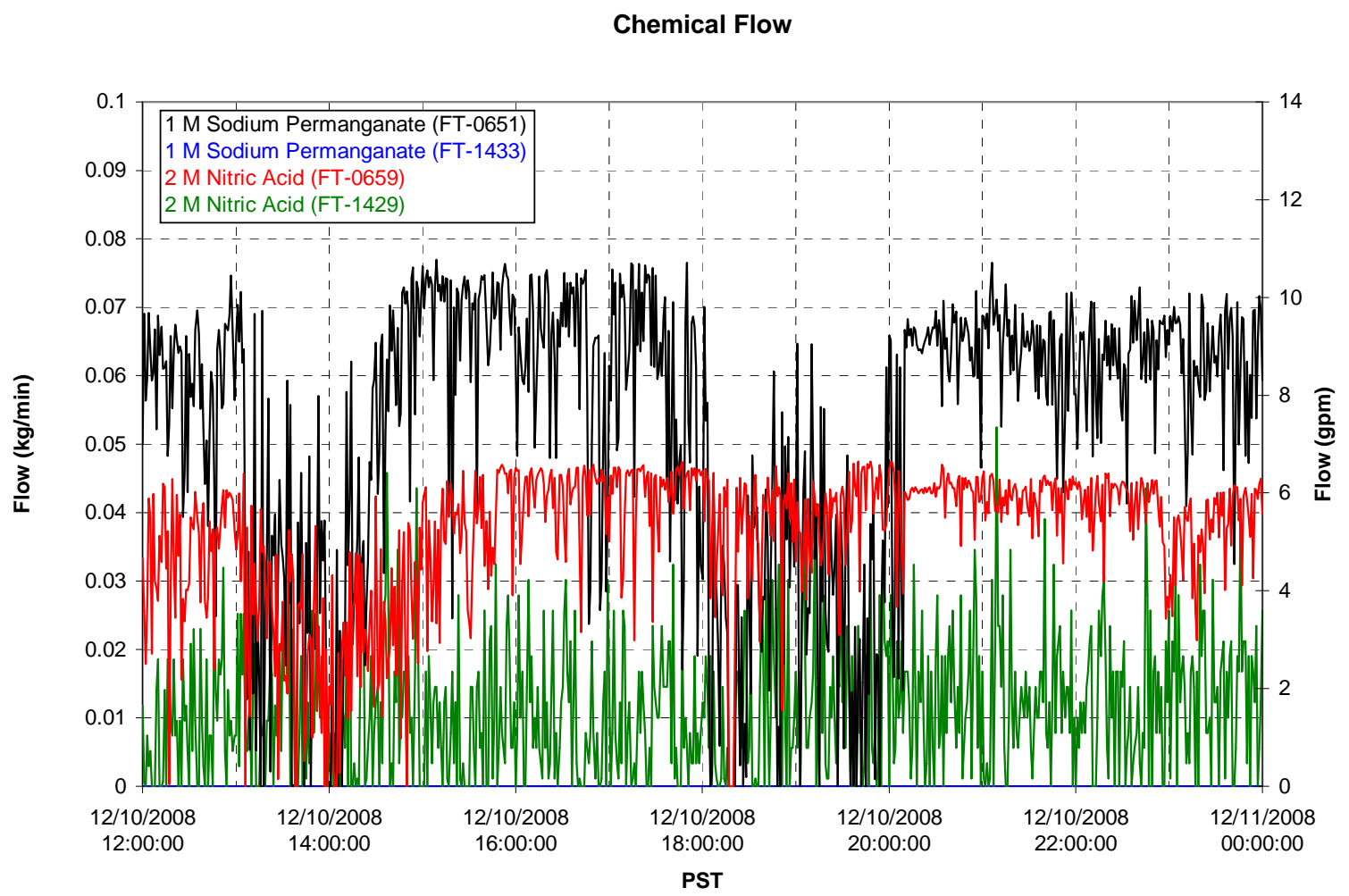

Air Flows

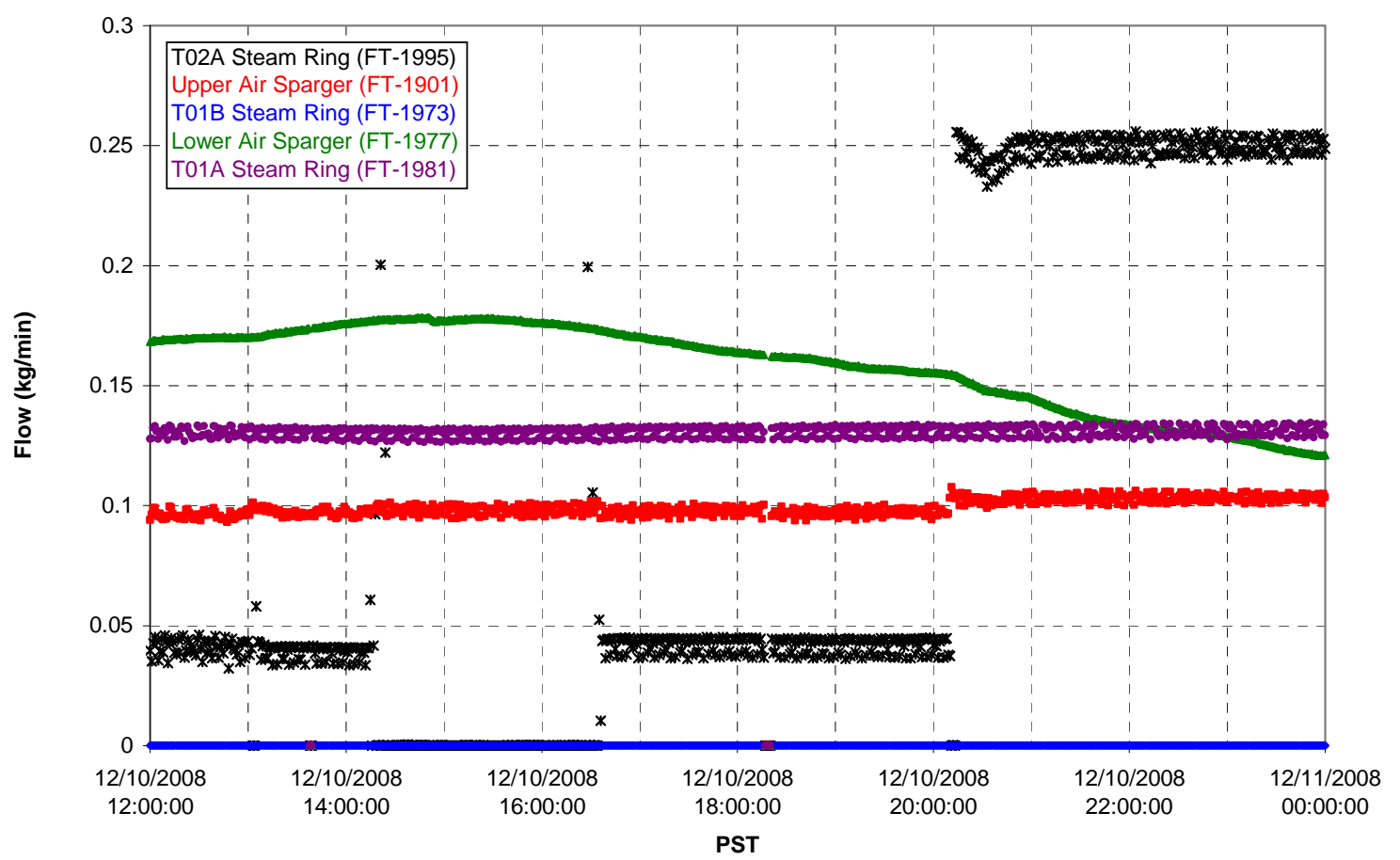


T02A Steam

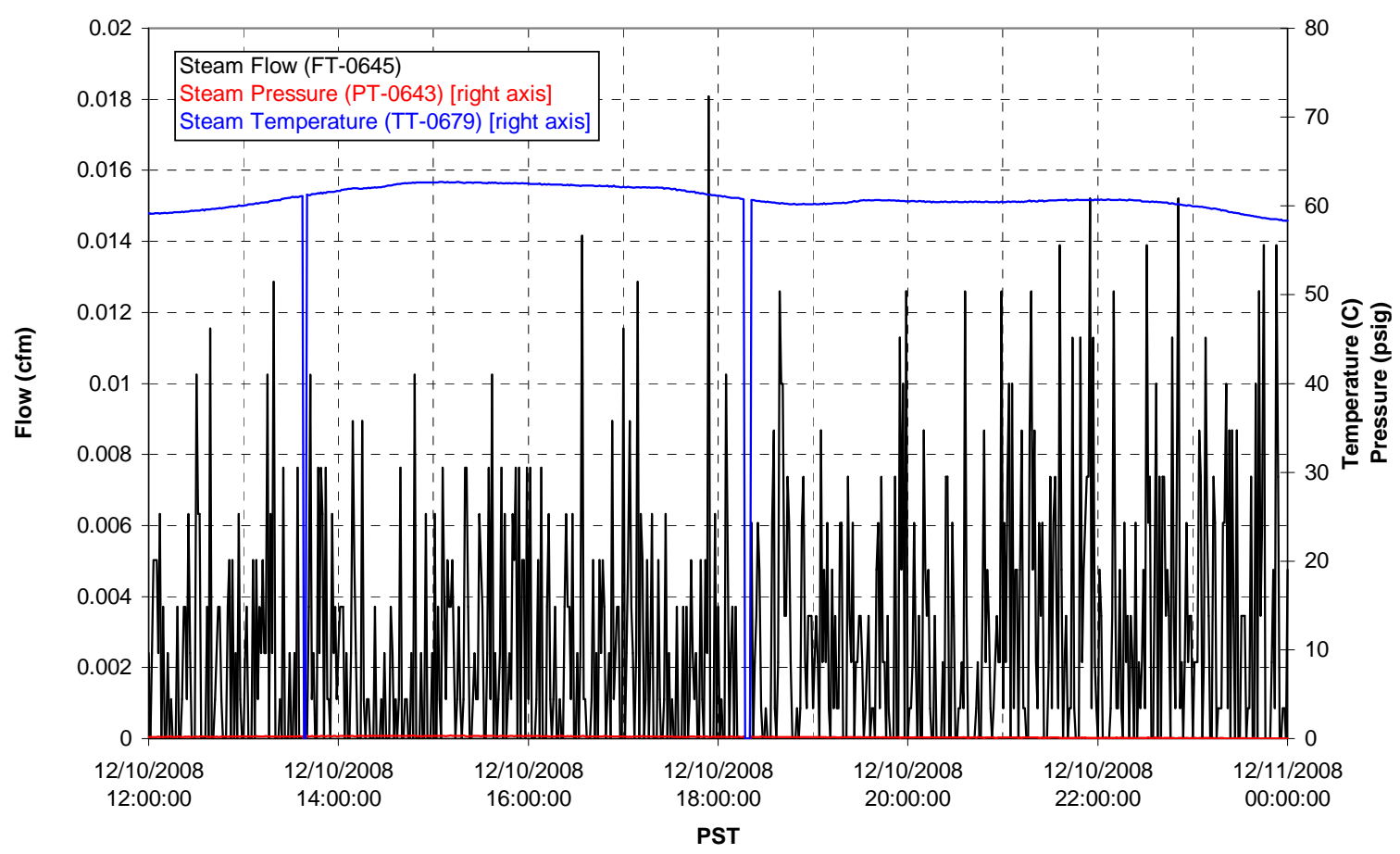

T01A Steam

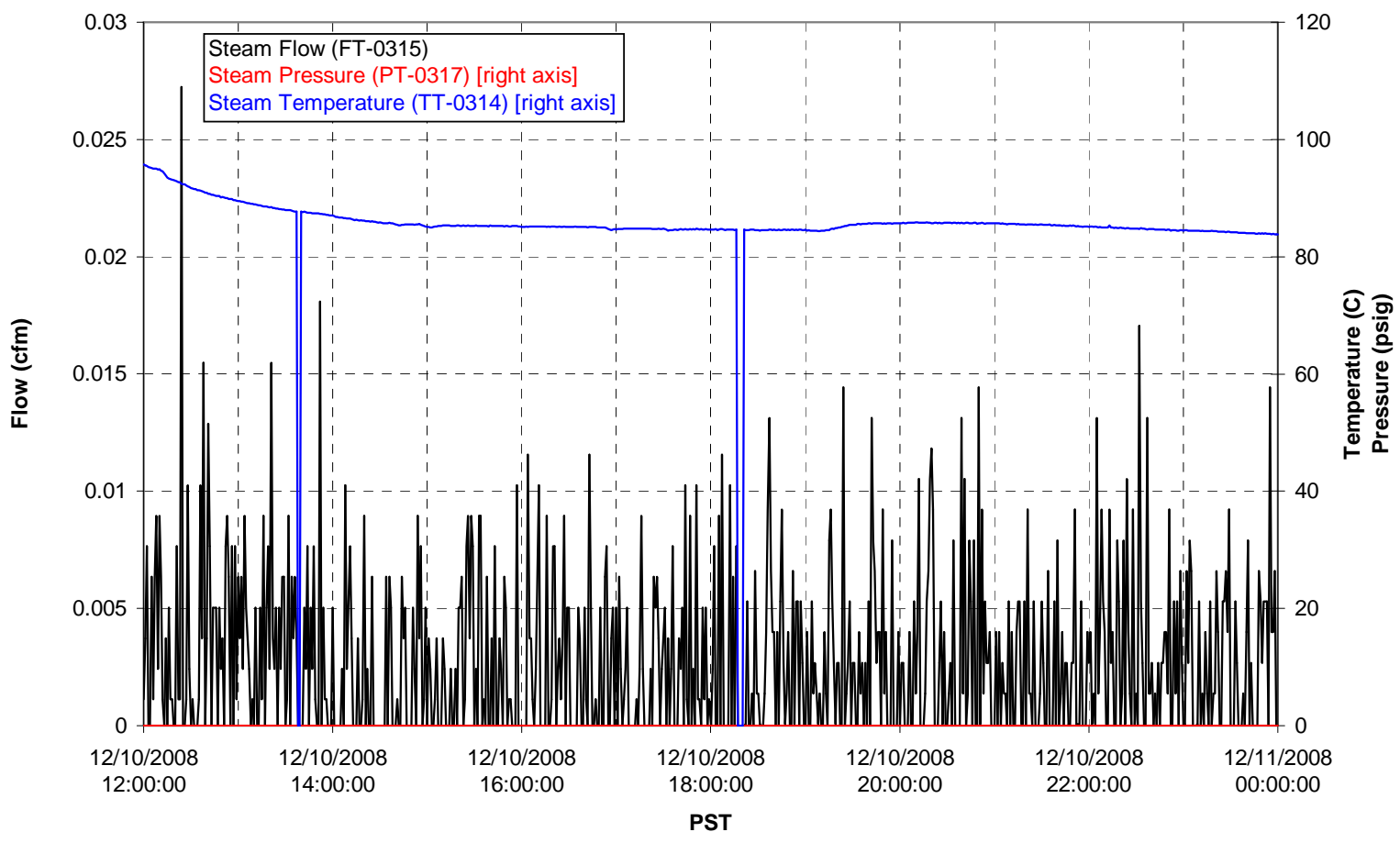


T01B Steam

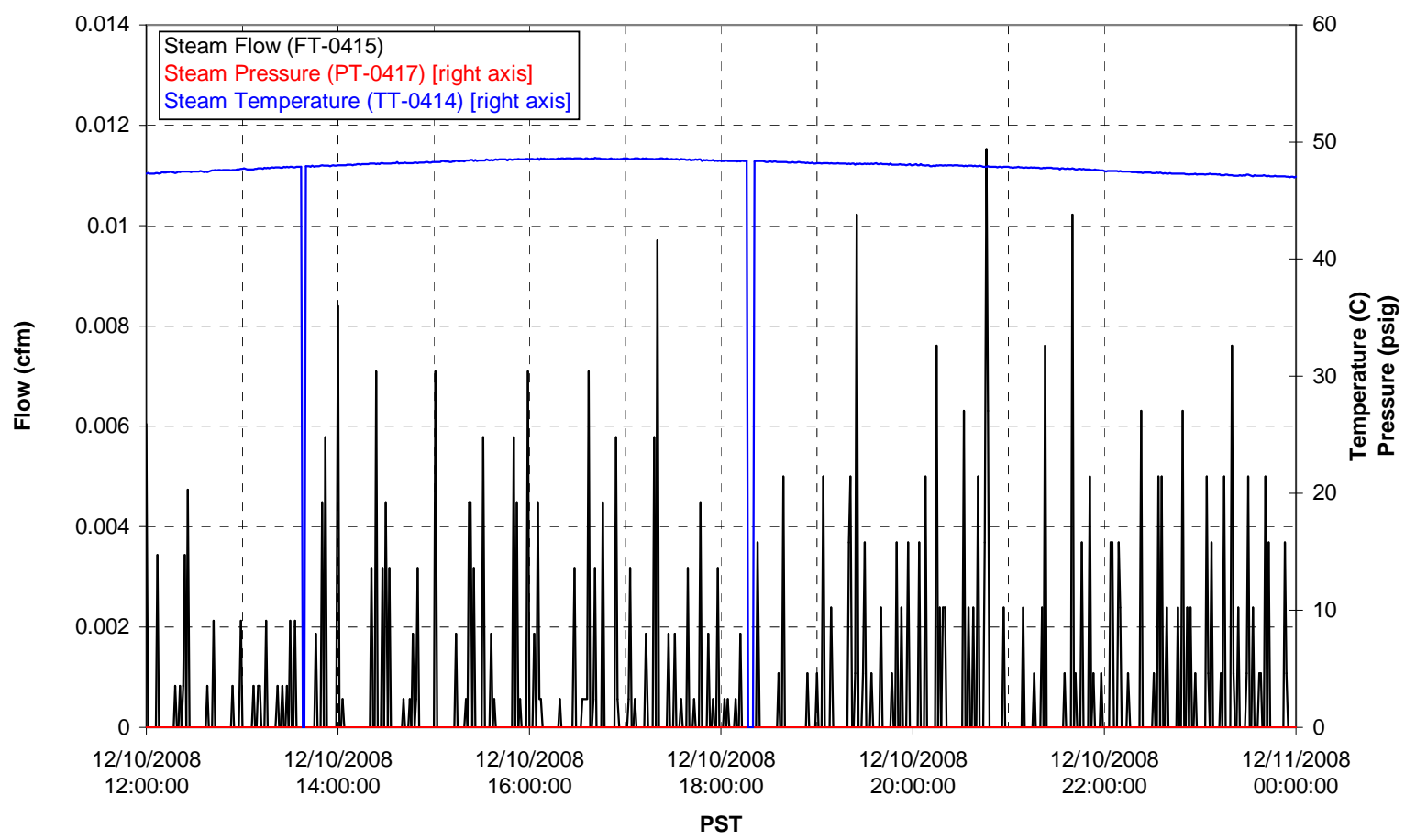


Functional Test Data Plots 12/20/08 00:00 - 12/20/08 12:00 
T01A level

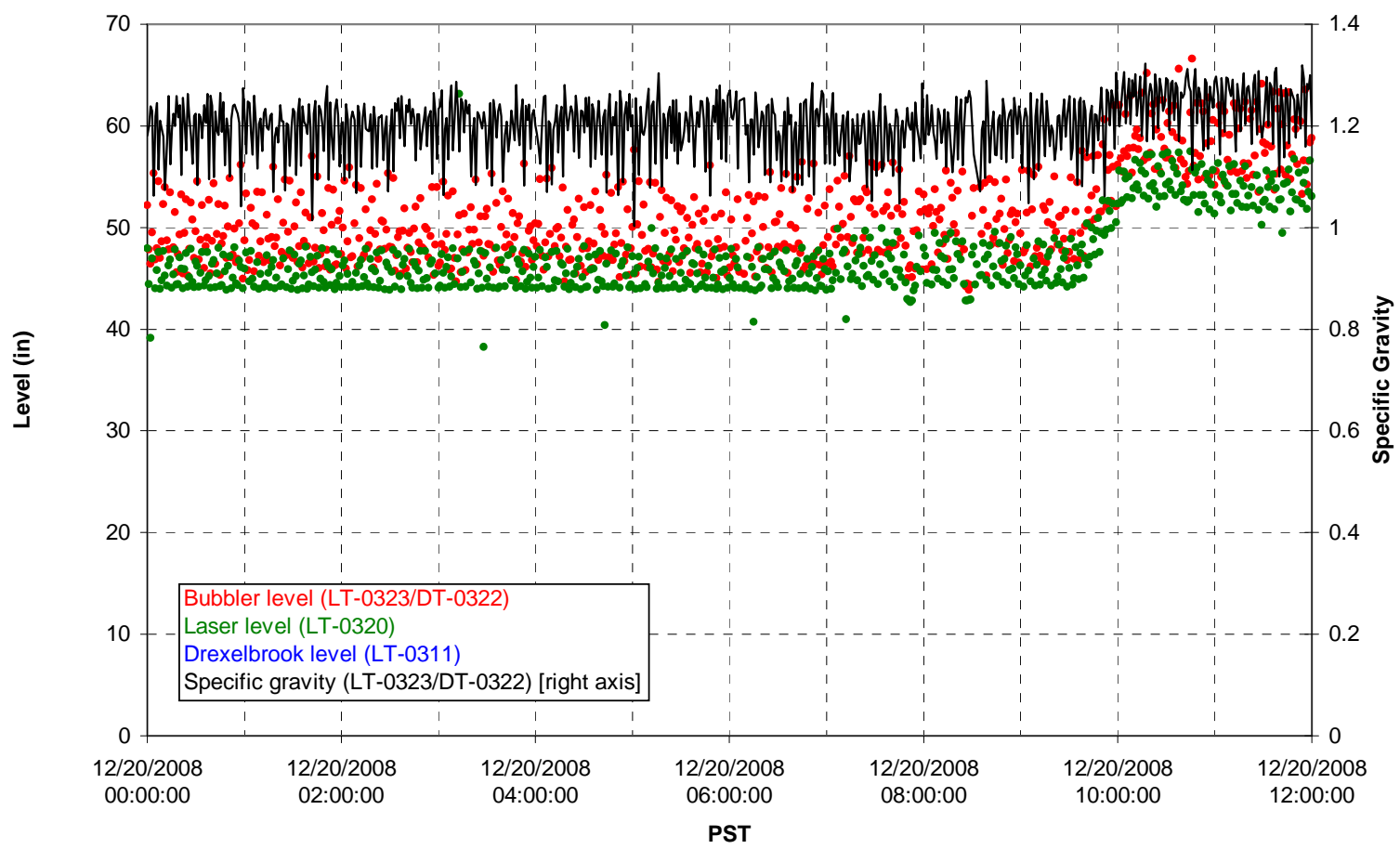

T01A temperatures

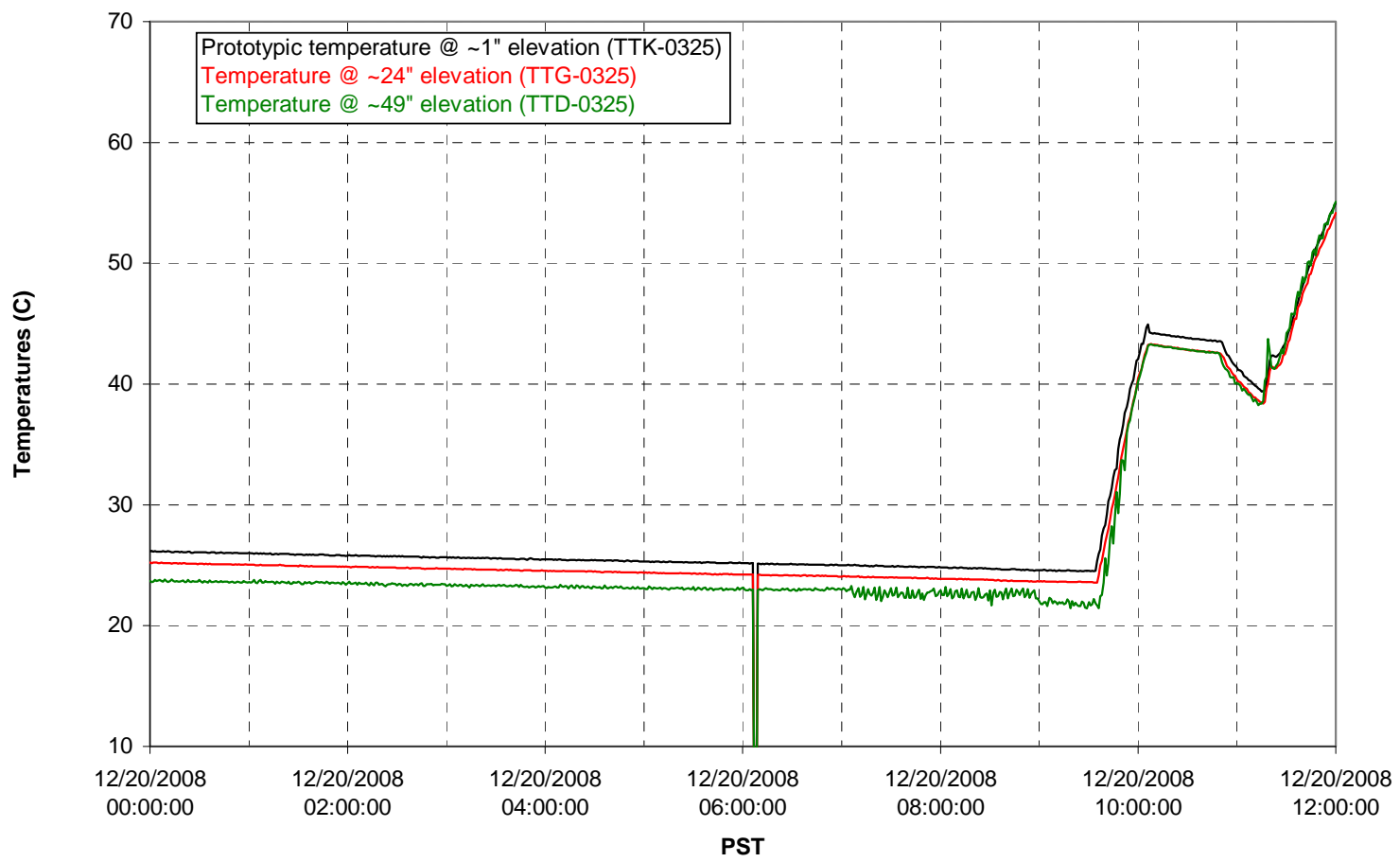


T01B level

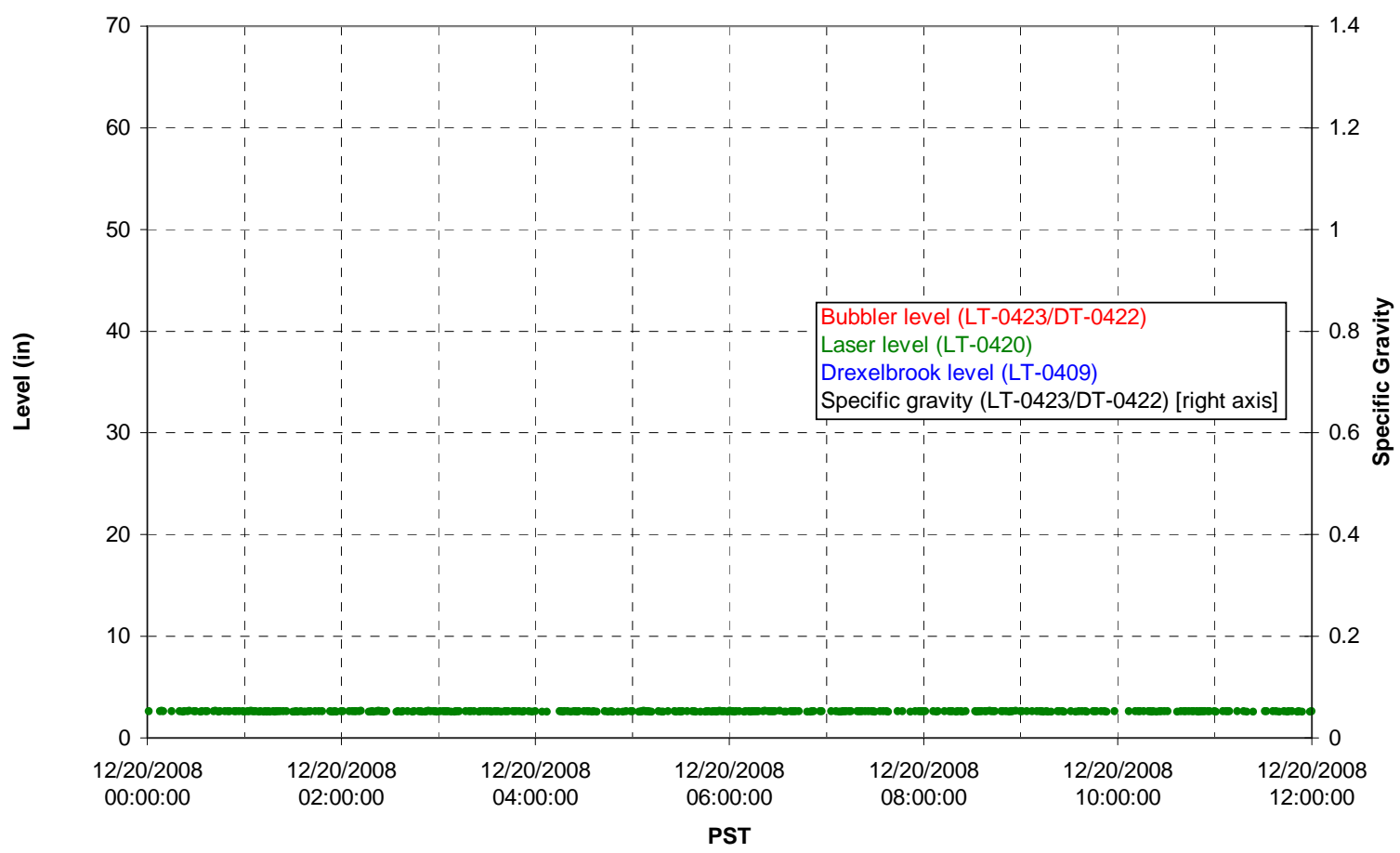

T01B temperatures

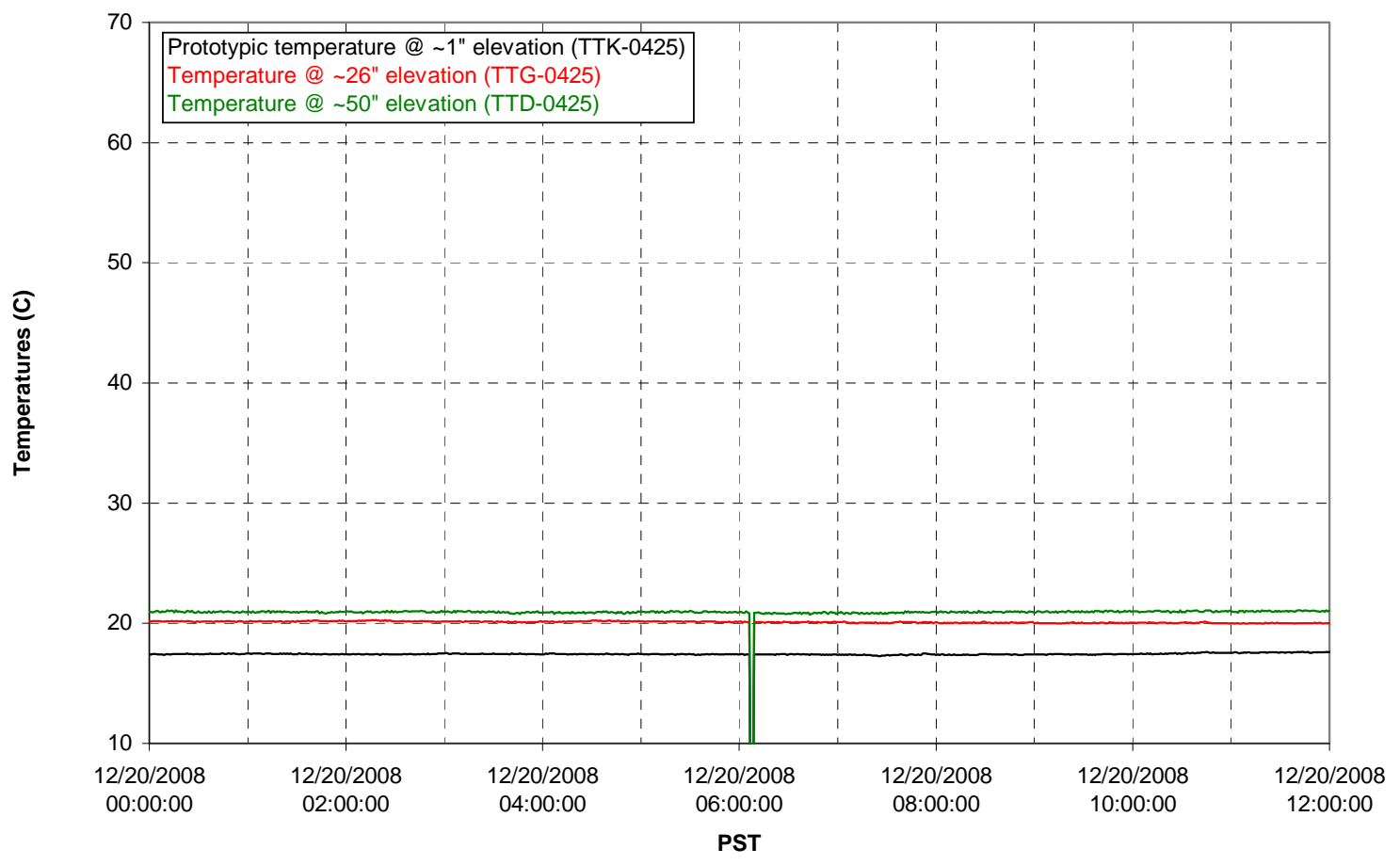


T02A level

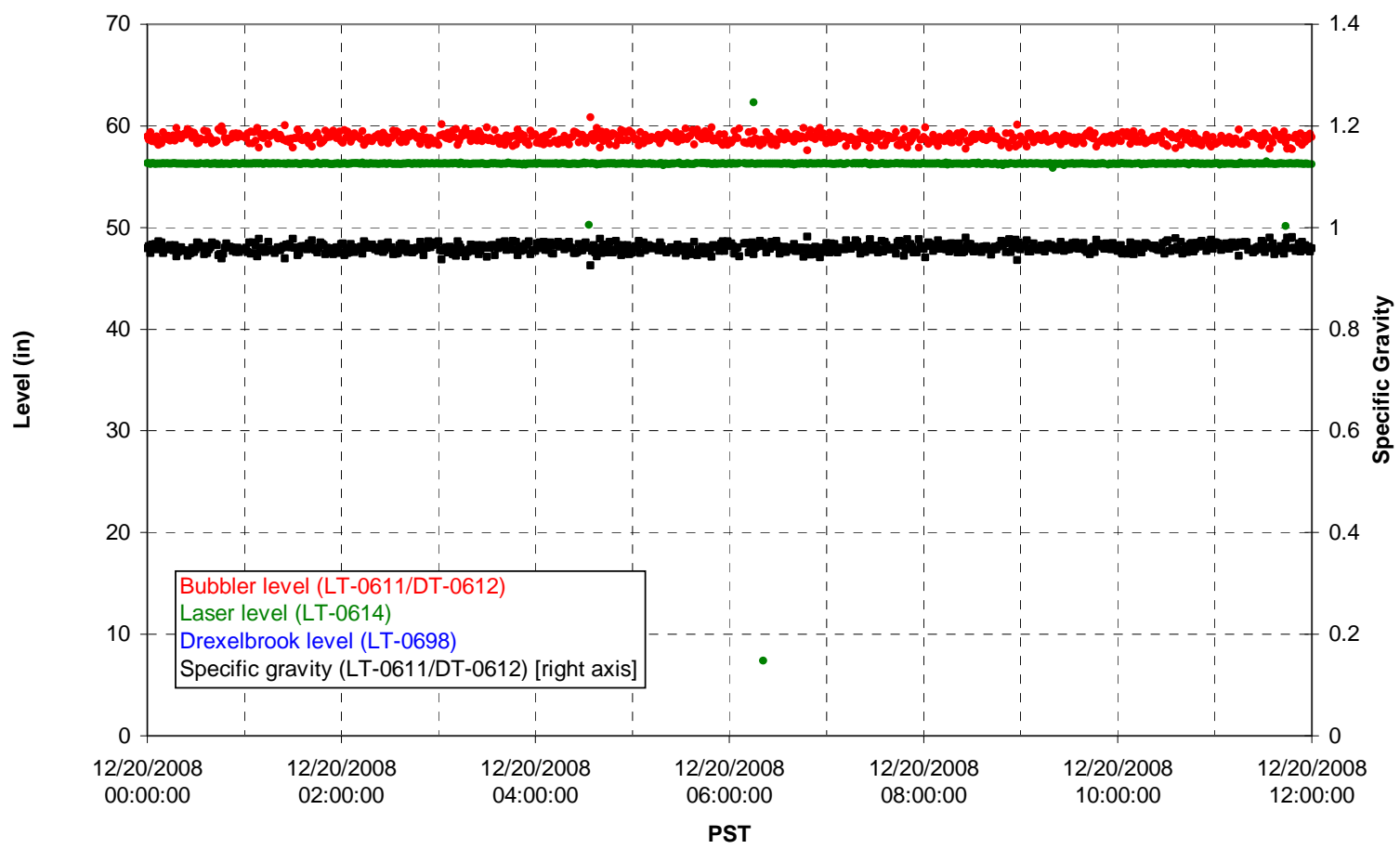

T02A temperatures

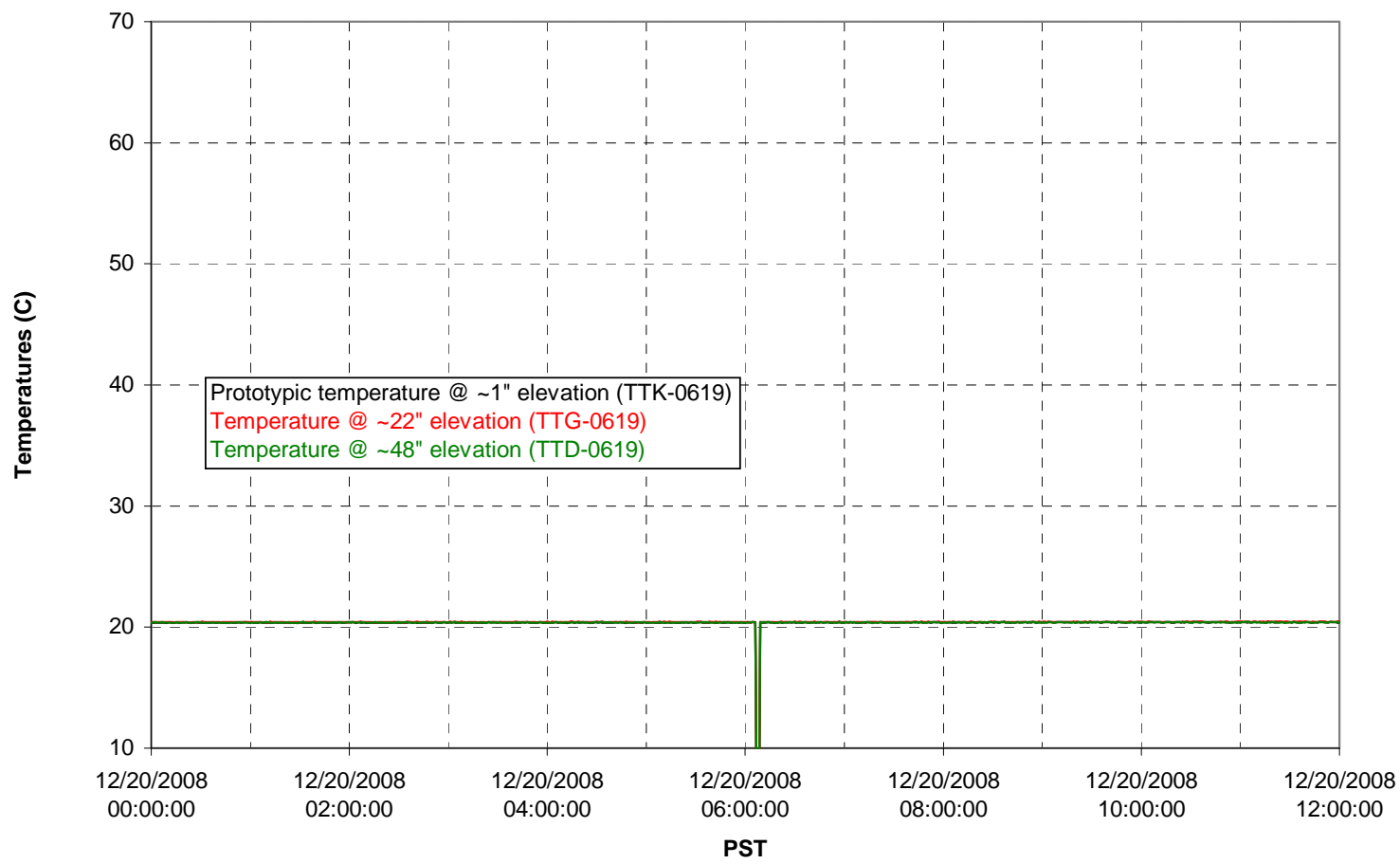


T02A and filter loop temperatures

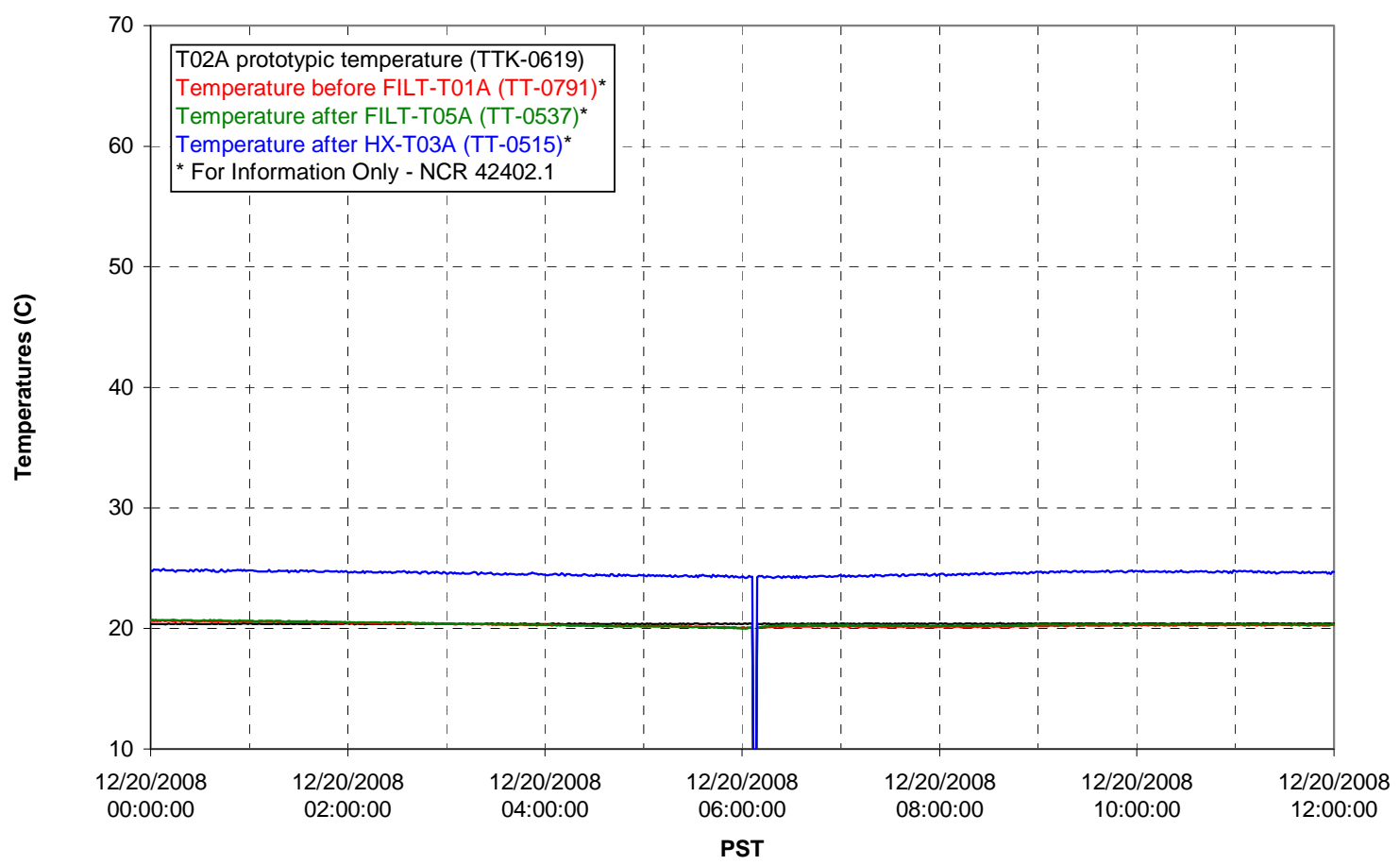

Pump Pressures and Flow

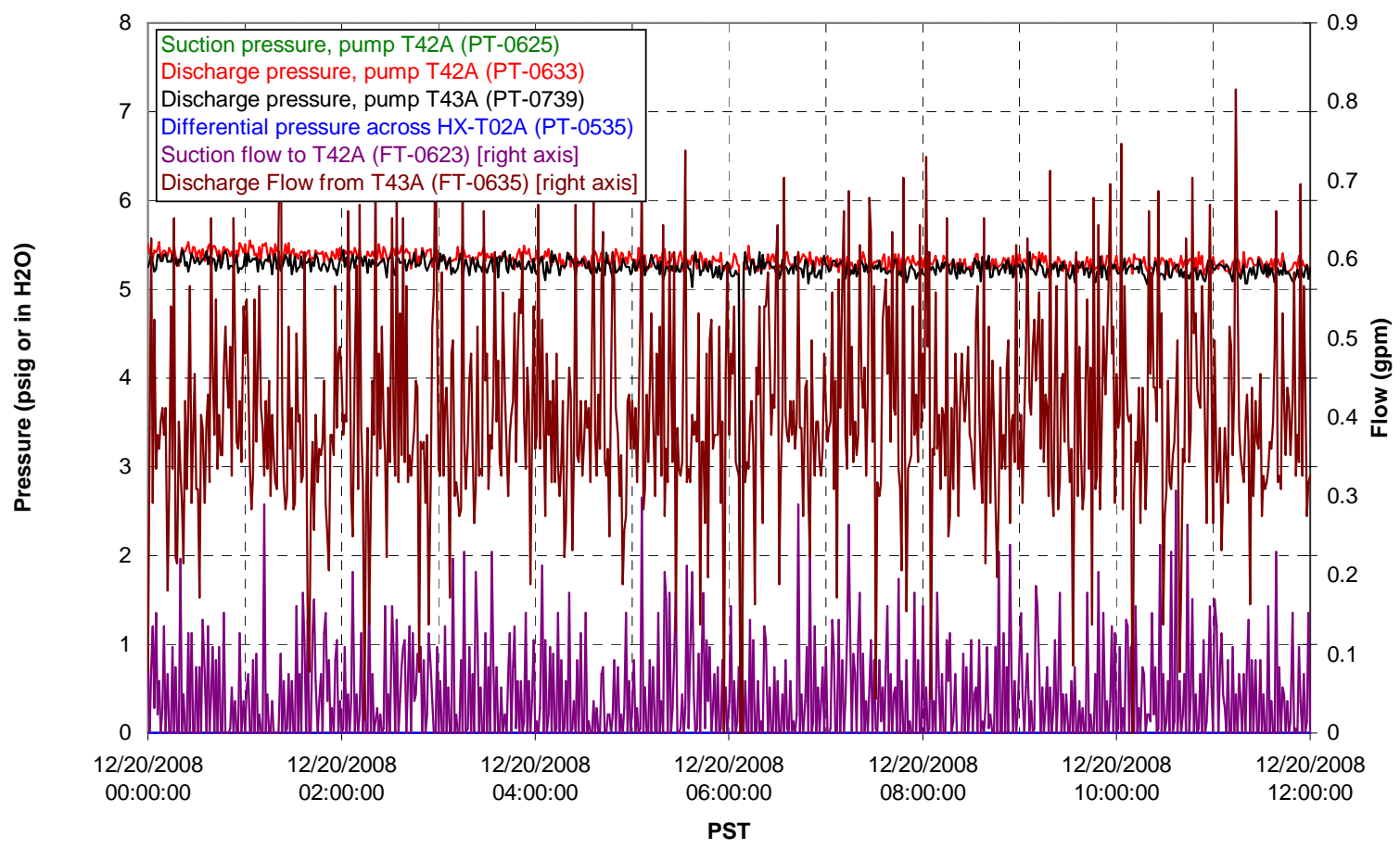


Axial pressure drop

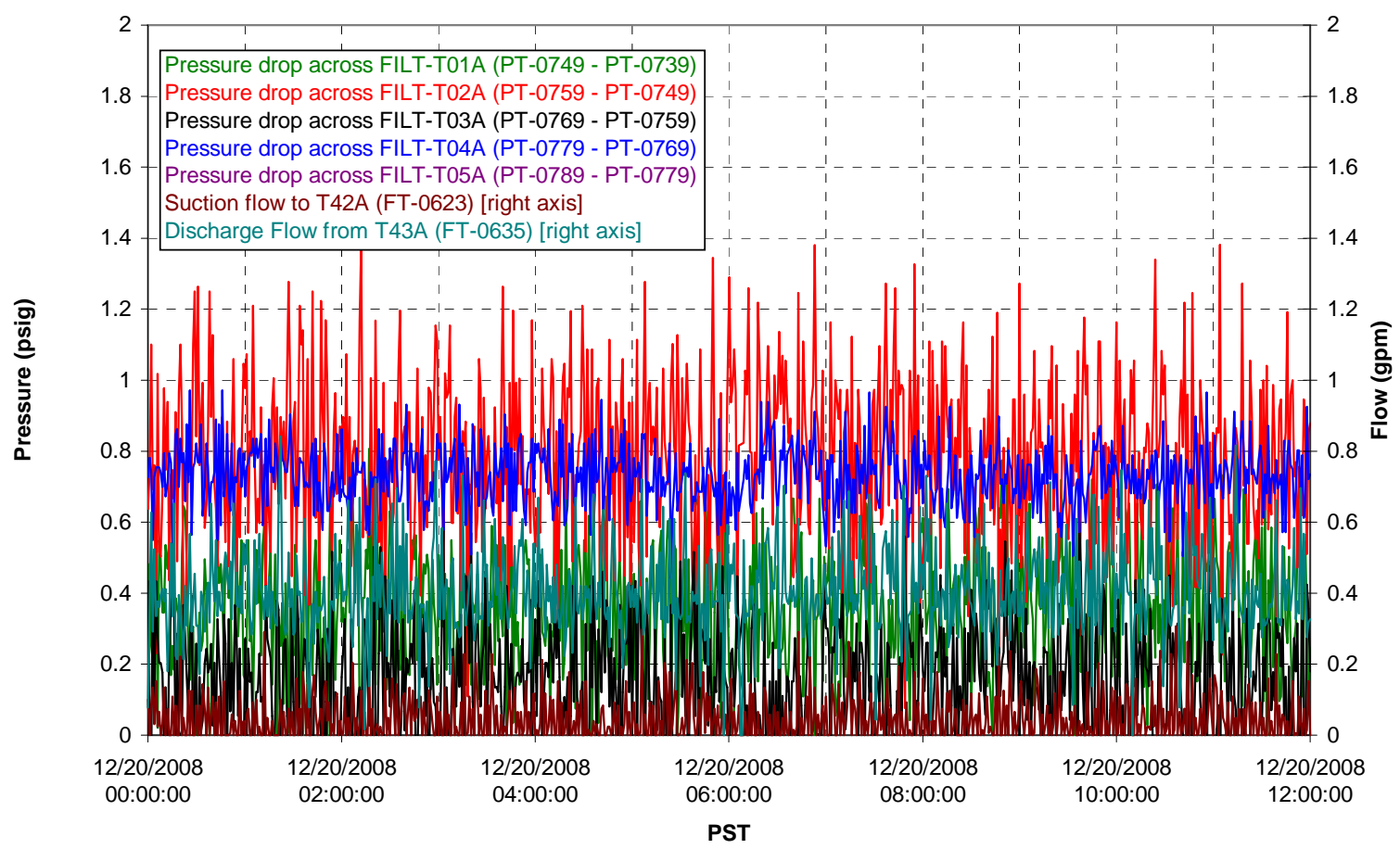

Permeate flow rates

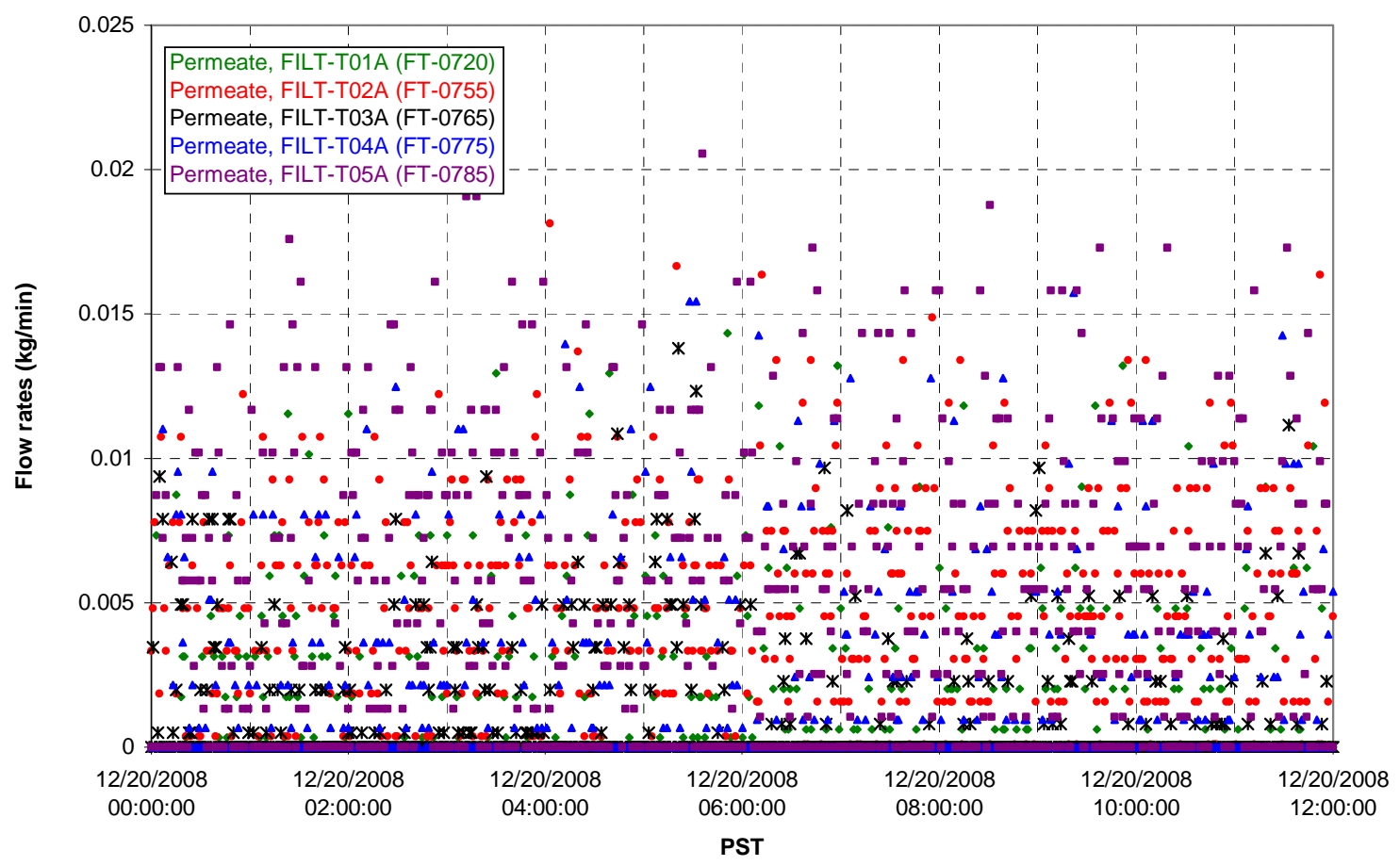


T02A Inner Temperature Tree

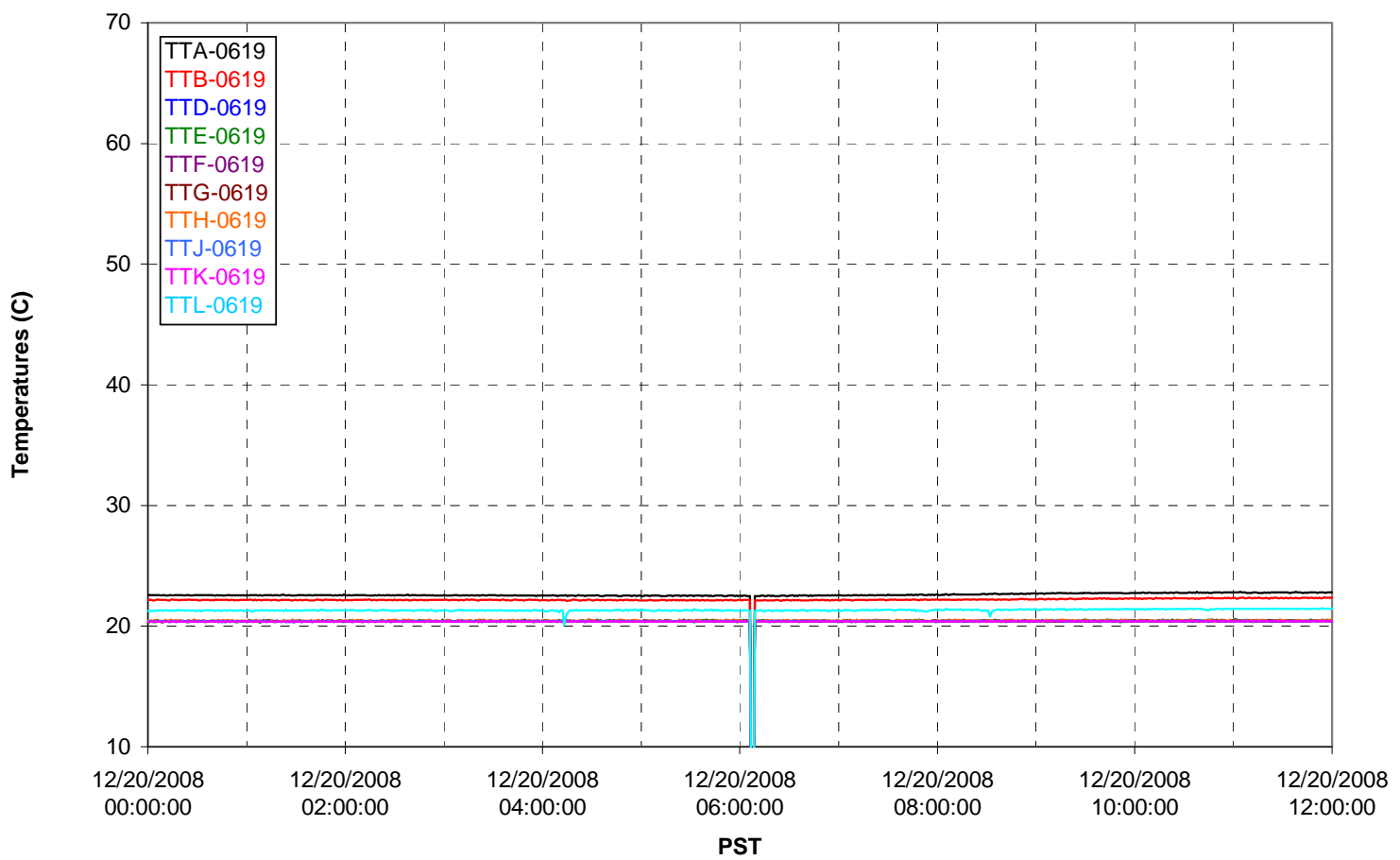

T02A Outer Temperature Tree

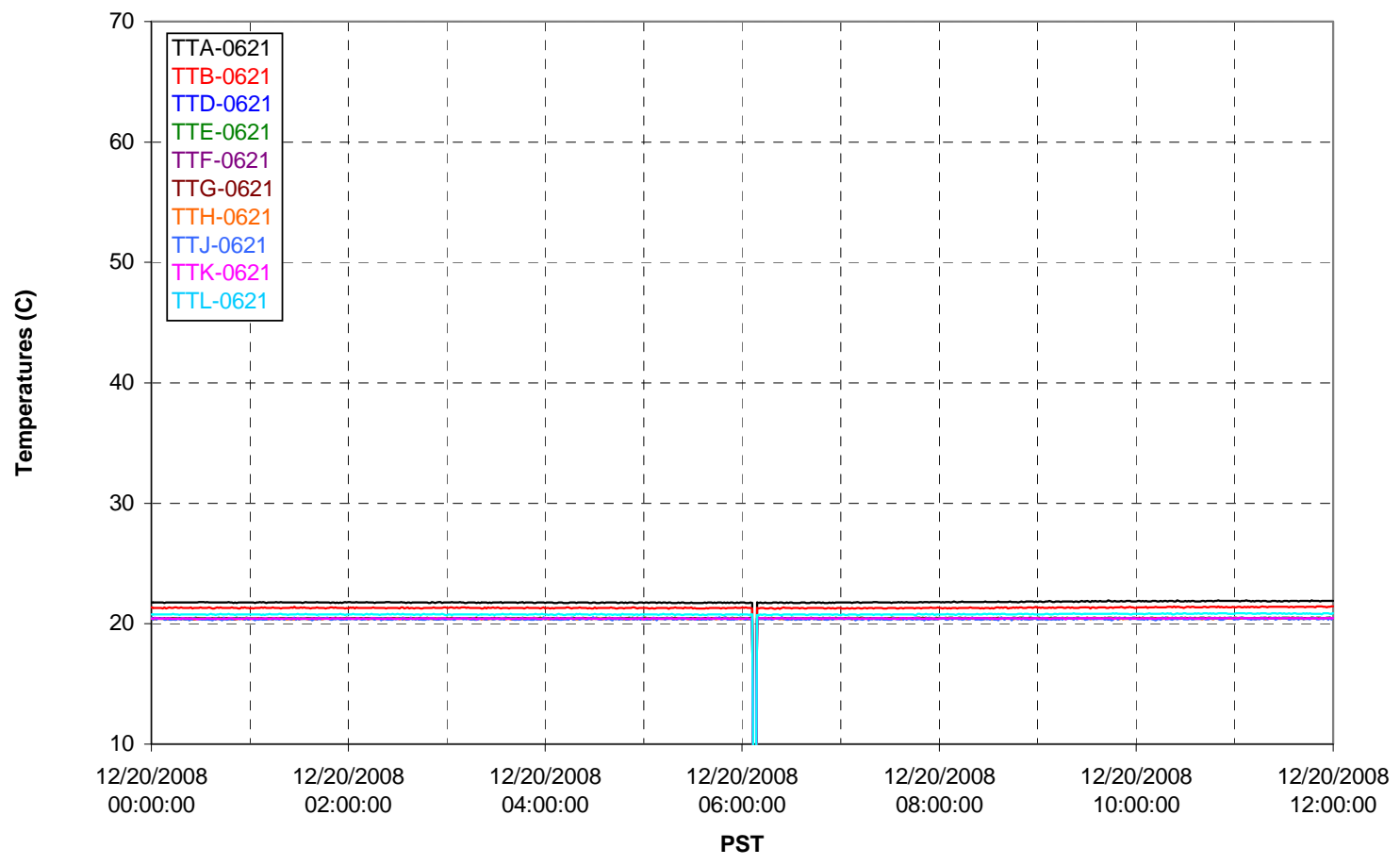


T02A temperatures

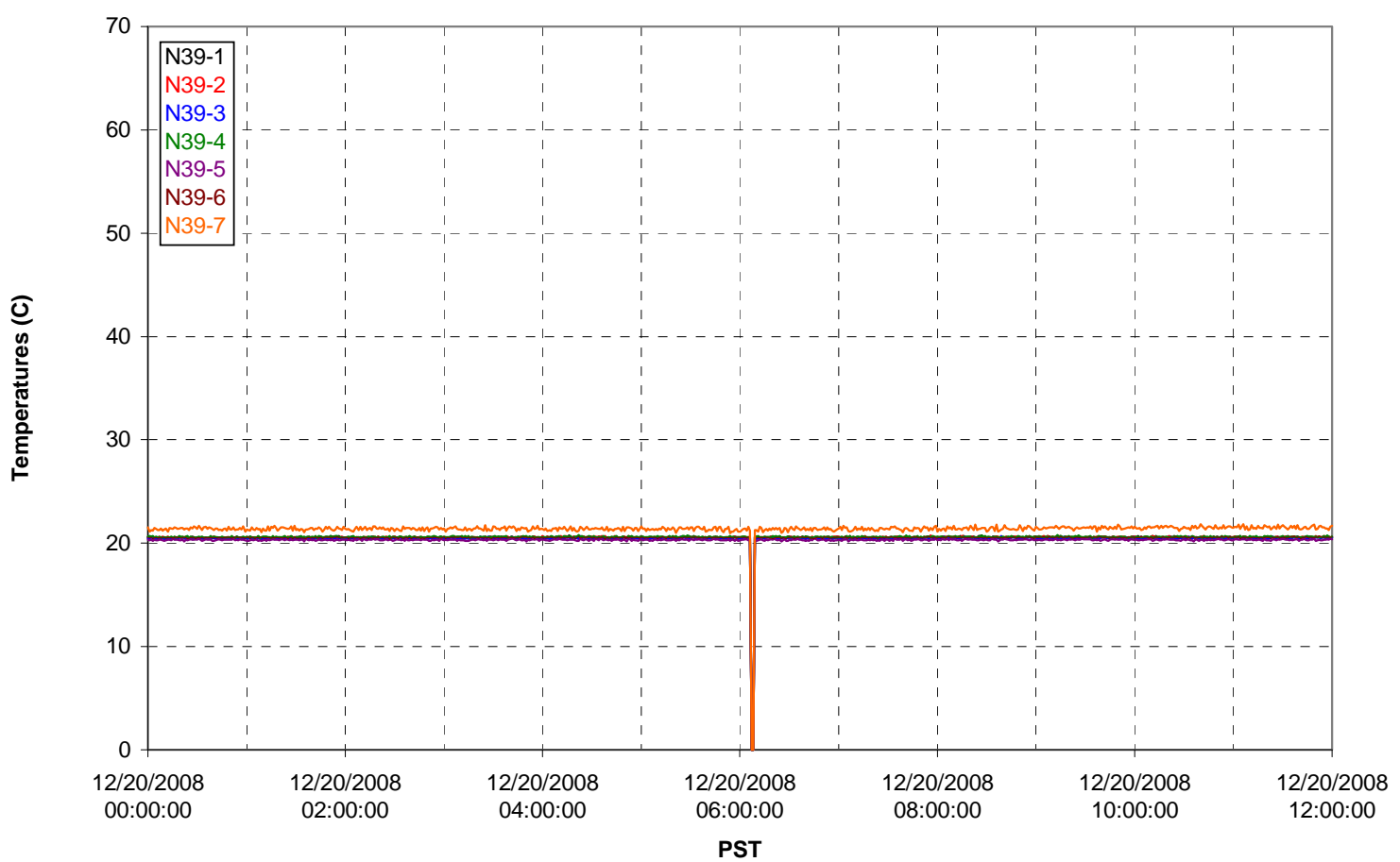

T02A temperatures

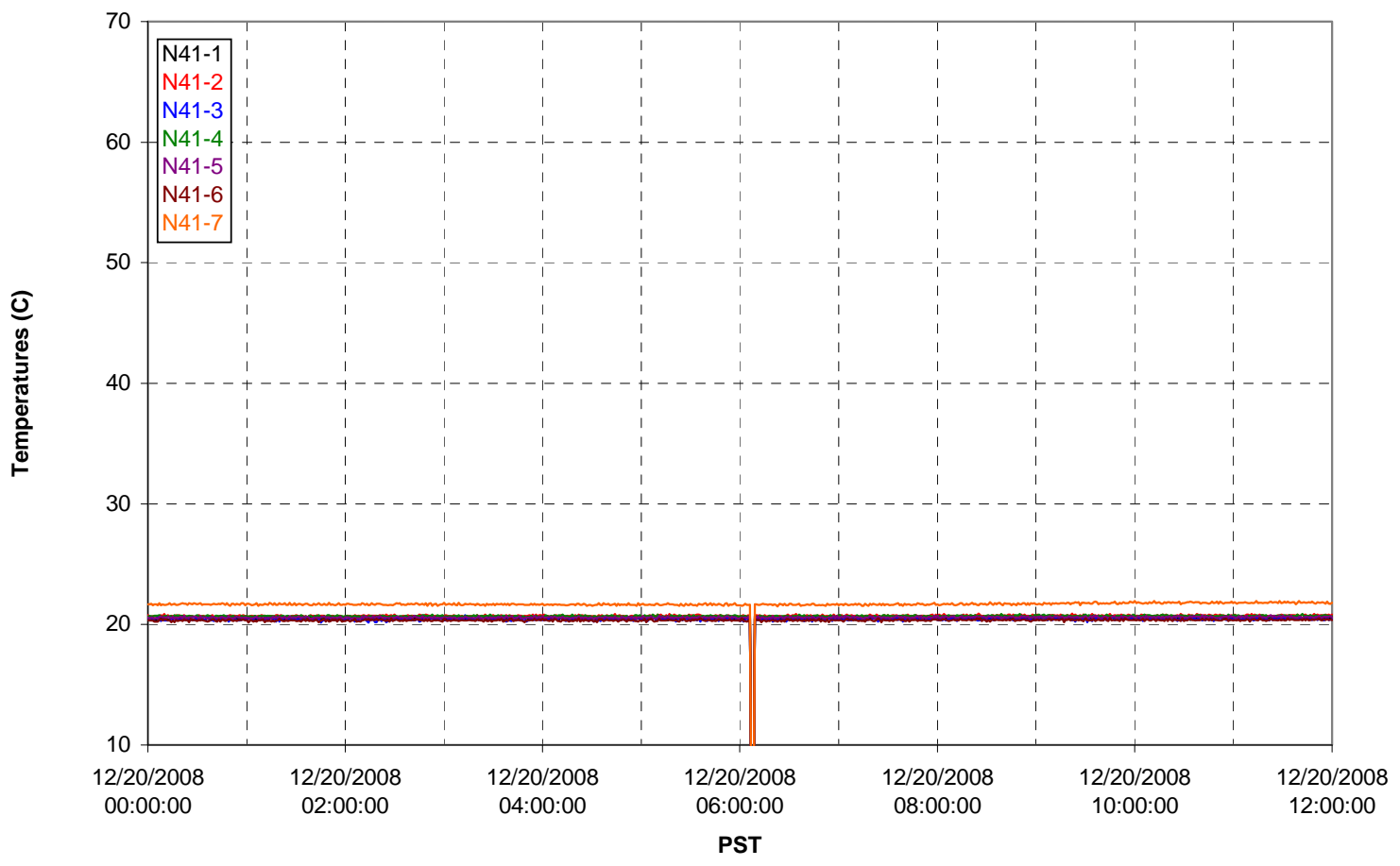


T02A temperatures

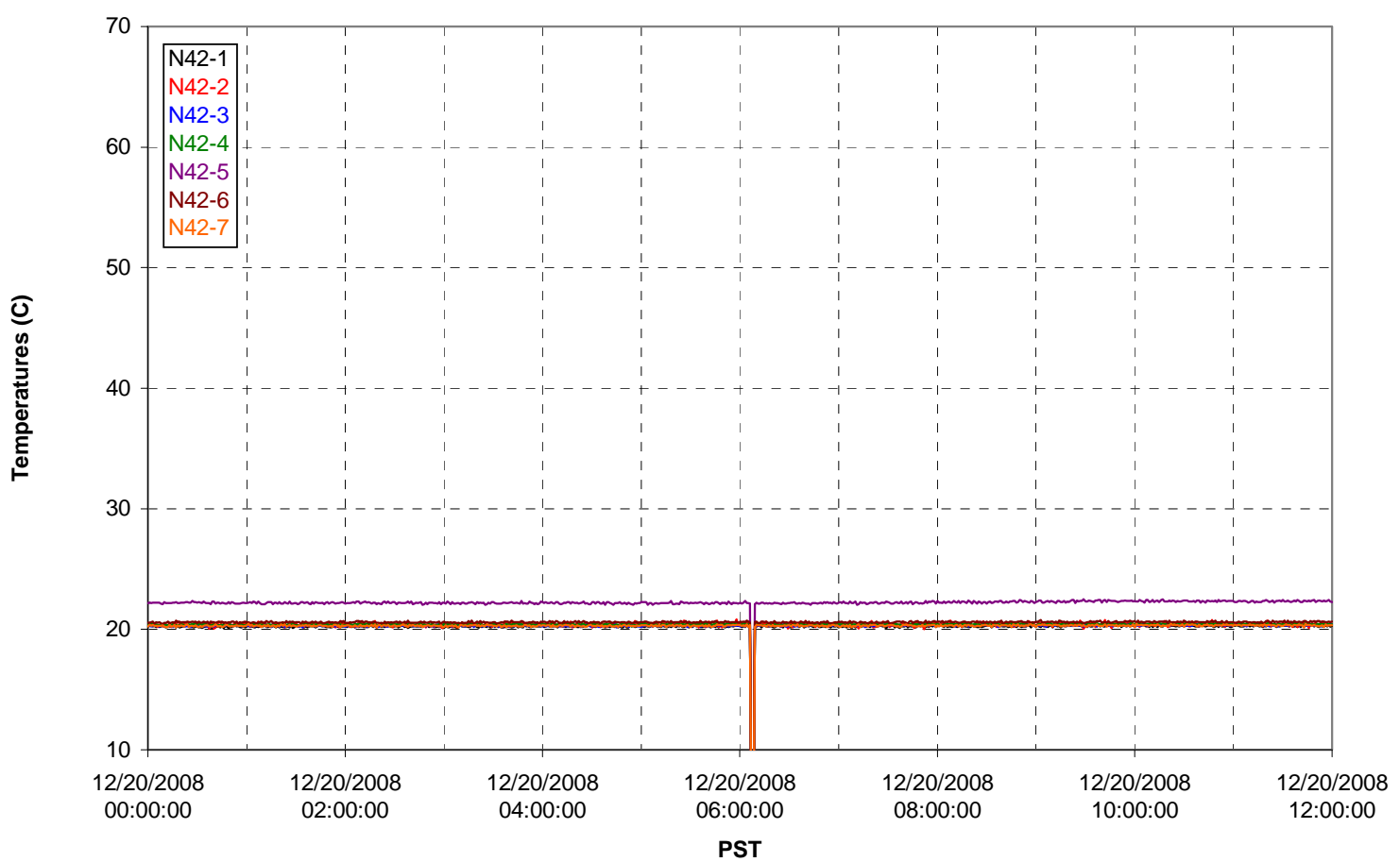

T02A temperatures

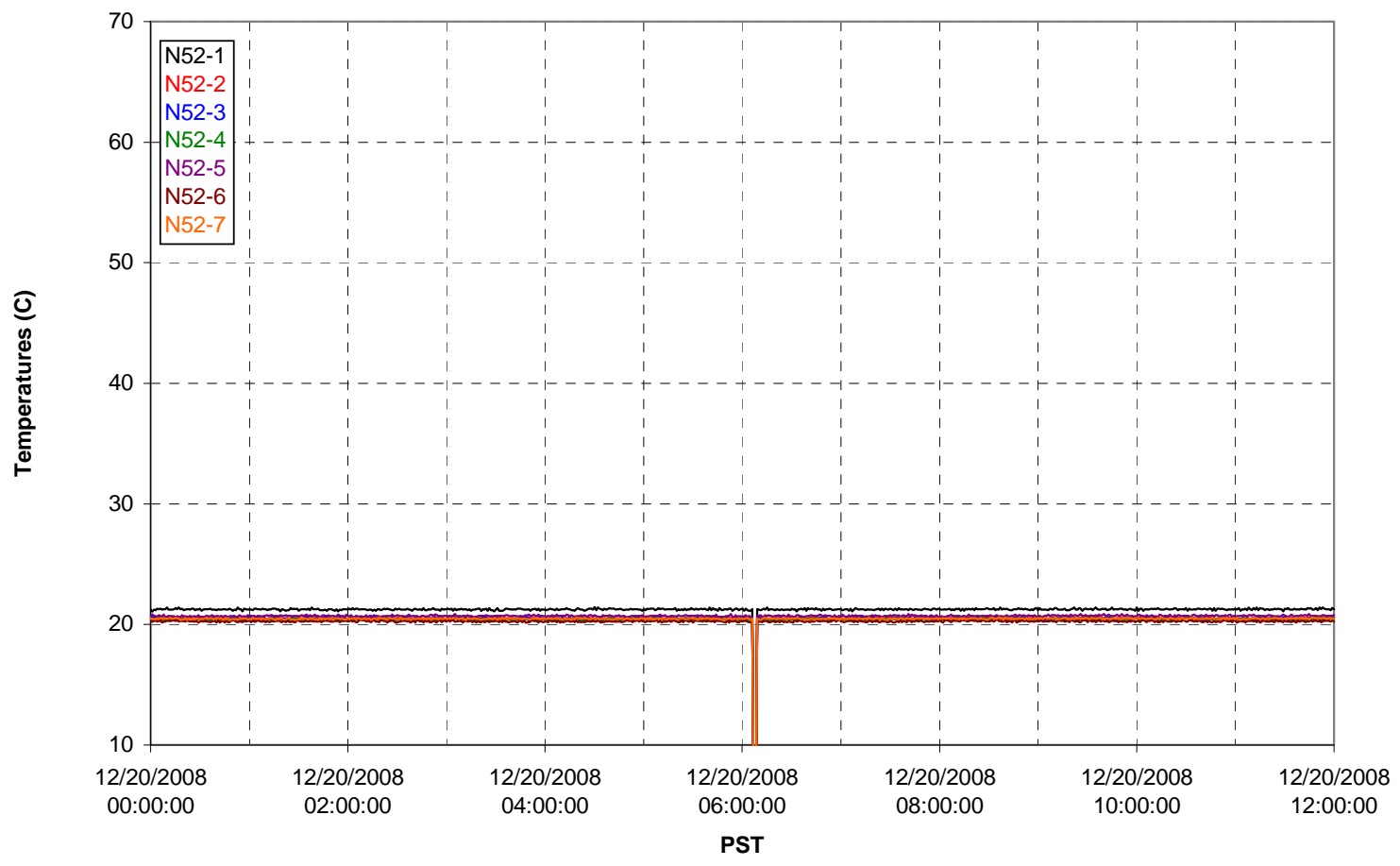


T02A Heating and Cooling

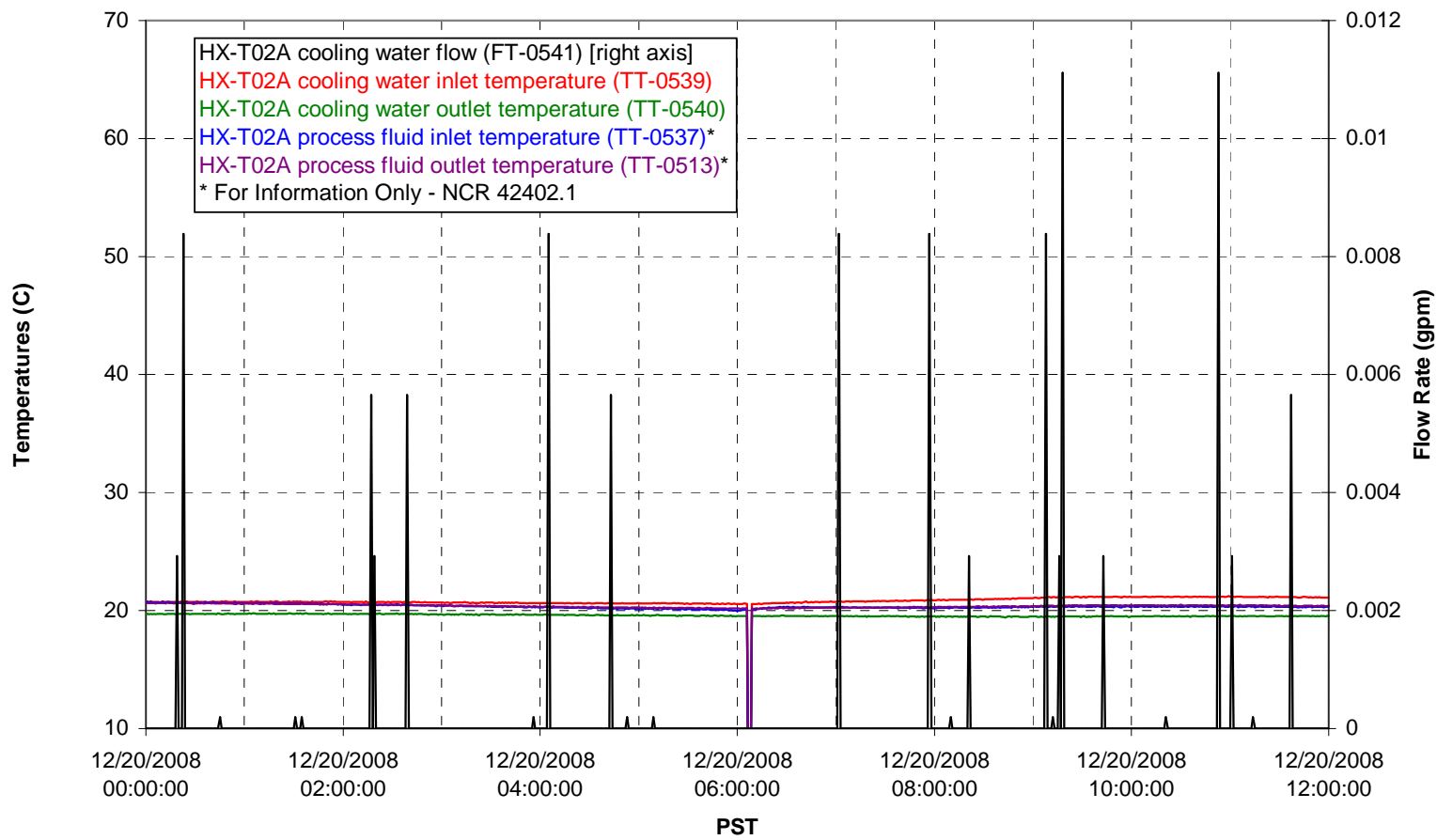

Pump Operation

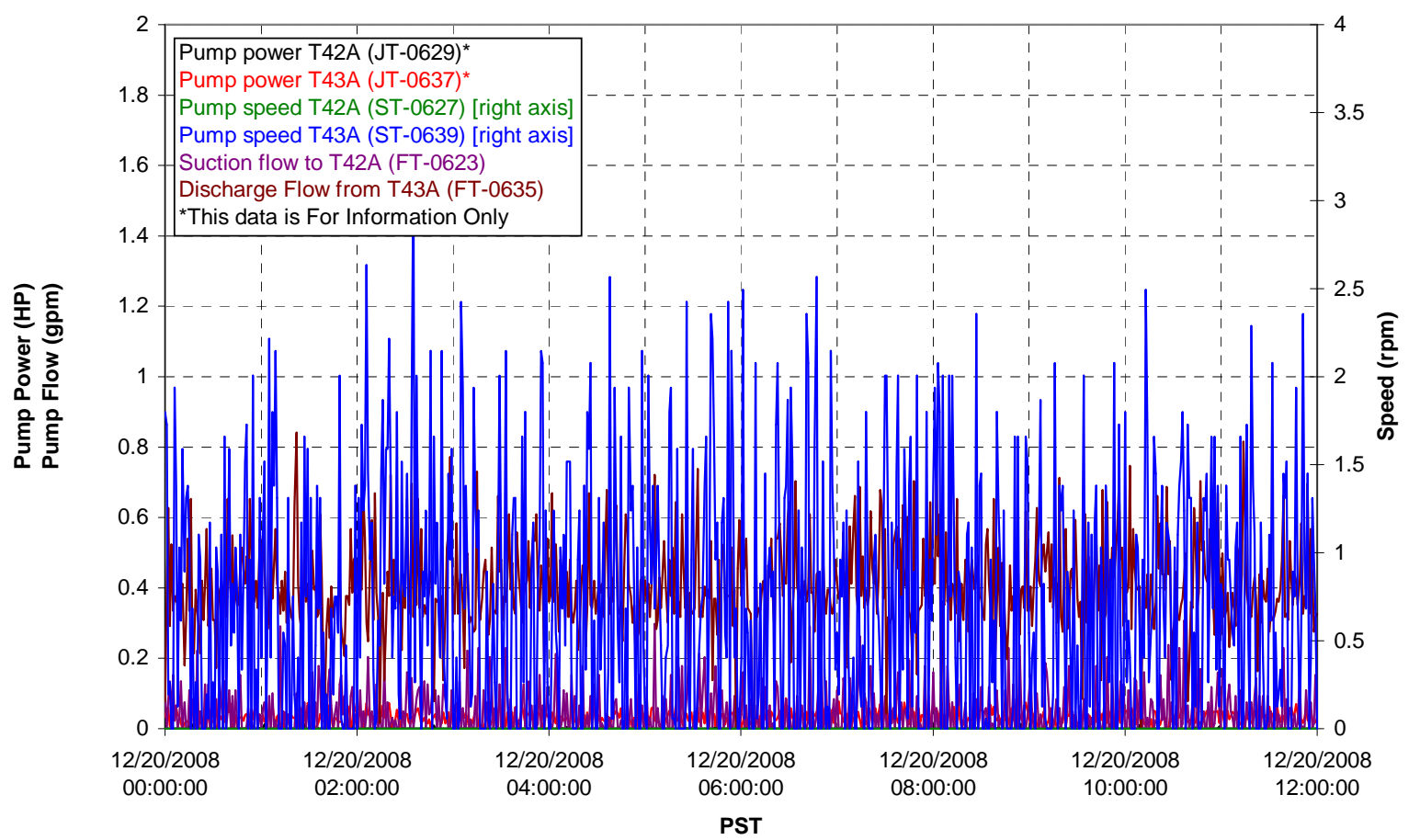


Pulsepot UFP-PP-T01A

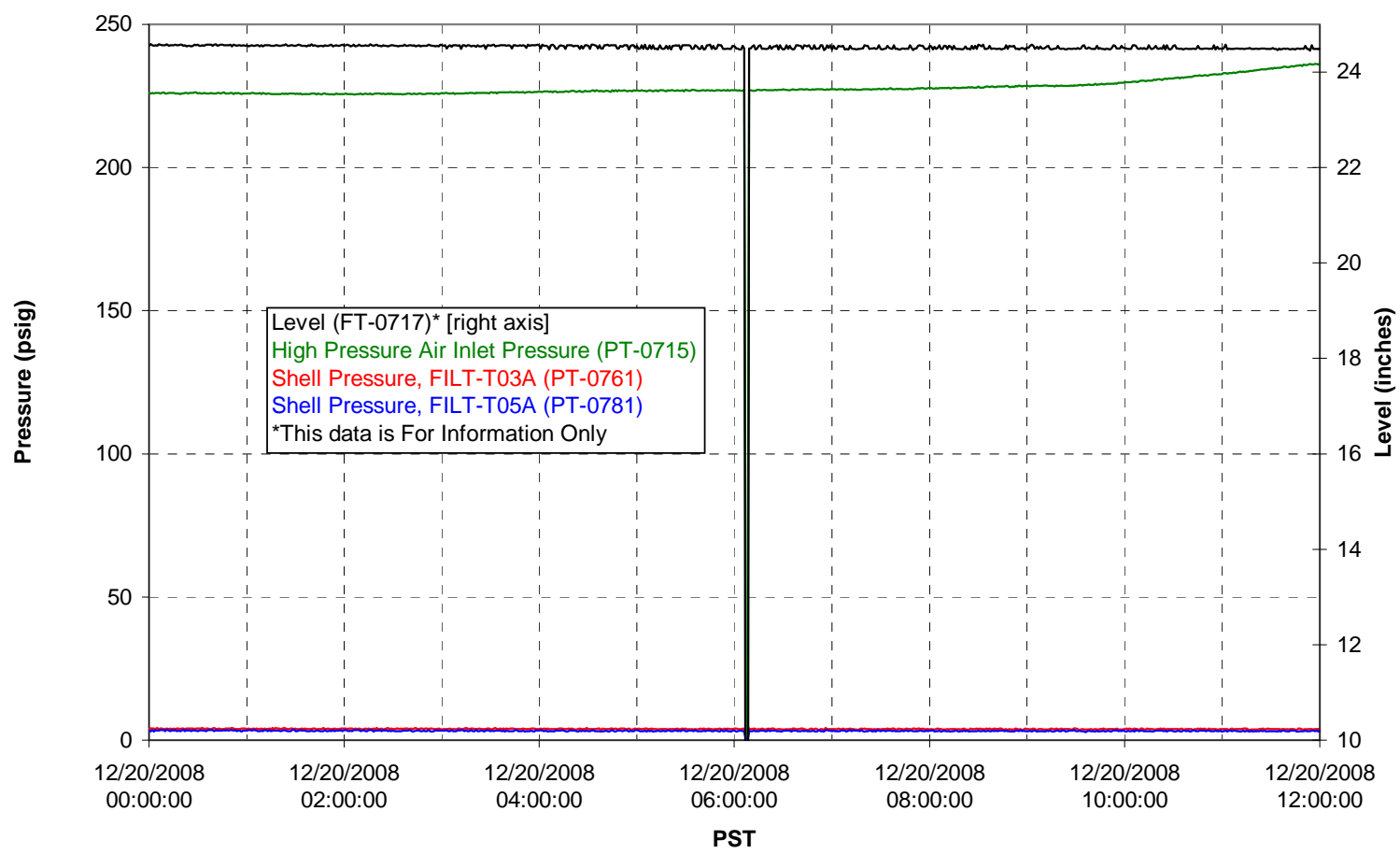

Pulsepot UFP-PP-T02A

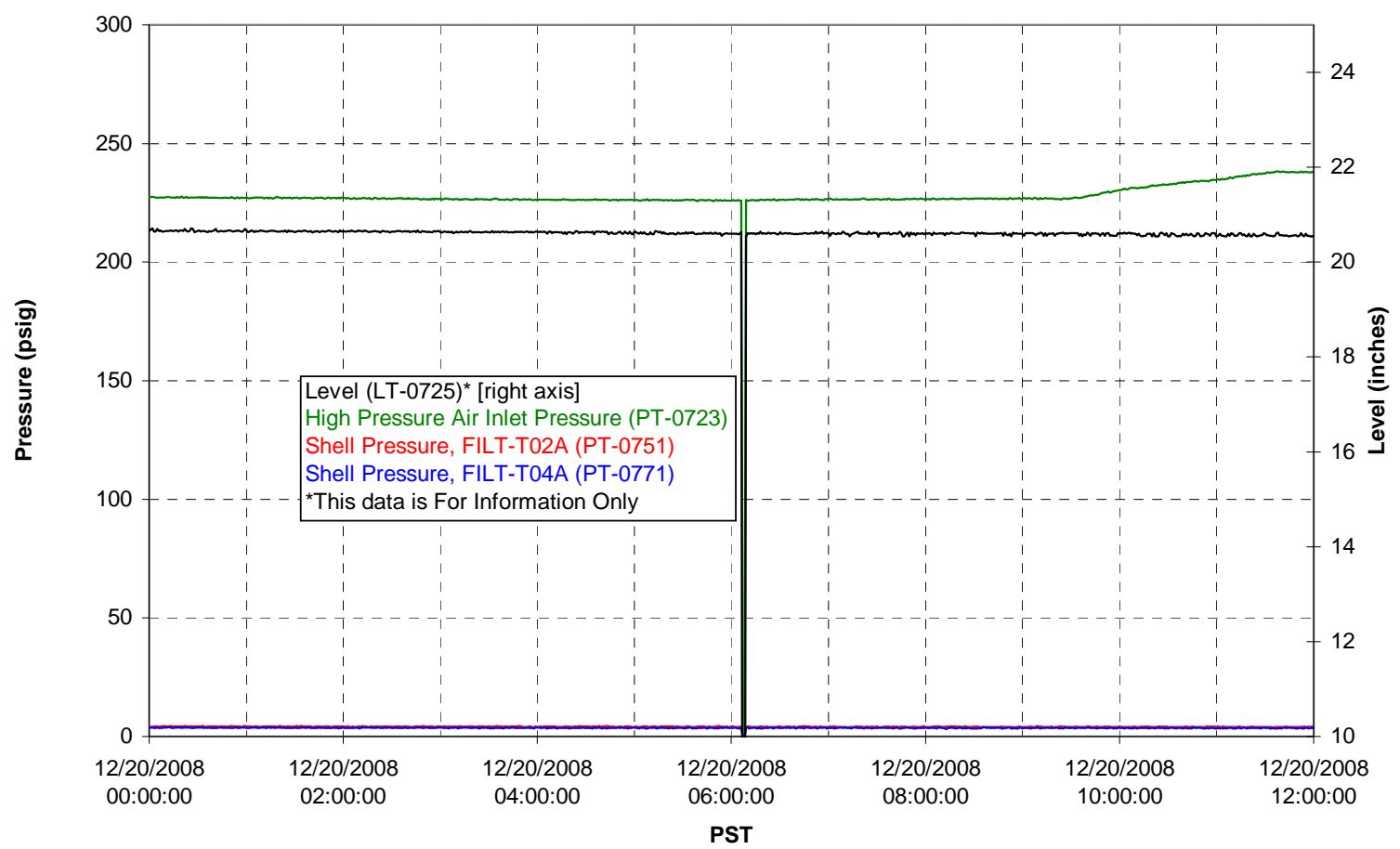


Pulsepot UFP-PP-T03A

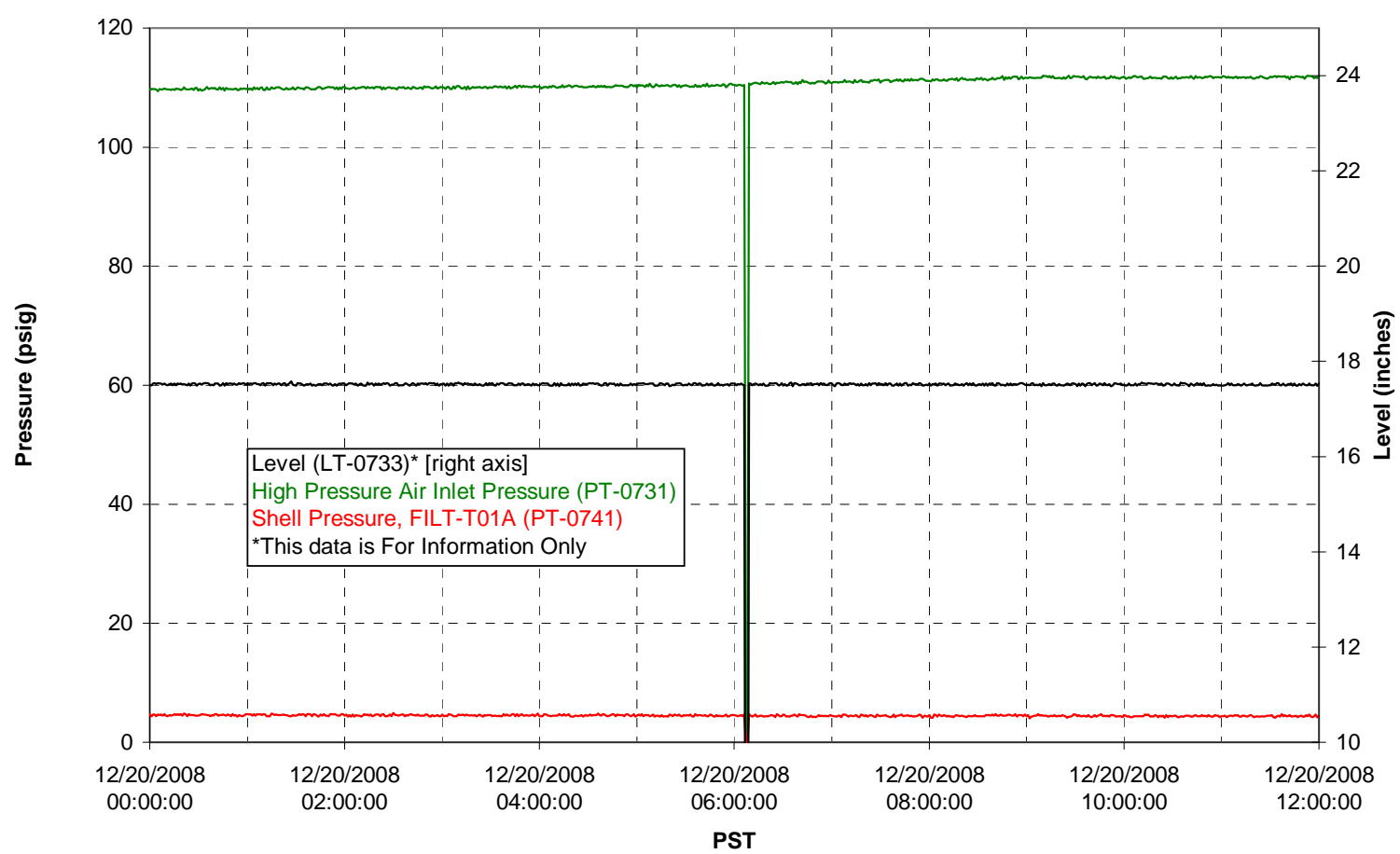

Pulsepot Levels

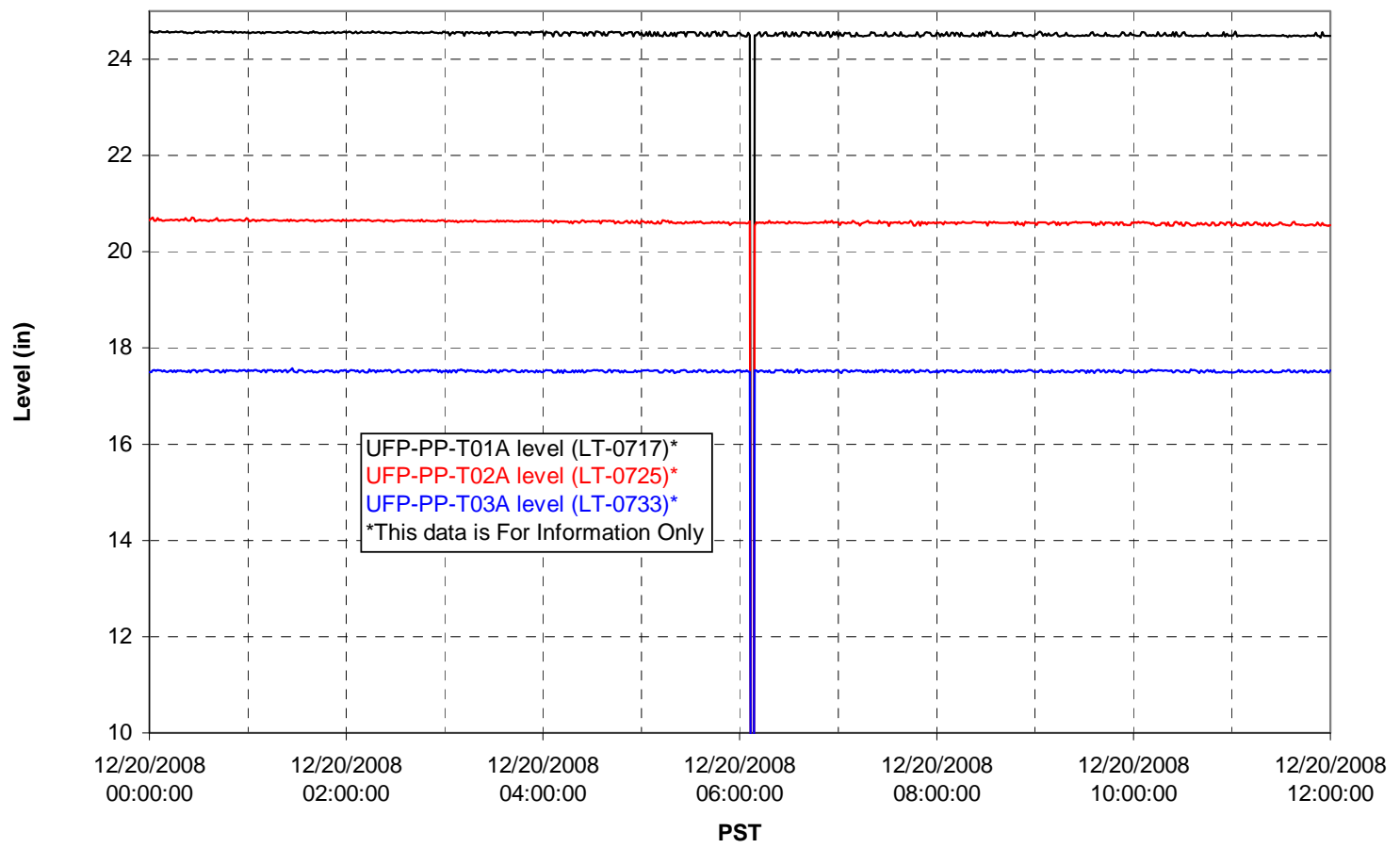


Filter UFP-FILT-T01A

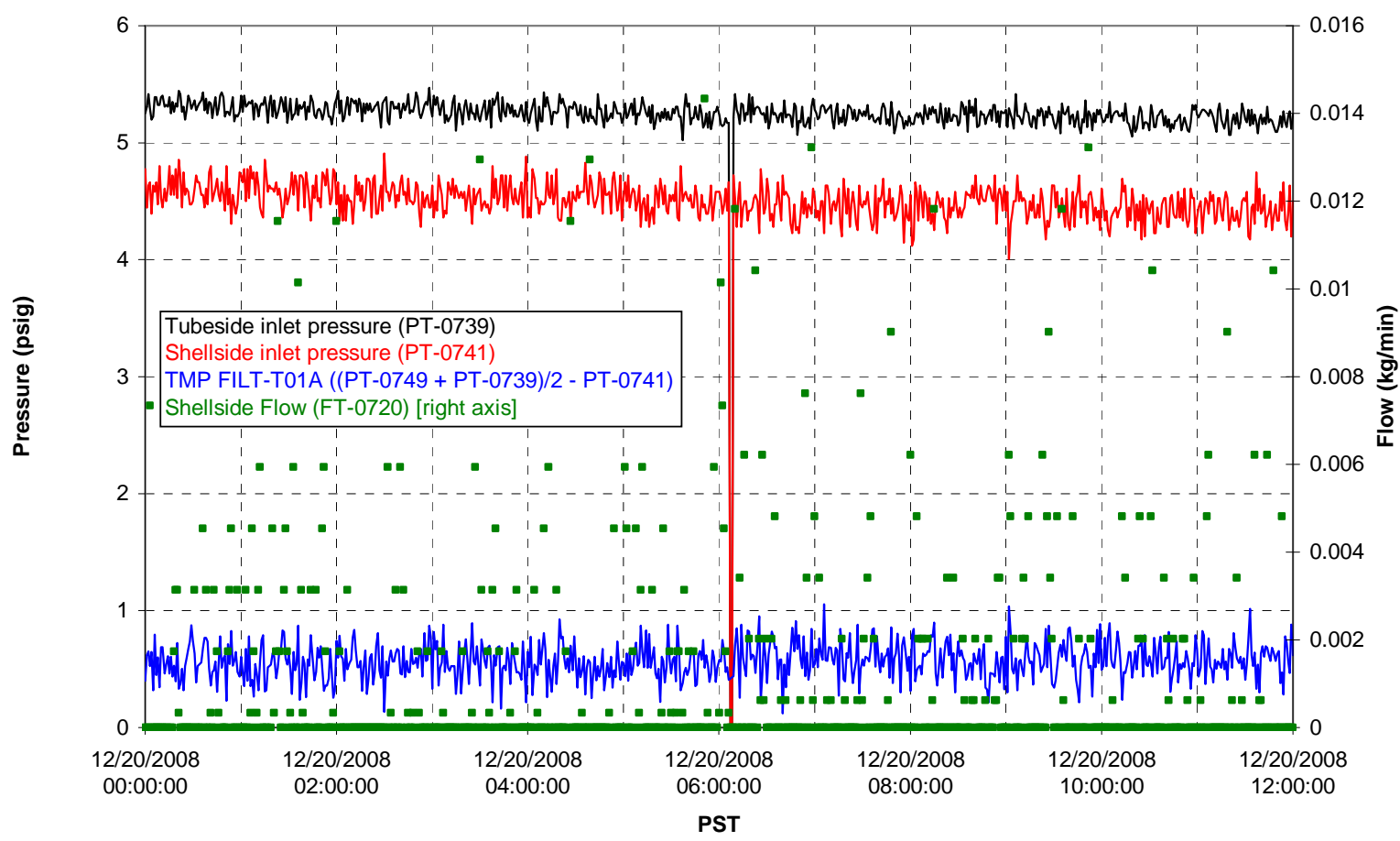

Filter UFP-FILT-T02A

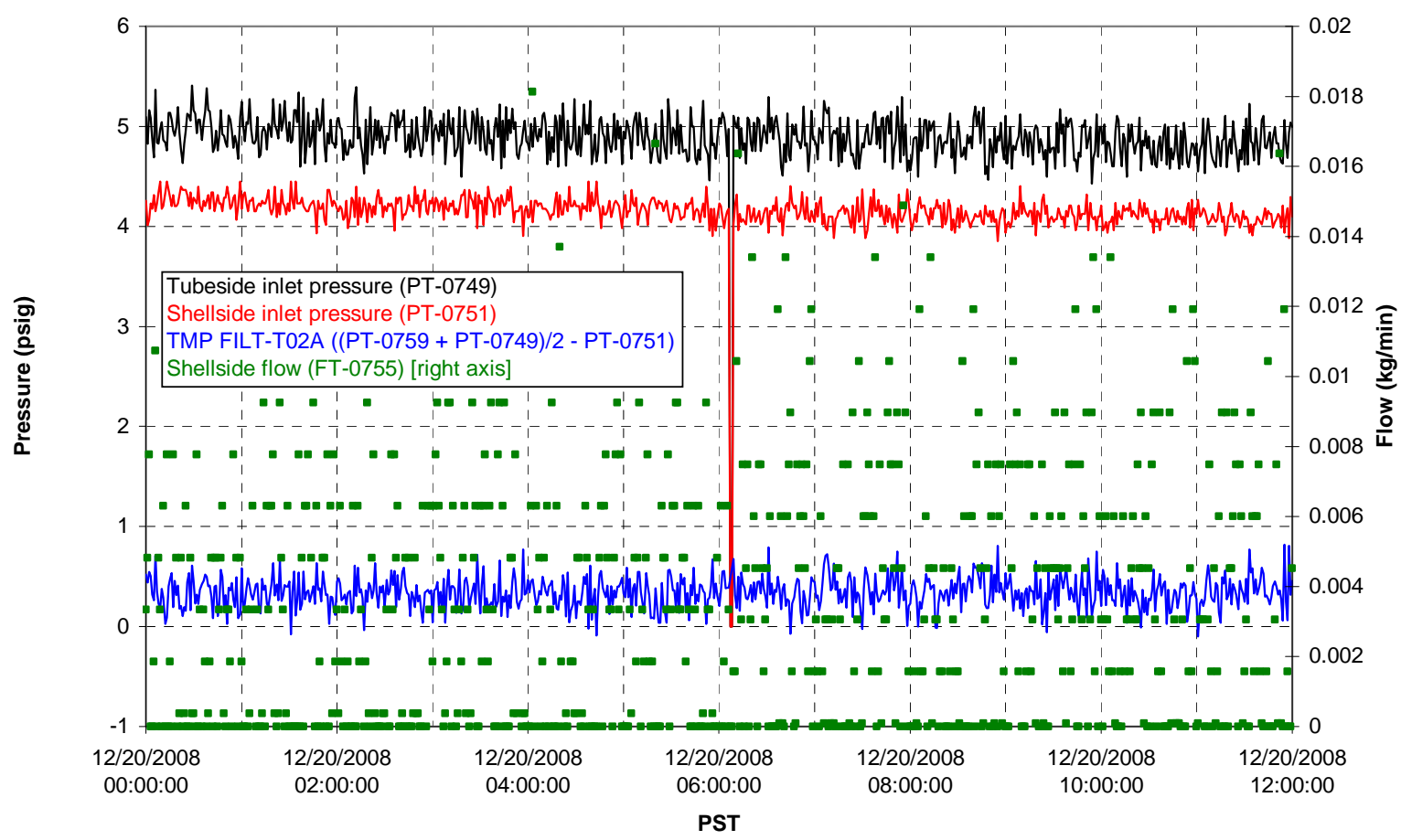


Filter UFP-FILT-T03A

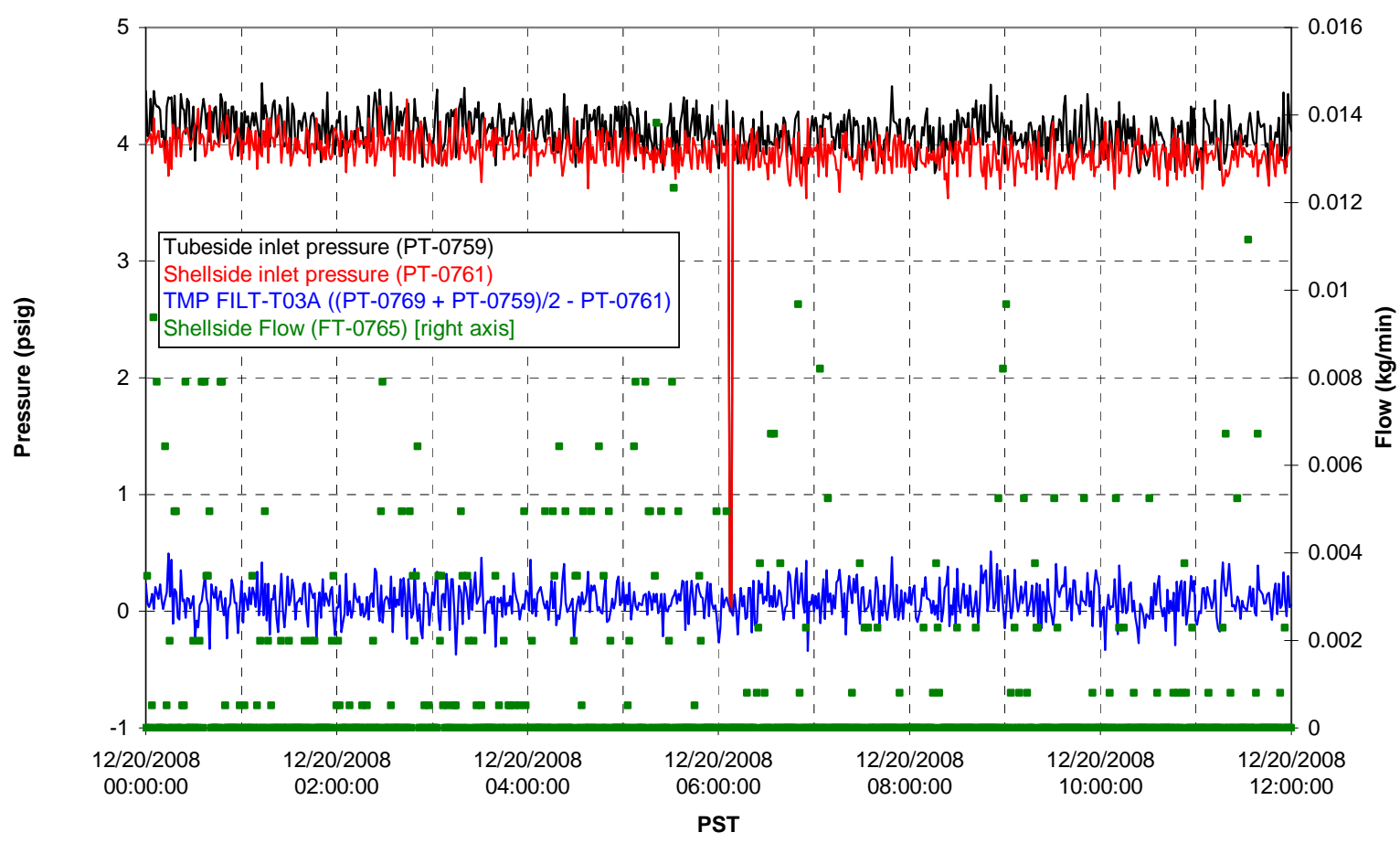

Filter UFP-FILT-T04A

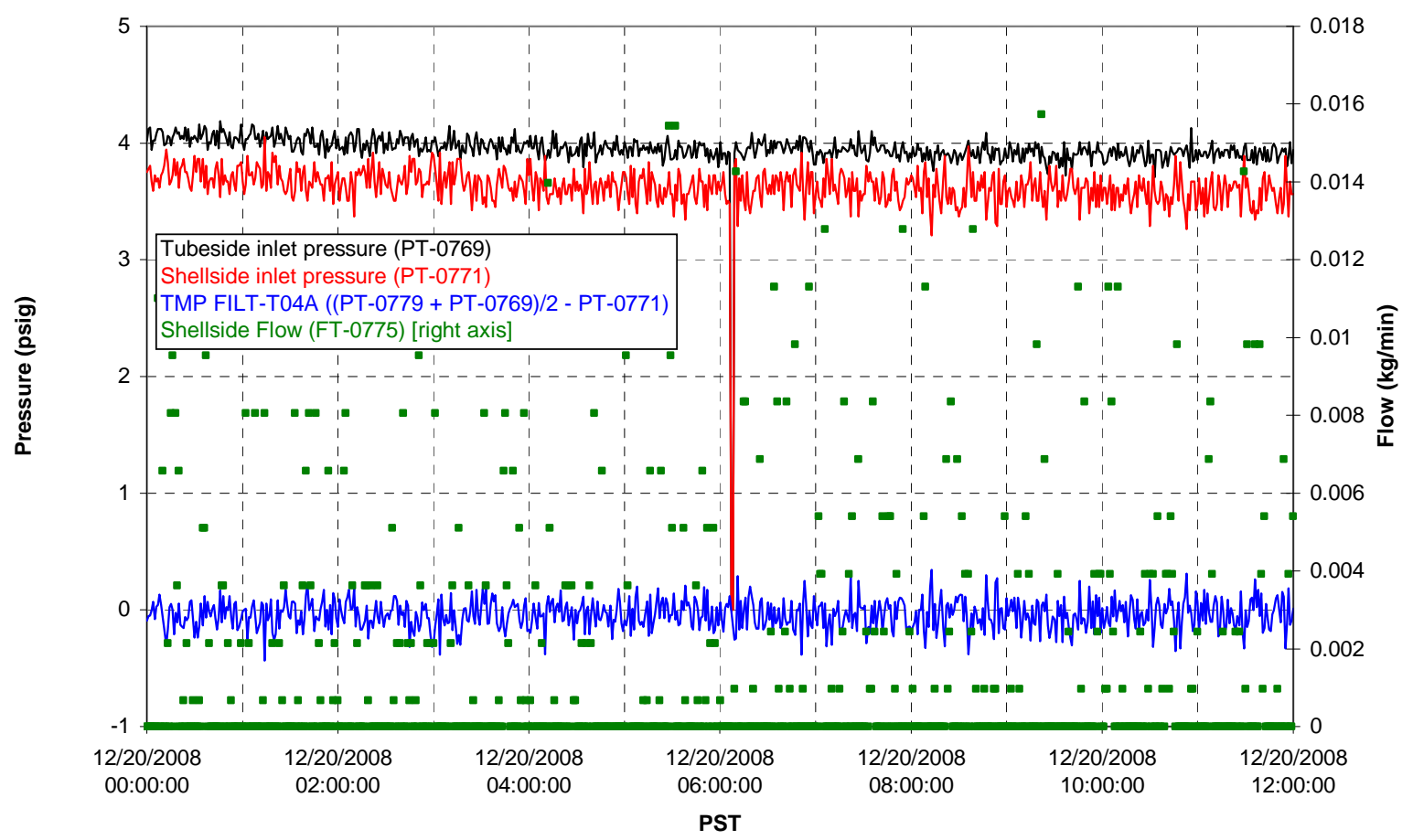


Filter UFP-FILT-T05A

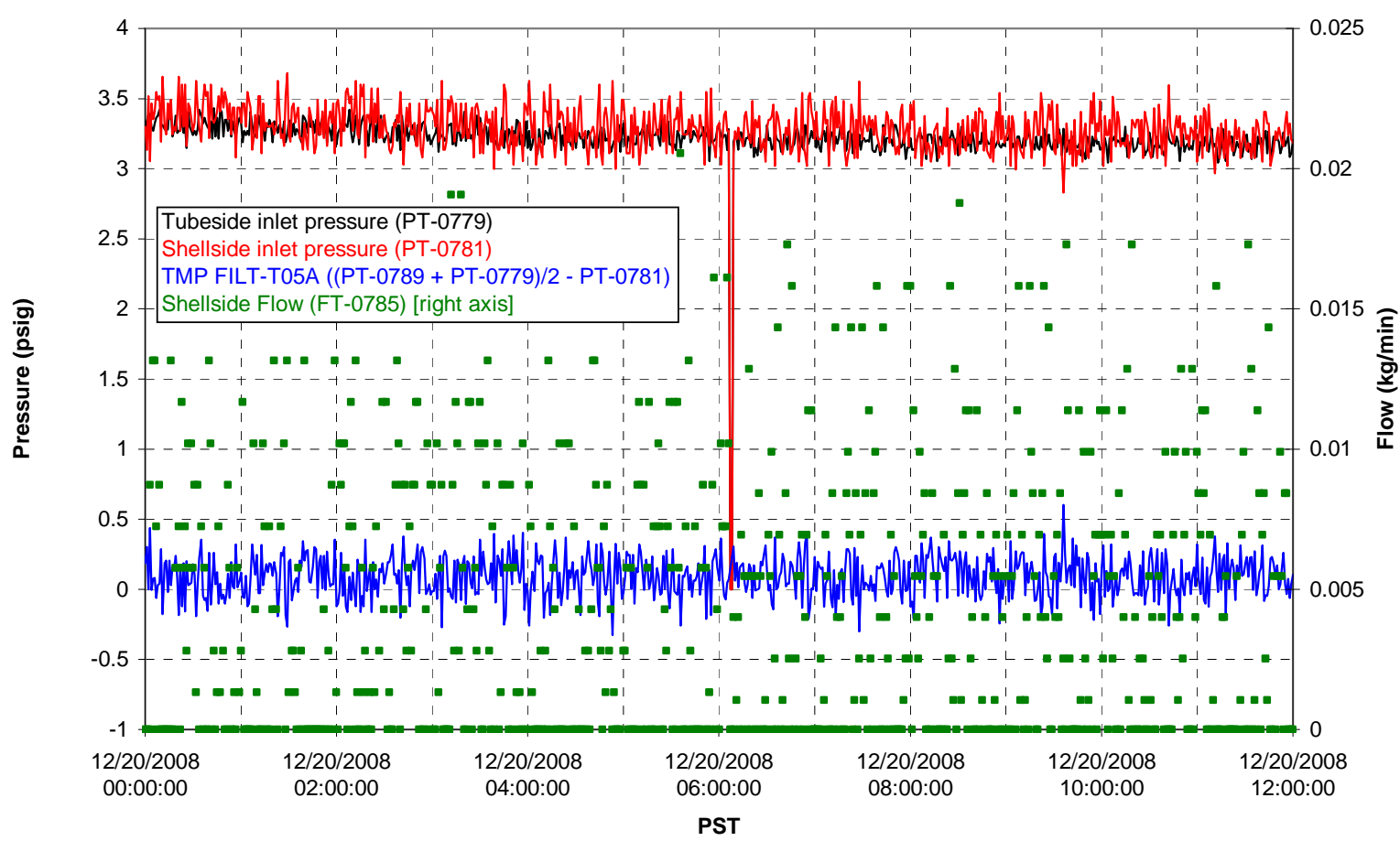

Chemical Flow

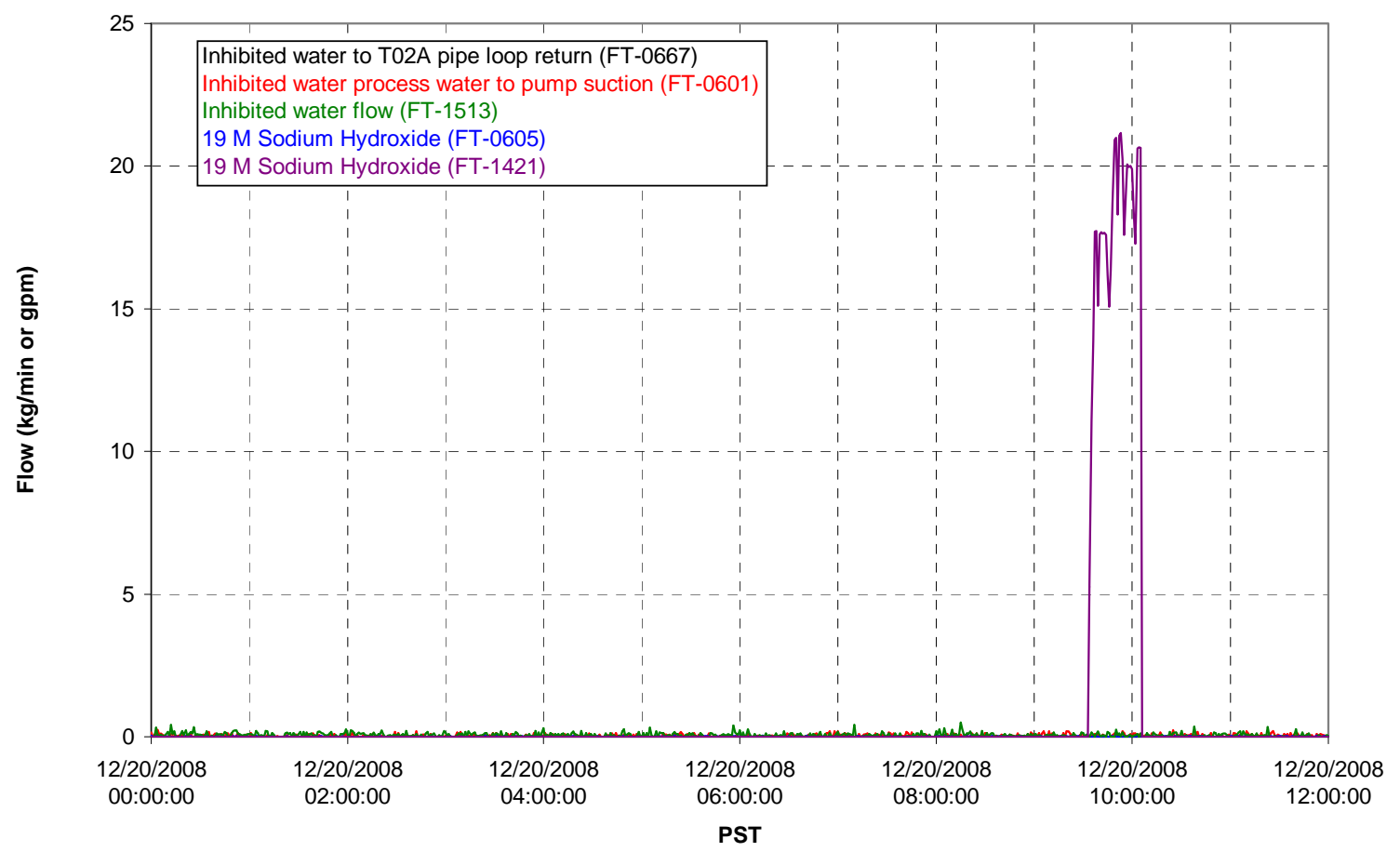




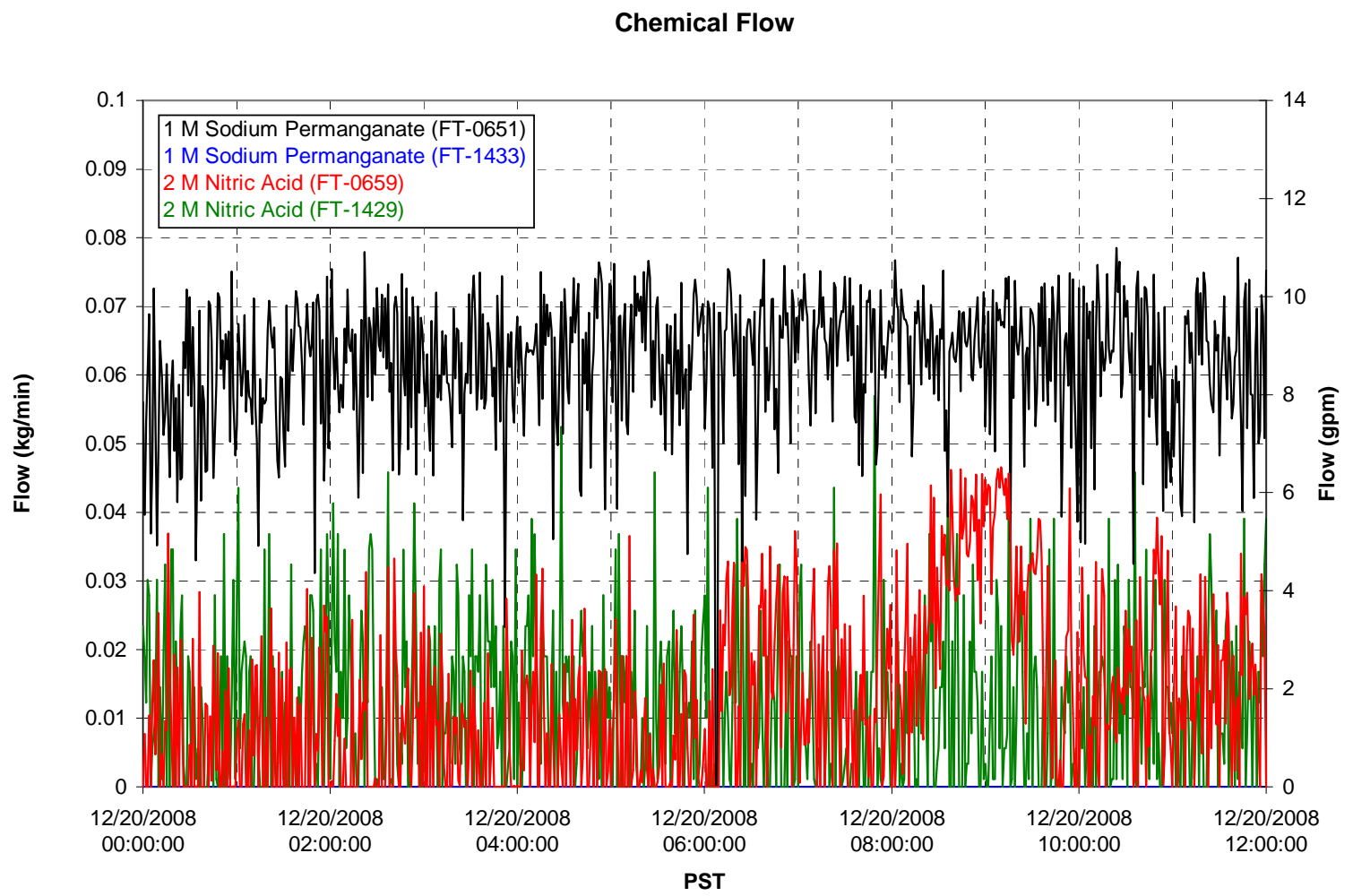

Air Flows

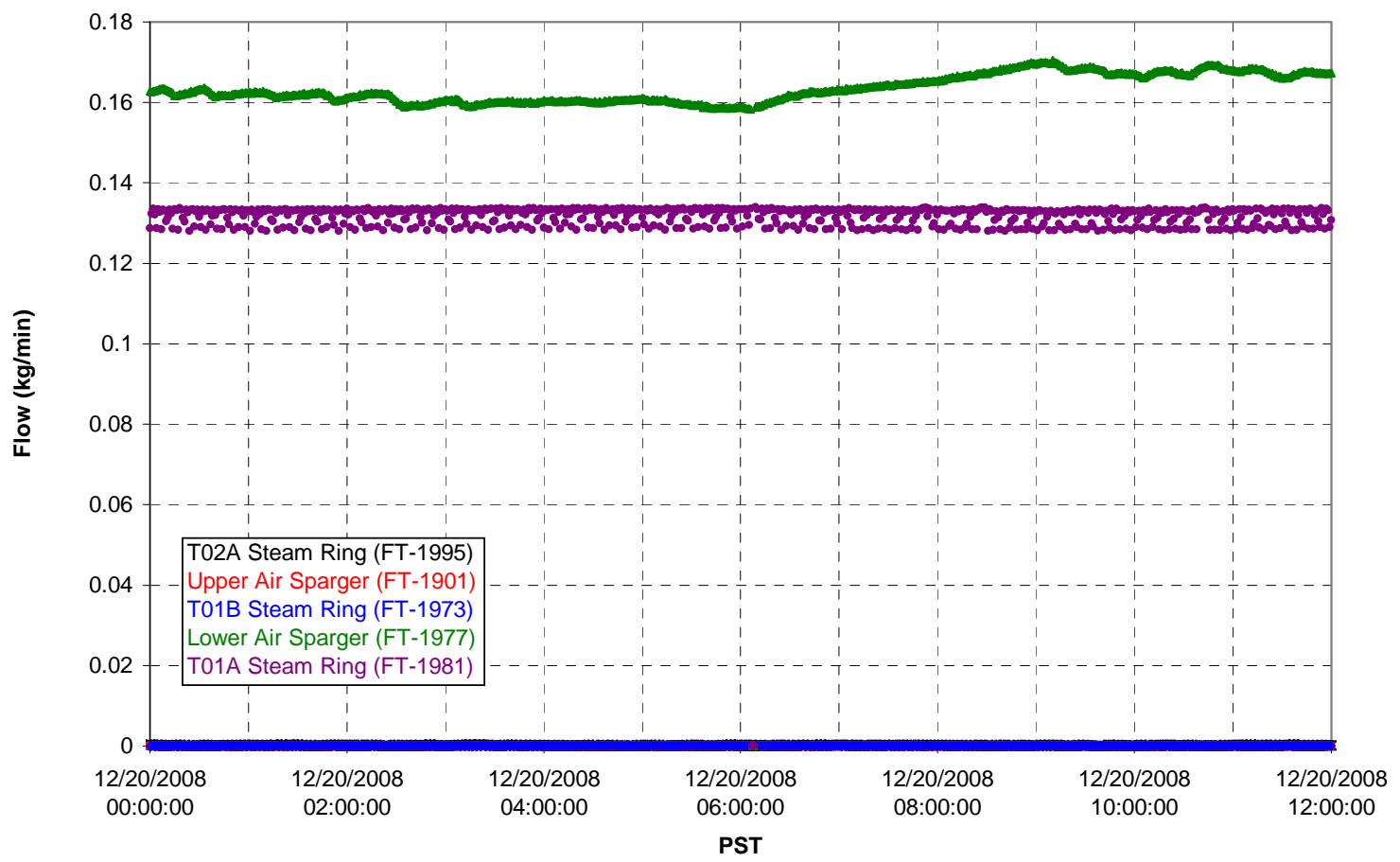


T02A Steam

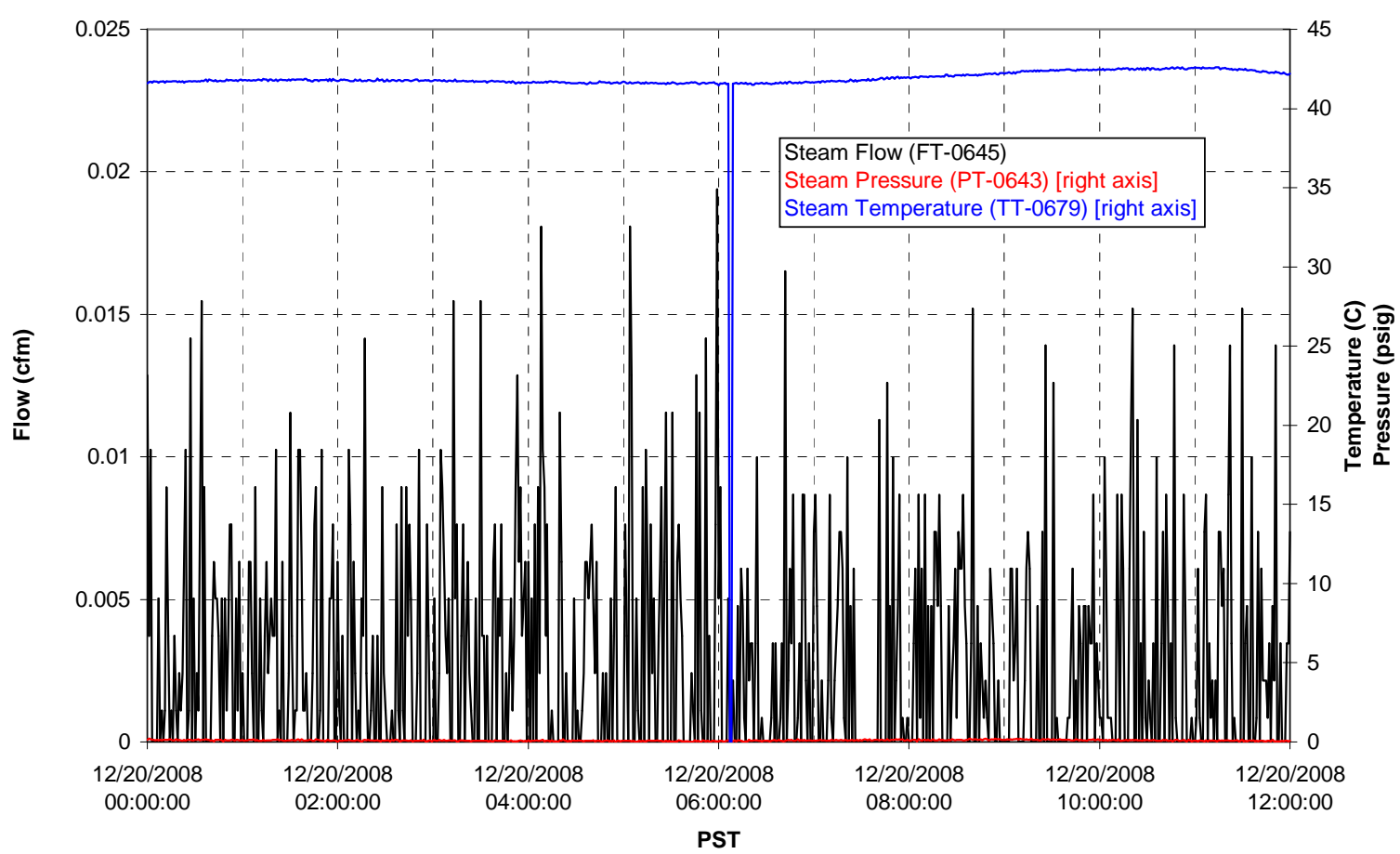

T01A Steam

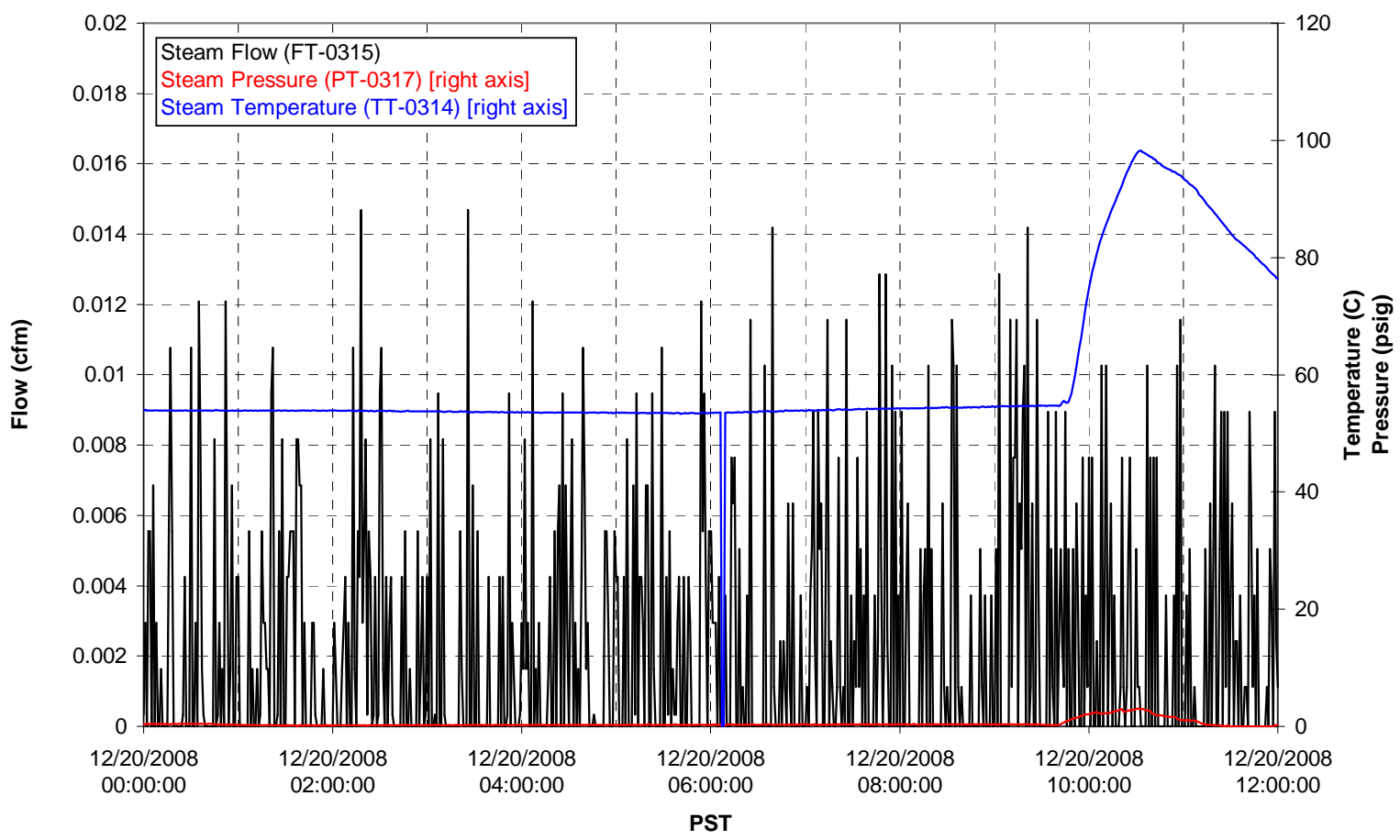


T01B Steam

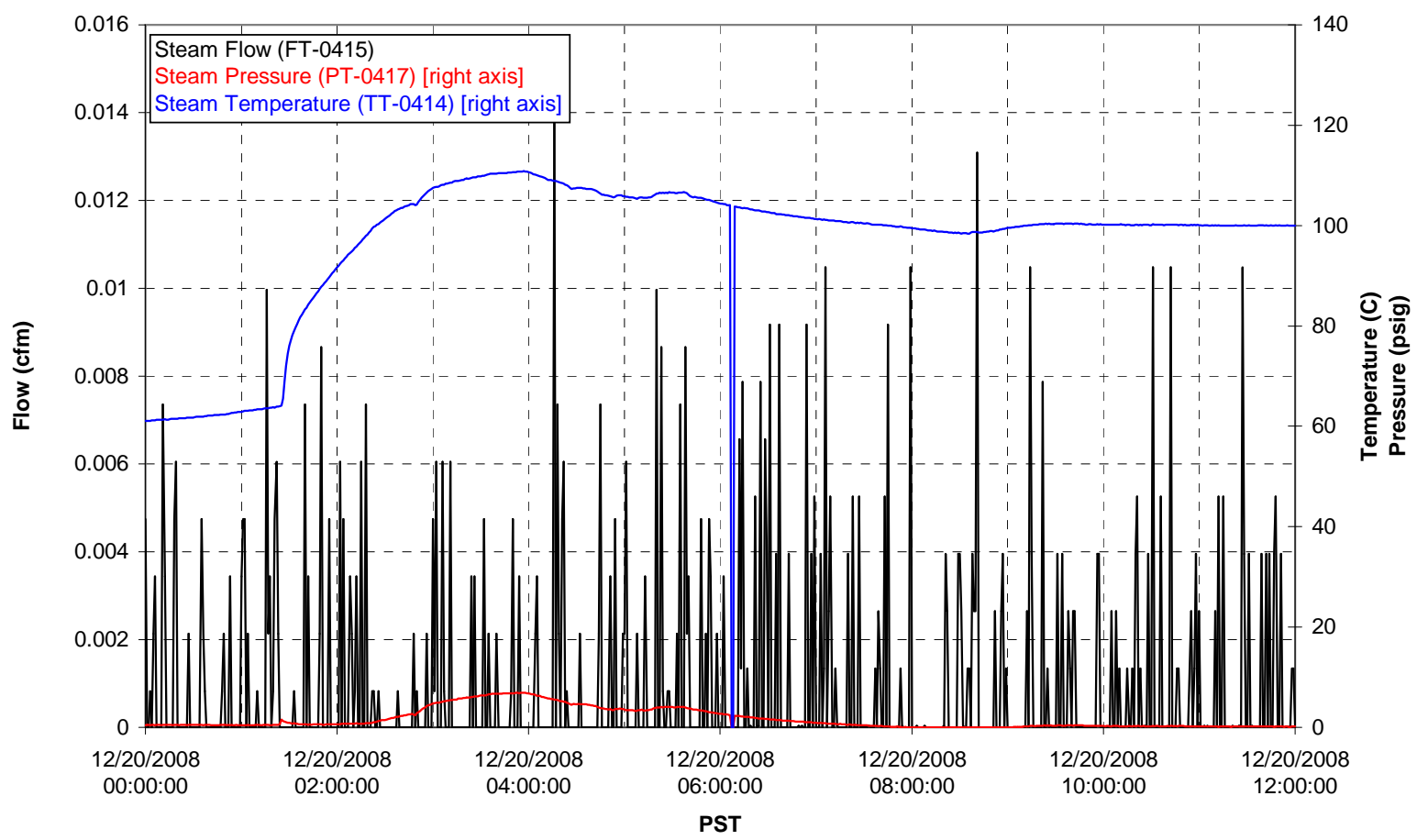


Functional Test Data Plots 12/20/08 12:00 - 12/21/08 00:00 
T01A level

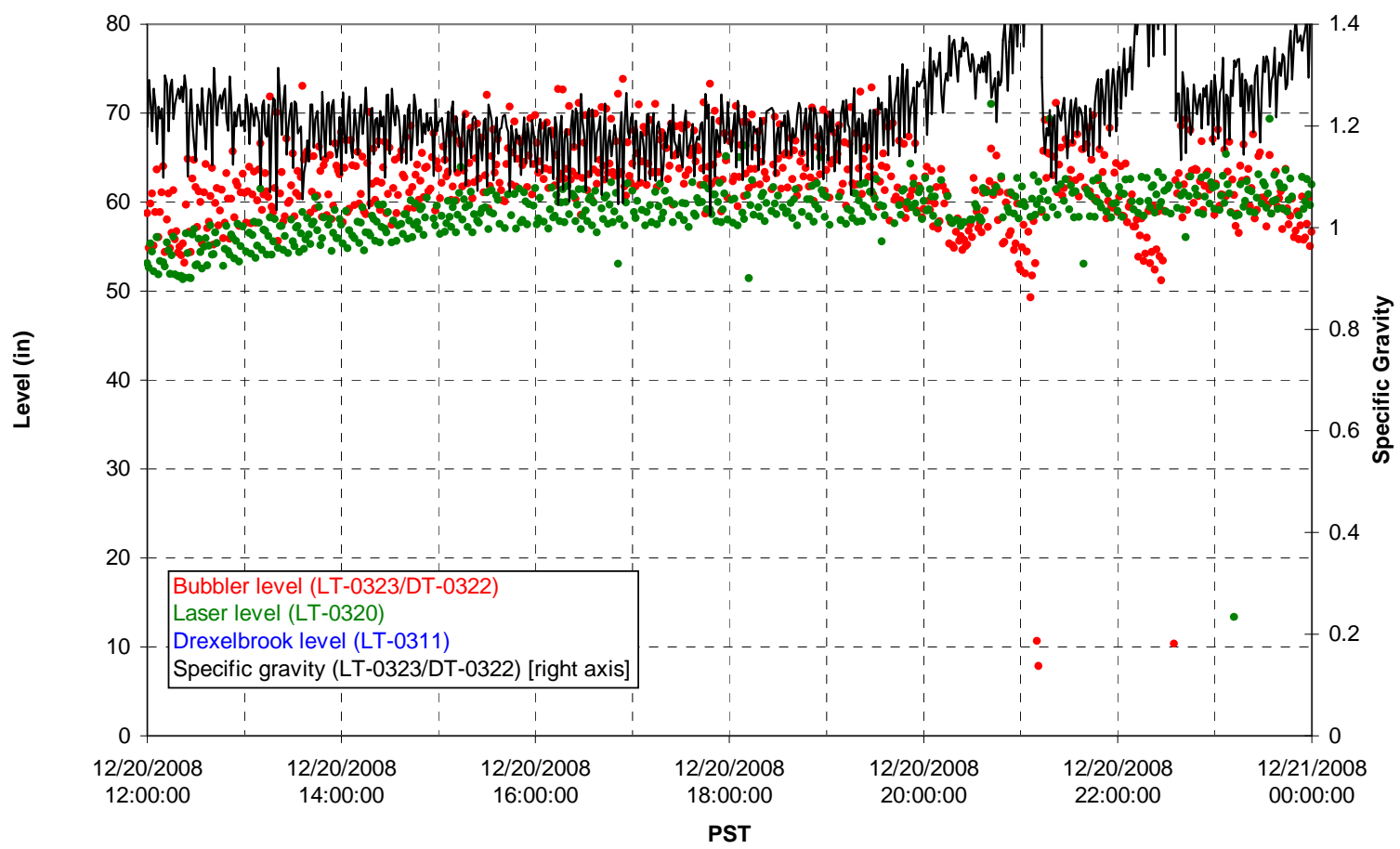

T01A temperatures

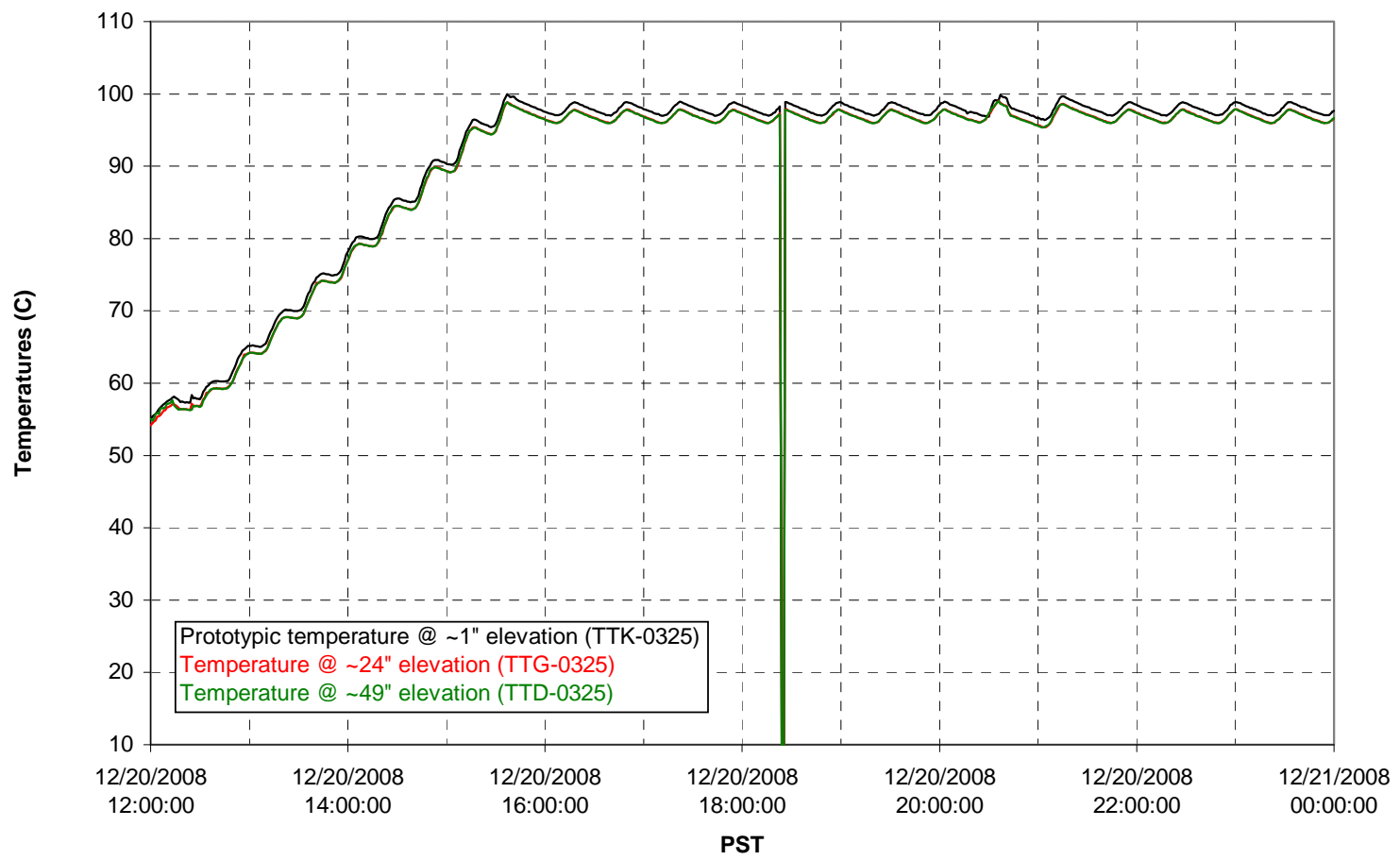


T01B level

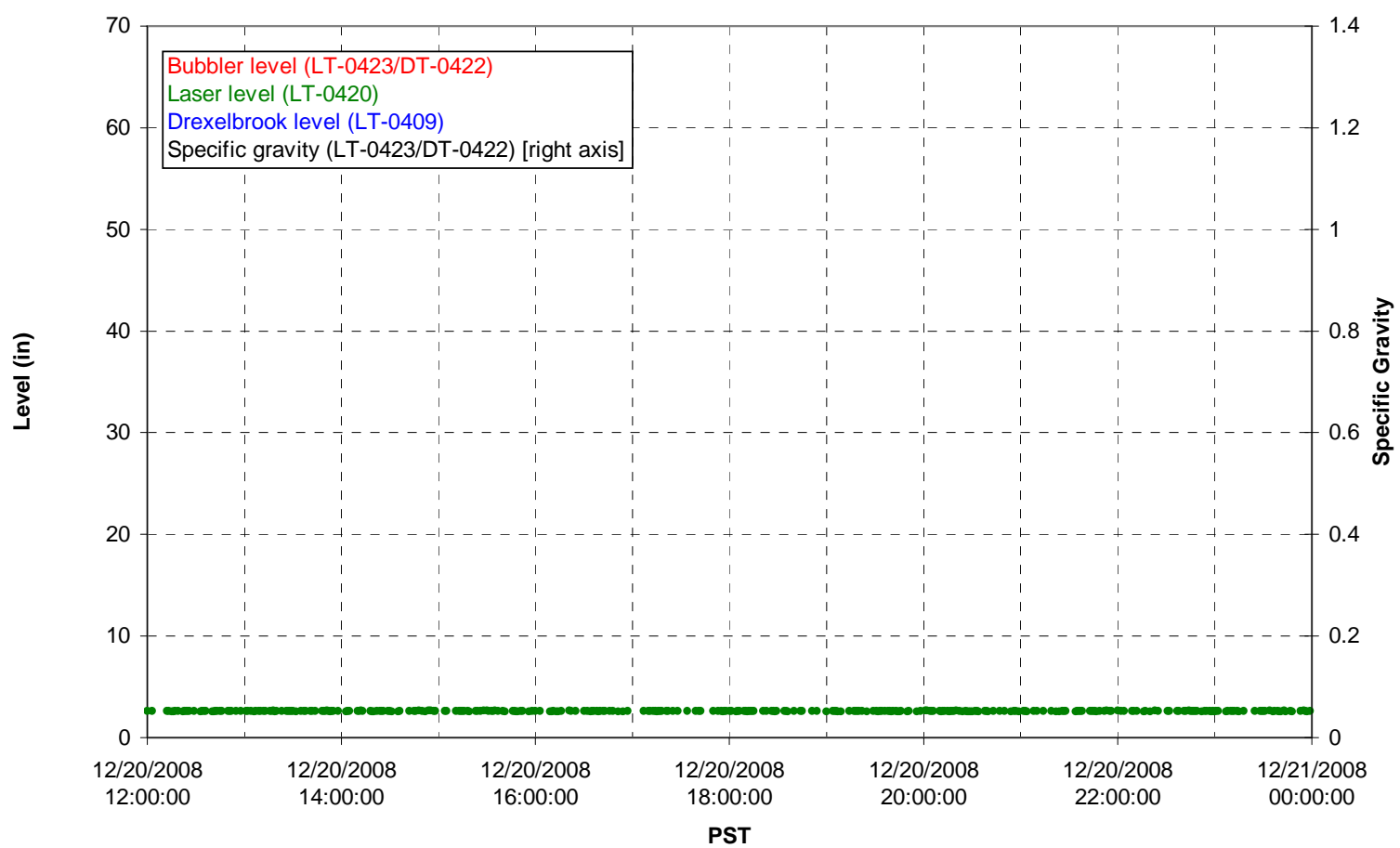

T01B temperatures

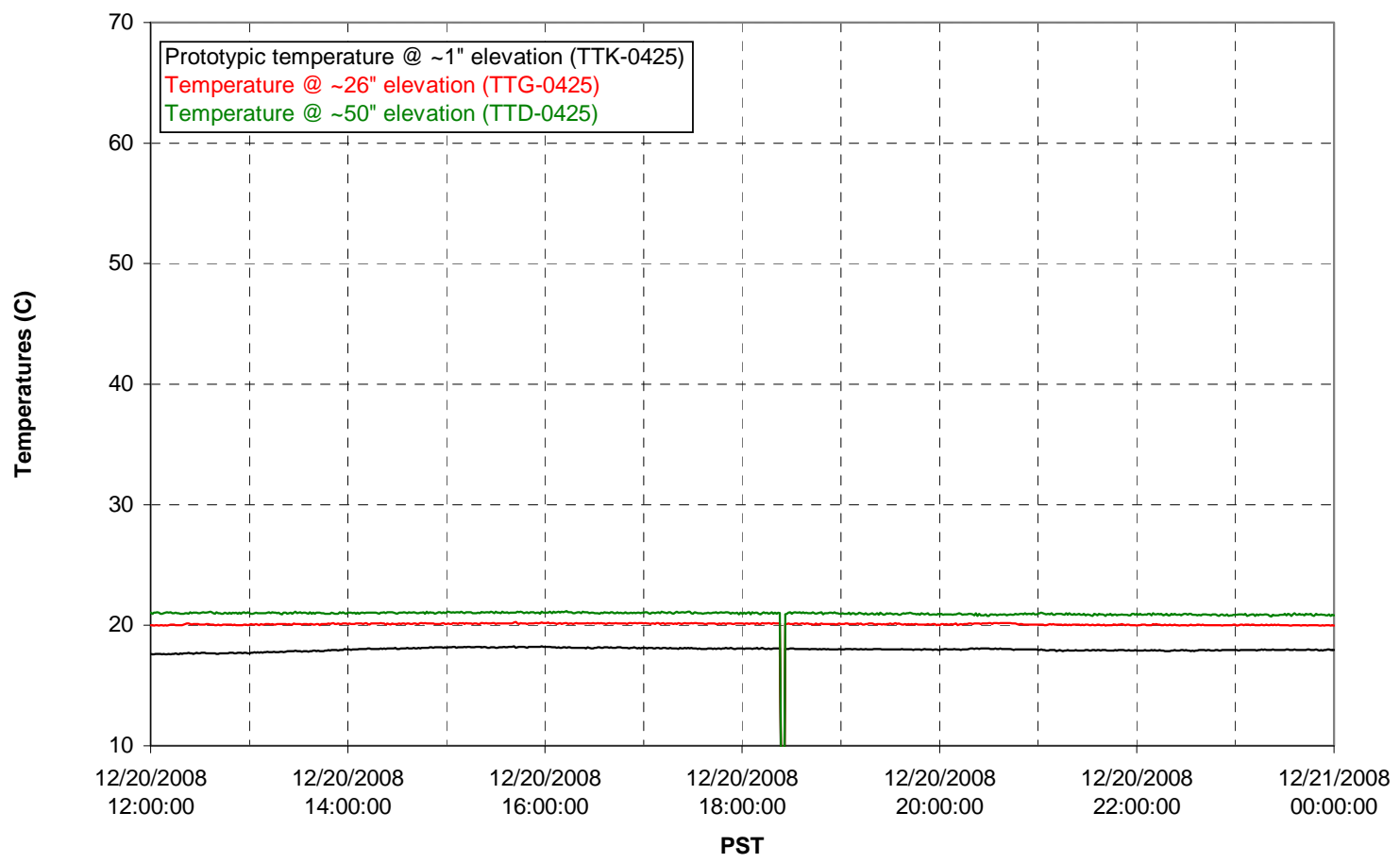


T02A level

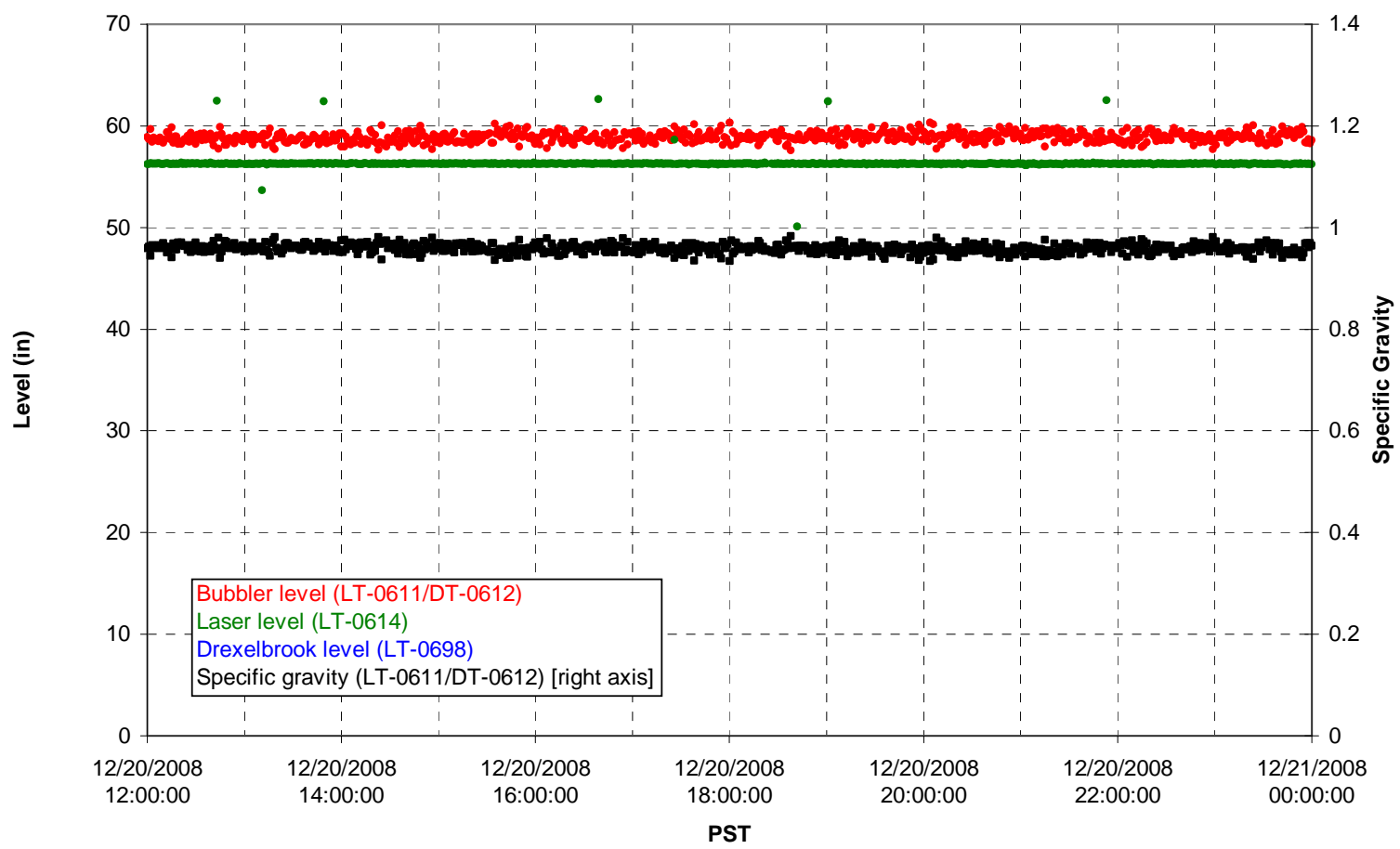

T02A temperatures

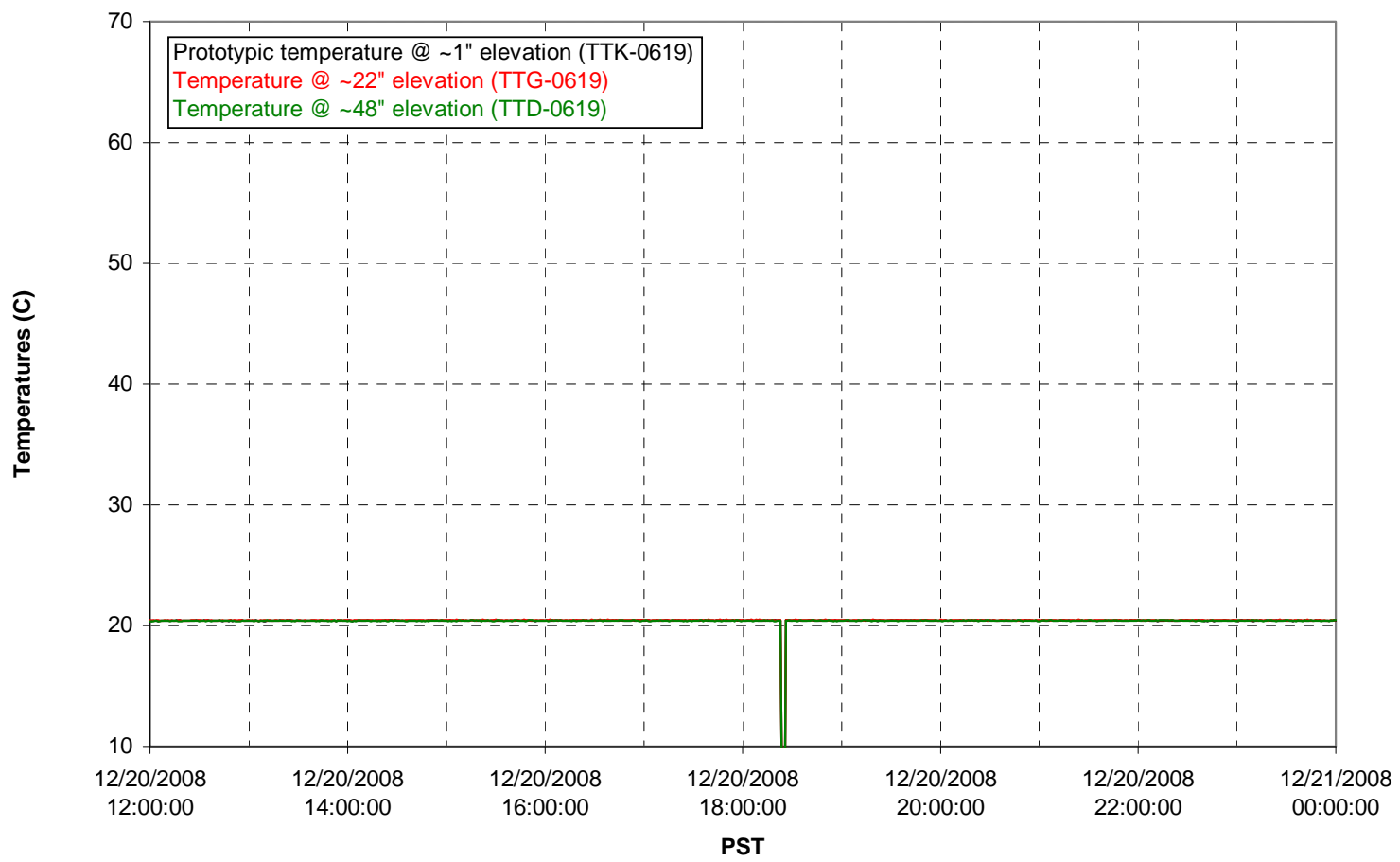


T02A and filter loop temperatures

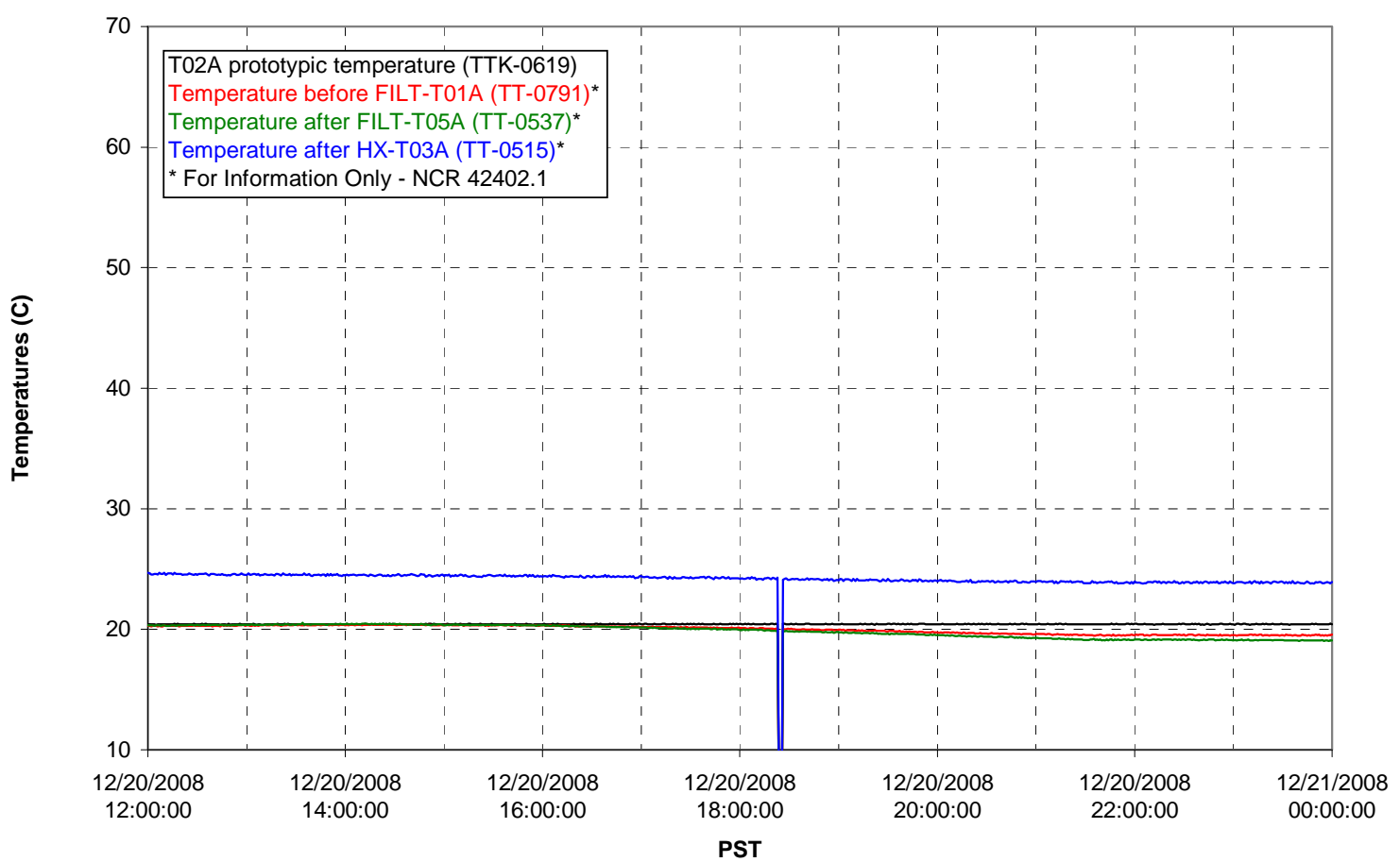

Pump Pressures and Flow

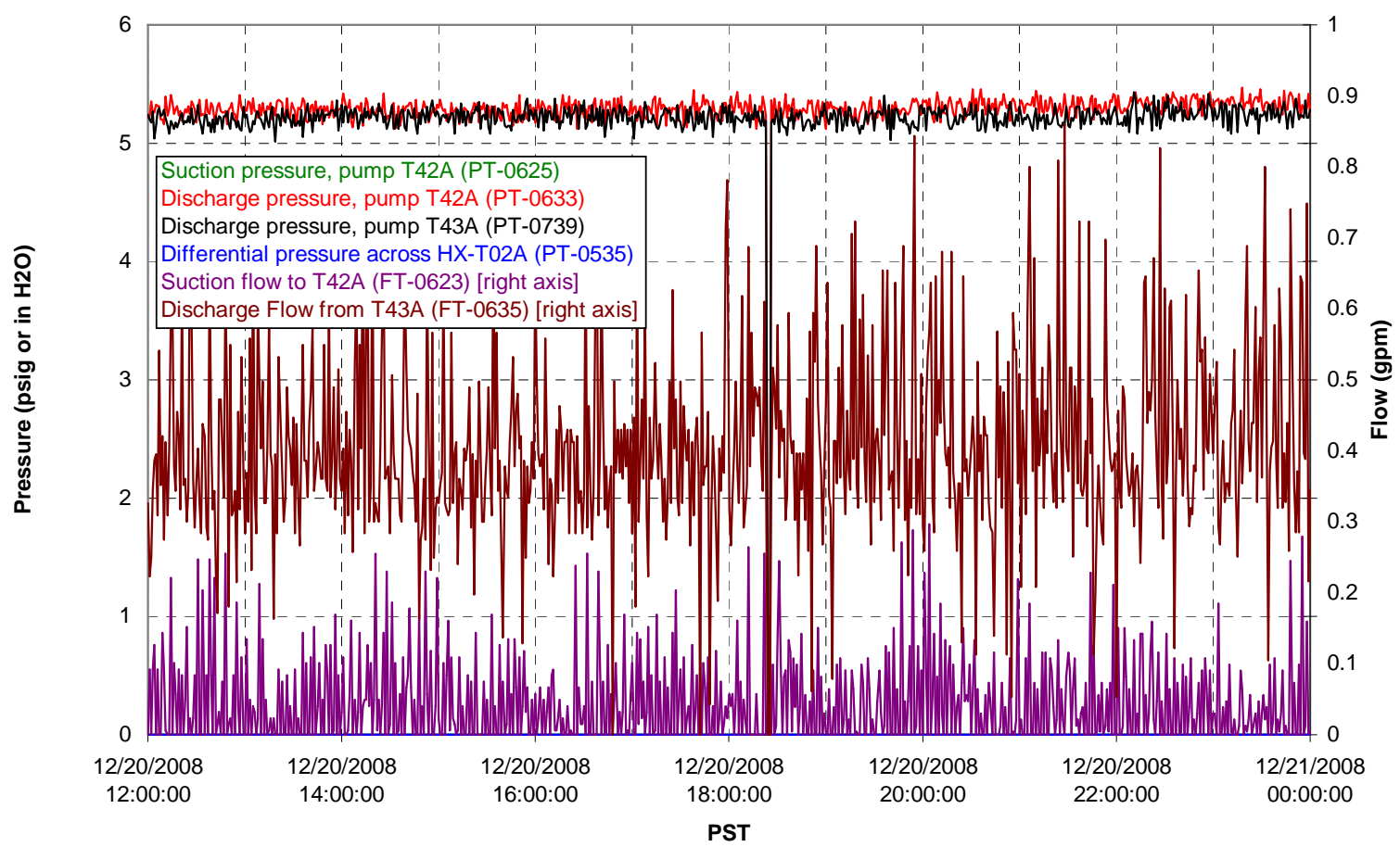


Axial pressure drop

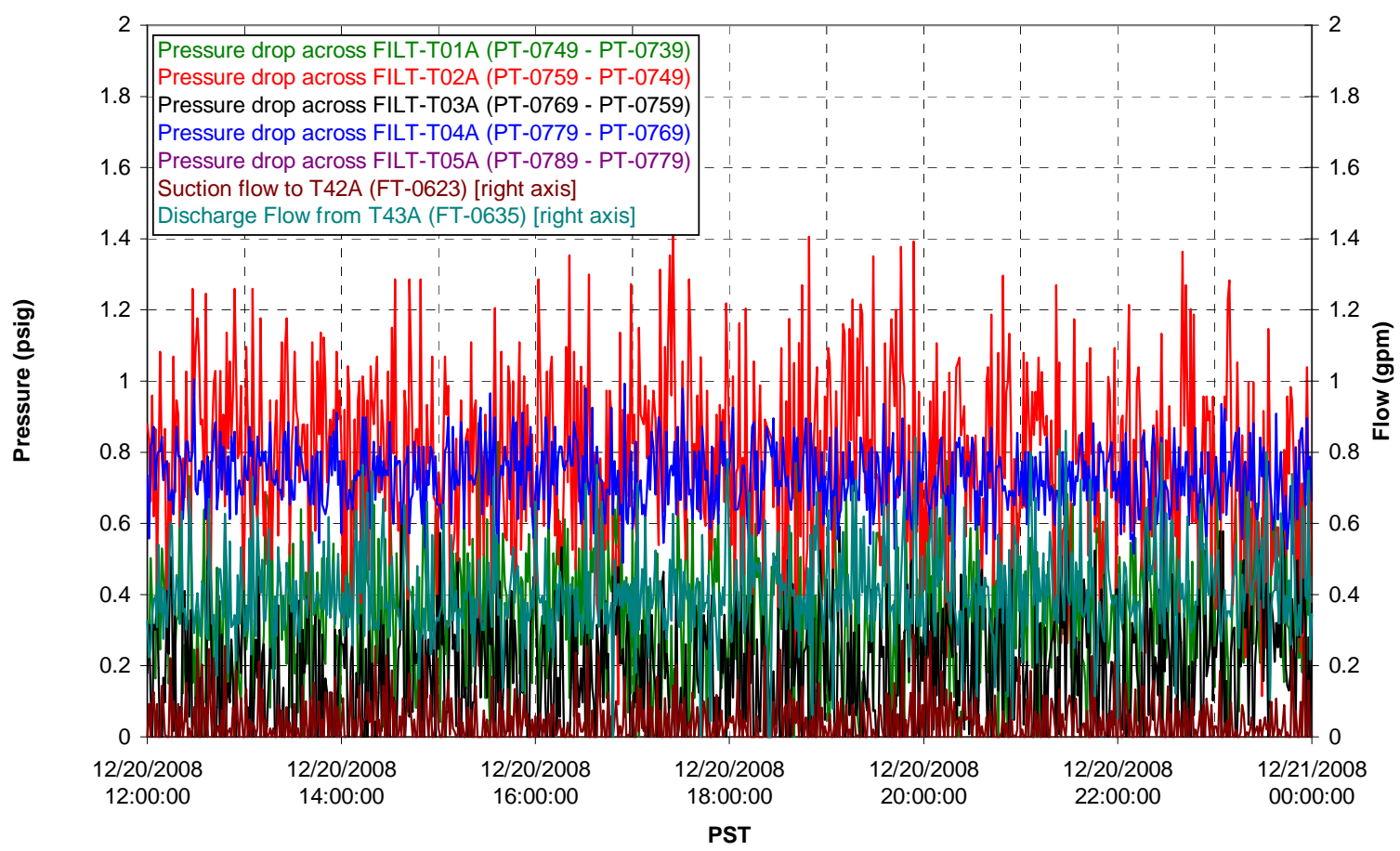

Permeate flow rates

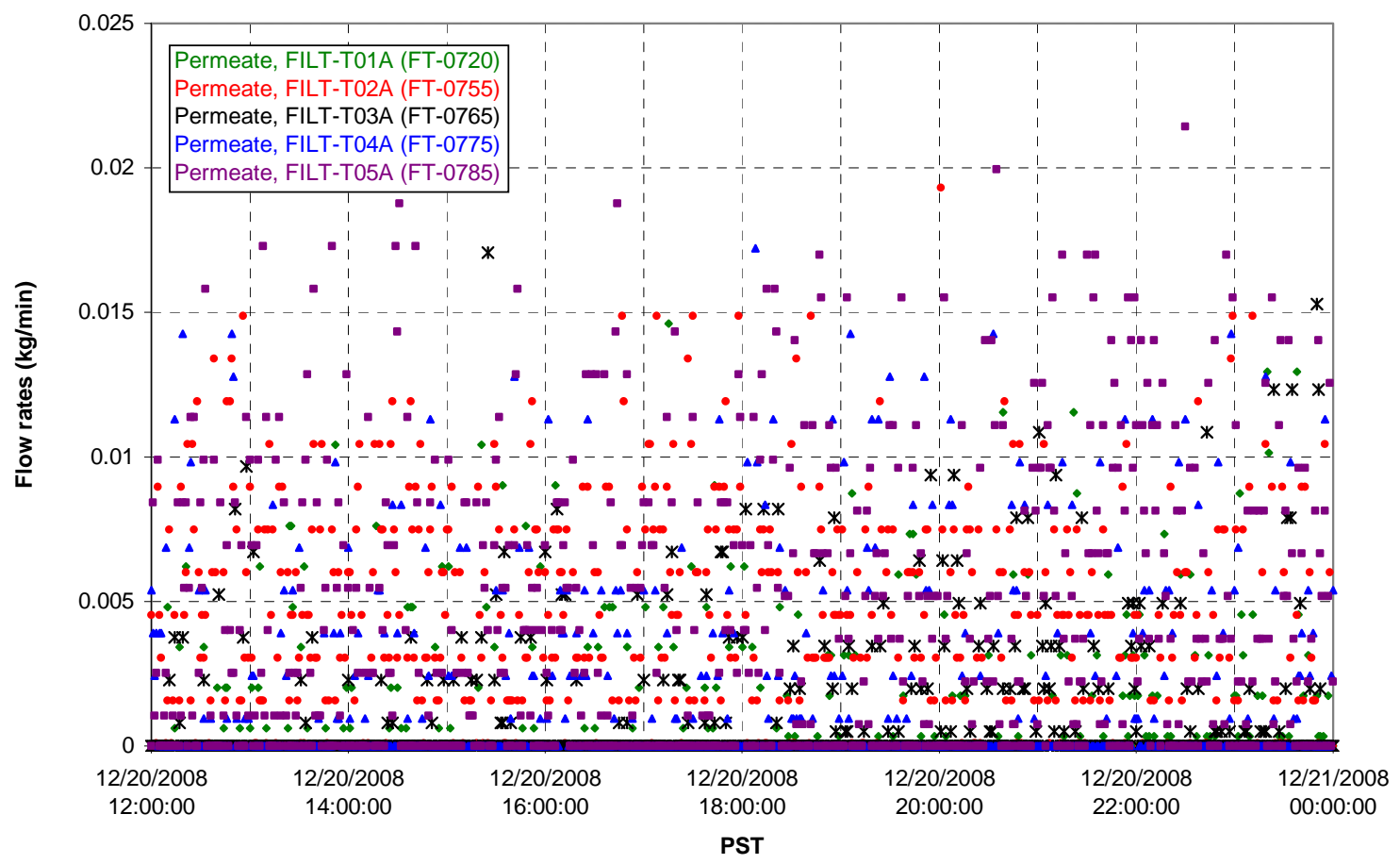


T02A Inner Temperature Tree

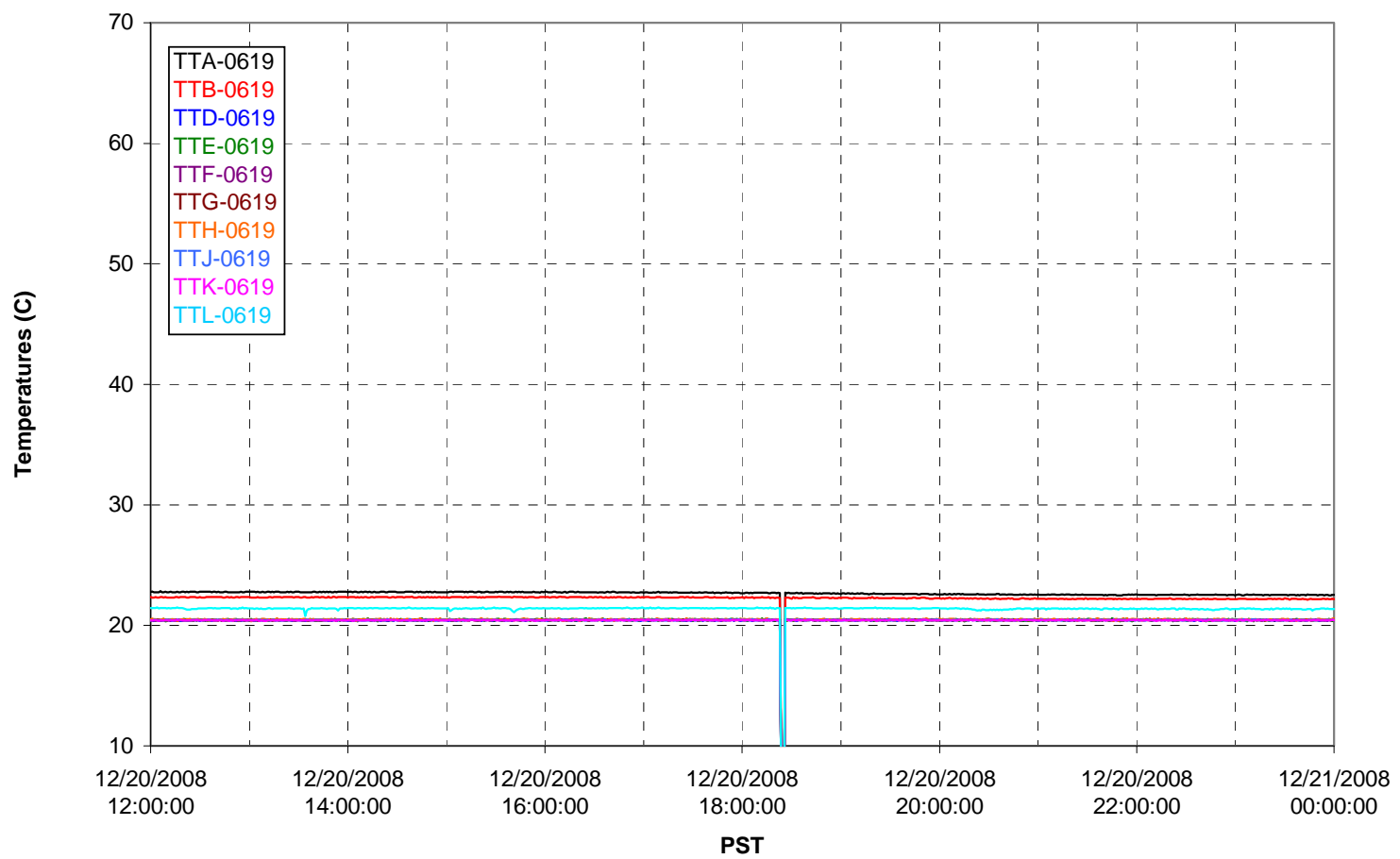

T02A Outer Temperature Tree

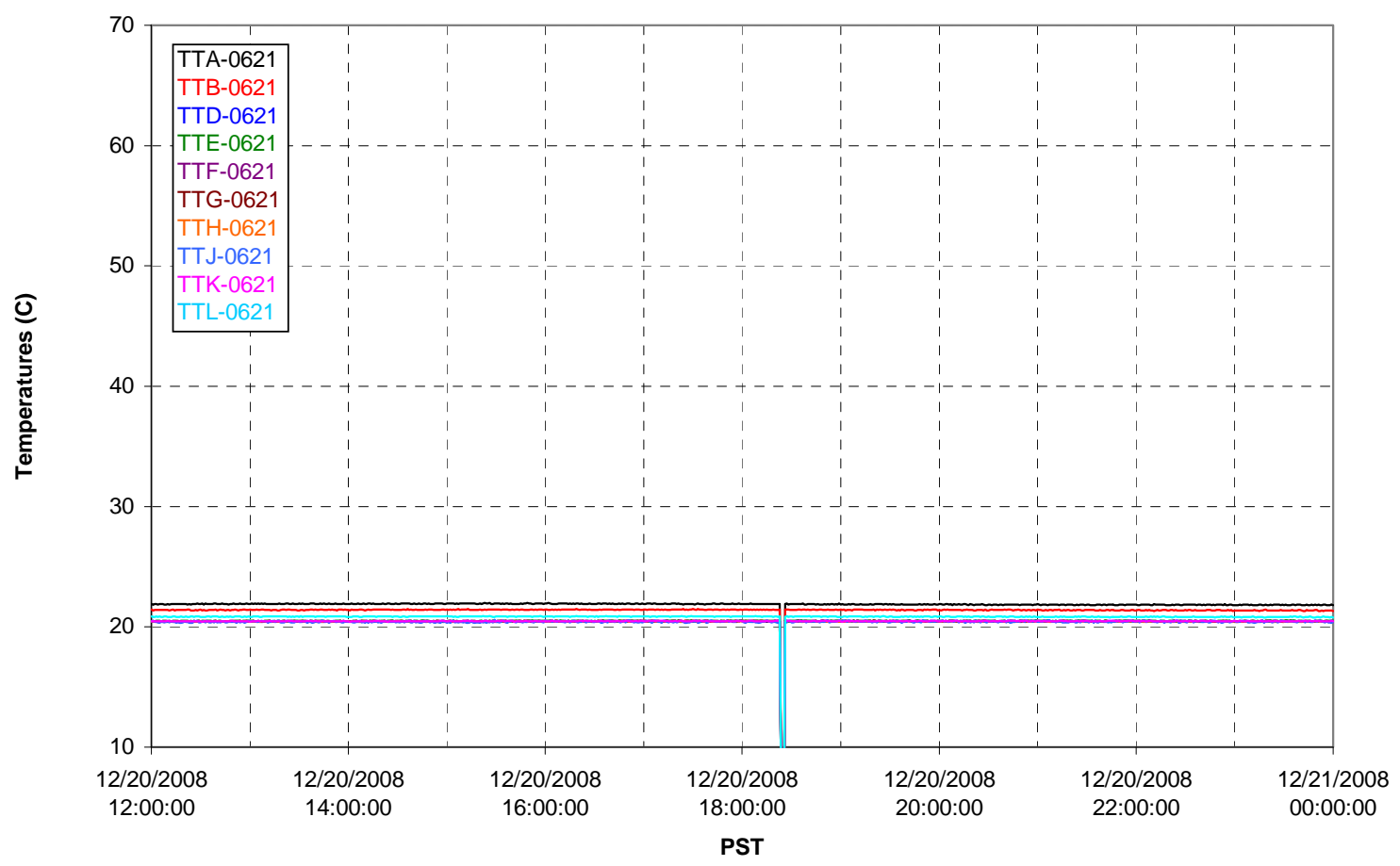


T02A temperatures

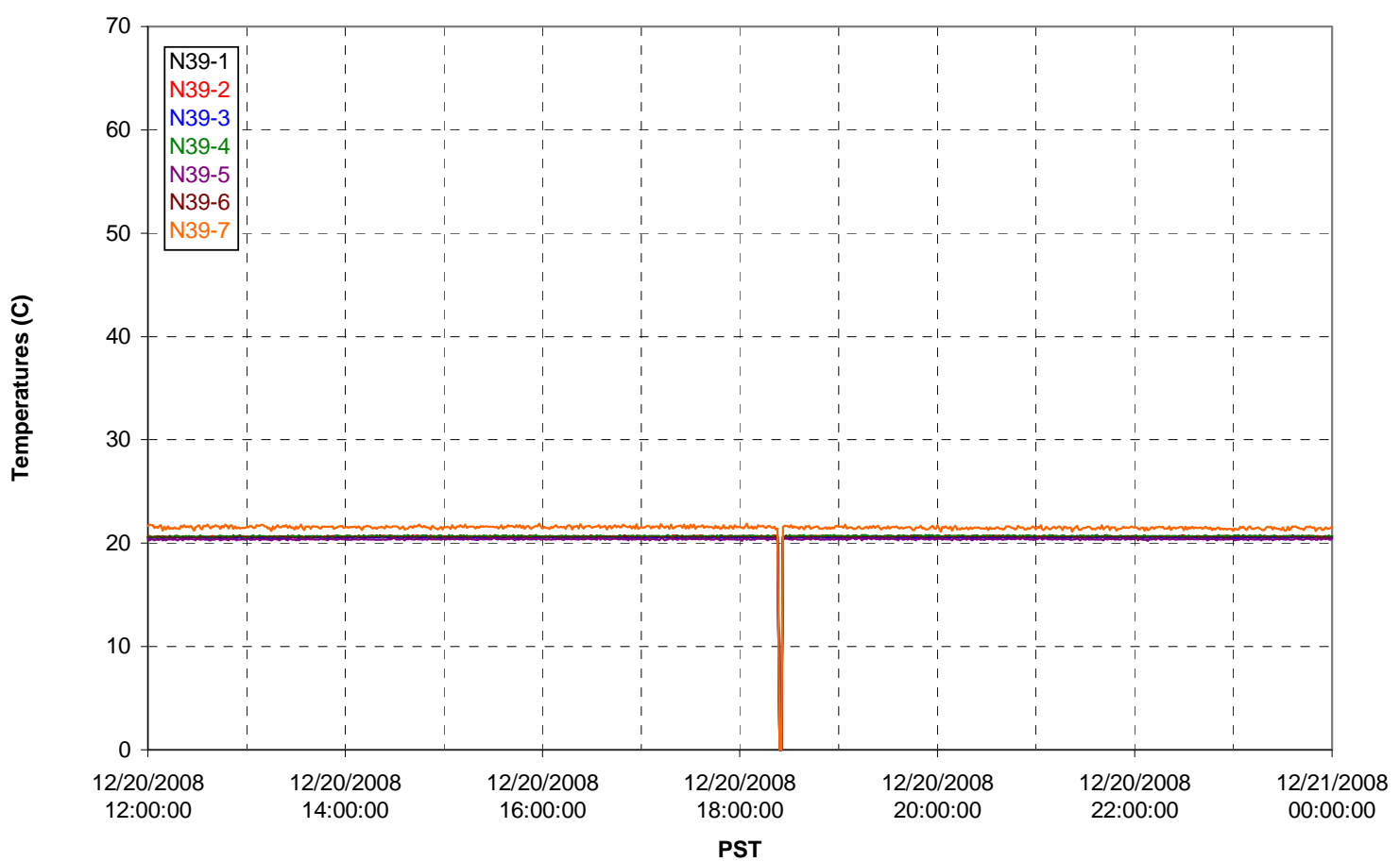

T02A temperatures

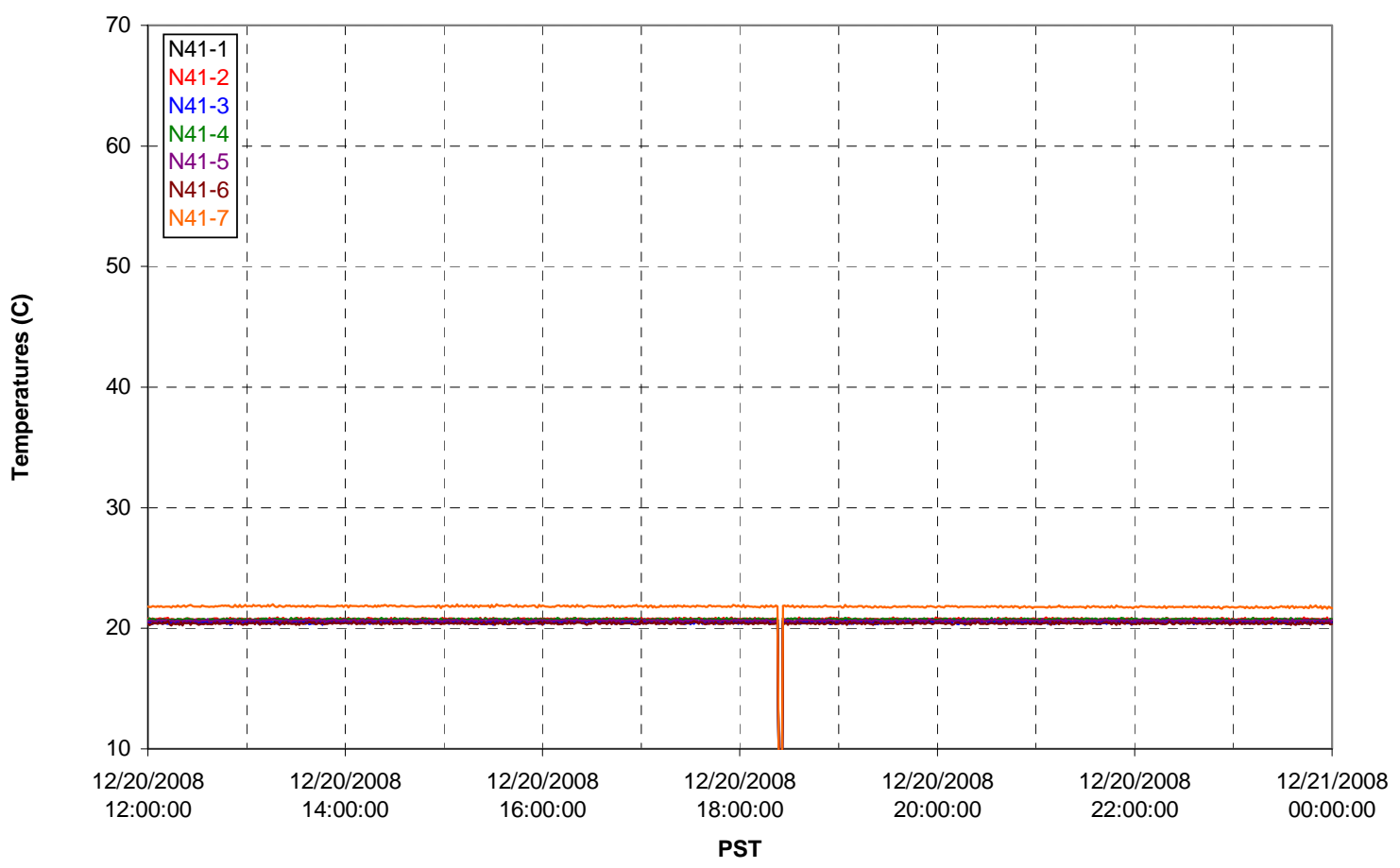


T02A temperatures

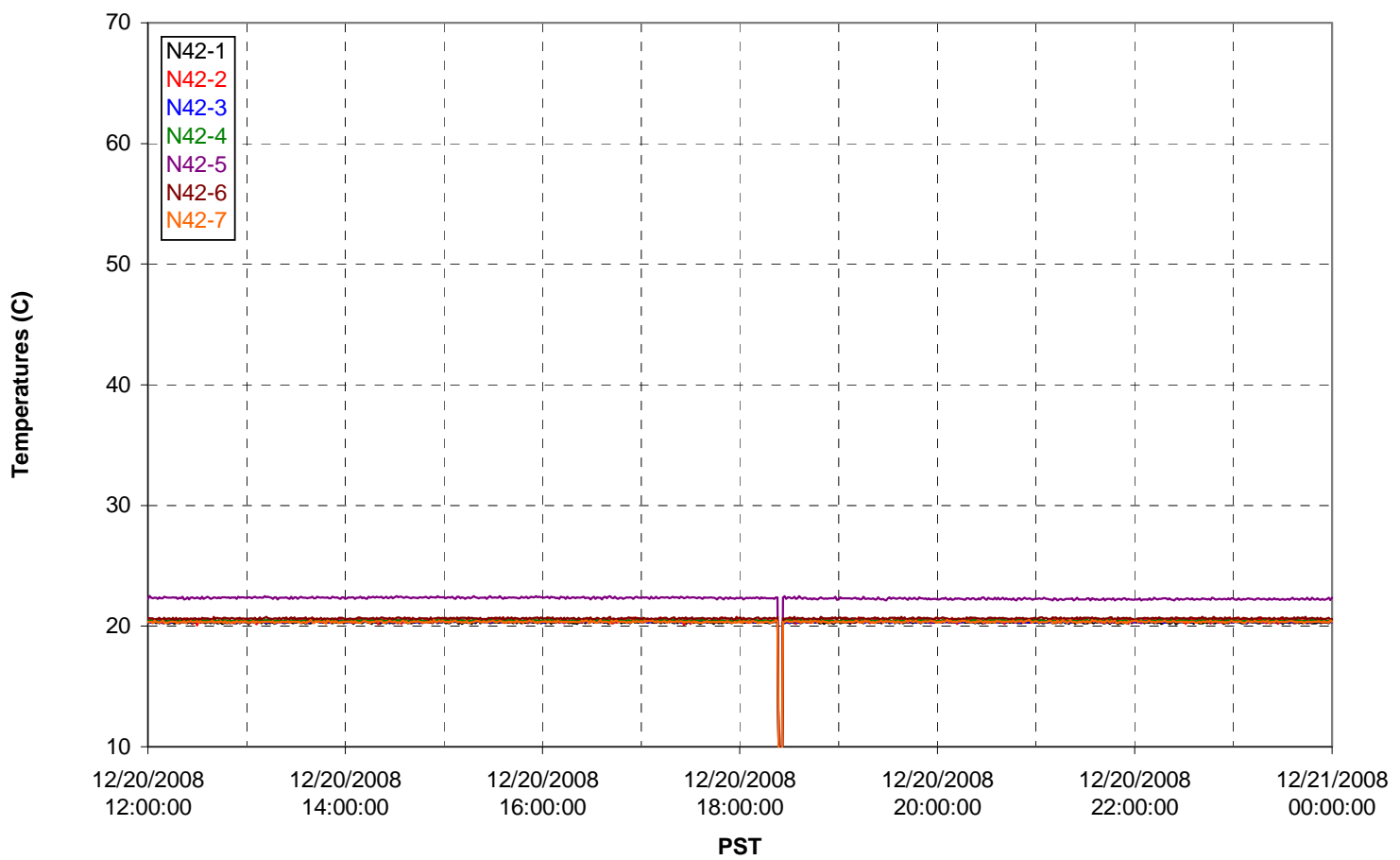

T02A temperatures

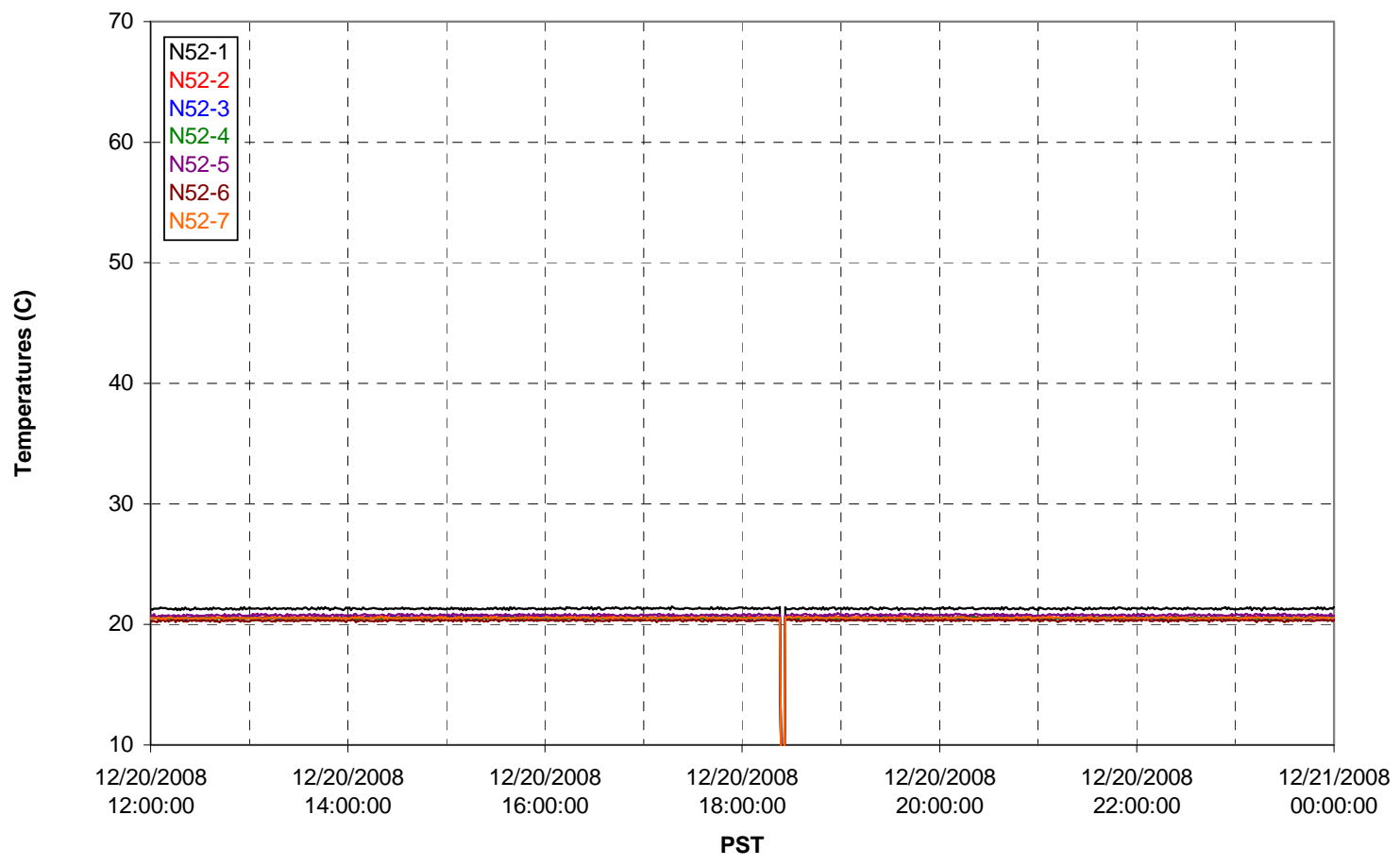


T02A Heating and Cooling

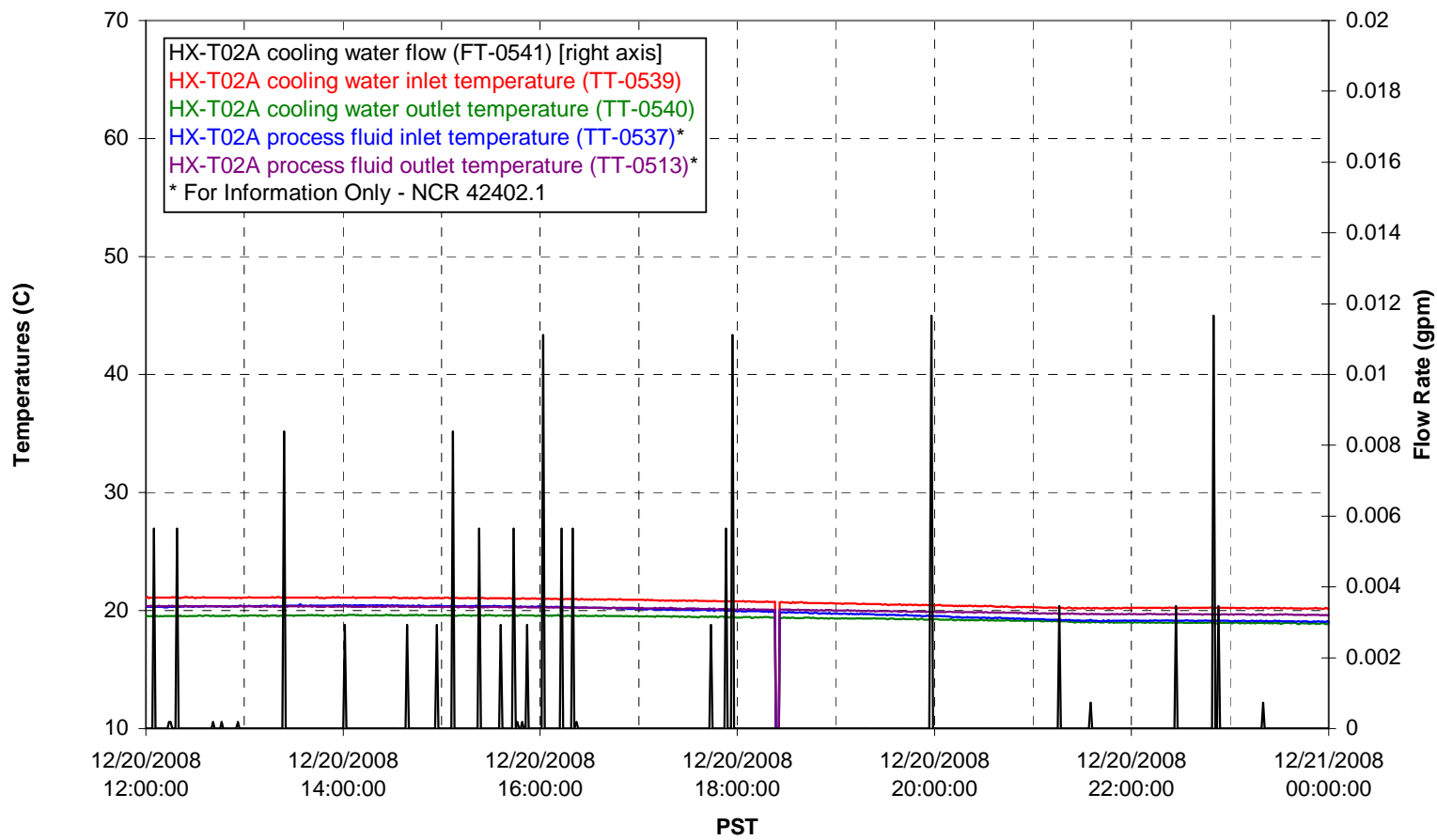

Pump Operation

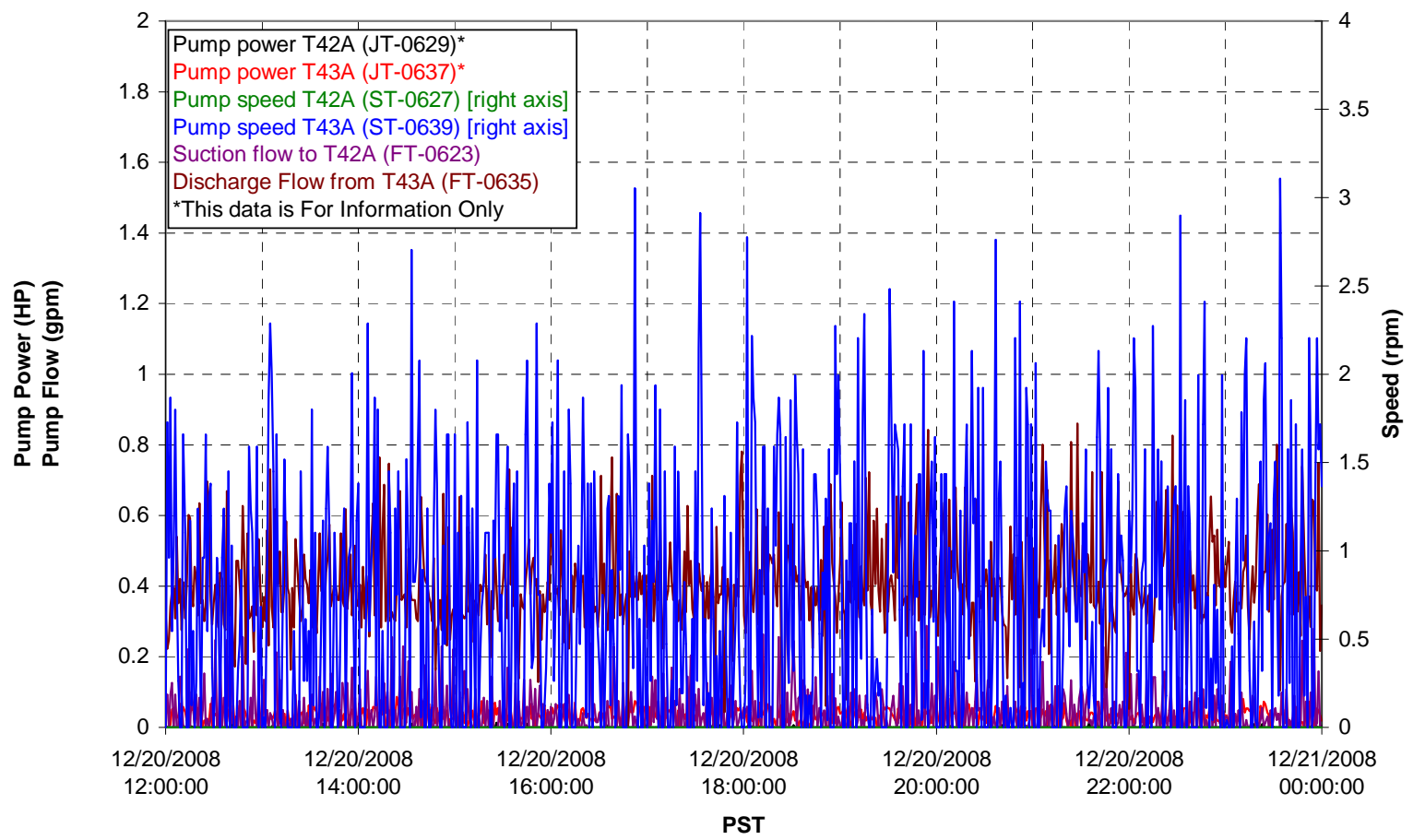


Pulsepot UFP-PP-T01A

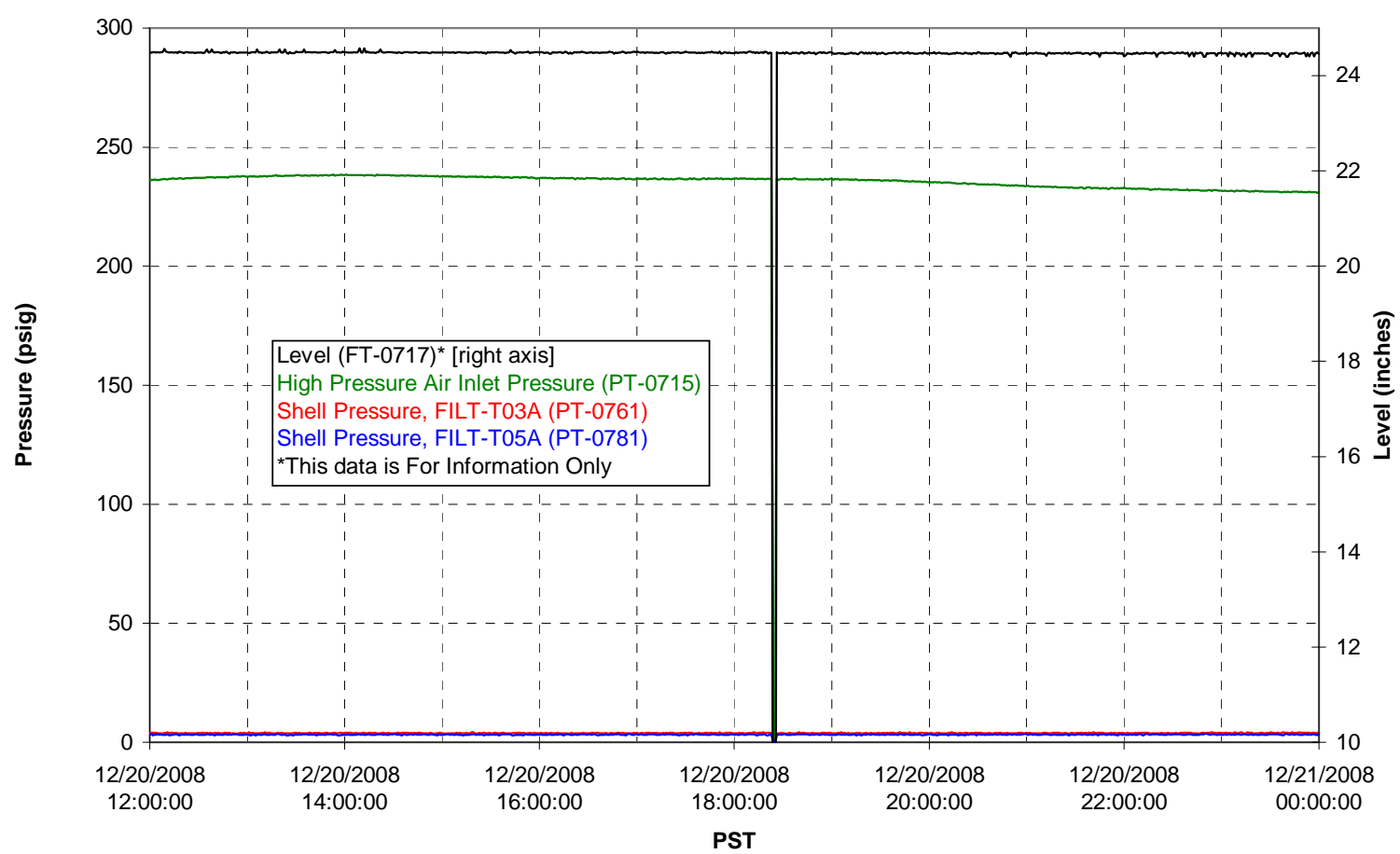

Pulsepot UFP-PP-T02A

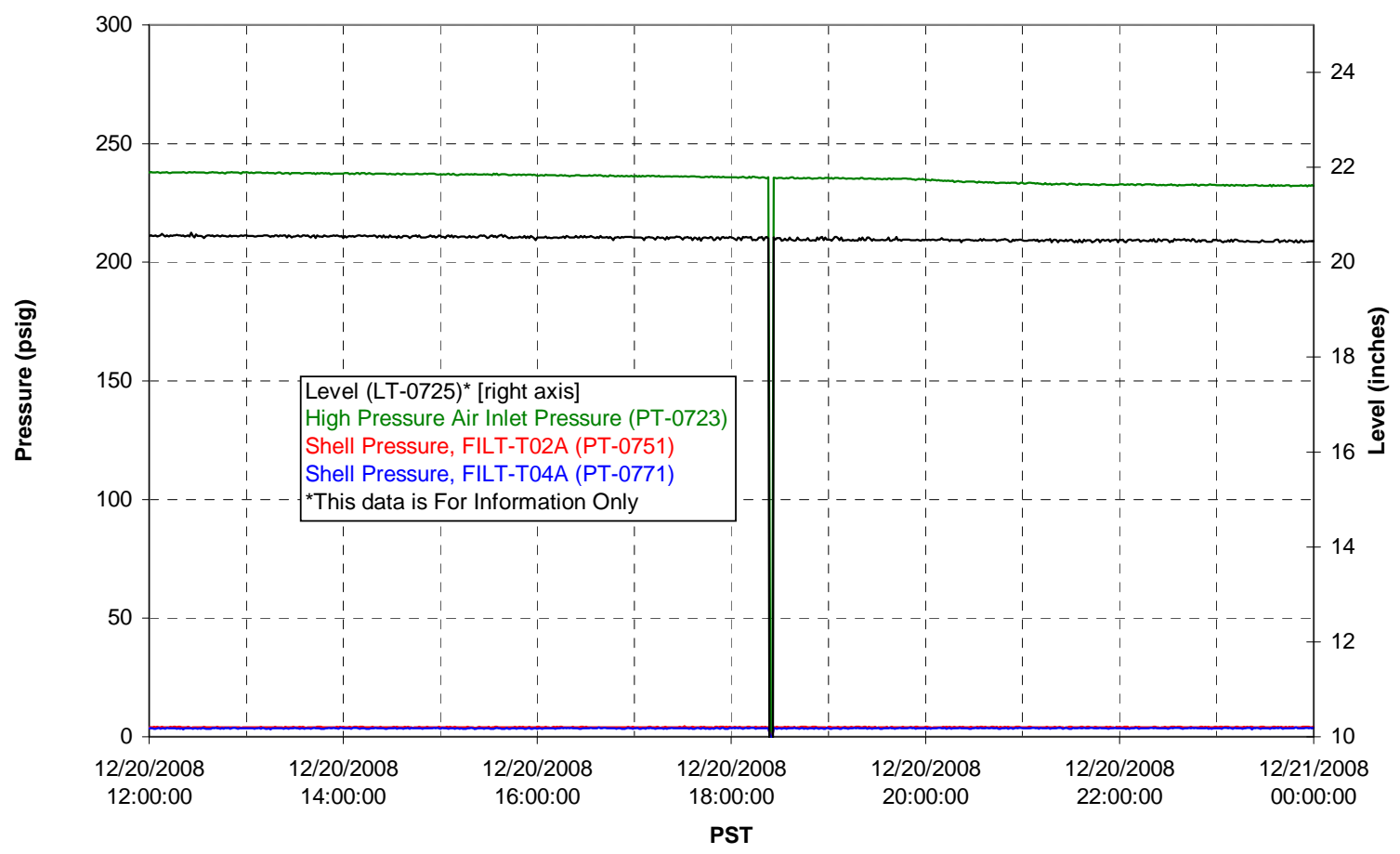


Pulsepot UFP-PP-T03A

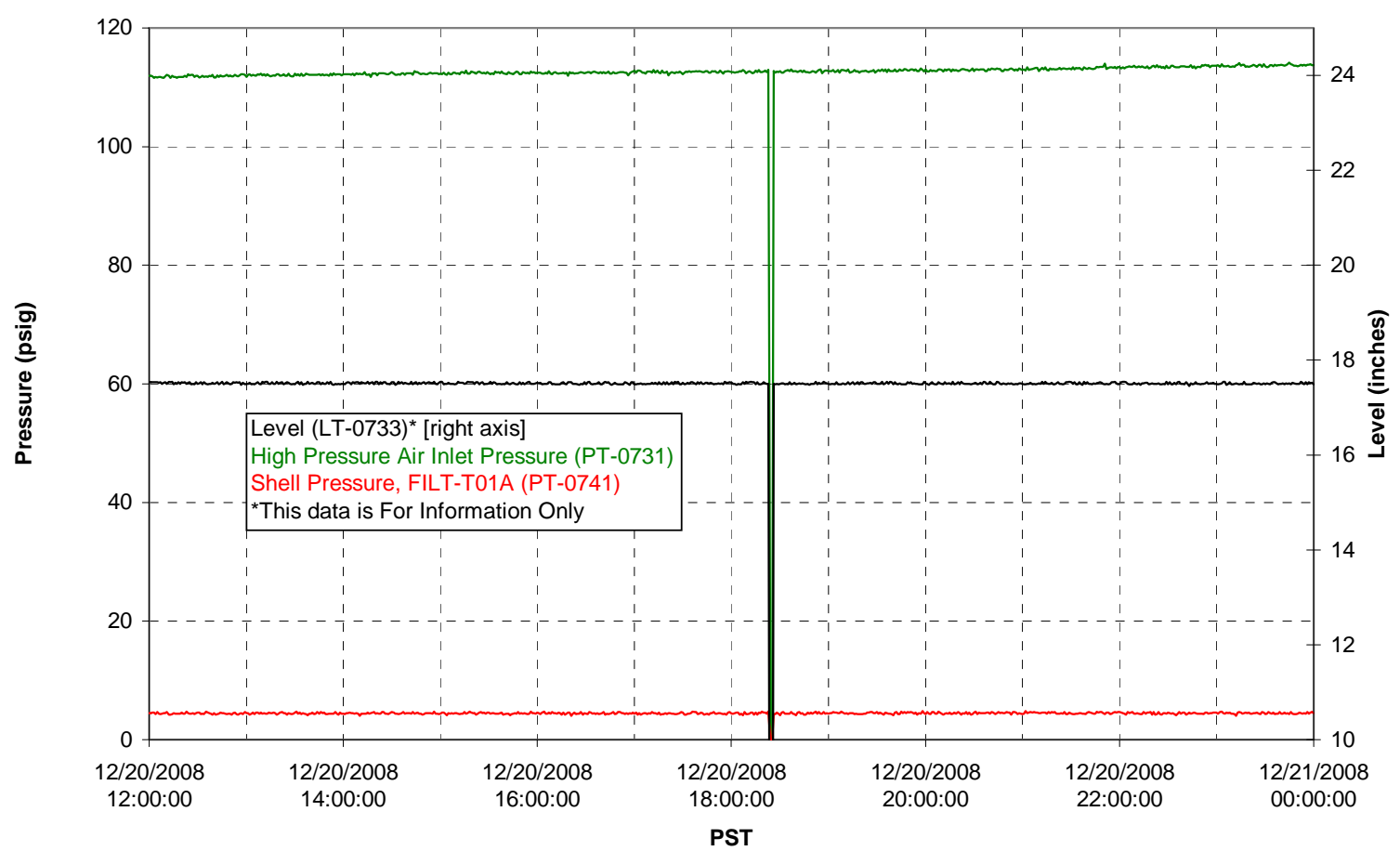

Pulsepot Levels

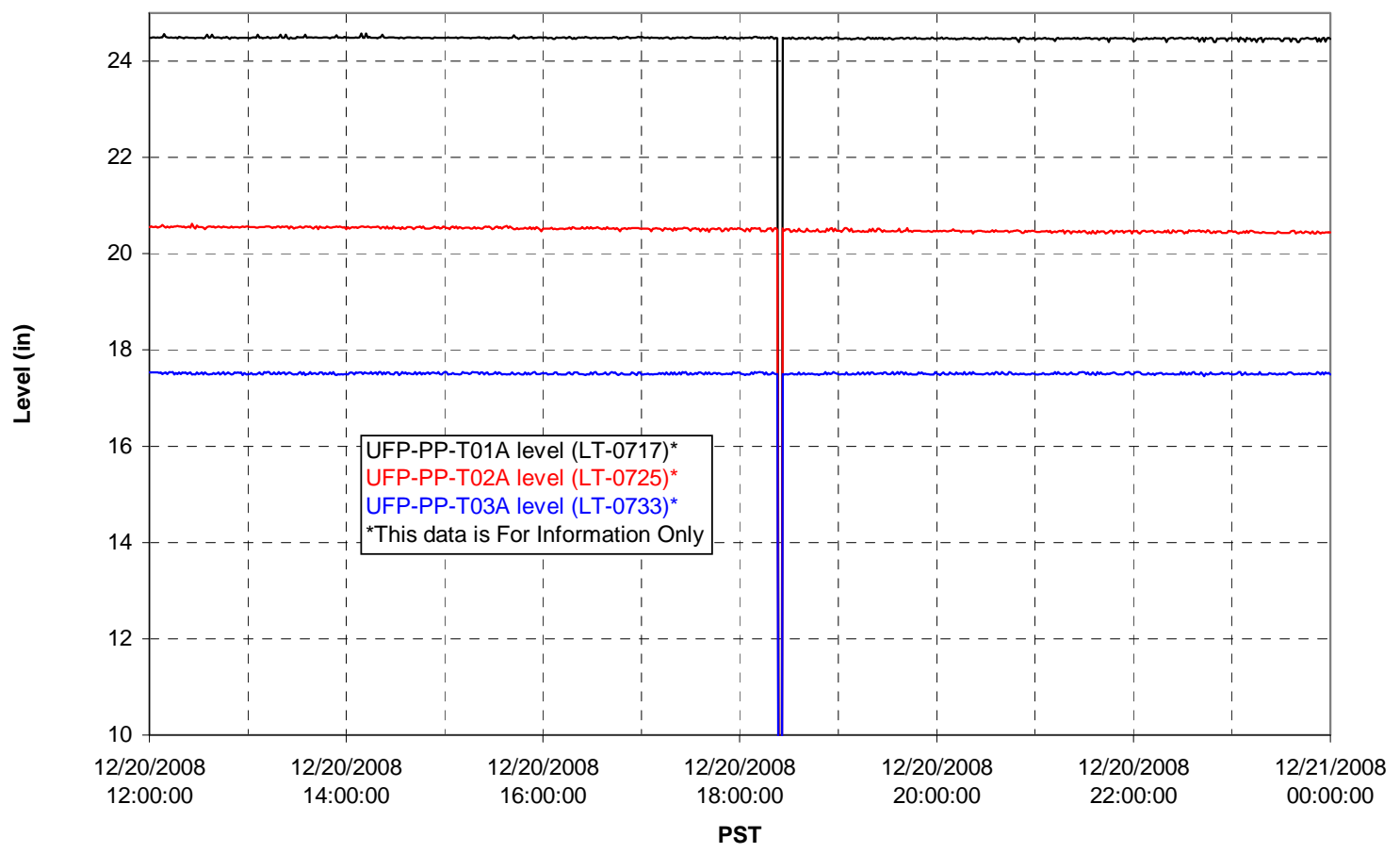


Filter UFP-FILT-T01A

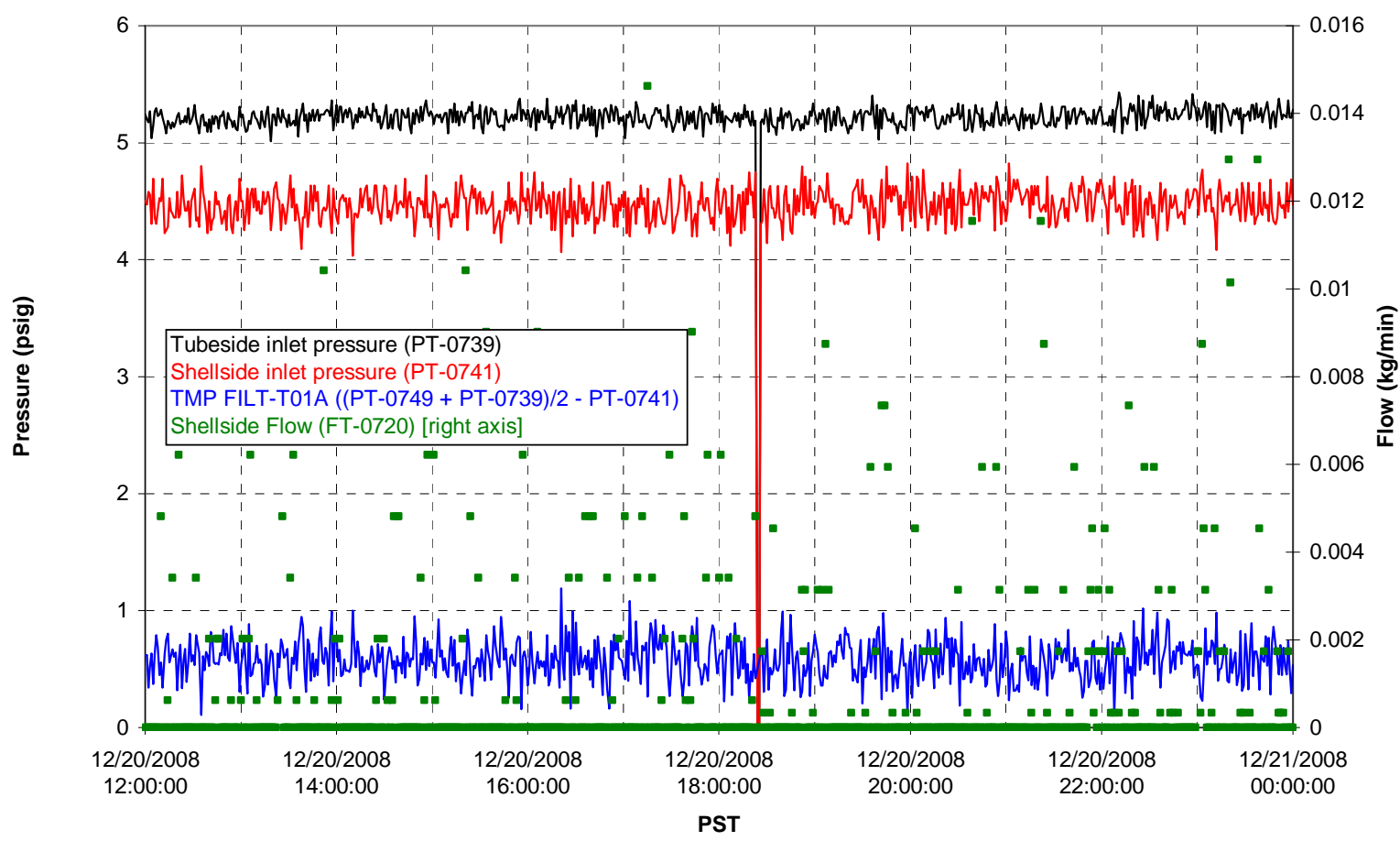

Filter UFP-FILT-T02A

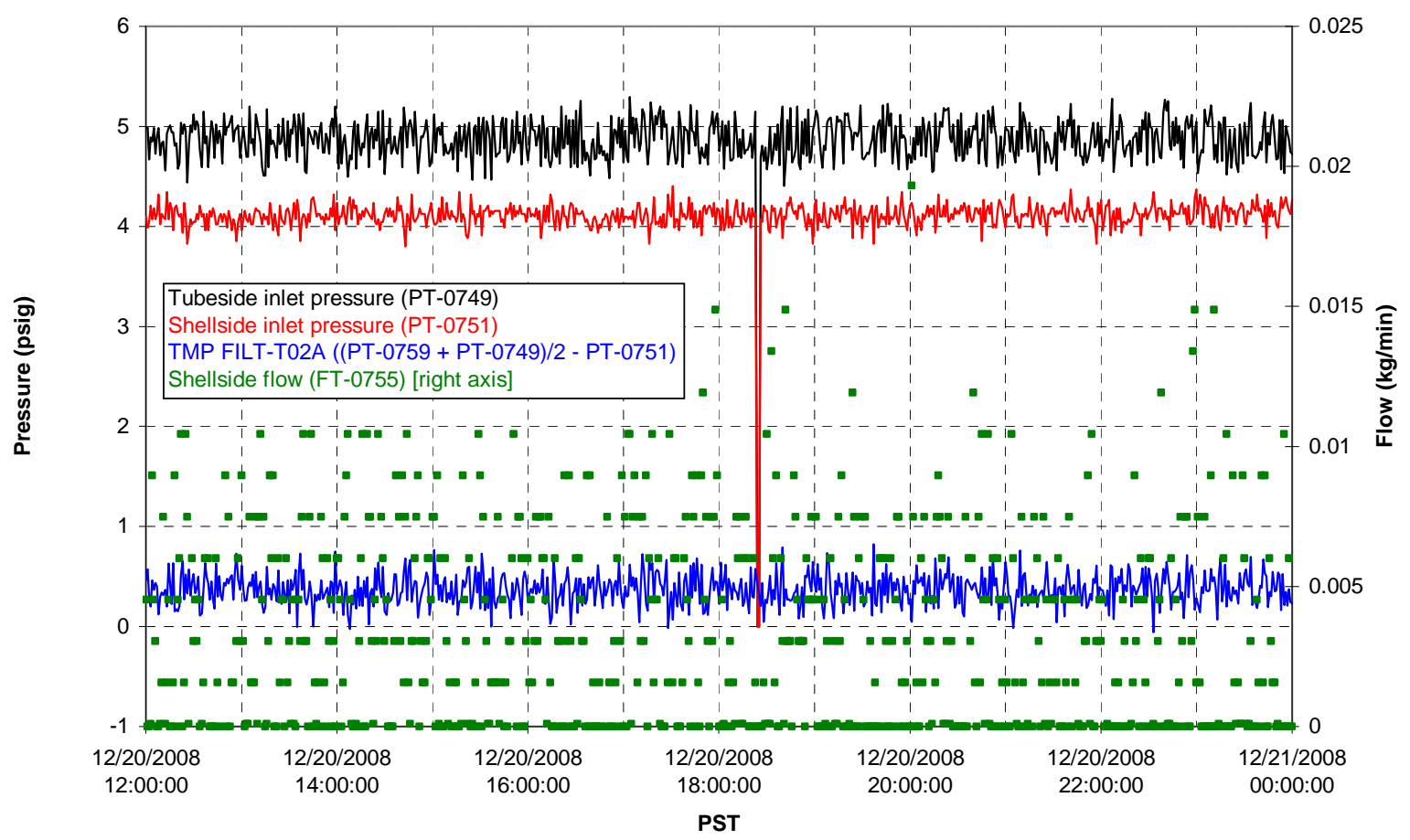


Filter UFP-FILT-T03A

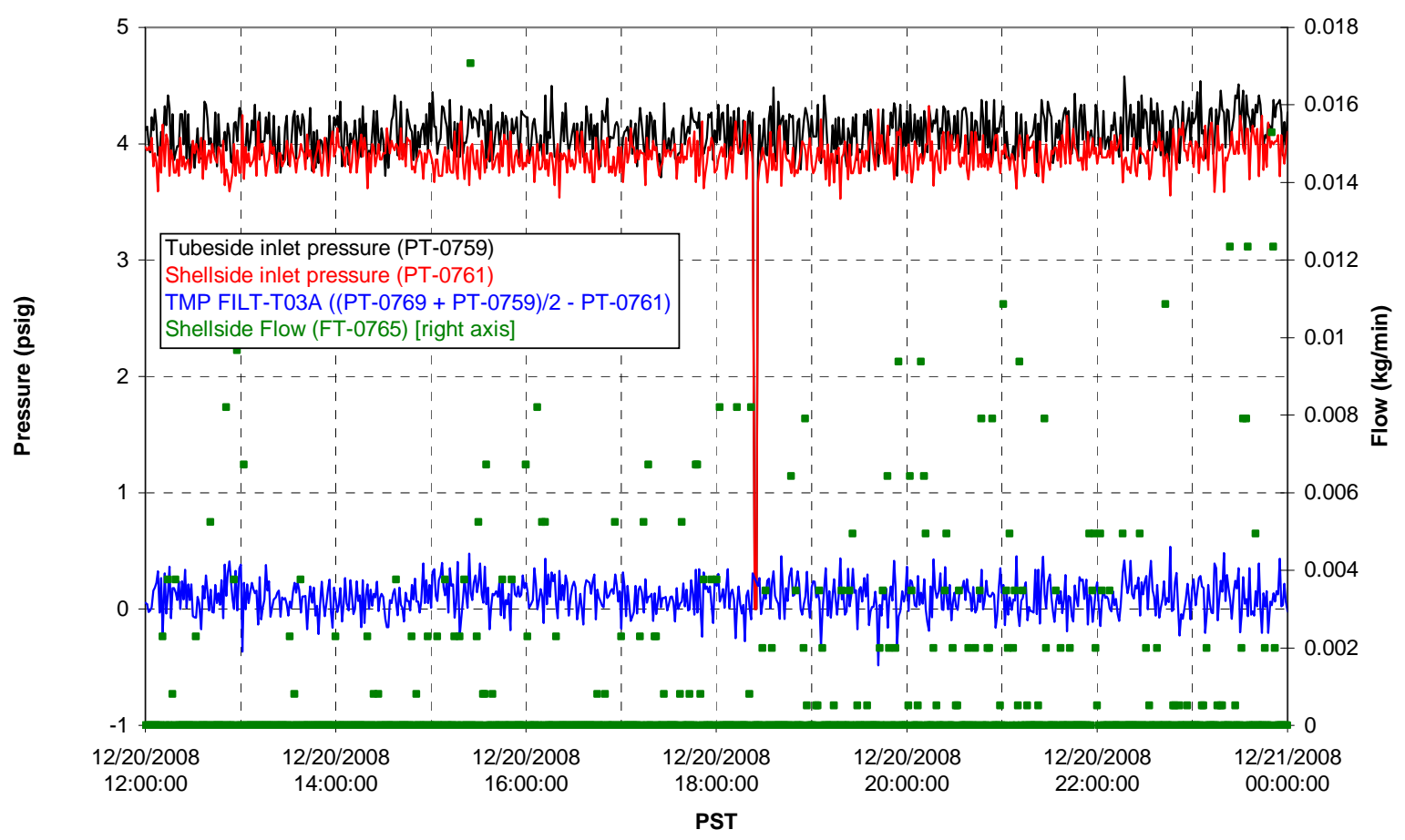

Filter UFP-FILT-T04A

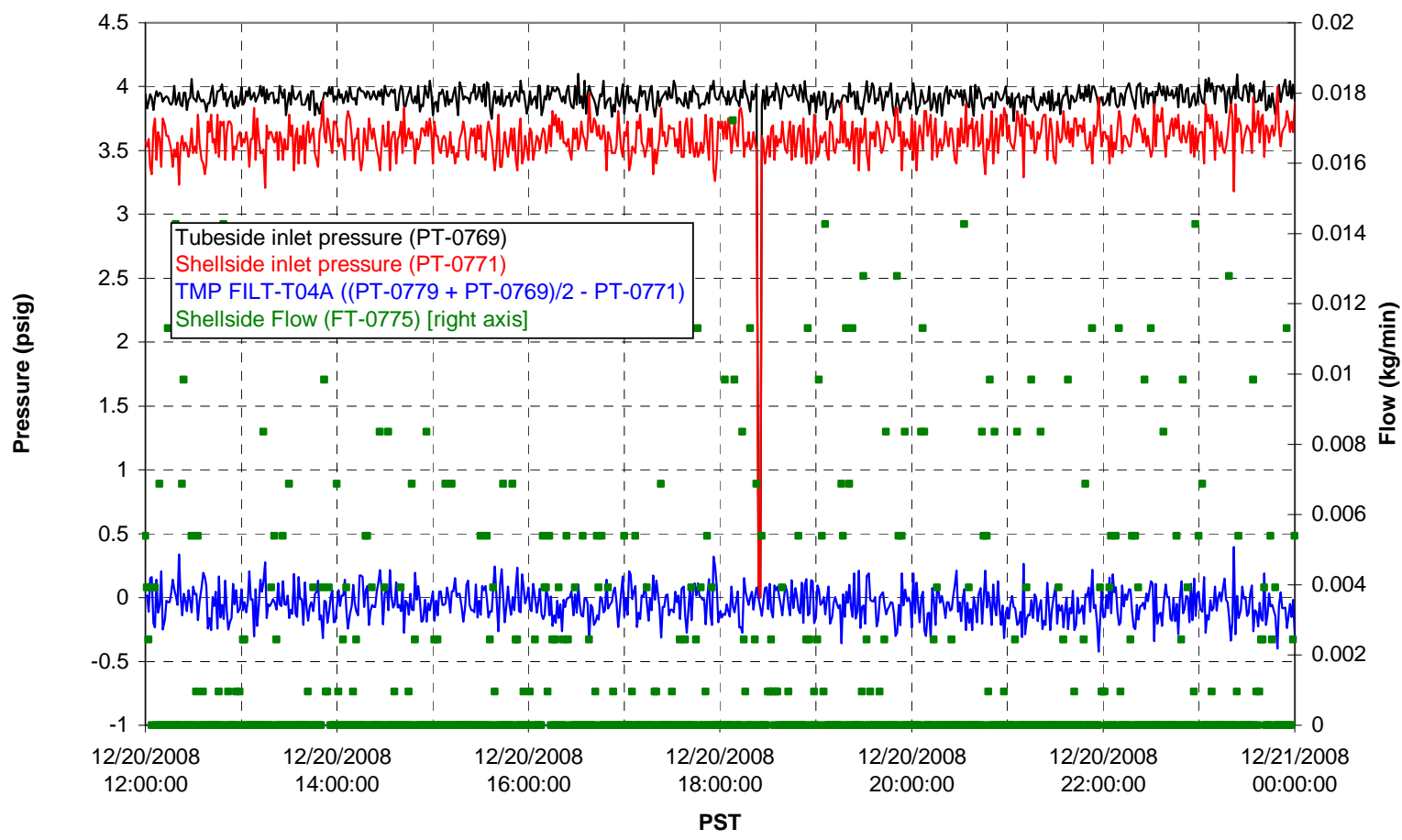


Filter UFP-FILT-T05A

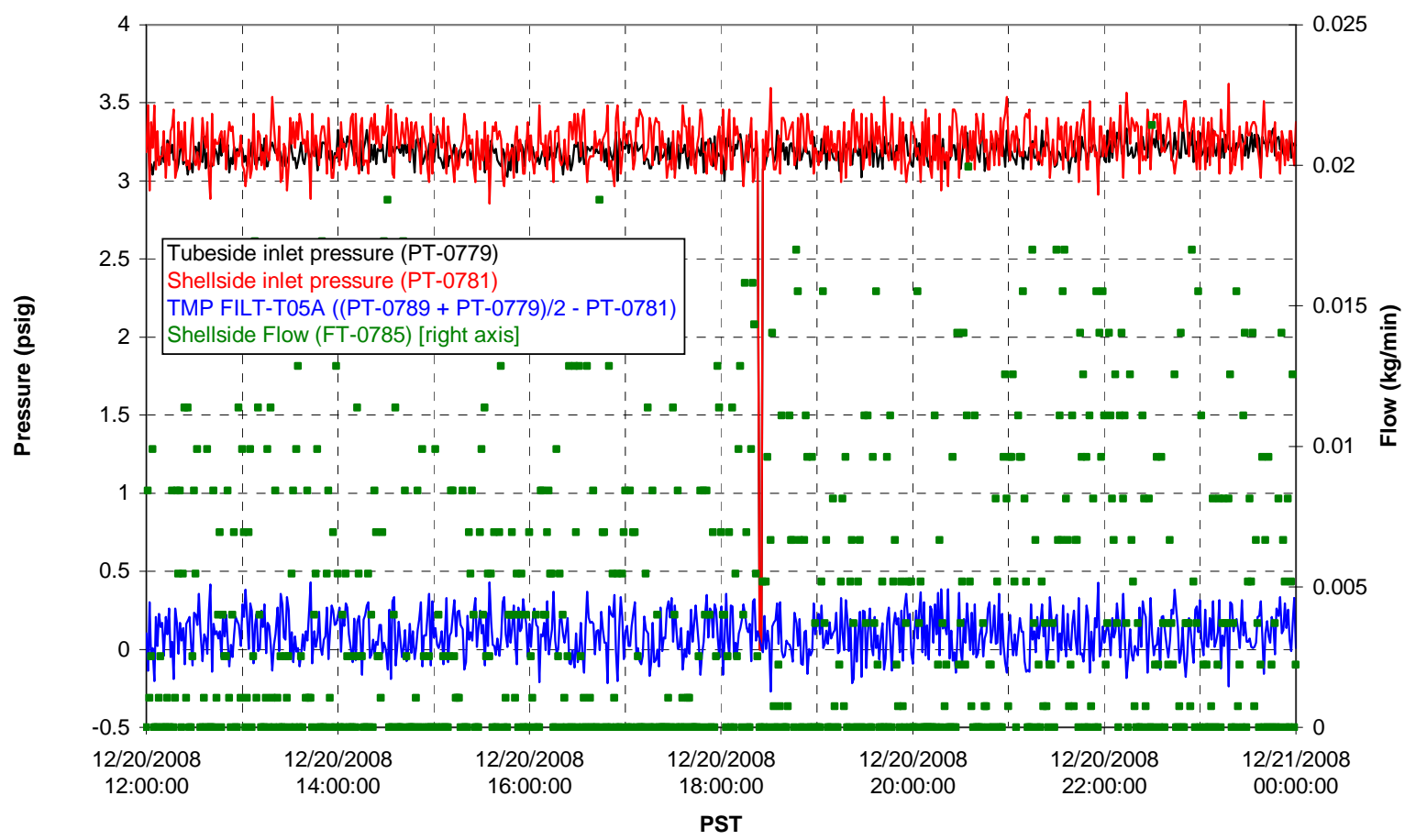

Chemical Flow

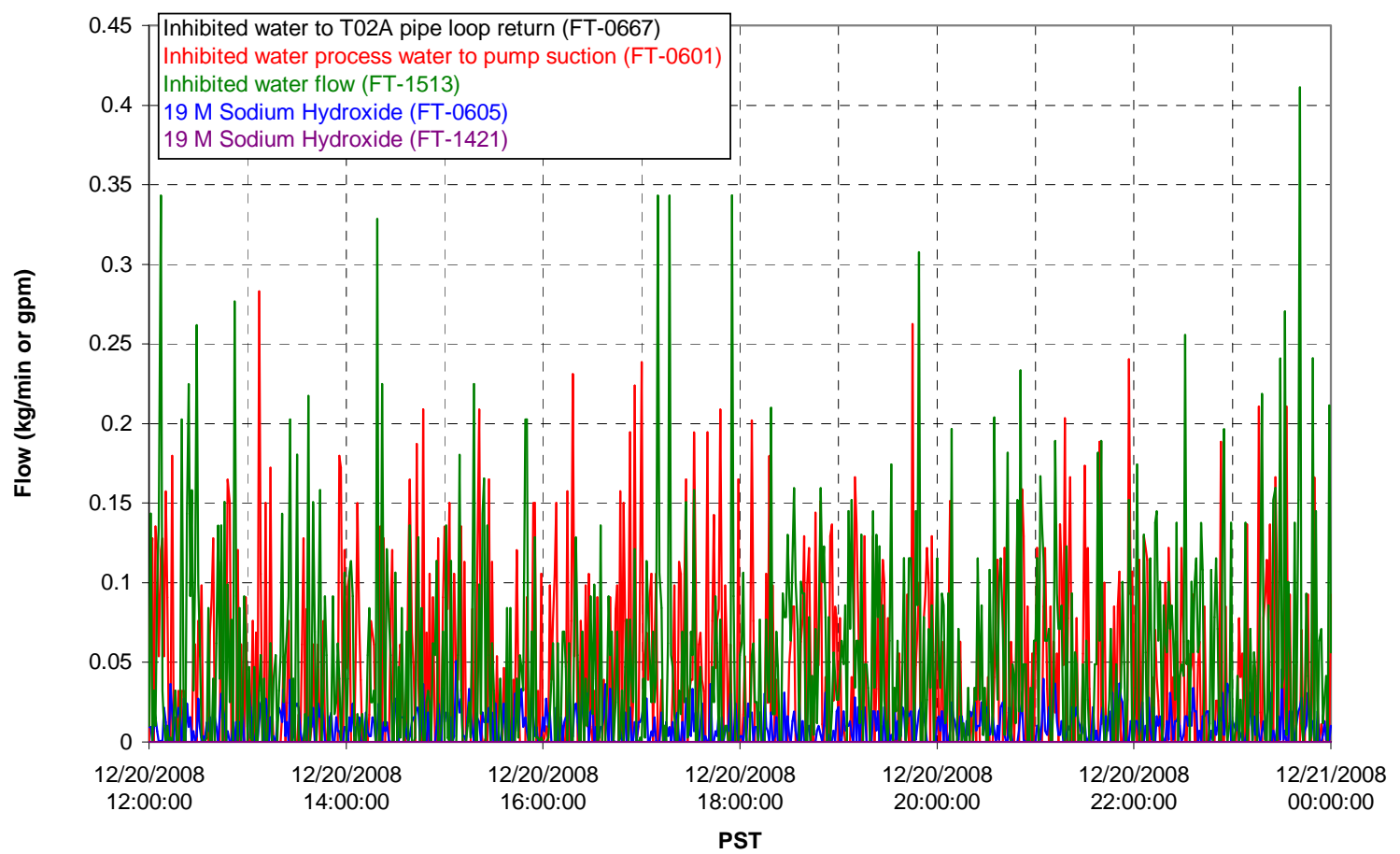




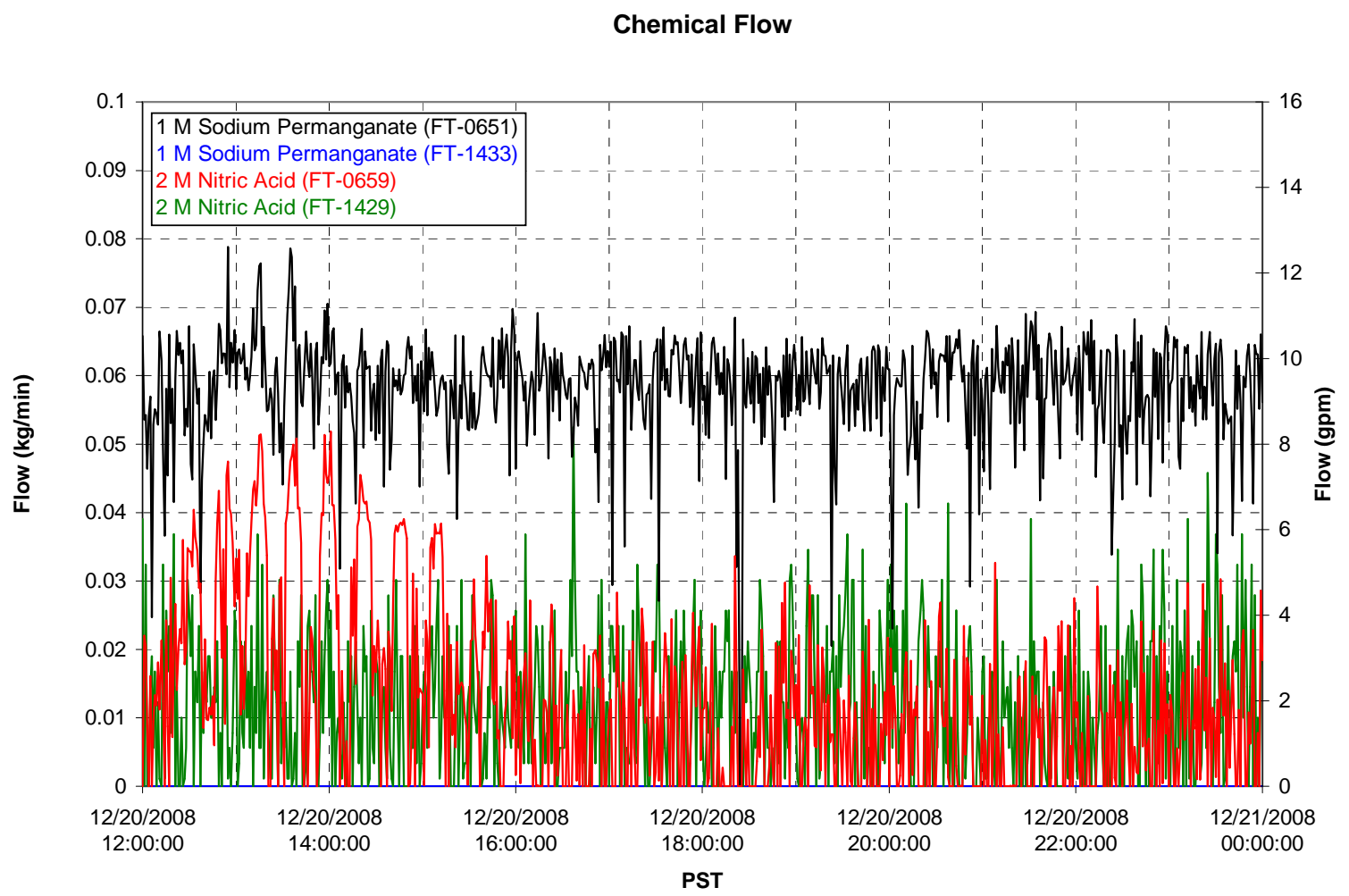

Air Flows
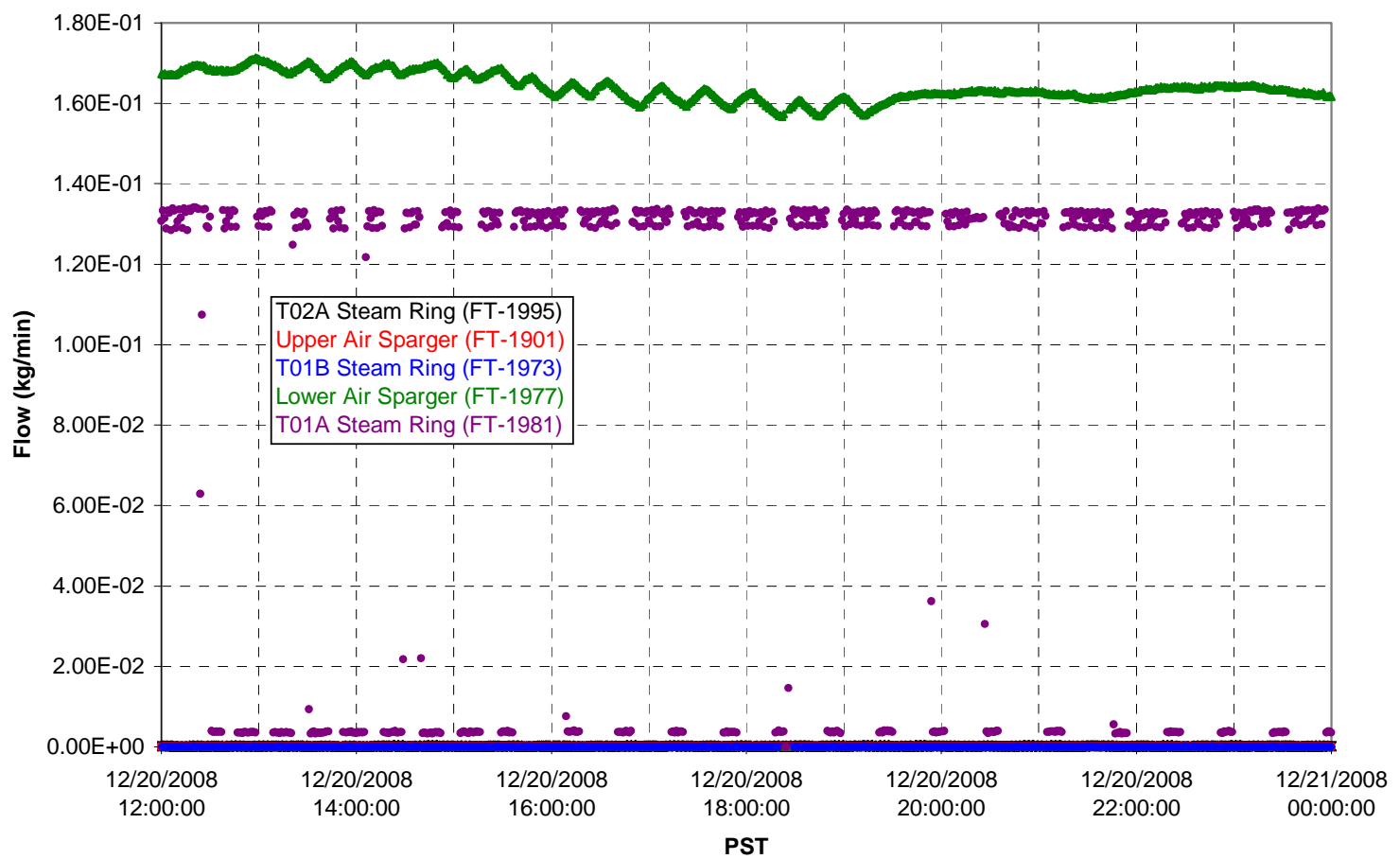
T02A Steam

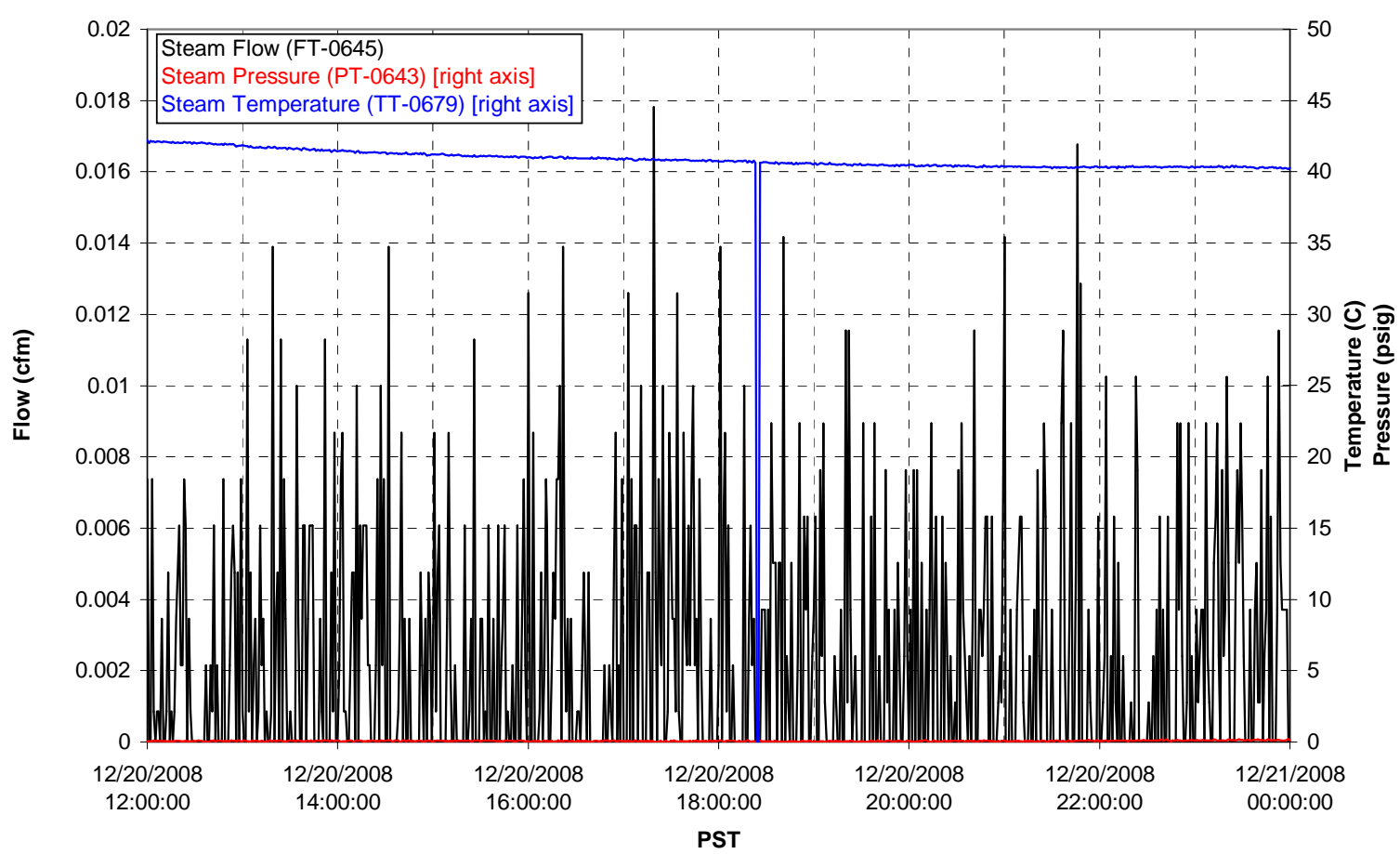

T01A Steam

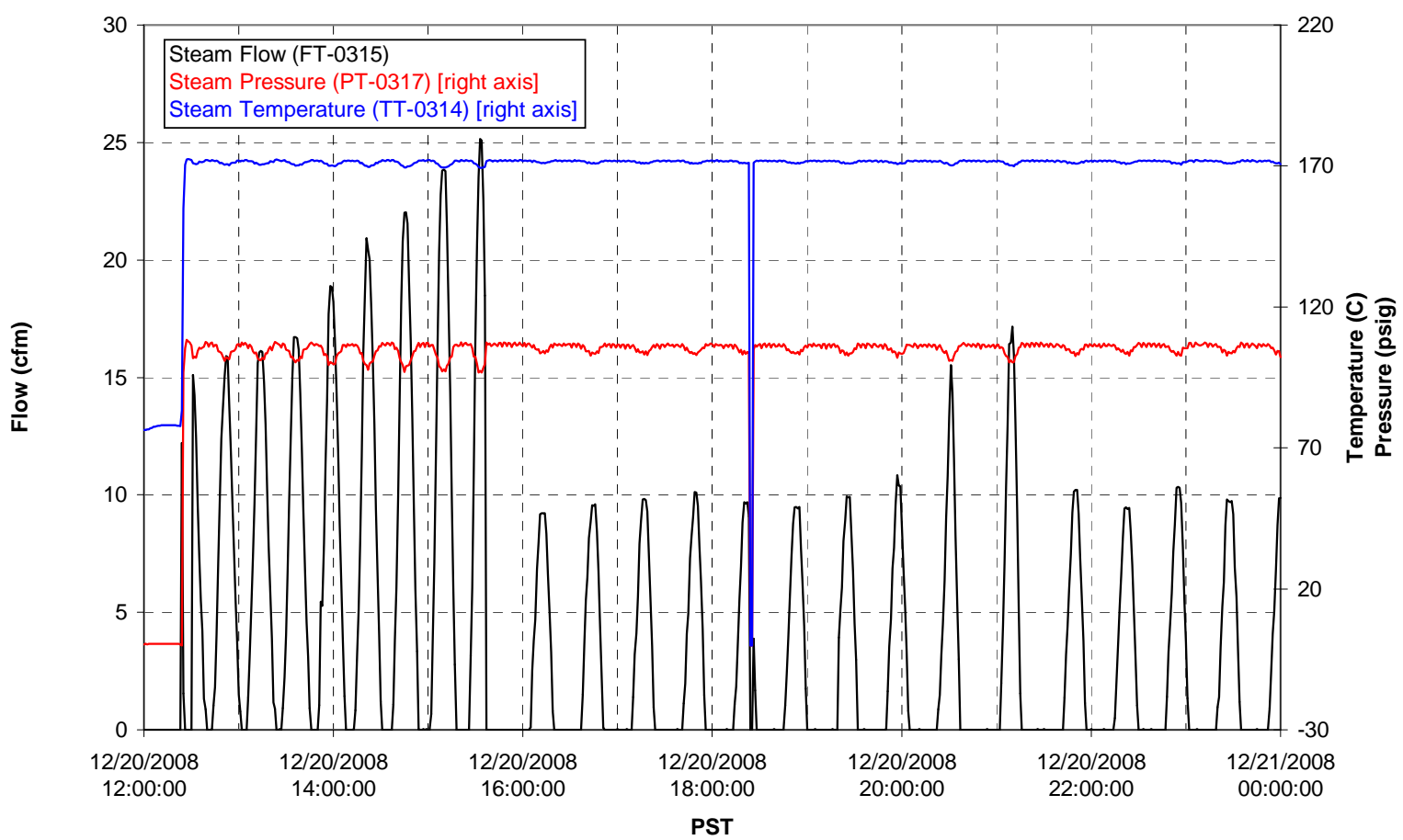


T01B Steam

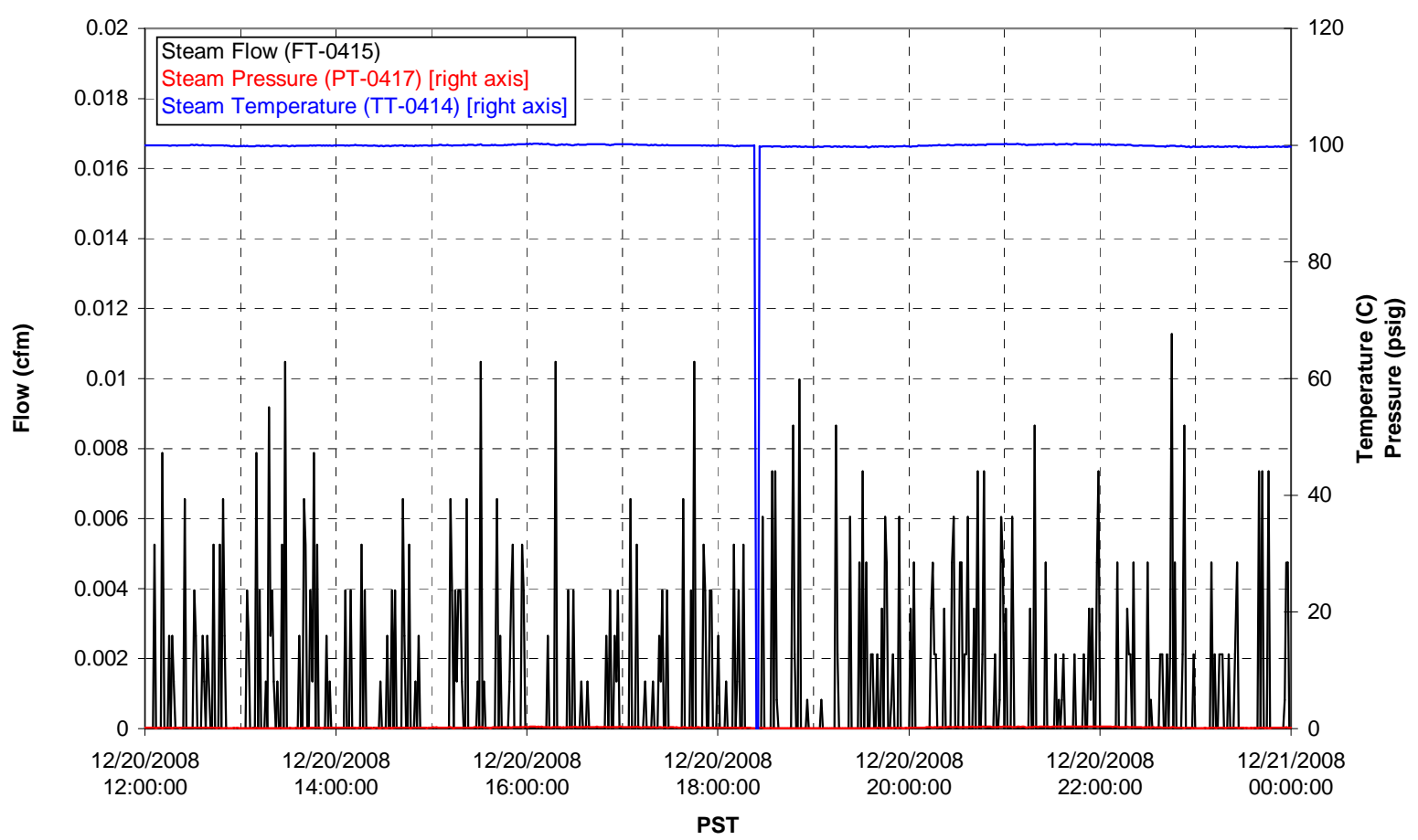


Functional Test Data Plots

12/21/08 00:00 - 12/21/08 12:00 
T01A level

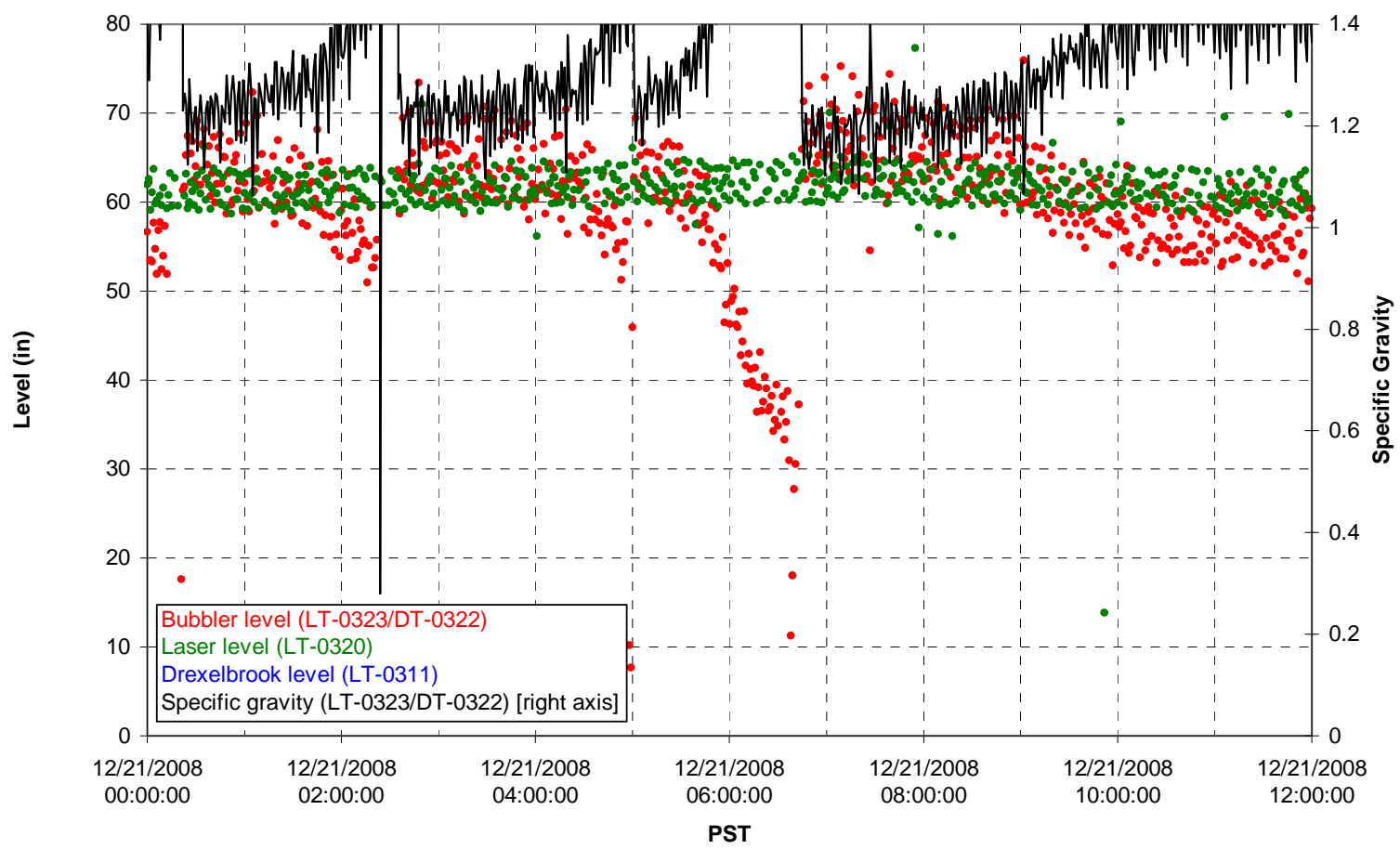

T01A temperatures

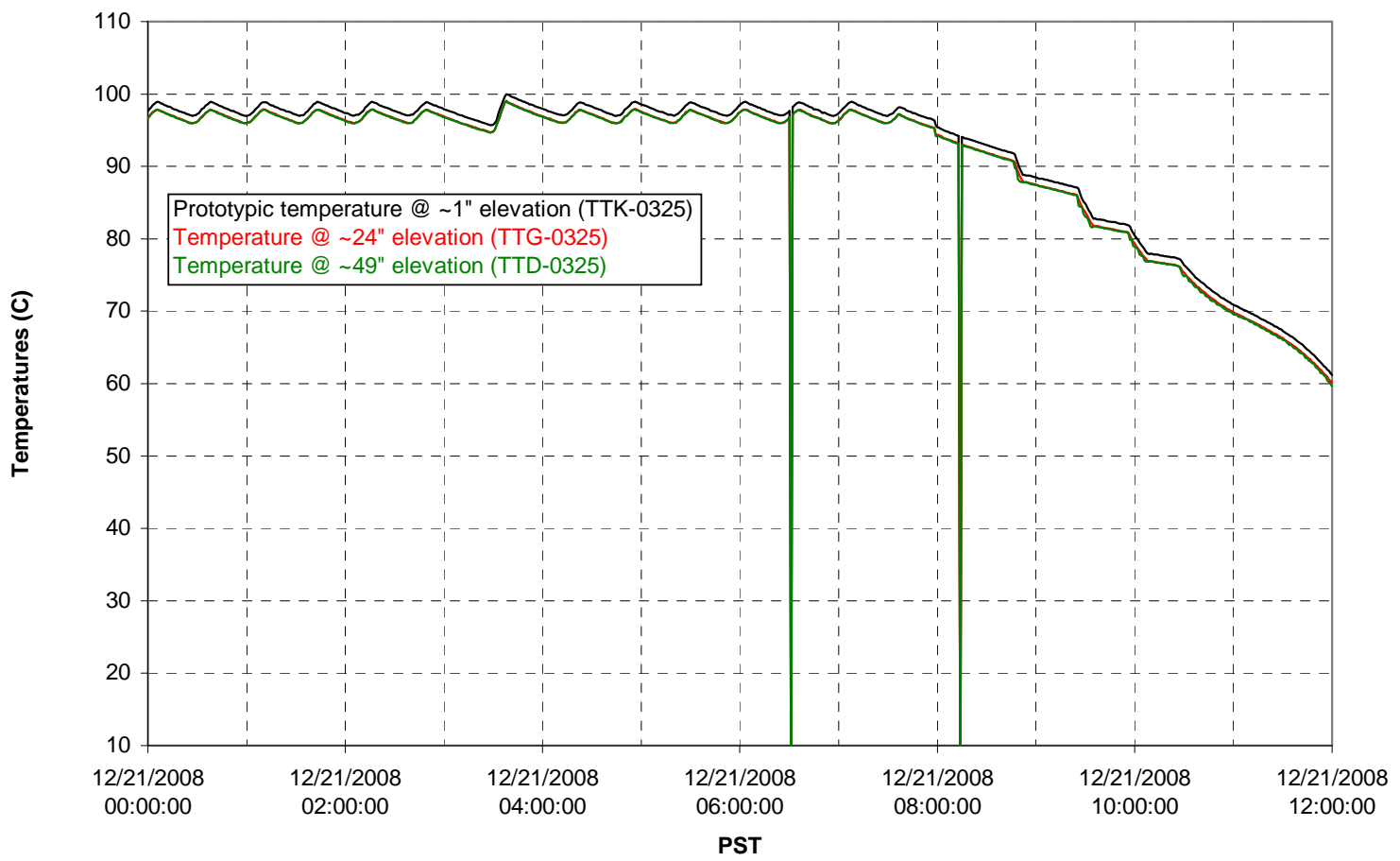


T01B level

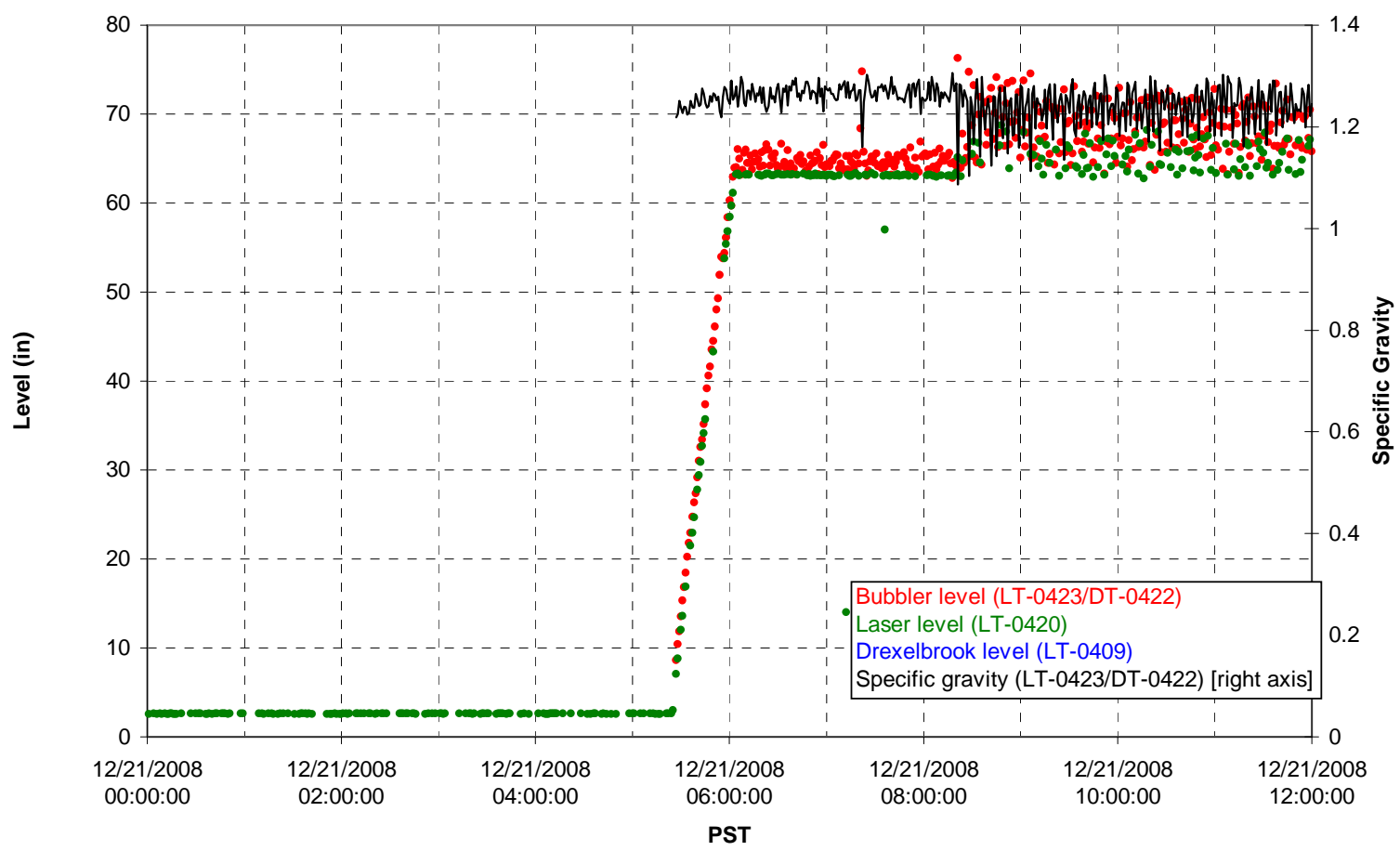

T01B temperatures

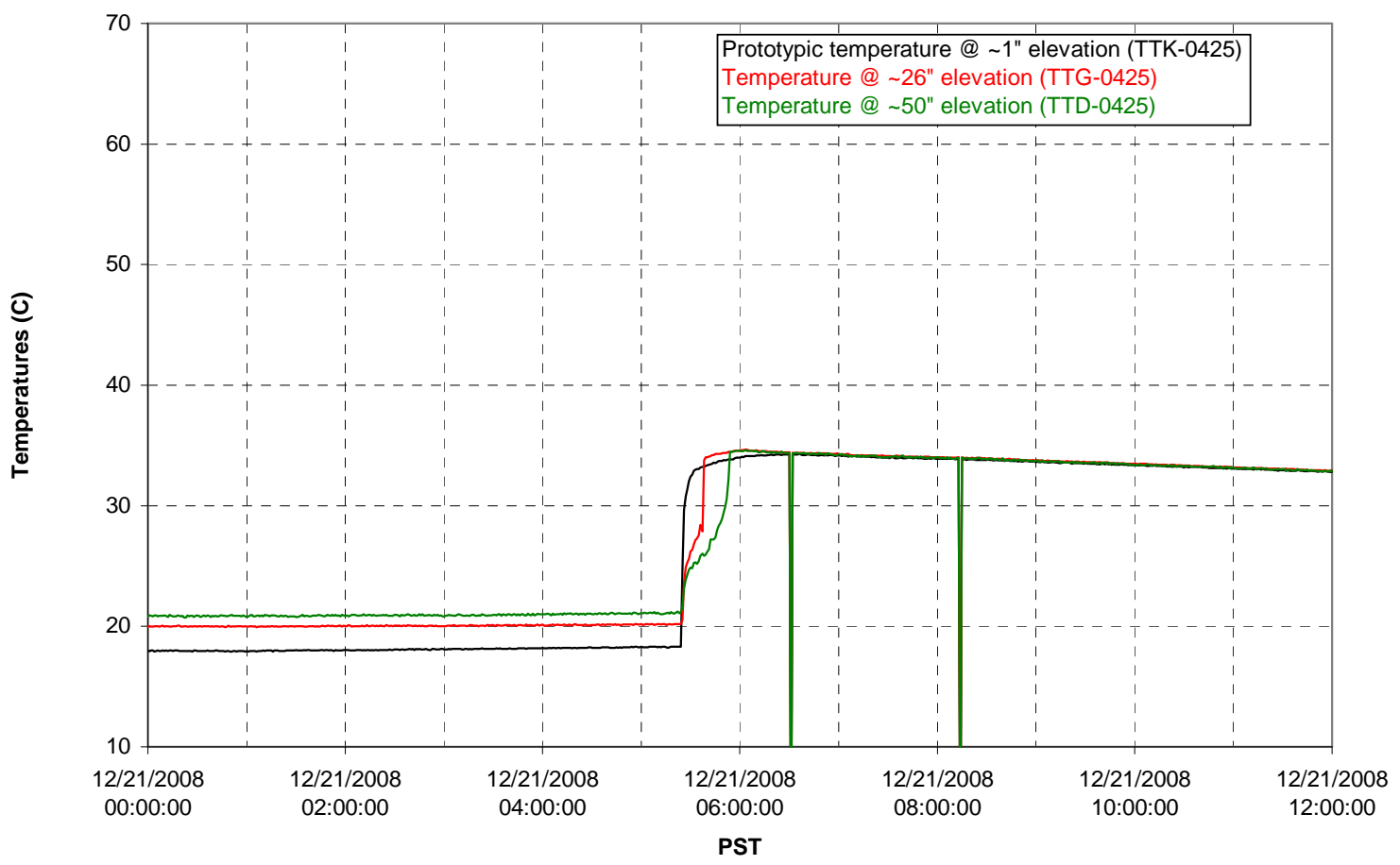


T02A level

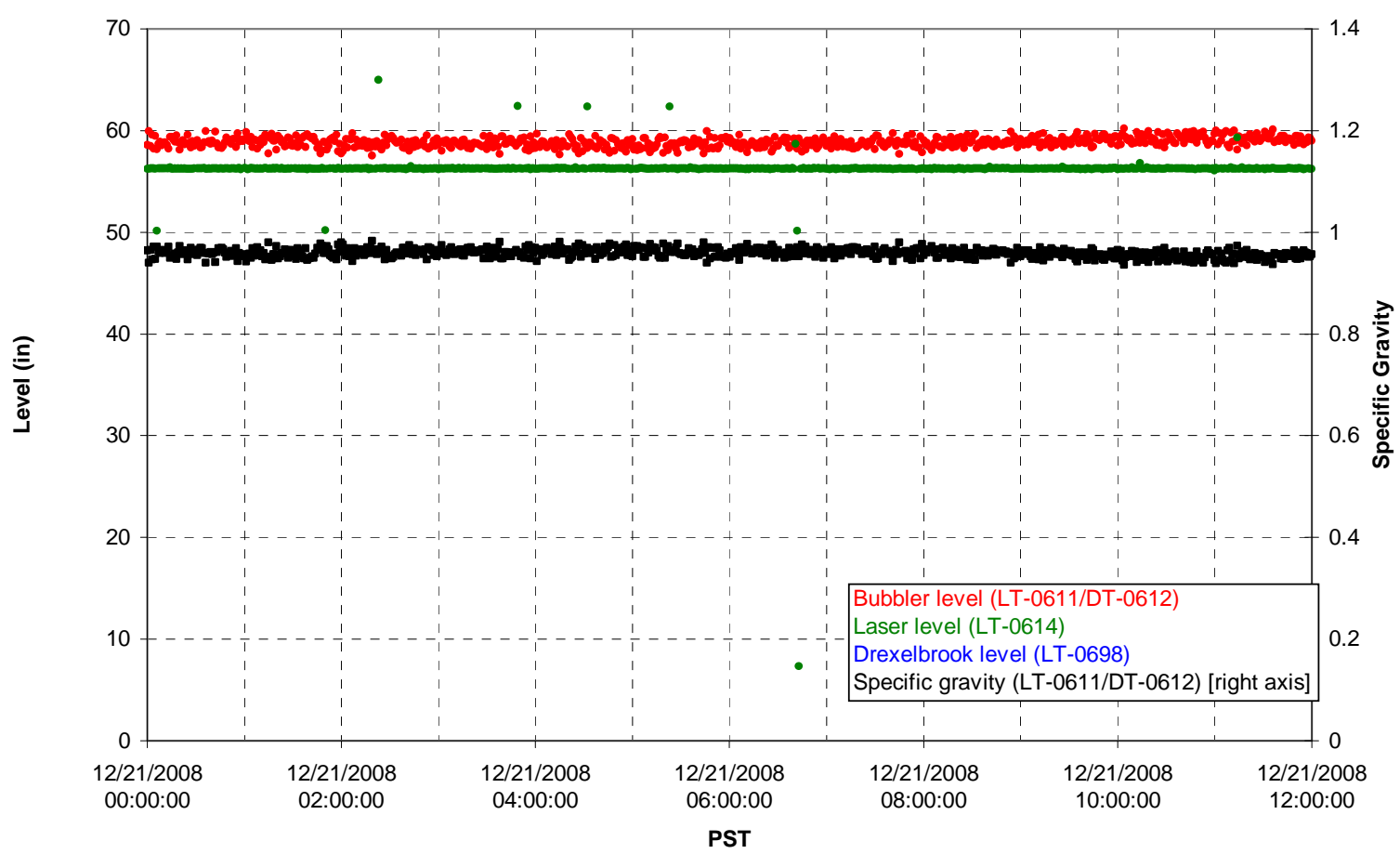

T02A temperatures

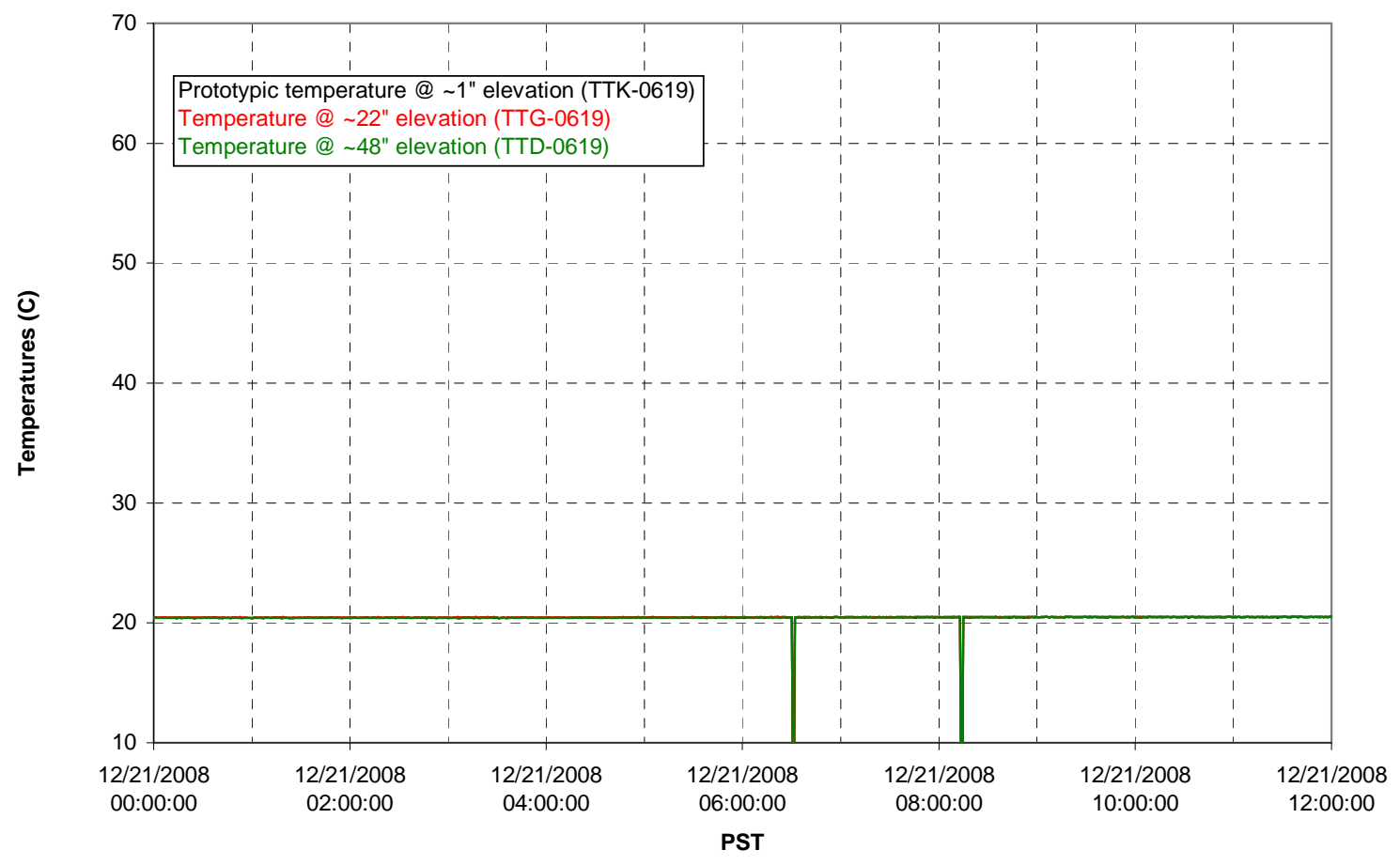


T02A and filter loop temperatures

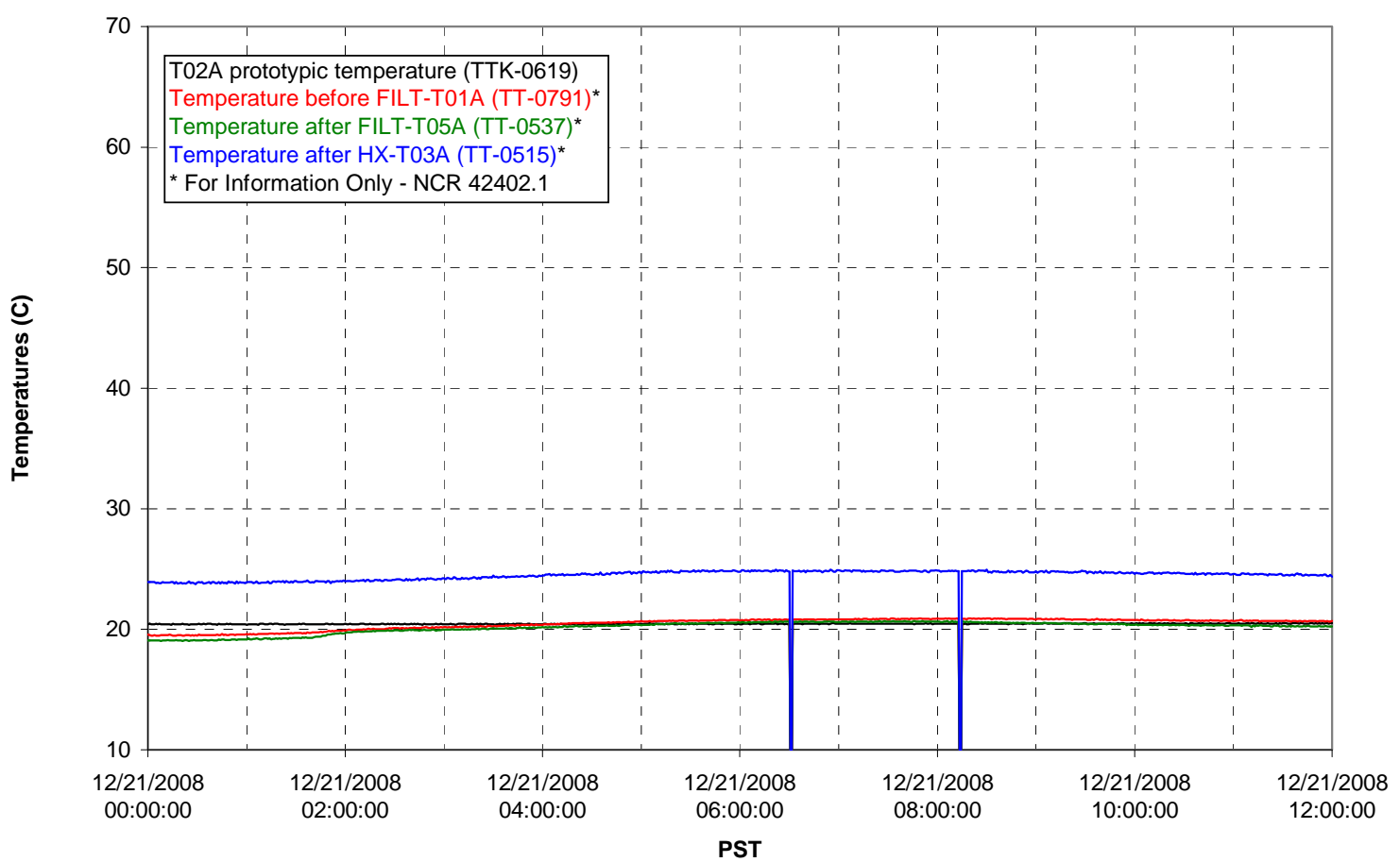

Pump Pressures and Flow

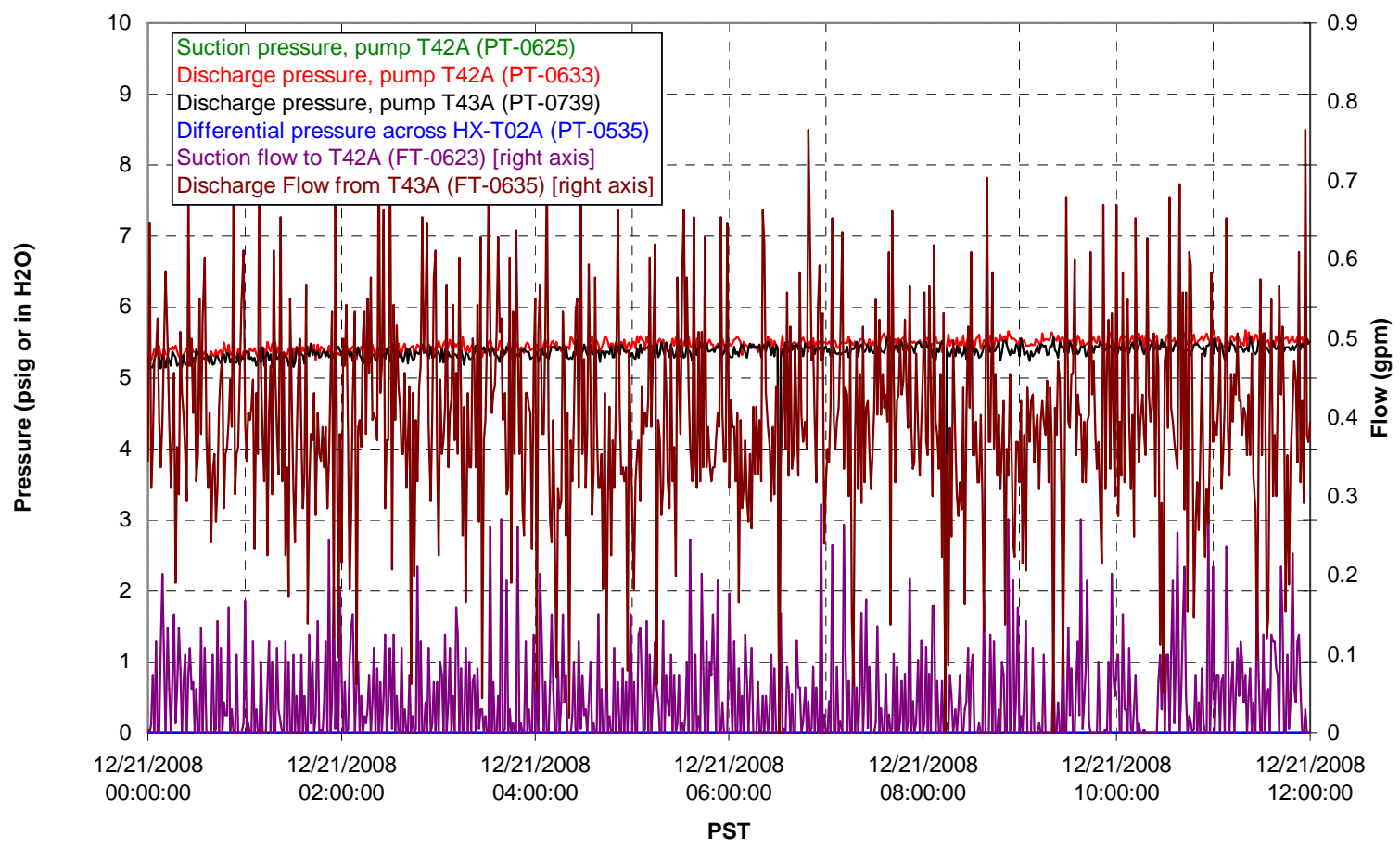


Axial pressure drop

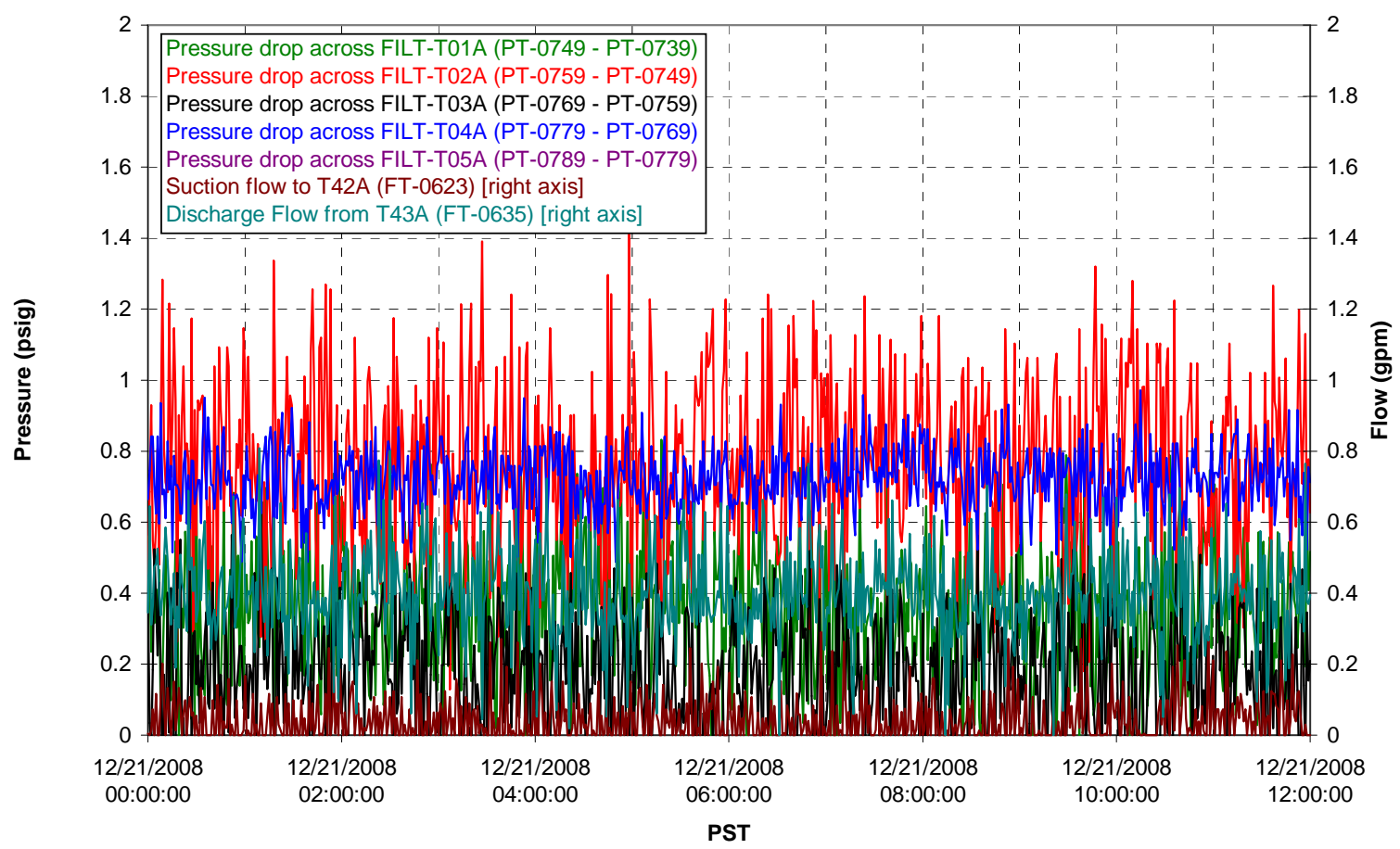

Permeate flow rates

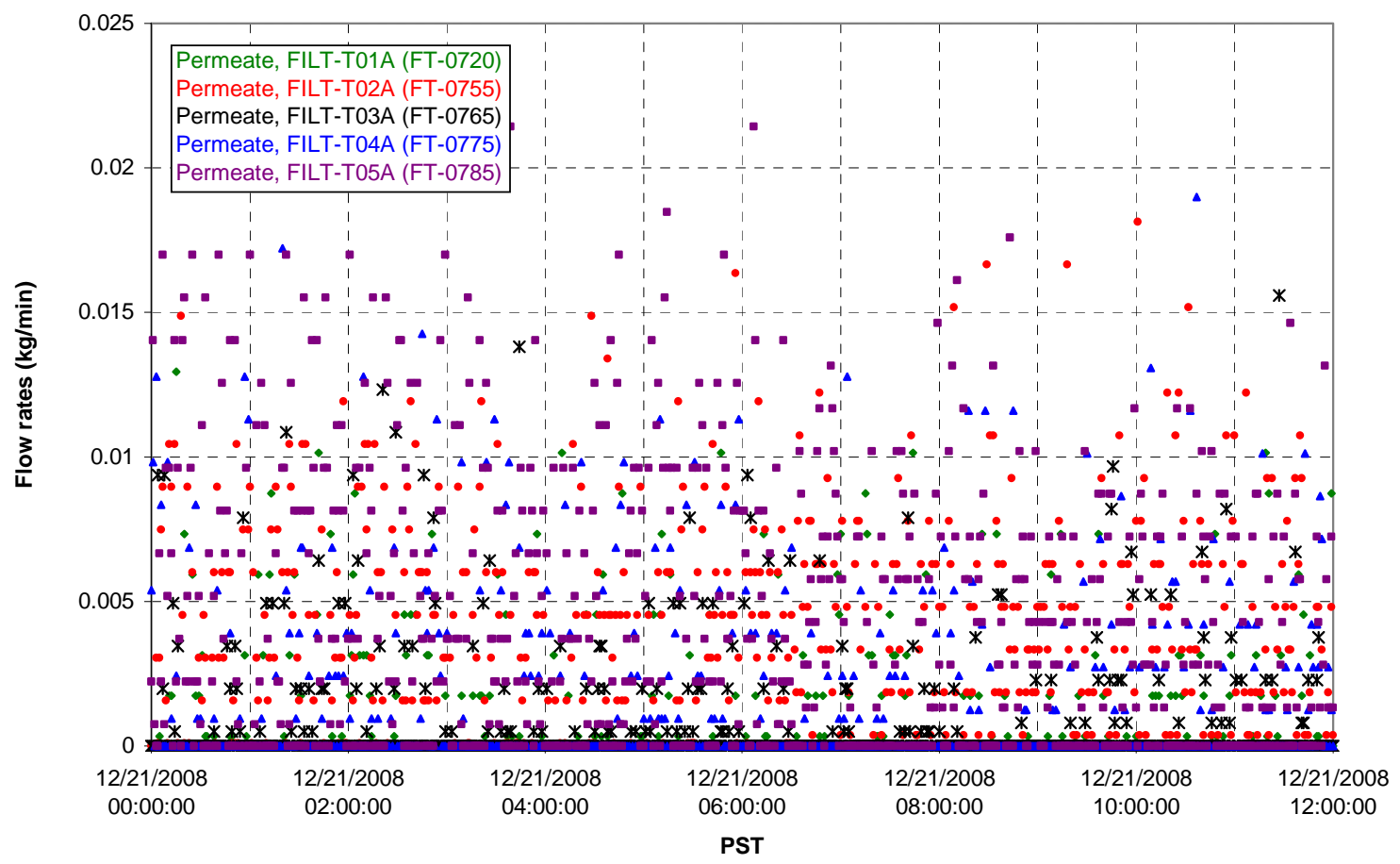


T02A Inner Temperature Tree

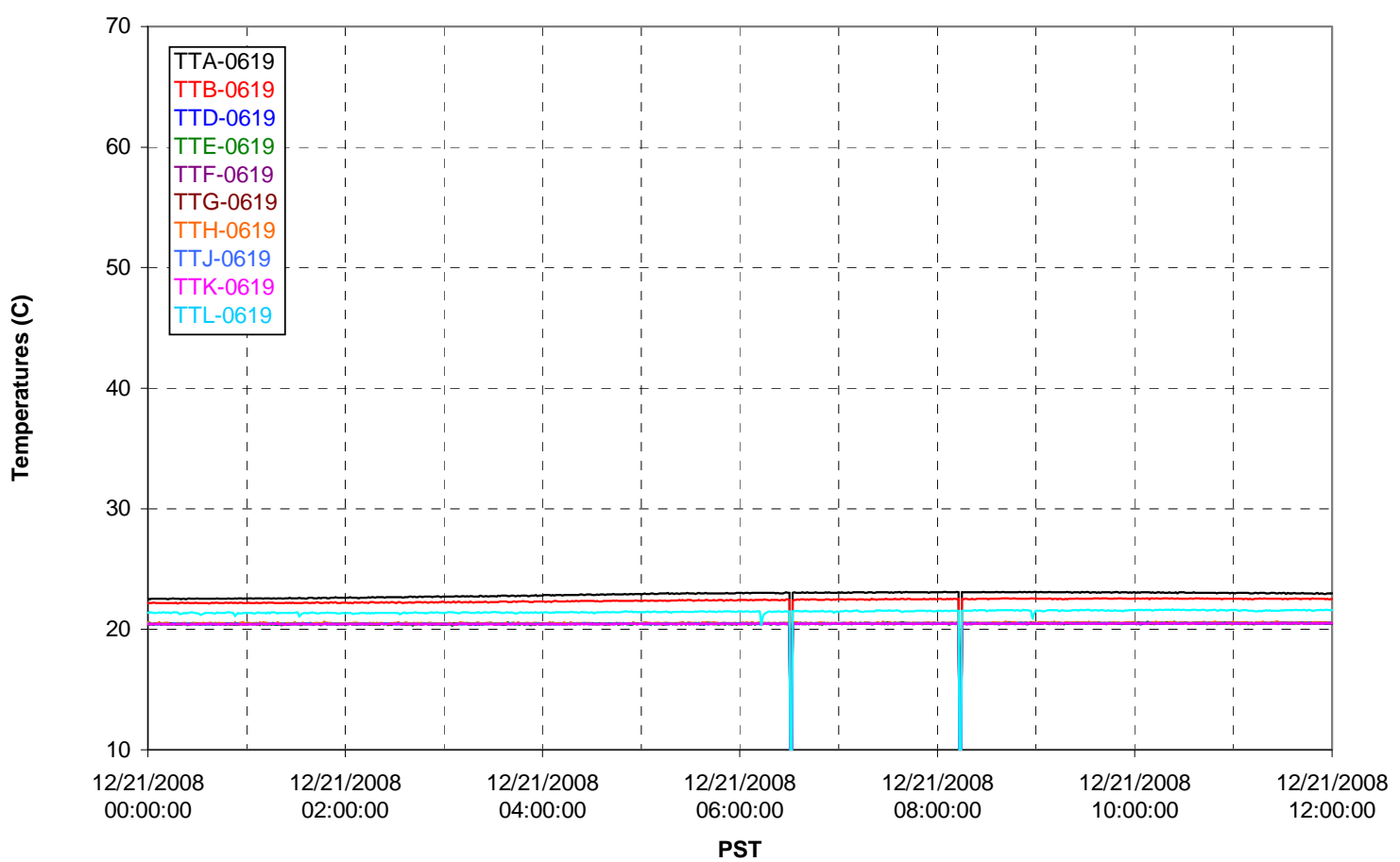

T02A Outer Temperature Tree

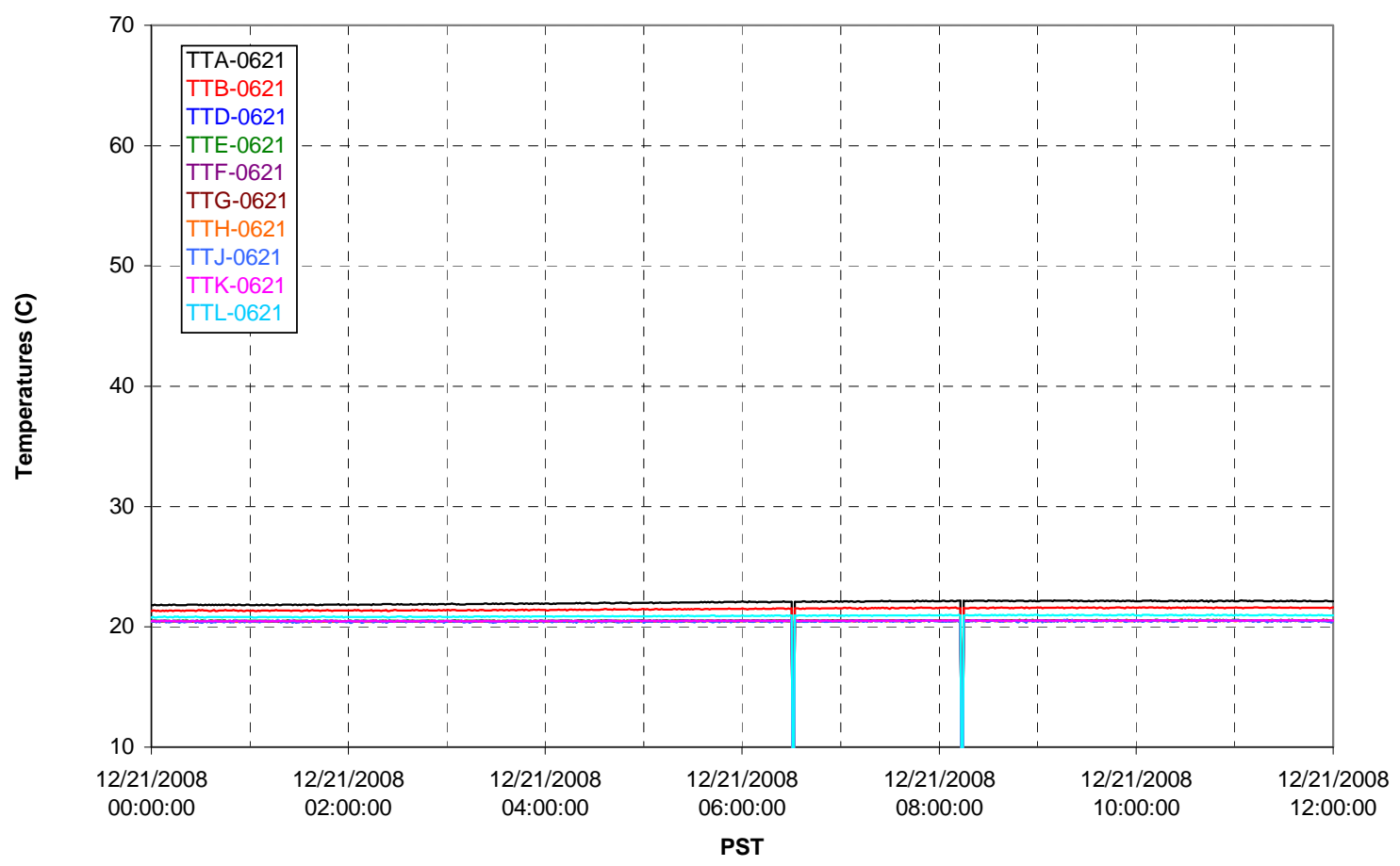


T02A temperatures

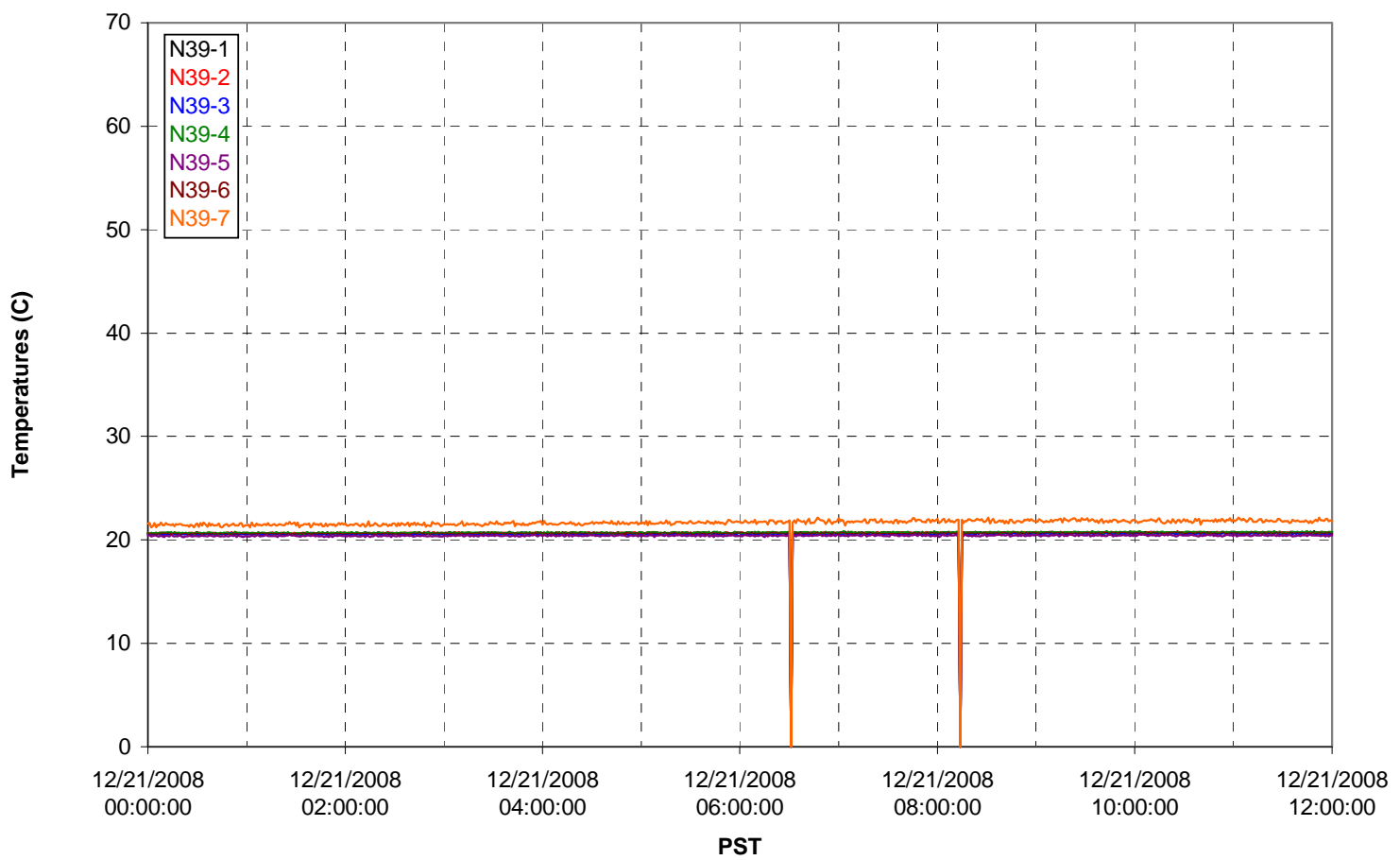

T02A temperatures

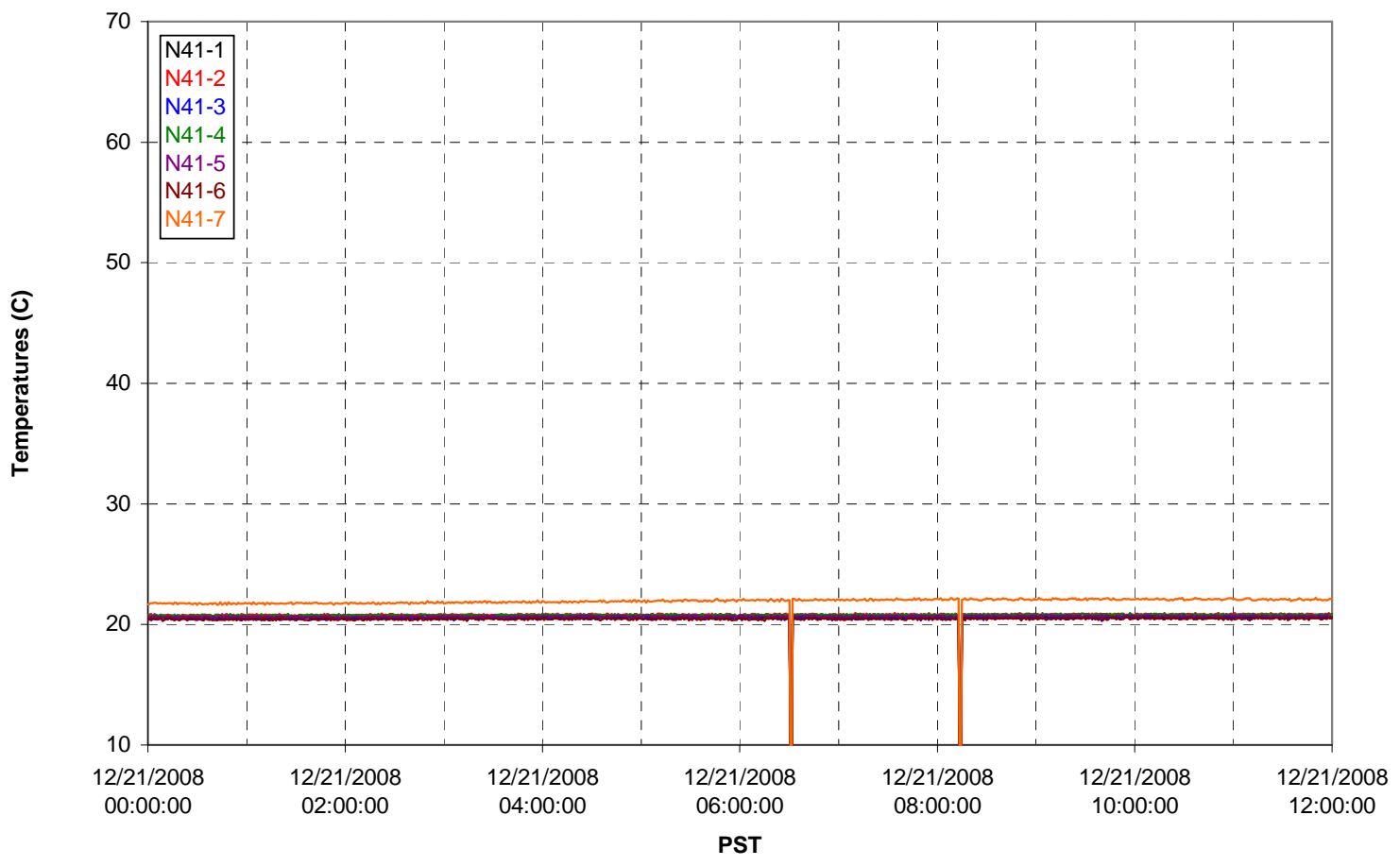


T02A temperatures

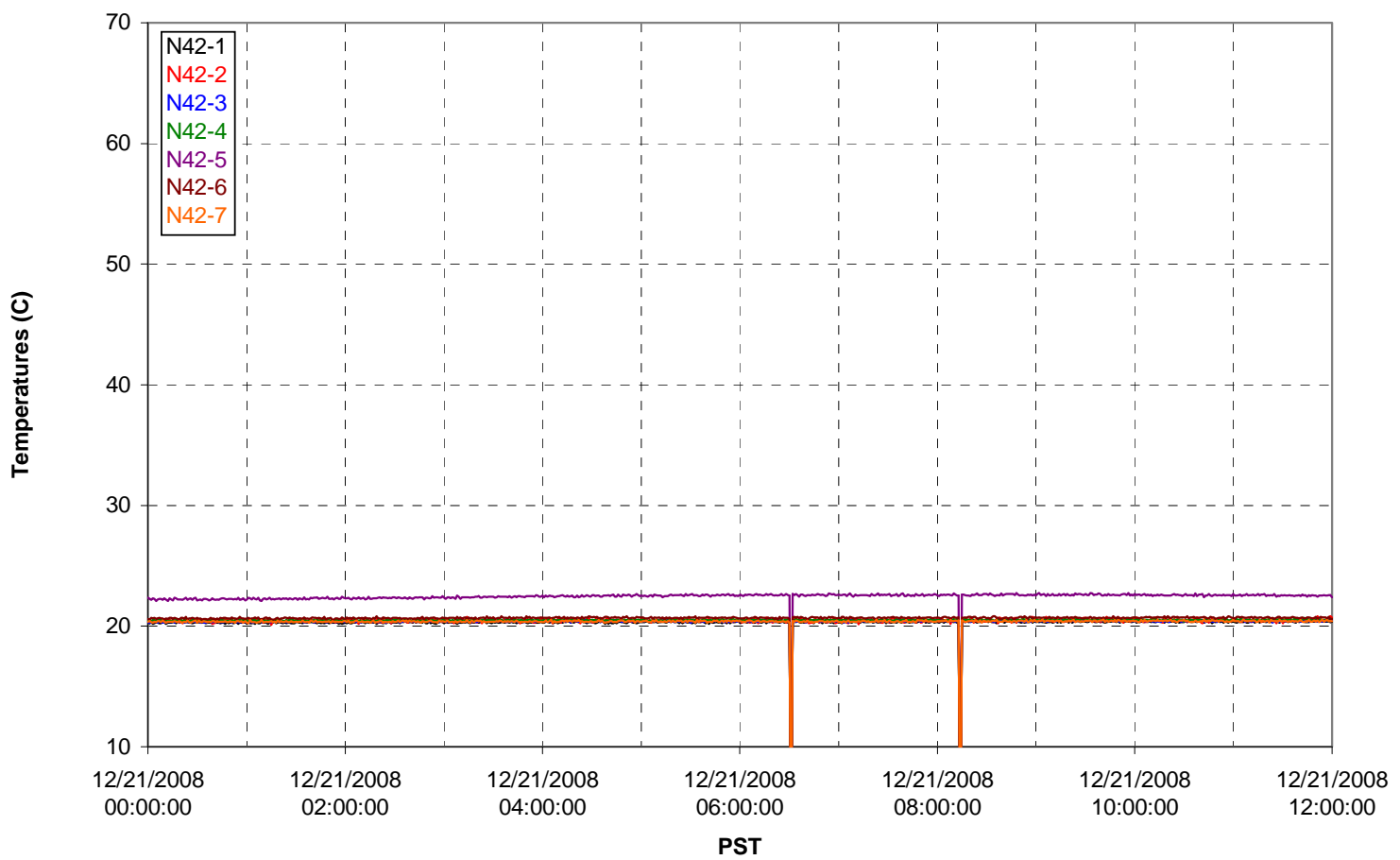

T02A temperatures

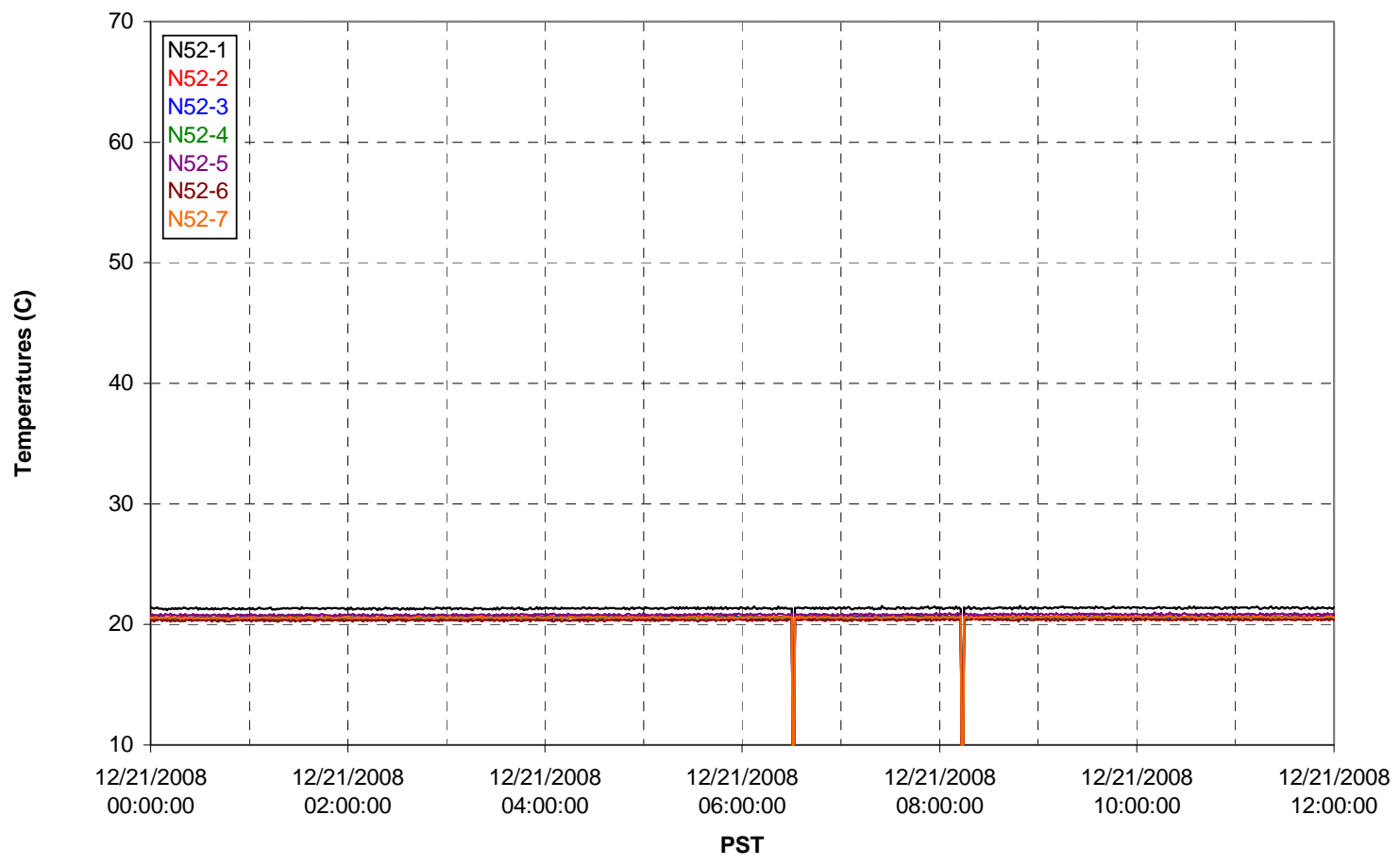


T02A Heating and Cooling

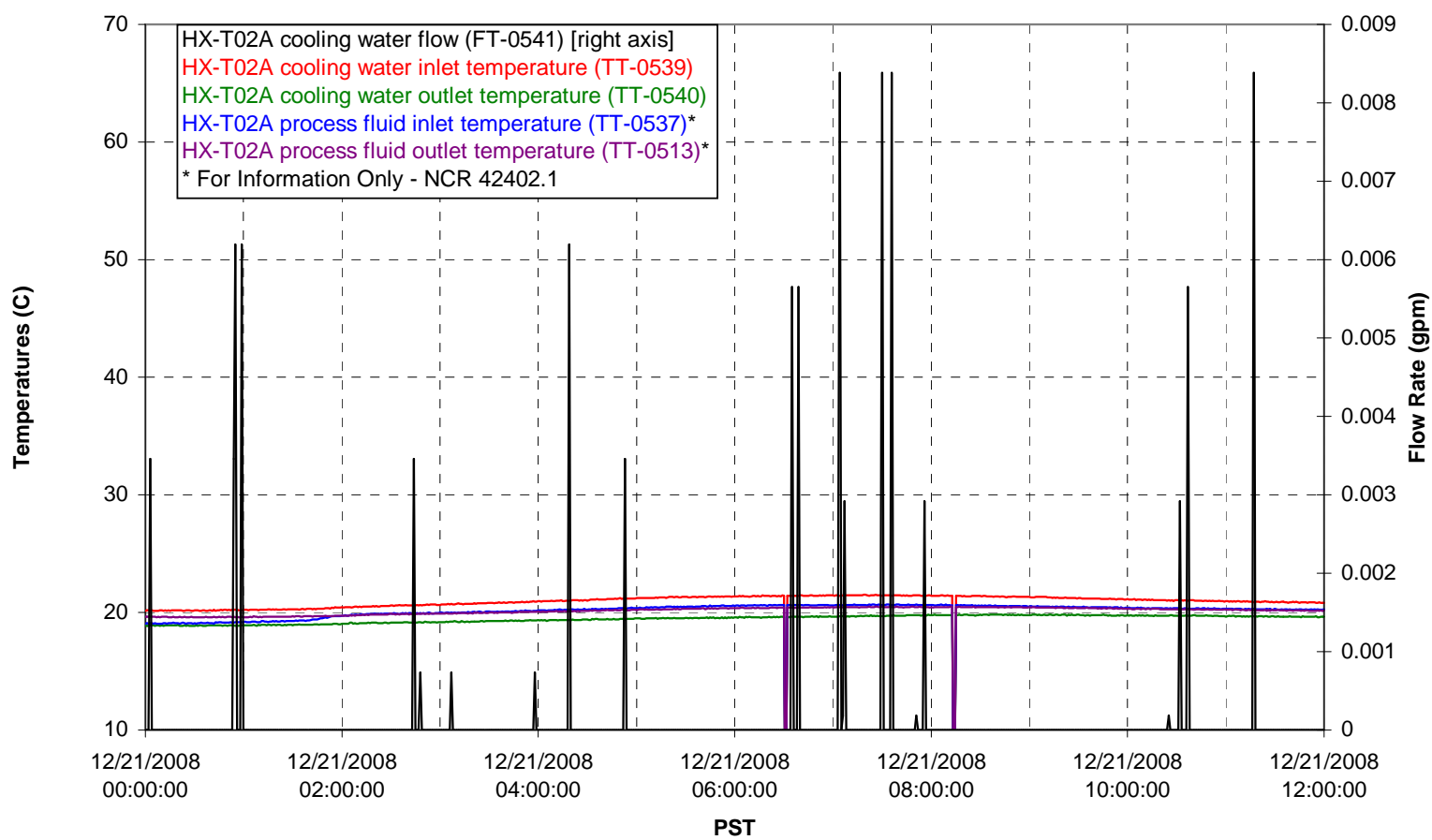

Pump Operation

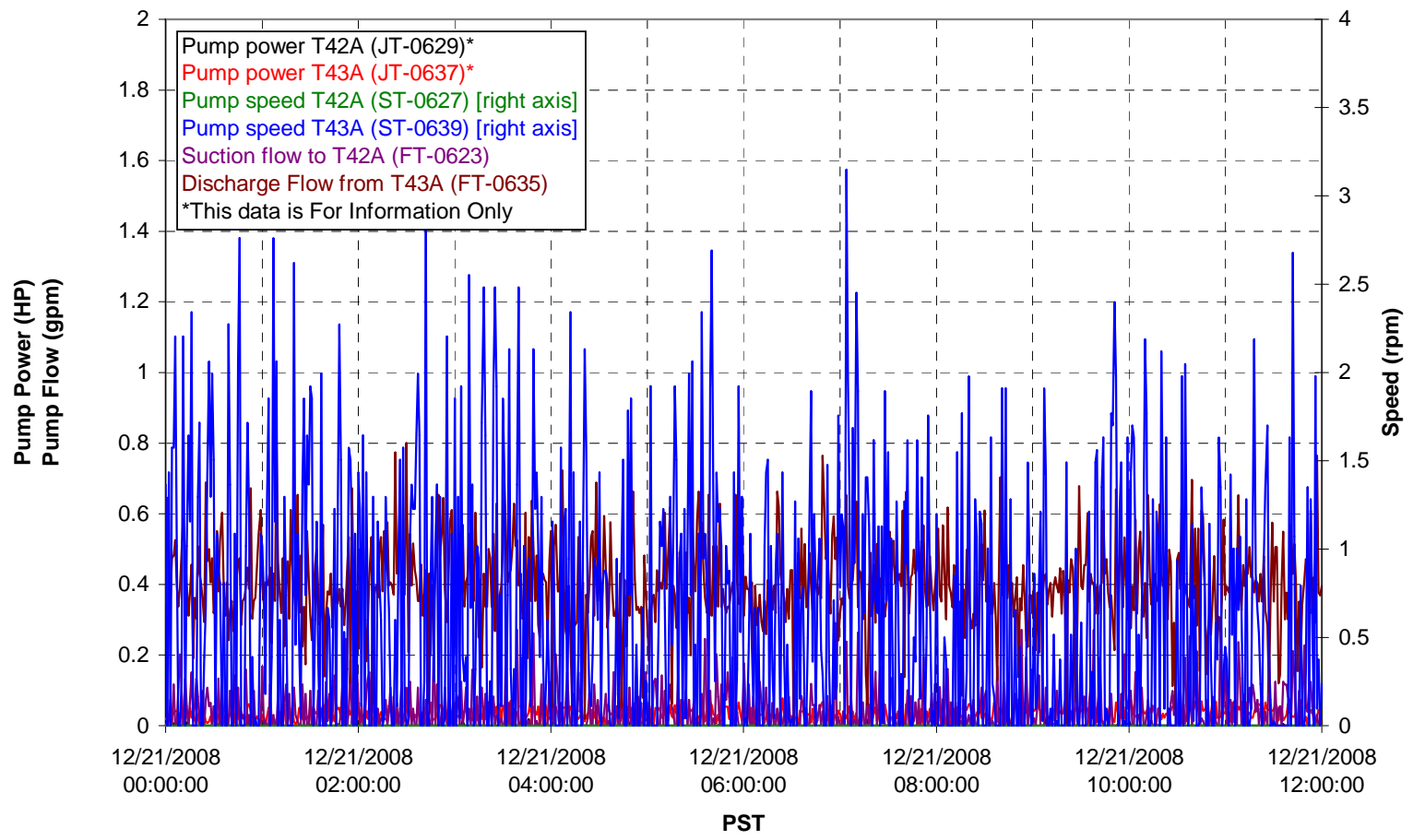


Pulsepot UFP-PP-T01A

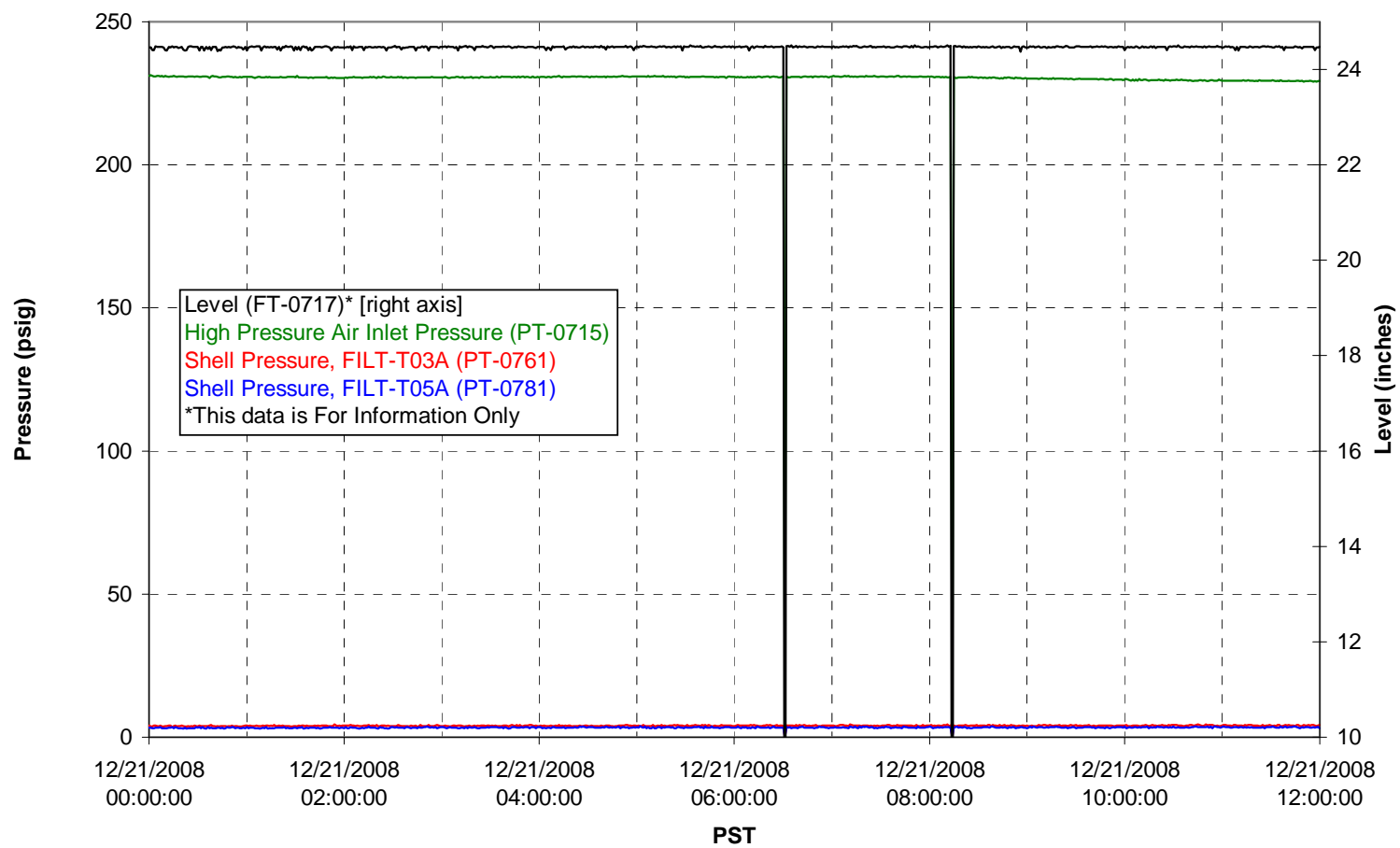

Pulsepot UFP-PP-T02A

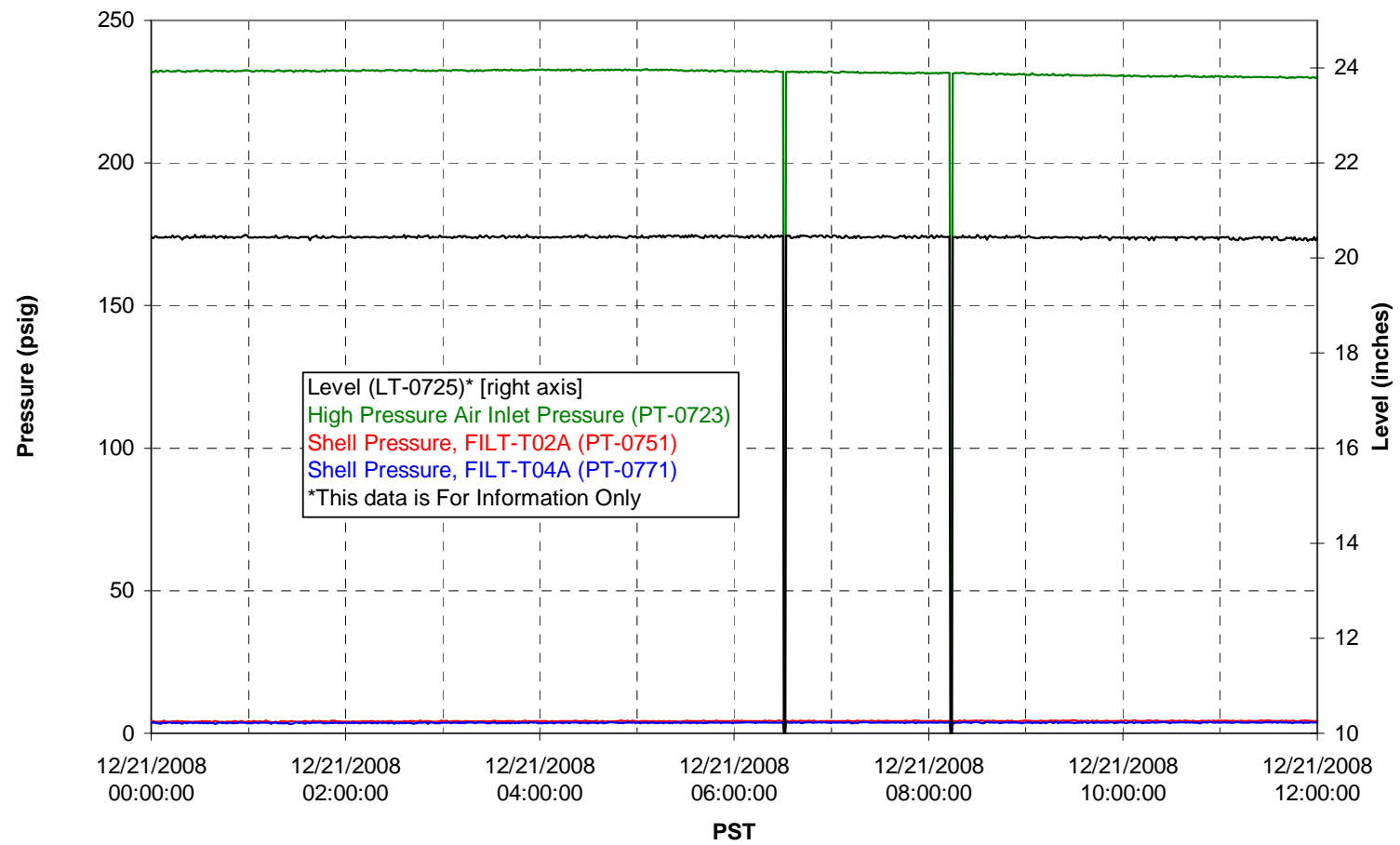


Pulsepot UFP-PP-T03A

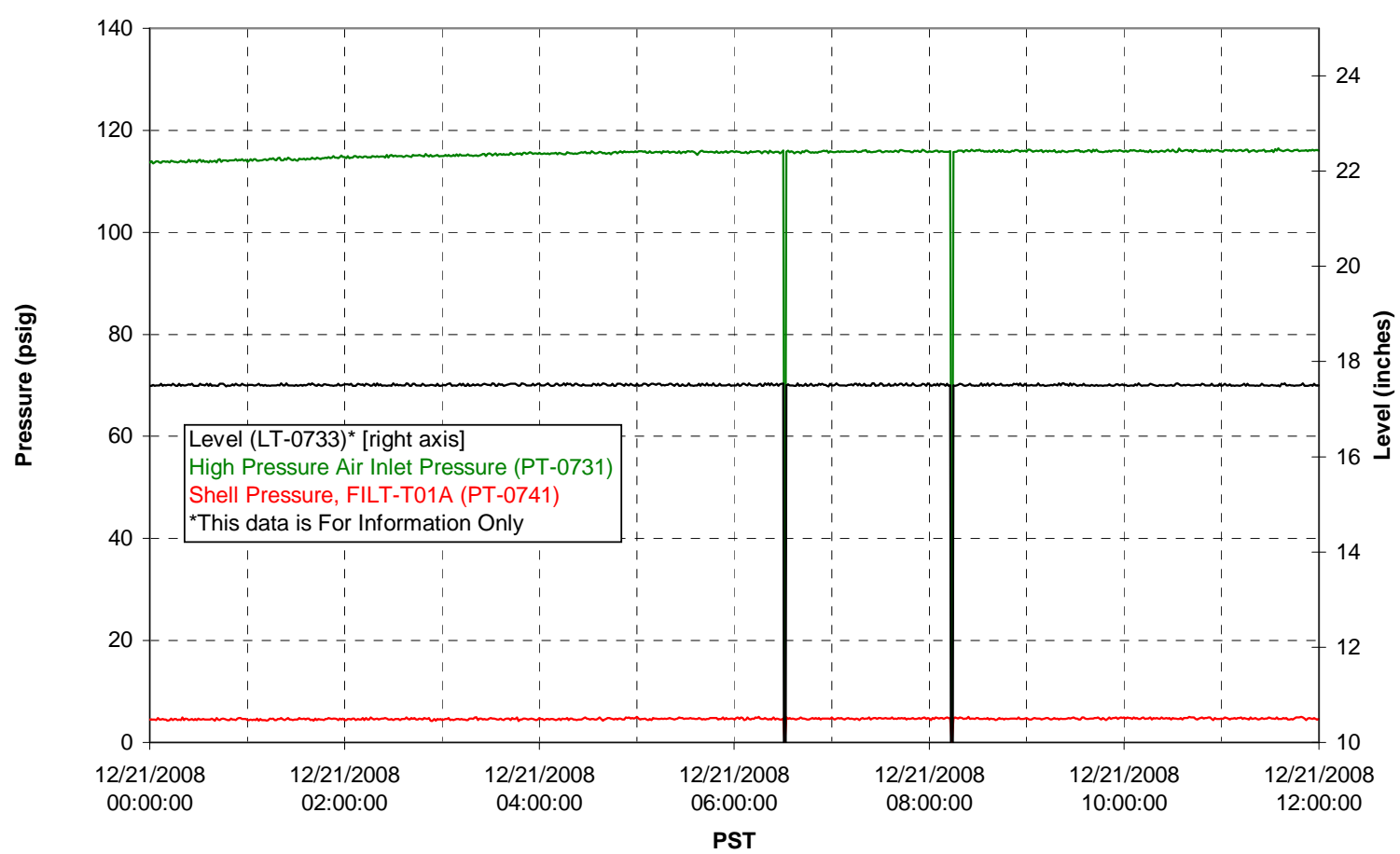

Pulsepot Levels

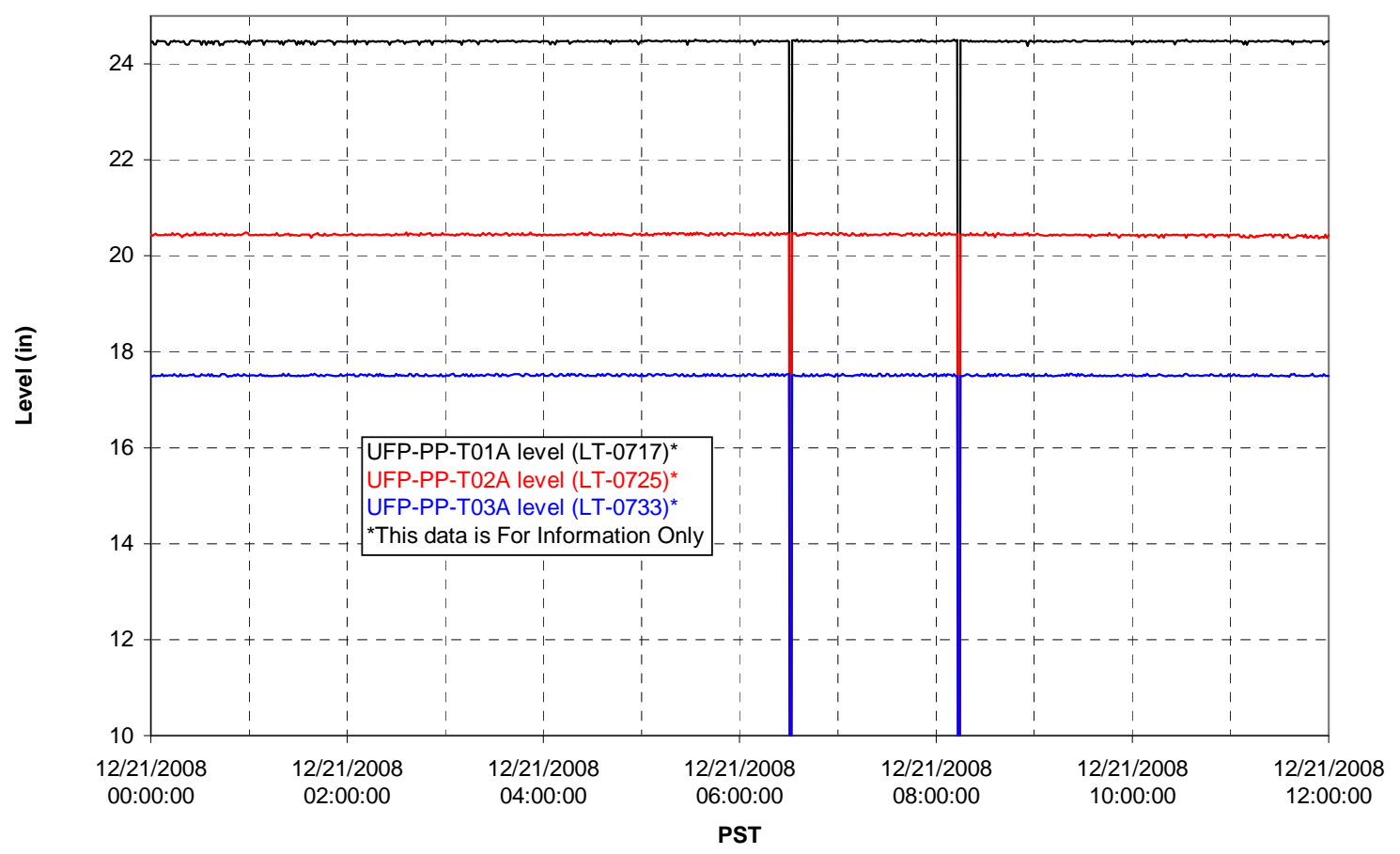


Filter UFP-FILT-T01A

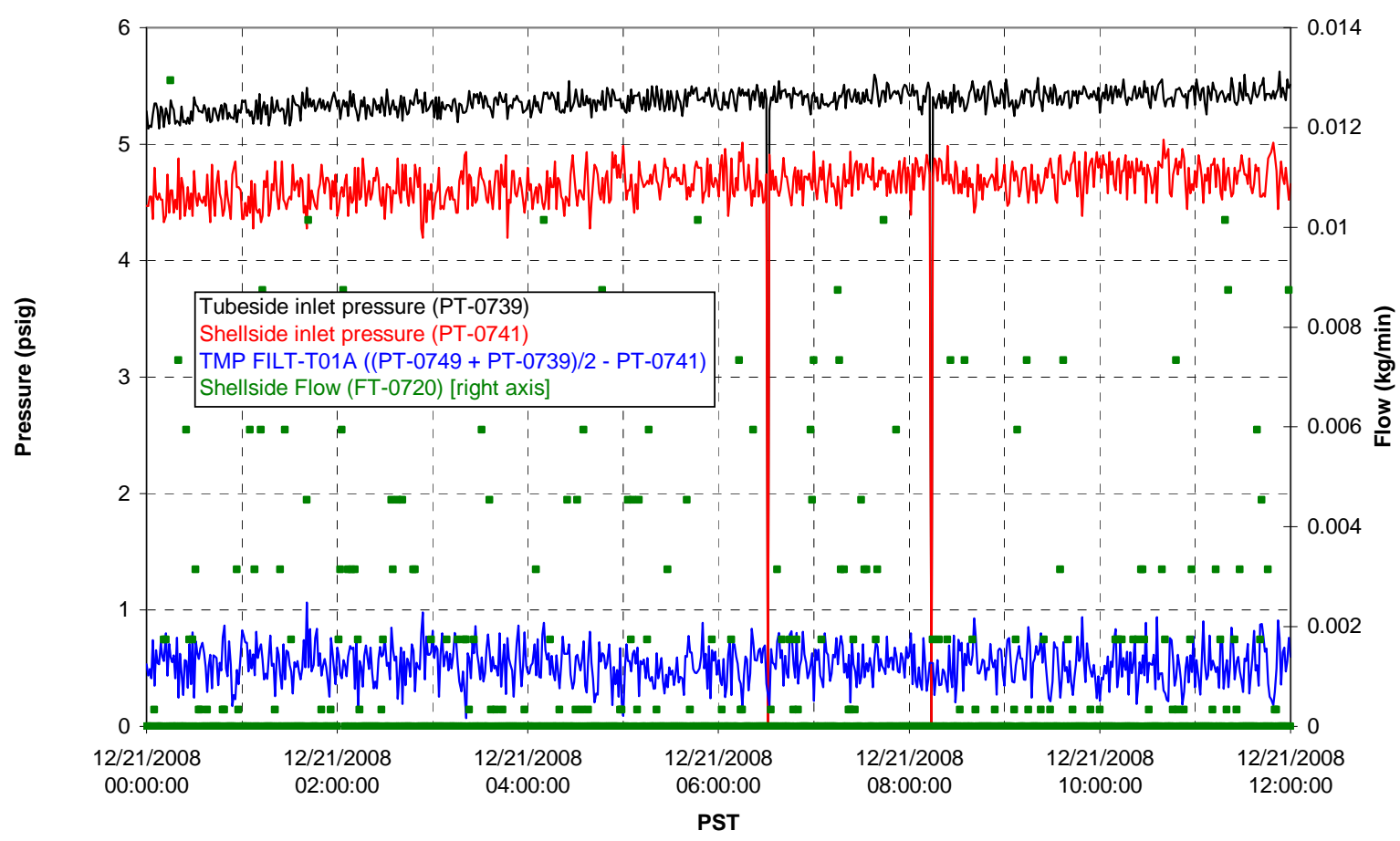

Filter UFP-FILT-T02A

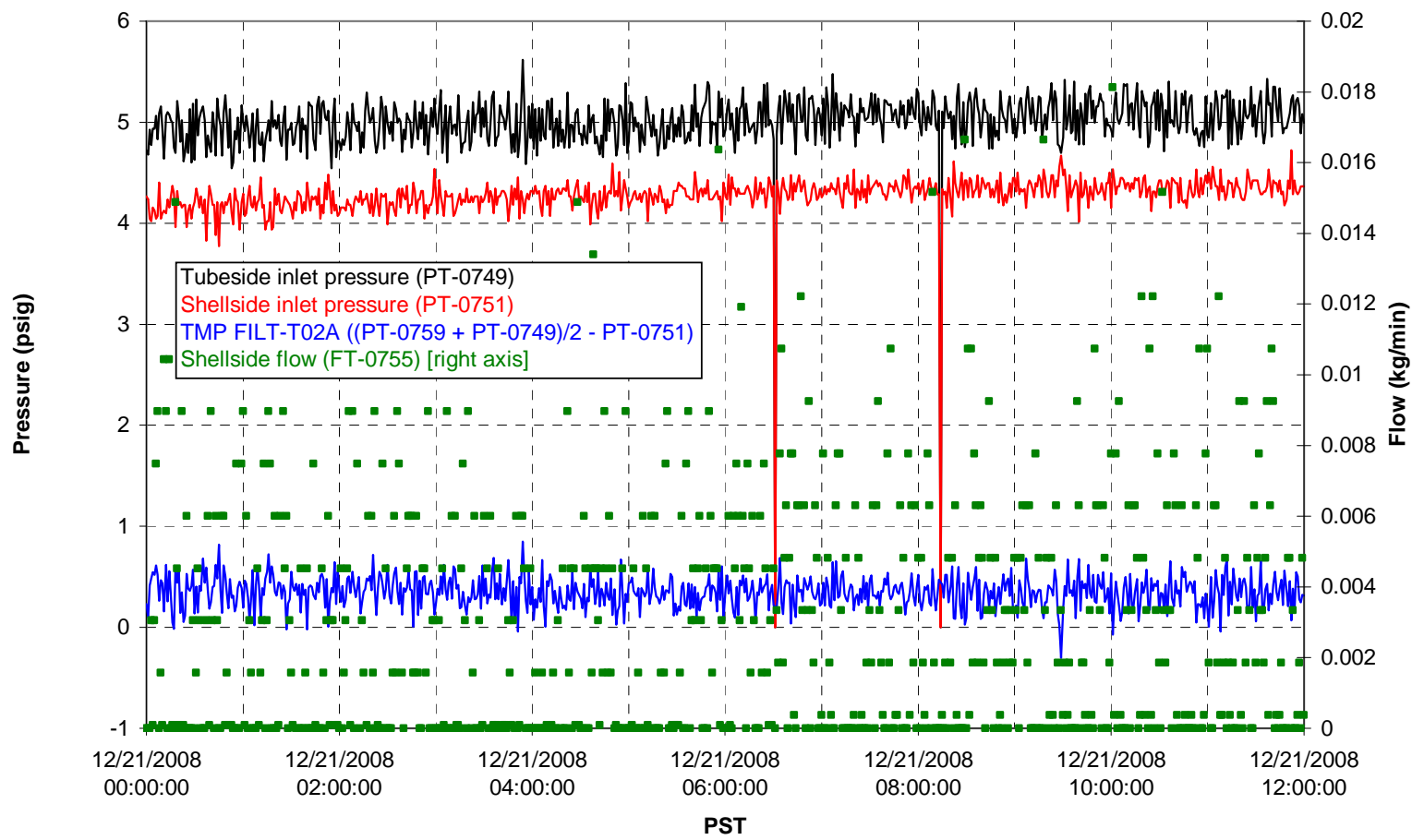


Filter UFP-FILT-T03A

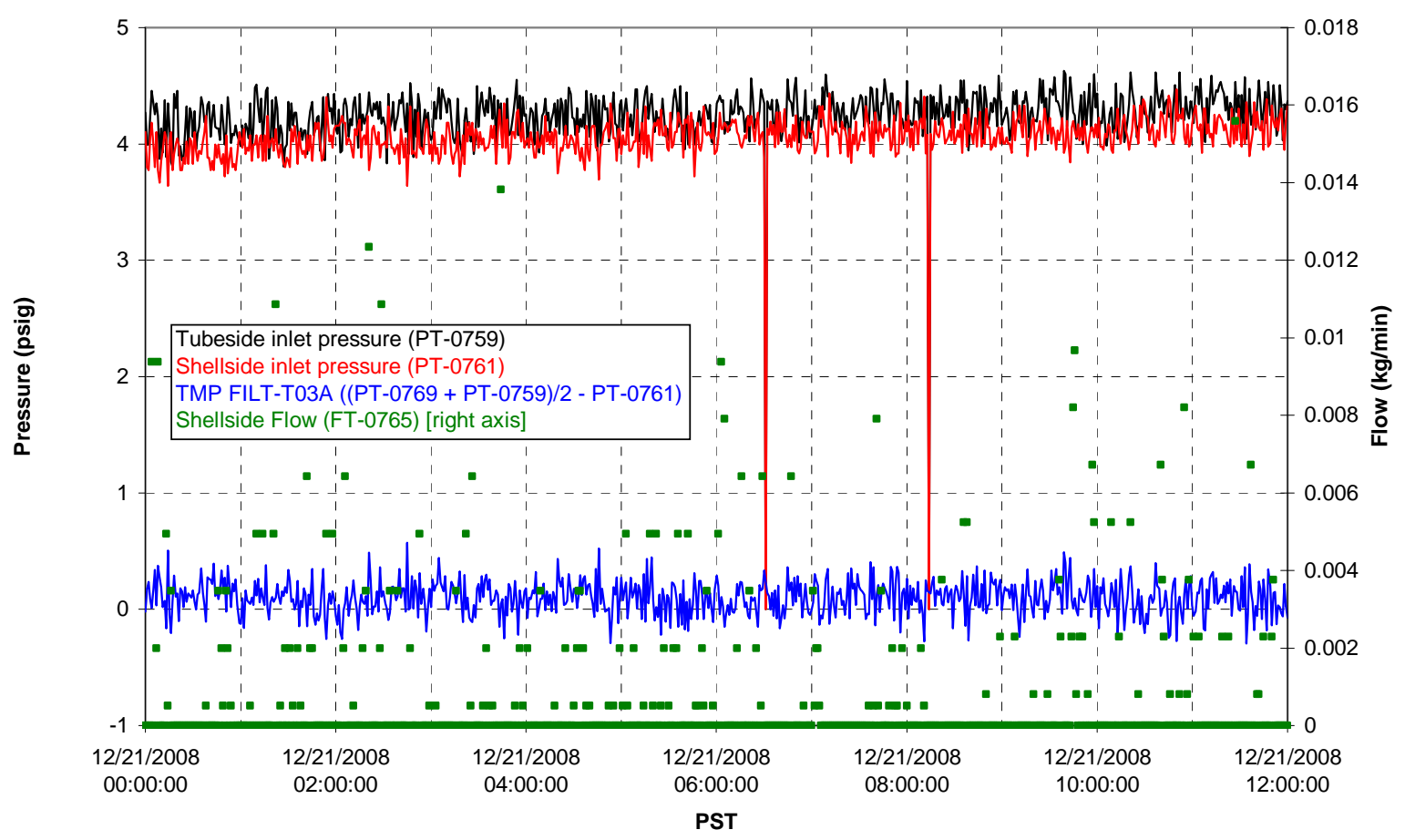

Filter UFP-FILT-T04A

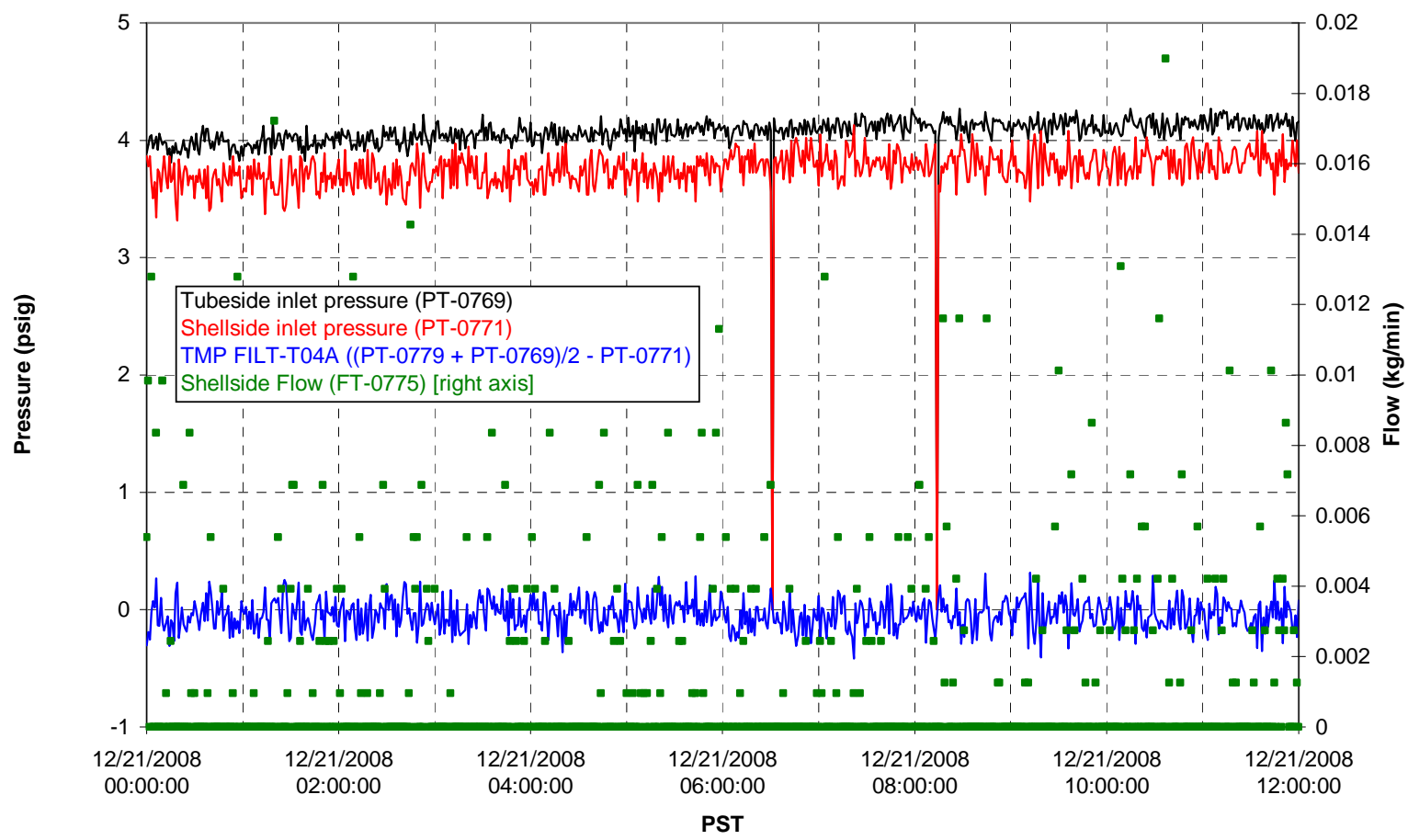


Filter UFP-FILT-T05A

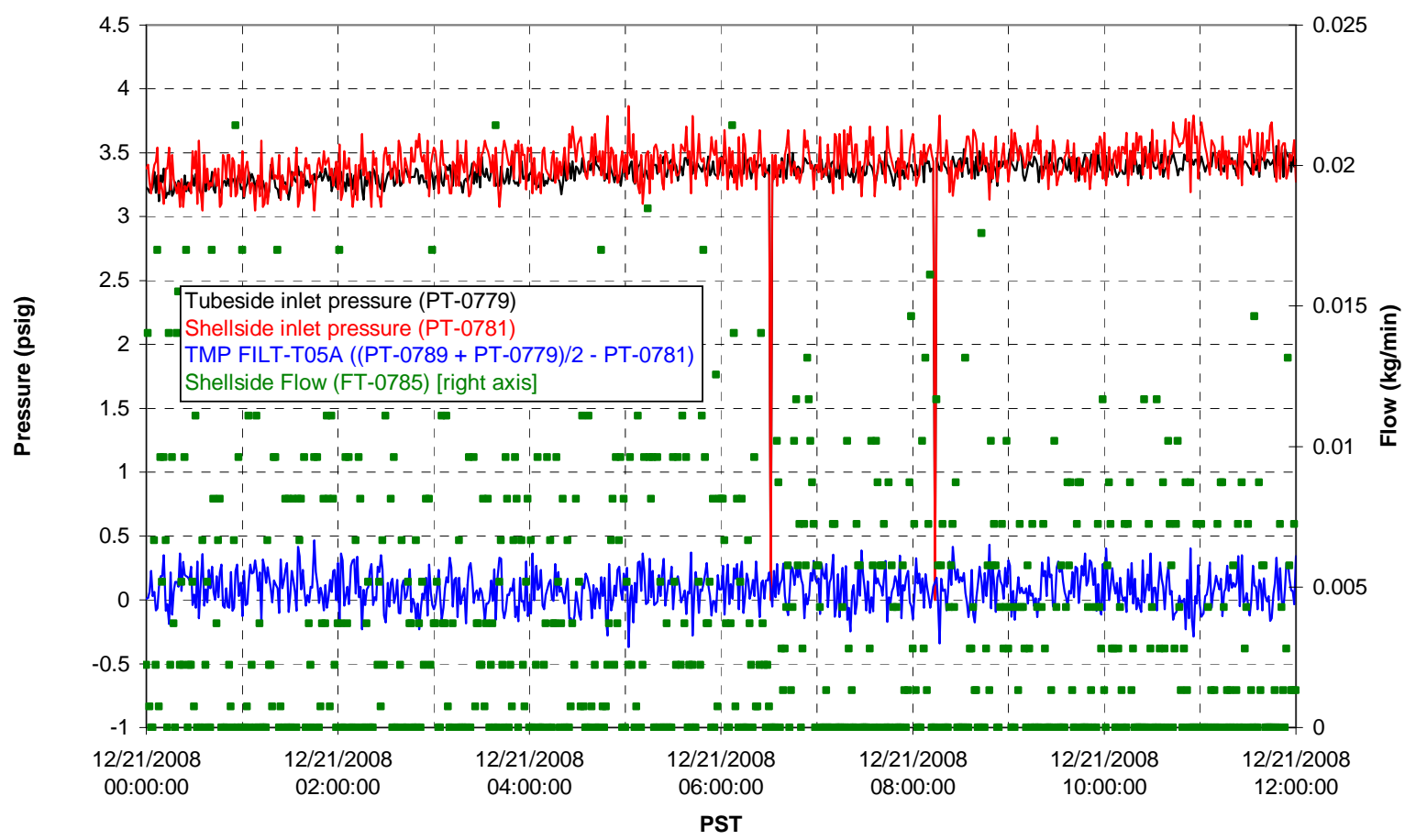

Chemical Flow

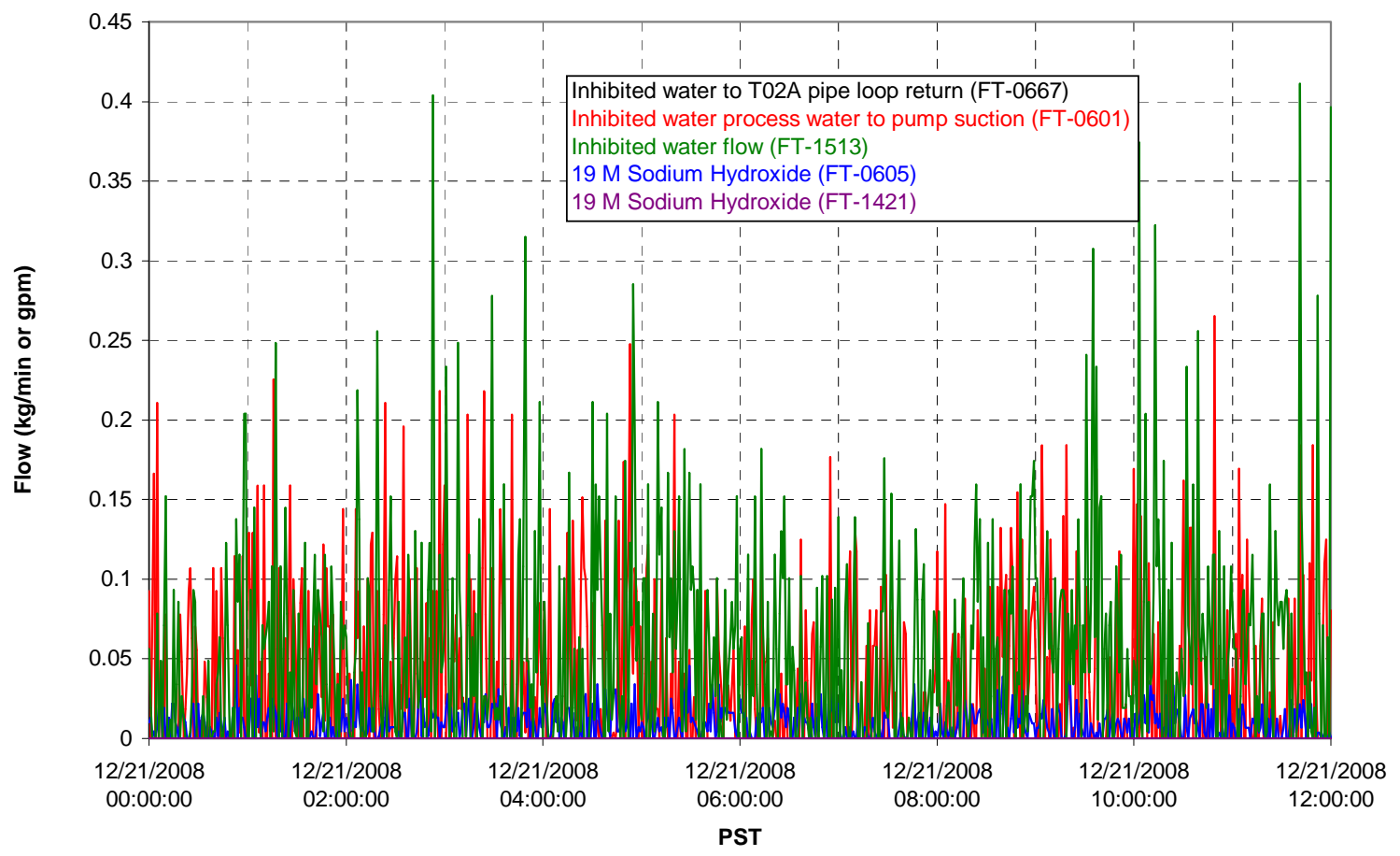




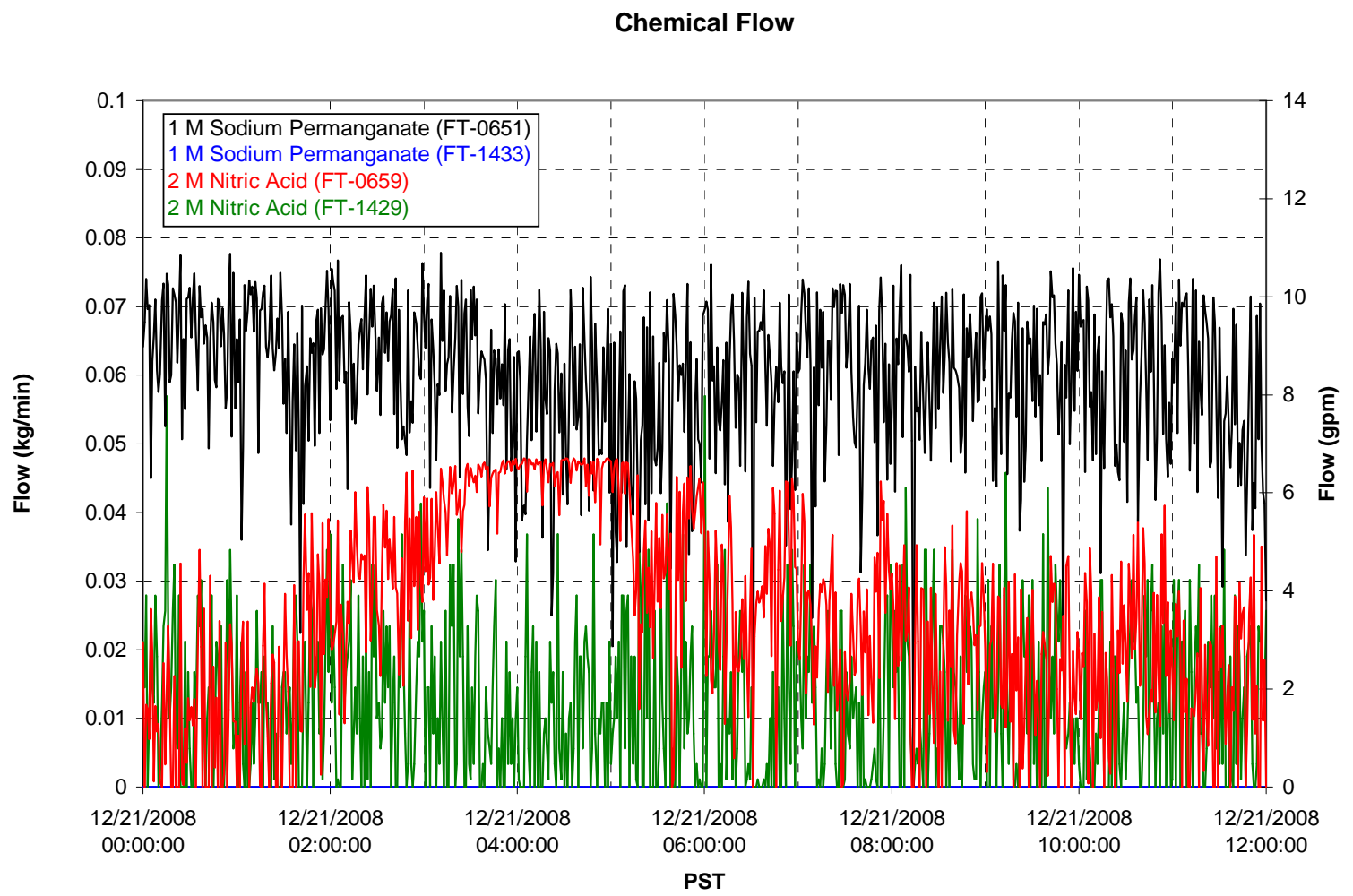

Air Flows

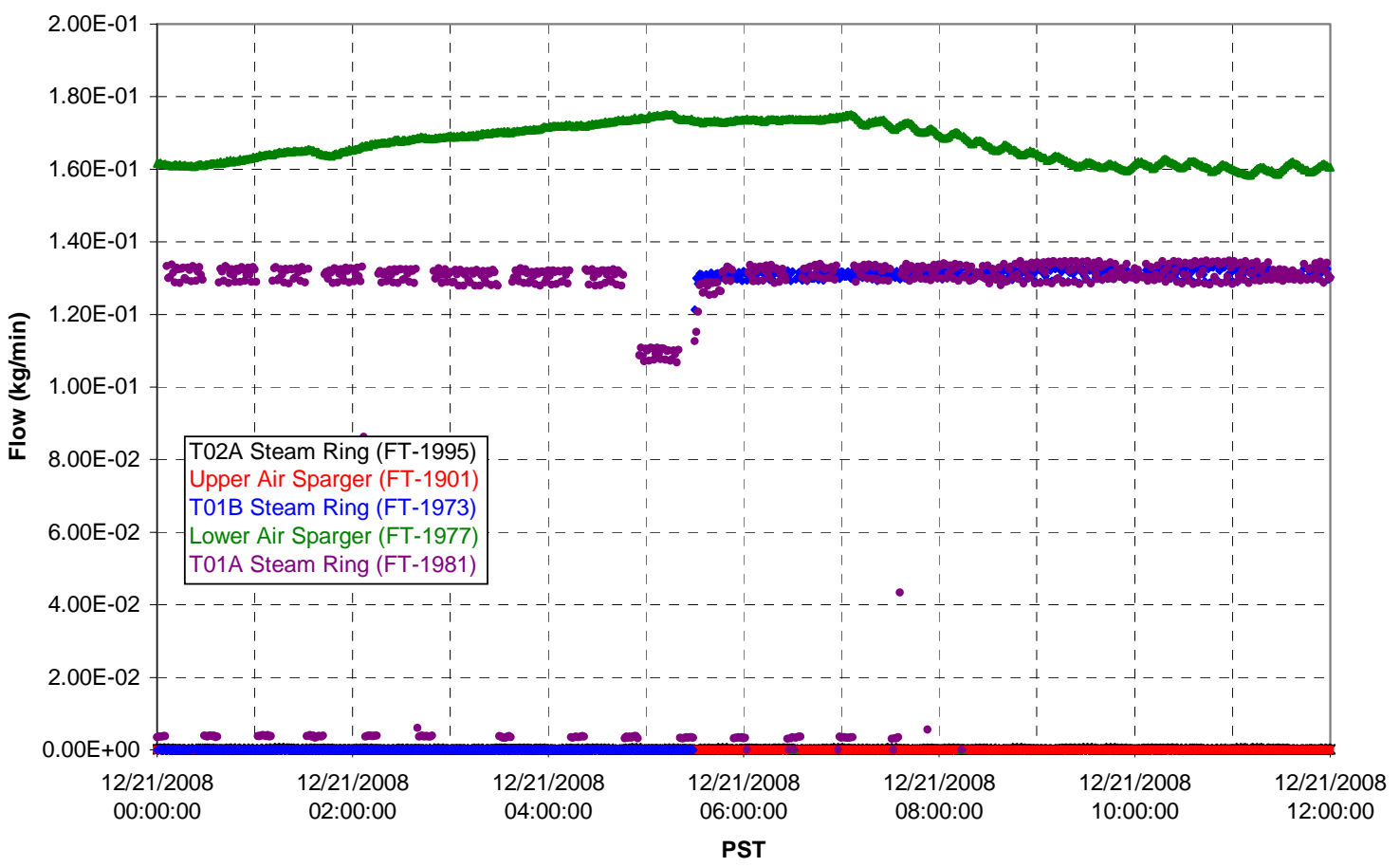


T02A Steam

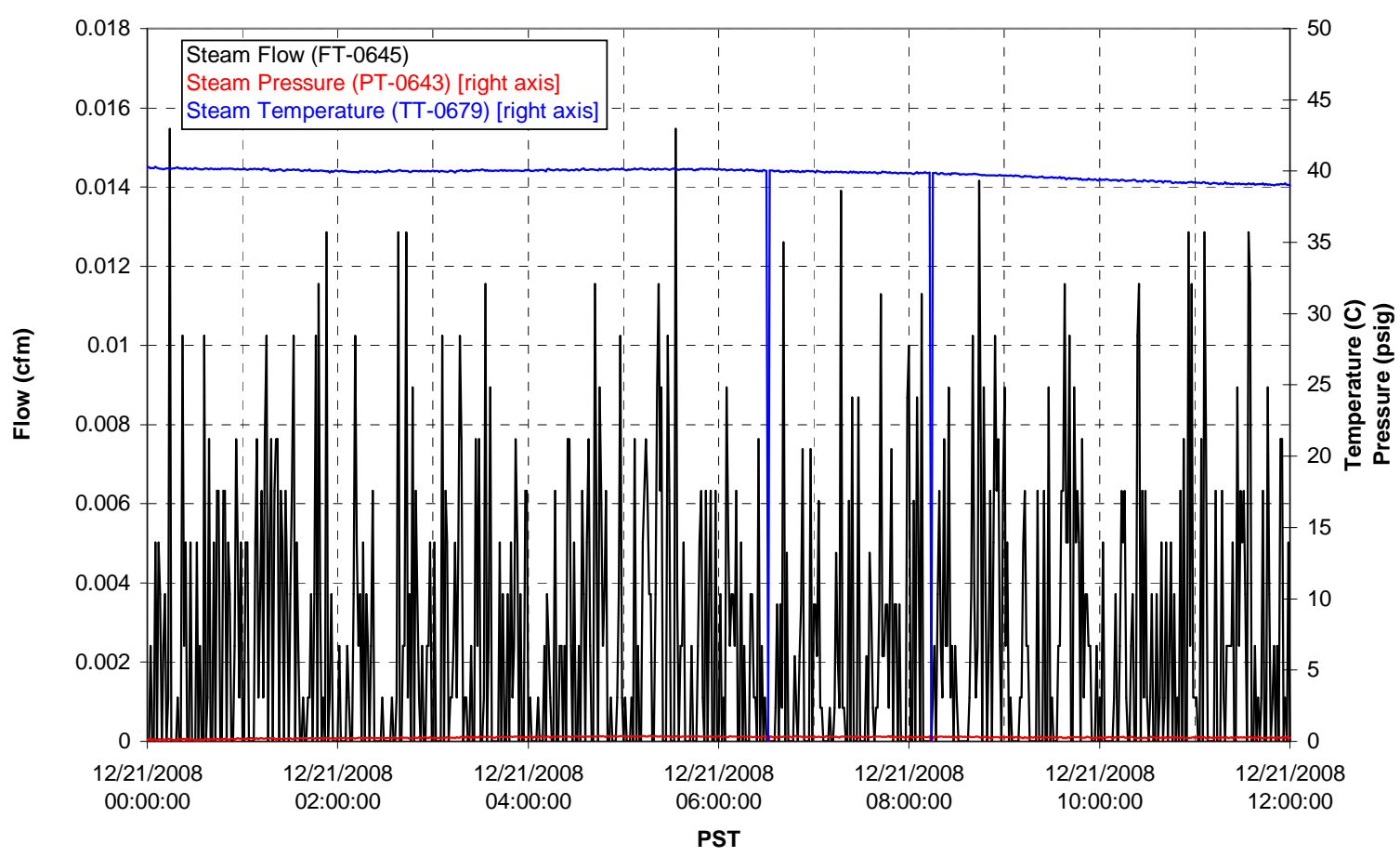

T01A Steam

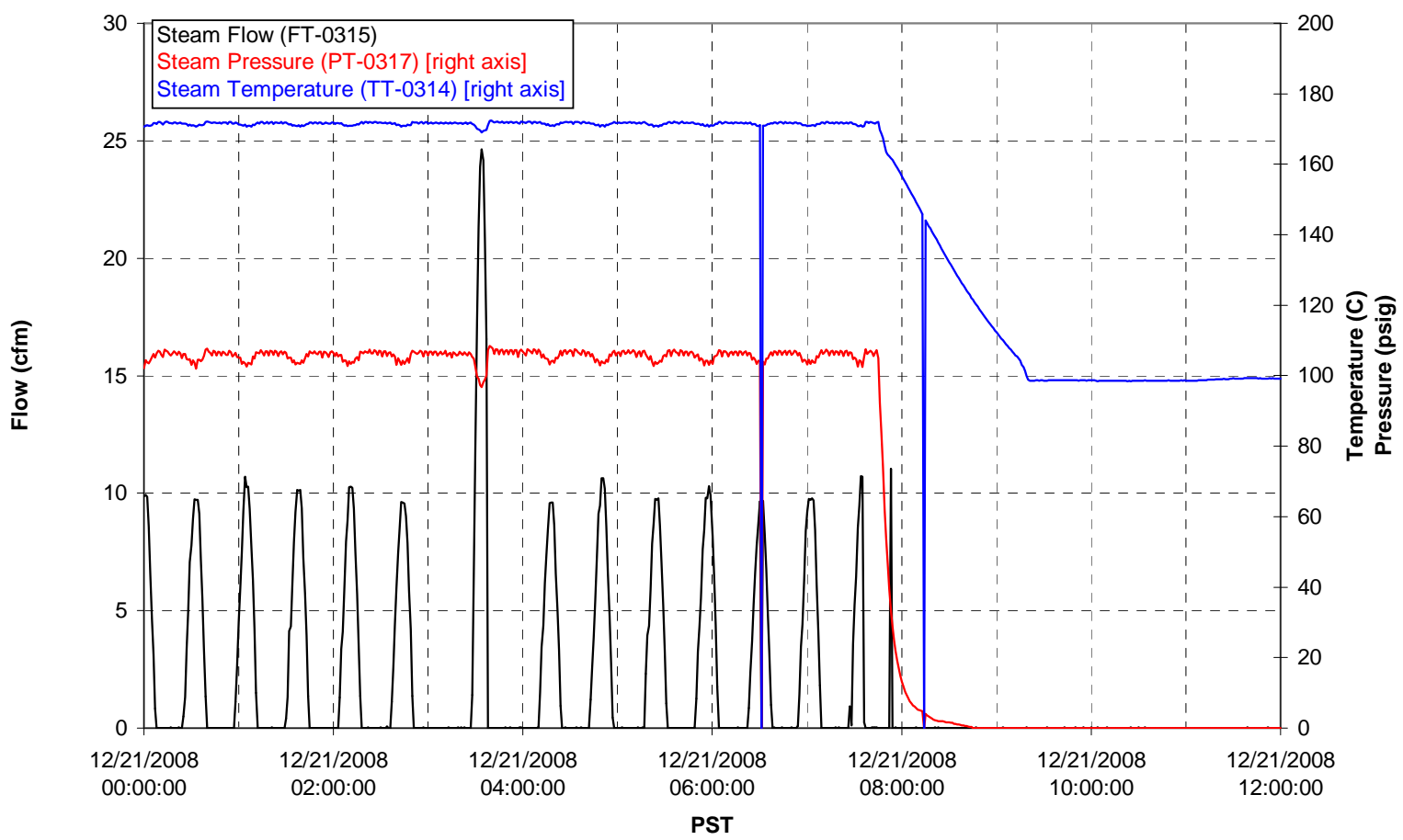


T01B Steam

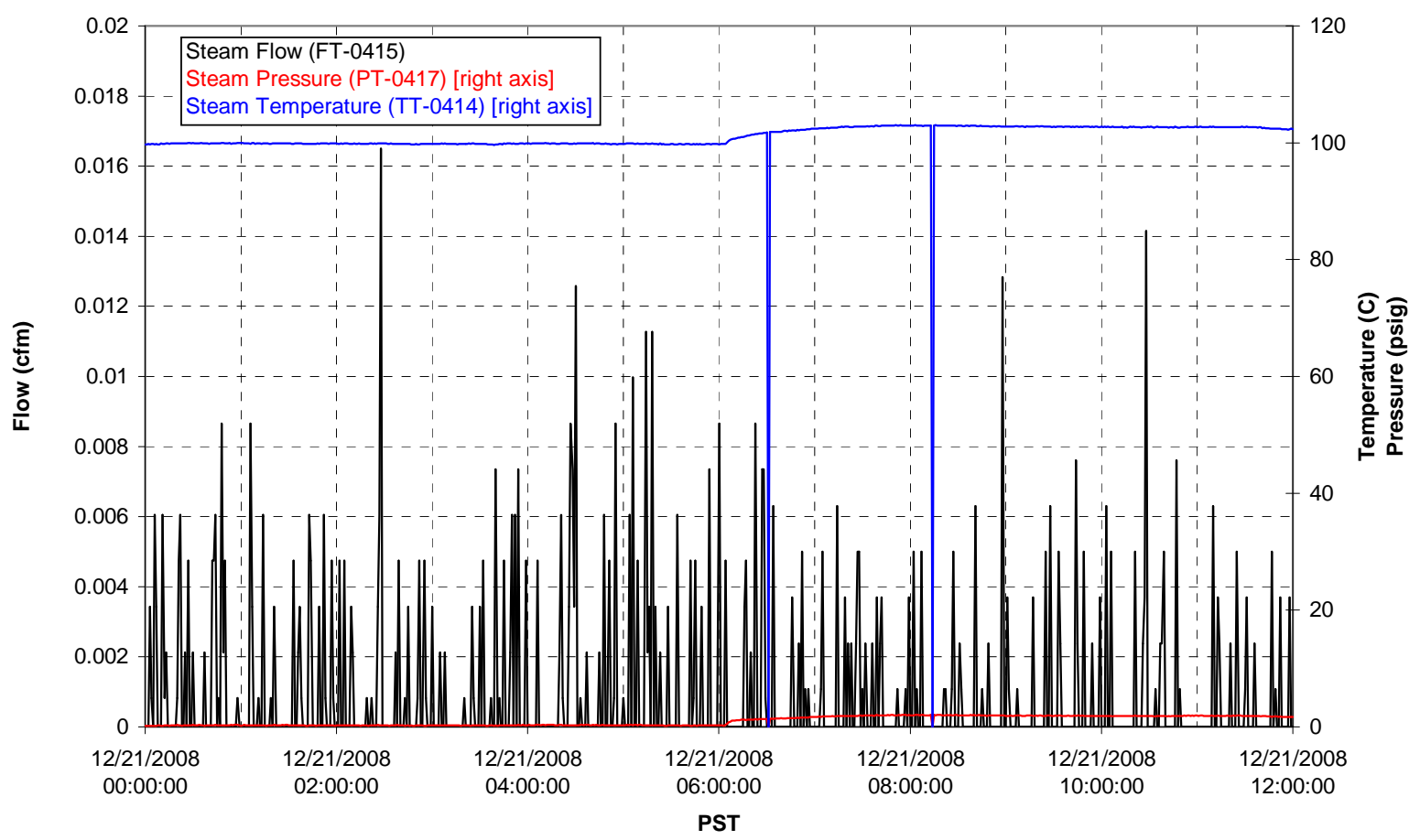


Functional Test Data Plots 12/21/08 12:00 - 12/22/08 00:00 
T01A level

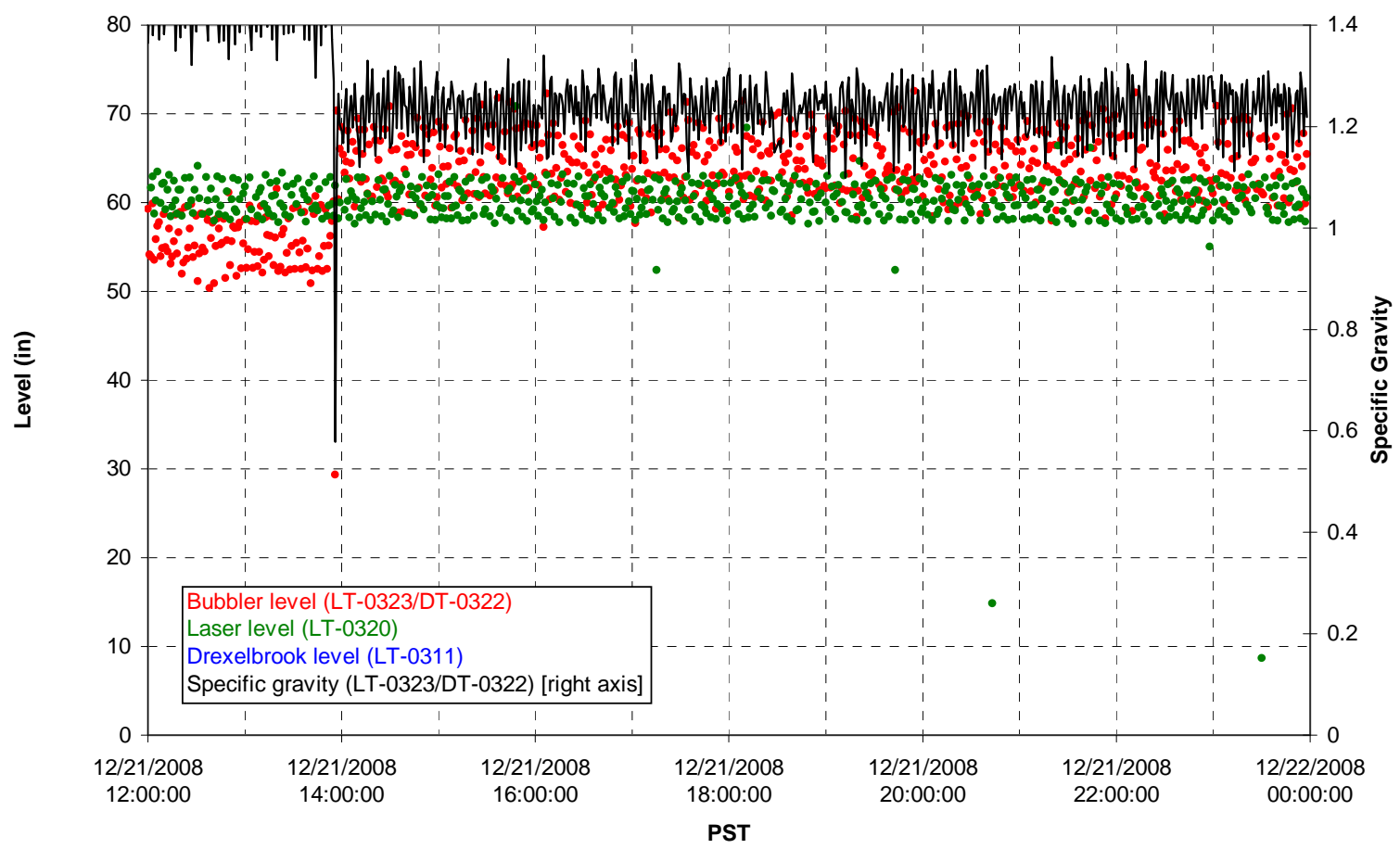

T01A temperatures

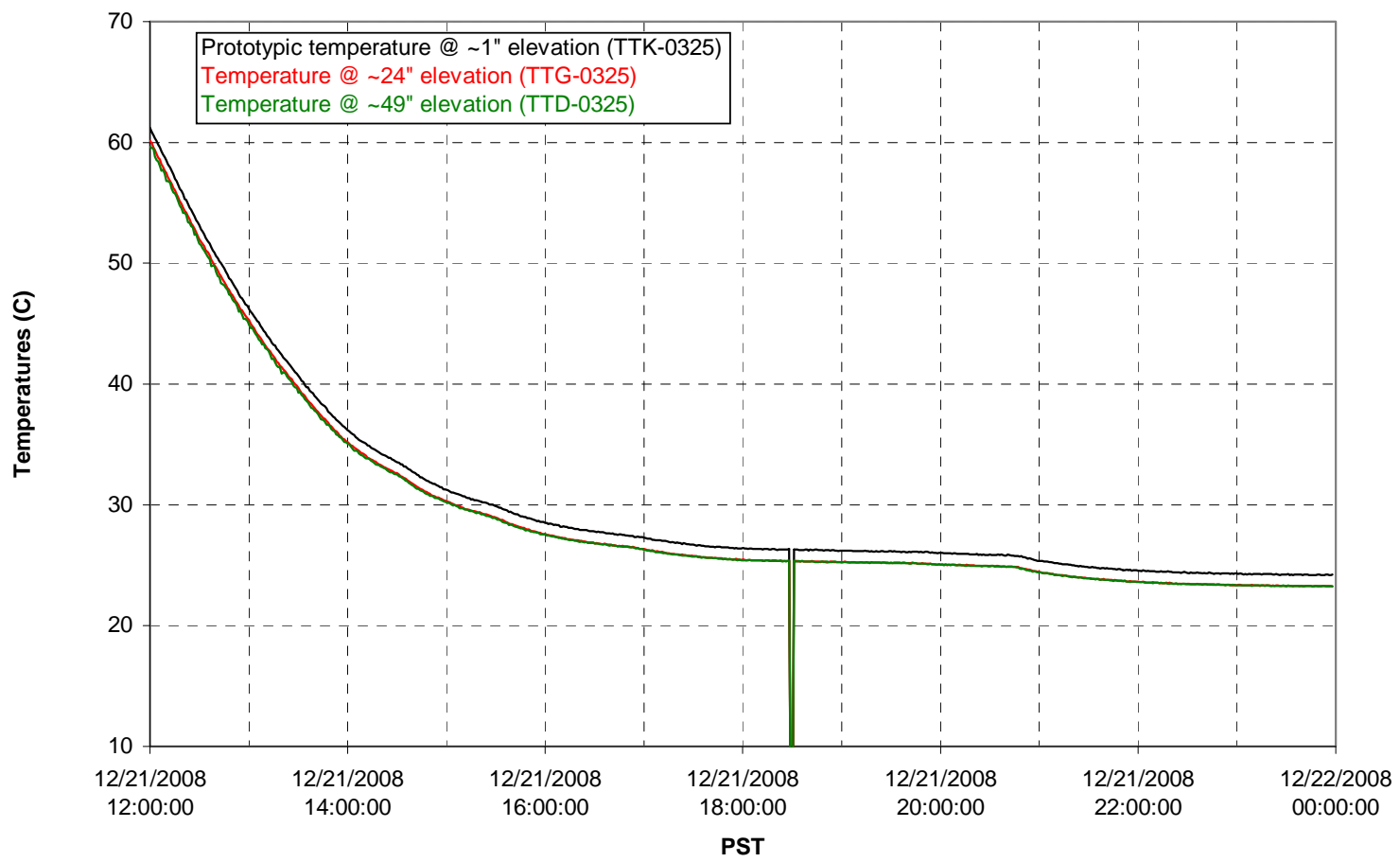


T01B level

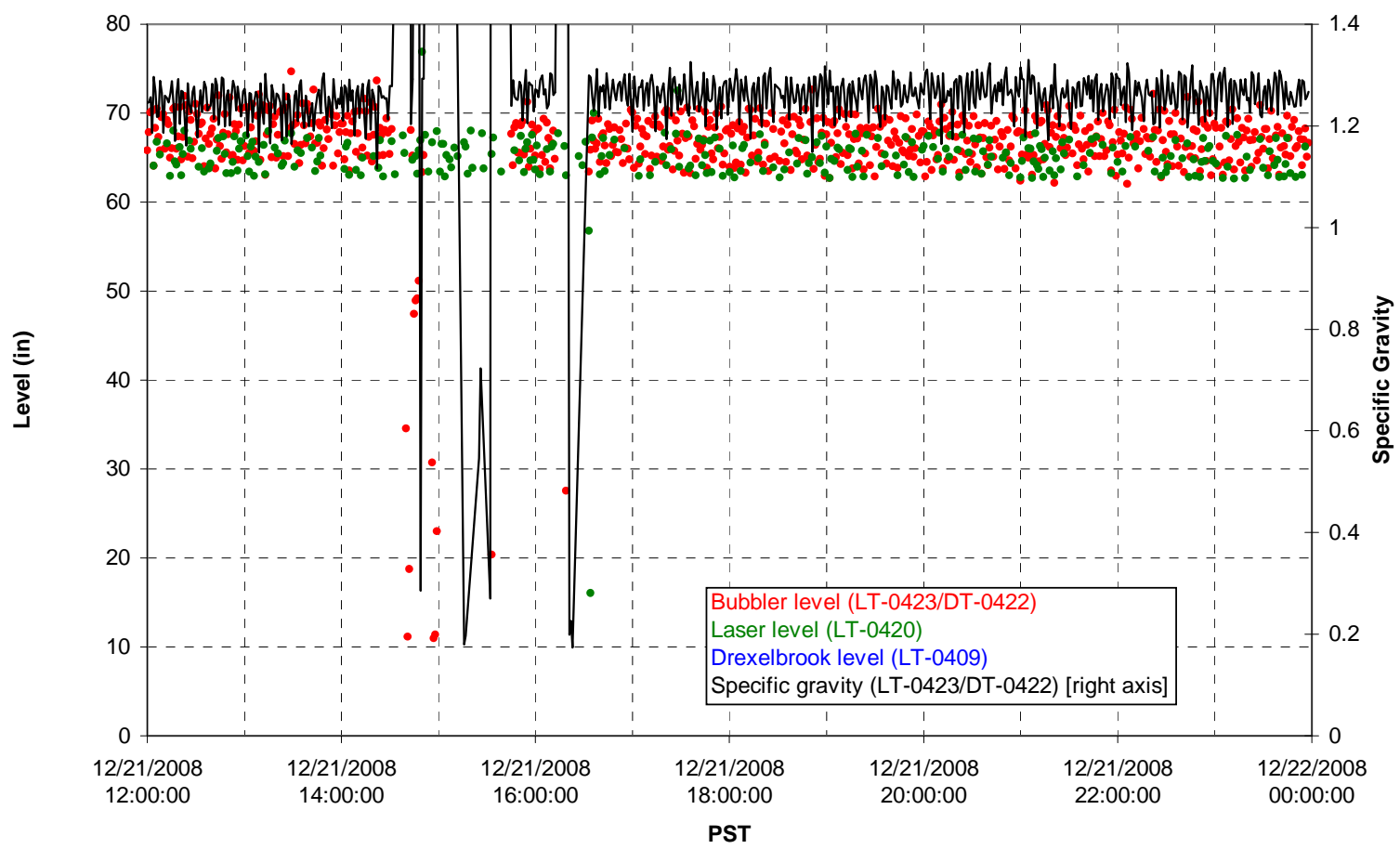

T01B temperatures

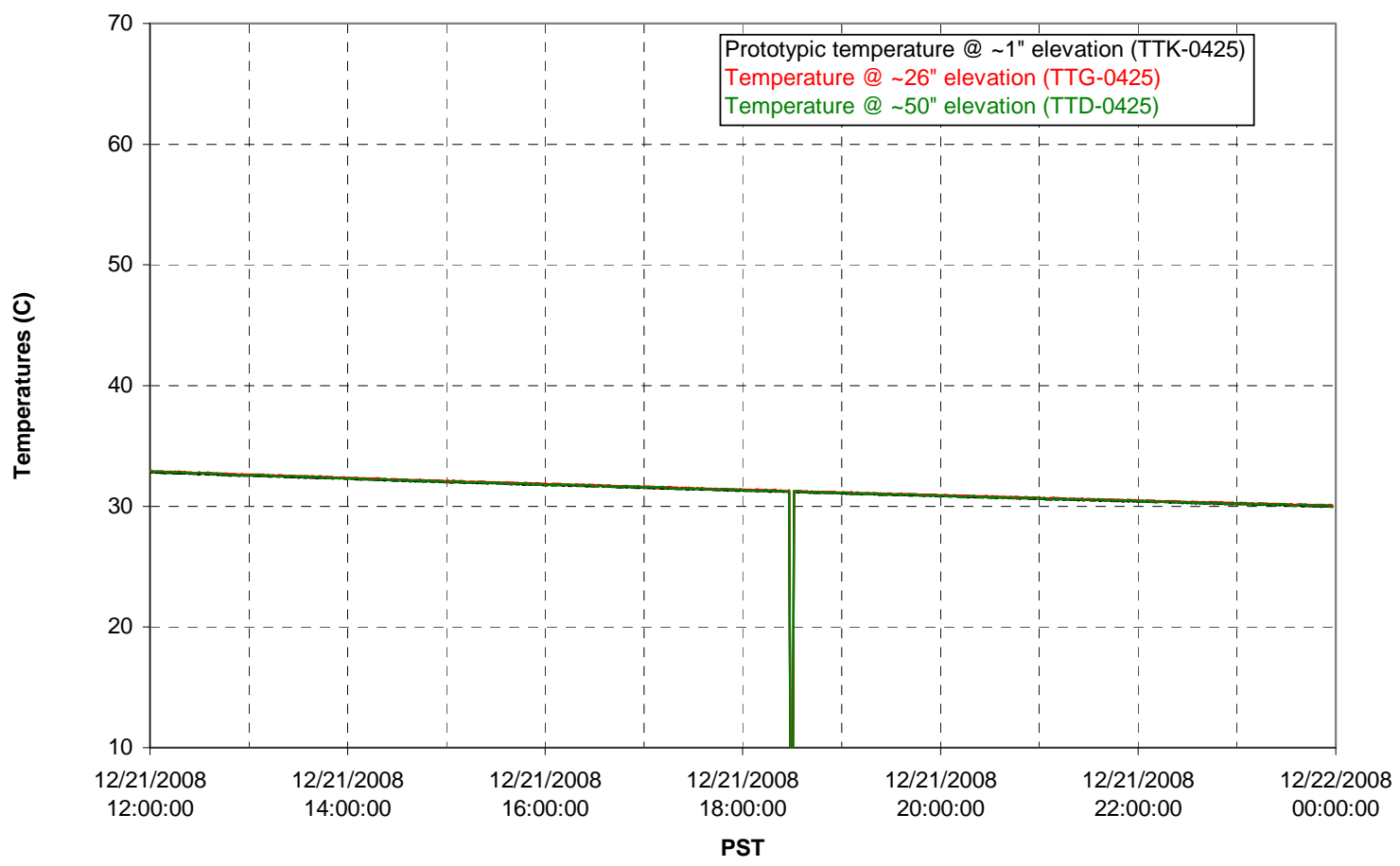


T02A level

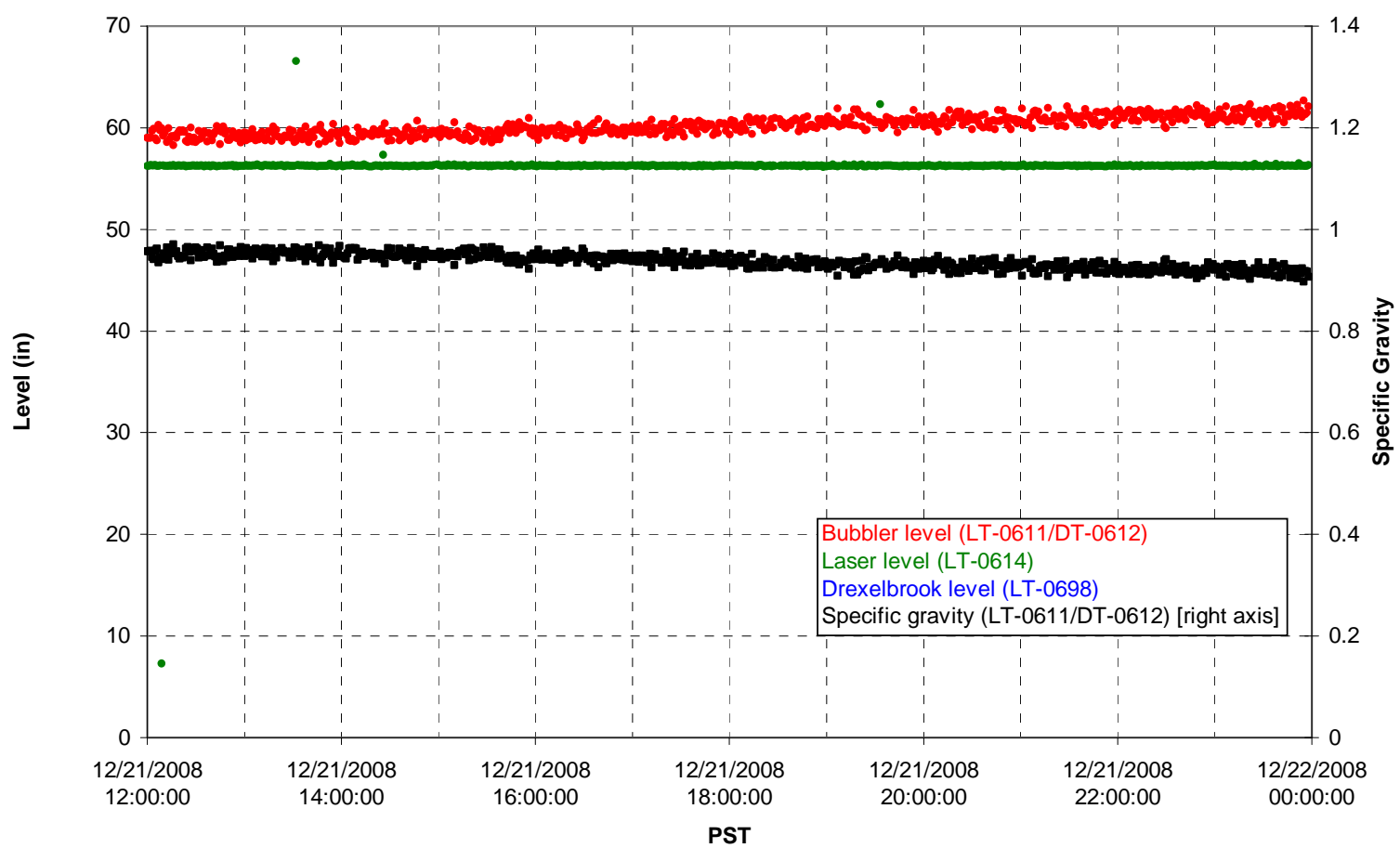

T02A temperatures

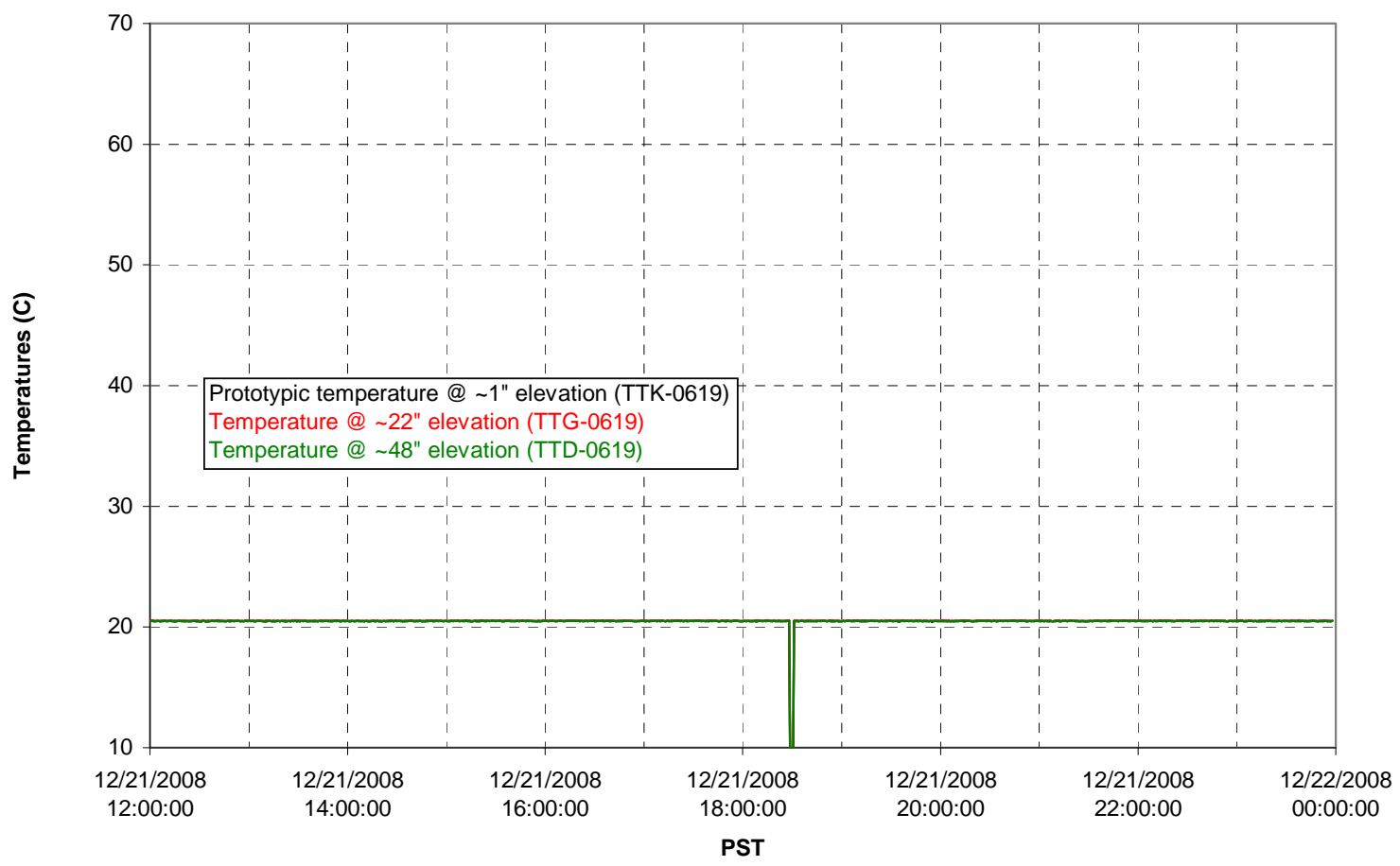


T02A and filter loop temperatures

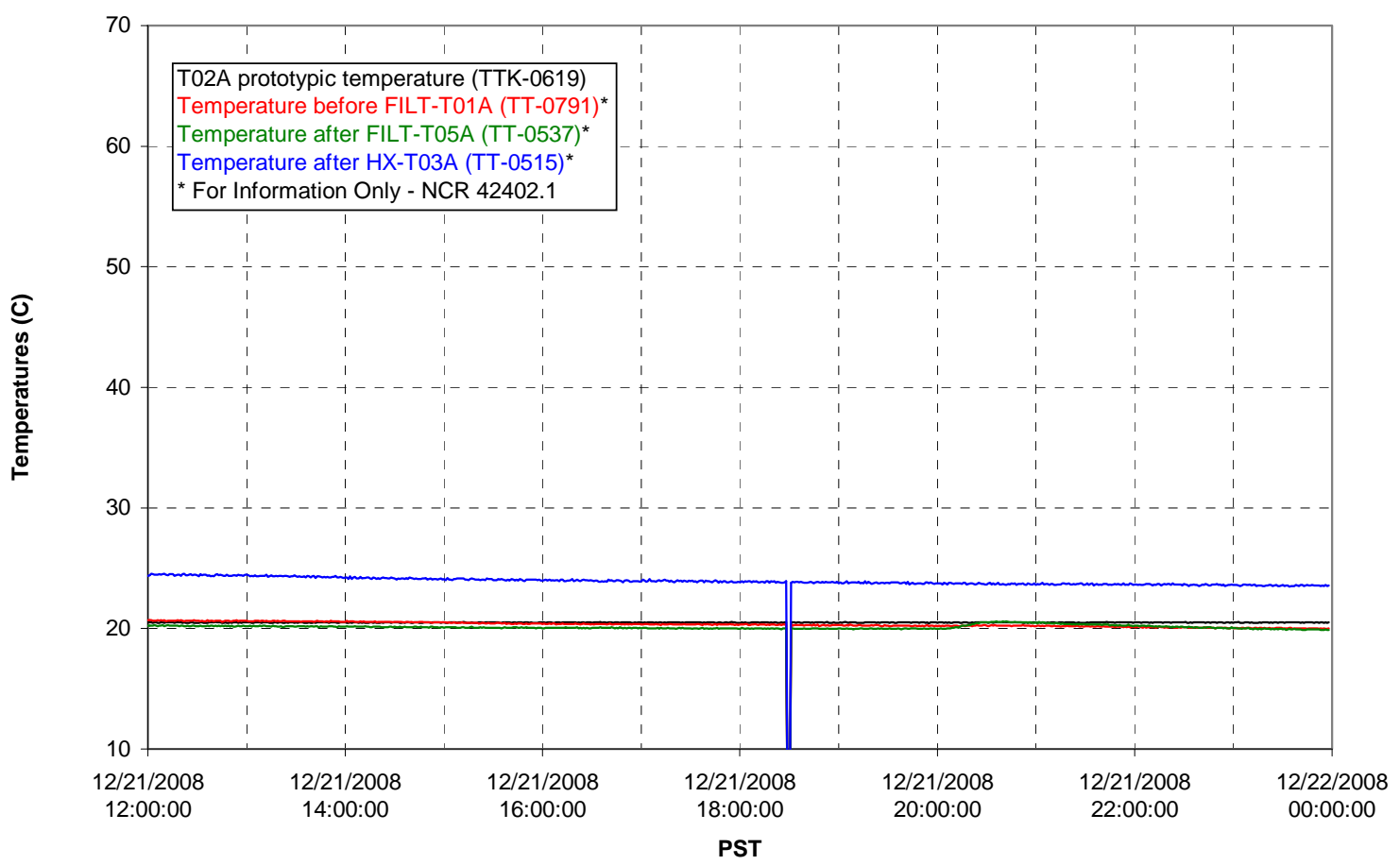

Pump Pressures and Flow

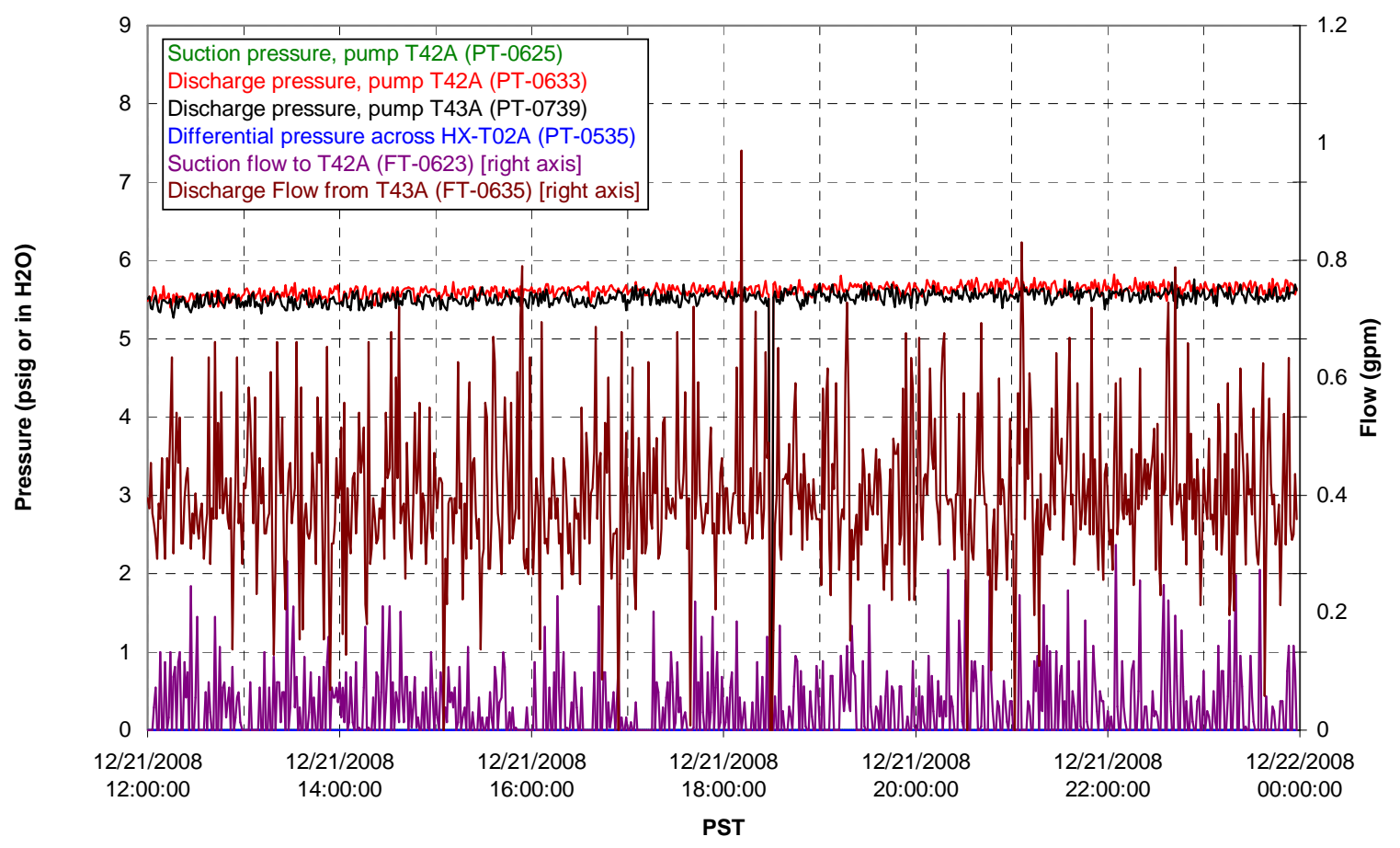


Axial pressure drop

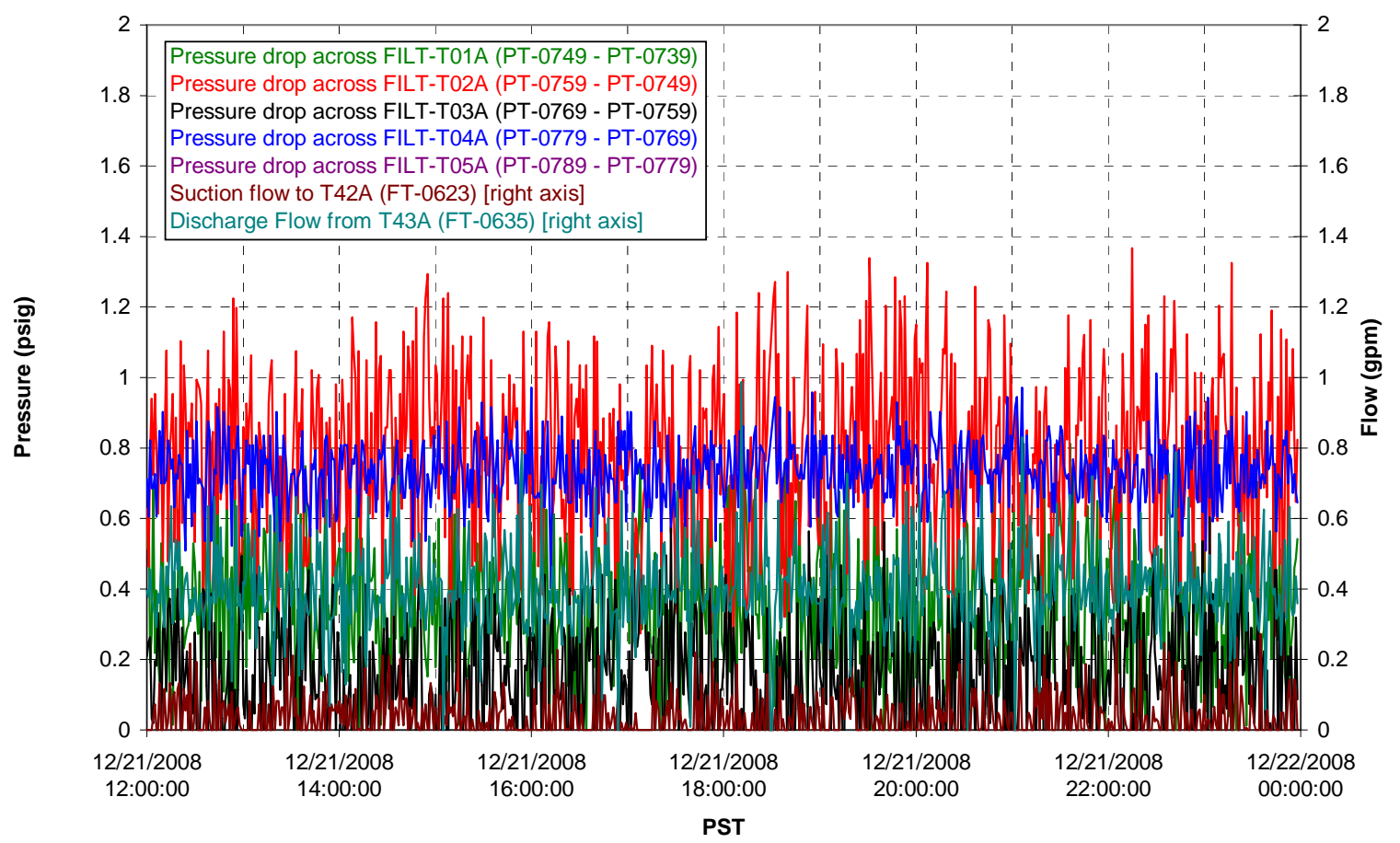

Permeate flow rates

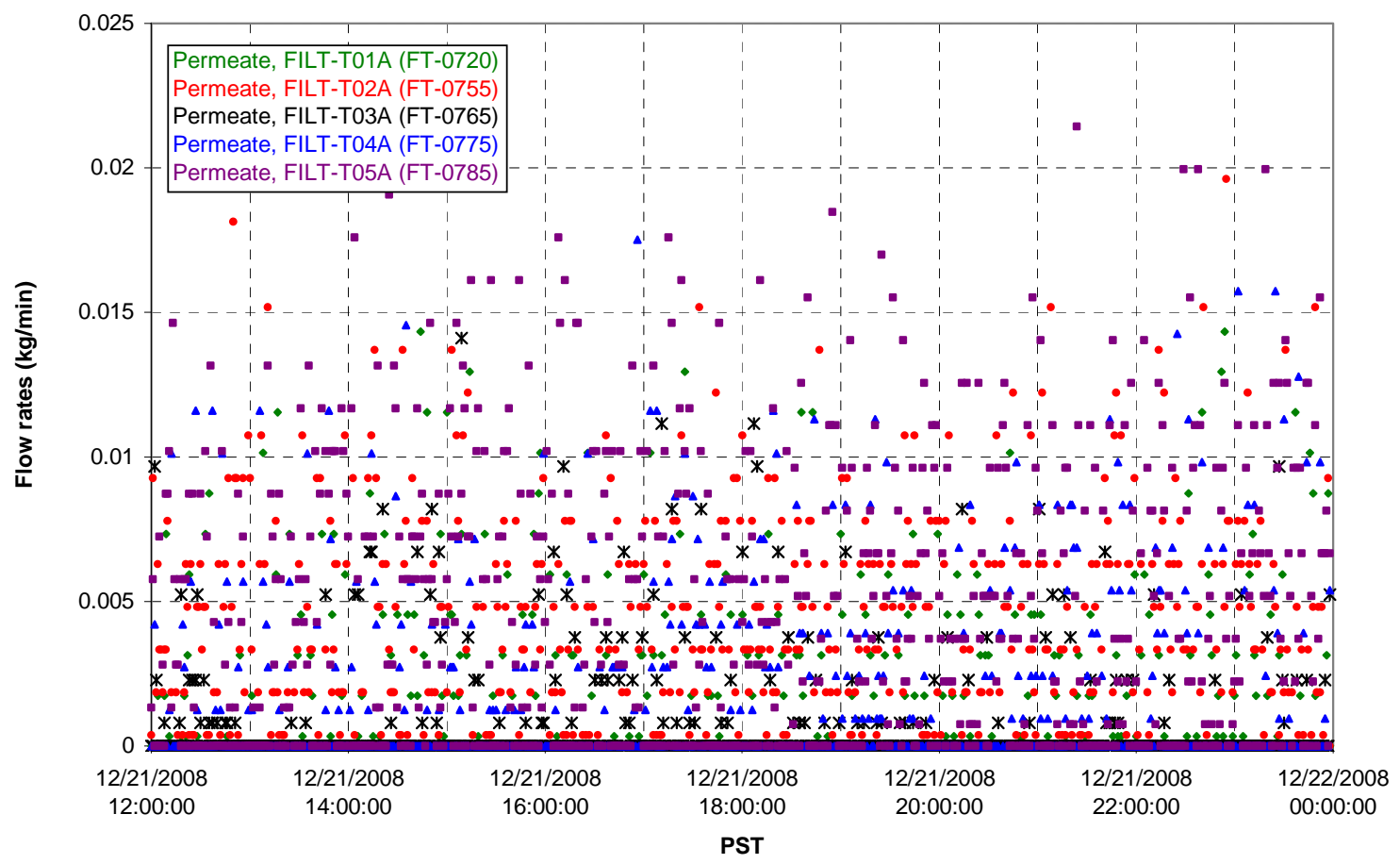


T02A Inner Temperature Tree

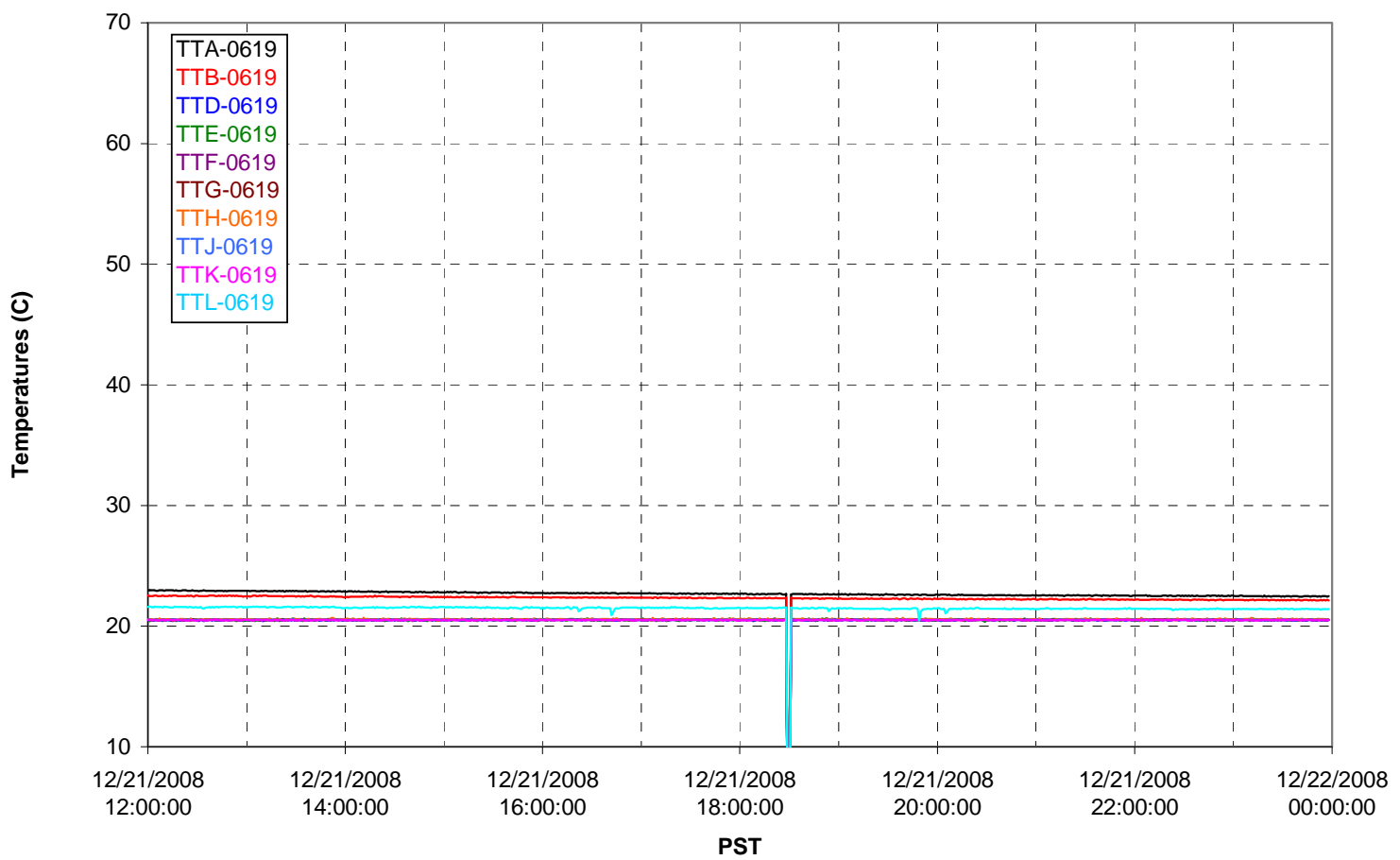

T02A Outer Temperature Tree

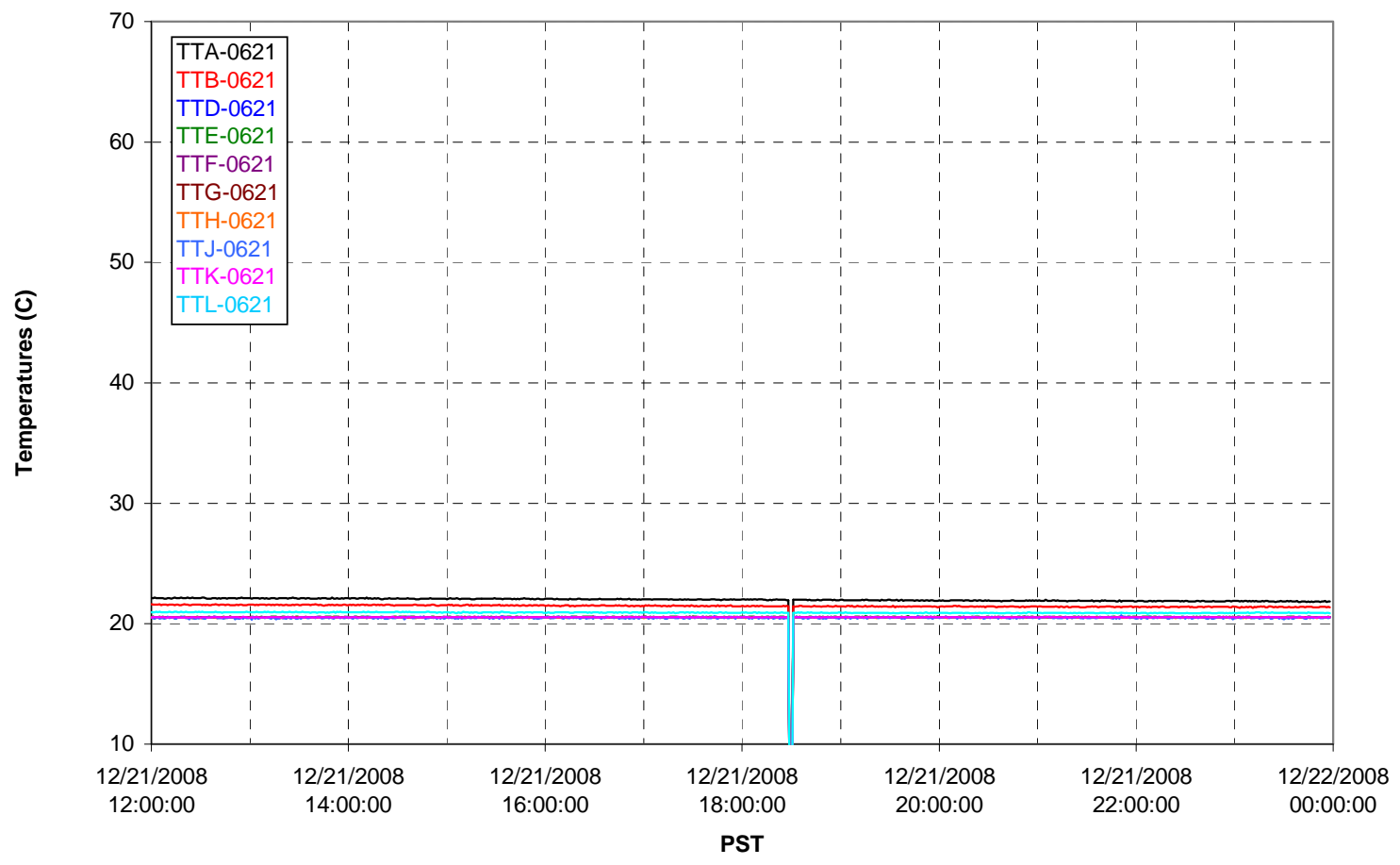


T02A temperatures

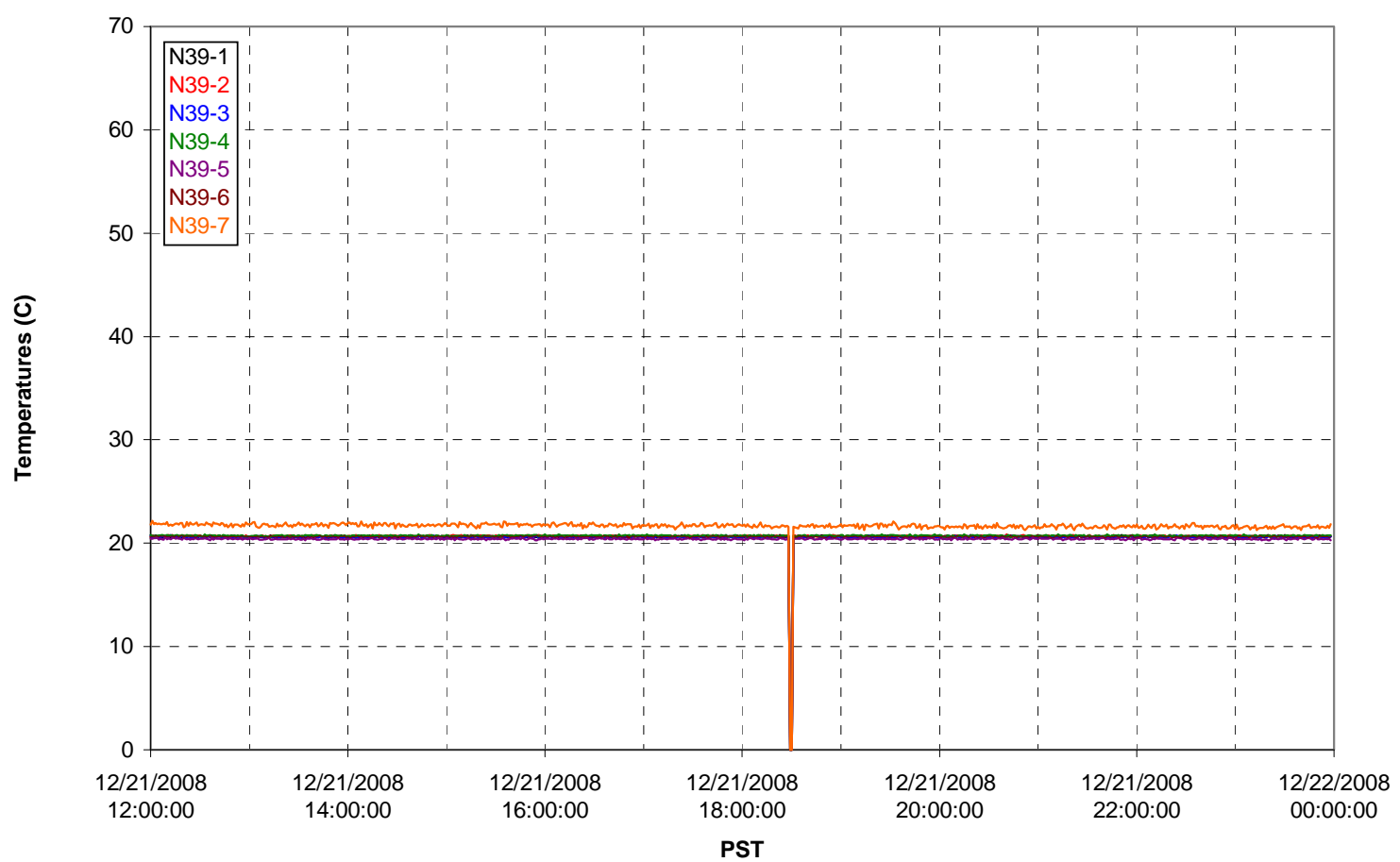

T02A temperatures

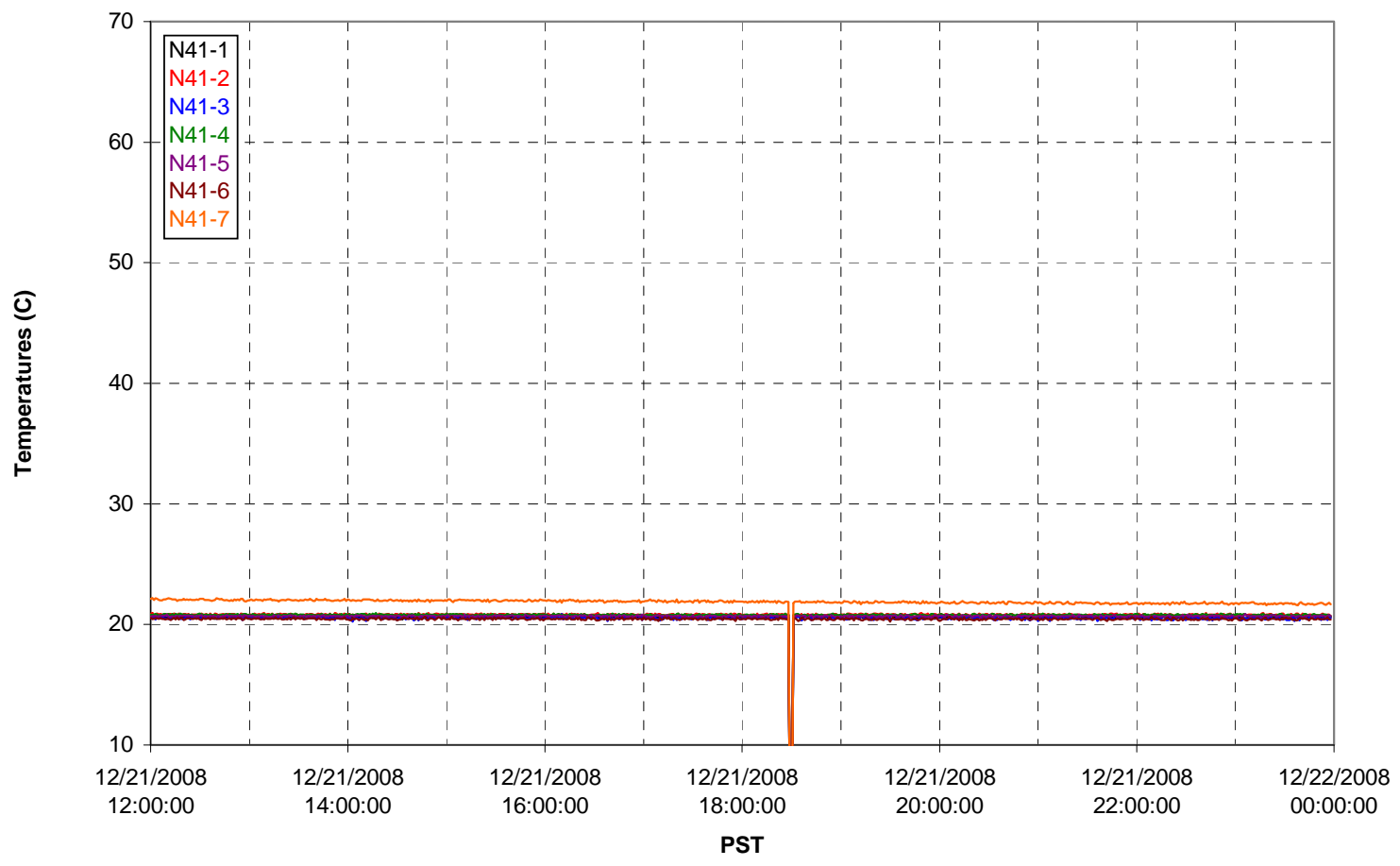


T02A temperatures

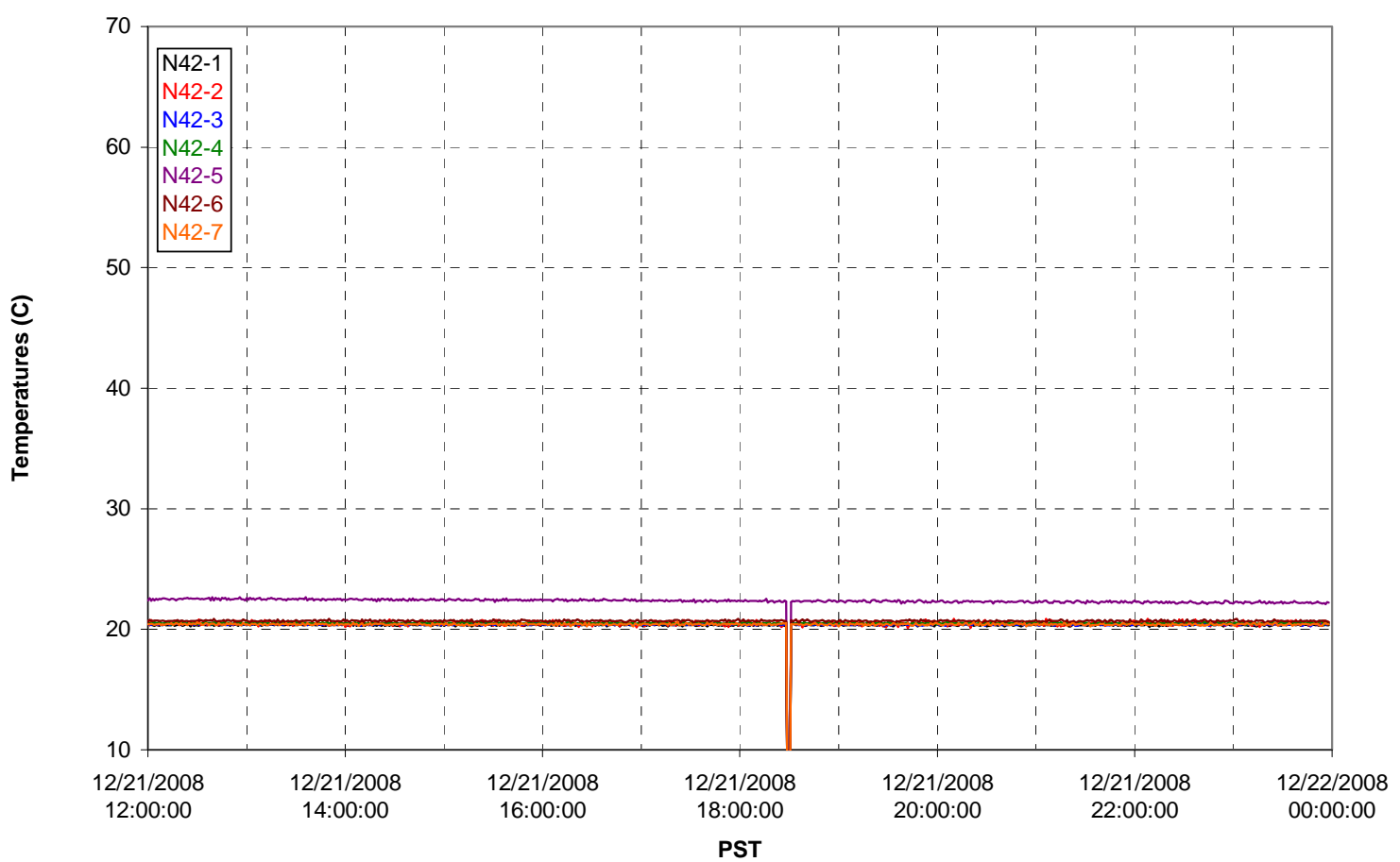

T02A temperatures

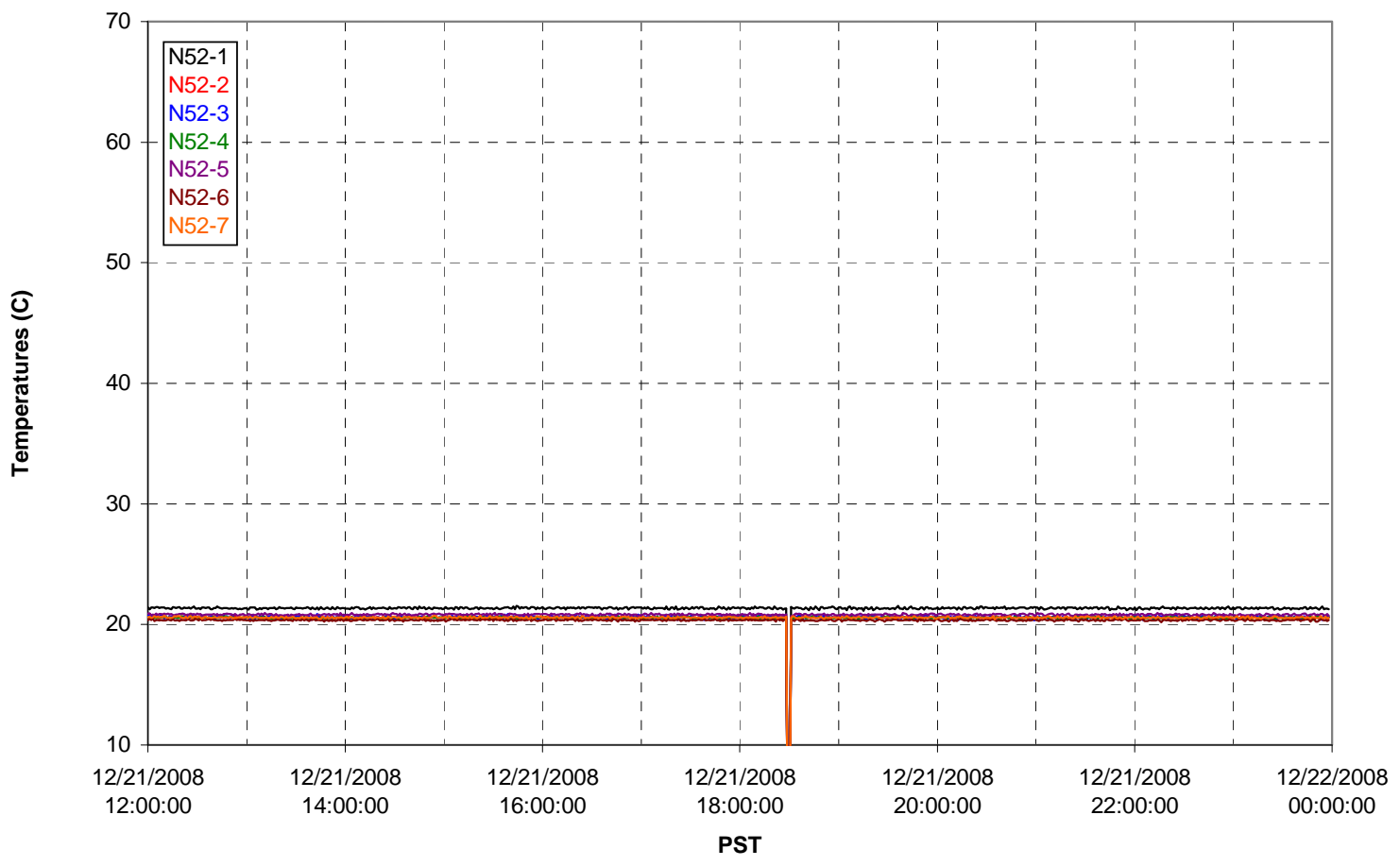


T02A Heating and Cooling

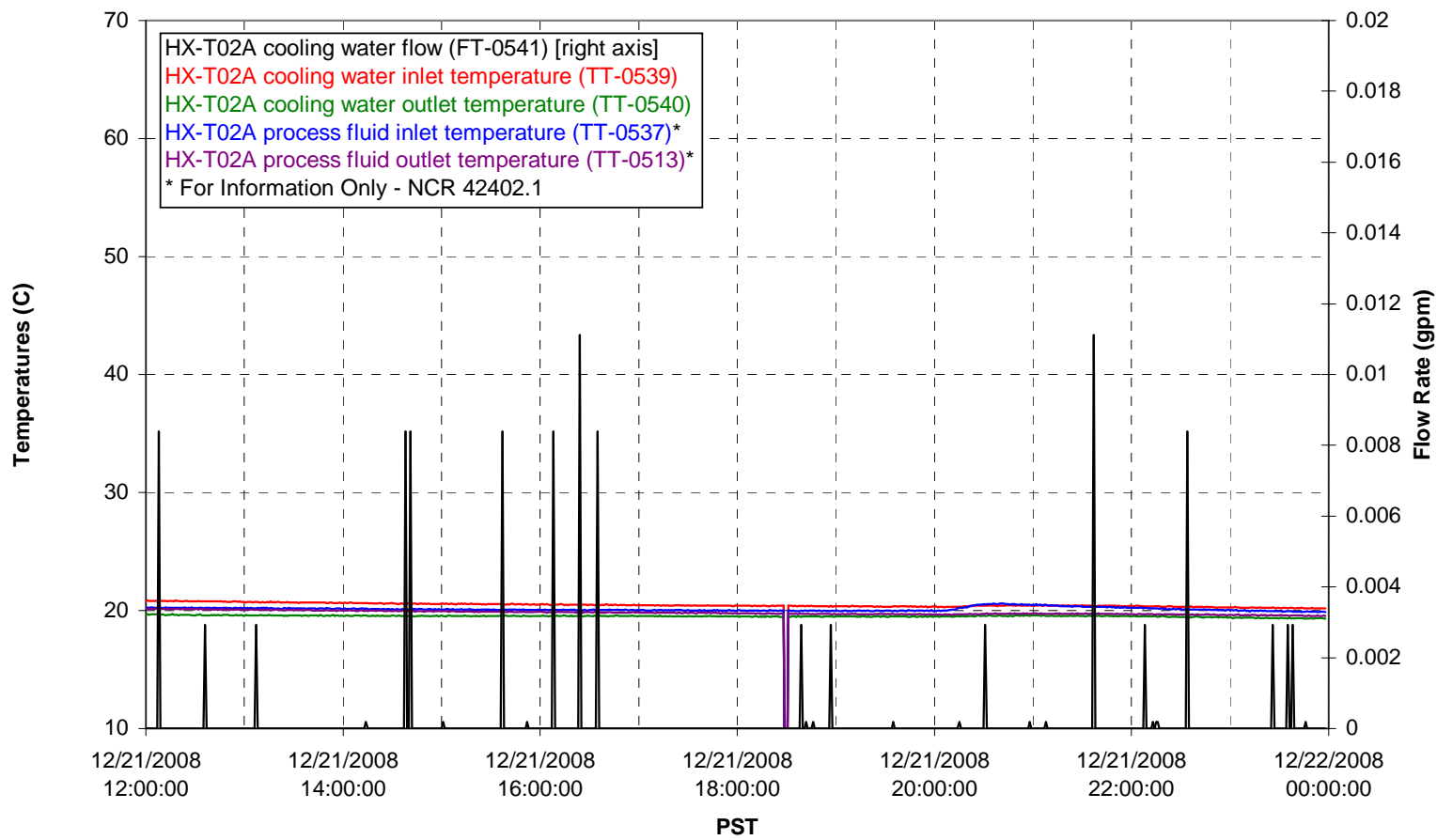

Pump Operation

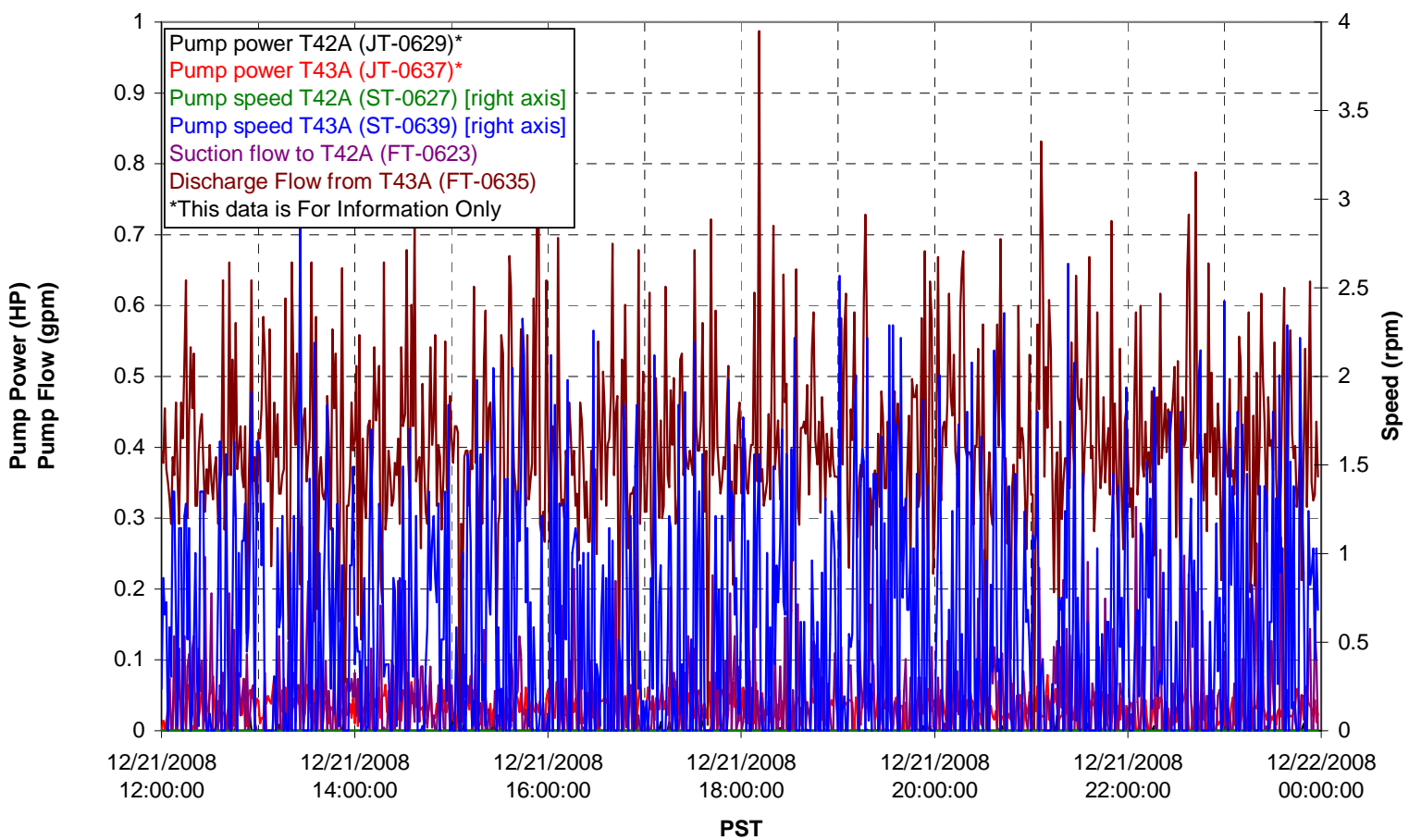


Pulsepot UFP-PP-T01A

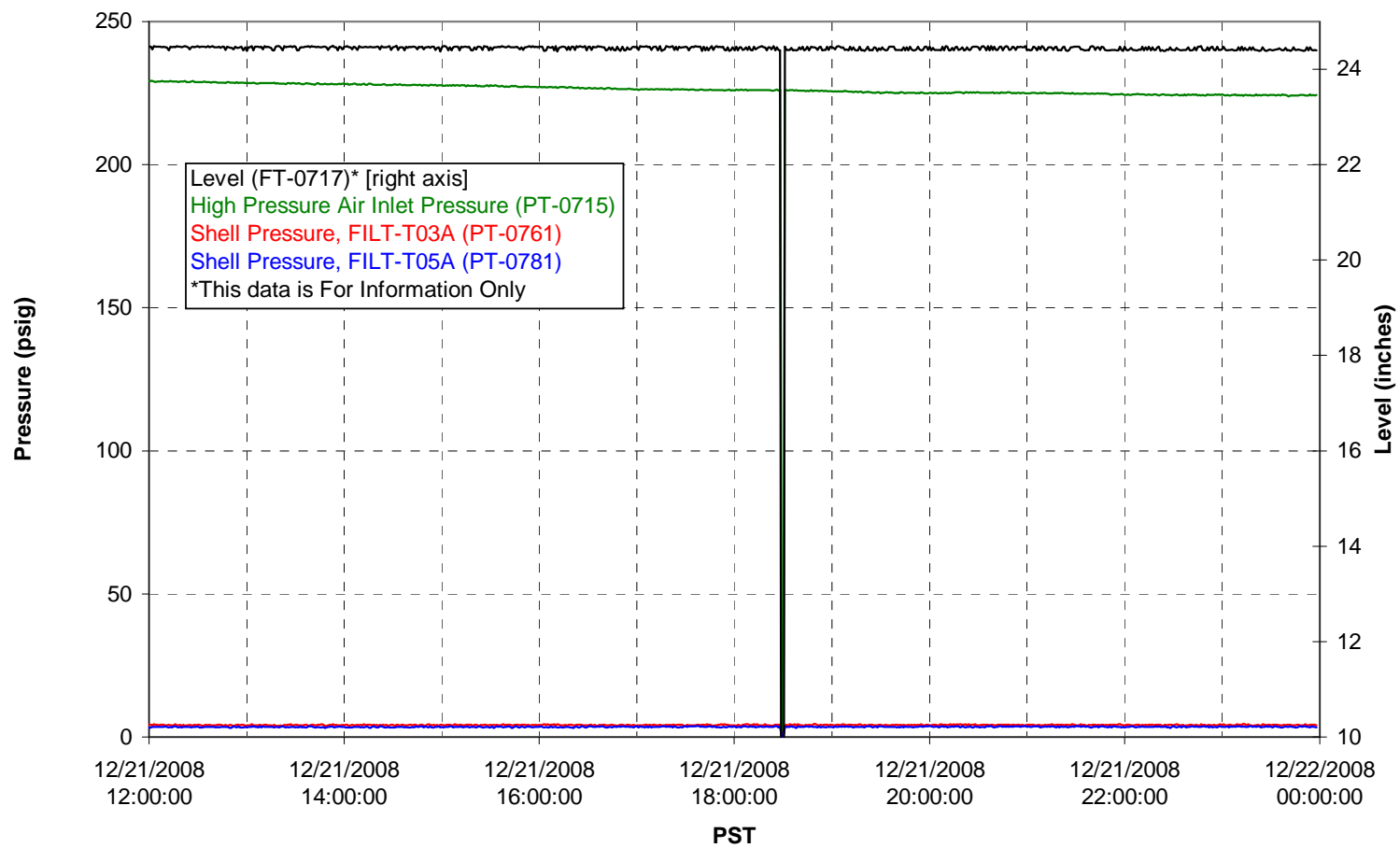

Pulsepot UFP-PP-T02A

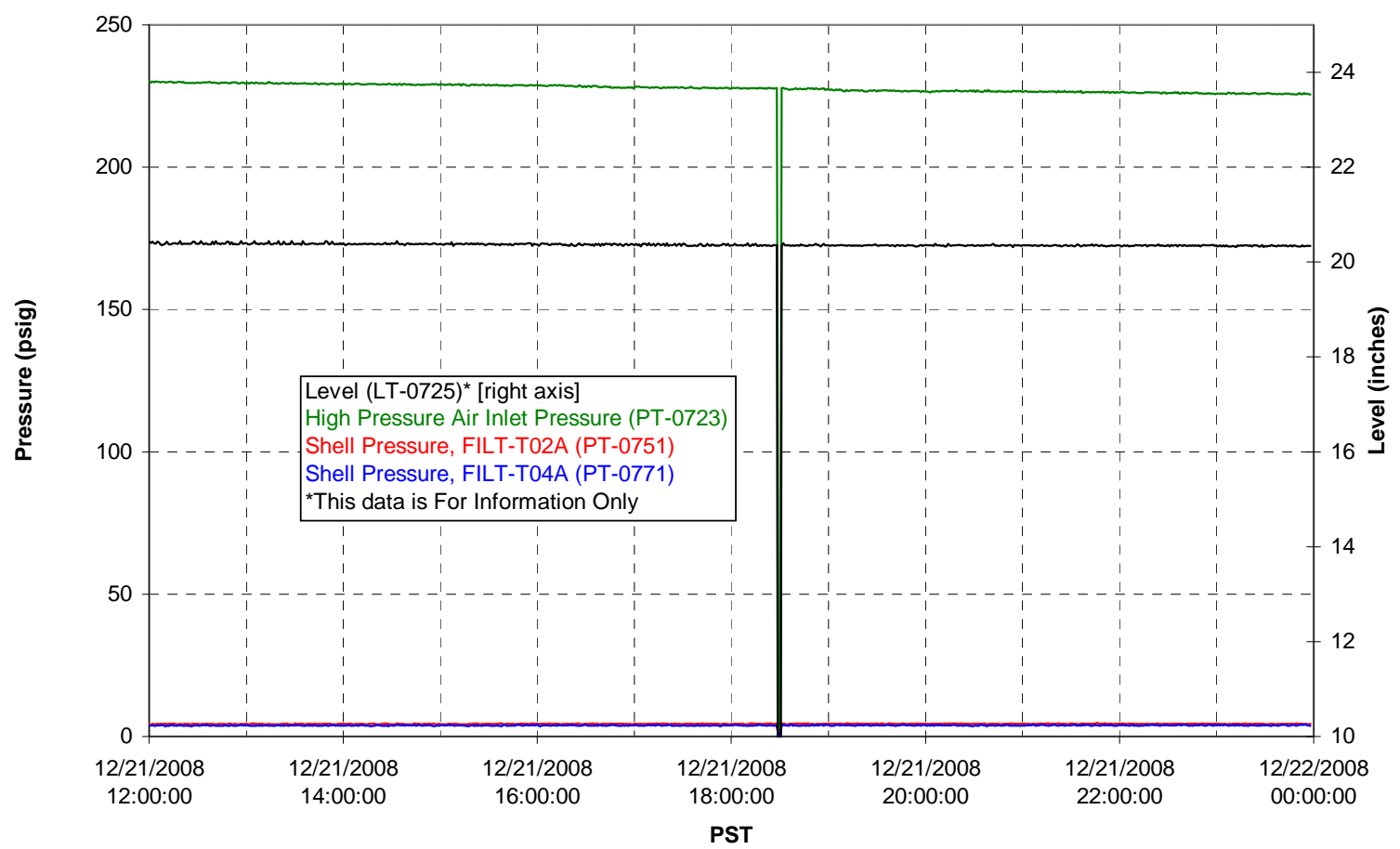


Pulsepot UFP-PP-T03A

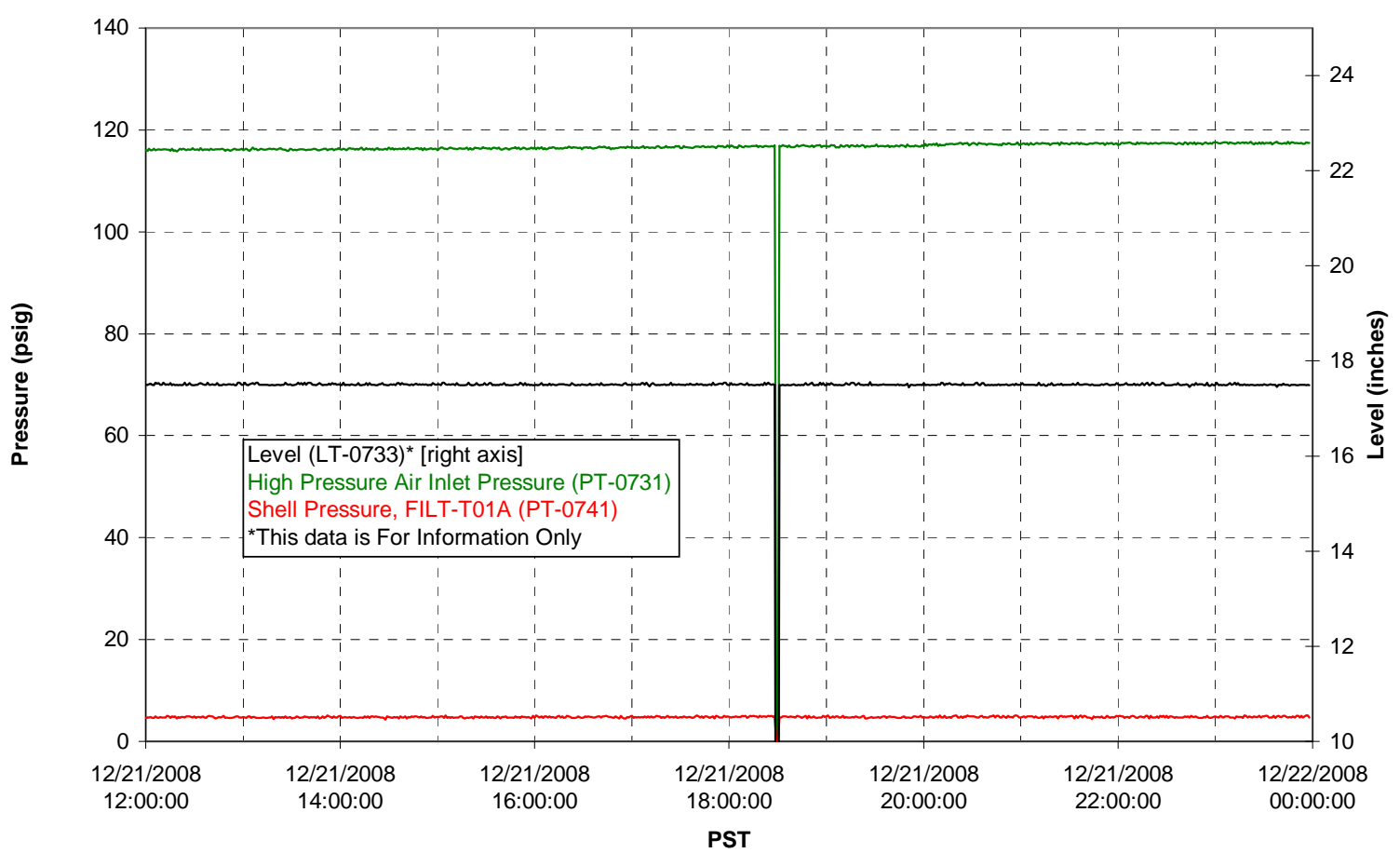

Pulsepot Levels

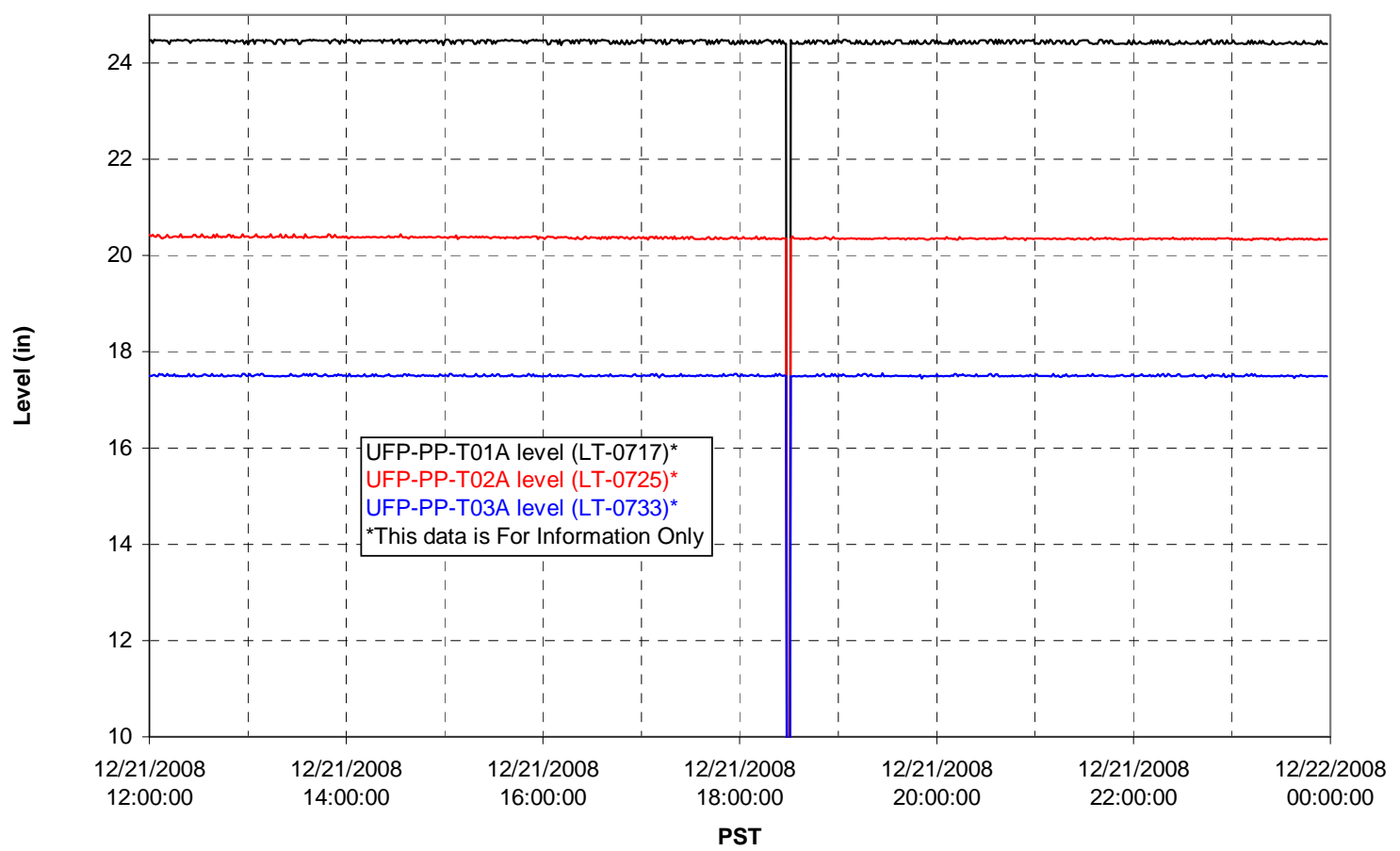


Filter UFP-FILT-T01A

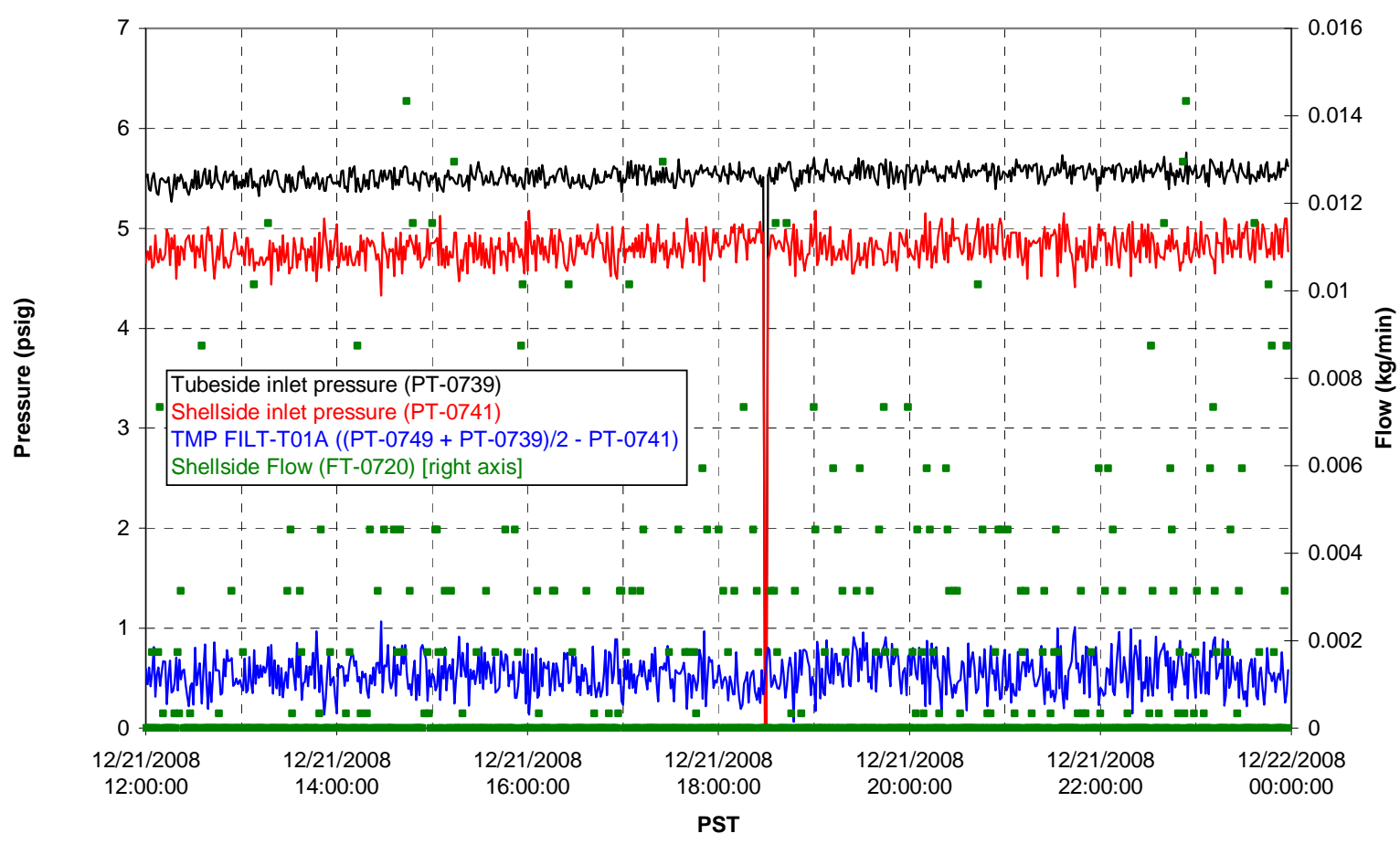

Filter UFP-FILT-T02A

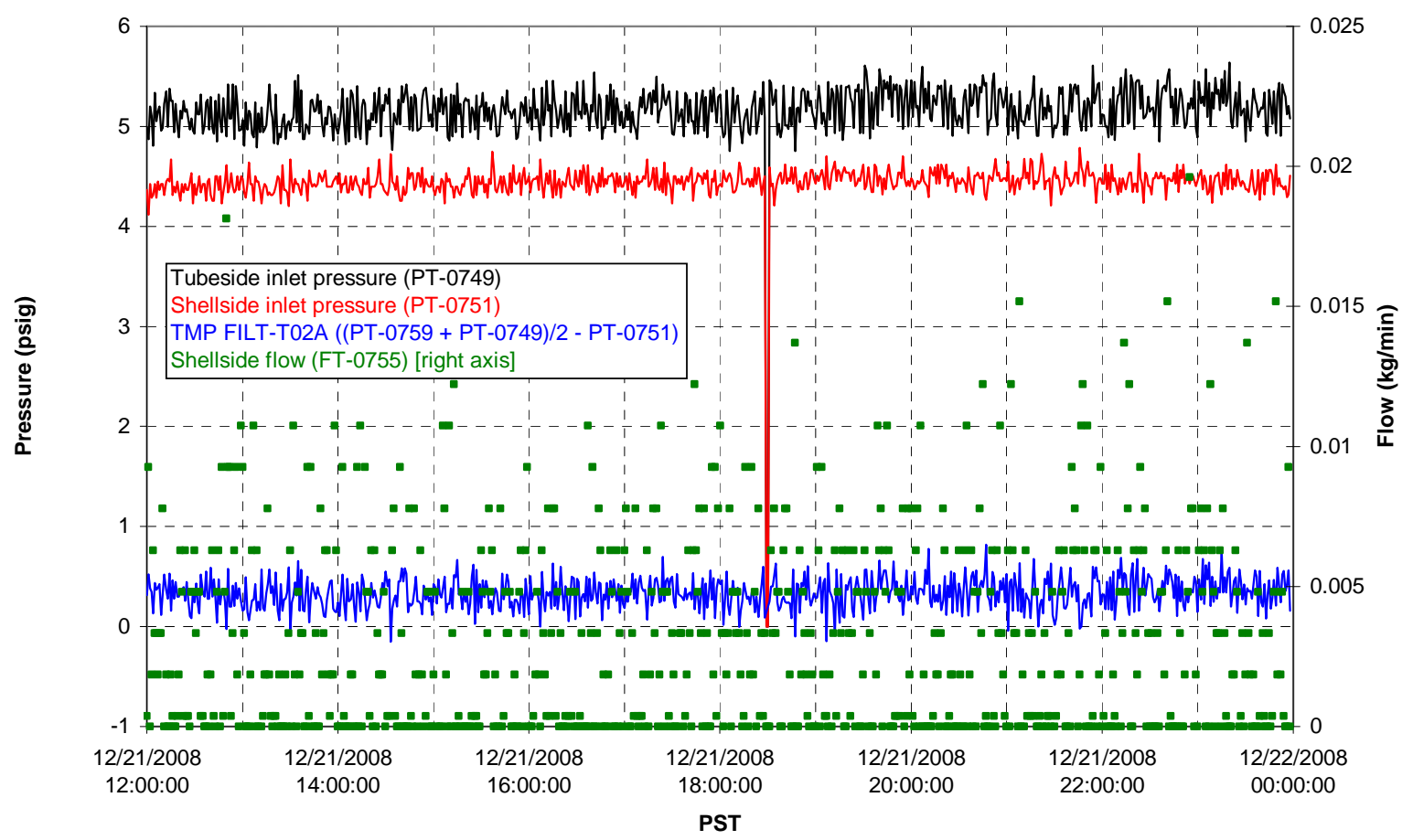


Filter UFP-FILT-T03A

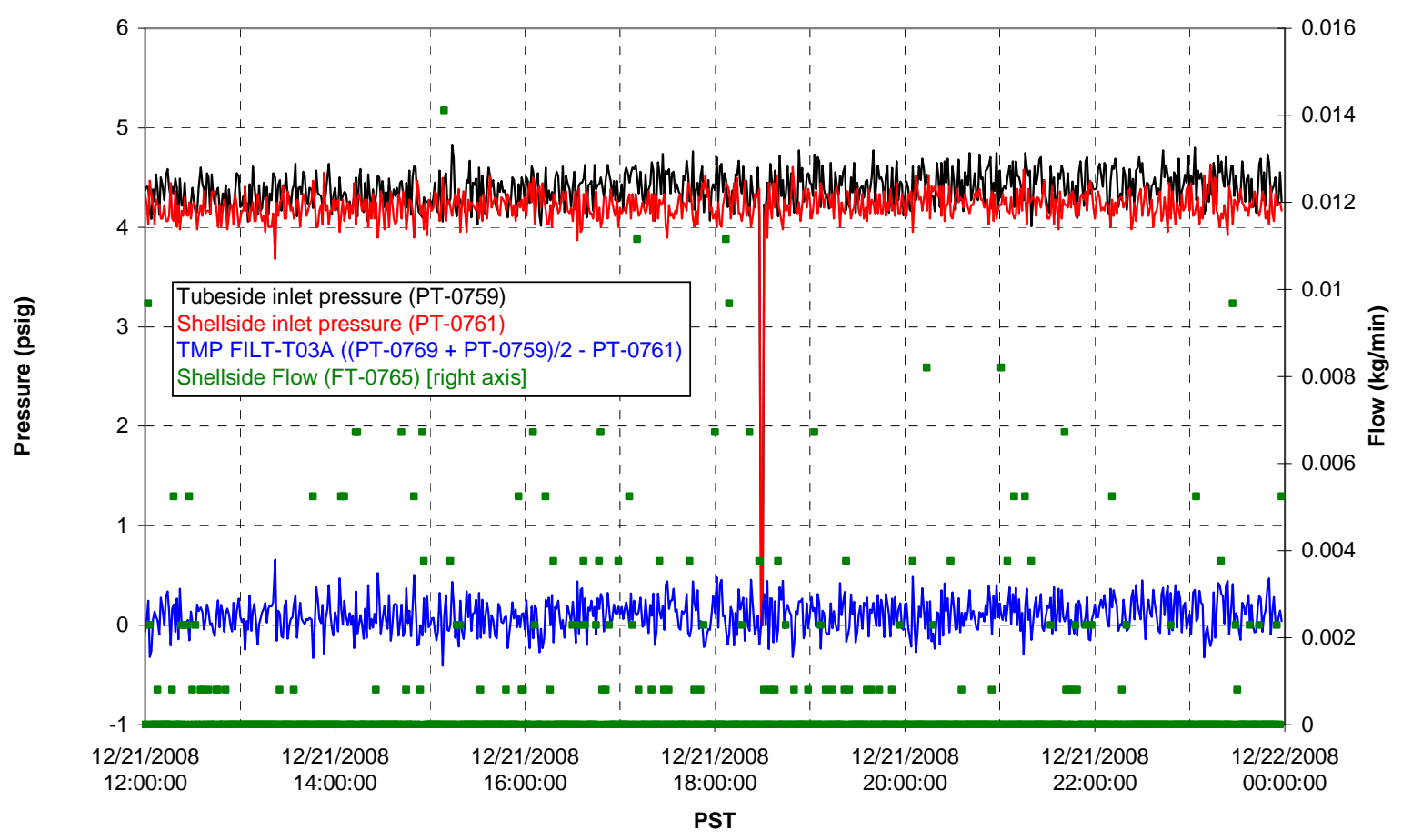

Filter UFP-FILT-T04A

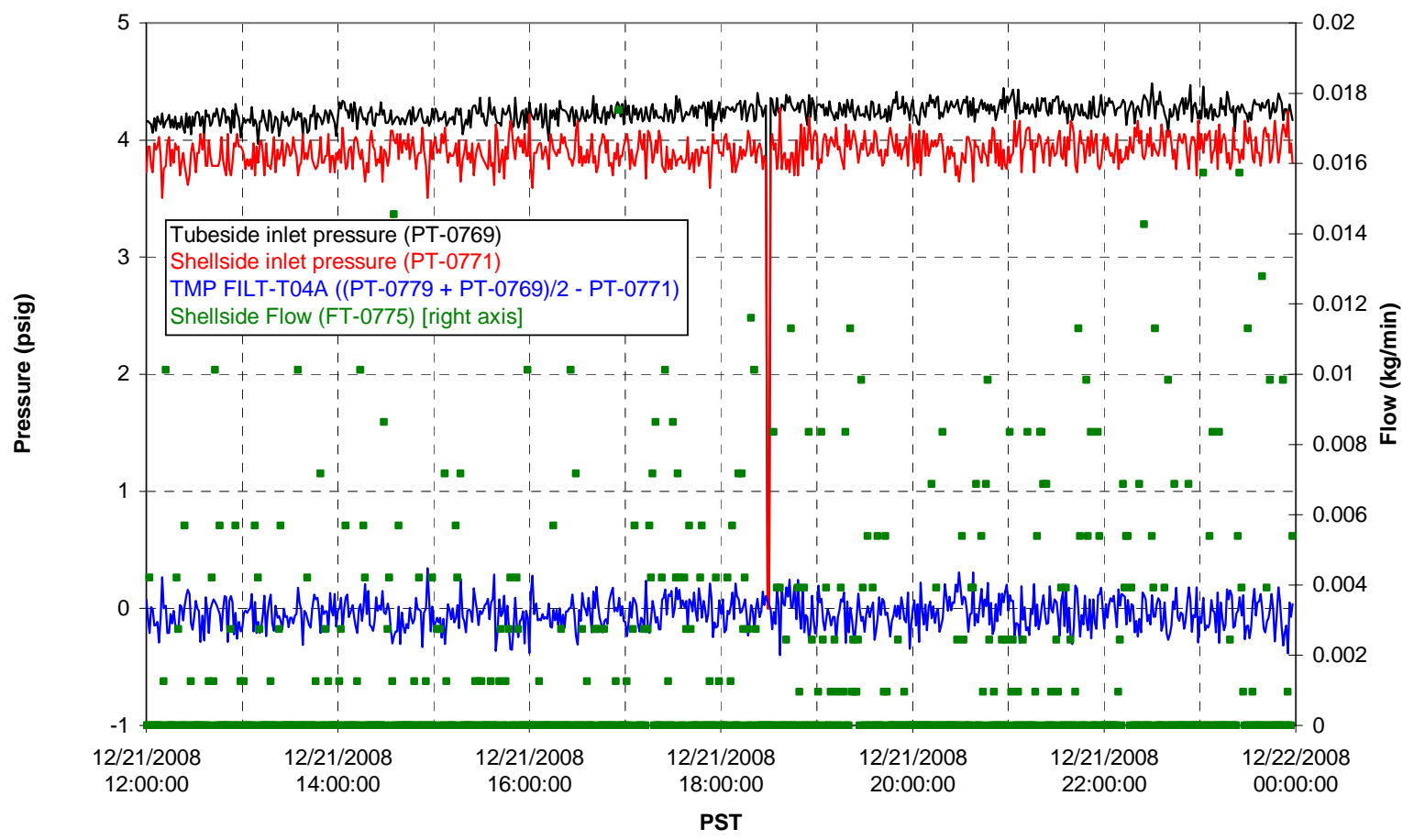


Filter UFP-FILT-T05A

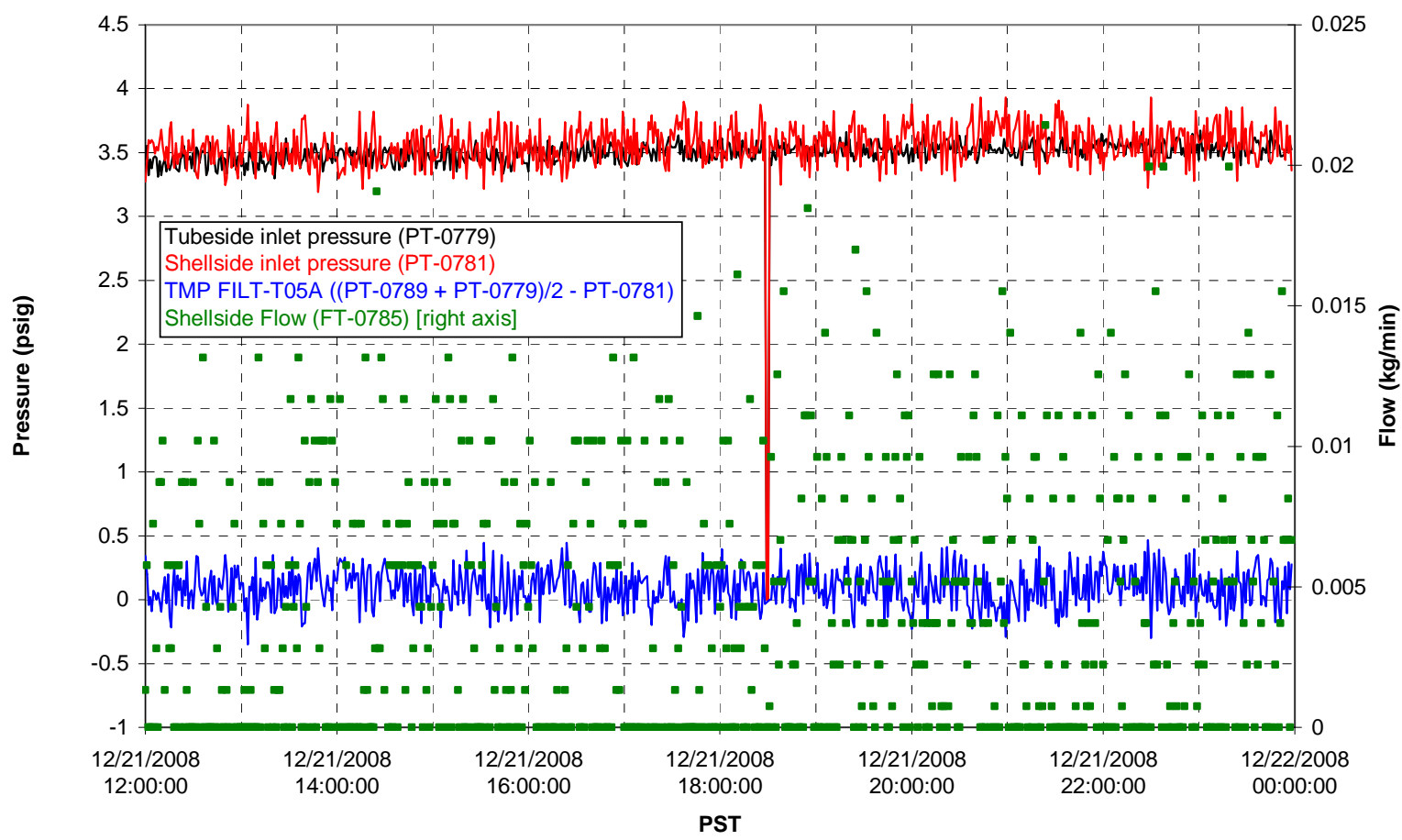

Chemical Flow

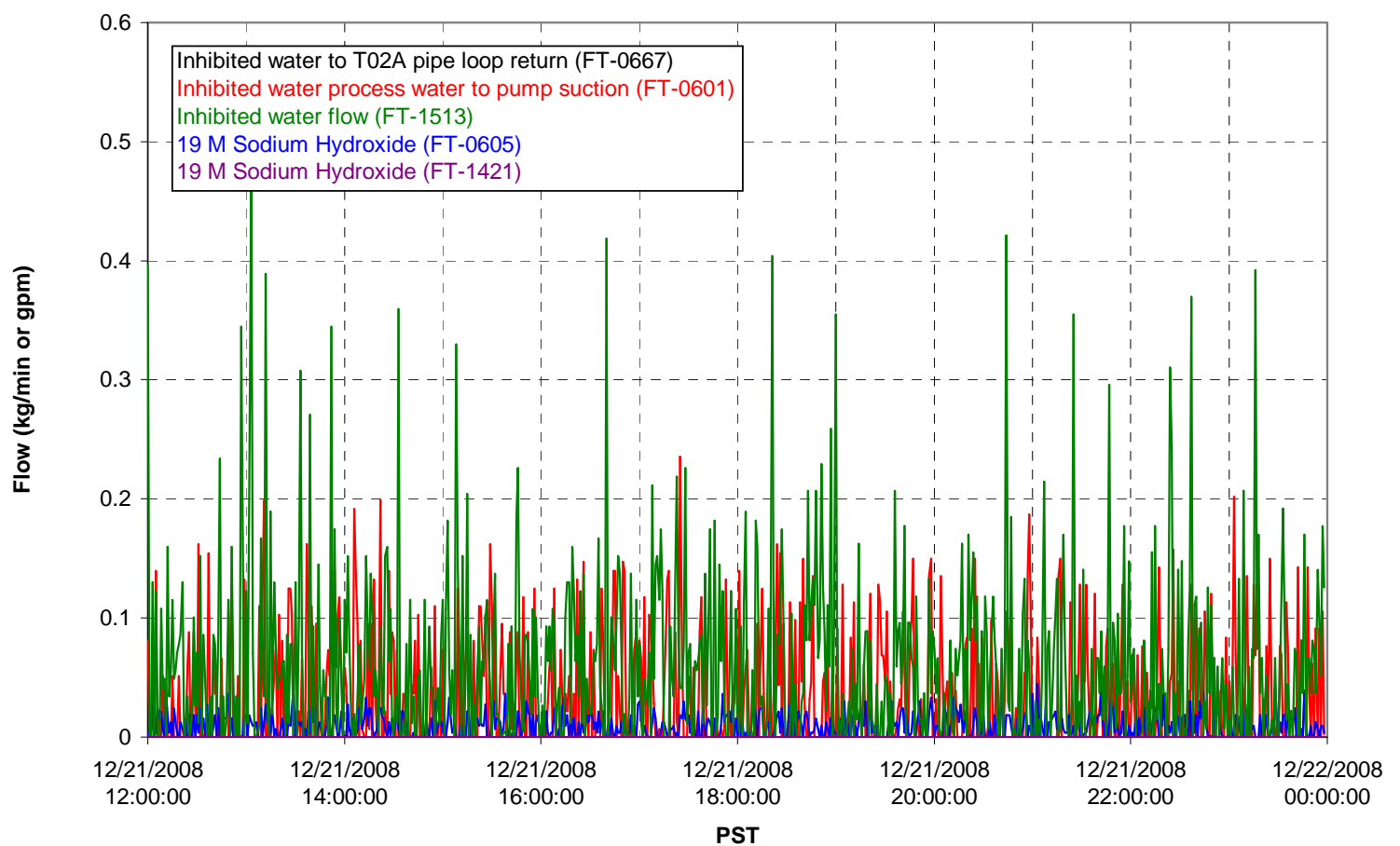




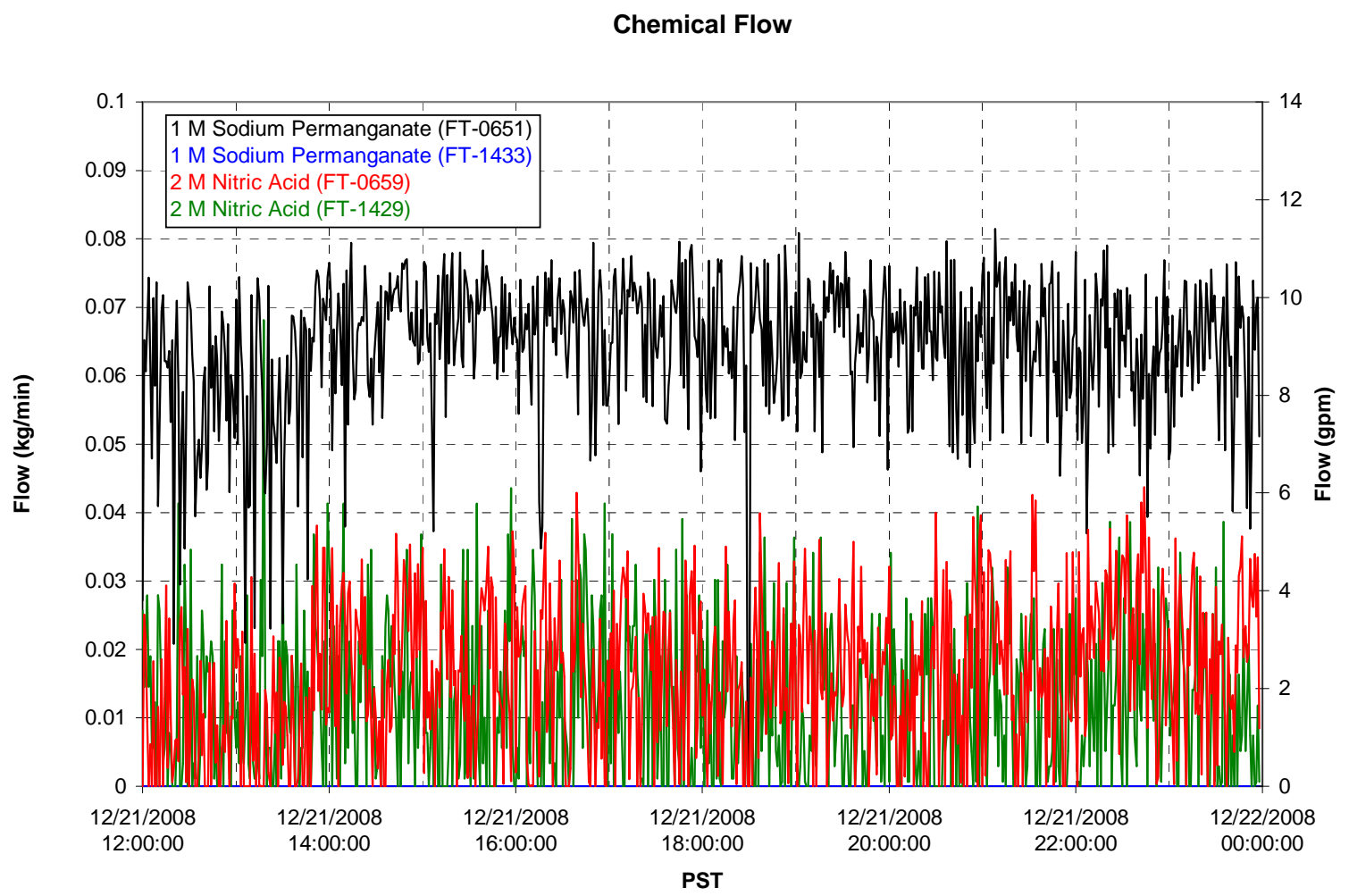

Air Flows

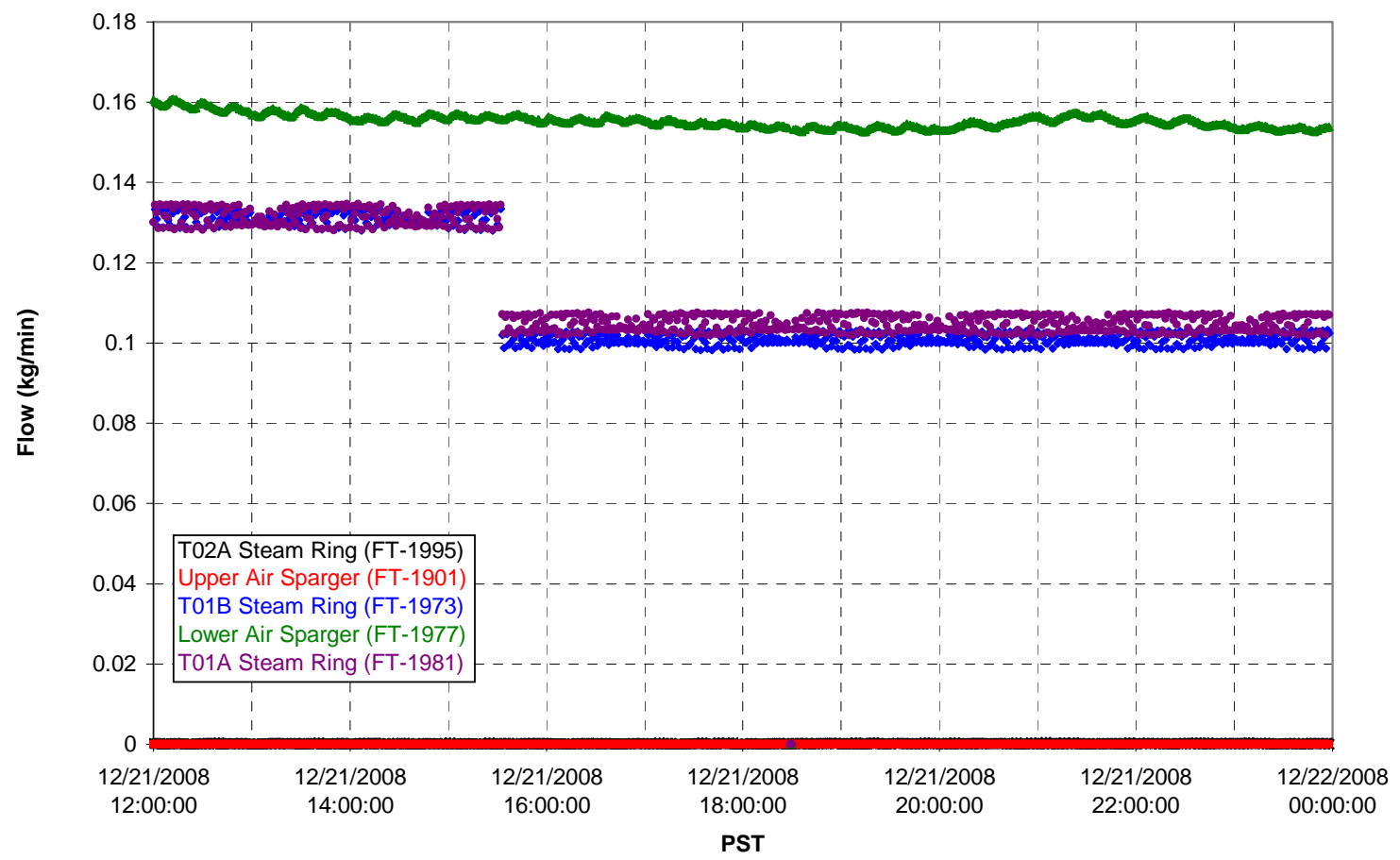


T02A Steam

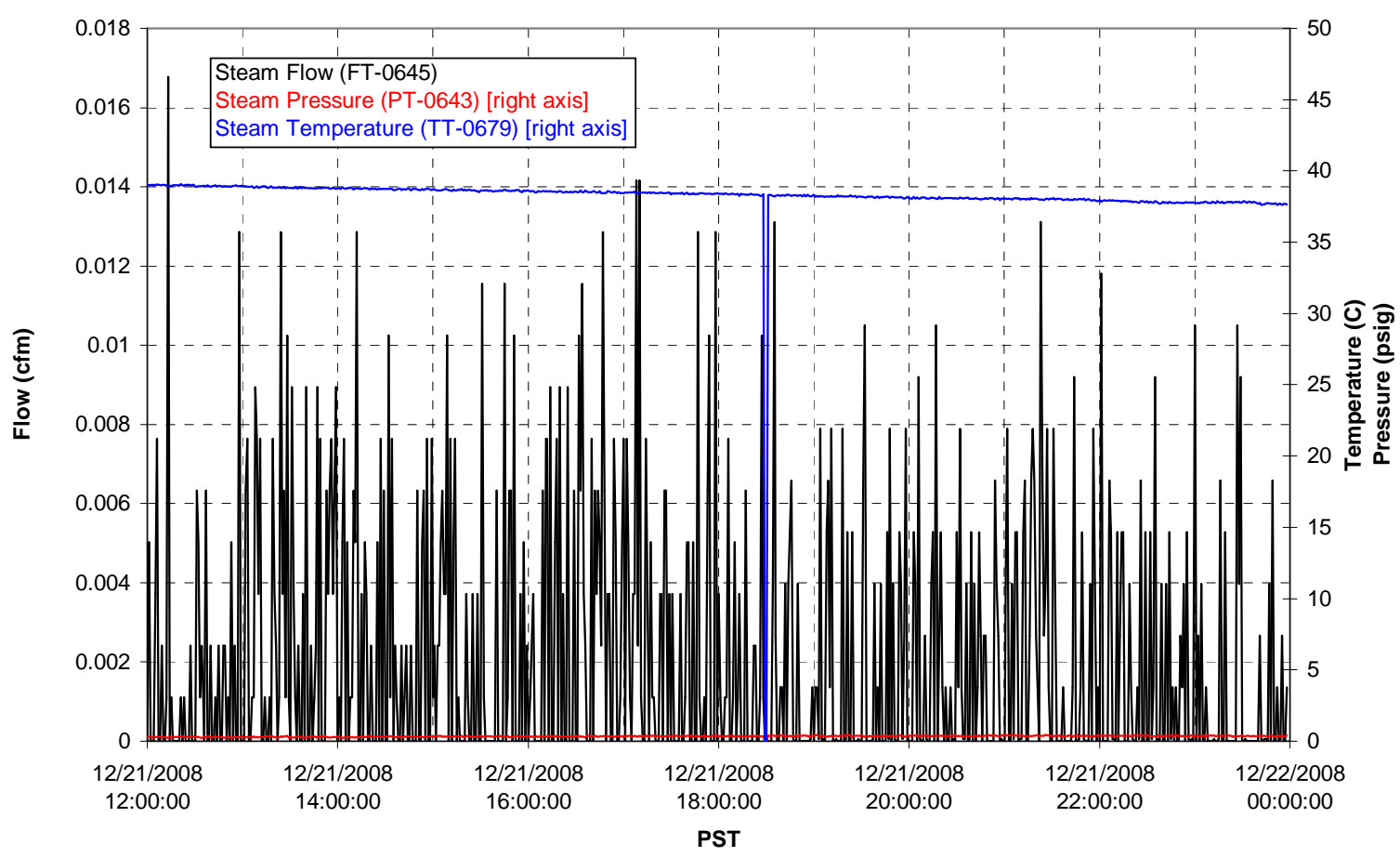

T01A Steam

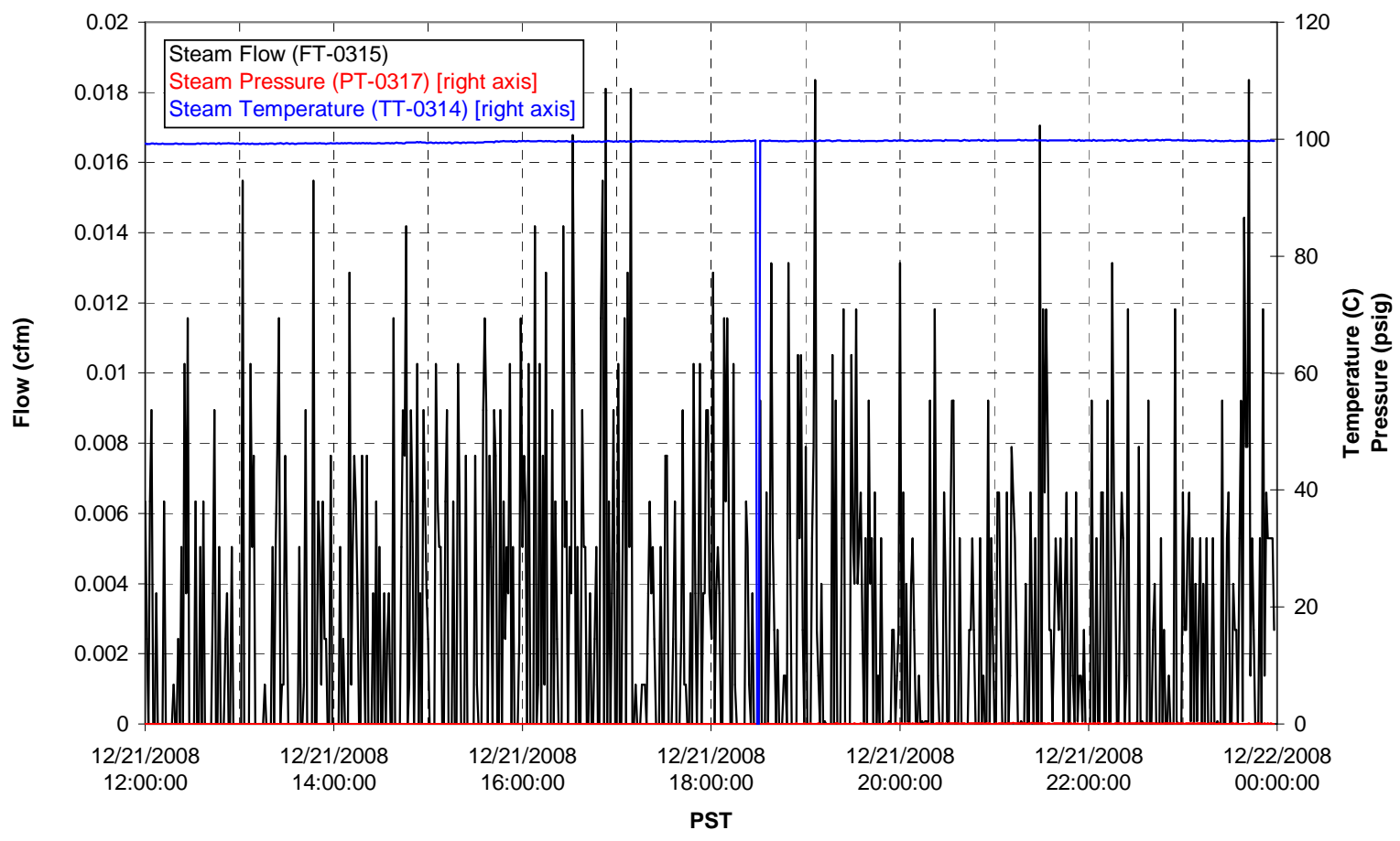


T01B Steam

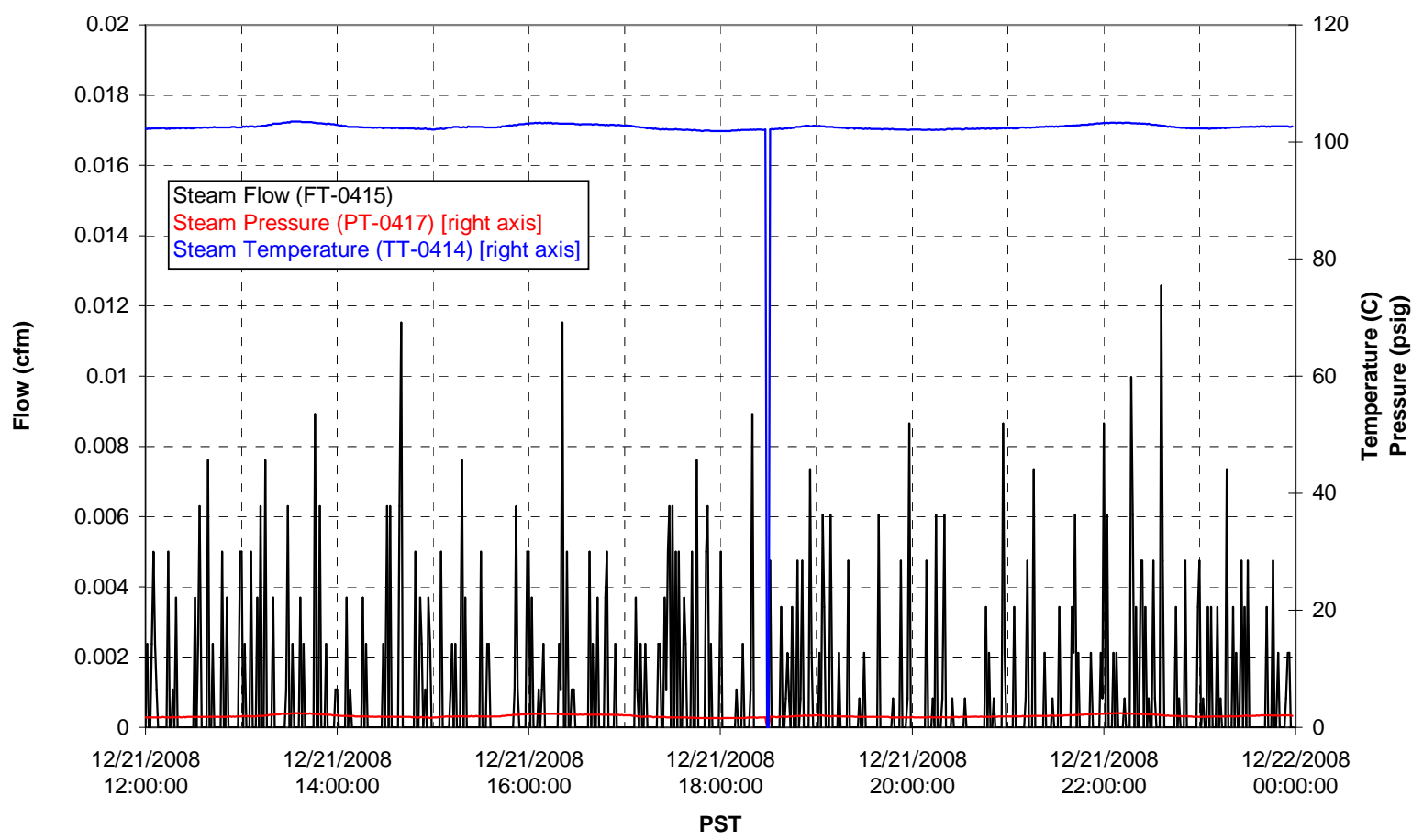


Functional Test Data Plots

12/22/08 00:00 - 12/22/08 12:00 
T01A level

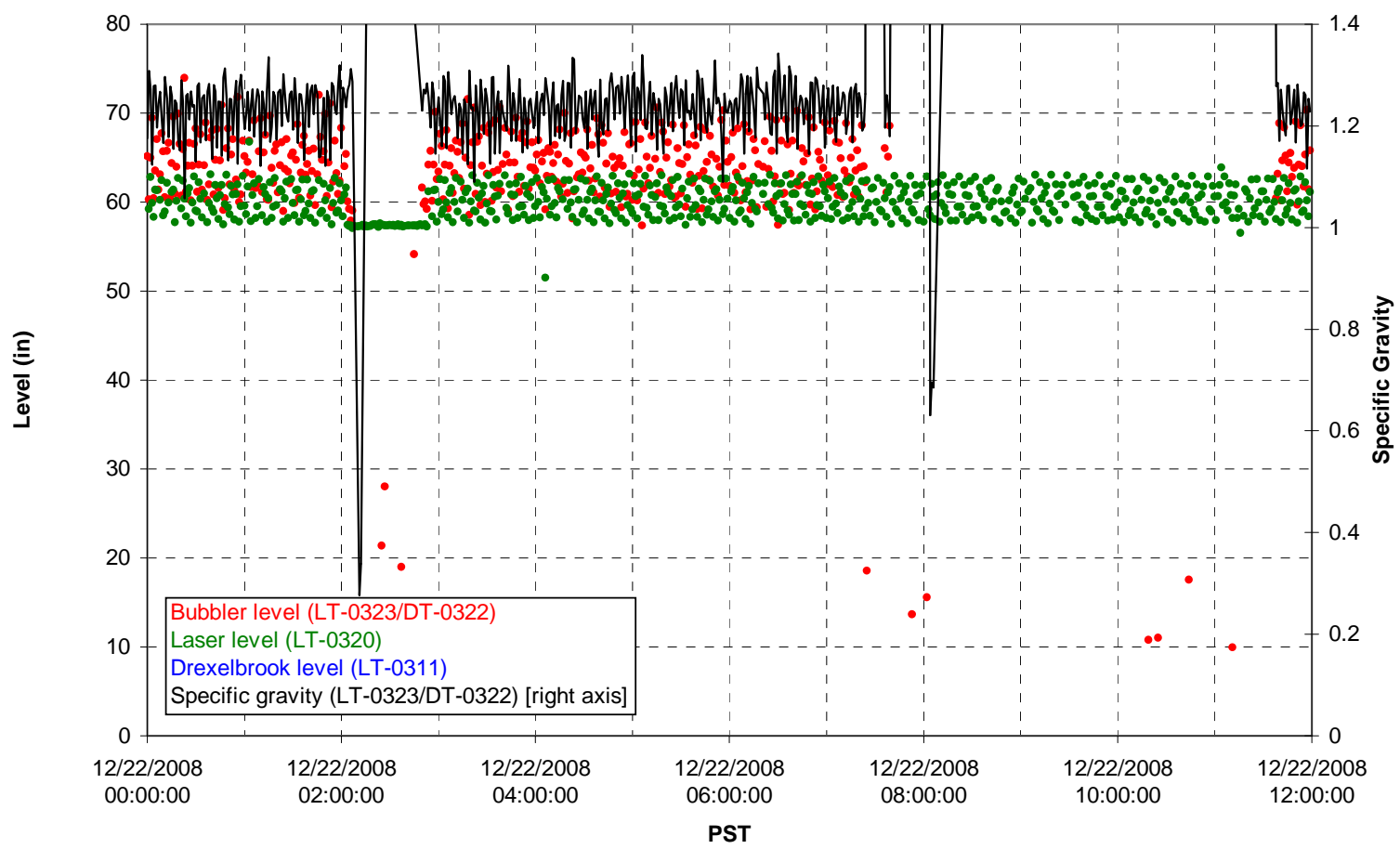

T01A temperatures

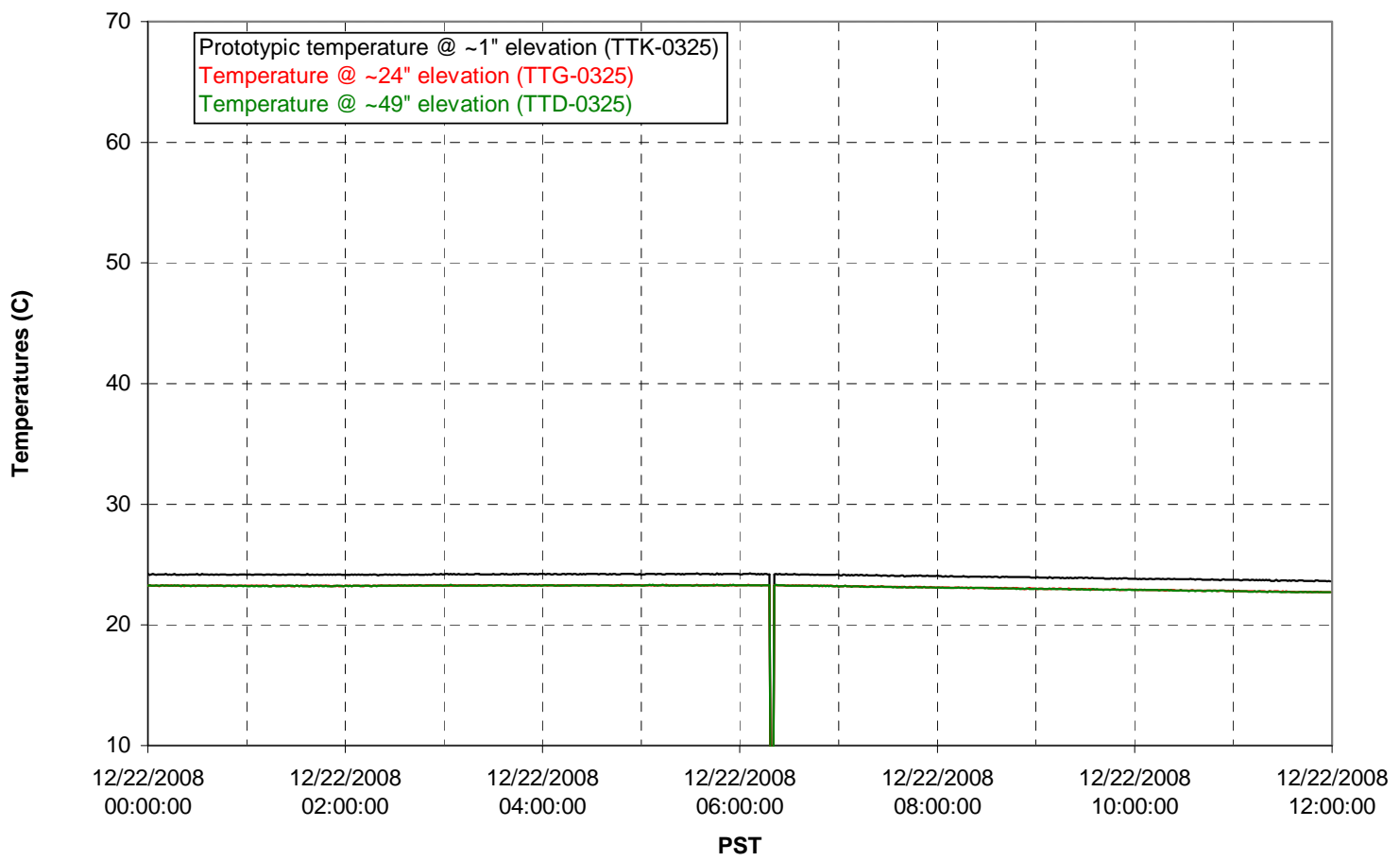


T01B level

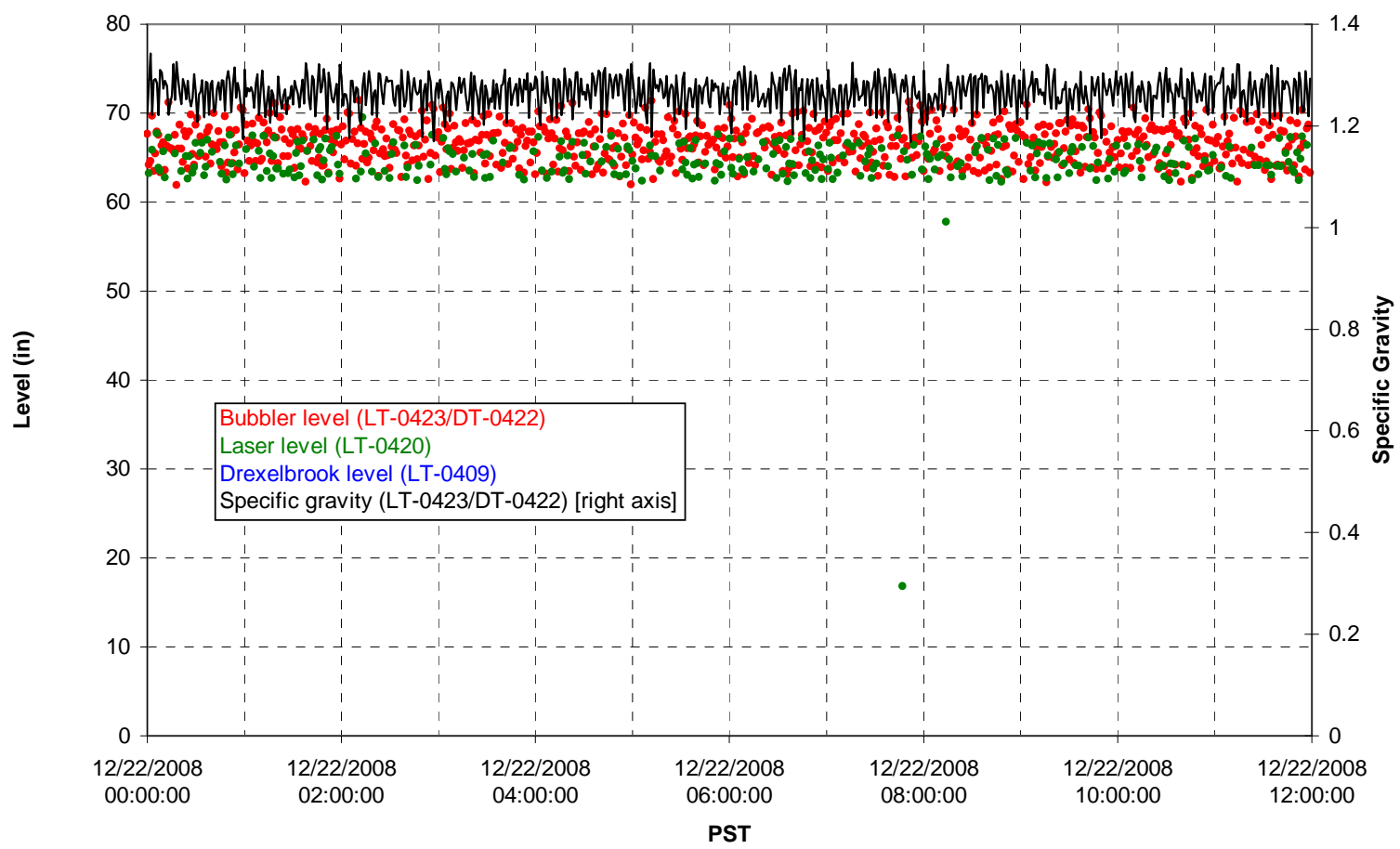

T01B temperatures

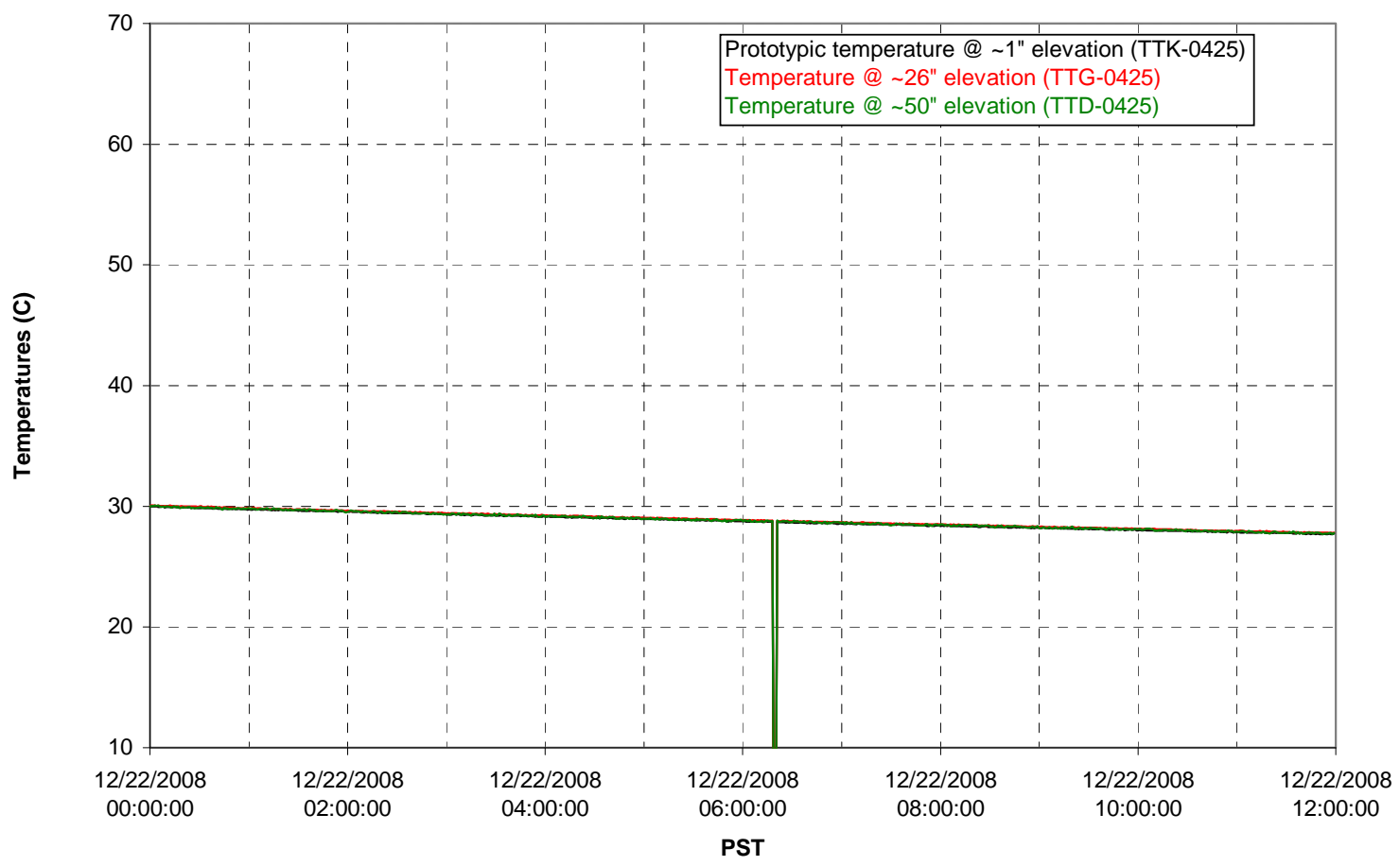


T02A level

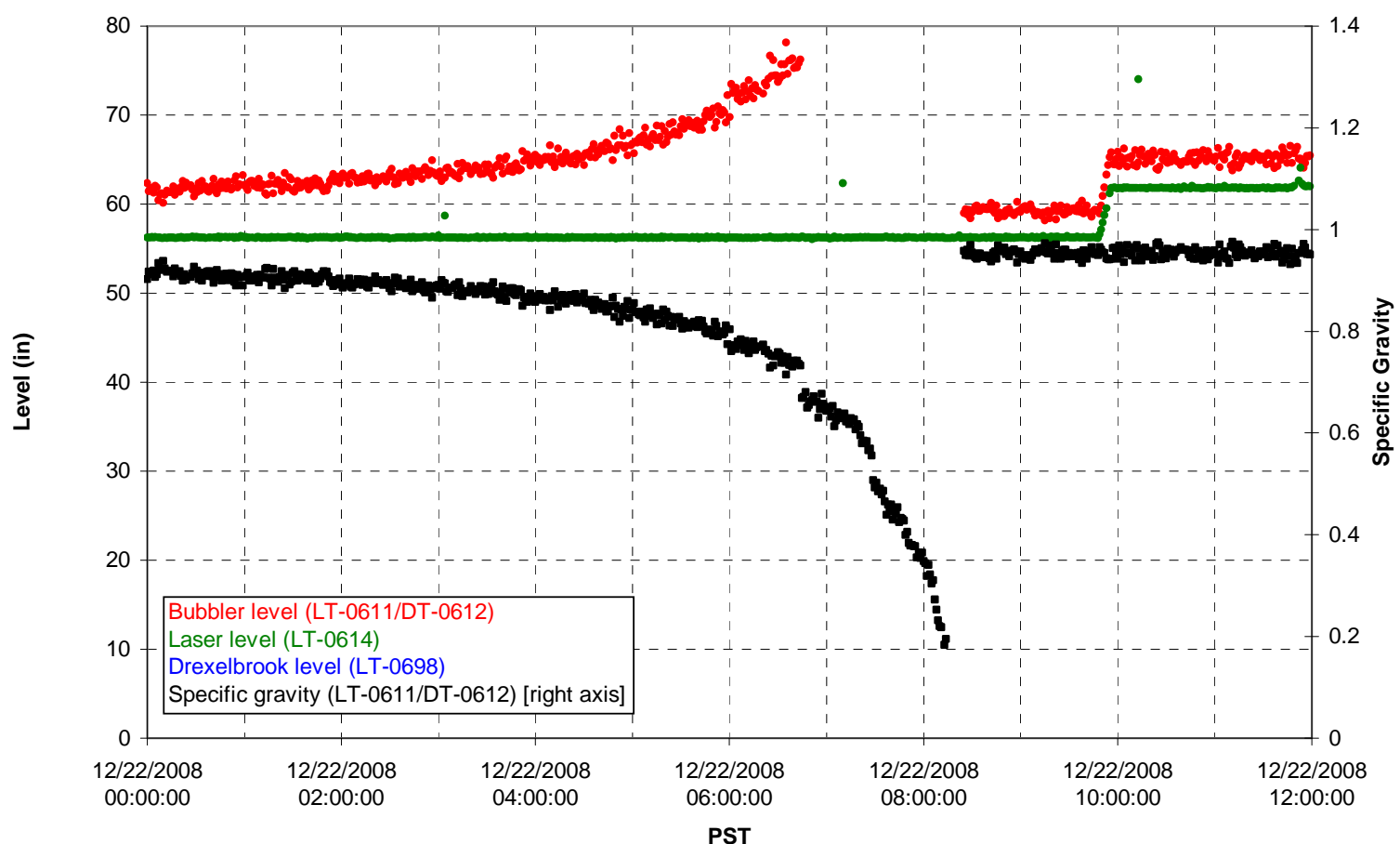

T02A temperatures

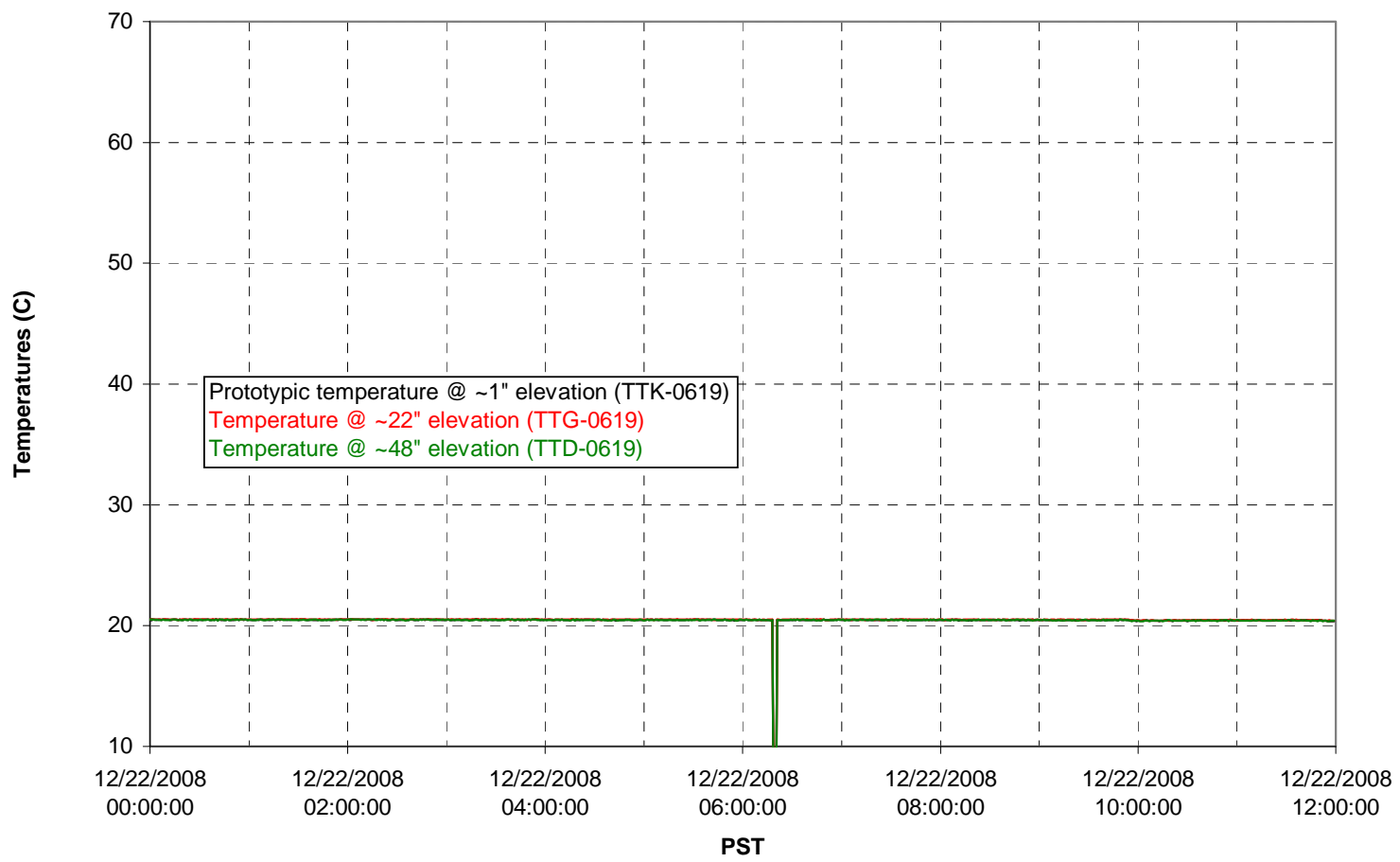


T02A and filter loop temperatures

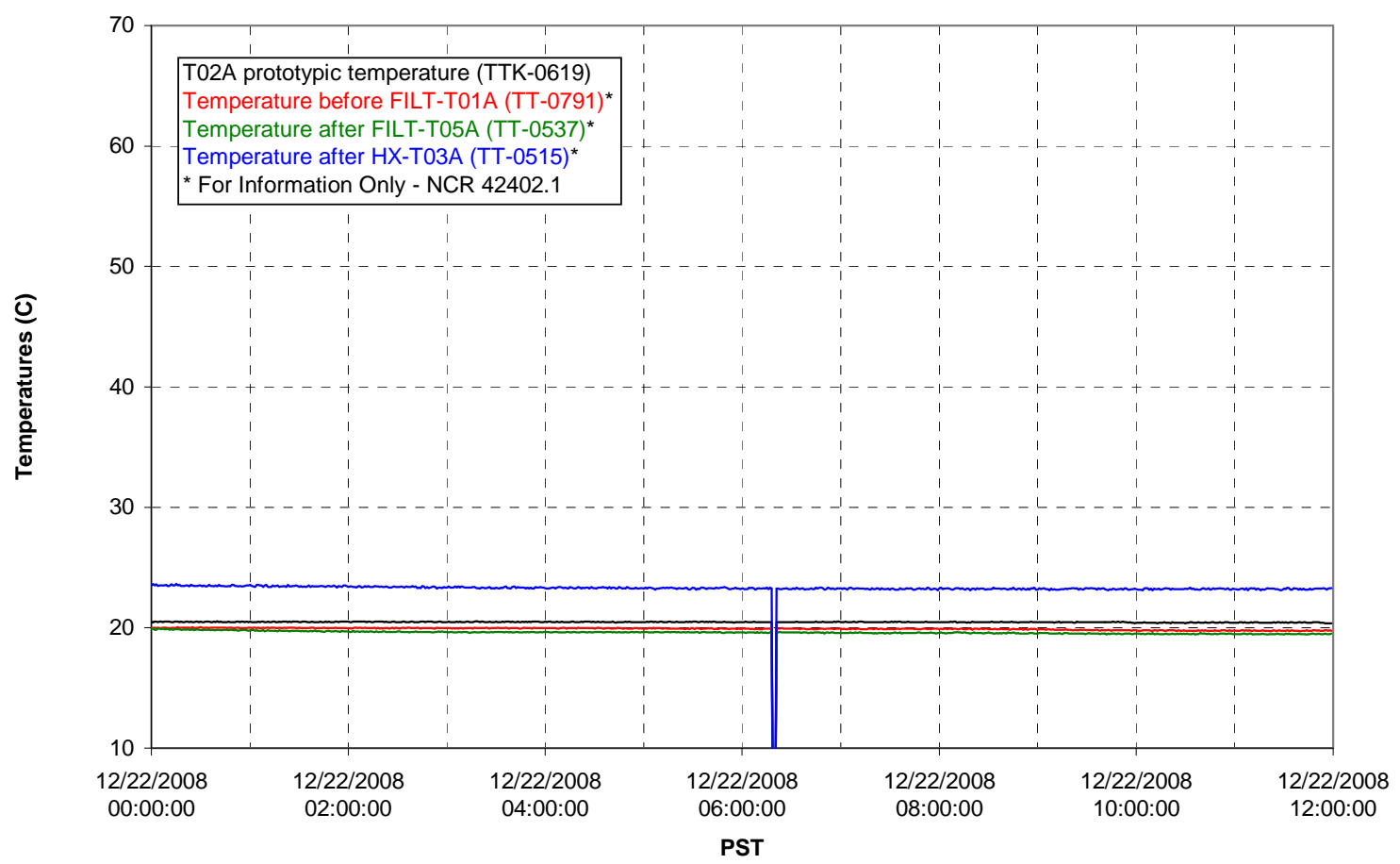

Pump Pressures and Flow

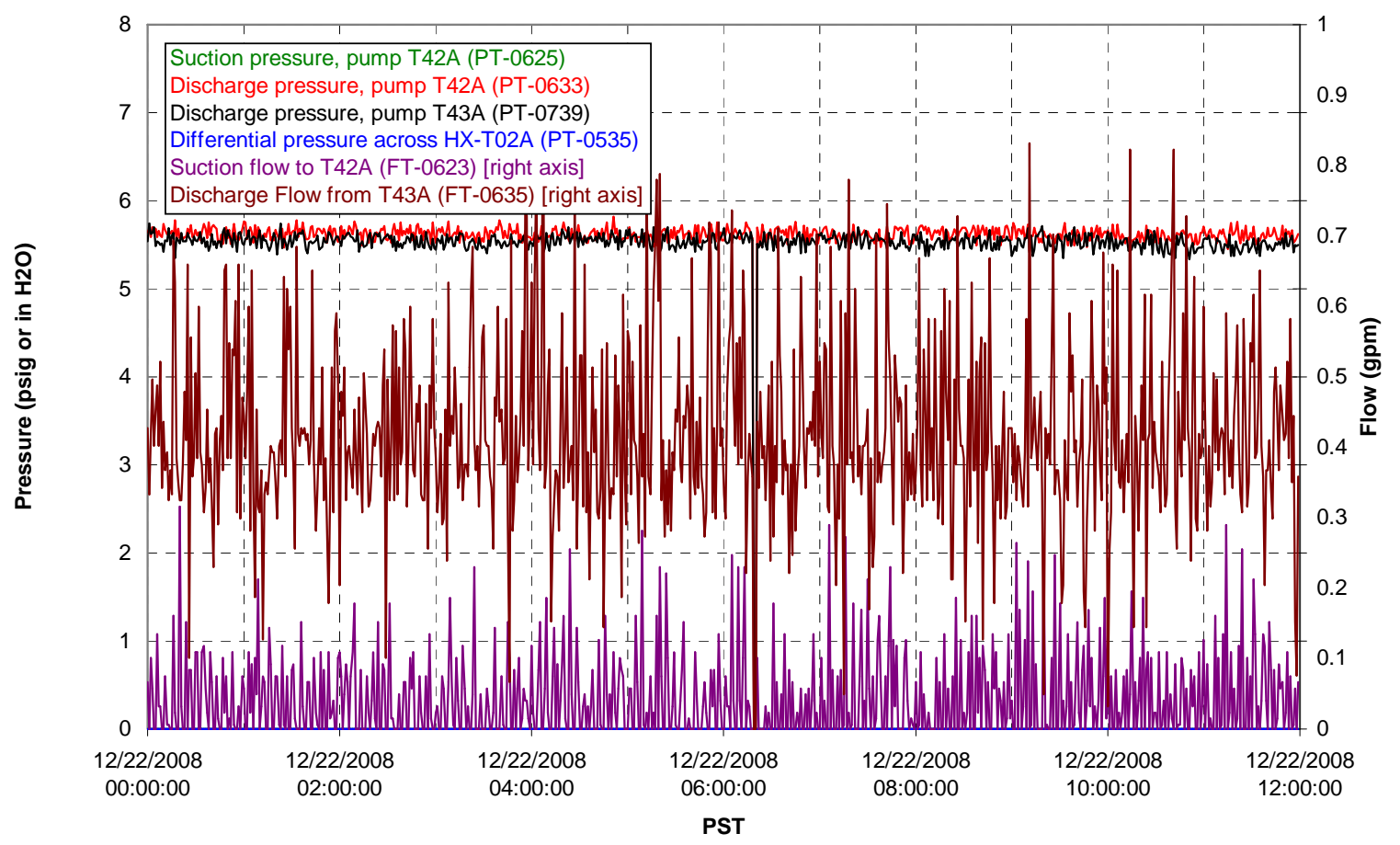


Axial pressure drop

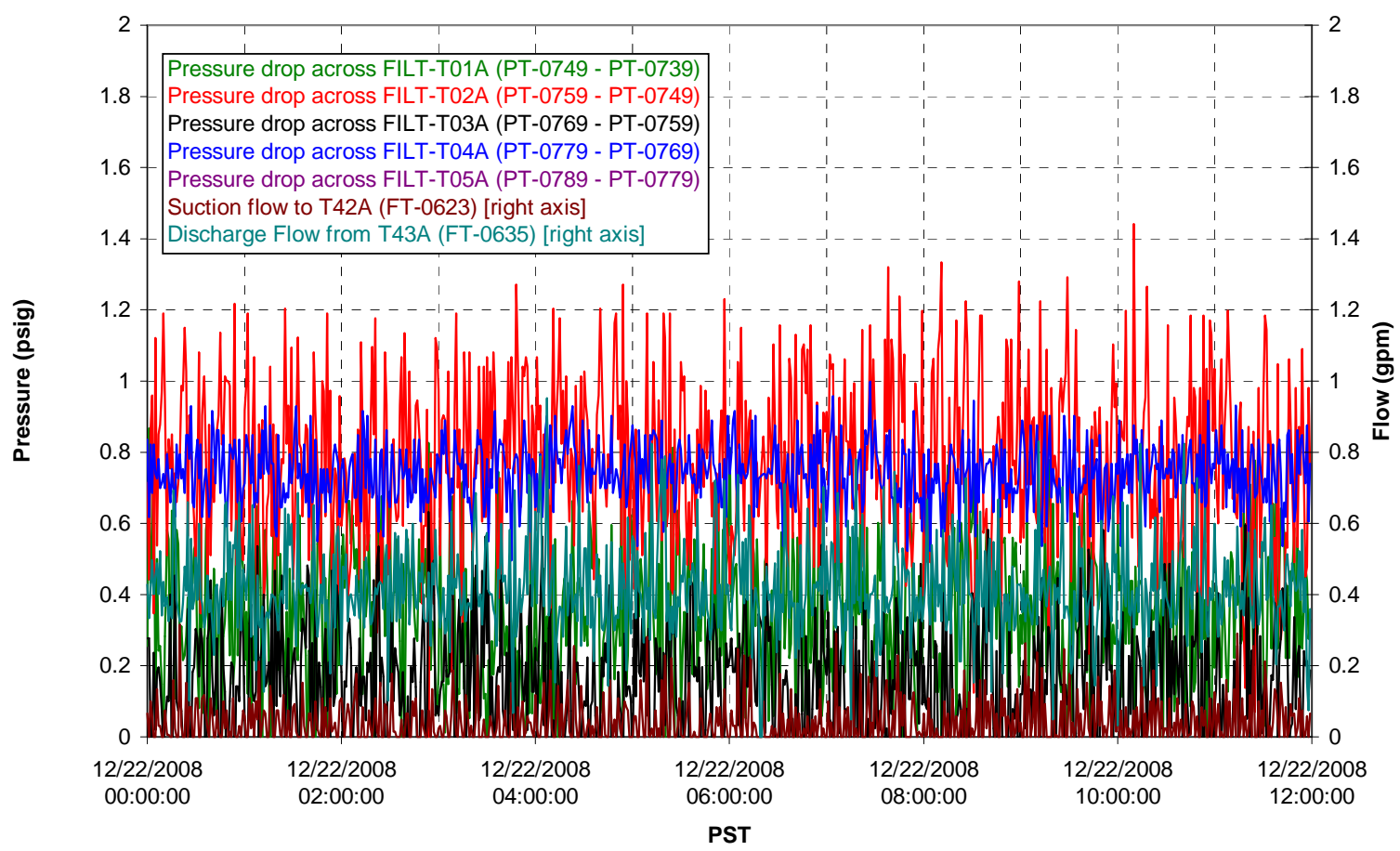

Permeate flow rates

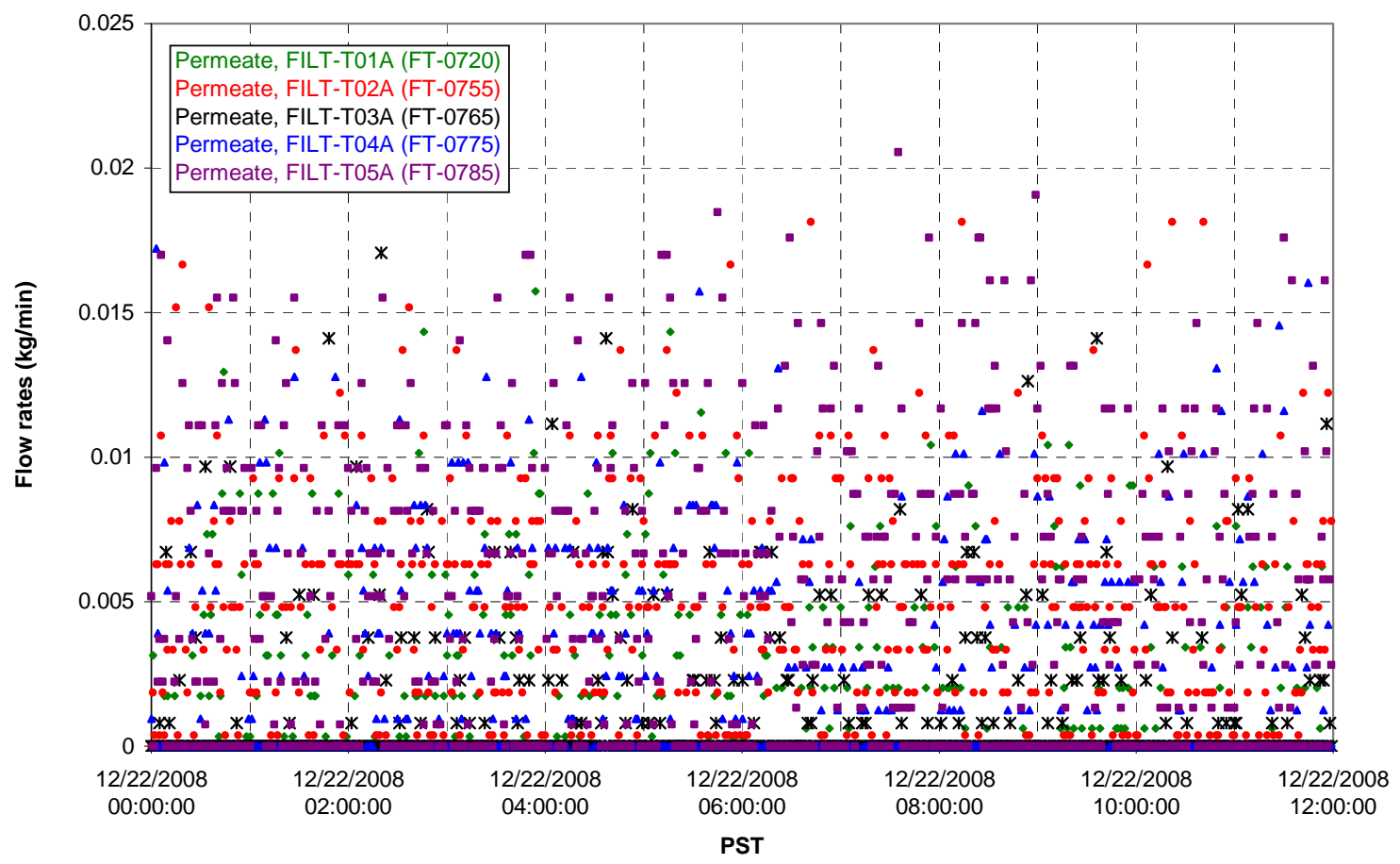


T02A Inner Temperature Tree

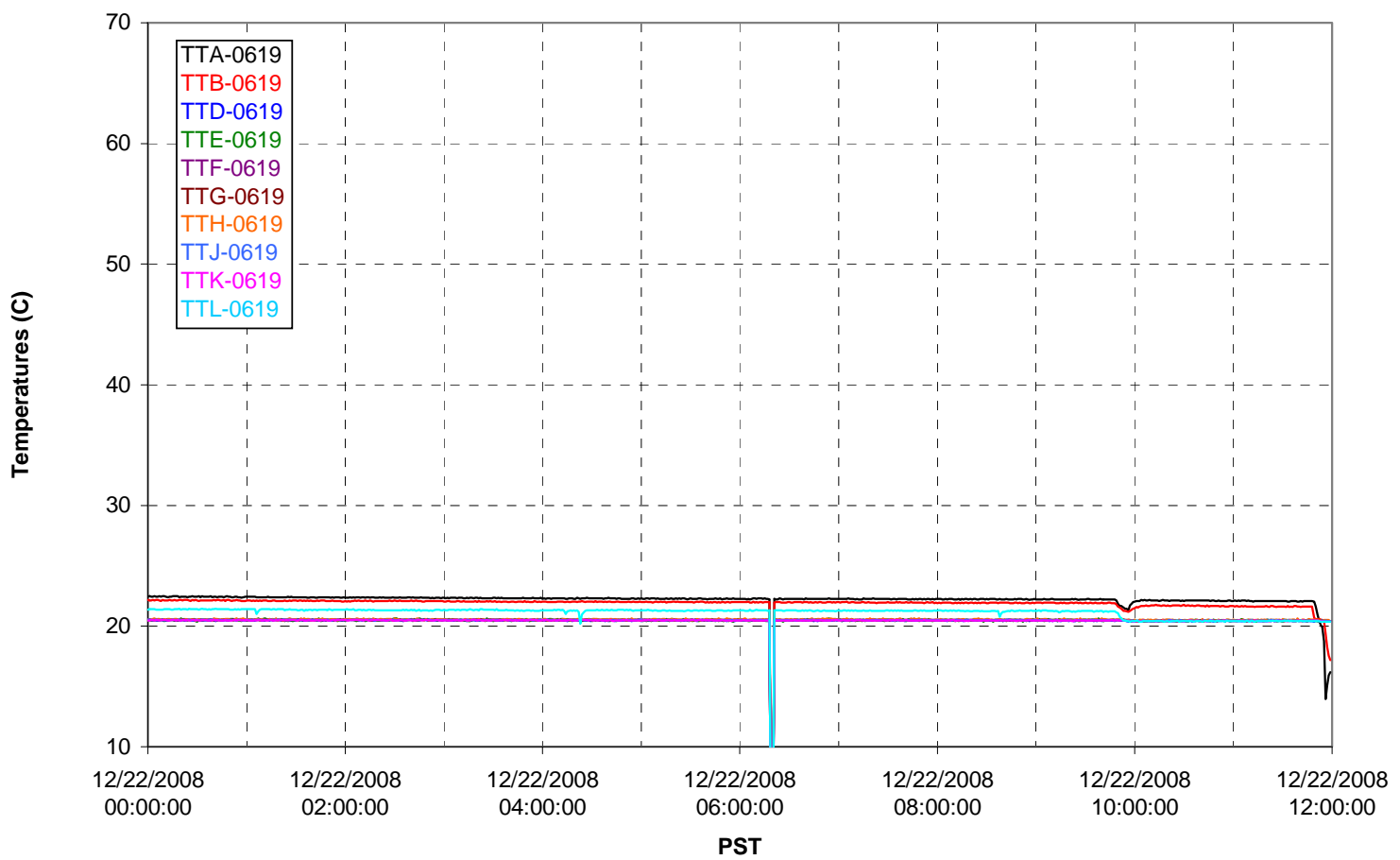

T02A Outer Temperature Tree

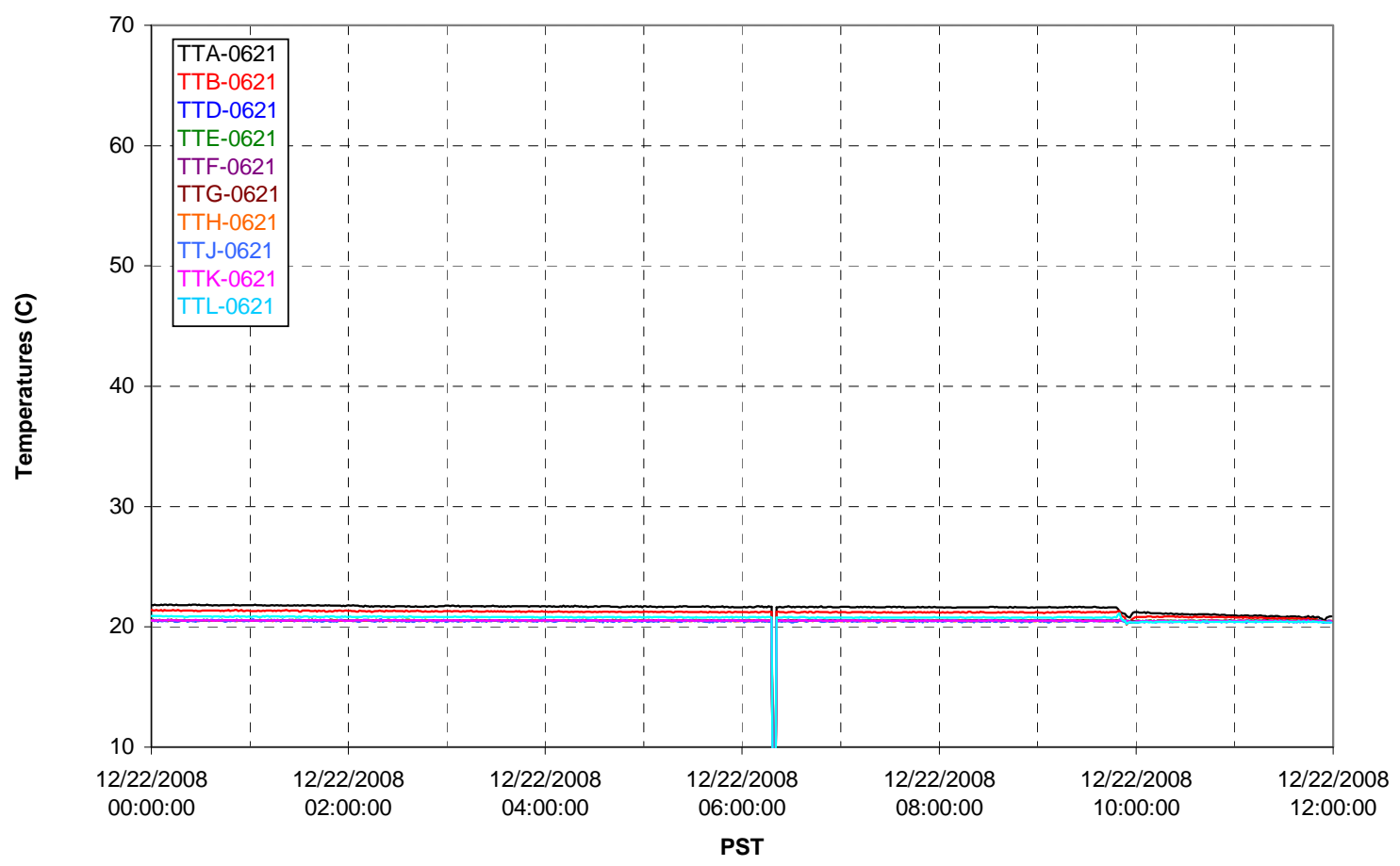


T02A temperatures

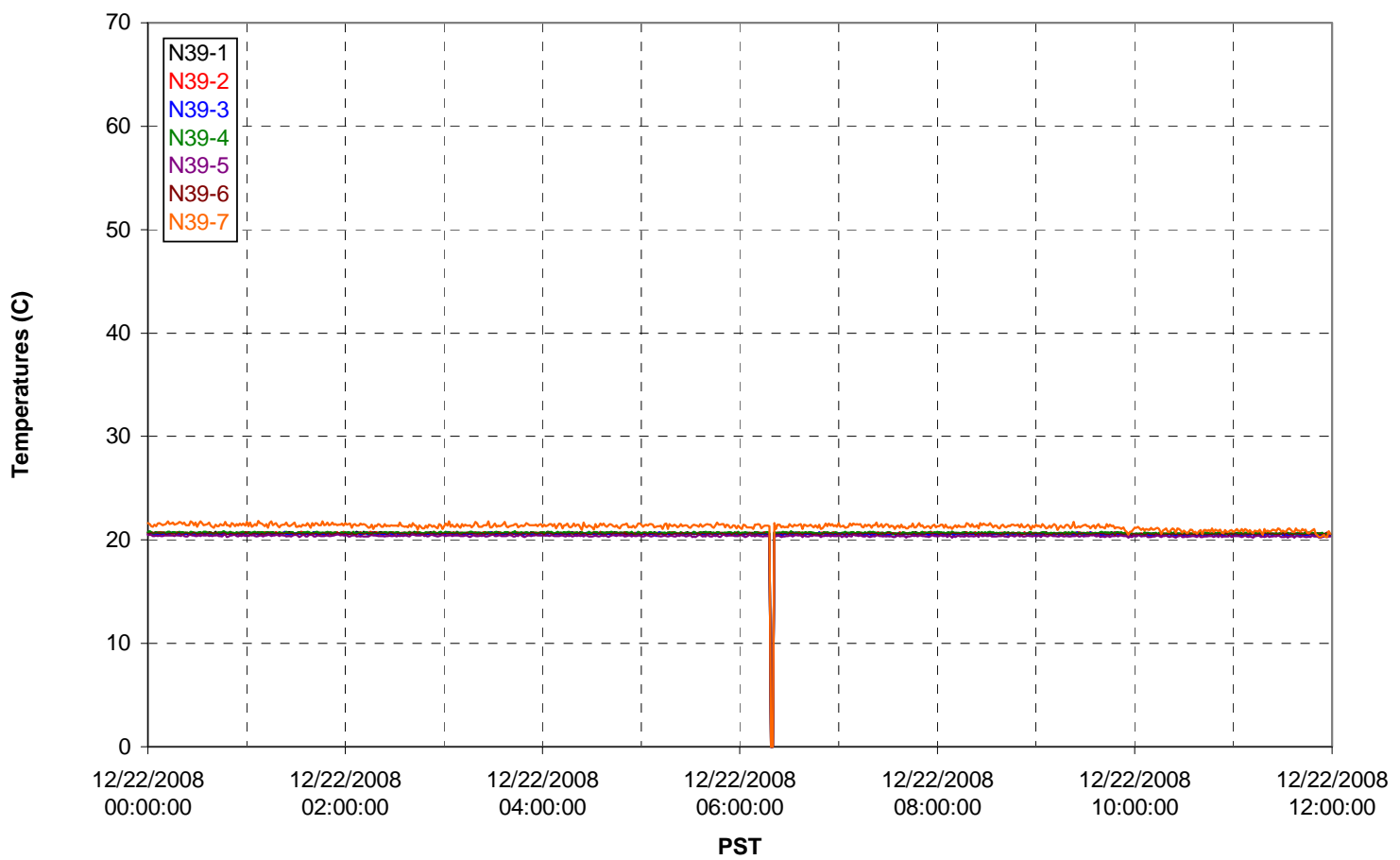

T02A temperatures

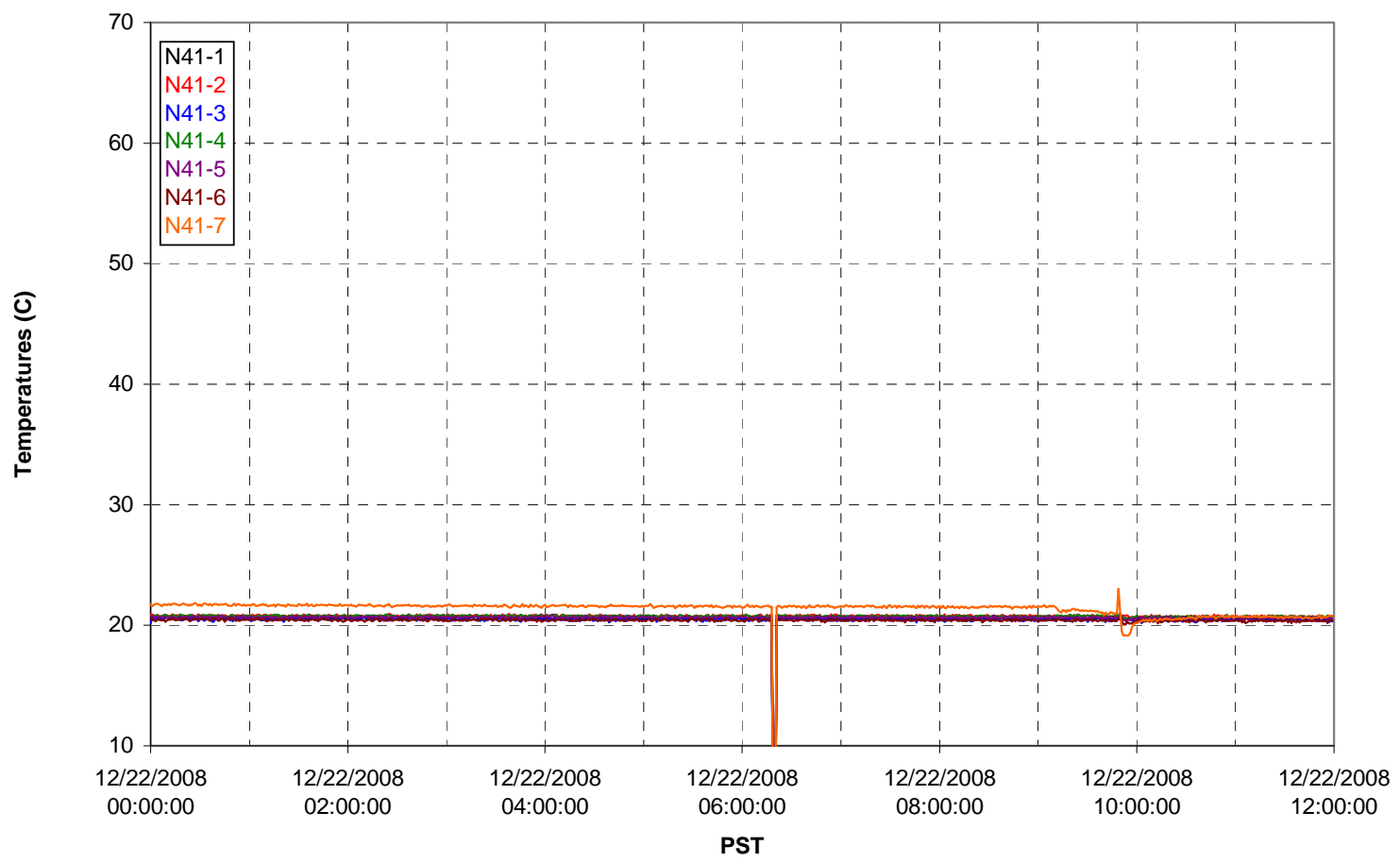


T02A temperatures

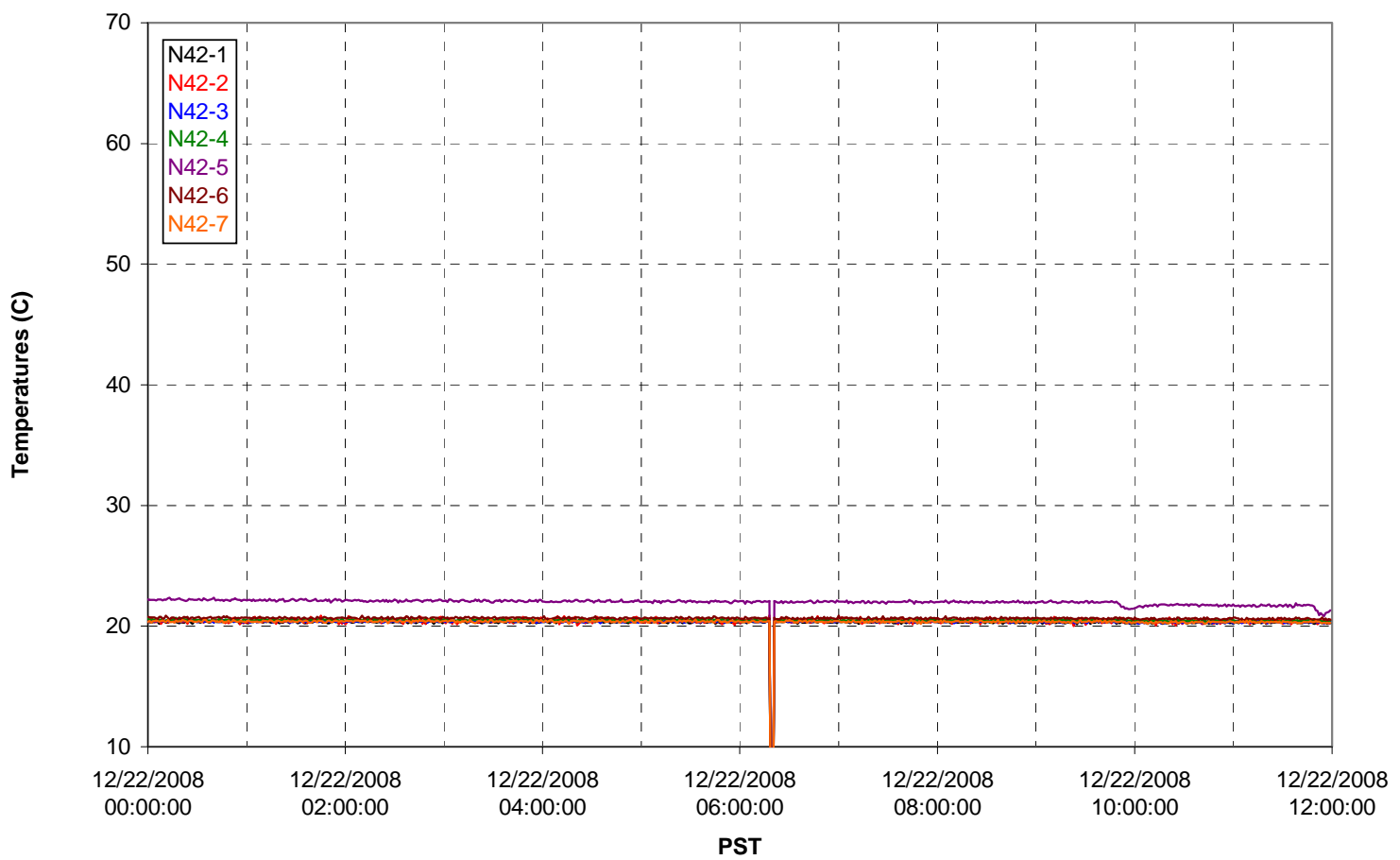

T02A temperatures

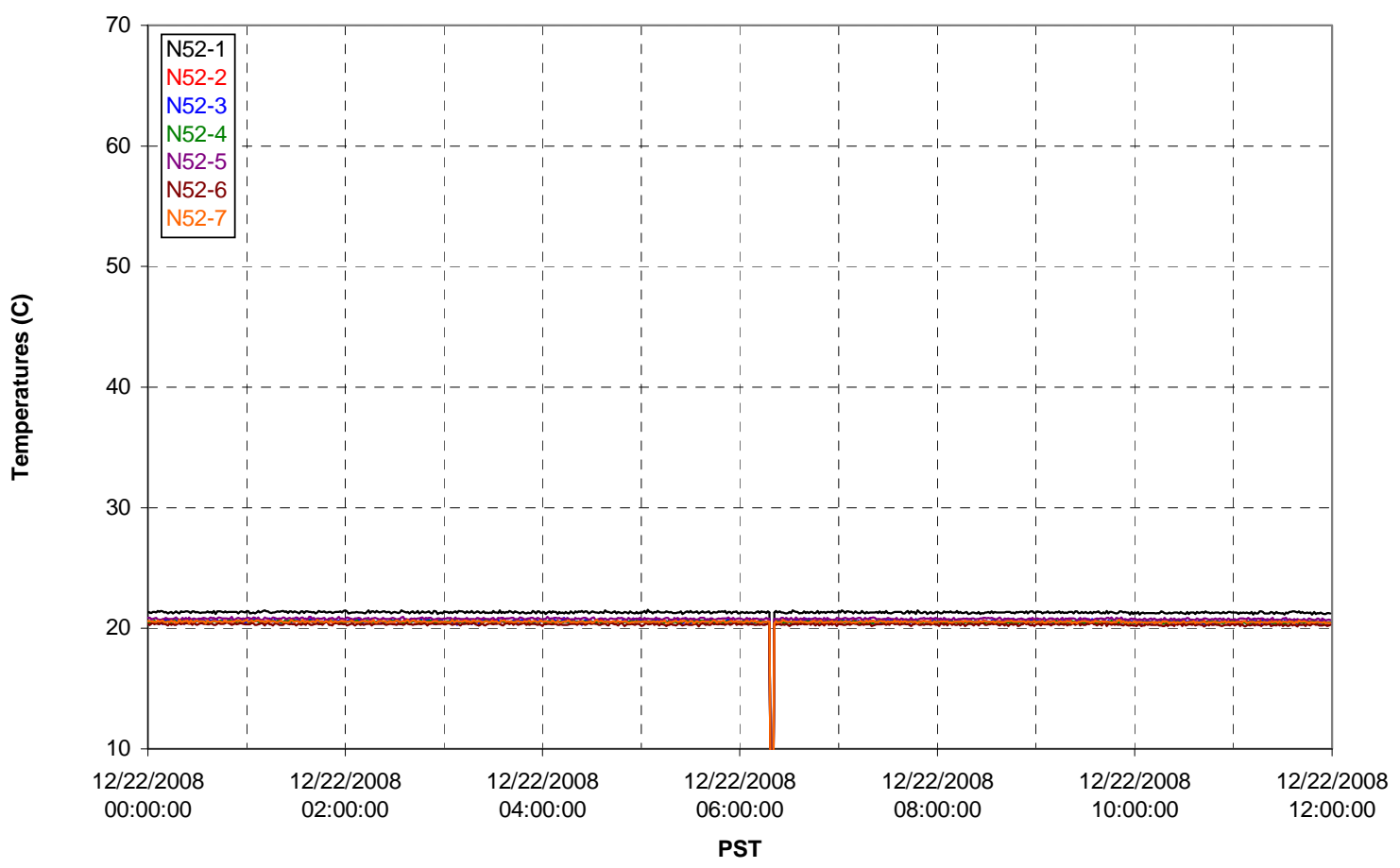


T02A Heating and Cooling

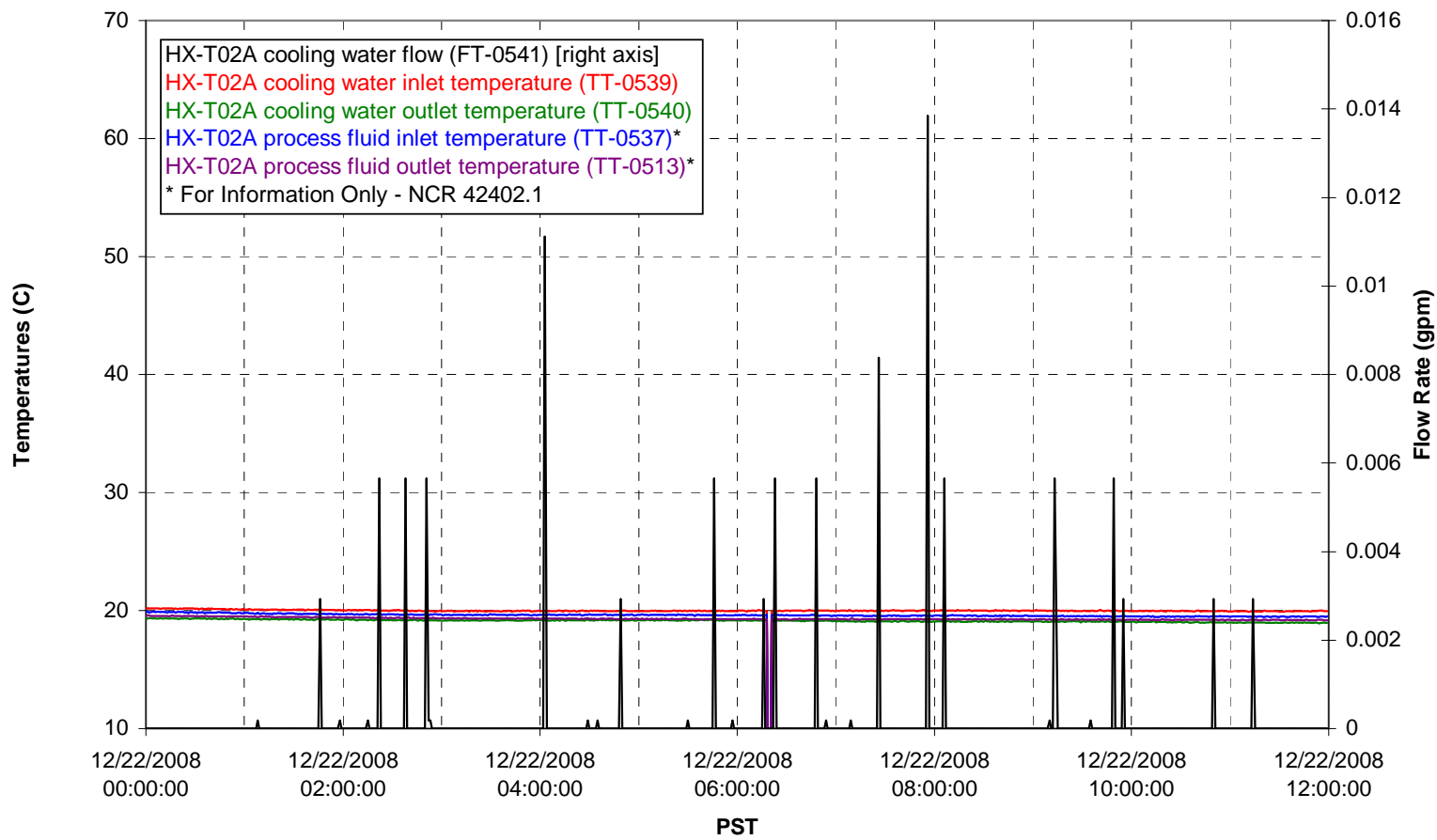

Pump Operation

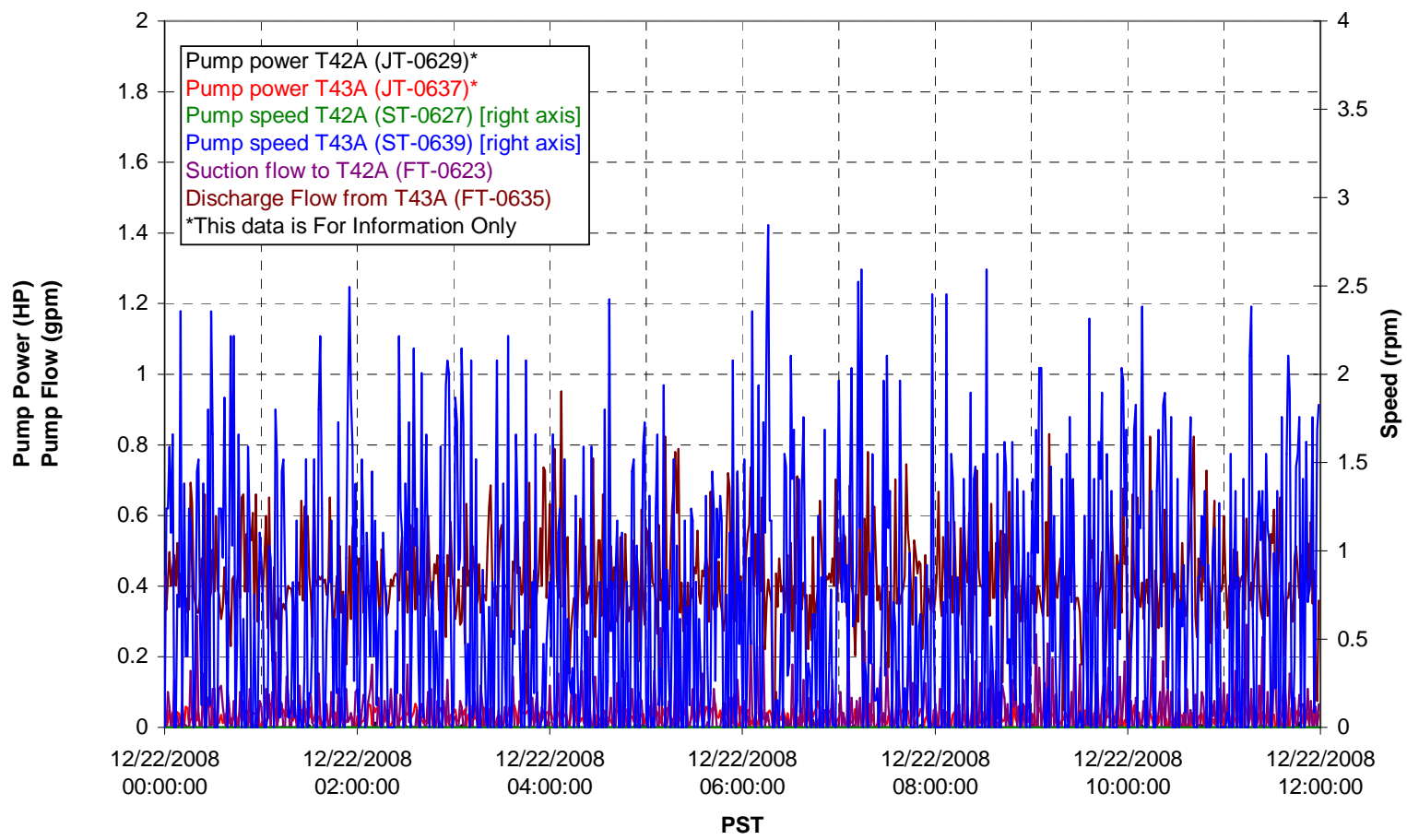




\section{Pulsepot UFP-PP-T01A}

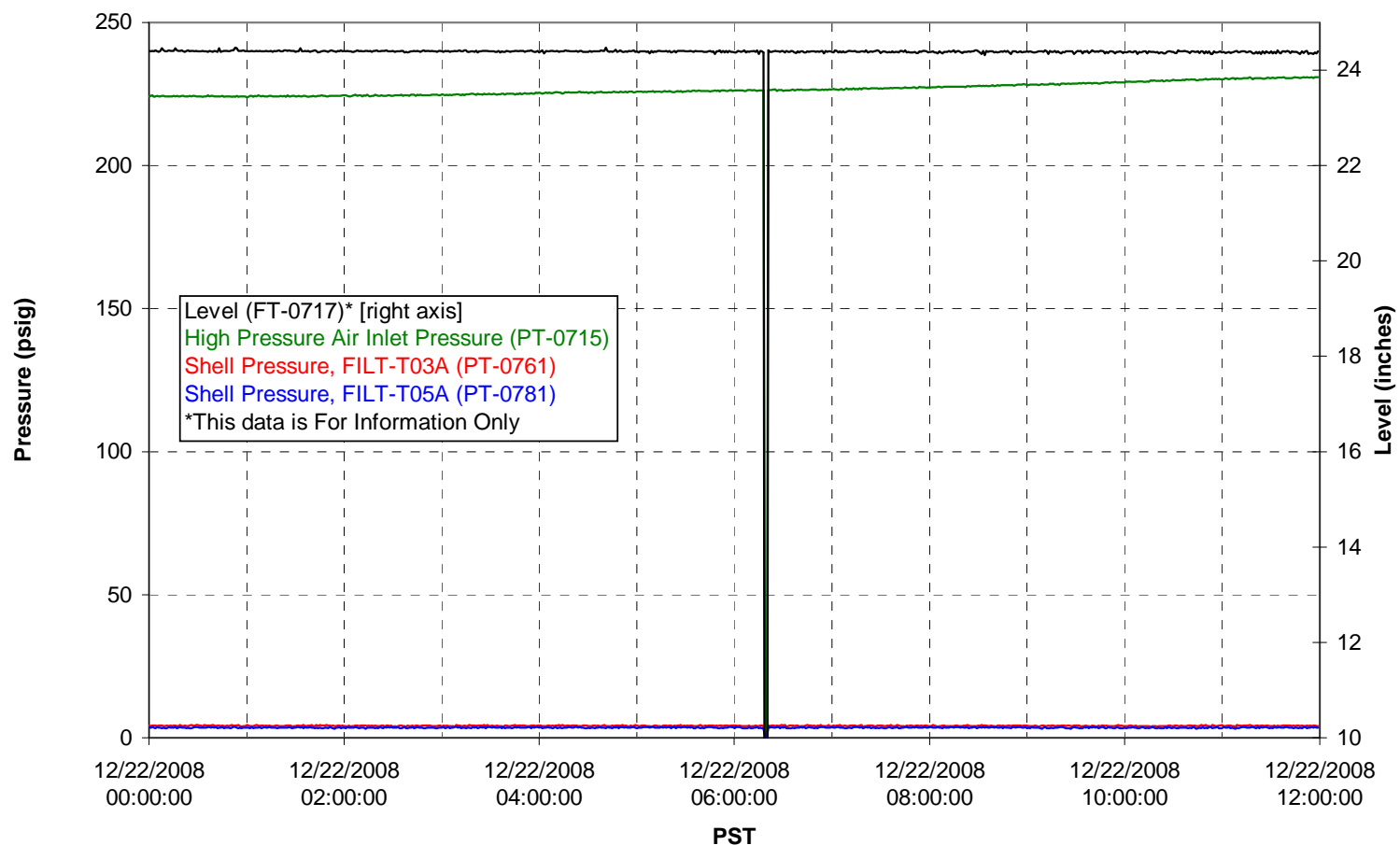

Pulsepot UFP-PP-T02A

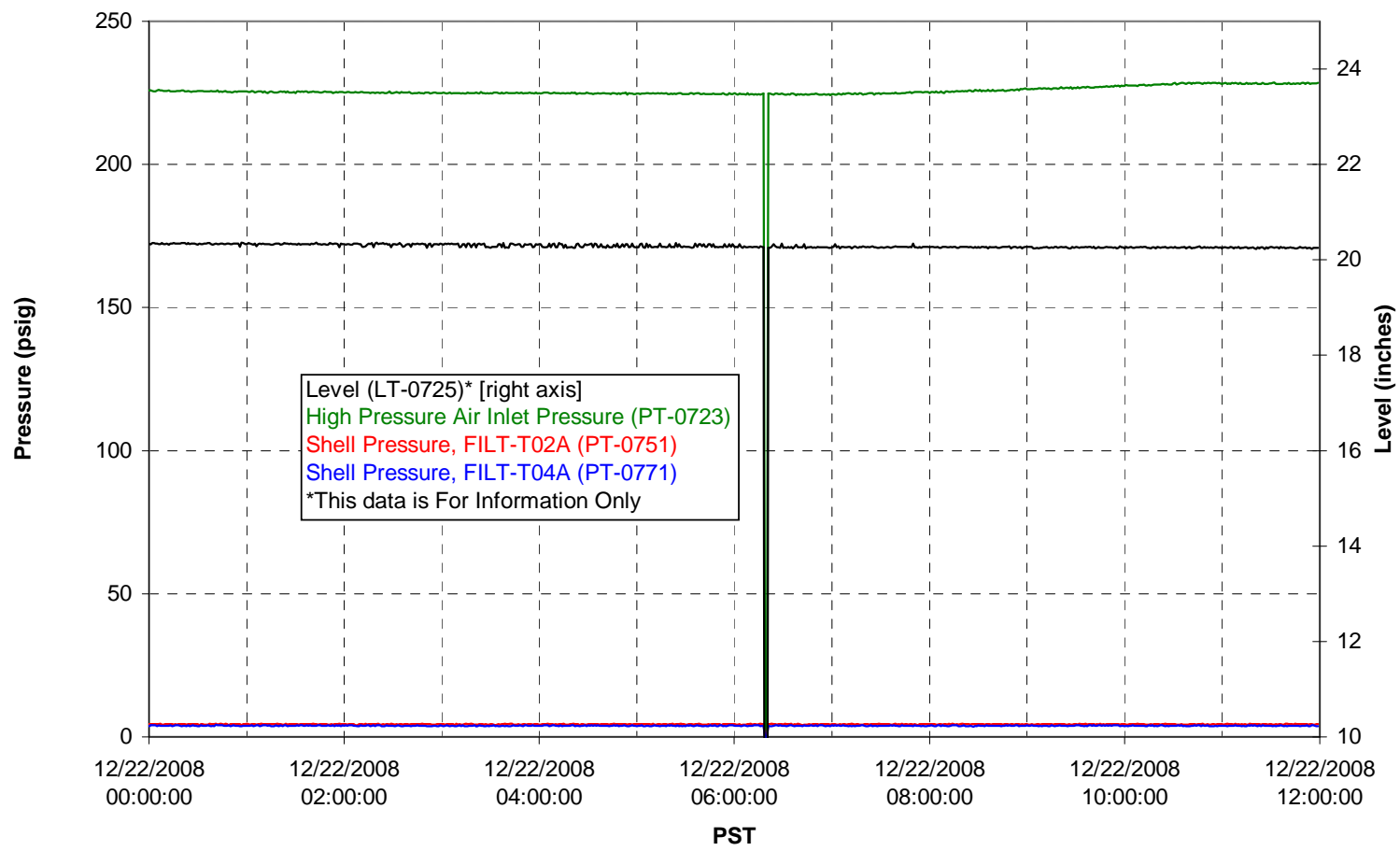


Pulsepot UFP-PP-T03A

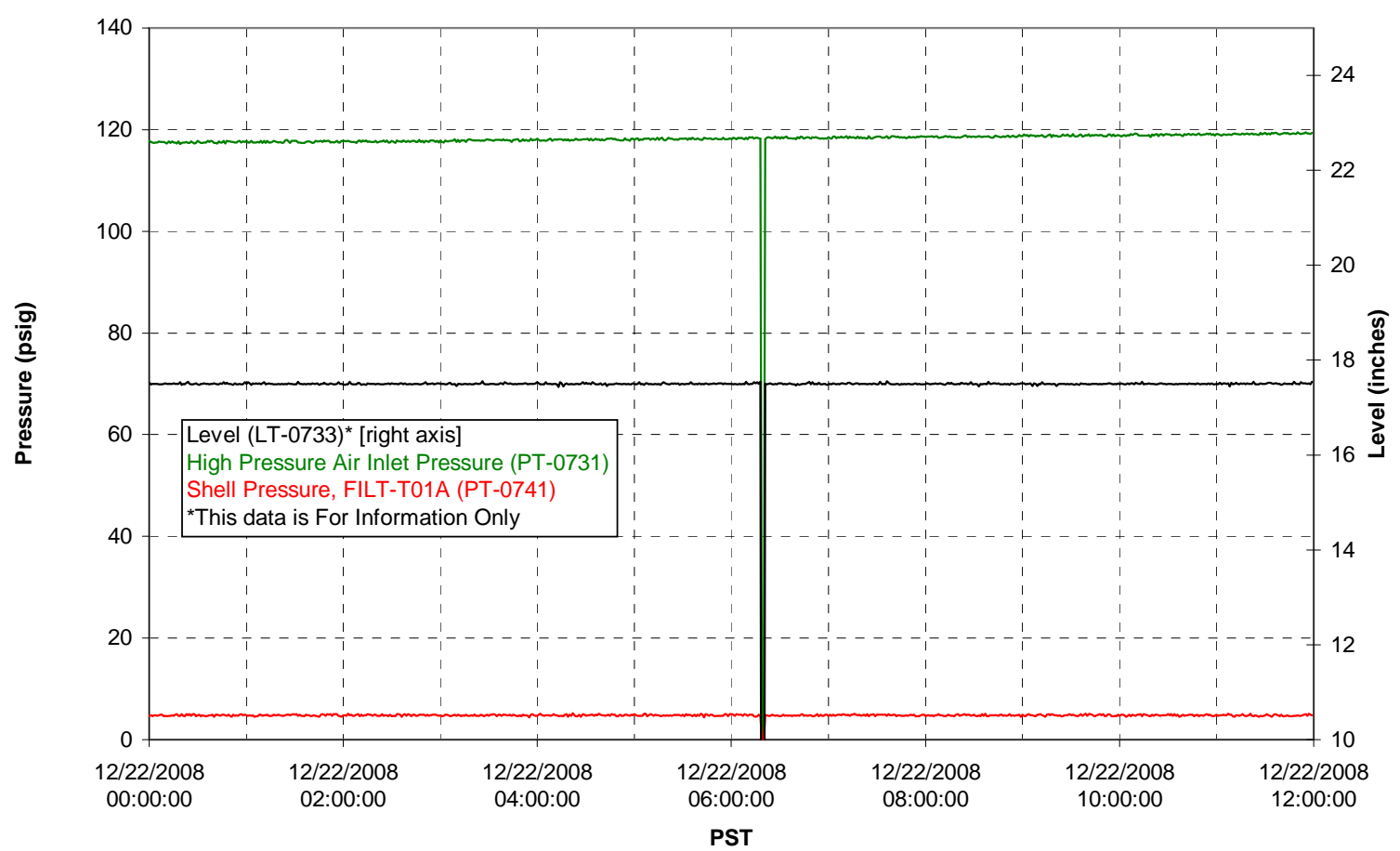

Pulsepot Levels

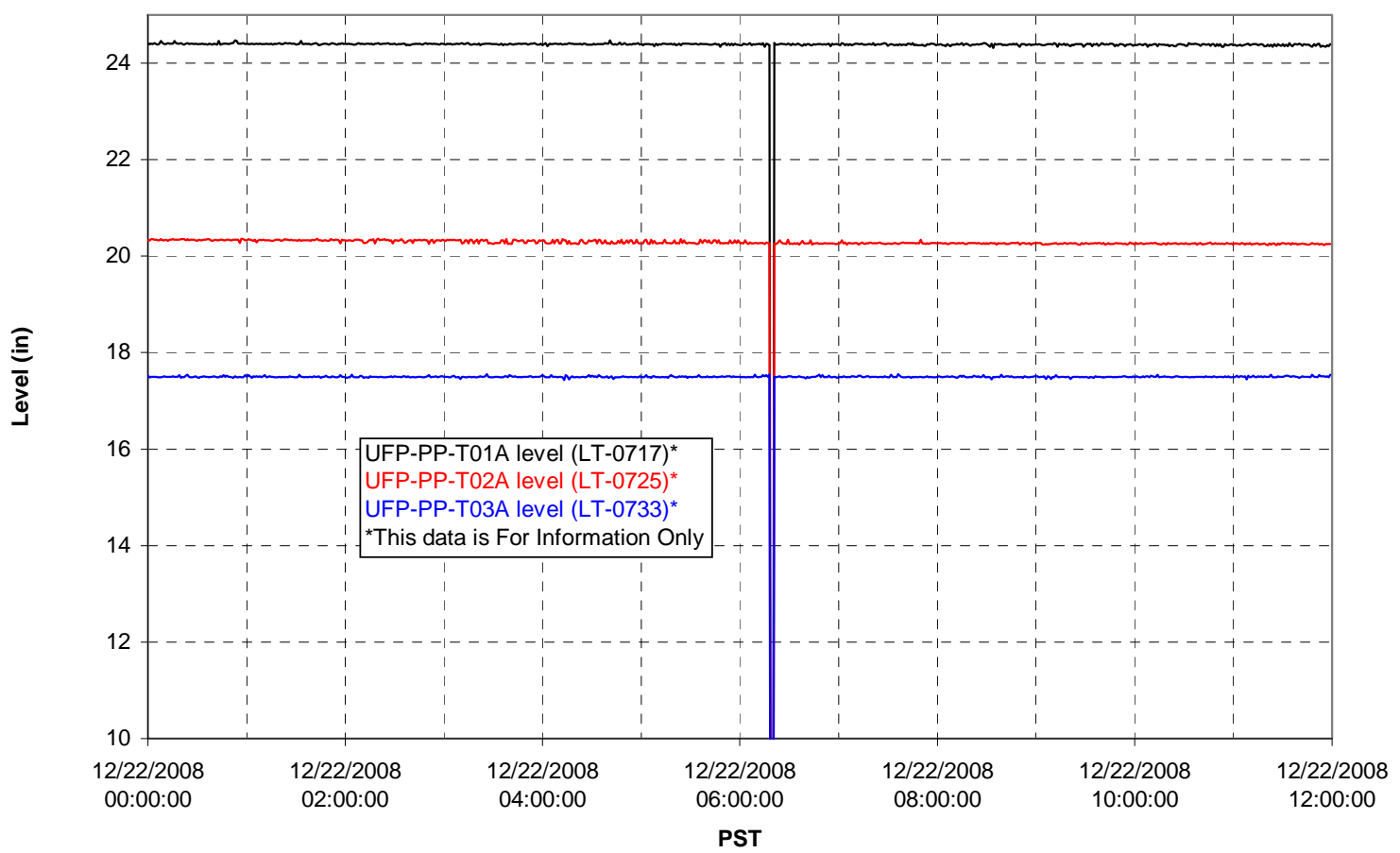


Filter UFP-FILT-T01A

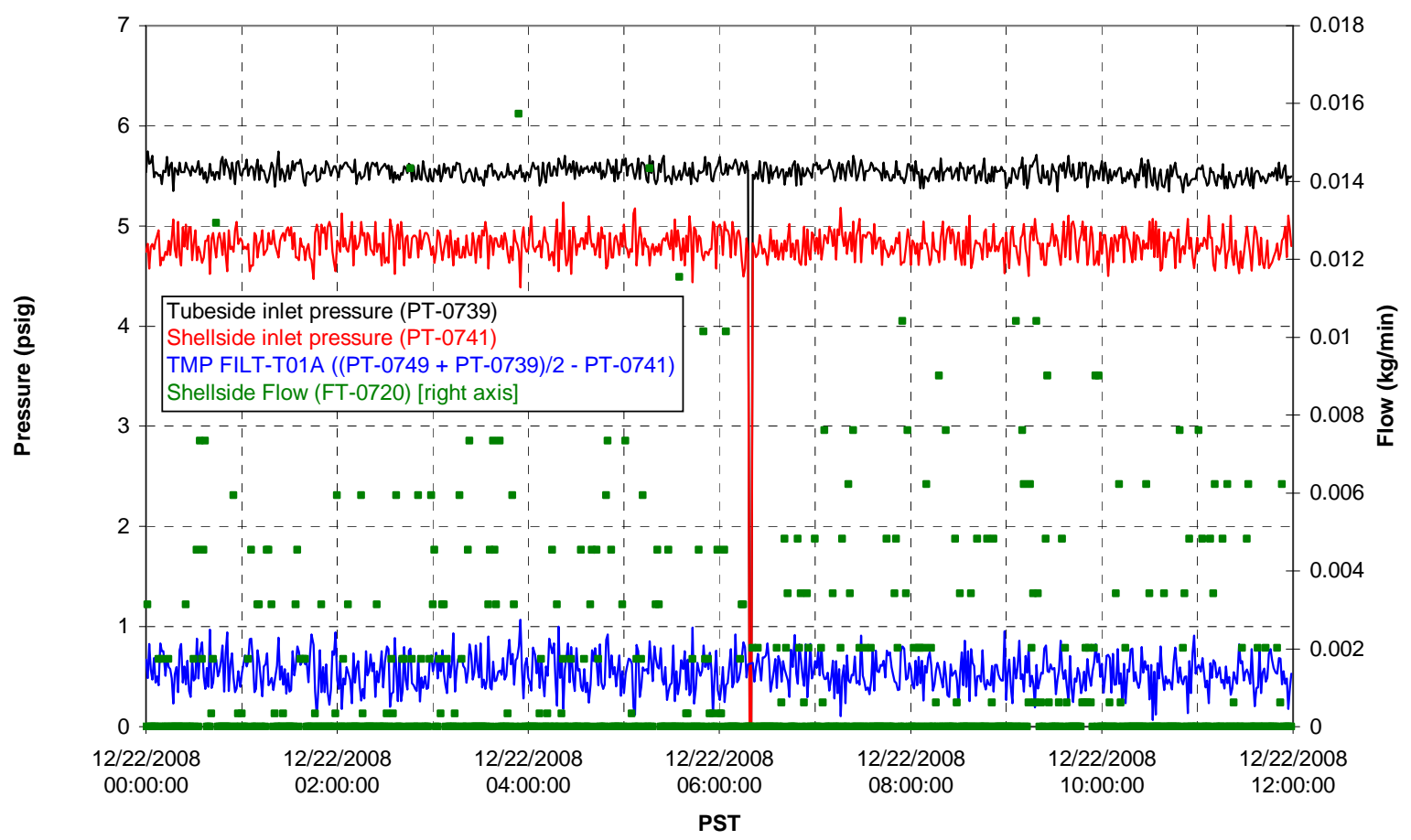

Filter UFP-FILT-T02A

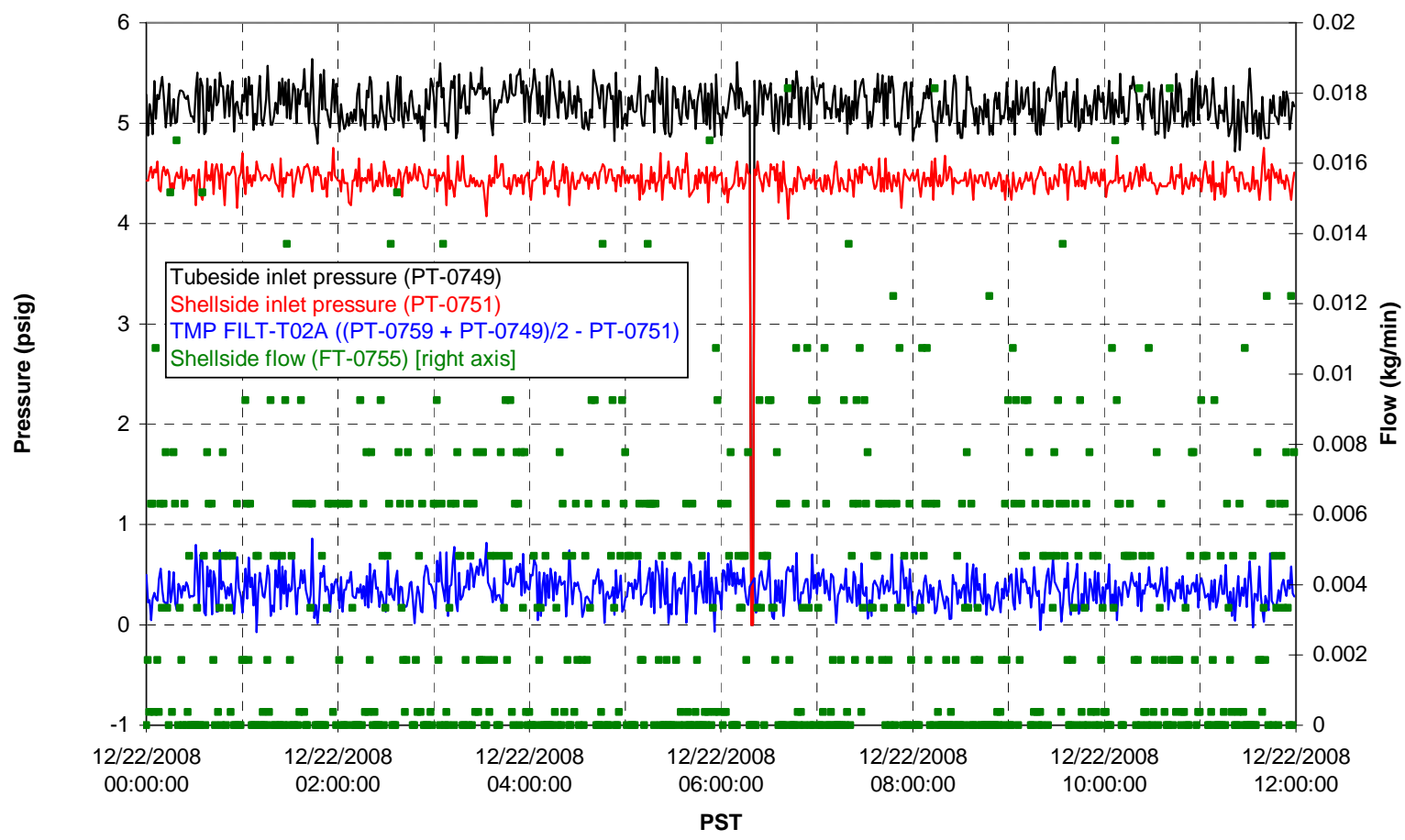


Filter UFP-FILT-T03A

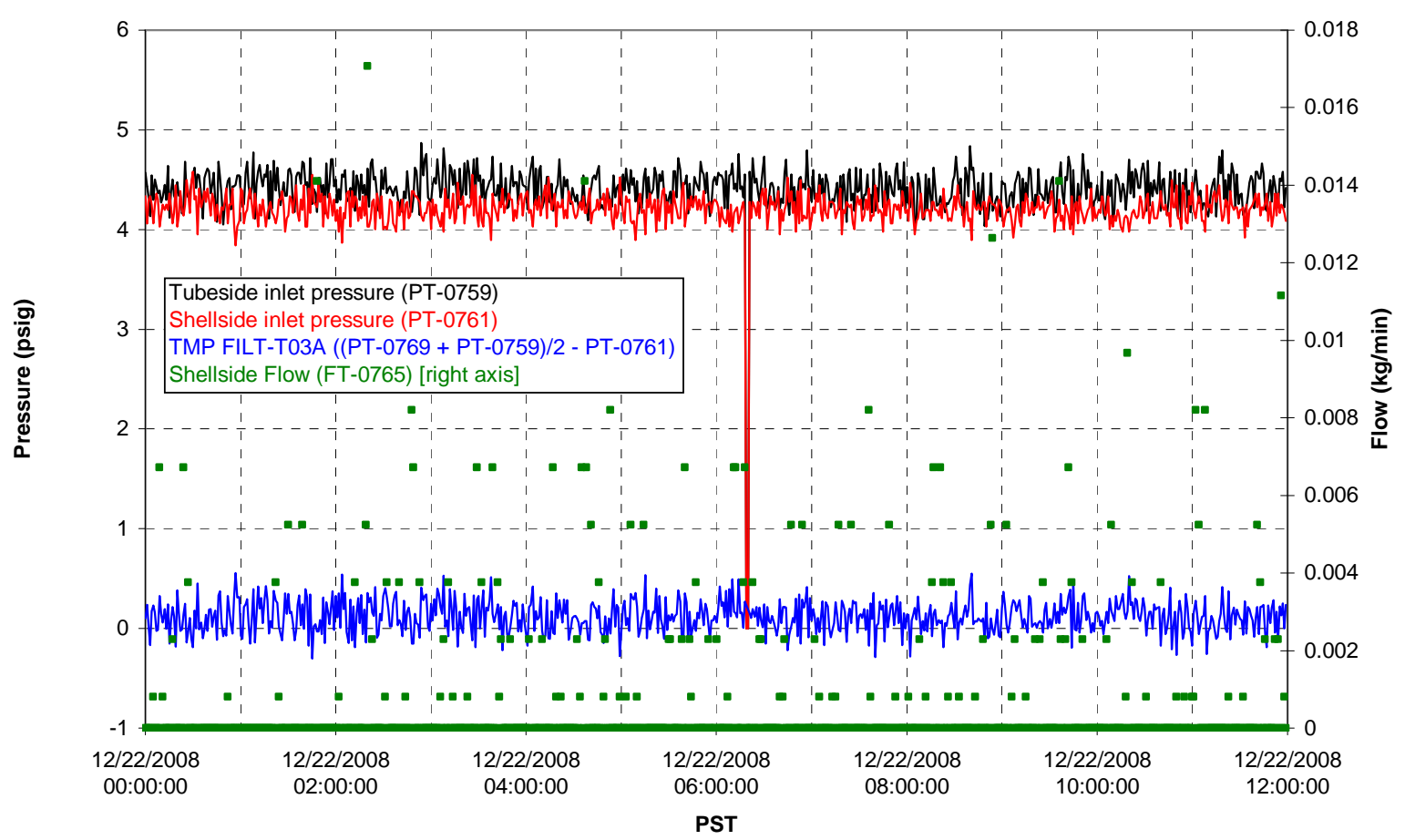

Filter UFP-FILT-T04A

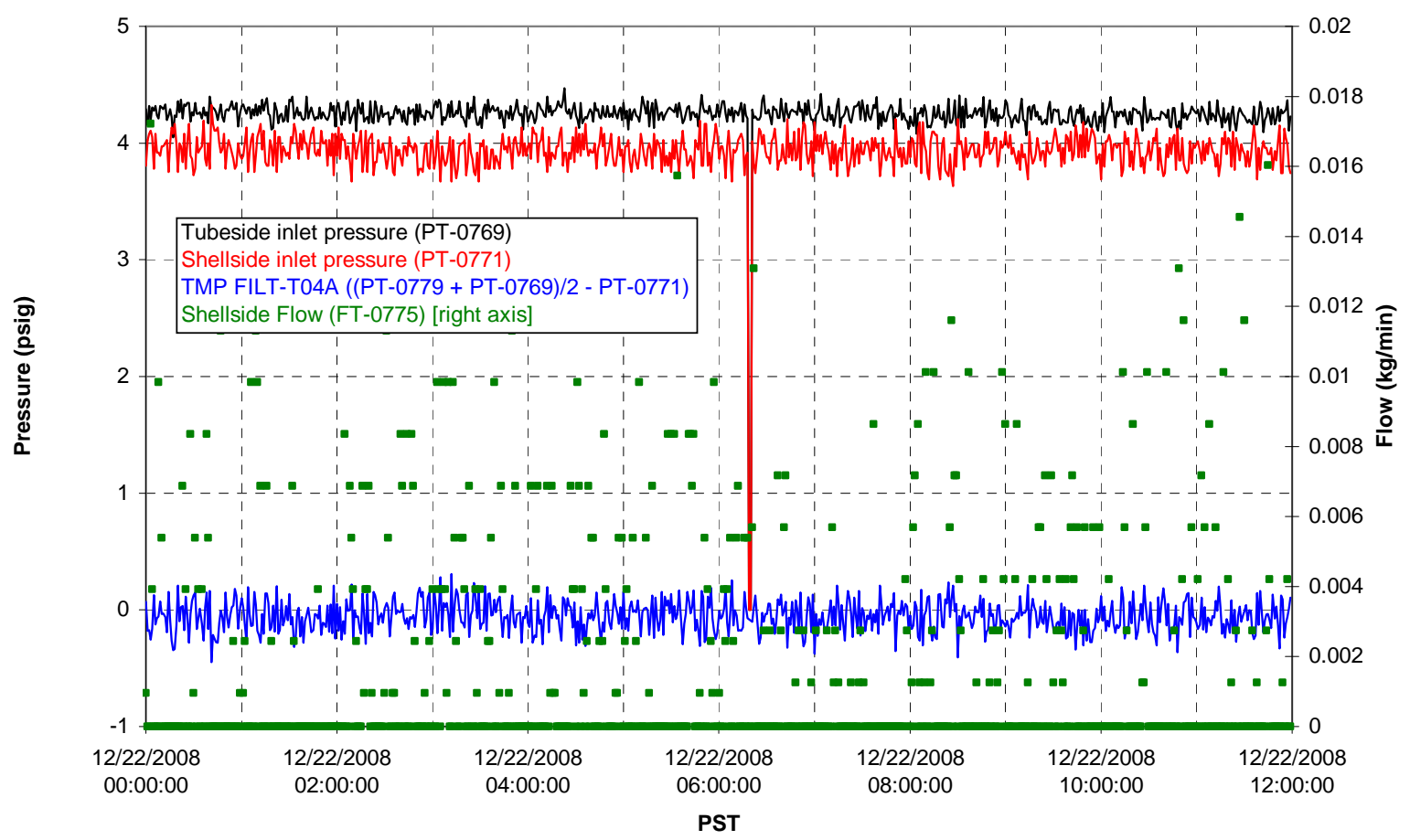


Filter UFP-FILT-T05A

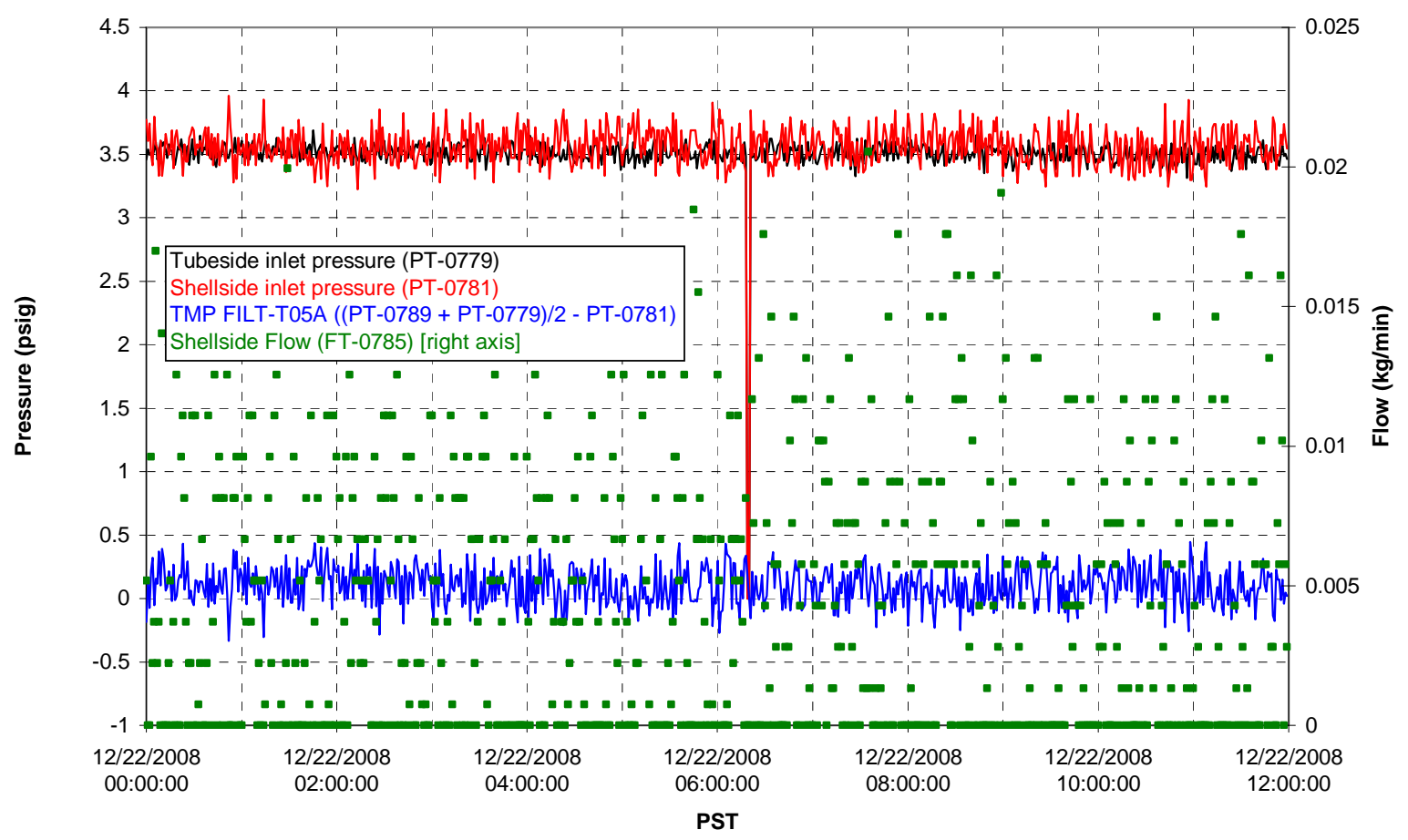

Chemical Flow

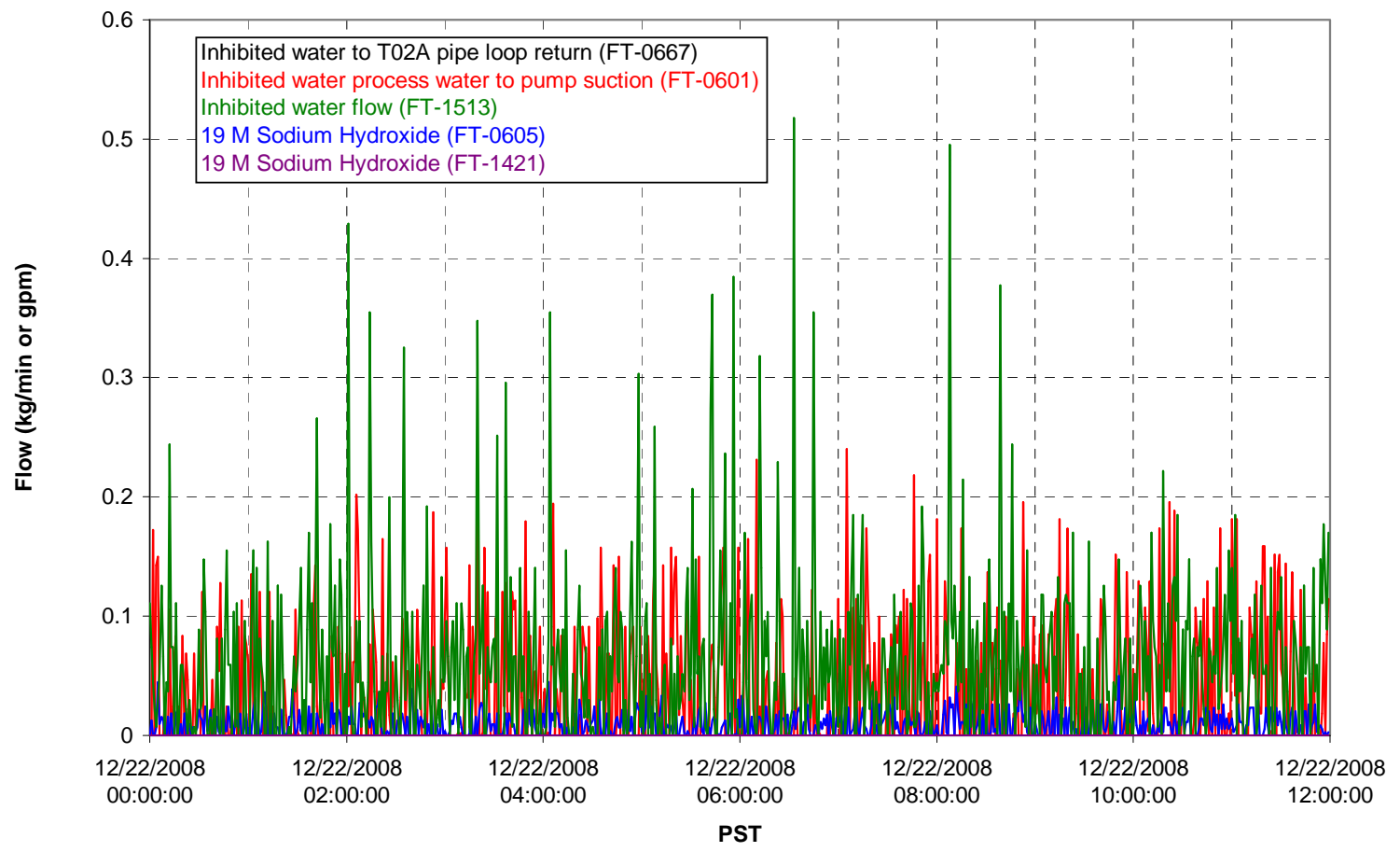




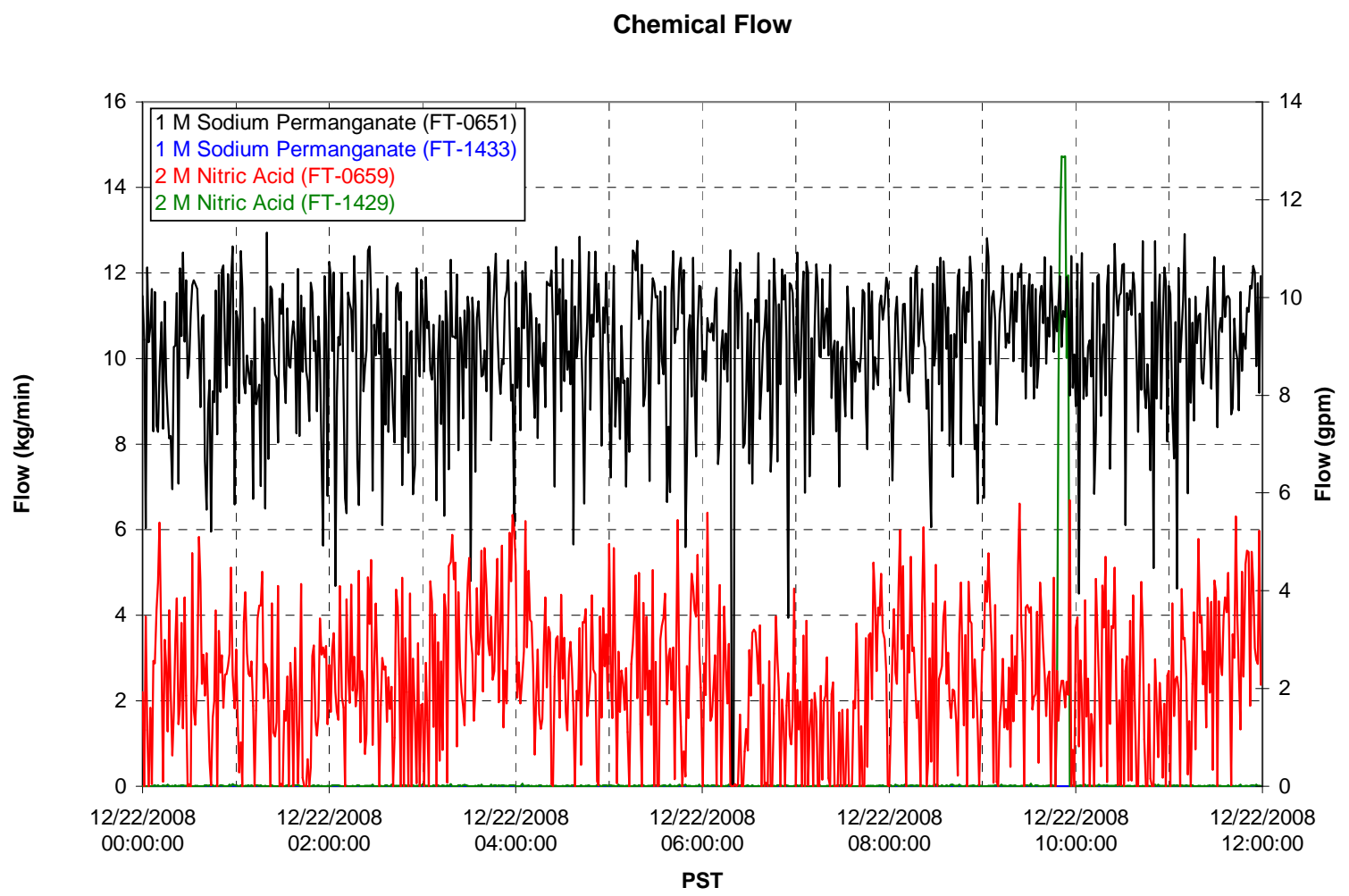

Air Flows

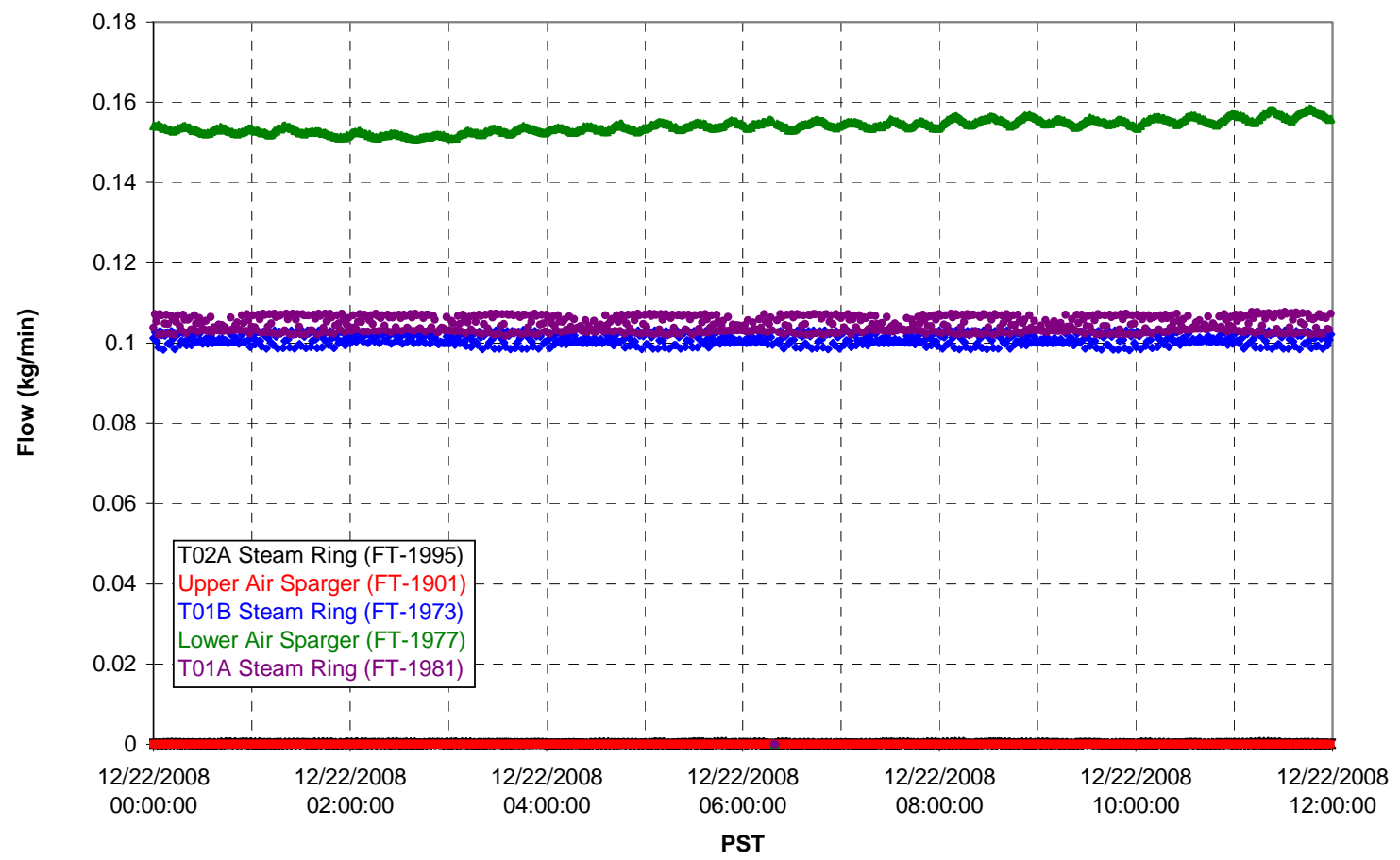


T02A Steam

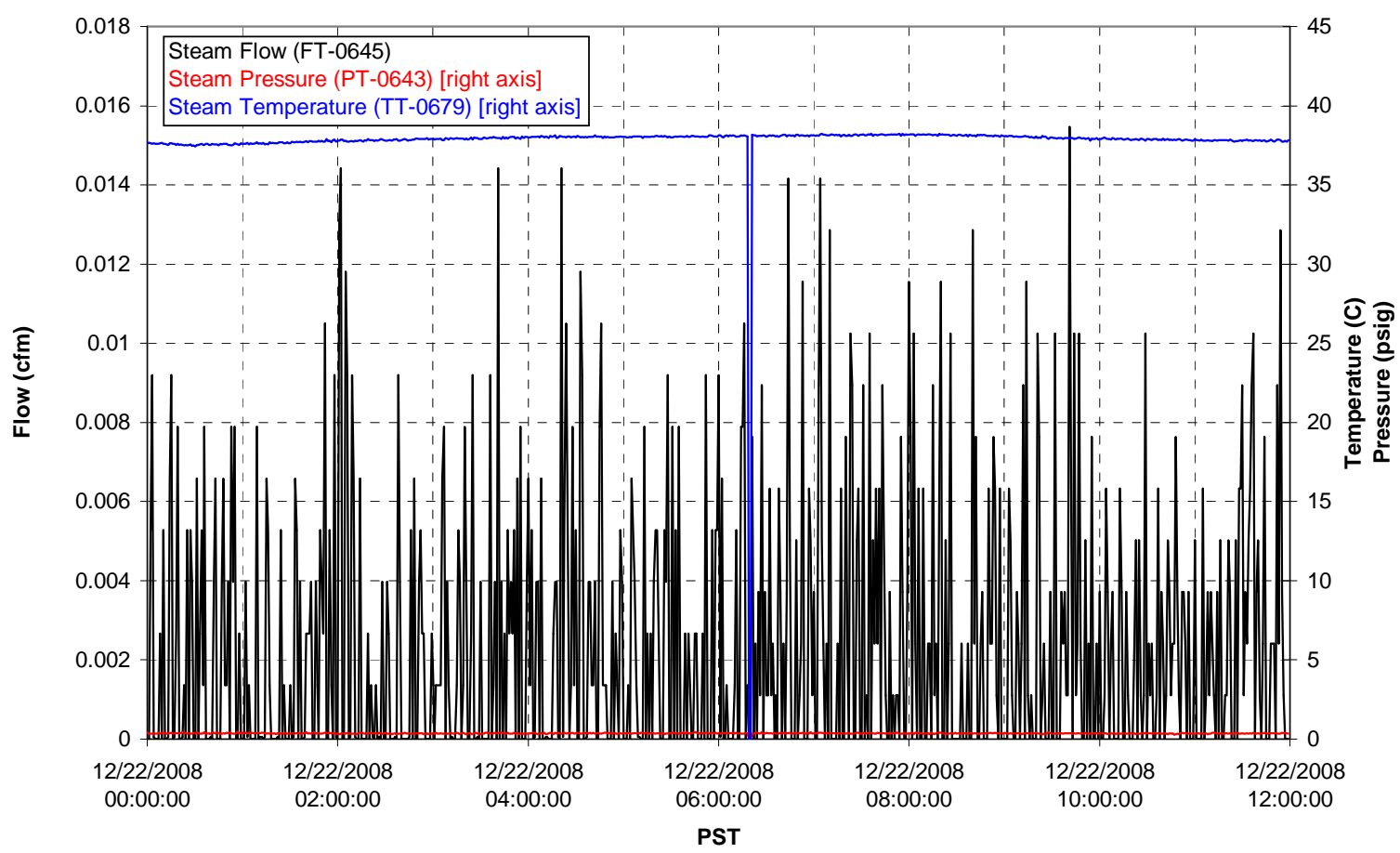

T01A Steam

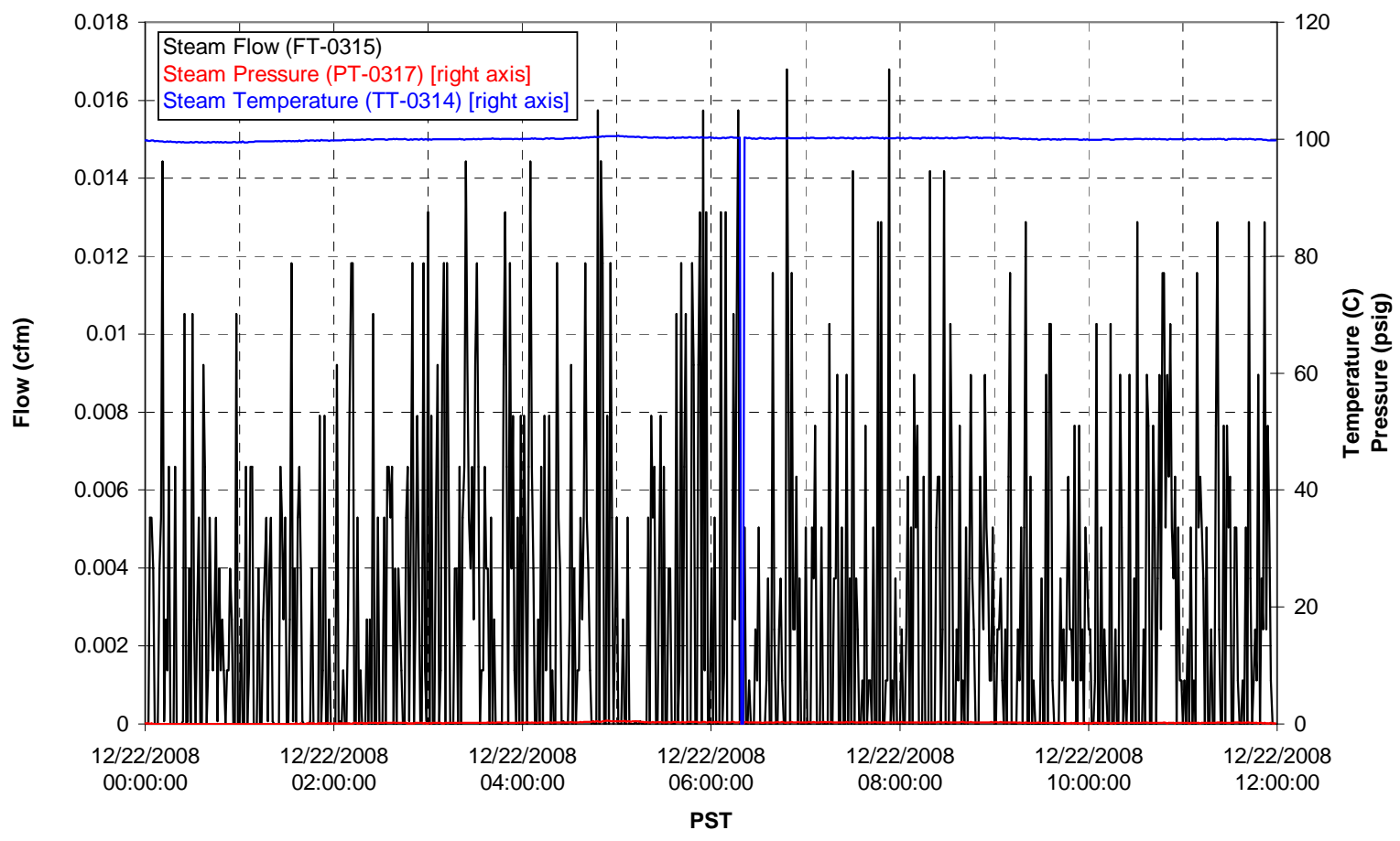


T01B Steam

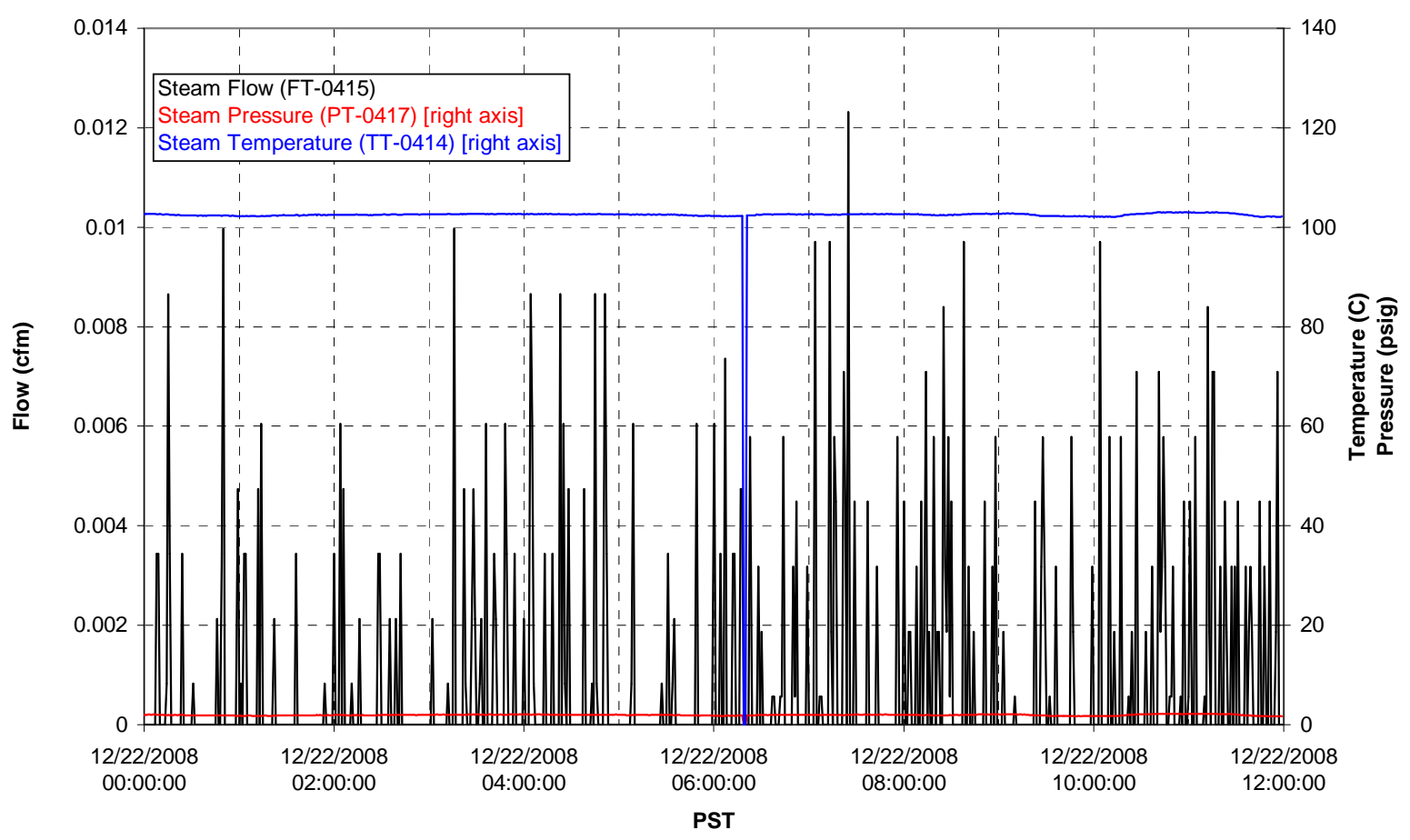


Functional Test Data Plots 12/22/08 12:00 - 12/23/08 00:00 
T01A level

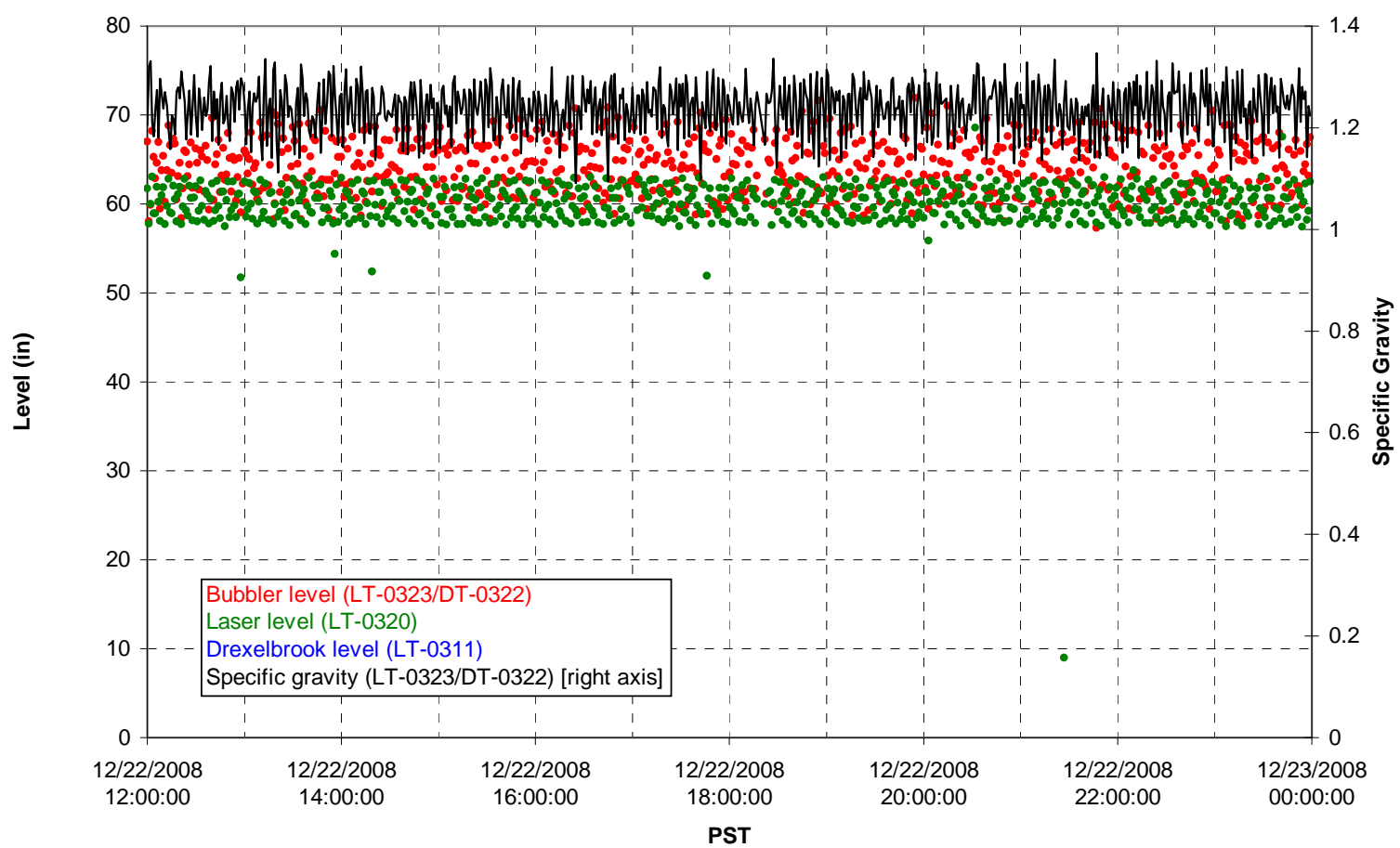

T01A temperatures

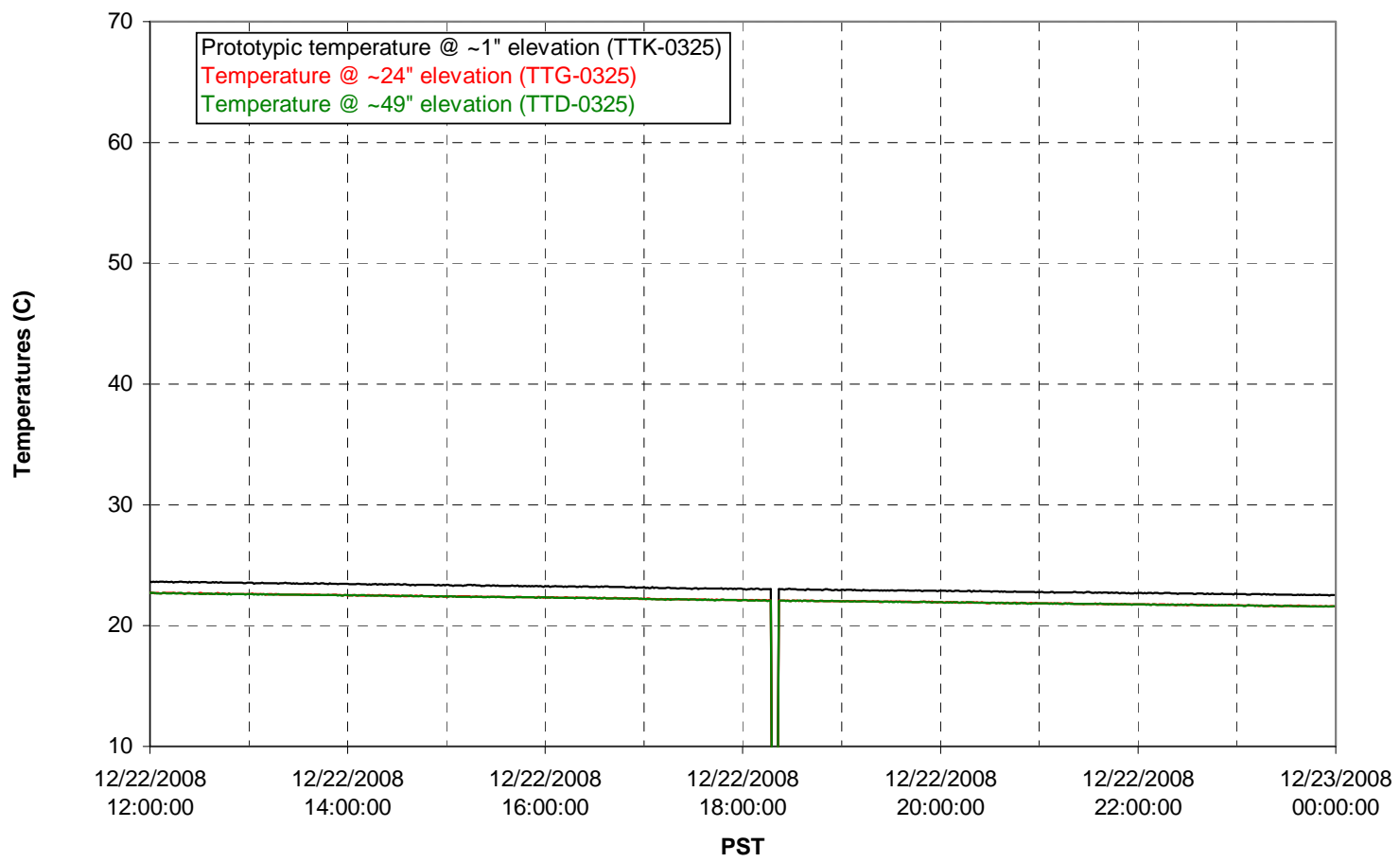


T01B level

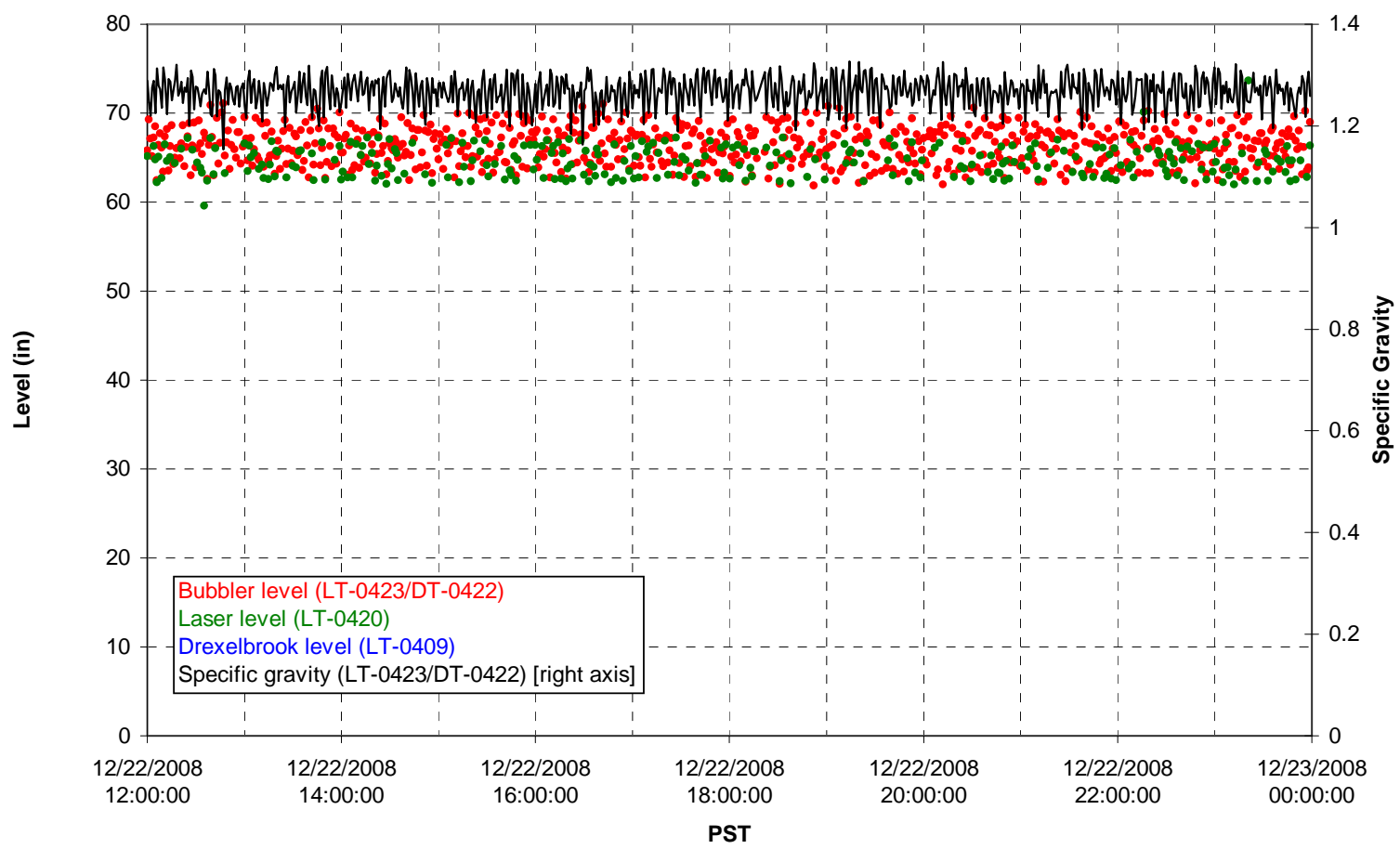

T01B temperatures

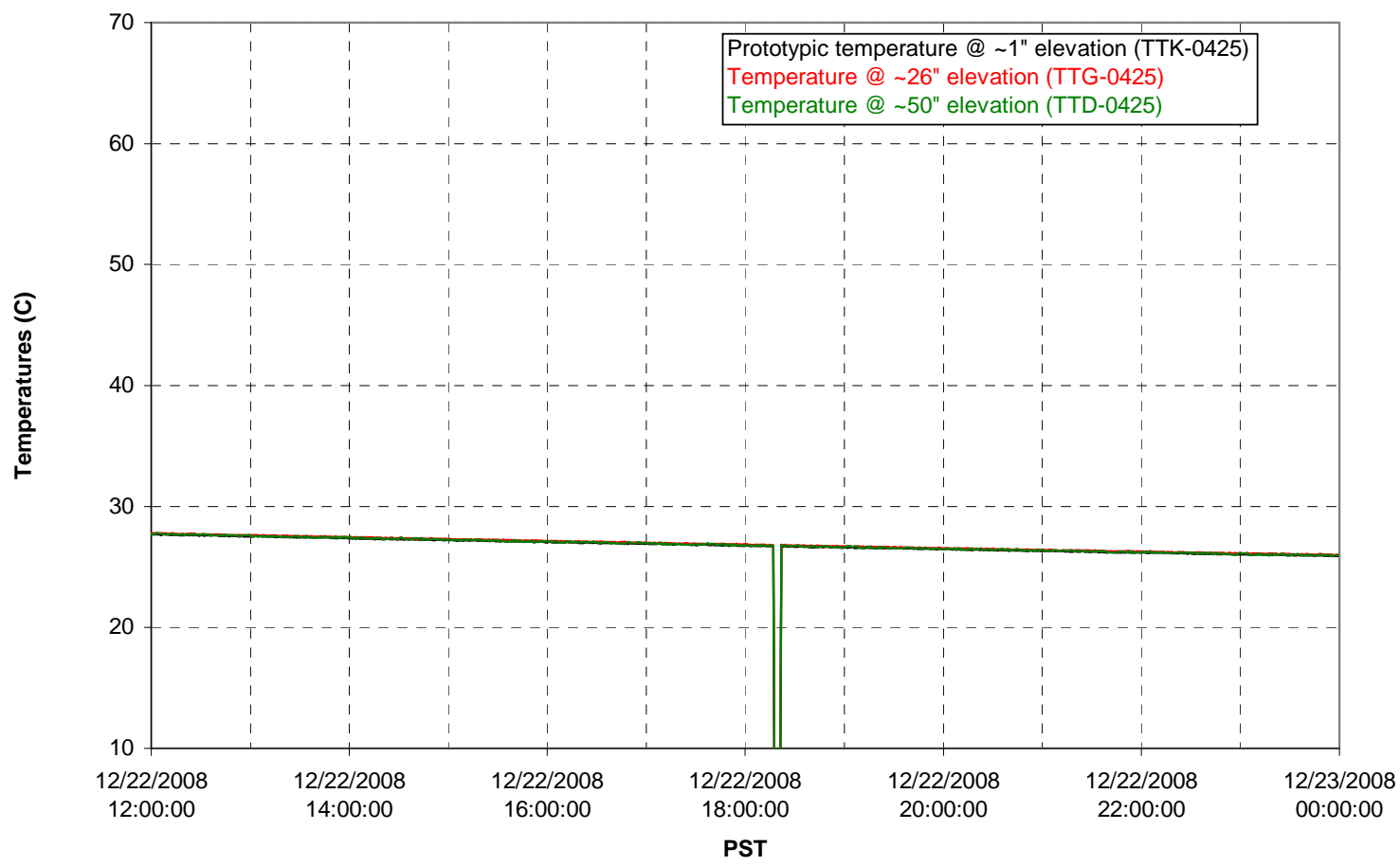


T02A level

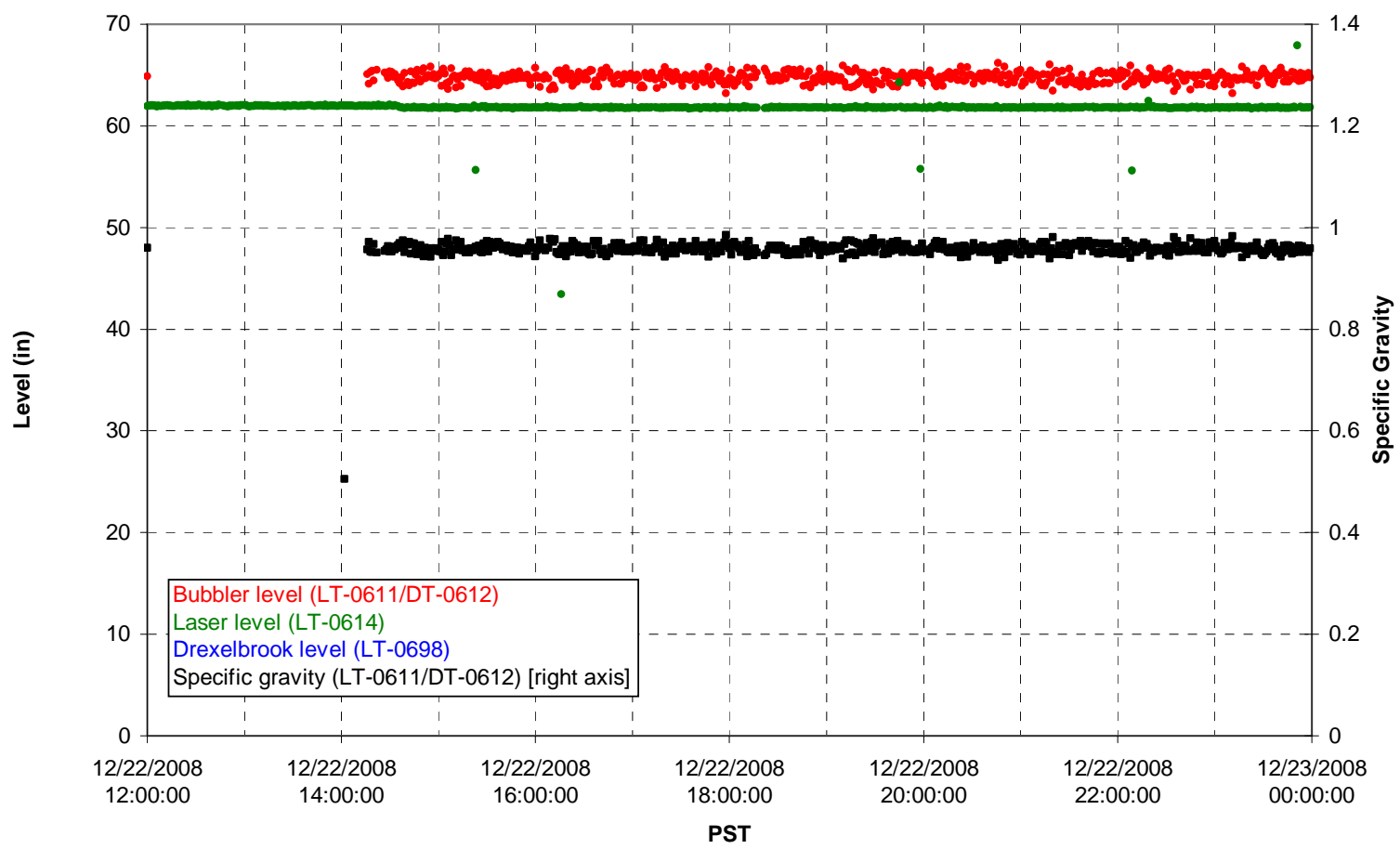

T02A temperatures

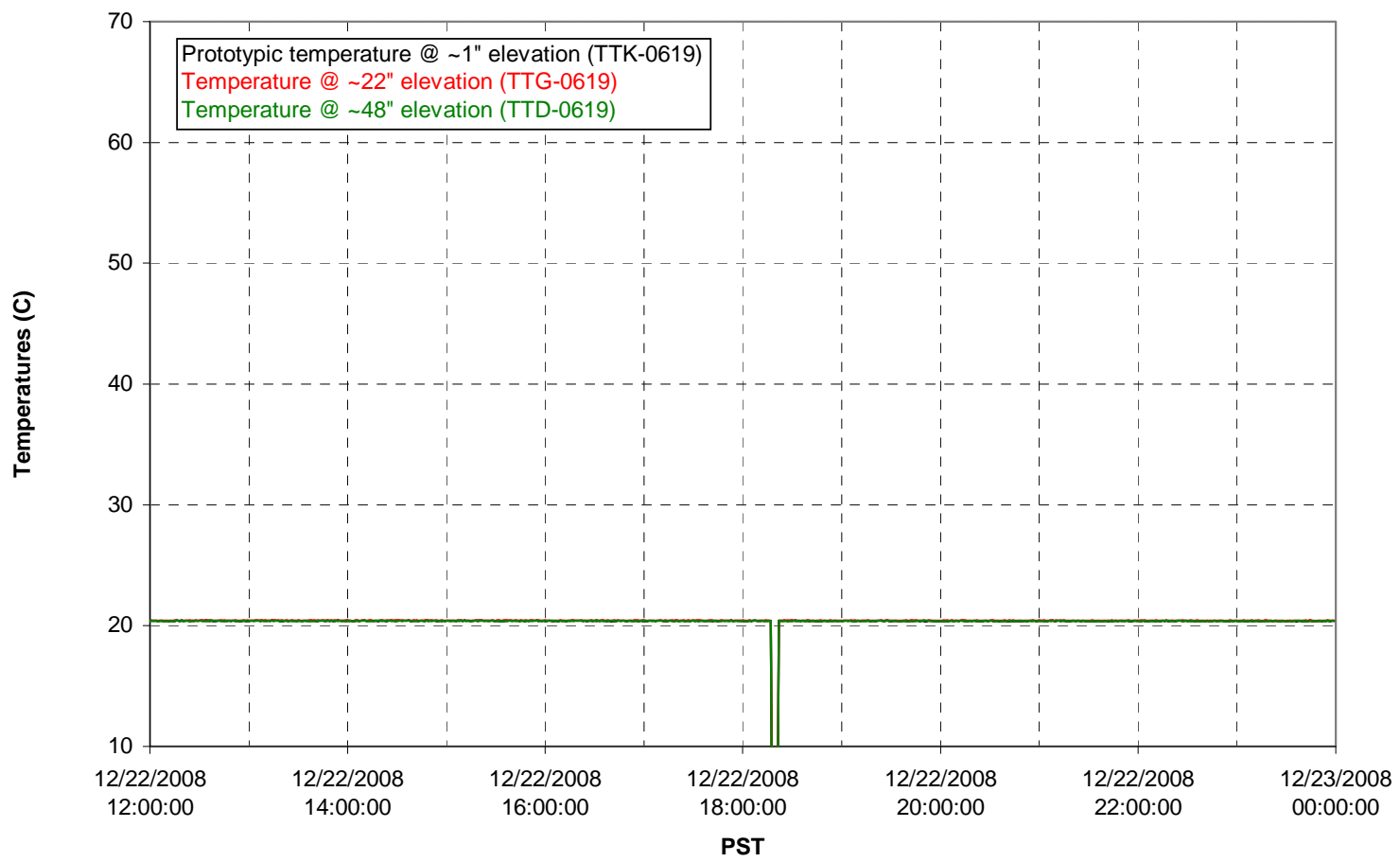


T02A and filter loop temperatures

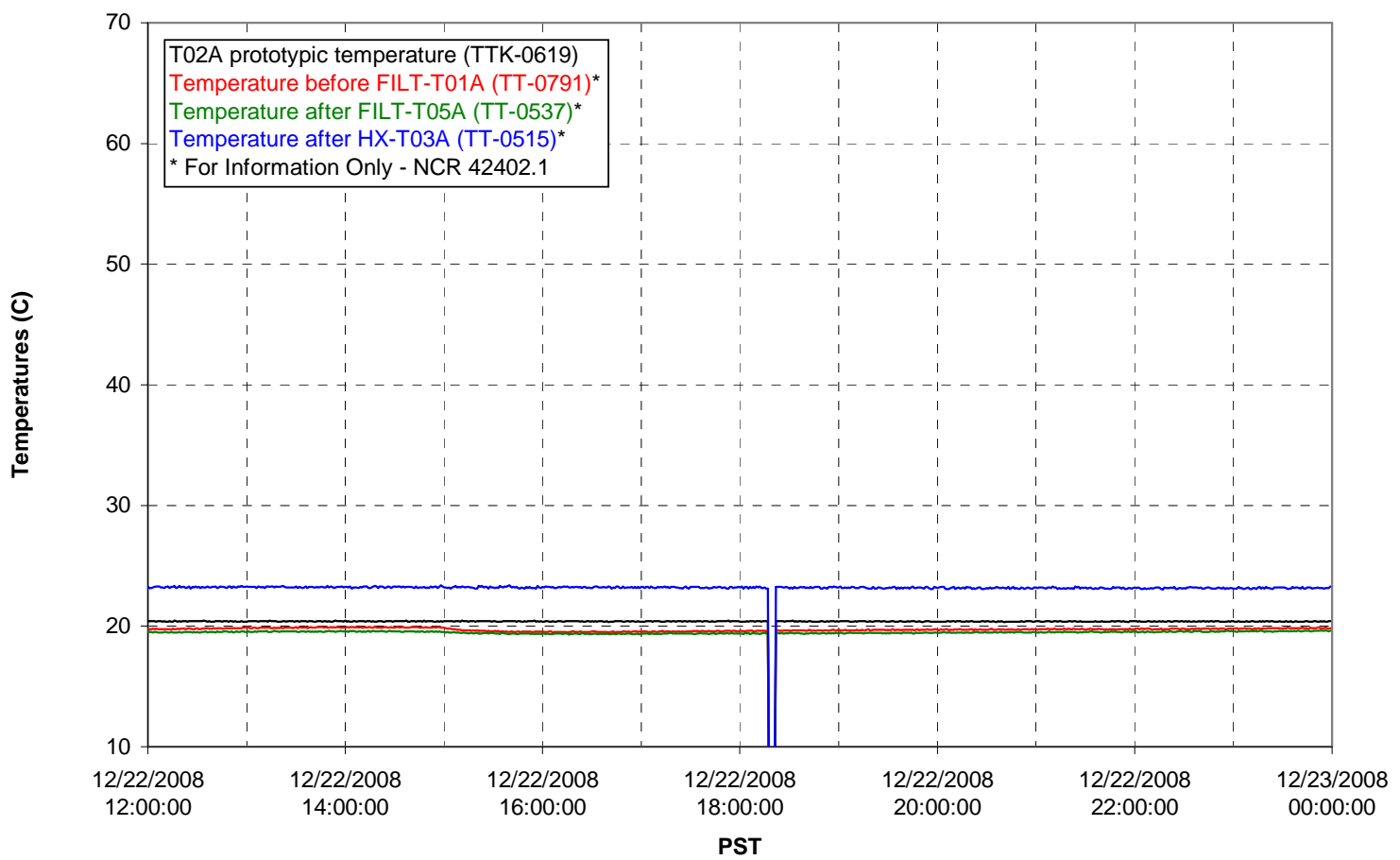

Pump Pressures and Flow

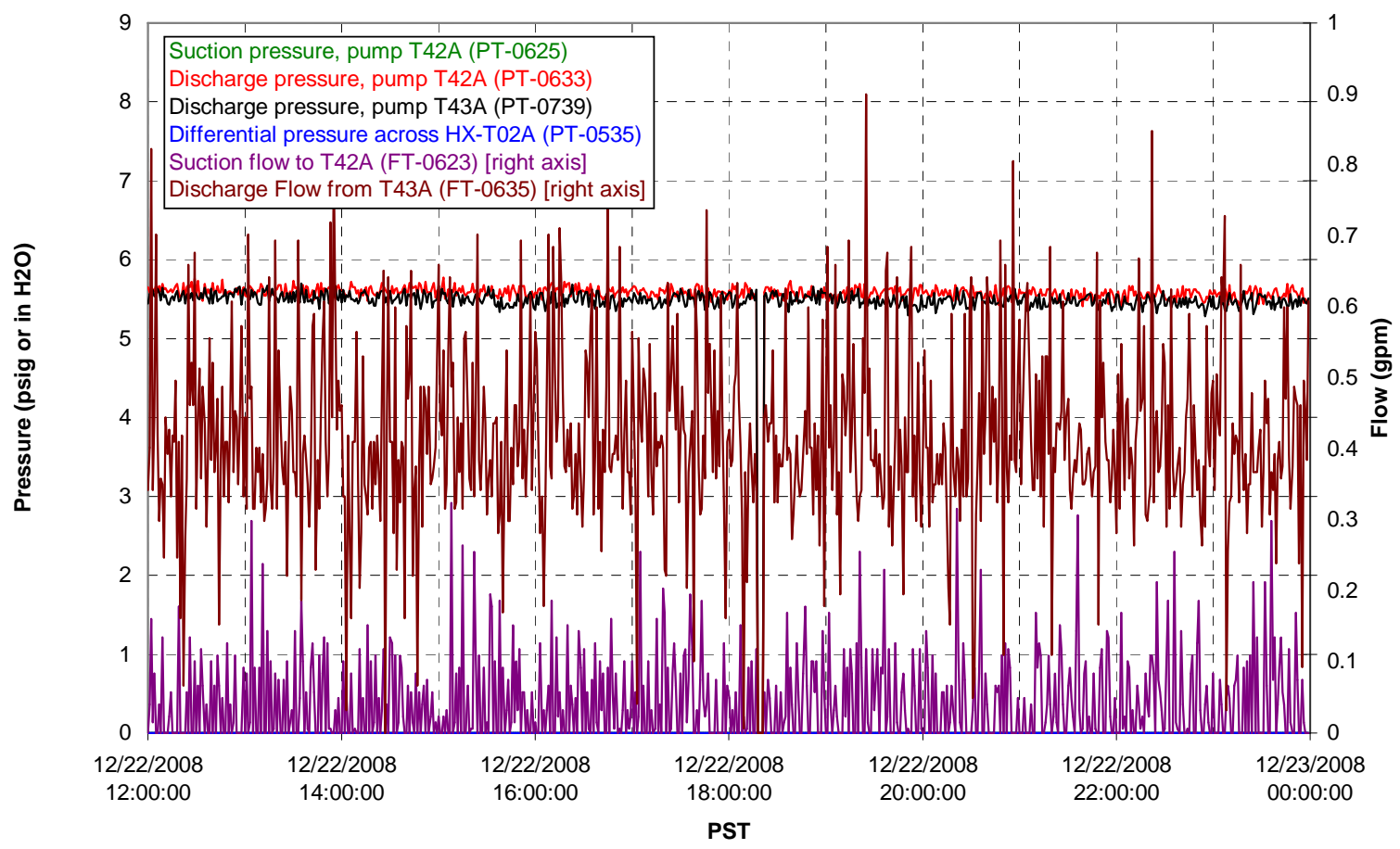


Axial pressure drop

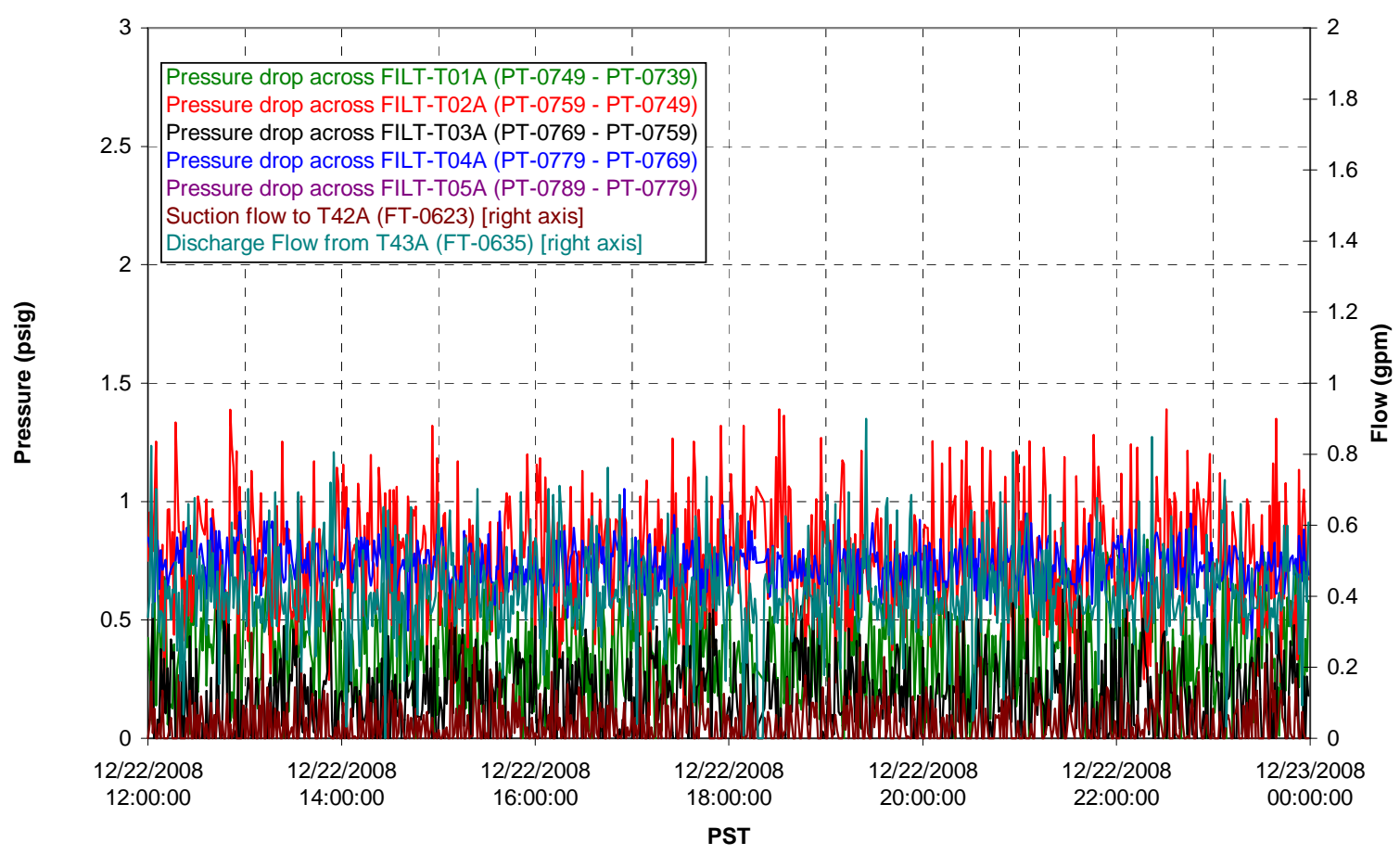

Permeate flow rates

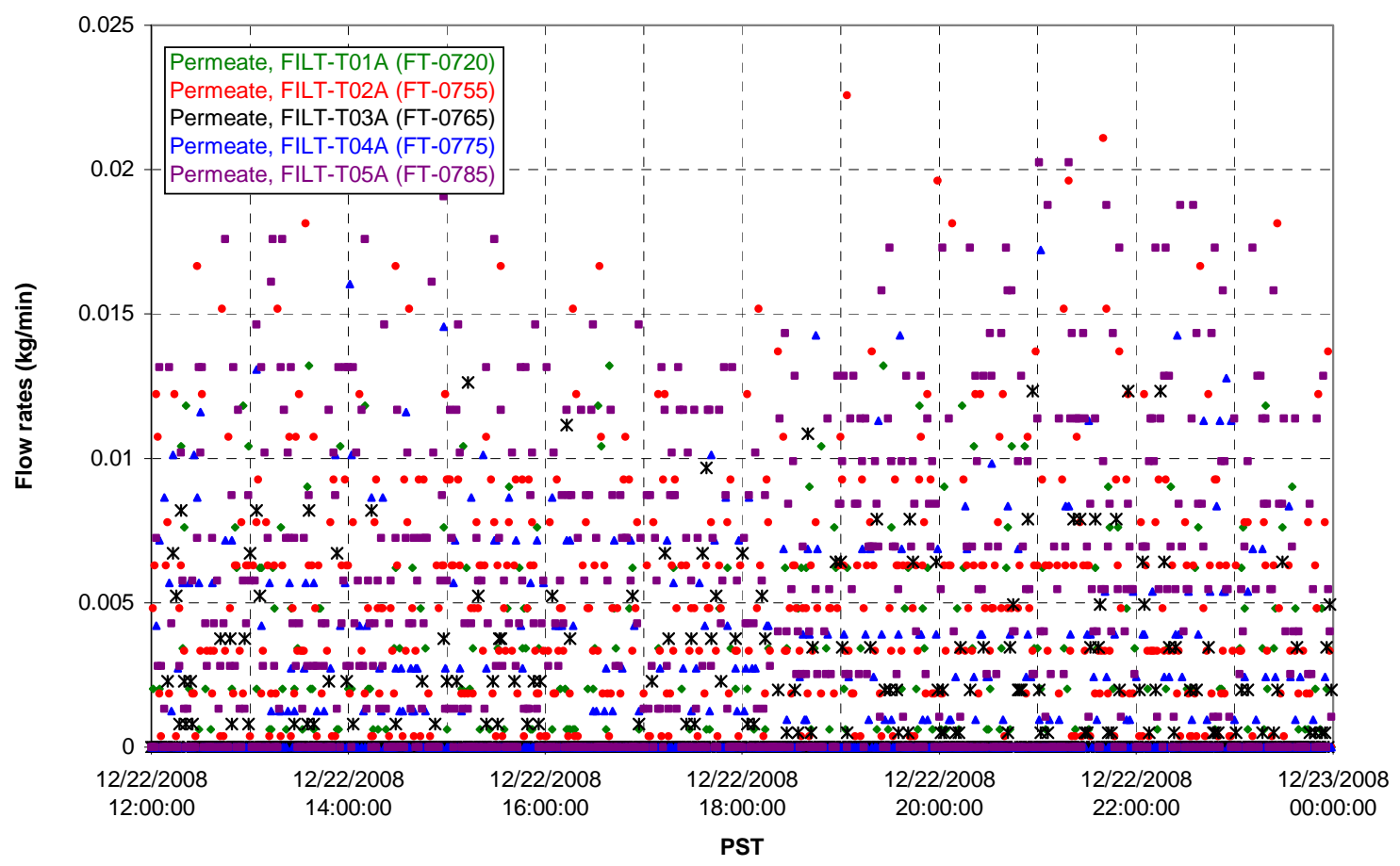


T02A Inner Temperature Tree

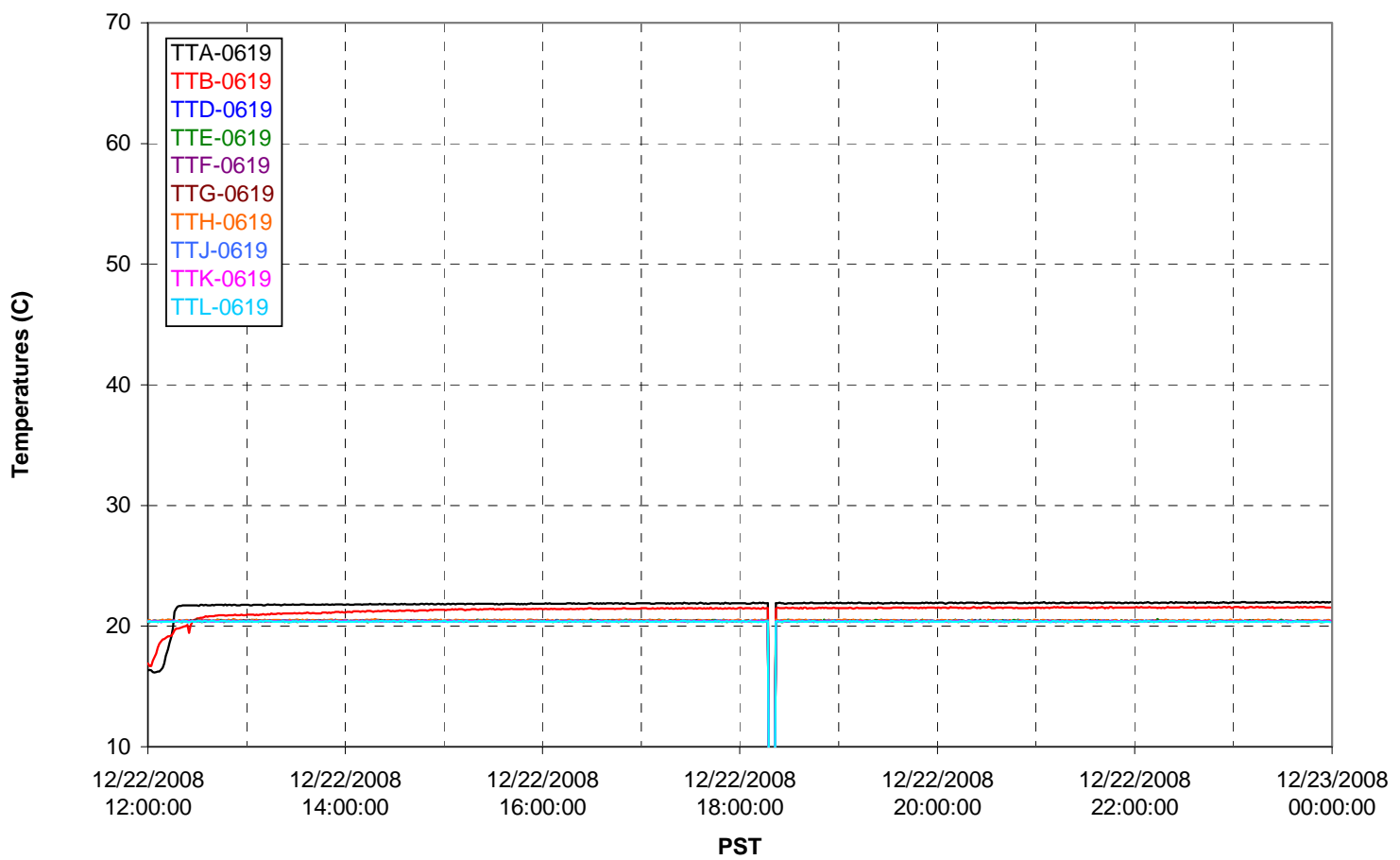

T02A Outer Temperature Tree

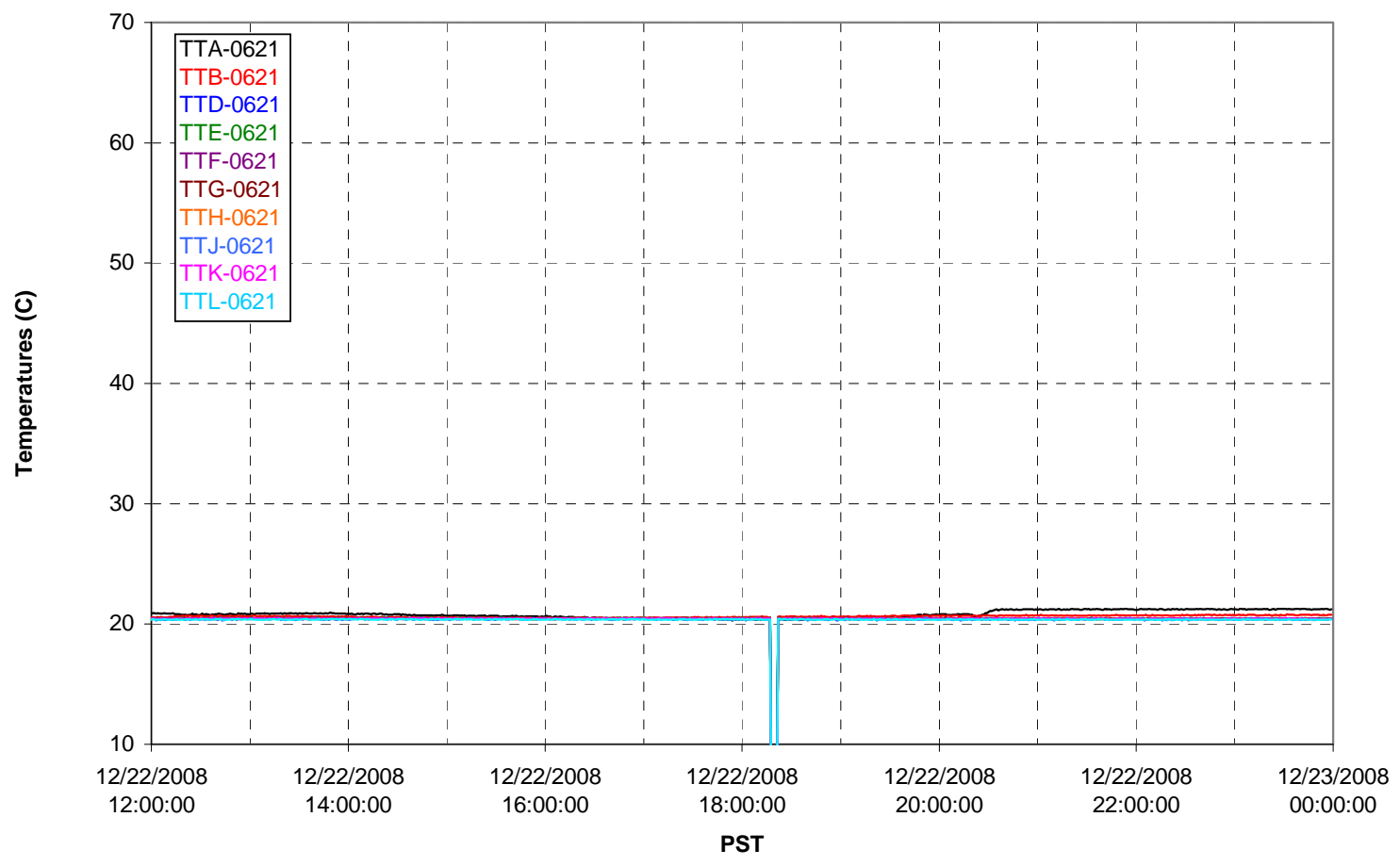


T02A temperatures

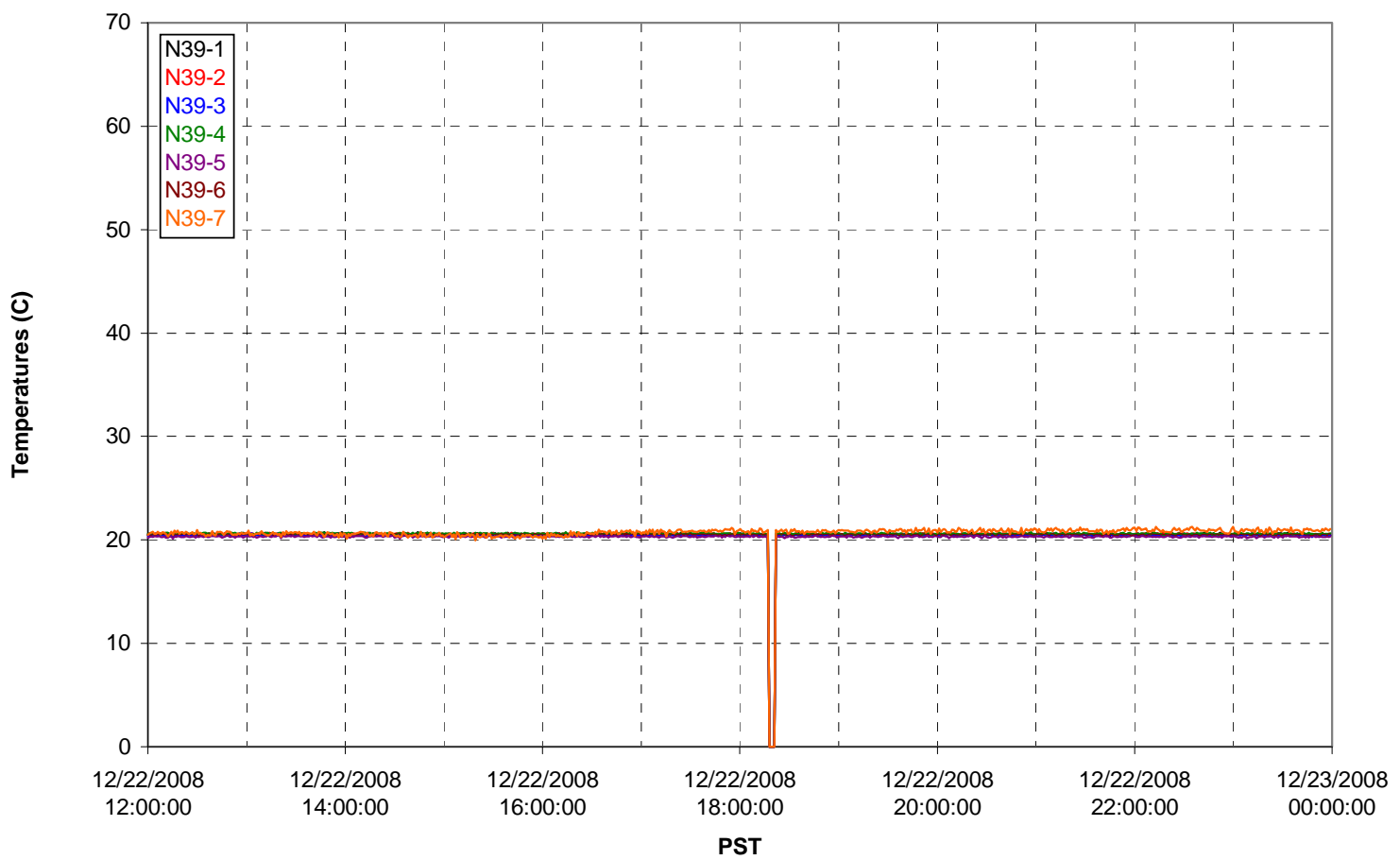

T02A temperatures

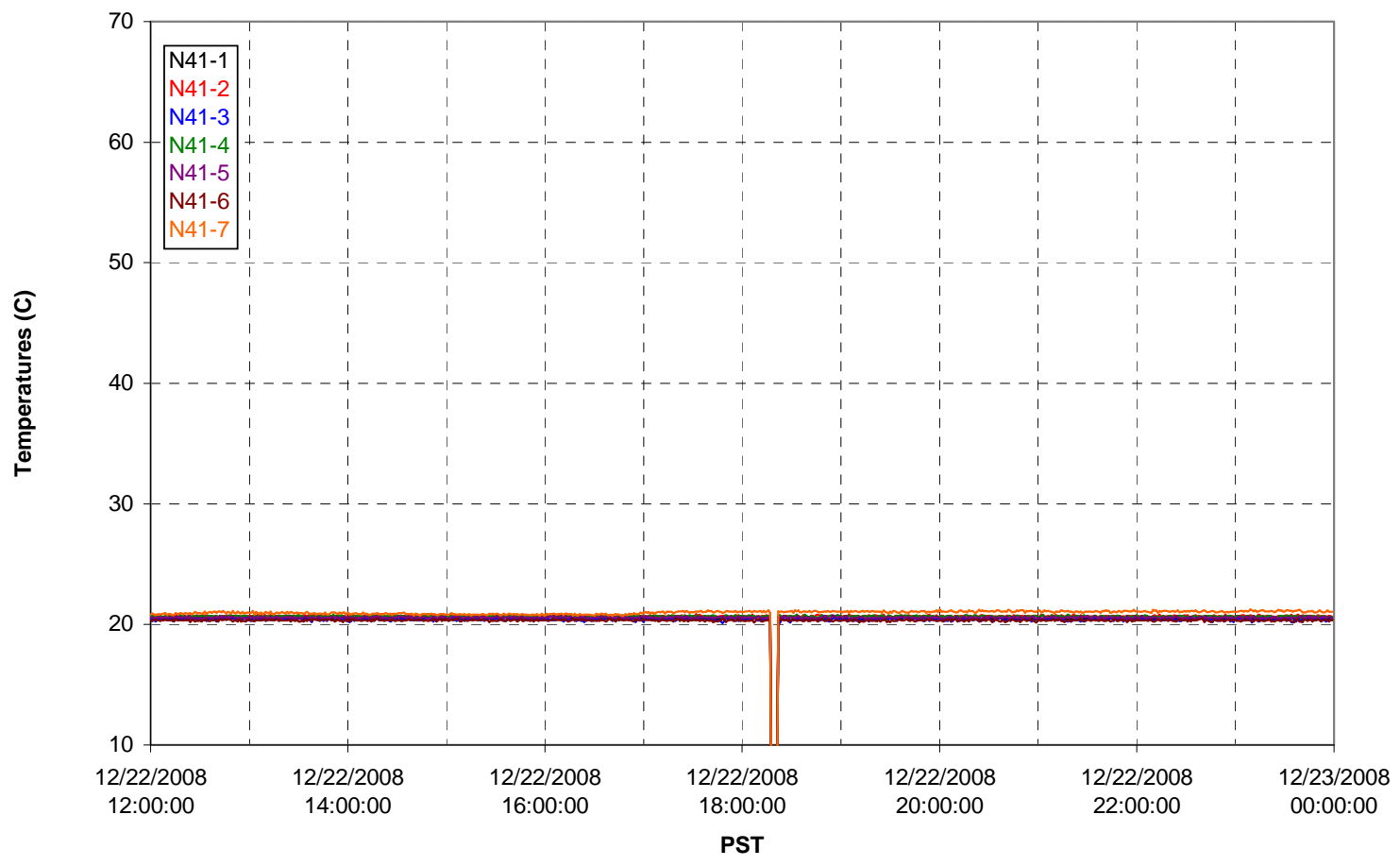


T02A temperatures

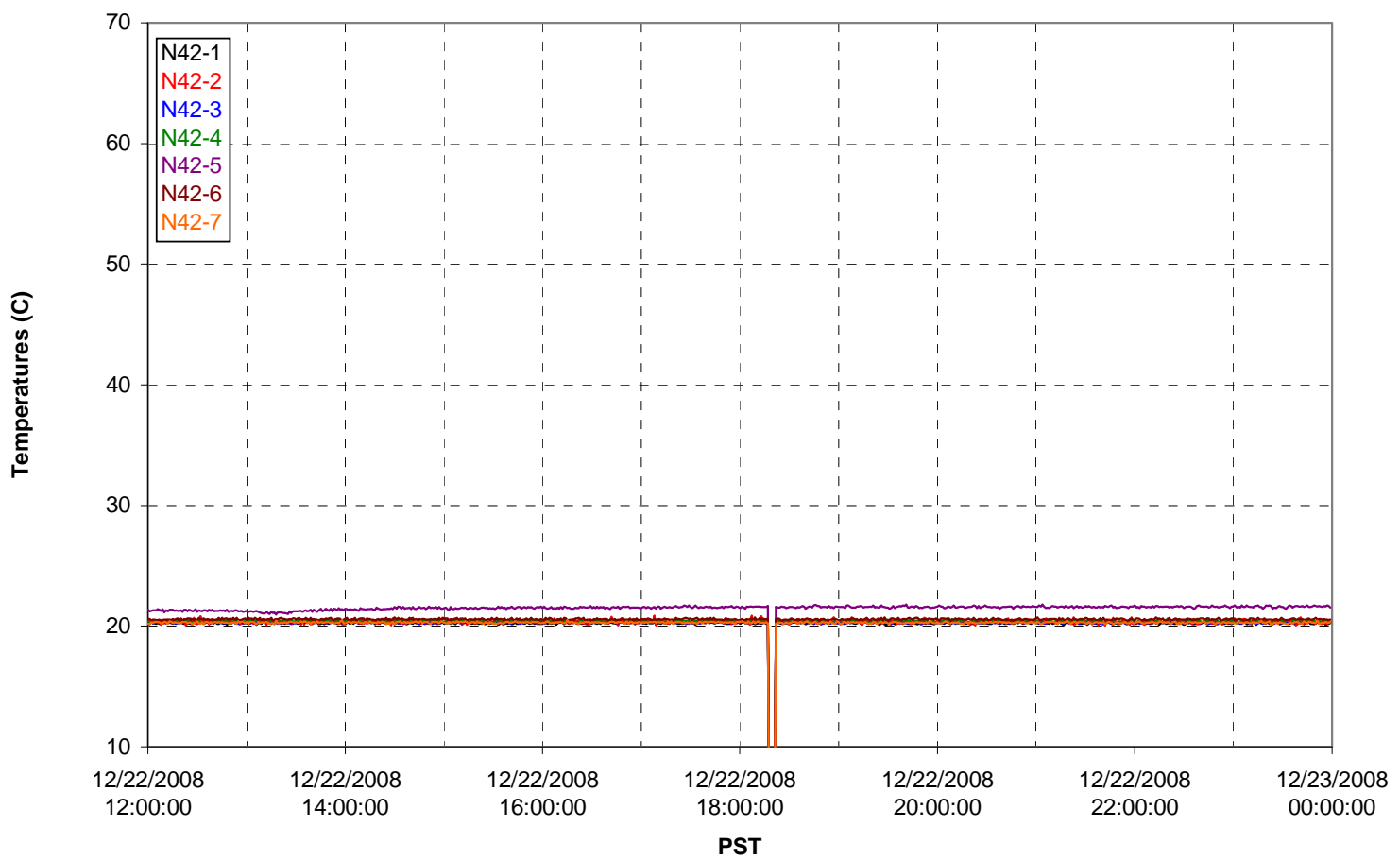

T02A temperatures

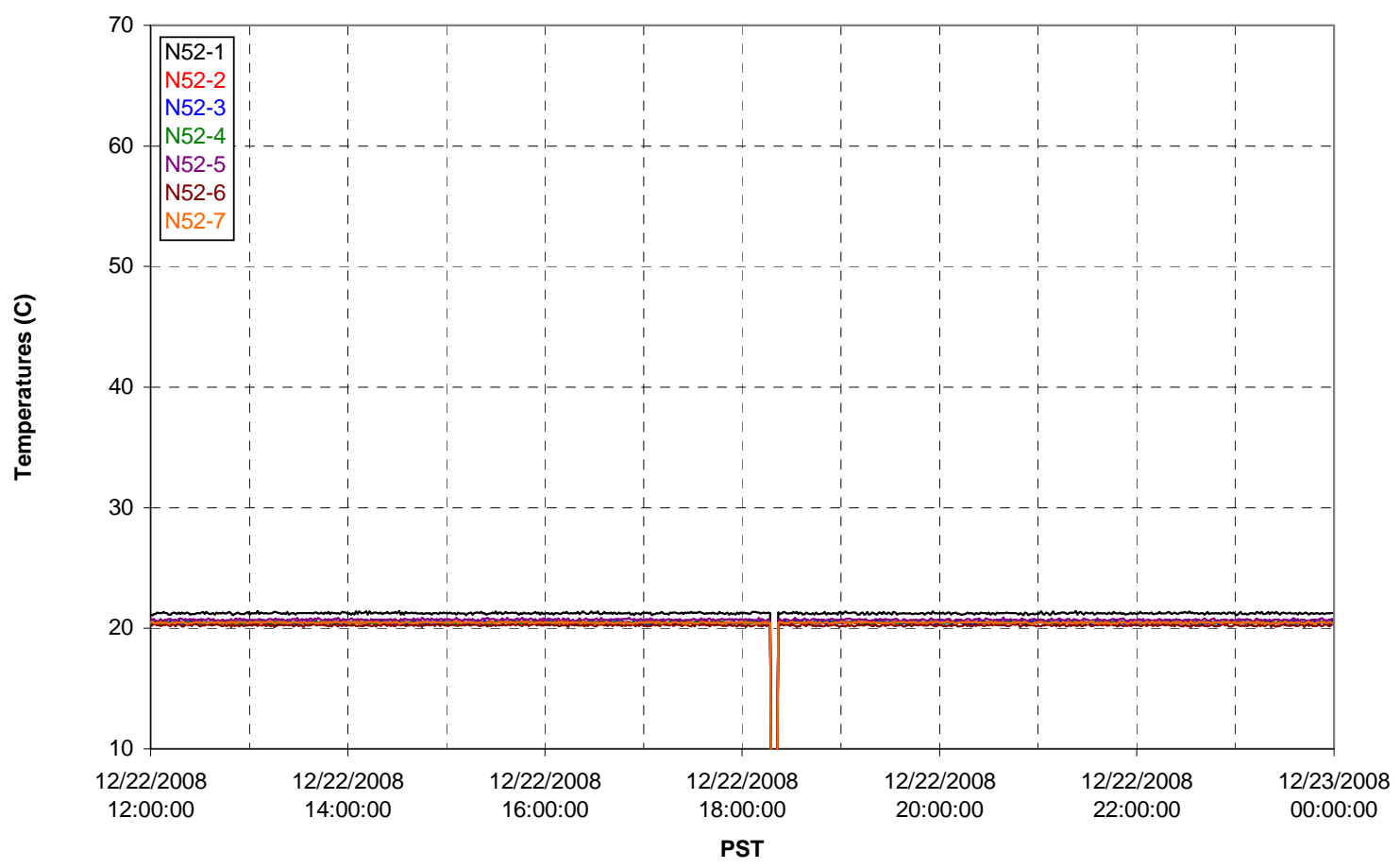


T02A Heating and Cooling

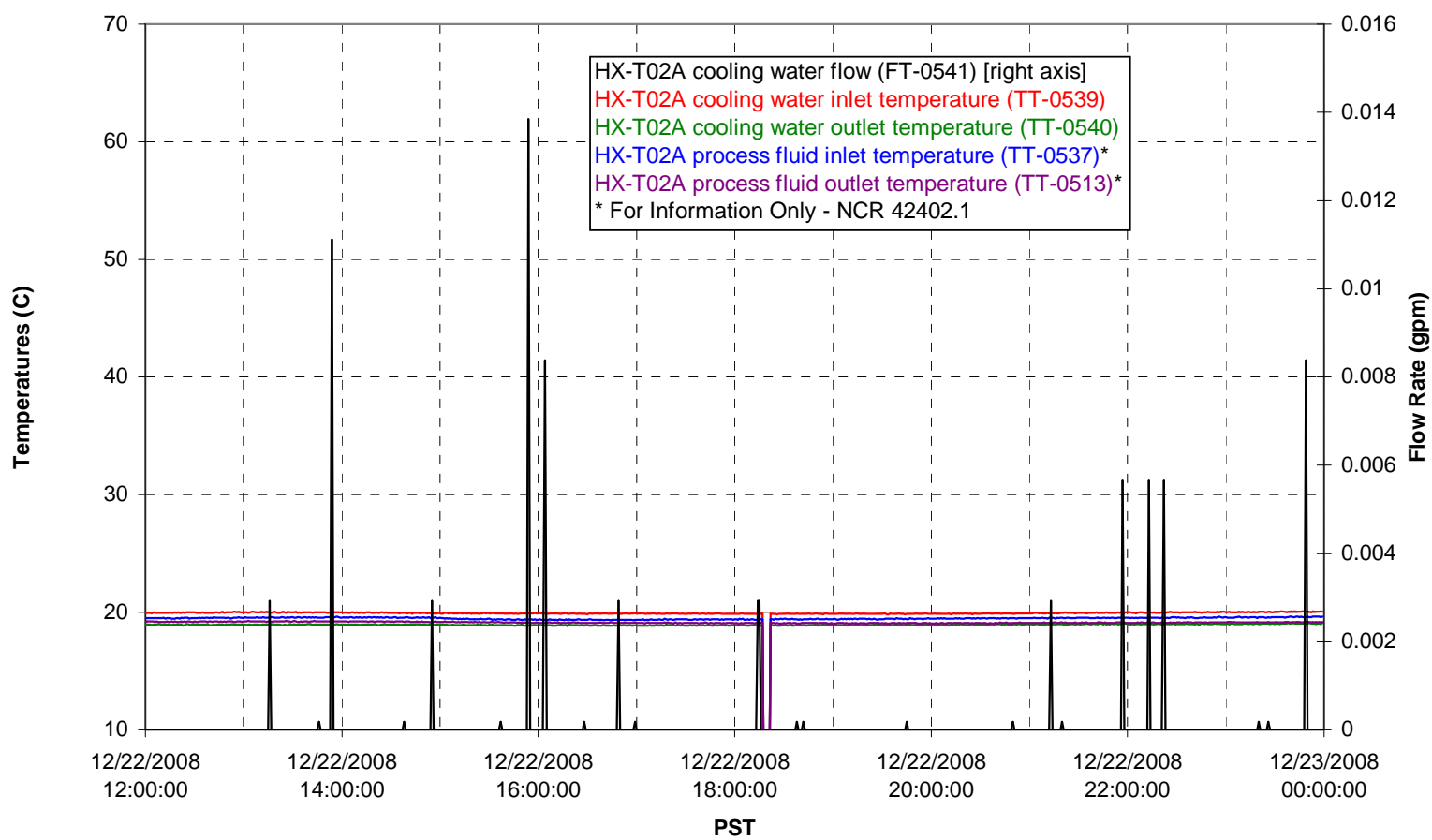

Pump Operation

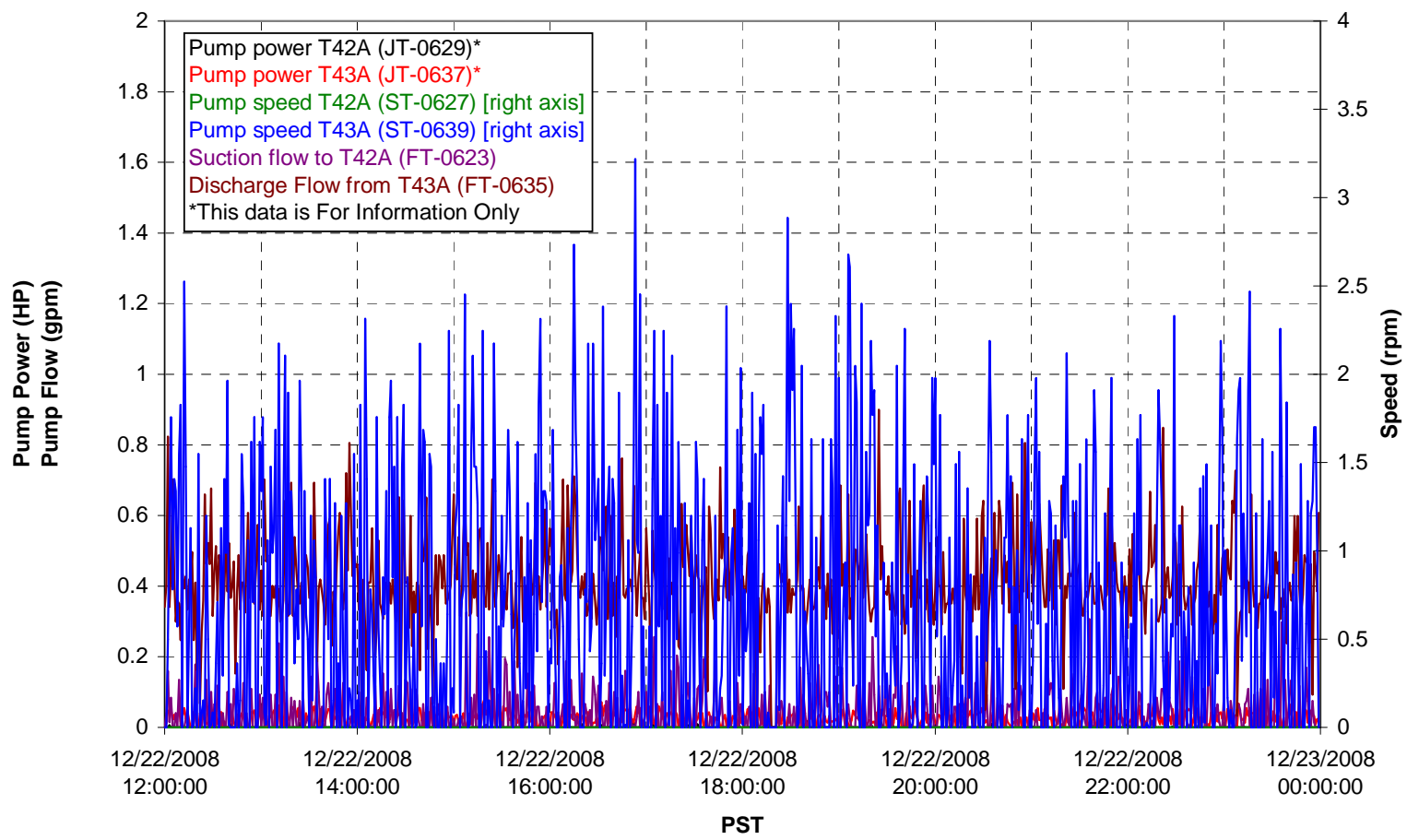


Pulsepot UFP-PP-T01A

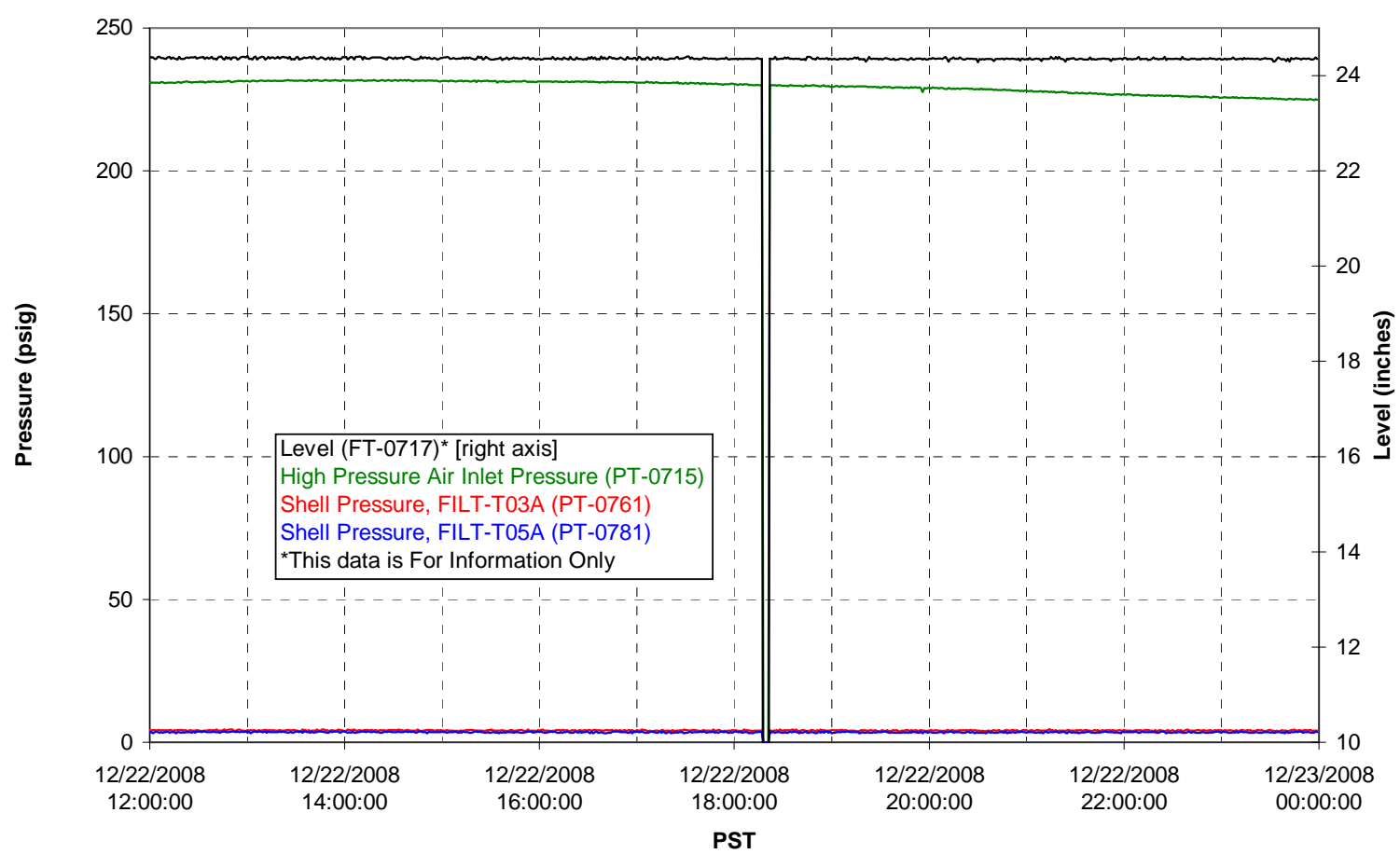

Pulsepot UFP-PP-T02A

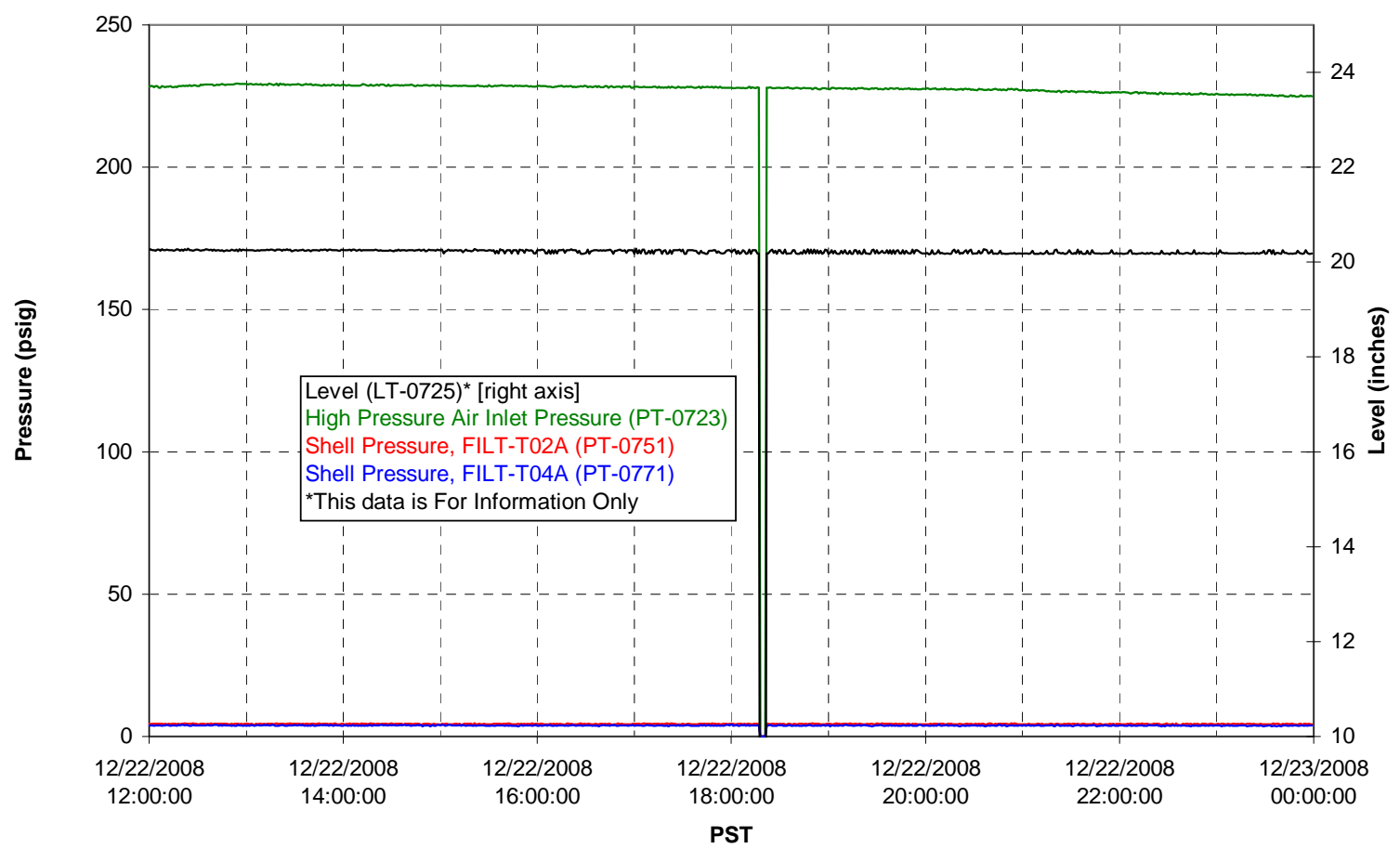


Pulsepot UFP-PP-T03A

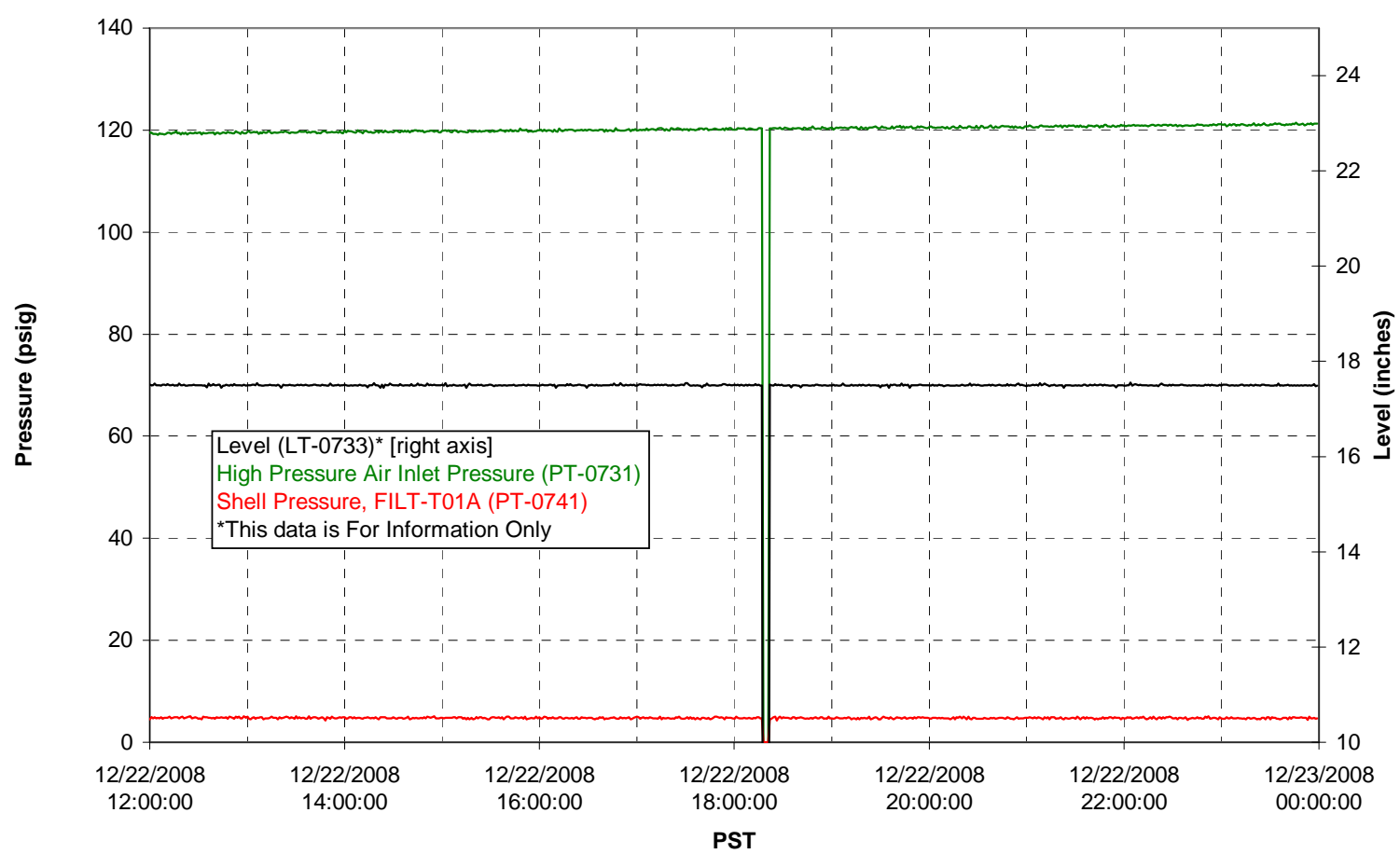

Pulsepot Levels

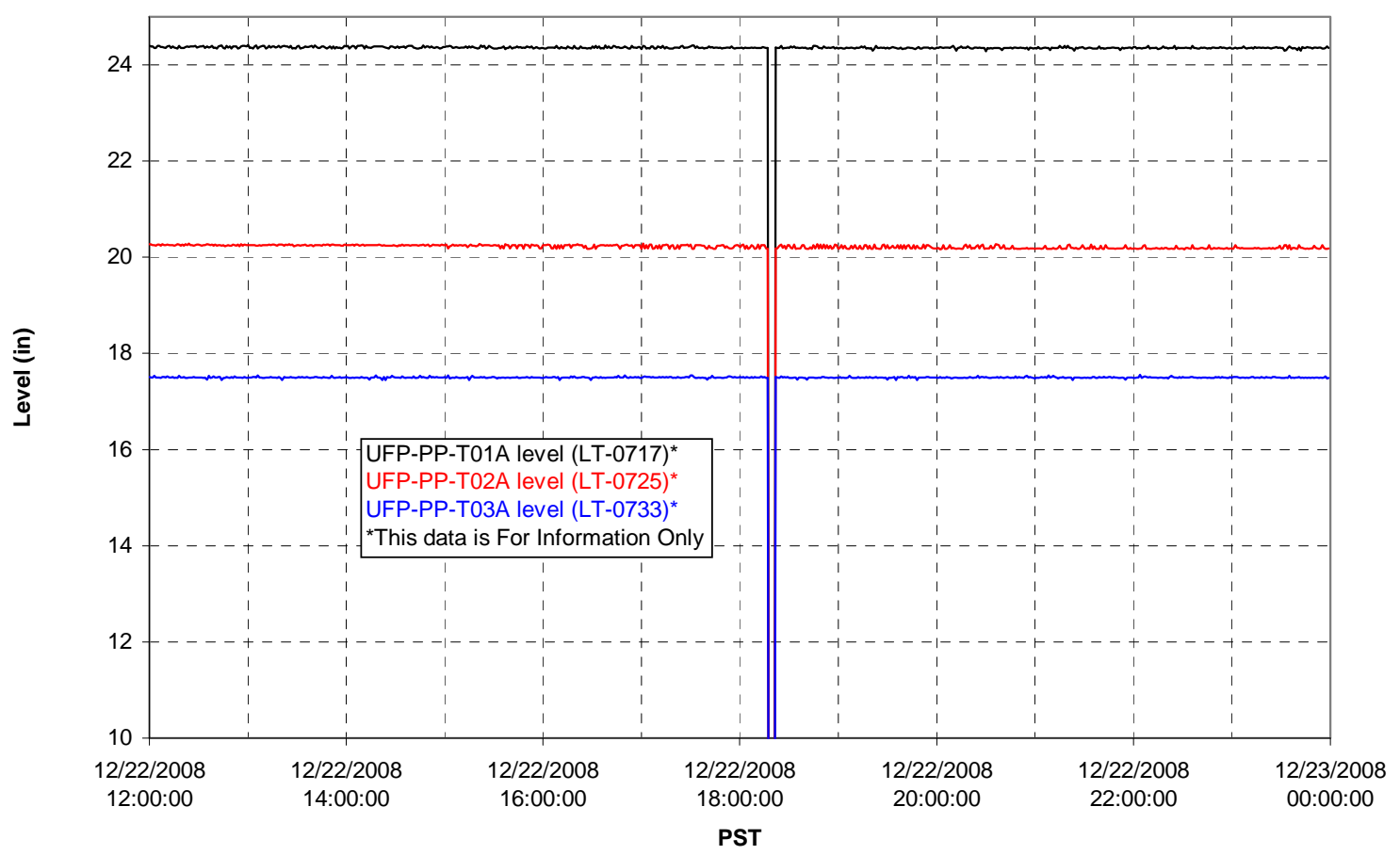


Filter UFP-FILT-T01A

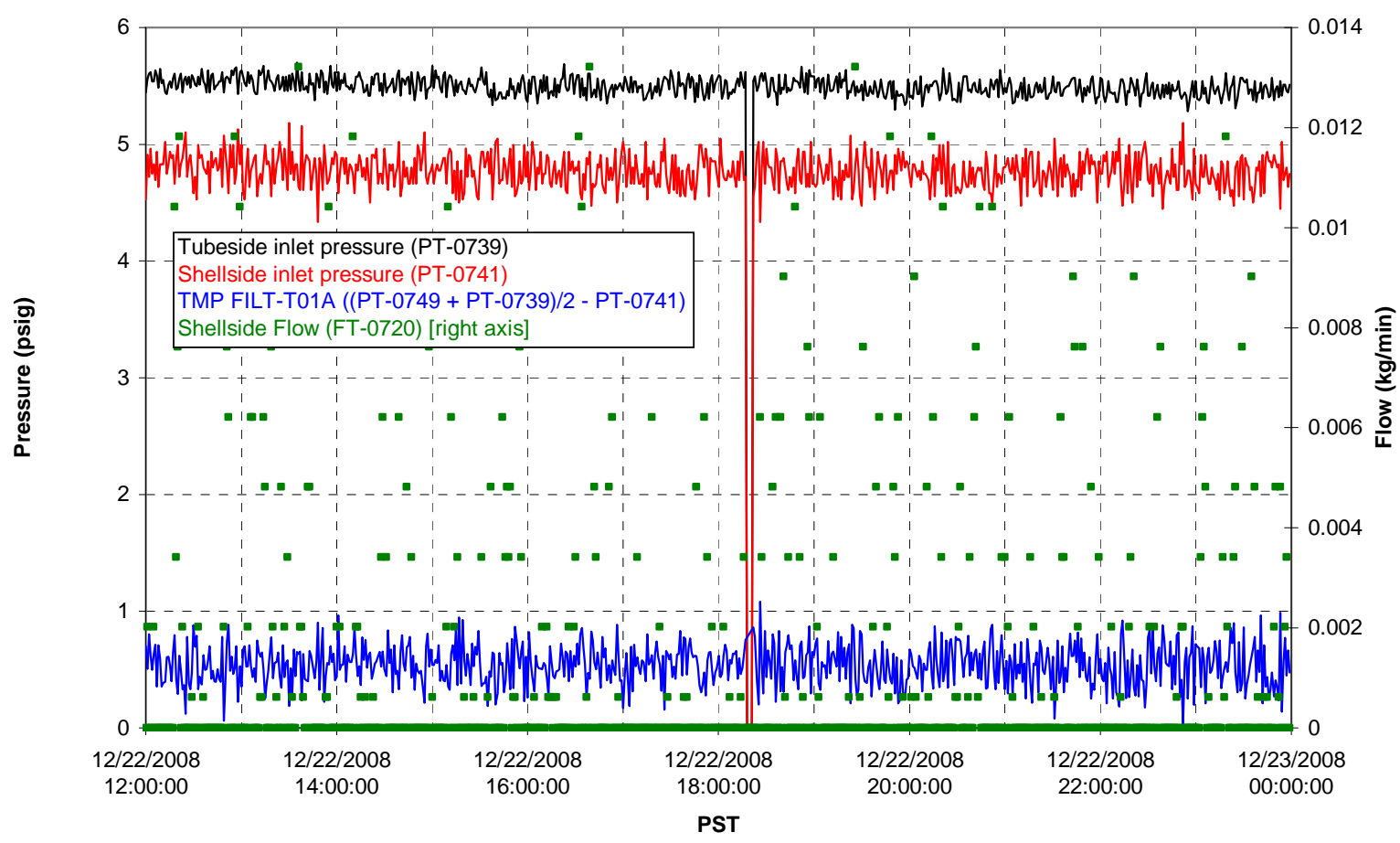

Filter UFP-FILT-T02A

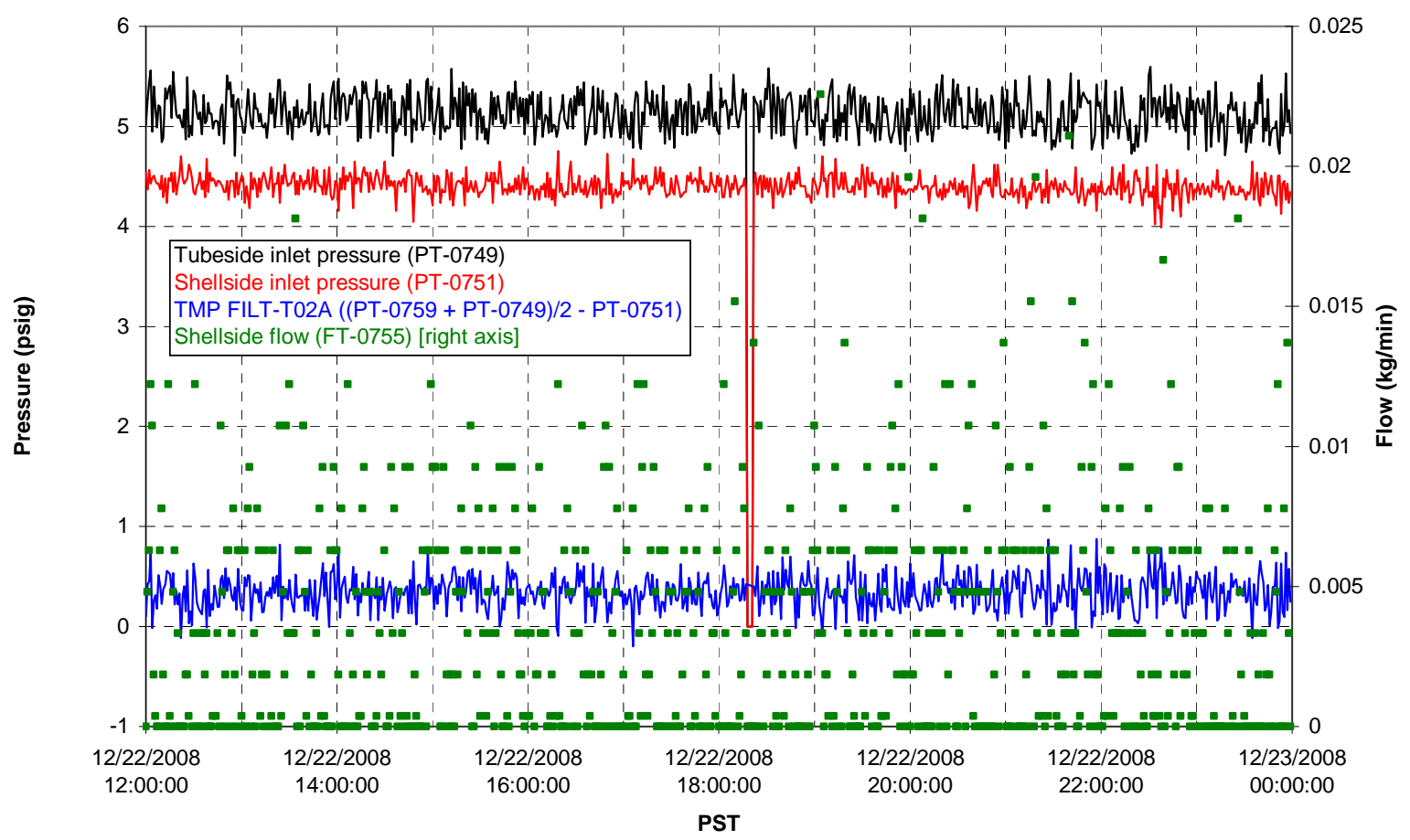


Filter UFP-FILT-T03A

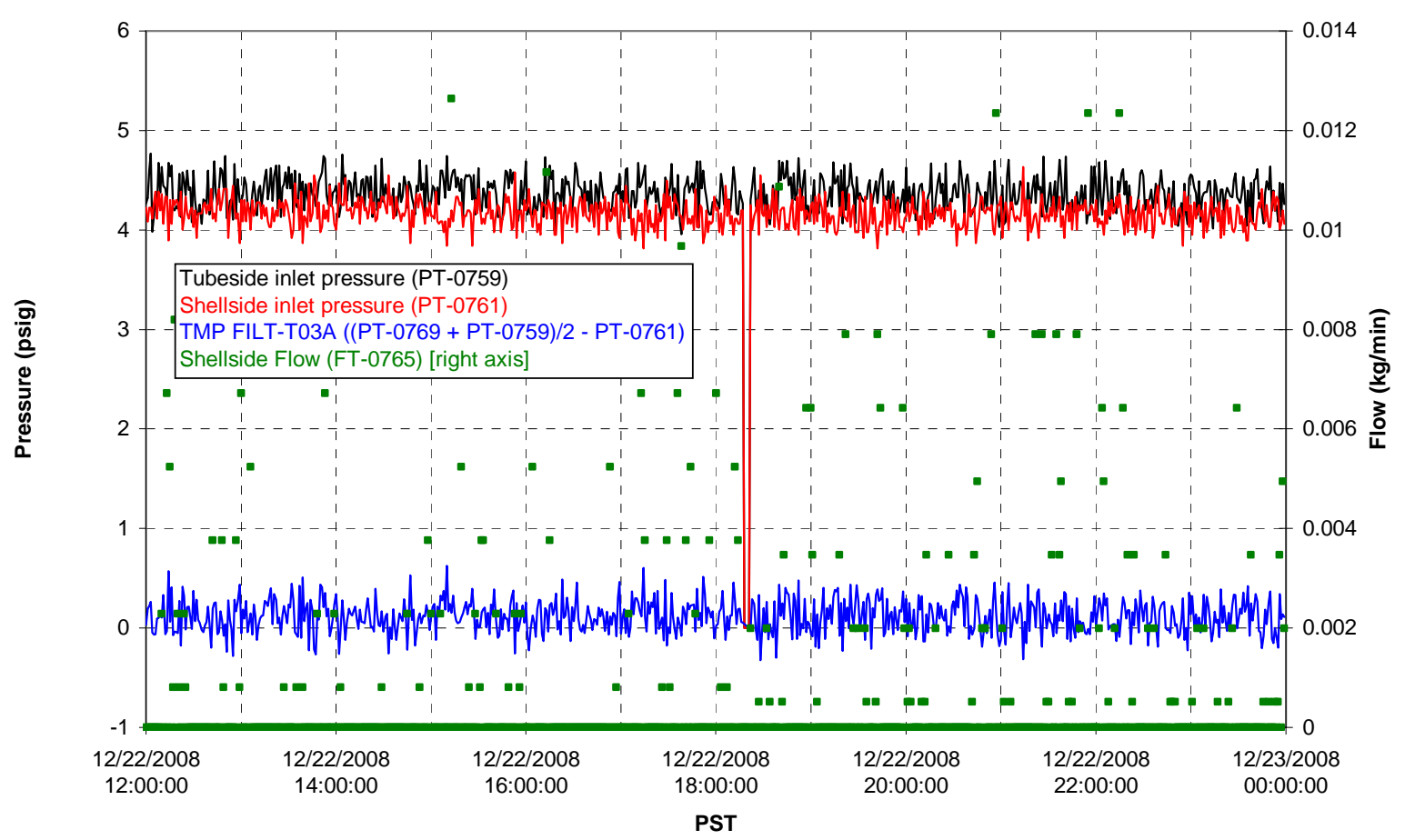

Filter UFP-FILT-T04A

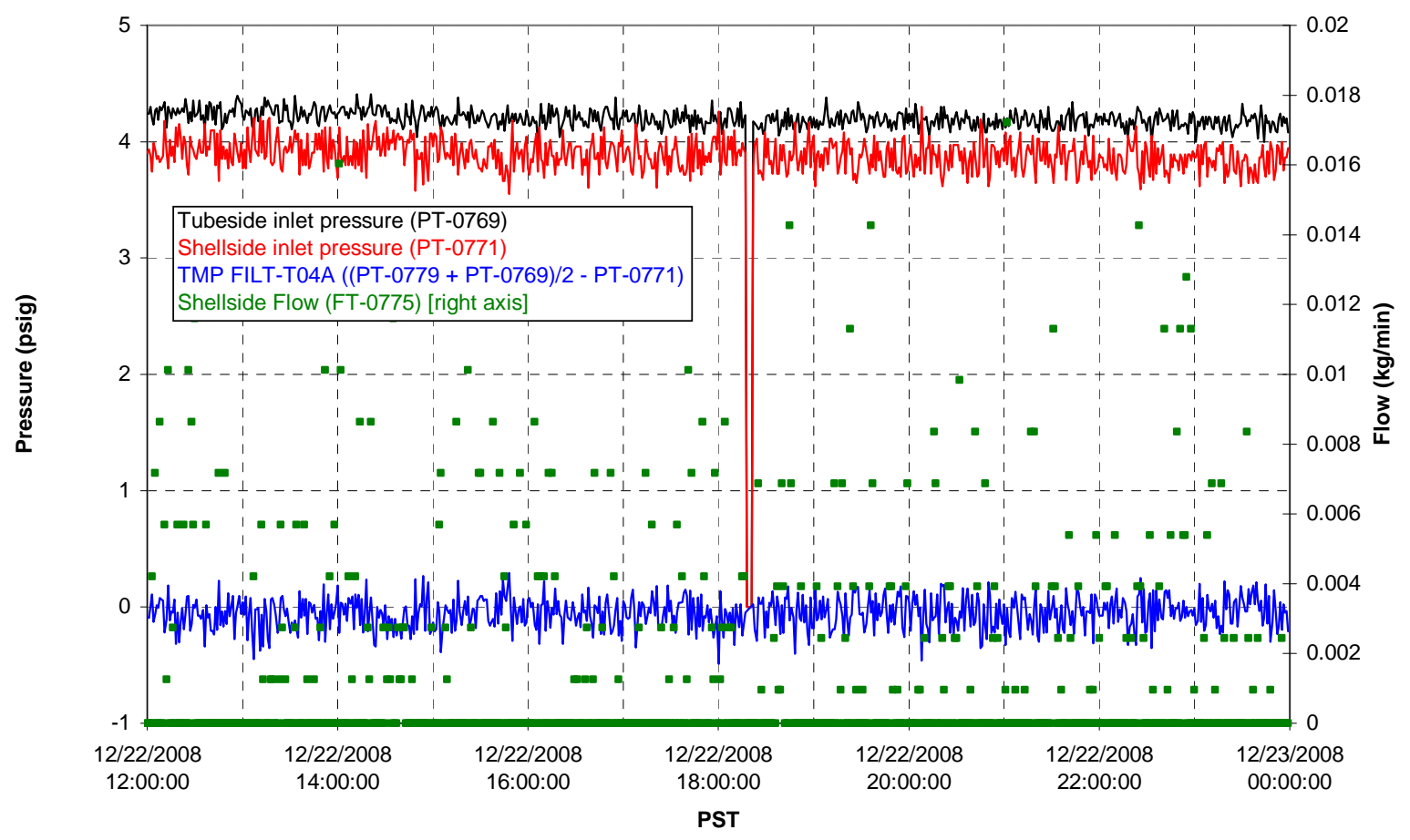


Filter UFP-FILT-T05A

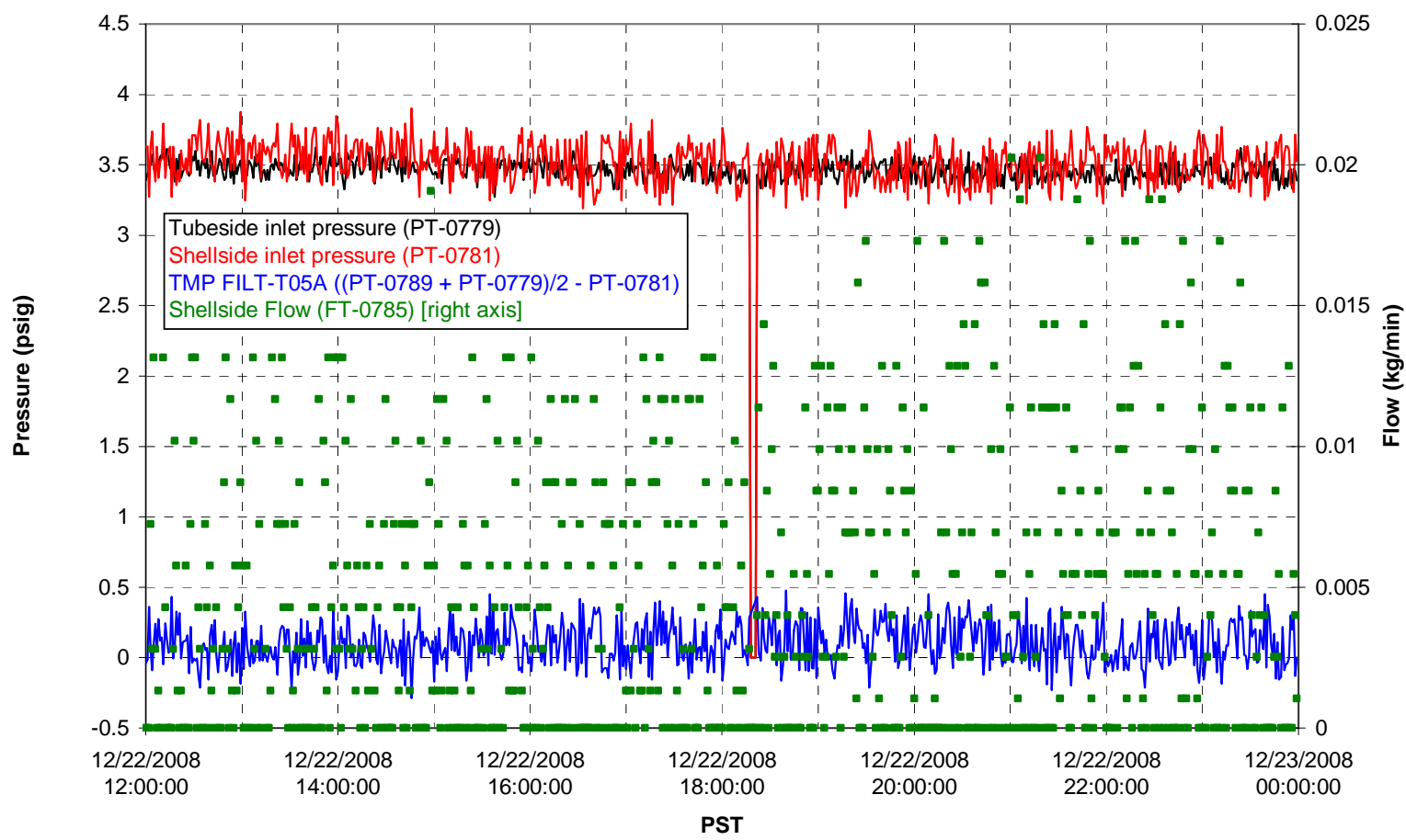

Chemical Flow

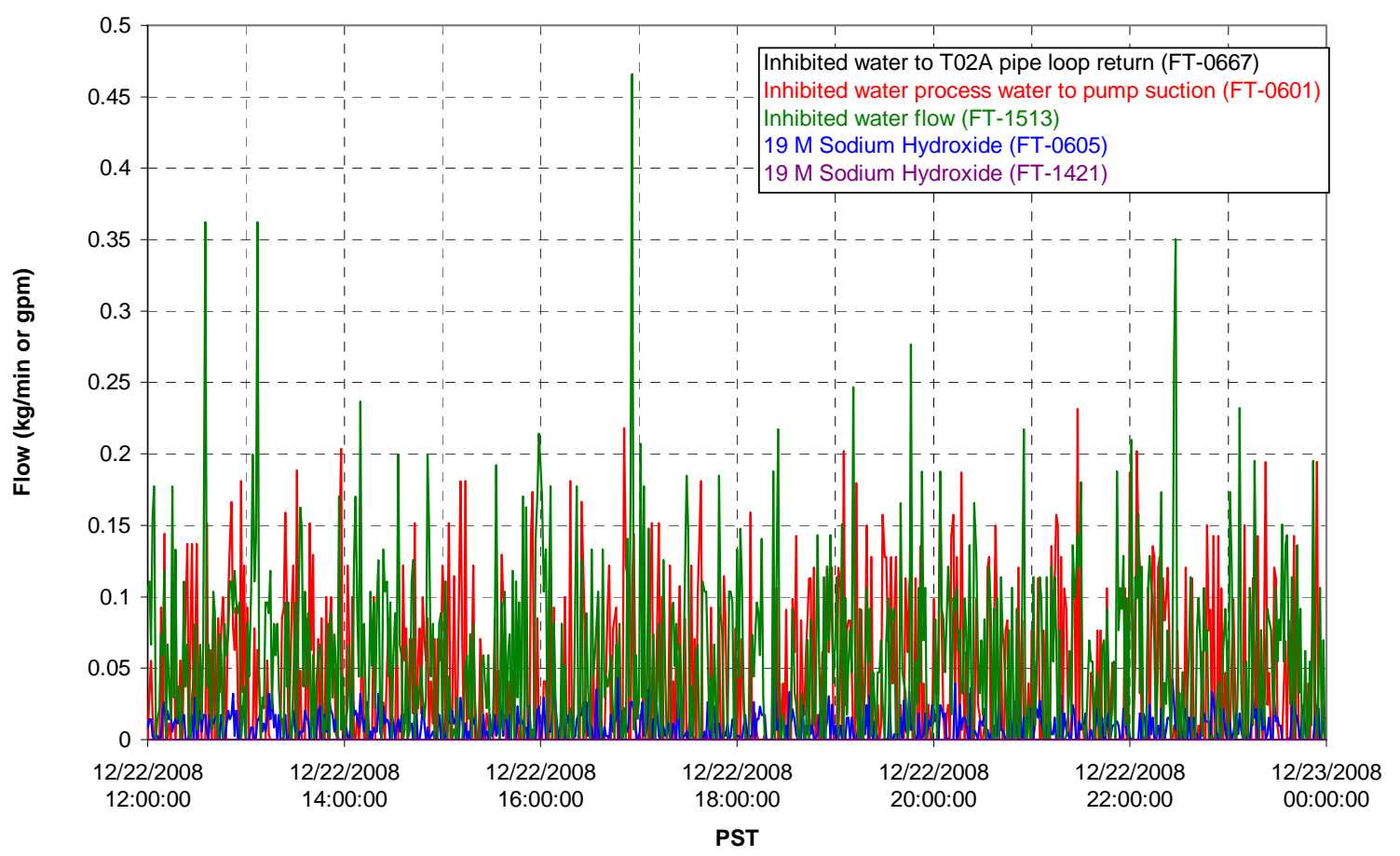




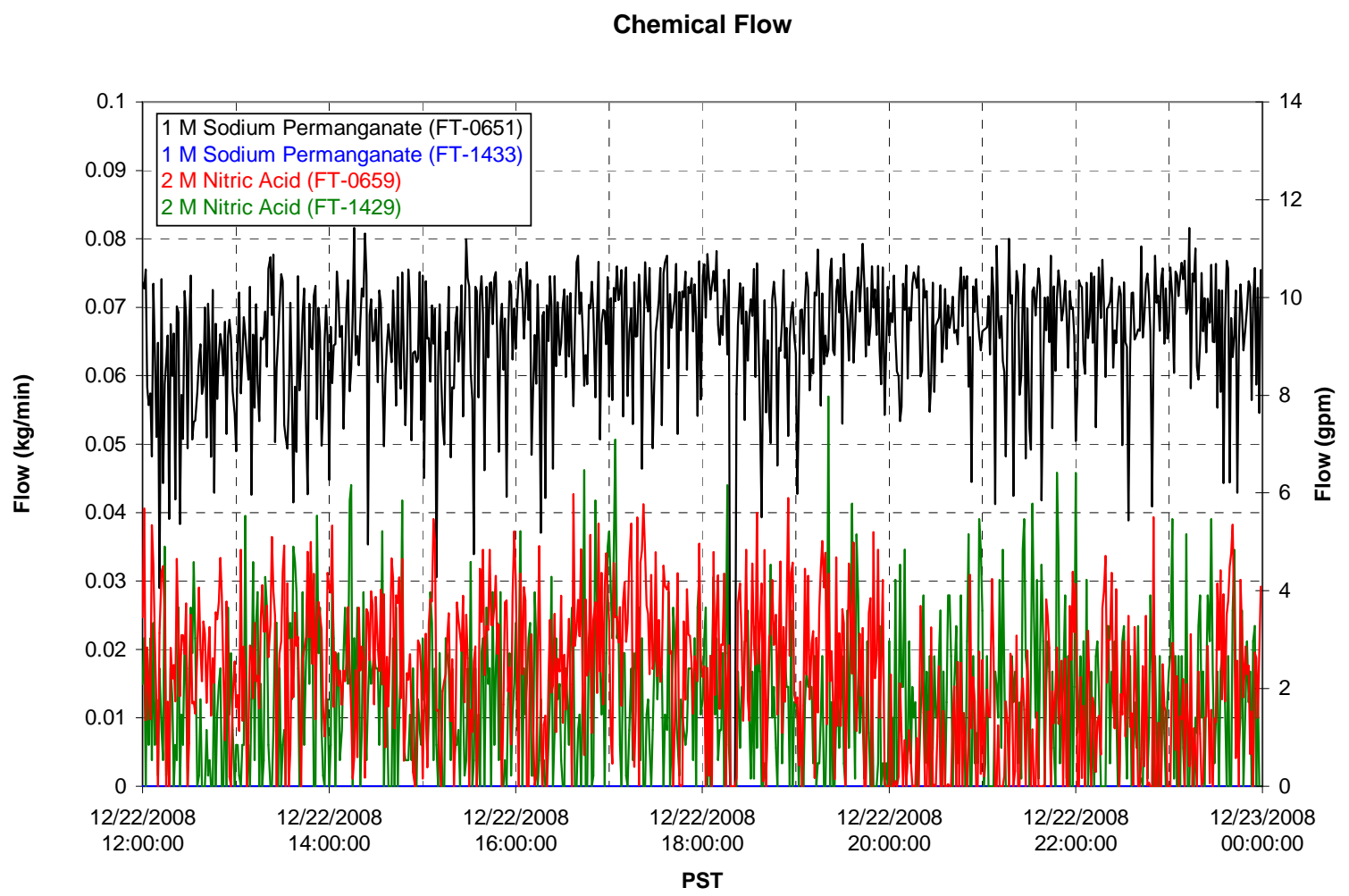

Air Flows

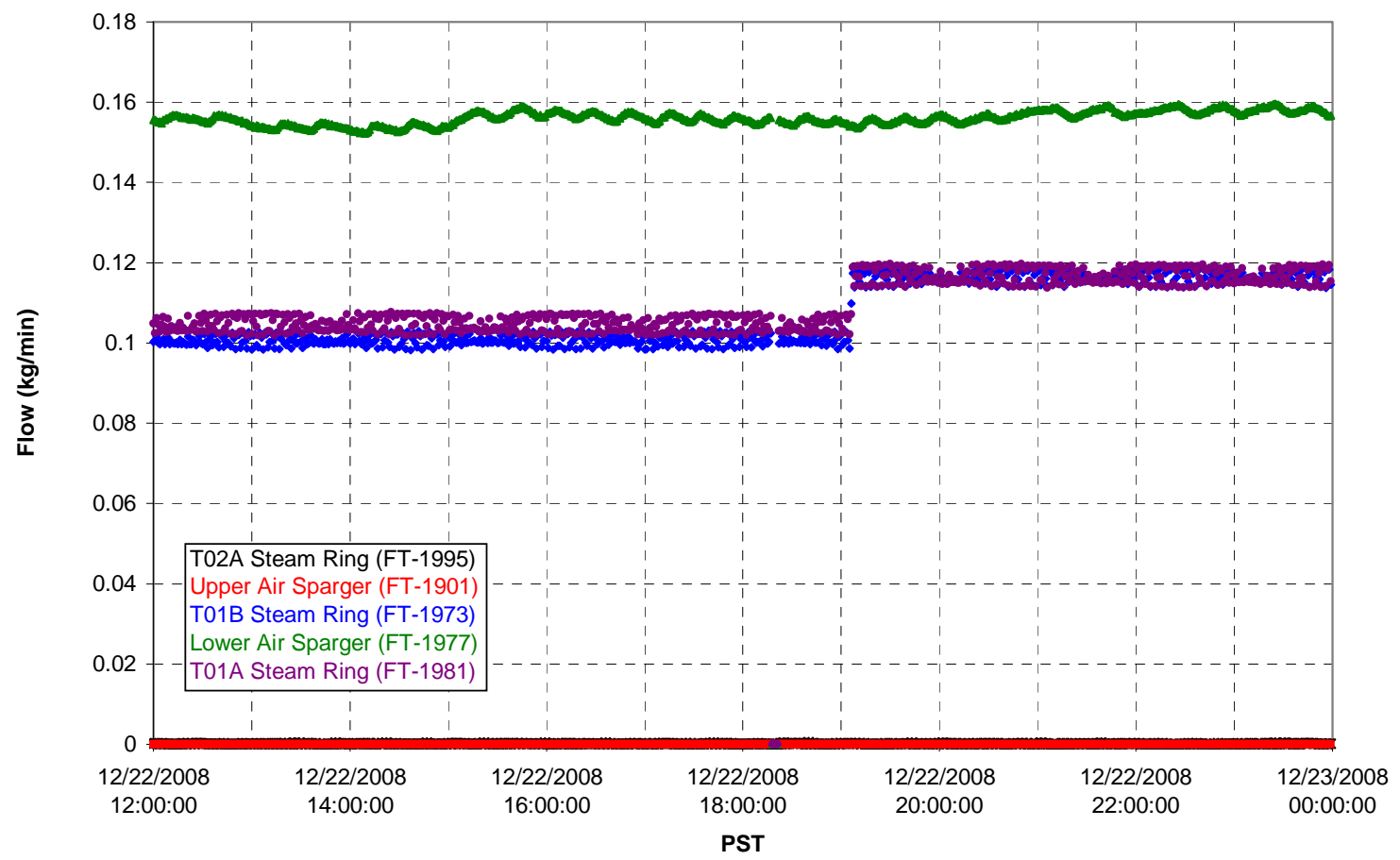


T02A Steam

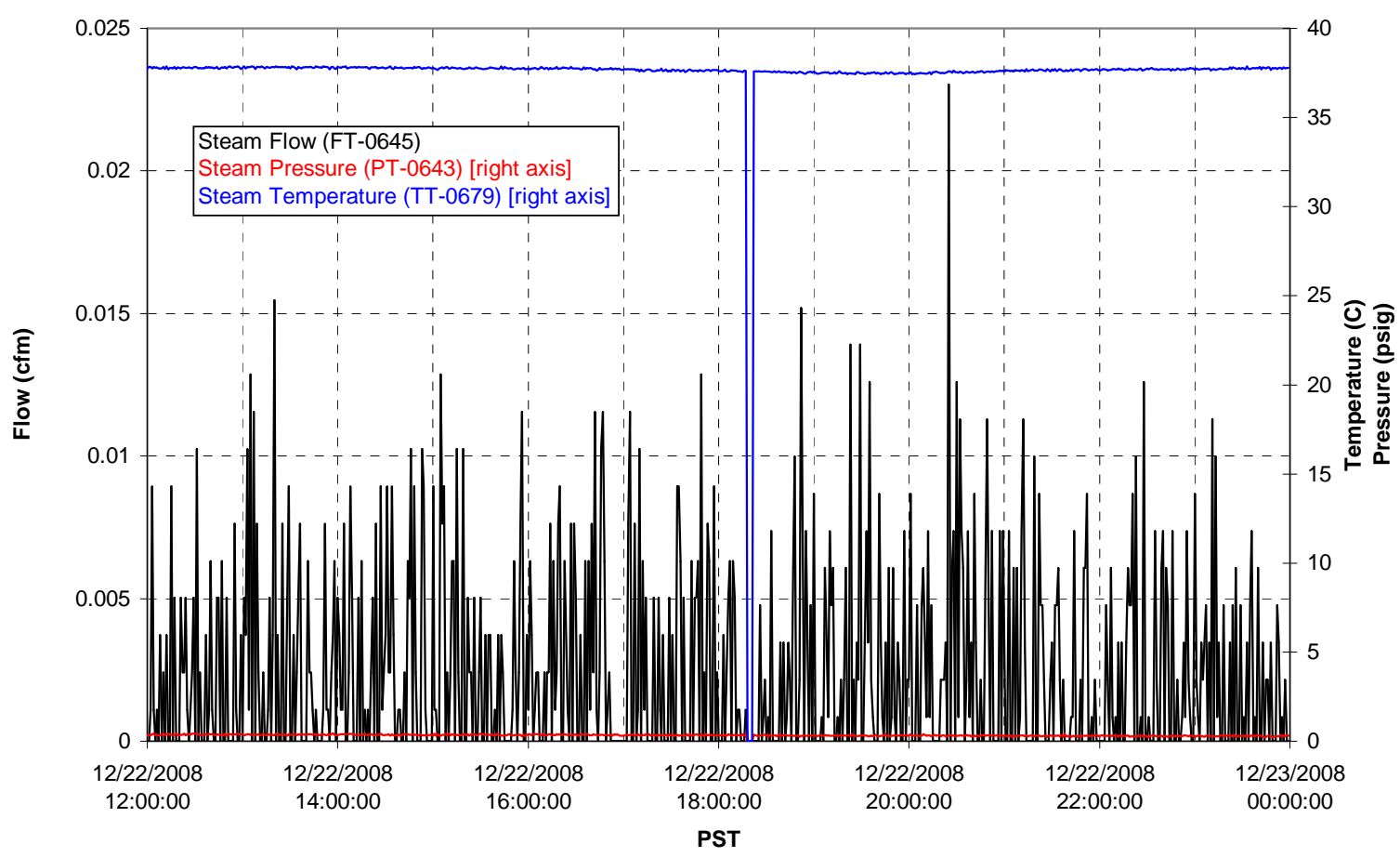

T01A Steam

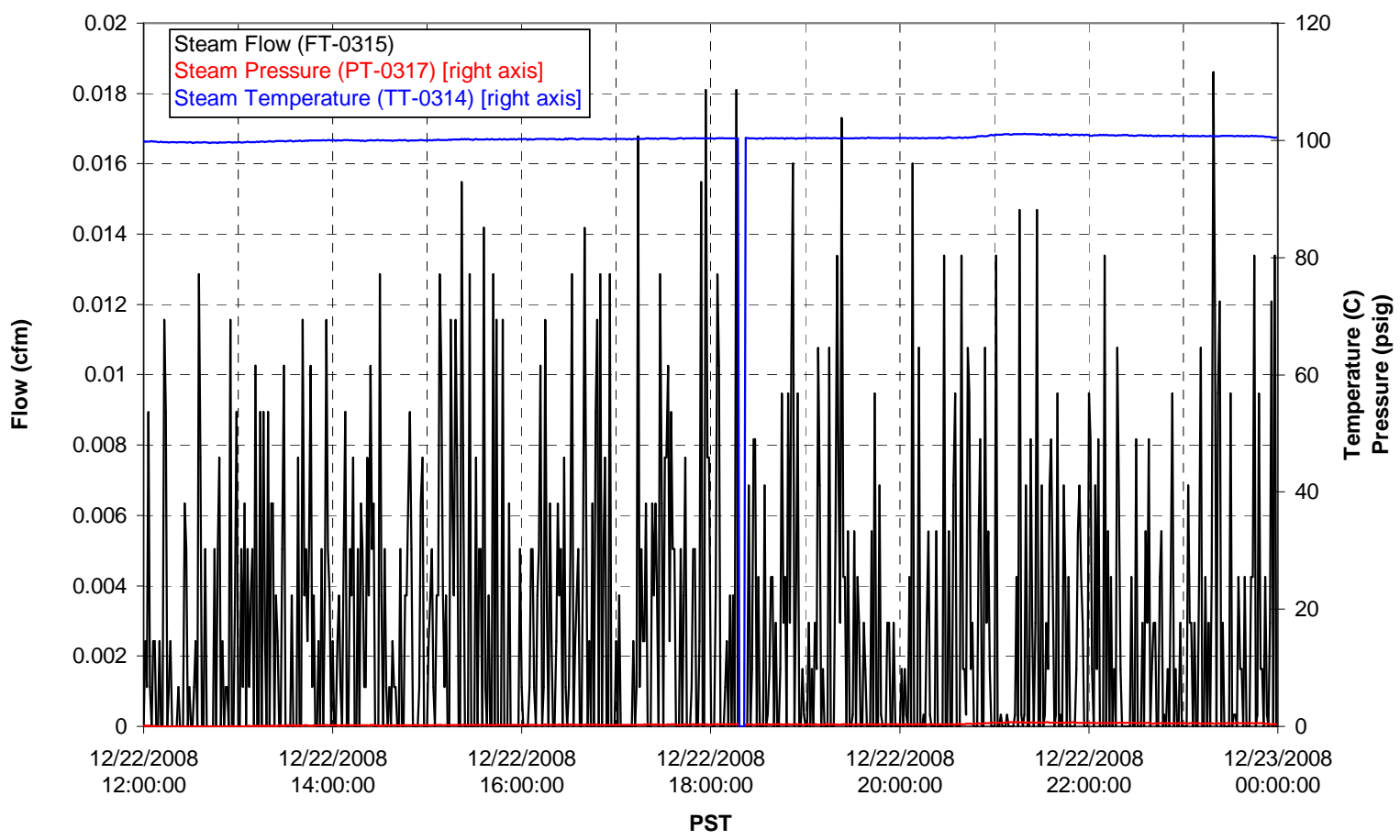


T01B Steam

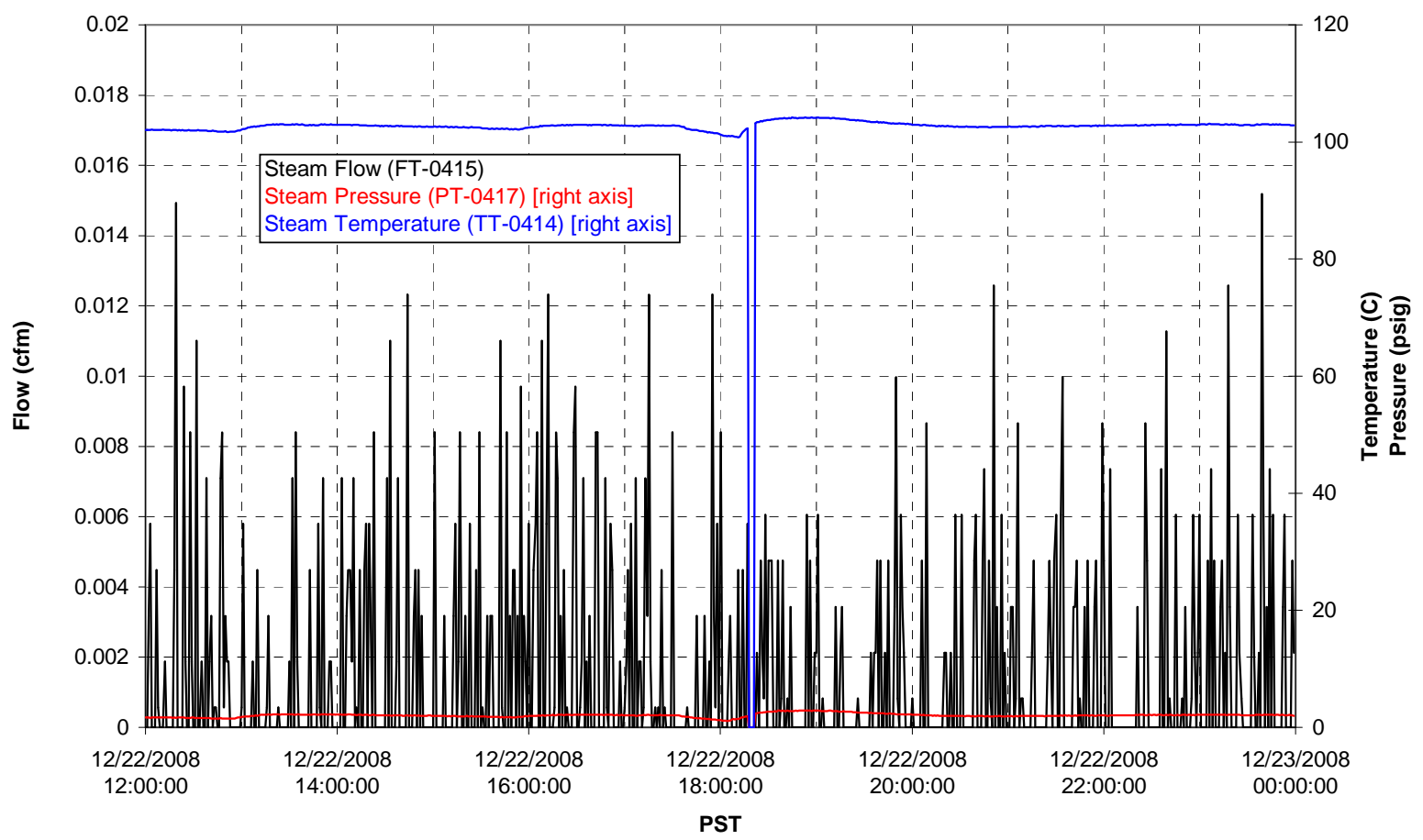


Functional Test Data Plots

12/23/08 00:00 - 12/23/08 12:00 
T01A level

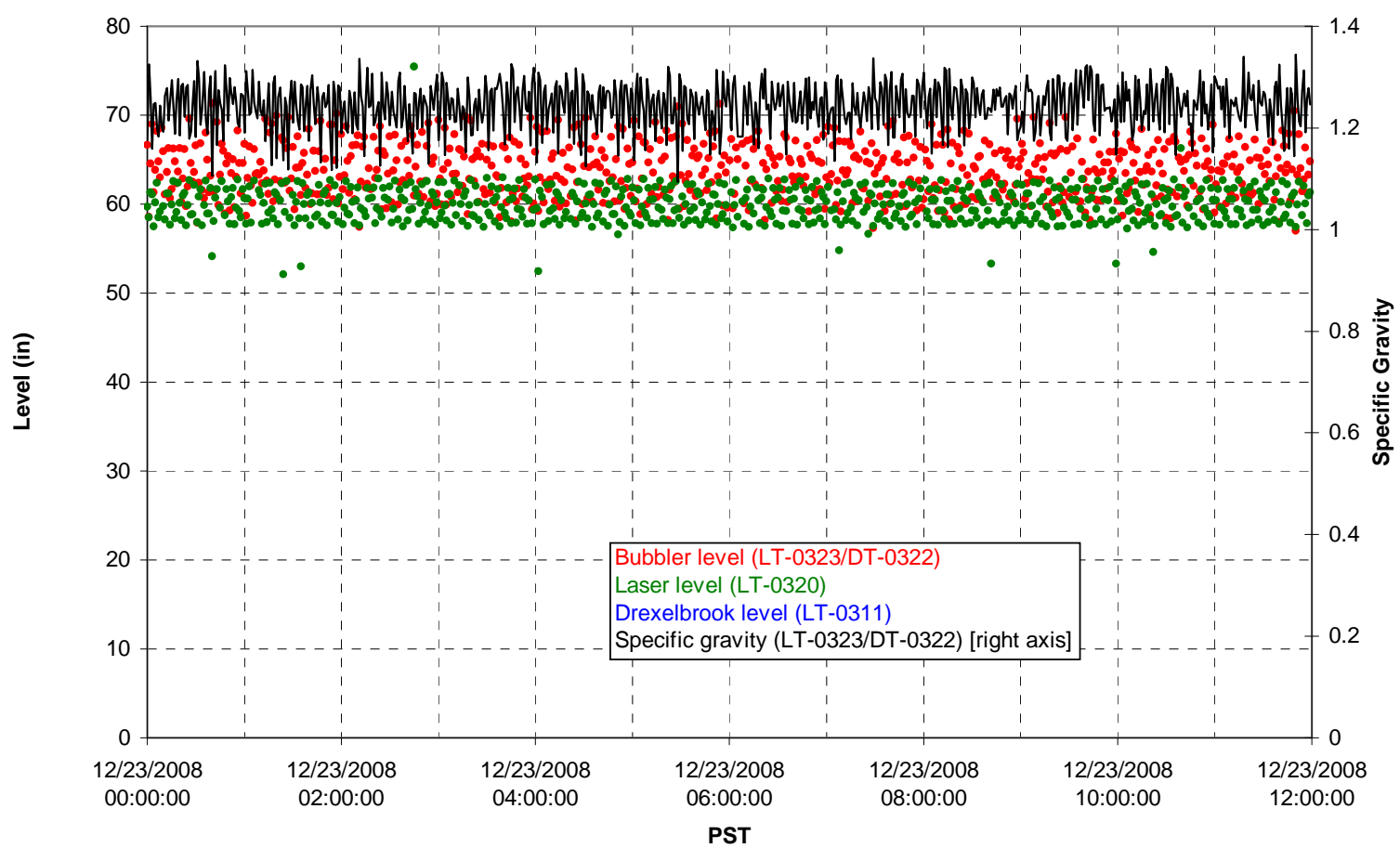

T01A temperatures

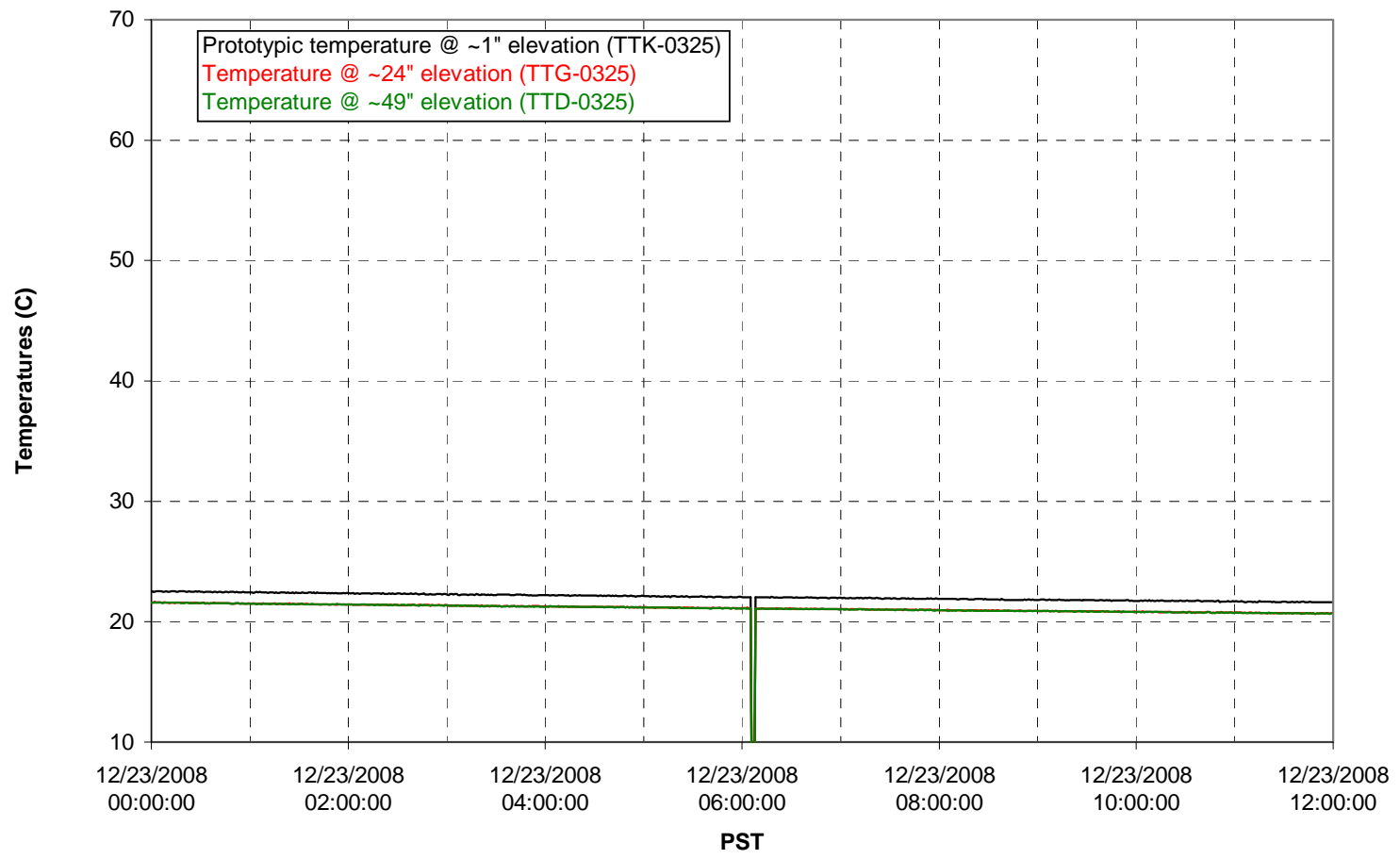


T01B level

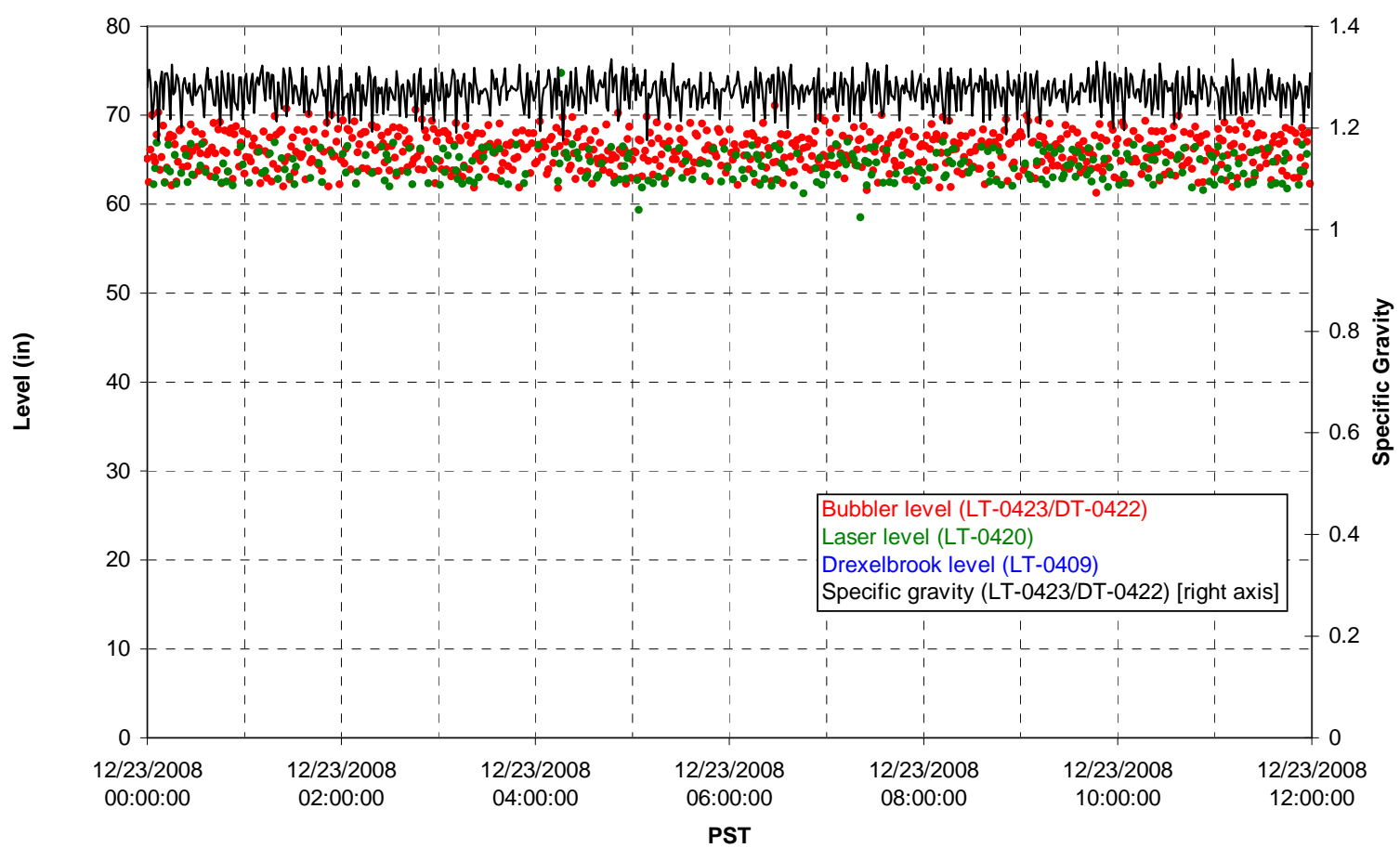

T01B temperatures

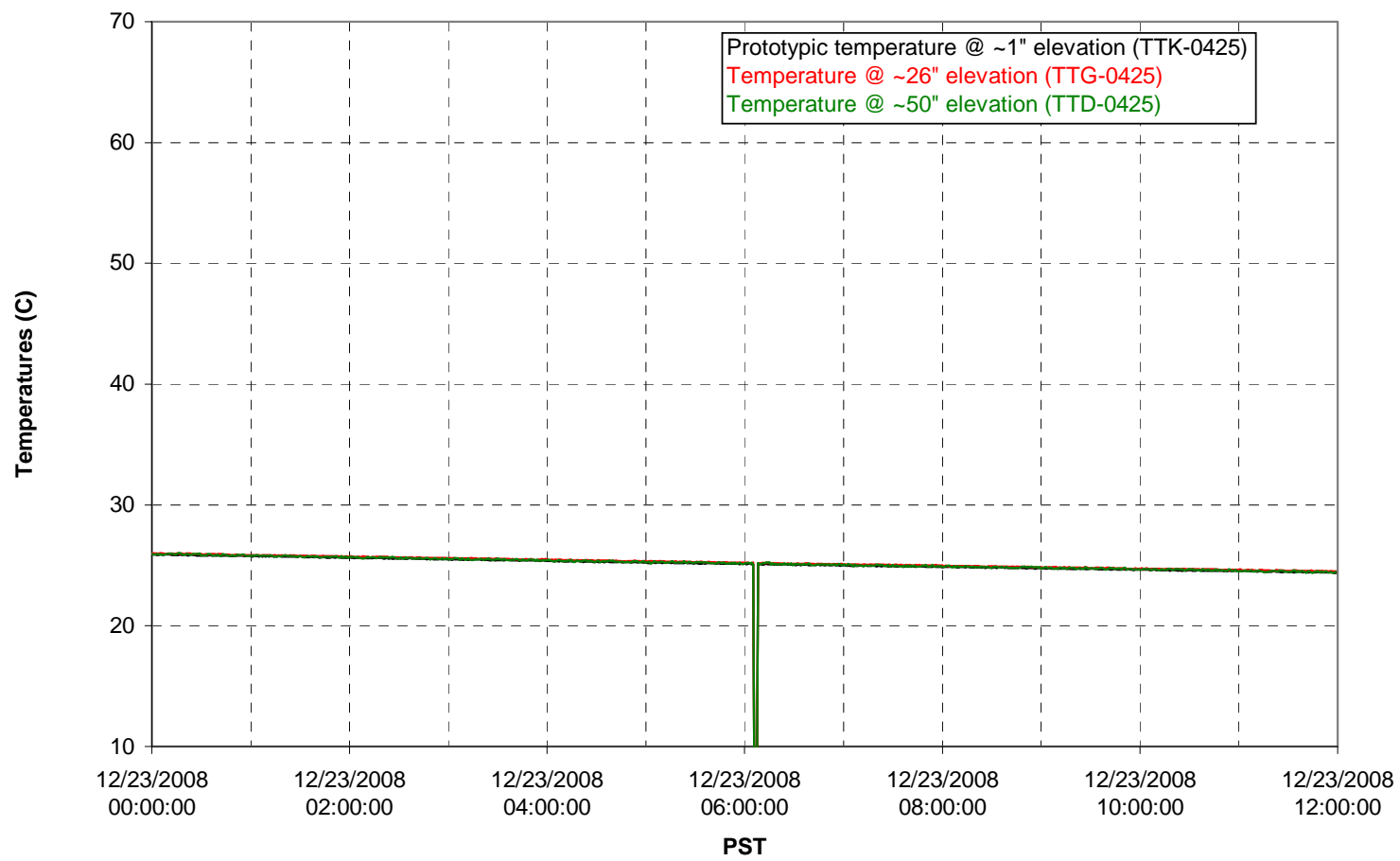


T02A level

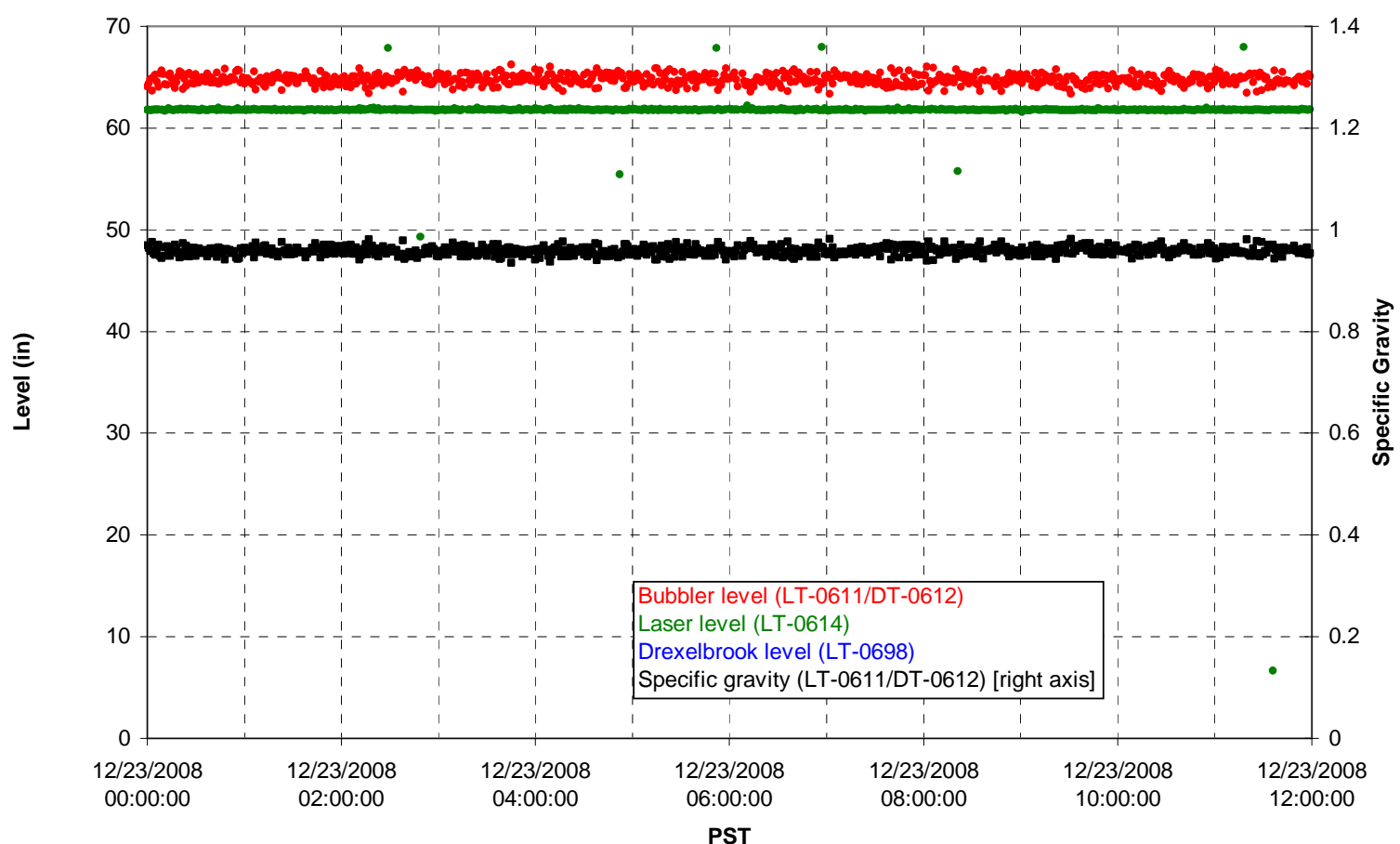

T02A temperatures

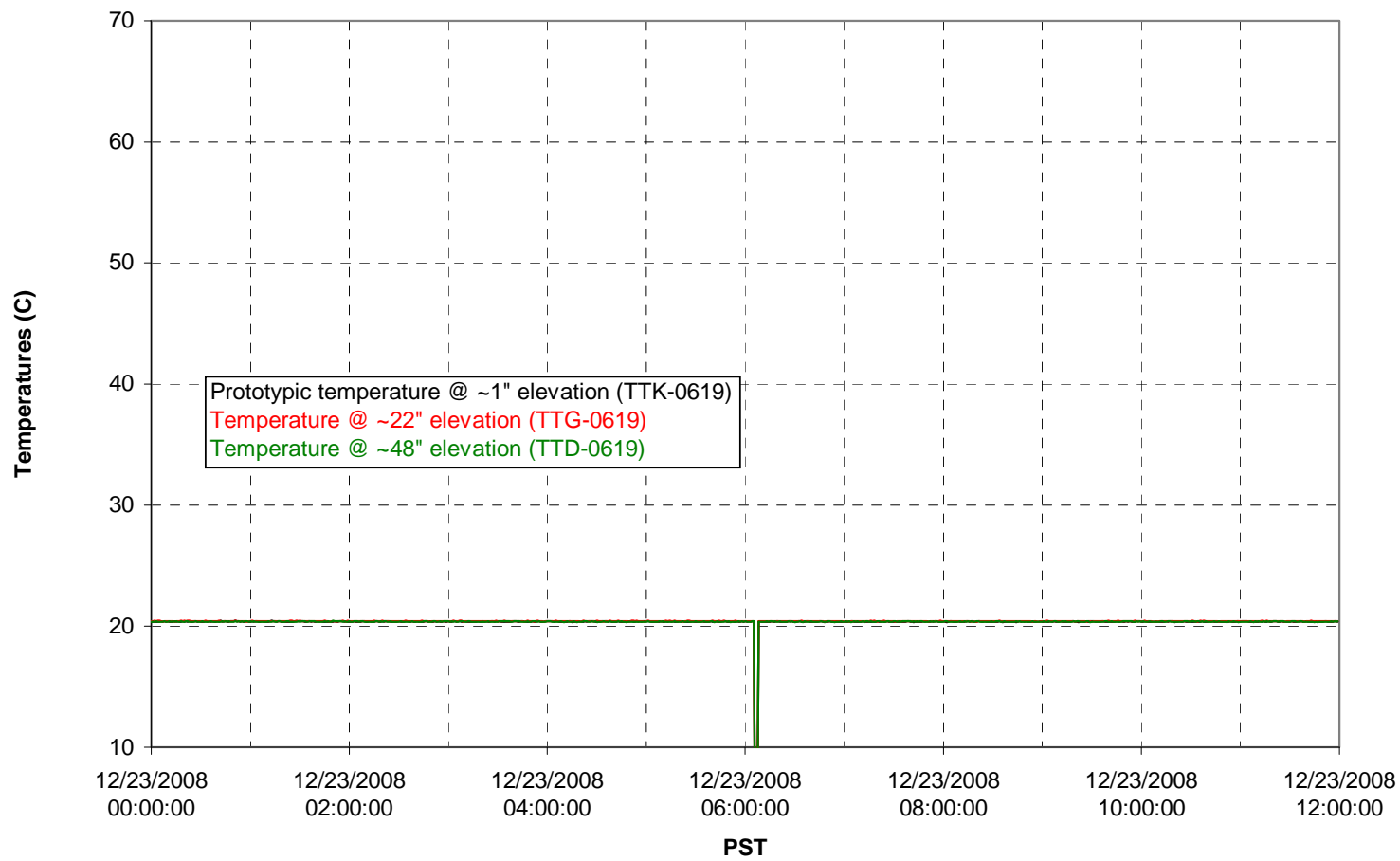


T02A and filter loop temperatures

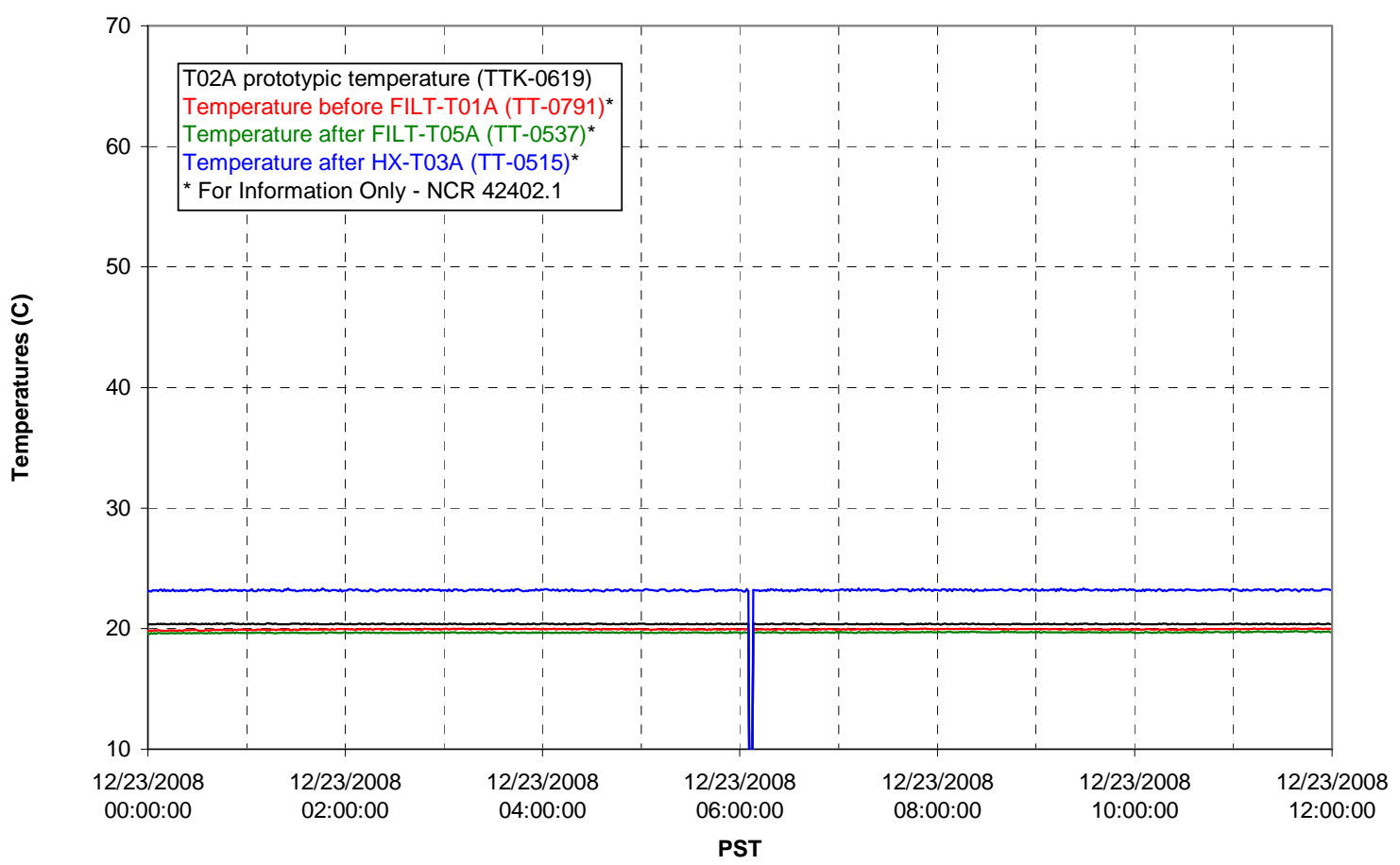

Pump Pressures and Flow

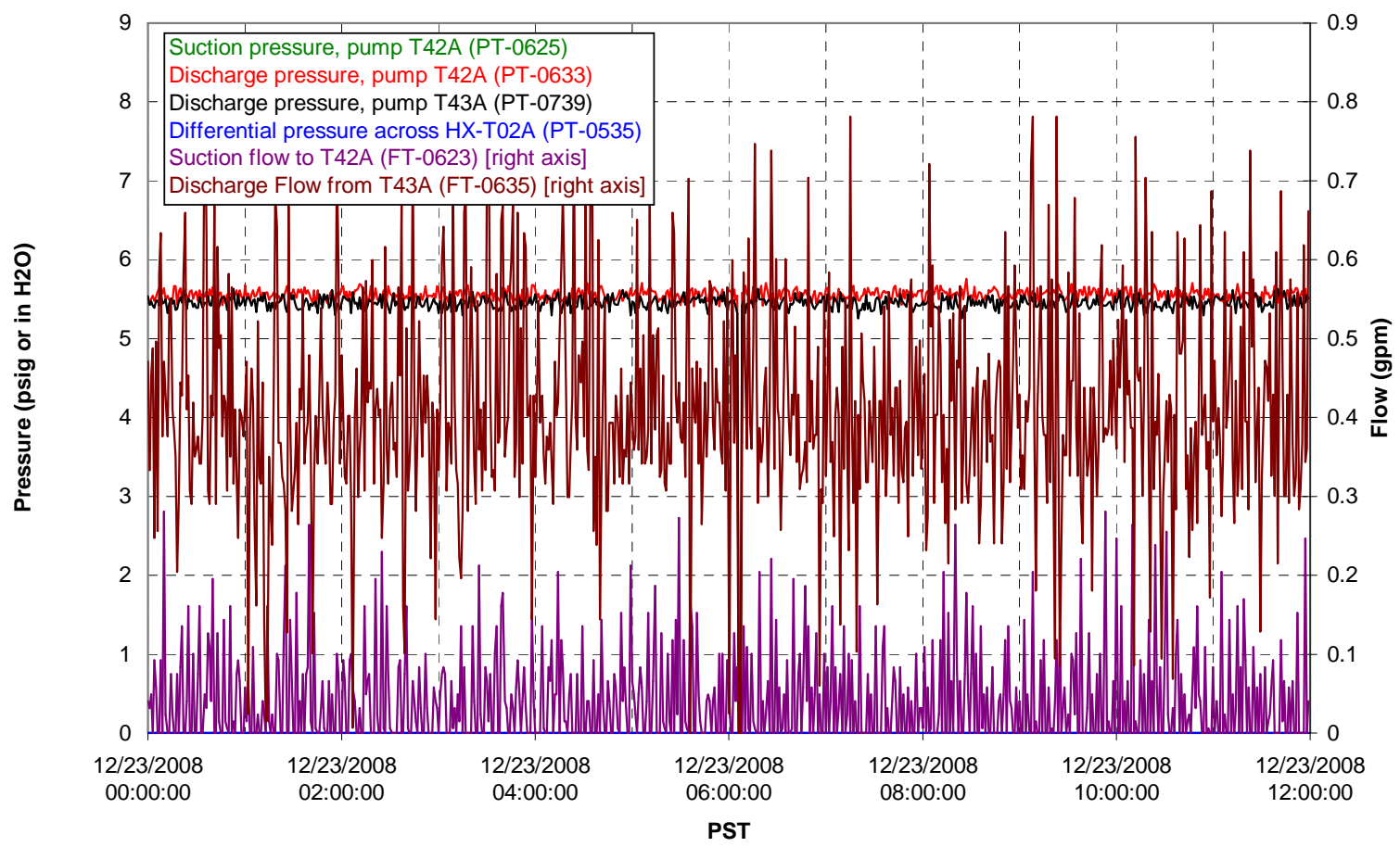


Axial pressure drop

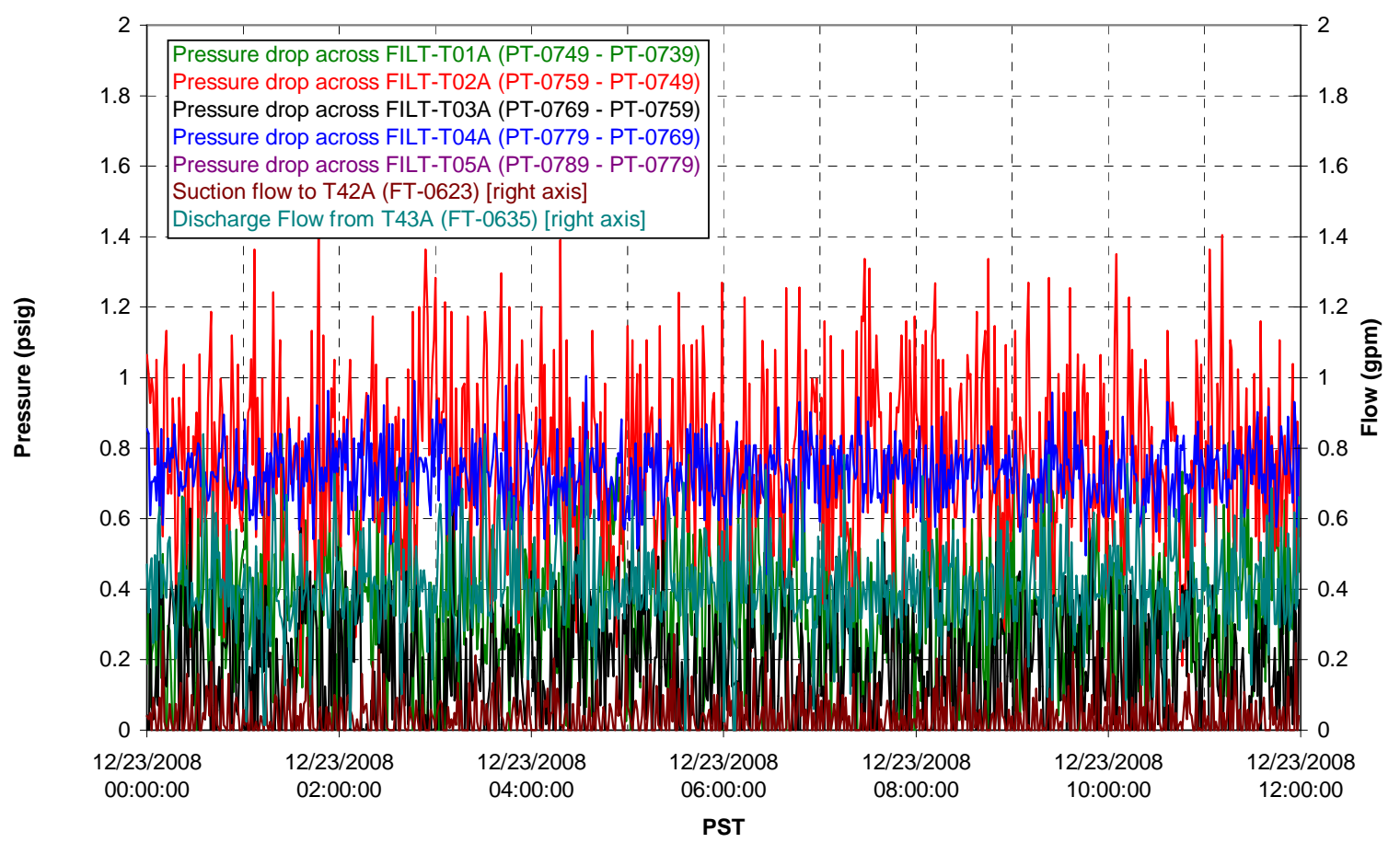

Permeate flow rates

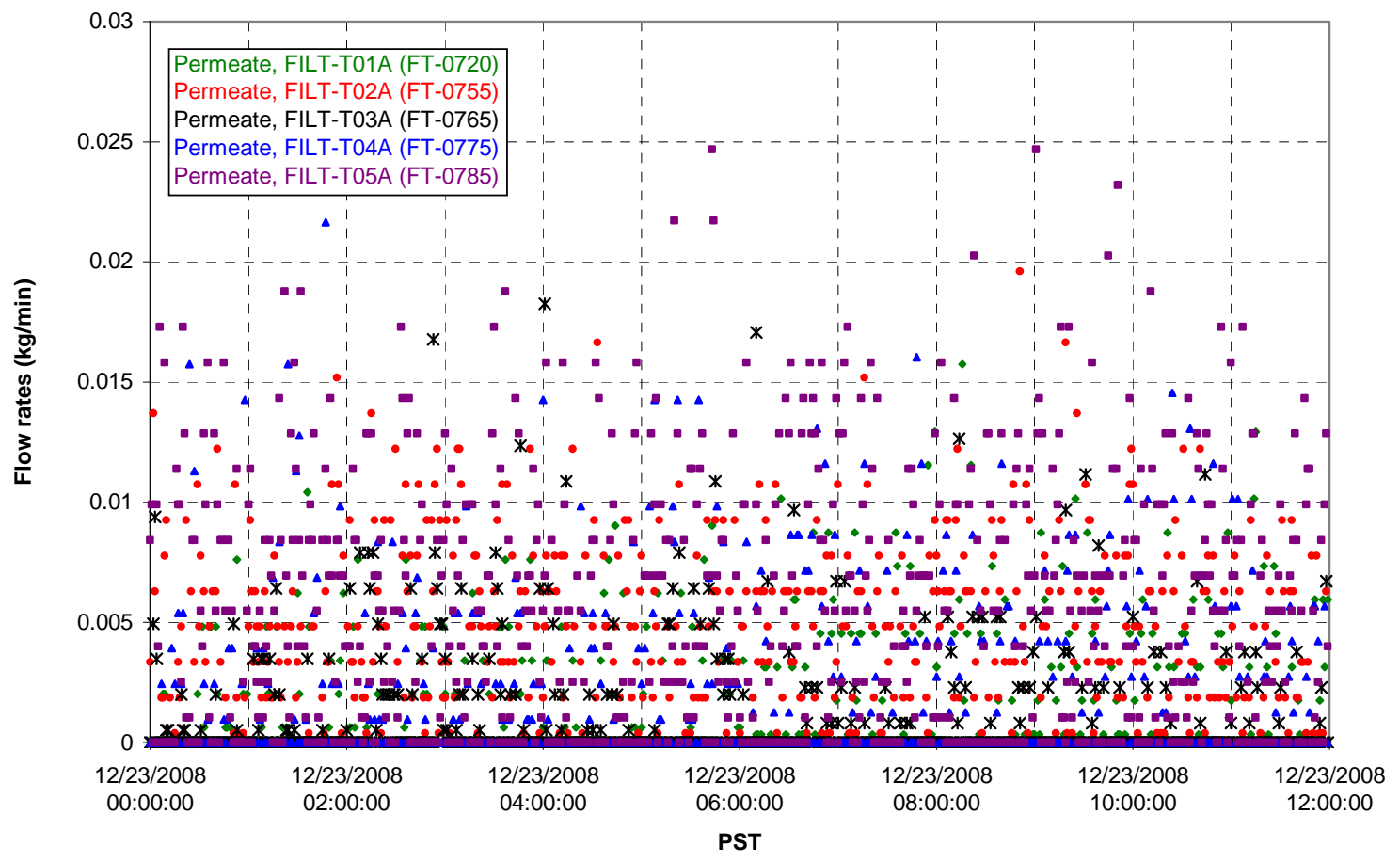


T02A Inner Temperature Tree

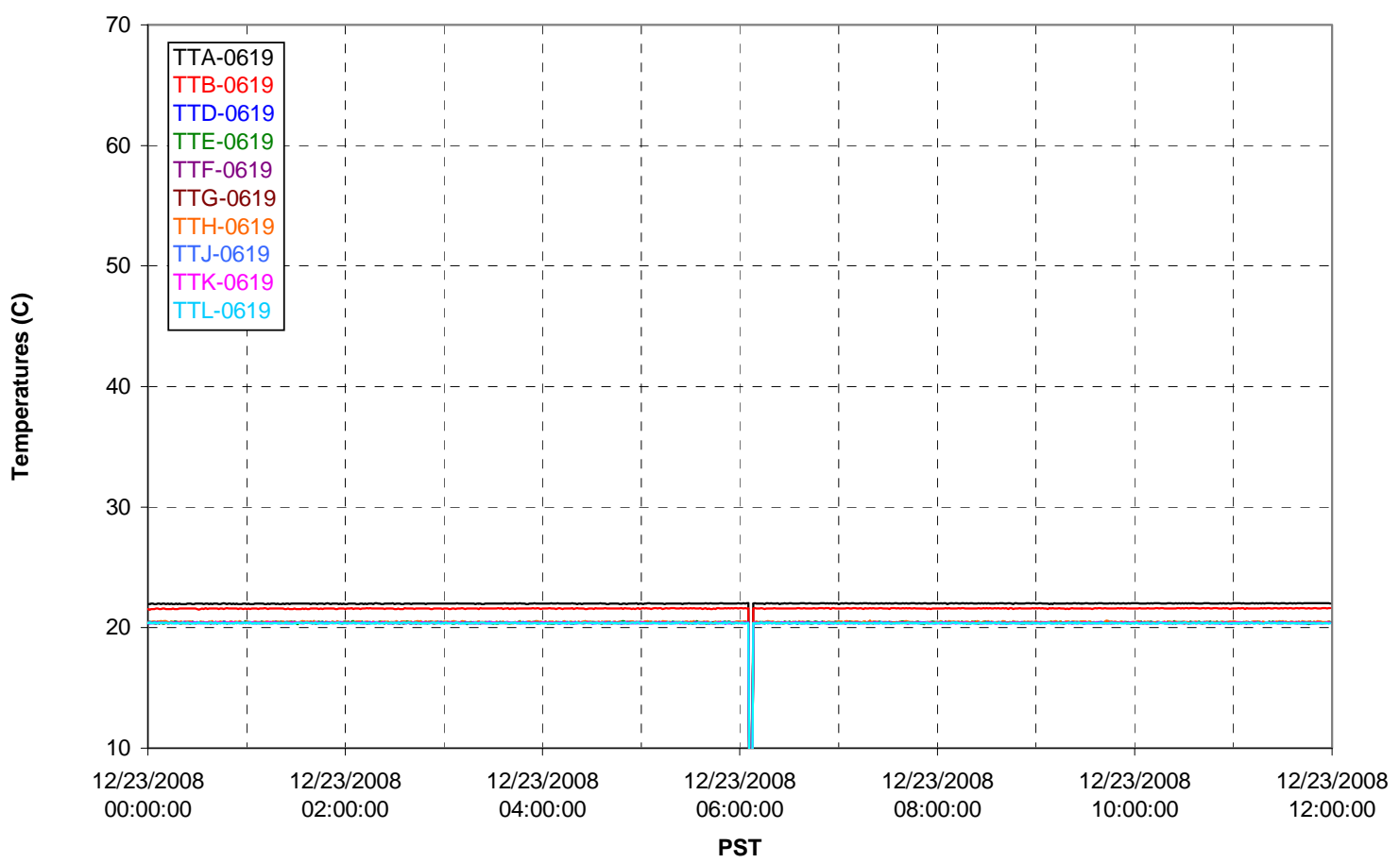

T02A Outer Temperature Tree

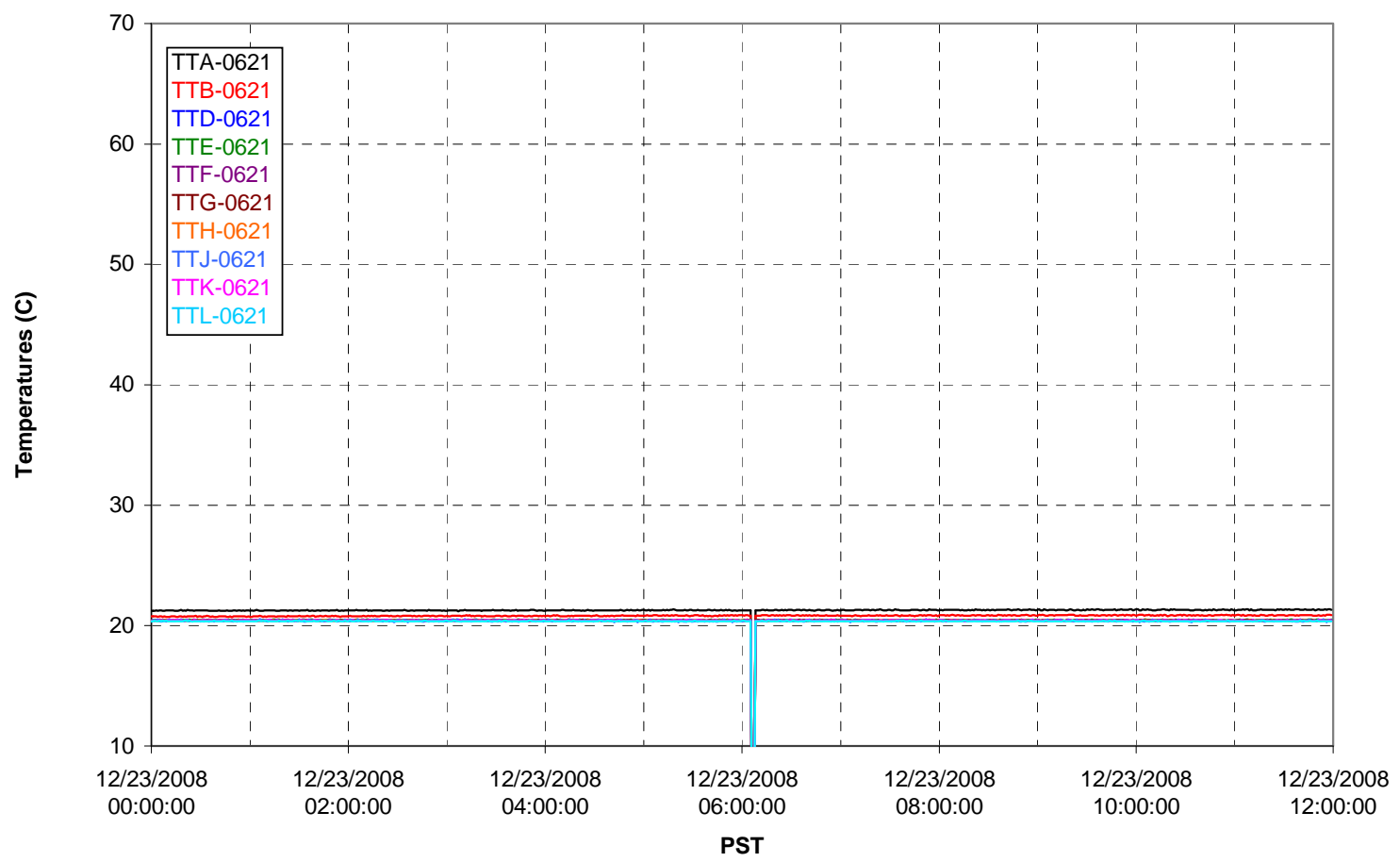


T02A temperatures

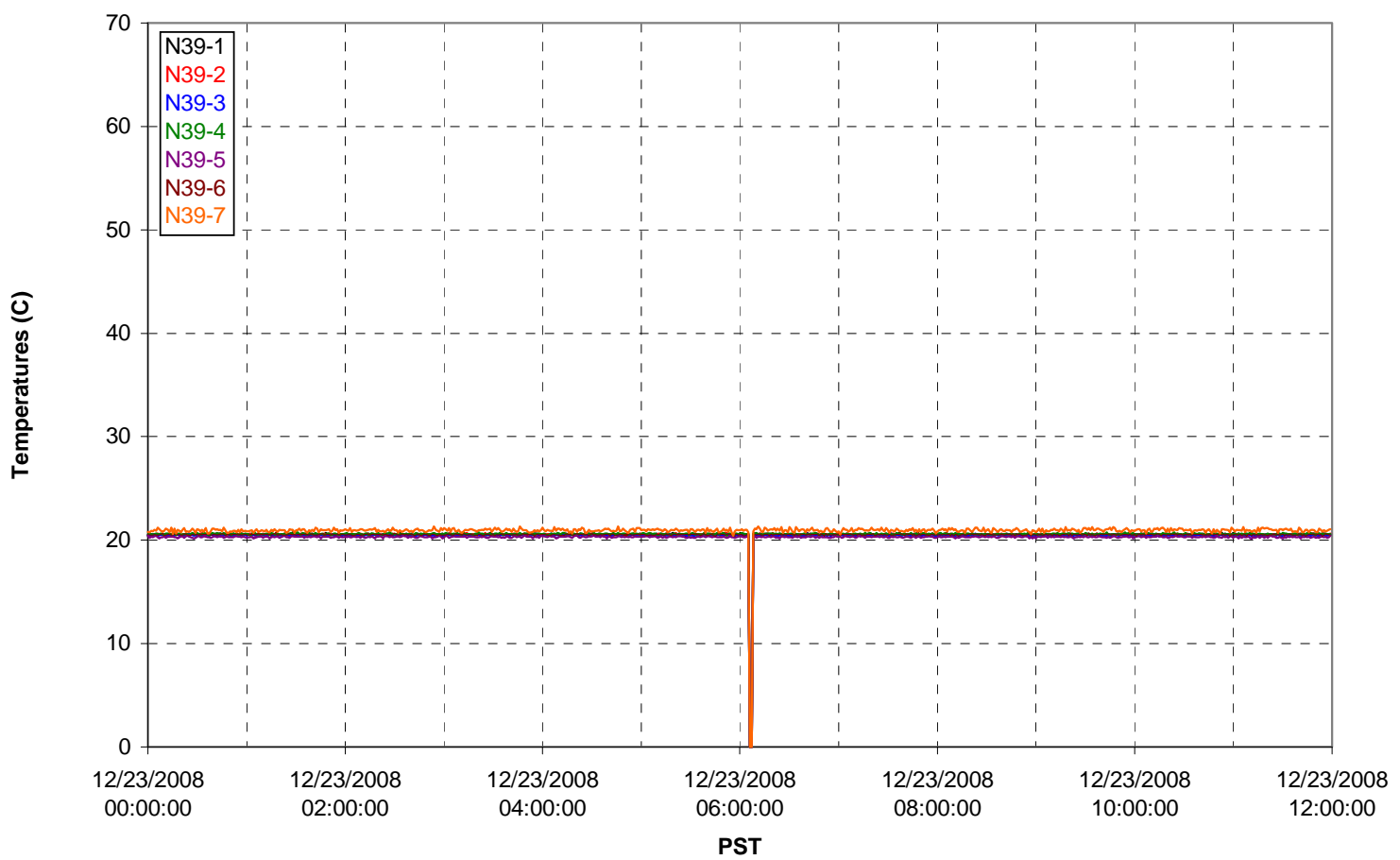

T02A temperatures

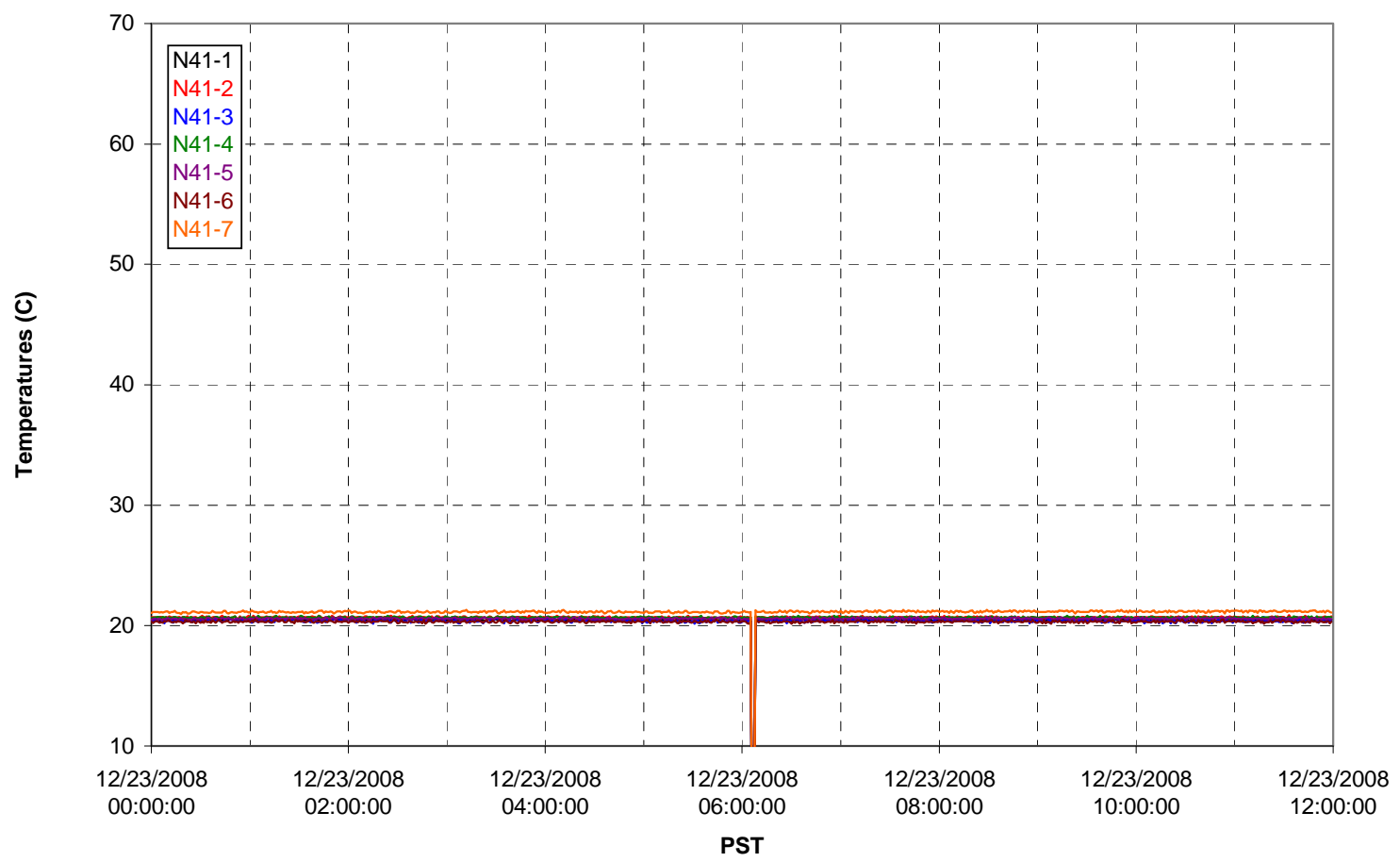


T02A temperatures

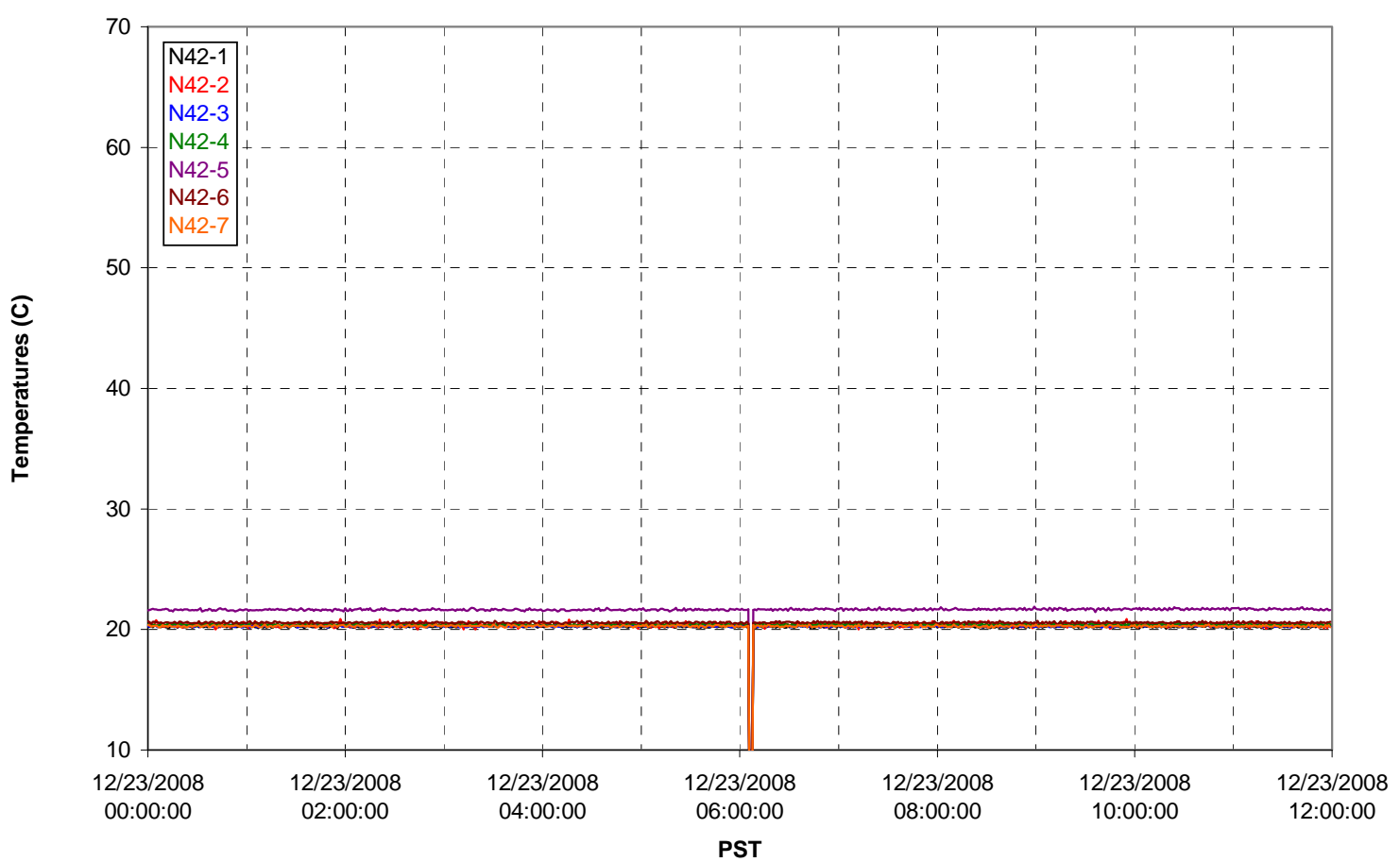

T02A temperatures

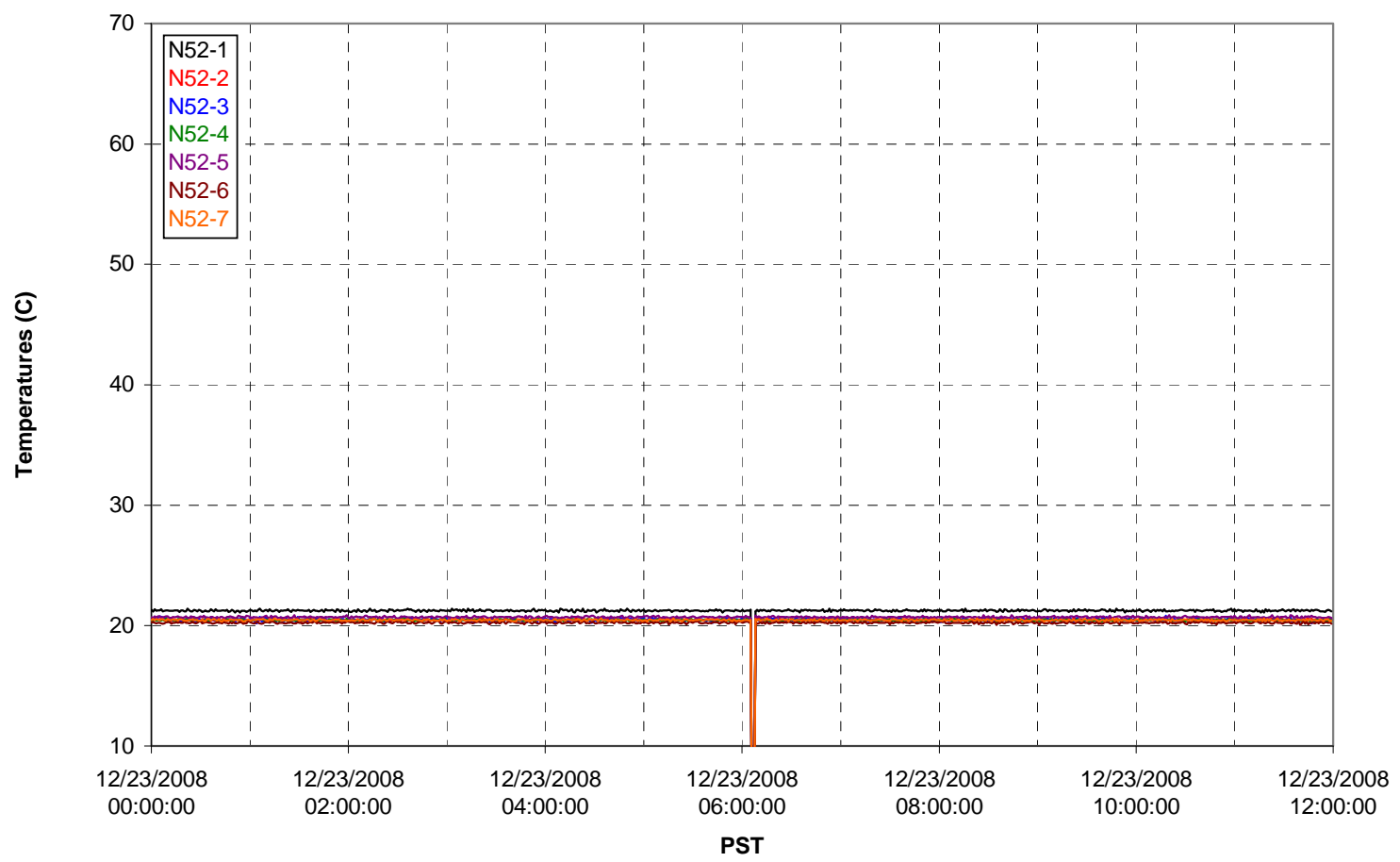


T02A Heating and Cooling

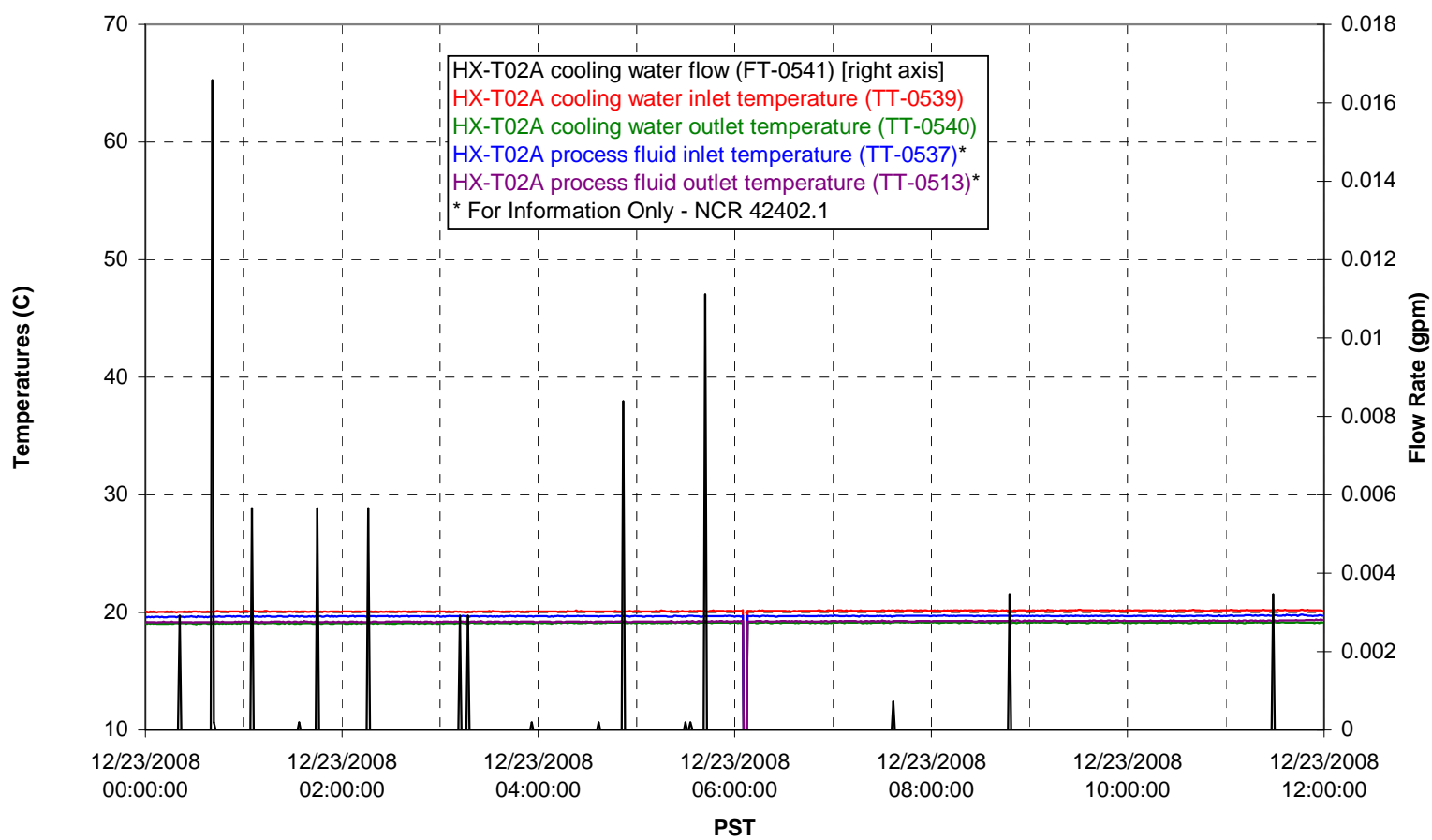

Pump Operation

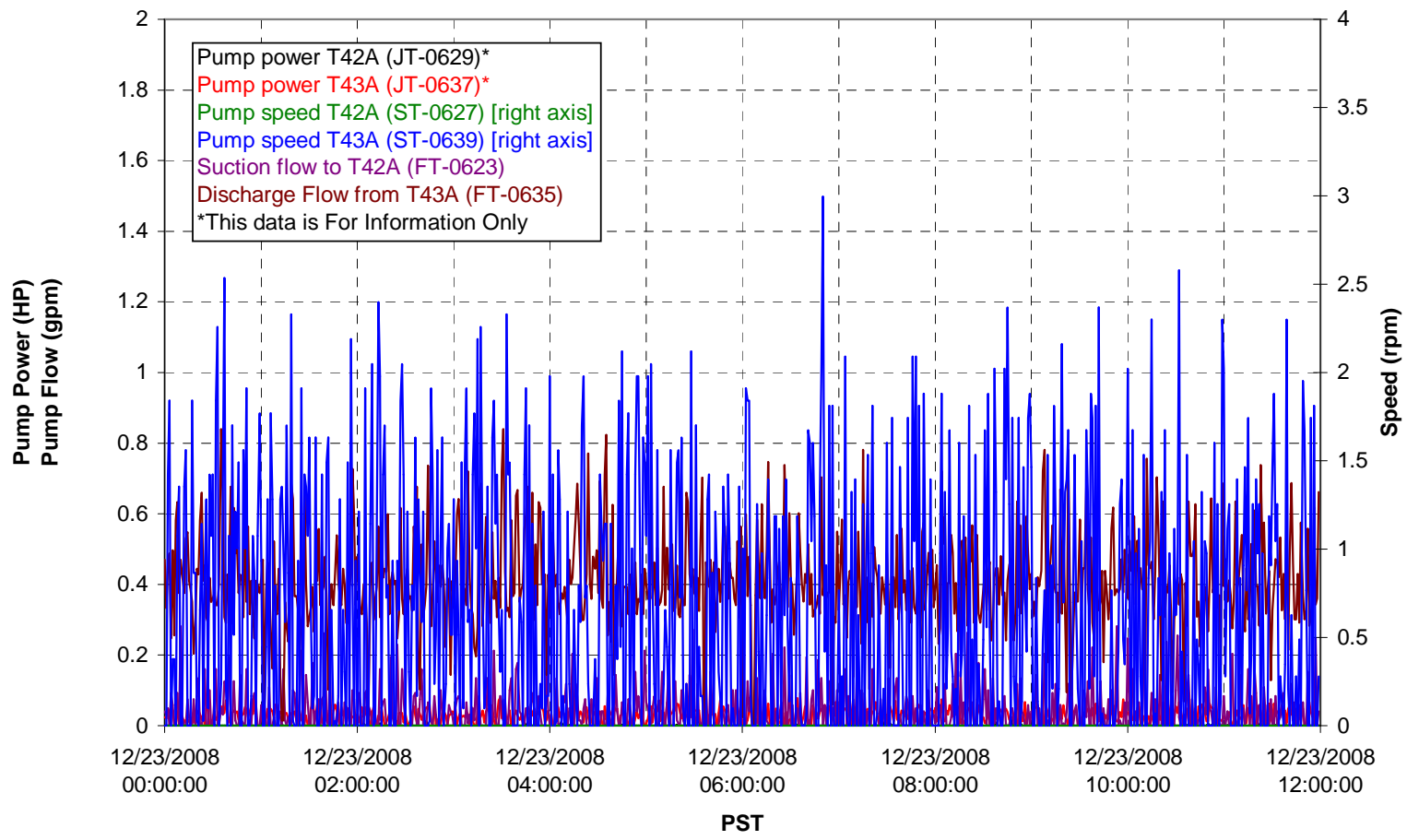


Pulsepot UFP-PP-T01A

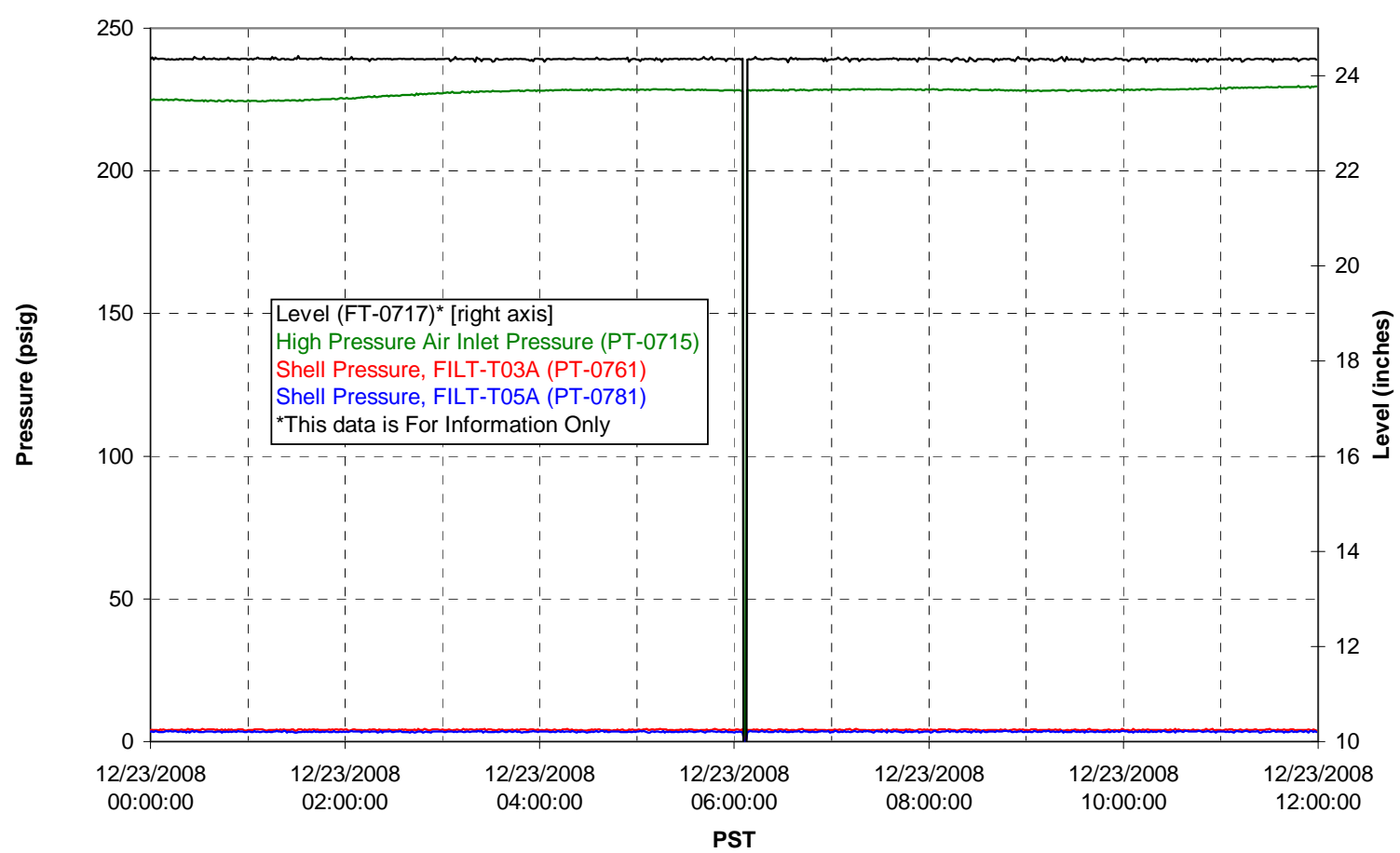

Pulsepot UFP-PP-T02A

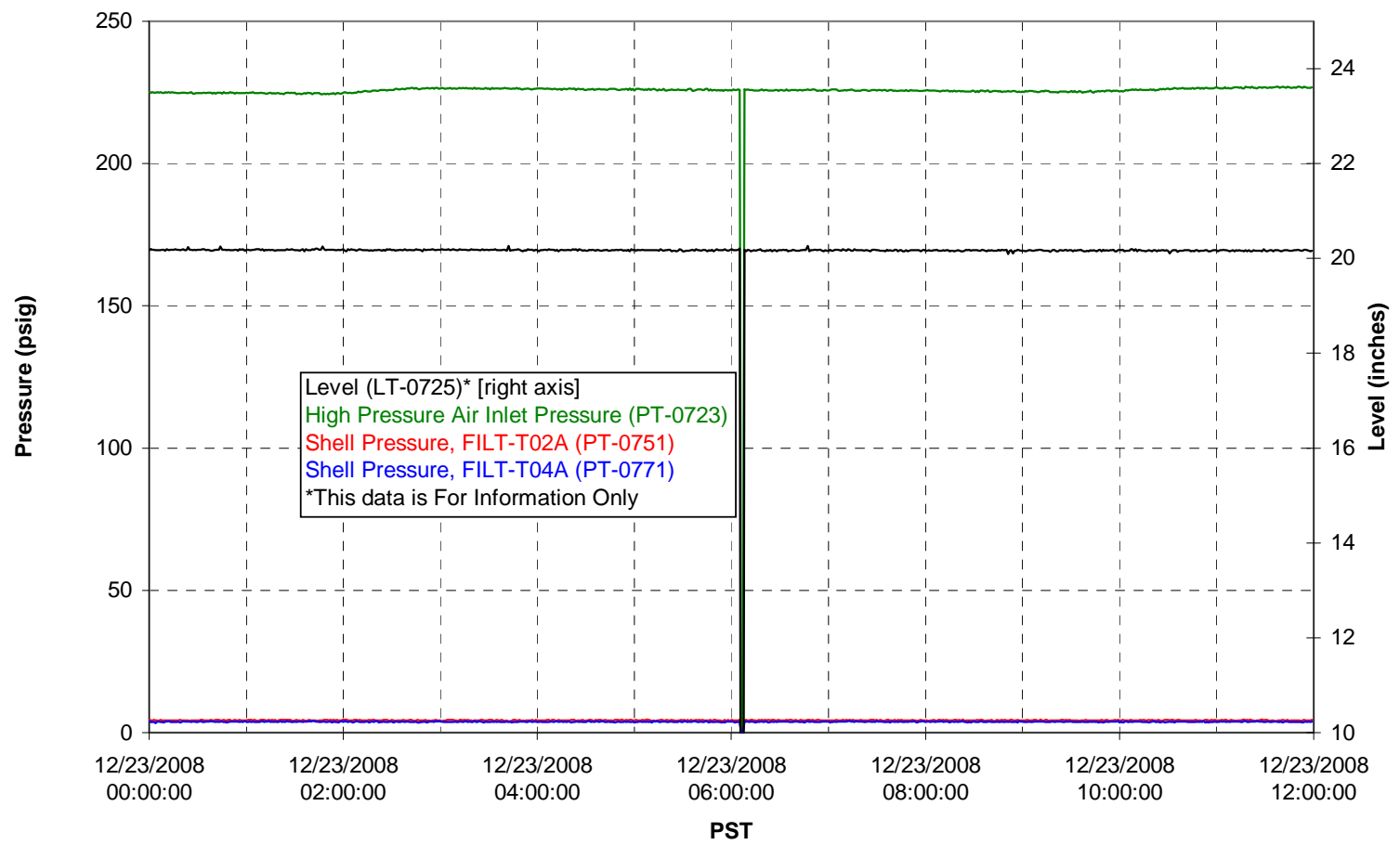


Pulsepot UFP-PP-T03A

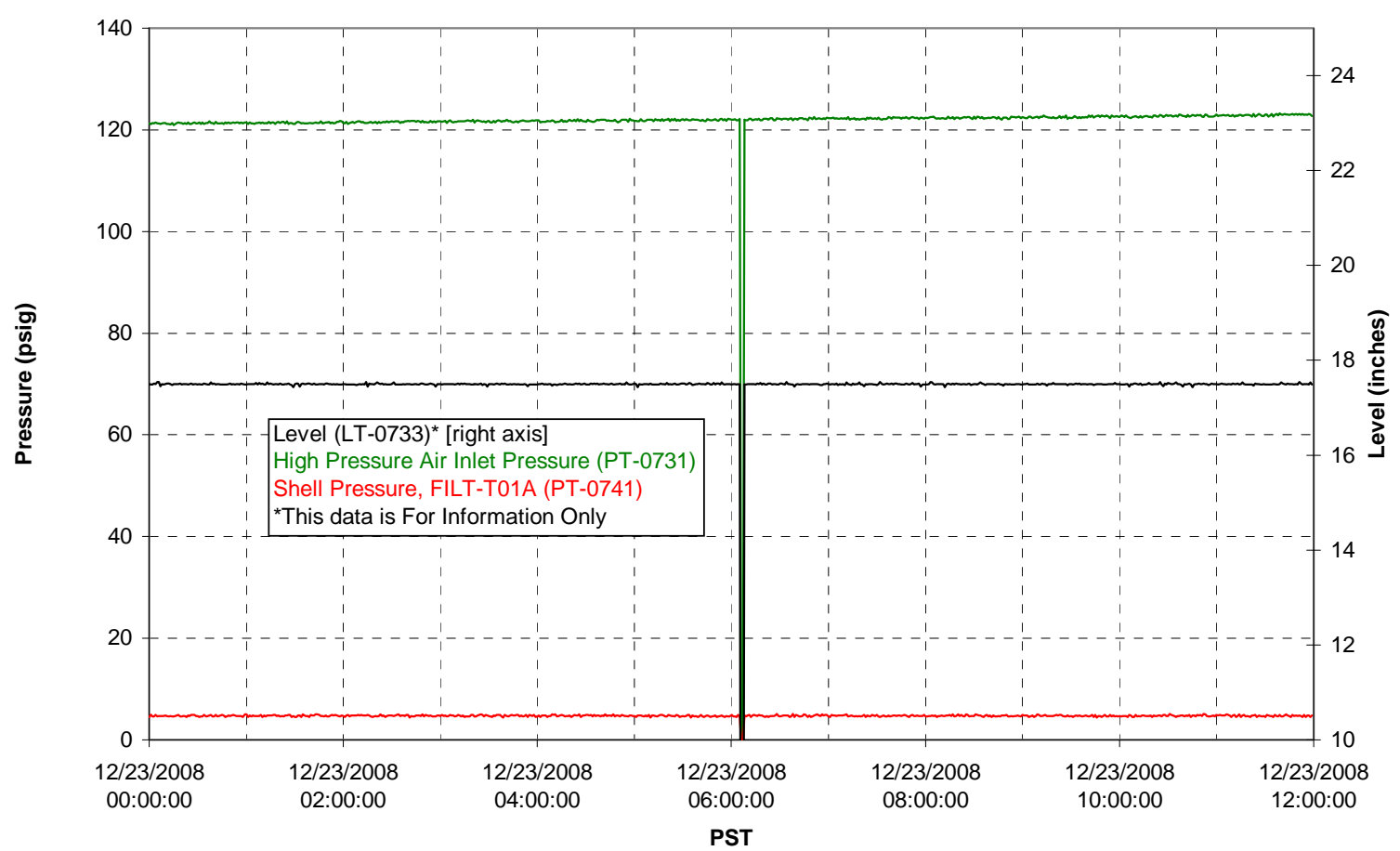

Pulsepot Levels

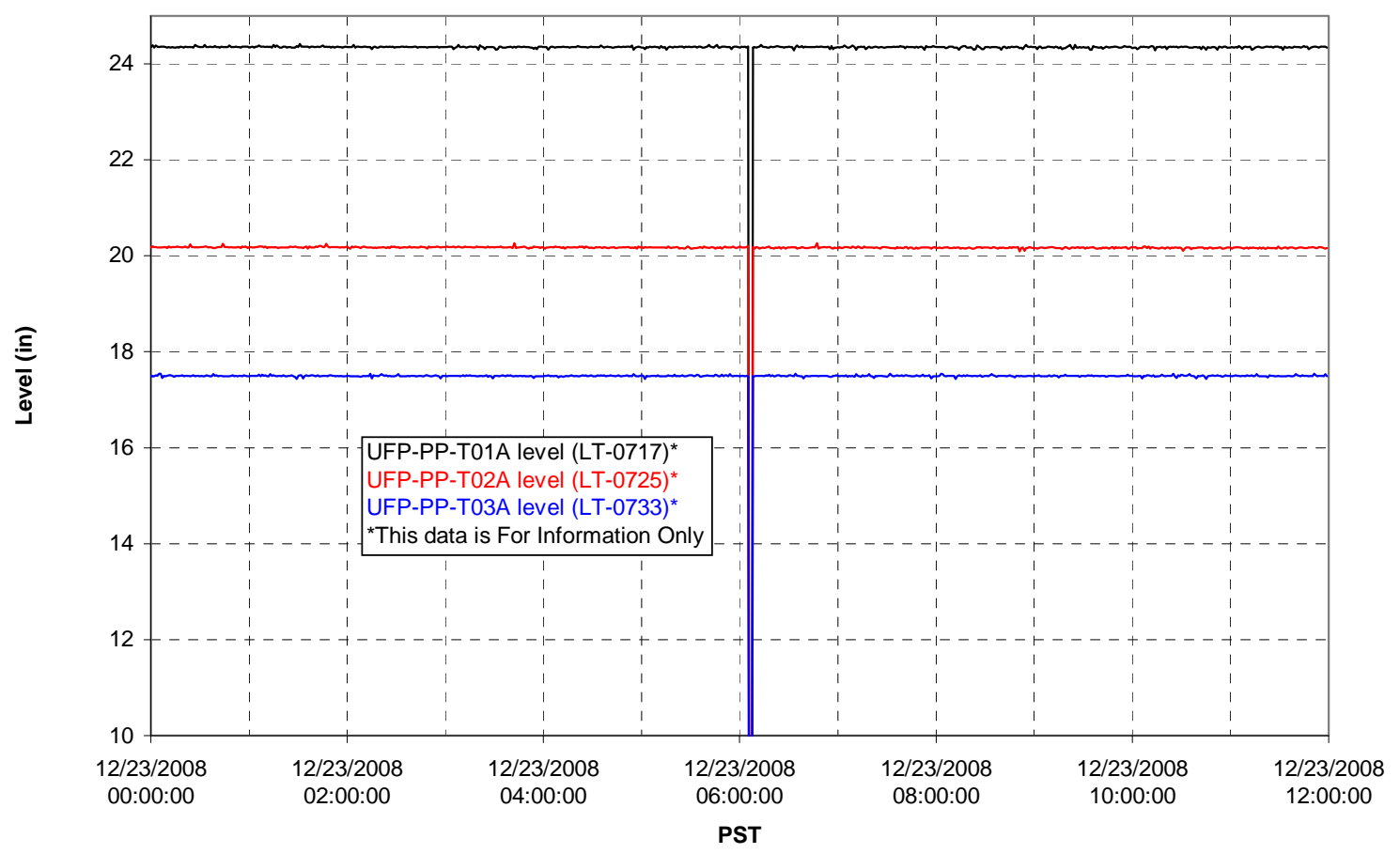


Filter UFP-FILT-T01A

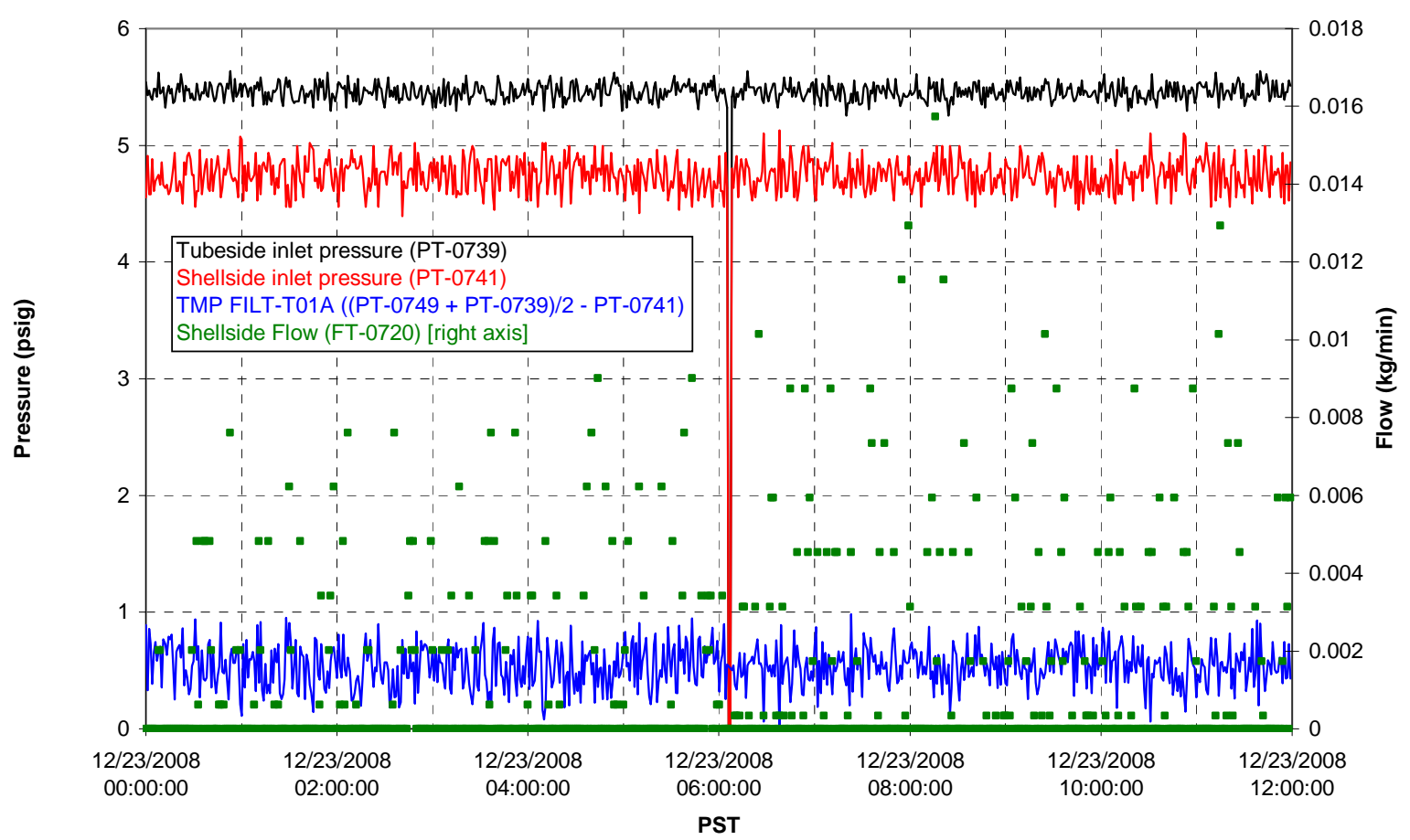

Filter UFP-FILT-T02A

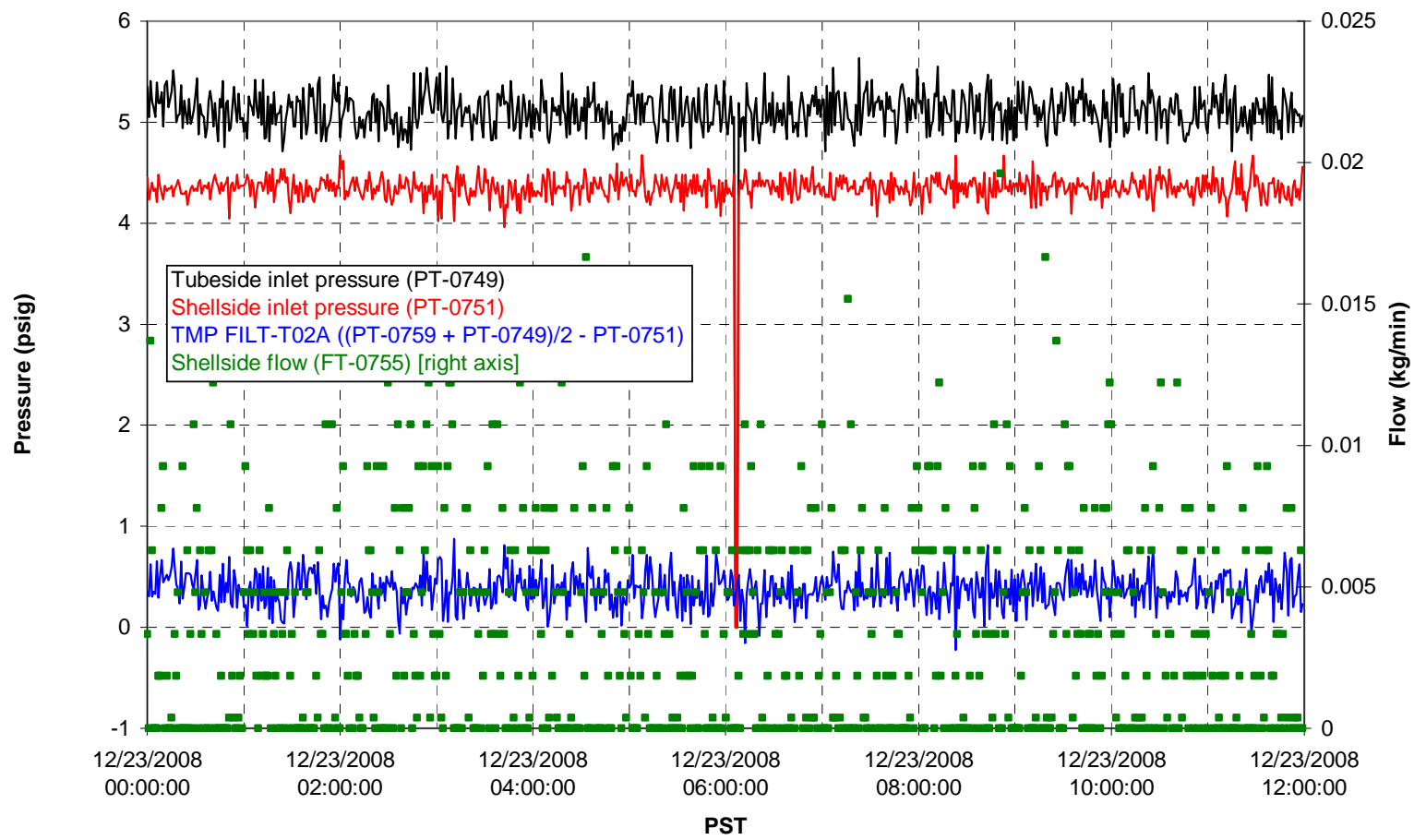


Filter UFP-FILT-T03A

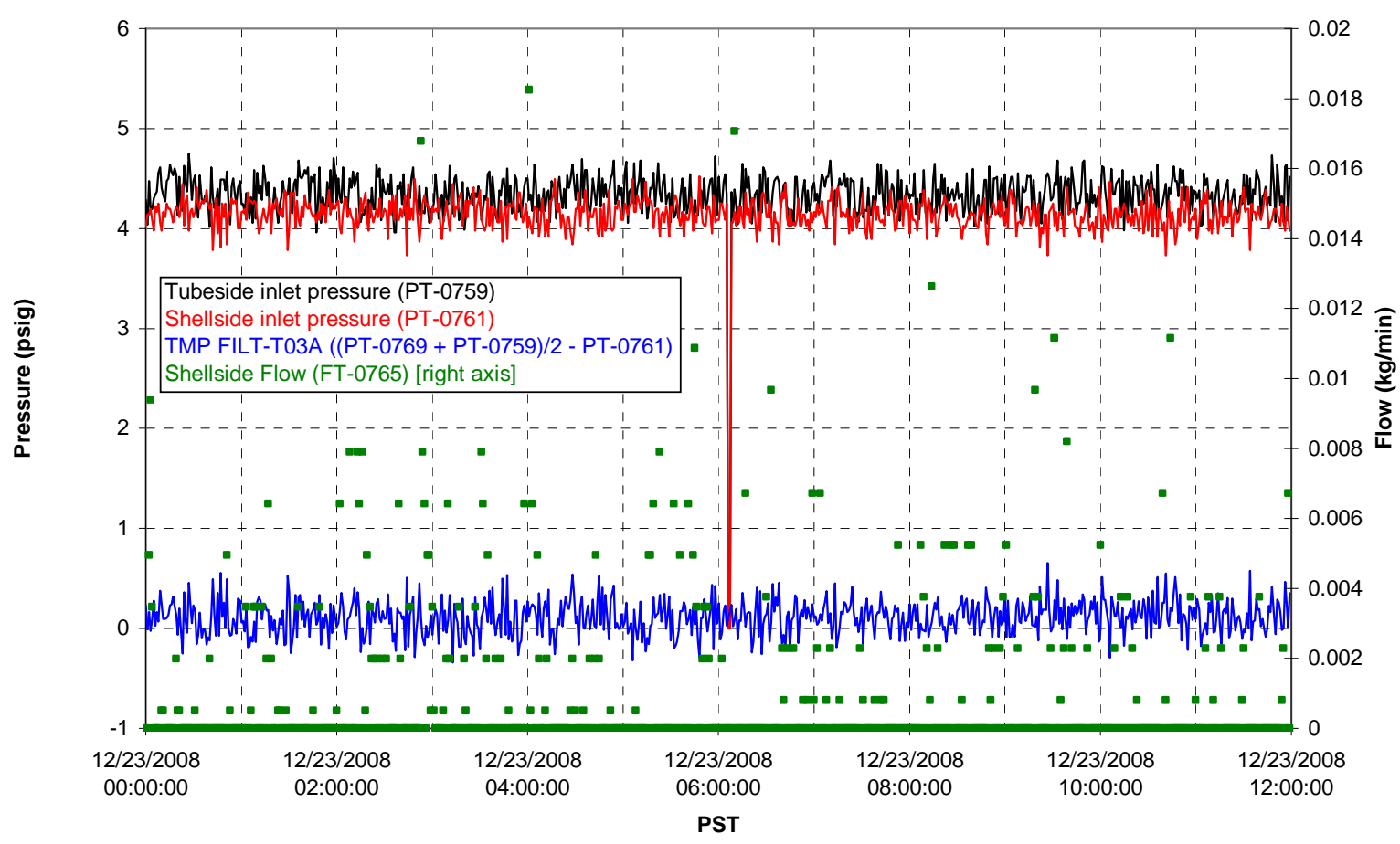

Filter UFP-FILT-T04A

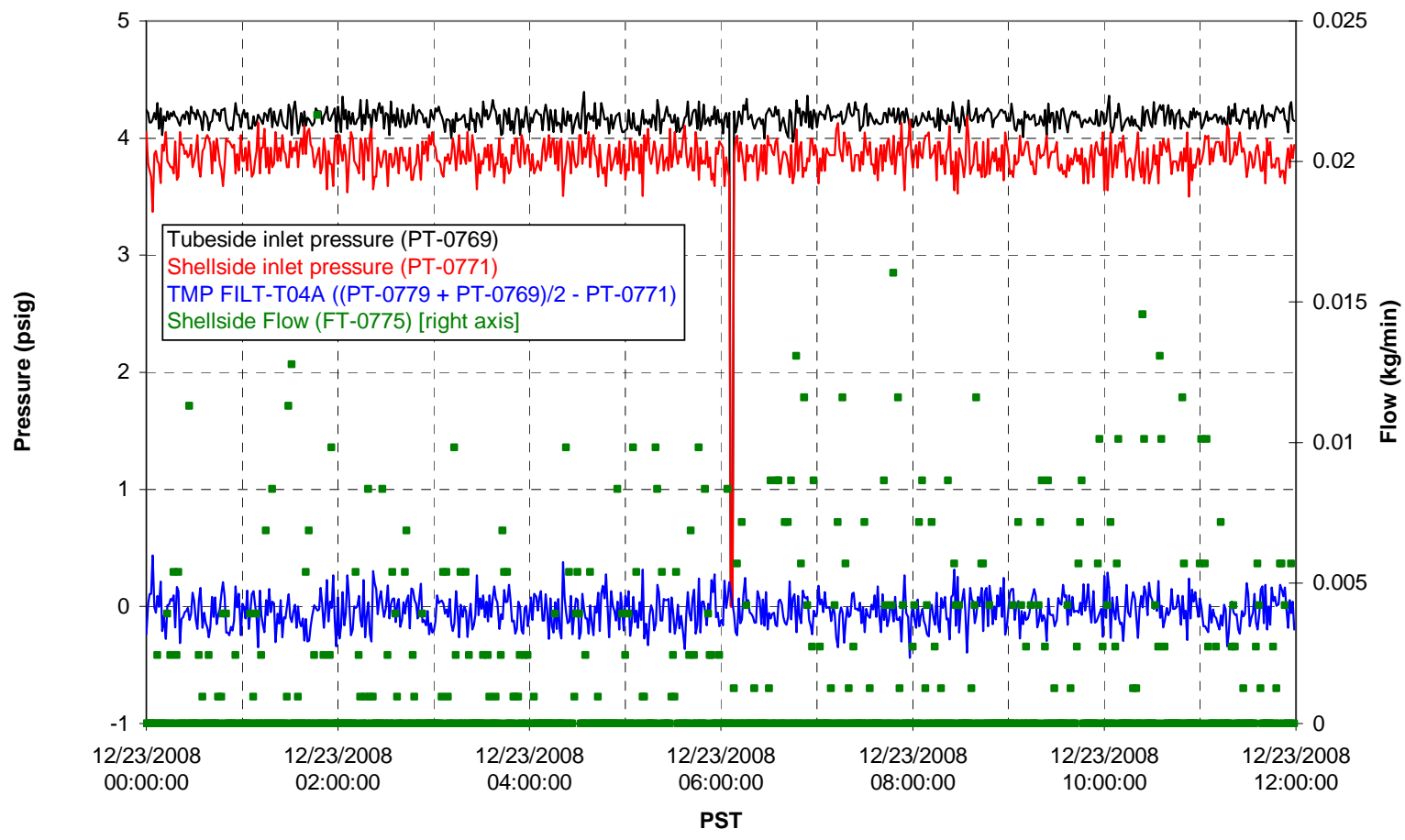


Filter UFP-FILT-T05A

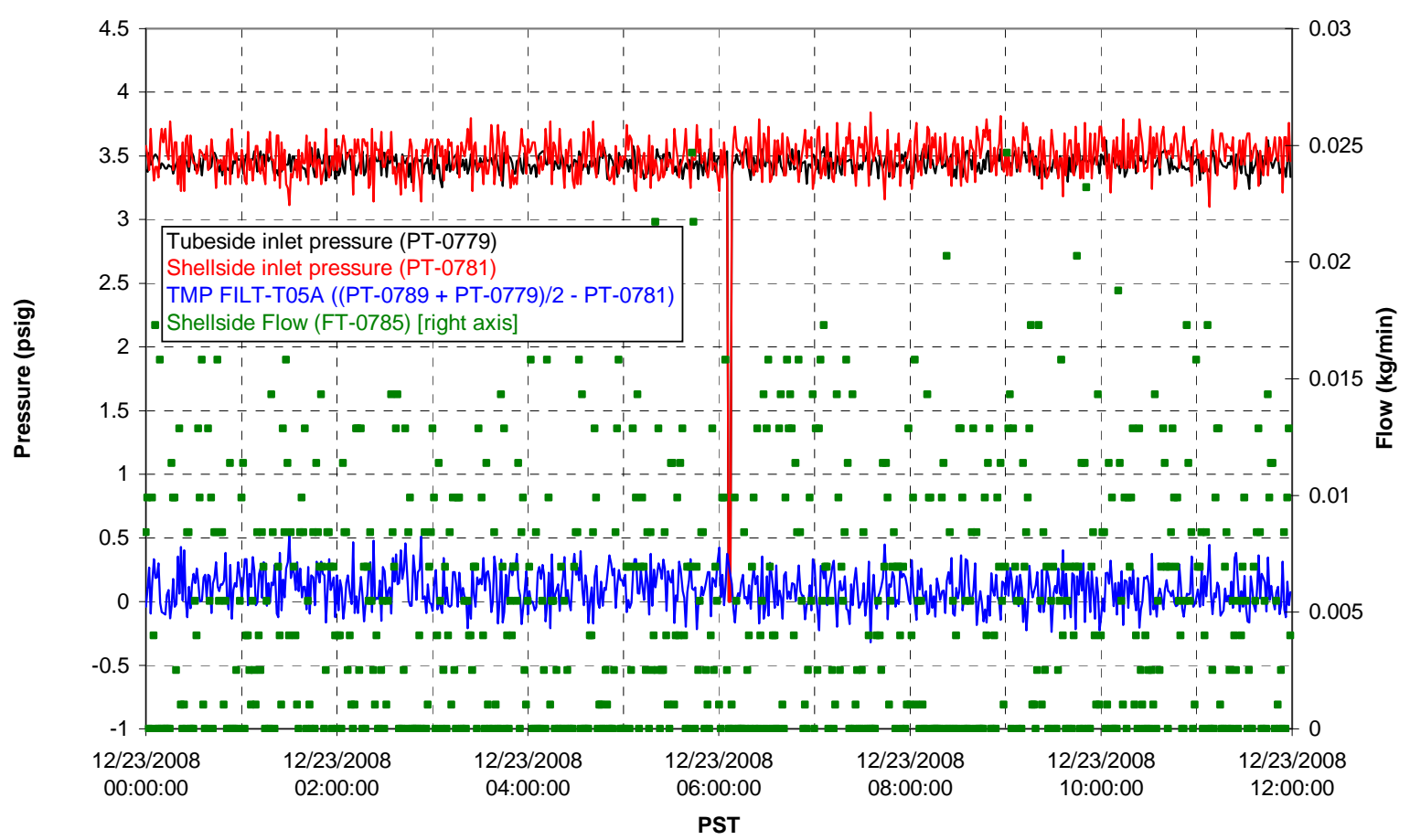

Chemical Flow

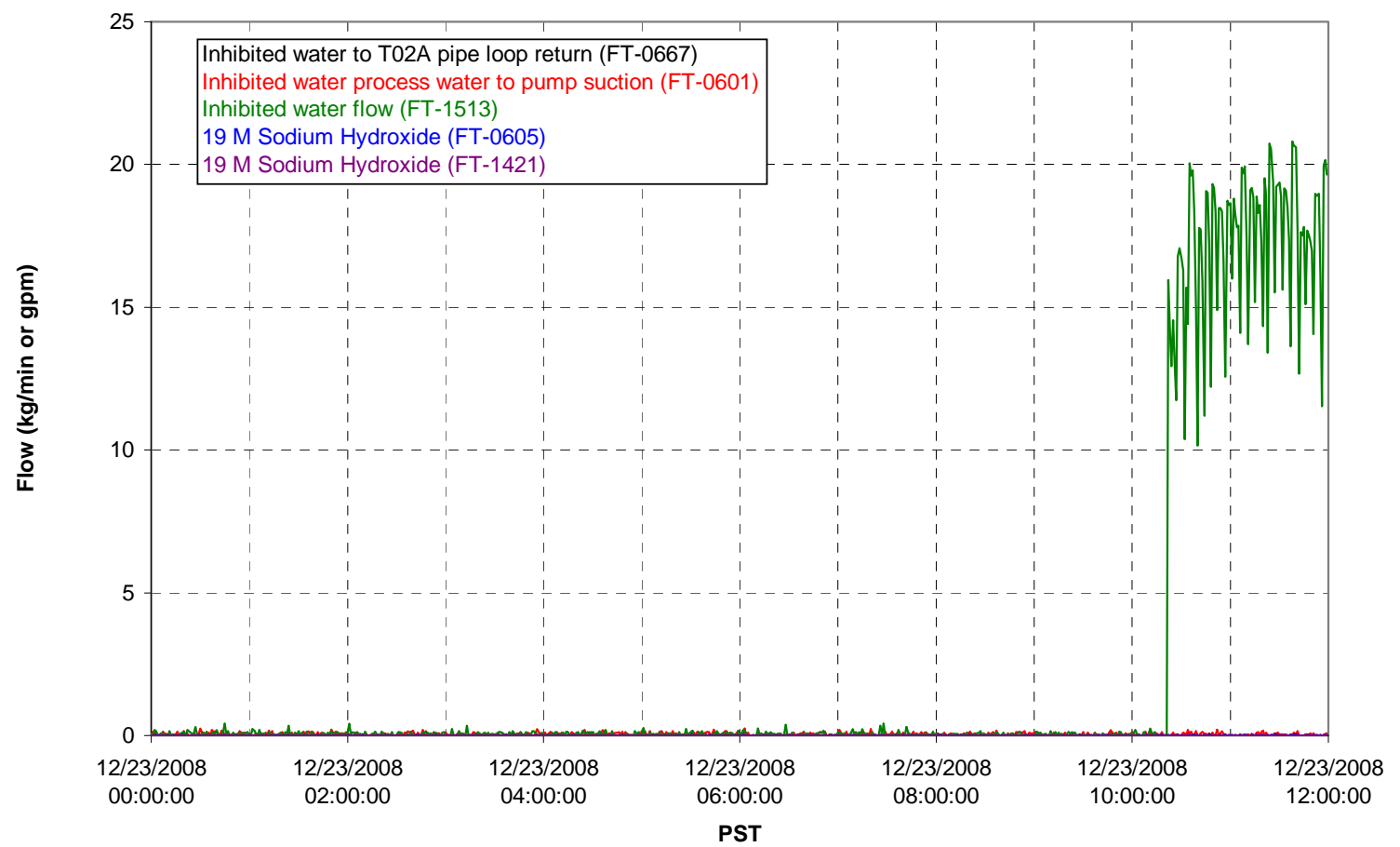




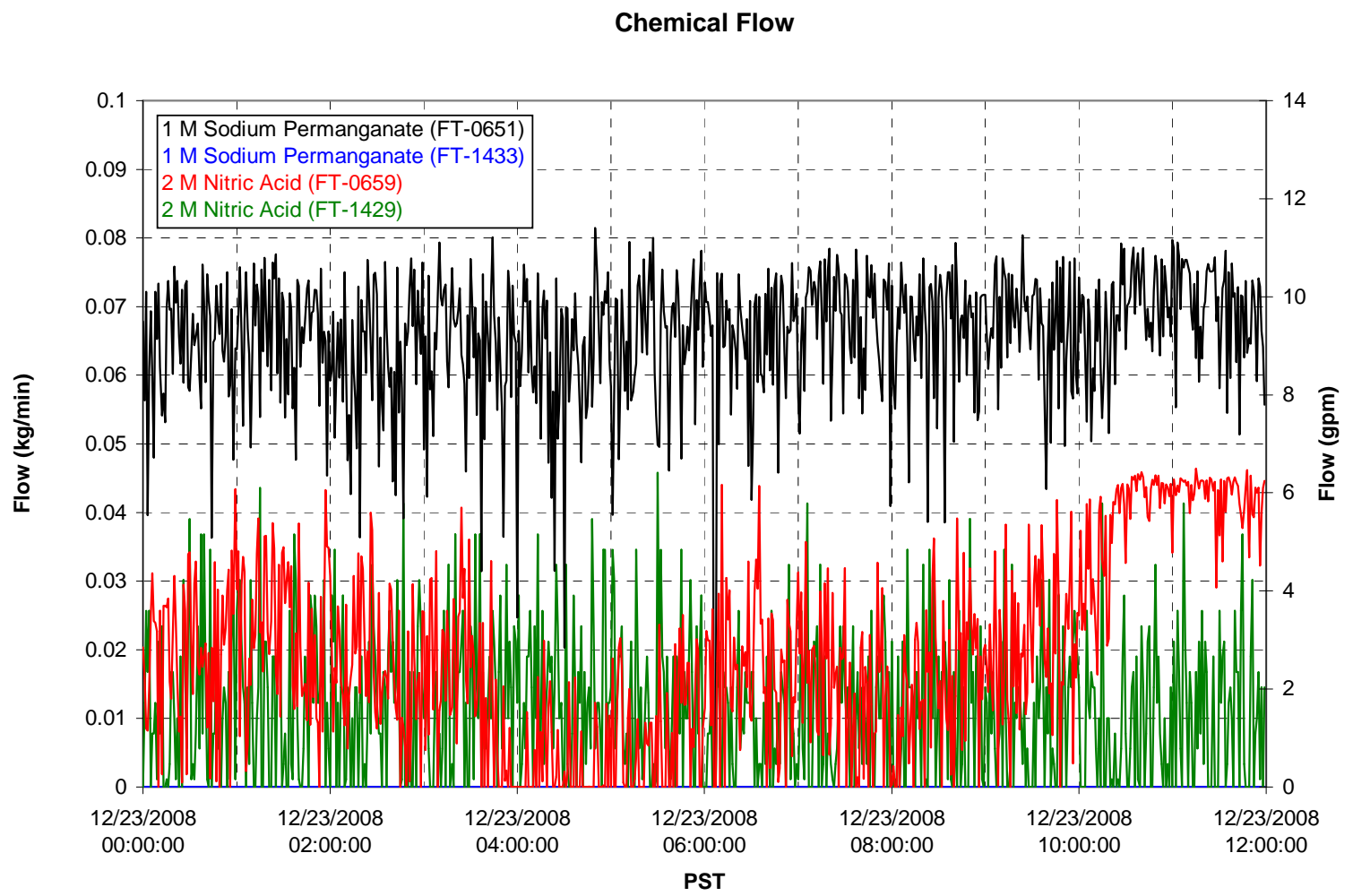

Air Flows

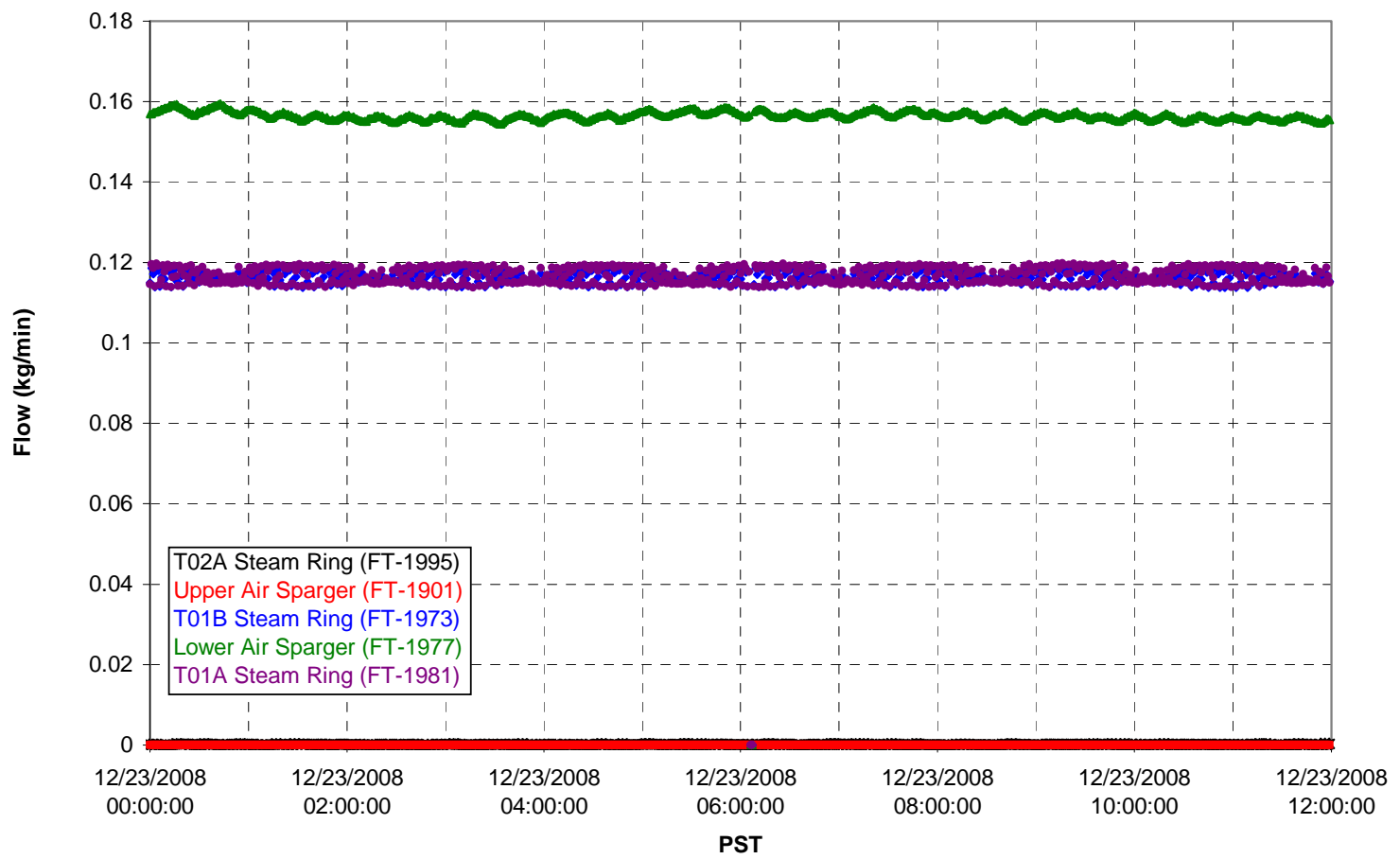


T02A Steam

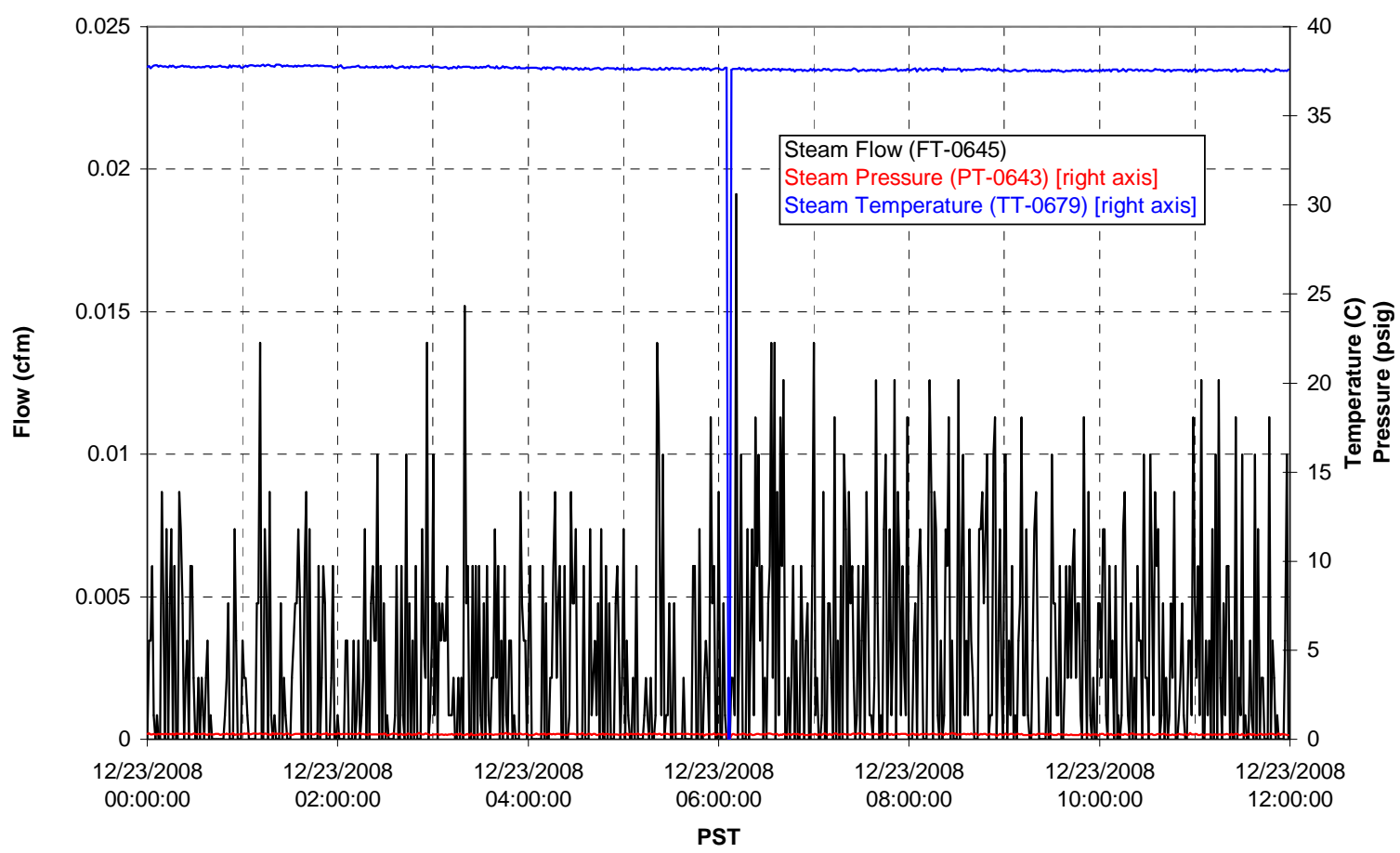

T01A Steam

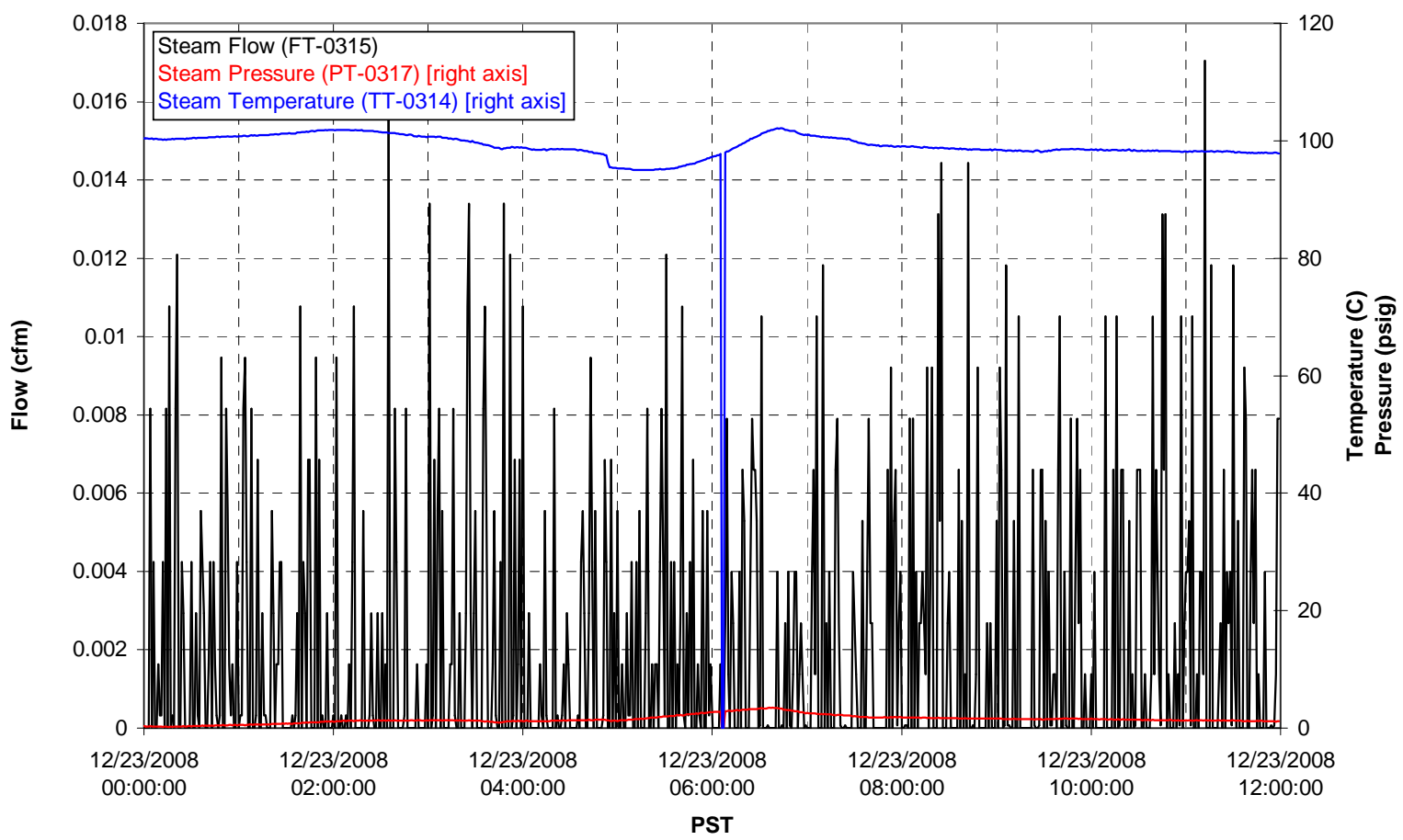


T01B Steam

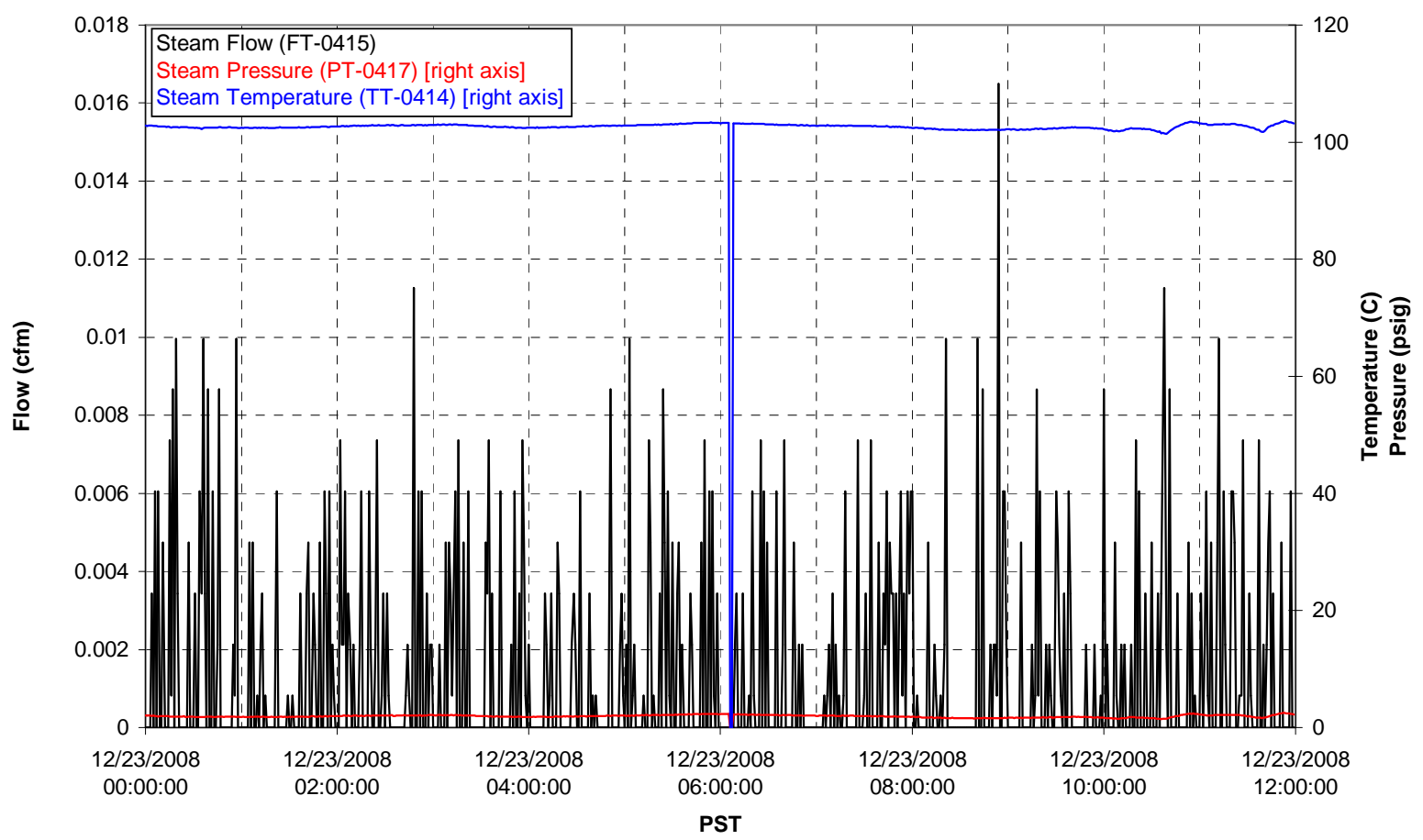


Functional Test Data Plots

12/30/08 00:00 - 12/30/08 12:00 
T01A level

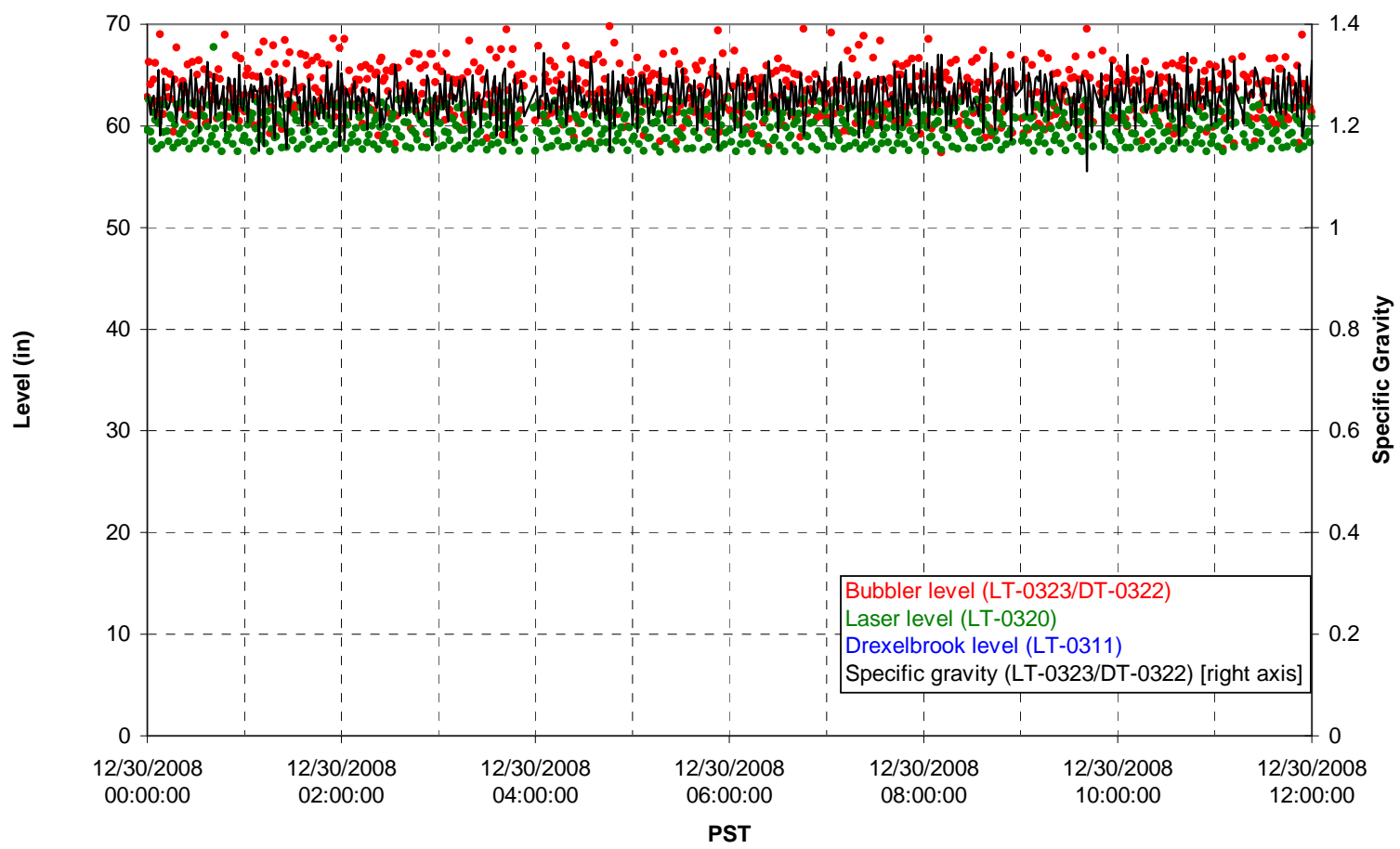

T01A temperatures

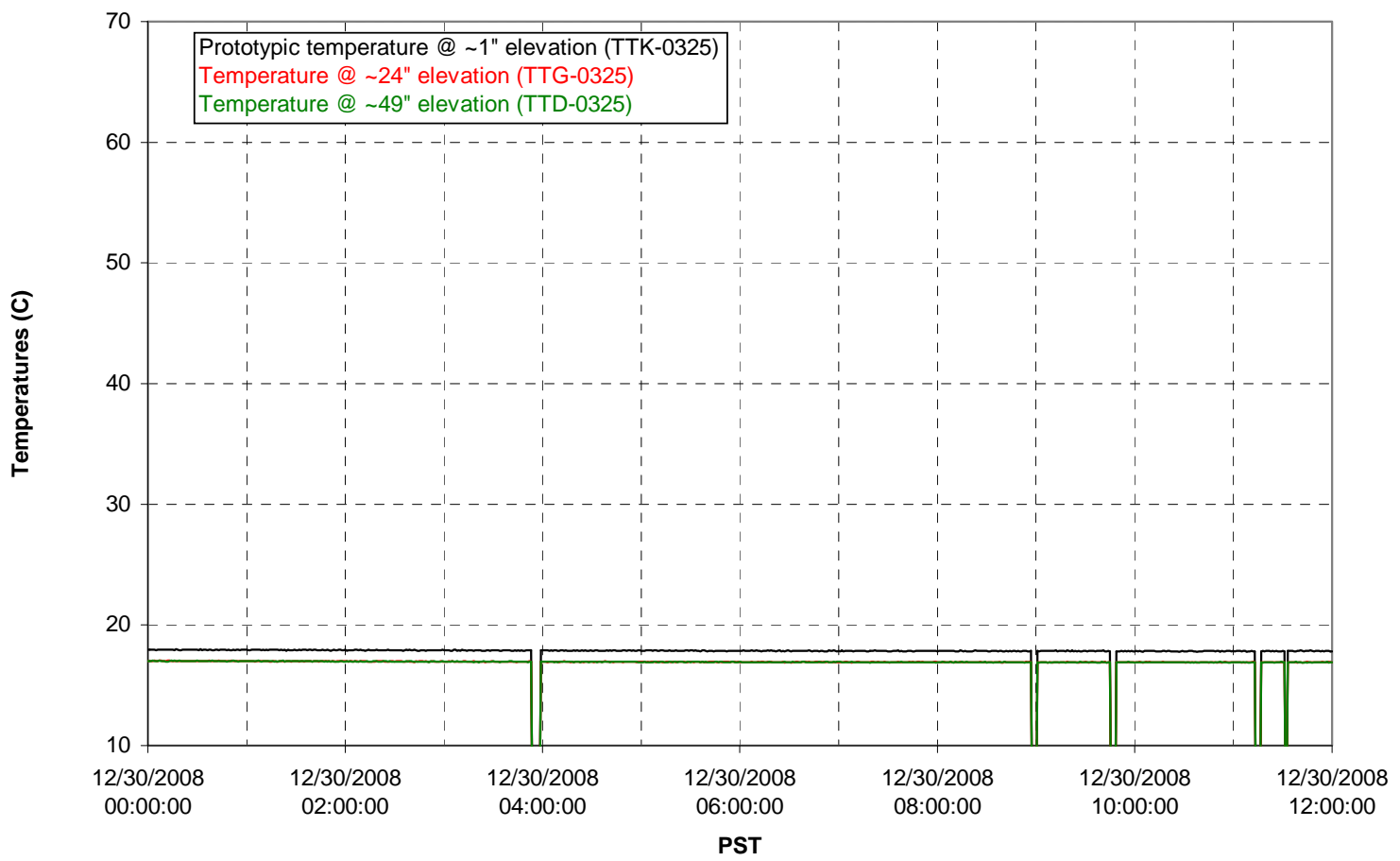


T01B level

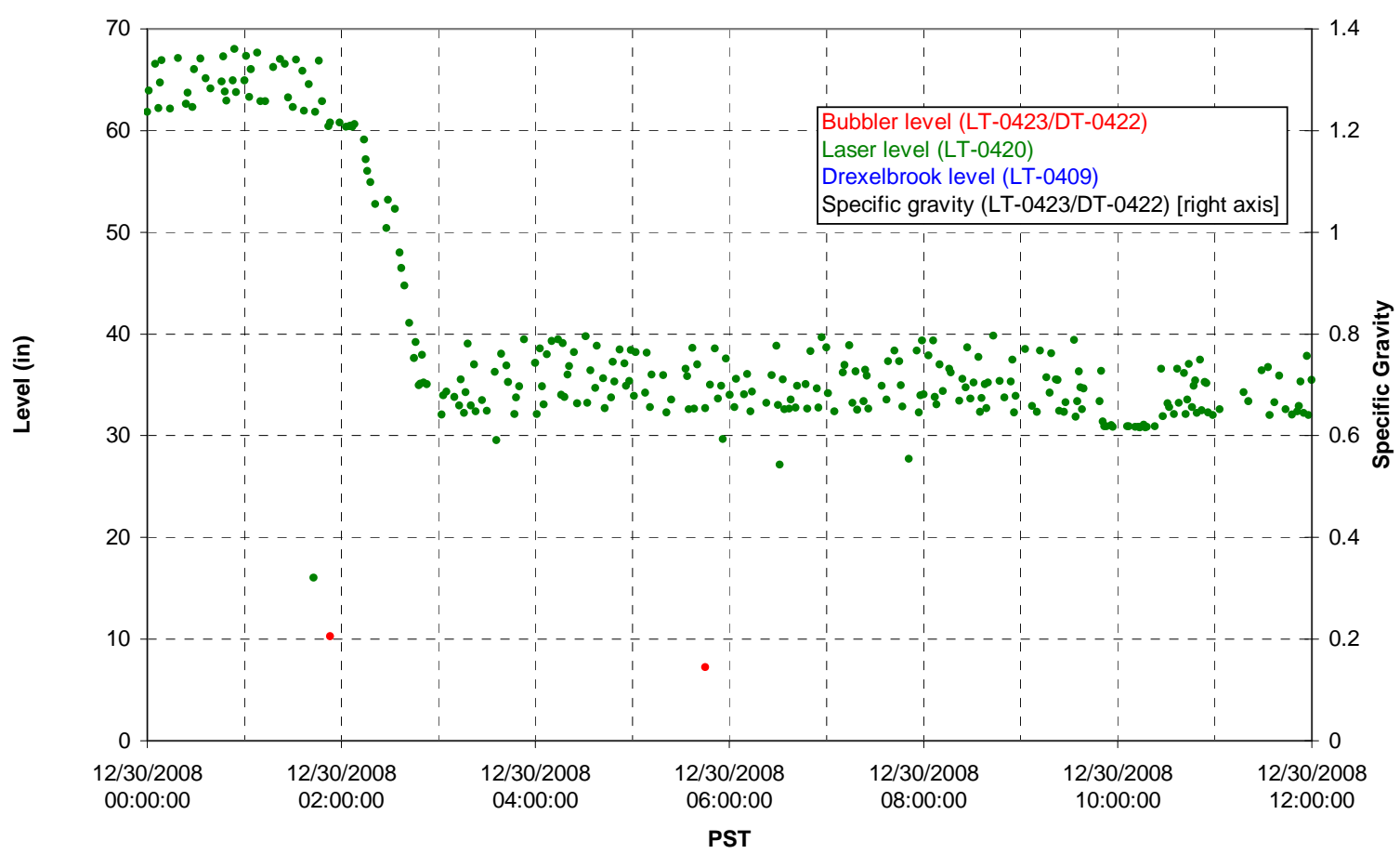

T01B temperatures

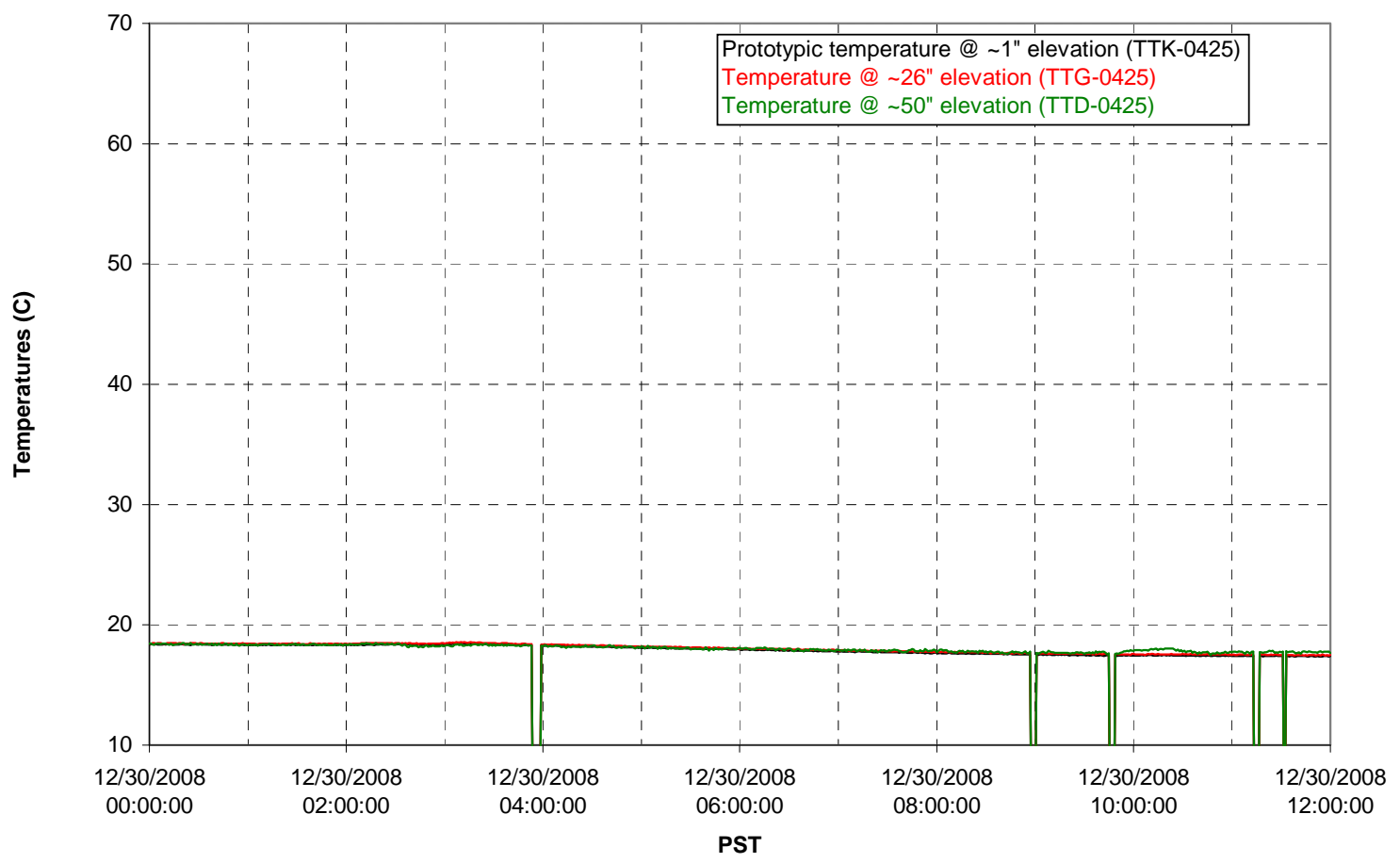


T02A level

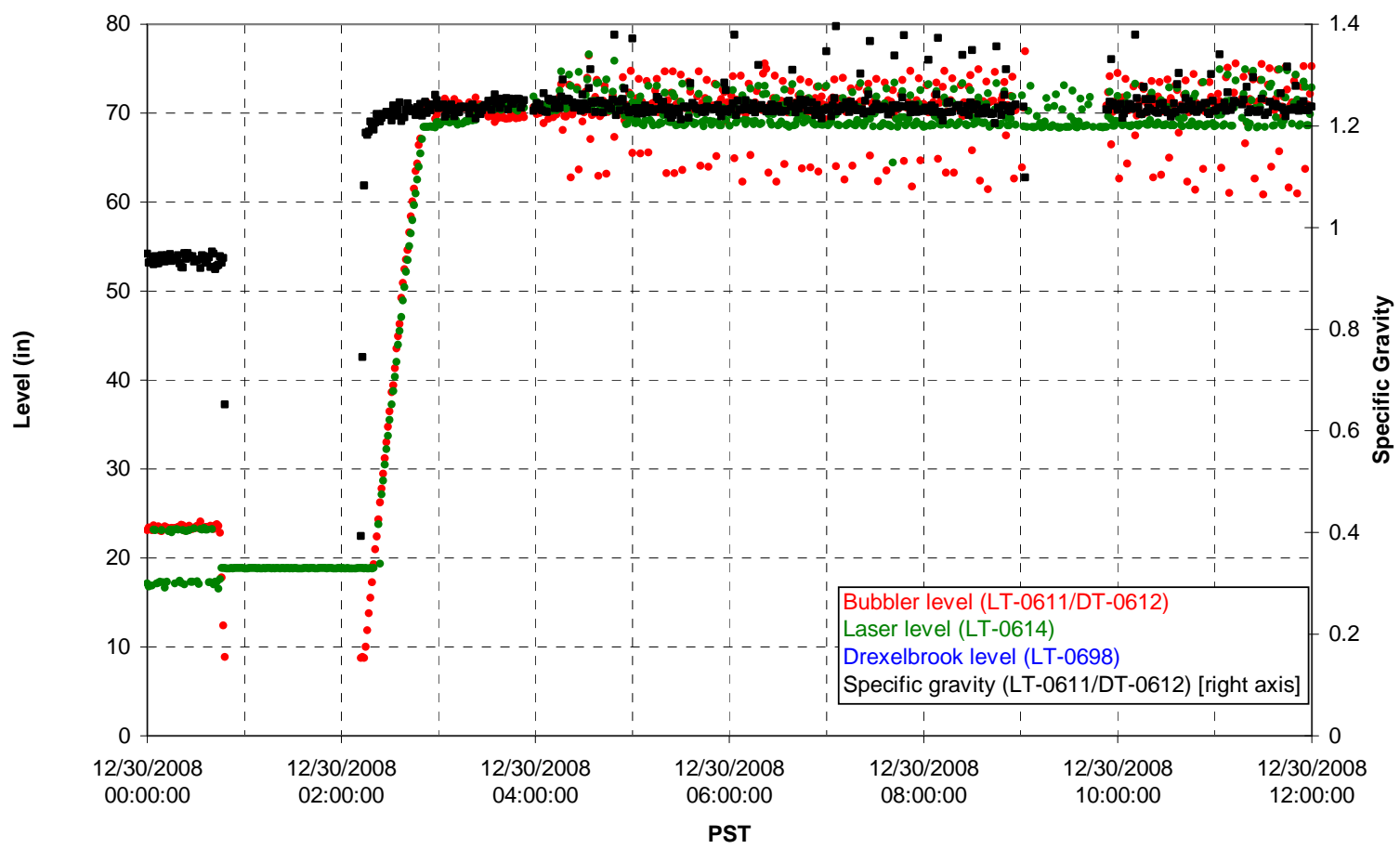

T02A temperatures

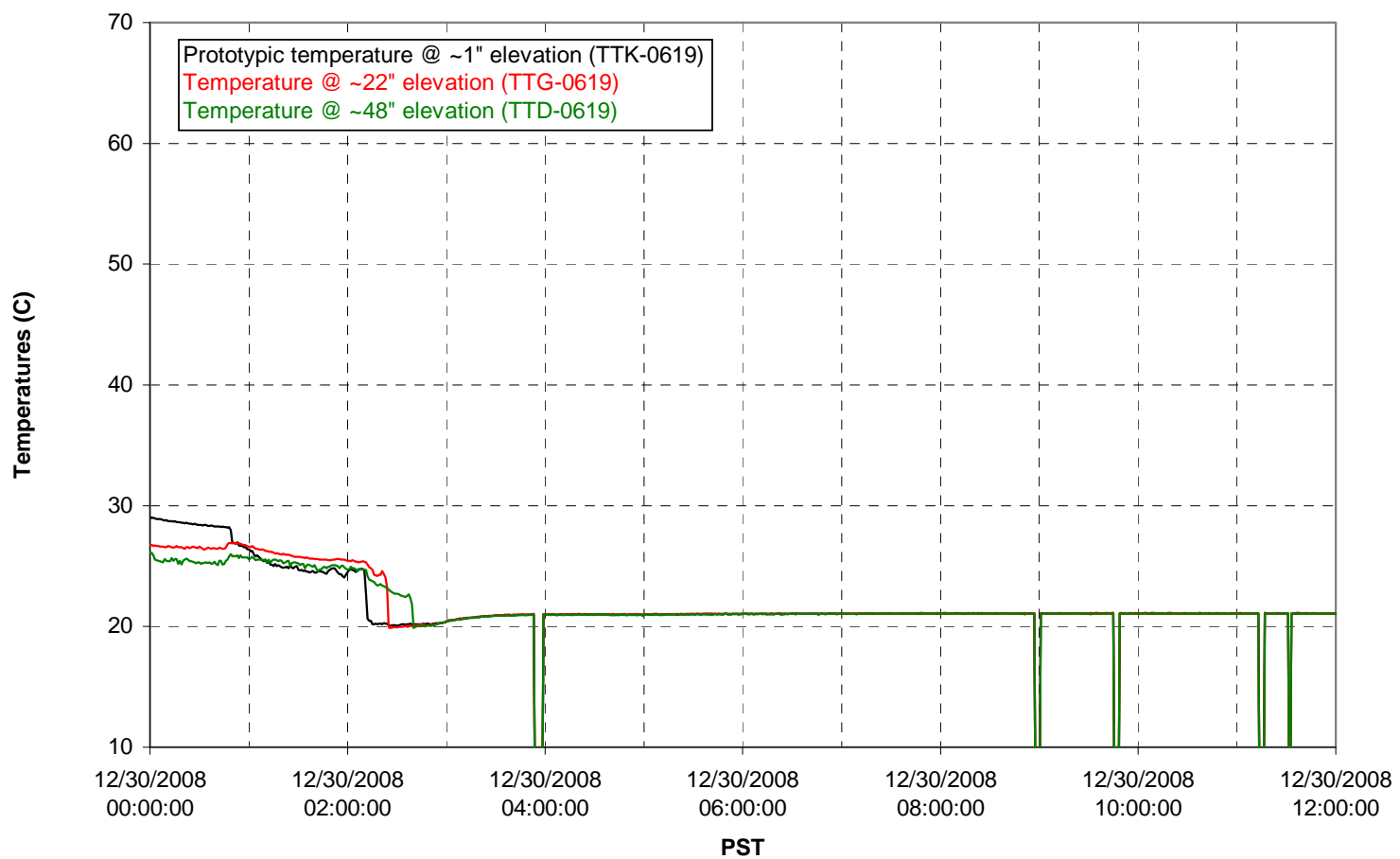


T02A and filter loop temperatures

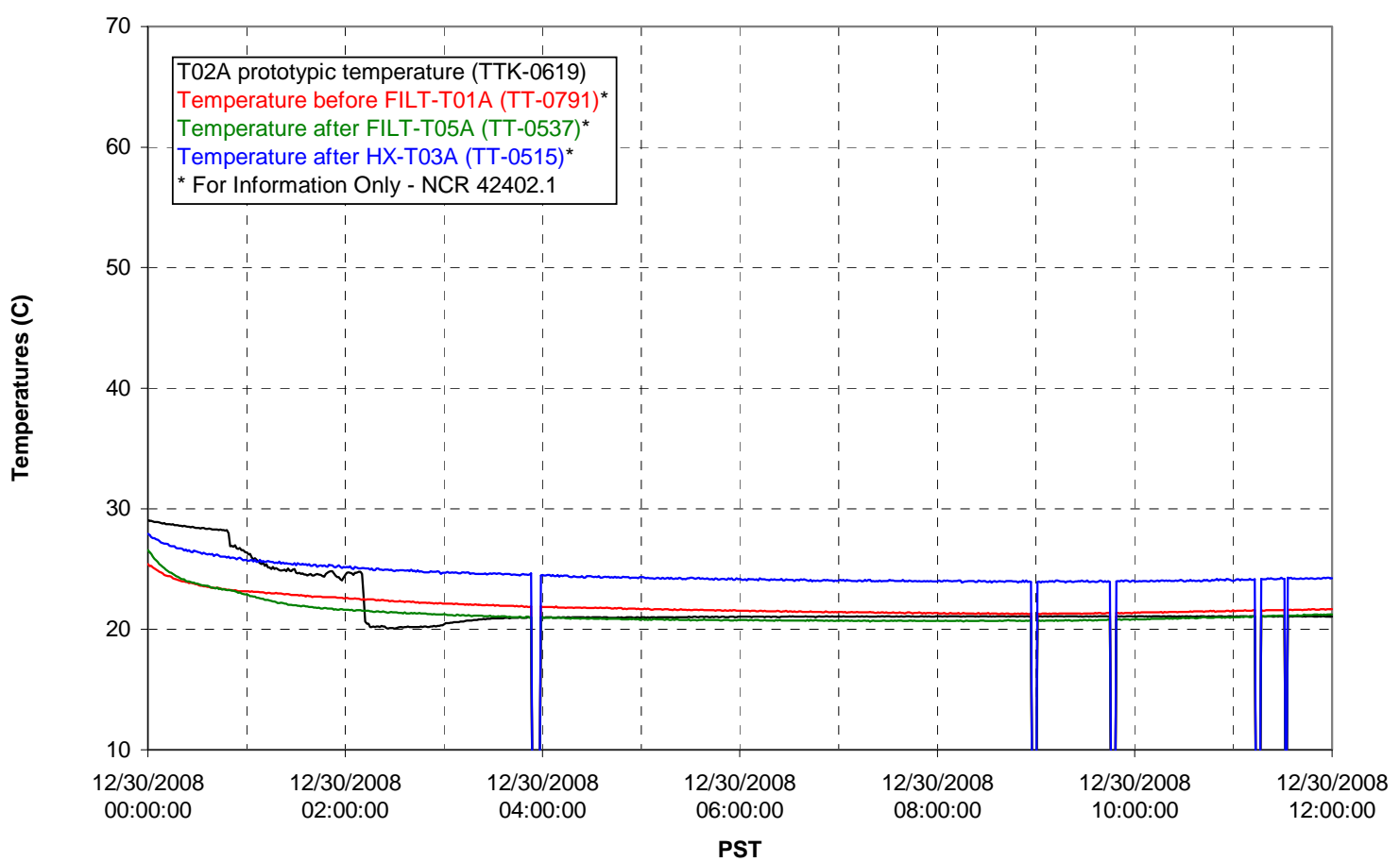

Pump Pressures and Flow

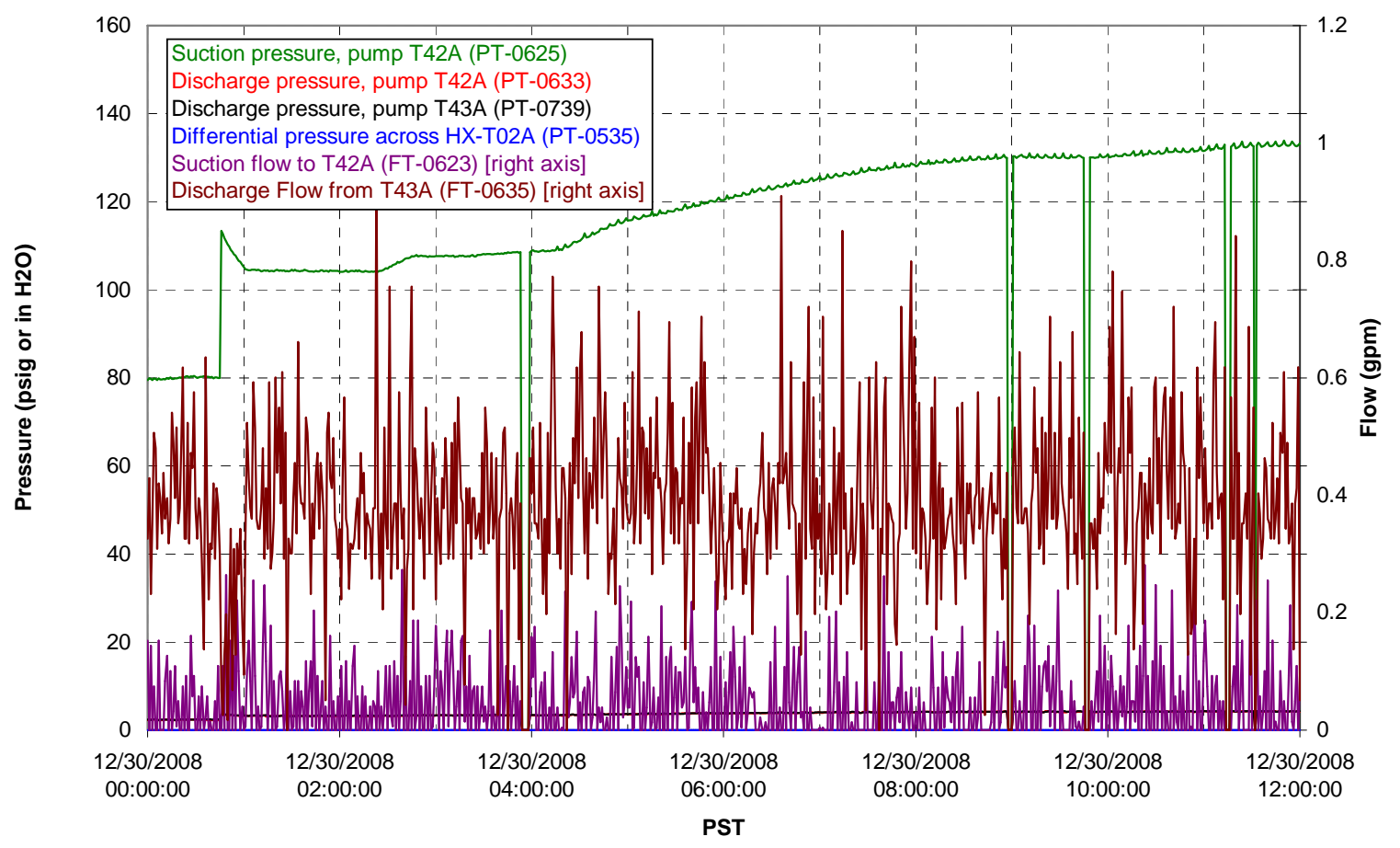


Axial pressure drop

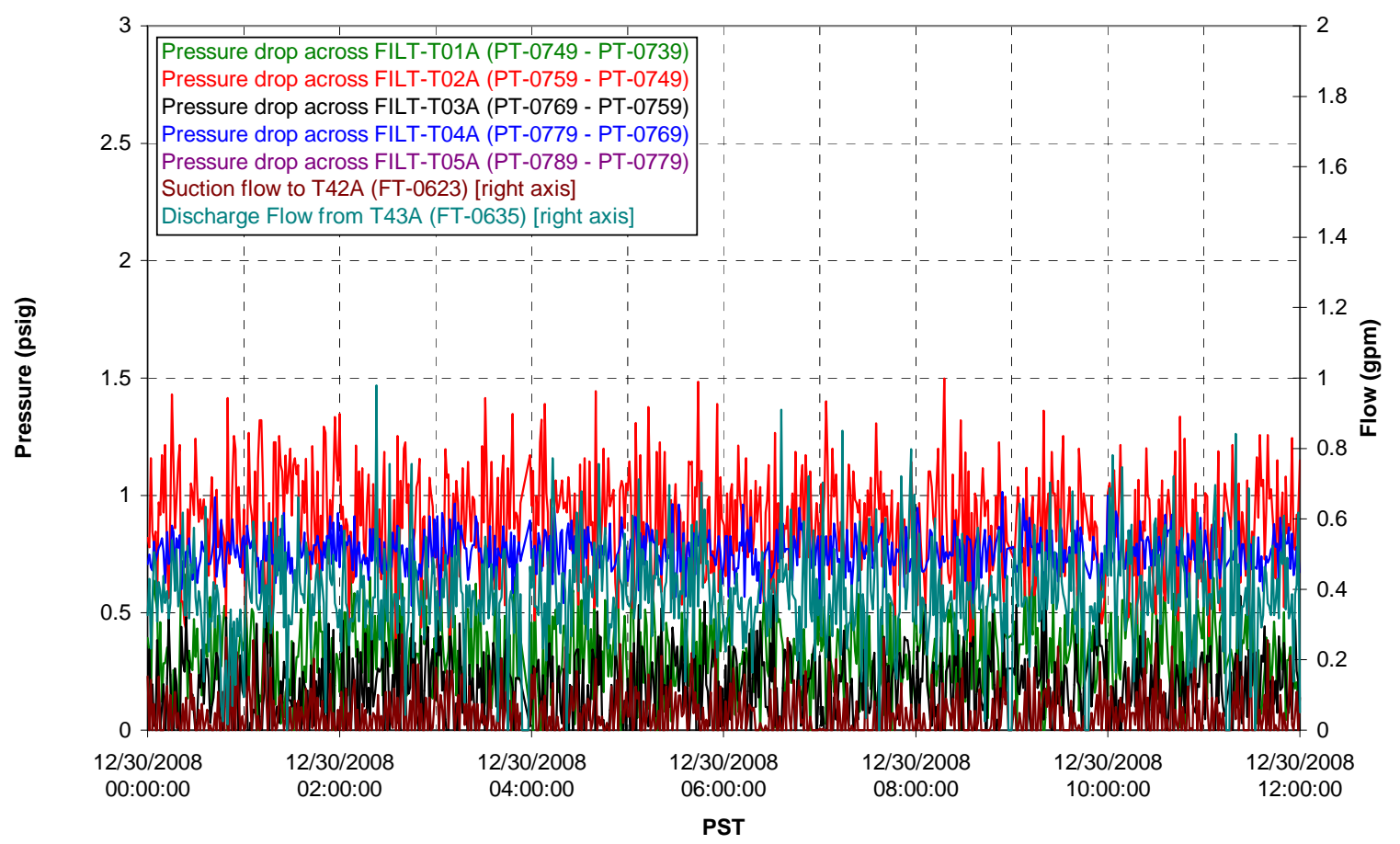

Permeate flow rates

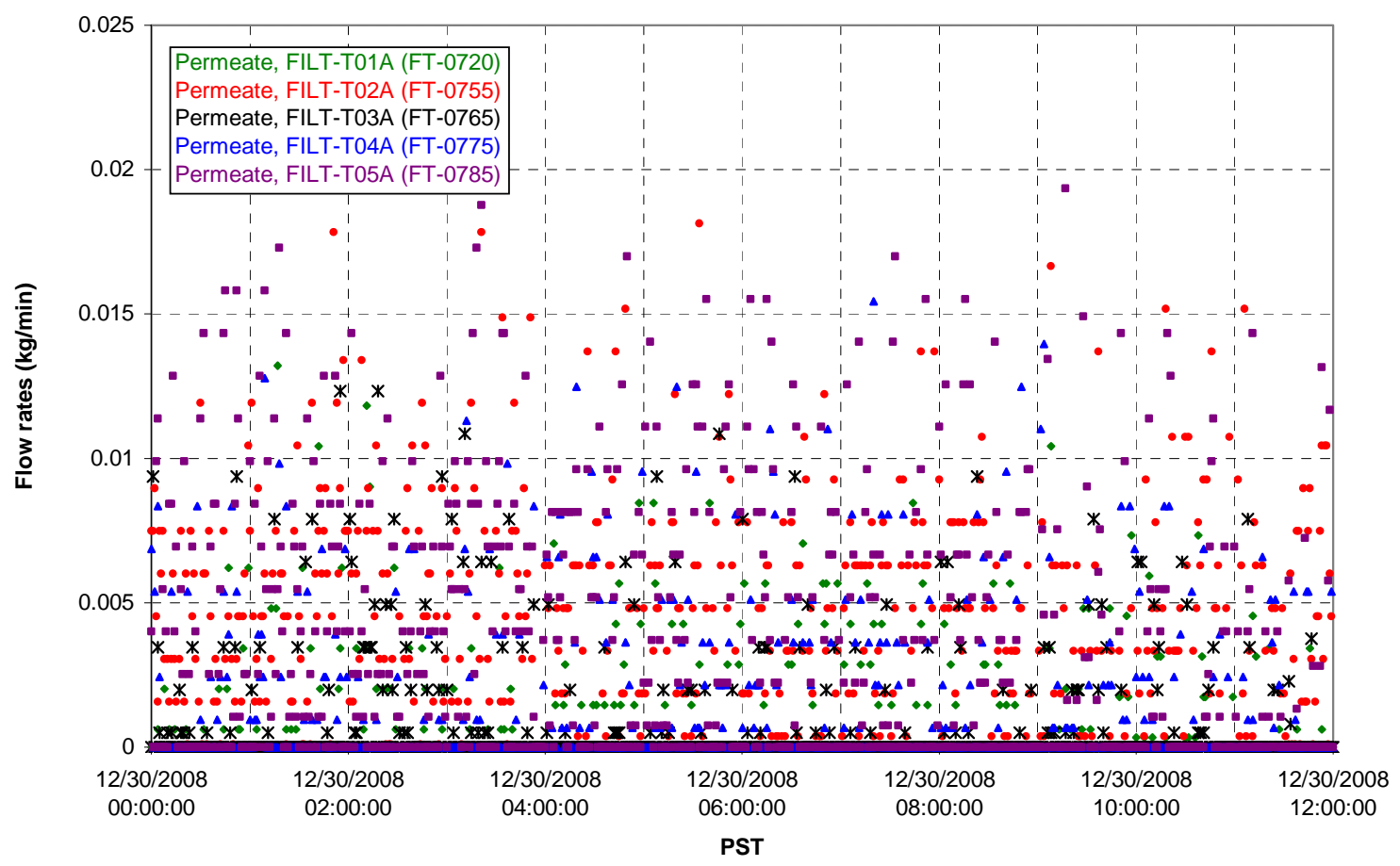


T02A Inner Temperature Tree

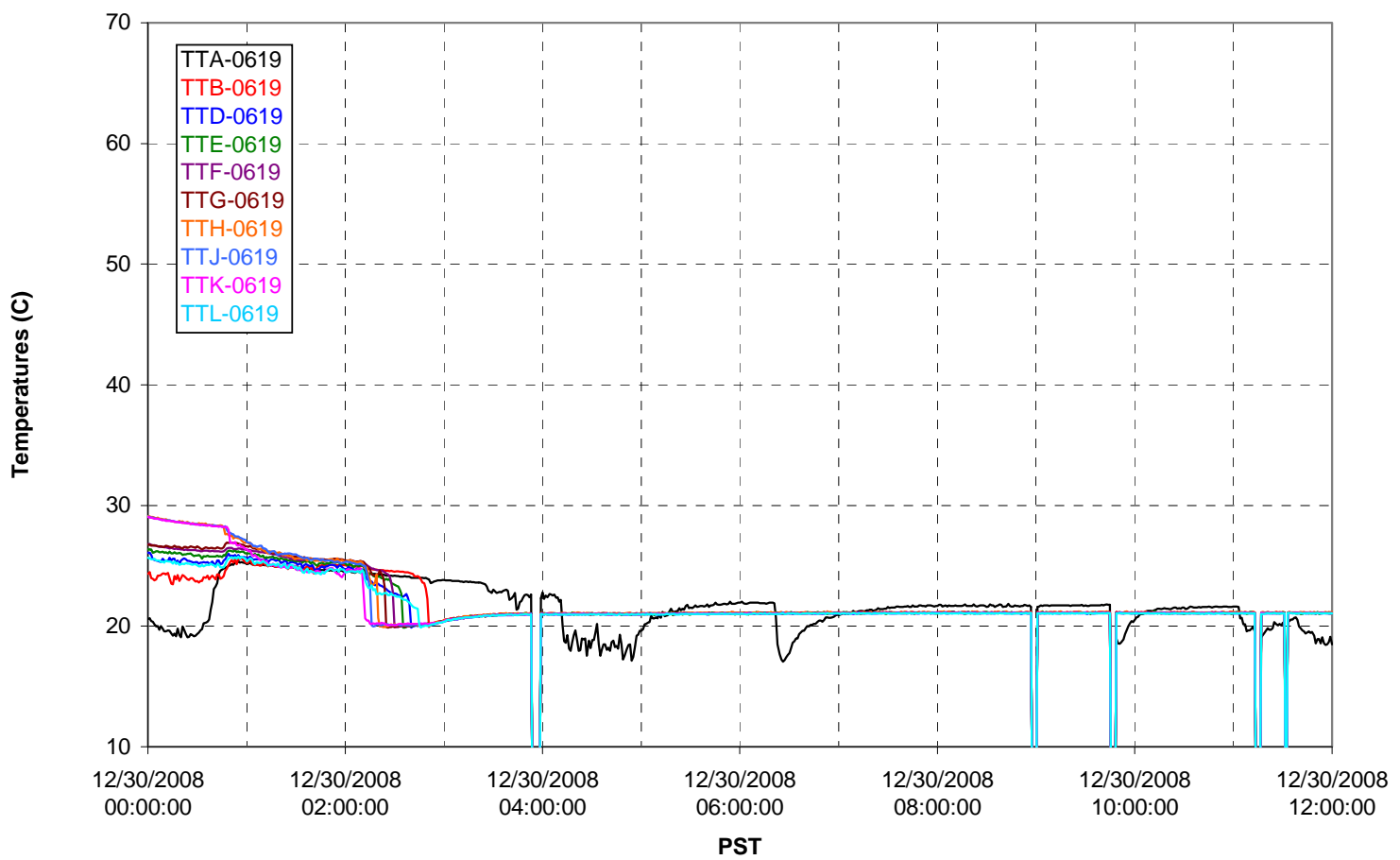

T02A Outer Temperature Tree

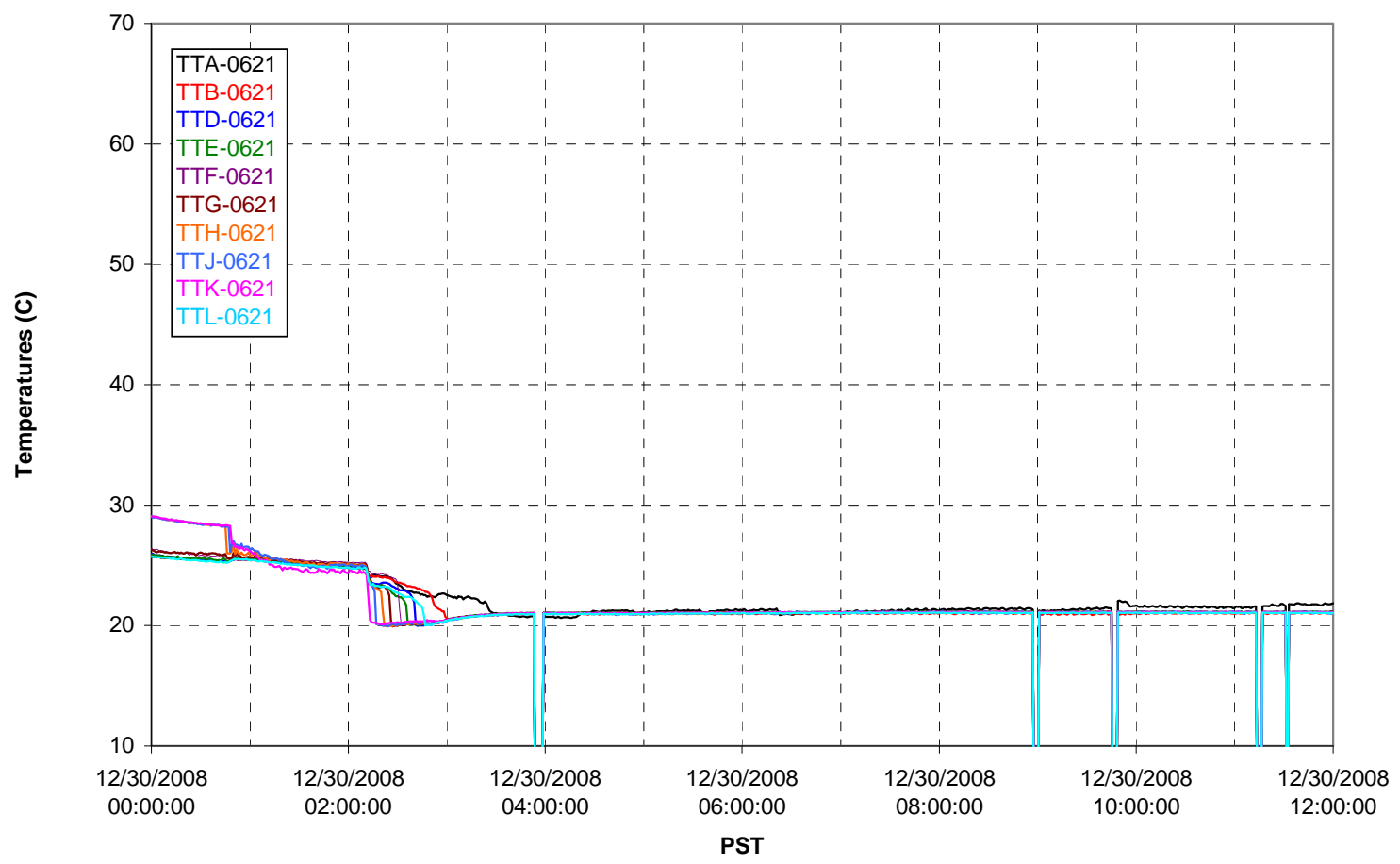


T02A temperatures

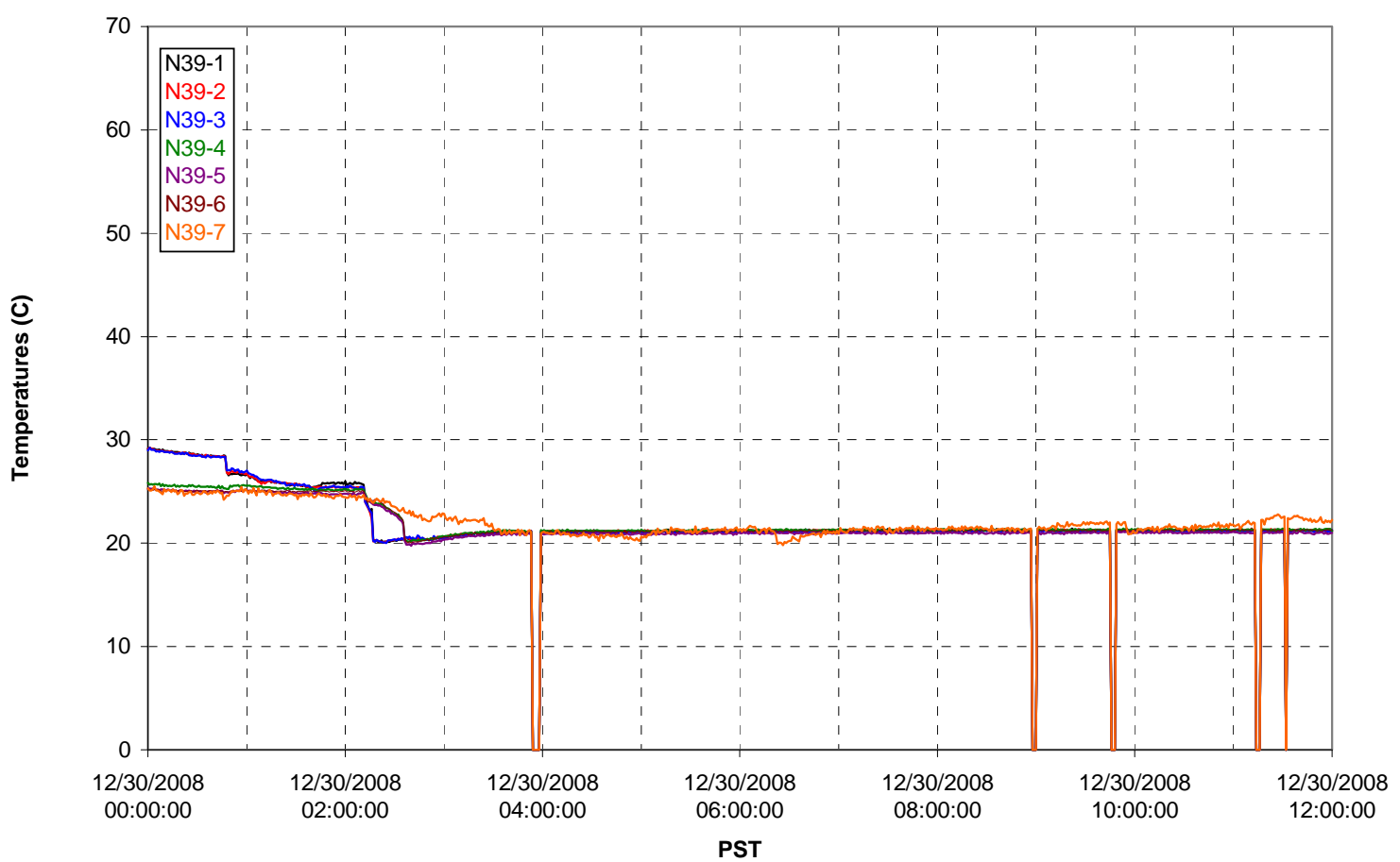

T02A temperatures

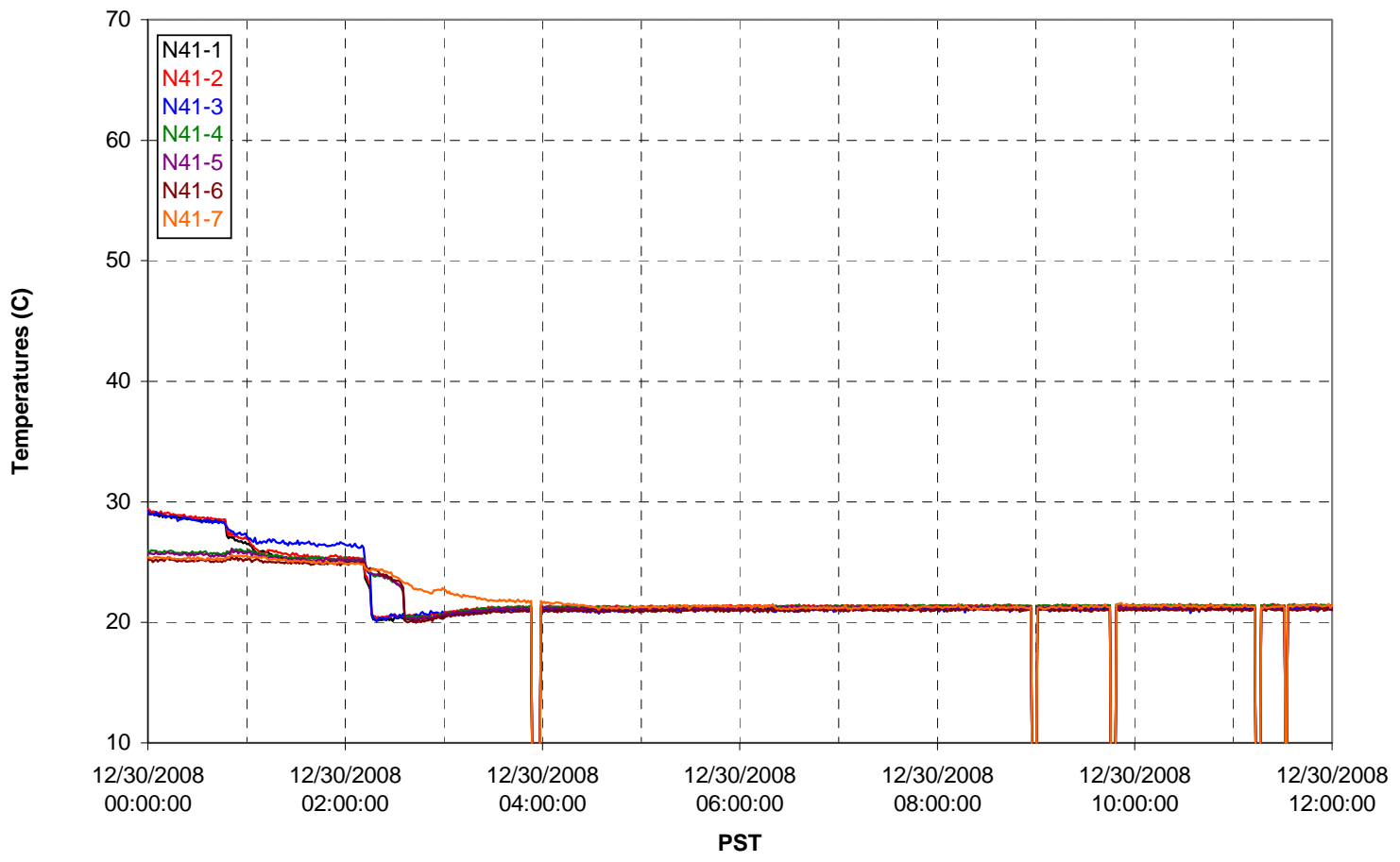


T02A temperatures

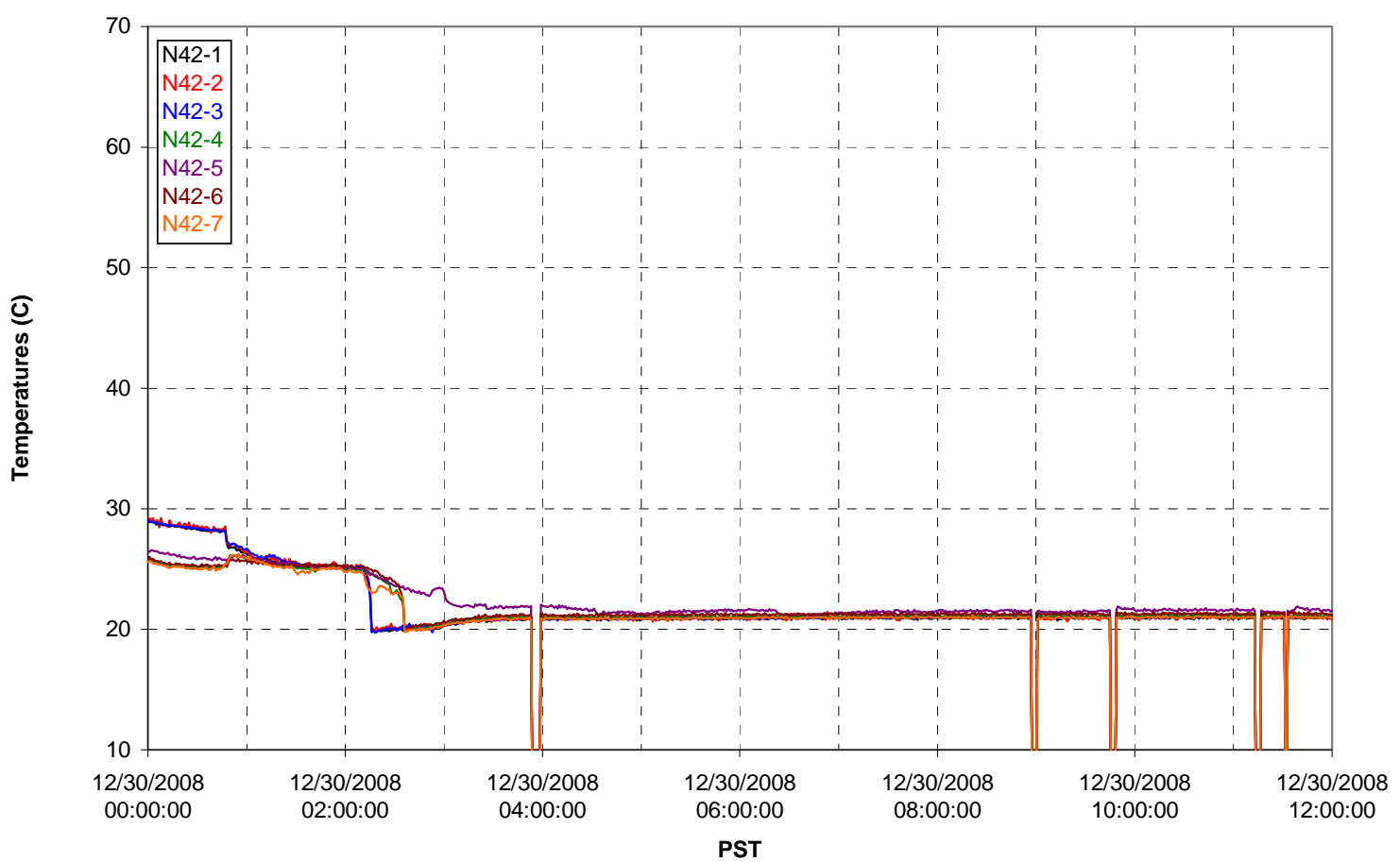

T02A temperatures

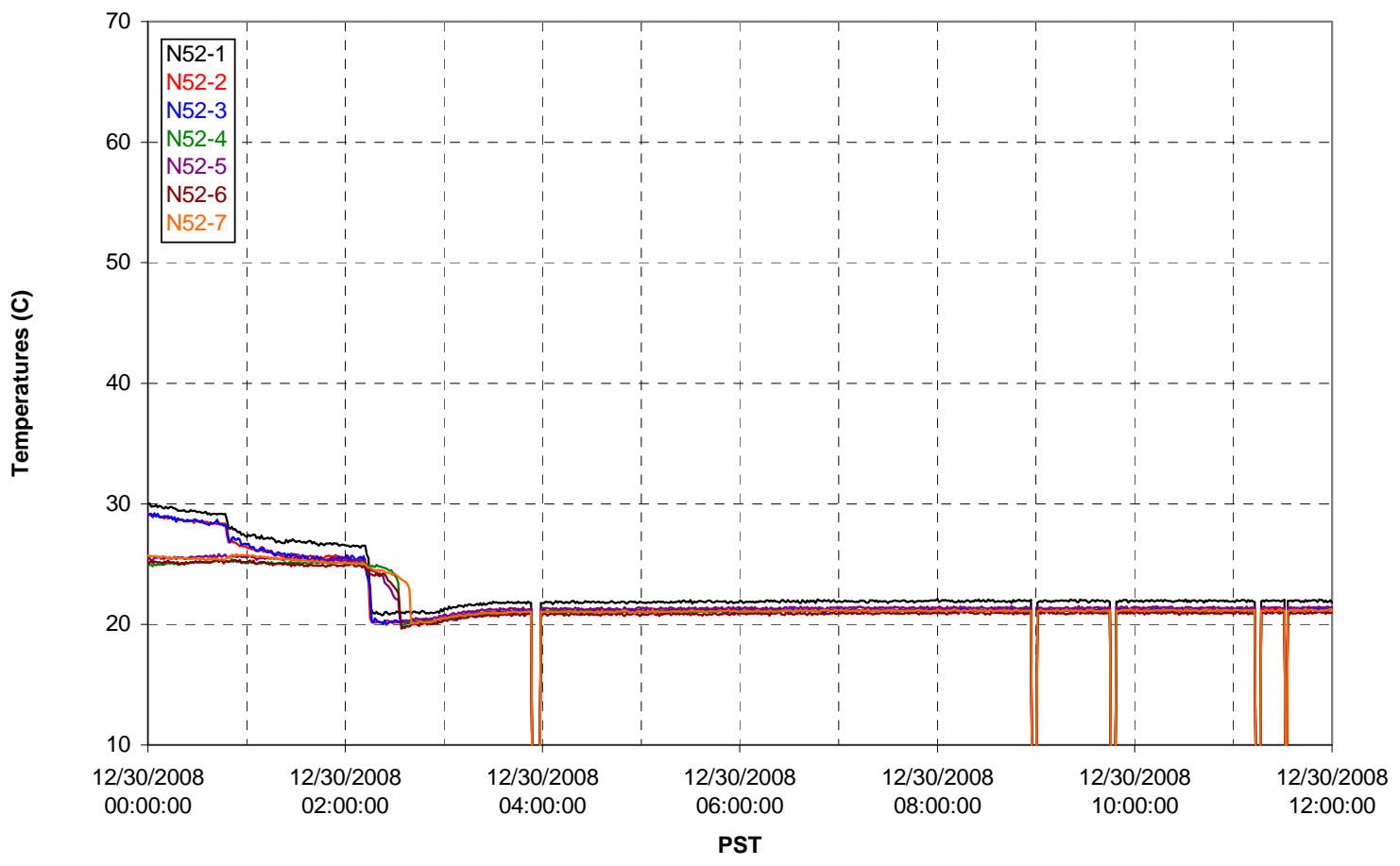


T02A Heating and Cooling

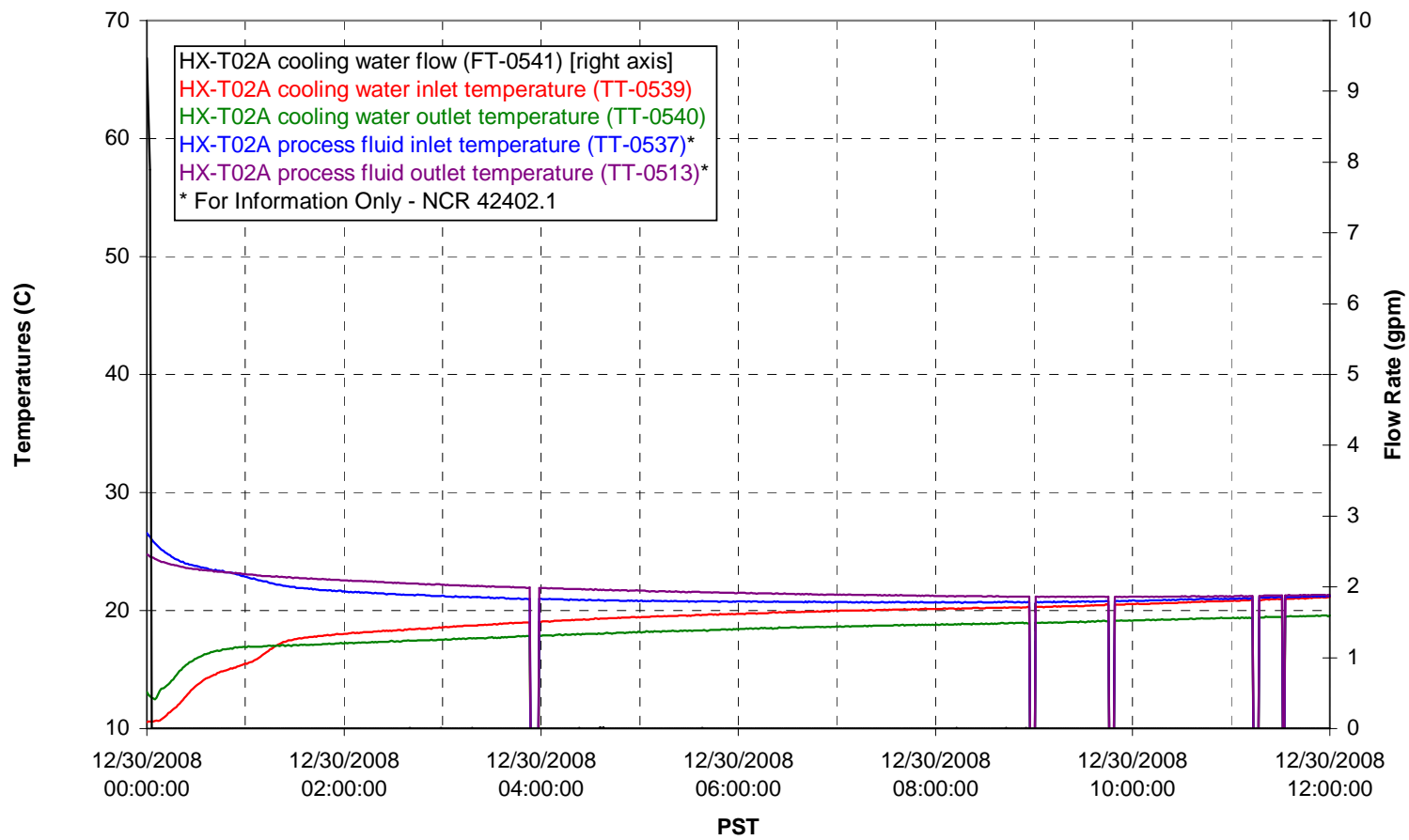

Pump Operation

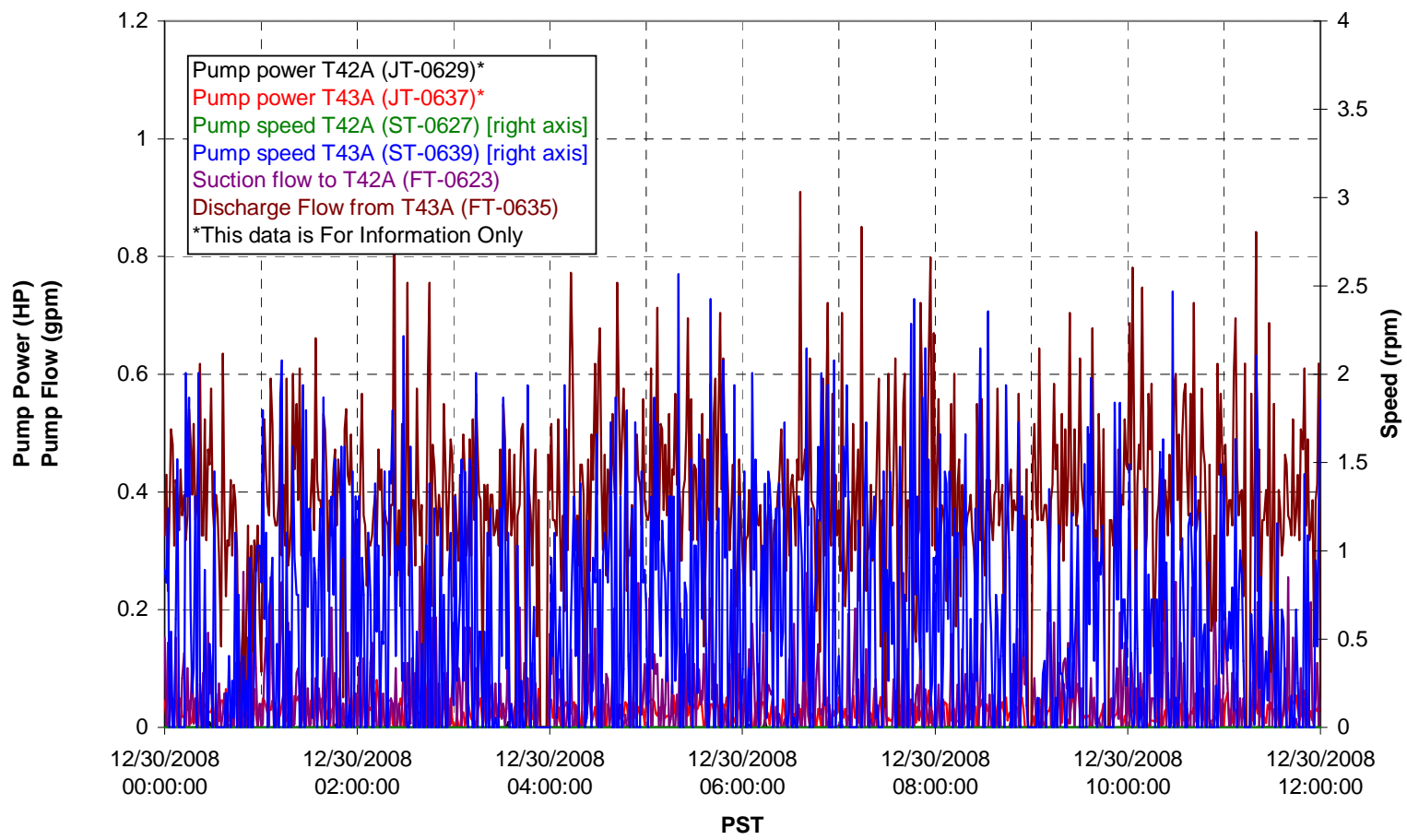


Pulsepot UFP-PP-T01A

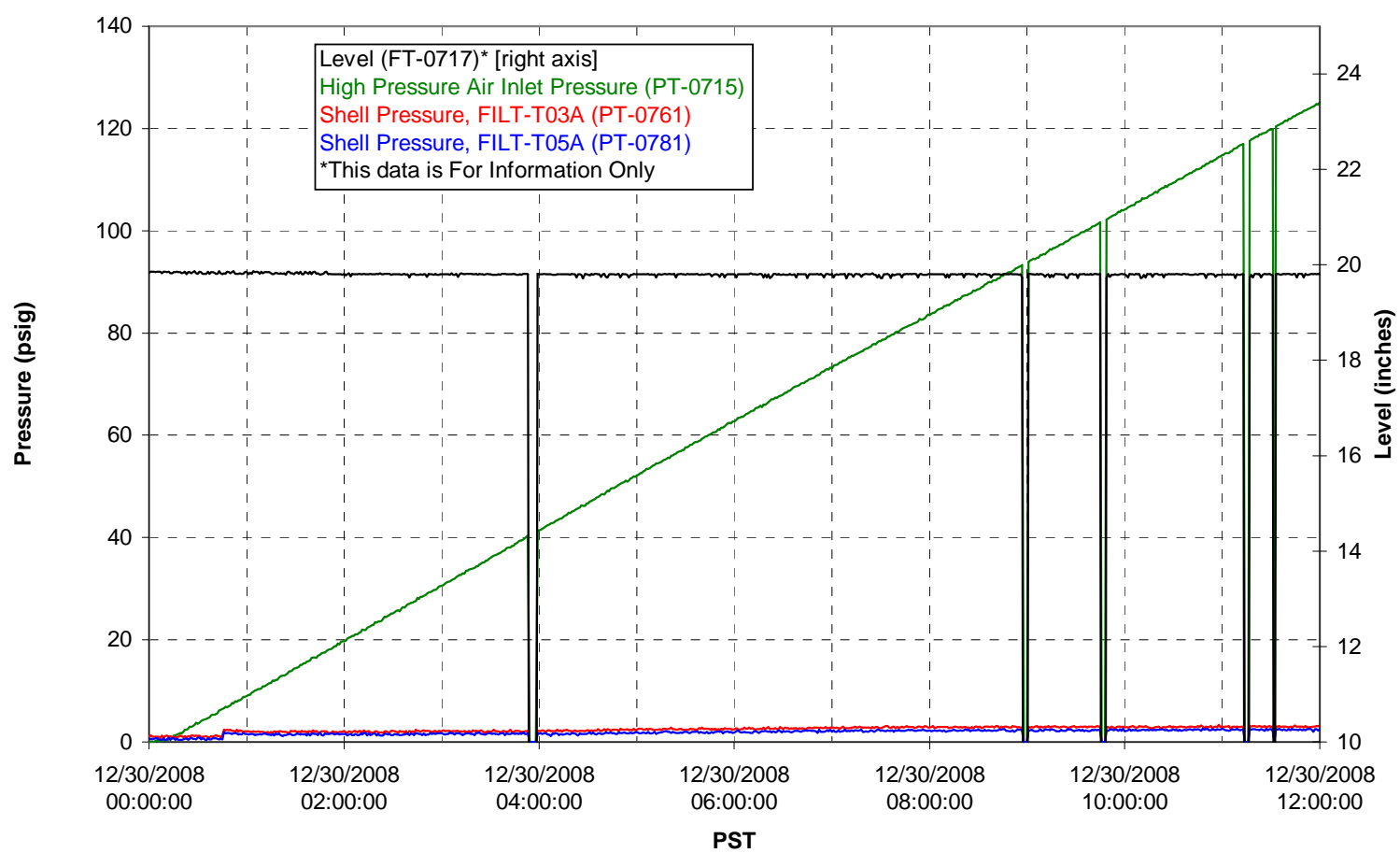

Pulsepot UFP-PP-T02A

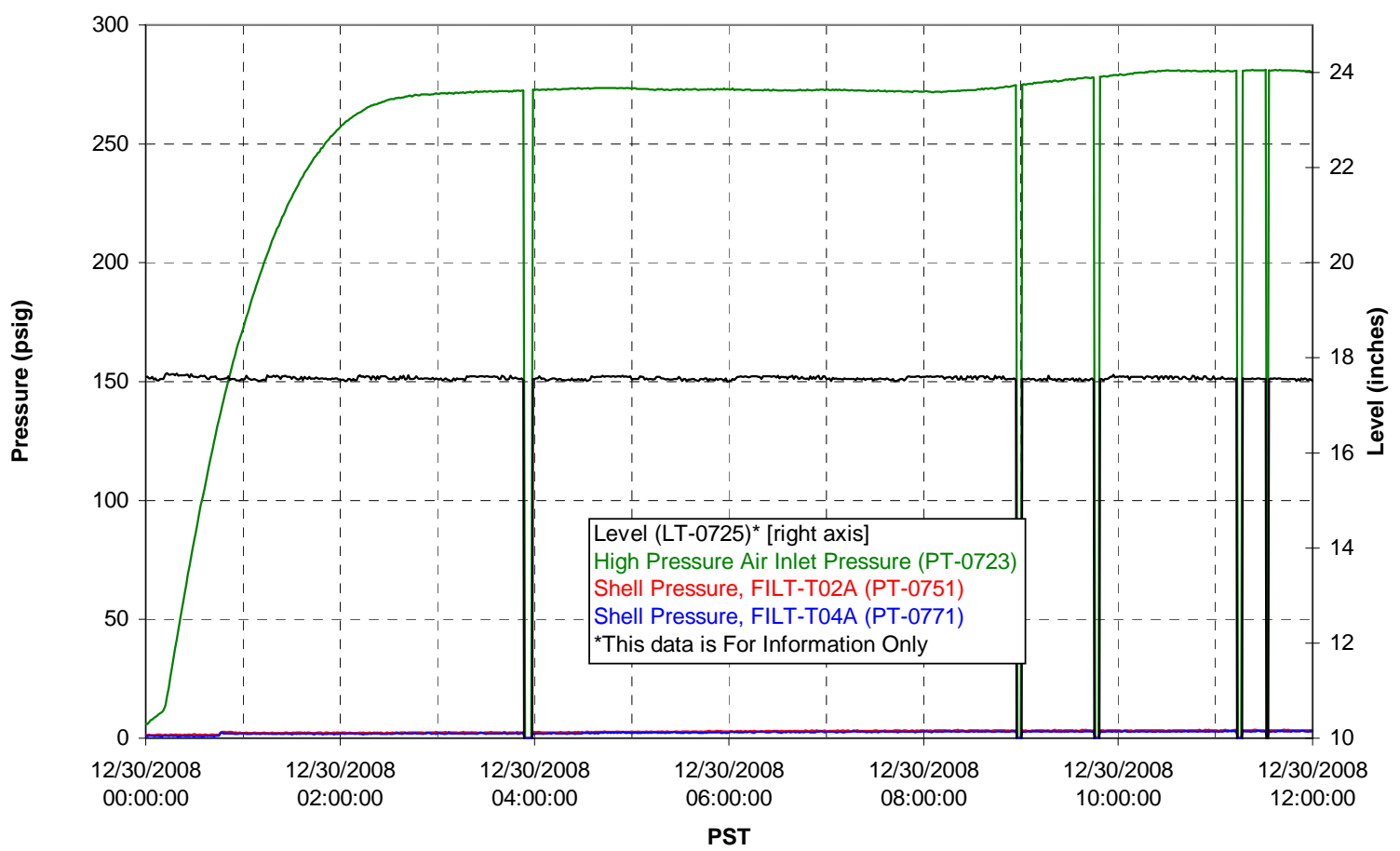


Pulsepot UFP-PP-T03A

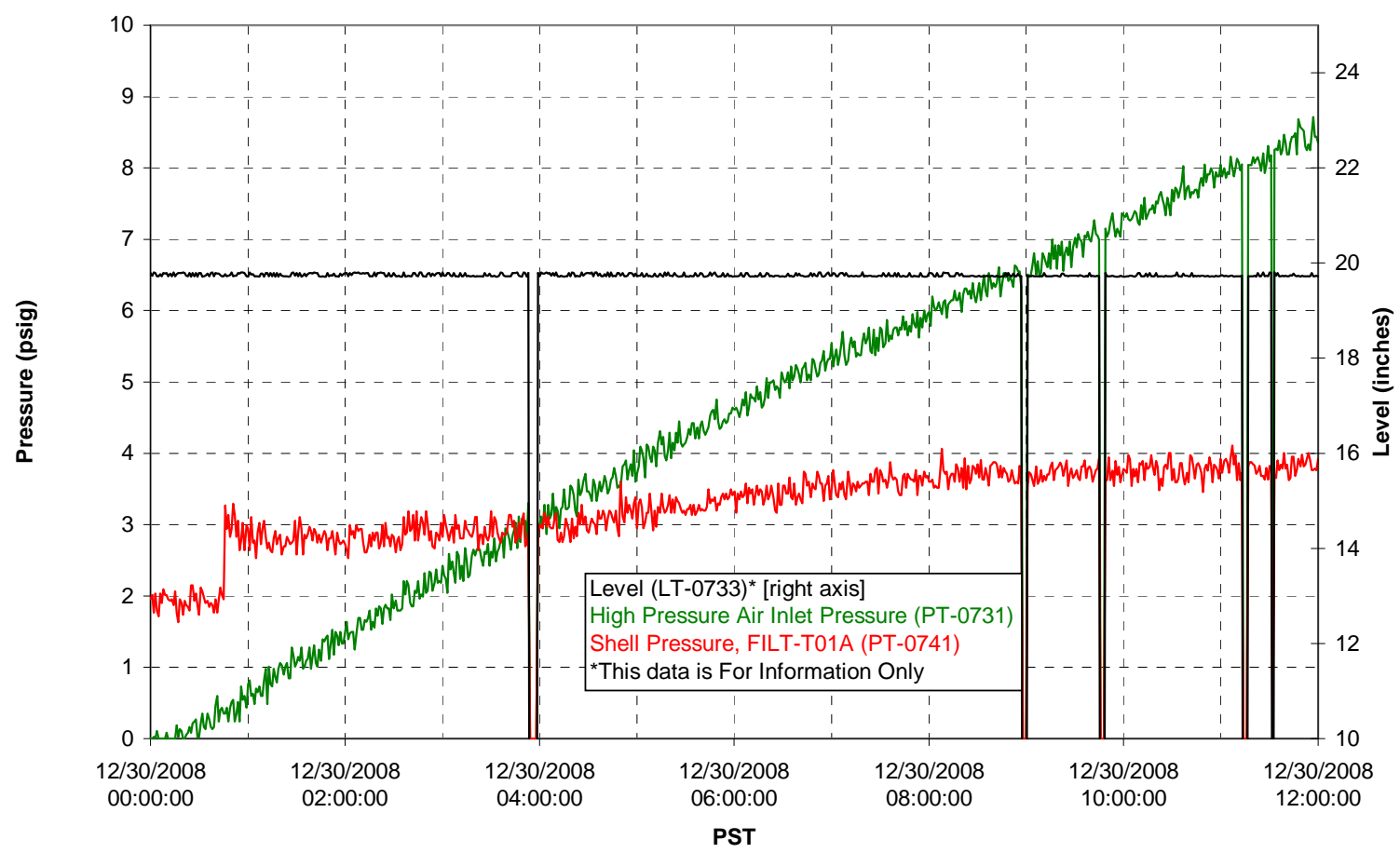

Pulsepot Levels

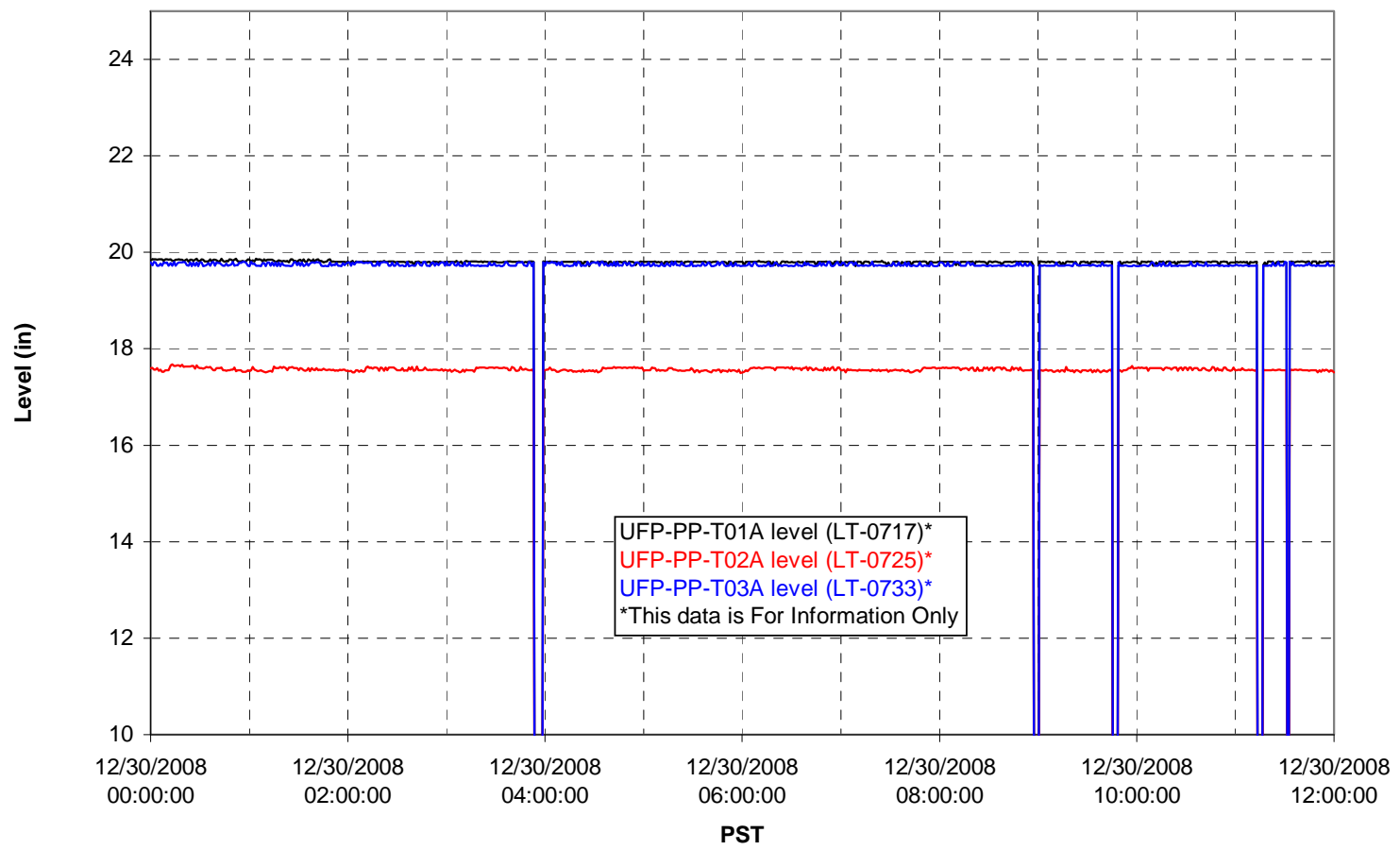


Filter UFP-FILT-T01A

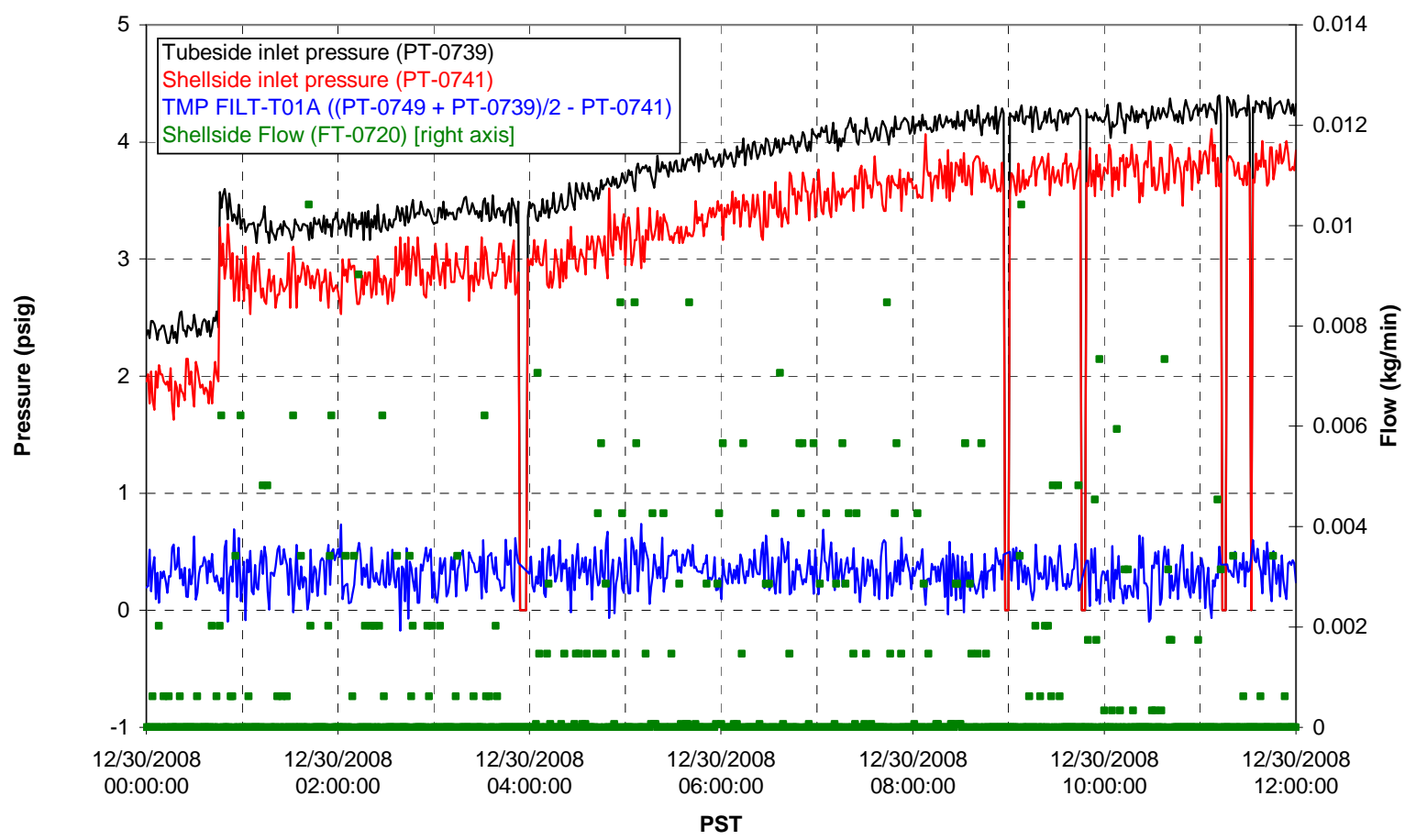

Filter UFP-FILT-T02A

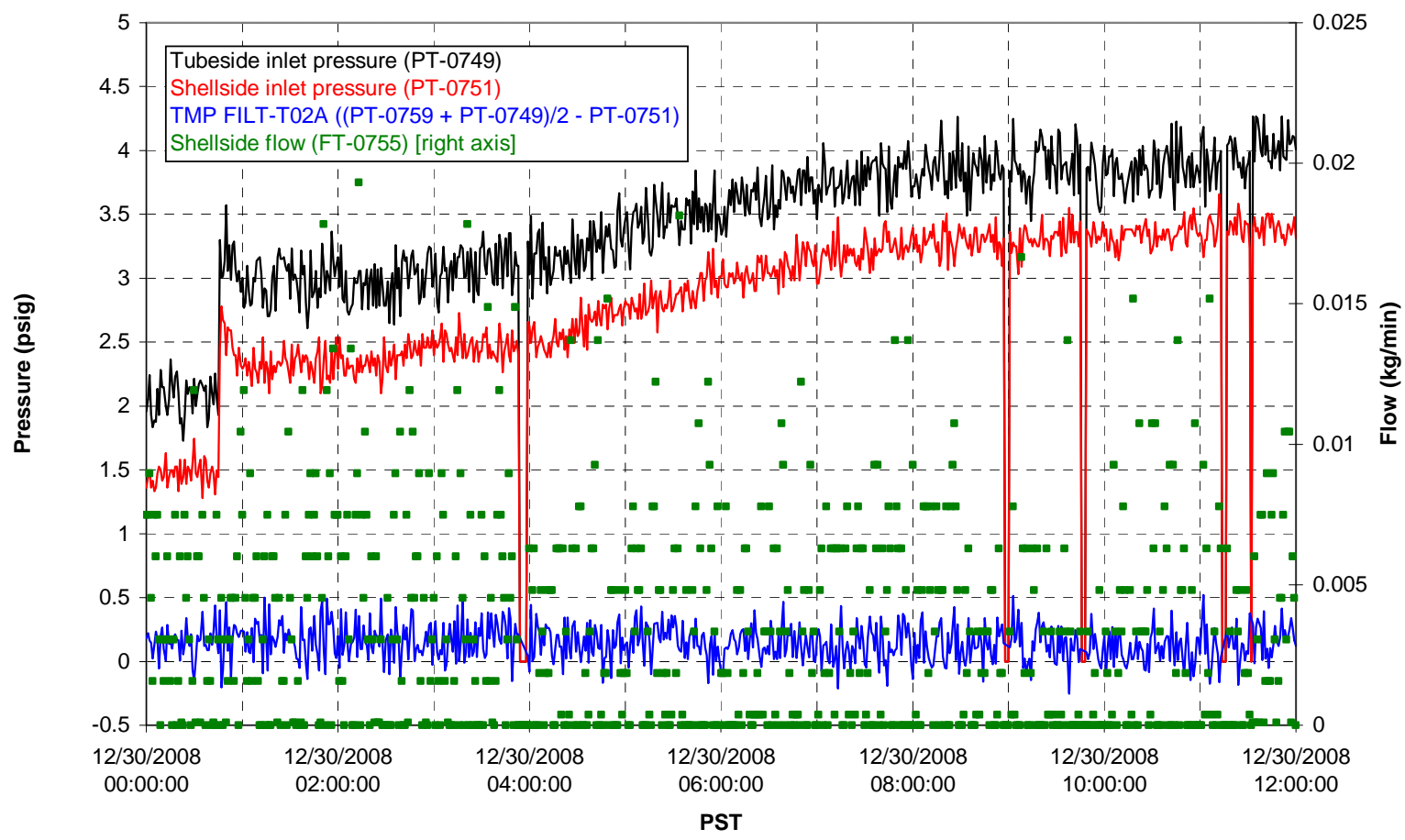


Filter UFP-FILT-T03A

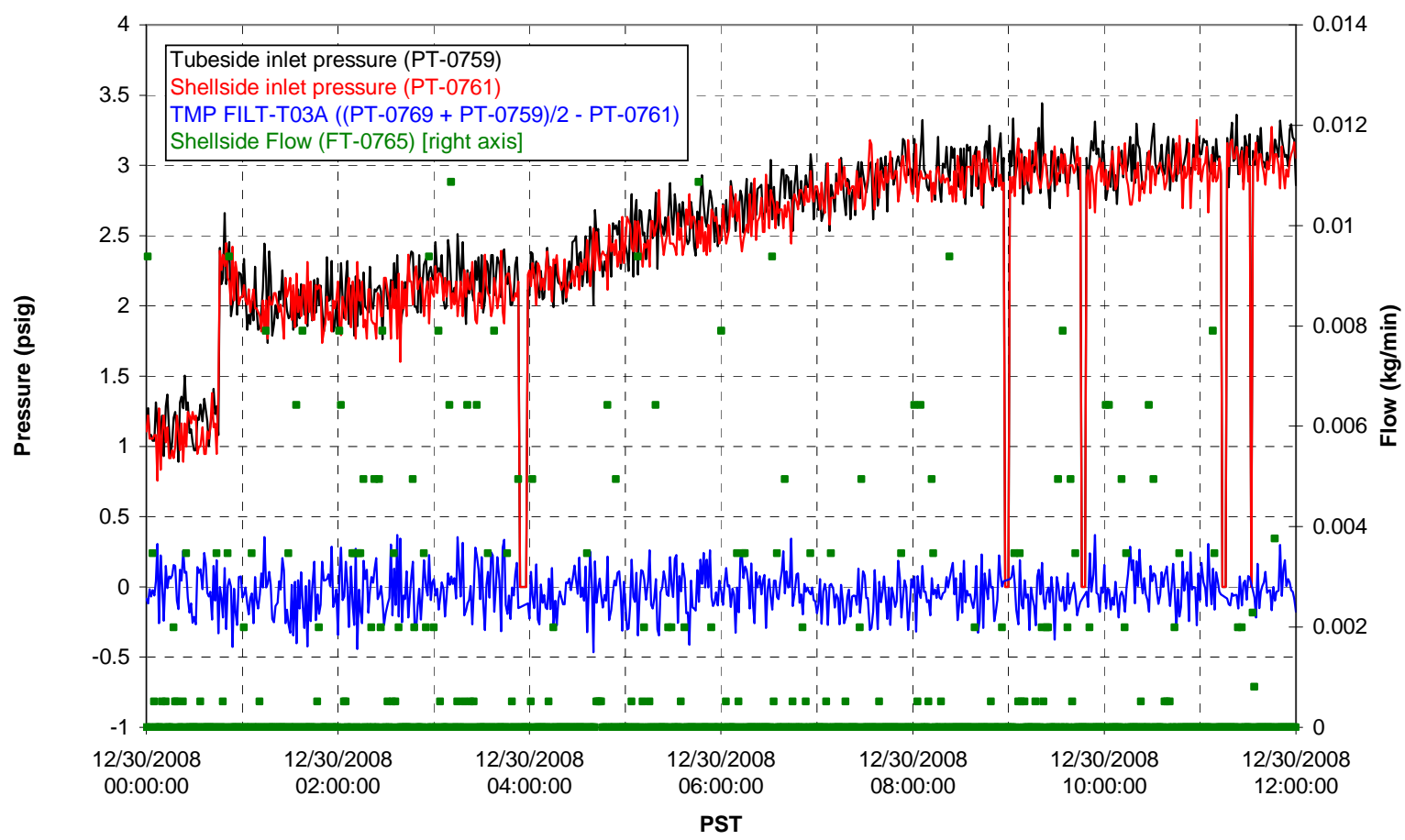

Filter UFP-FILT-T04A

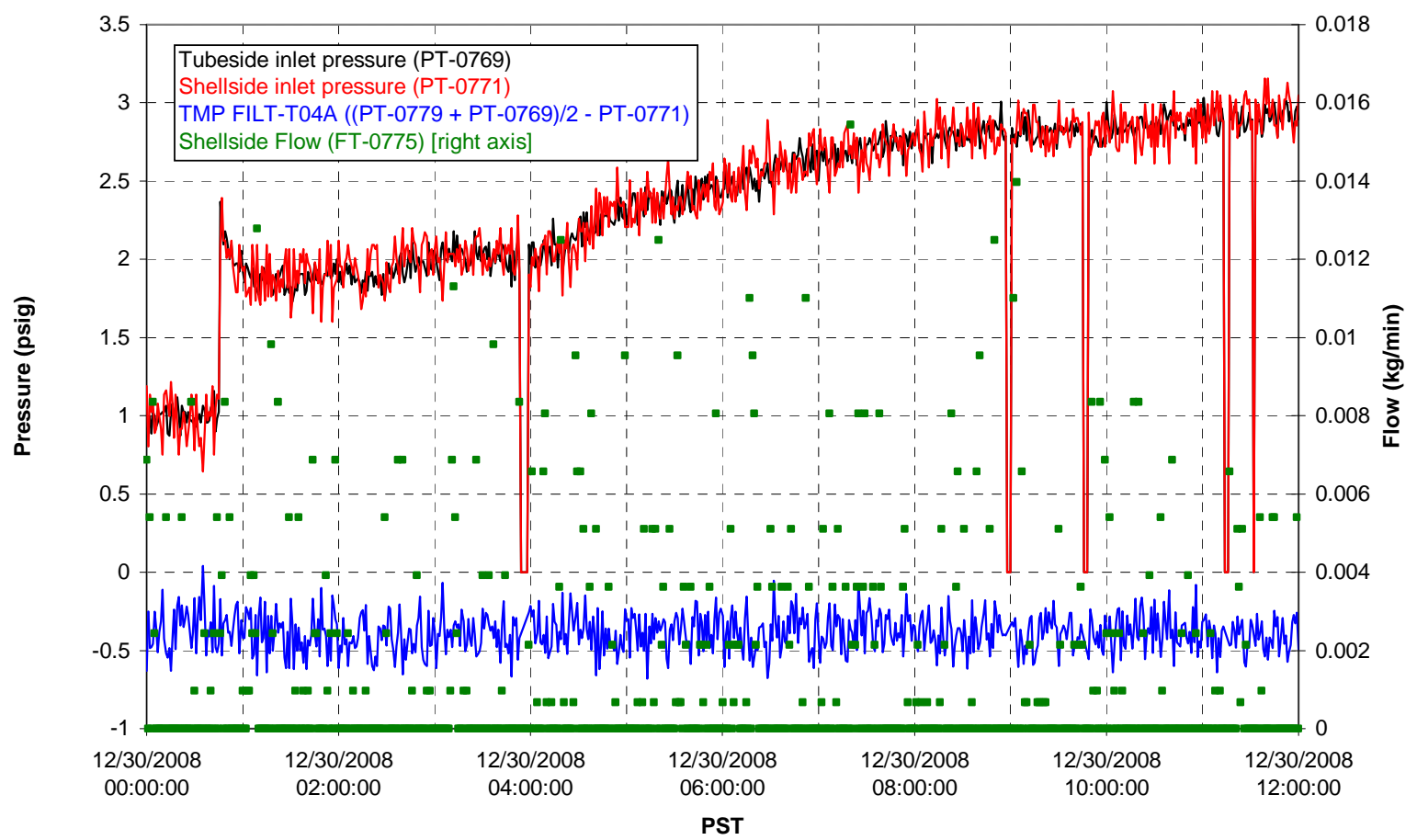


Filter UFP-FILT-T05A

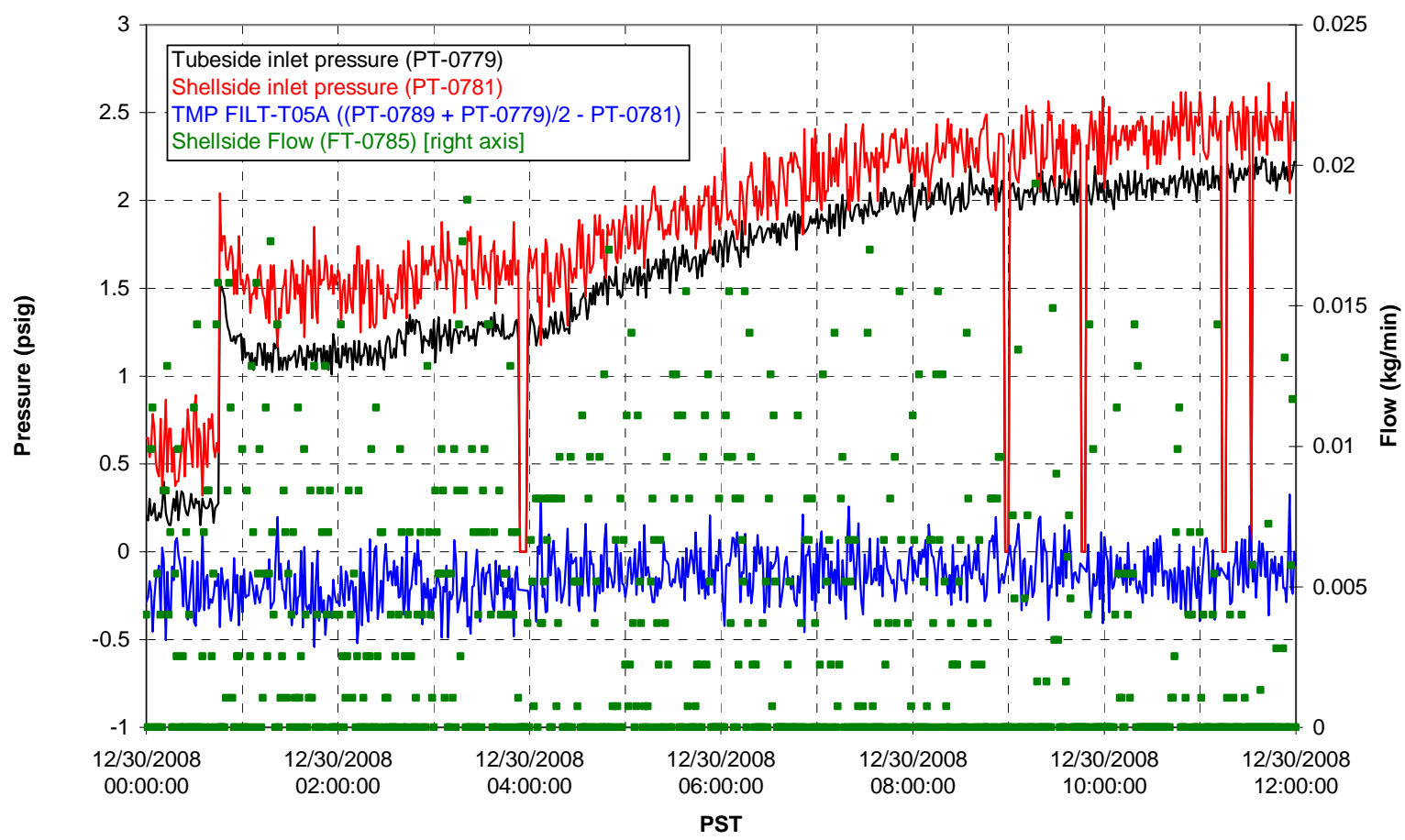

Chemical Flow

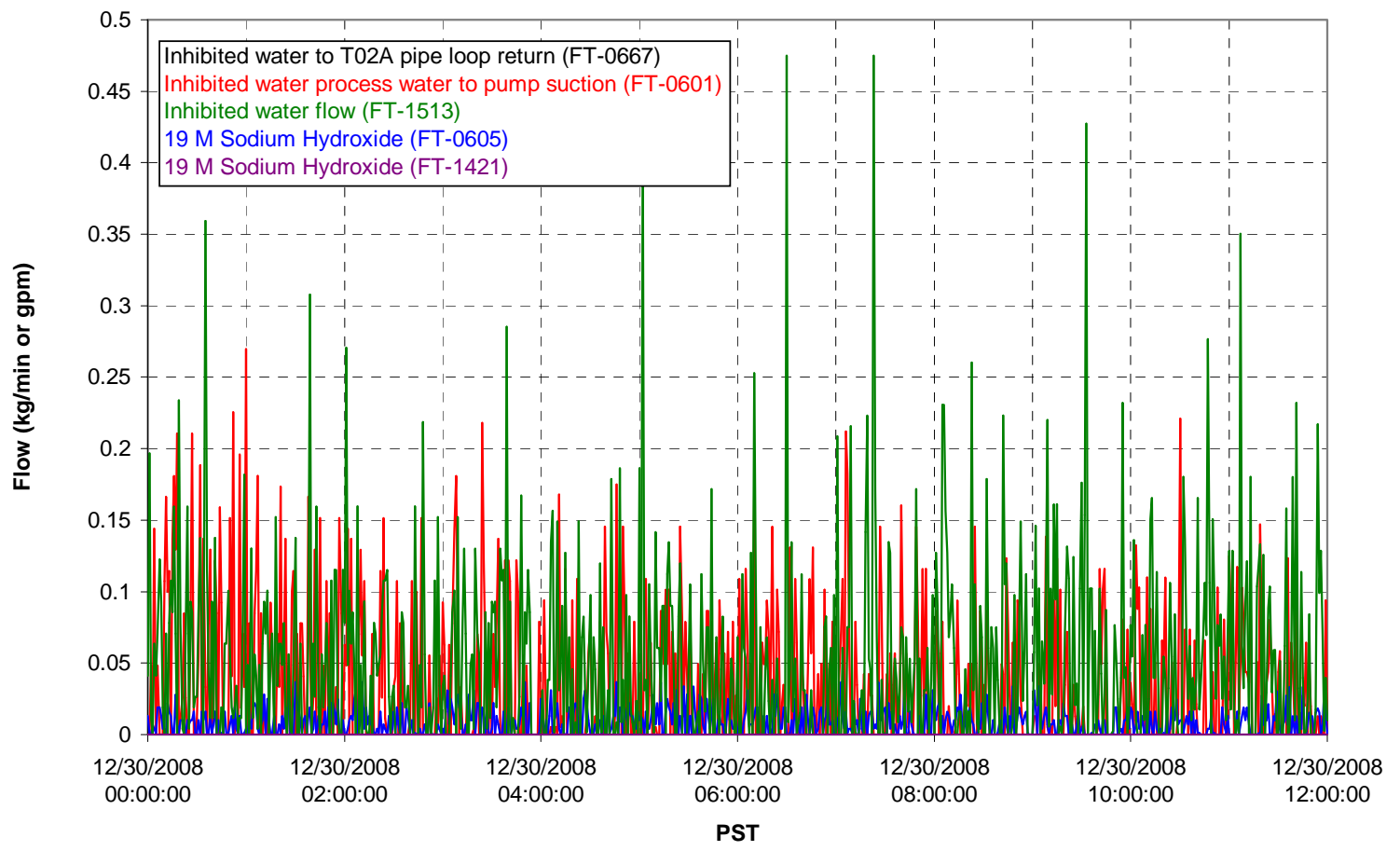




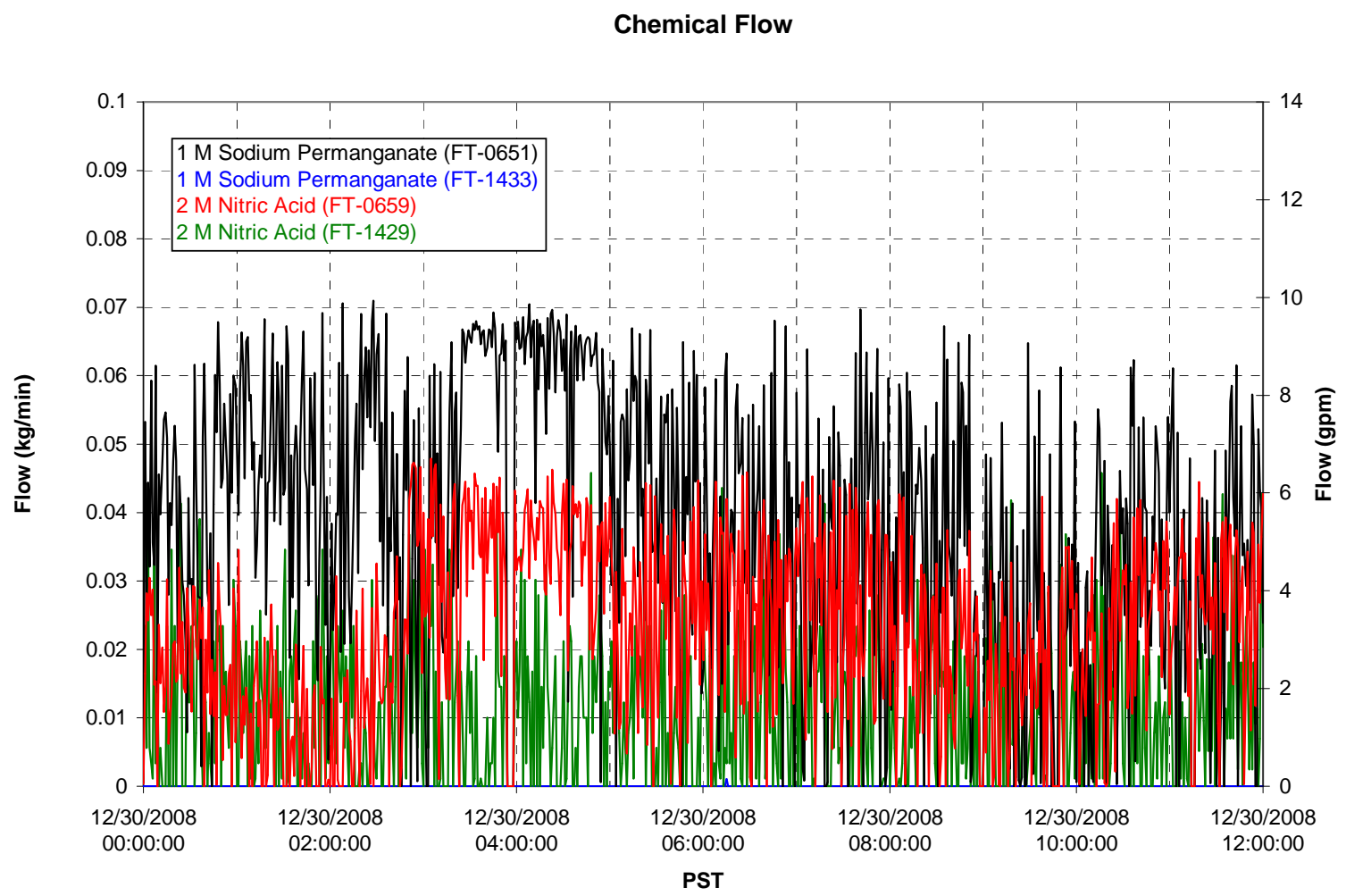

Air Flows

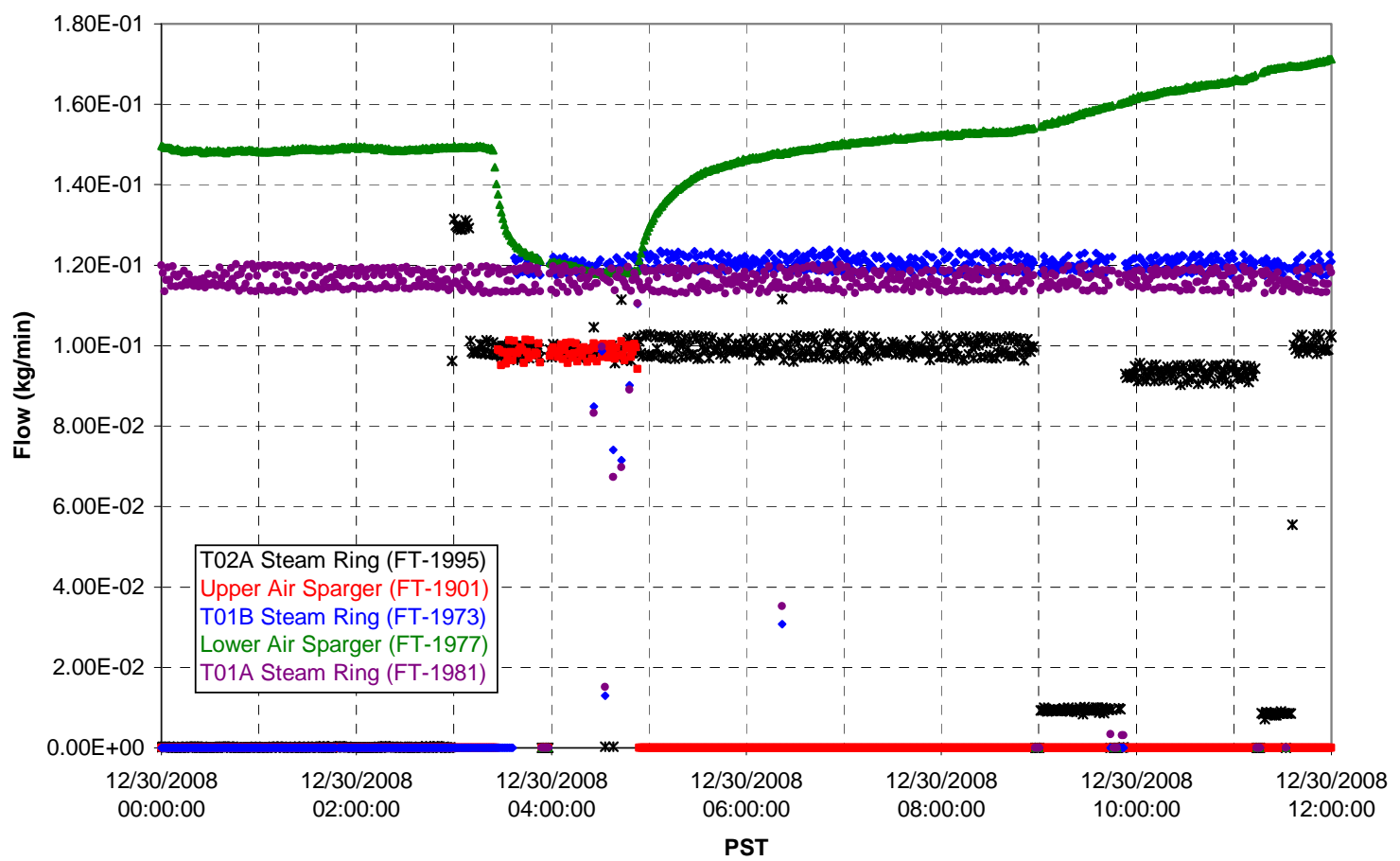


T02A Steam

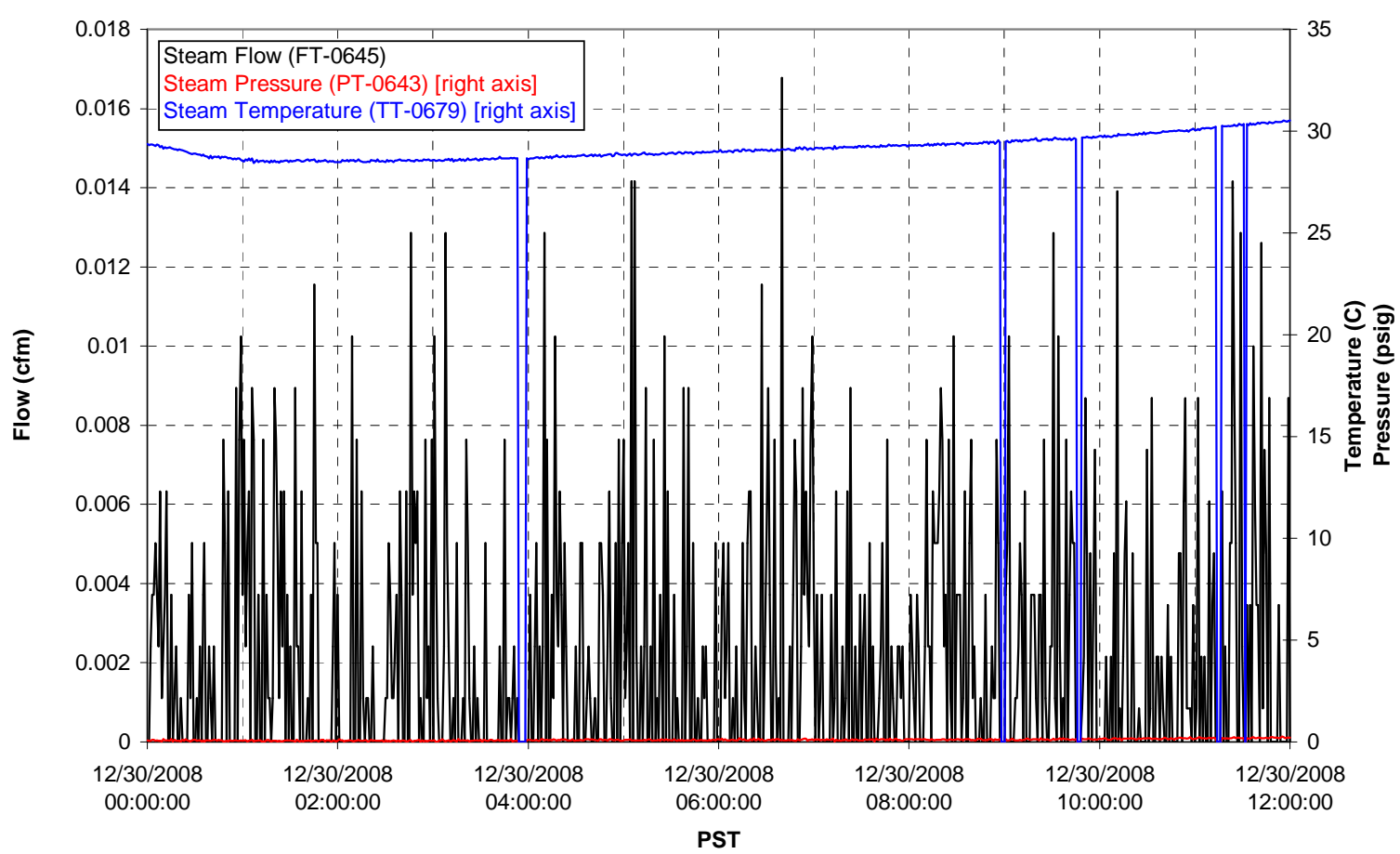

T01A Steam

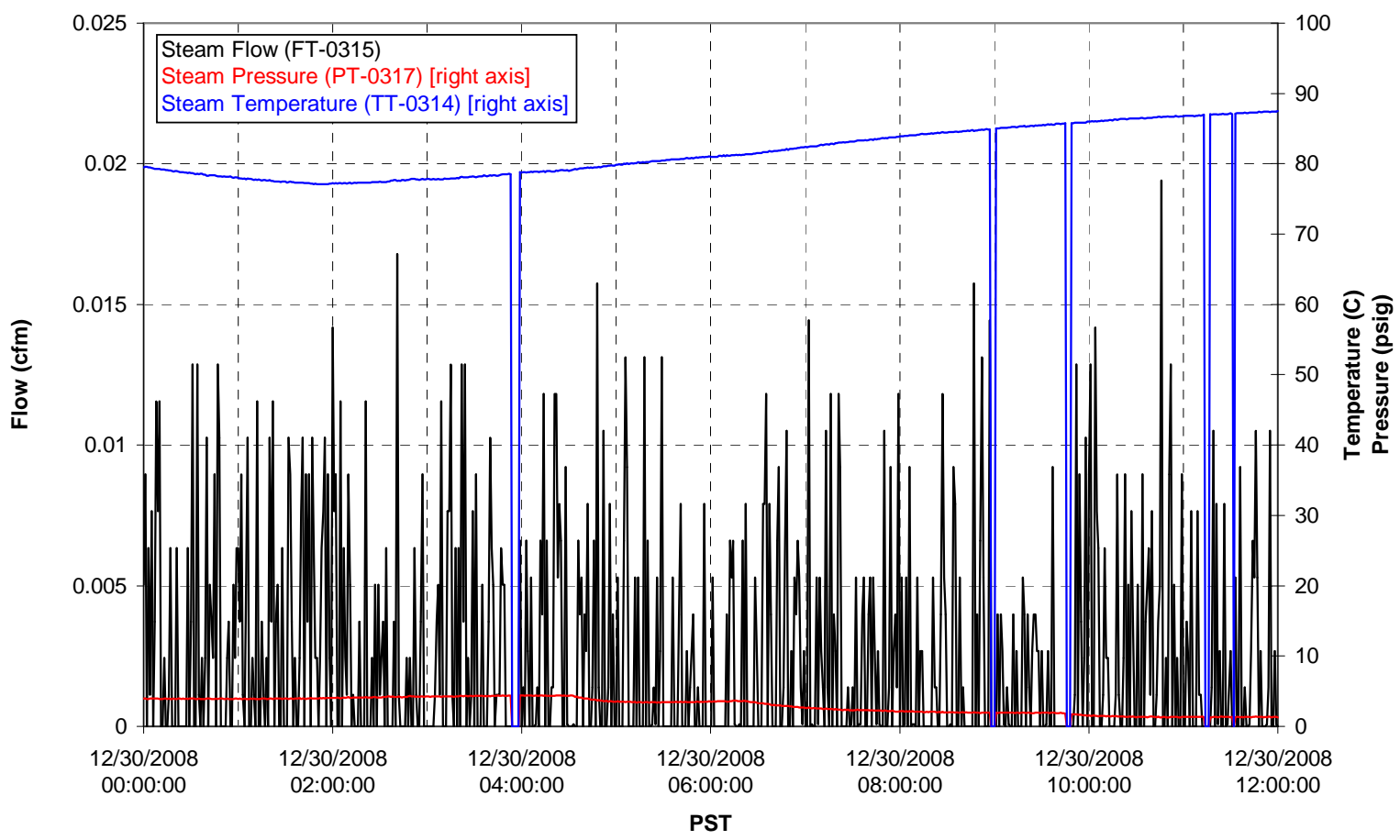


T01B Steam

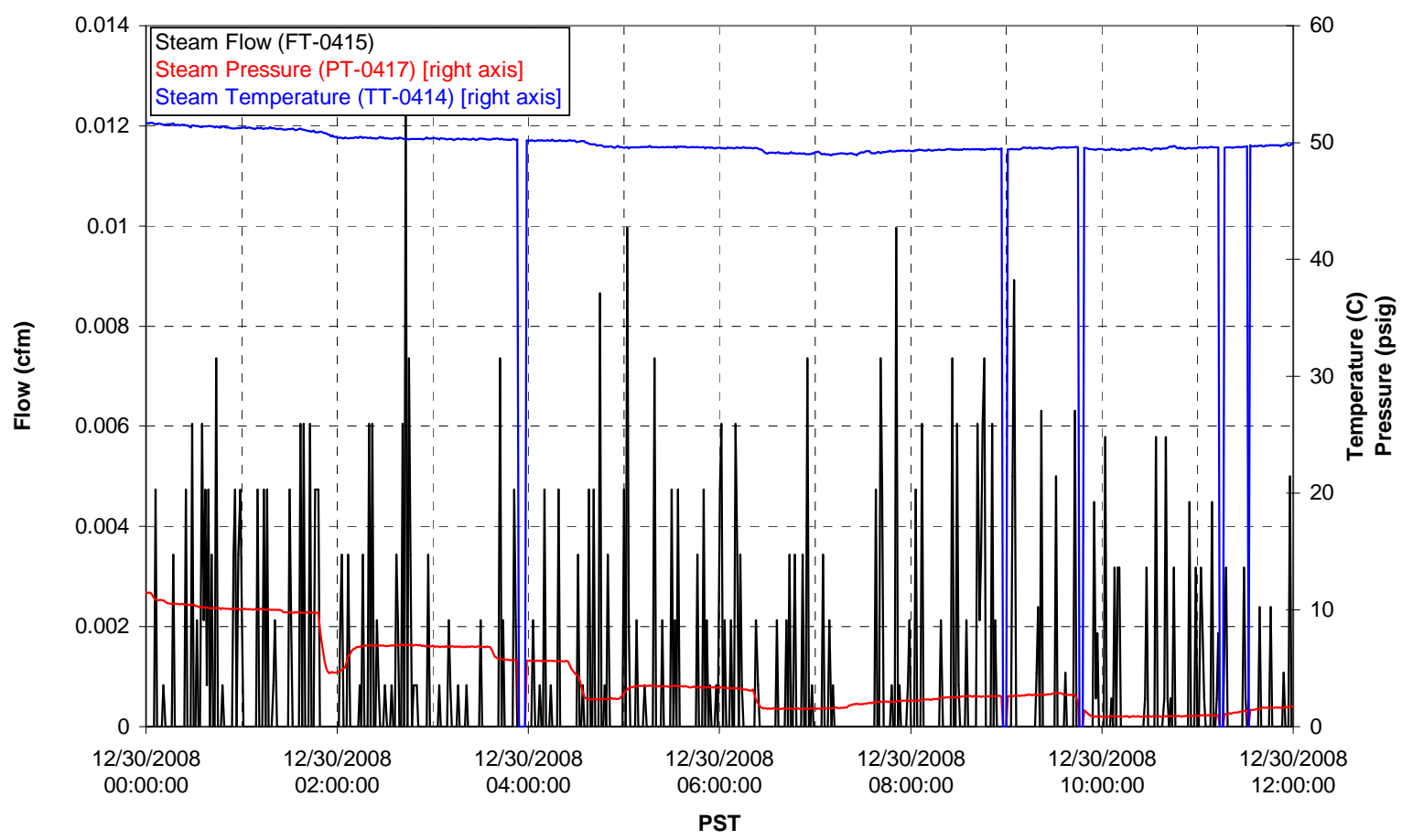


Functional Test Data Plots 12/30/08 12:00 - 12/31/08 00:00 
T01A level

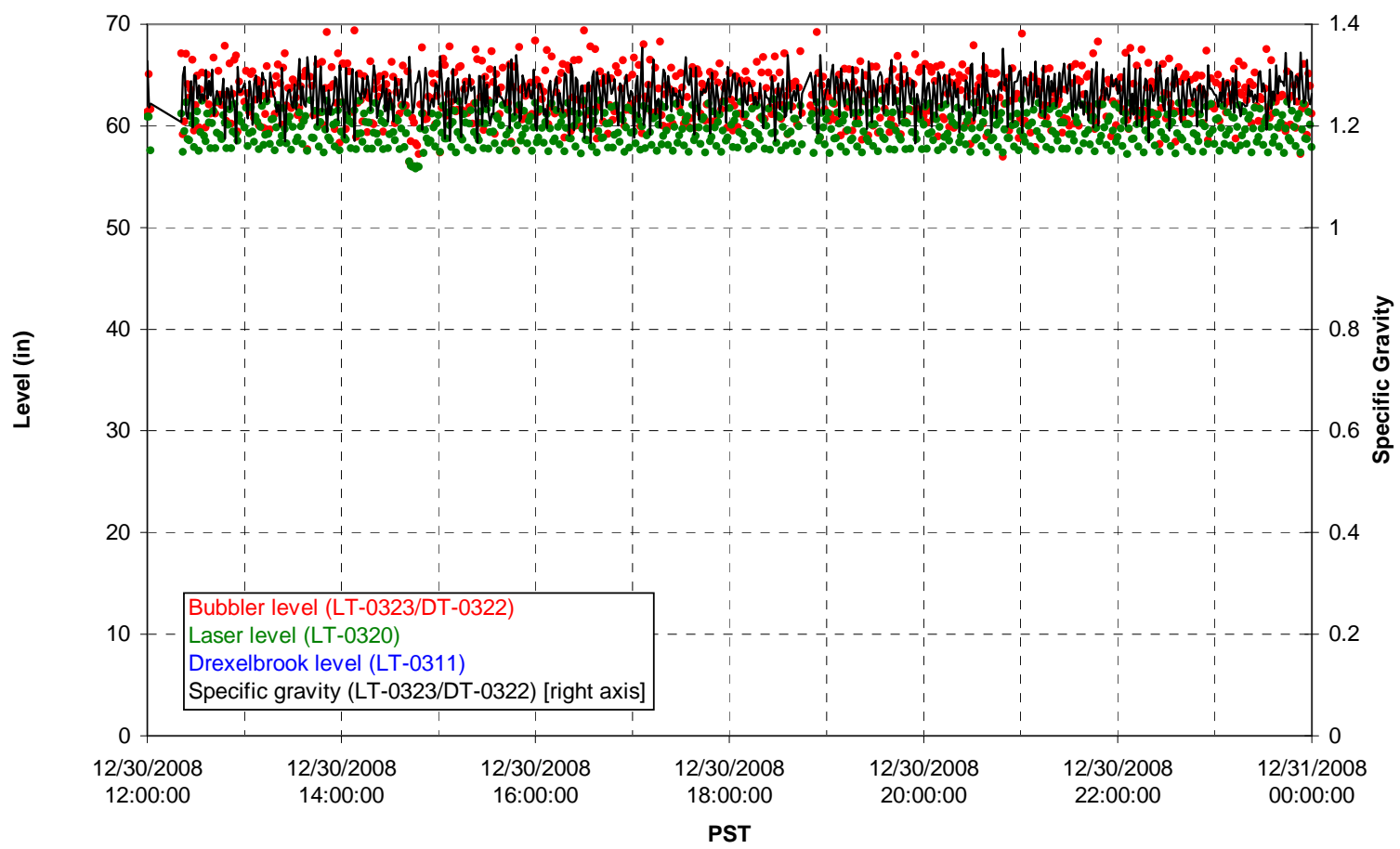

T01A temperatures

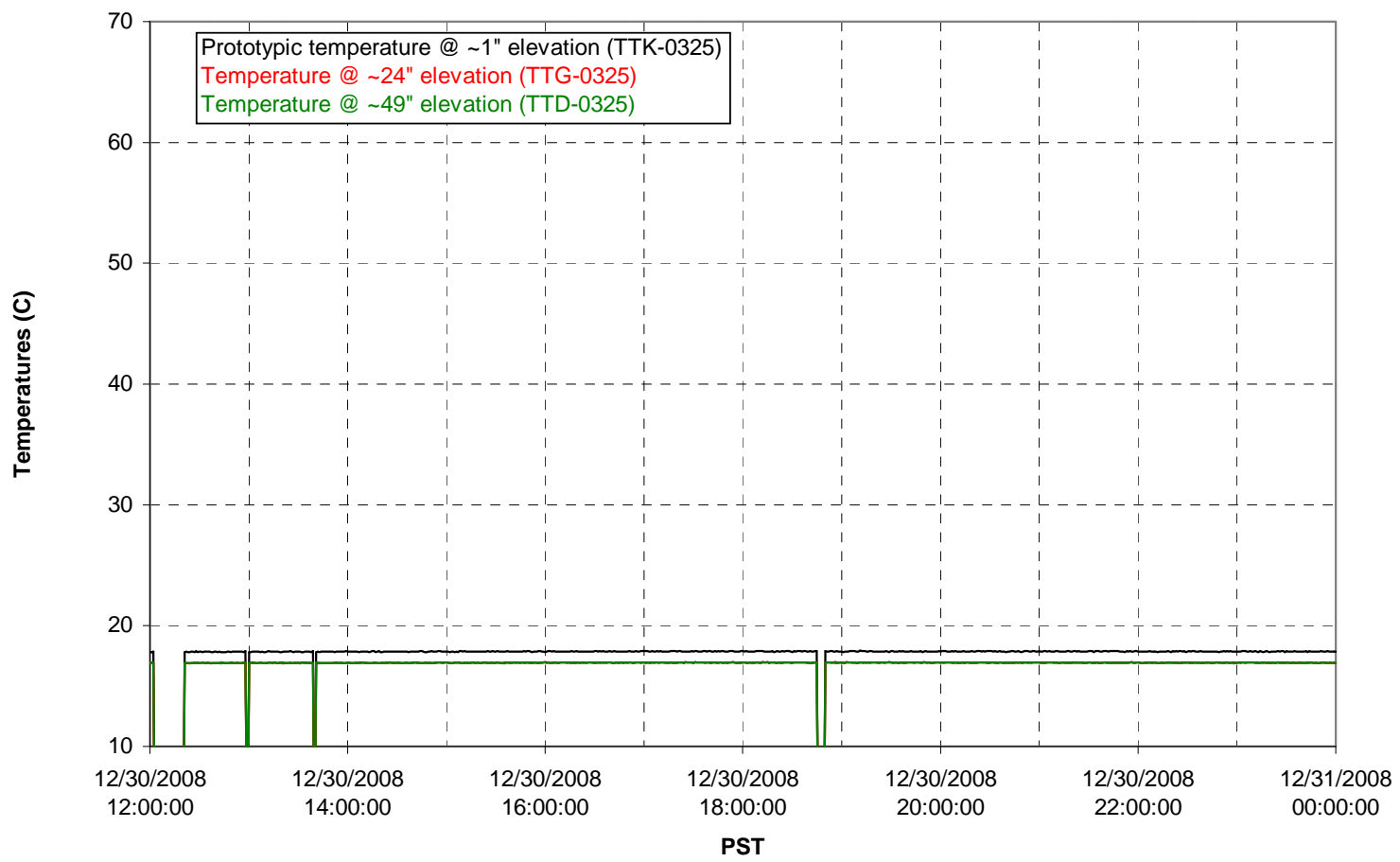


T01B level

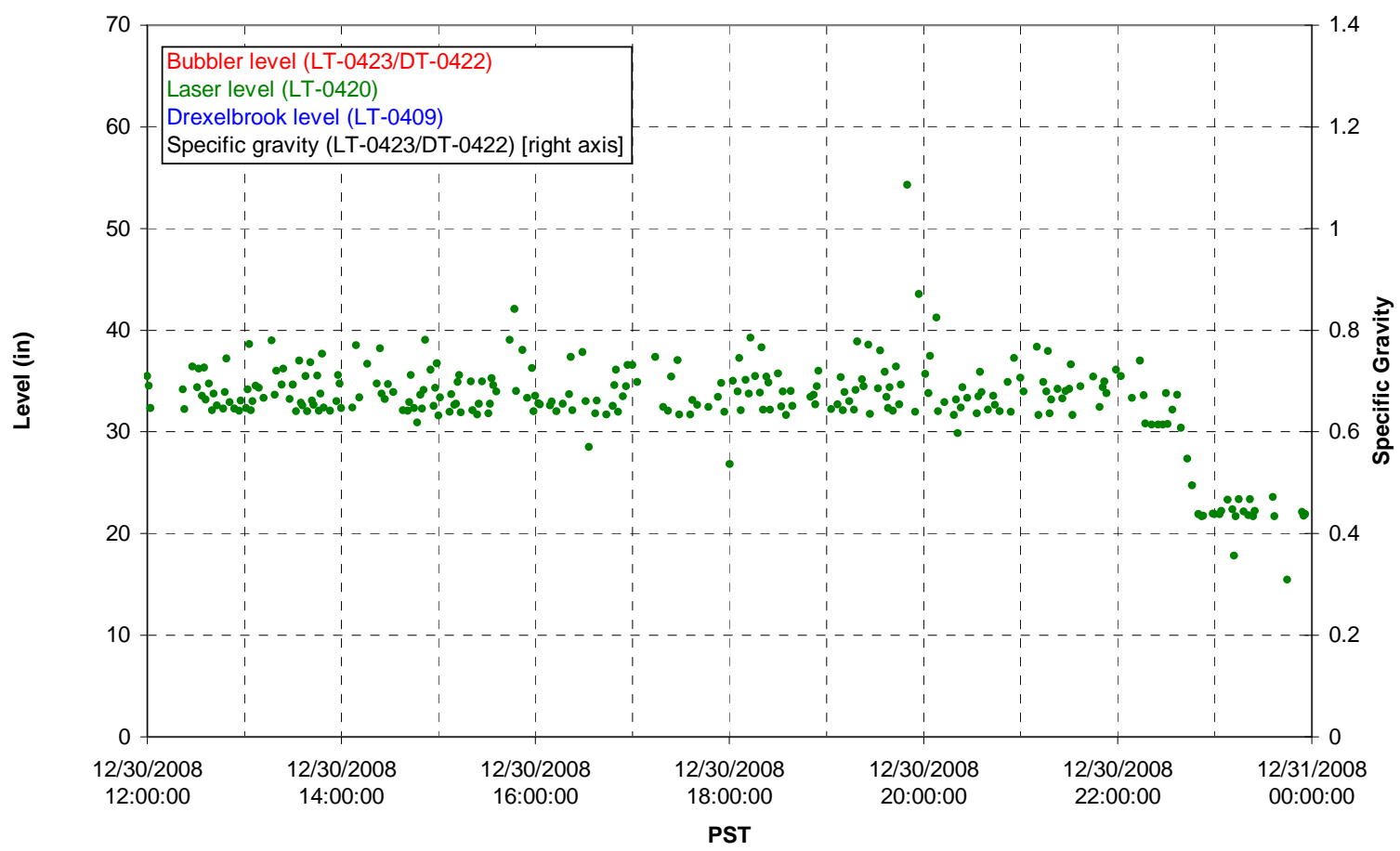

T01B temperatures

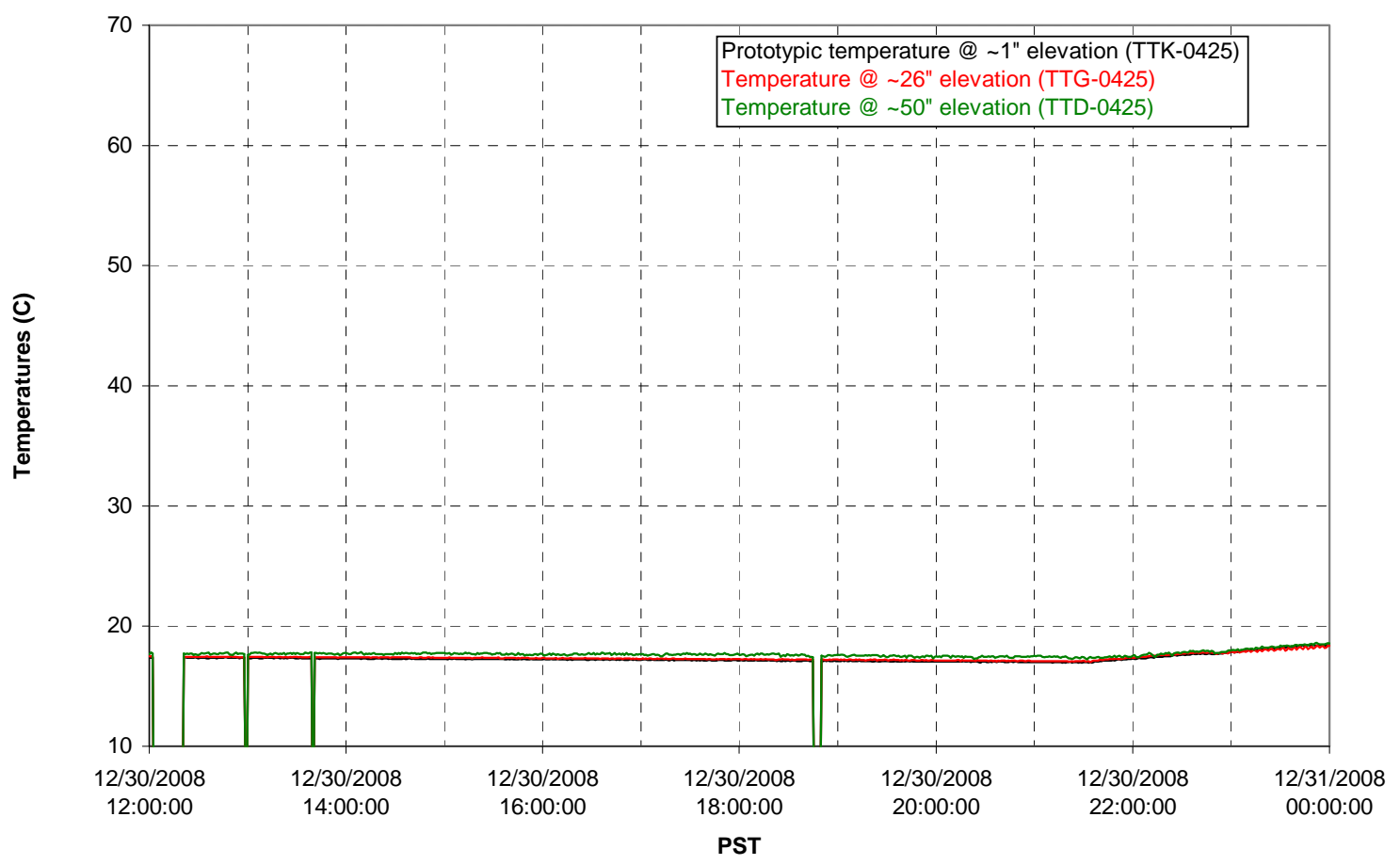


T02A level

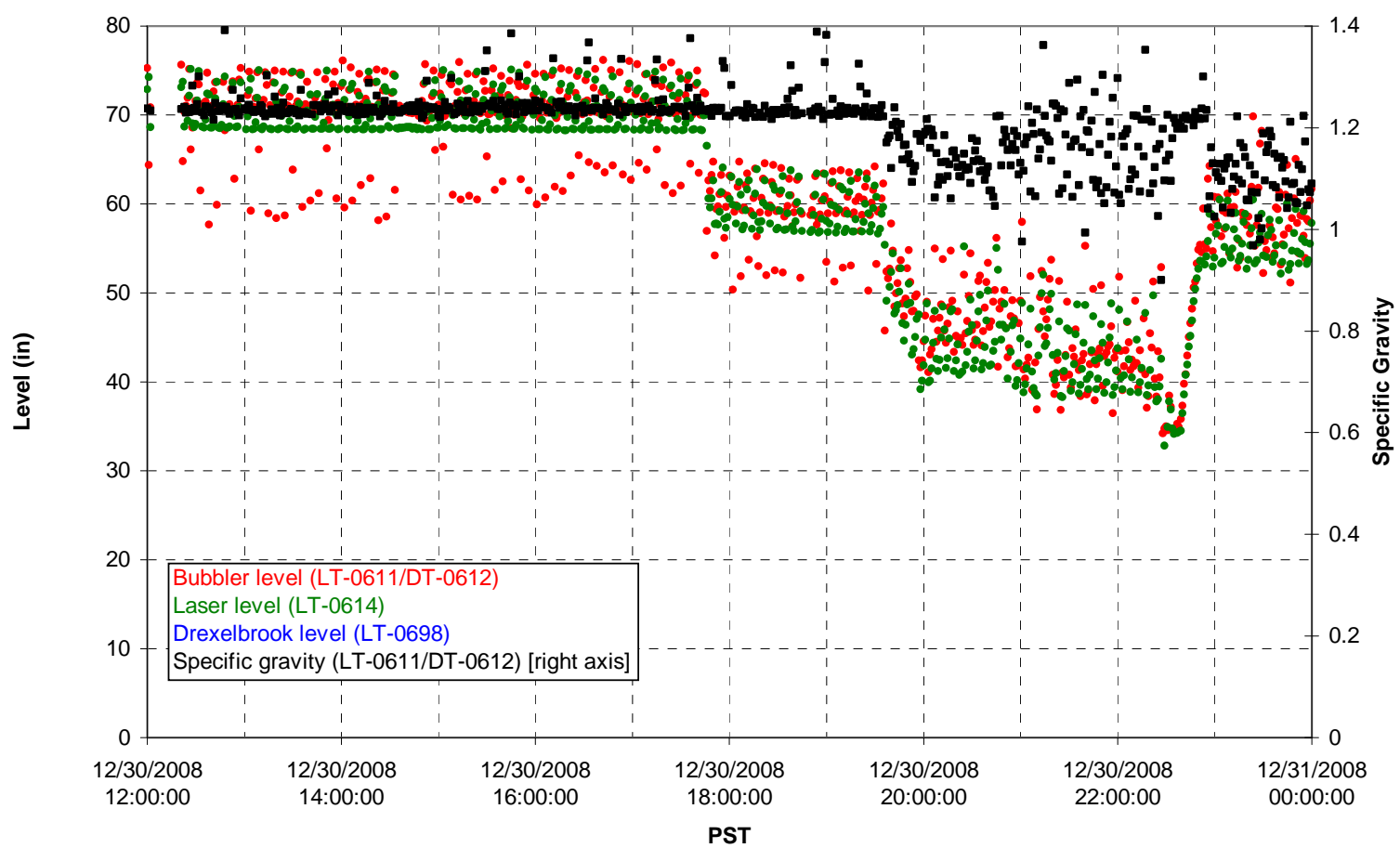

T02A temperatures

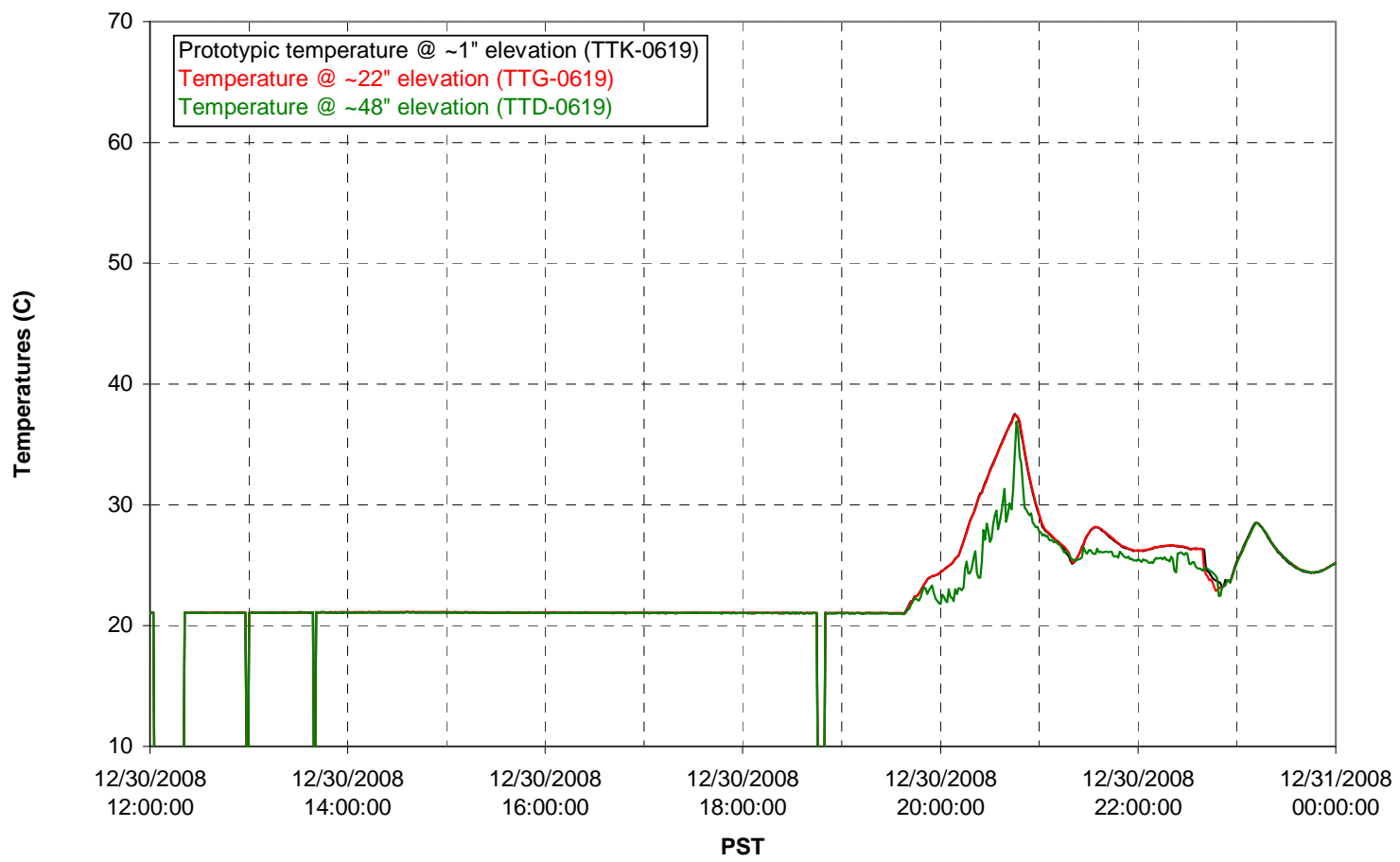


T02A and filter loop temperatures

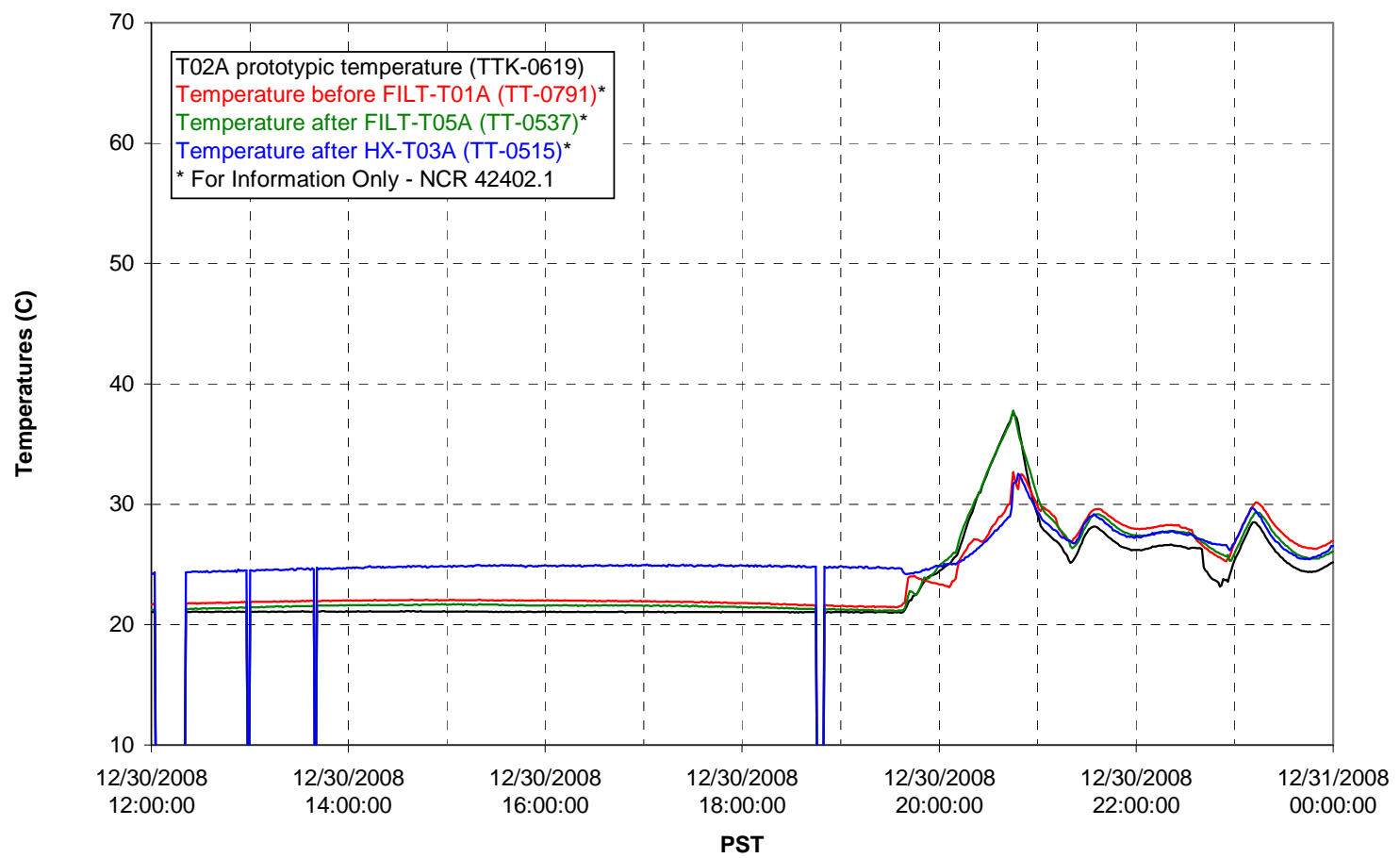

Pump Pressures and Flow

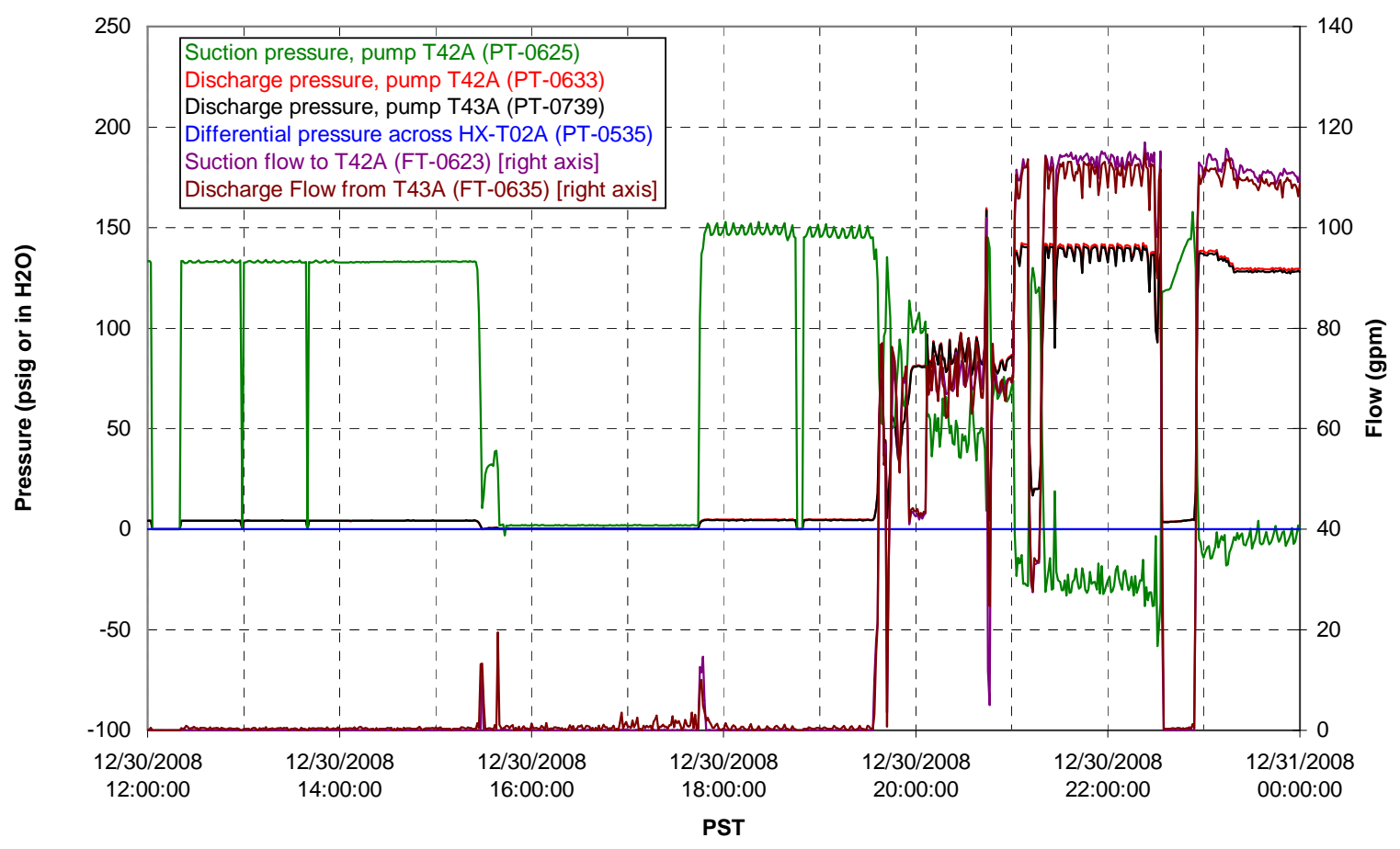




\section{Axial pressure drop}

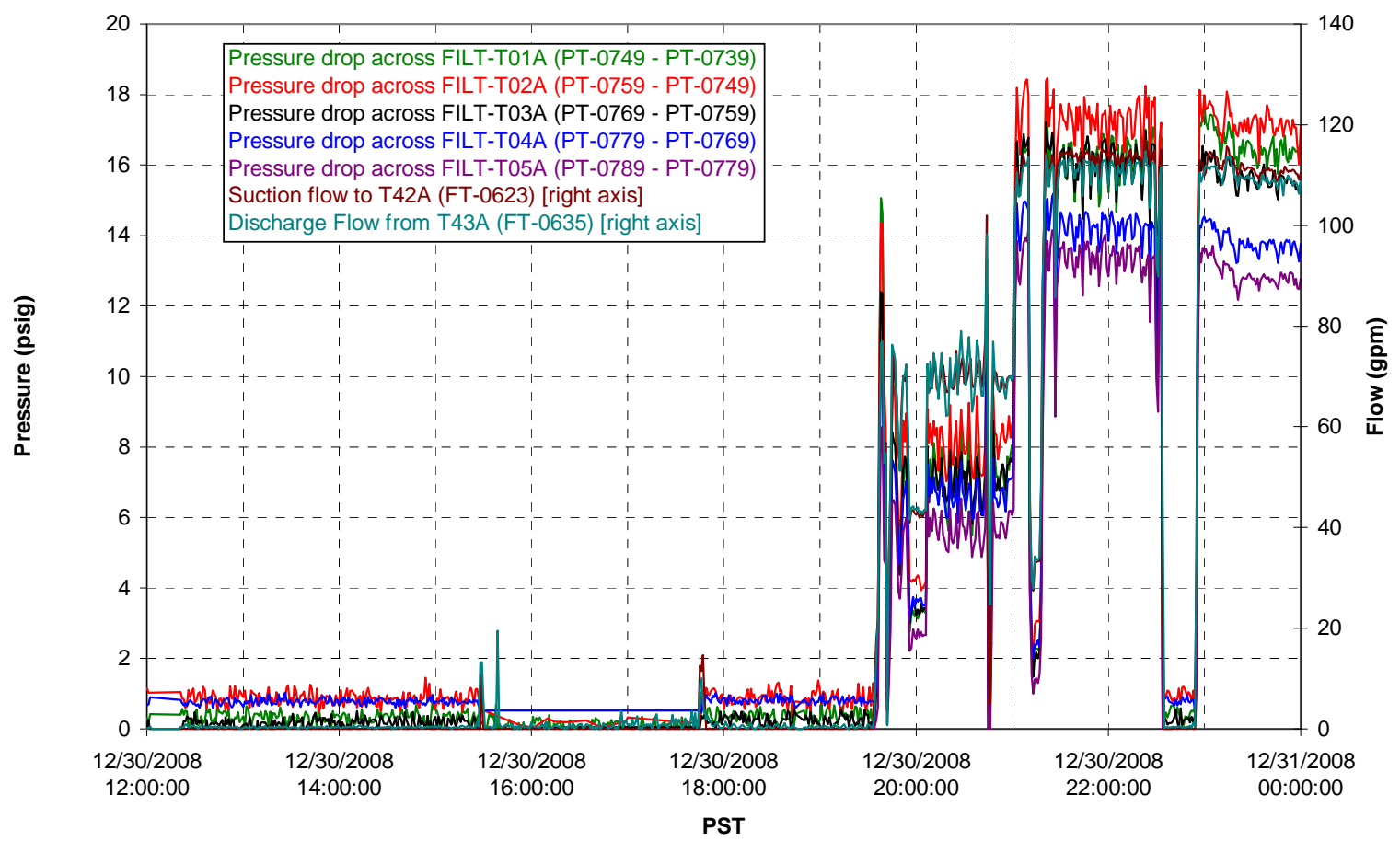

Permeate flow rates

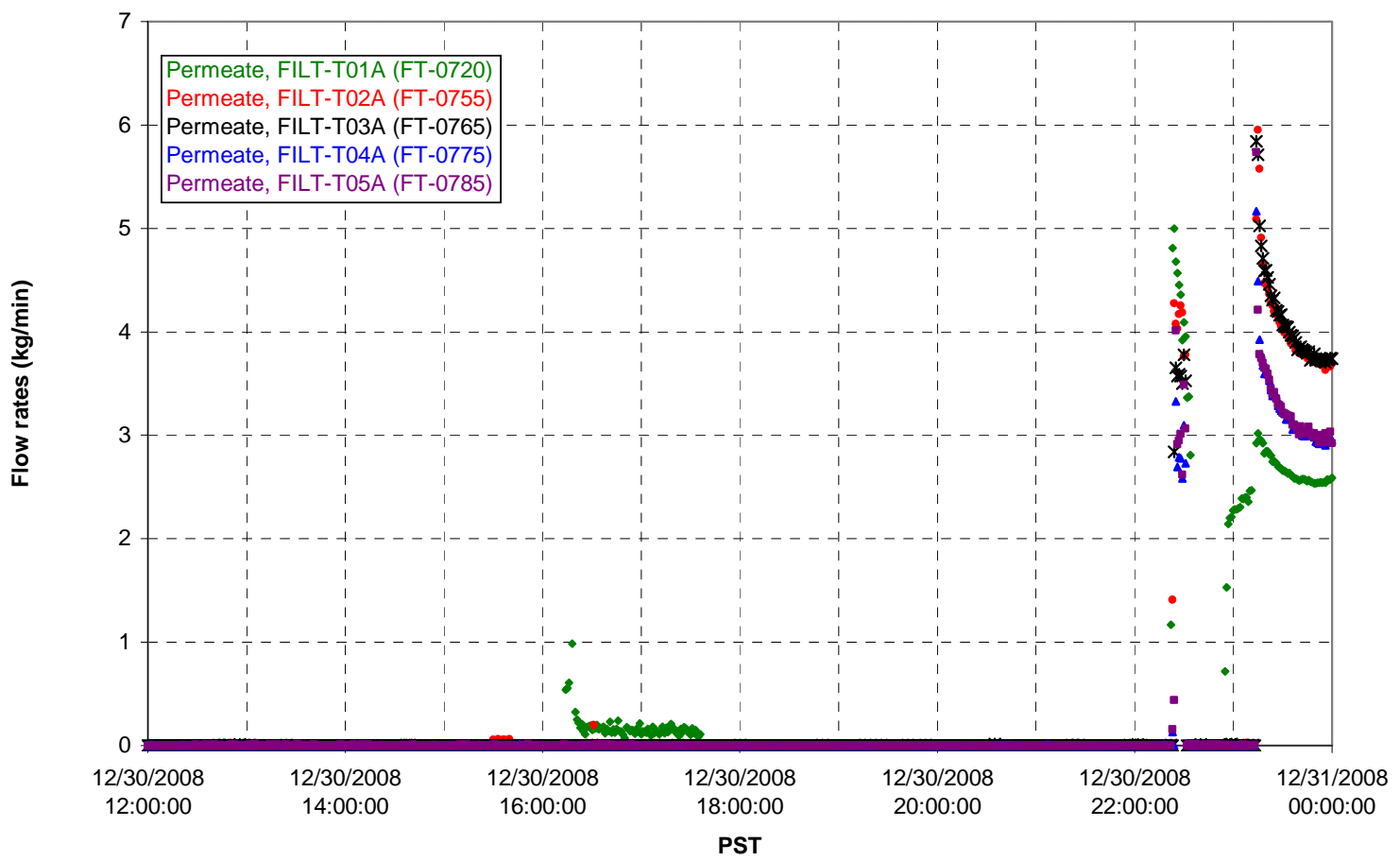


T02A Inner Temperature Tree

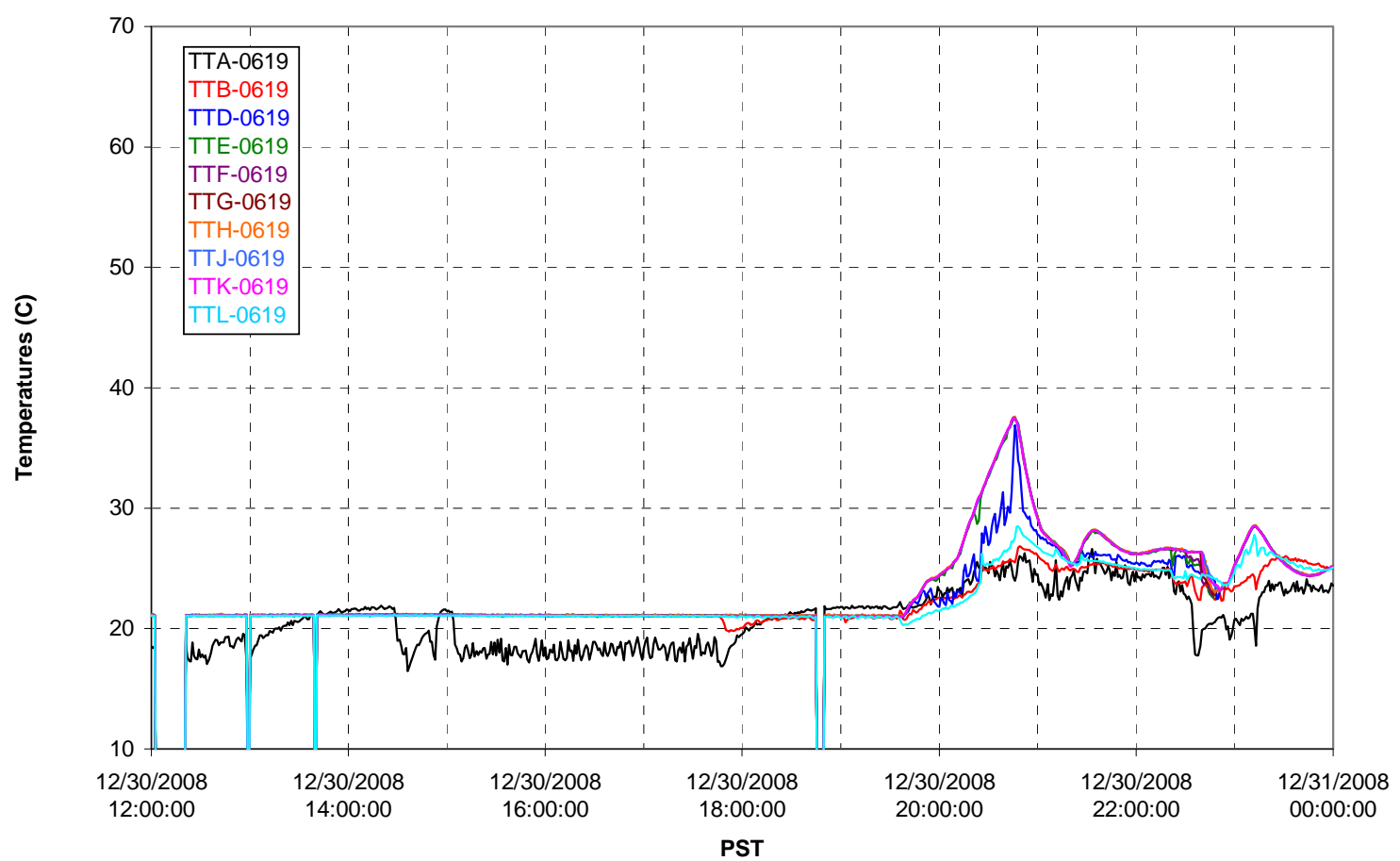

T02A Outer Temperature Tree

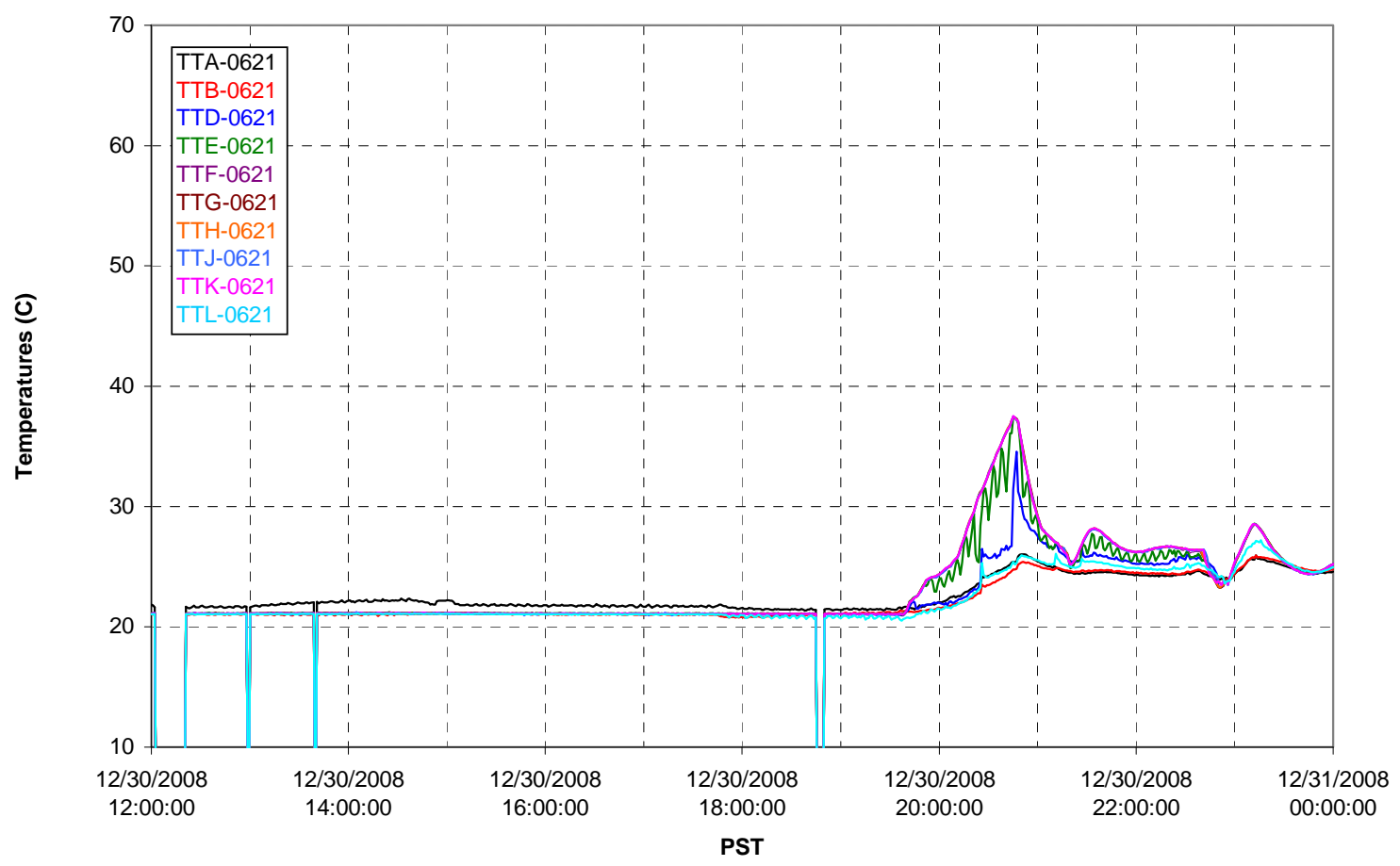


T02A temperatures

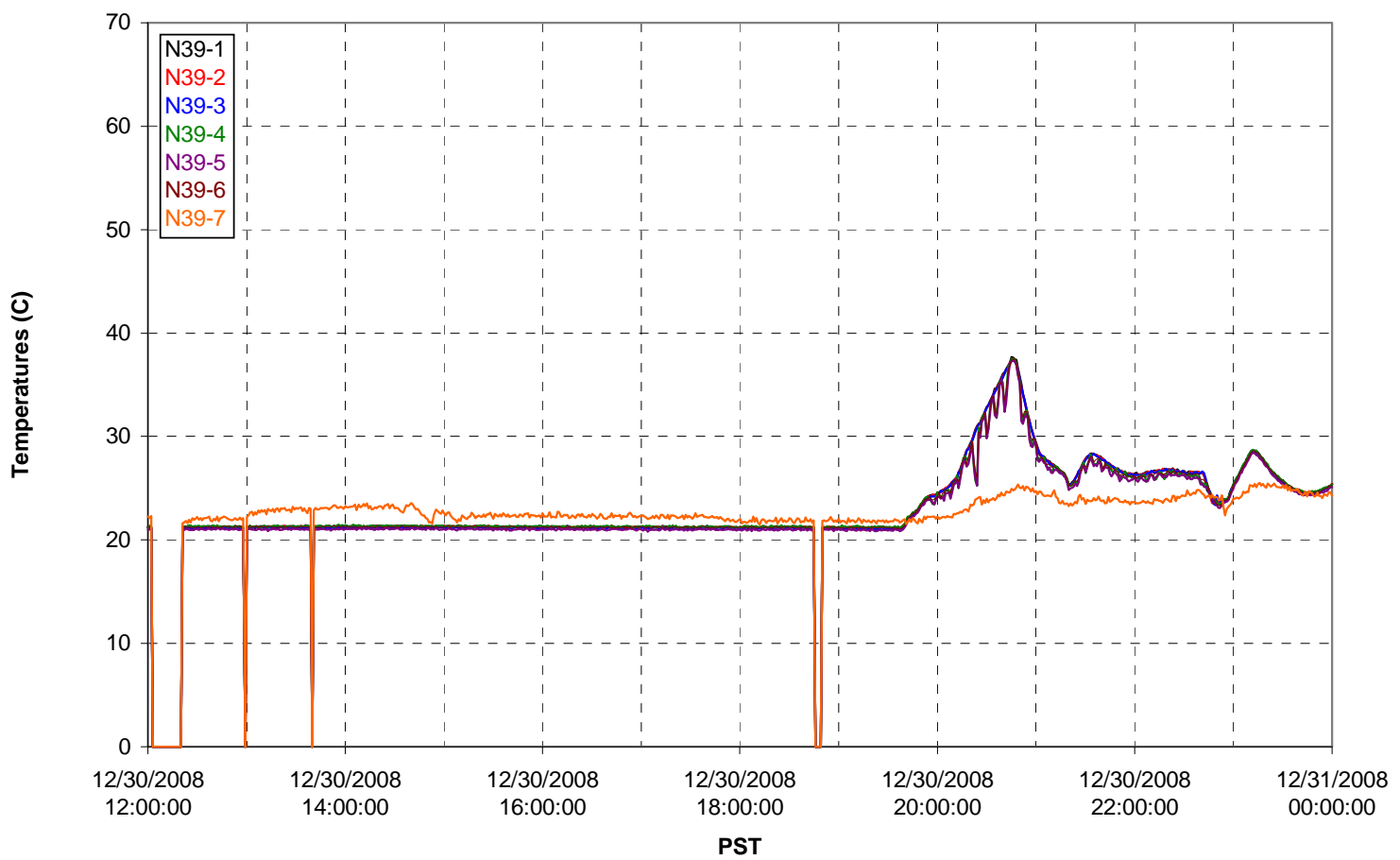

T02A temperatures

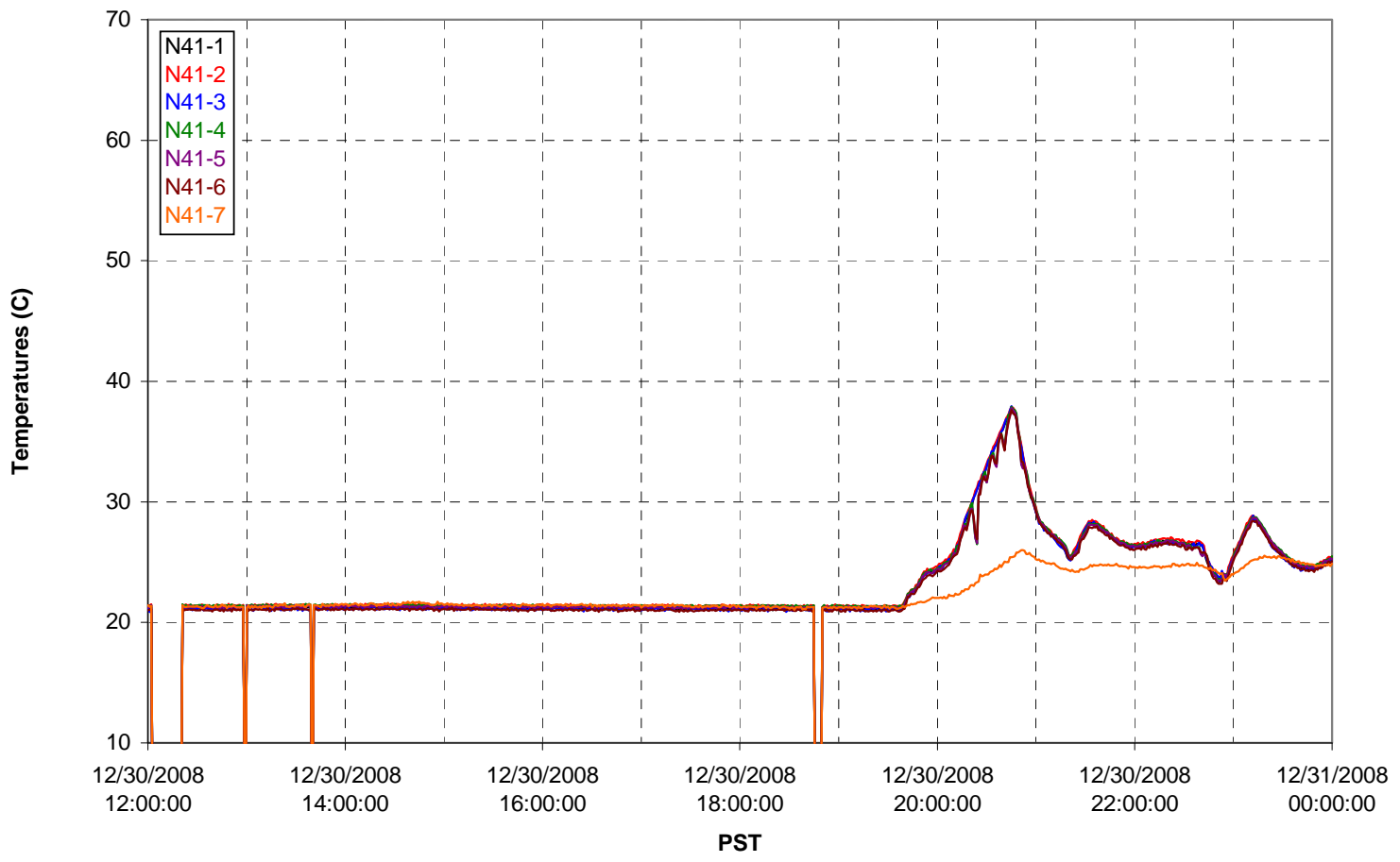


T02A temperatures

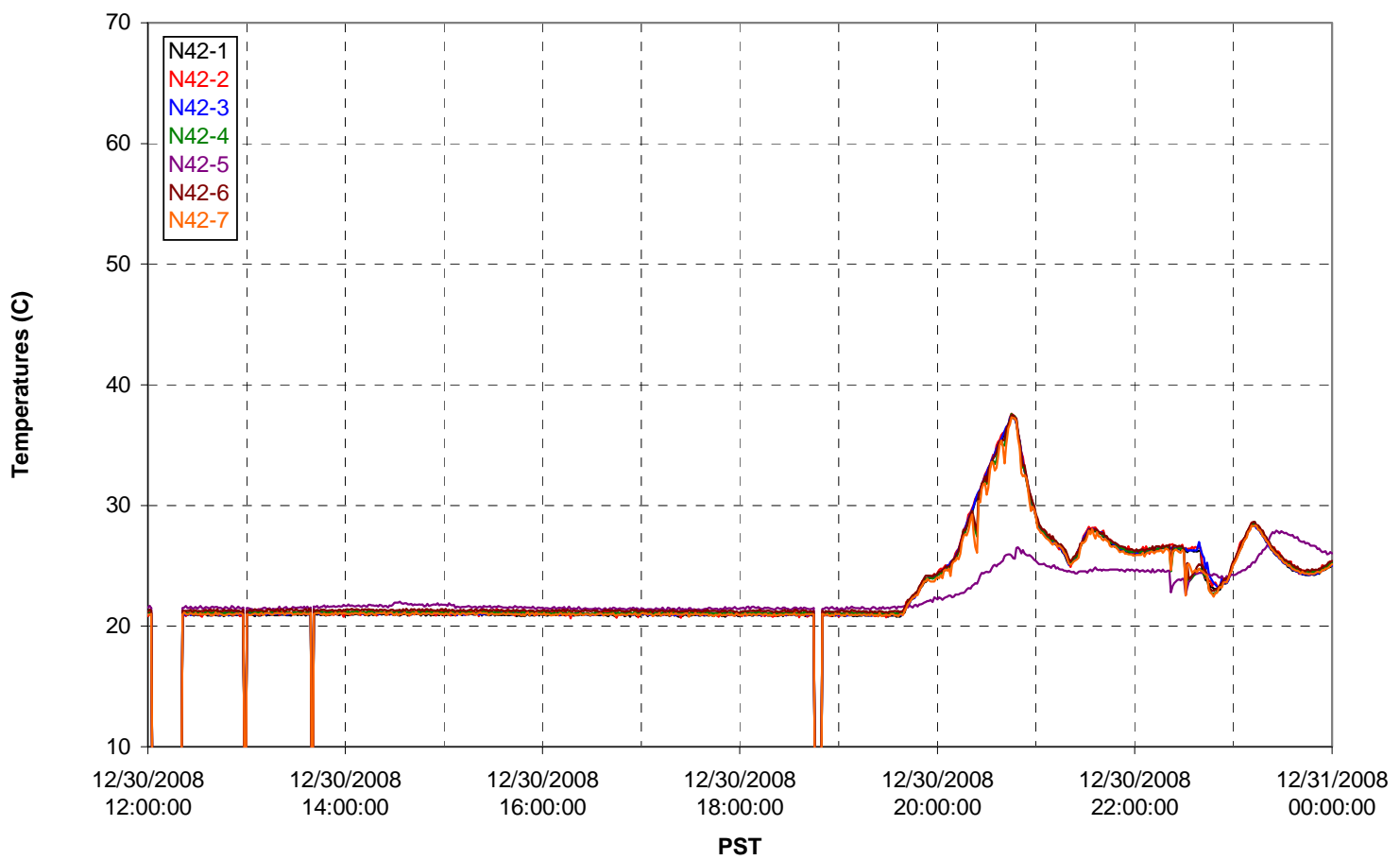

T02A temperatures

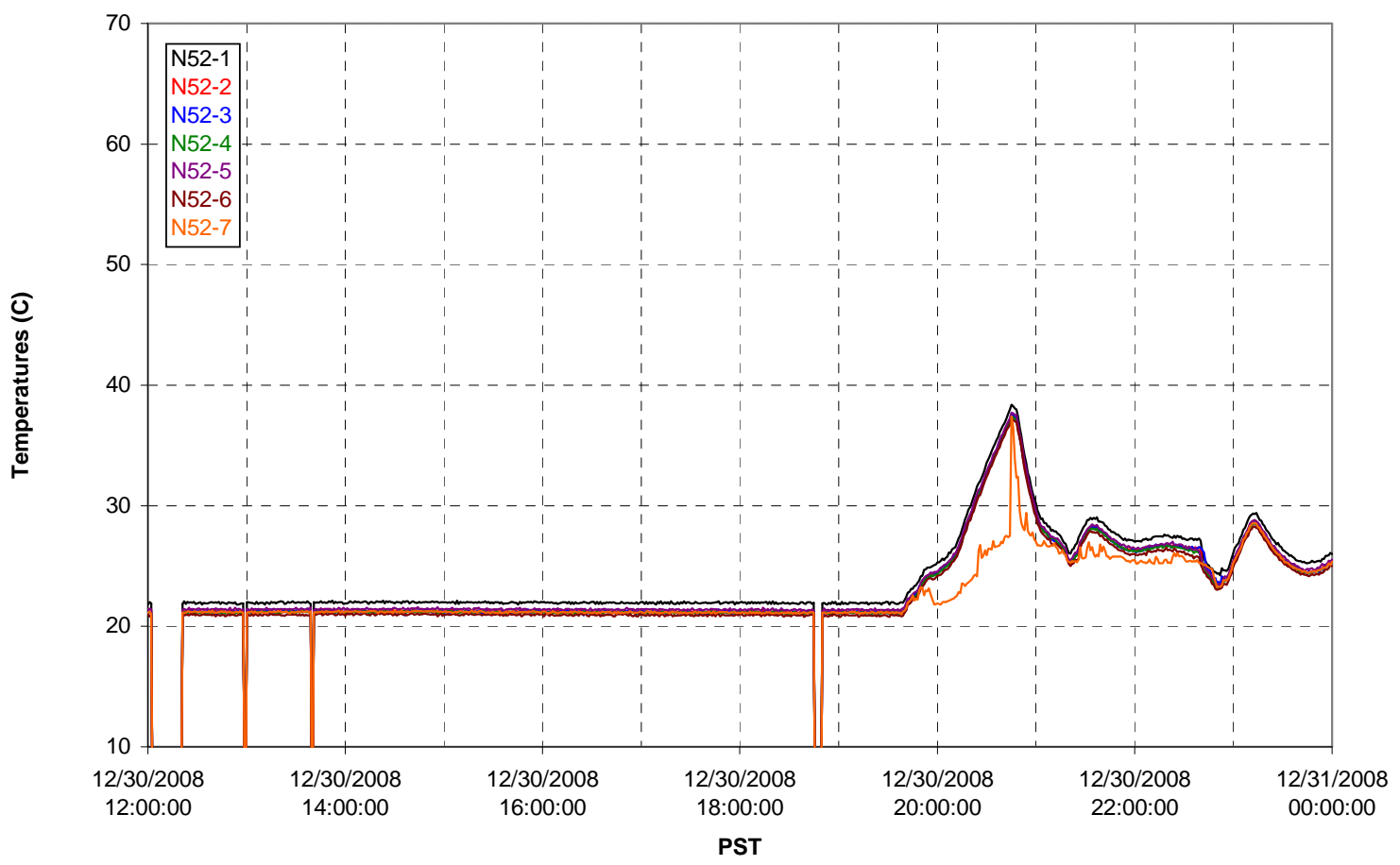


T02A Heating and Cooling

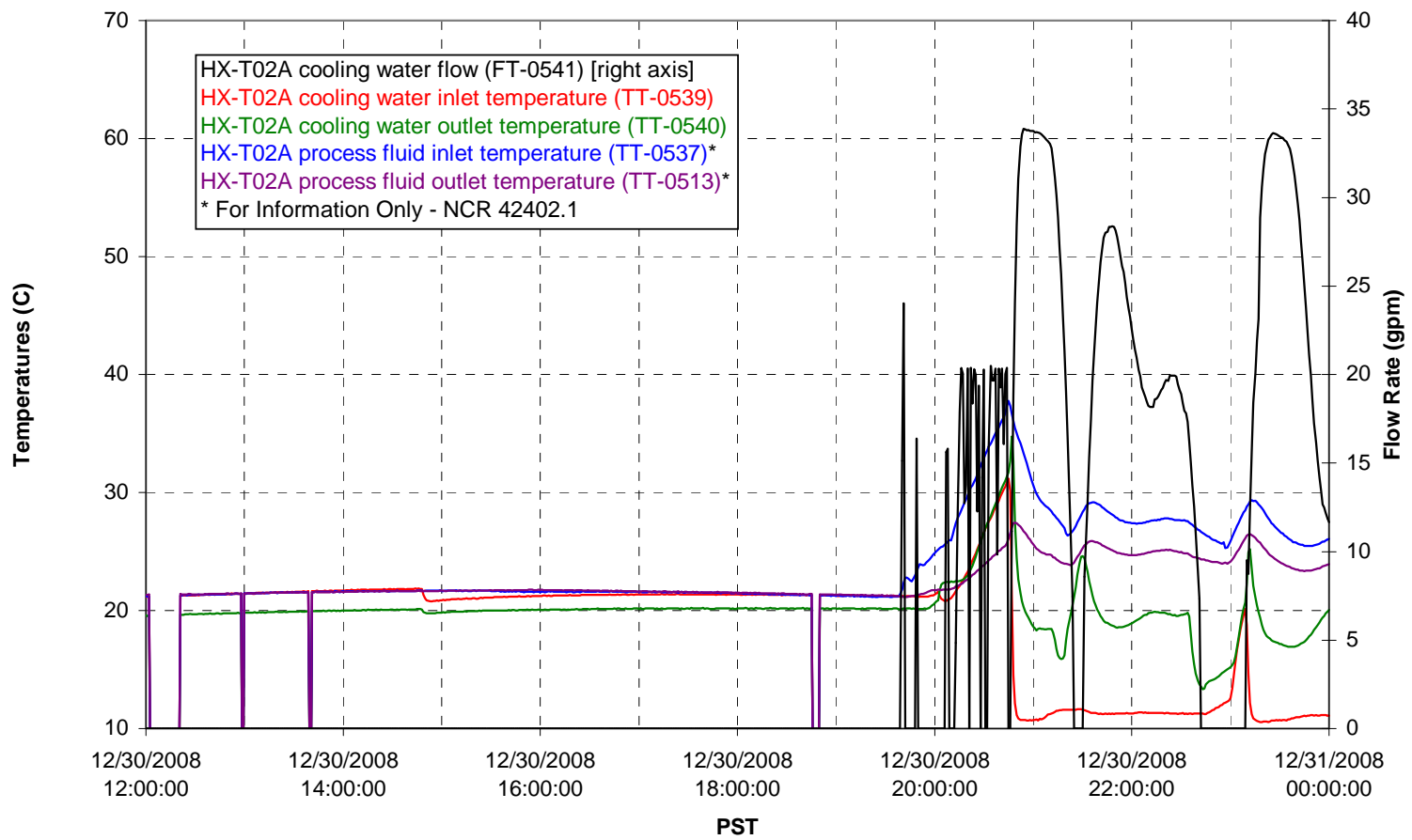

Pump Operation

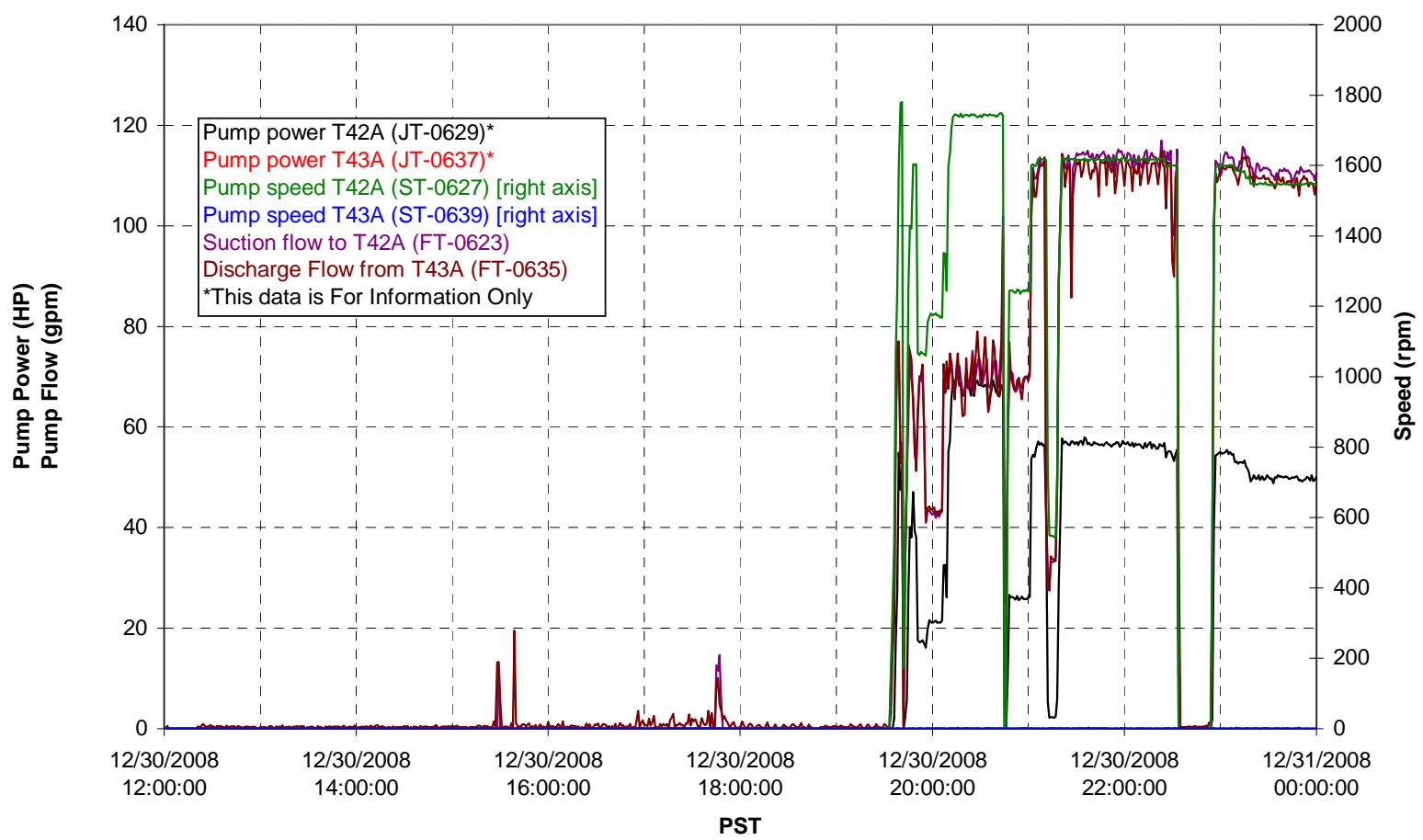


Pulsepot UFP-PP-T01A

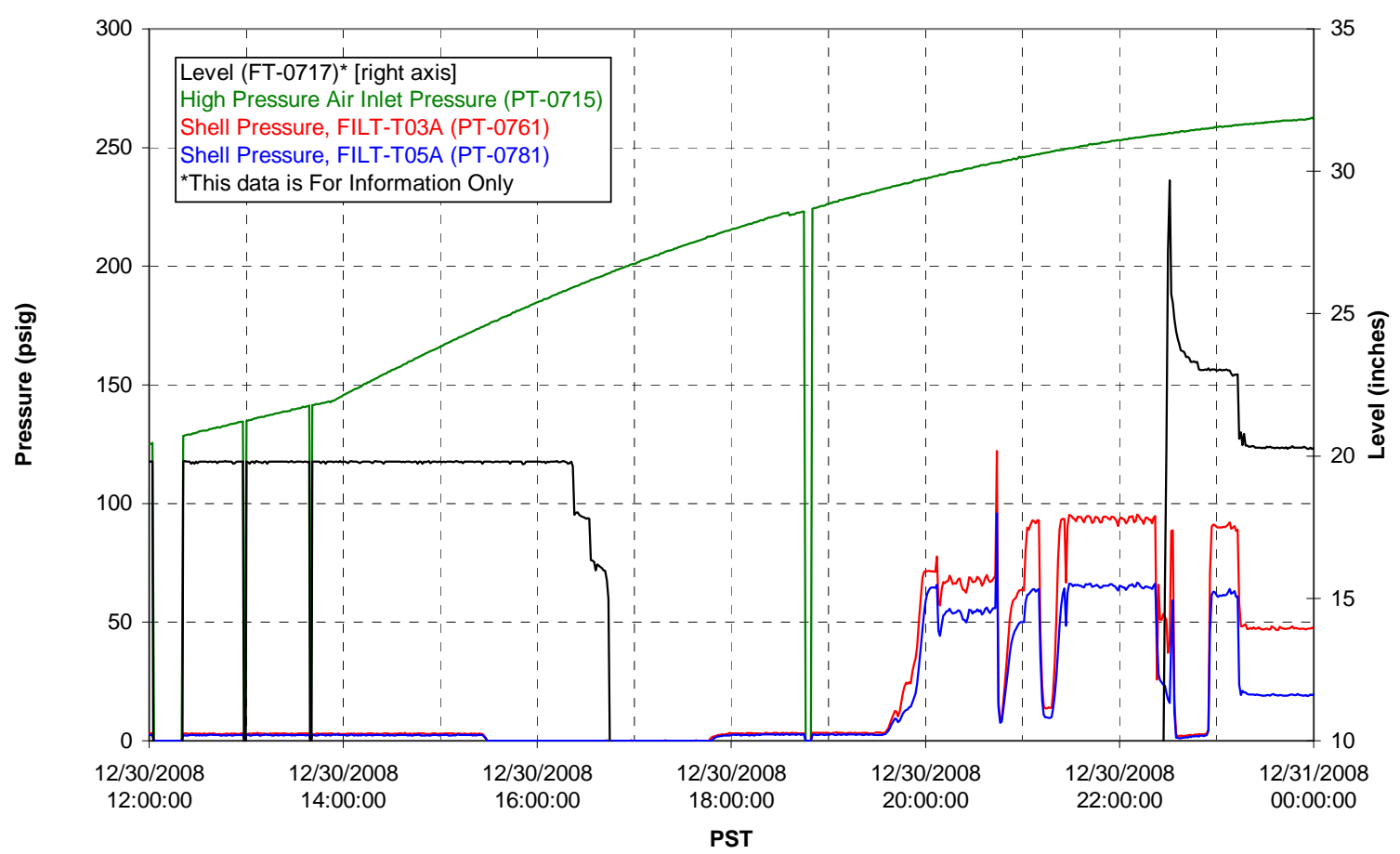

Pulsepot UFP-PP-T02A

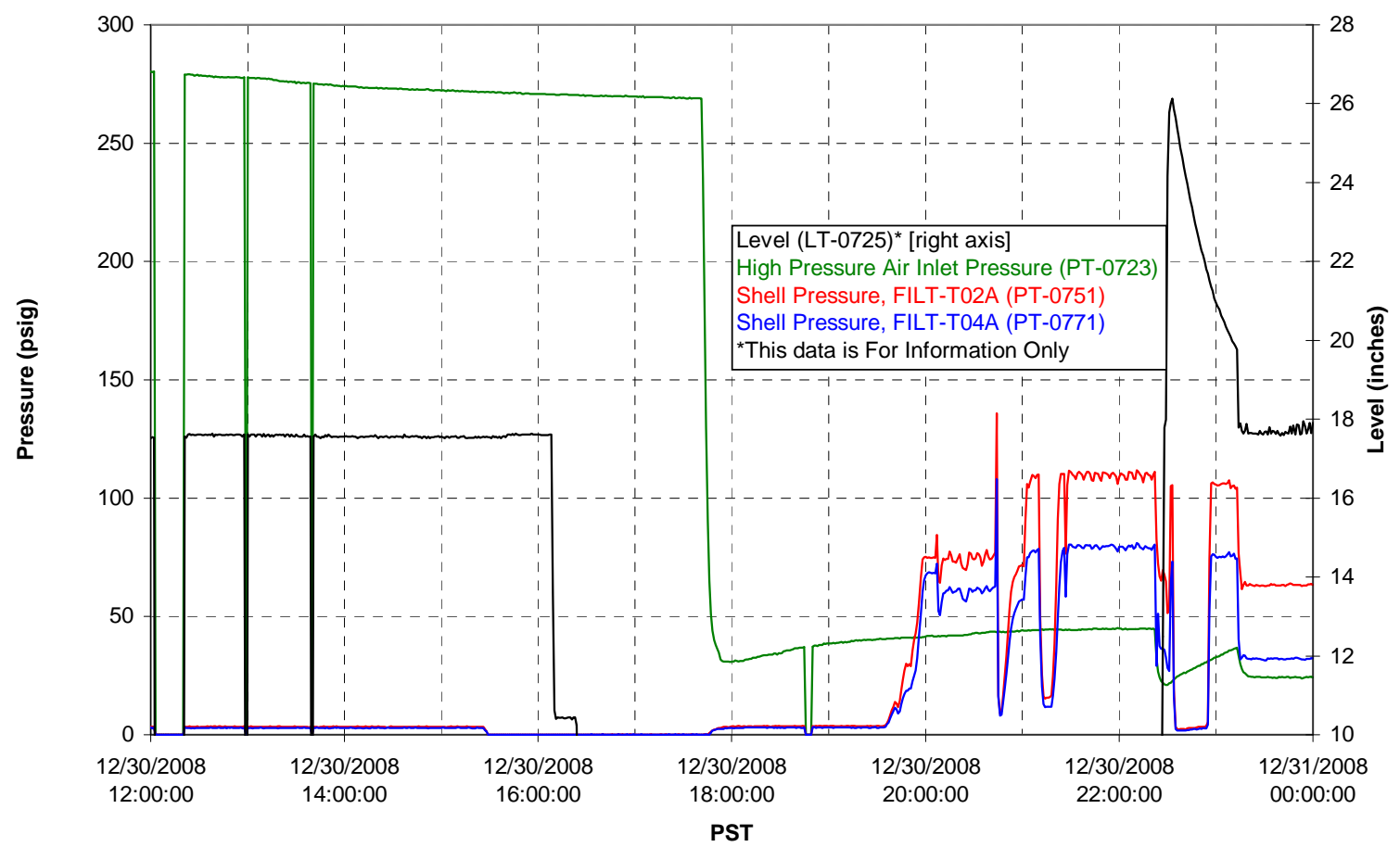


Pulsepot UFP-PP-T03A

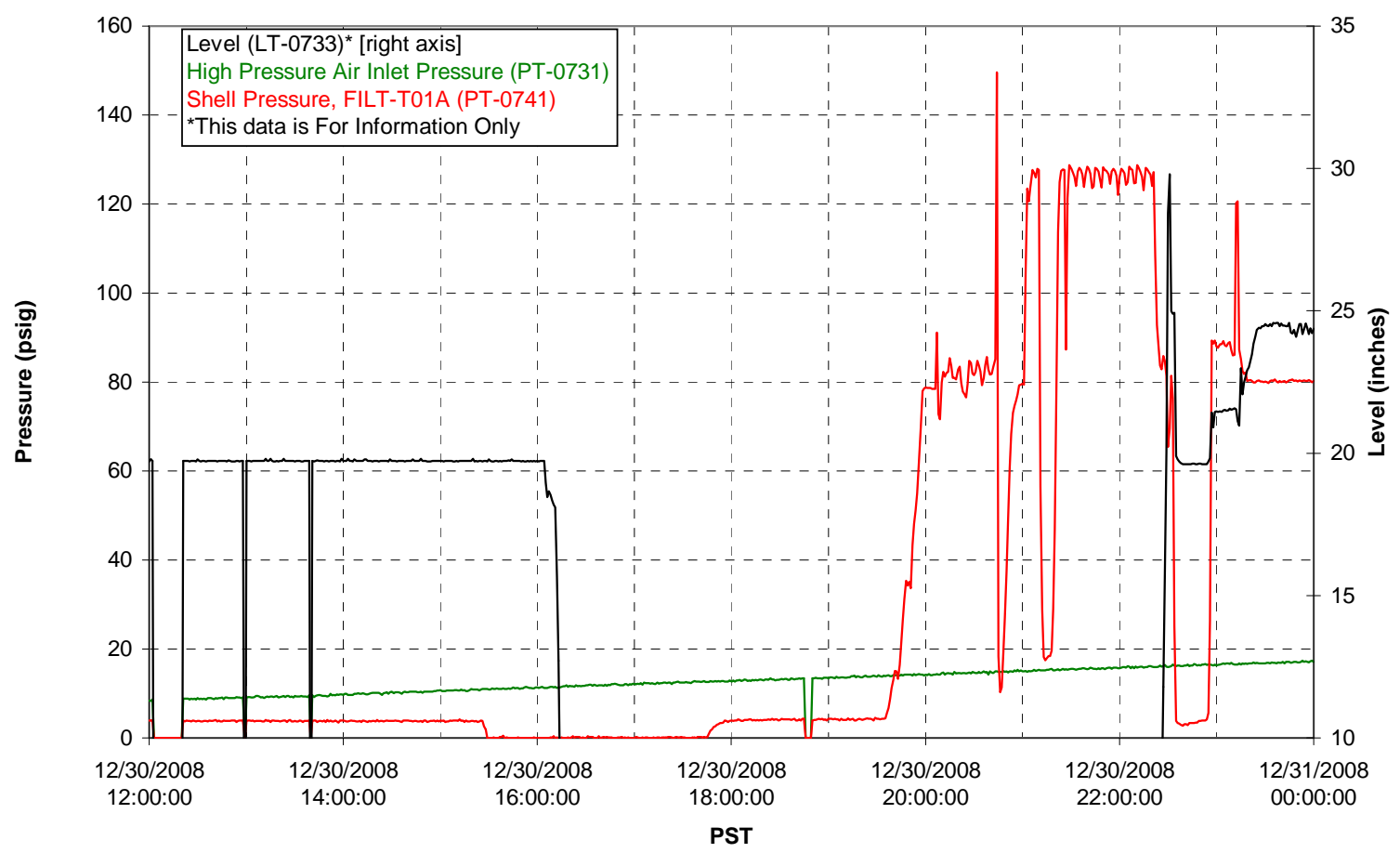

Pulsepot Levels

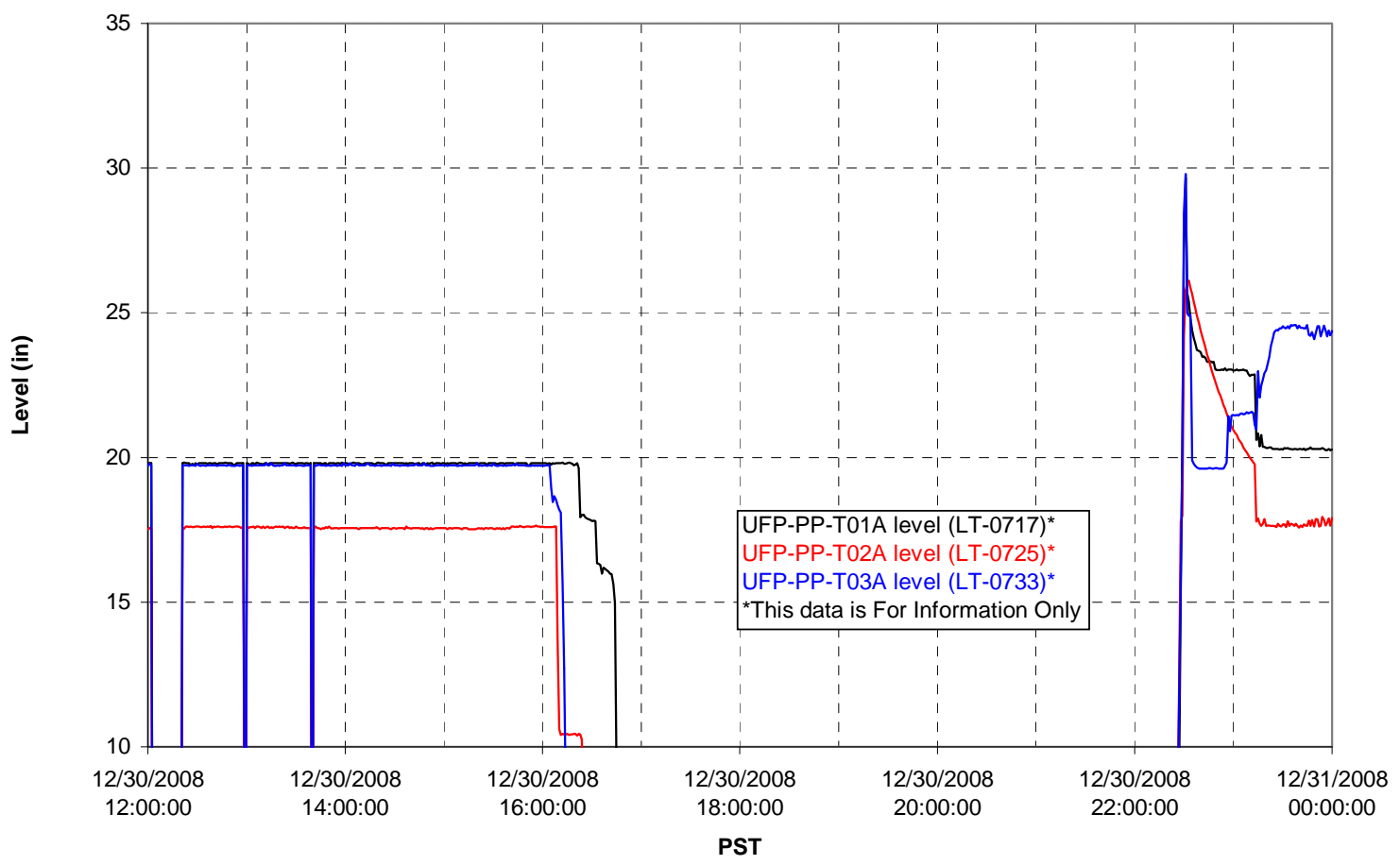


Filter UFP-FILT-T01A

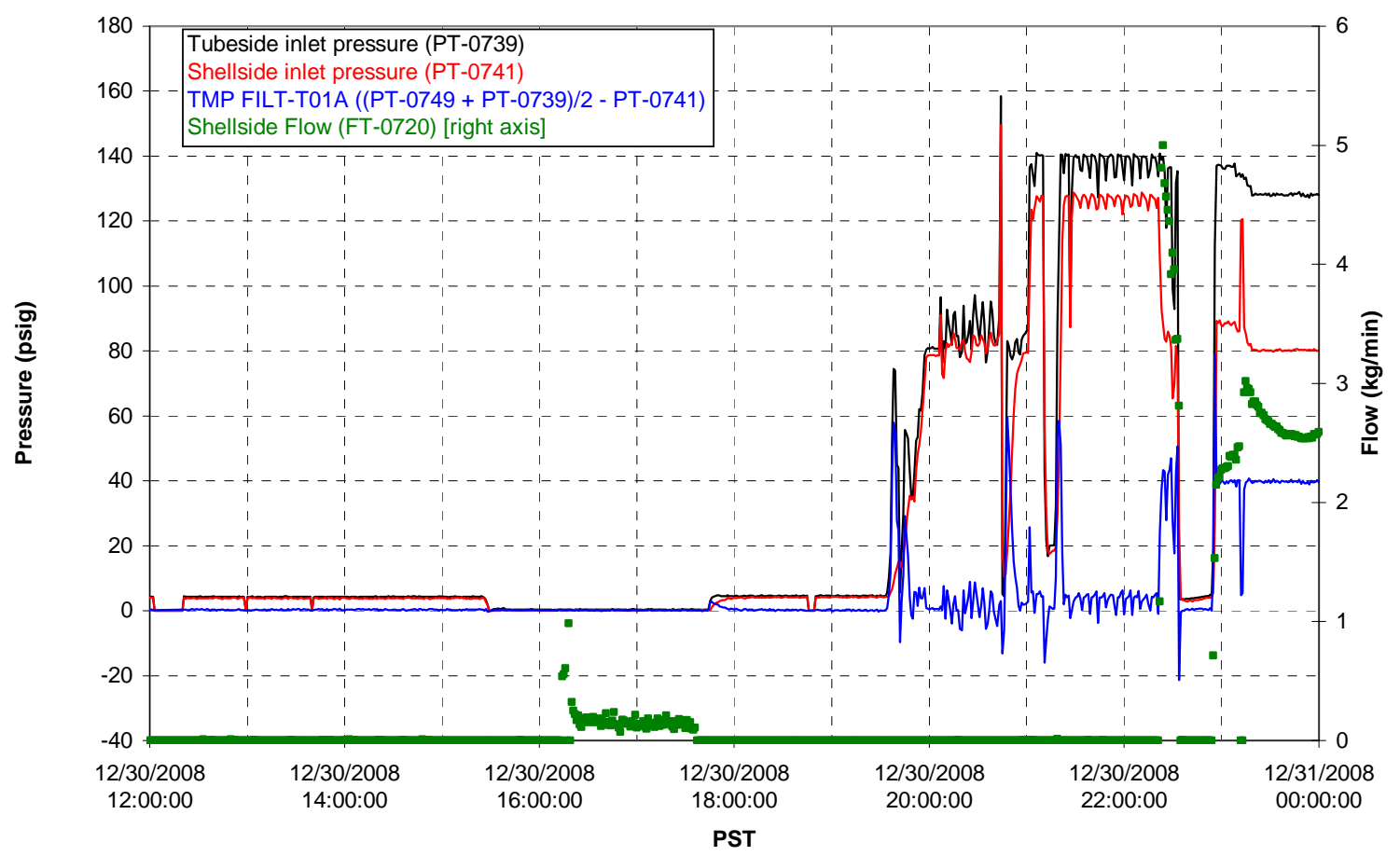

Filter UFP-FILT-T02A

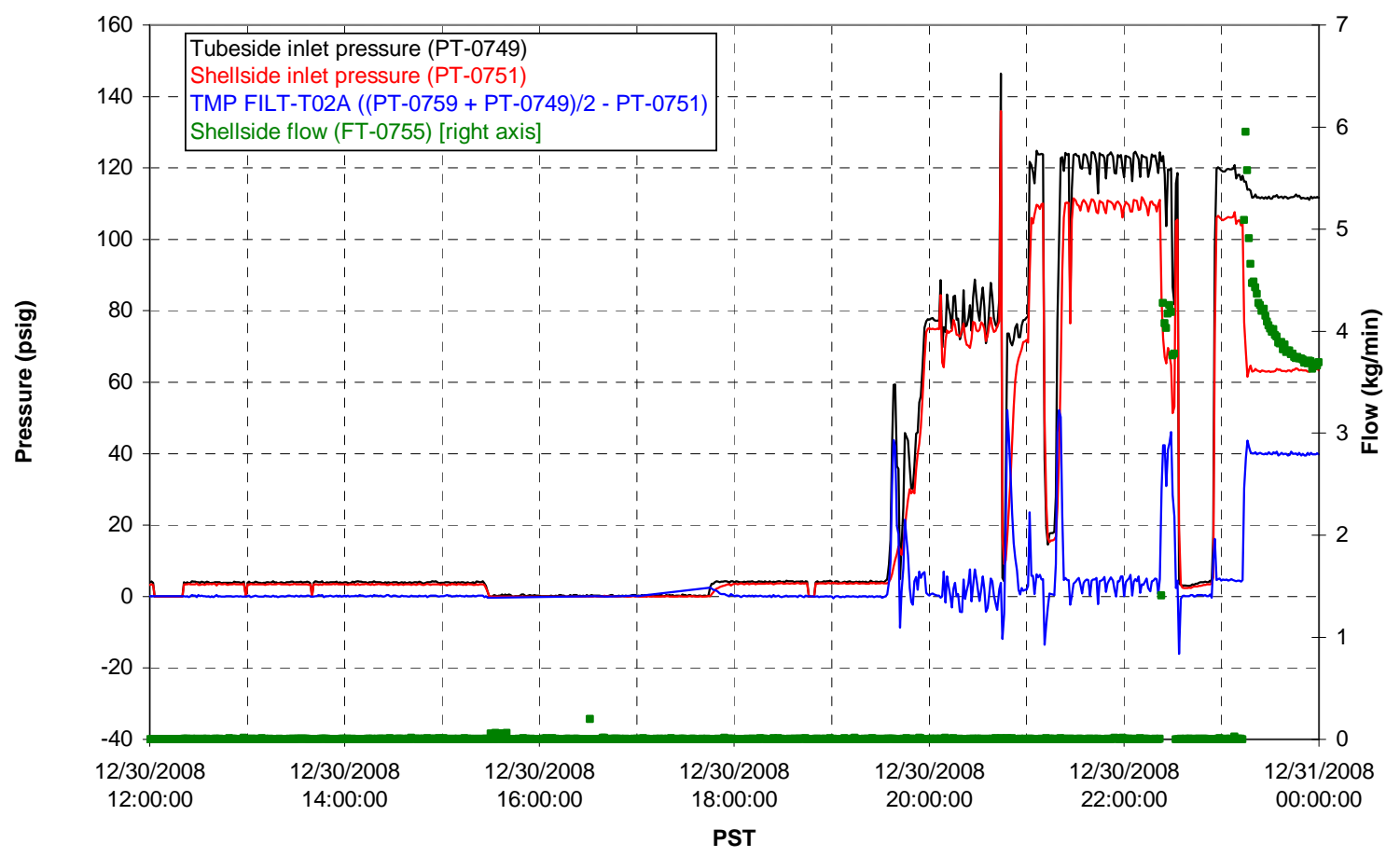


Filter UFP-FILT-T03A

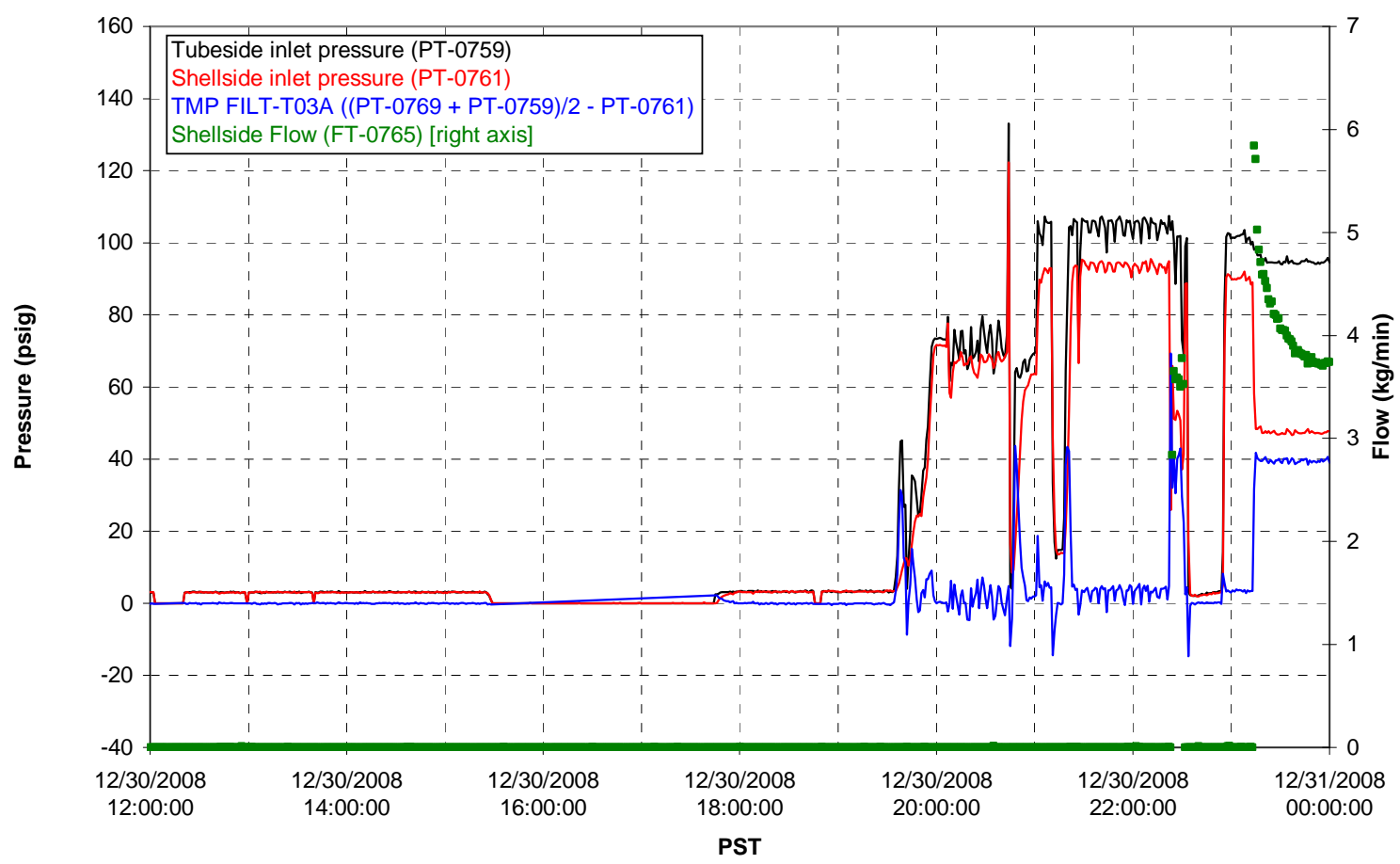

Filter UFP-FILT-T04A

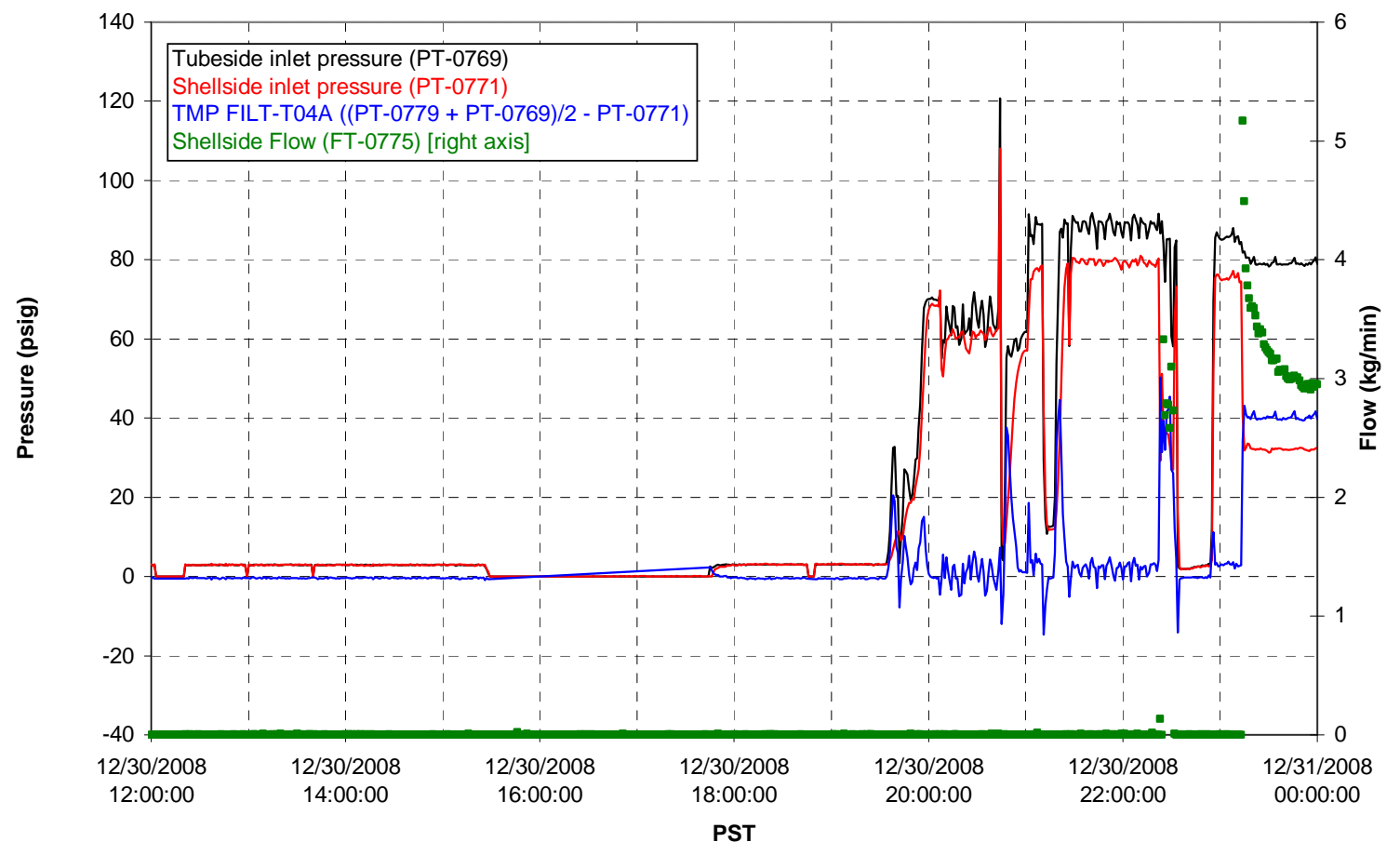


Filter UFP-FILT-T05A

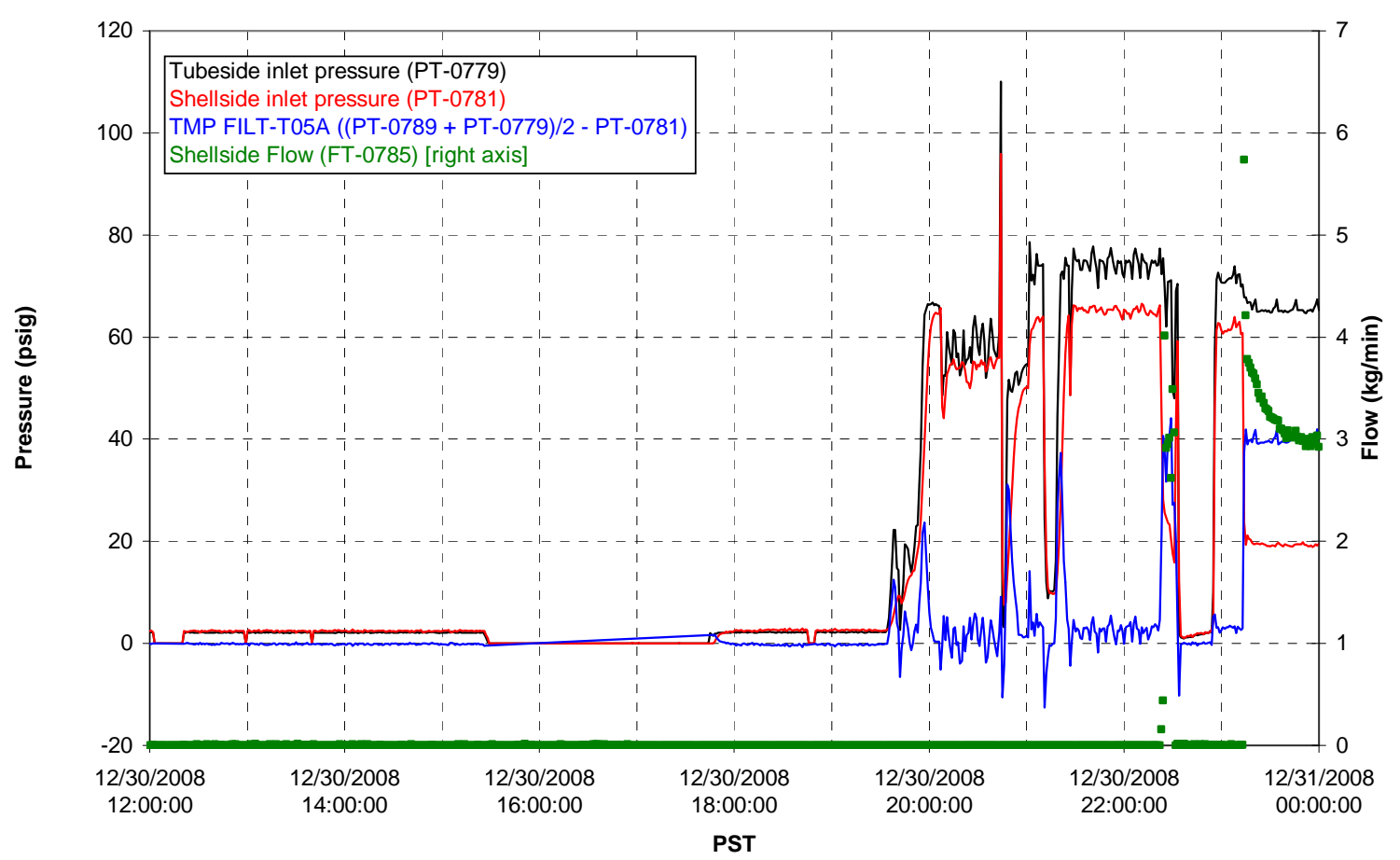

Chemical Flow

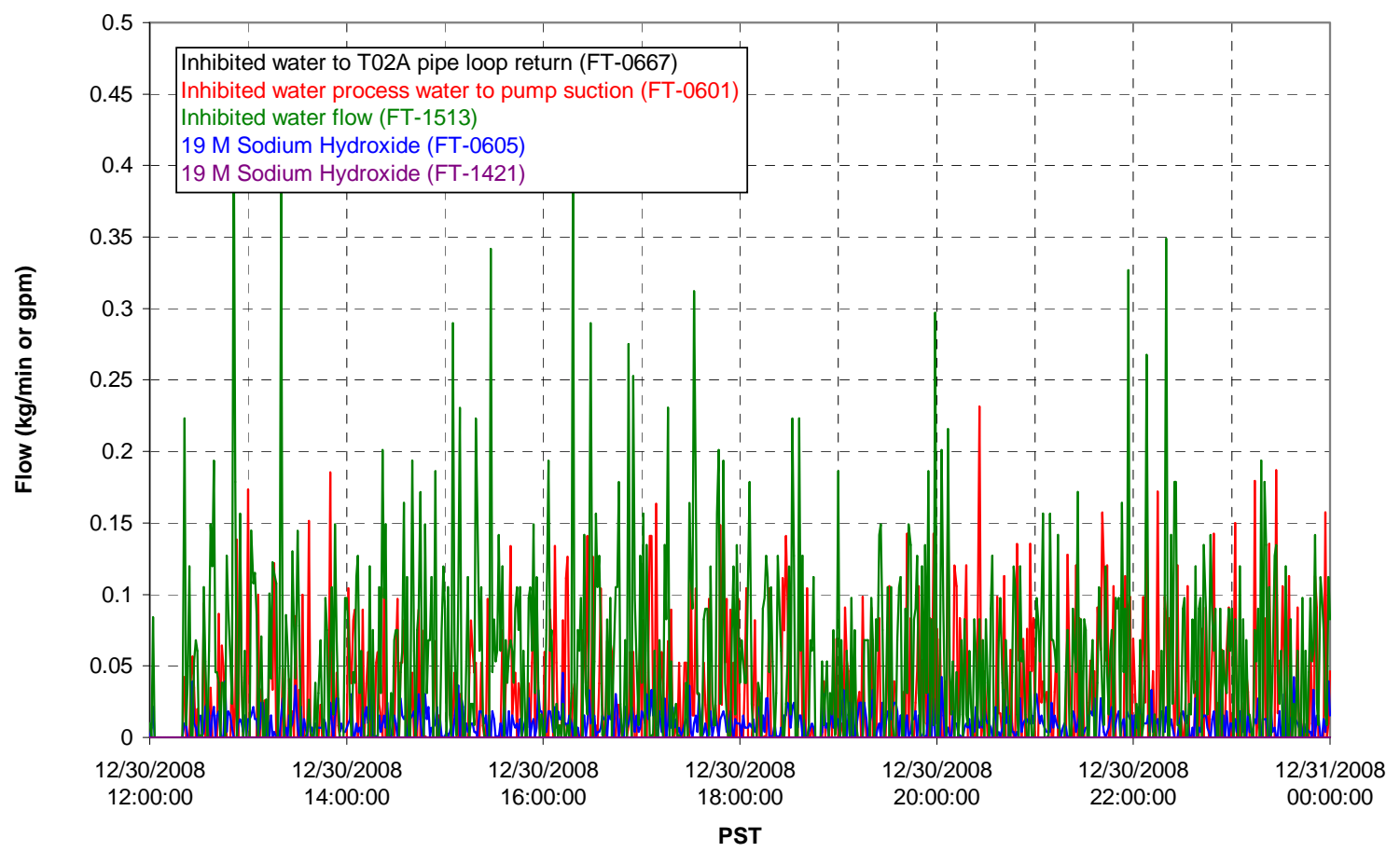




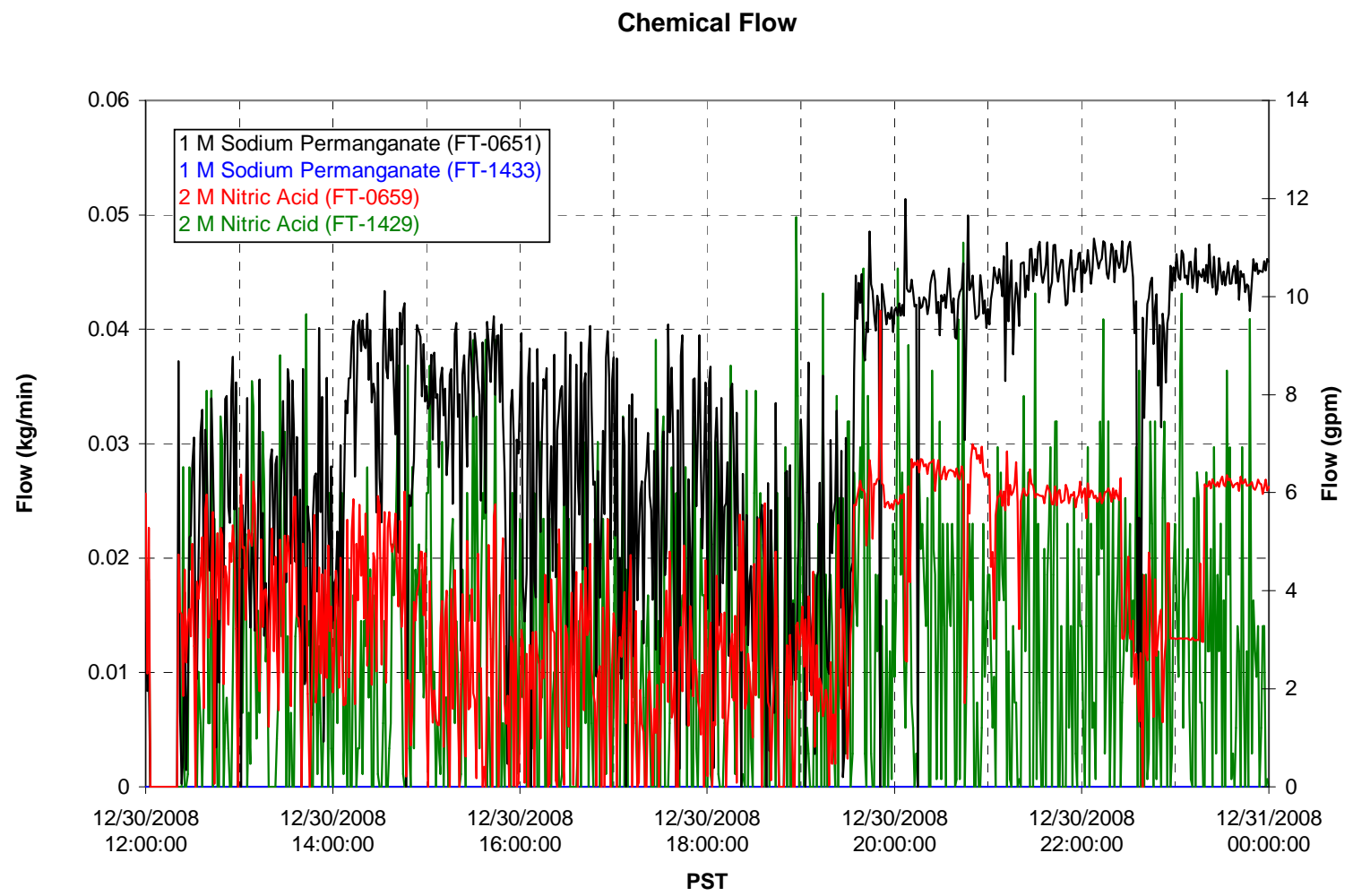

Air Flows

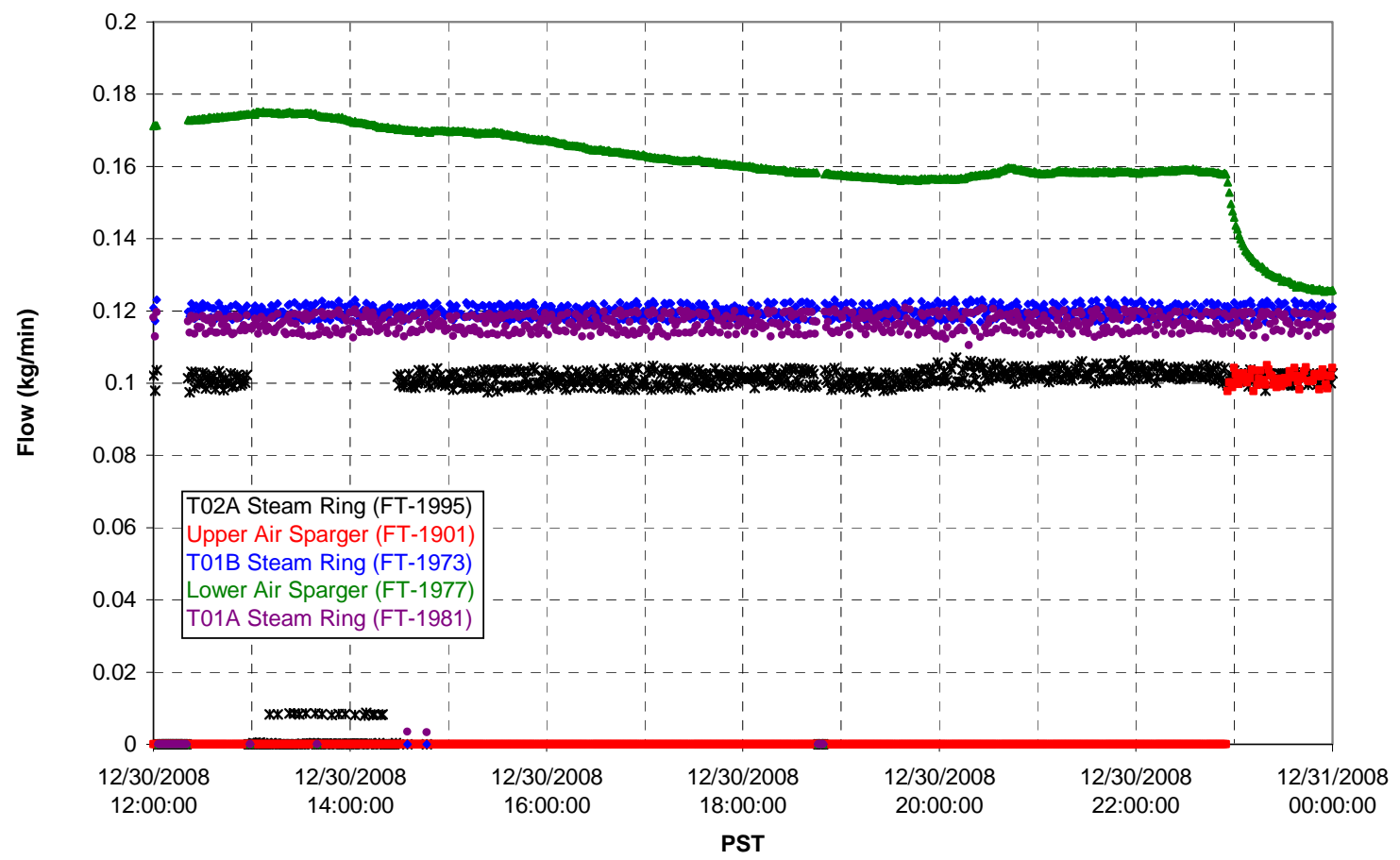


T02A Steam

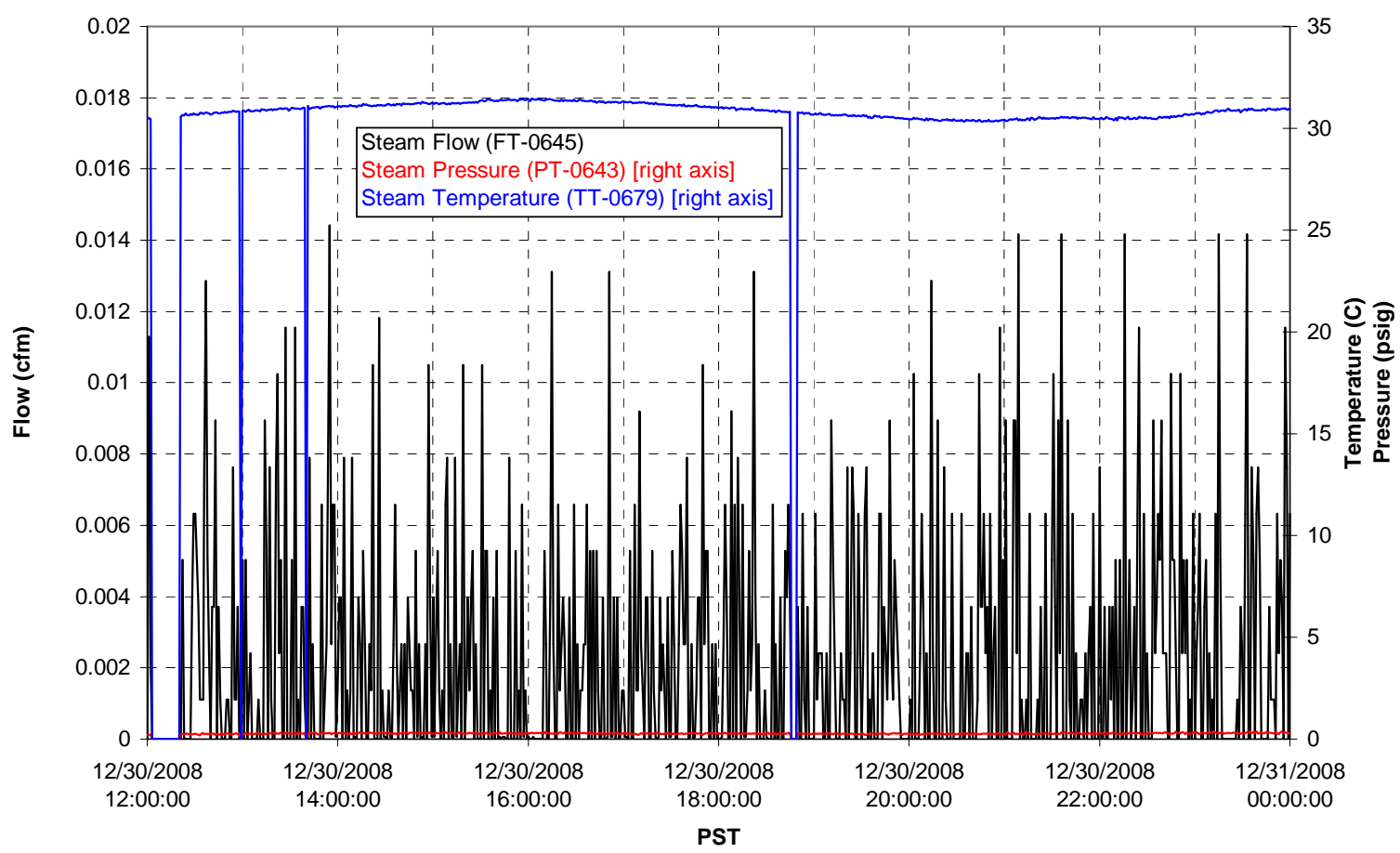

T01A Steam

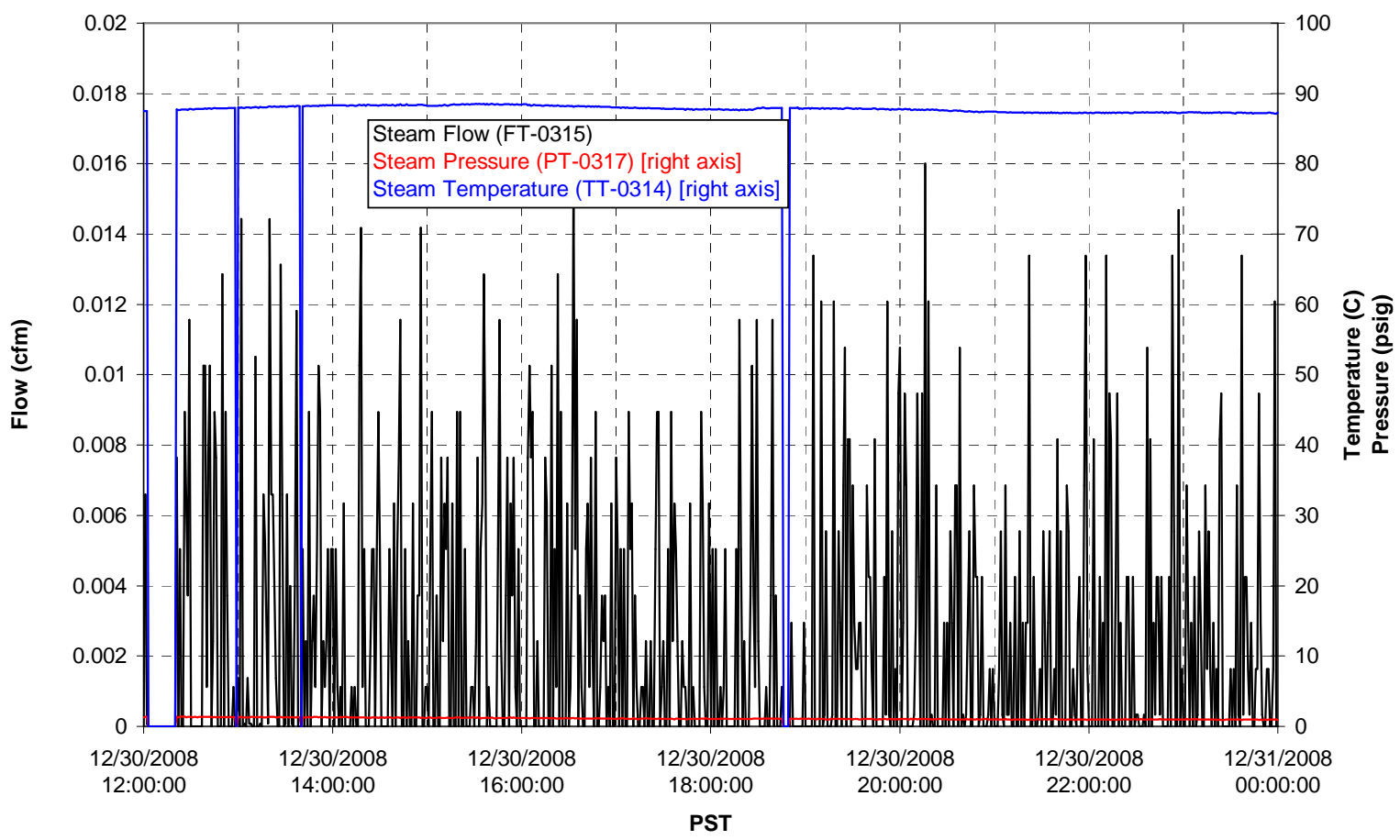


T01B Steam

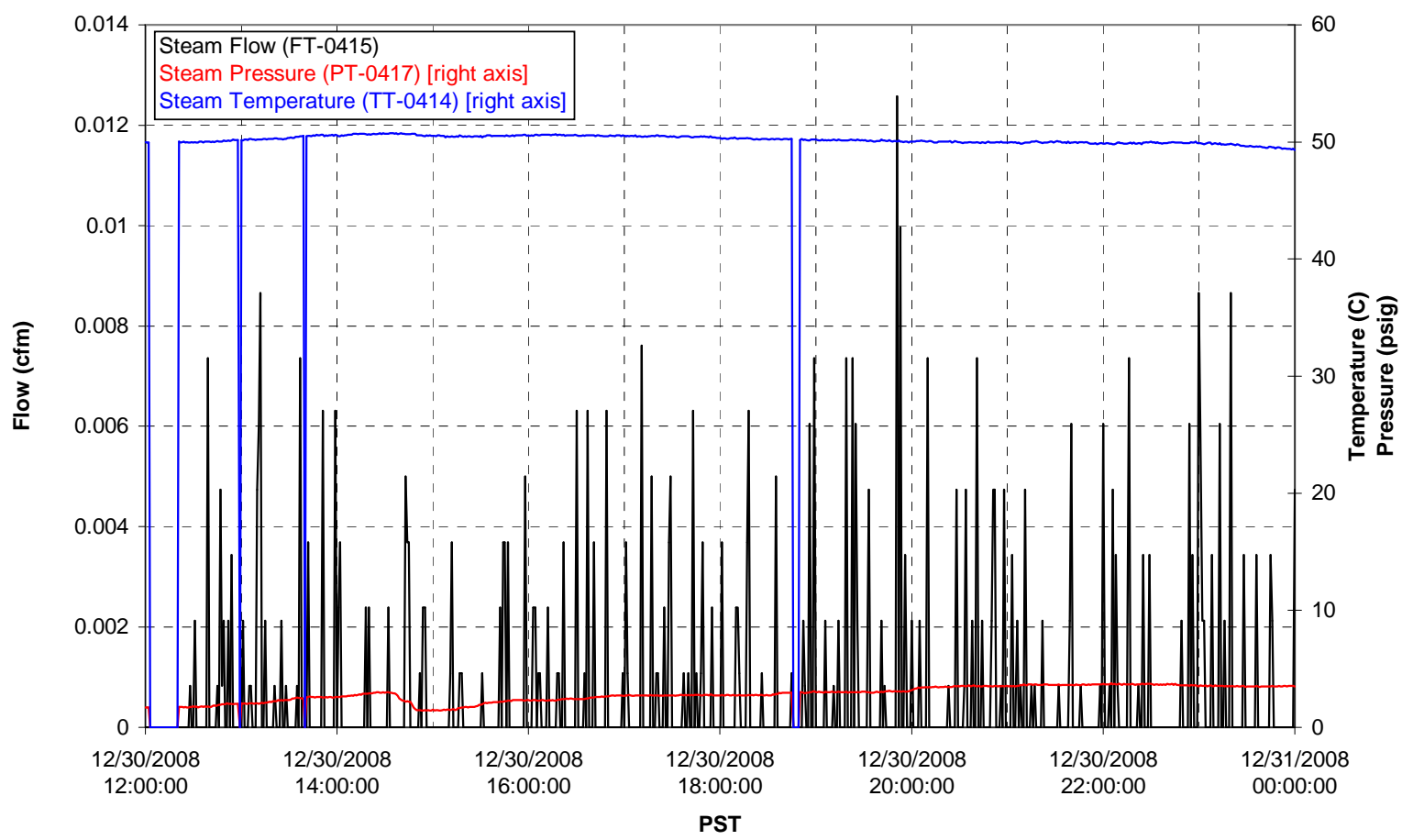


Functional Test Data Plots

12/31/08 00:00 - 12/31/08 12:00 
T01A level

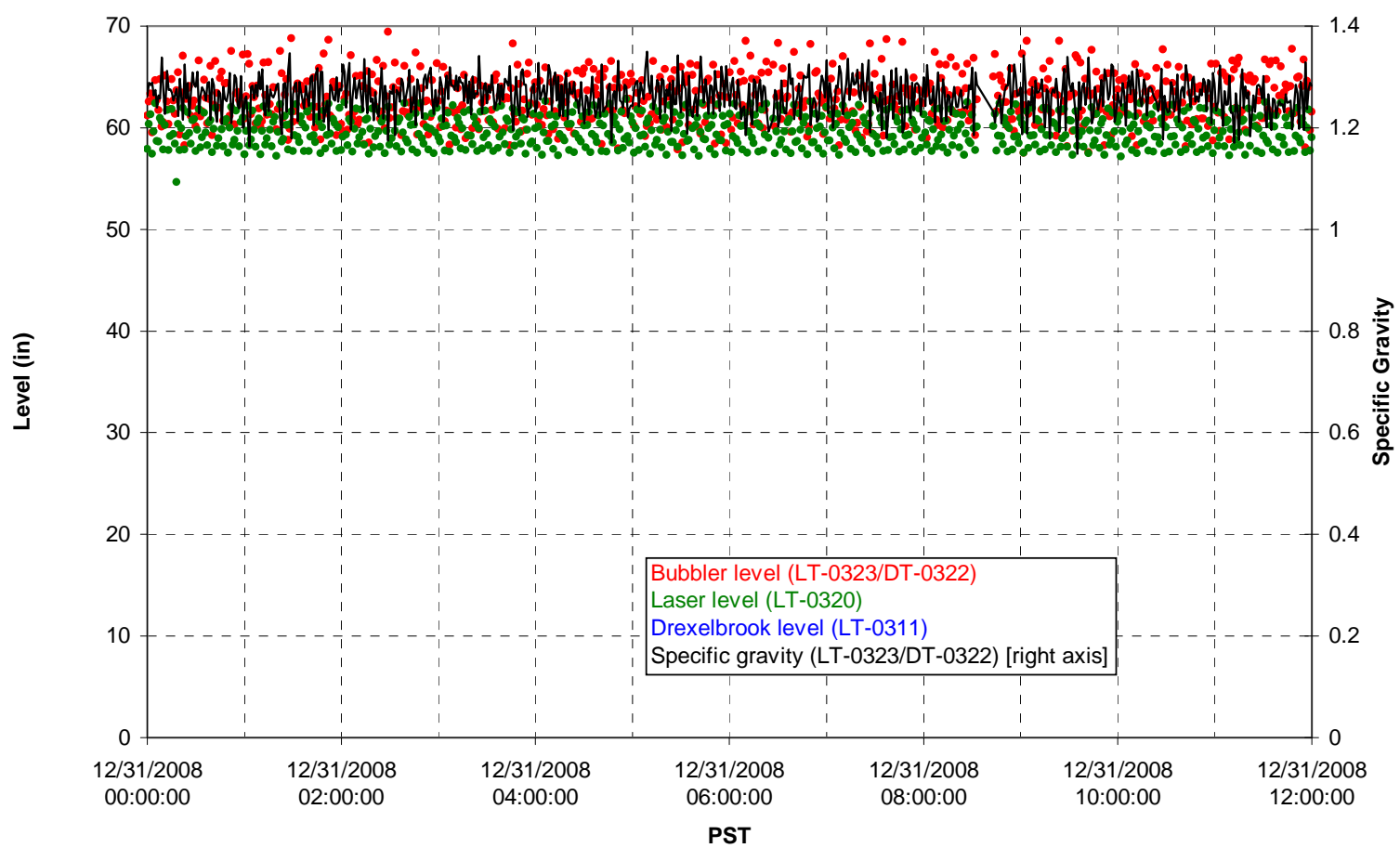

T01A temperatures

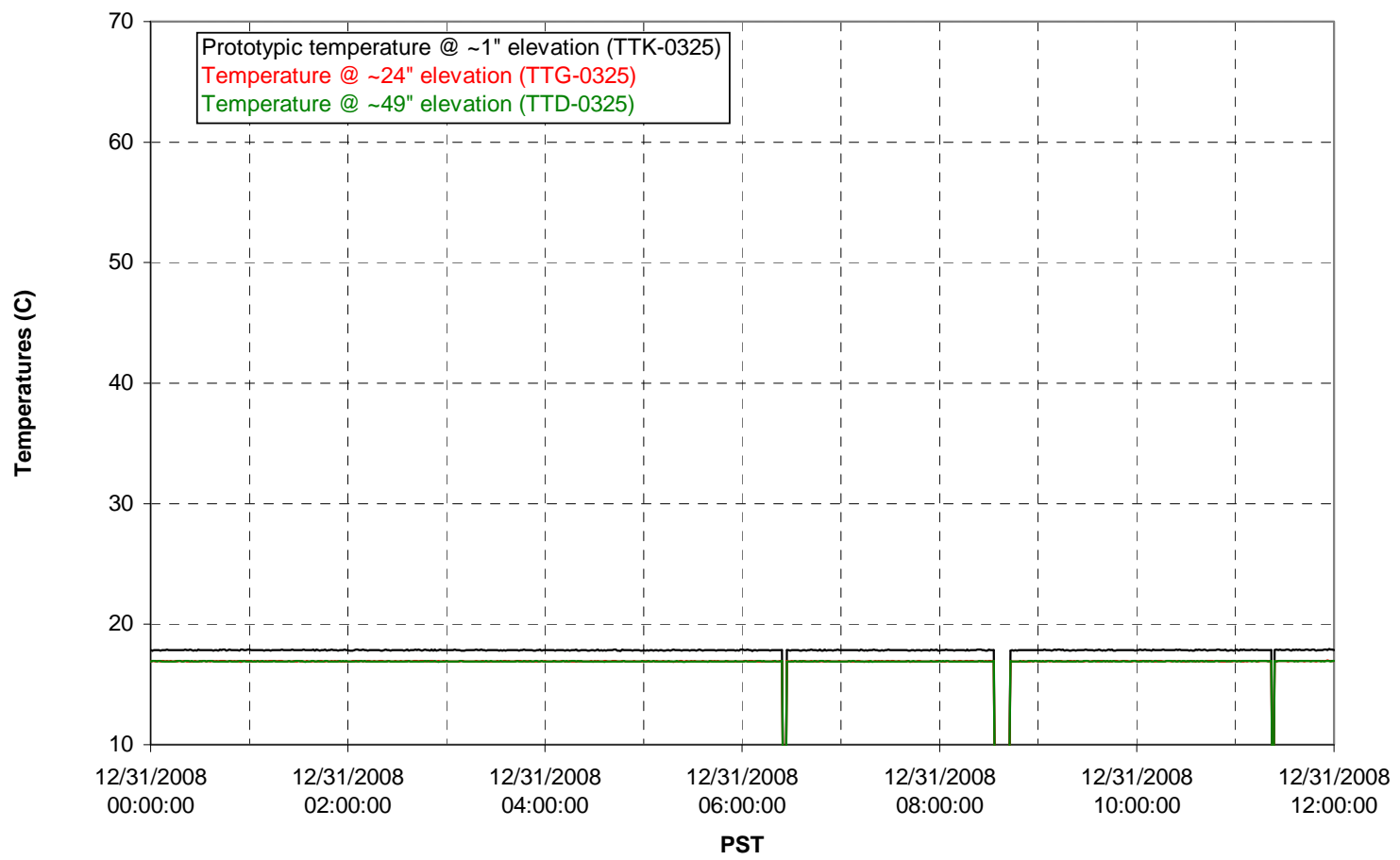


T01B level

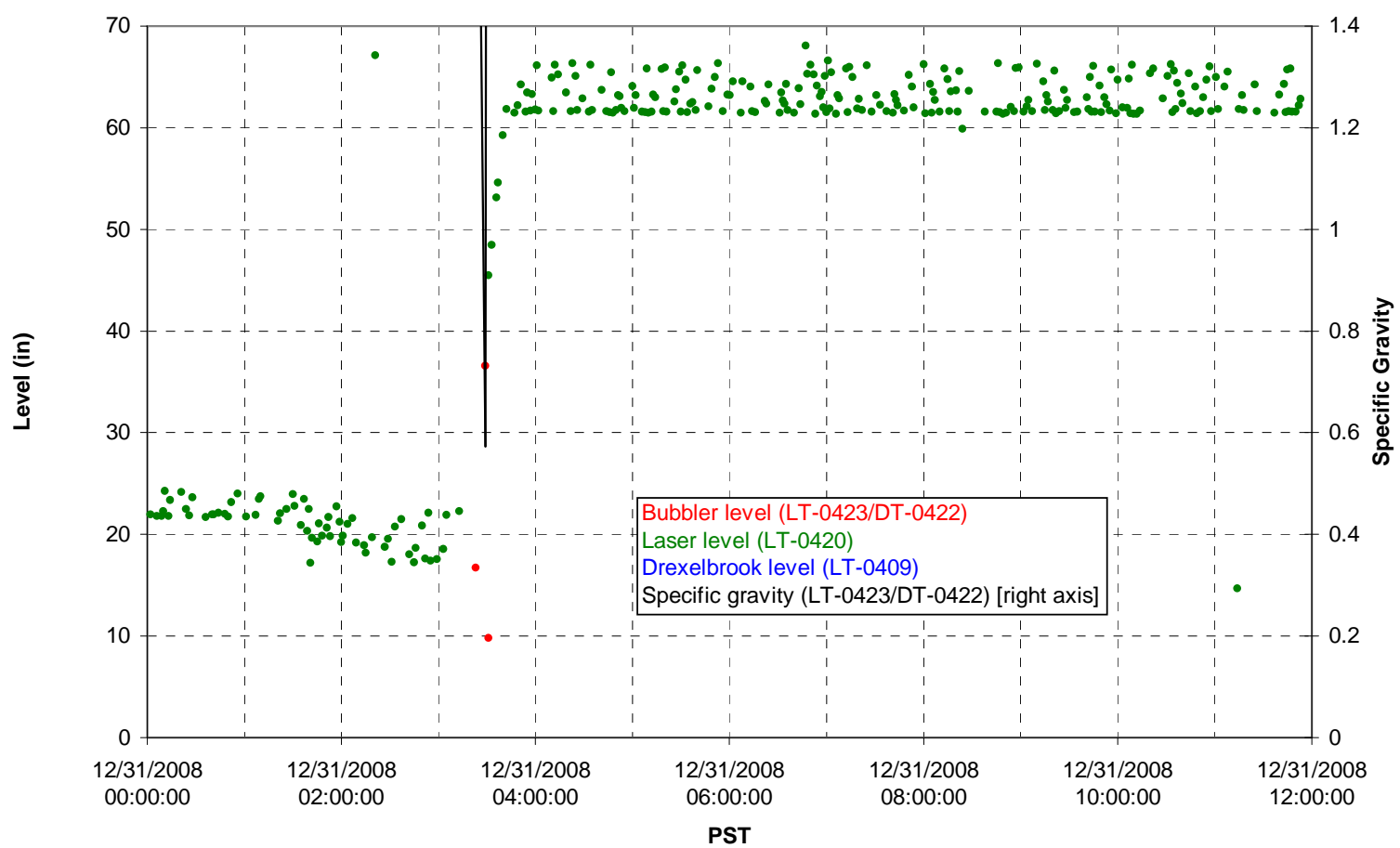

T01B temperatures

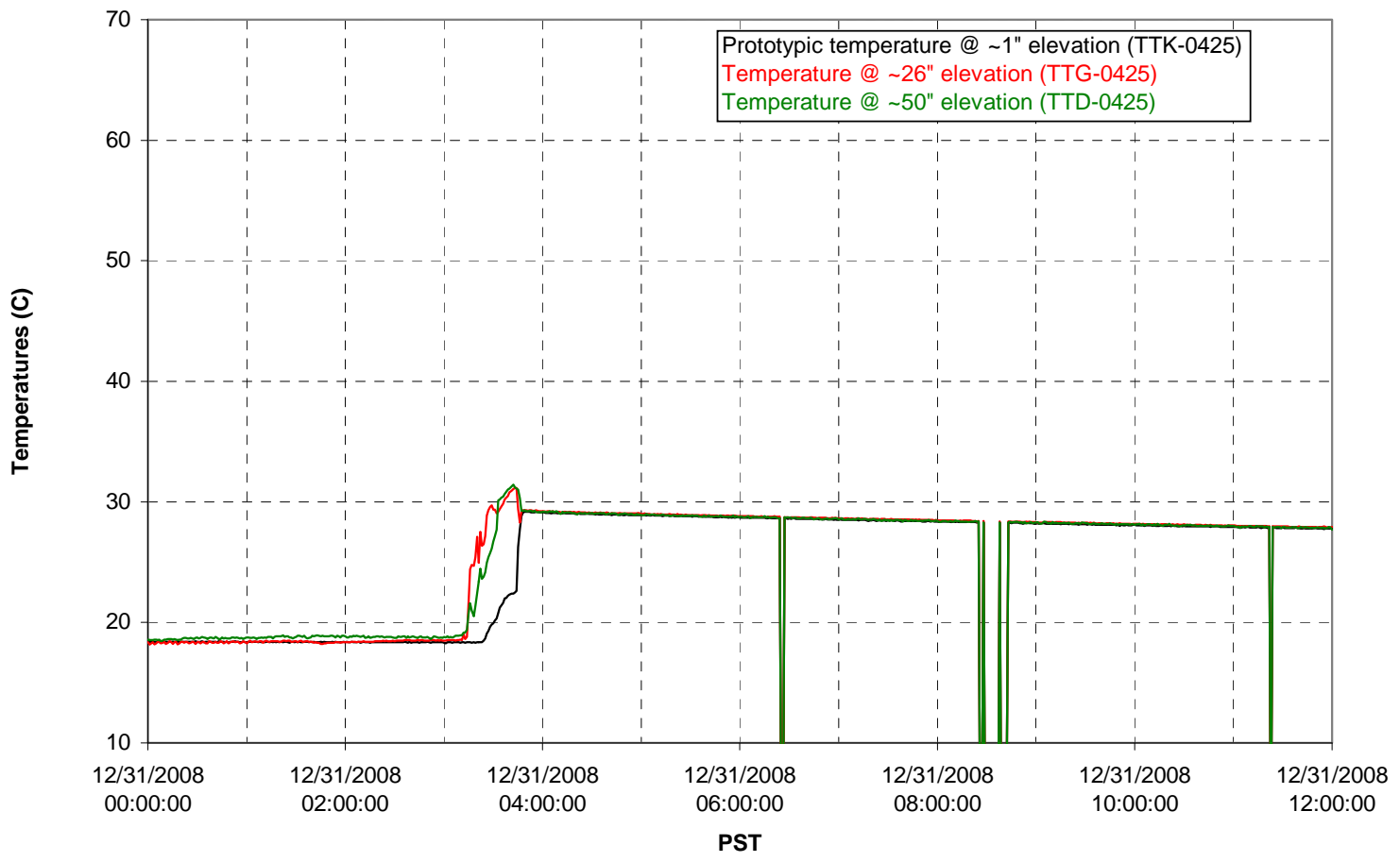


T02A level

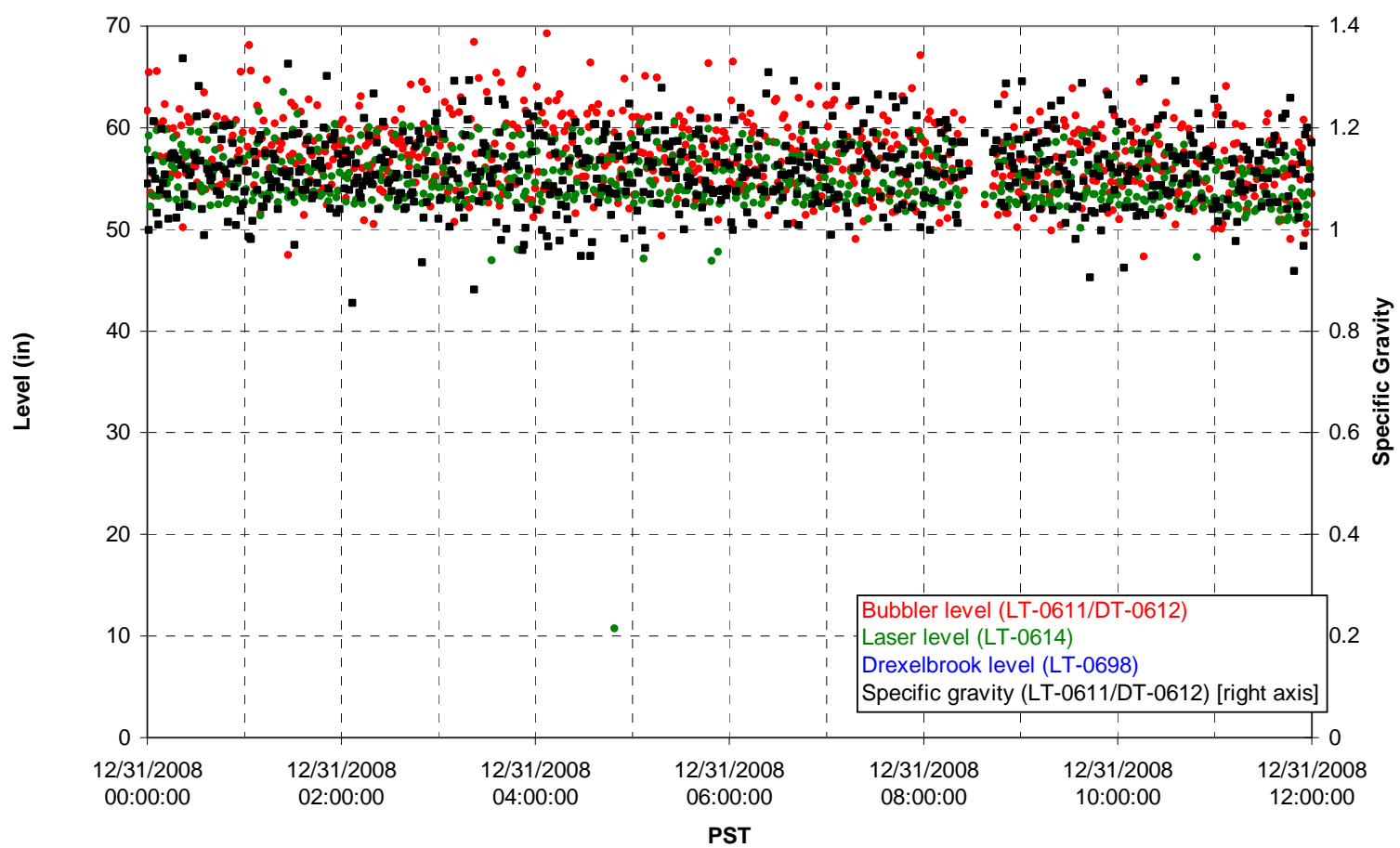

T02A temperatures

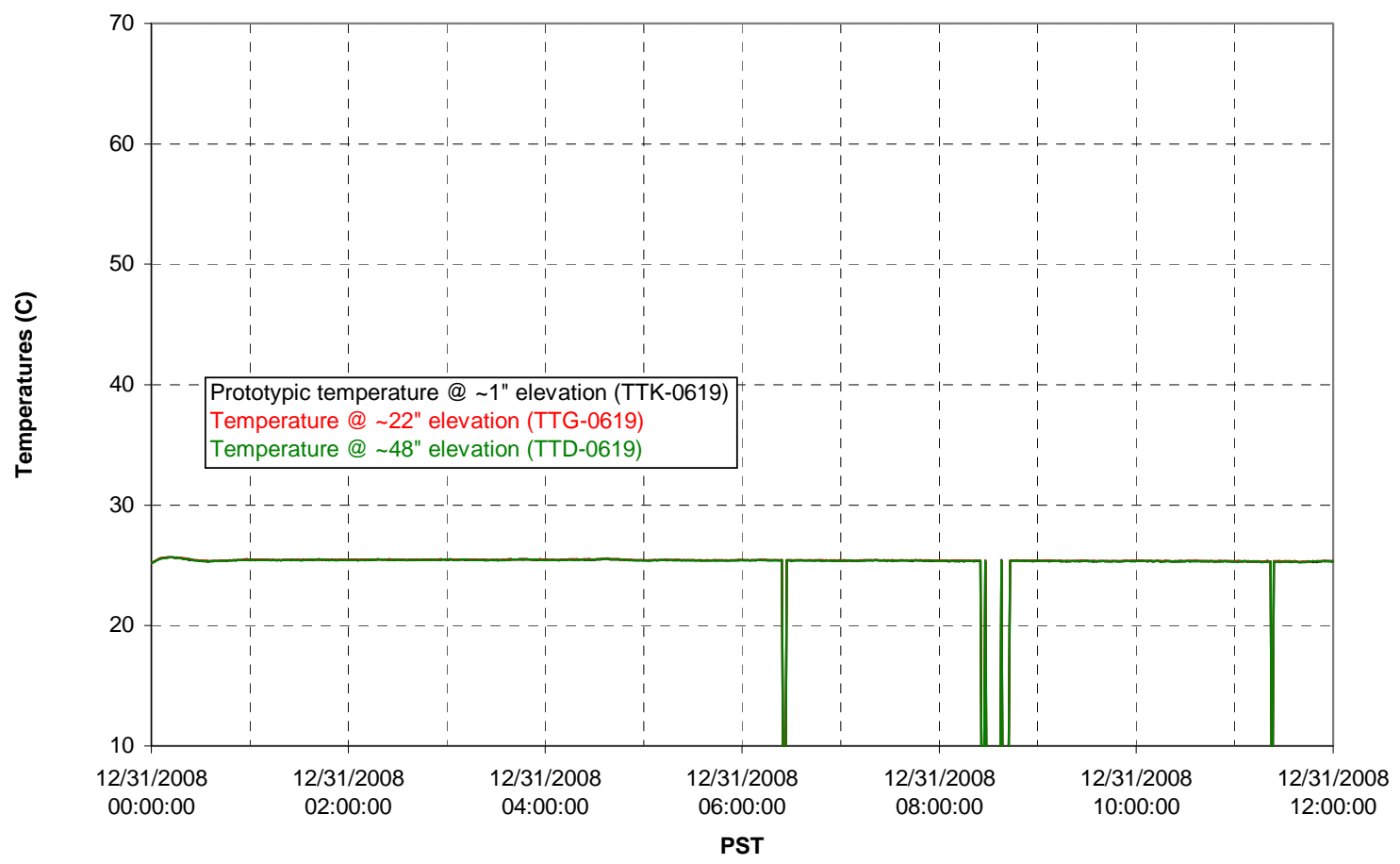


T02A and filter loop temperatures

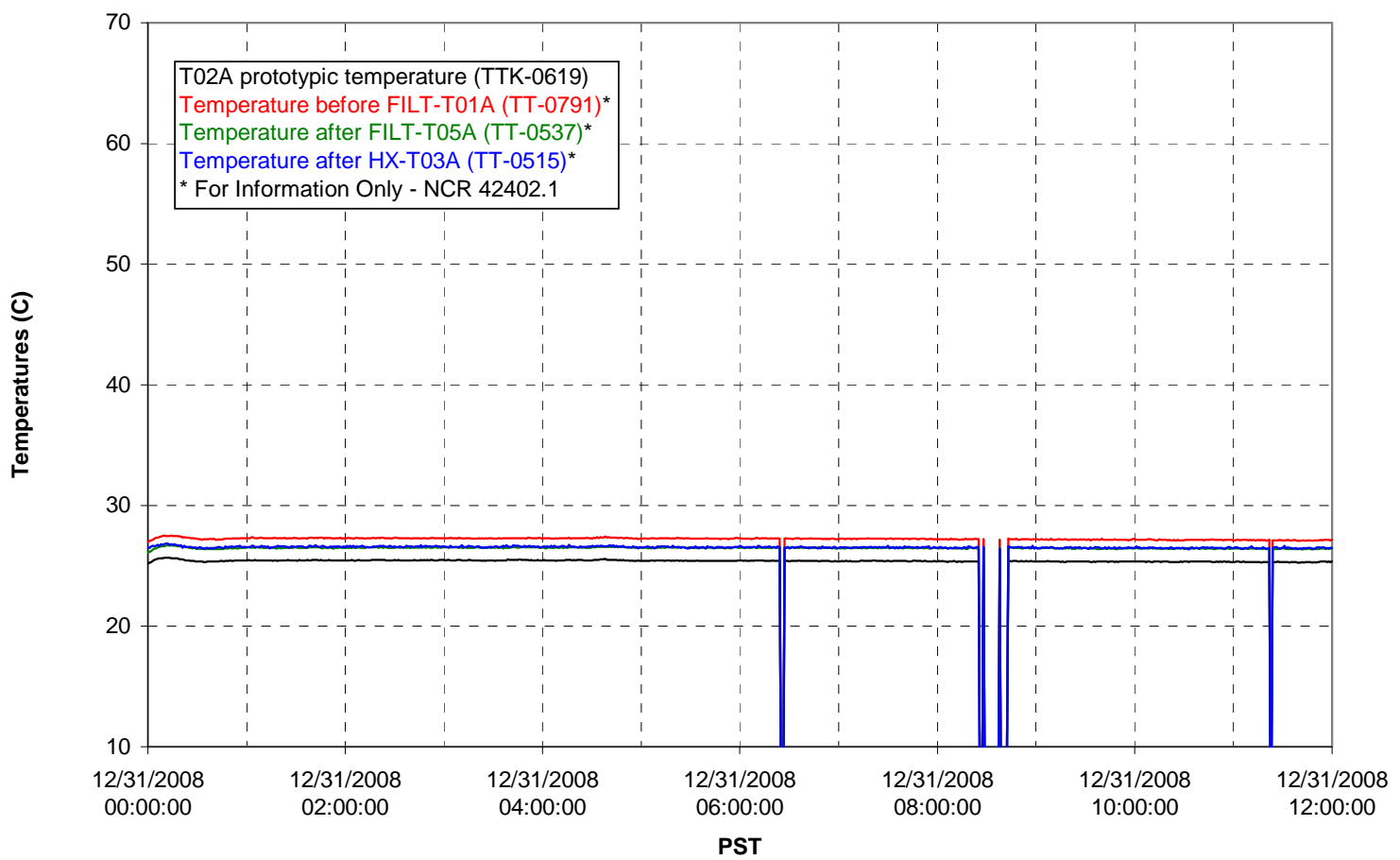

Pump Pressures and Flow

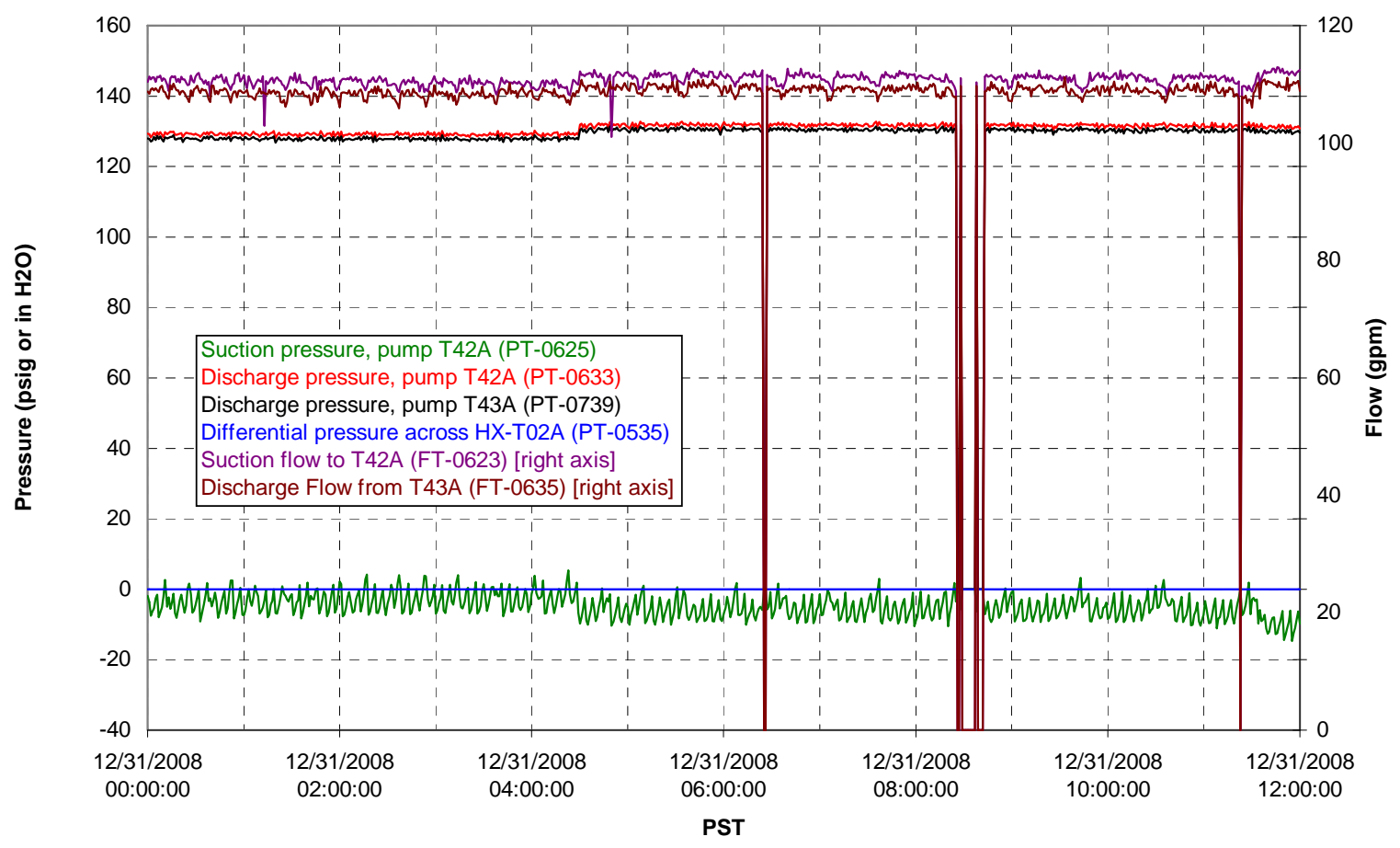


Axial pressure drop

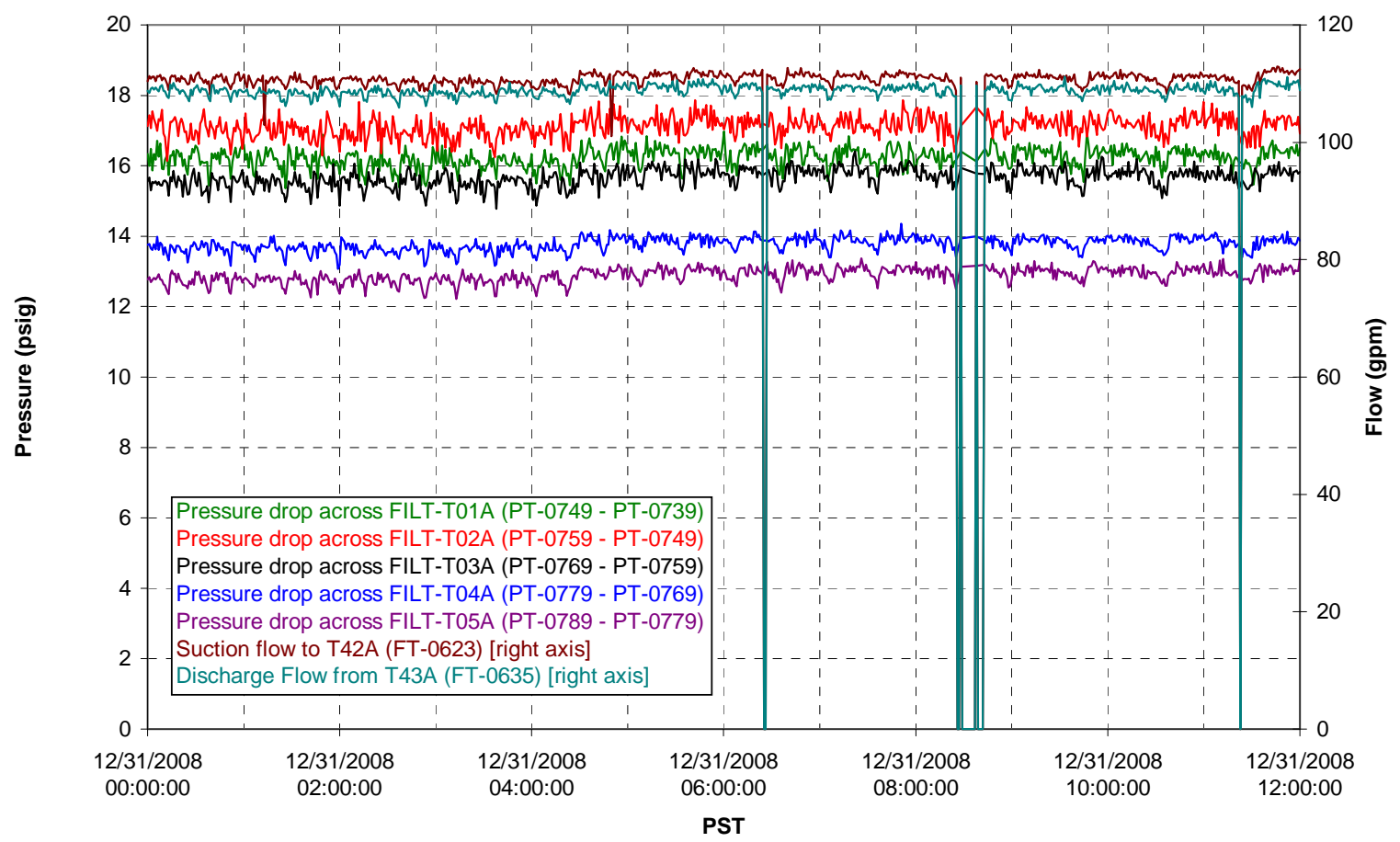

Permeate flow rates

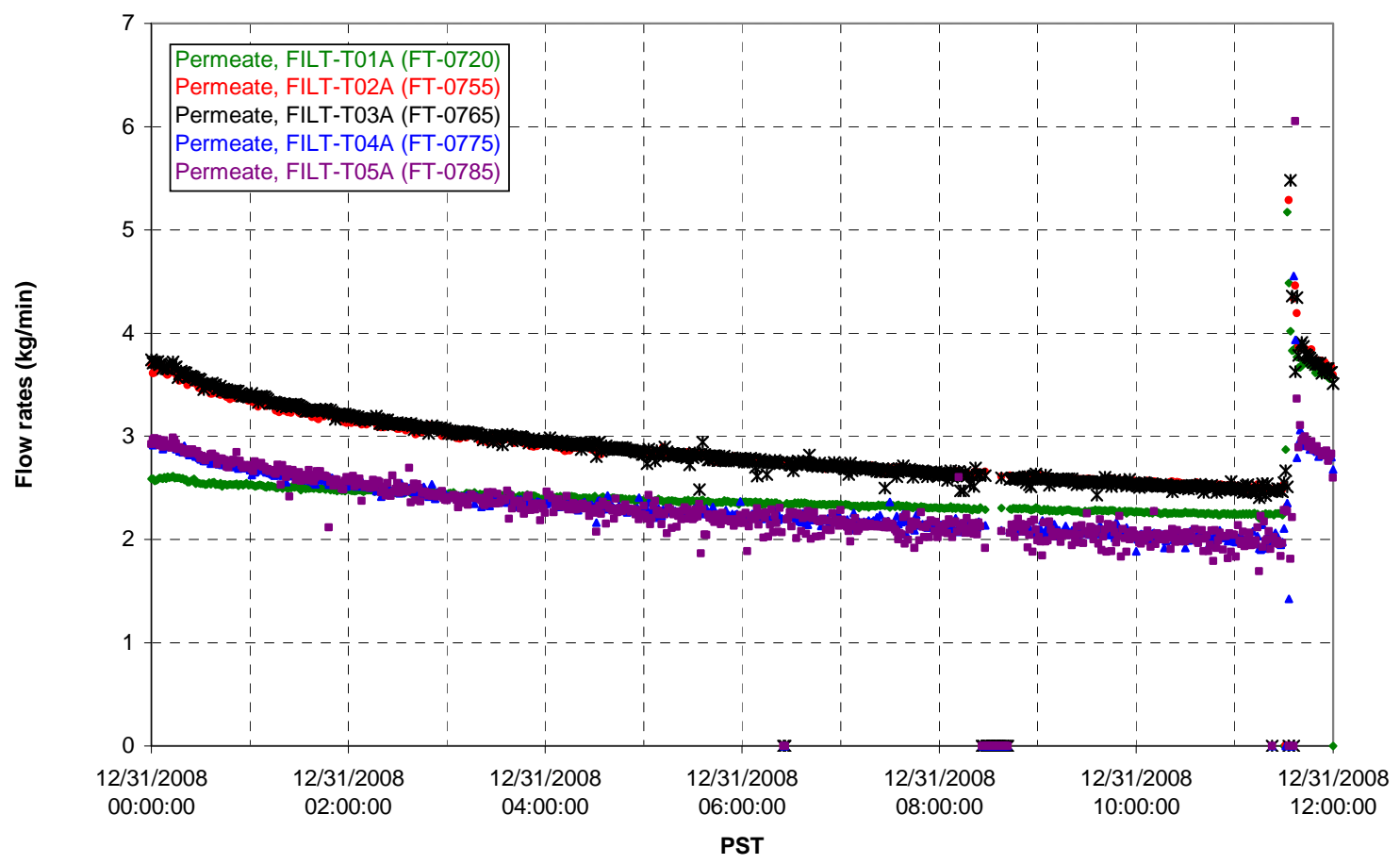


T02A Inner Temperature Tree

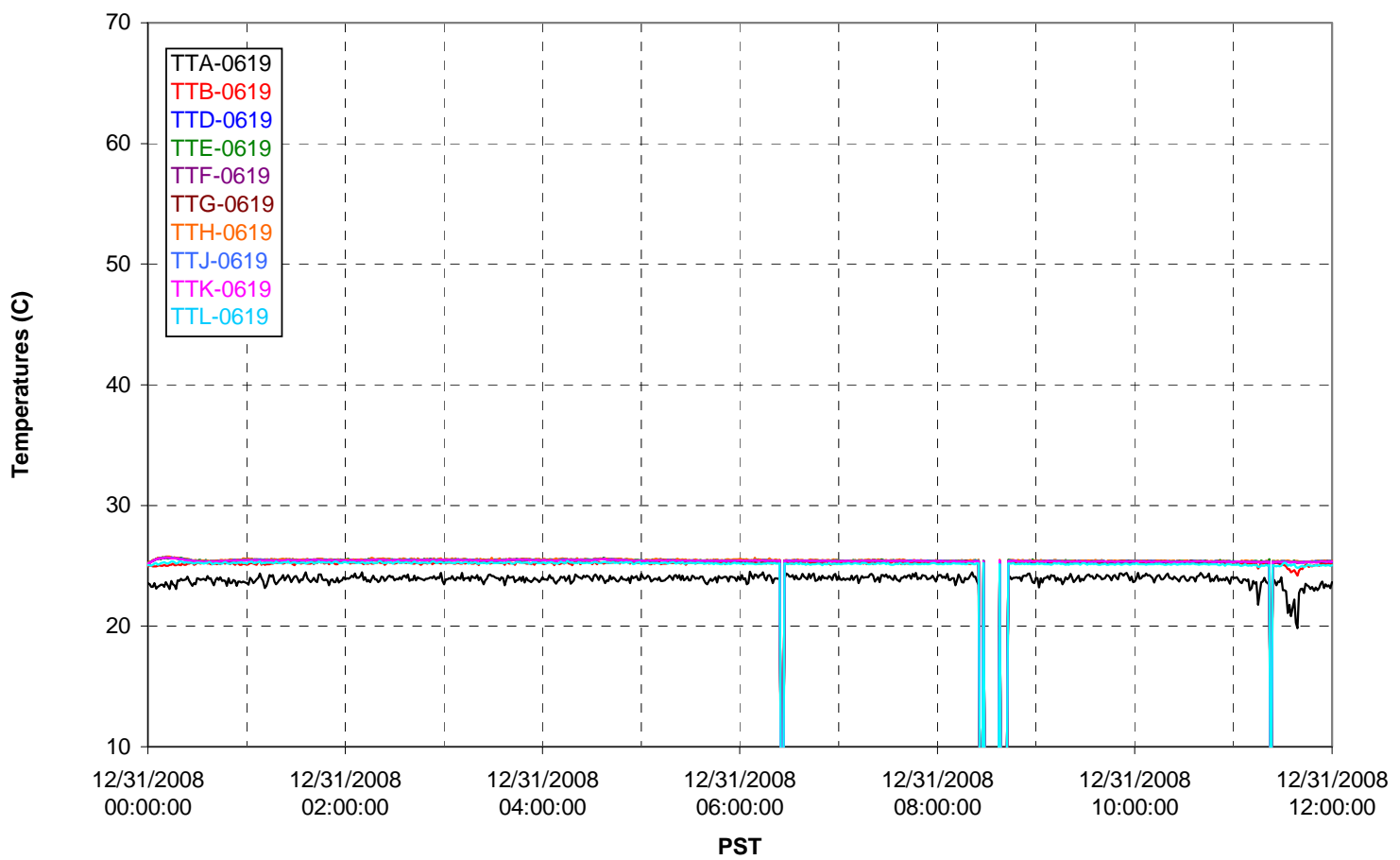

T02A Outer Temperature Tree

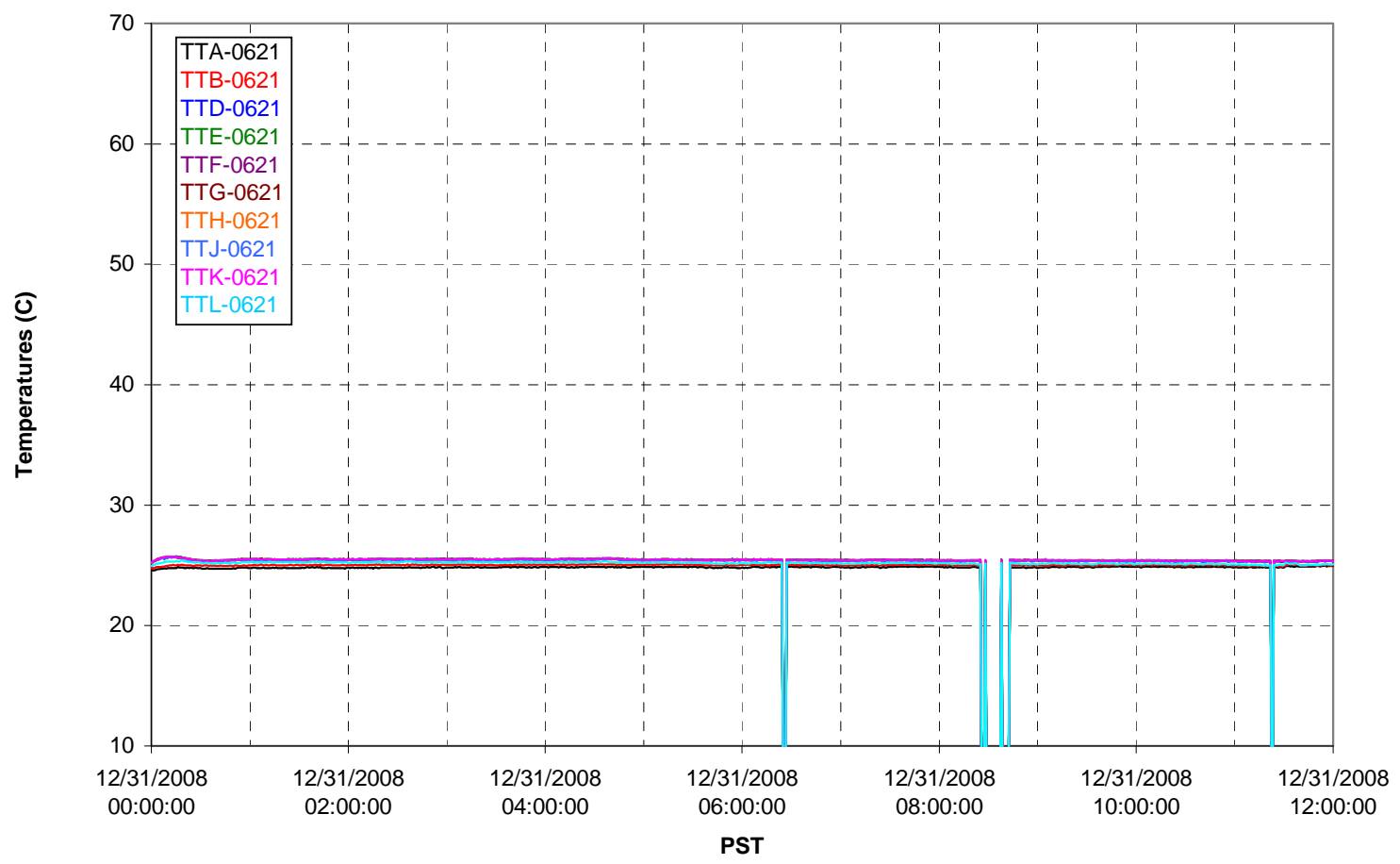


T02A temperatures

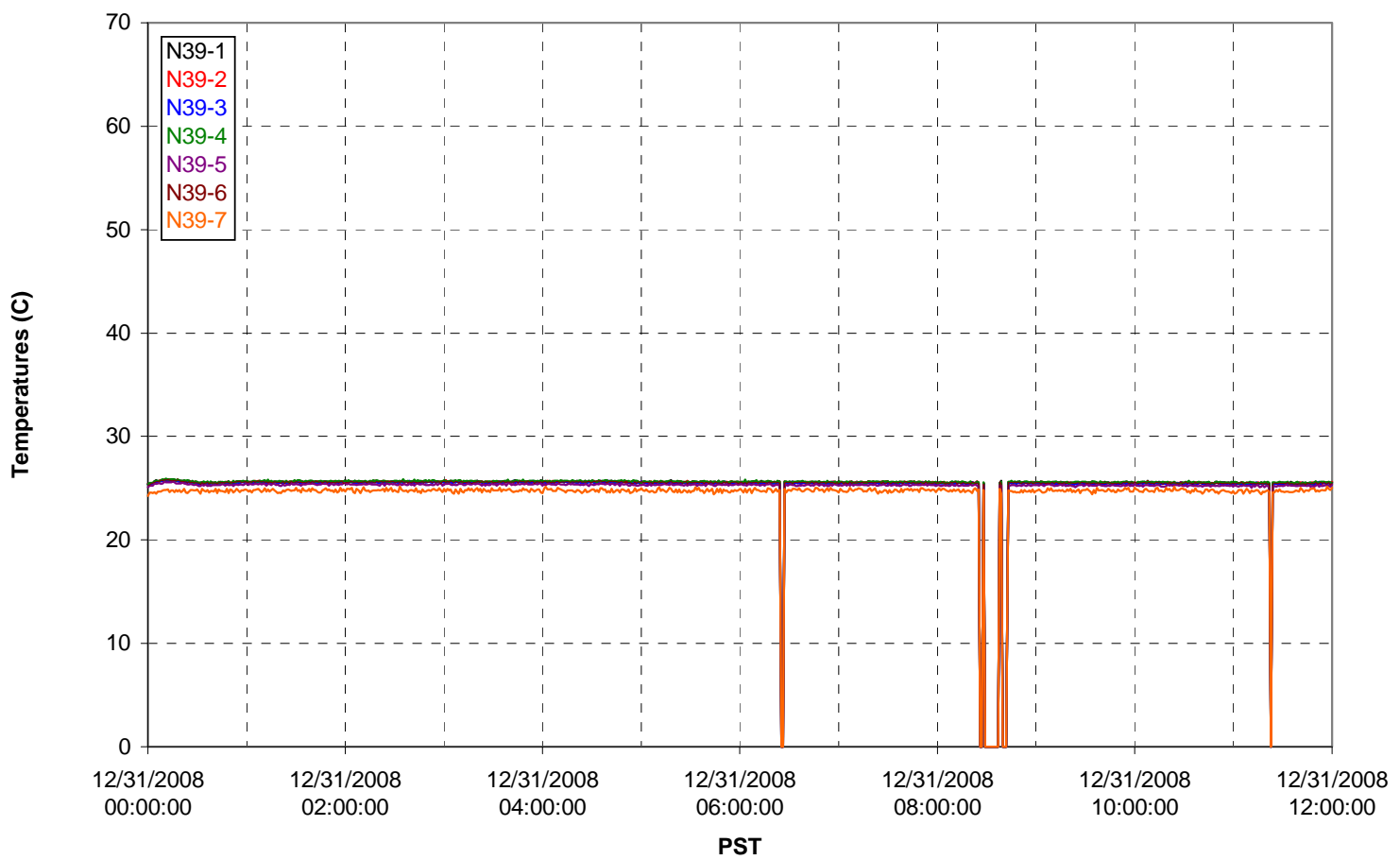

T02A temperatures

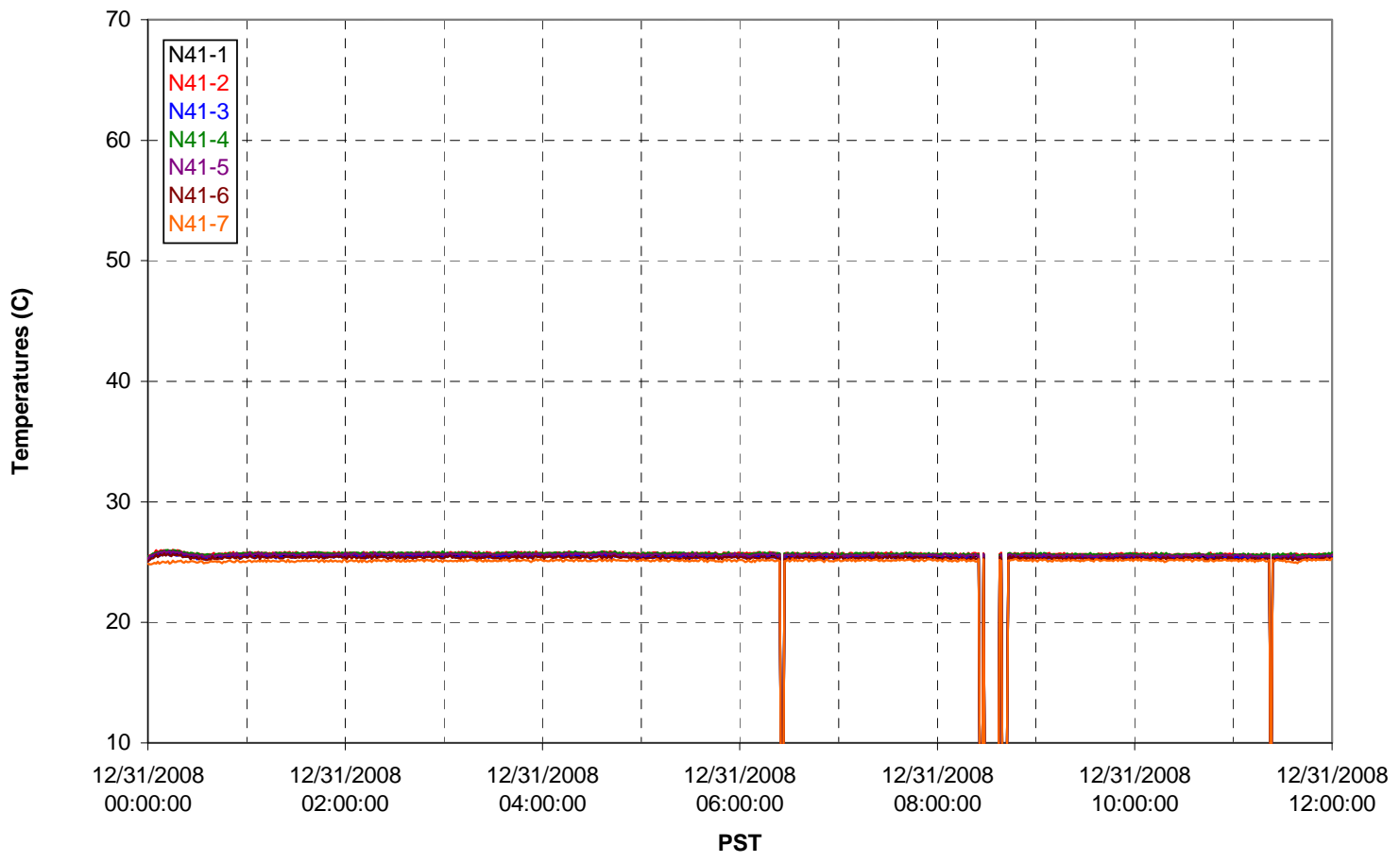


T02A temperatures

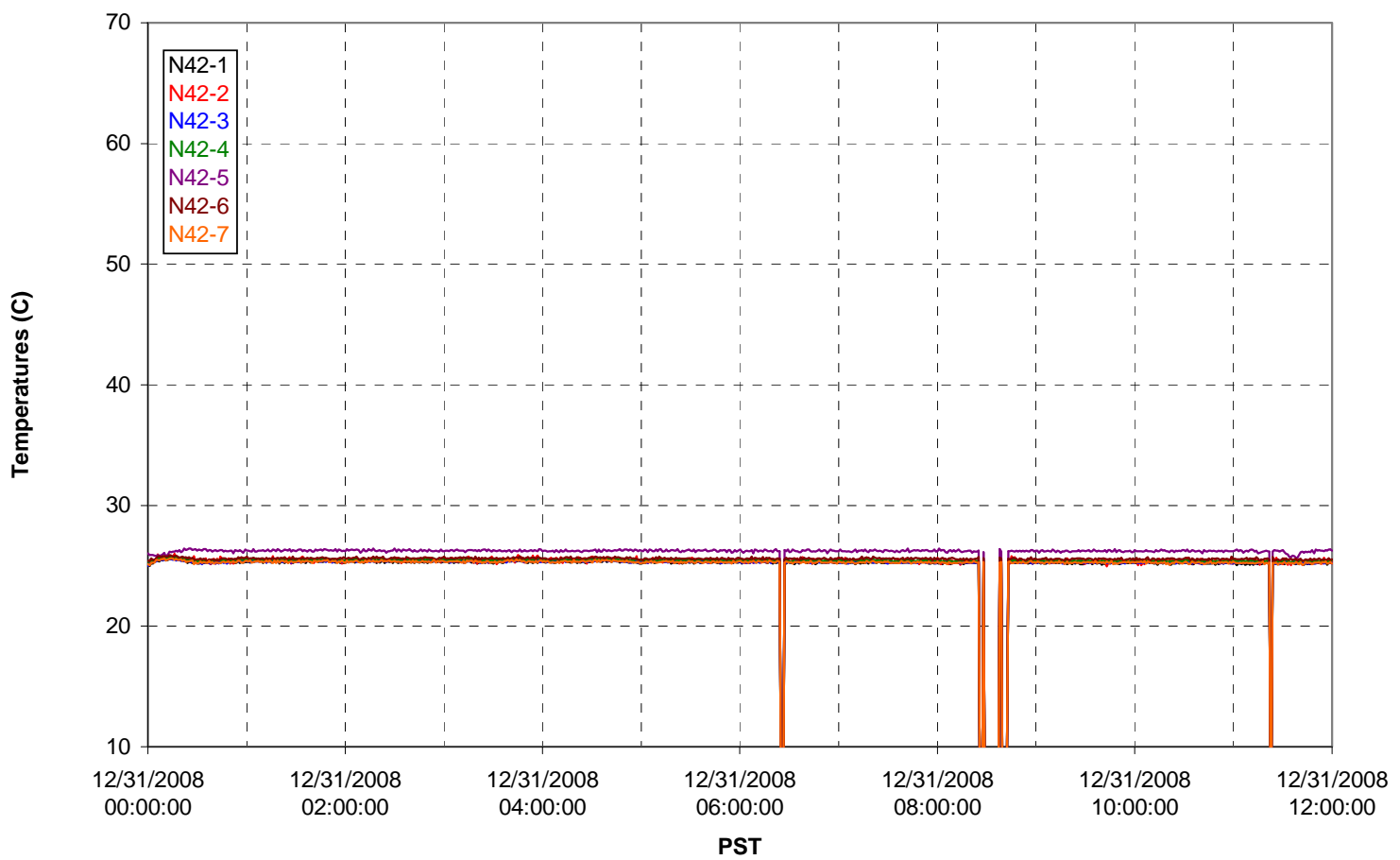

T02A temperatures

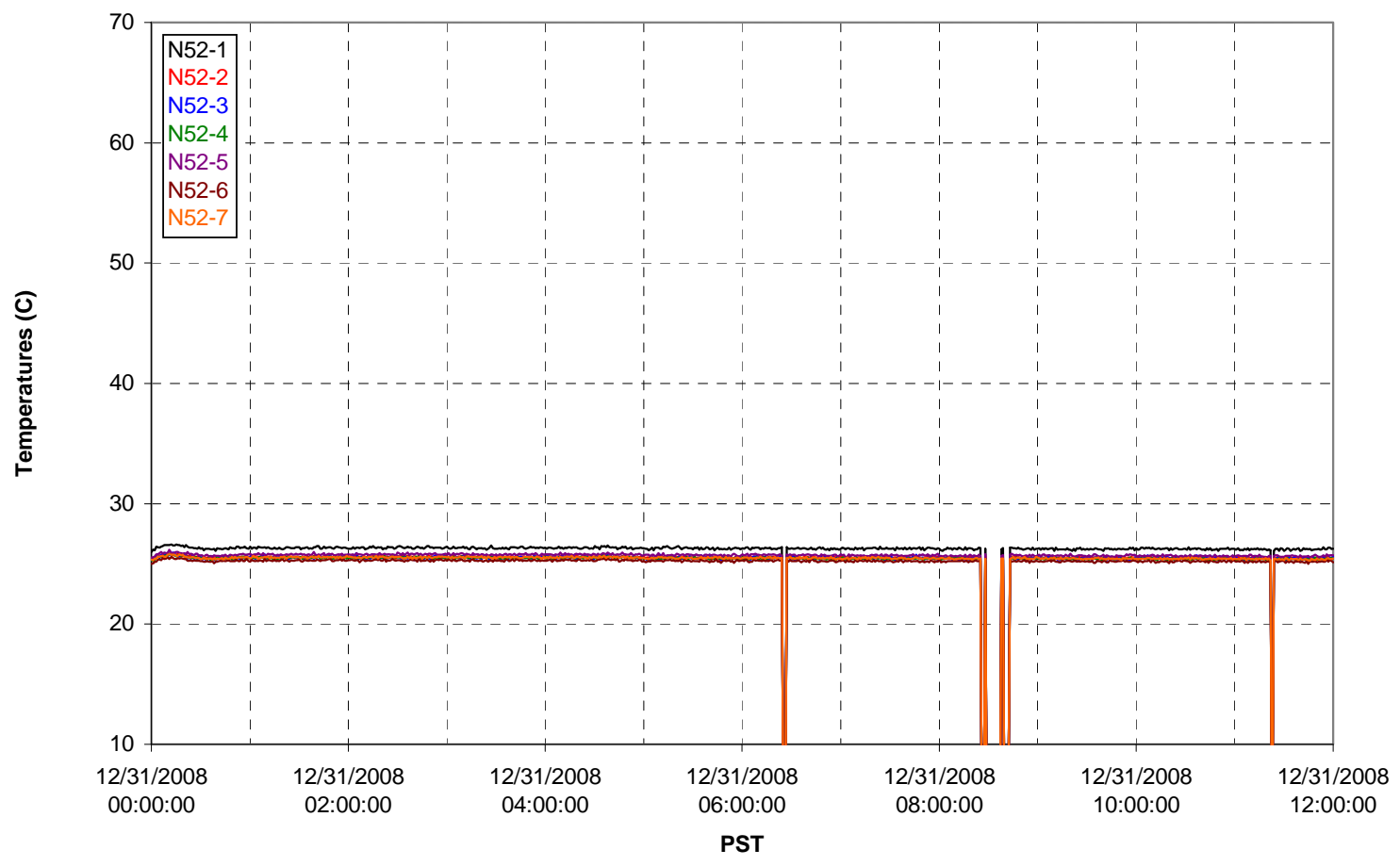


T02A Heating and Cooling

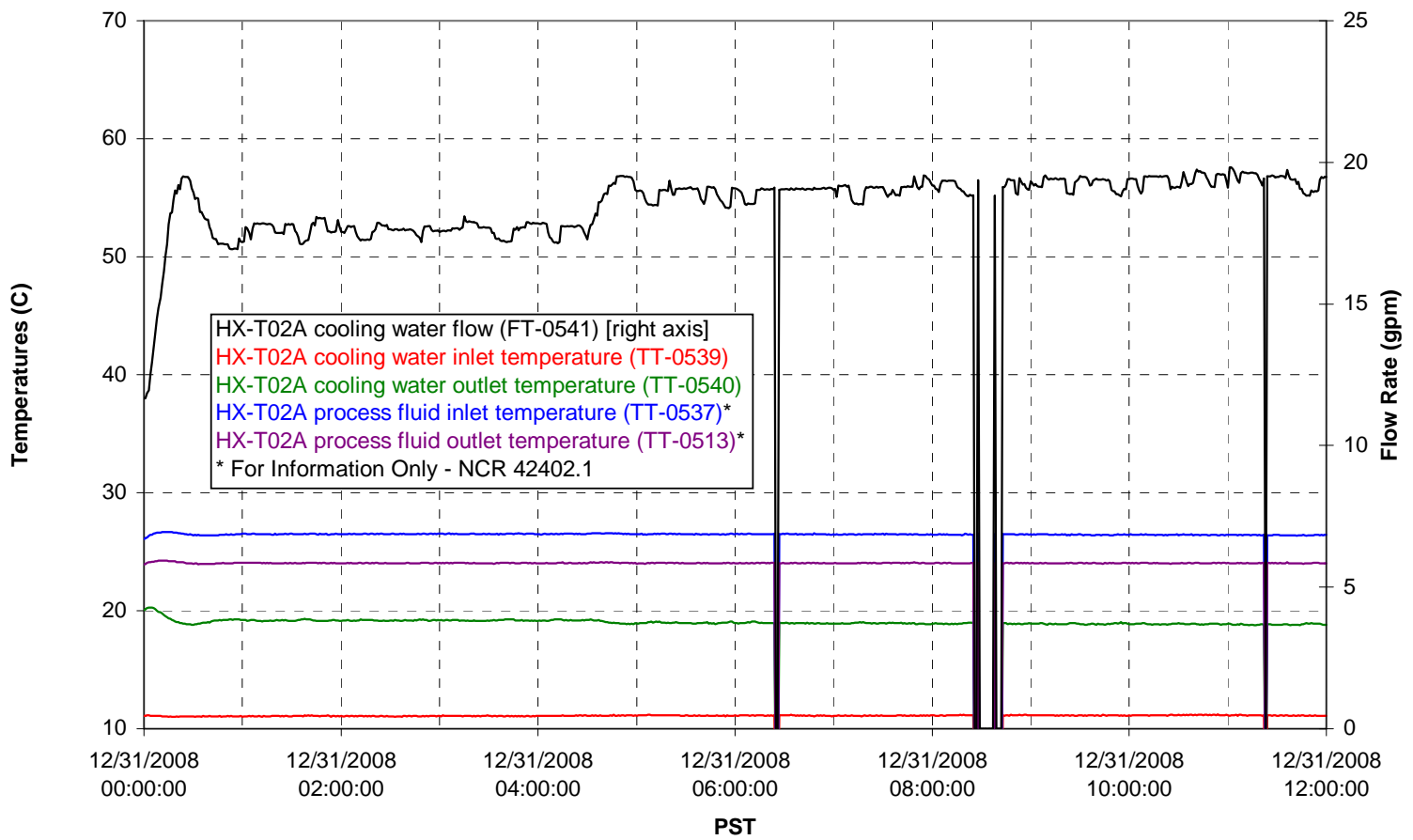

Pump Operation

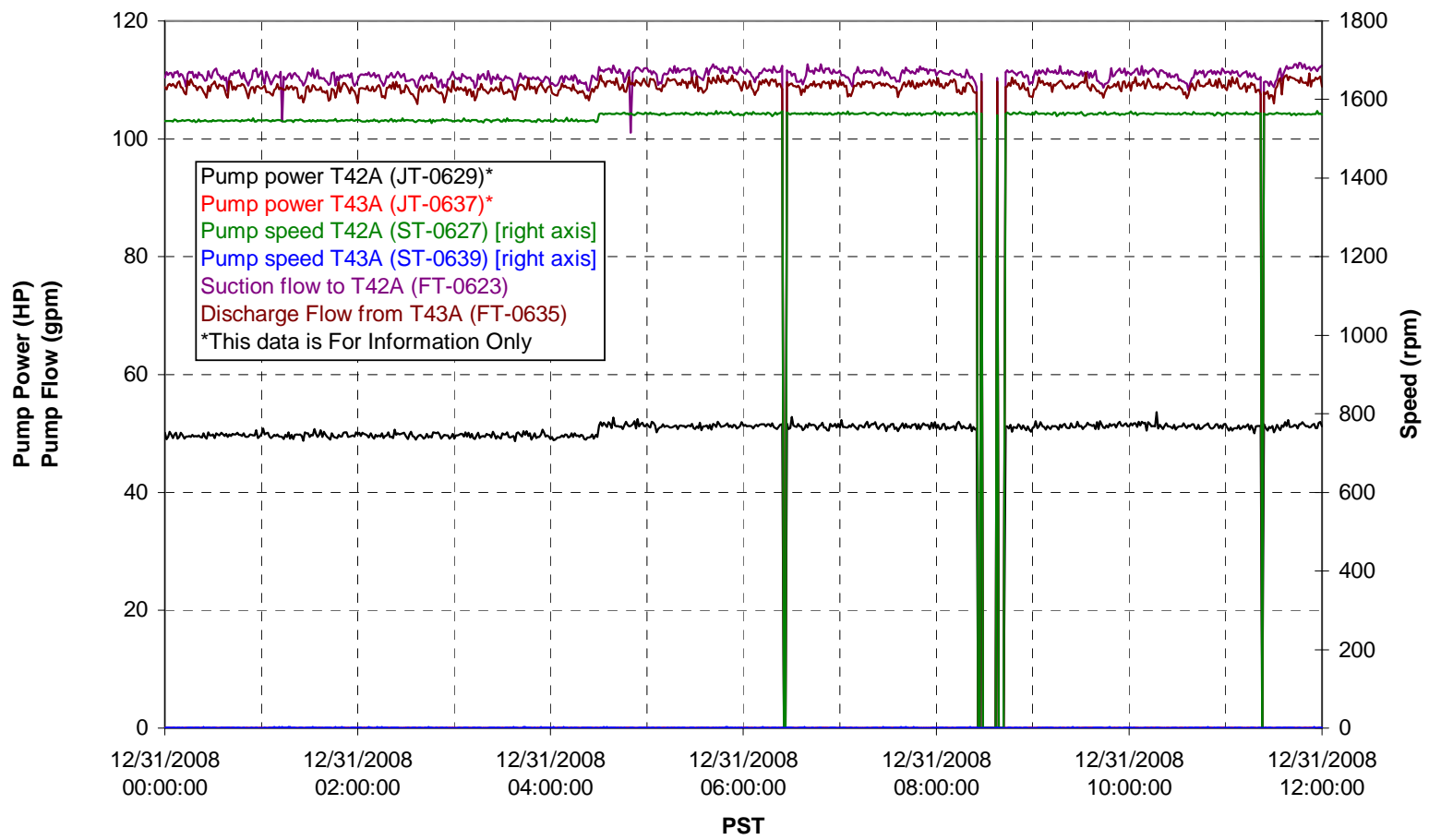


Pulsepot UFP-PP-T01A

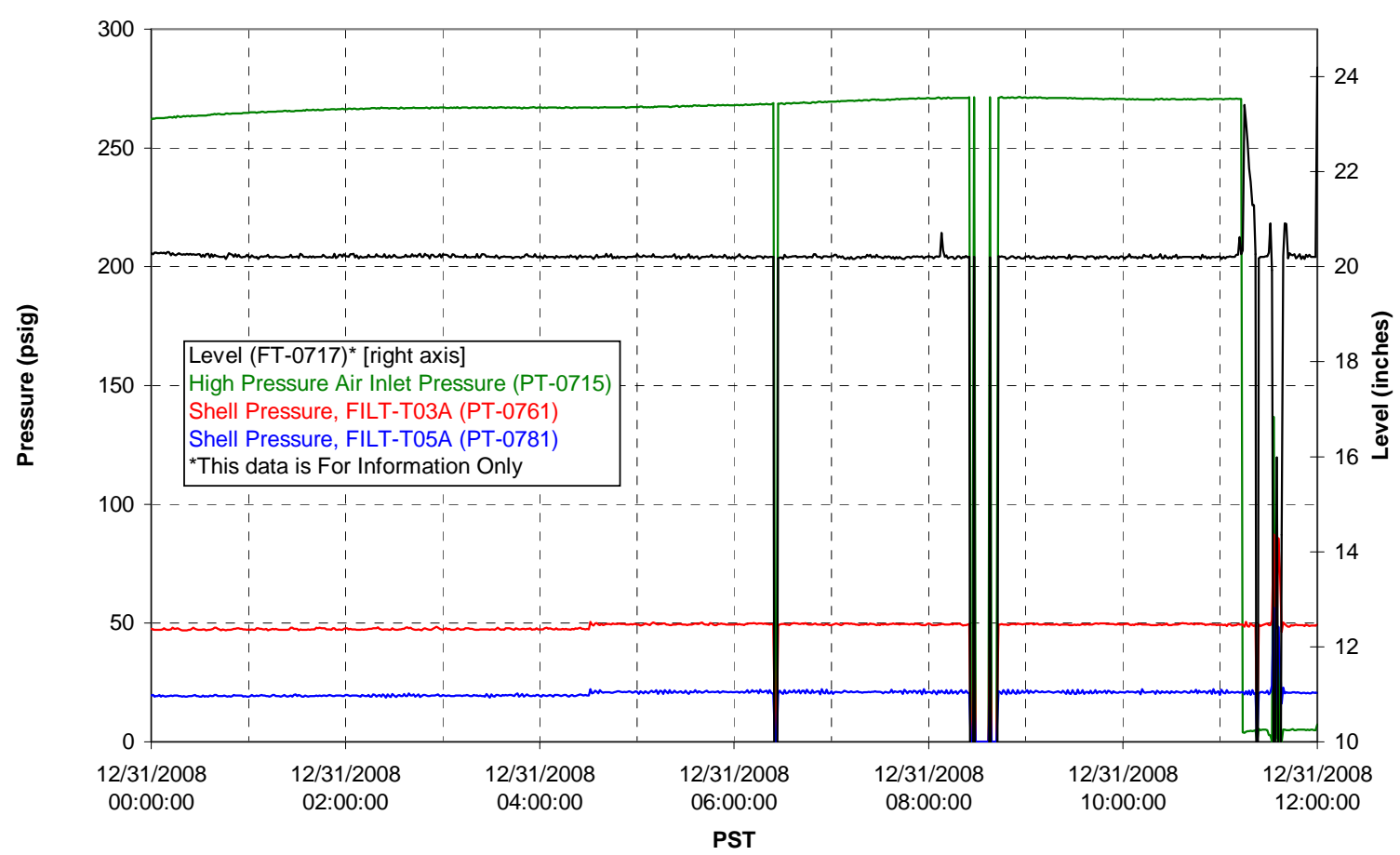

Pulsepot UFP-PP-T02A

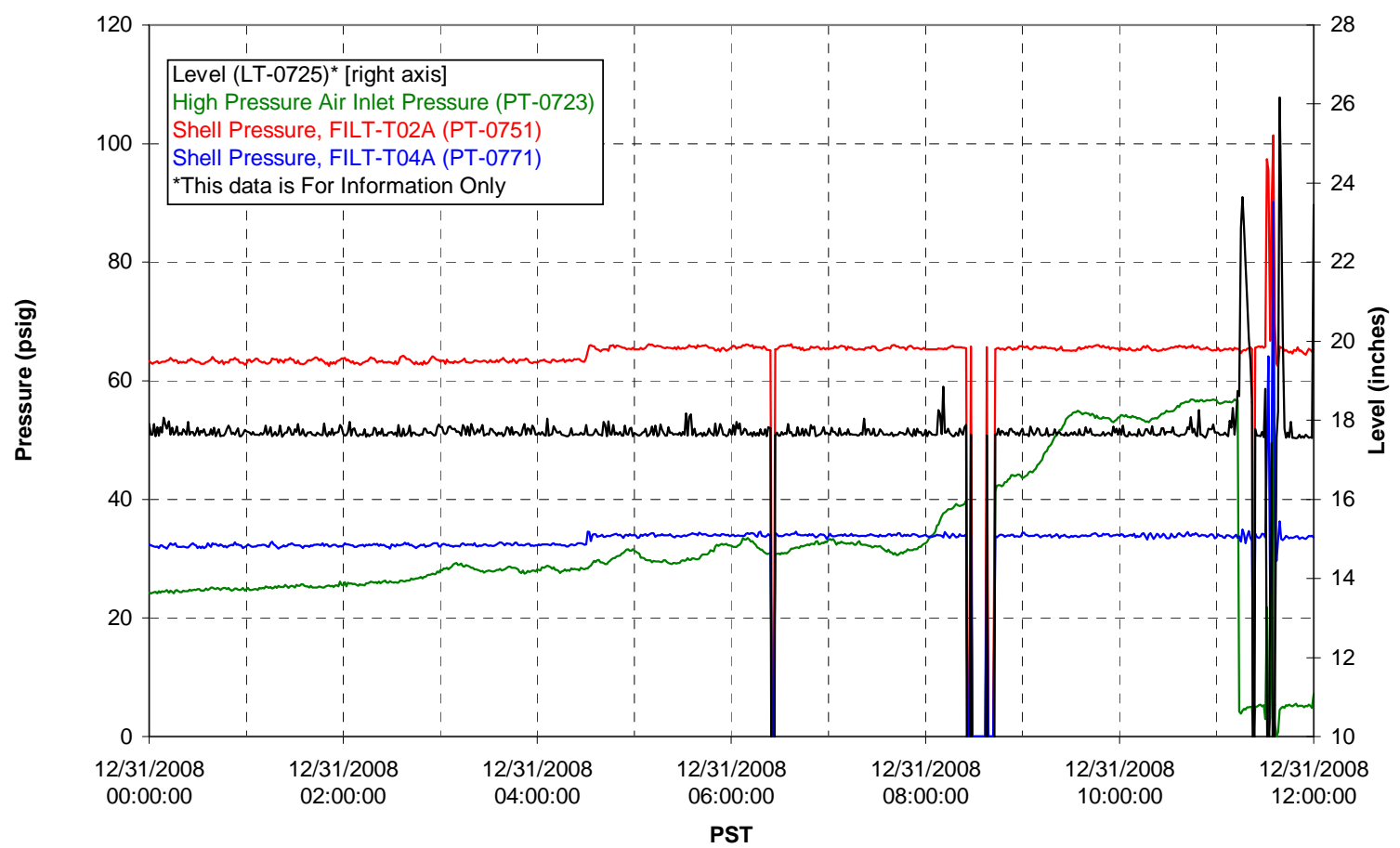


Pulsepot UFP-PP-T03A

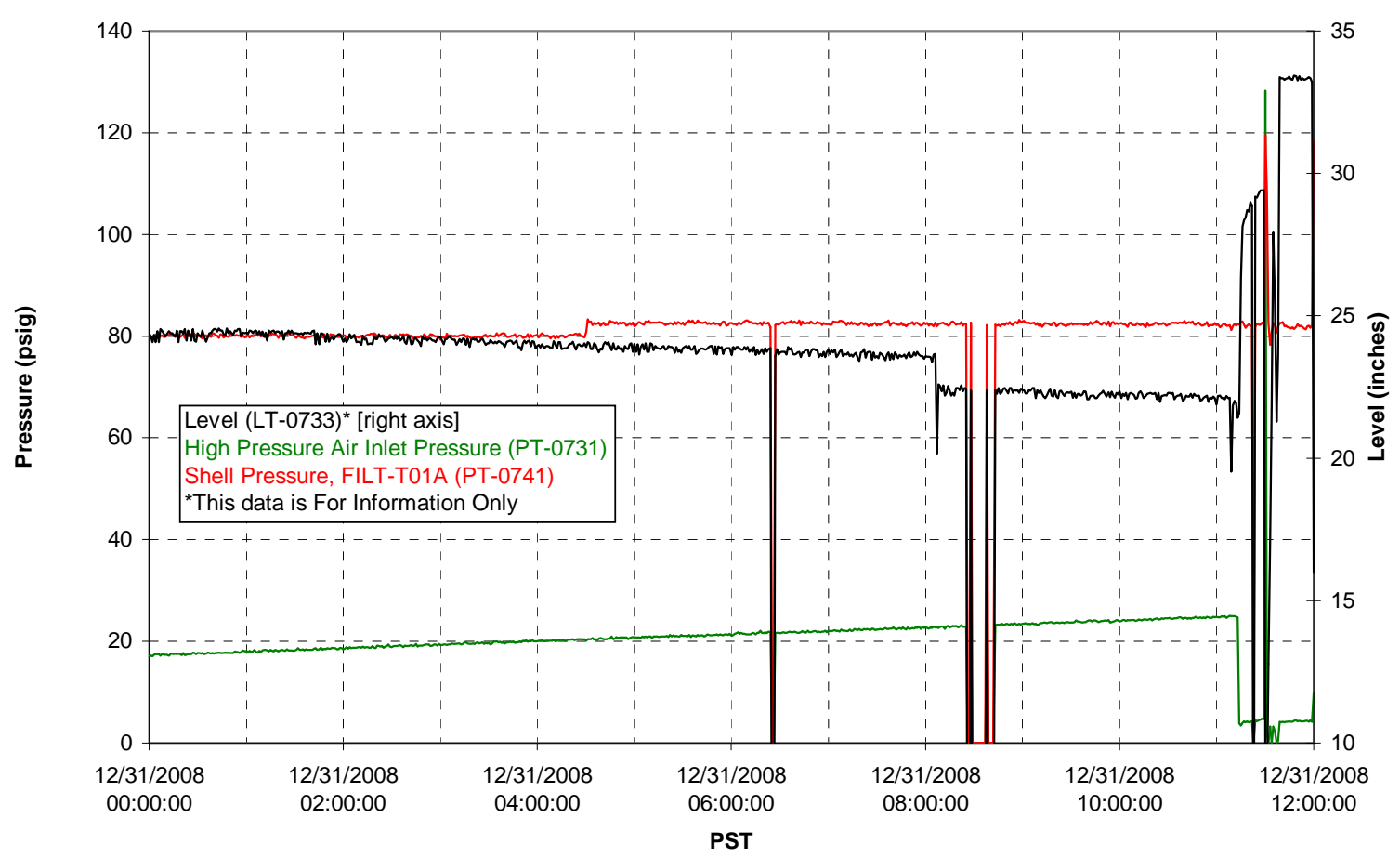

Pulsepot Levels

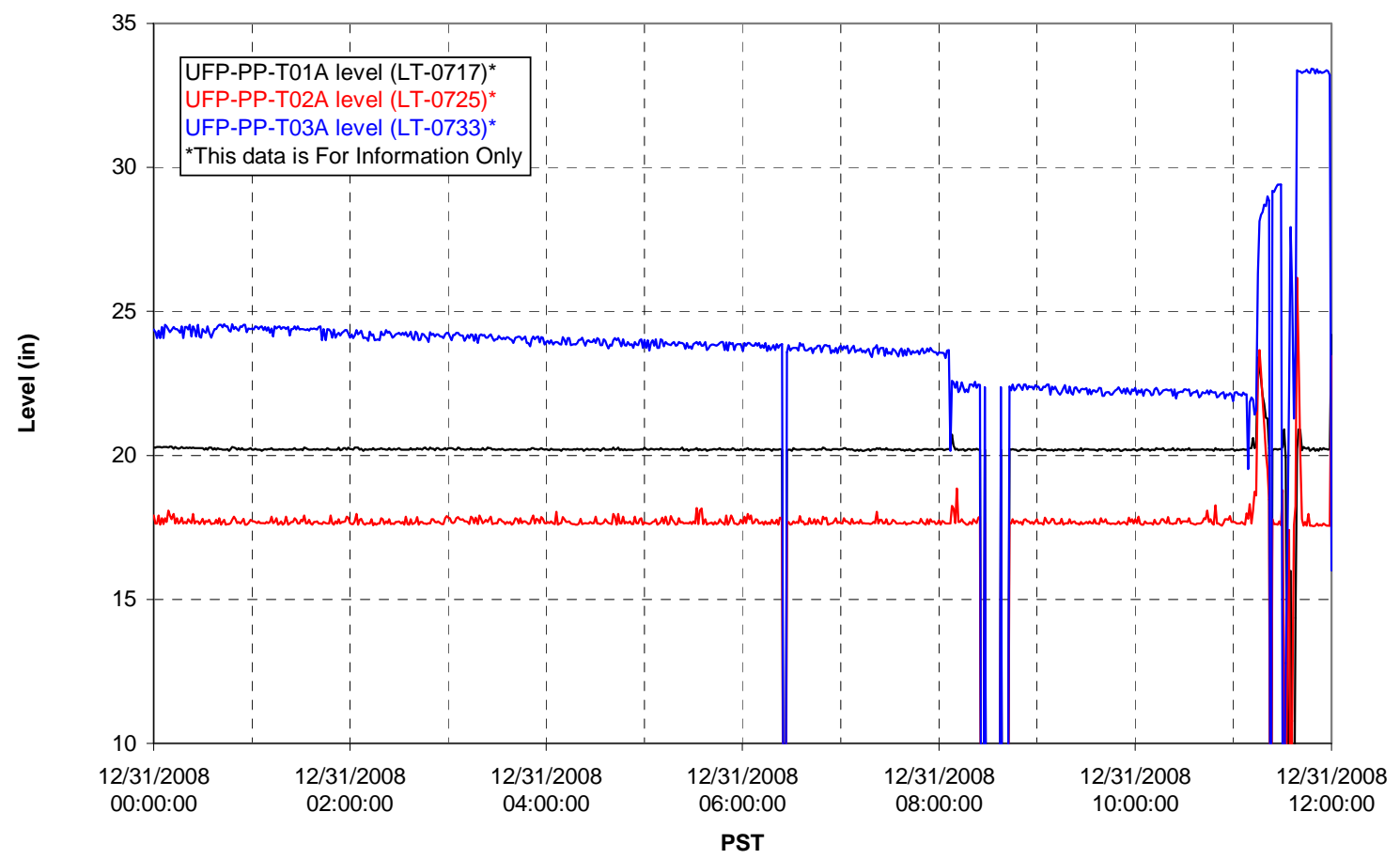


Filter UFP-FILT-T01A

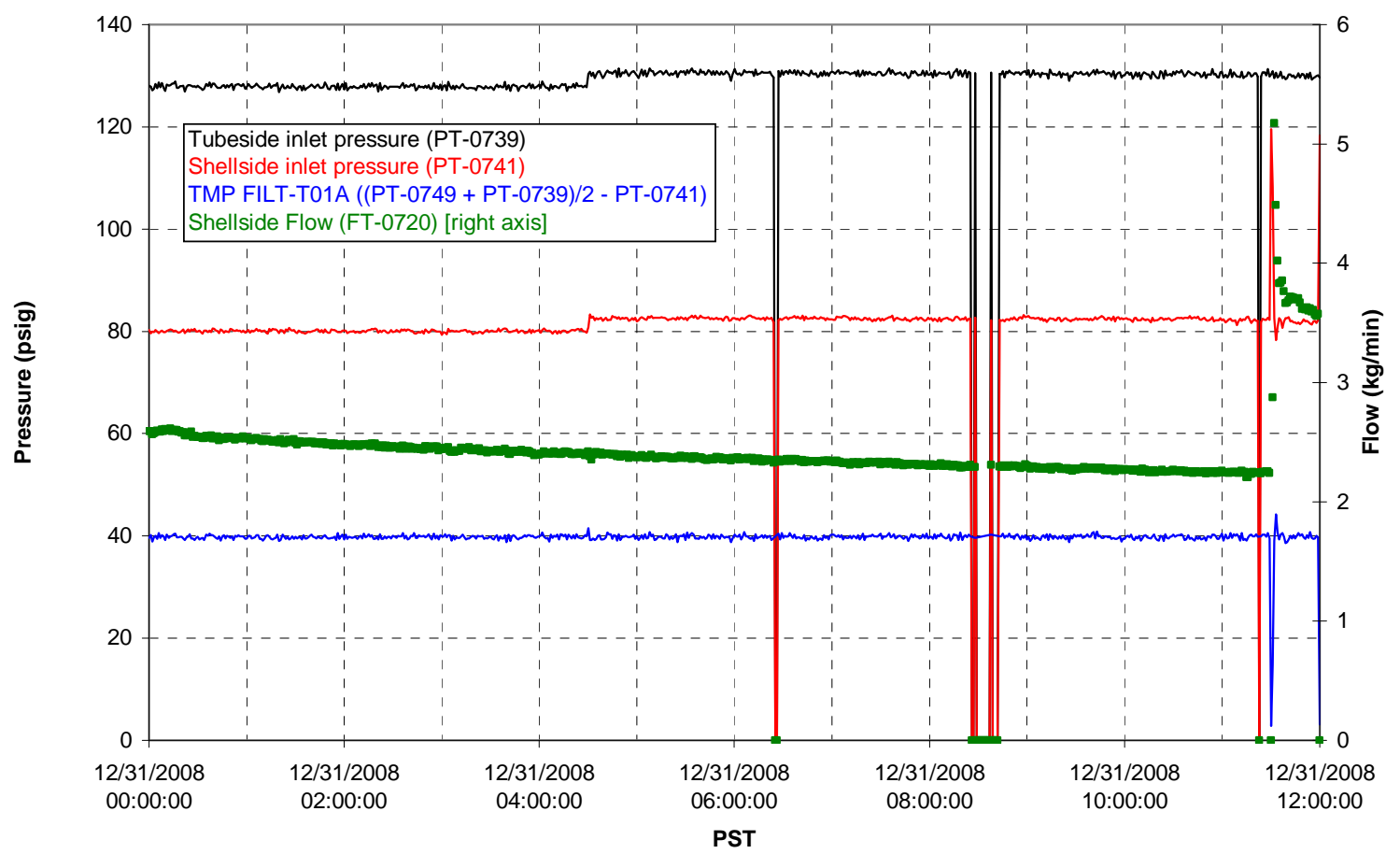

Filter UFP-FILT-T02A

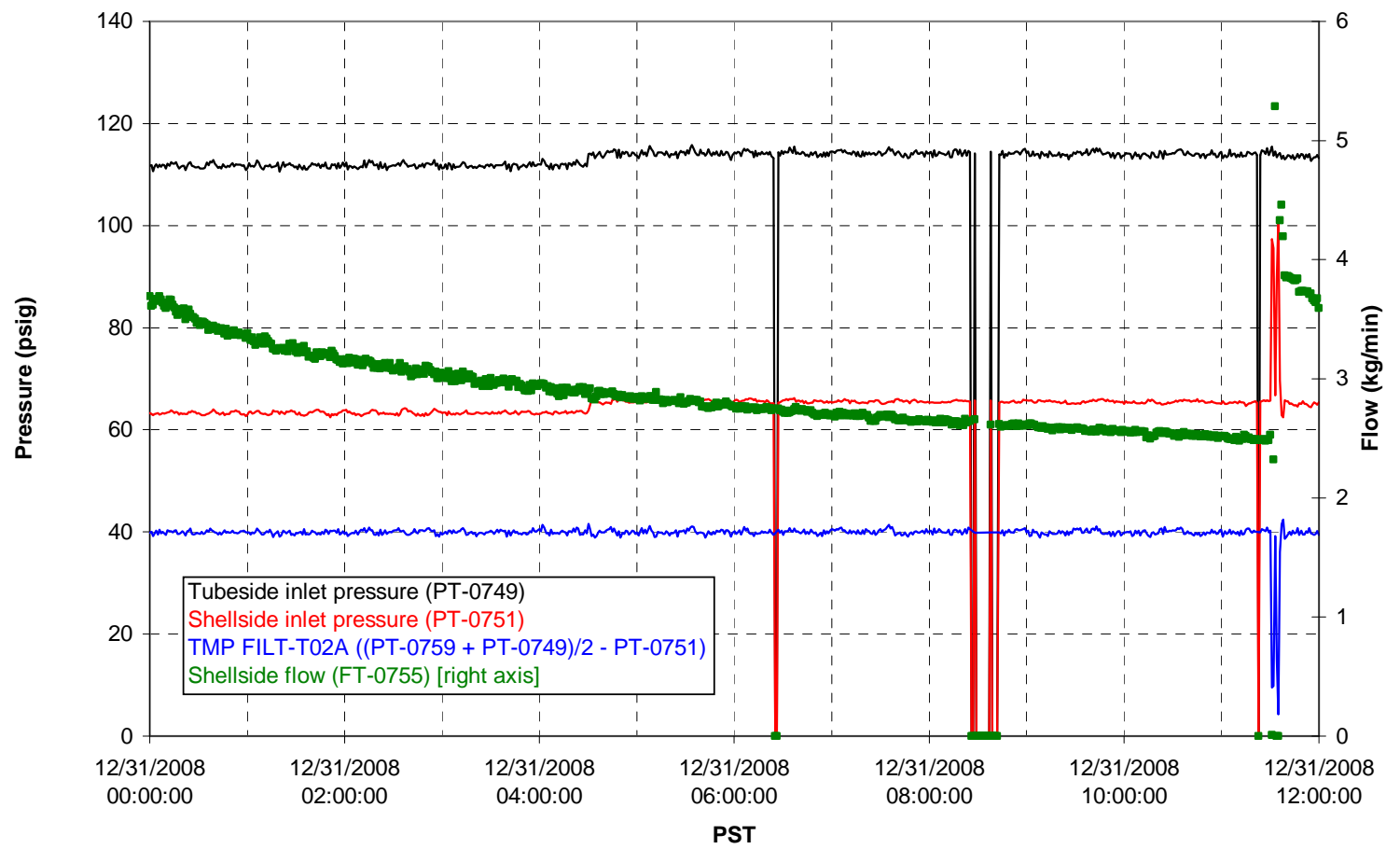


Filter UFP-FILT-T03A

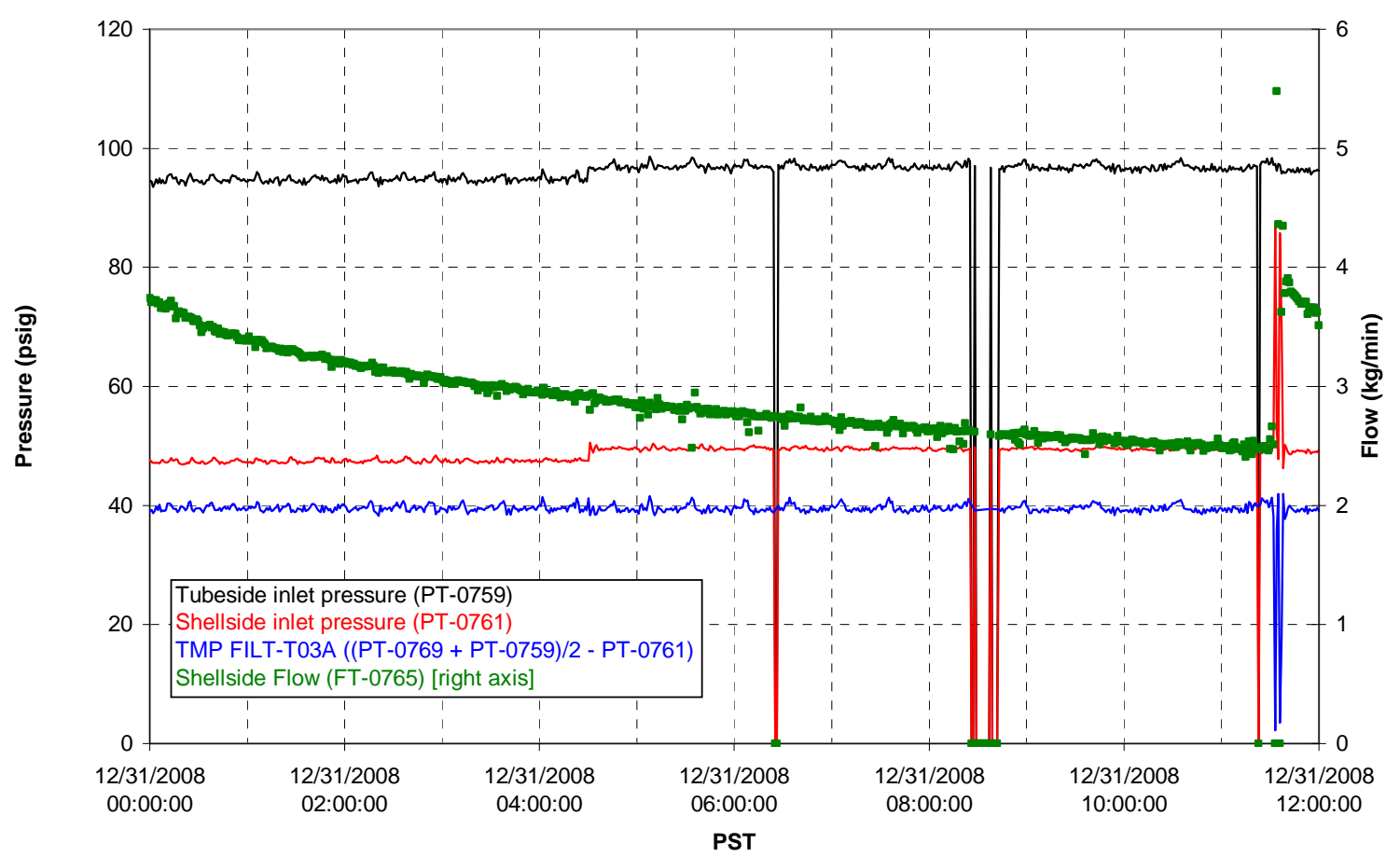

Filter UFP-FILT-T04A

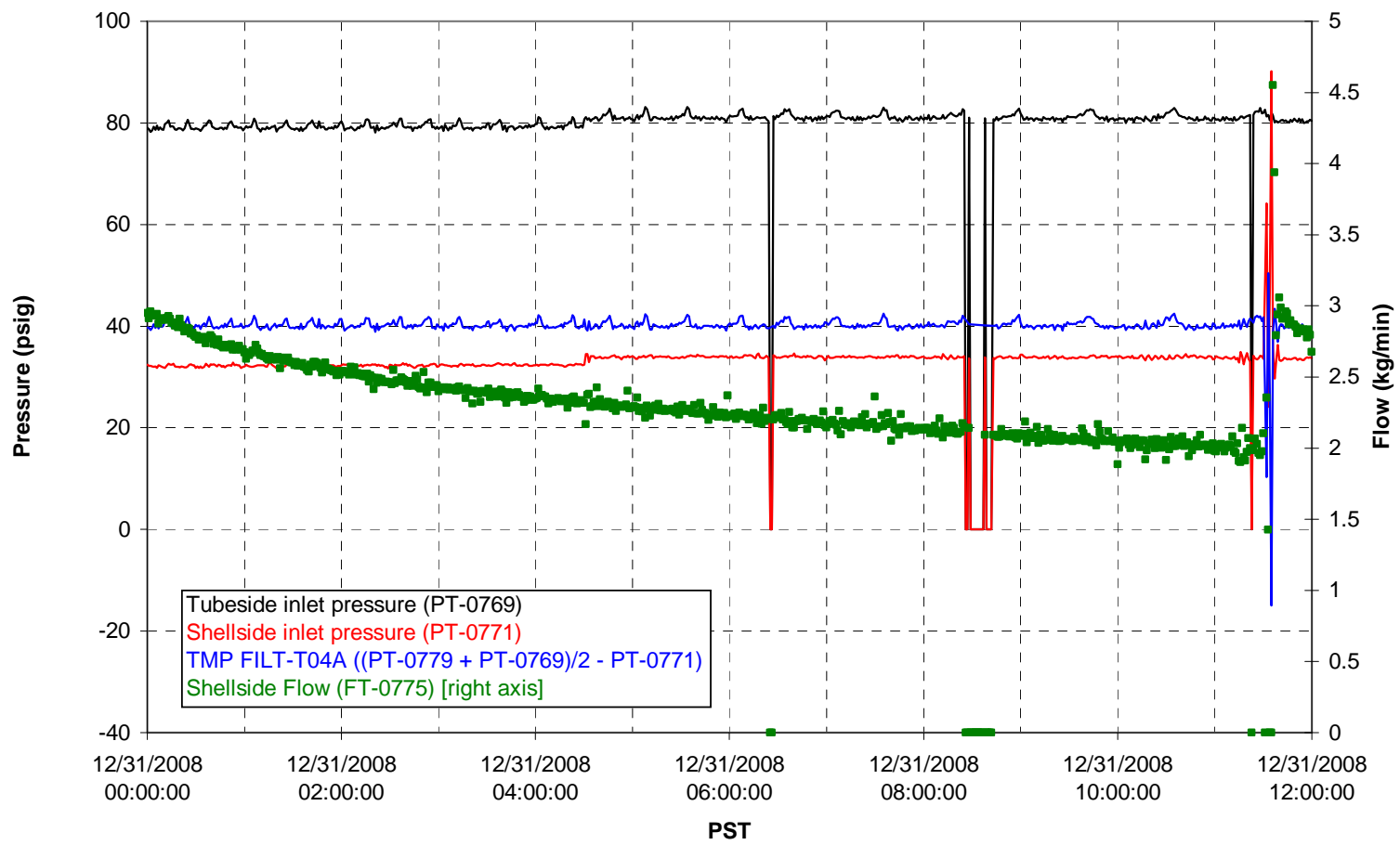


Filter UFP-FILT-T05A

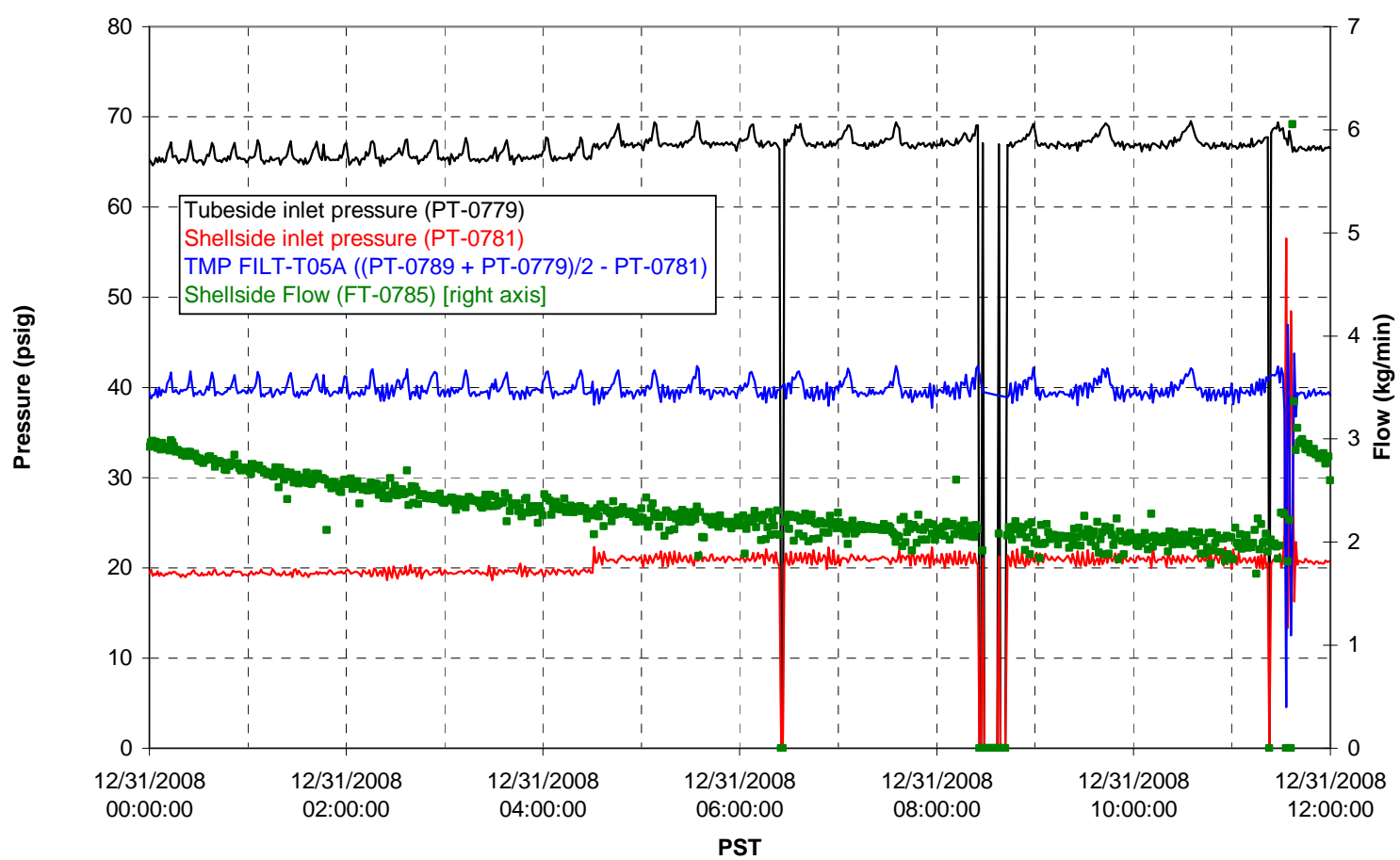

Chemical Flow

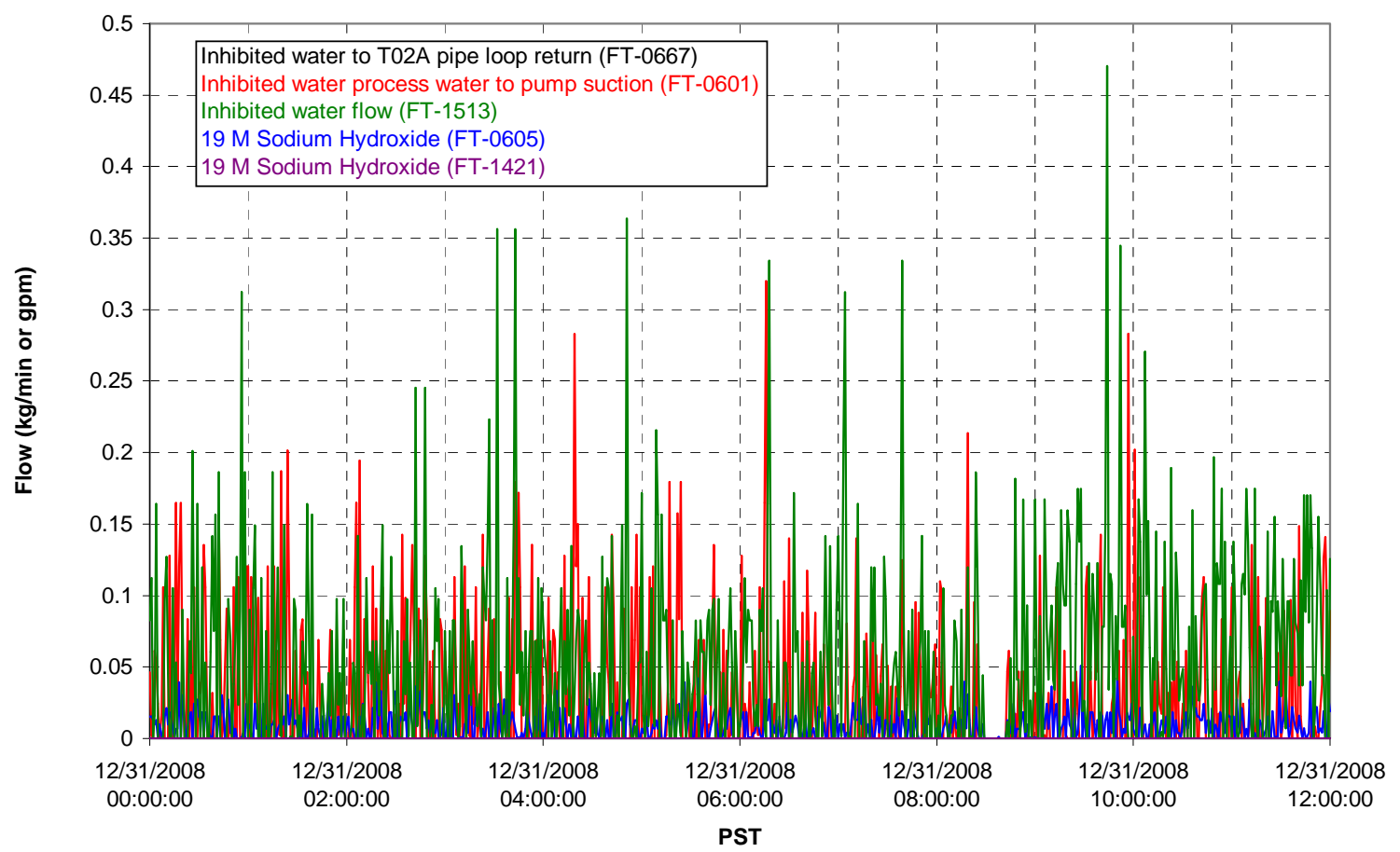


Chemical Flow

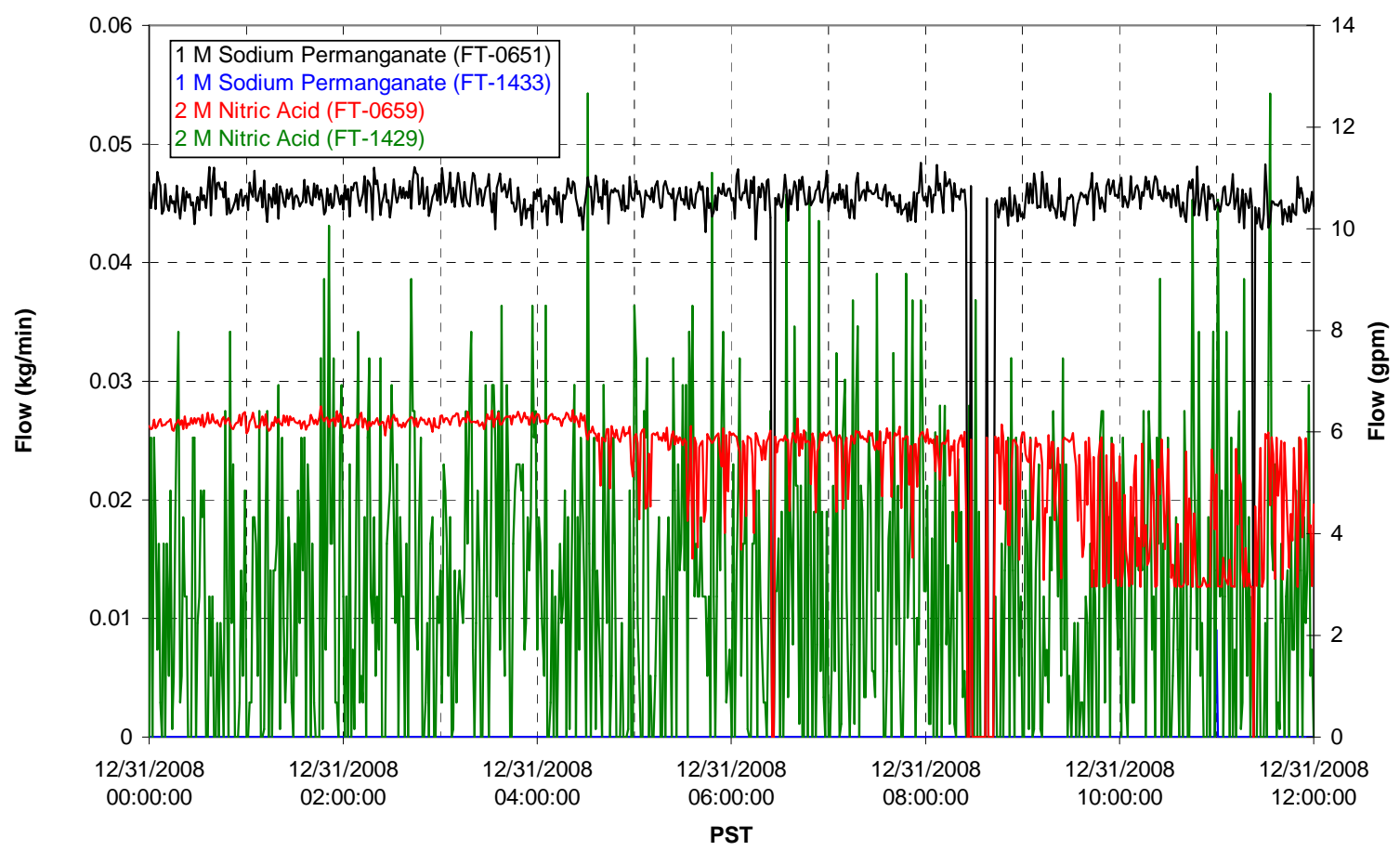

Air Flows

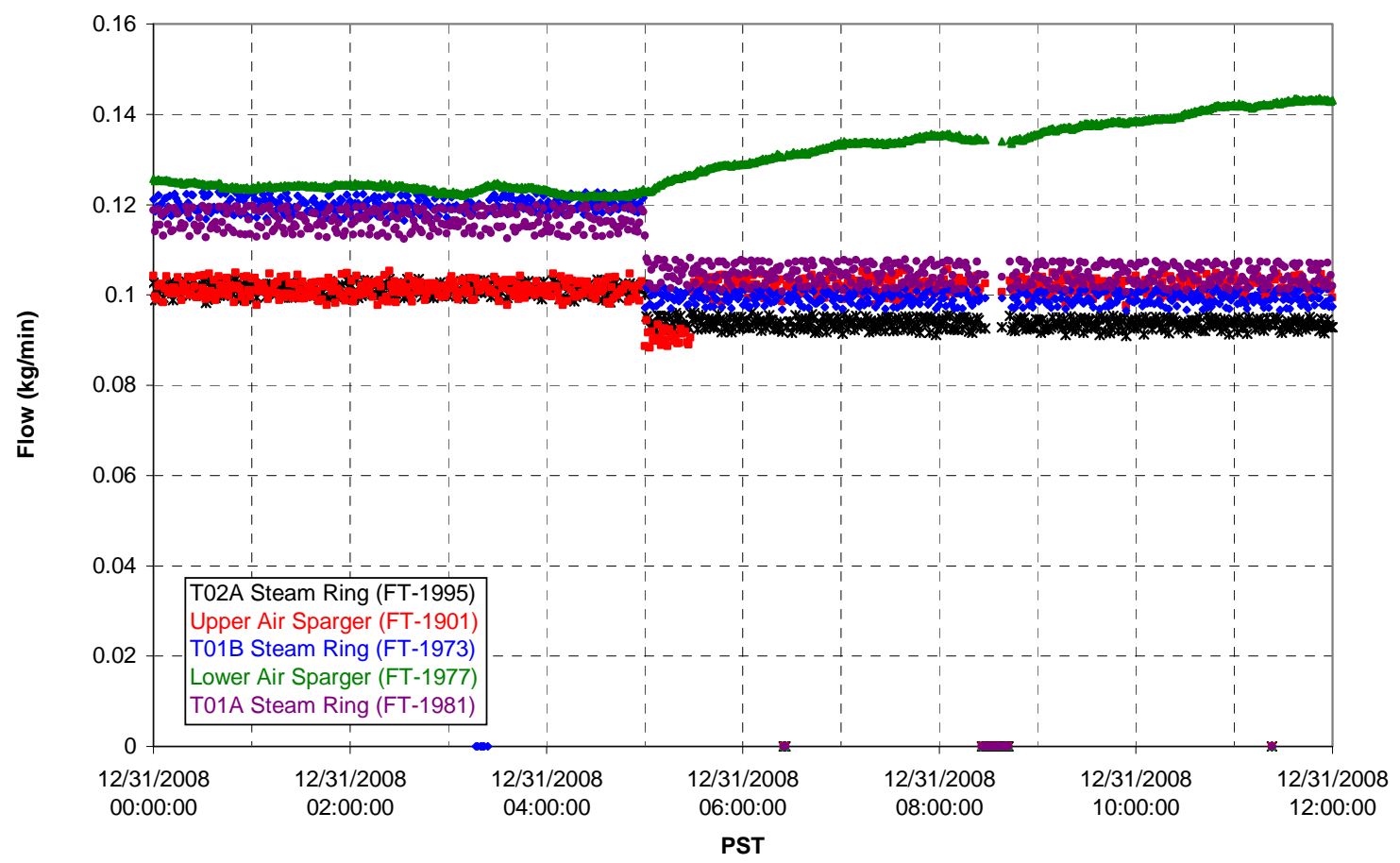


T02A Steam

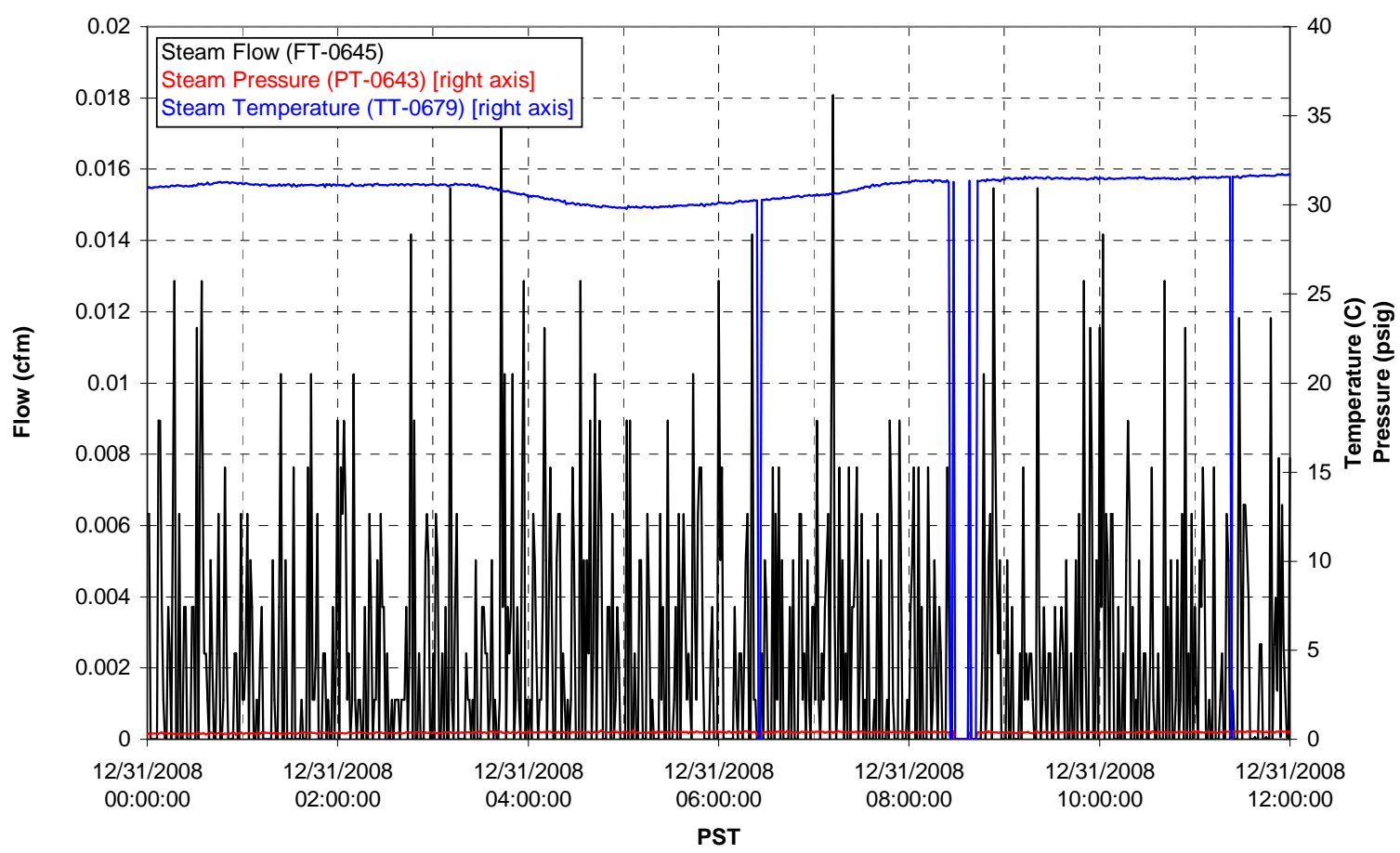

T01A Steam

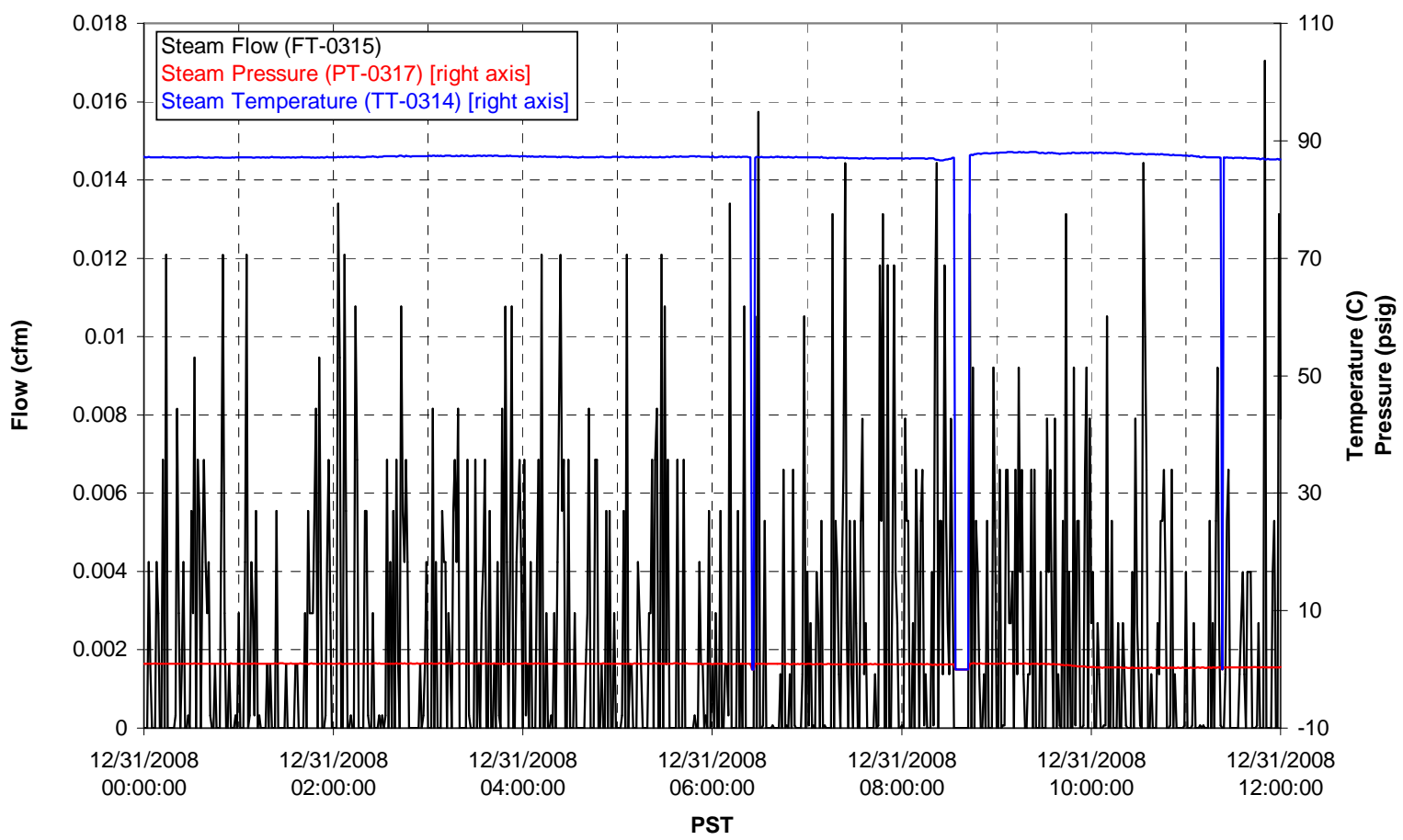


T01B Steam

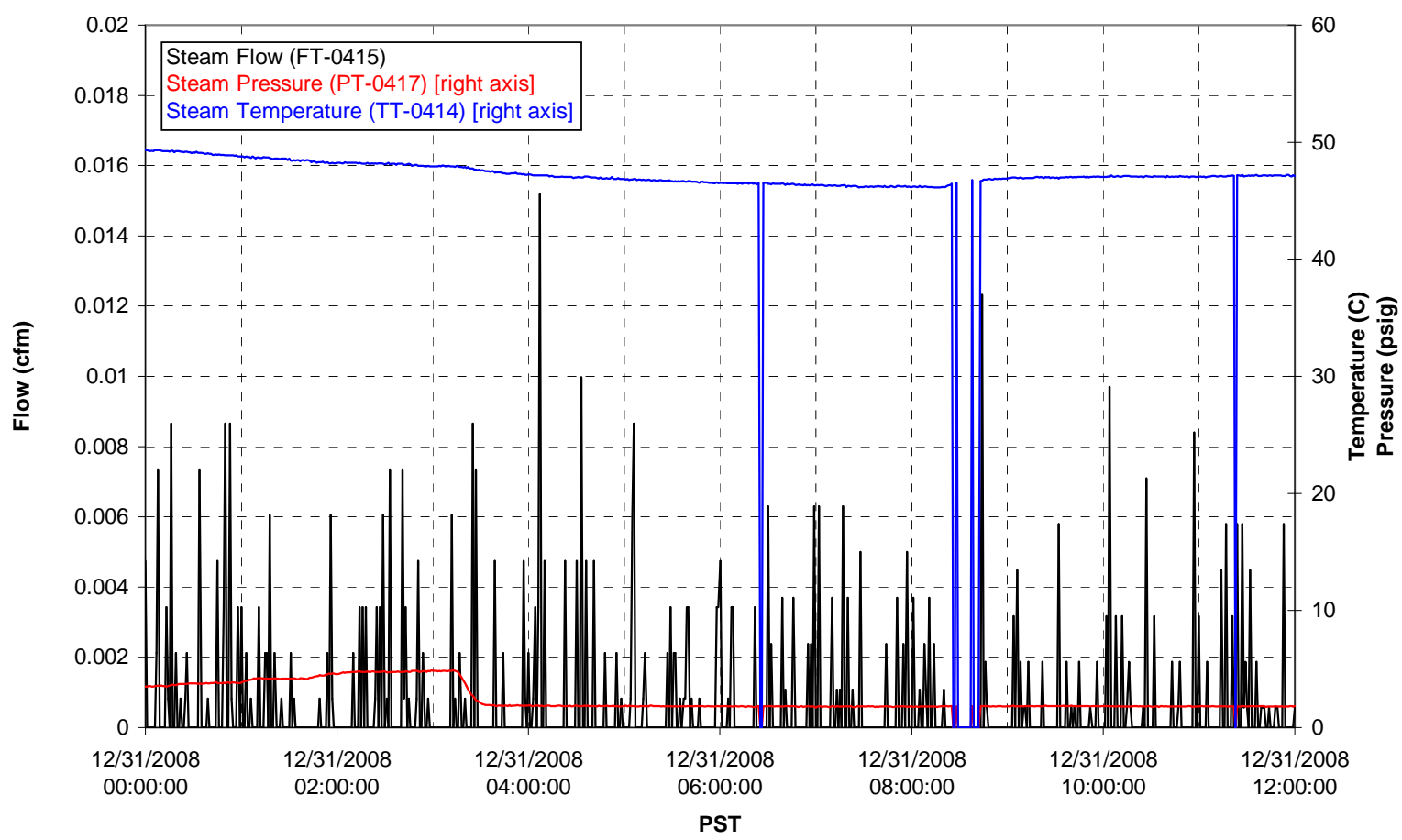


Functional Test Data Plots

12/31/2008 12:00 - 01/01/2009 00:00 
T01A level

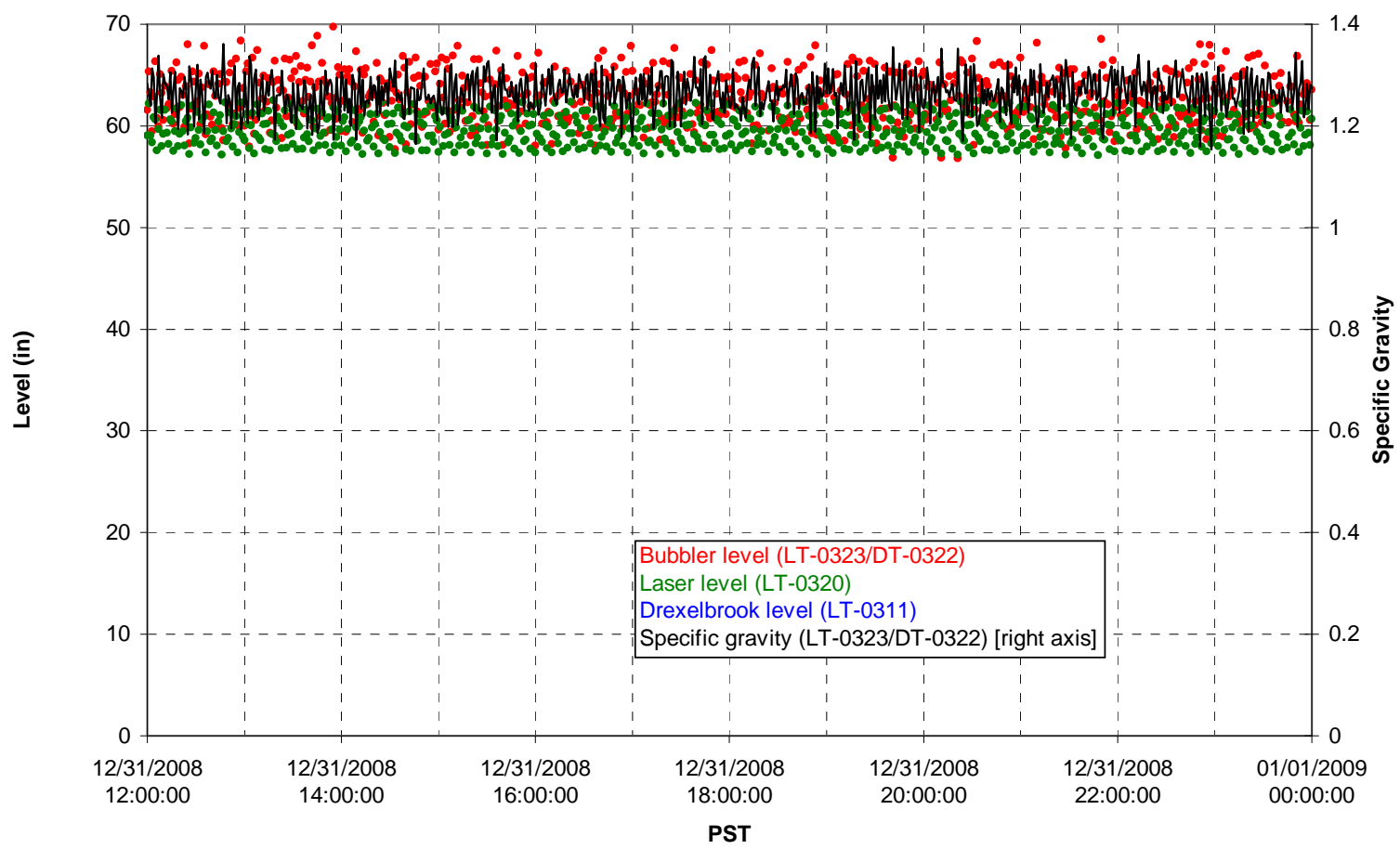

T01A temperatures

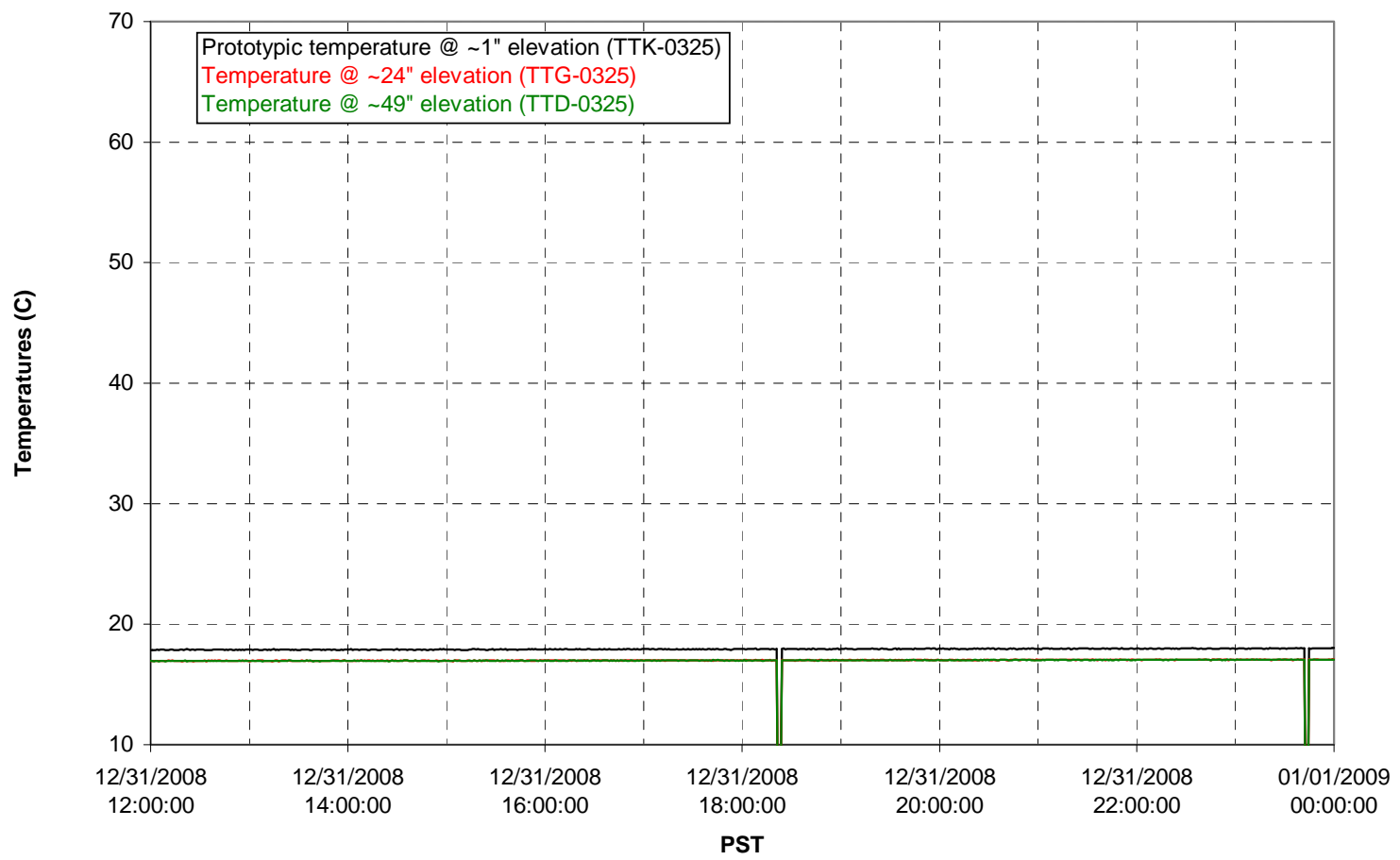


T01B level

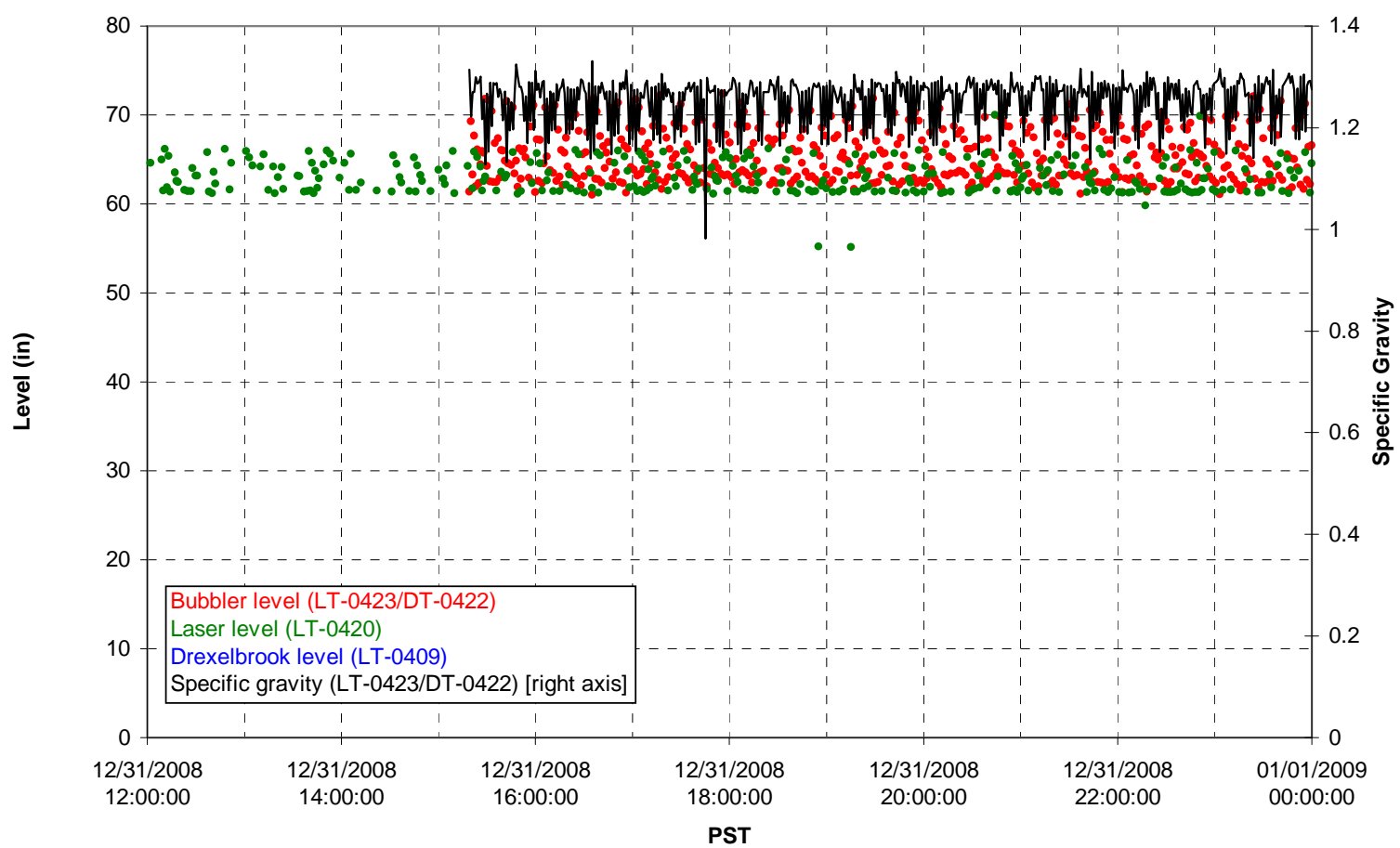

T01B temperatures

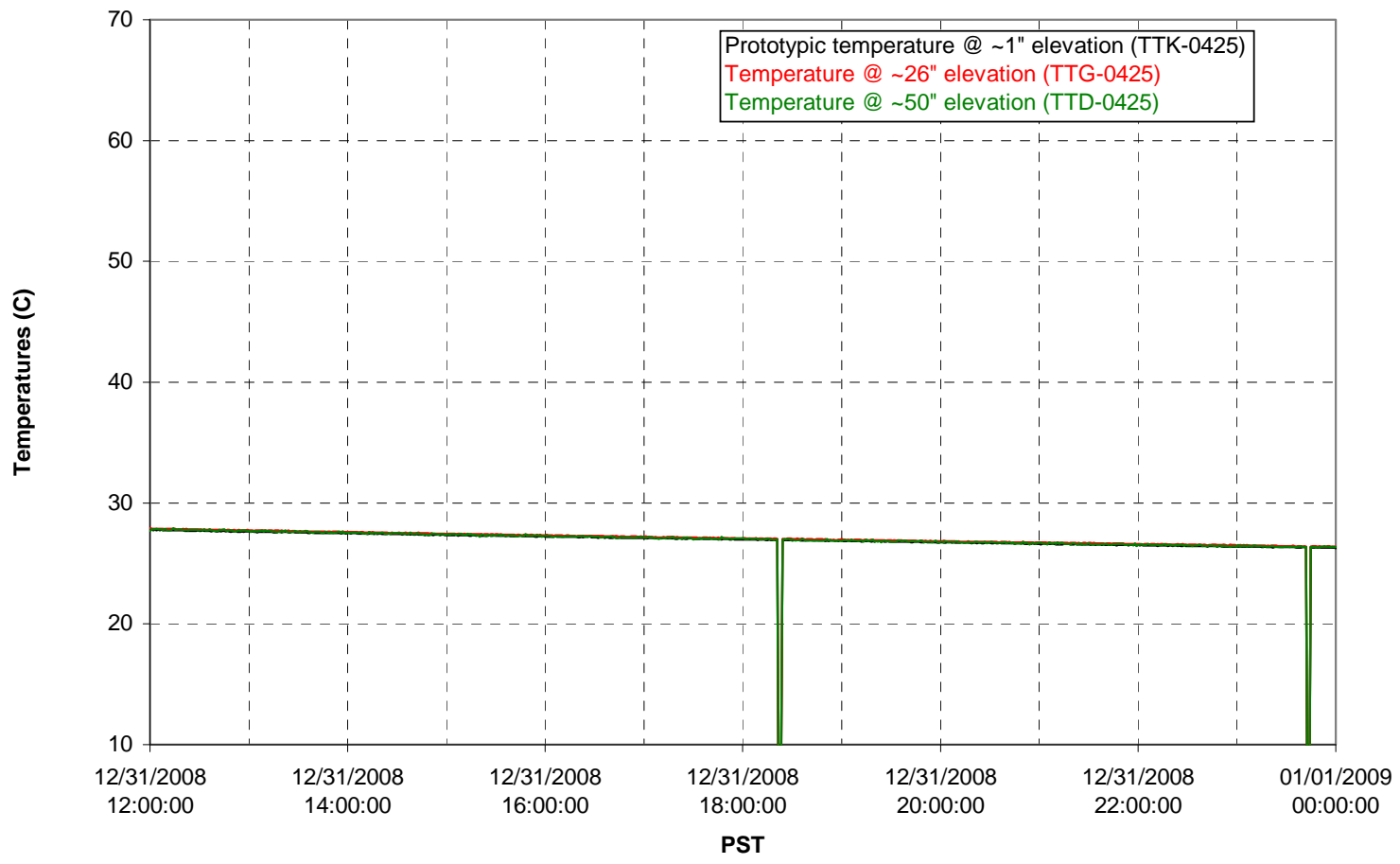


T02A level

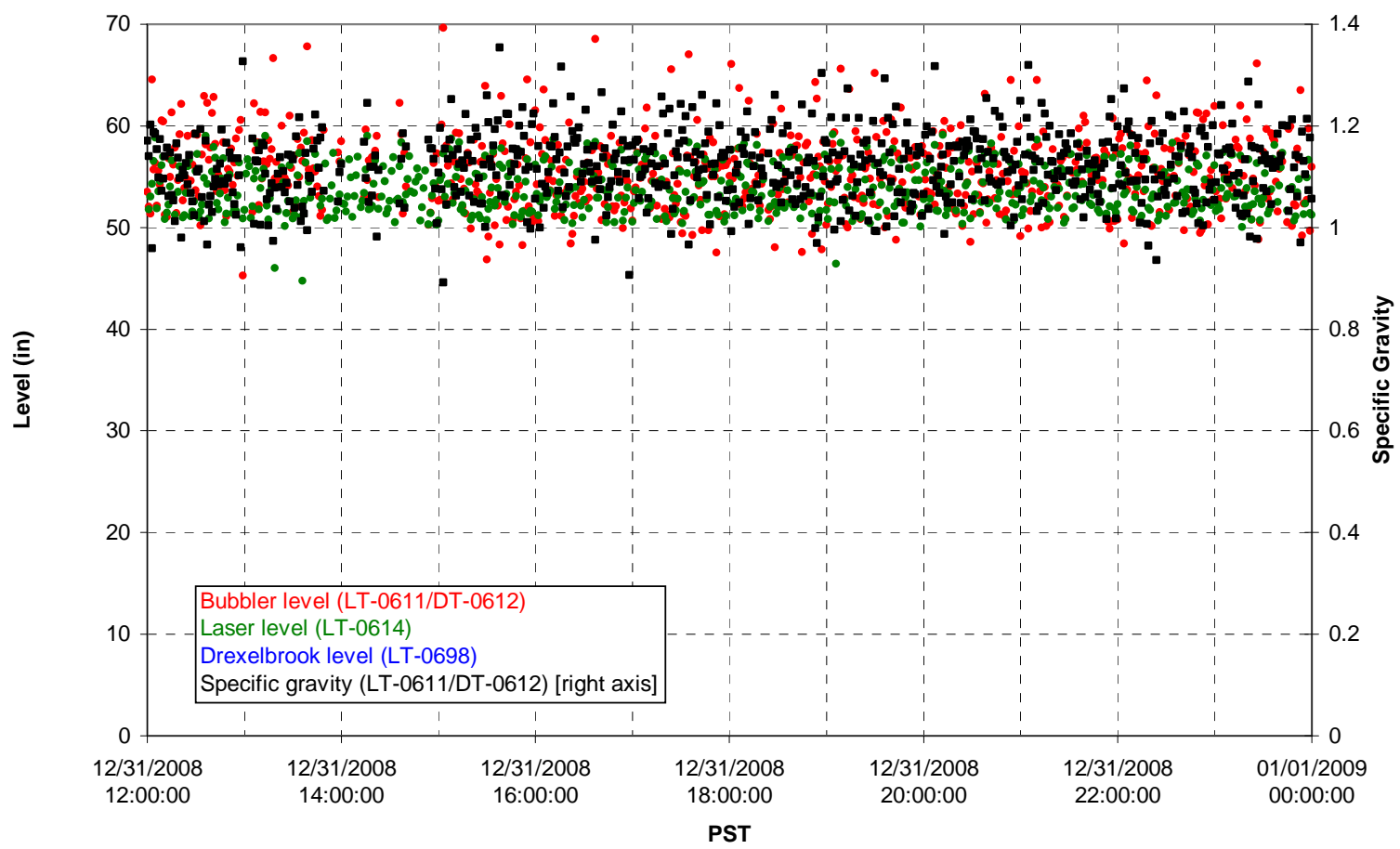

T02A temperatures

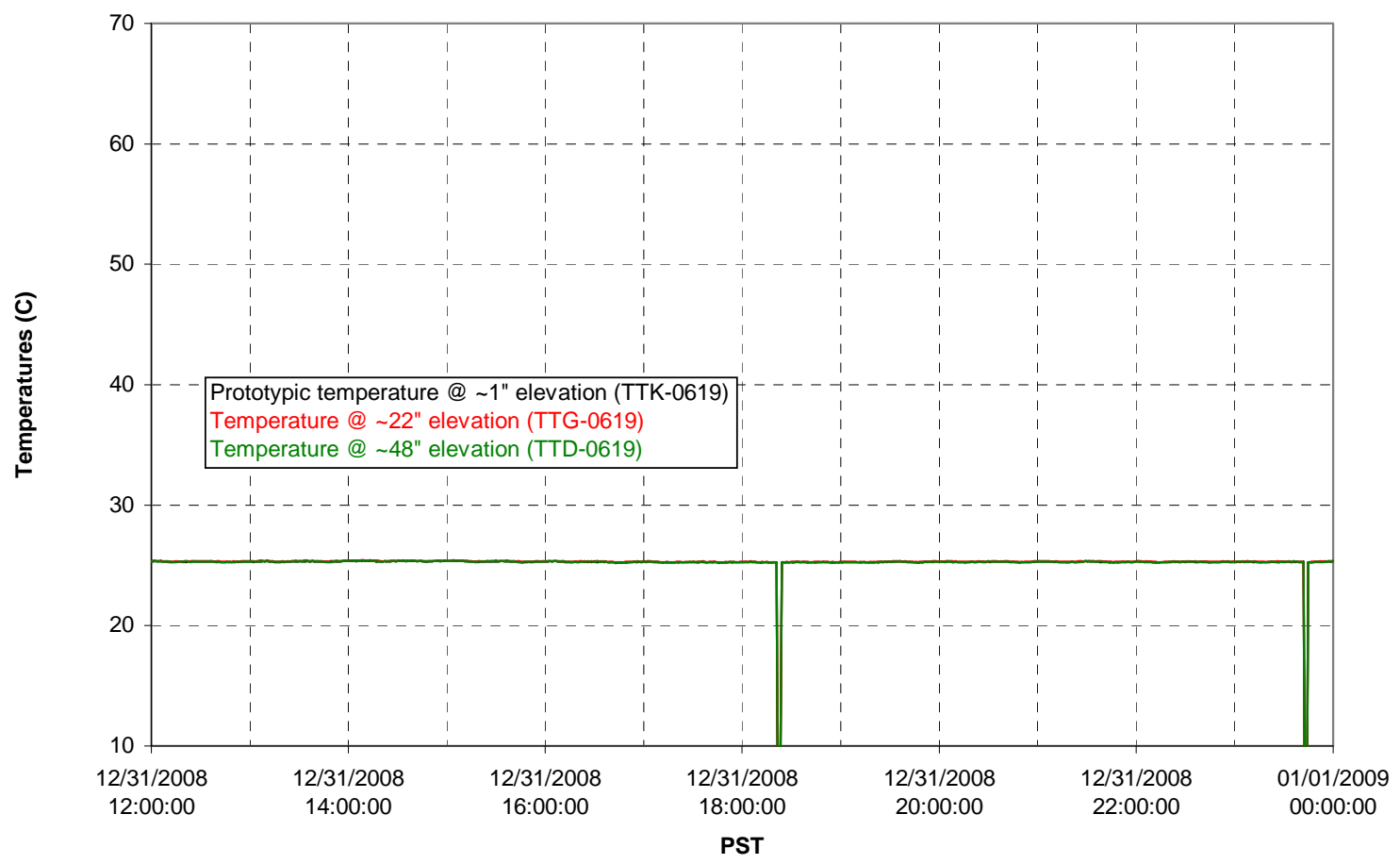


T02A and filter loop temperatures

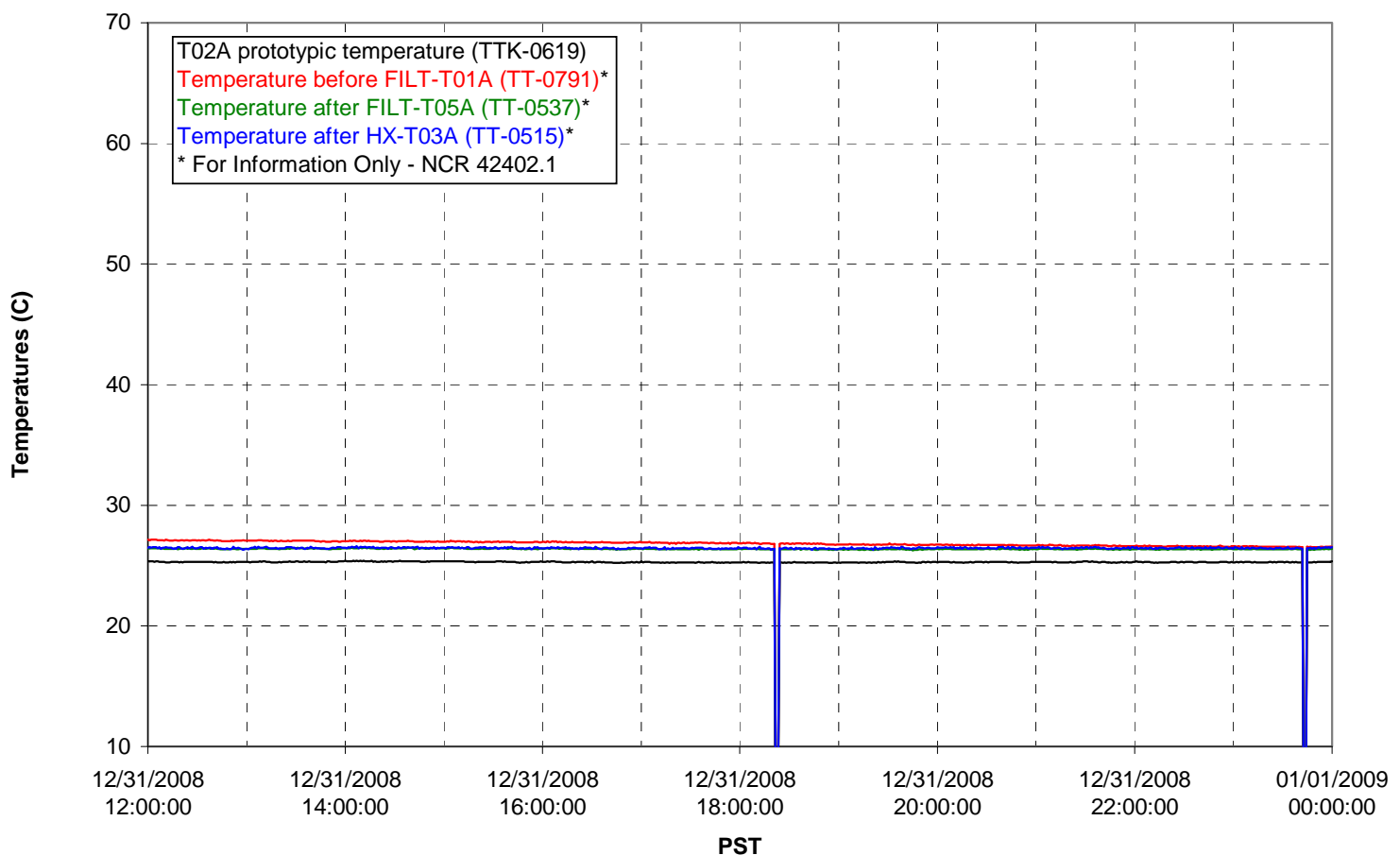

Pump Pressures and Flow

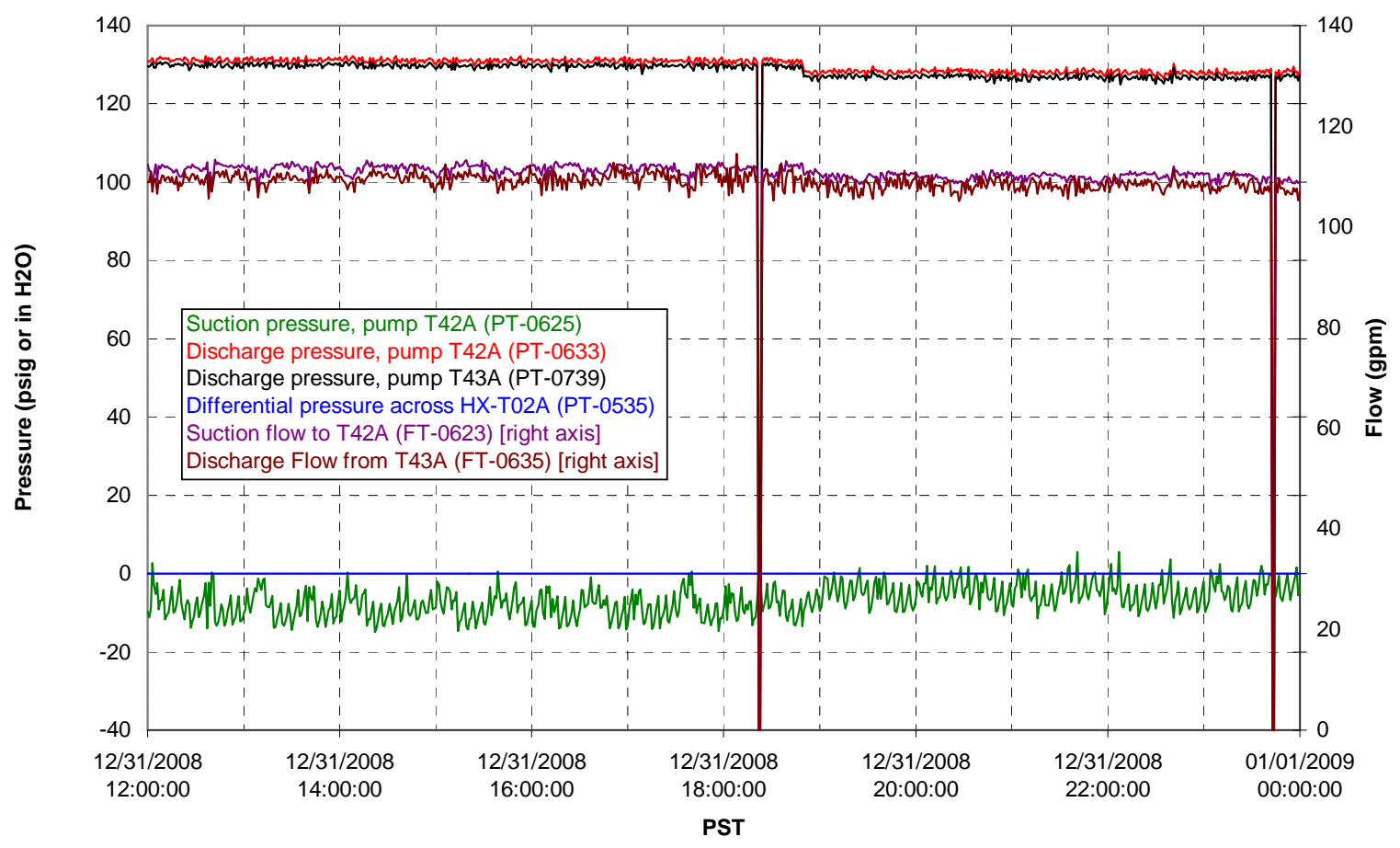


Axial pressure drop

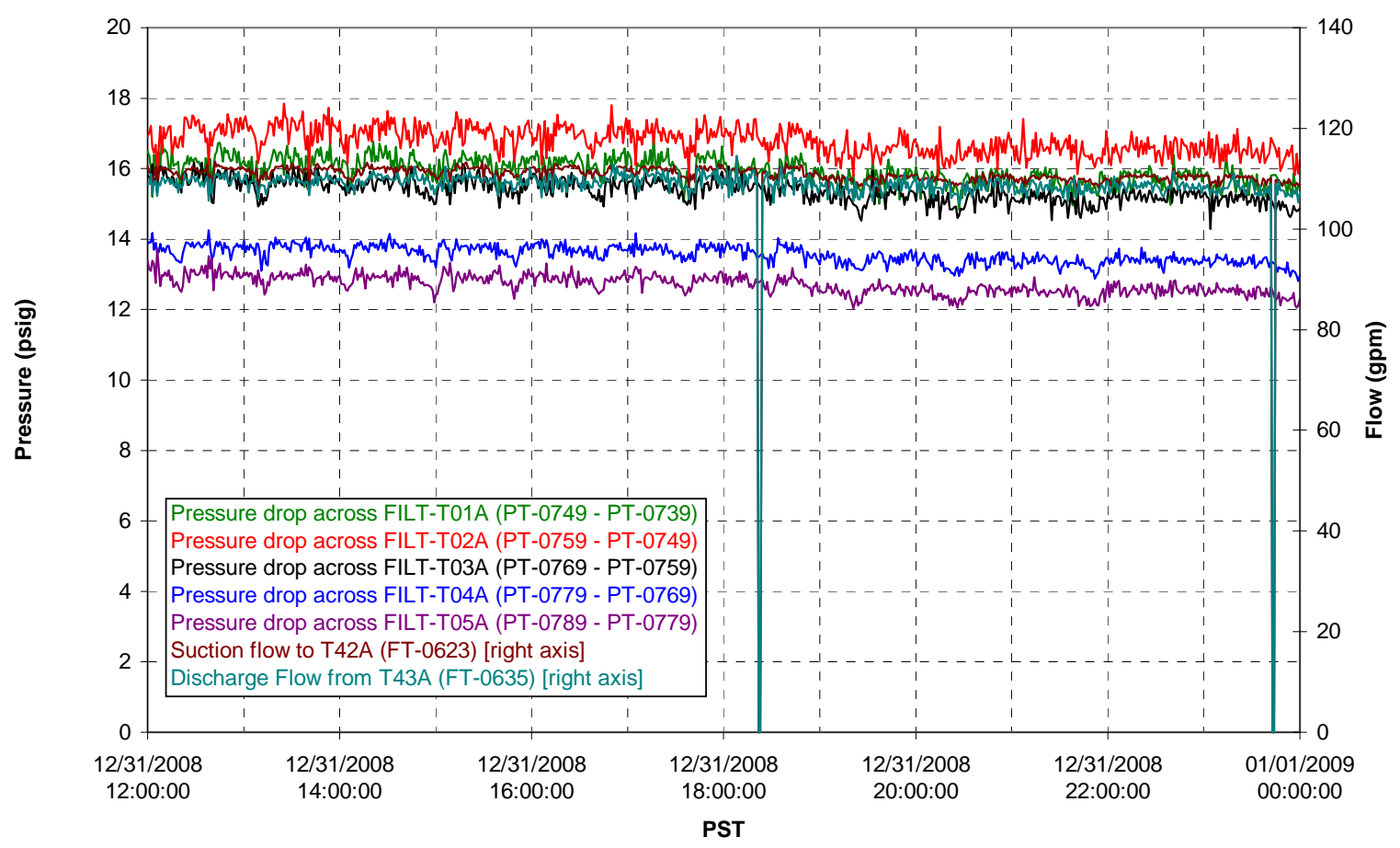

Permeate flow rates

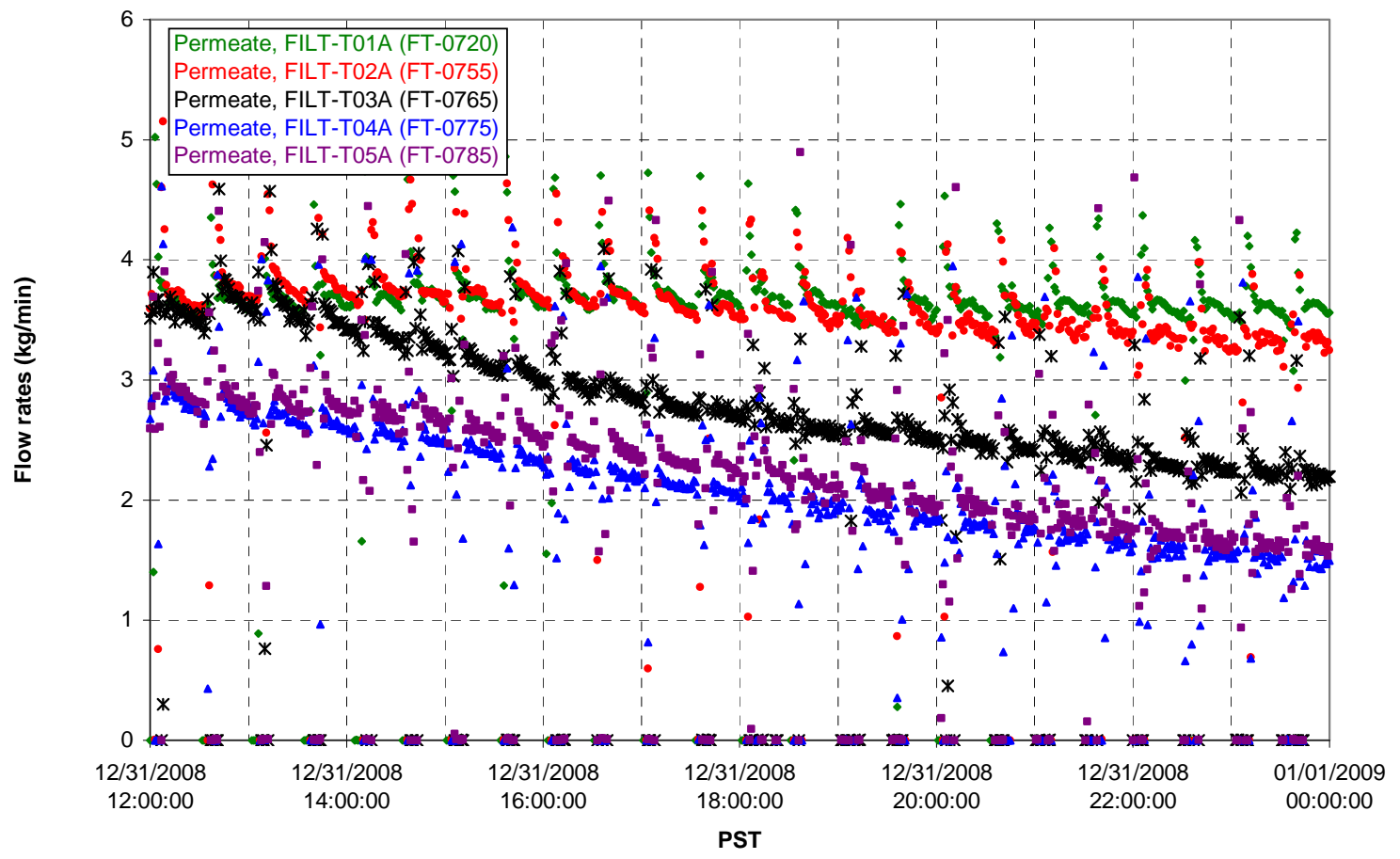


T02A Inner Temperature Tree

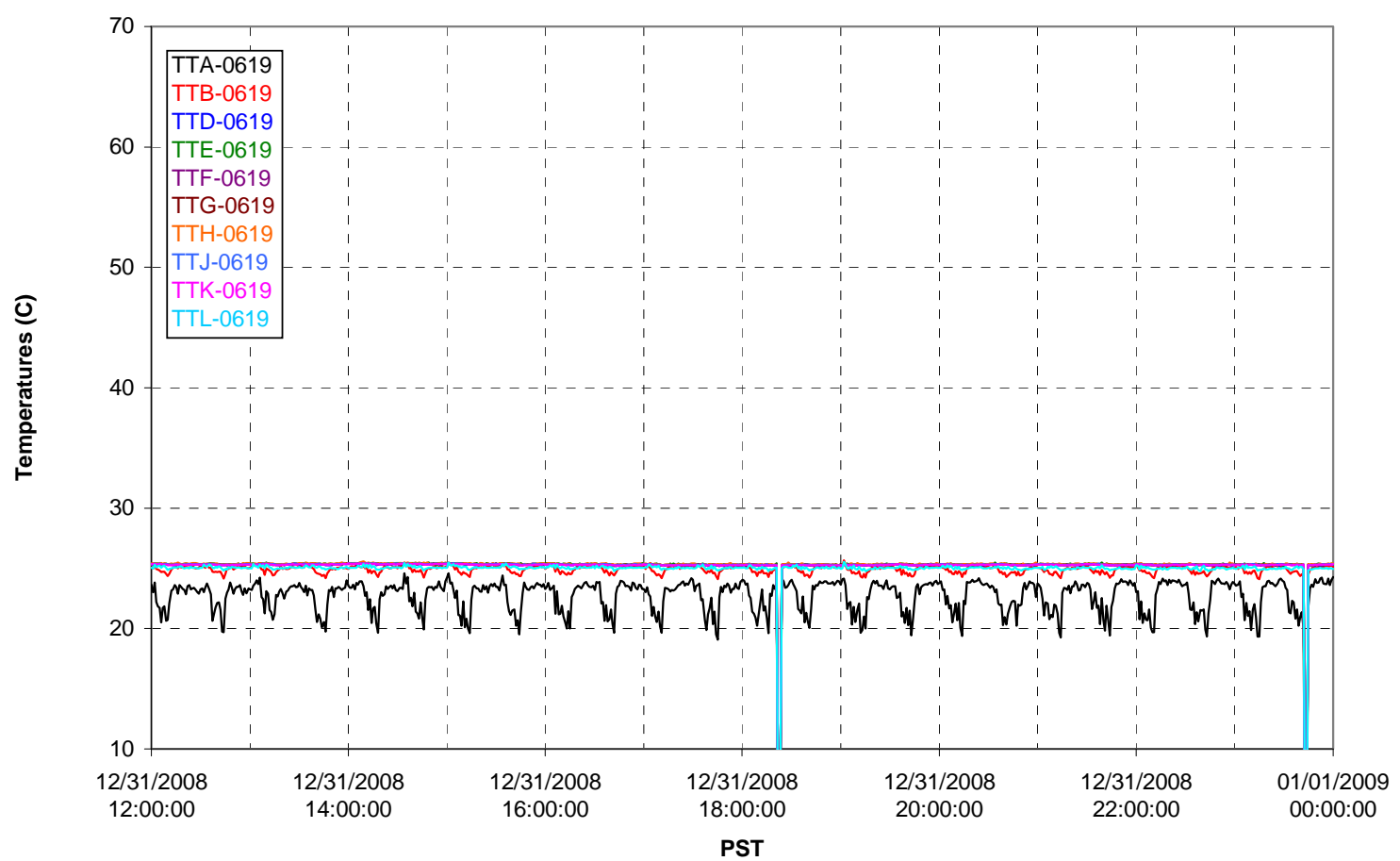

T02A Outer Temperature Tree

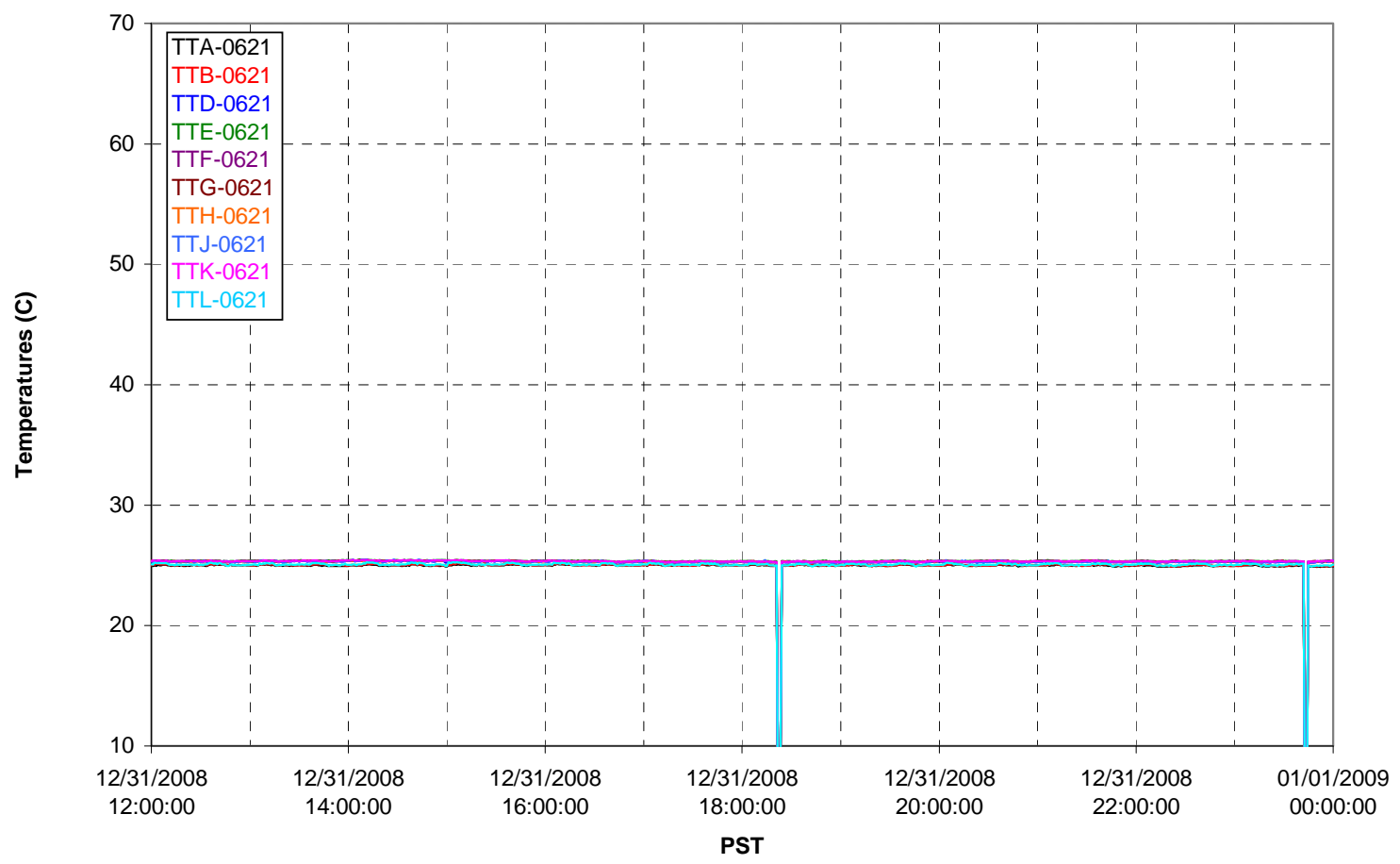


T02A temperatures

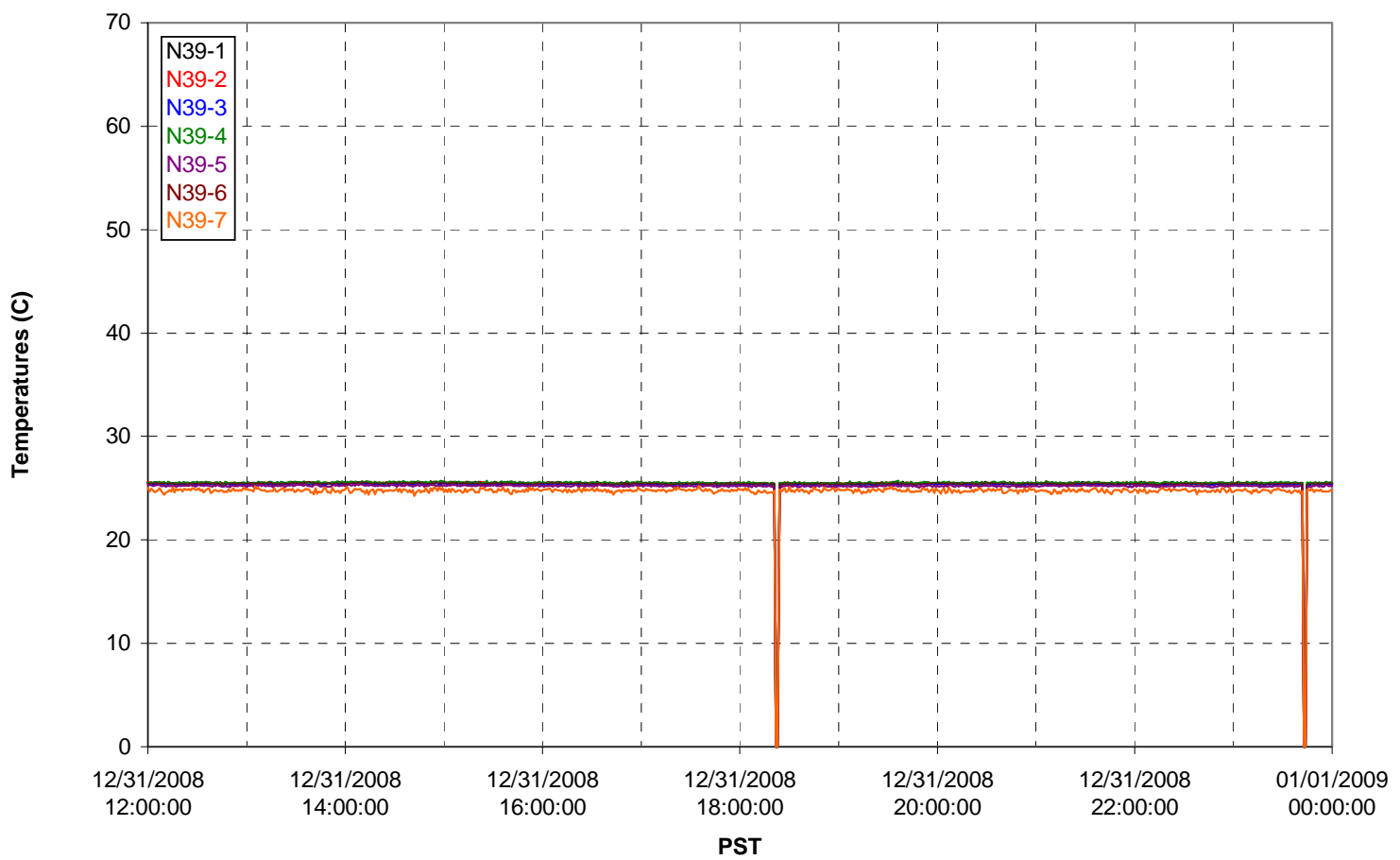

T02A temperatures

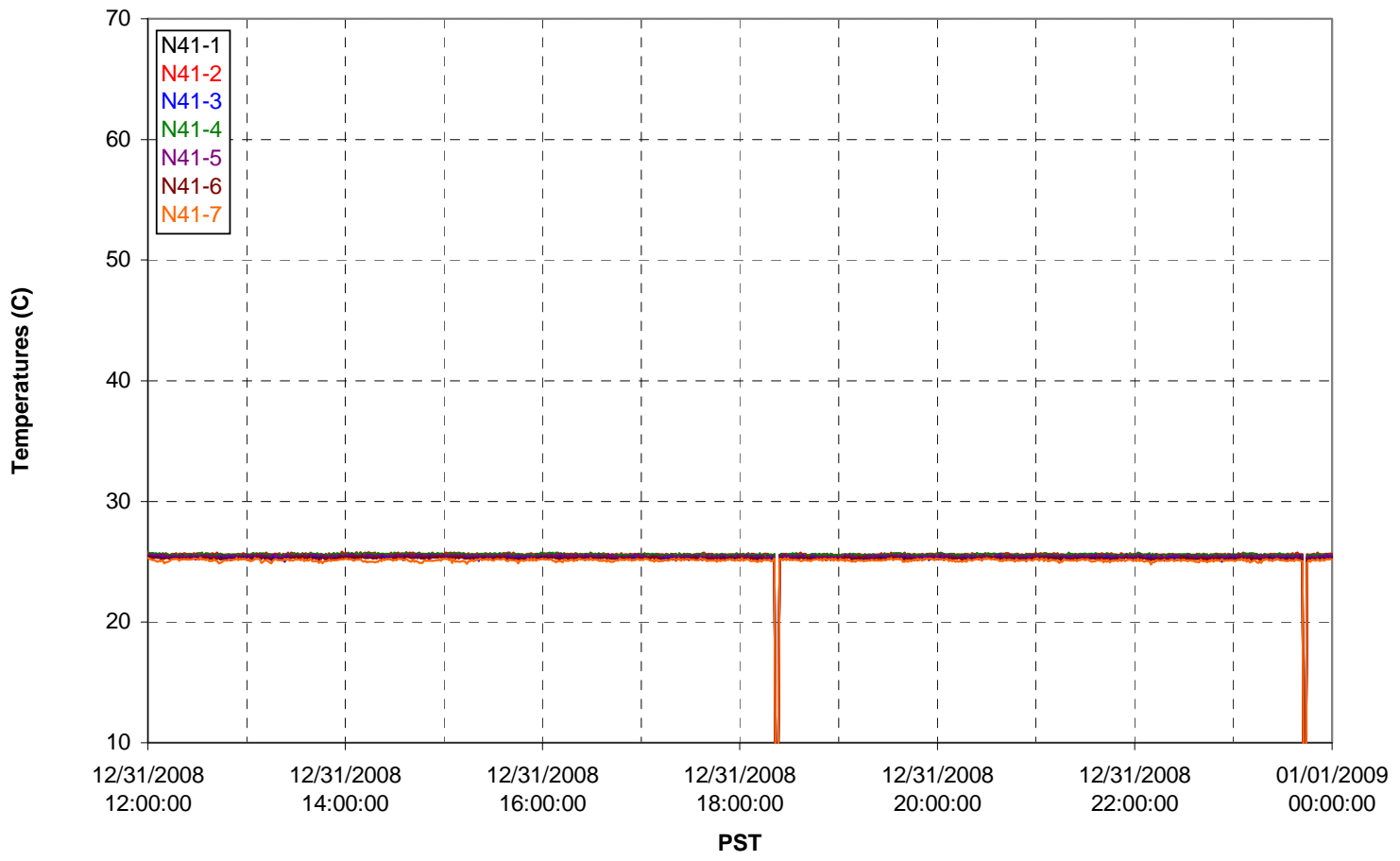


T02A temperatures

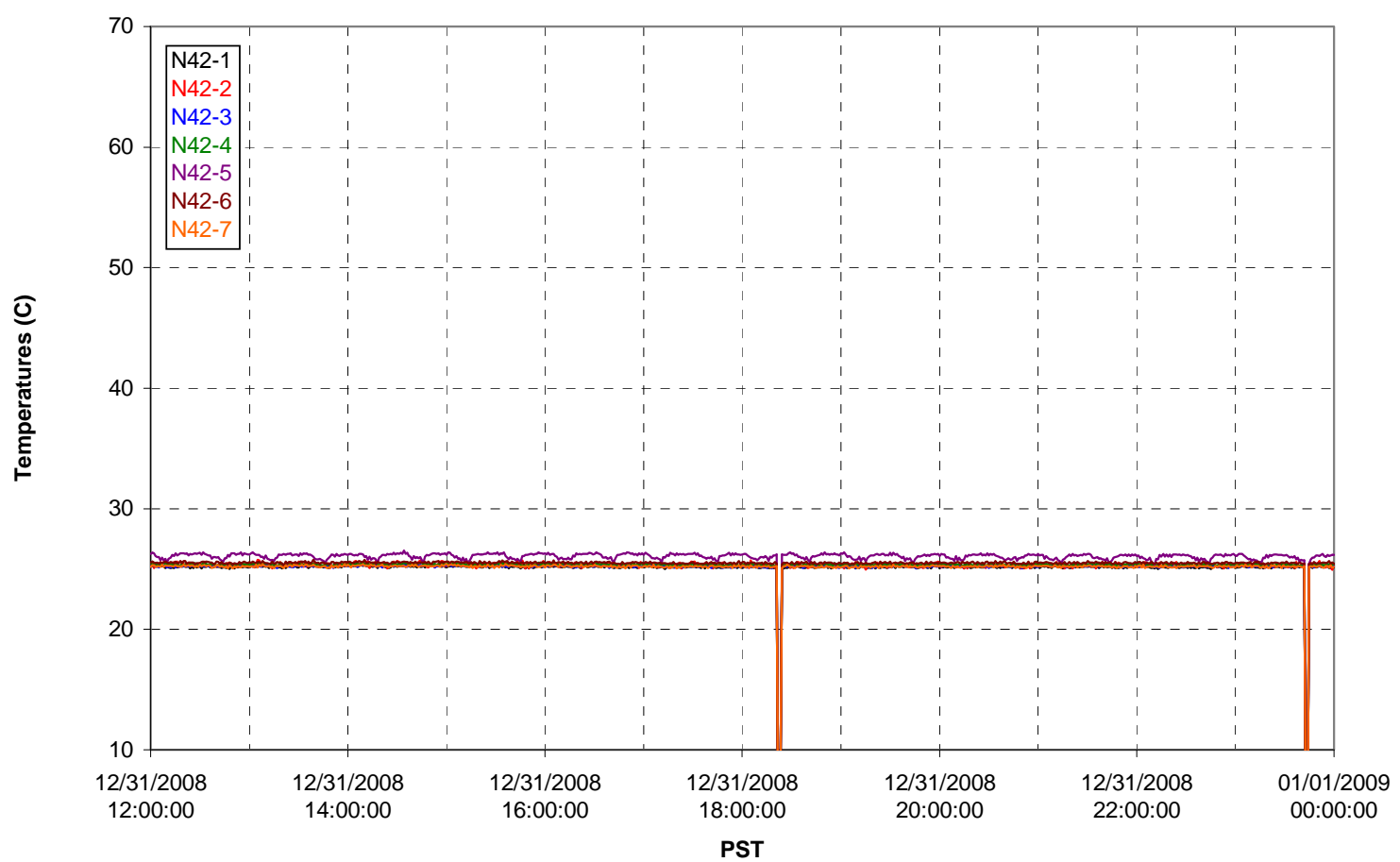

T02A temperatures

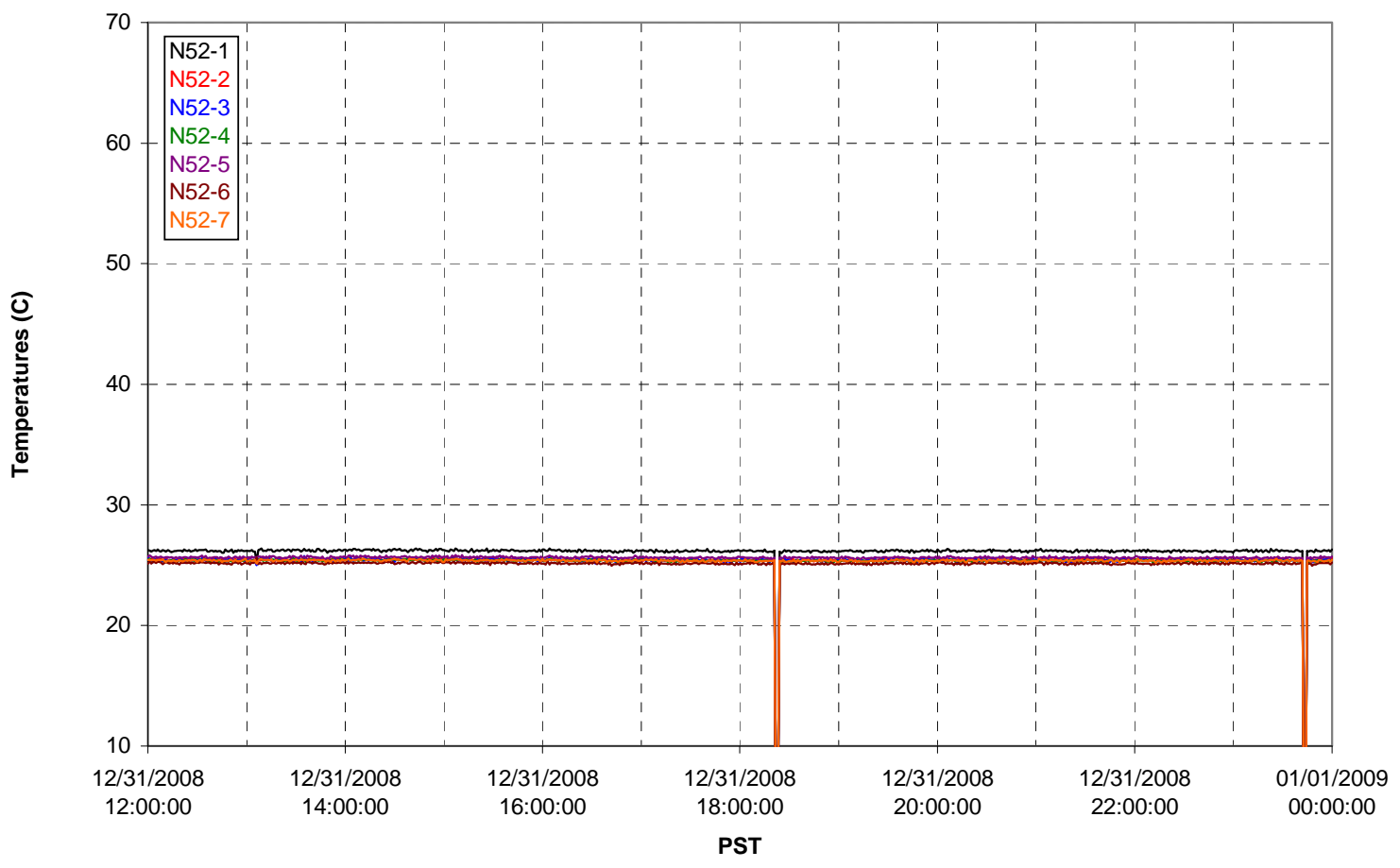


T02A Heating and Cooling

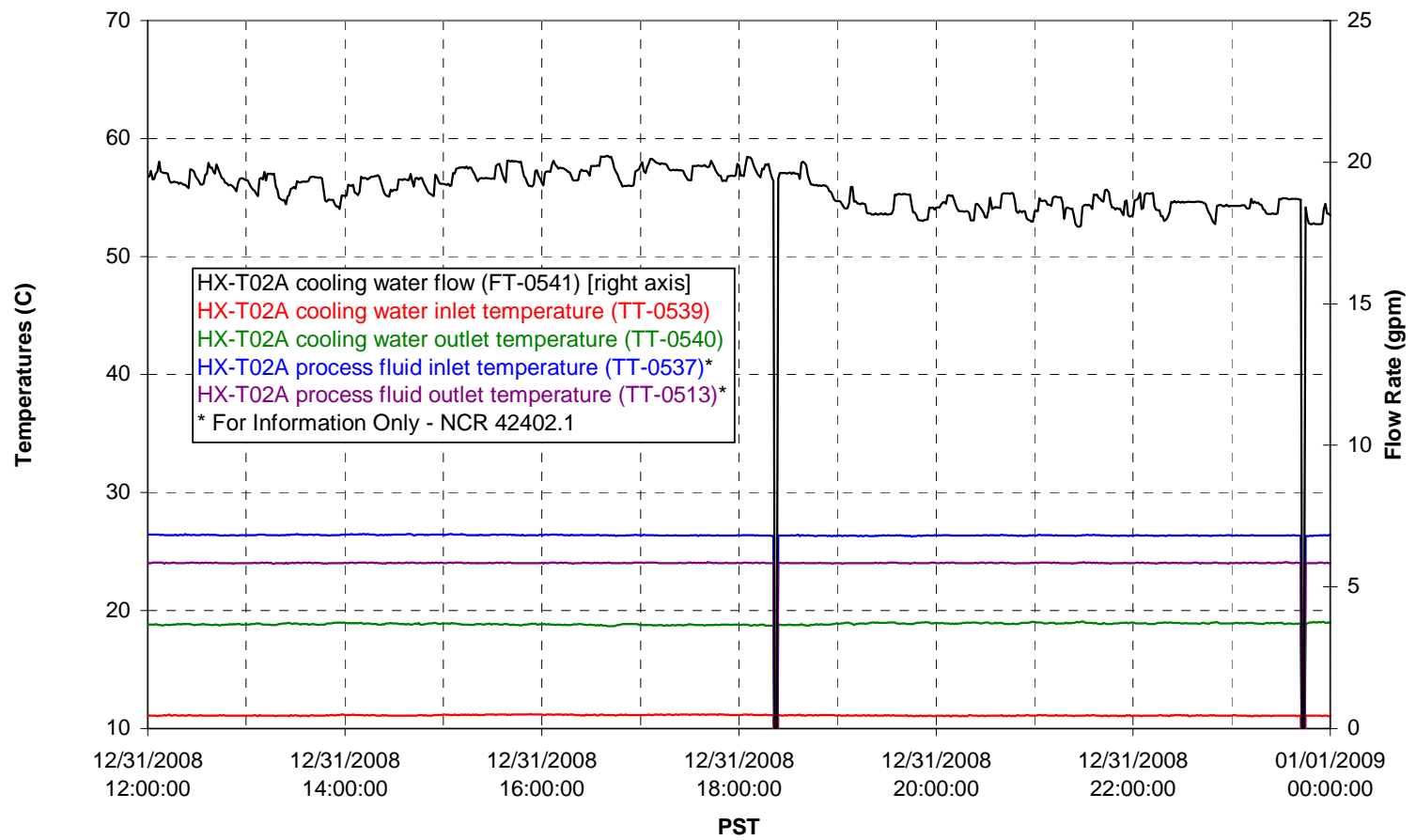

Pump Operation

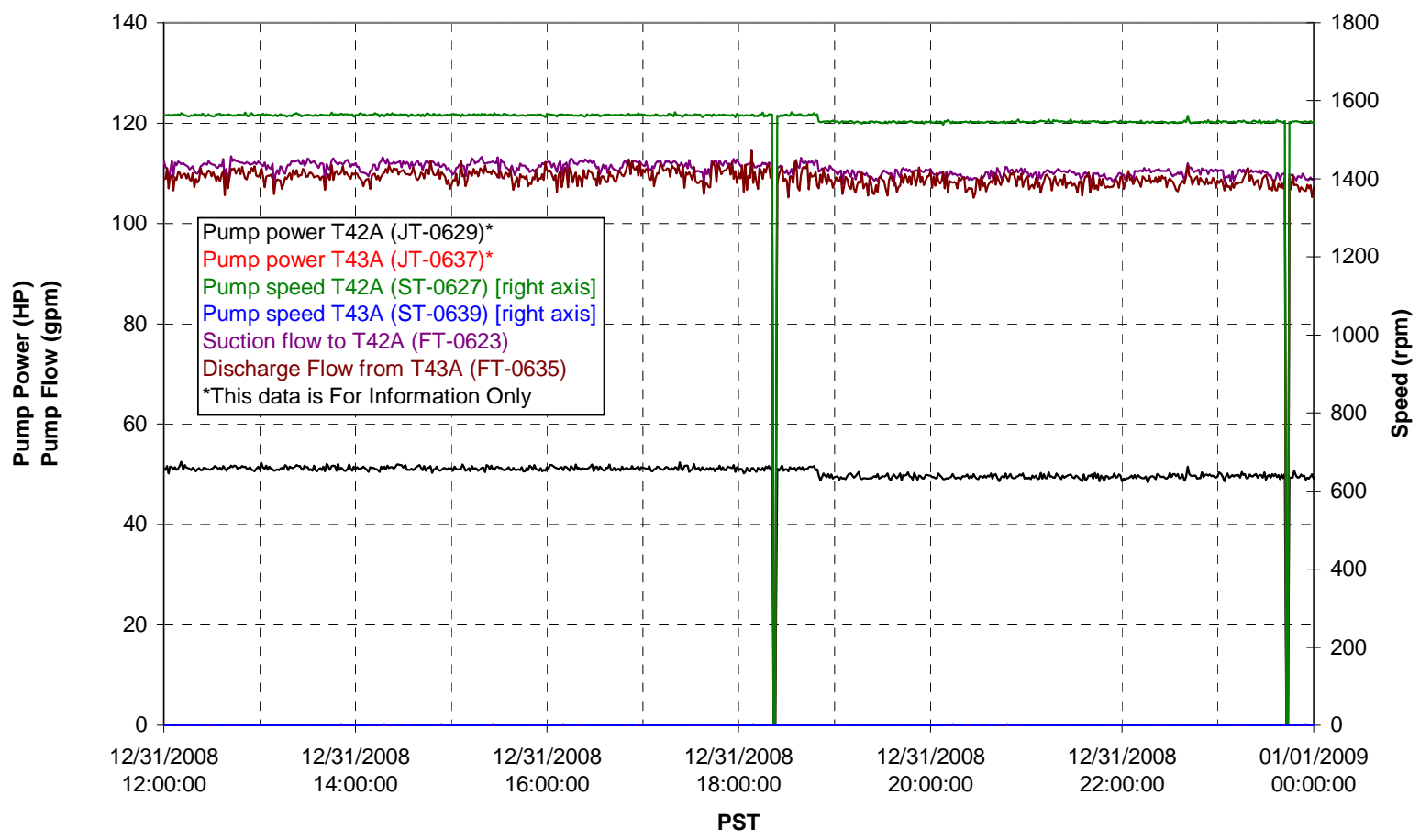


Pulsepot UFP-PP-T01A

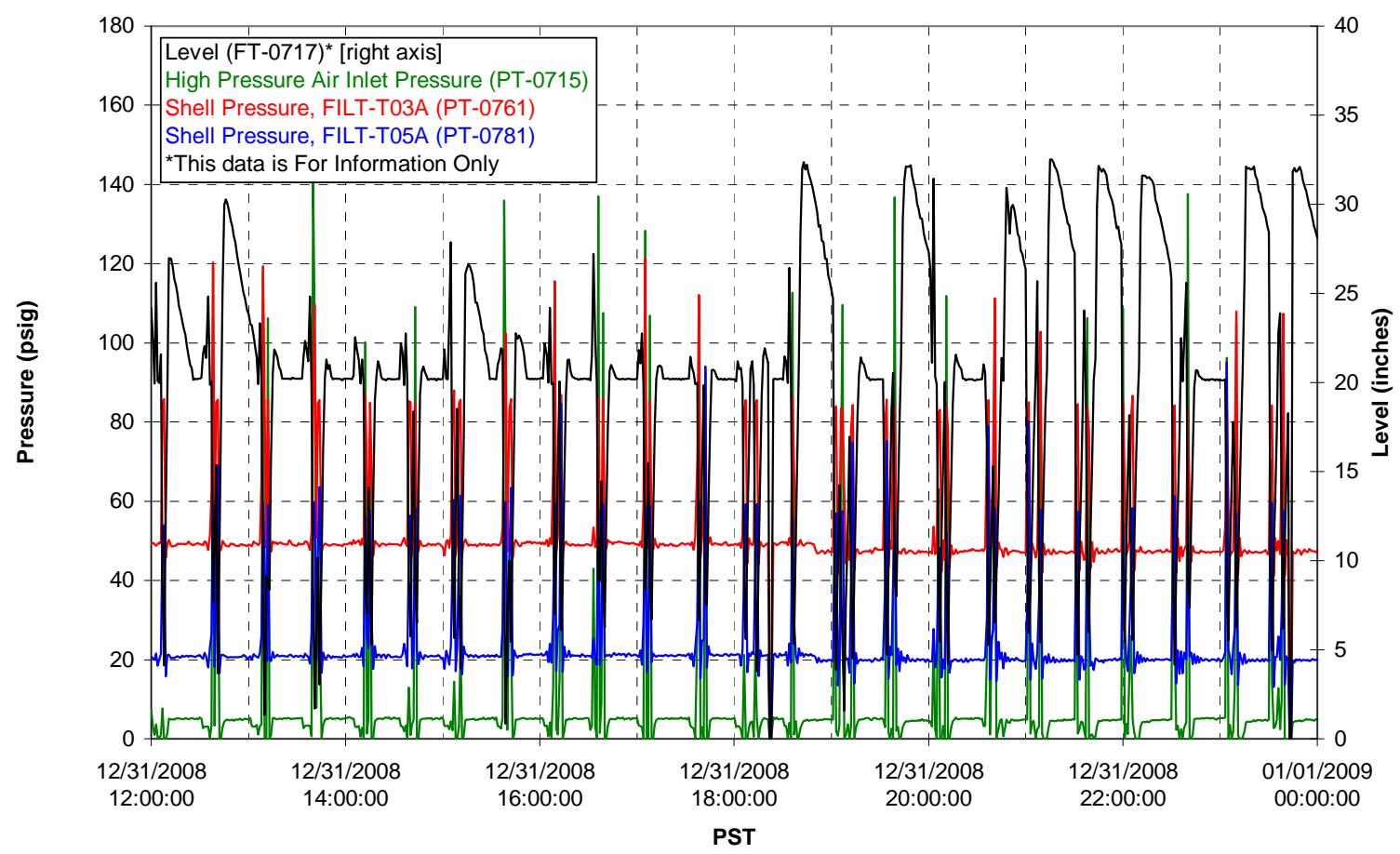

Pulsepot UFP-PP-T02A

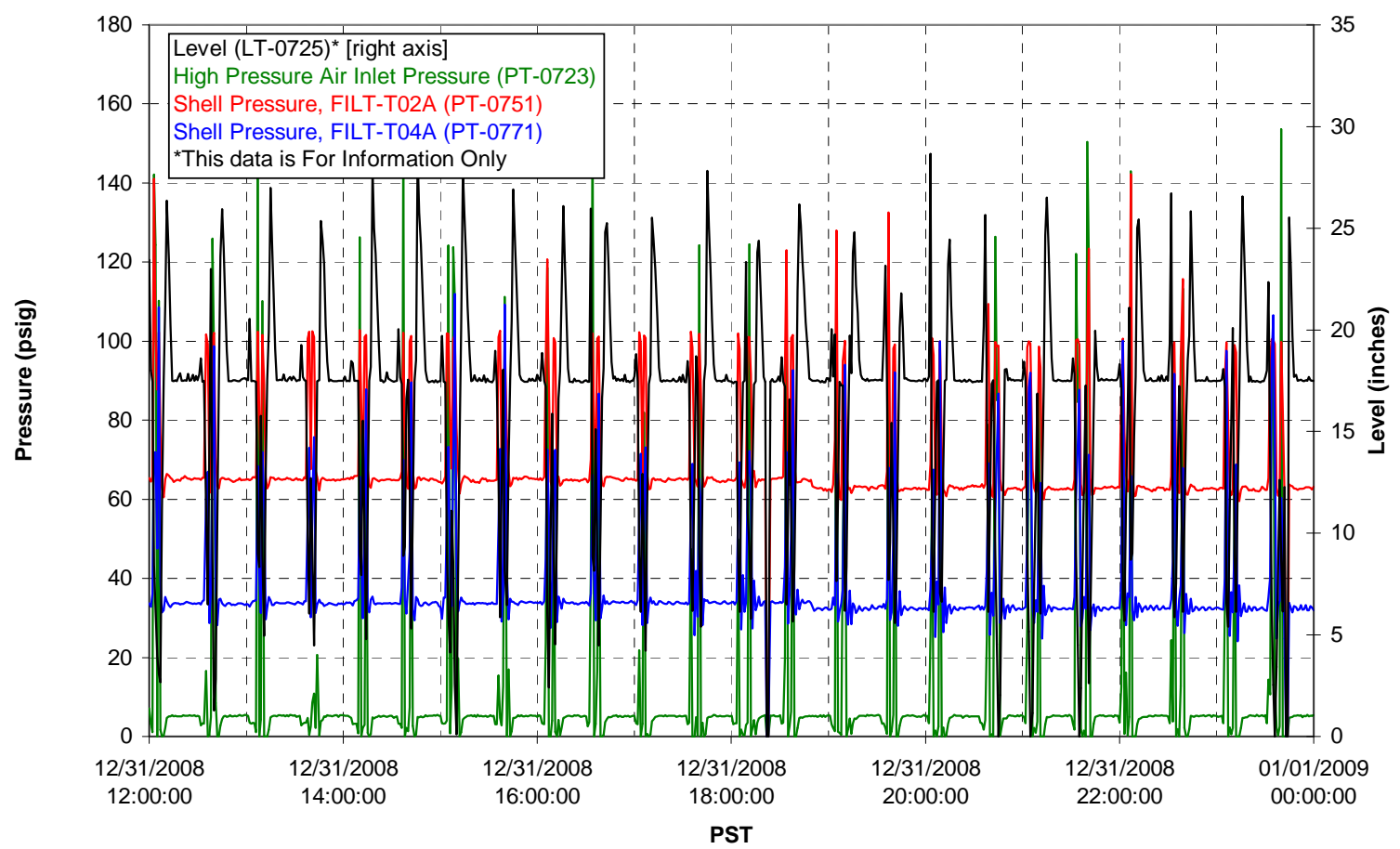


Pulsepot UFP-PP-T03A

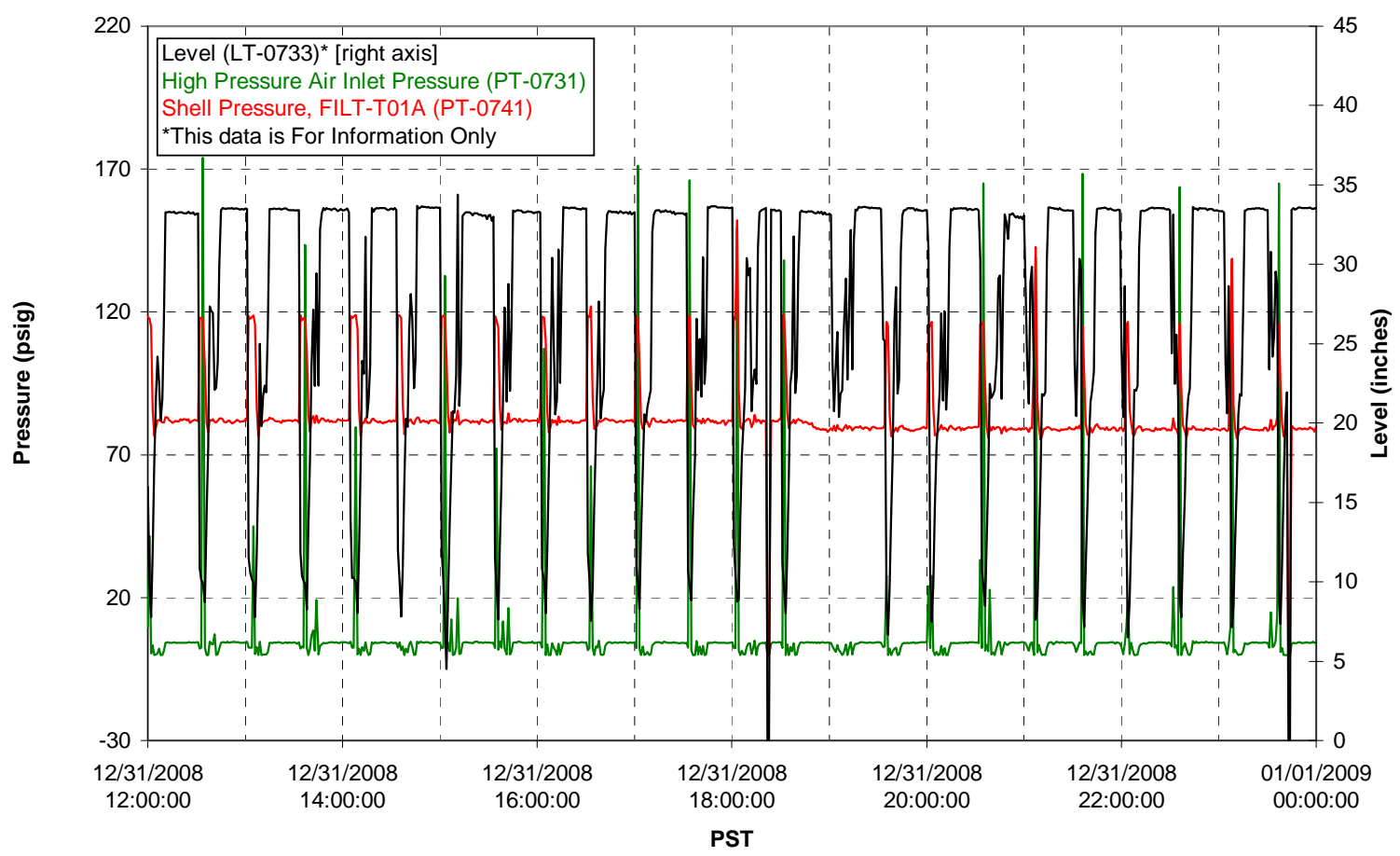

Pulsepot Levels

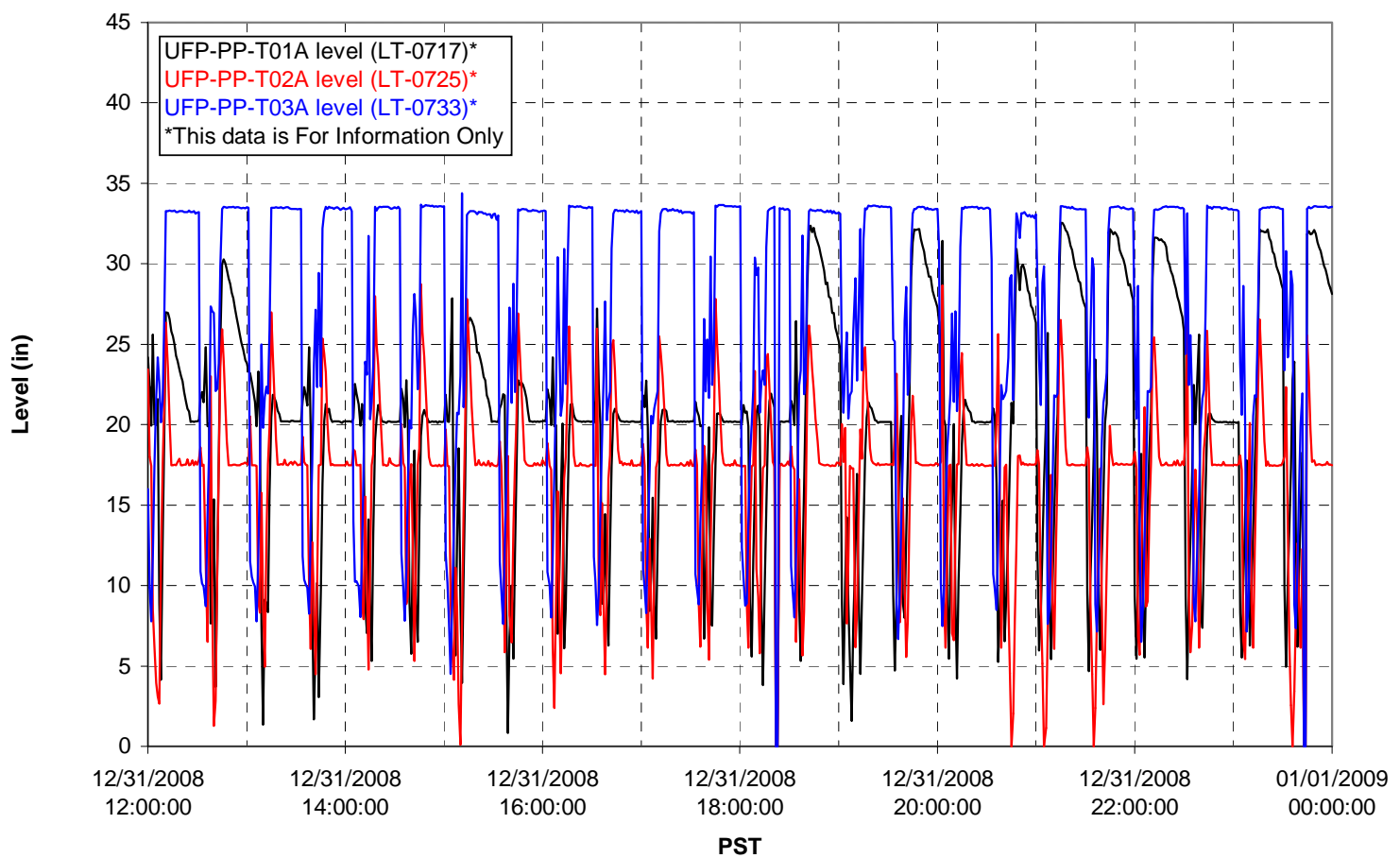


Filter UFP-FILT-T01A

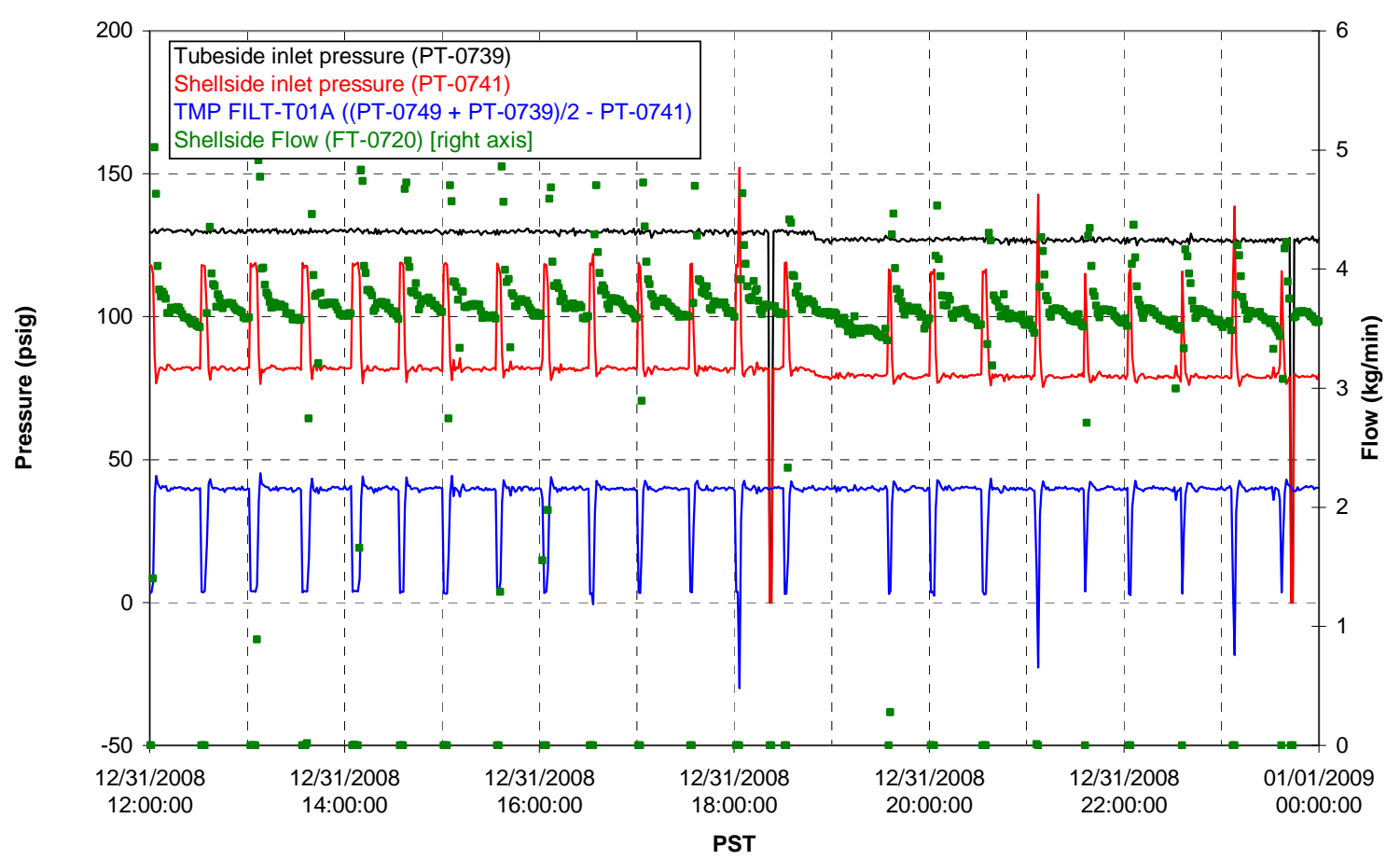

Filter UFP-FILT-T02A

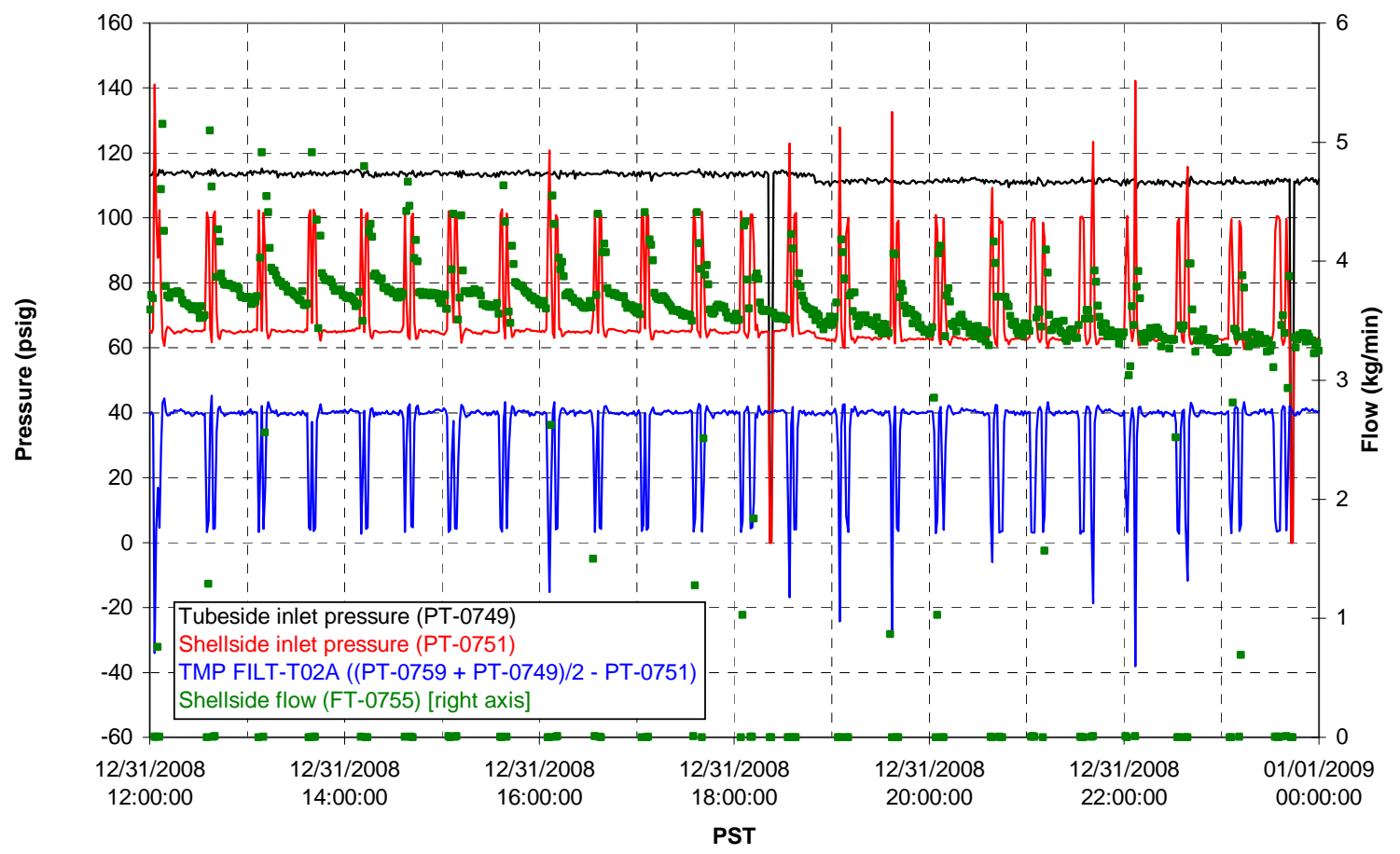


Filter UFP-FILT-T03A

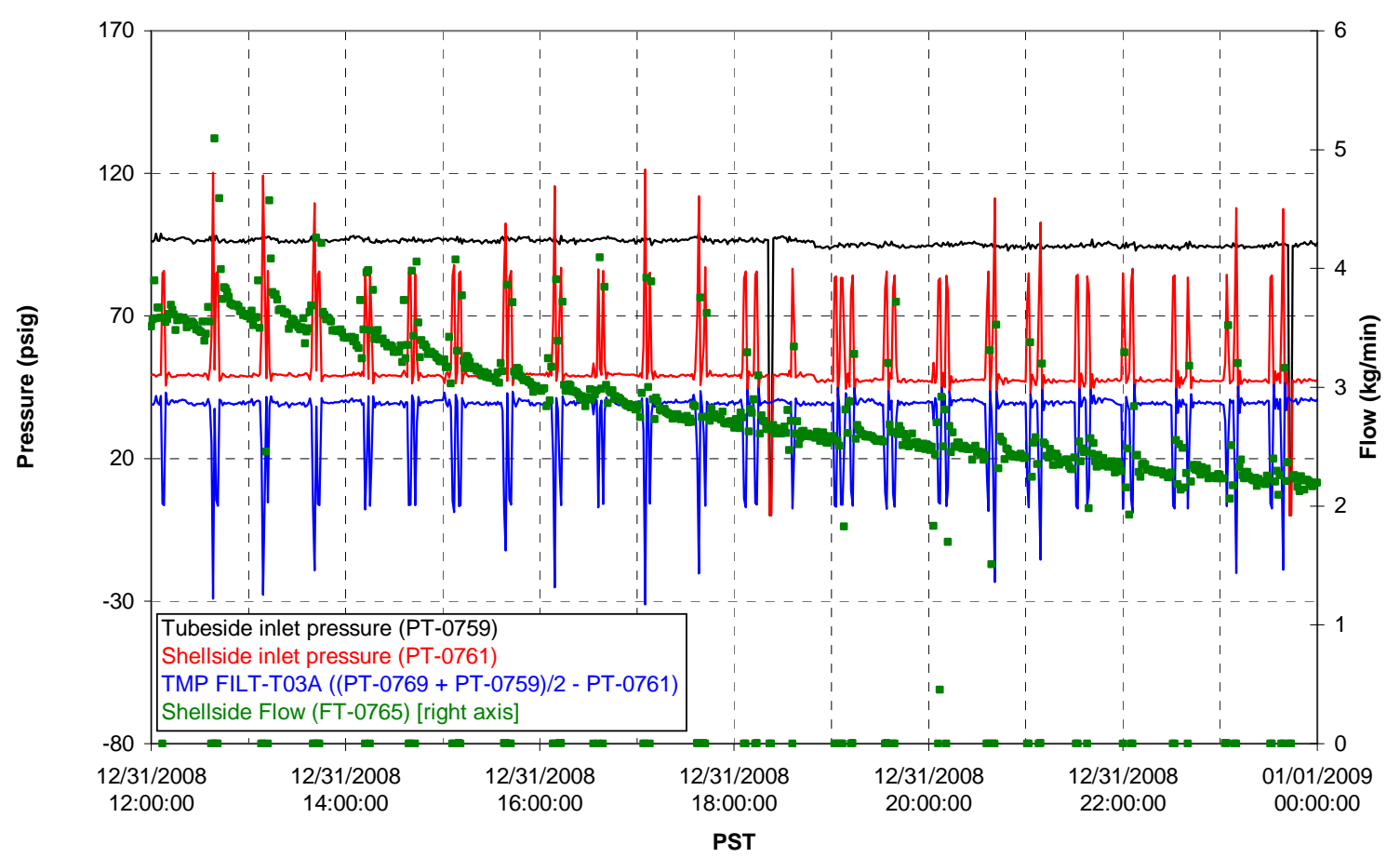

Filter UFP-FILT-T04A

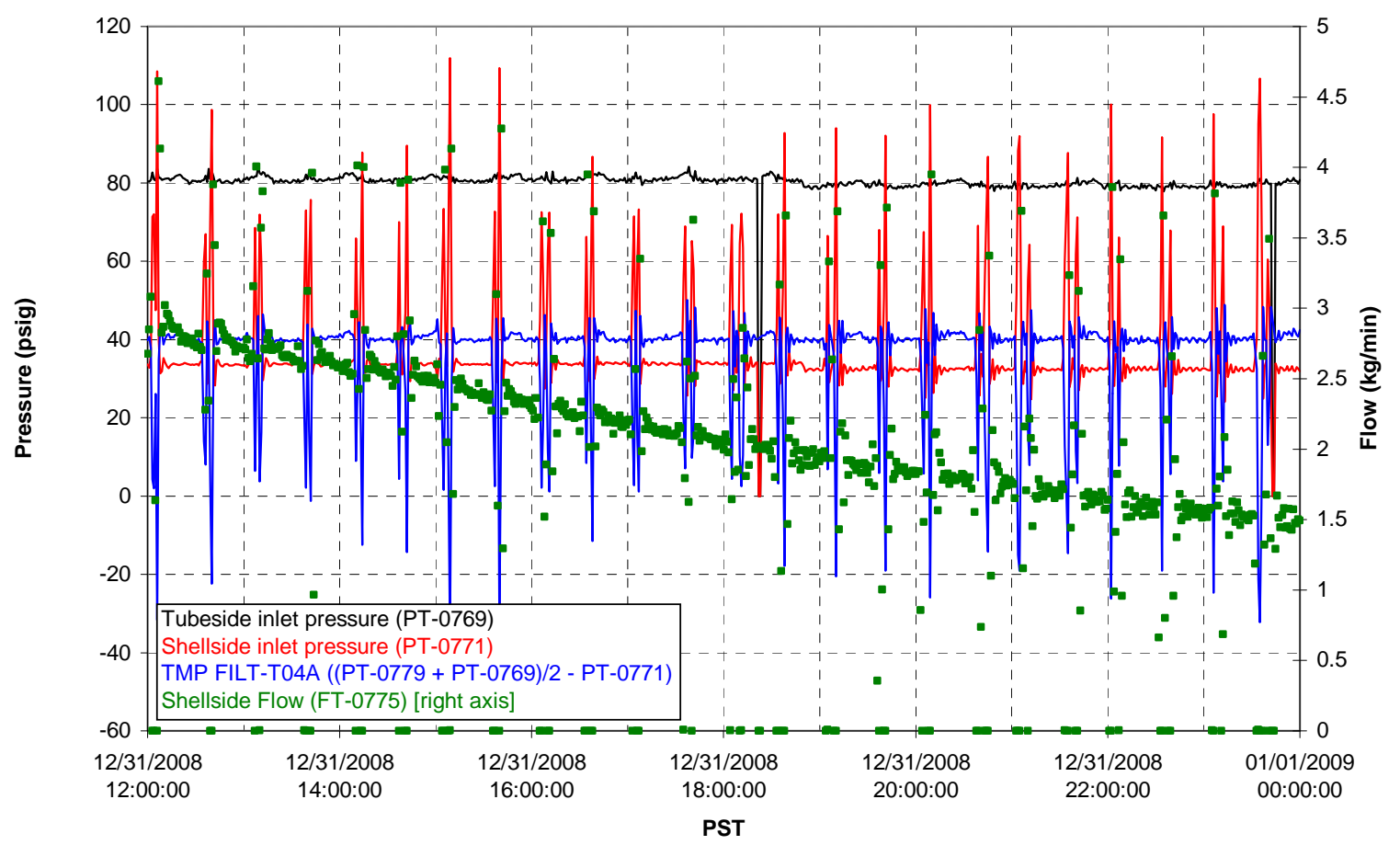


Filter UFP-FILT-T05A

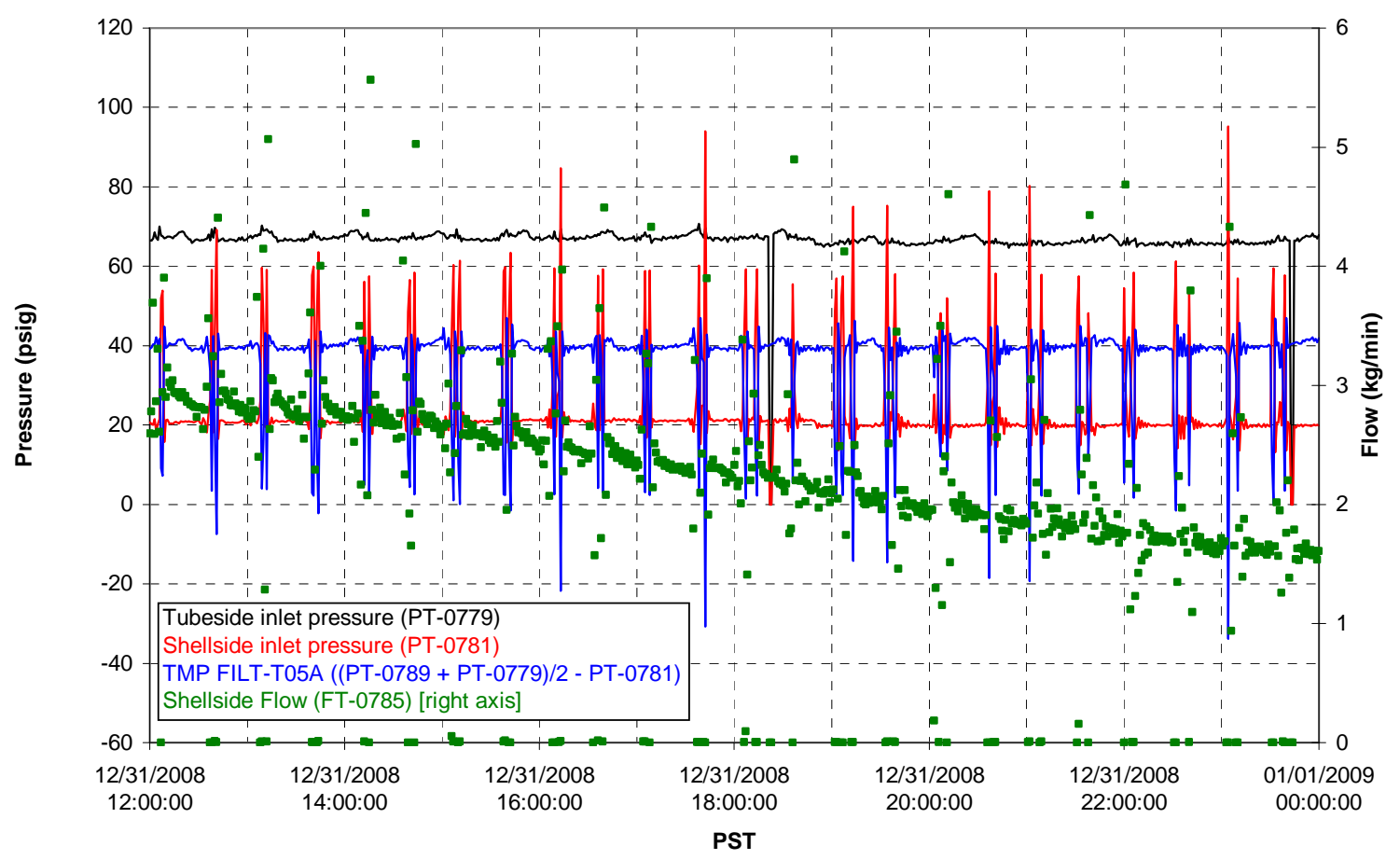

Chemical Flow

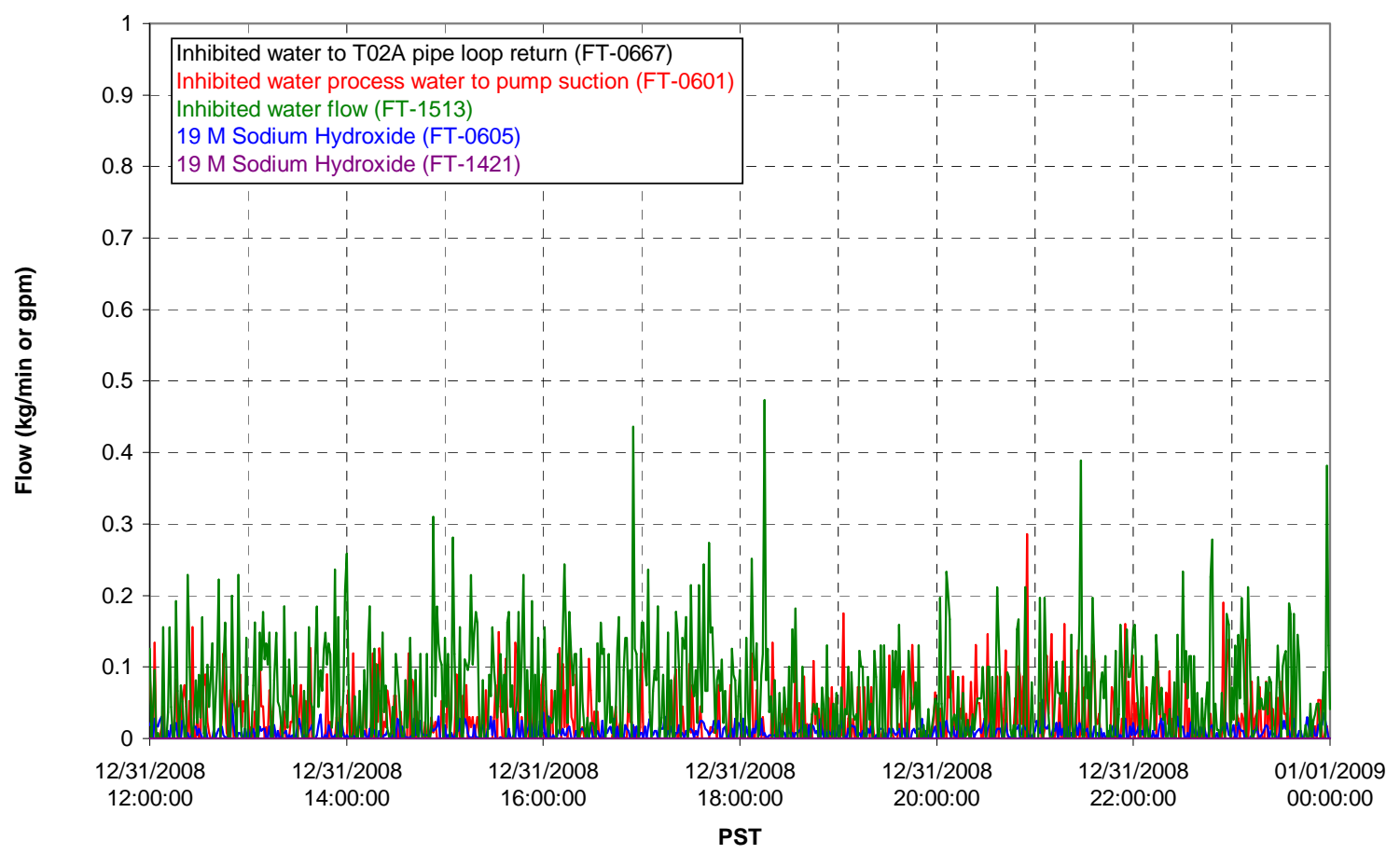


Chemical Flow

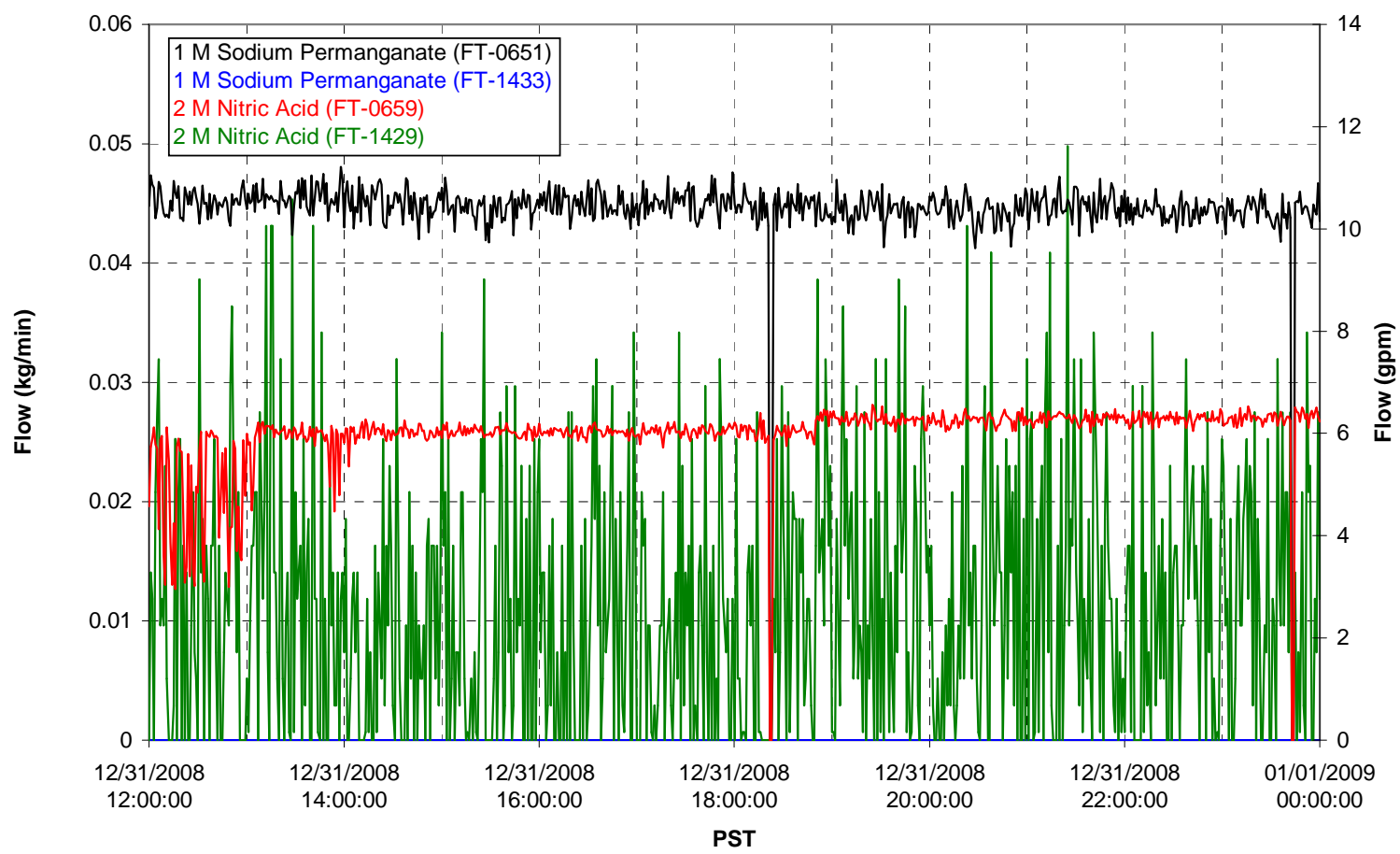

Air Flows

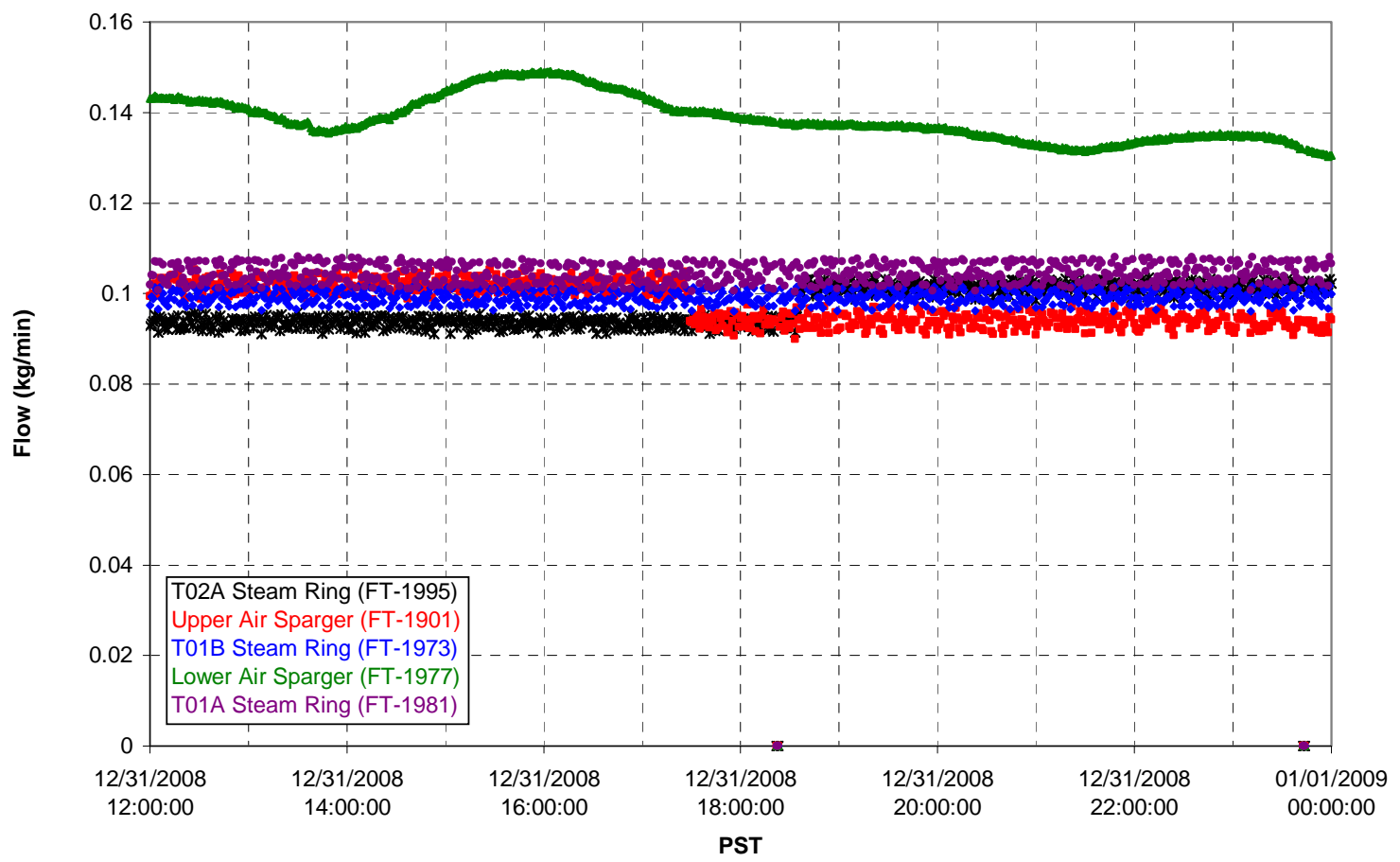


T02A Steam

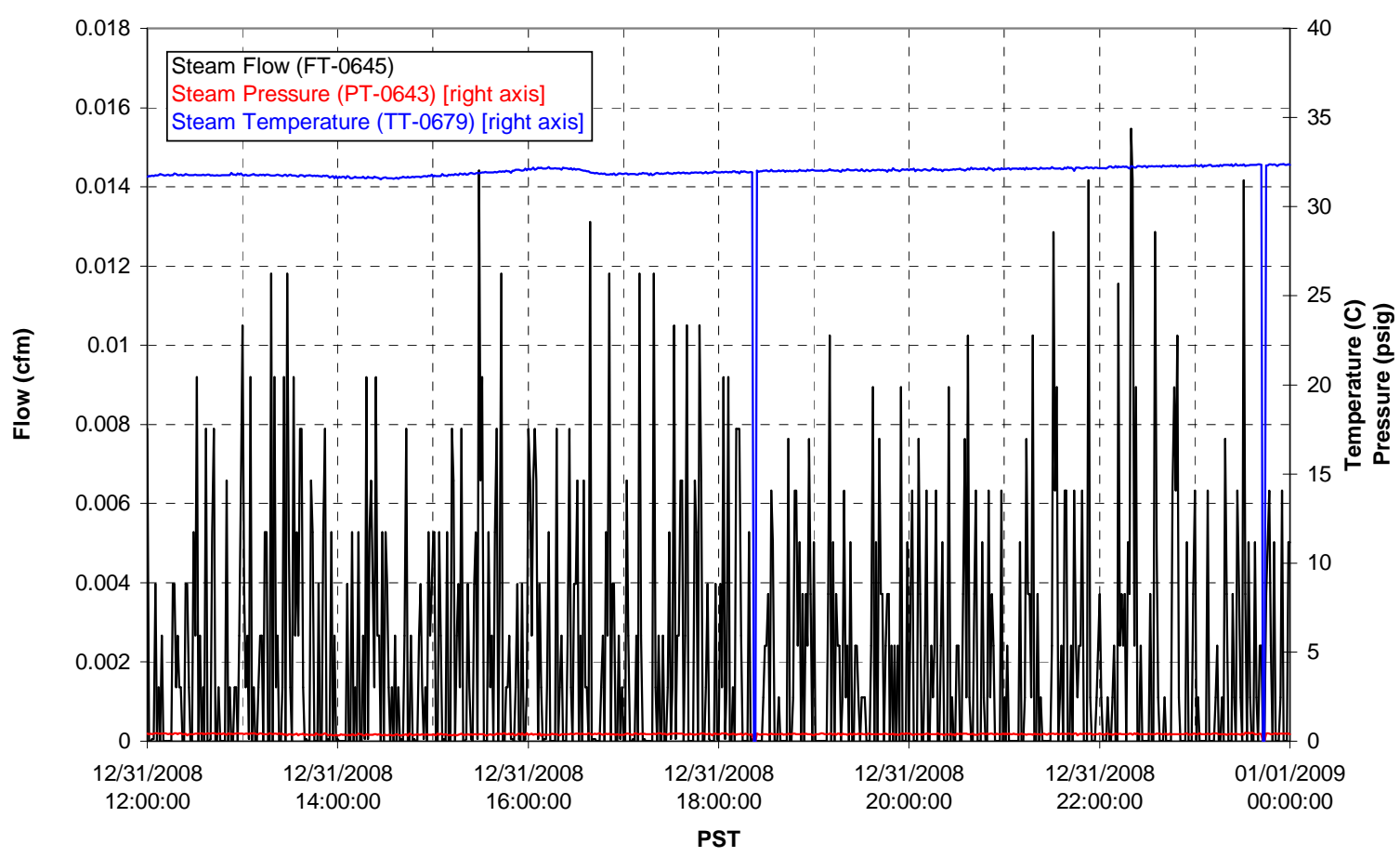

T01A Steam

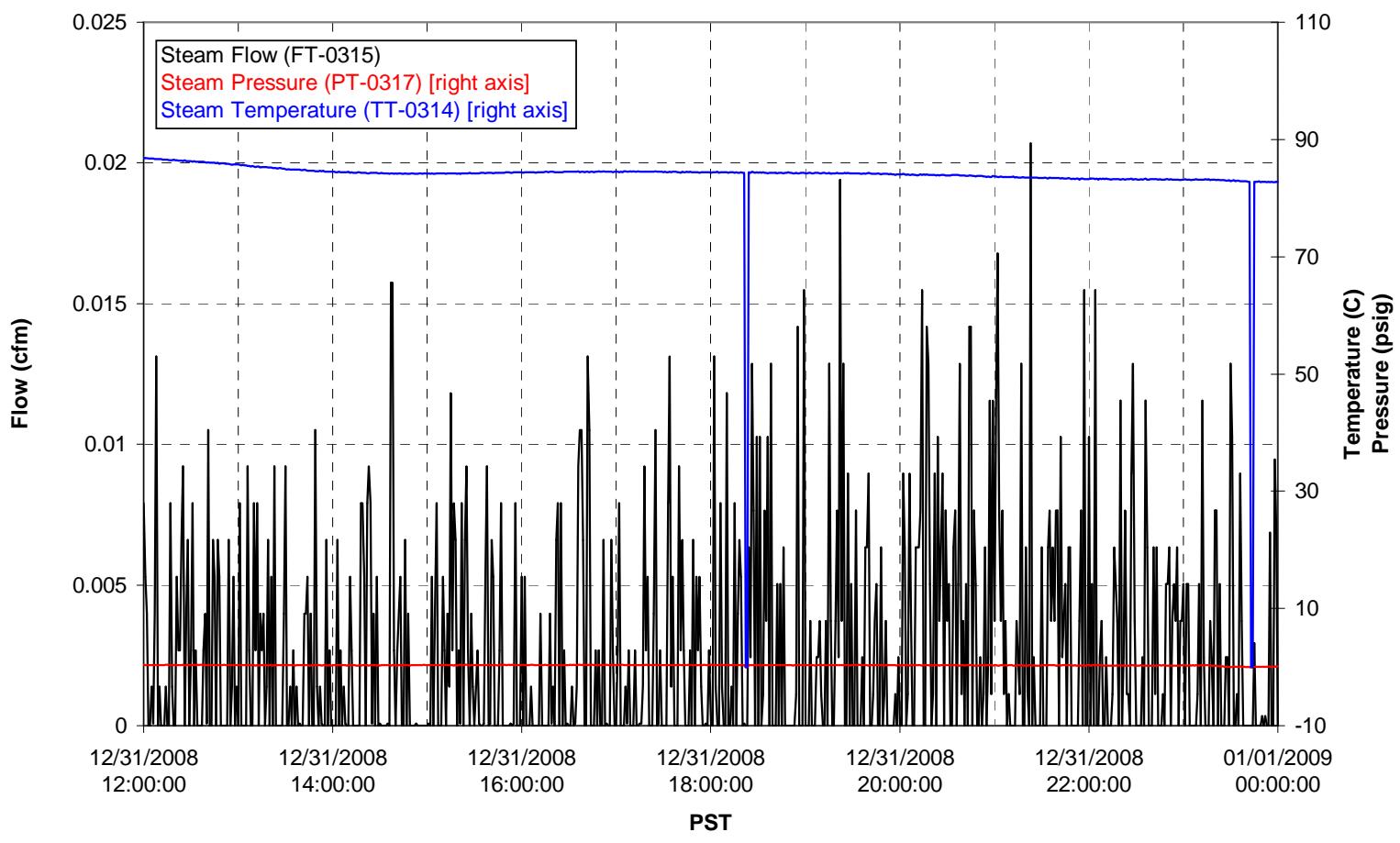


T01B Steam

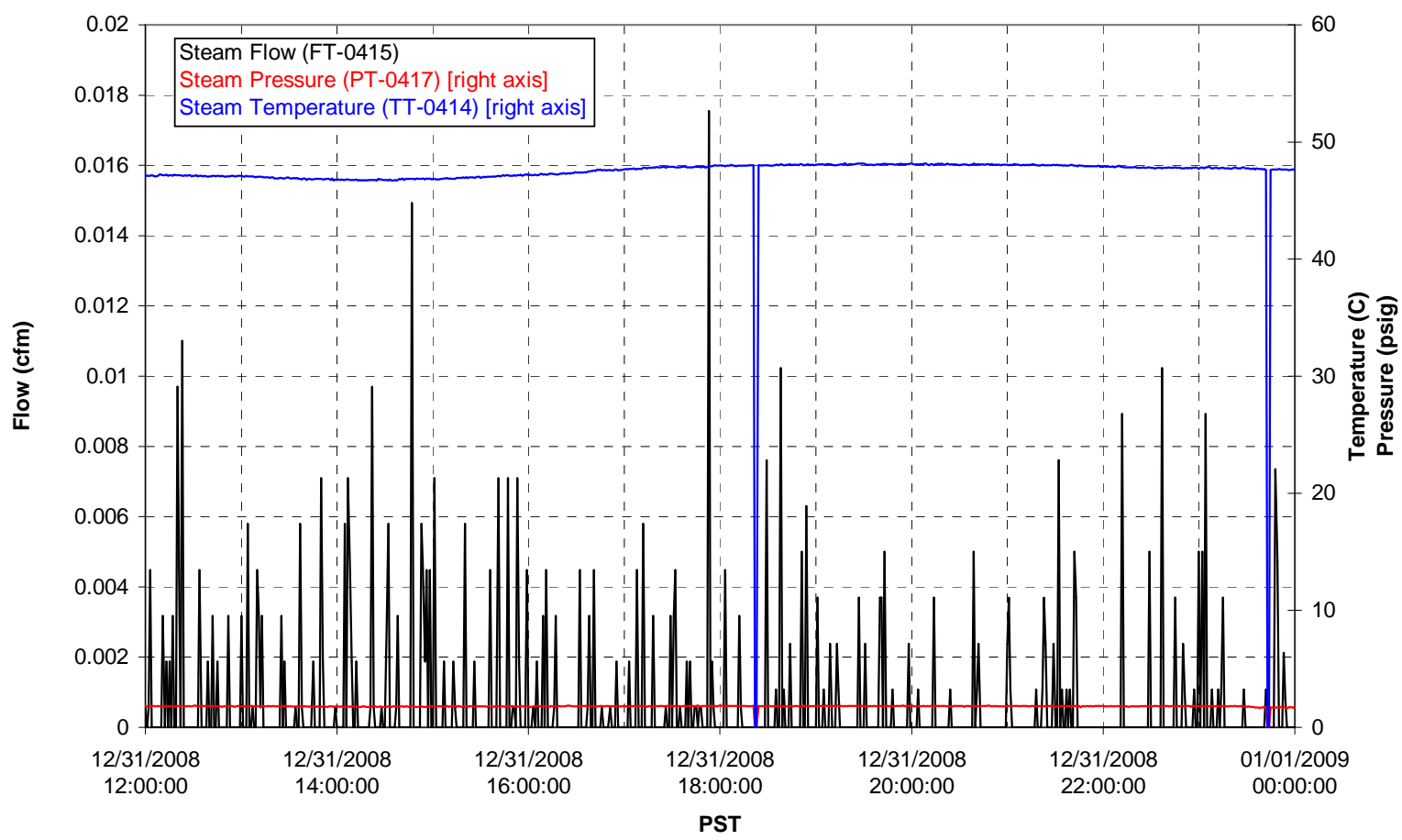


Functional Test Data Plots

01/01/2009 00:00 - 01/01/2009 12:00 
T01A level

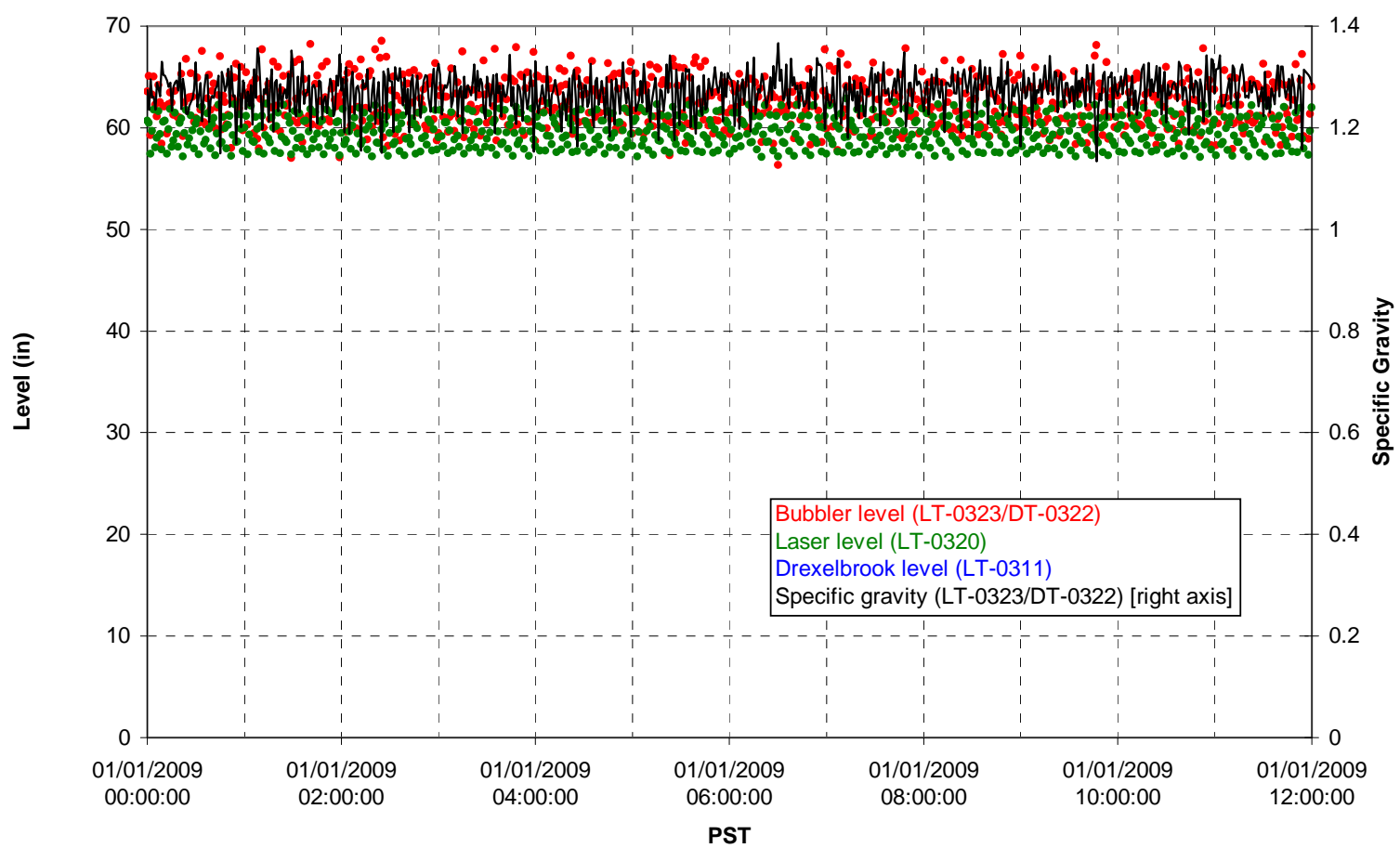

T01A temperatures

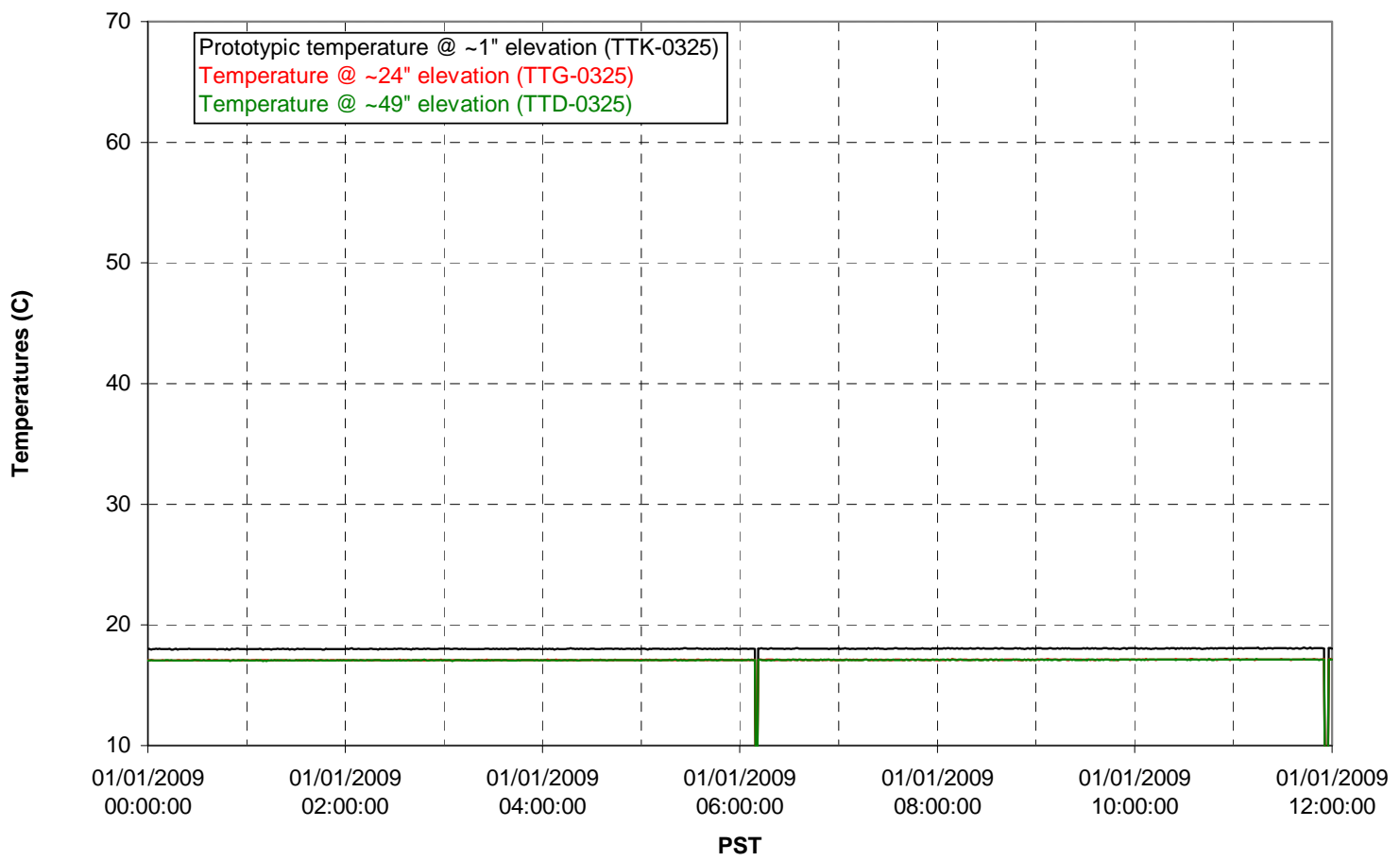


T01B level

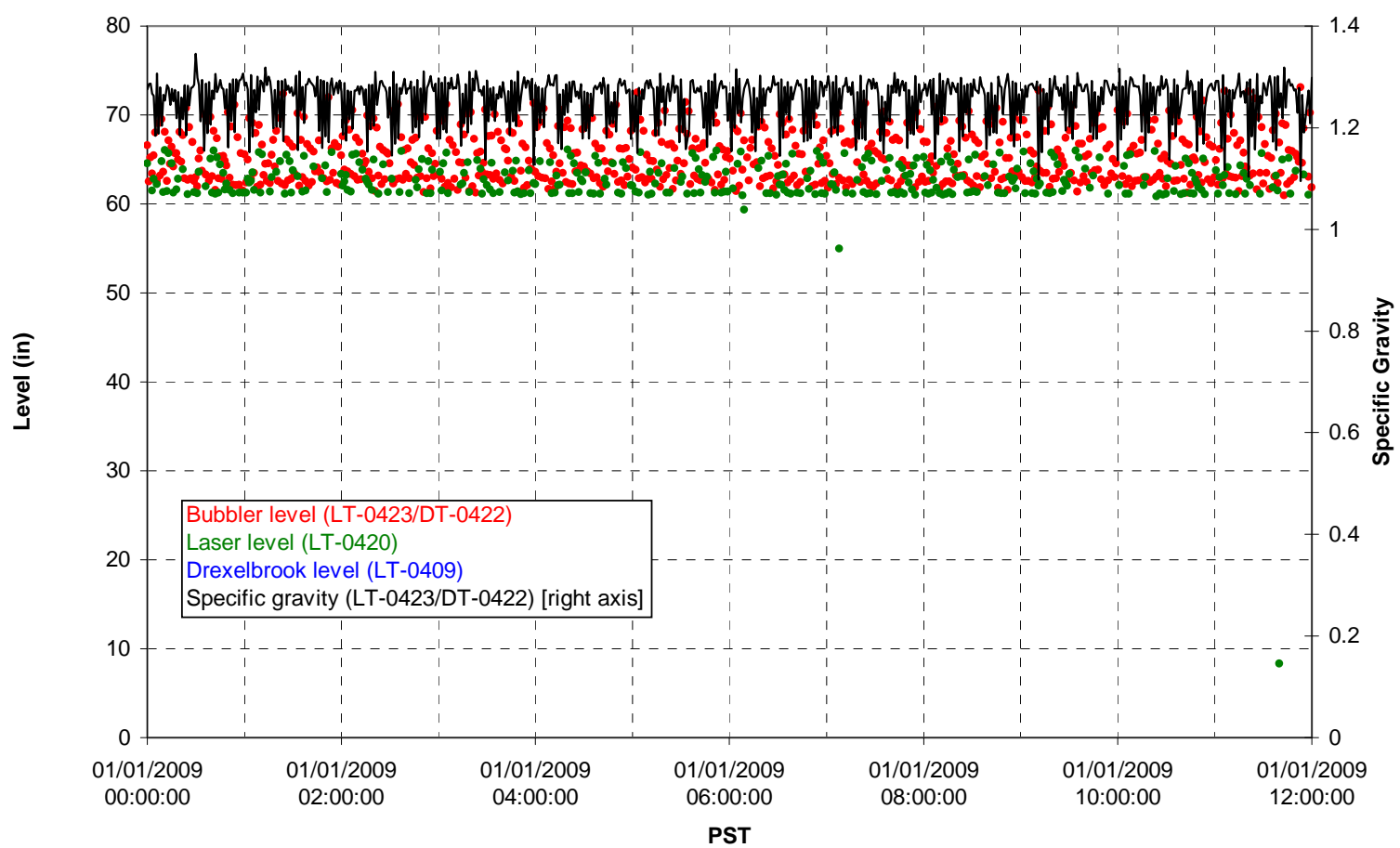

T01B temperatures

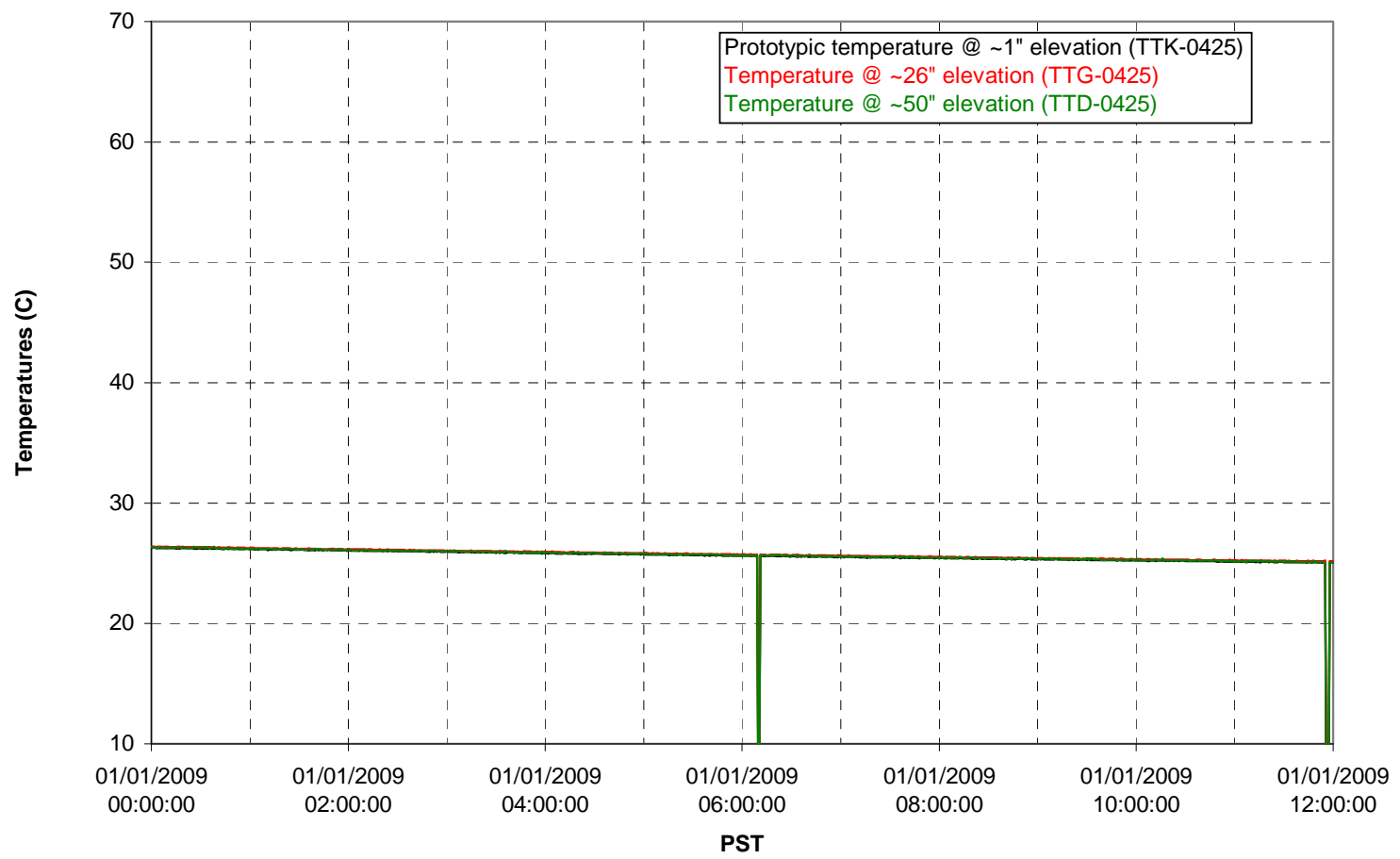


T02A level

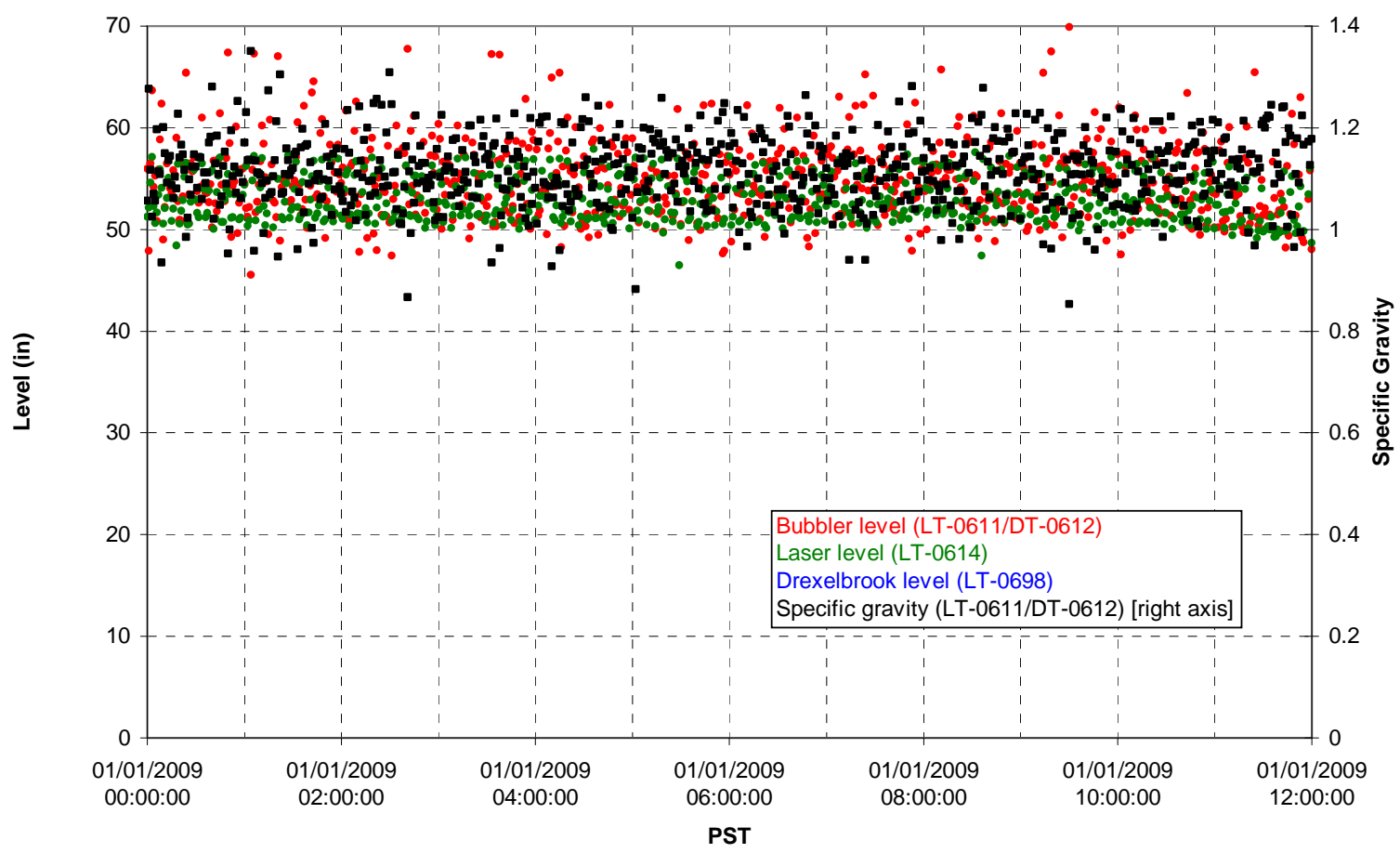

T02A temperatures

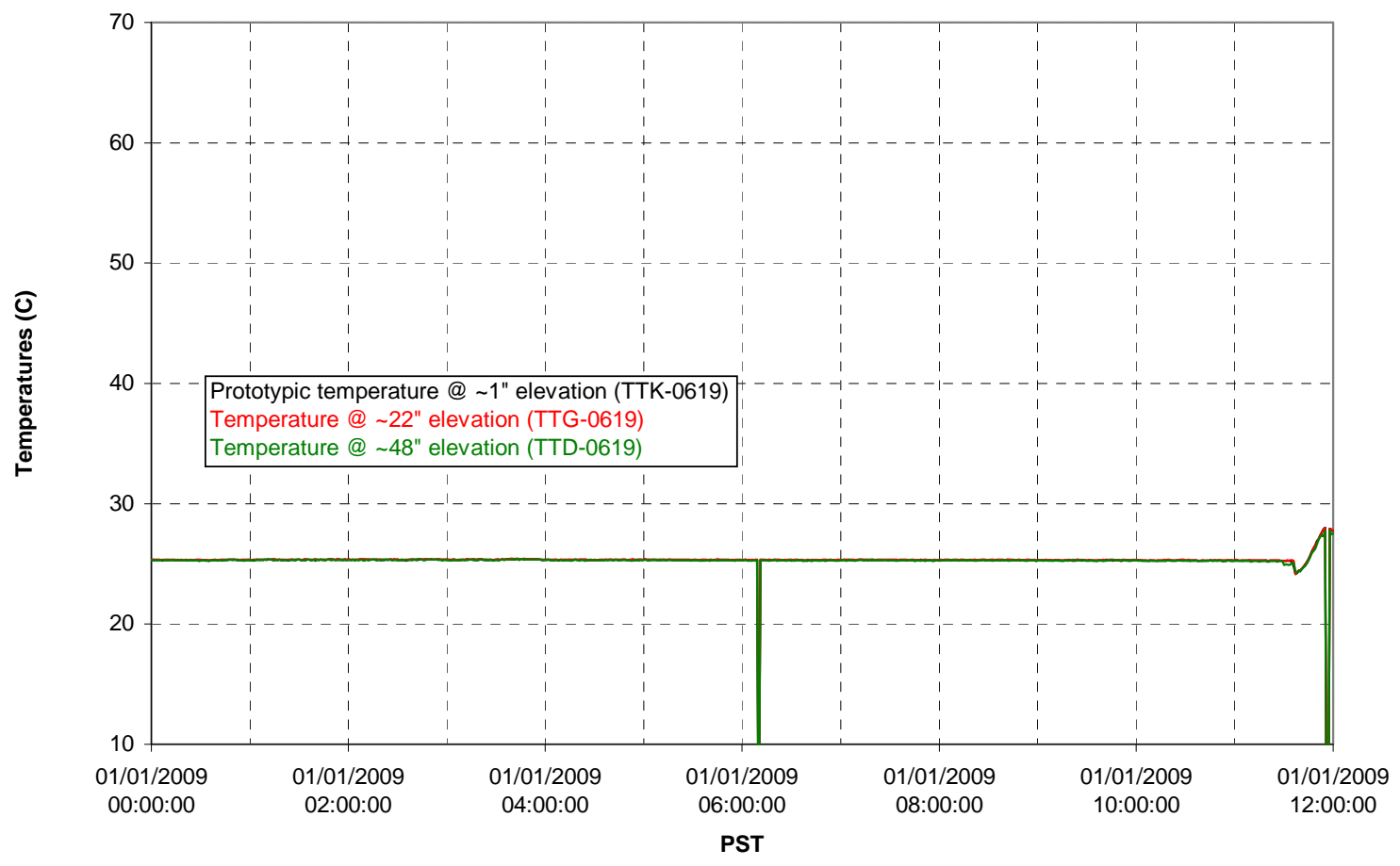


T02A and filter loop temperatures

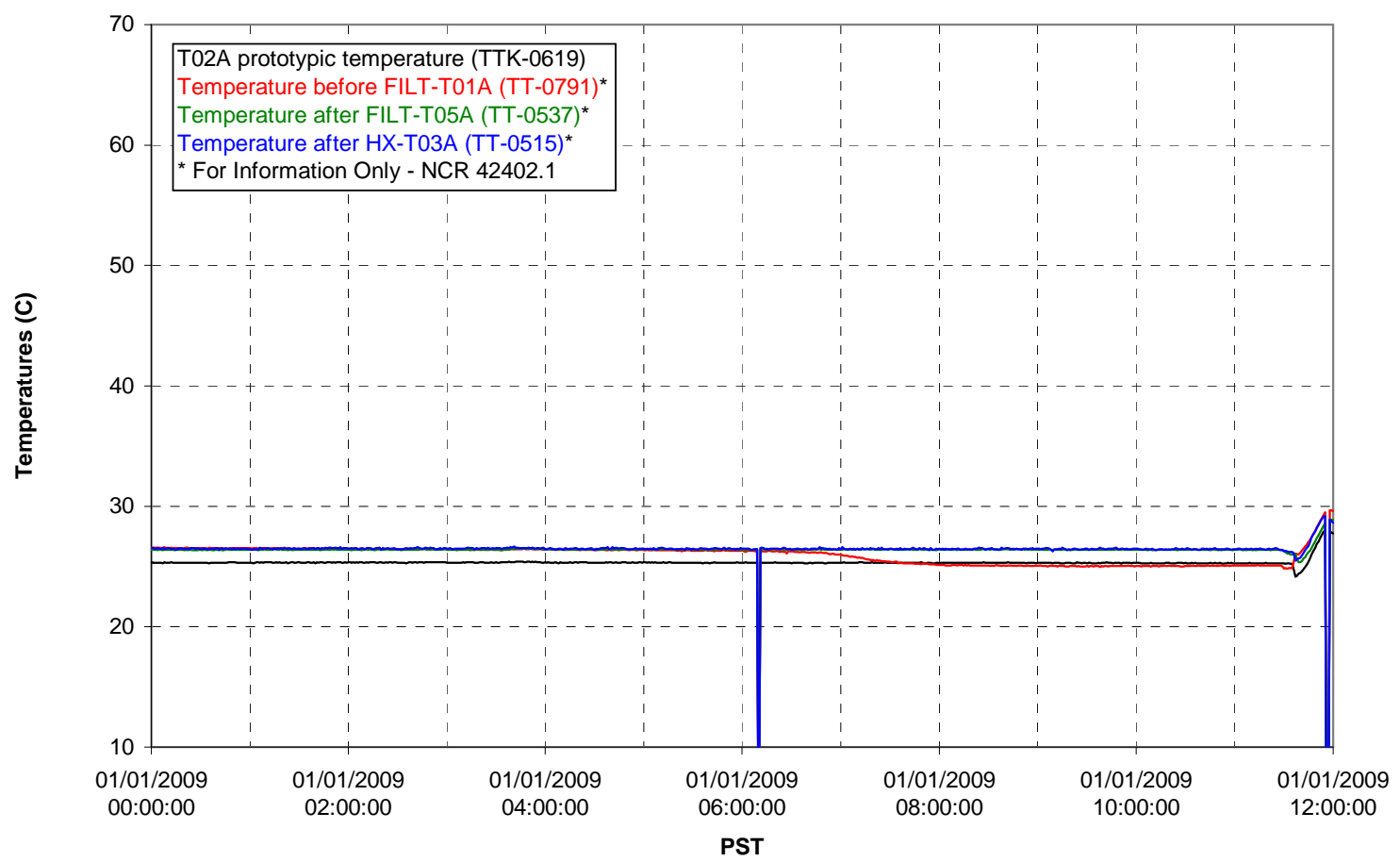

Pump Pressures and Flow

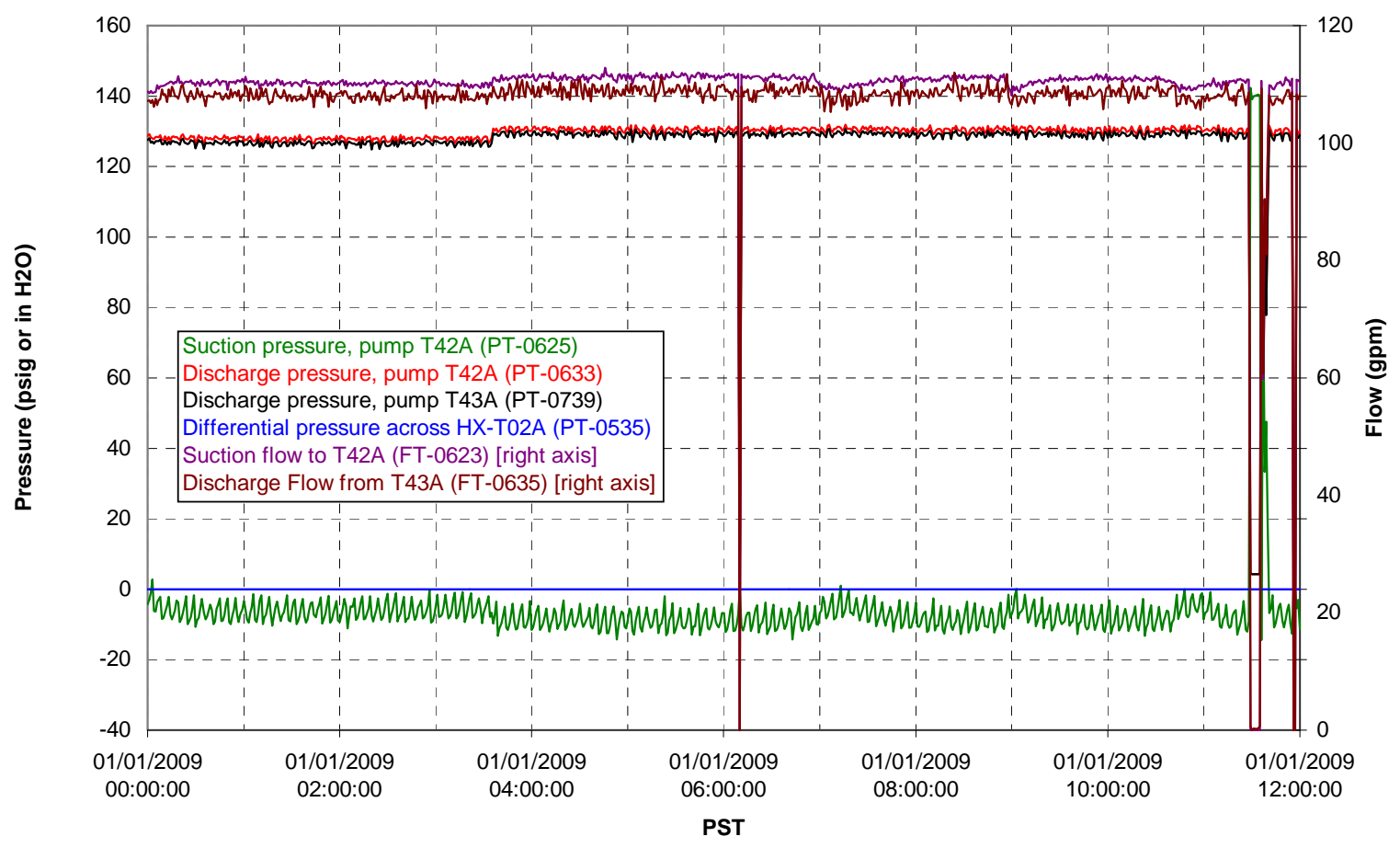


Axial pressure drop

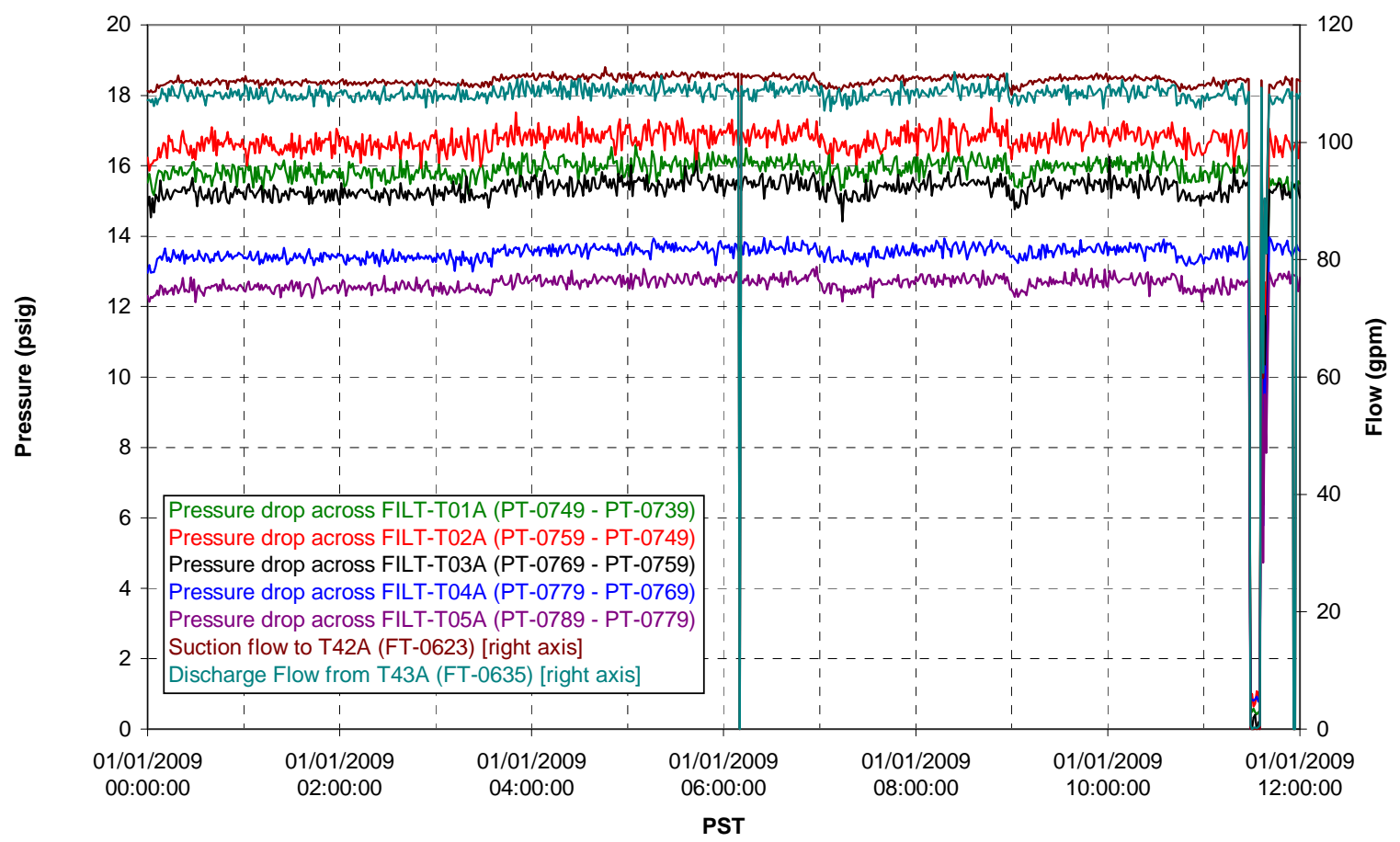

Permeate flow rates

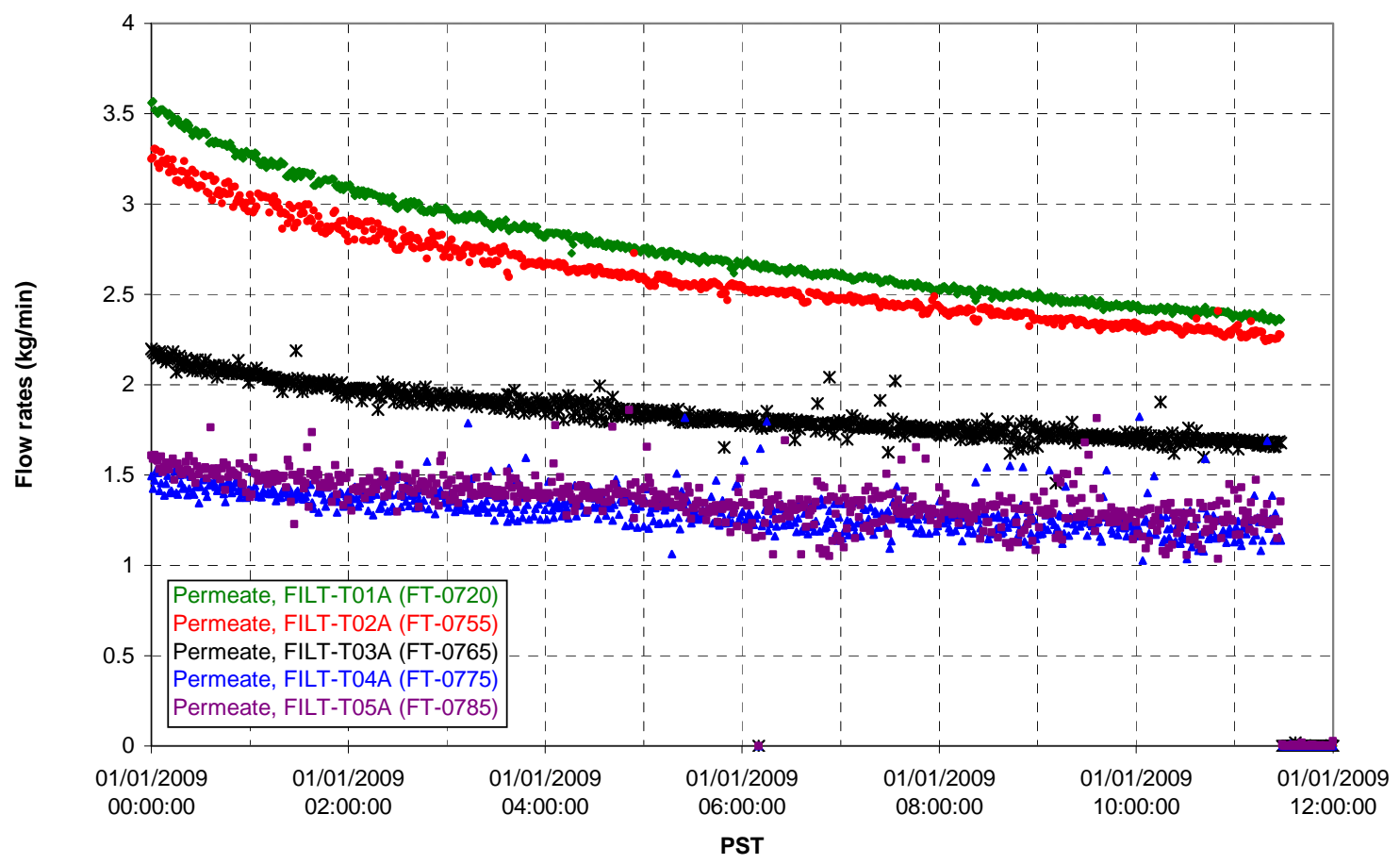


T02A Inner Temperature Tree

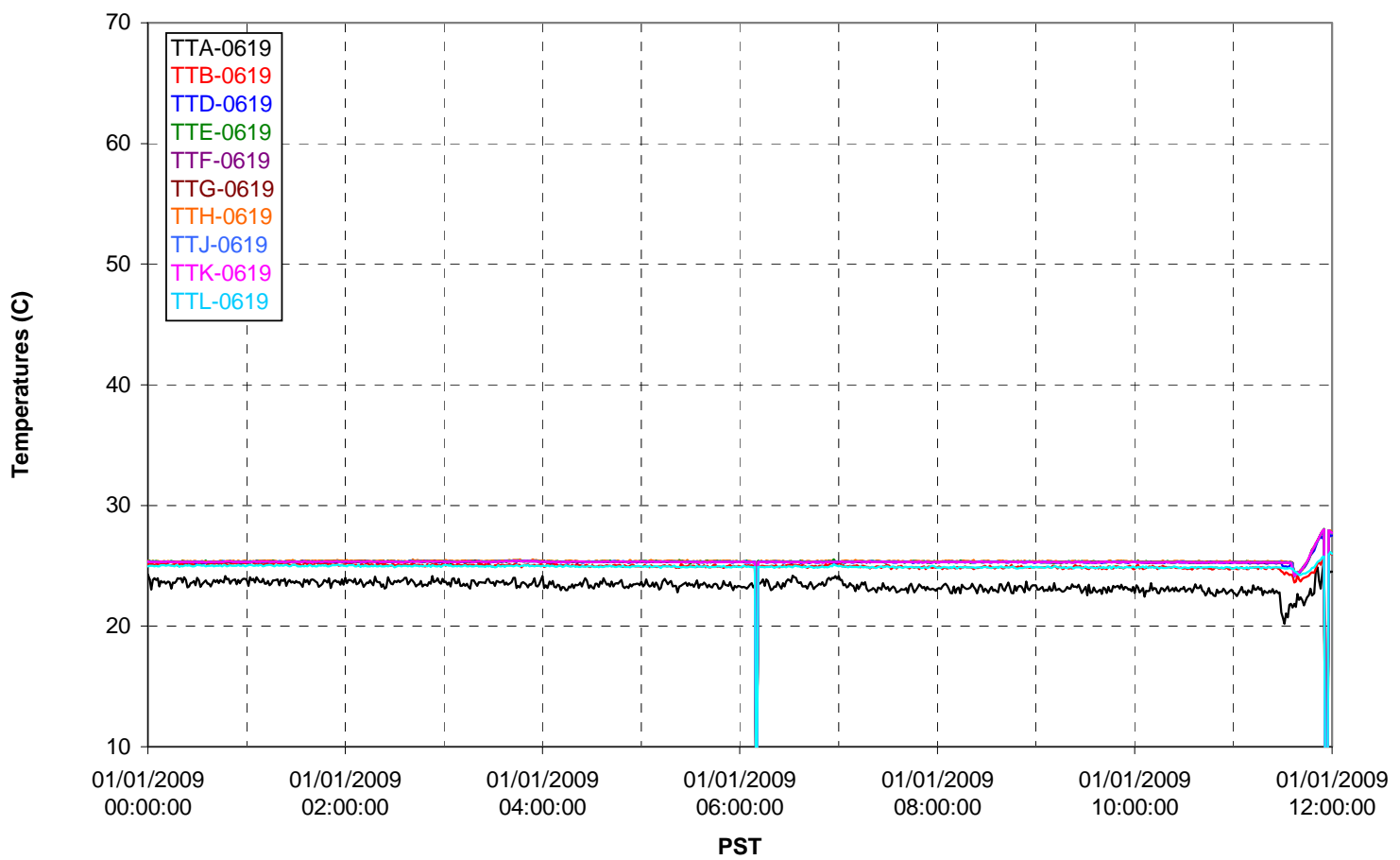

T02A Outer Temperature Tree

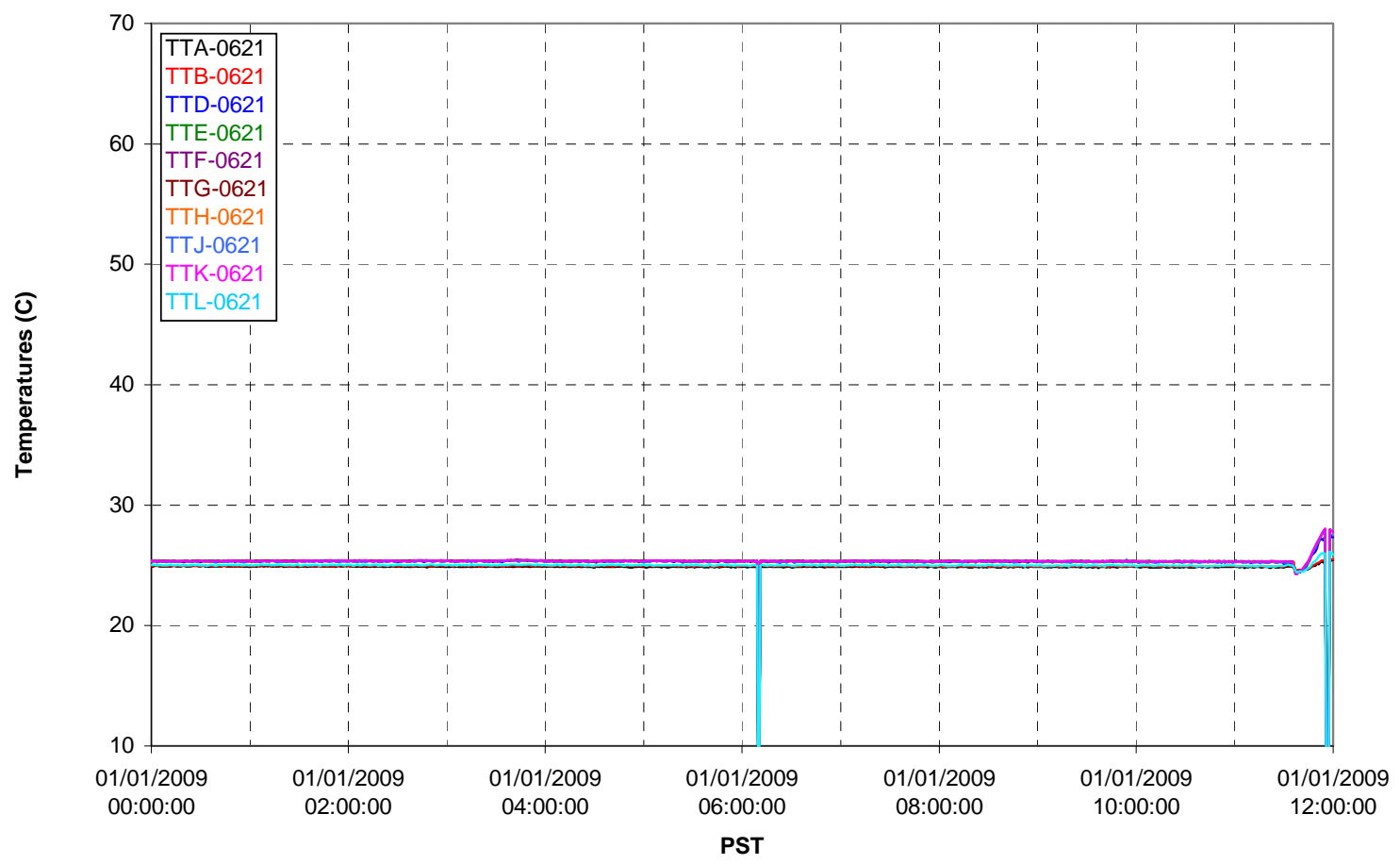


T02A temperatures

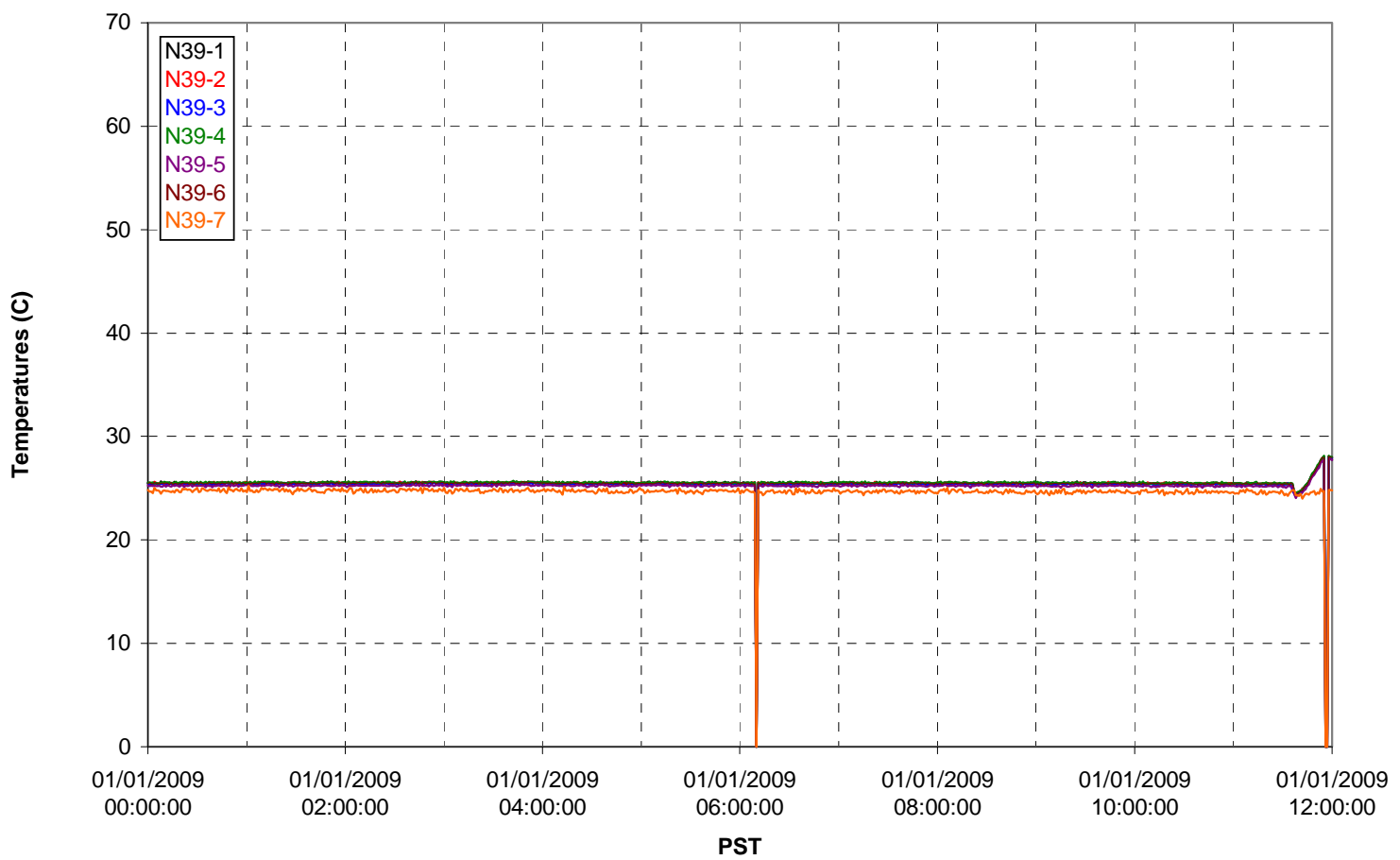

T02A temperatures

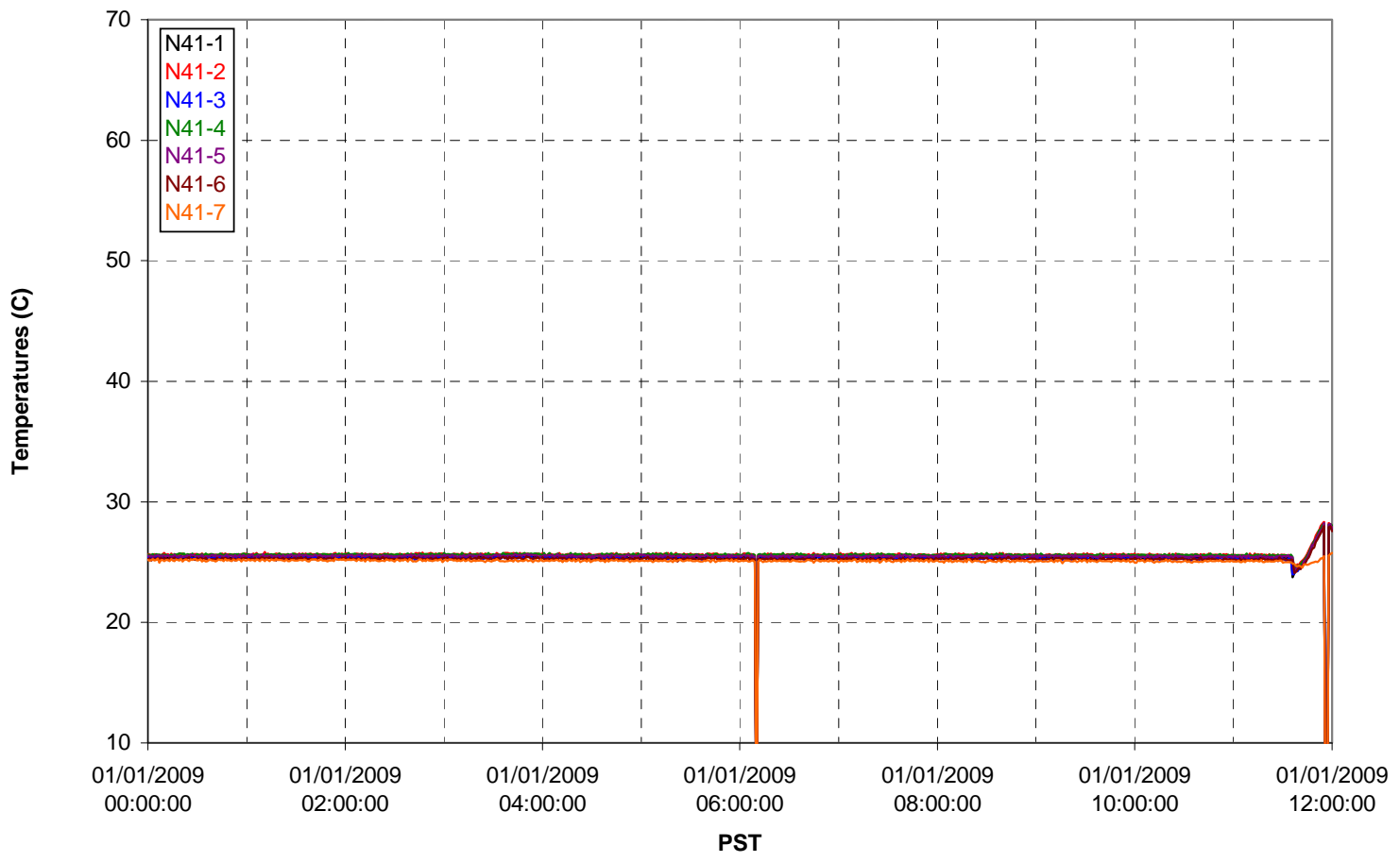


T02A temperatures

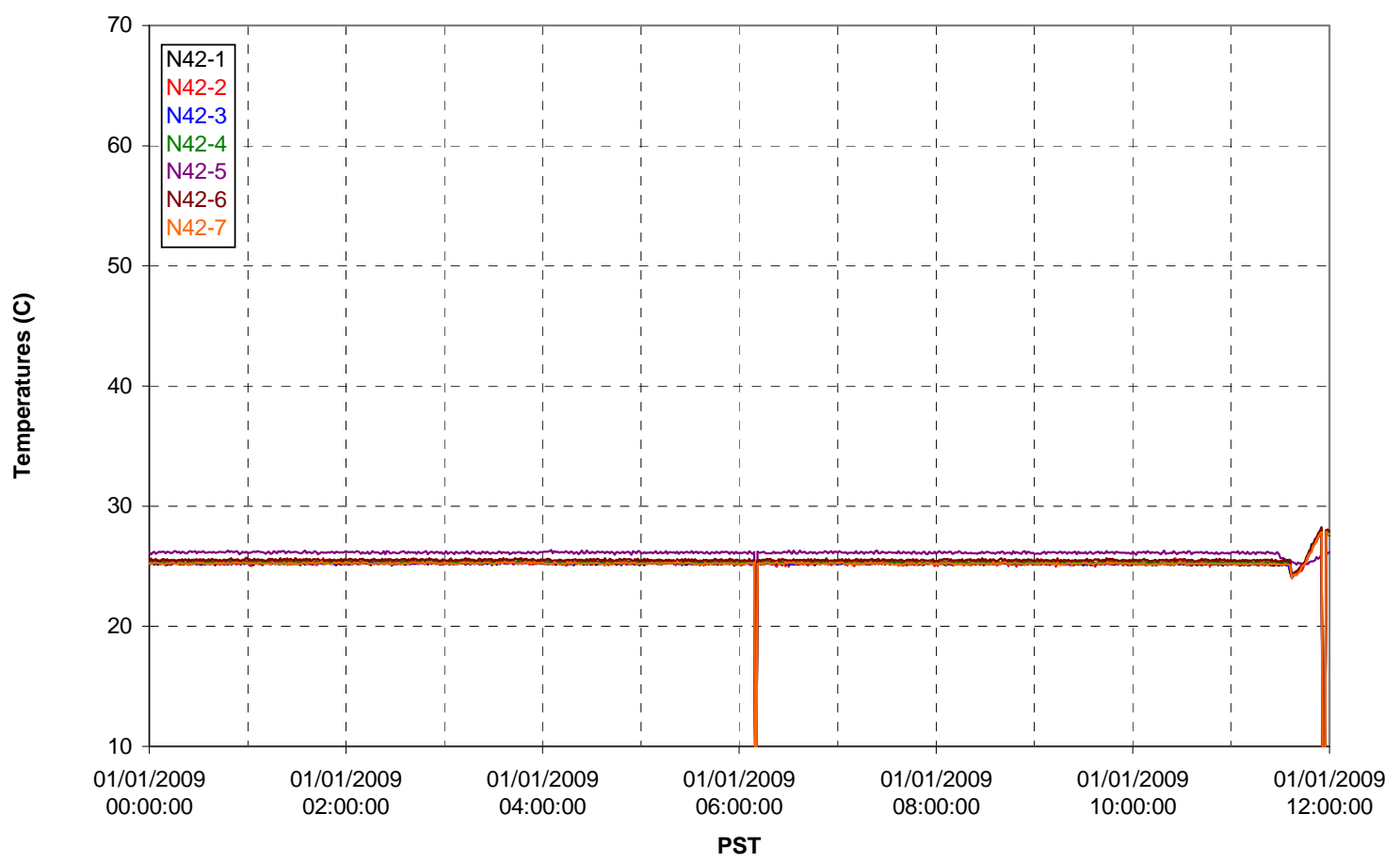

T02A temperatures

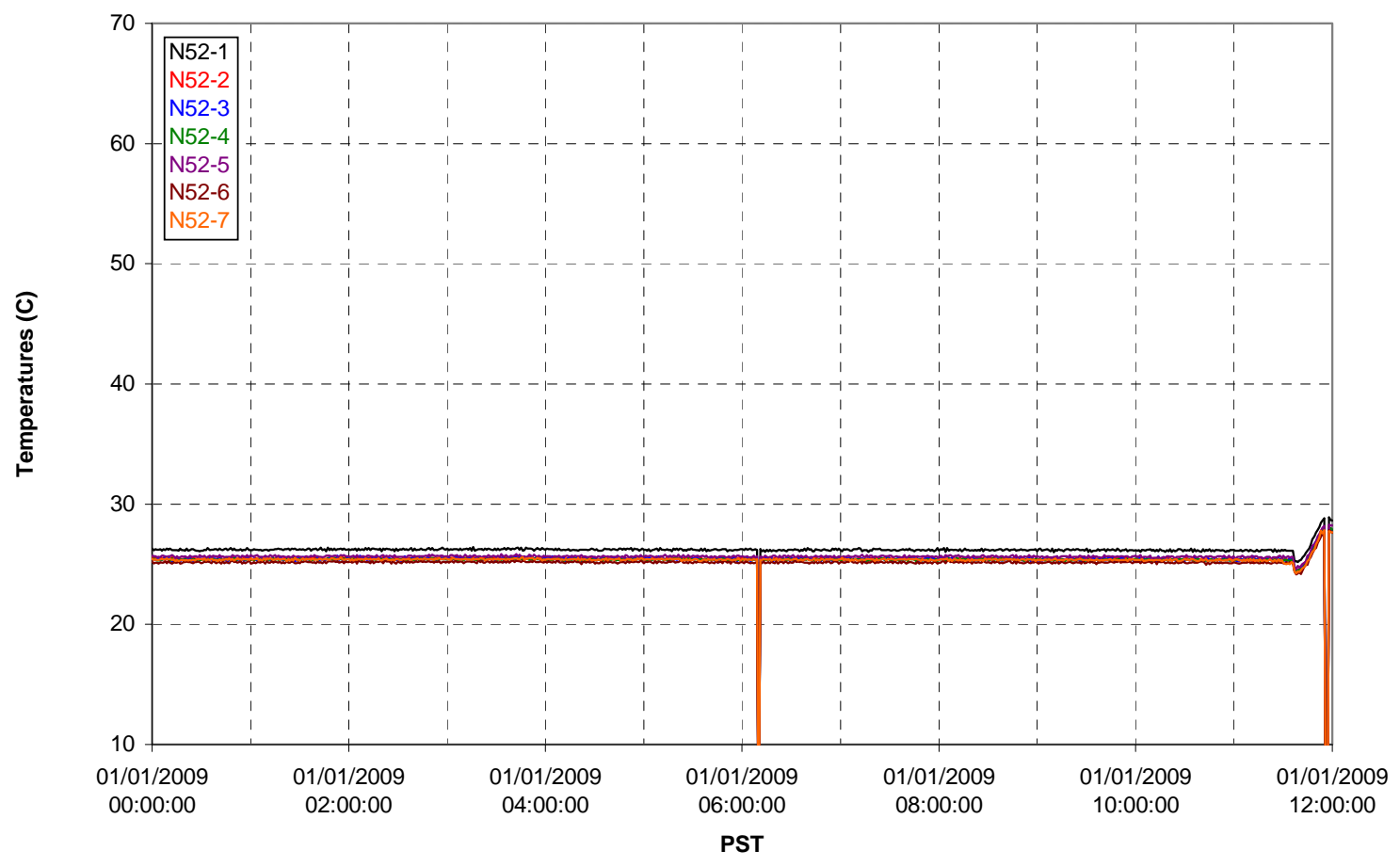


T02A Heating and Cooling

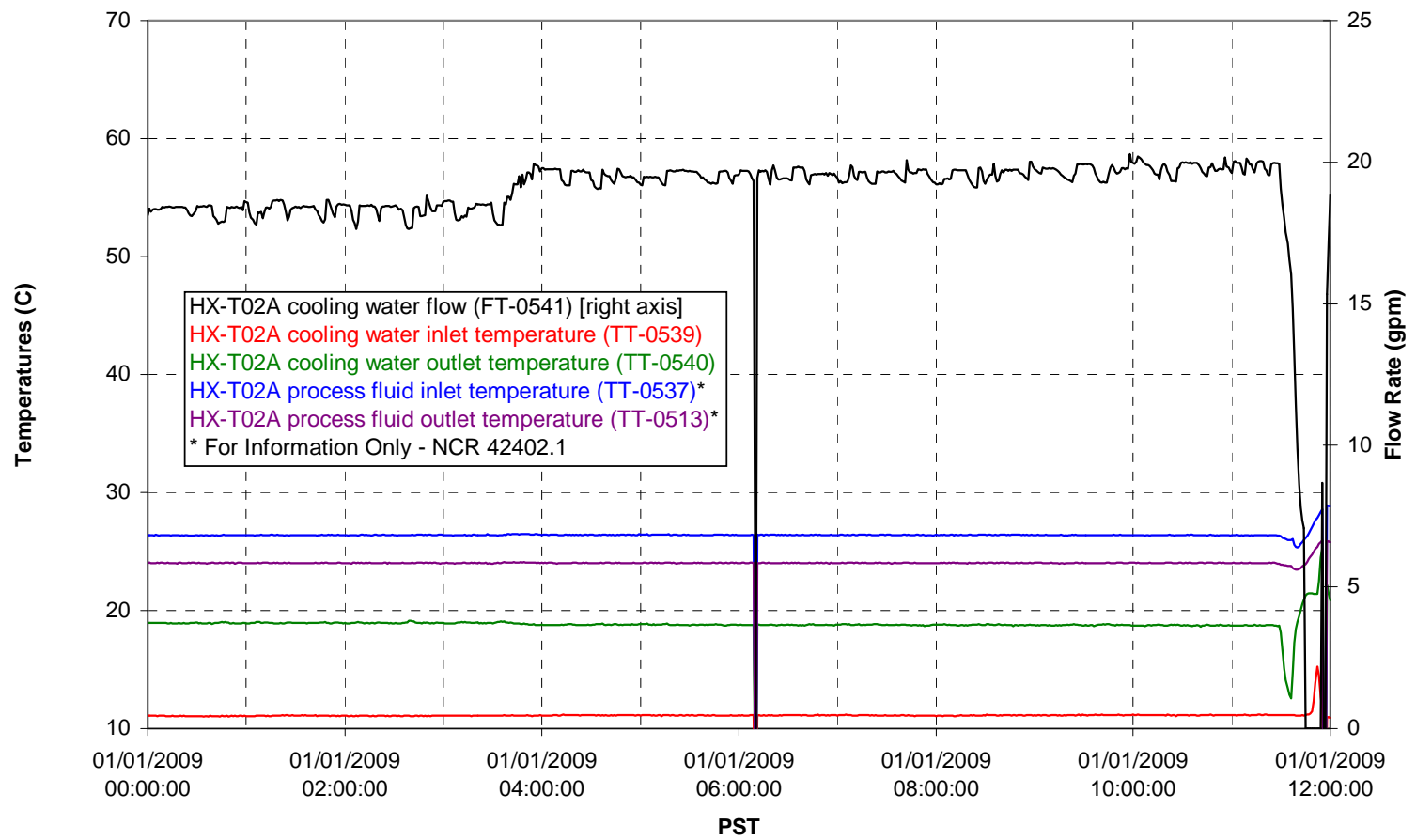

Pump Operation

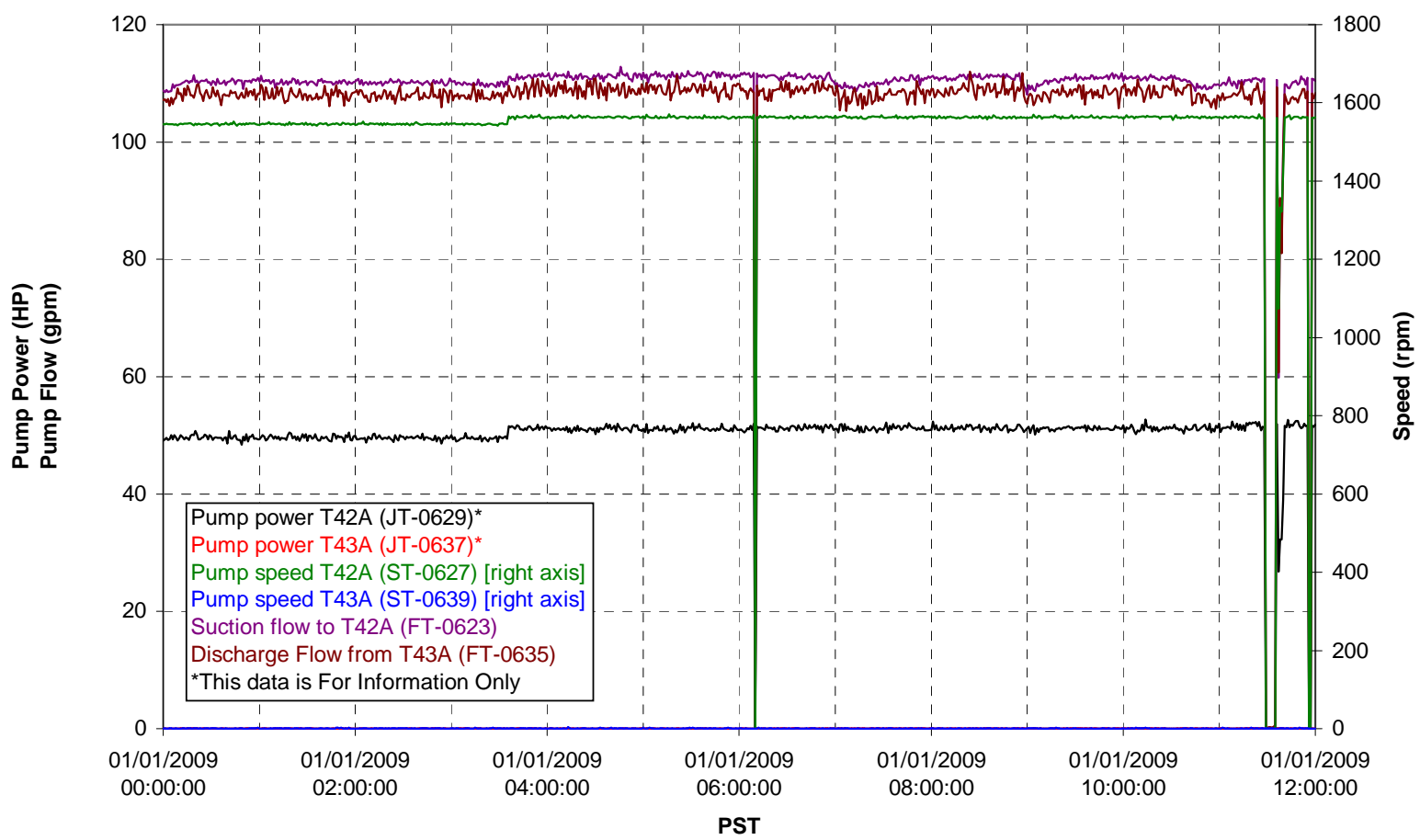


Pulsepot UFP-PP-T01A

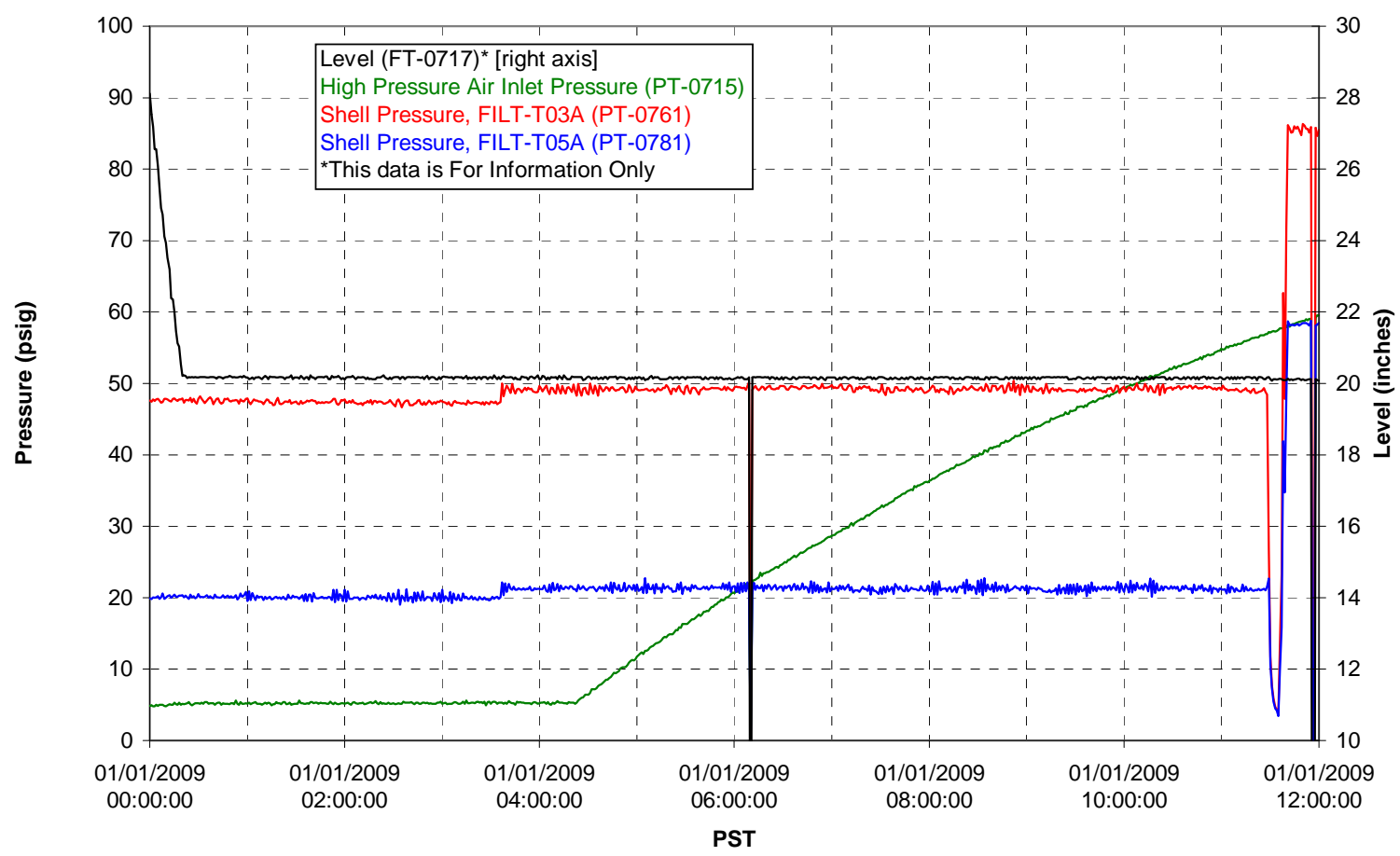

Pulsepot UFP-PP-T02A

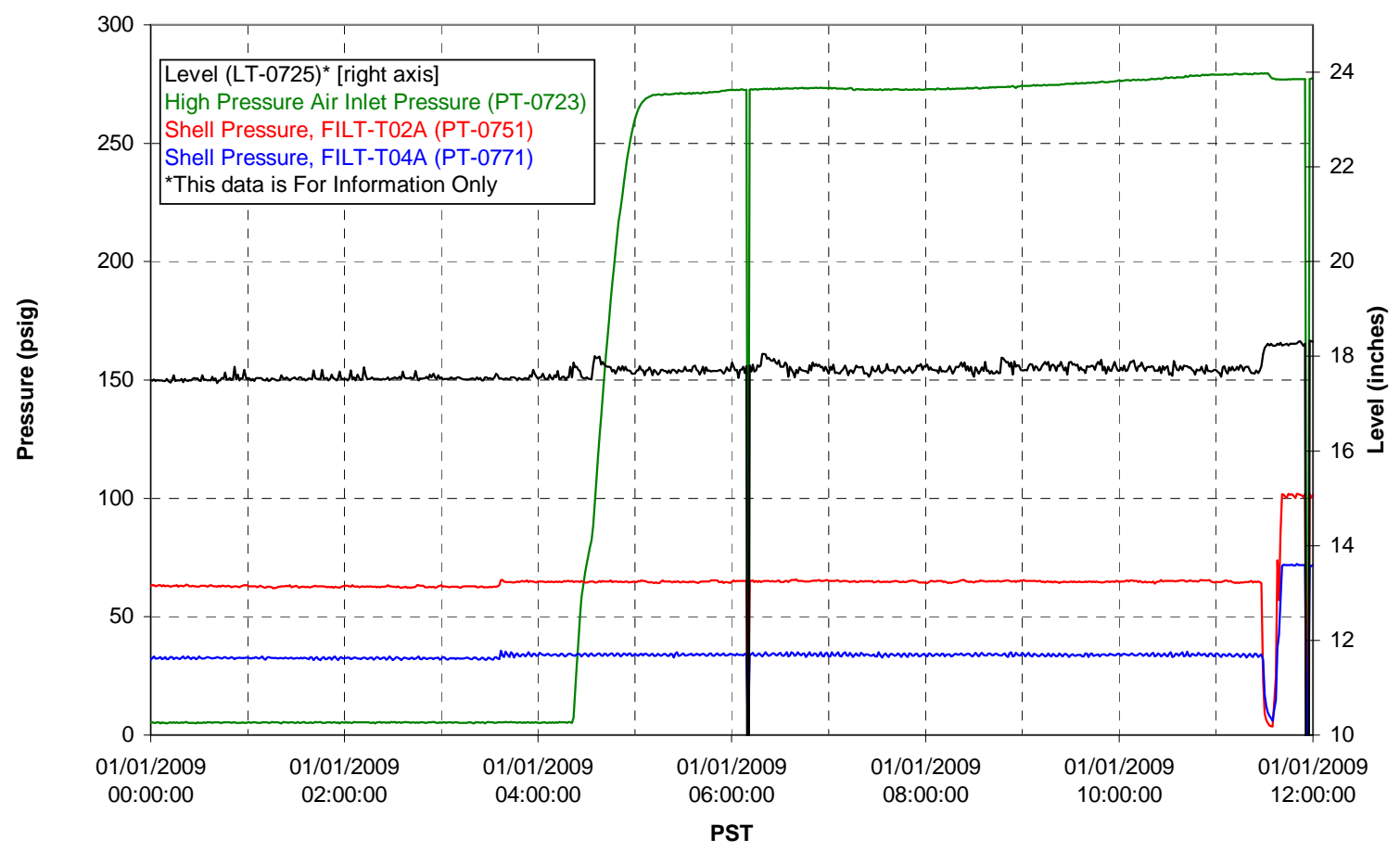


Pulsepot UFP-PP-T03A

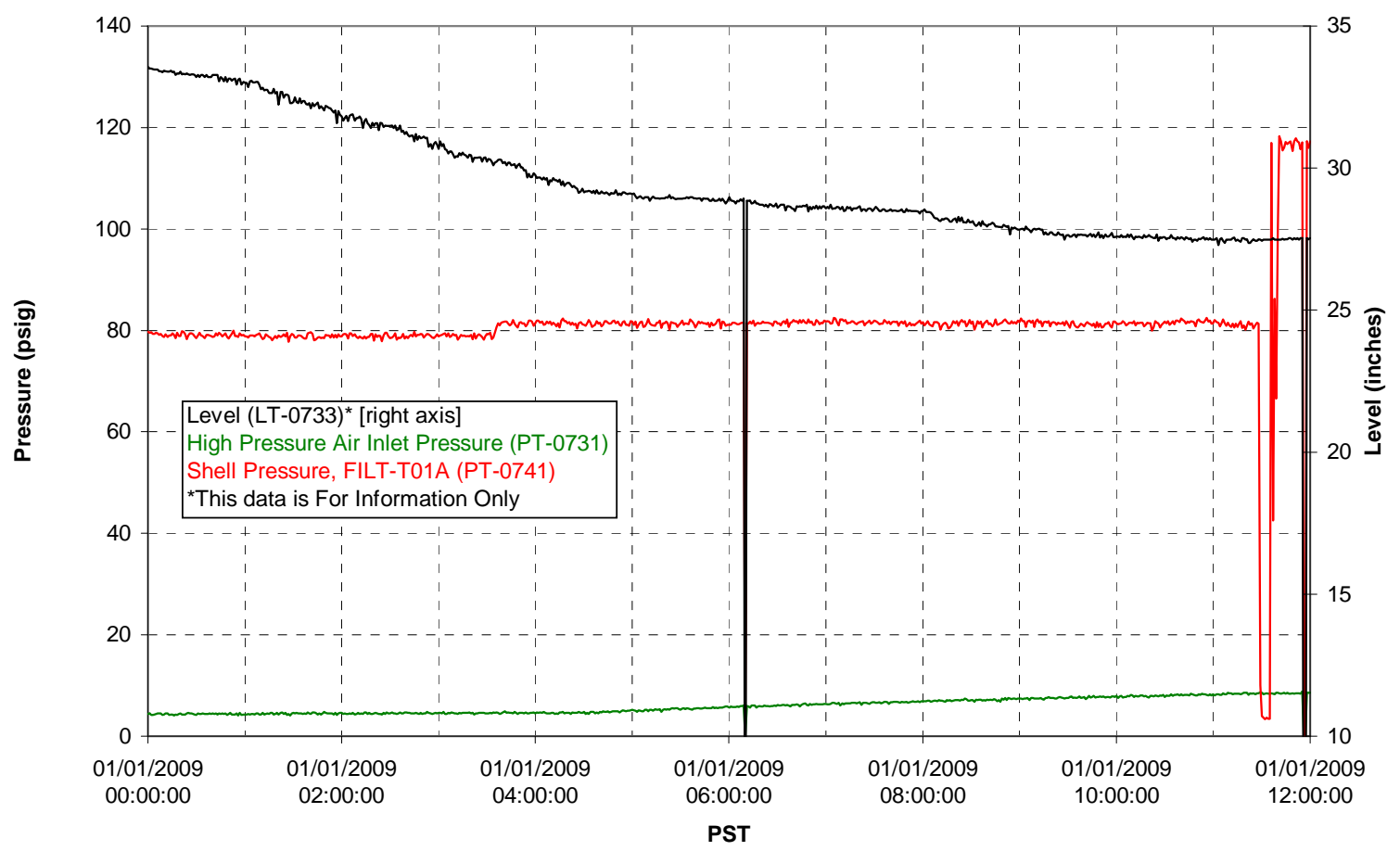

Pulsepot Levels

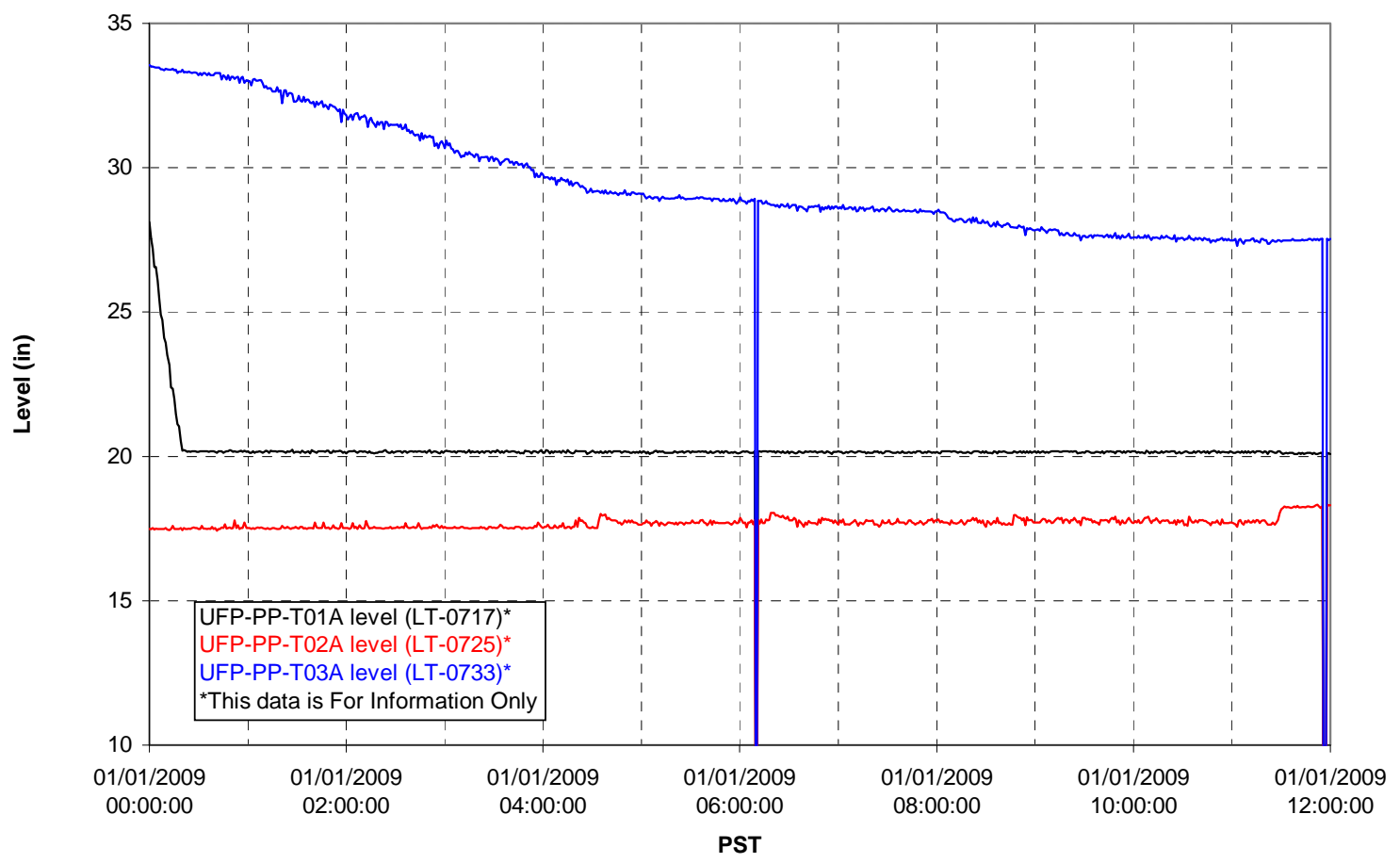


Filter UFP-FILT-T01A

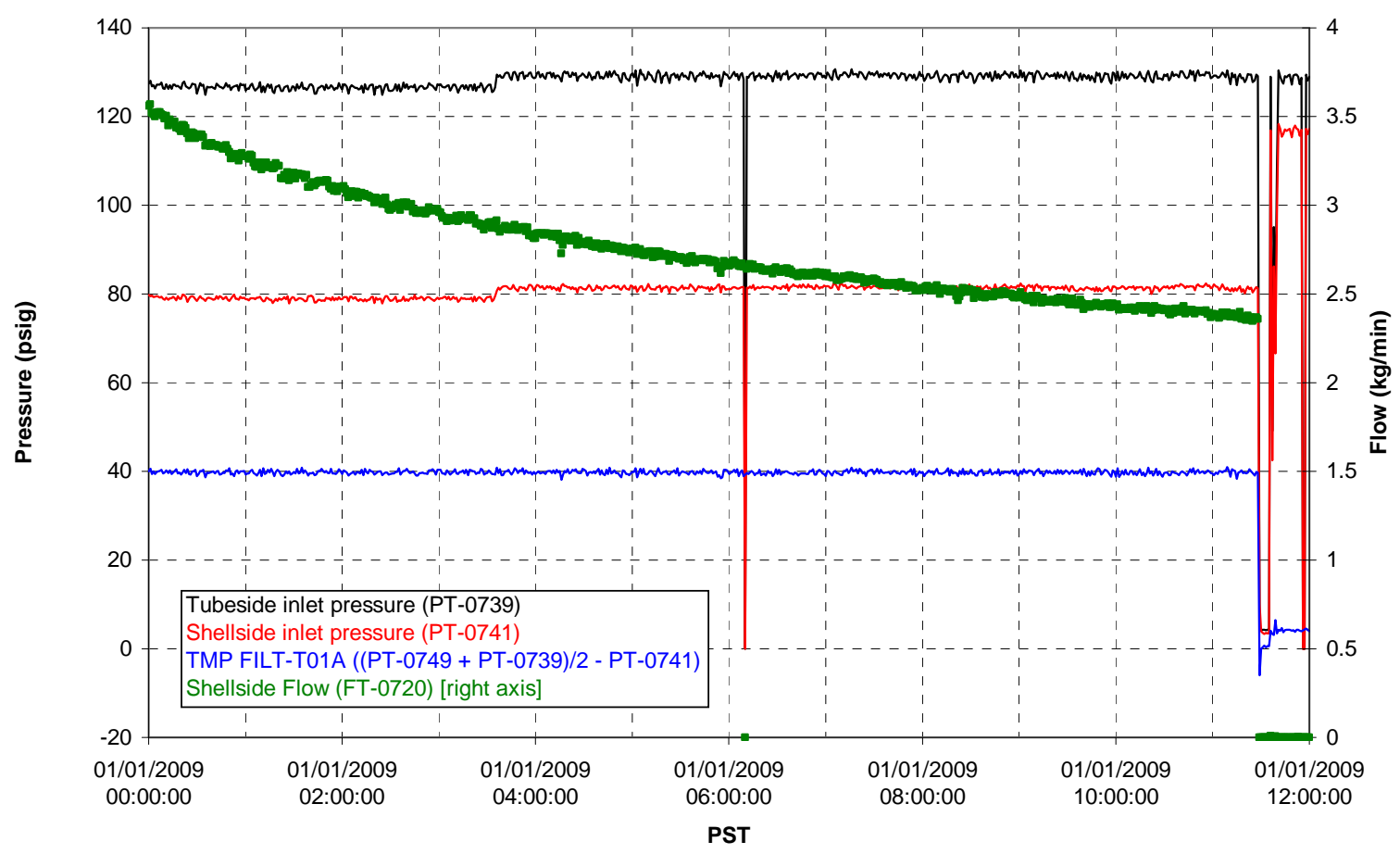

Filter UFP-FILT-T02A

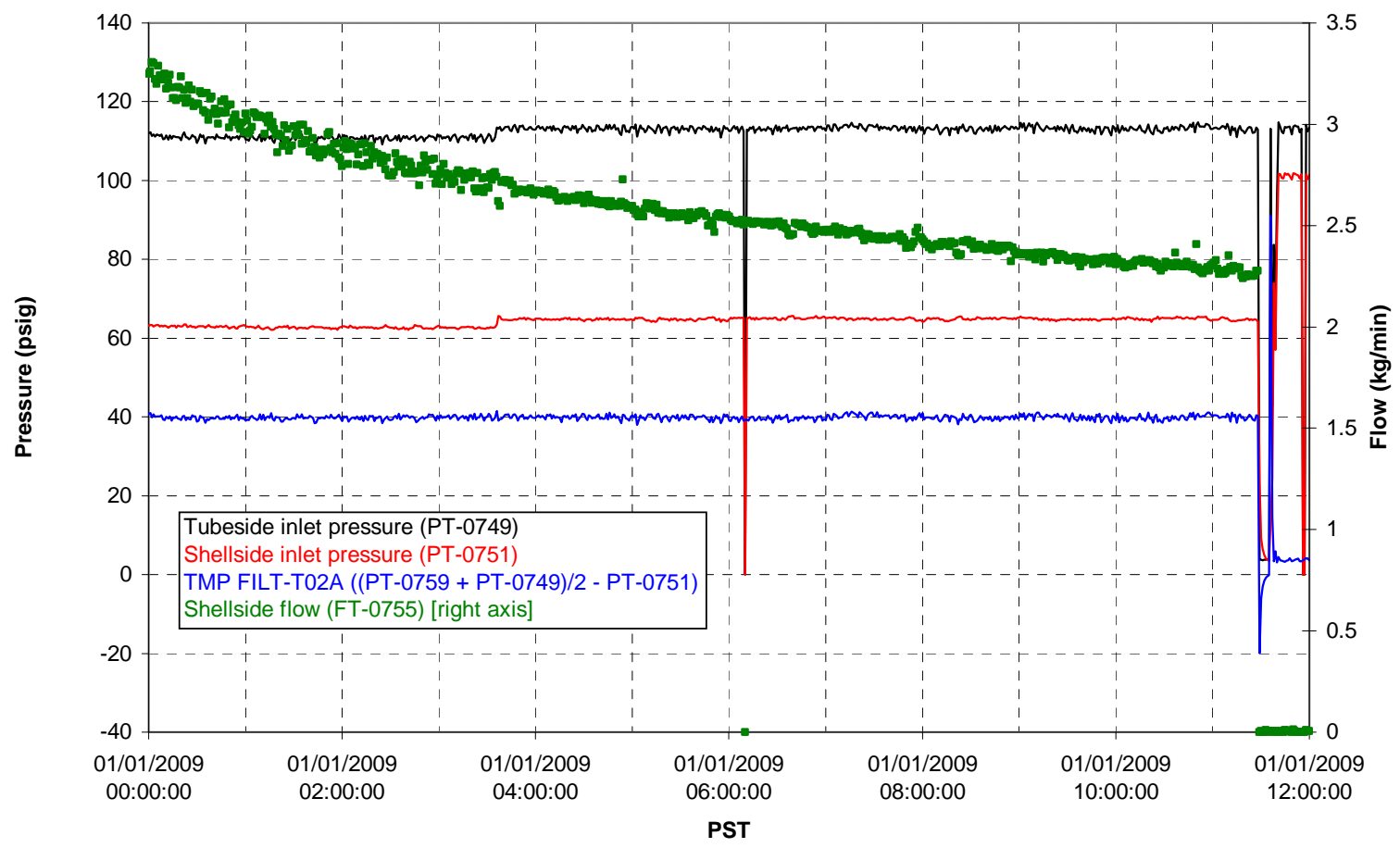


Filter UFP-FILT-T03A

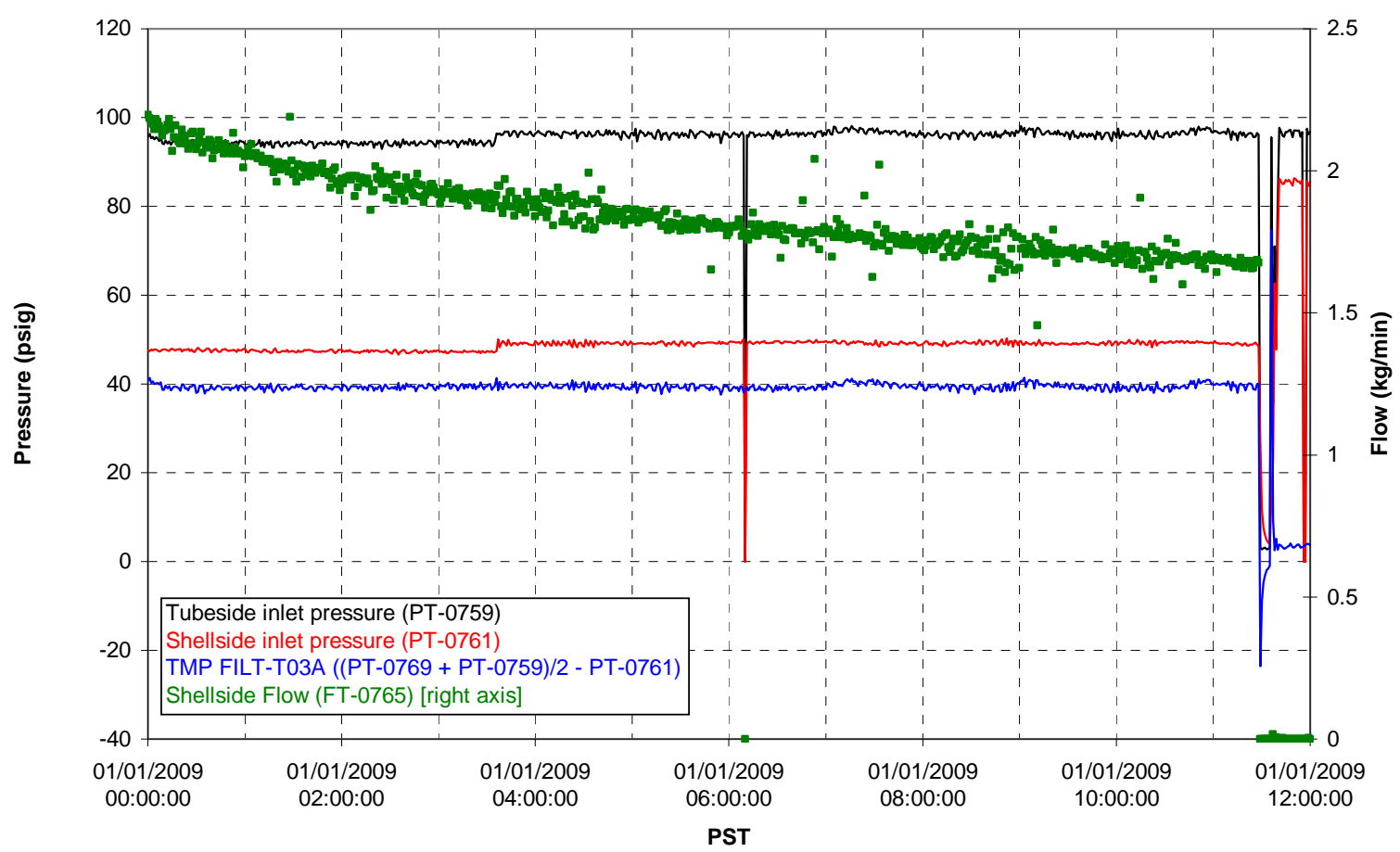

Filter UFP-FILT-T04A

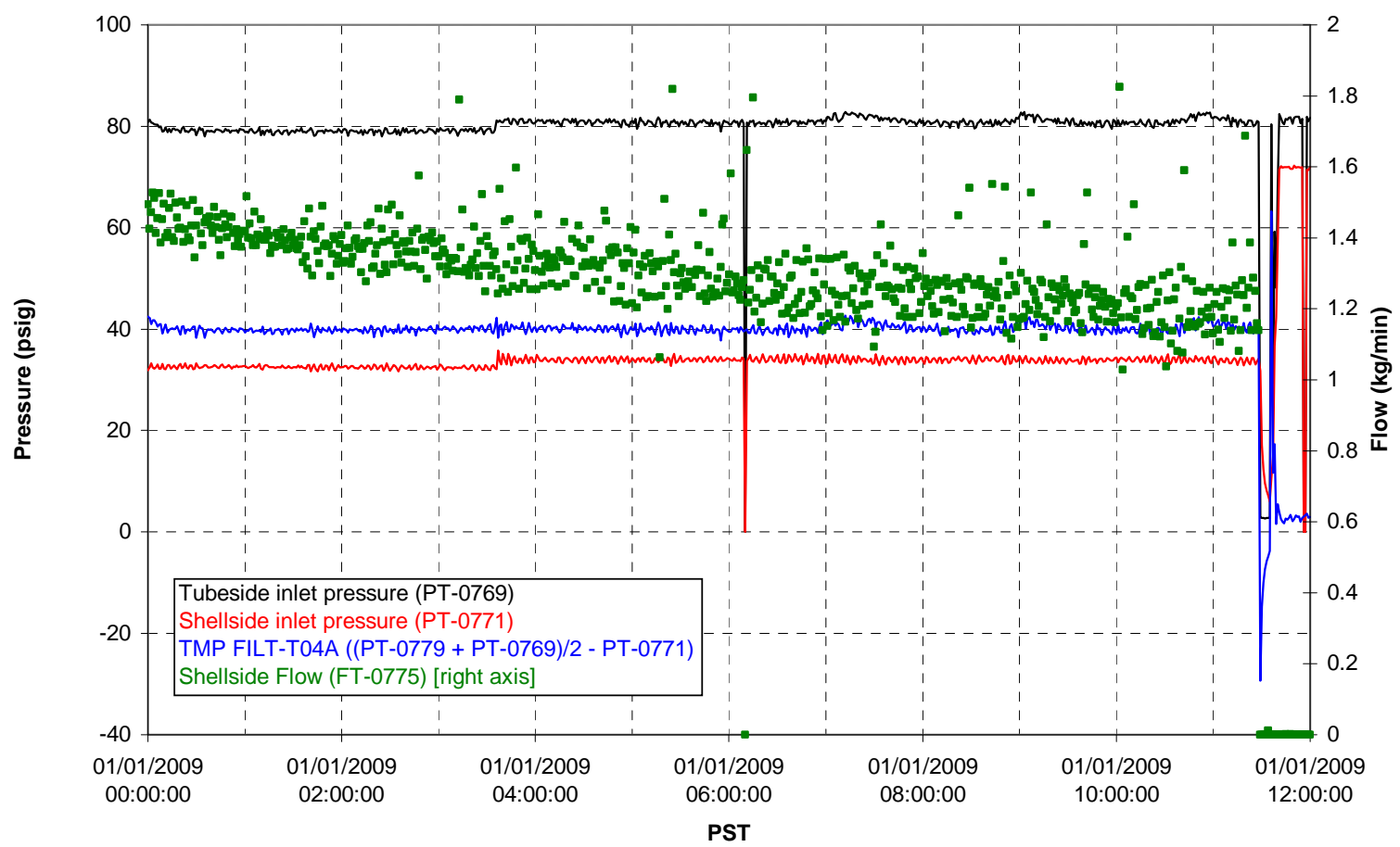


Filter UFP-FILT-T05A

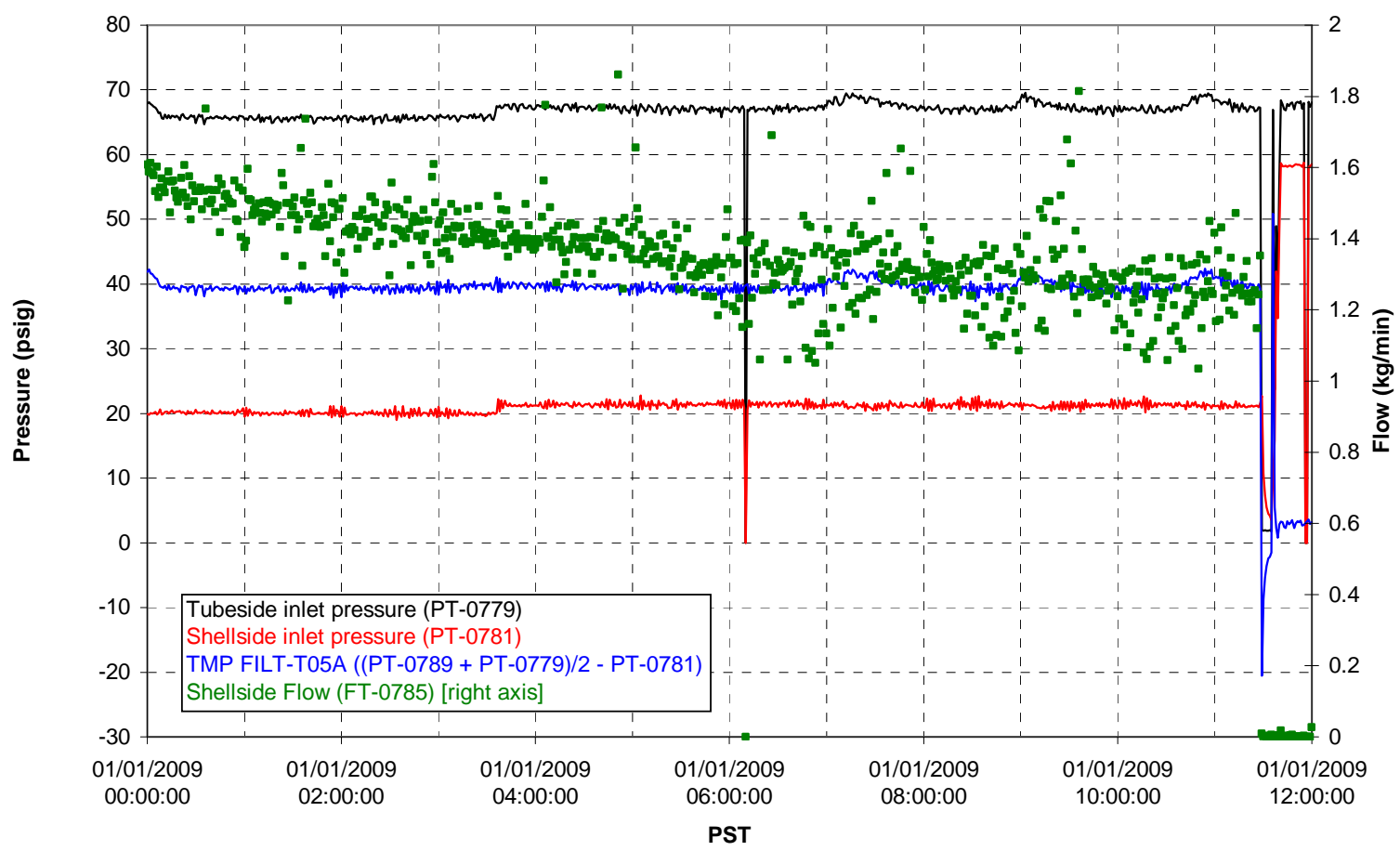

Chemical Flow

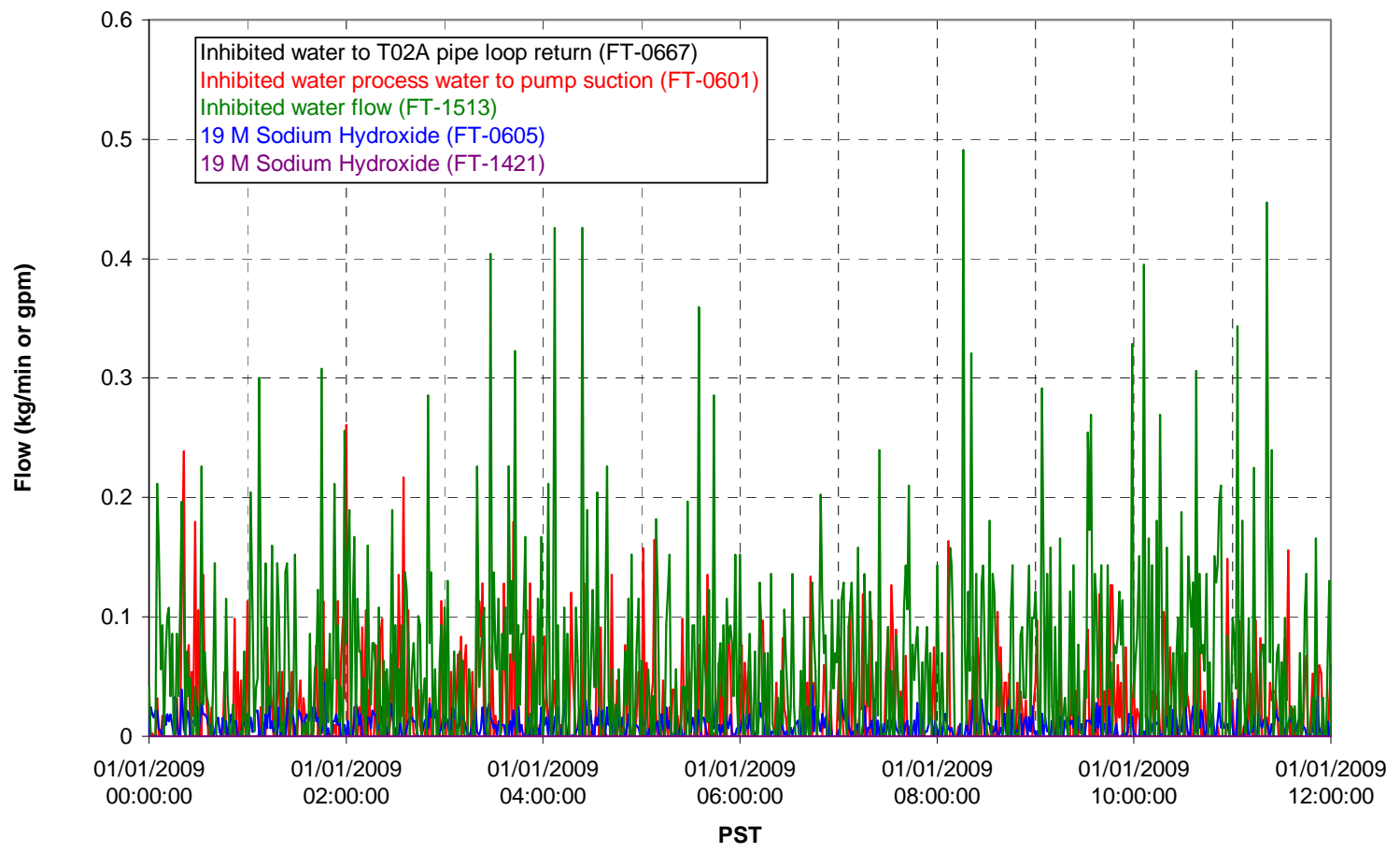


Chemical Flow

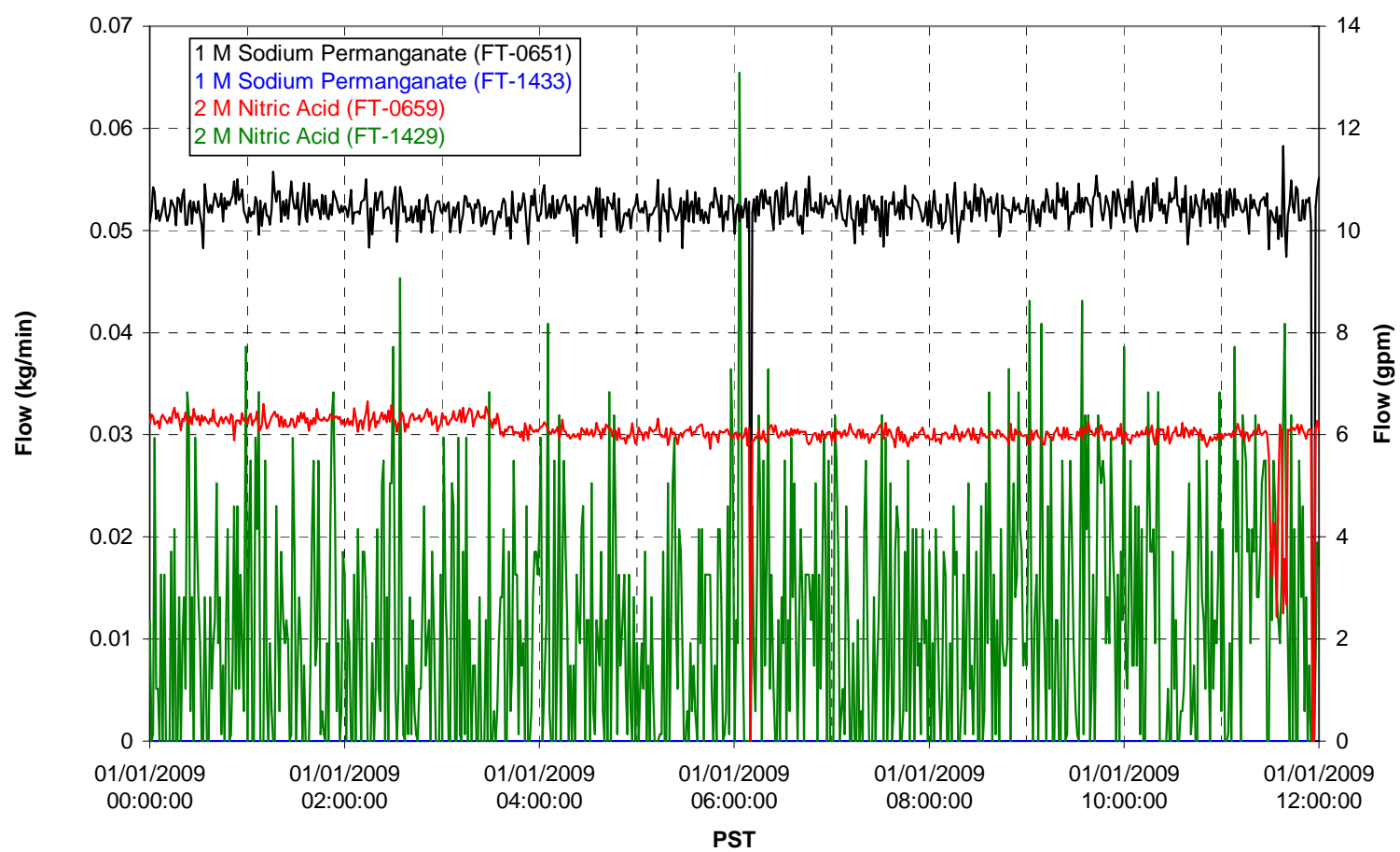

Air Flows

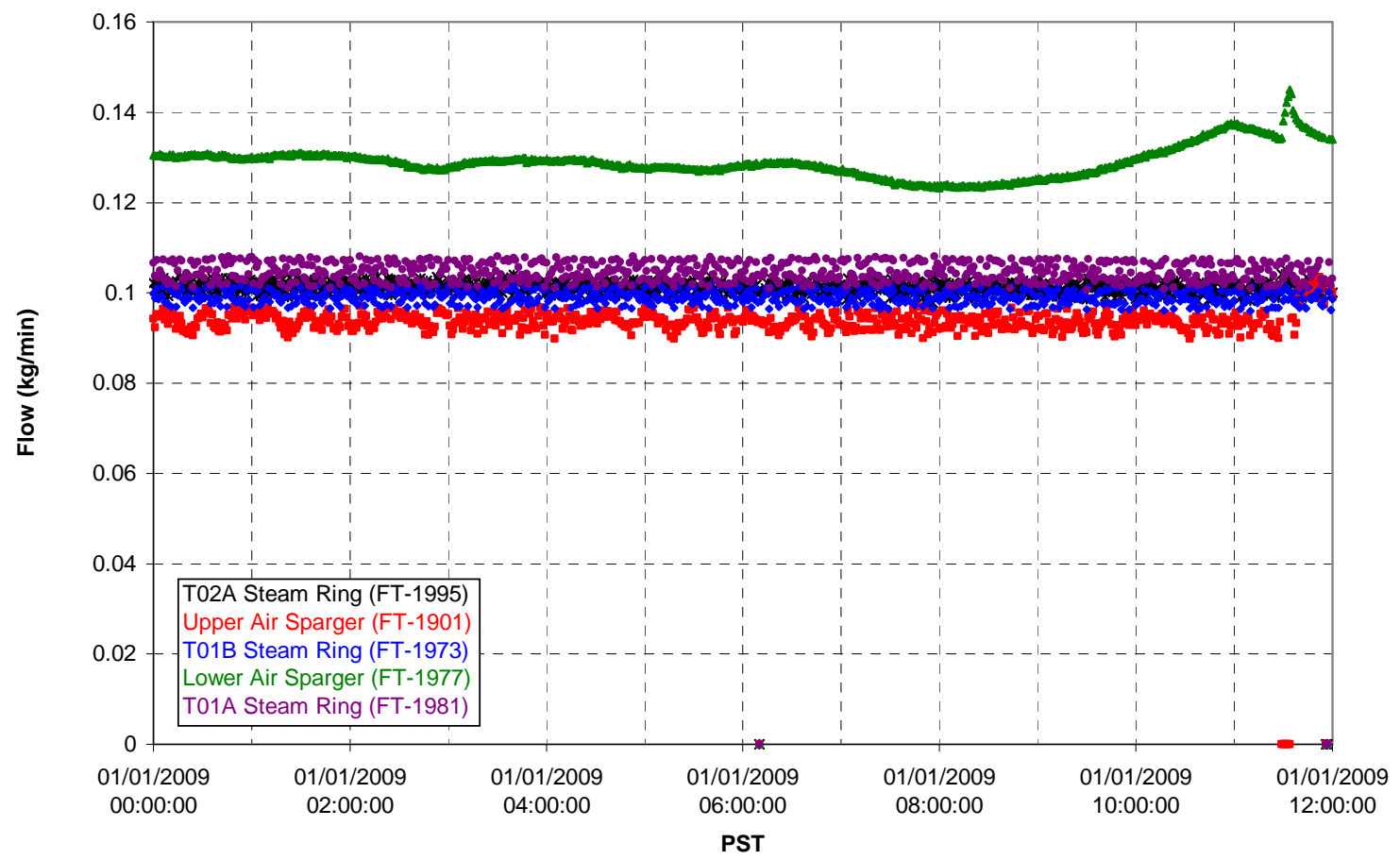


T02A Steam

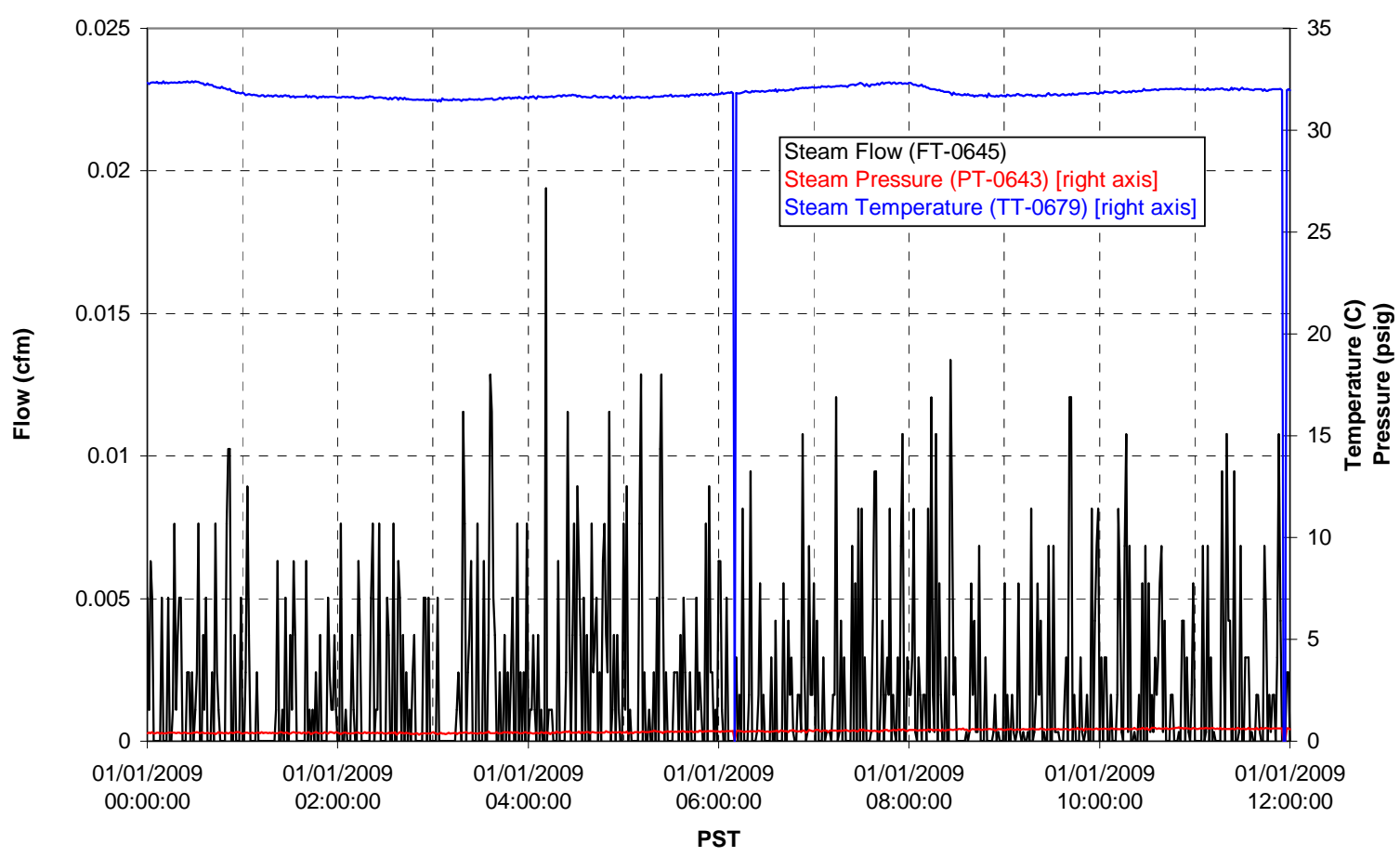

T01A Steam

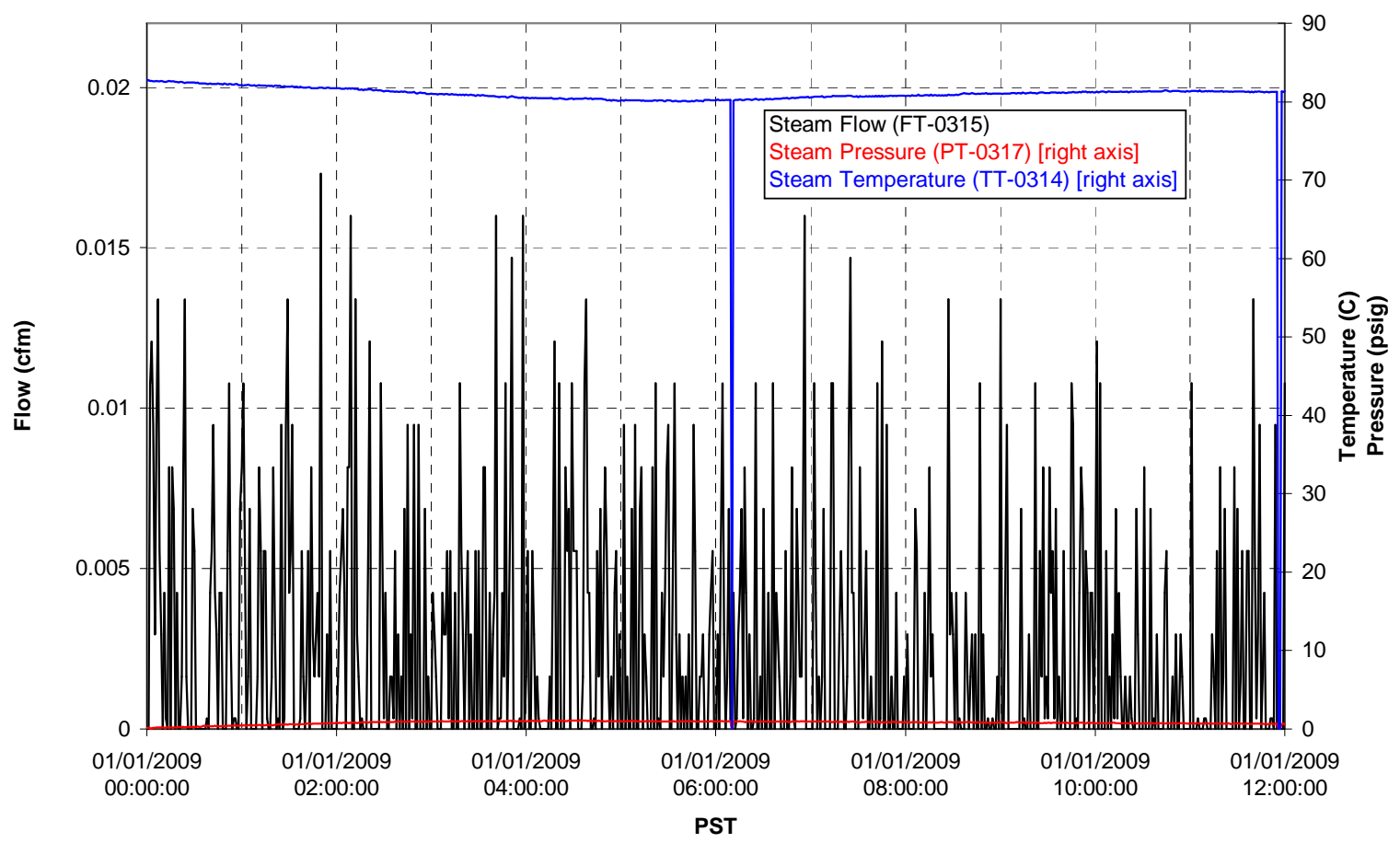


T01B Steam

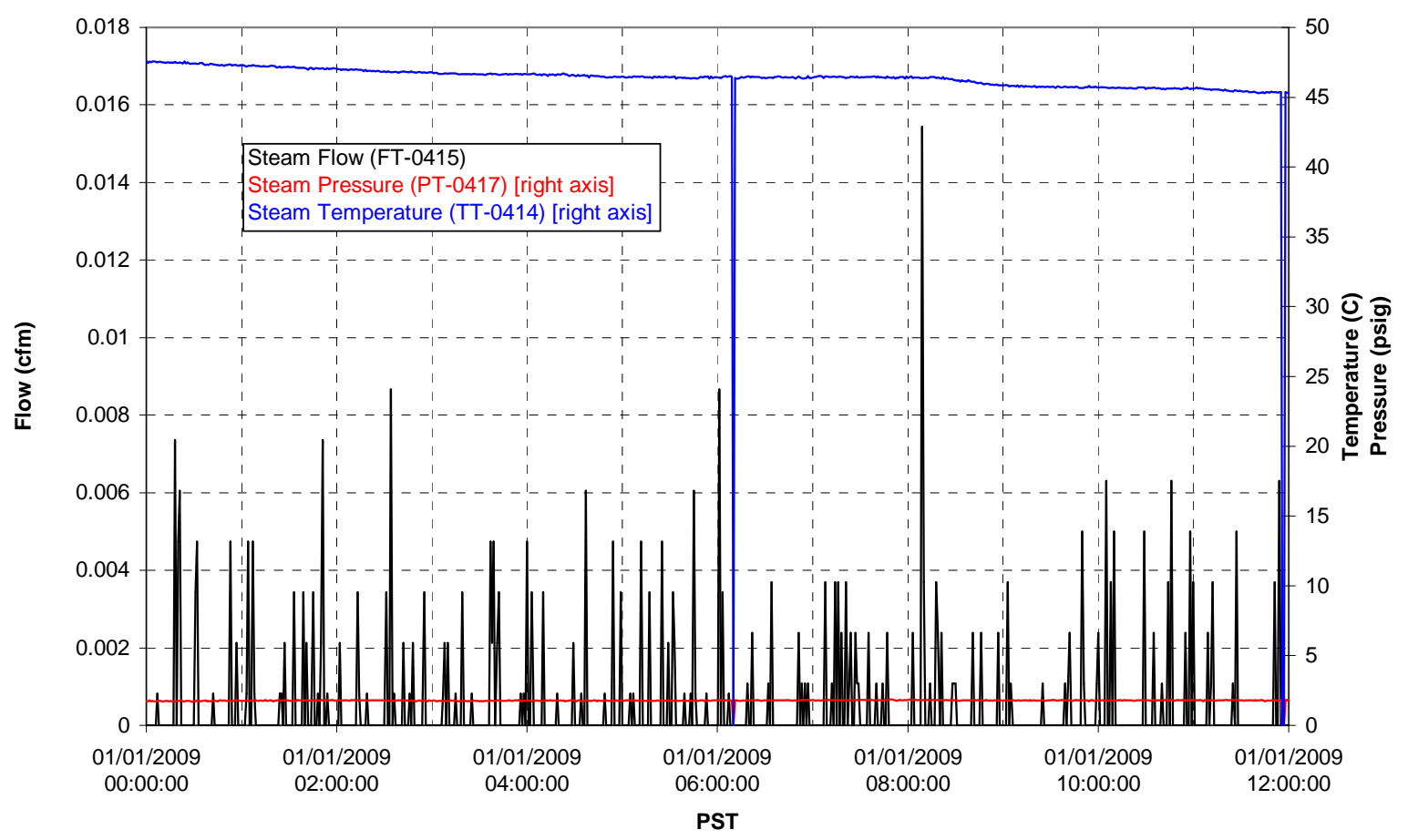


Functional Test Data Plots

01/01/2009 12:00 - 01/02/2009 00:00 
T01A level

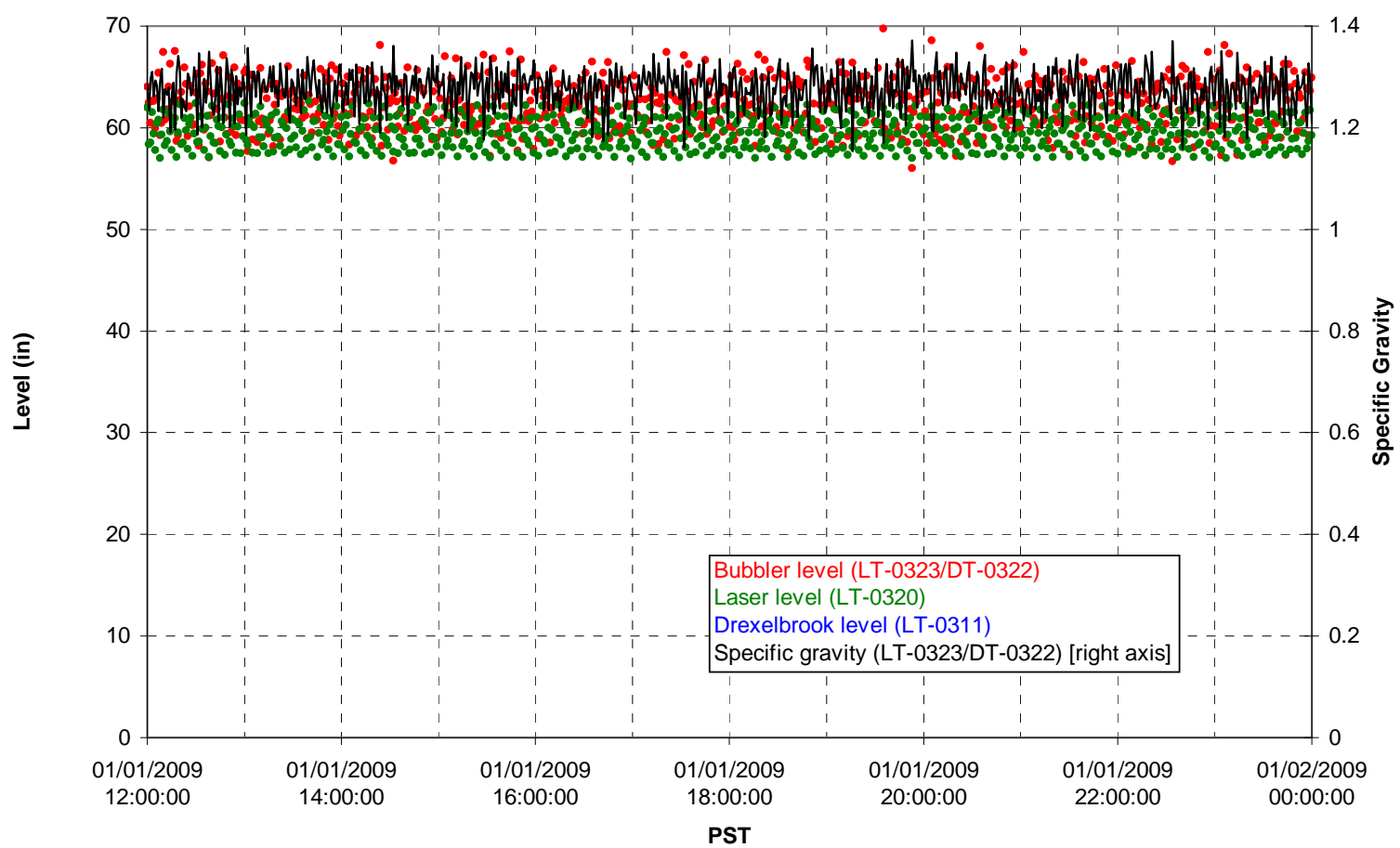

T01A temperatures

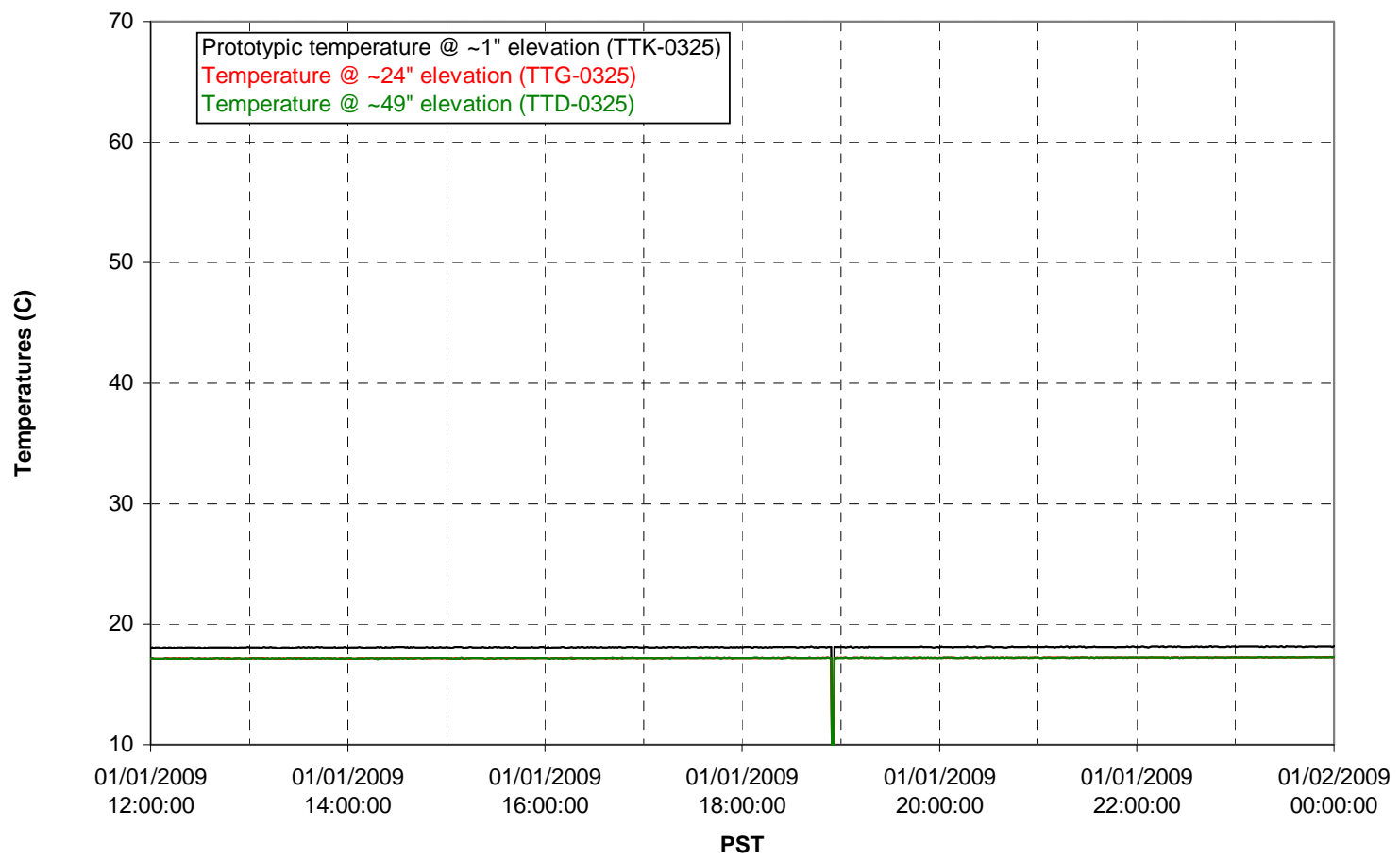


T01B level

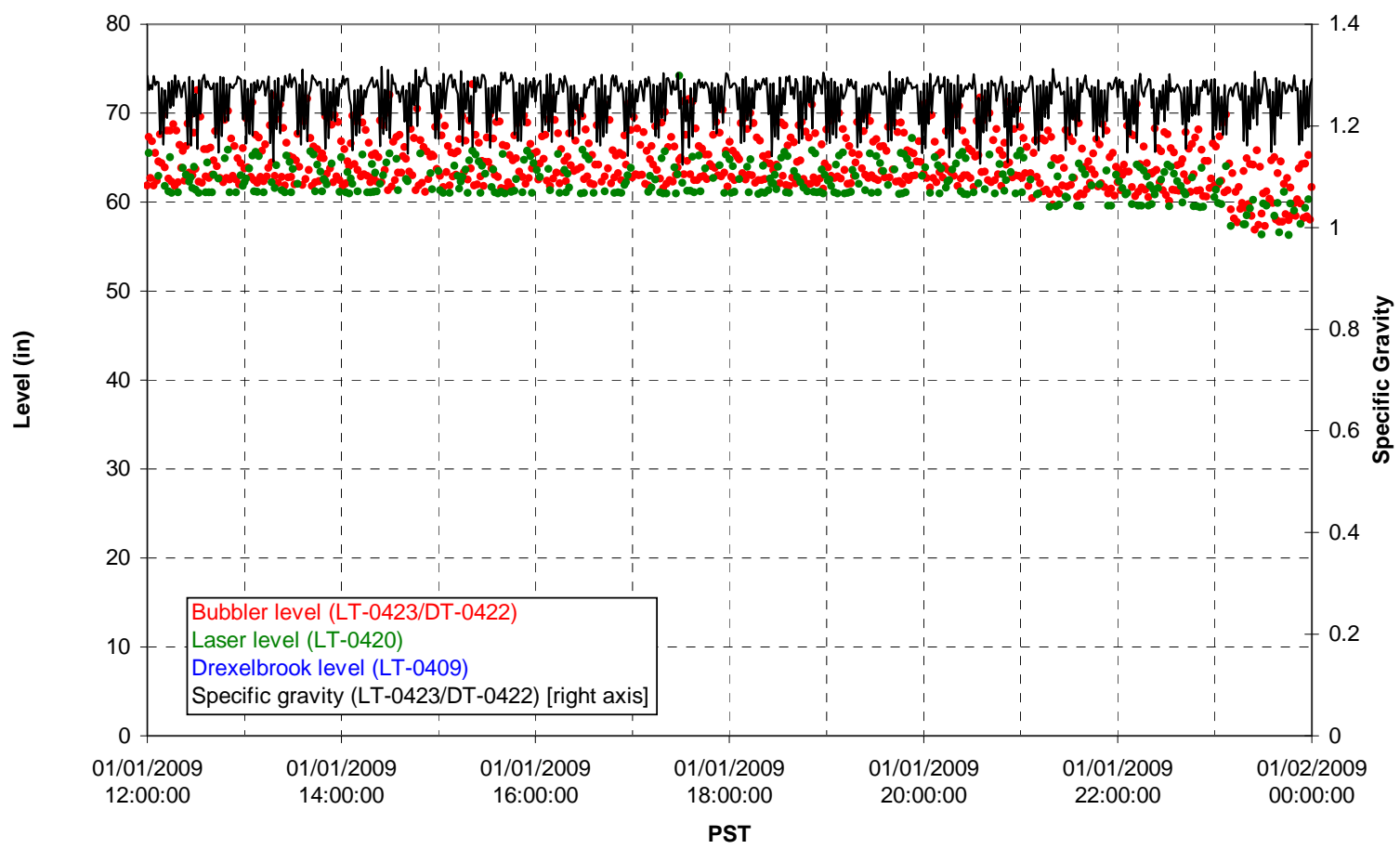

T01B temperatures

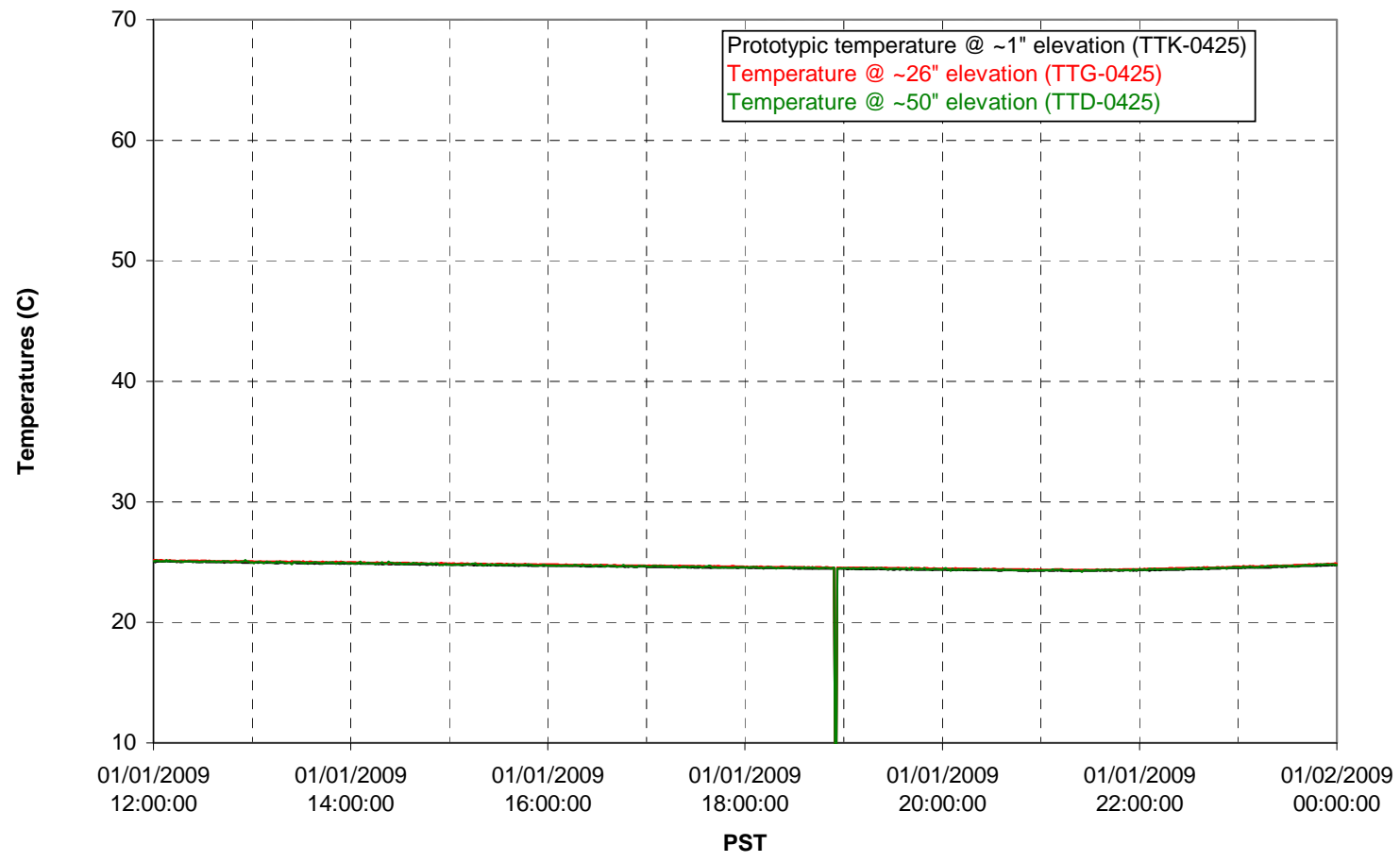


T02A level

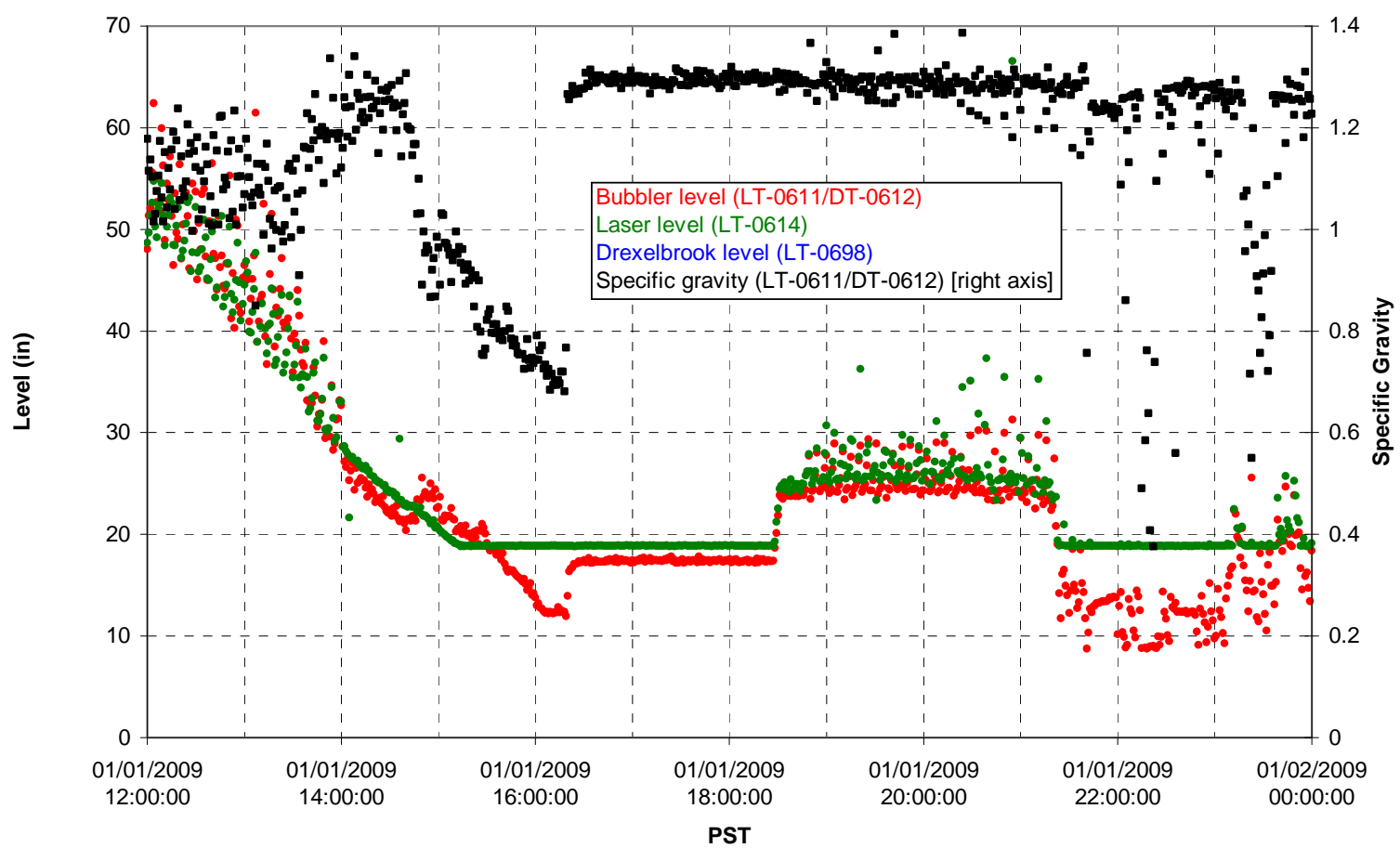

T02A temperatures

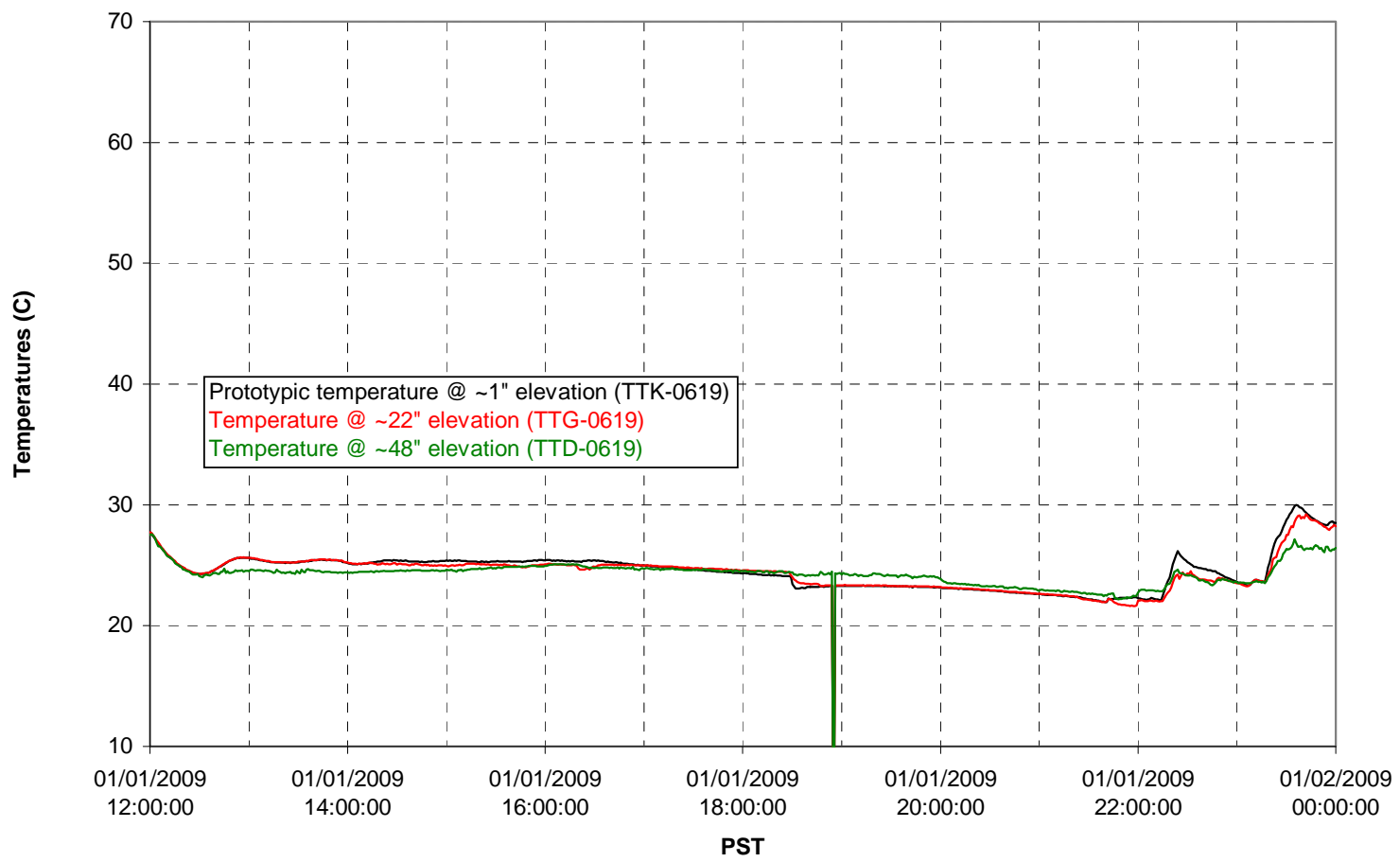


T02A and filter loop temperatures

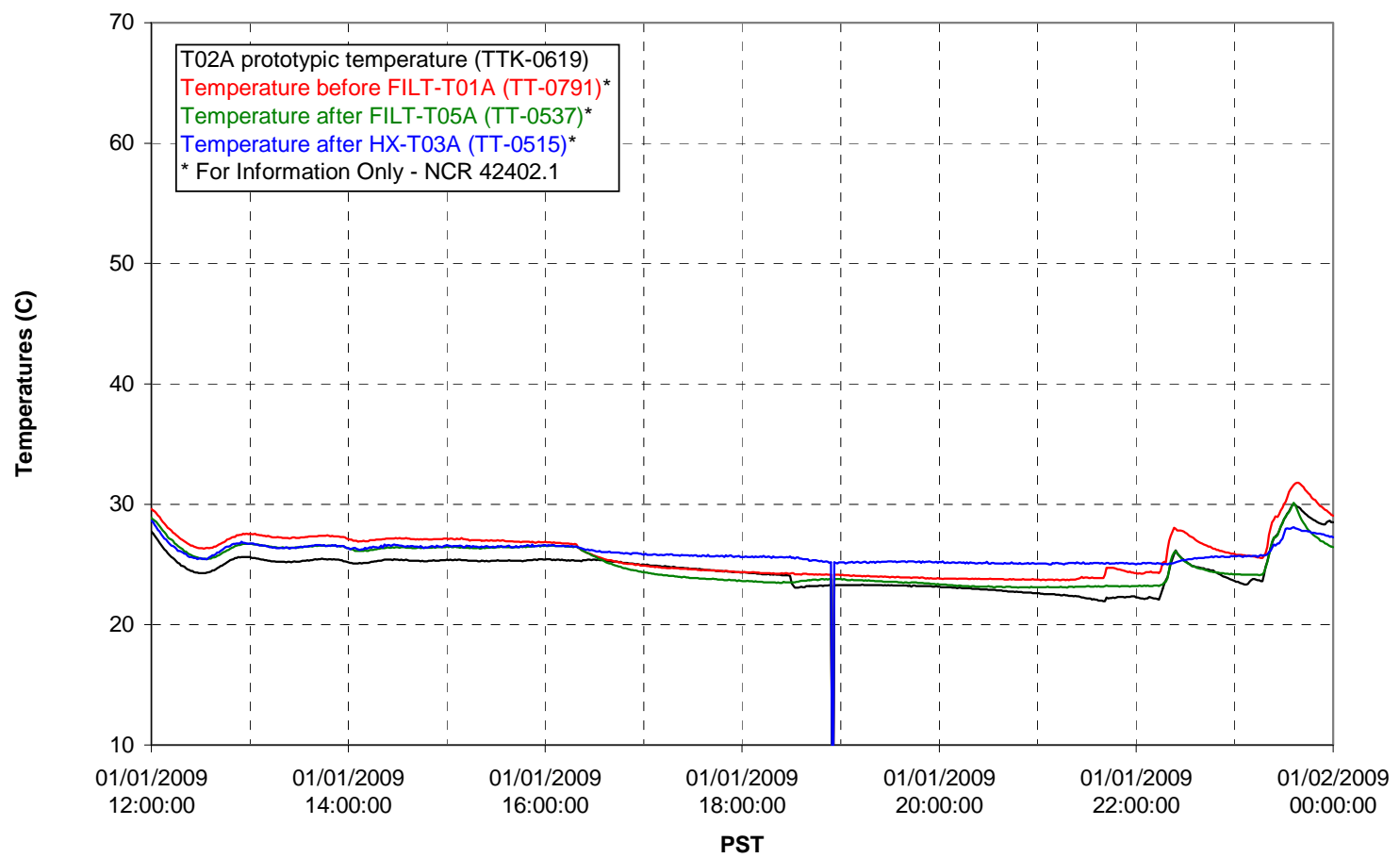

Pump Pressures and Flow

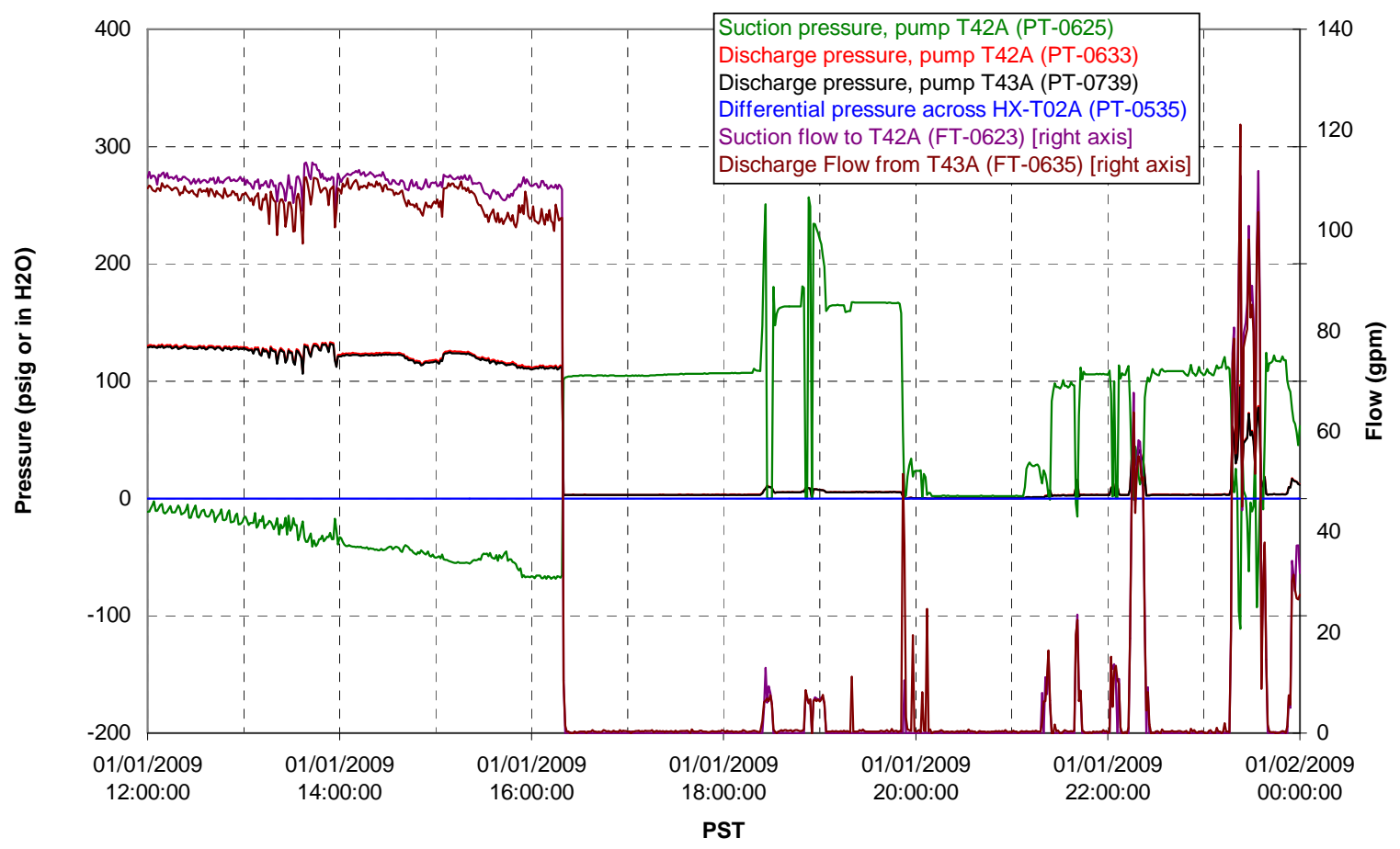


Axial pressure drop

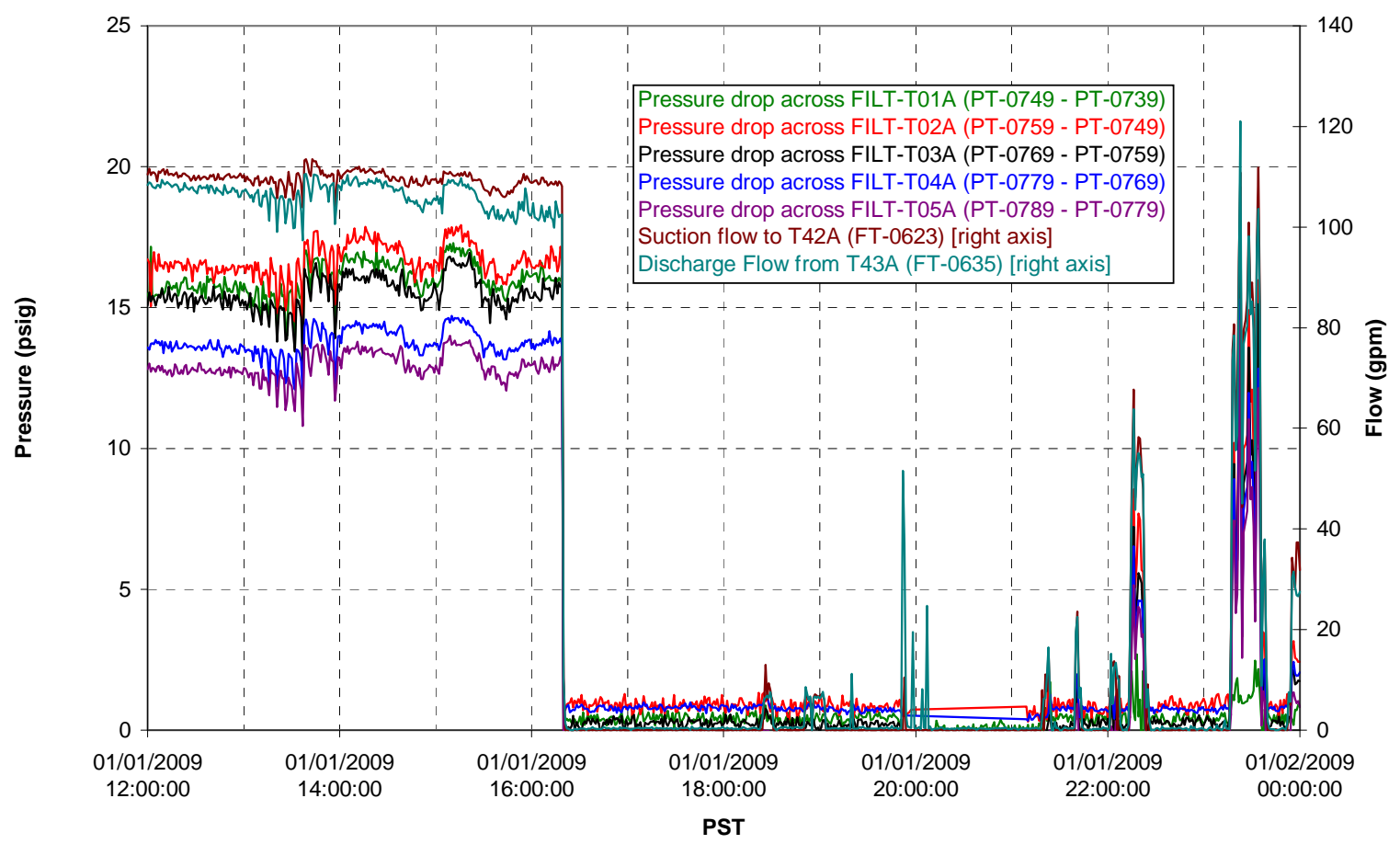

Permeate flow rates

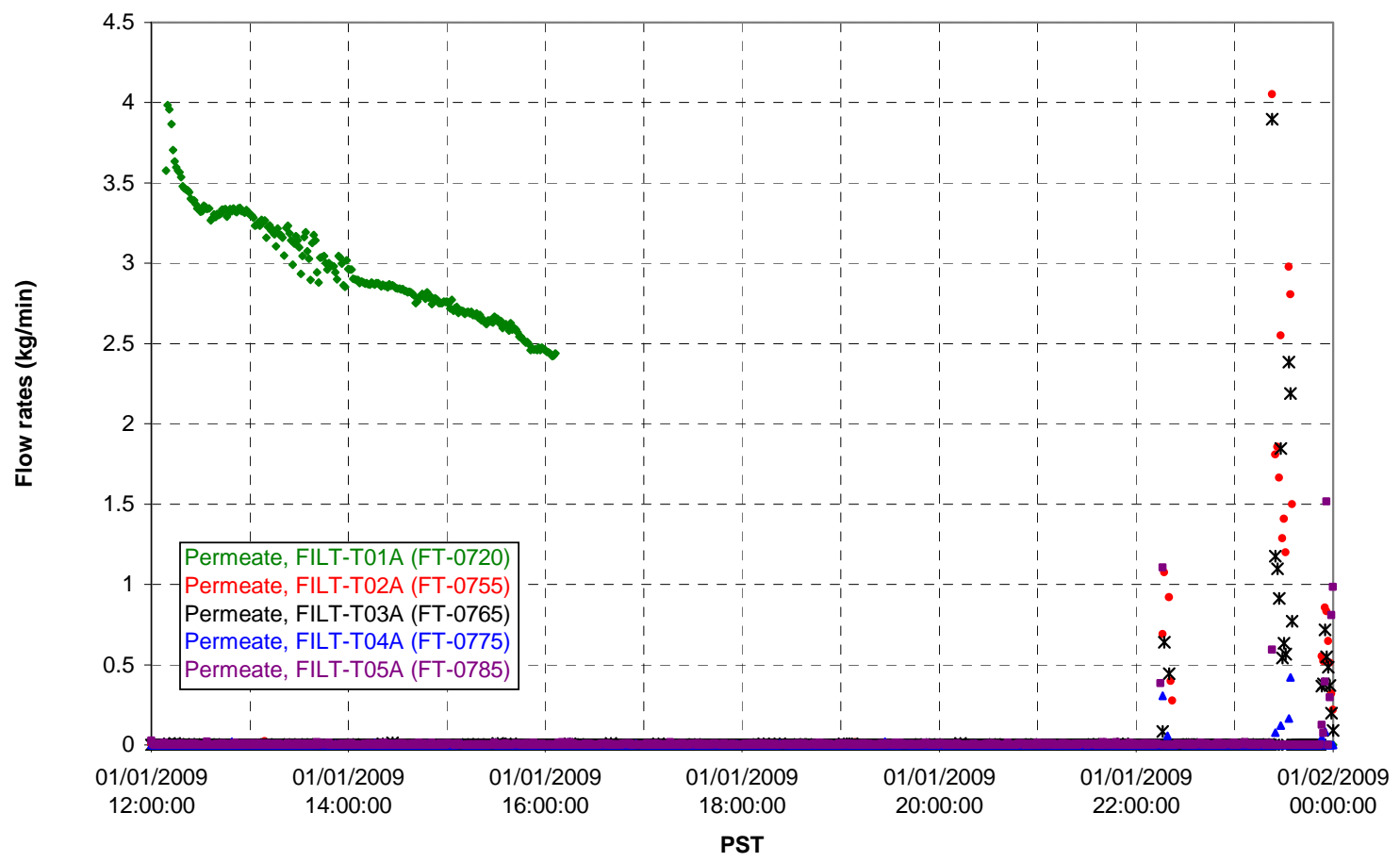


T02A Inner Temperature Tree

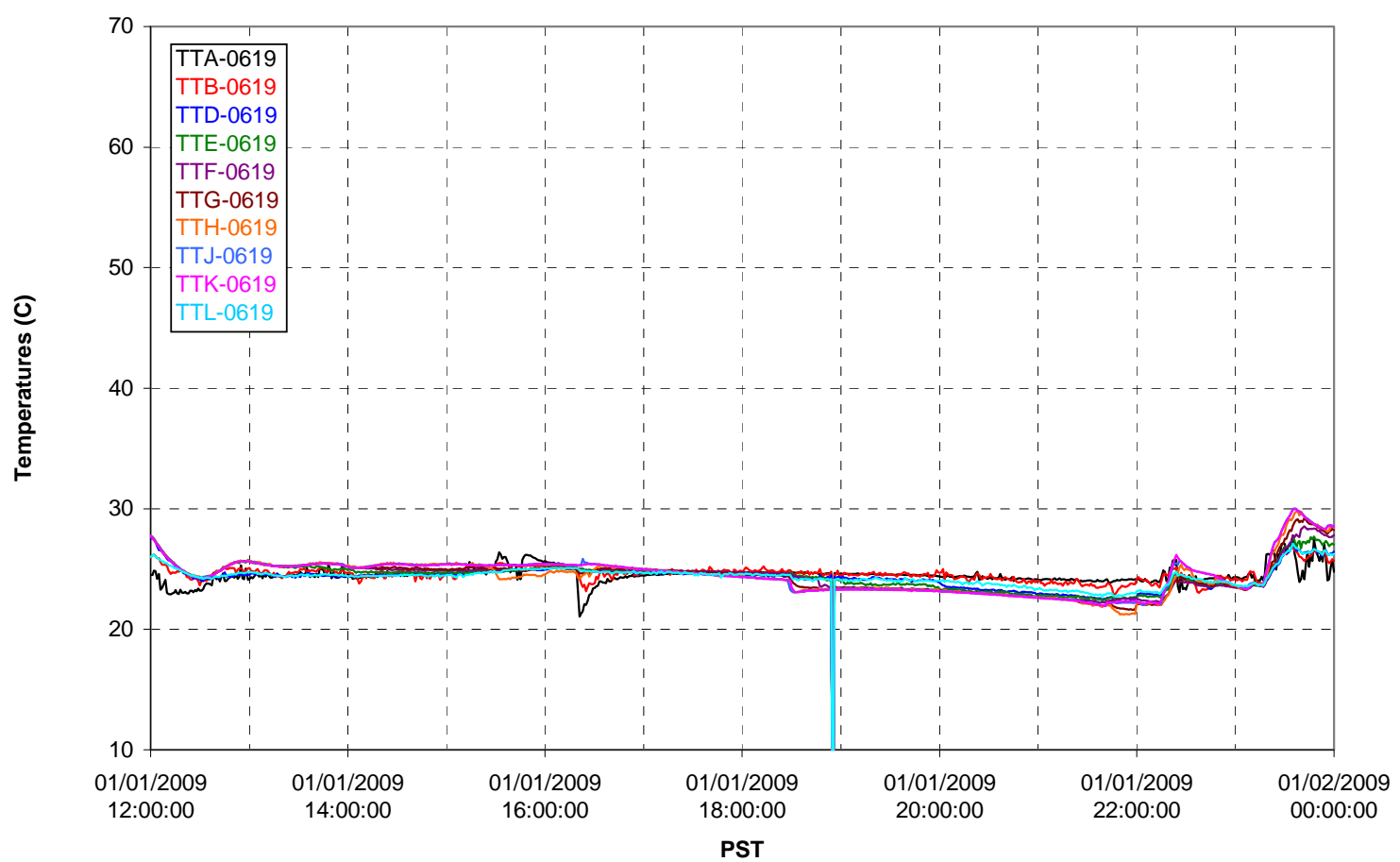

T02A Outer Temperature Tree

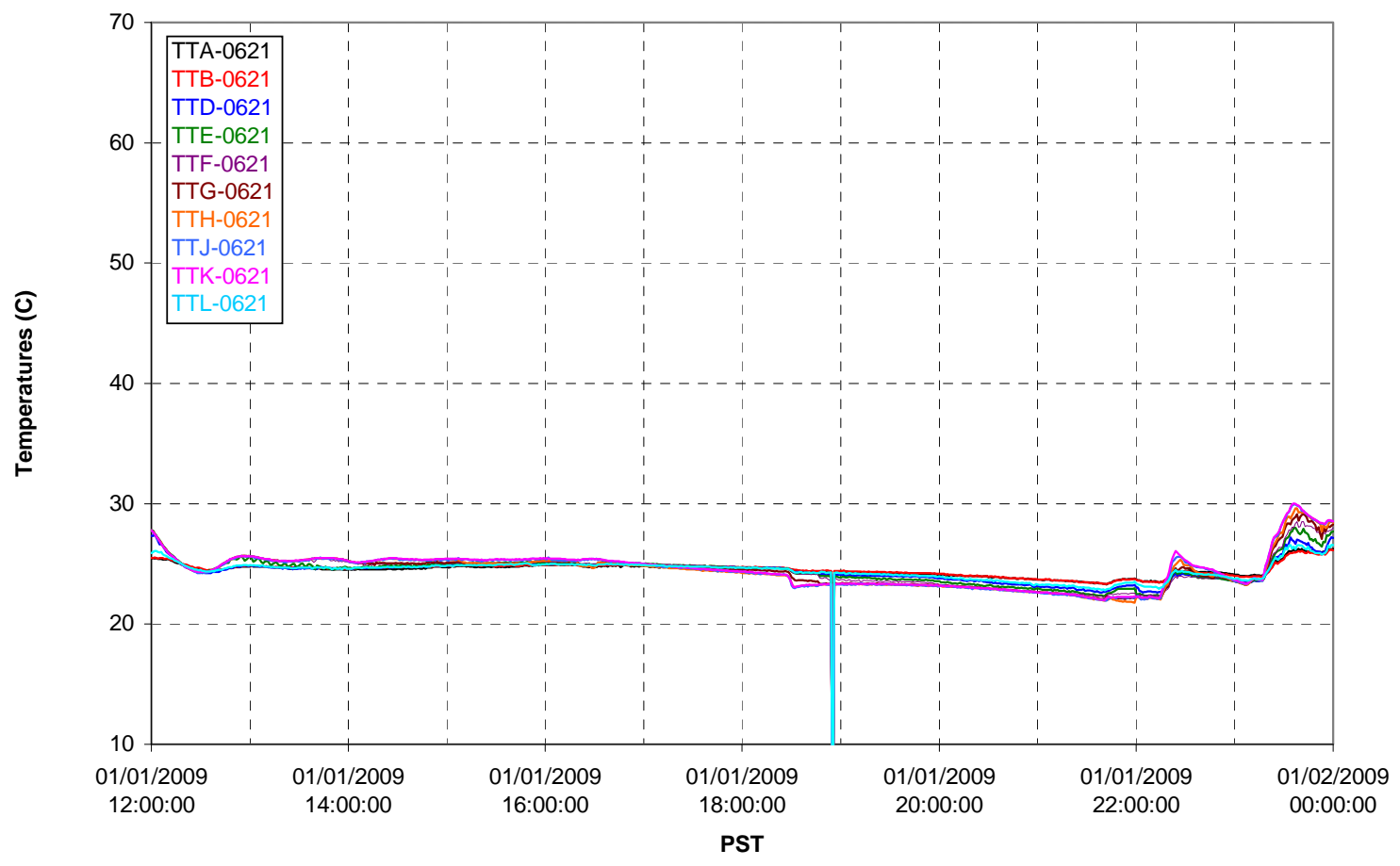


T02A temperatures

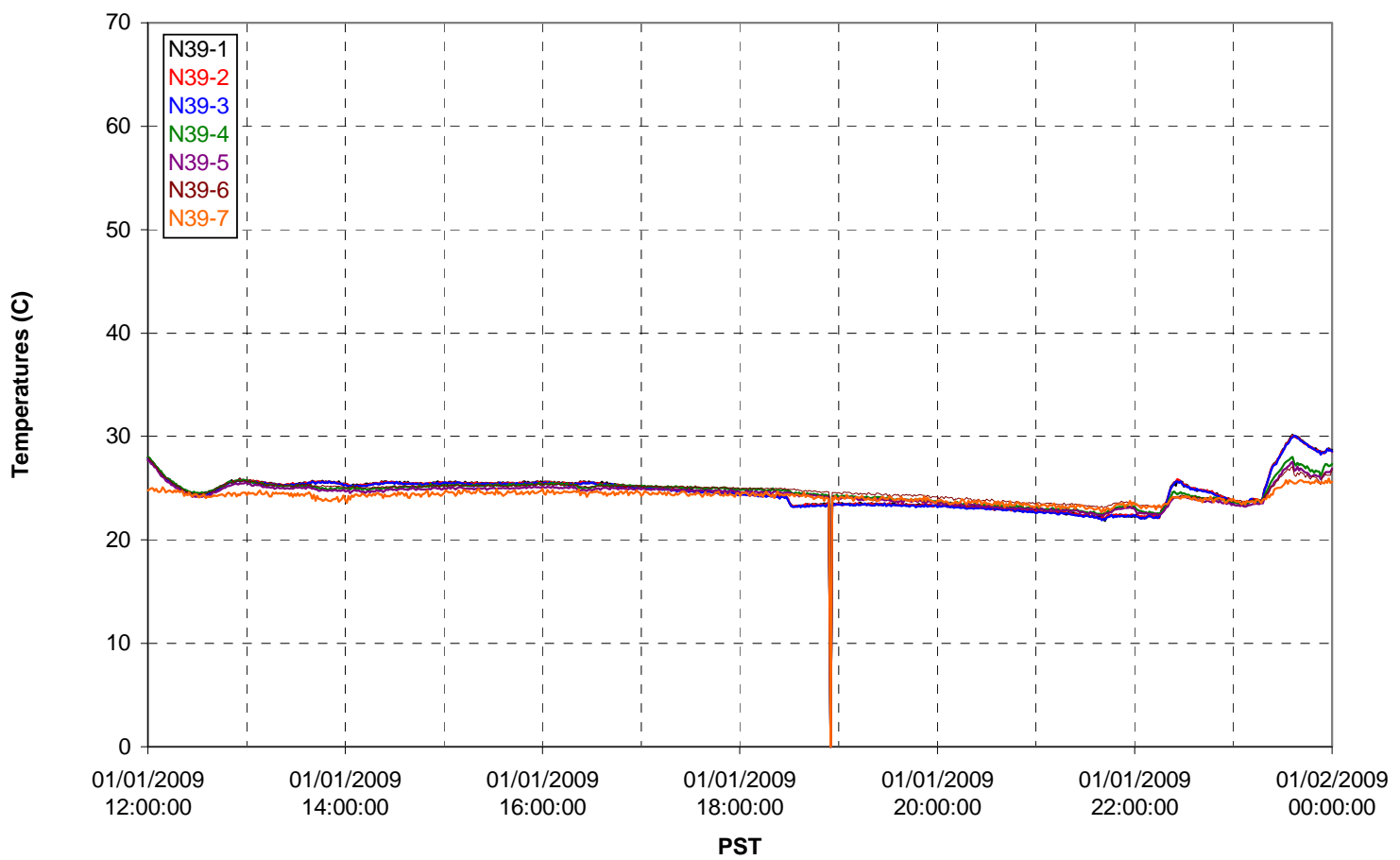

T02A temperatures

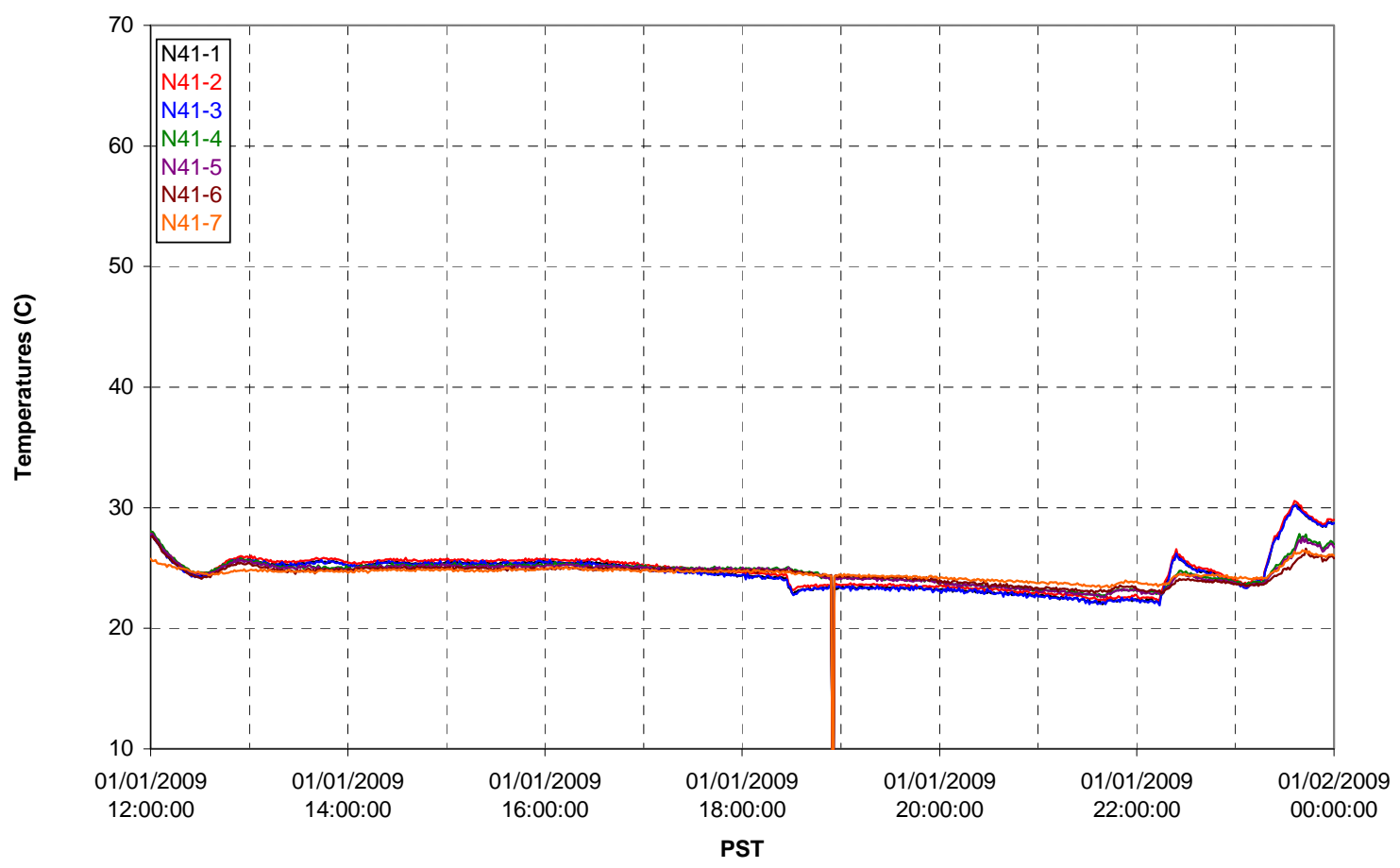


T02A temperatures

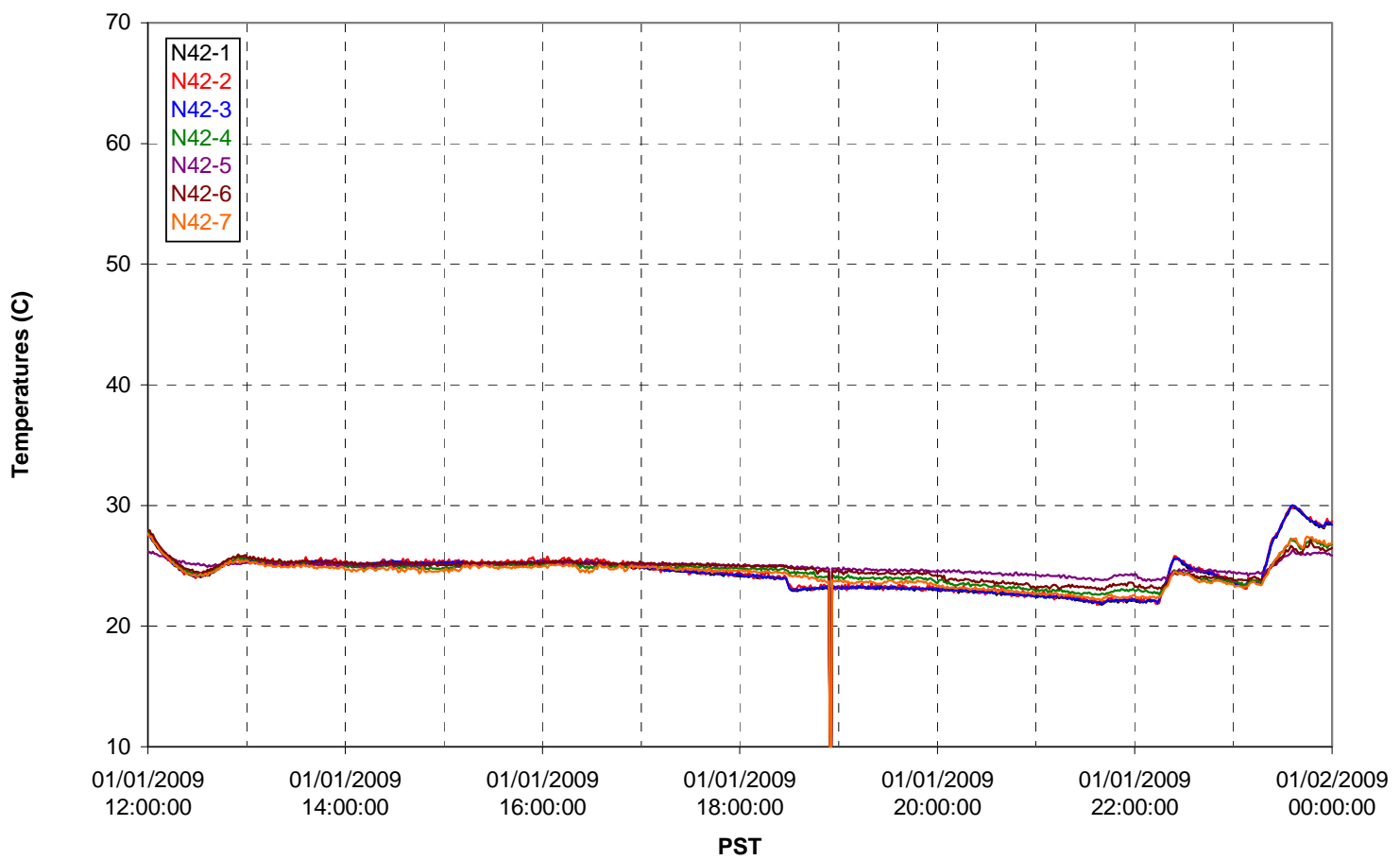

T02A temperatures

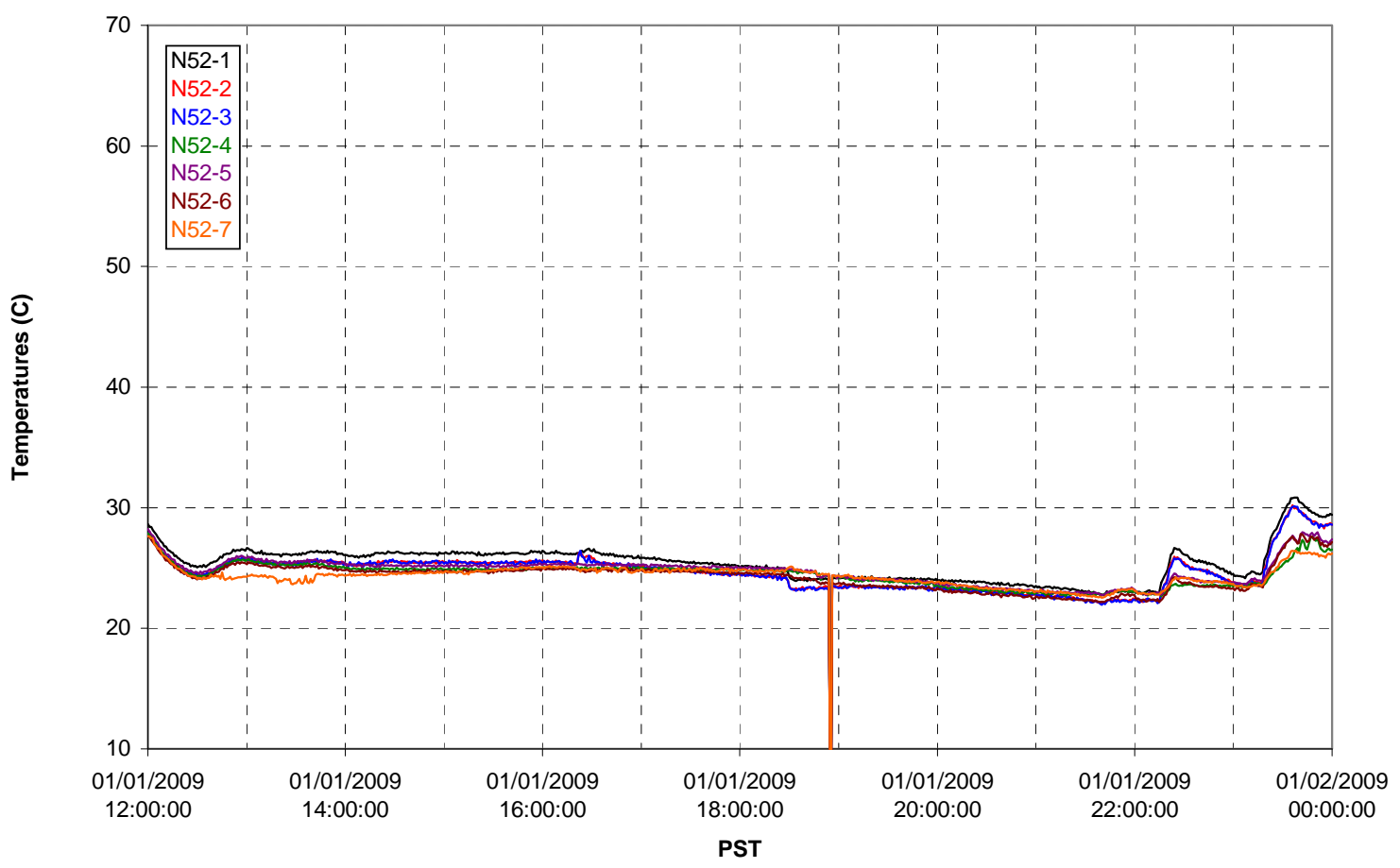


T02A Heating and Cooling

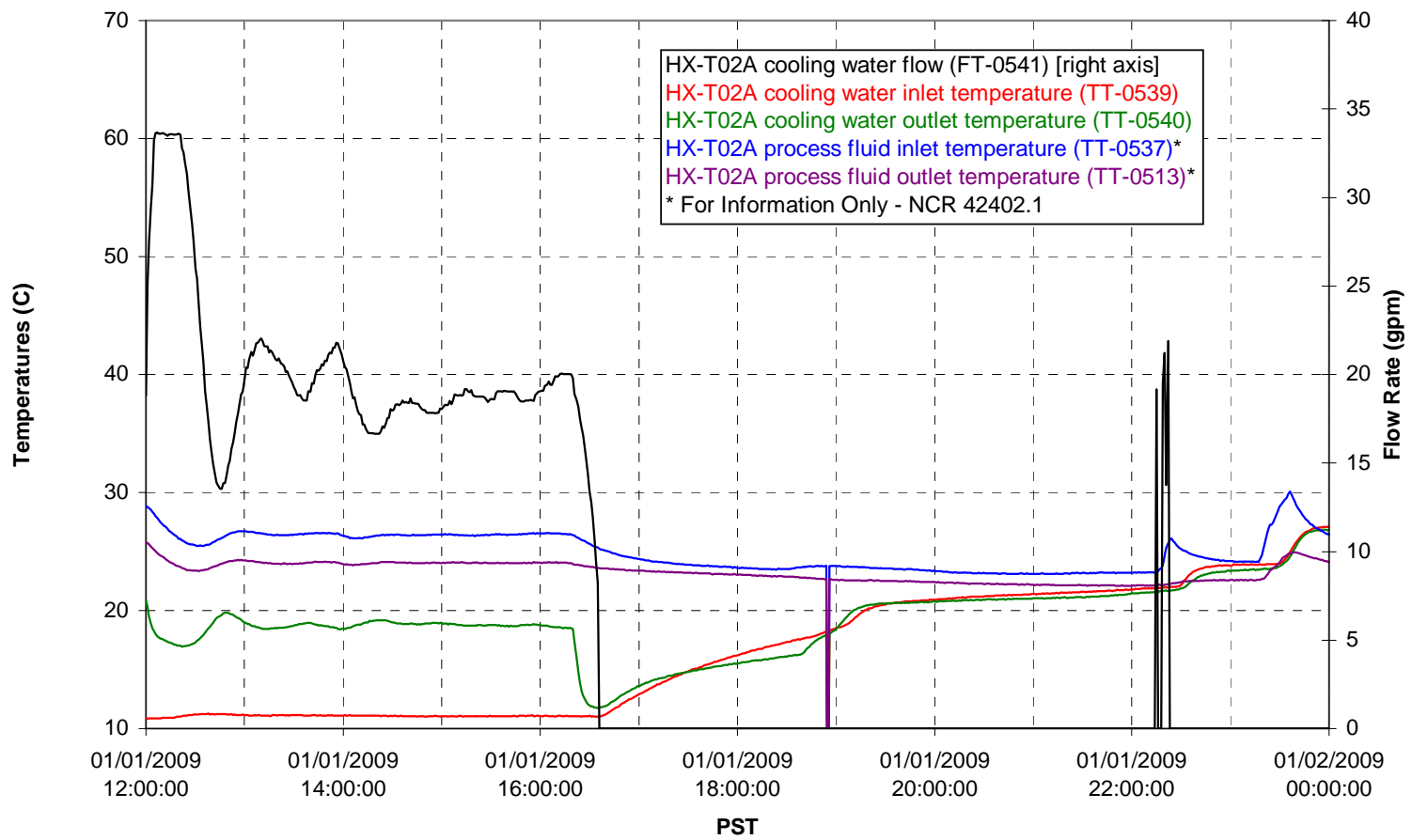

Pump Operation

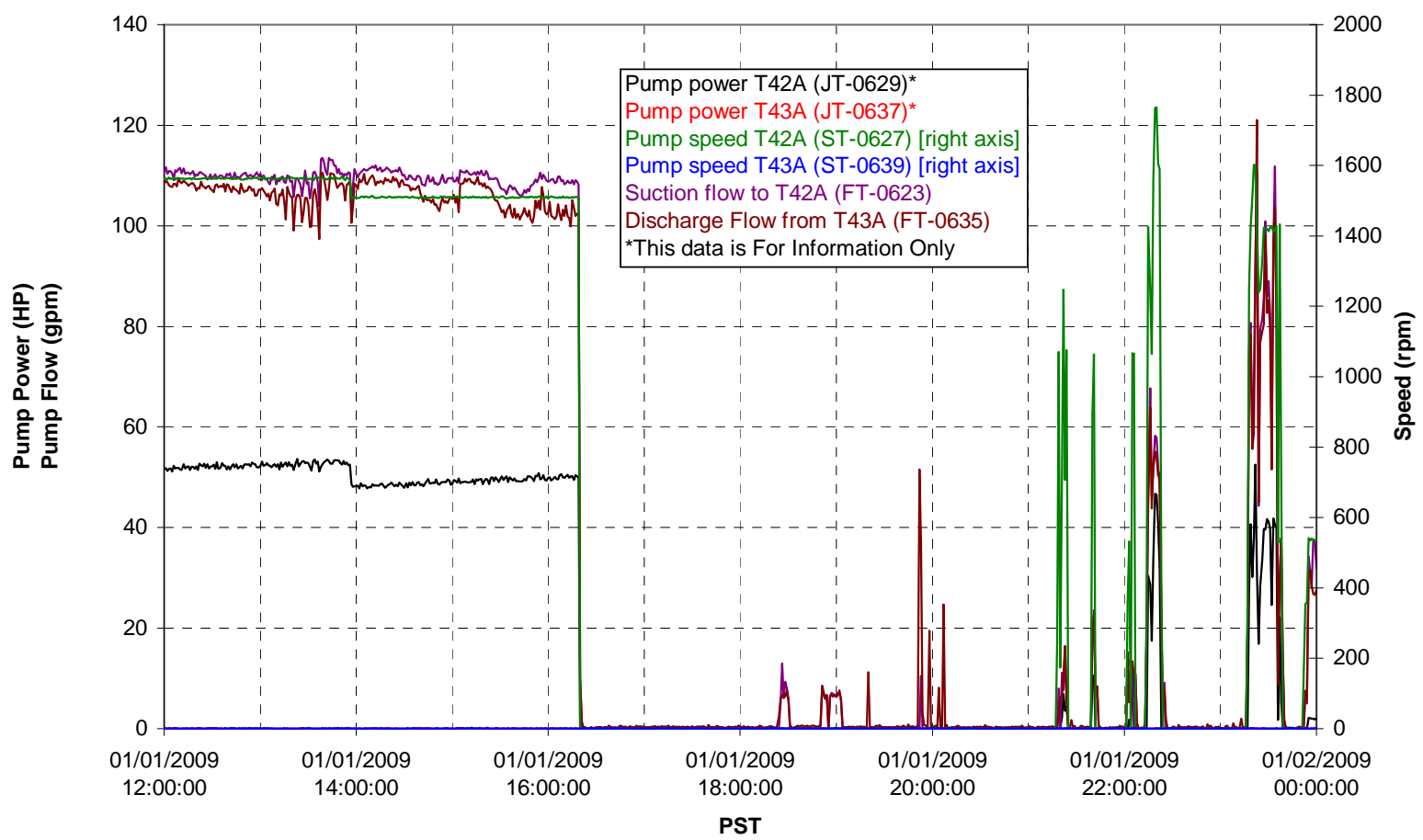


Pulsepot UFP-PP-T01A

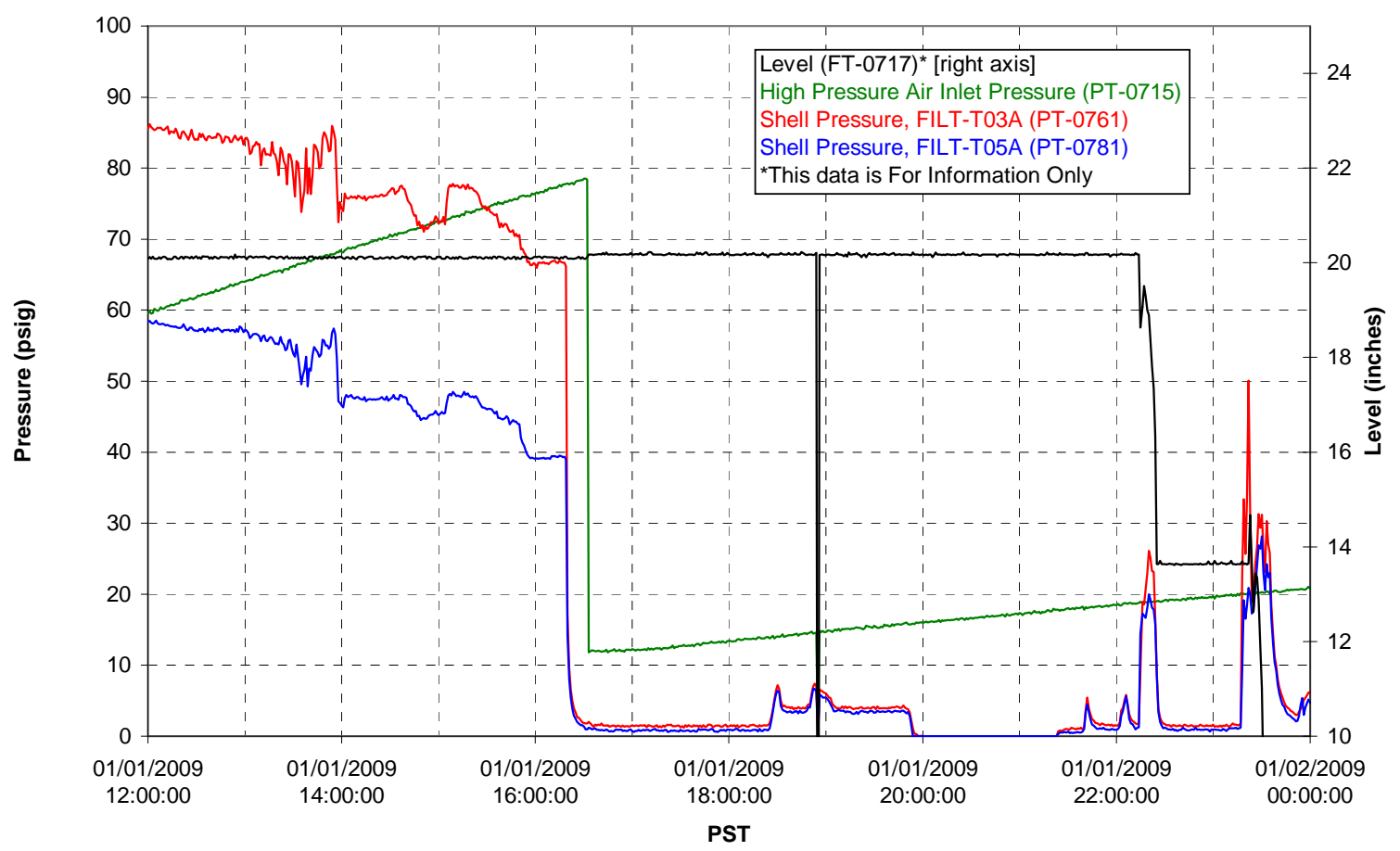

Pulsepot UFP-PP-T02A

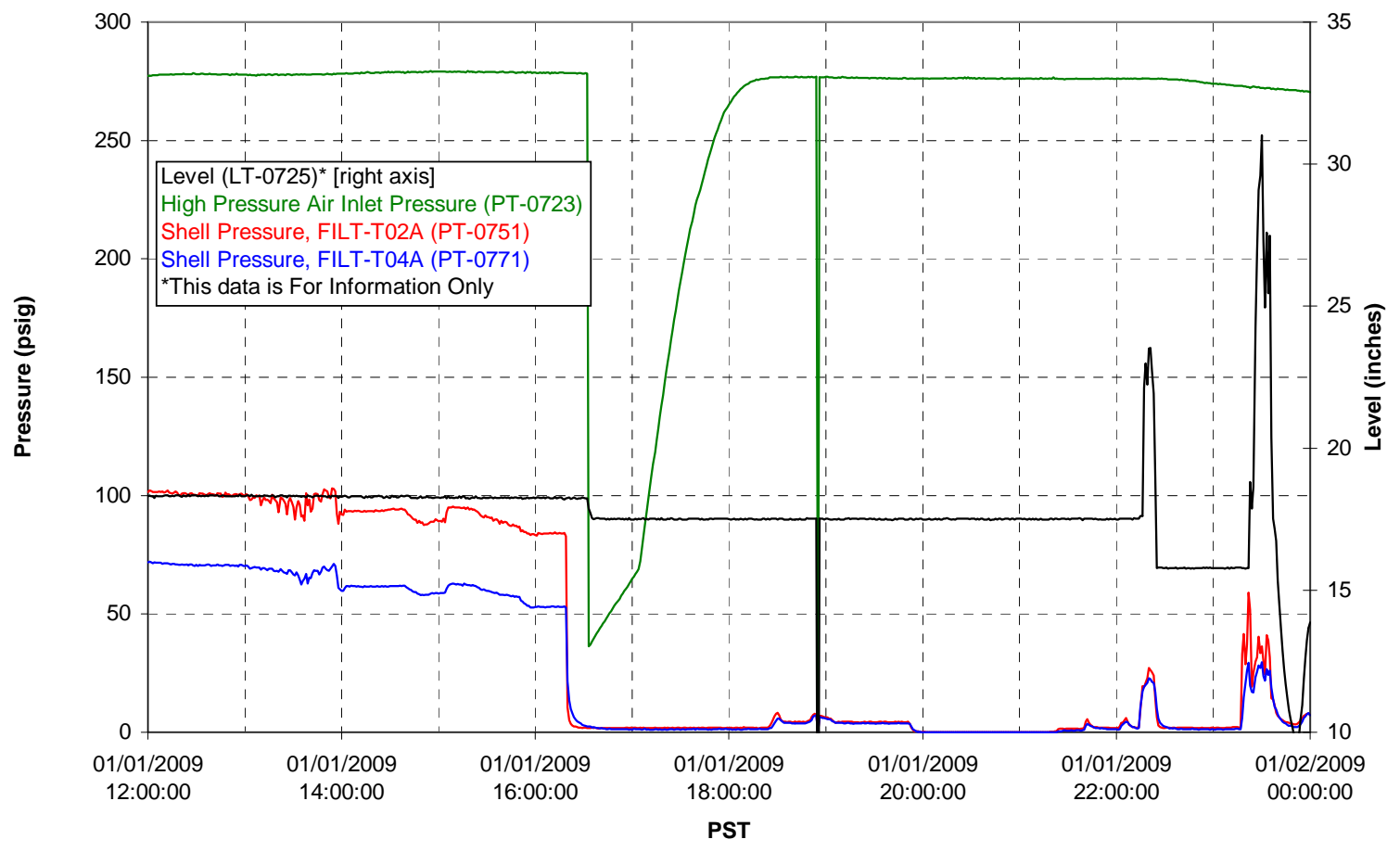


Pulsepot UFP-PP-T03A

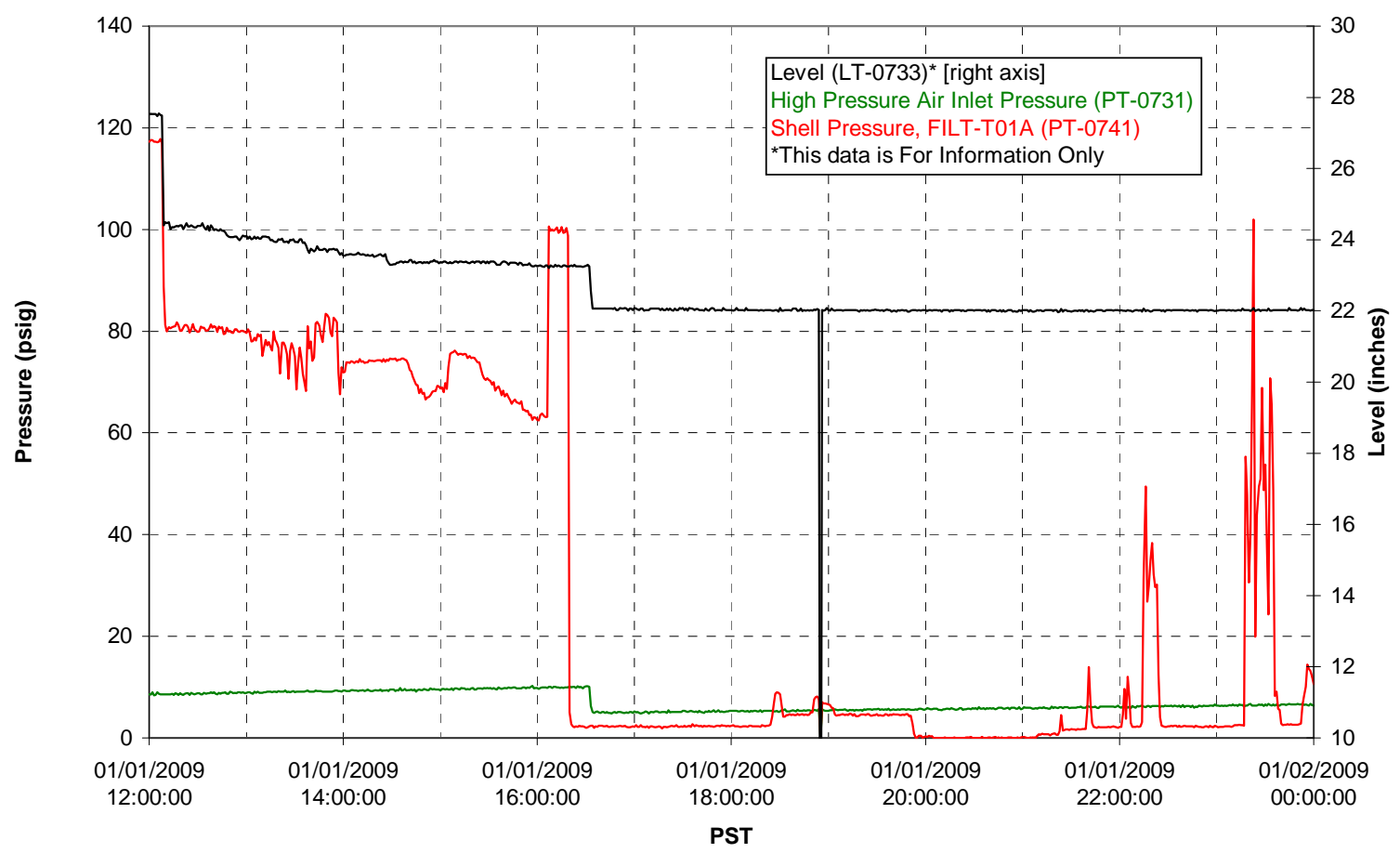

Pulsepot Levels

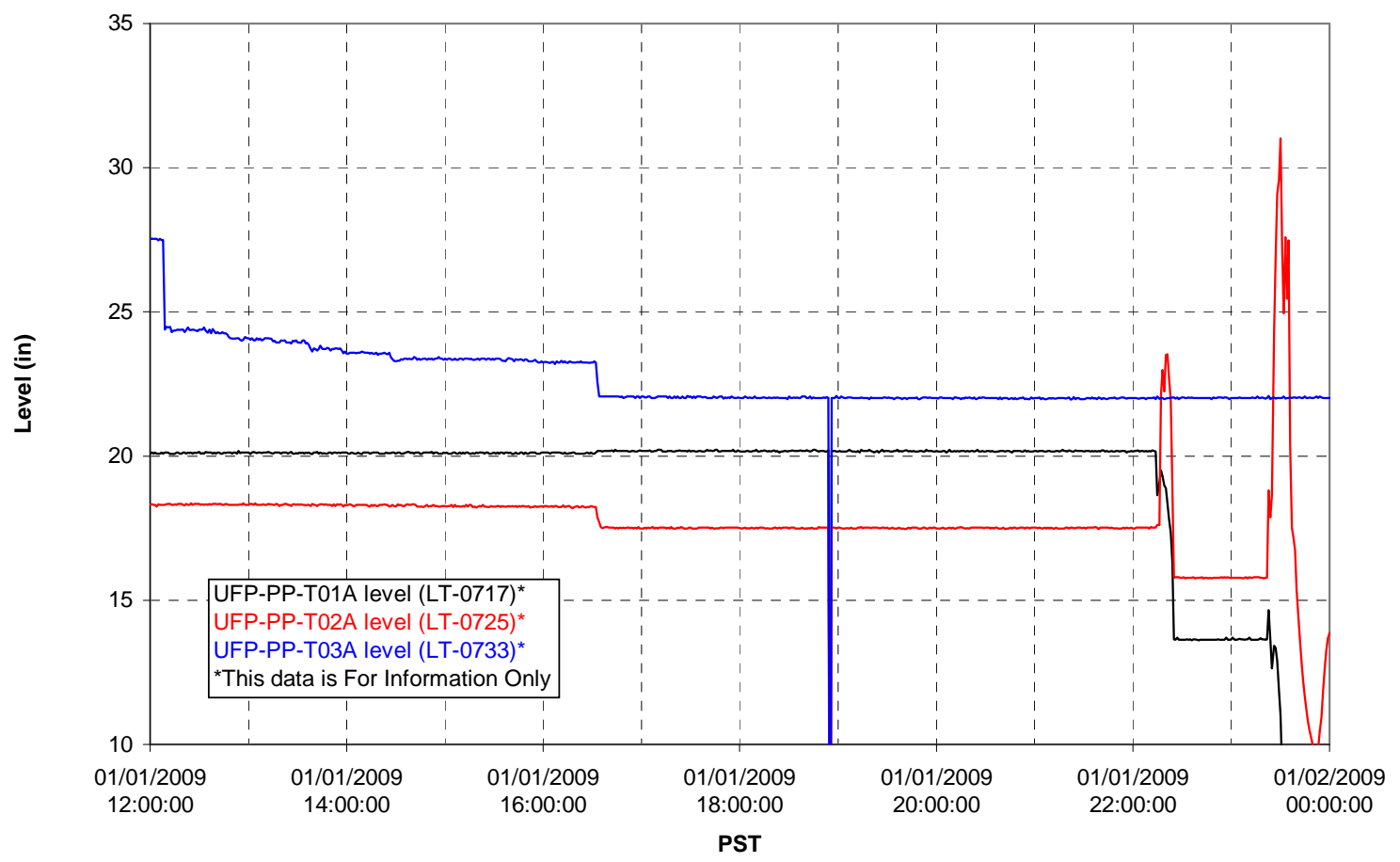


Filter UFP-FILT-T01A

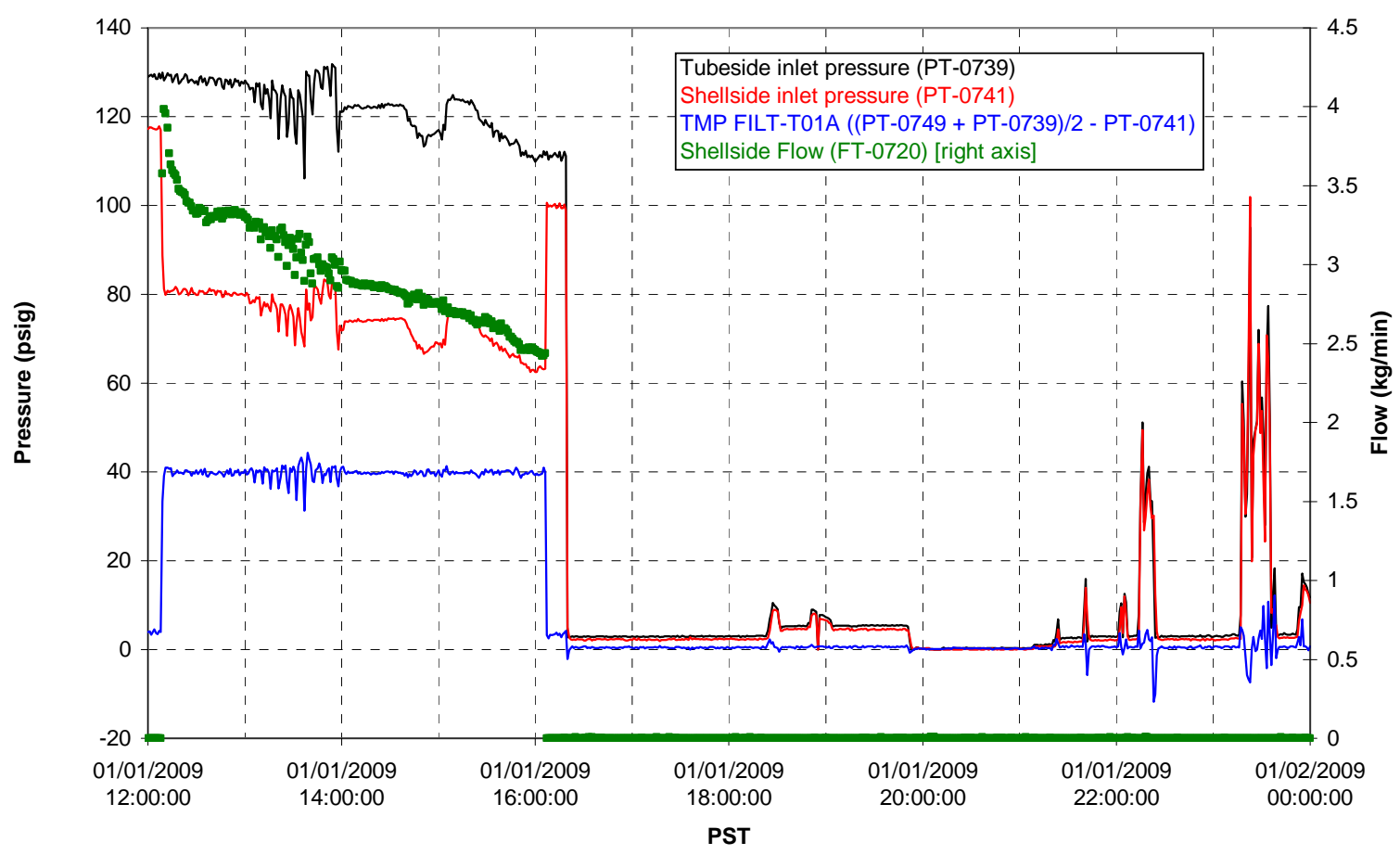

Filter UFP-FILT-T02A

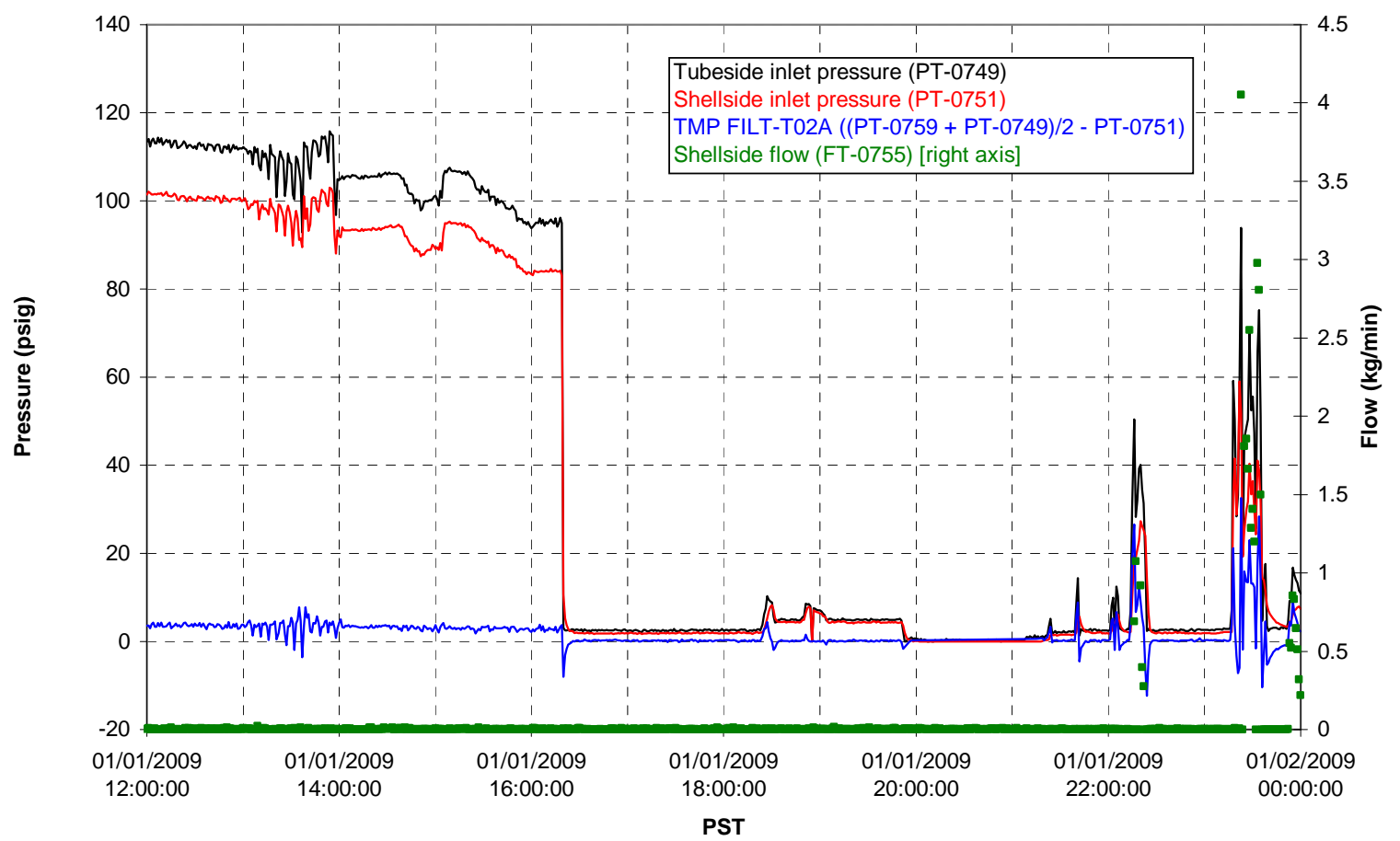


Filter UFP-FILT-T03A

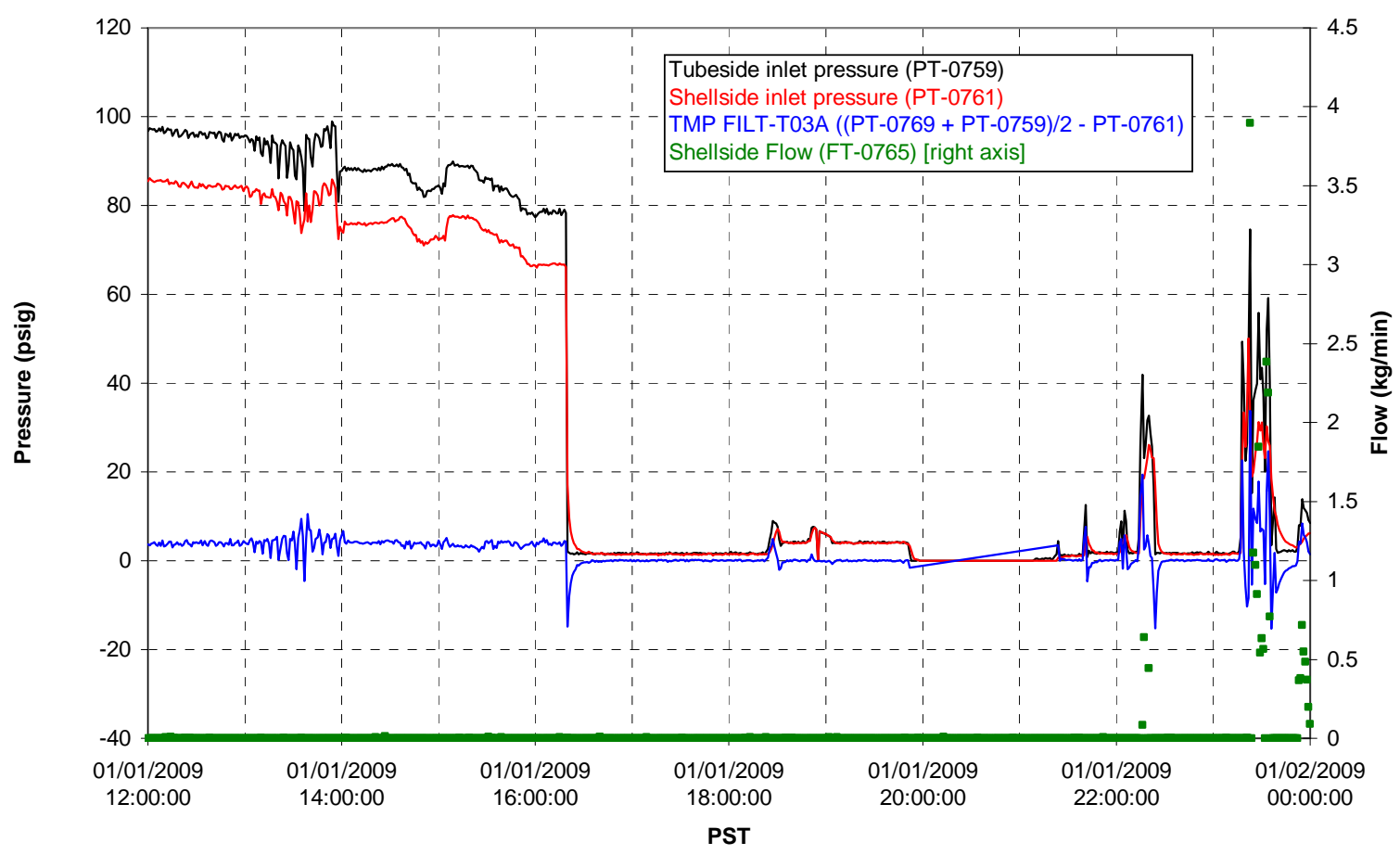

Filter UFP-FILT-T04A

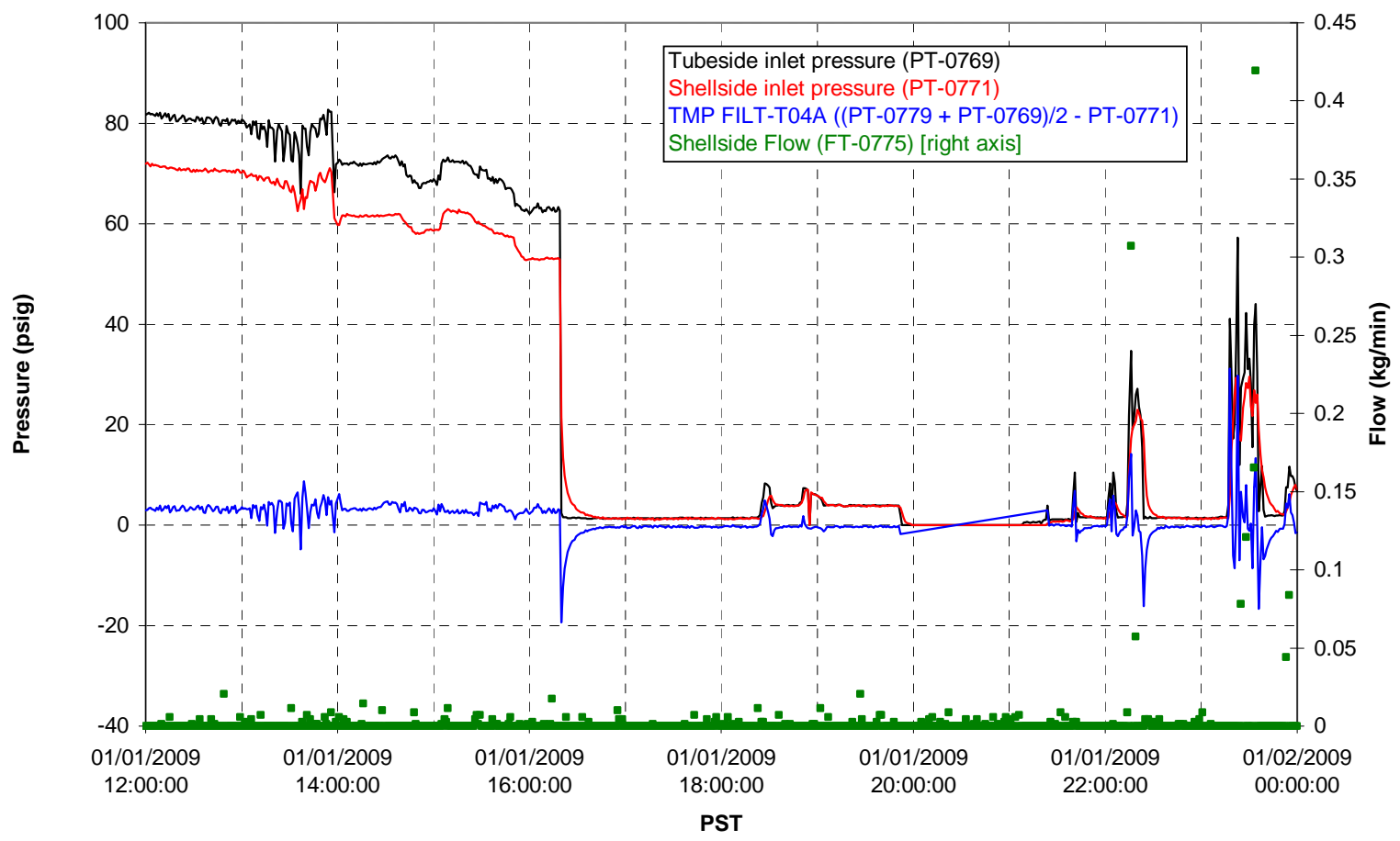


Filter UFP-FILT-T05A

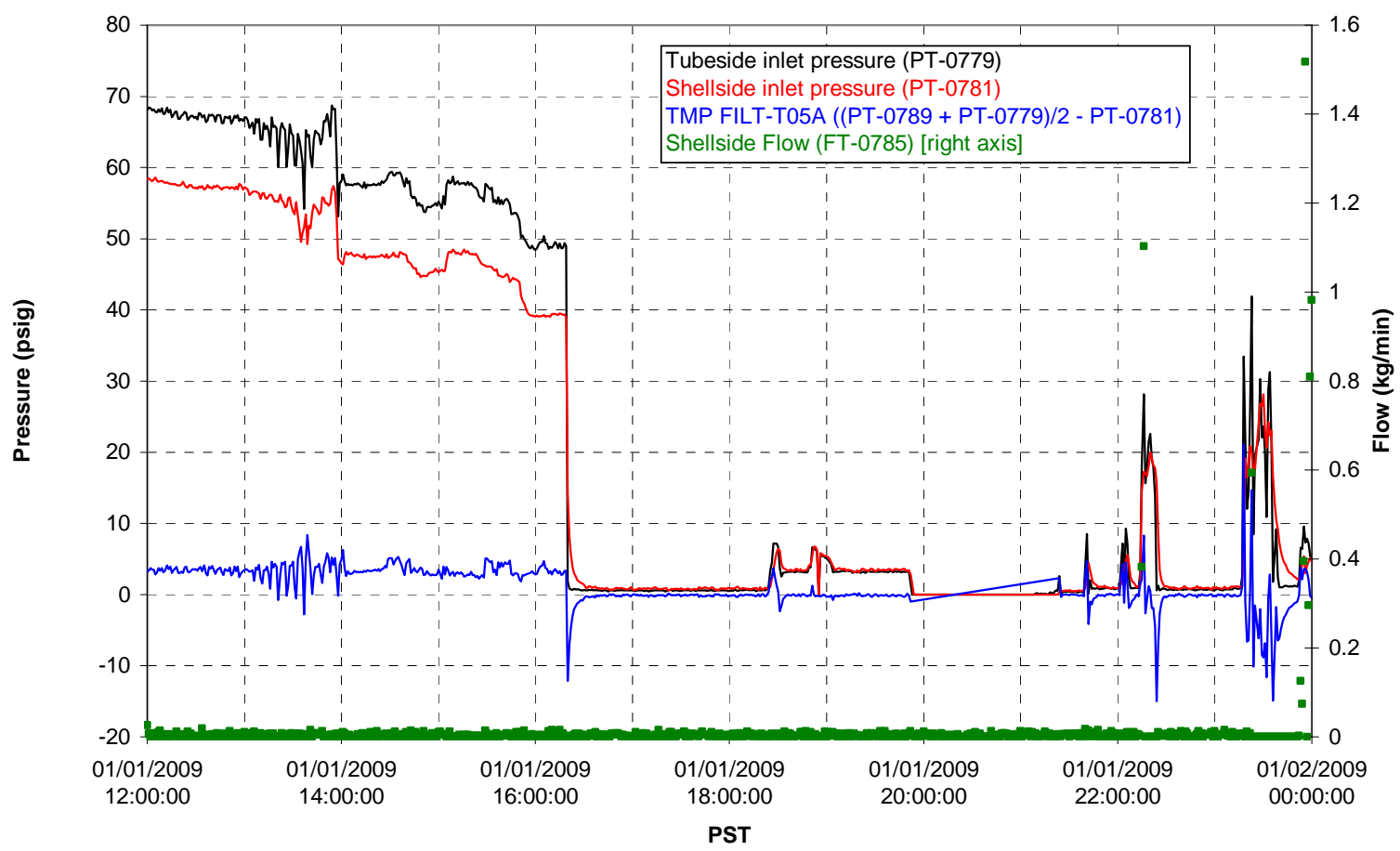

Chemical Flow

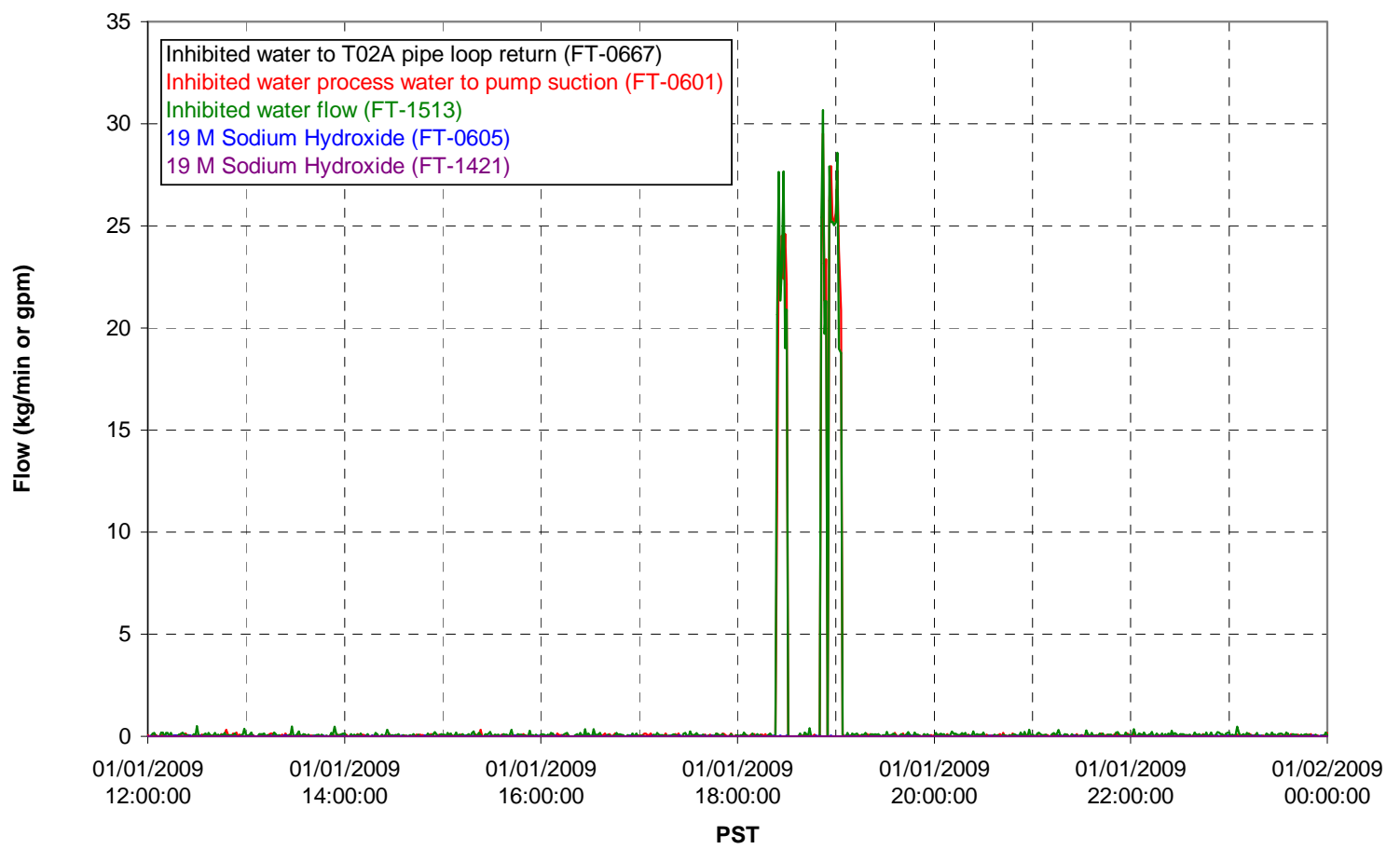




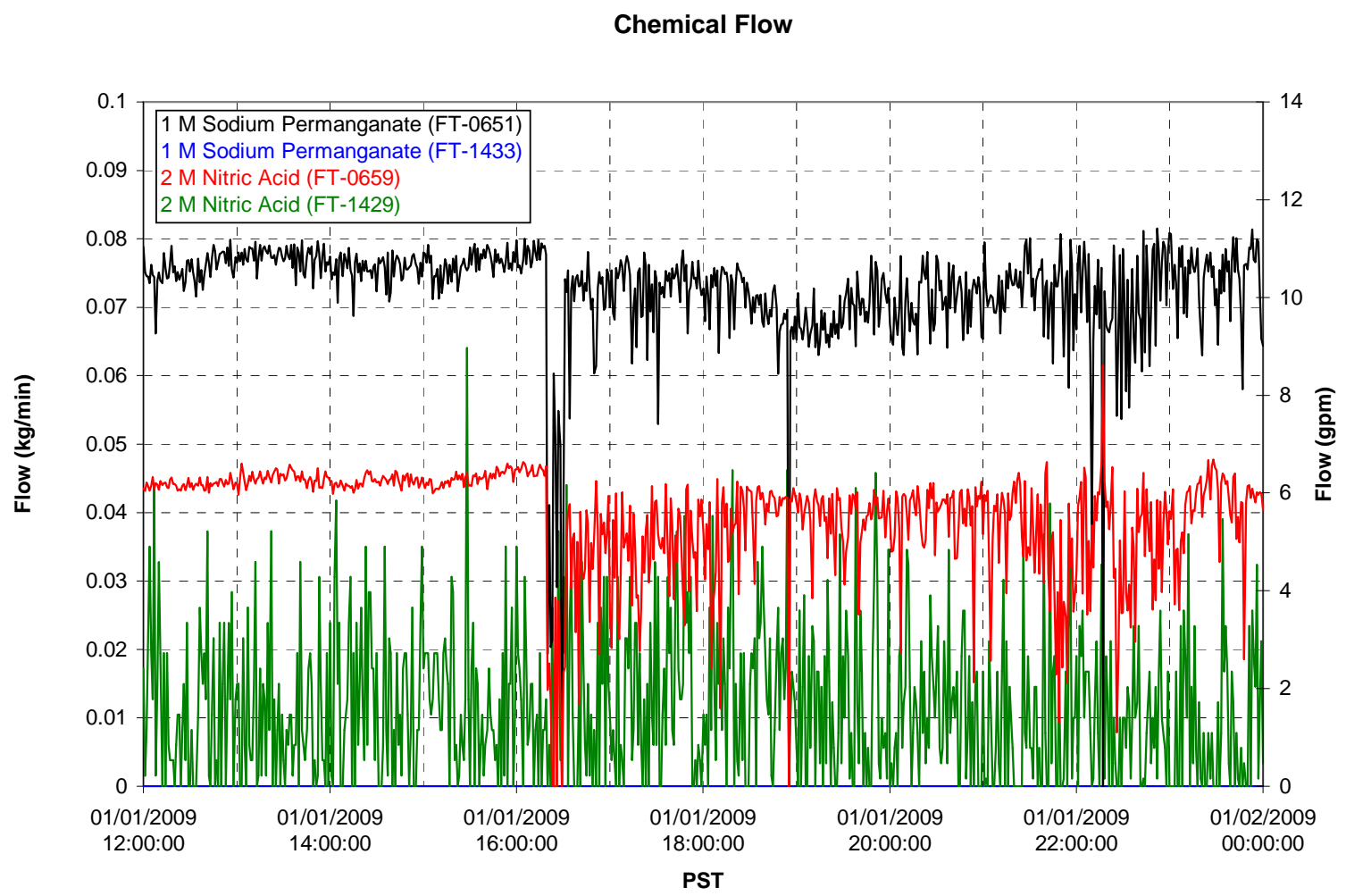

Air Flows

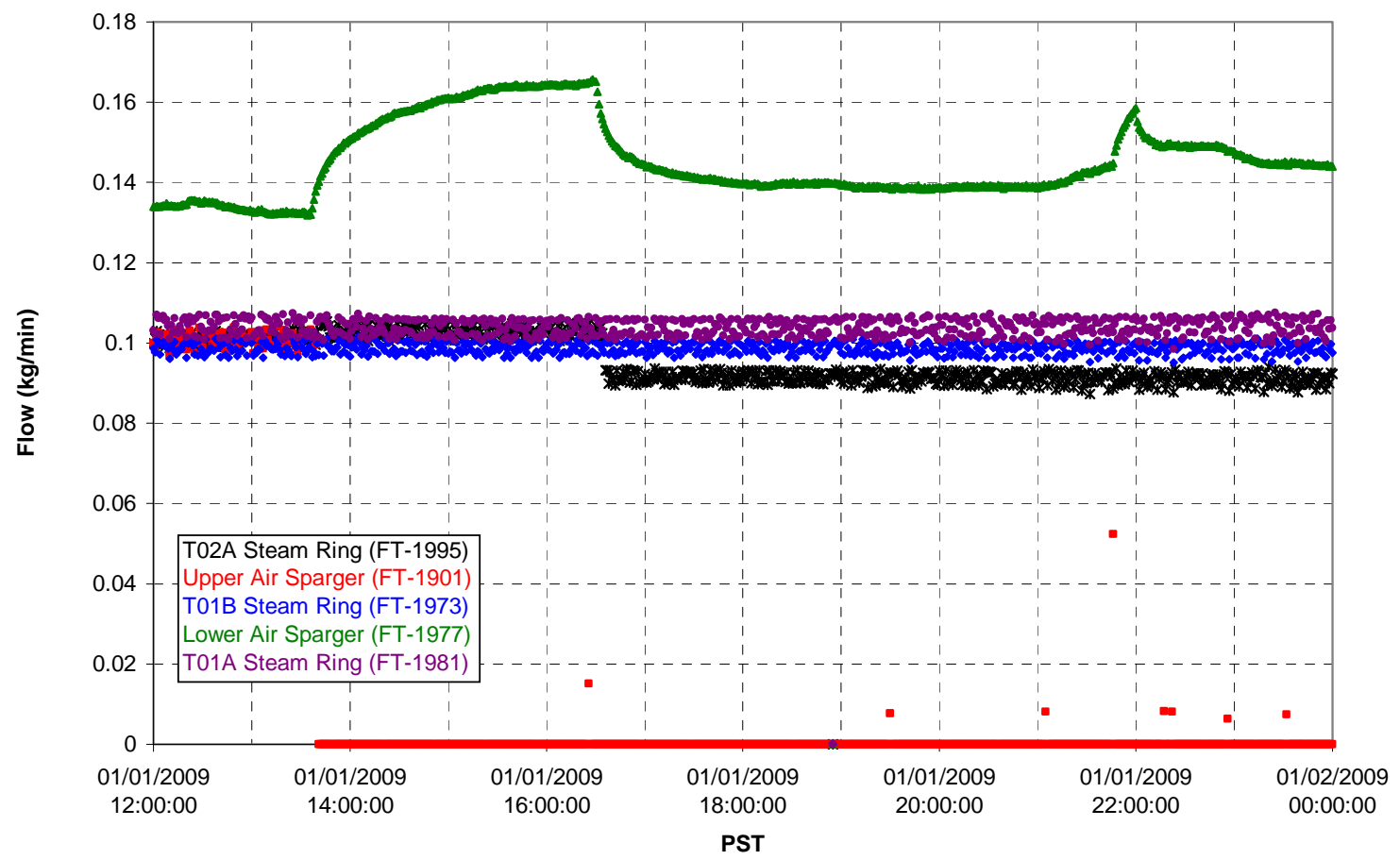


T02A Steam

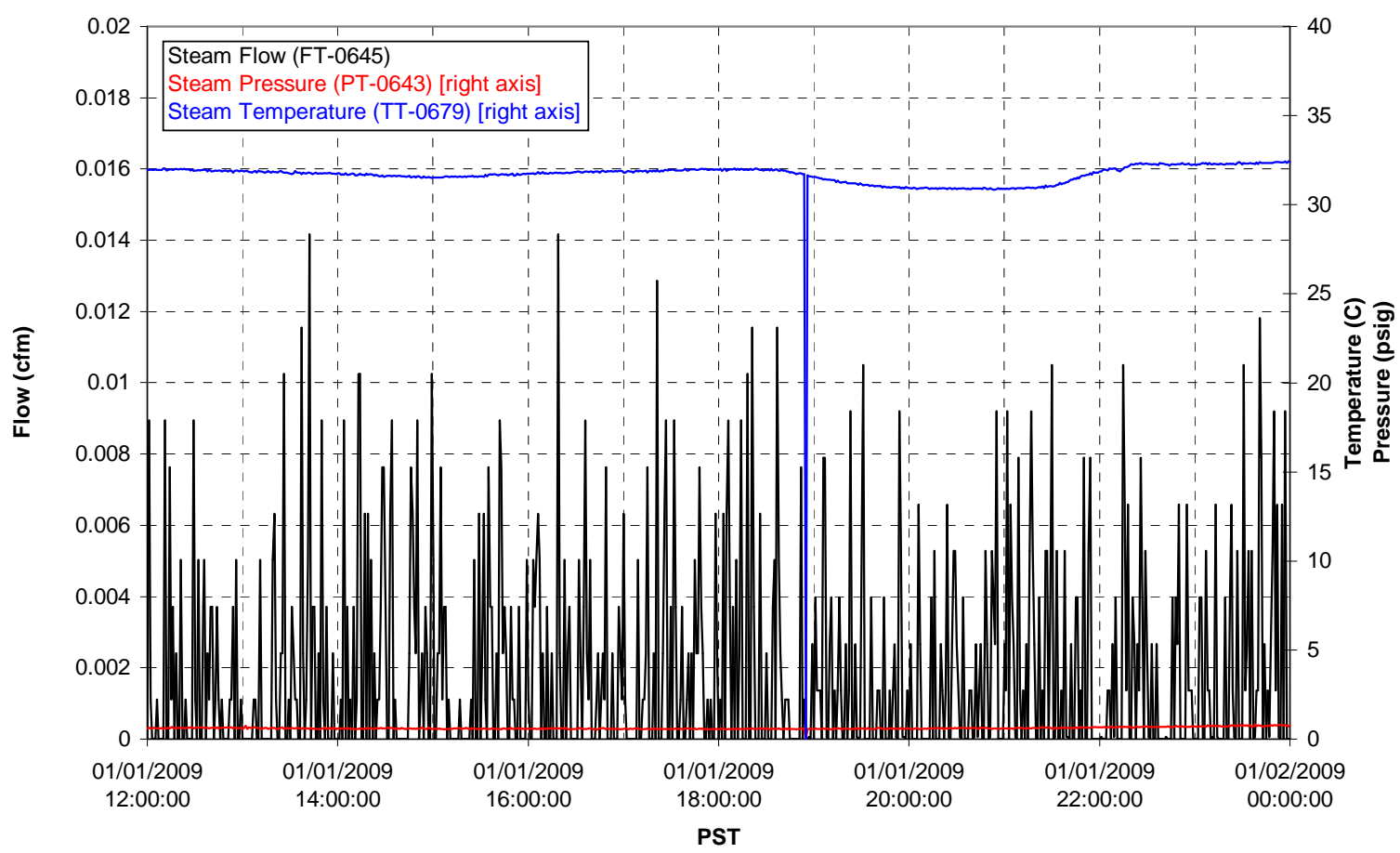

T01A Steam

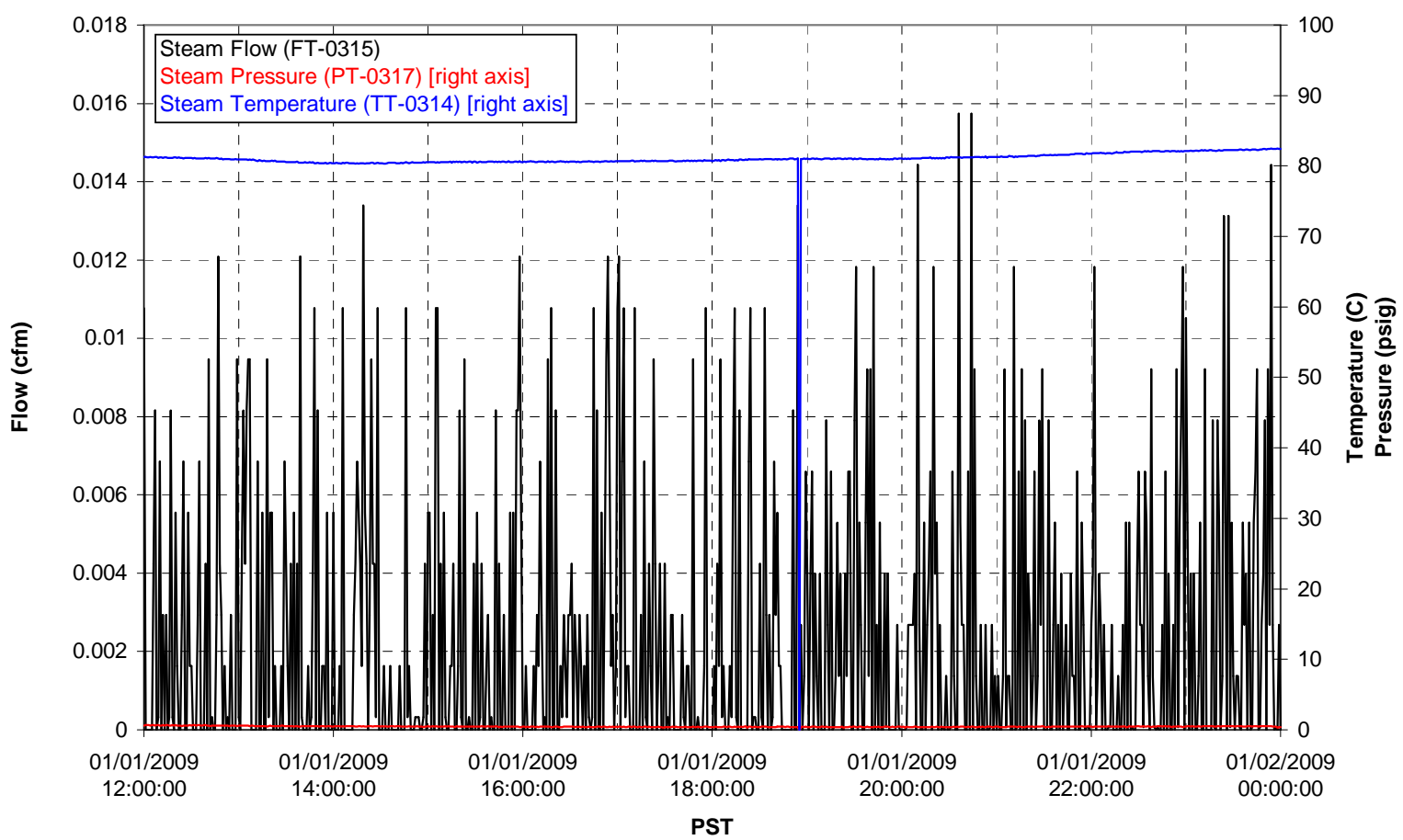


T01B Steam

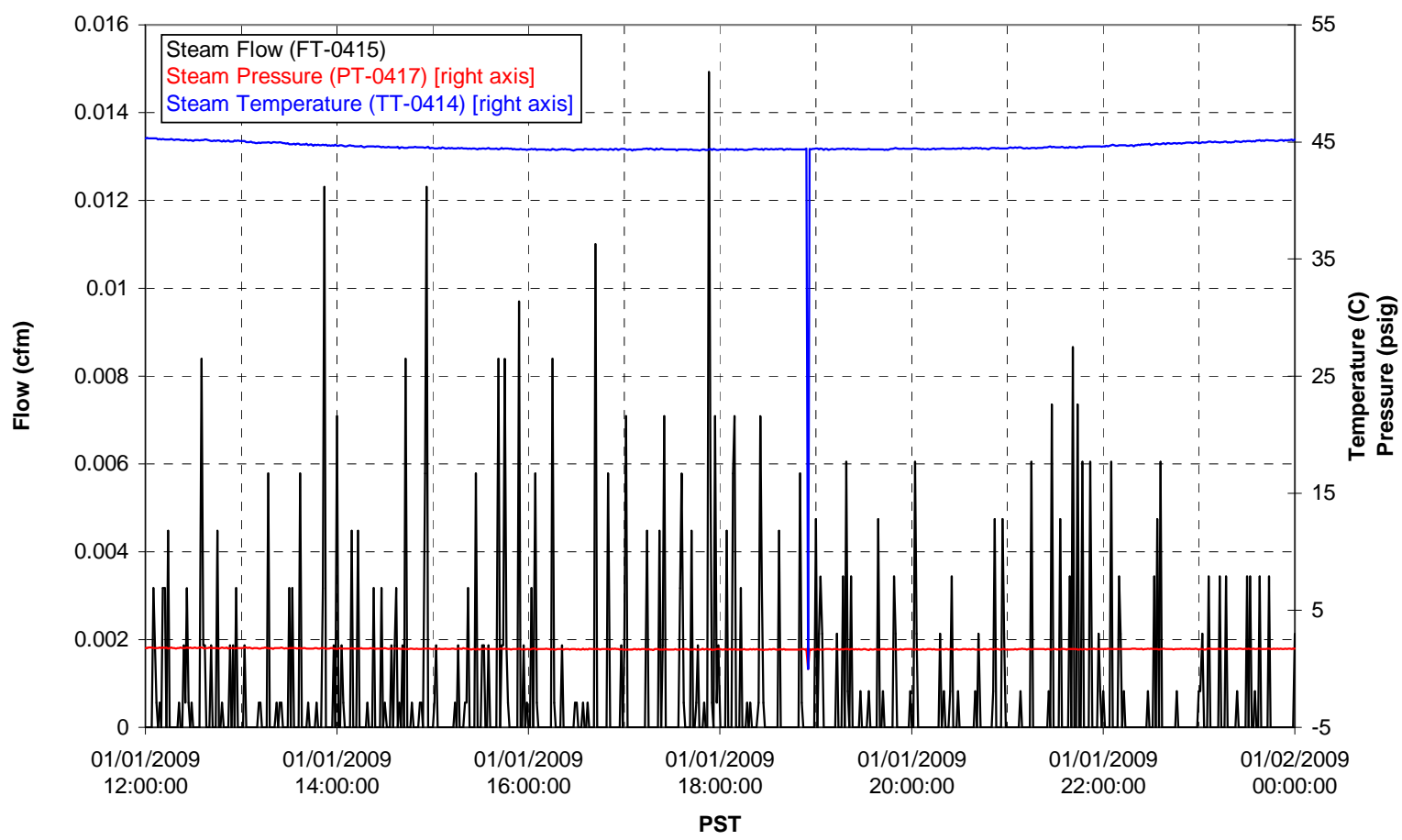


Functional Test Data Plots

01/02/2009 00:00 - 01/02/2009 12:00 
T01A level

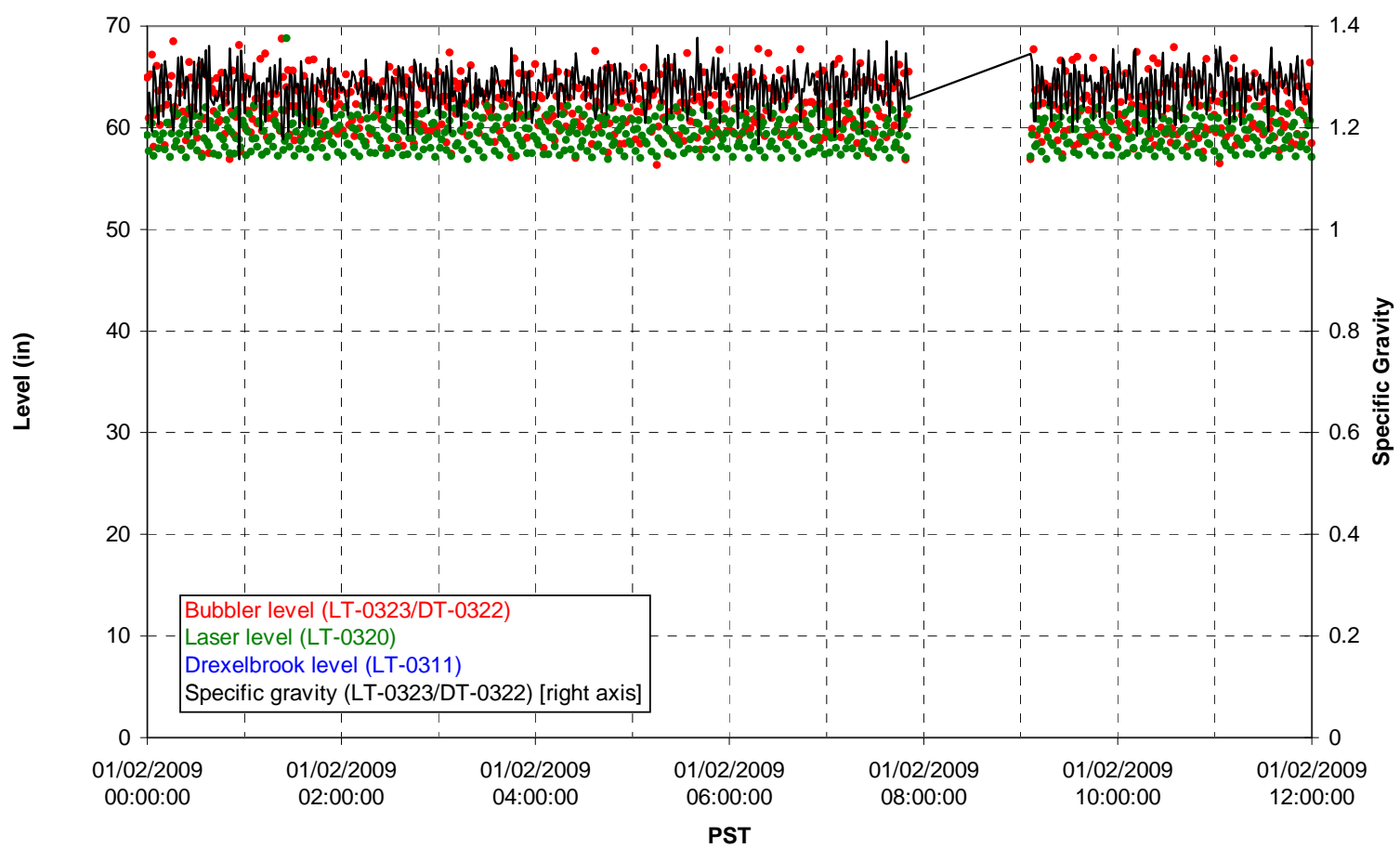

T01A temperatures

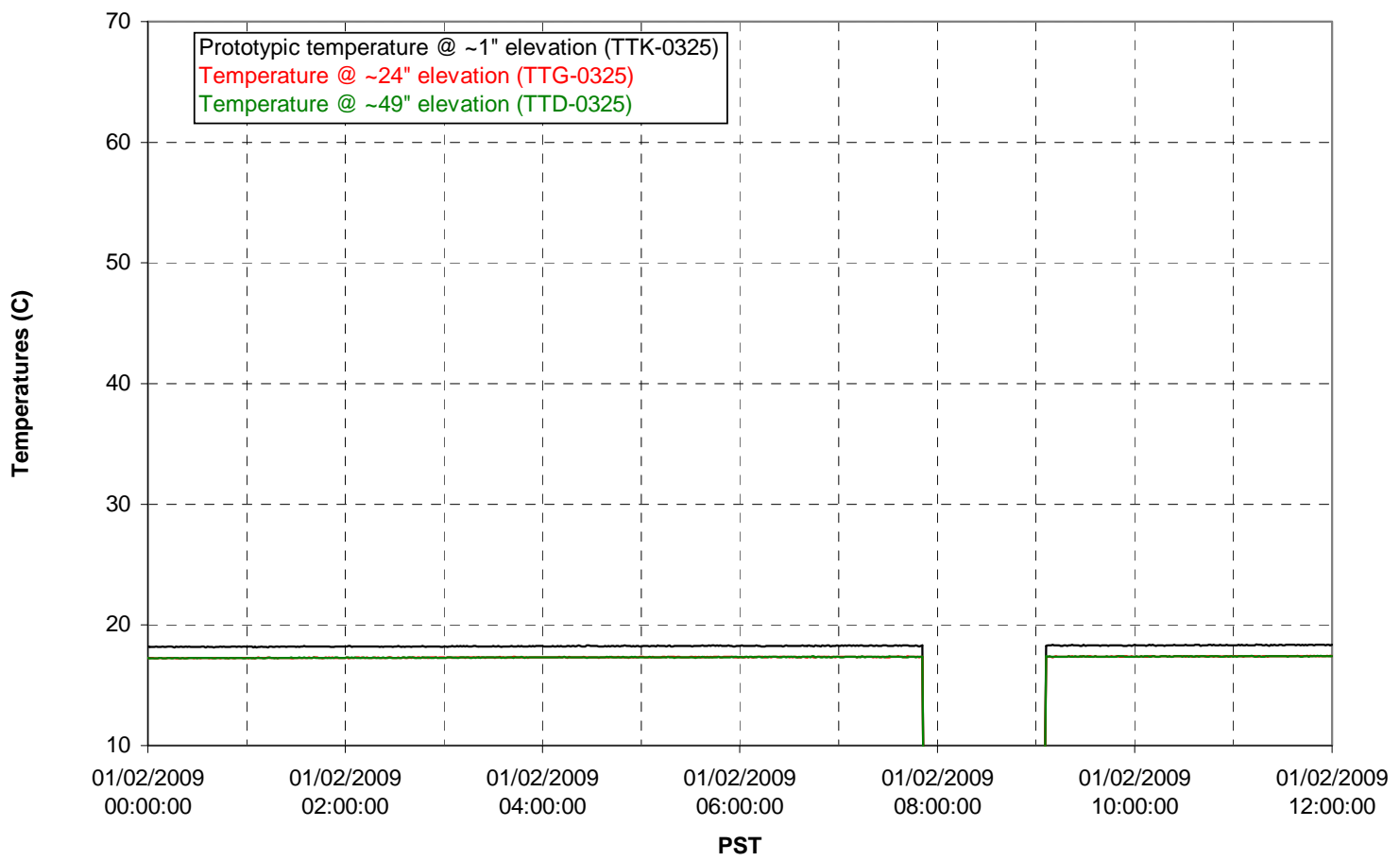


T01B level

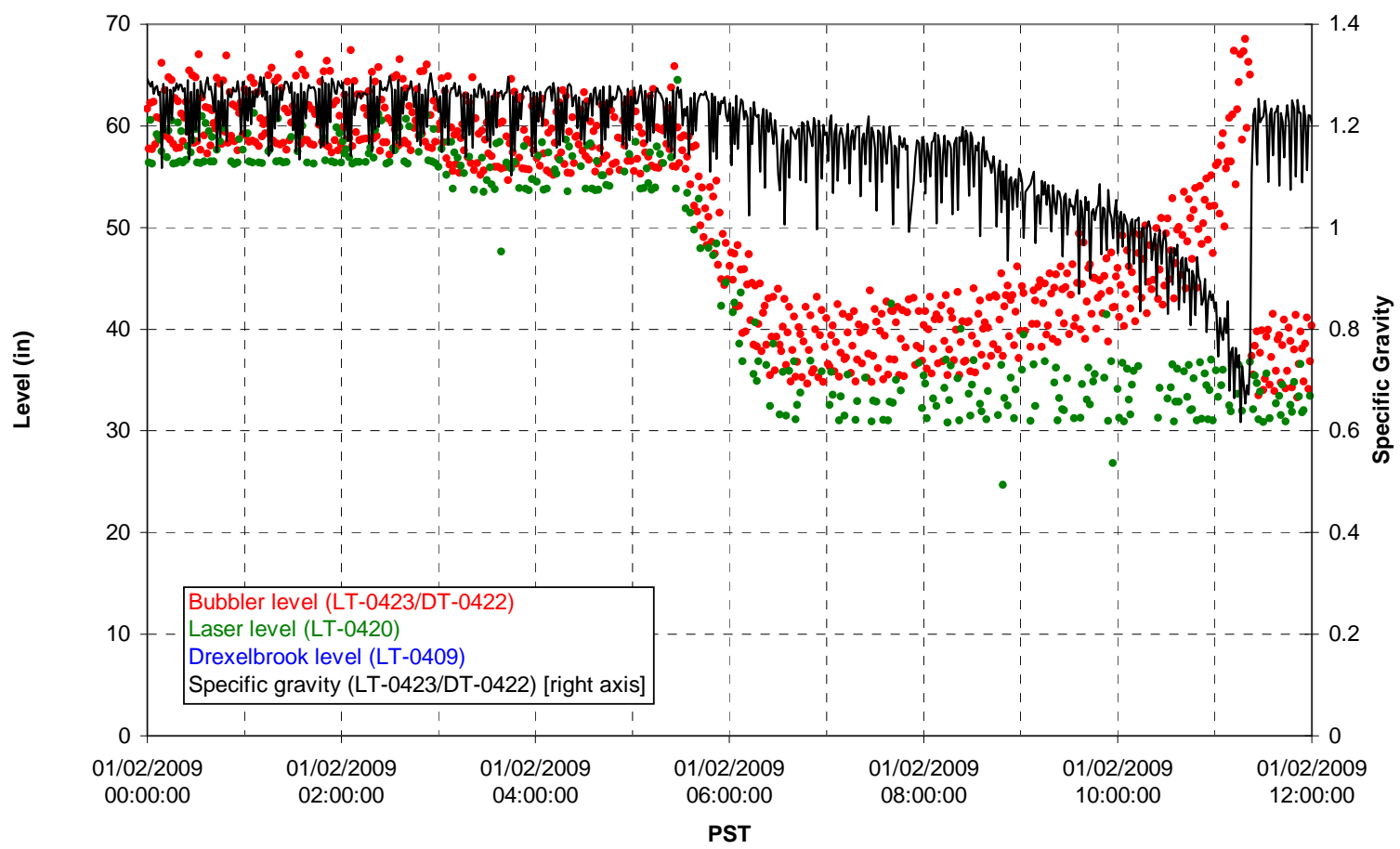

T01B temperatures

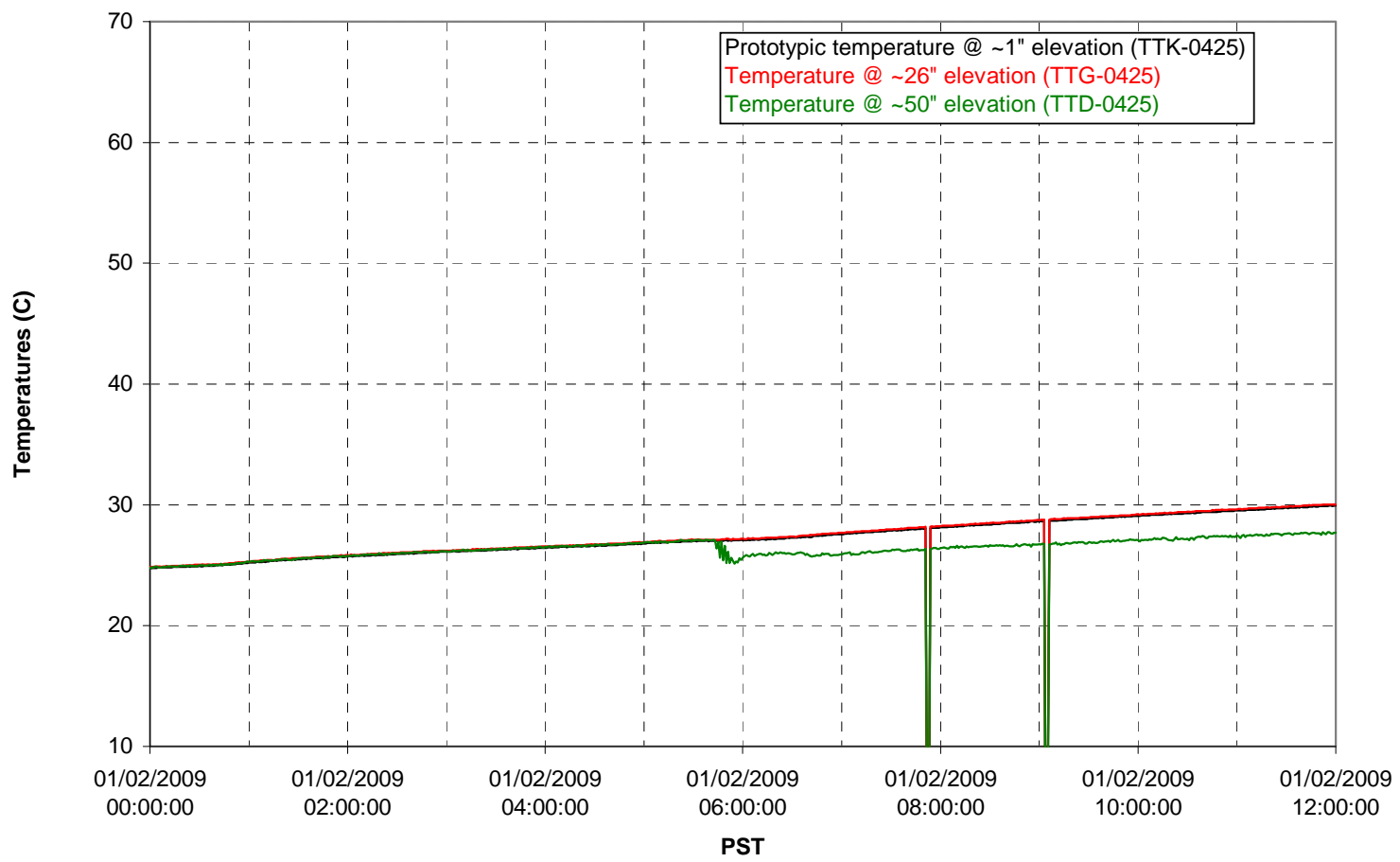


T02A level

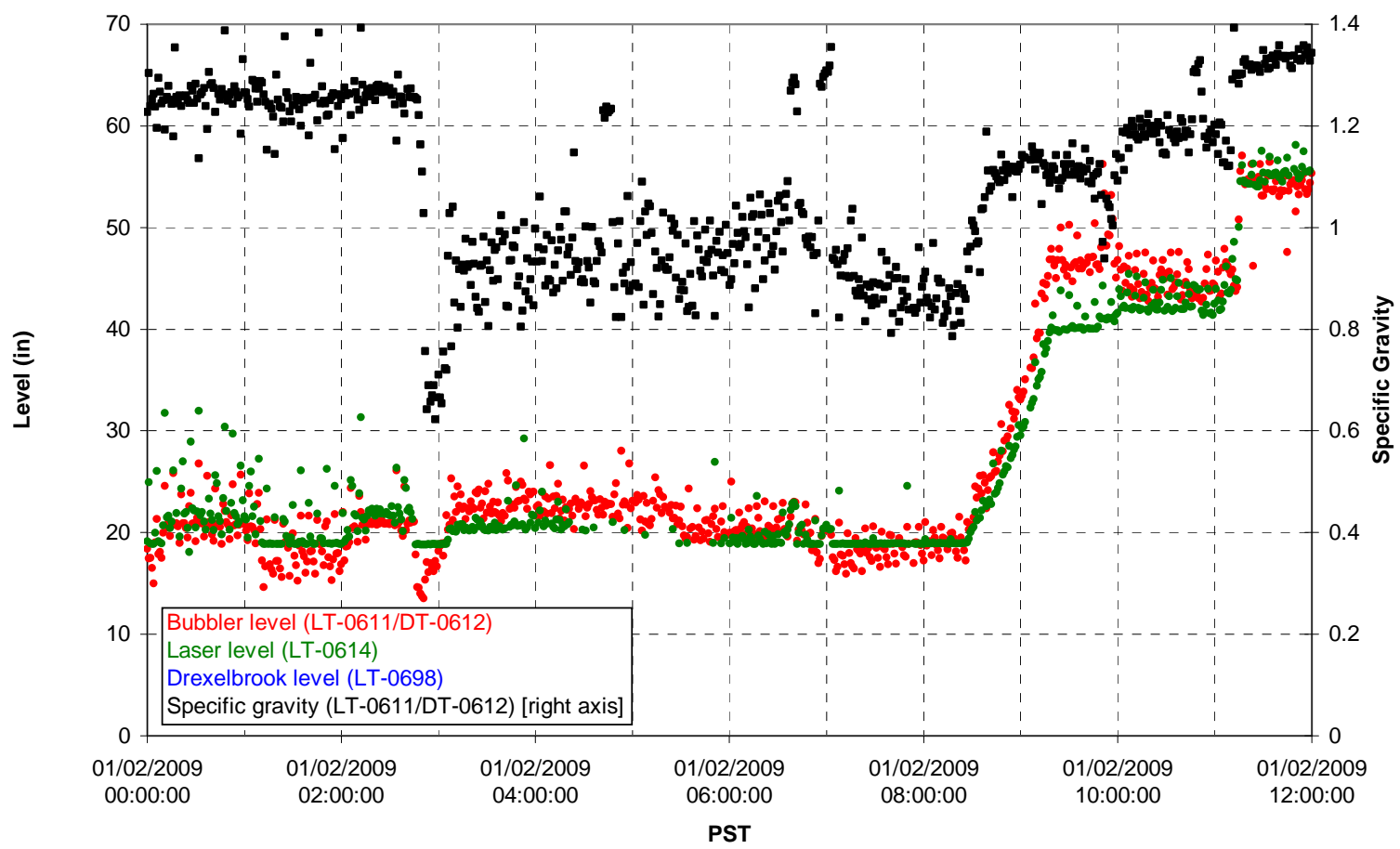

T02A temperatures

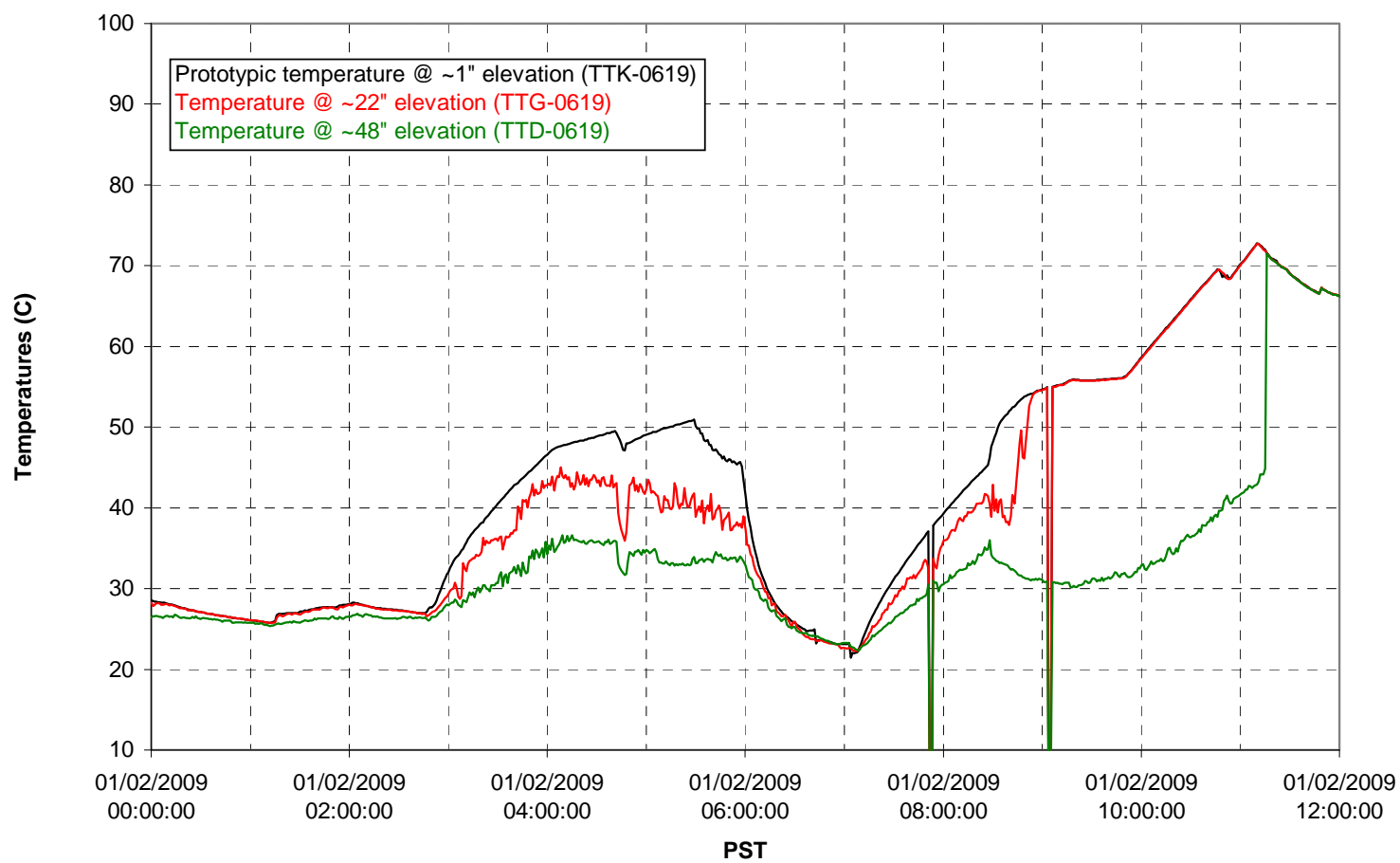


T02A and filter loop temperatures

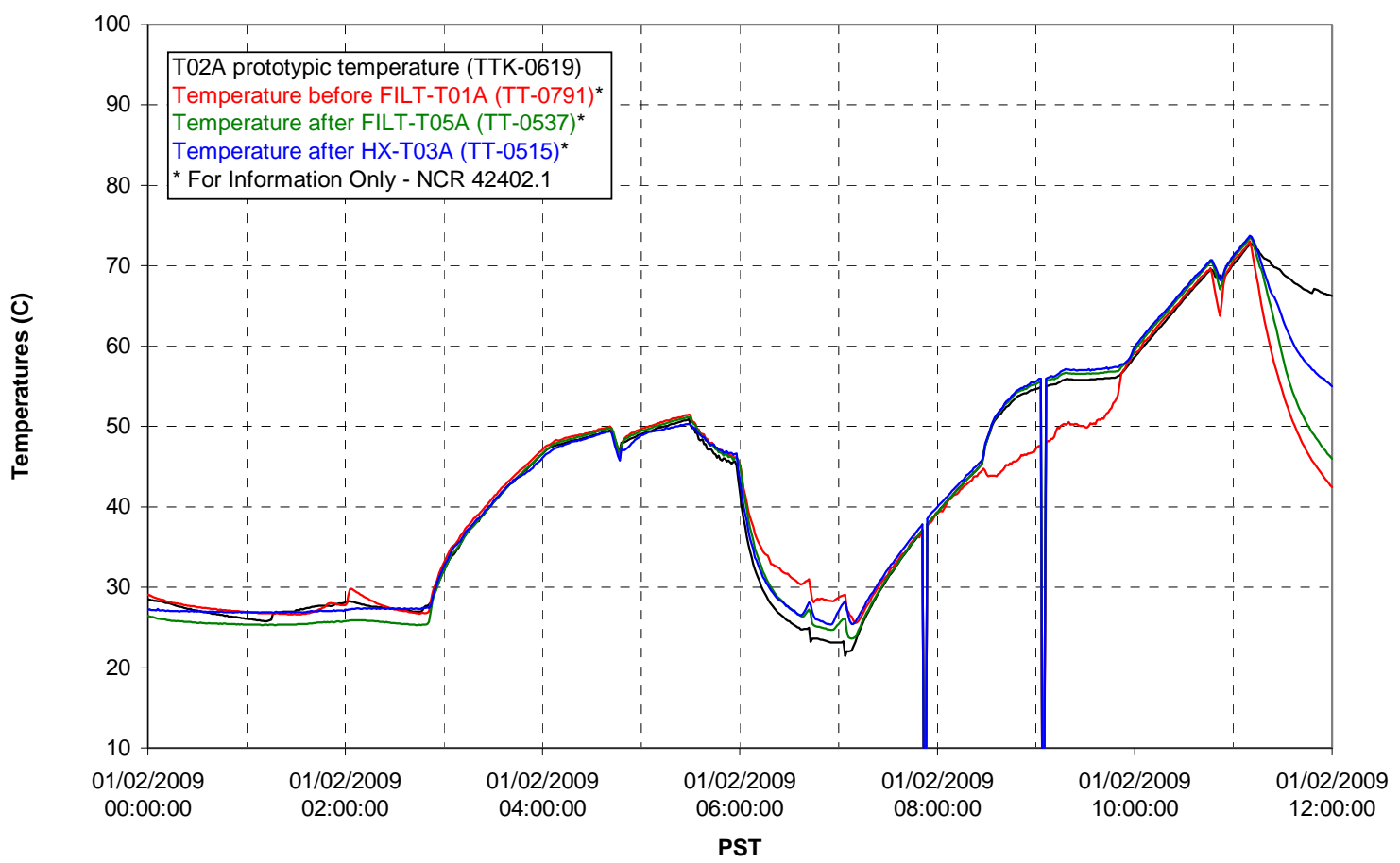

Pump Pressures and Flow

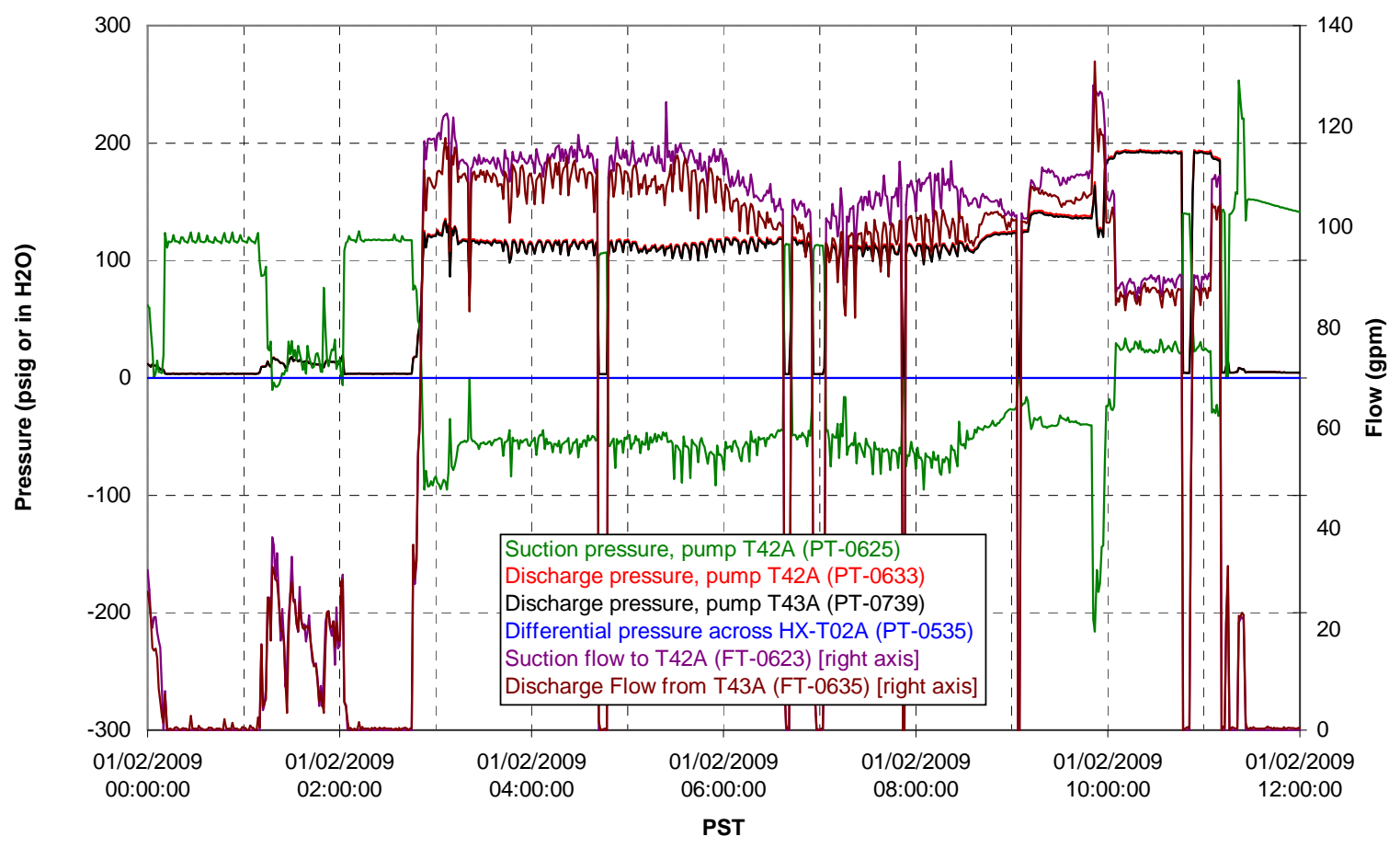




\section{Axial pressure drop}

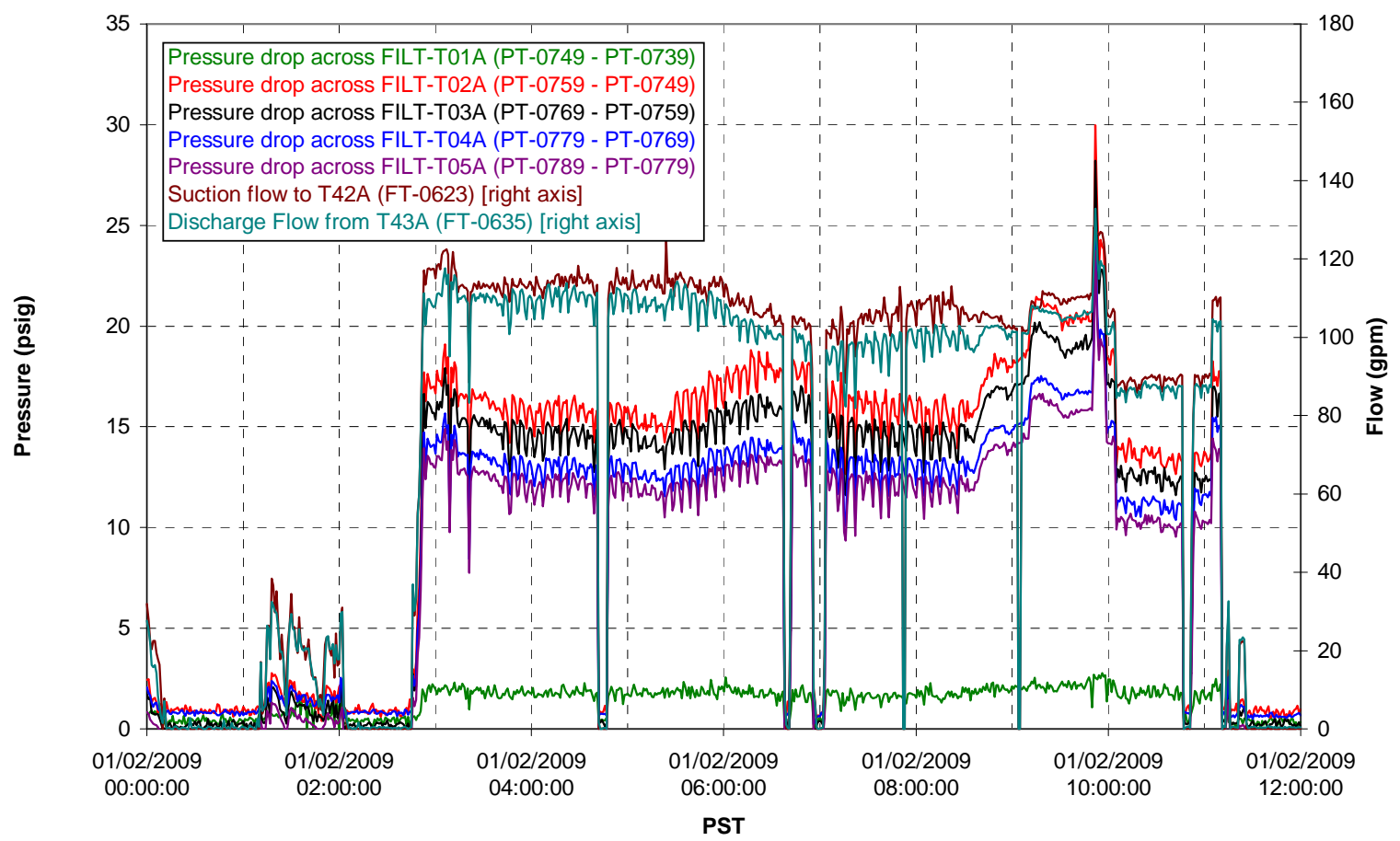

Permeate flow rates

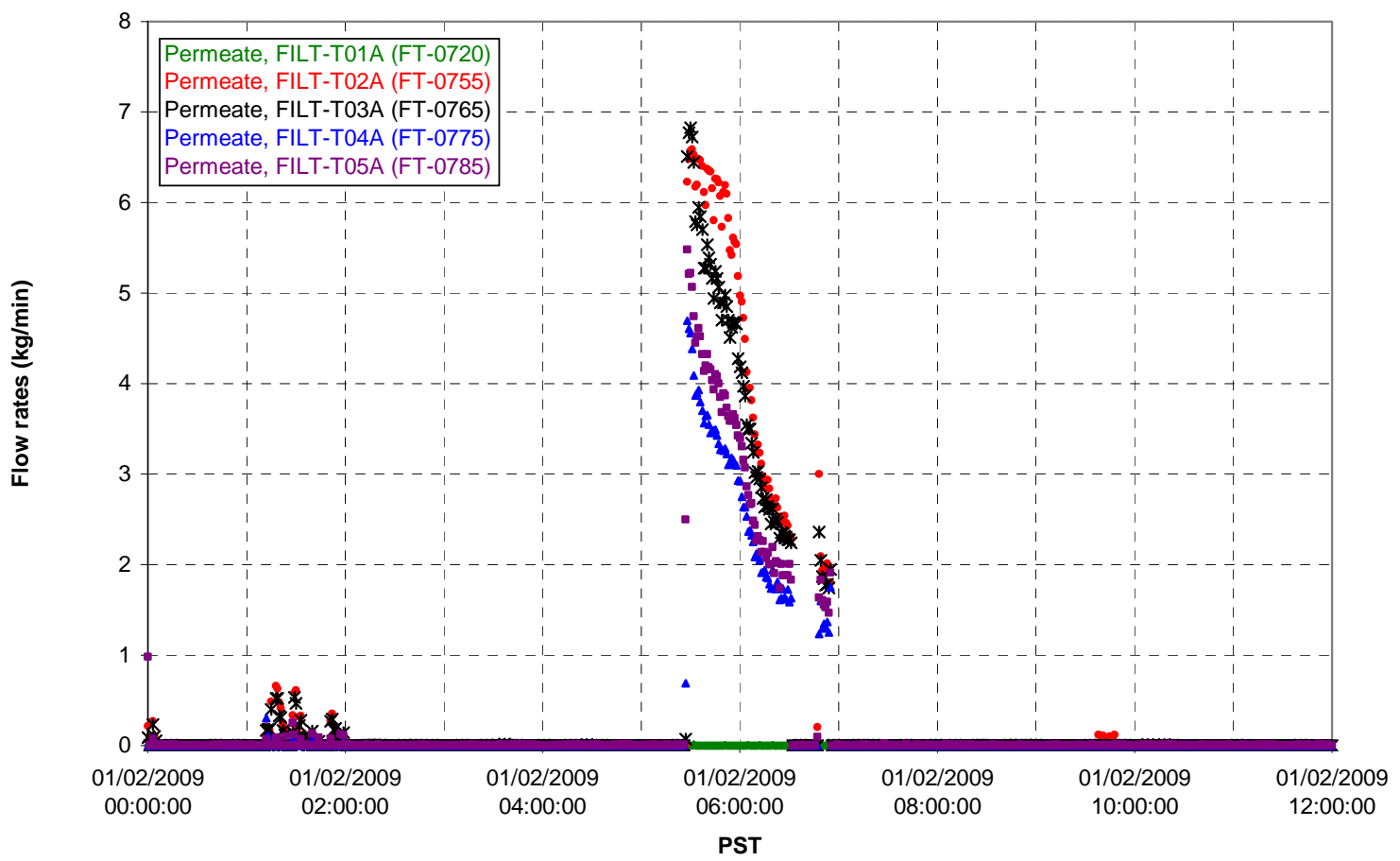


T02A Inner Temperature Tree

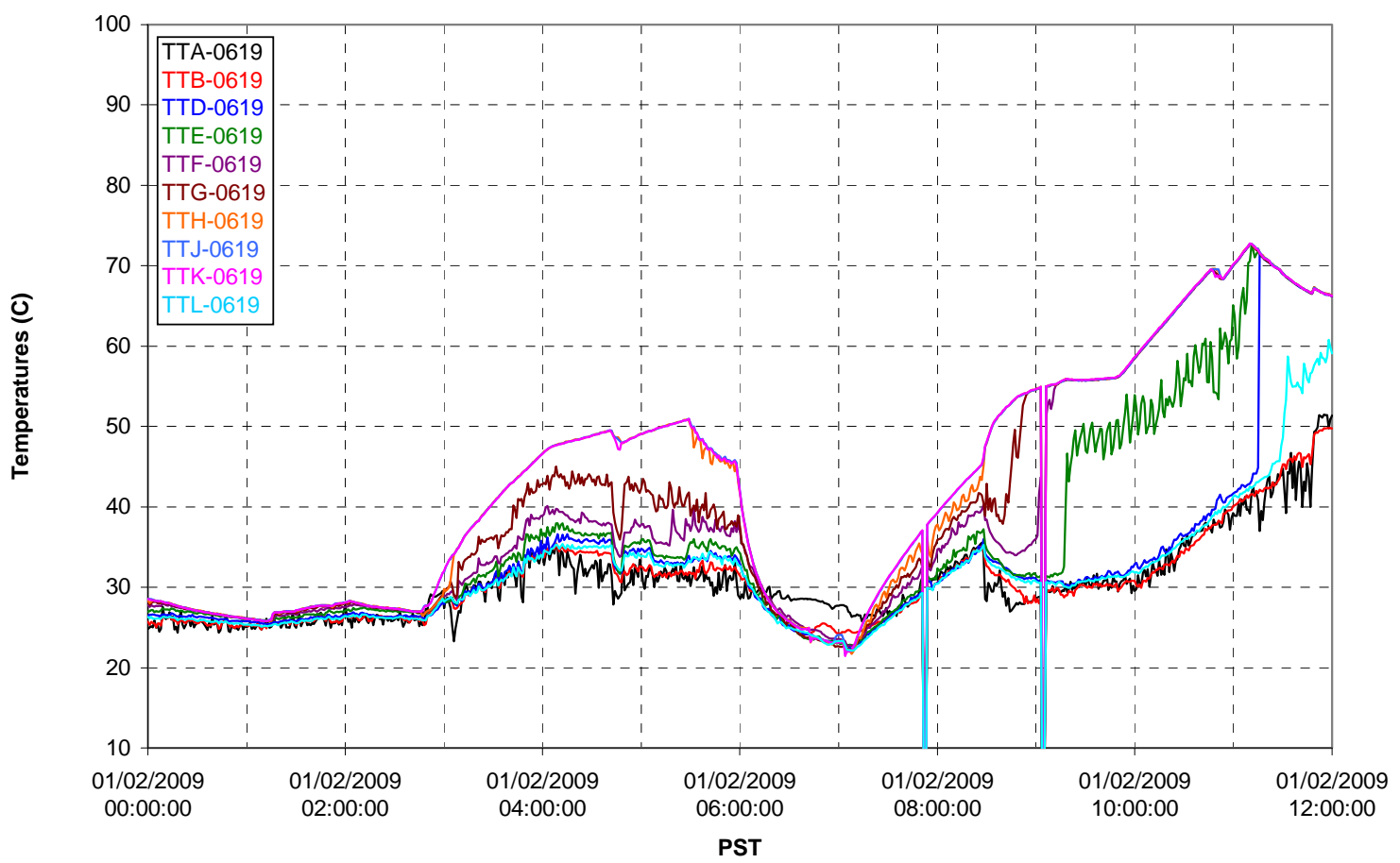

T02A Outer Temperature Tree

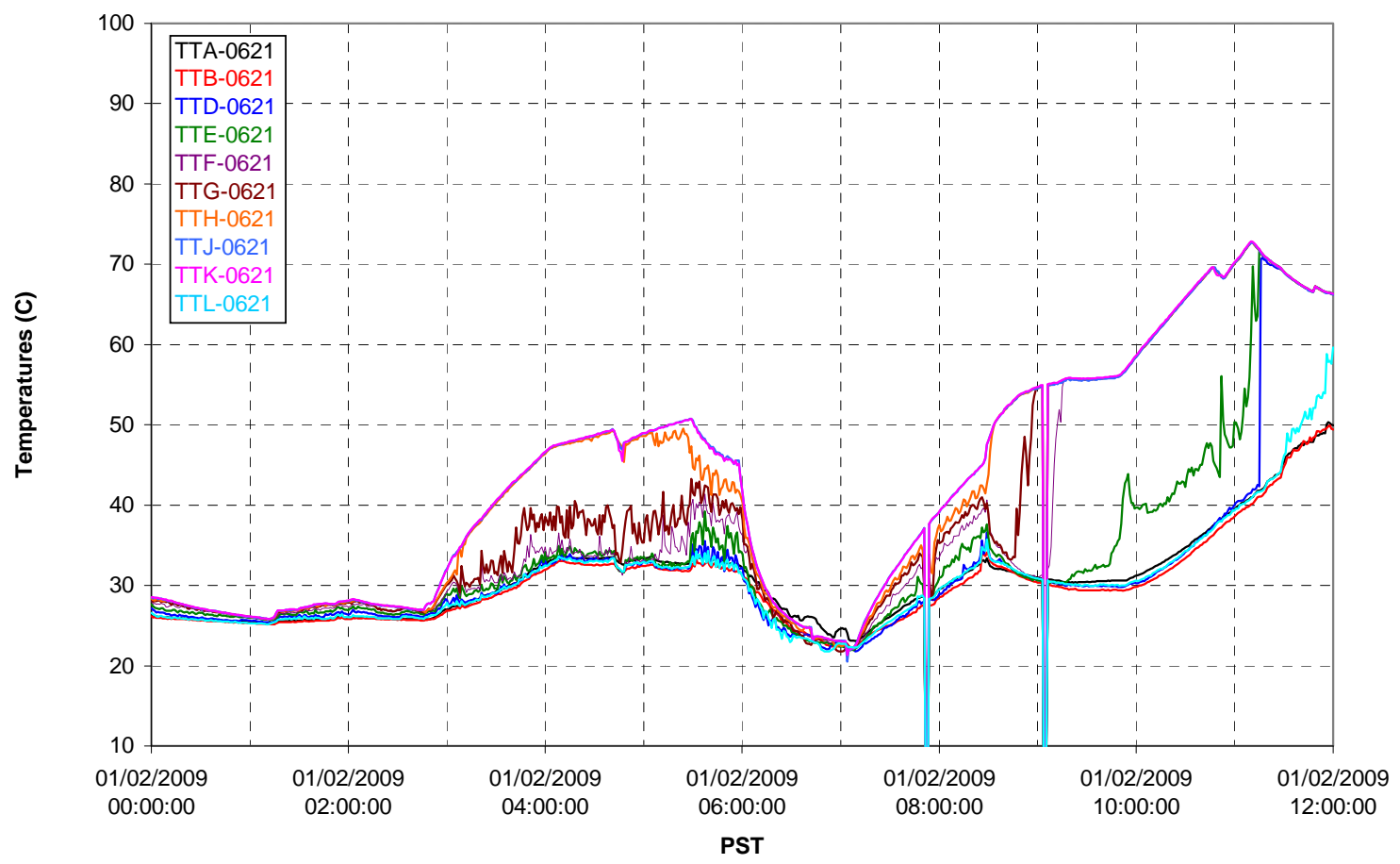


T02A temperatures

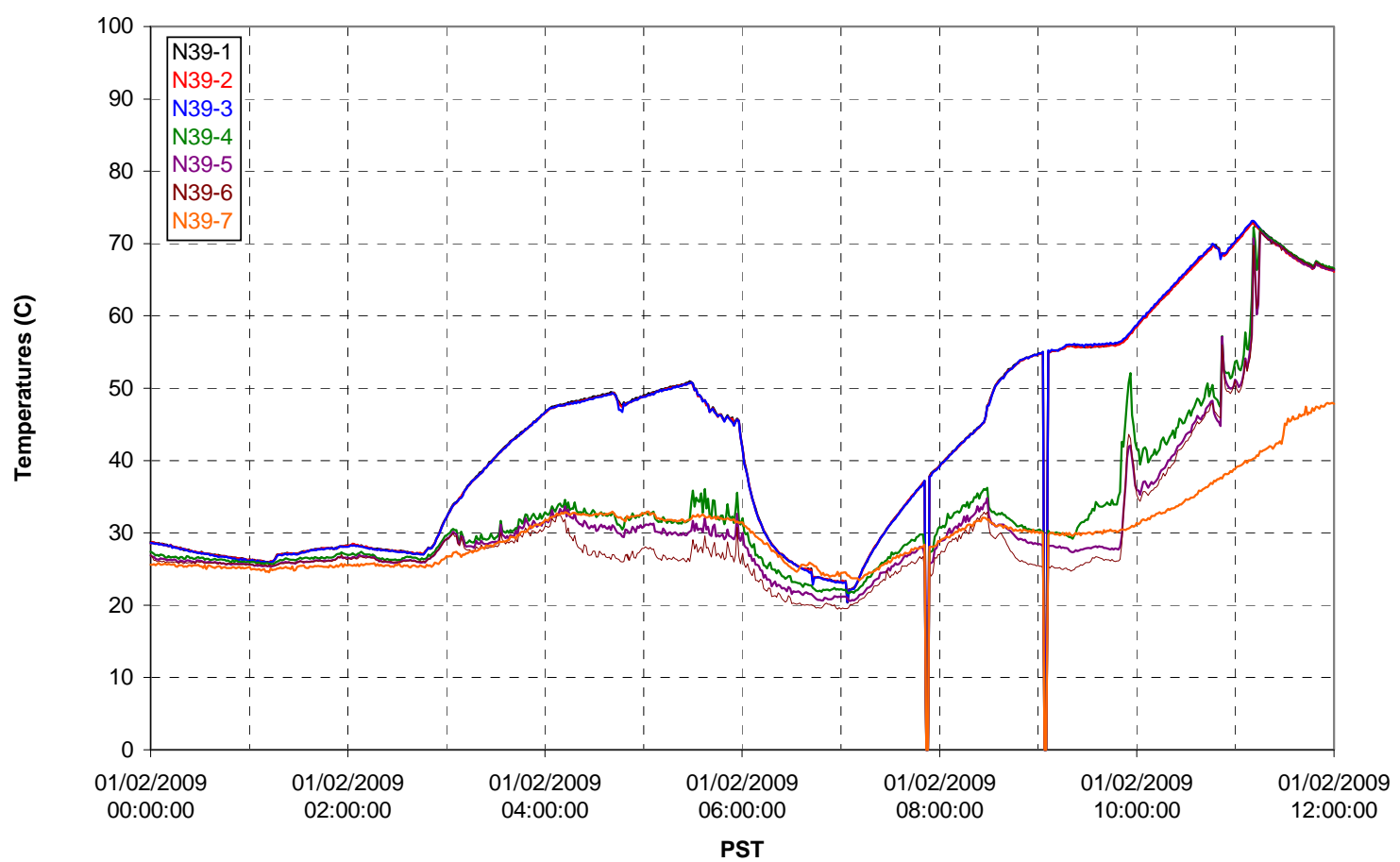

T02A temperatures

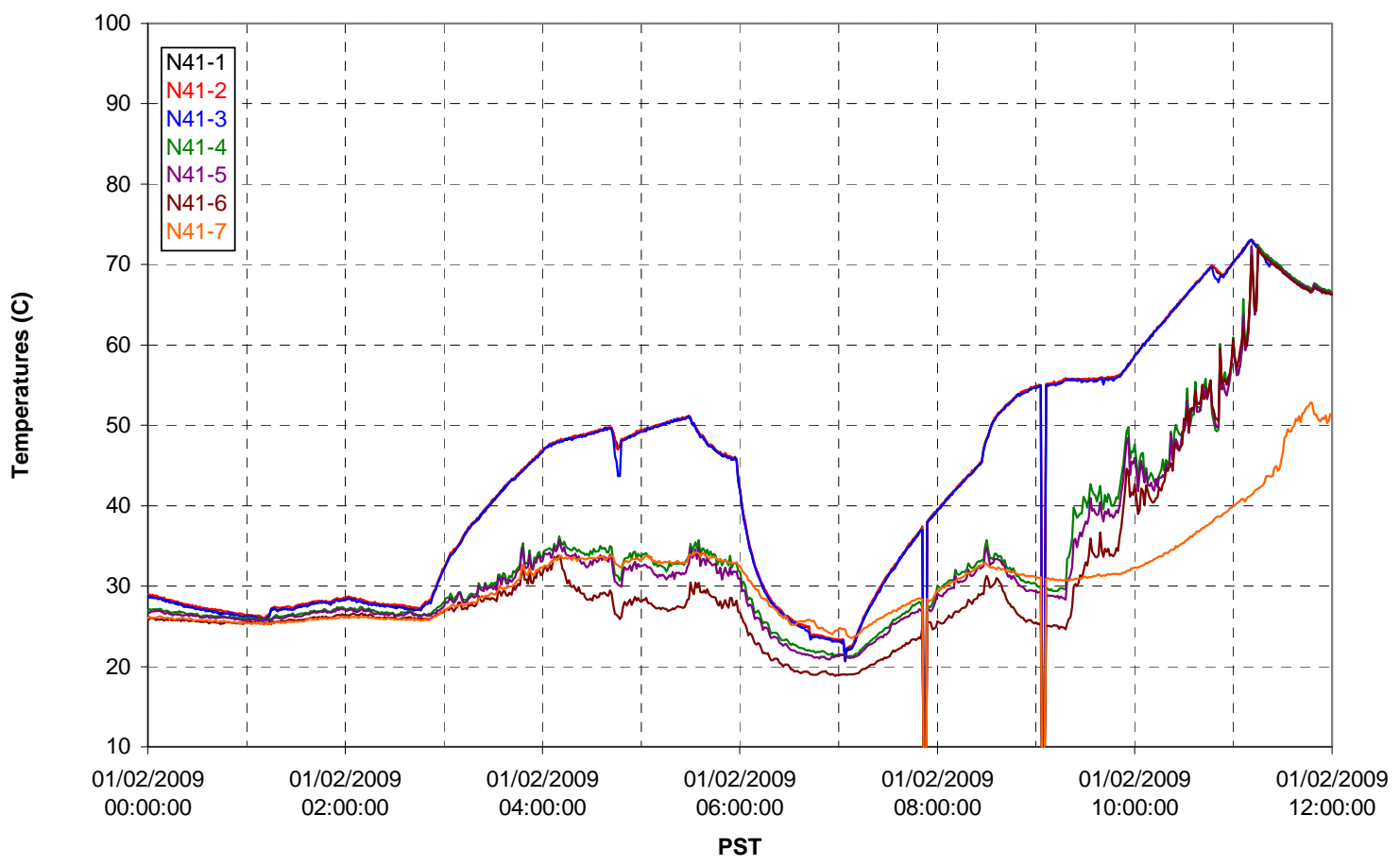


T02A temperatures

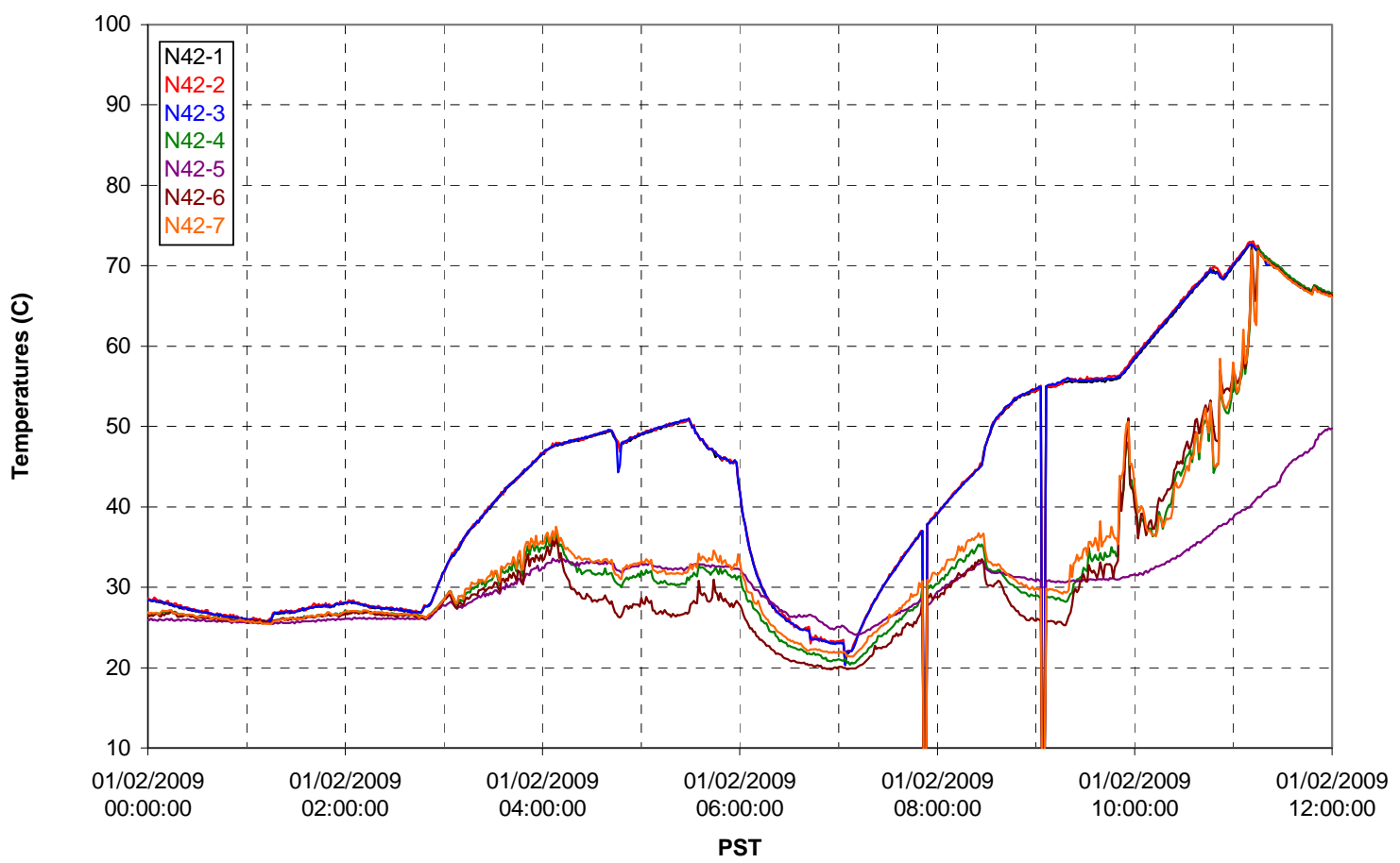

T02A temperatures

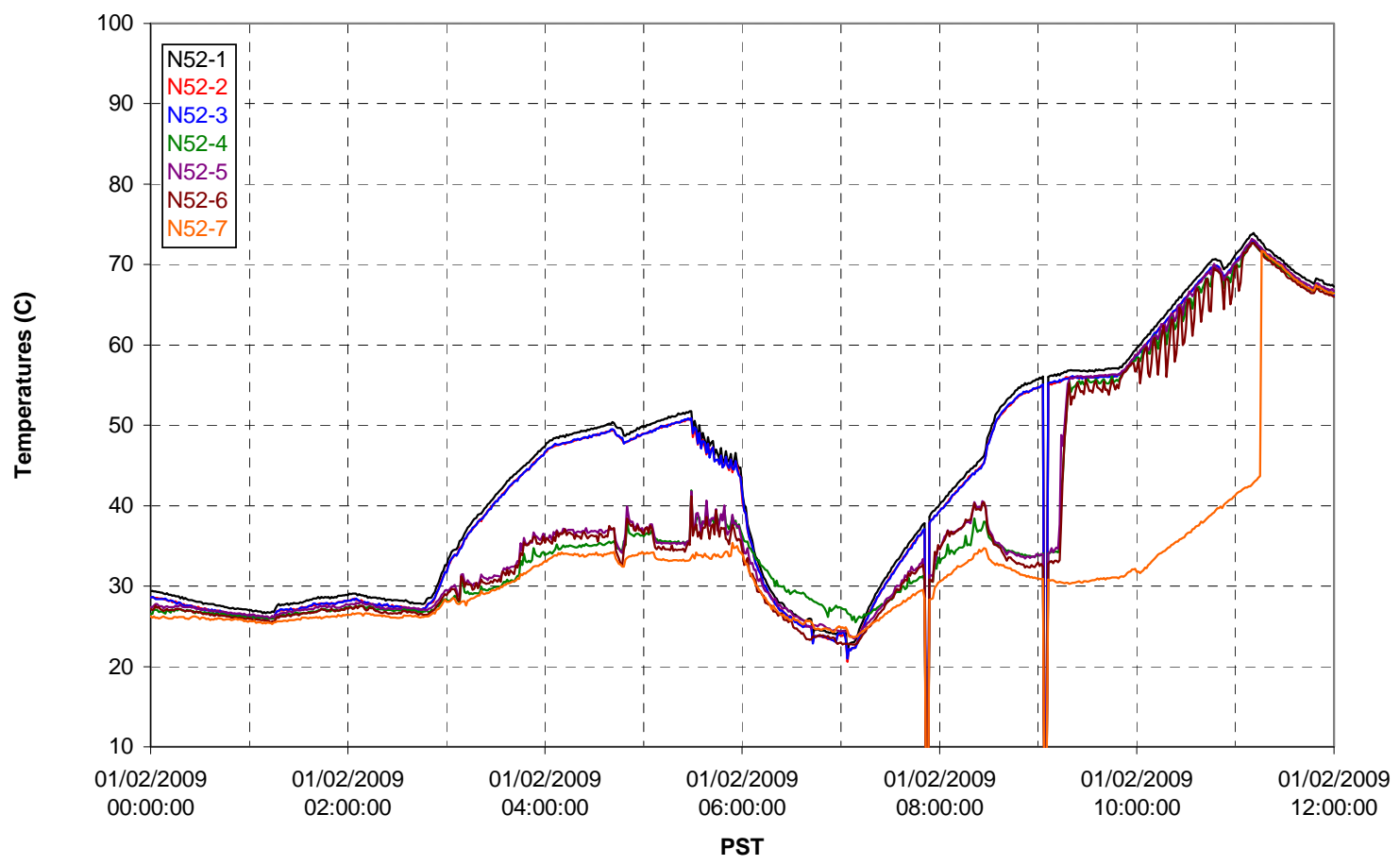


T02A Heating and Cooling

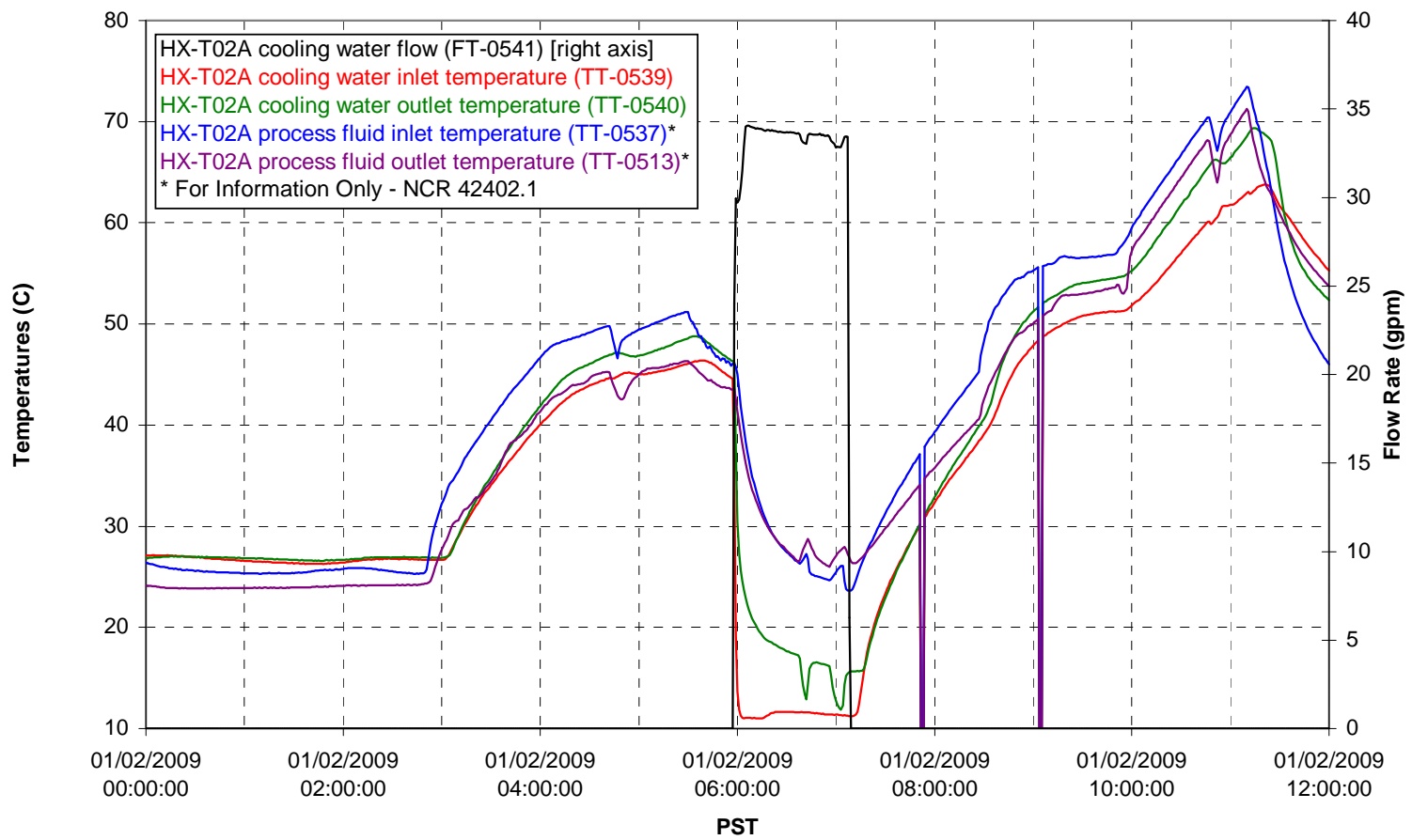

Pump Operation

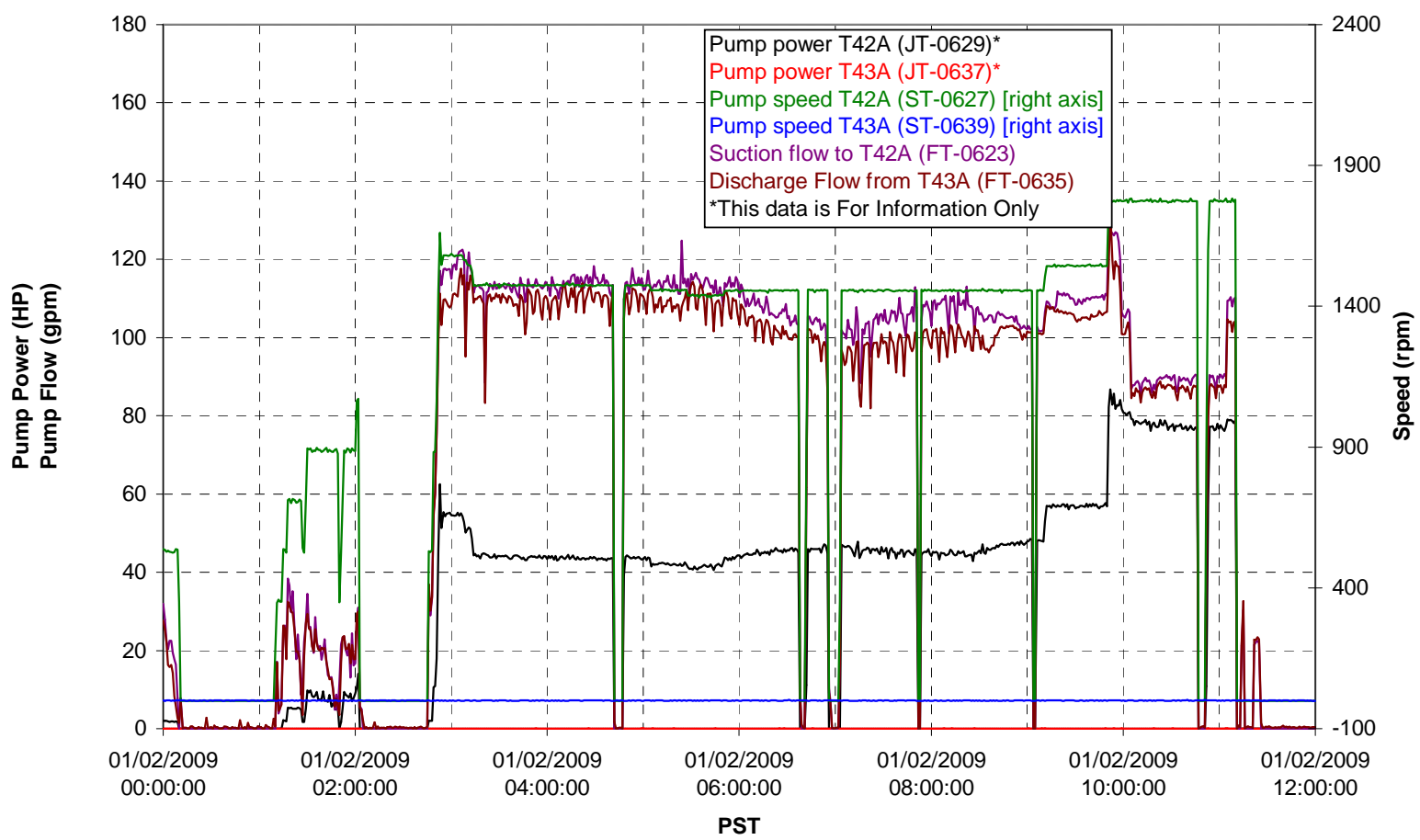


Pulsepot UFP-PP-T01A

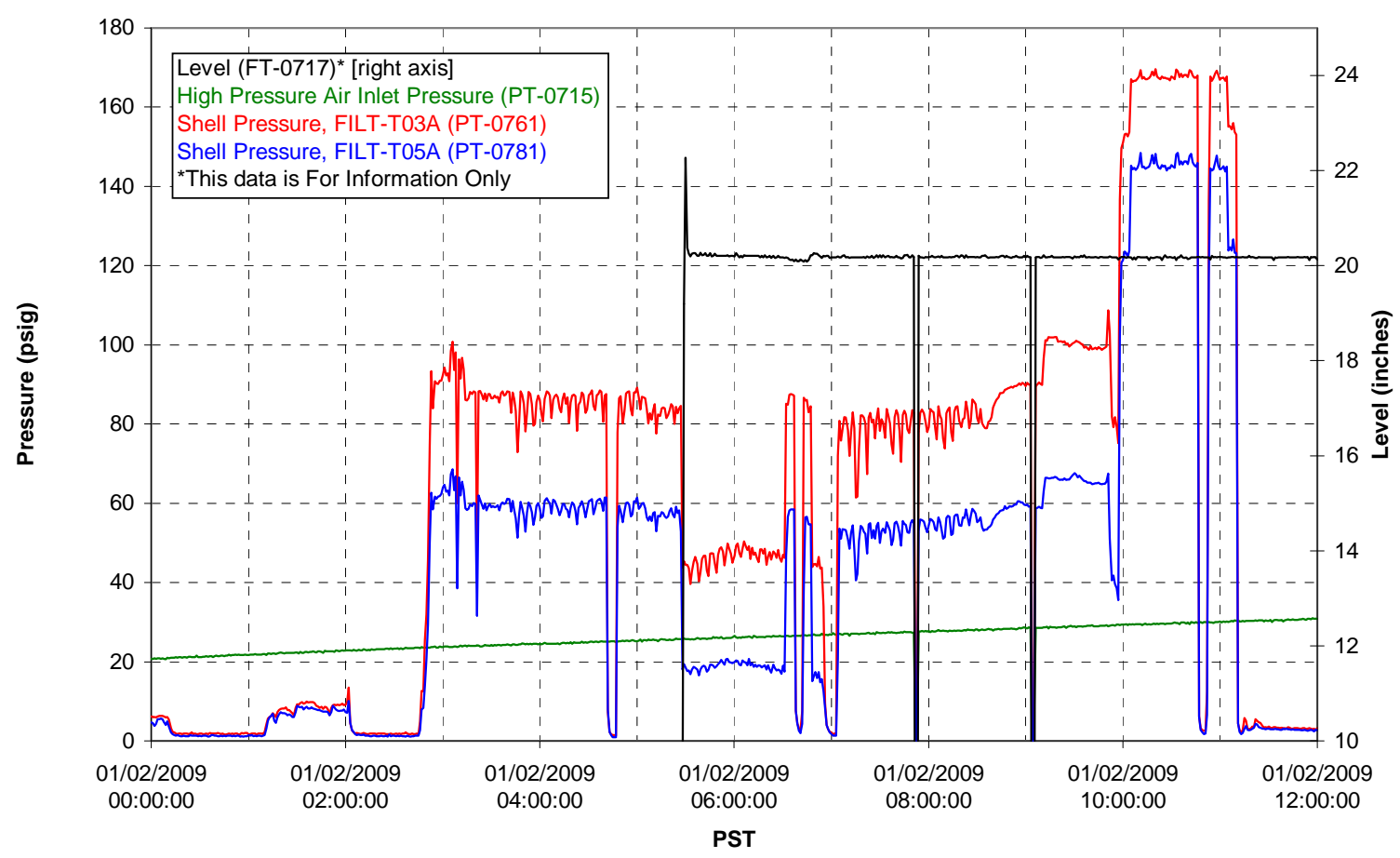

Pulsepot UFP-PP-T02A

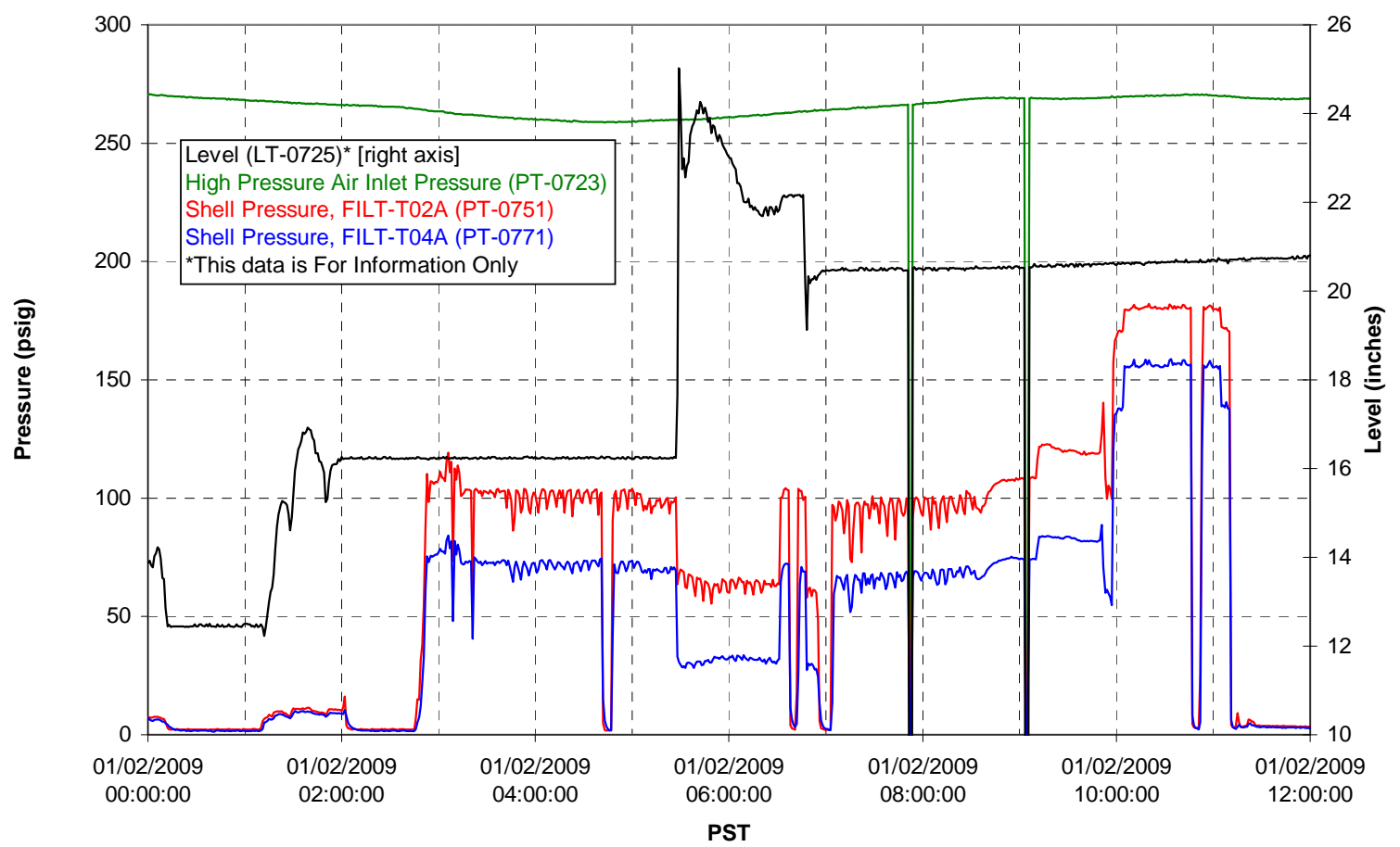


Pulsepot UFP-PP-T03A

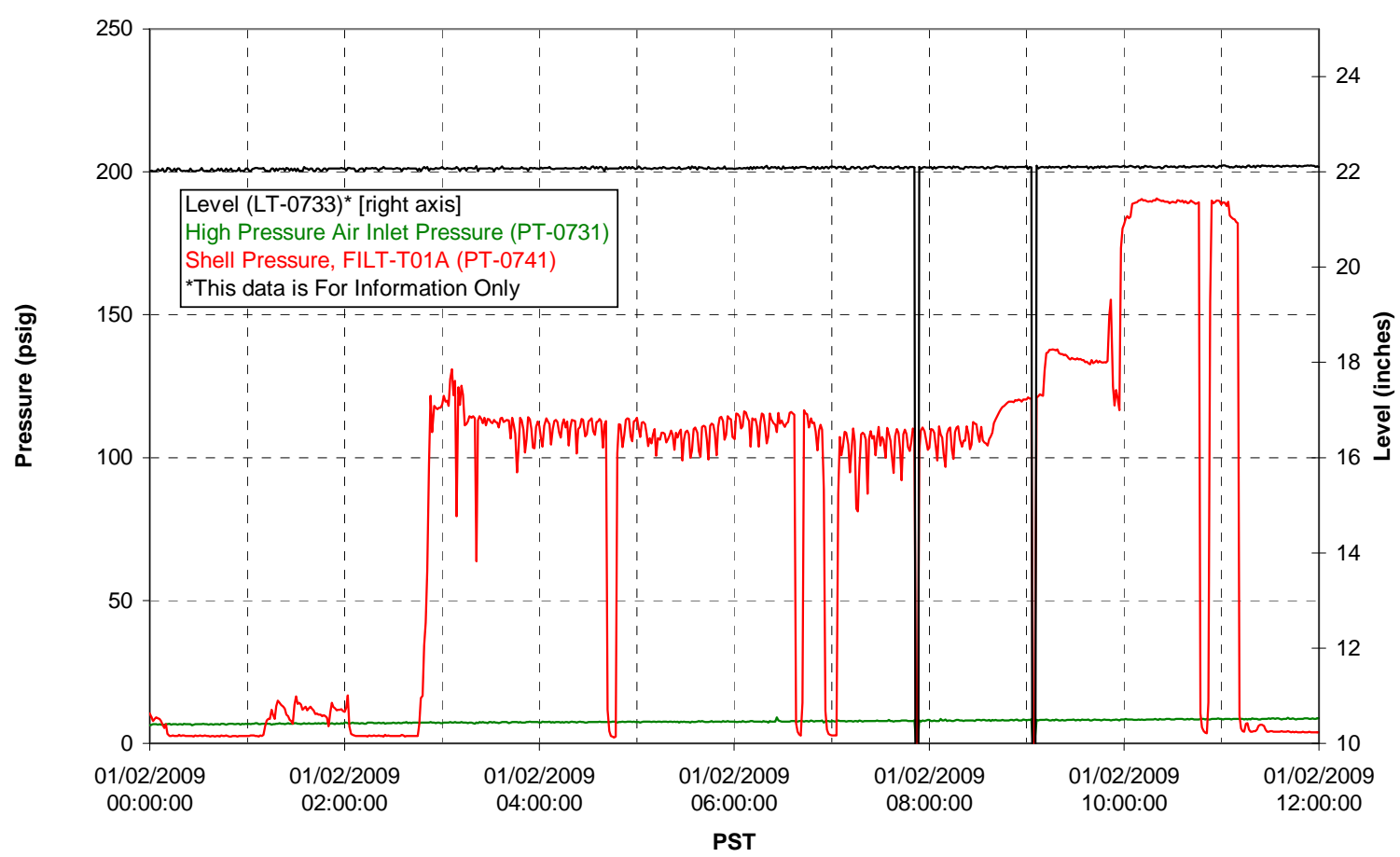

Pulsepot Levels

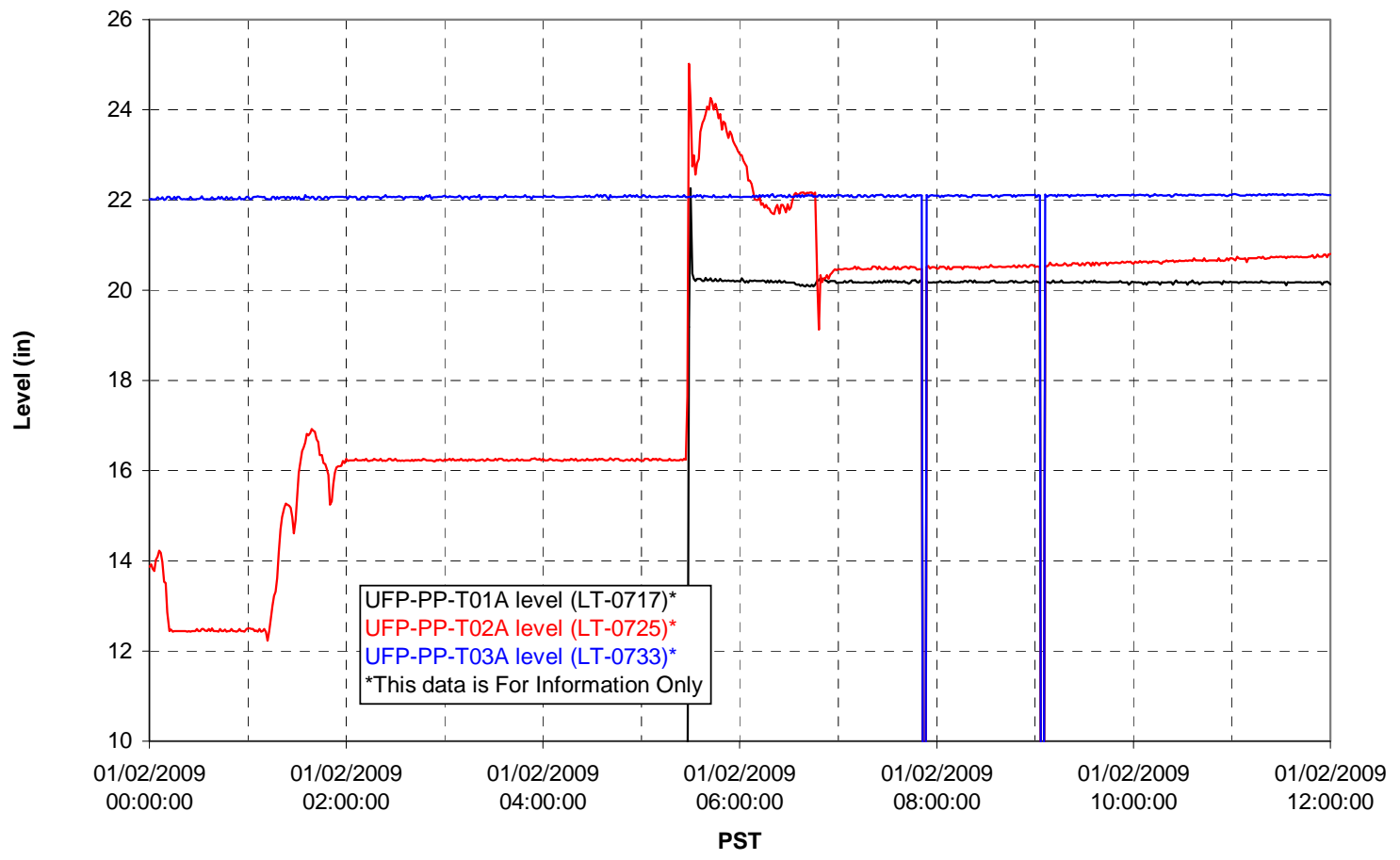


Filter UFP-FILT-T01A

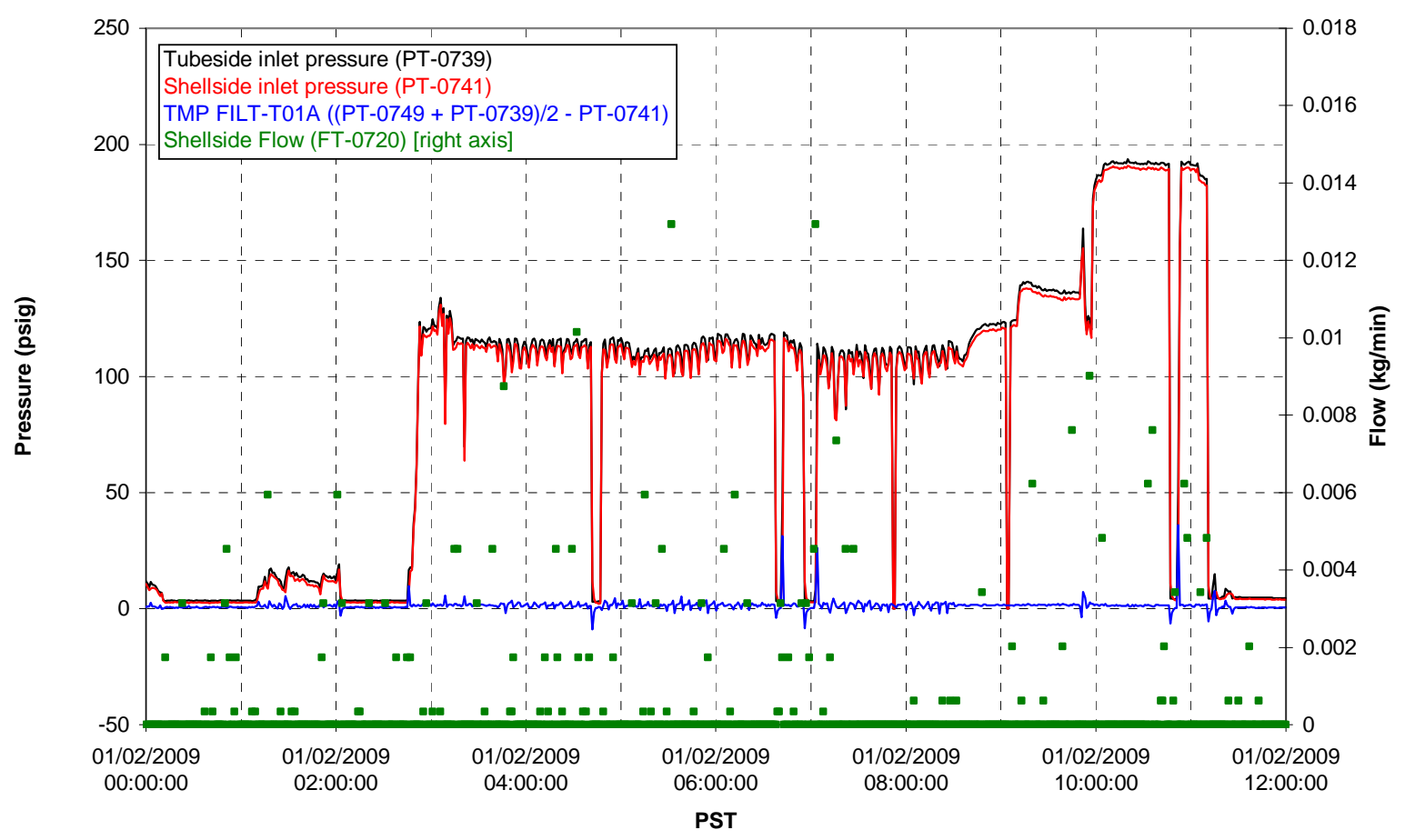

Filter UFP-FILT-T02A

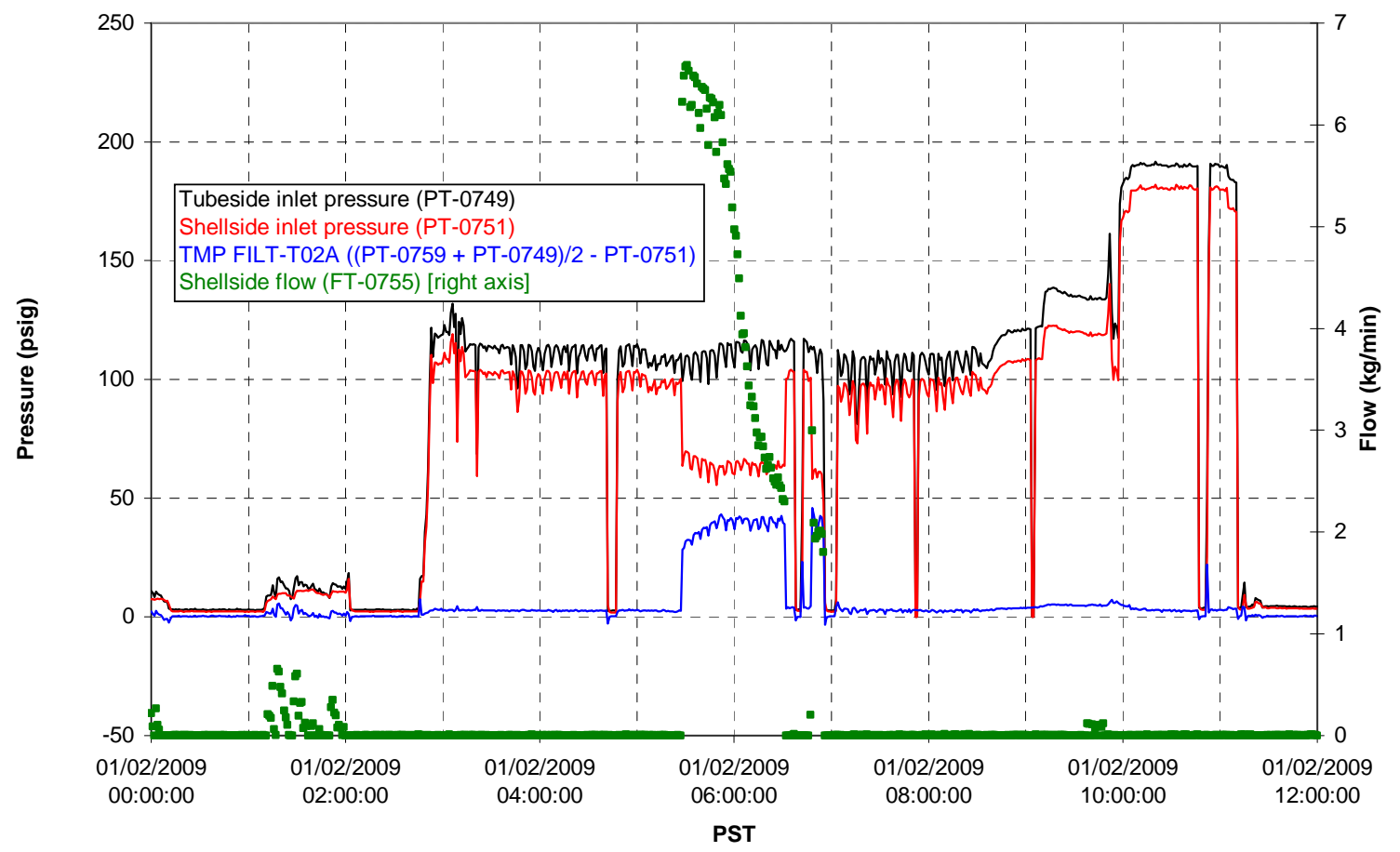


Filter UFP-FILT-T03A

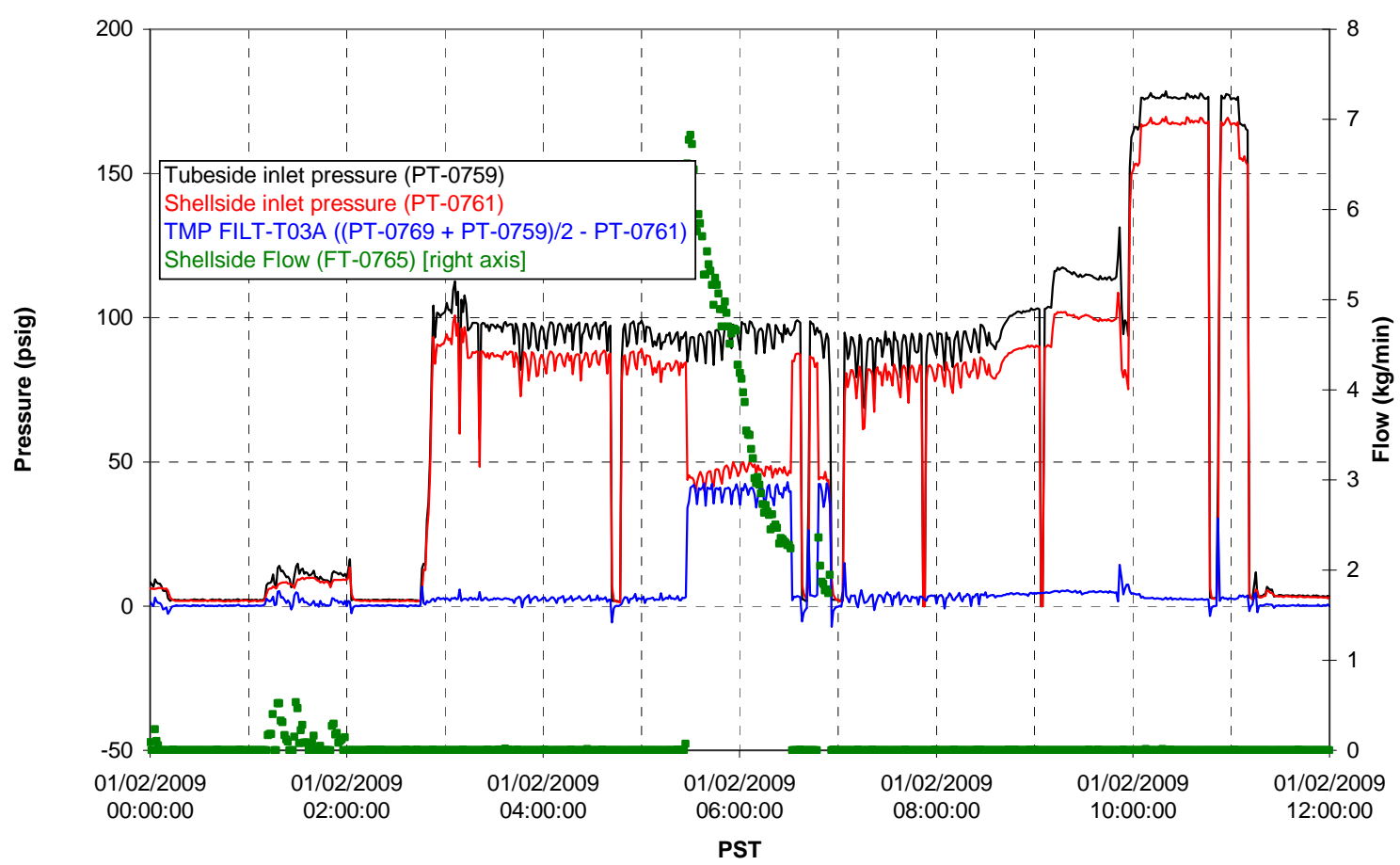

Filter UFP-FILT-T04A

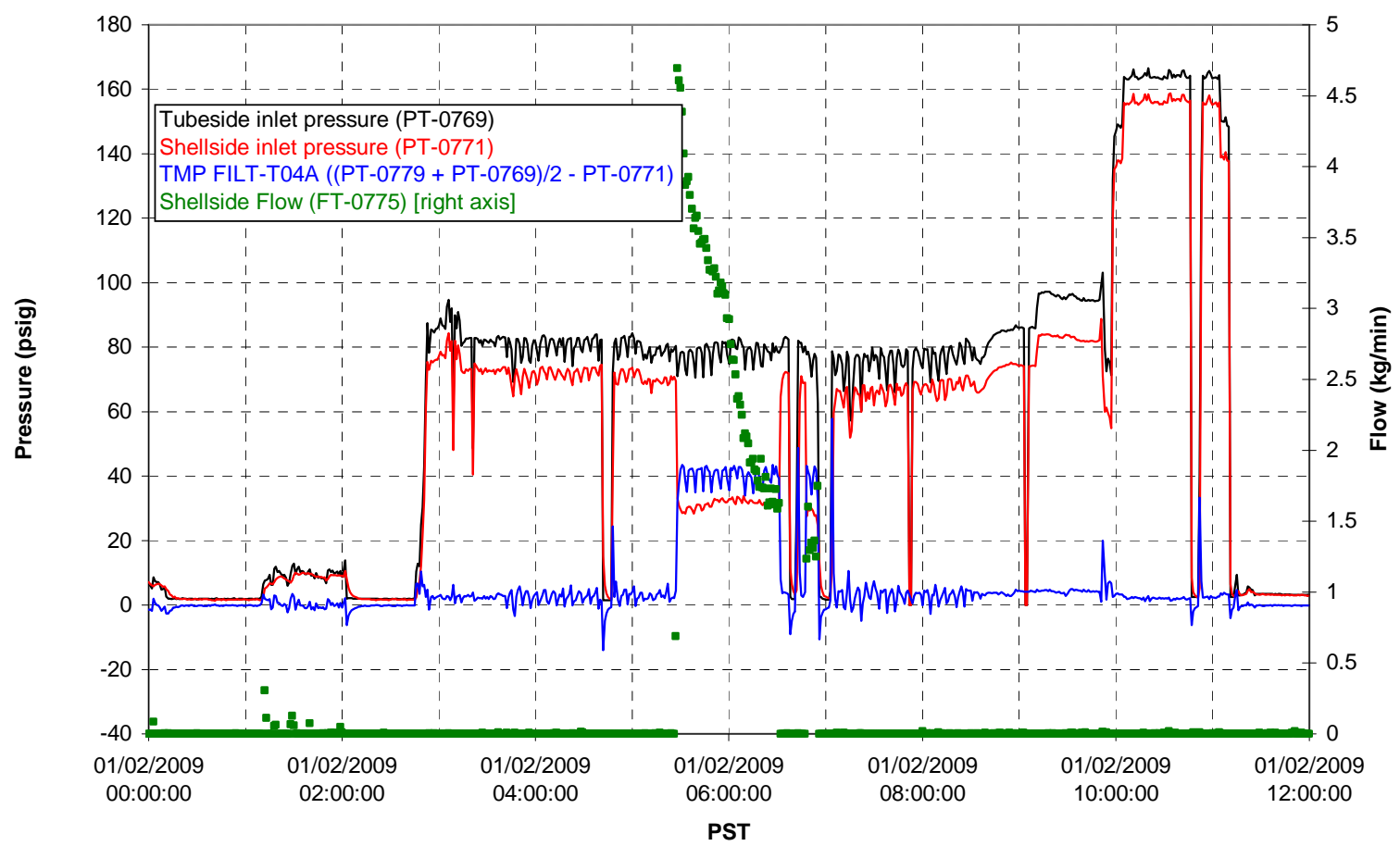


Filter UFP-FILT-T05A

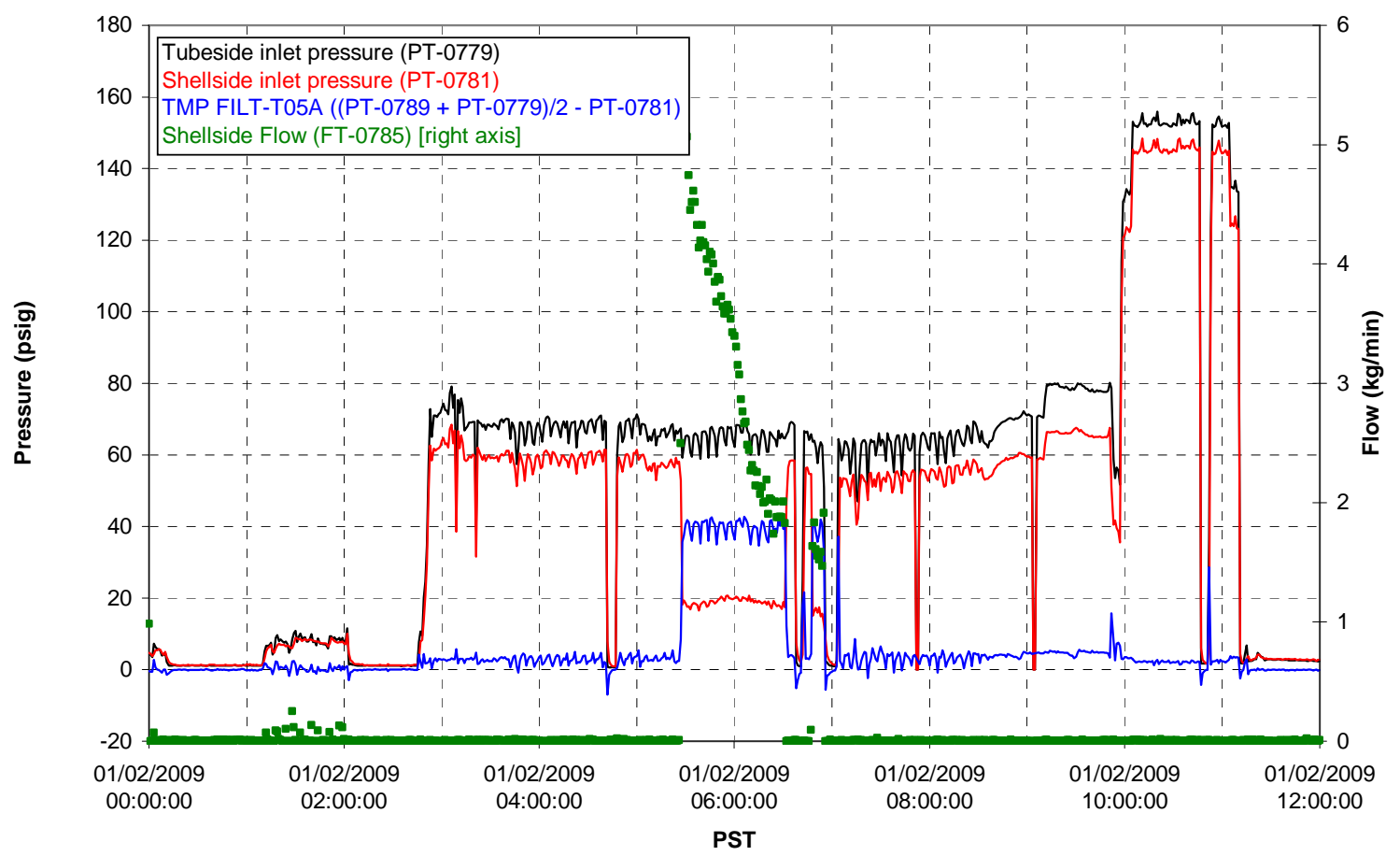

Chemical Flow

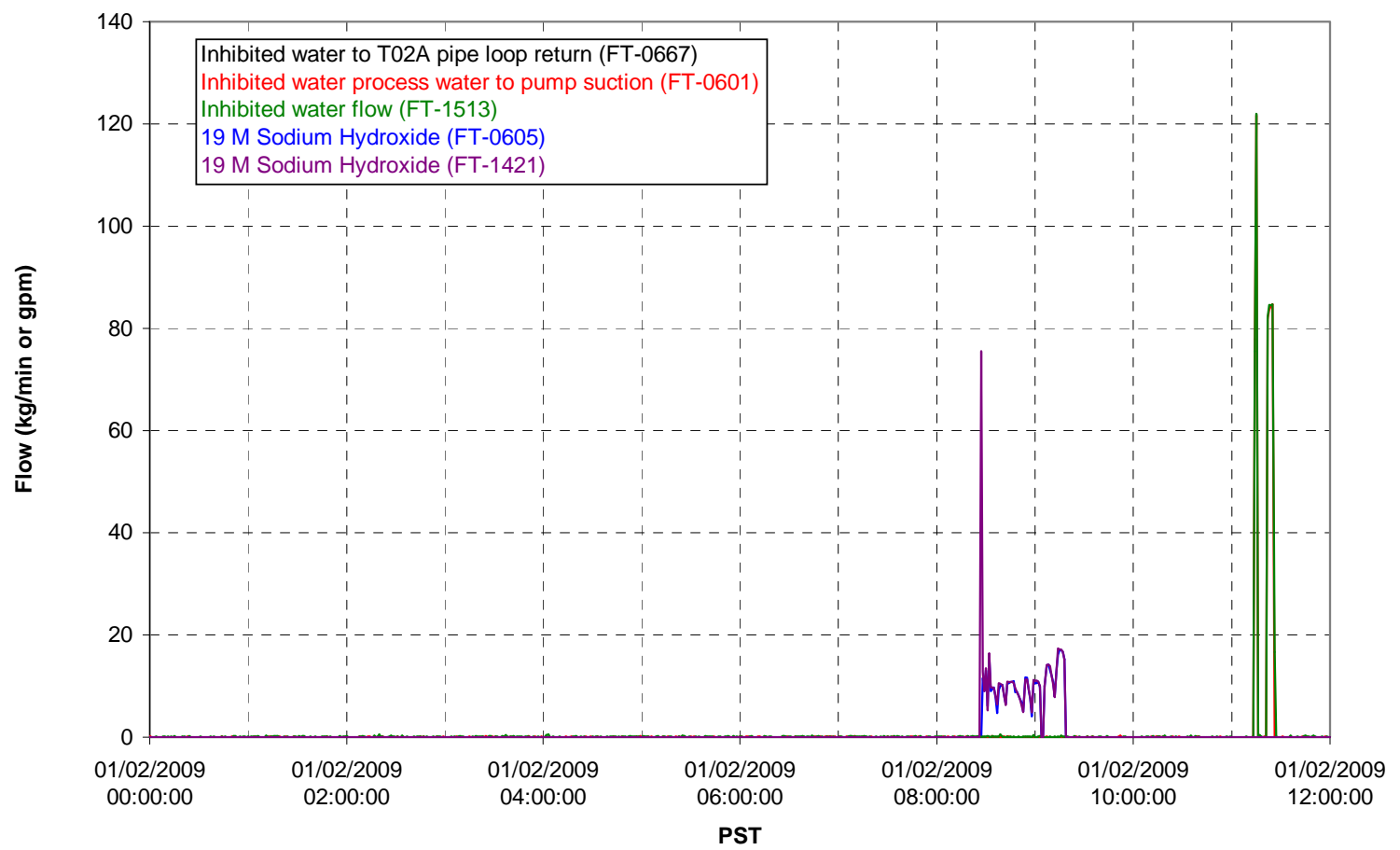


Chemical Flow

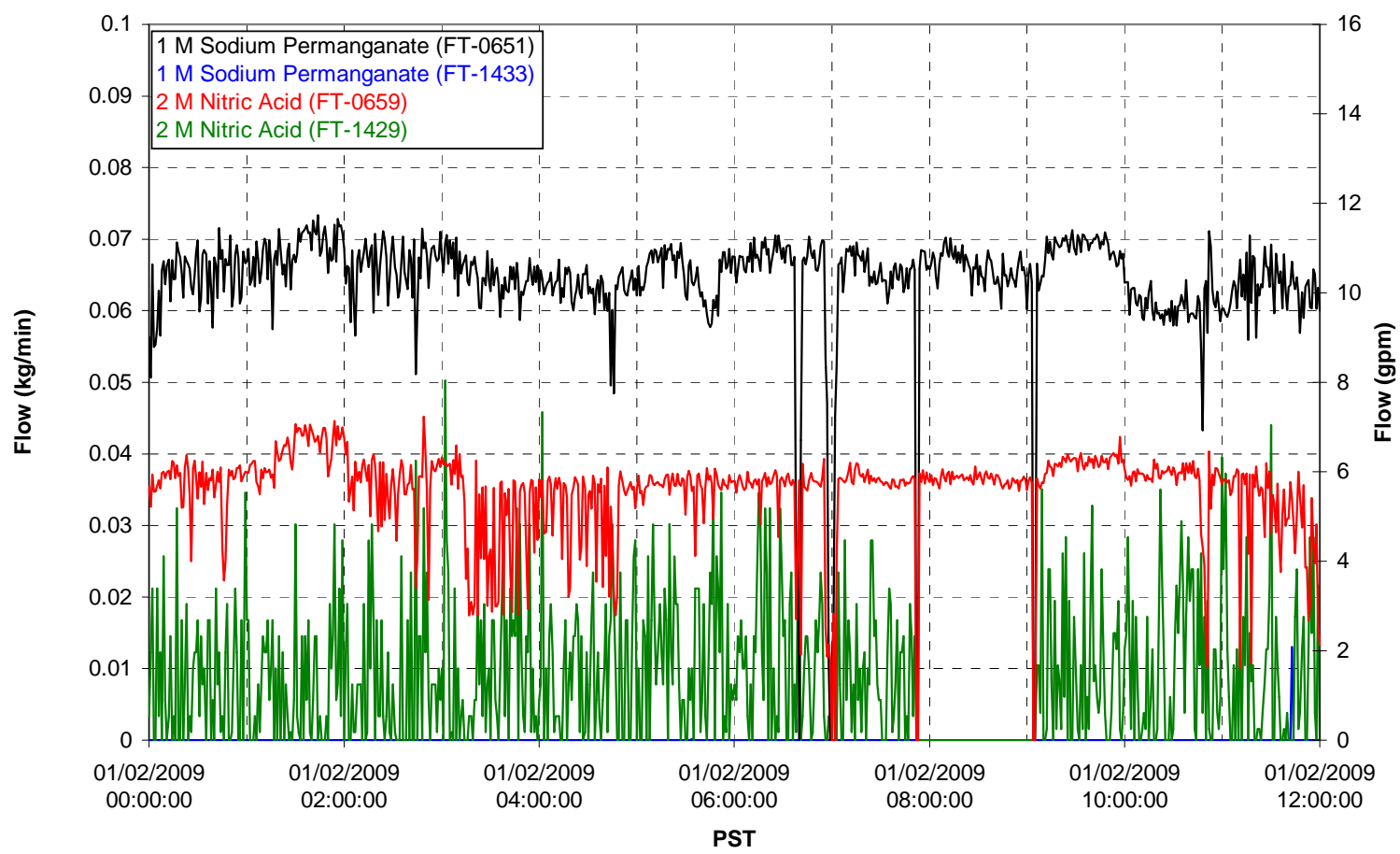

Air Flows

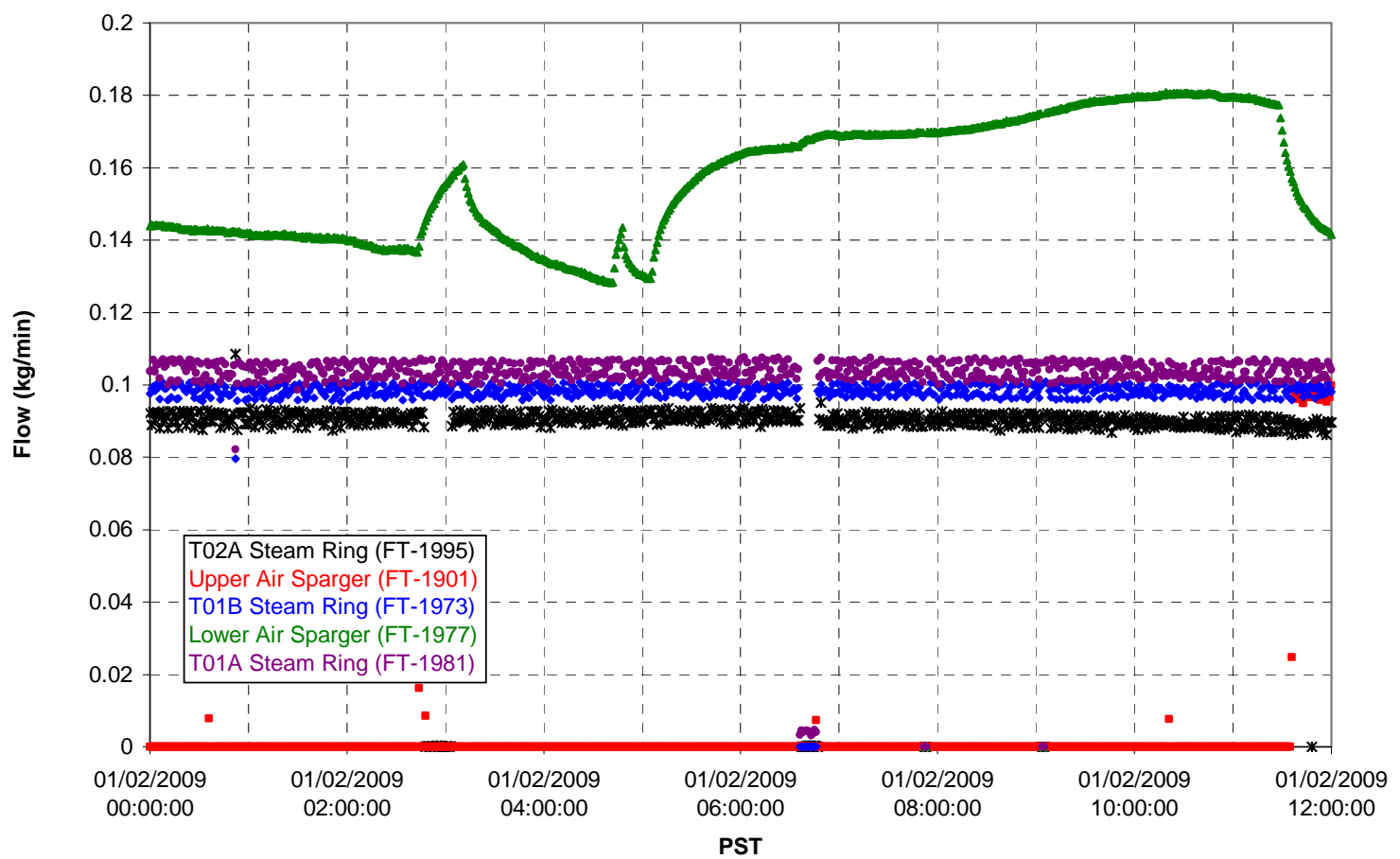


T02A Steam

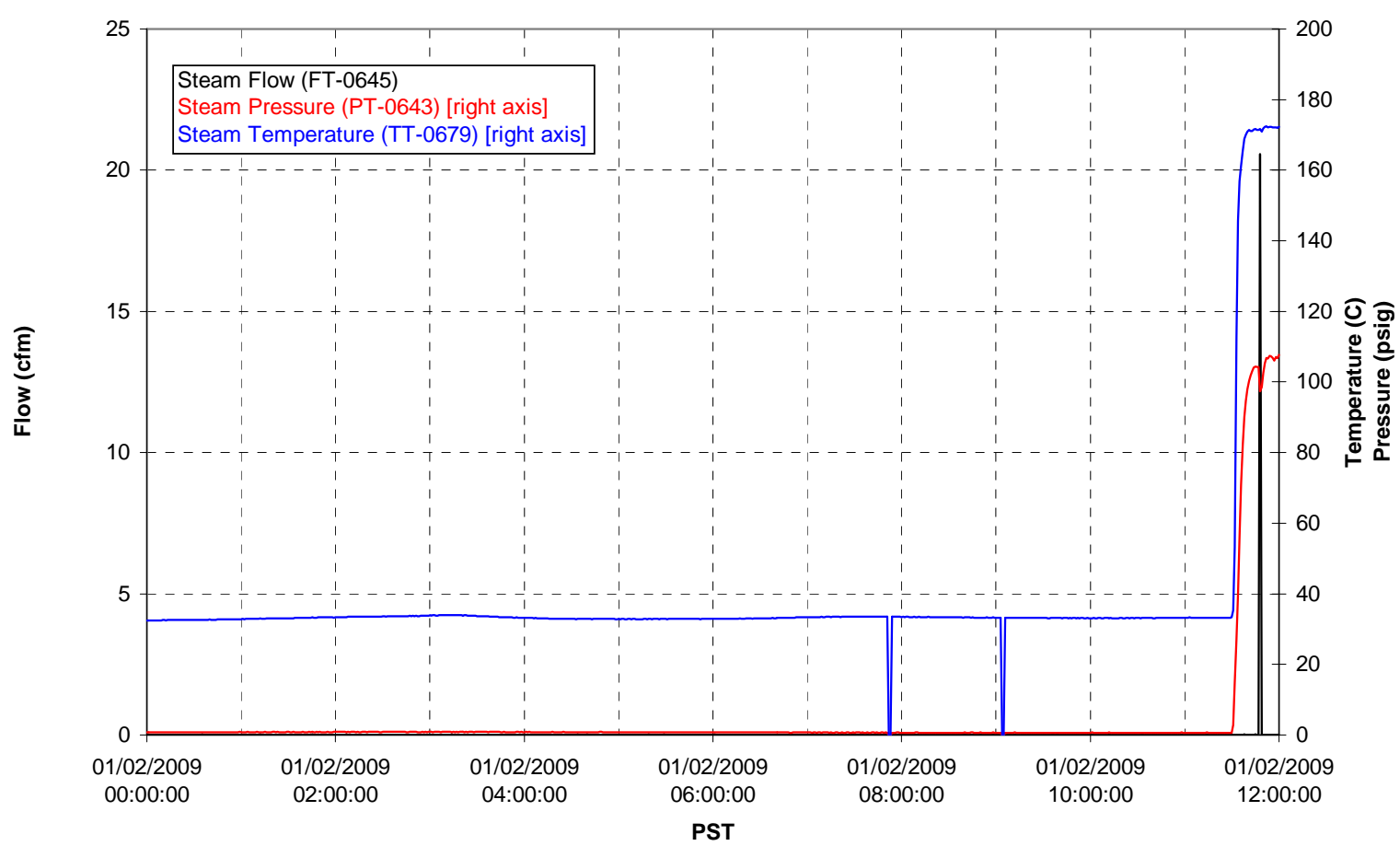

T01A Steam

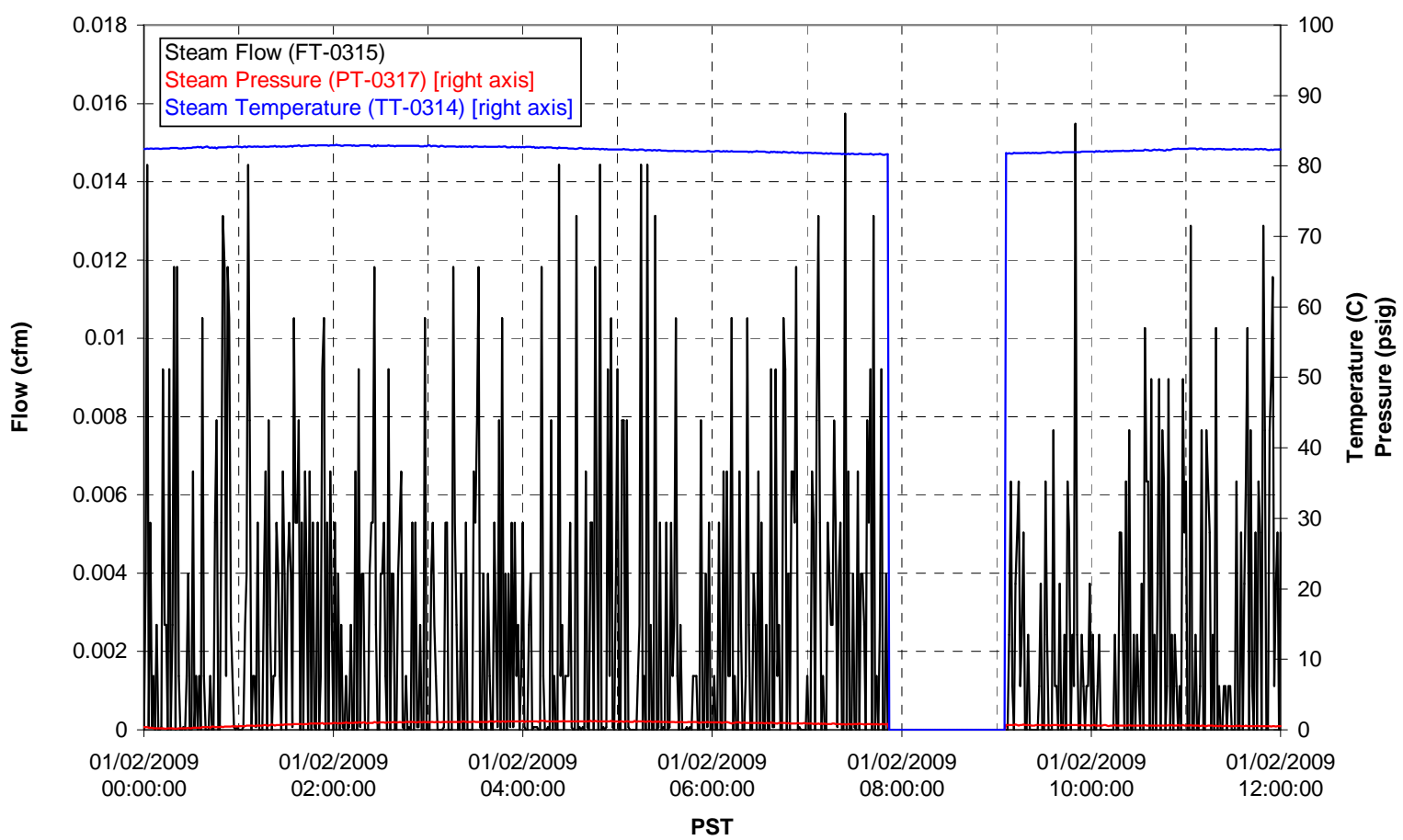


T01B Steam

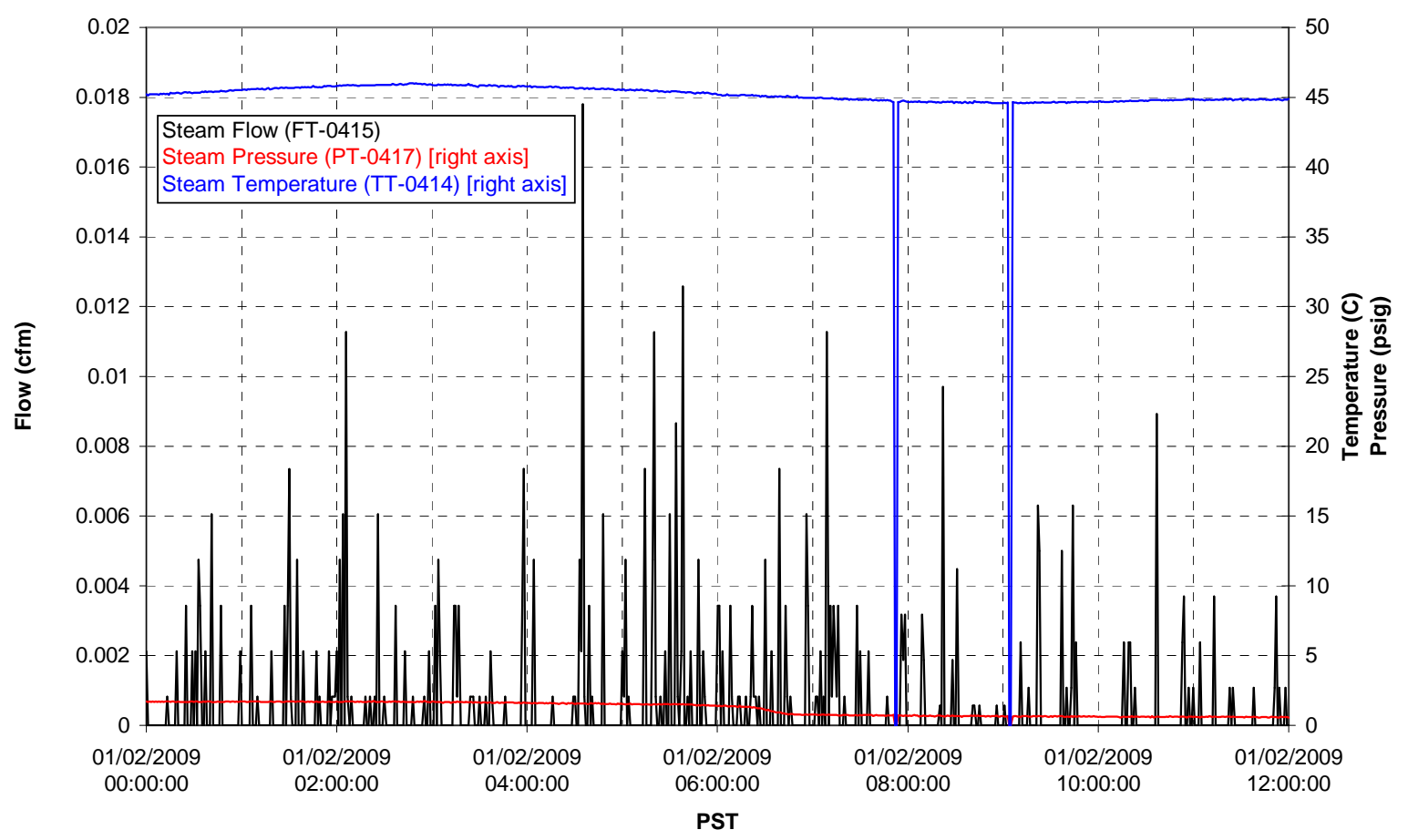


Functional Test Data Plots

01/02/2009 12:00 - 01/03/2009 00:00 
T01A level

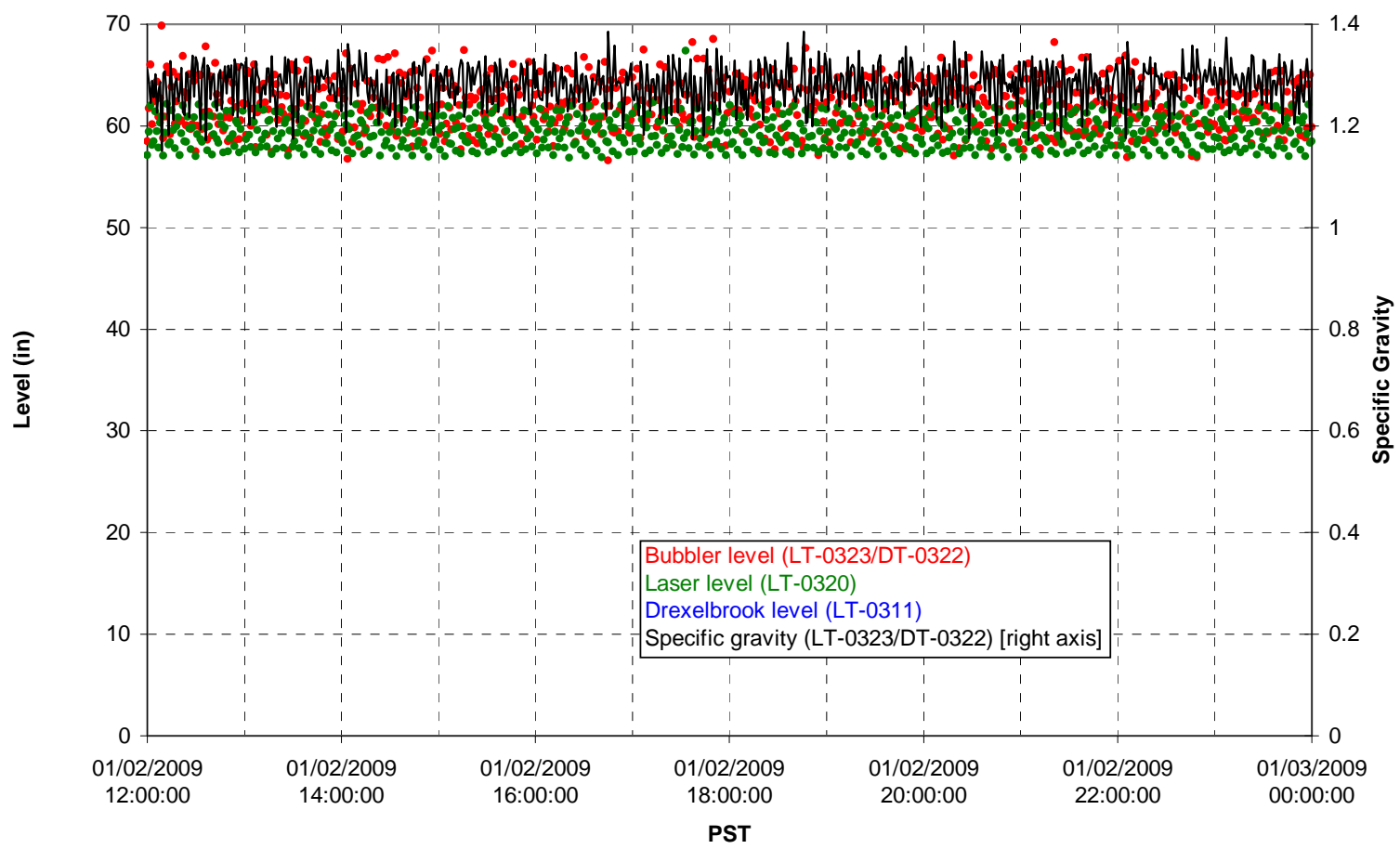

T01A temperatures

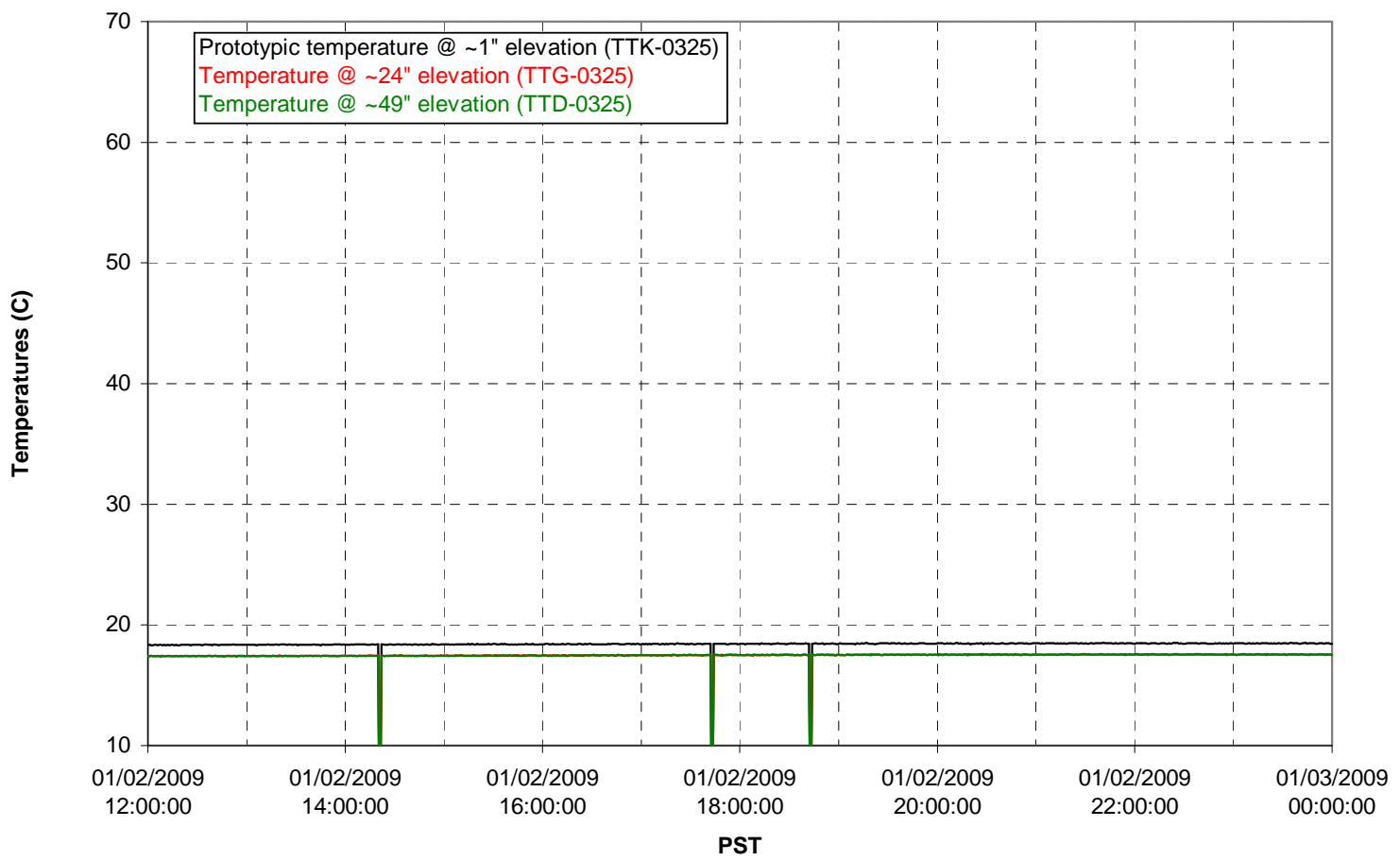


T01B level

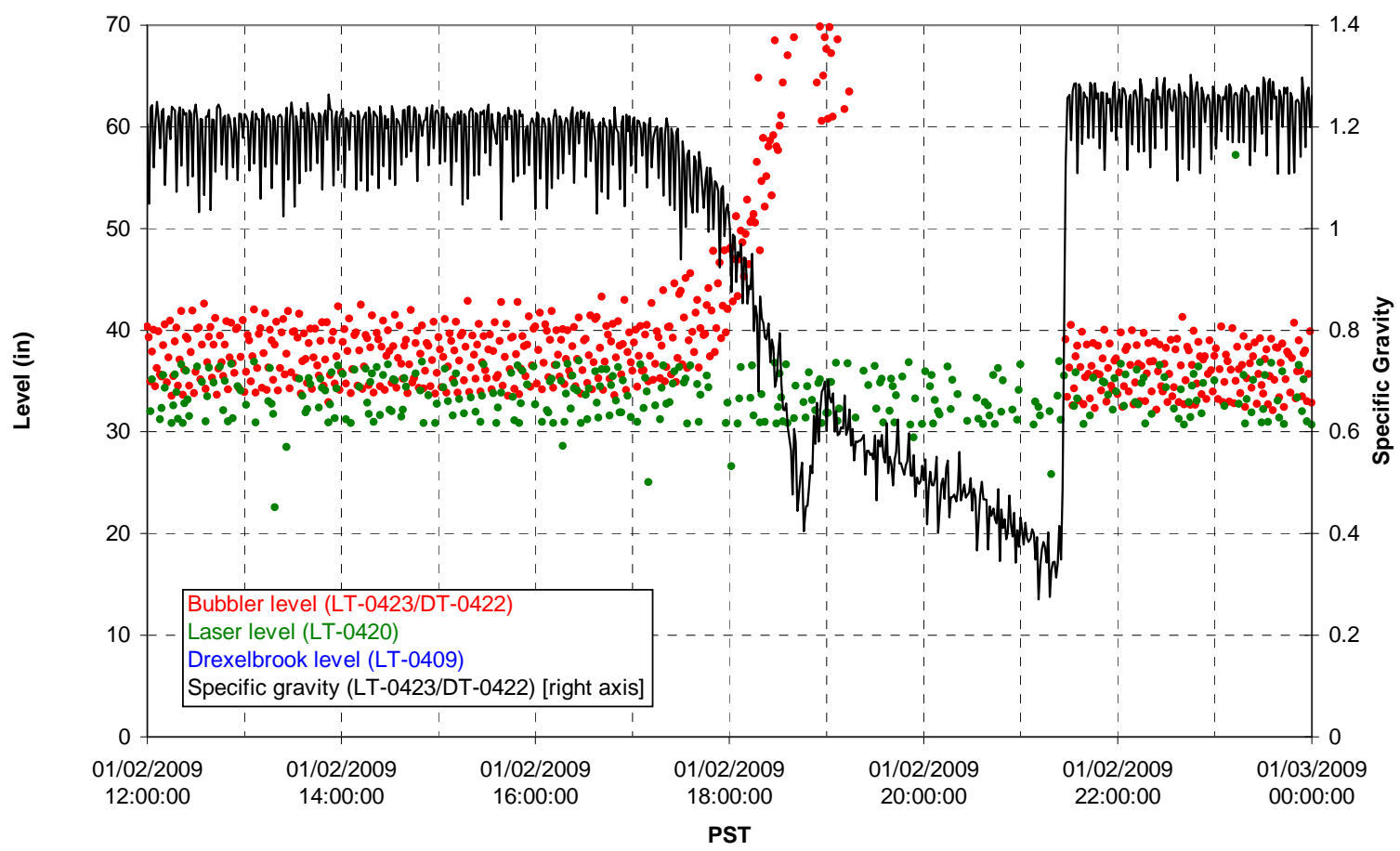

T01B temperatures

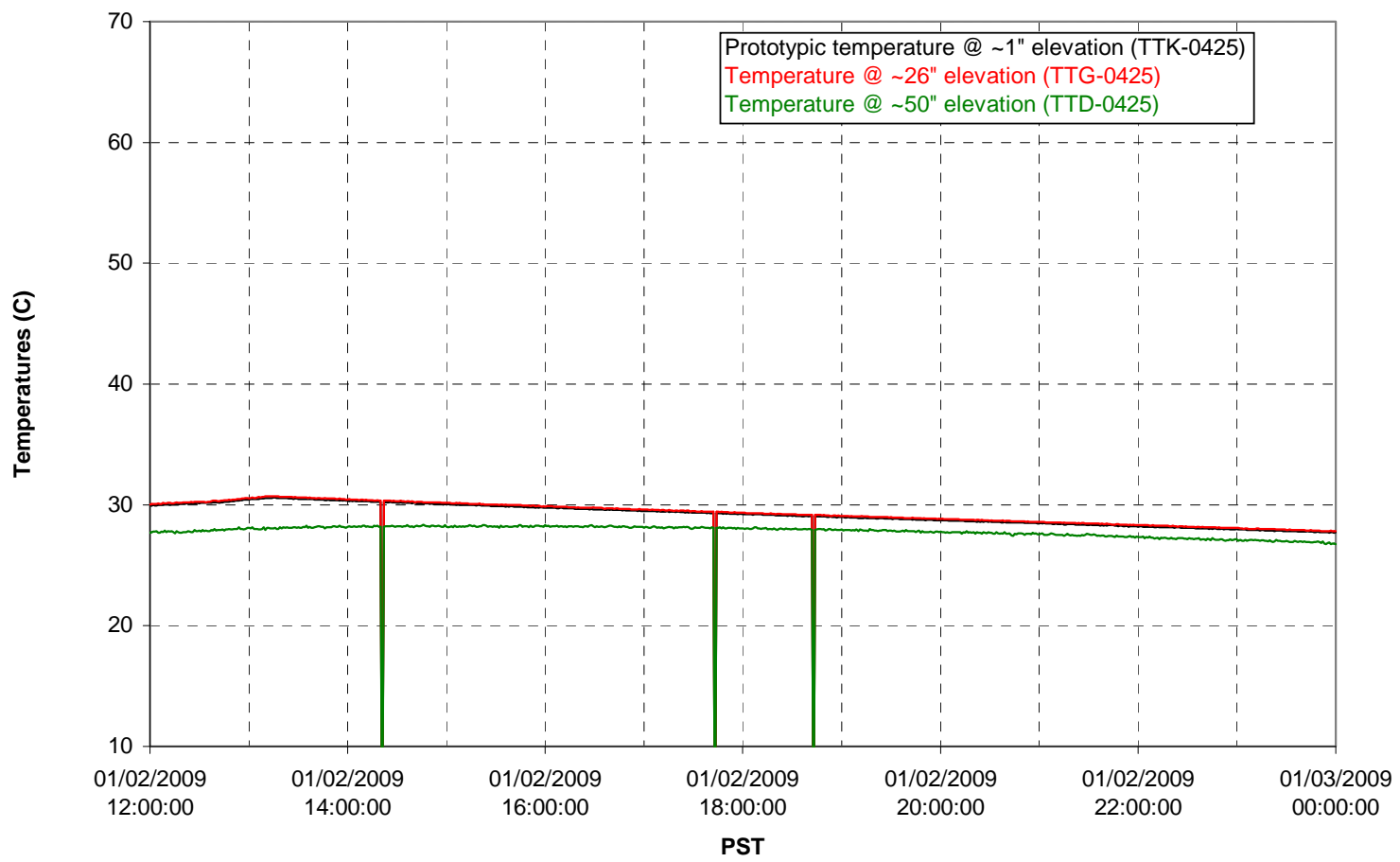


T02A level

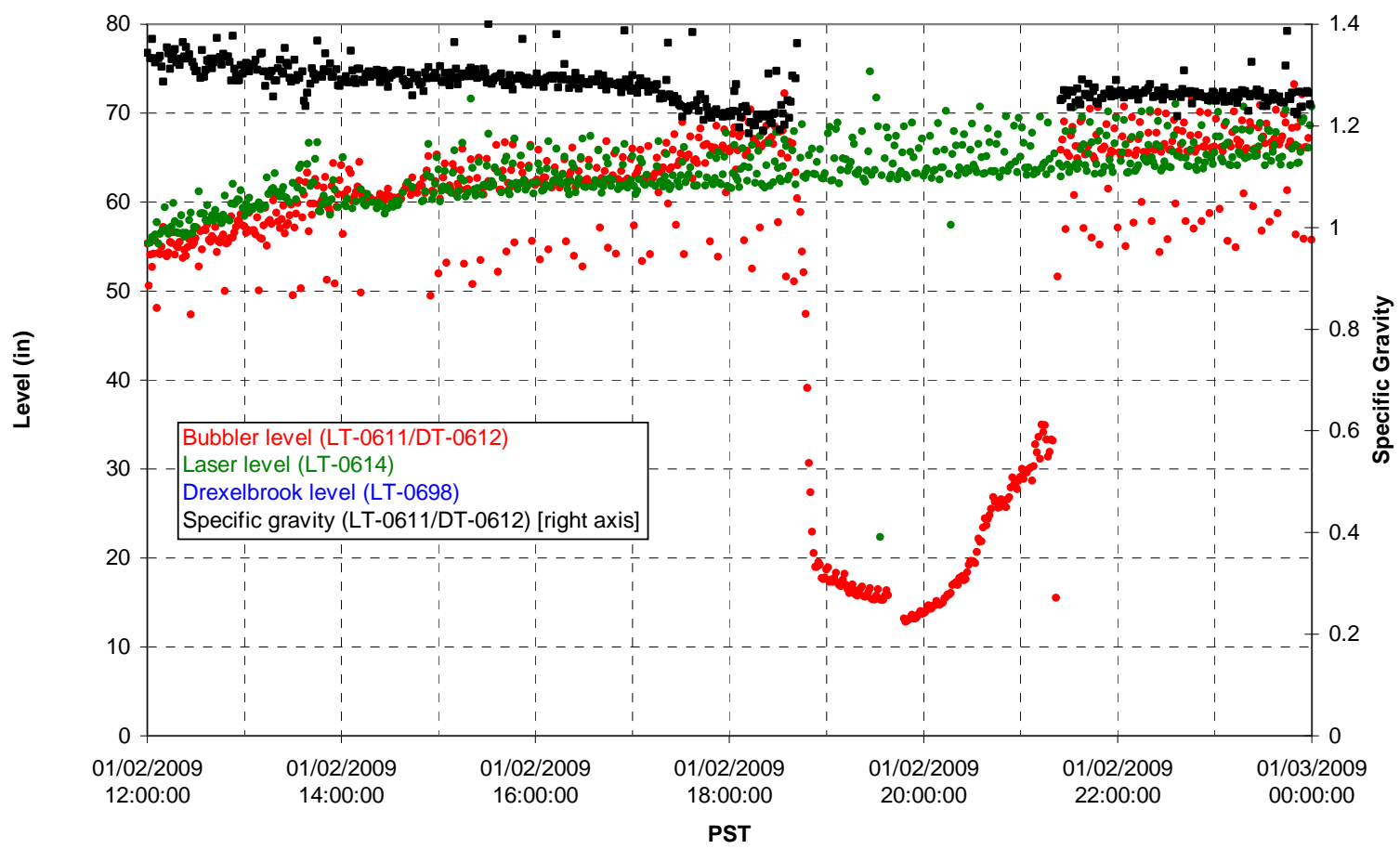

T02A temperatures

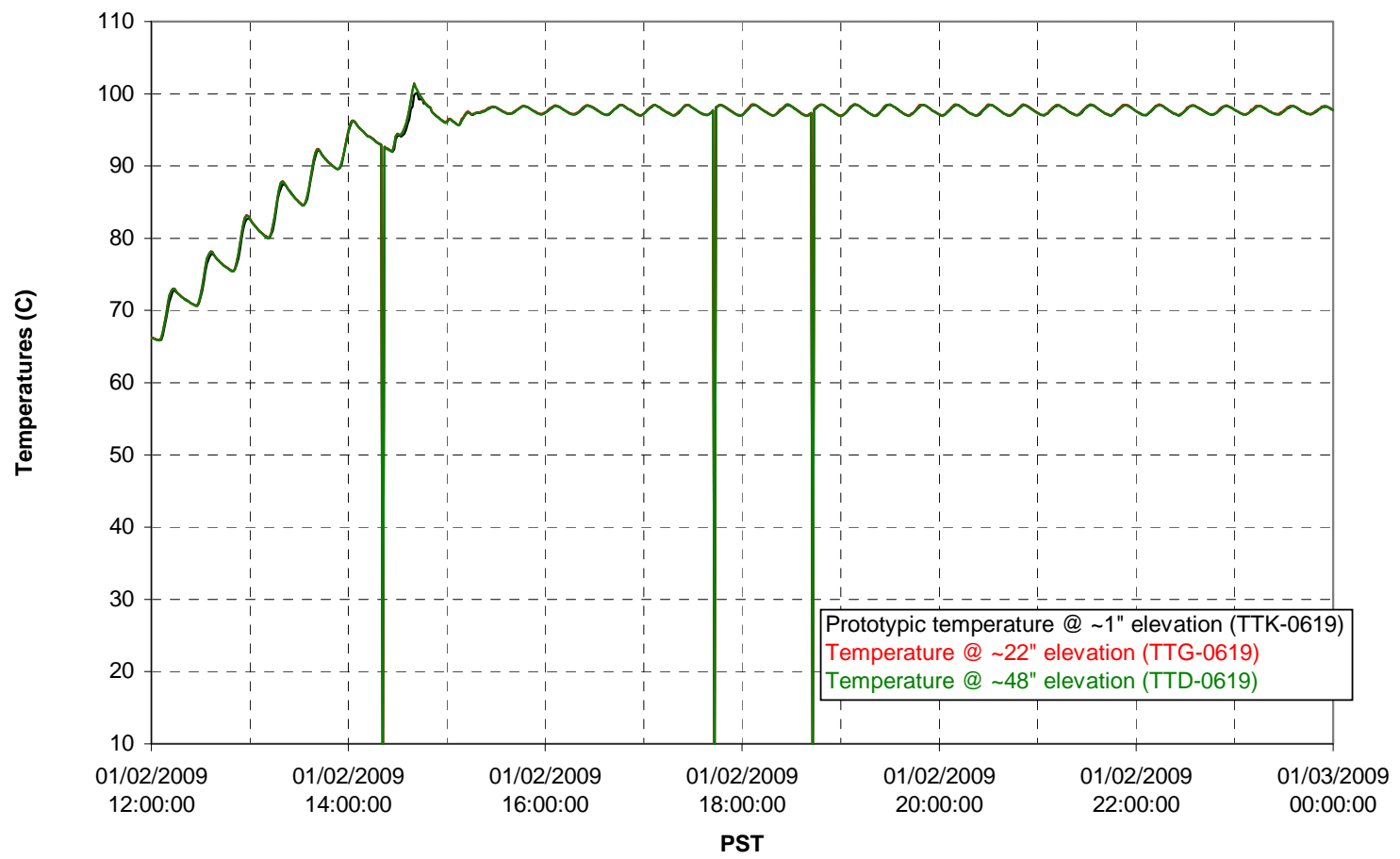


T02A and filter loop temperatures

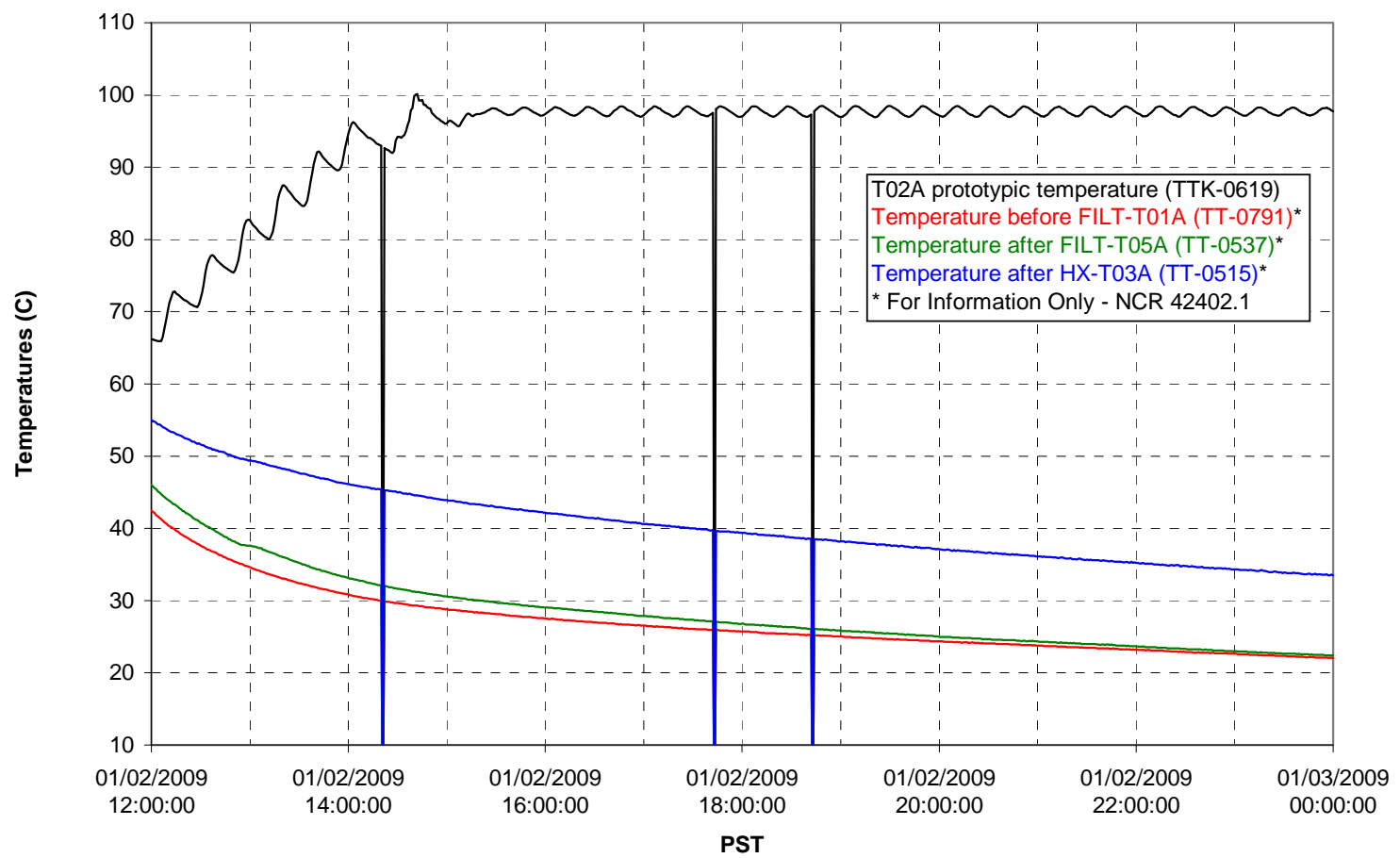

Pump Pressures and Flow

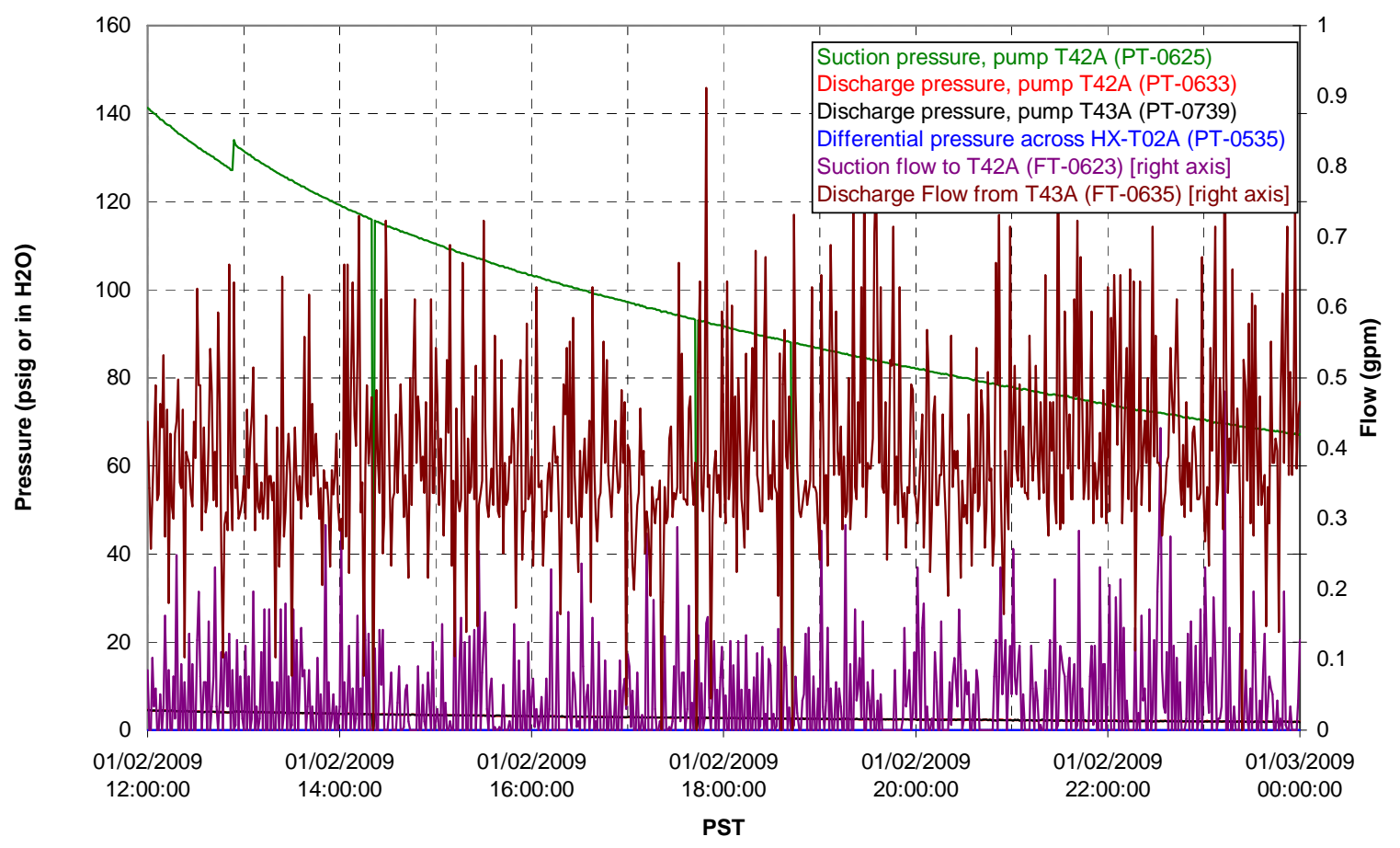


Axial pressure drop

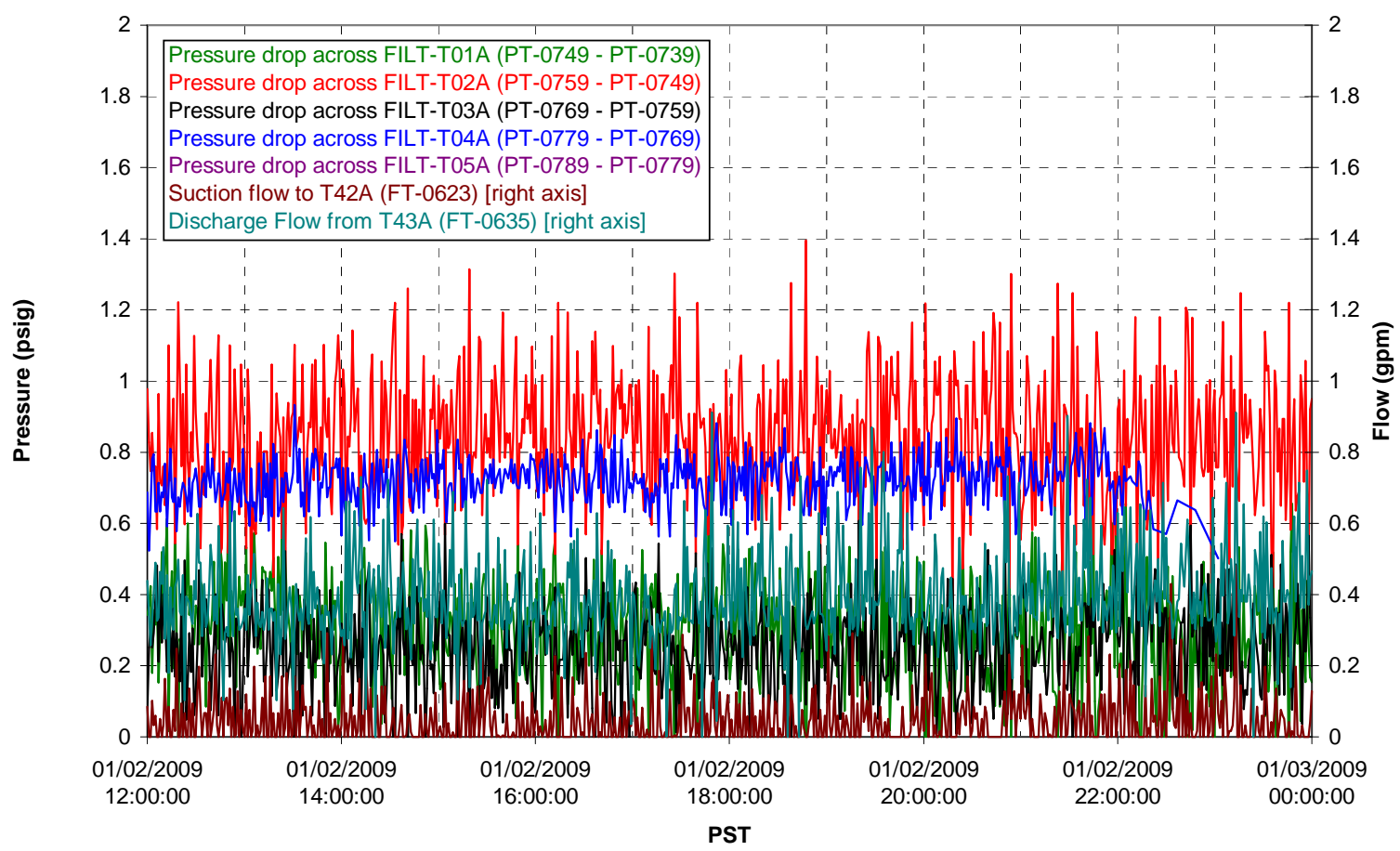

Permeate flow rates

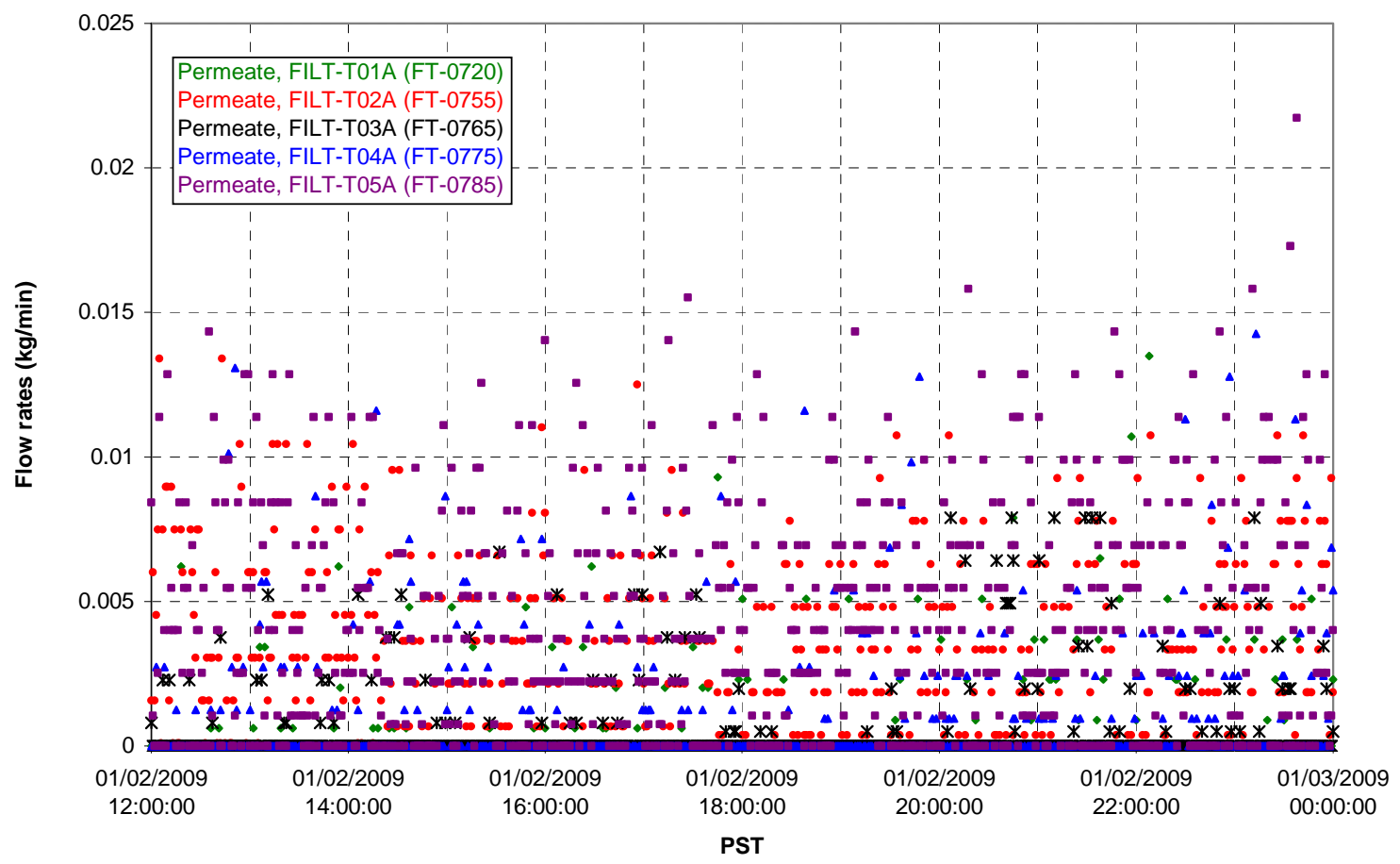


T02A Inner Temperature Tree

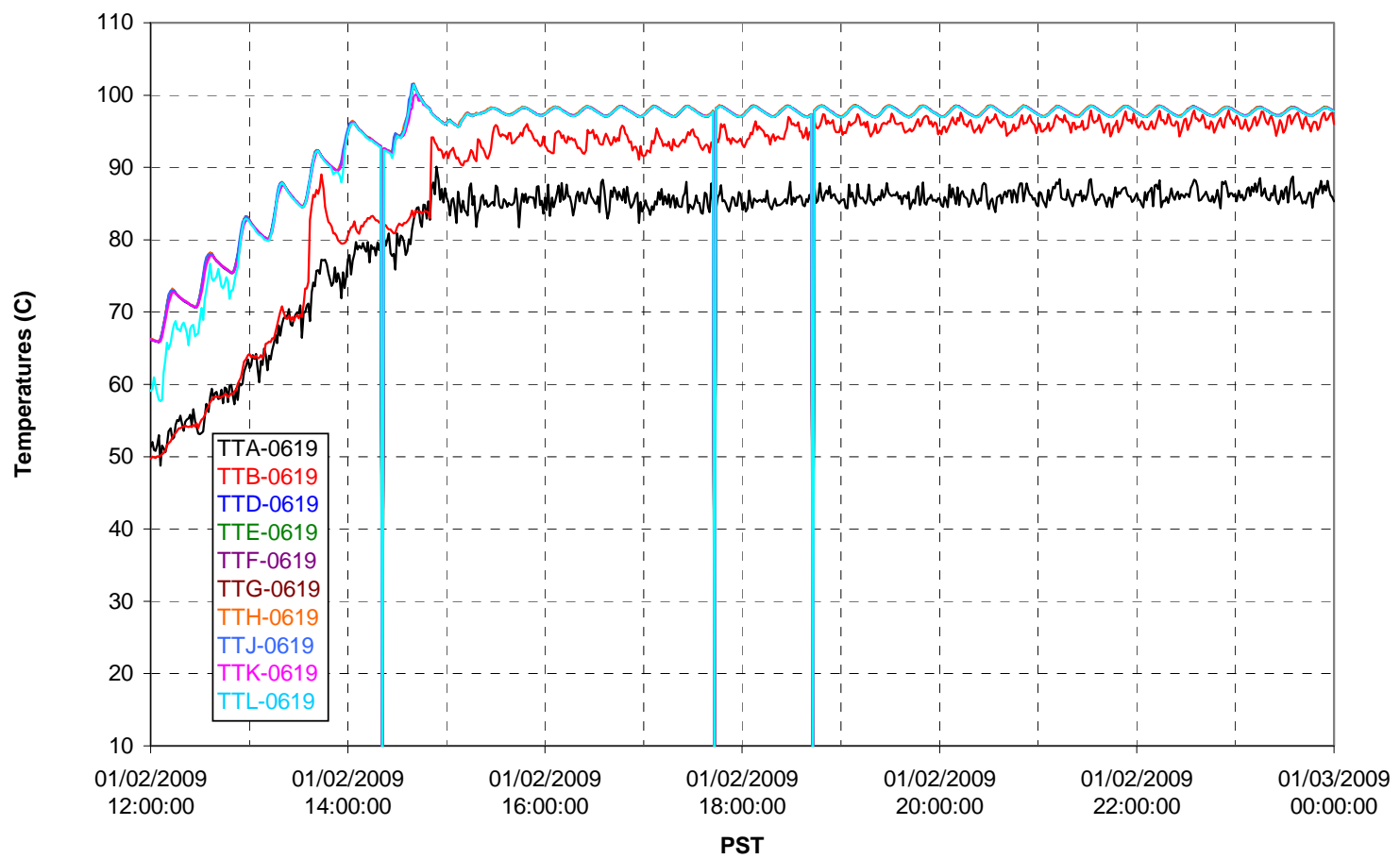

T02A Outer Temperature Tree

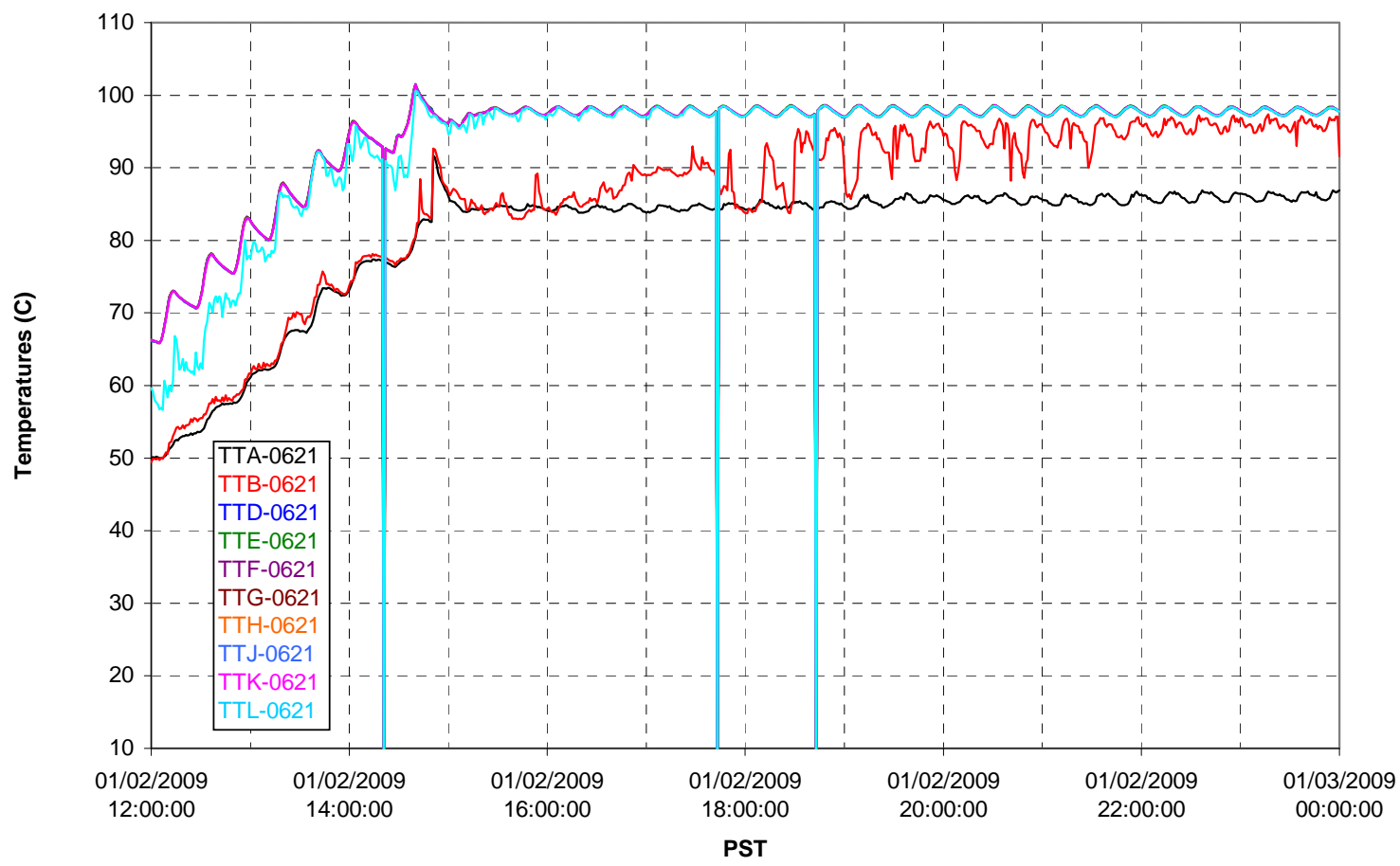


T02A temperatures

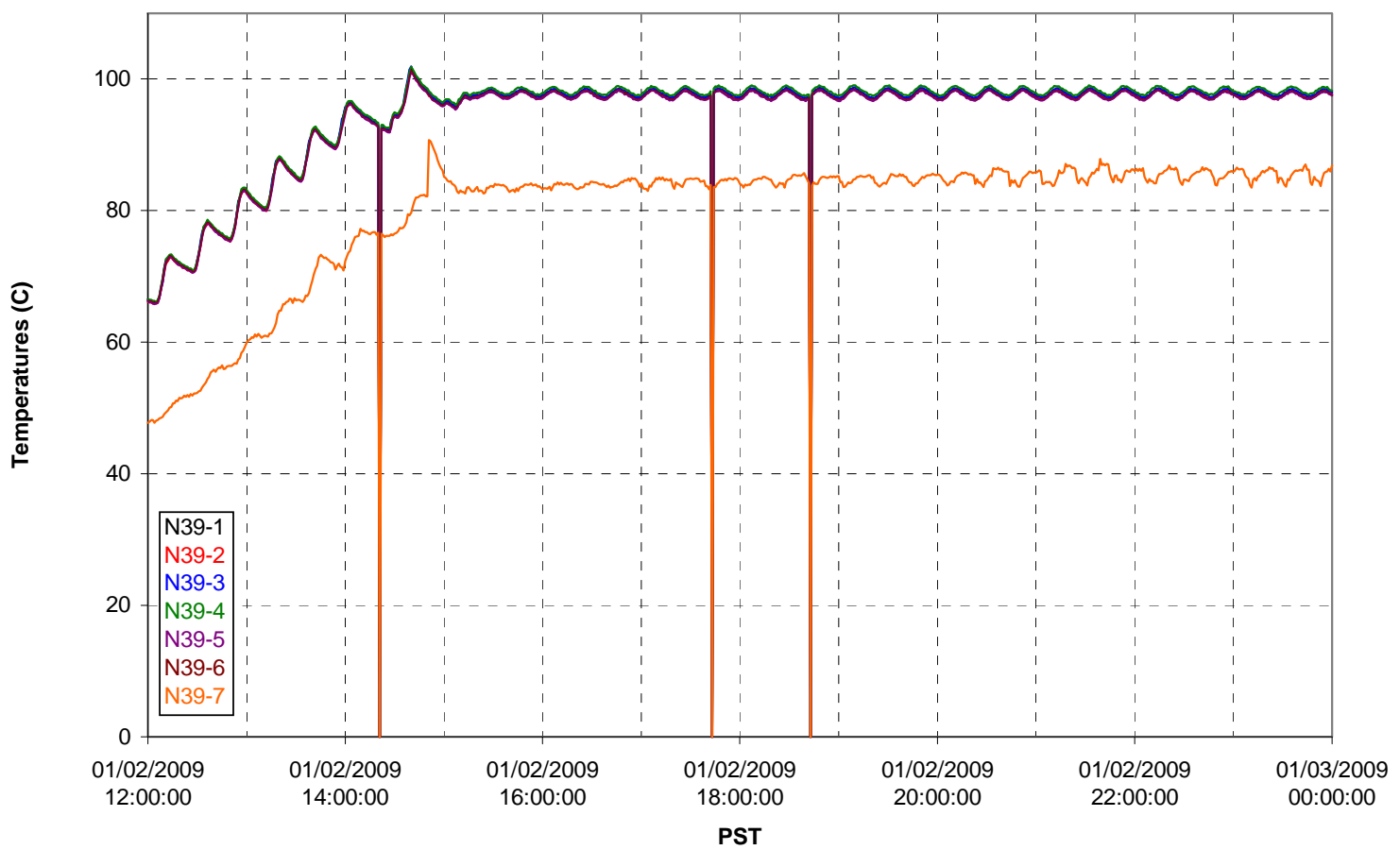

T02A temperatures

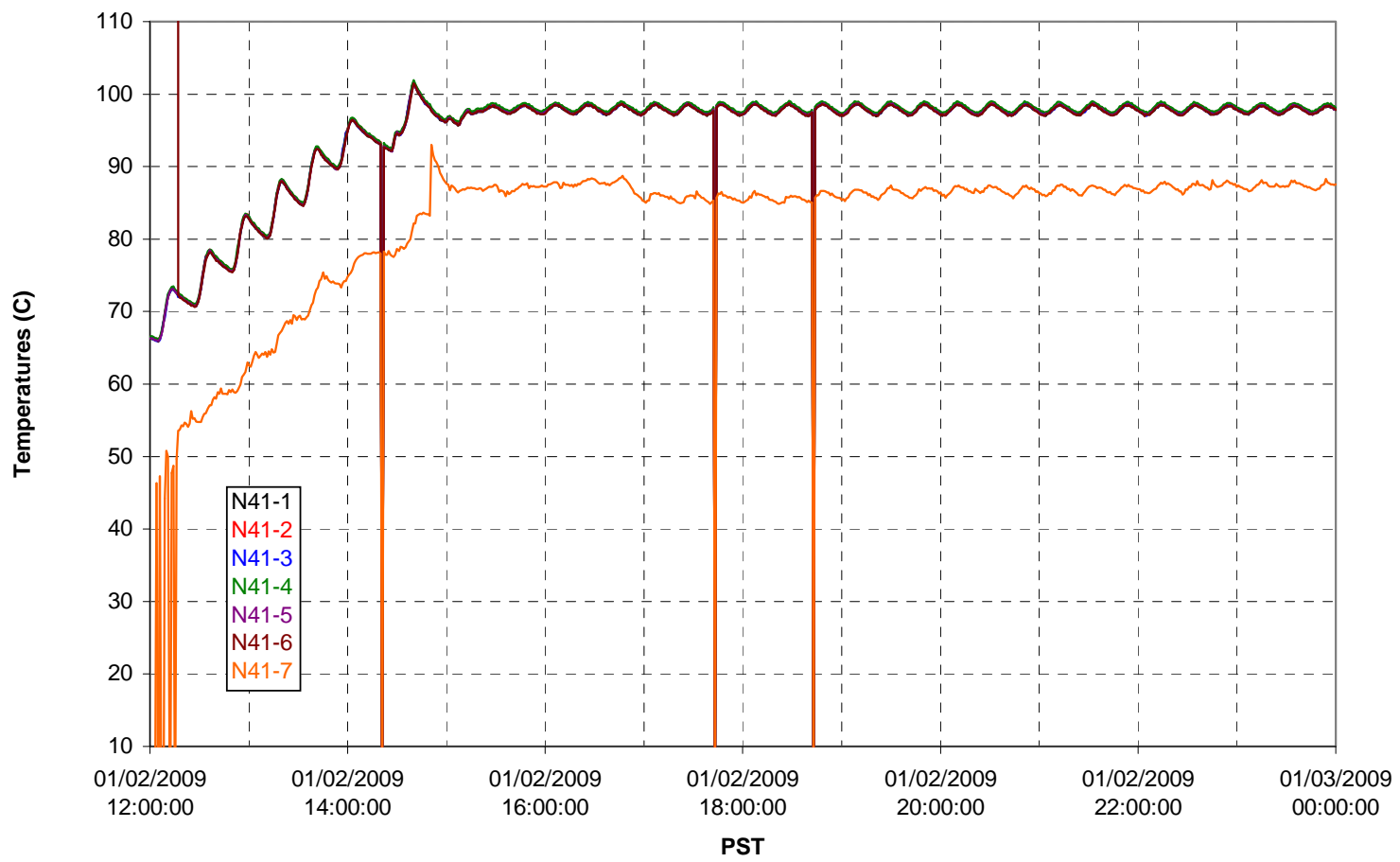


T02A temperatures

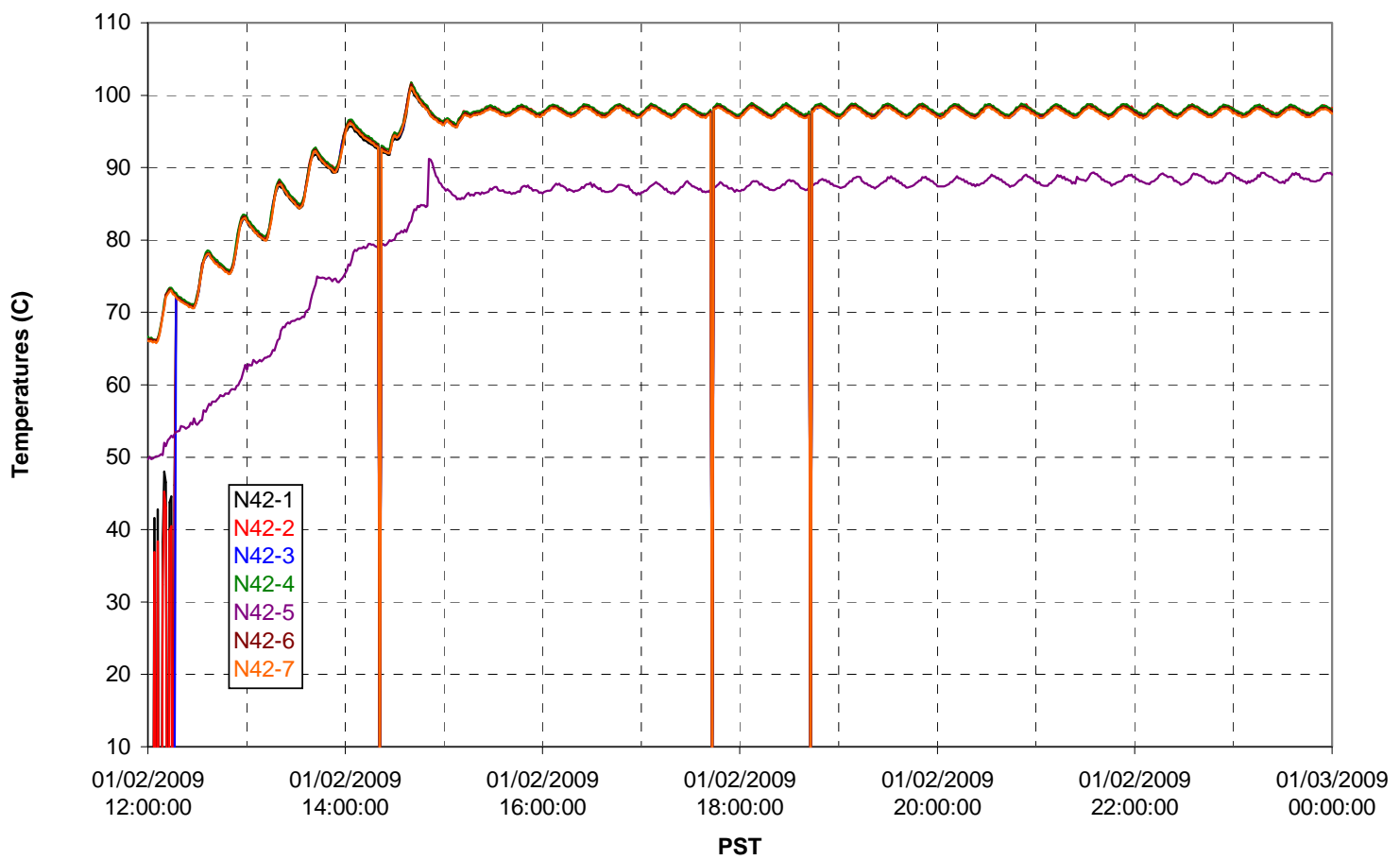

T02A temperatures

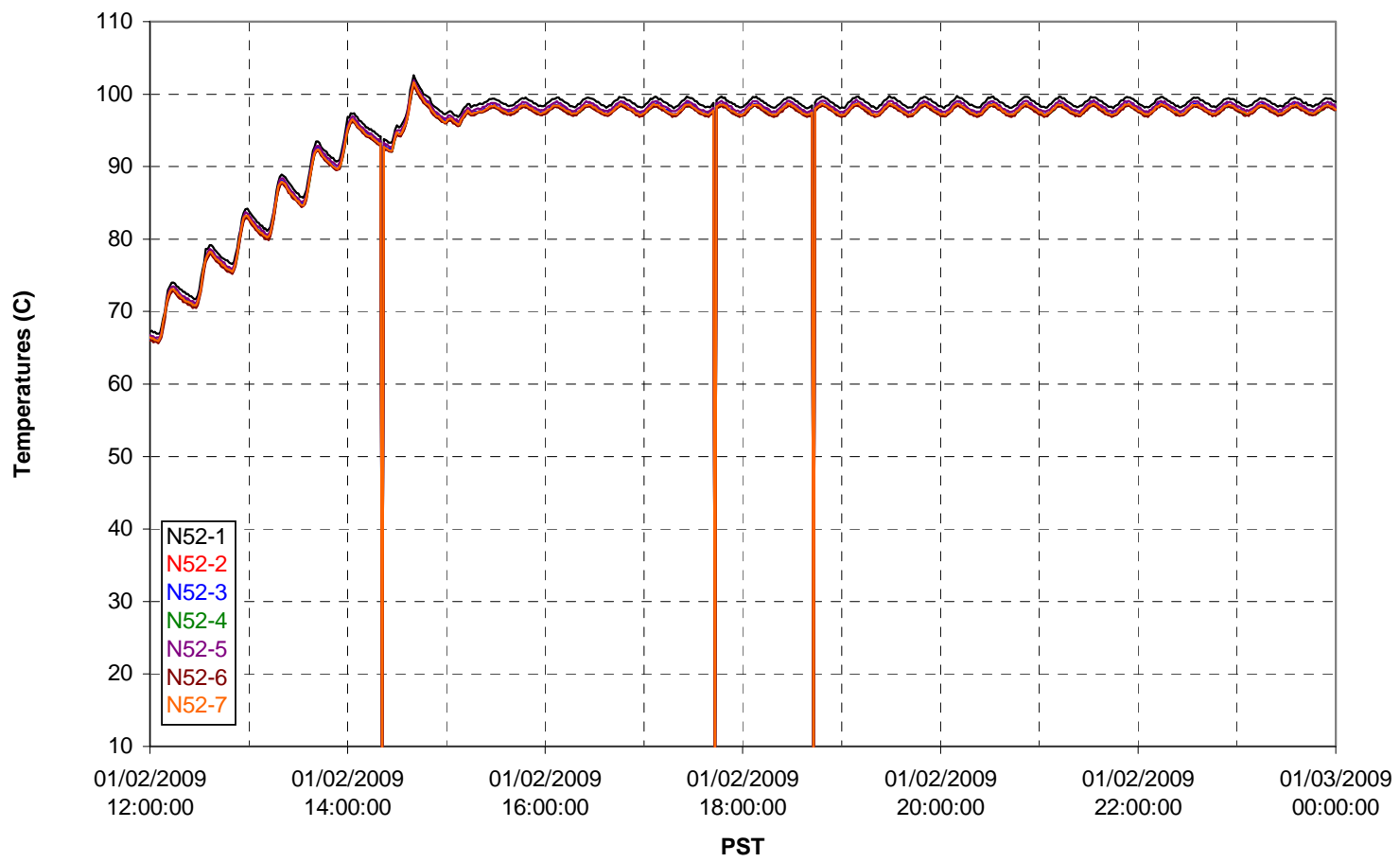


T02A Heating and Cooling

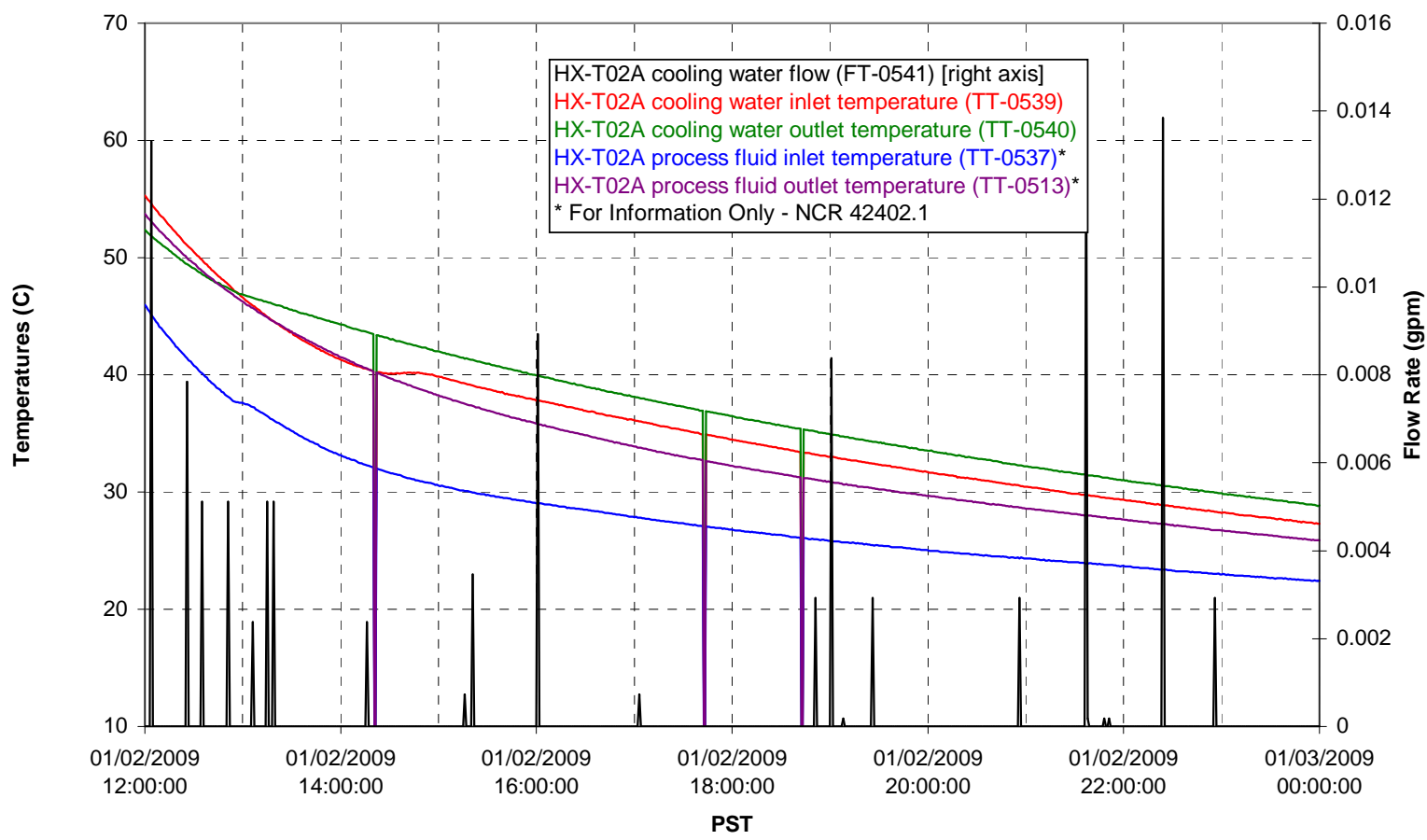

Pump Operation

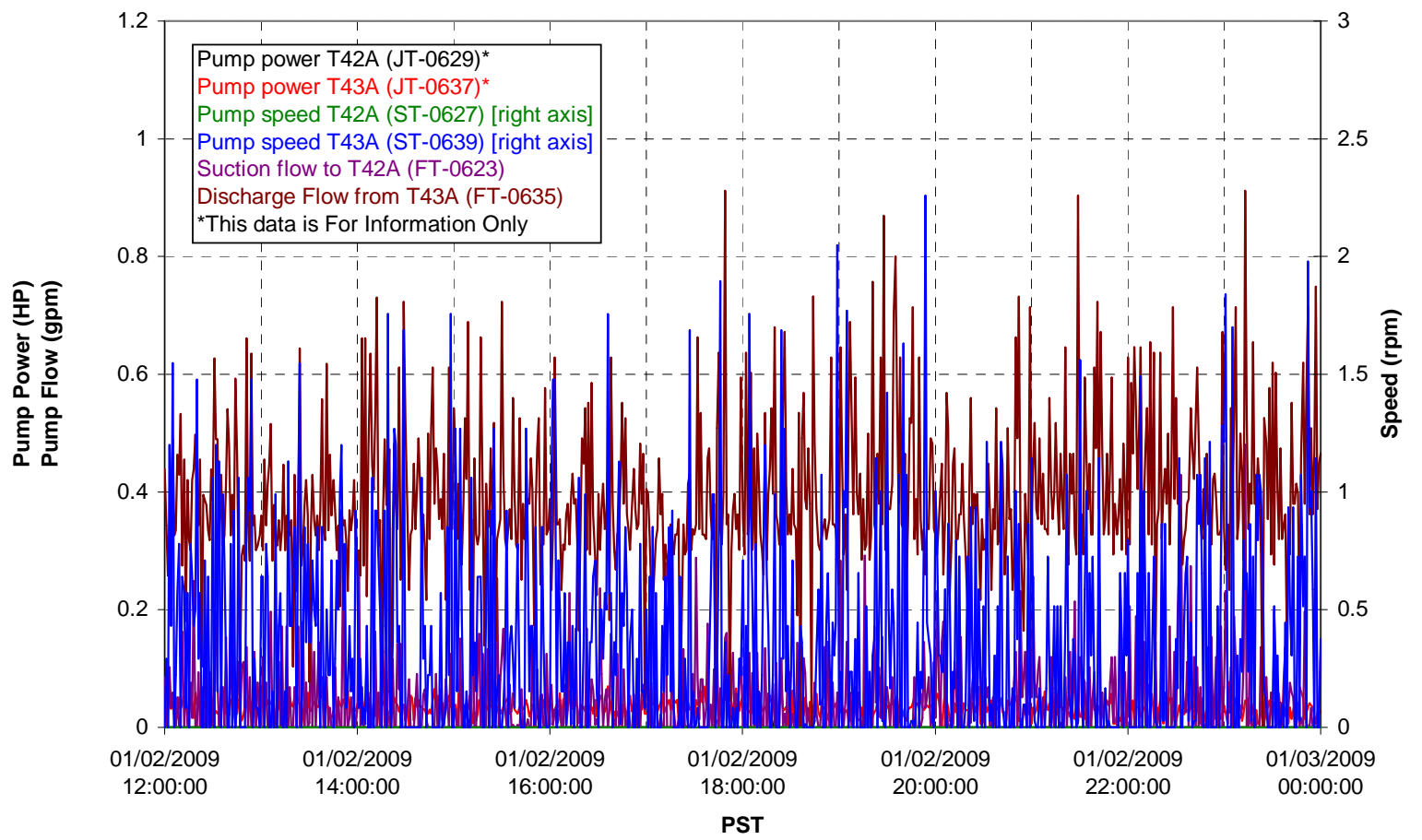


Pulsepot UFP-PP-T01A

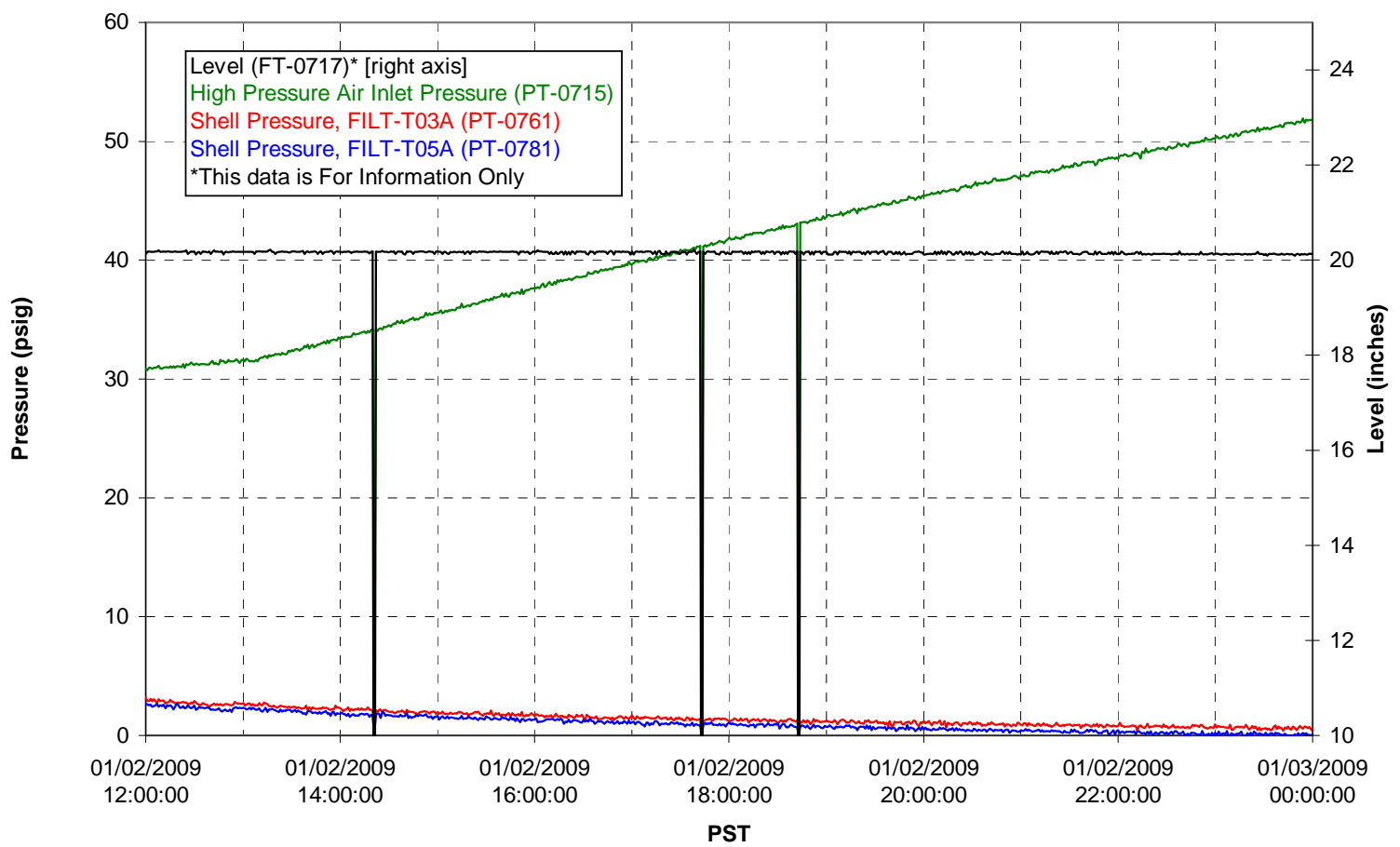

Pulsepot UFP-PP-T02A

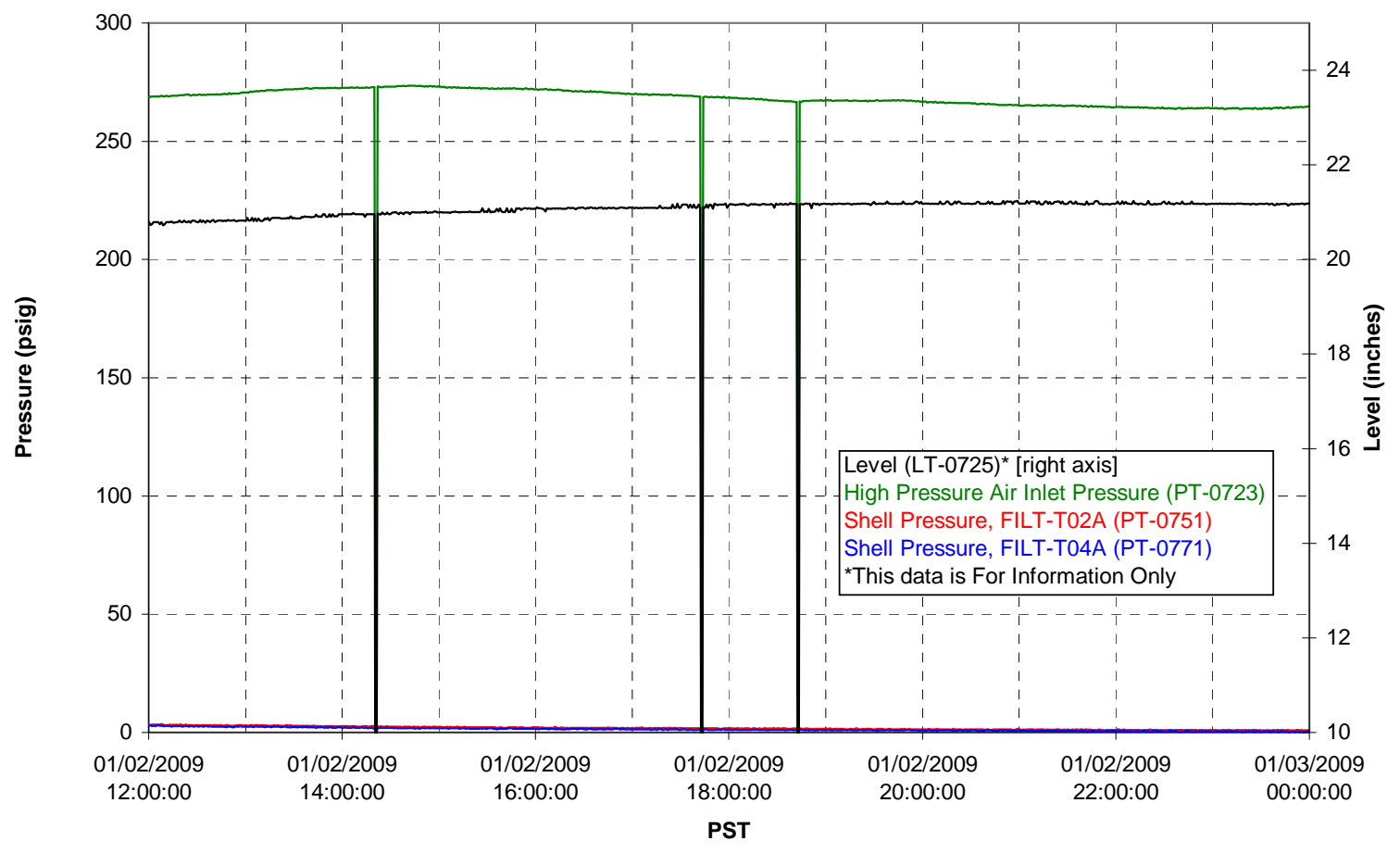


Pulsepot UFP-PP-T03A

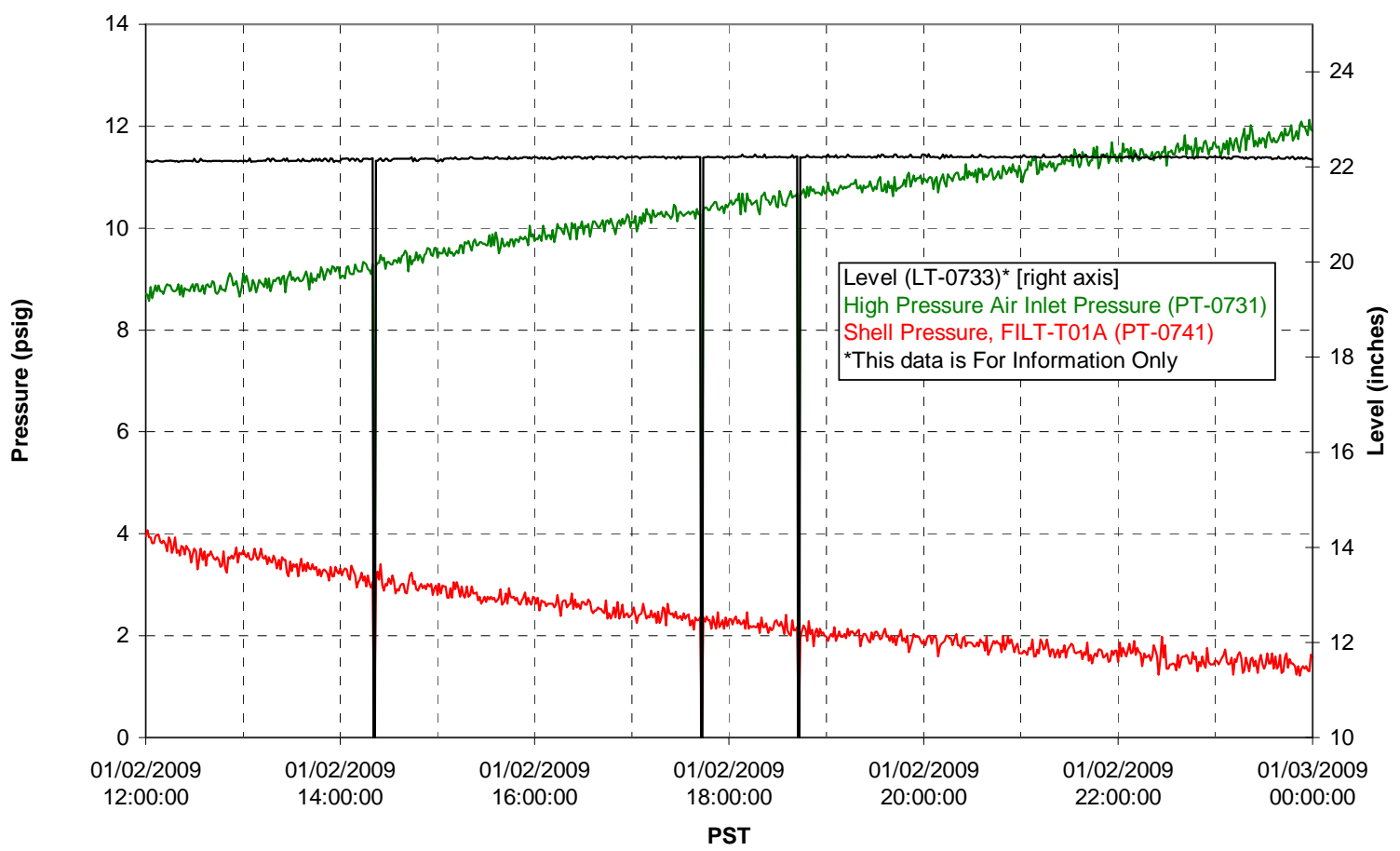

Pulsepot Levels

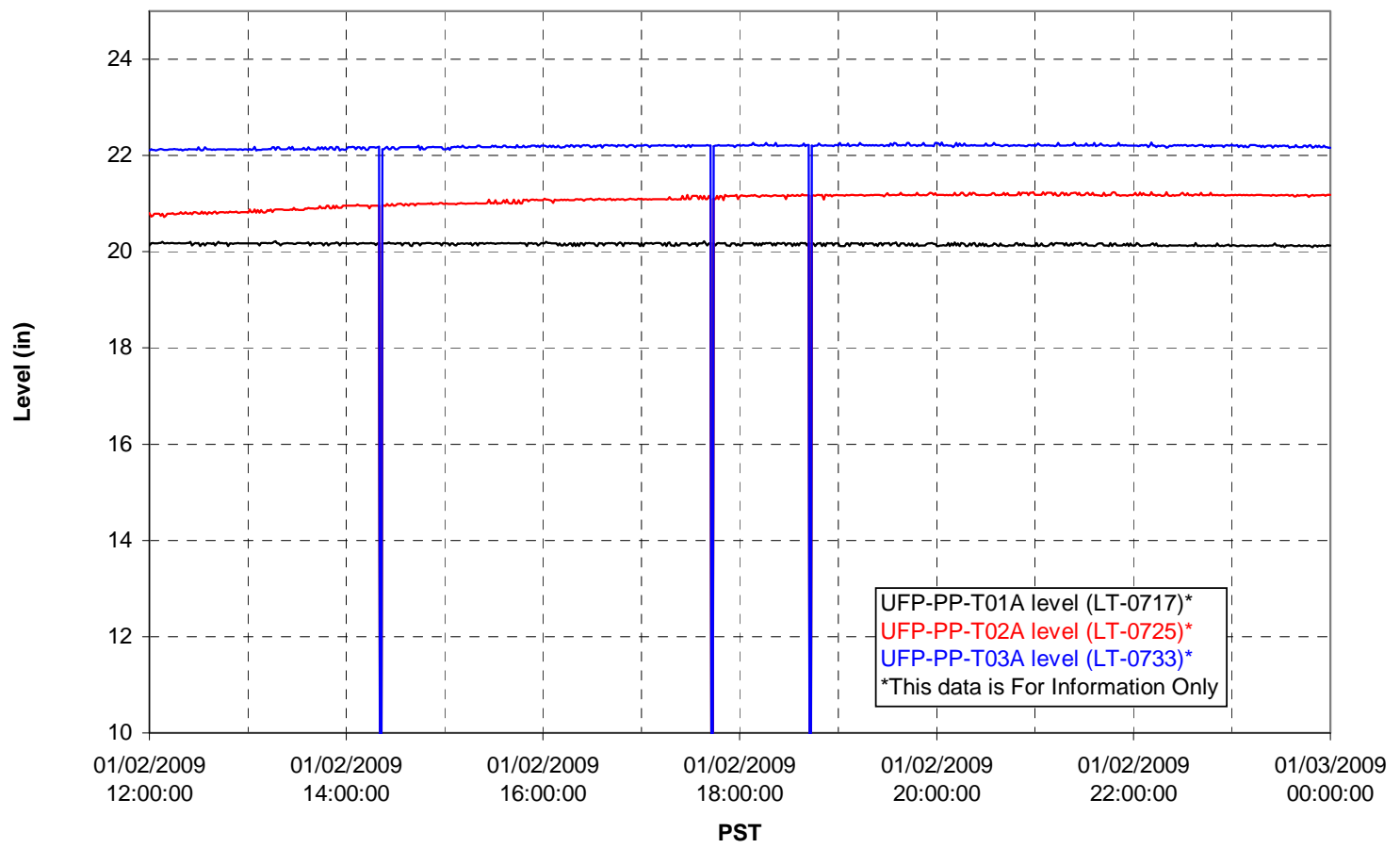


Filter UFP-FILT-T01A

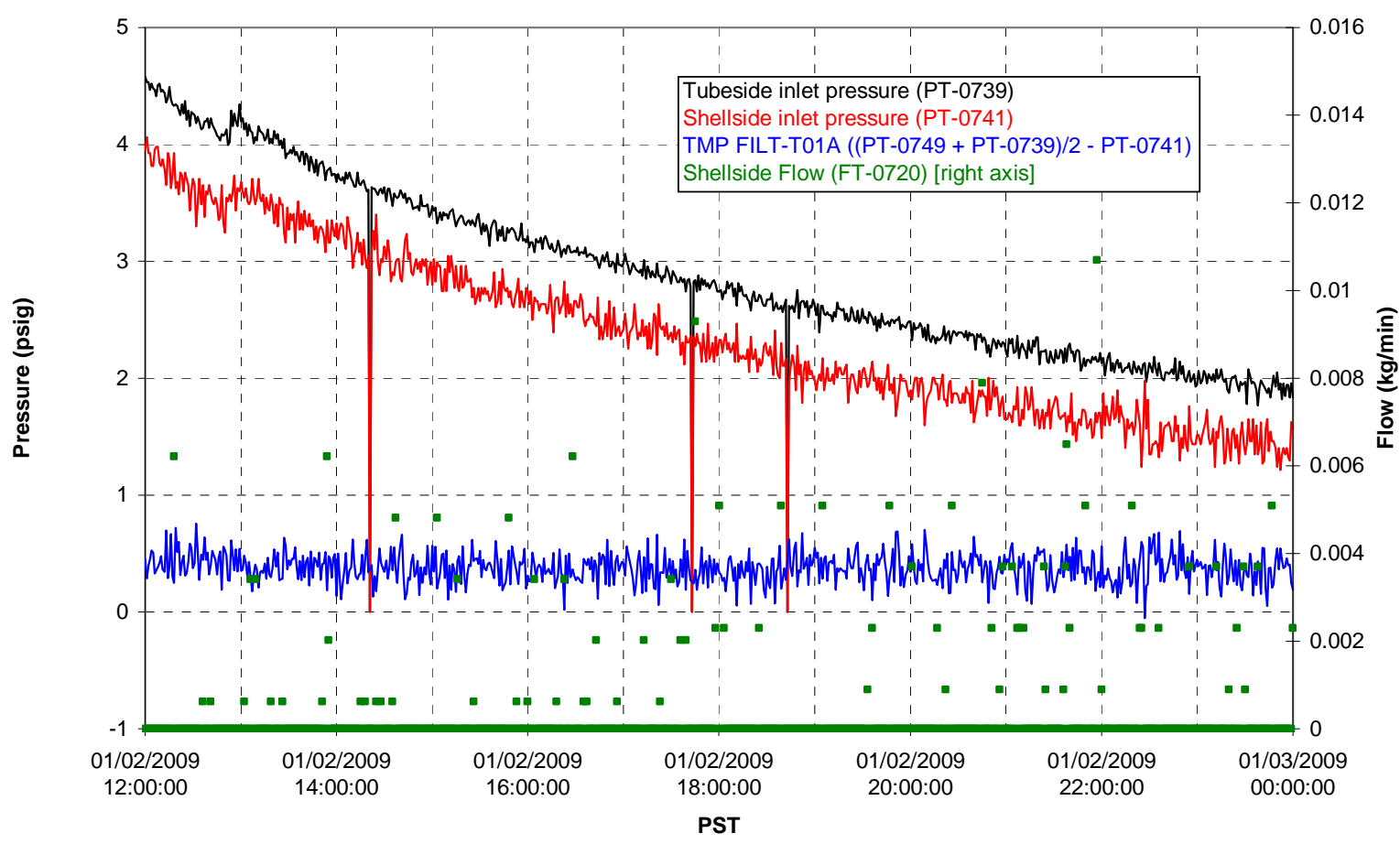

Filter UFP-FILT-T02A

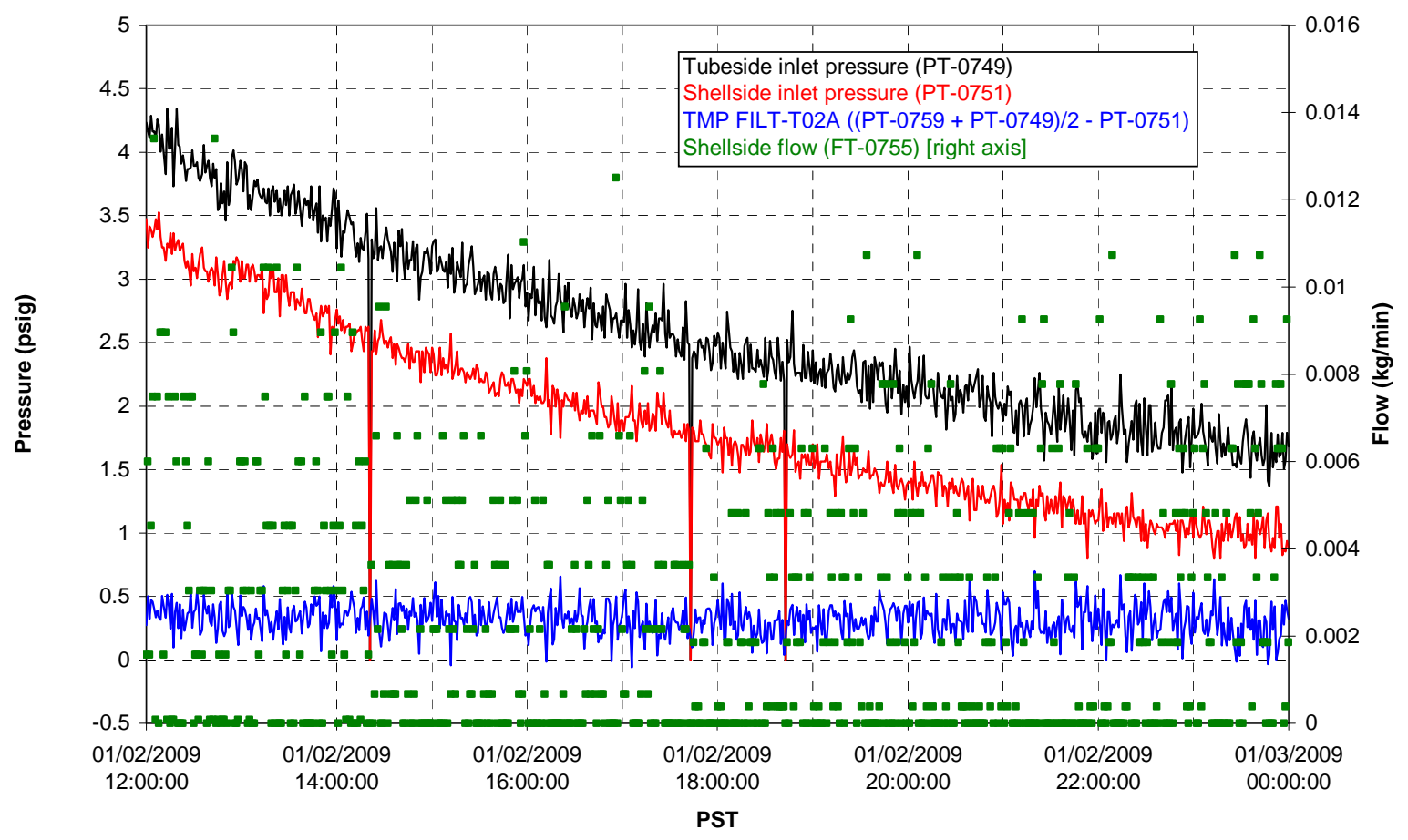


Filter UFP-FILT-T03A

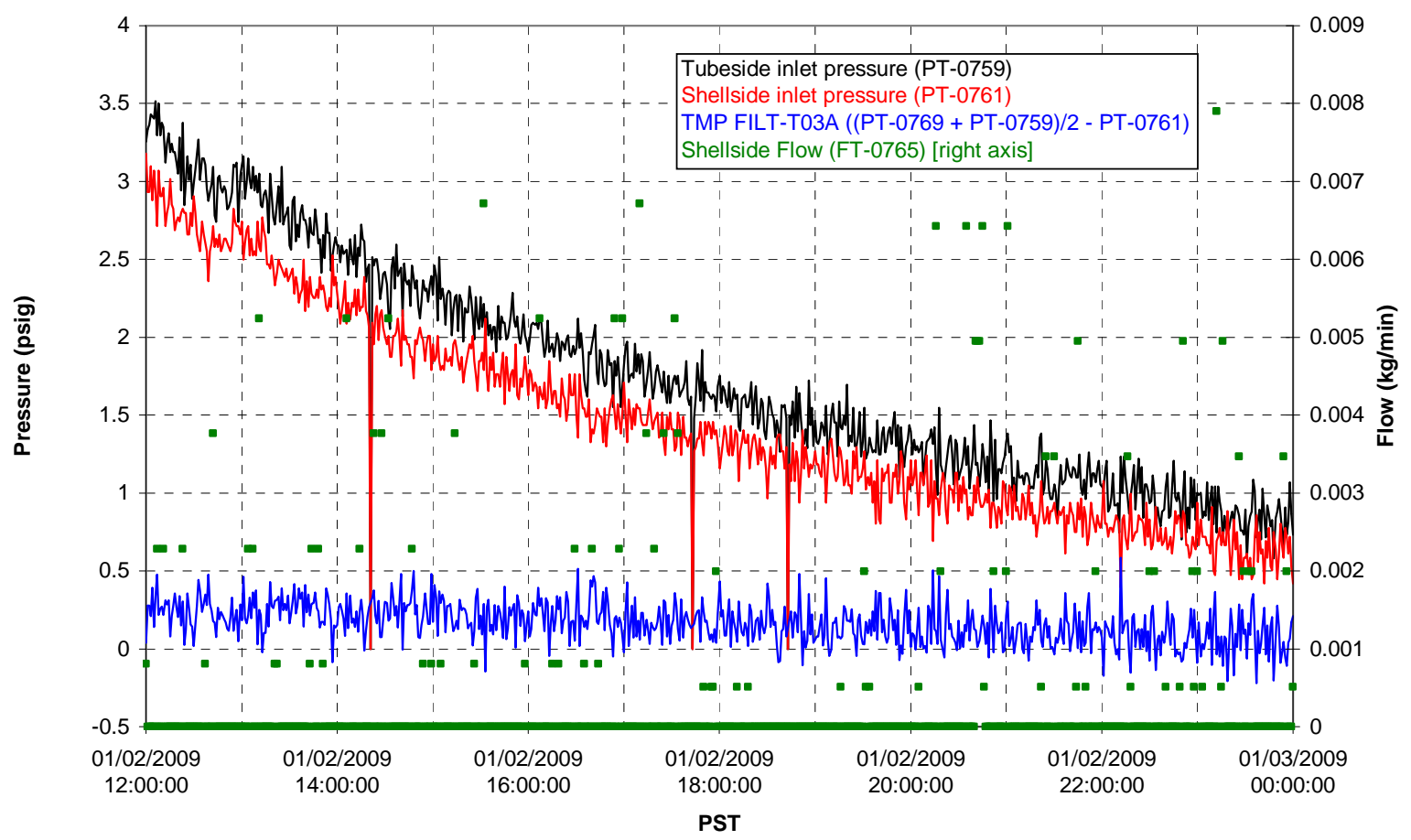

Filter UFP-FILT-T04A

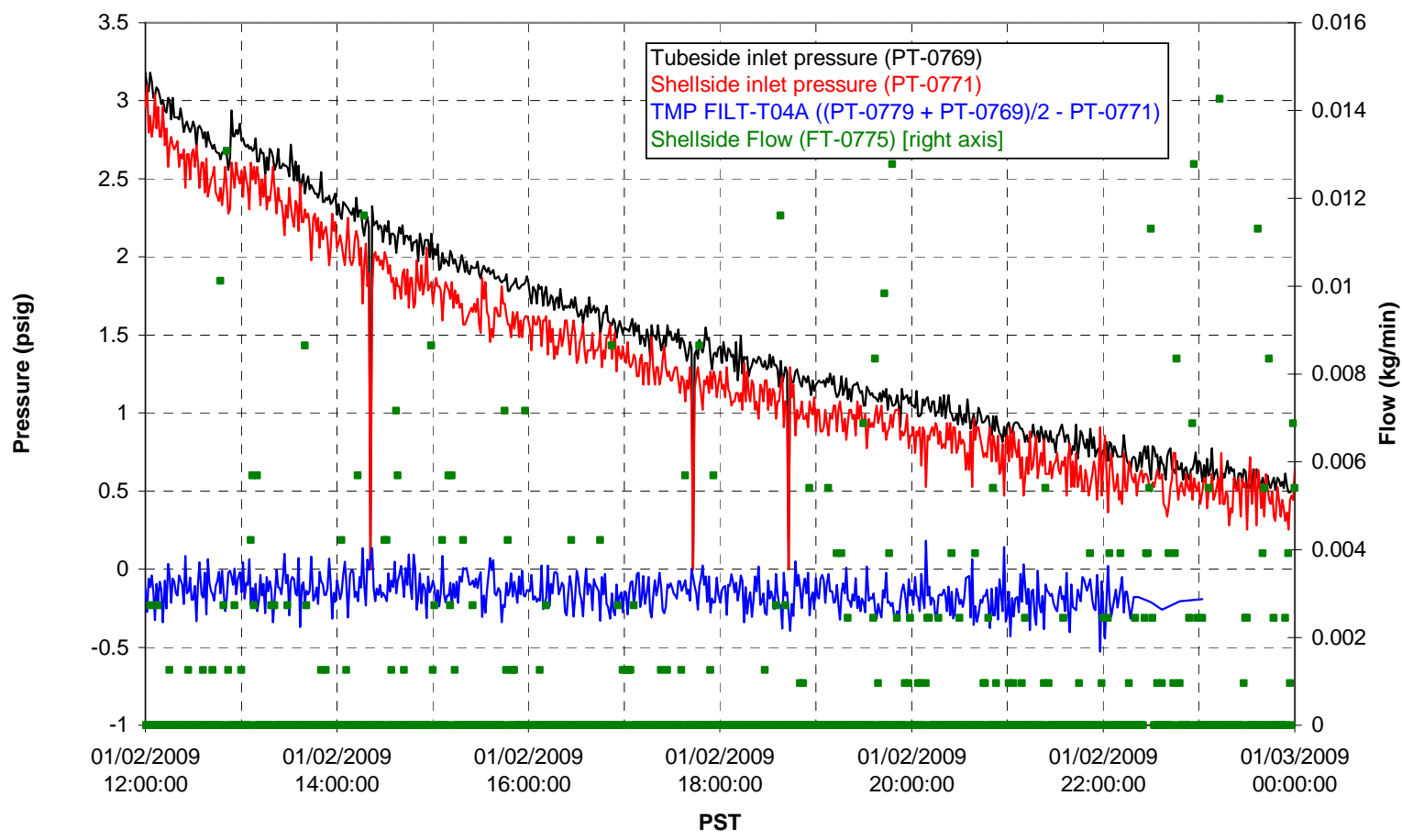


Filter UFP-FILT-T05A

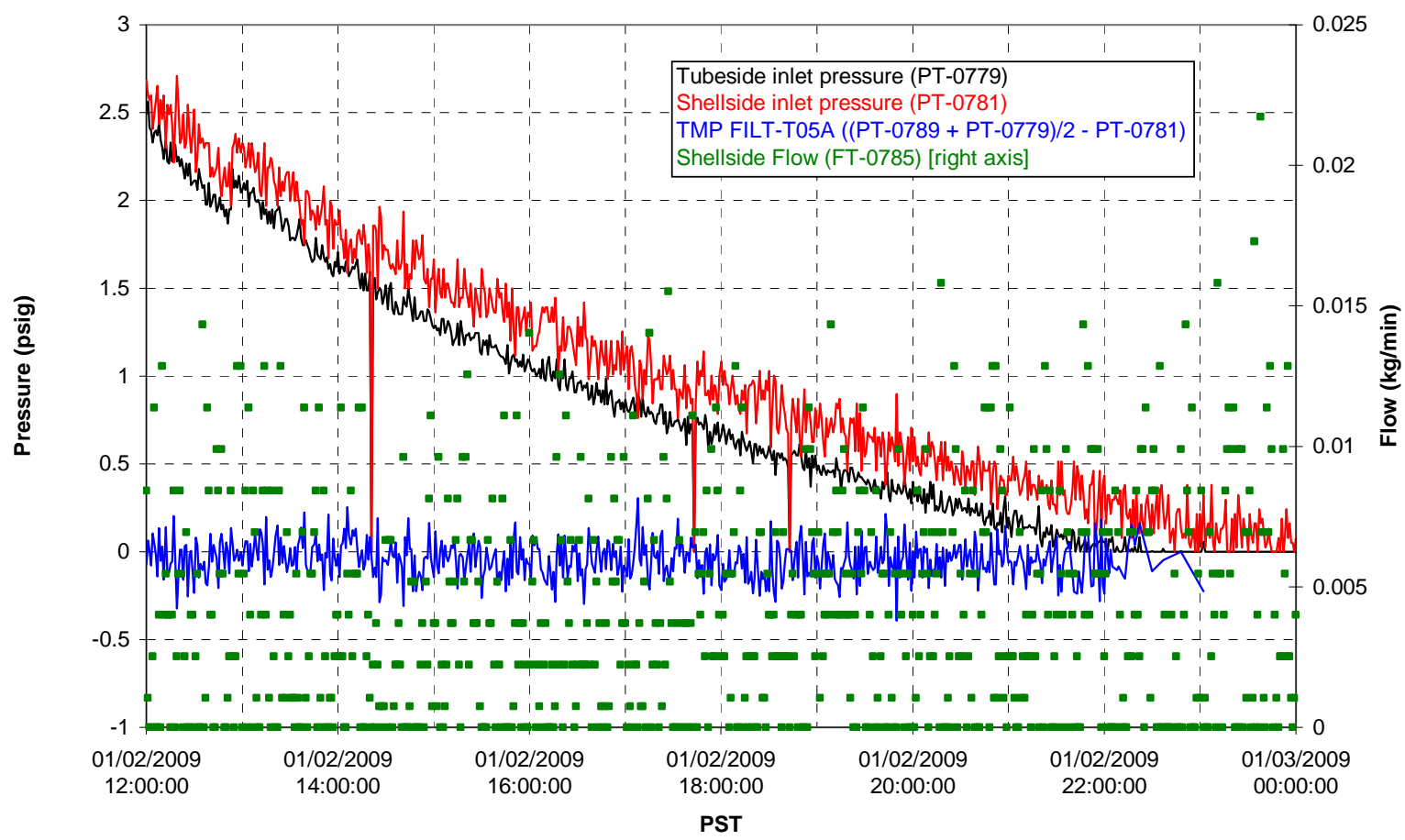

Chemical Flow

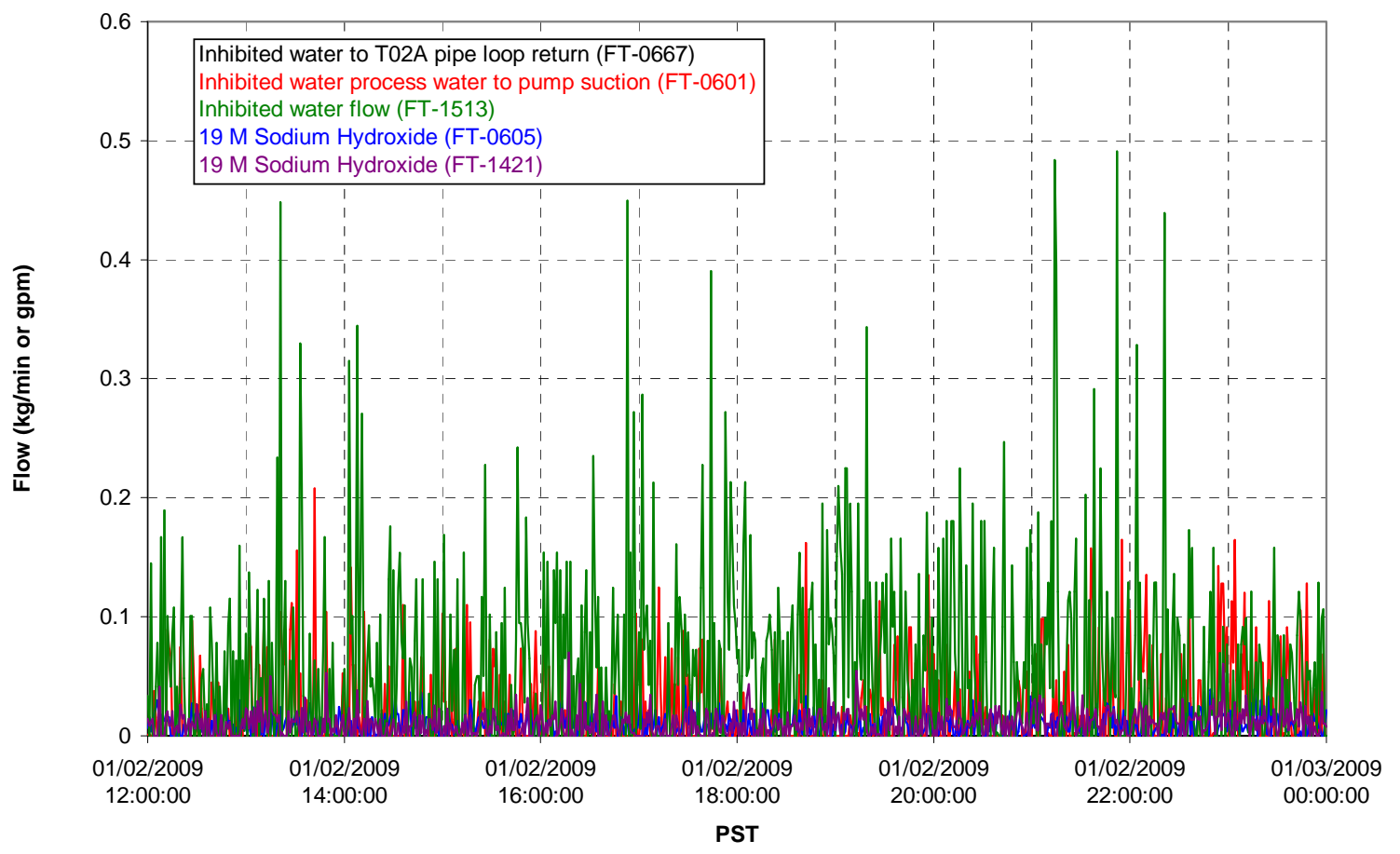


Chemical Flow

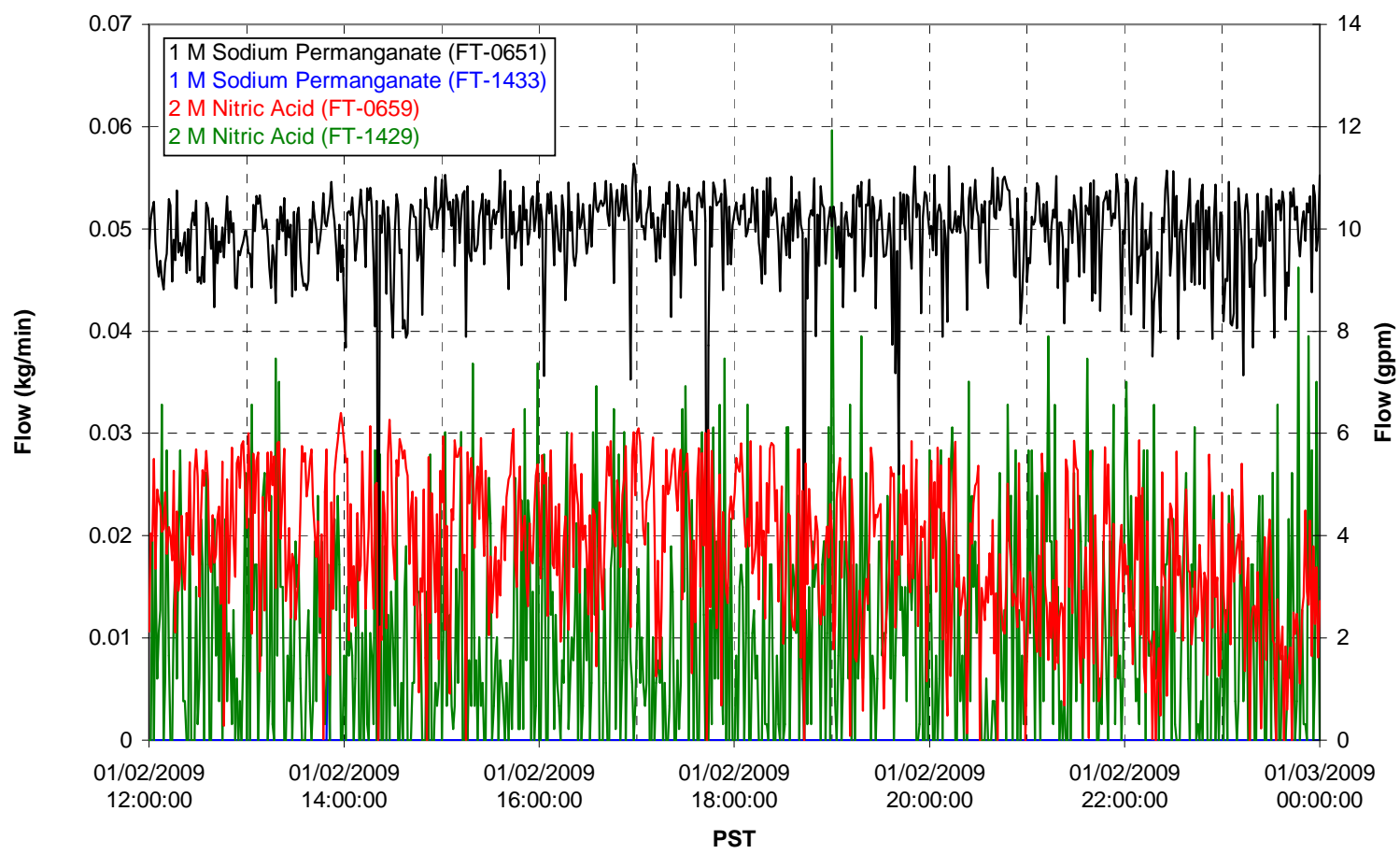

Air Flows

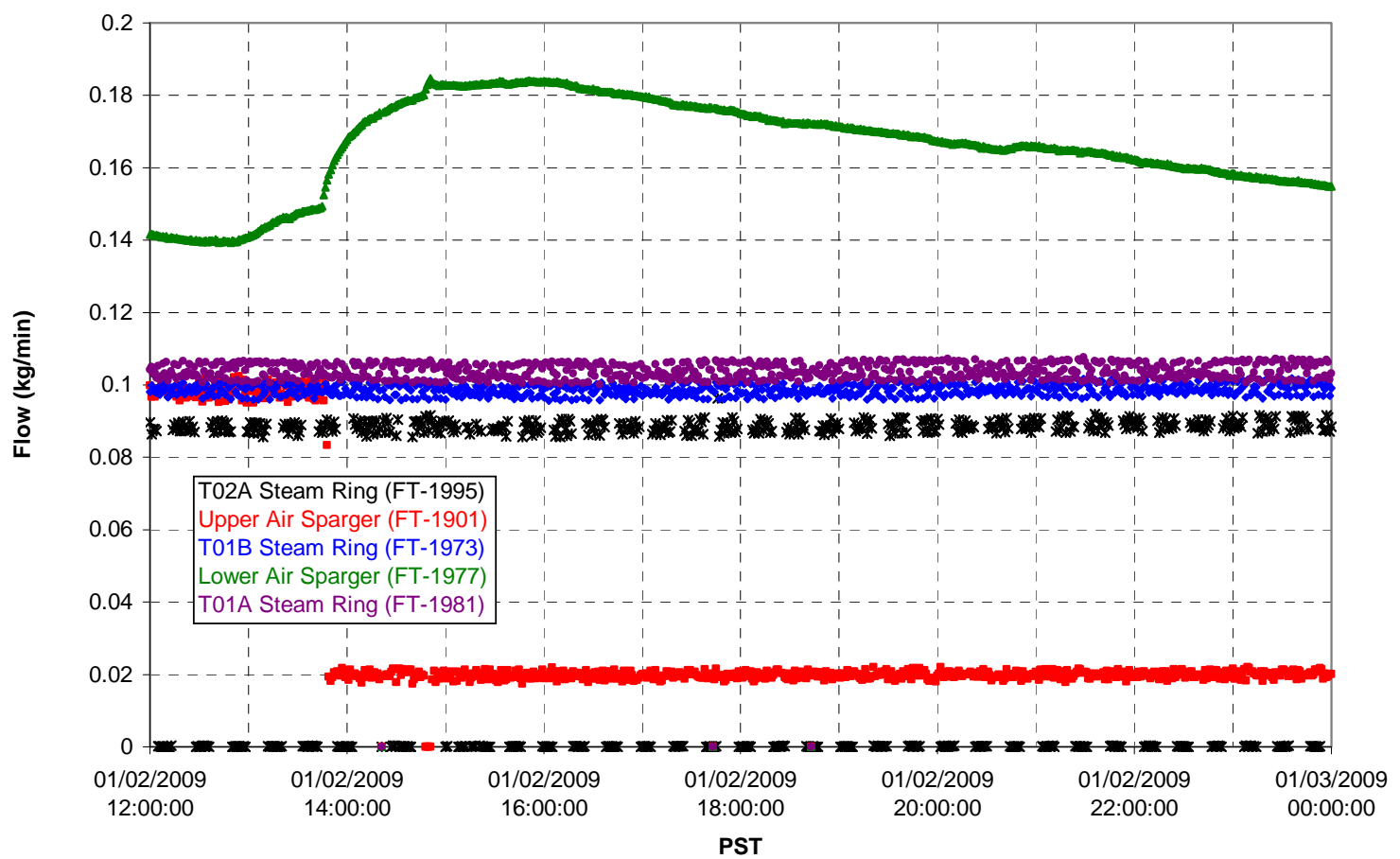


T02A Steam

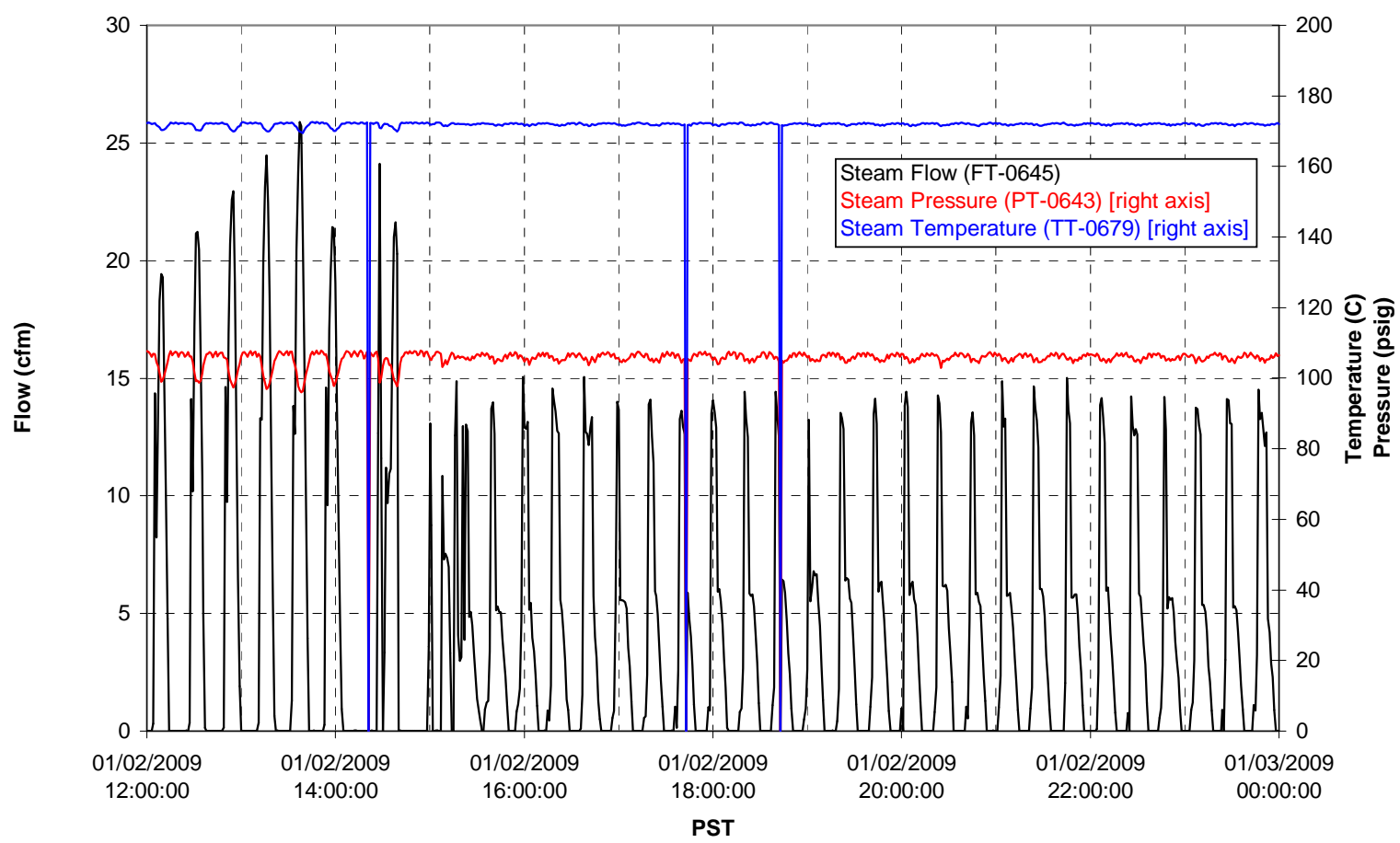

T01A Steam

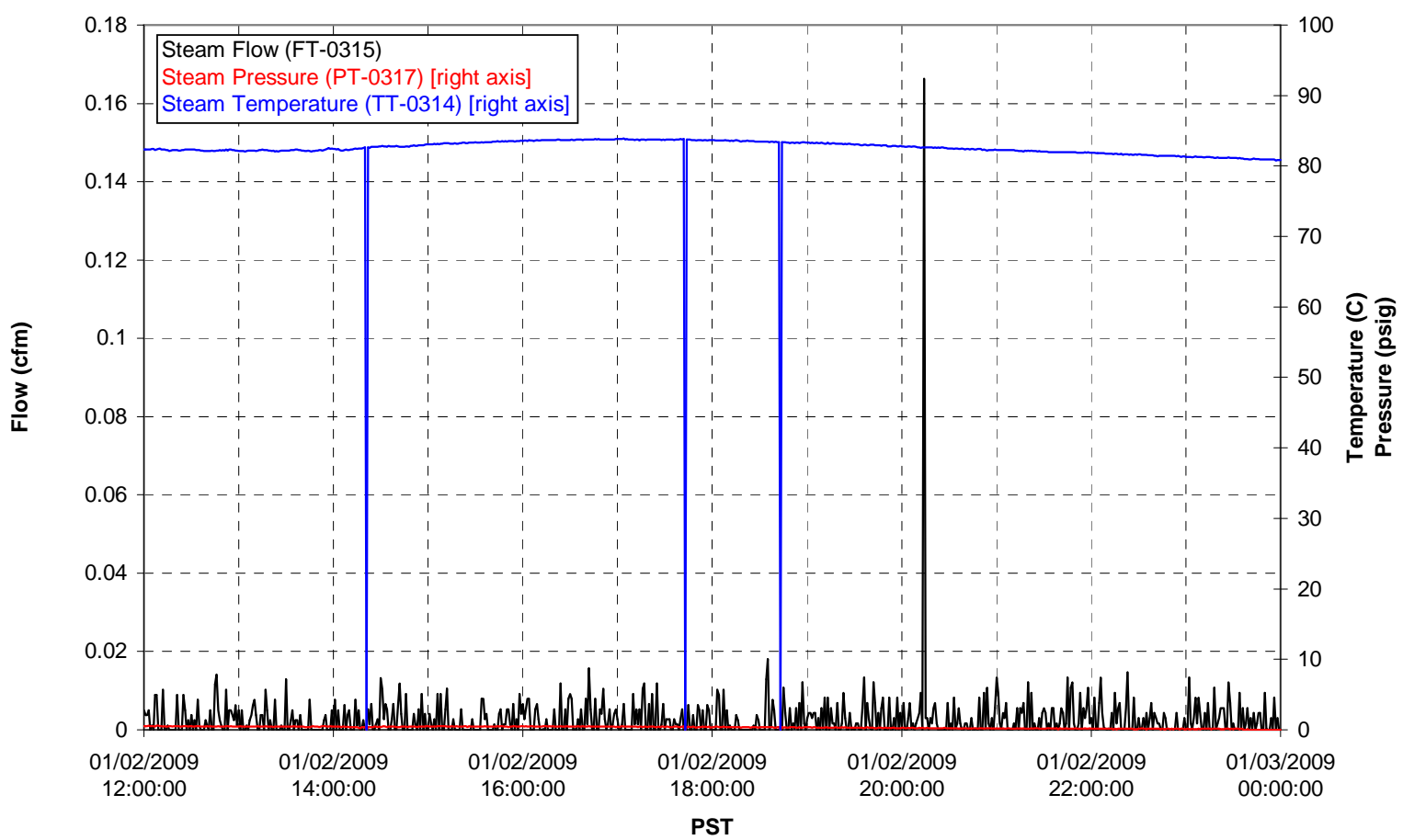


T01B Steam

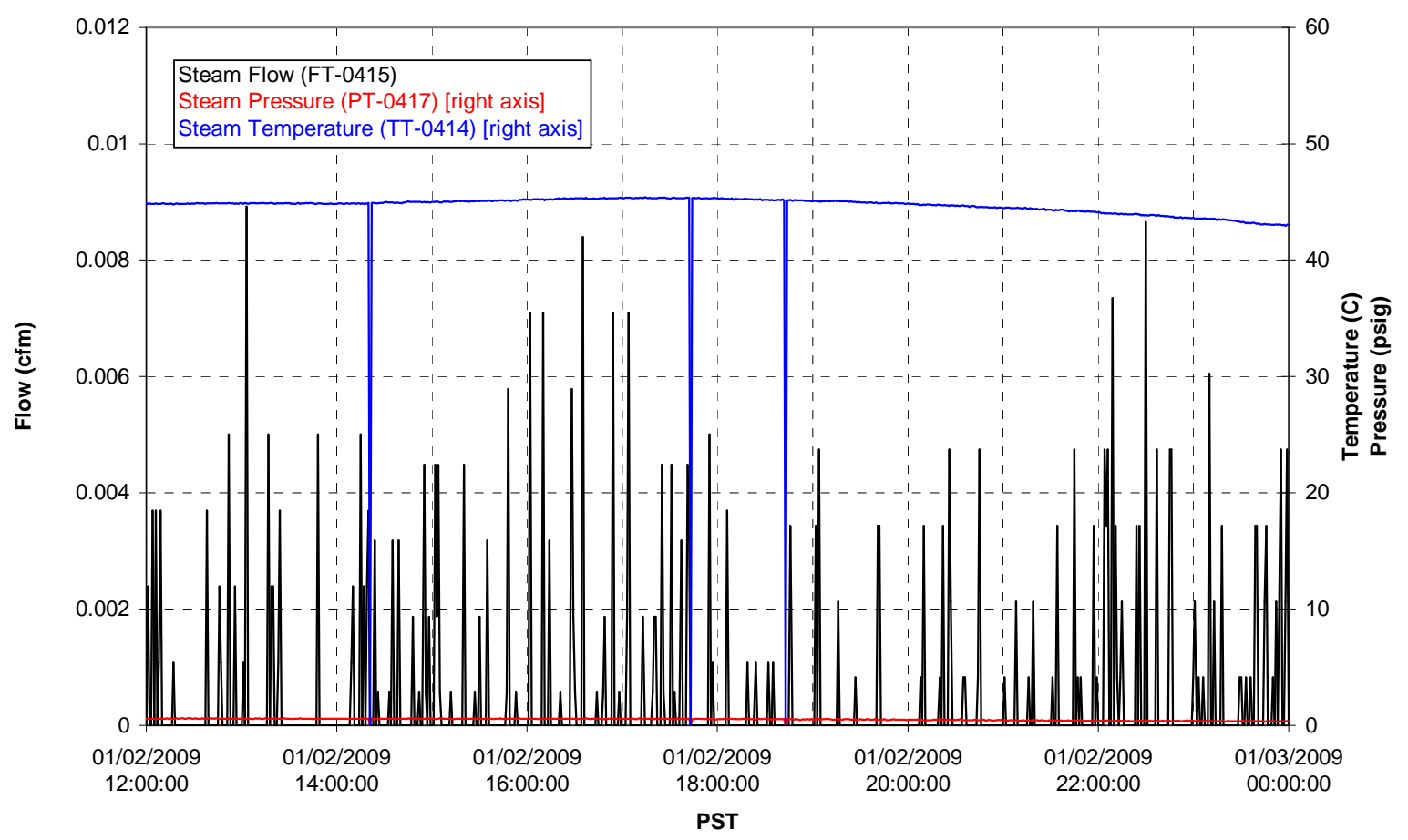


Functional Test Data Plots

01/03/2009 00:00 - 01/03/2009 12:00 
T01A level

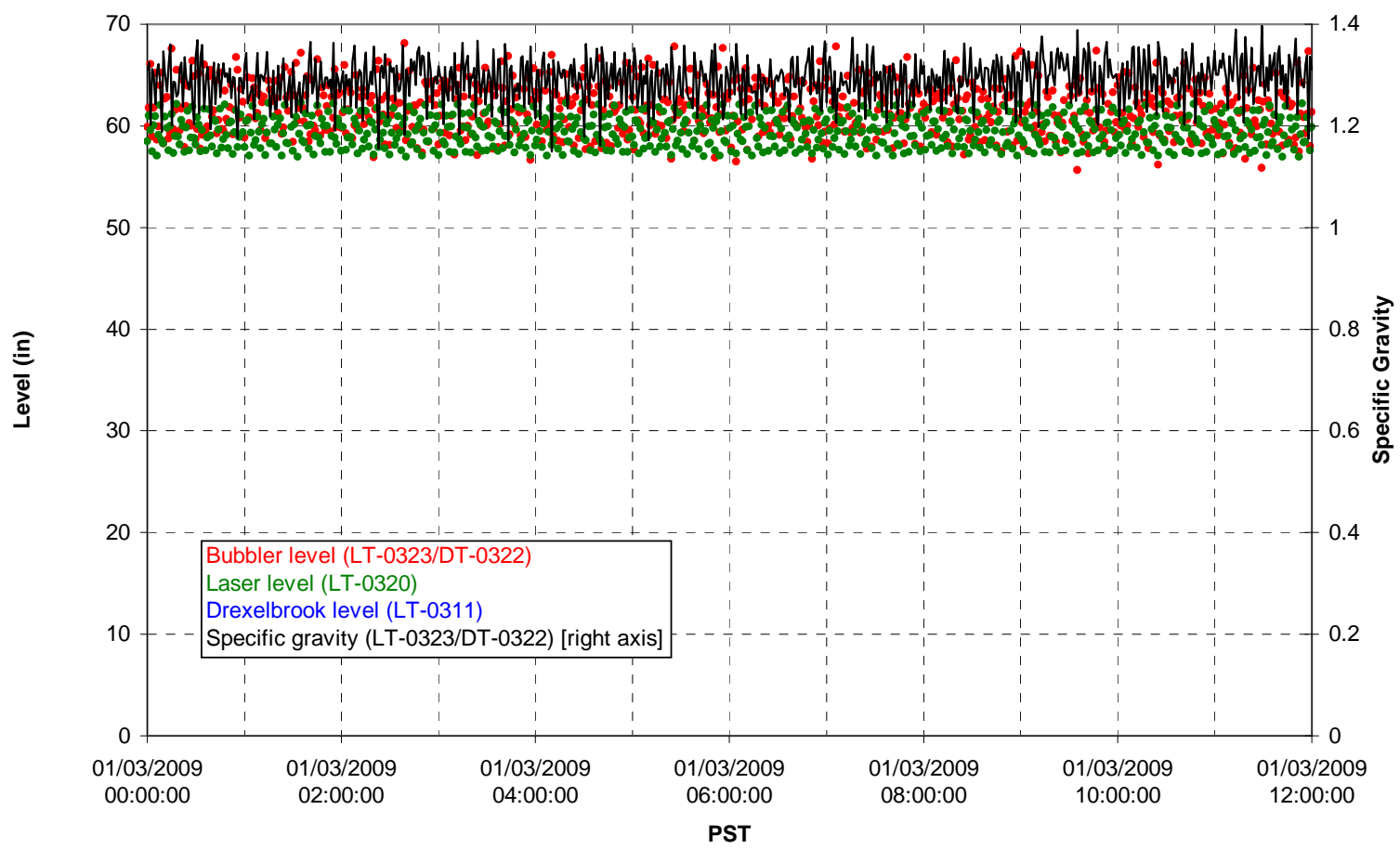

T01A temperatures

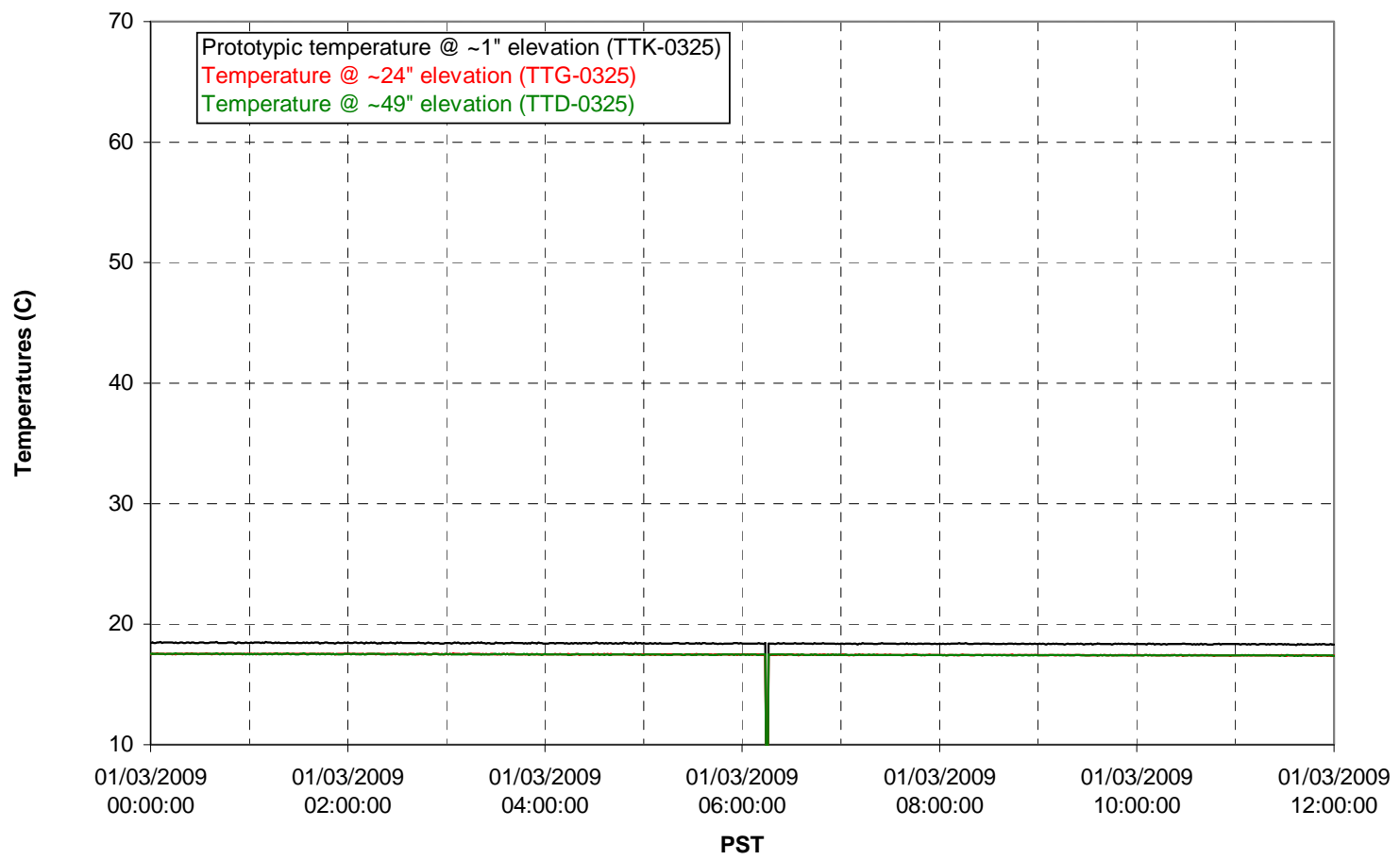


T01B level

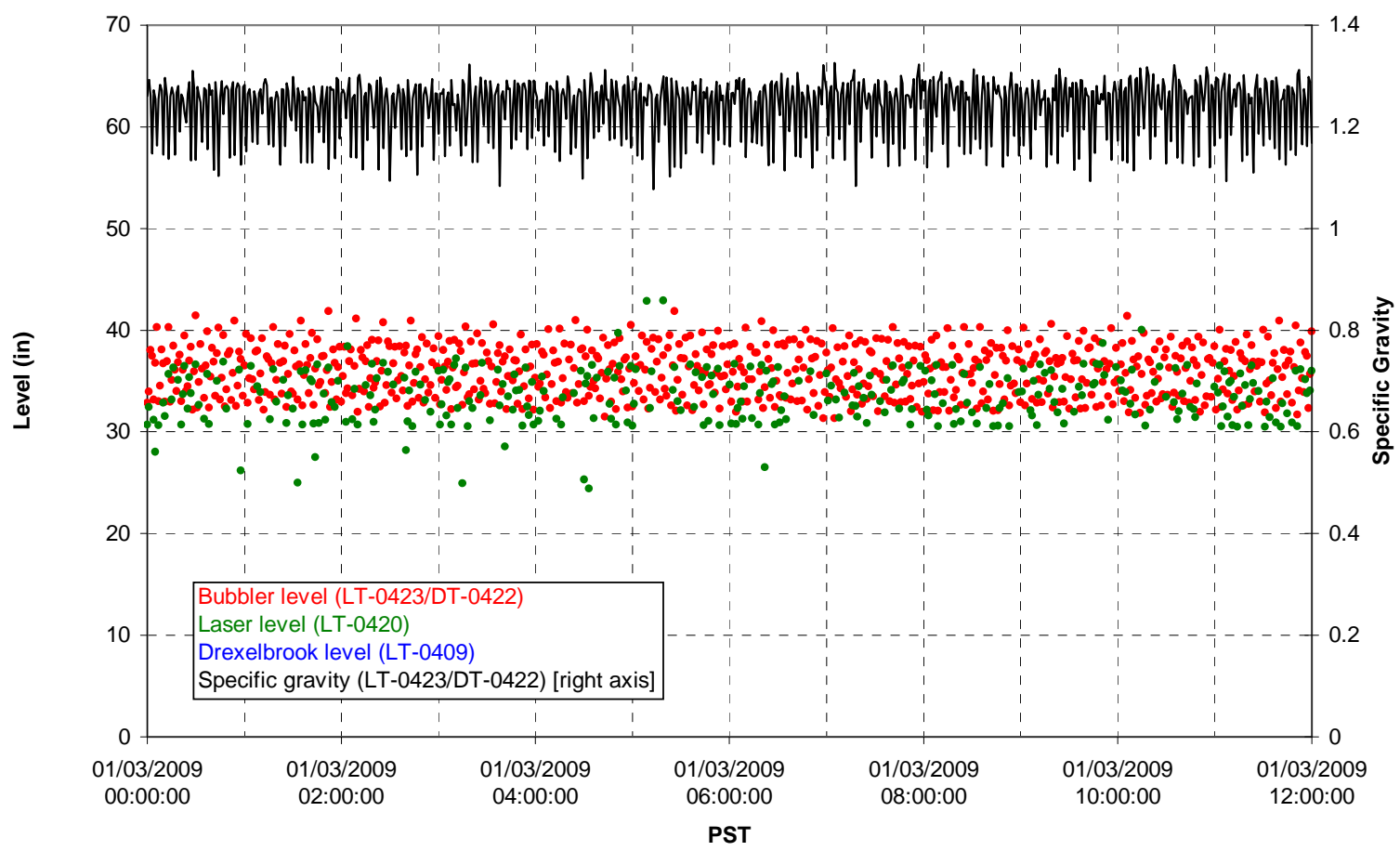

T01B temperatures

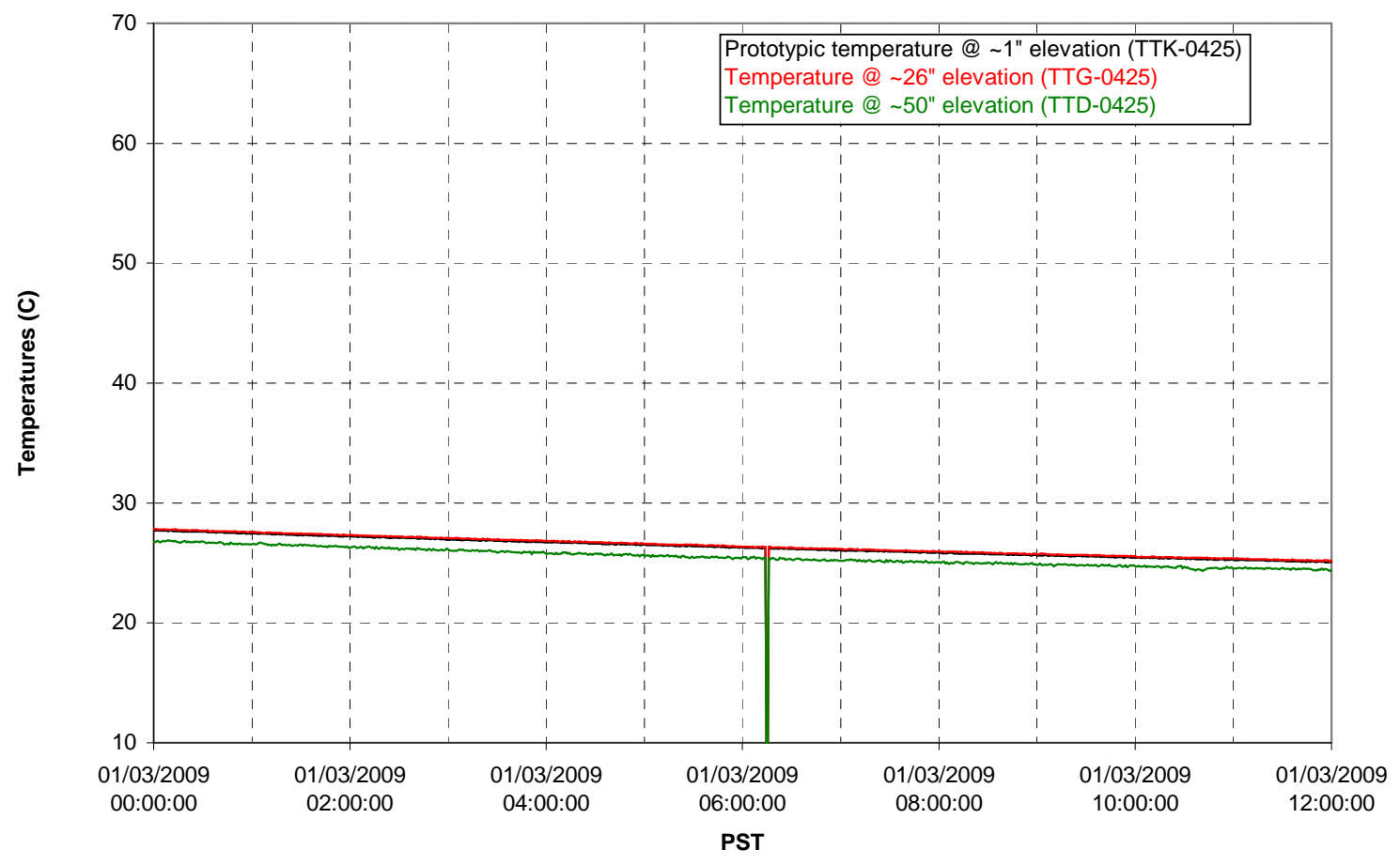


T02A level

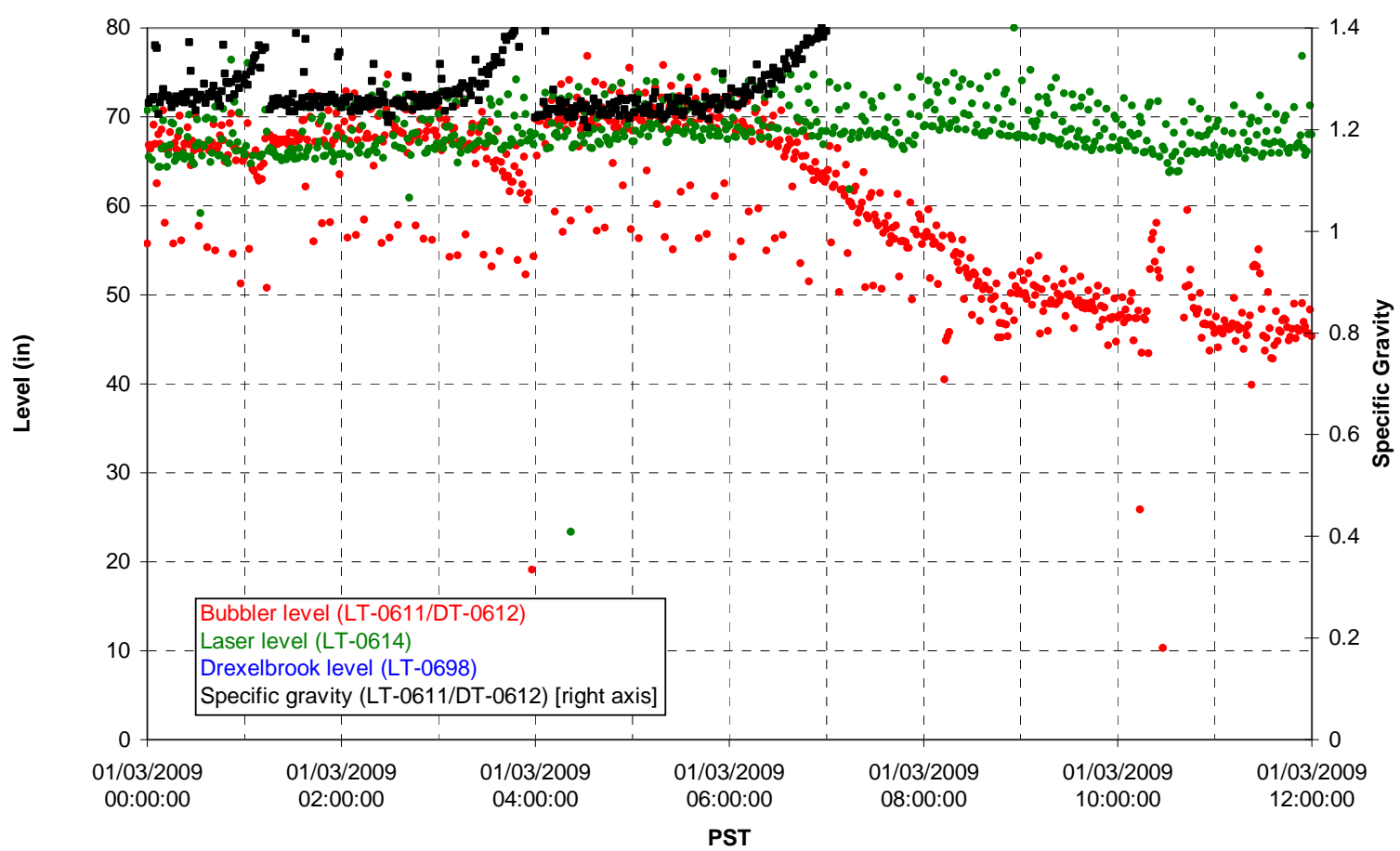

T02A temperatures

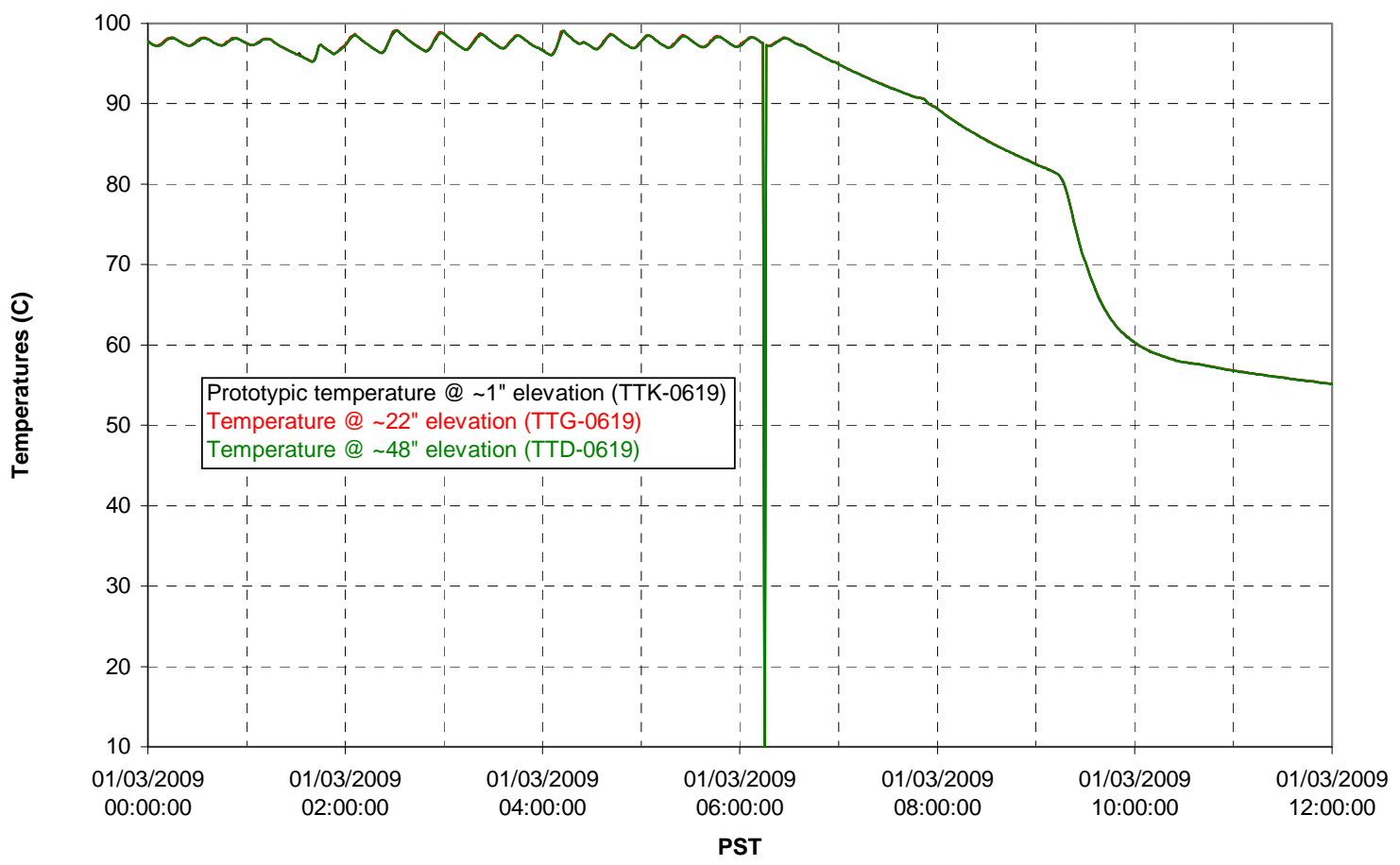


T02A and filter loop temperatures

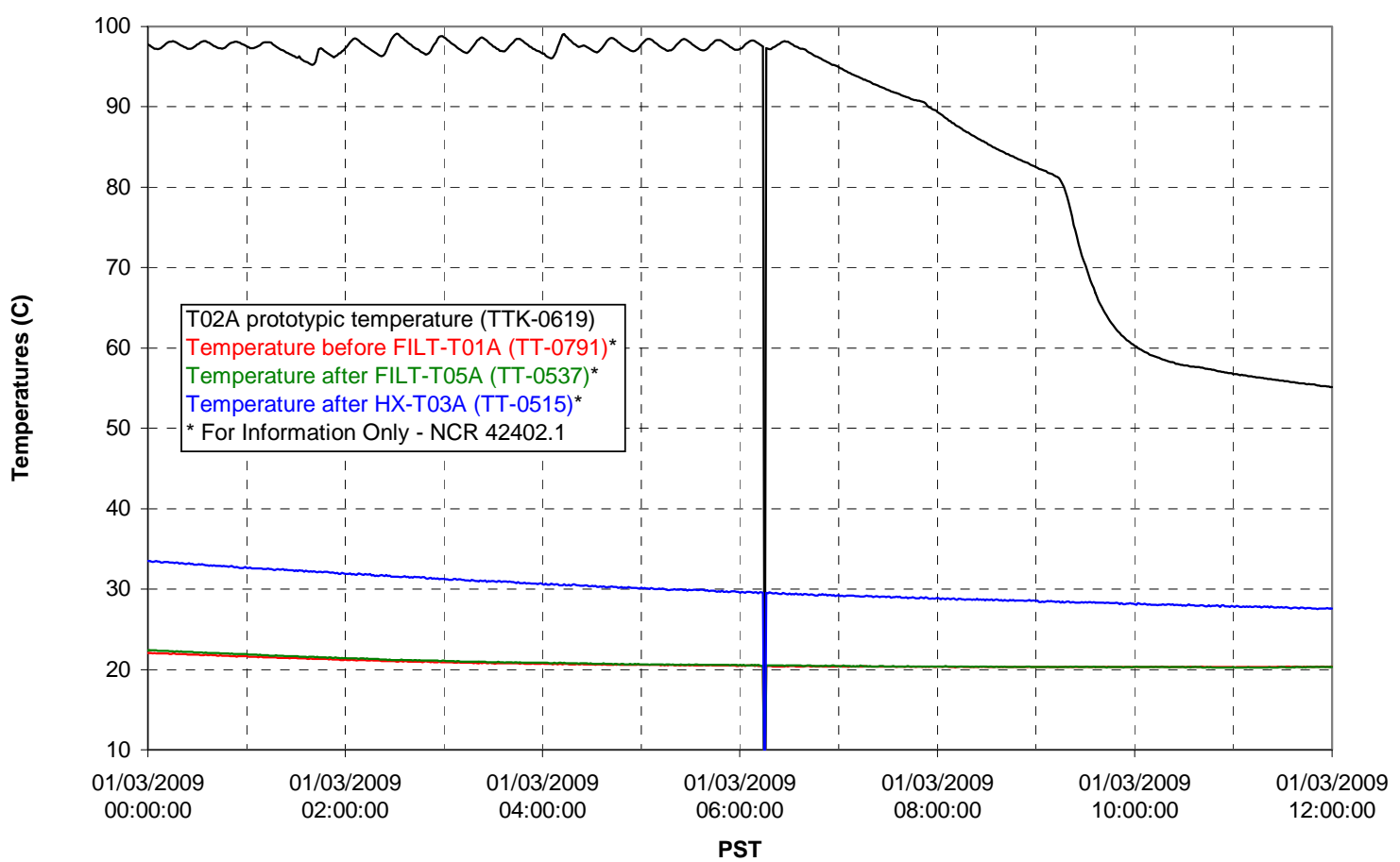

Pump Pressures and Flow

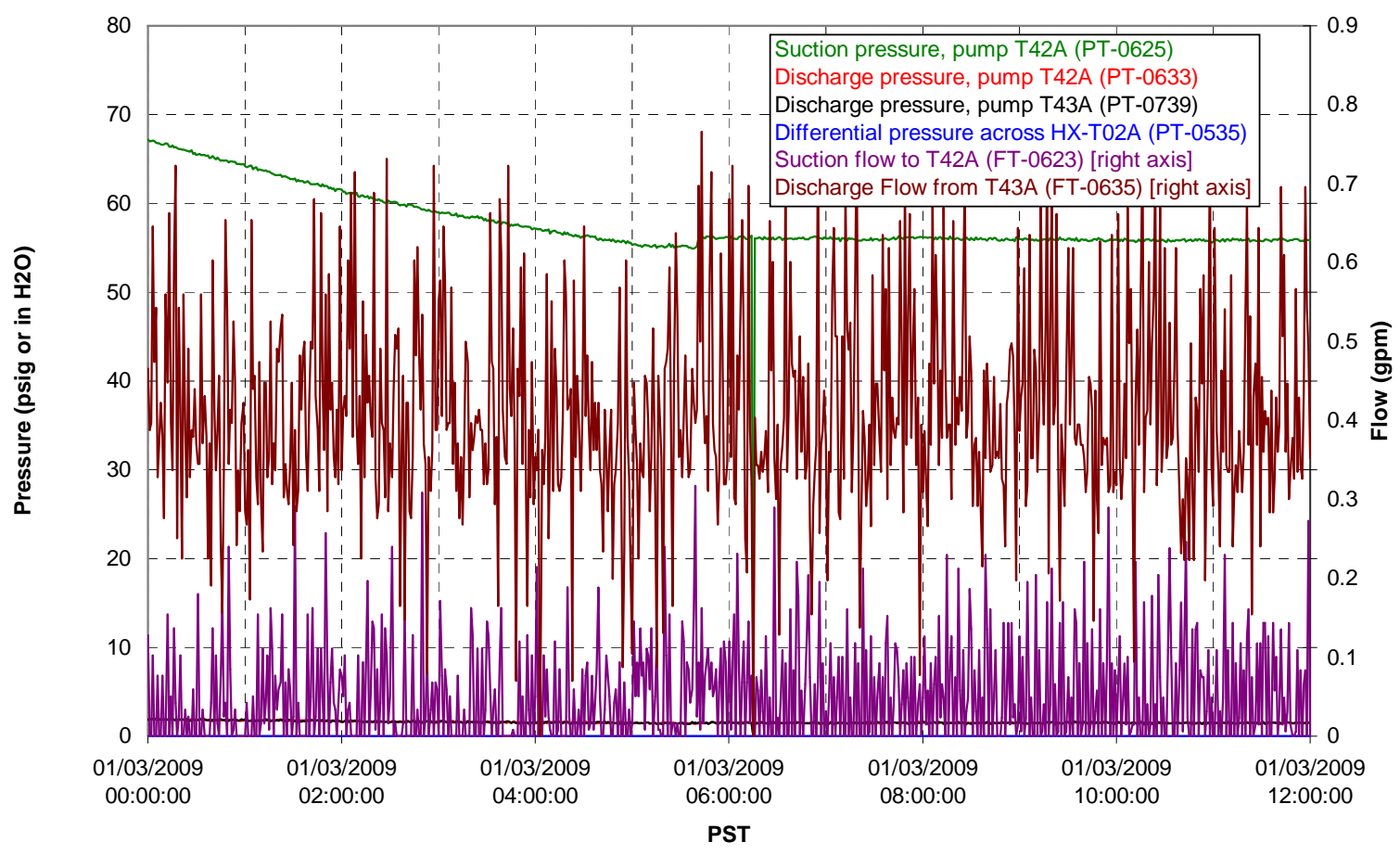


Axial pressure drop

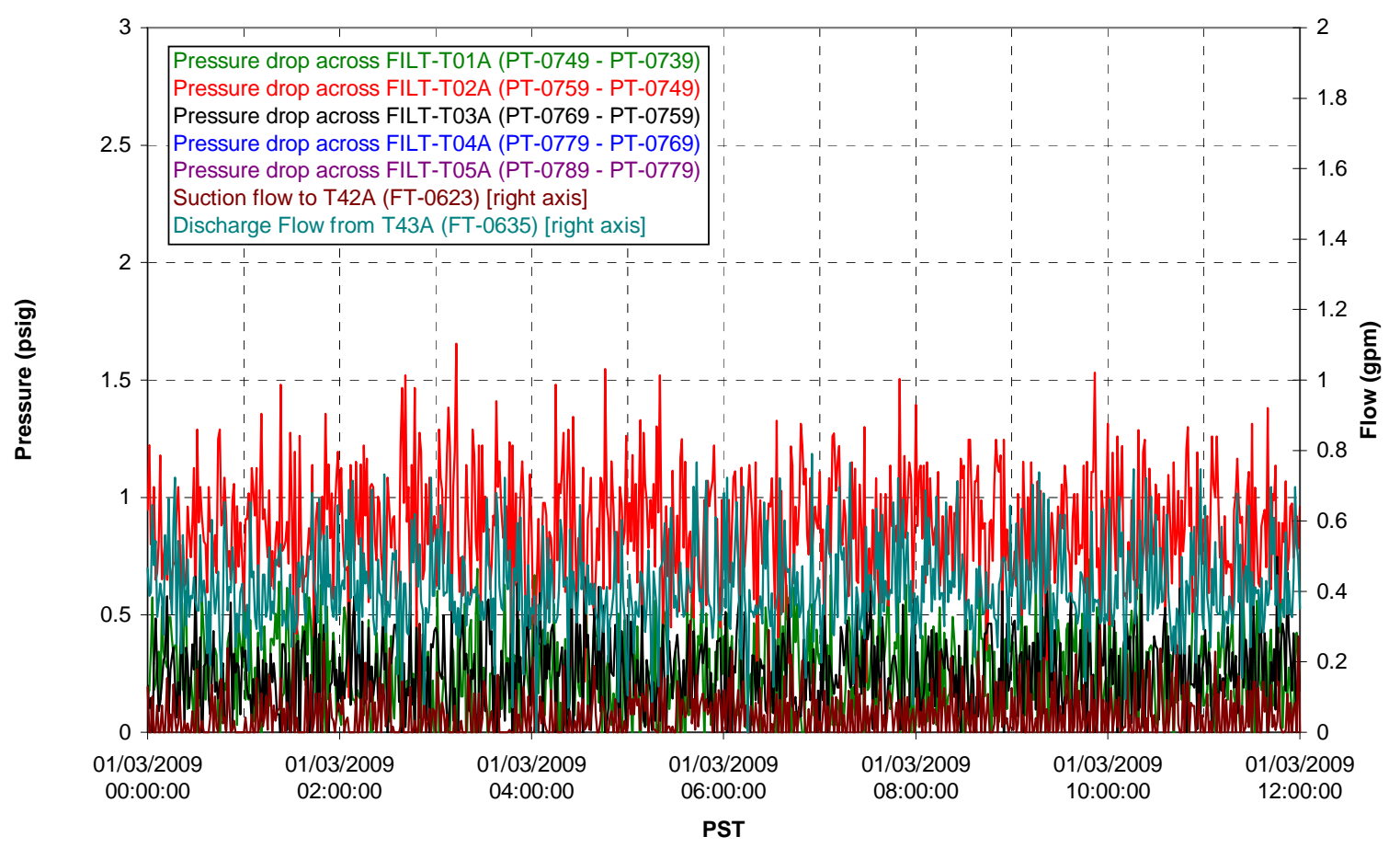

Permeate flow rates

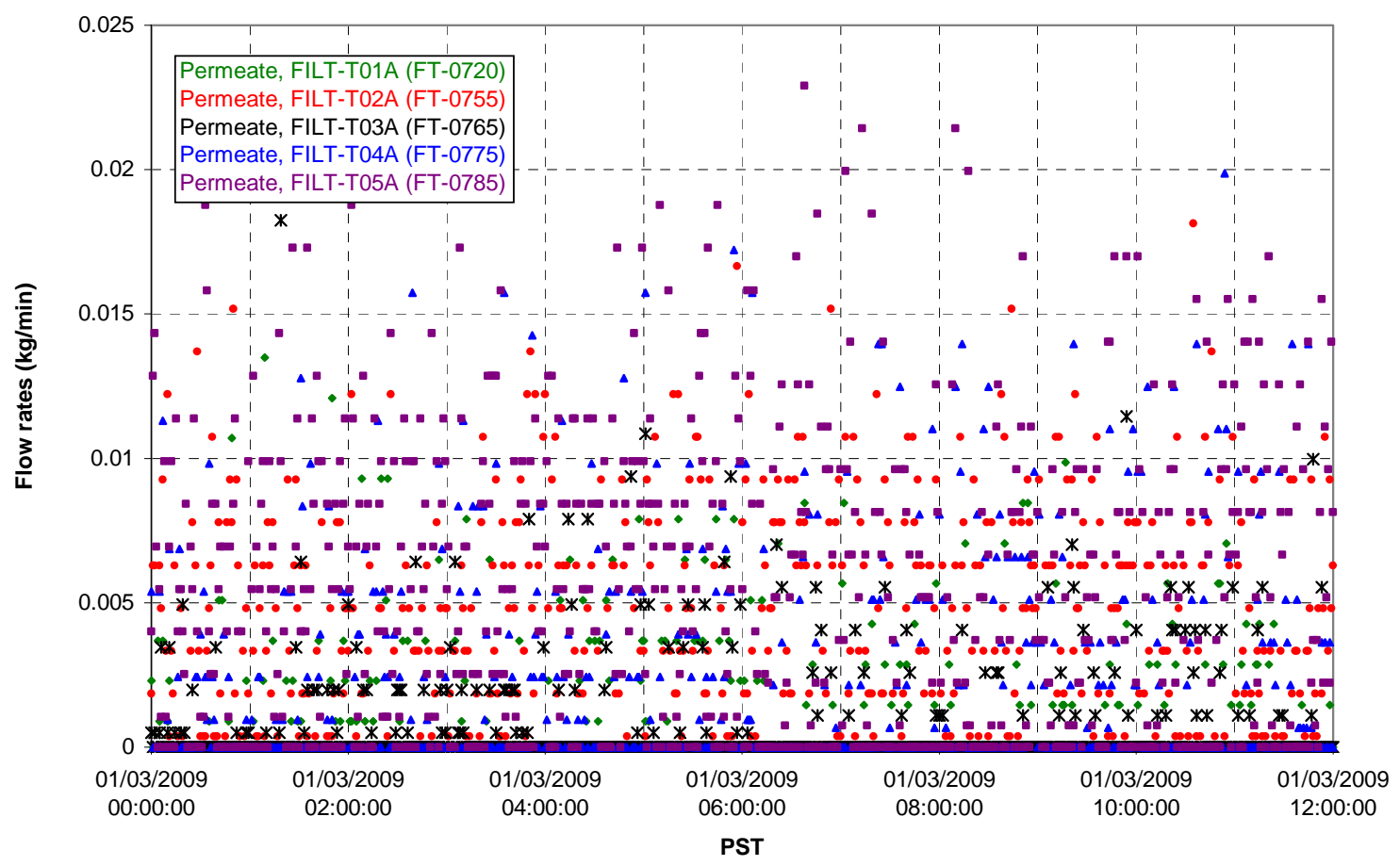


T02A Inner Temperature Tree

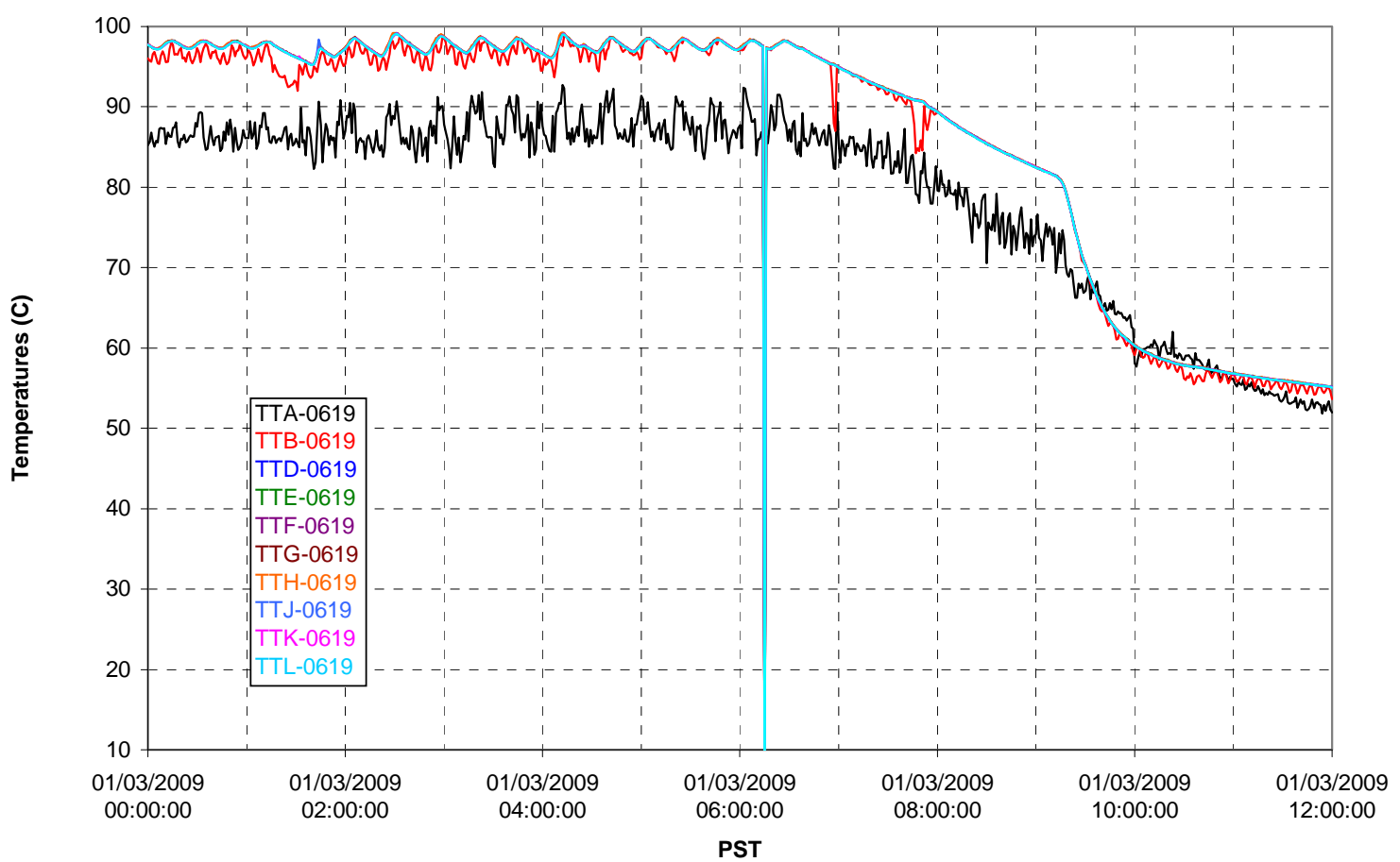

T02A Outer Temperature Tree

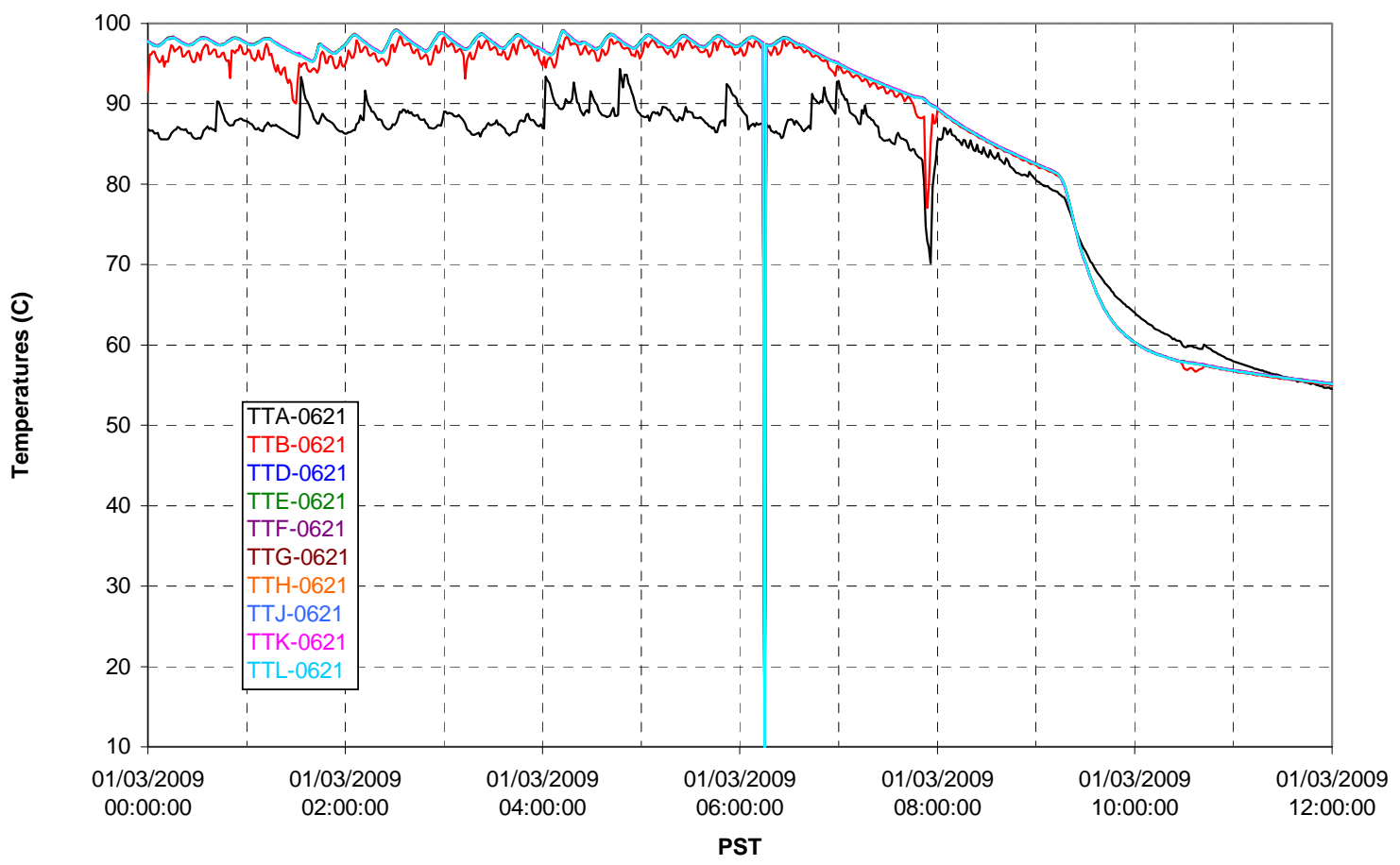


T02A temperatures

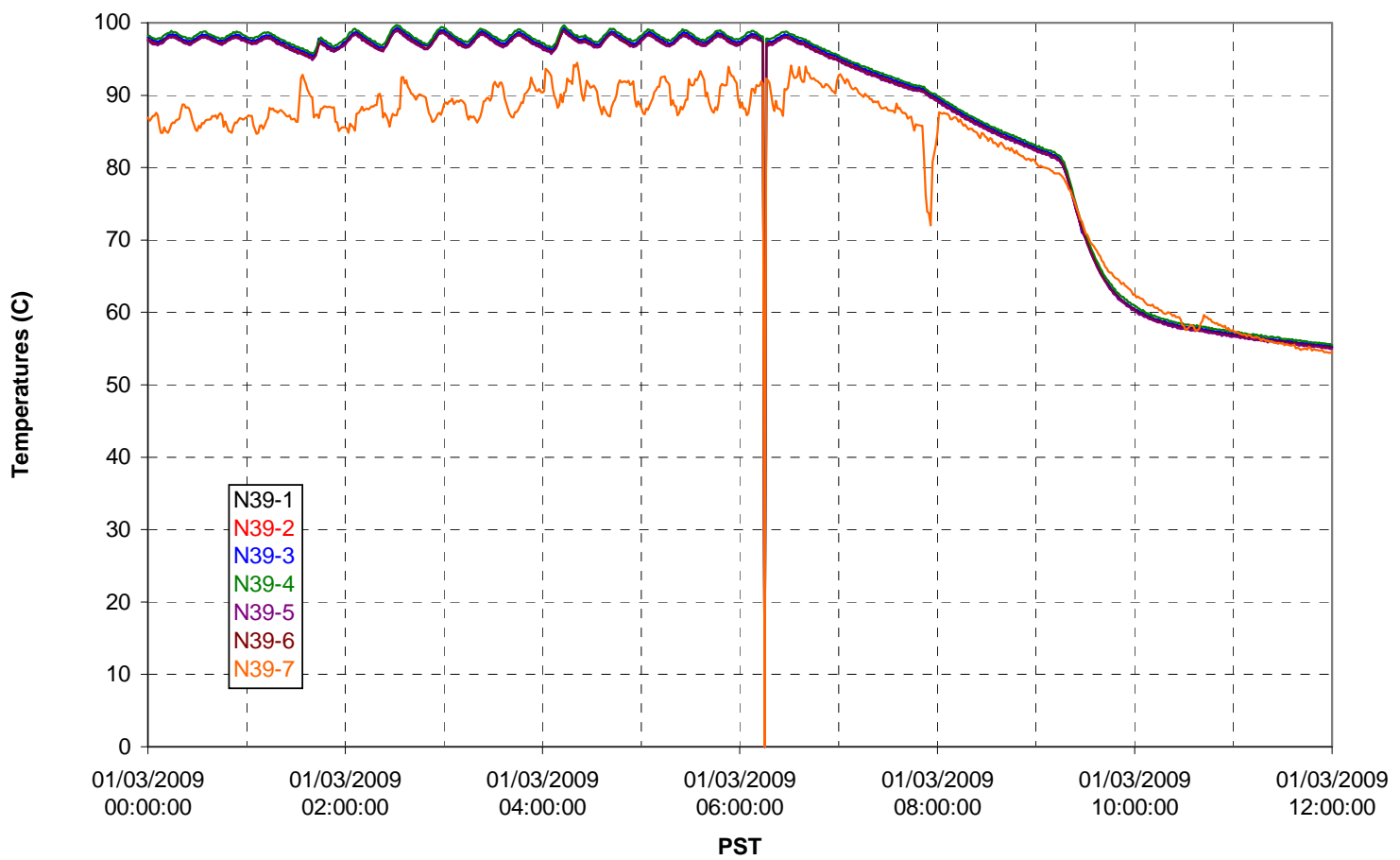

T02A temperatures

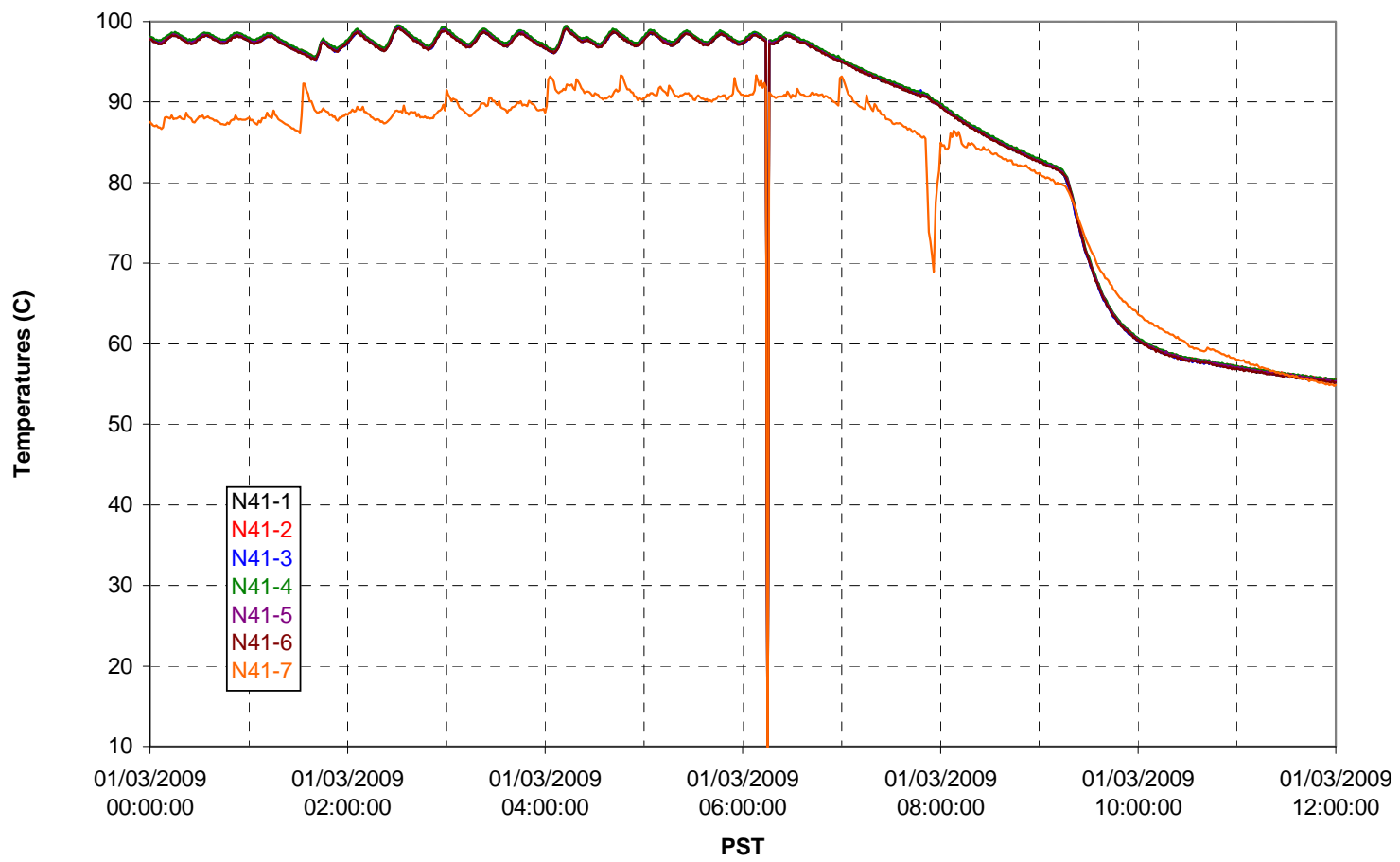


T02A temperatures

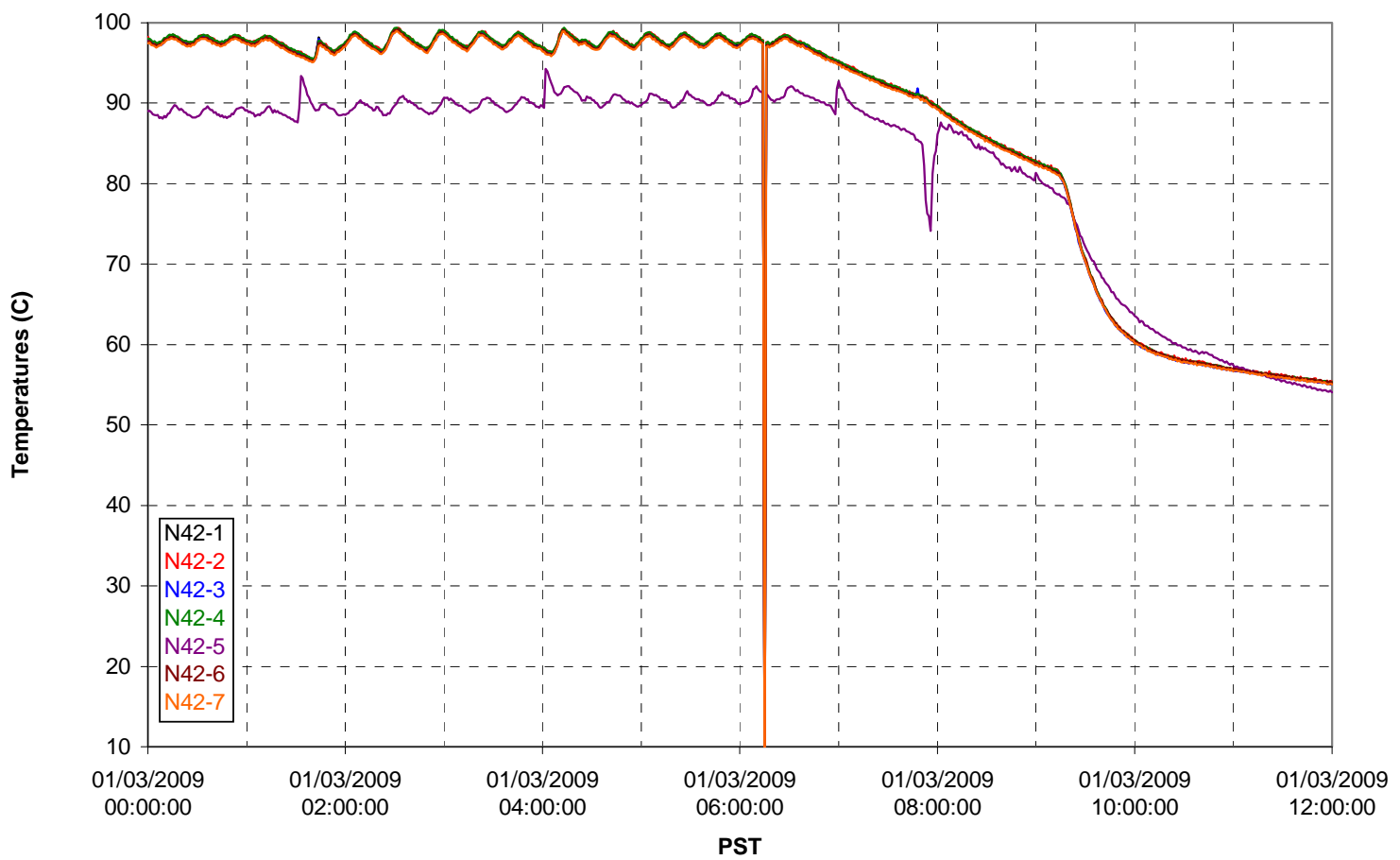

T02A temperatures

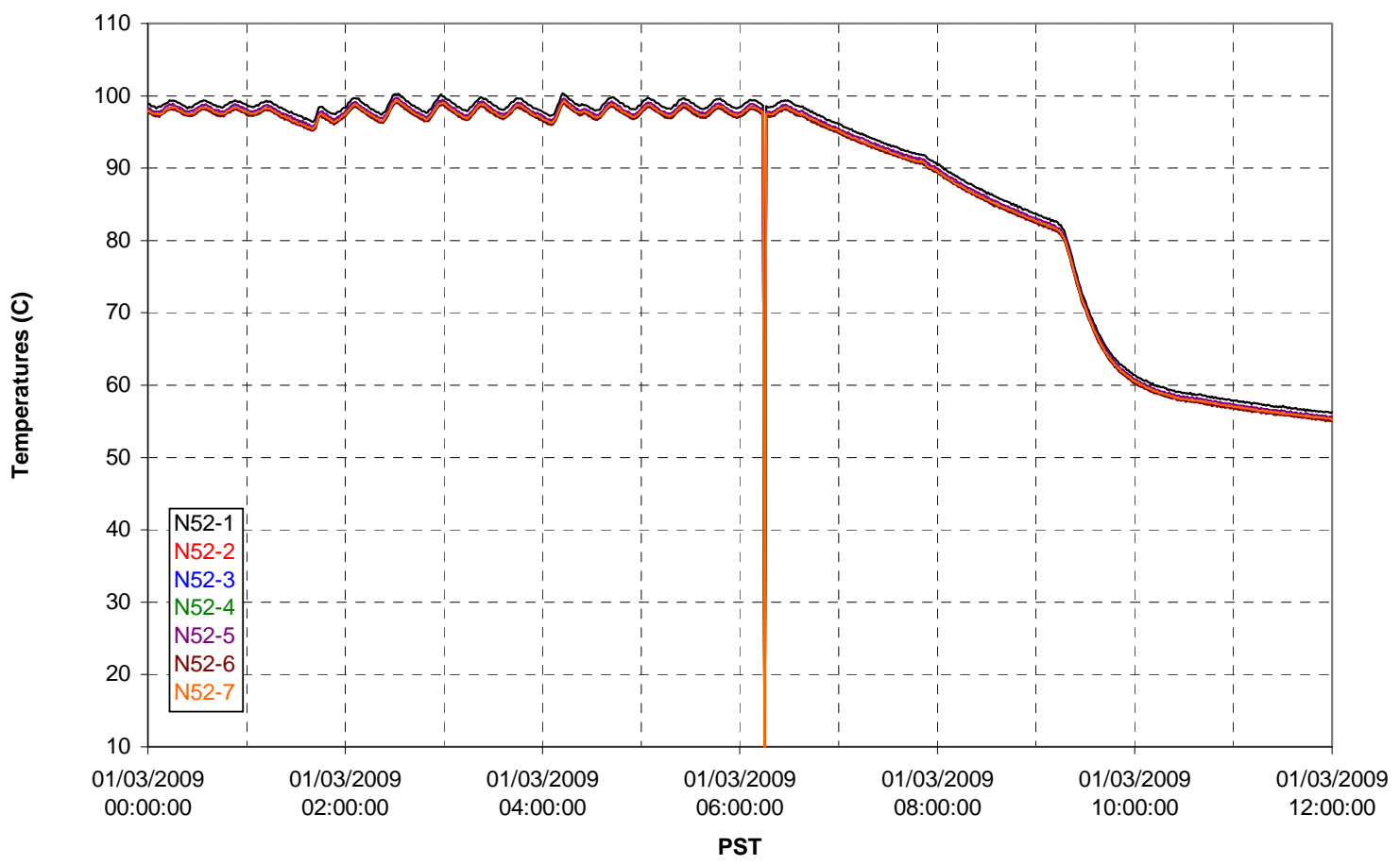


T02A Heating and Cooling

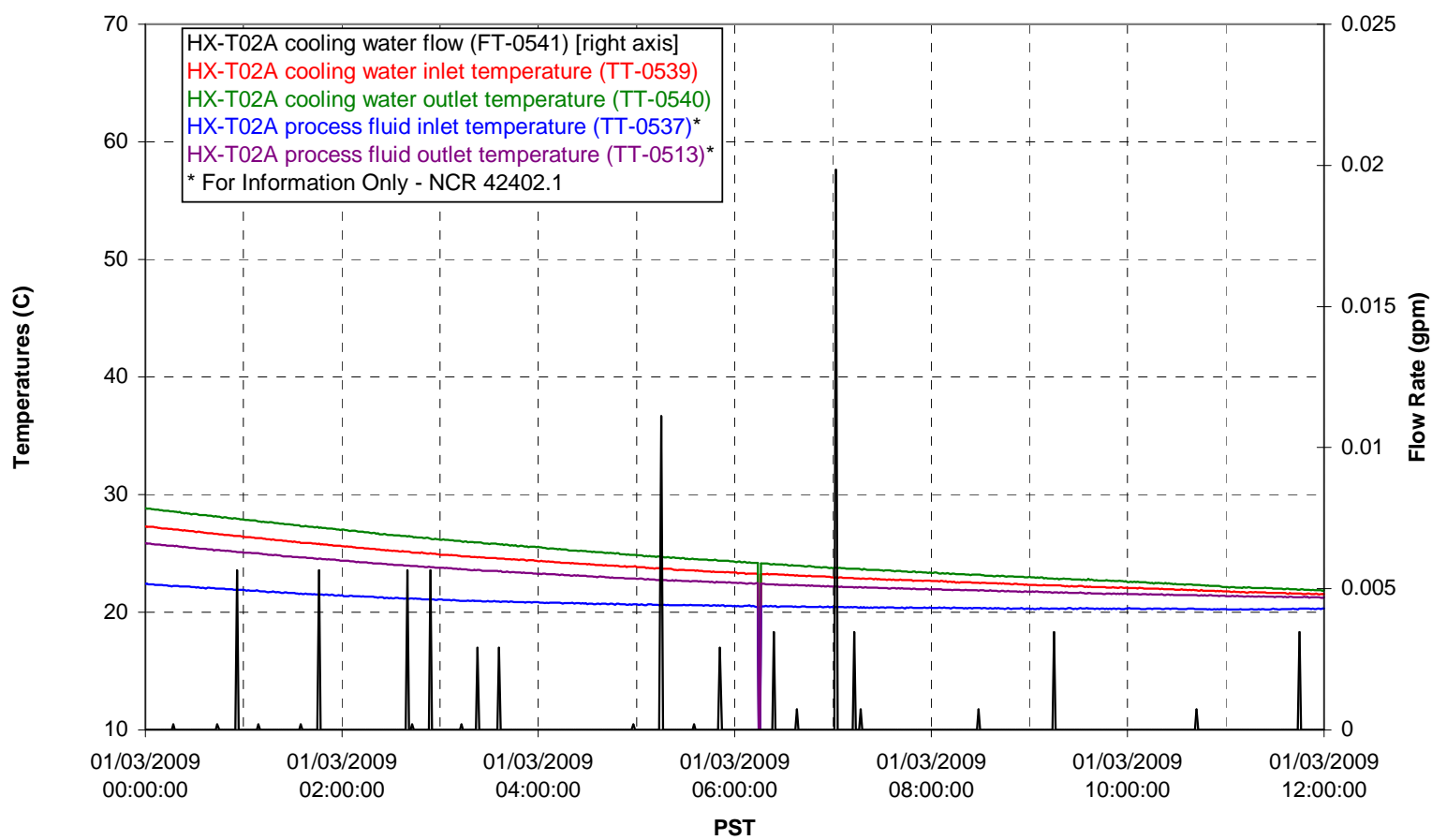

Pump Operation

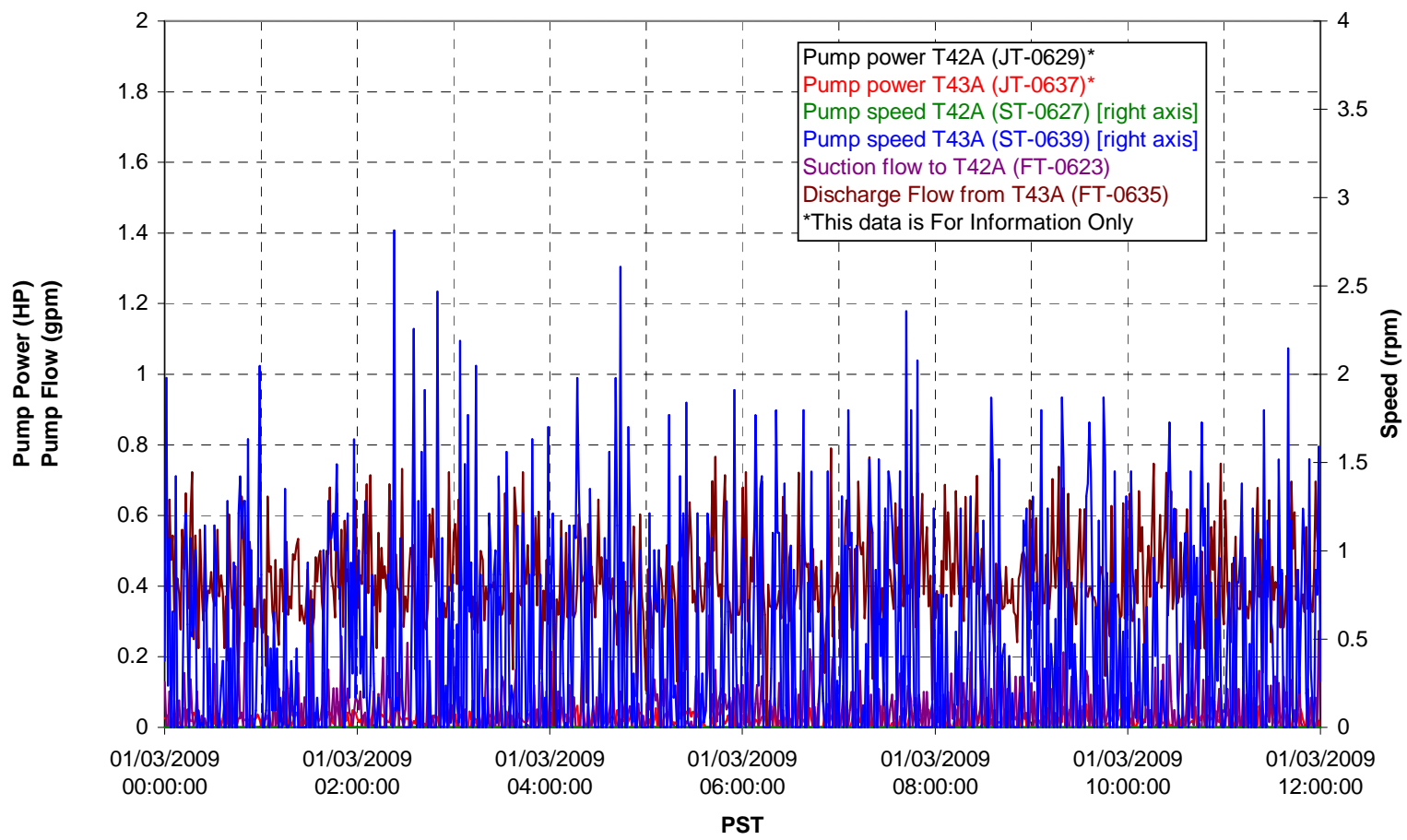


Pulsepot UFP-PP-T01A

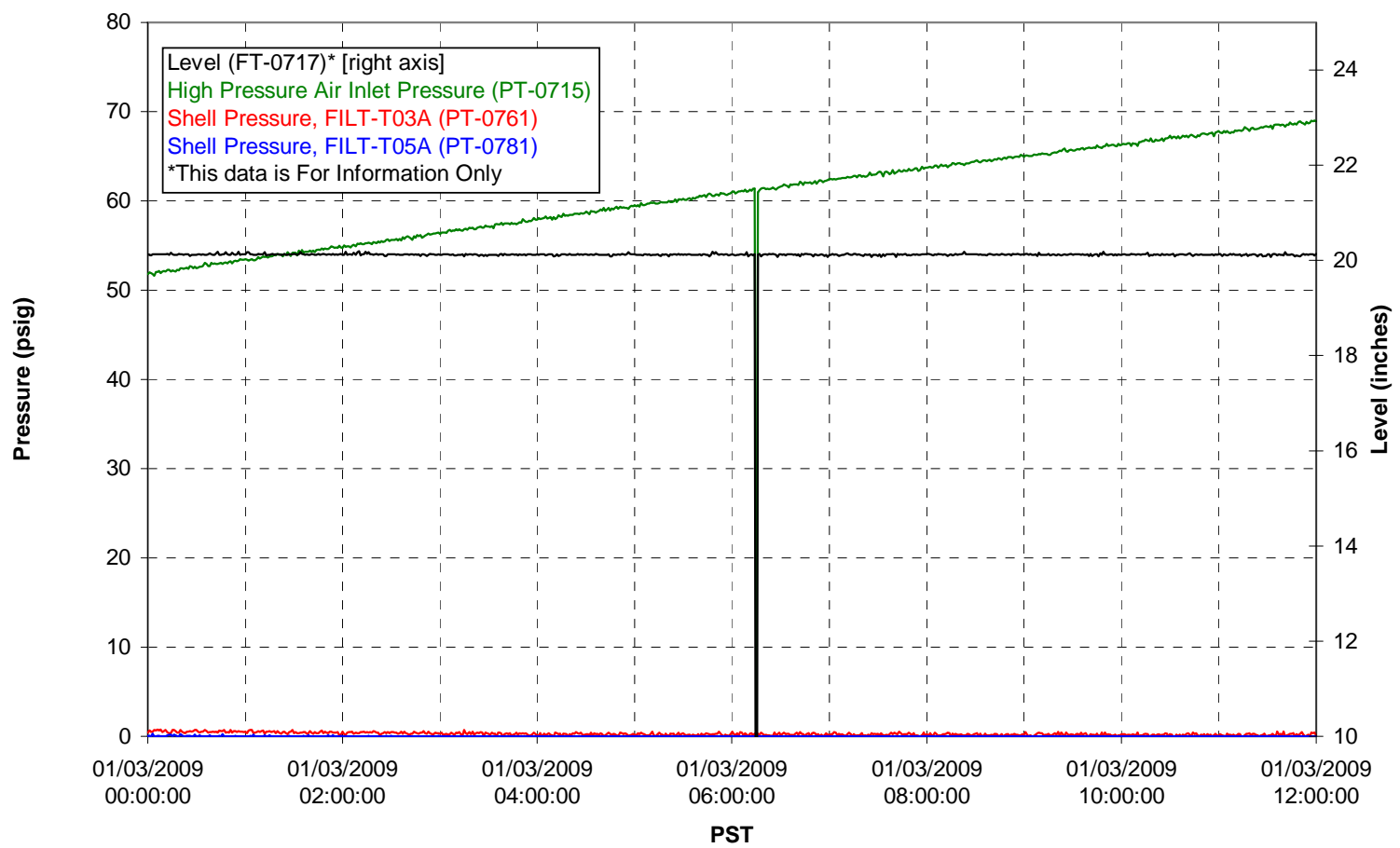

Pulsepot UFP-PP-T02A

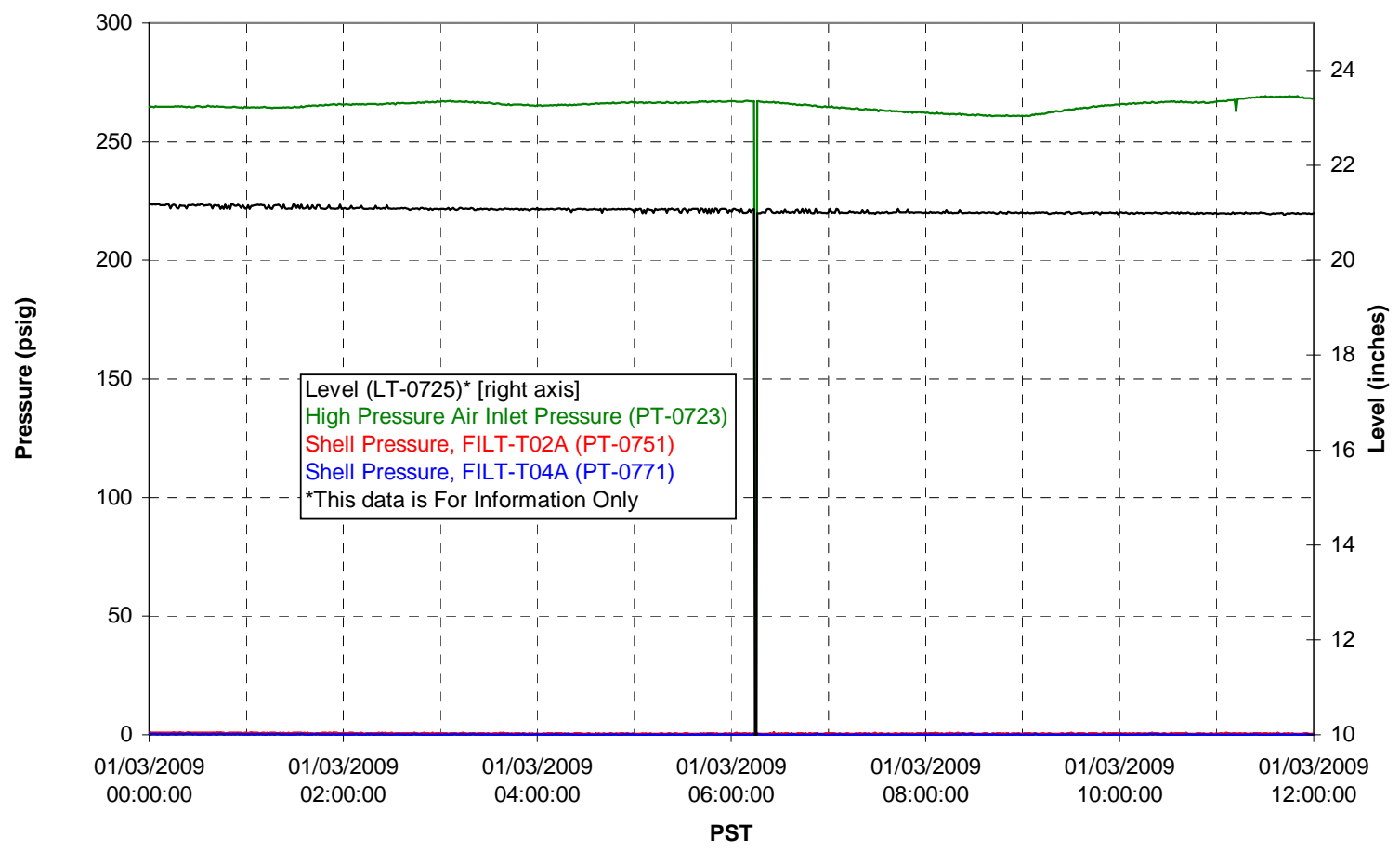


Pulsepot UFP-PP-T03A

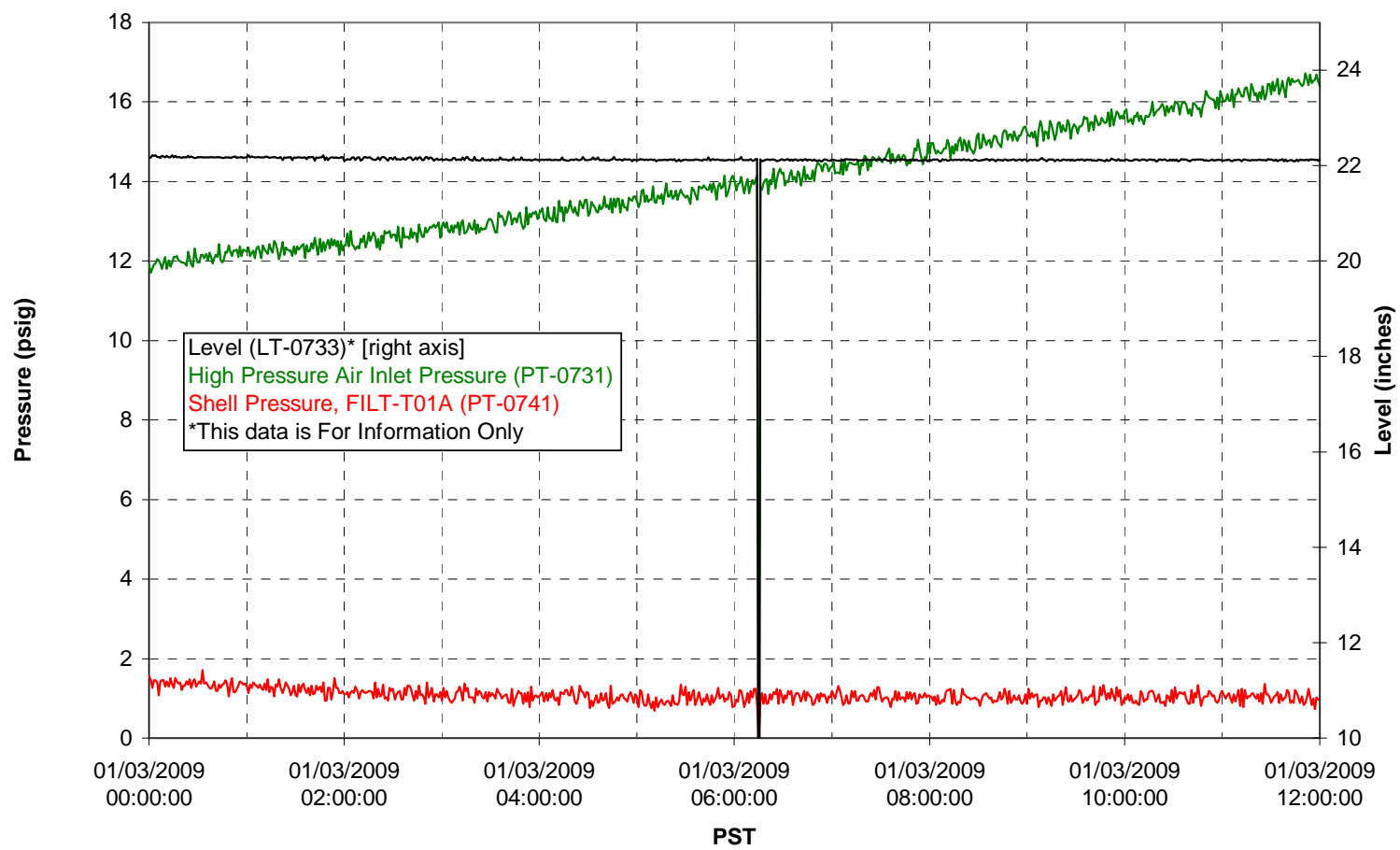

Pulsepot Levels

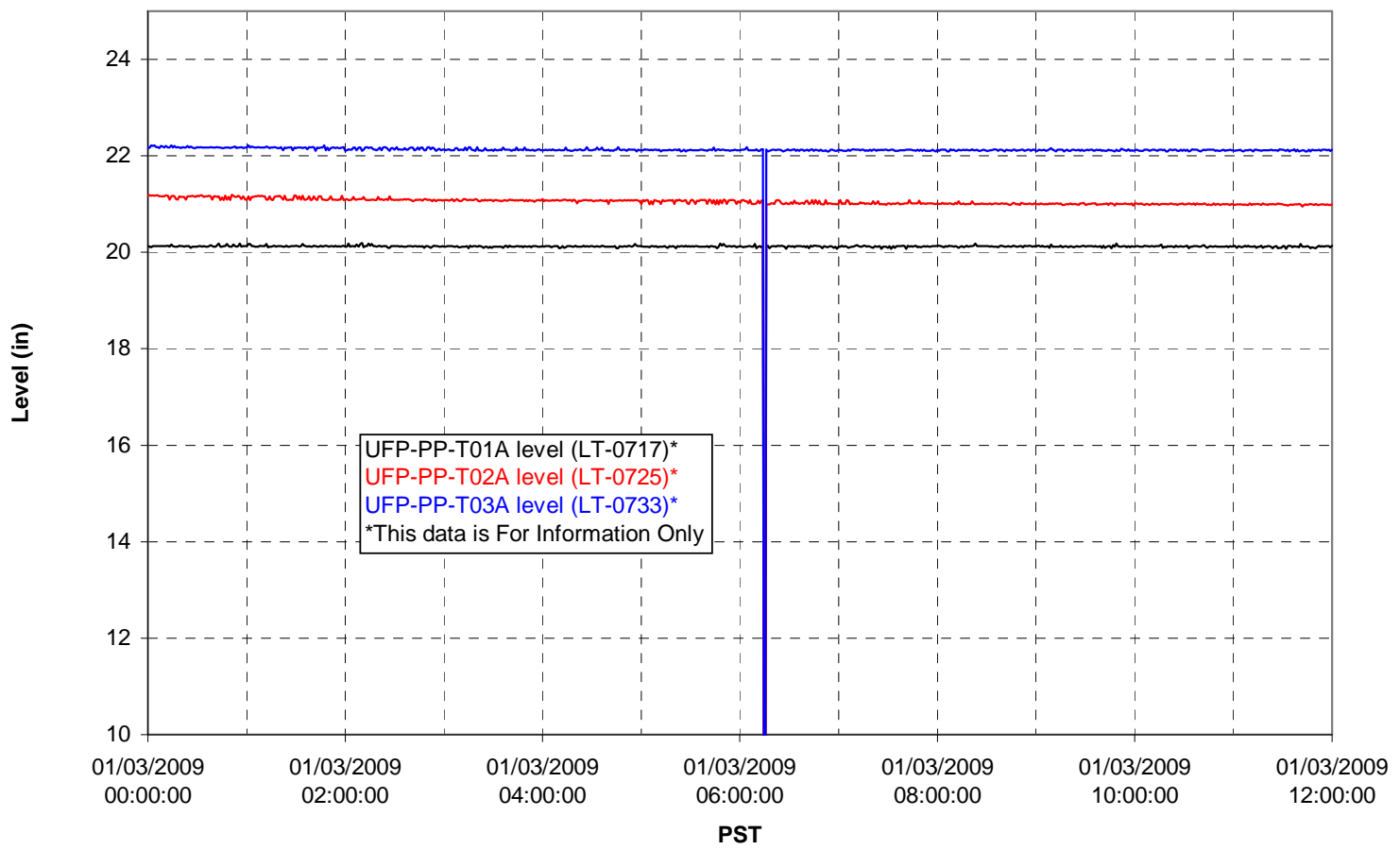


Filter UFP-FILT-T01A

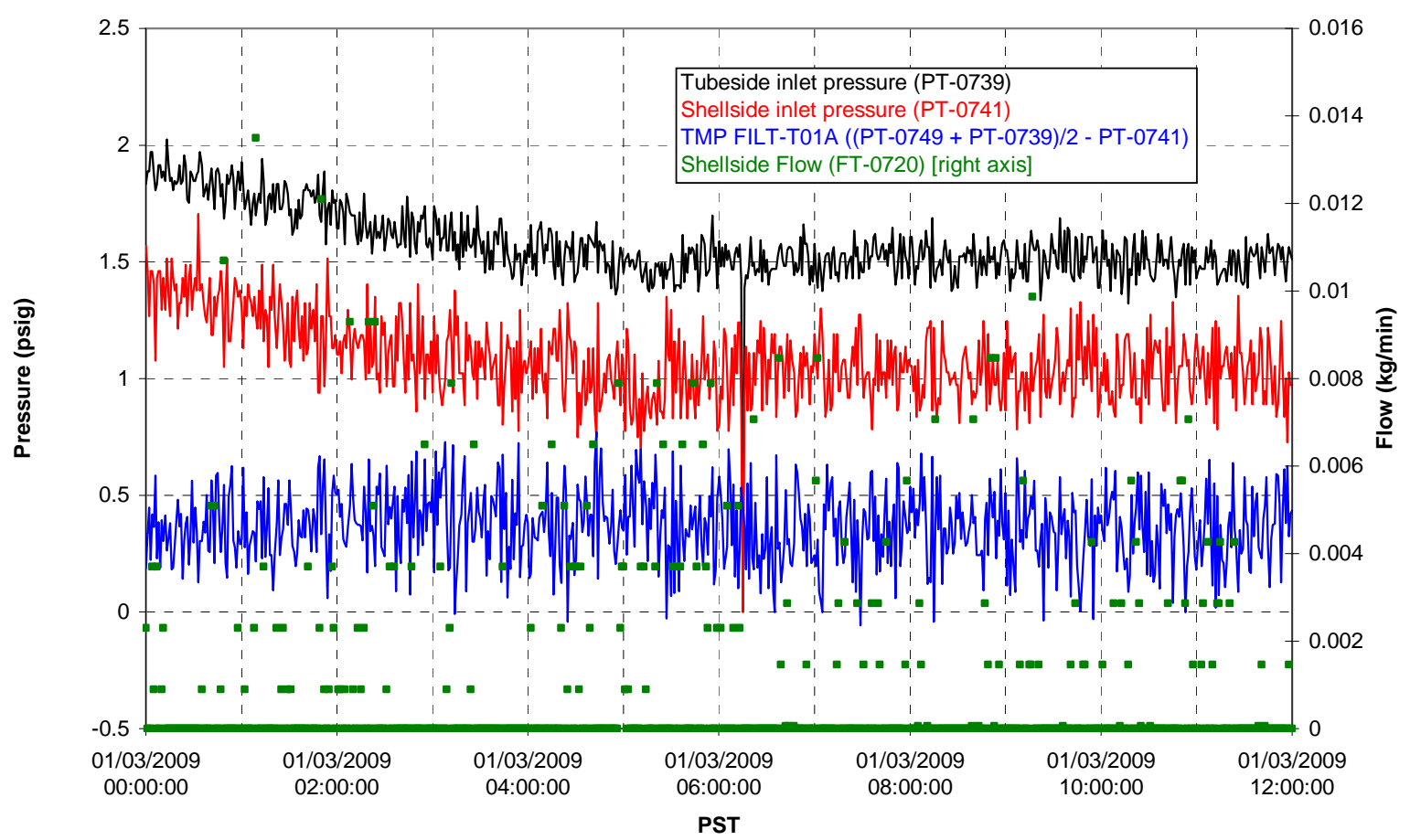

Filter UFP-FILT-T02A

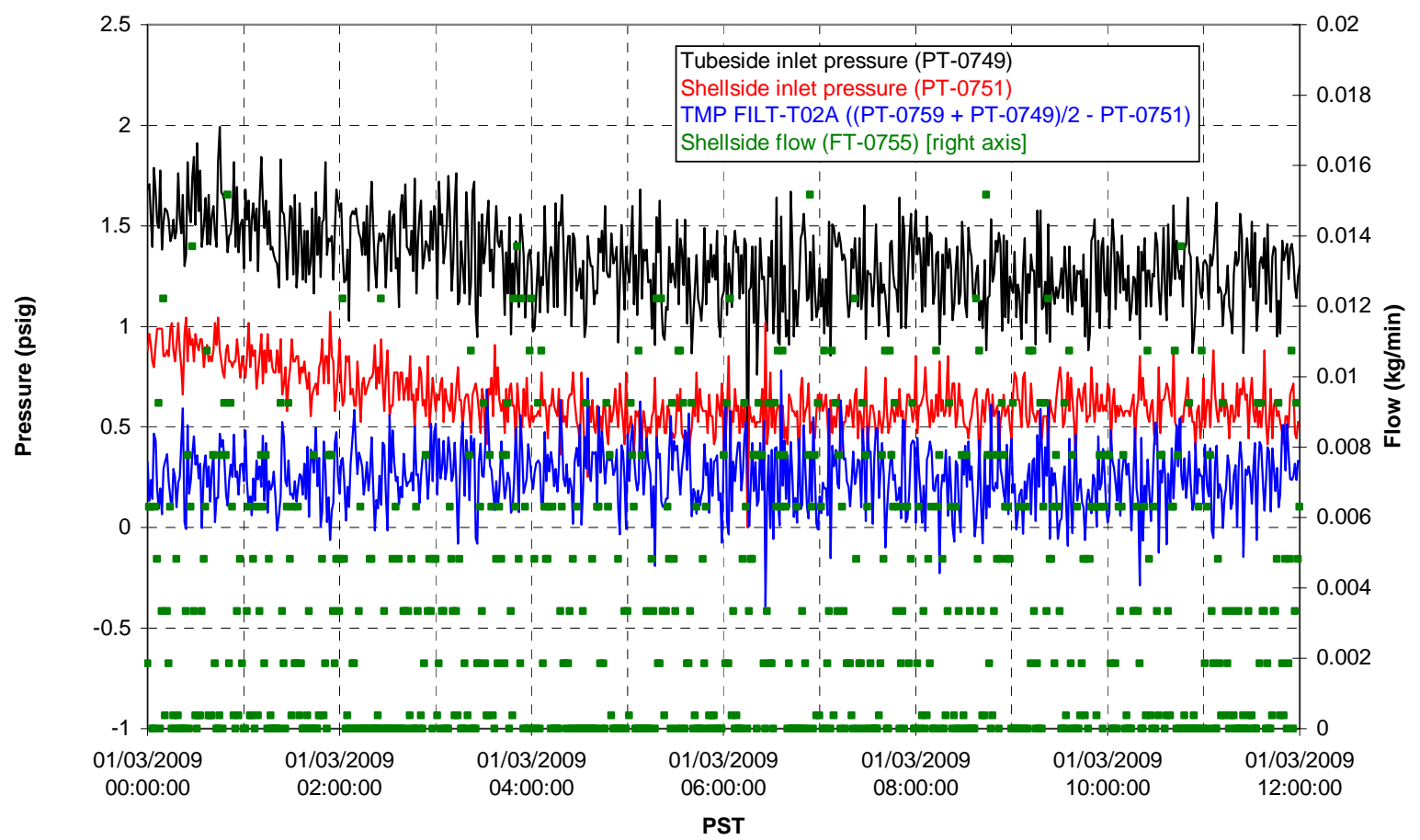


Filter UFP-FILT-T03A

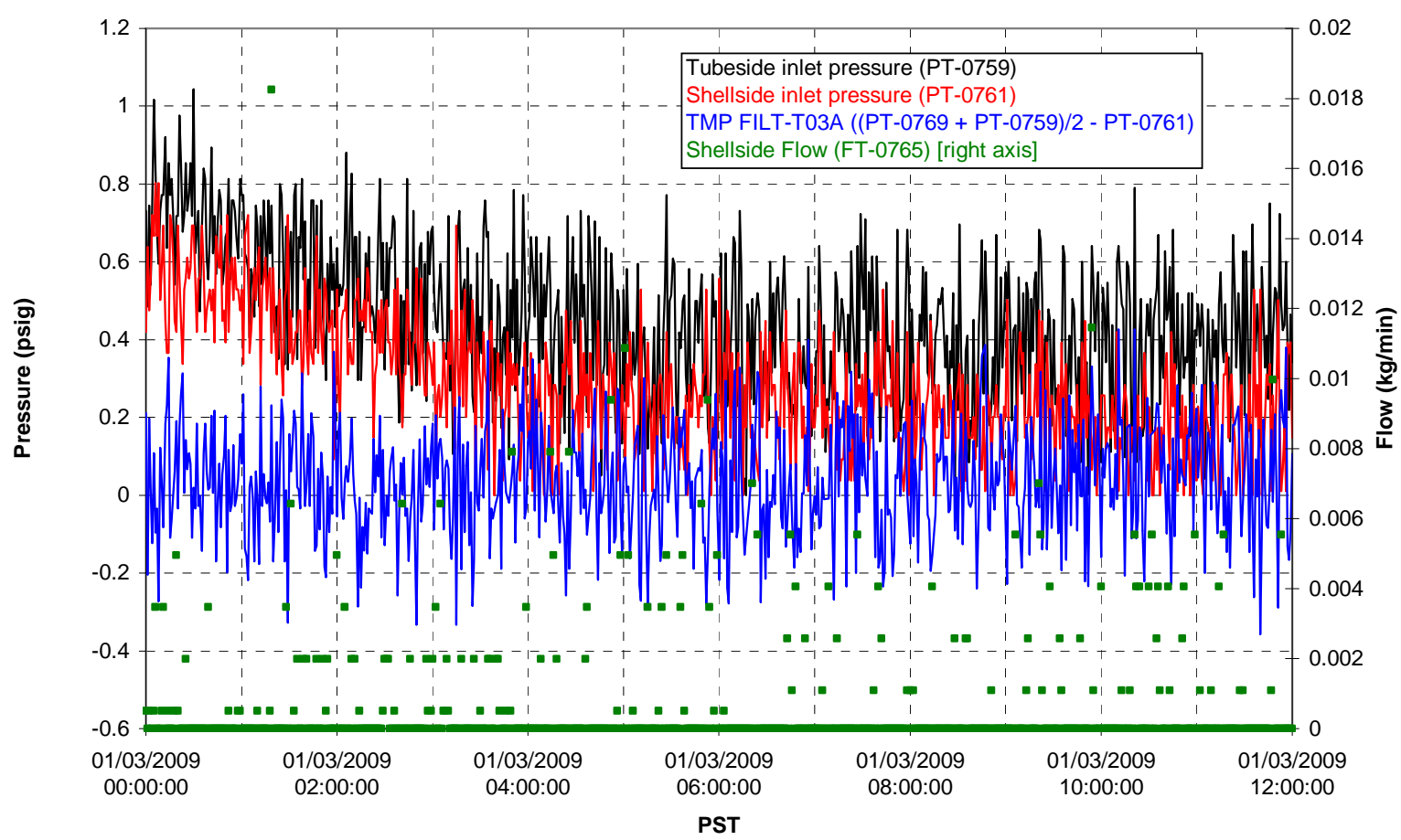

Filter UFP-FILT-T04A

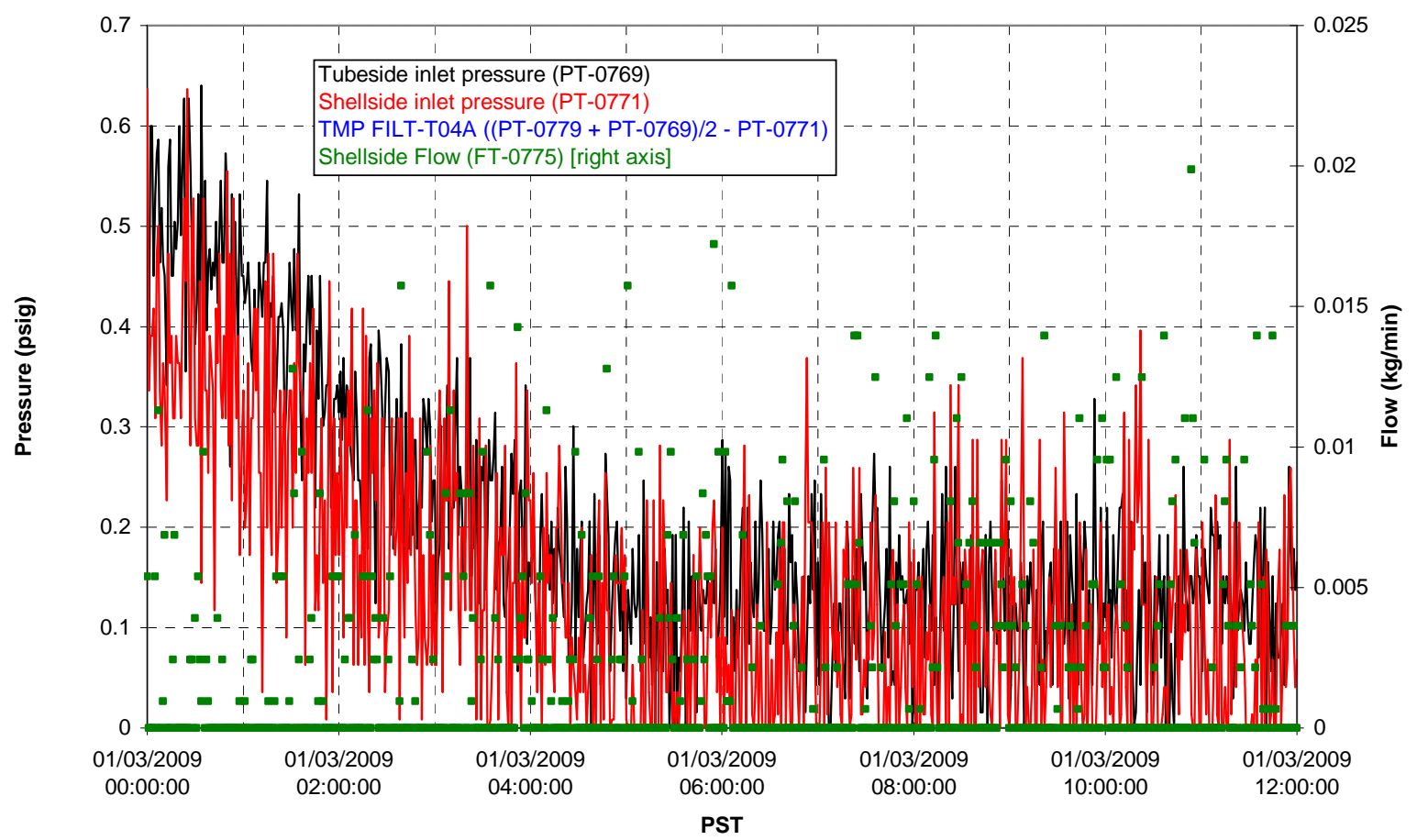


Filter UFP-FILT-T05A

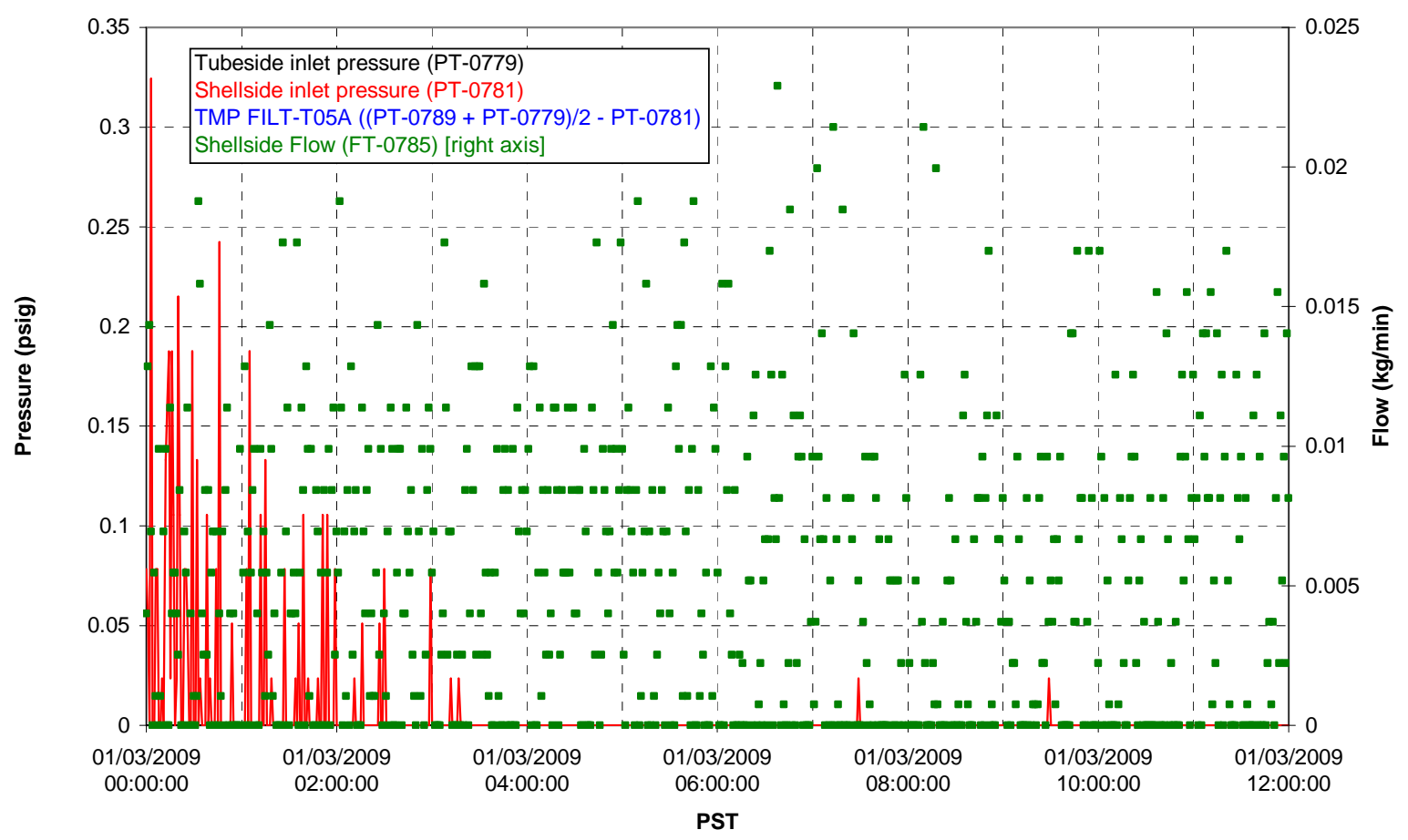

Chemical Flow

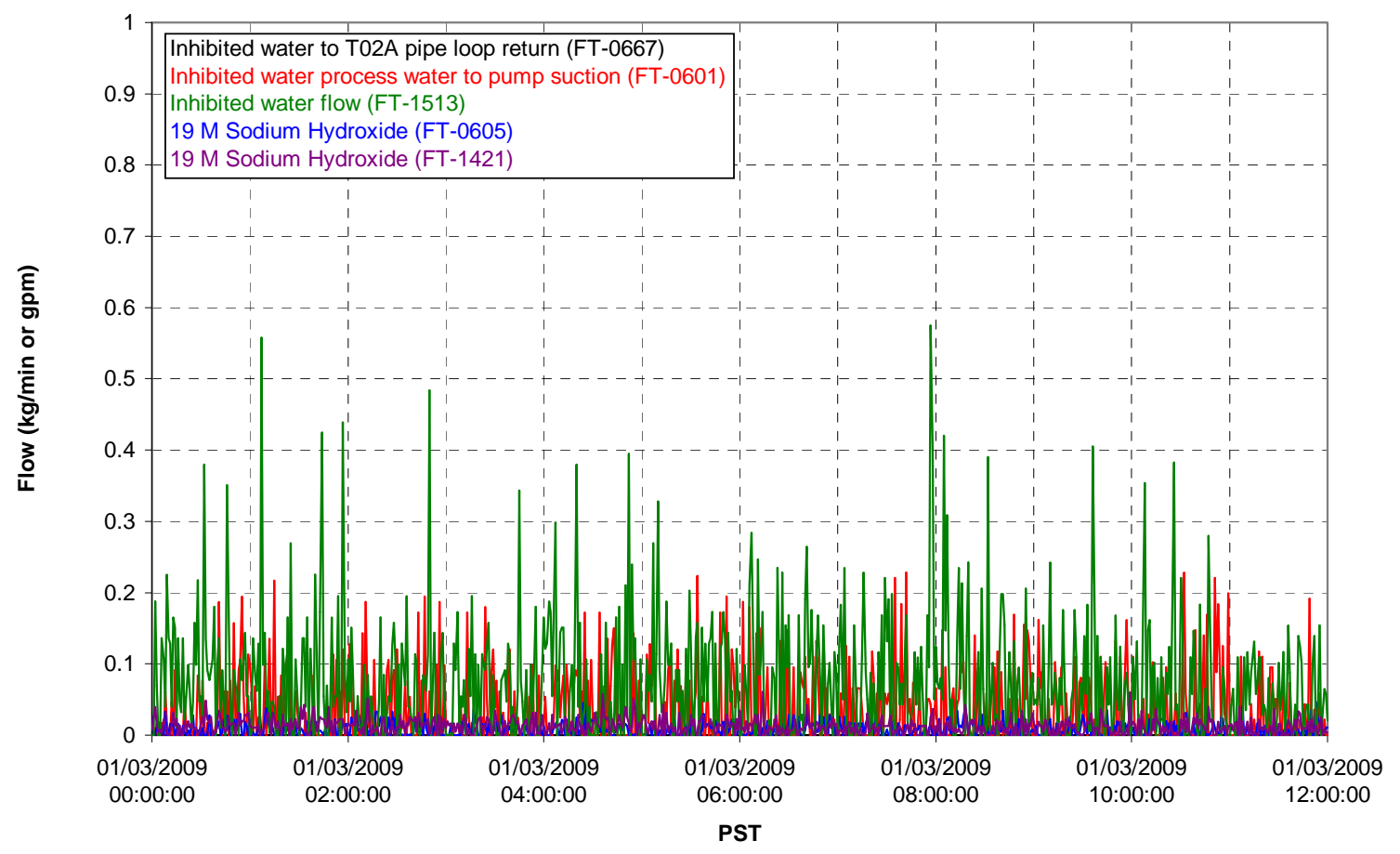


Chemical Flow

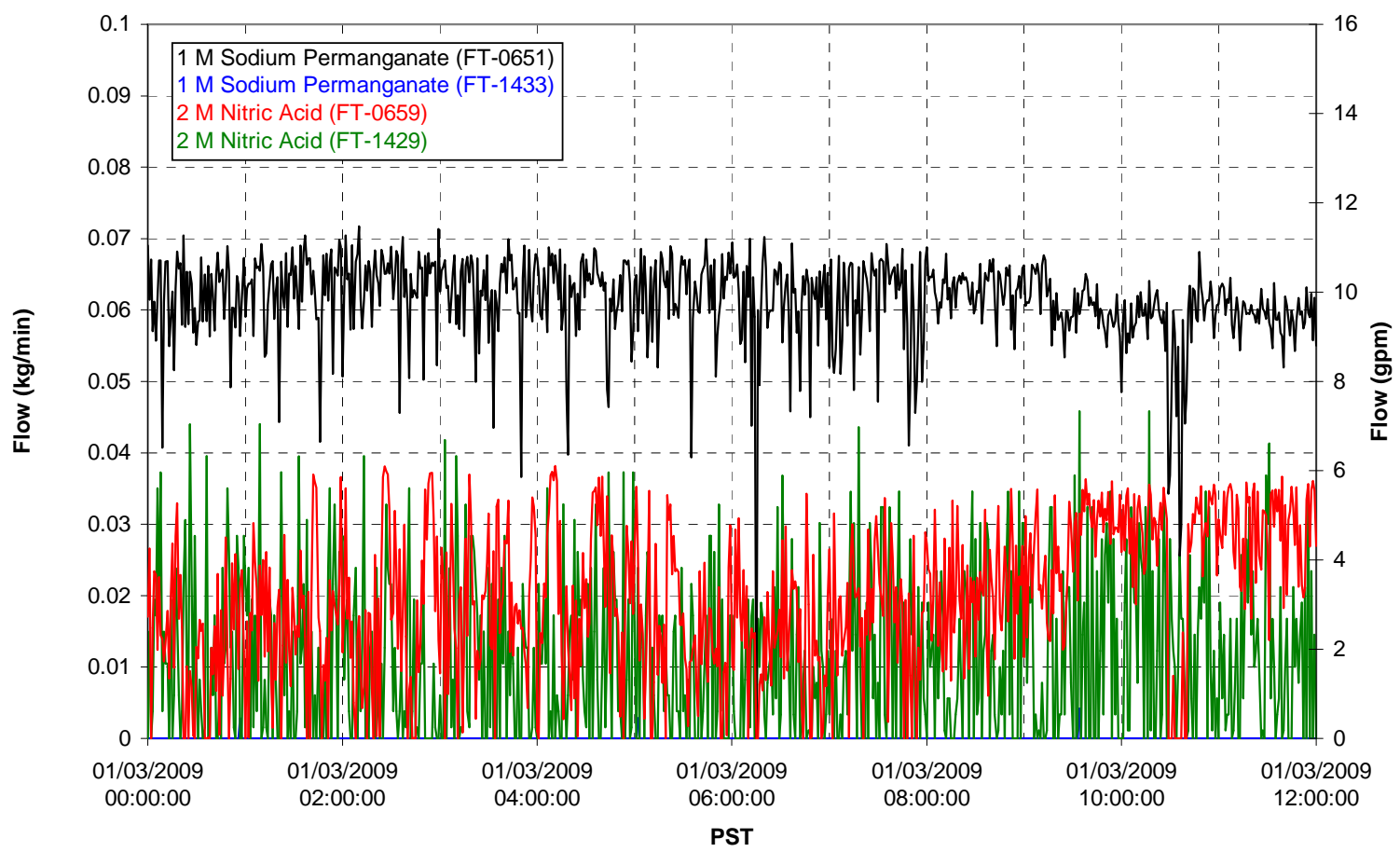

Air Flows

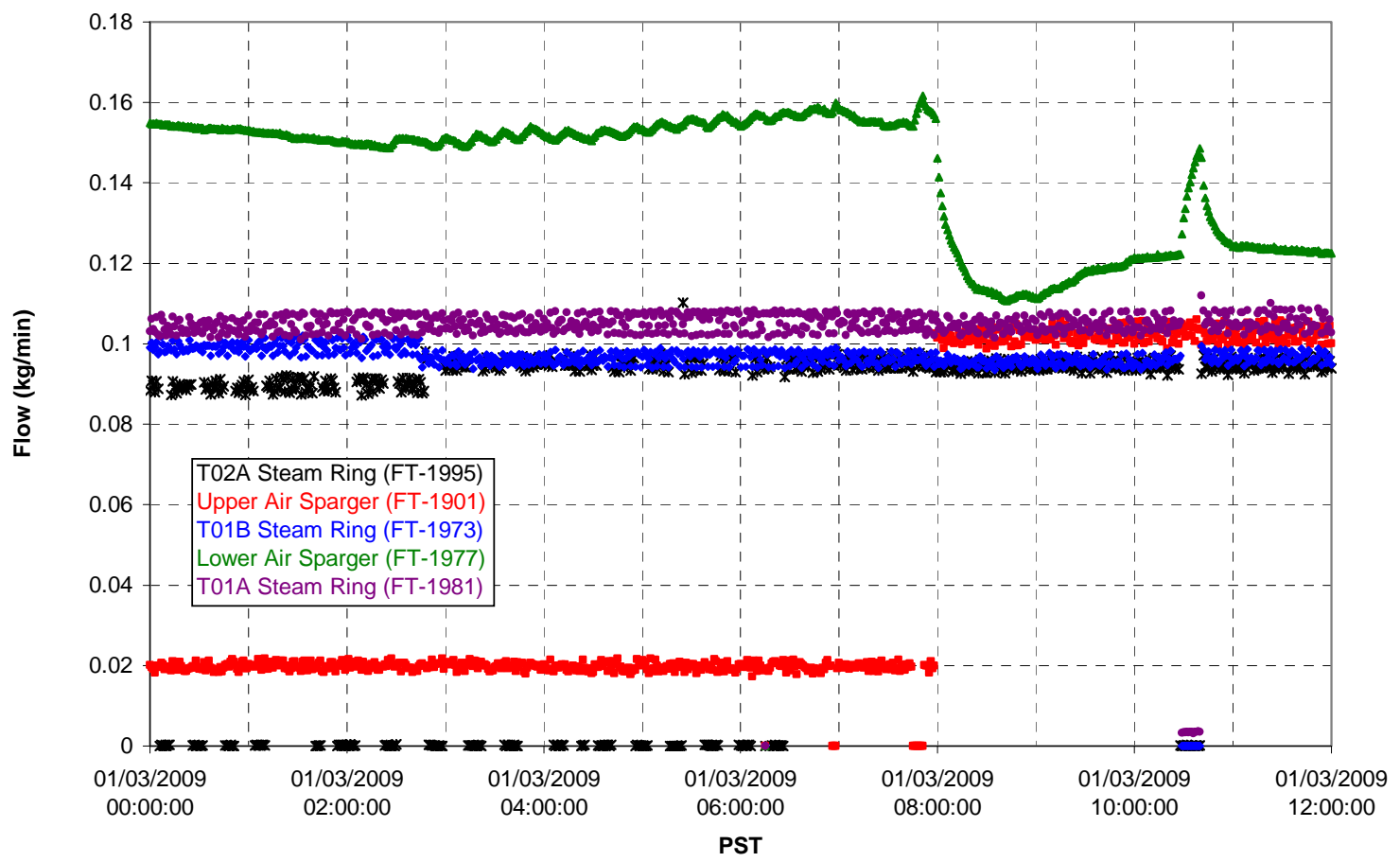


T02A Steam

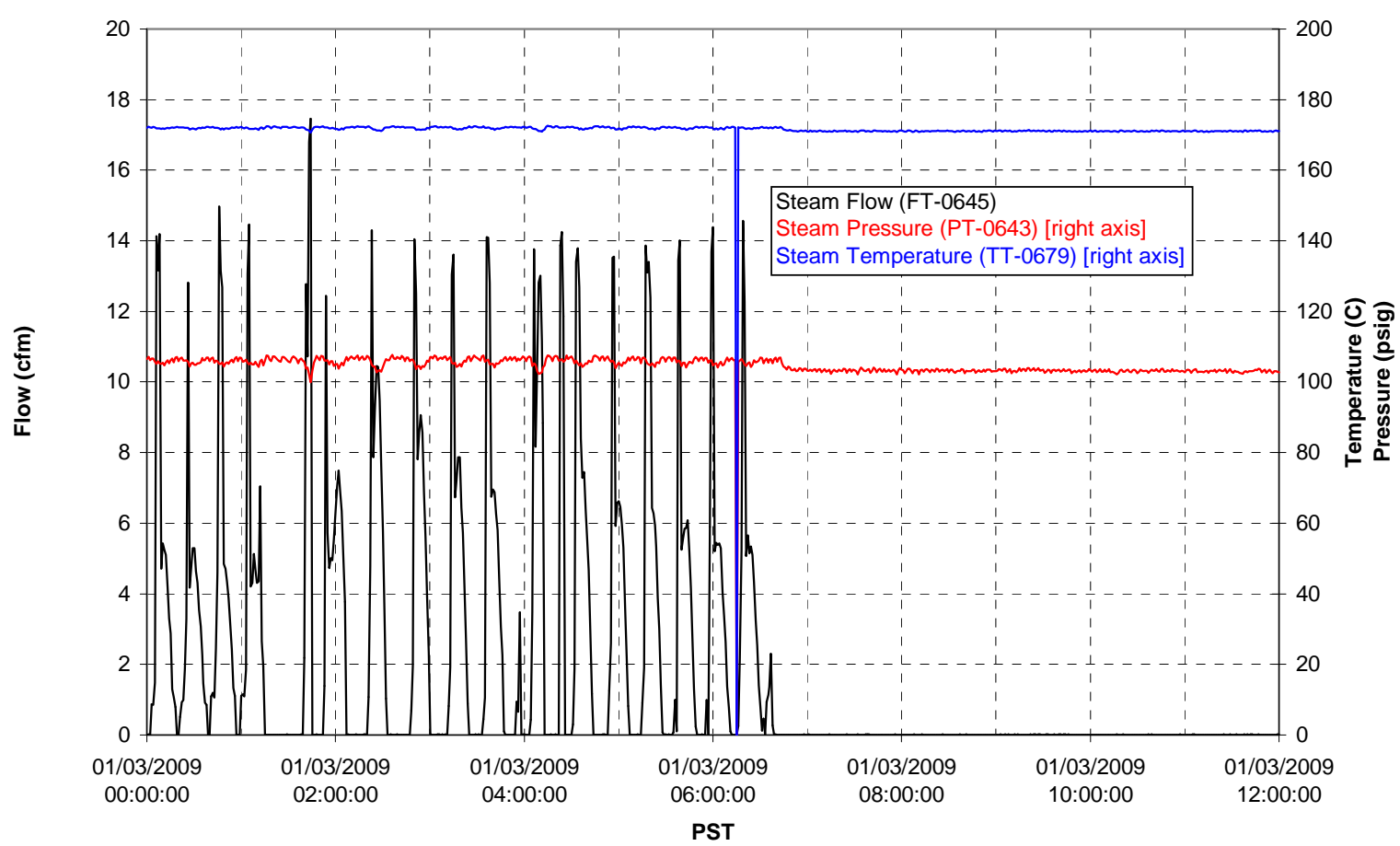

T01A Steam

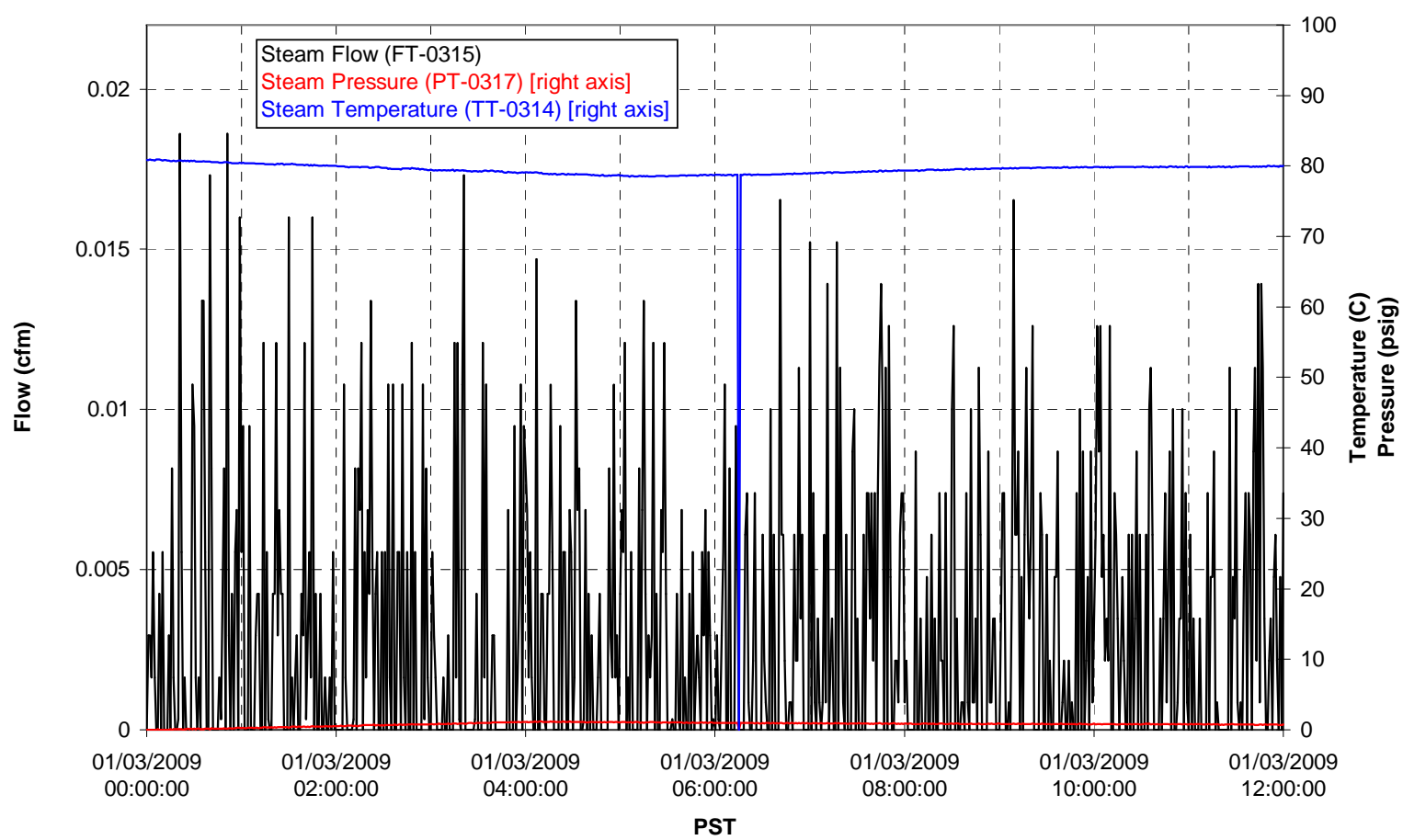


T01B Steam

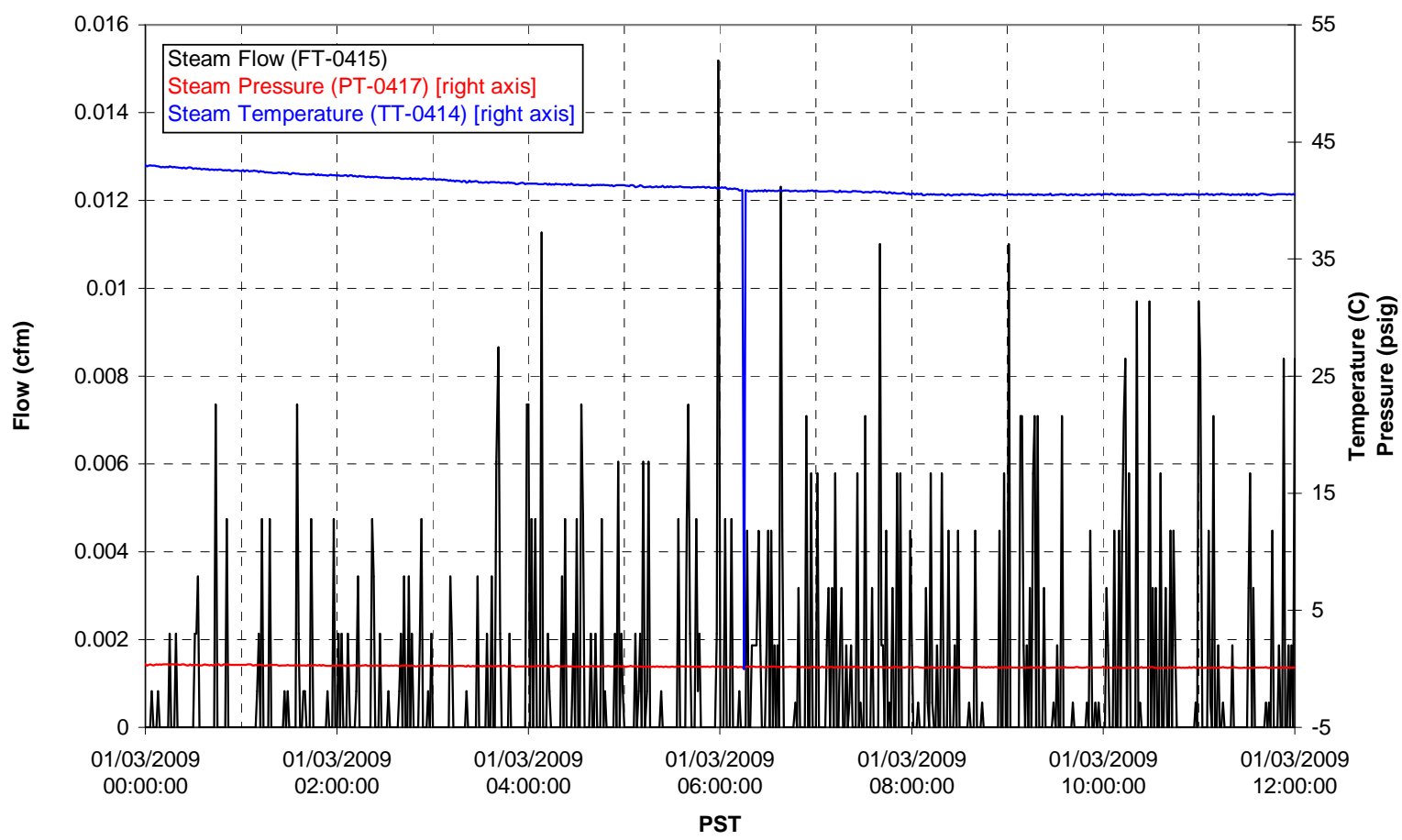


Functional Test Data Plots

01/03/2009 12:00 - 01/04/2009 00:00 
T01A level

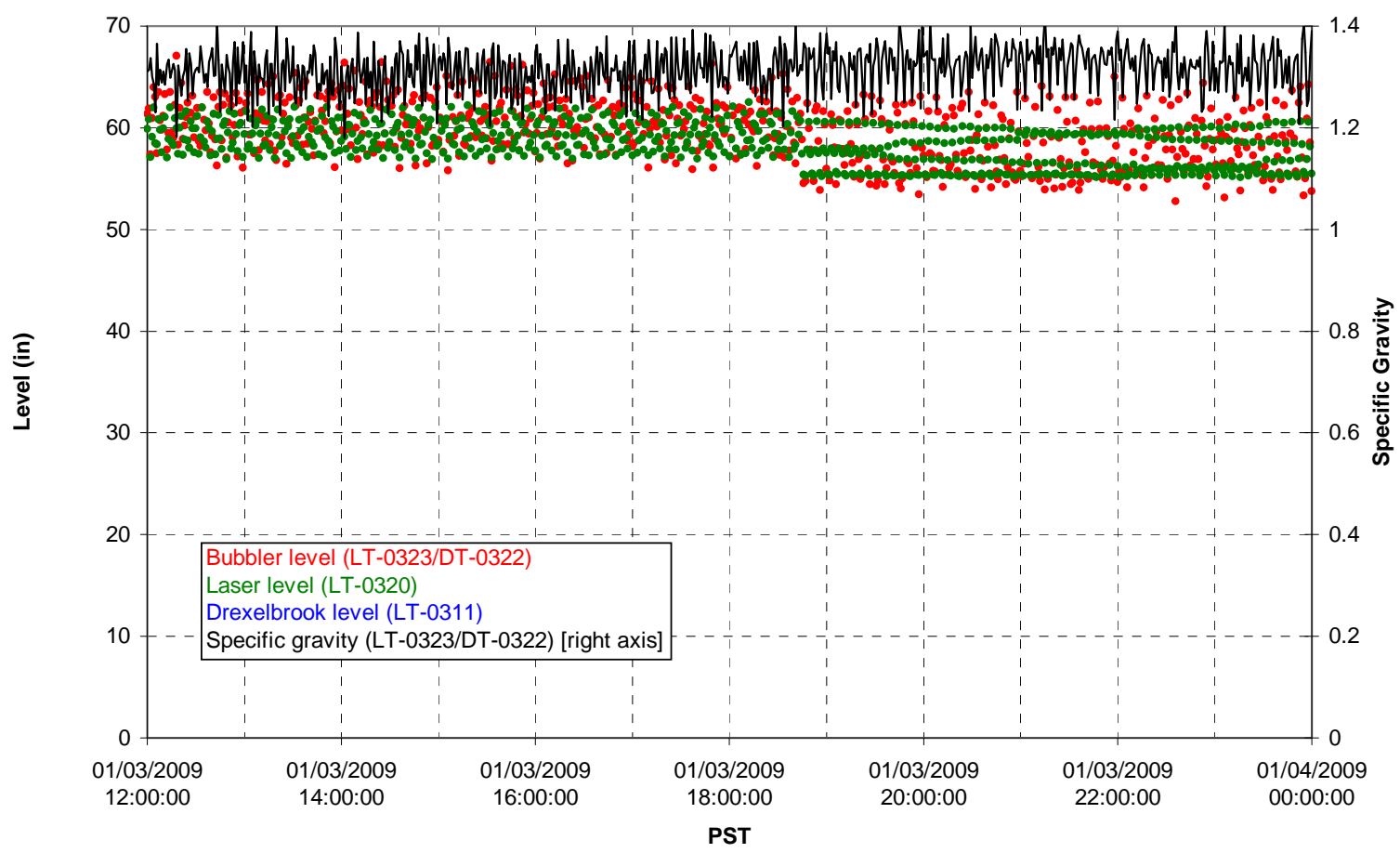

T01A temperatures

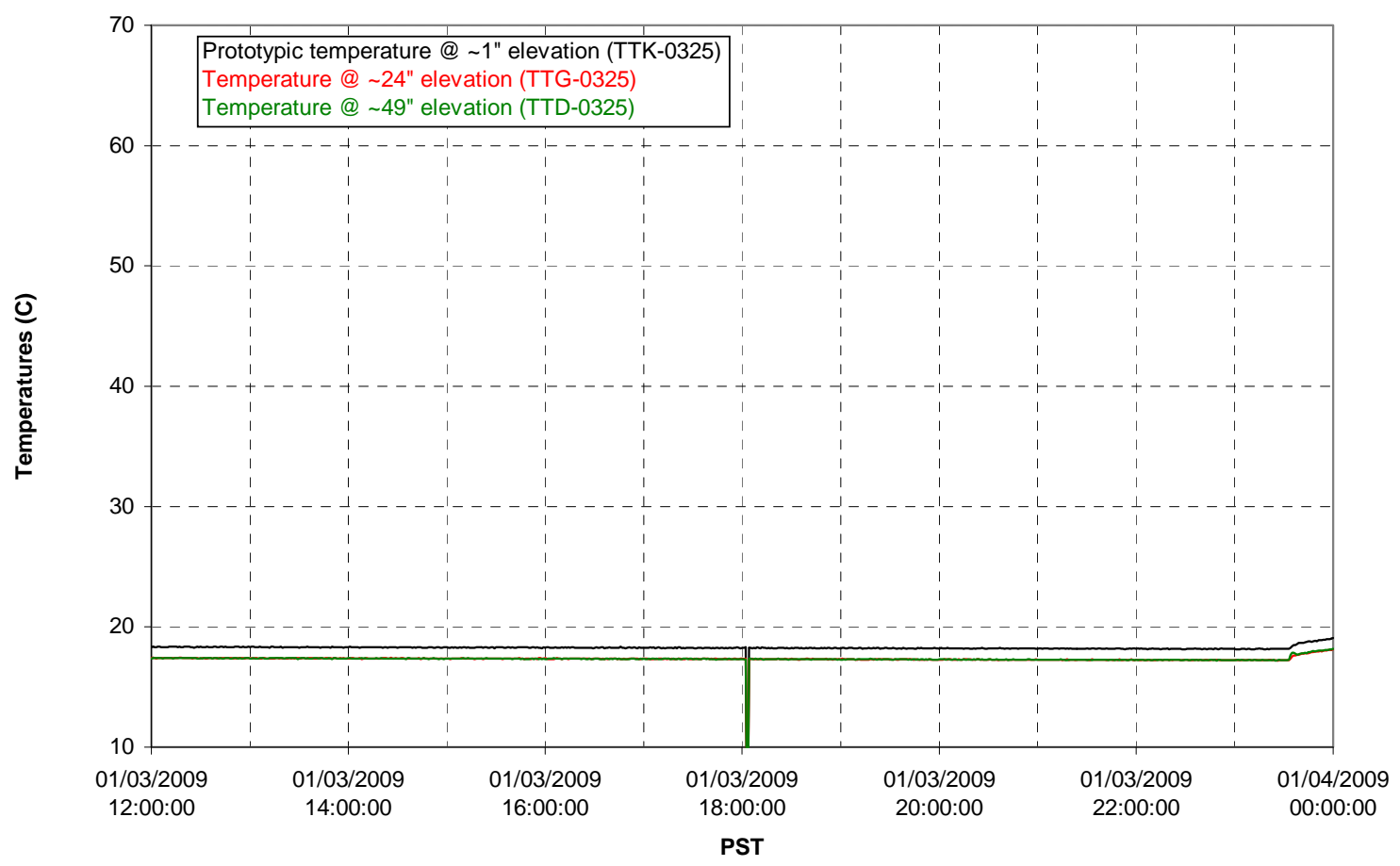


T01B level

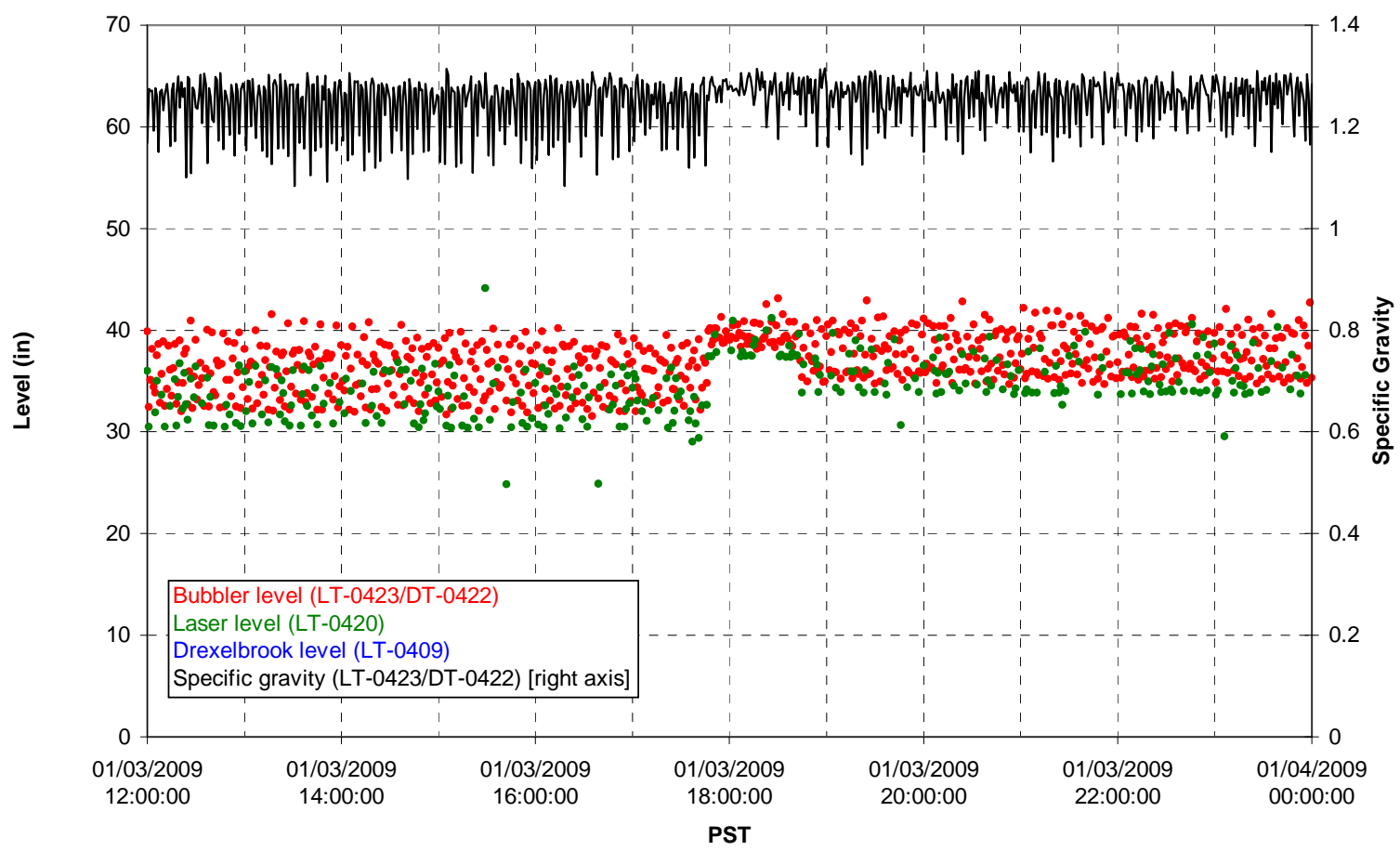

T01B temperatures

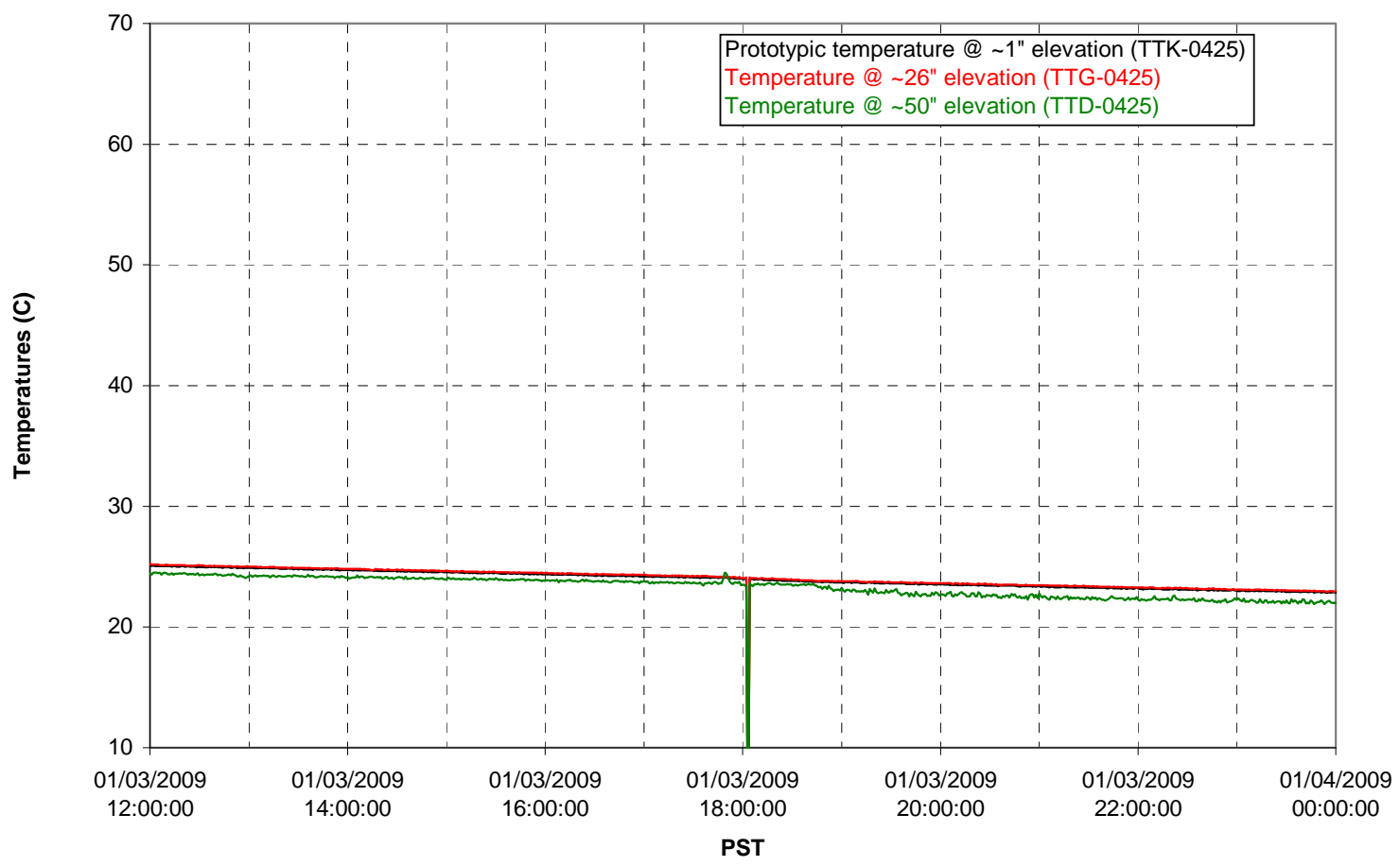


T02A level

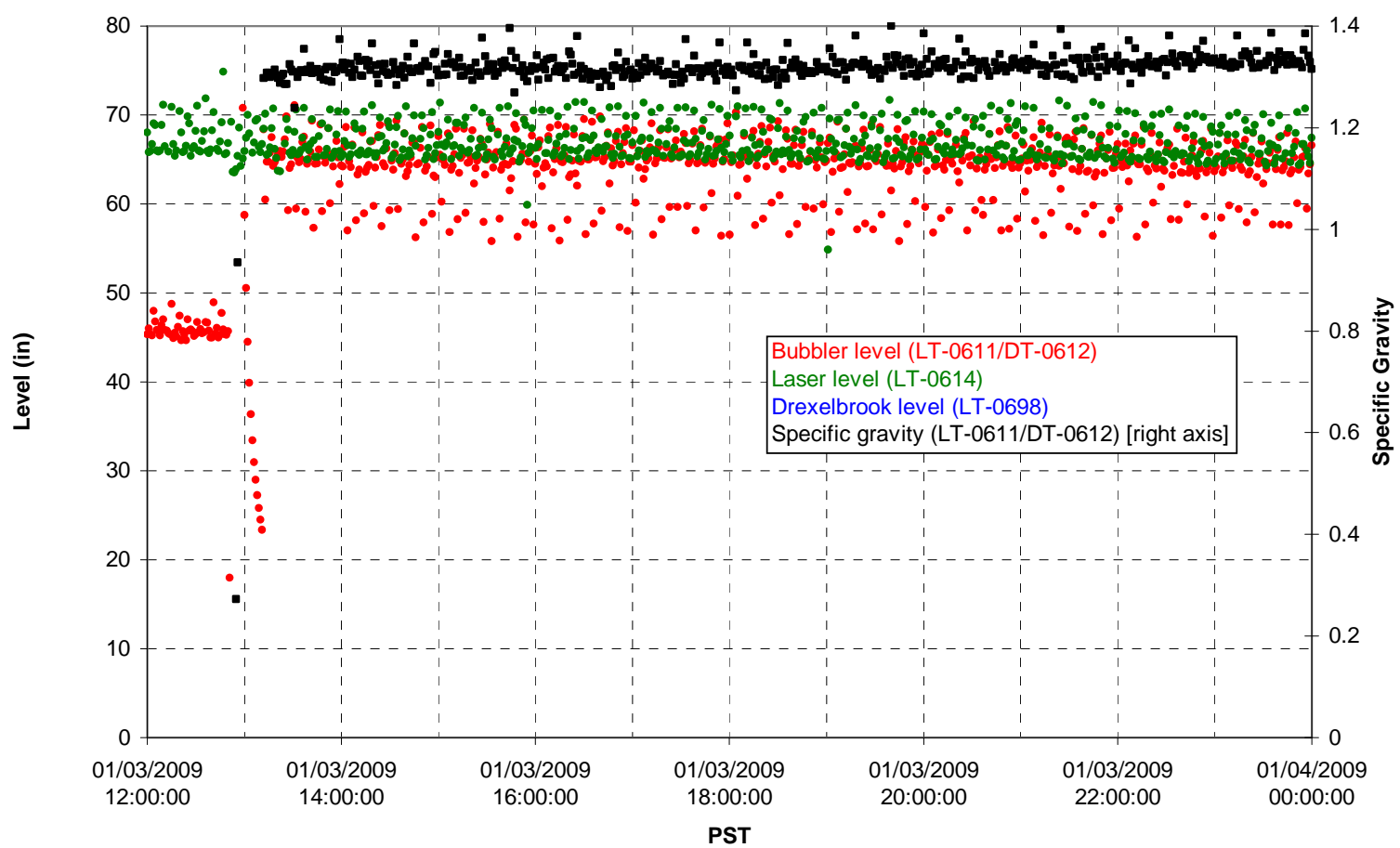

T02A temperatures

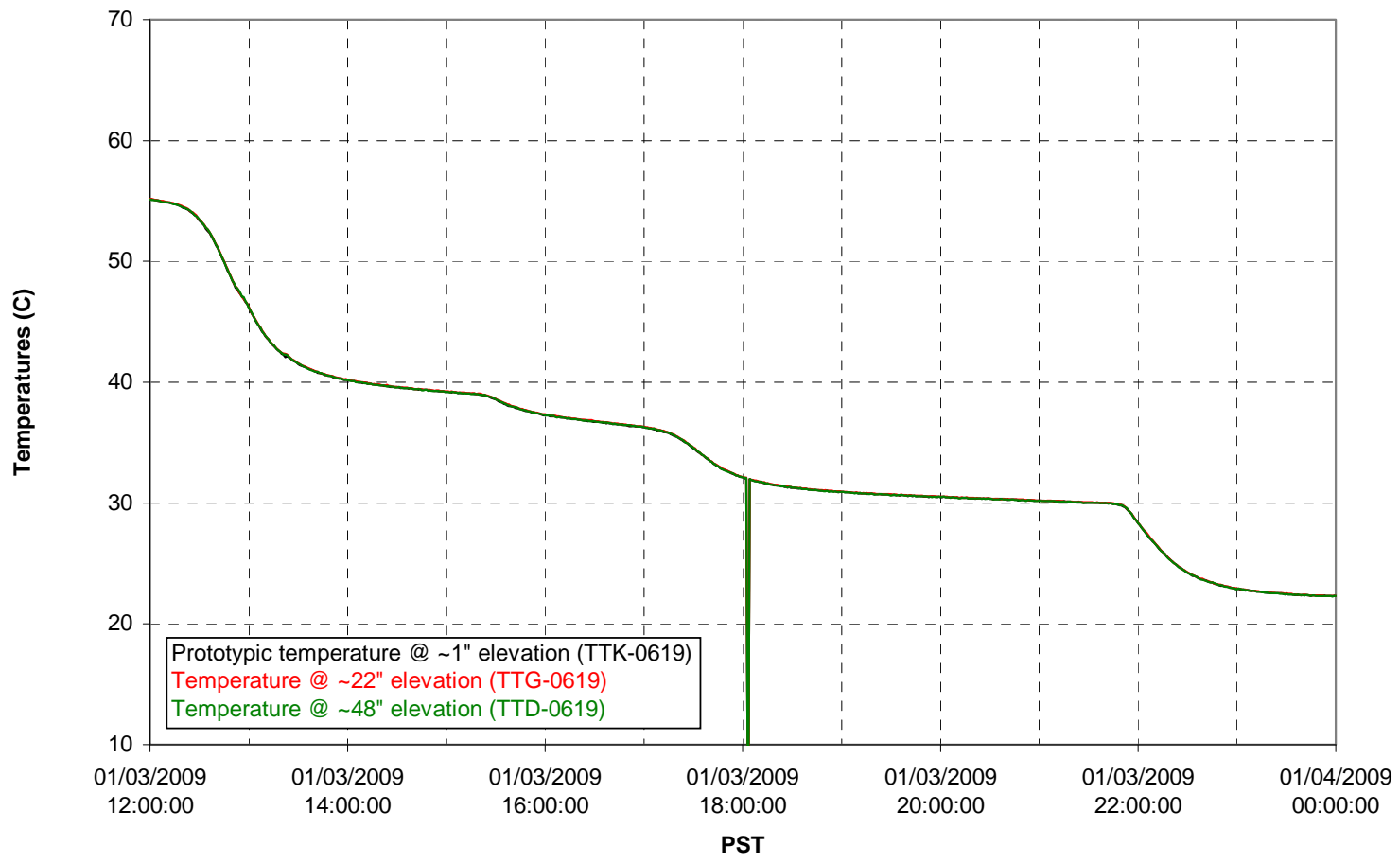


T02A and filter loop temperatures

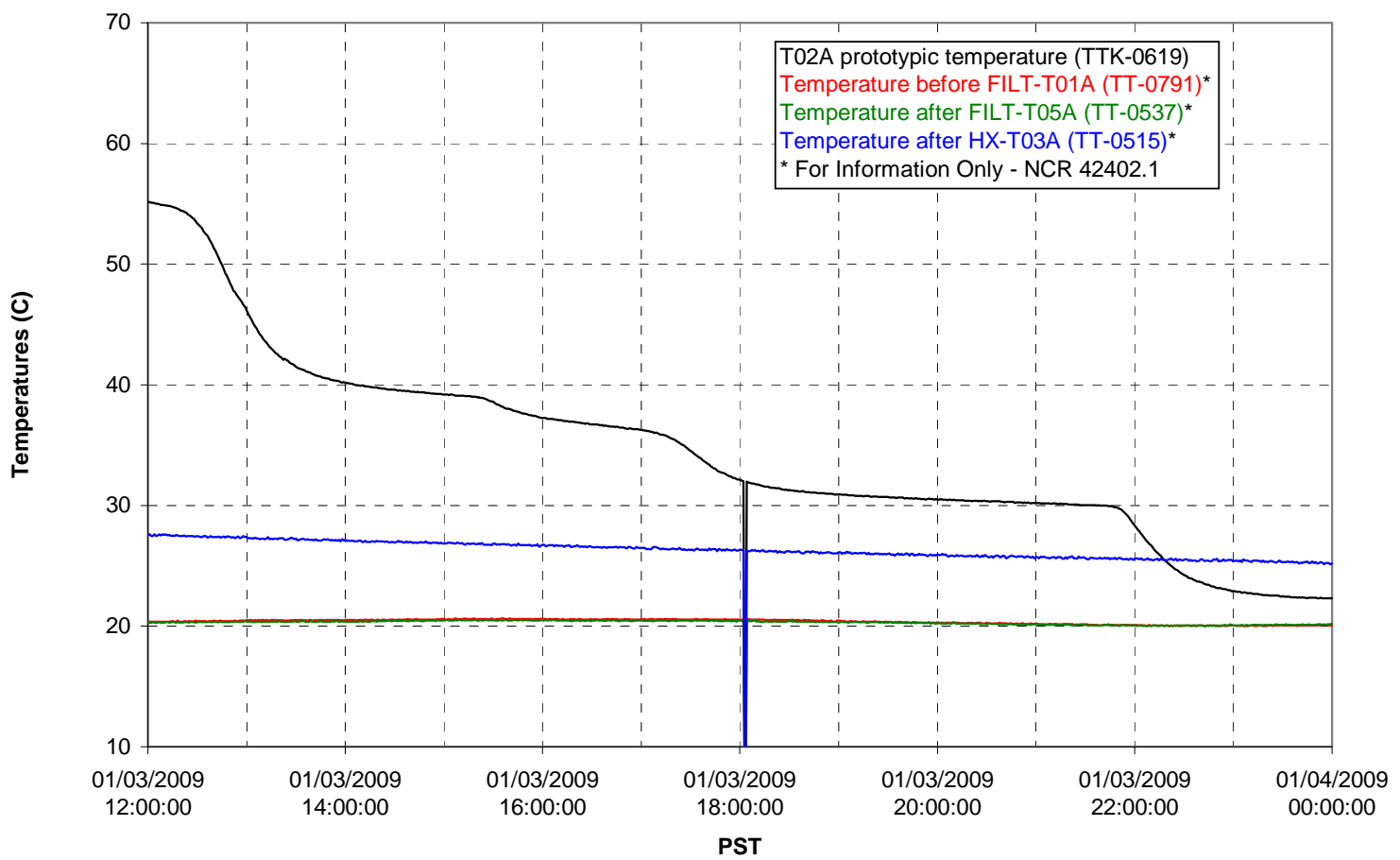

Pump Pressures and Flow

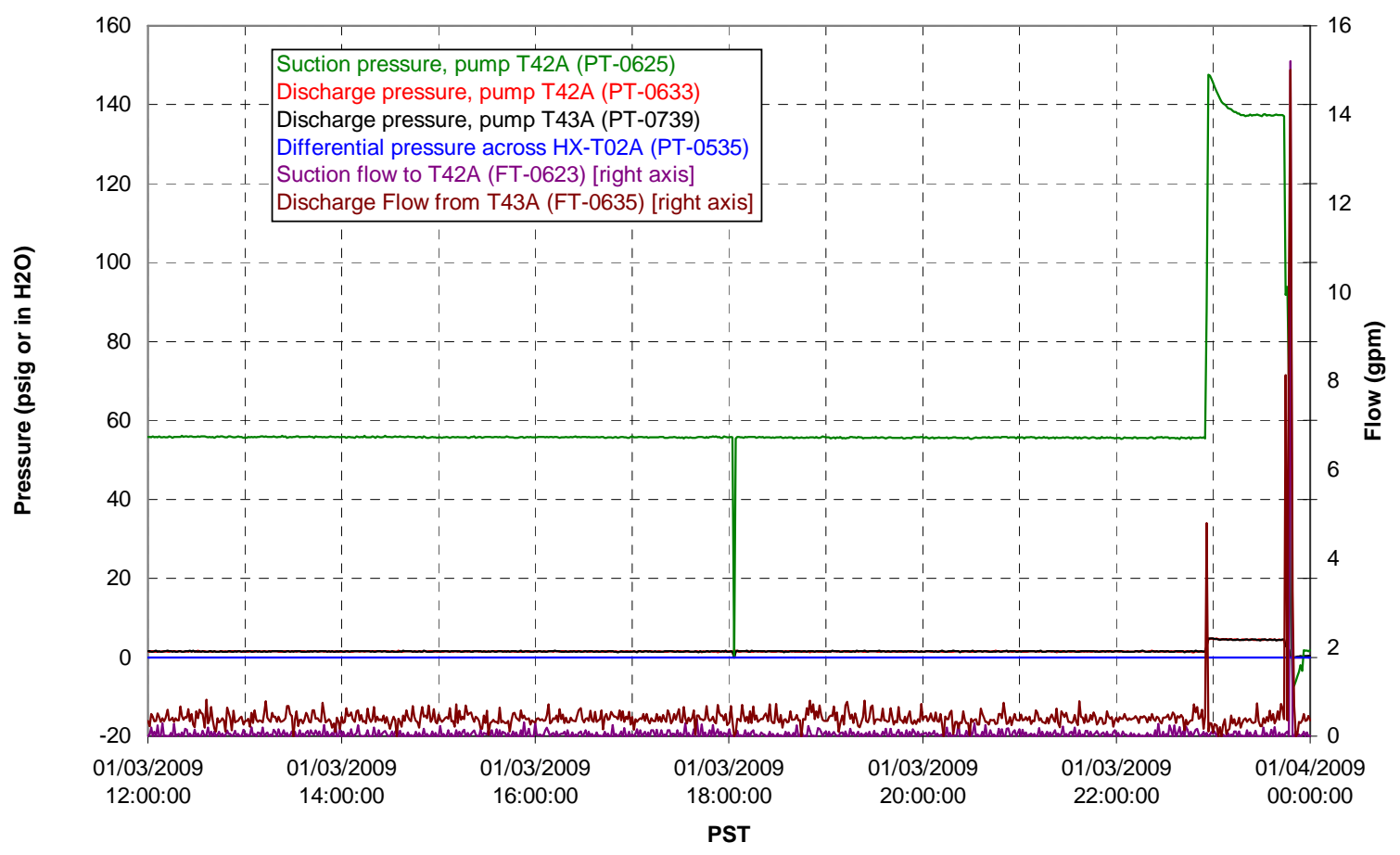


Axial pressure drop

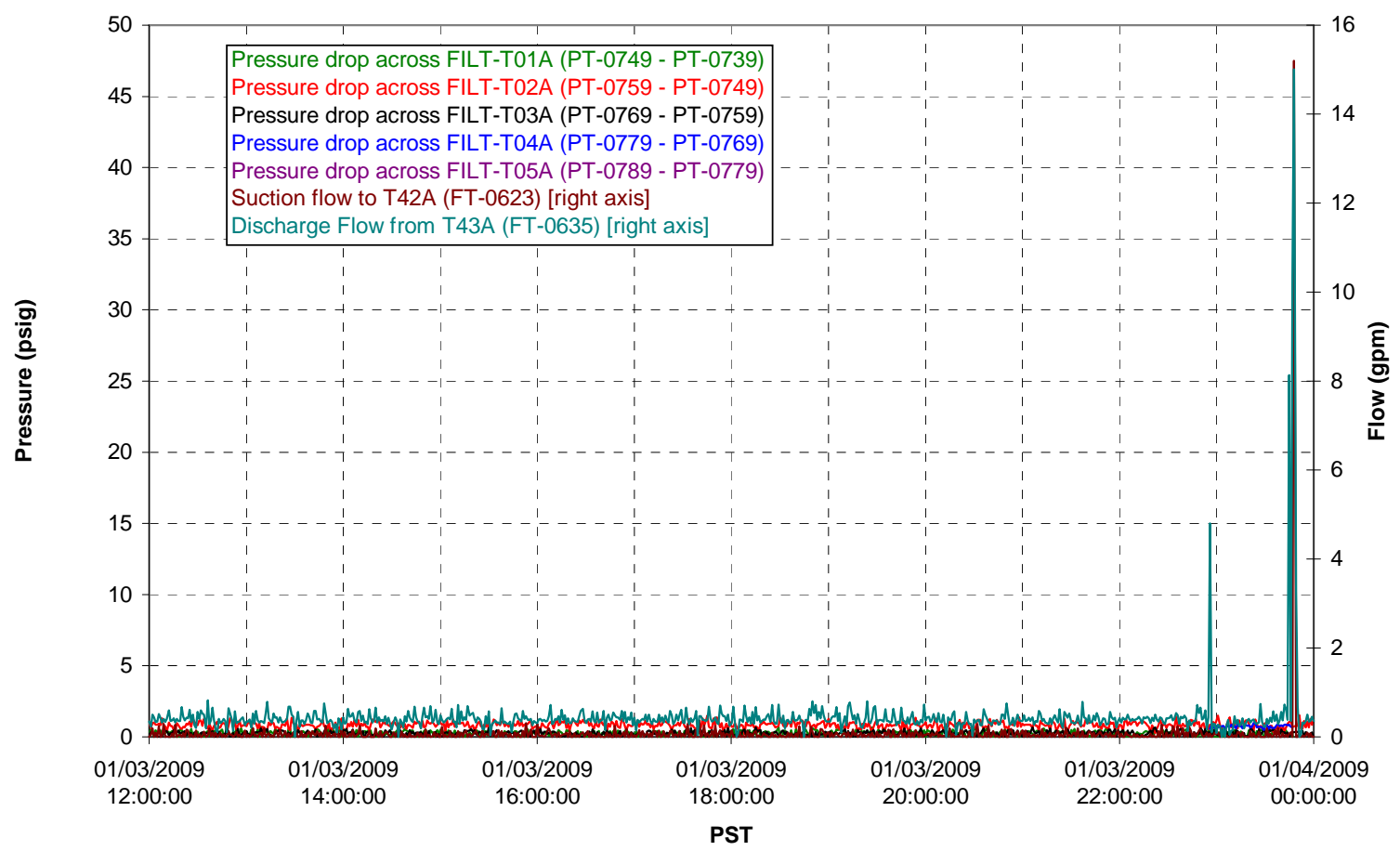

Permeate flow rates

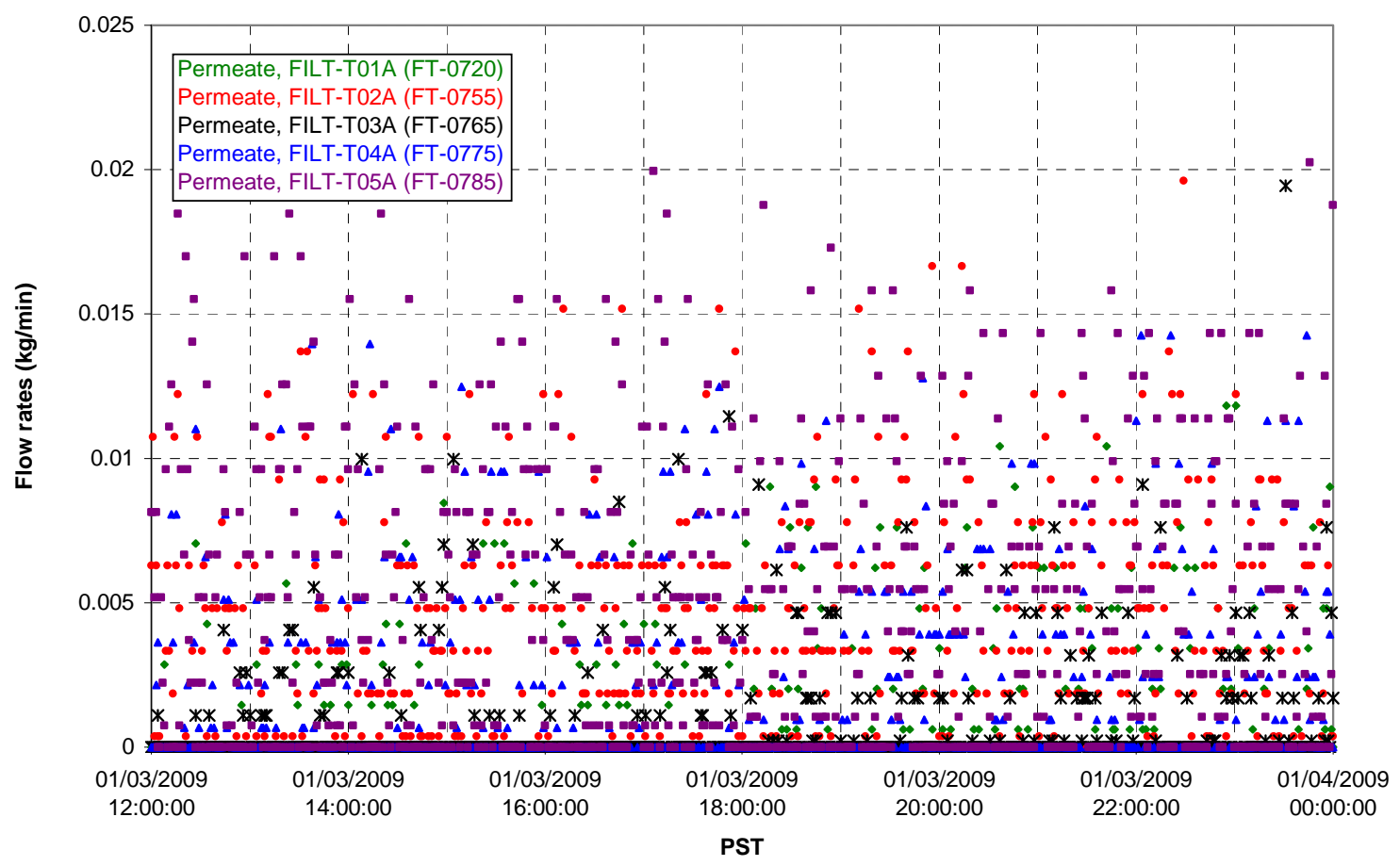


T02A Inner Temperature Tree

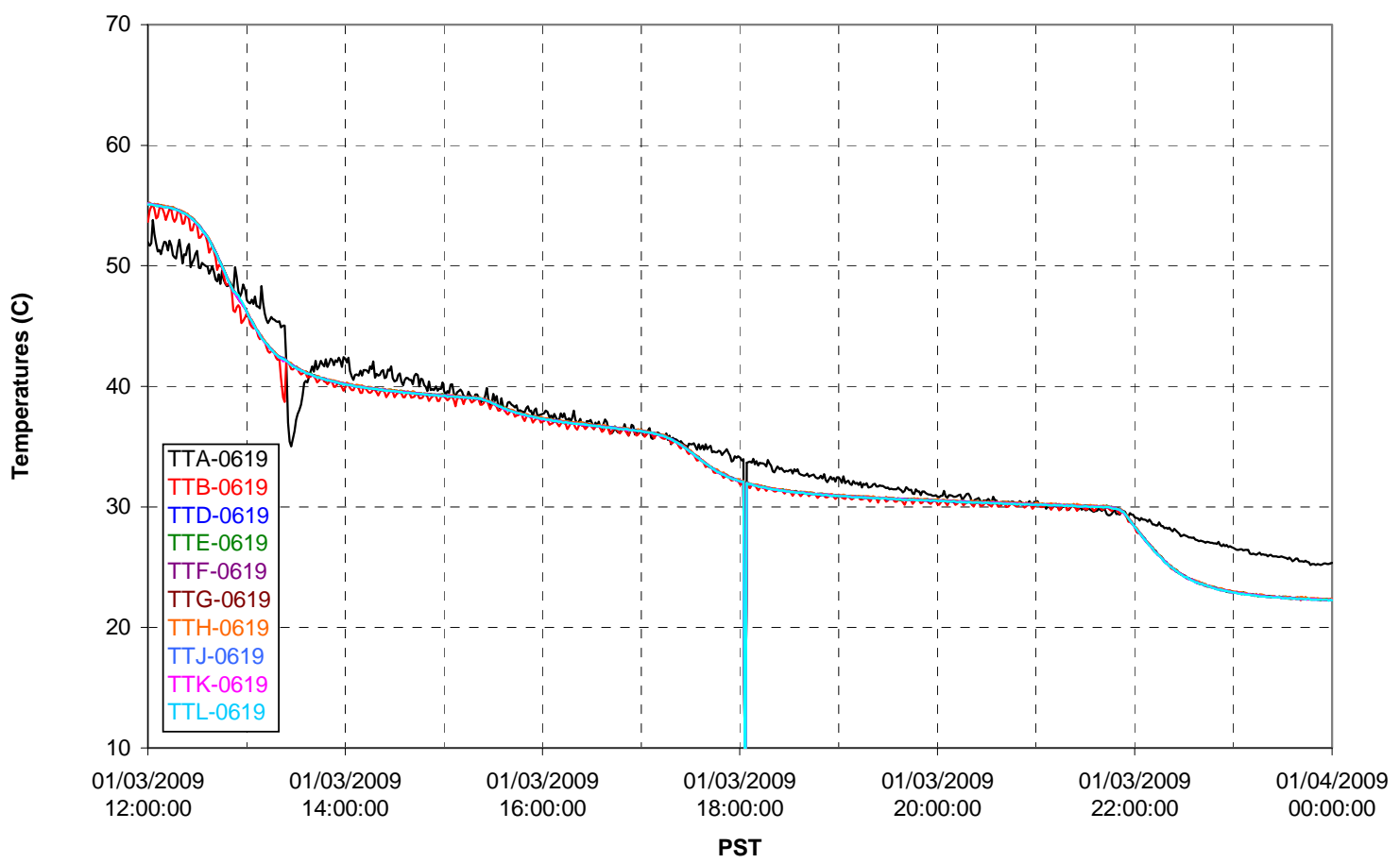

T02A Outer Temperature Tree

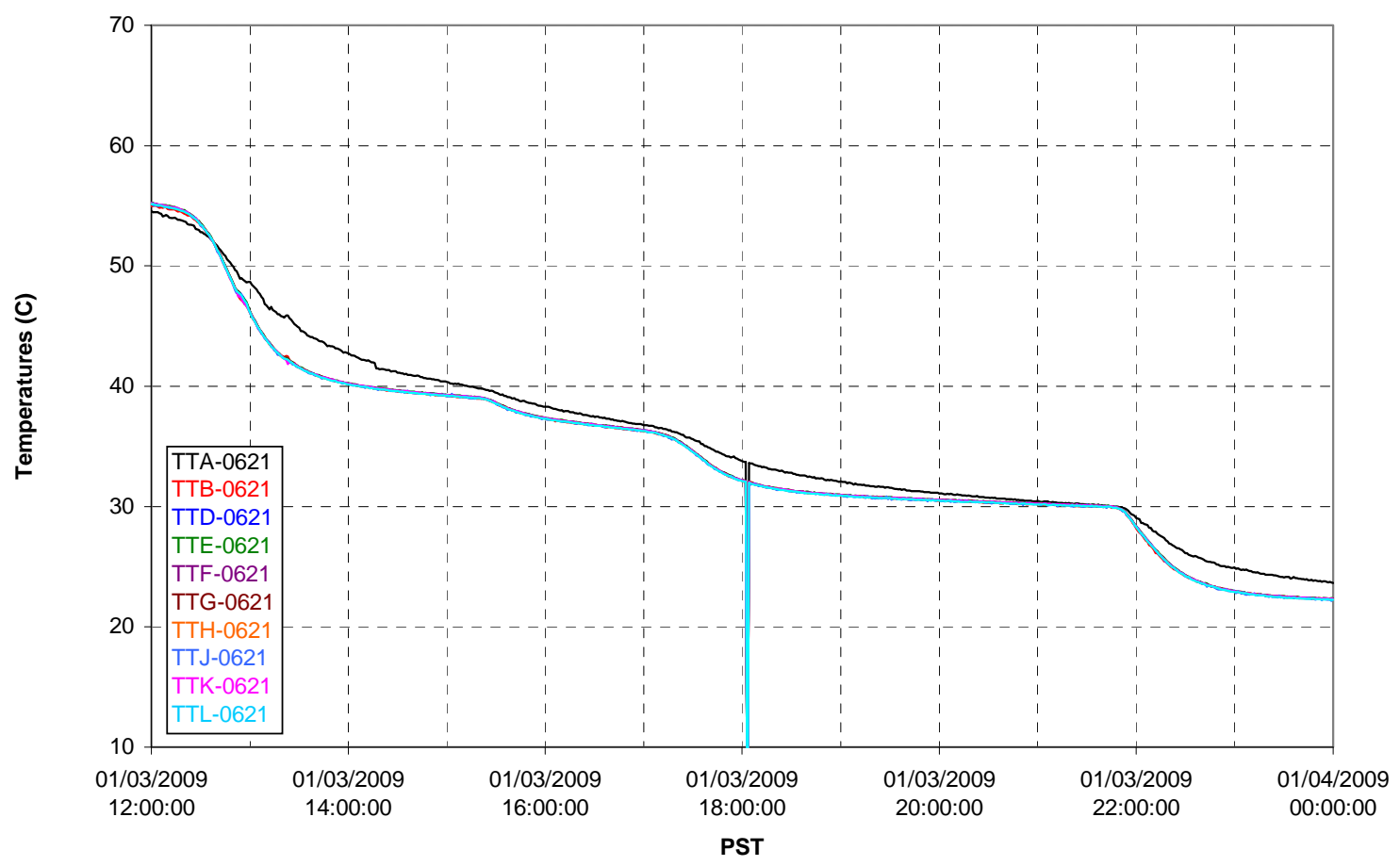


T02A temperatures

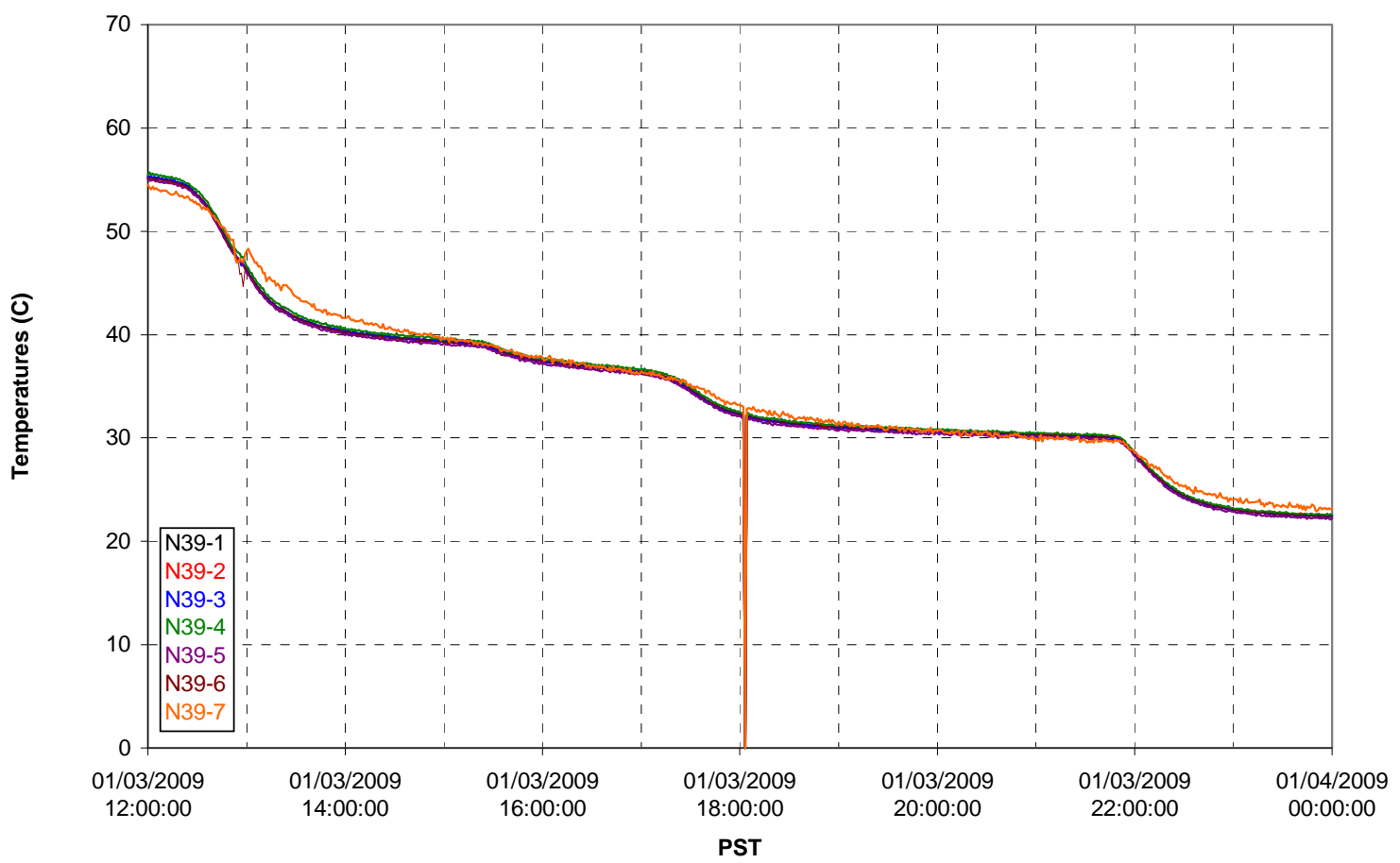

T02A temperatures

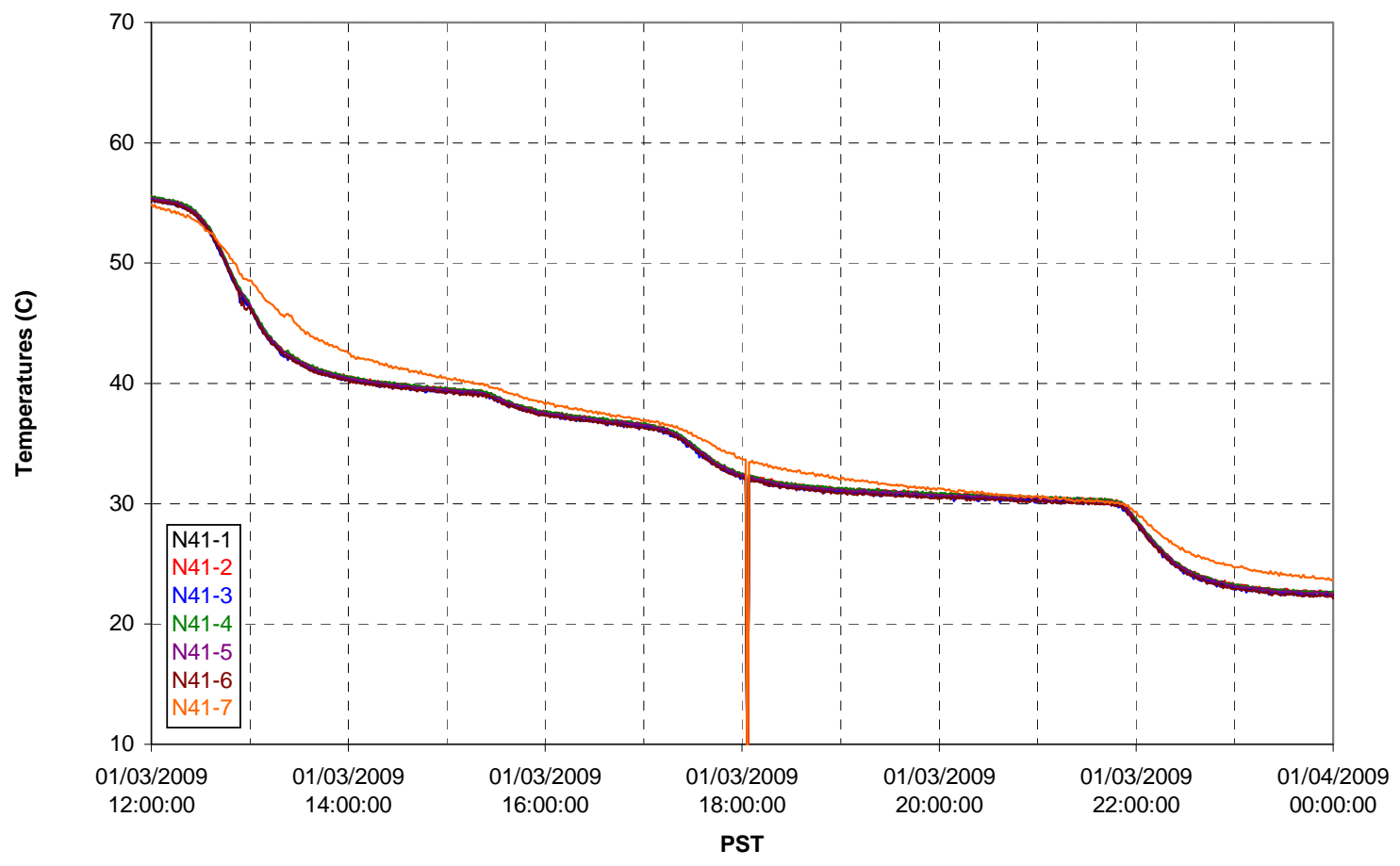


T02A temperatures

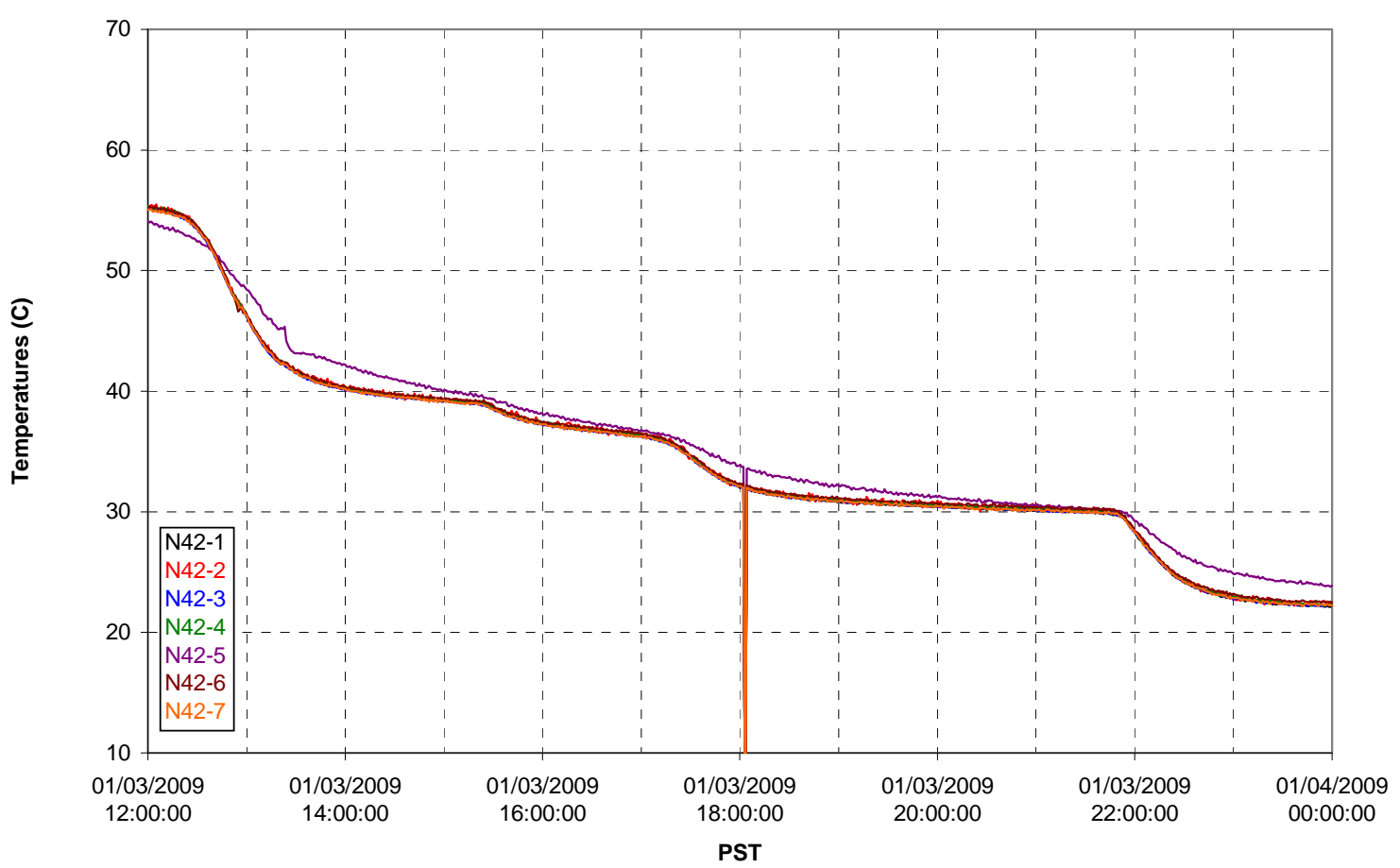

T02A temperatures

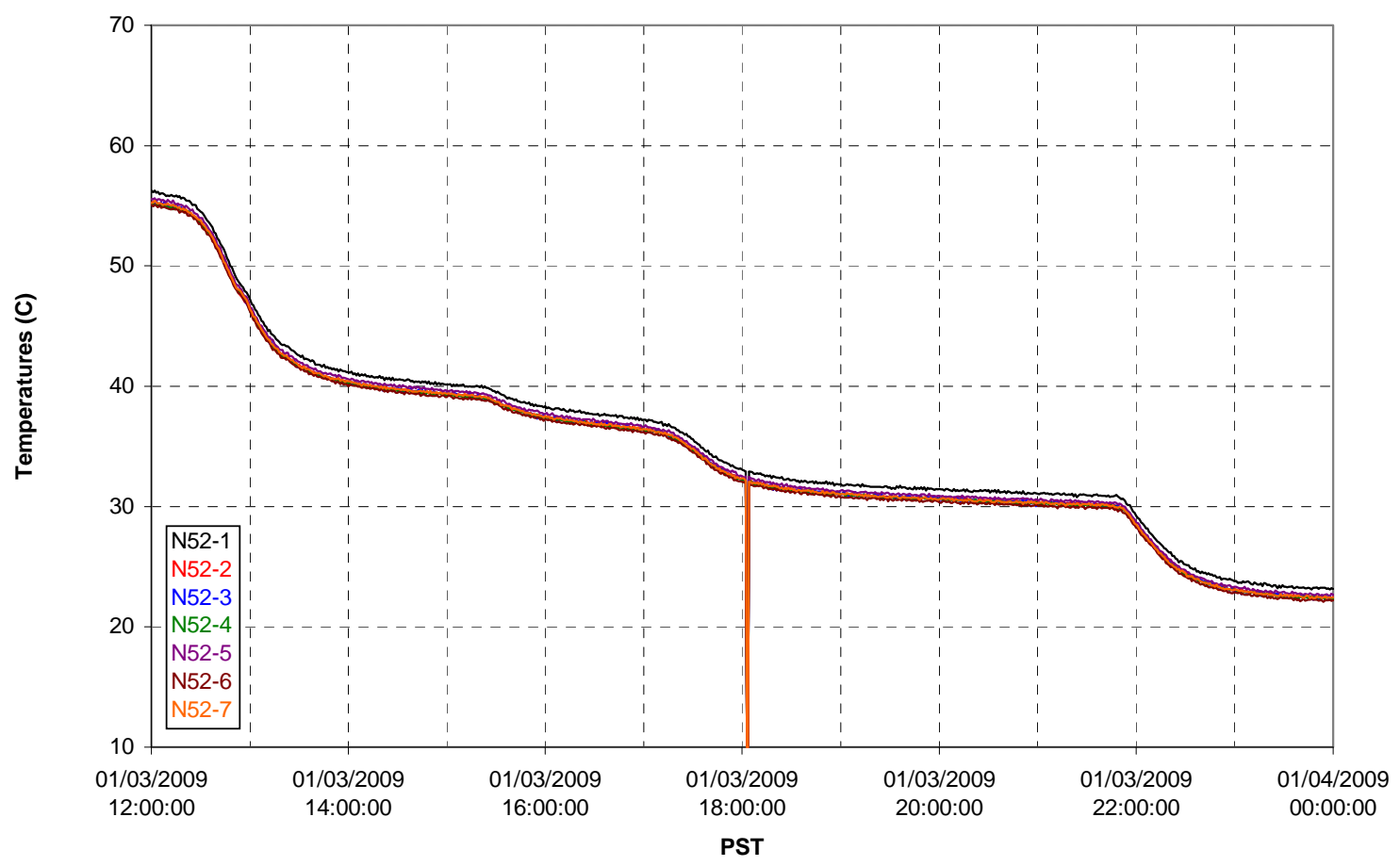


T02A Heating and Cooling

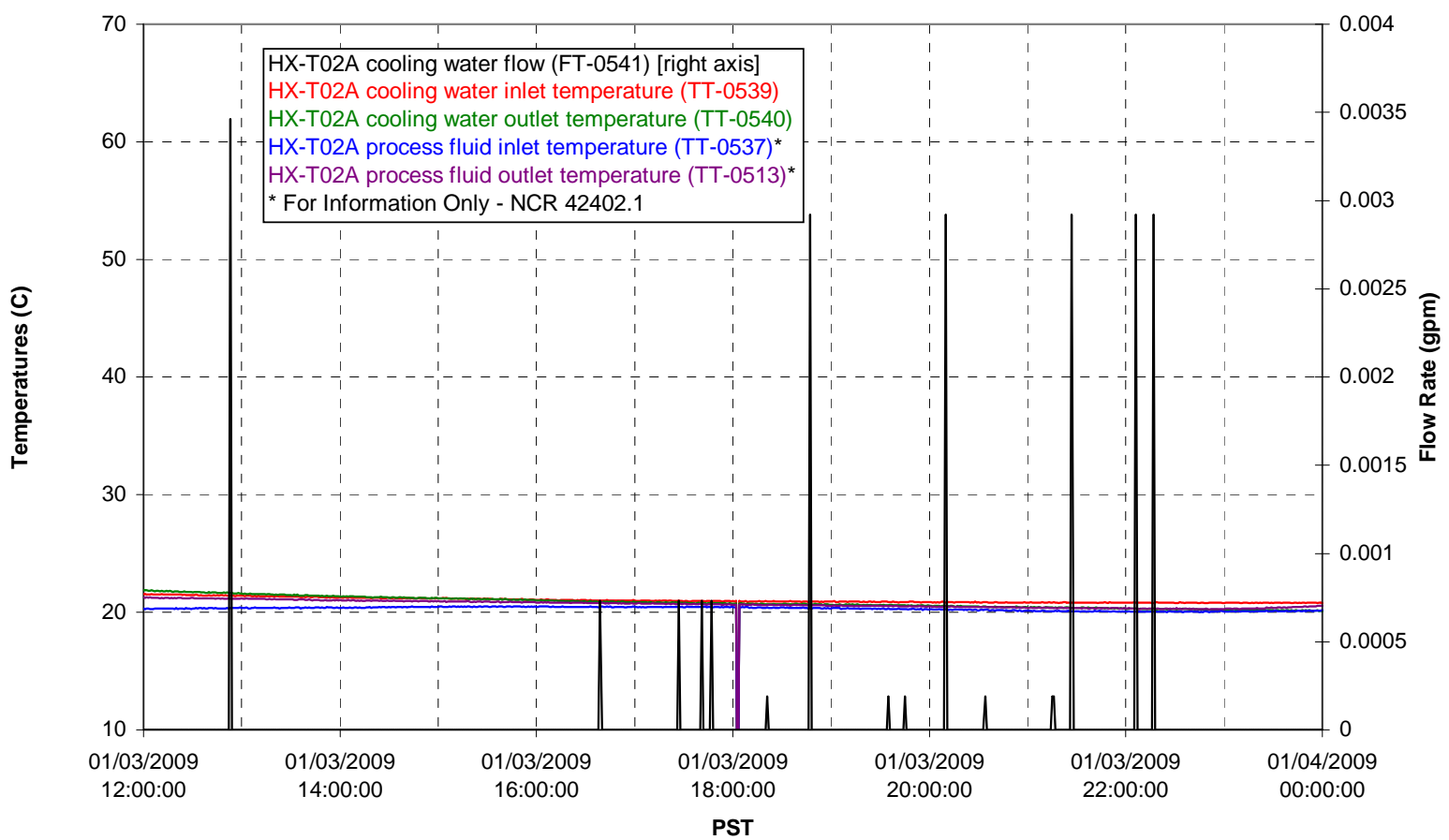

Pump Operation

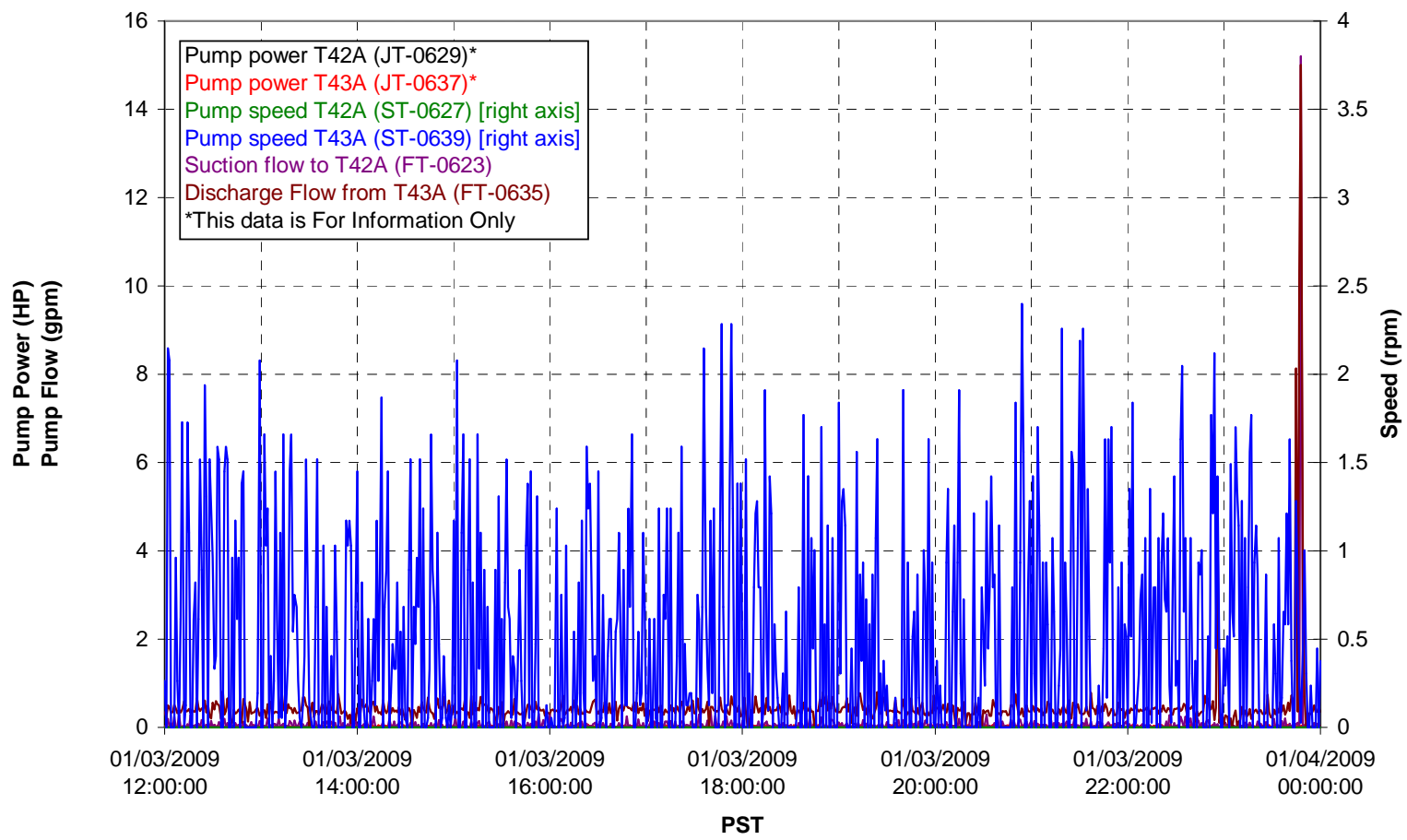


Pulsepot UFP-PP-T01A

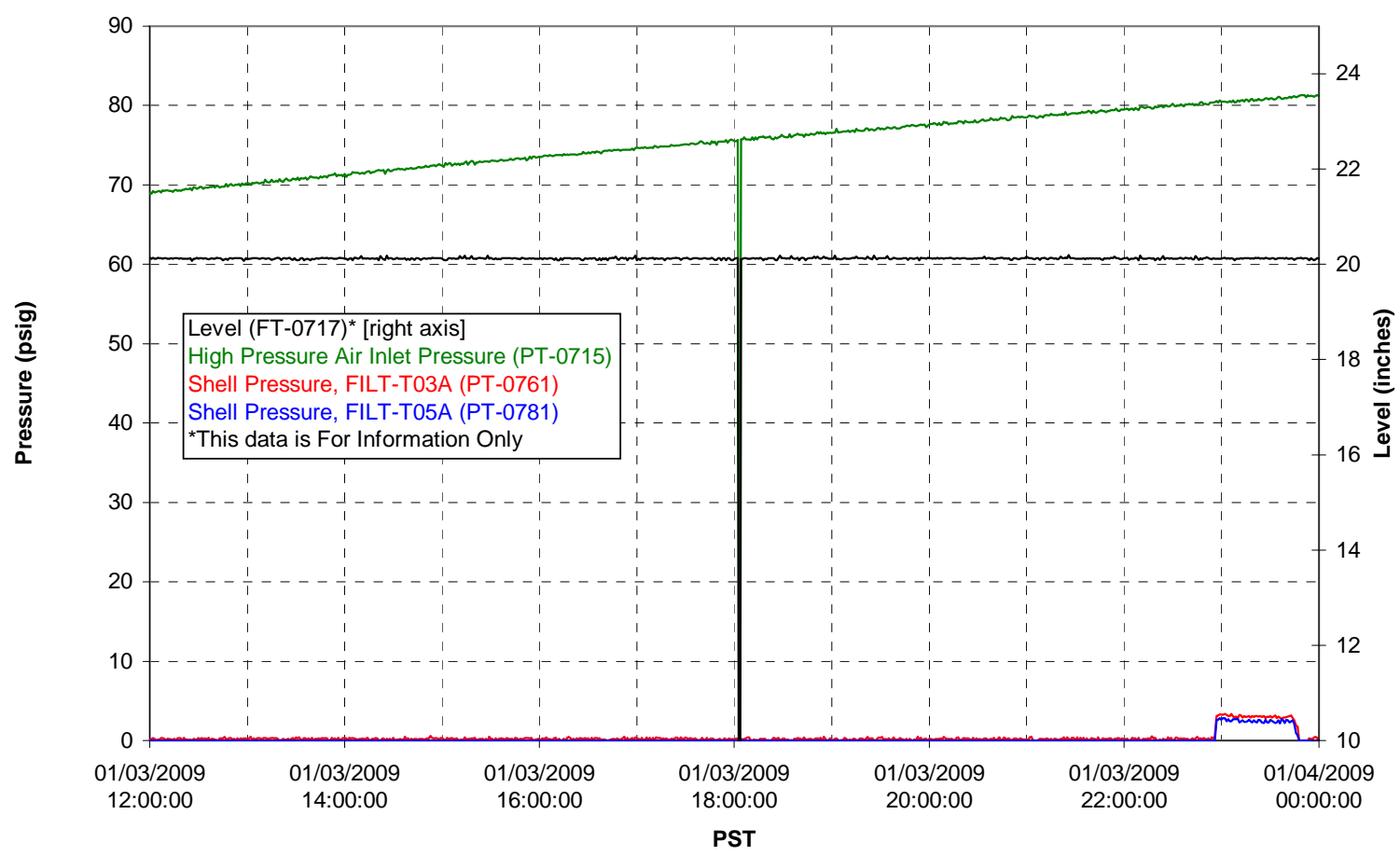

Pulsepot UFP-PP-T02A

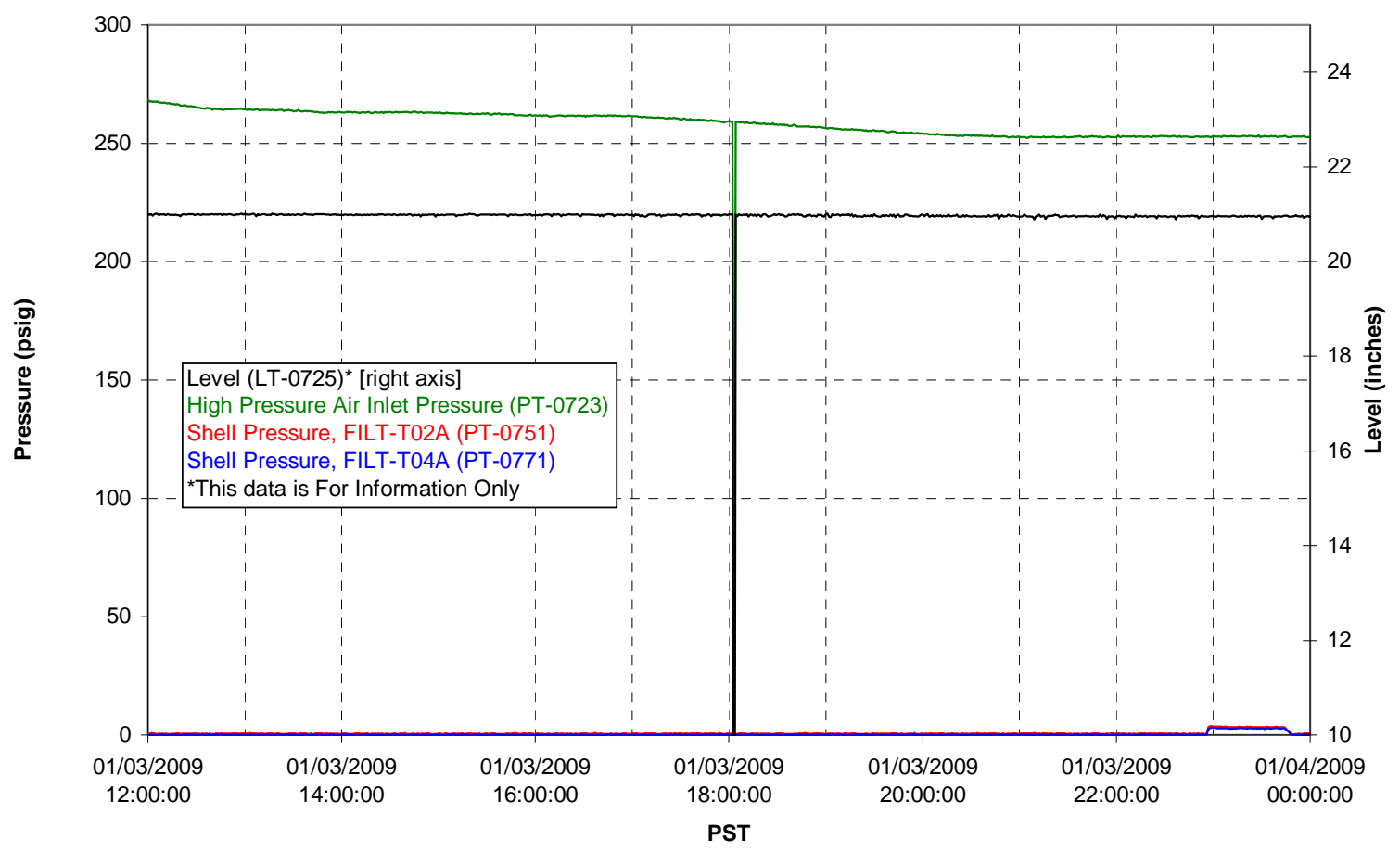


Pulsepot UFP-PP-T03A

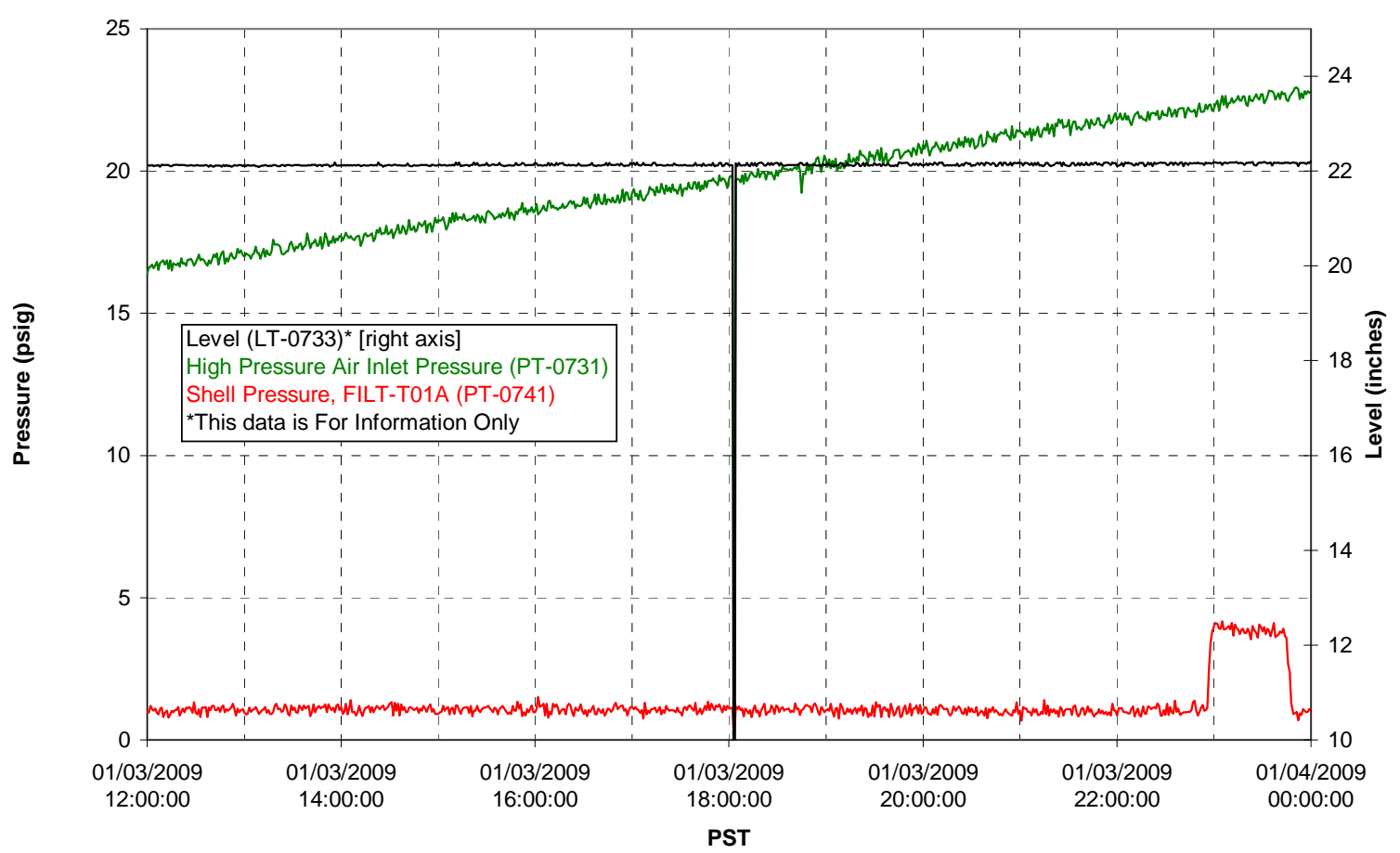

Pulsepot Levels

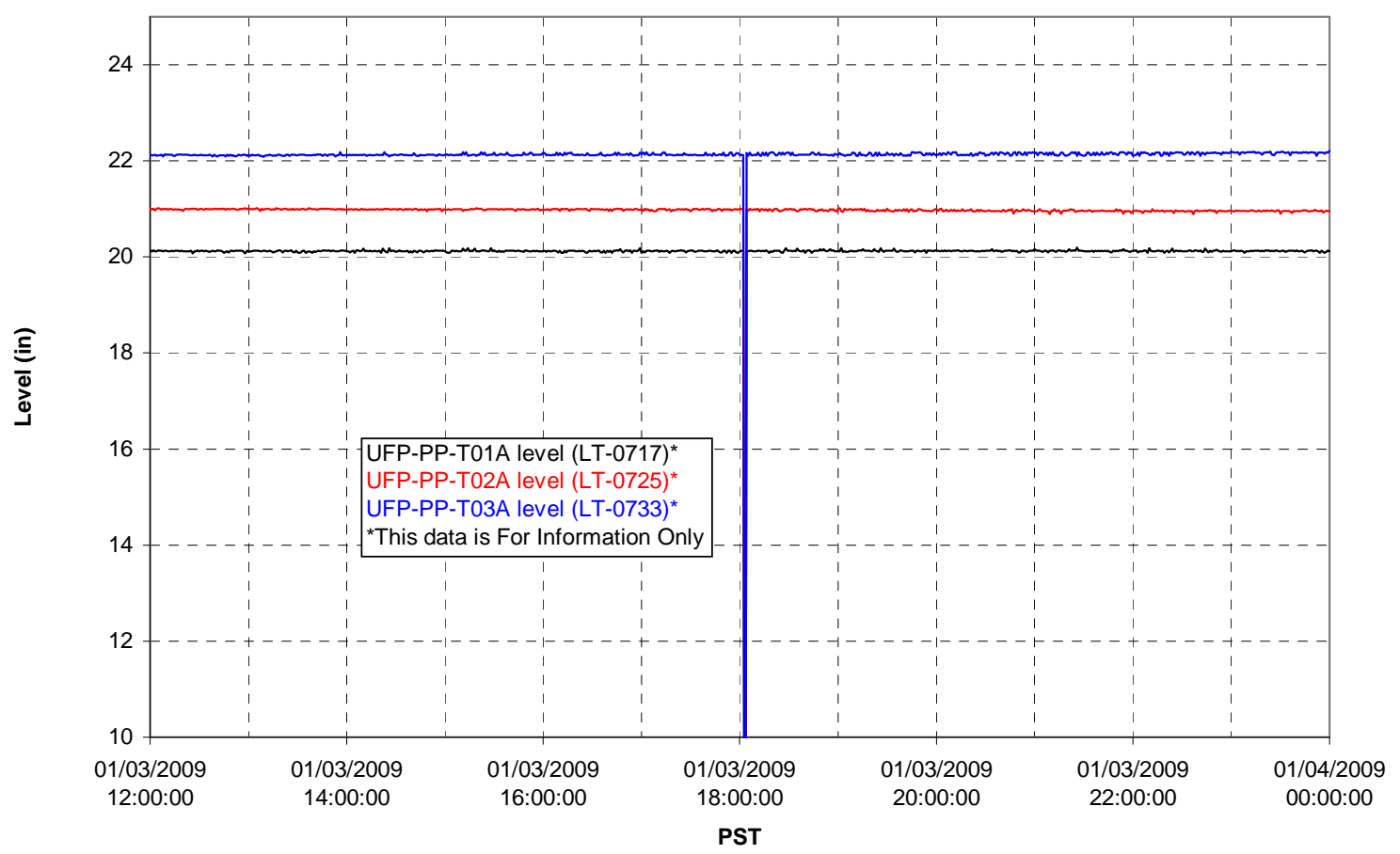


Filter UFP-FILT-T01A

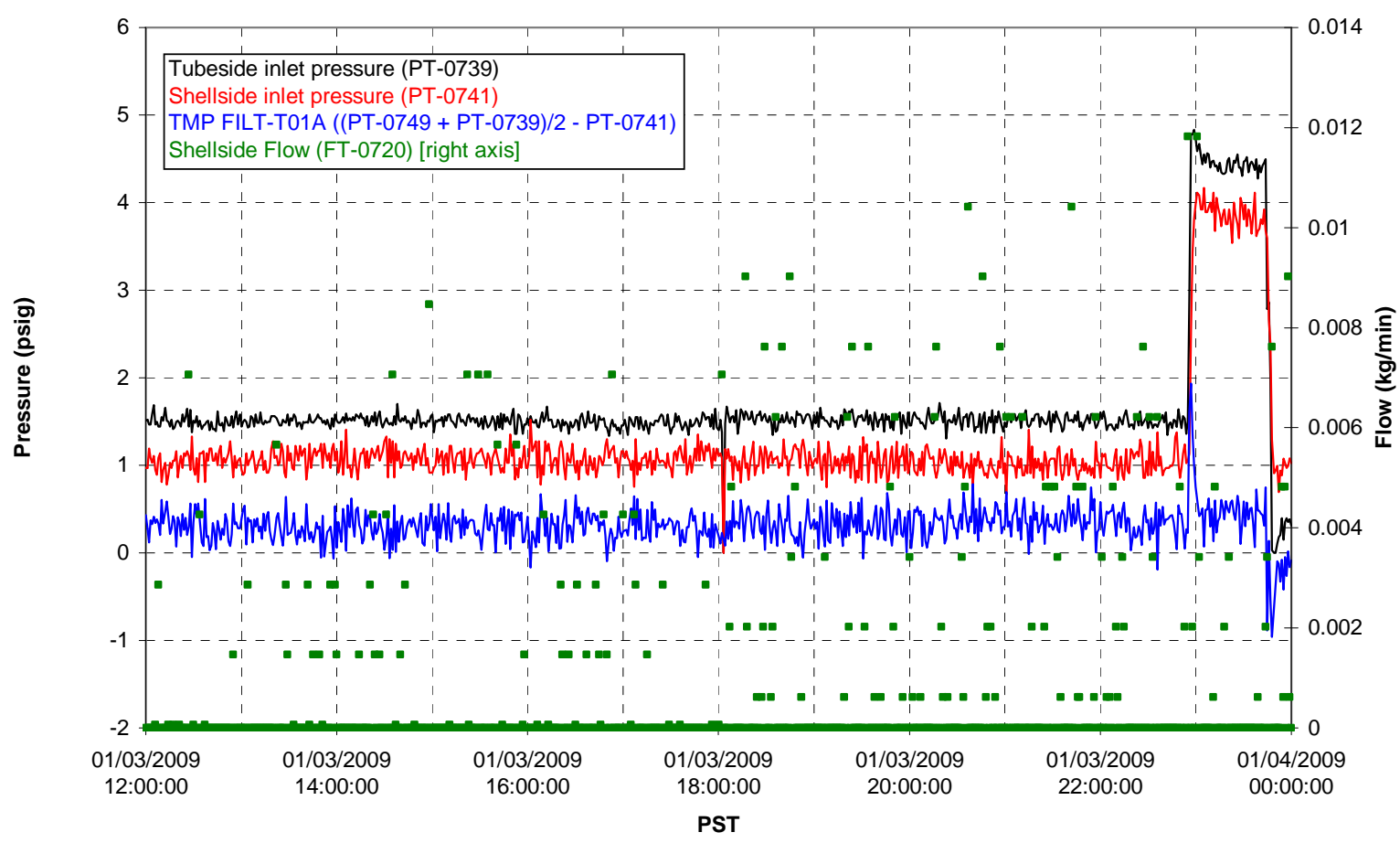

Filter UFP-FILT-T02A

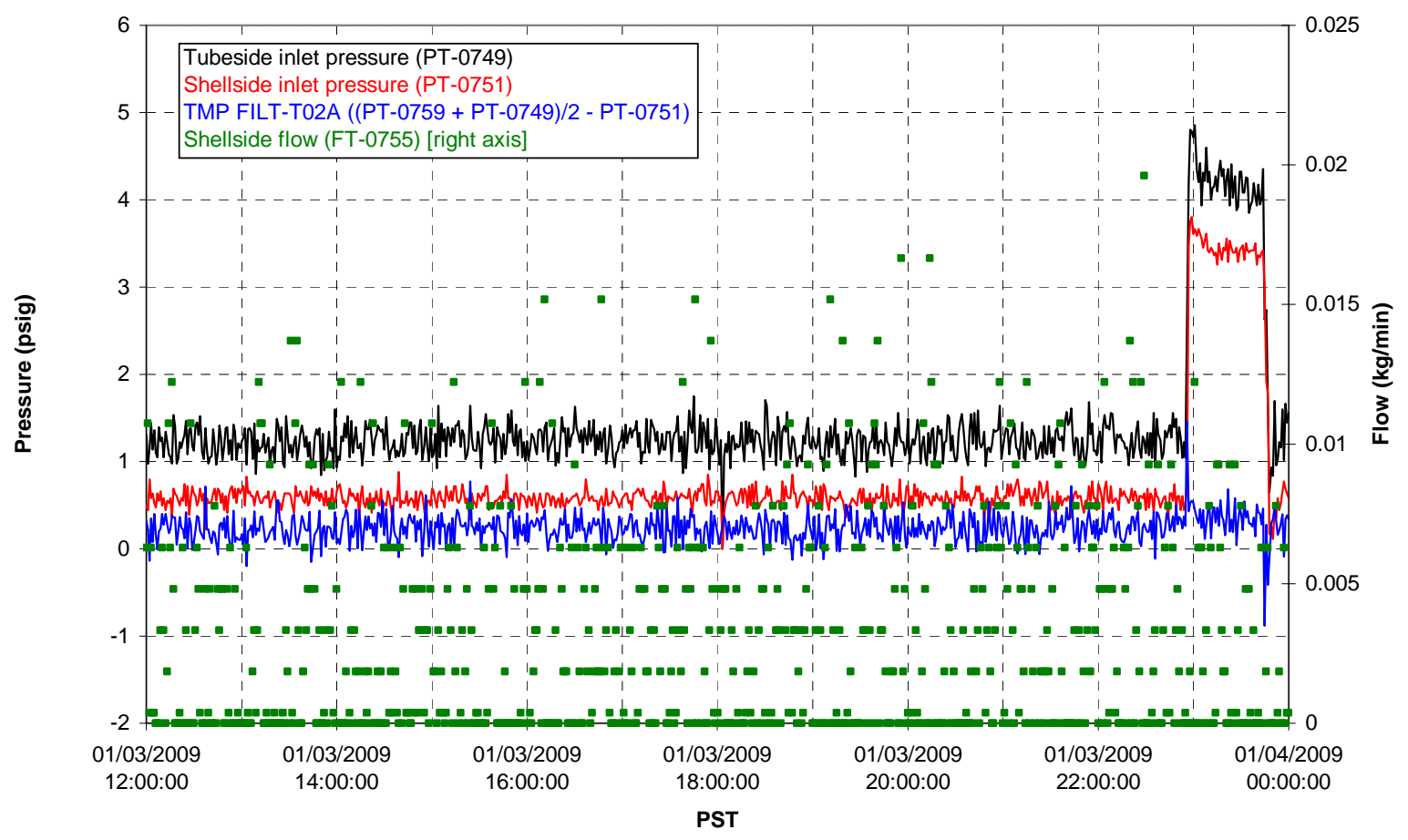


Filter UFP-FILT-T03A

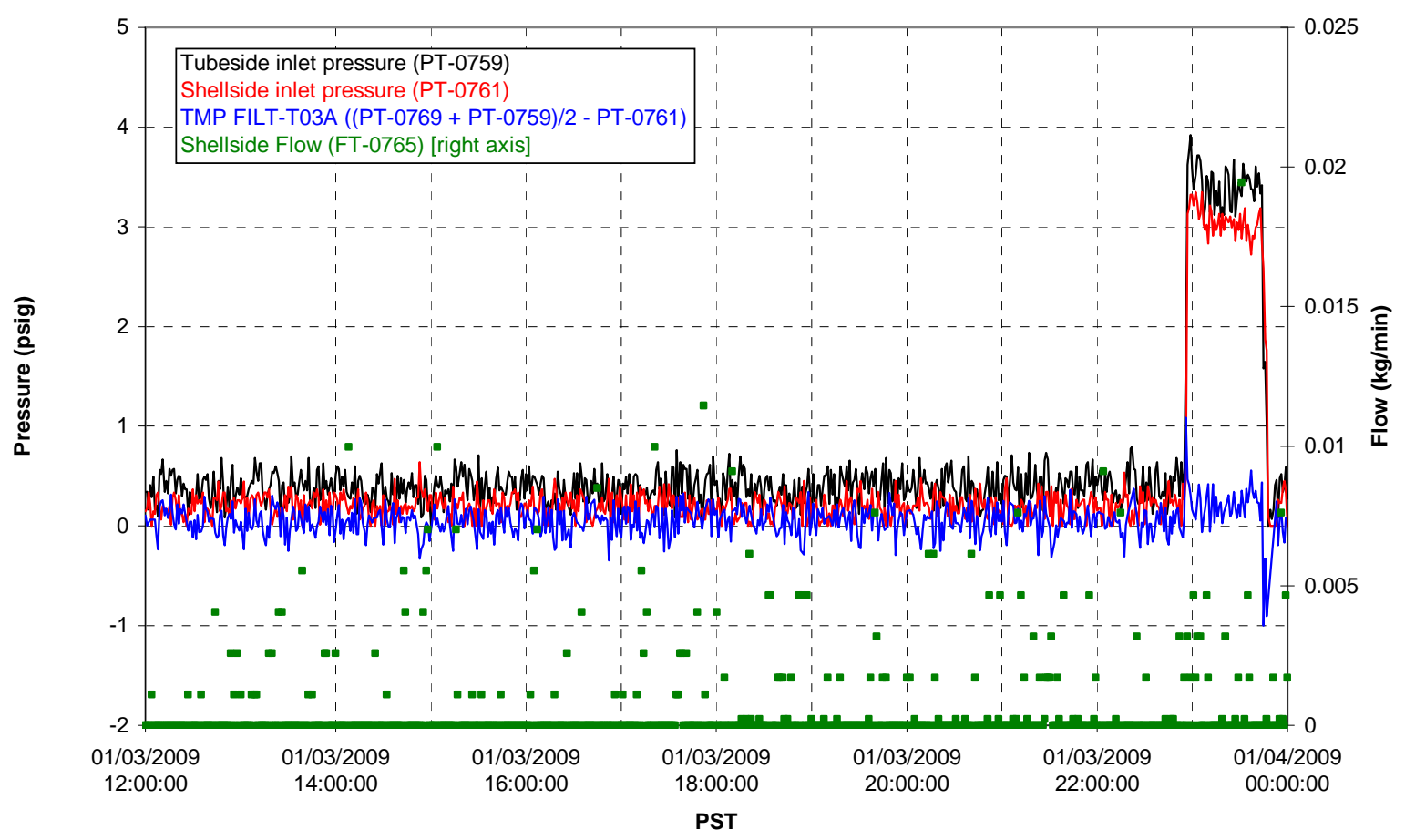

Filter UFP-FILT-T04A

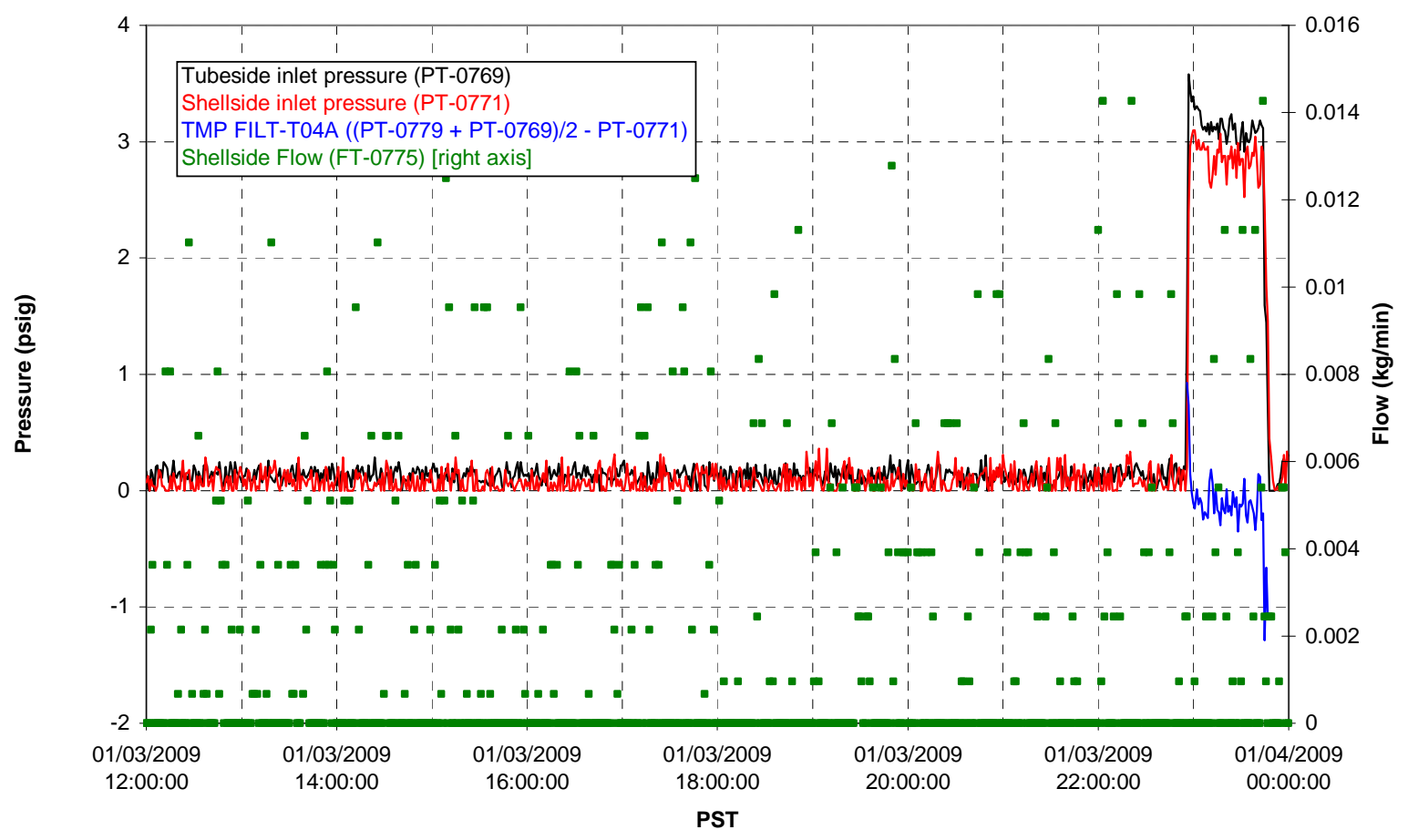


Filter UFP-FILT-T05A

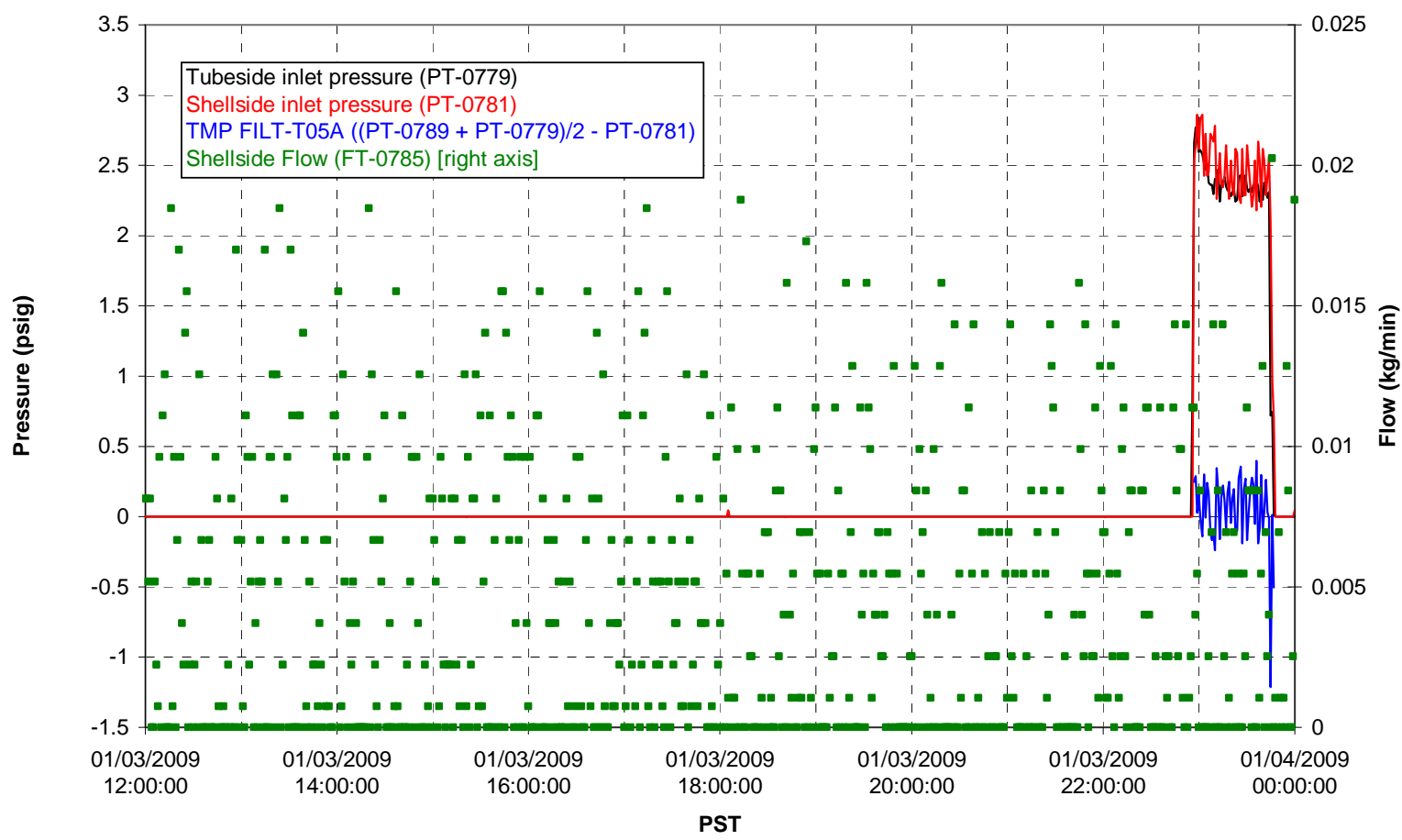

Chemical Flow

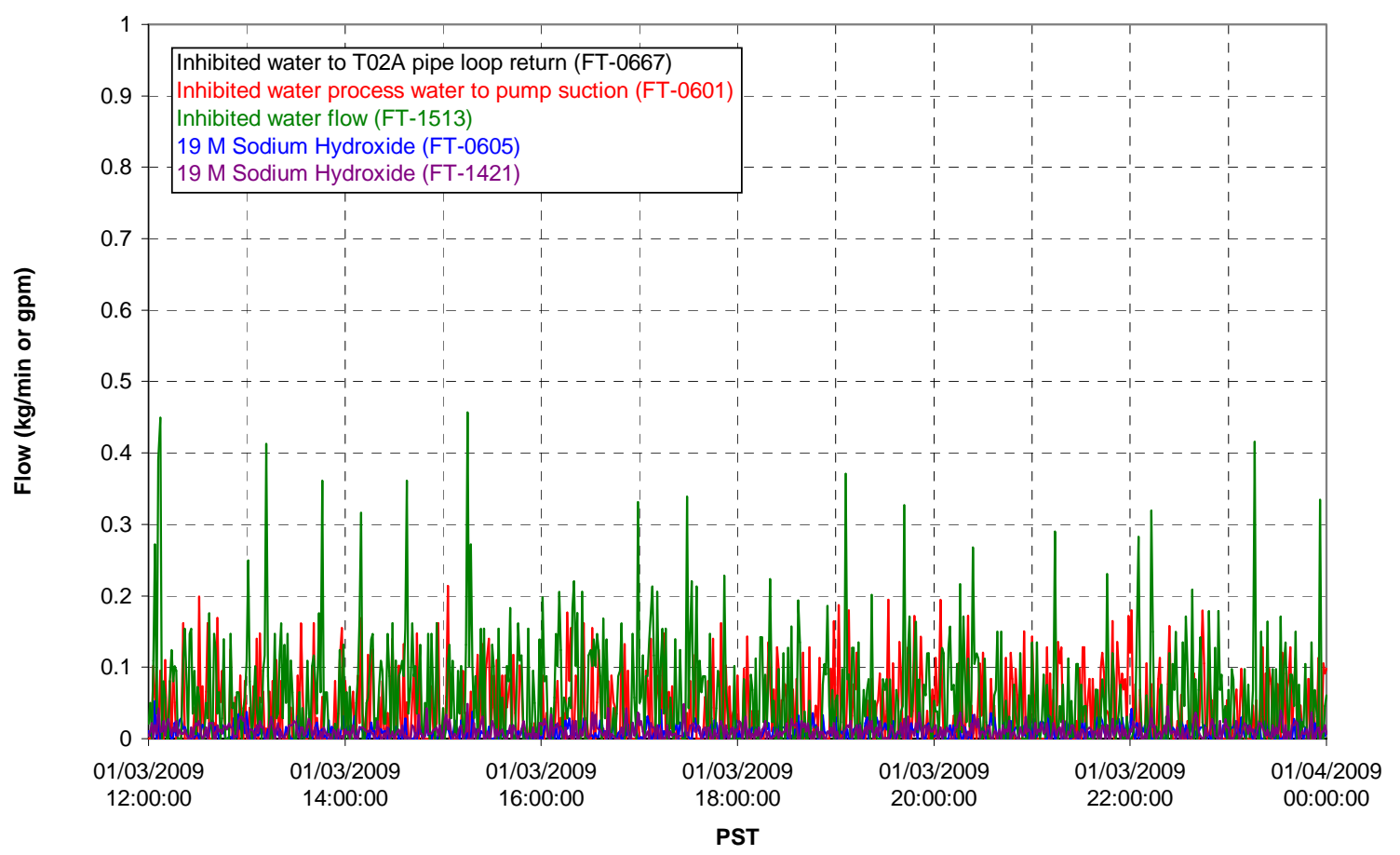




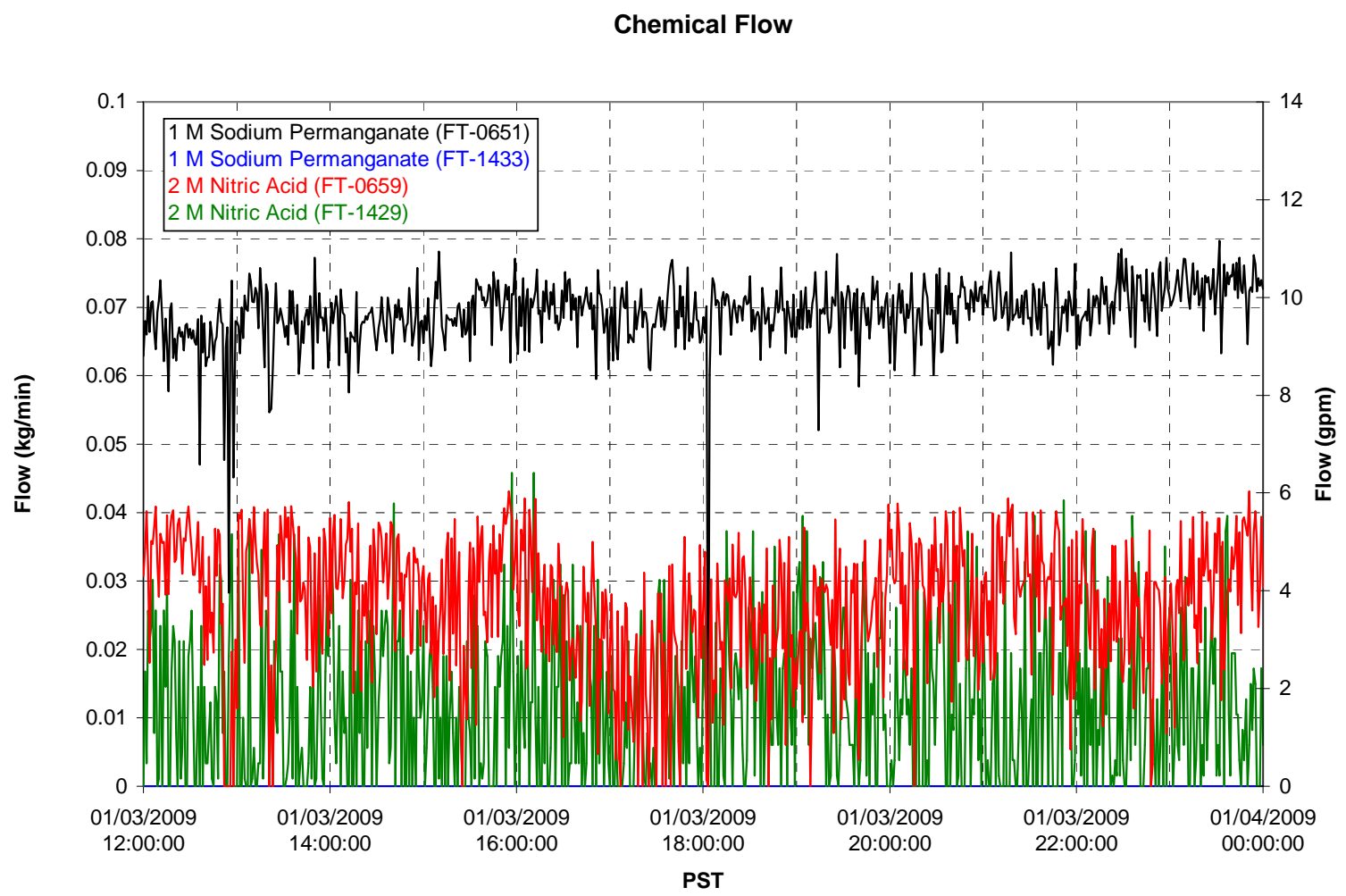

Air Flows

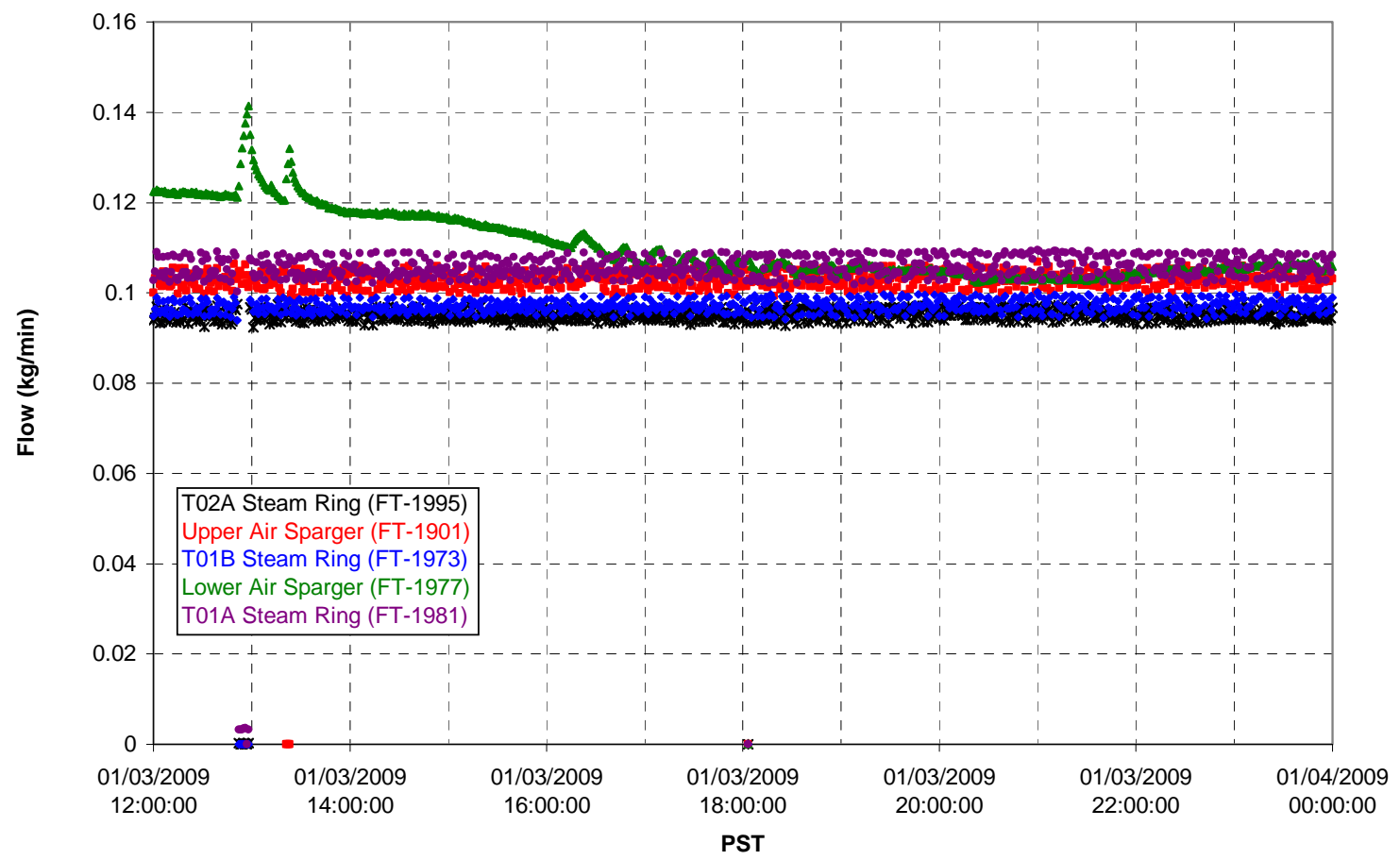


T02A Steam

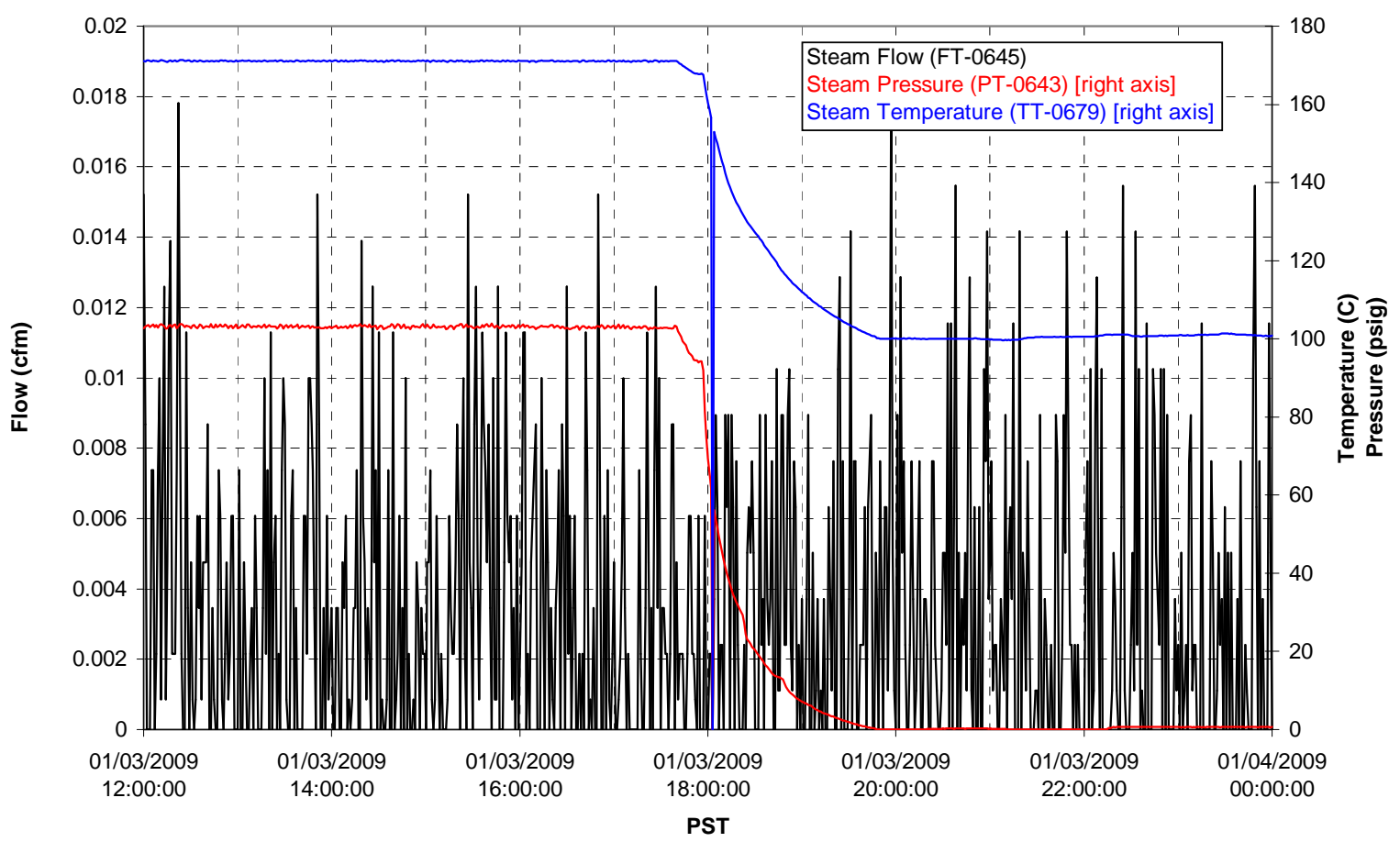

T01A Steam

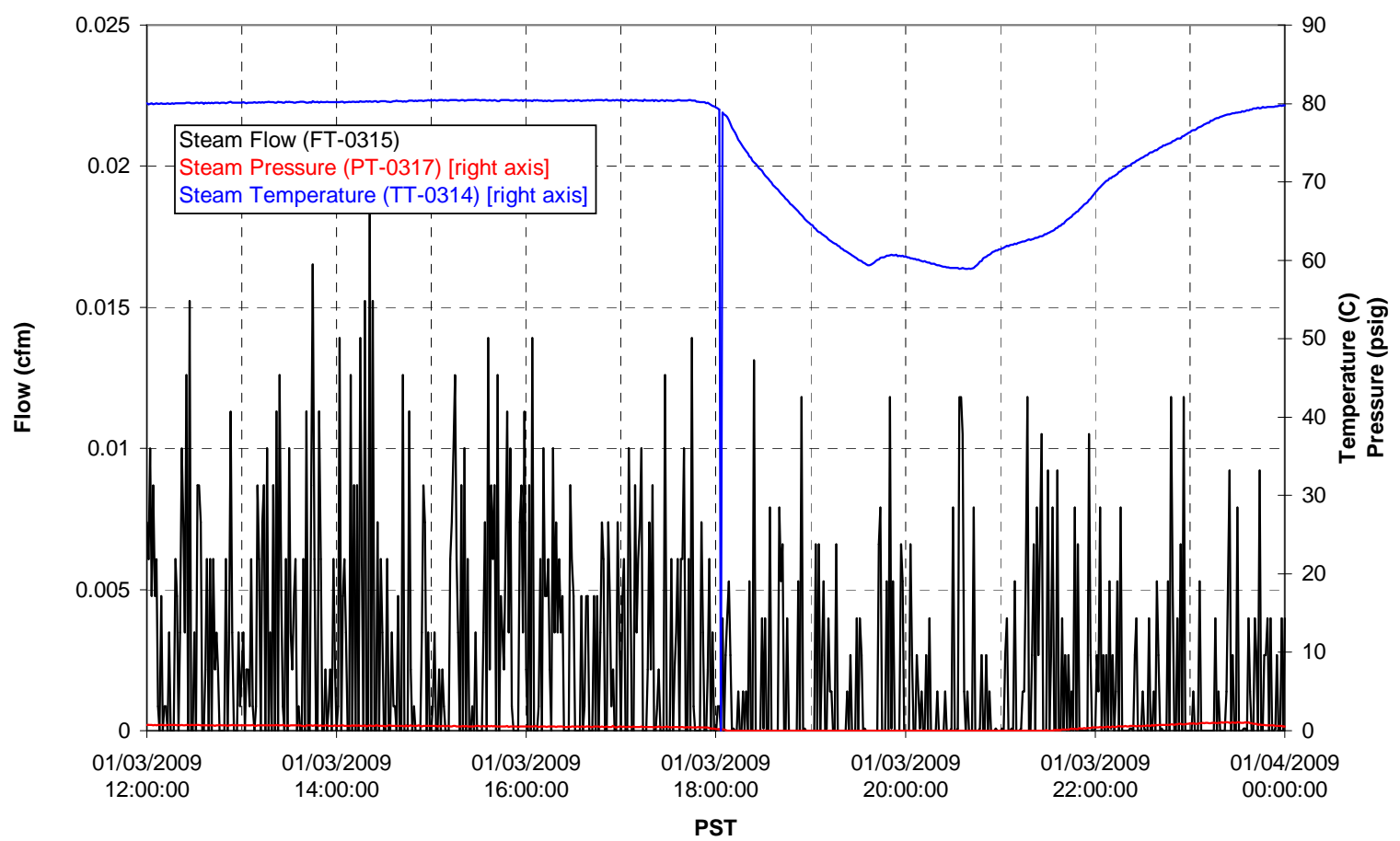


T01B Steam

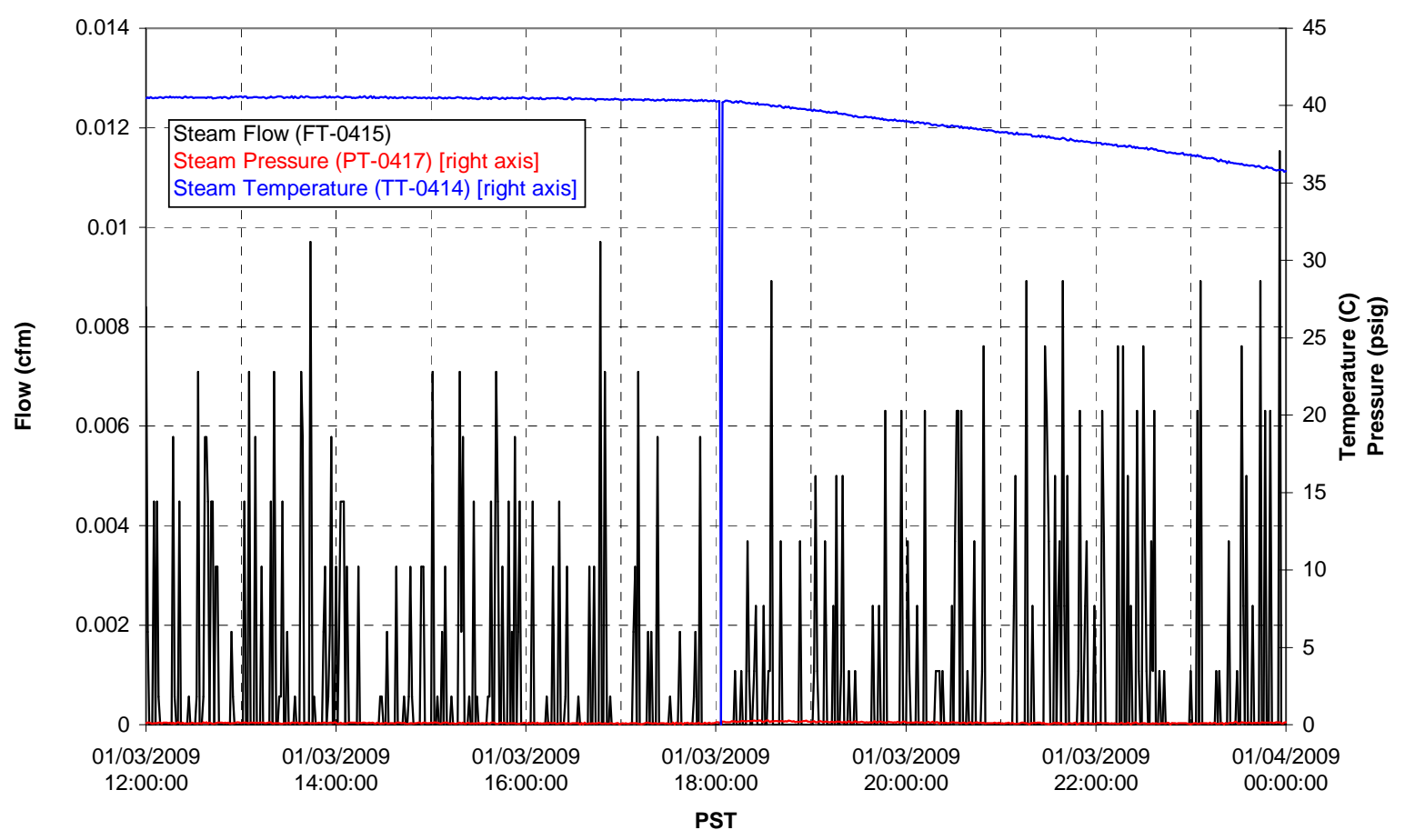


Functional Test Data Plots

01/04/2009 00:00 - 01/04/2009 12:00 
T01A level

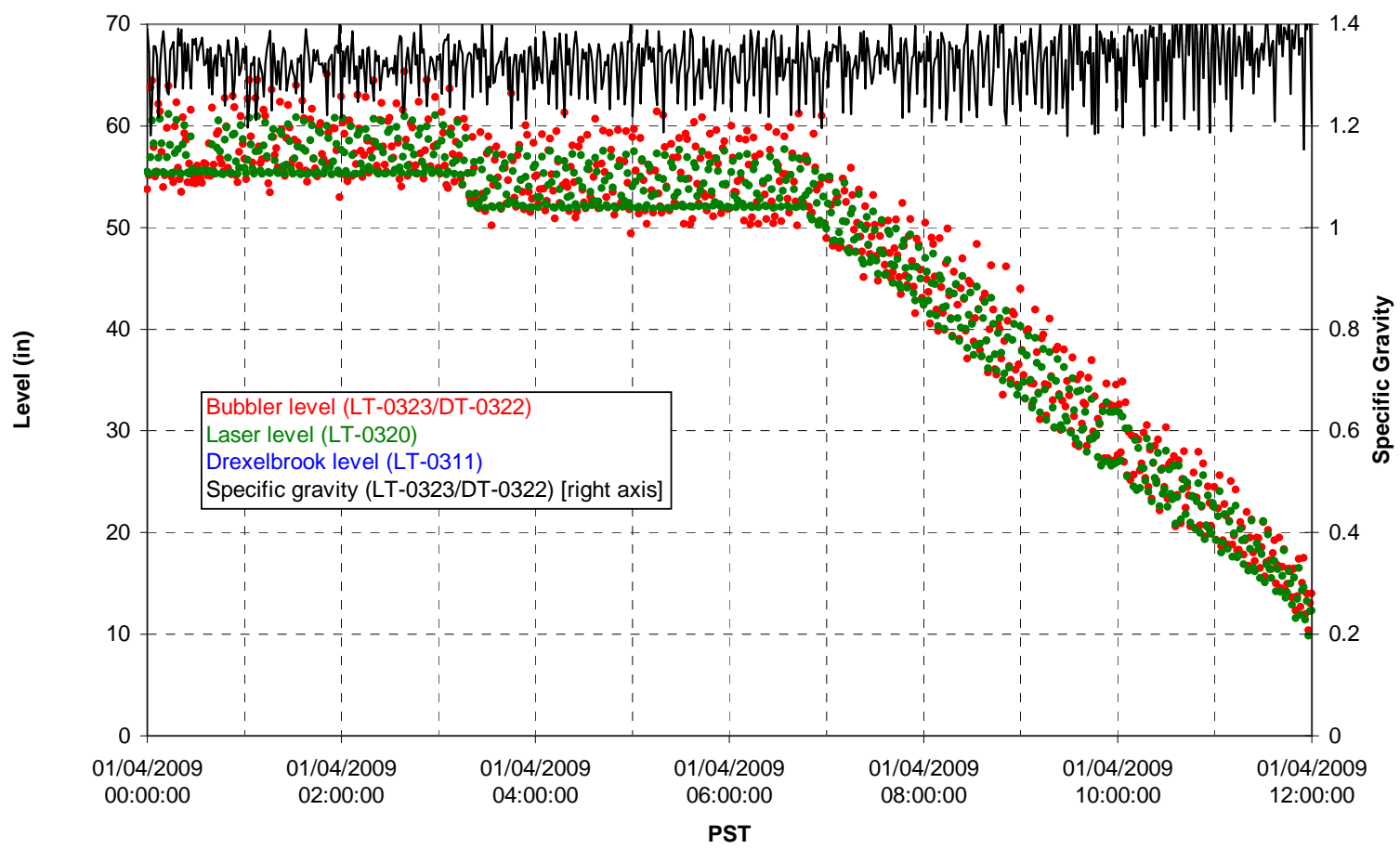

T01A temperatures

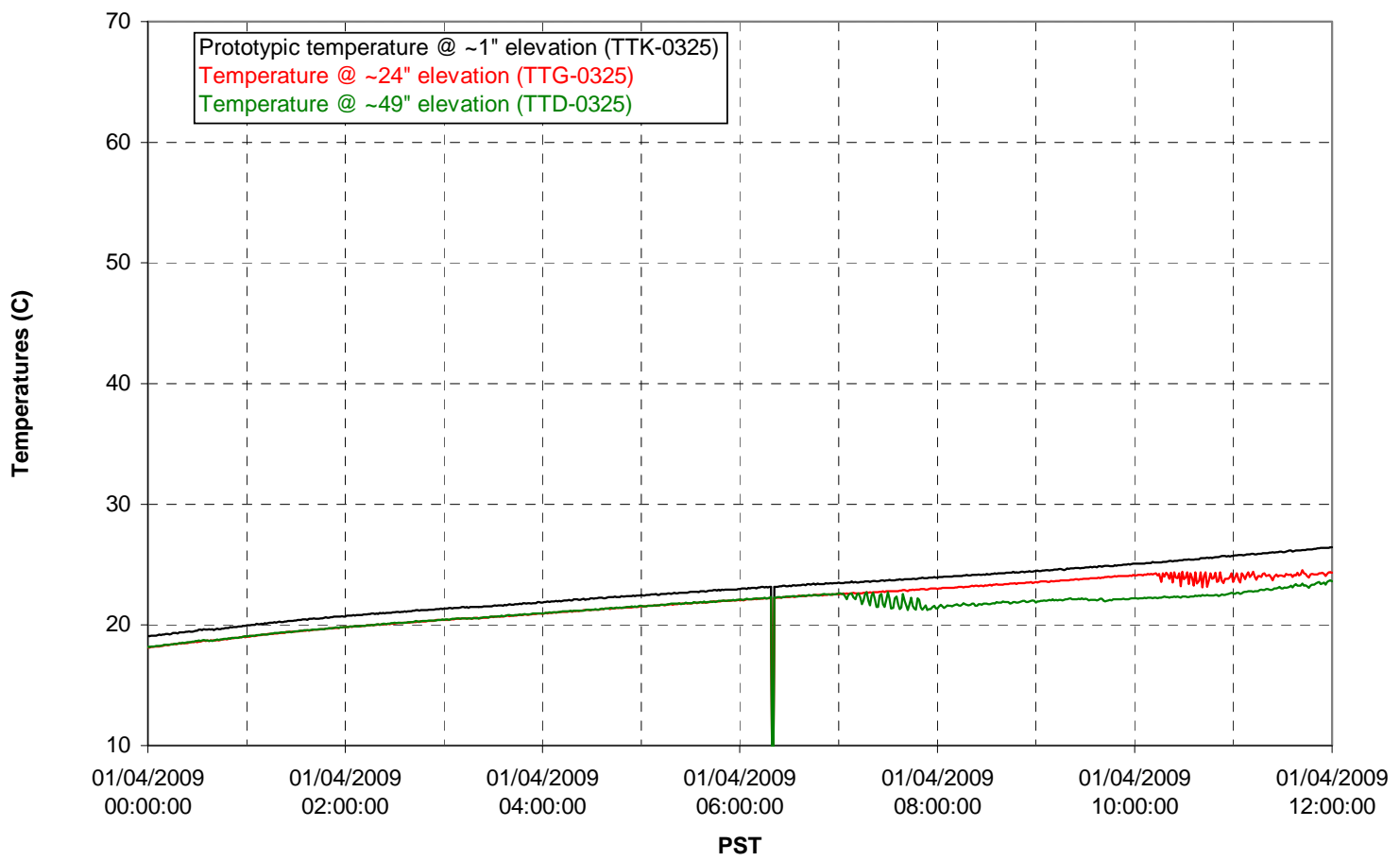


T01B level

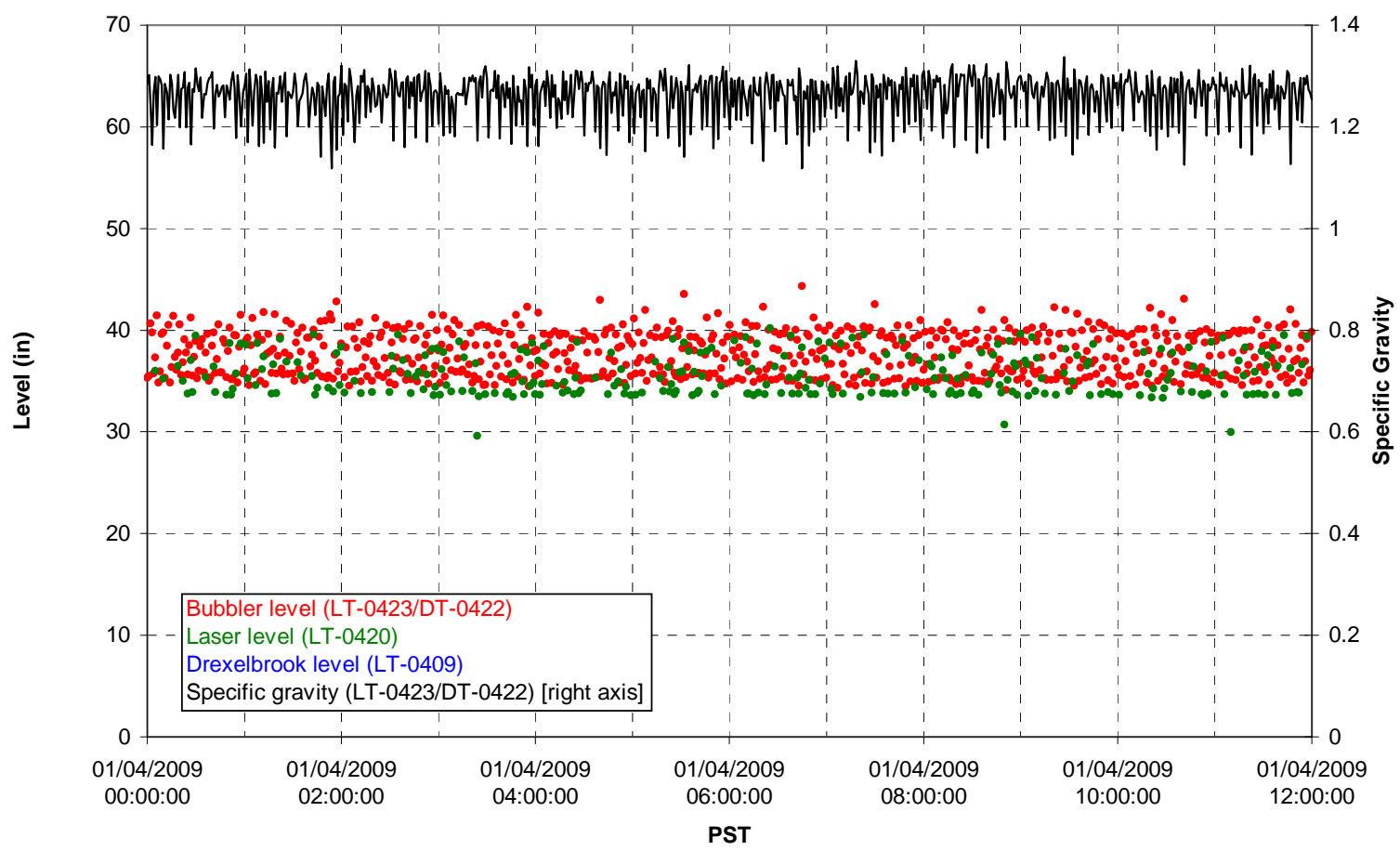

T01B temperatures

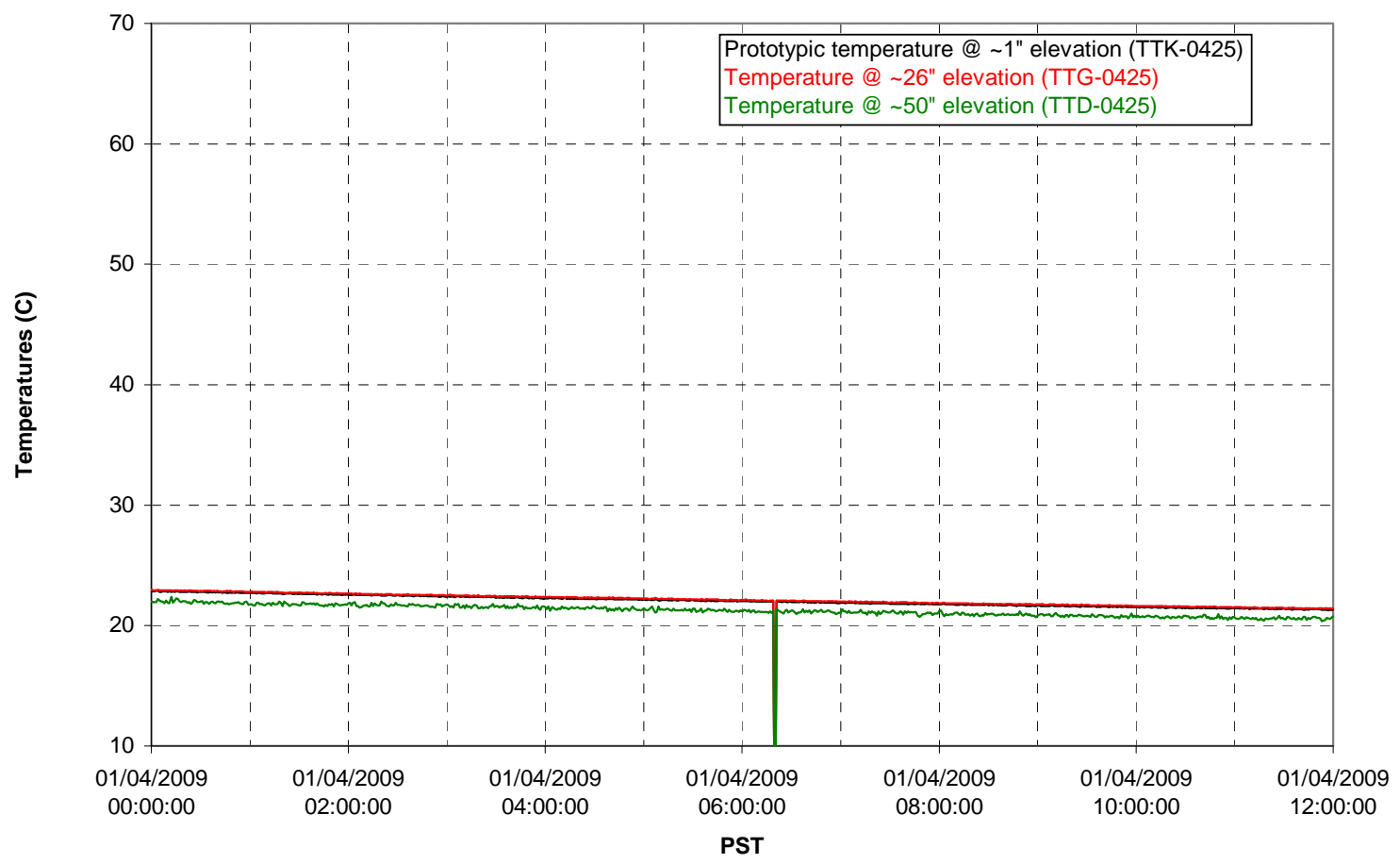


T02A level

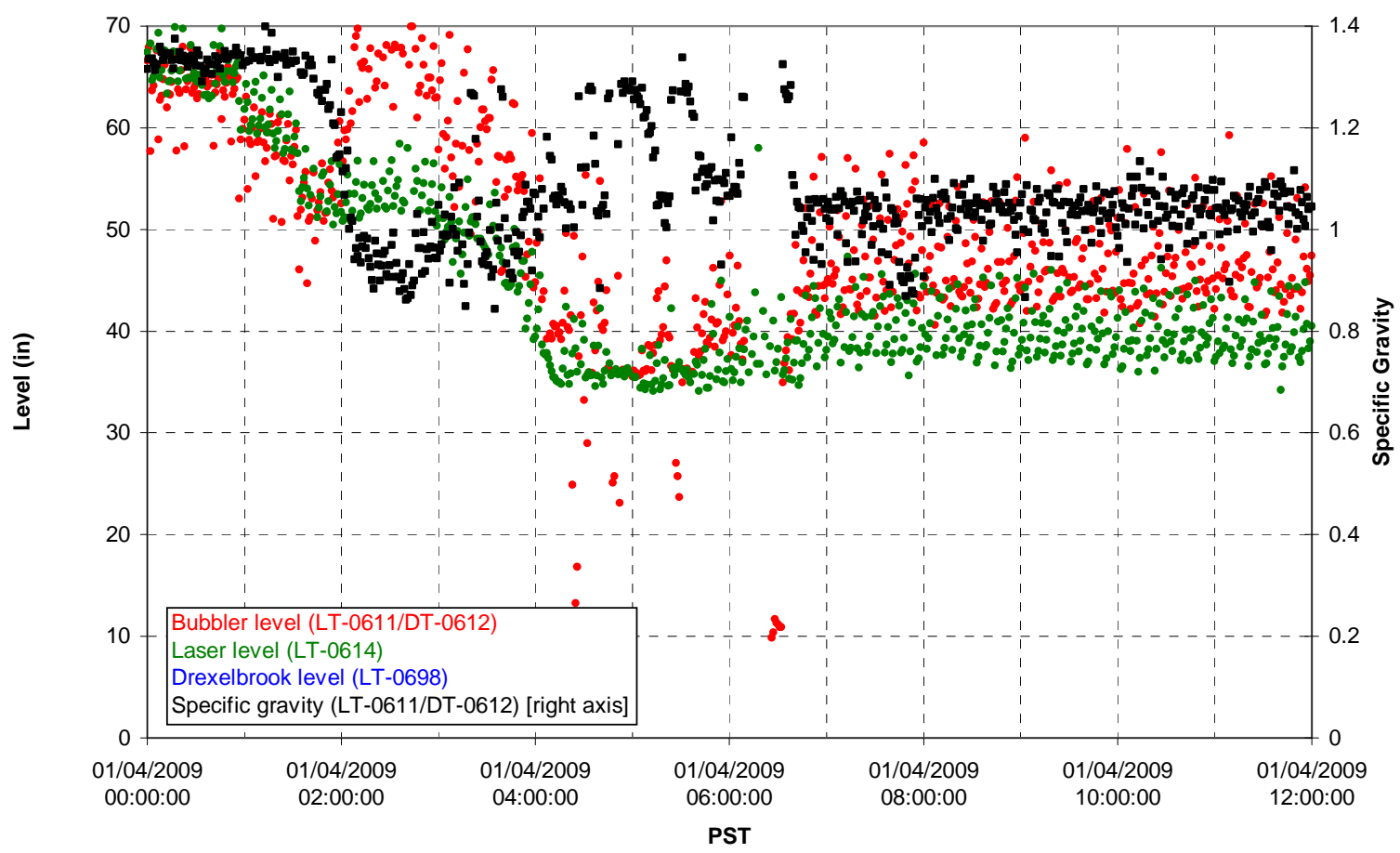

T02A temperatures

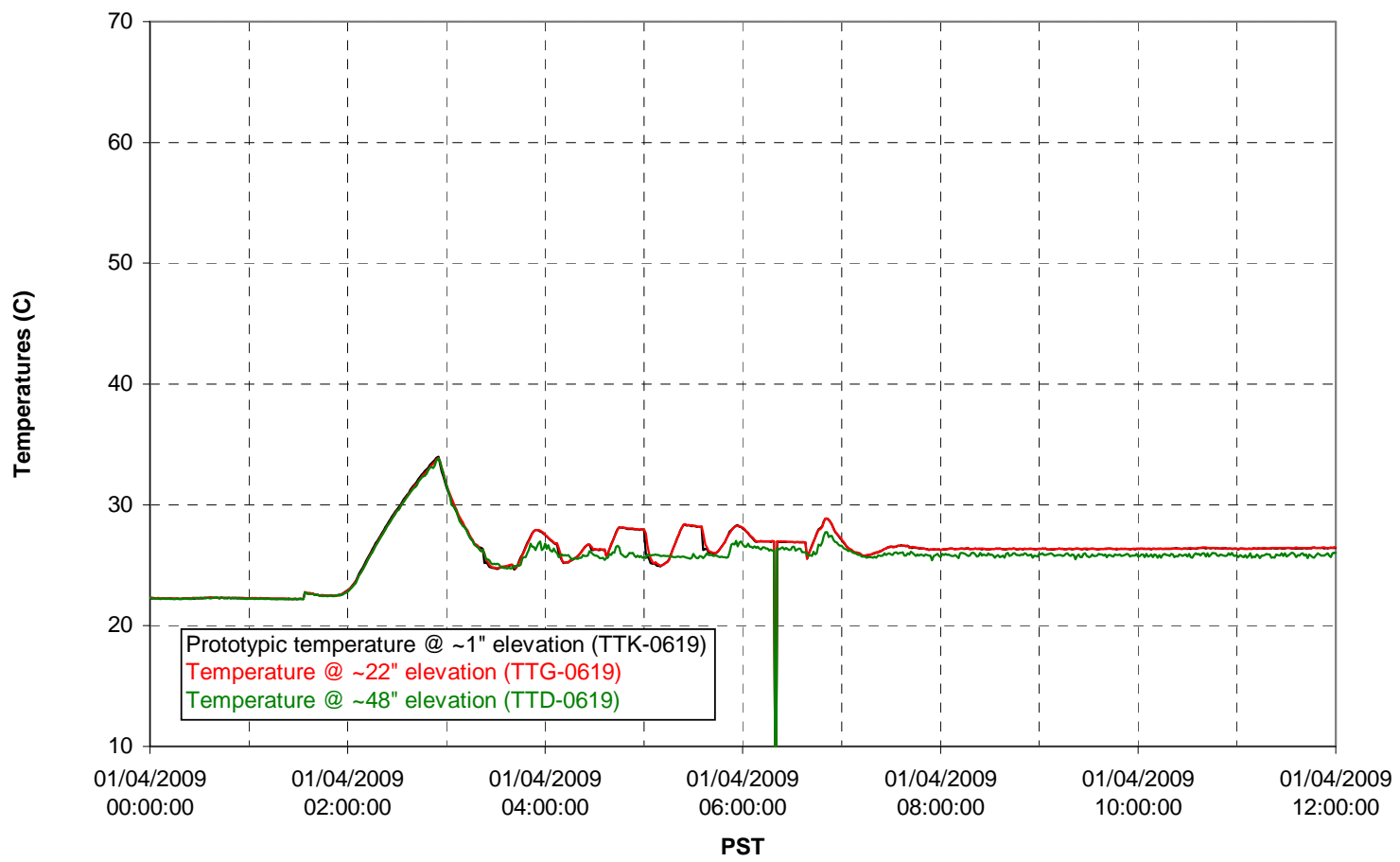


T02A and filter loop temperatures

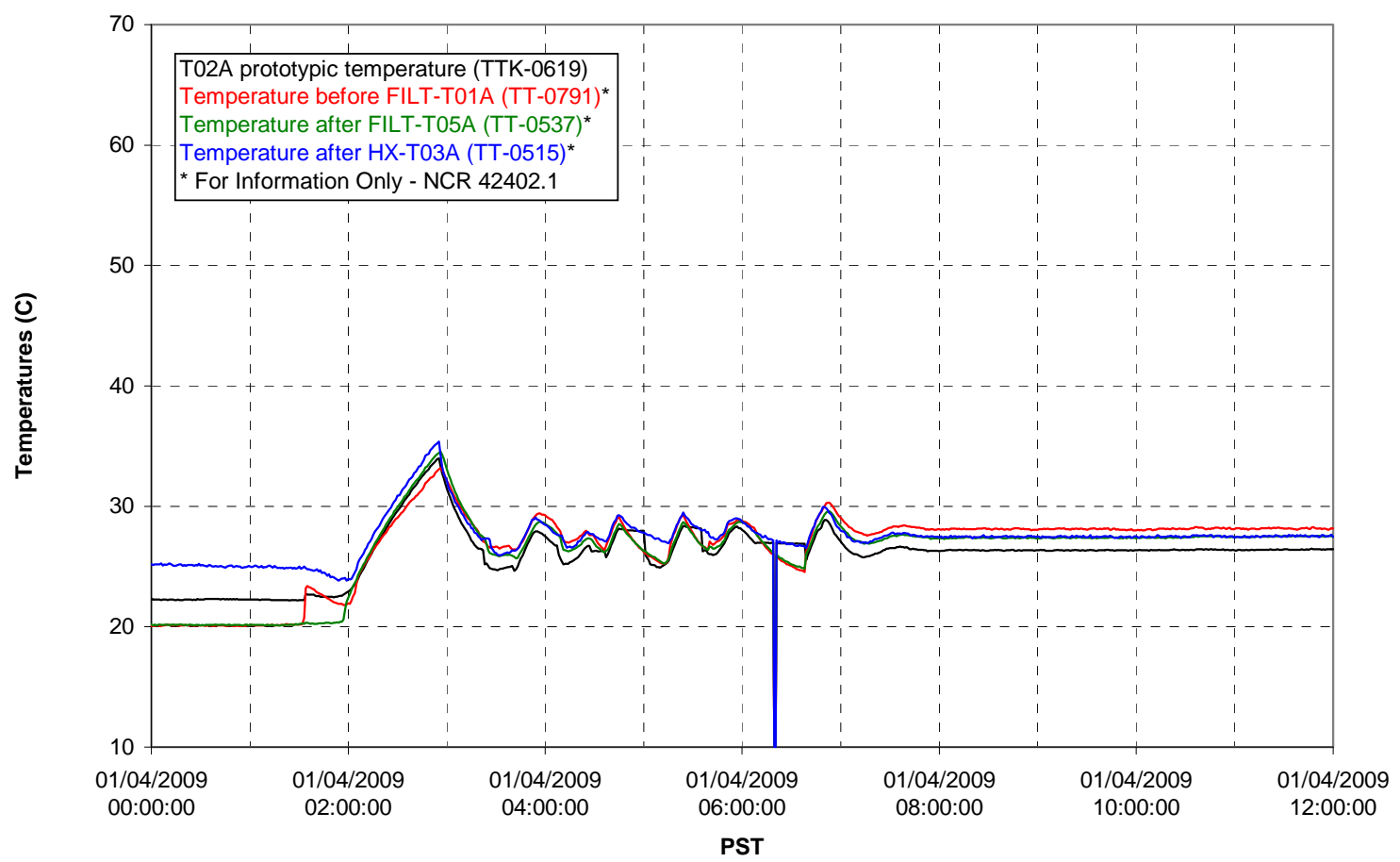

Pump Pressures and Flow

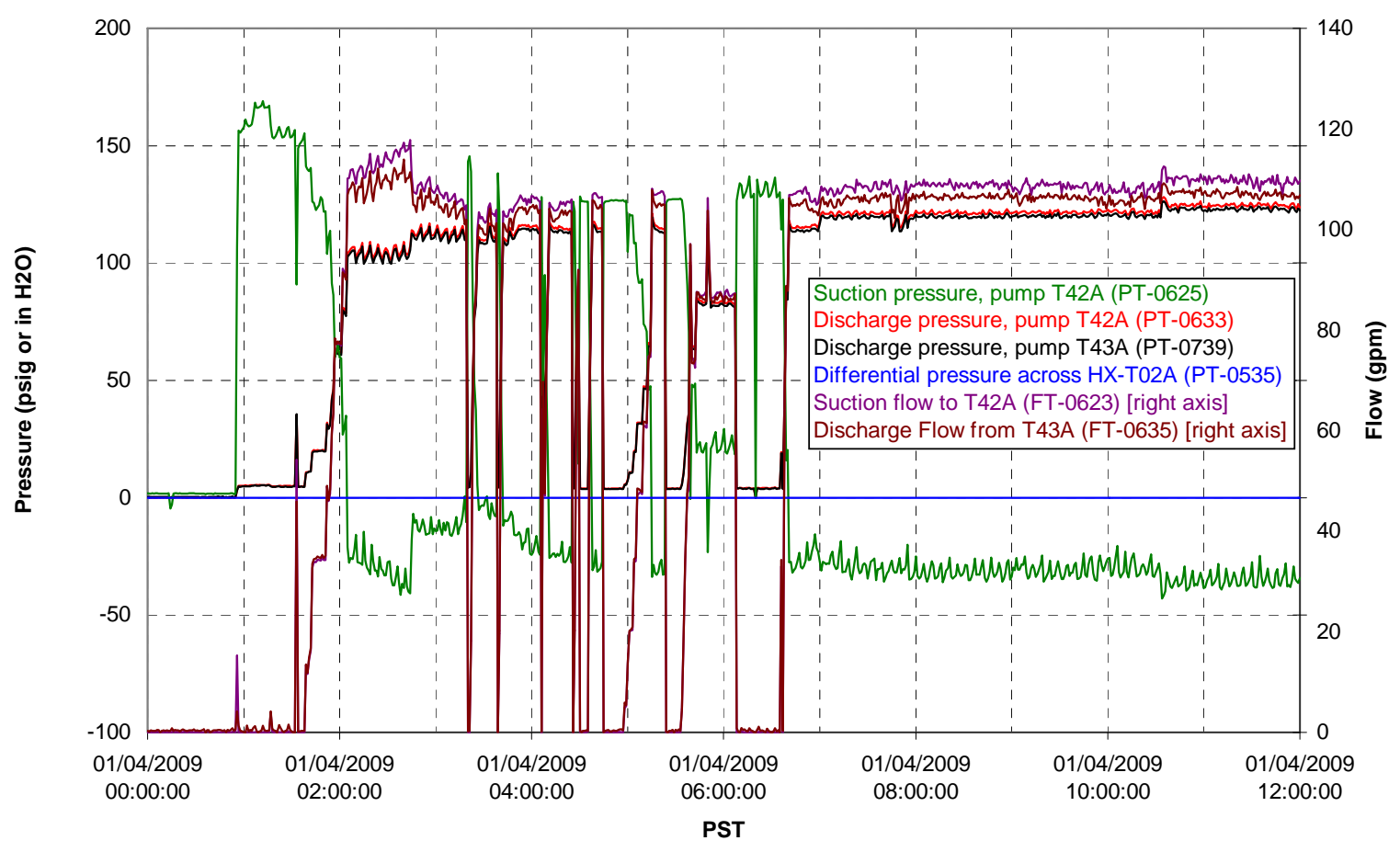


Axial pressure drop

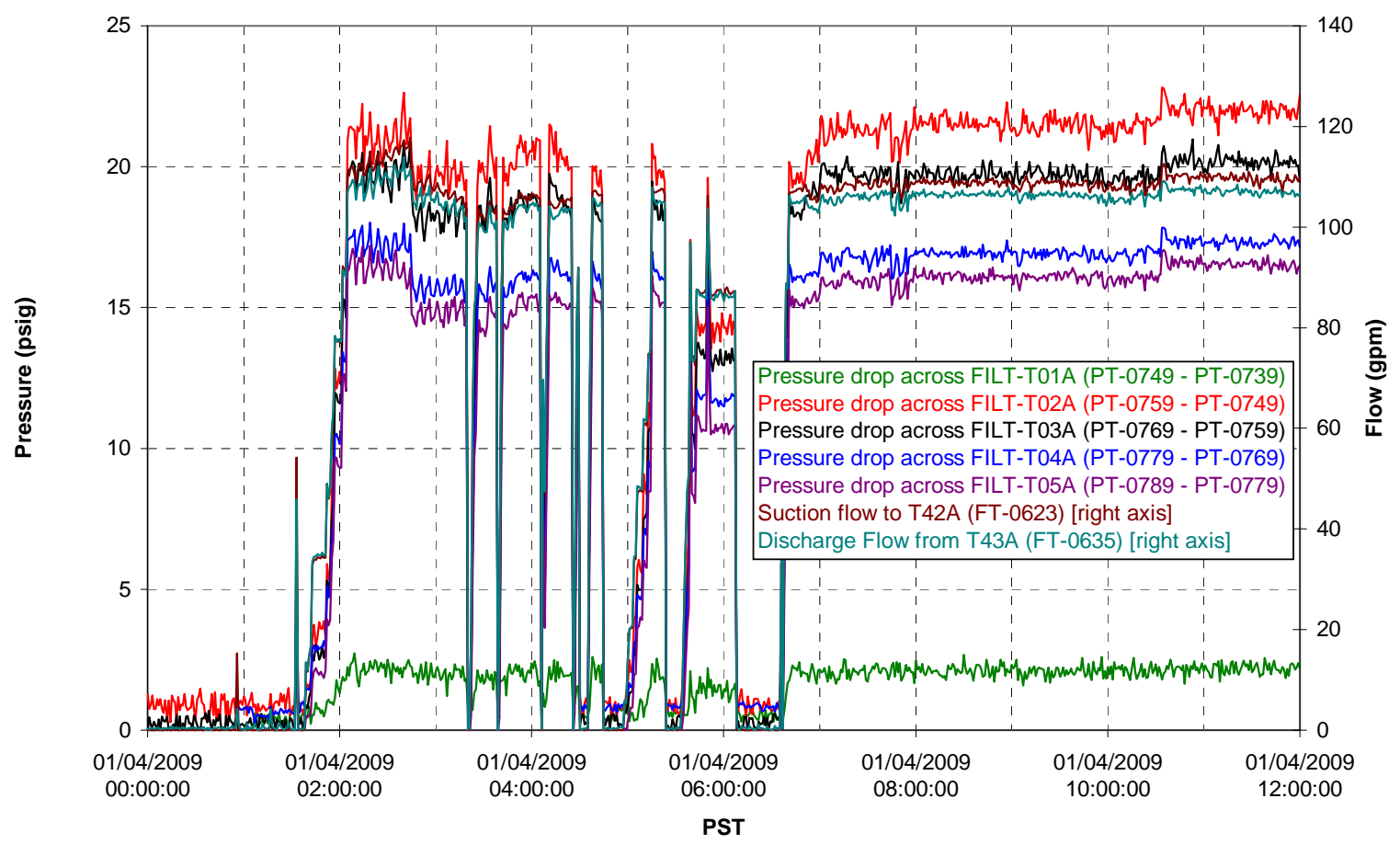

Permeate flow rates

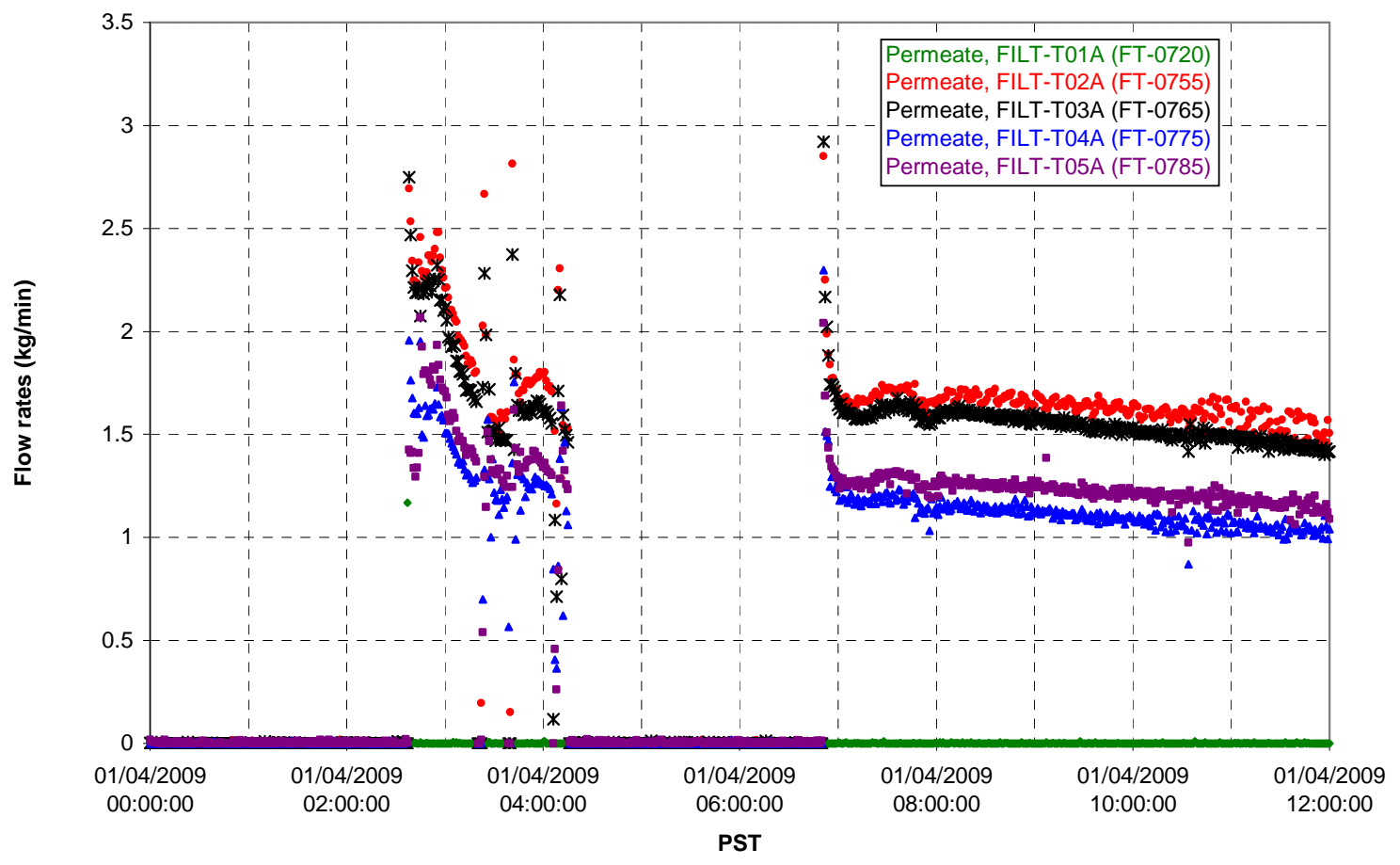


T02A Inner Temperature Tree

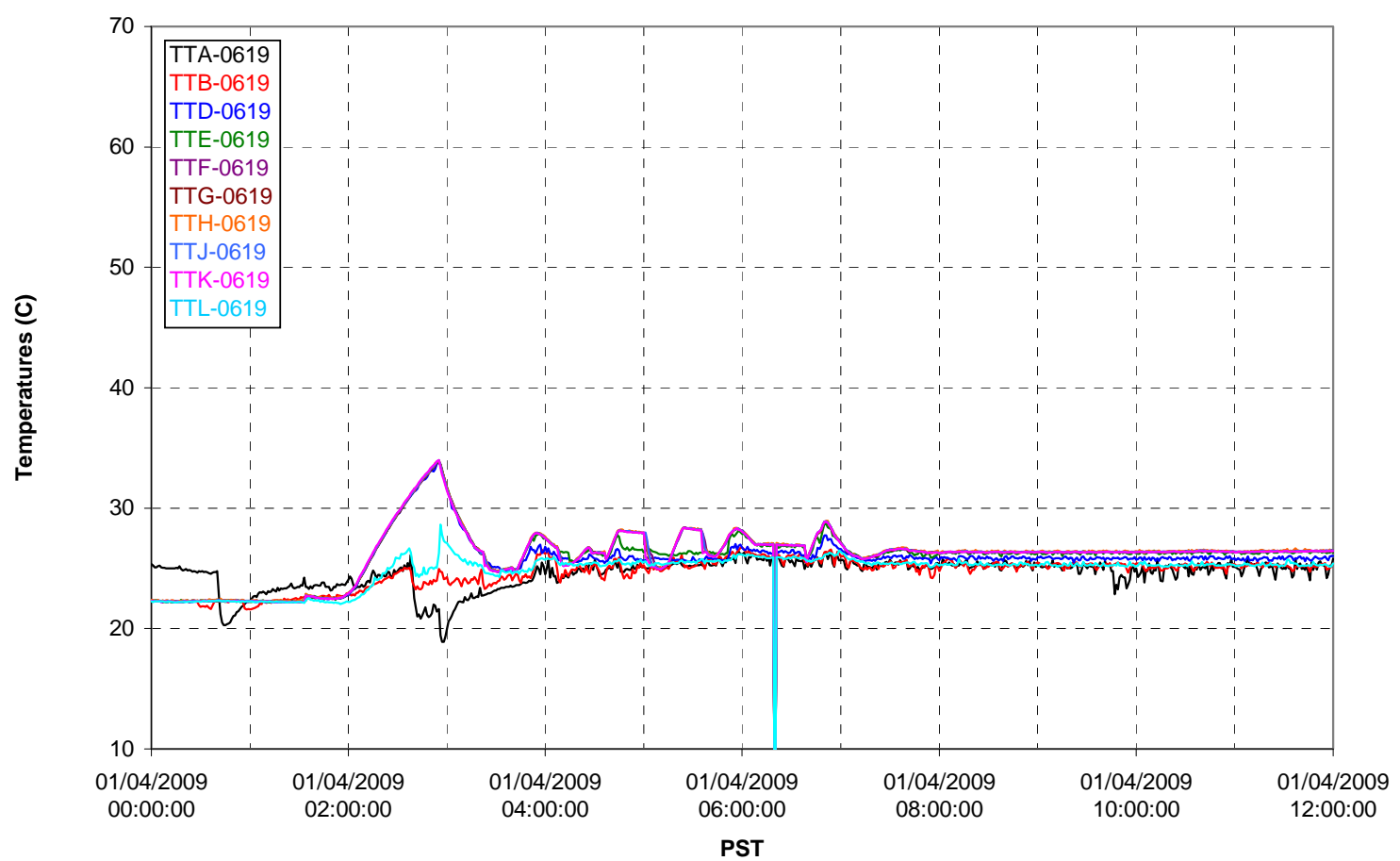

T02A Outer Temperature Tree

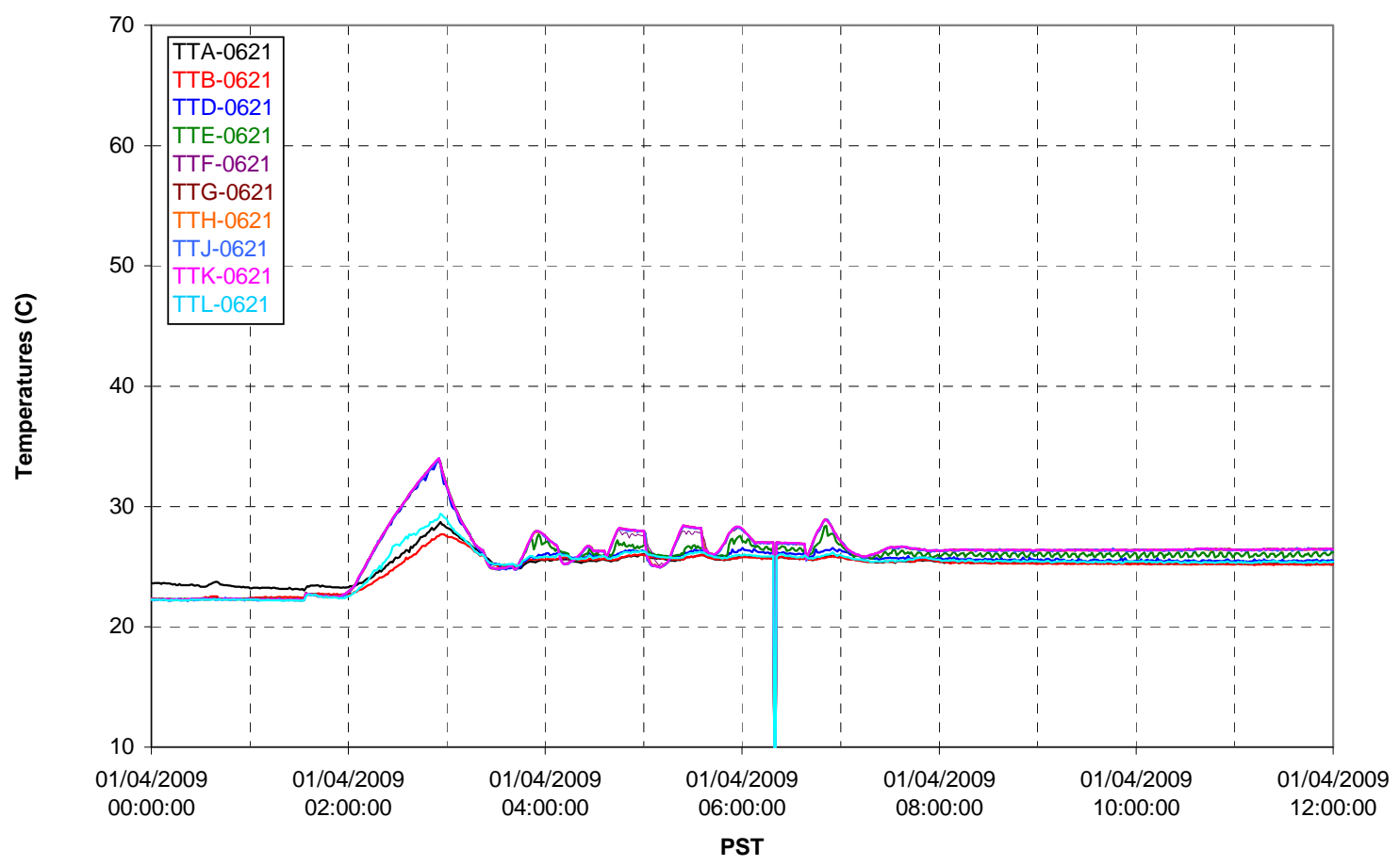


T02A temperatures

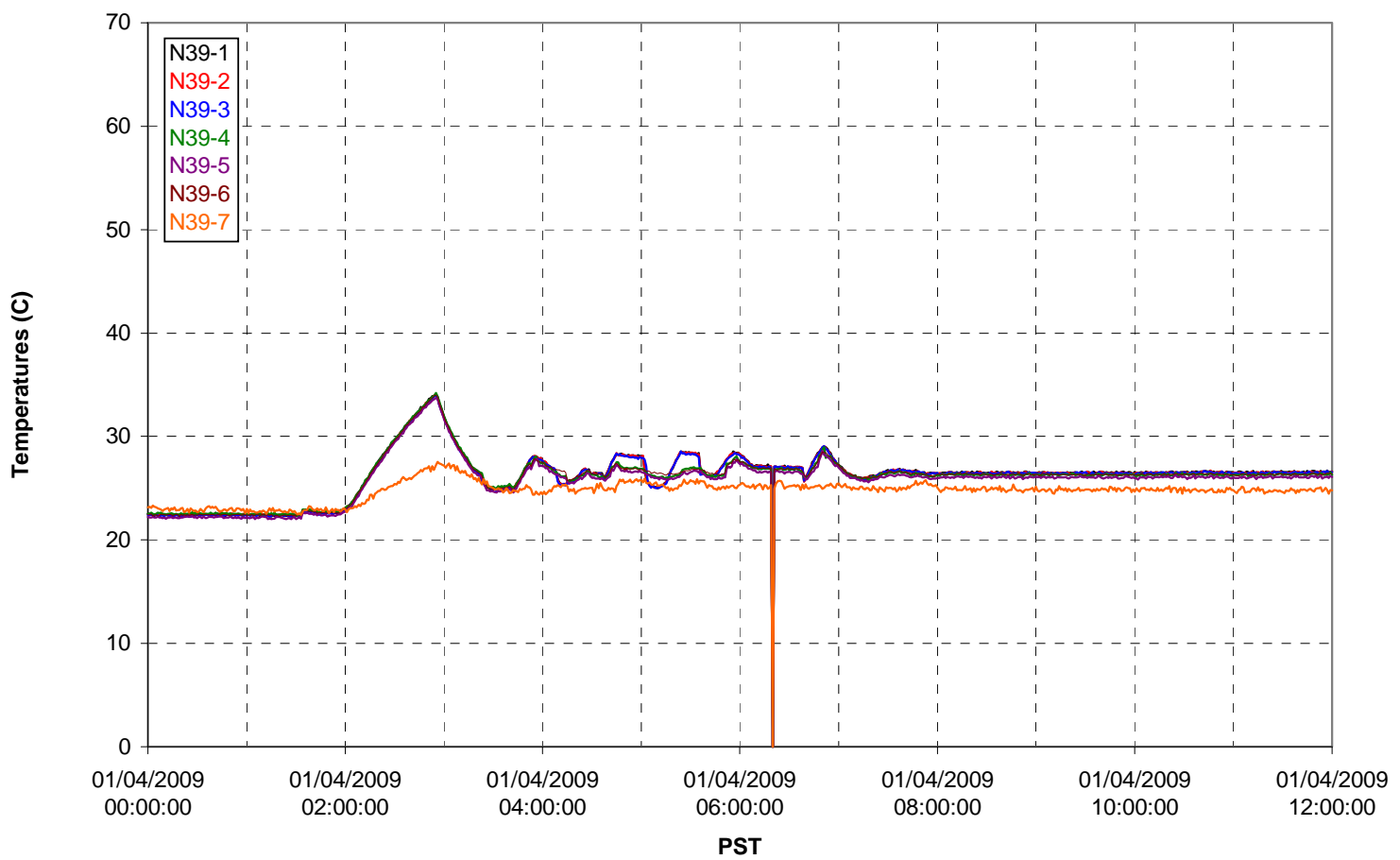

T02A temperatures

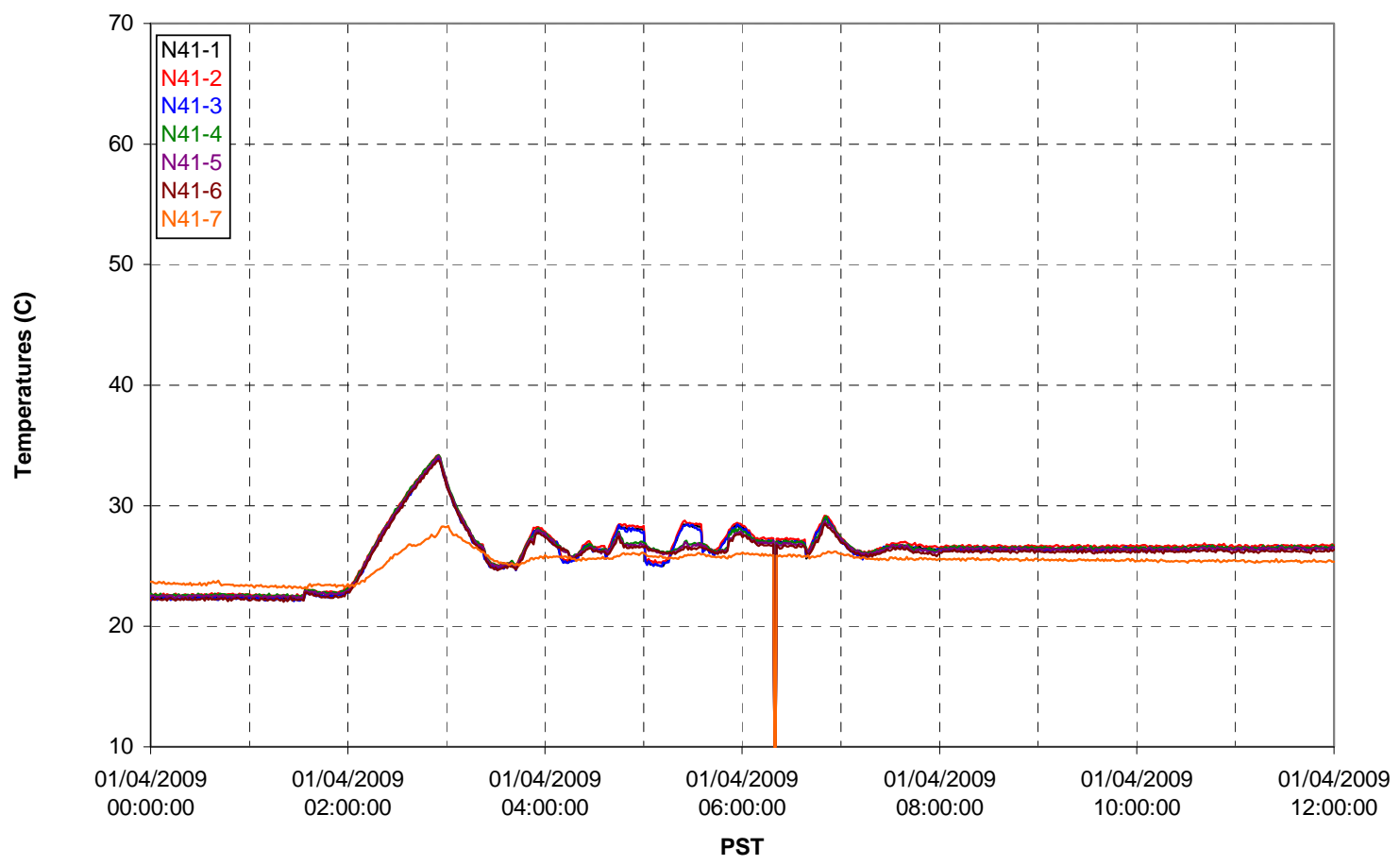


T02A temperatures

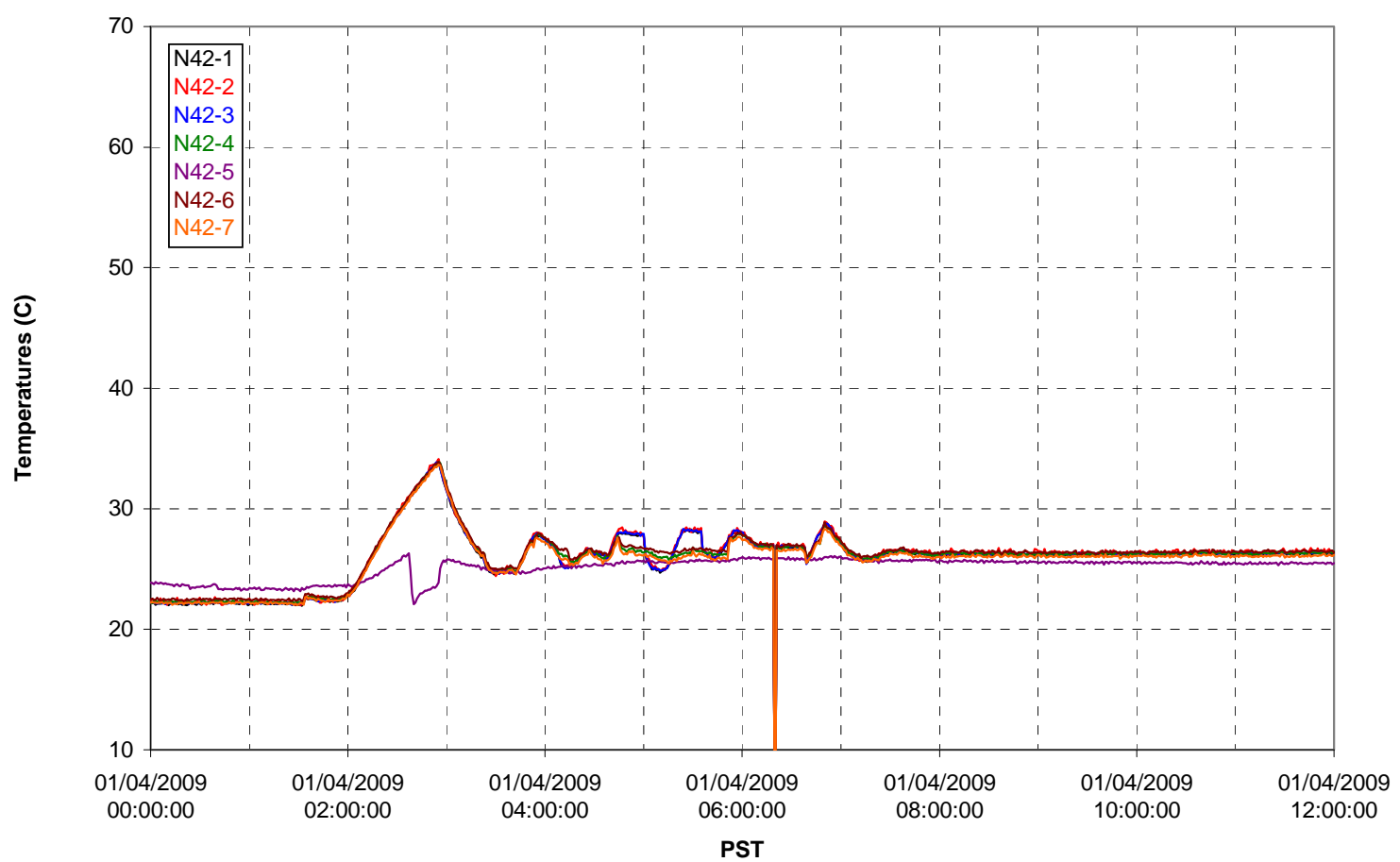

T02A temperatures

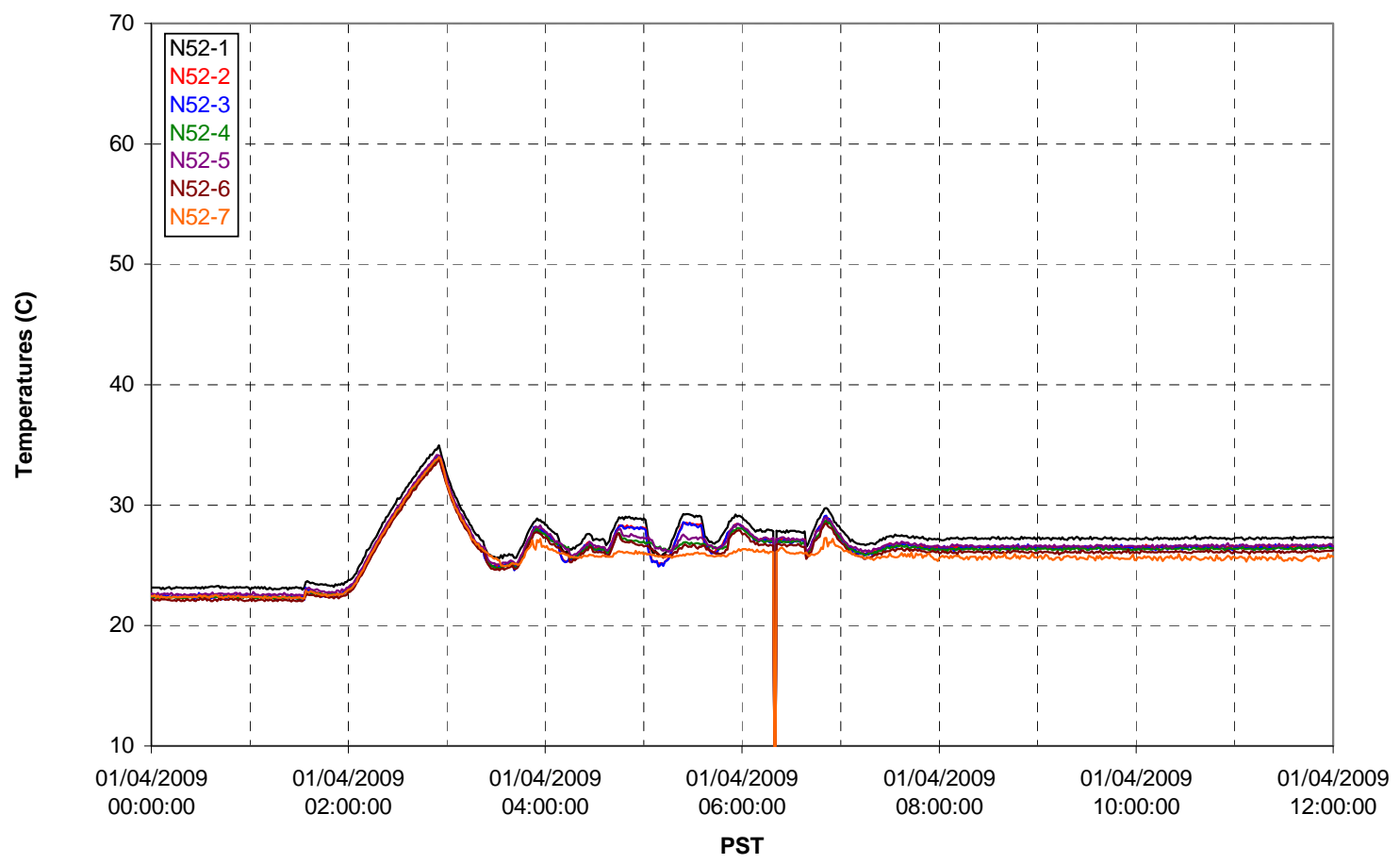


T02A Heating and Cooling

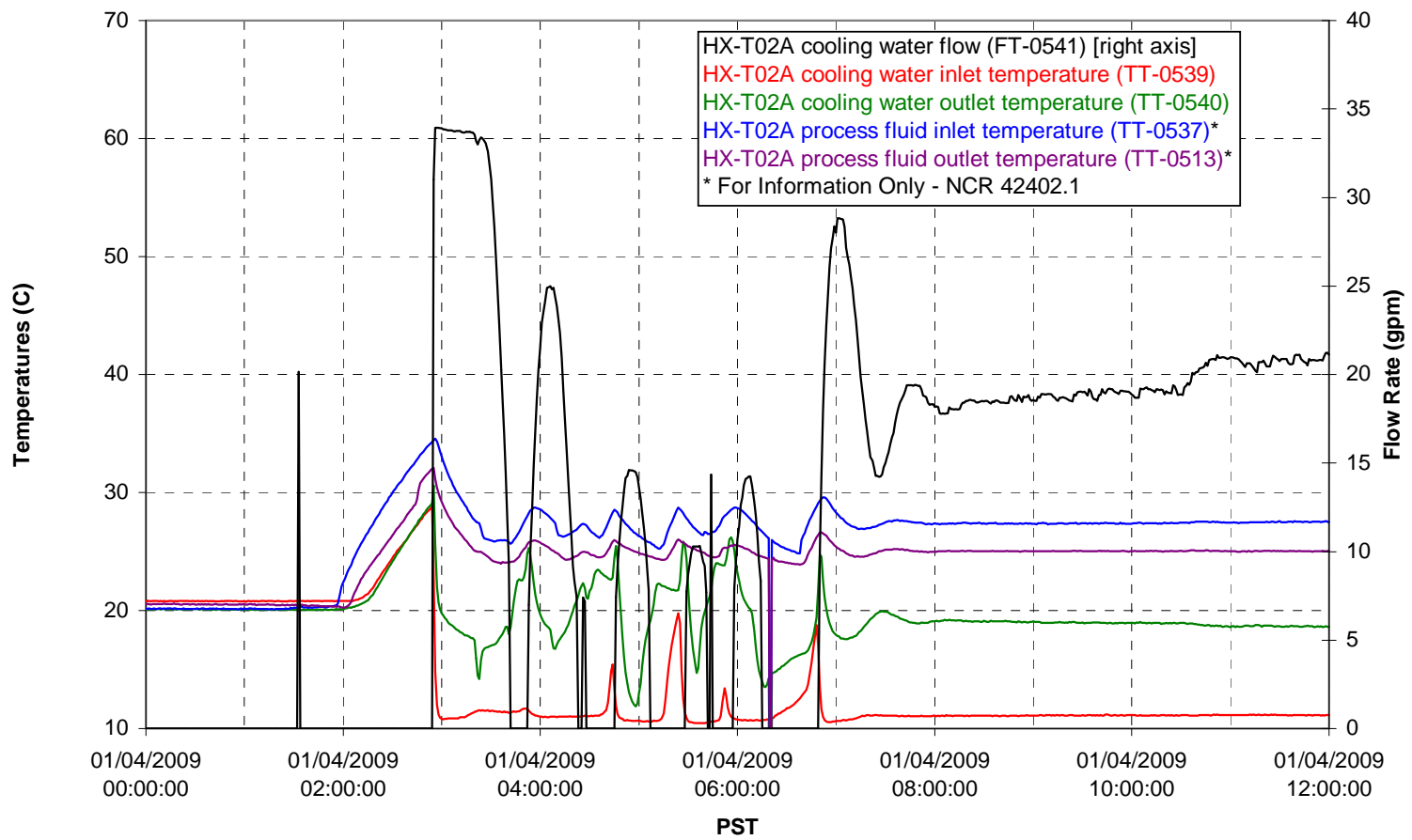

Pump Operation

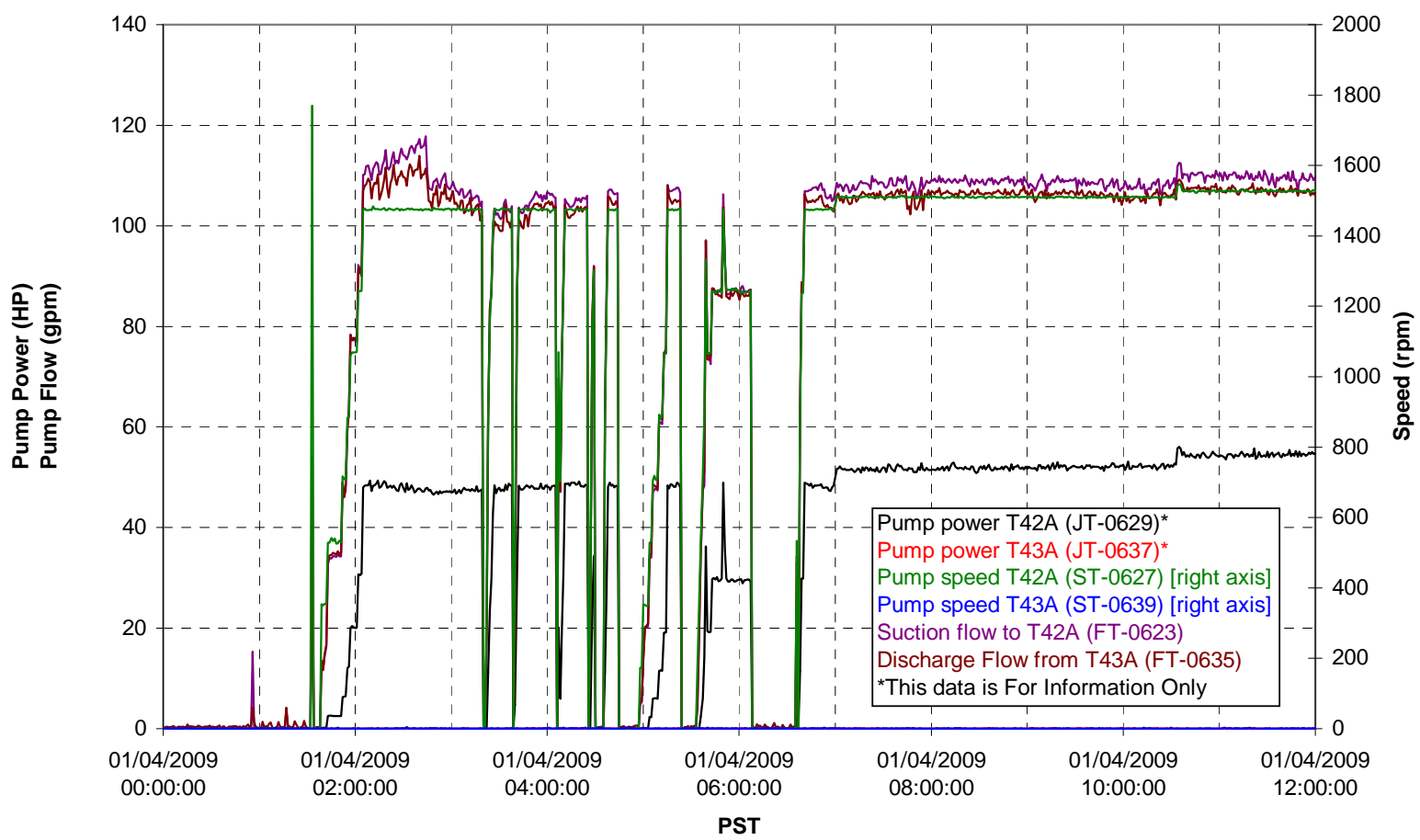


Pulsepot UFP-PP-T01A

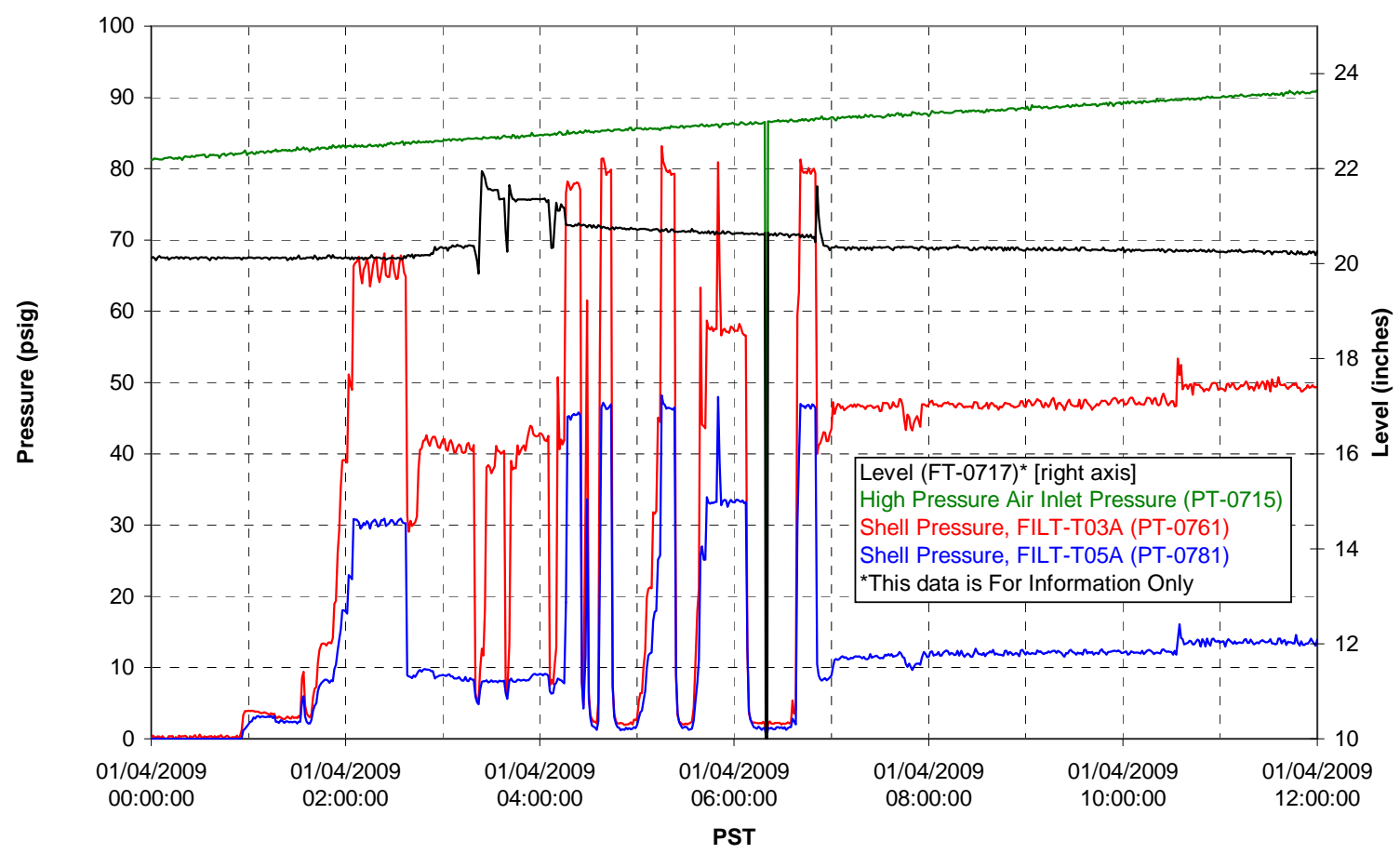

Pulsepot UFP-PP-T02A

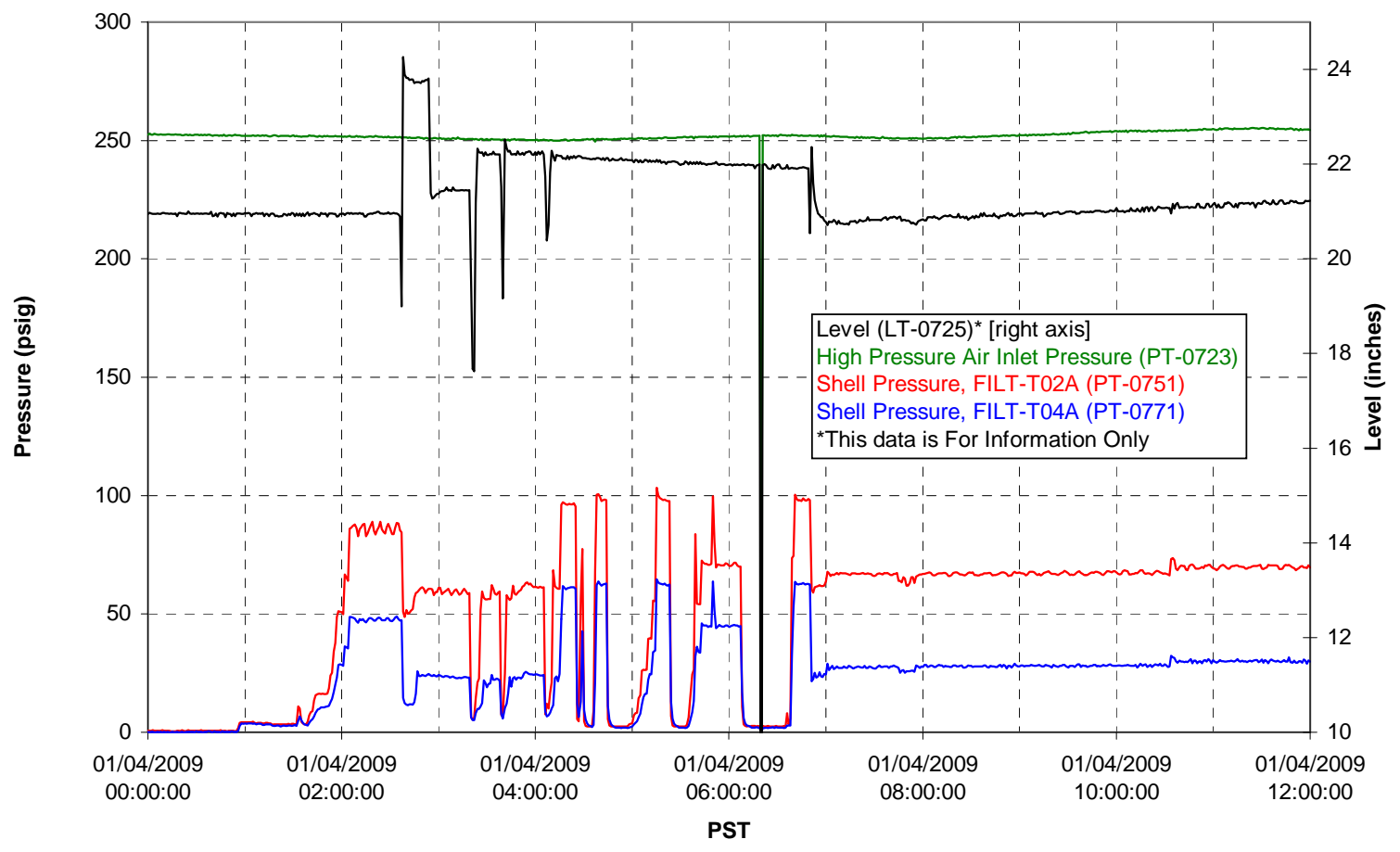


Pulsepot UFP-PP-T03A

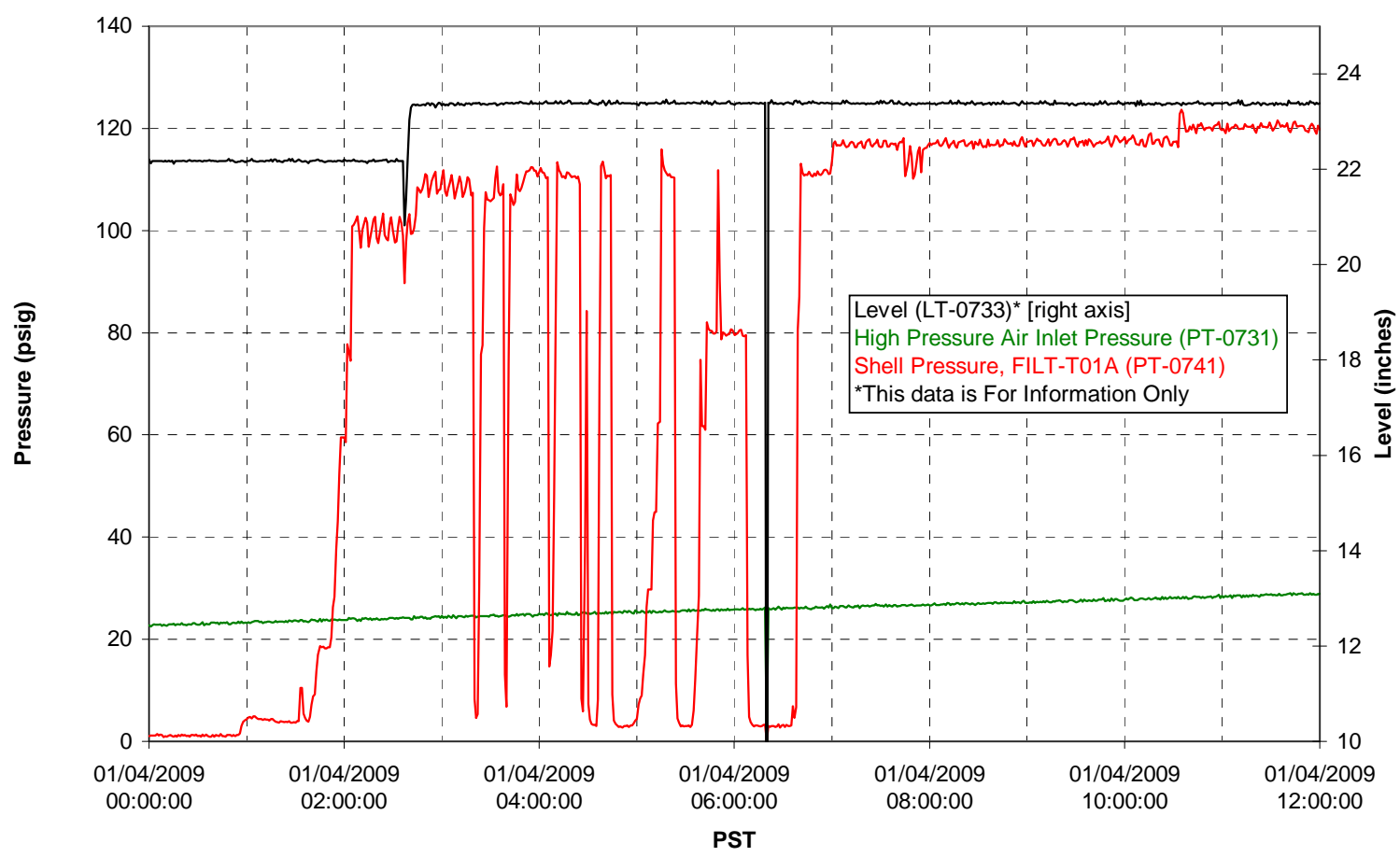

Pulsepot Levels

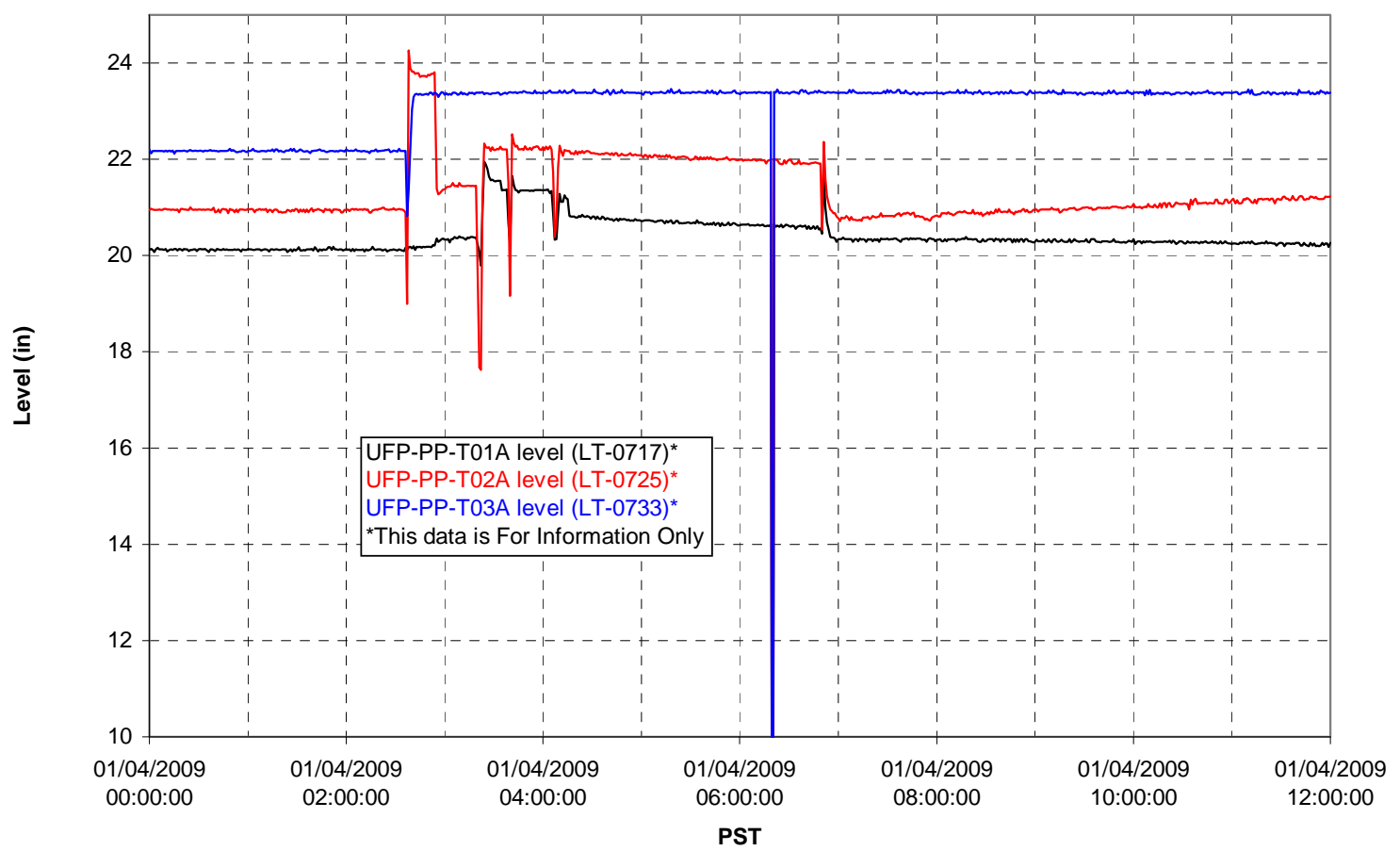


Filter UFP-FILT-T01A

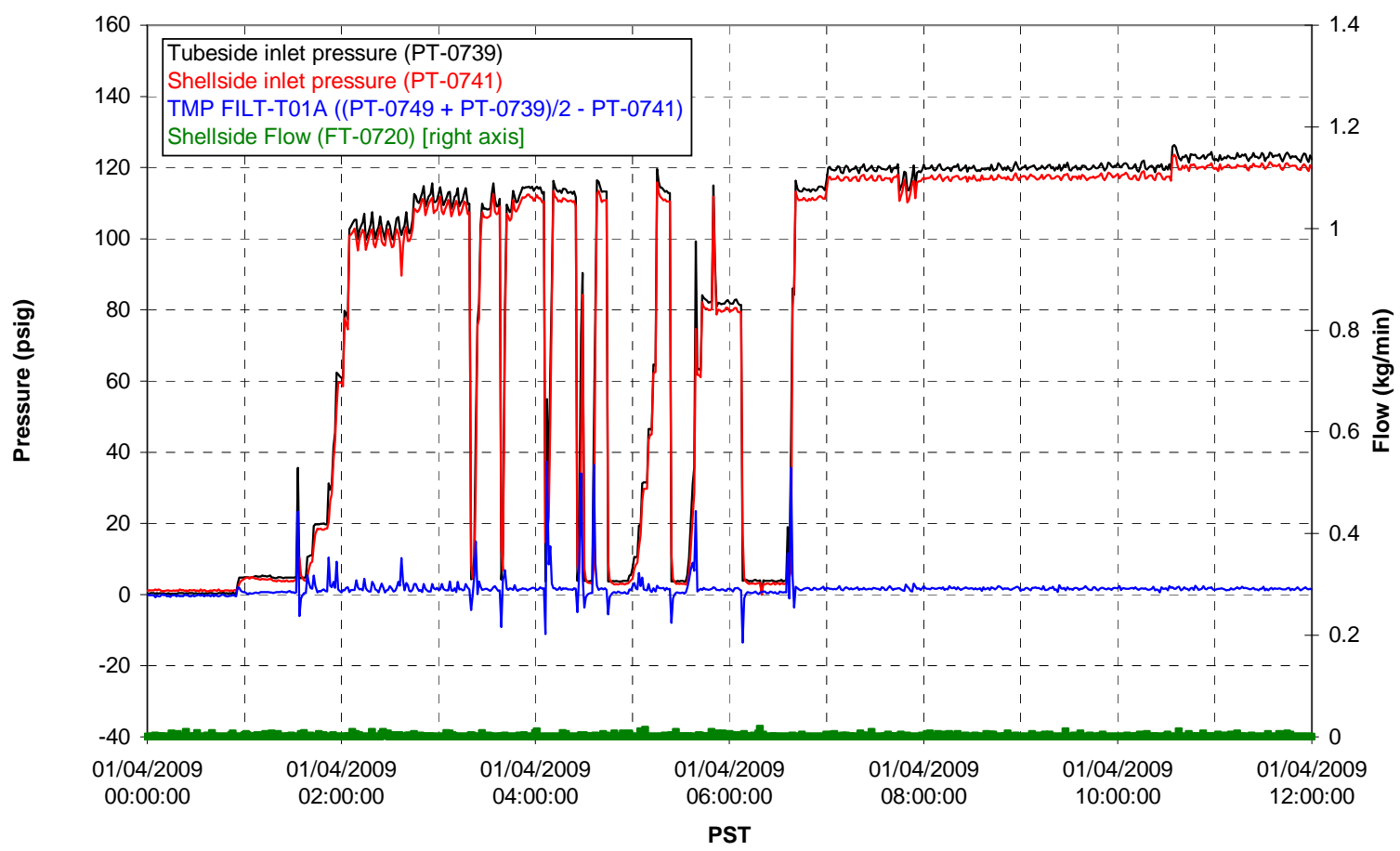

Filter UFP-FILT-T02A

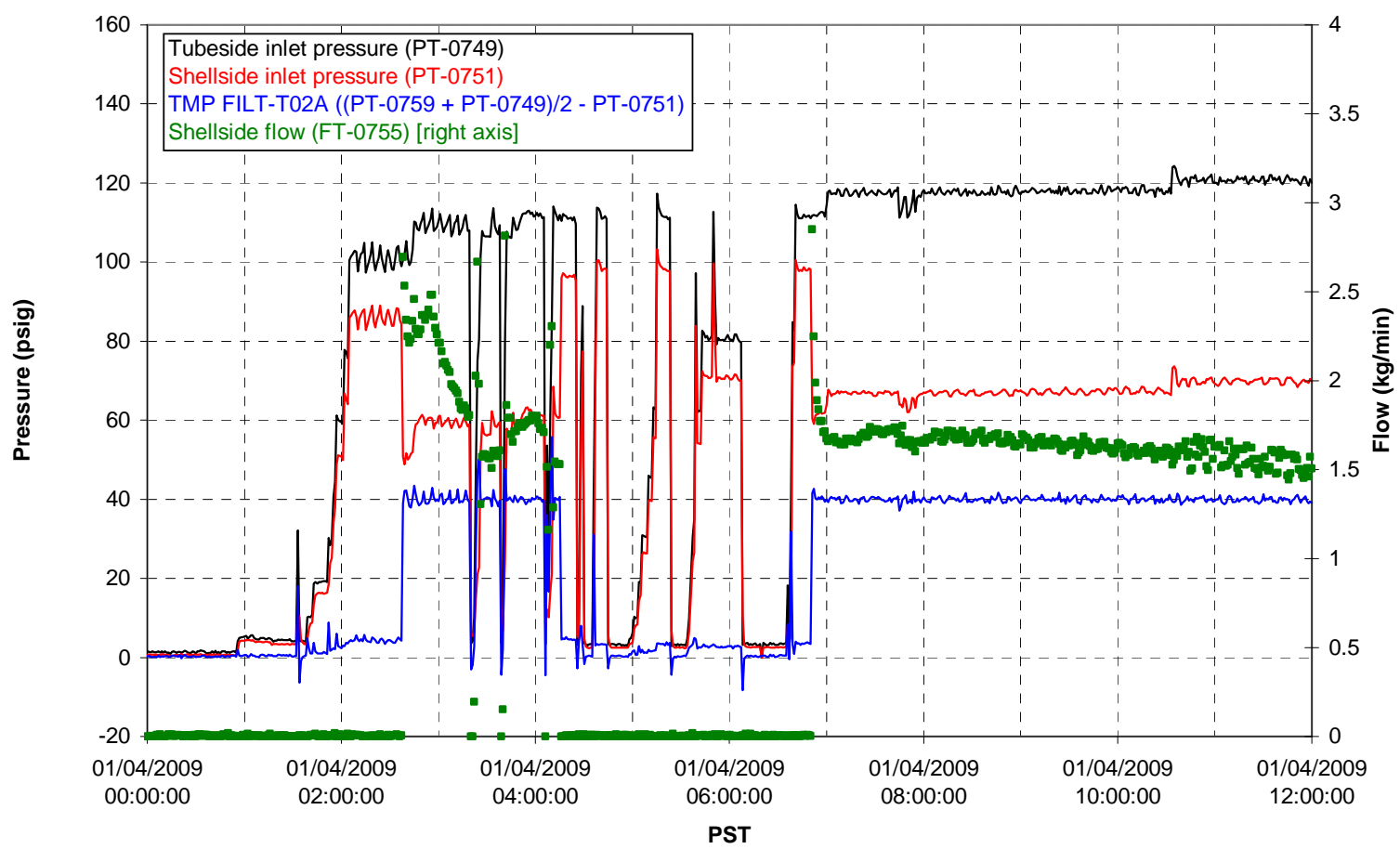


Filter UFP-FILT-T03A

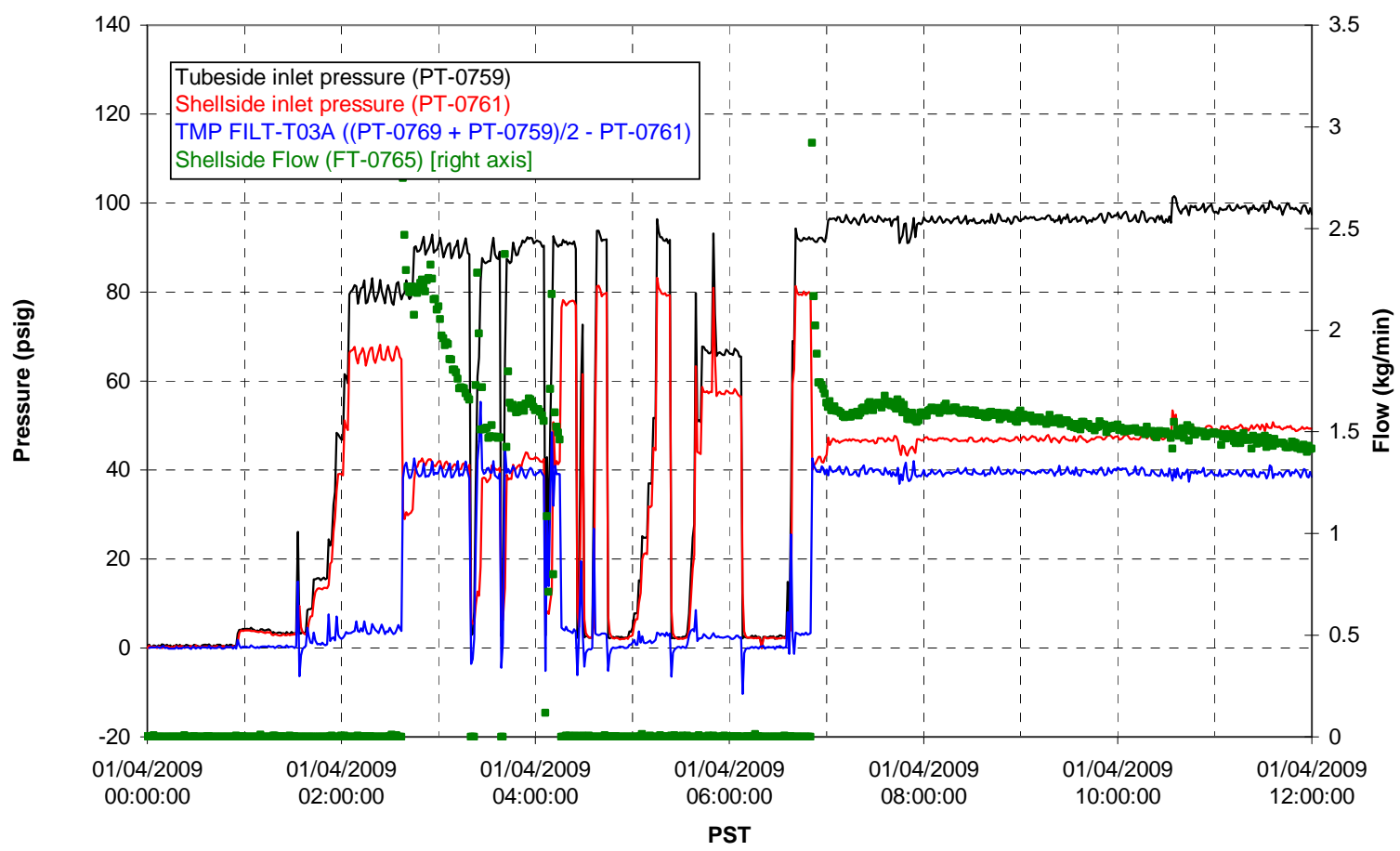

Filter UFP-FILT-T04A

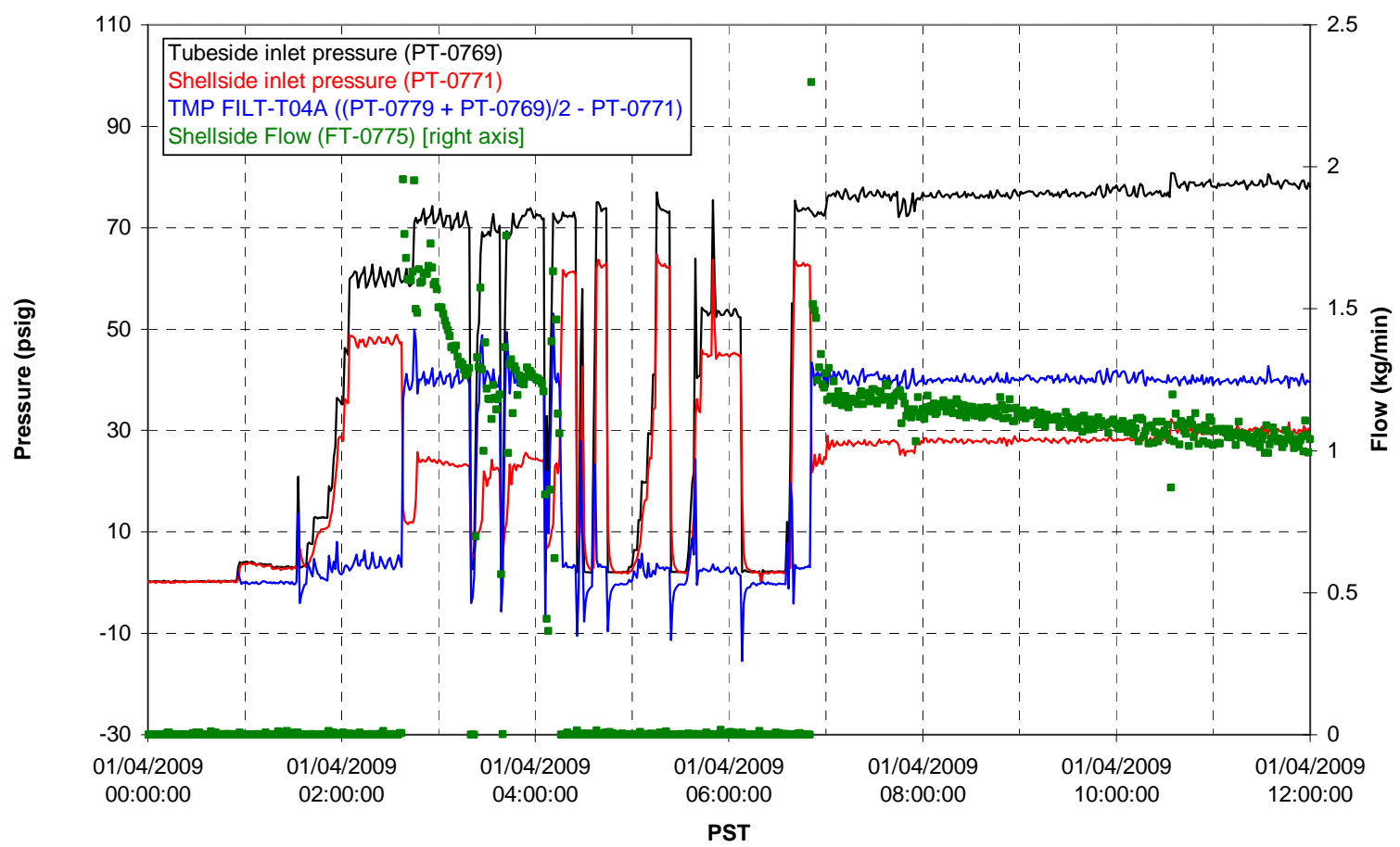


Filter UFP-FILT-T05A

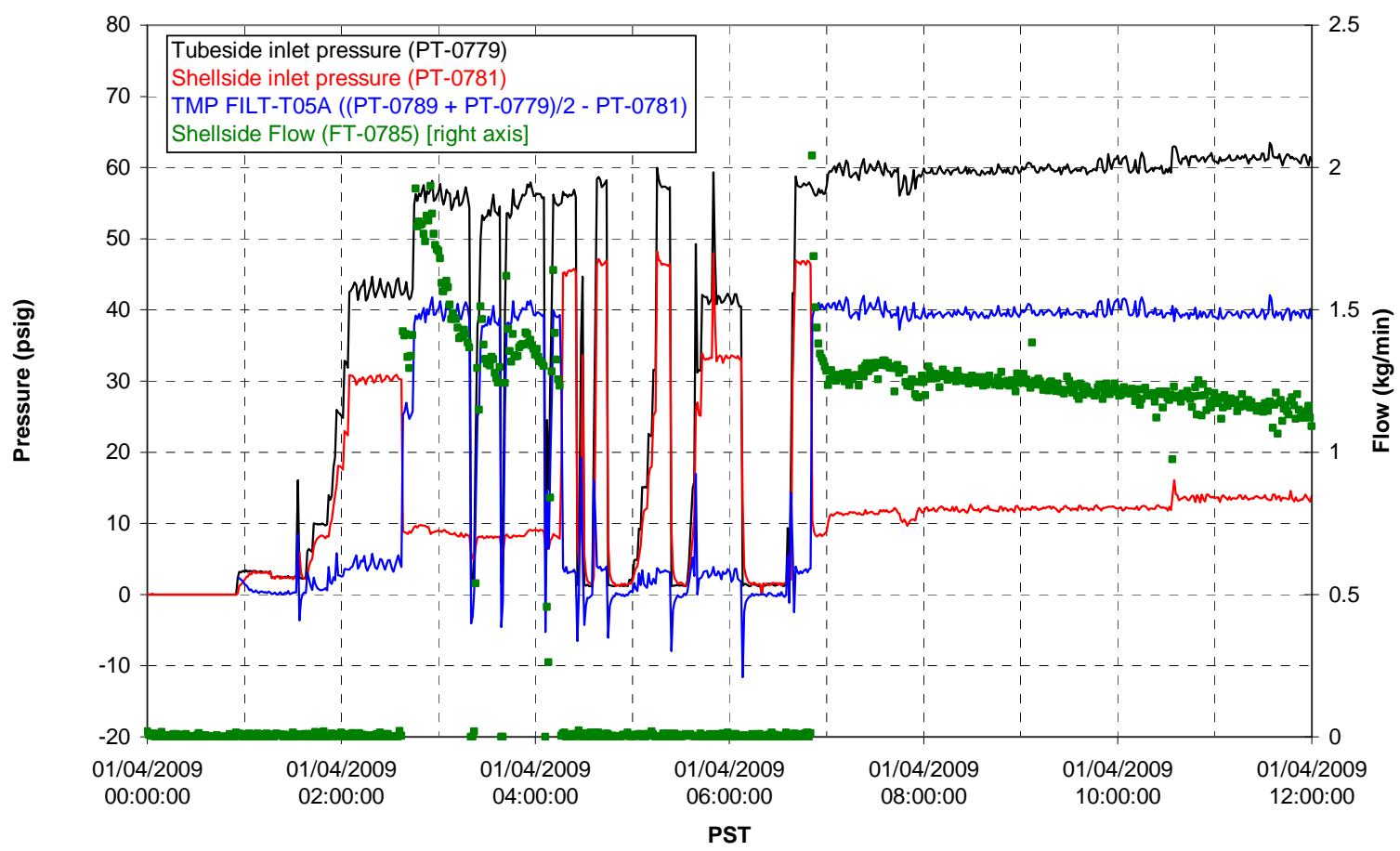

Chemical Flow

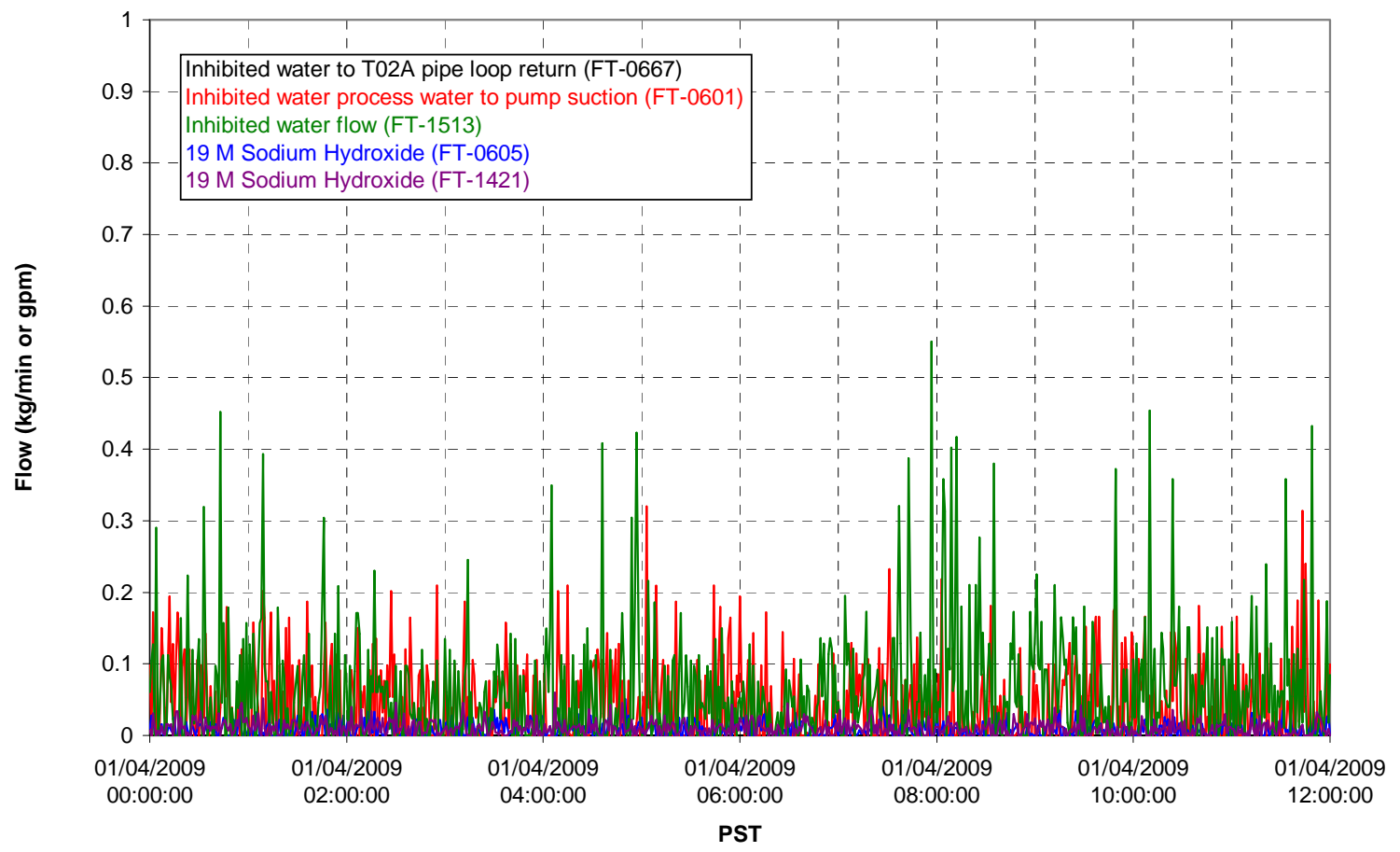


Chemical Flow

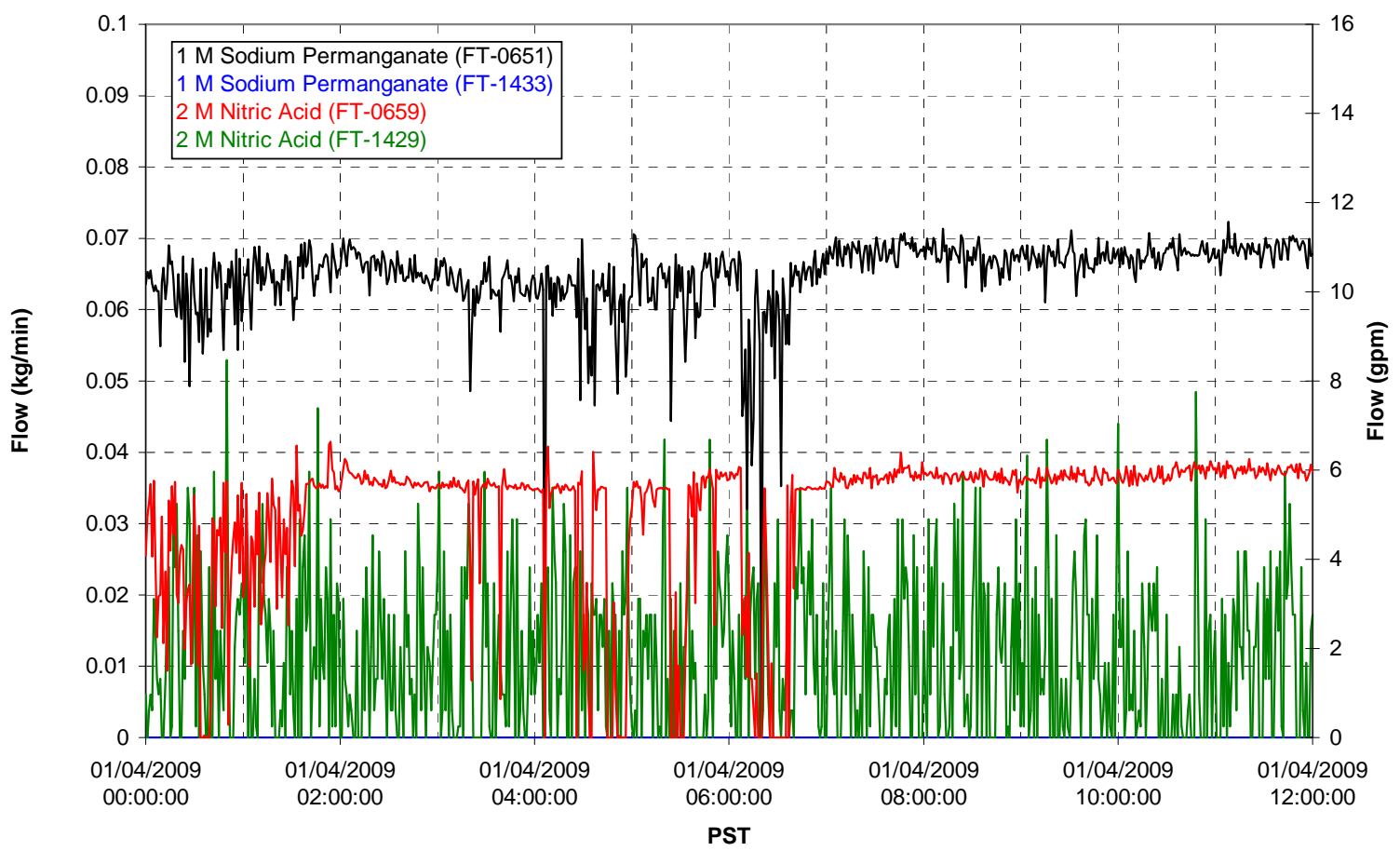

Air Flows

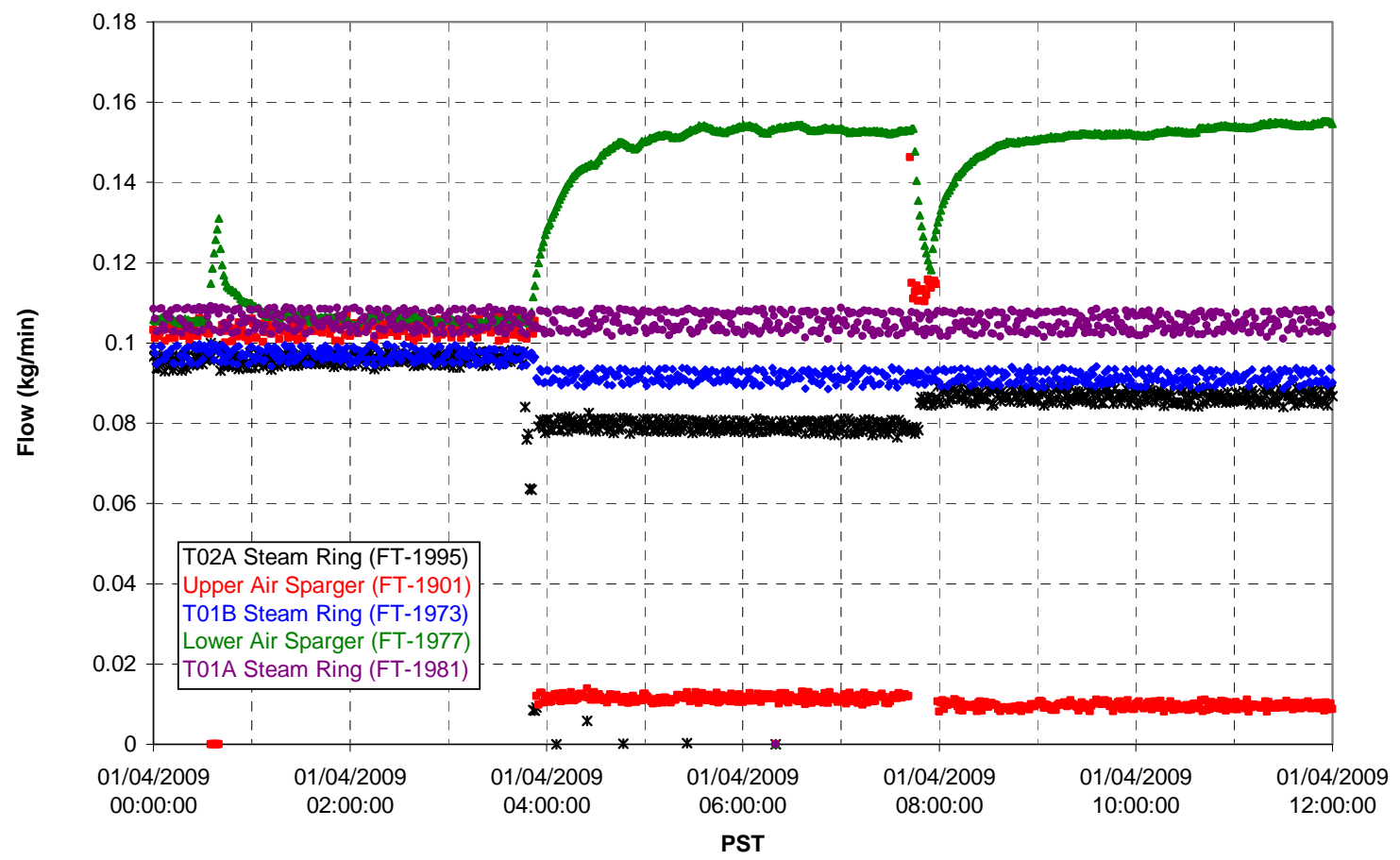


T02A Steam

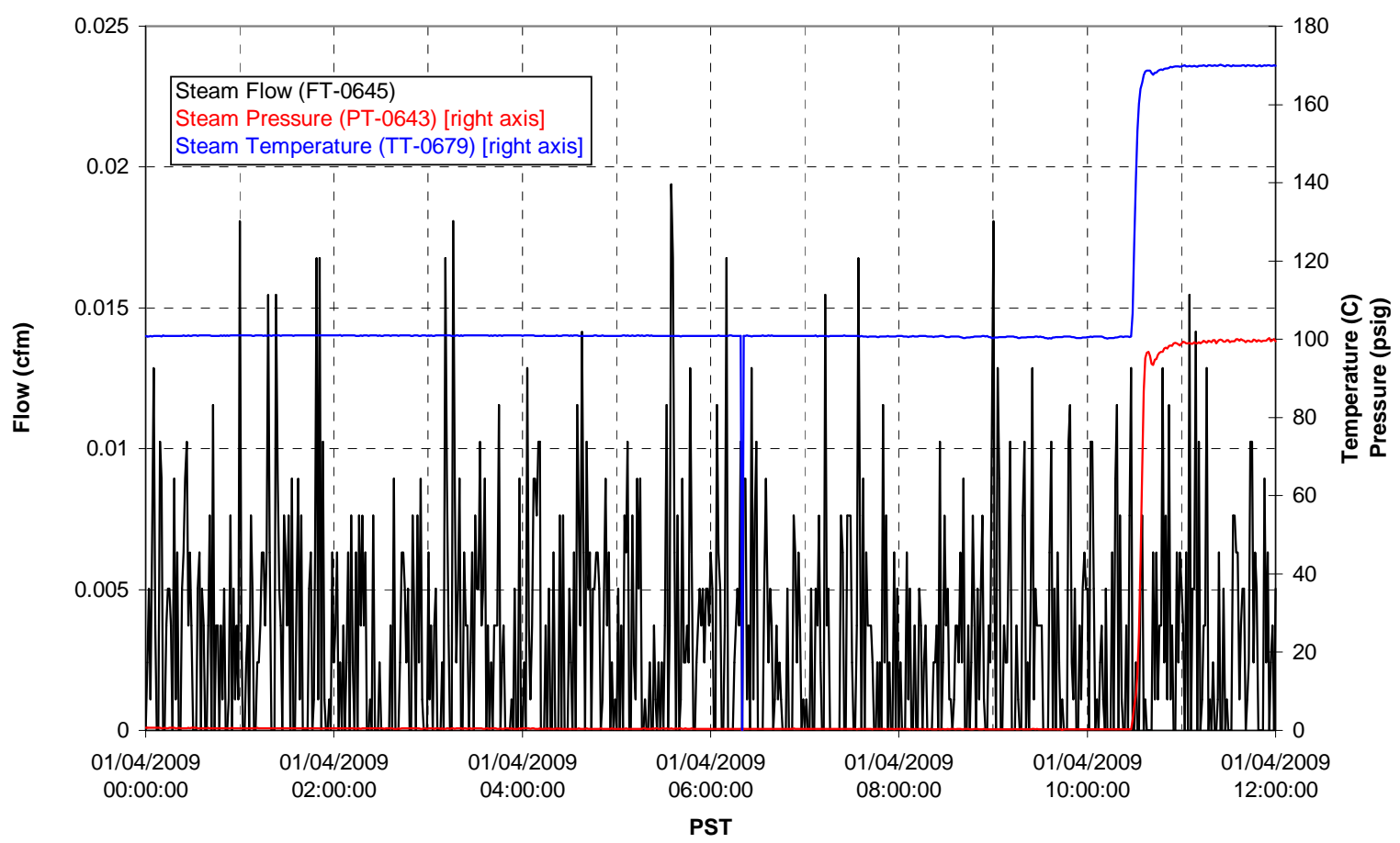

T01A Steam

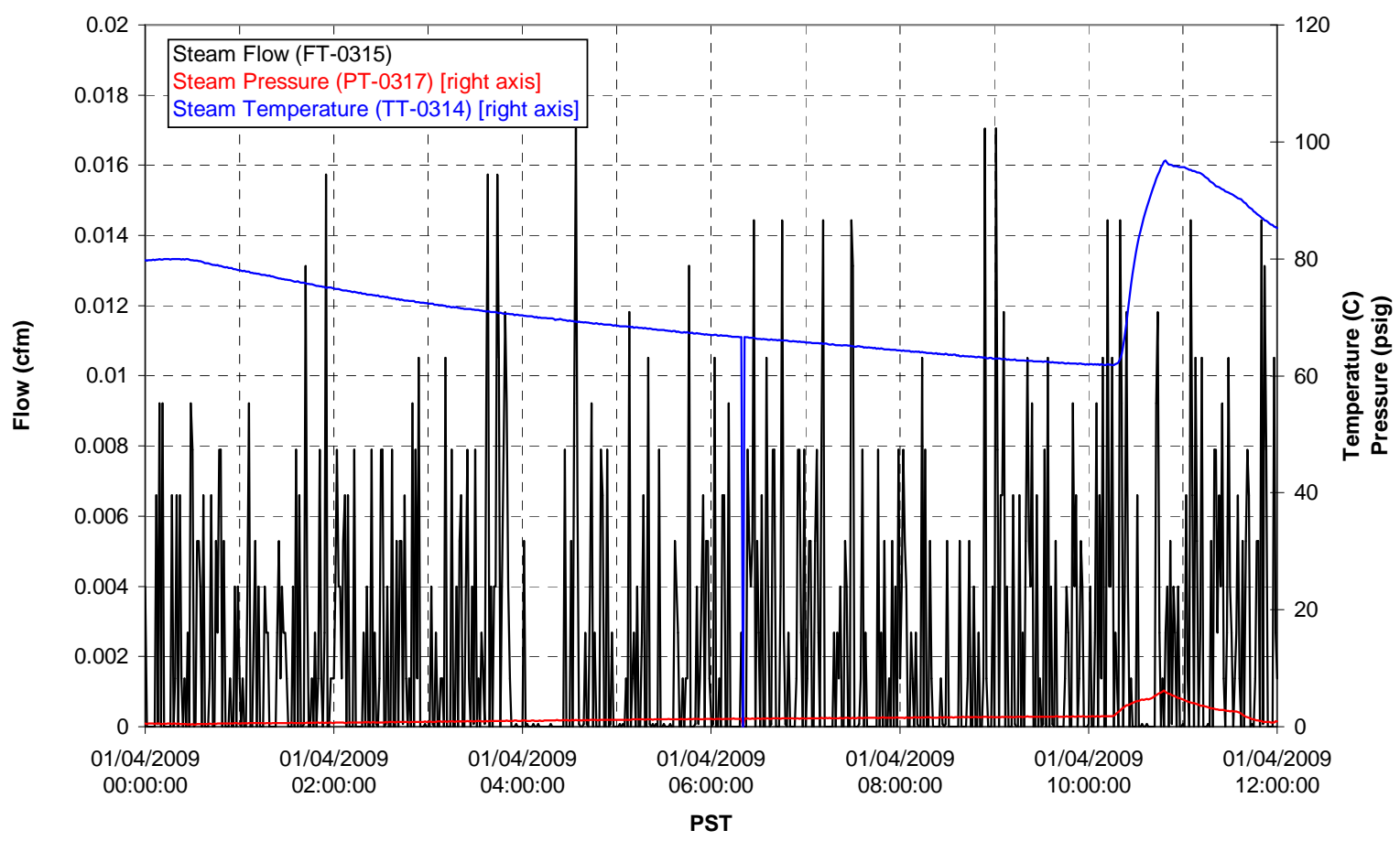


T01B Steam

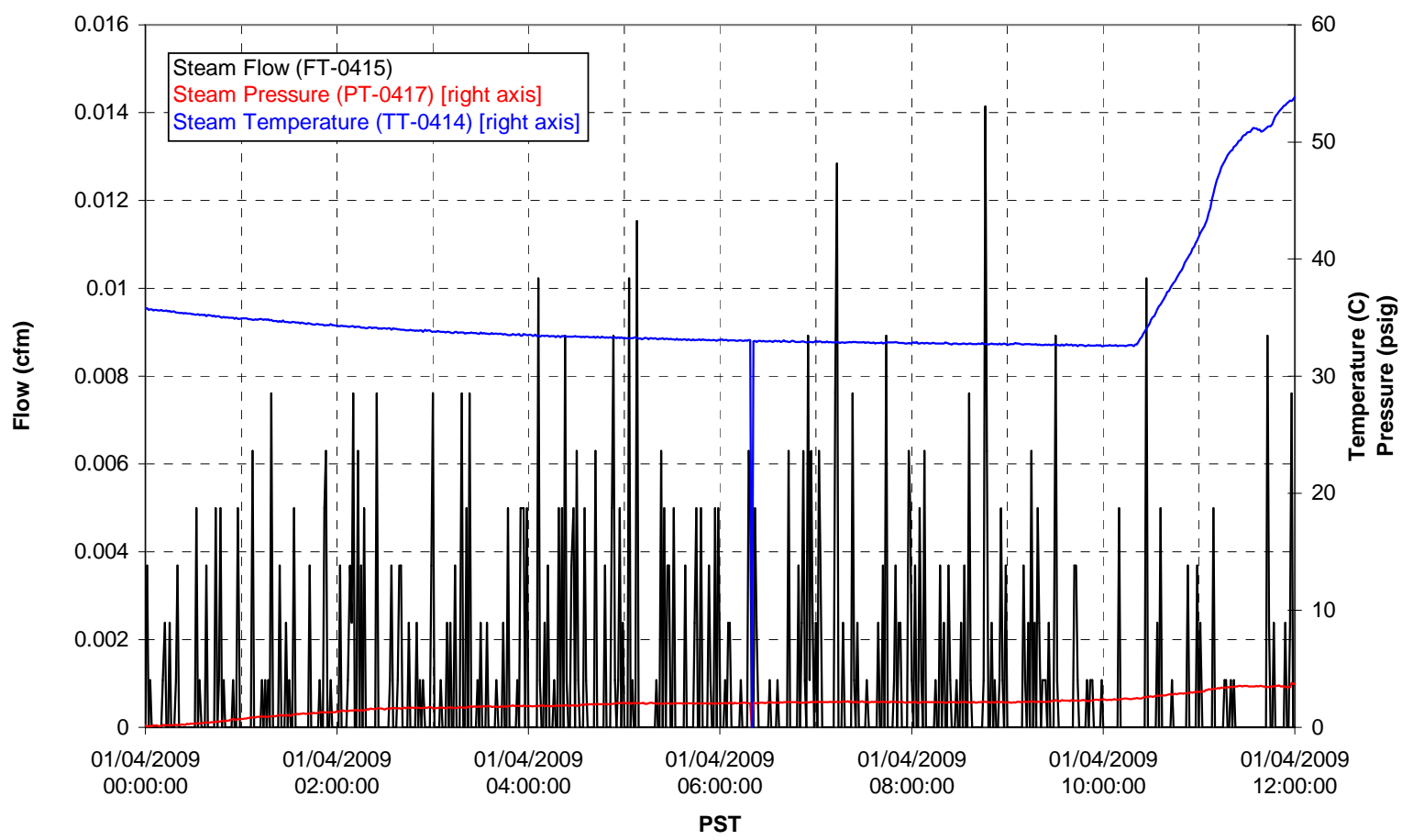


Functional Test Data Plots

01/04/2009 12:00 - 01/05/2009 00:00 
T01A level

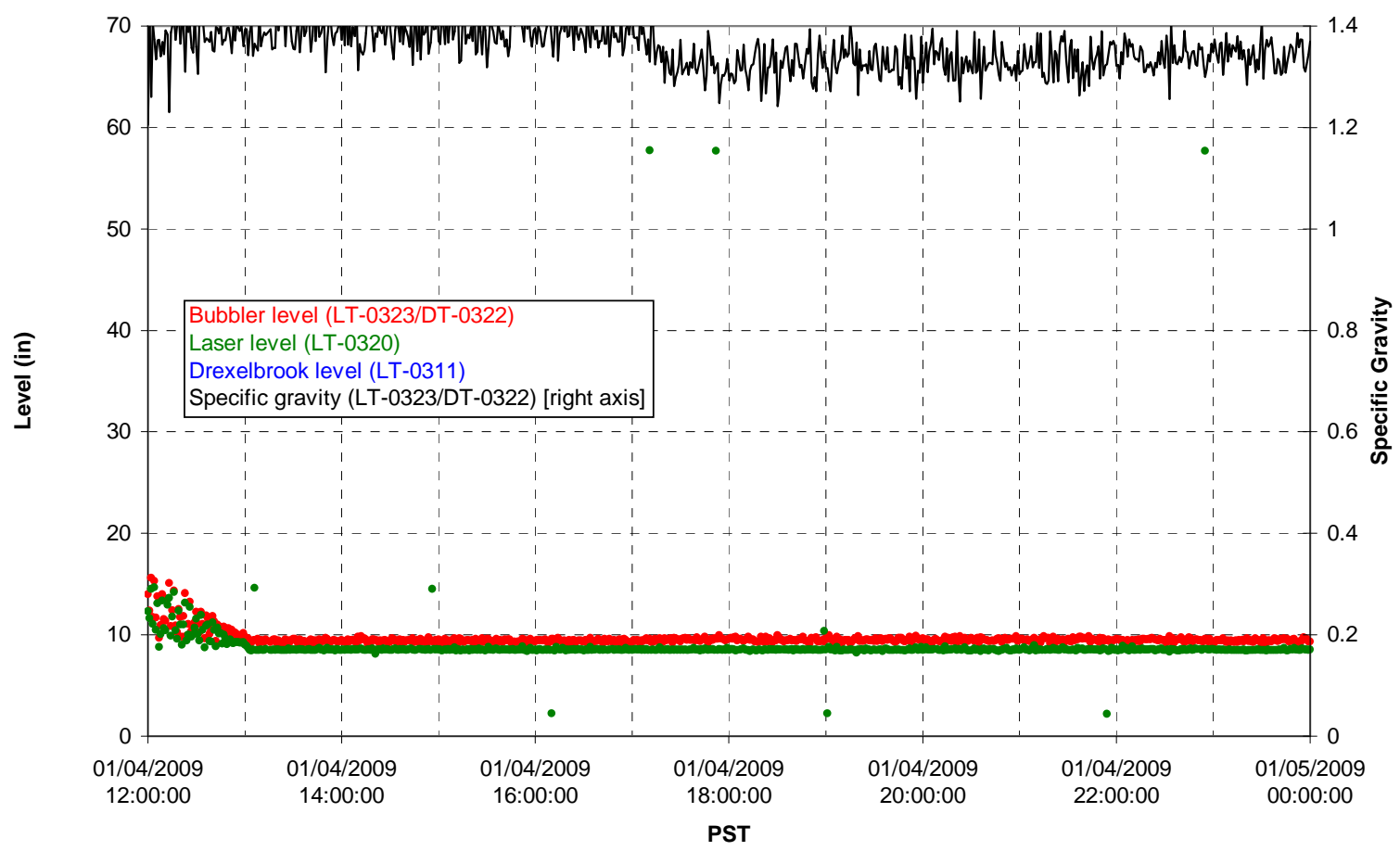

T01A temperatures

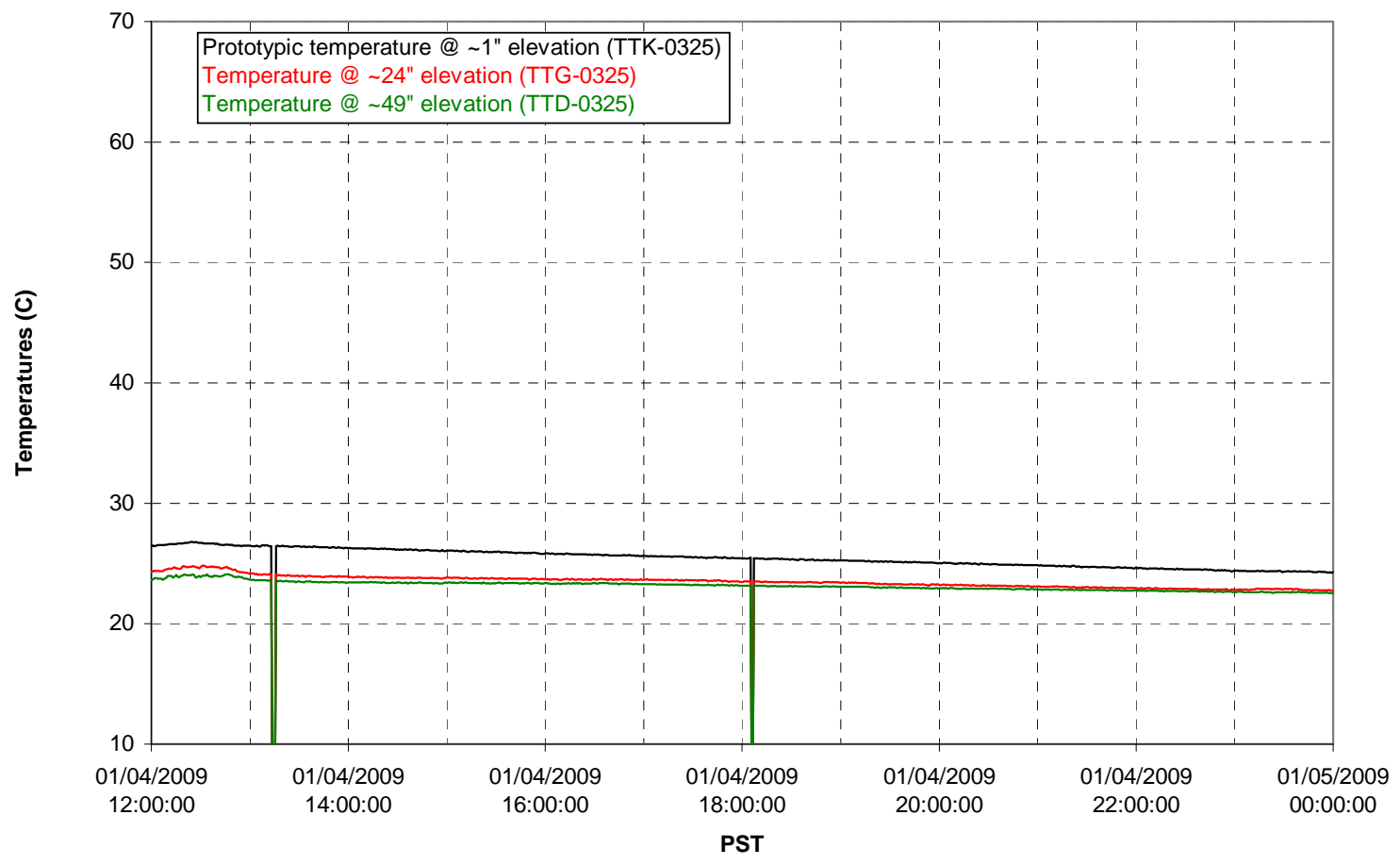


T01B level

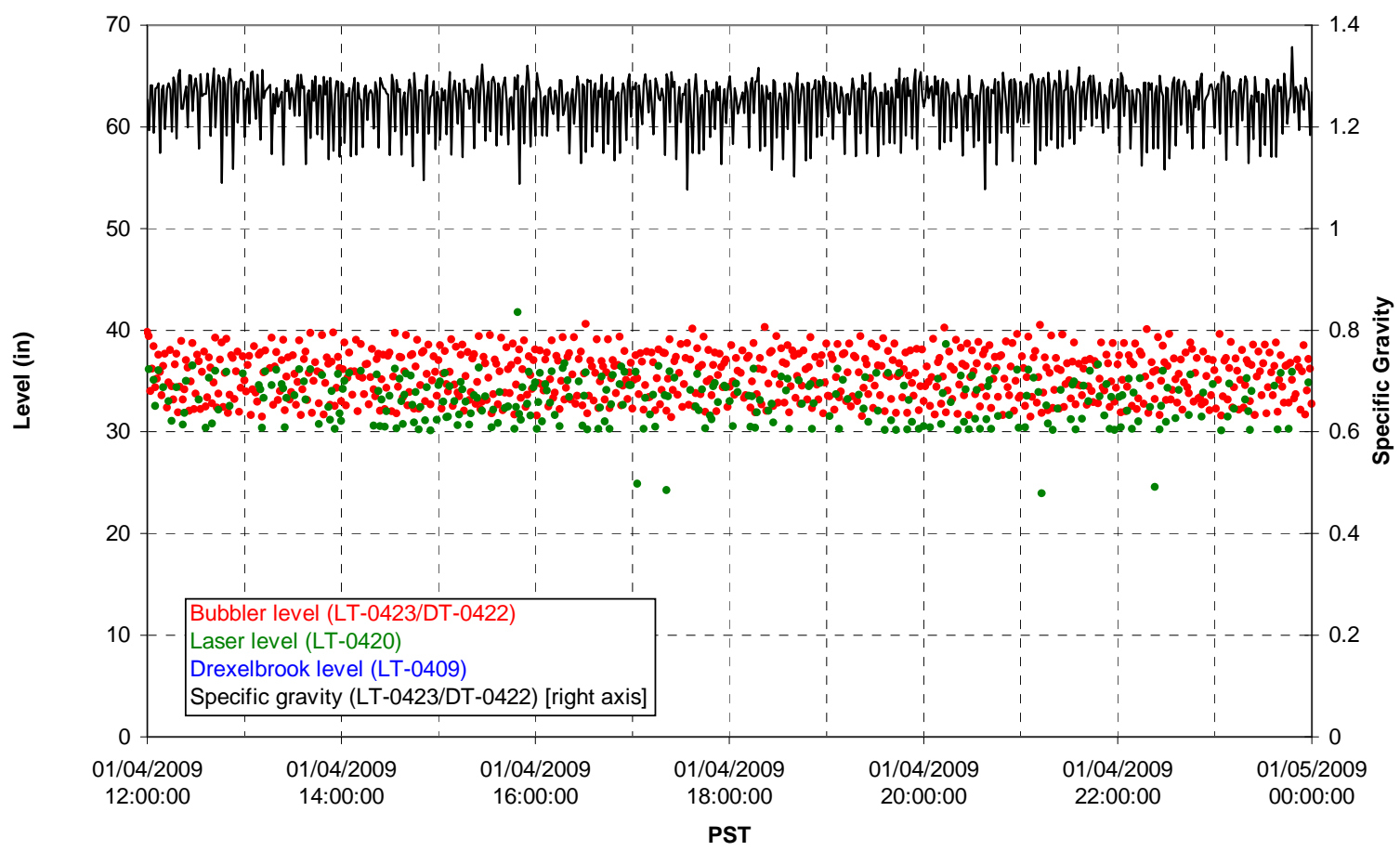

T01B temperatures

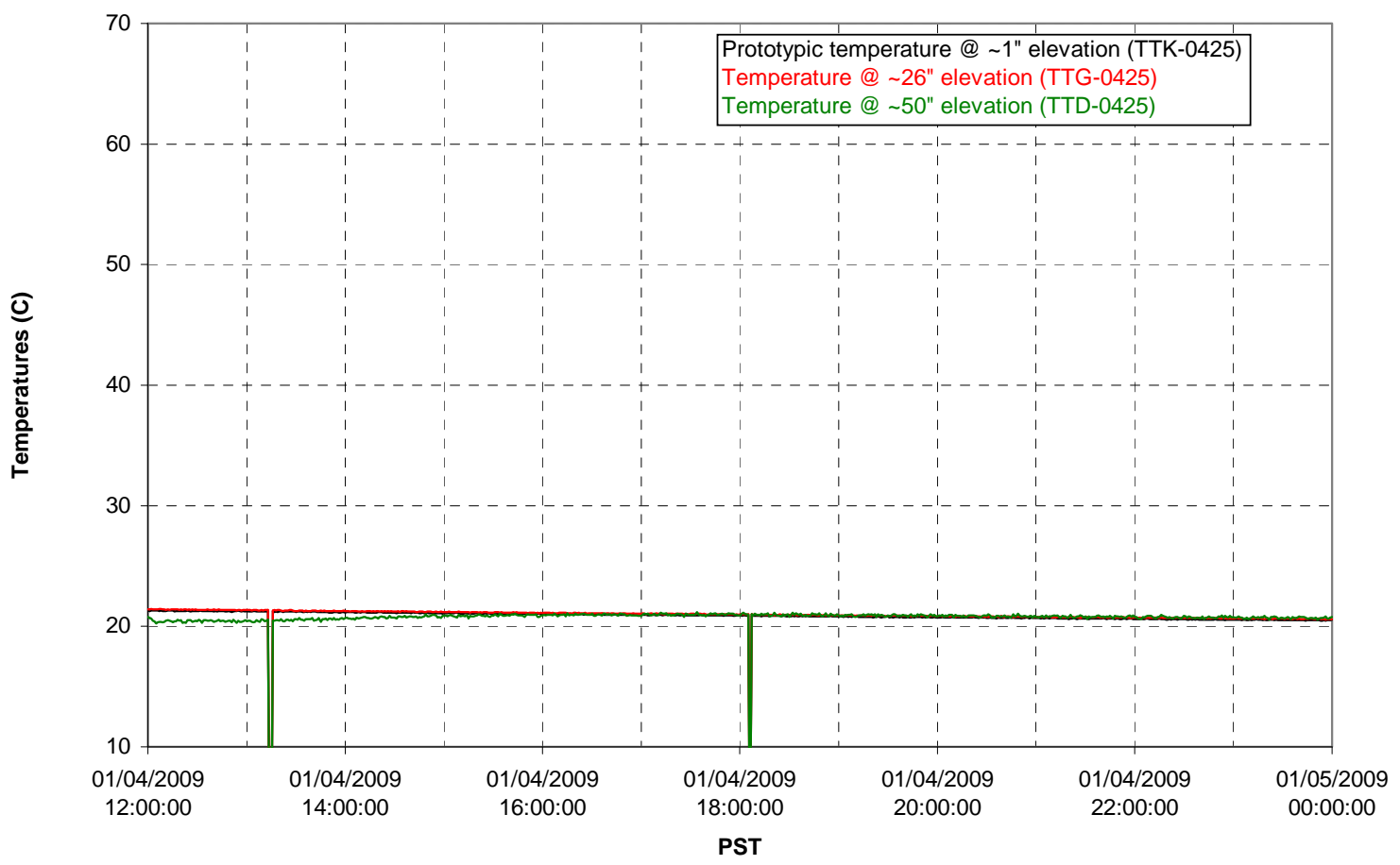




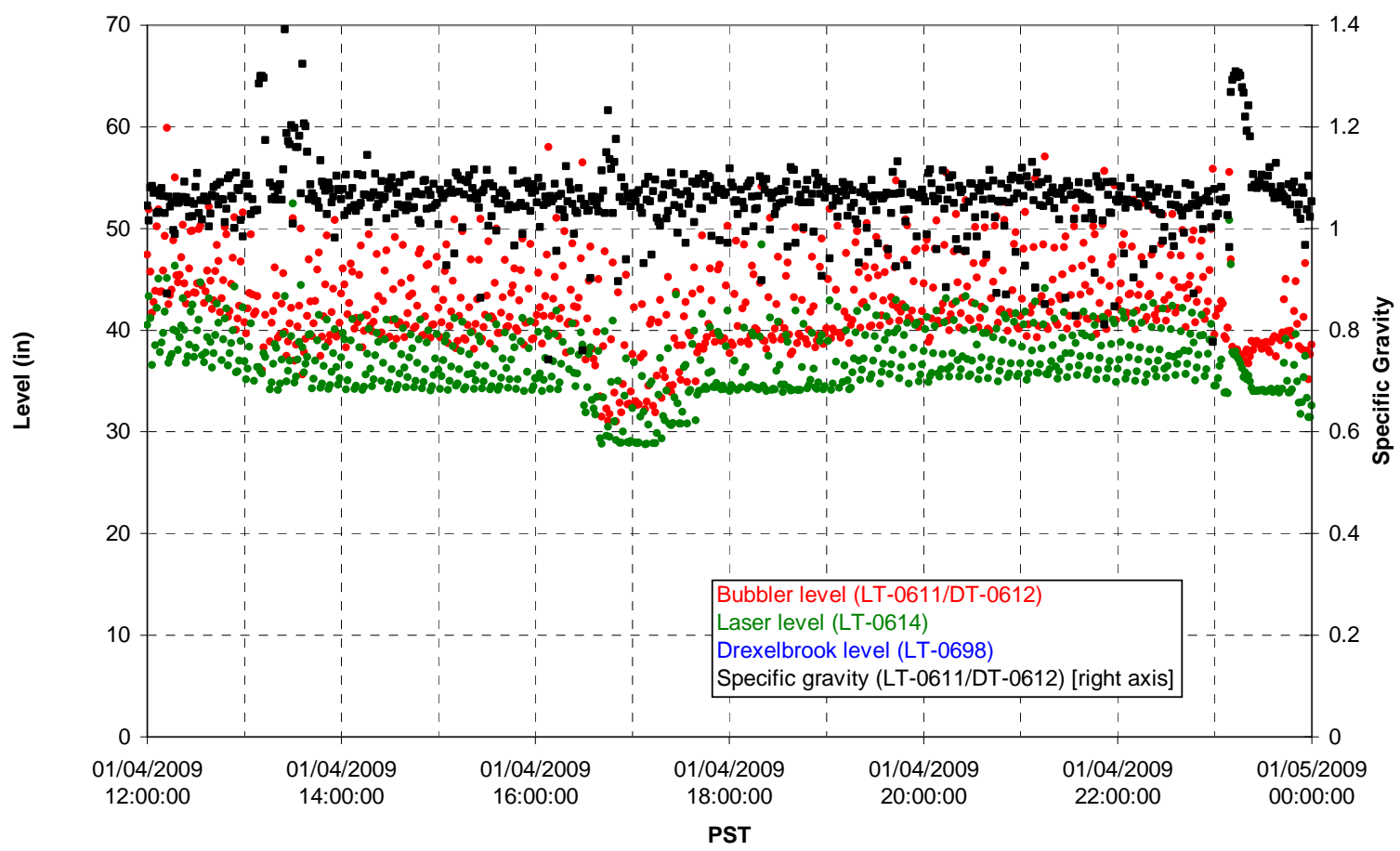

T02A temperatures

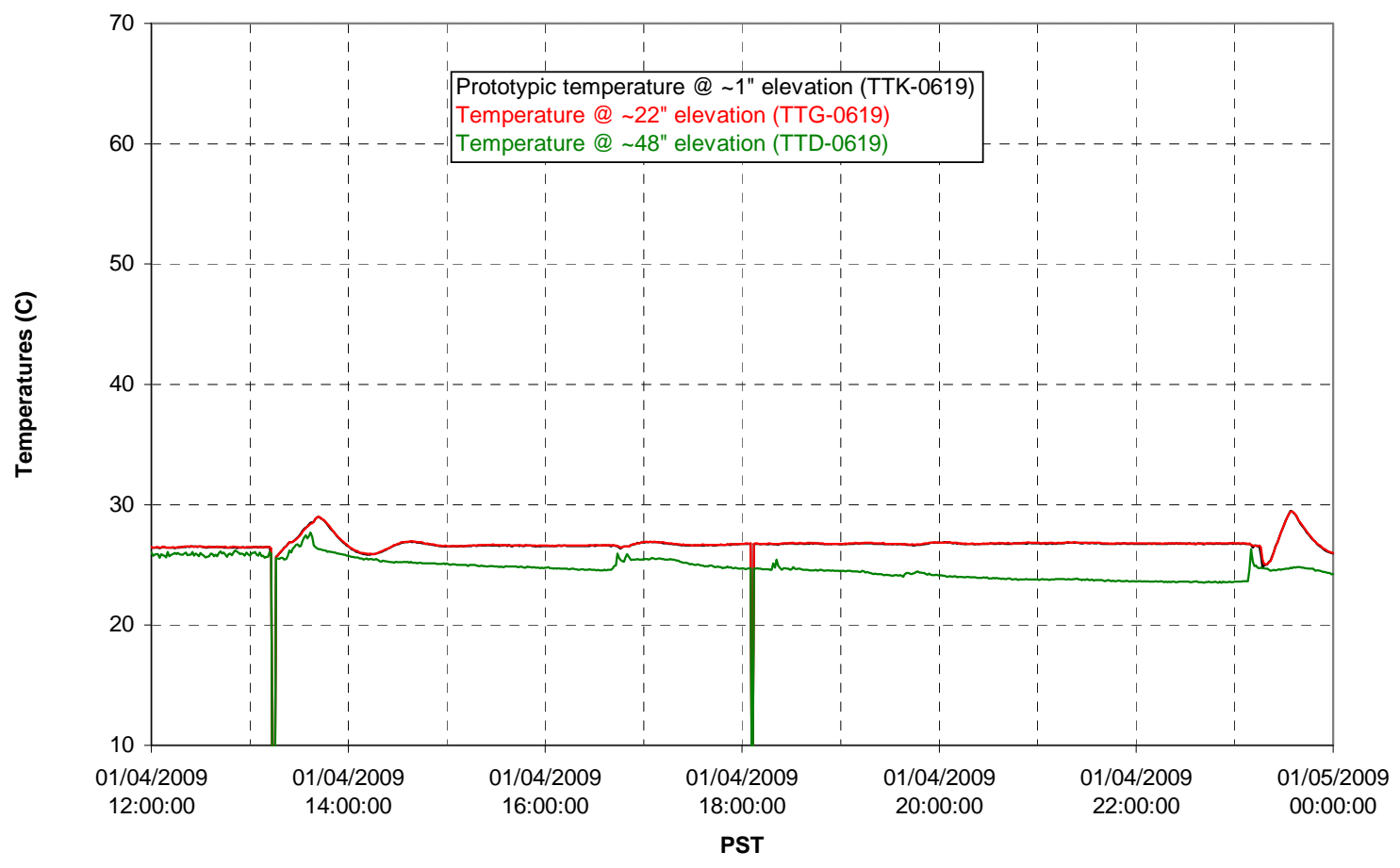


T02A and filter loop temperatures

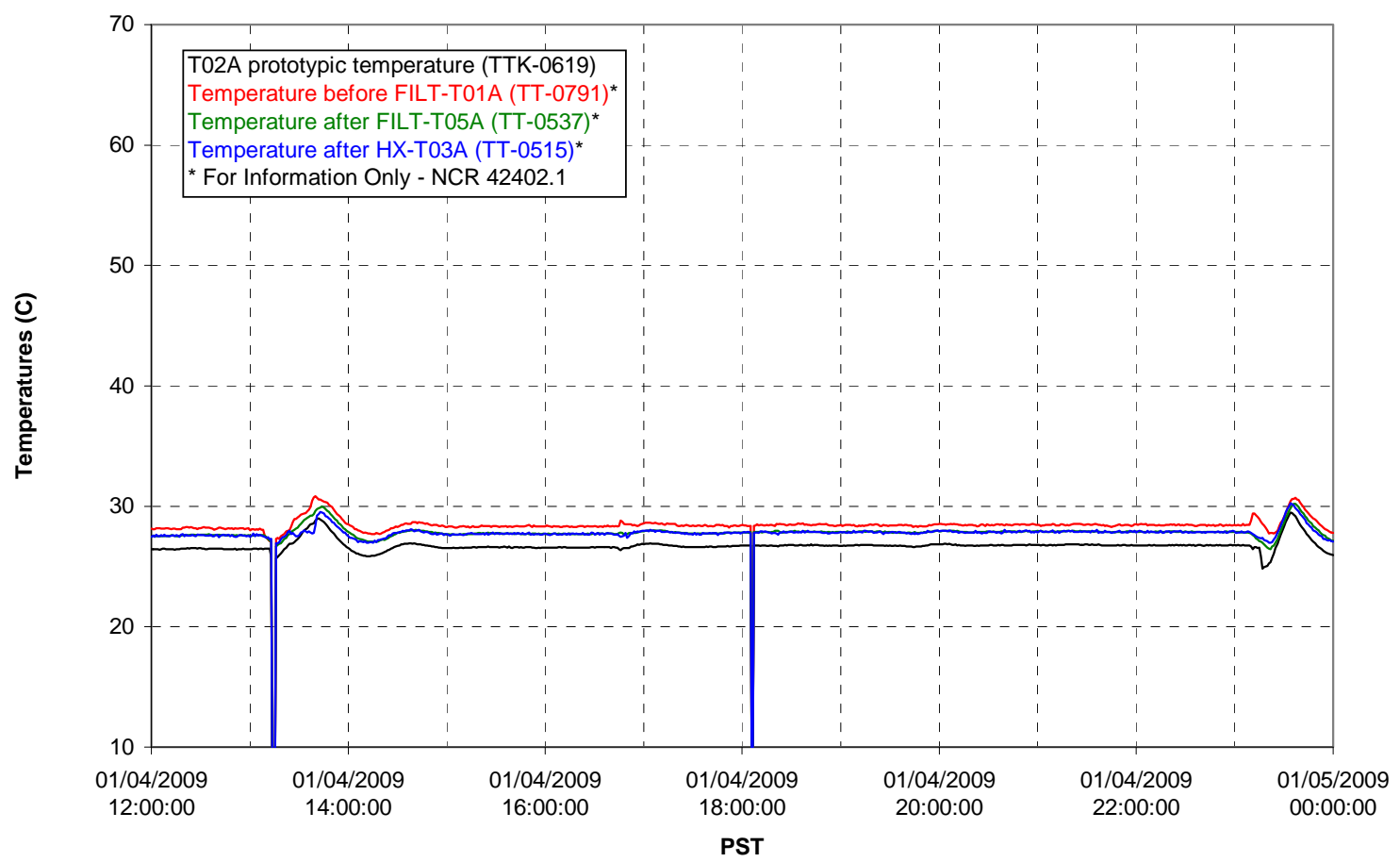

Pump Pressures and Flow

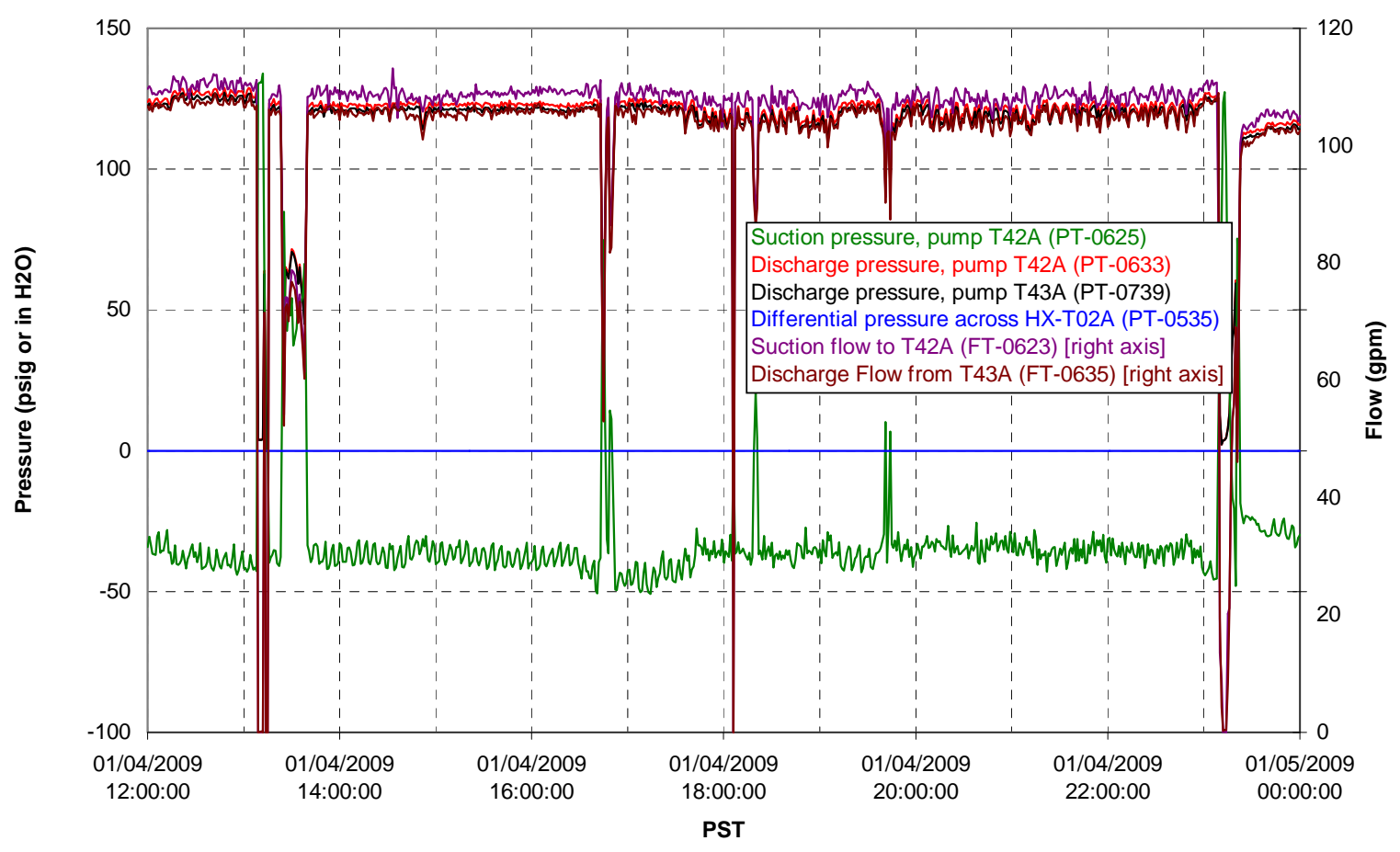


Axial pressure drop

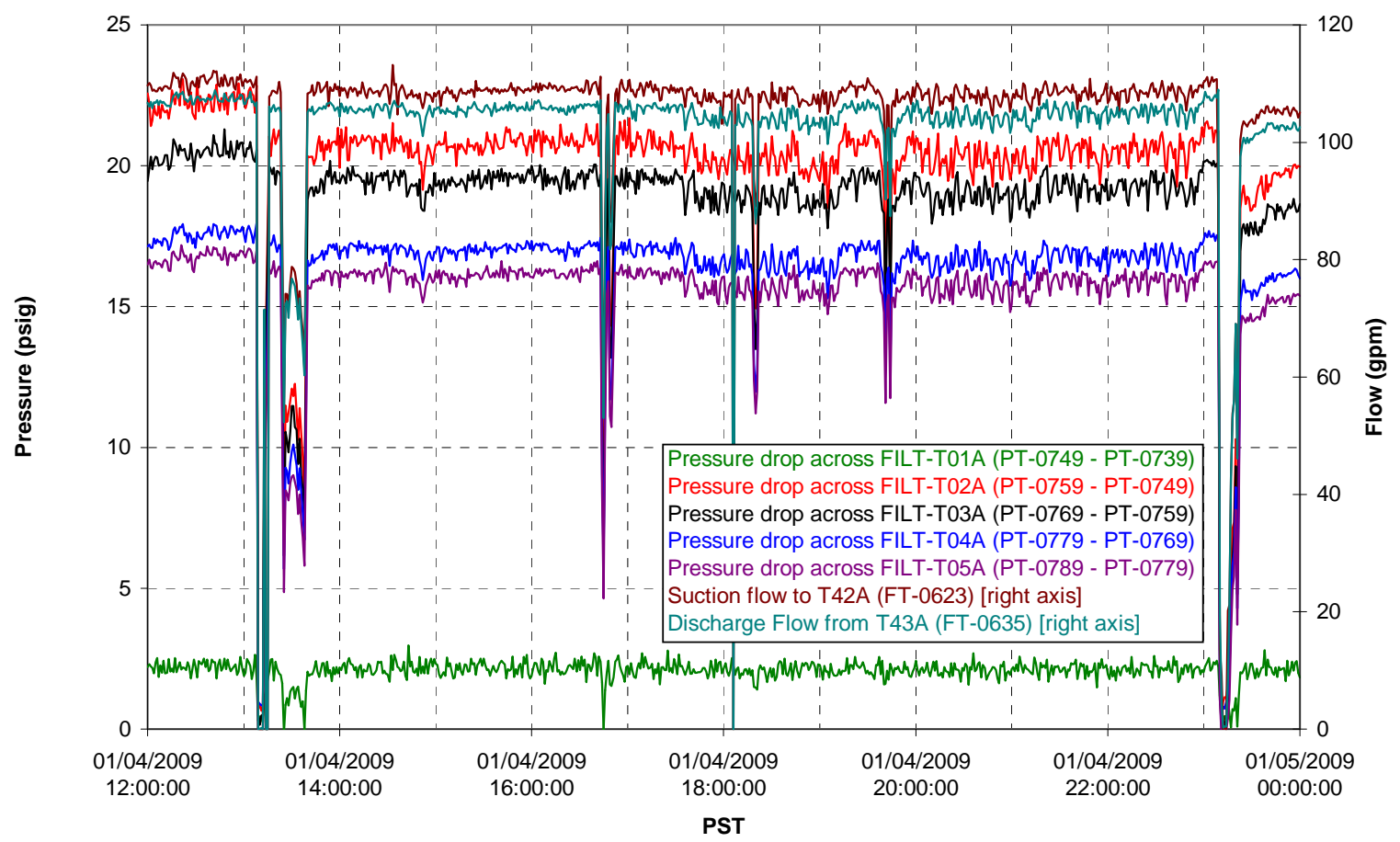

Permeate flow rates

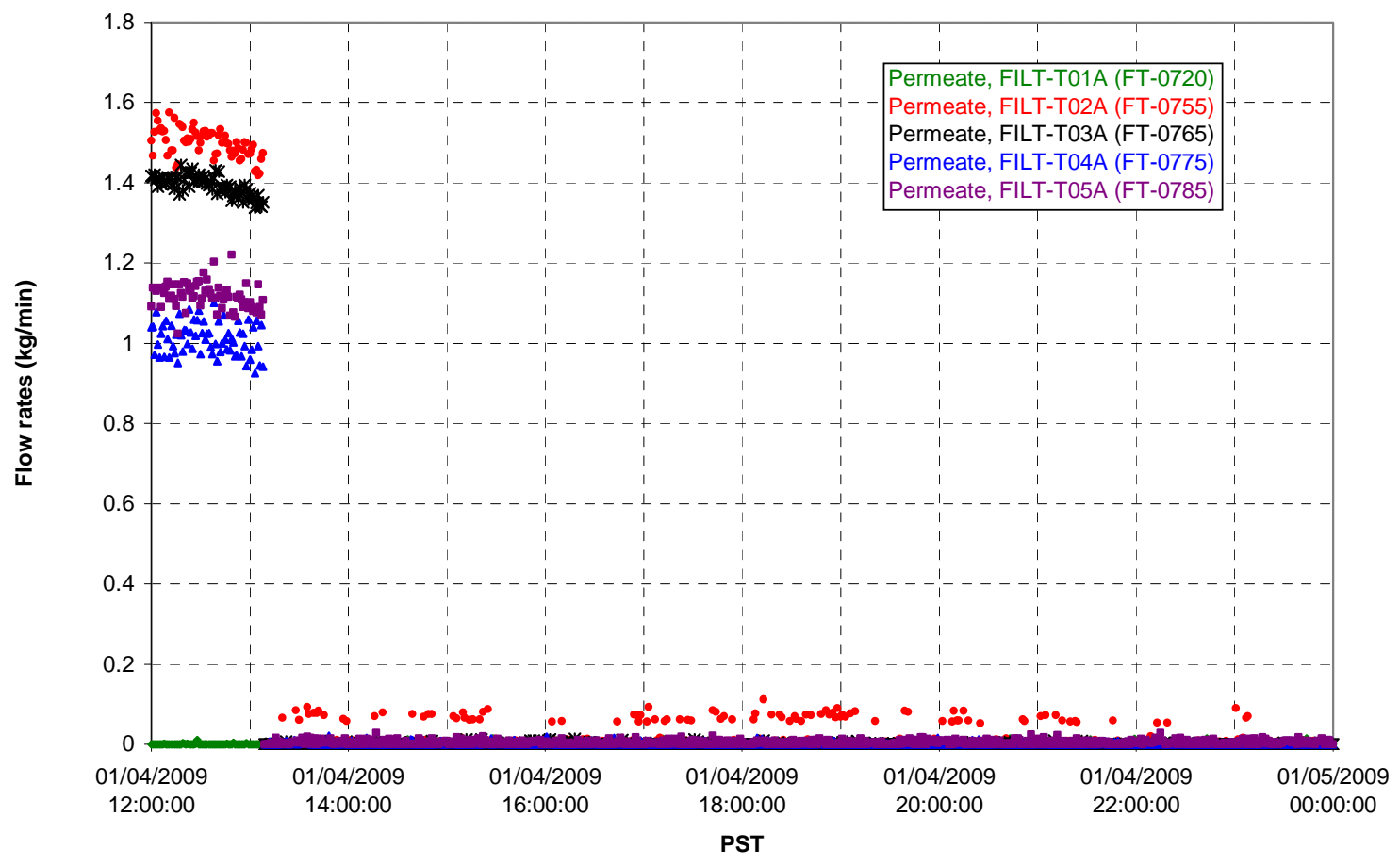


T02A Inner Temperature Tree

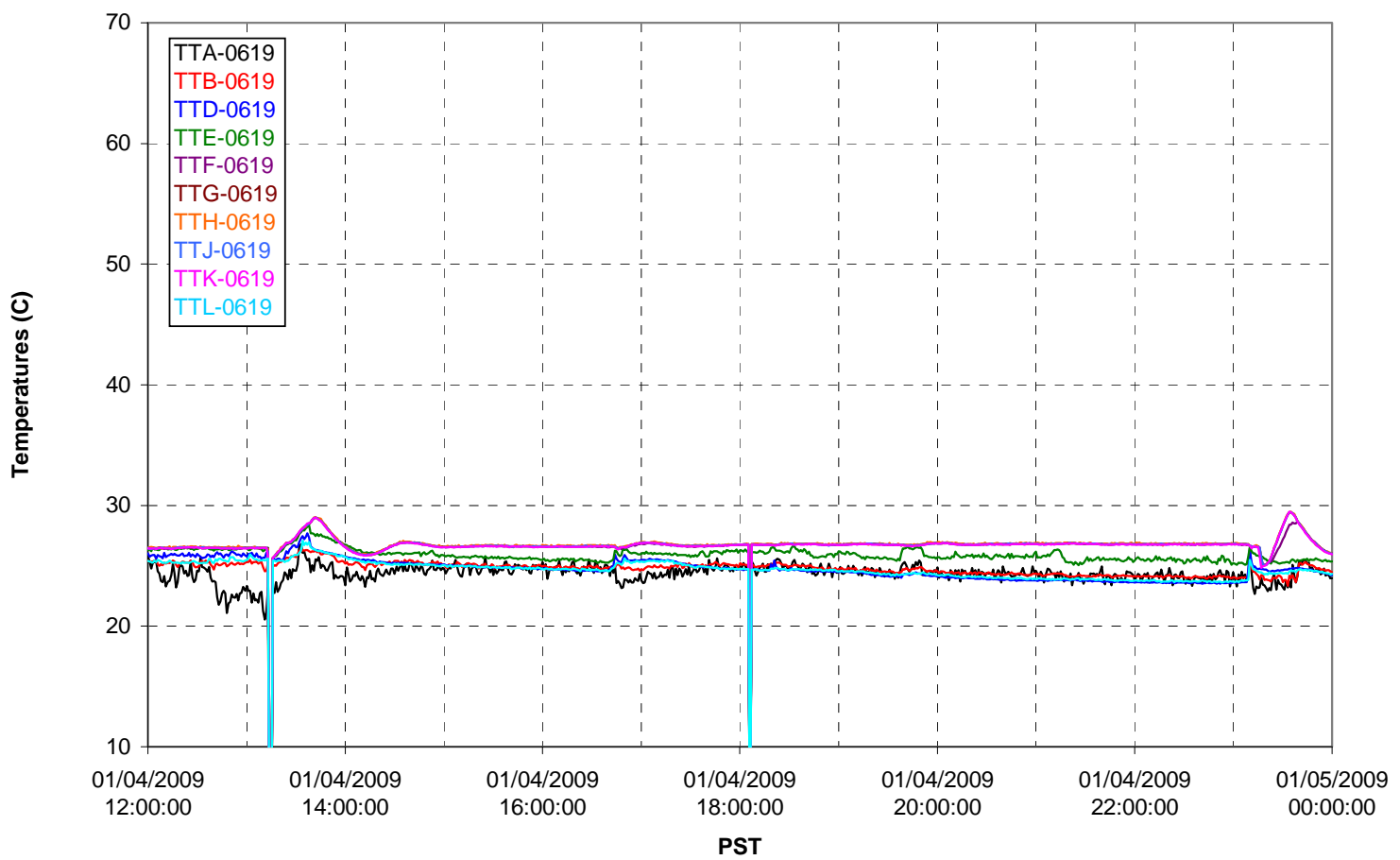

T02A Outer Temperature Tree

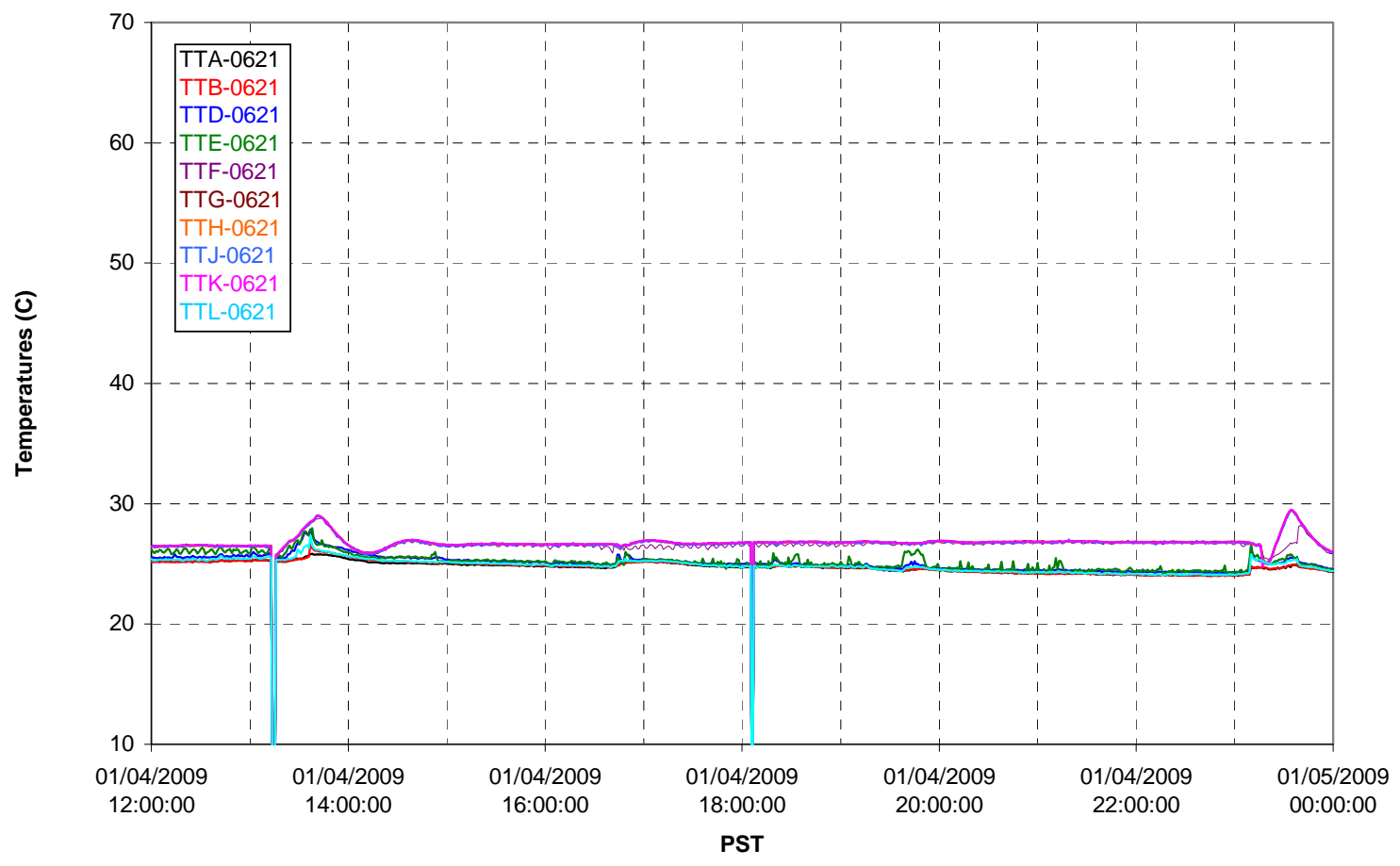


T02A temperatures

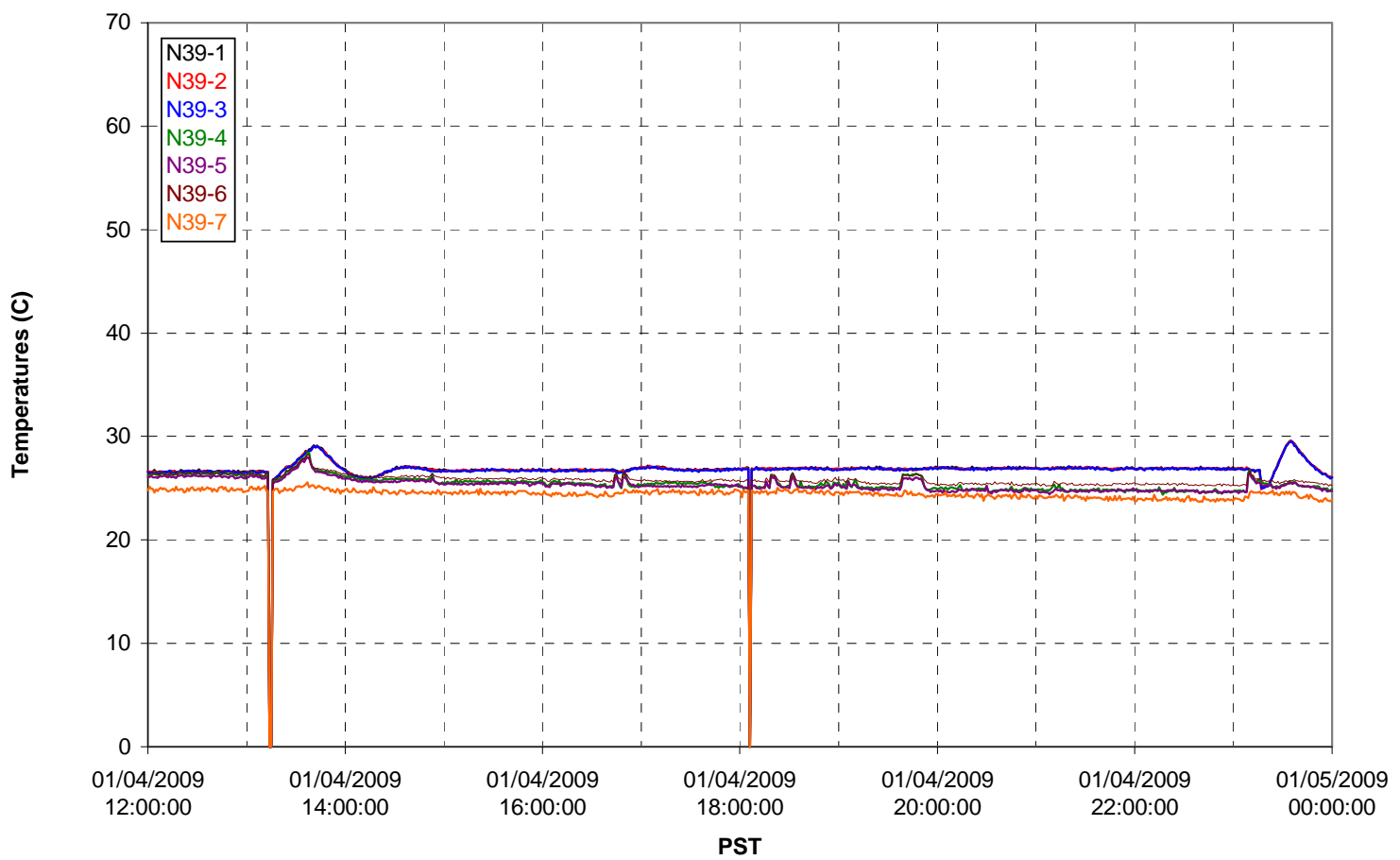

T02A temperatures

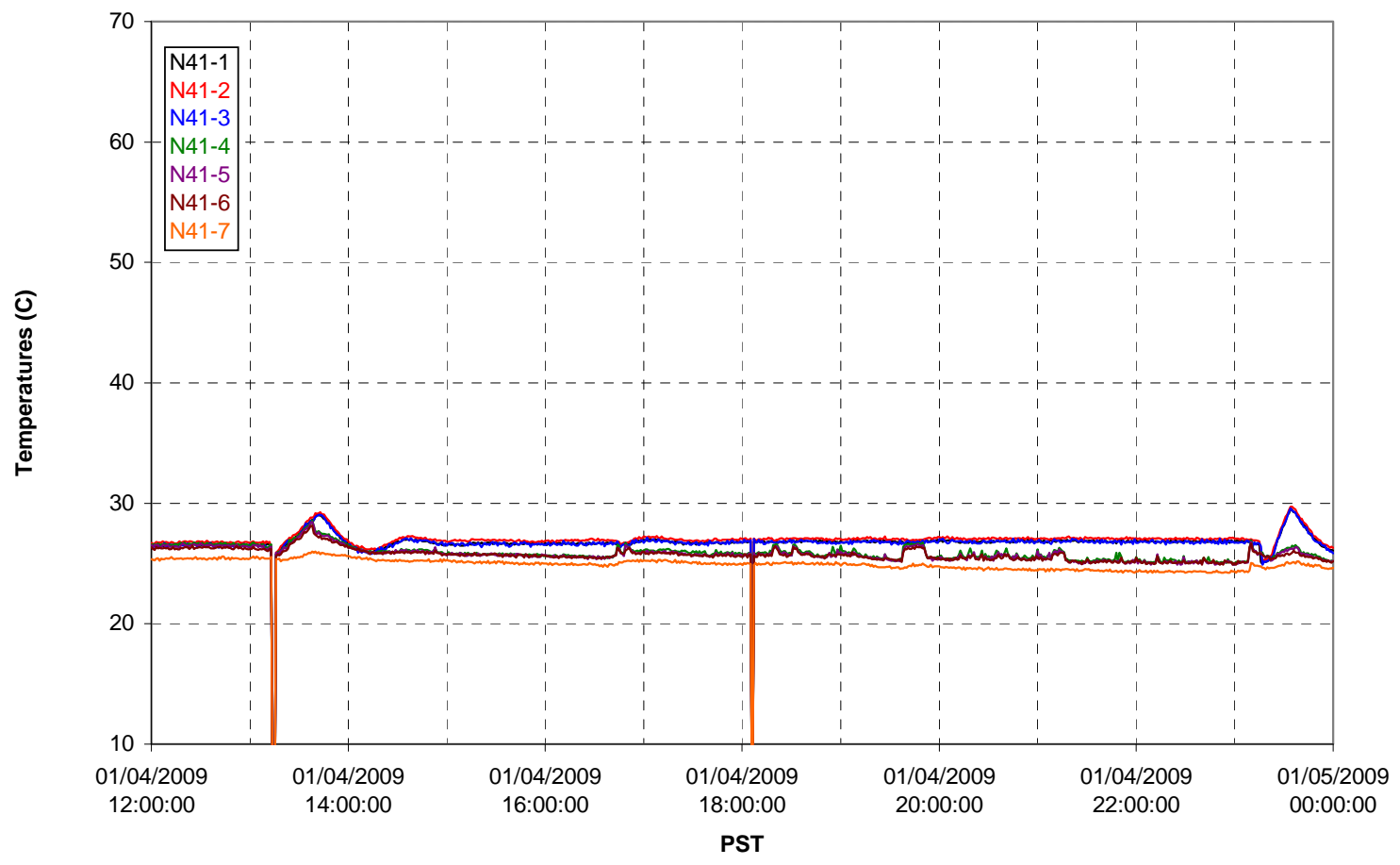


T02A temperatures

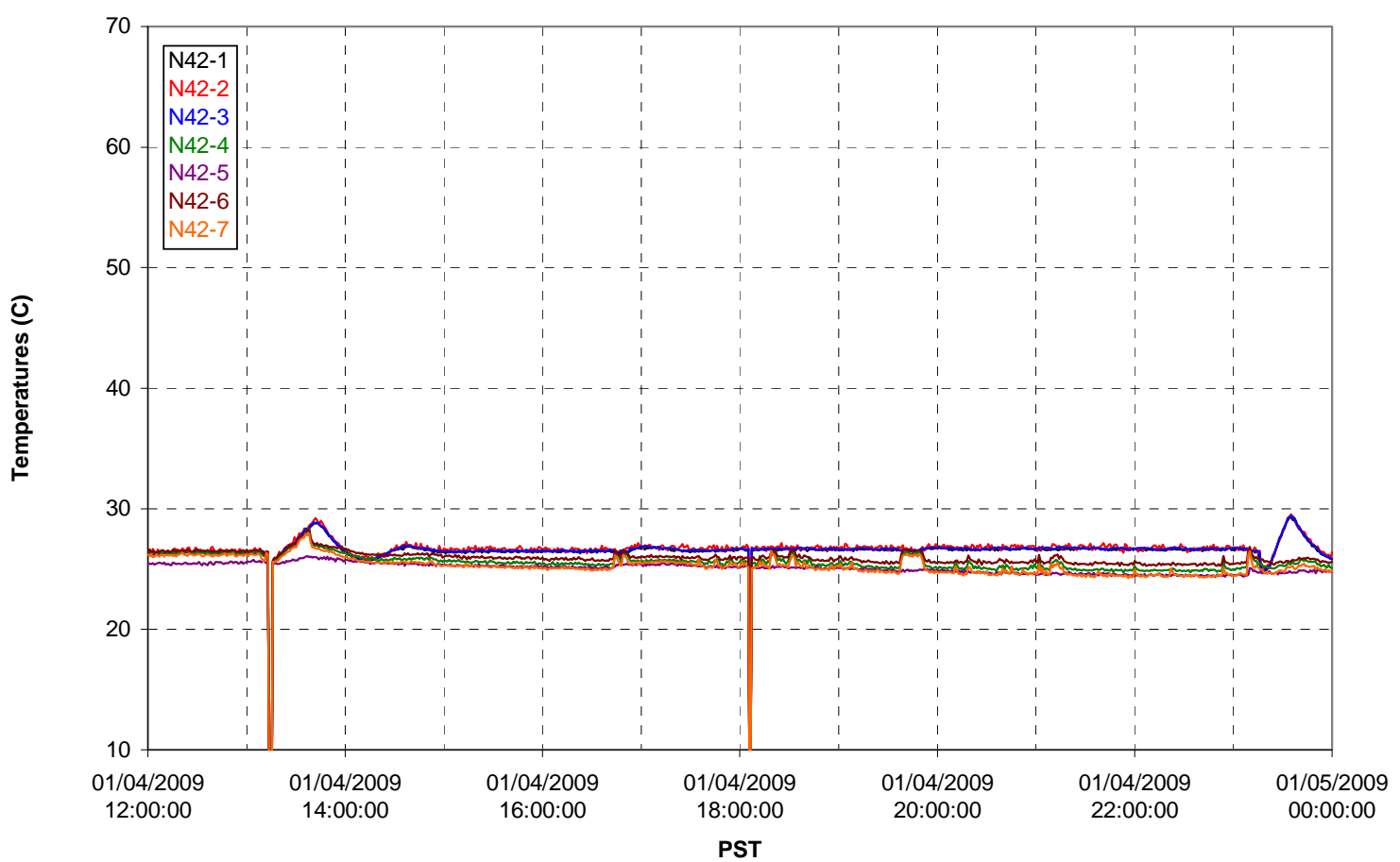

T02A temperatures

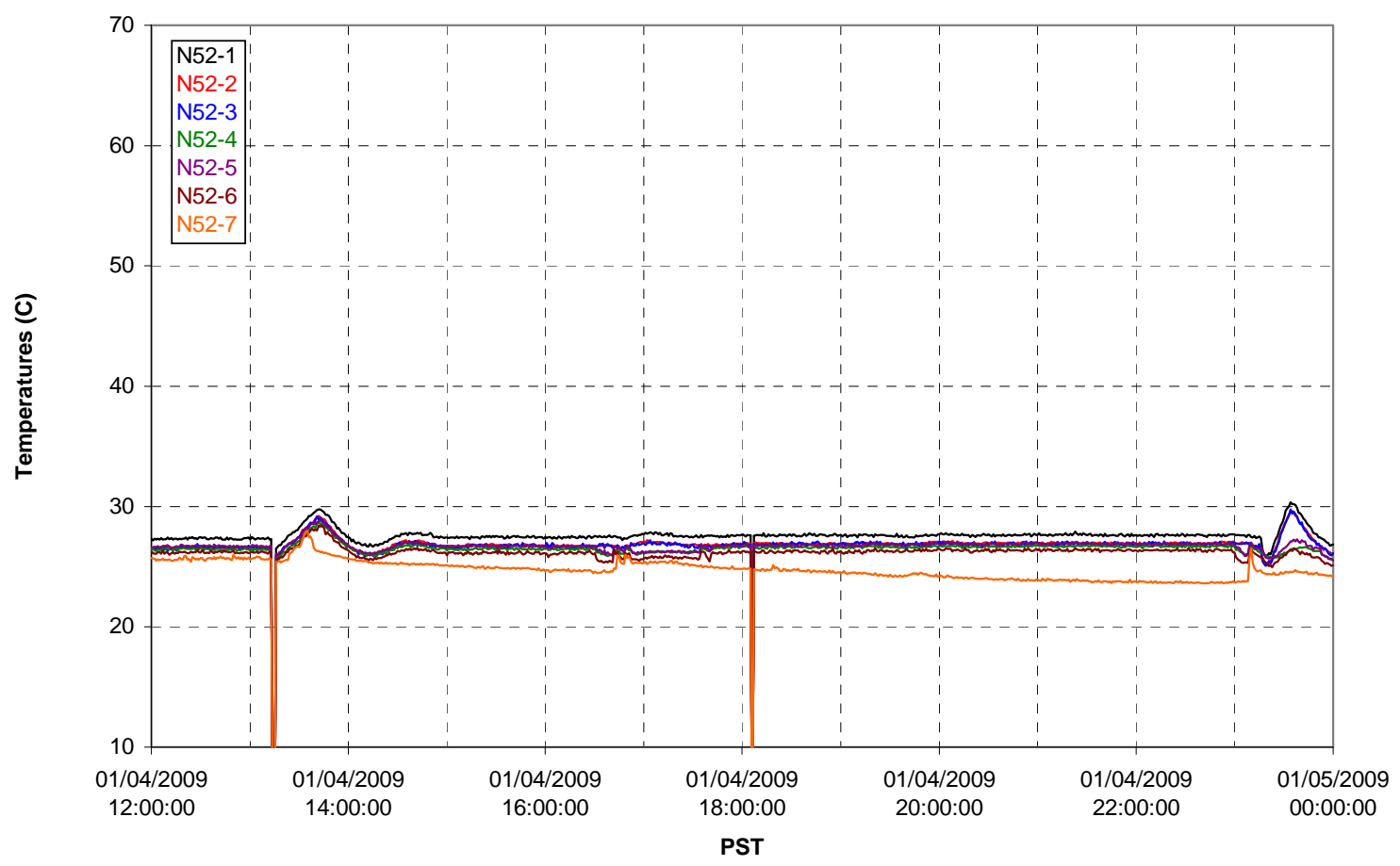


T02A Heating and Cooling

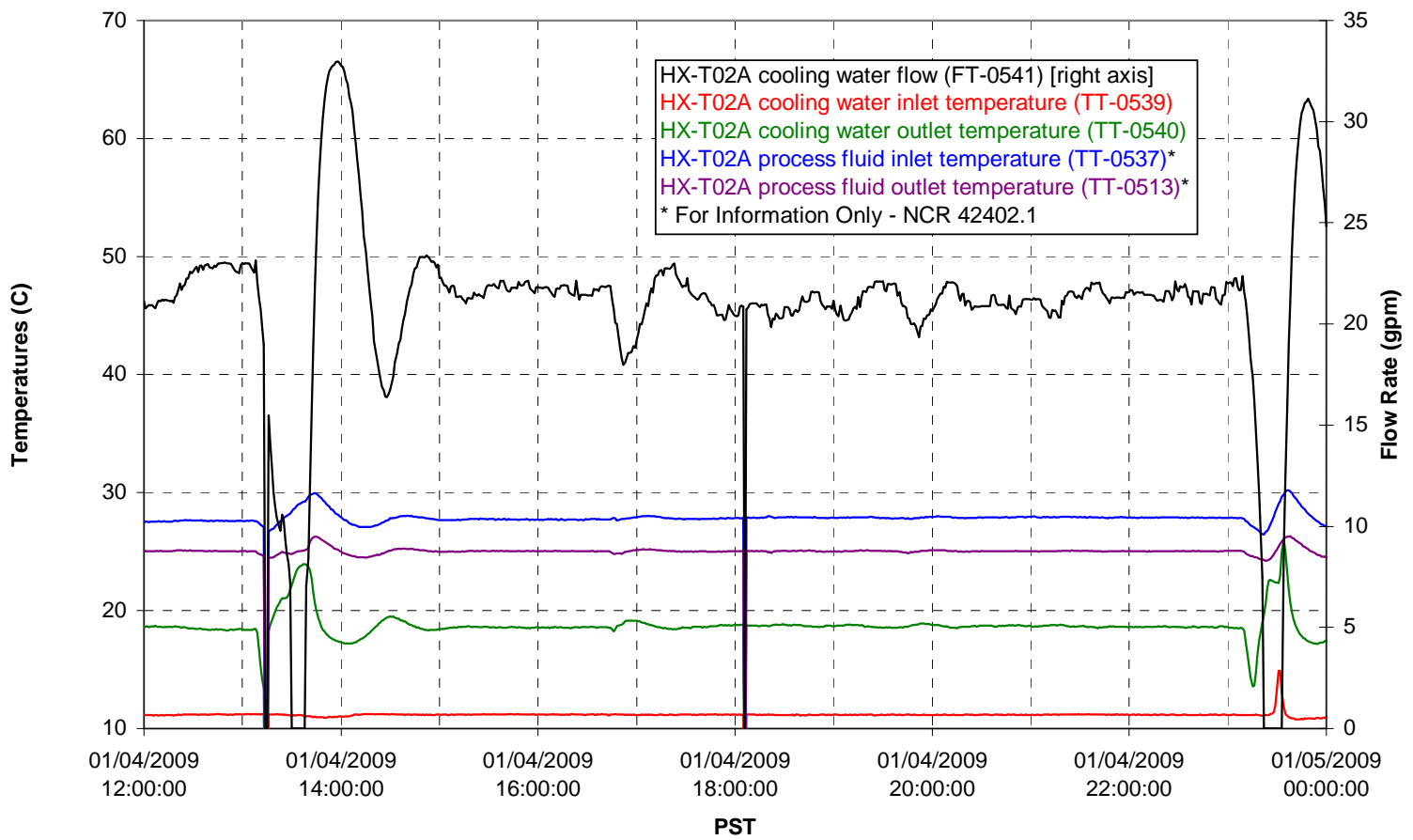

Pump Operation

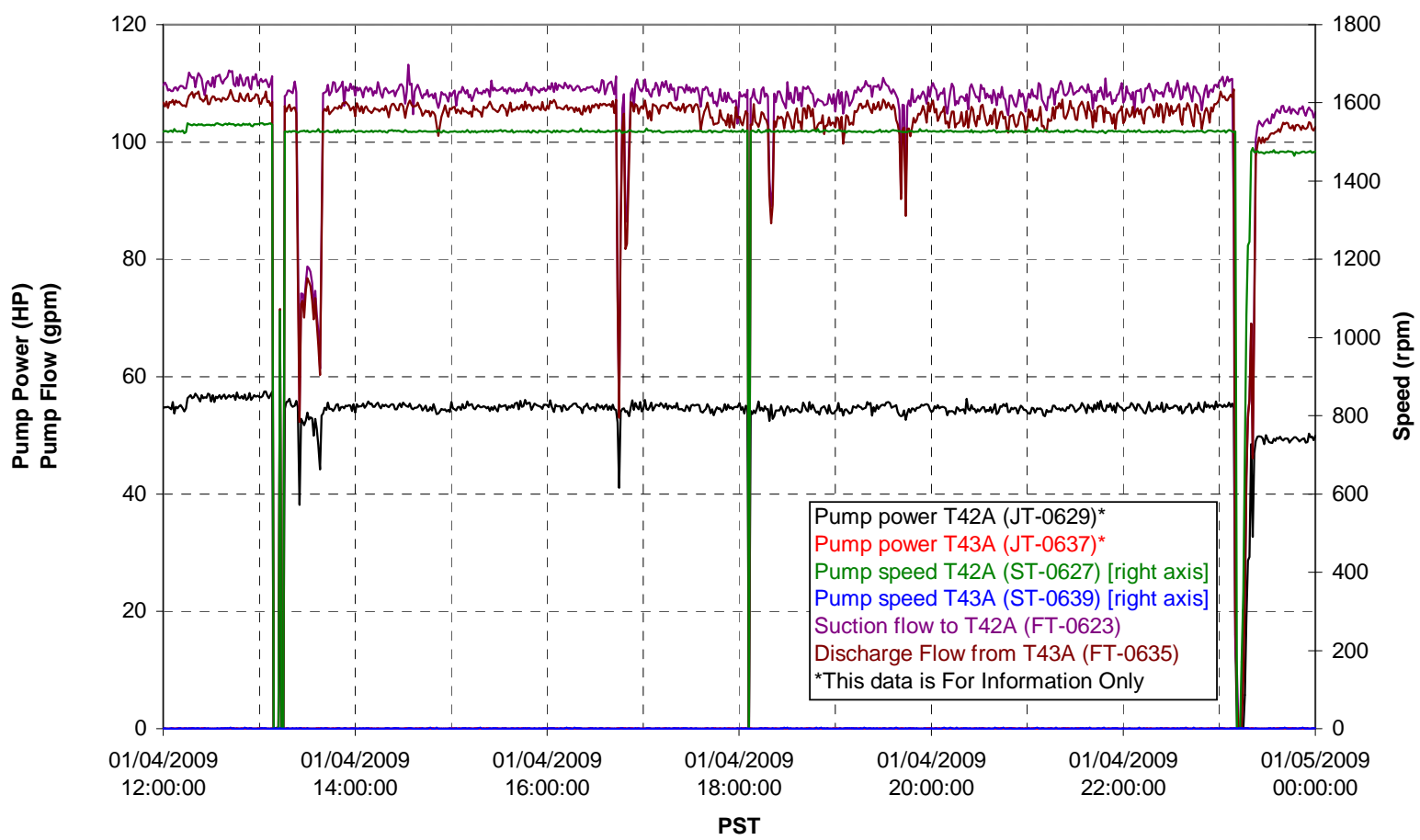


Pulsepot UFP-PP-T01A

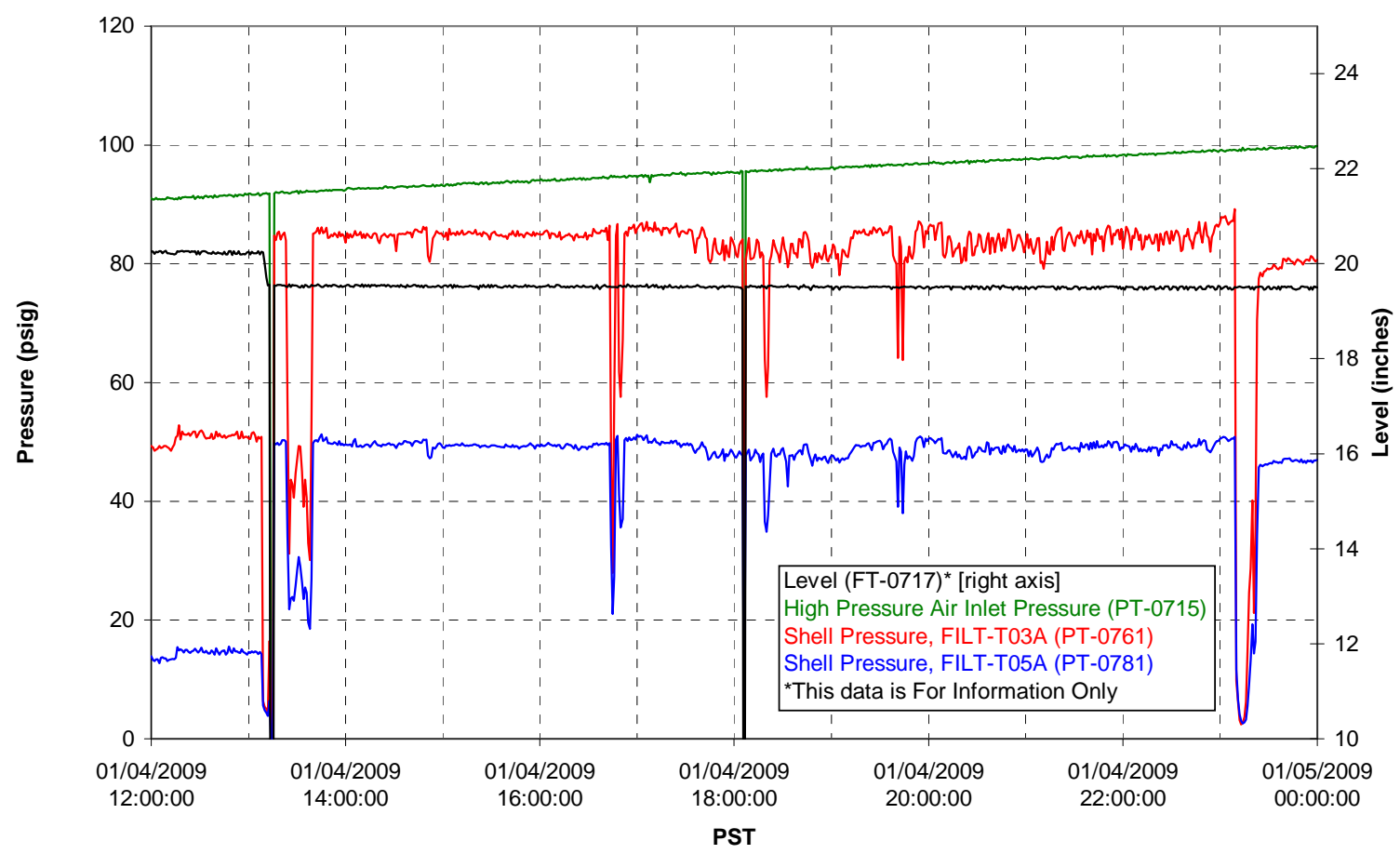

Pulsepot UFP-PP-T02A

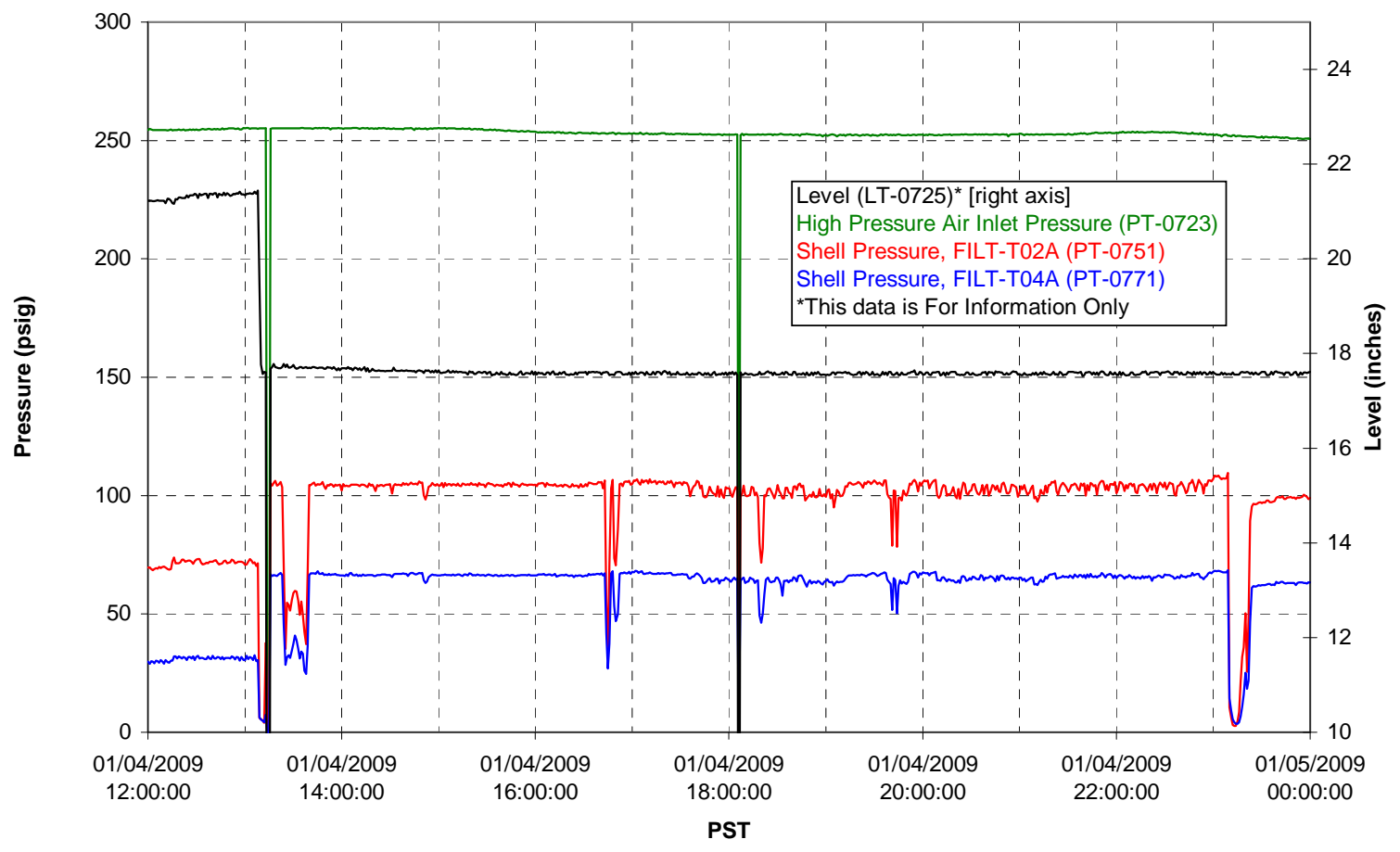


Pulsepot UFP-PP-T03A

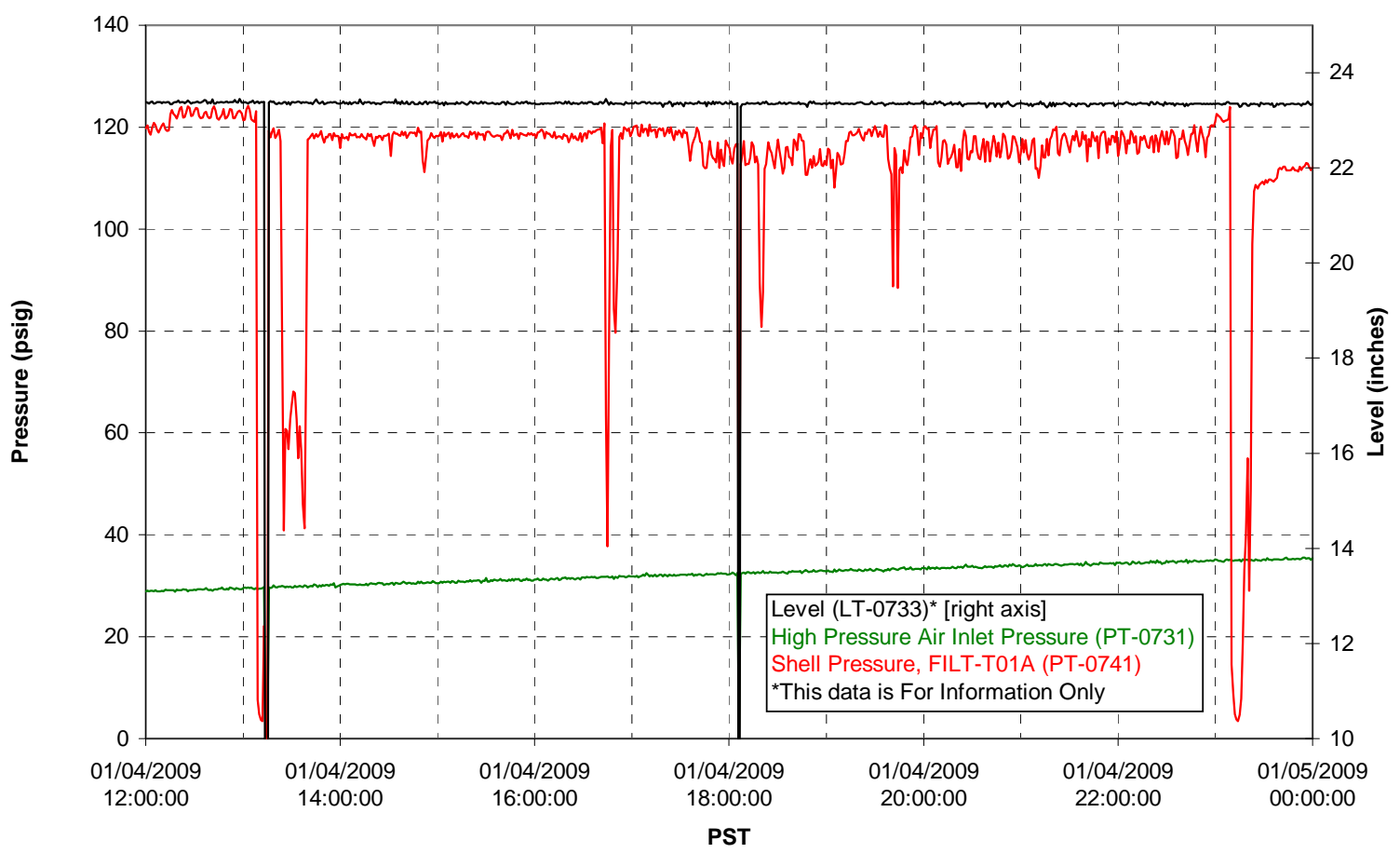

Pulsepot Levels

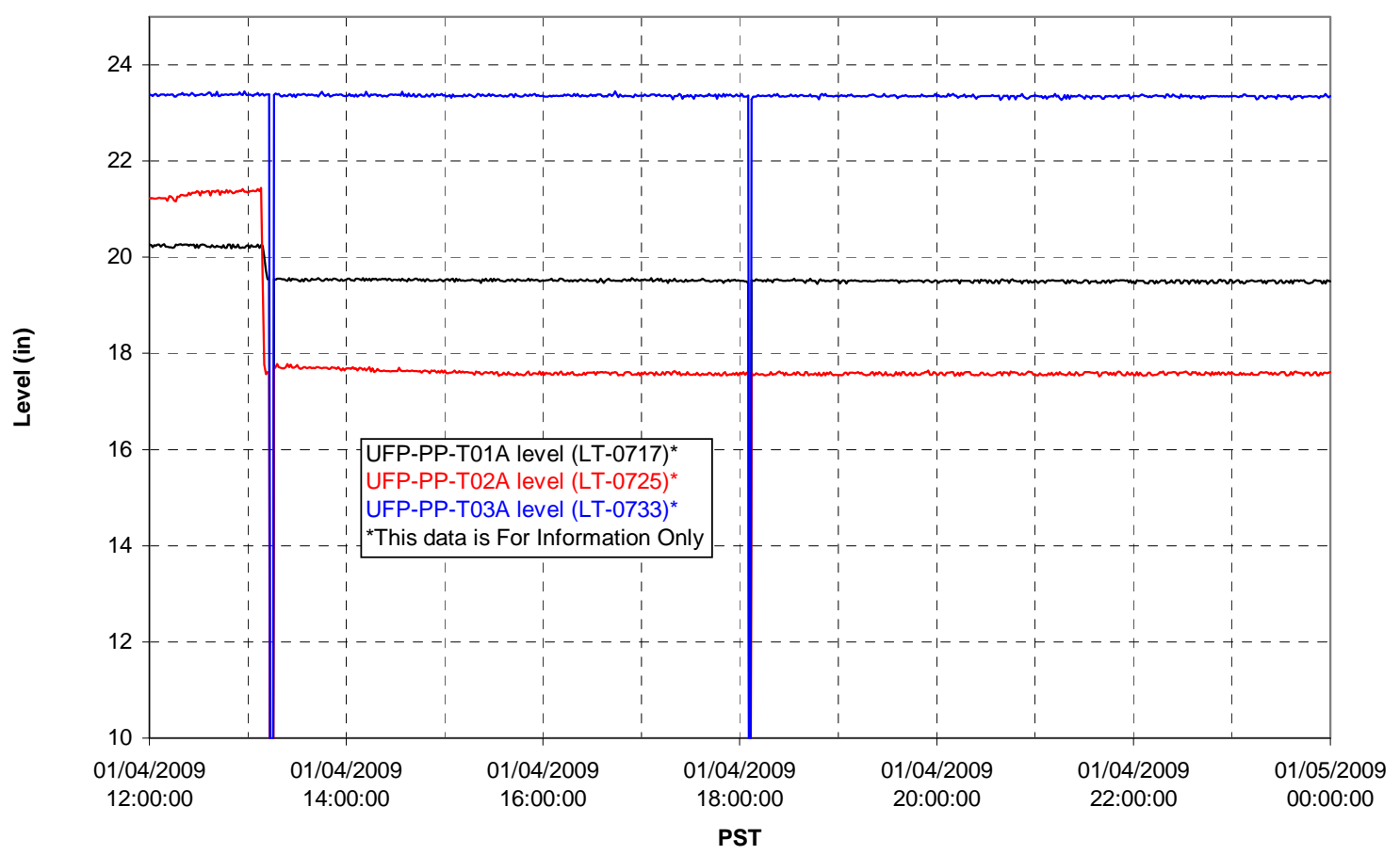


Filter UFP-FILT-T01A

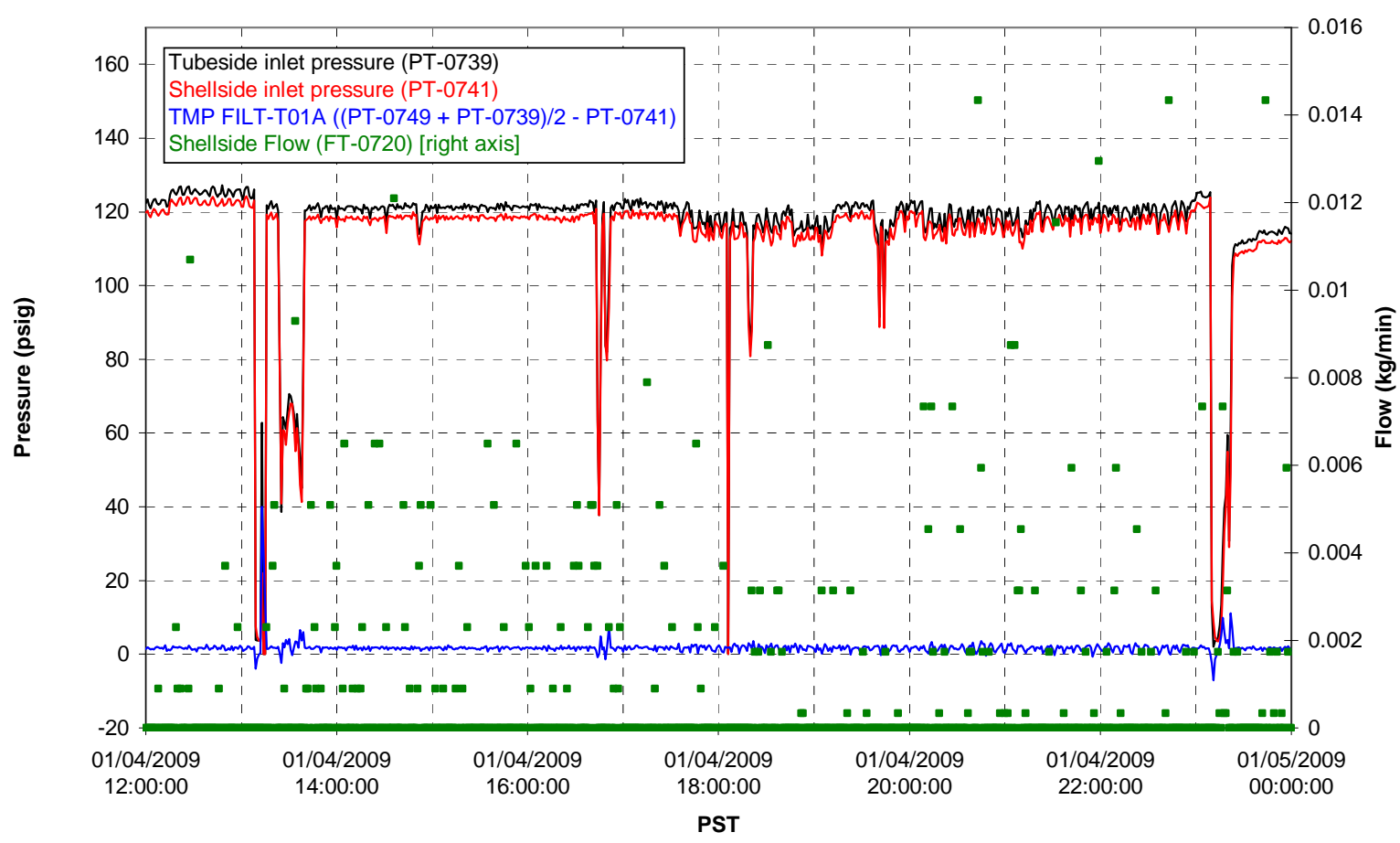

Filter UFP-FILT-T02A

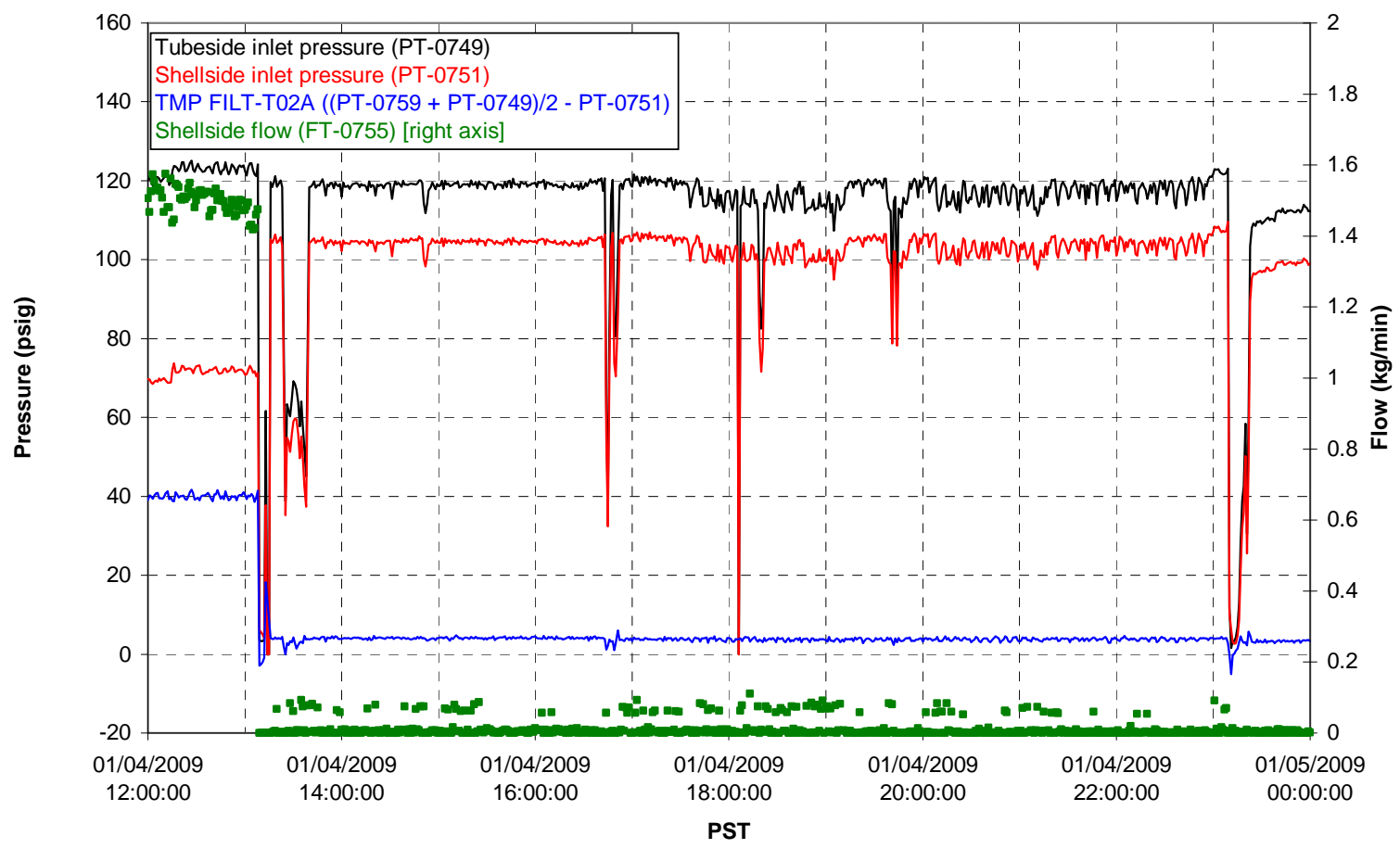


Filter UFP-FILT-T03A

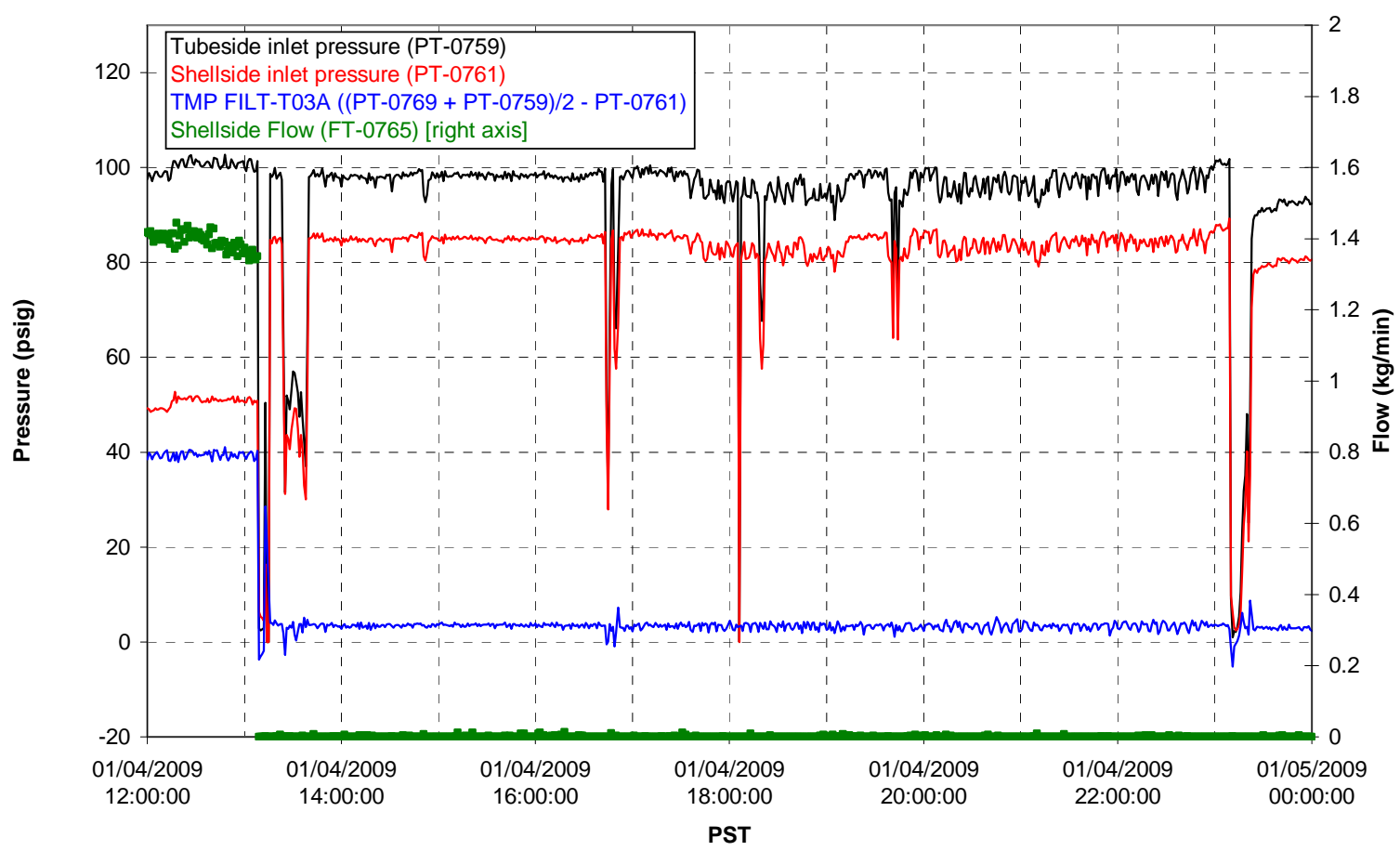

Filter UFP-FILT-T04A

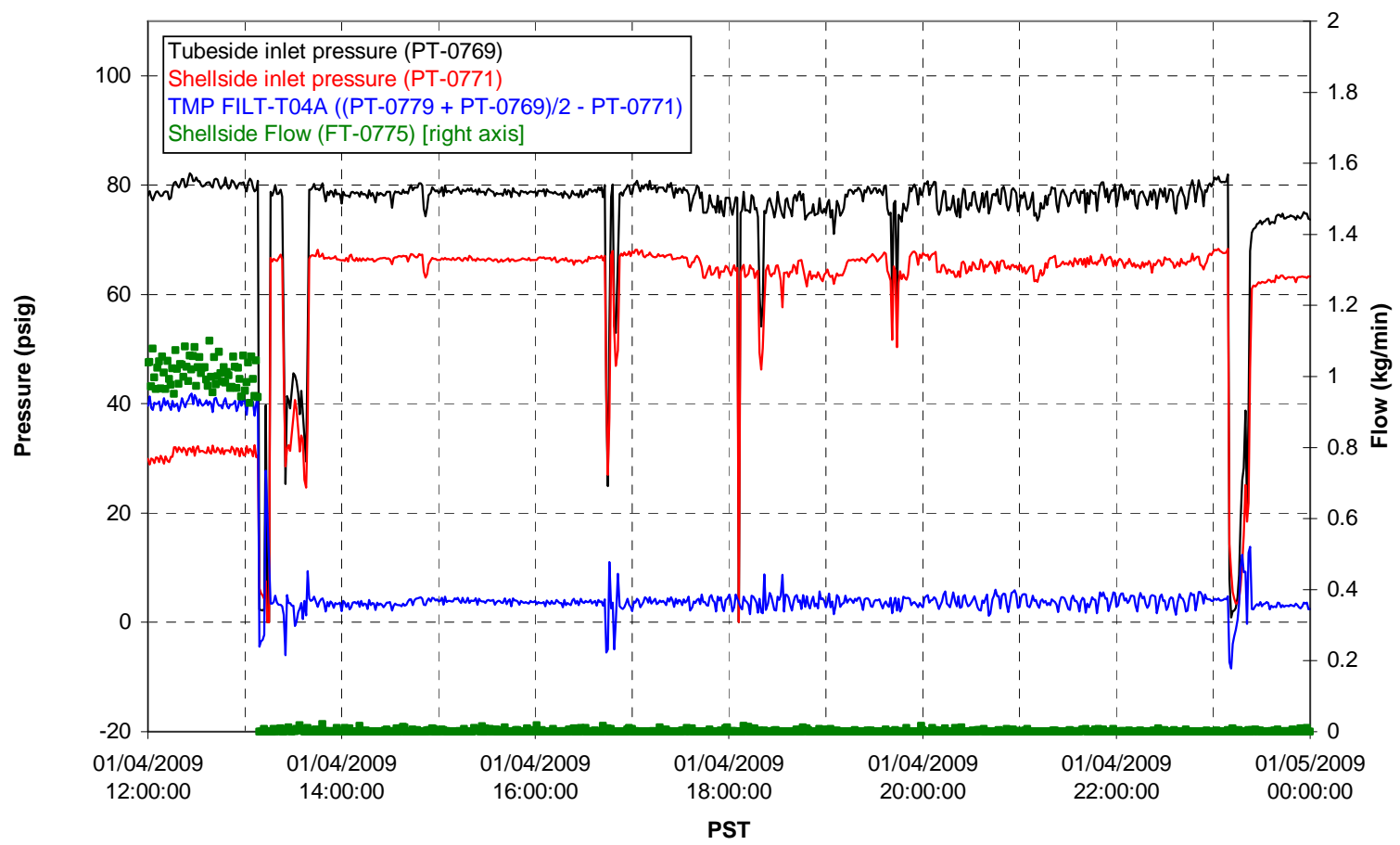


Filter UFP-FILT-T05A

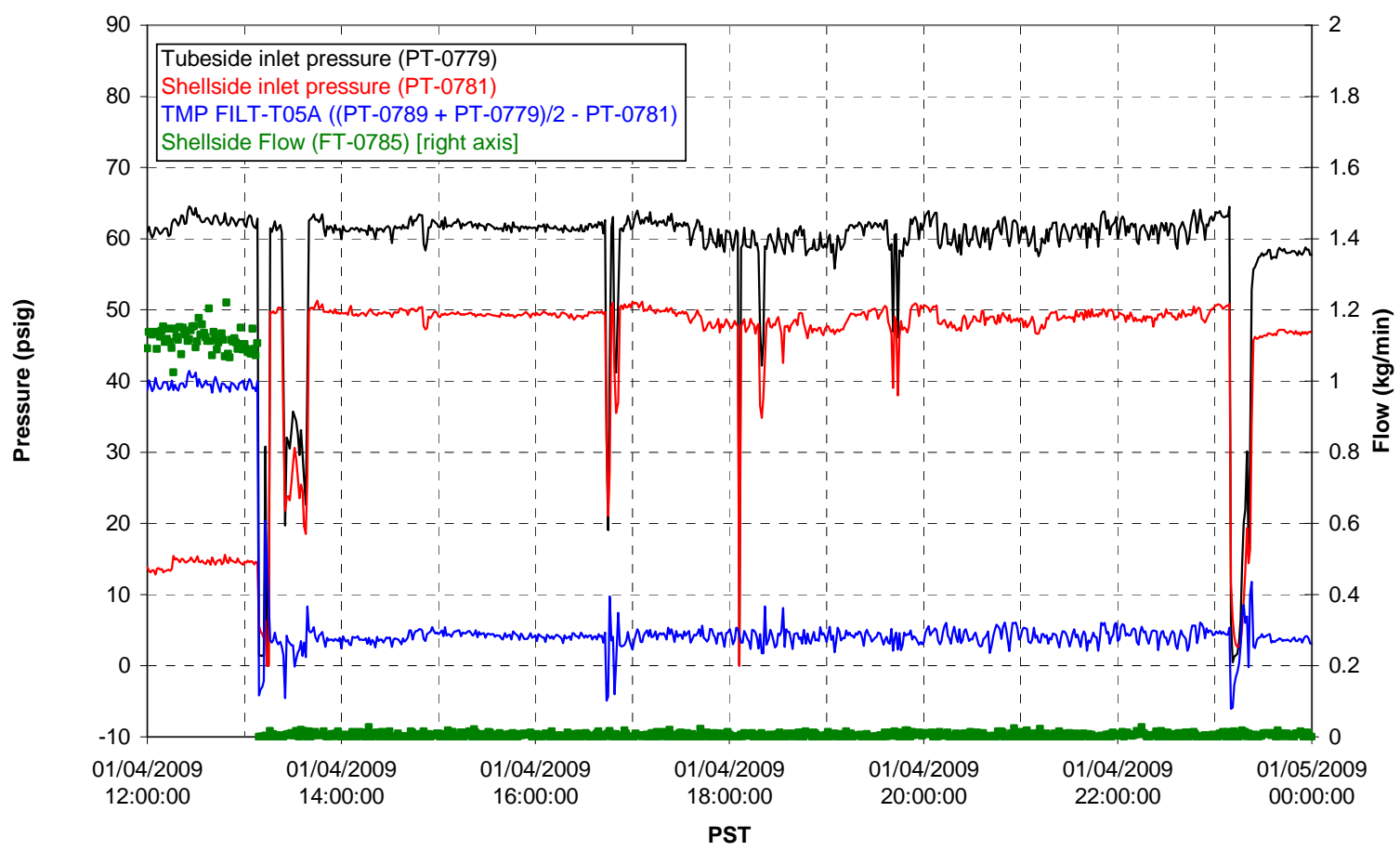

Chemical Flow

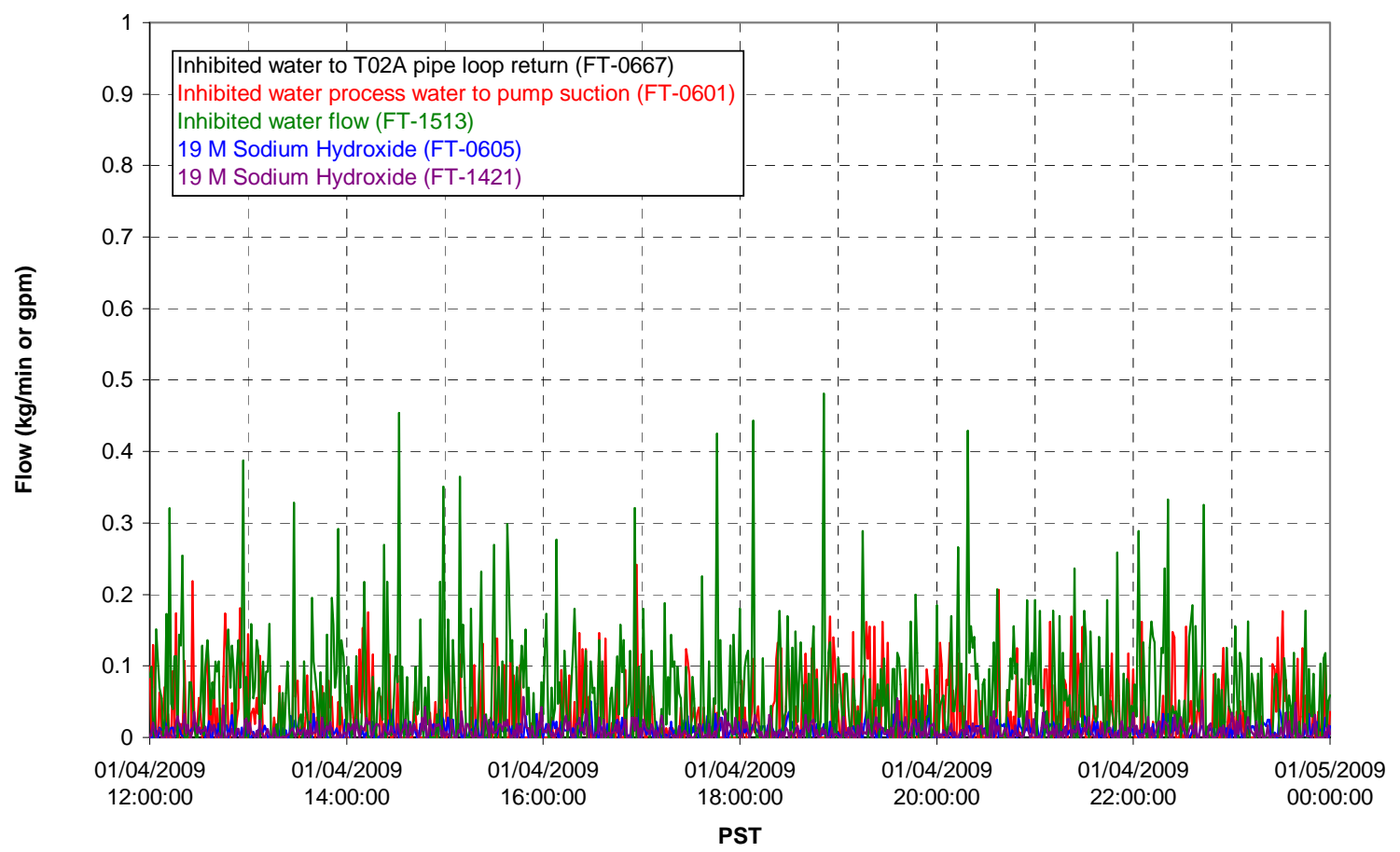


Chemical Flow

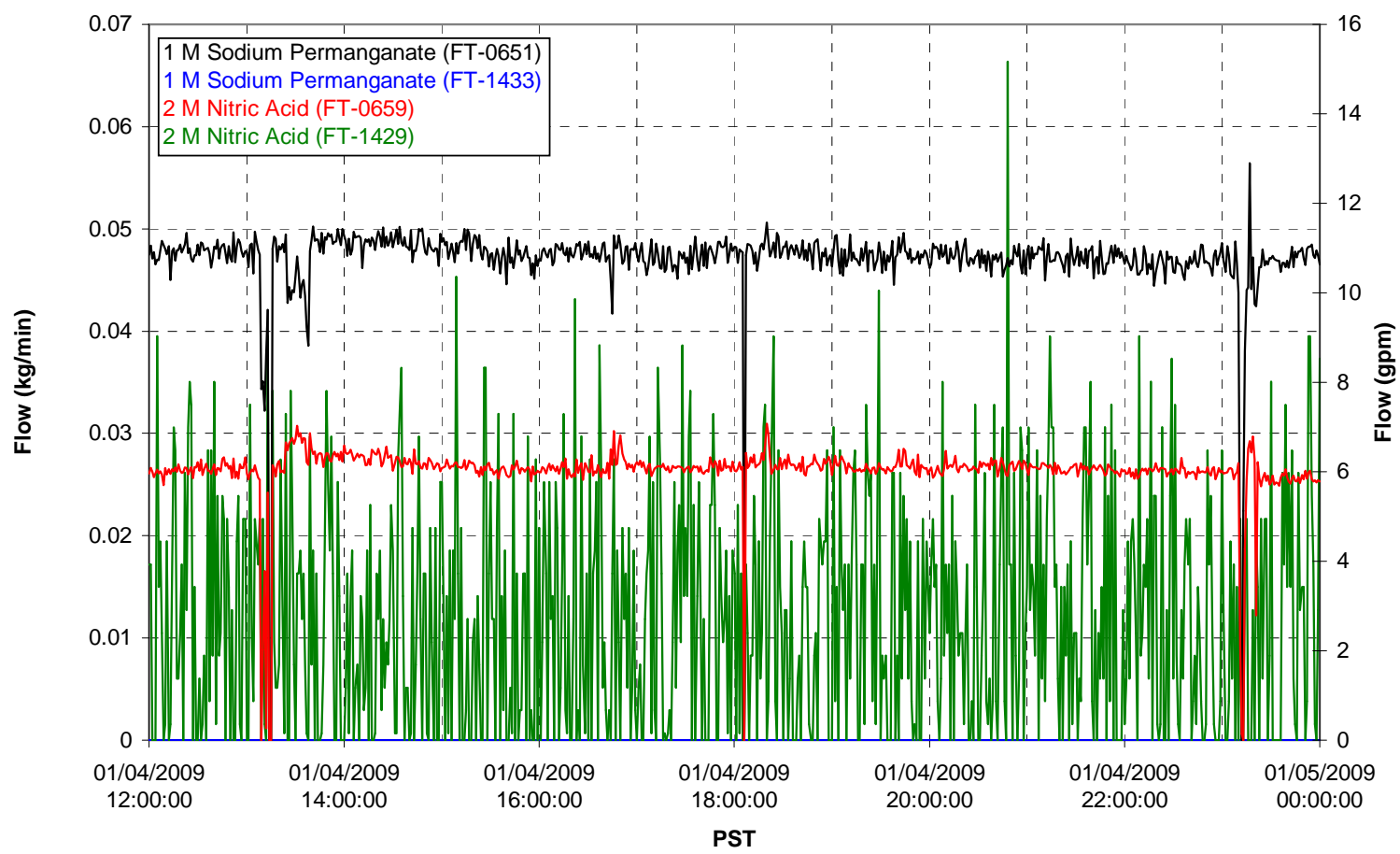

Air Flows

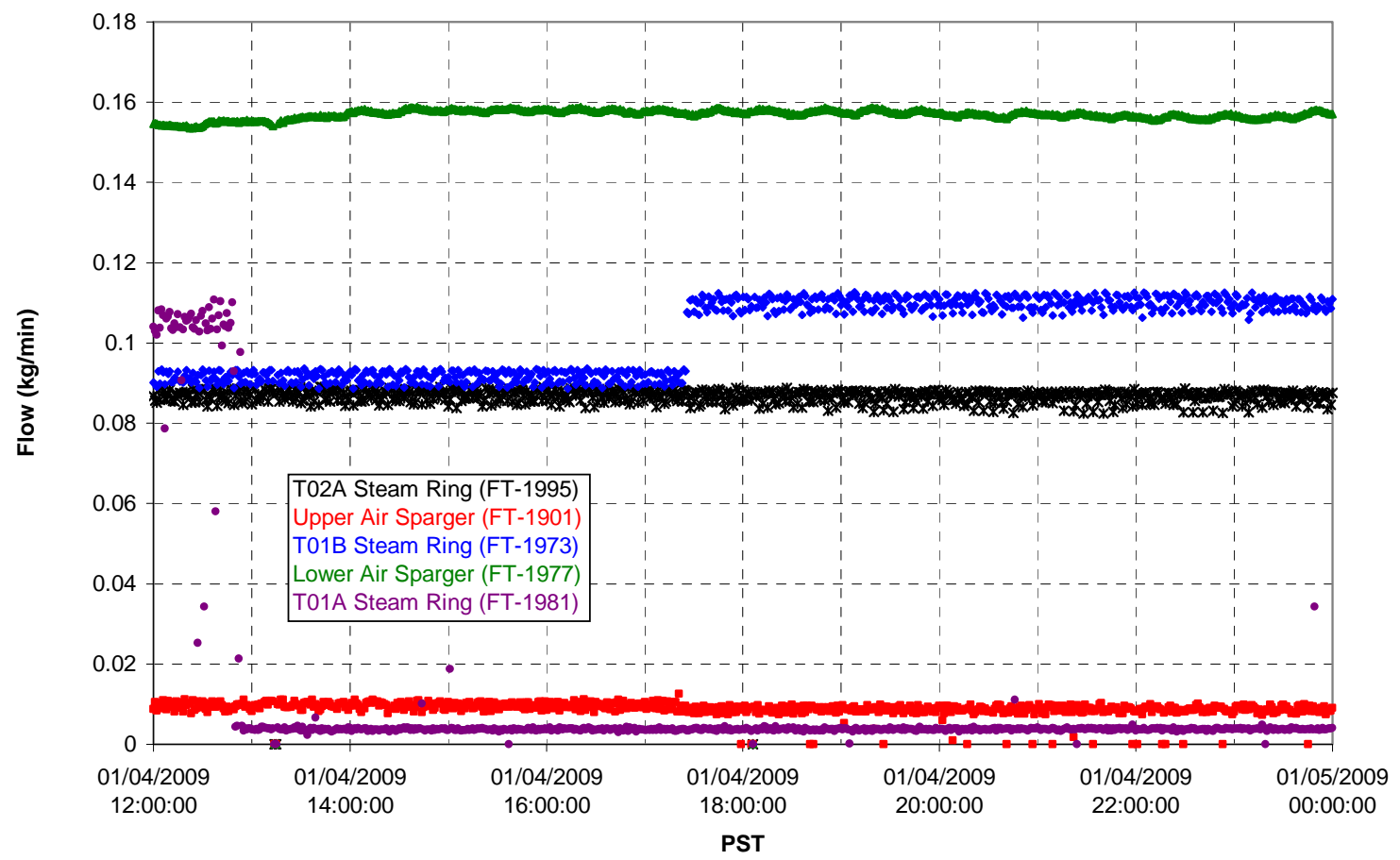


T02A Steam

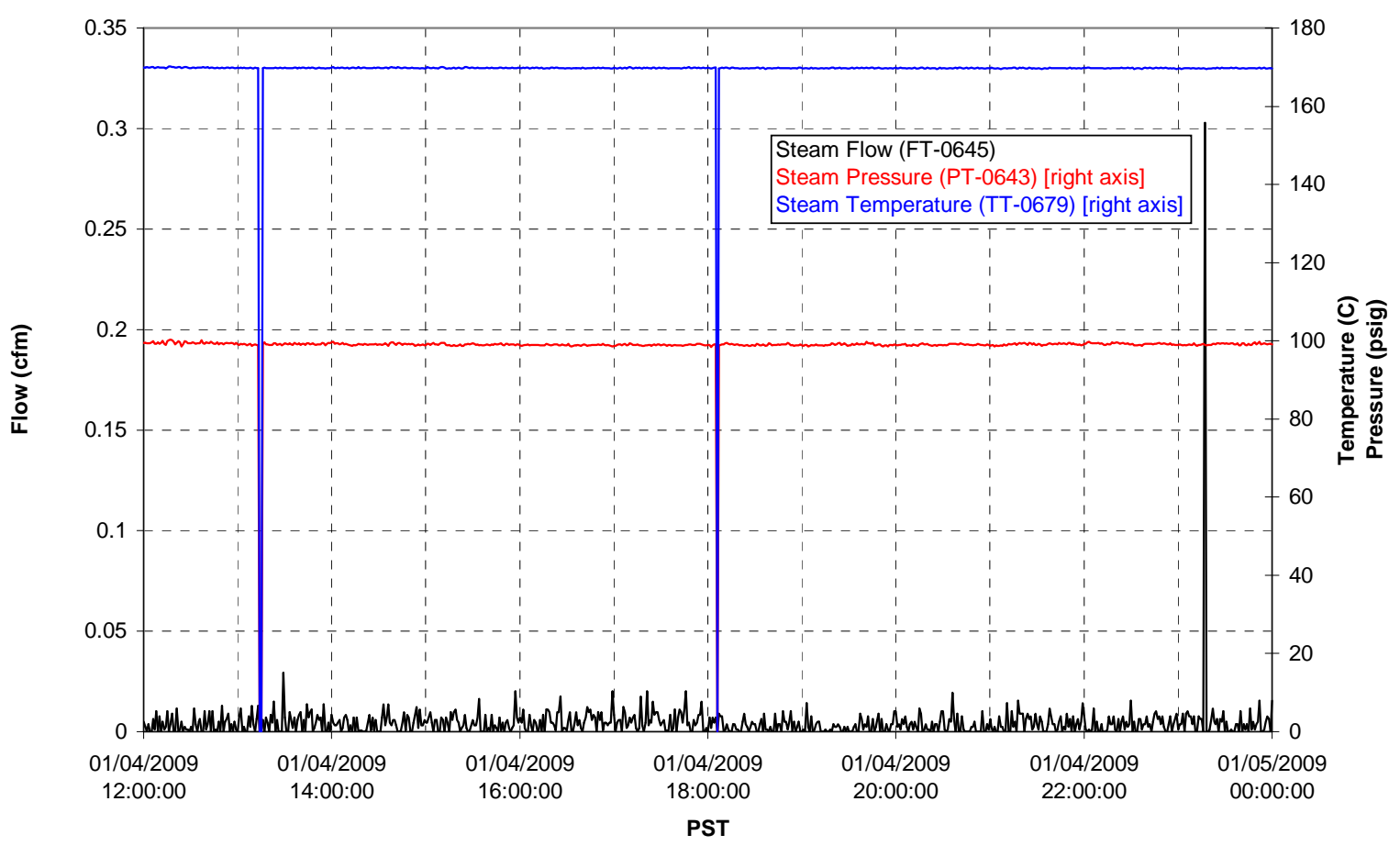

T01A Steam

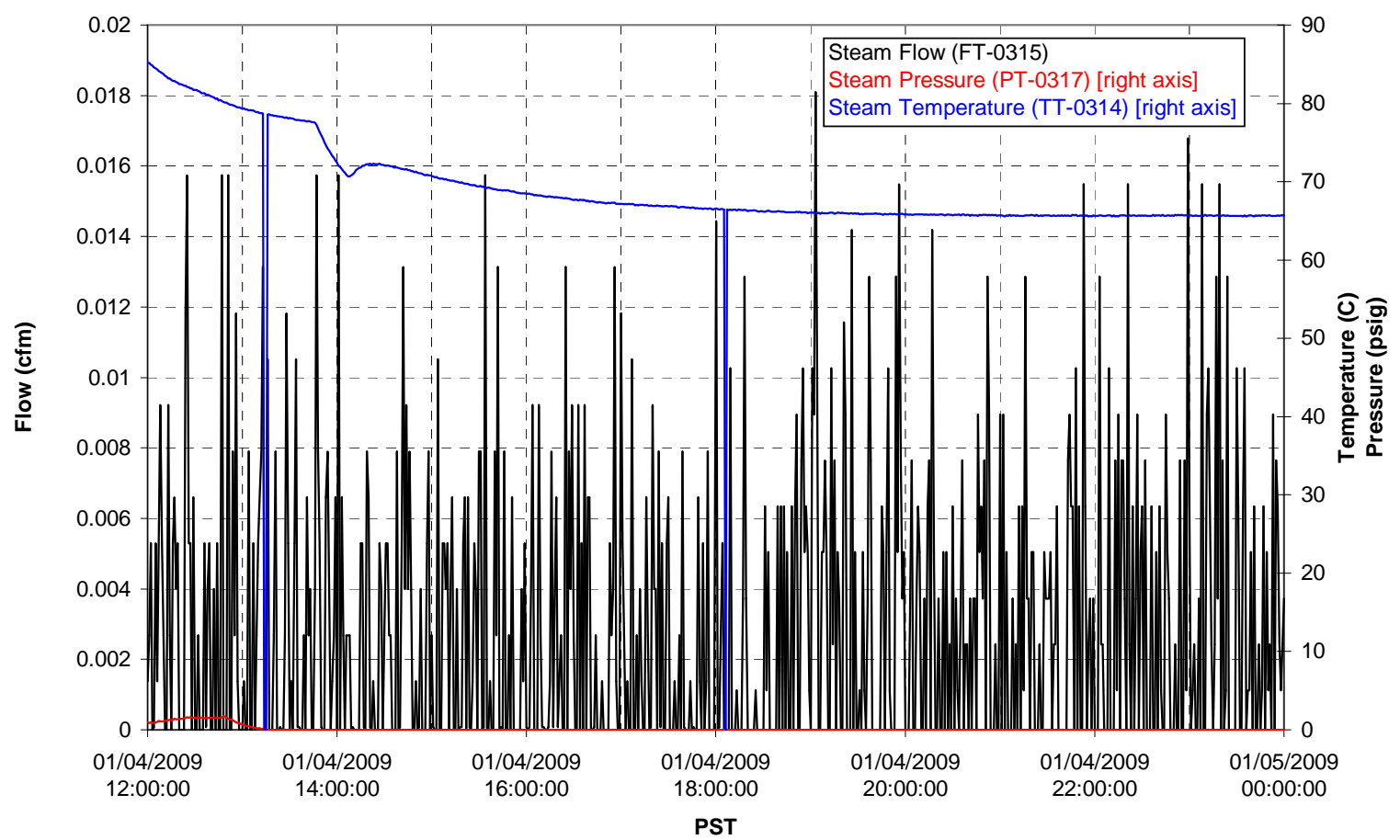


T01B Steam

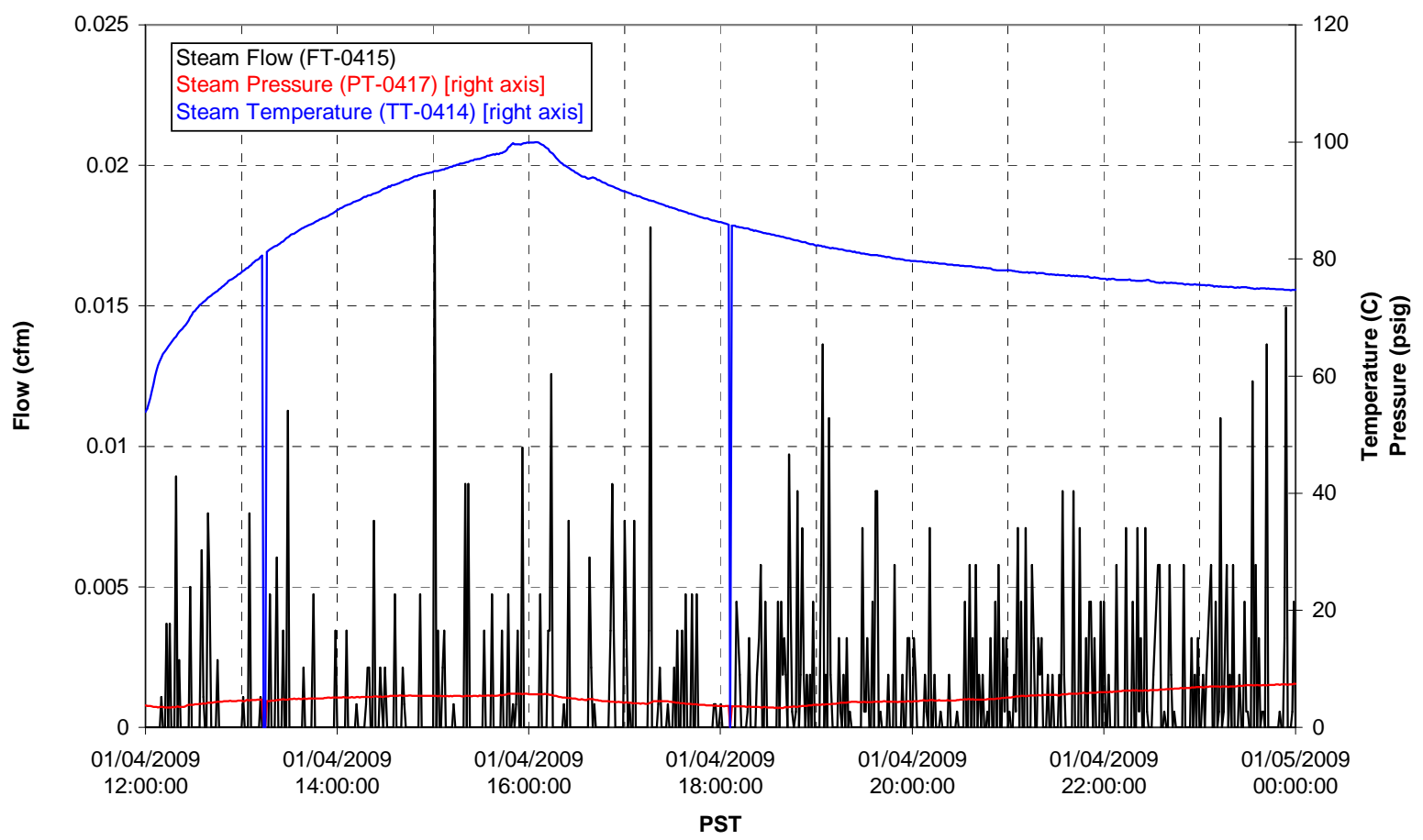


Functional Test Data Plots

01/05/2009 00:00 - 01/05/2009 12:00 
T01A level

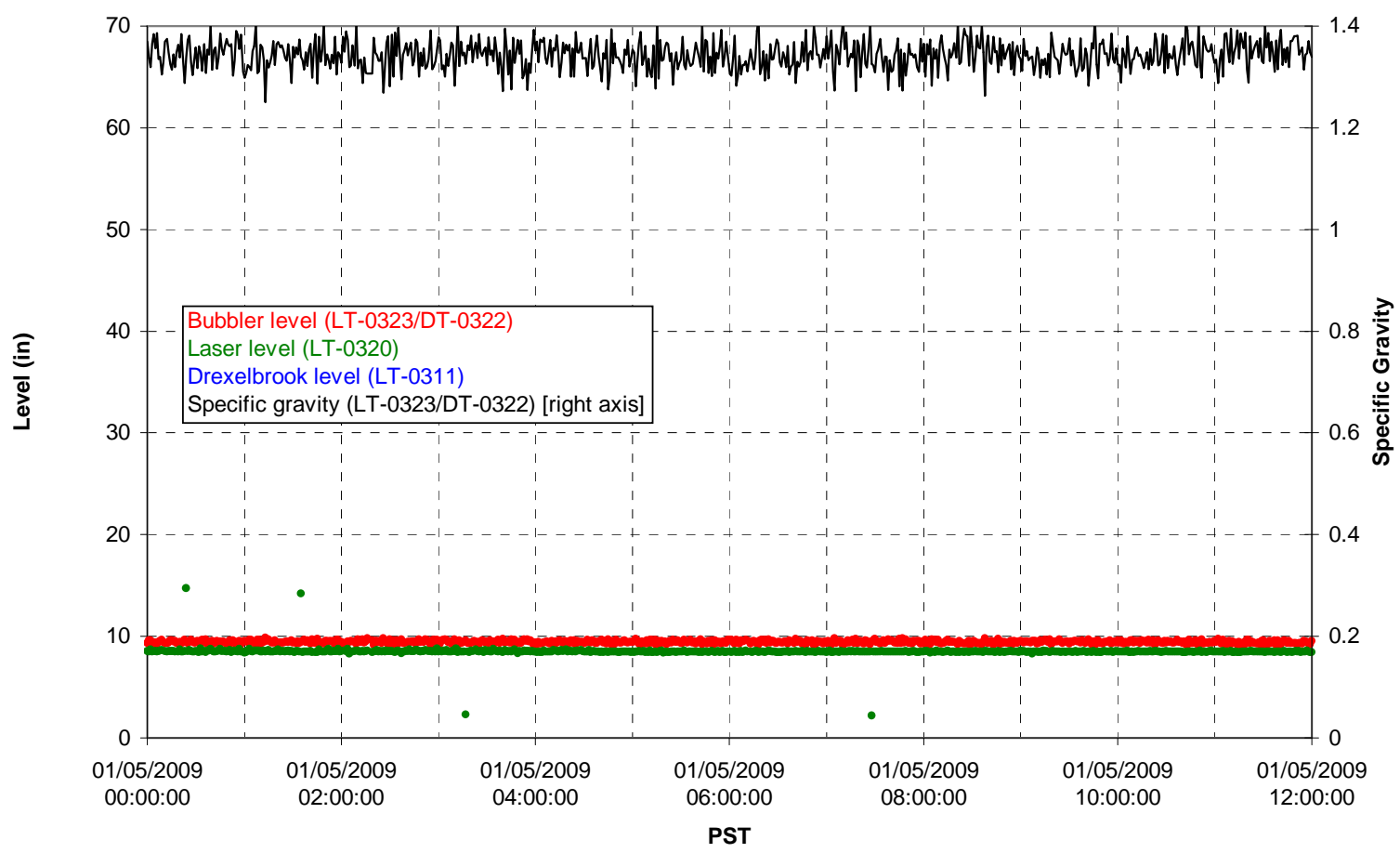

T01A temperatures

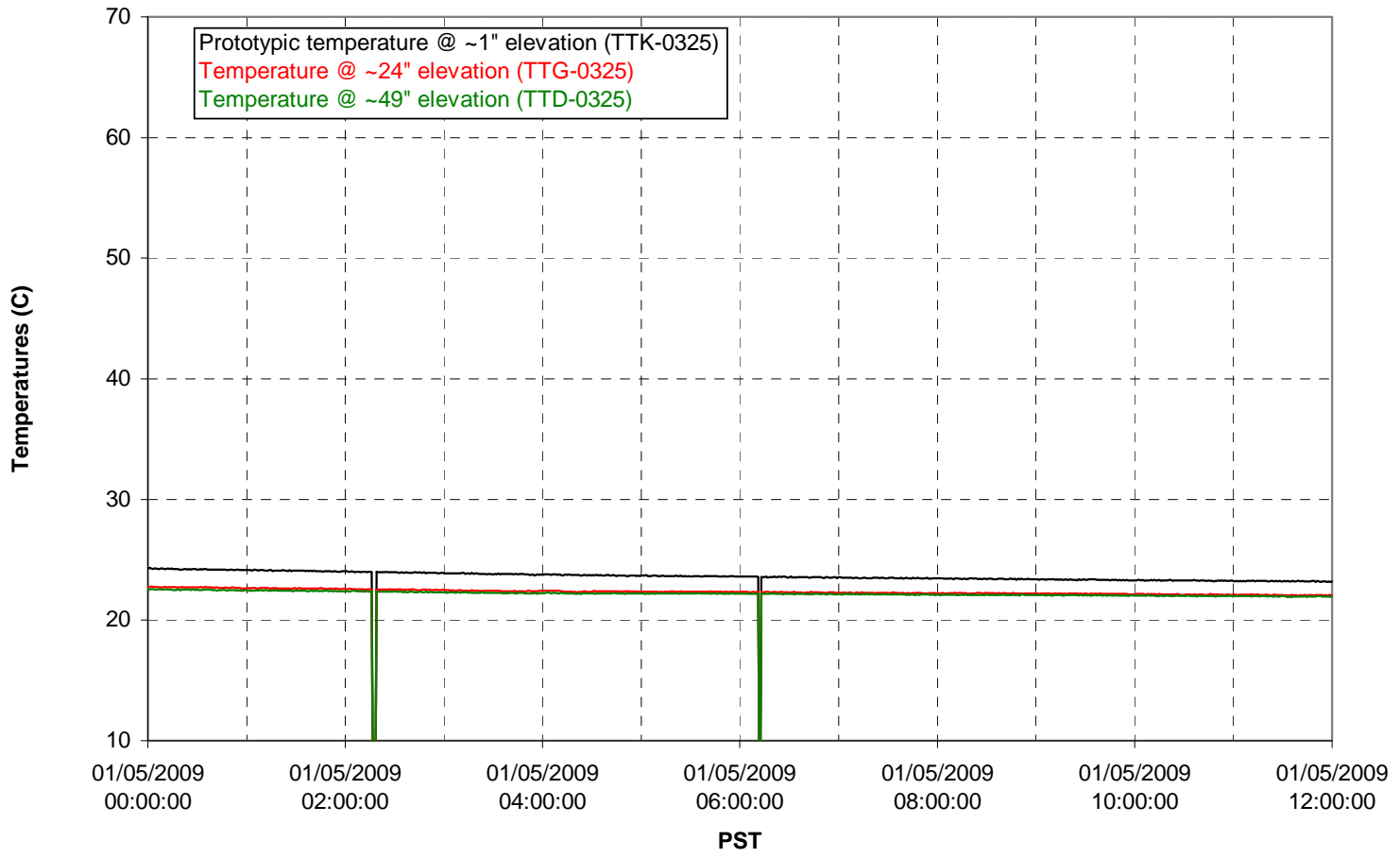


T01B level

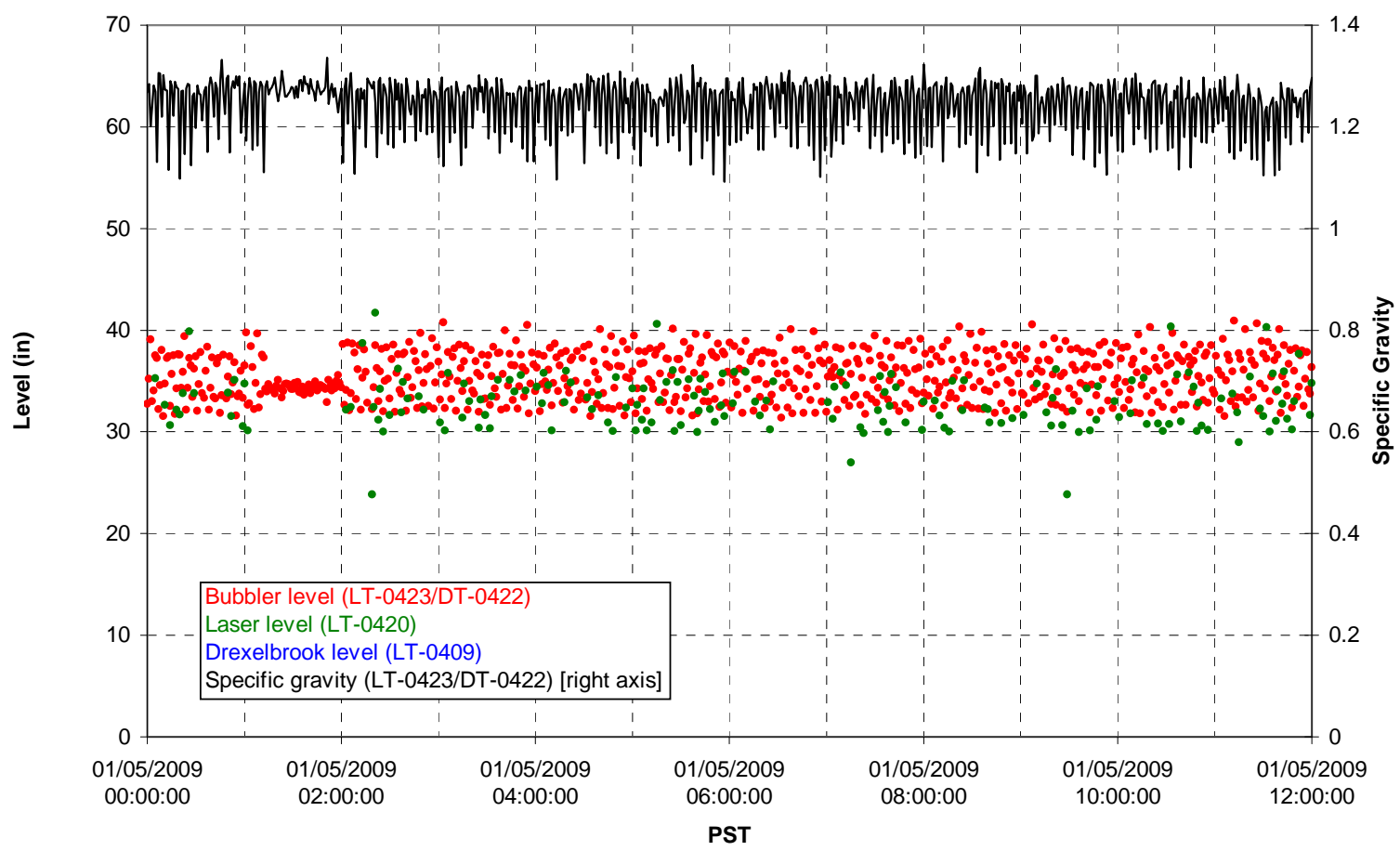

T01B temperatures

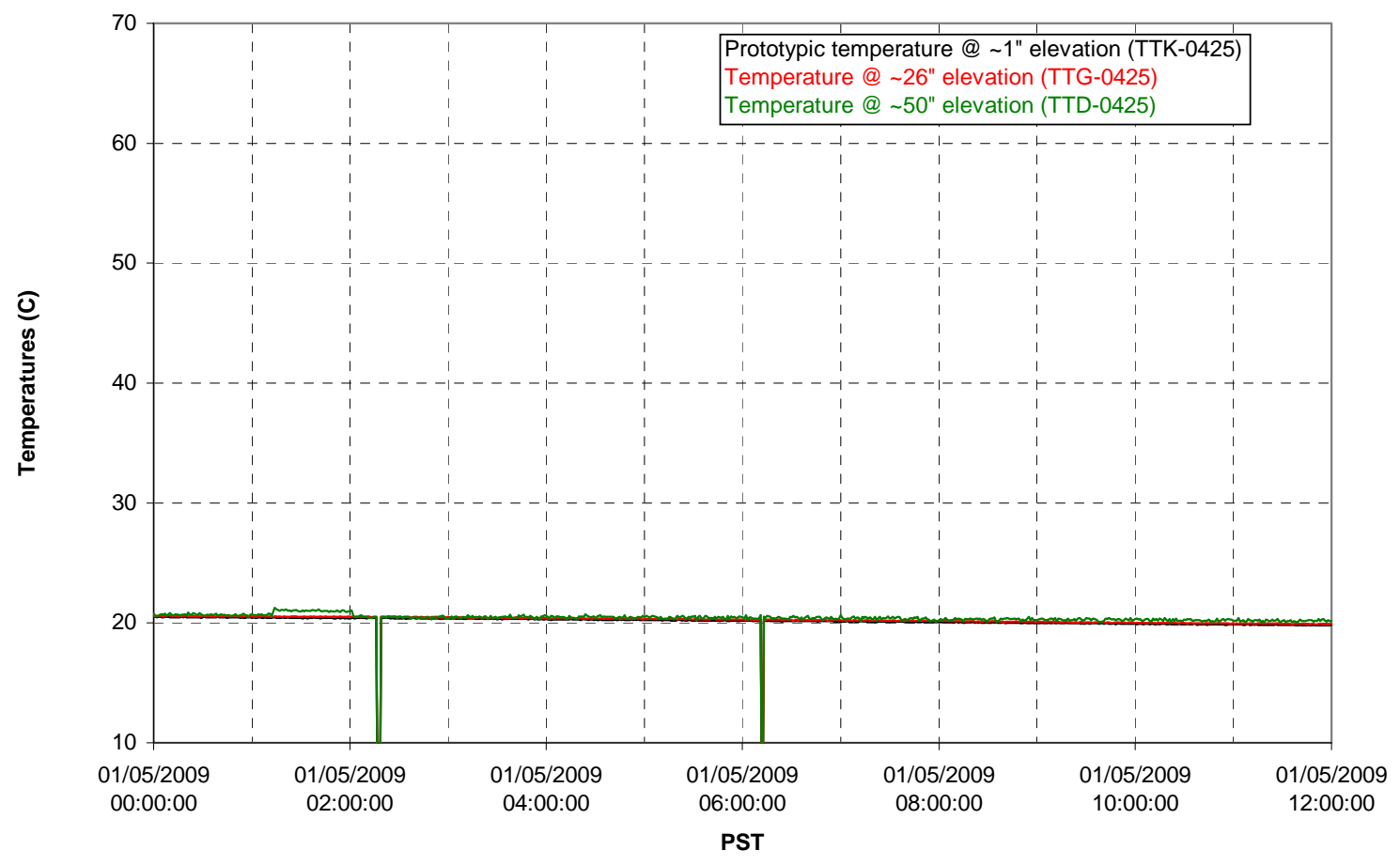


T02A level

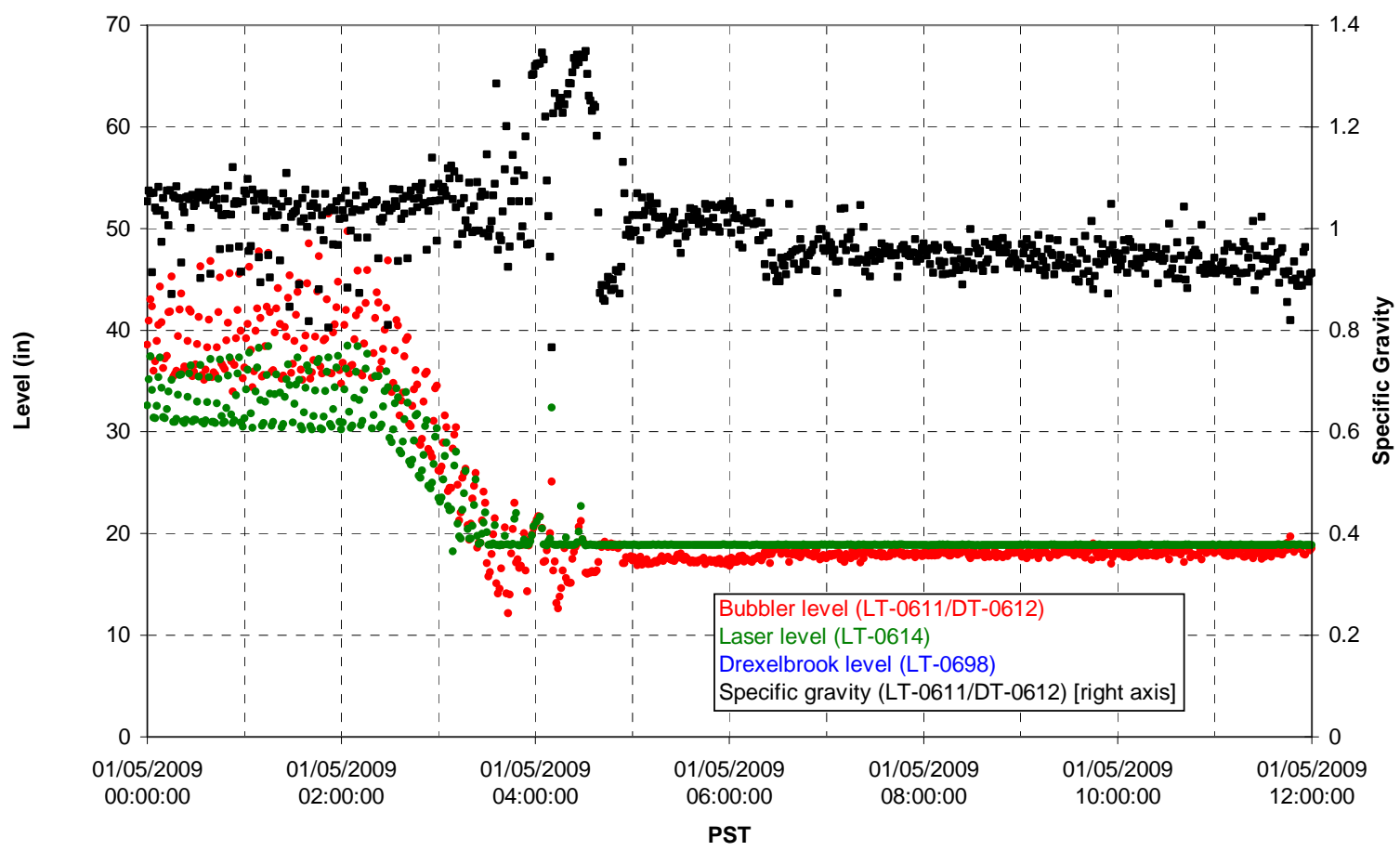

T02A temperatures

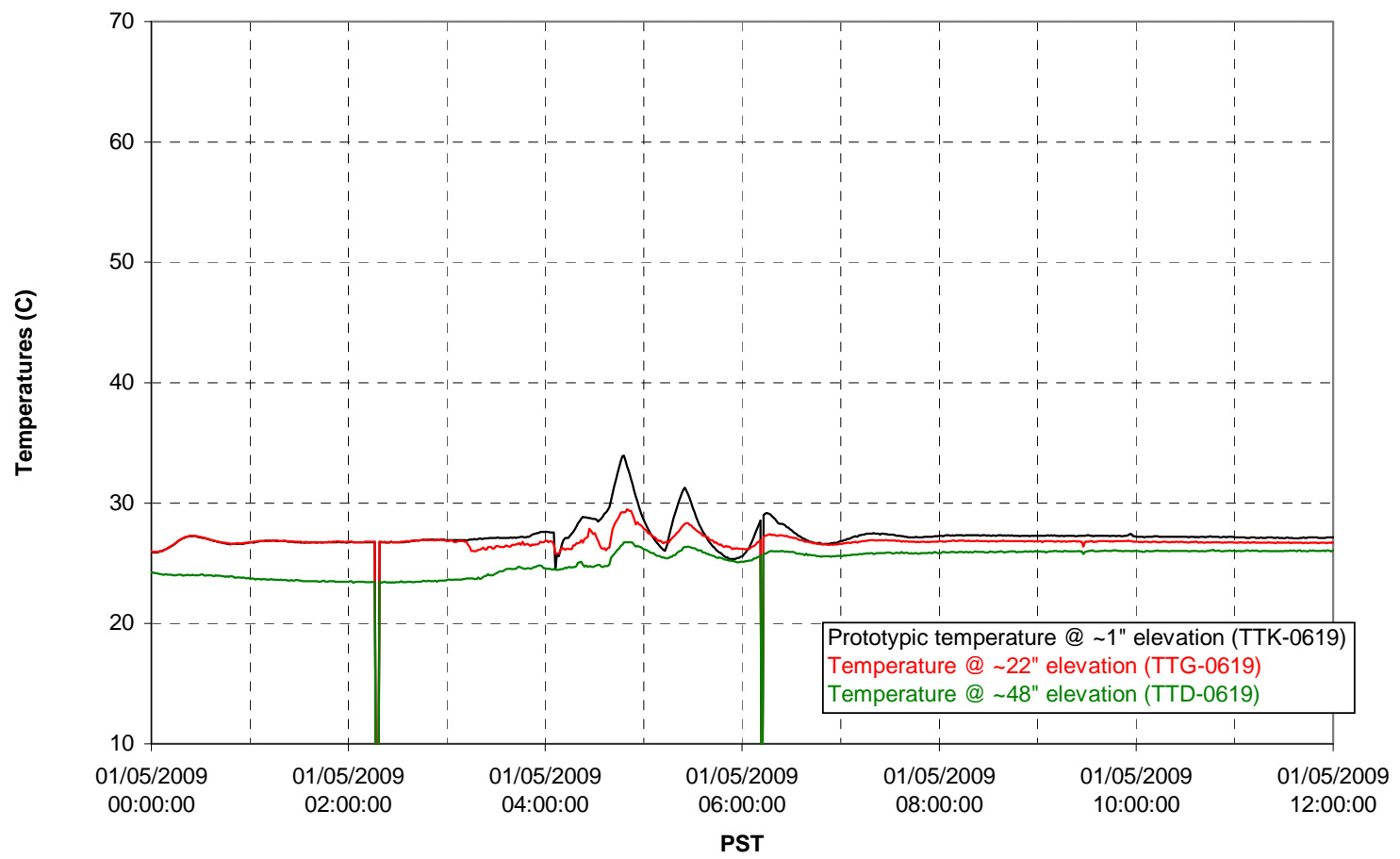


T02A and filter loop temperatures

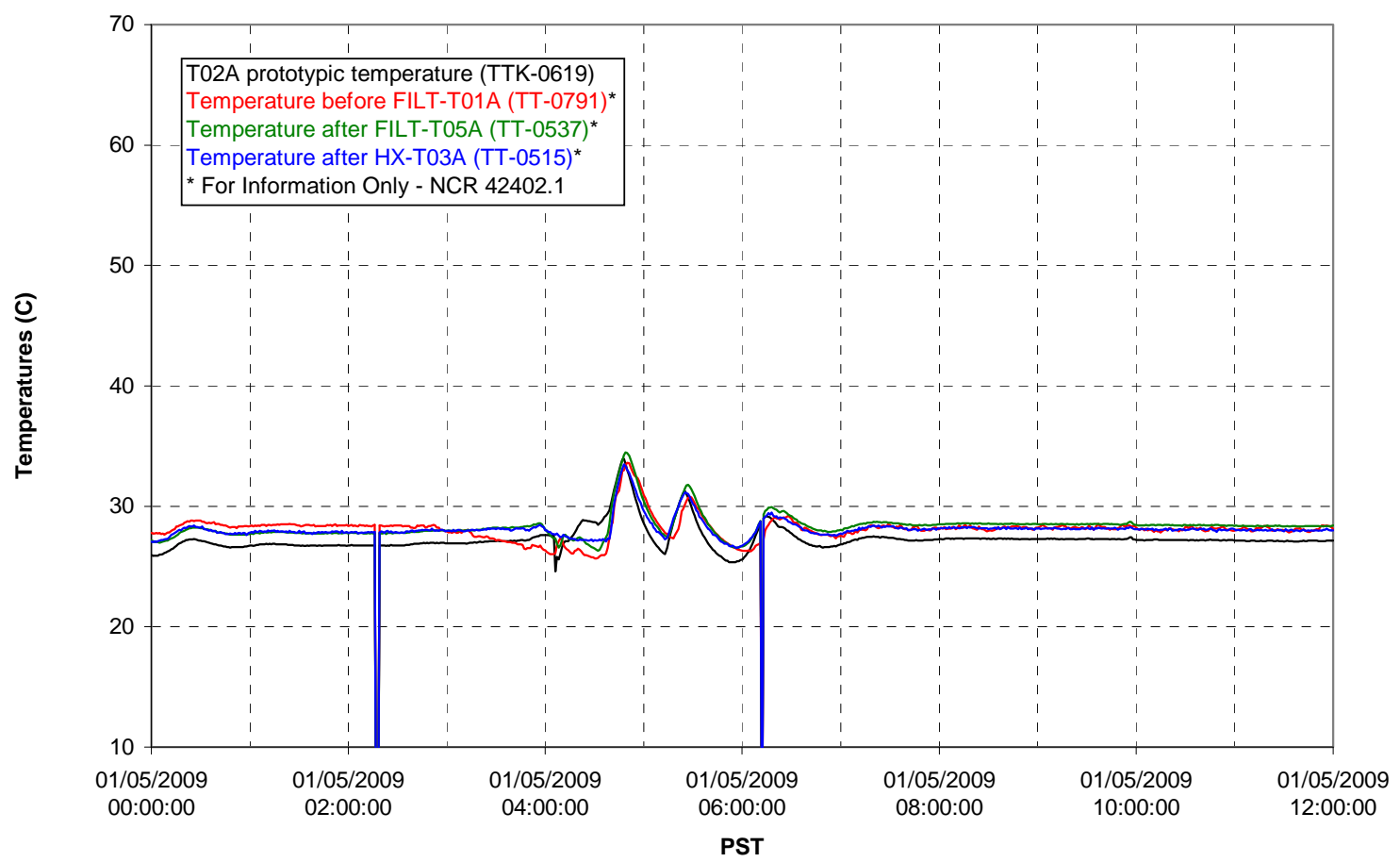

Pump Pressures and Flow

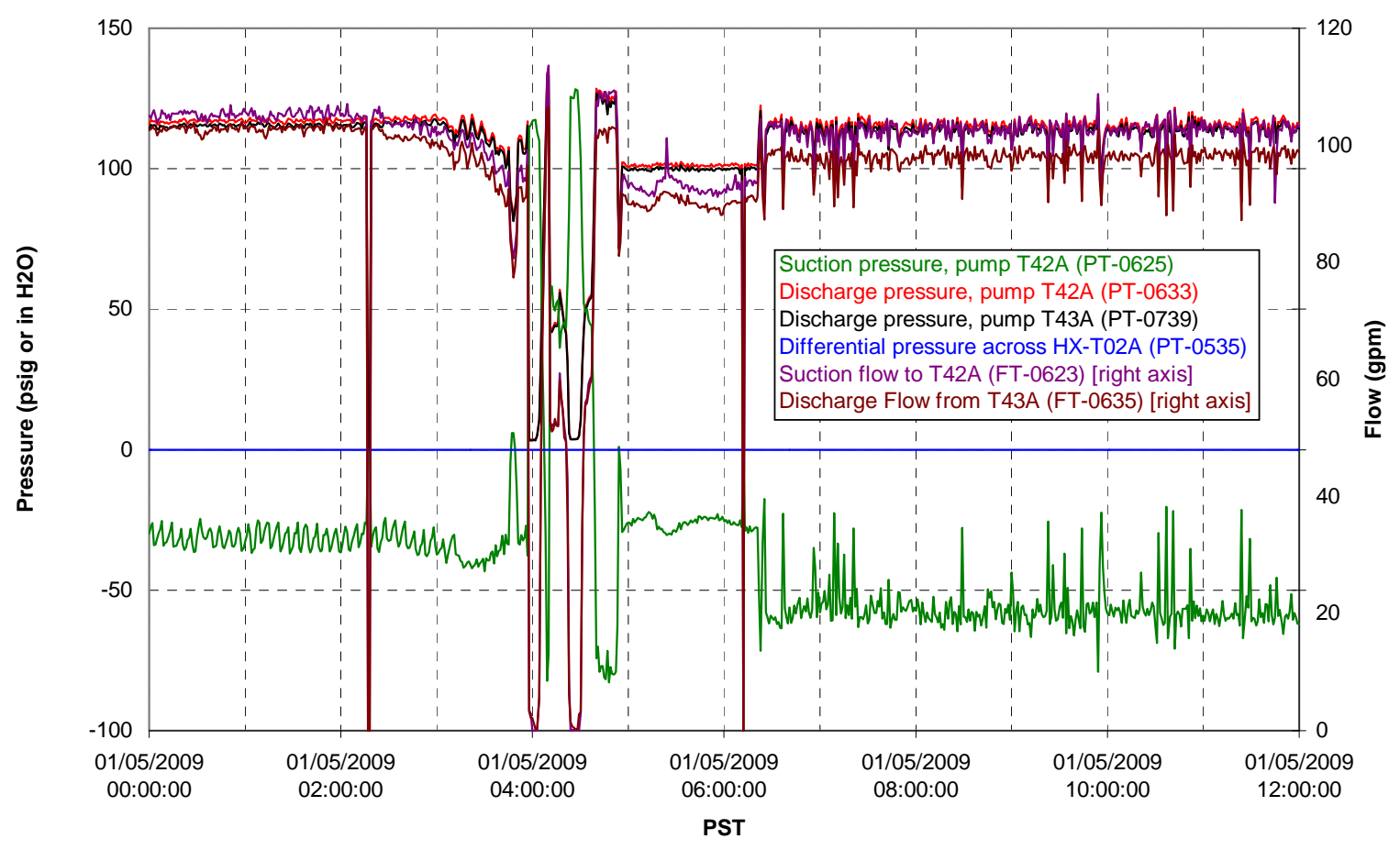


Axial pressure drop

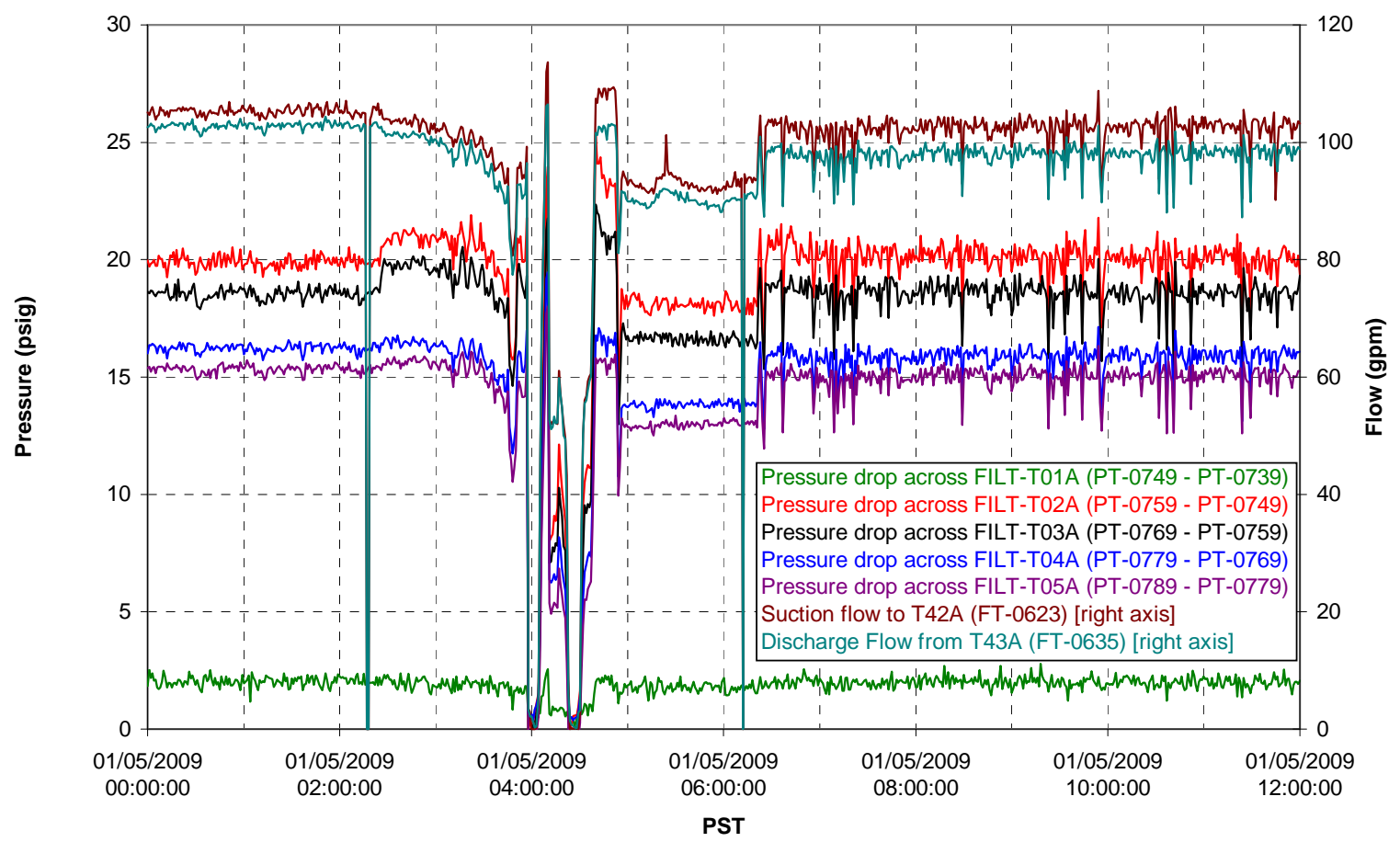

Permeate flow rates

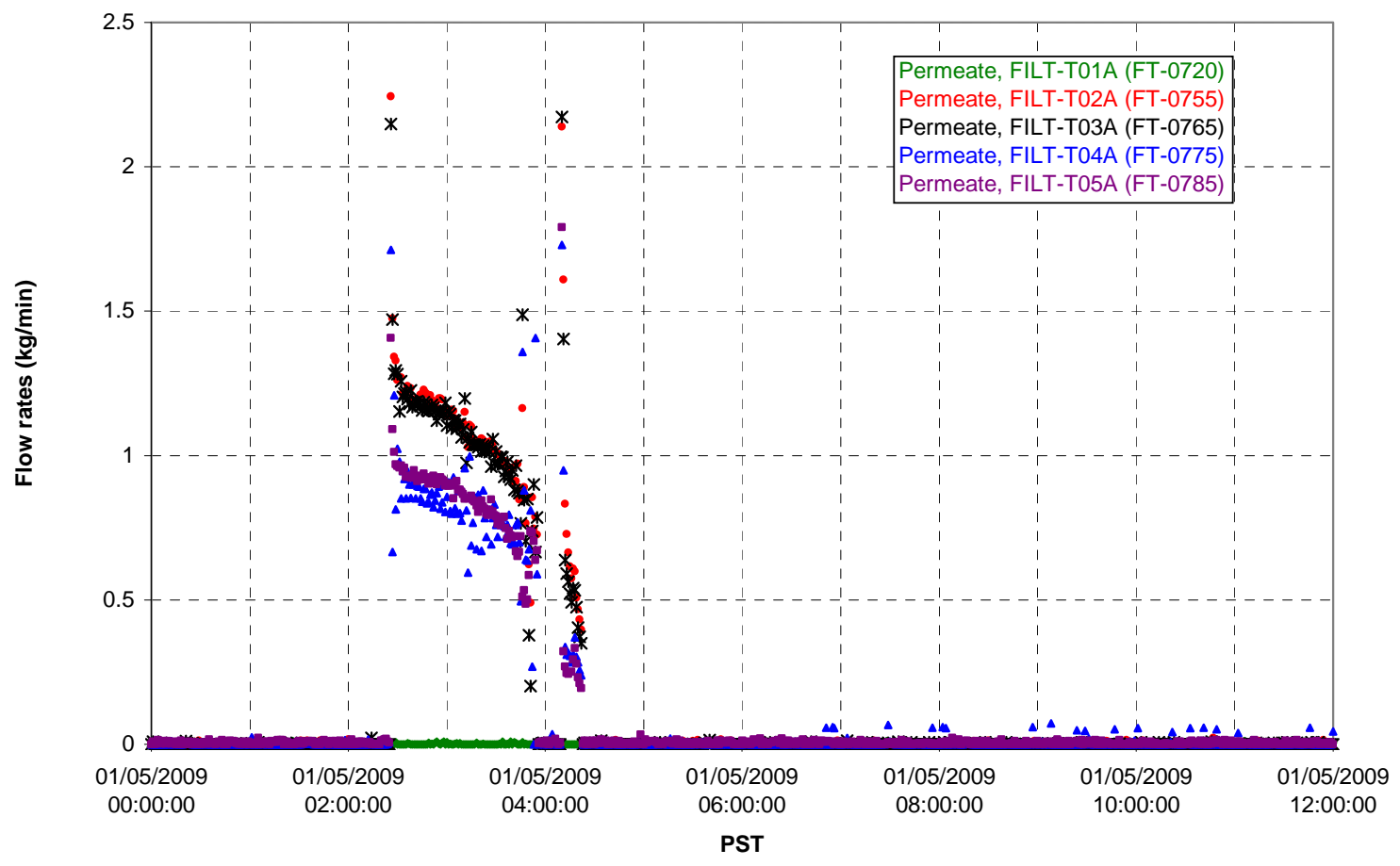


T02A Inner Temperature Tree

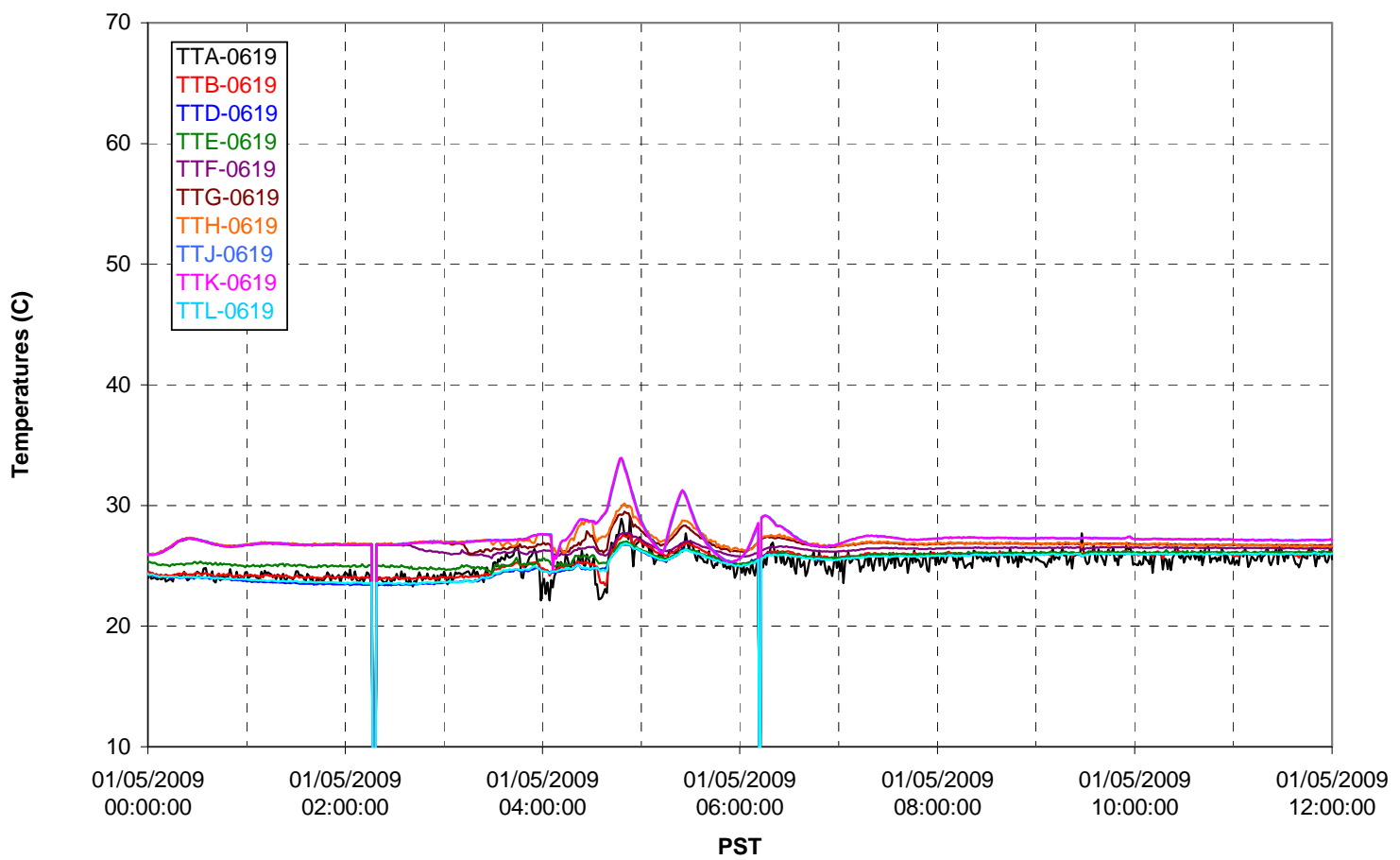

T02A Outer Temperature Tree

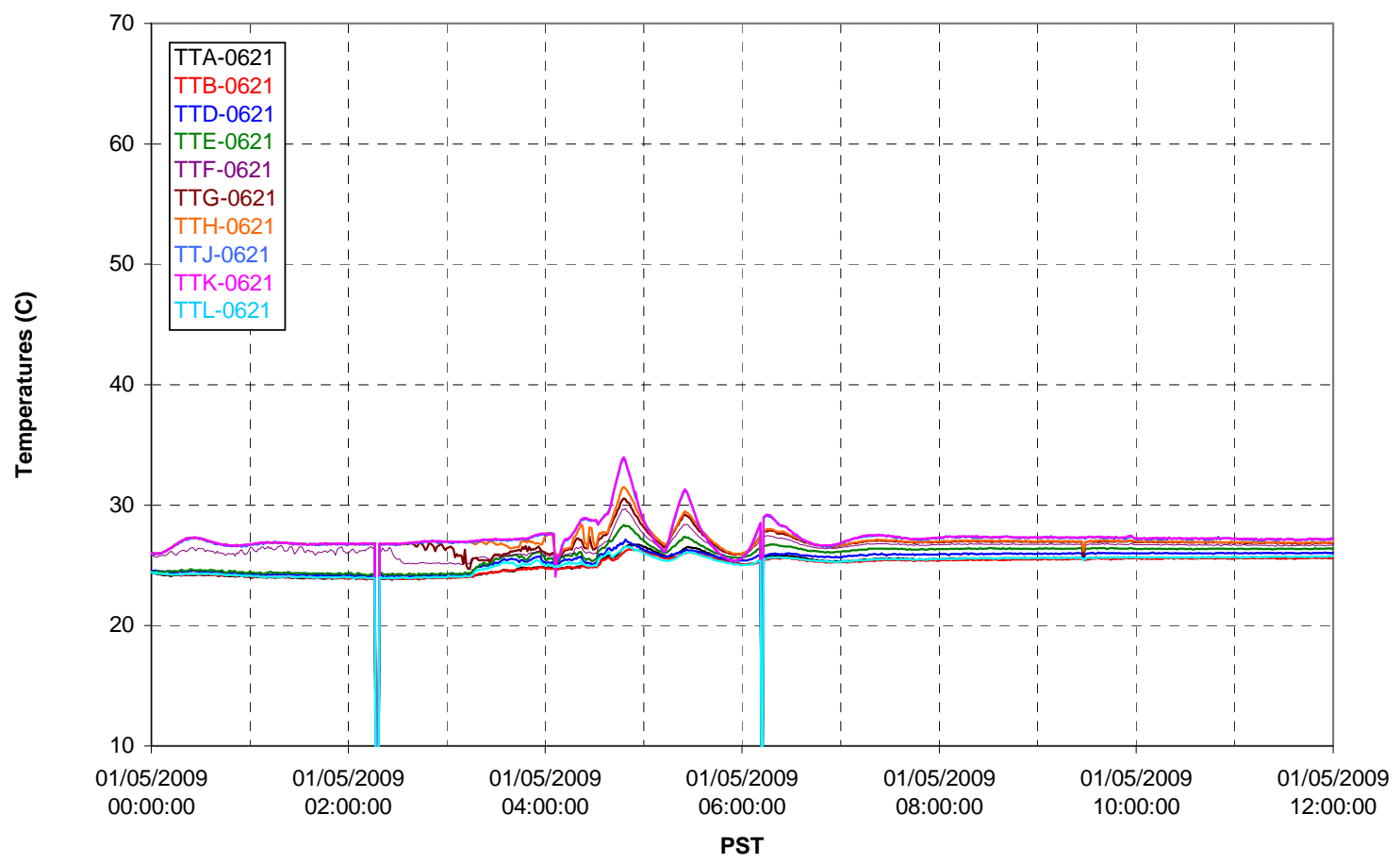


T02A temperatures

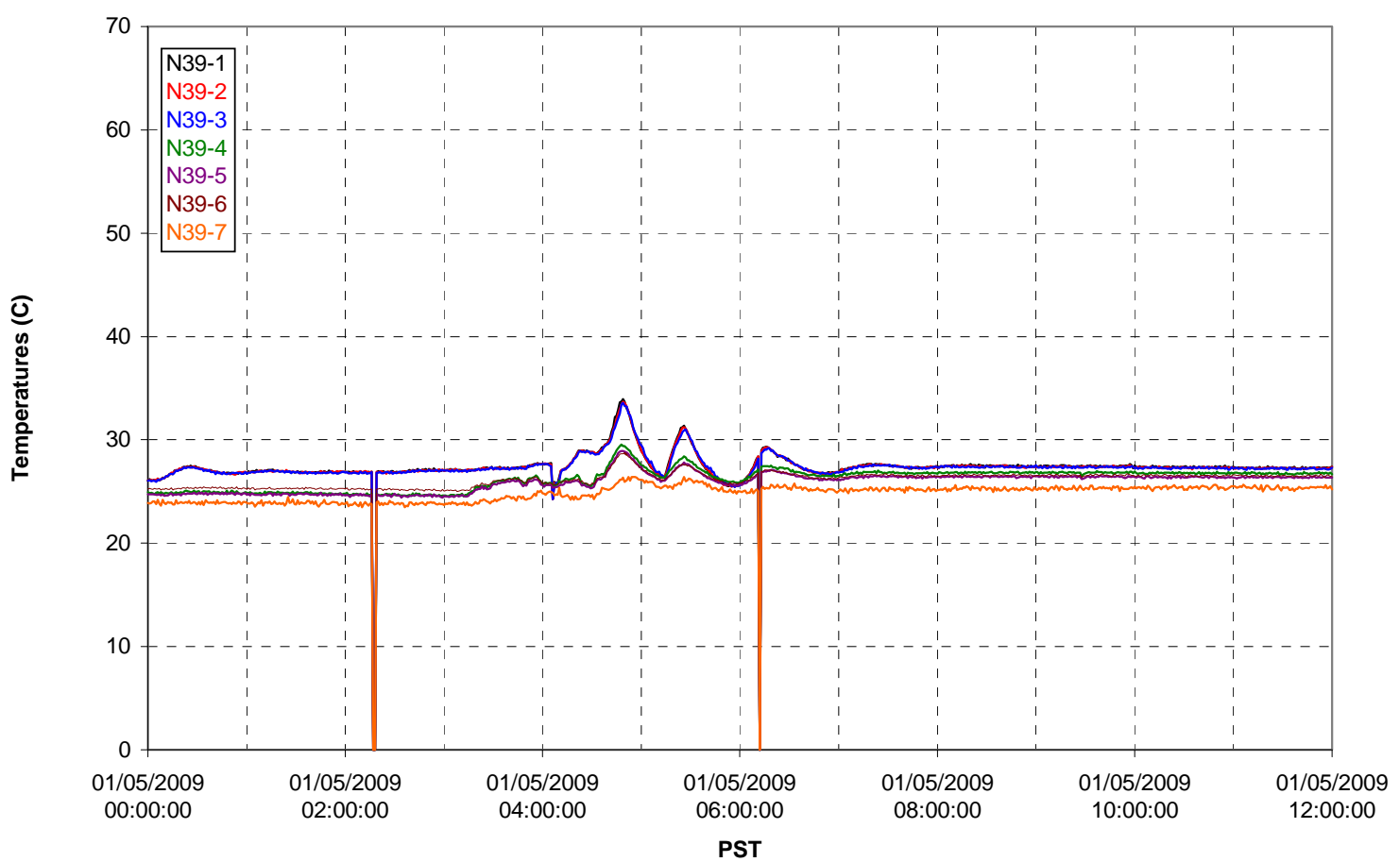

T02A temperatures

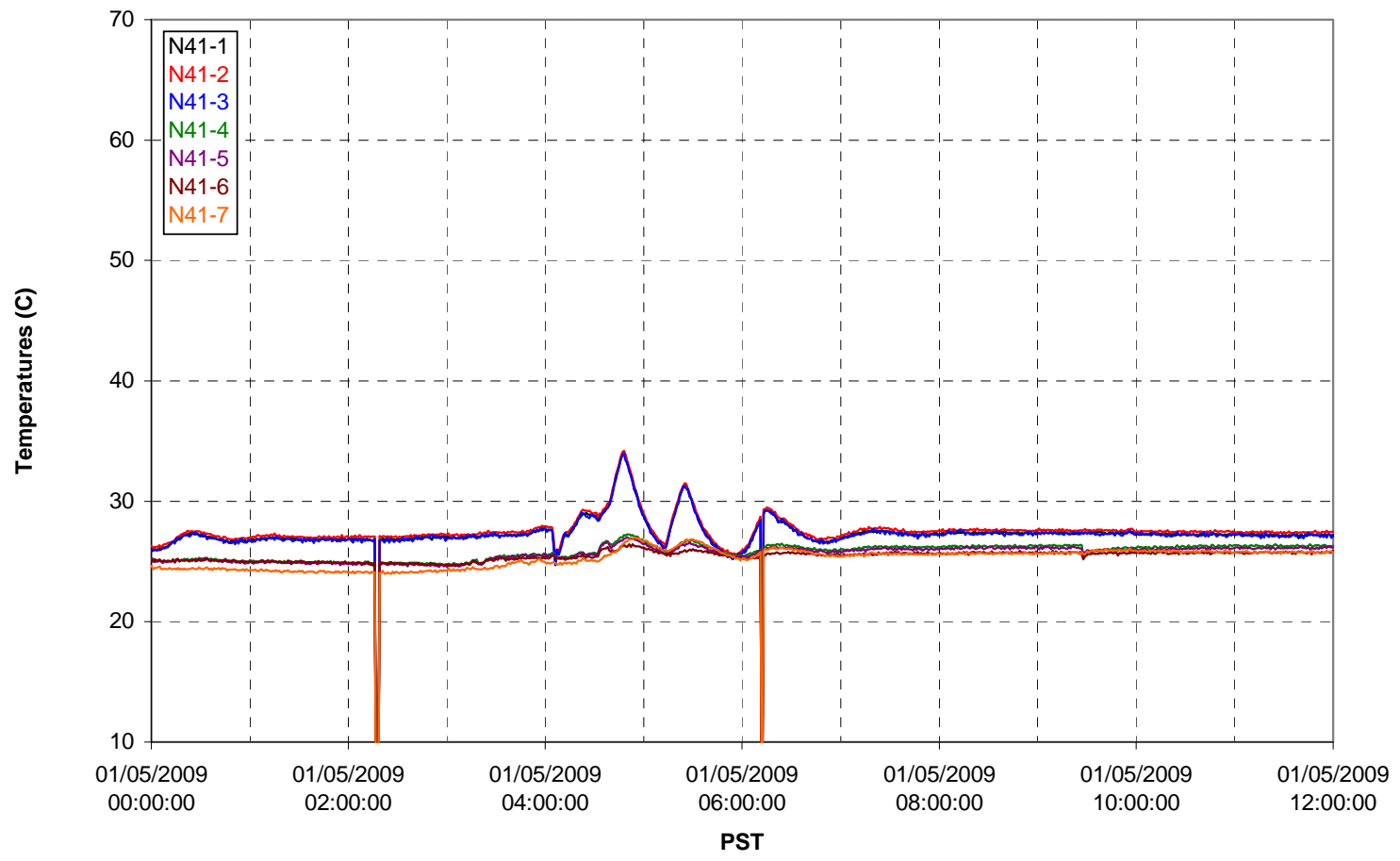


T02A temperatures

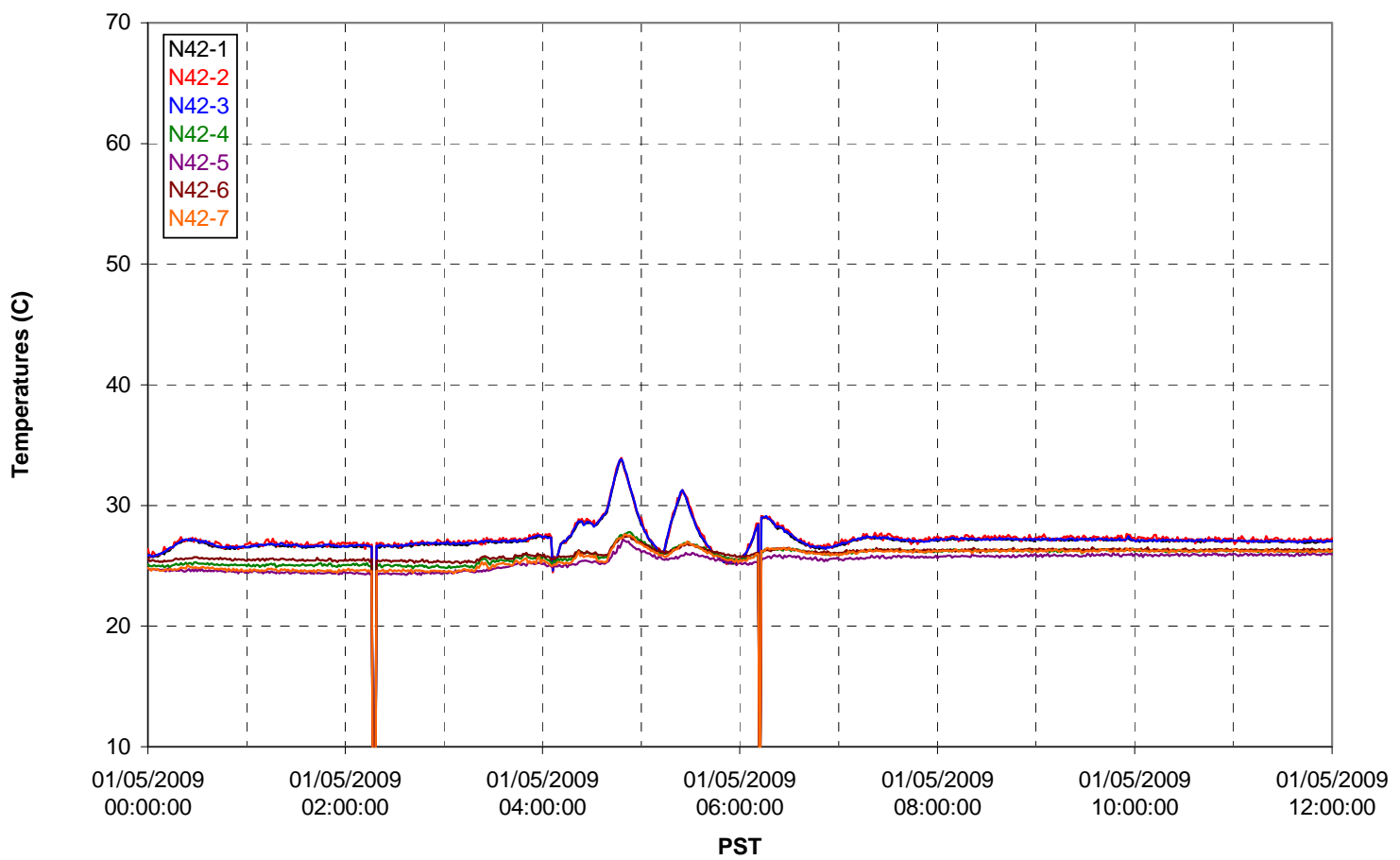

T02A temperatures

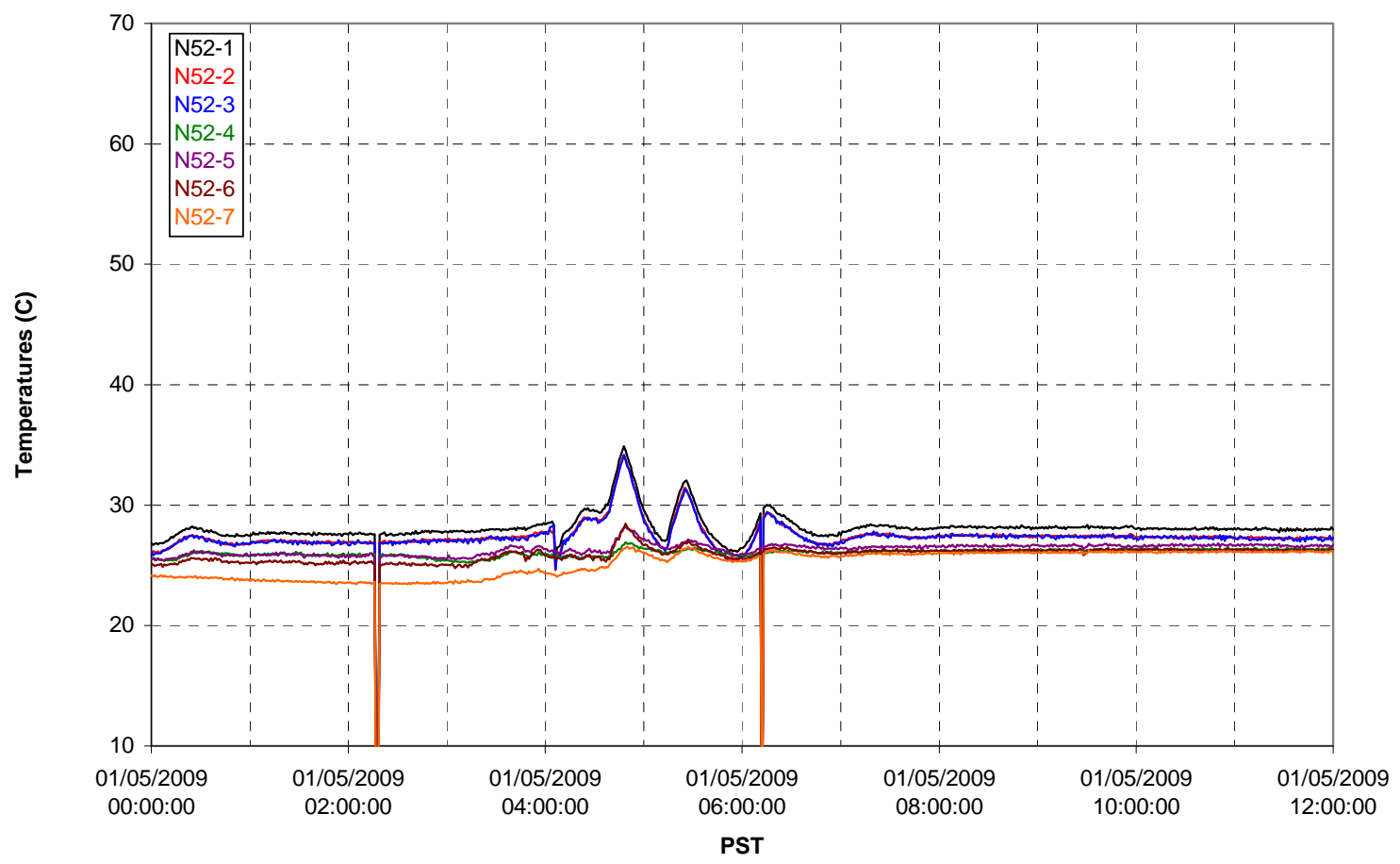


T02A Heating and Cooling

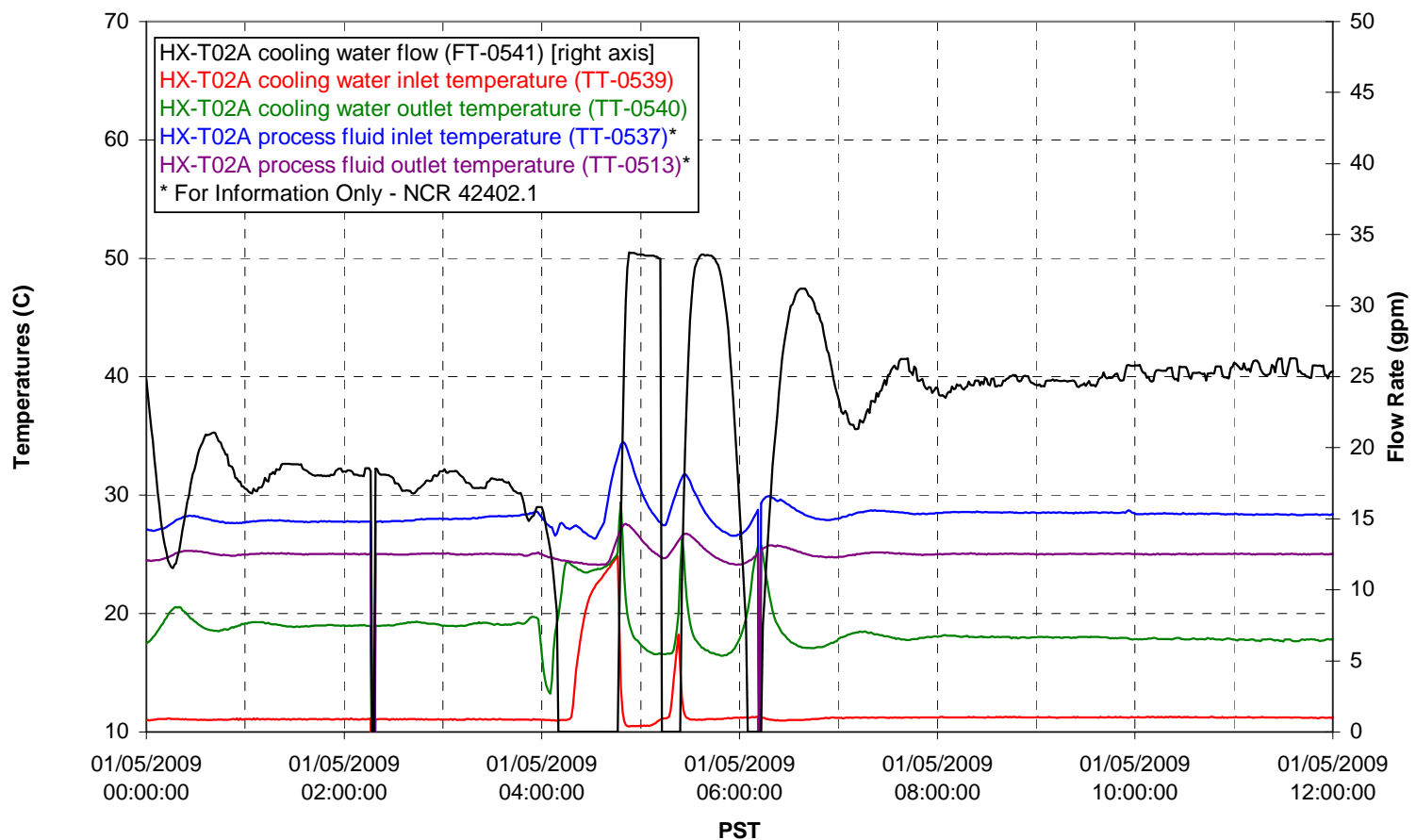

Pump Operation

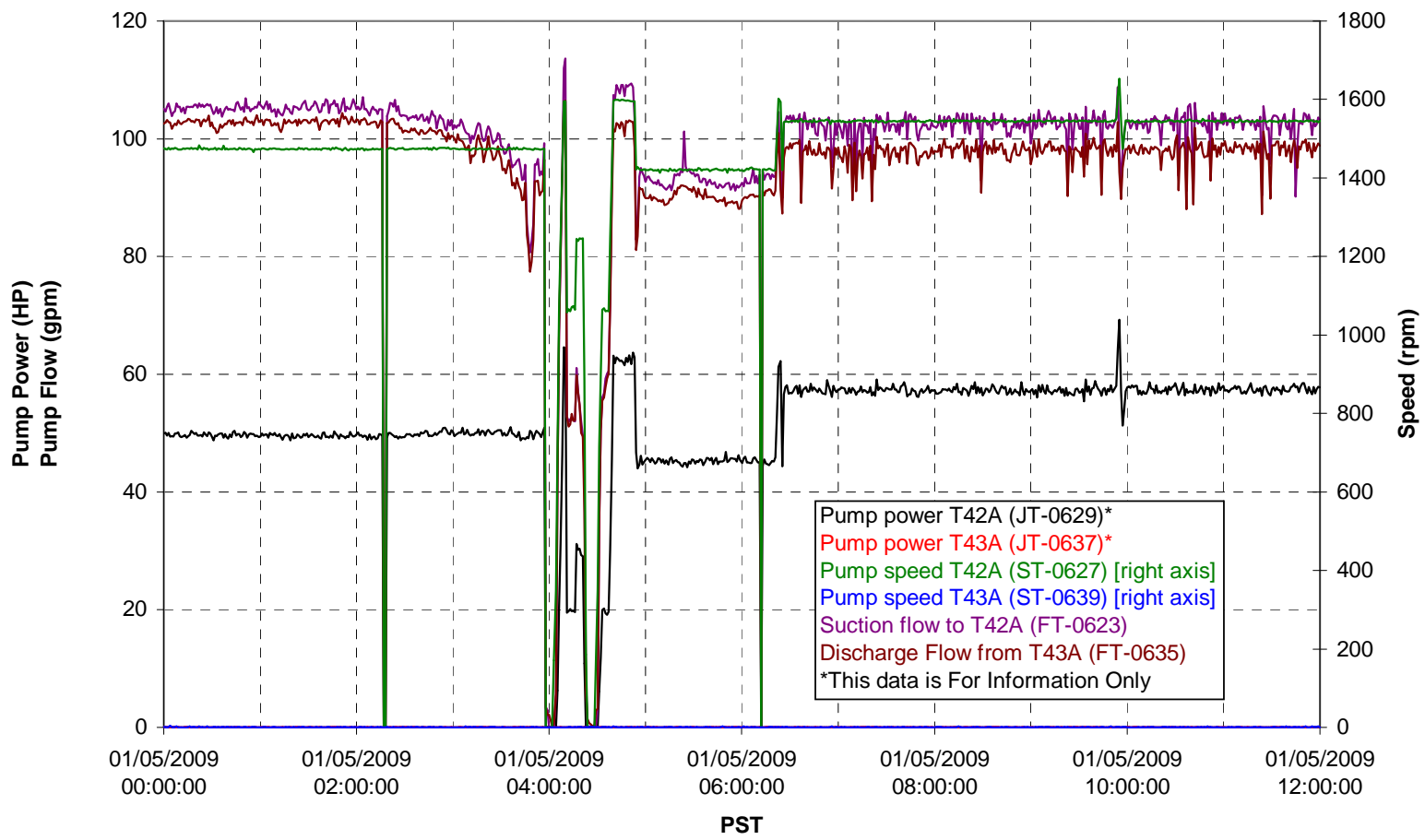


Pulsepot UFP-PP-T01A

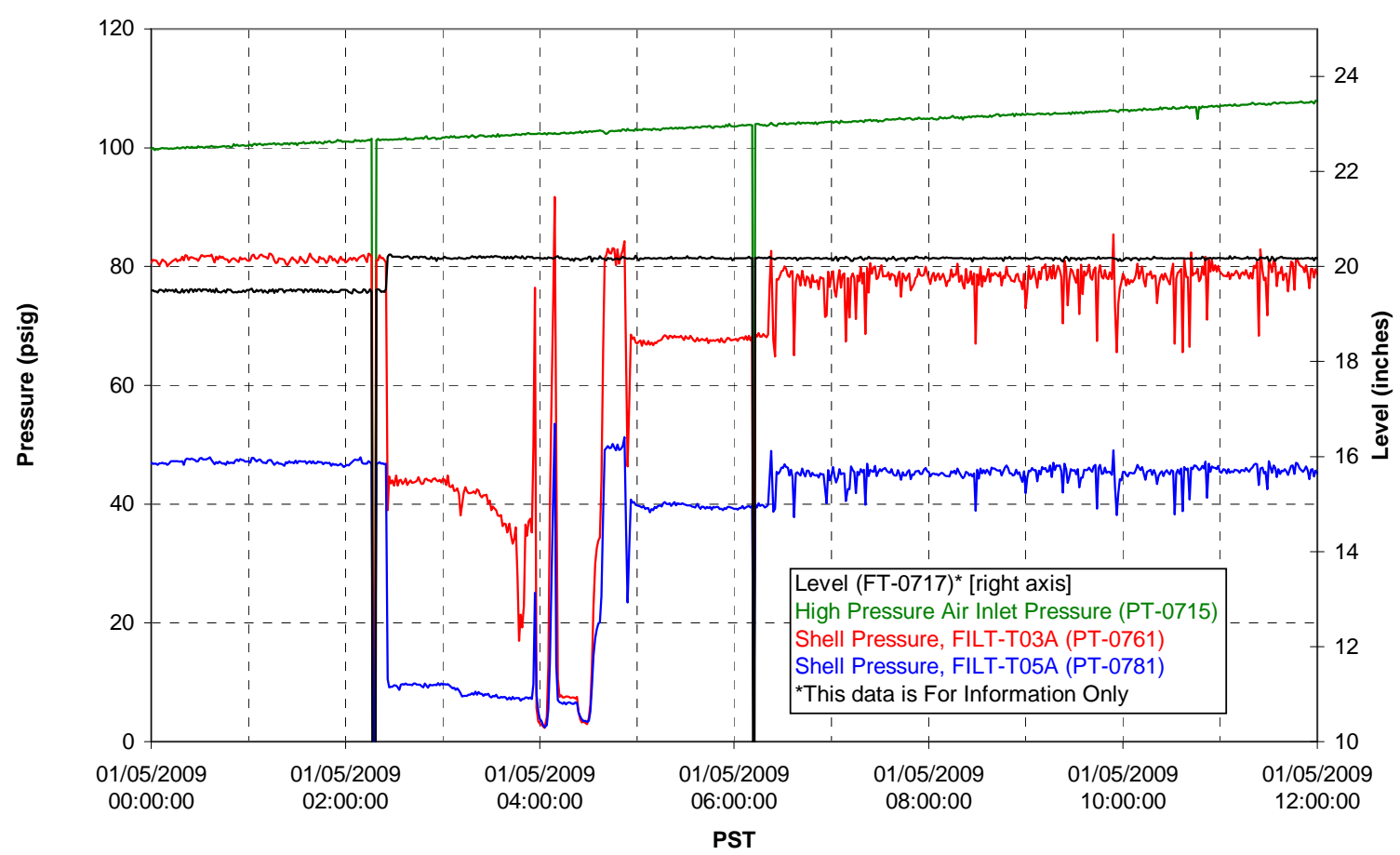

Pulsepot UFP-PP-T02A

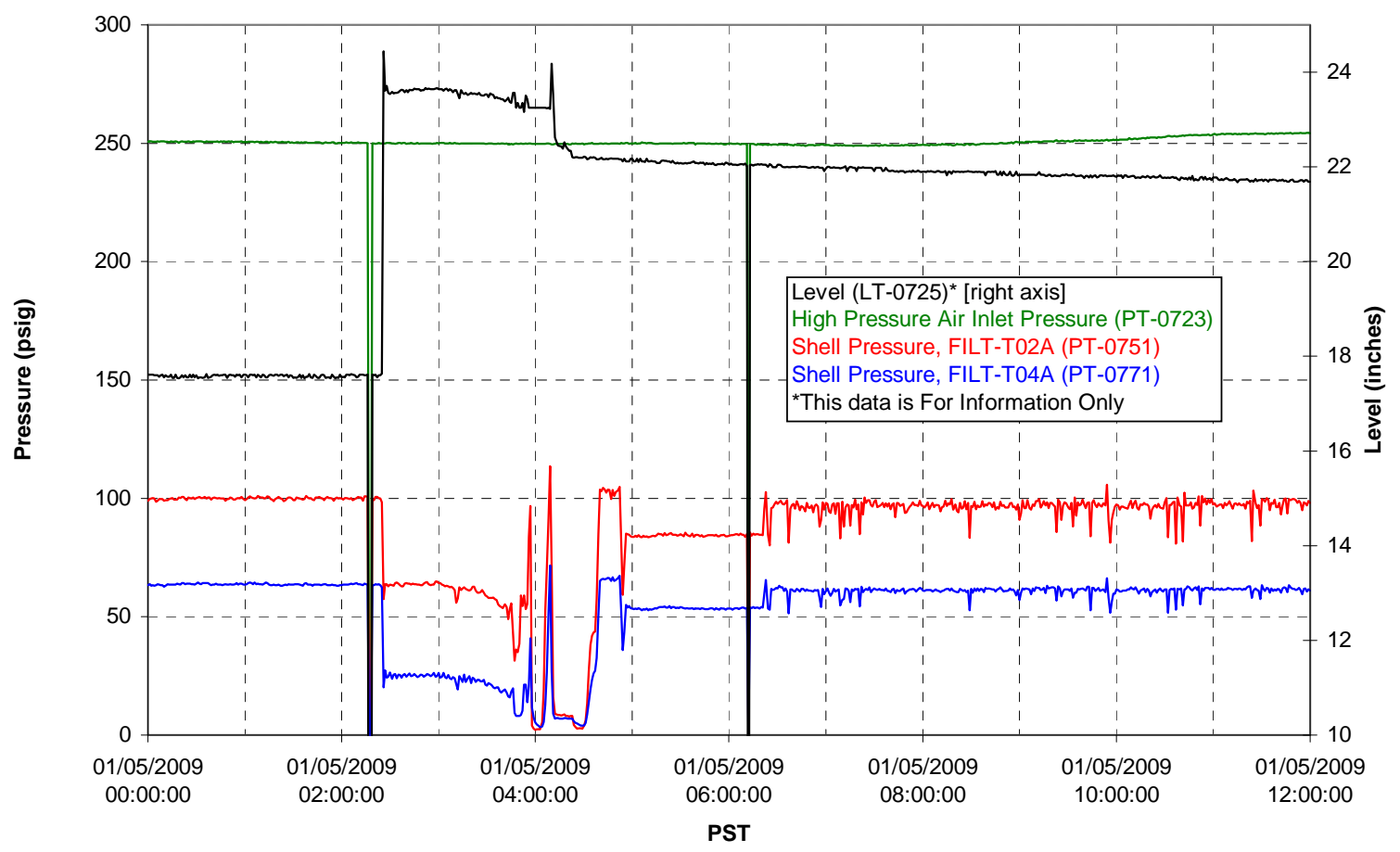


Pulsepot UFP-PP-T03A

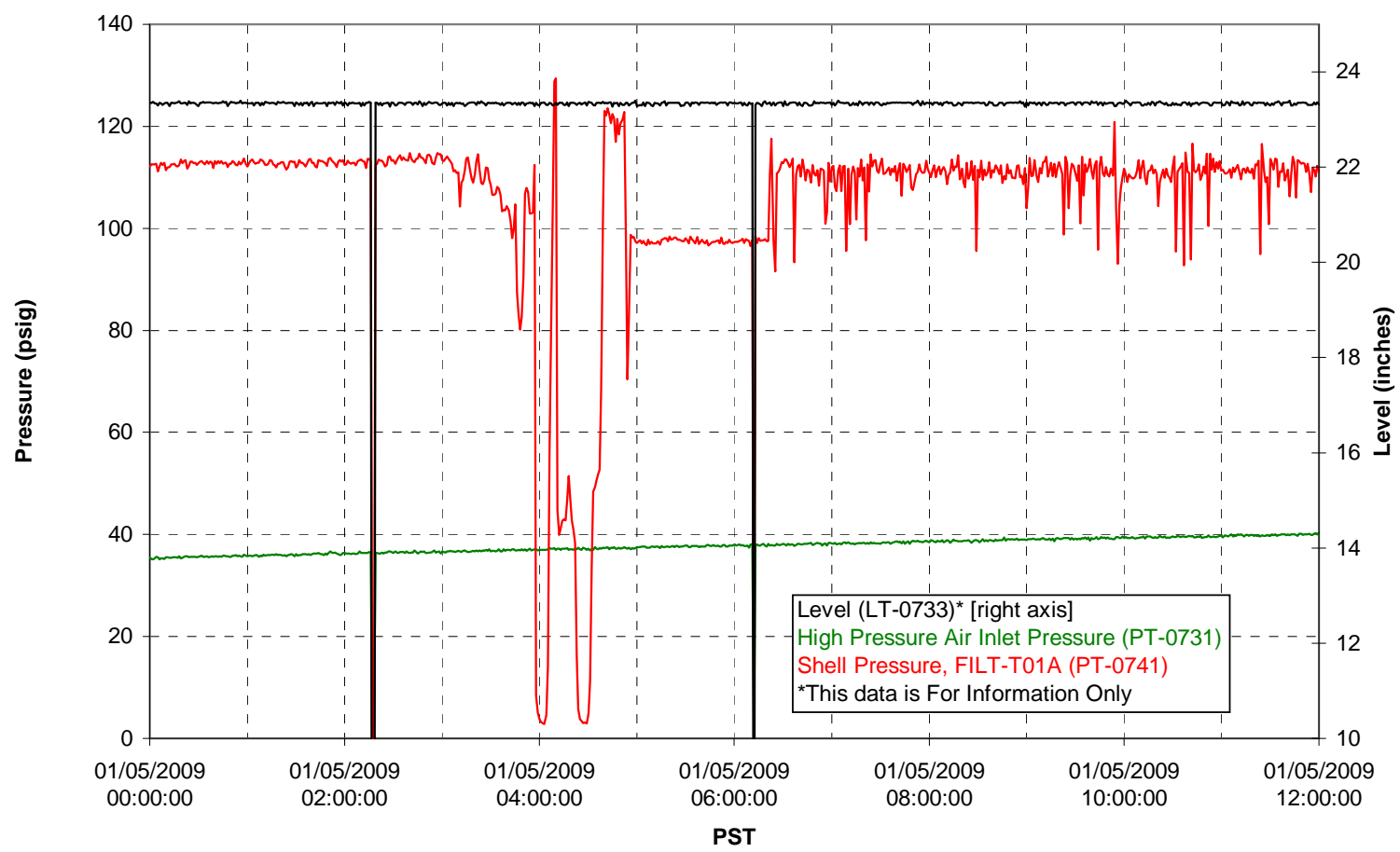

Pulsepot Levels

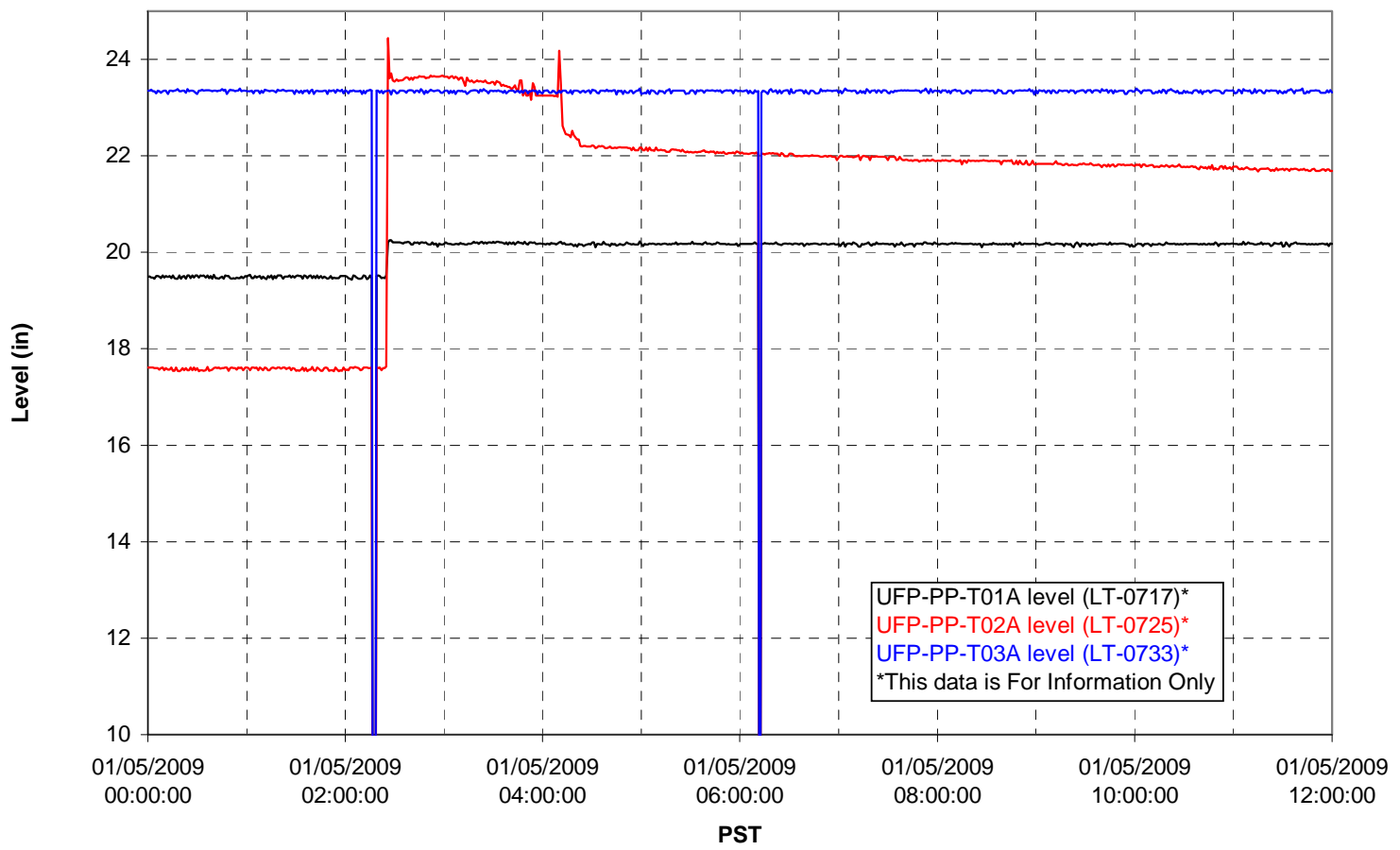


Filter UFP-FILT-T01A

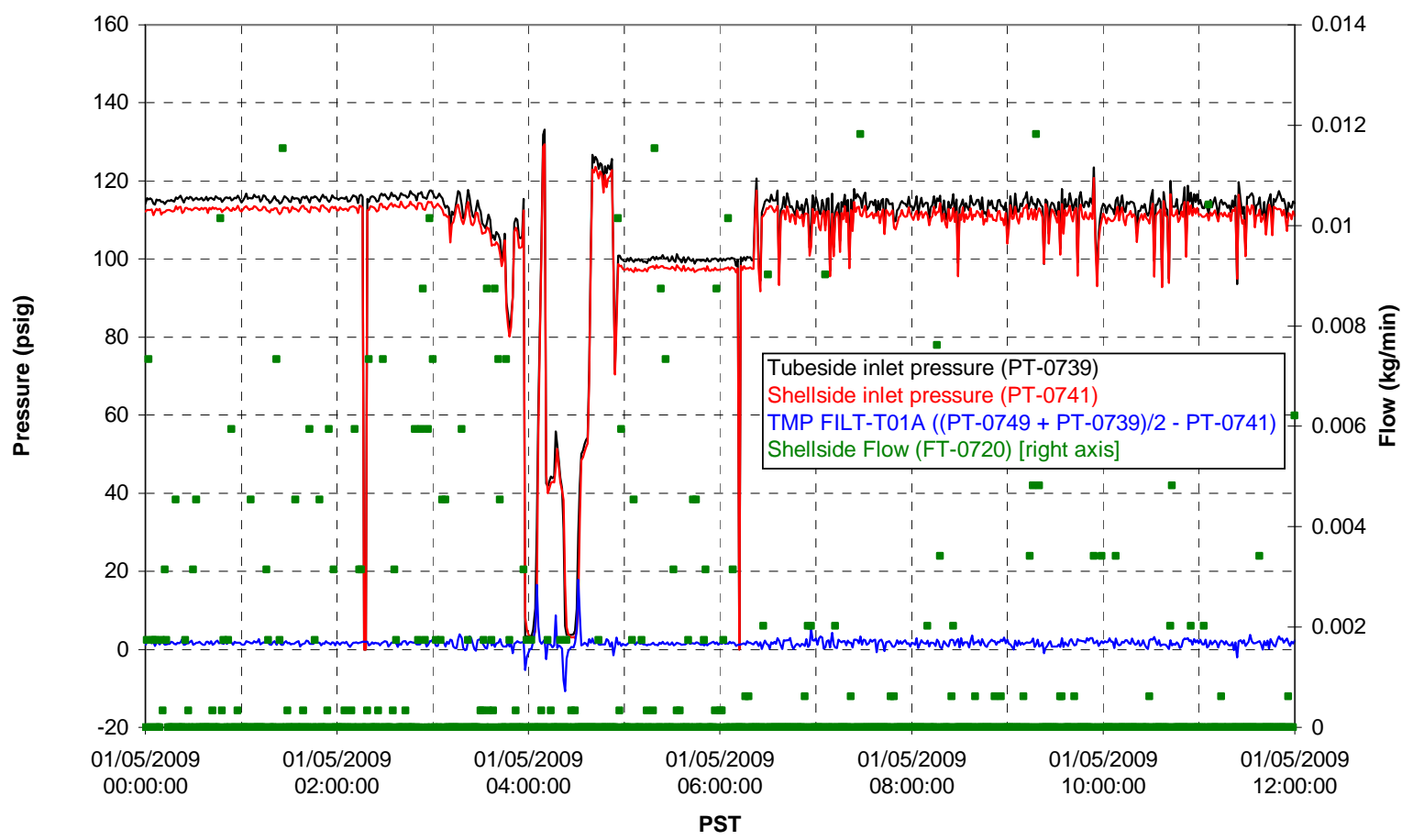

Filter UFP-FILT-T02A

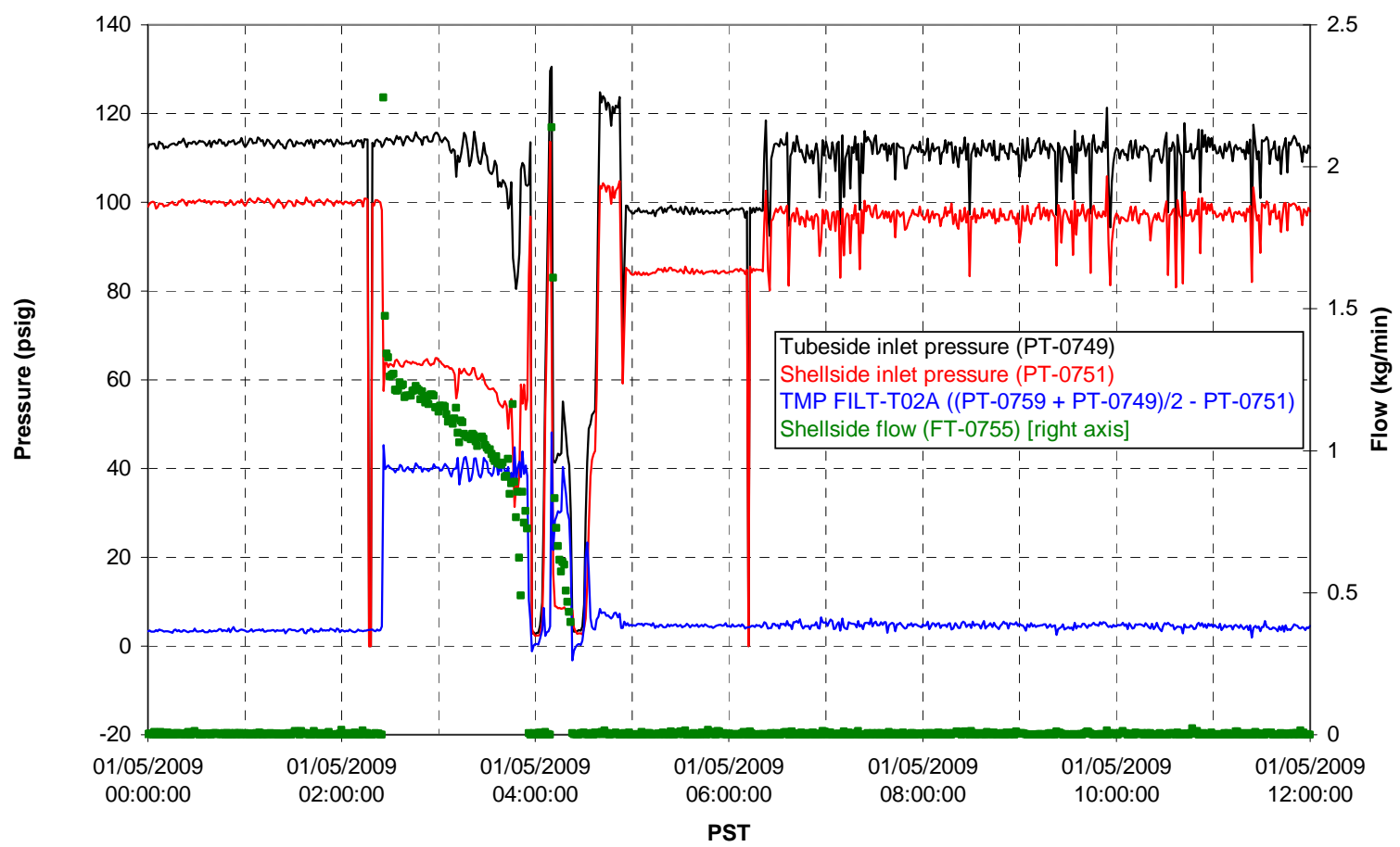


Filter UFP-FILT-T03A

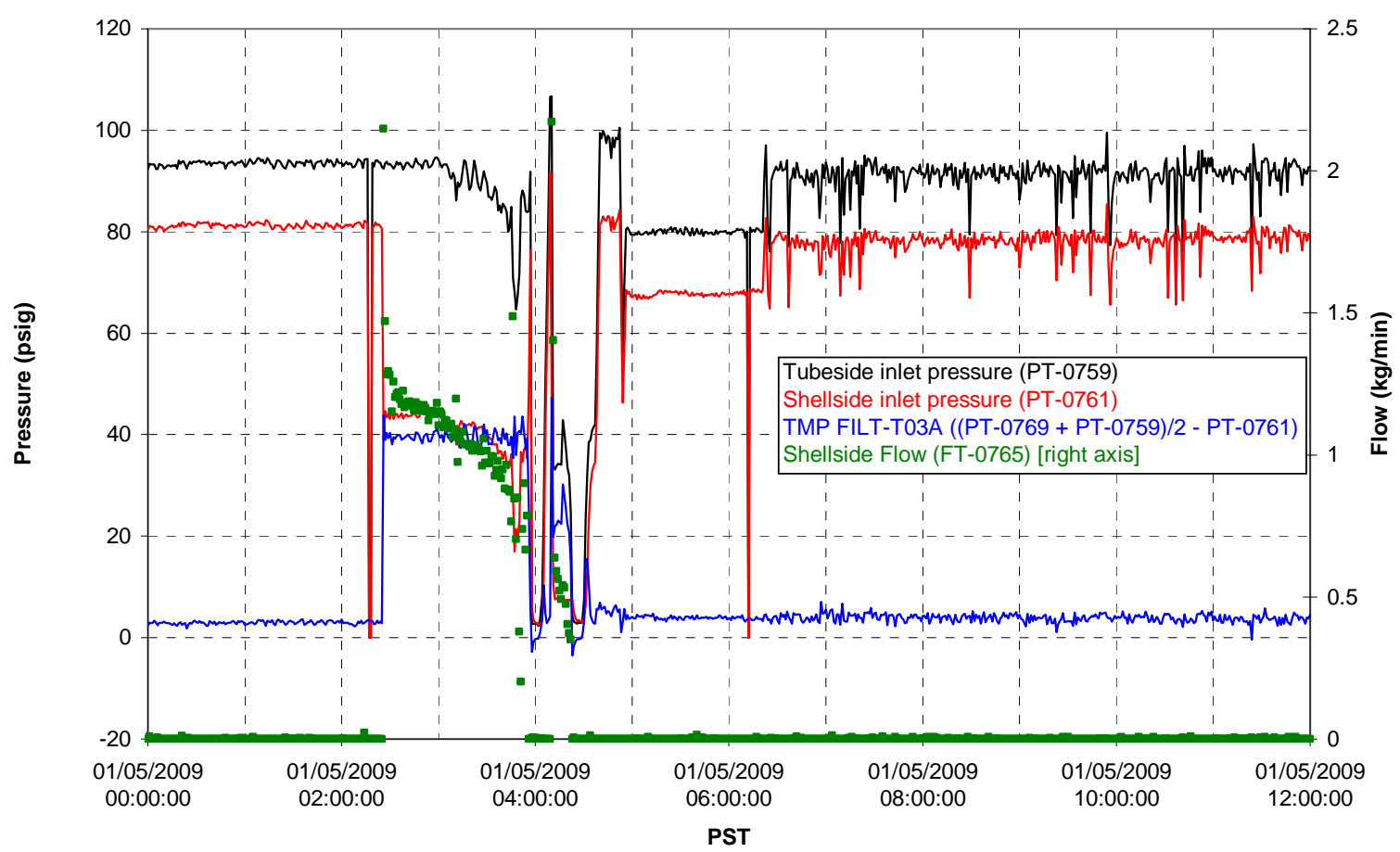

Filter UFP-FILT-T04A

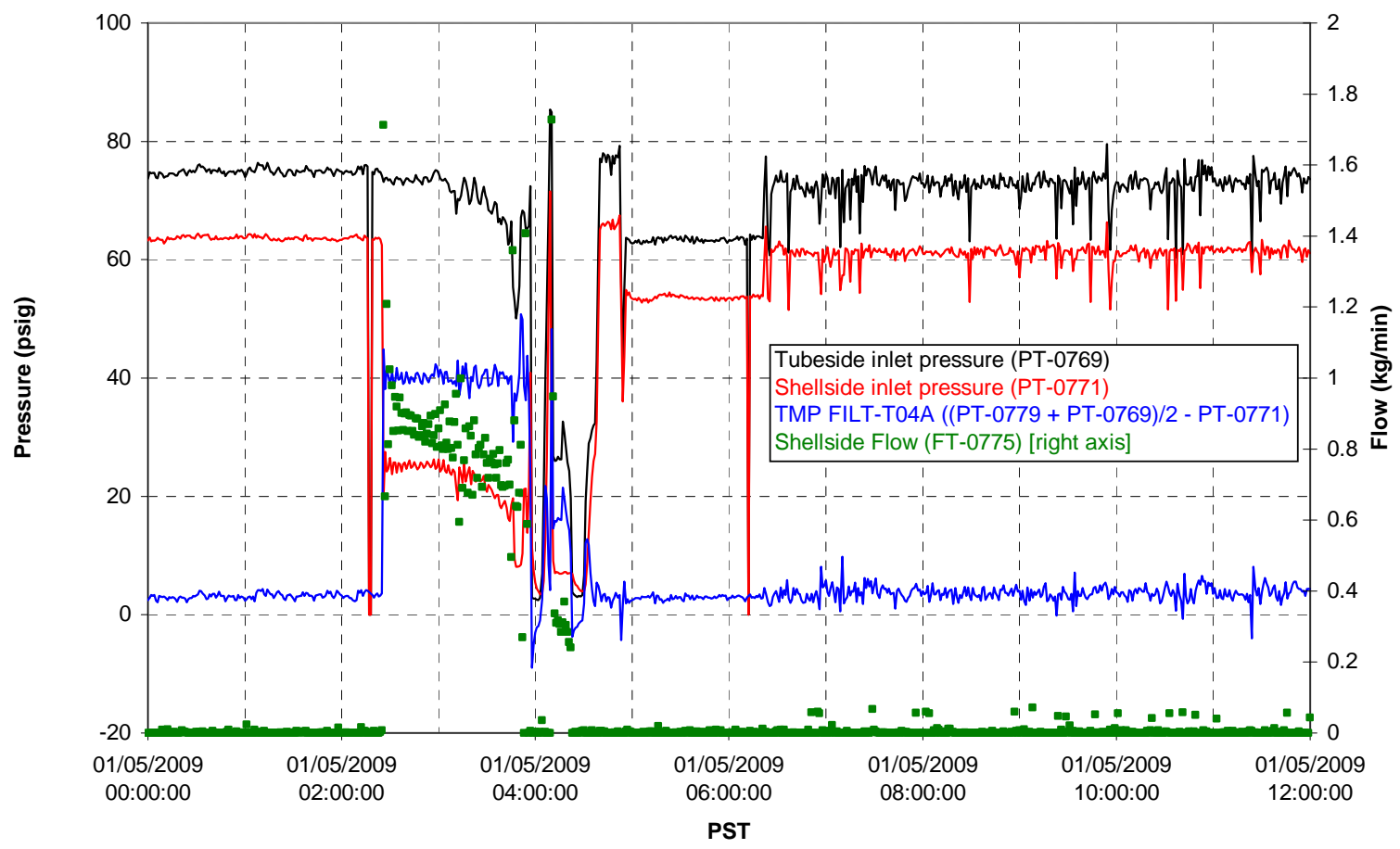


Filter UFP-FILT-T05A

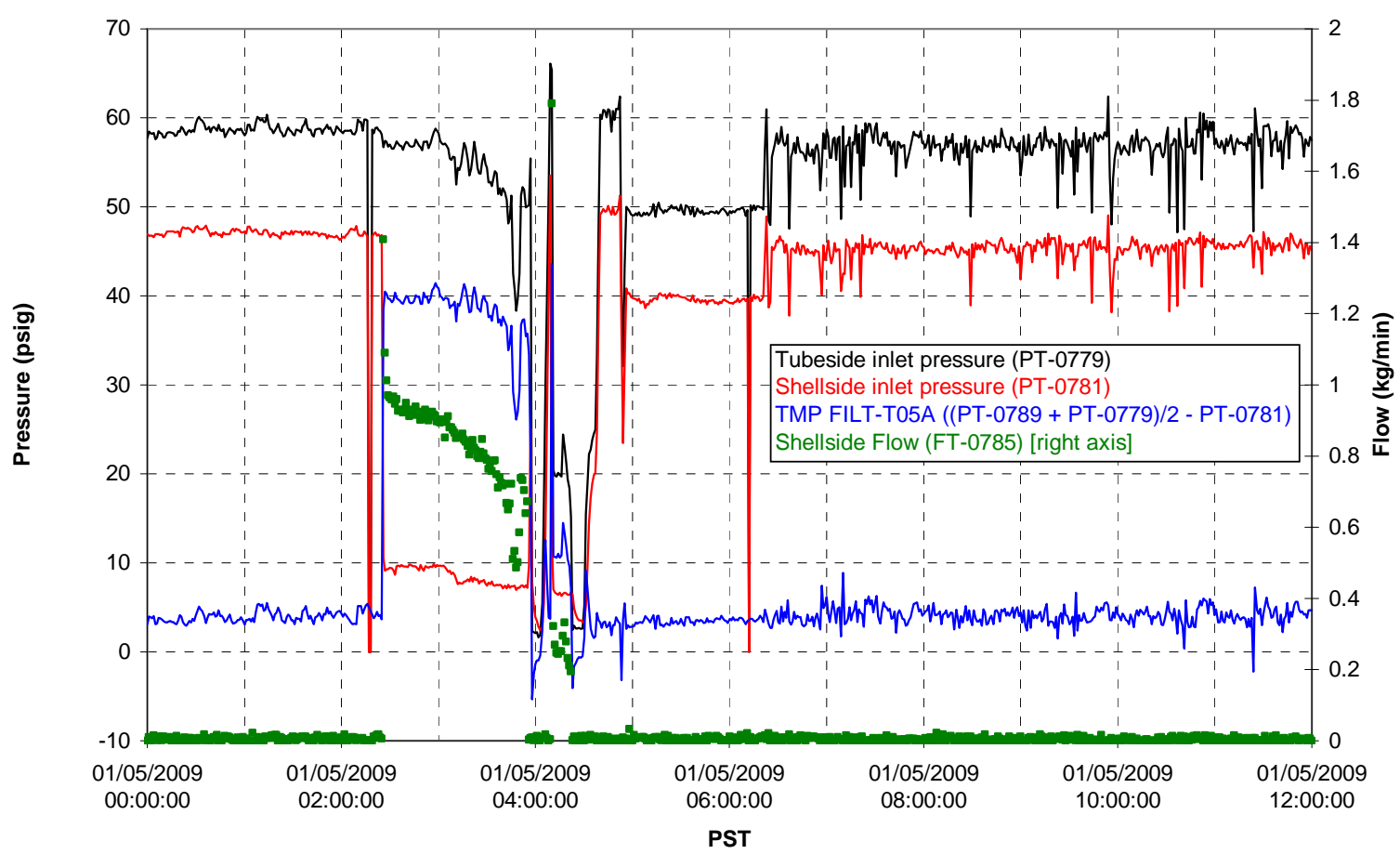

Chemical Flow

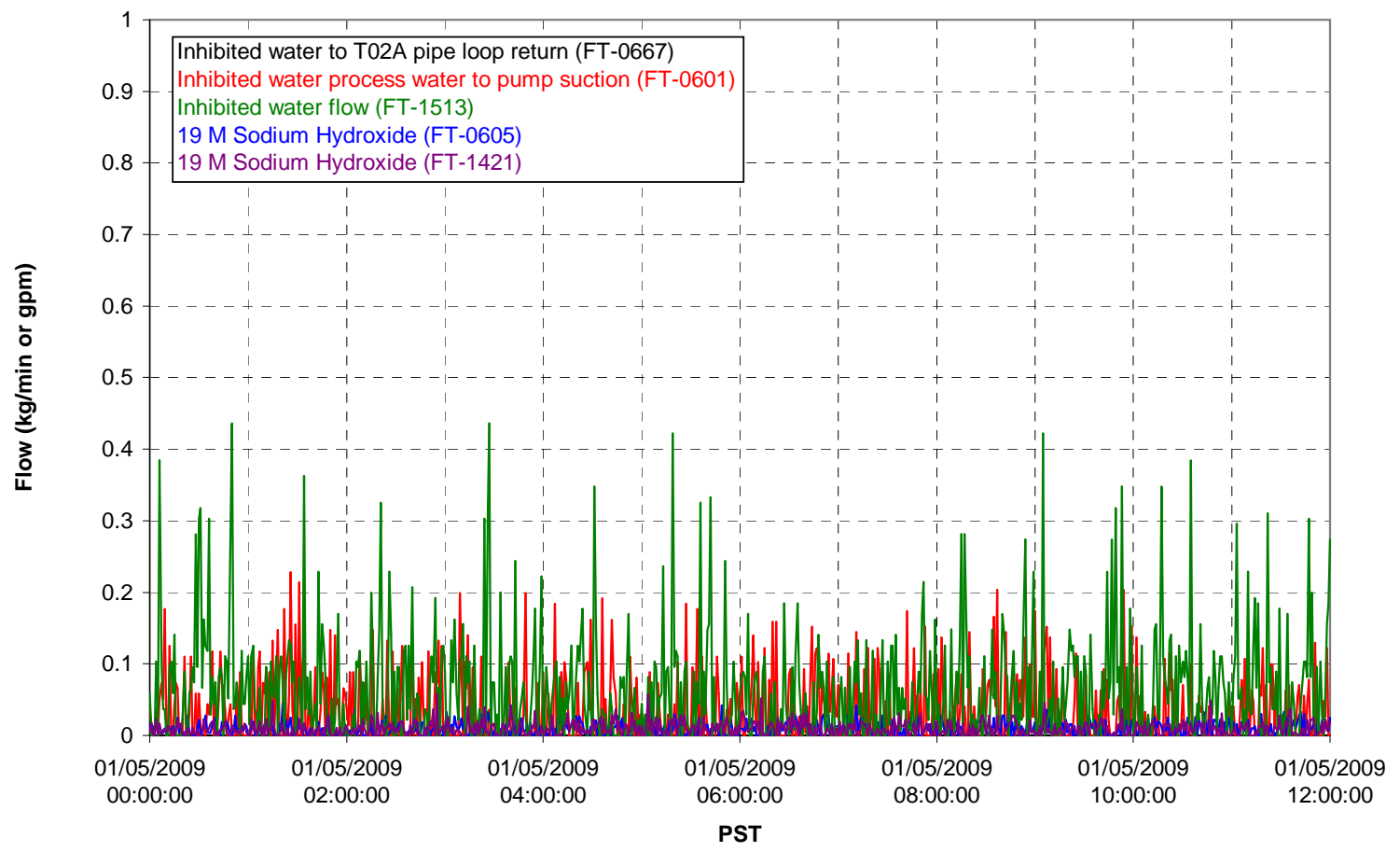


Chemical Flow

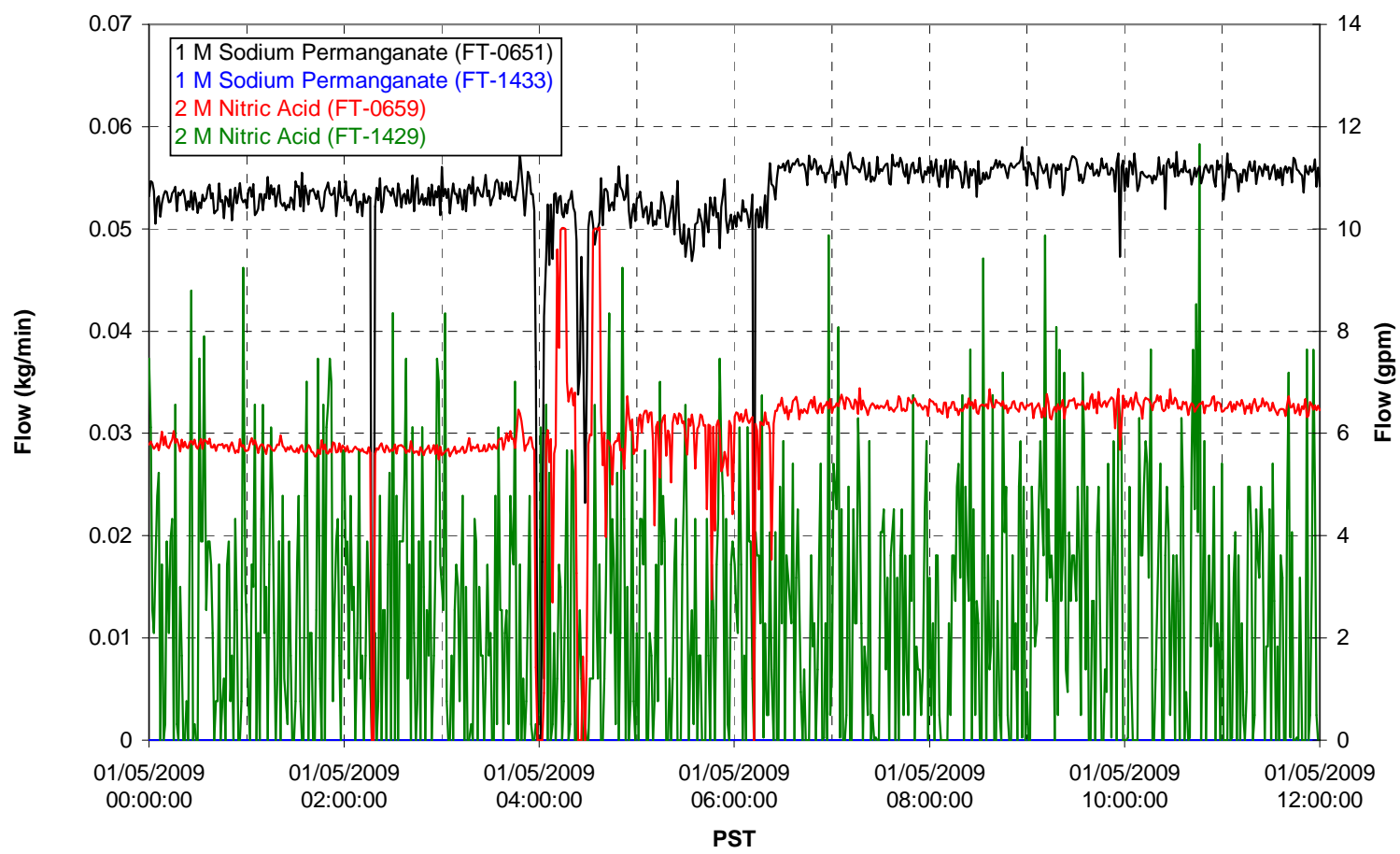

Air Flows

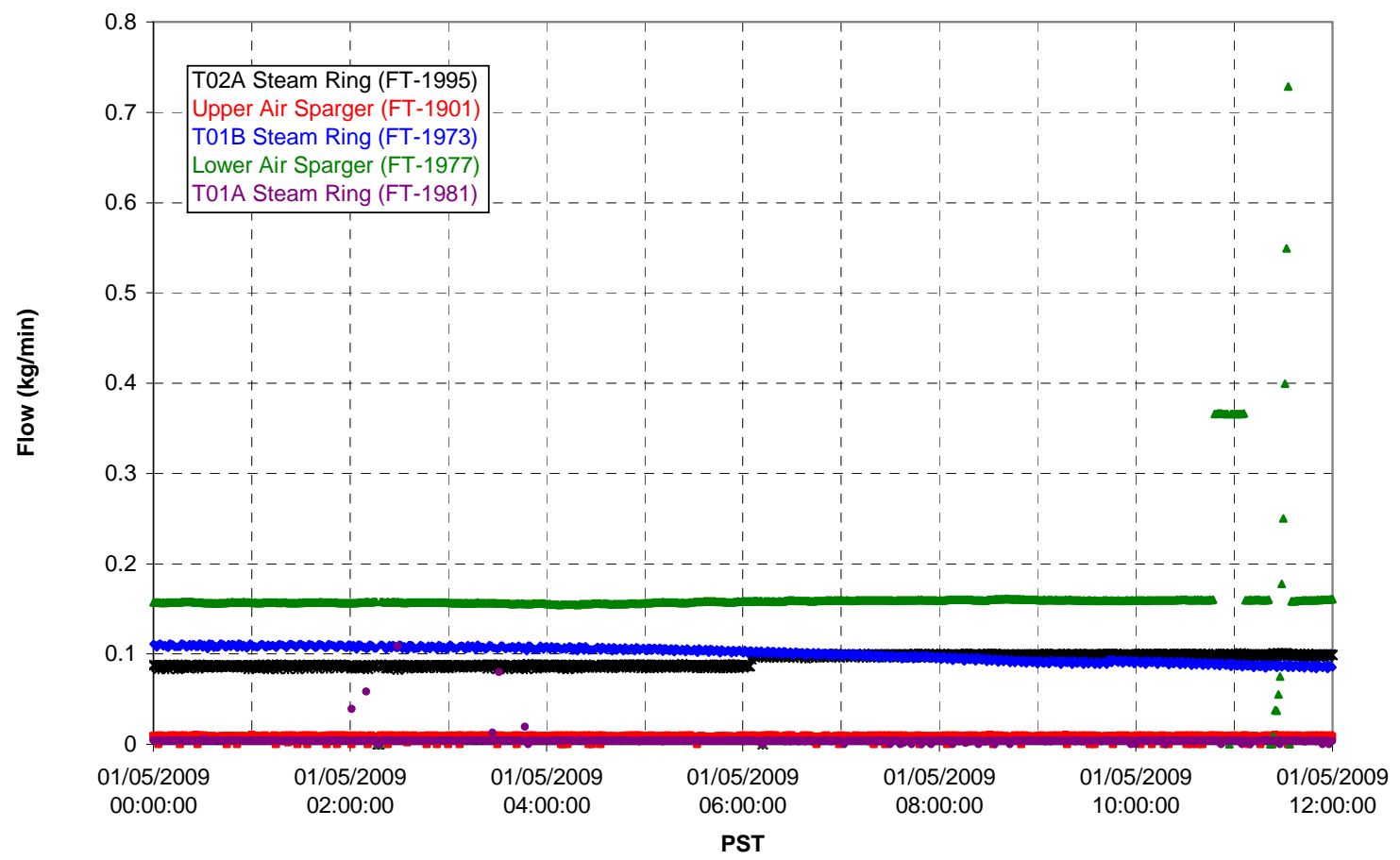


T02A Steam

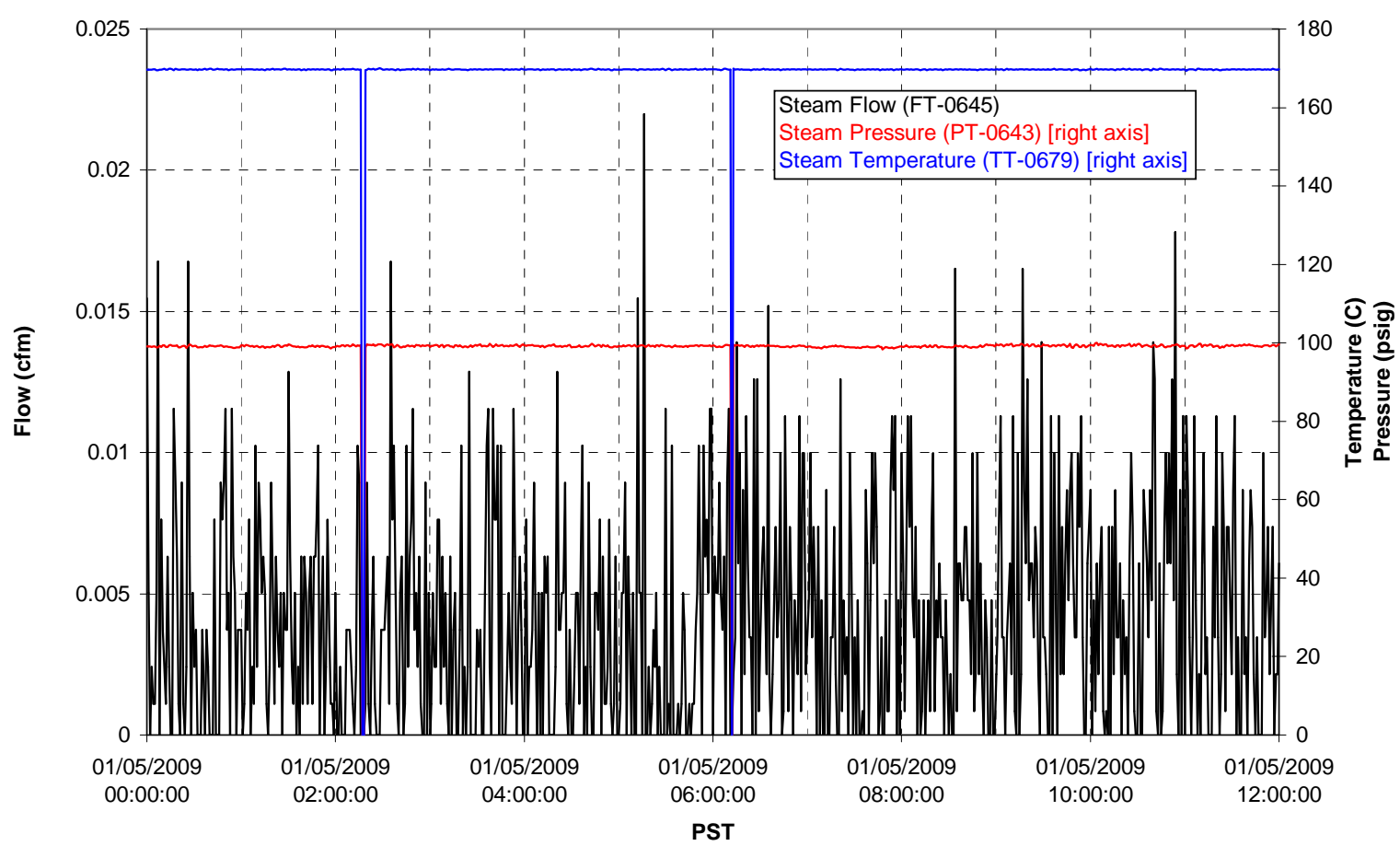

T01A Steam

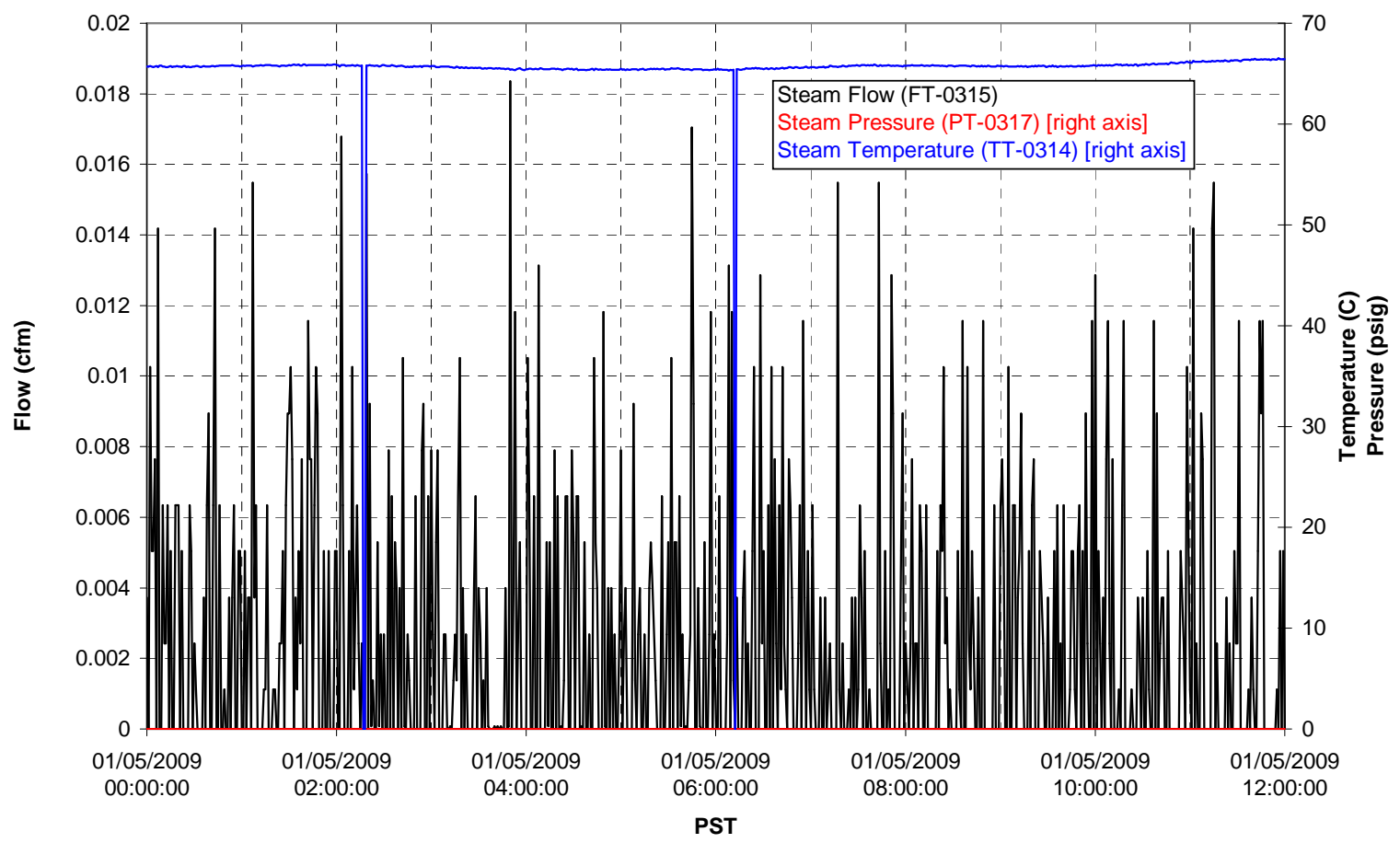


T01B Steam

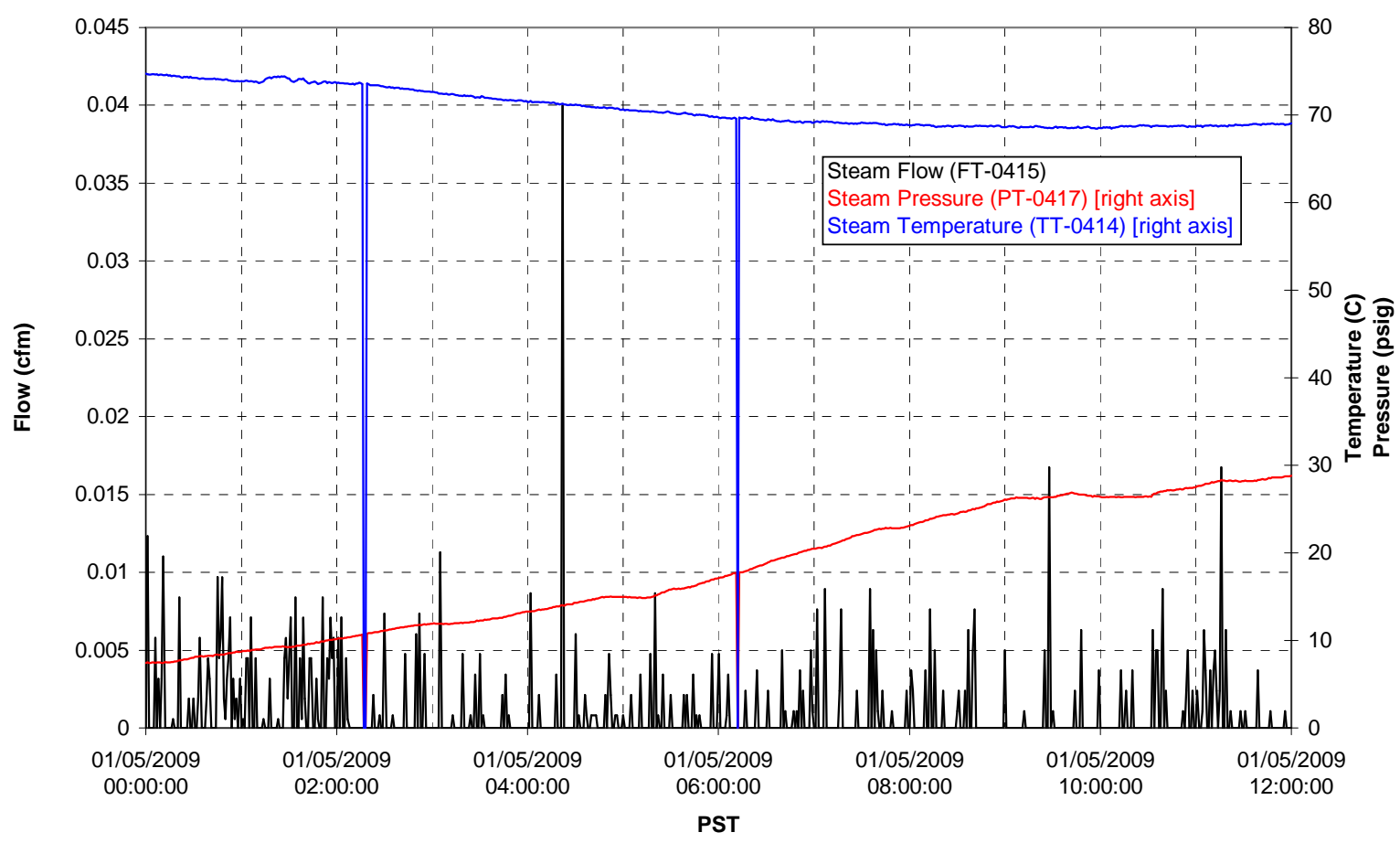


Functional Test Data Plots

01/05/2009 12:00 - 01/06/2009 00:00 
T01A level

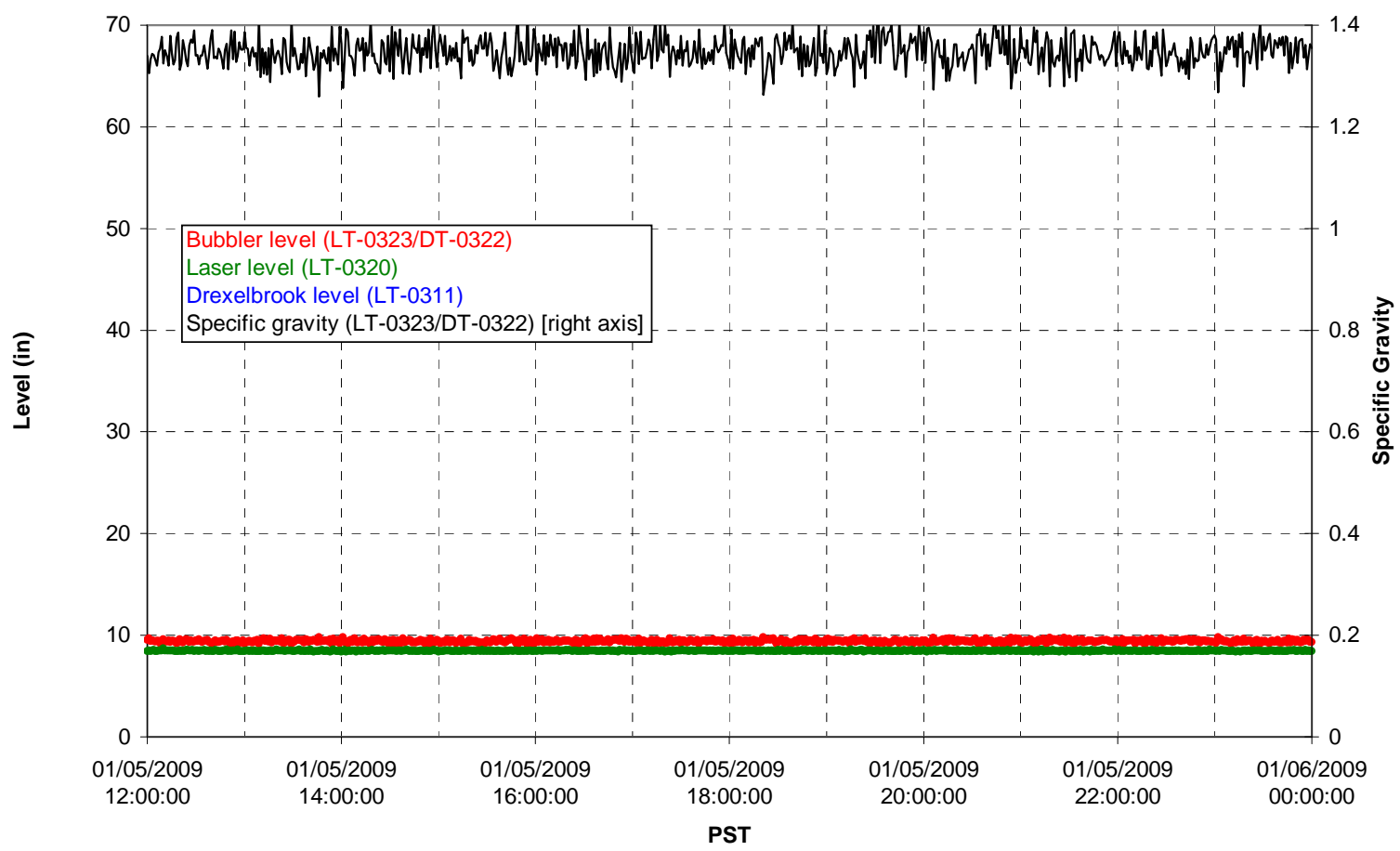

T01A temperatures

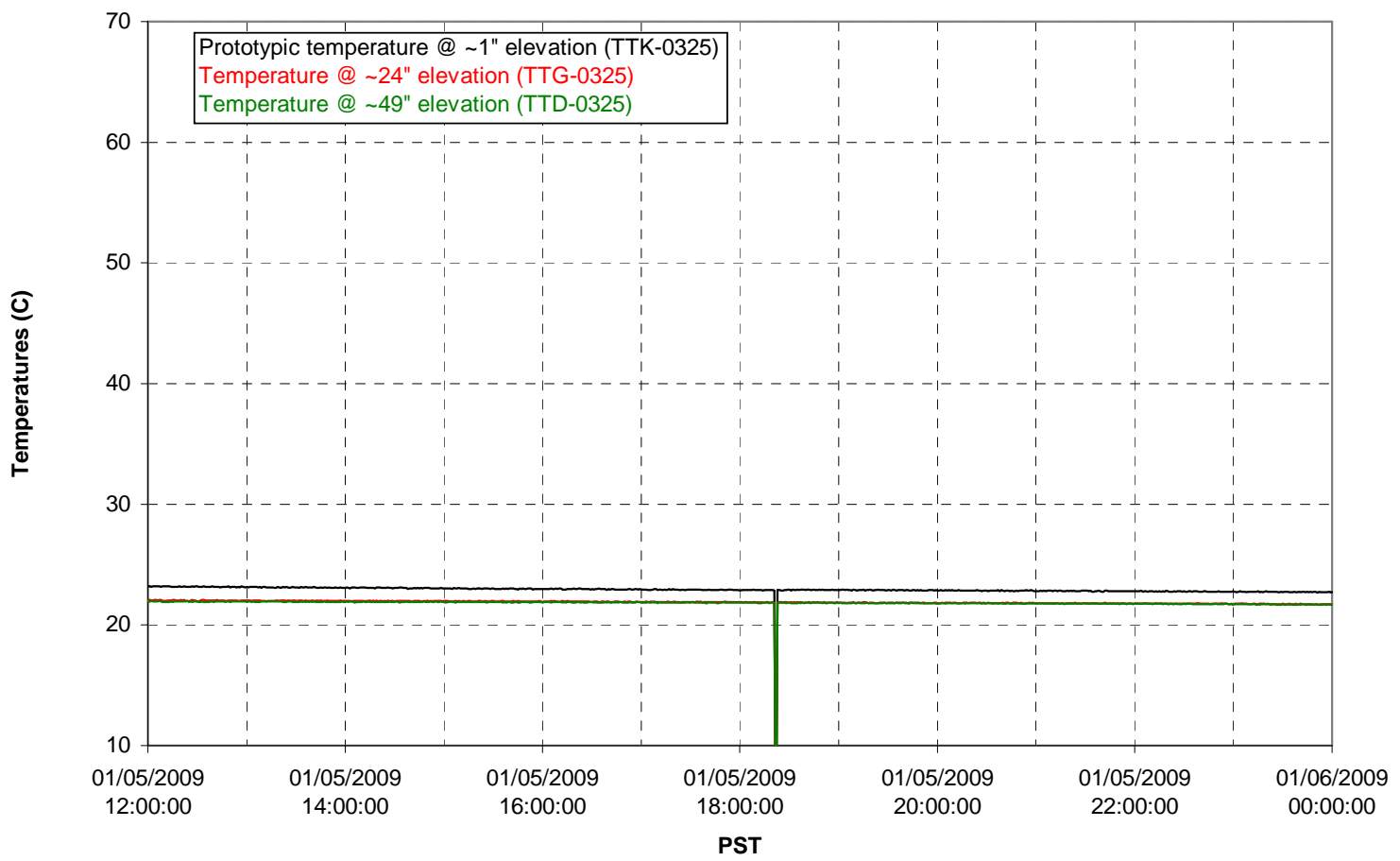


T01B level

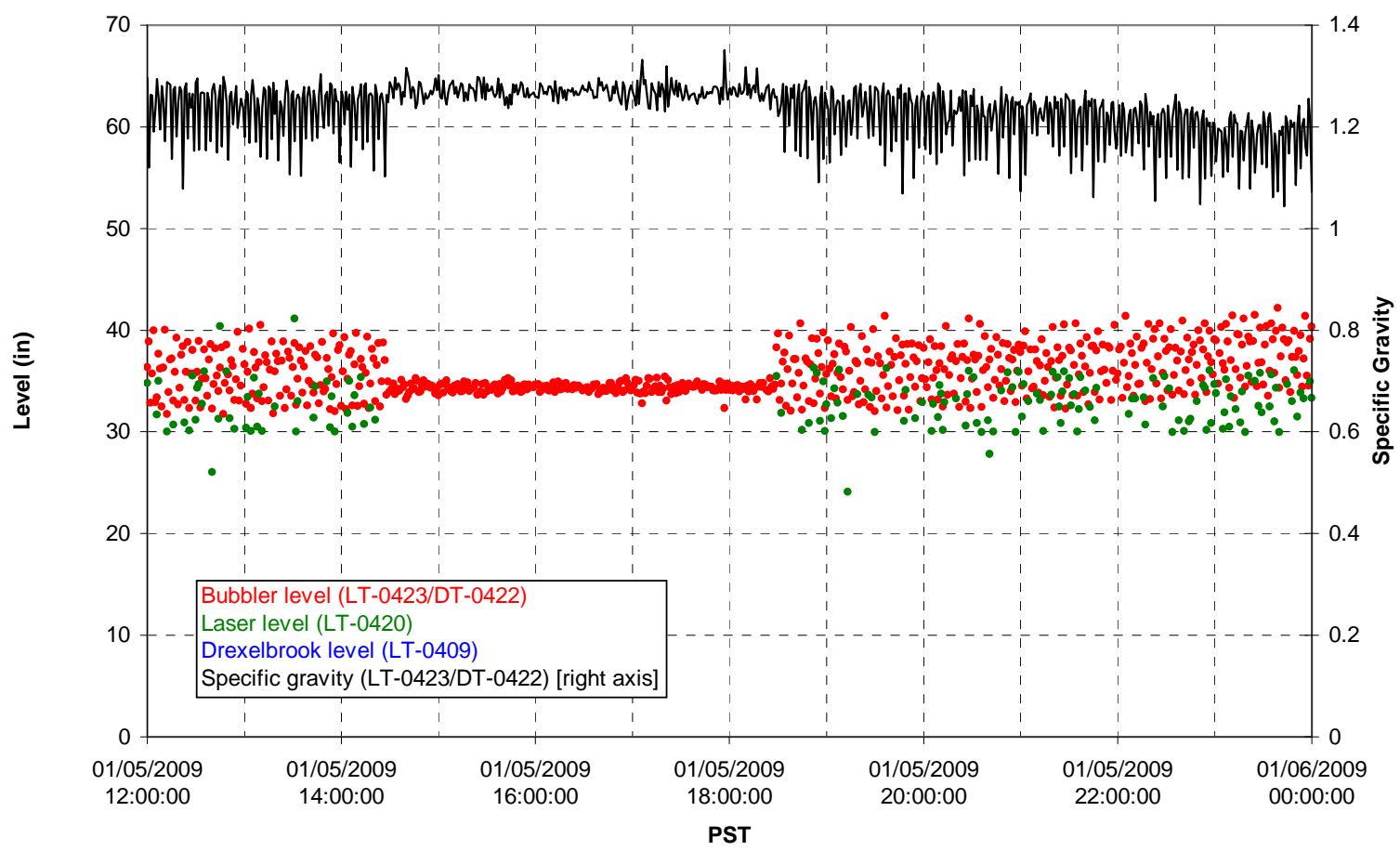

T01B temperatures

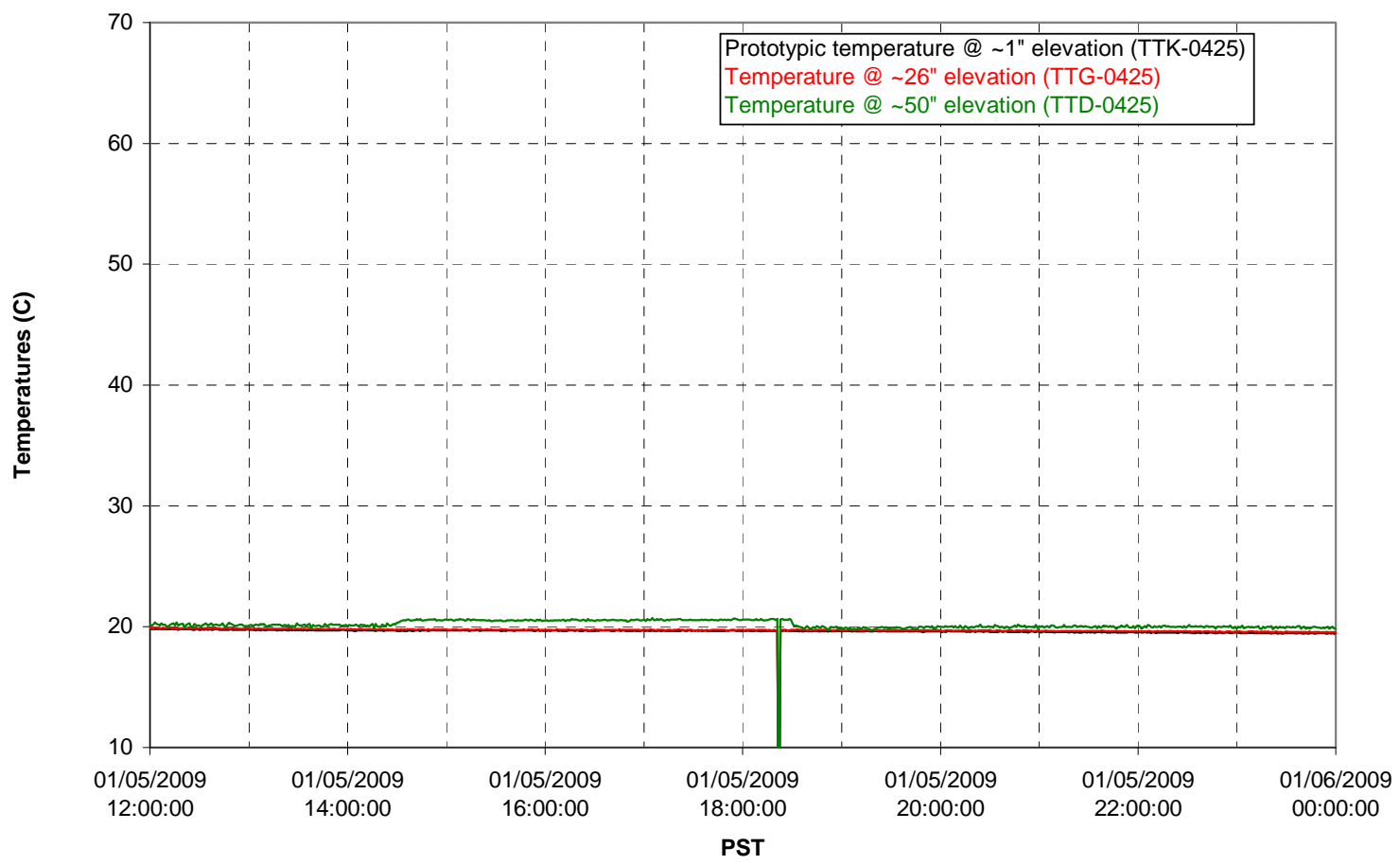


T02A level

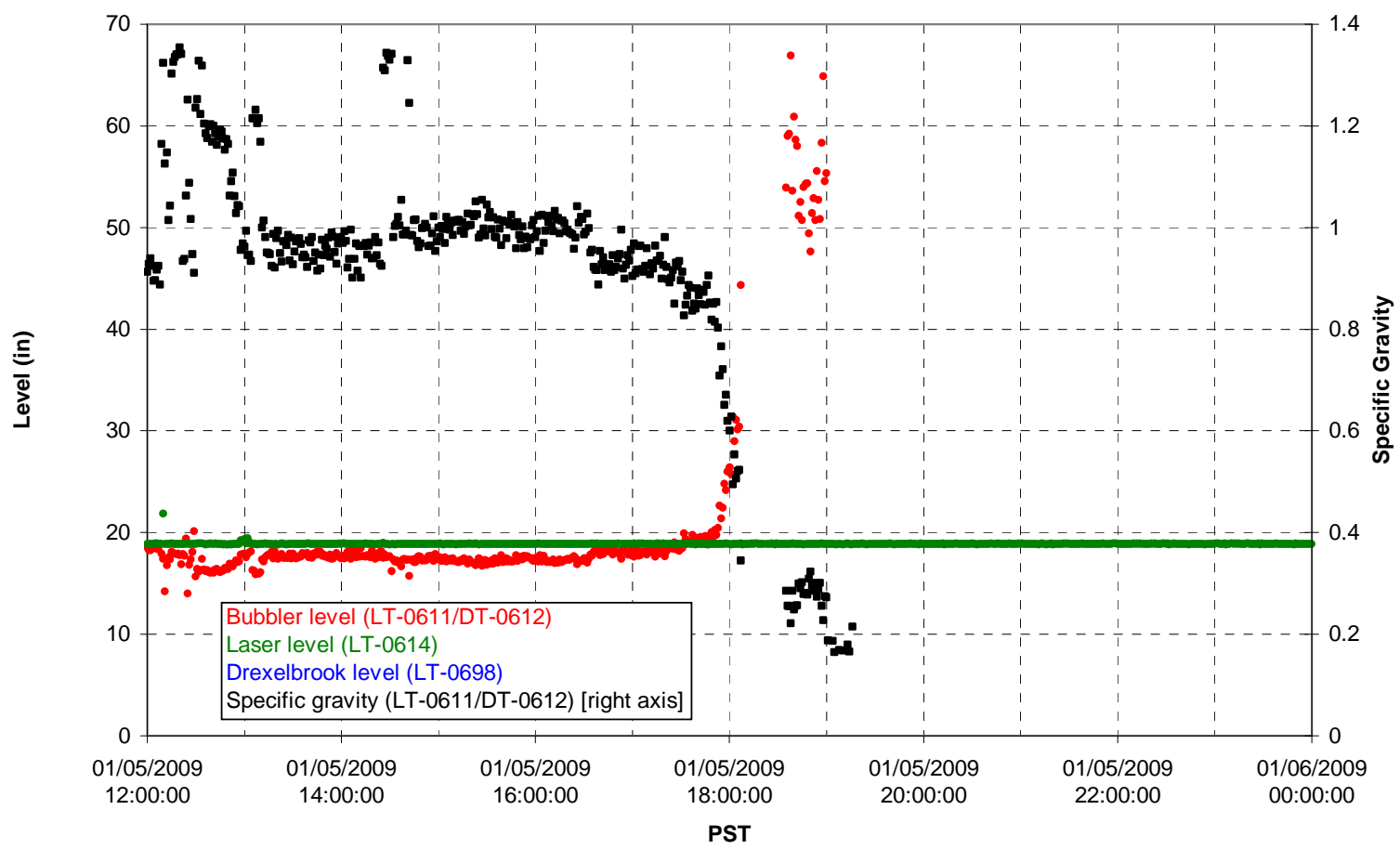

T02A temperatures

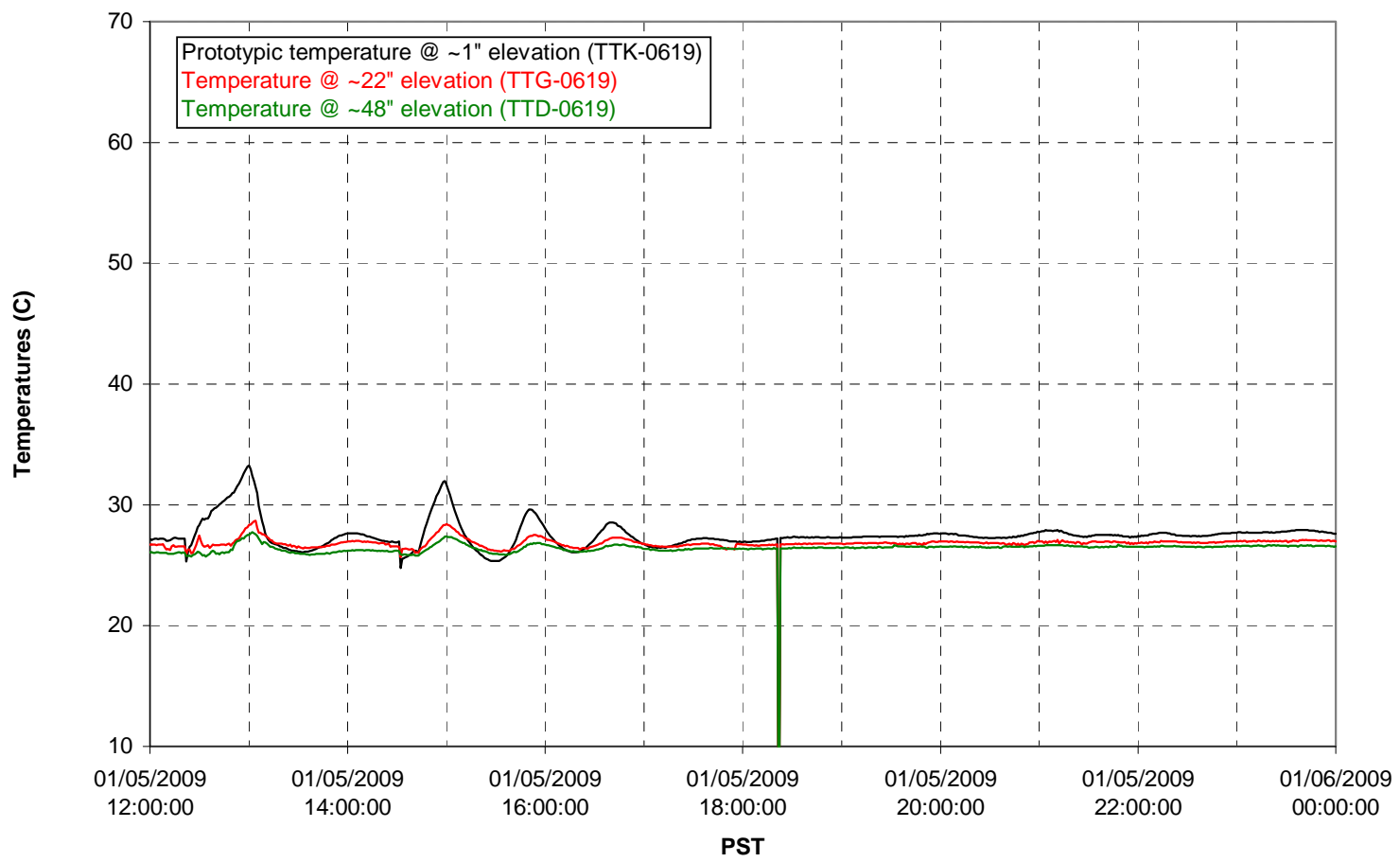


T02A and filter loop temperatures

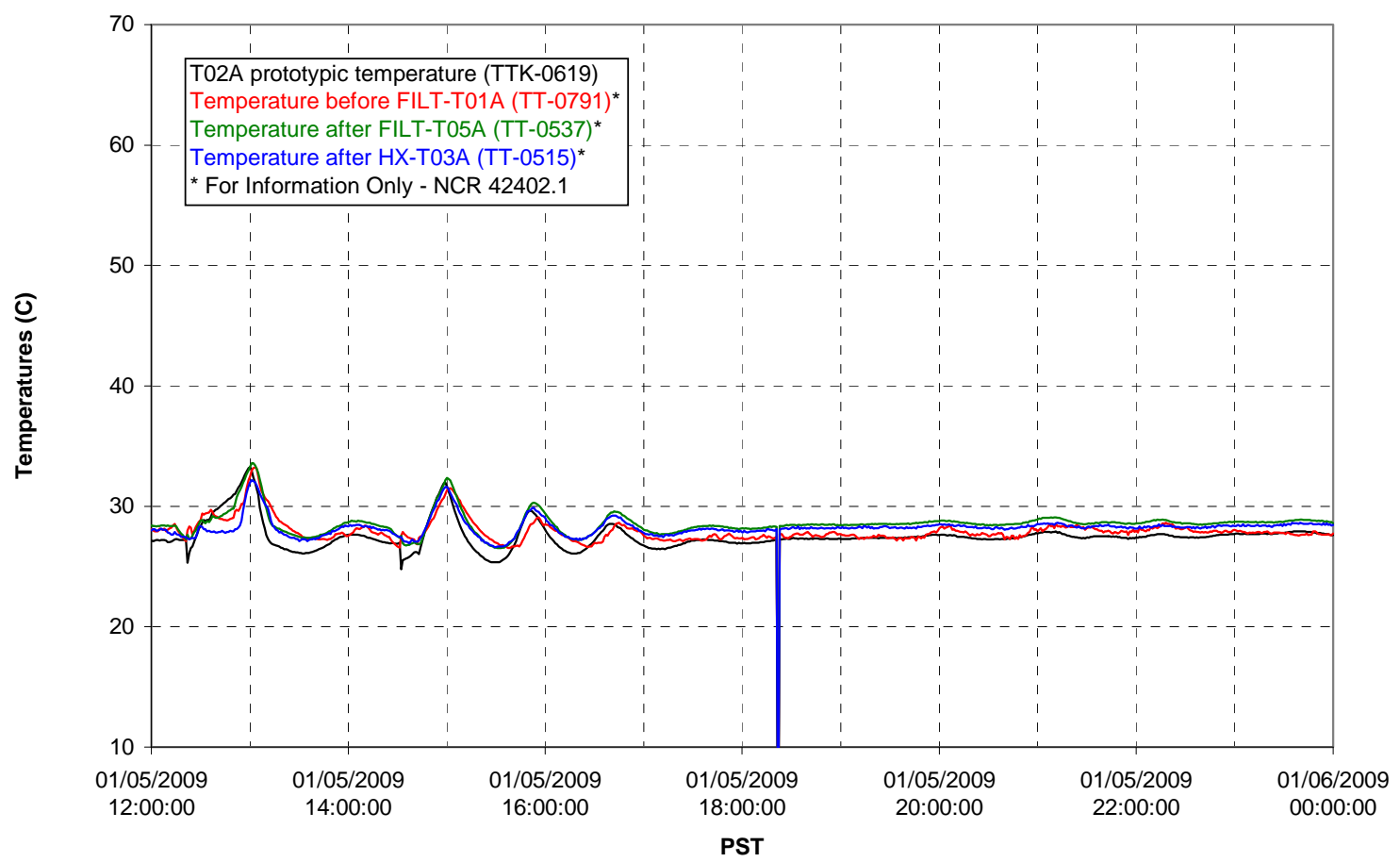

Pump Pressures and Flow

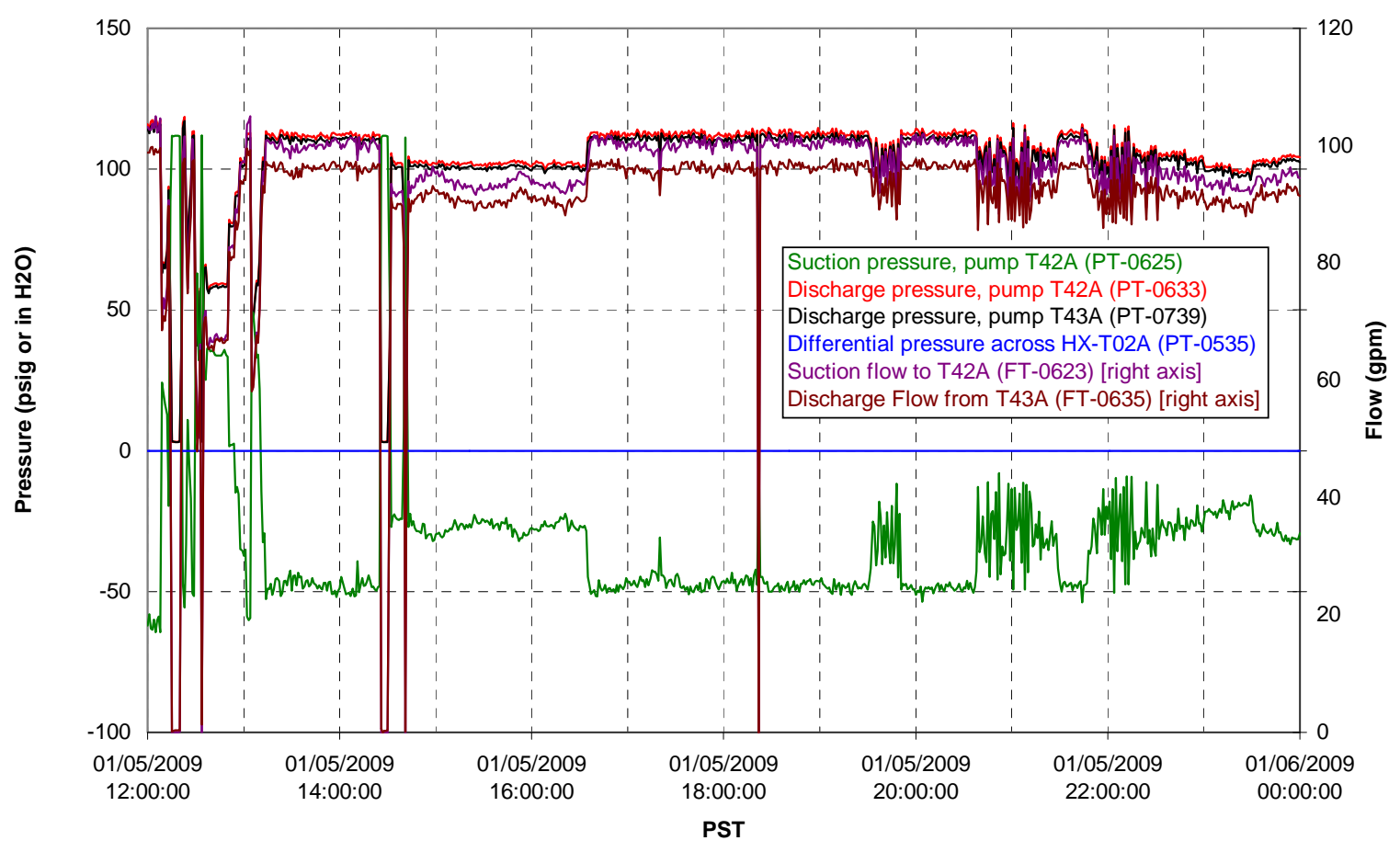


Axial pressure drop

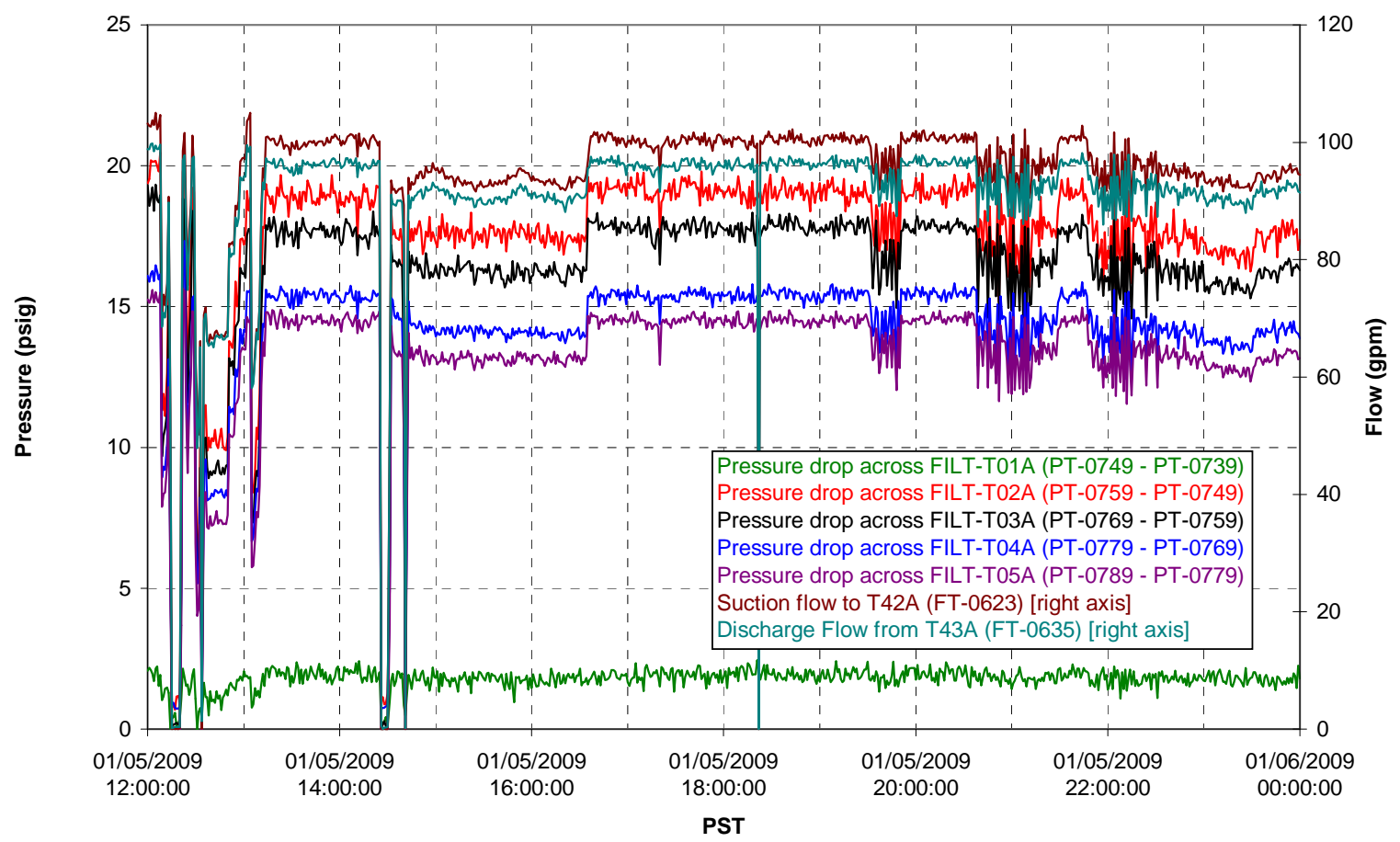

Permeate flow rates

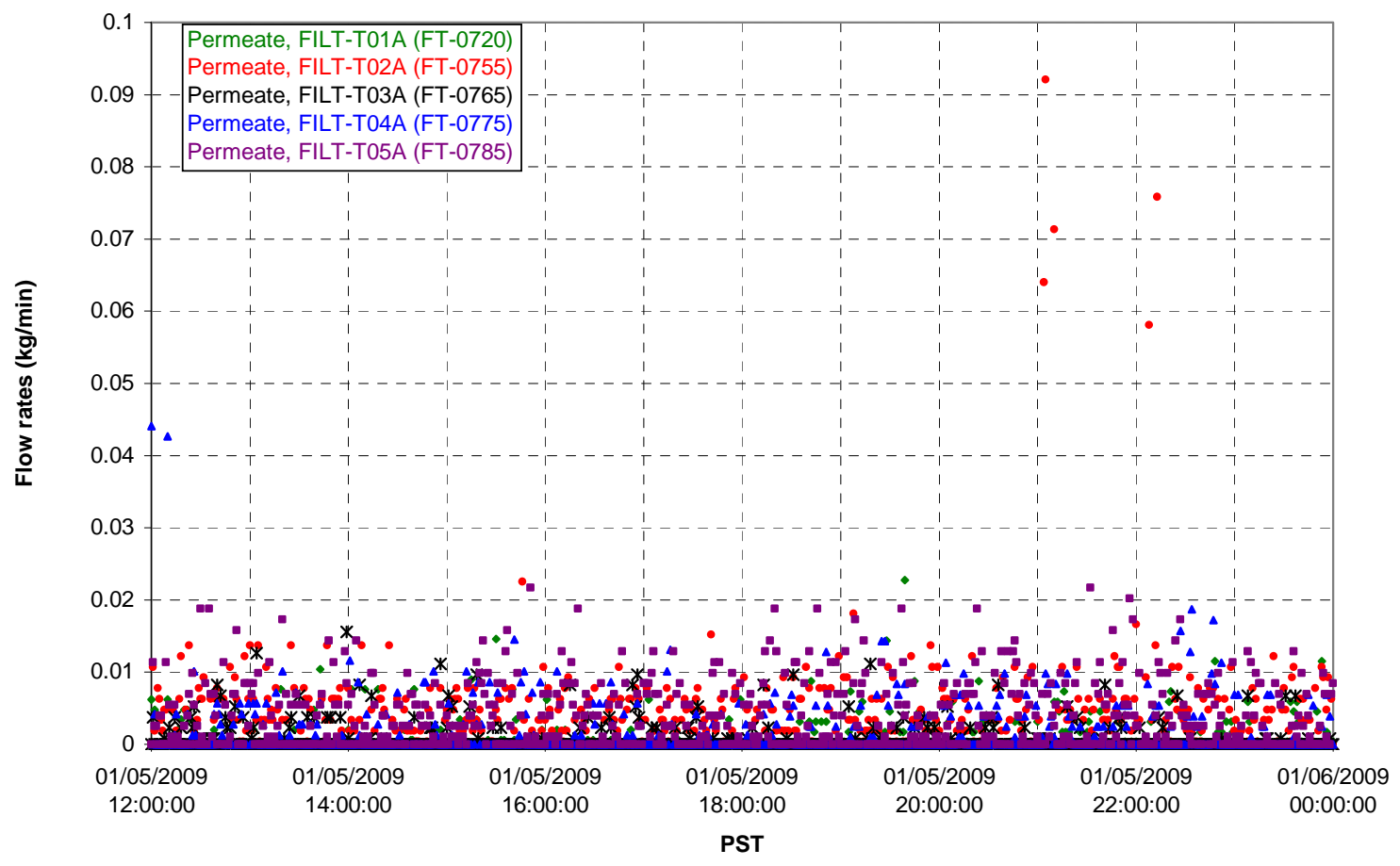


T02A Inner Temperature Tree

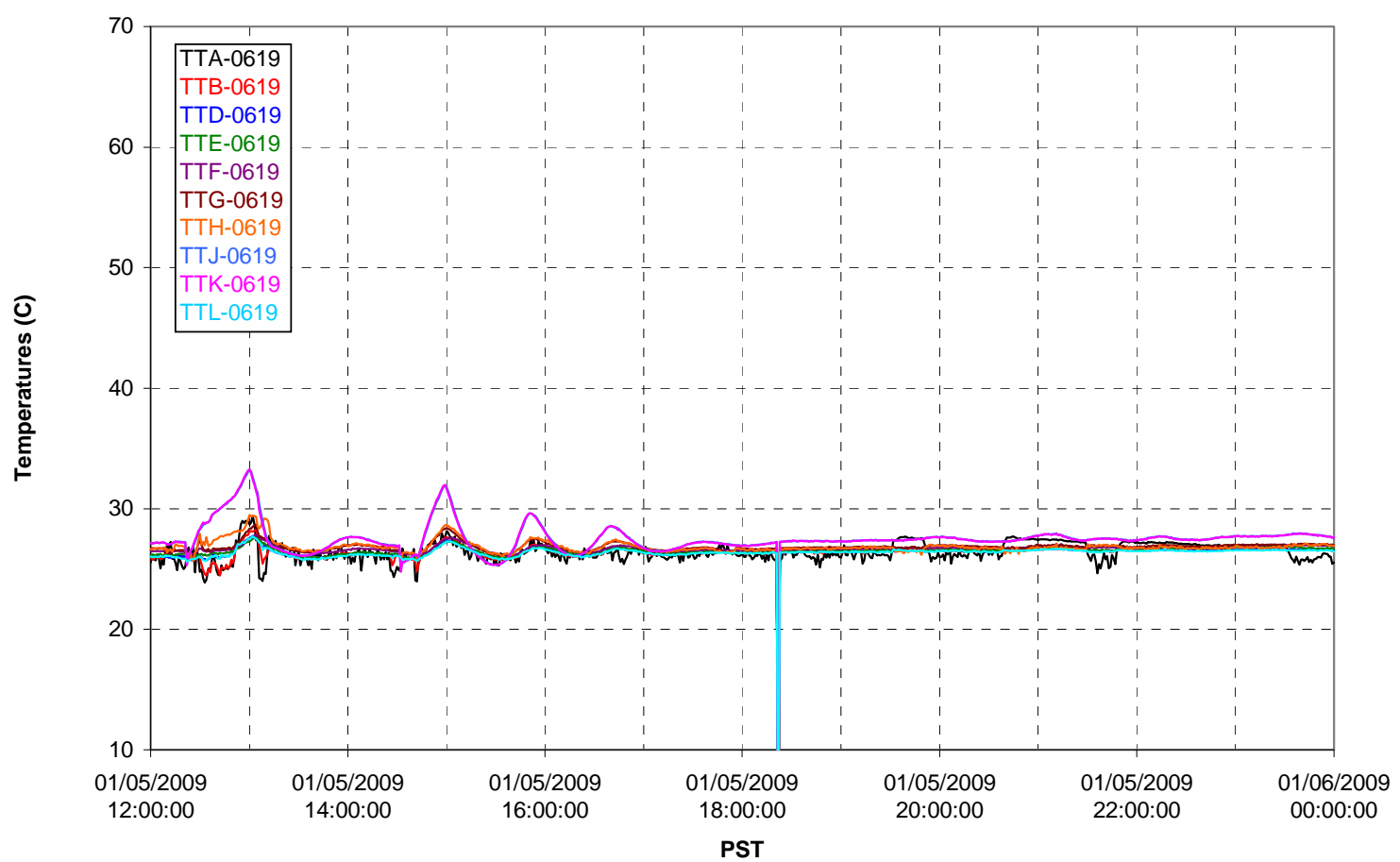

T02A Outer Temperature Tree

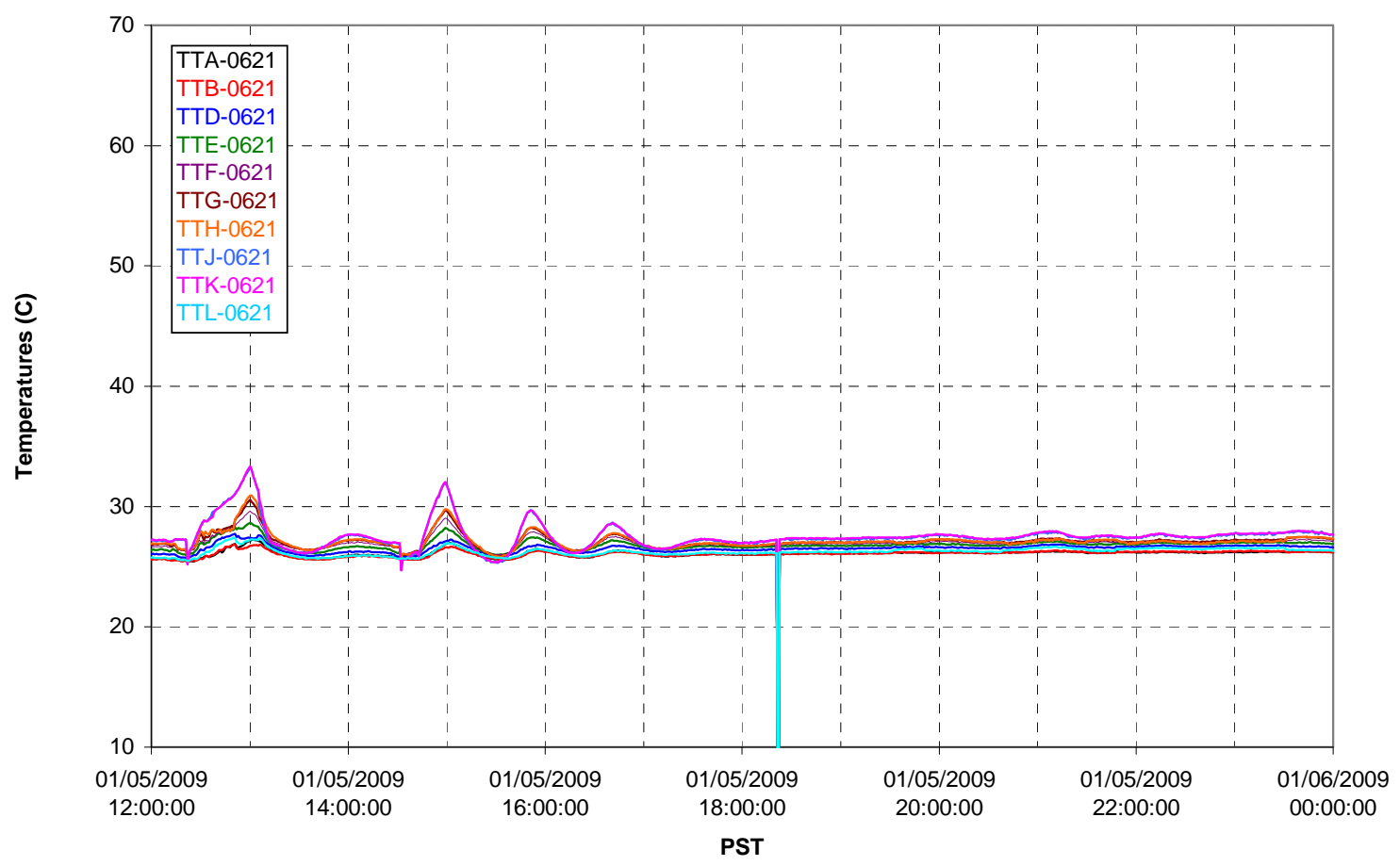


T02A temperatures

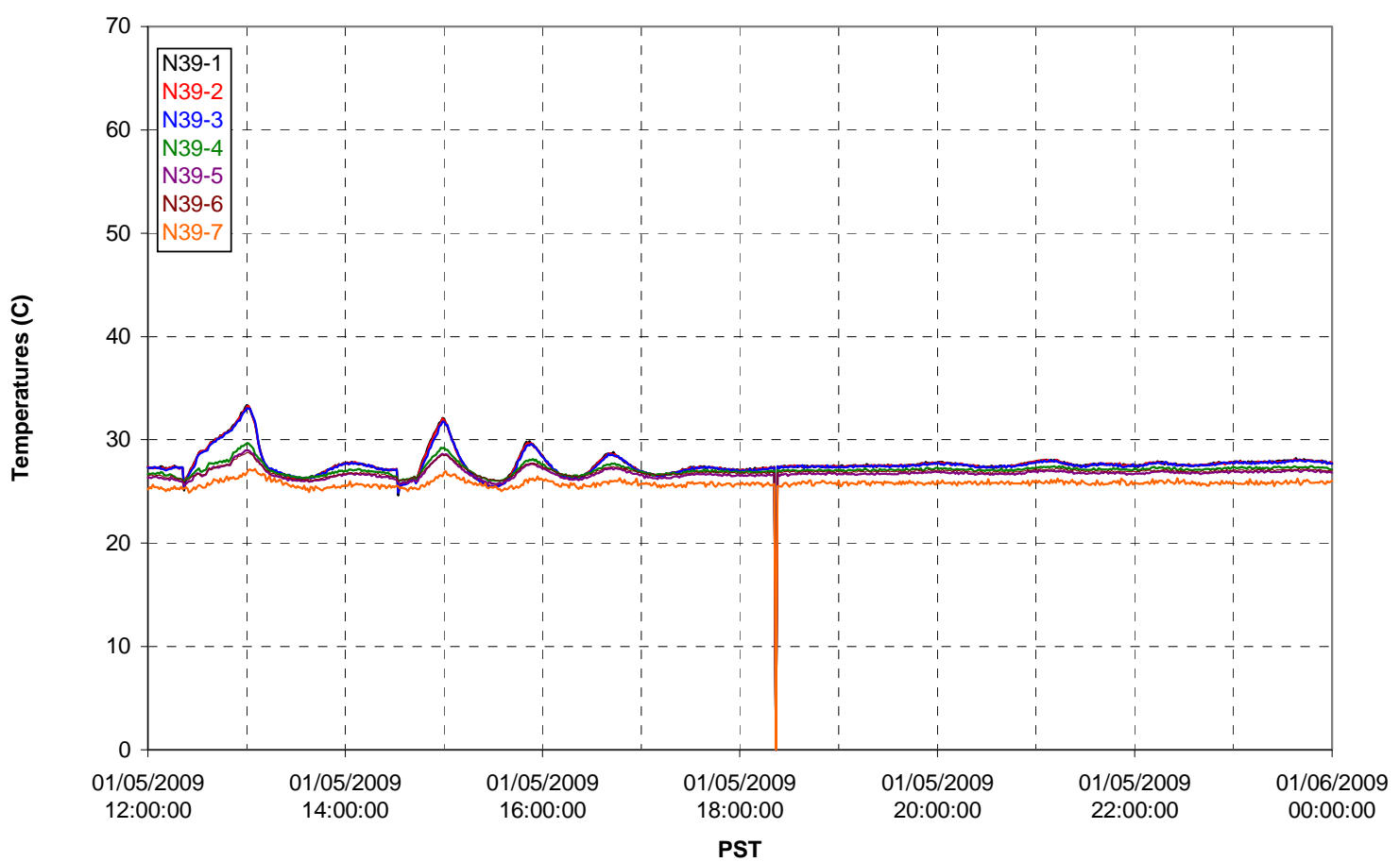

T02A temperatures

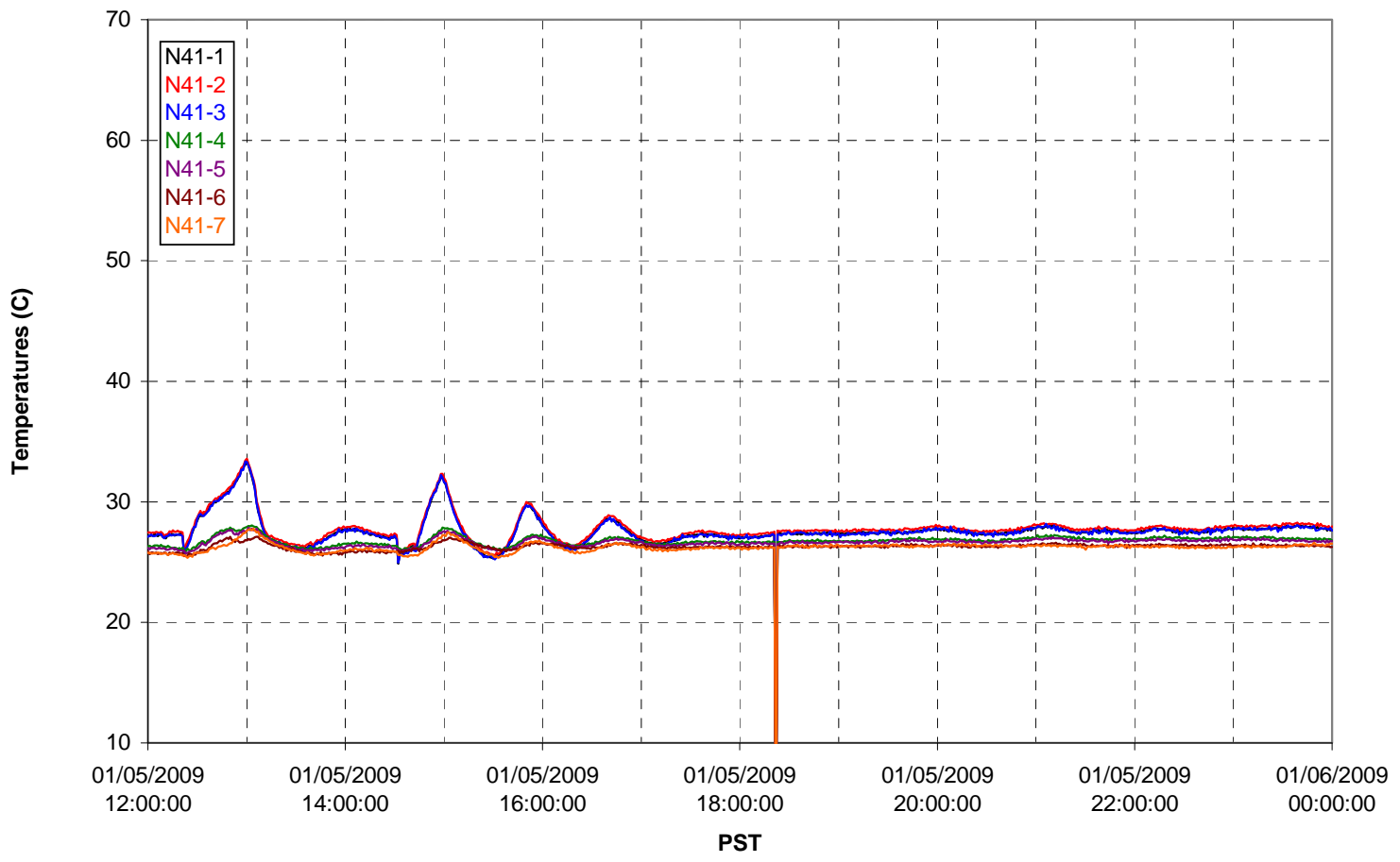


T02A temperatures

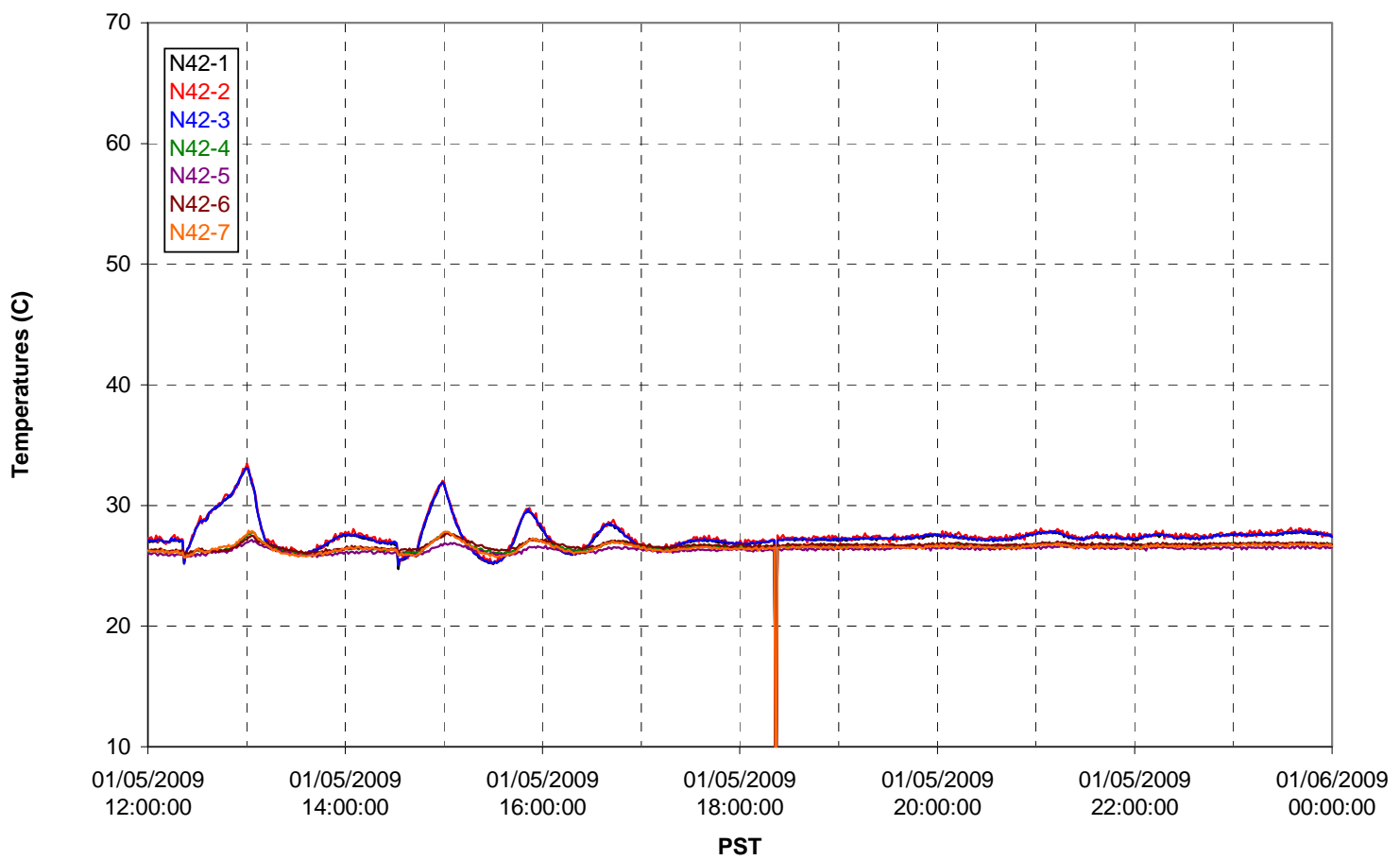

T02A temperatures

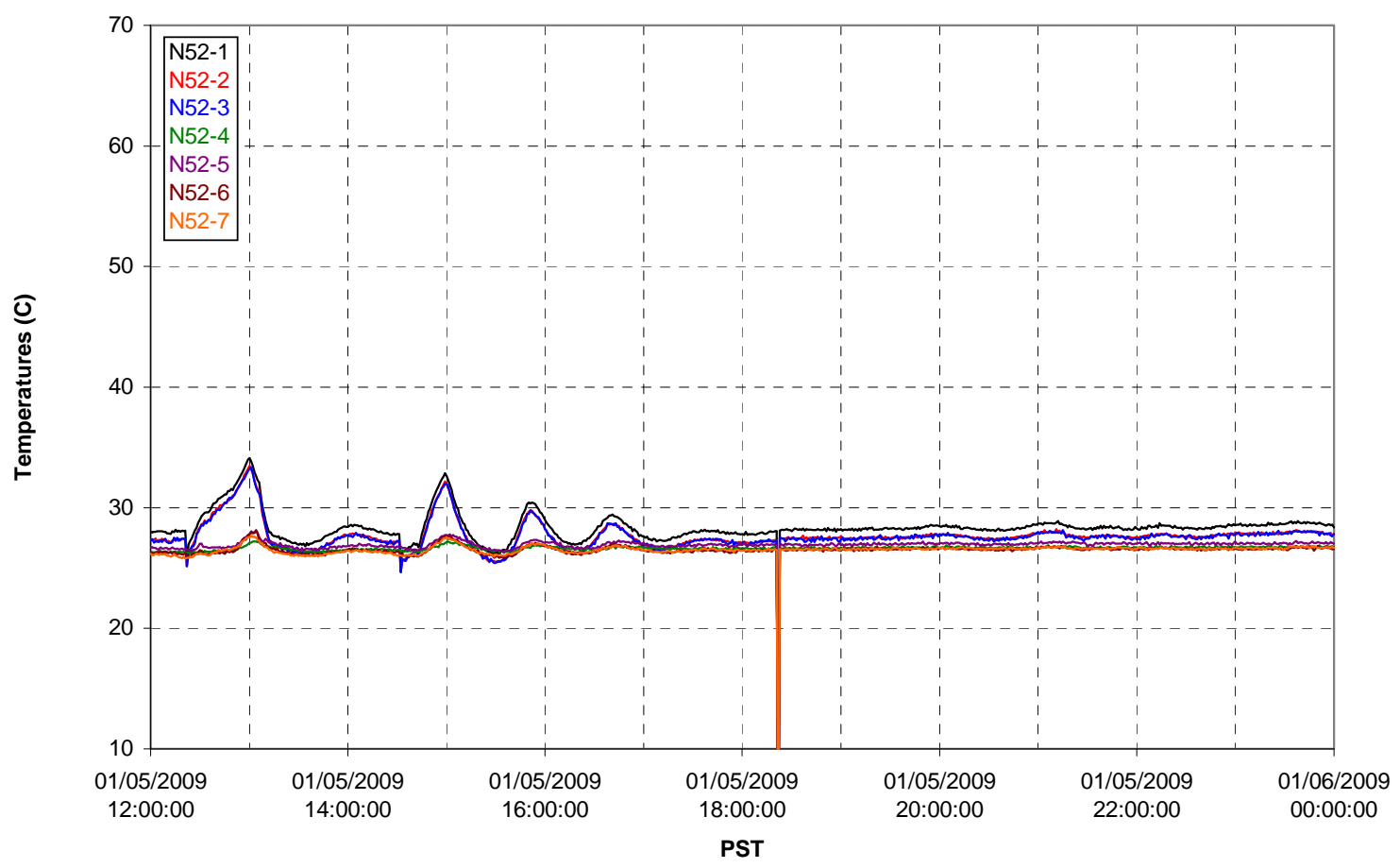


T02A Heating and Cooling

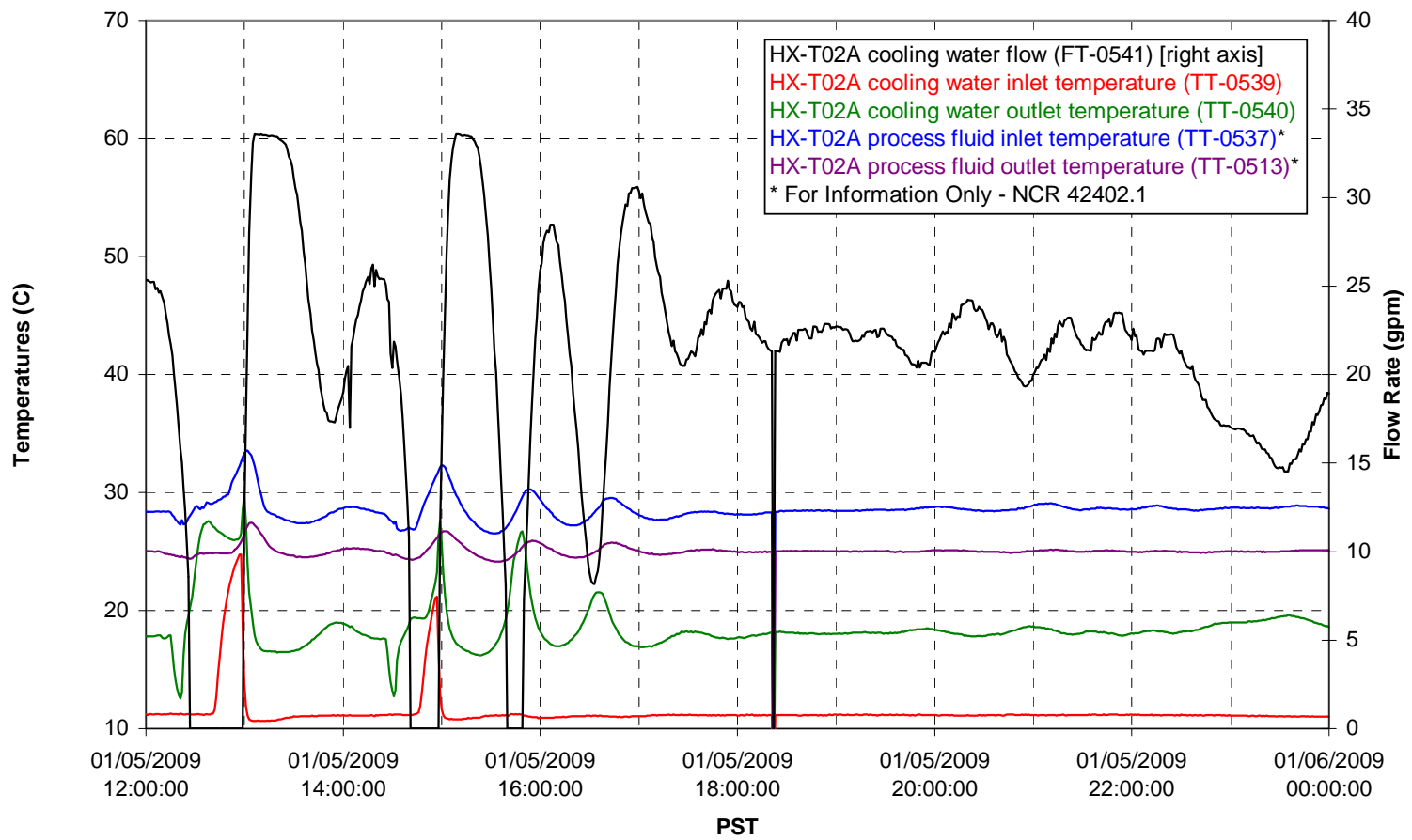

Pump Operation

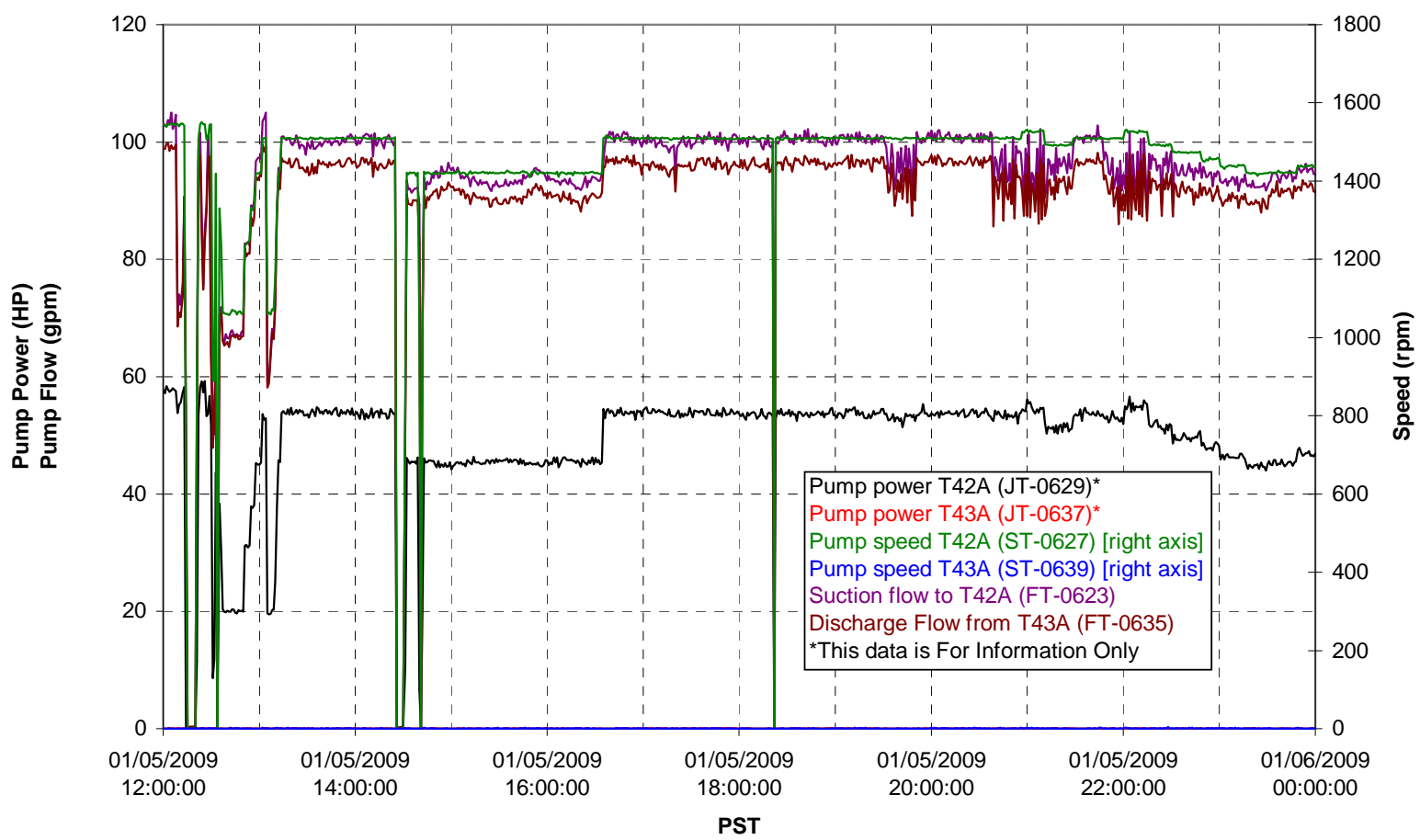


Pulsepot UFP-PP-T01A

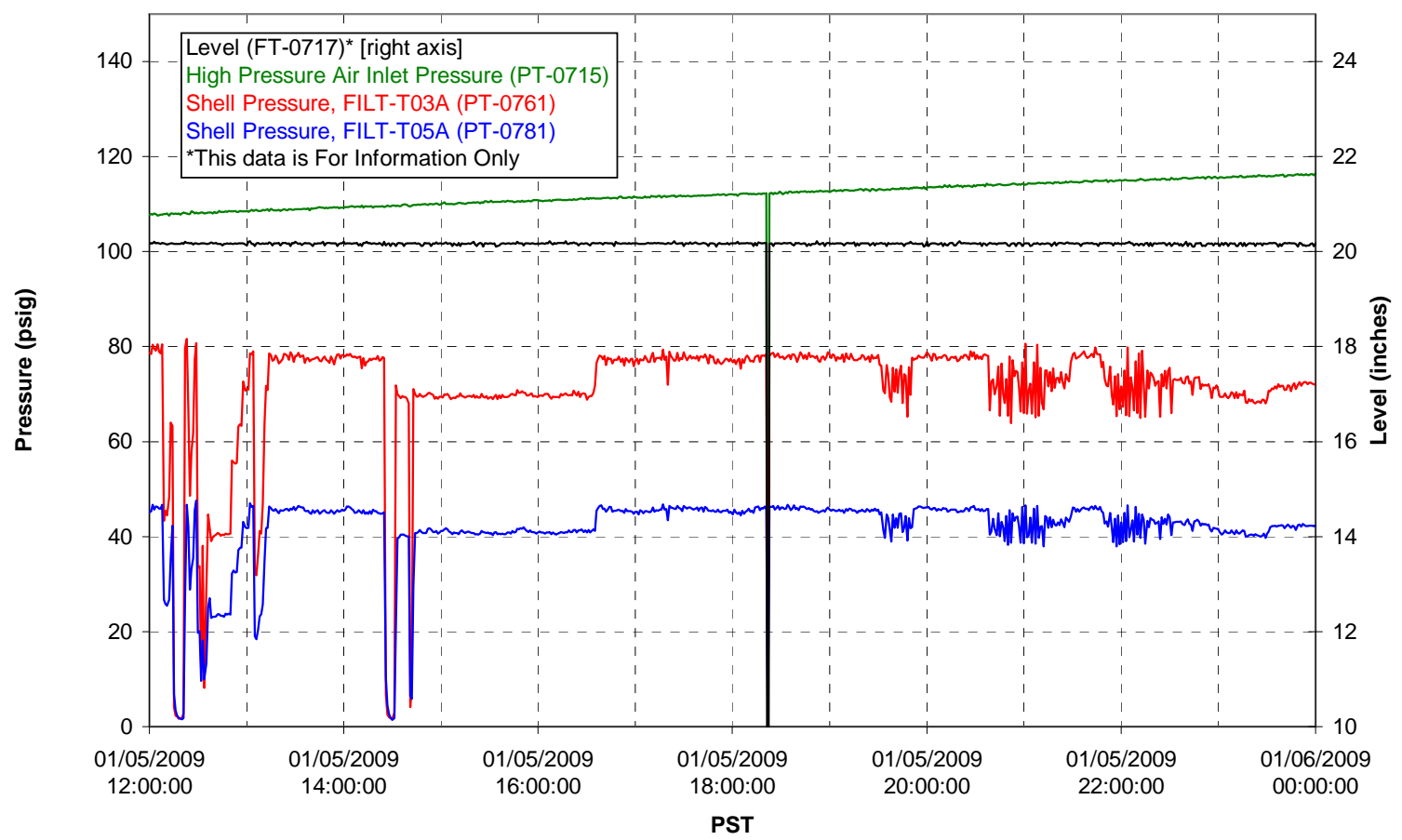

Pulsepot UFP-PP-T02A

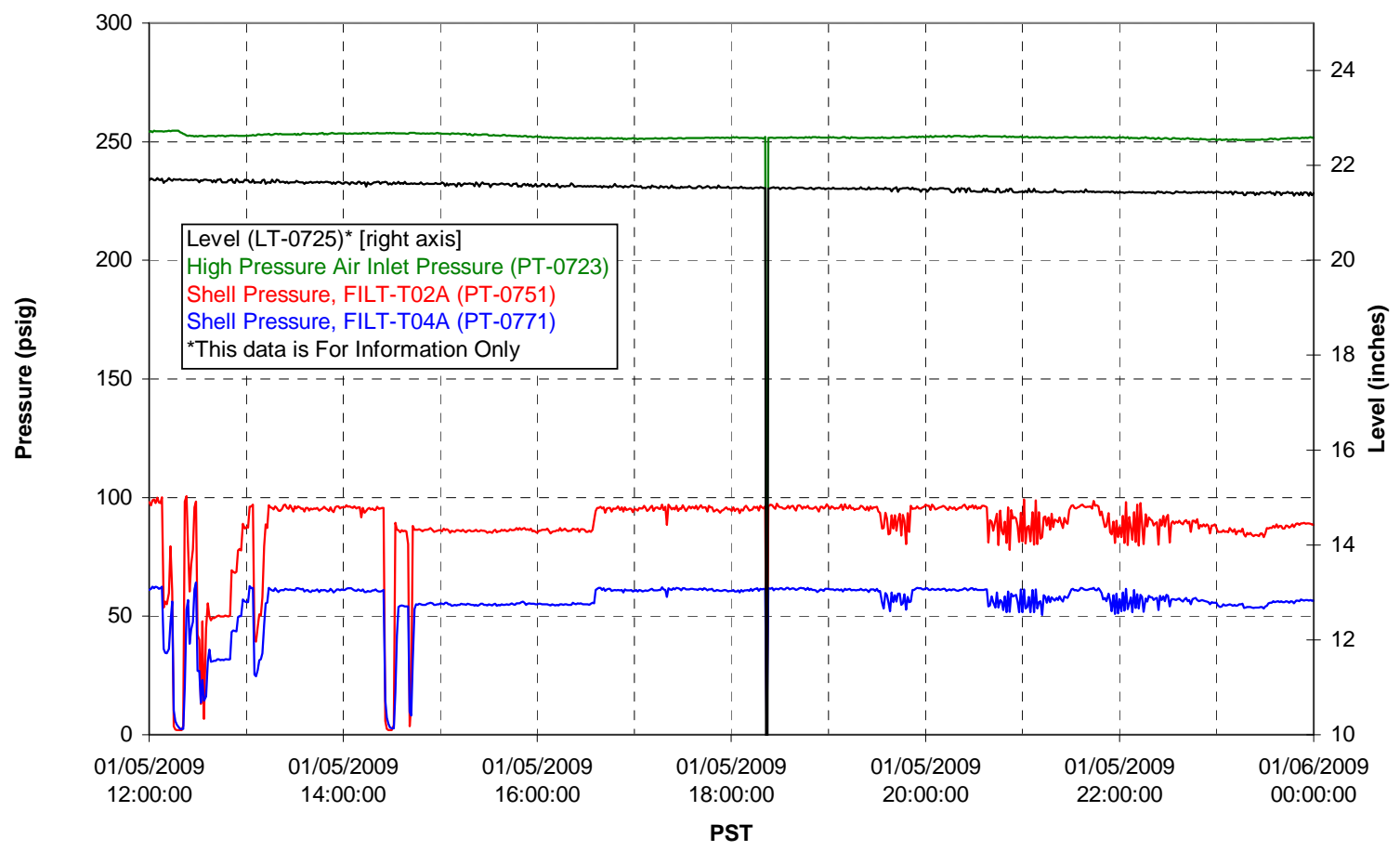


Pulsepot UFP-PP-T03A

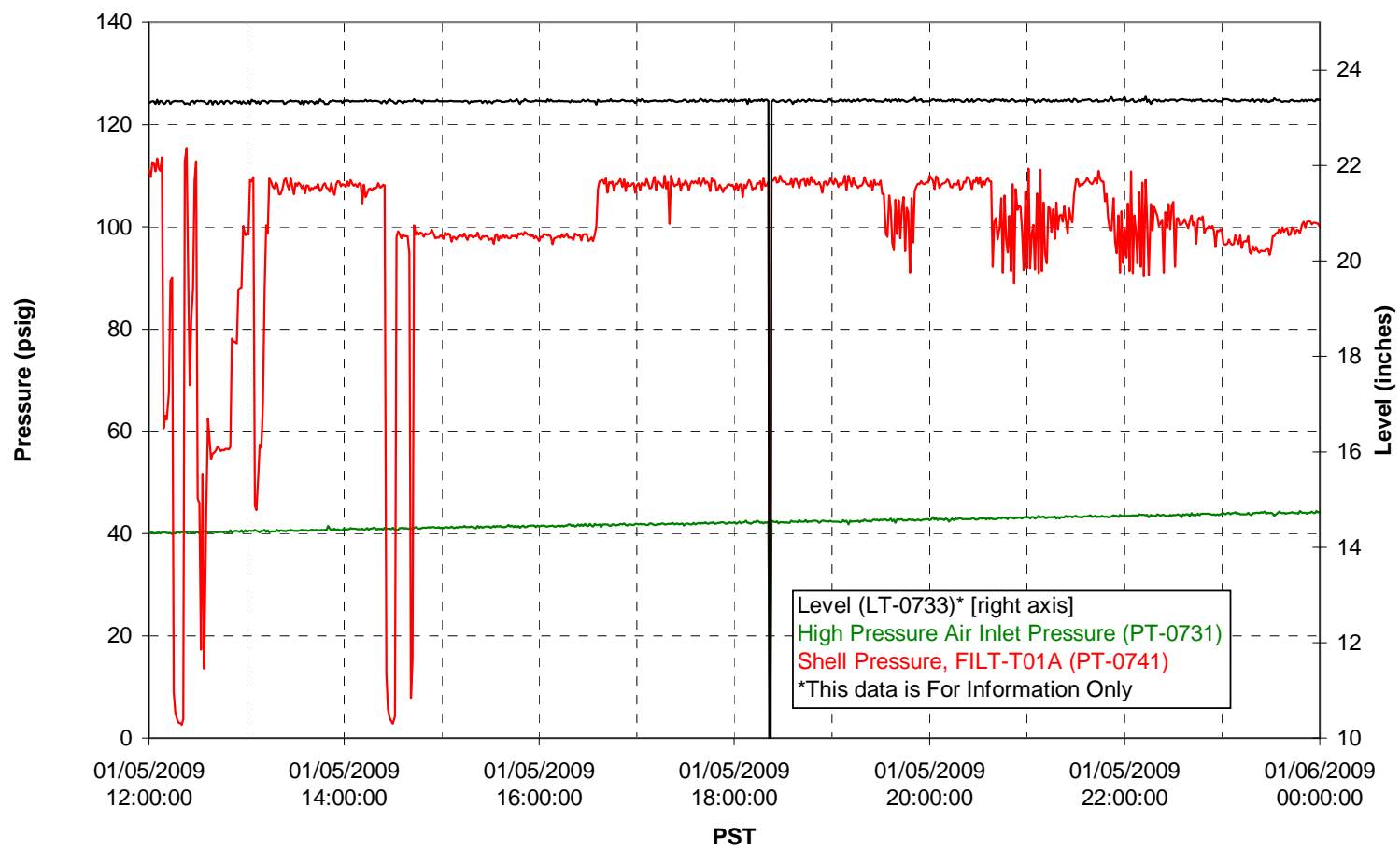

Pulsepot Levels

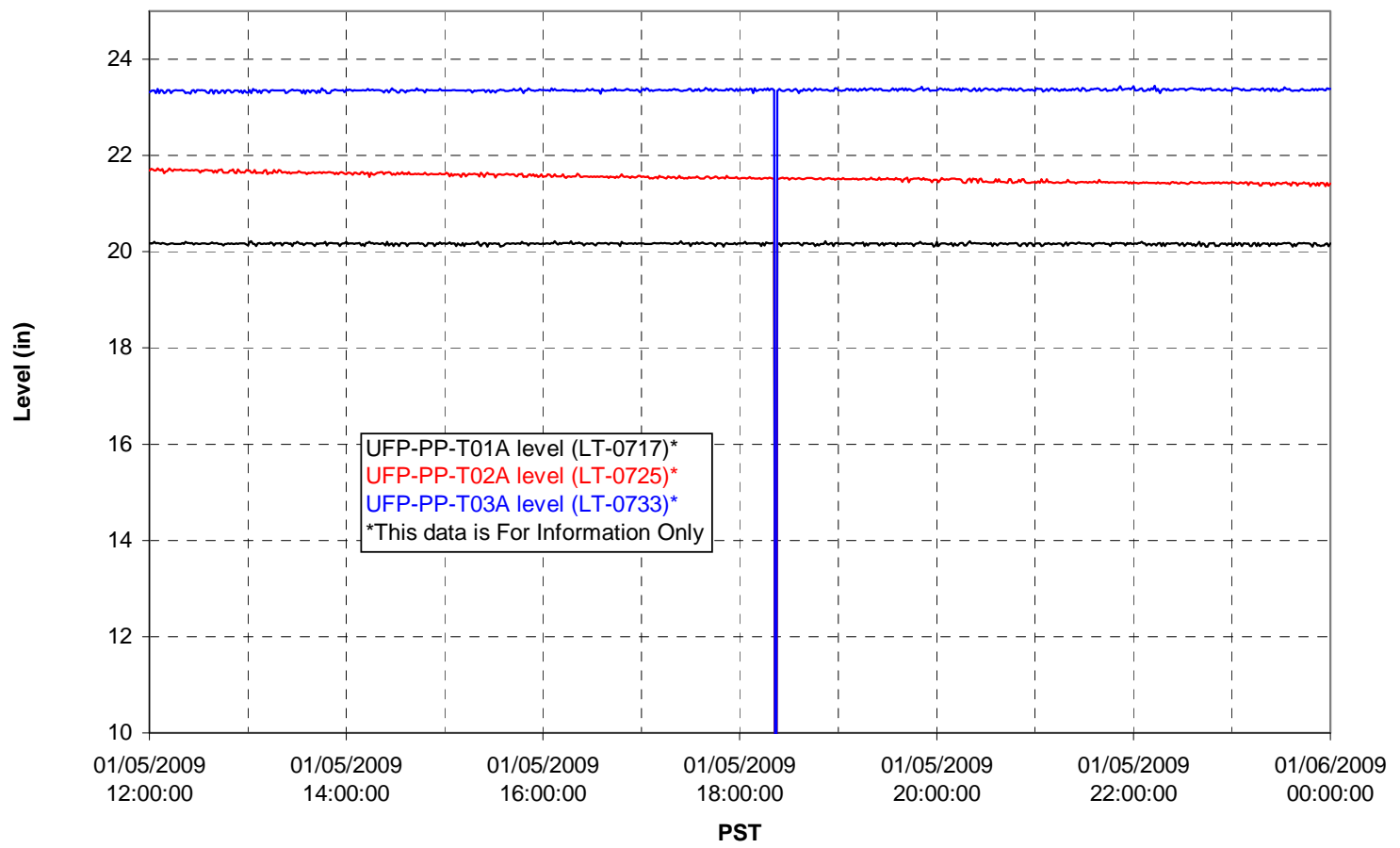


Filter UFP-FILT-T01A

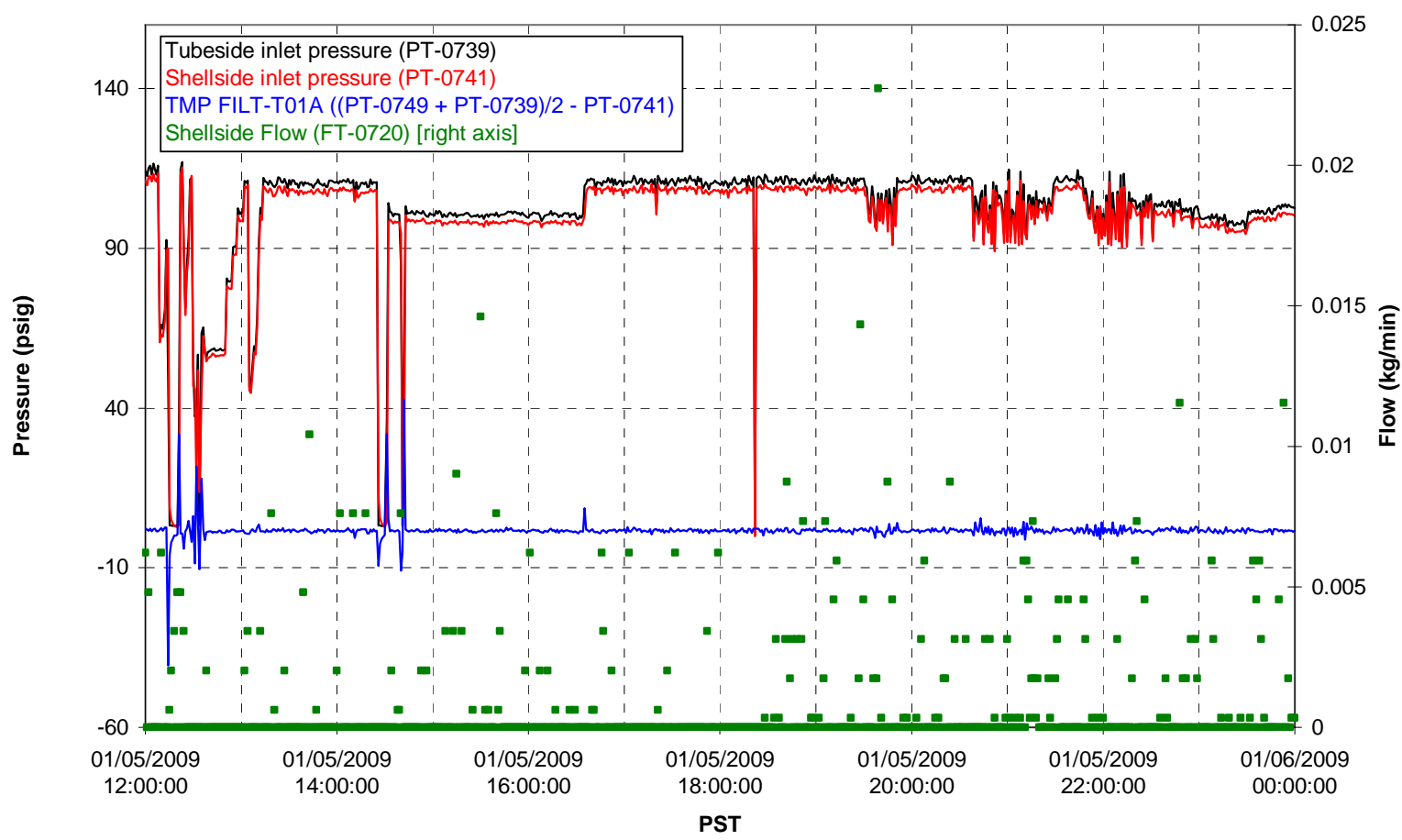

Filter UFP-FILT-T02A

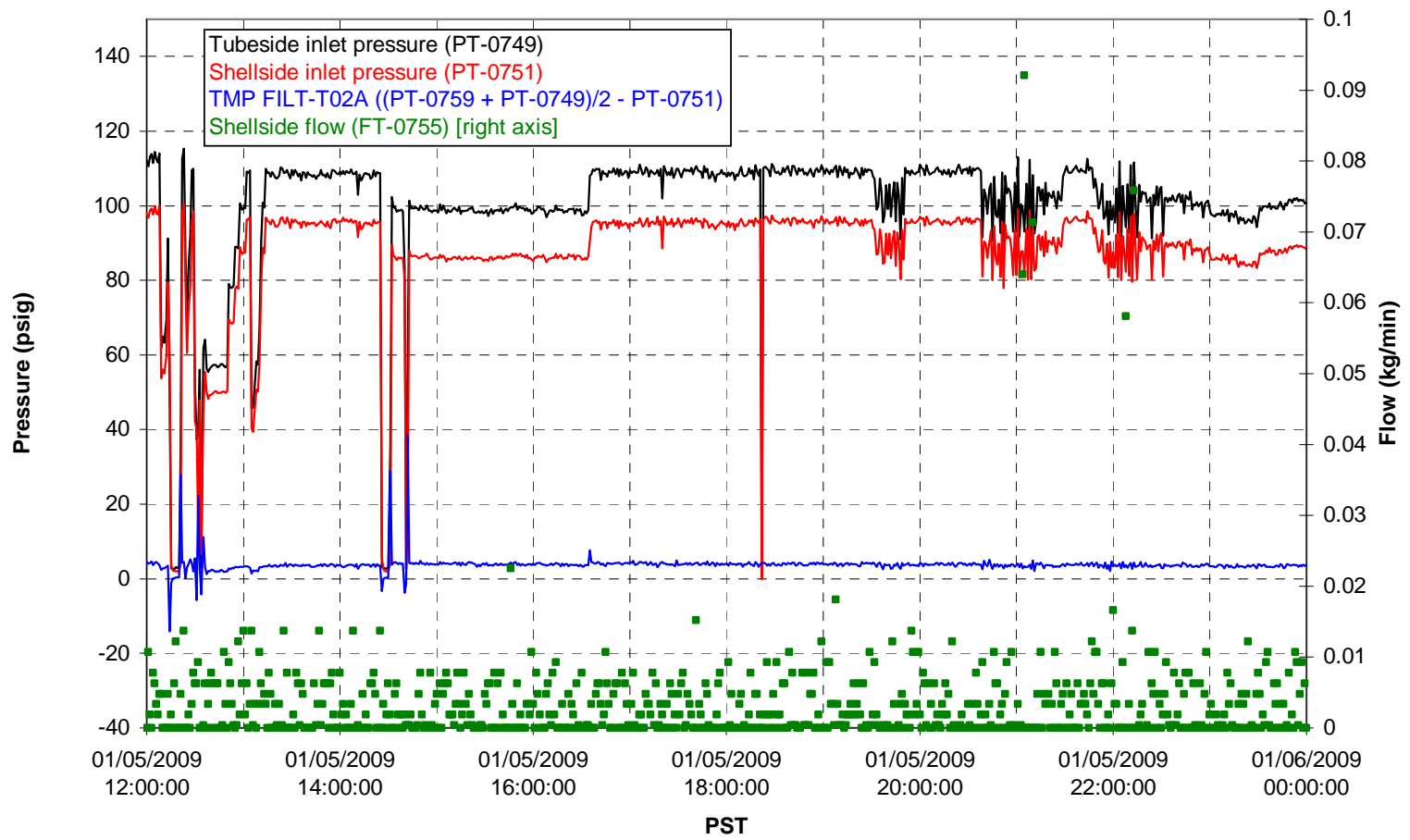


Filter UFP-FILT-T03A

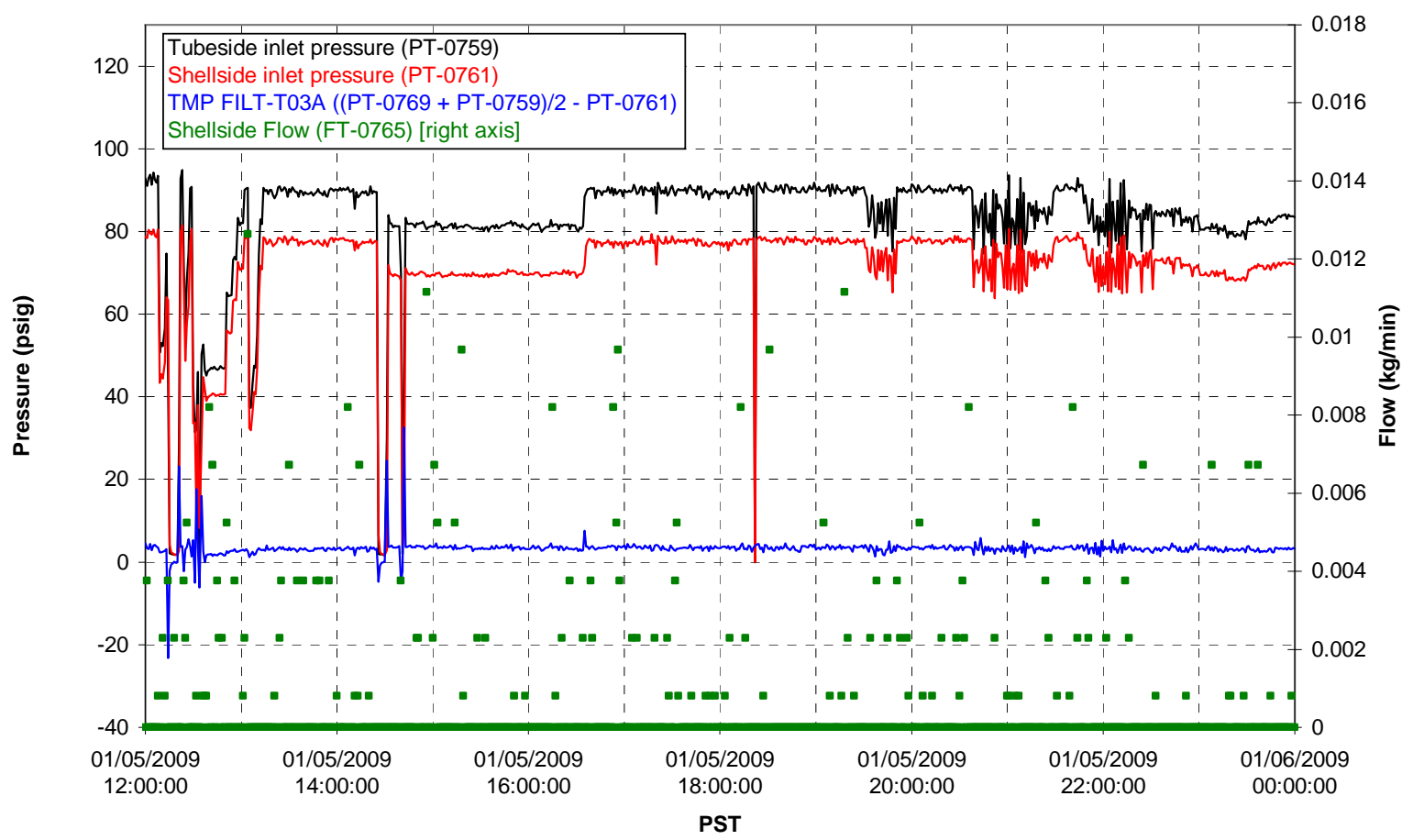

Filter UFP-FILT-T04A

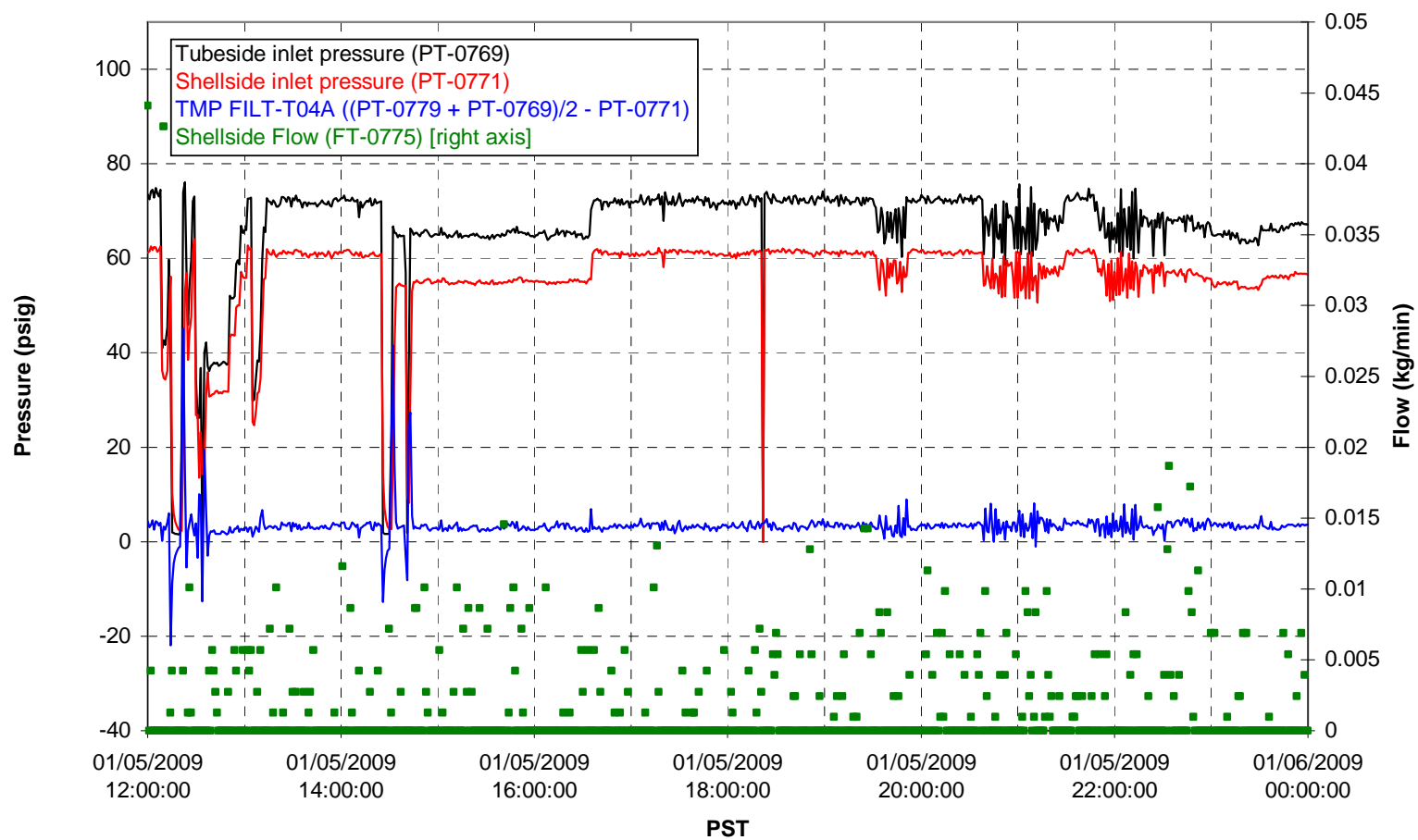


Filter UFP-FILT-T05A

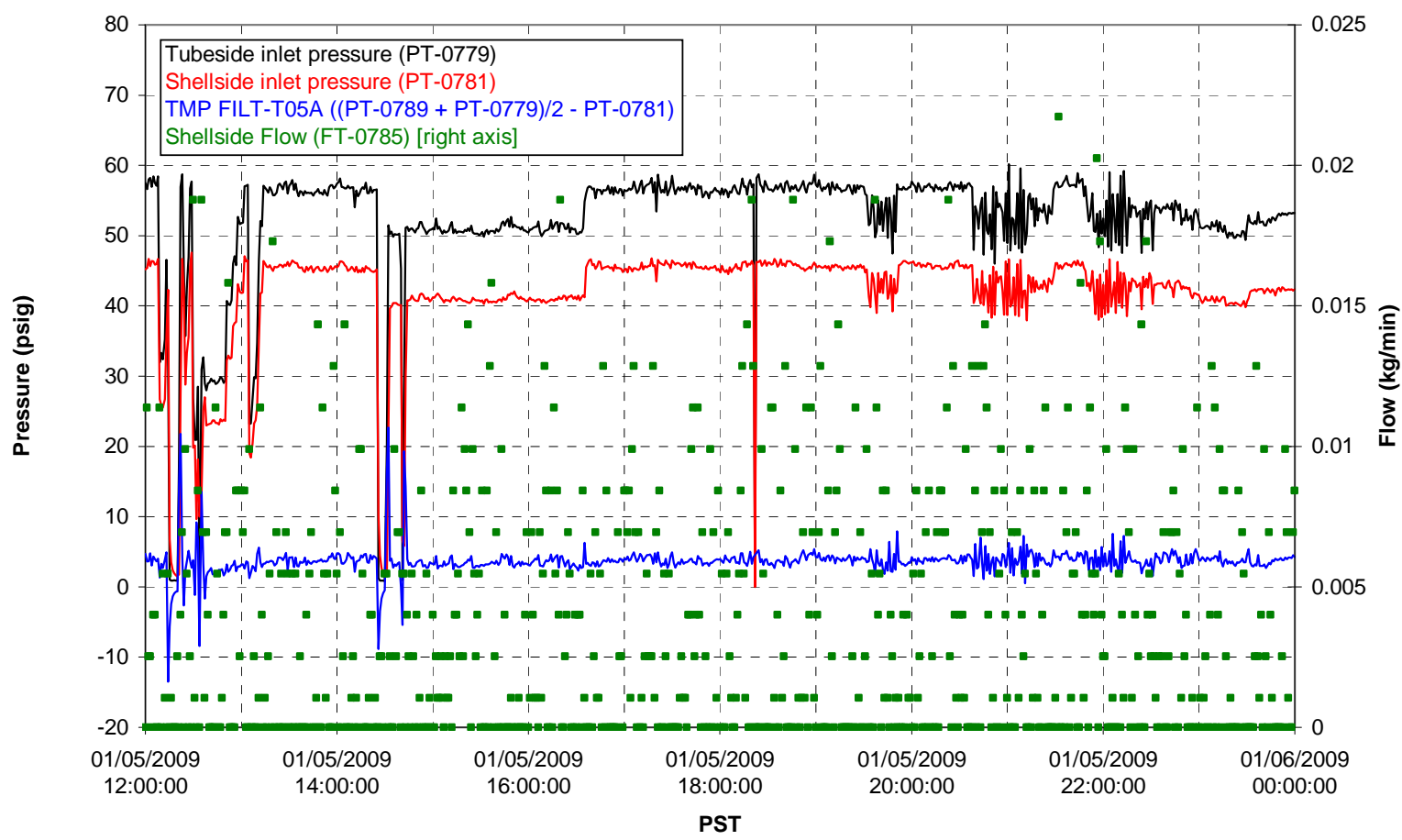

Chemical Flow

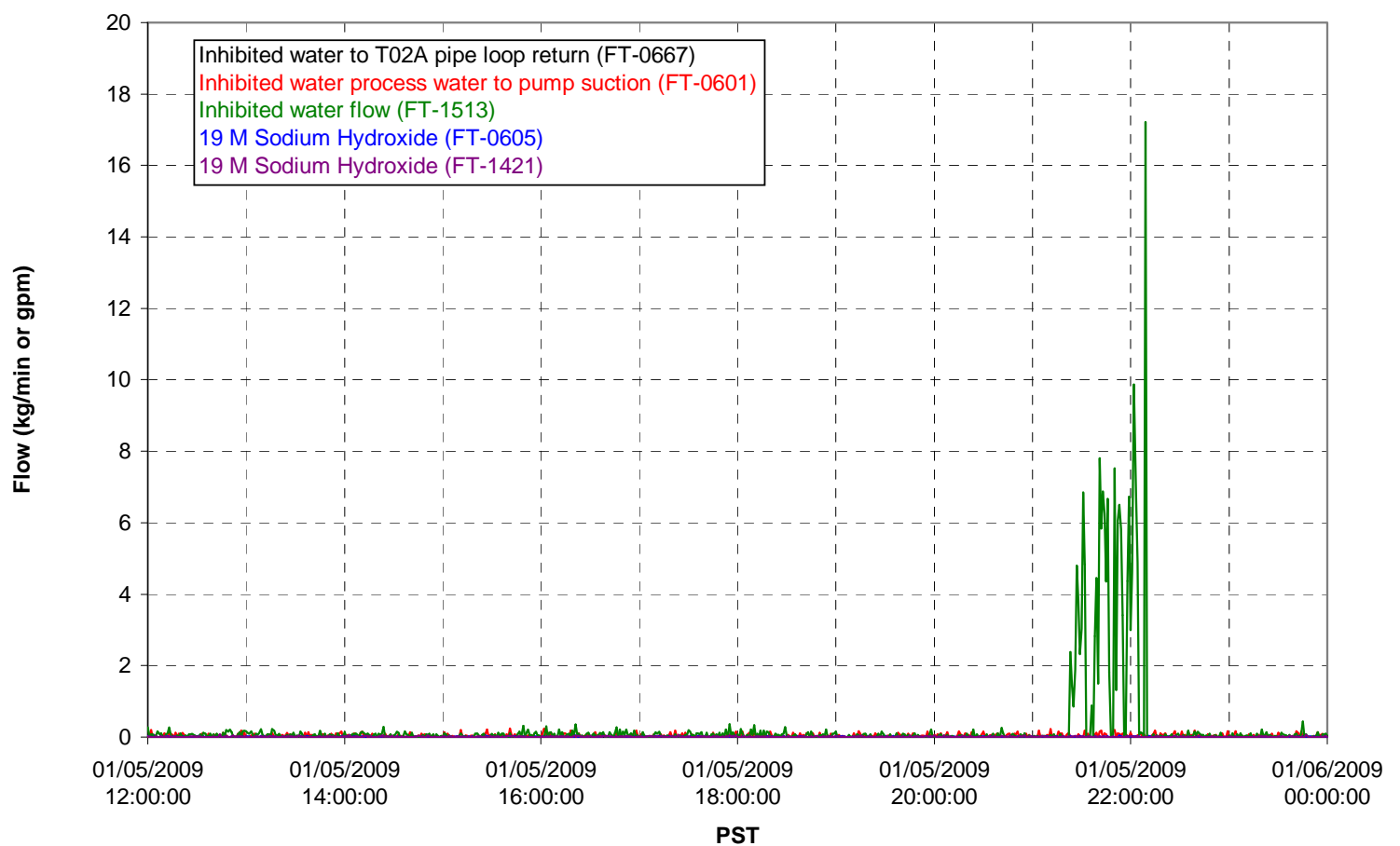


Chemical Flow

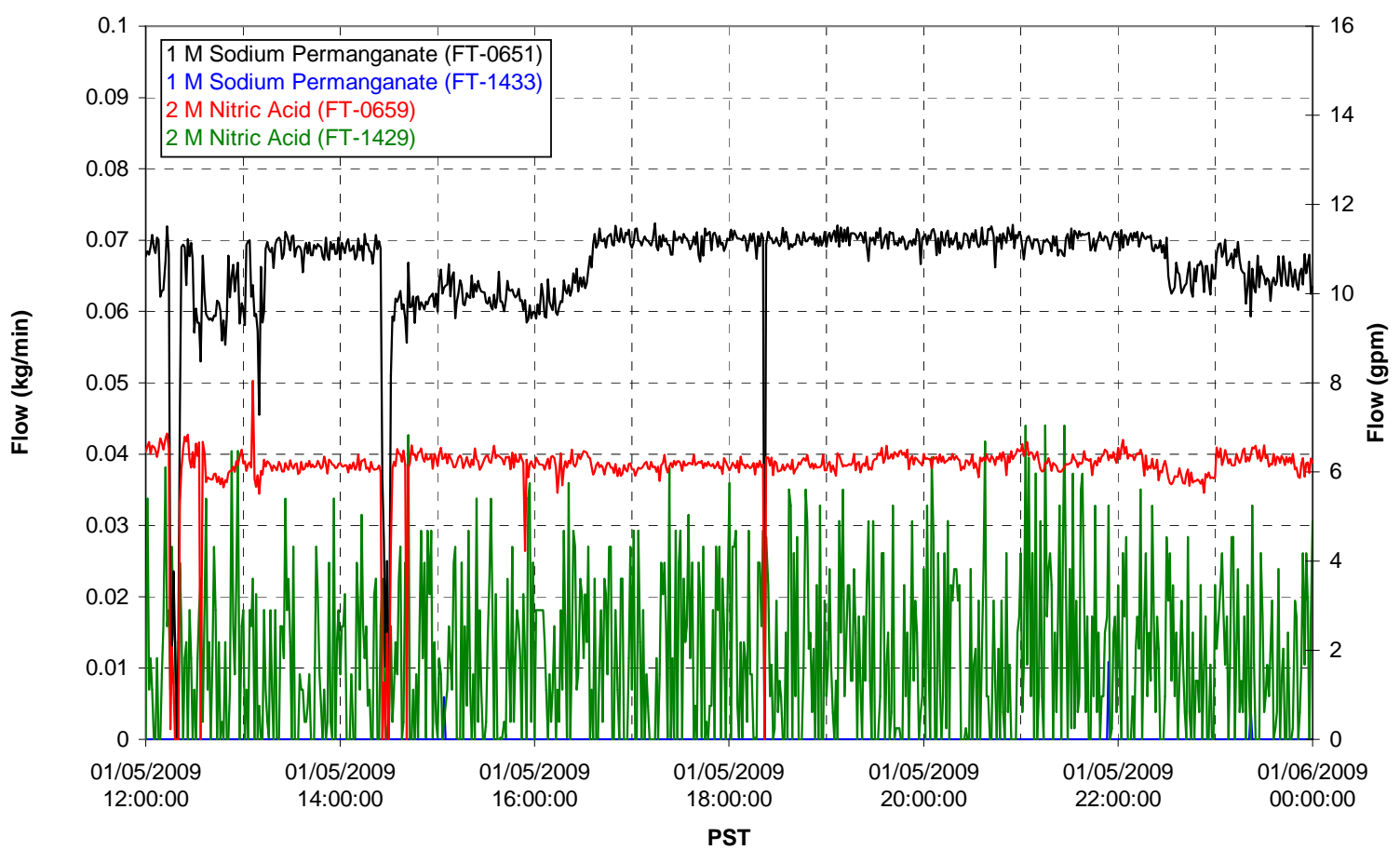

Air Flows

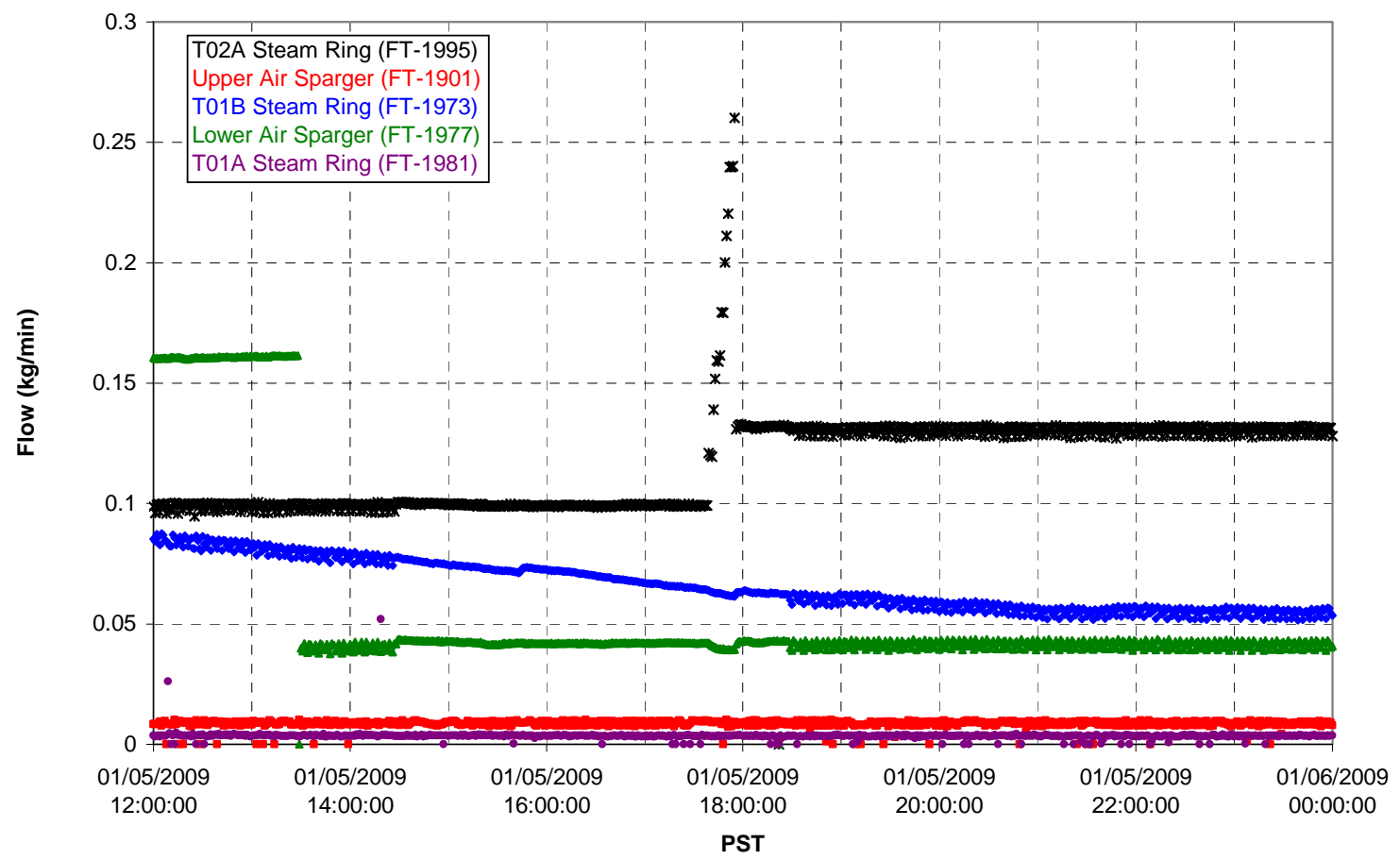


T02A Steam

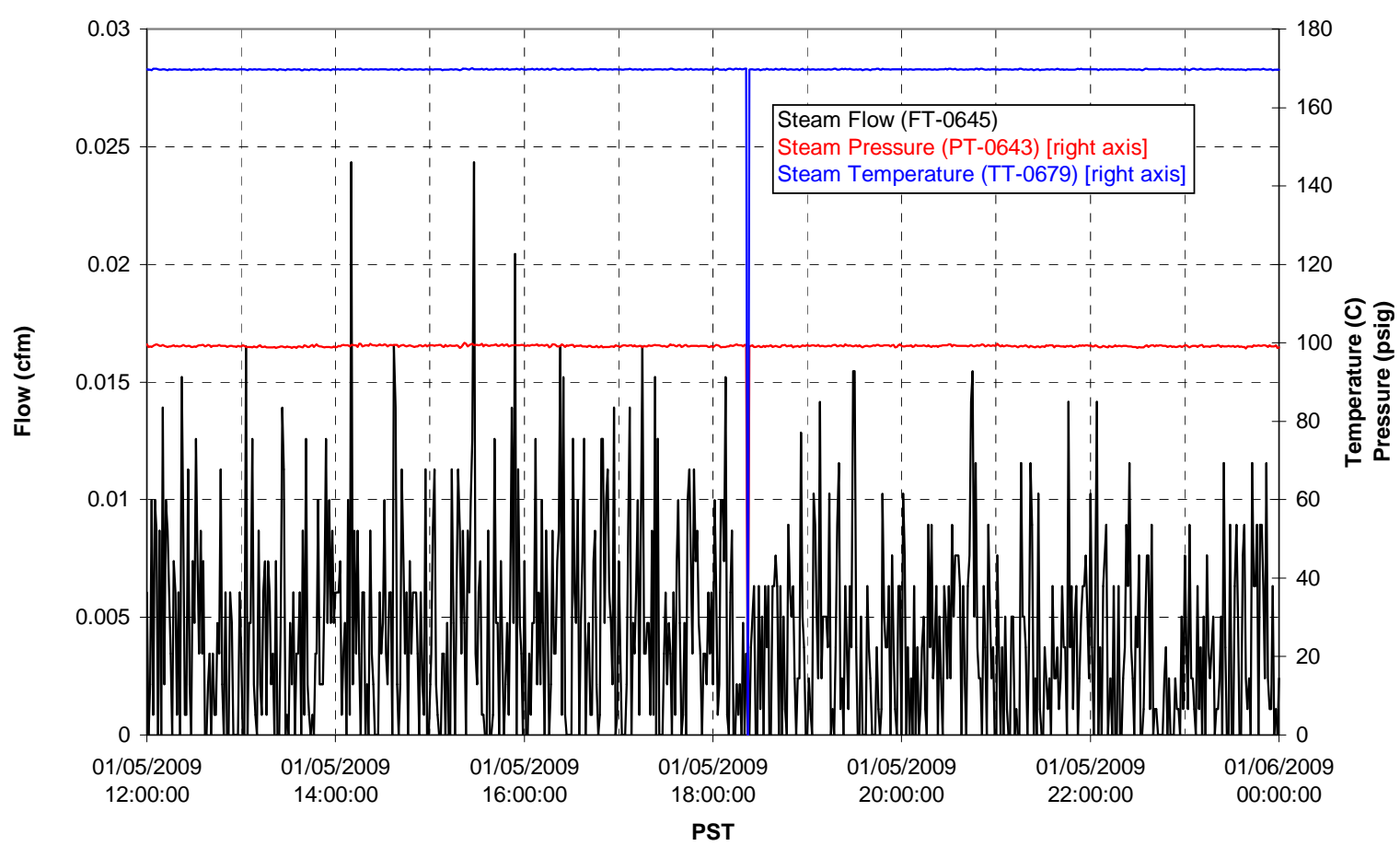

T01A Steam

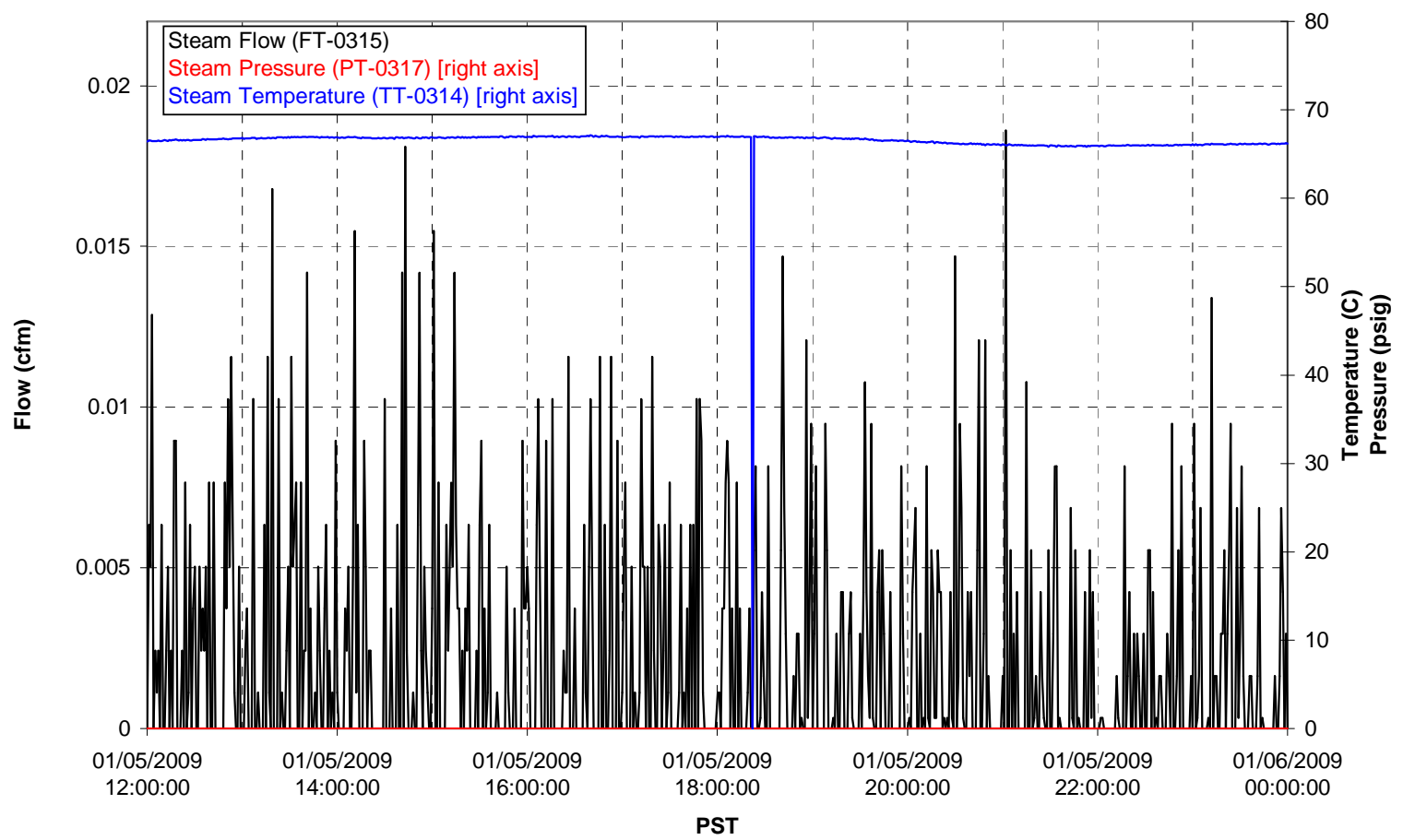


T01B Steam

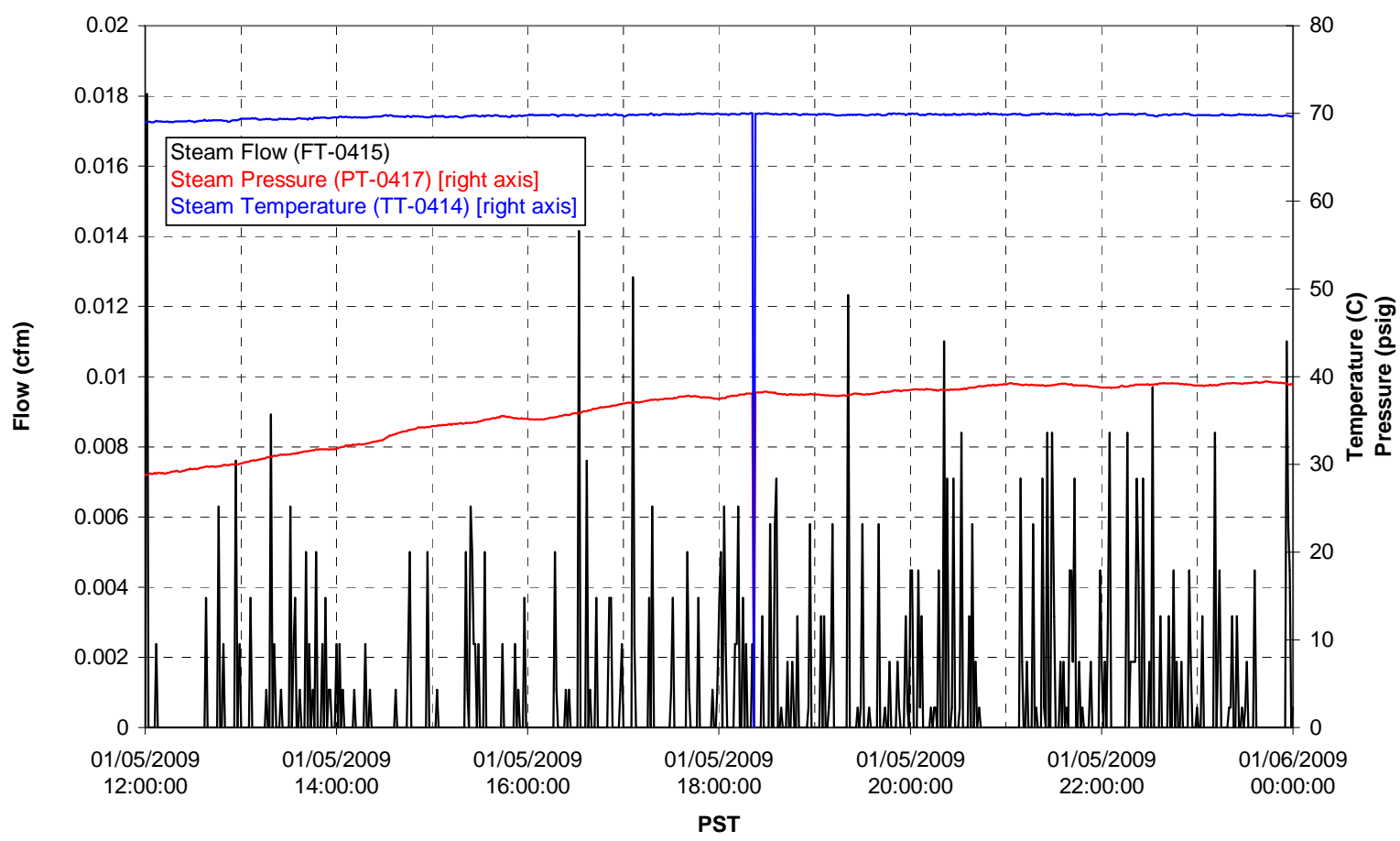


Functional Test Data Plots

01/06/2009 00:00 - 01/06/2009 12:00 
T01A level

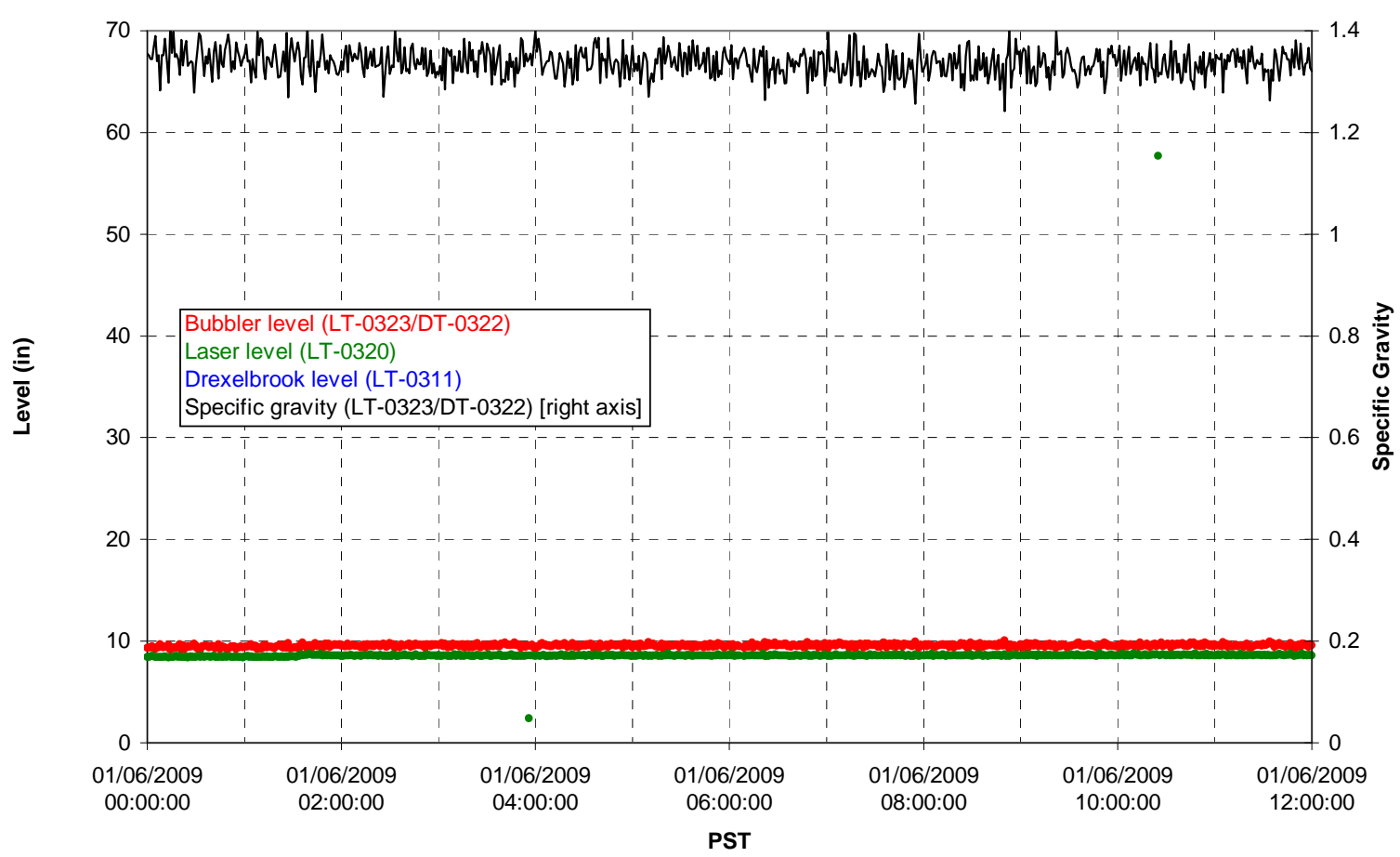

T01A temperatures

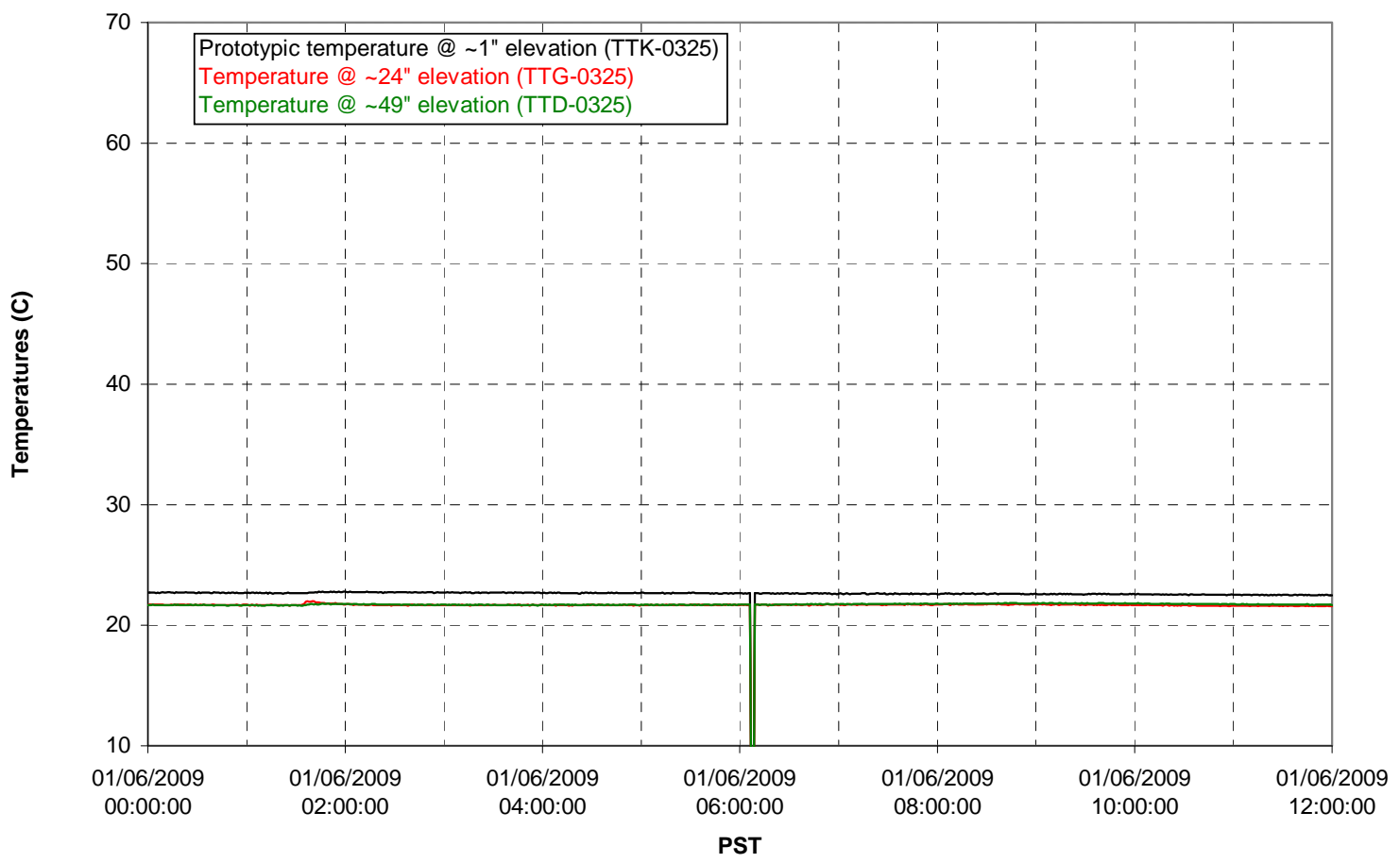


T01B level

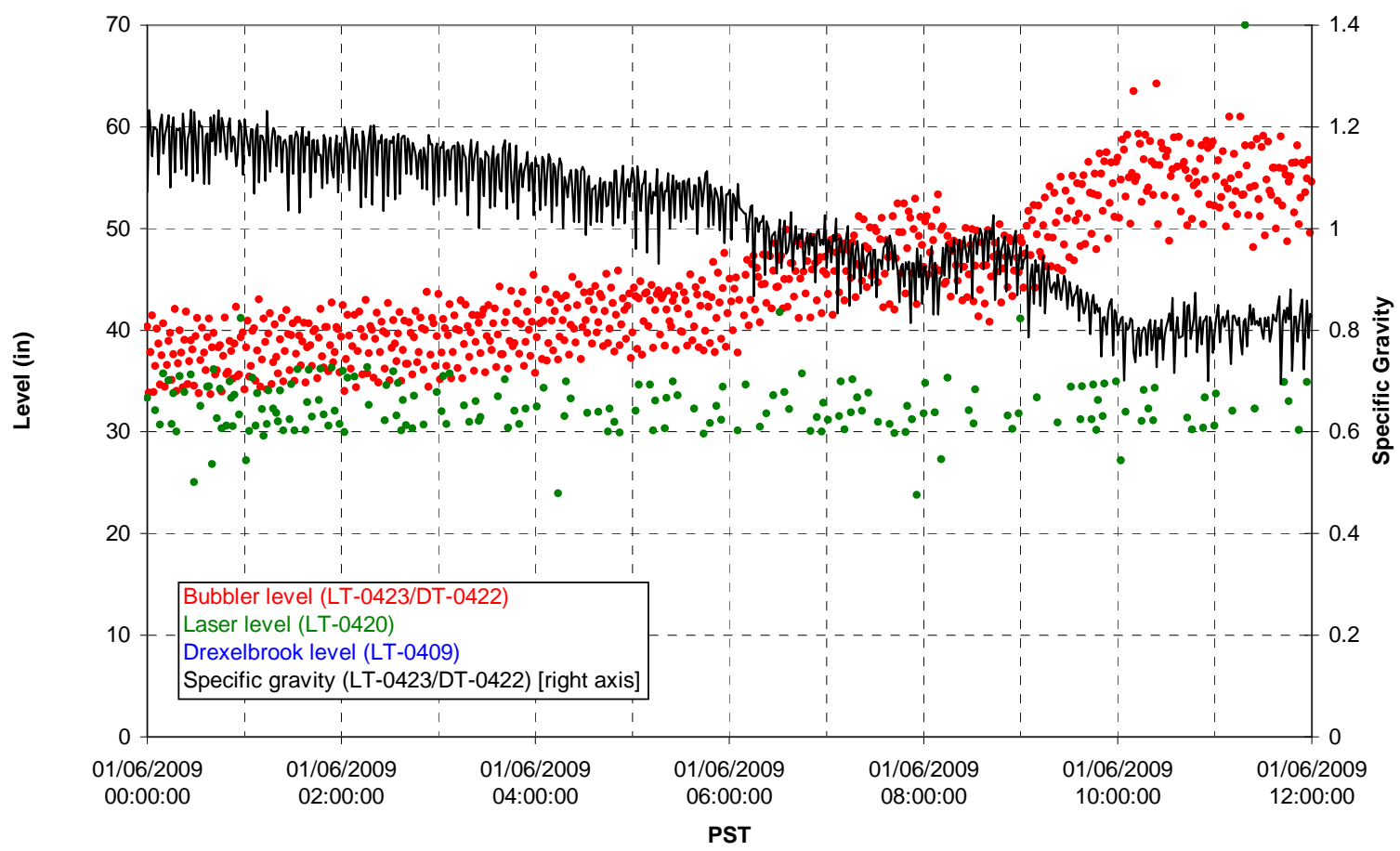

T01B temperatures

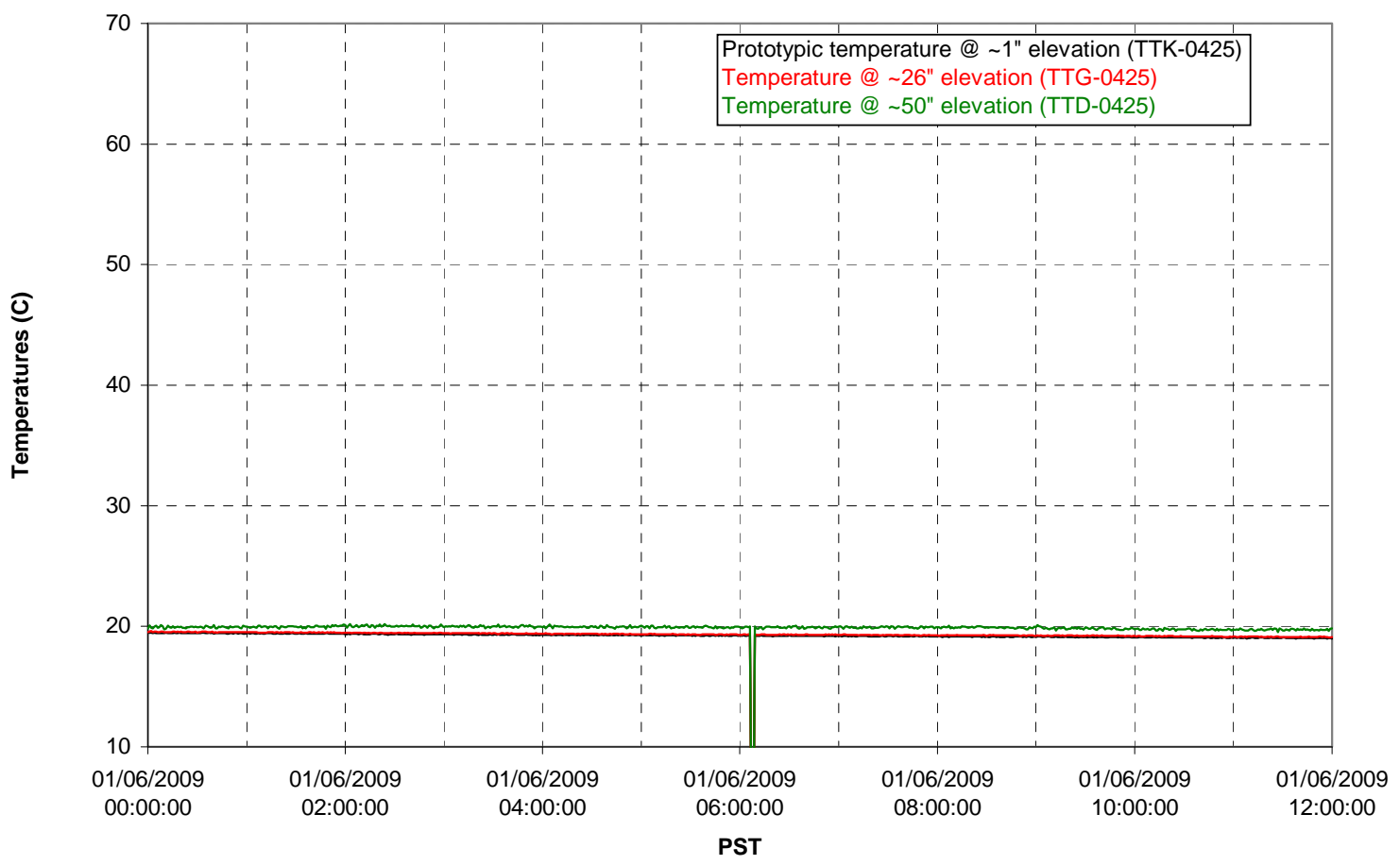


T02A level

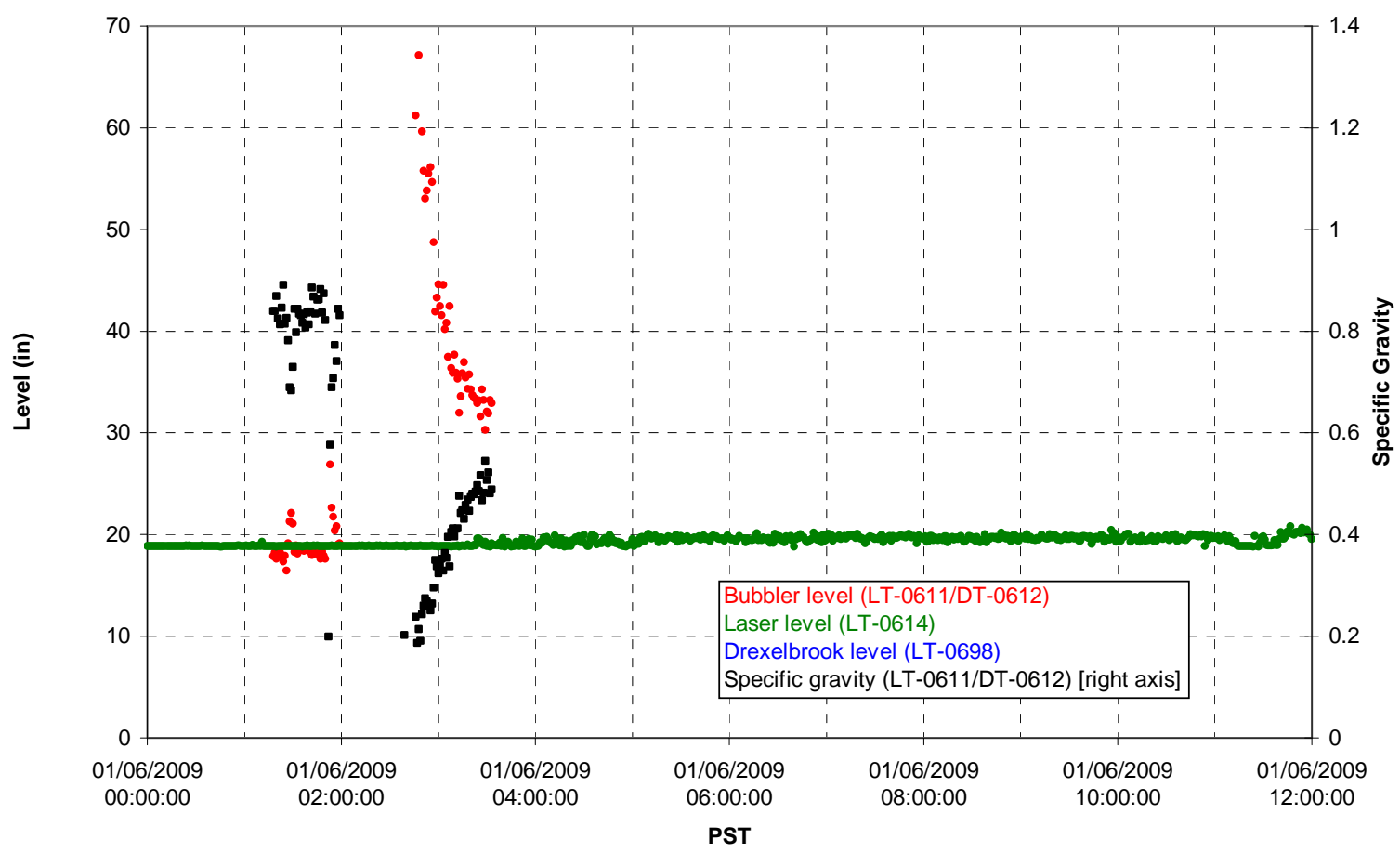

T02A temperatures

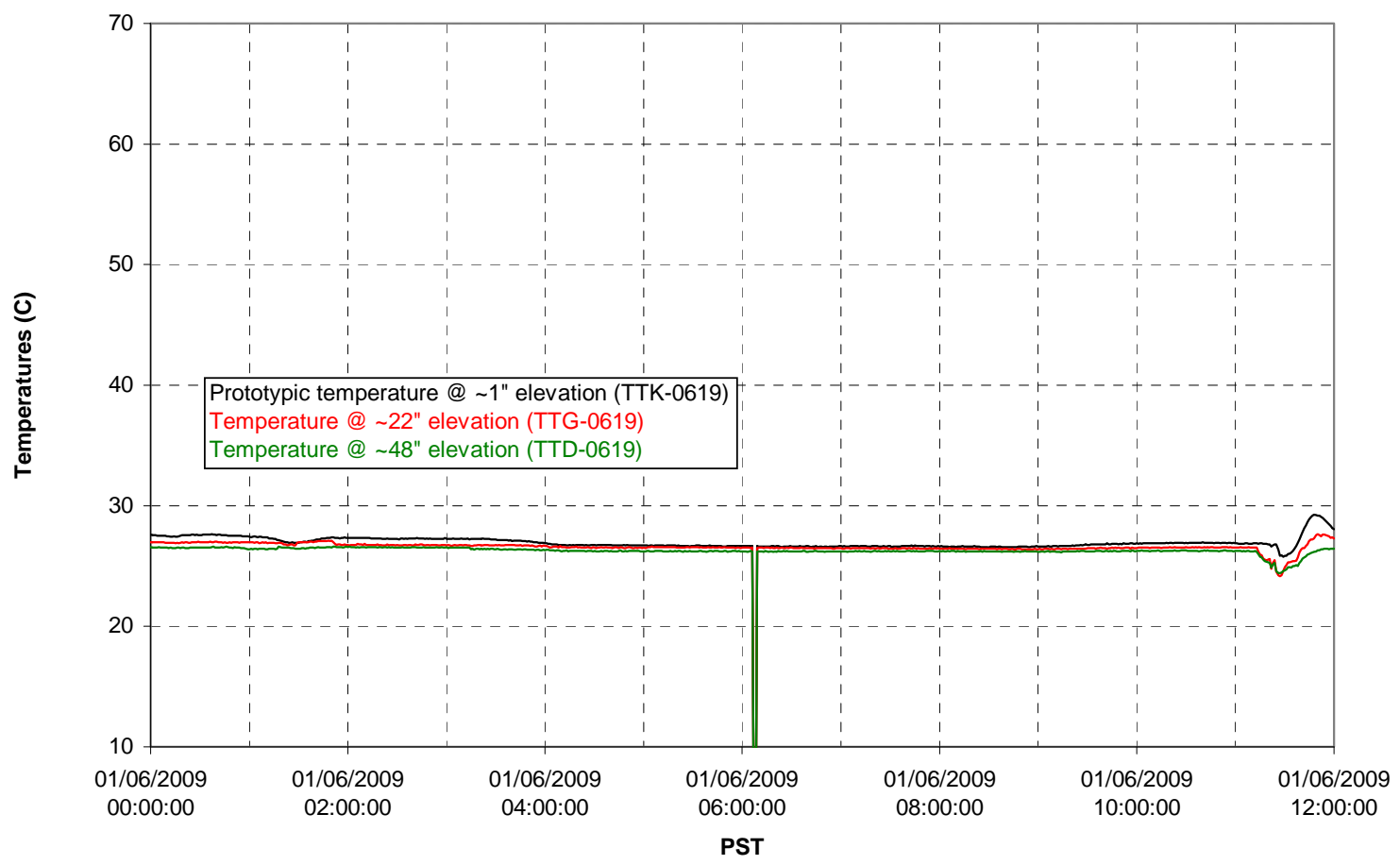


T02A and filter loop temperatures

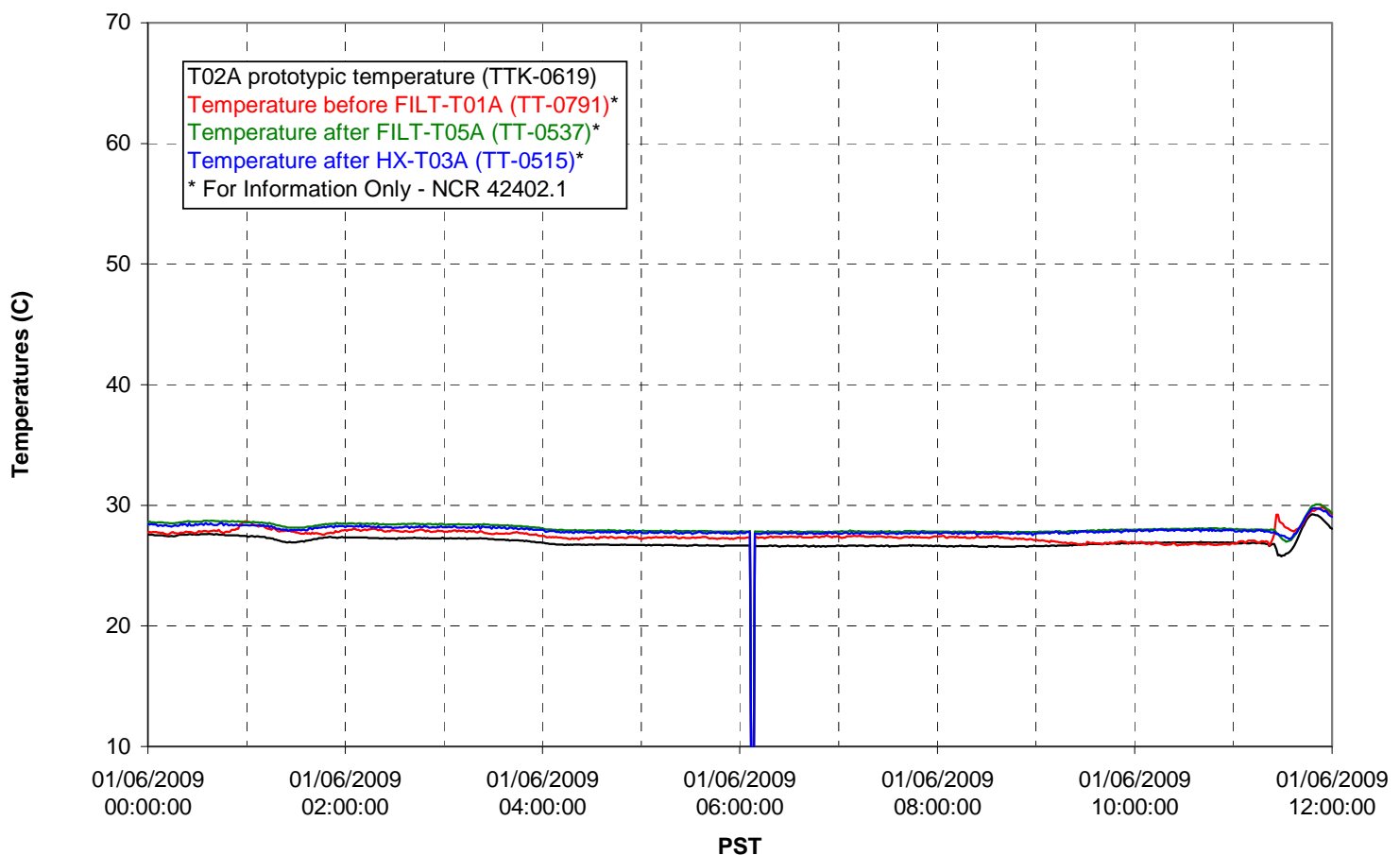

Pump Pressures and Flow

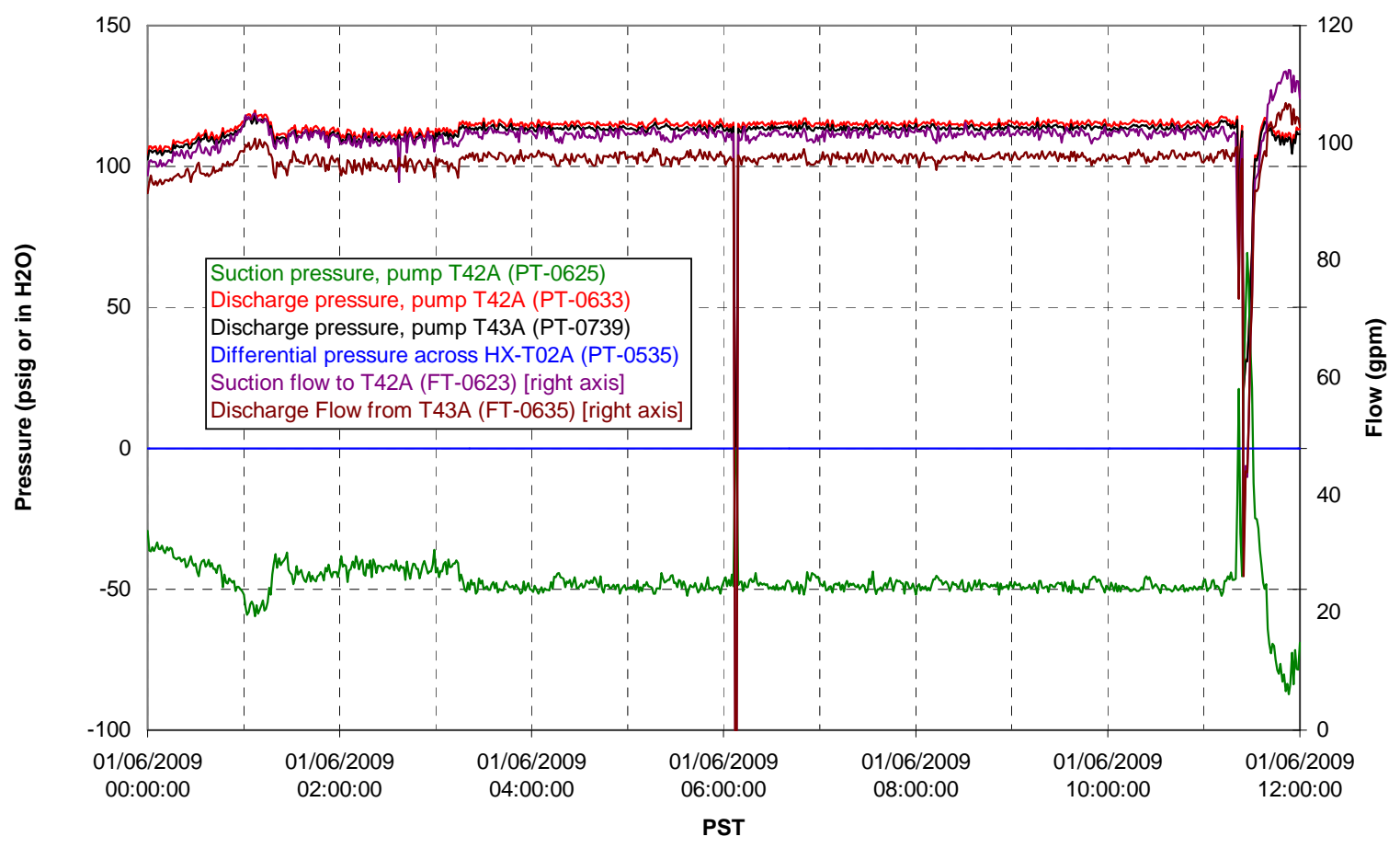


Axial pressure drop

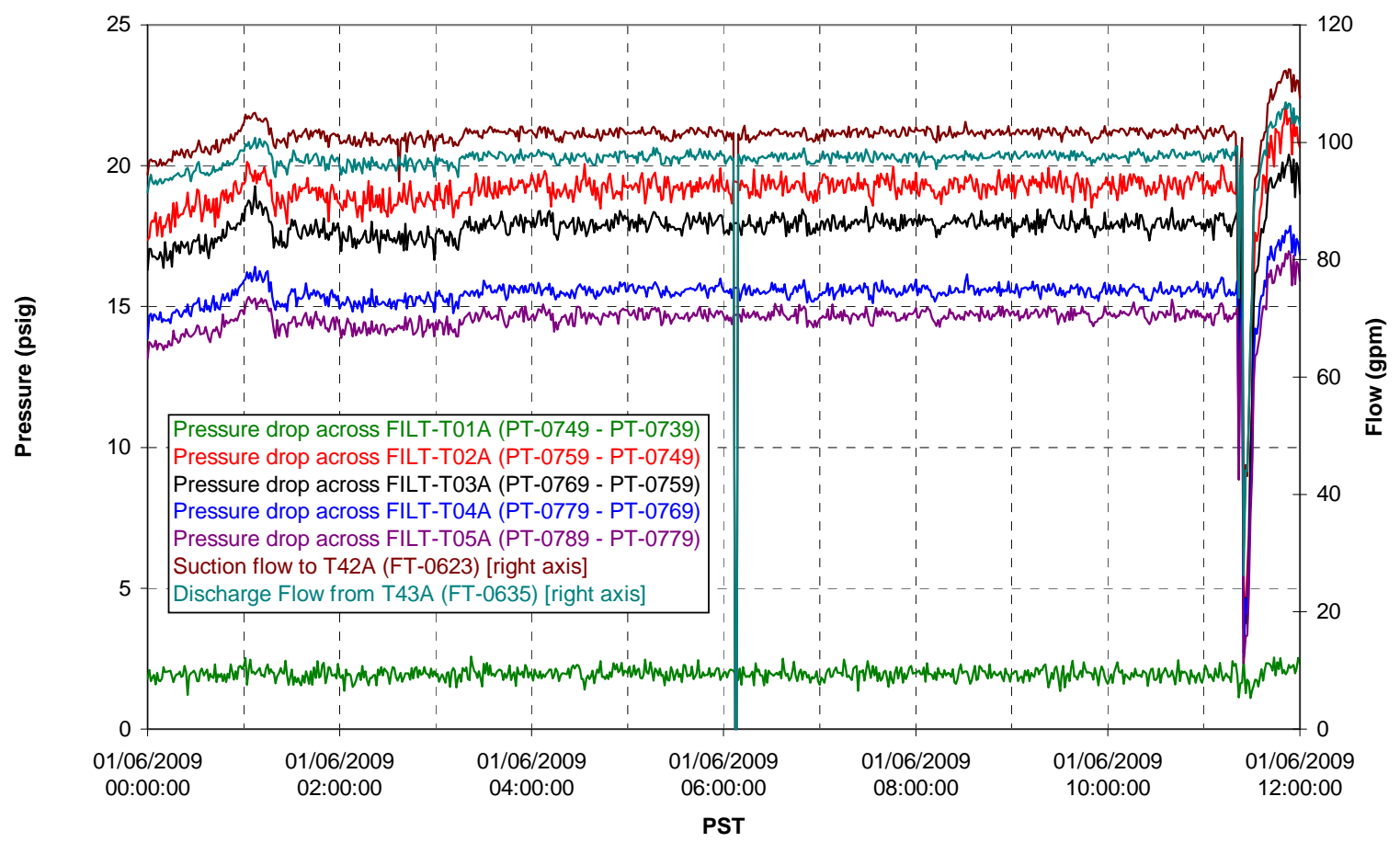

Permeate flow rates

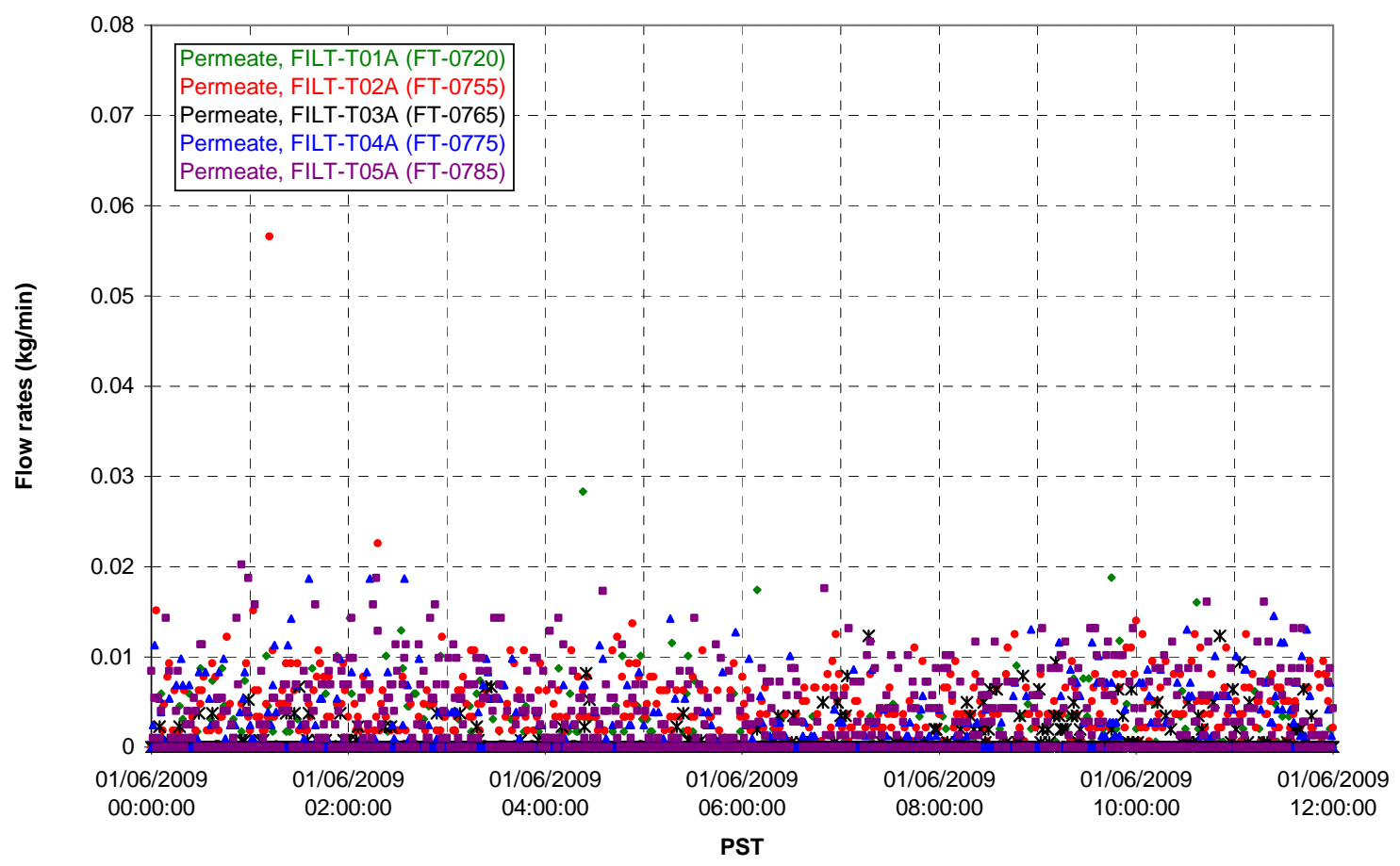


T02A Inner Temperature Tree

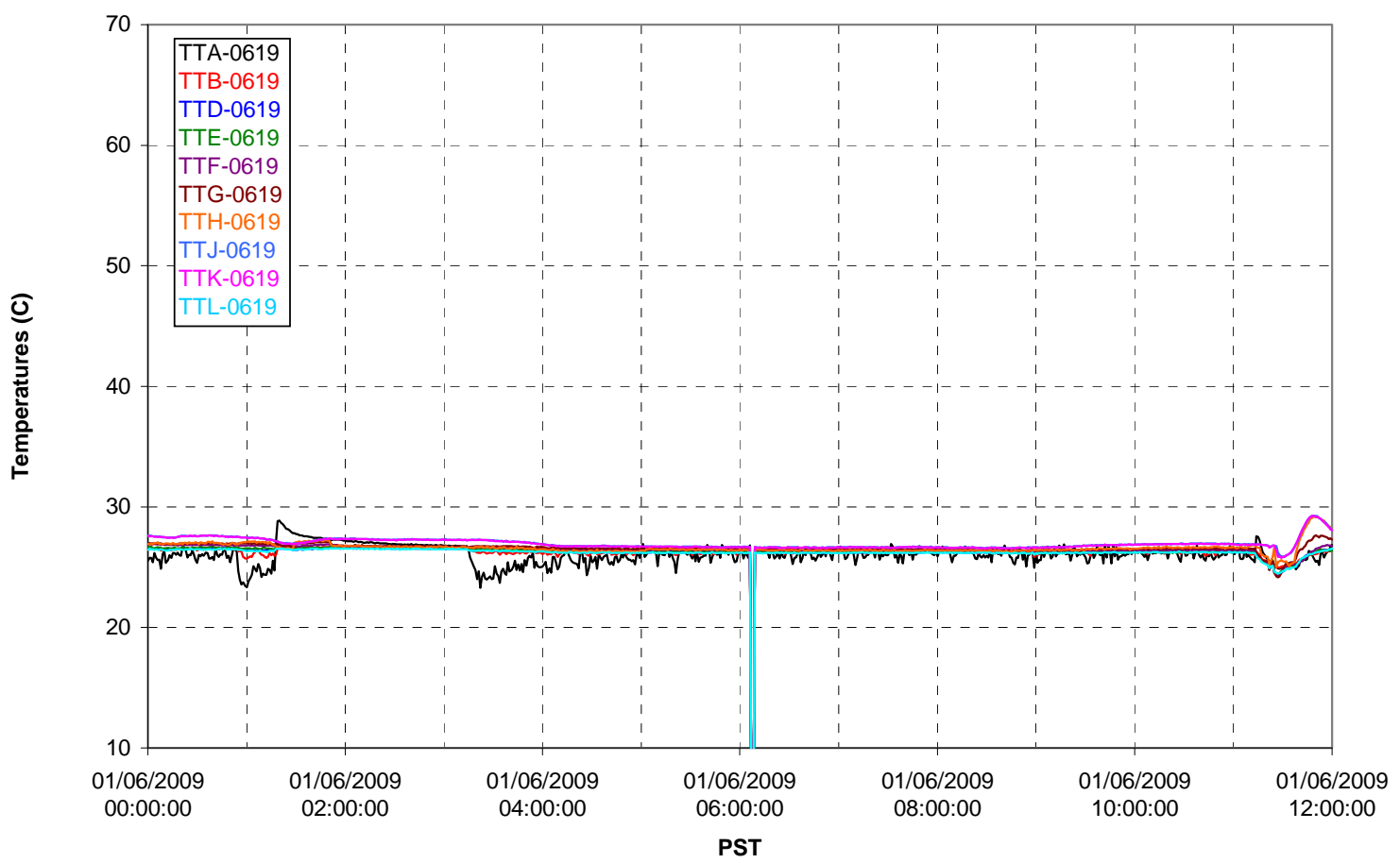

T02A Outer Temperature Tree

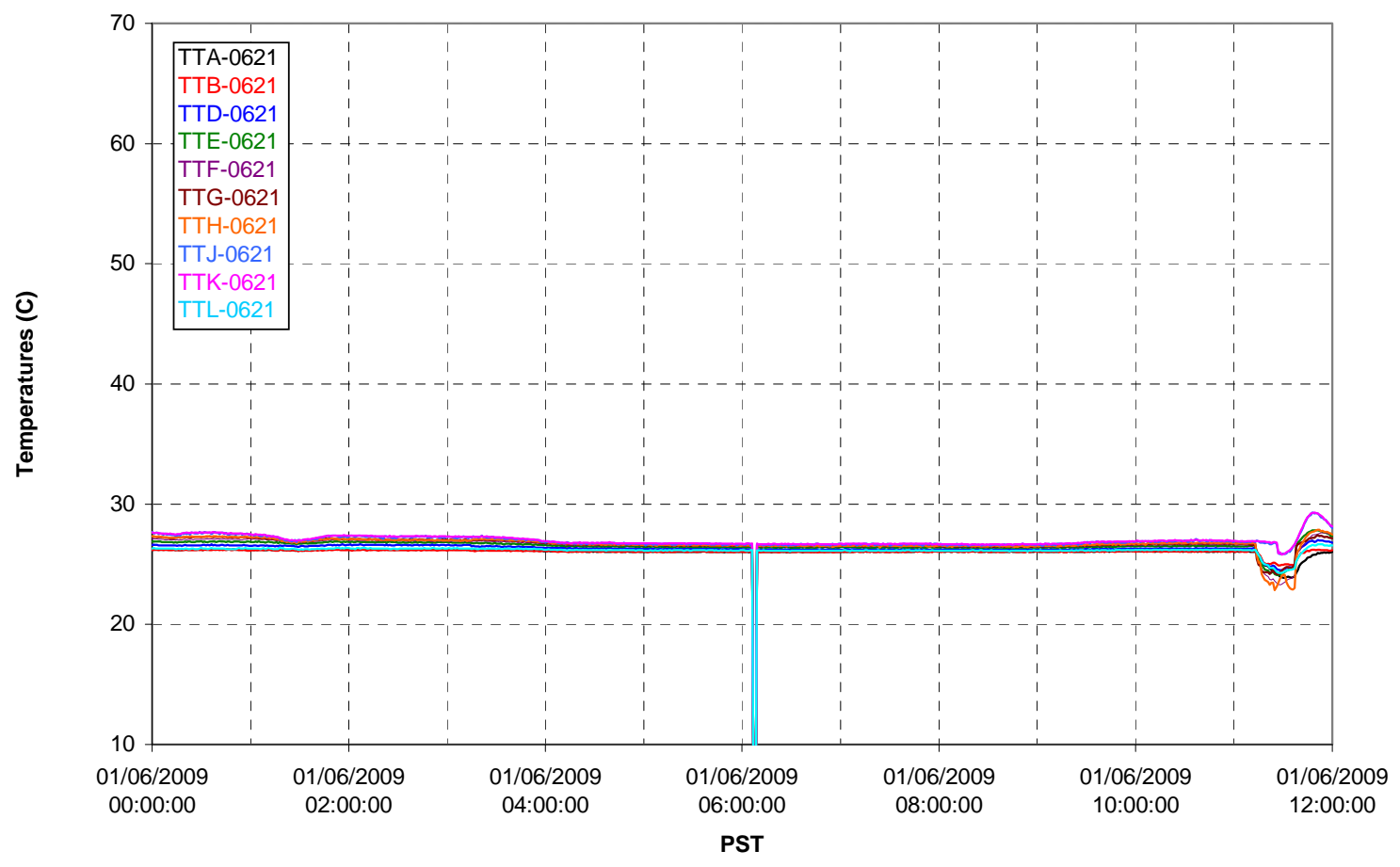


T02A temperatures

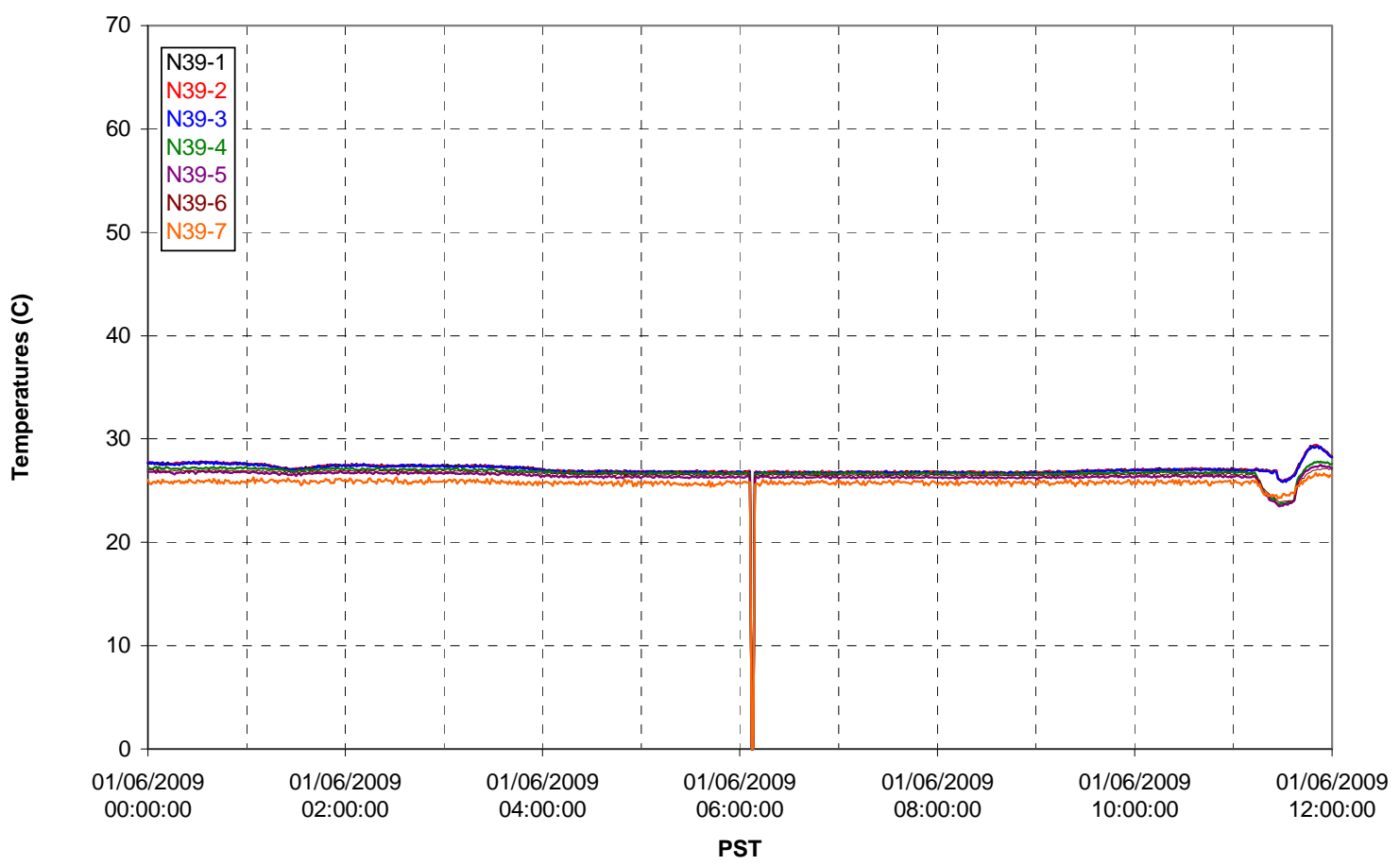

T02A temperatures

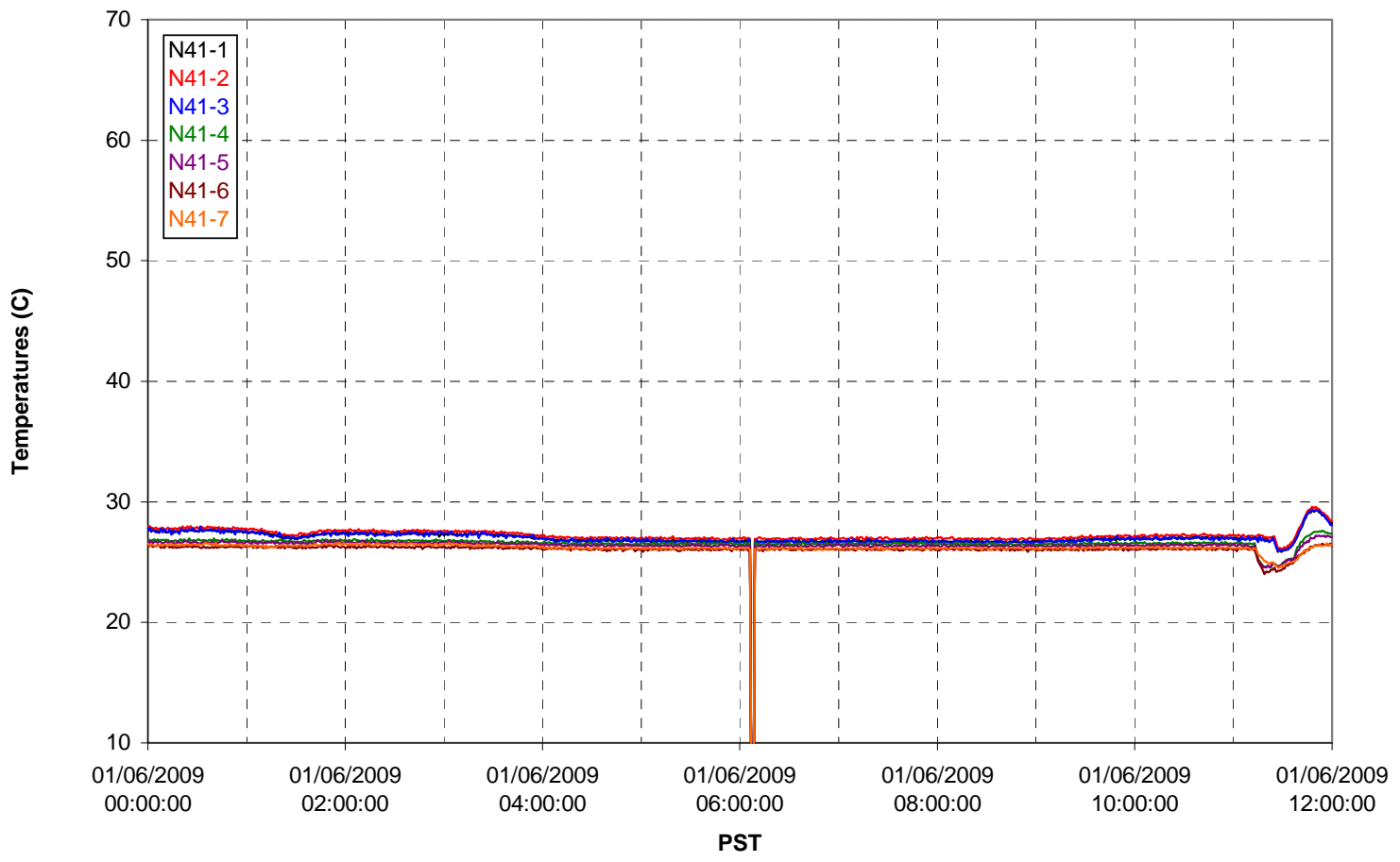


T02A temperatures

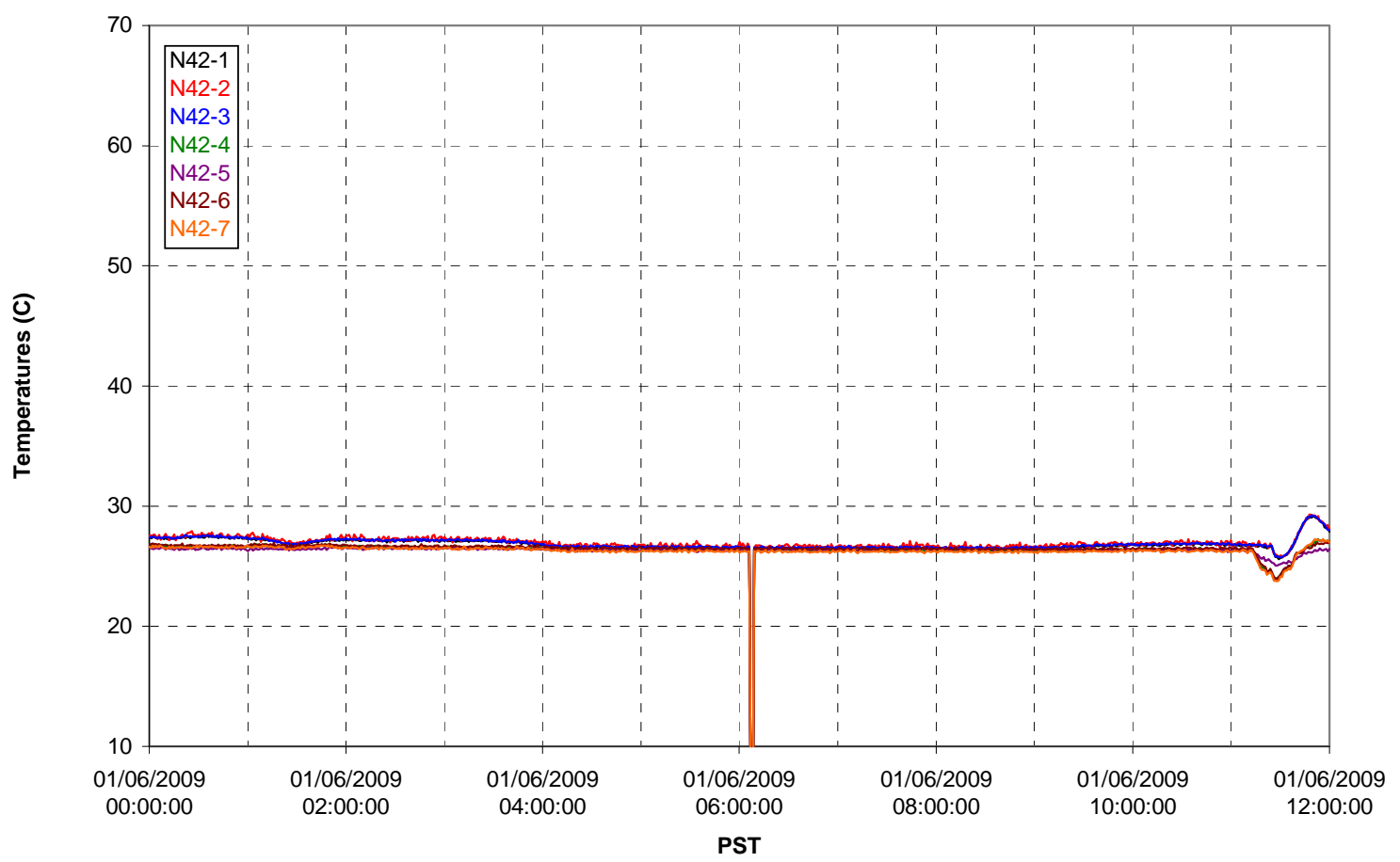

T02A temperatures

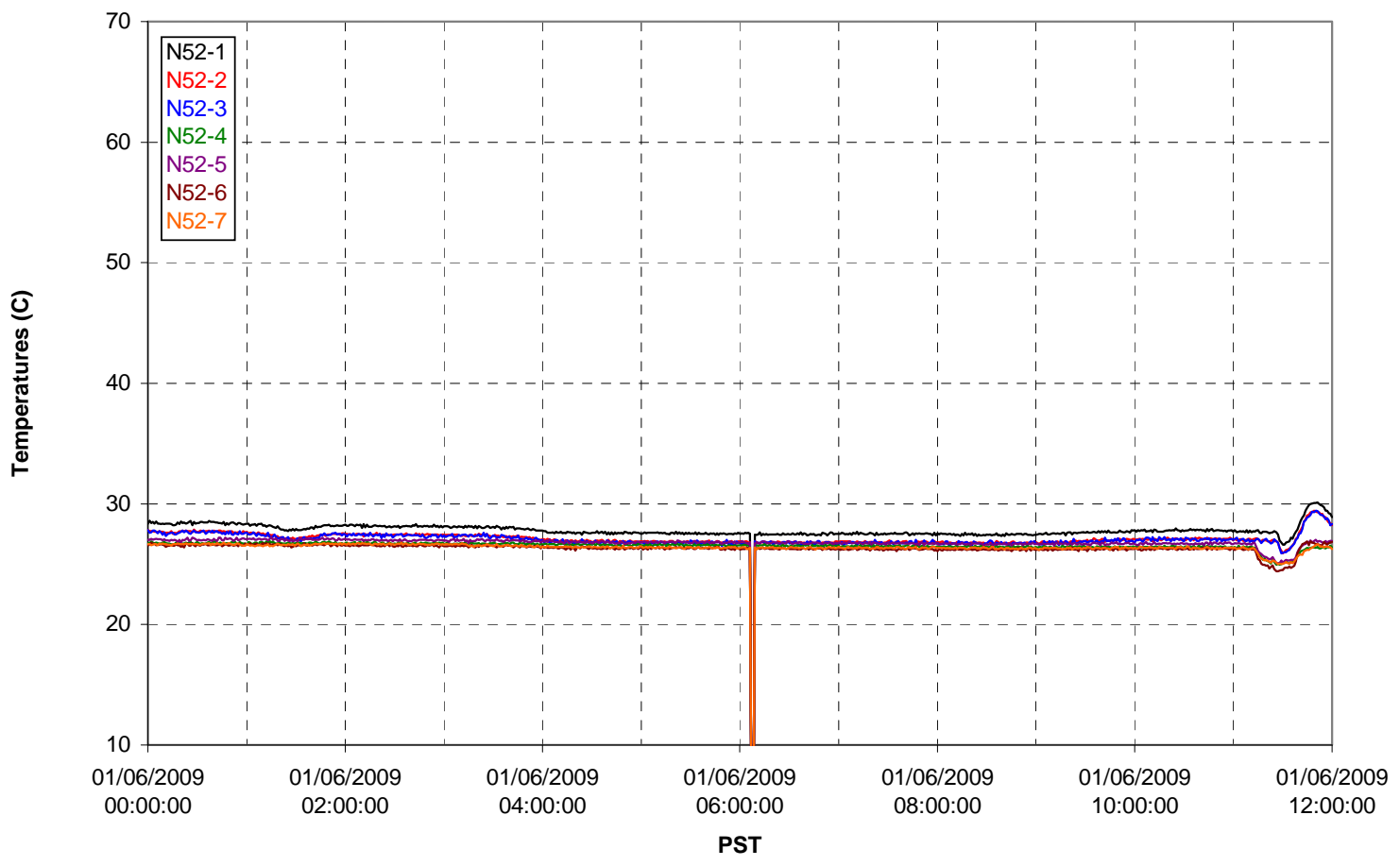


T02A Heating and Cooling

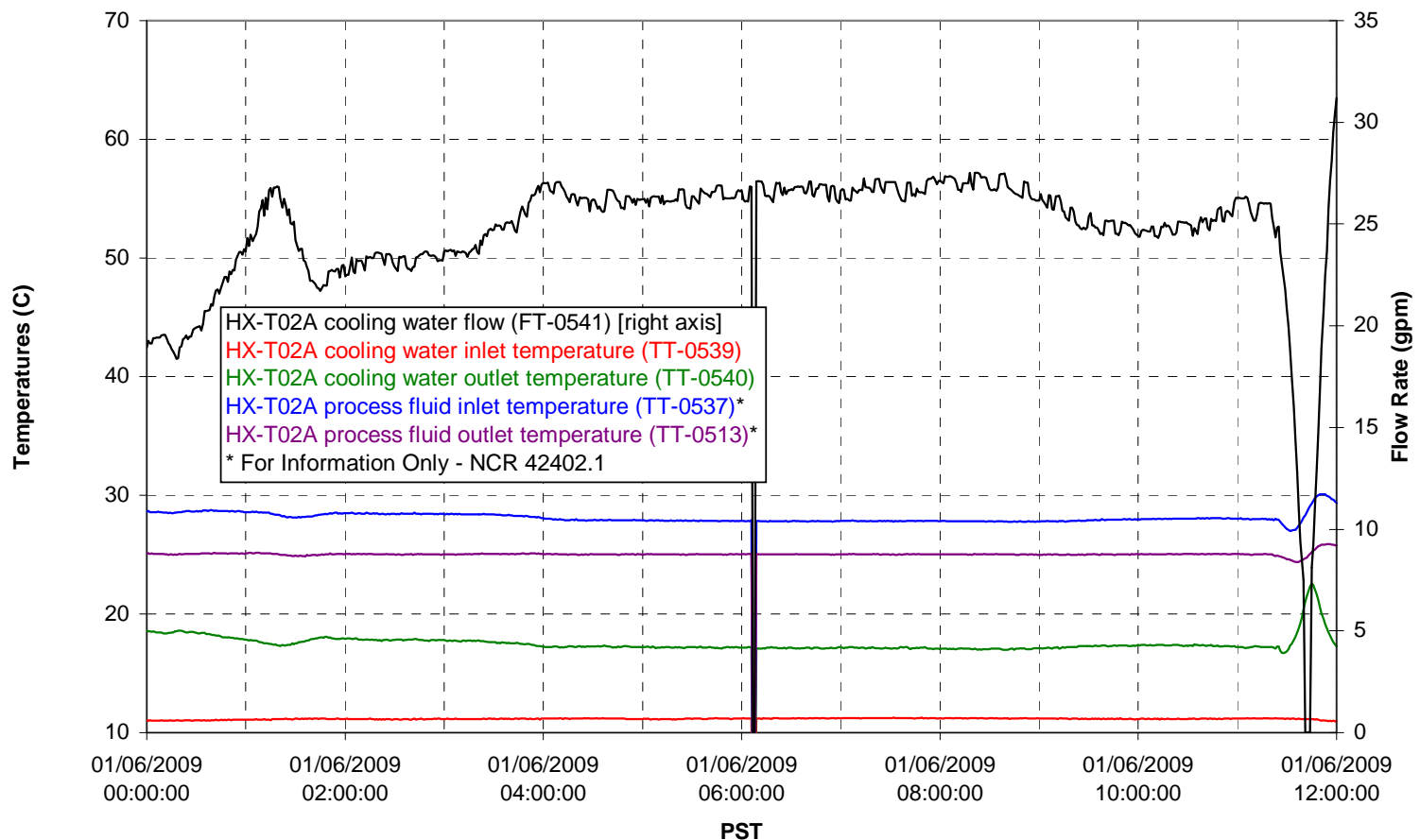

Pump Operation

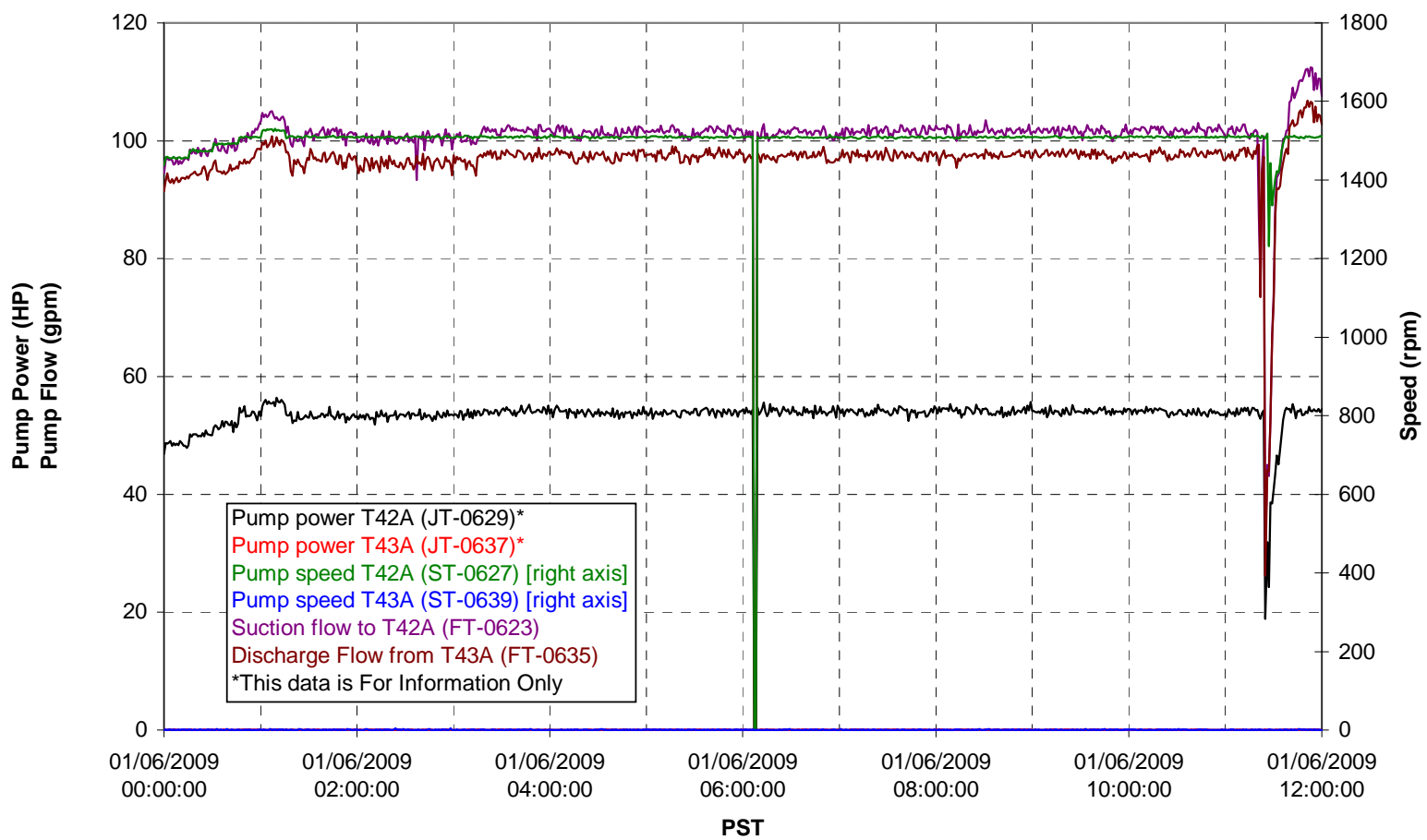


Pulsepot UFP-PP-T01A

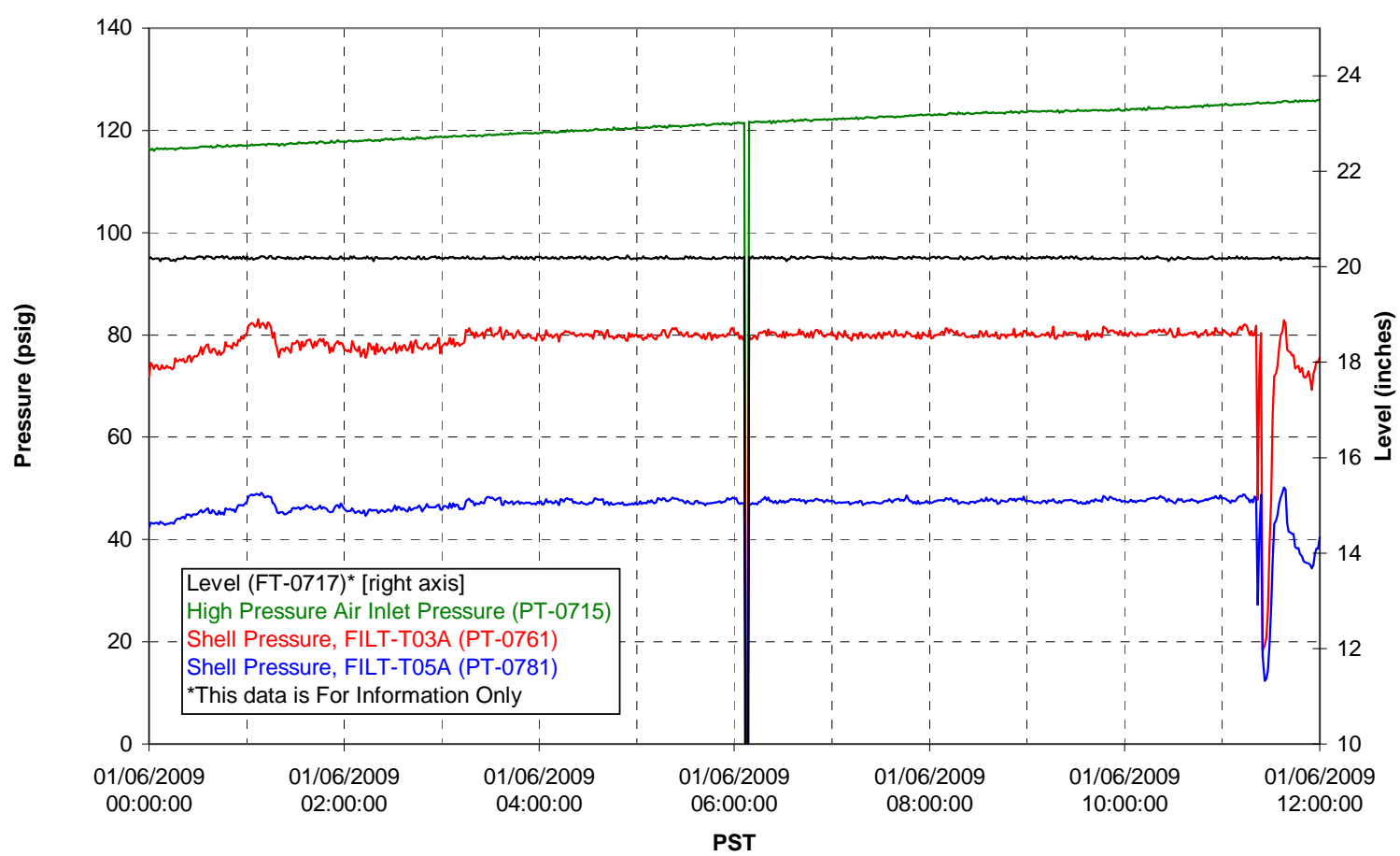

Pulsepot UFP-PP-T02A

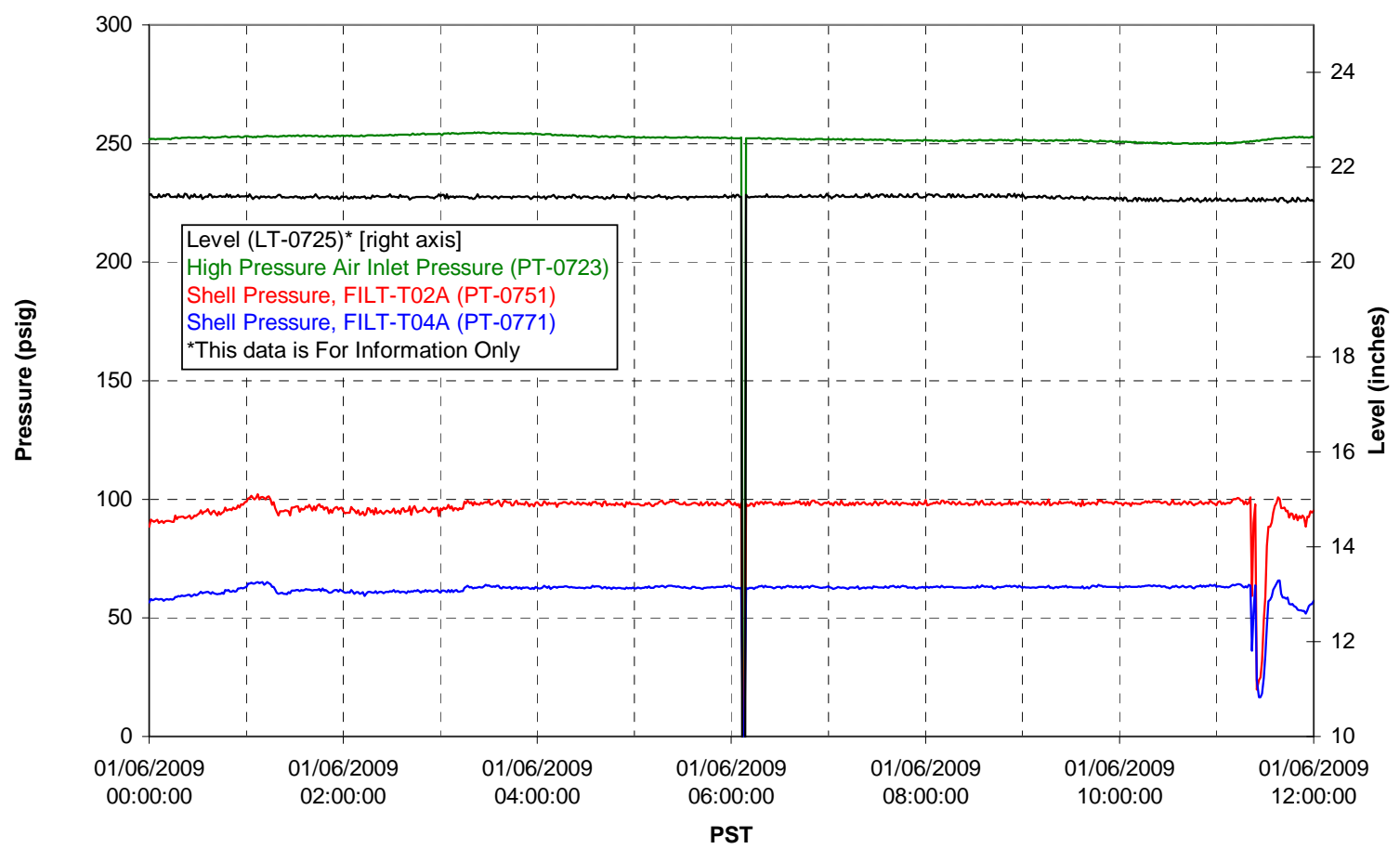


Pulsepot UFP-PP-T03A

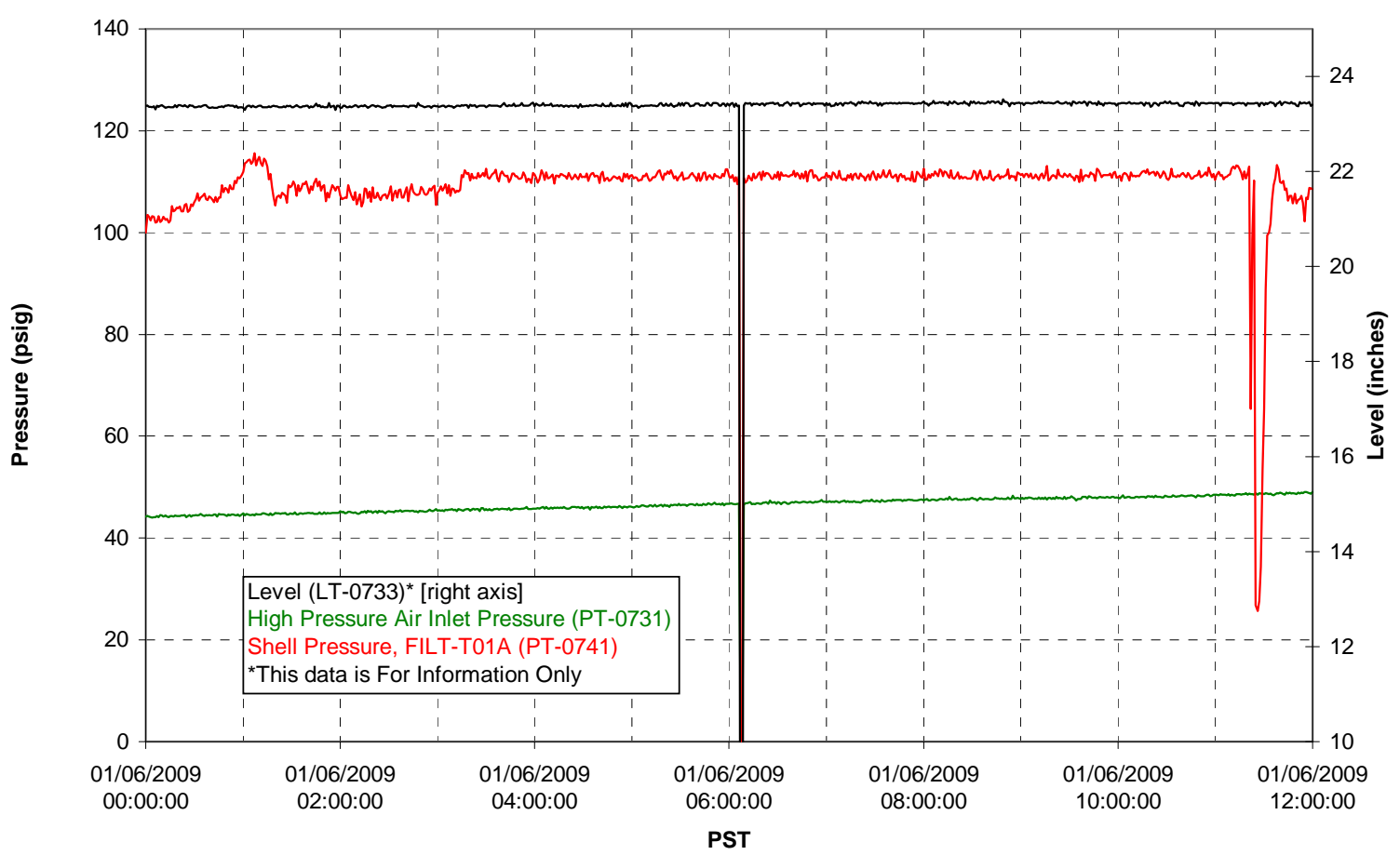

Pulsepot Levels

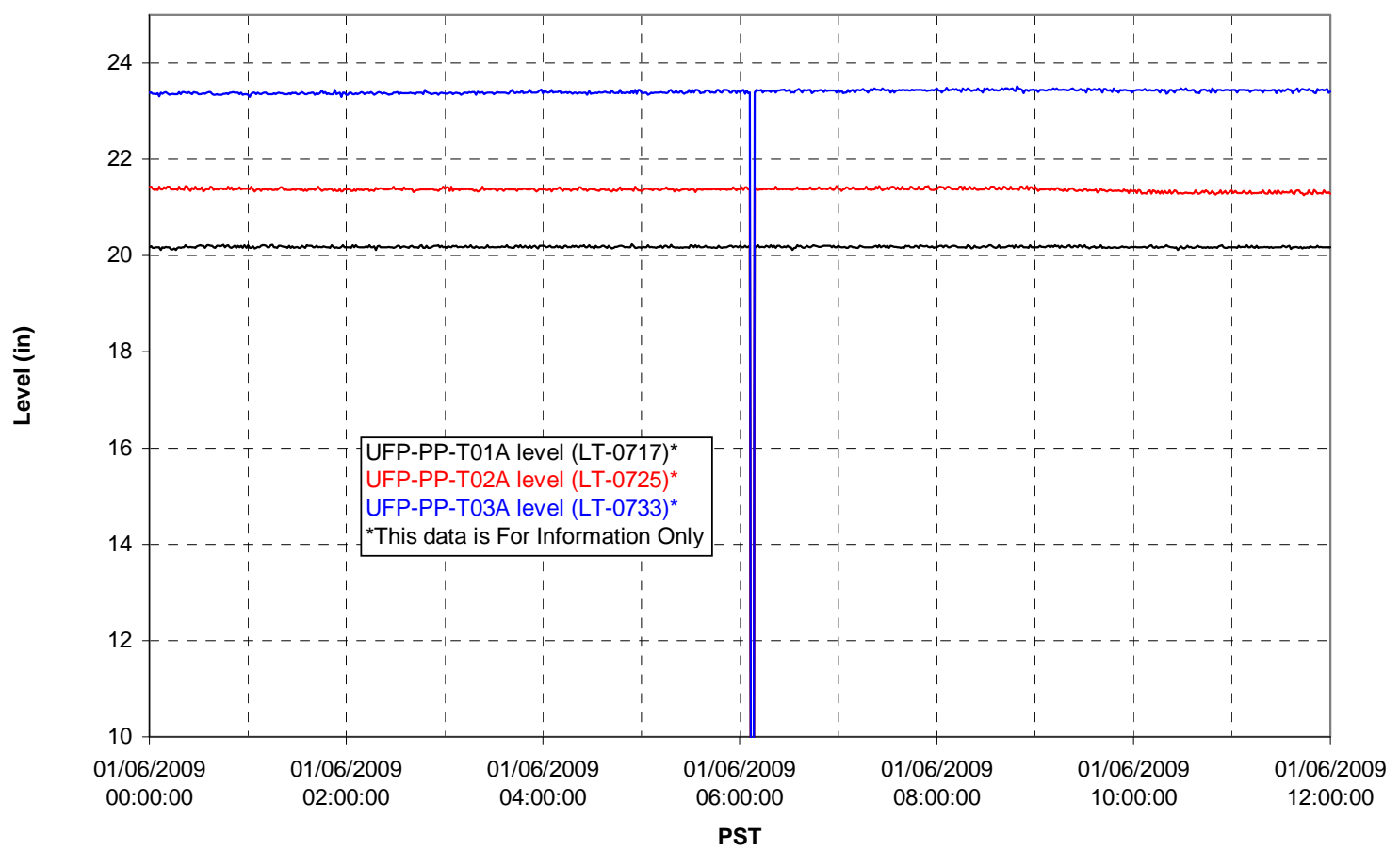


Filter UFP-FILT-T01A

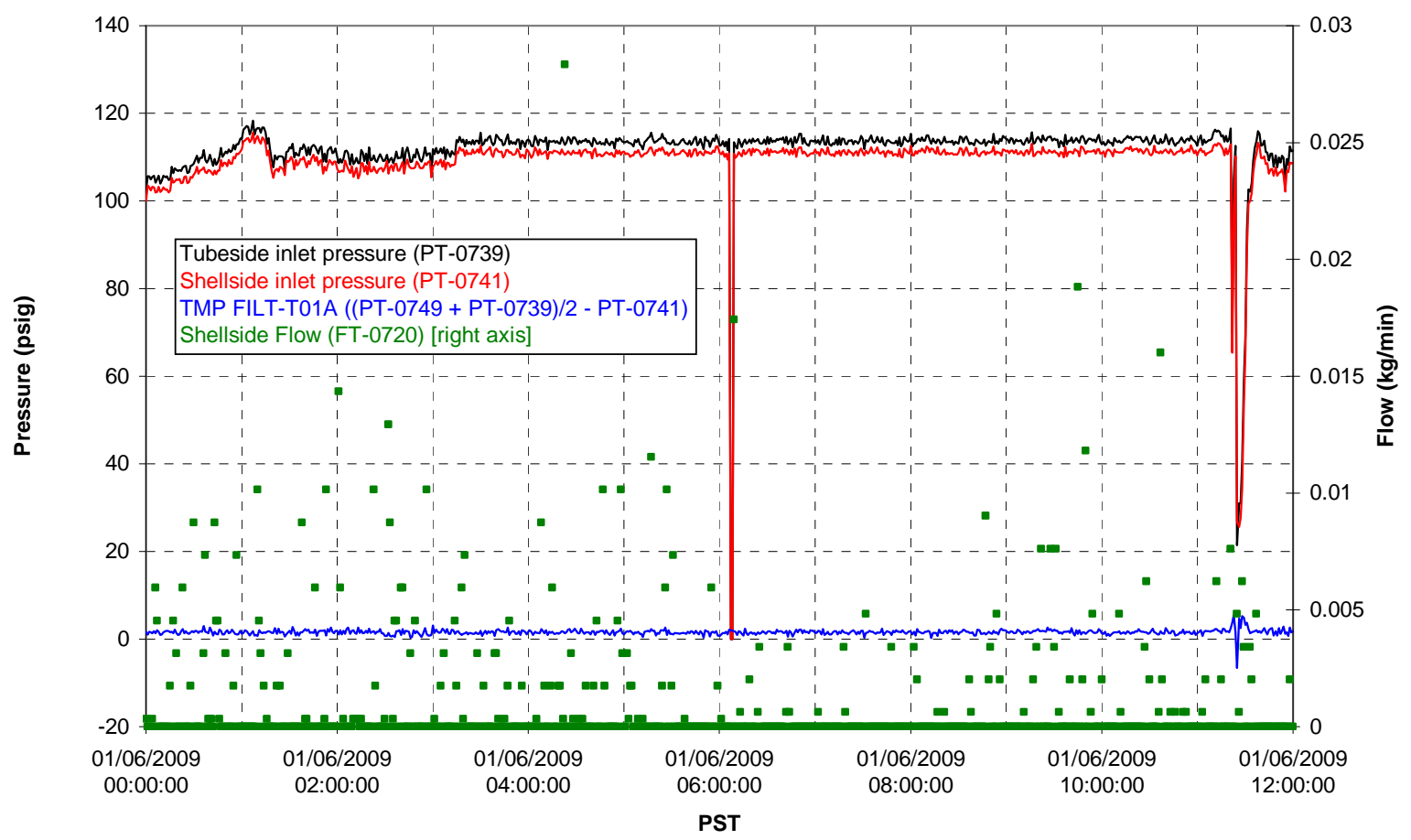

Filter UFP-FILT-T02A

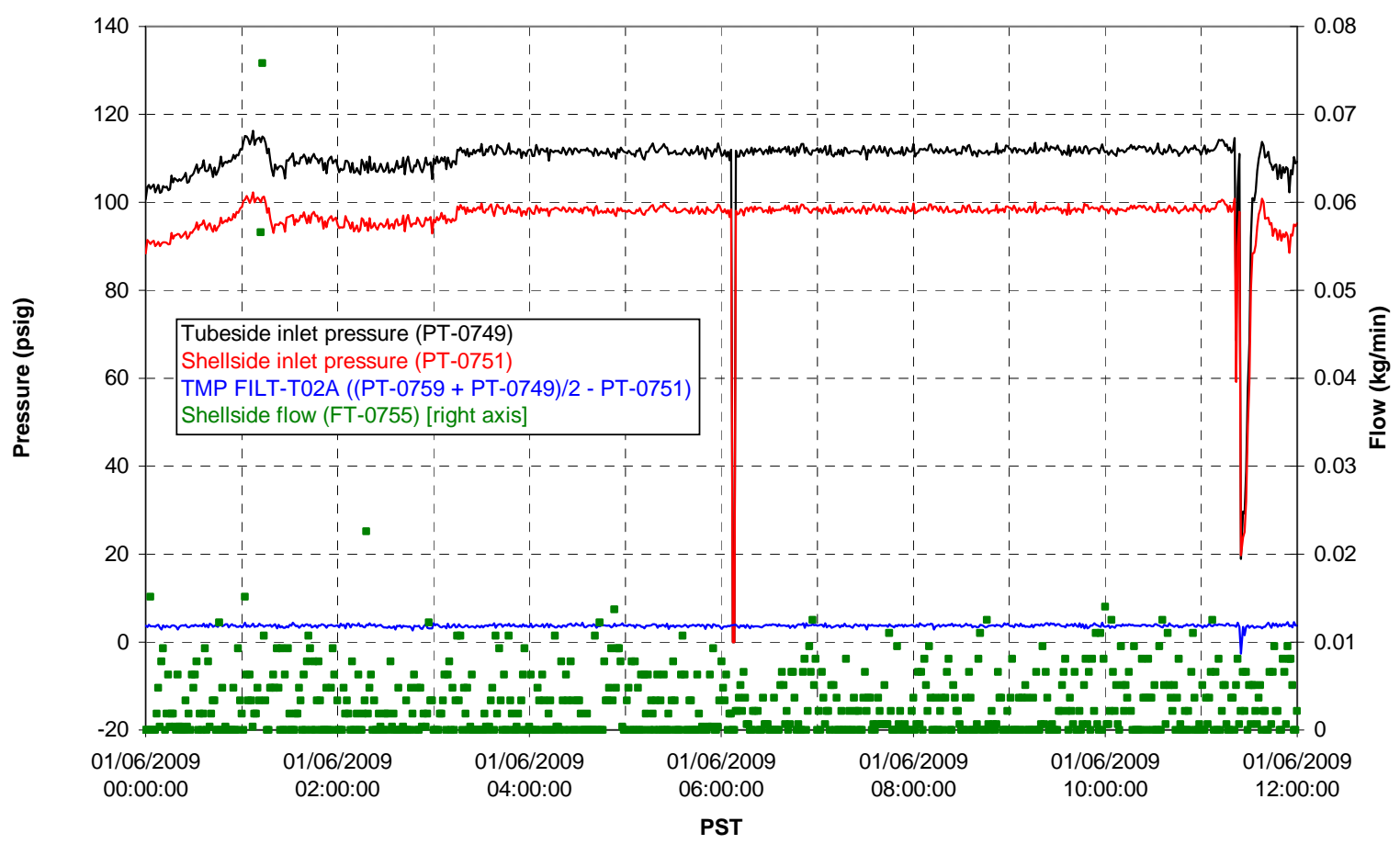


Filter UFP-FILT-T03A

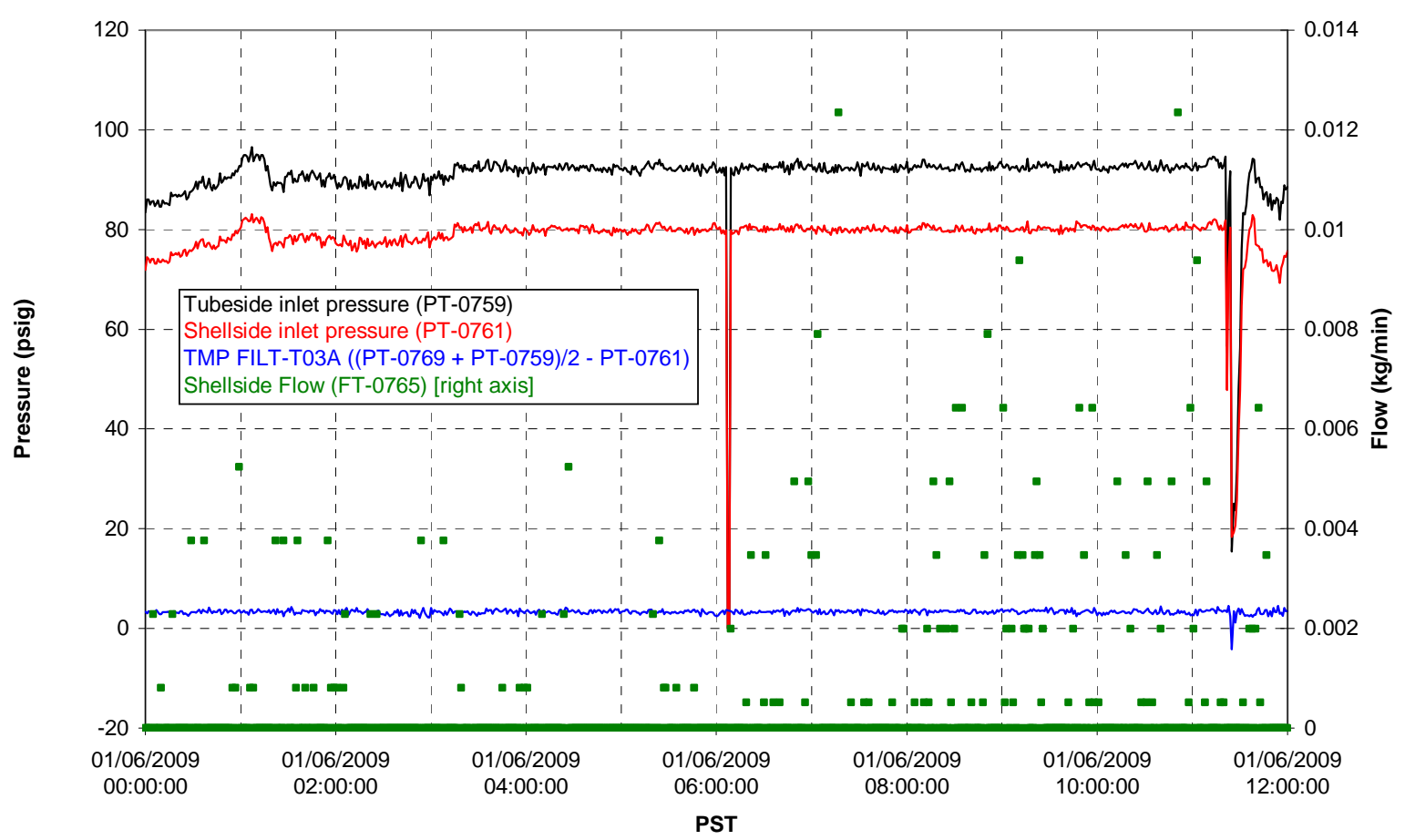

Filter UFP-FILT-T04A

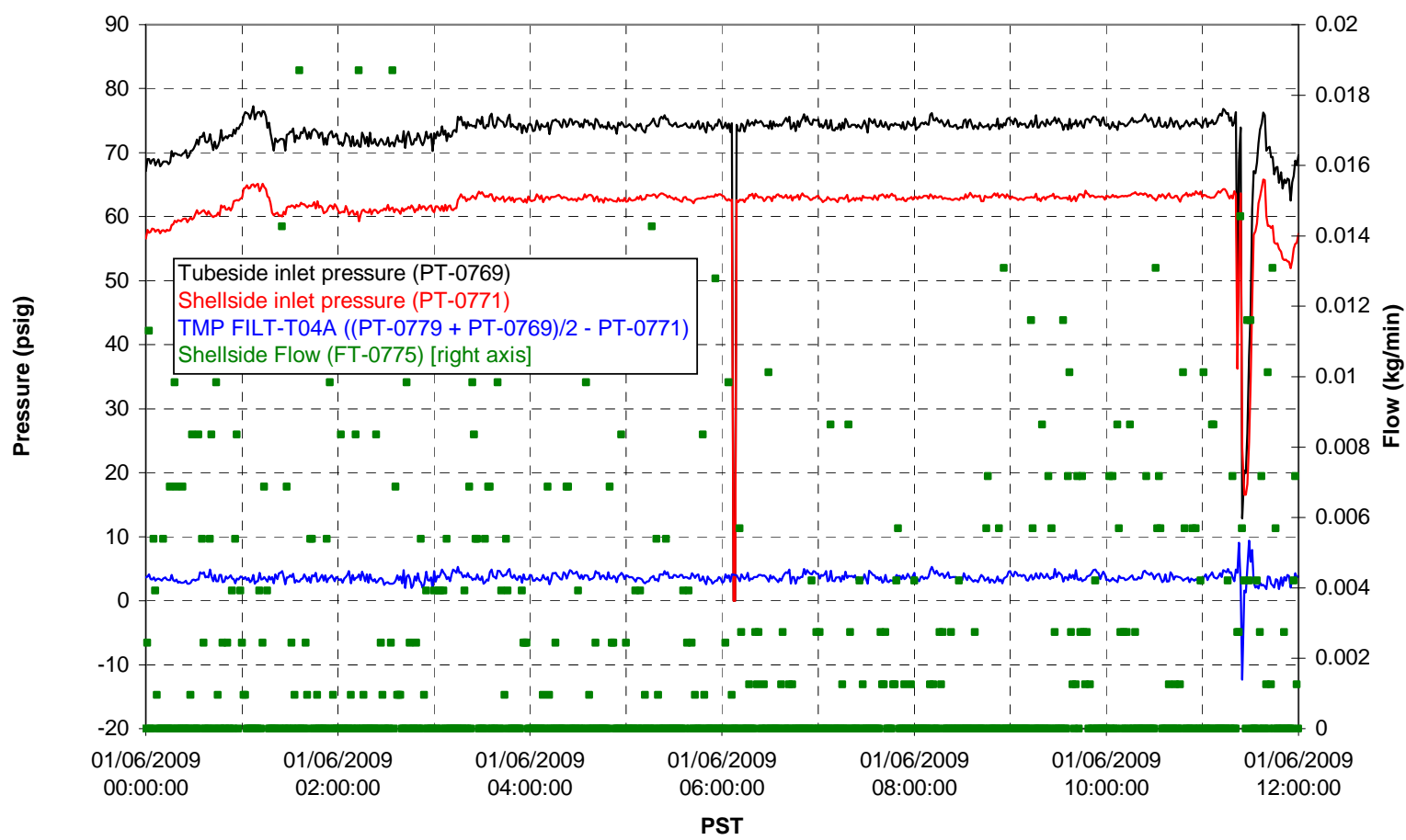


Filter UFP-FILT-T05A

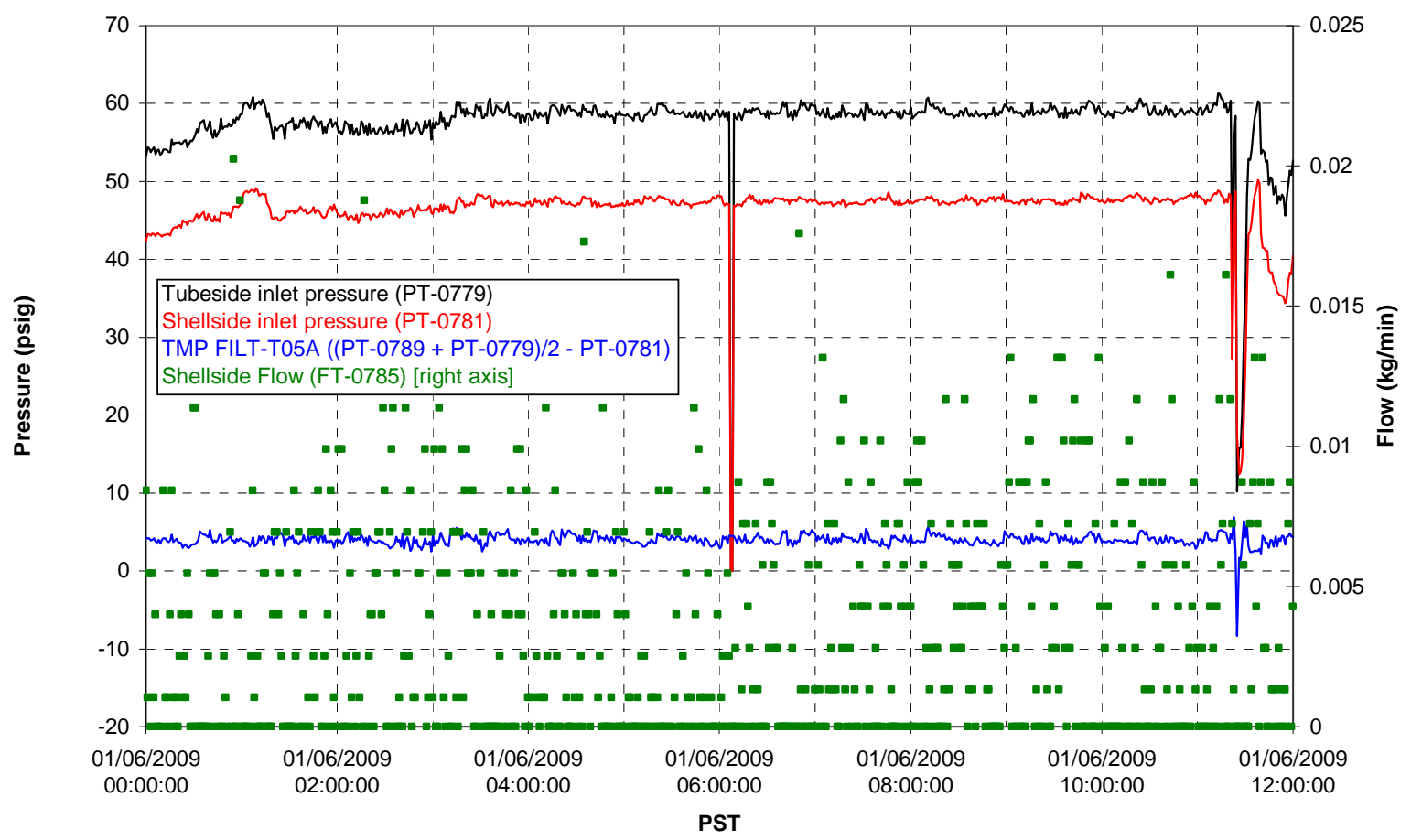

Chemical Flow

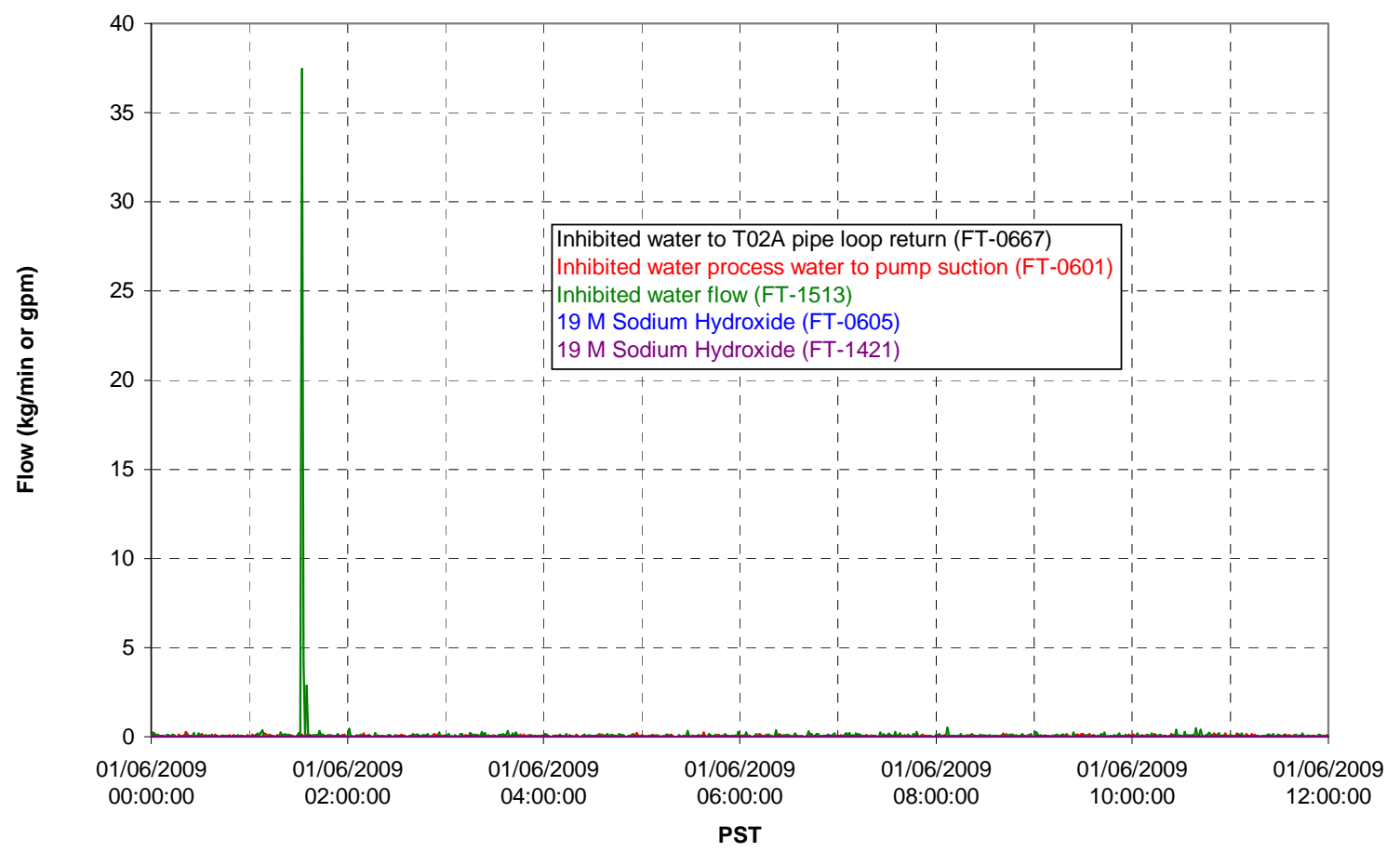


Chemical Flow

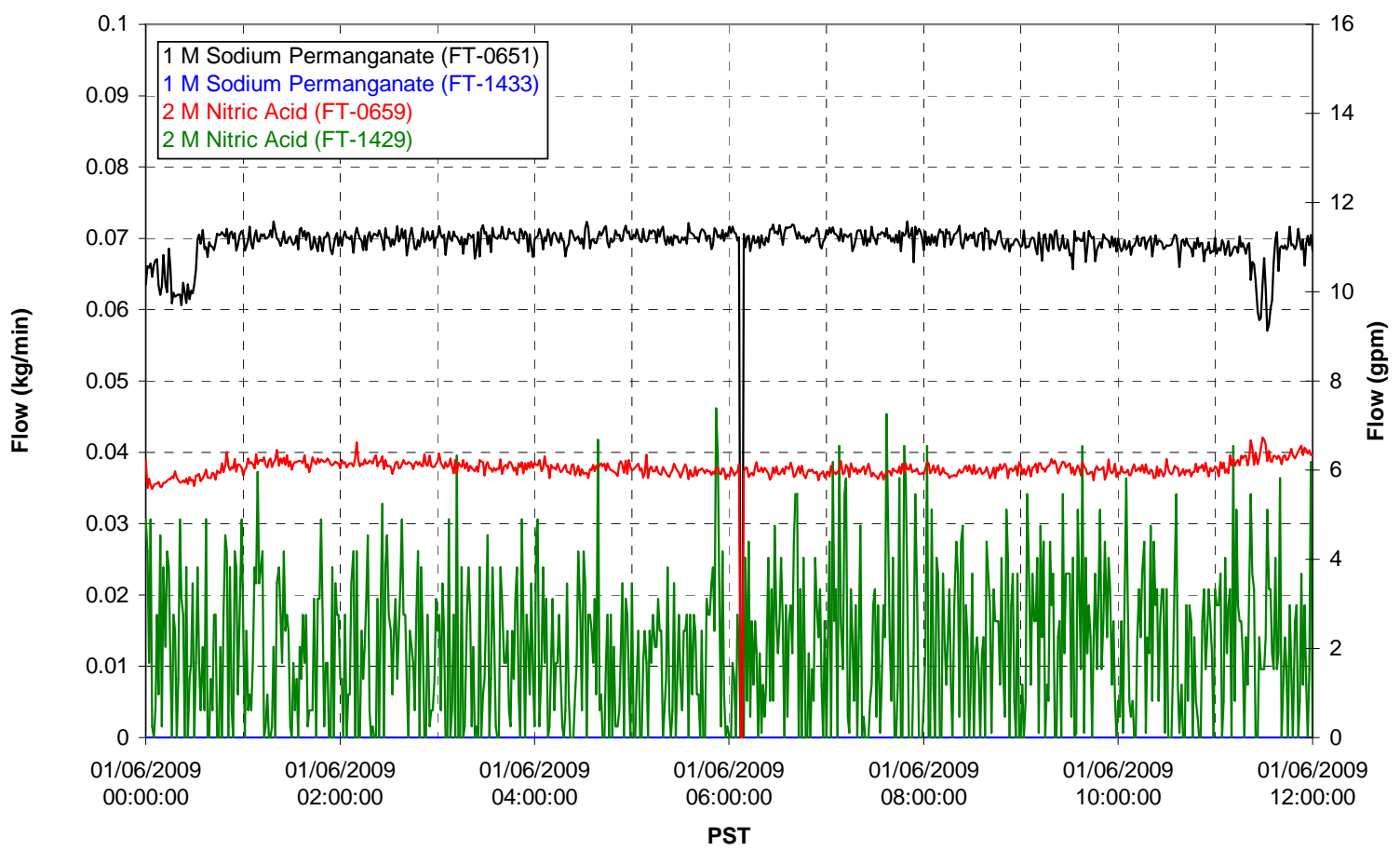

Air Flows

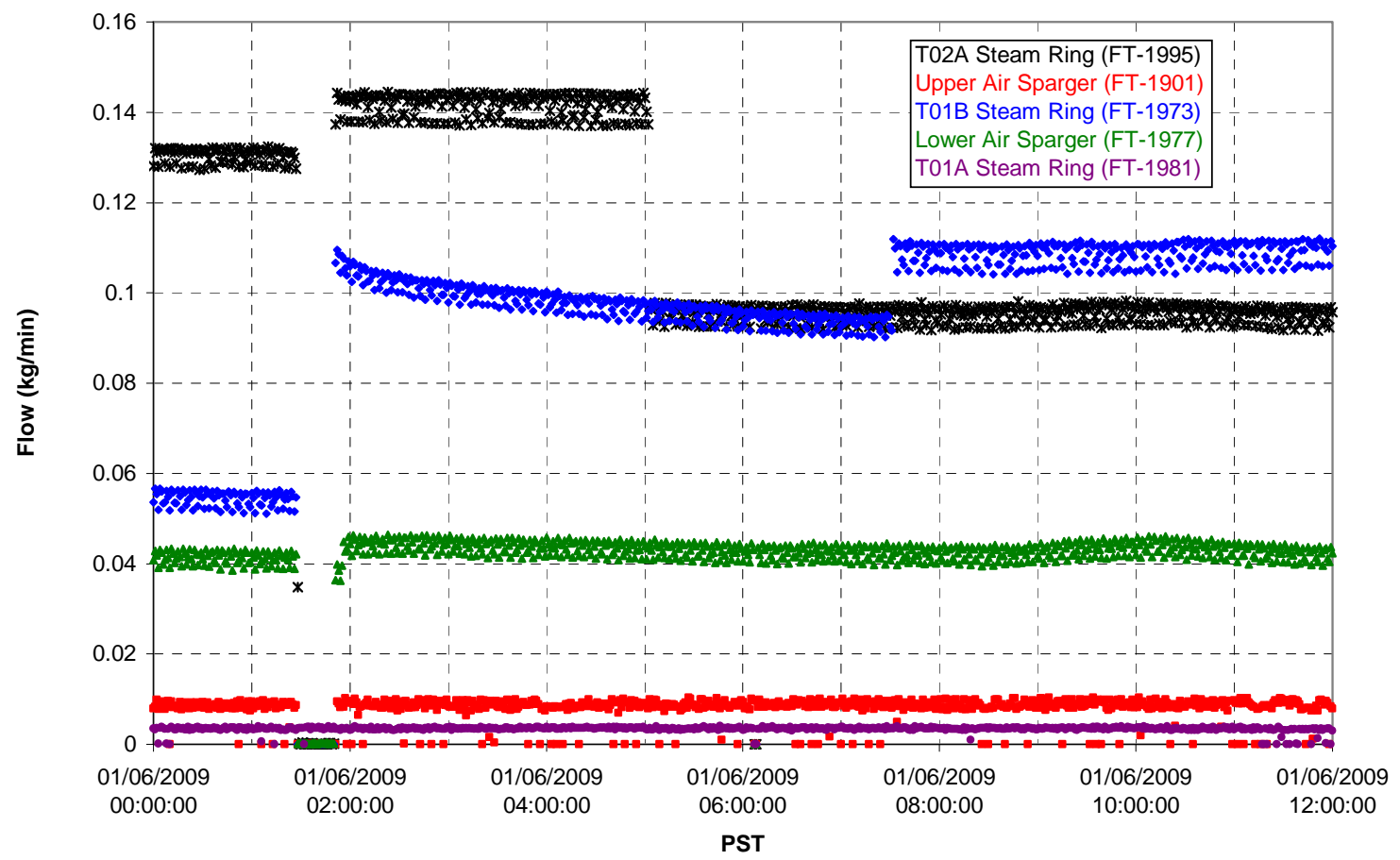


T02A Steam

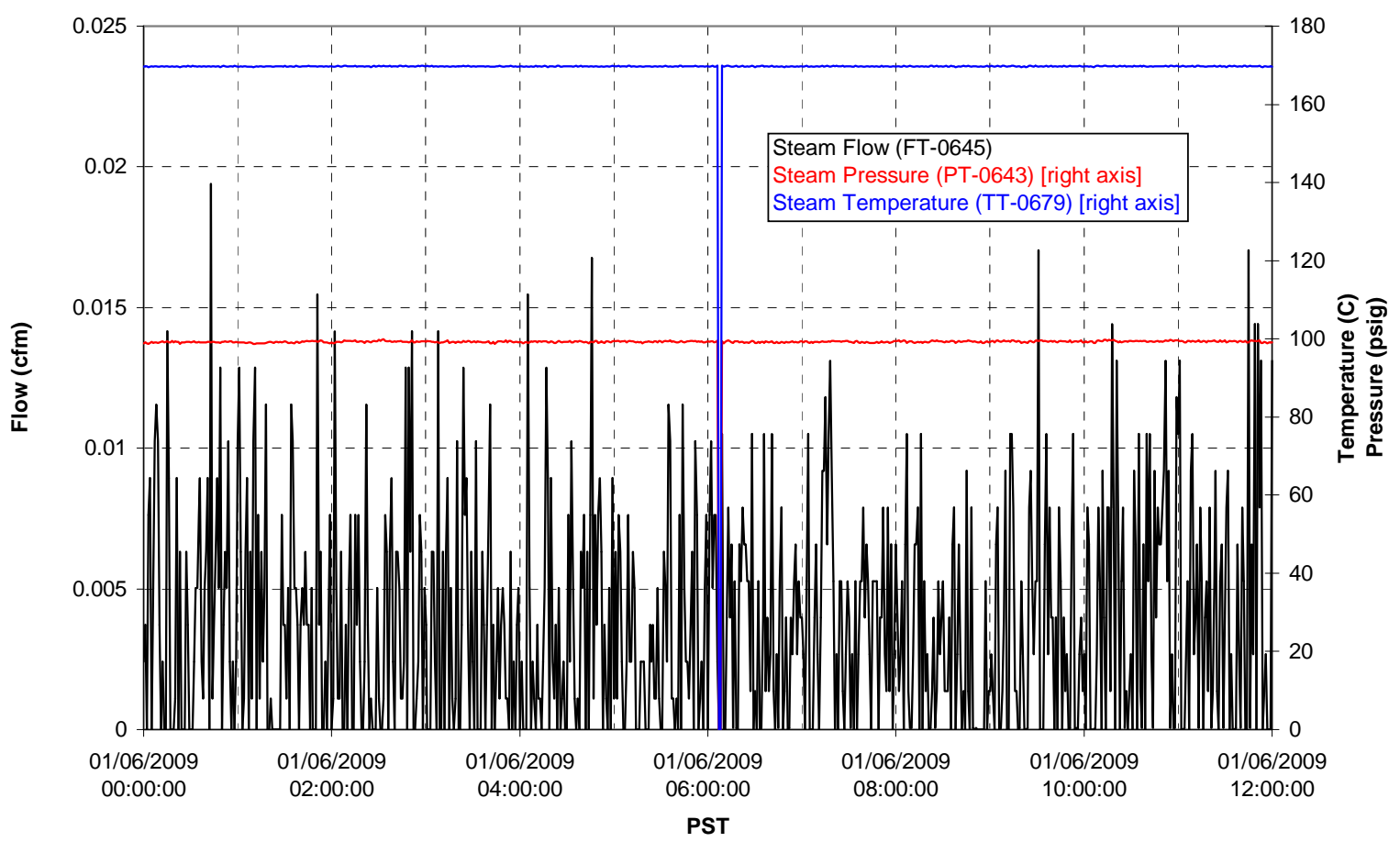

T01A Steam

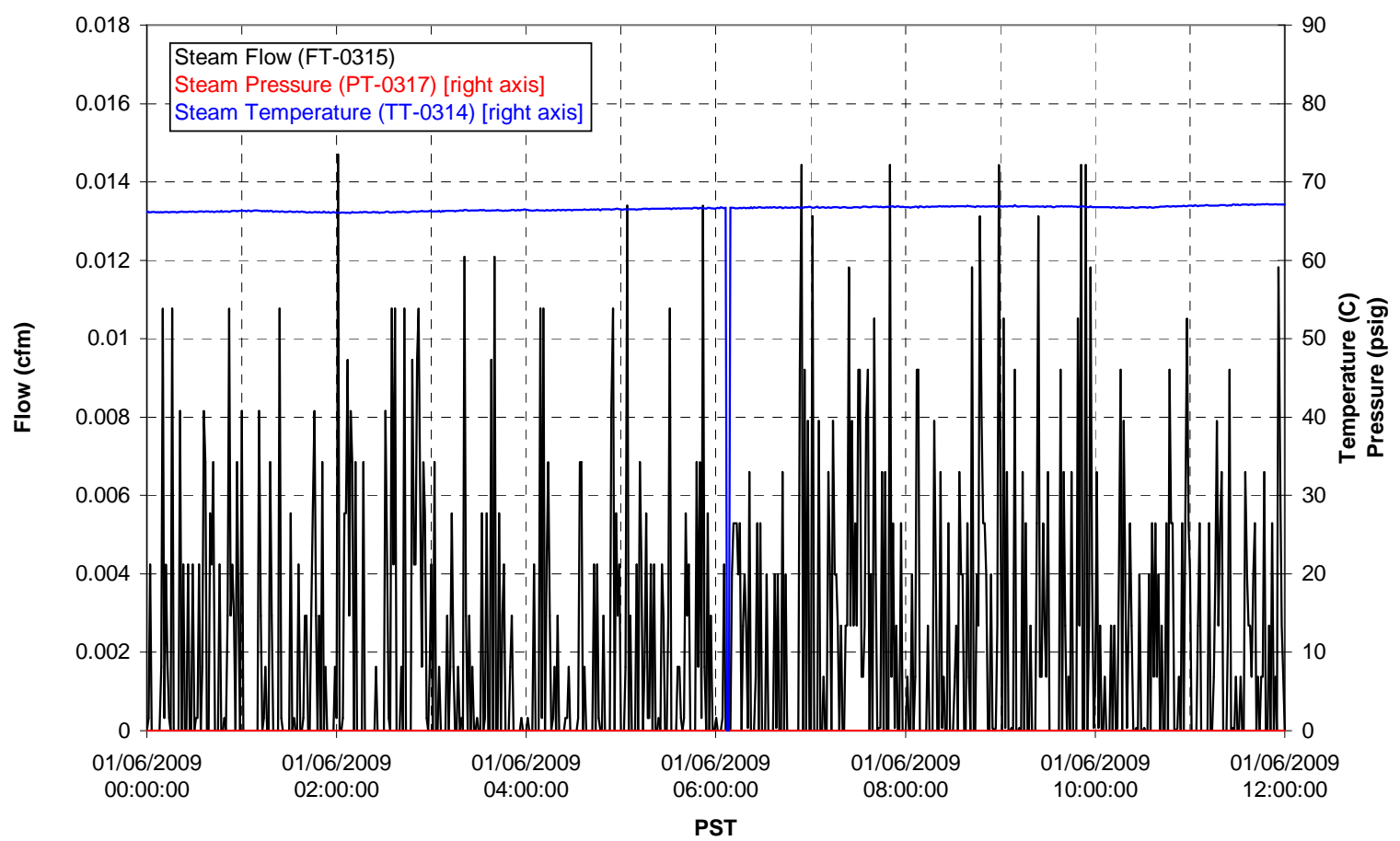


T01B Steam

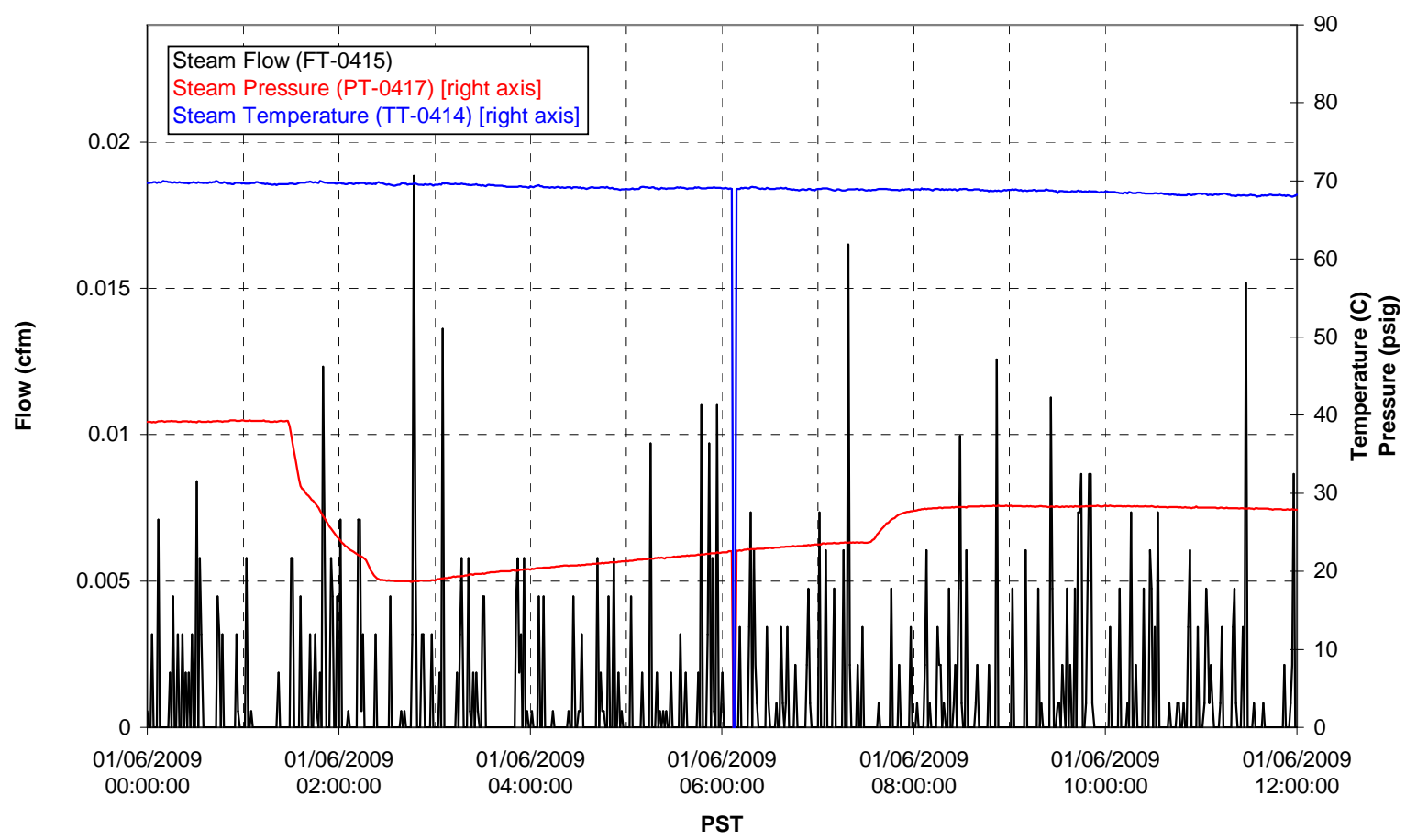


Functional Test Data Plots

01/06/2009 12:00 - 01/07/2009 00:00 
T01A level

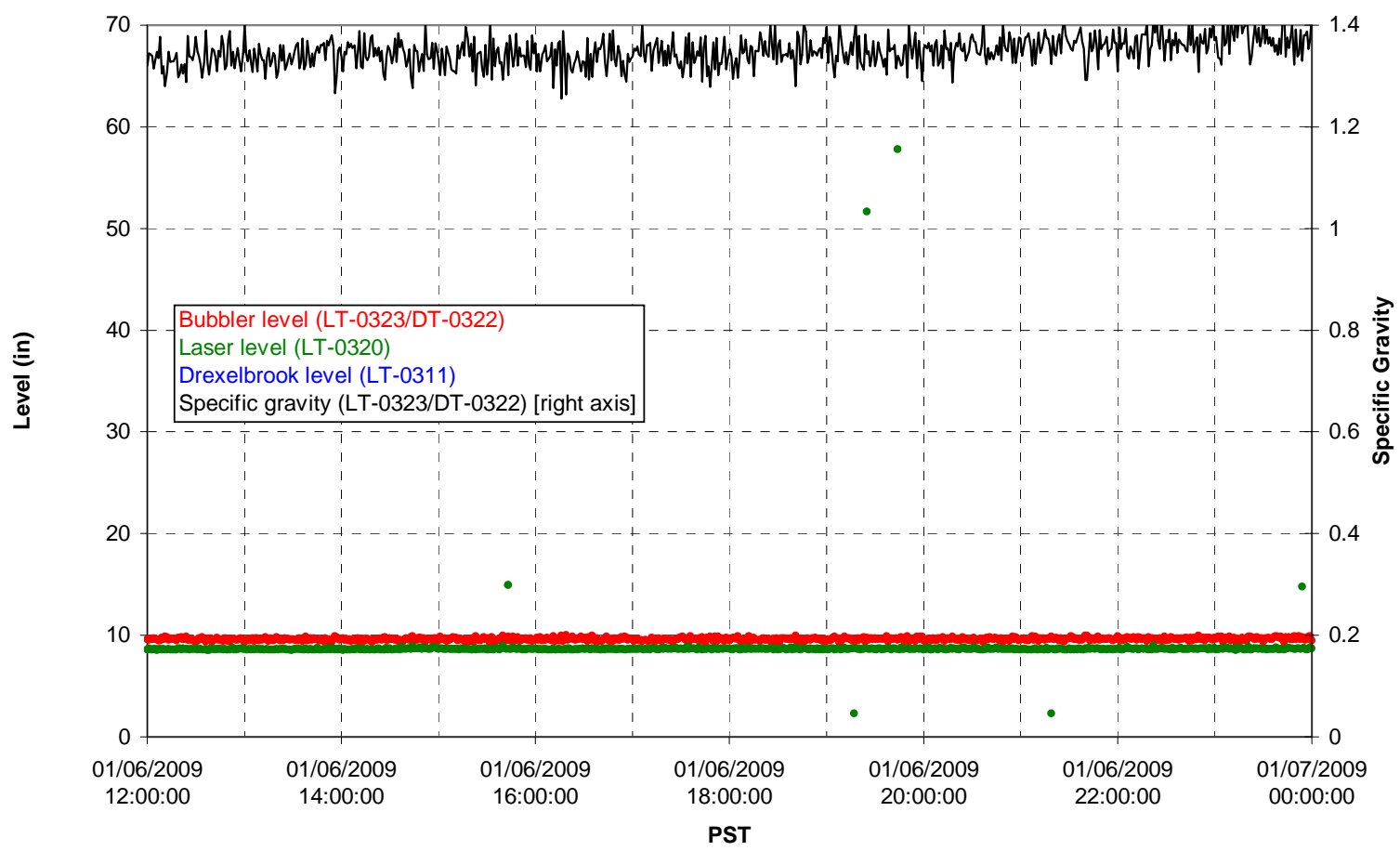

T01A temperatures

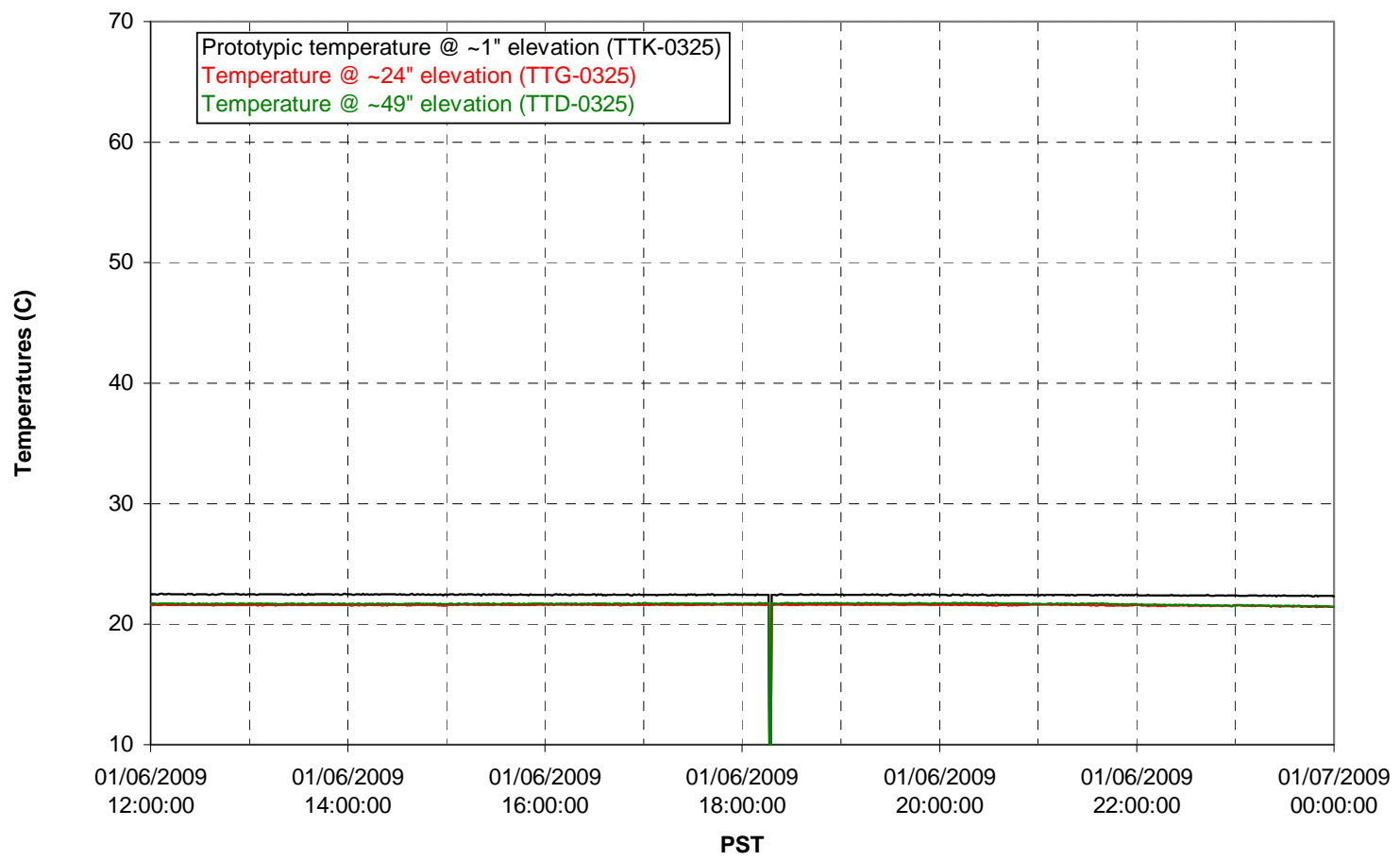


T01B level

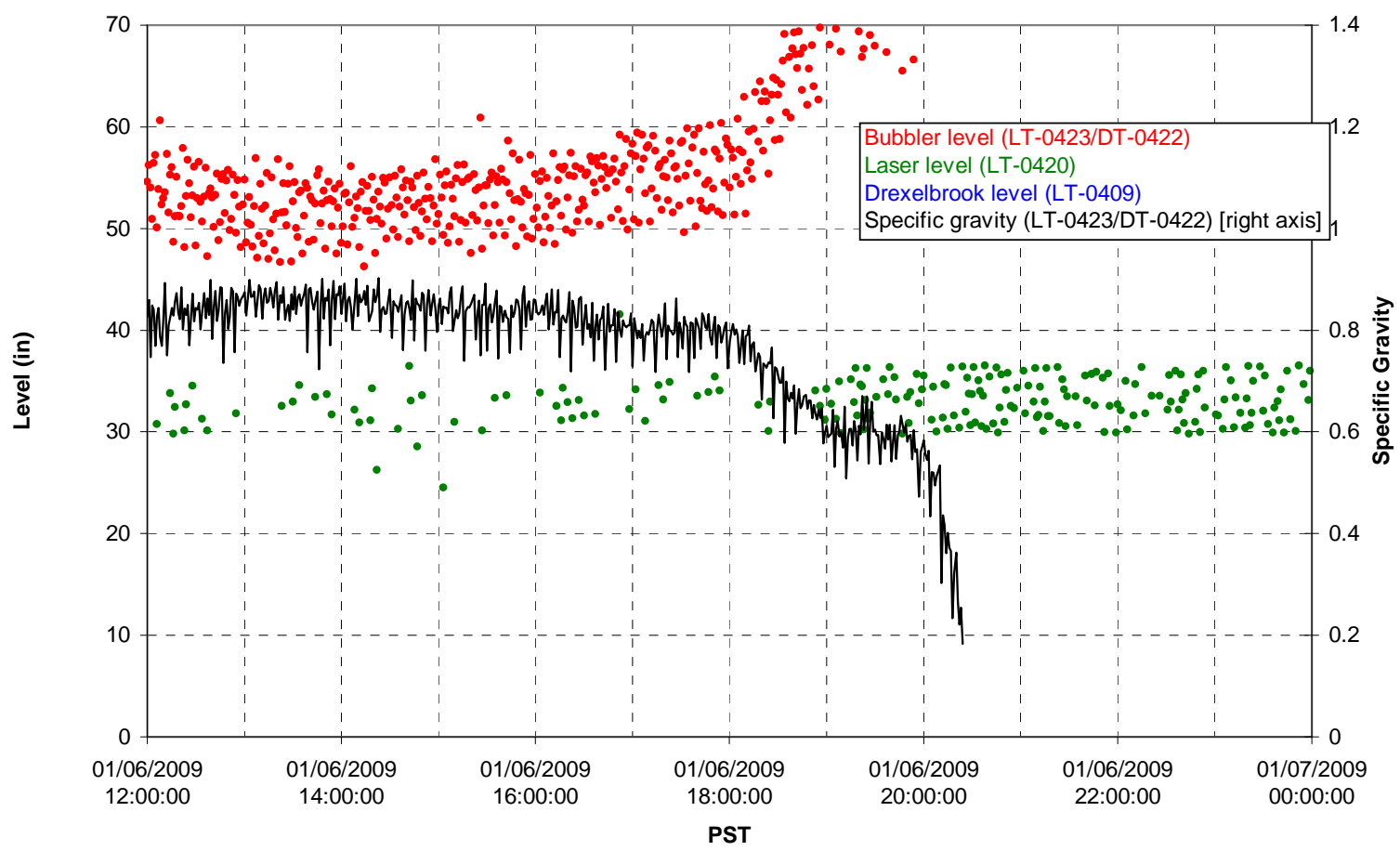

T01B temperatures

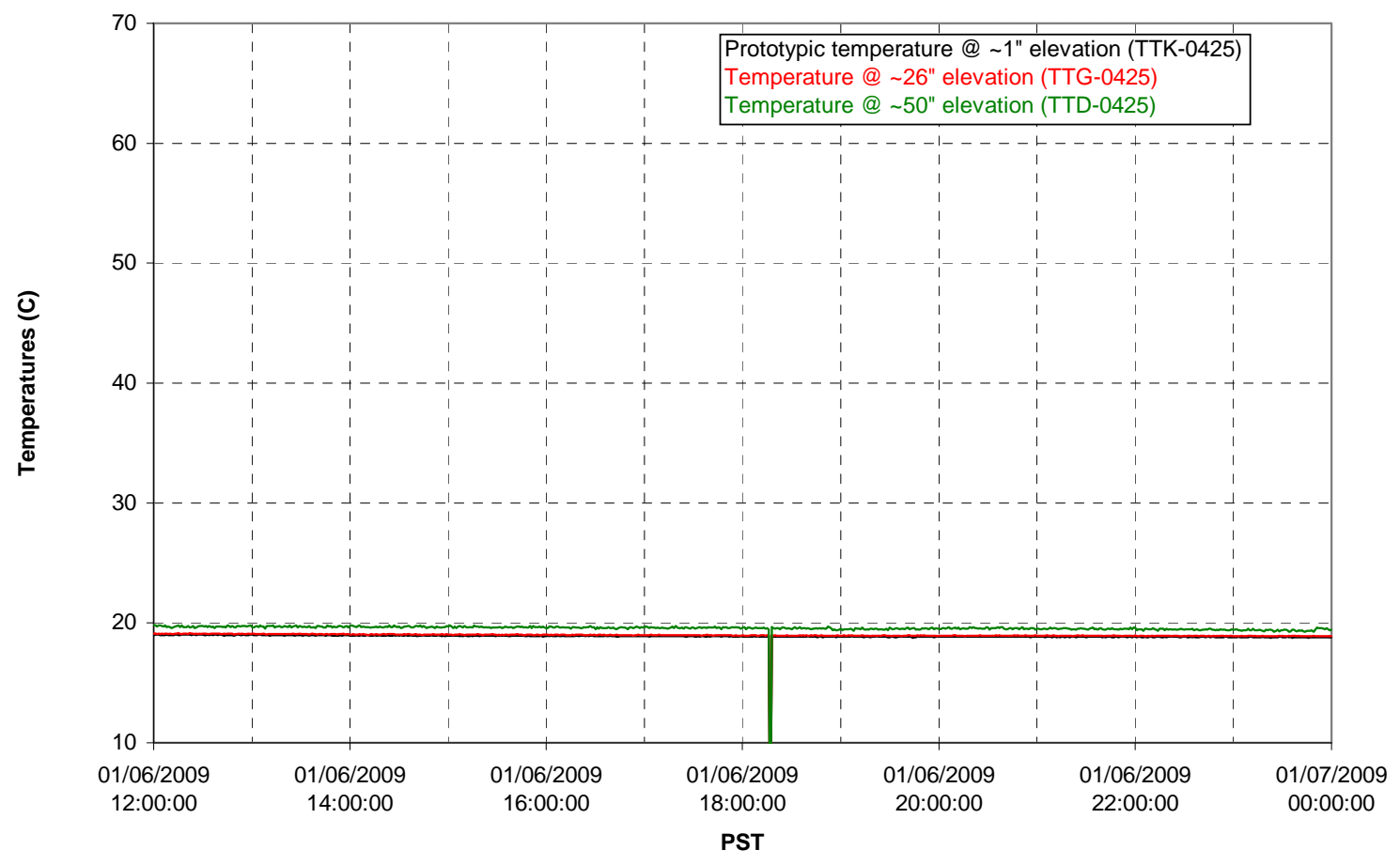


T02A level

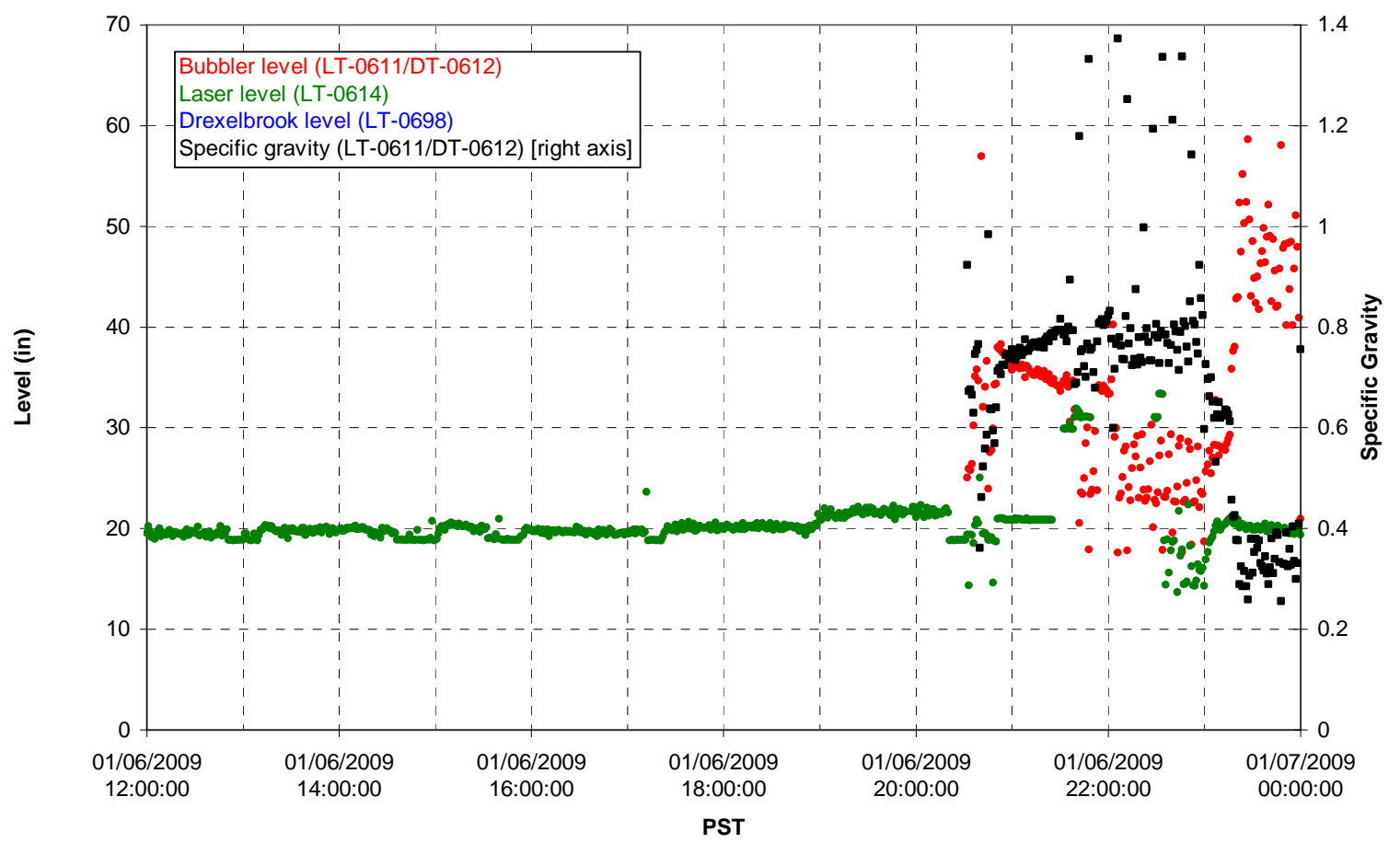

T02A temperatures

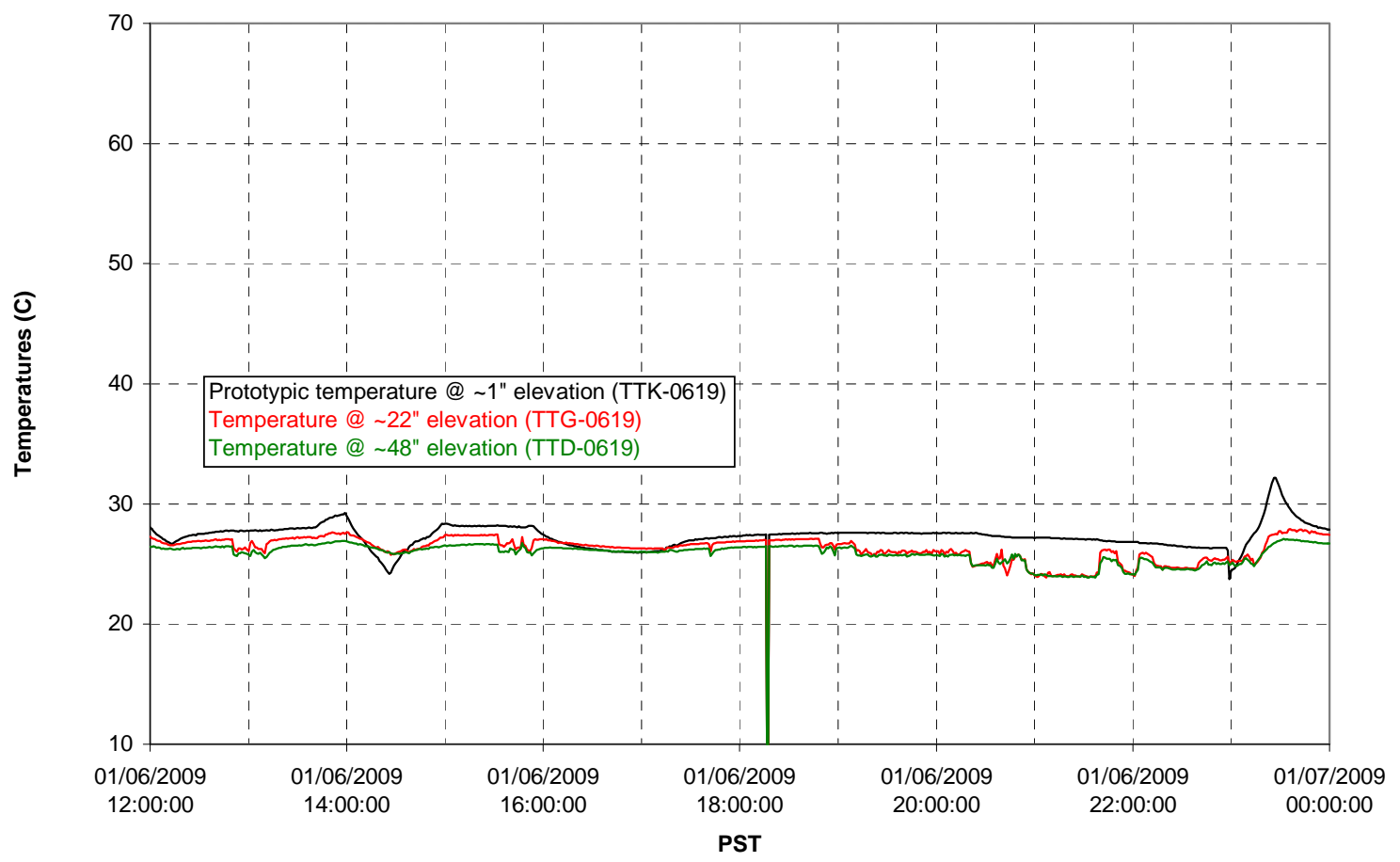


T02A and filter loop temperatures
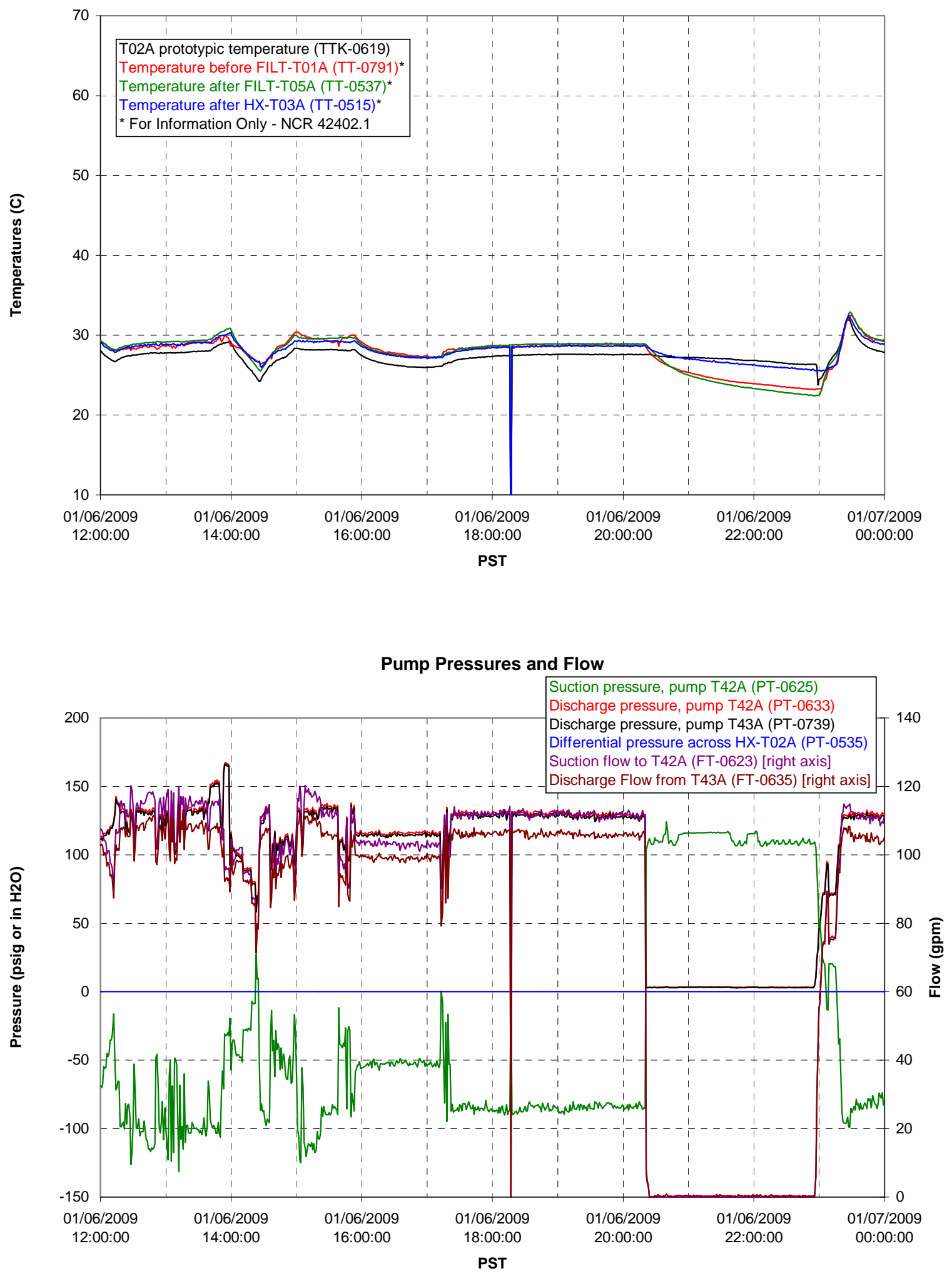
Axial pressure drop

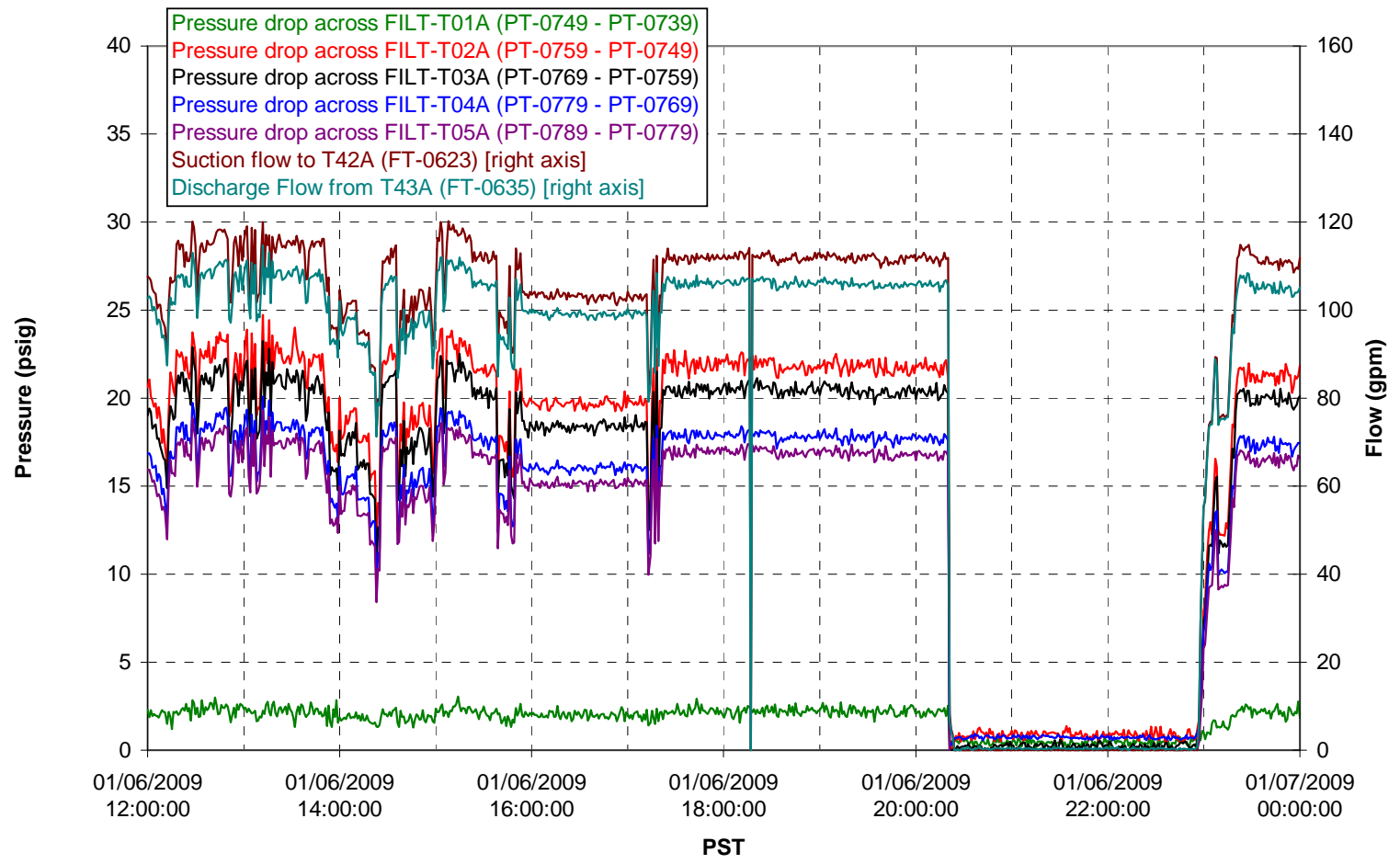

Permeate flow rates

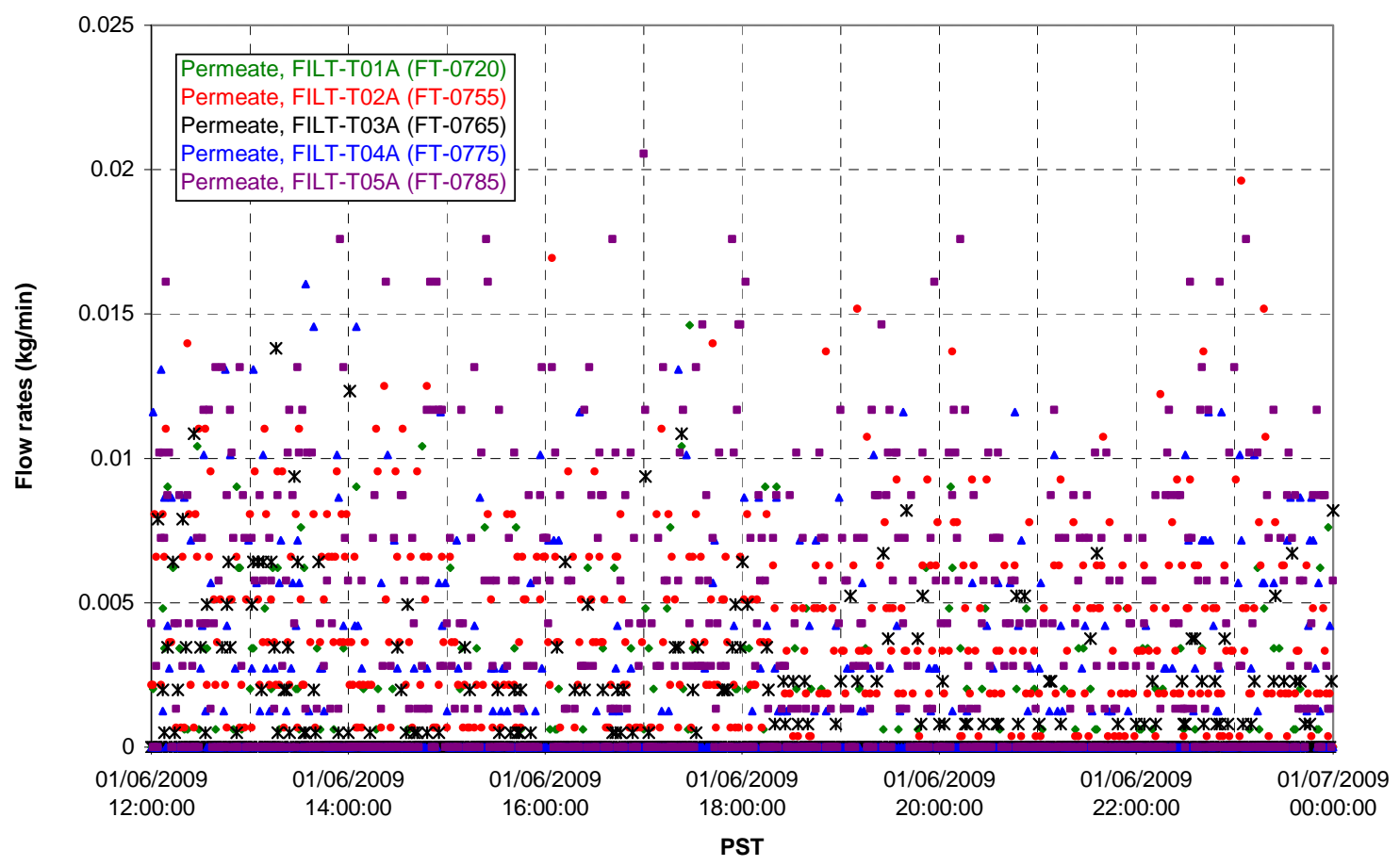


T02A Inner Temperature Tree

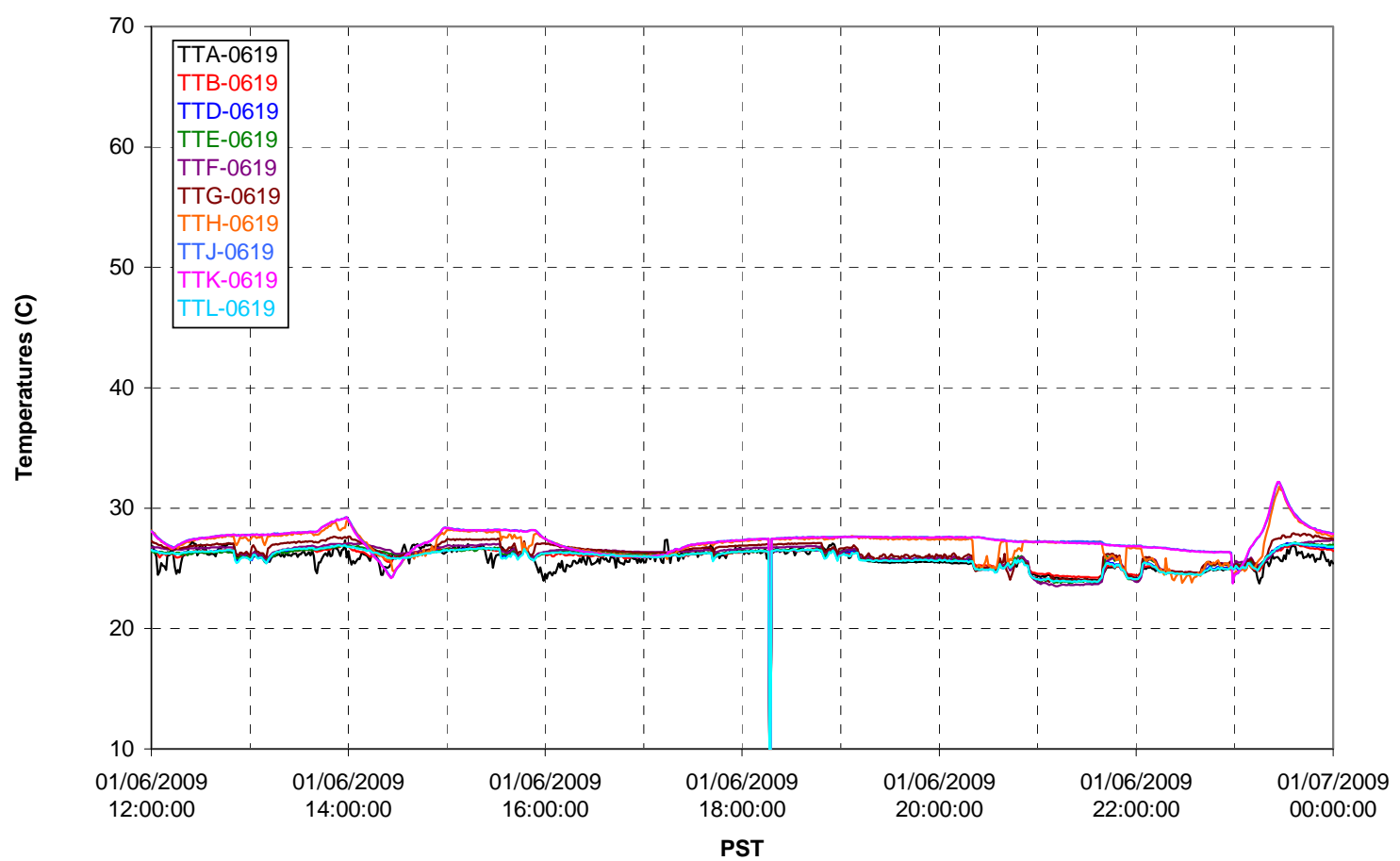

T02A Outer Temperature Tree

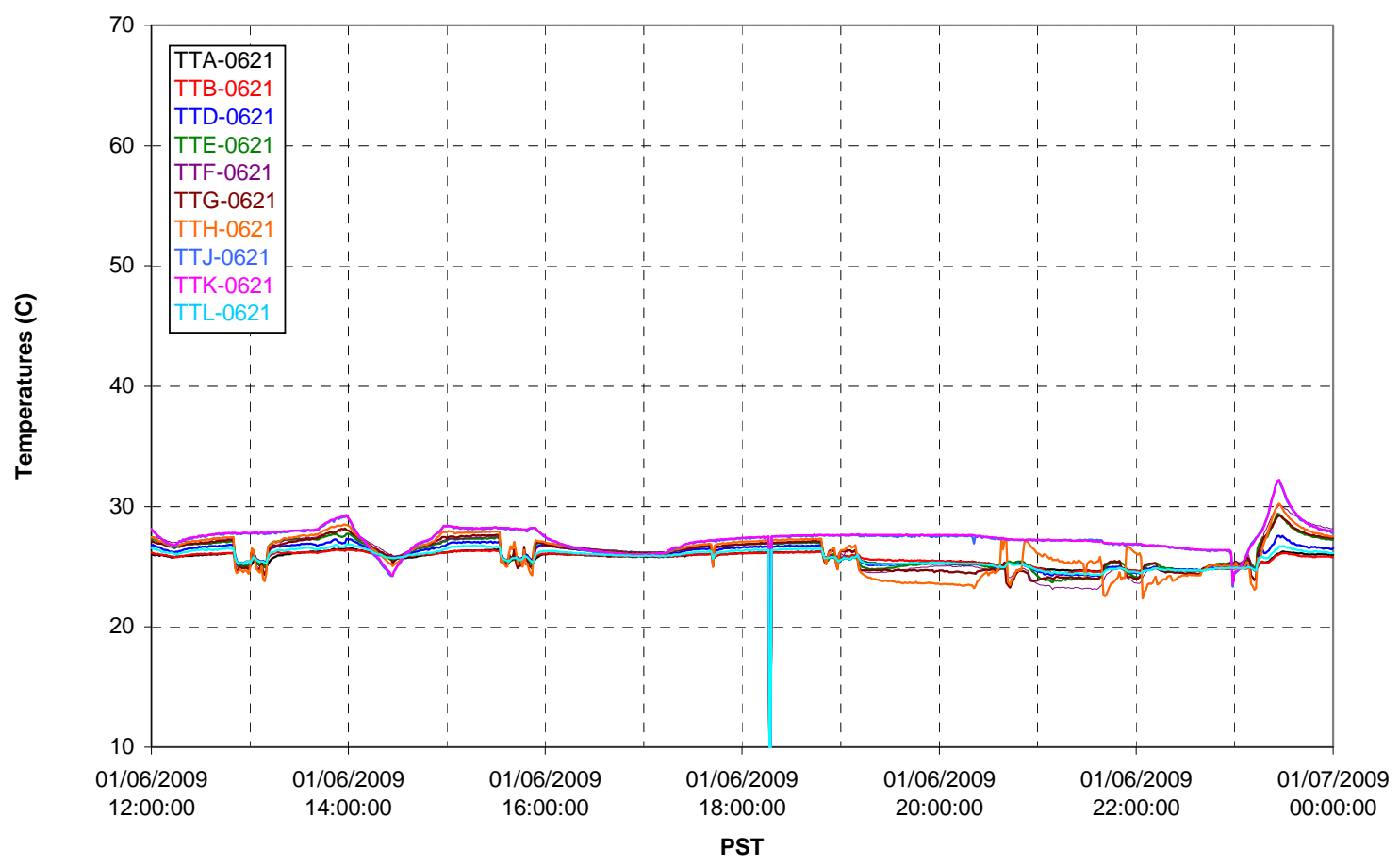


T02A temperatures

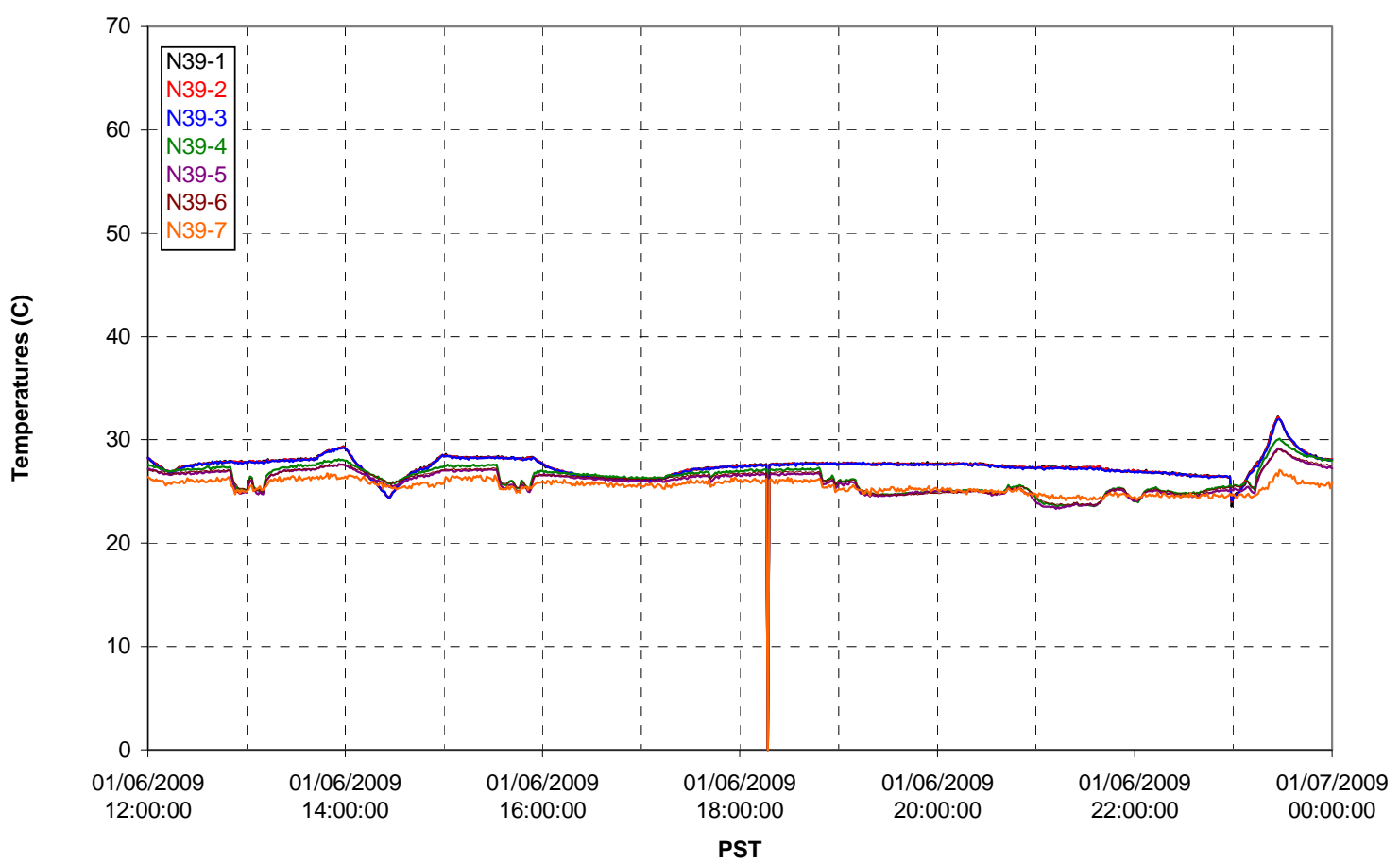

T02A temperatures

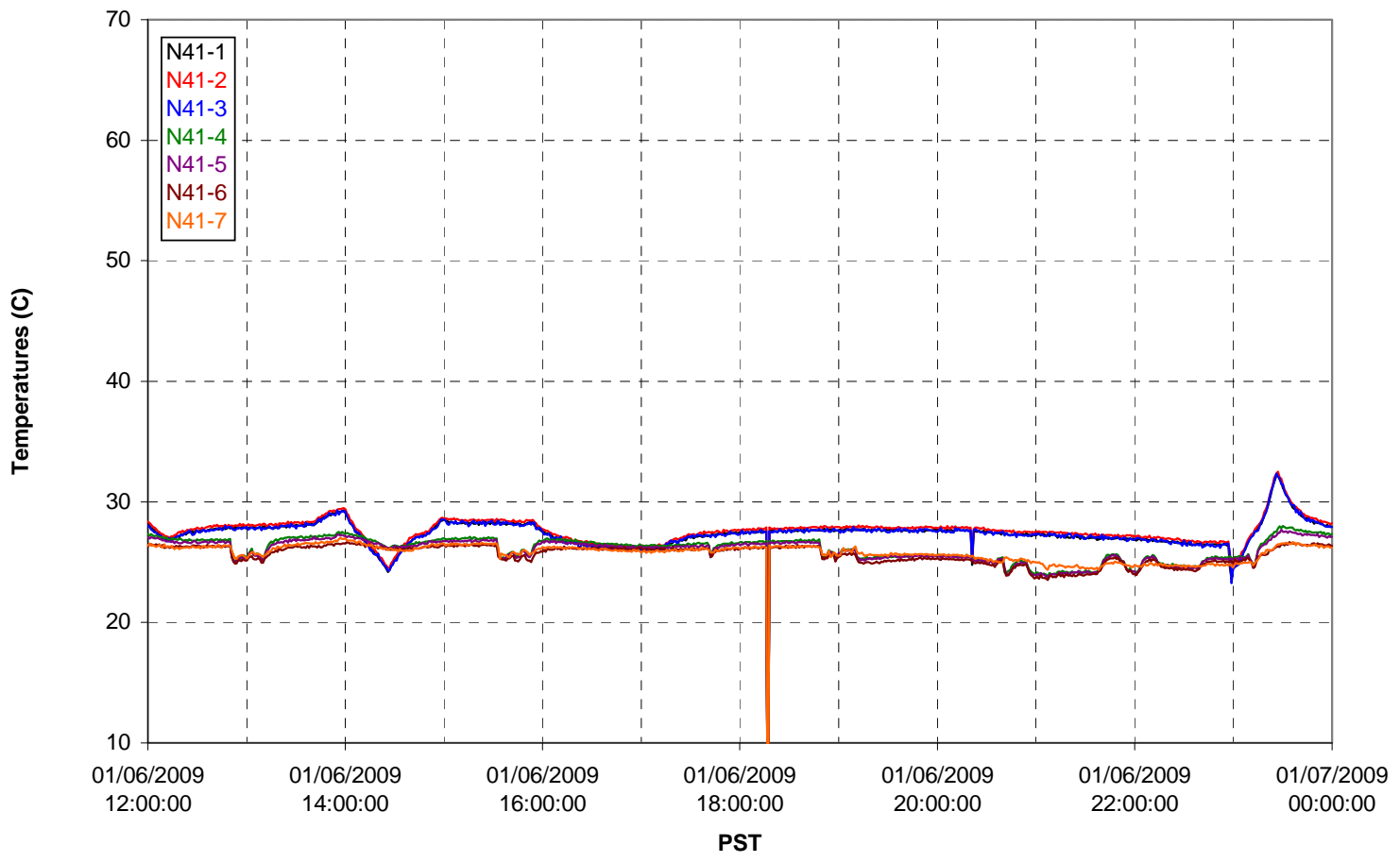


T02A temperatures

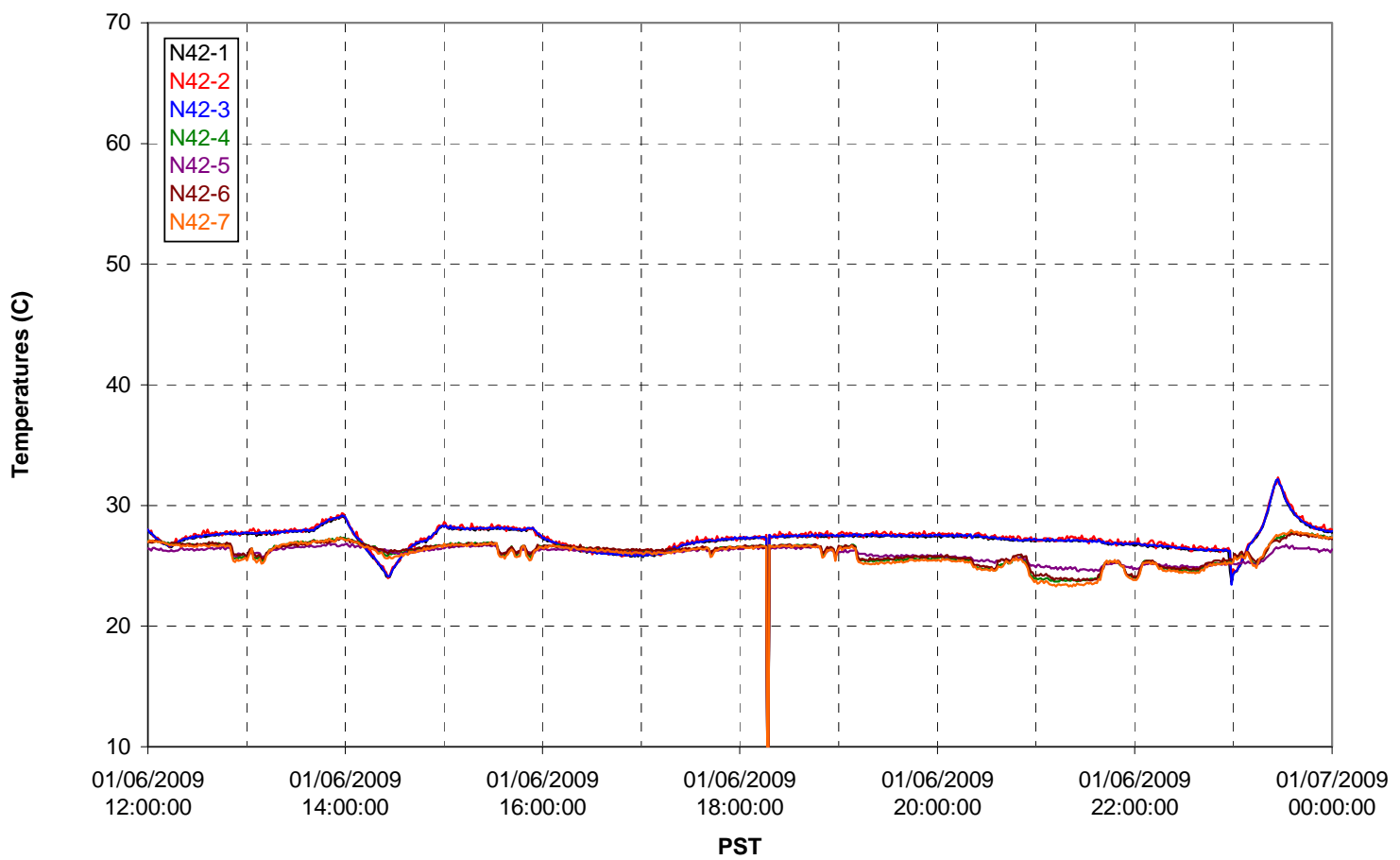

T02A temperatures

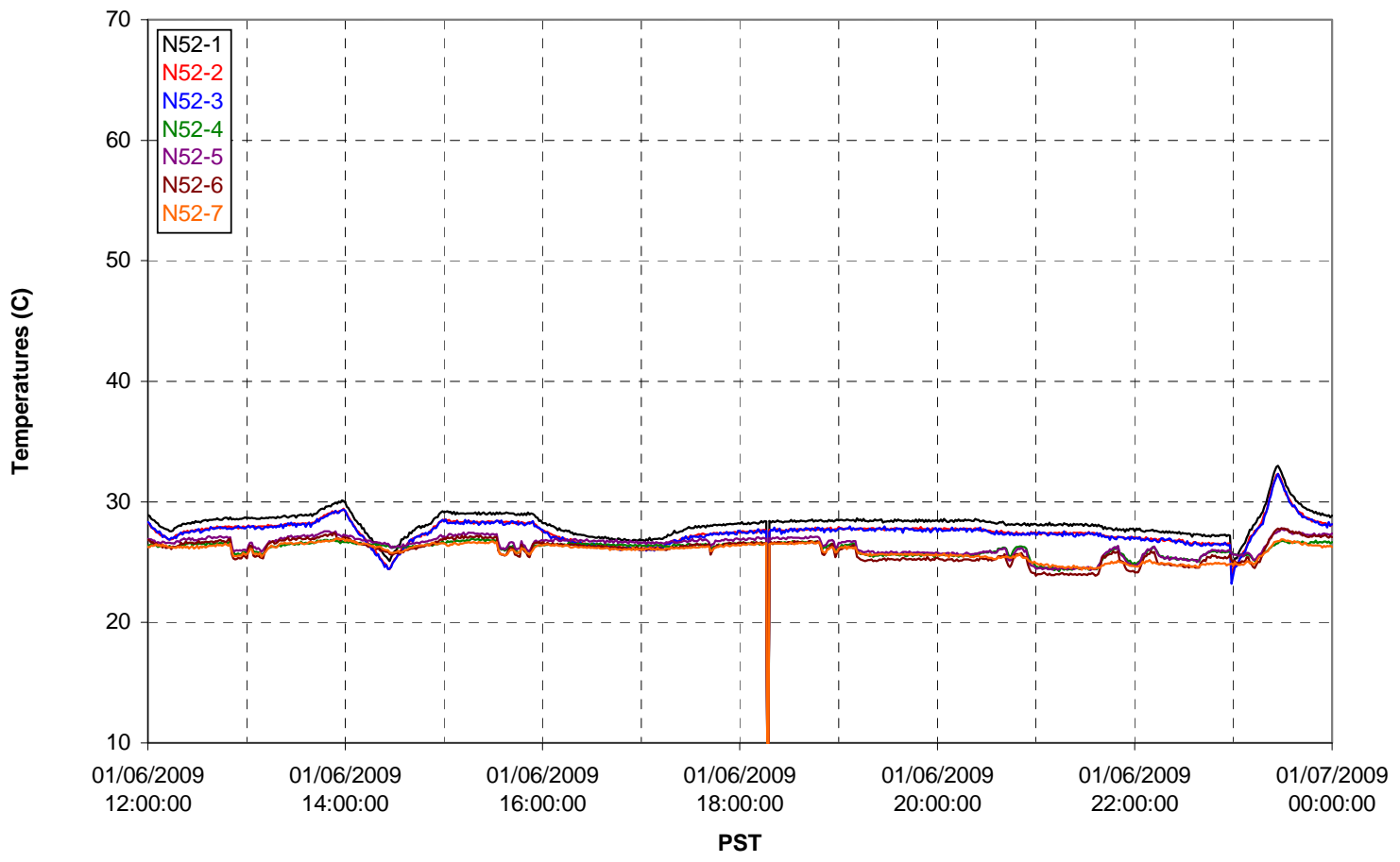


T02A Heating and Cooling
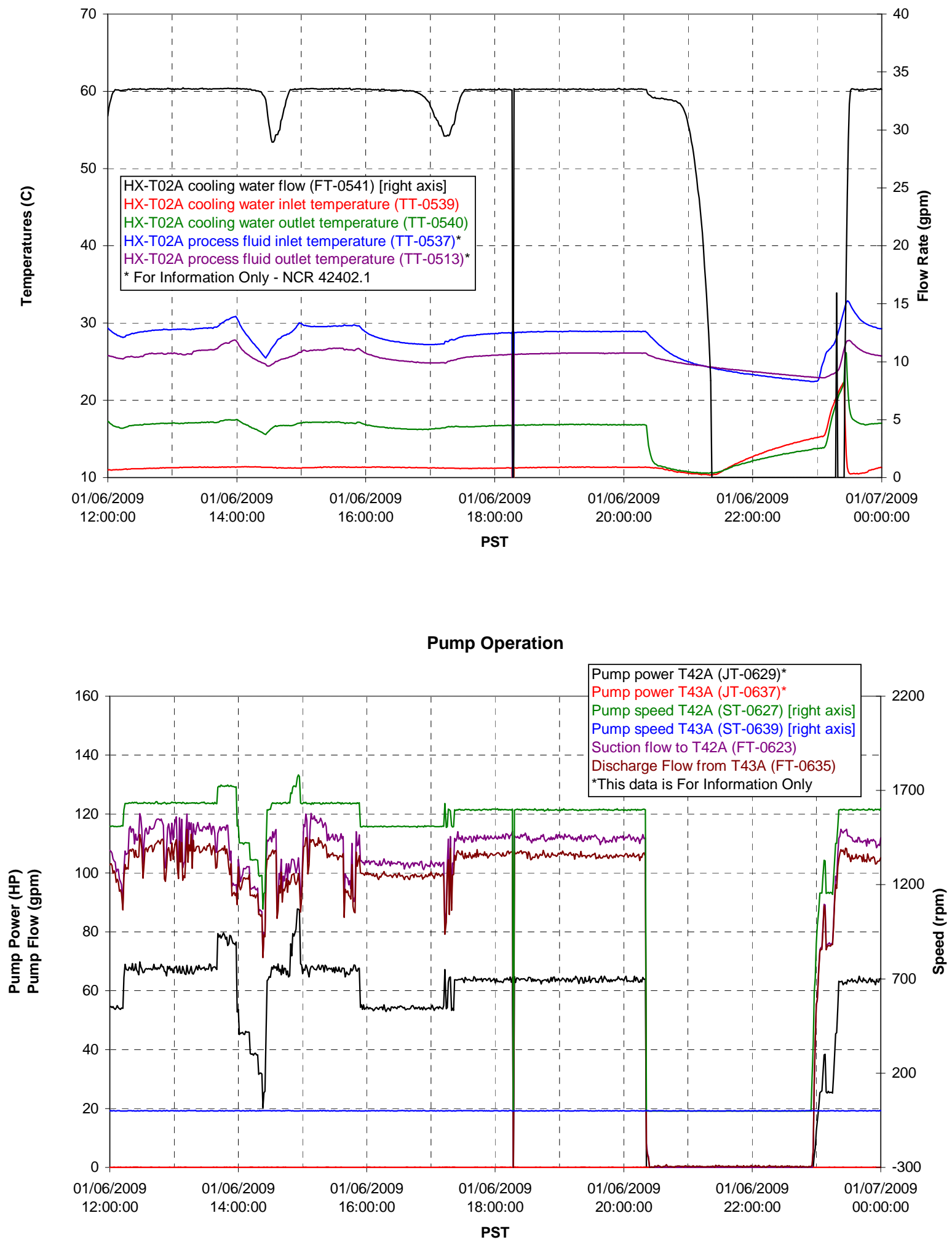
Pulsepot UFP-PP-T01A

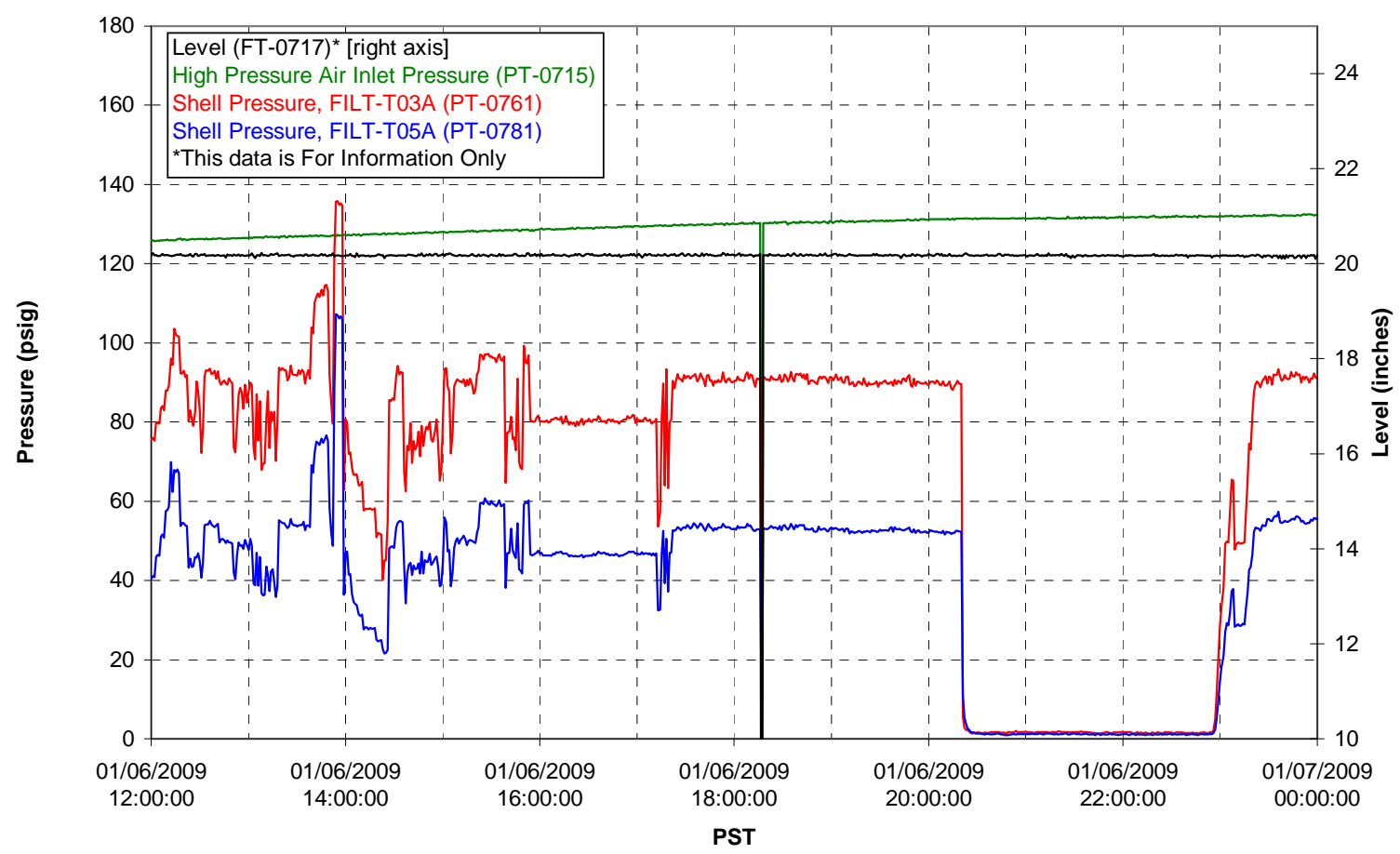

Pulsepot UFP-PP-T02A

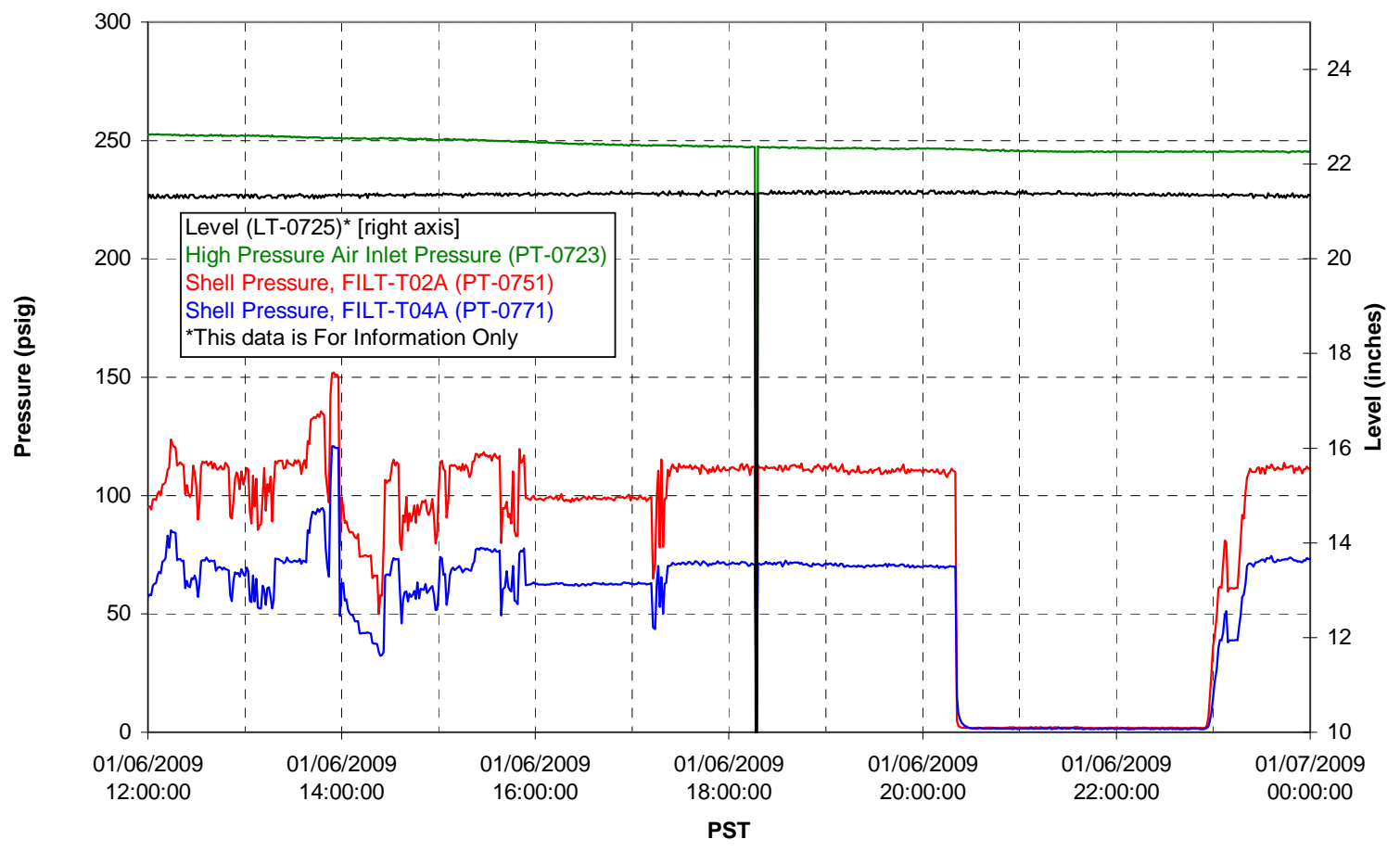


Pulsepot UFP-PP-T03A

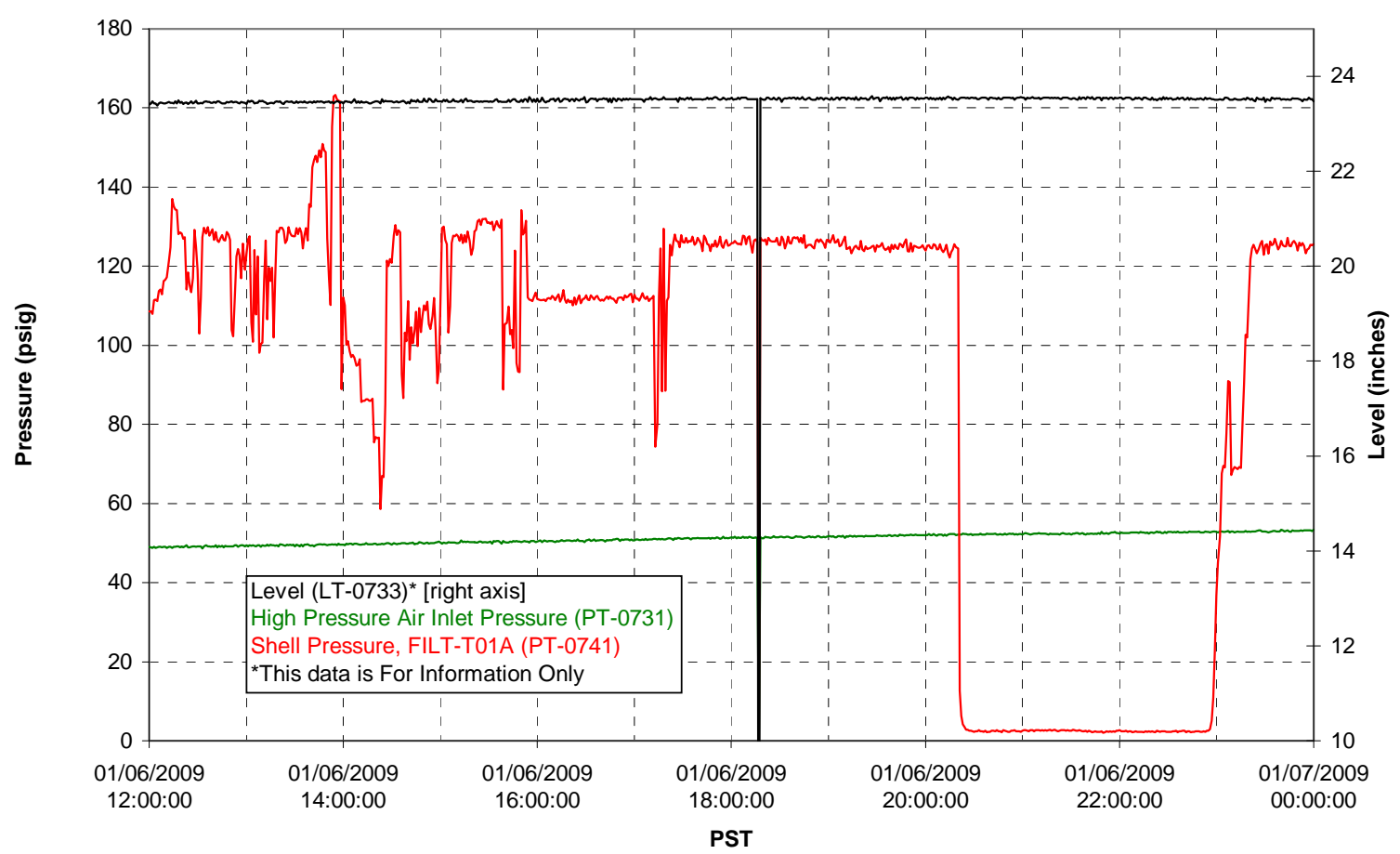

Pulsepot Levels

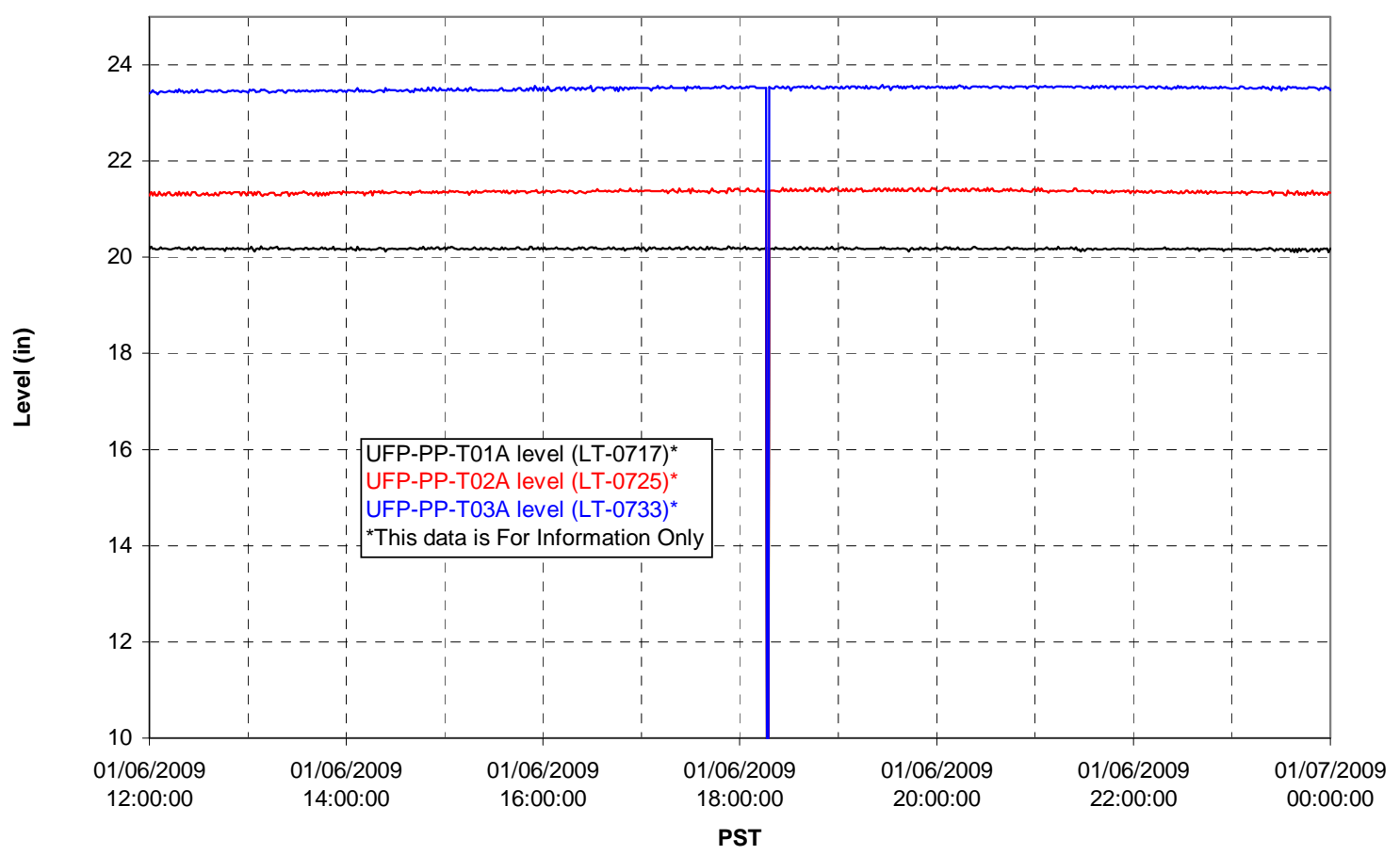


Filter UFP-FILT-T01A

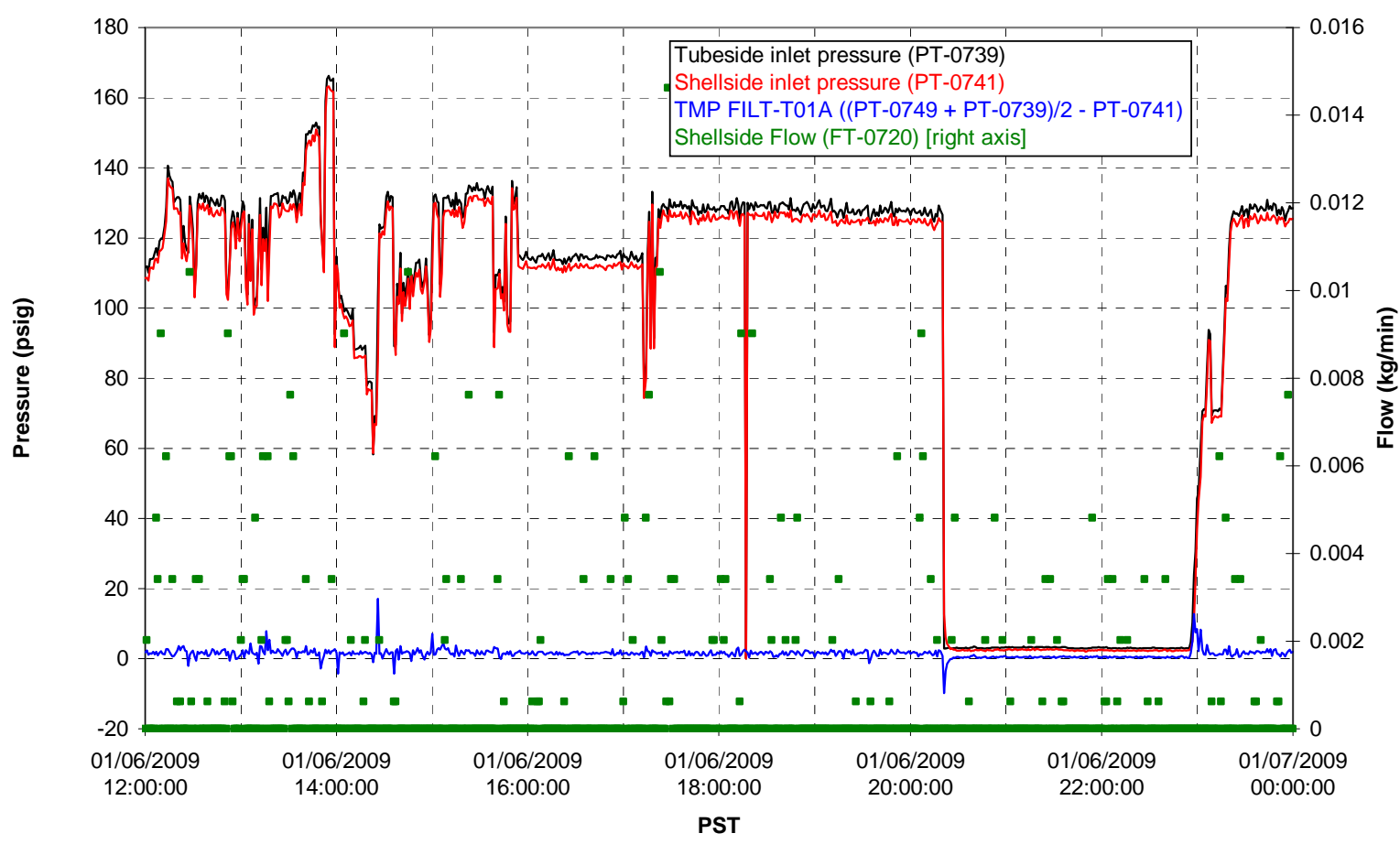

Filter UFP-FILT-T02A

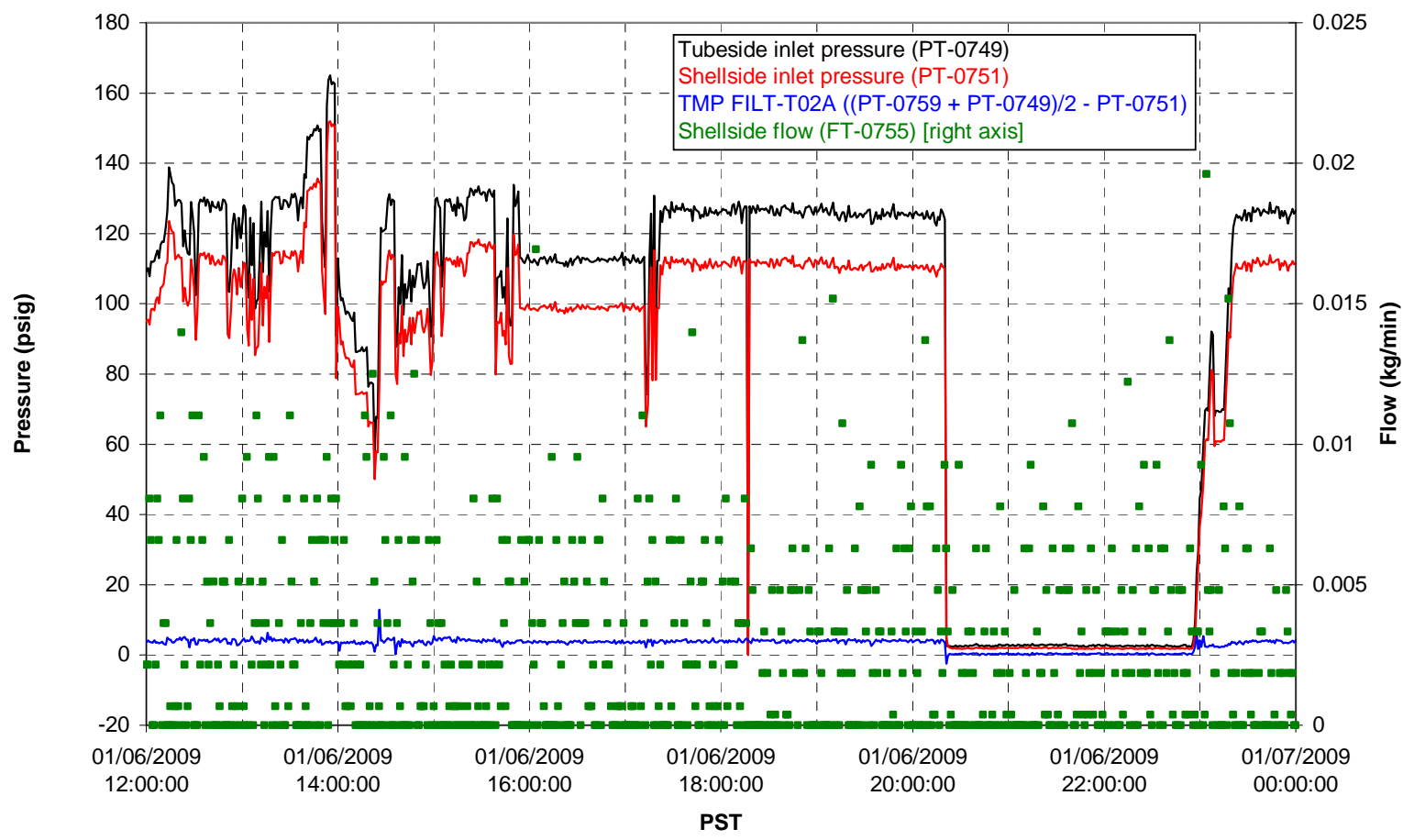


Filter UFP-FILT-T03A

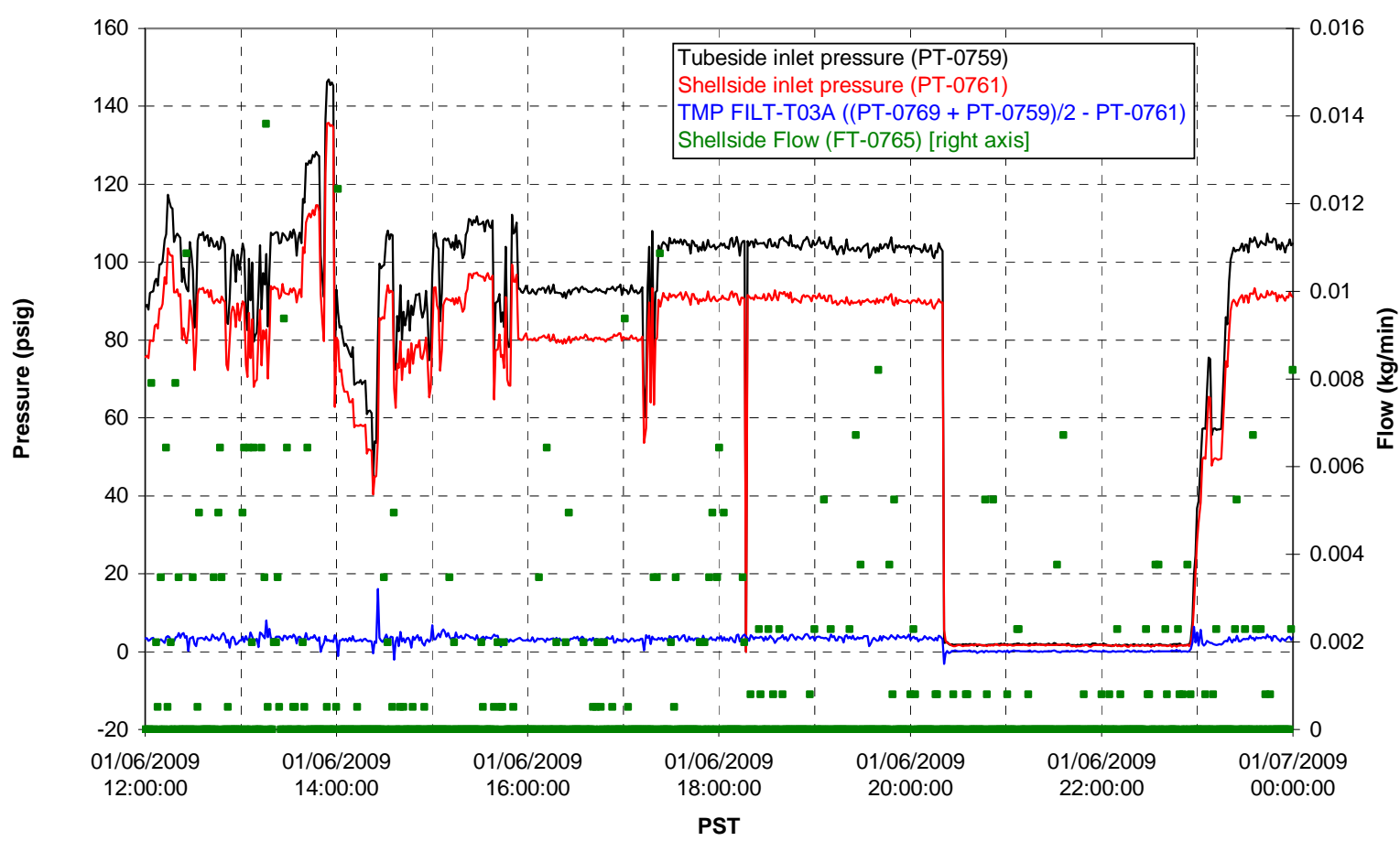

Filter UFP-FILT-T04A

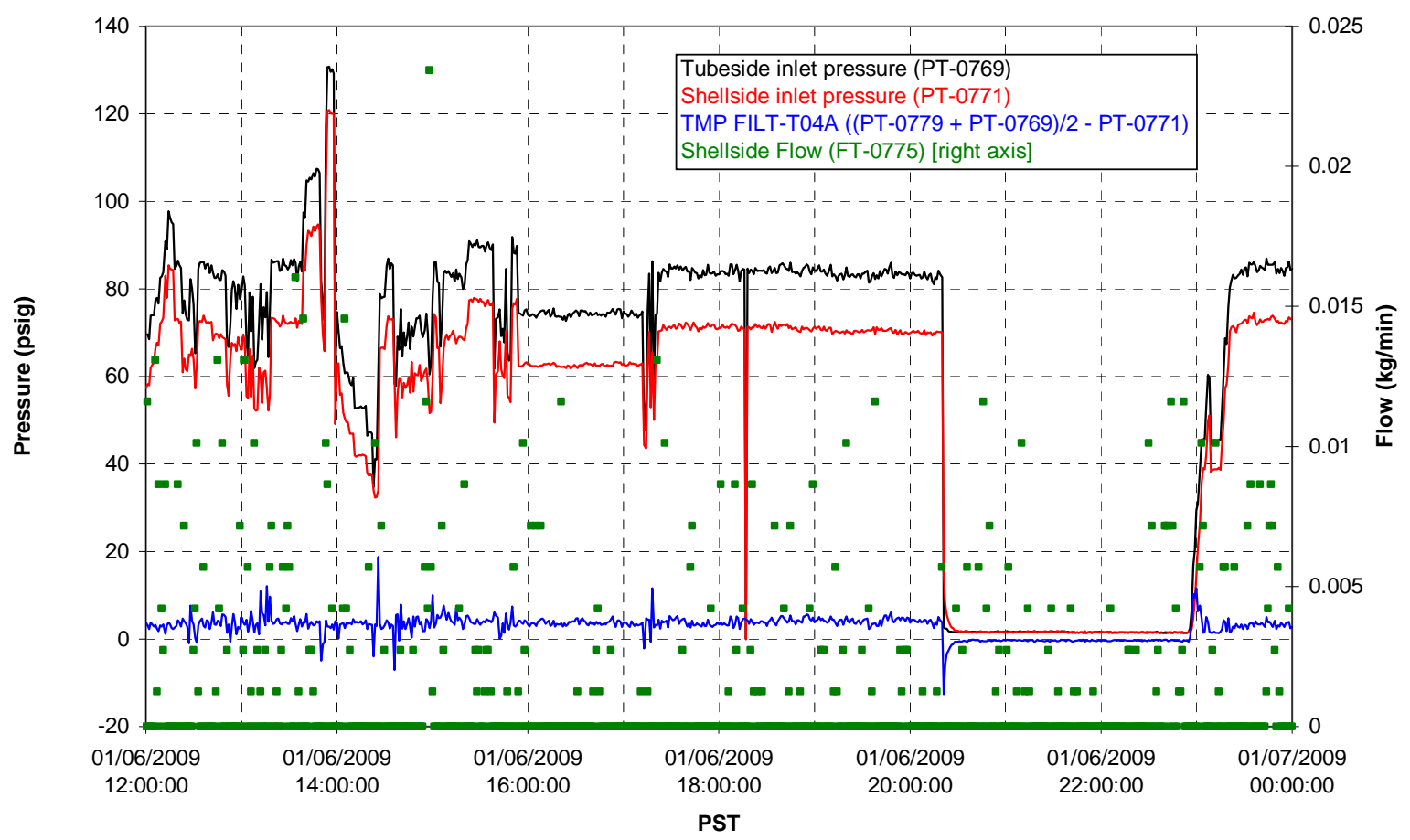


Filter UFP-FILT-T05A

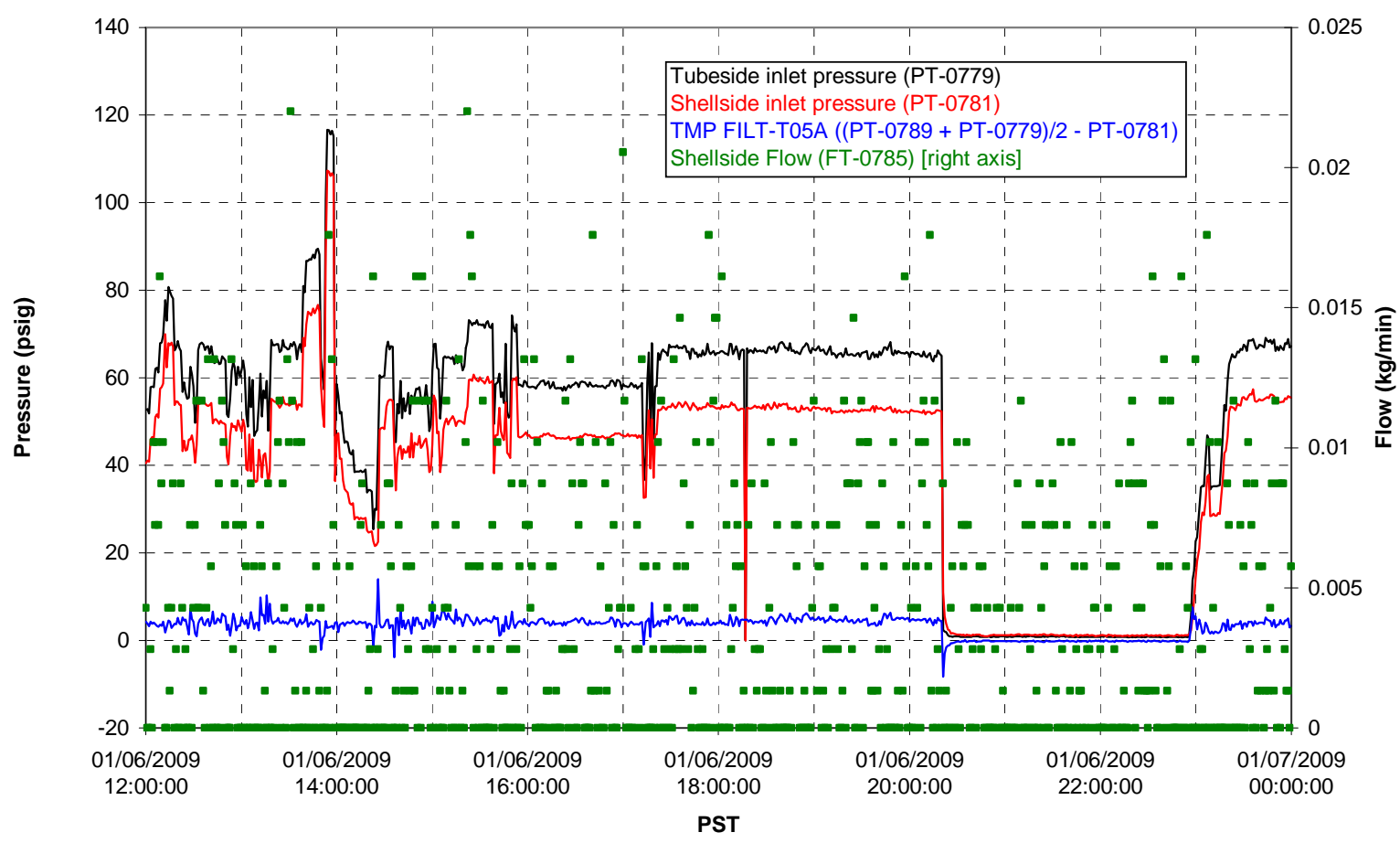

Chemical Flow

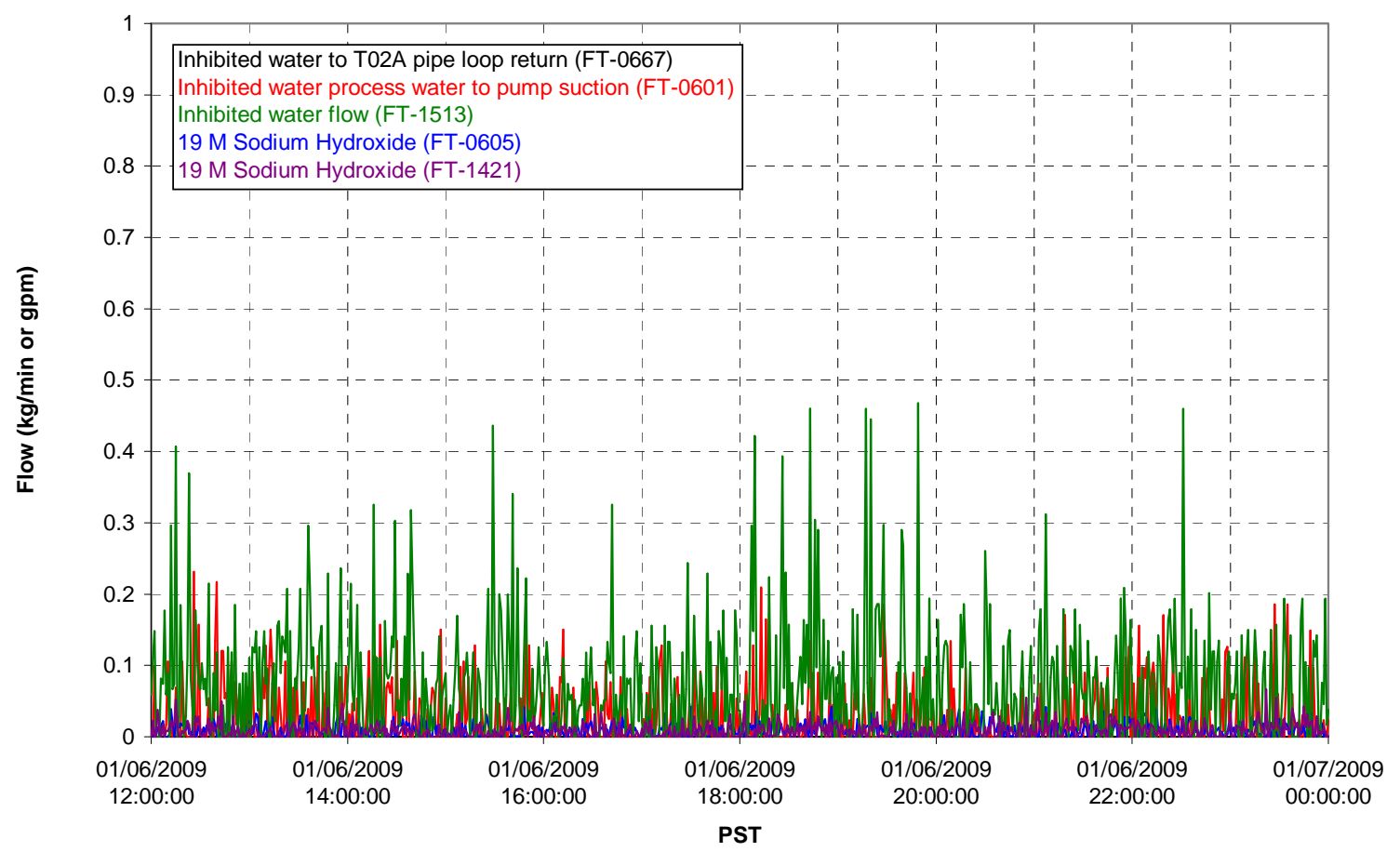


Chemical Flow

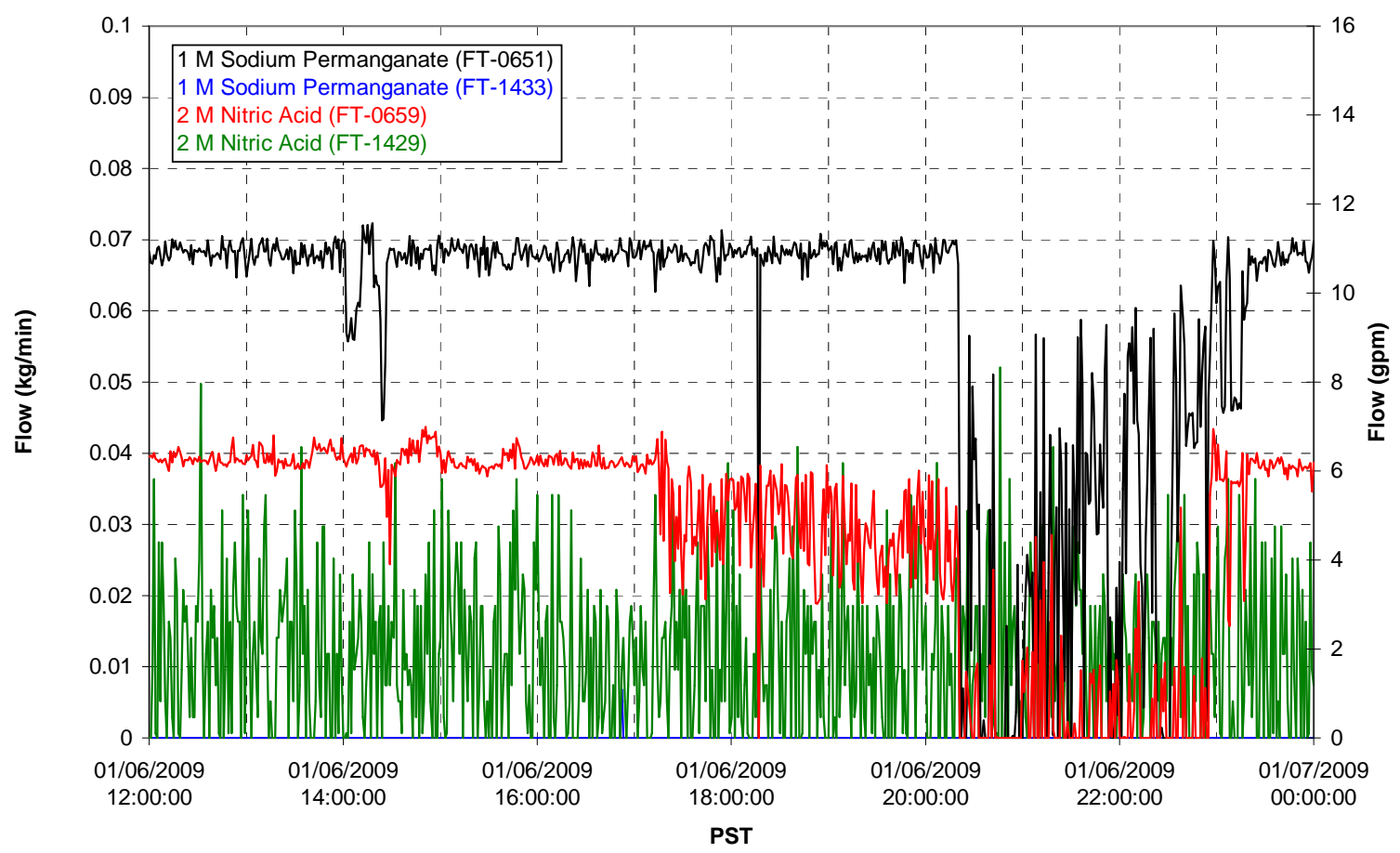

Air Flows

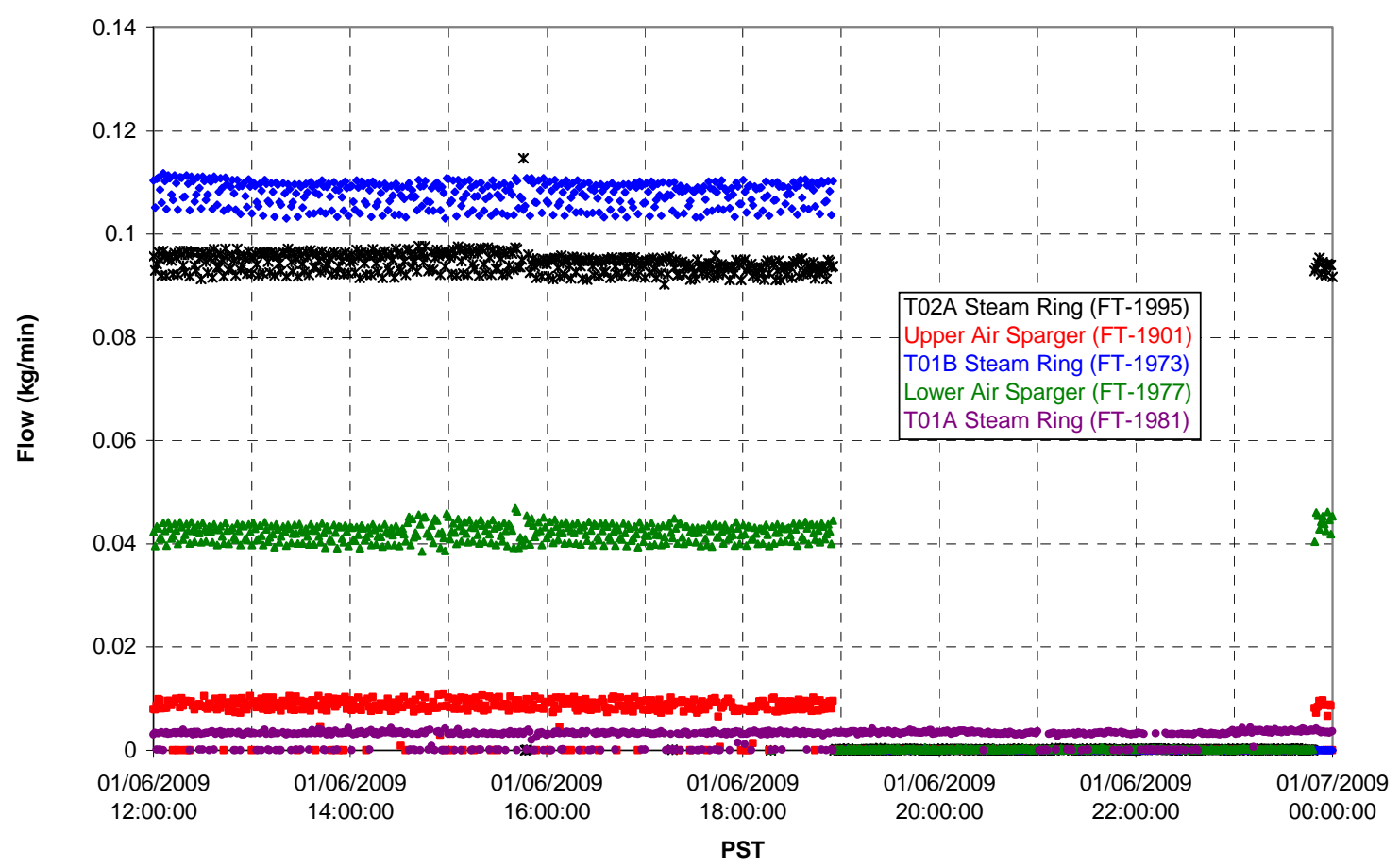


T02A Steam

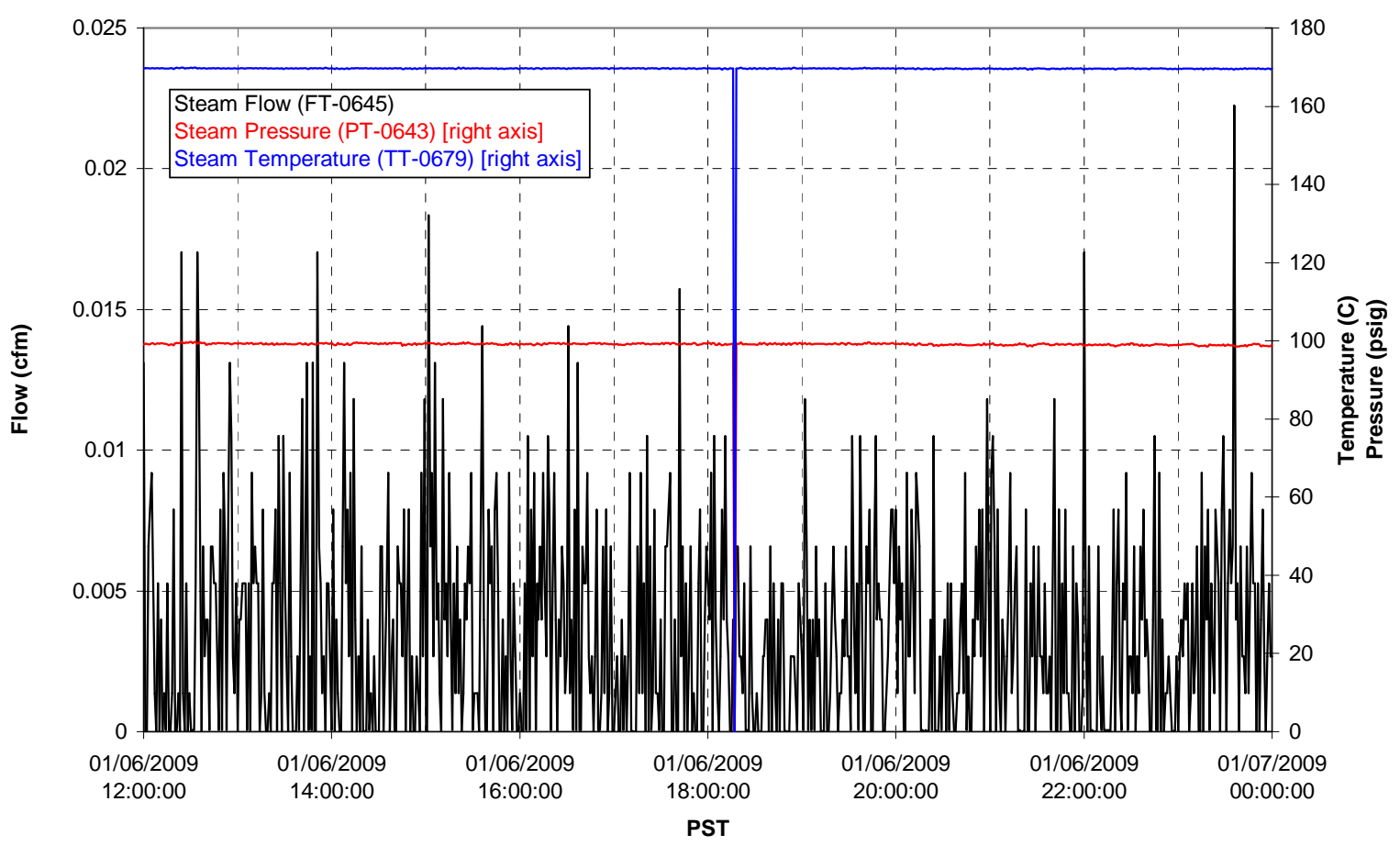

T01A Steam

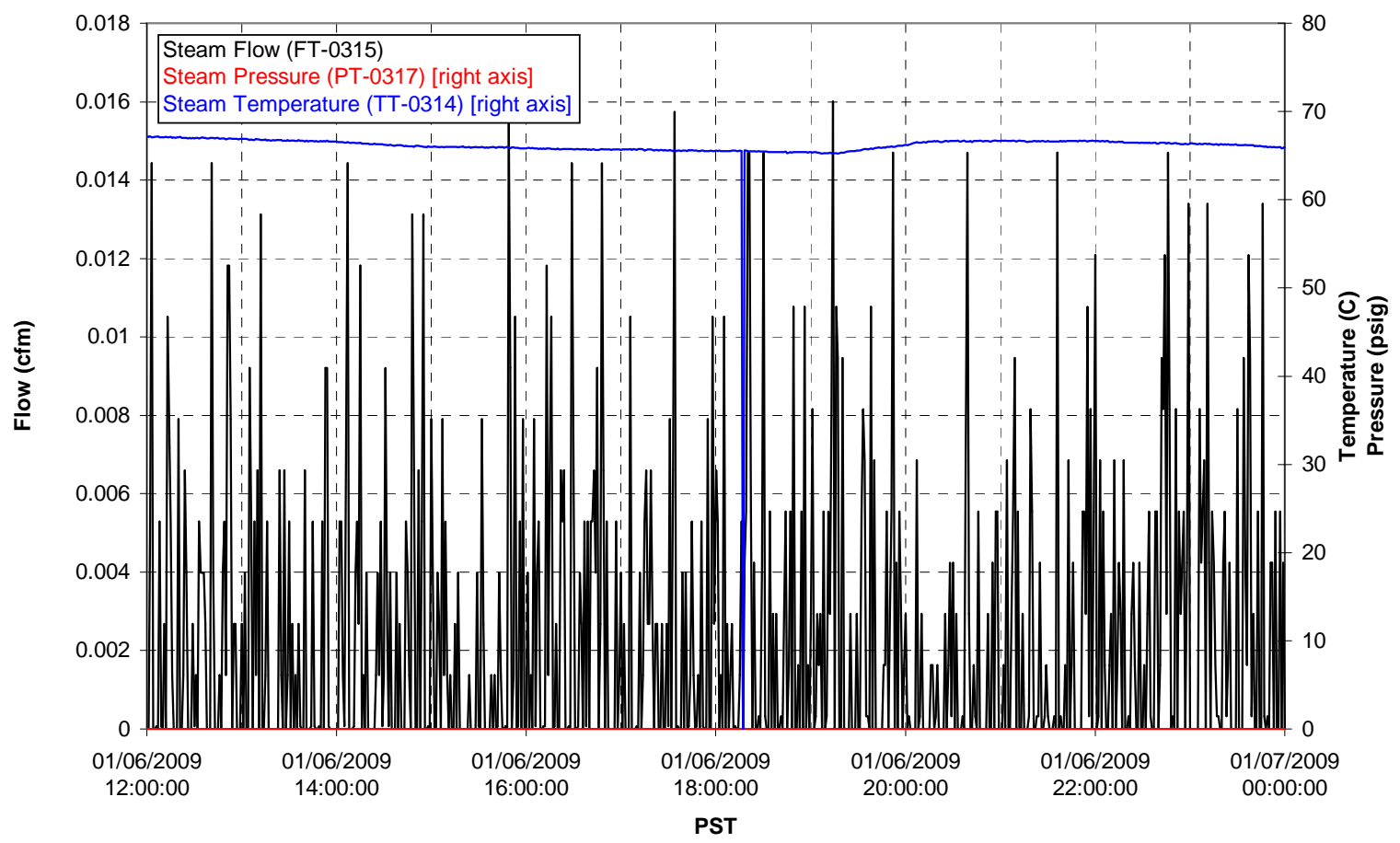


T01B Steam

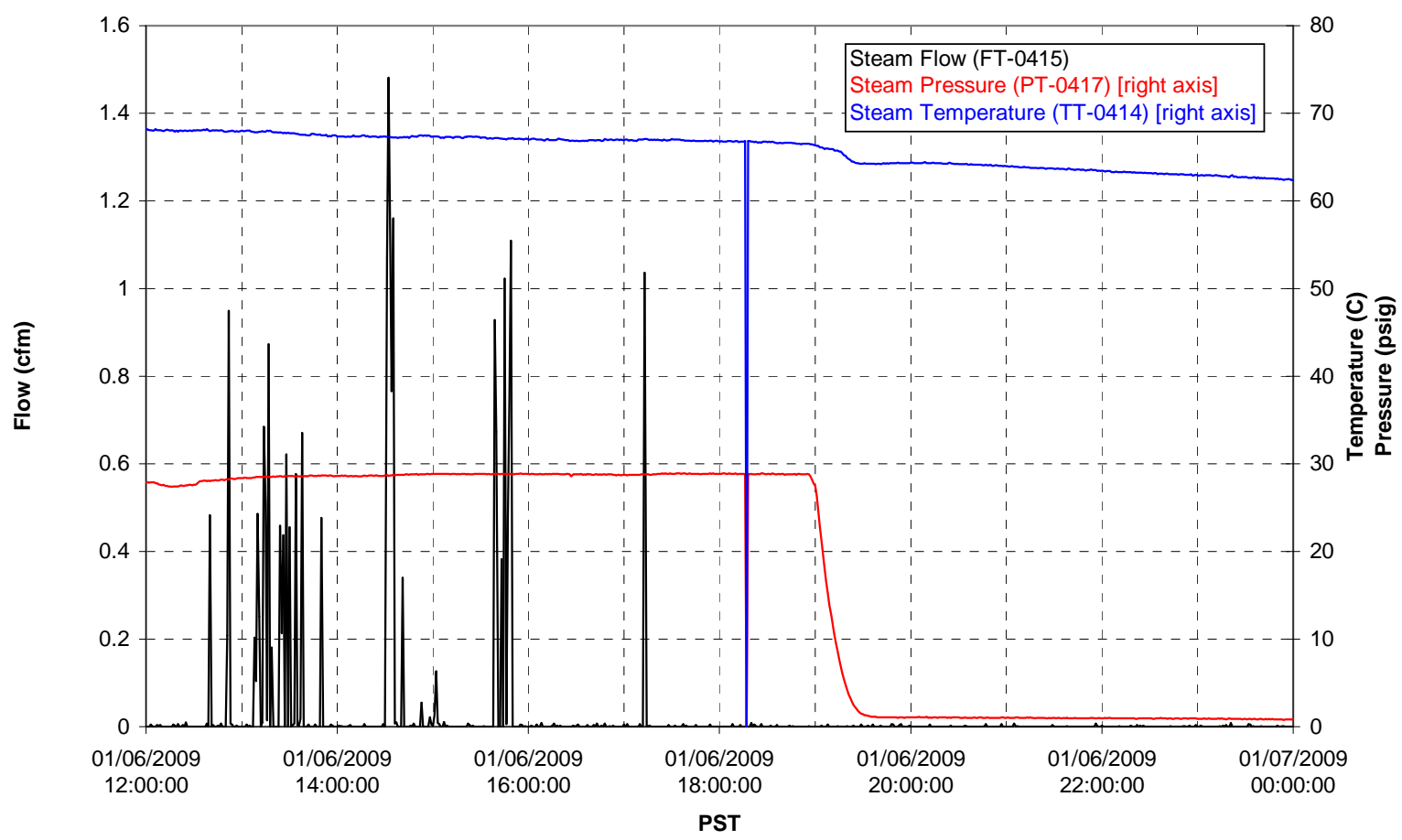


Functional Test Data Plots

01/07/2009 00:00 - 01/07/2009 12:00 
T01A level

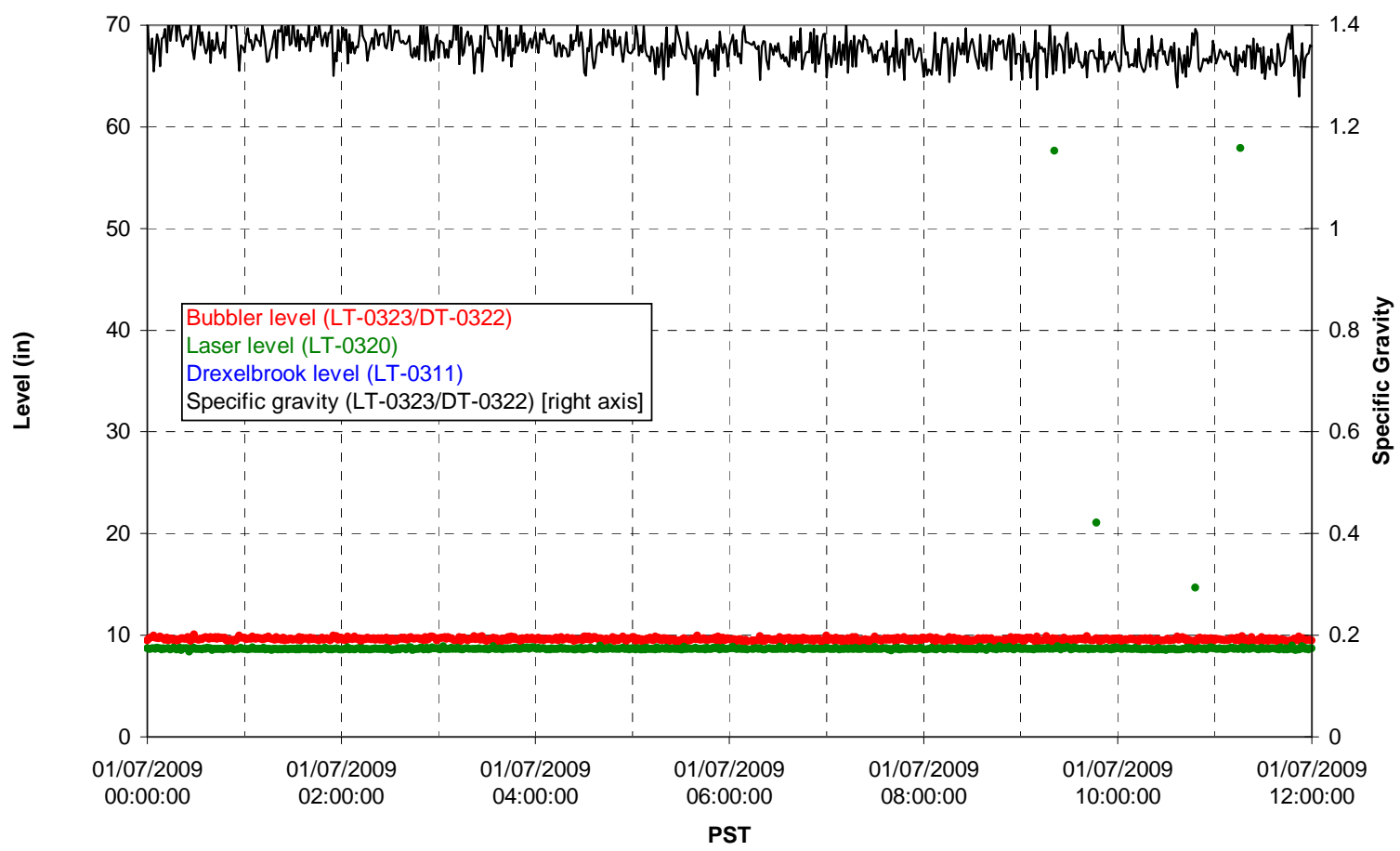

T01A temperatures

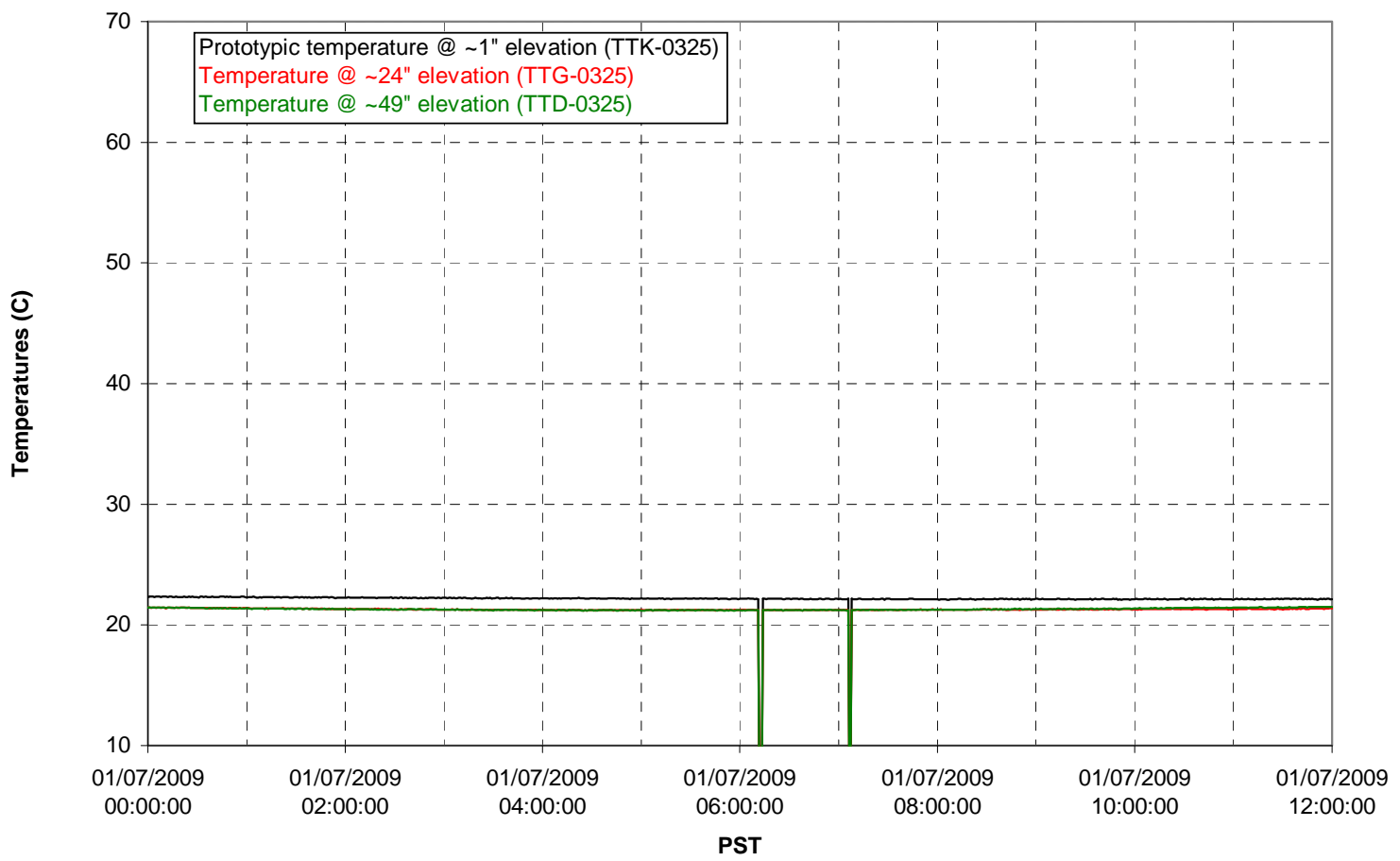


T01B level

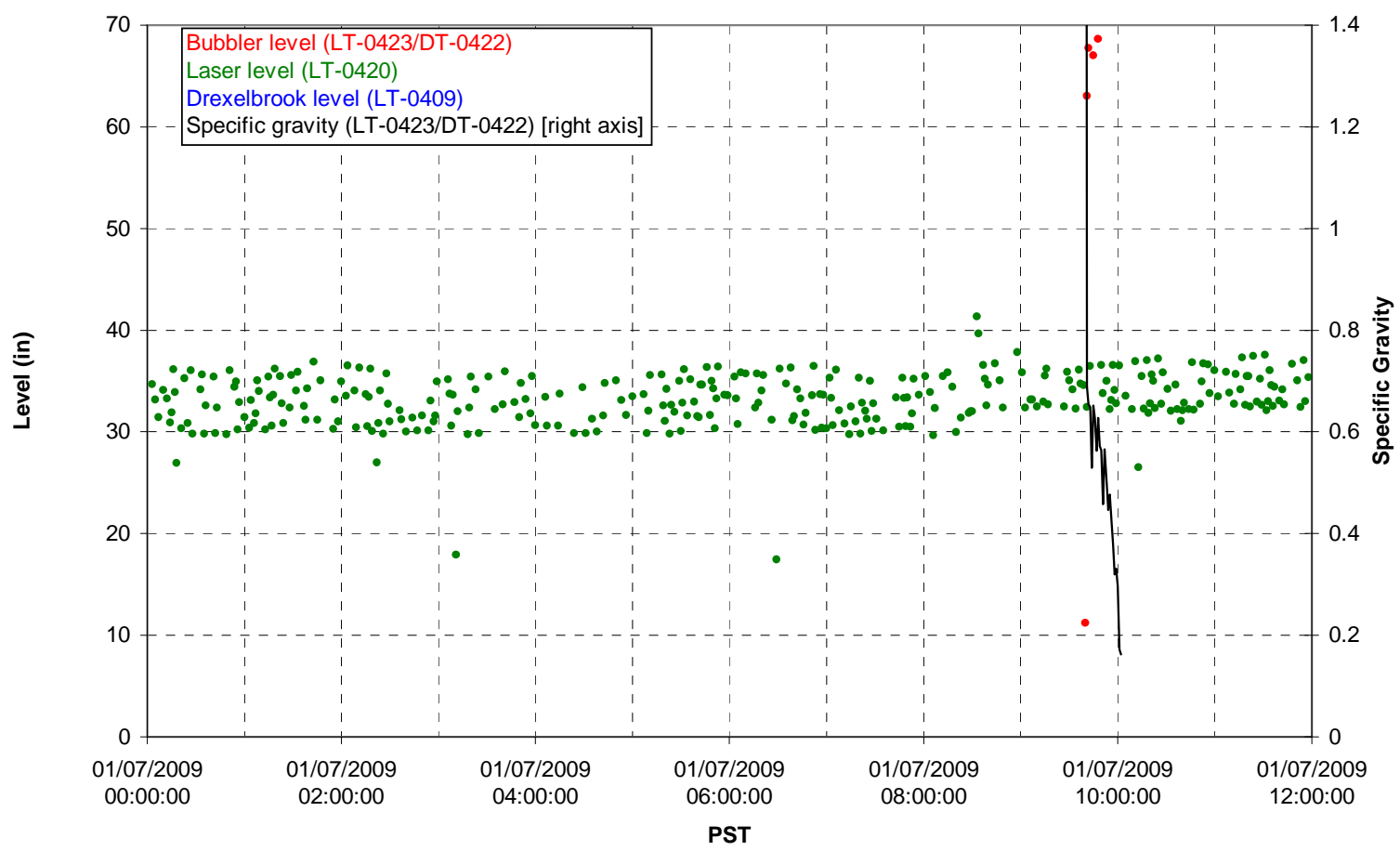

T01B temperatures

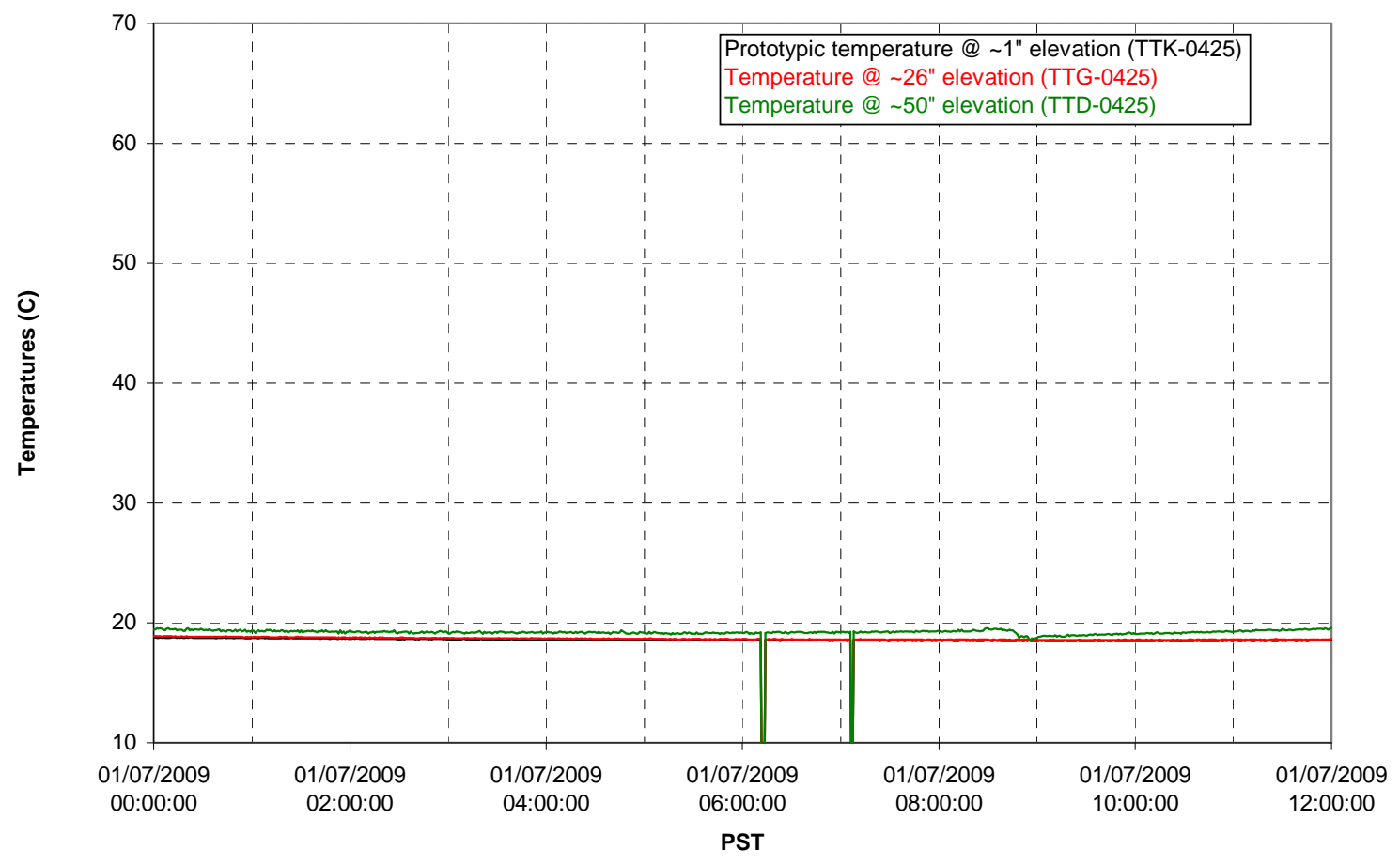


T02A level

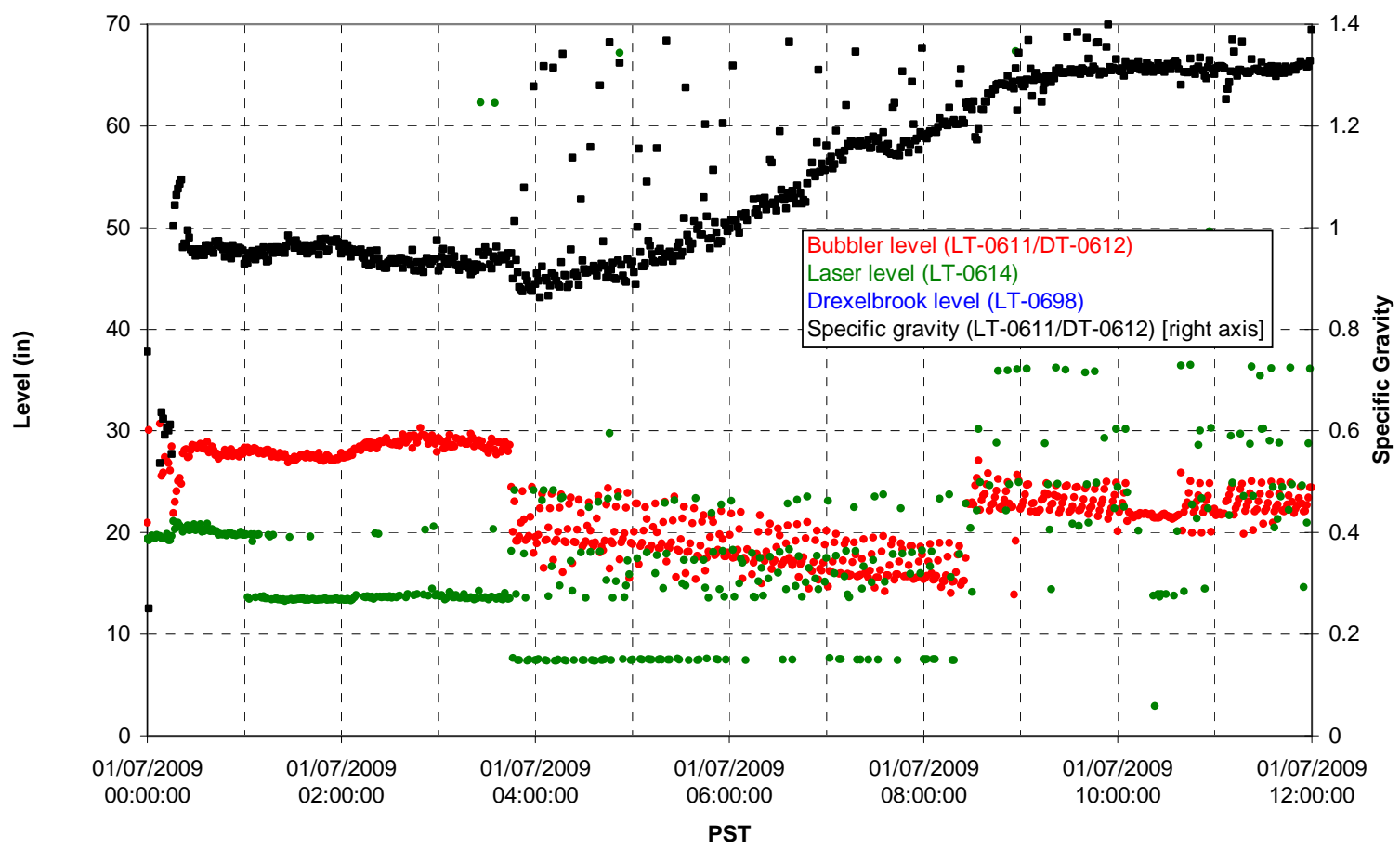

T02A temperatures

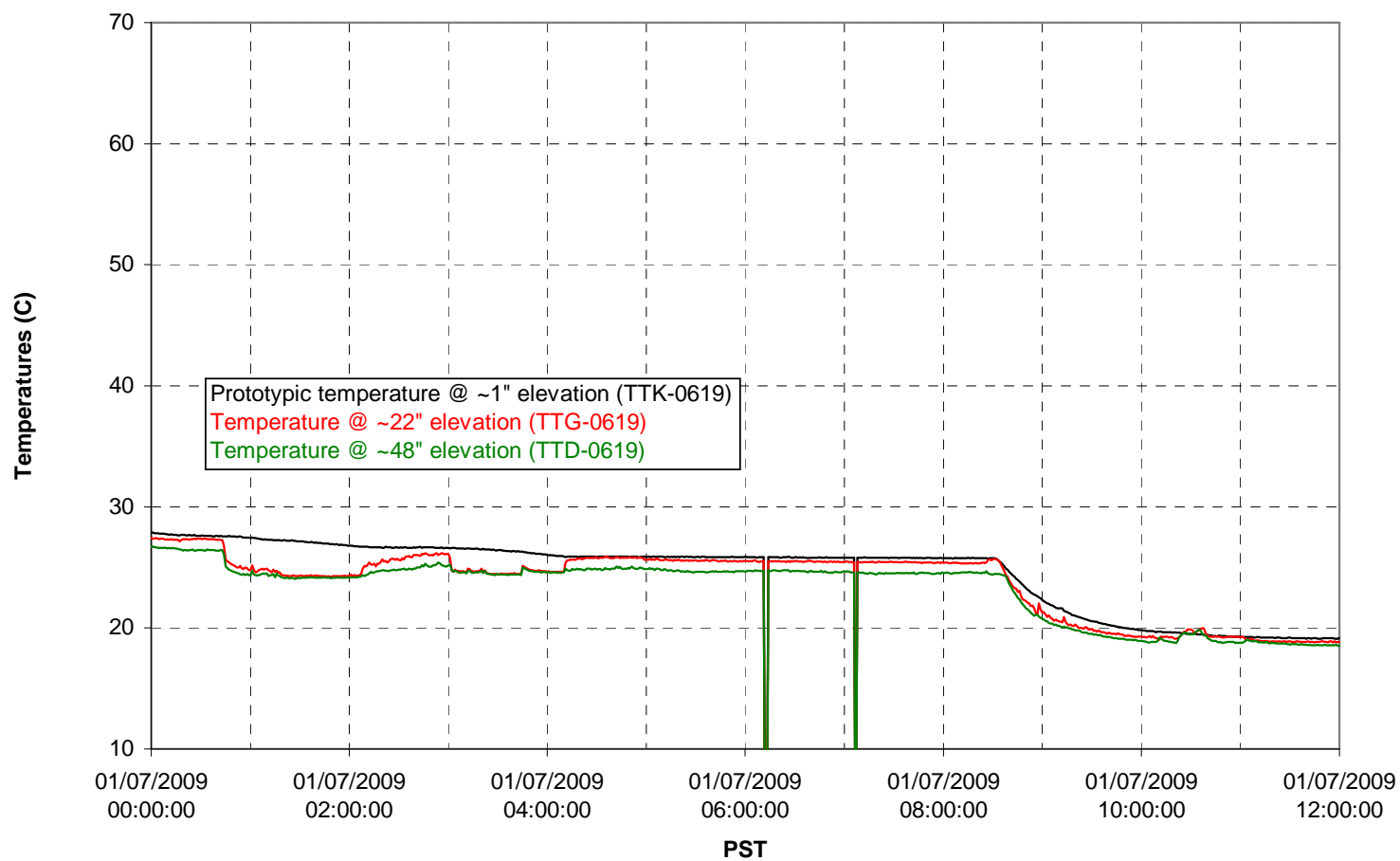


T02A and filter loop temperatures

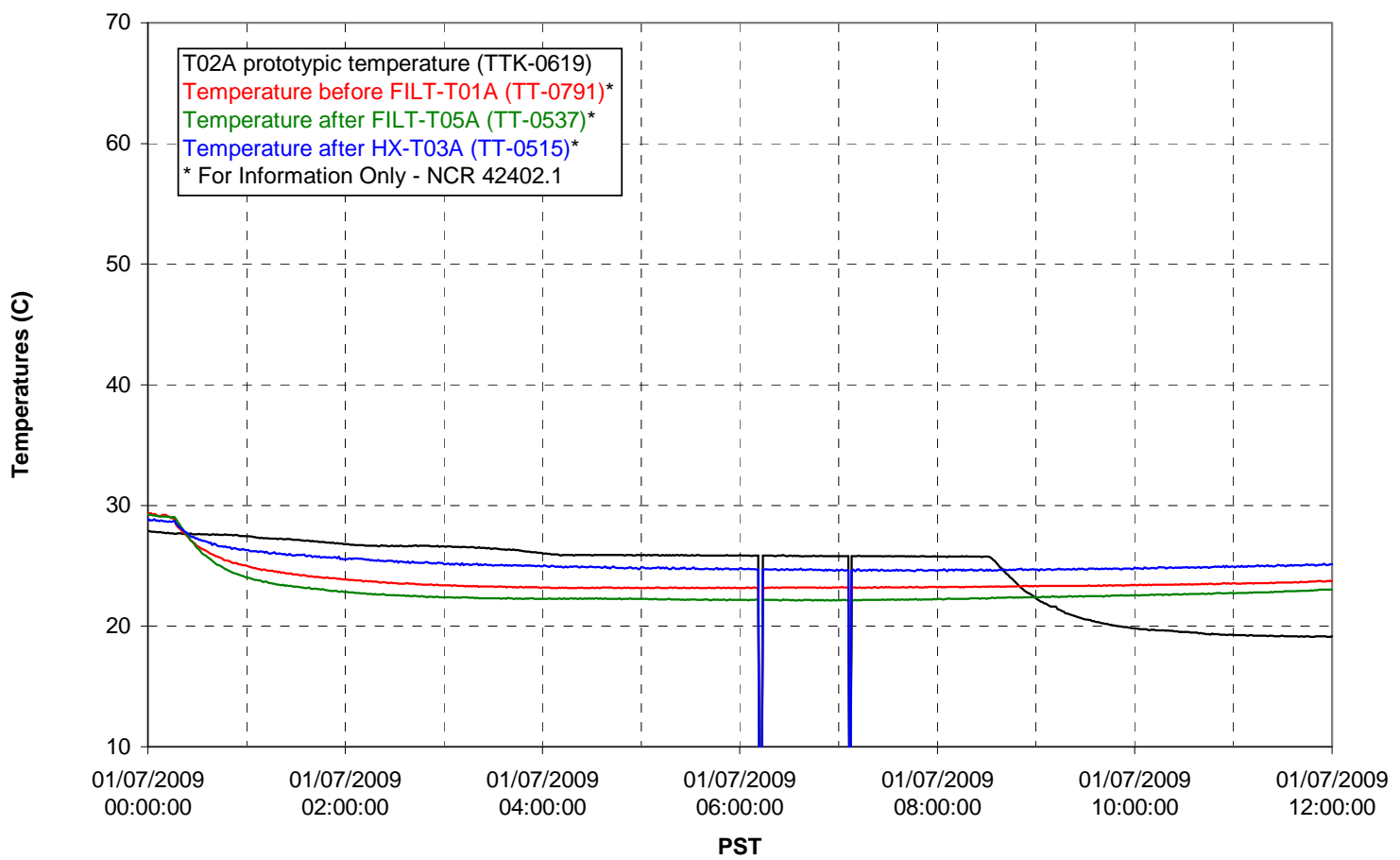

Pump Pressures and Flow

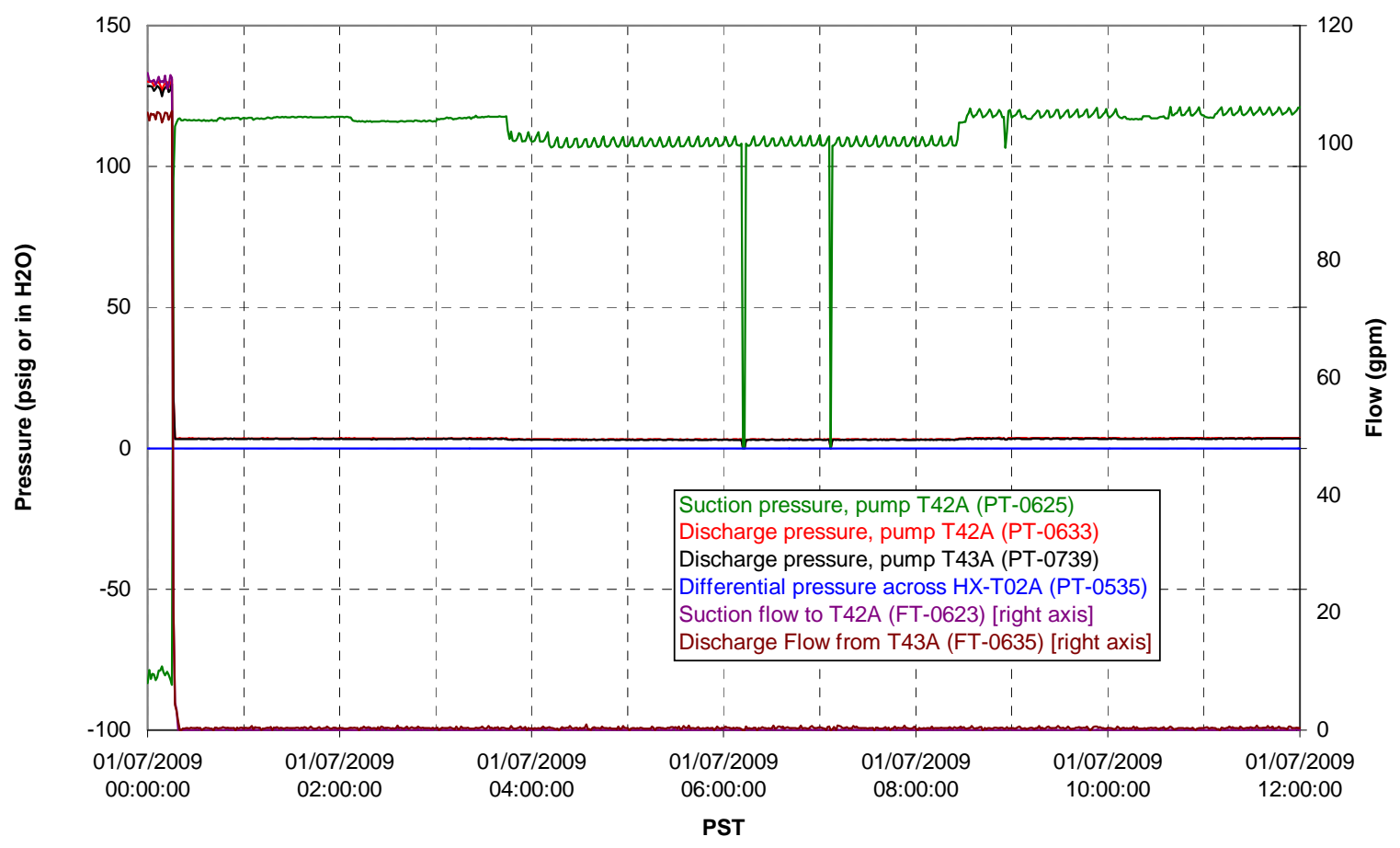


Axial pressure drop

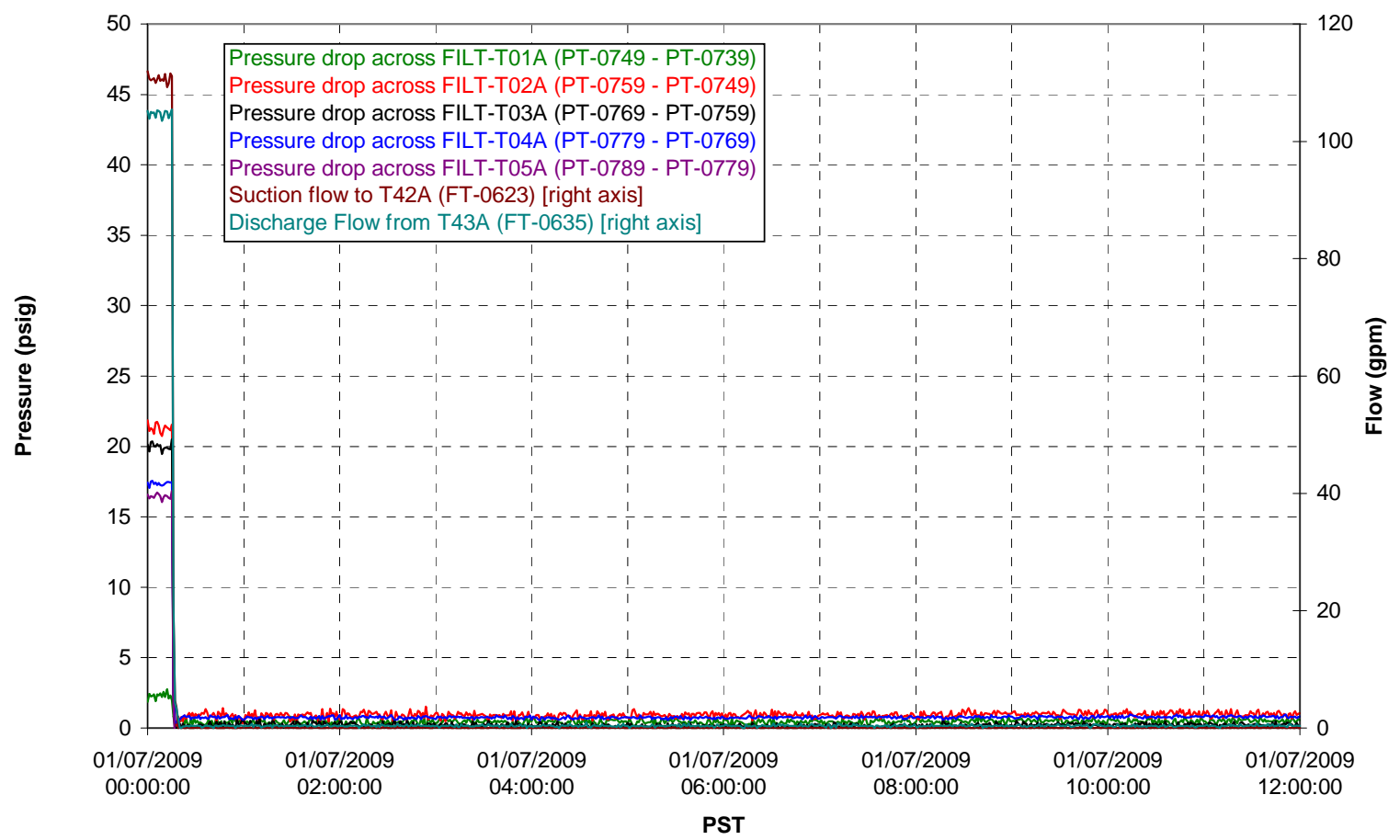

Permeate flow rates

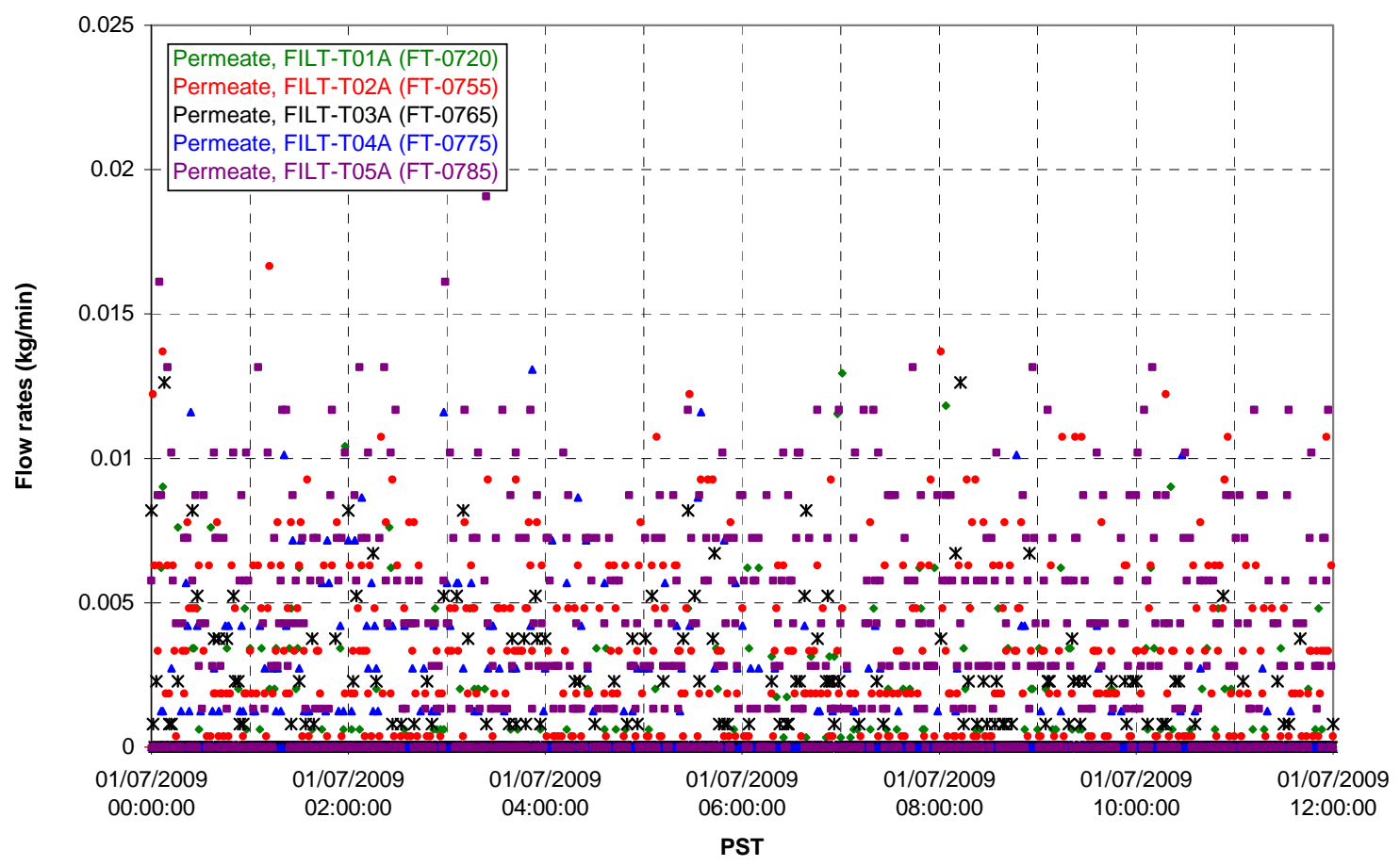


T02A Inner Temperature Tree

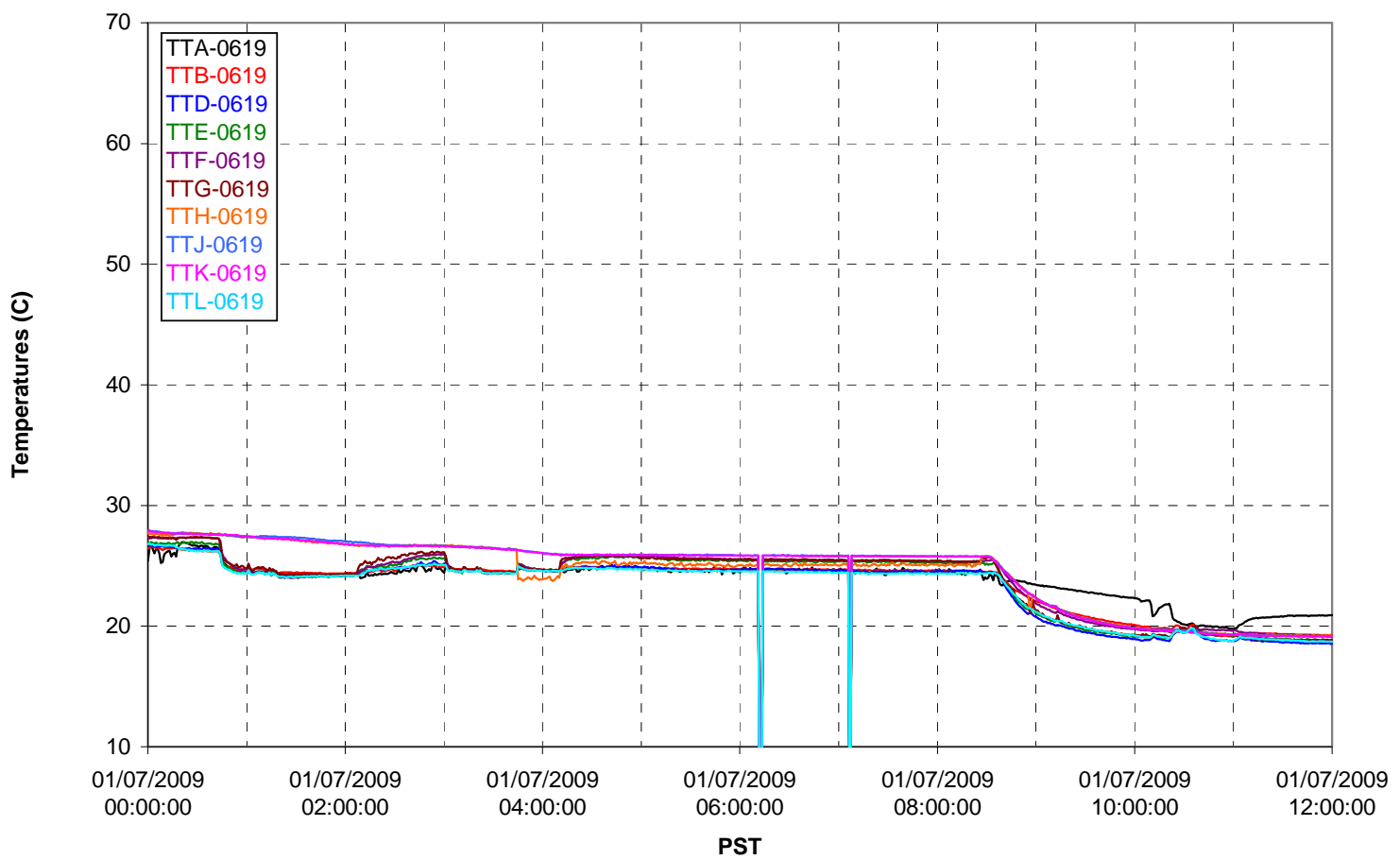

T02A Outer Temperature Tree

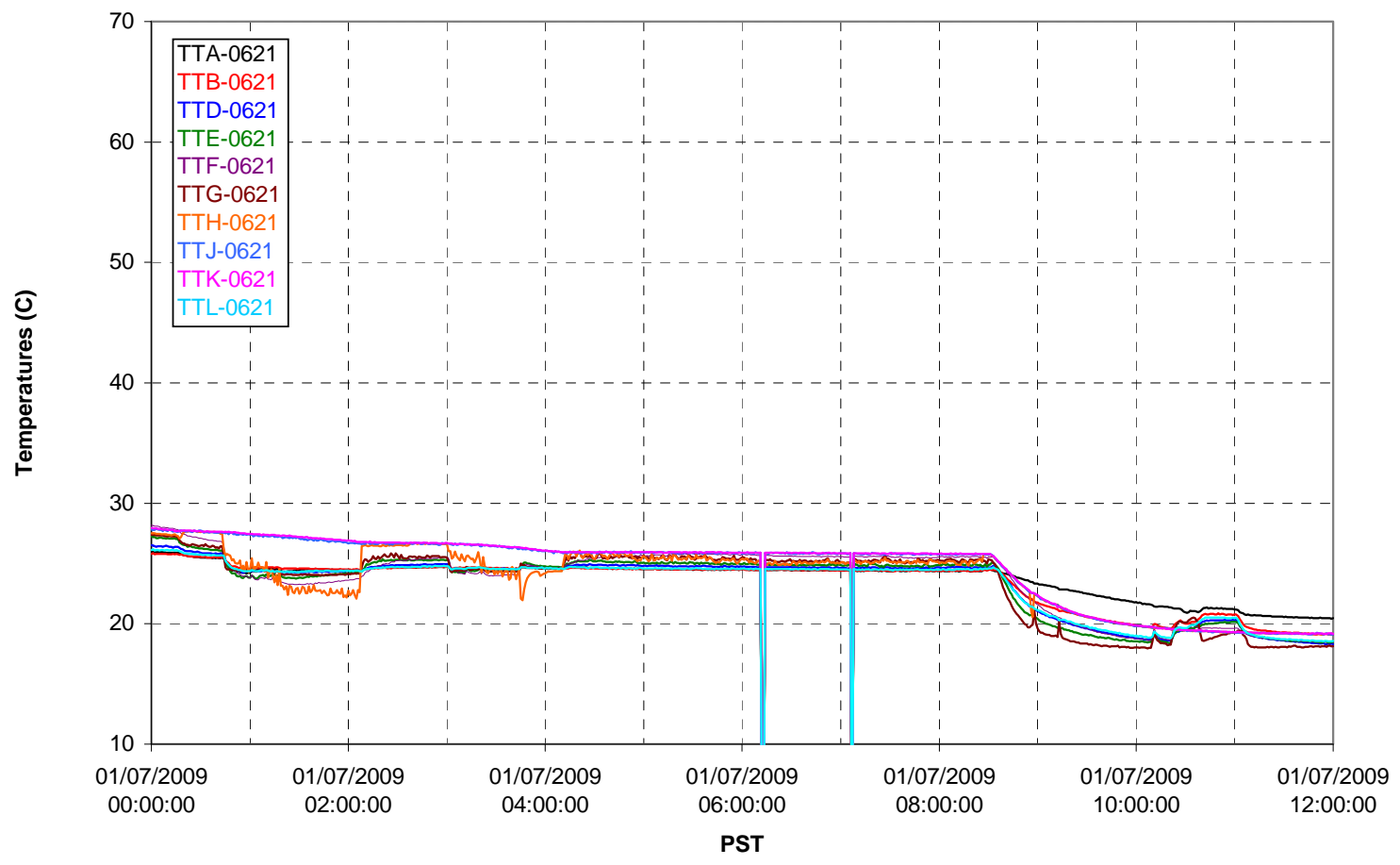


T02A temperatures

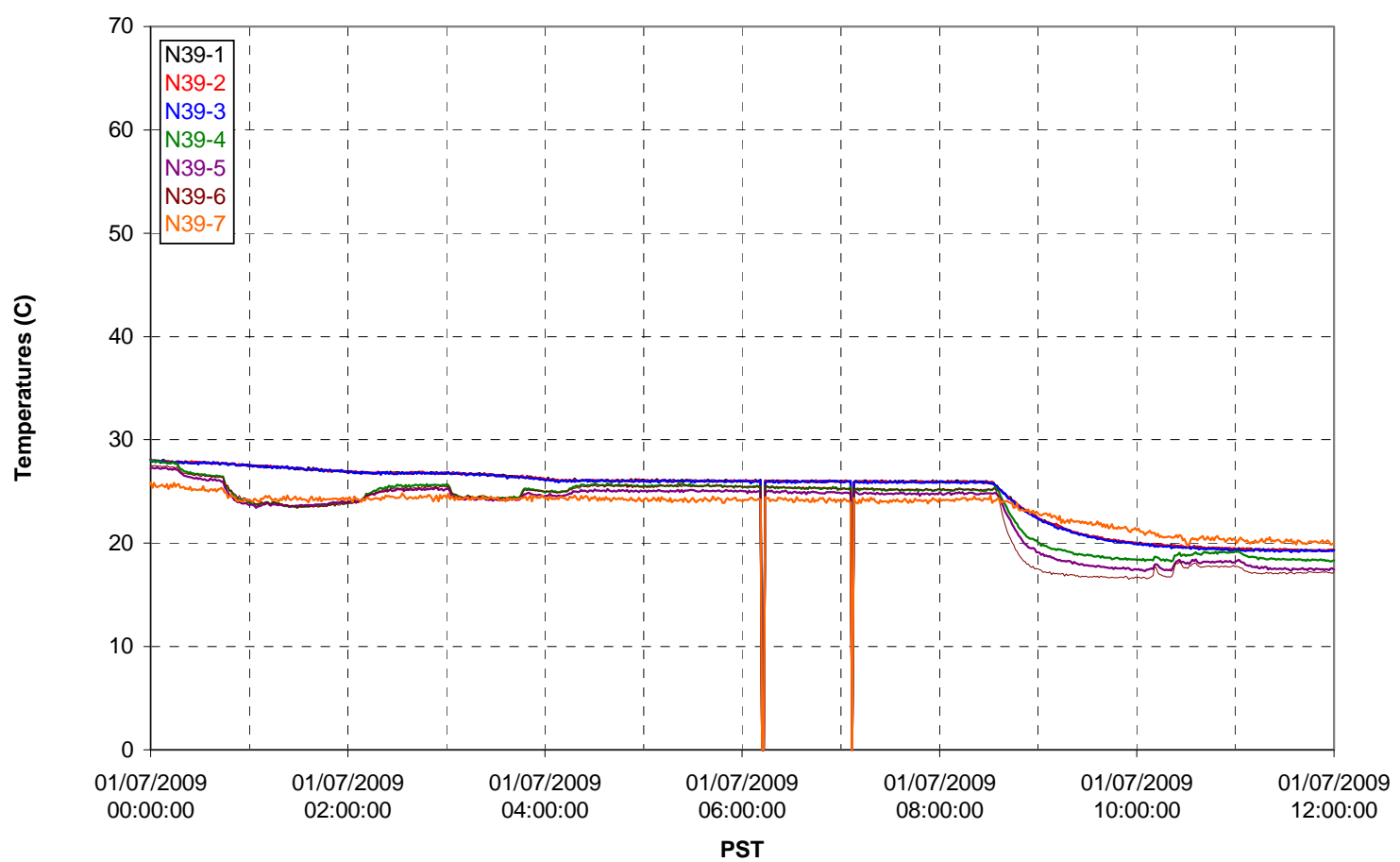

T02A temperatures

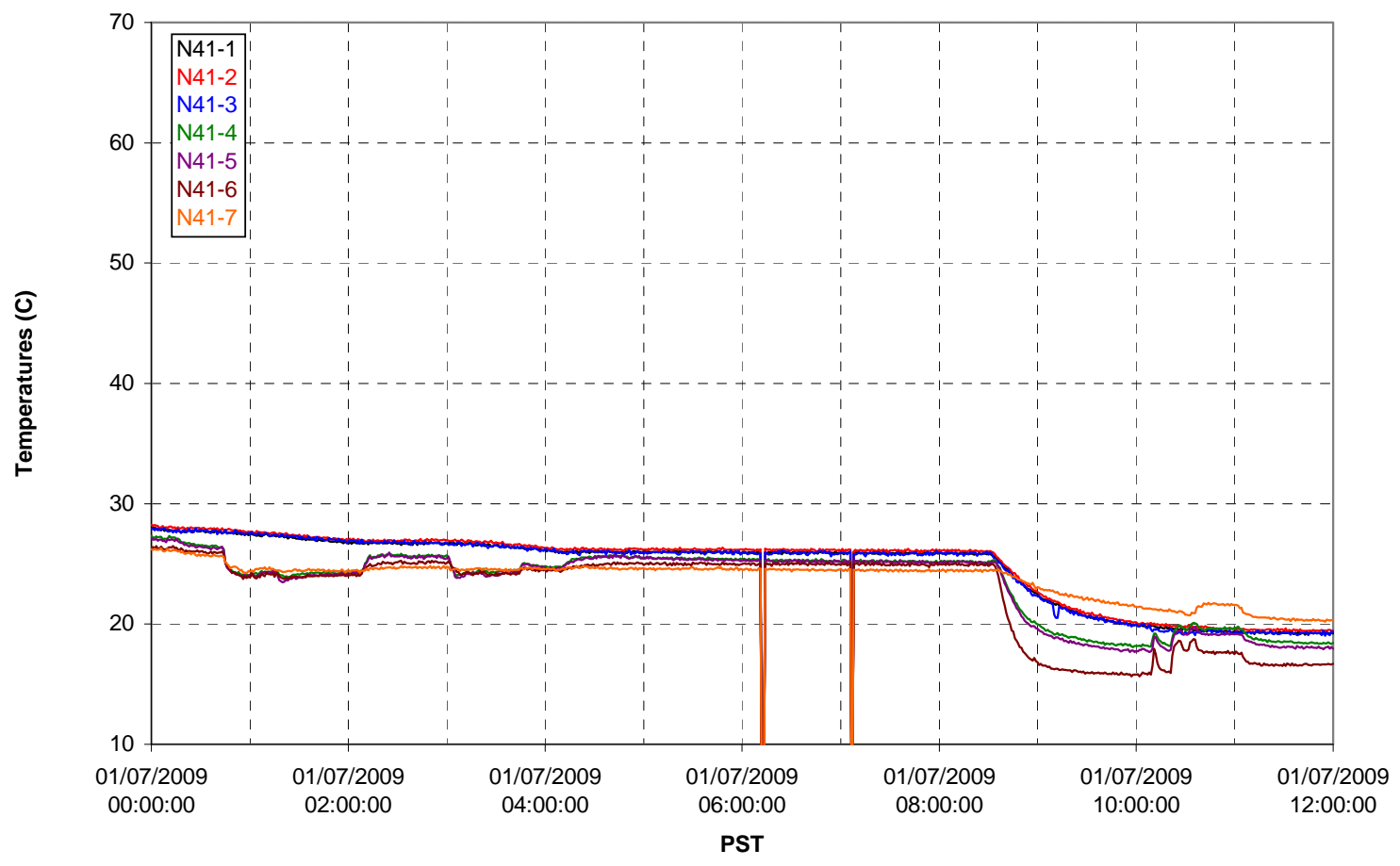


T02A temperatures

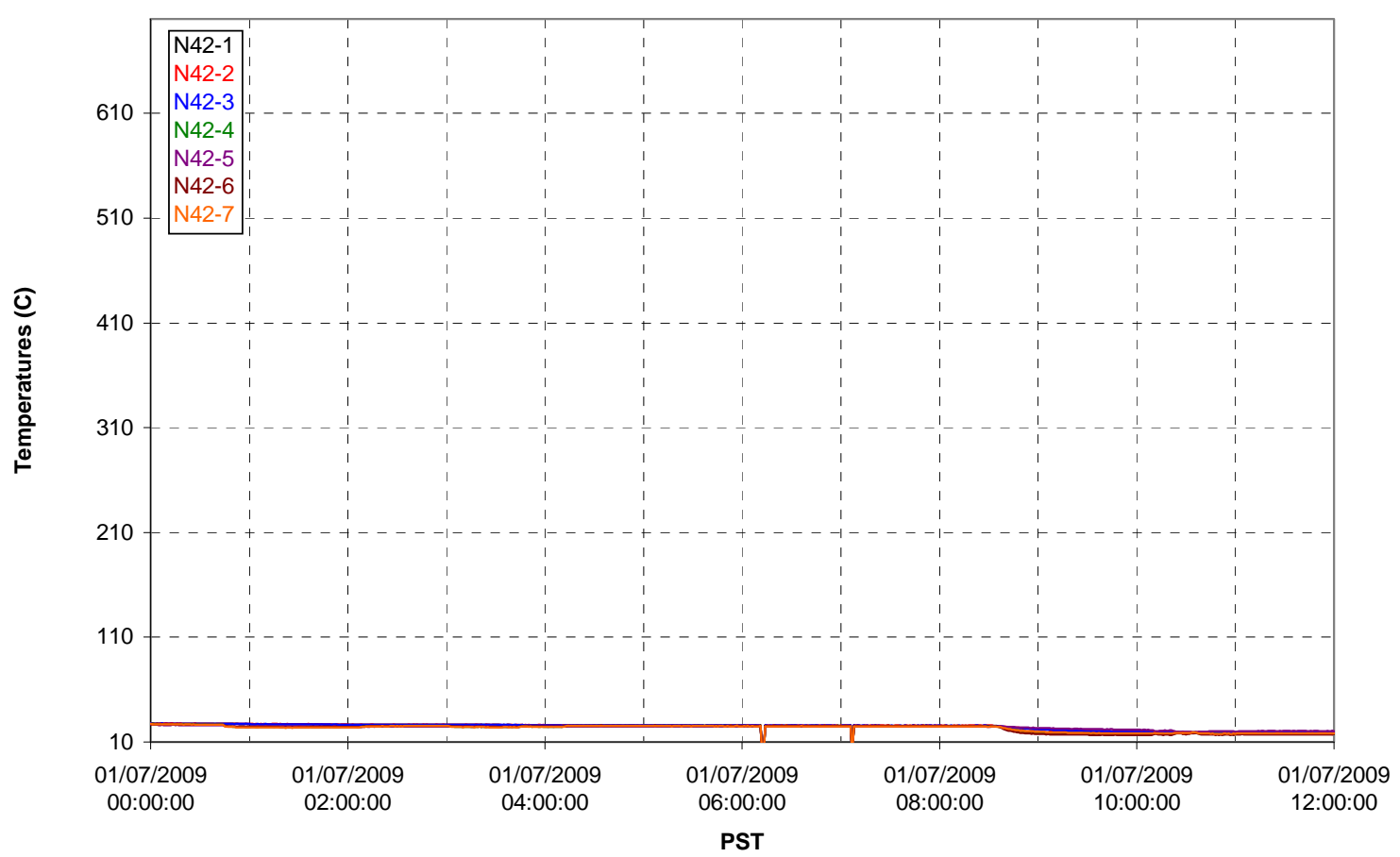

T02A temperatures

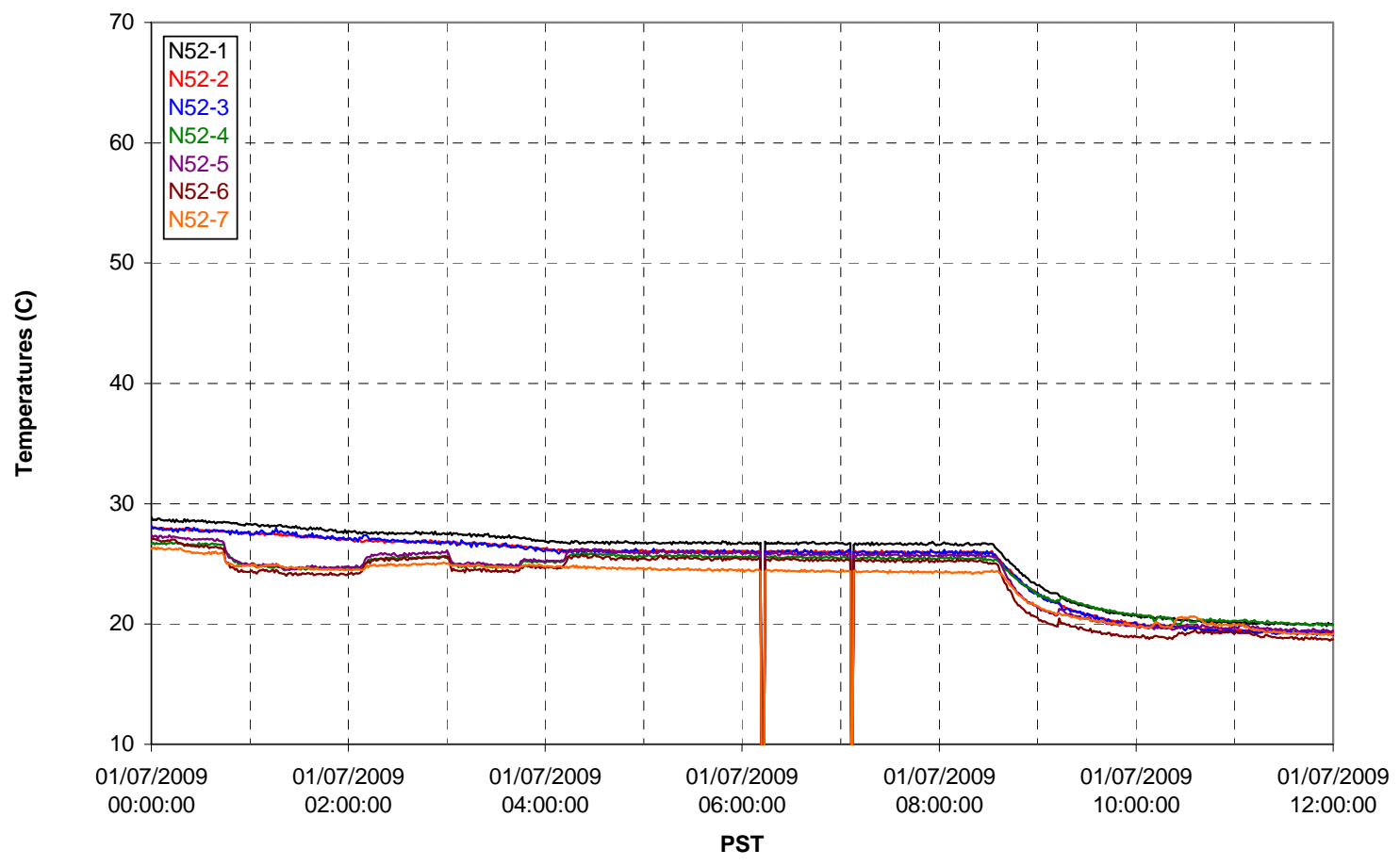


T02A Heating and Cooling

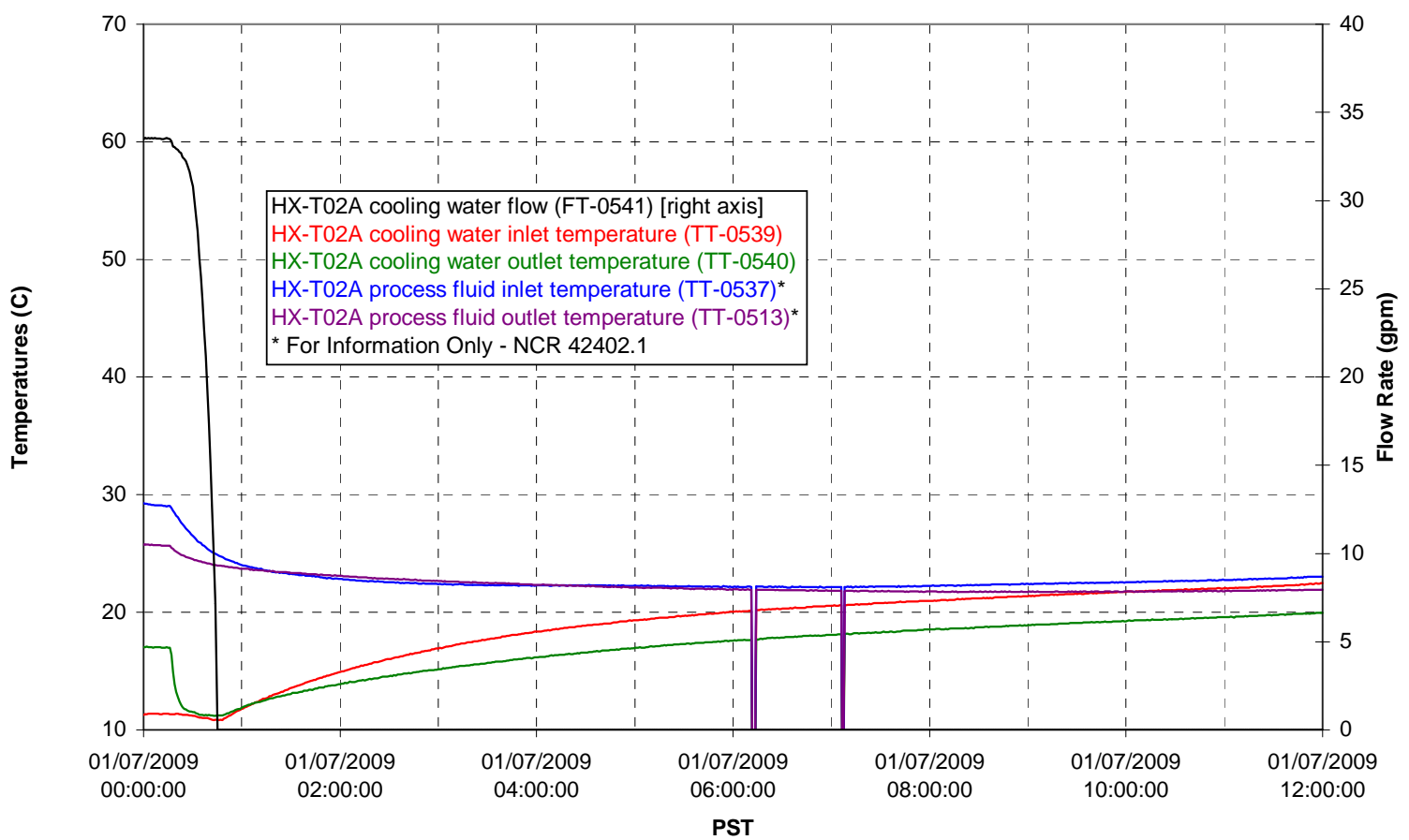

Pump Operation

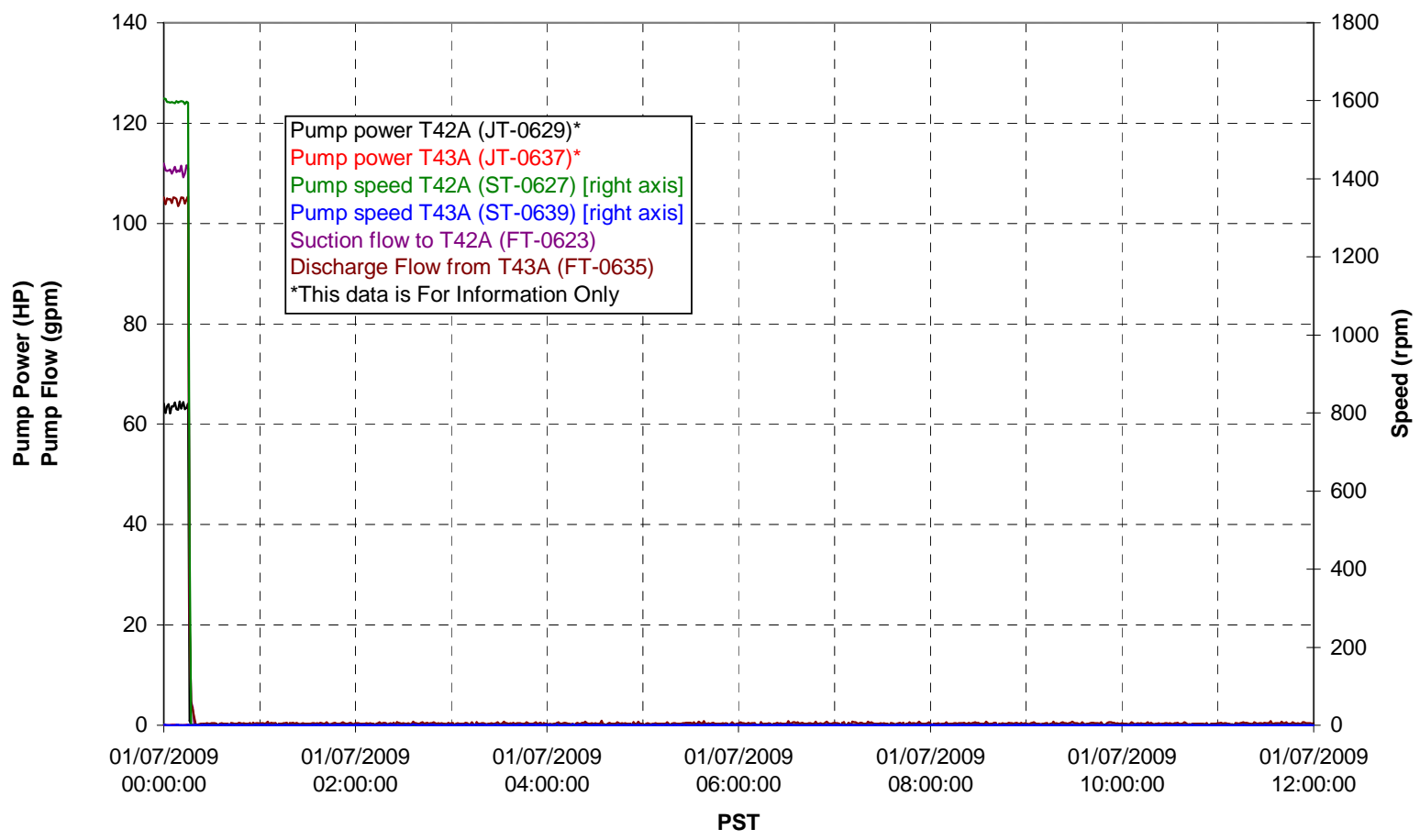


Pulsepot UFP-PP-T01A

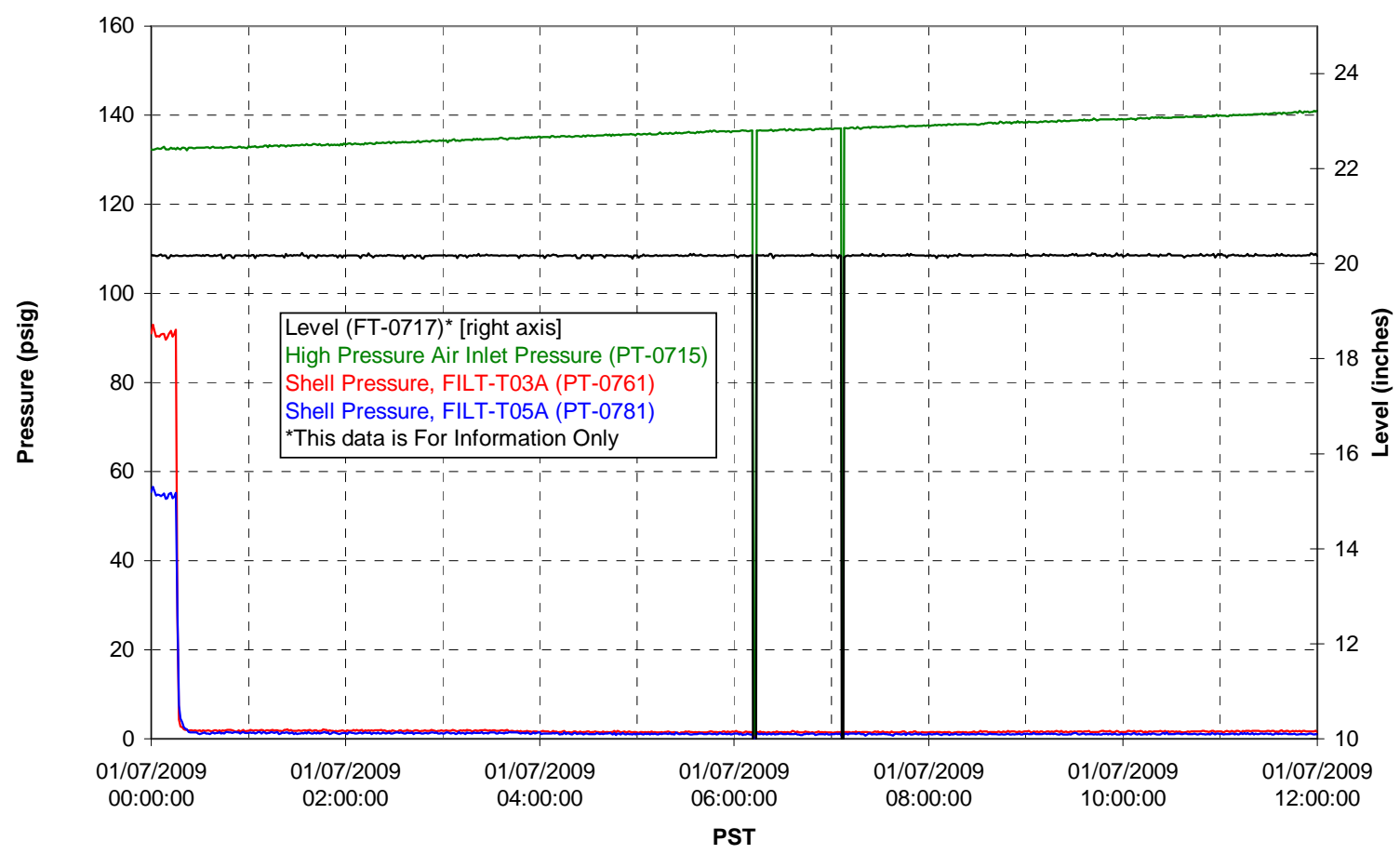

Pulsepot UFP-PP-T02A

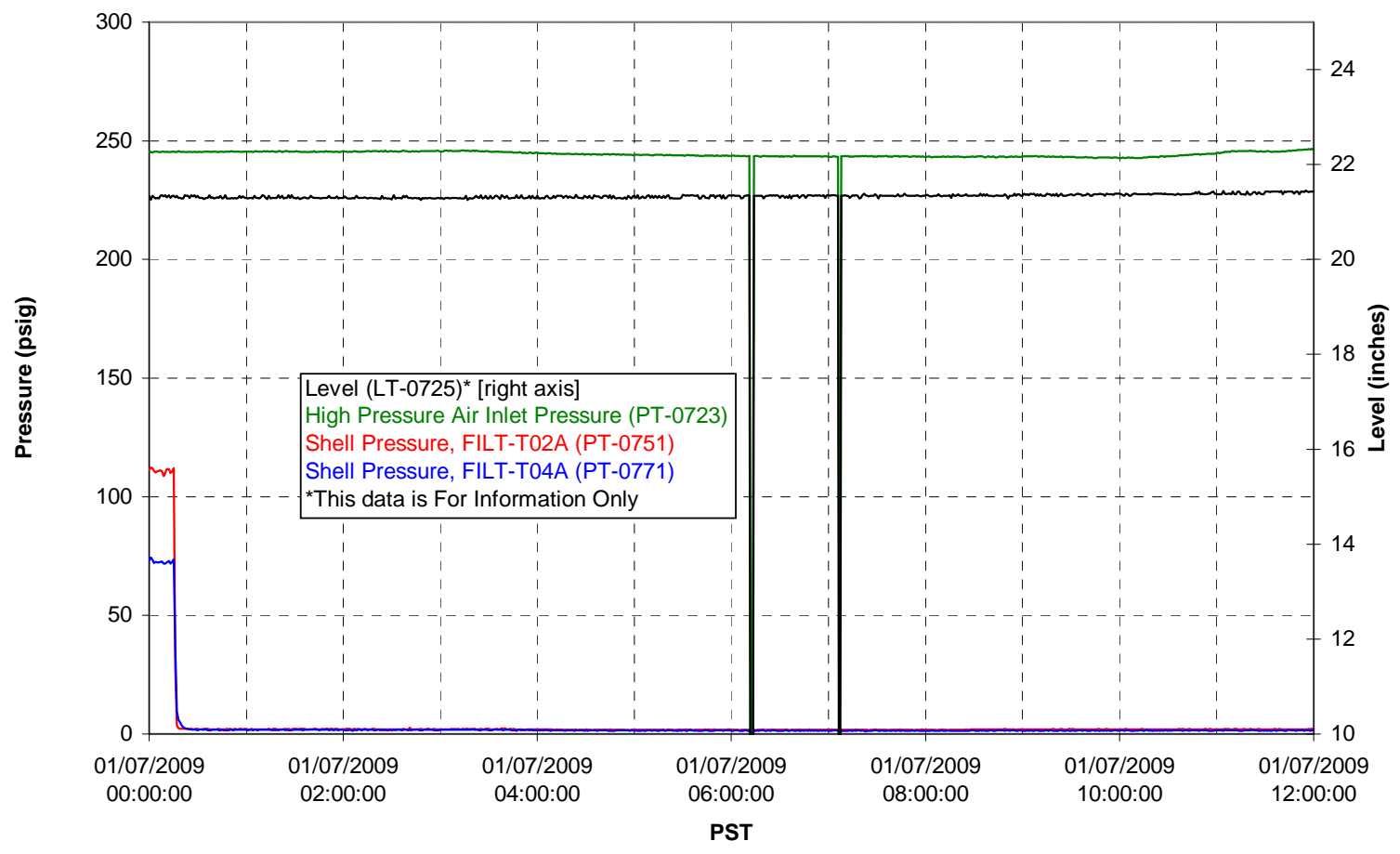


Pulsepot UFP-PP-T03A

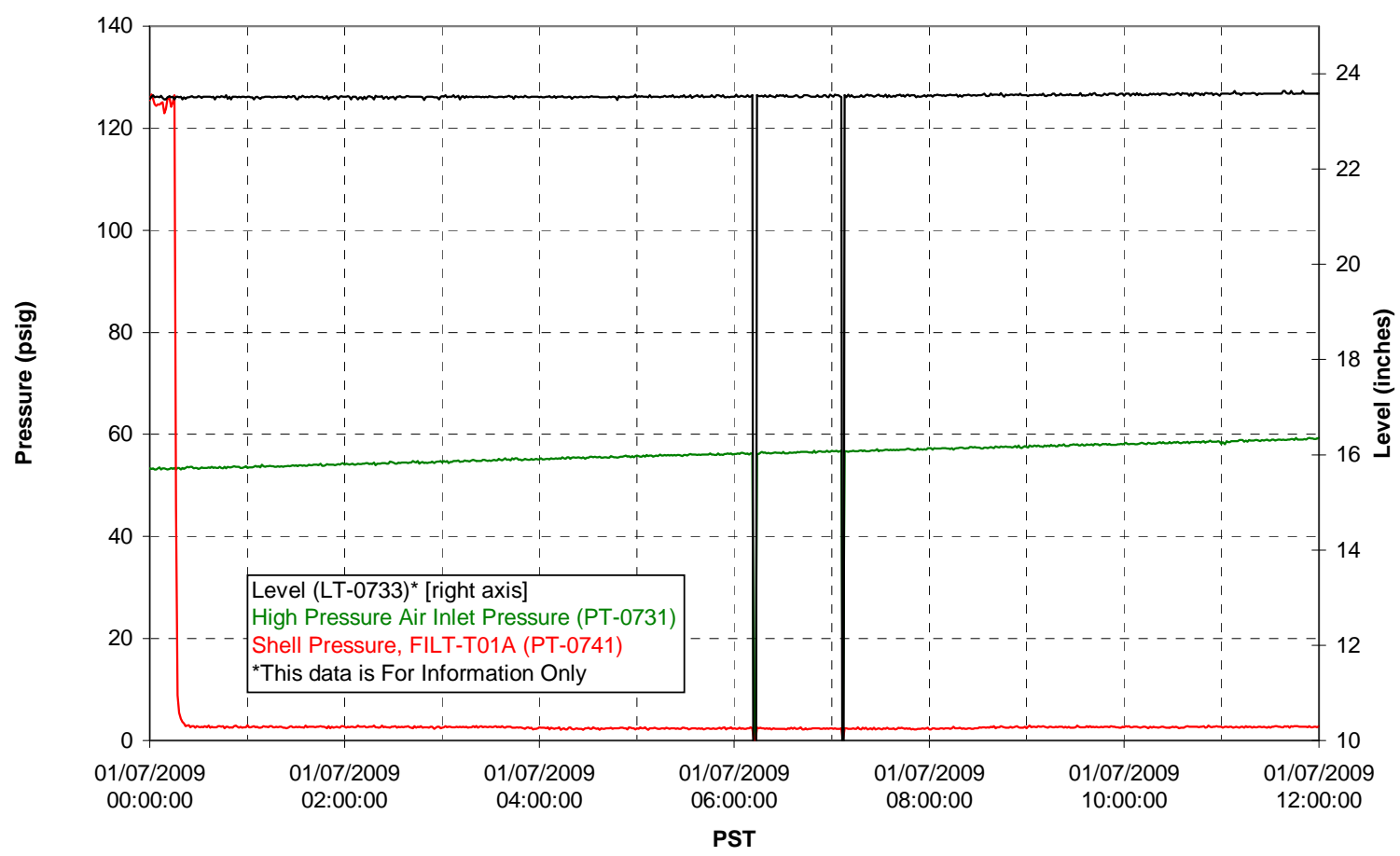

Pulsepot Levels

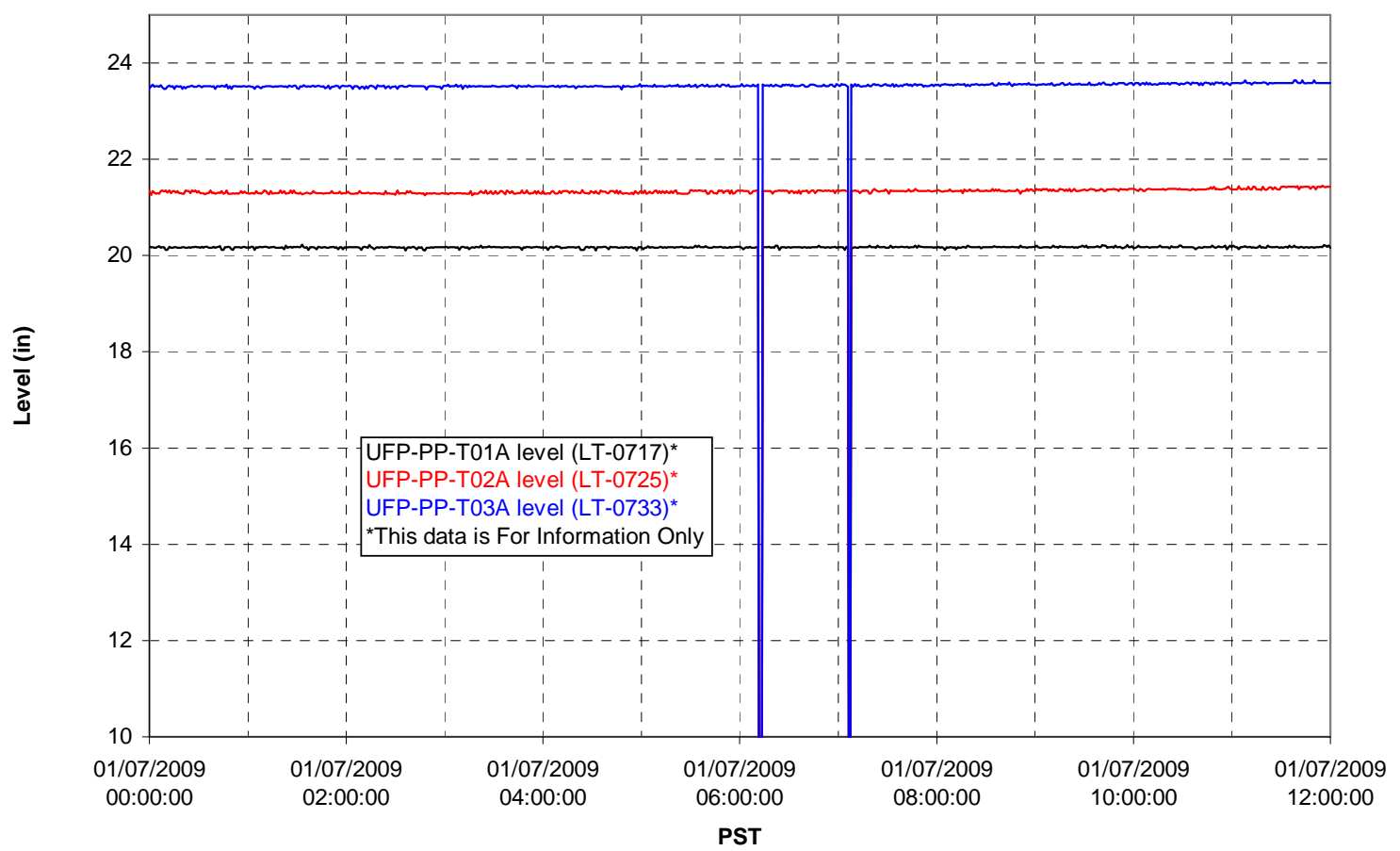


Filter UFP-FILT-T01A

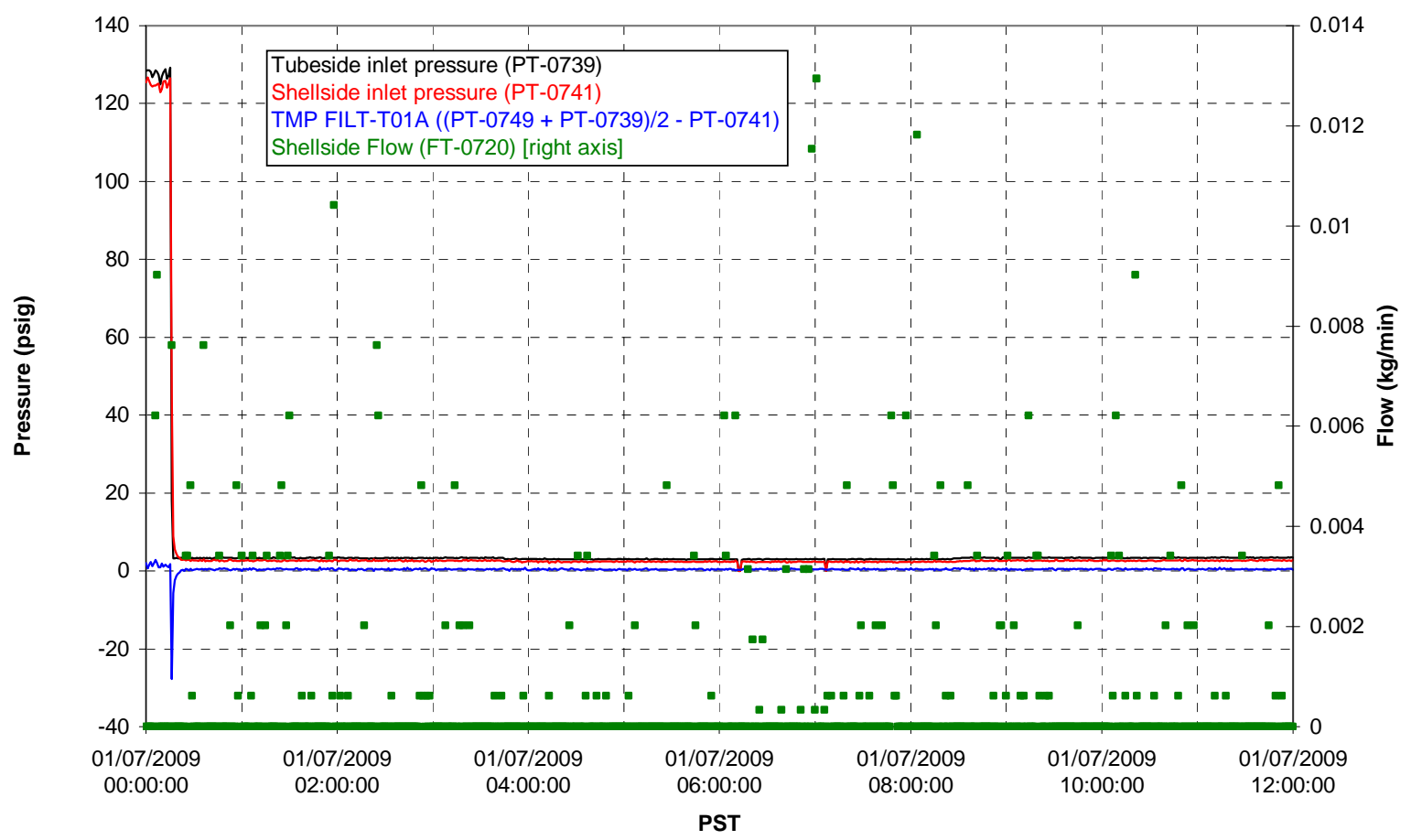

Filter UFP-FILT-T02A

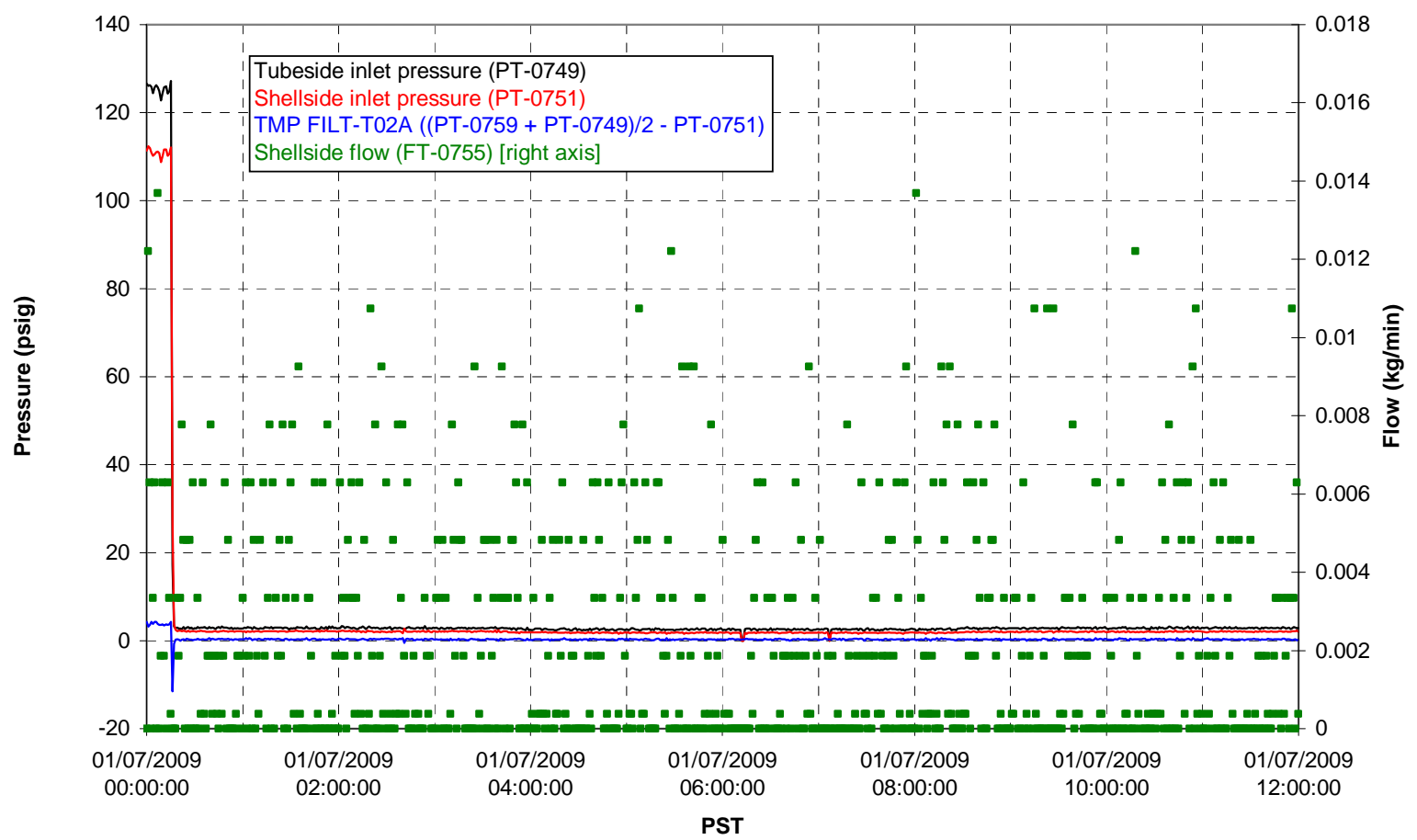


Filter UFP-FILT-T03A

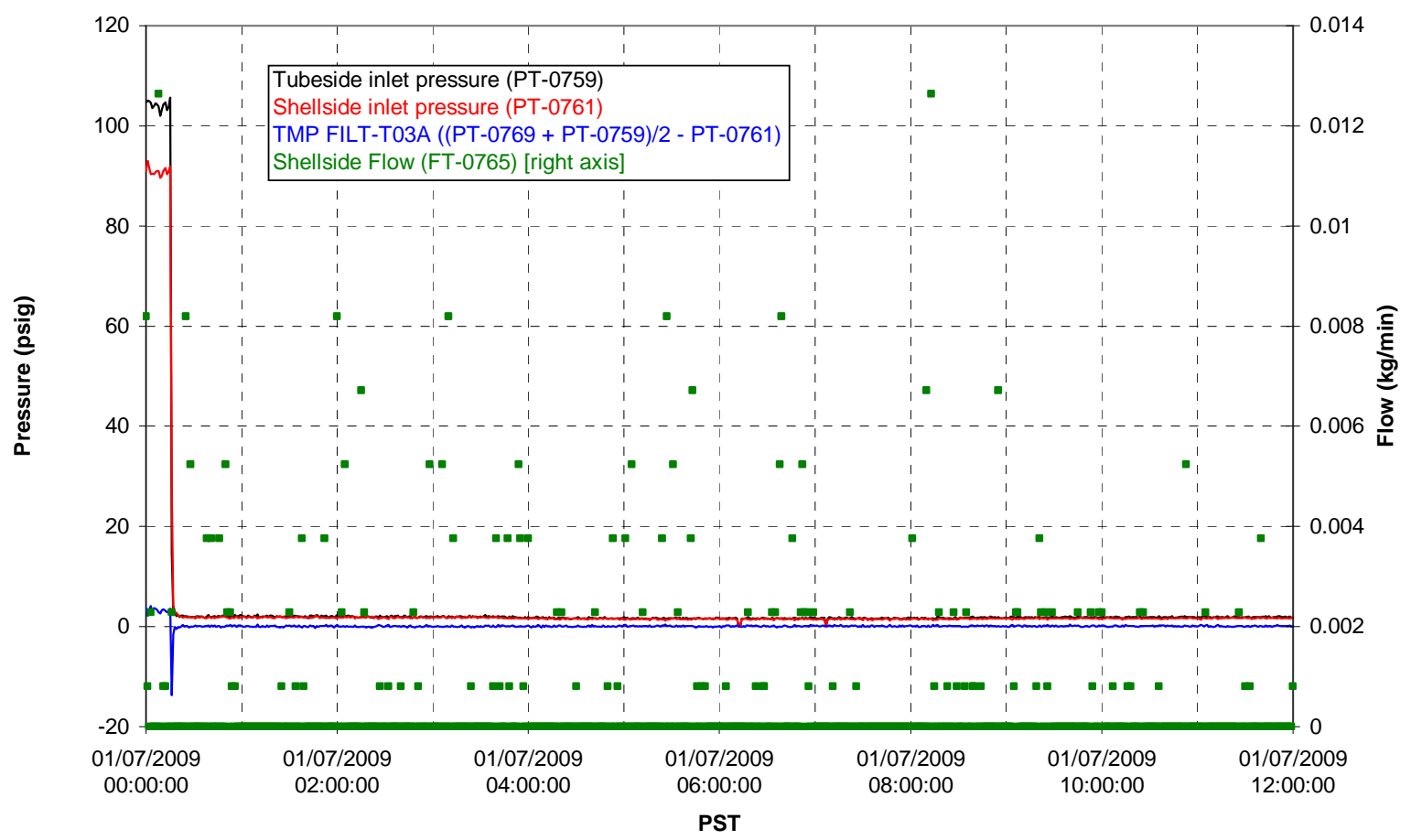

Filter UFP-FILT-T04A

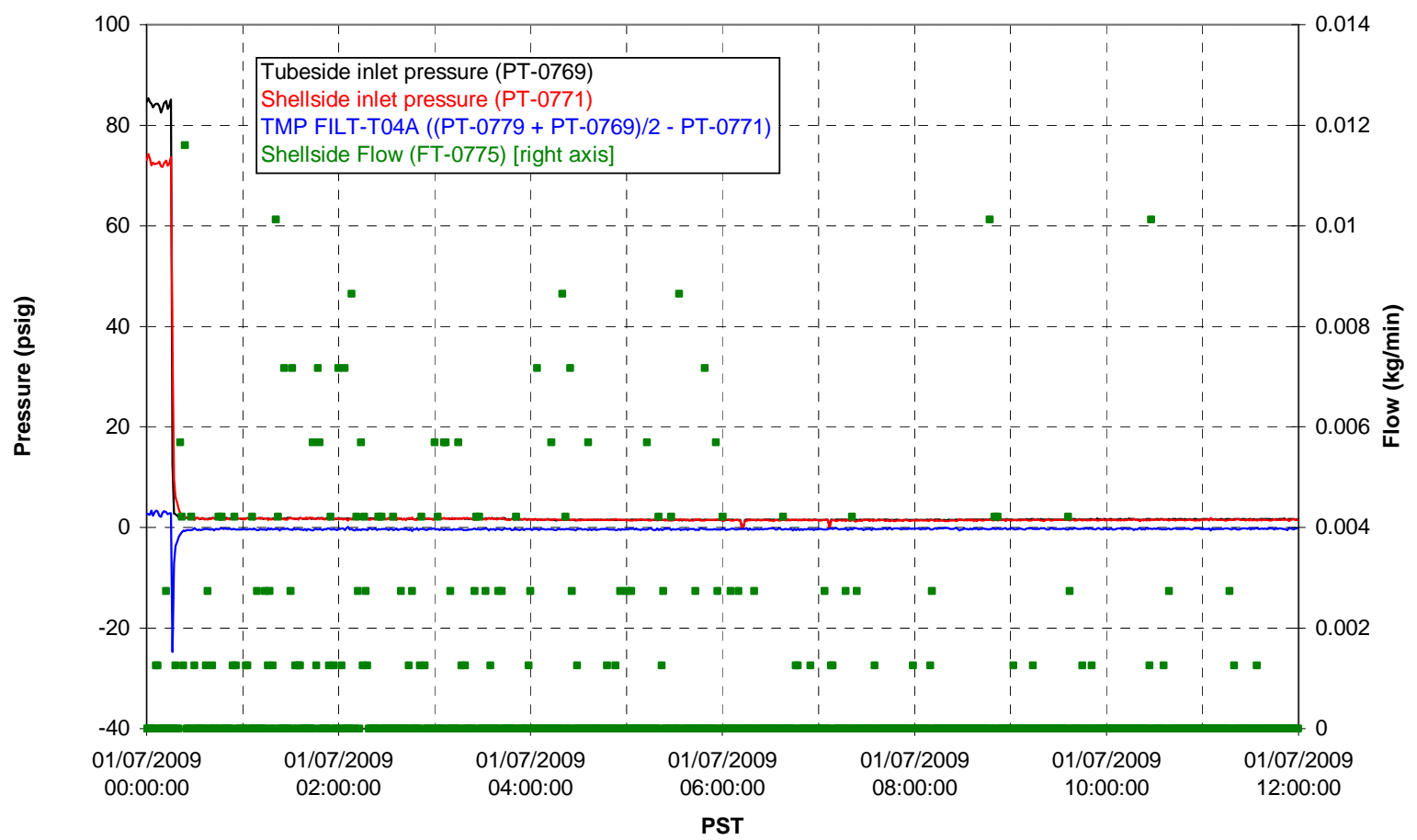


Filter UFP-FILT-T05A

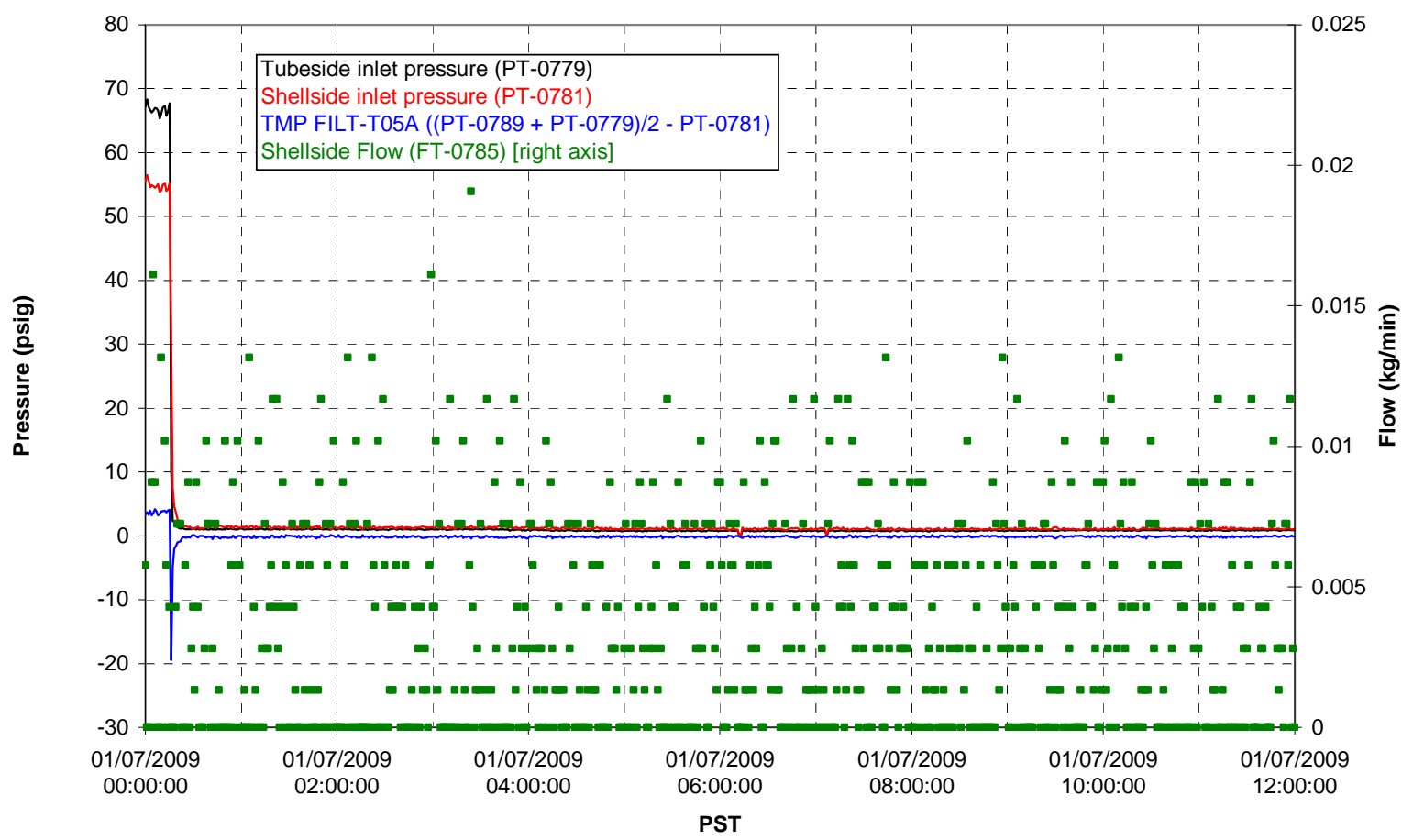

Chemical Flow

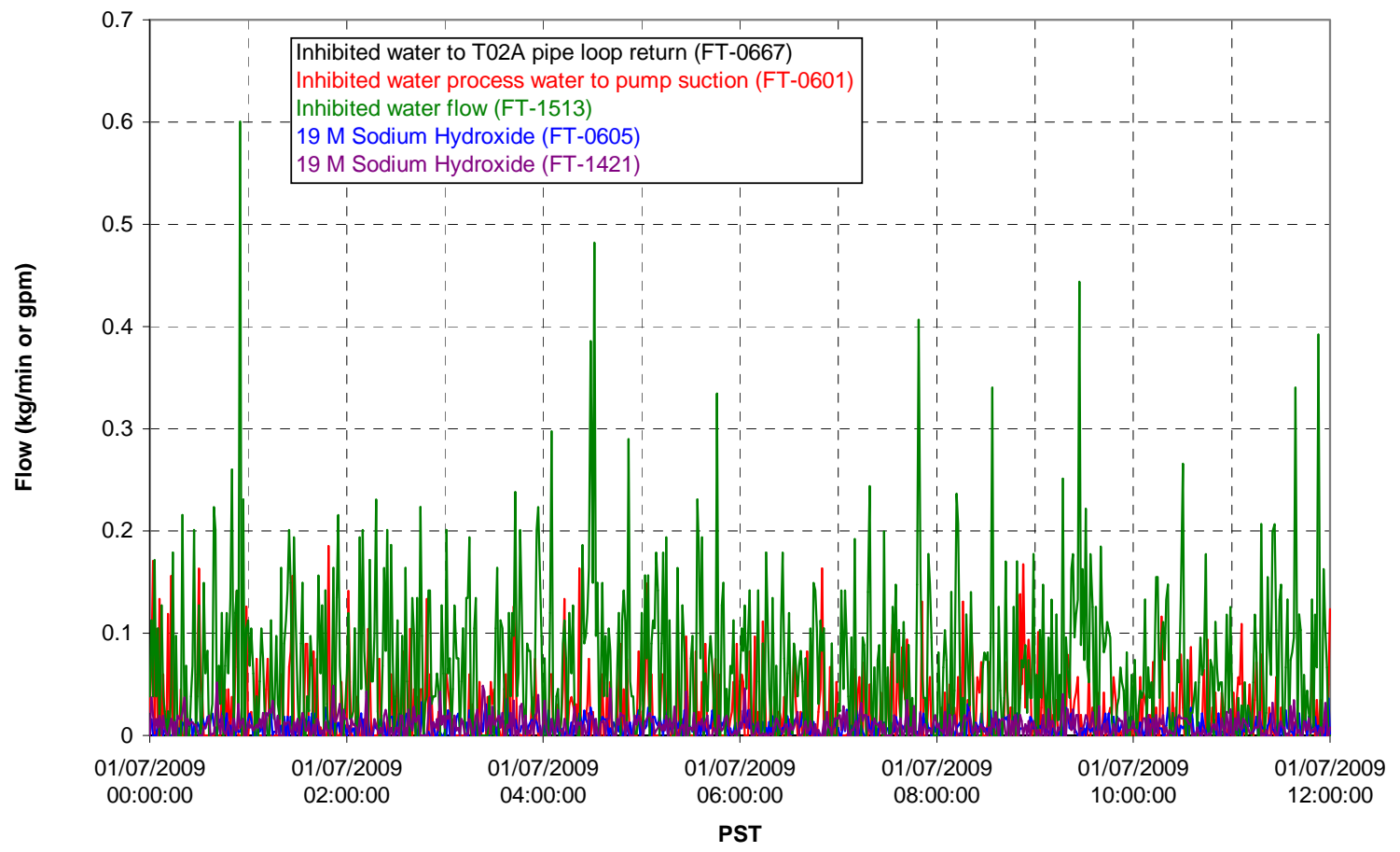




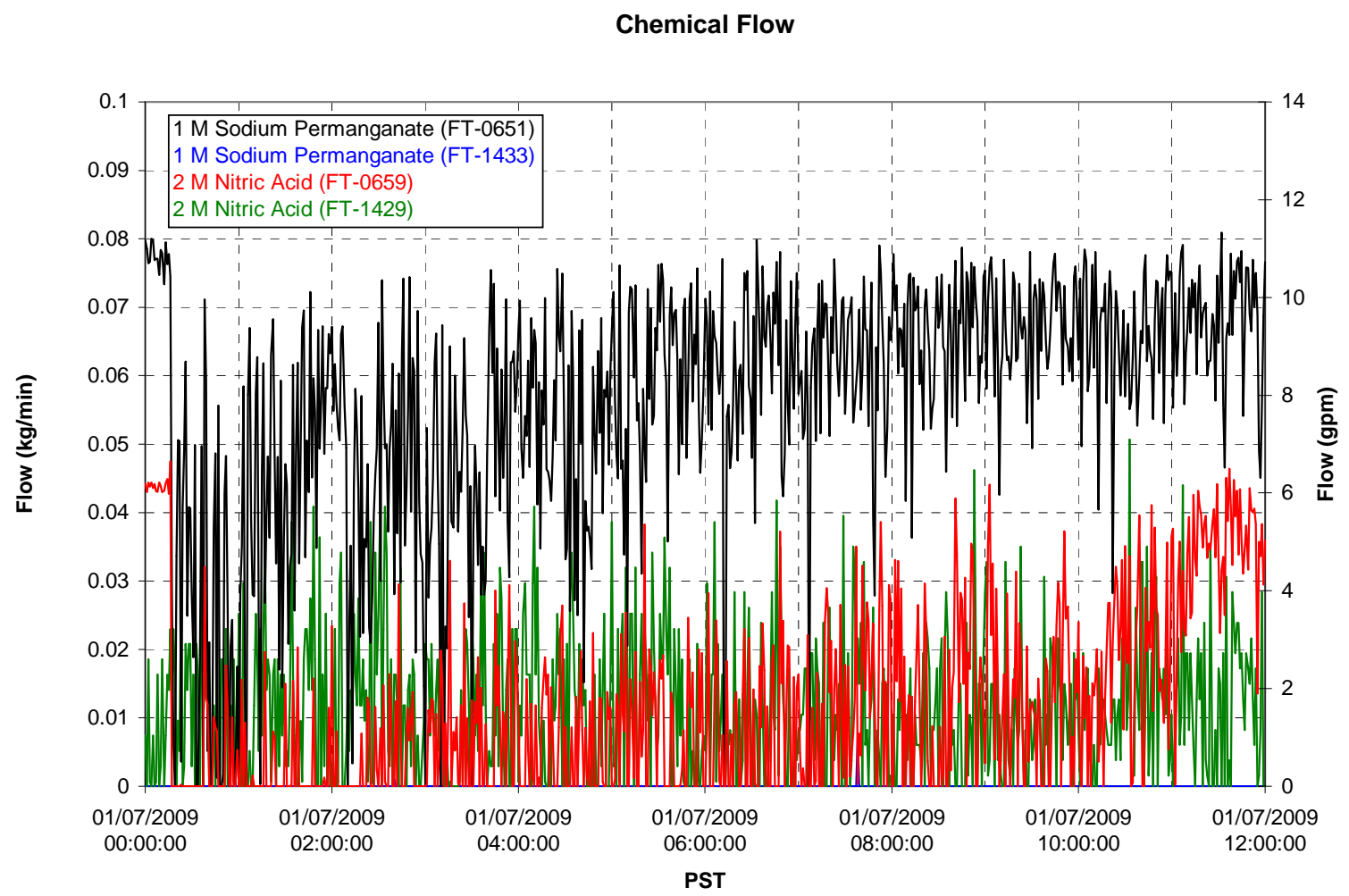

Air Flows

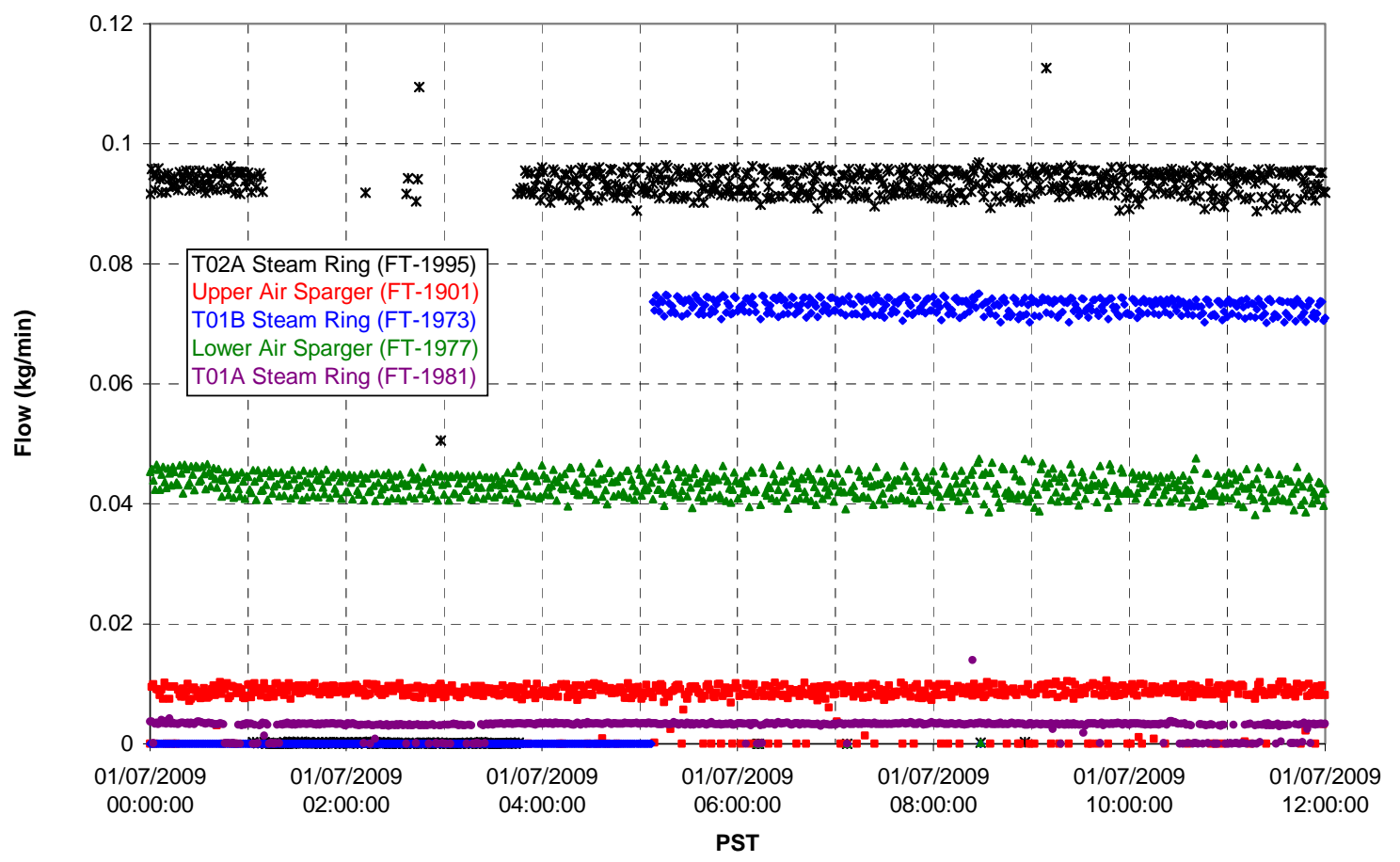


T02A Steam

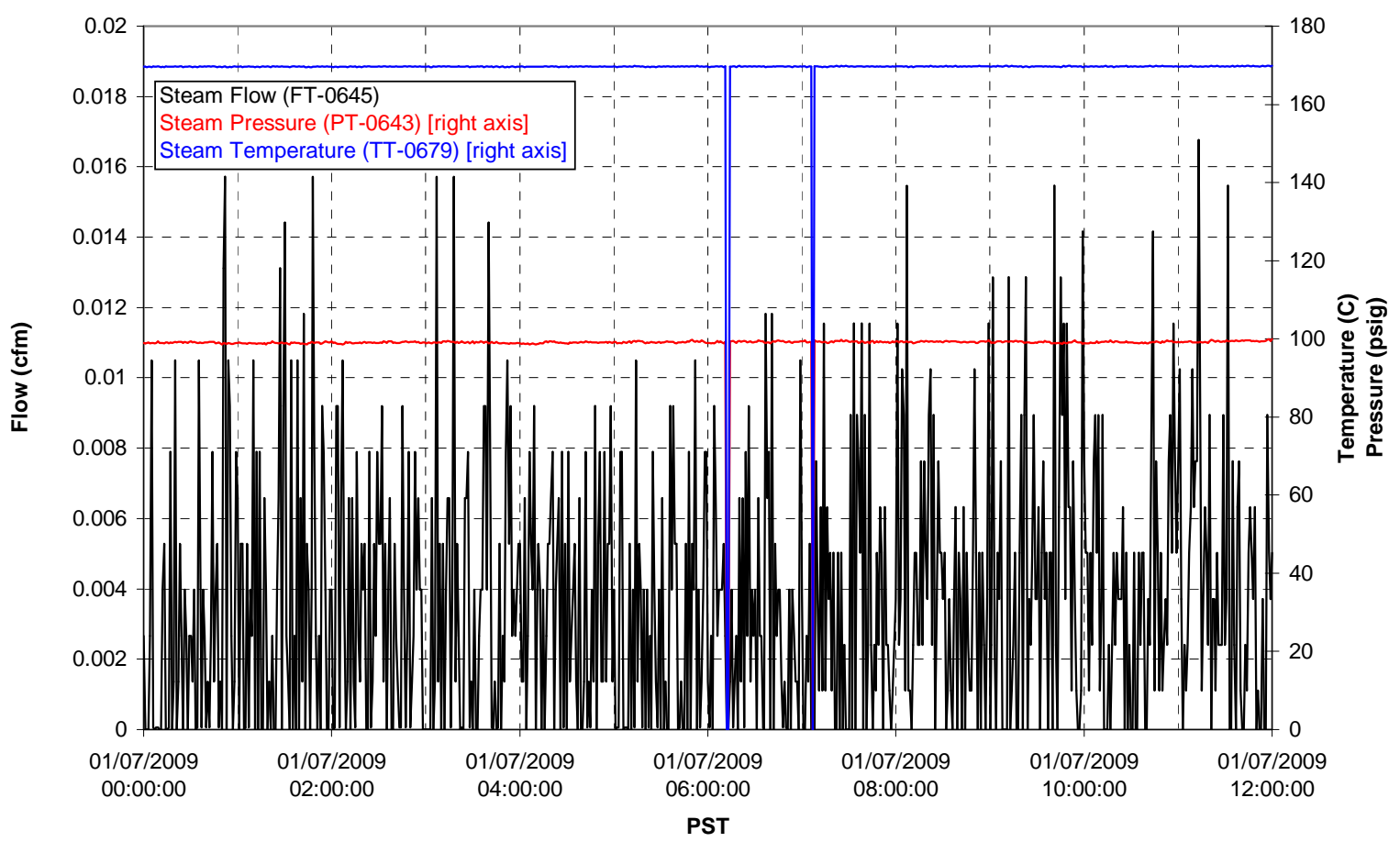

T01A Steam

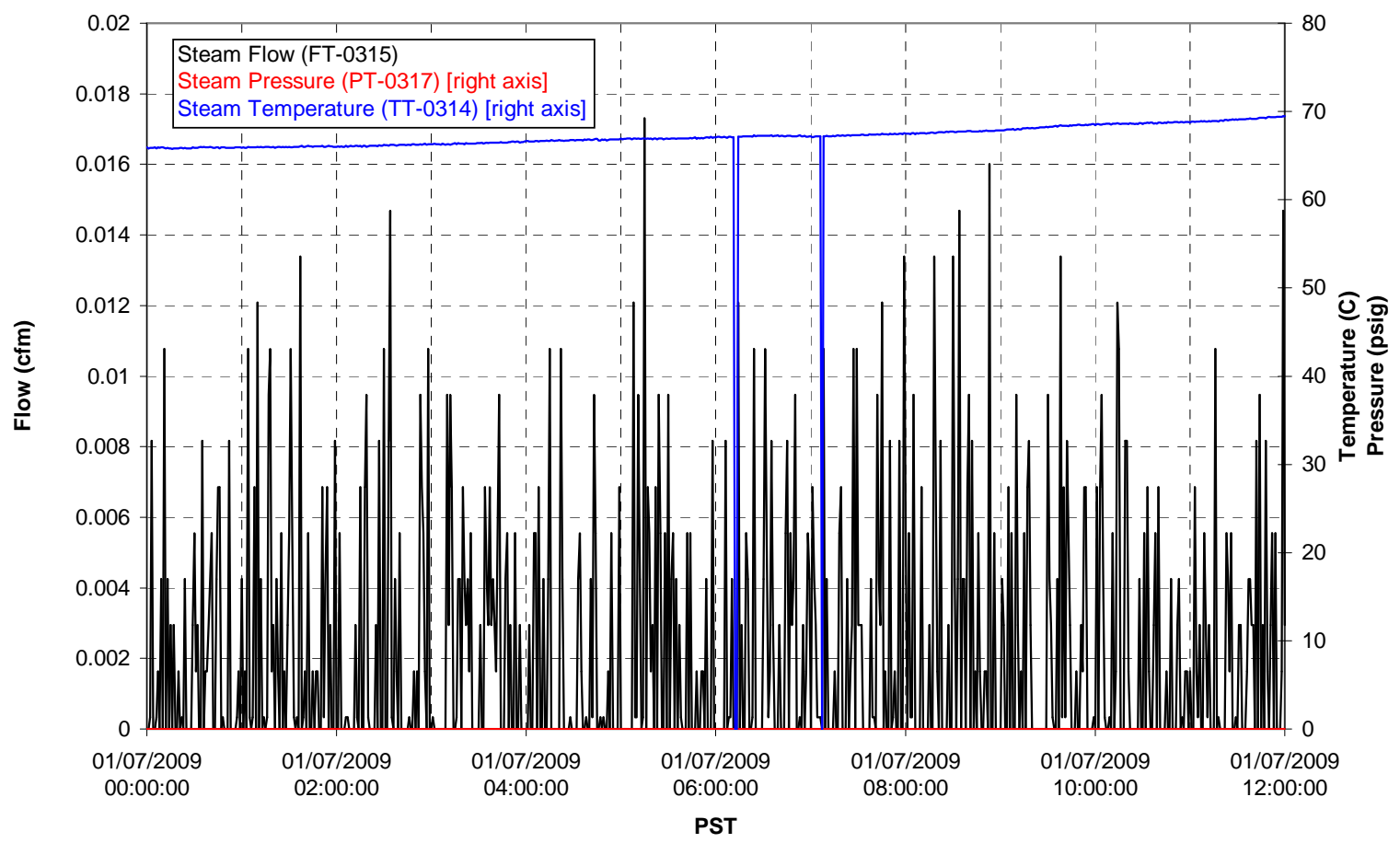


T01B Steam

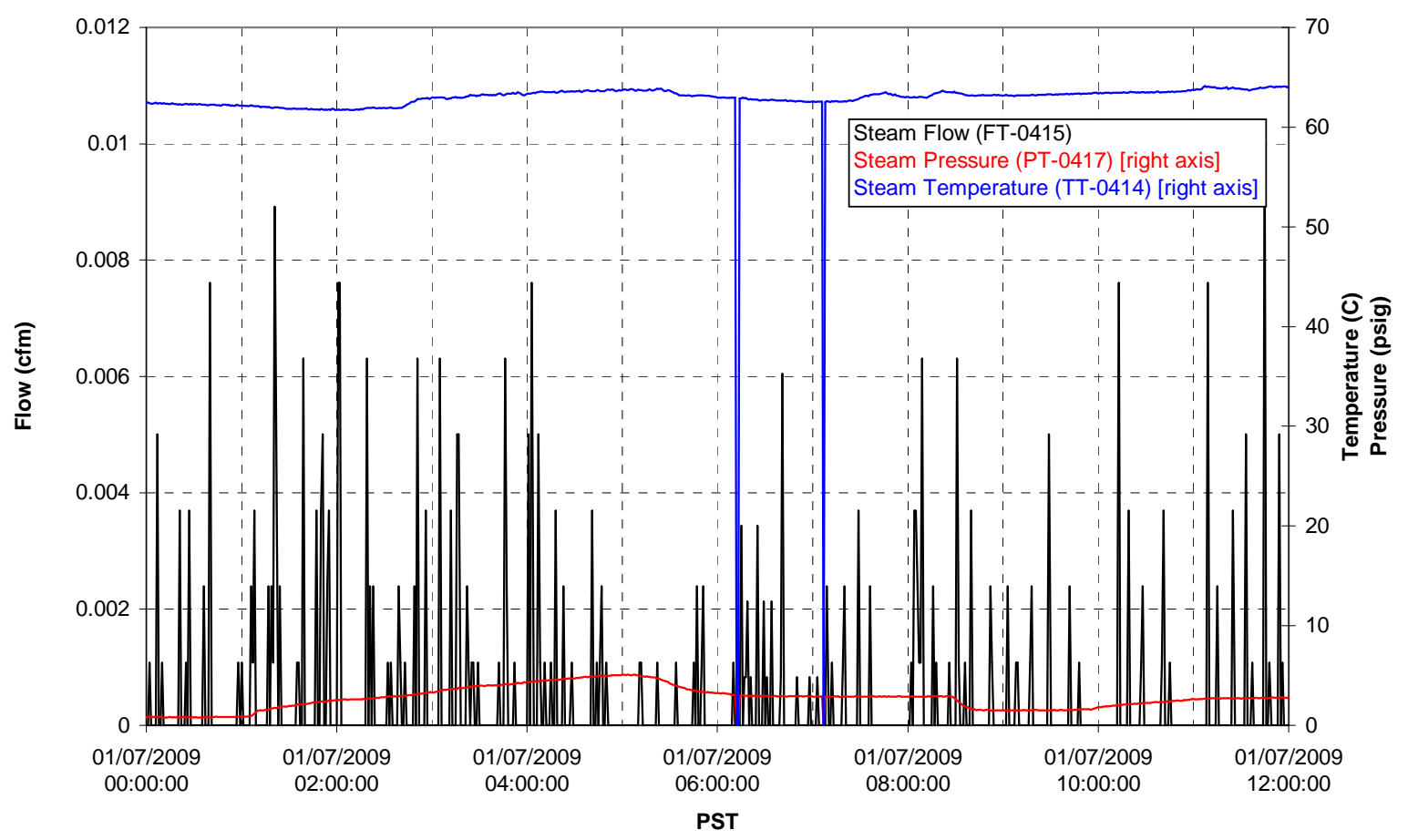


Functional Test Data Plots

01/07/2009 12:00 - 01/08/2009 00:00 
T01A level

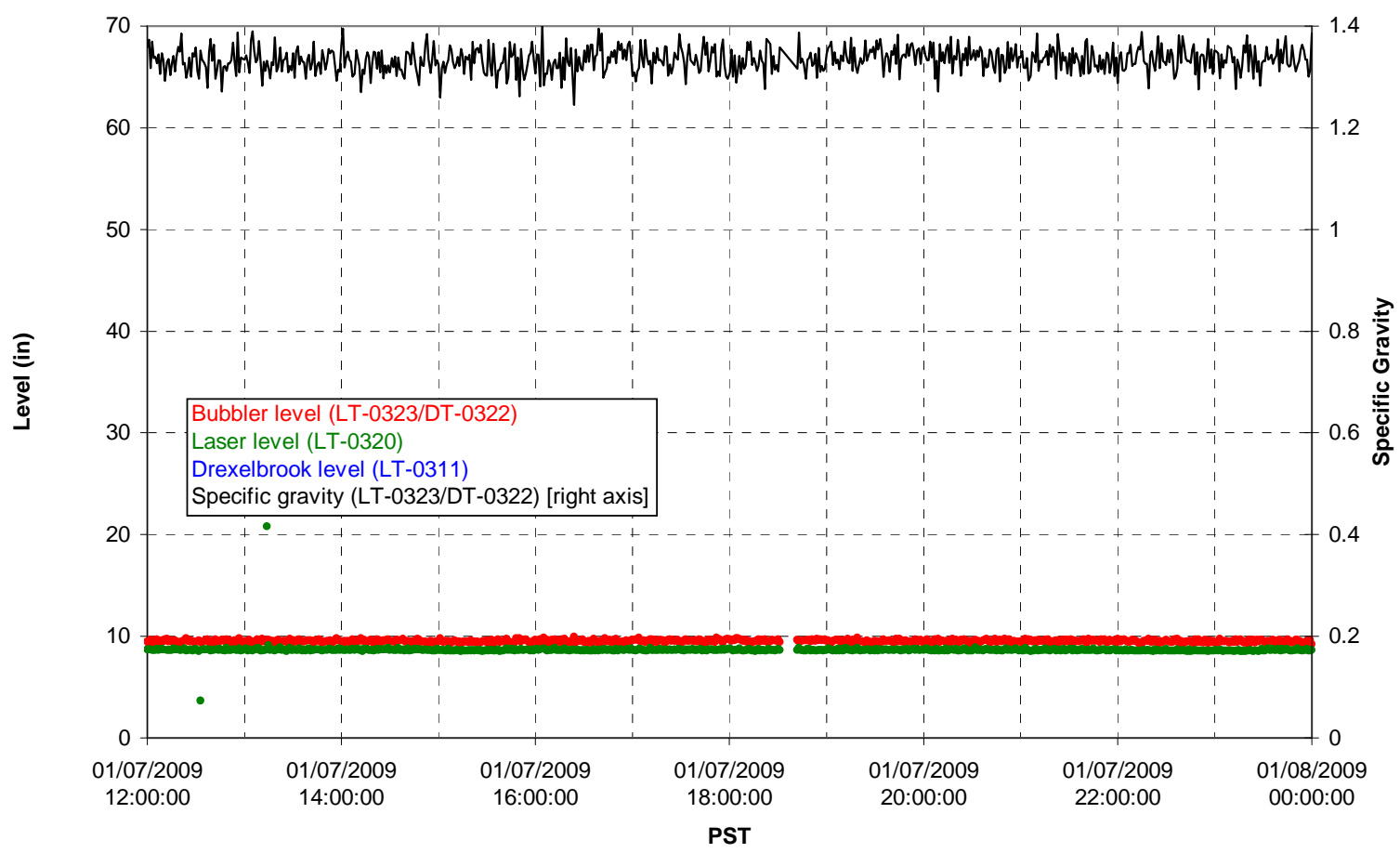

T01A temperatures

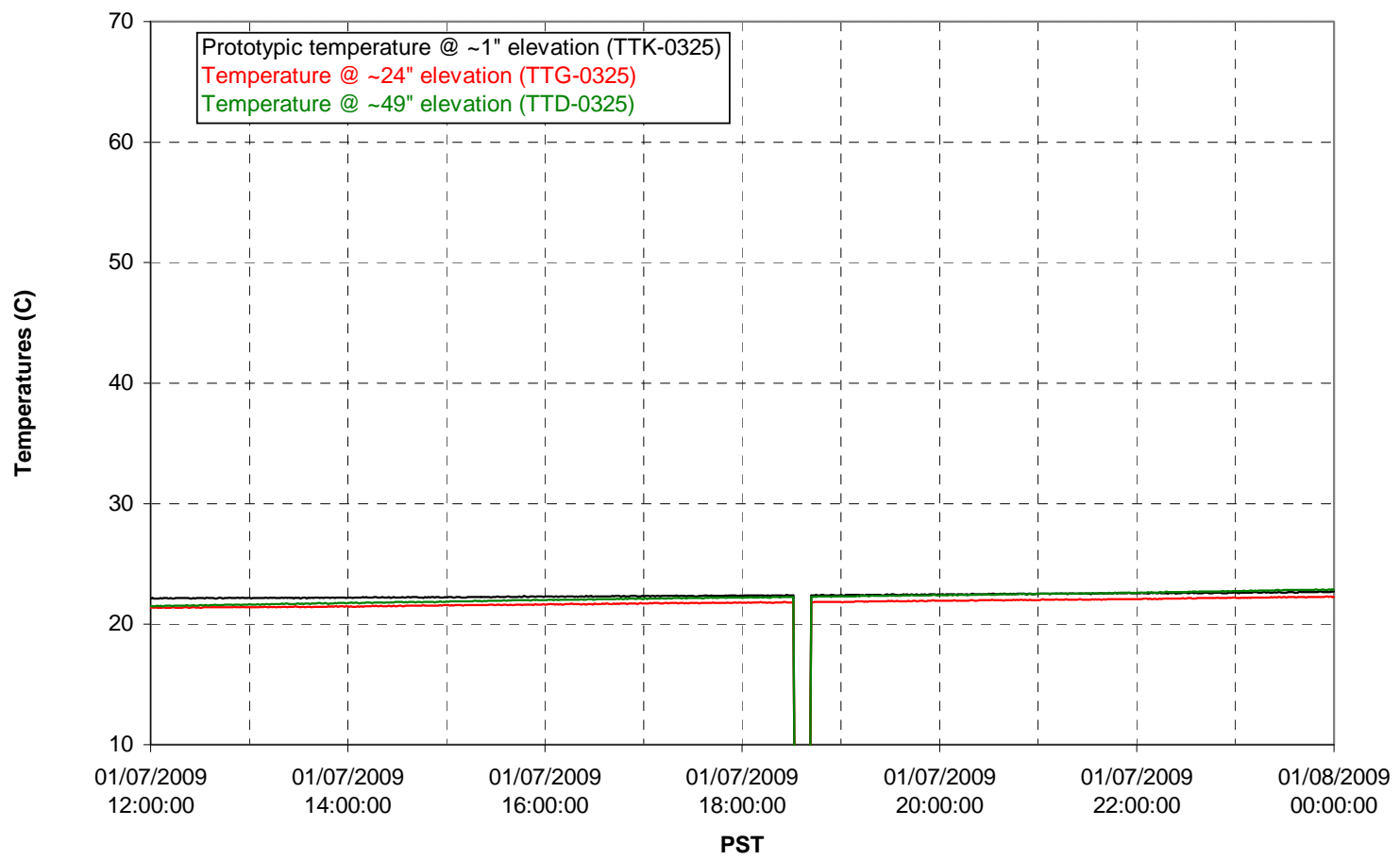


T01B level

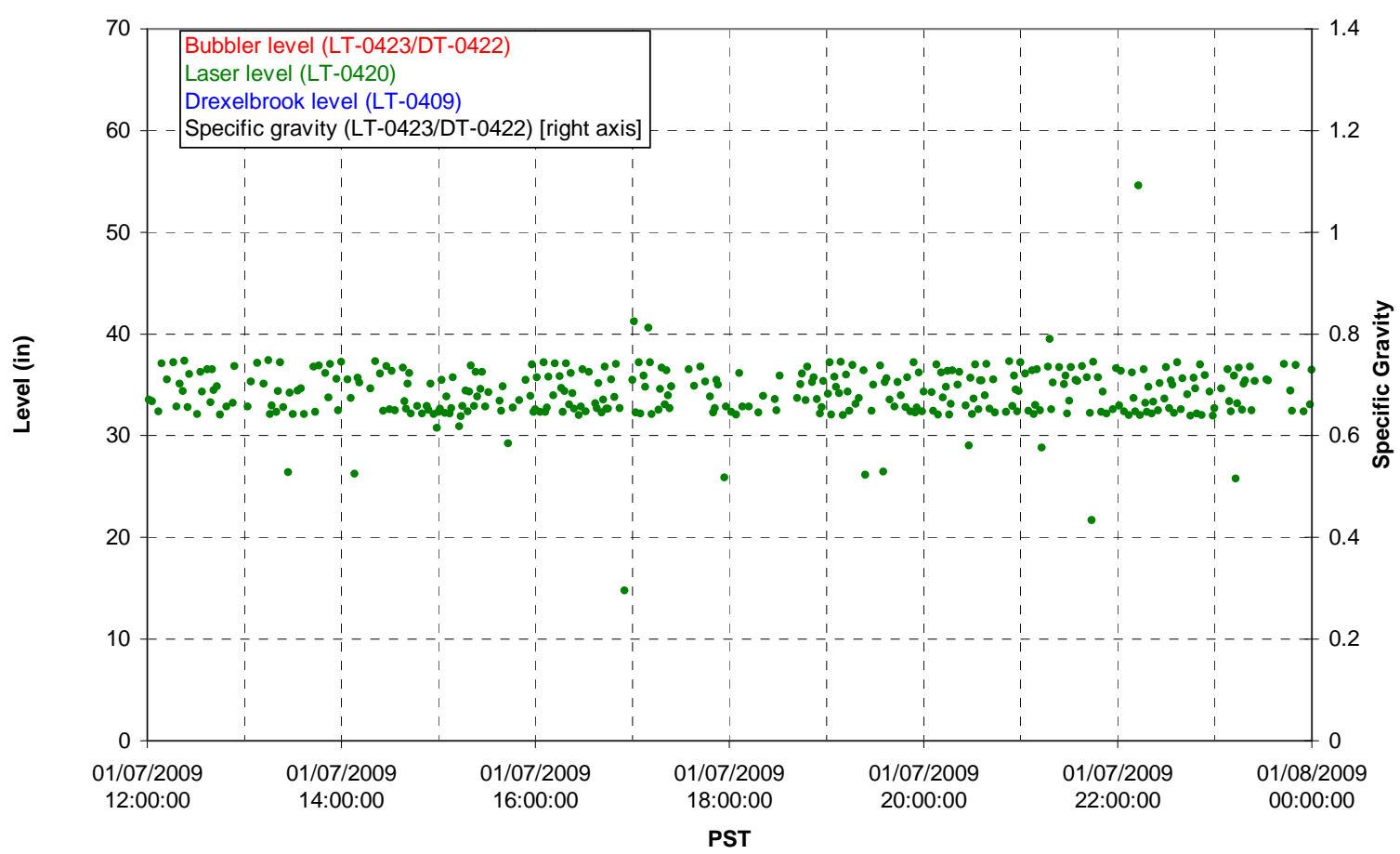

T01B temperatures

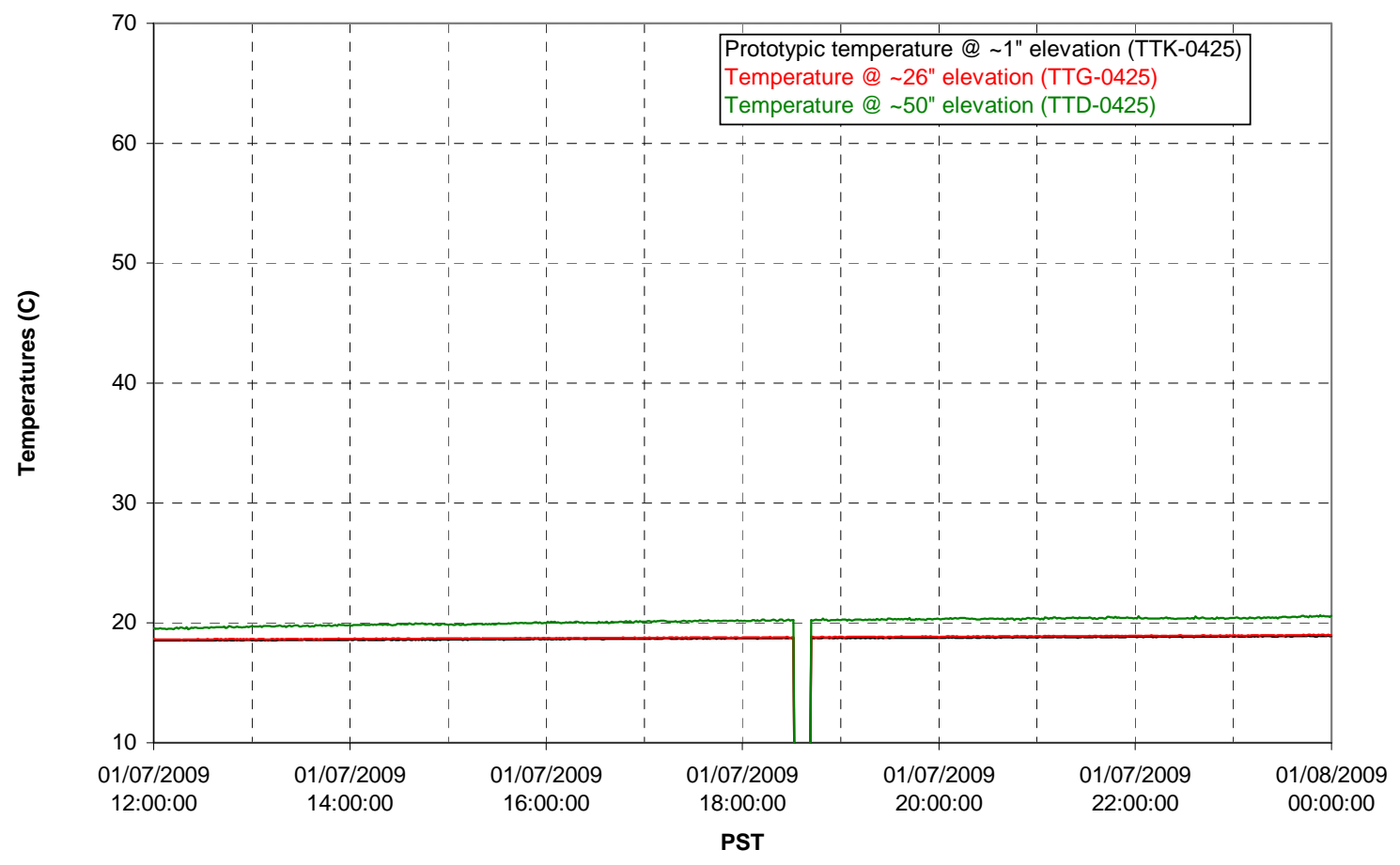


T02A level

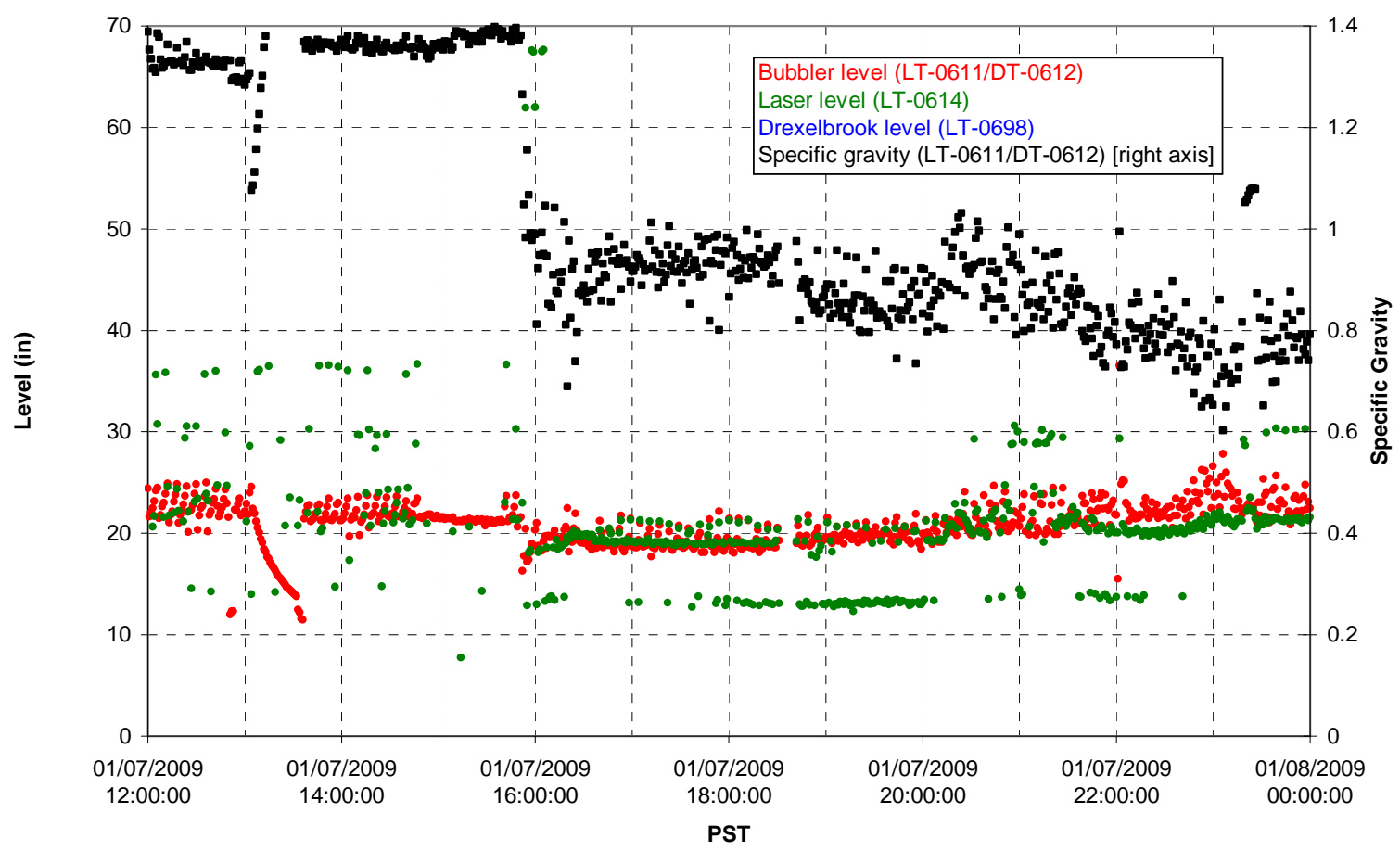

T02A temperatures

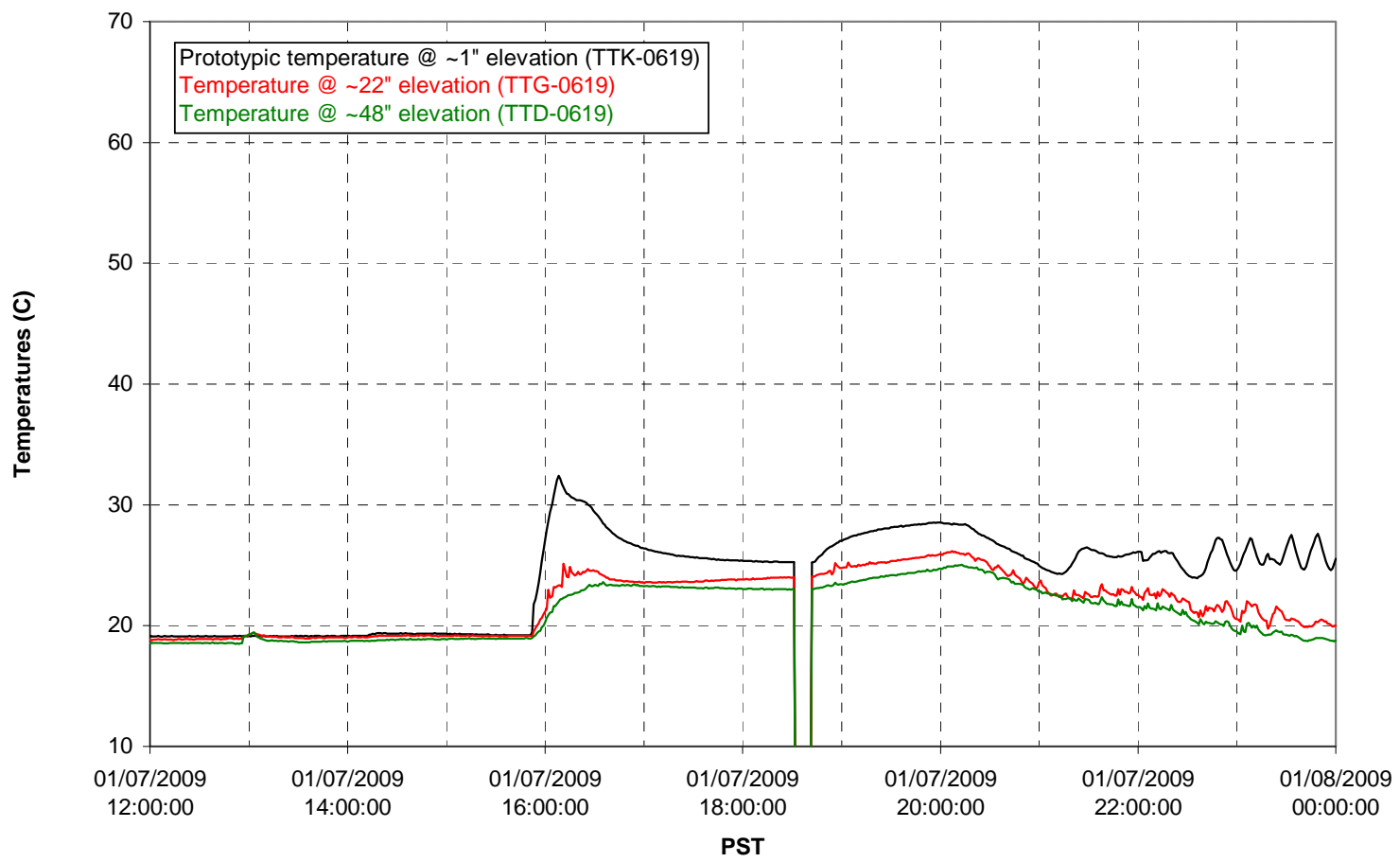


T02A and filter loop temperatures

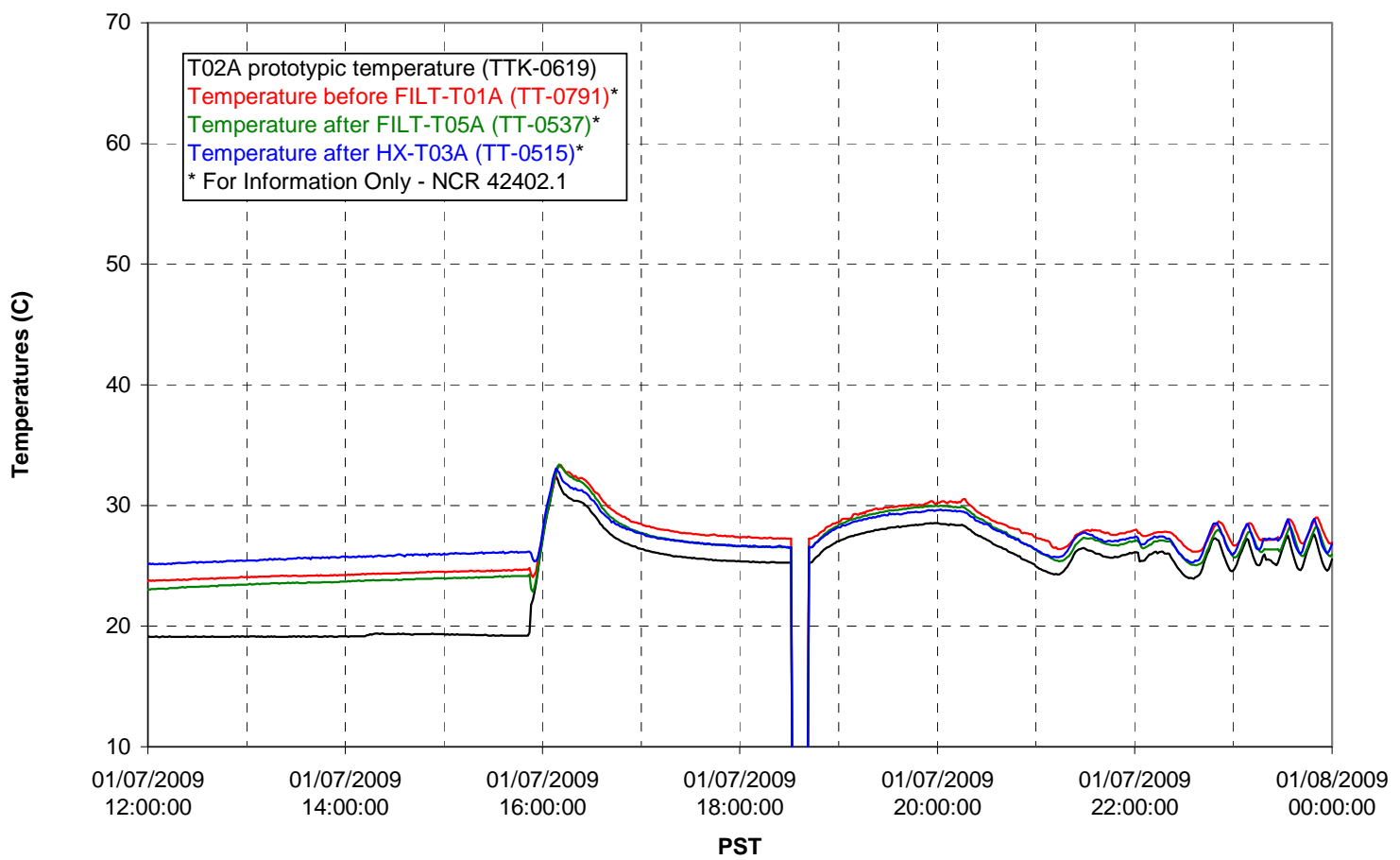

Pump Pressures and Flow

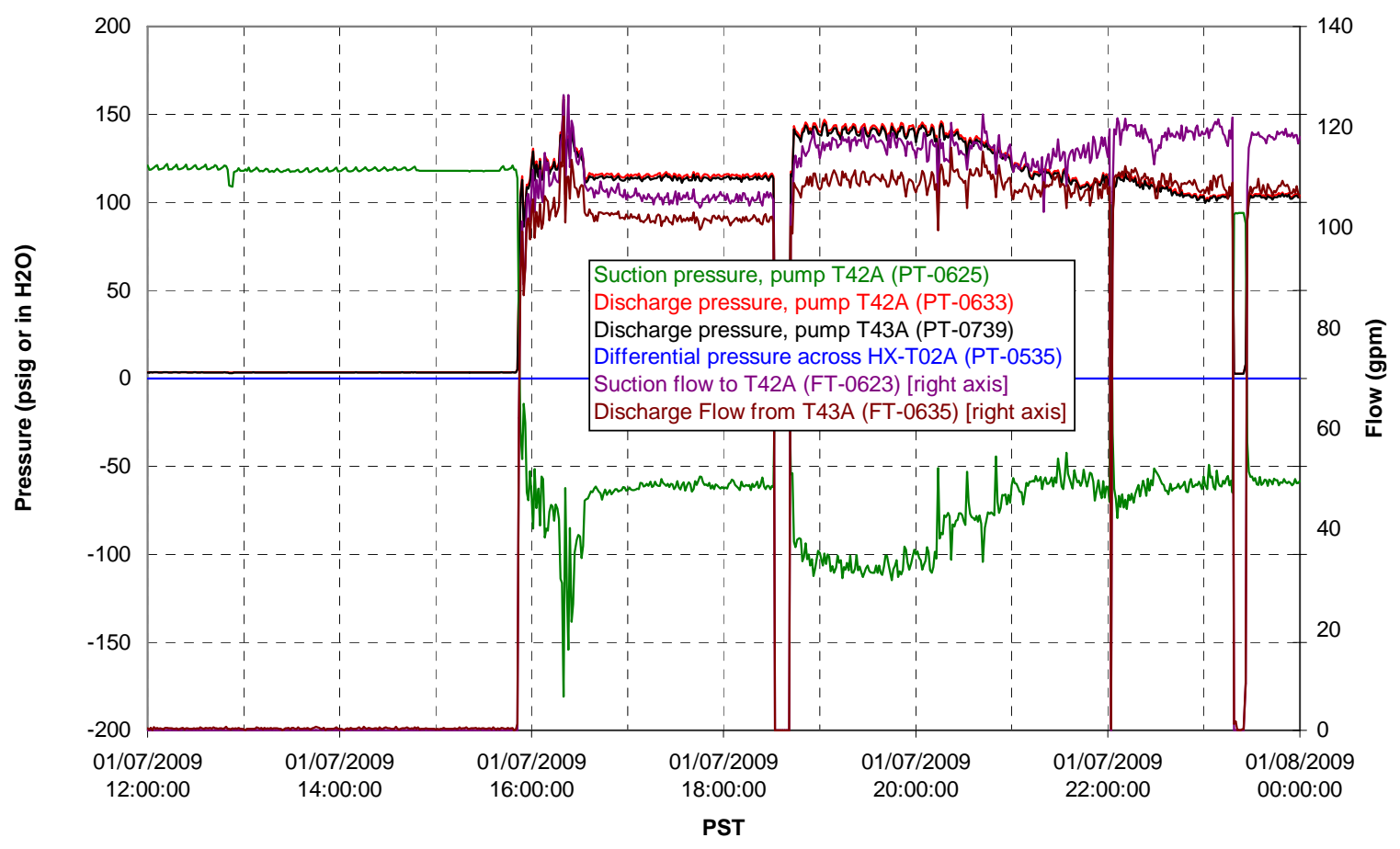




\section{Axial pressure drop}

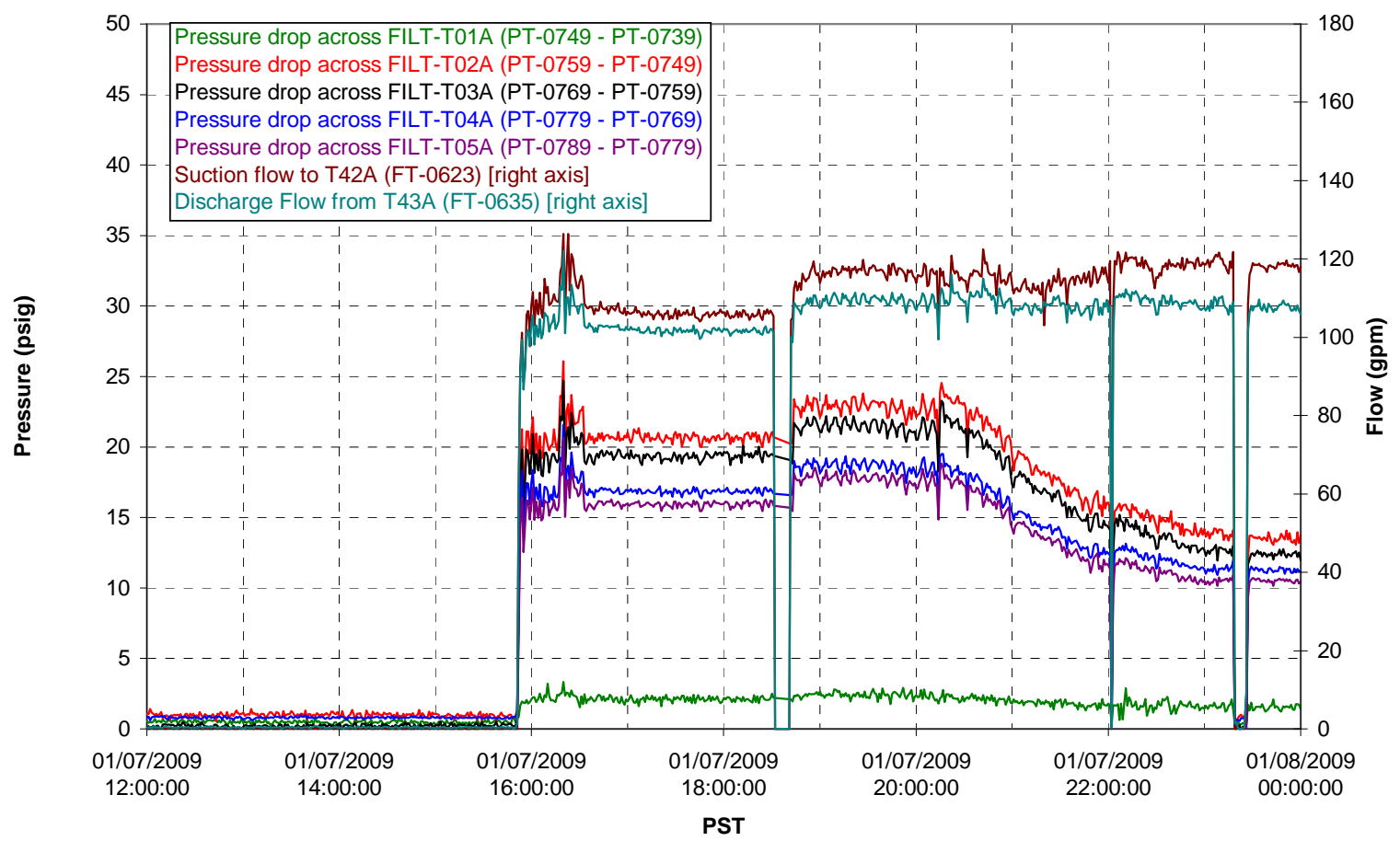

Permeate flow rates

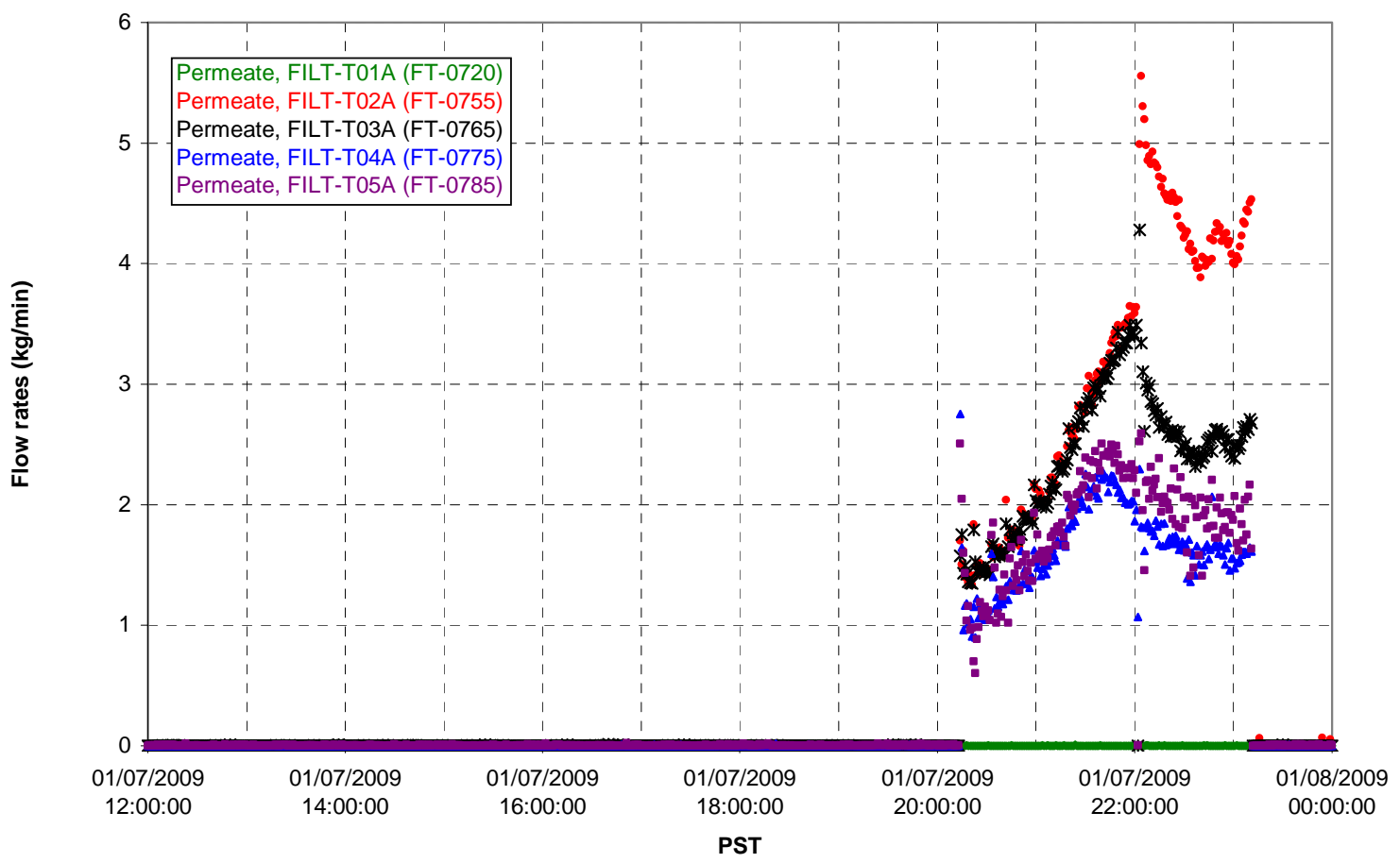


T02A Inner Temperature Tree

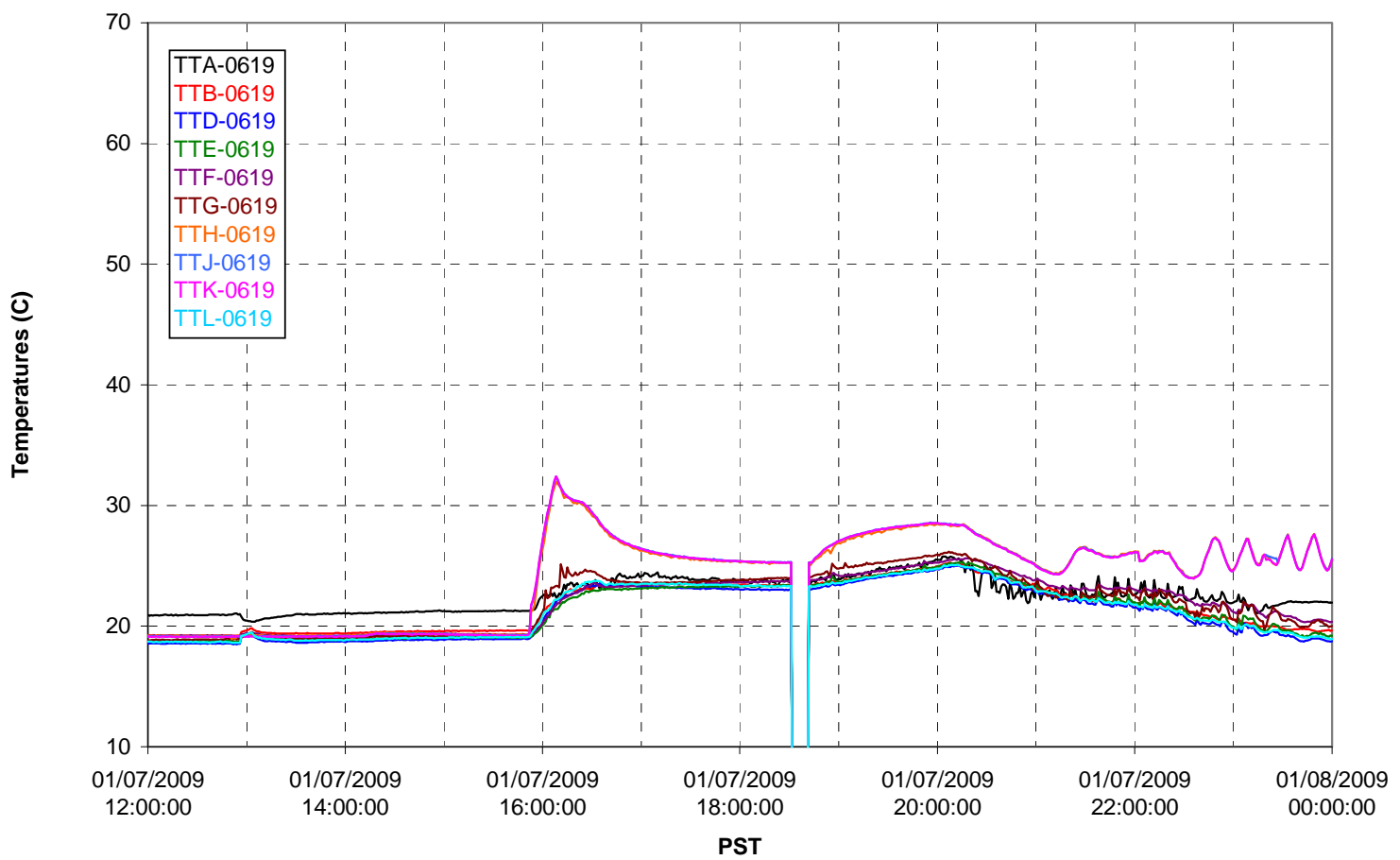

T02A Outer Temperature Tree

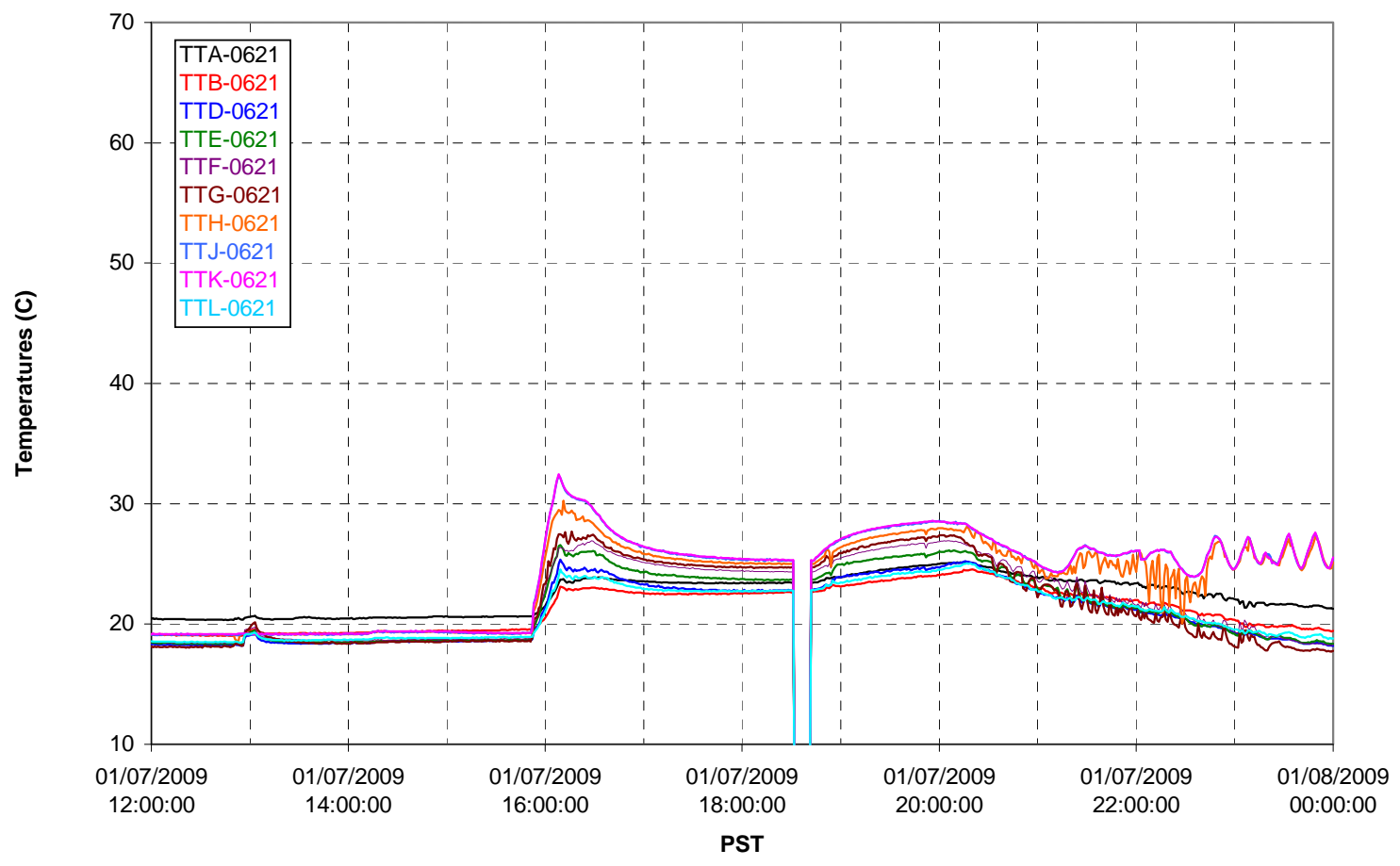


T02A temperatures

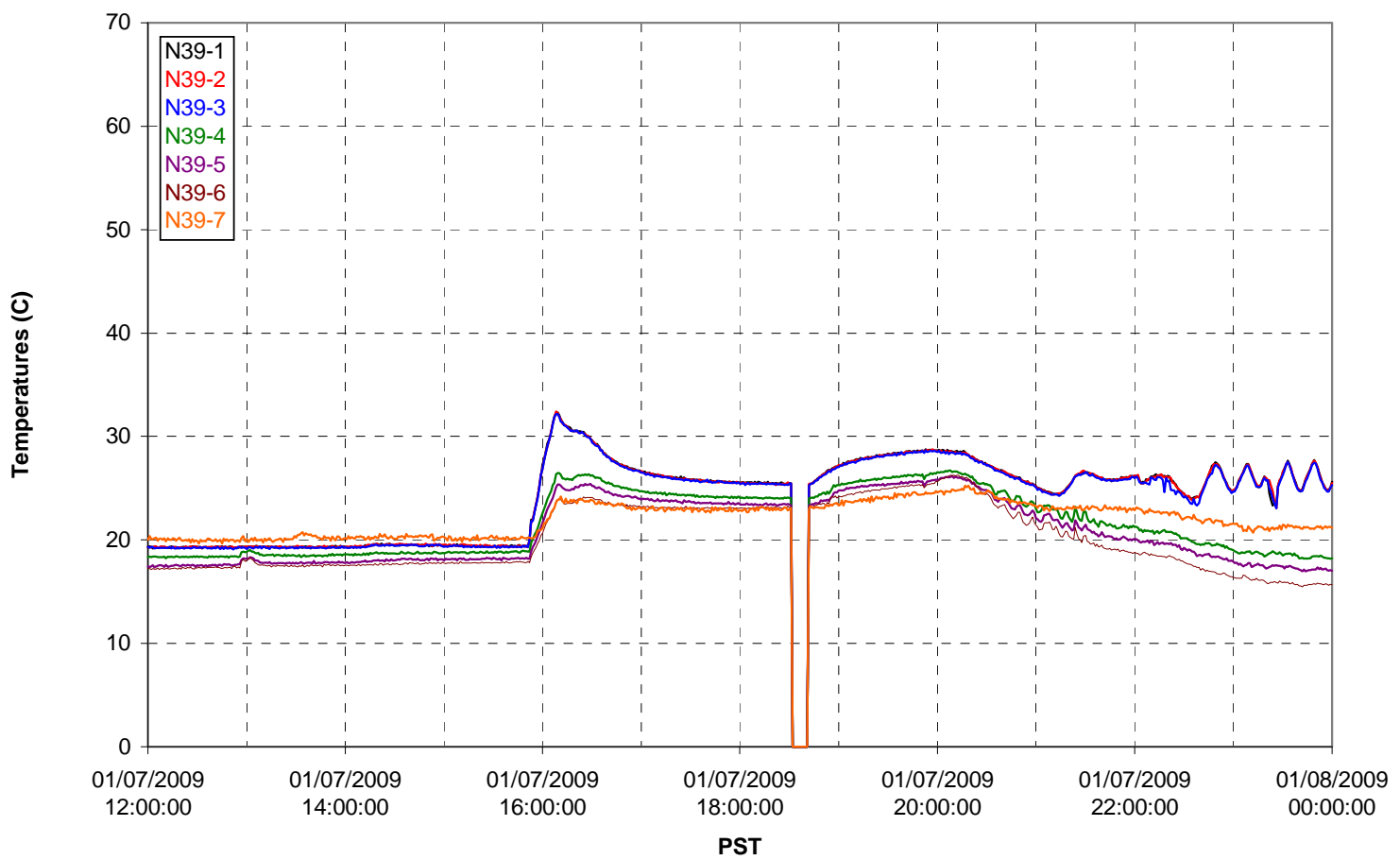

T02A temperatures

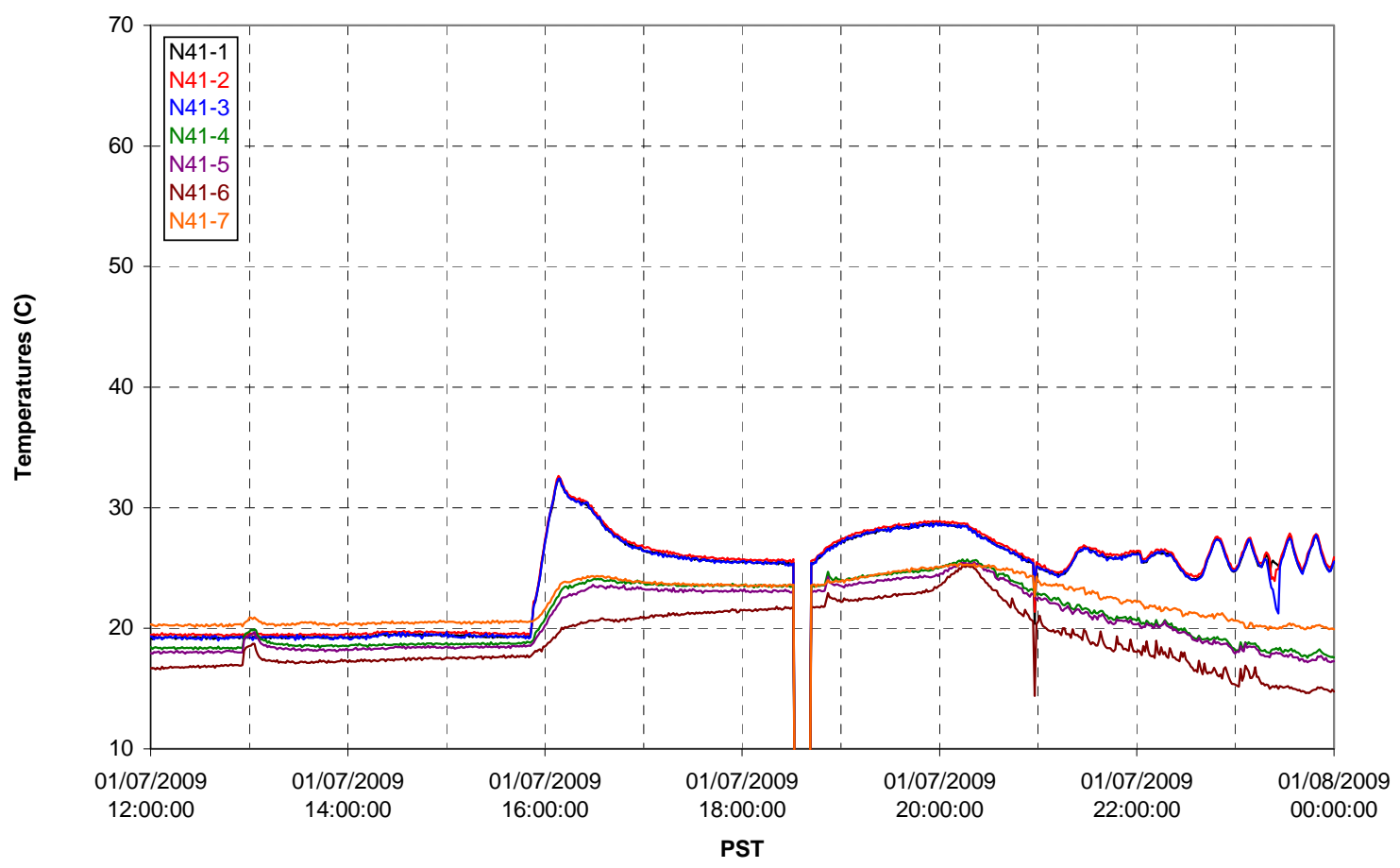


T02A temperatures

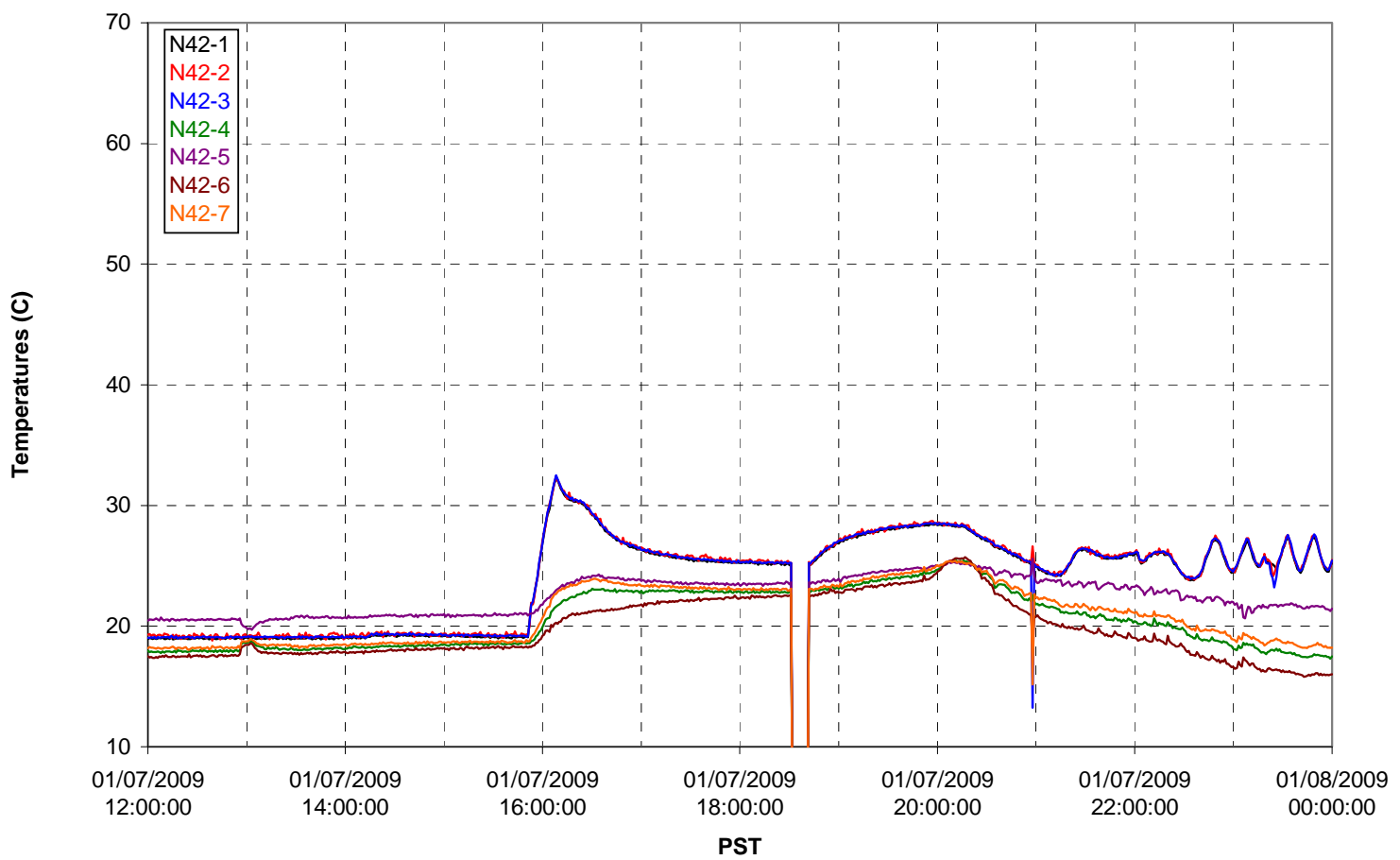

T02A temperatures

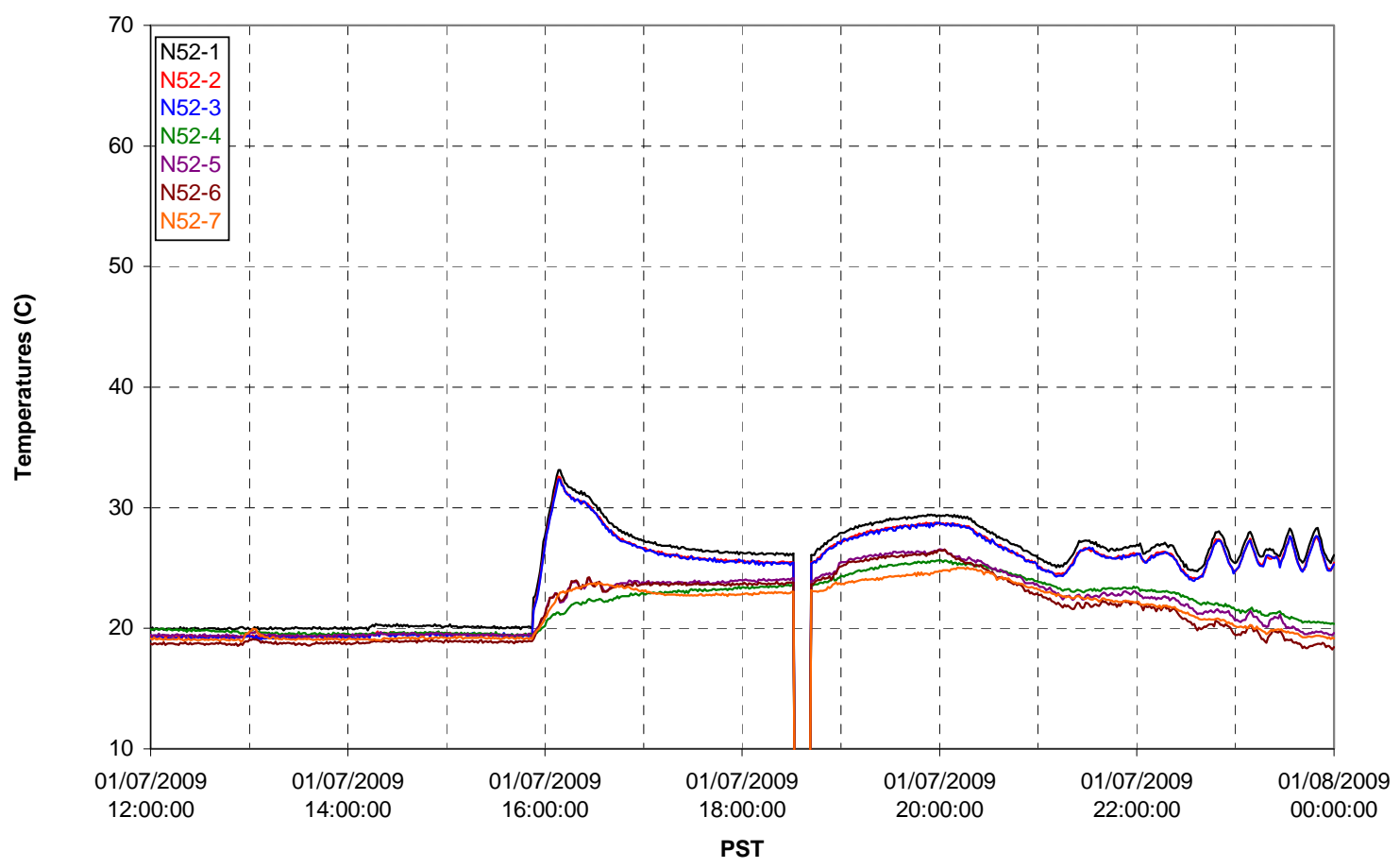


T02A Heating and Cooling

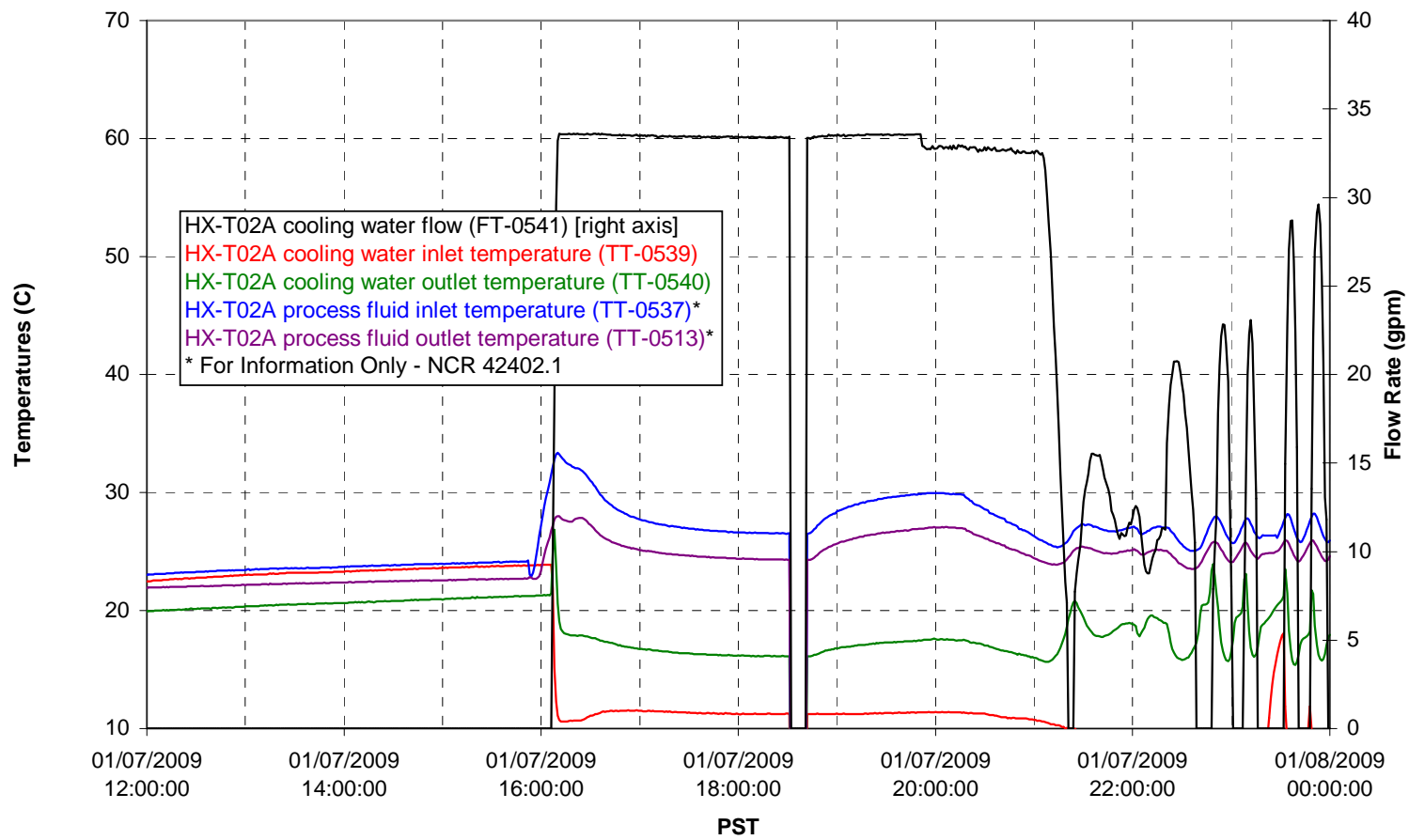

Pump Operation

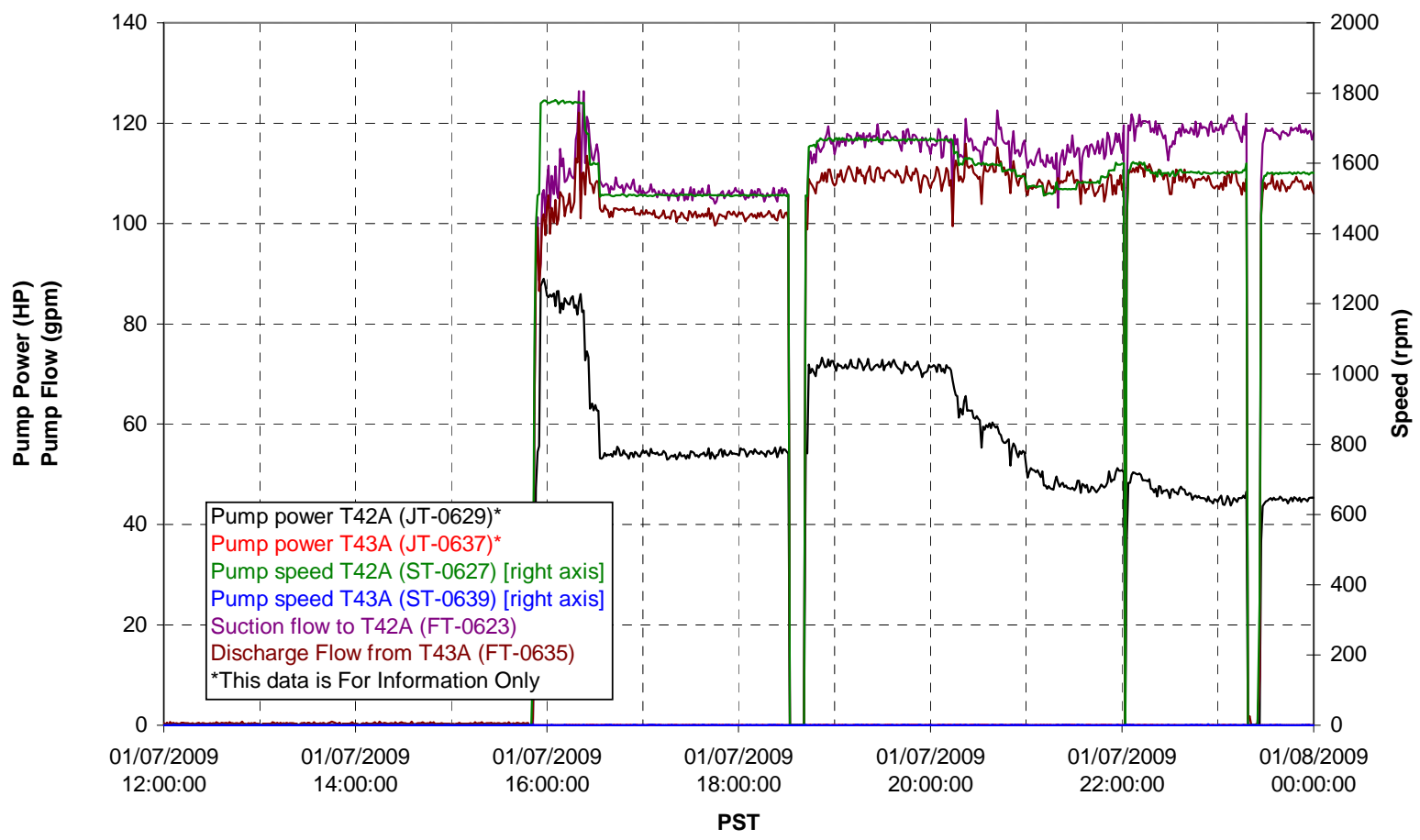


Pulsepot UFP-PP-T01A

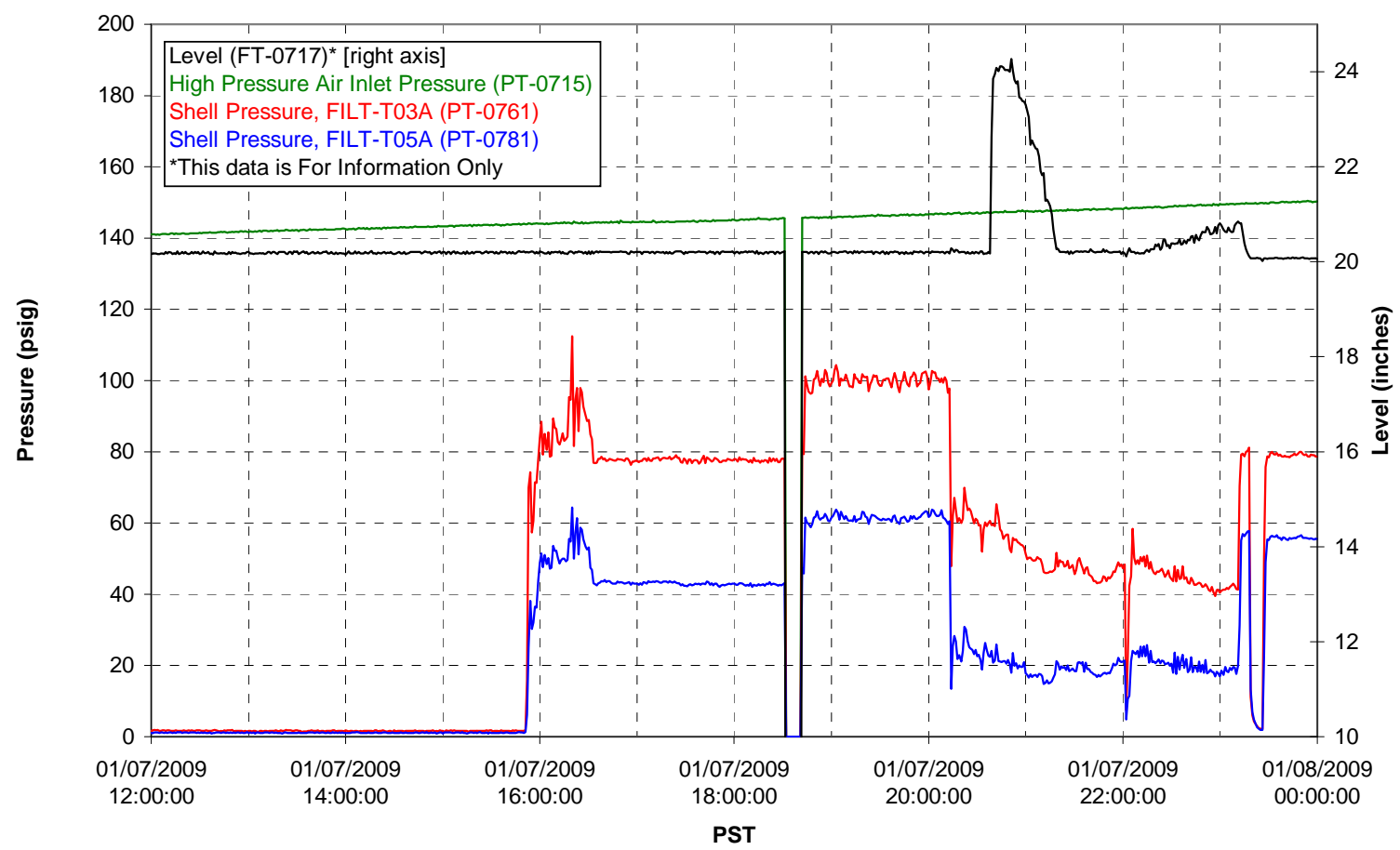

Pulsepot UFP-PP-T02A

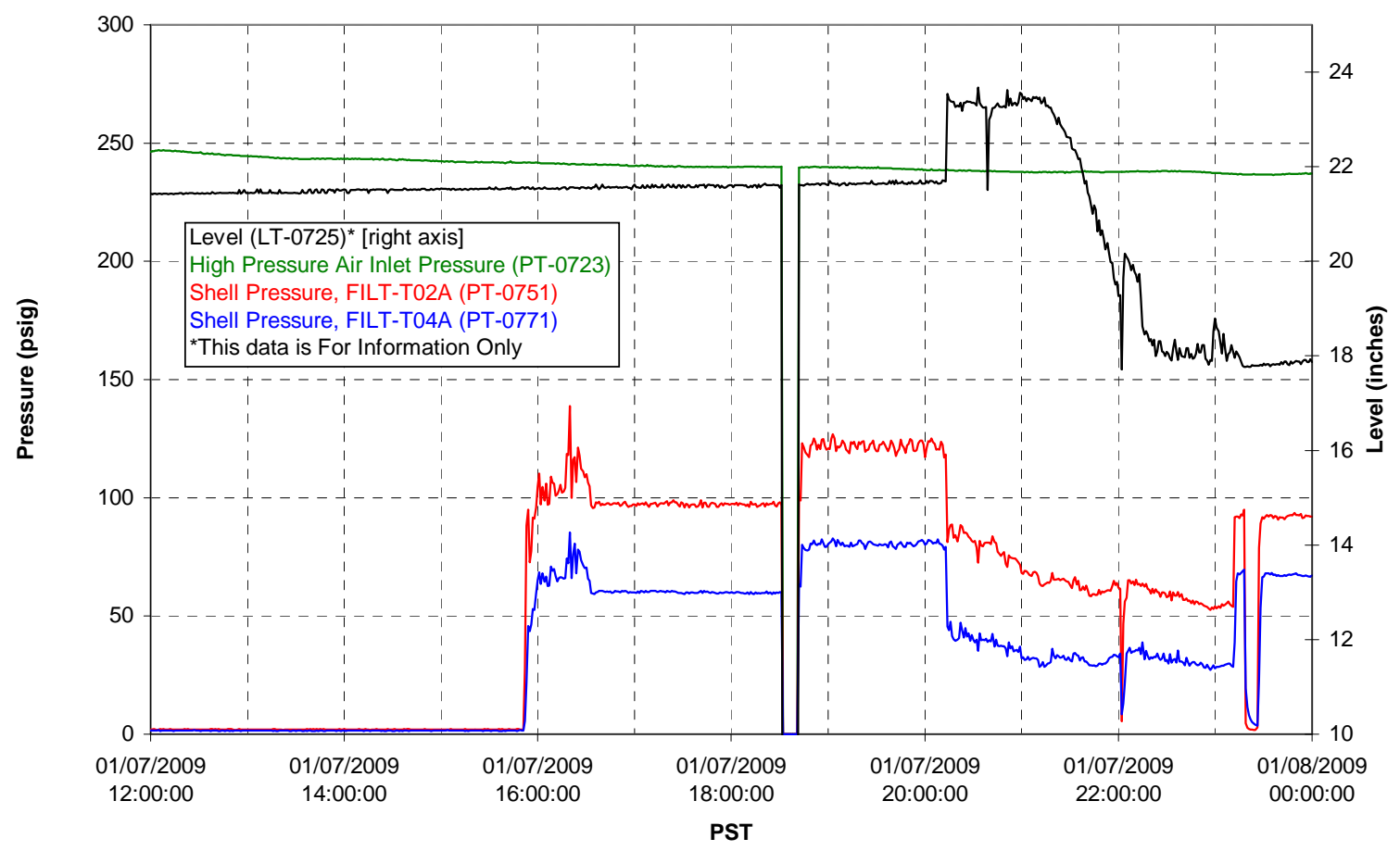


Pulsepot UFP-PP-T03A

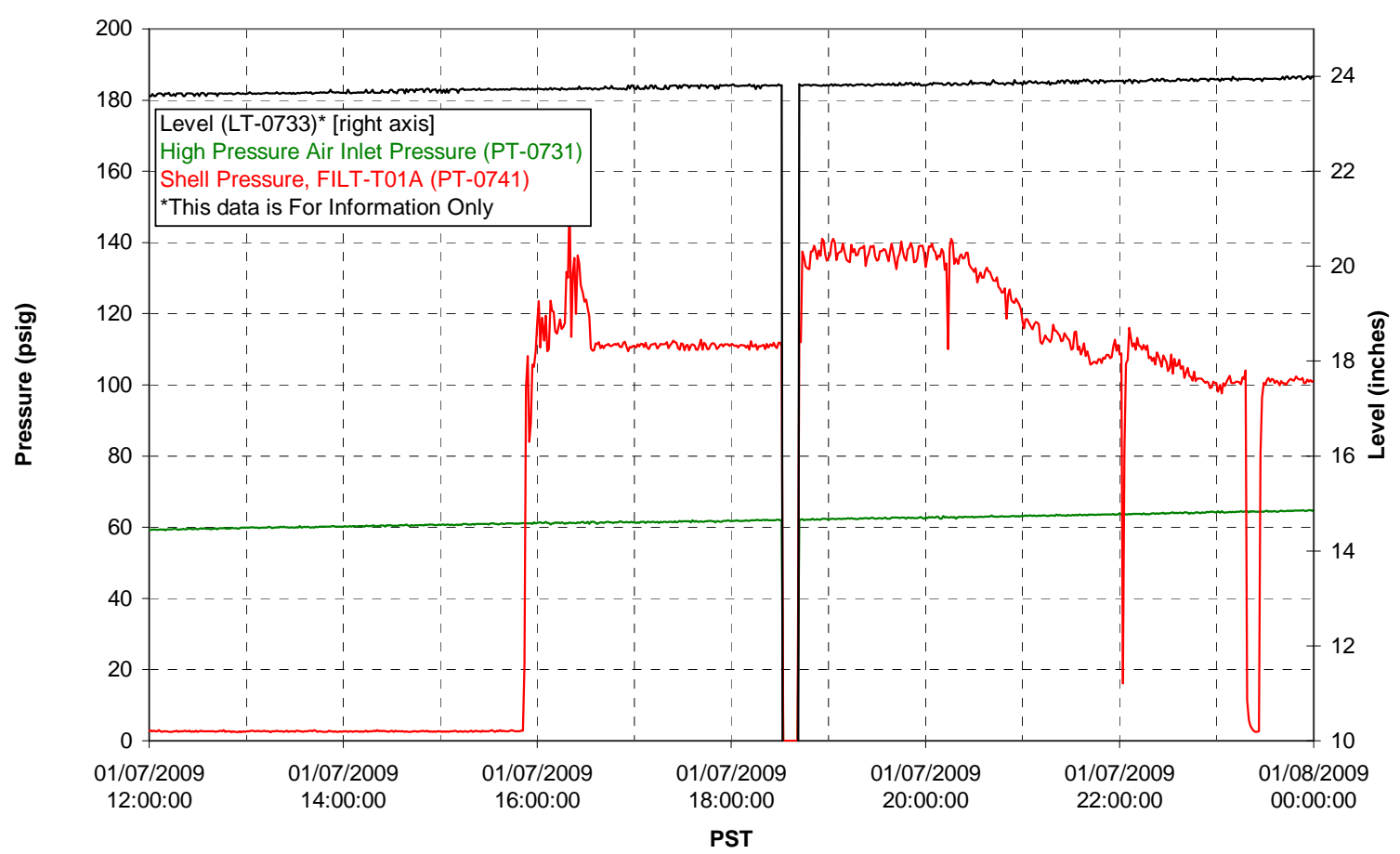

Pulsepot Levels

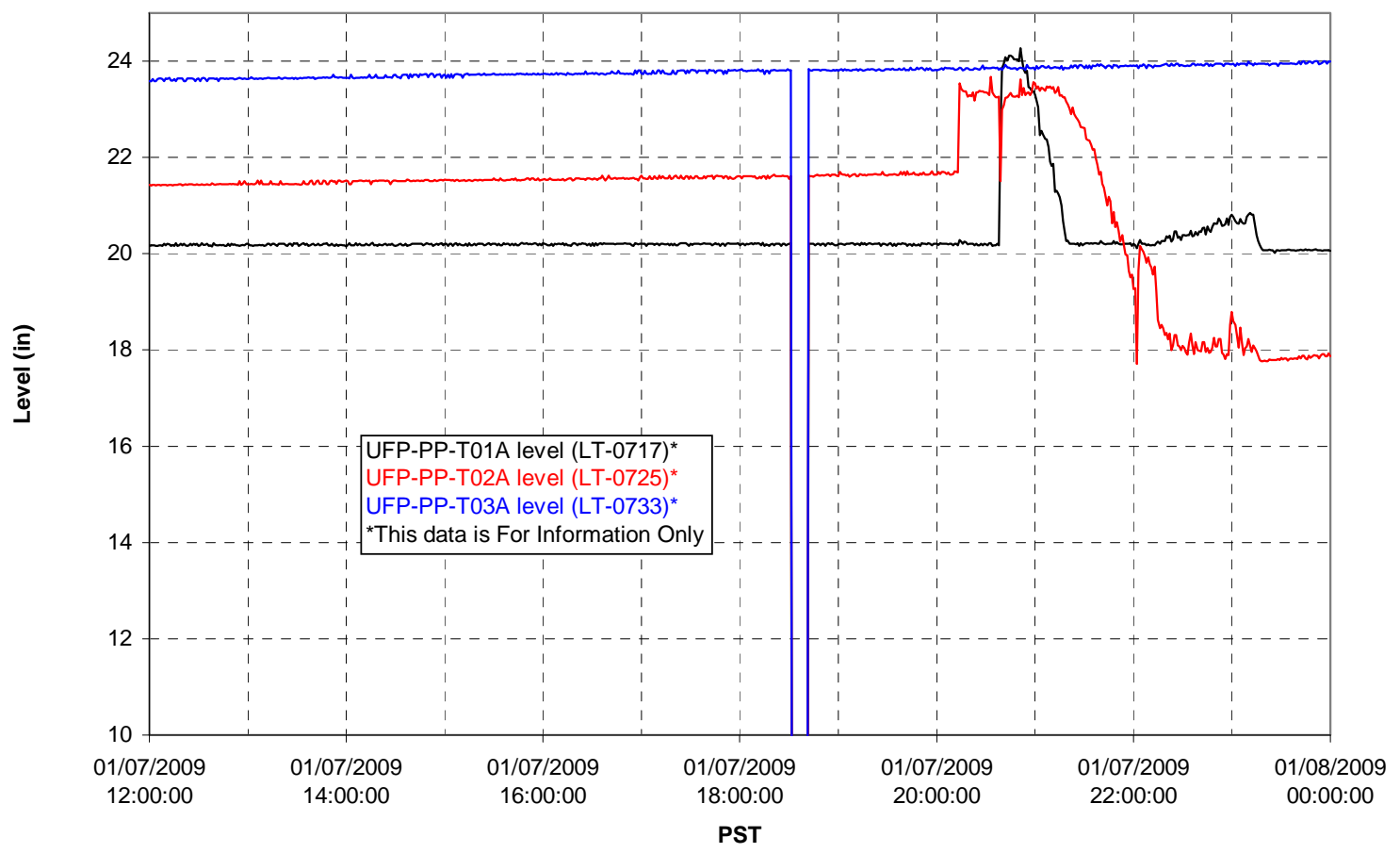


Filter UFP-FILT-T01A

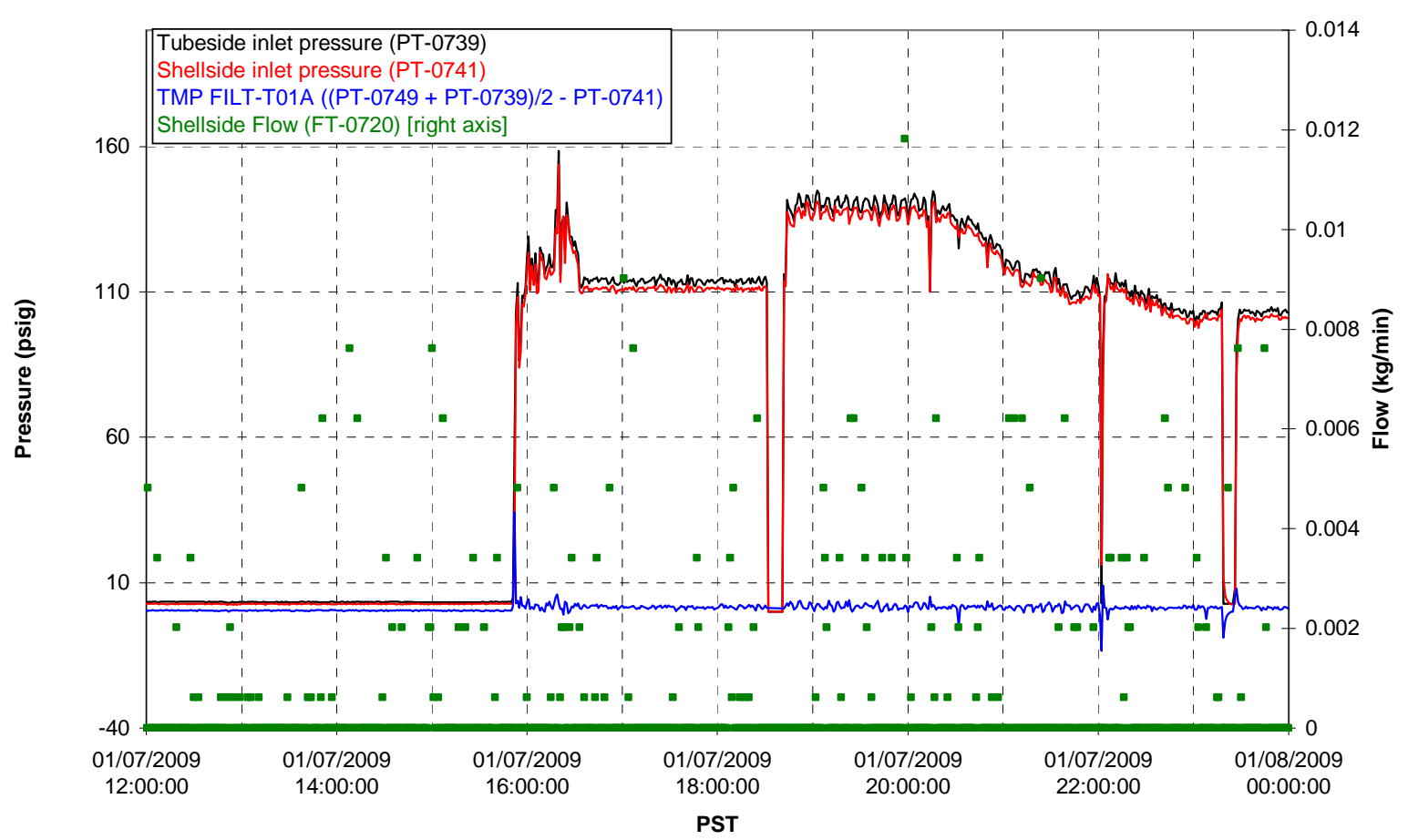

Filter UFP-FILT-T02A

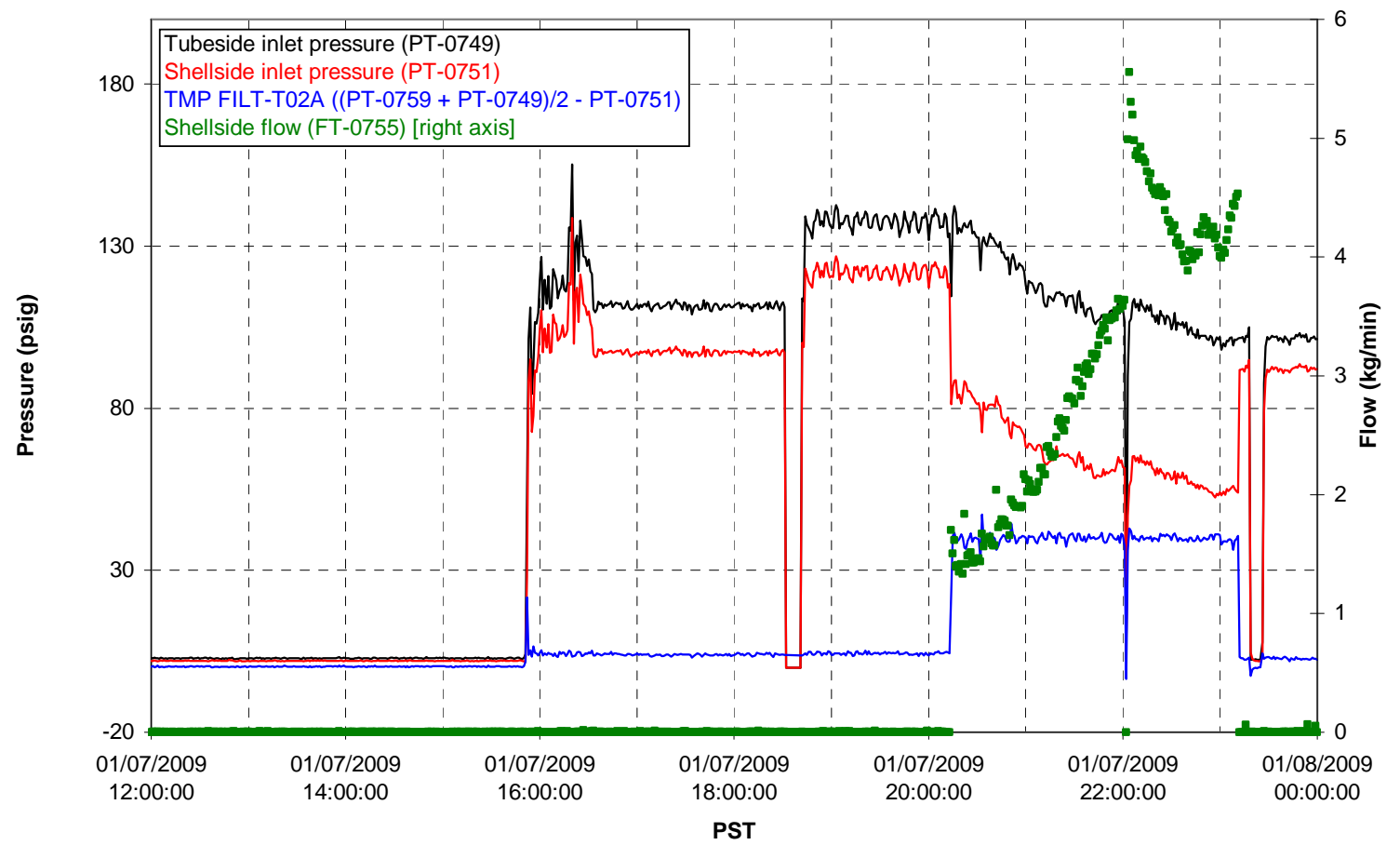


Filter UFP-FILT-T03A

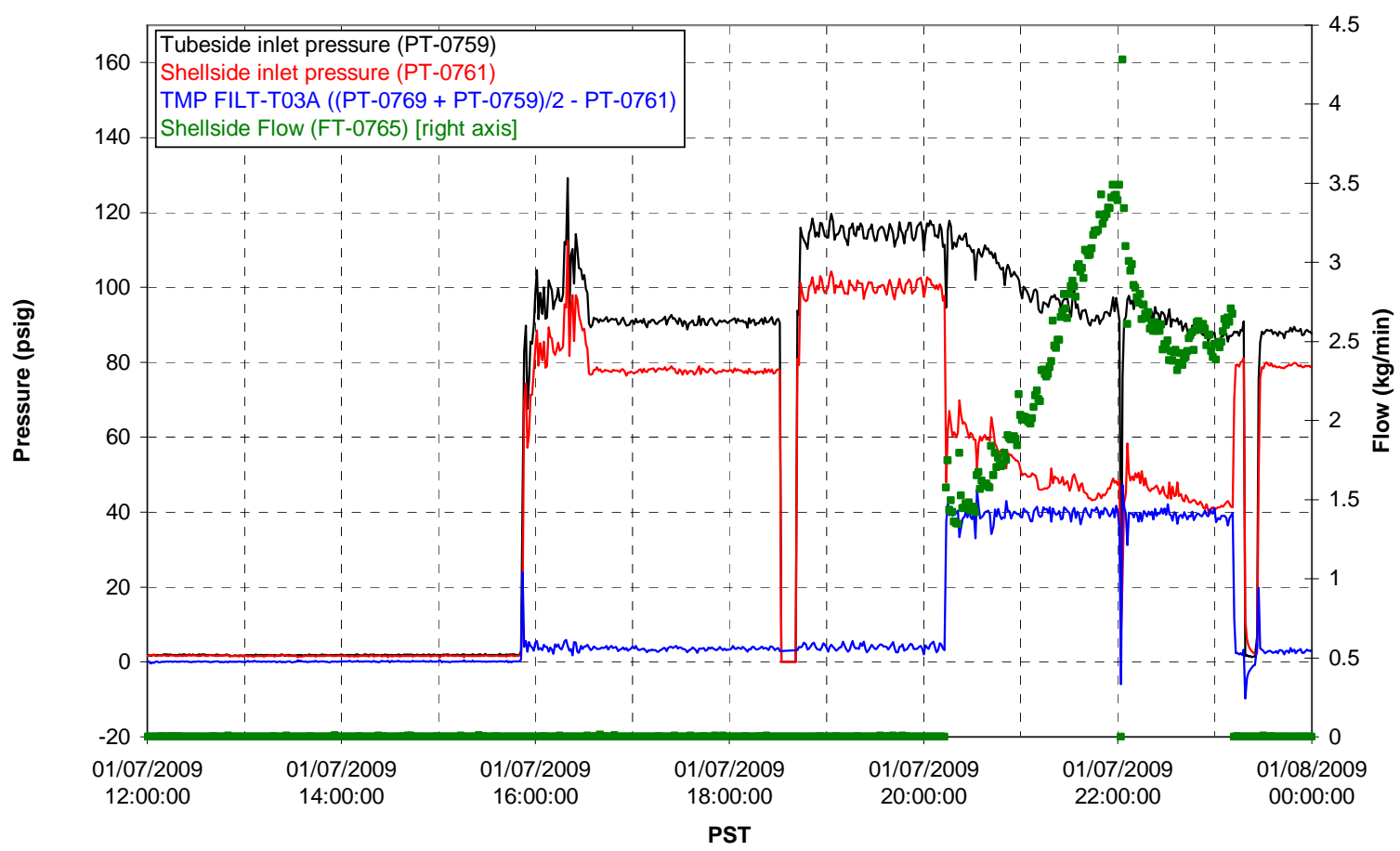

Filter UFP-FILT-T04A

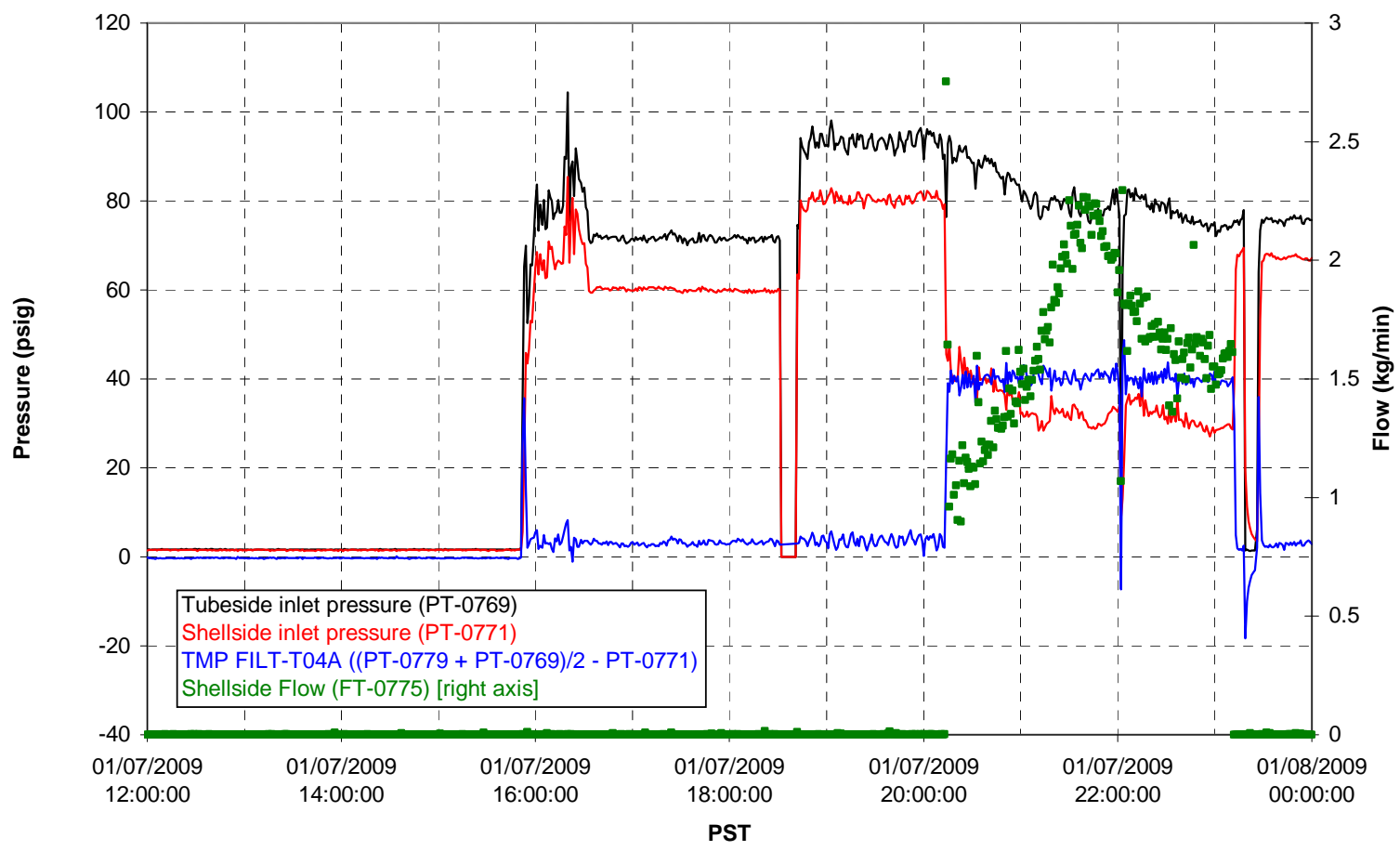


Filter UFP-FILT-T05A

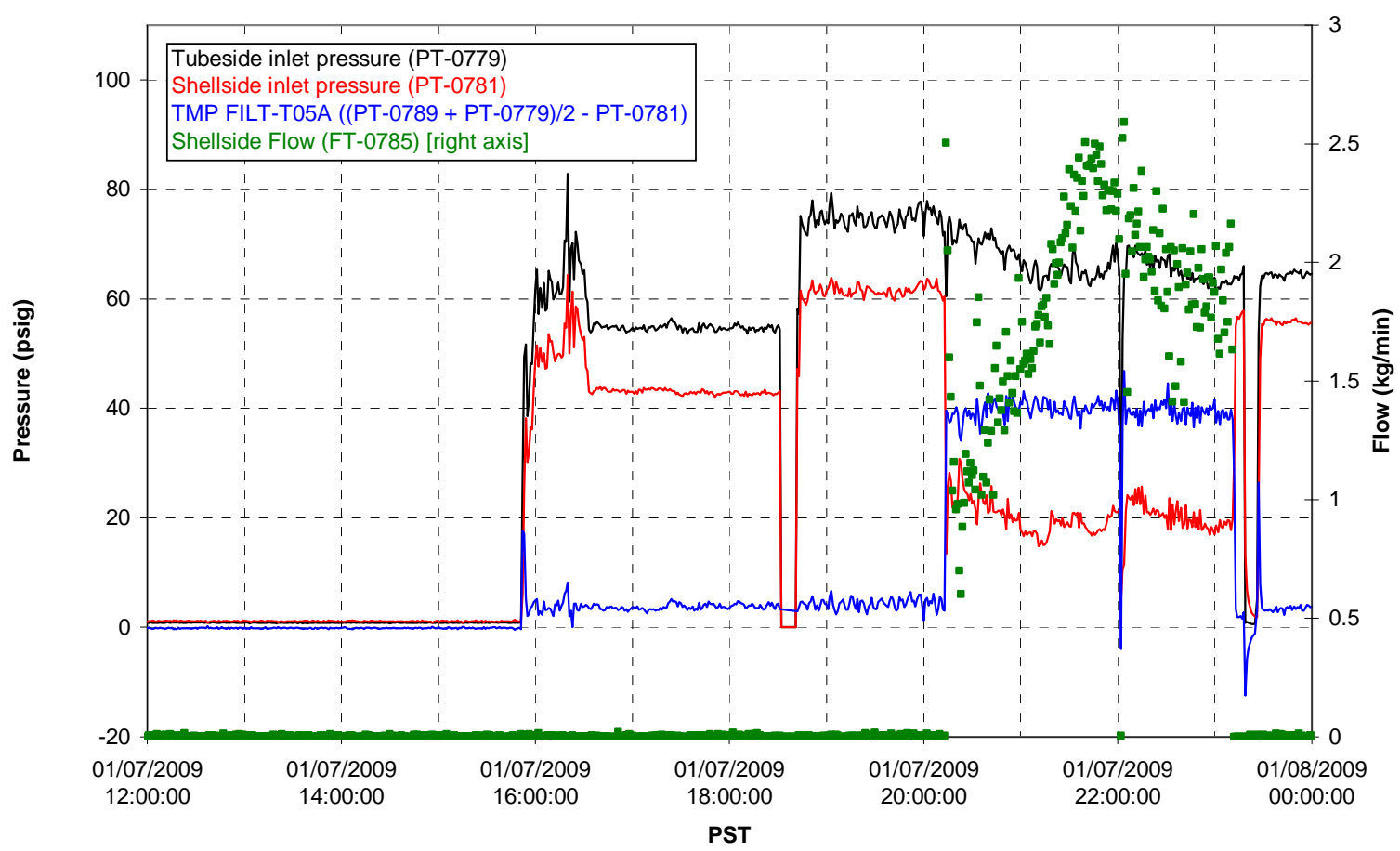

Chemical Flow

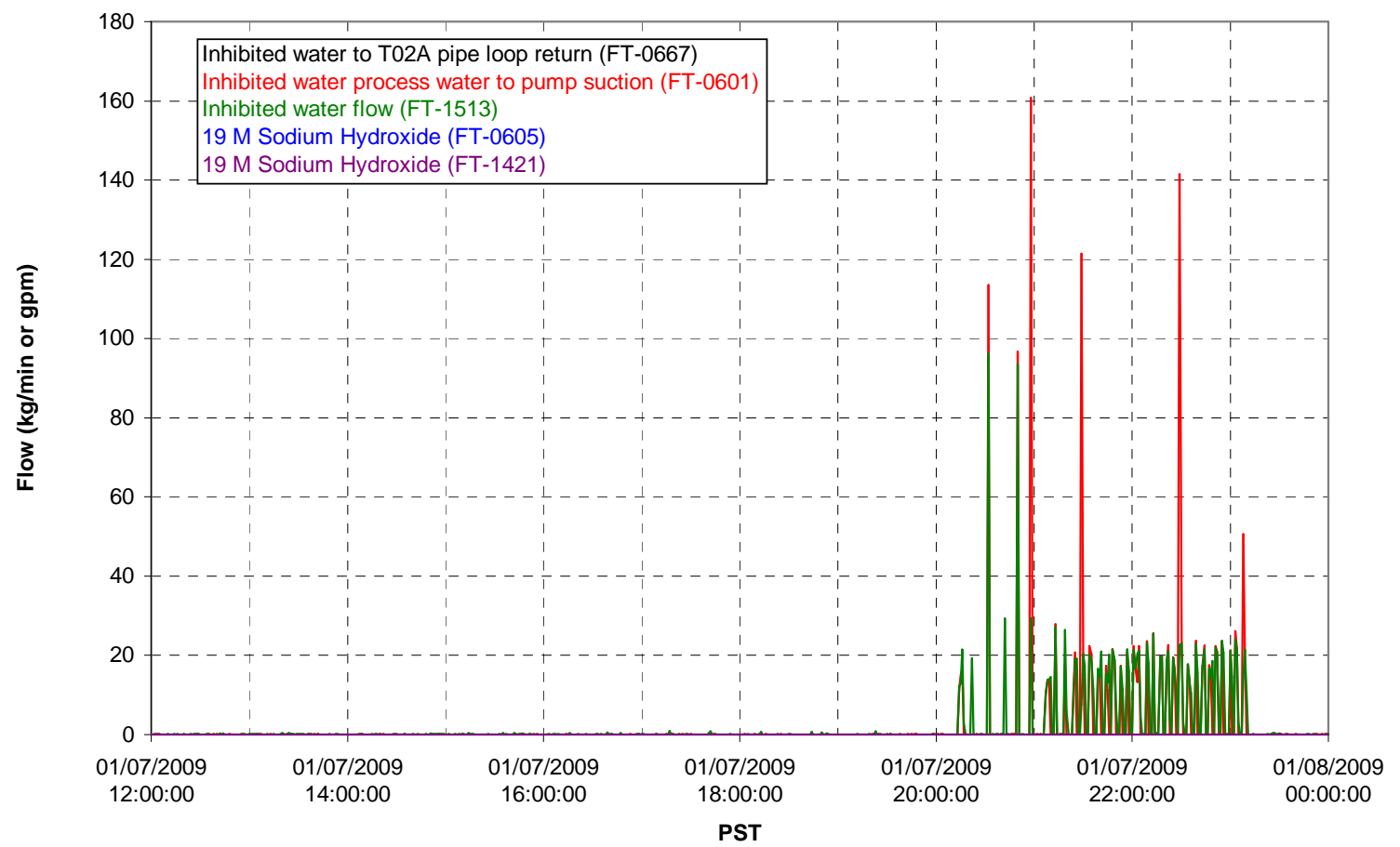


Chemical Flow

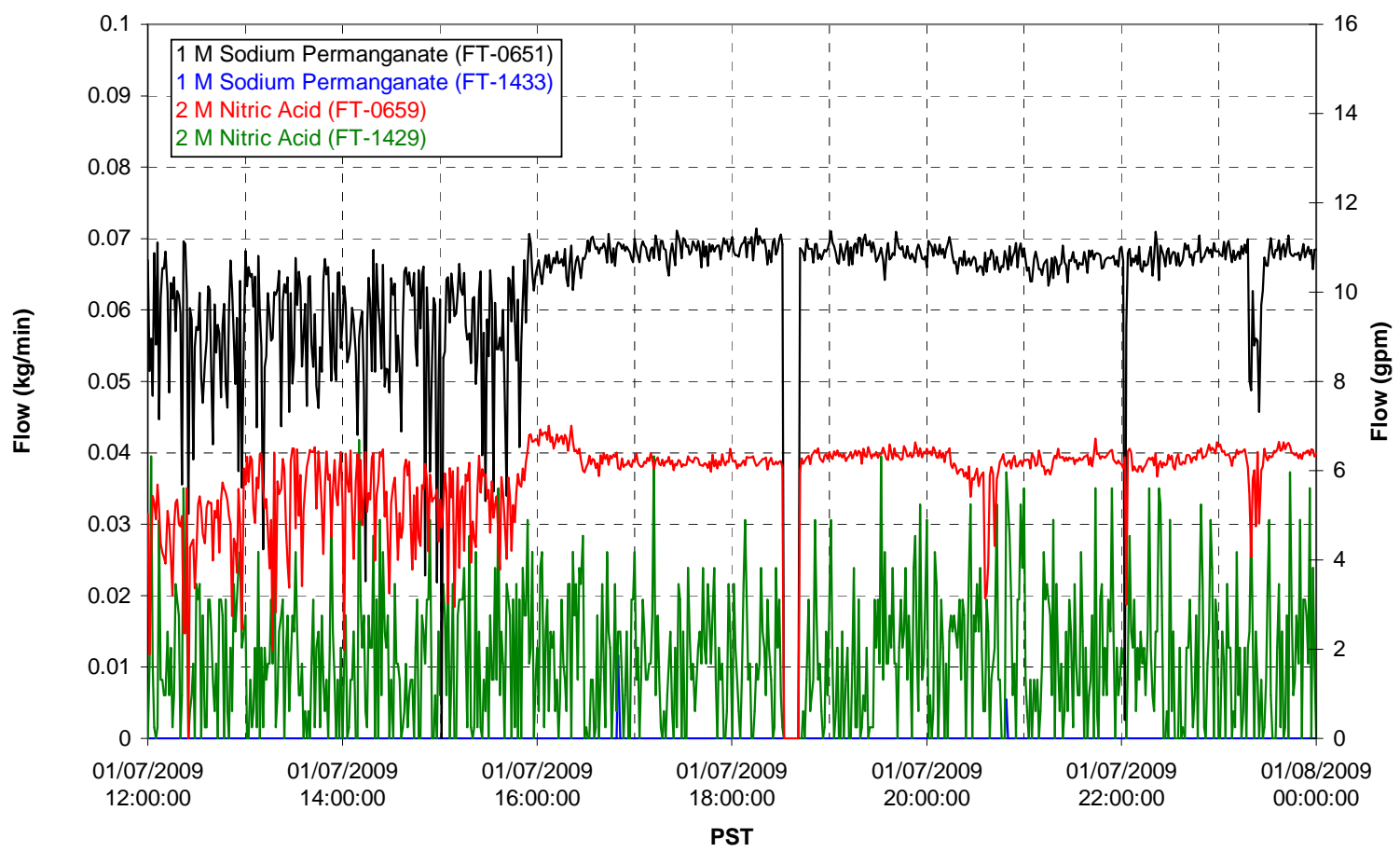

Air Flows

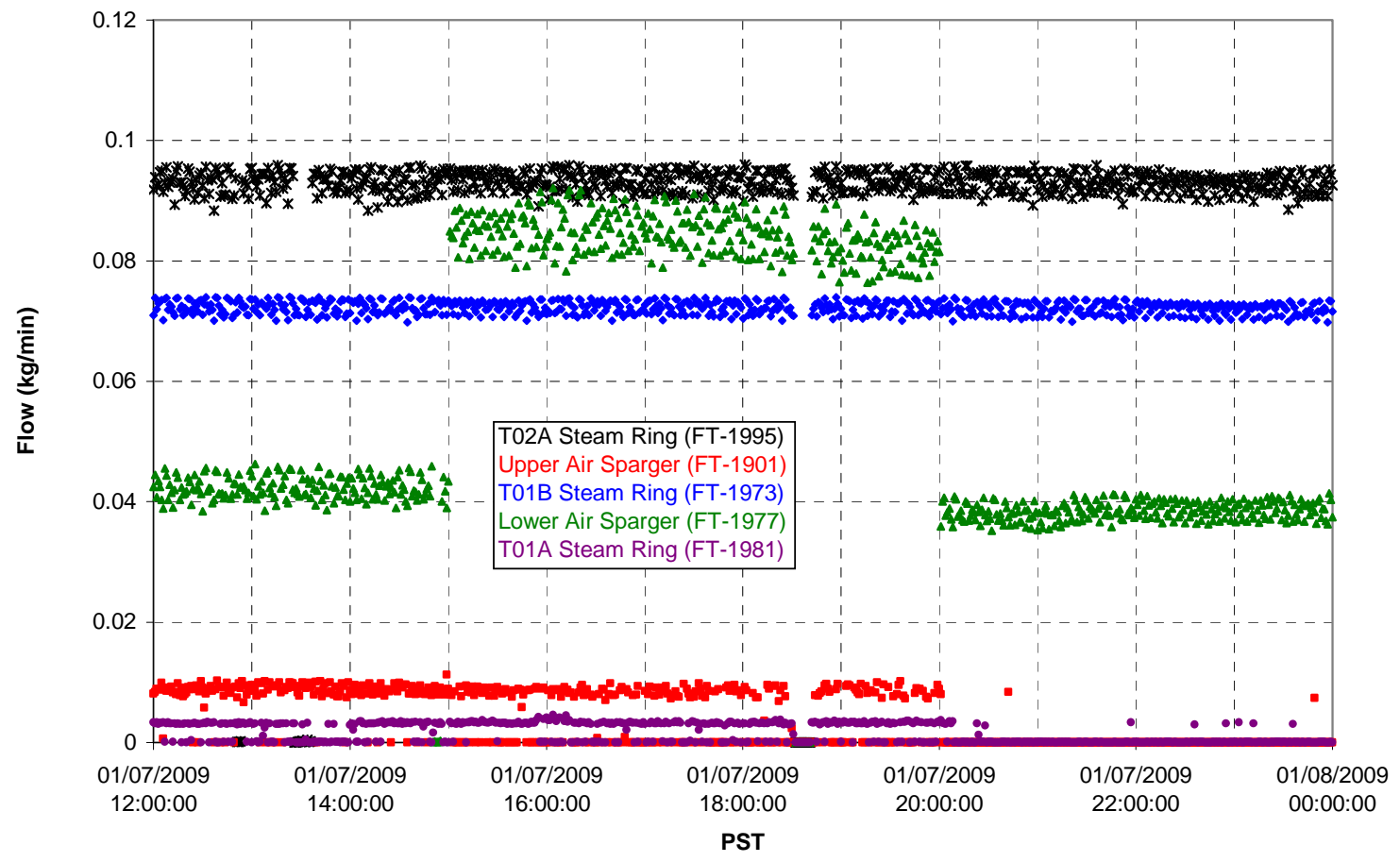


T02A Steam

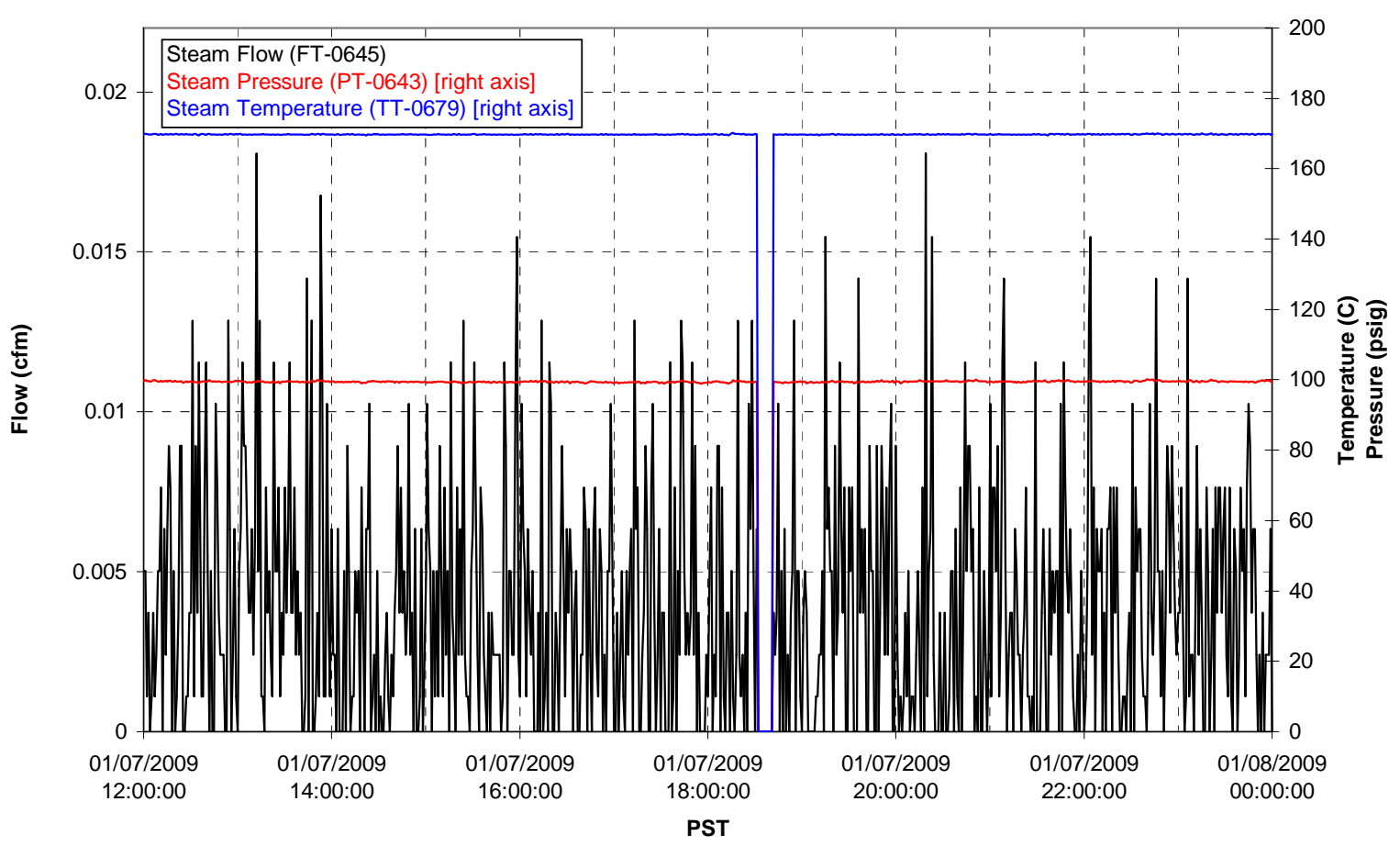

T01A Steam

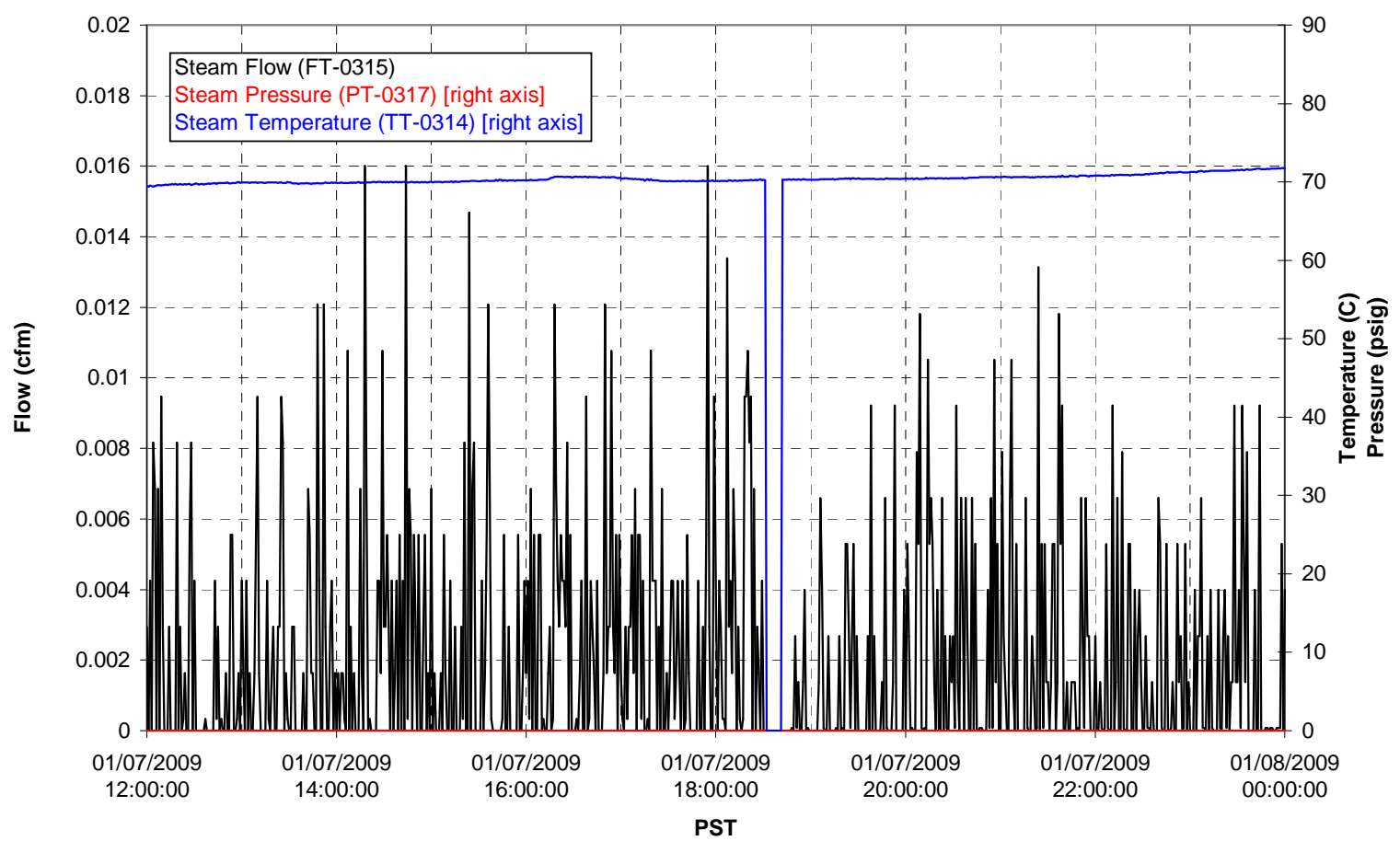


T01B Steam

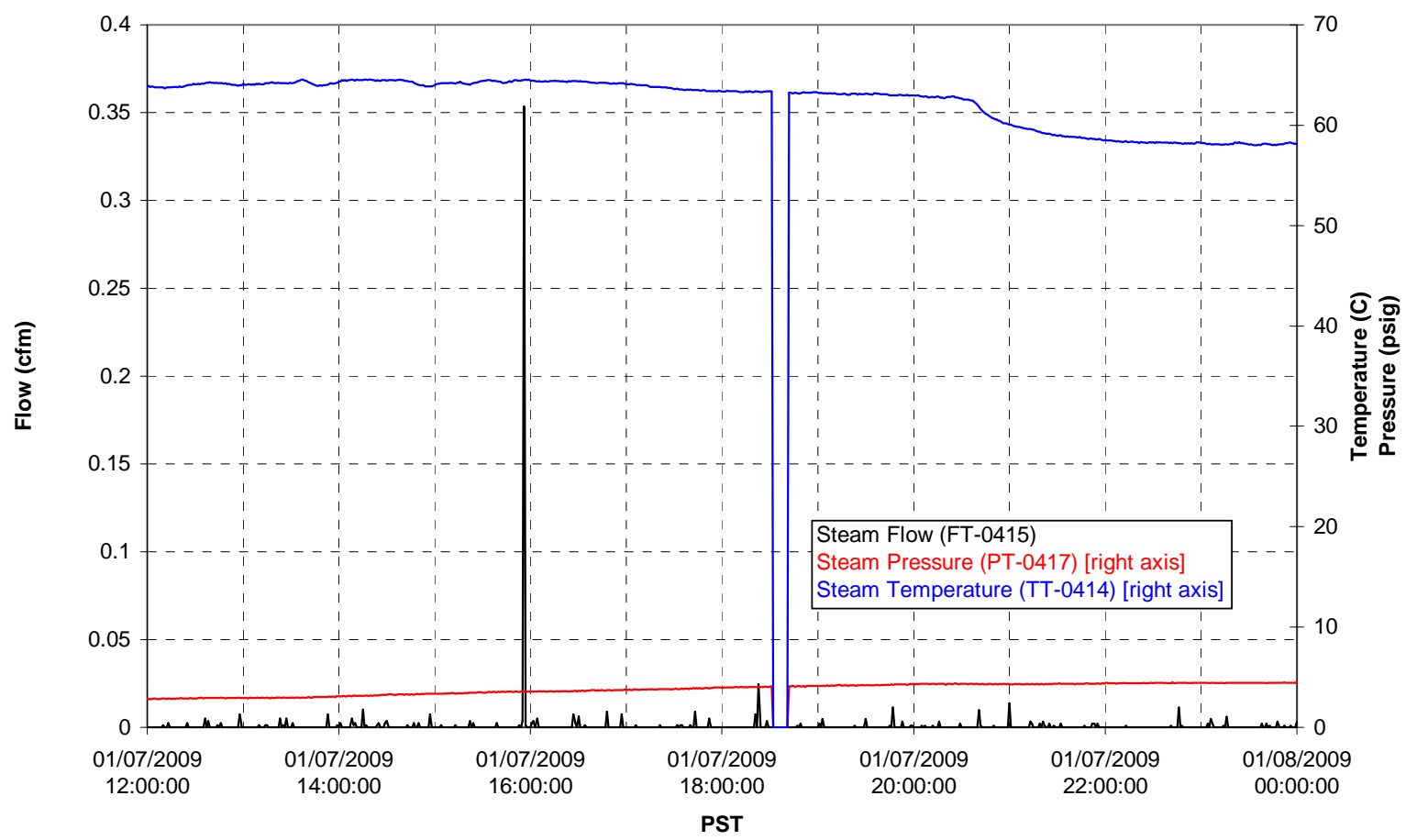


Functional Test Data Plots

01/08/2009 00:00 - 01/08/2009 12:00 
T01A level

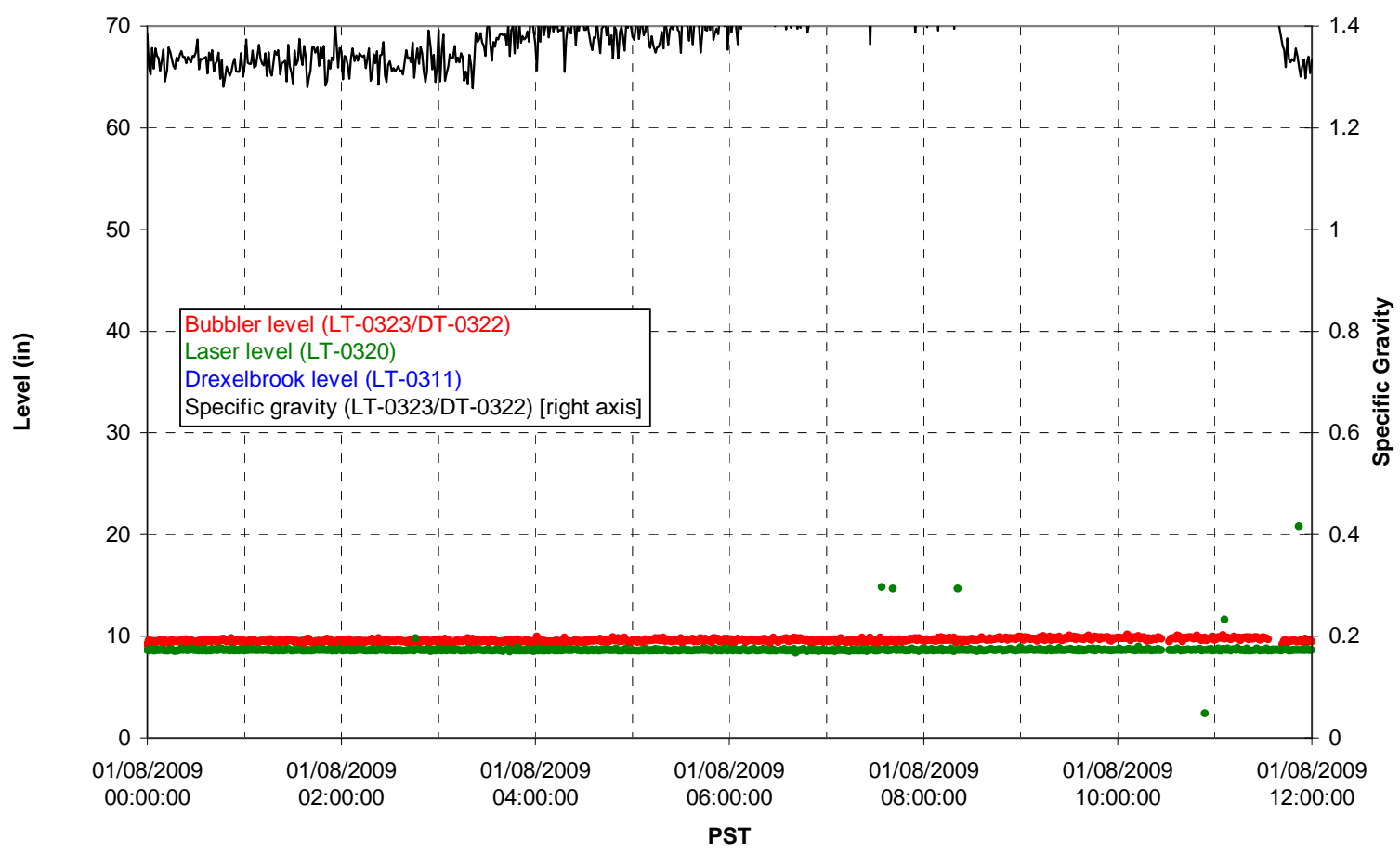

T01A temperatures

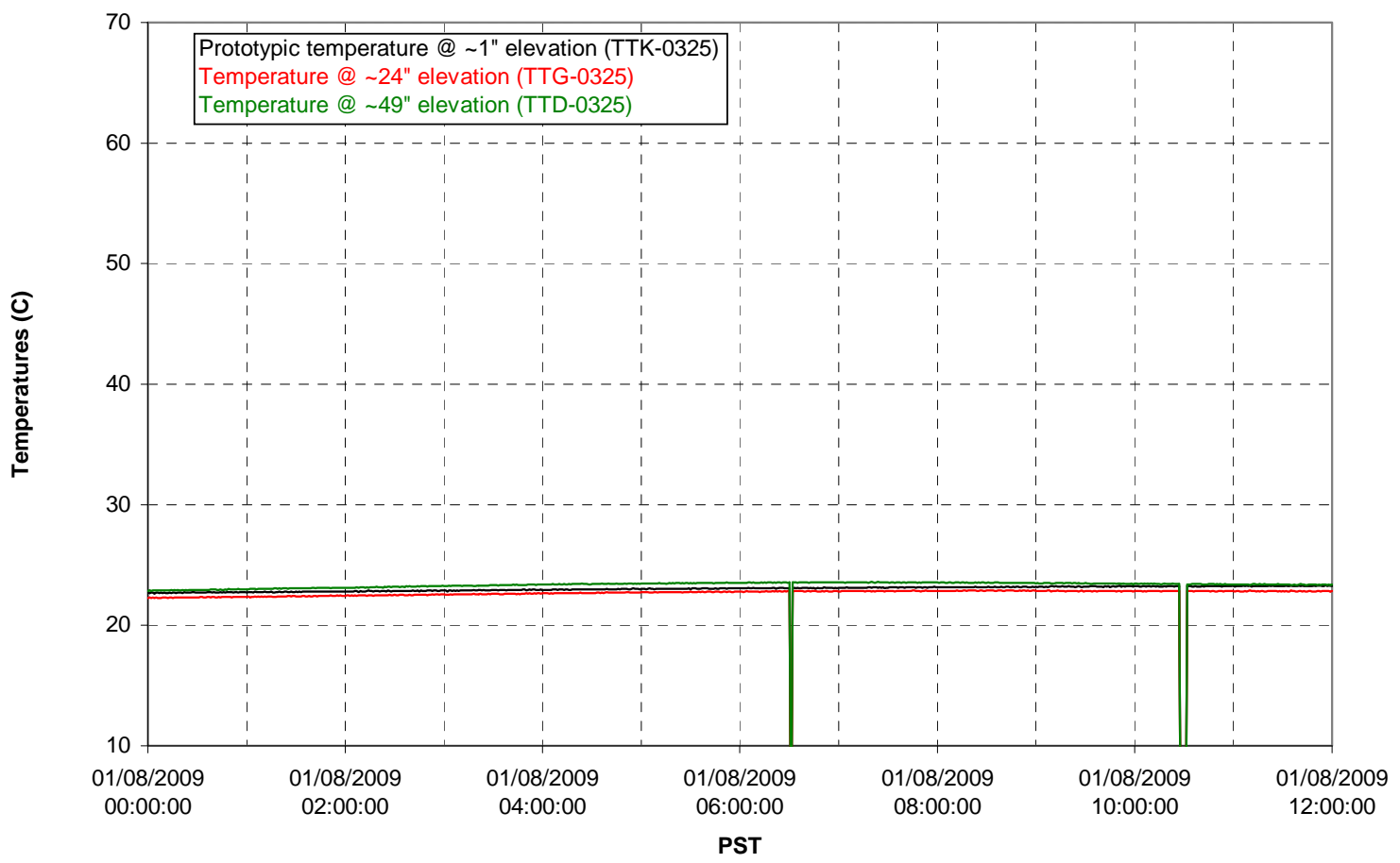


T01B level

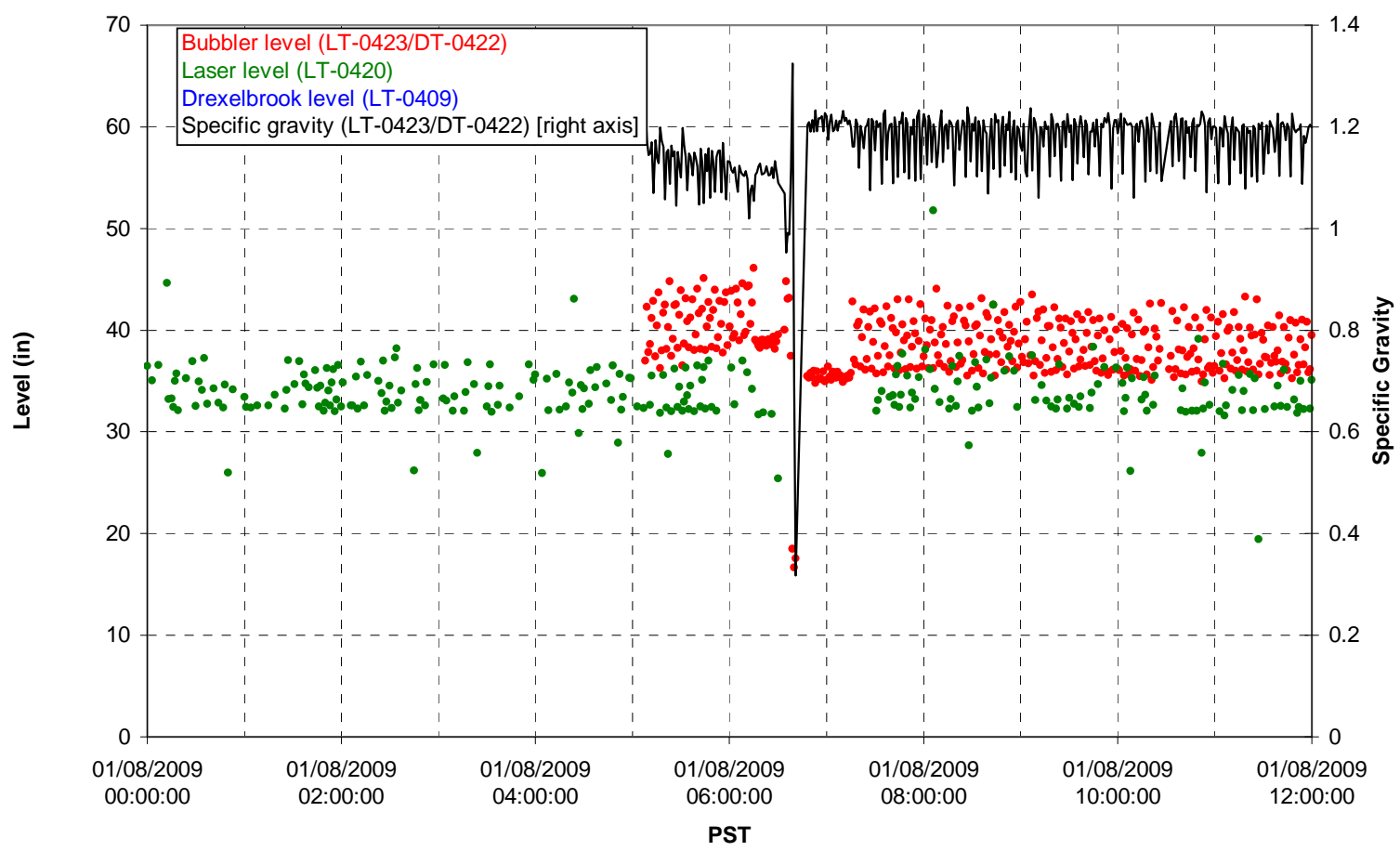

T01B temperatures

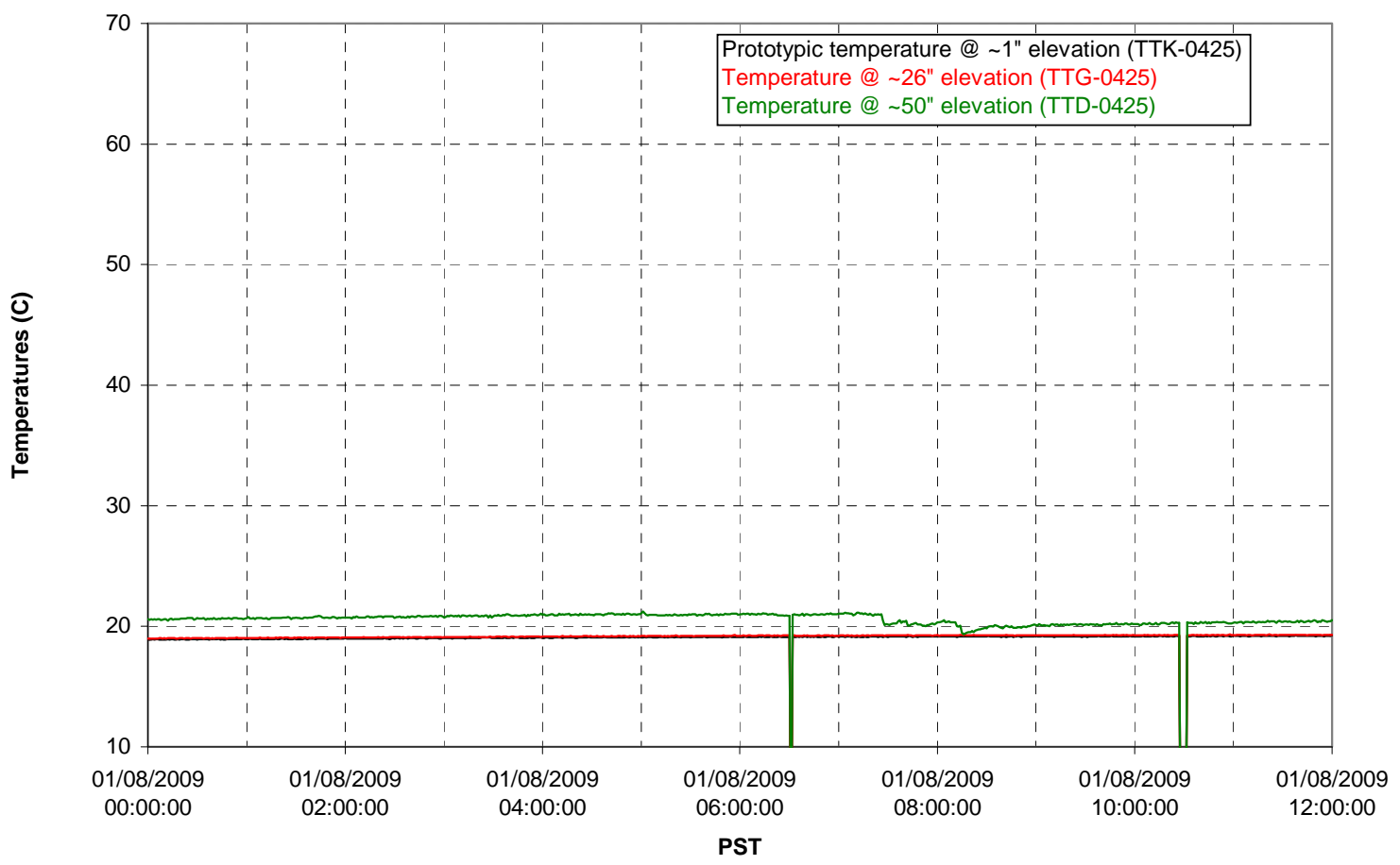


T02A level

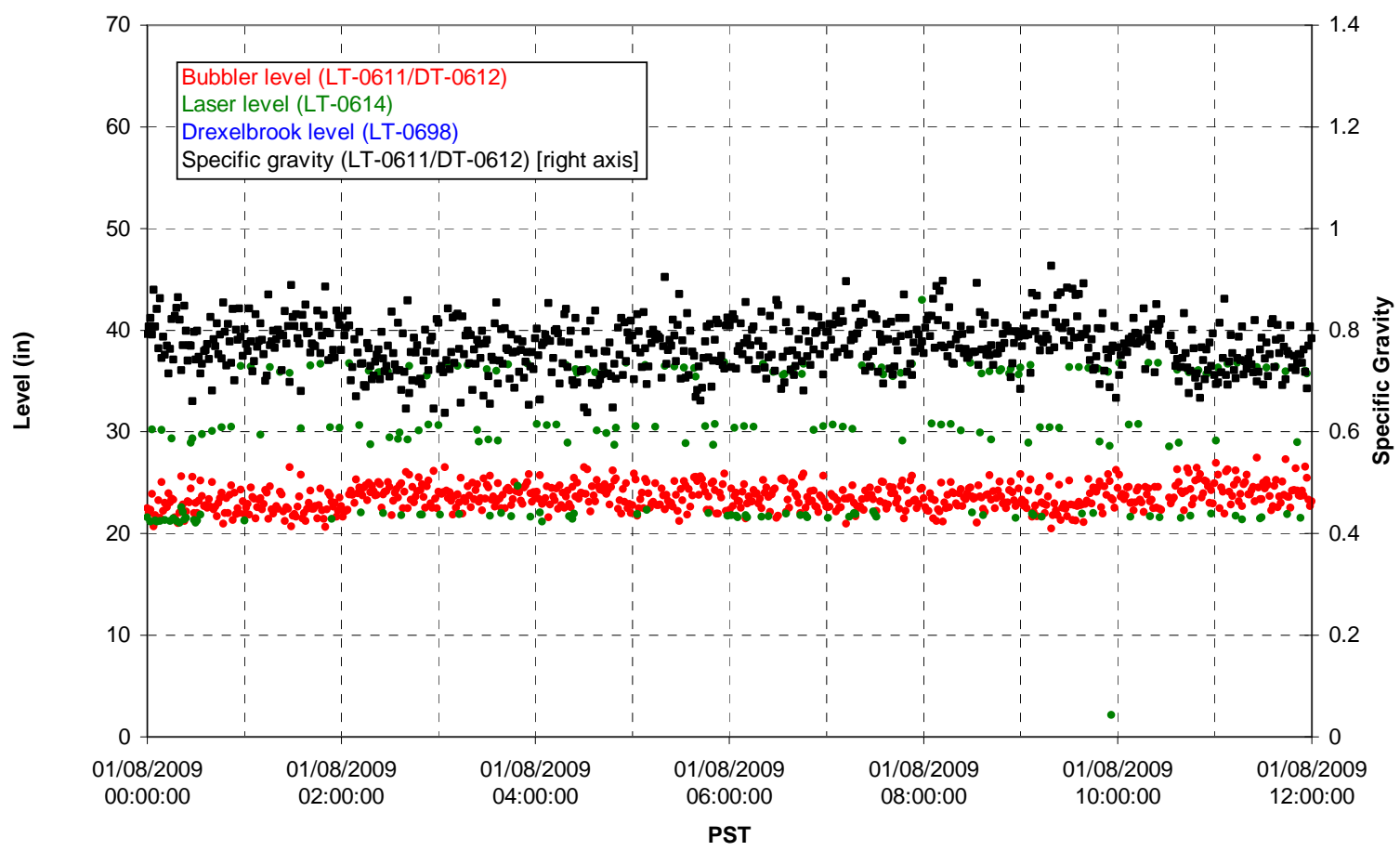

T02A temperatures

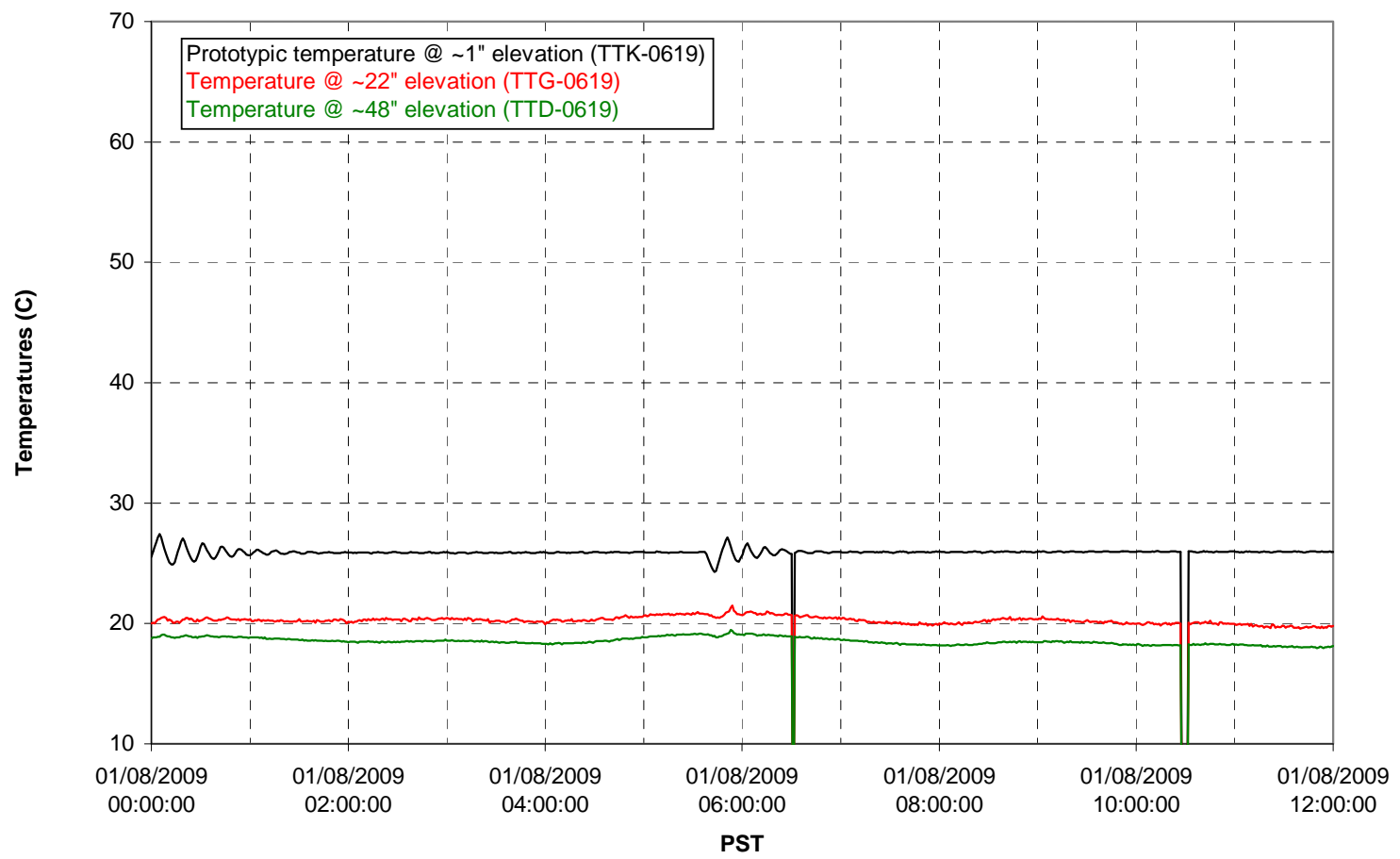


T02A and filter loop temperatures

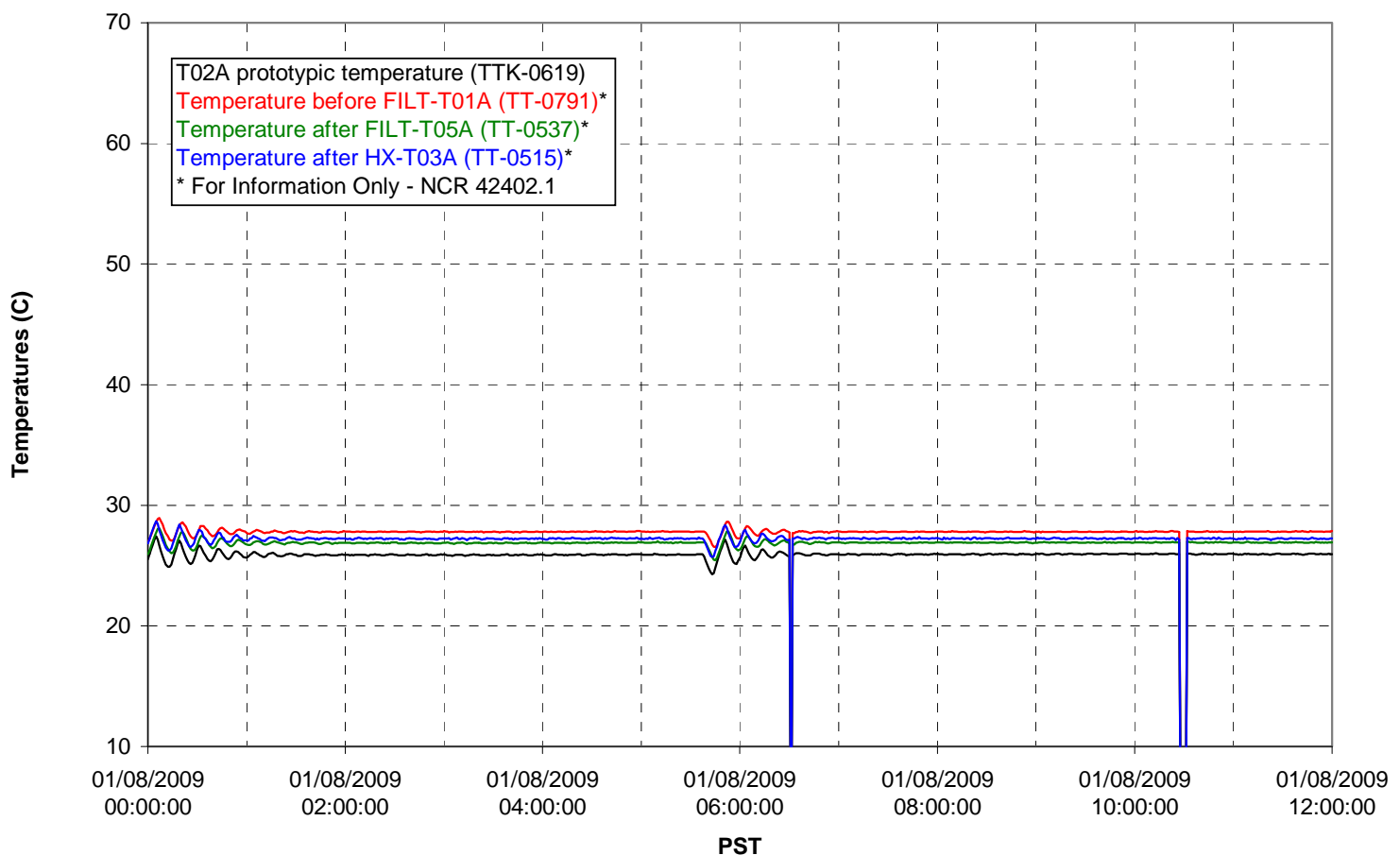

Pump Pressures and Flow

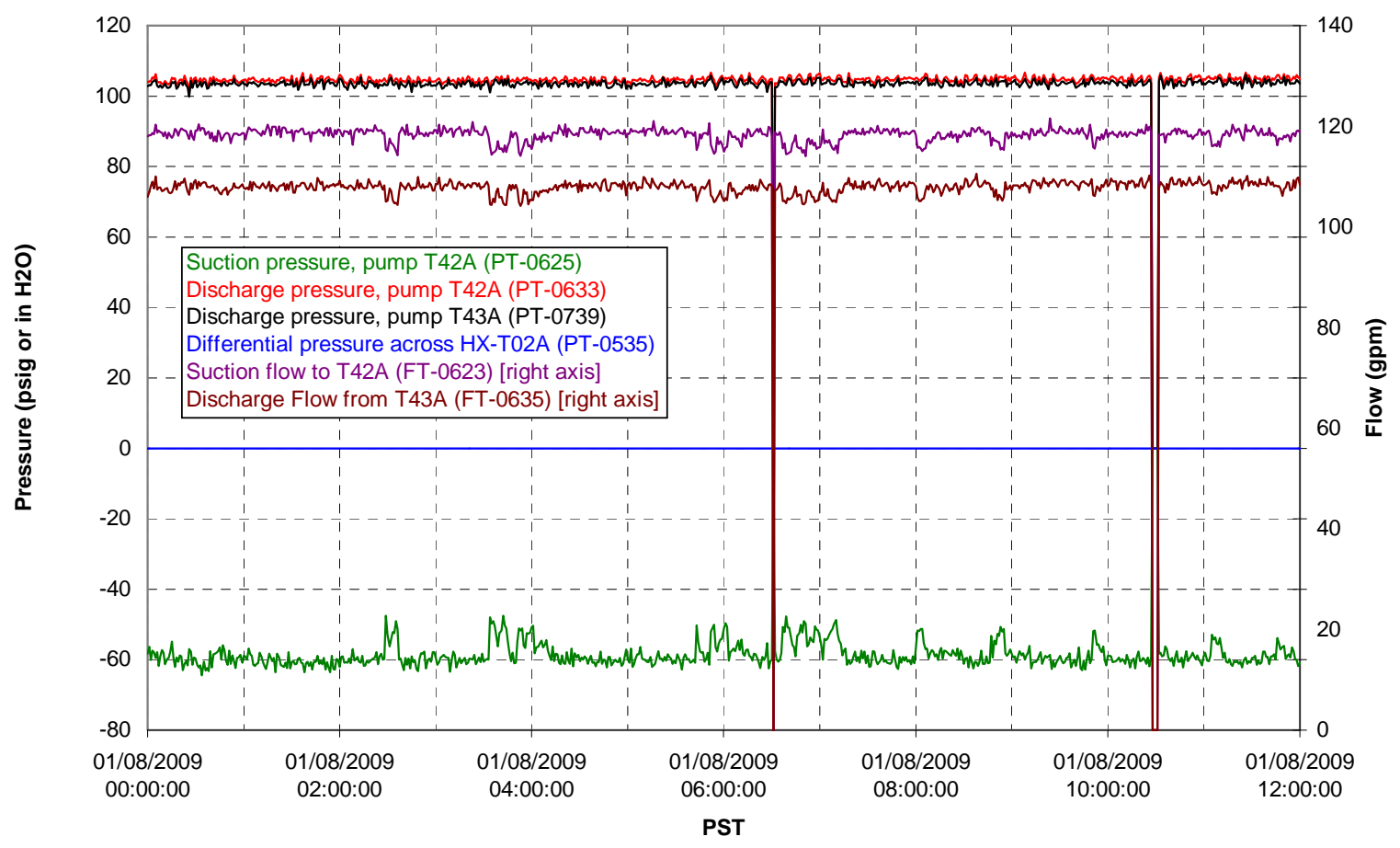


Axial pressure drop

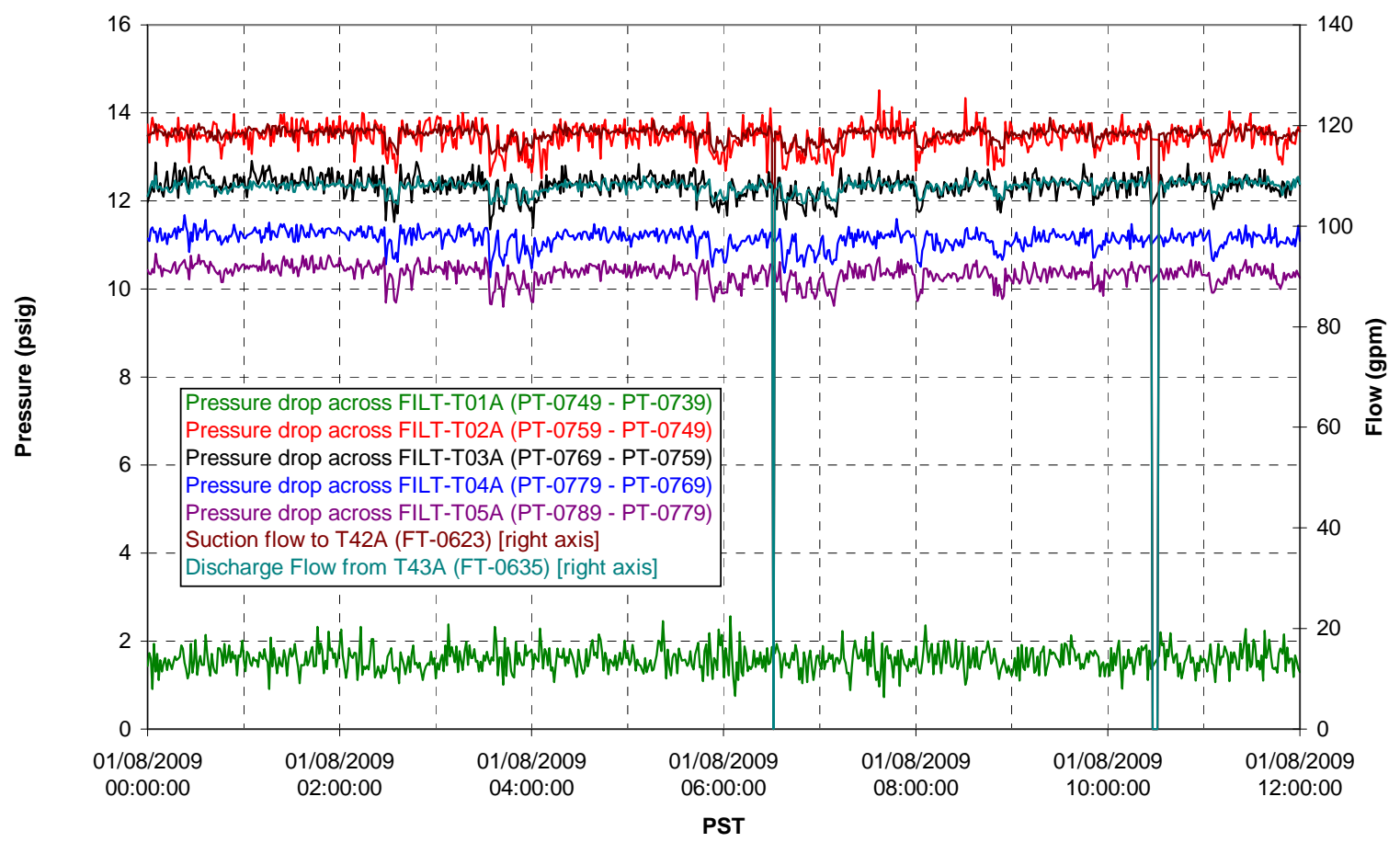

Permeate flow rates

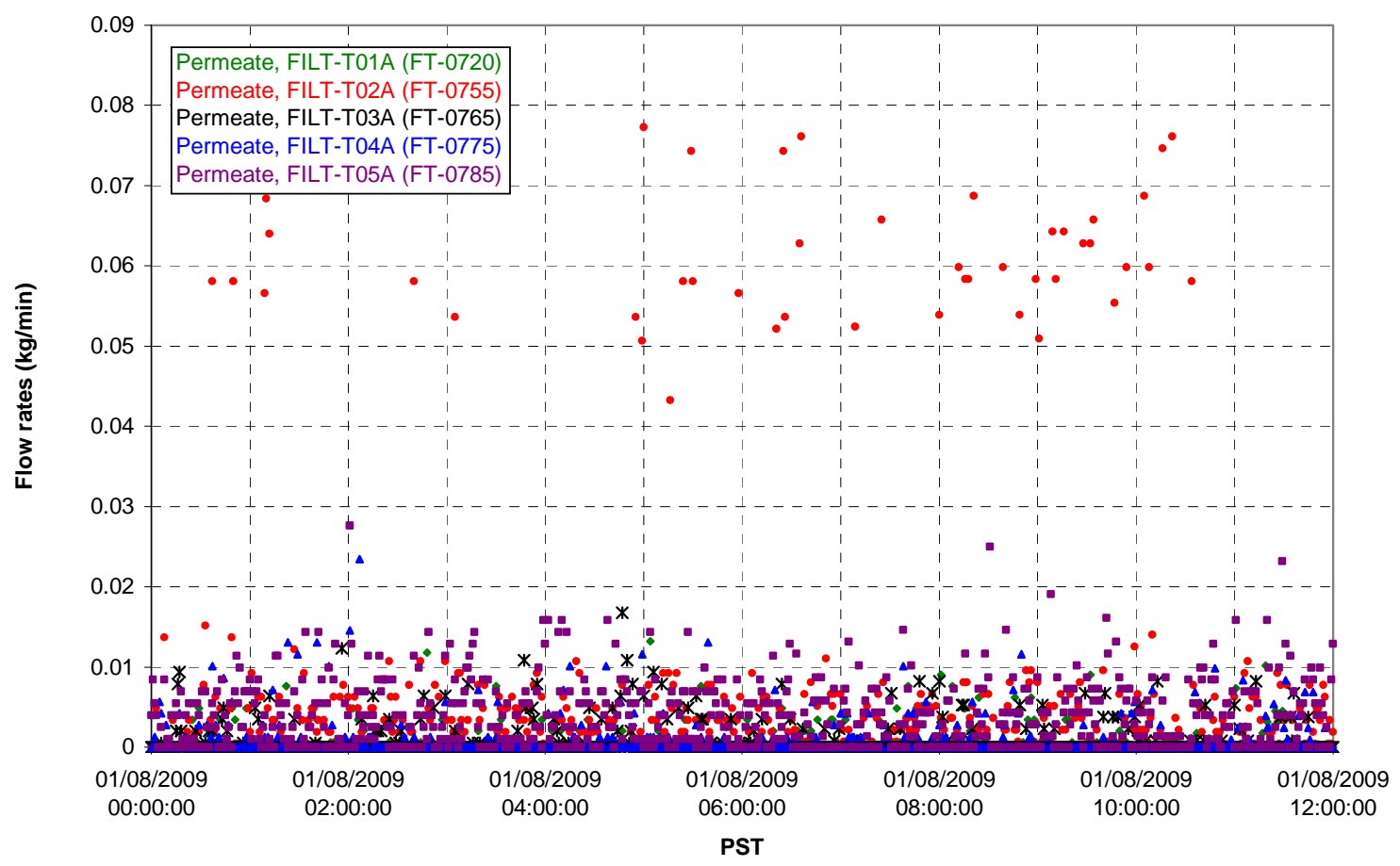


T02A Inner Temperature Tree

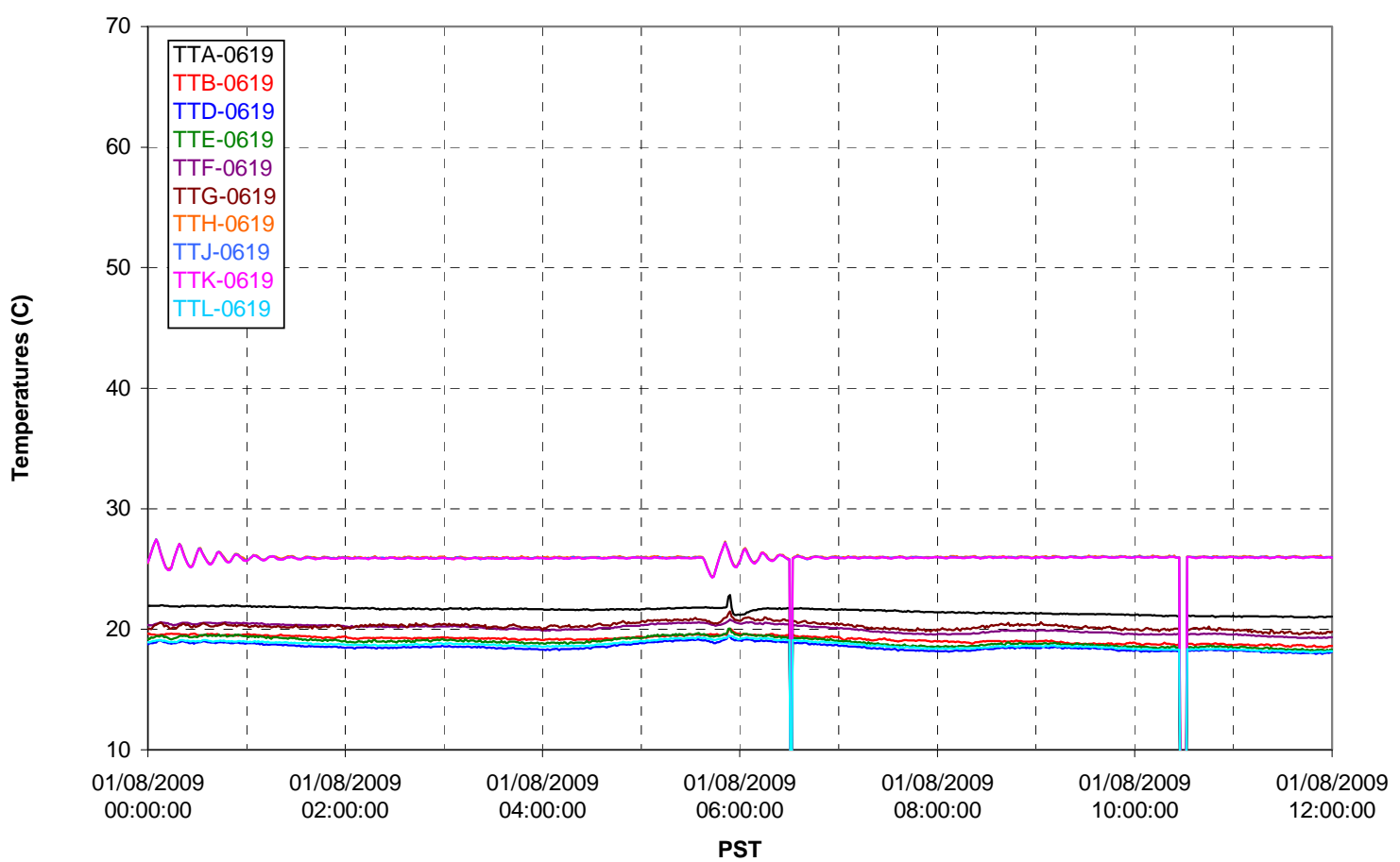

T02A Outer Temperature Tree

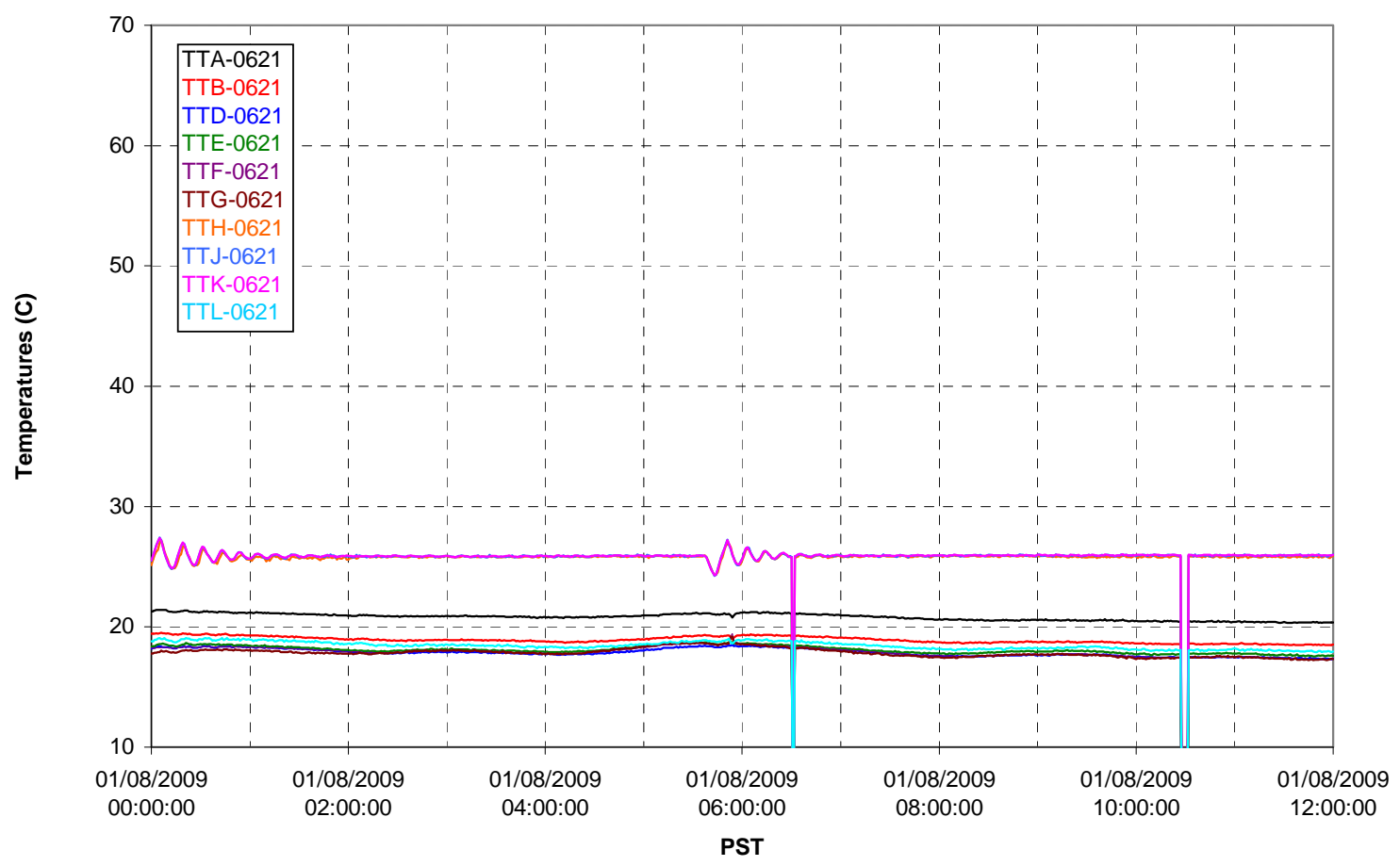


T02A temperatures

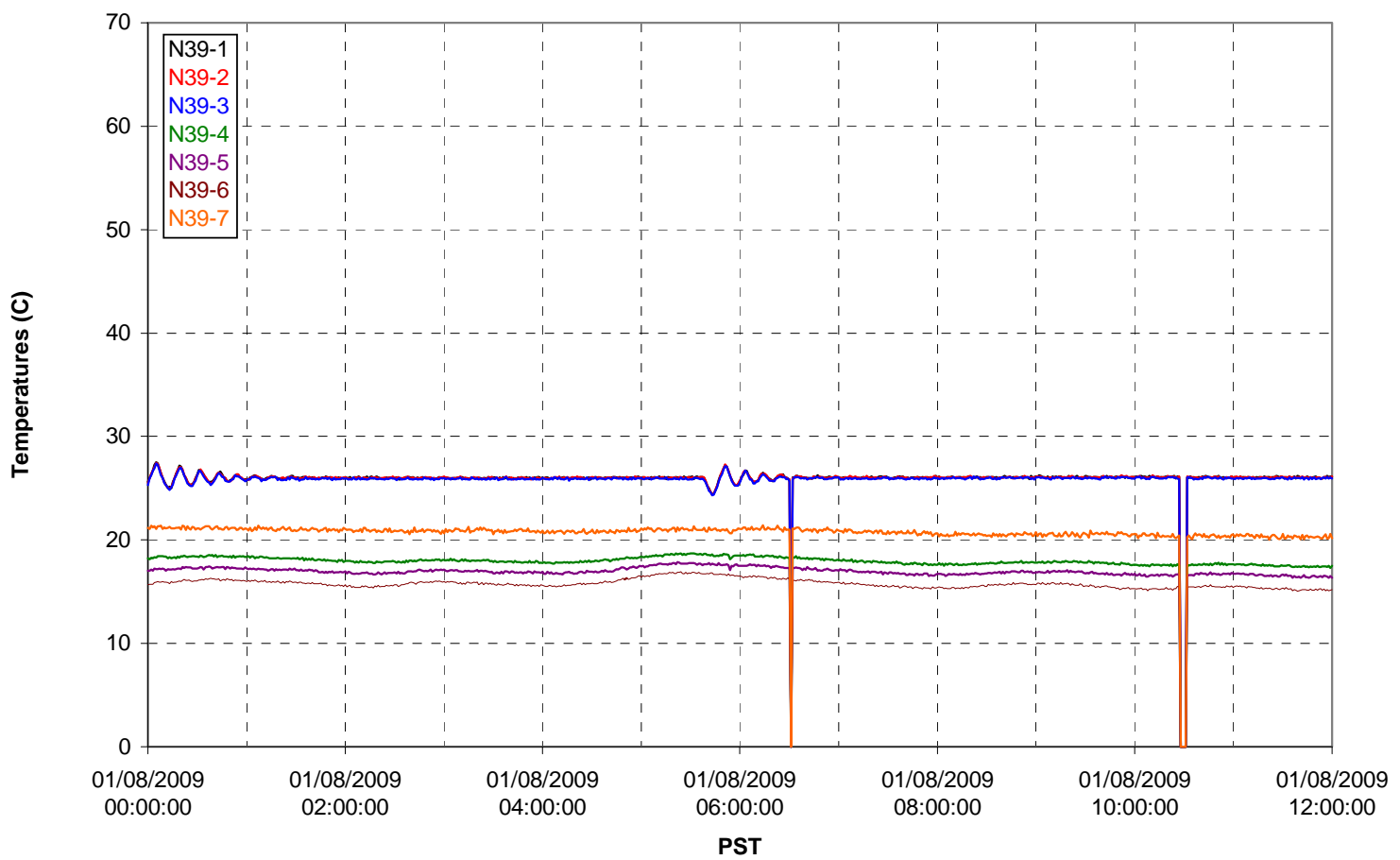

T02A temperatures

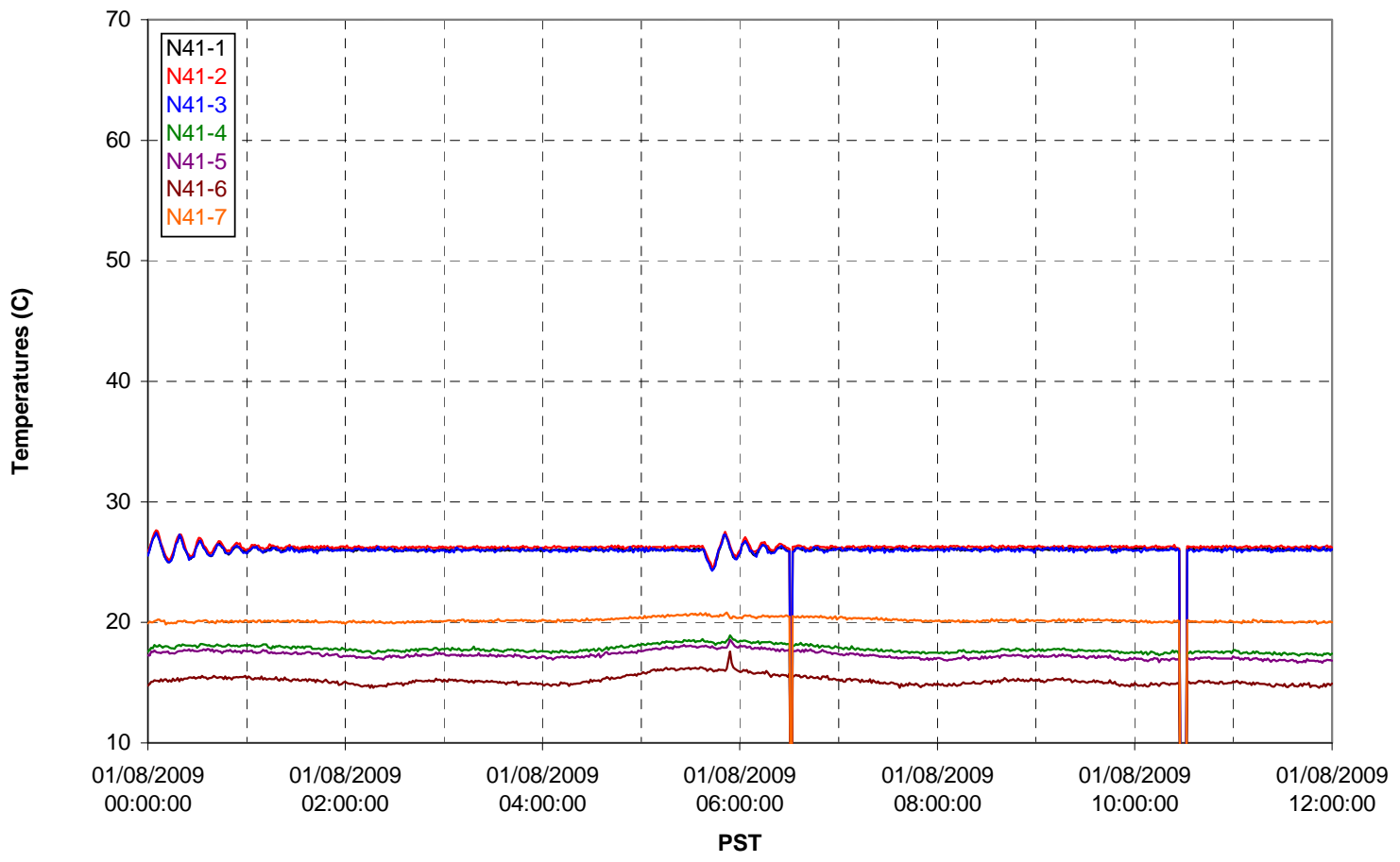


T02A temperatures

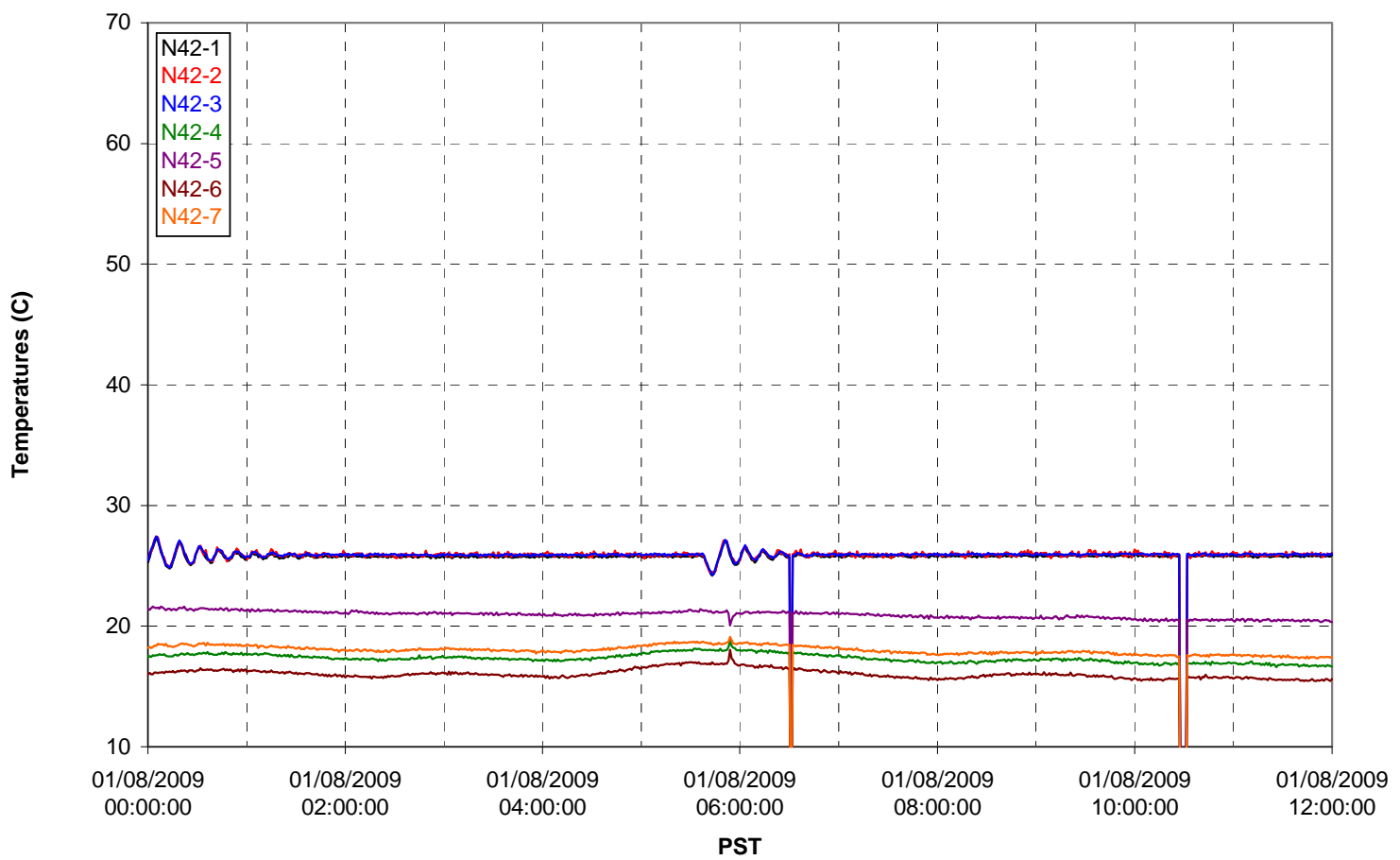

T02A temperatures

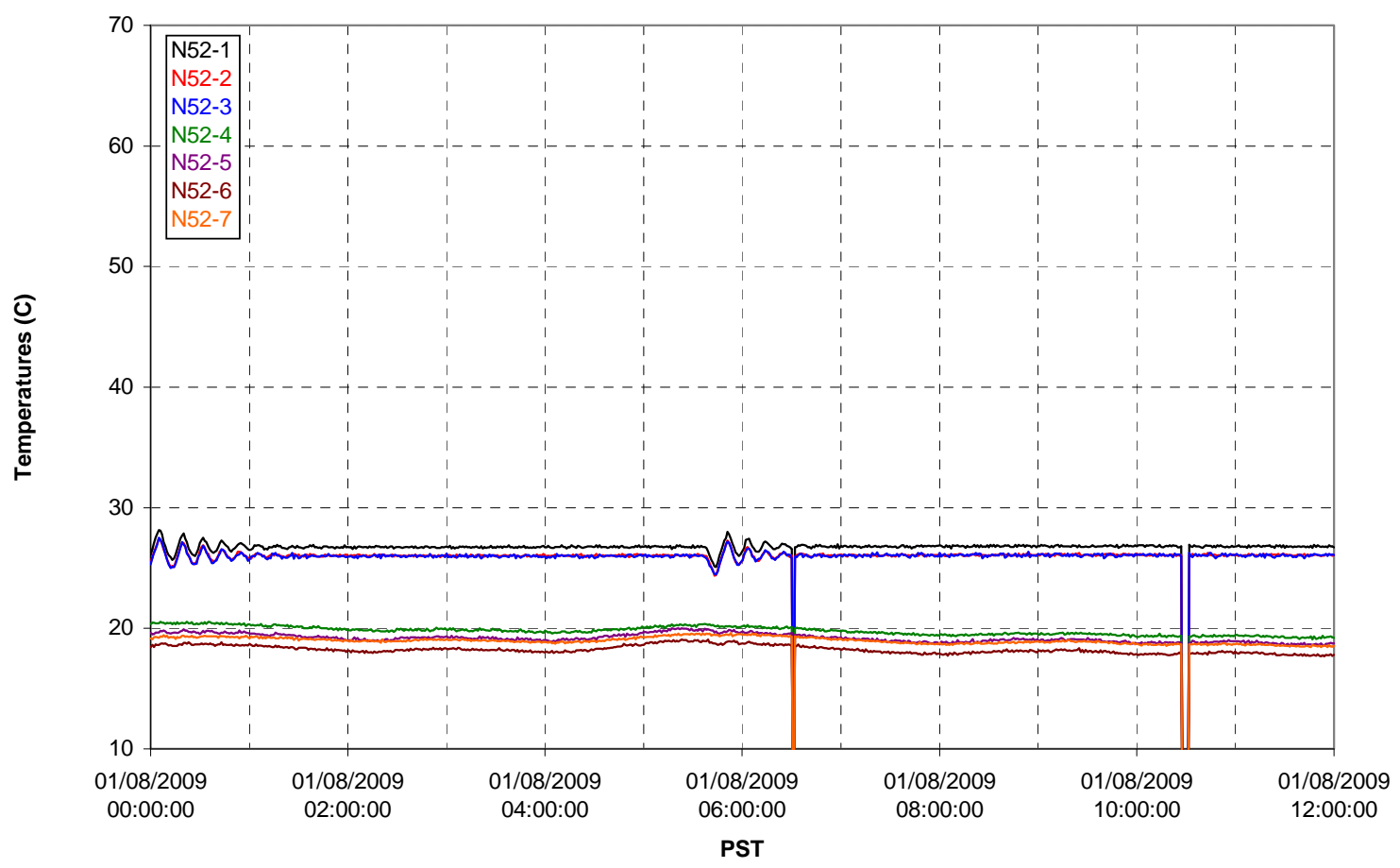


T02A Heating and Cooling

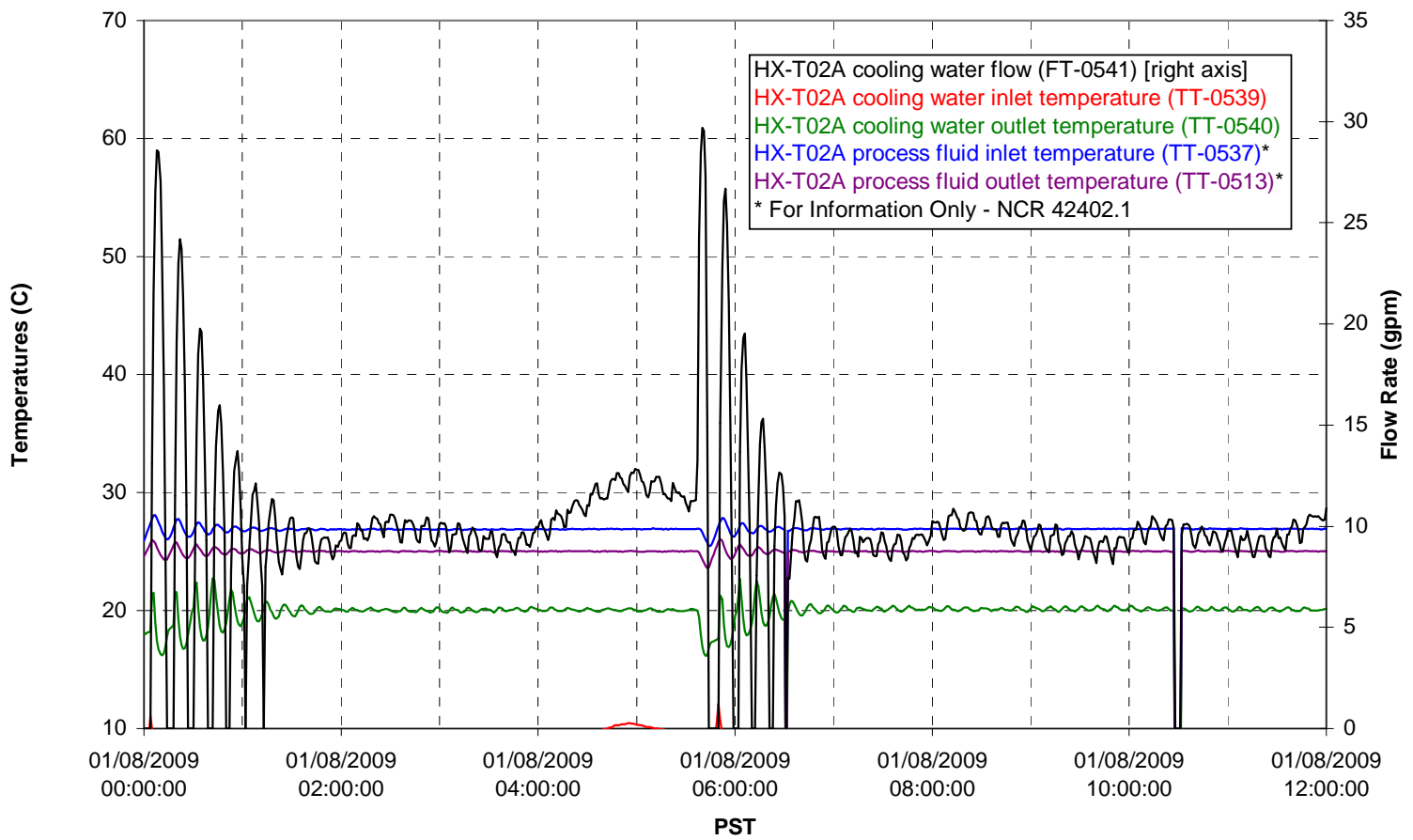

Pump Operation

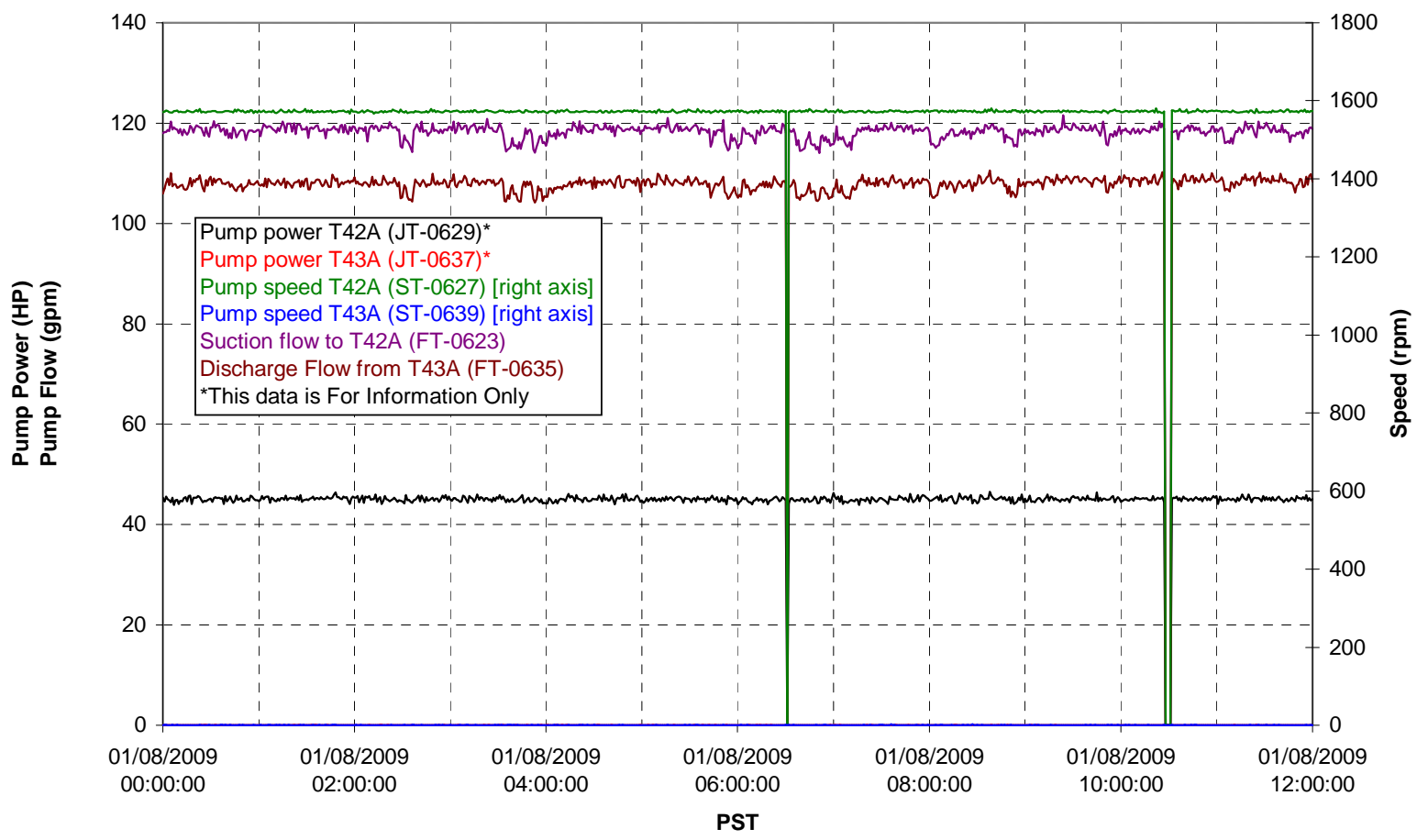


Pulsepot UFP-PP-T01A

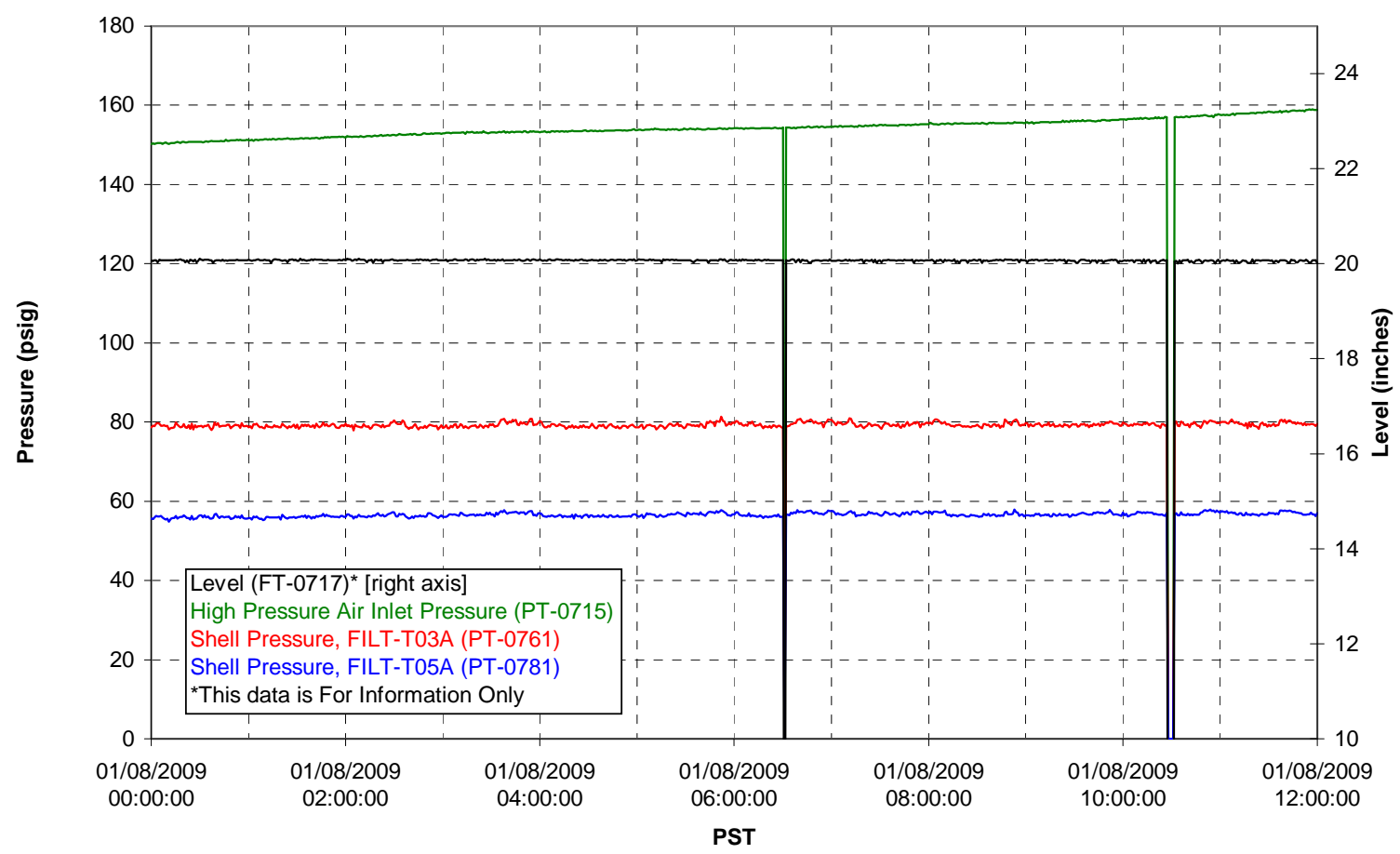

Pulsepot UFP-PP-T02A

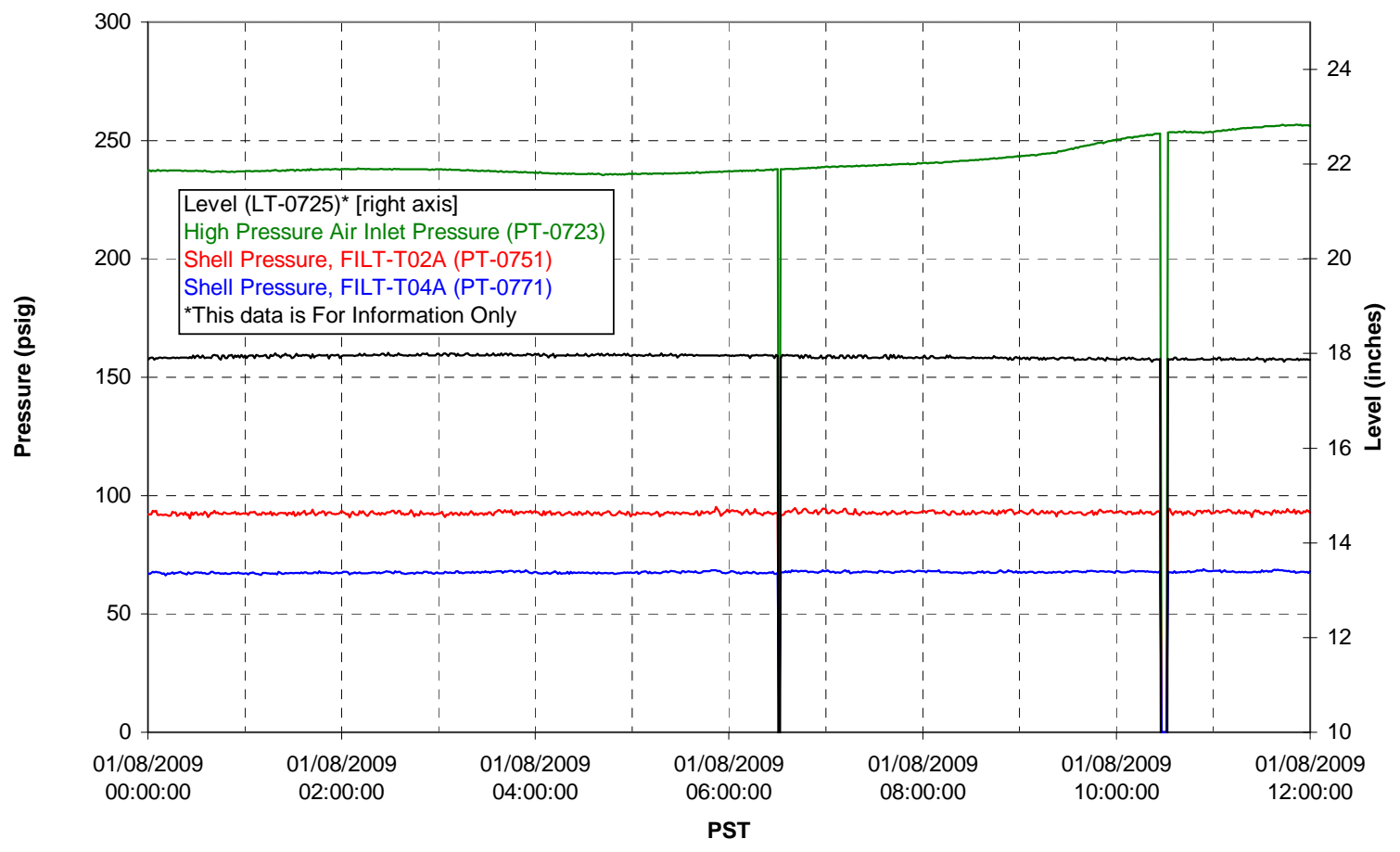


Pulsepot UFP-PP-T03A

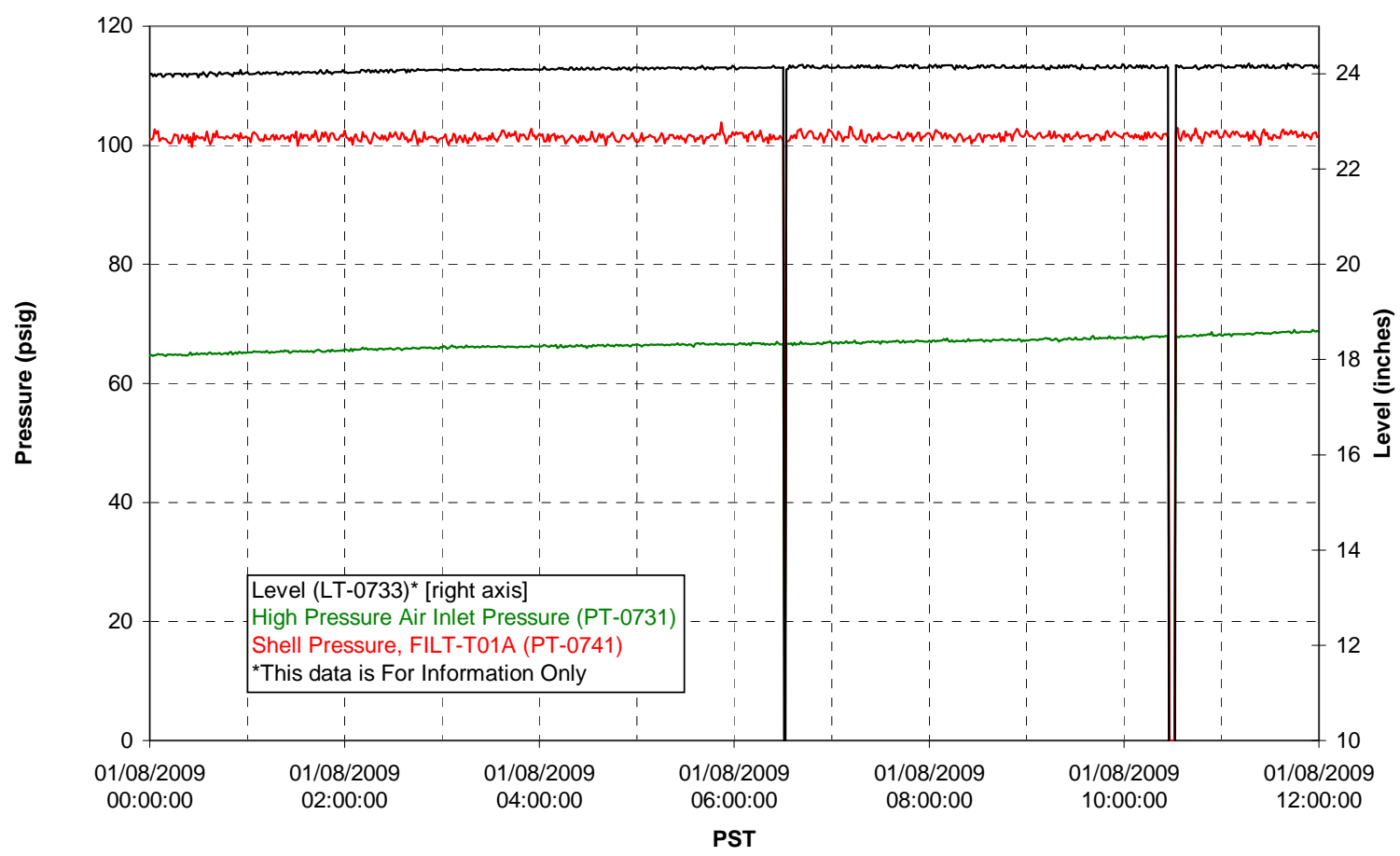

Pulsepot Levels

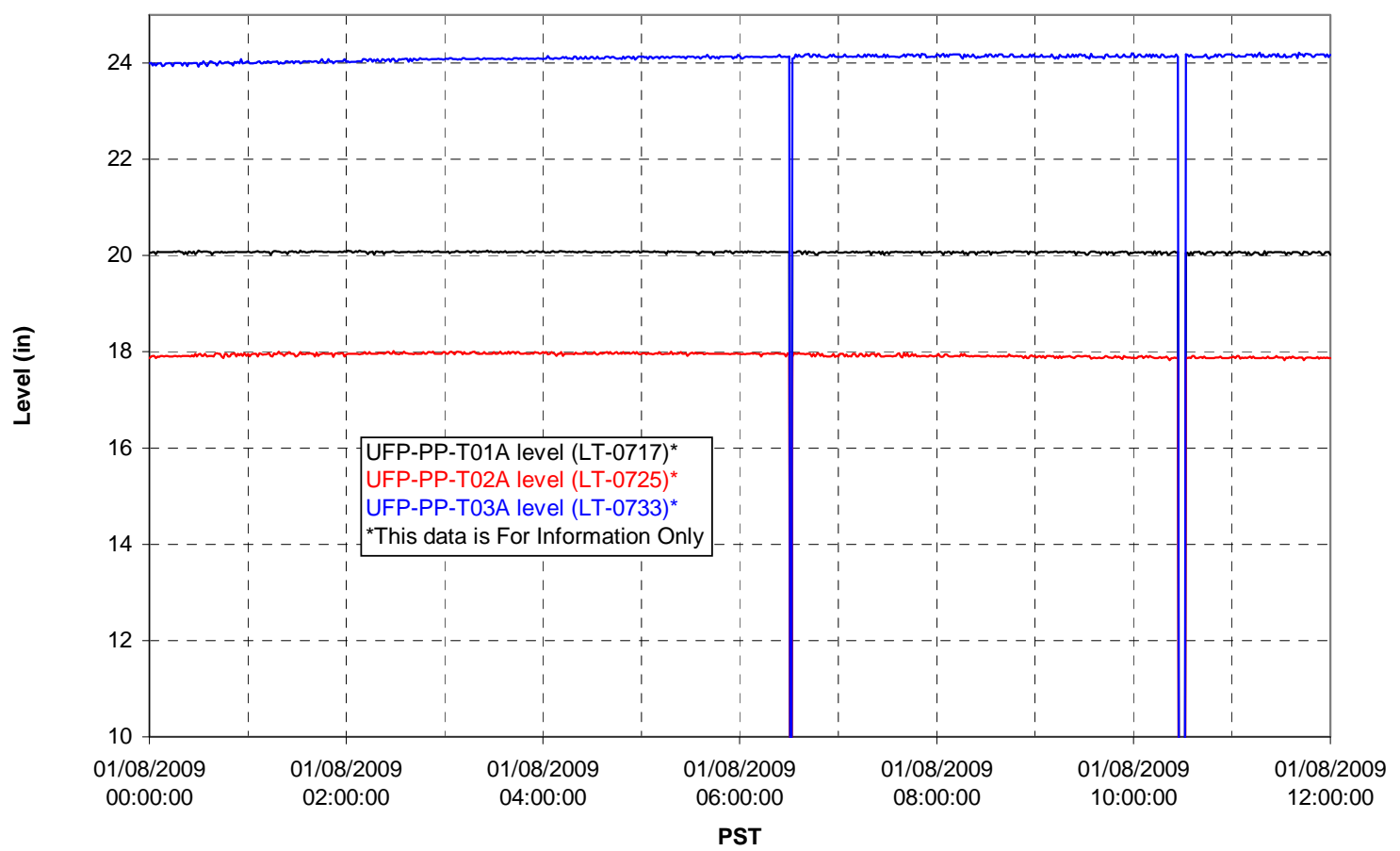


Filter UFP-FILT-T01A

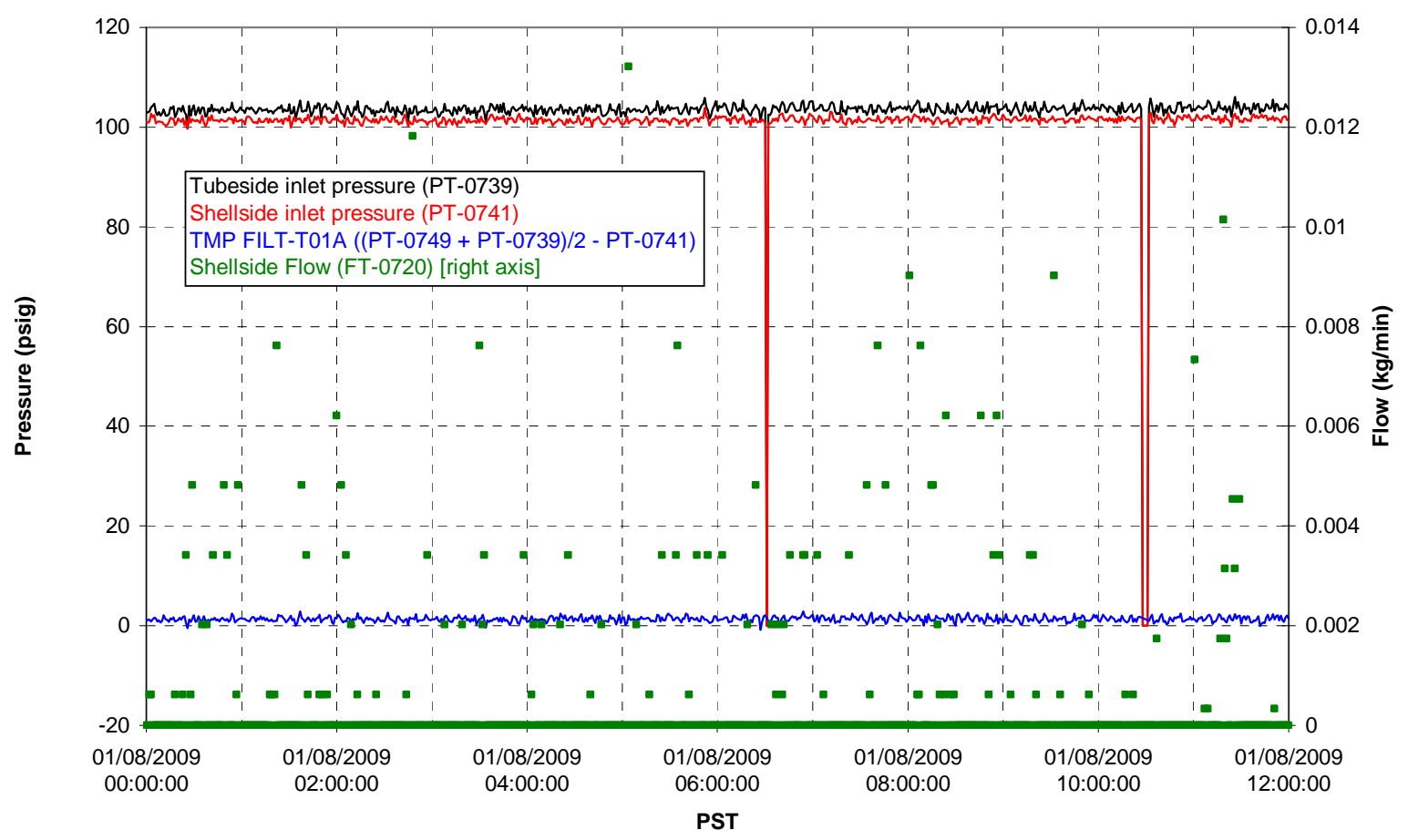

Filter UFP-FILT-T02A

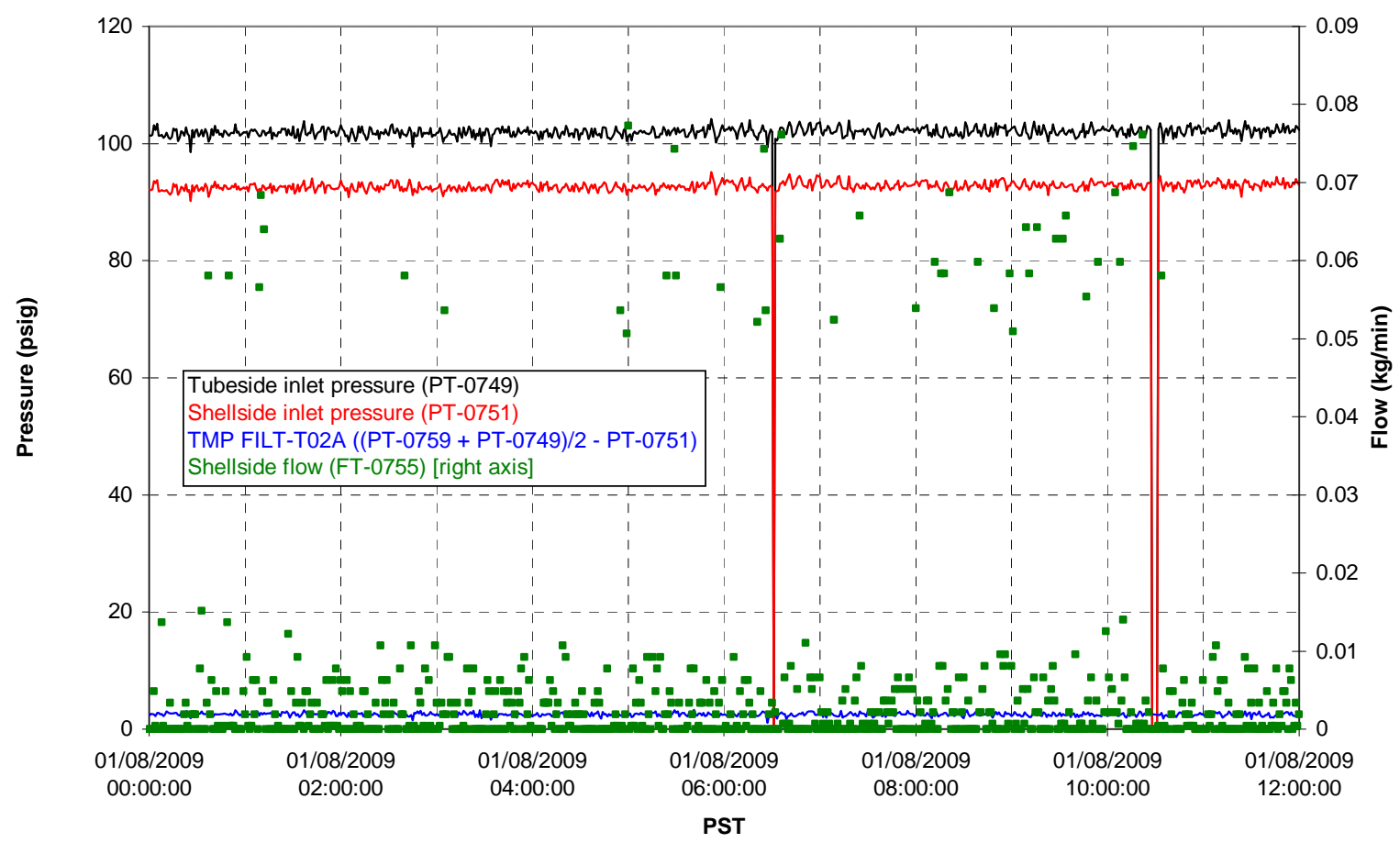


Filter UFP-FILT-T03A

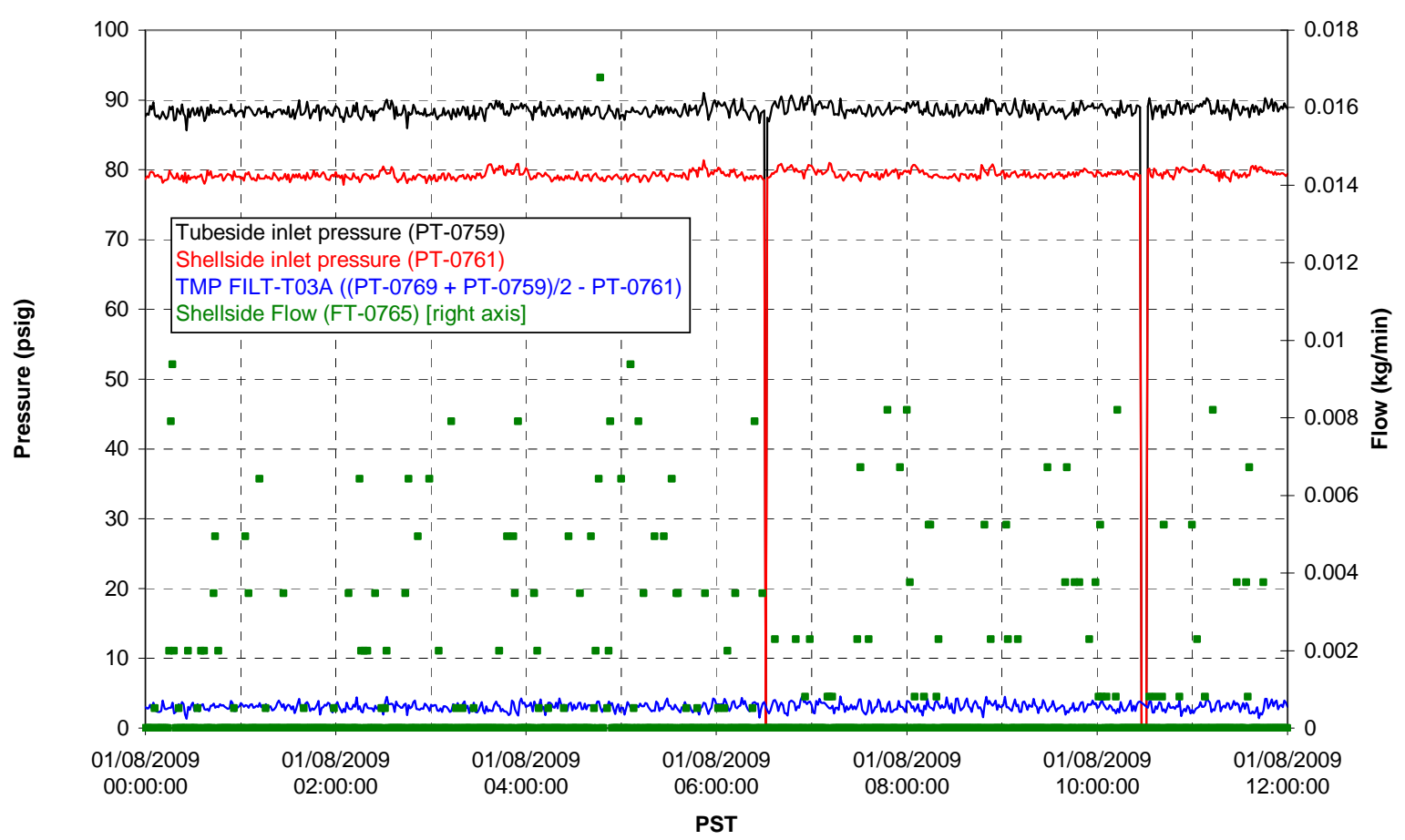

Filter UFP-FILT-T04A

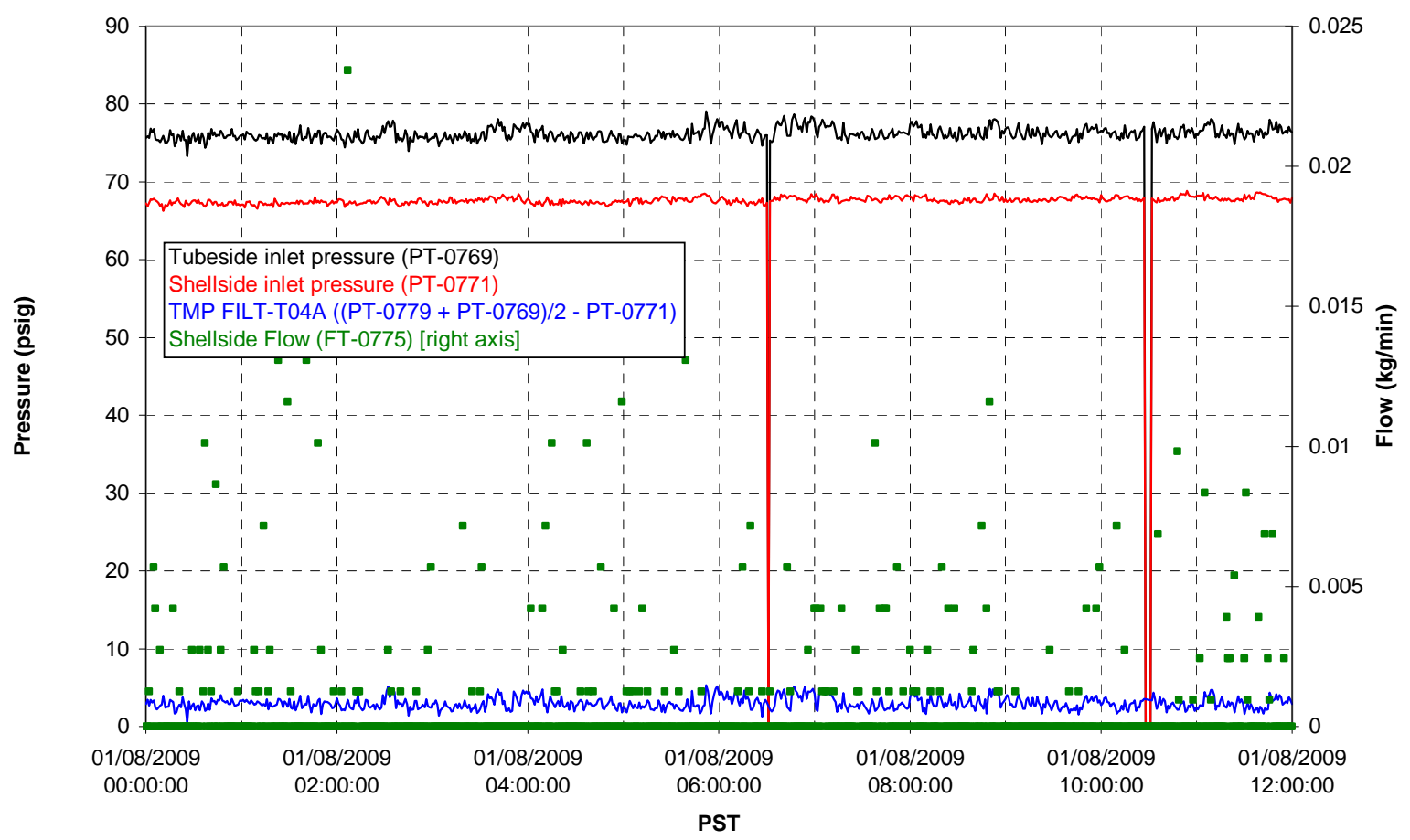


Filter UFP-FILT-T05A

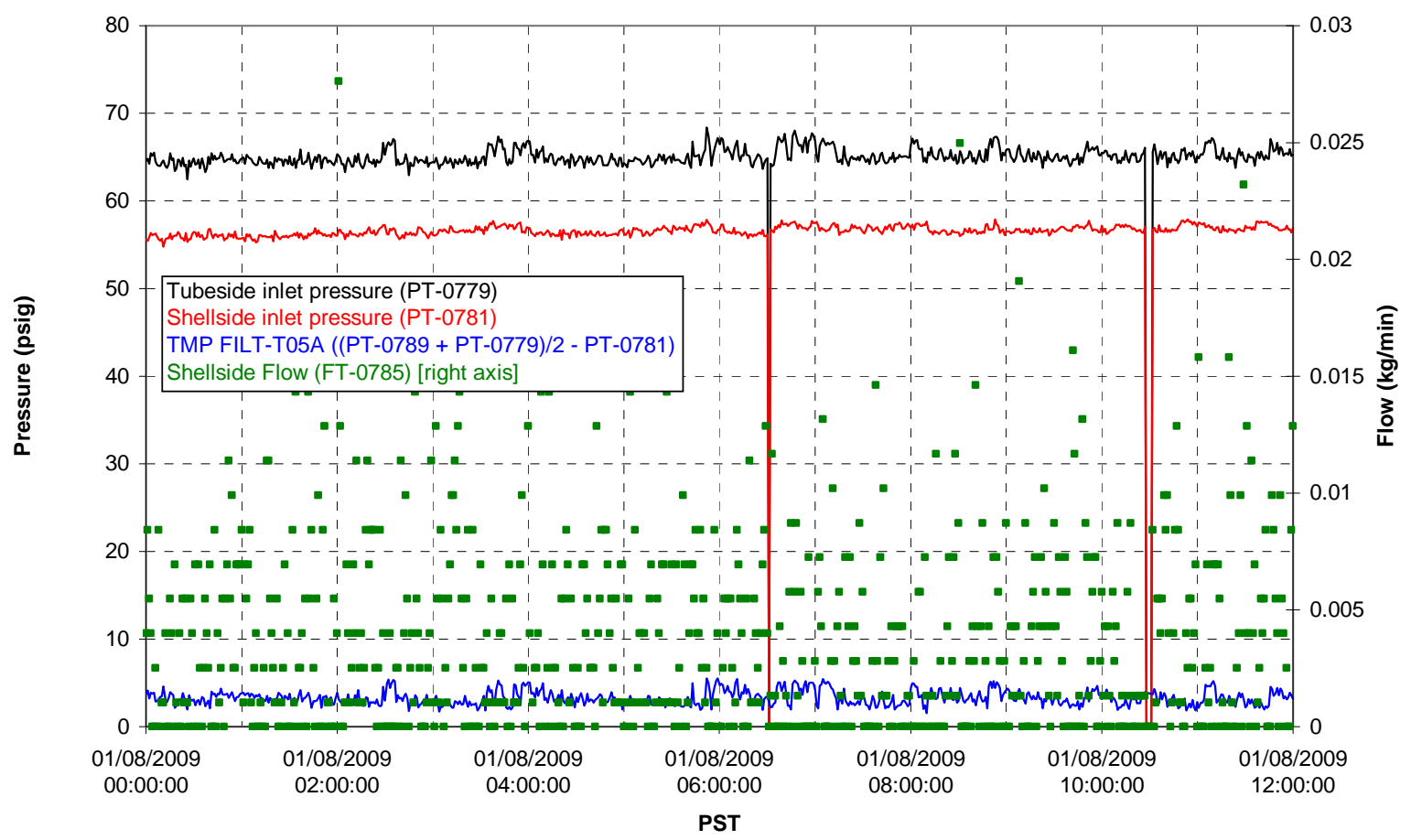

Chemical Flow

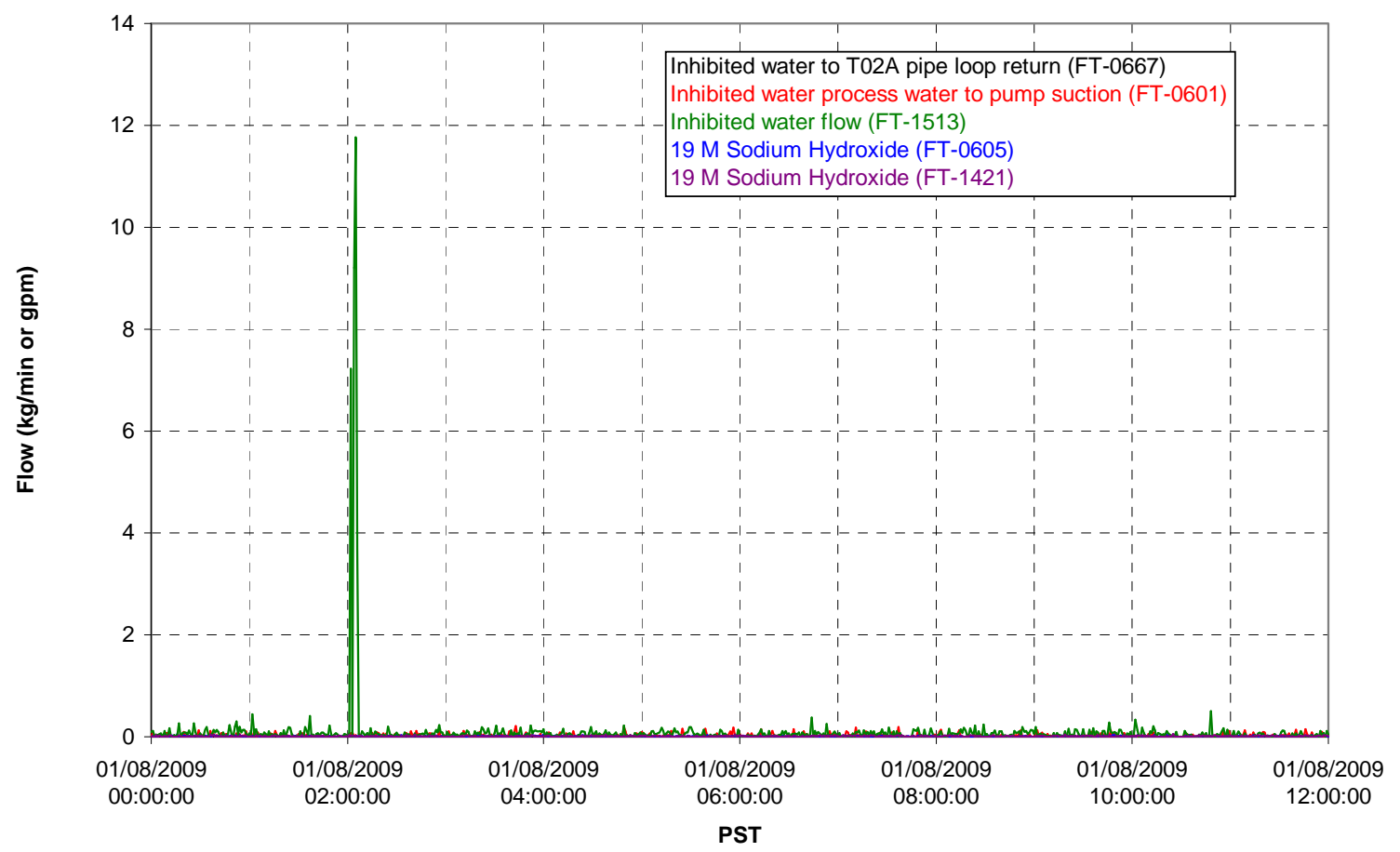


Chemical Flow

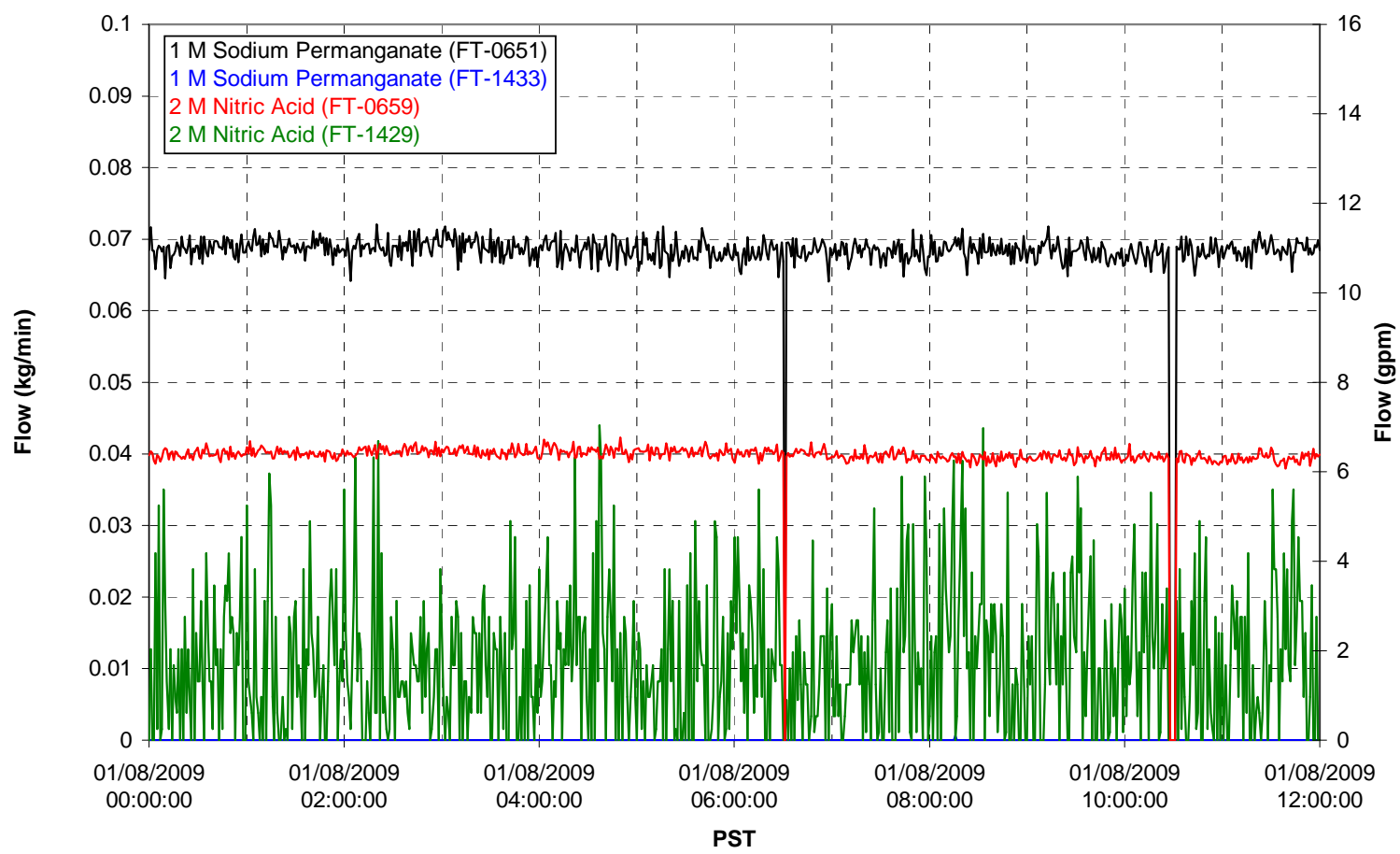

Air Flows

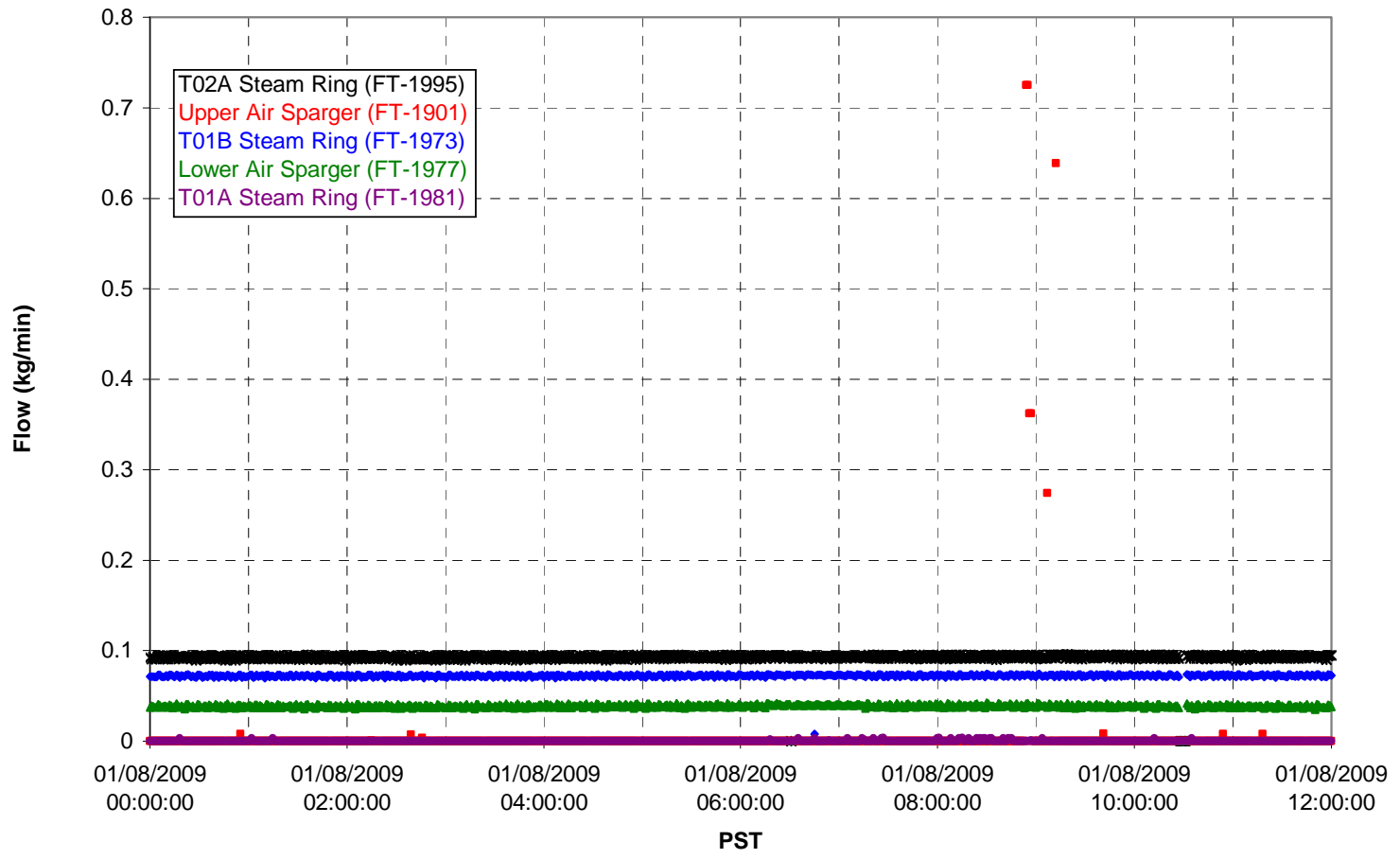


T02A Steam

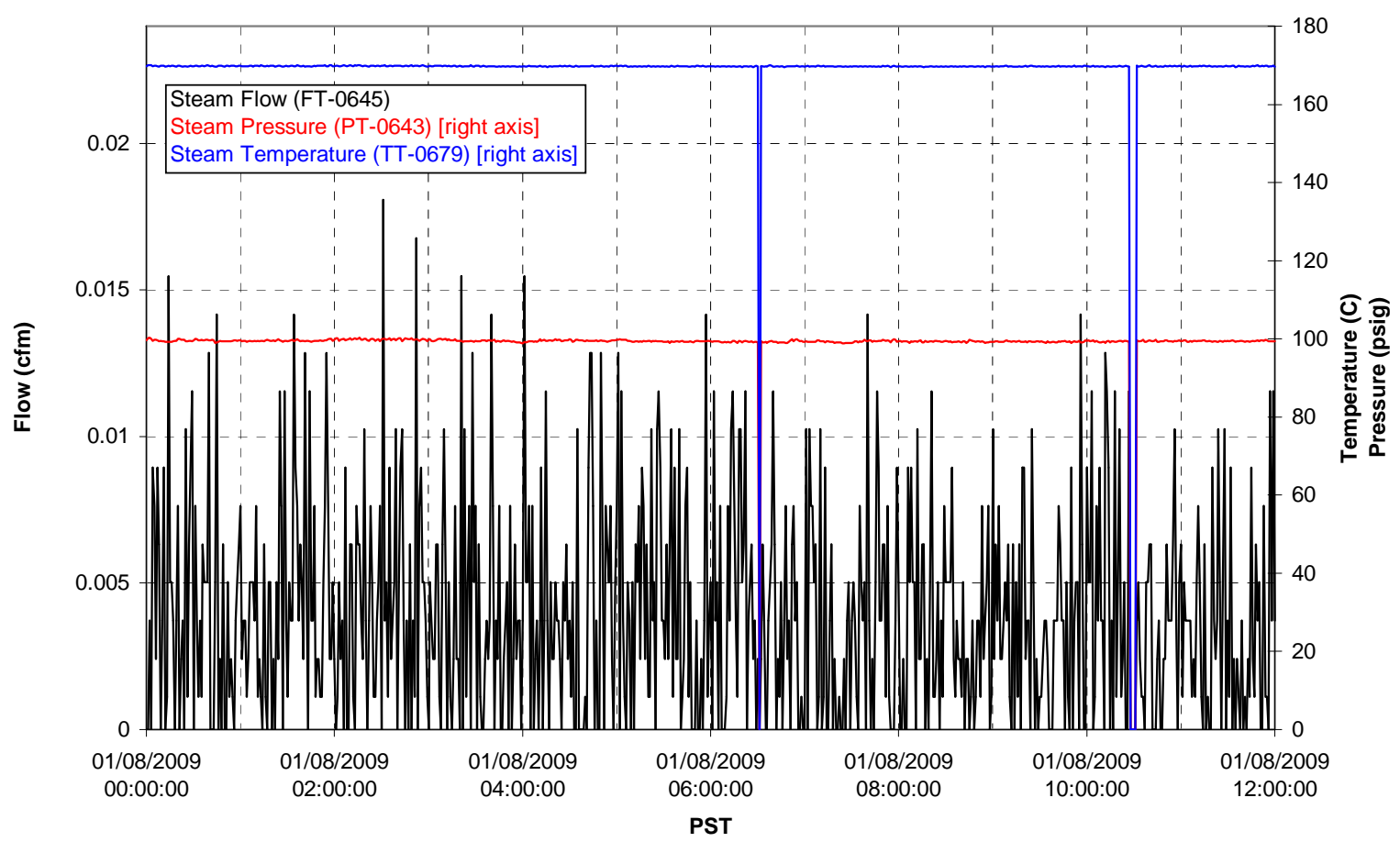

T01A Steam

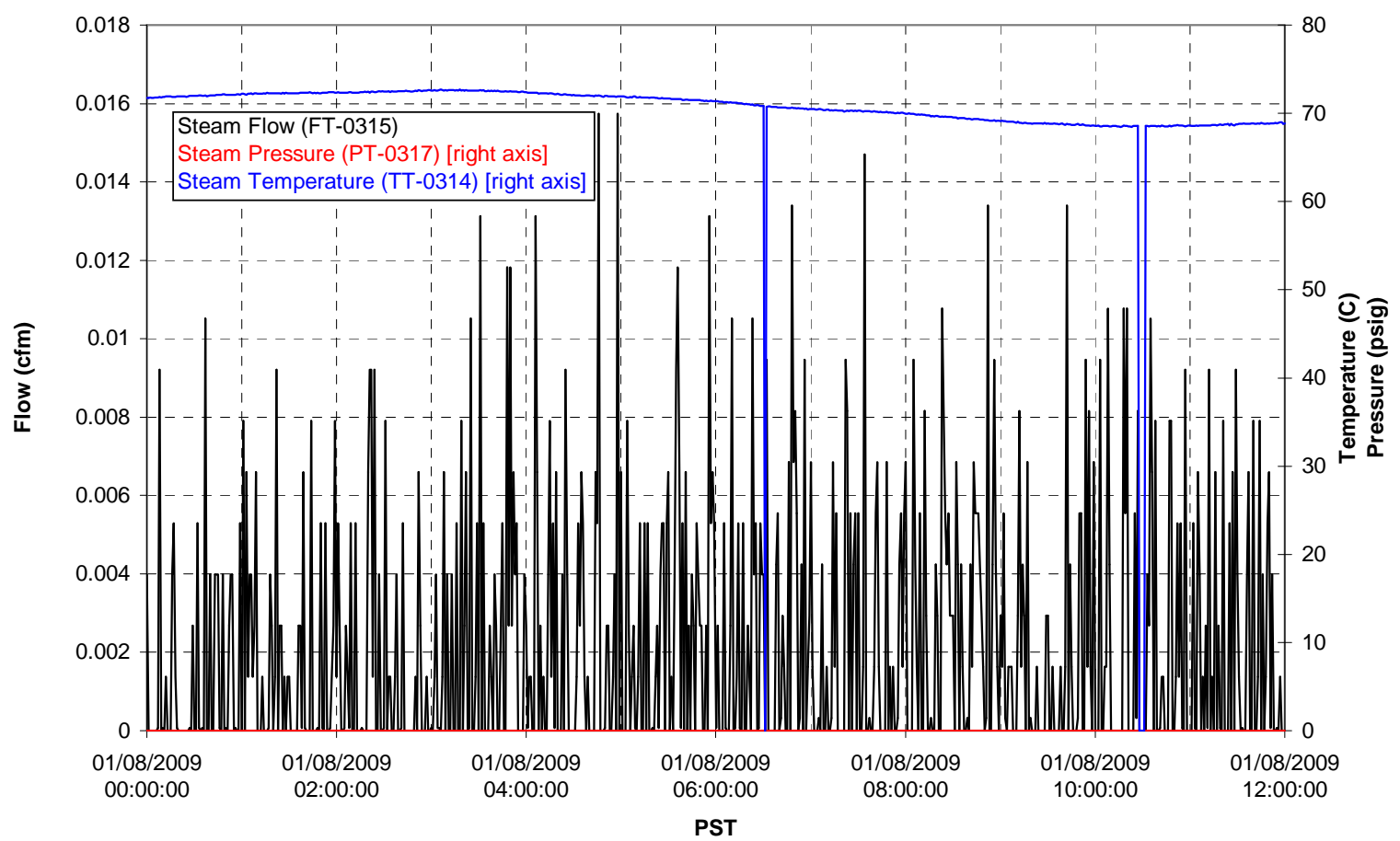


T01B Steam

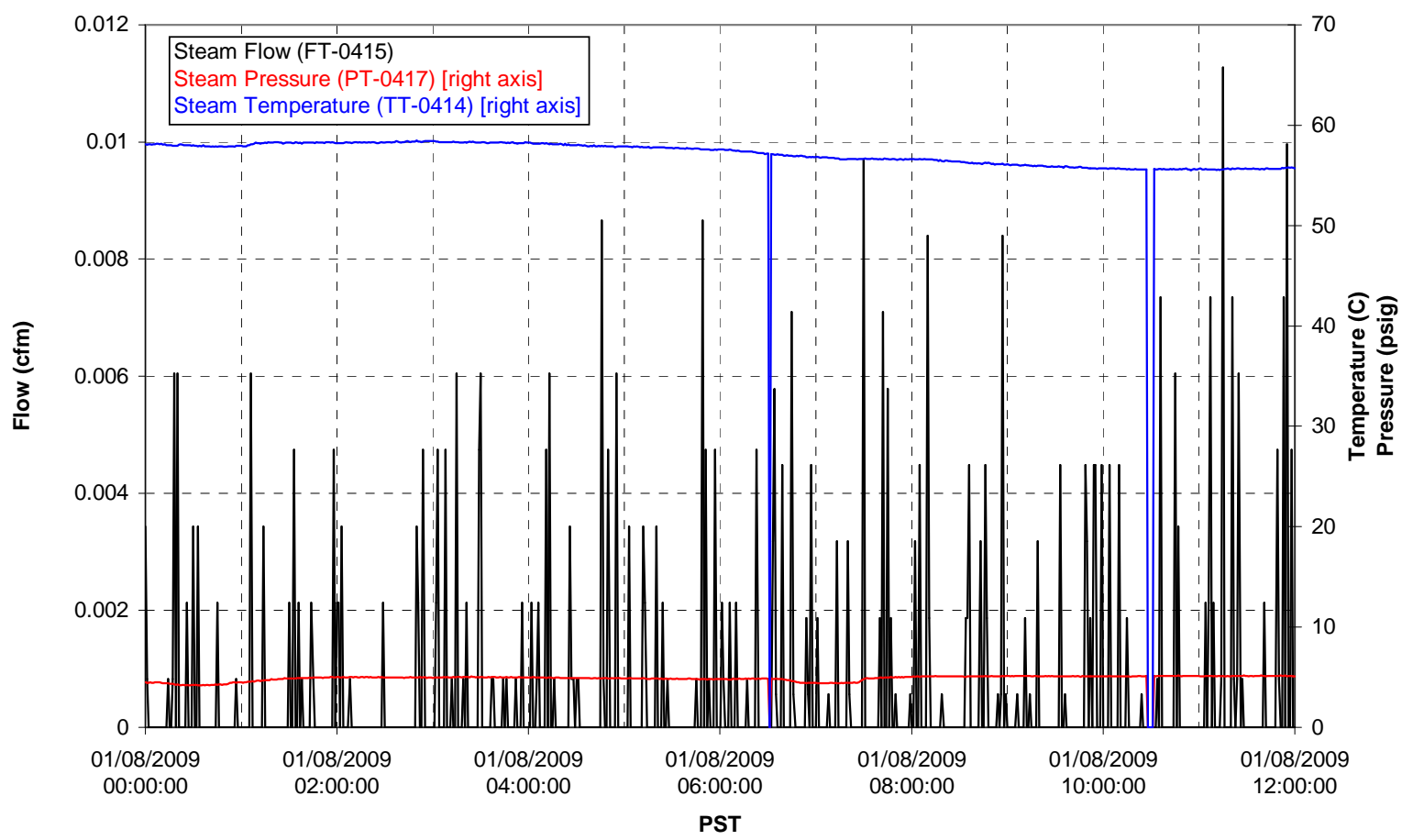


Functional Test Data Plots

01/08/2009 12:00 - 01/09/2009 00:00 
T01A level

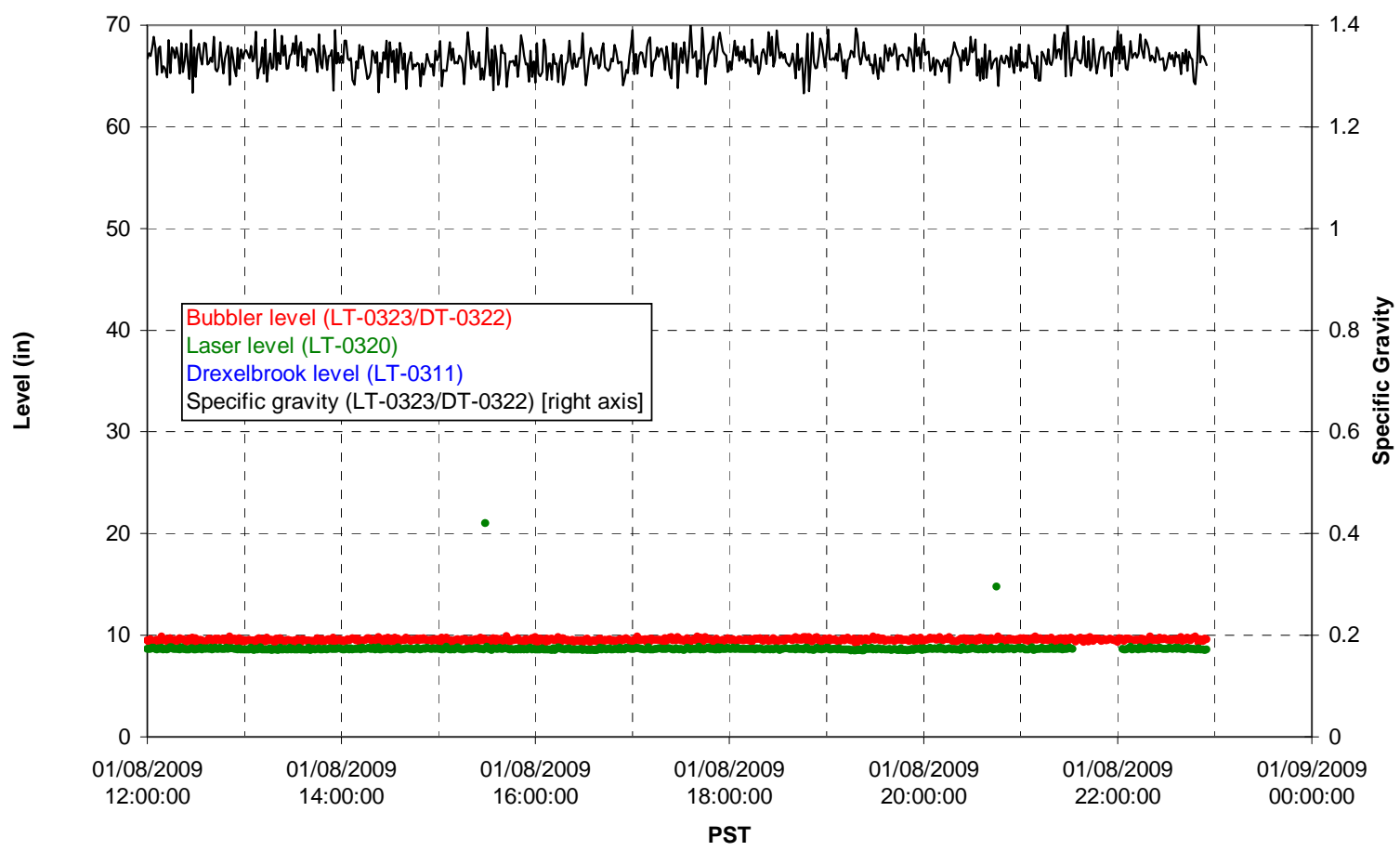

T01A temperatures

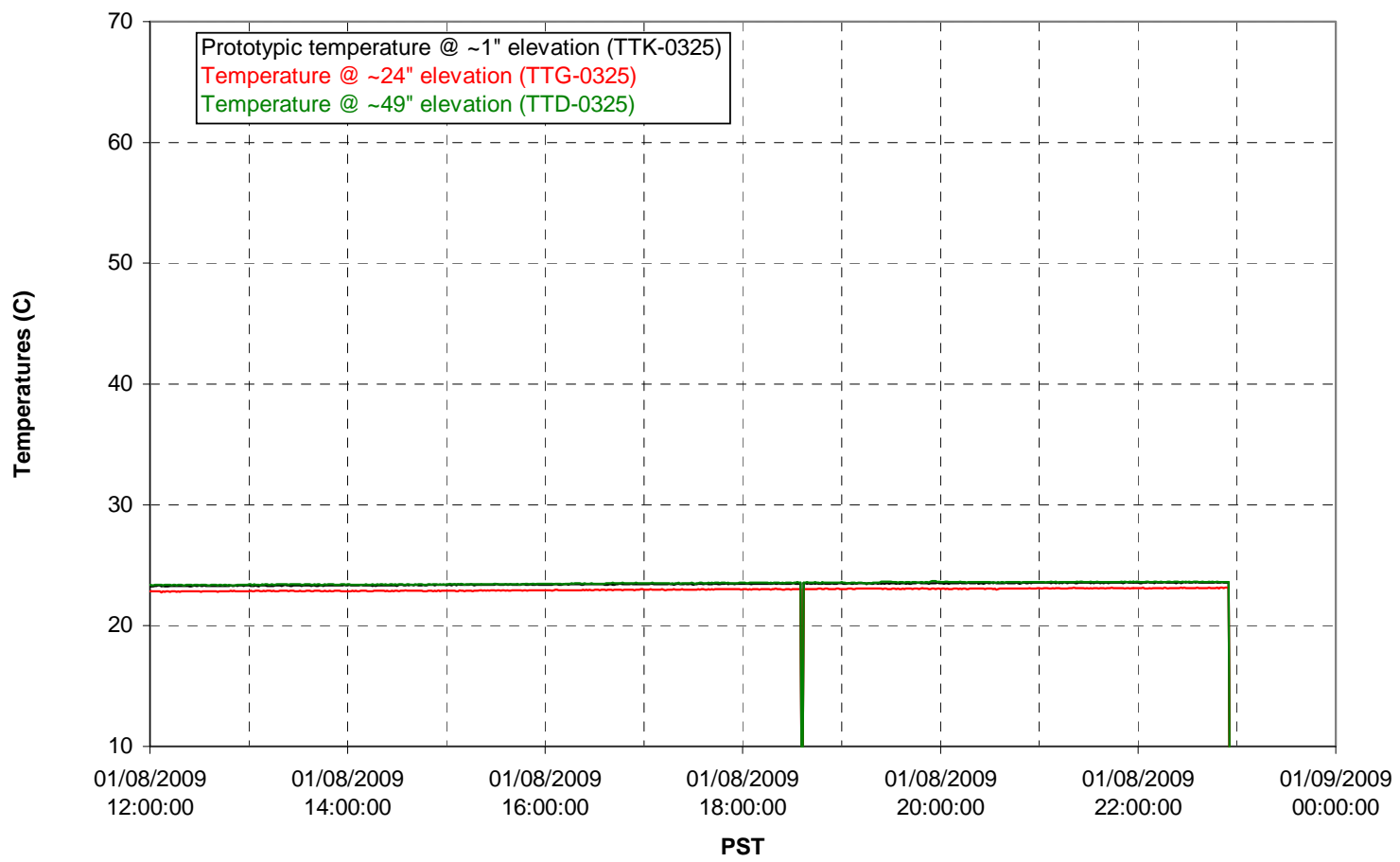


T01B level

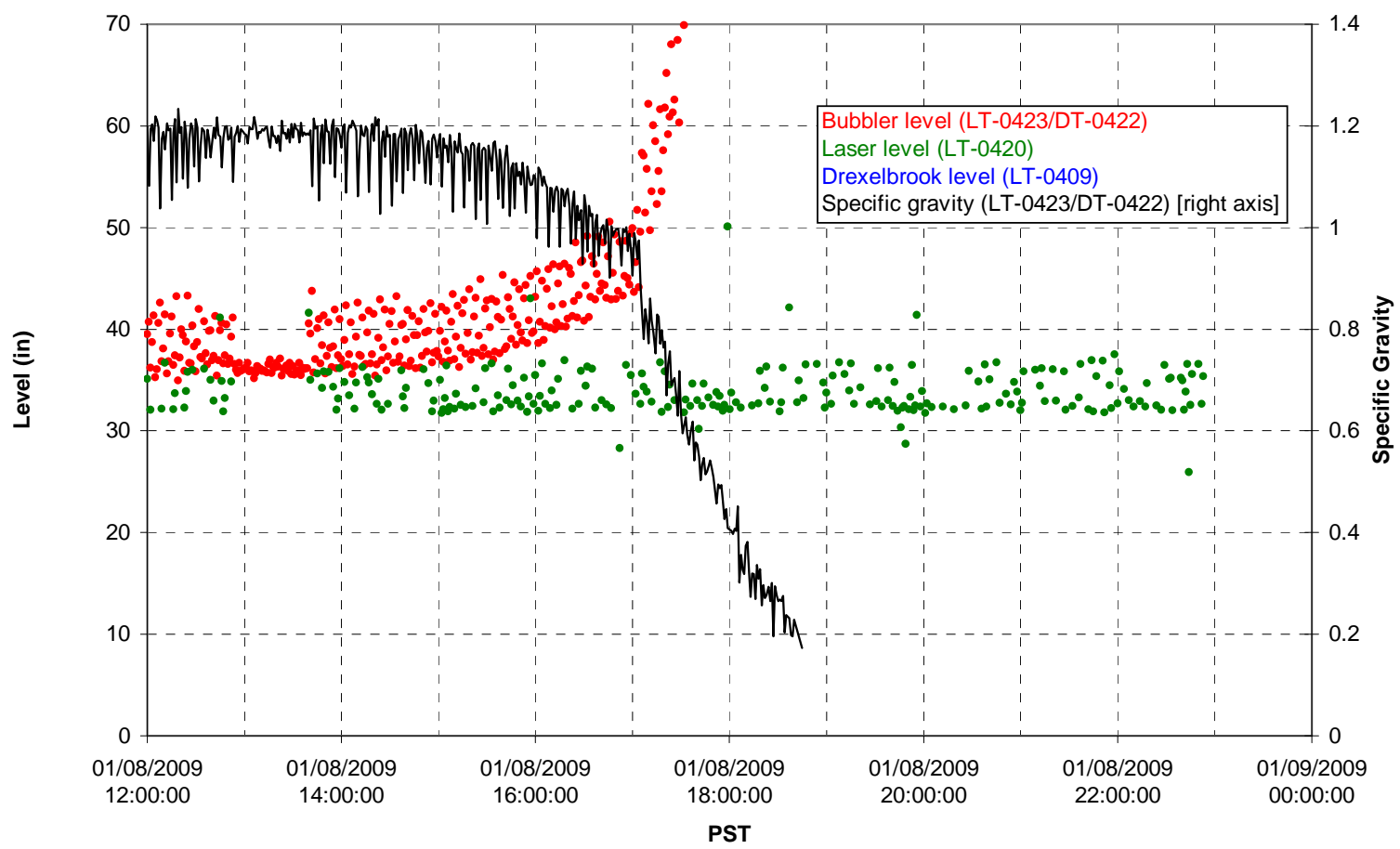

T01B temperatures

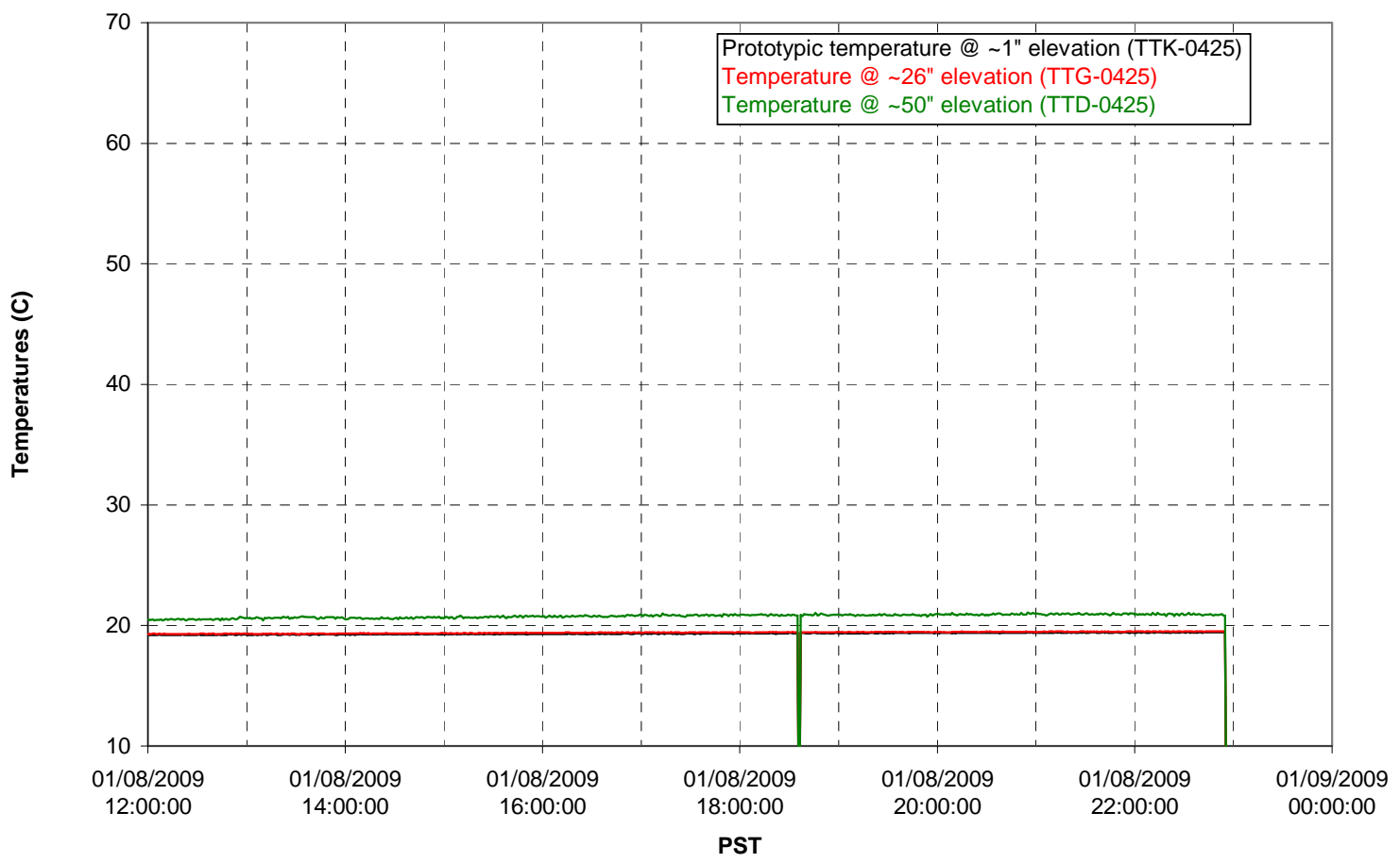


T02A level

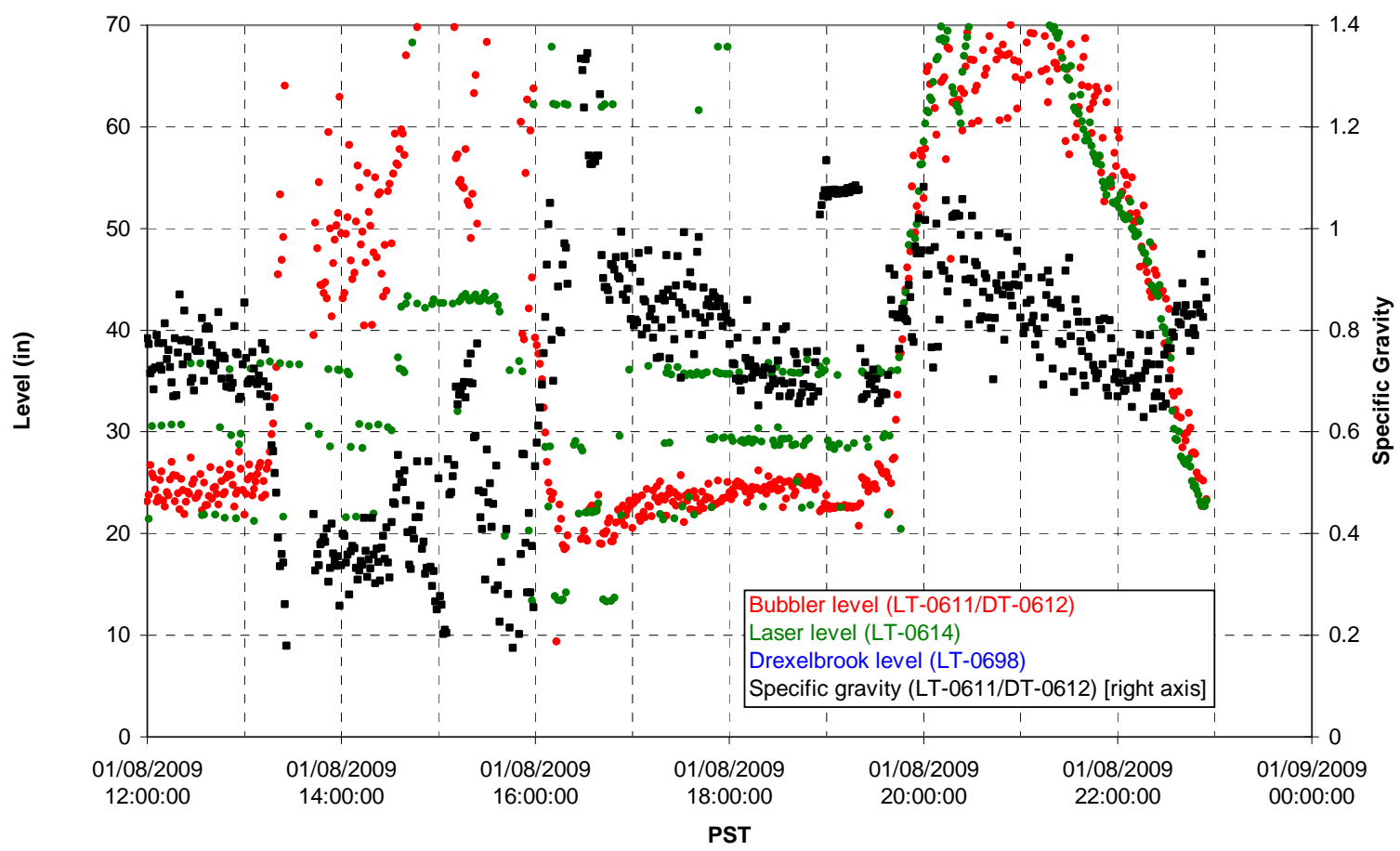

T02A temperatures

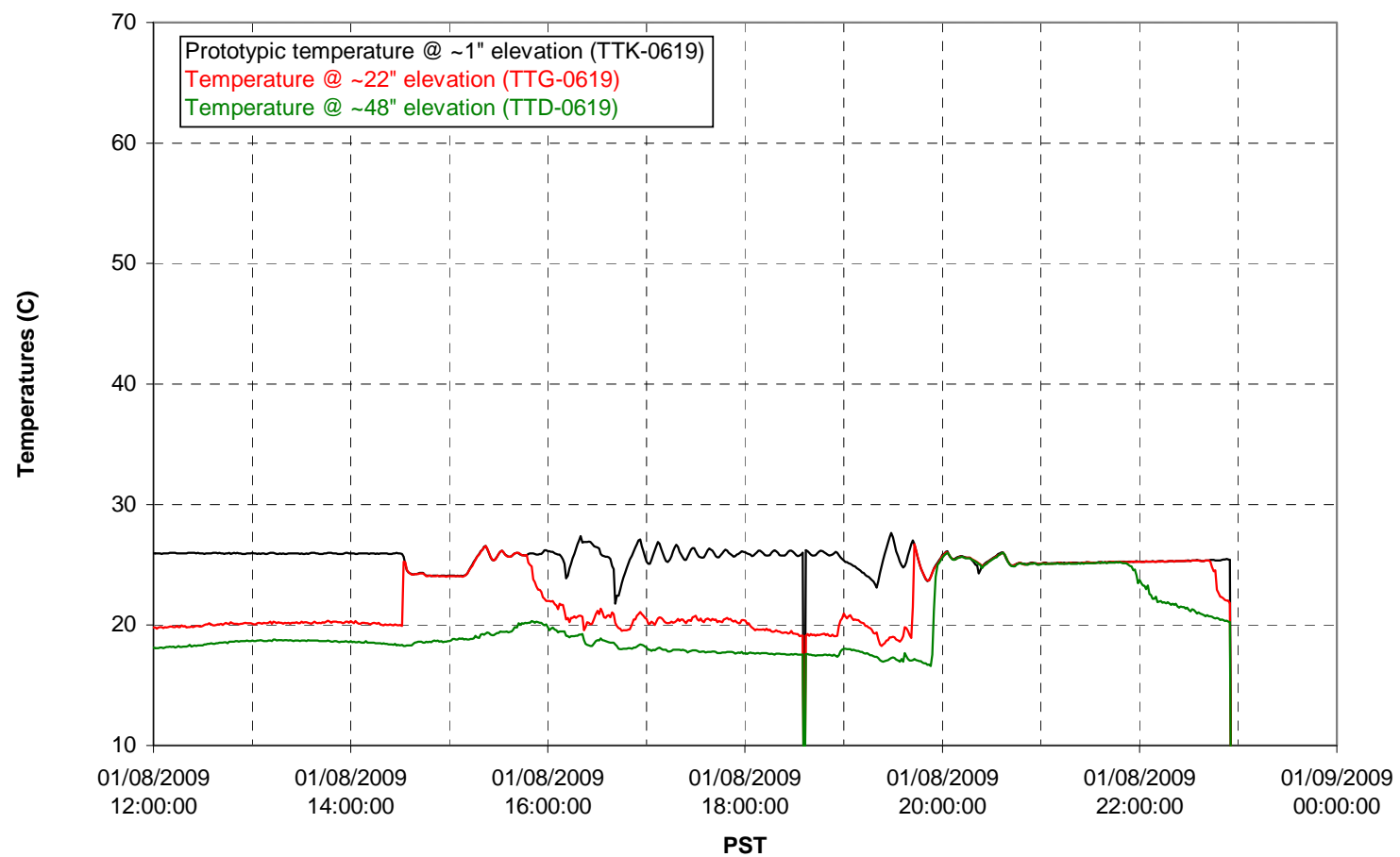


T02A and filter loop temperatures

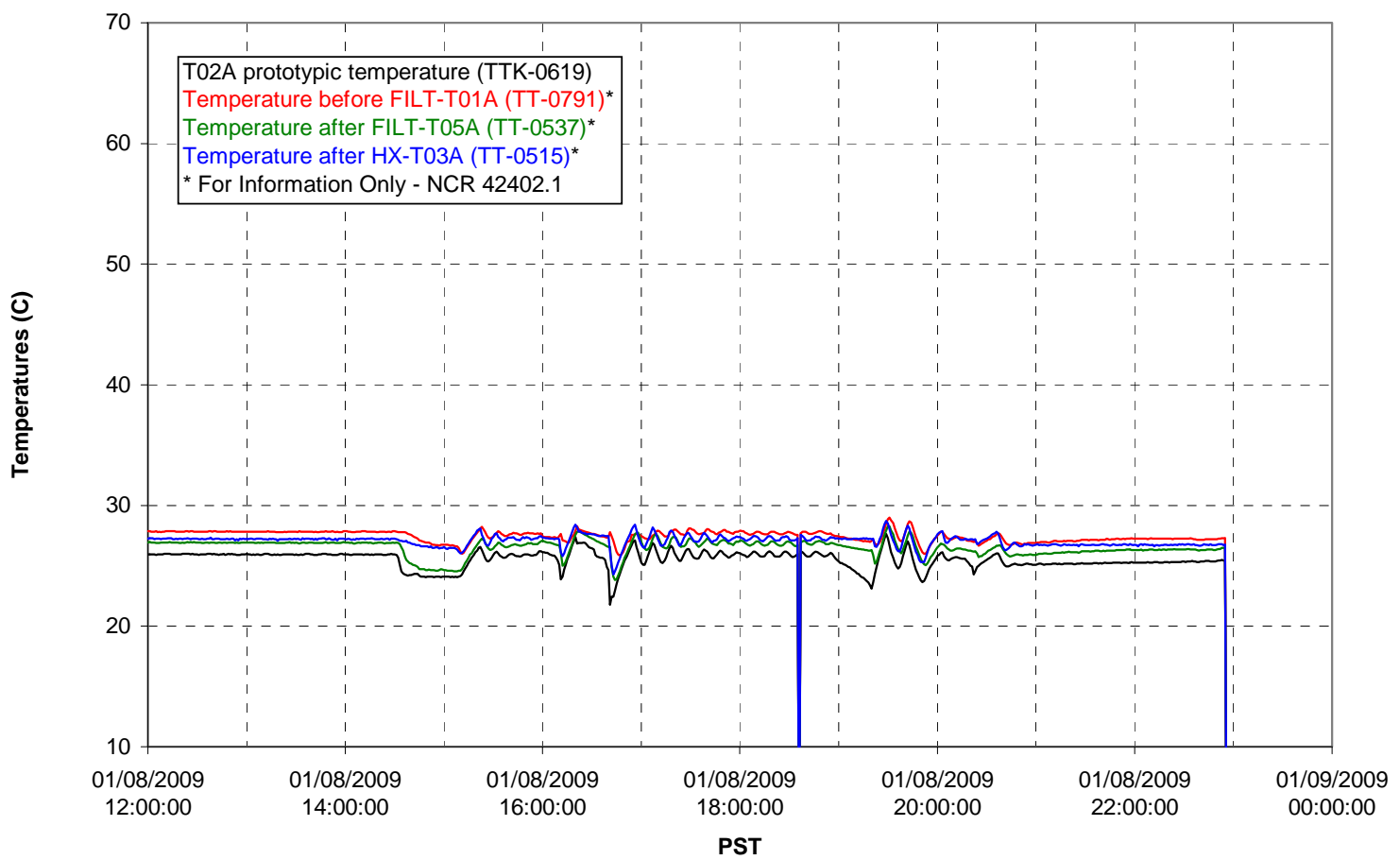

Pump Pressures and Flow

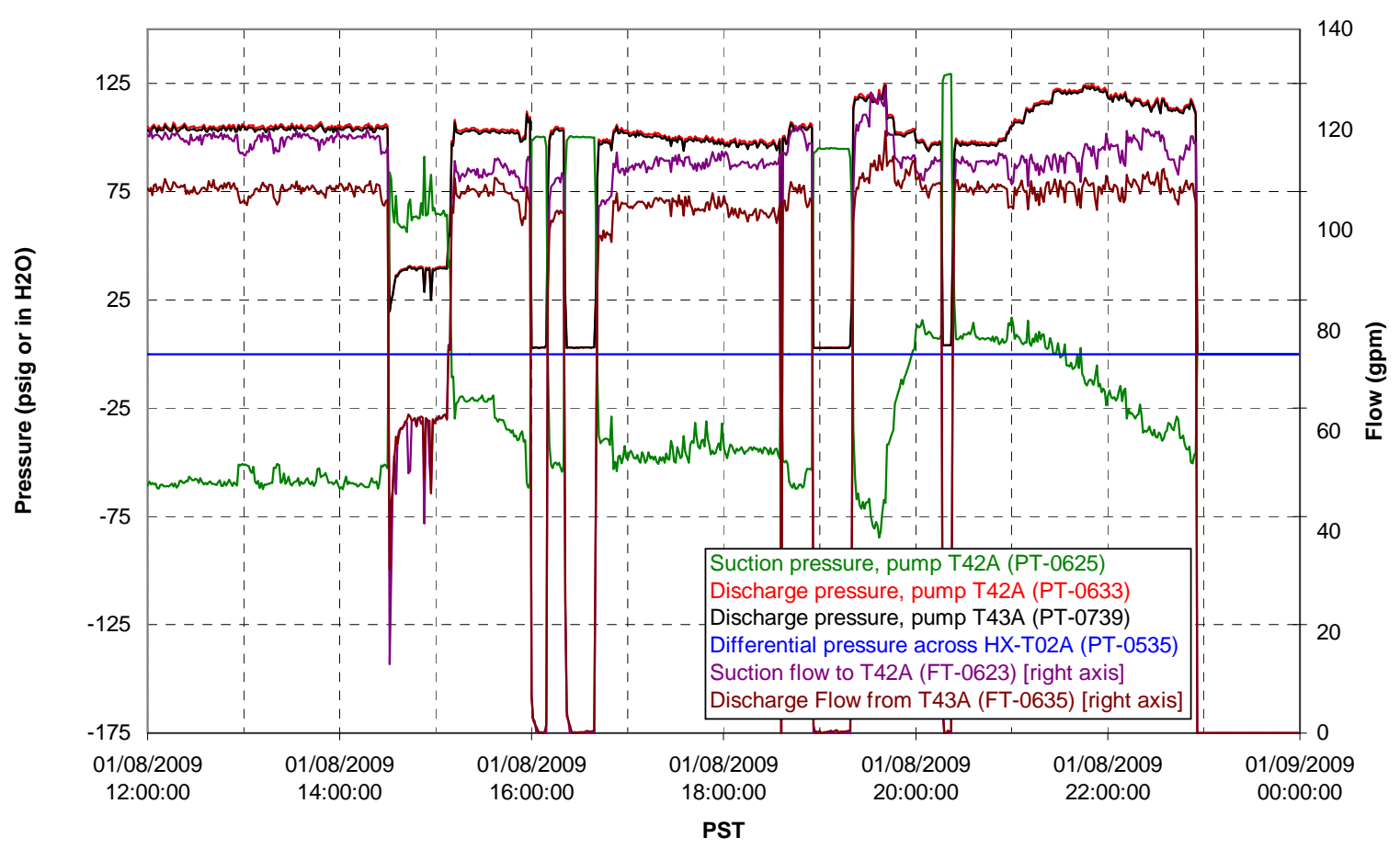


Axial pressure drop

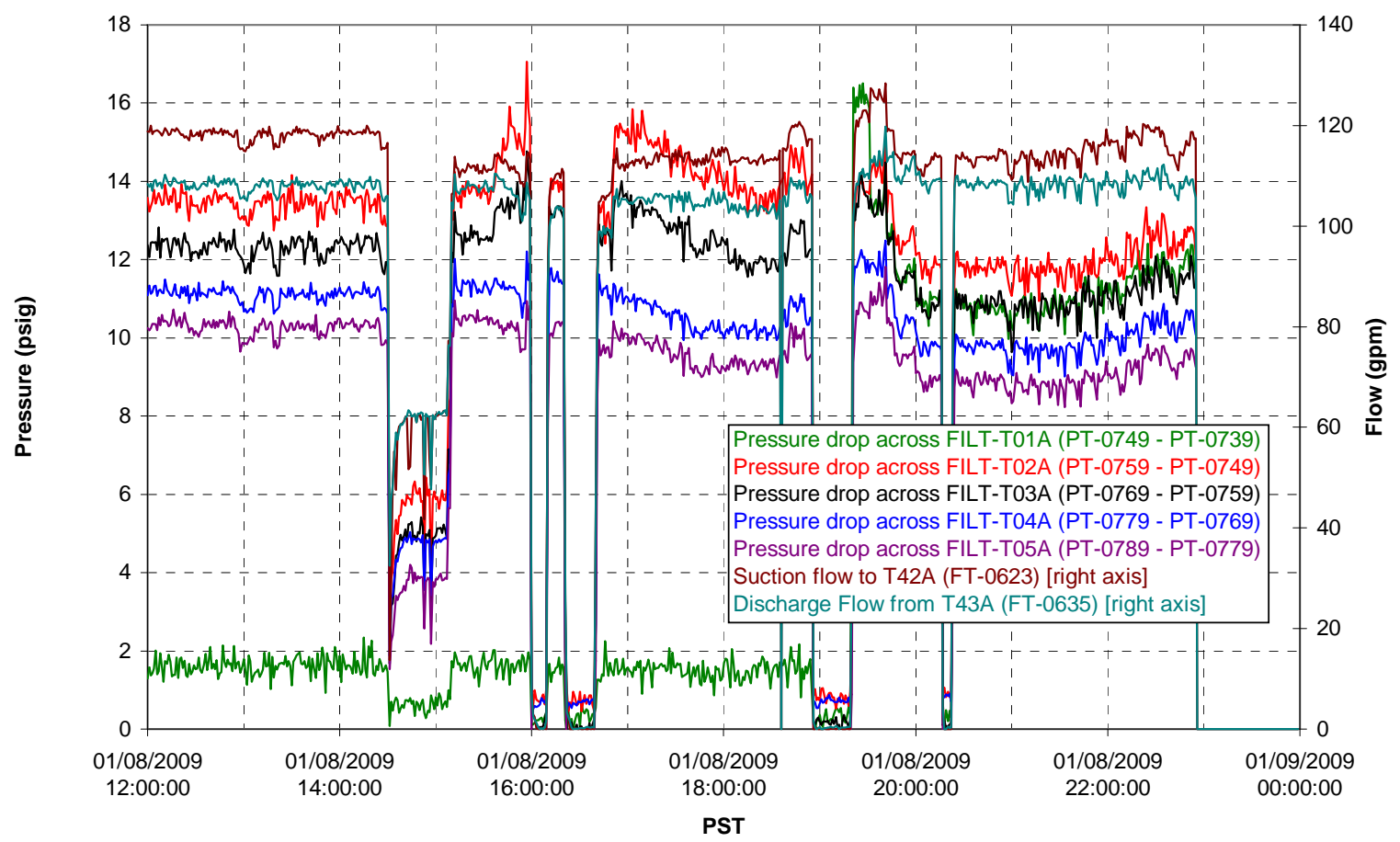

Permeate flow rates

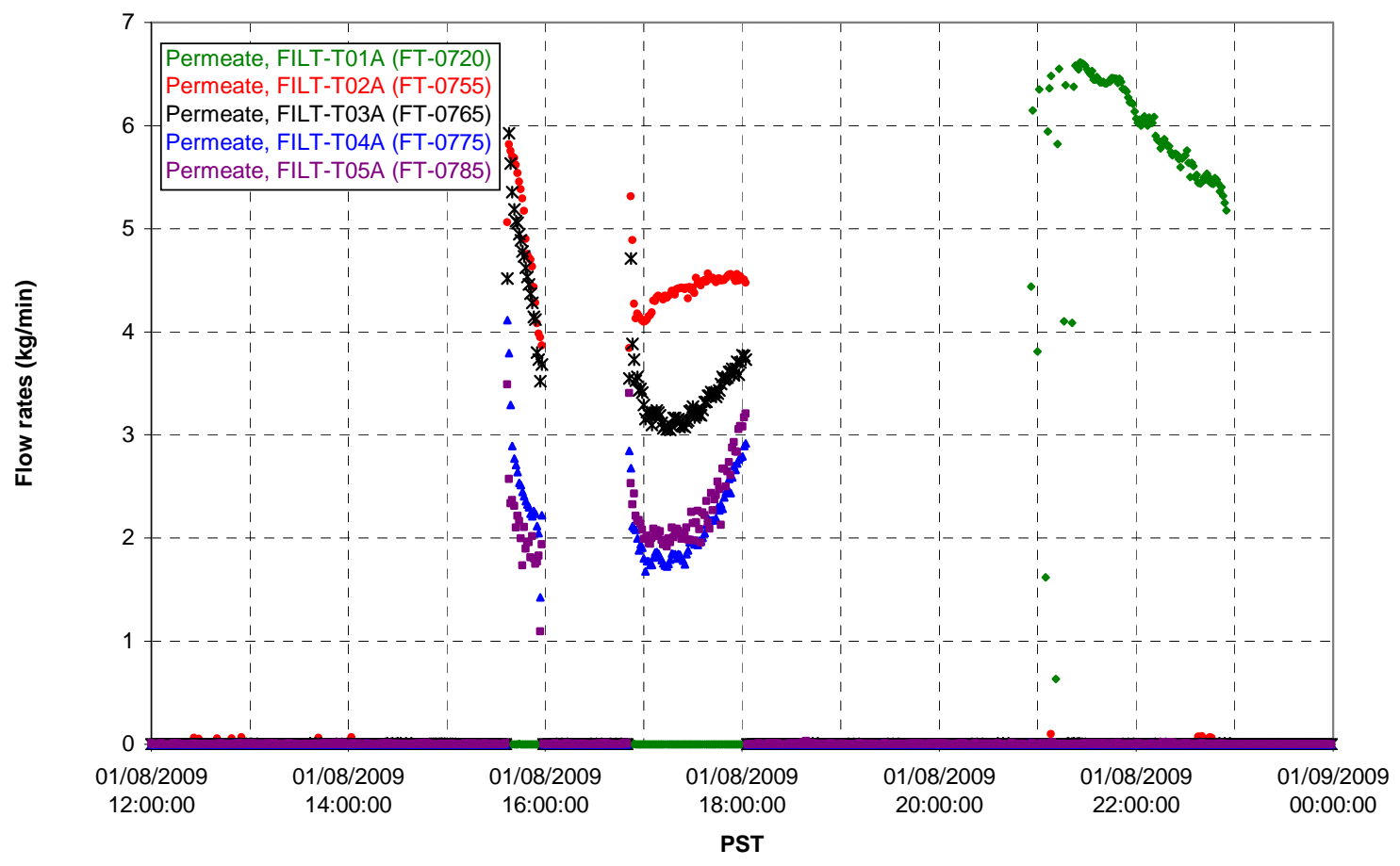


T02A Inner Temperature Tree

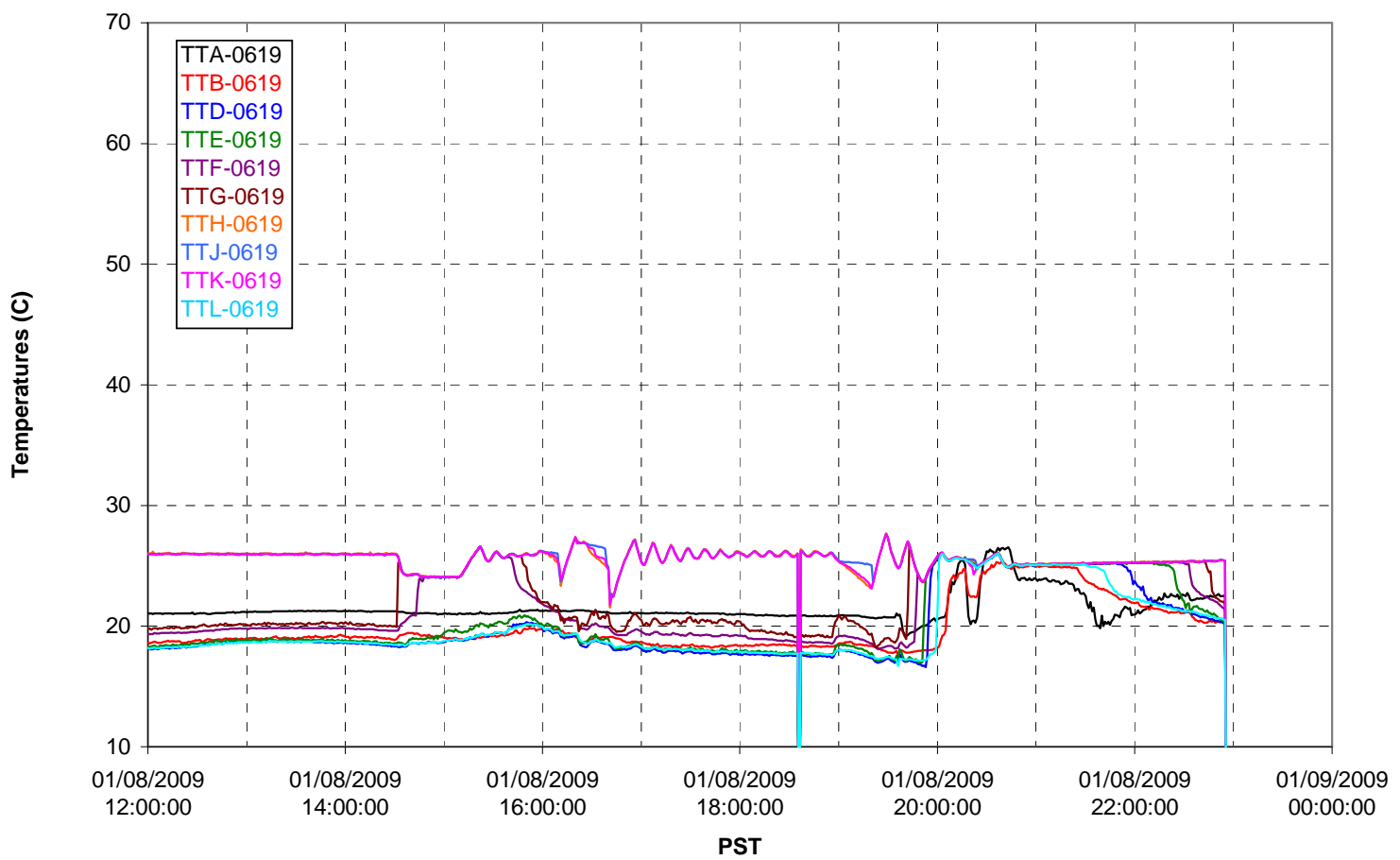

T02A Outer Temperature Tree

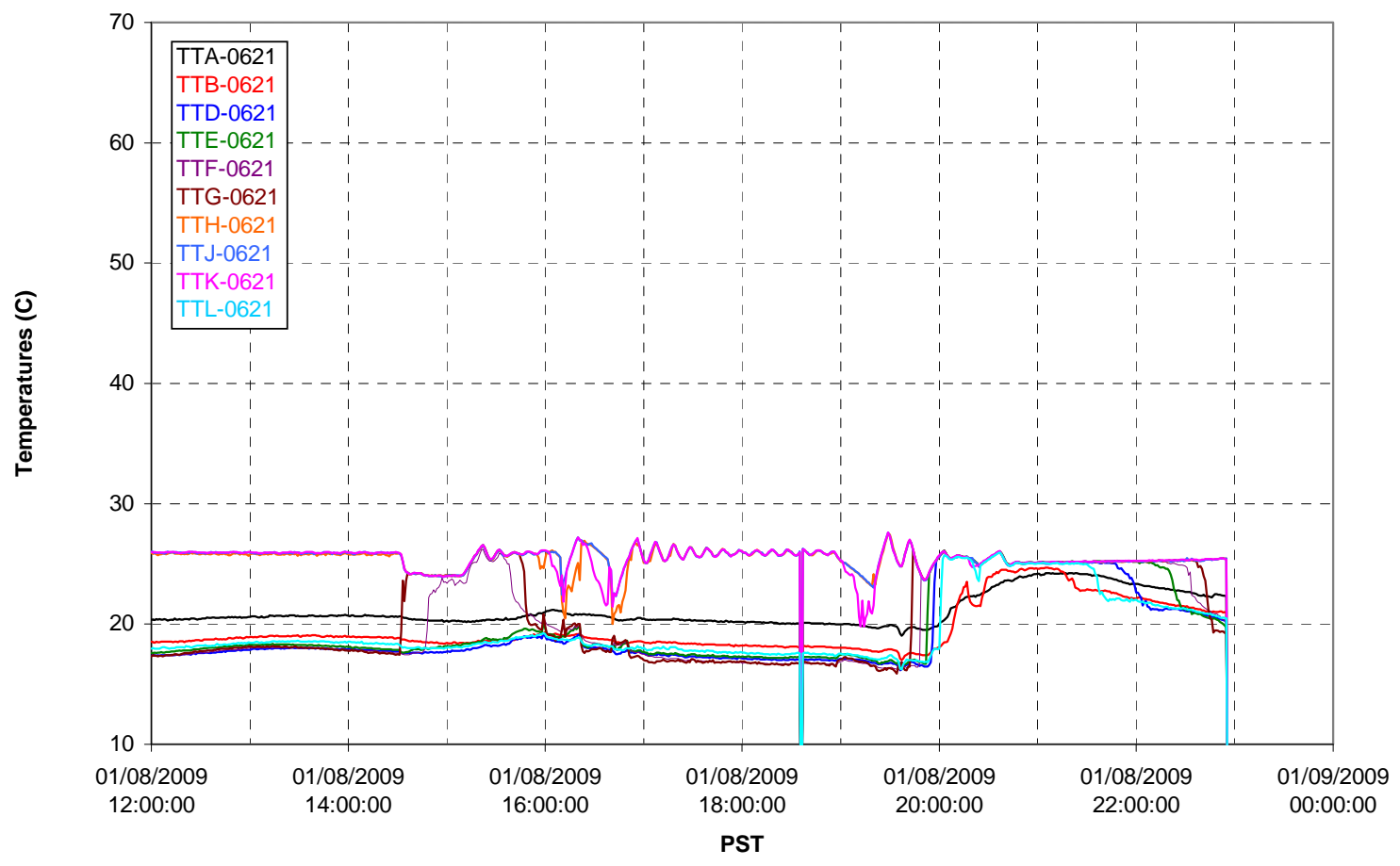


T02A temperatures

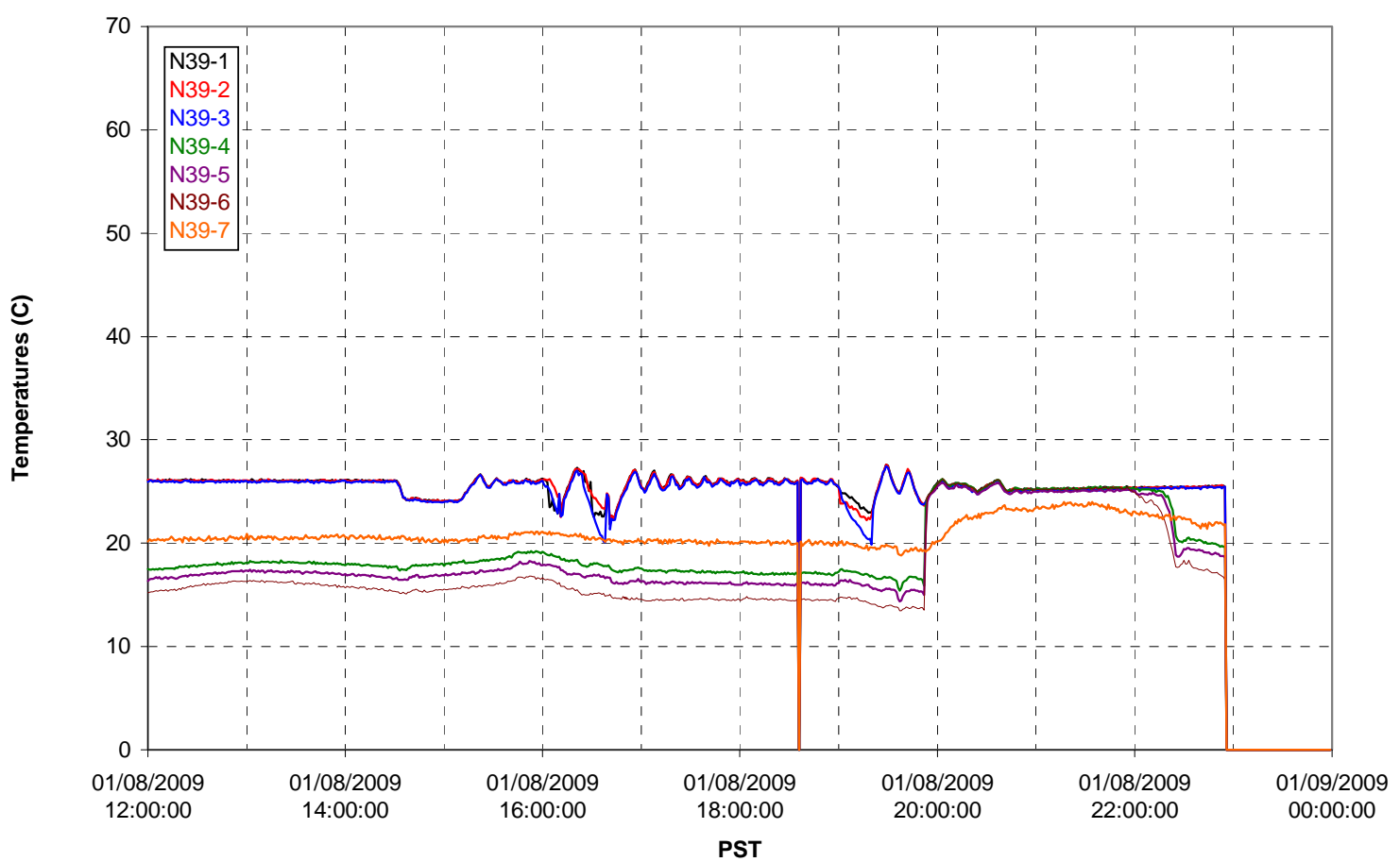

T02A temperatures

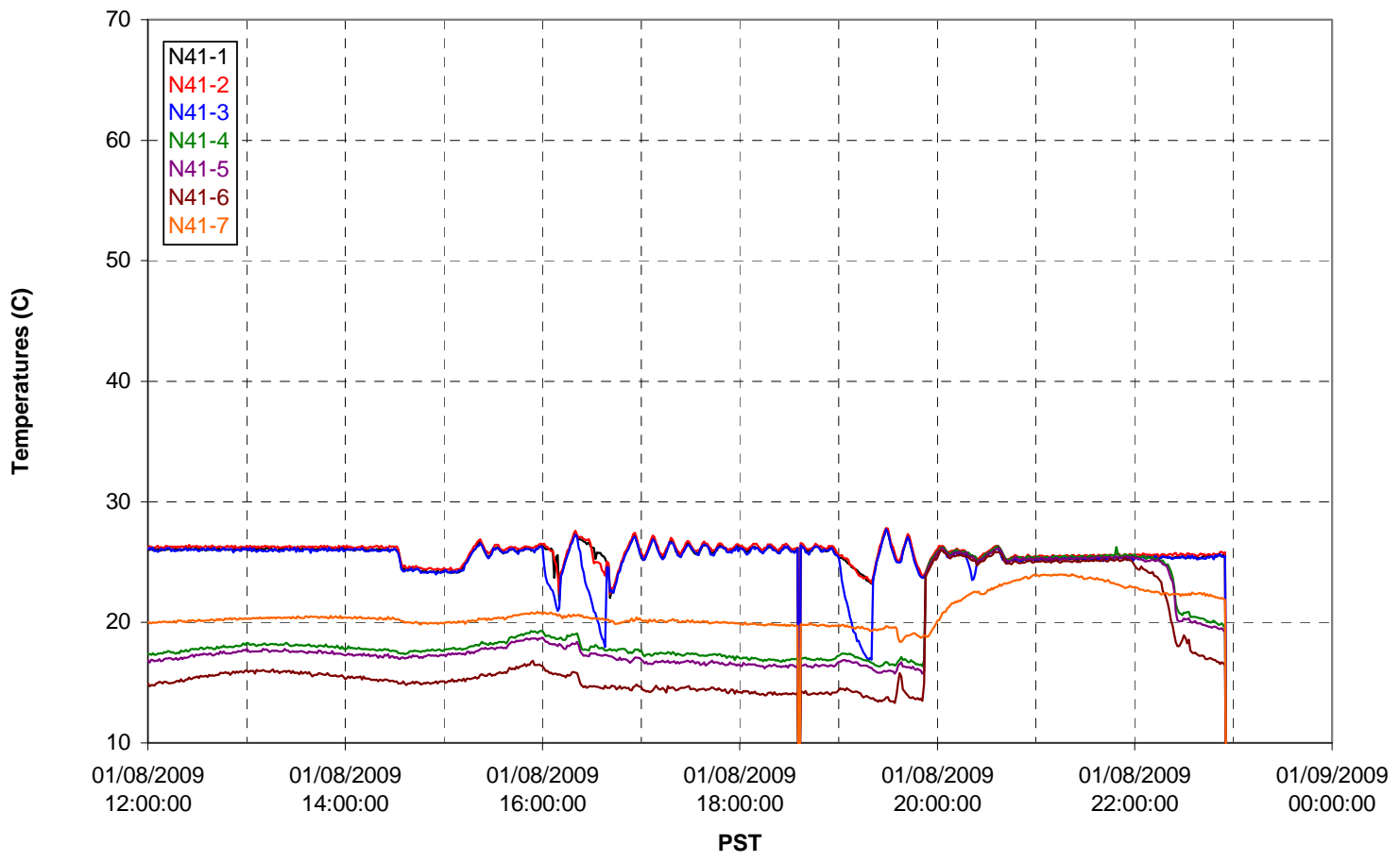


T02A temperatures

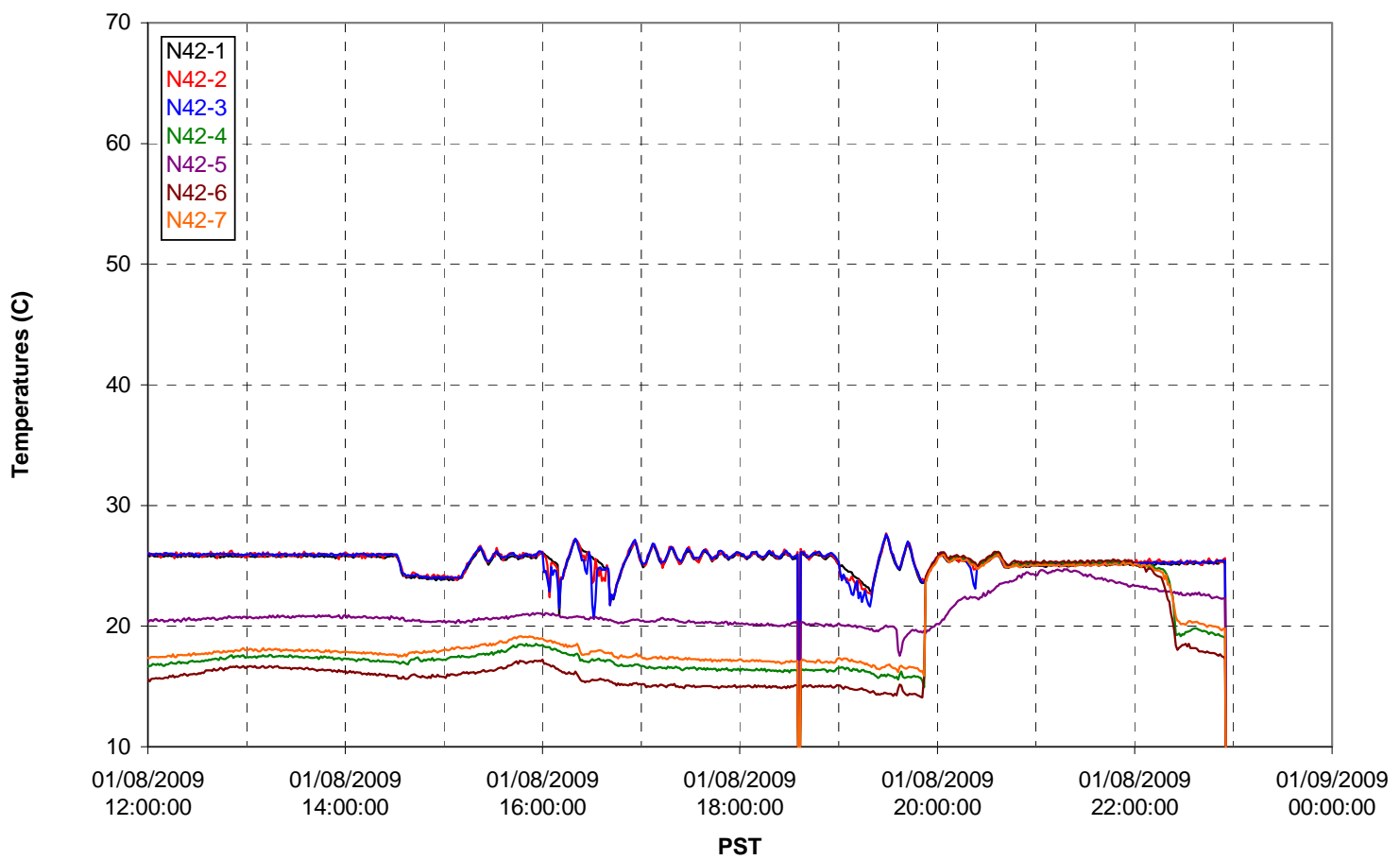

T02A temperatures

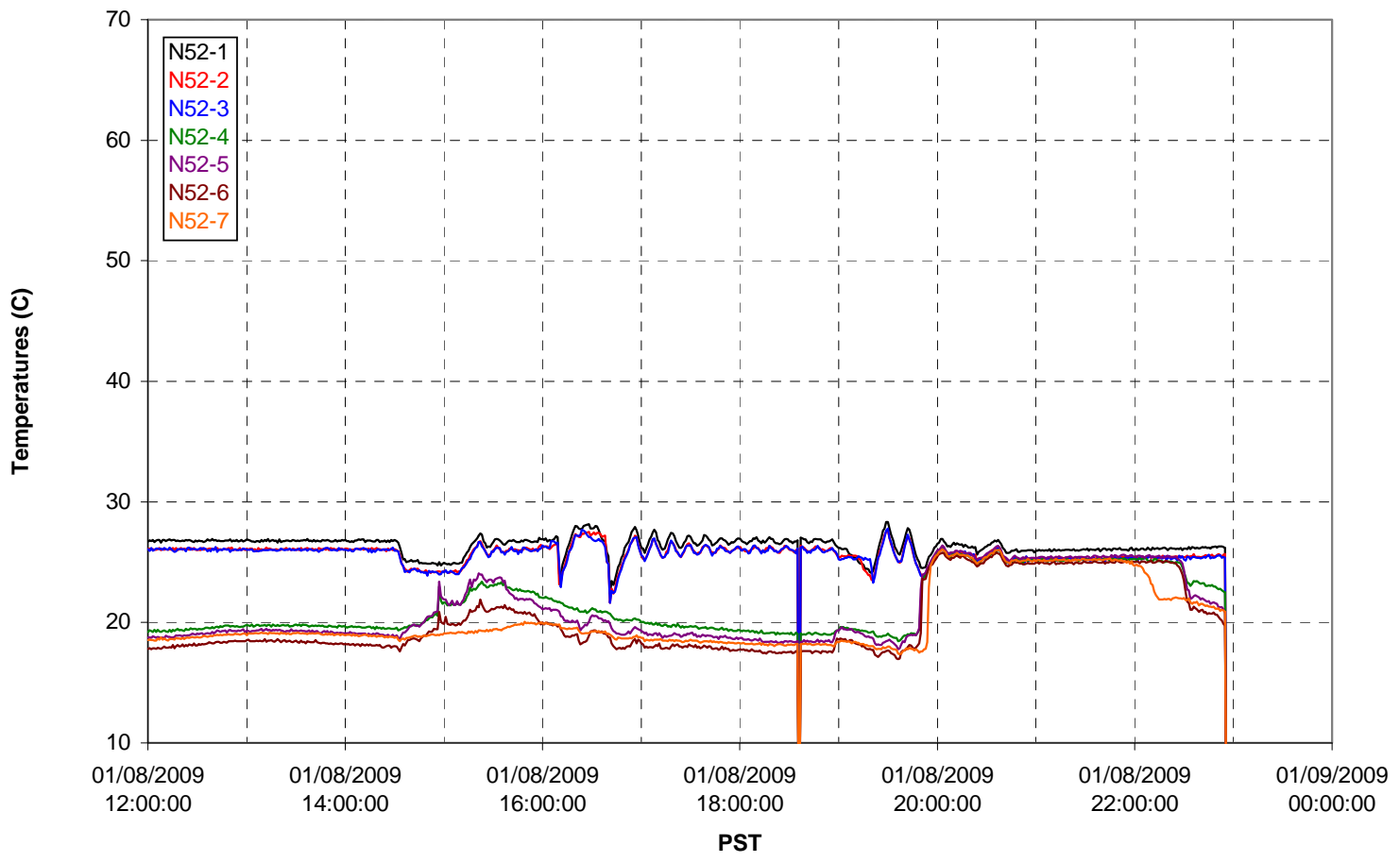


T02A Heating and Cooling

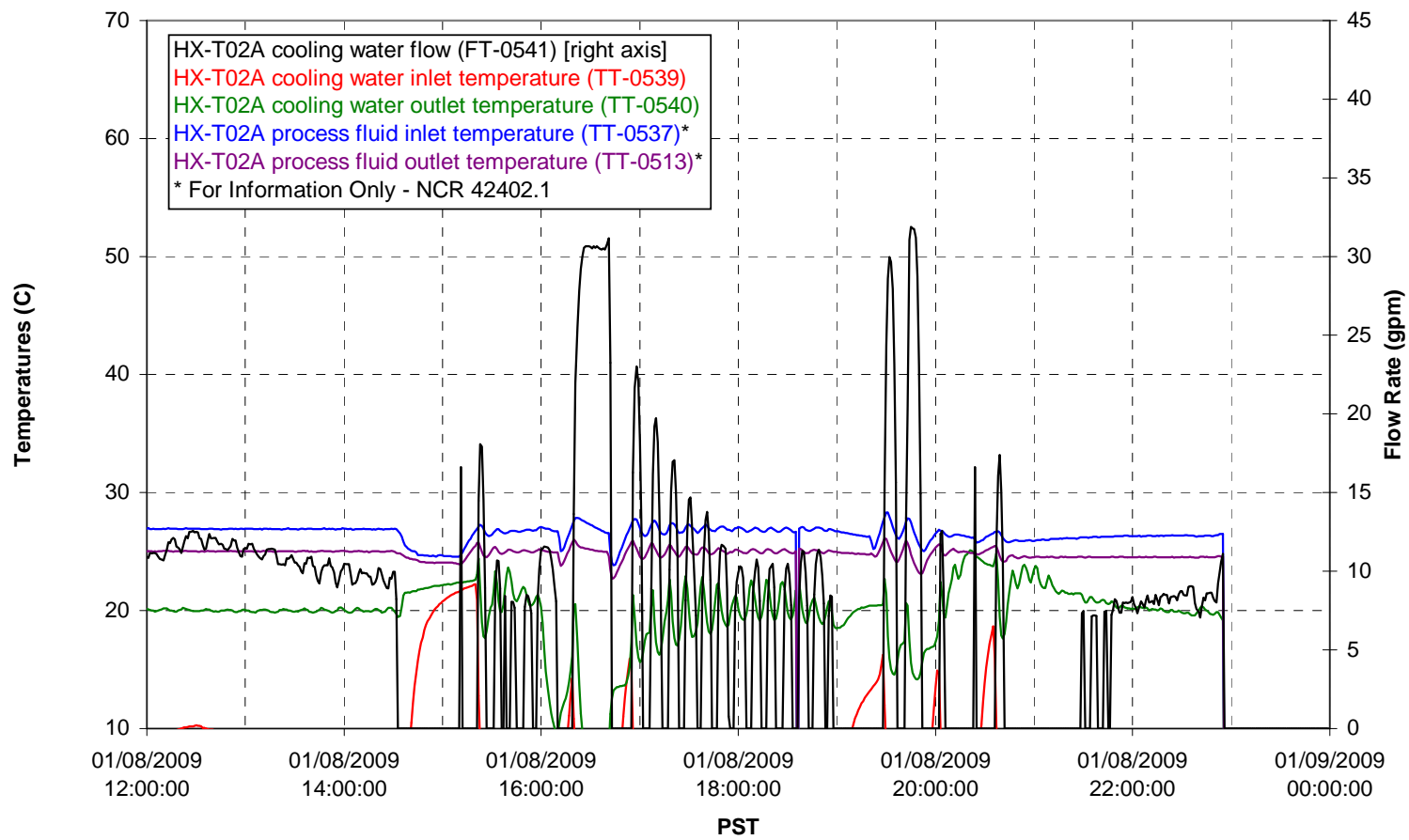

Pump Operation

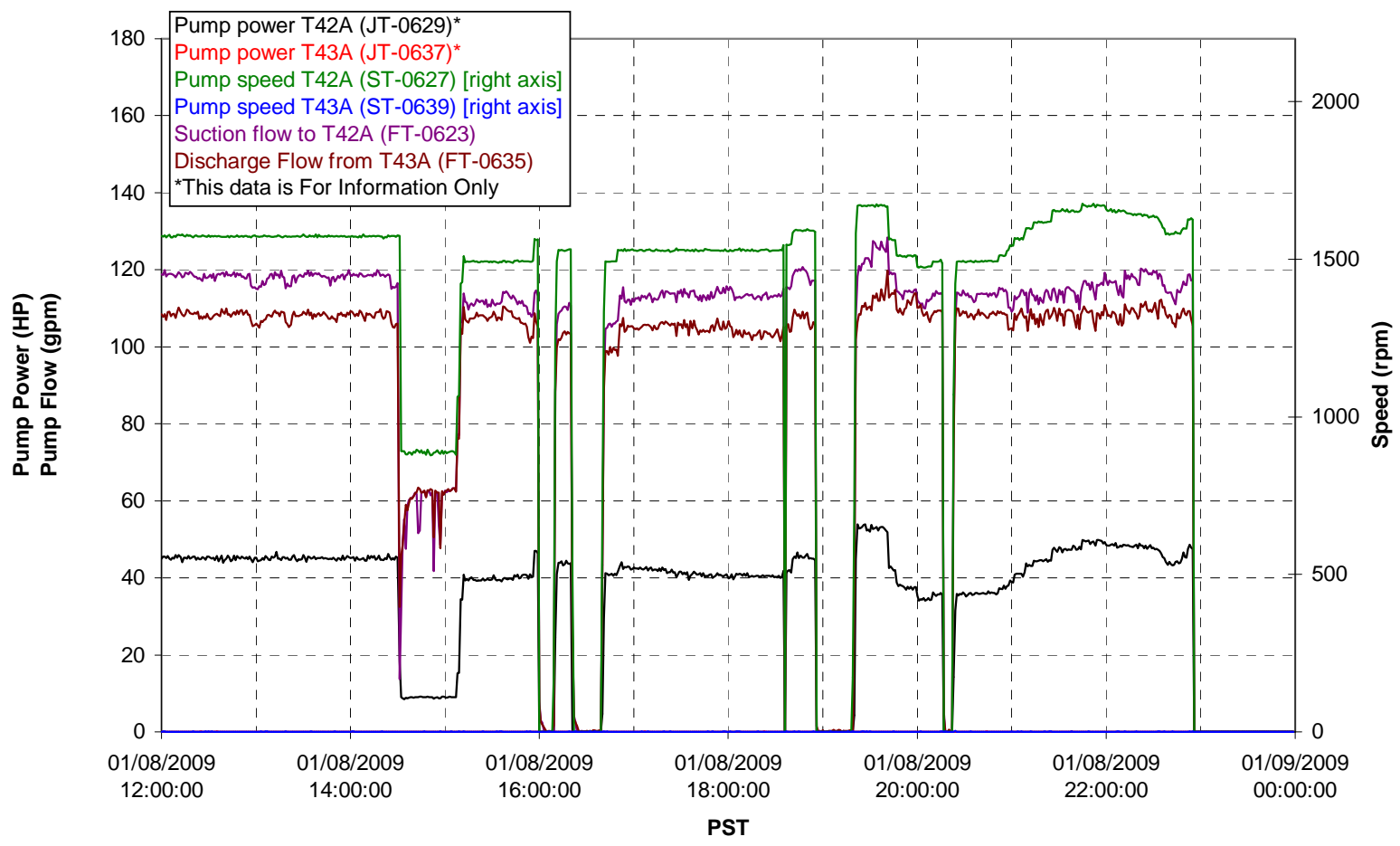


Pulsepot UFP-PP-T01A

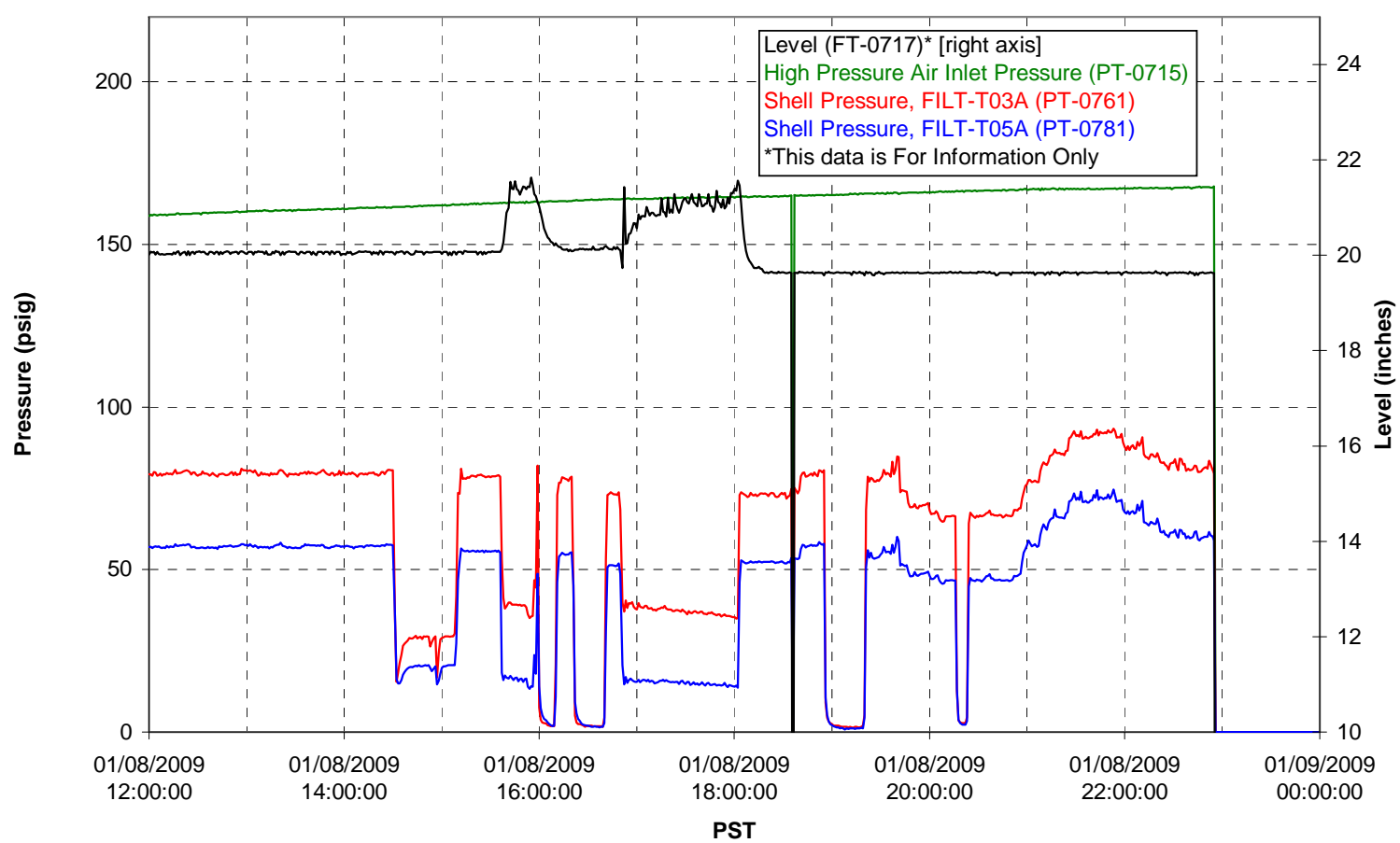

Pulsepot UFP-PP-T02A

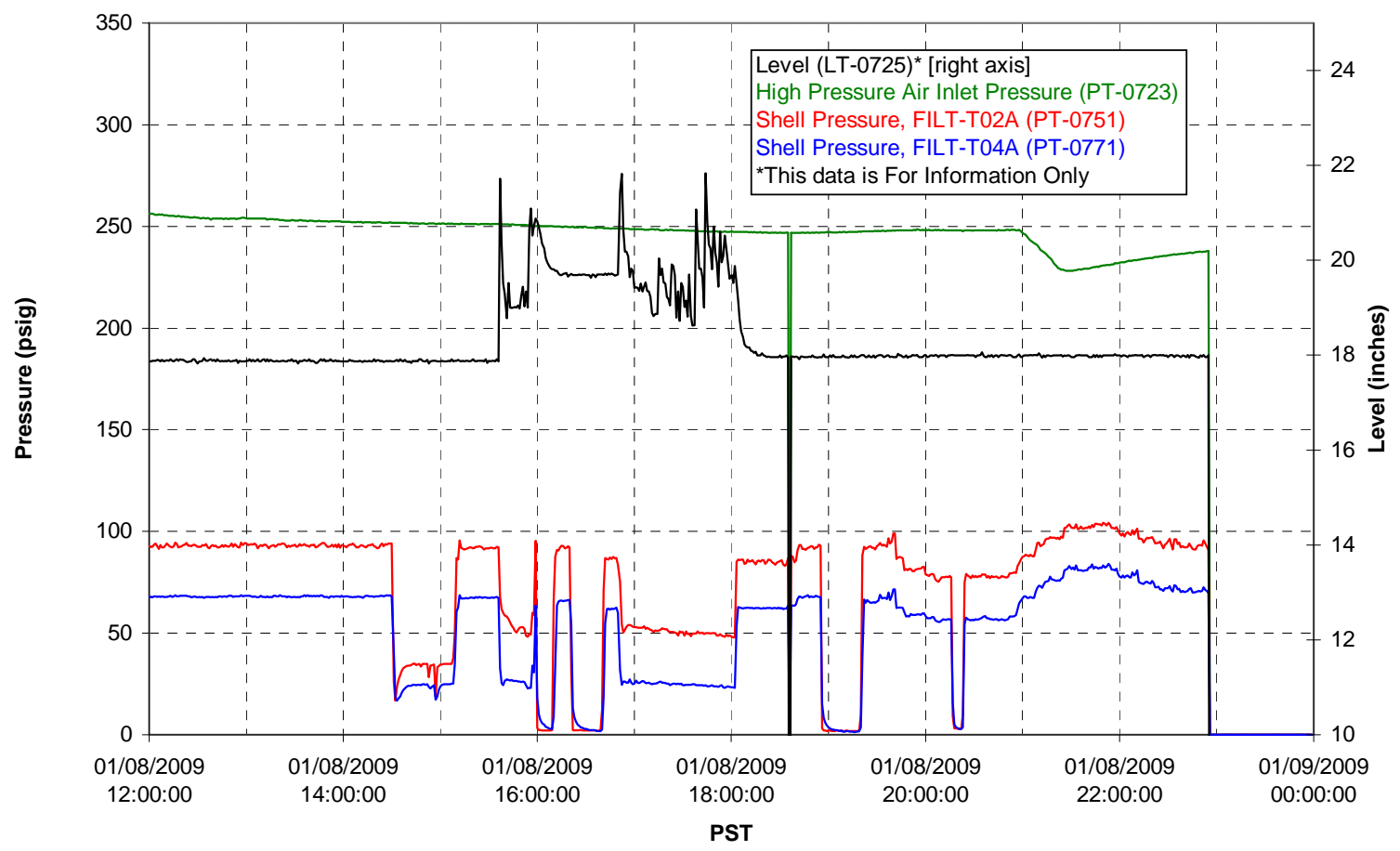


Pulsepot UFP-PP-T03A

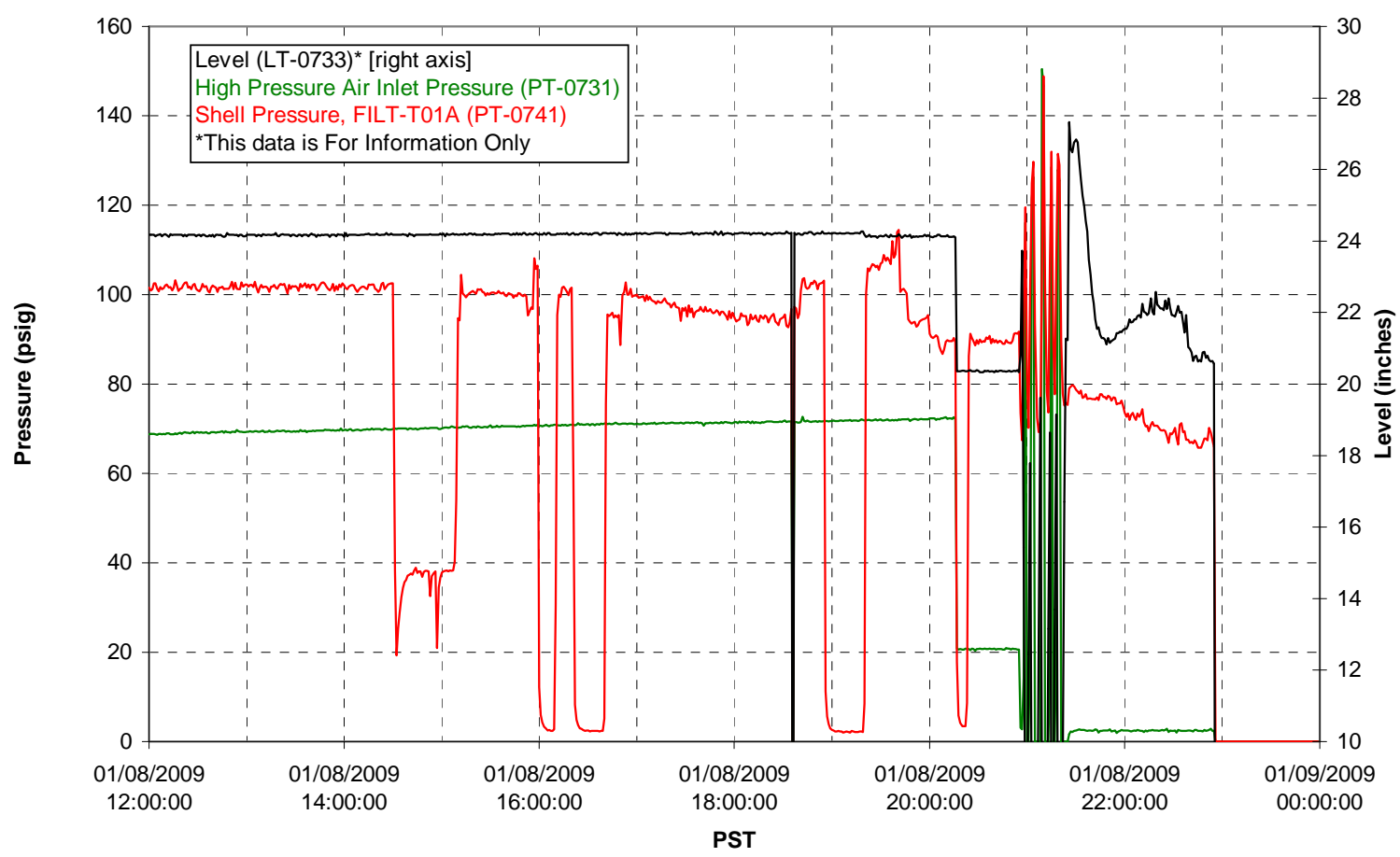

Pulsepot Levels

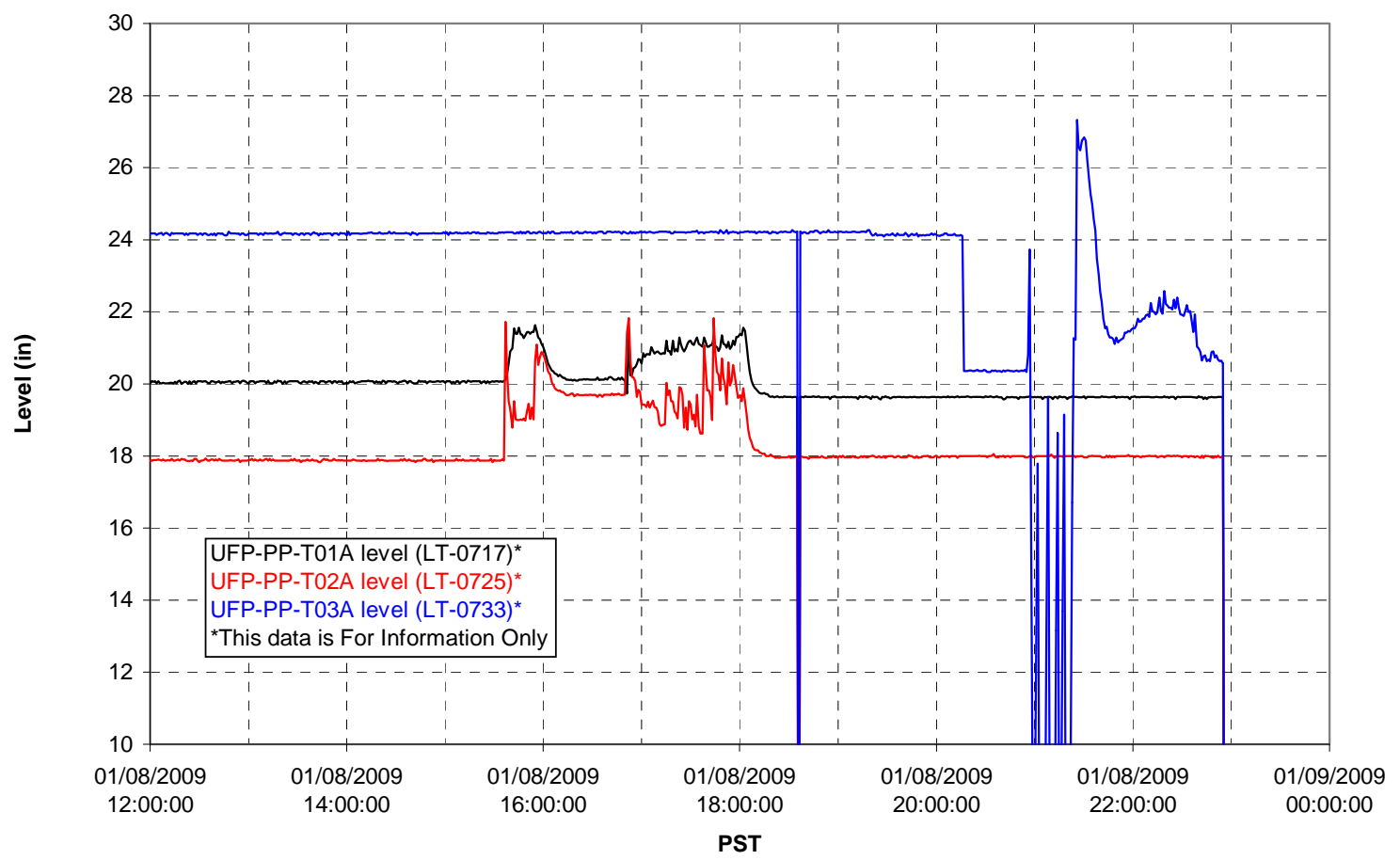


Filter UFP-FILT-T01A

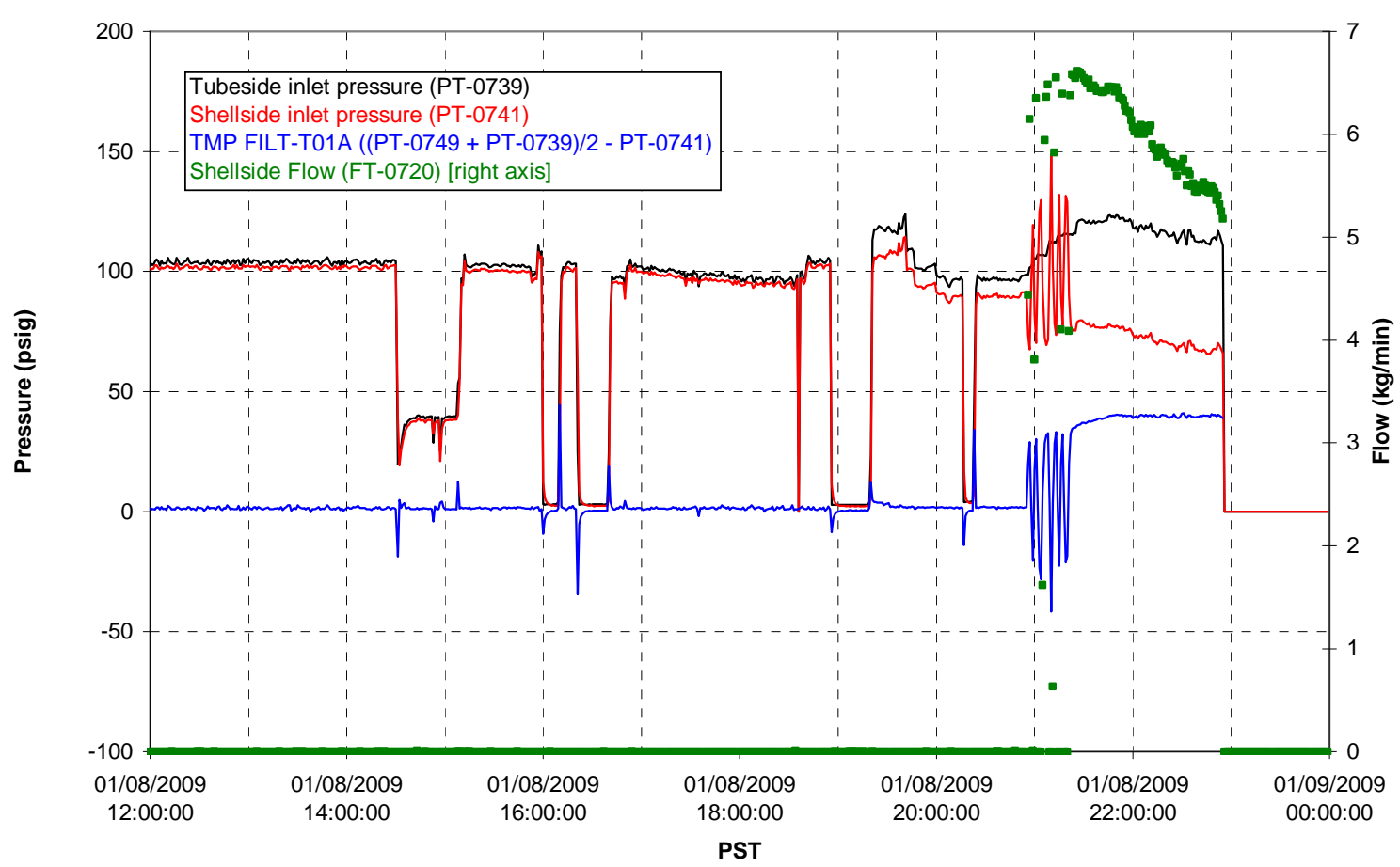

Filter UFP-FILT-T02A

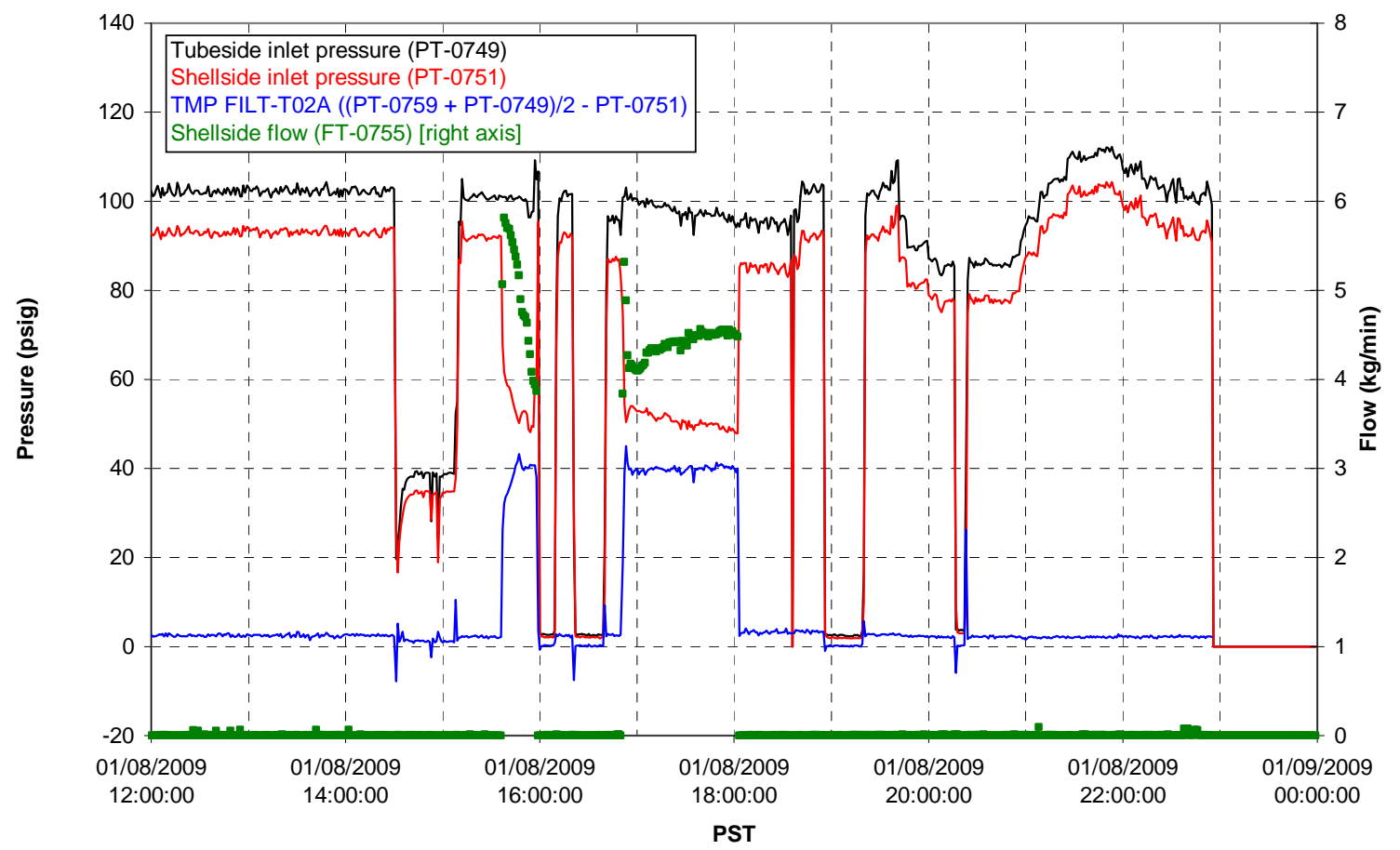


Filter UFP-FILT-T03A

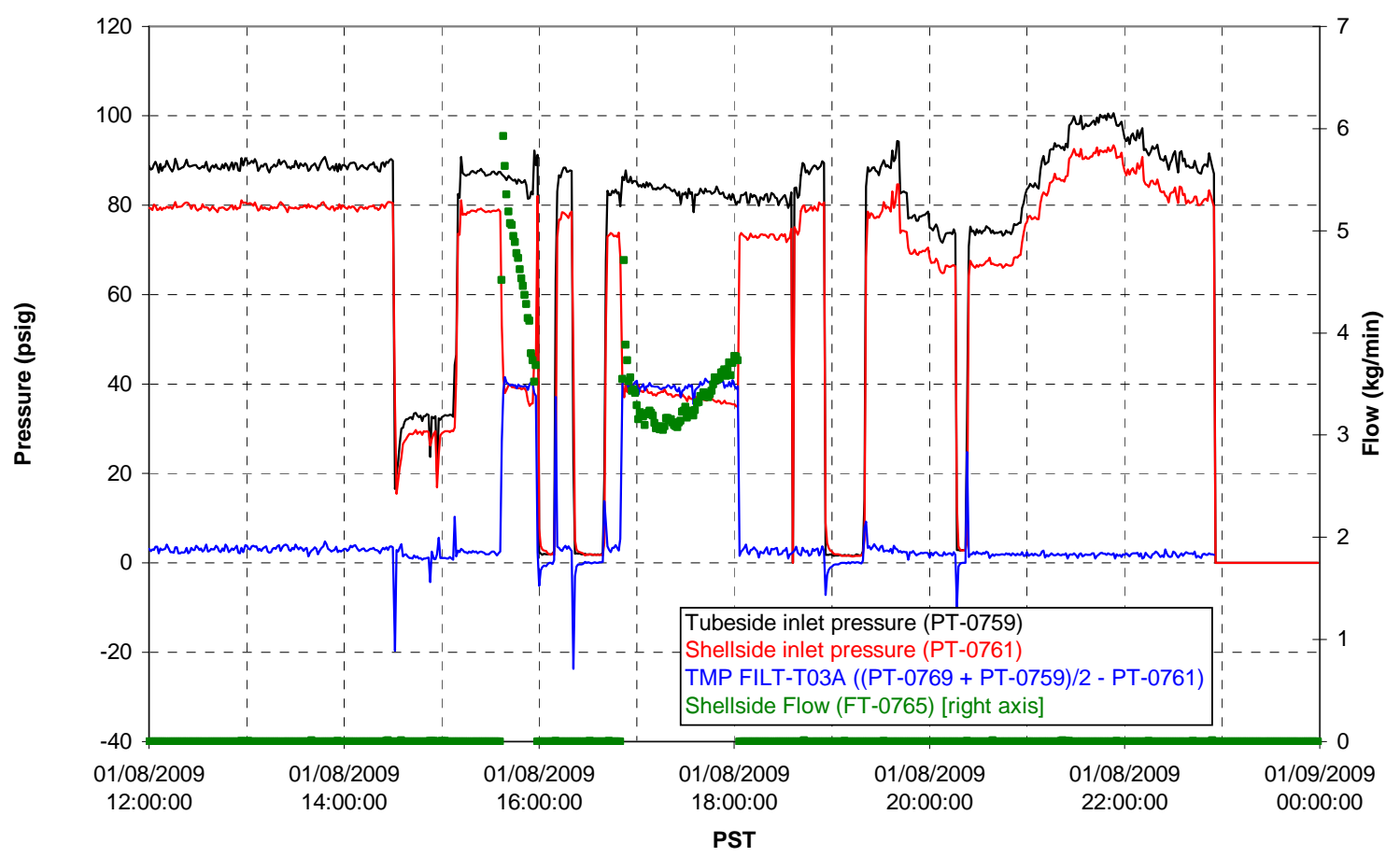

Filter UFP-FILT-T04A

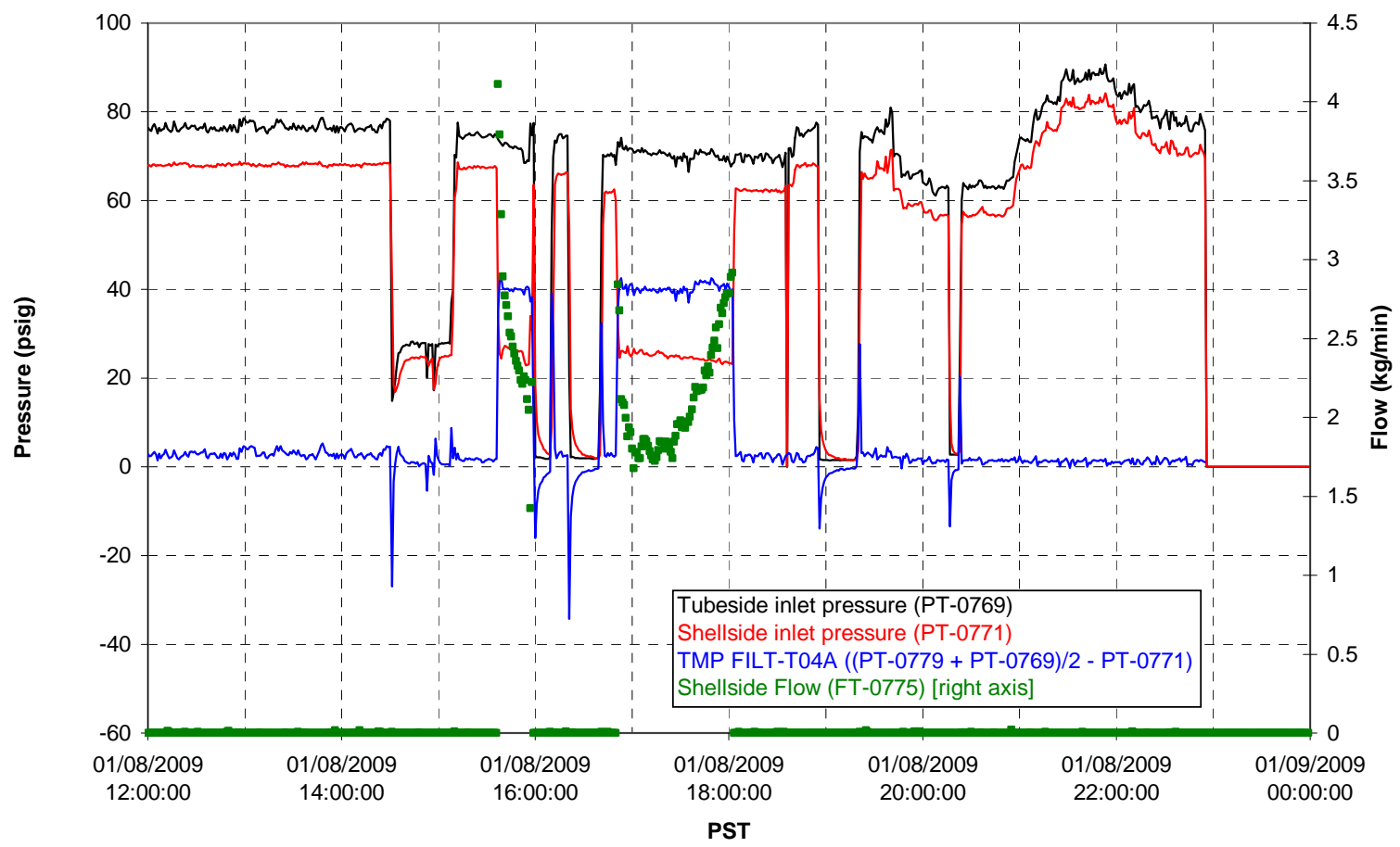


Filter UFP-FILT-T05A

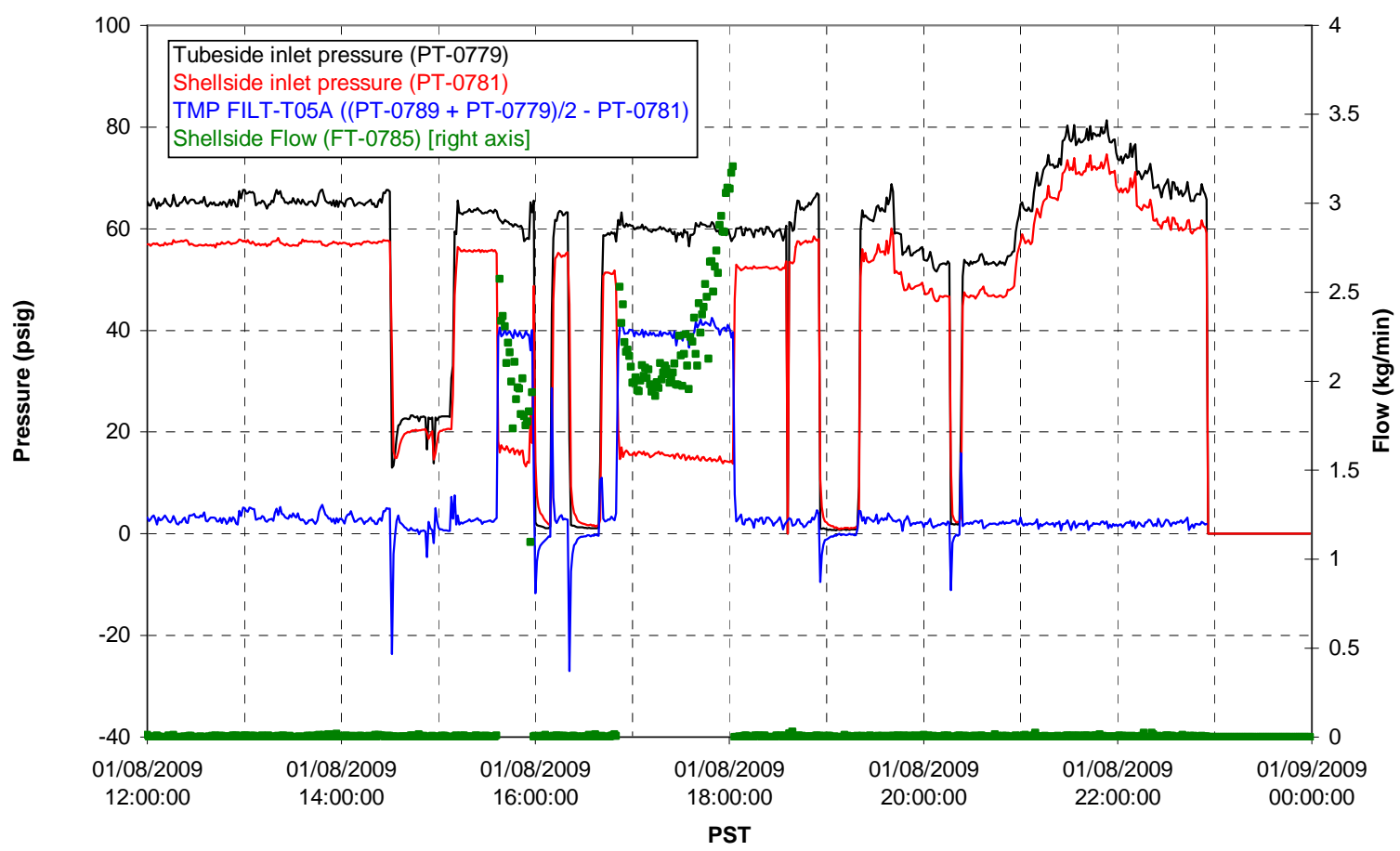

Chemical Flow

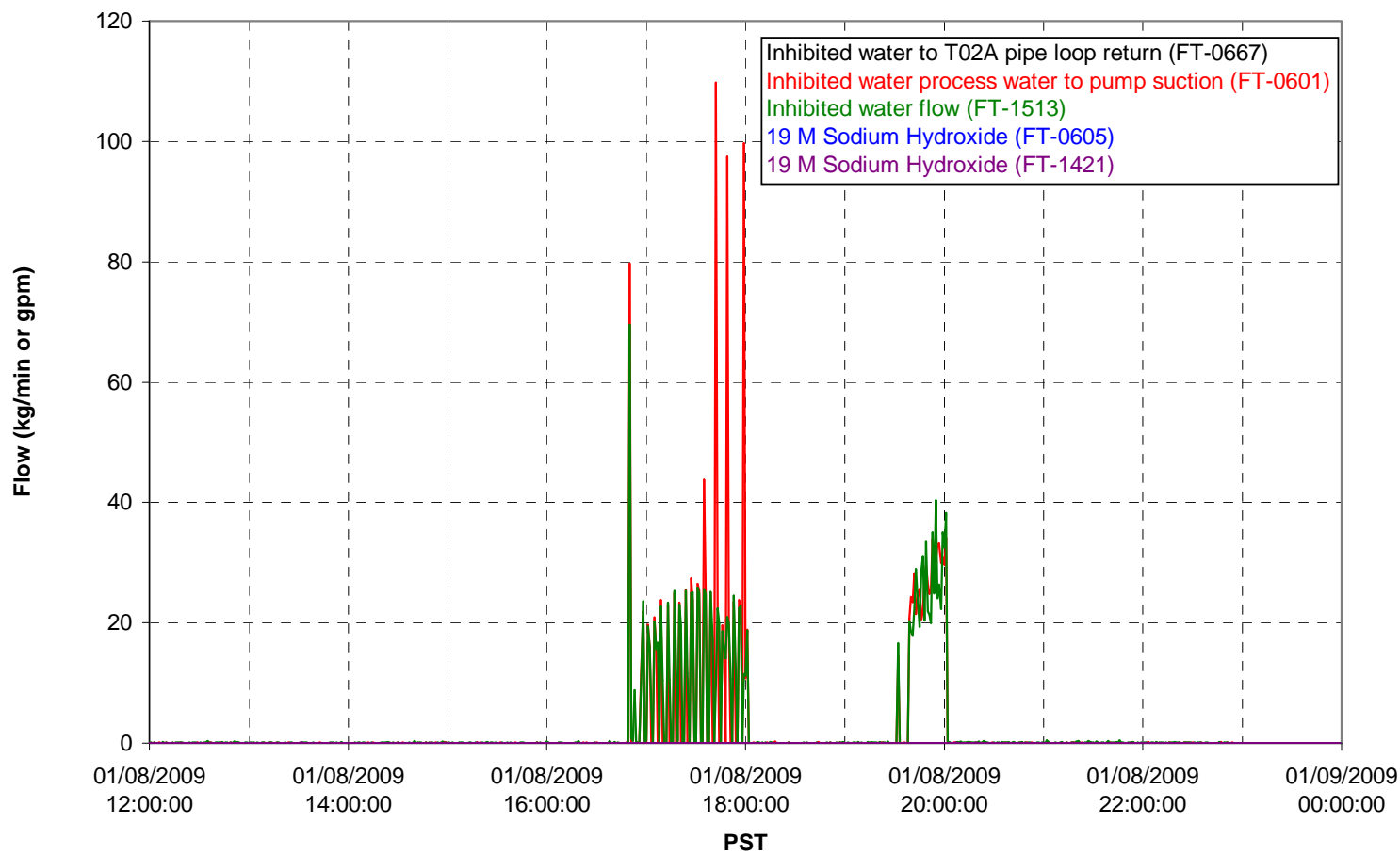


Chemical Flow

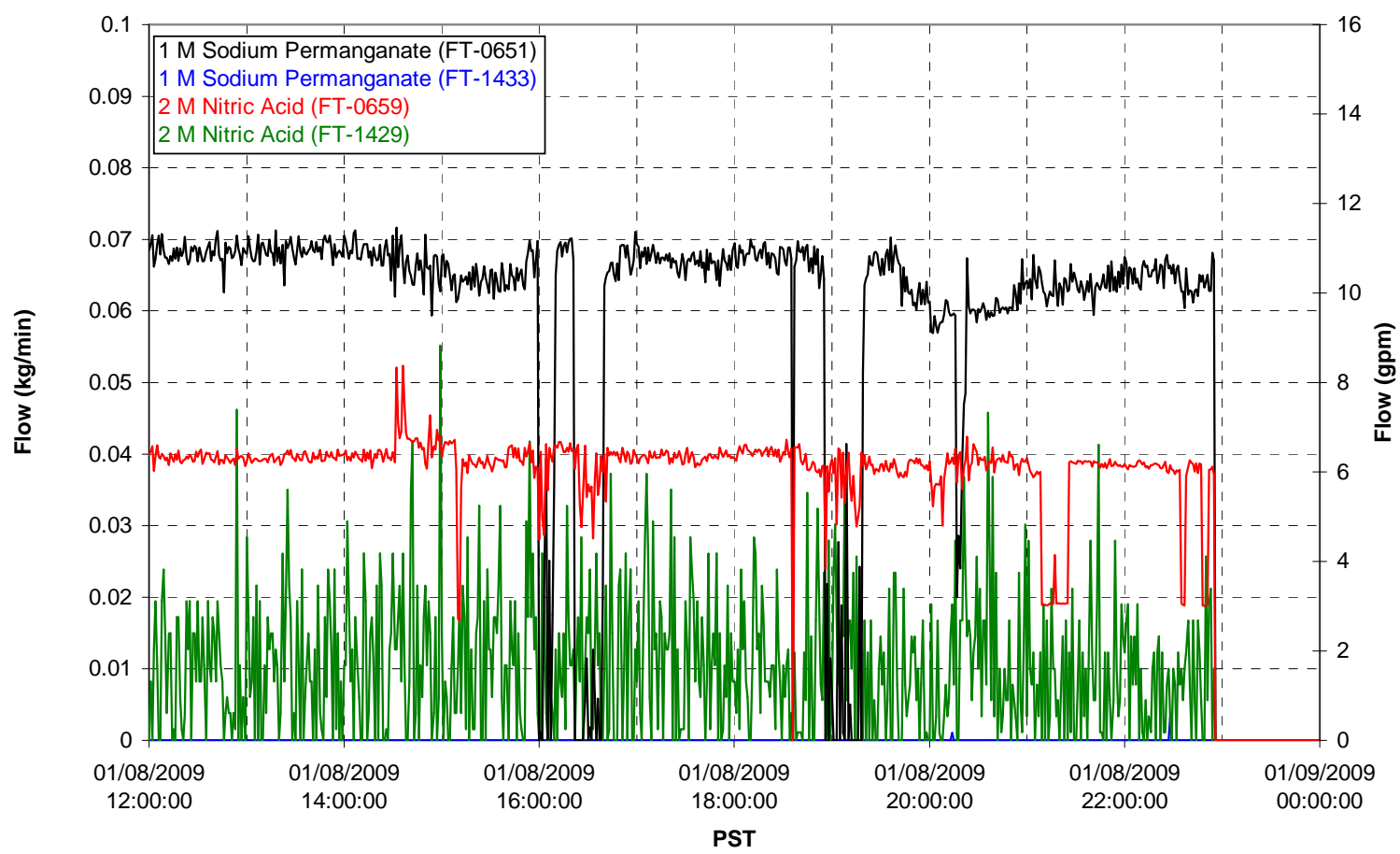

Air Flows

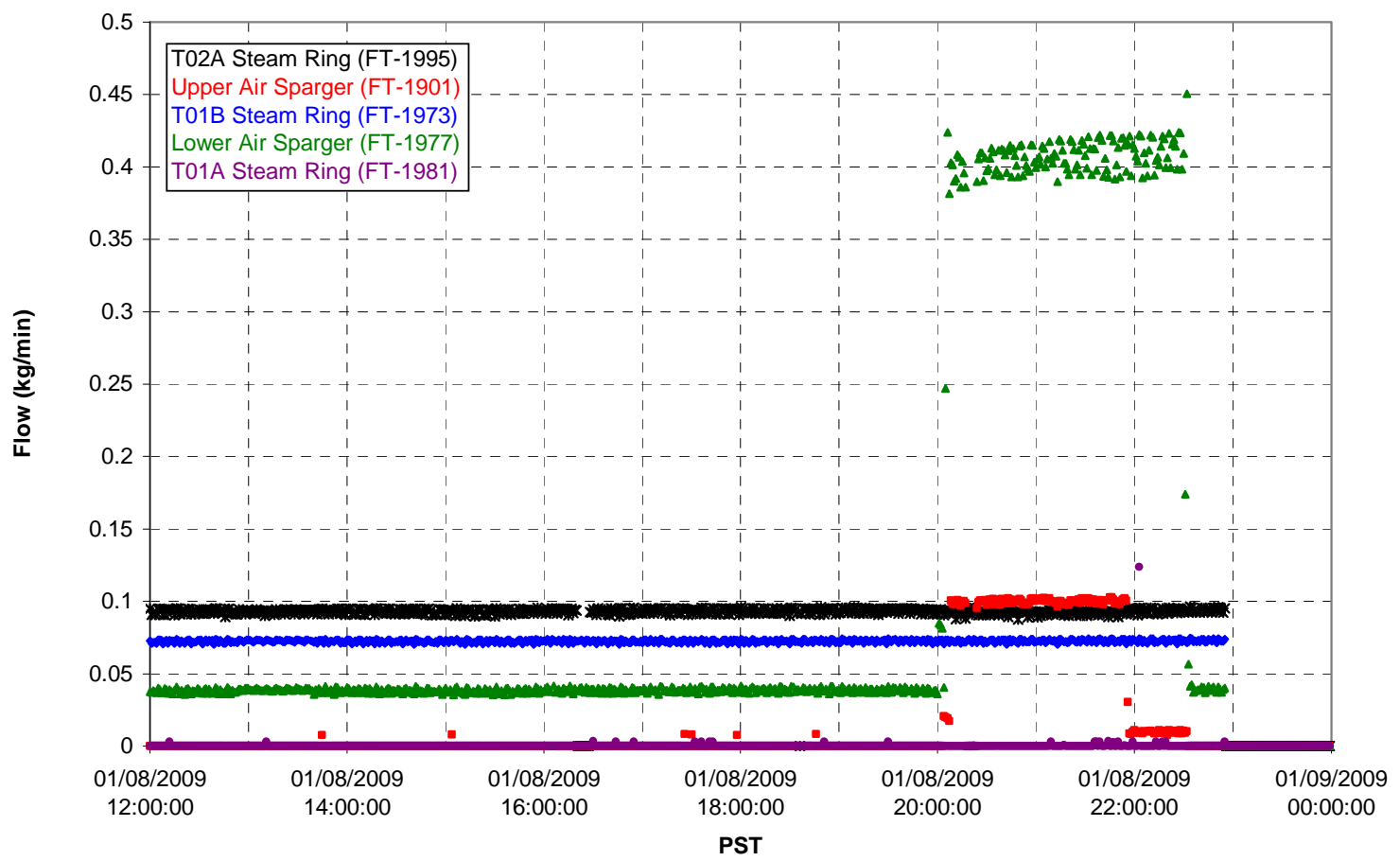


T02A Steam

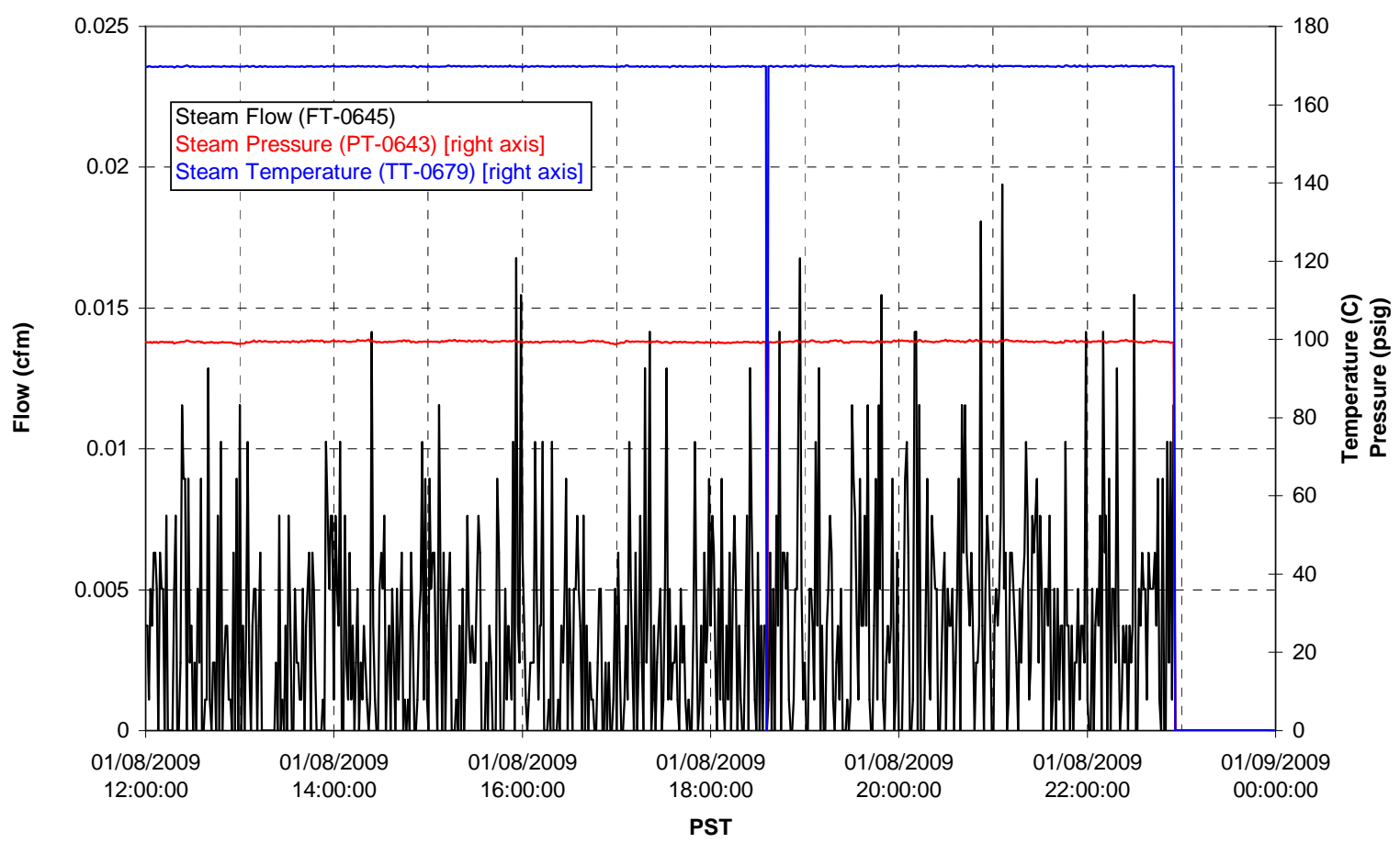

T01A Steam

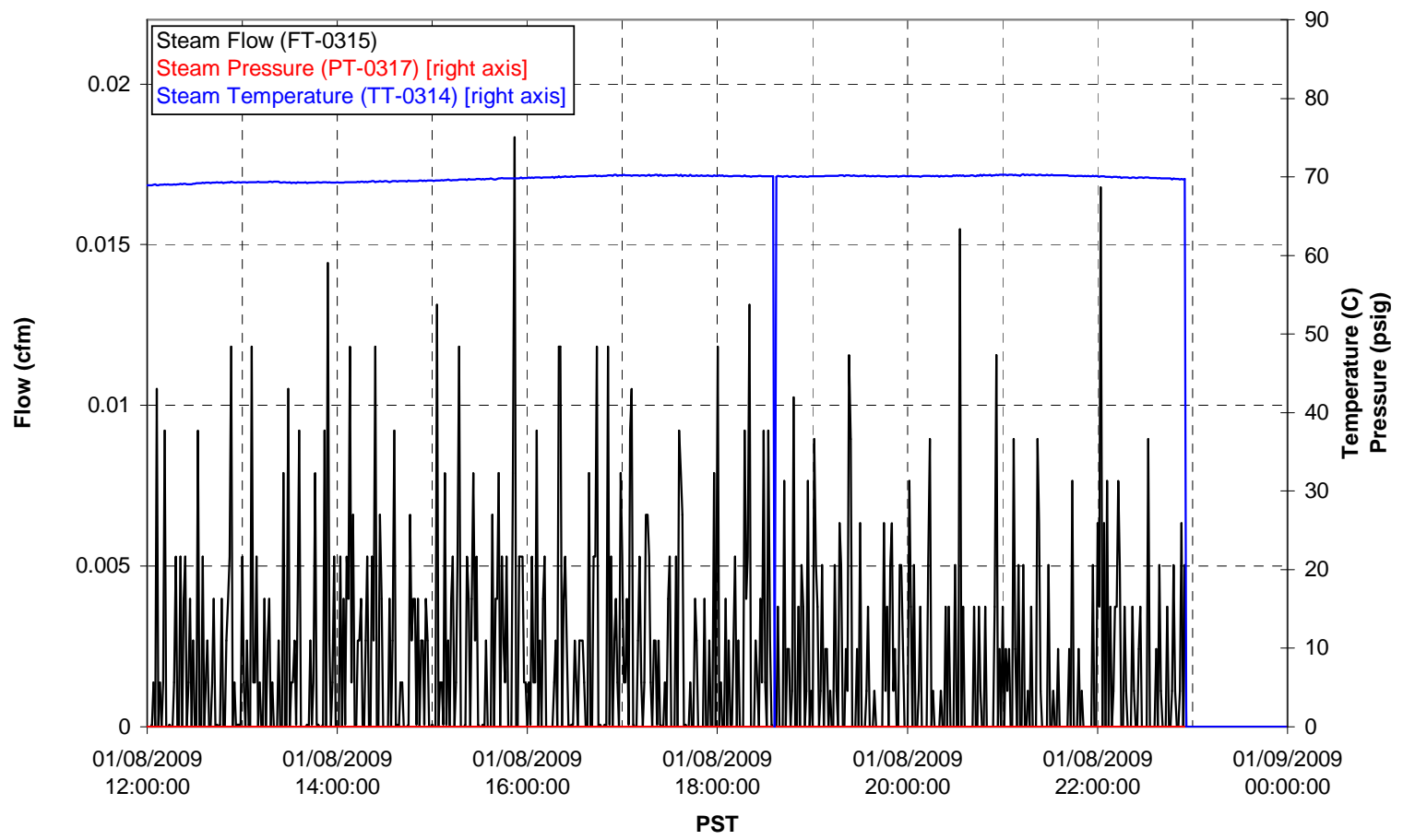


T01B Steam

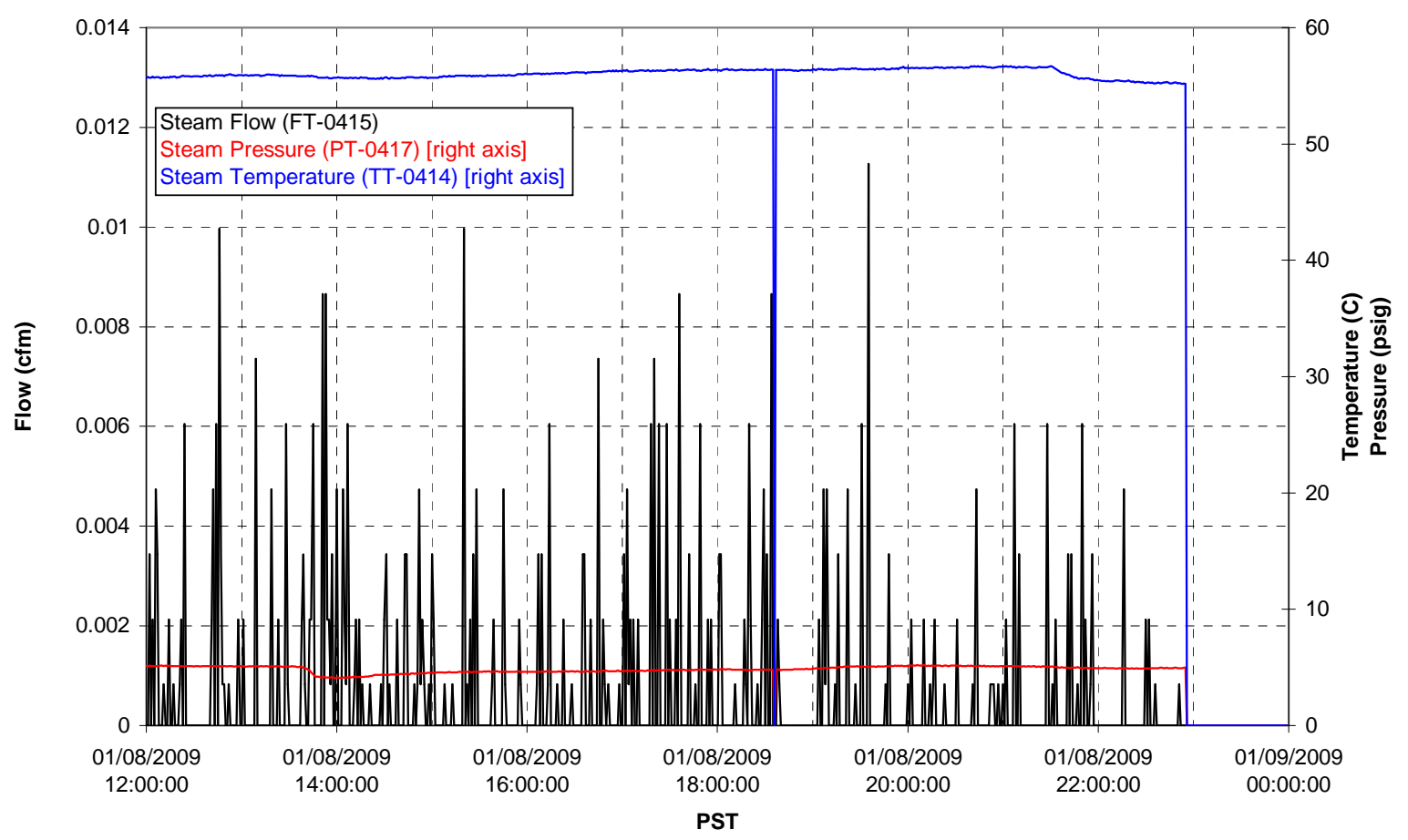


Functional Test Data Plots

01/09/2009 00:00 - 01/09/2009 12:00 
T01A level

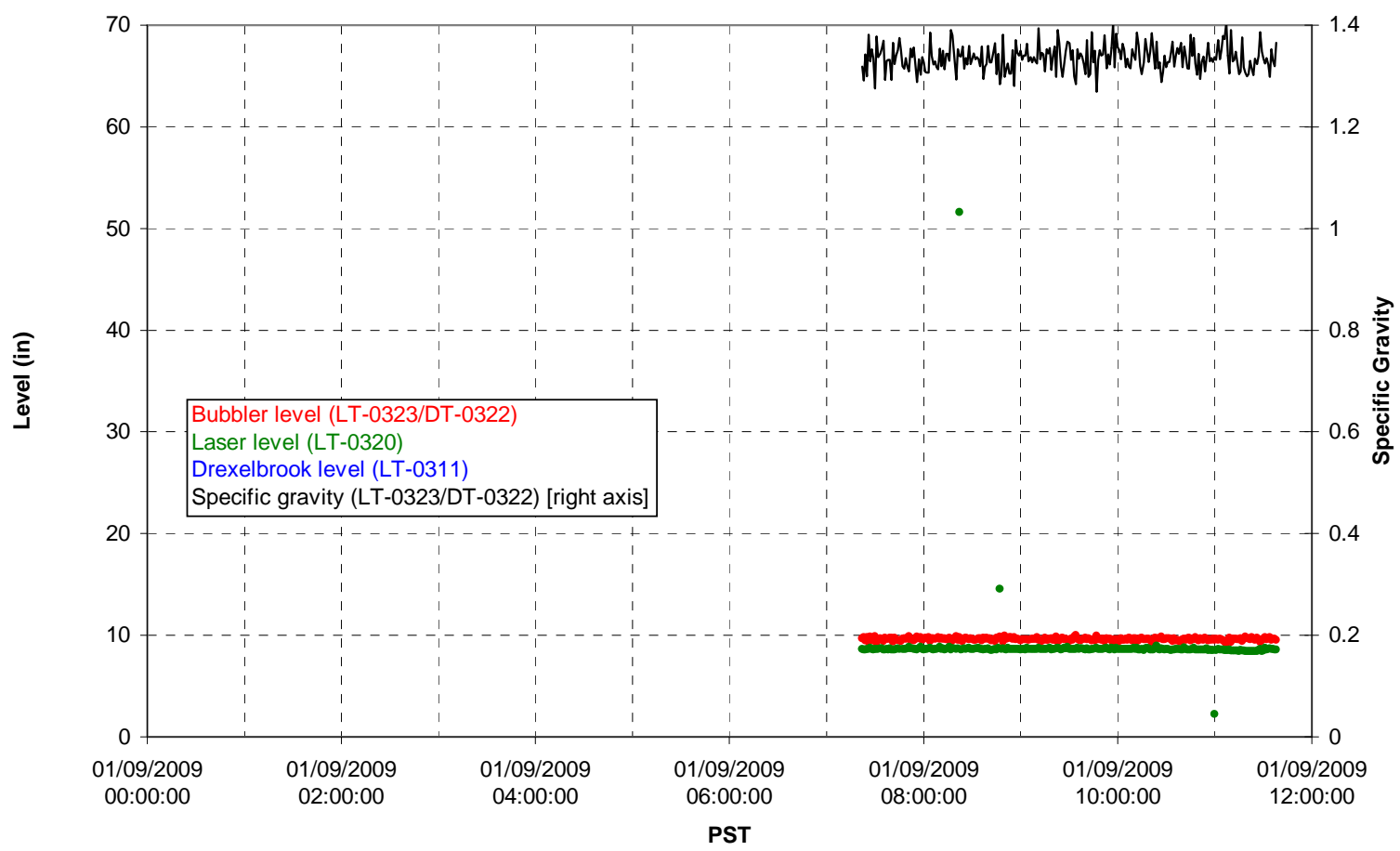

T01A temperatures

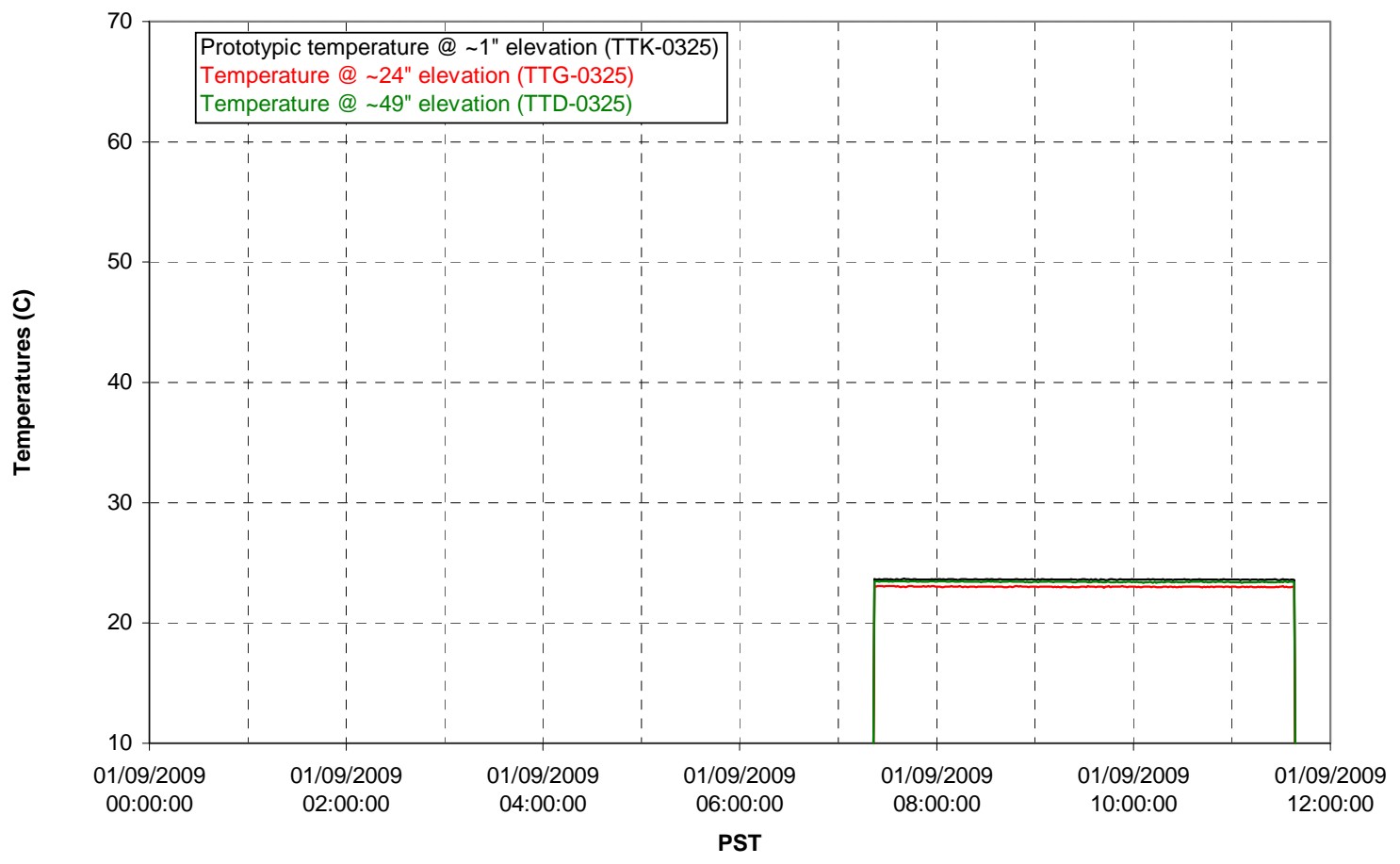


T01B level

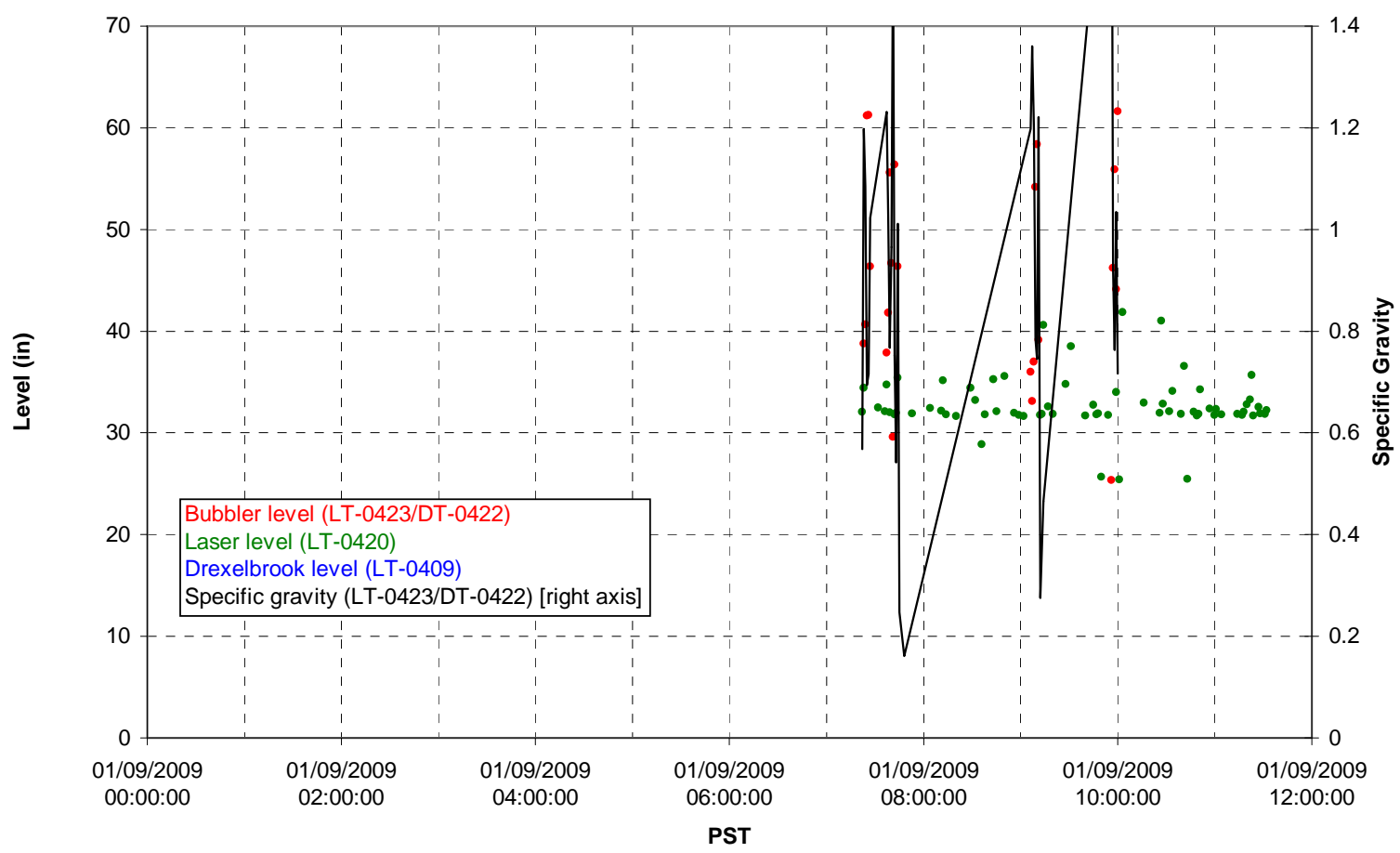

T01B temperatures

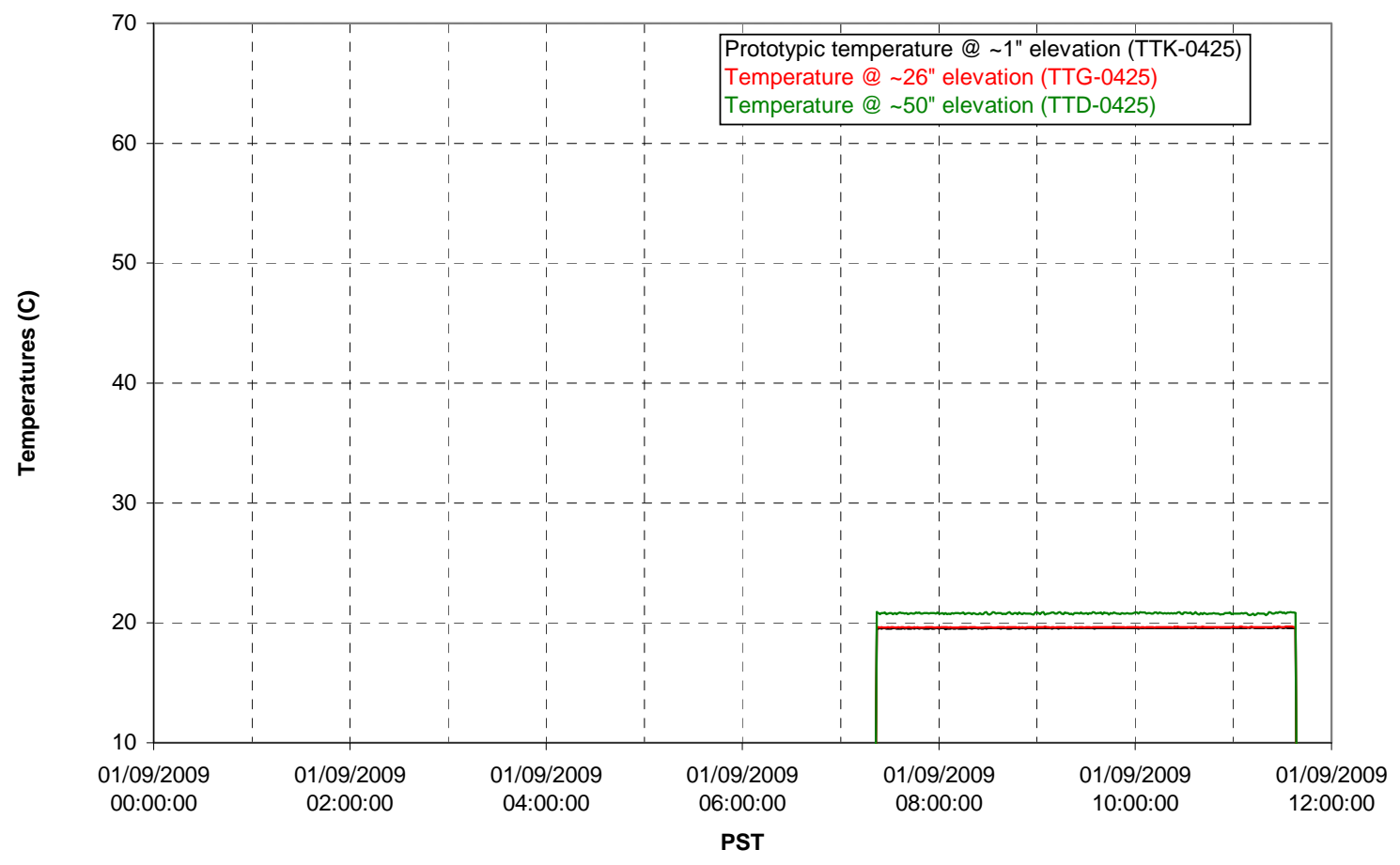


T02A level

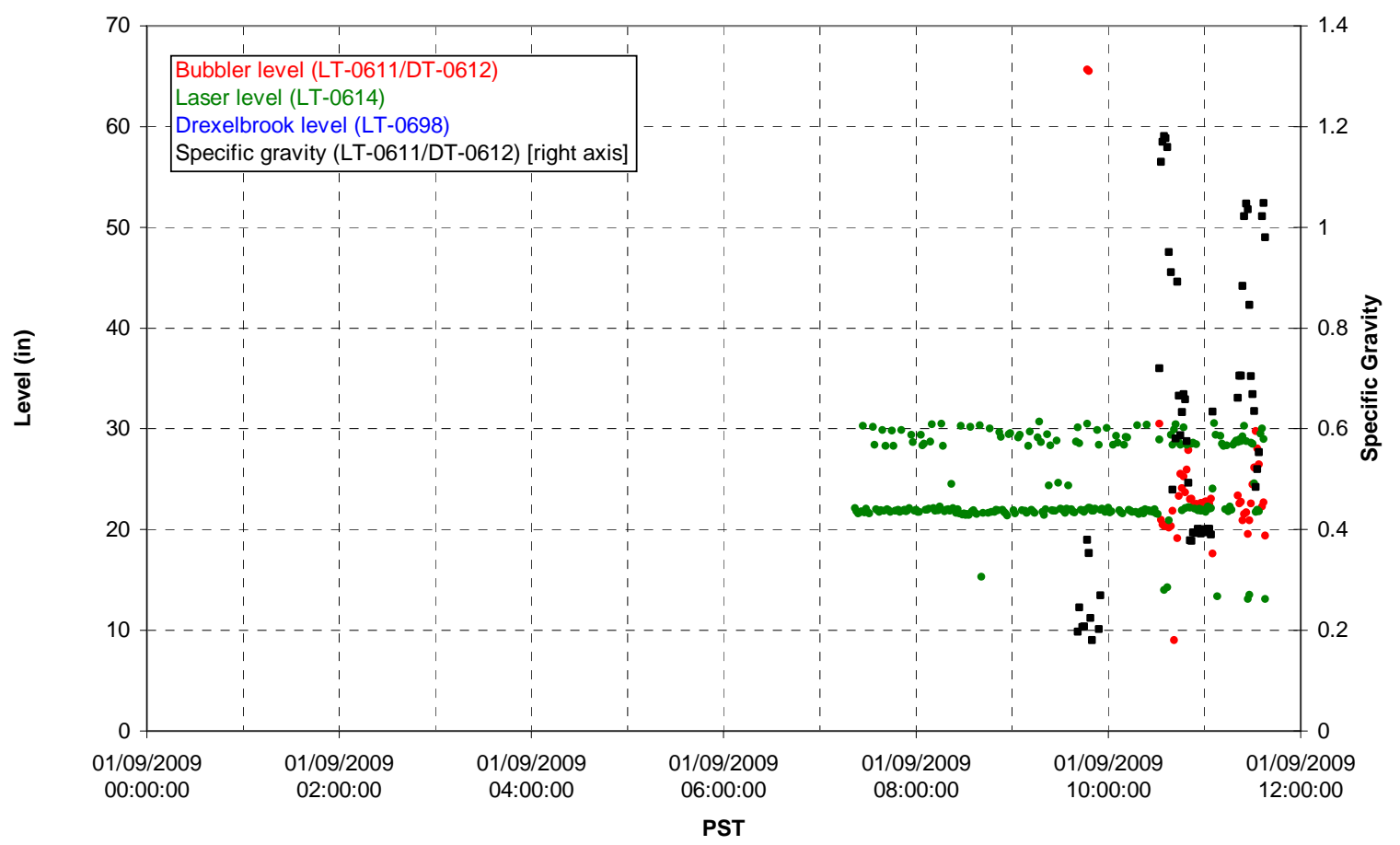

T02A temperatures

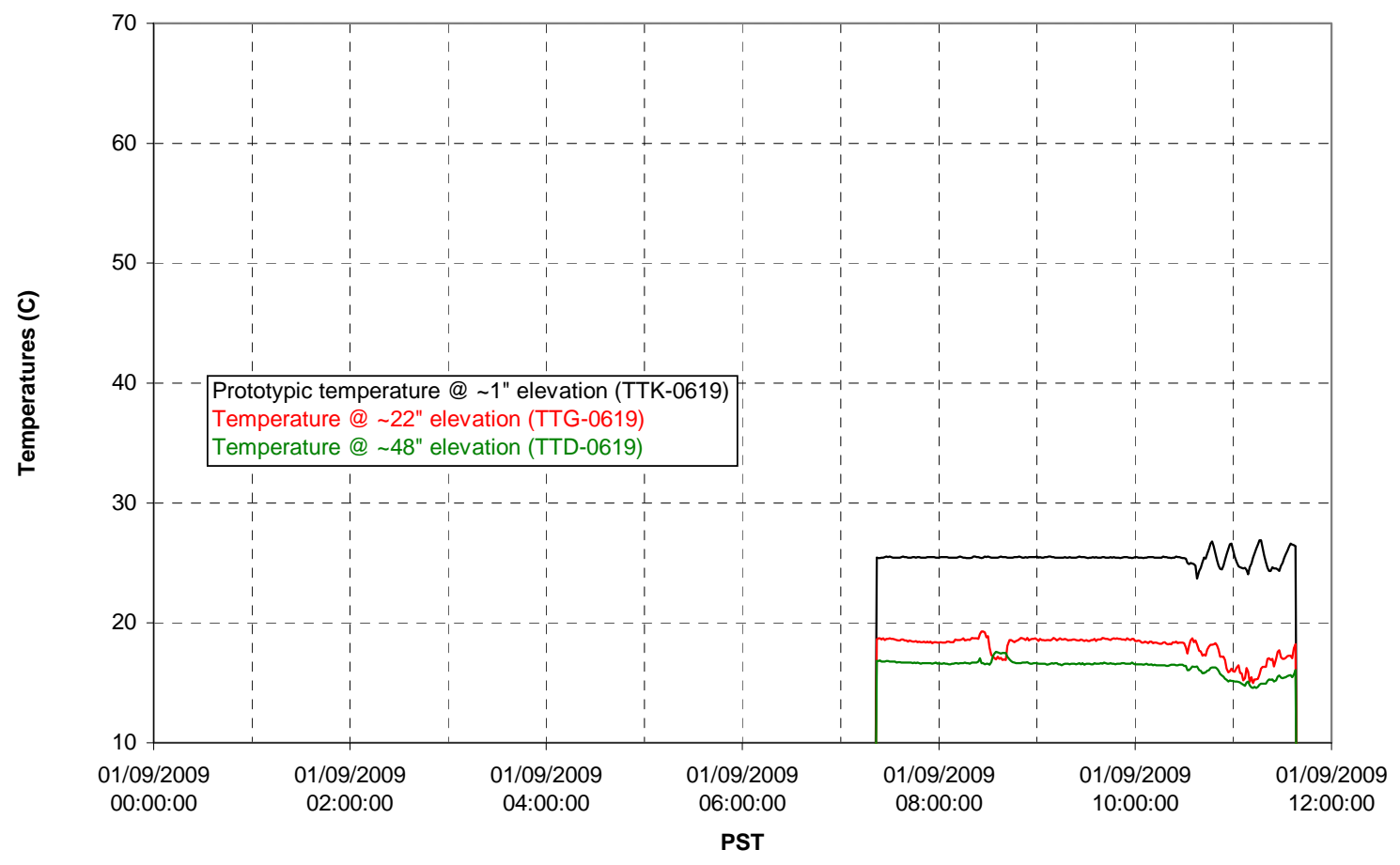


T02A and filter loop temperatures

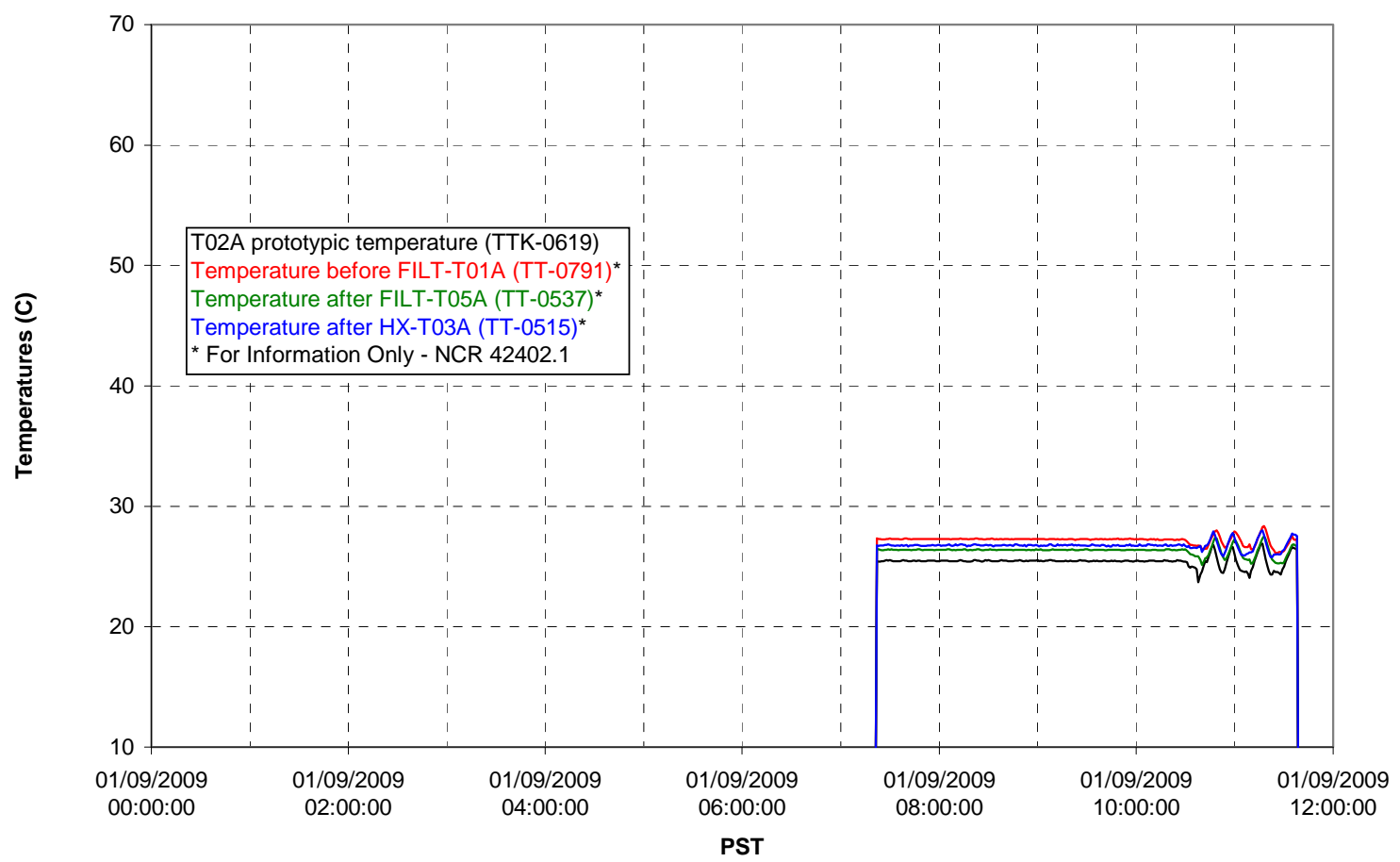

Pump Pressures and Flow

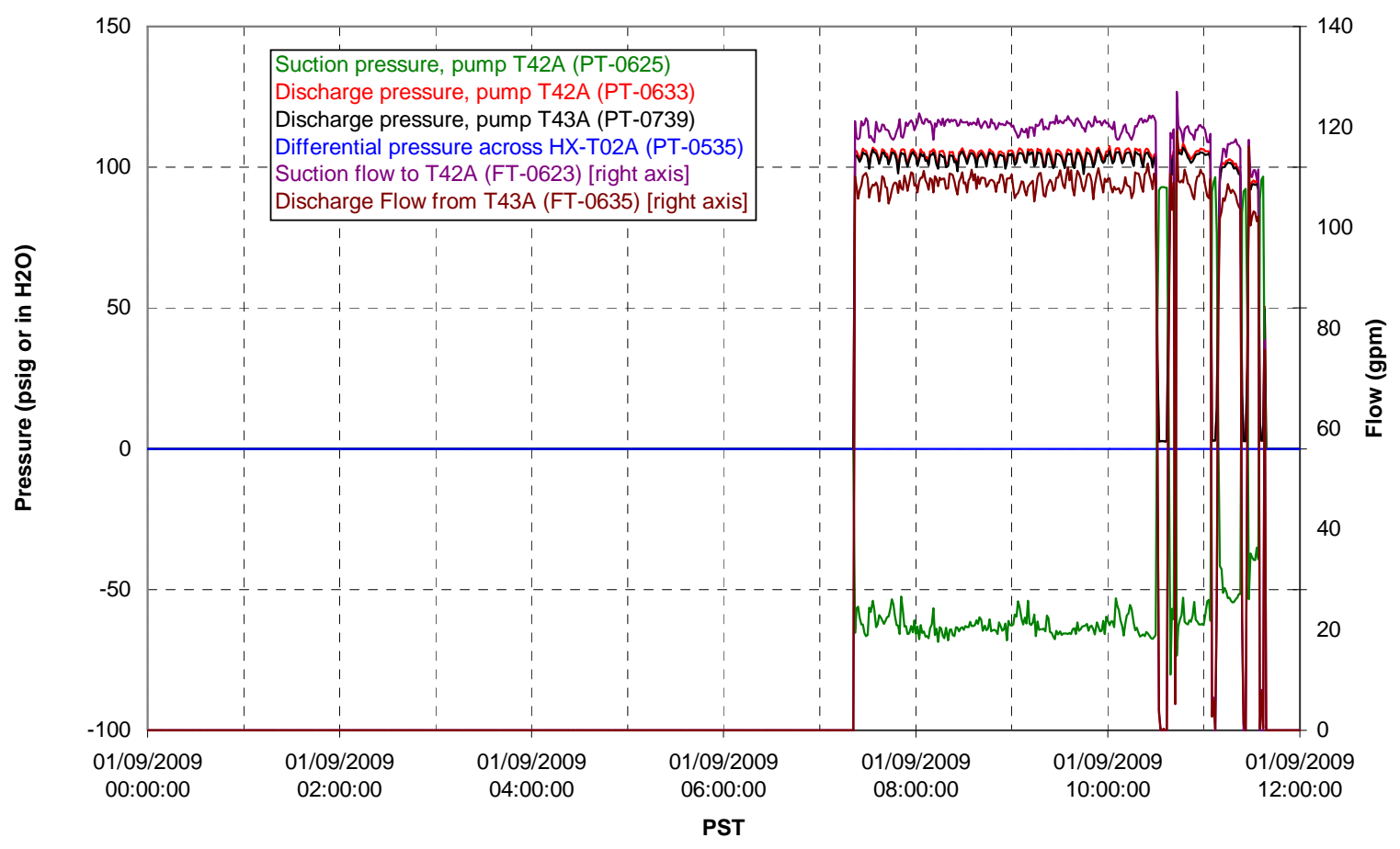




\section{Axial pressure drop}

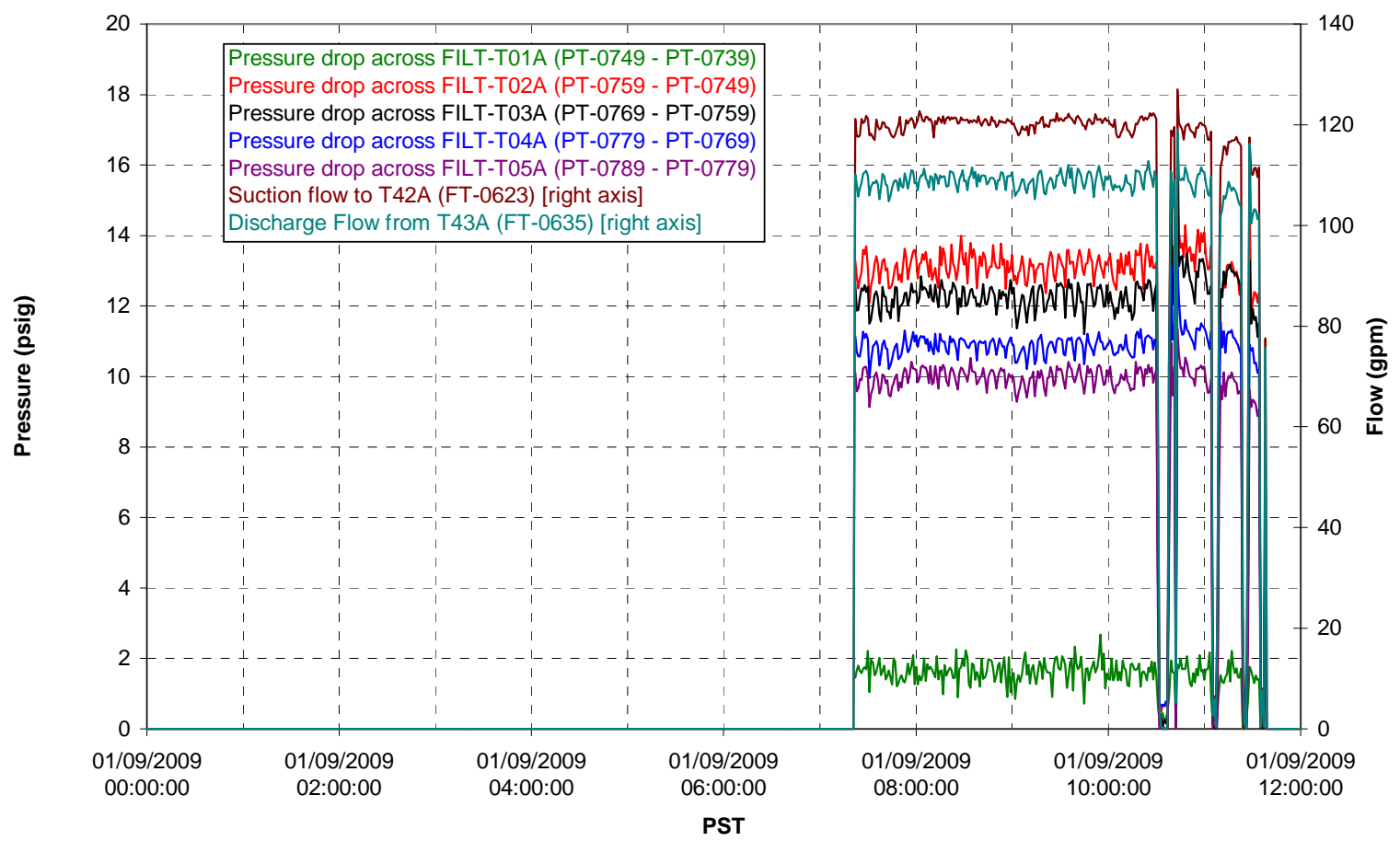

Permeate flow rates

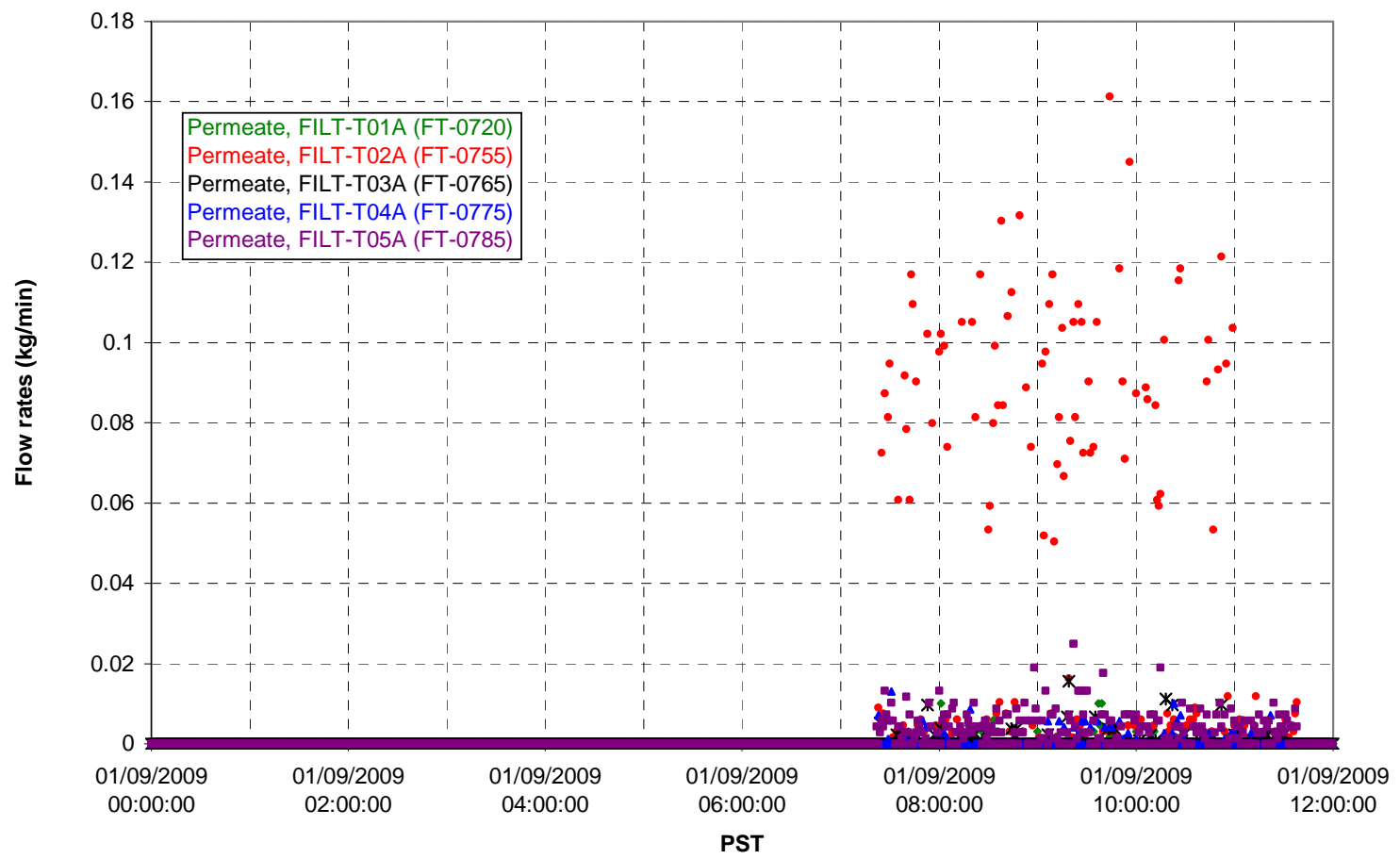


T02A Inner Temperature Tree

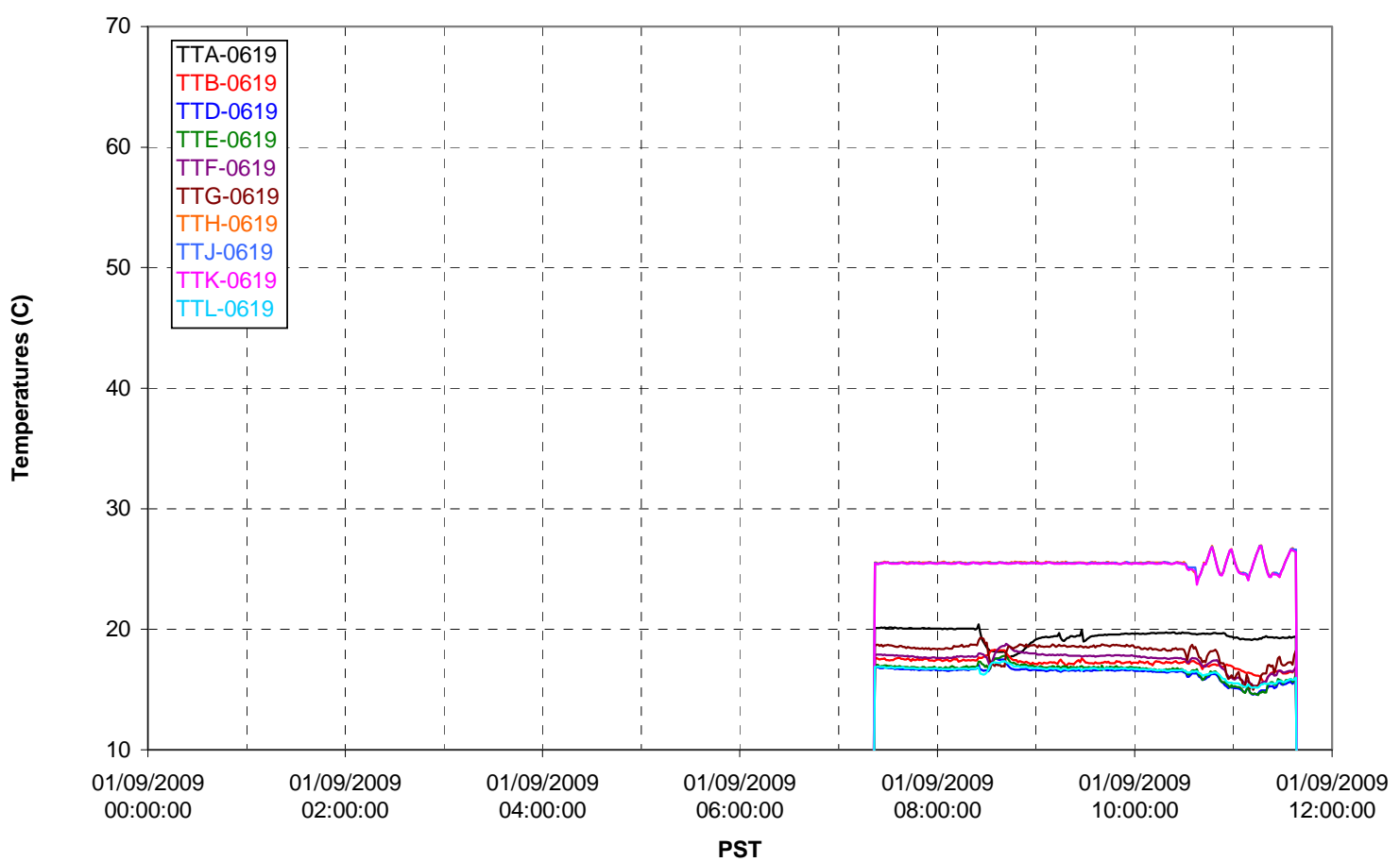

T02A Outer Temperature Tree

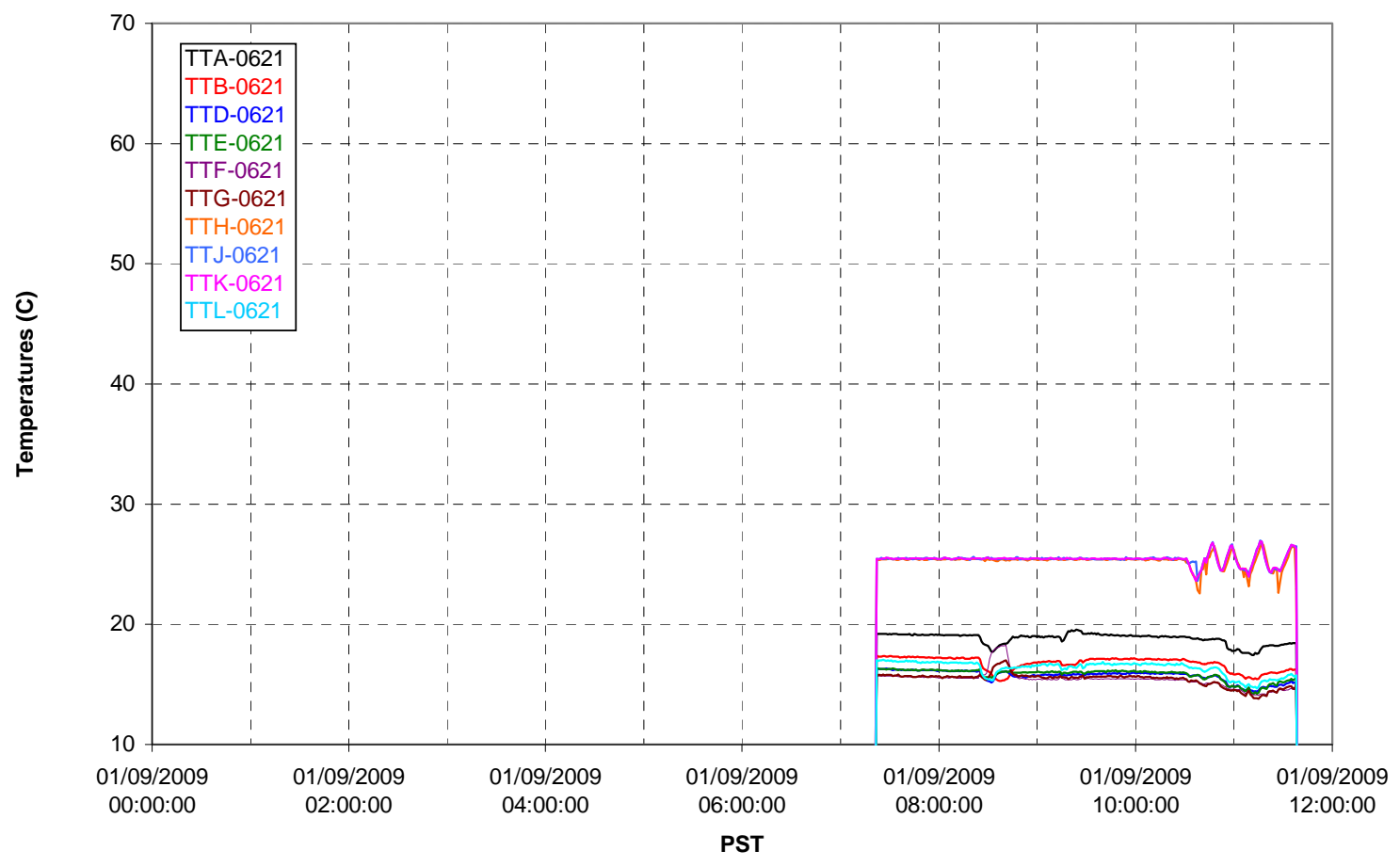


T02A temperatures

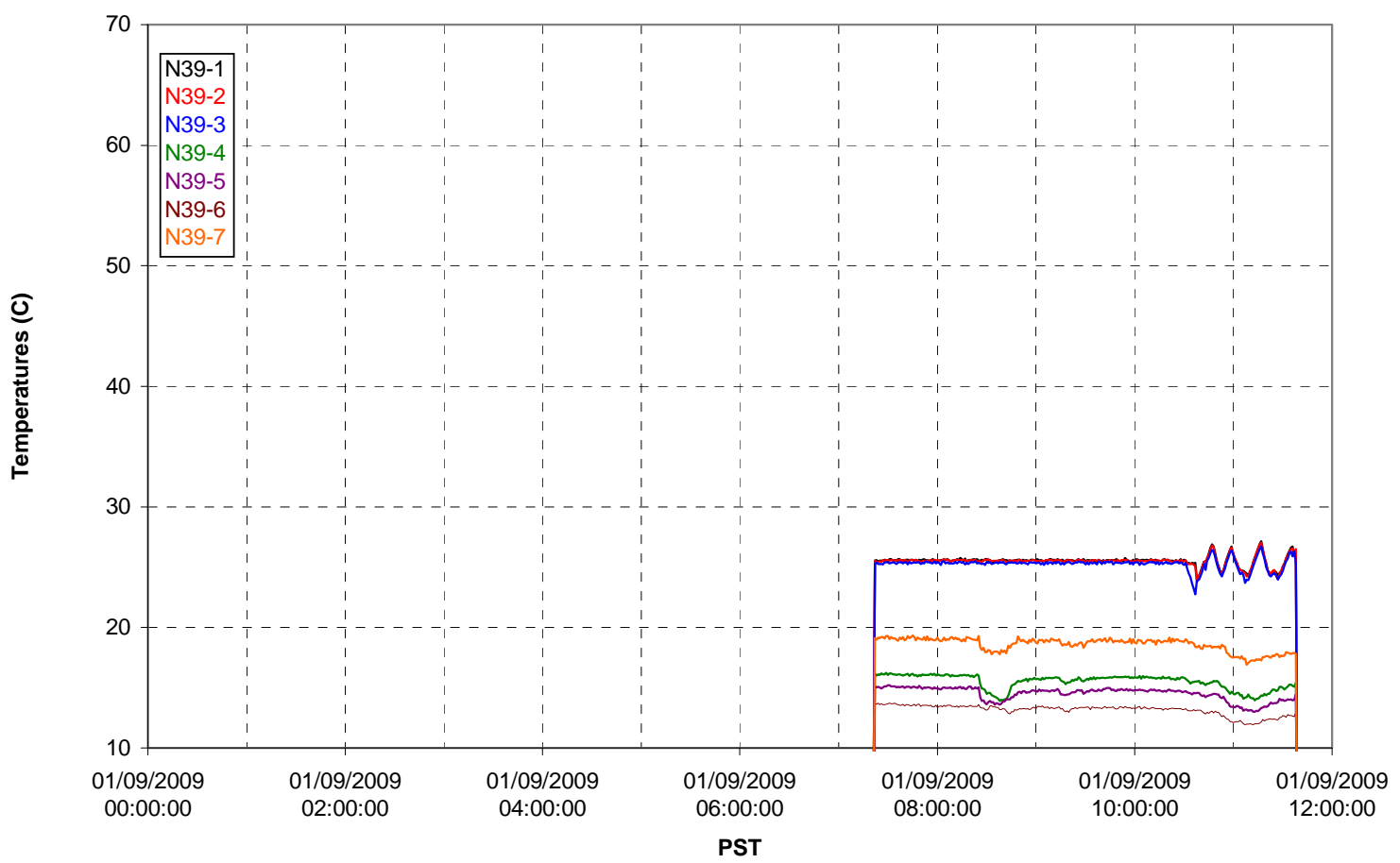

T02A temperatures

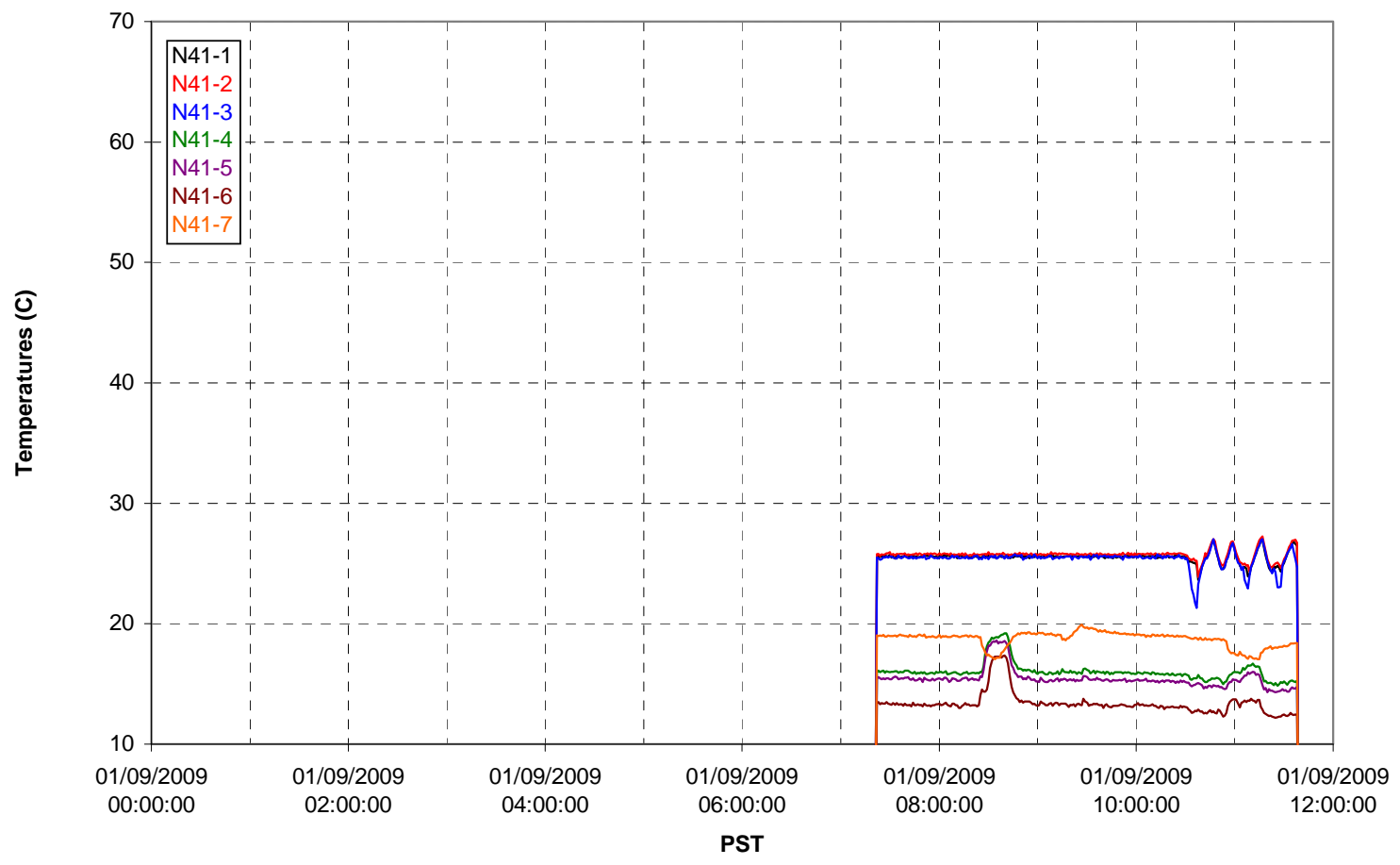


T02A temperatures

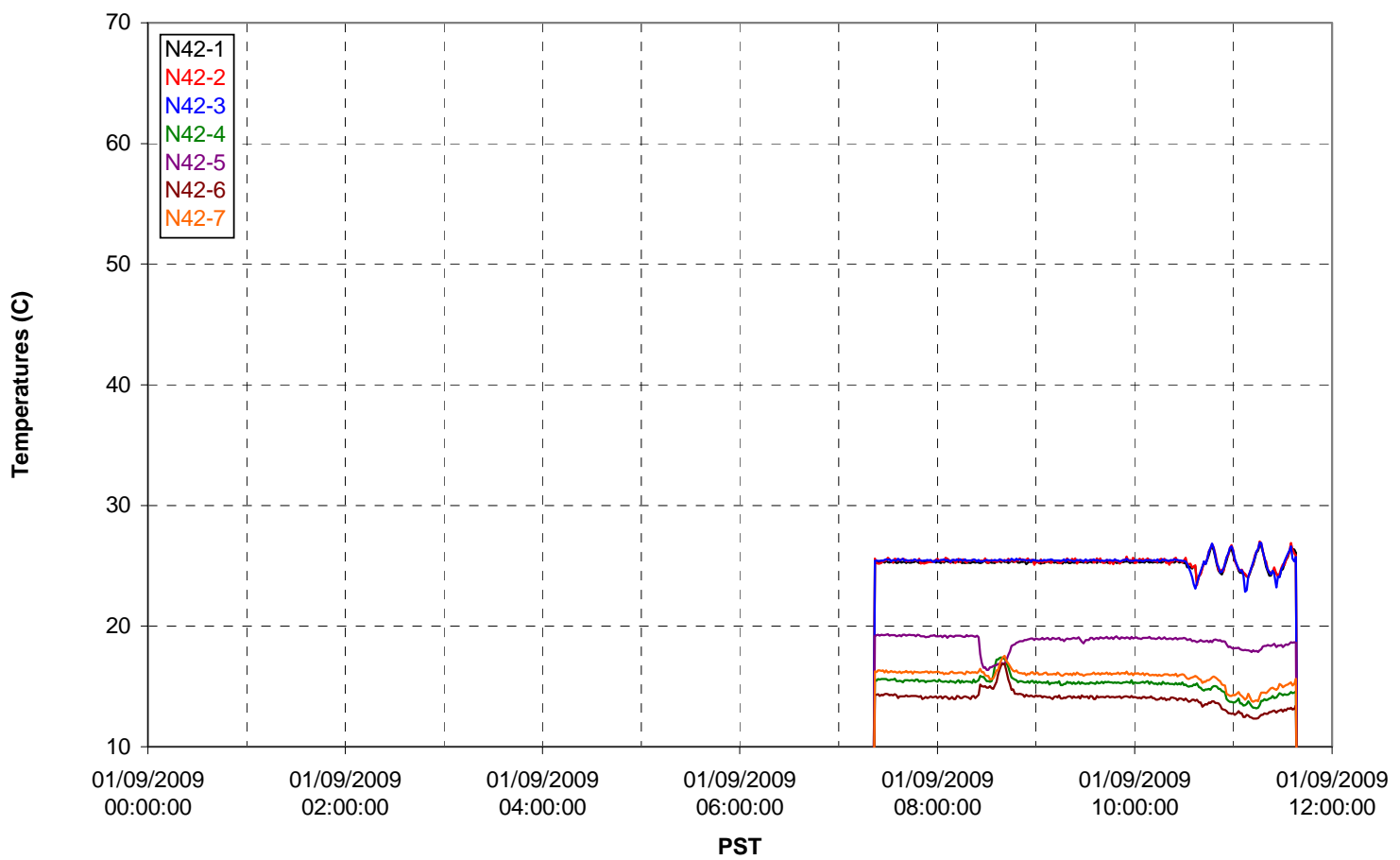

T02A temperatures

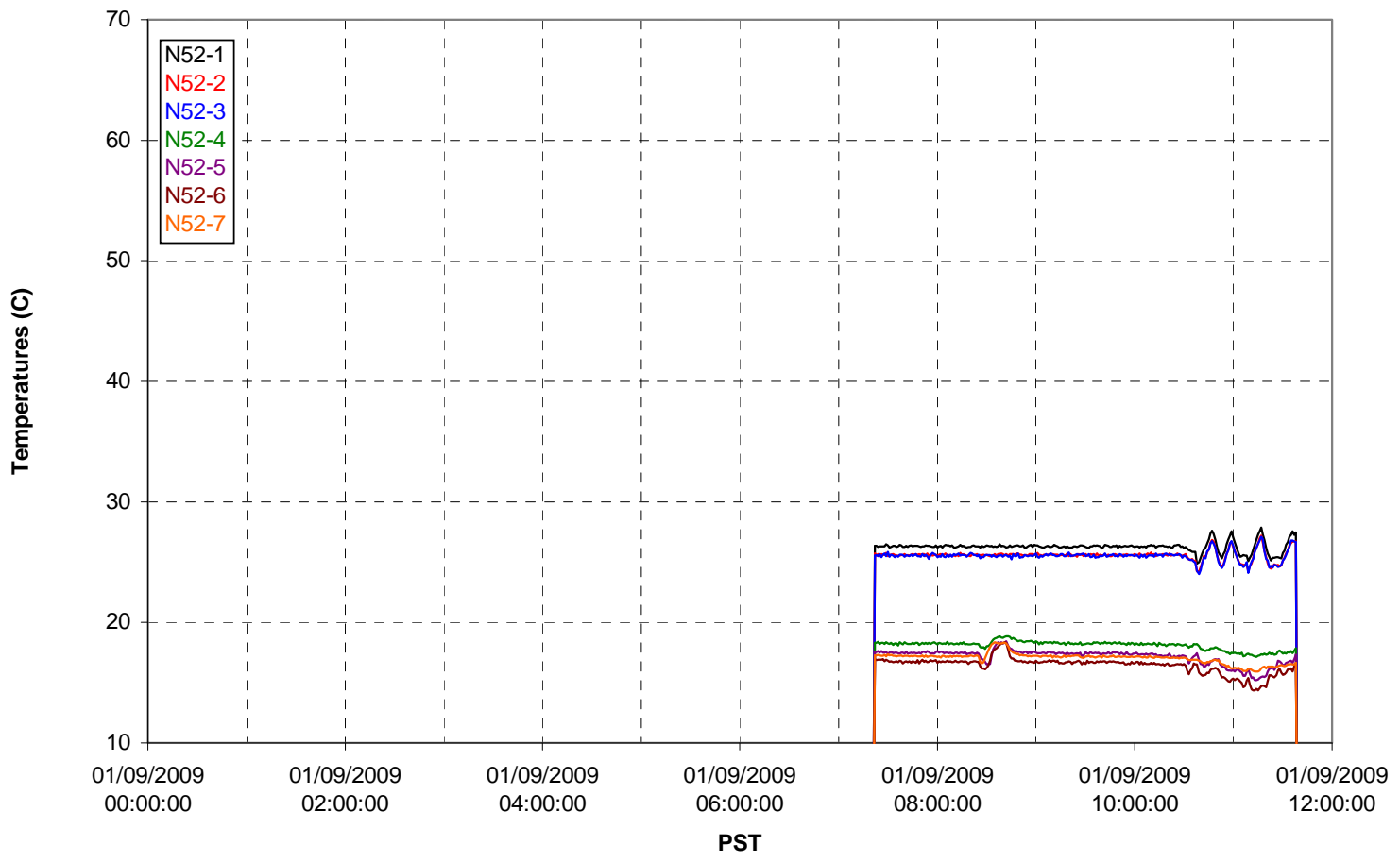


T02A Heating and Cooling

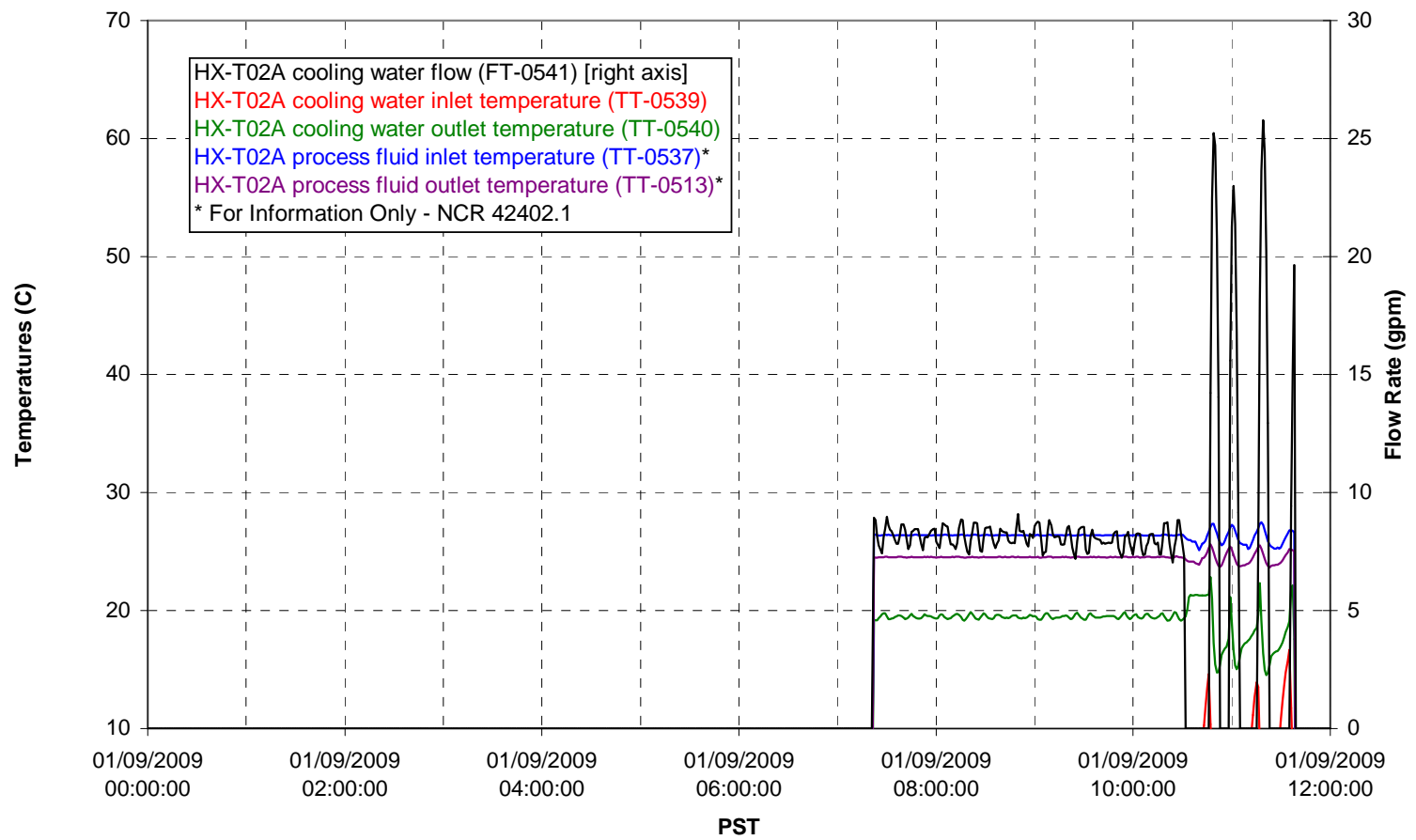

Pump Operation

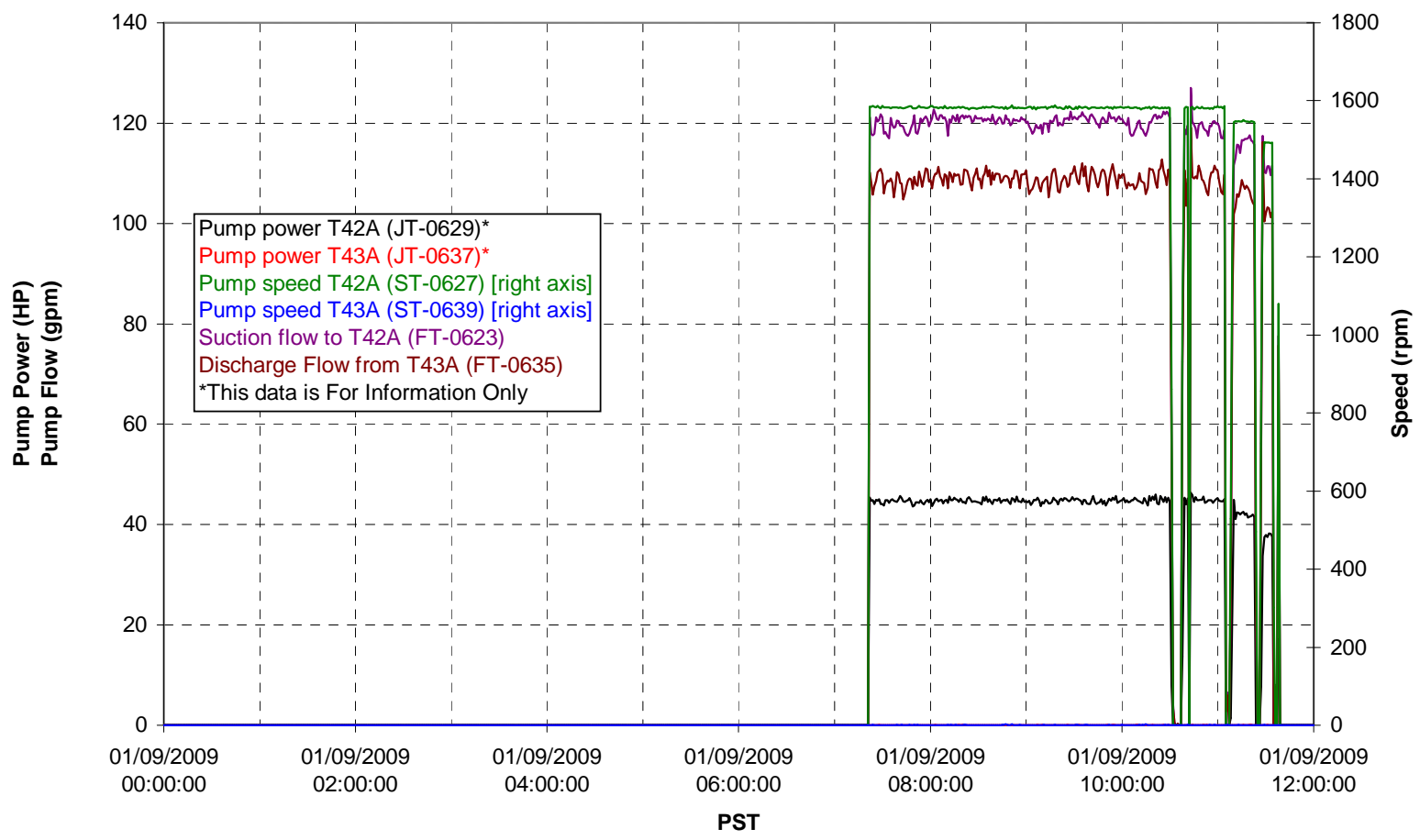


Pulsepot UFP-PP-T01A

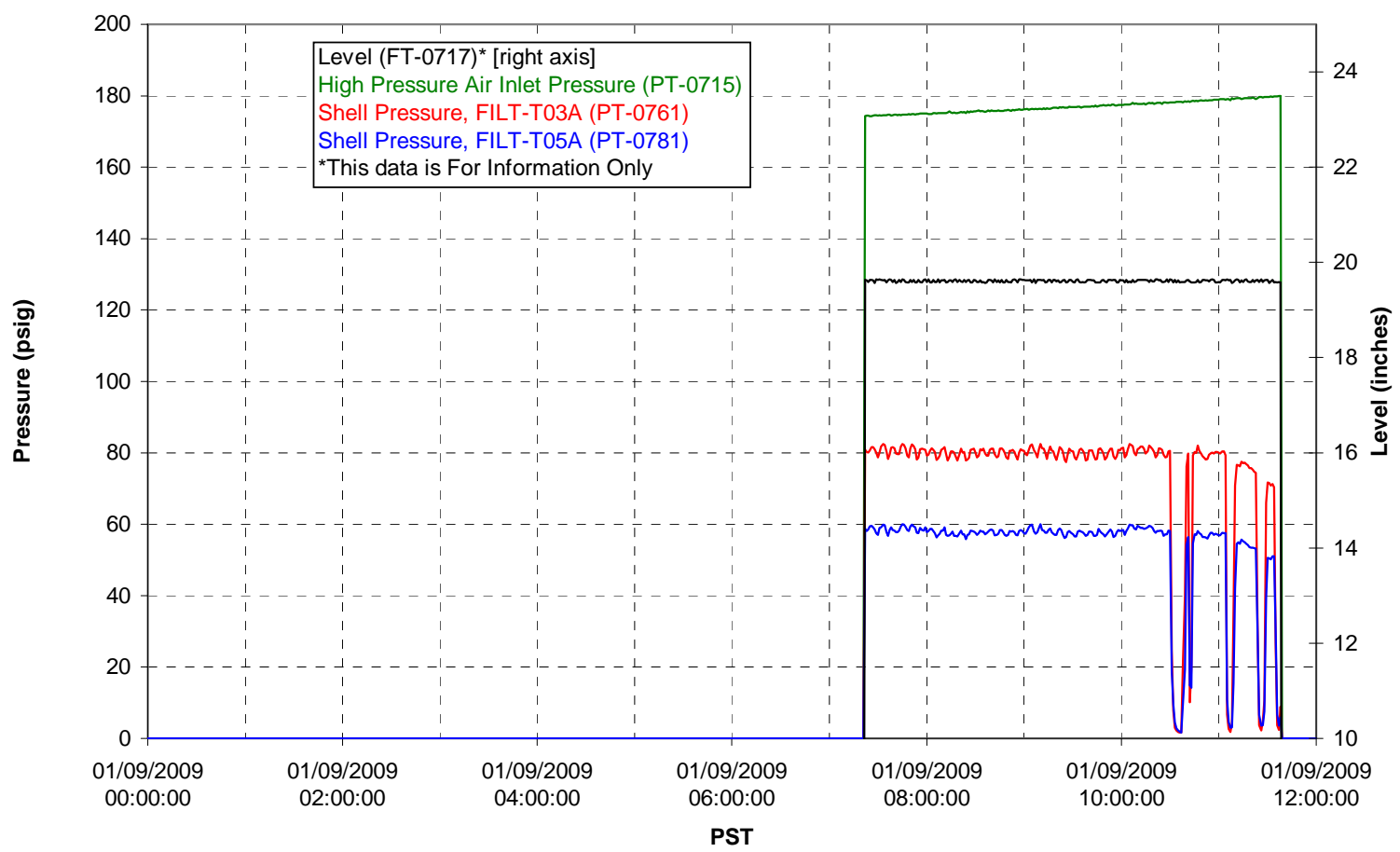

Pulsepot UFP-PP-T02A

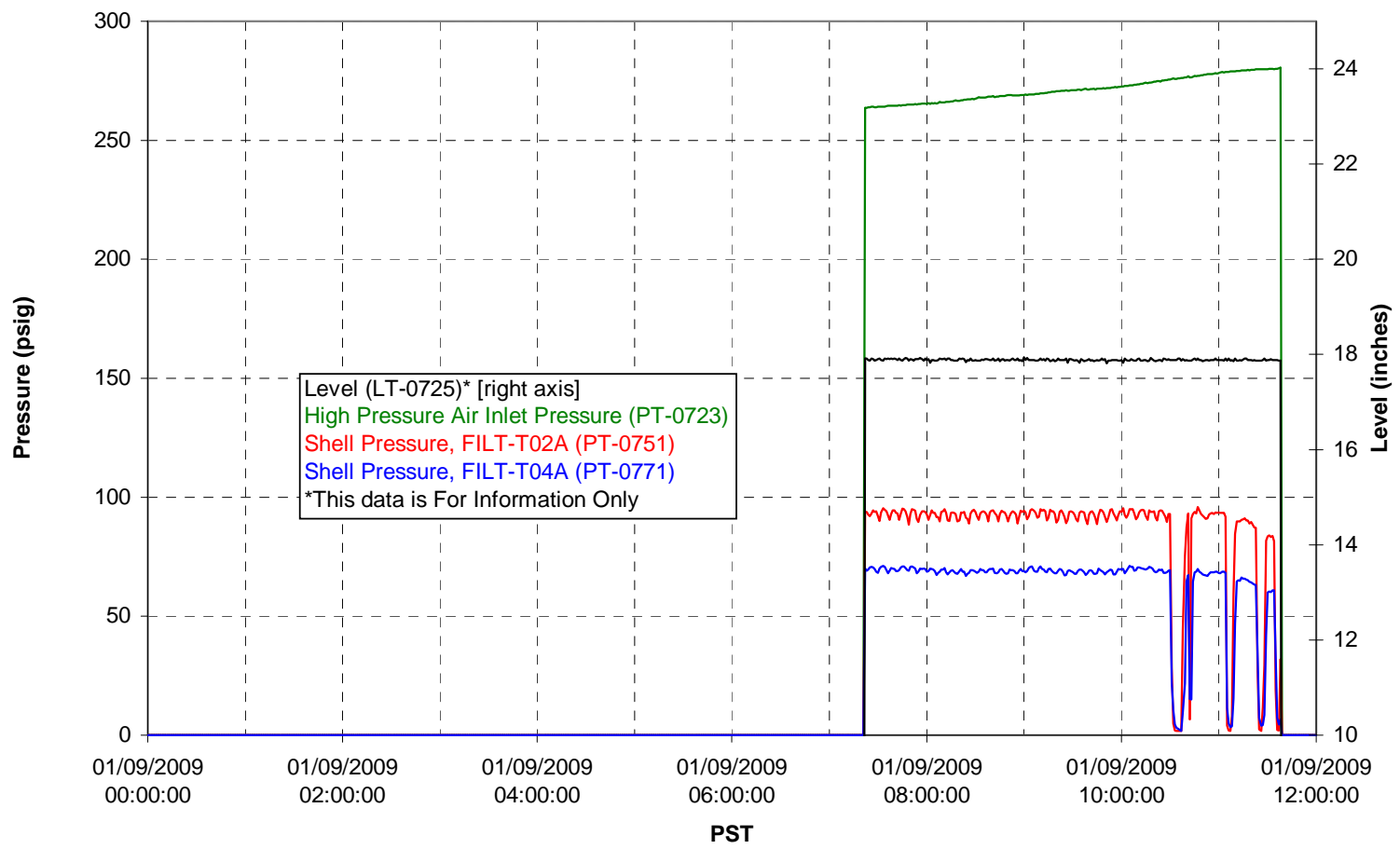


Pulsepot UFP-PP-T03A

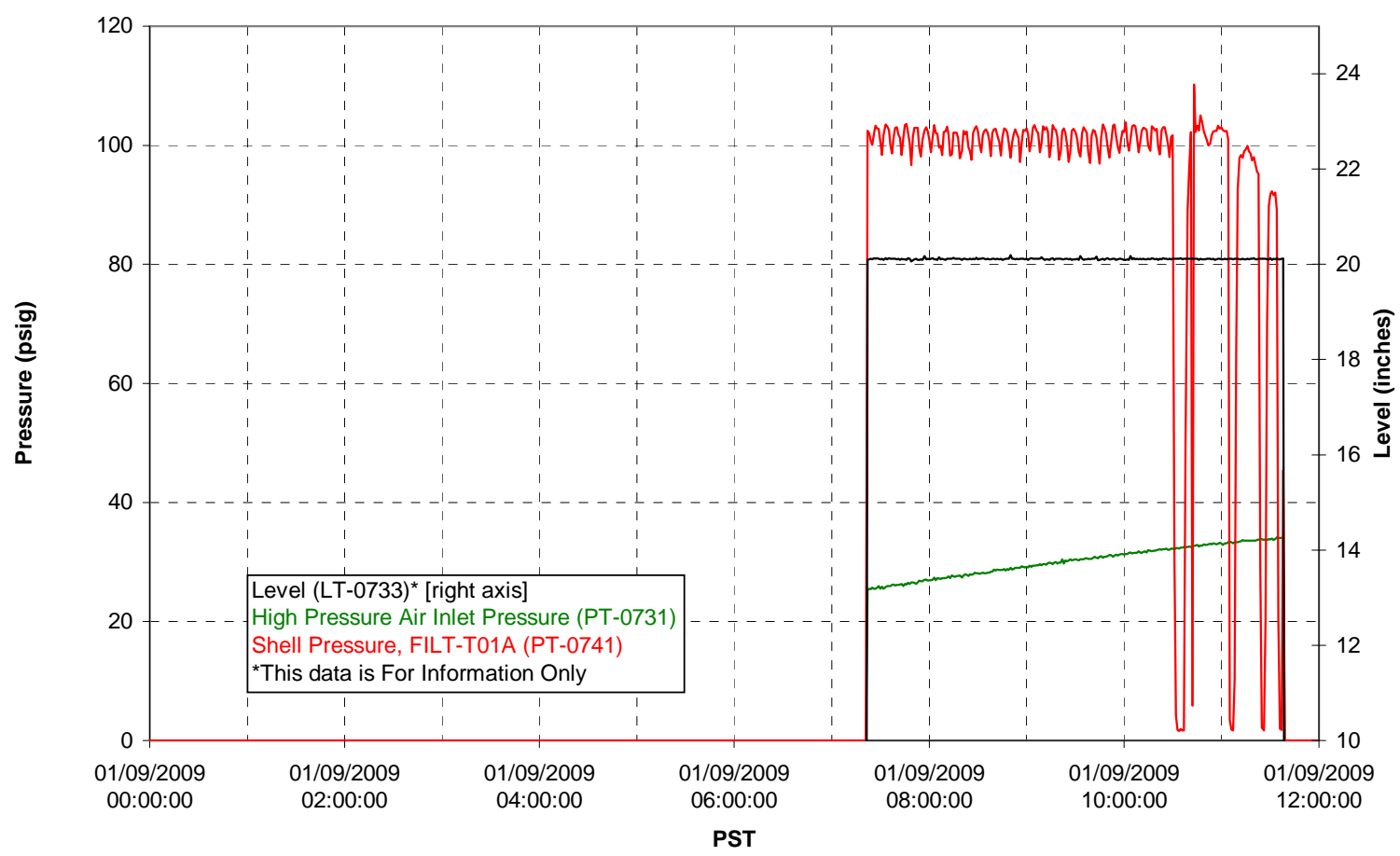

Pulsepot Levels

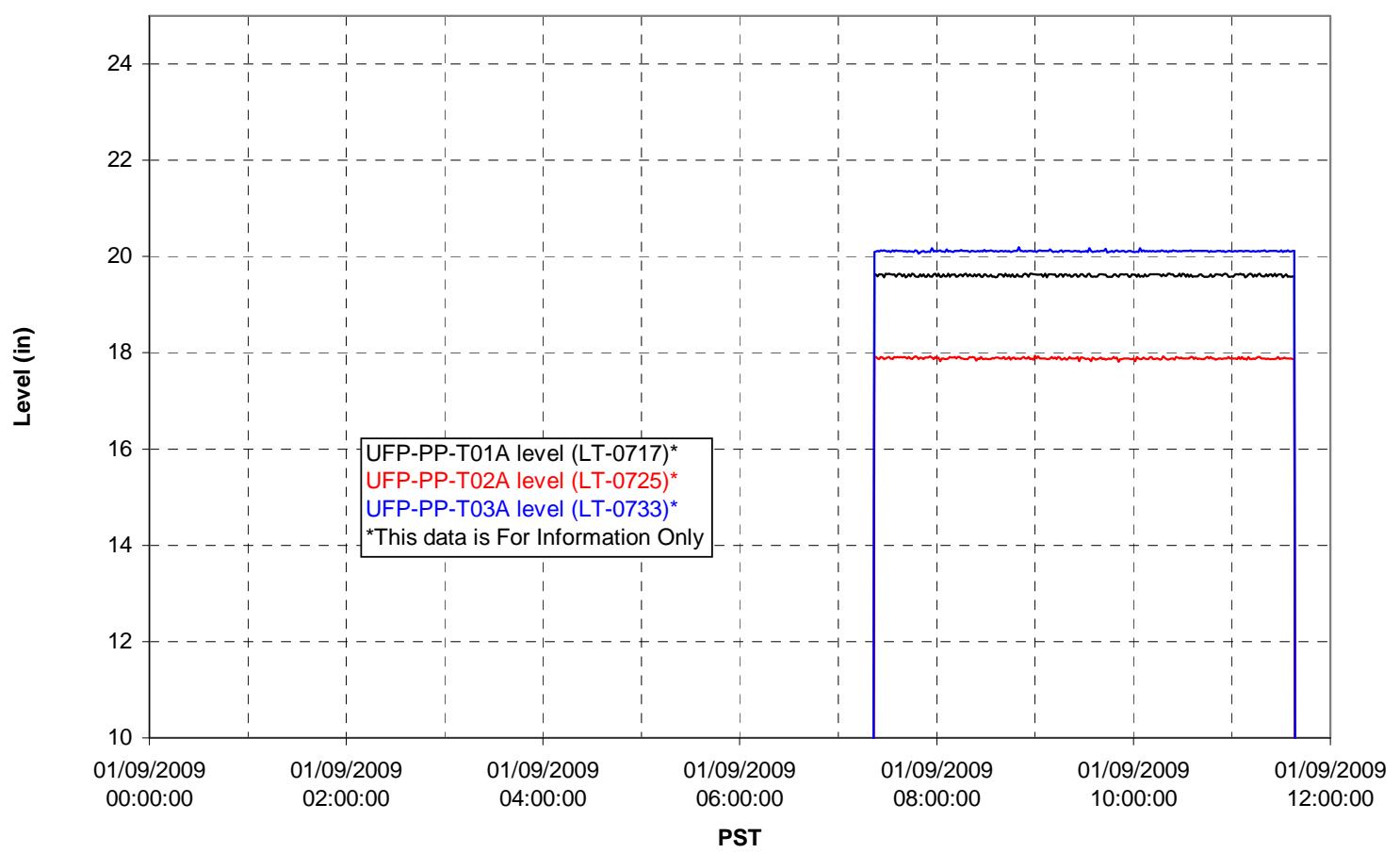


Filter UFP-FILT-T01A

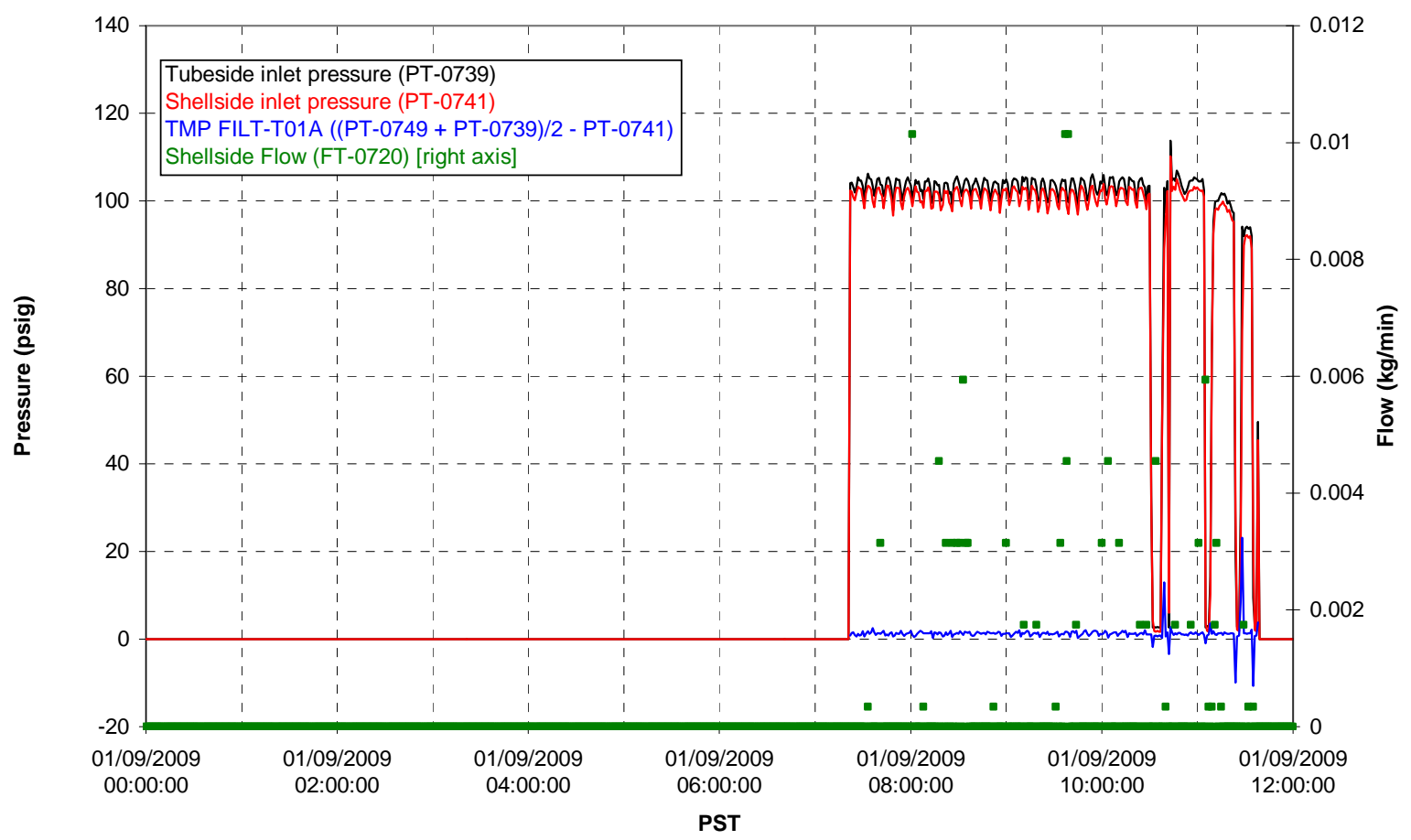

Filter UFP-FILT-T02A

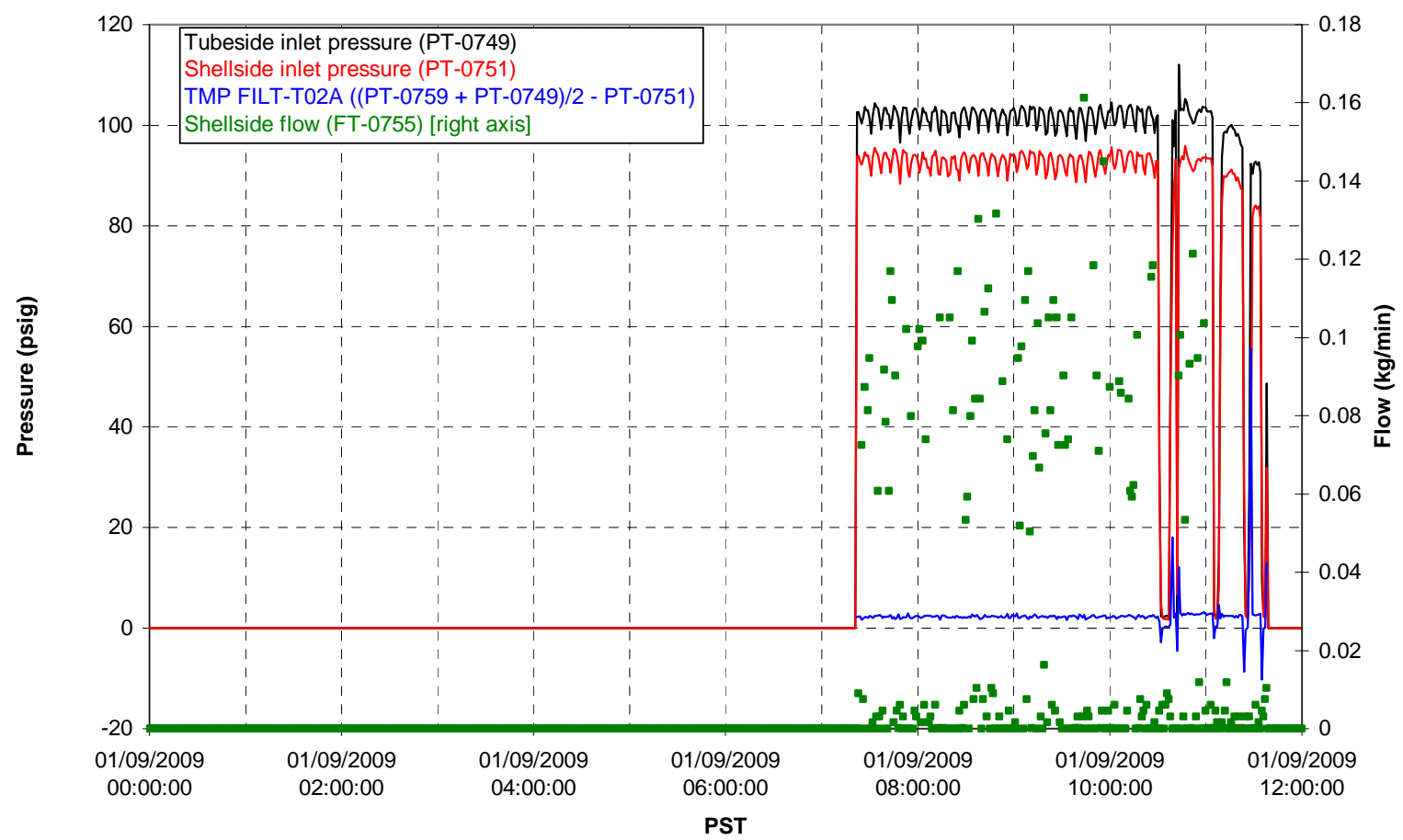


Filter UFP-FILT-T03A

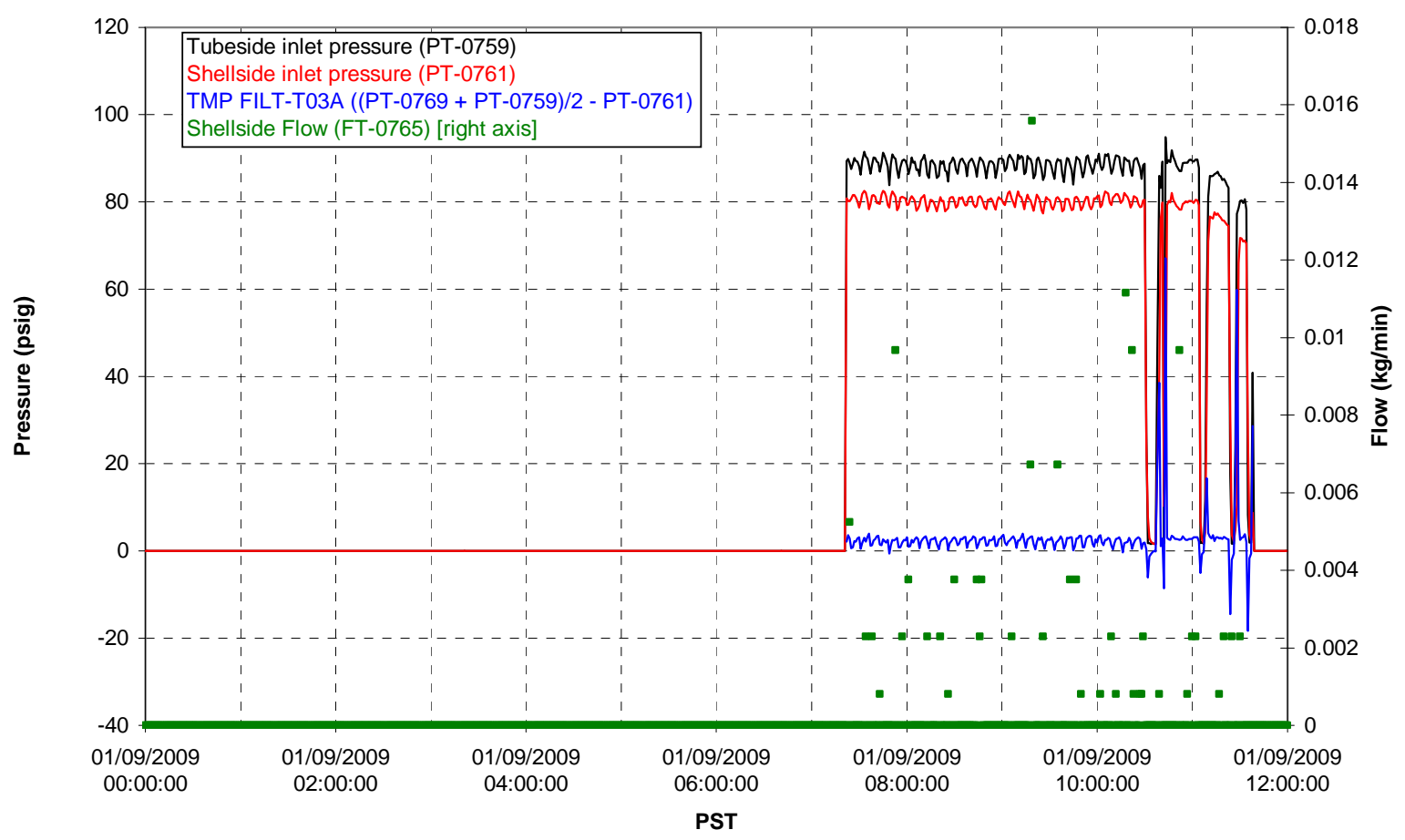

Filter UFP-FILT-T04A

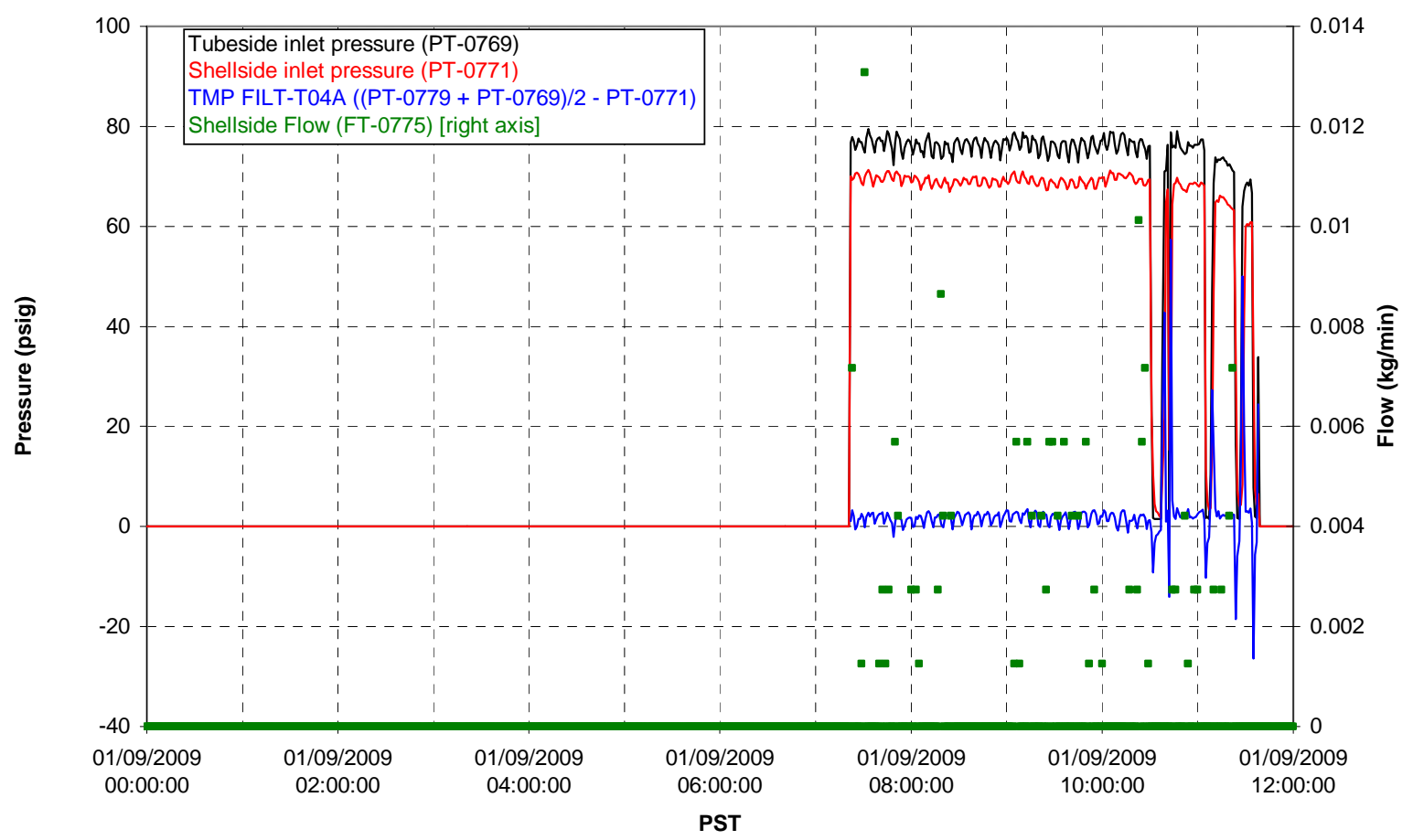


Filter UFP-FILT-T05A

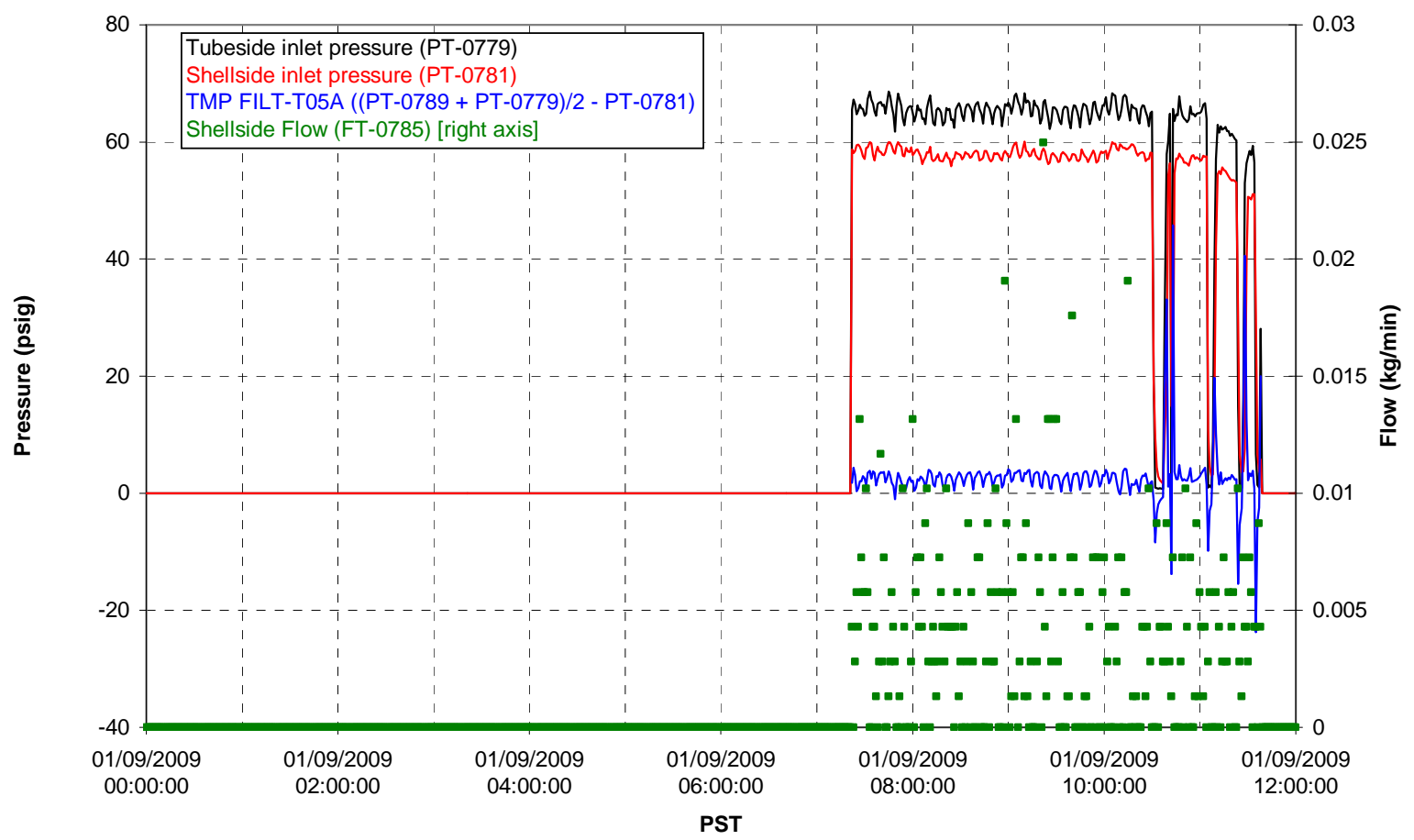

Chemical Flow

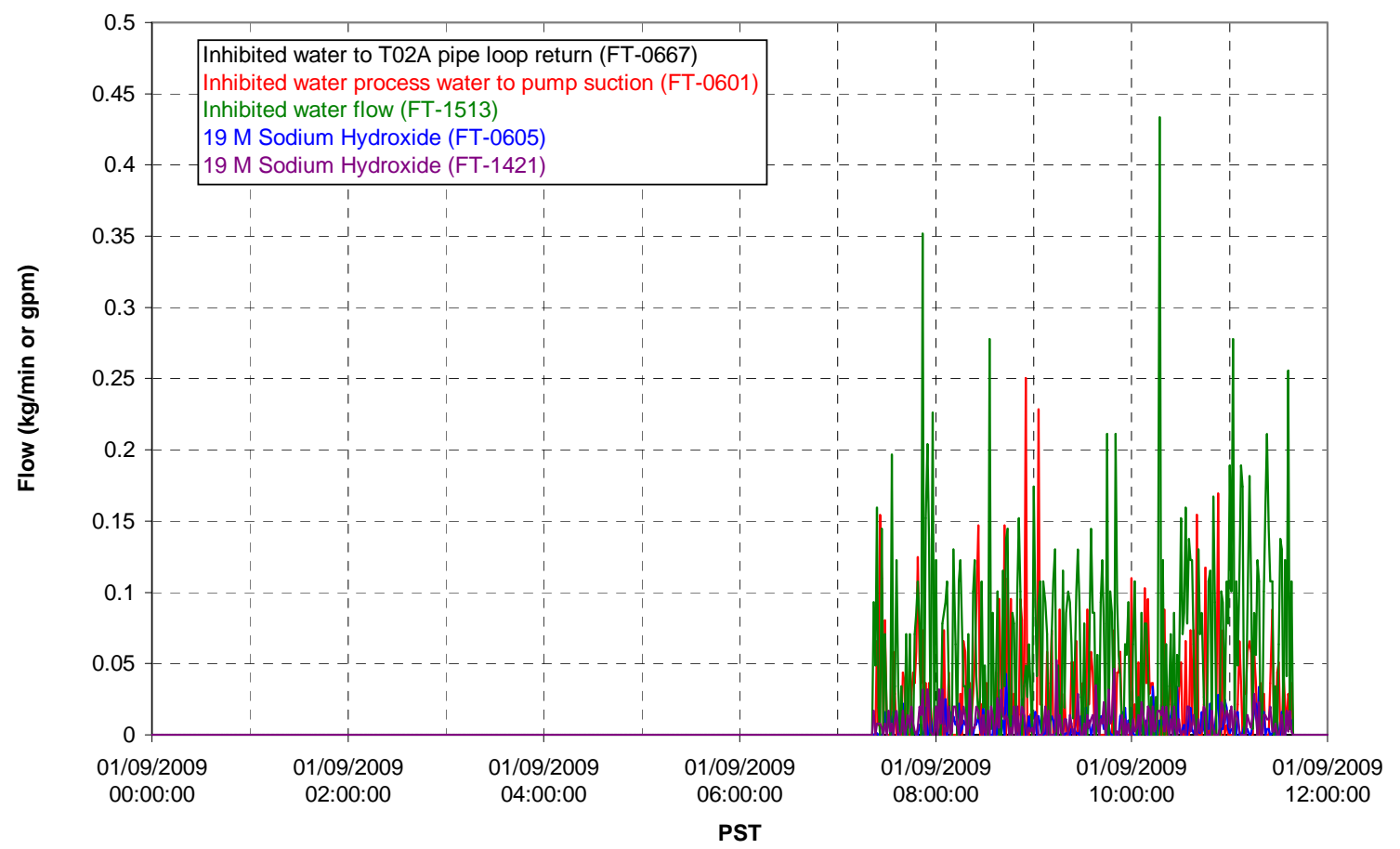



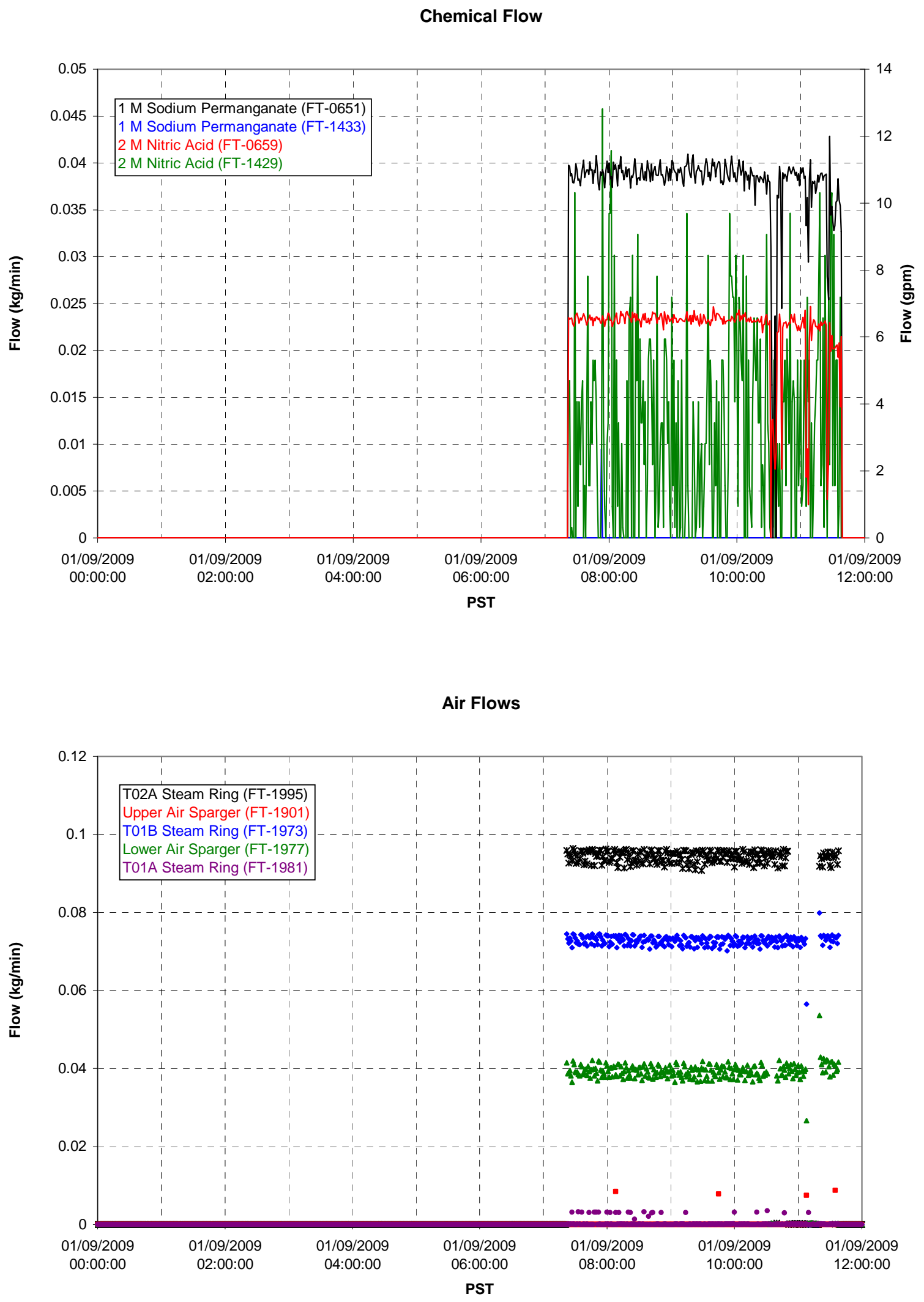
T02A Steam

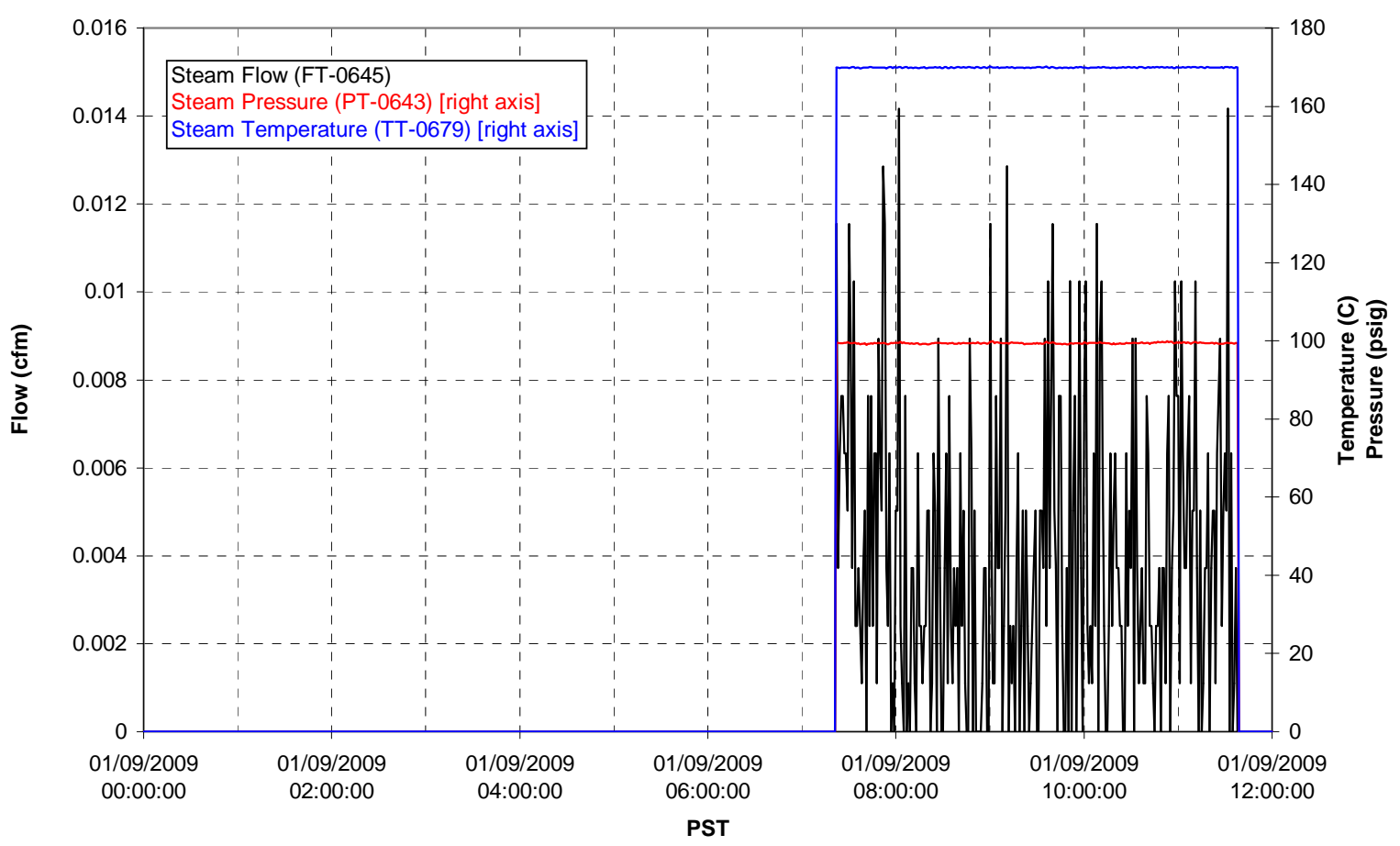

T01A Steam

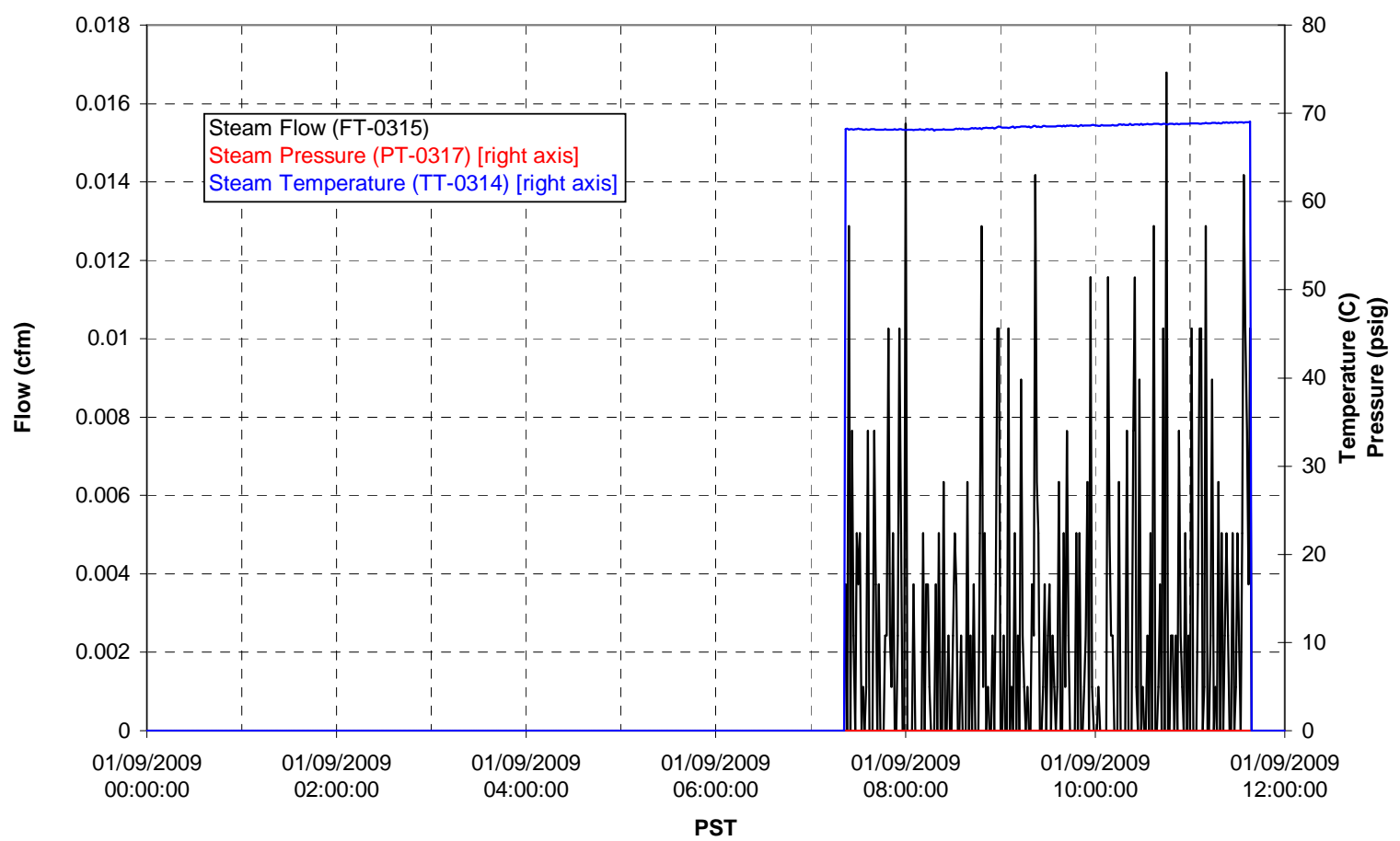


T01B Steam

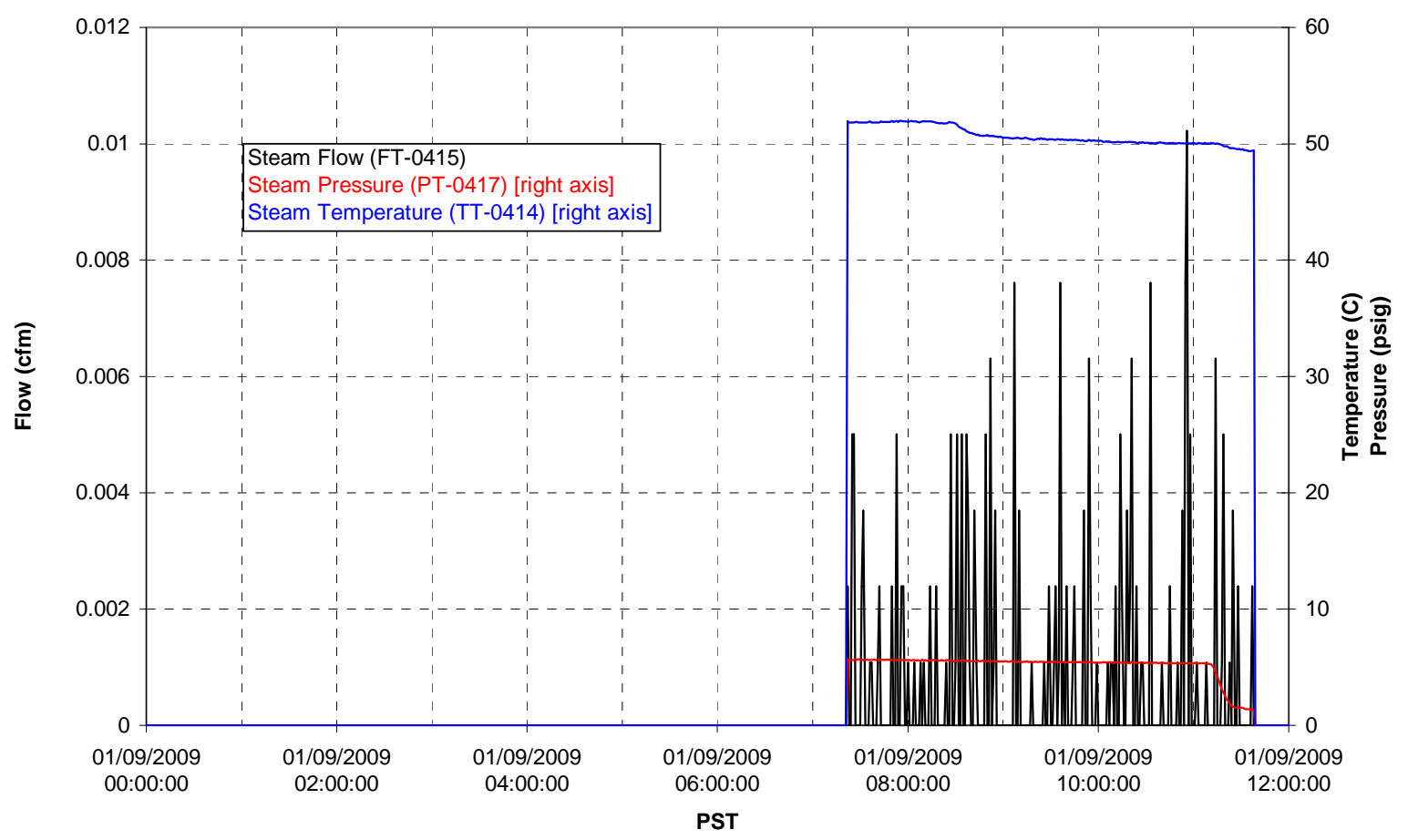


Functional Test Data Plots

01/09/2009 12:00 - 01/10/2009 00:00 
T01A level

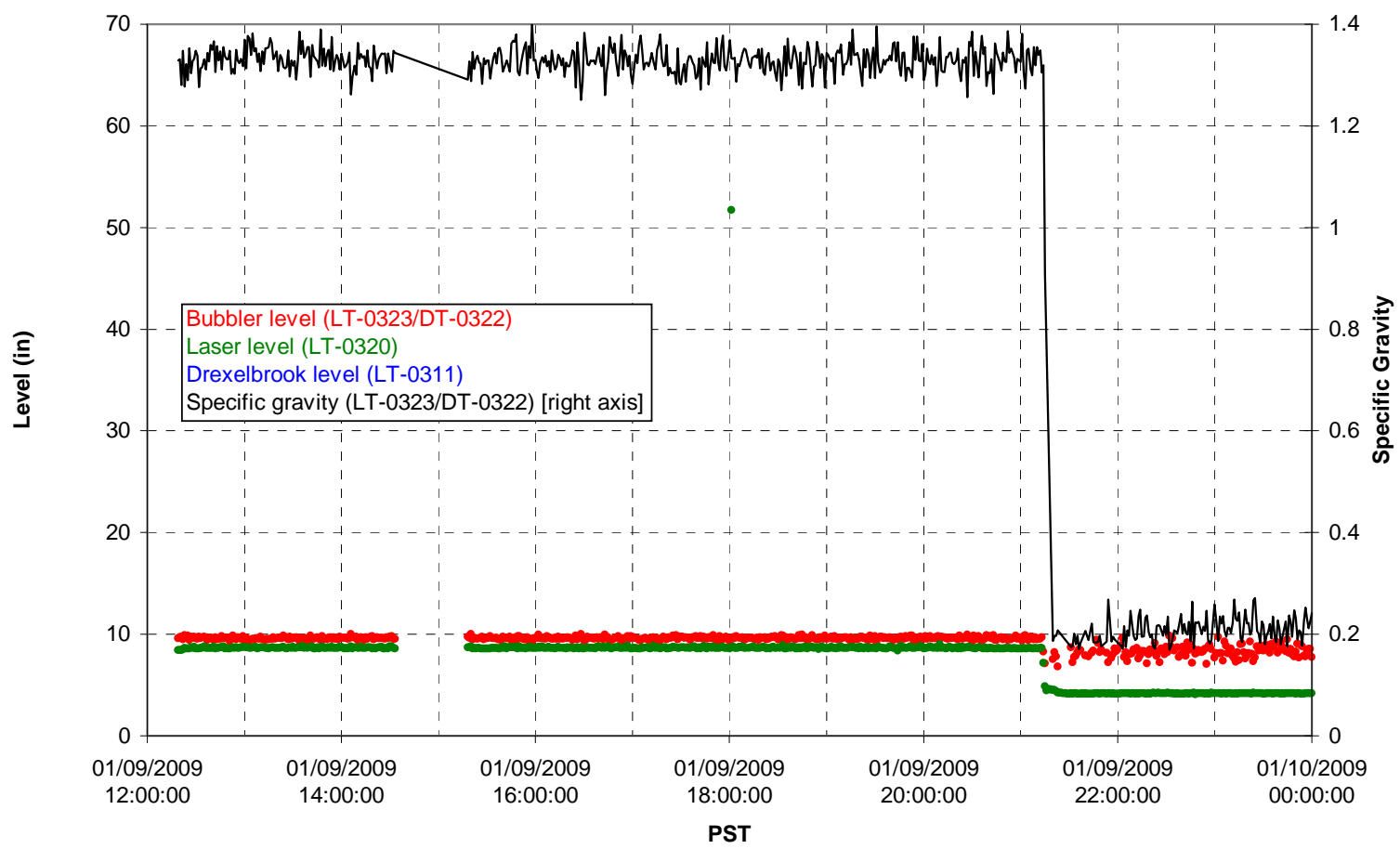

T01A temperatures

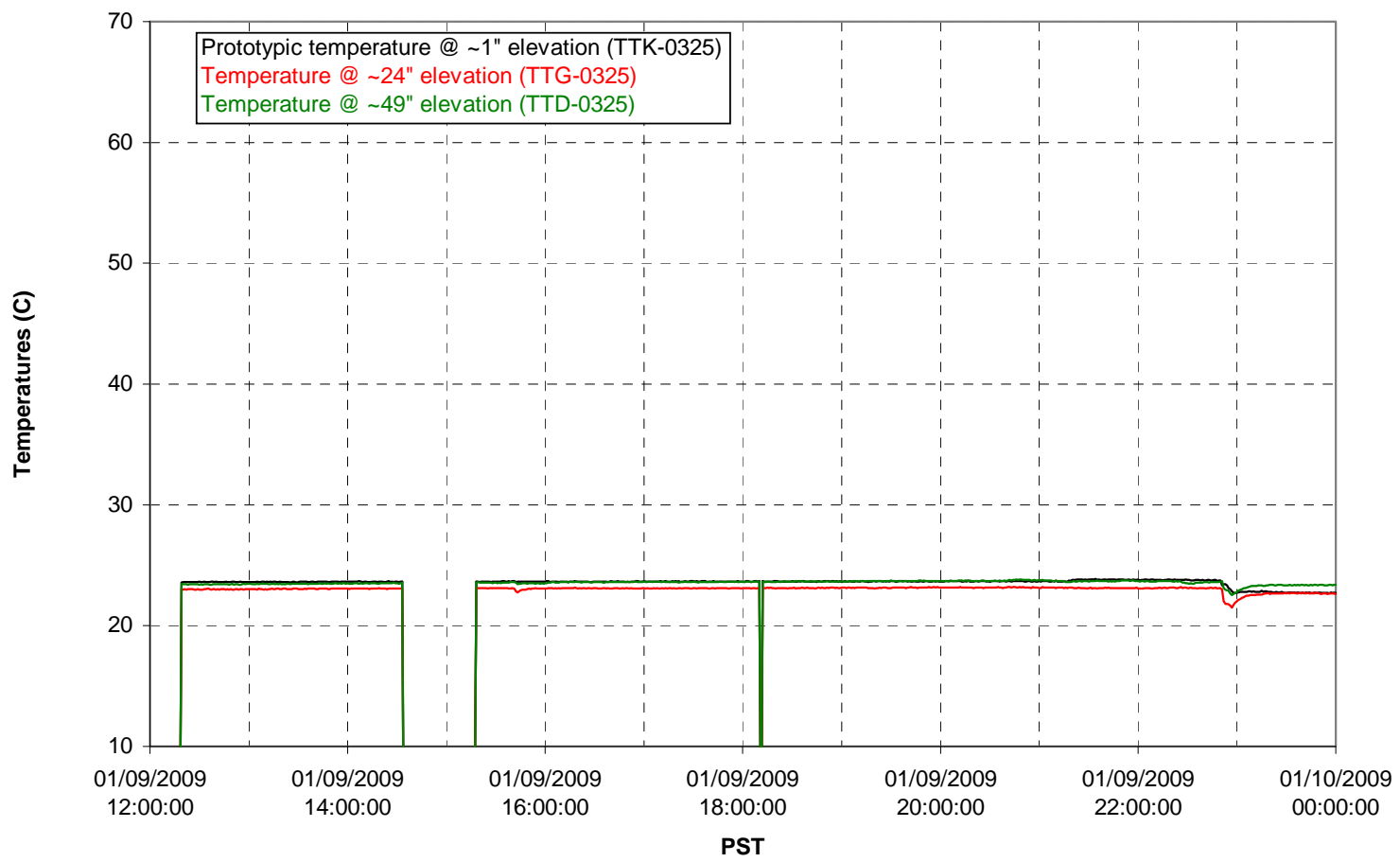


T01B level

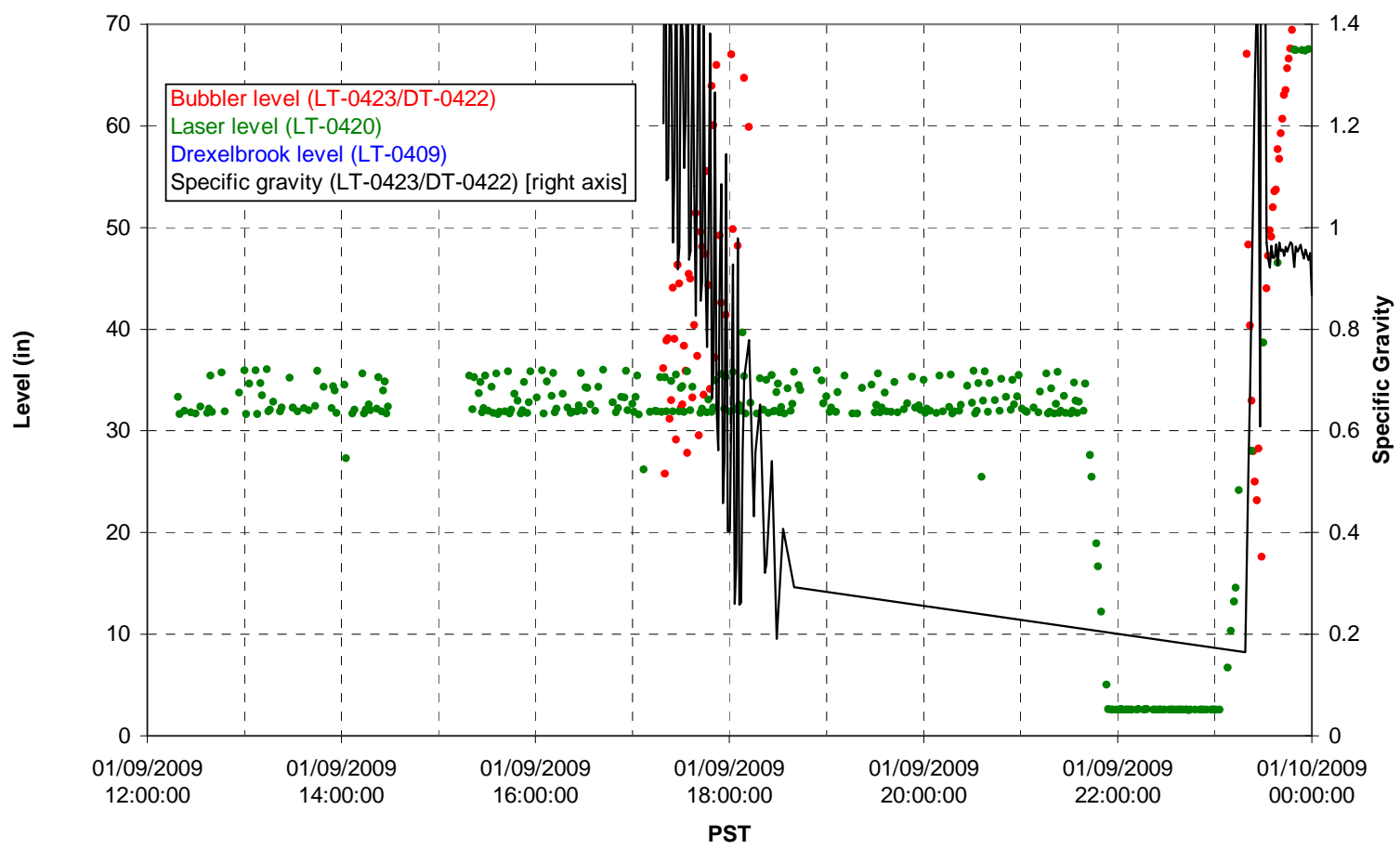

T01B temperatures

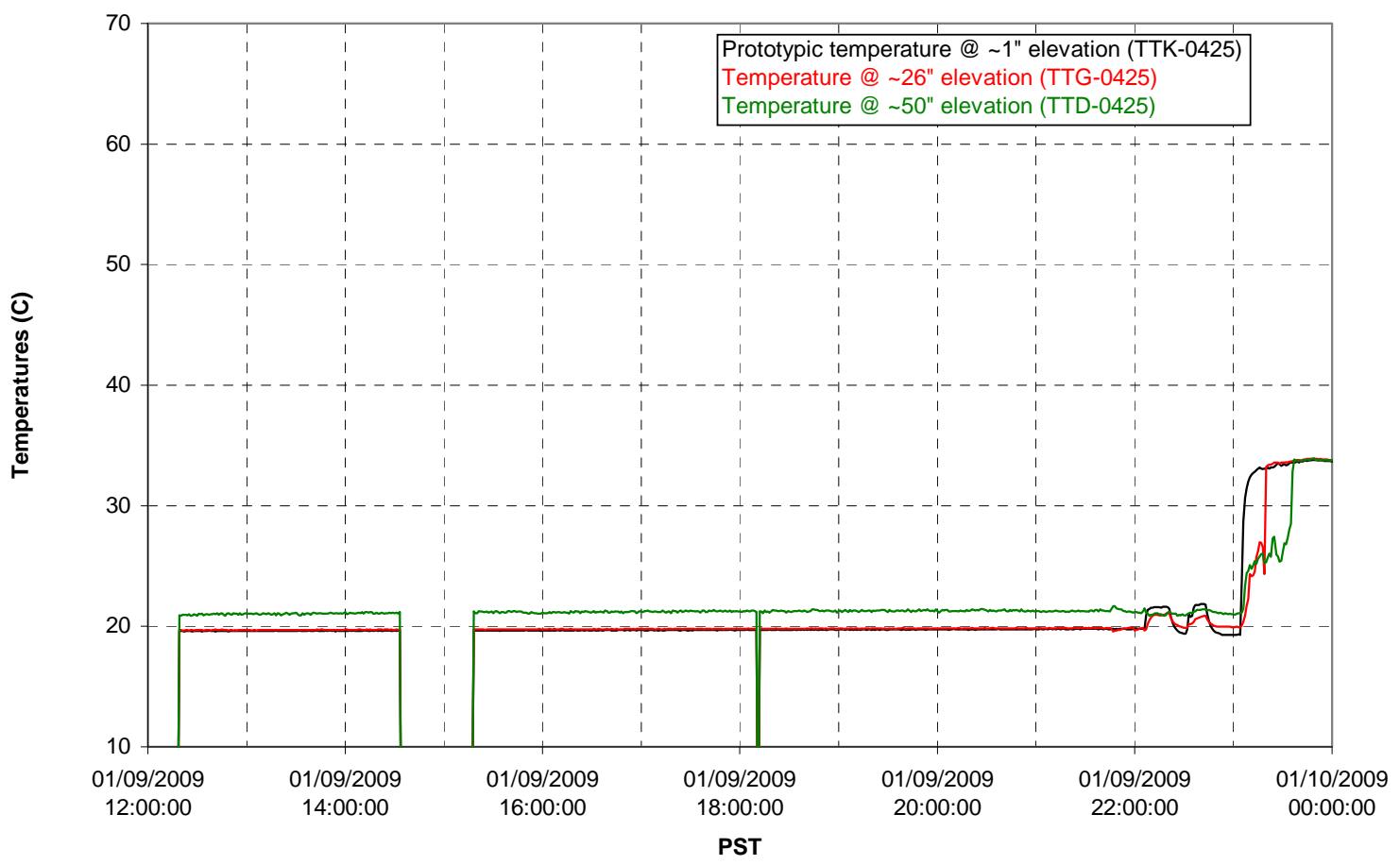


T02A level

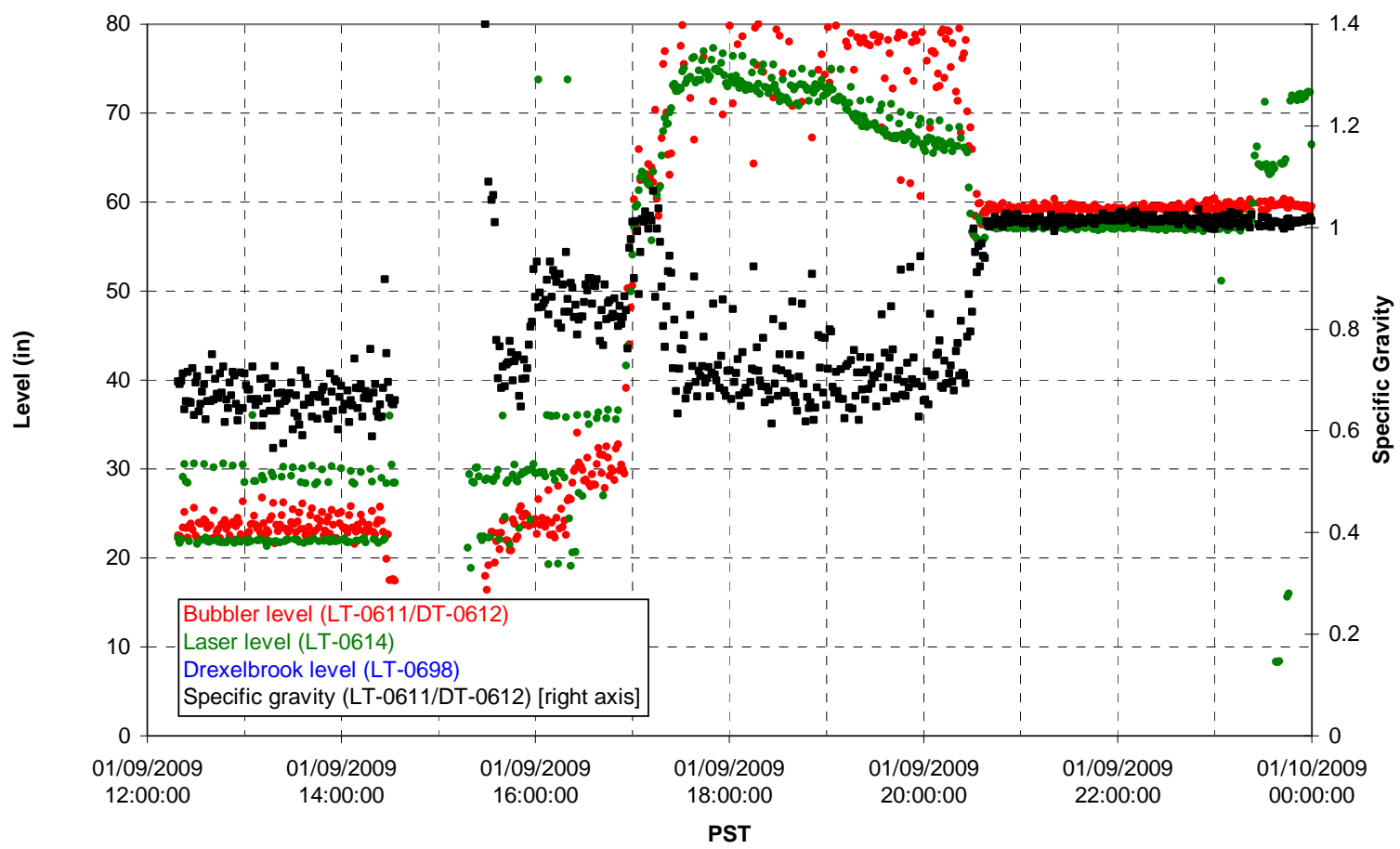

T02A temperatures

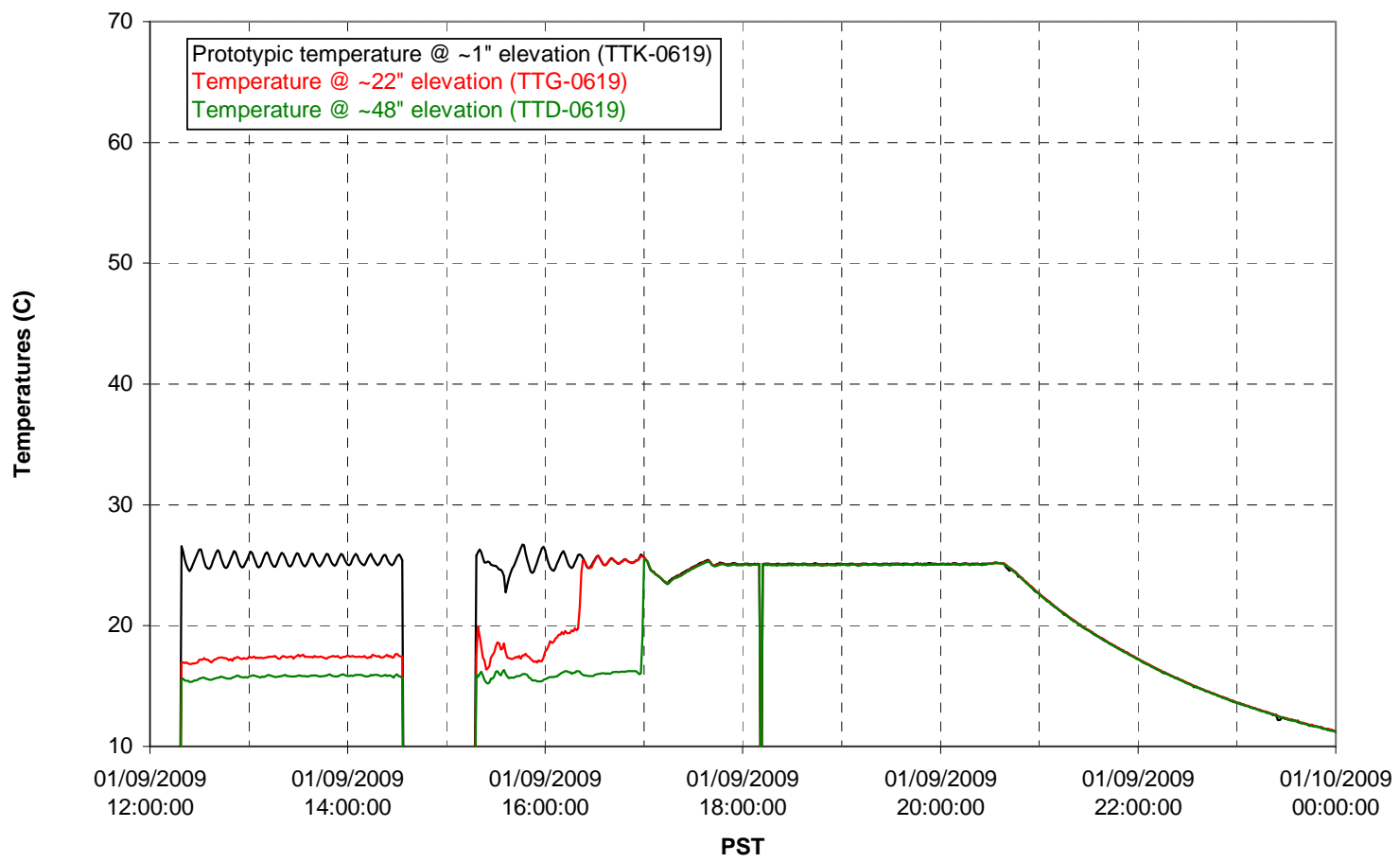


T02A and filter loop temperatures

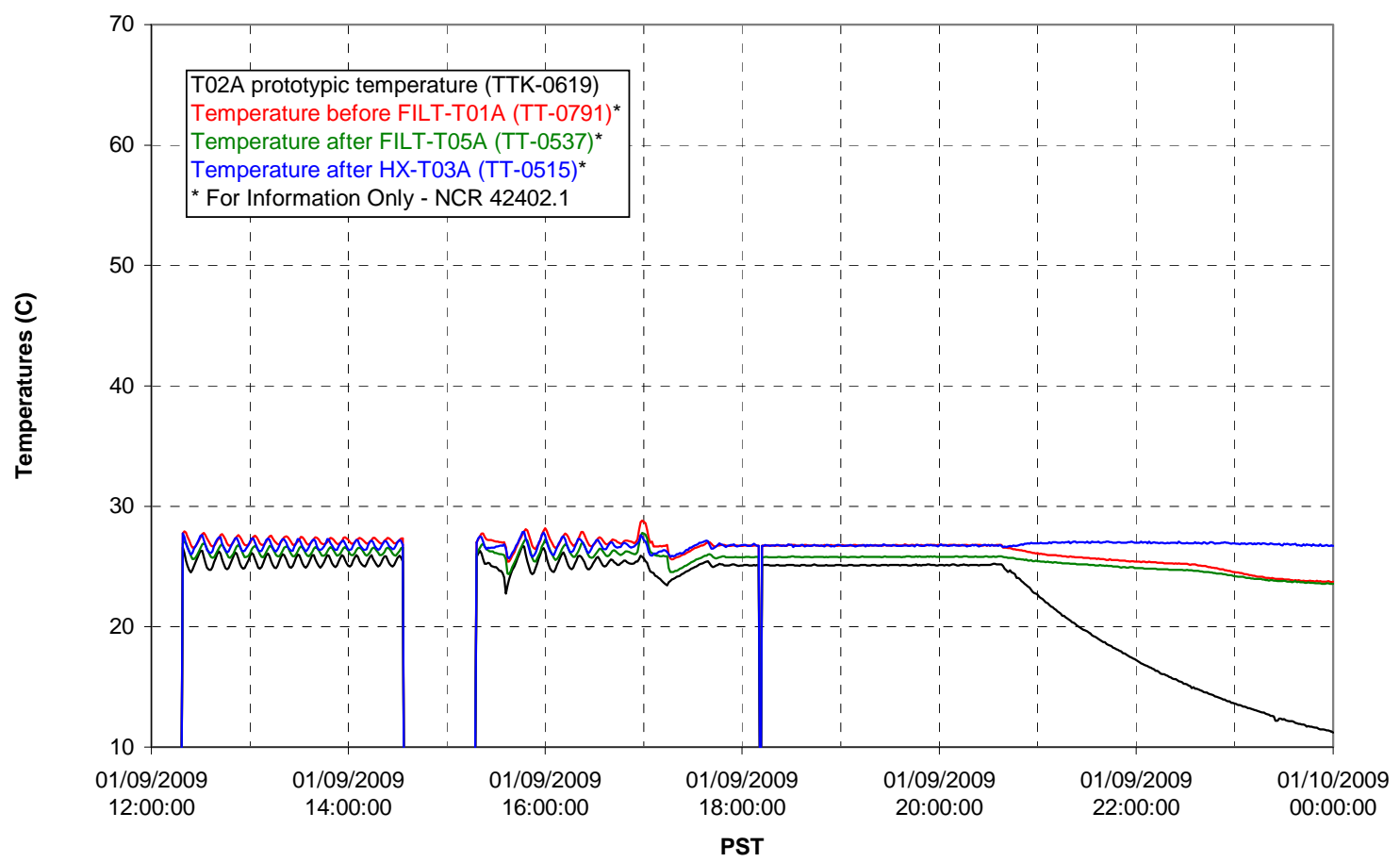

Pump Pressures and Flow

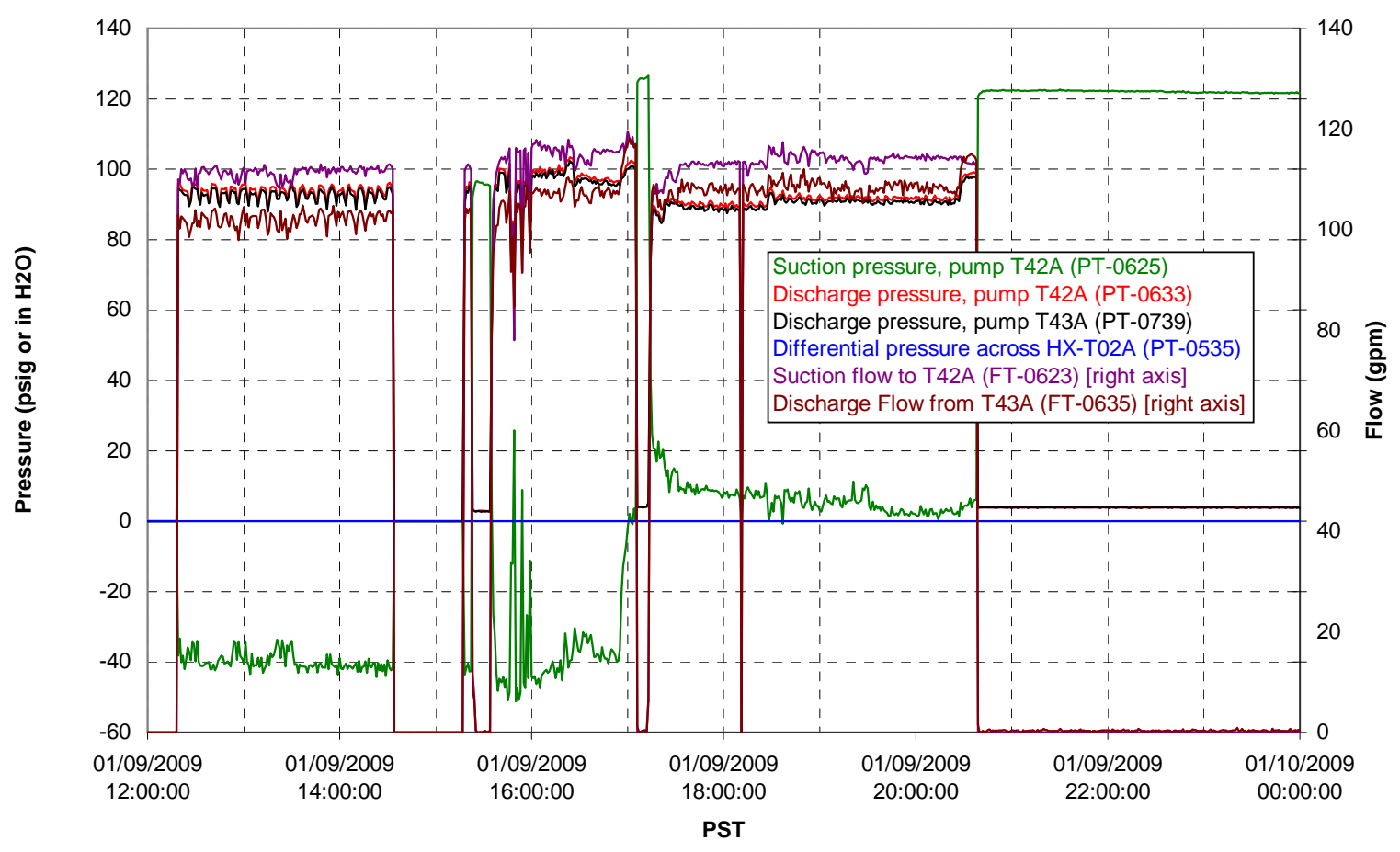


Axial pressure drop

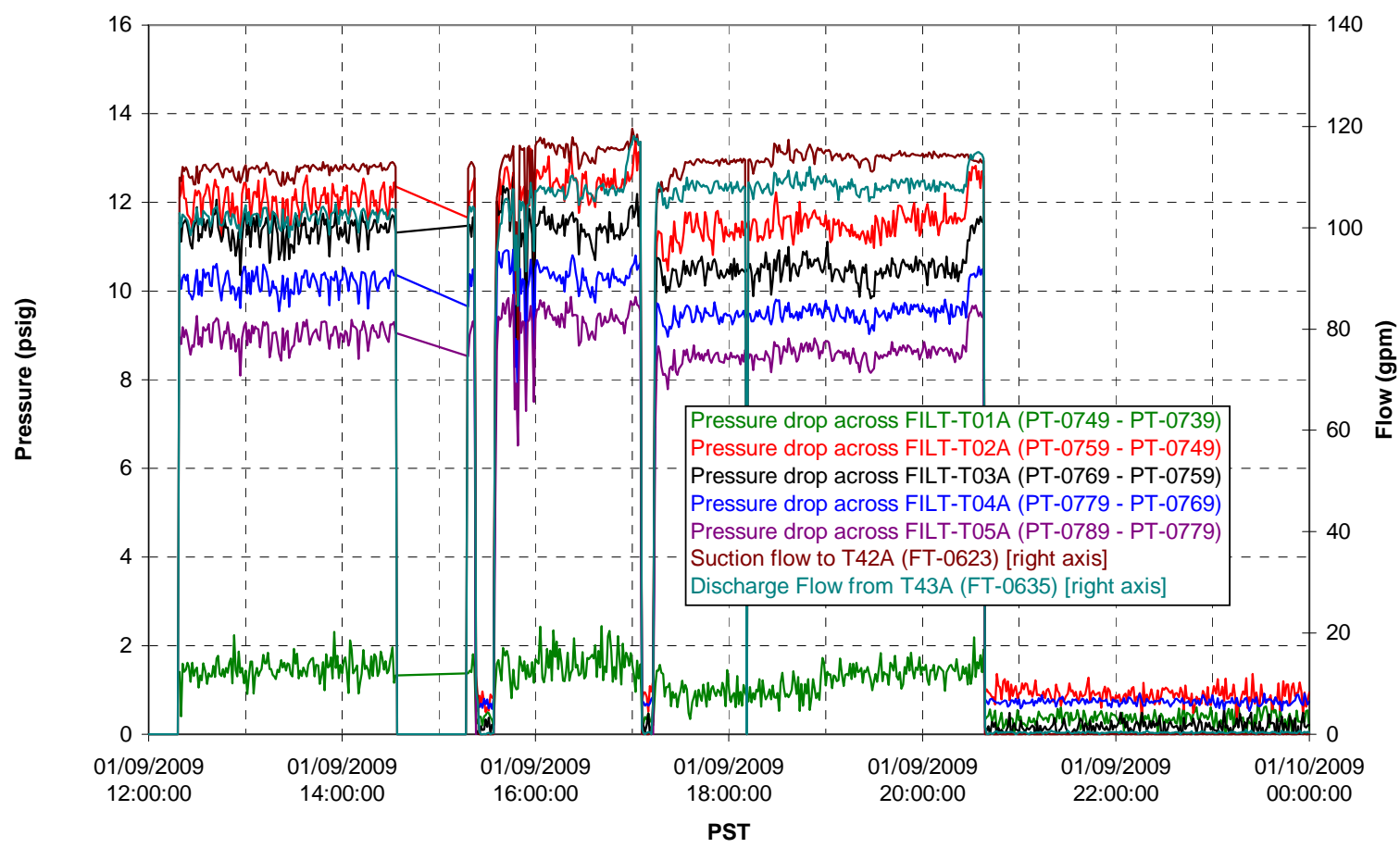

Permeate flow rates

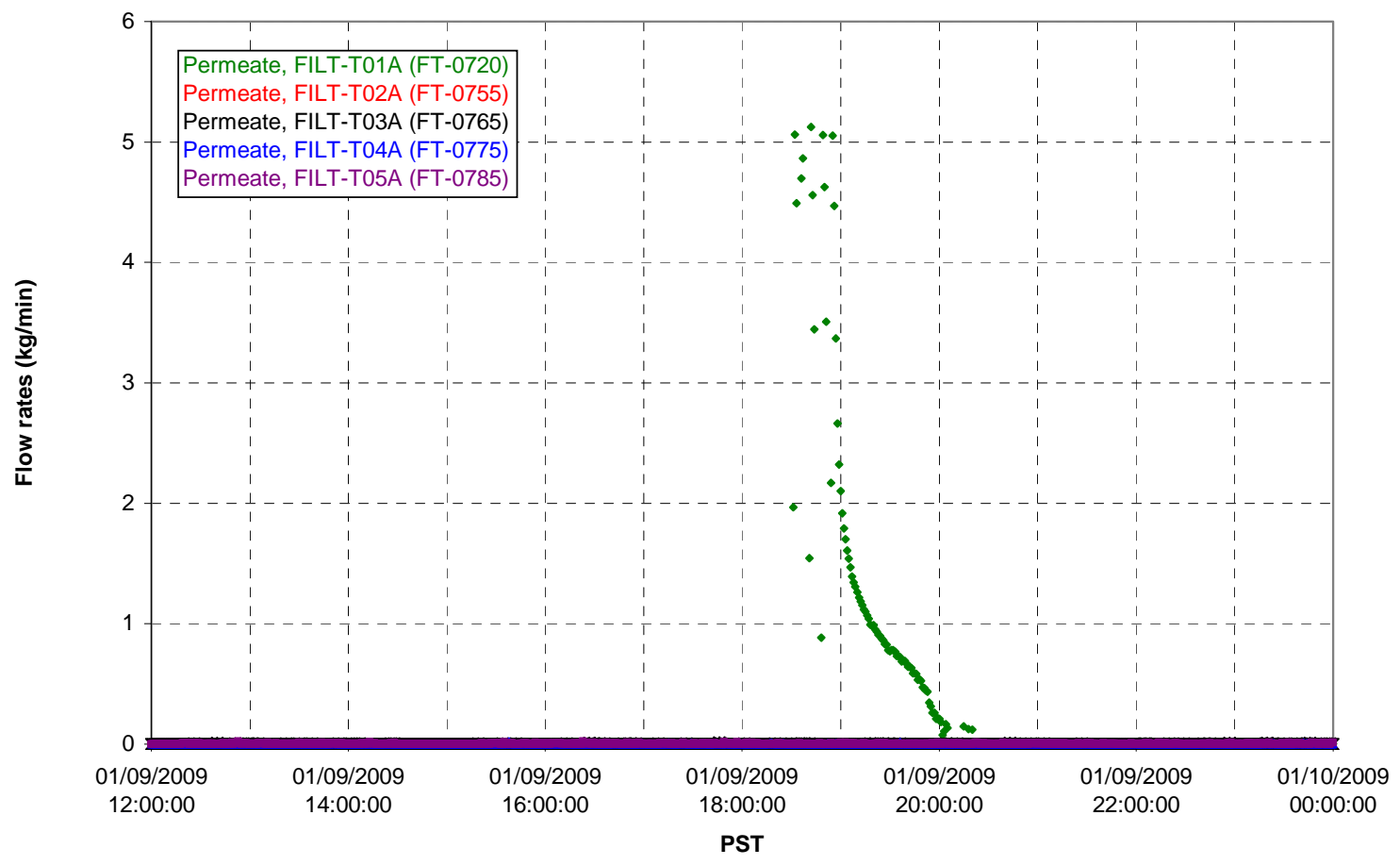


T02A Inner Temperature Tree

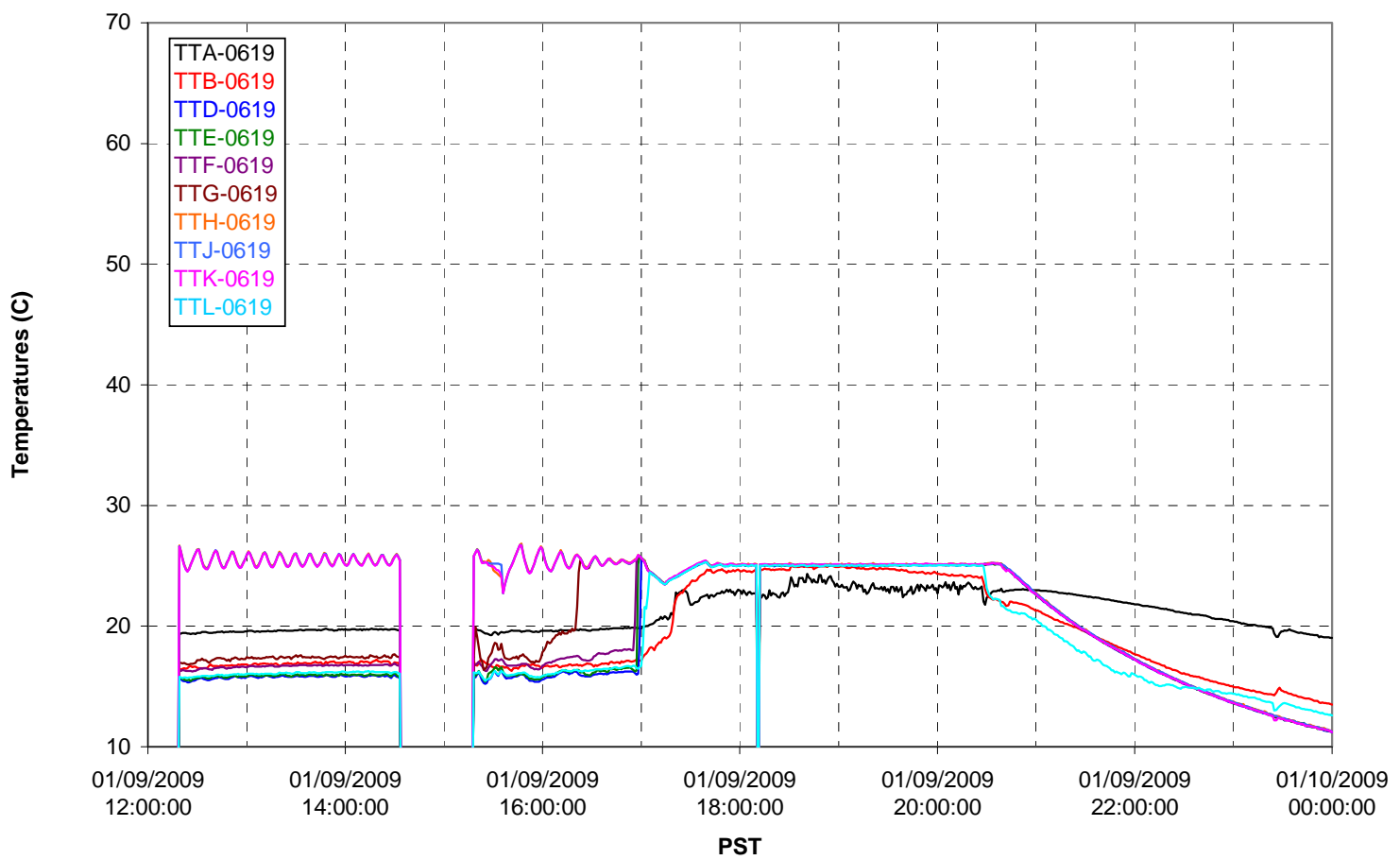

T02A Outer Temperature Tree

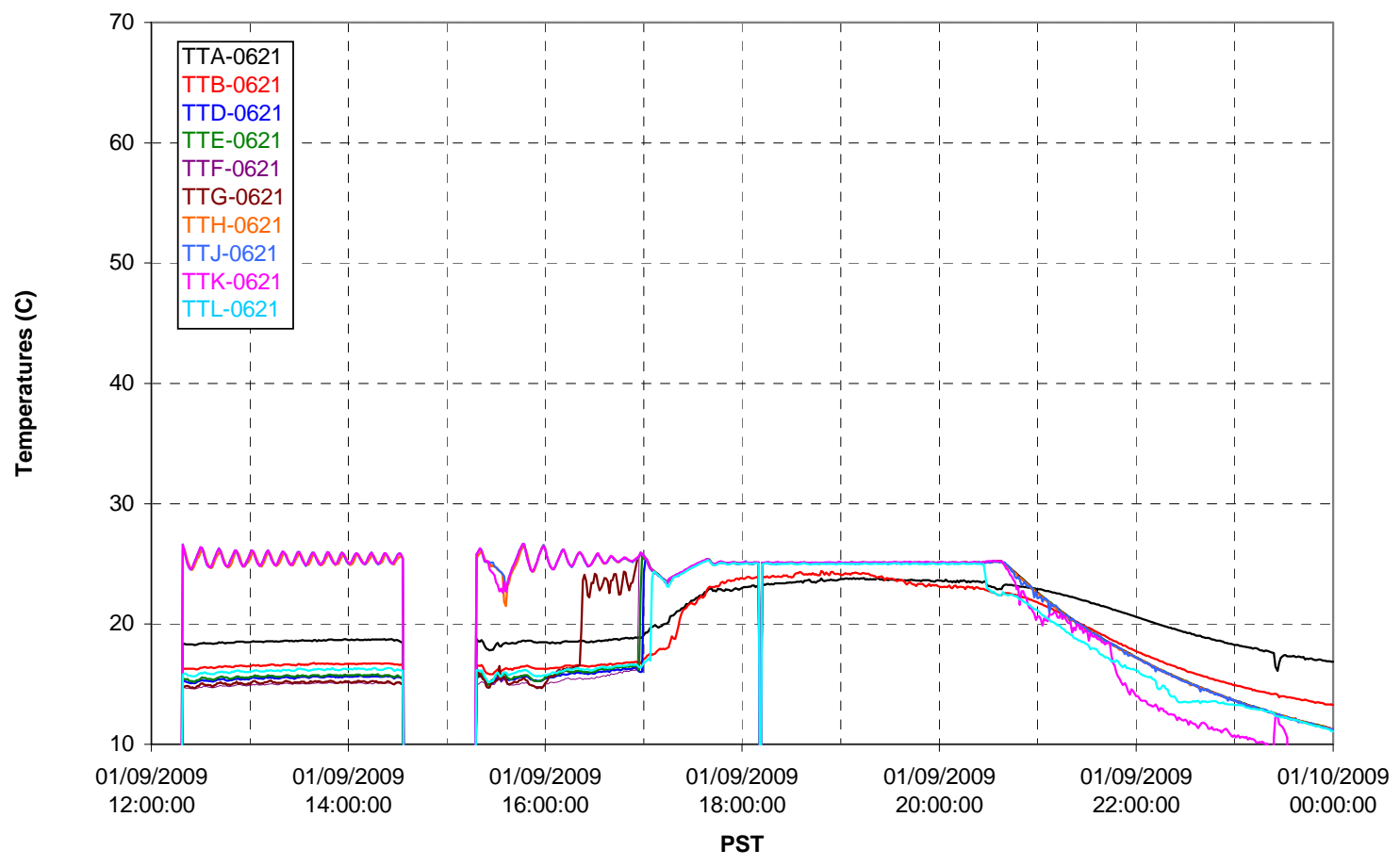


T02A temperatures

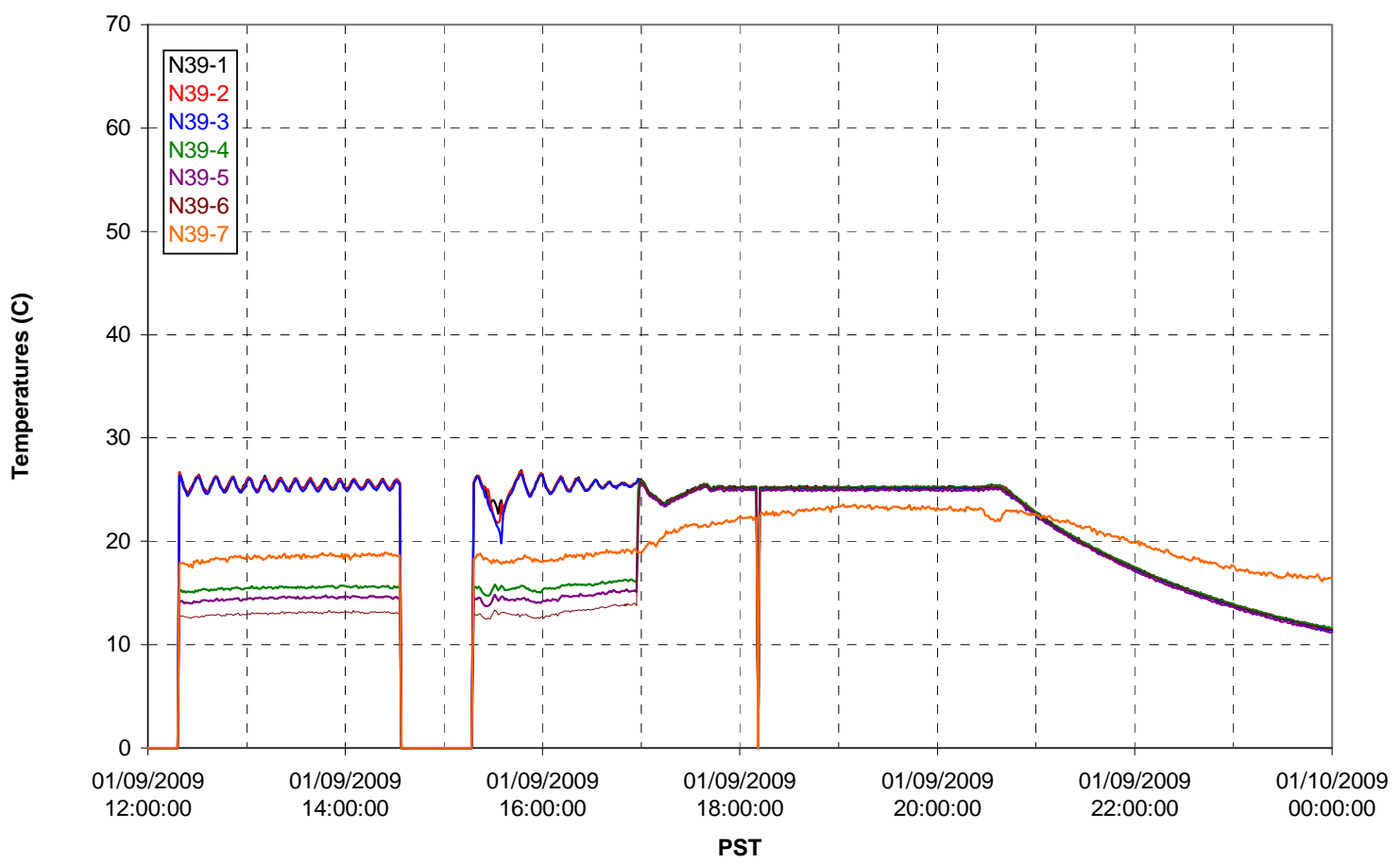

T02A temperatures

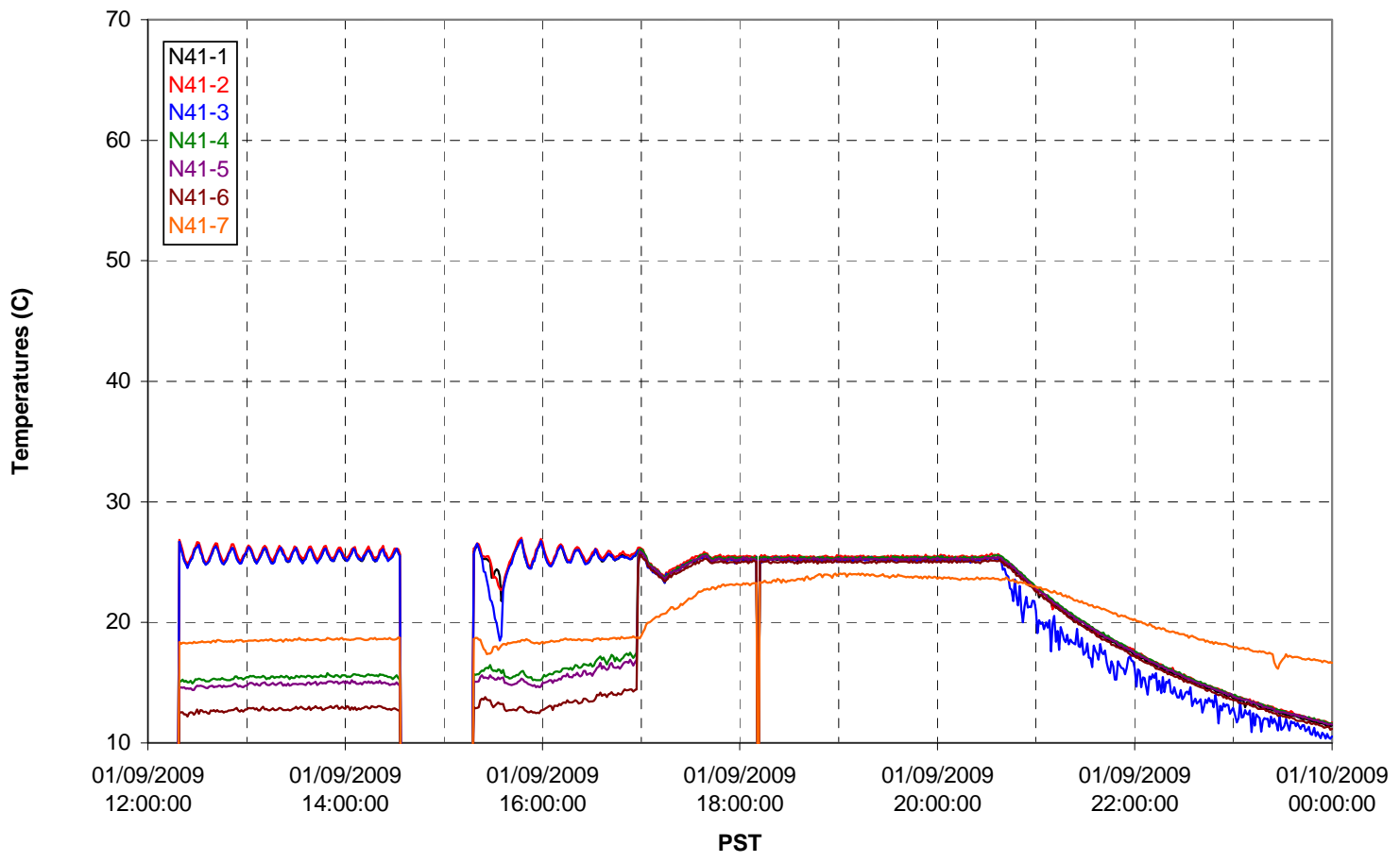


T02A temperatures

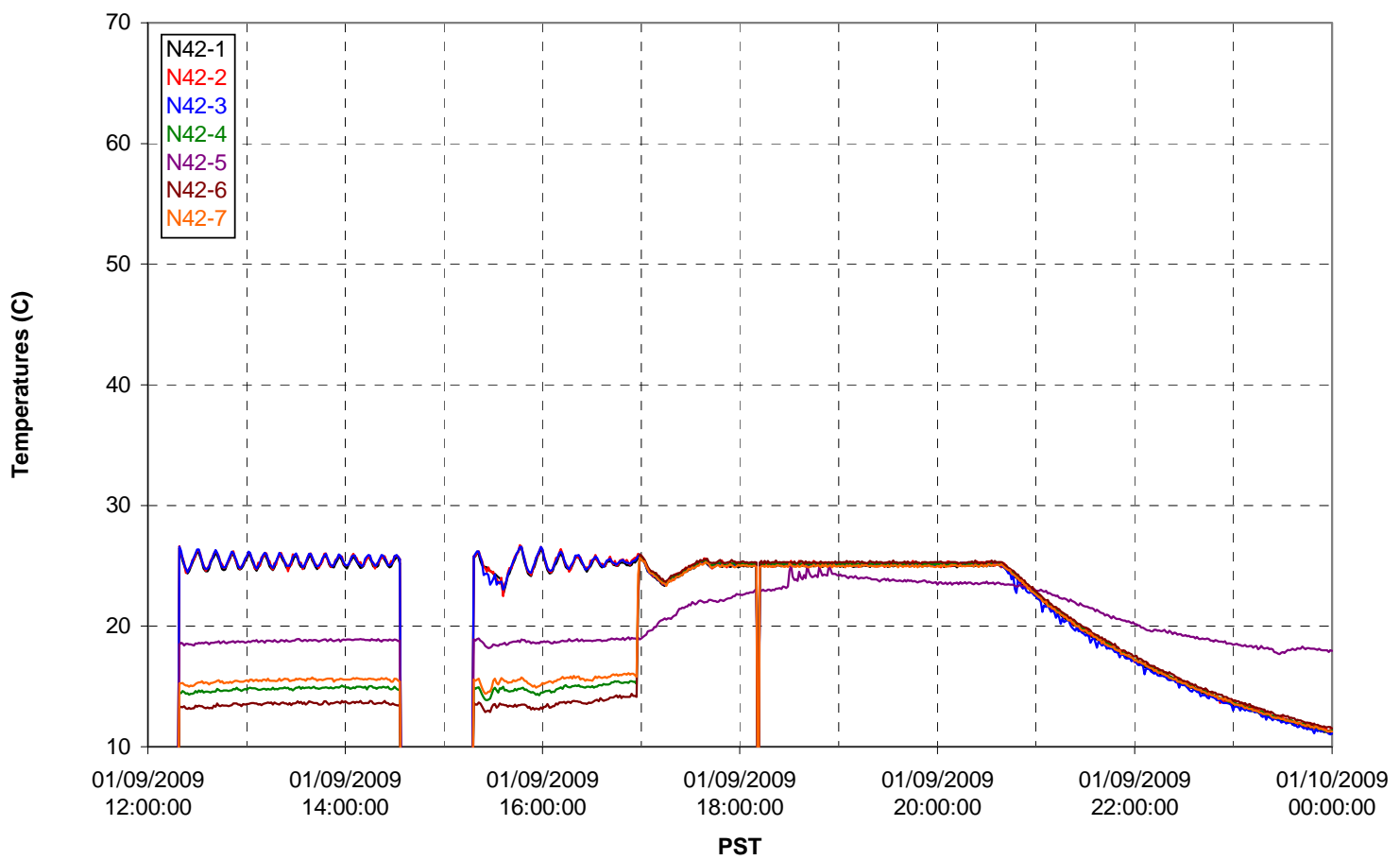

T02A temperatures

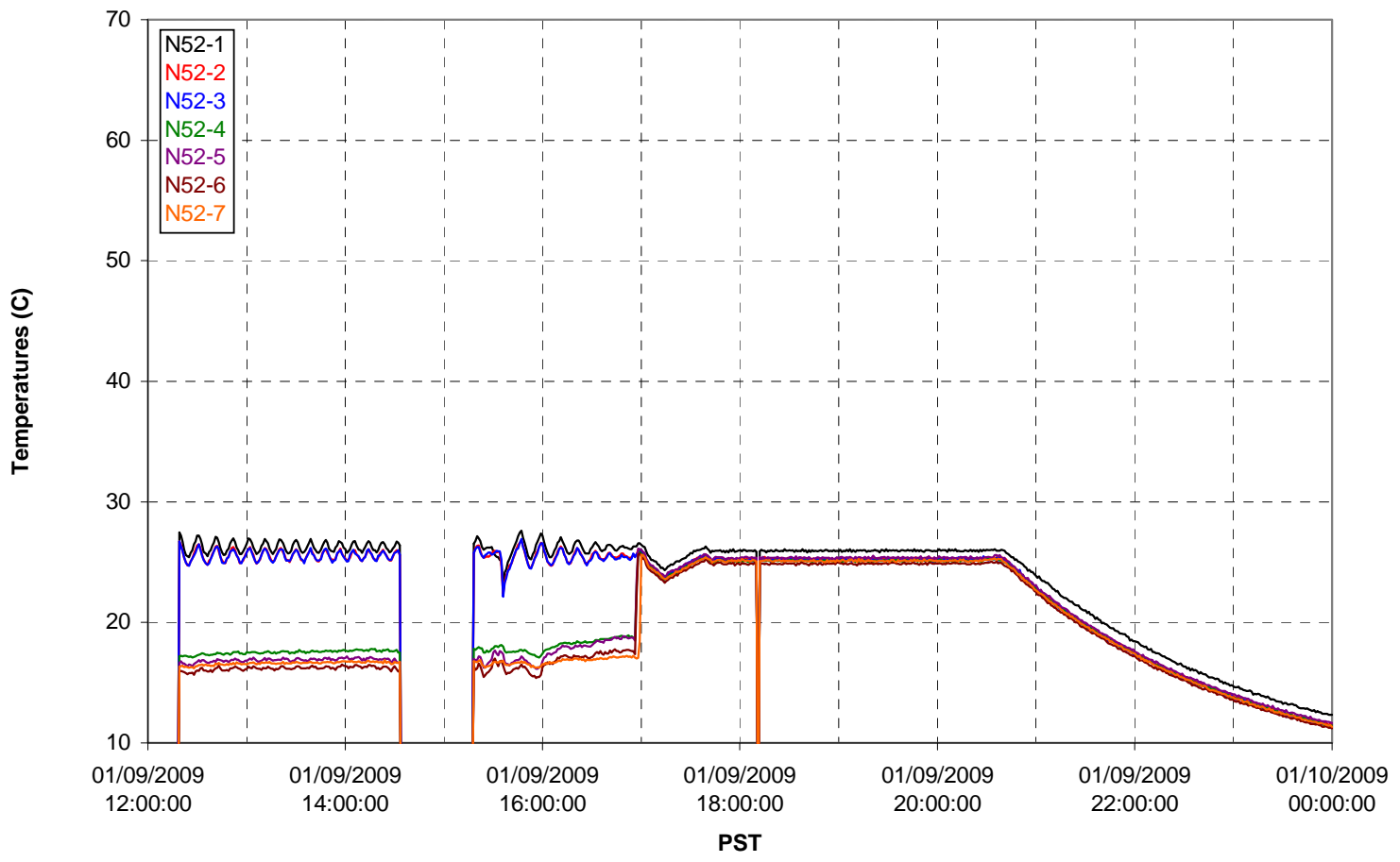


T02A Heating and Cooling

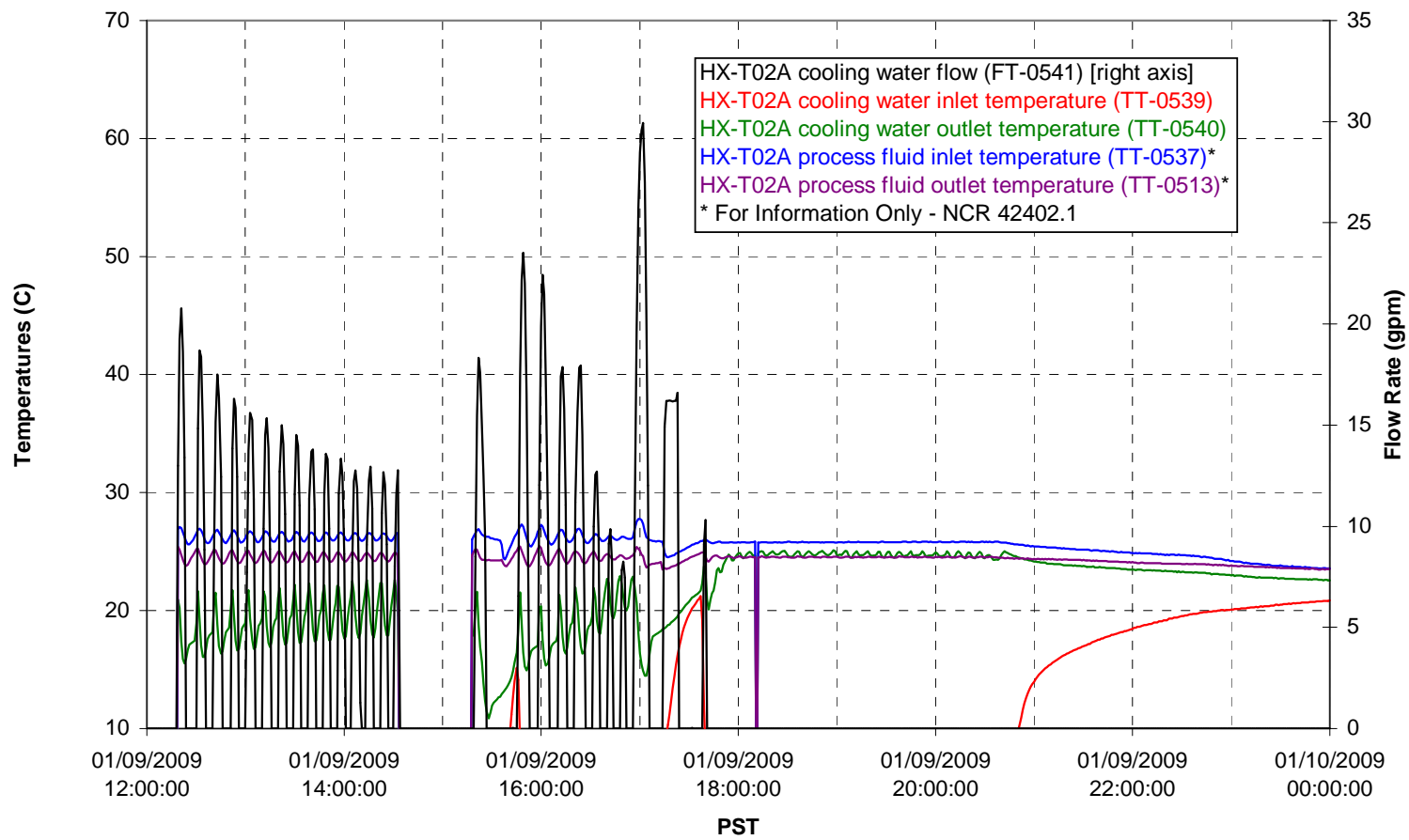

Pump Operation

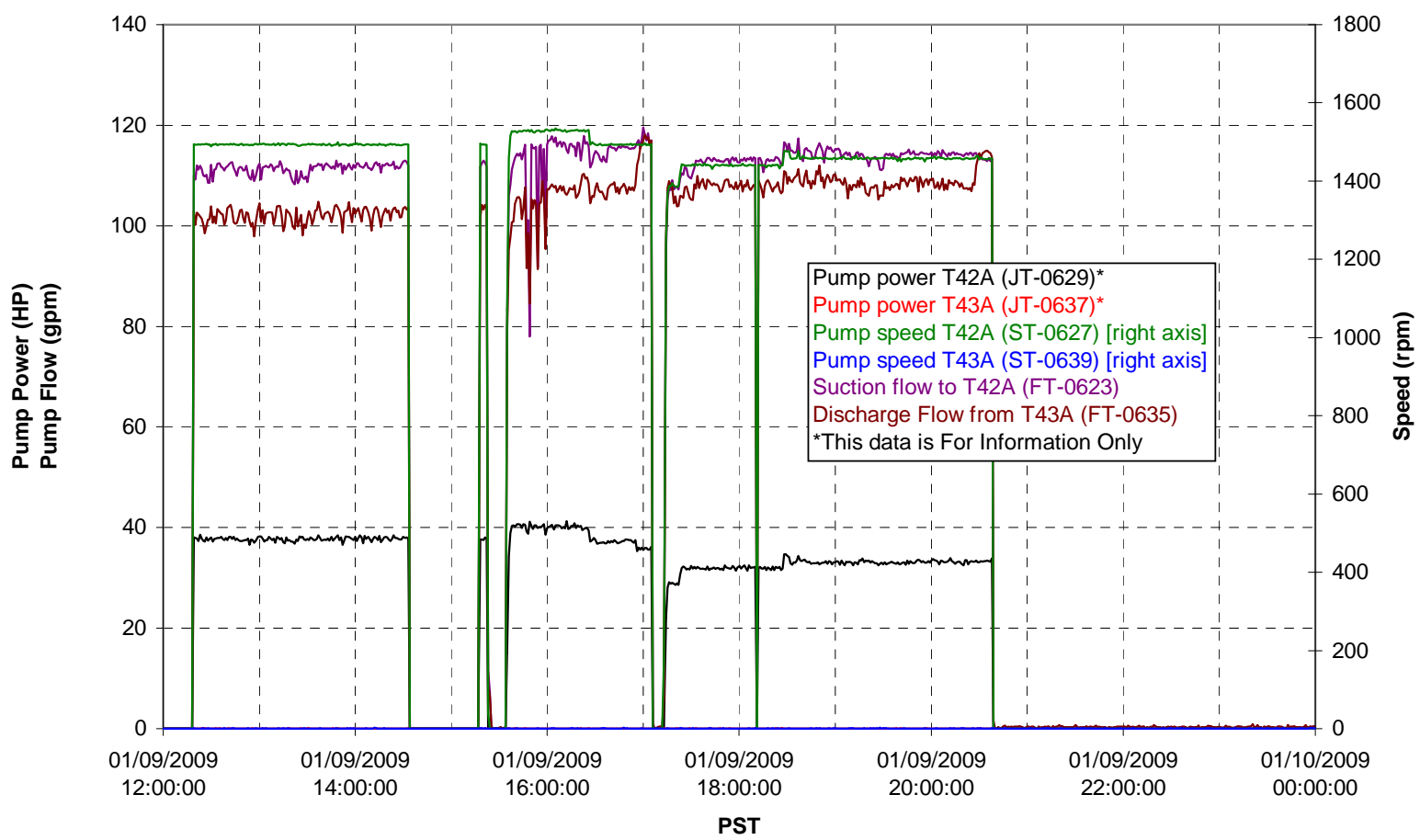


Pulsepot UFP-PP-T01A

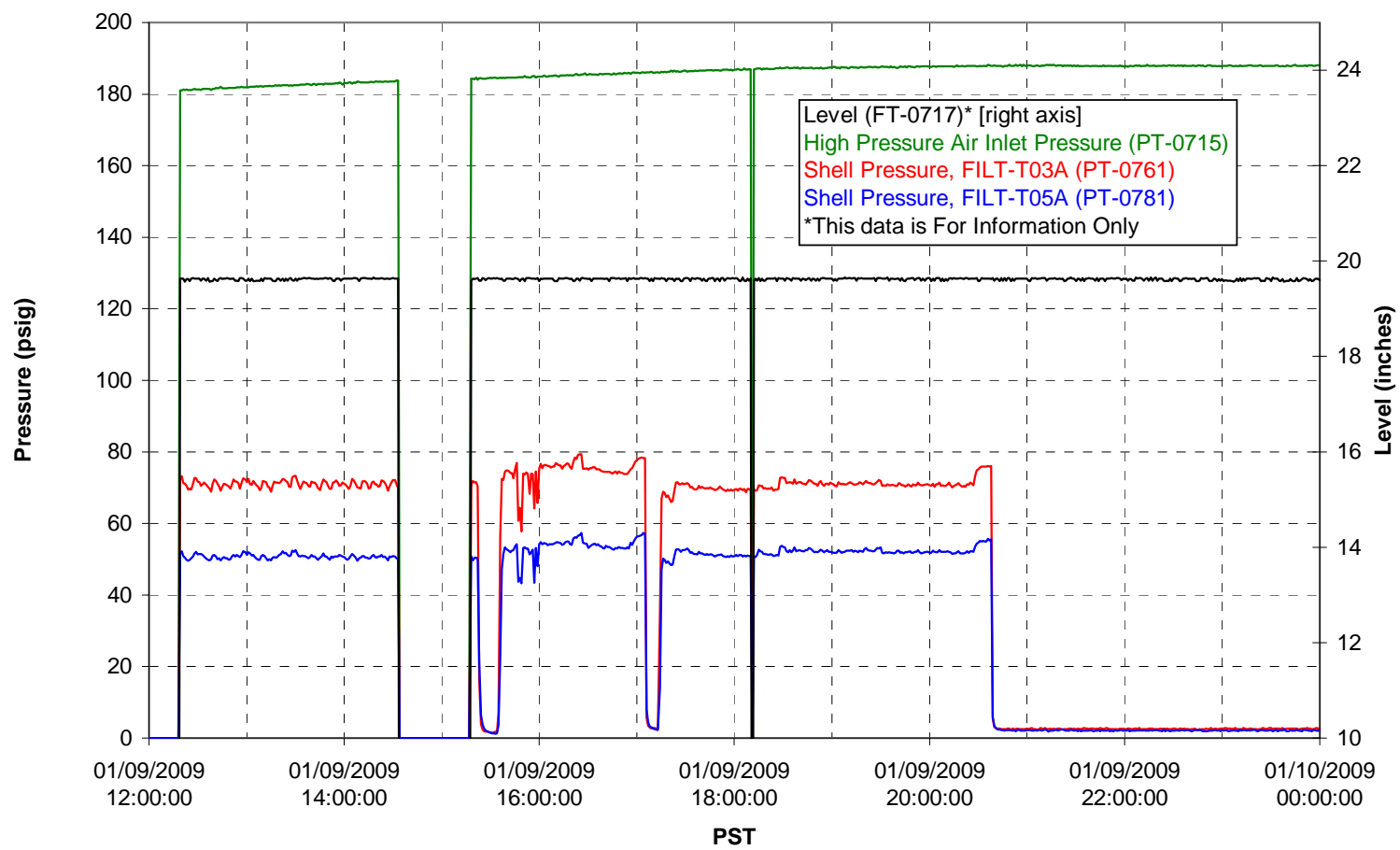

Pulsepot UFP-PP-T02A

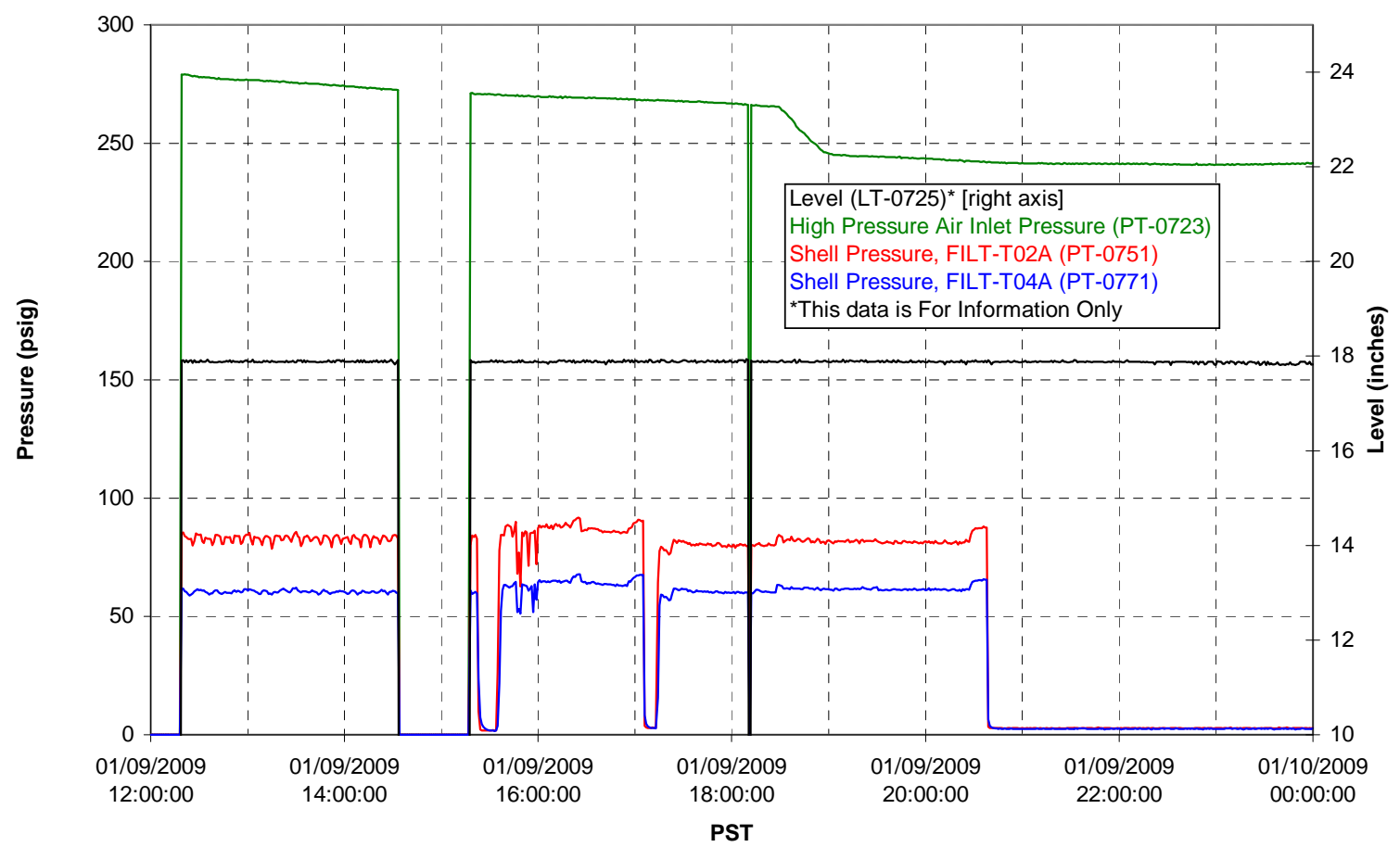


Pulsepot UFP-PP-T03A

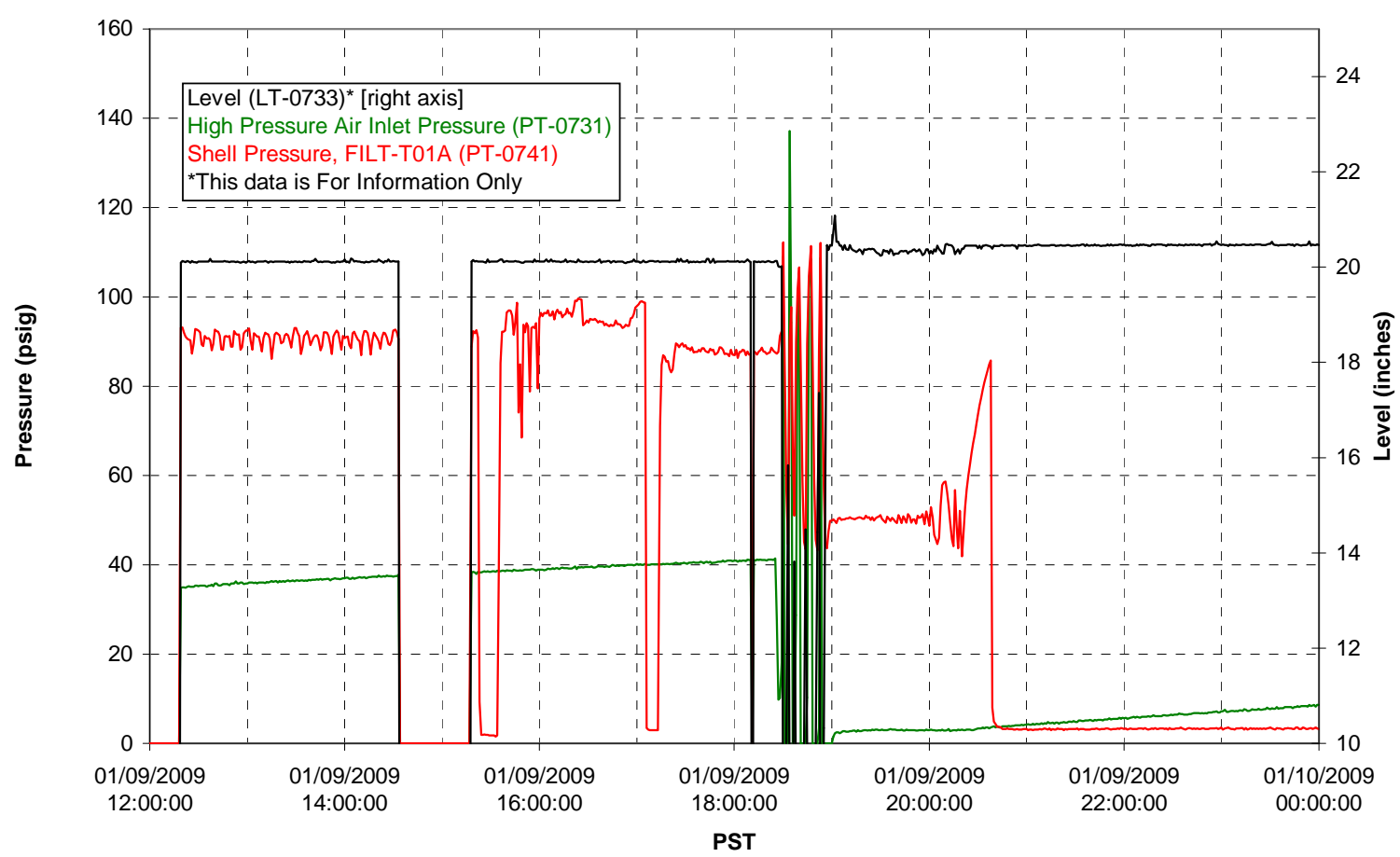

Pulsepot Levels

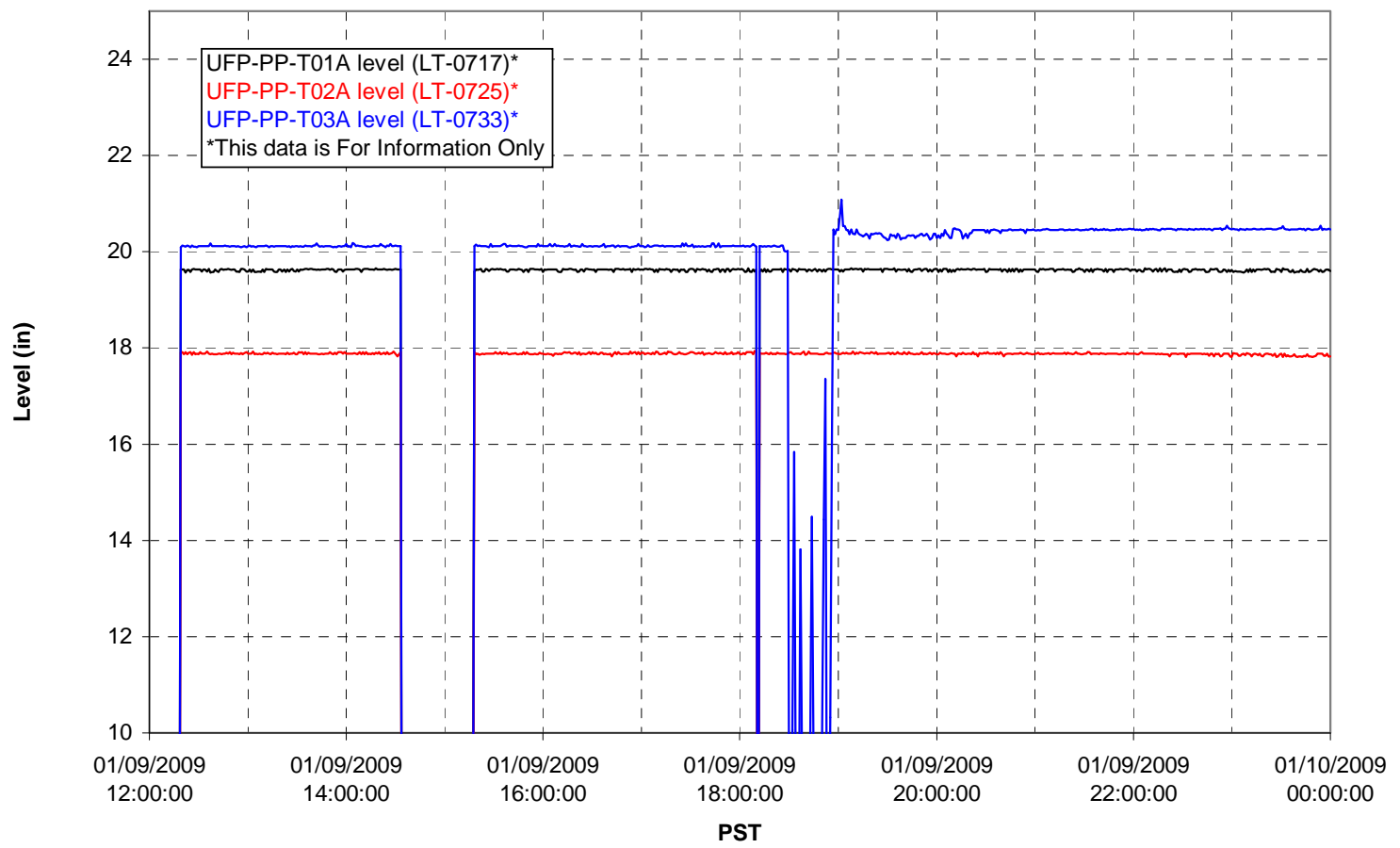


Filter UFP-FILT-T01A

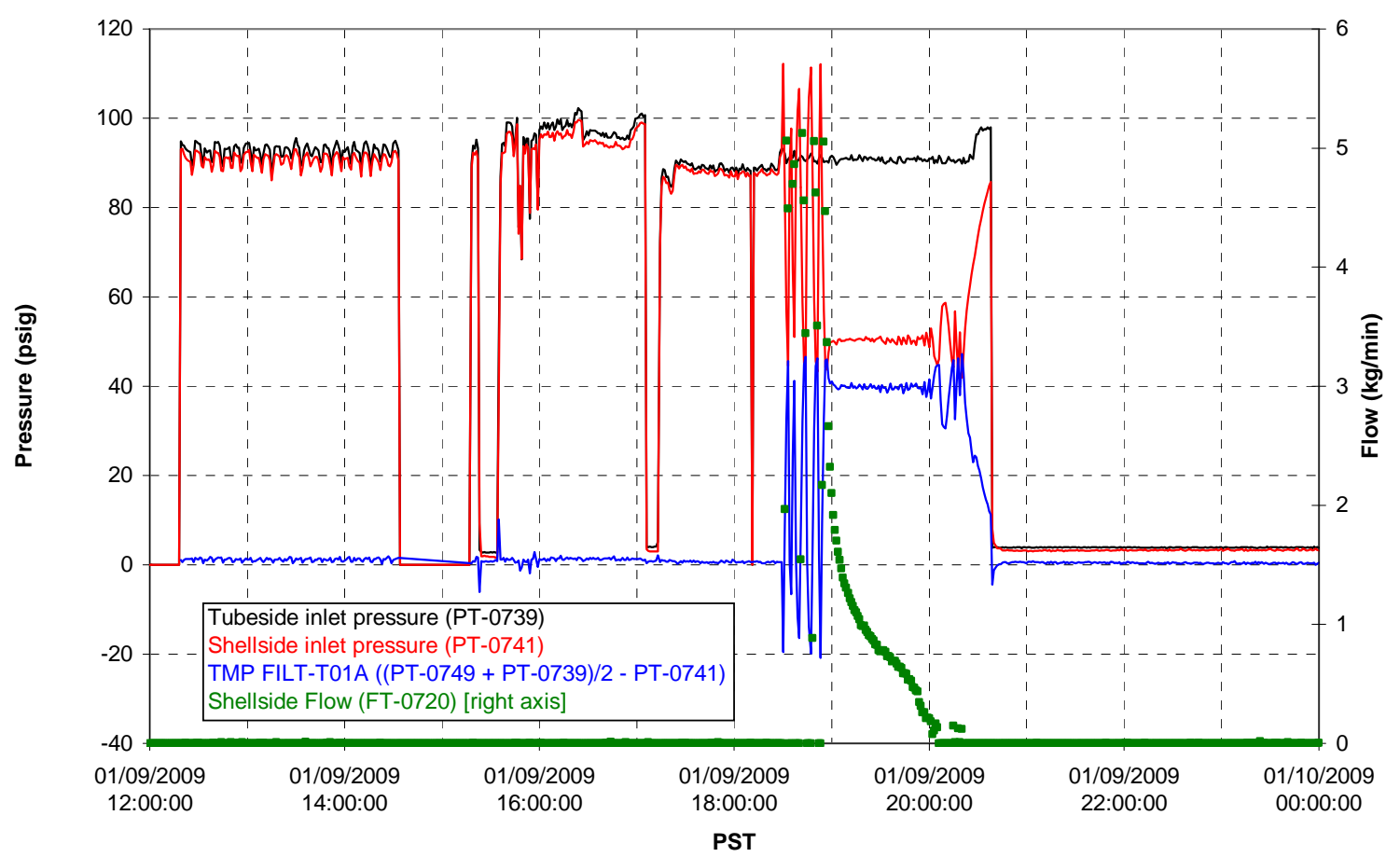

Filter UFP-FILT-T02A

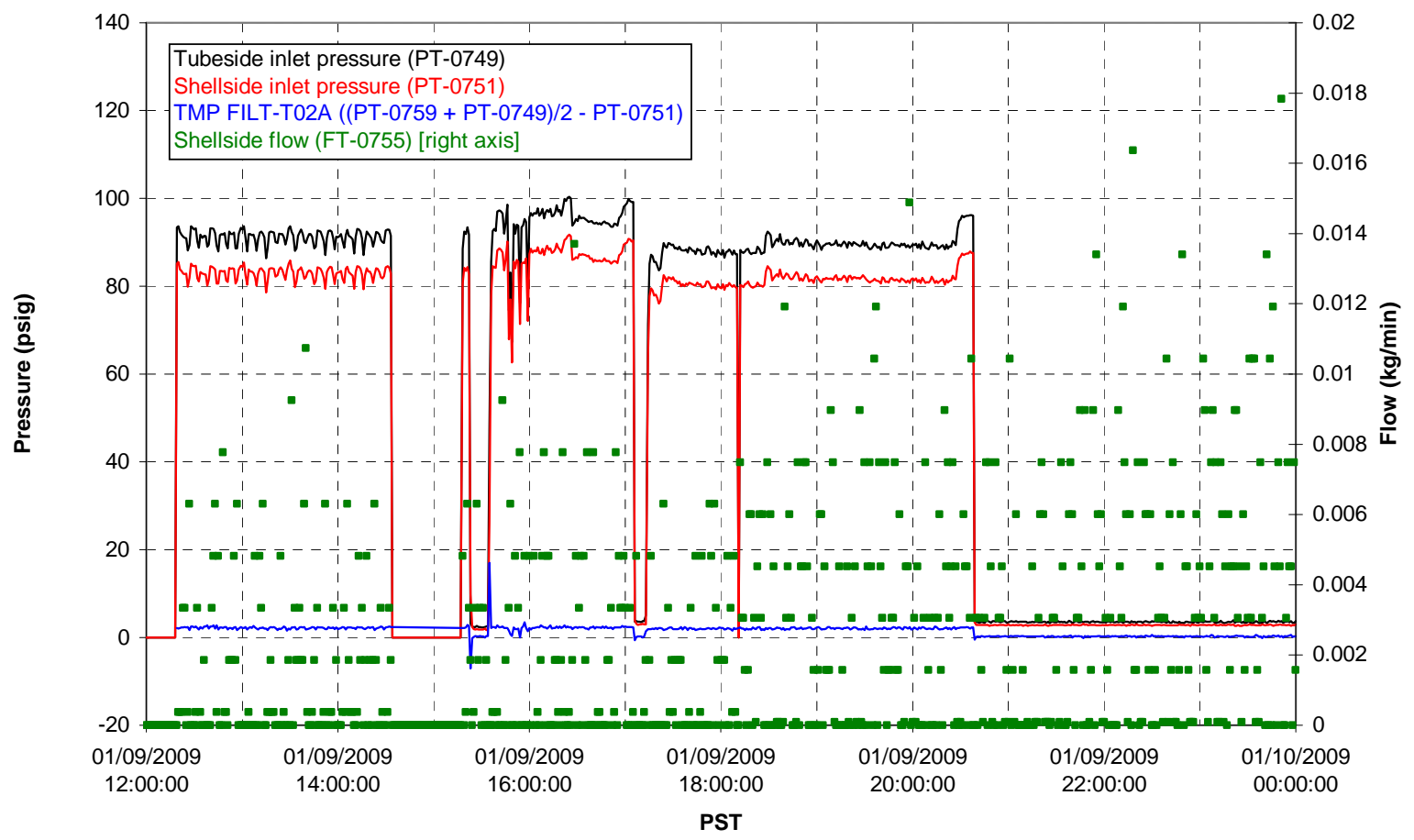


Filter UFP-FILT-T03A

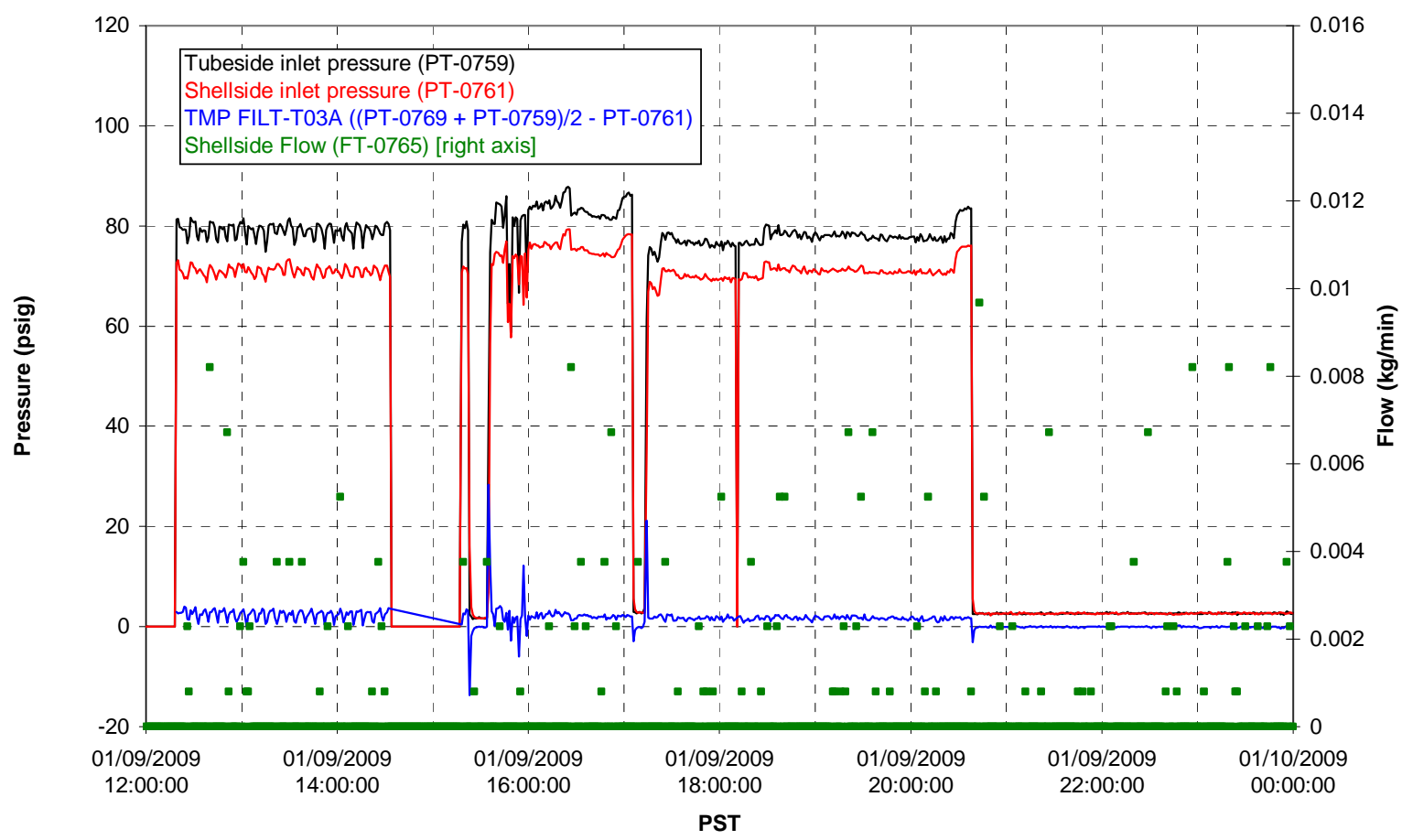

Filter UFP-FILT-T04A

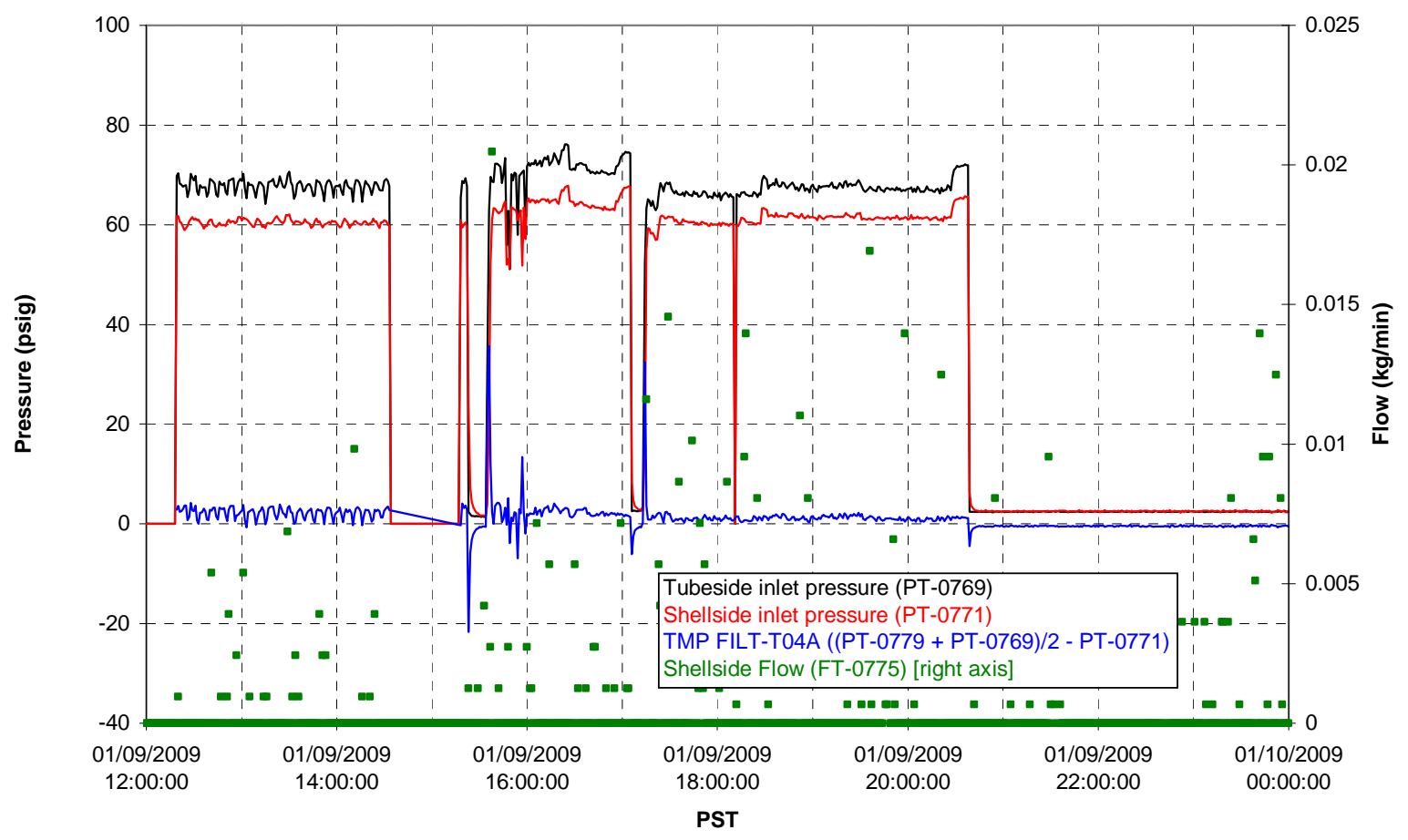


Filter UFP-FILT-T05A

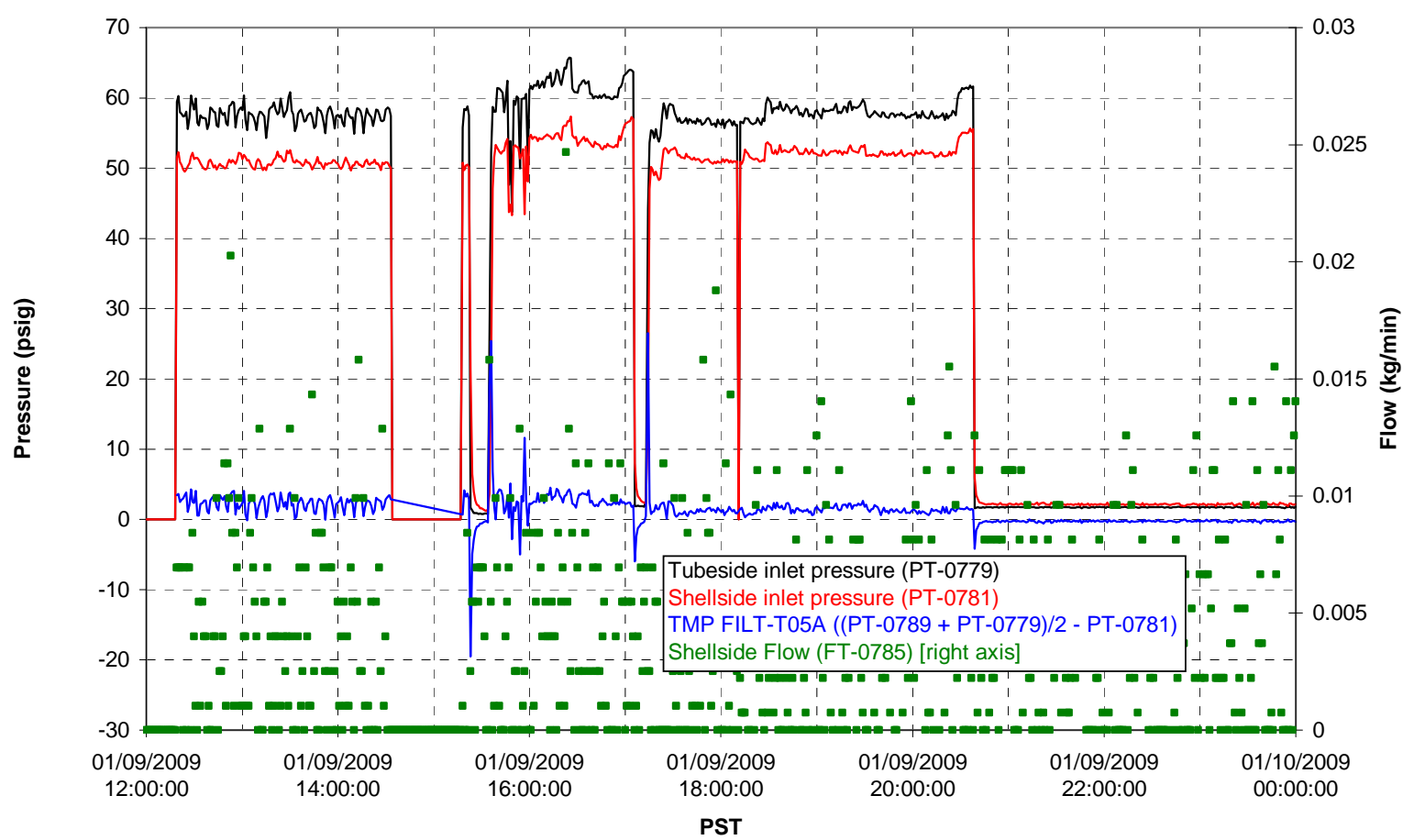

Chemical Flow

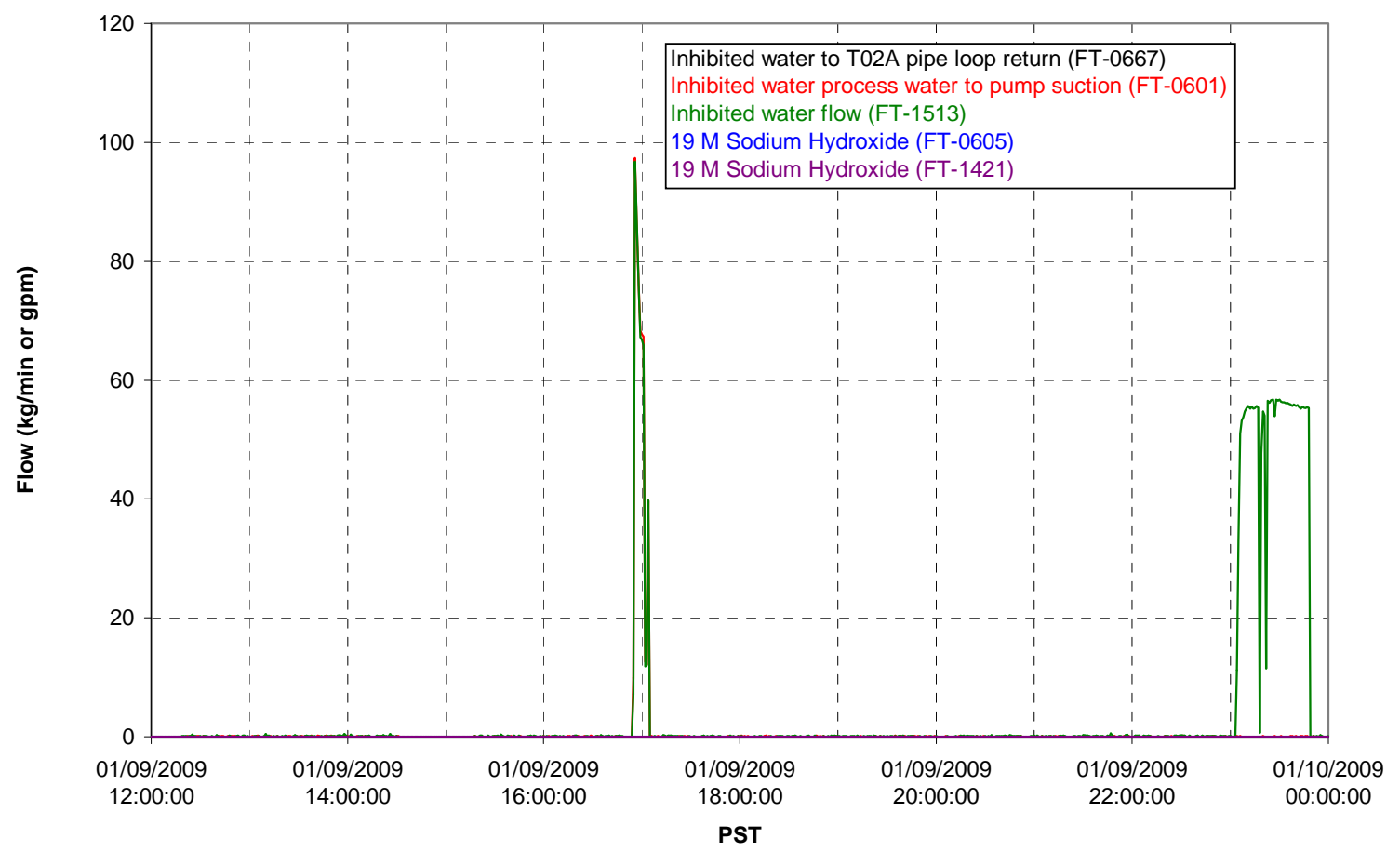




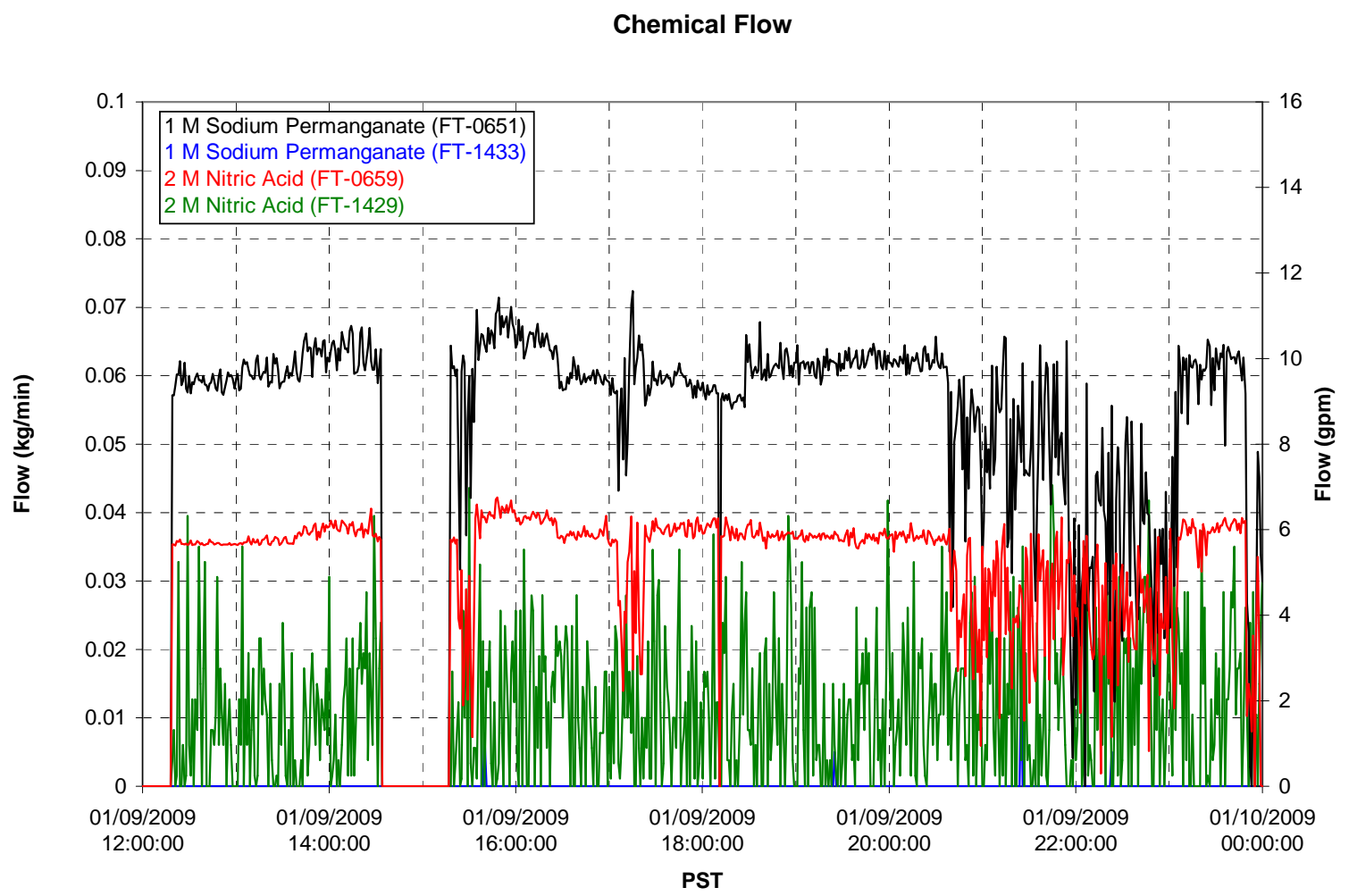

Air Flows

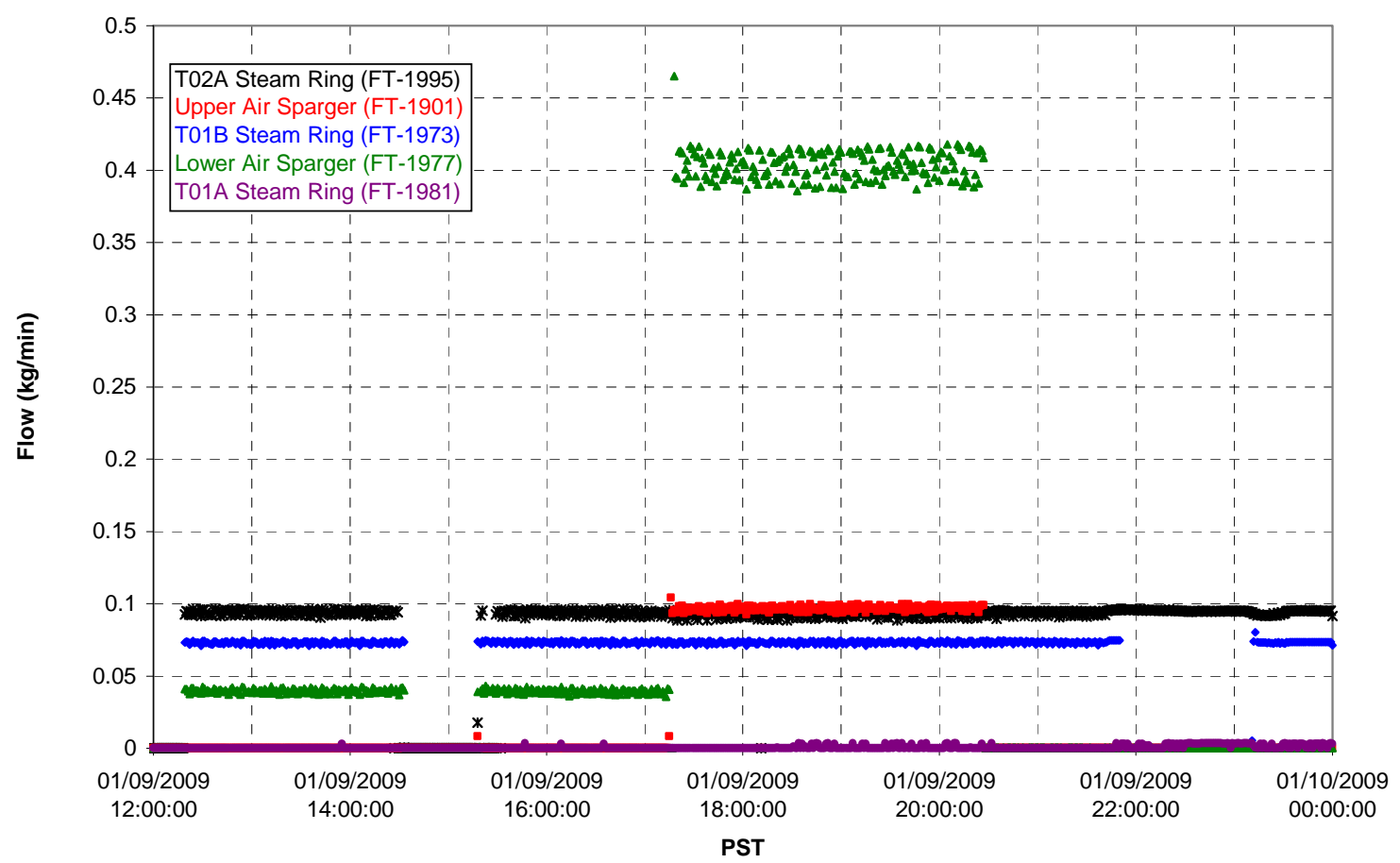


T02A Steam

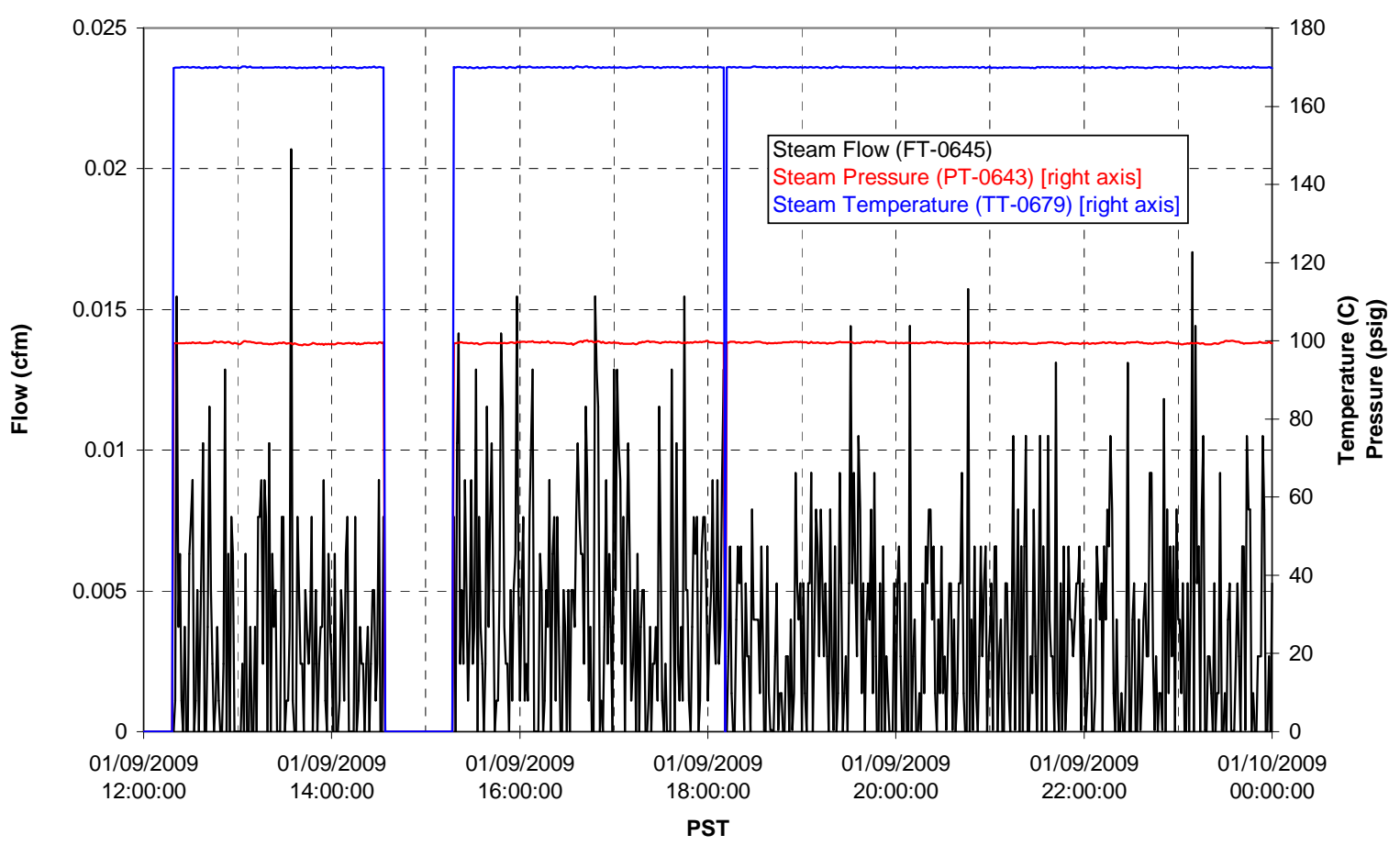

T01A Steam

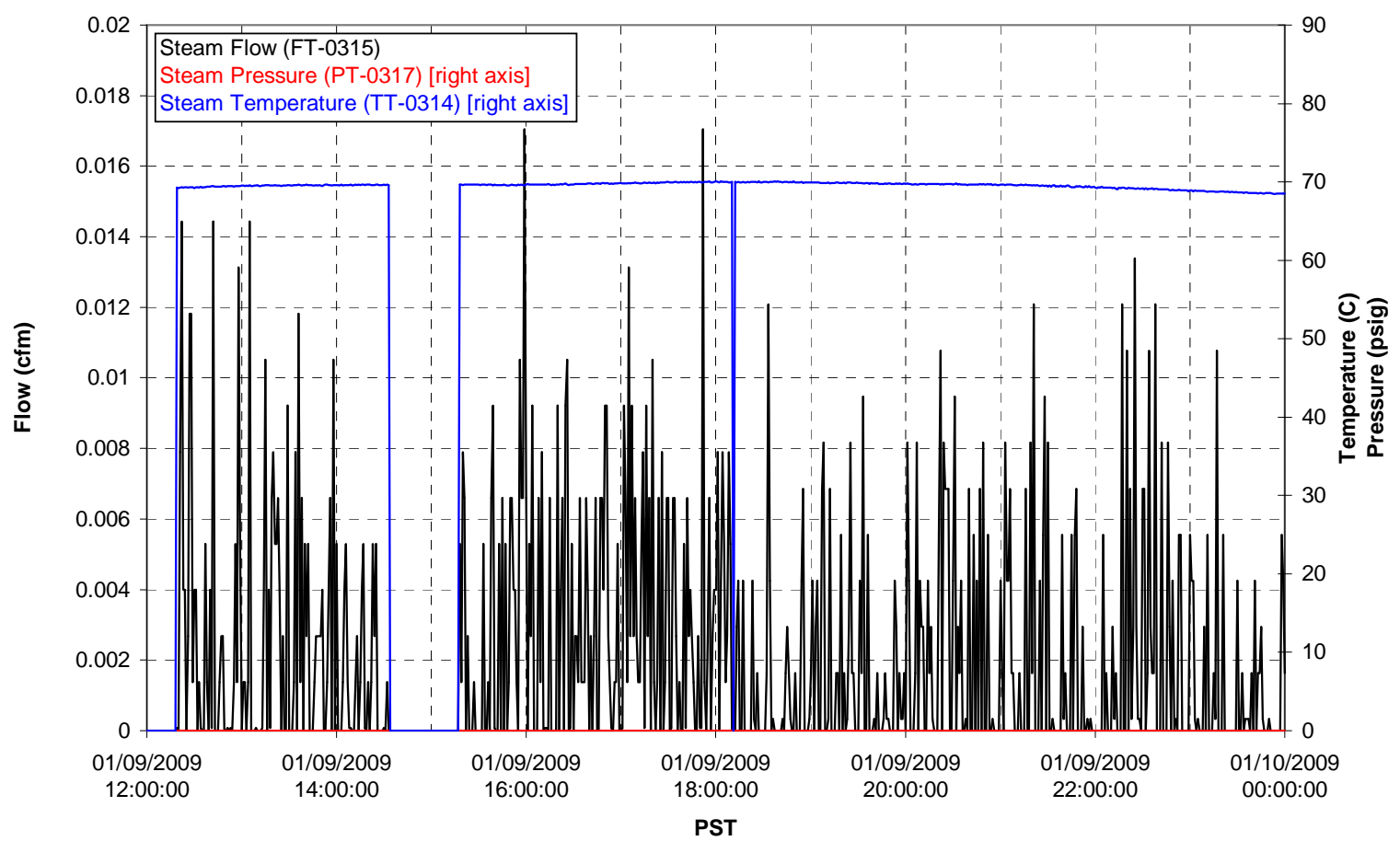


T01B Steam

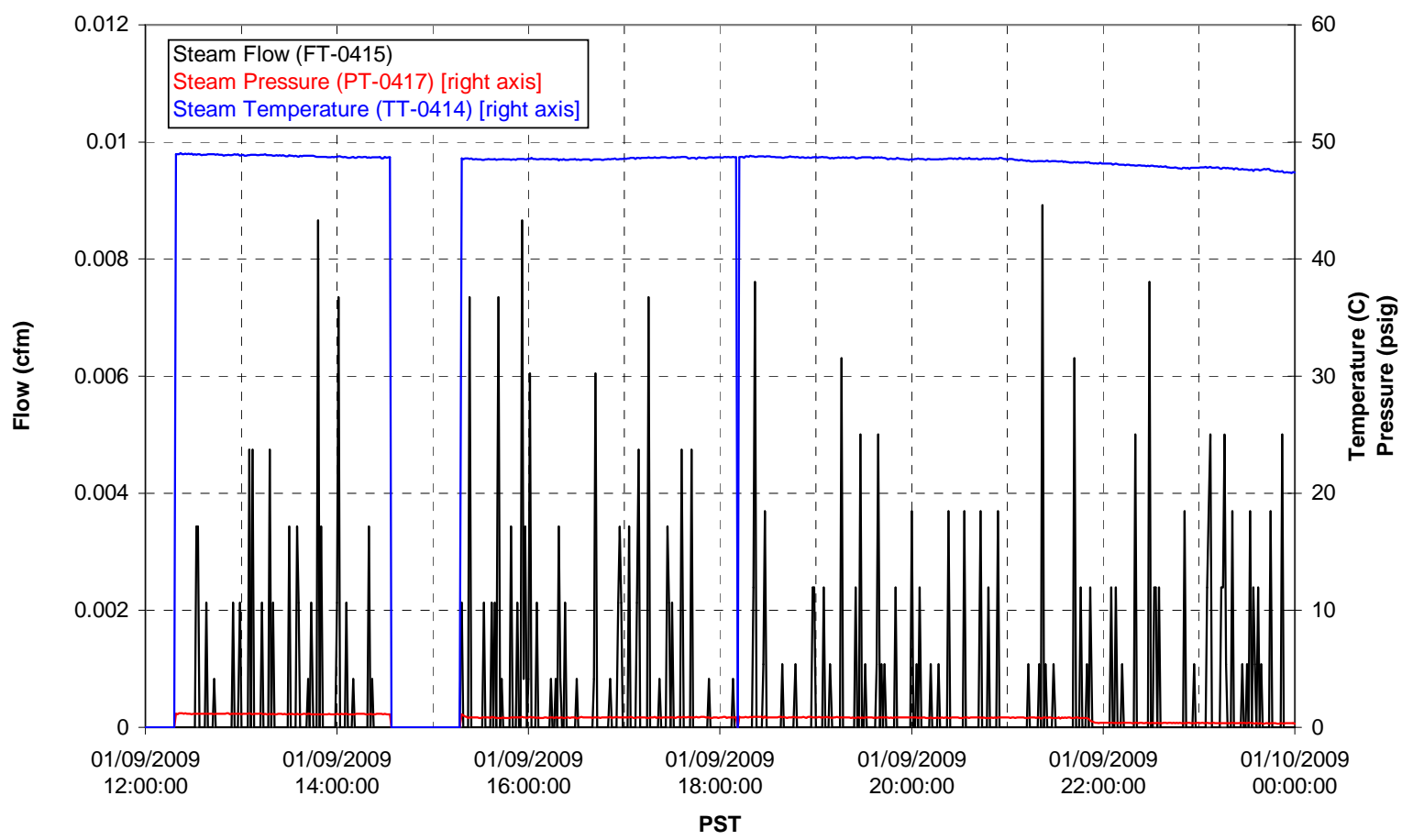


Functional Test Data Plots

01/10/2009 00:00 - 01/10/2009 12:00 
T01A level

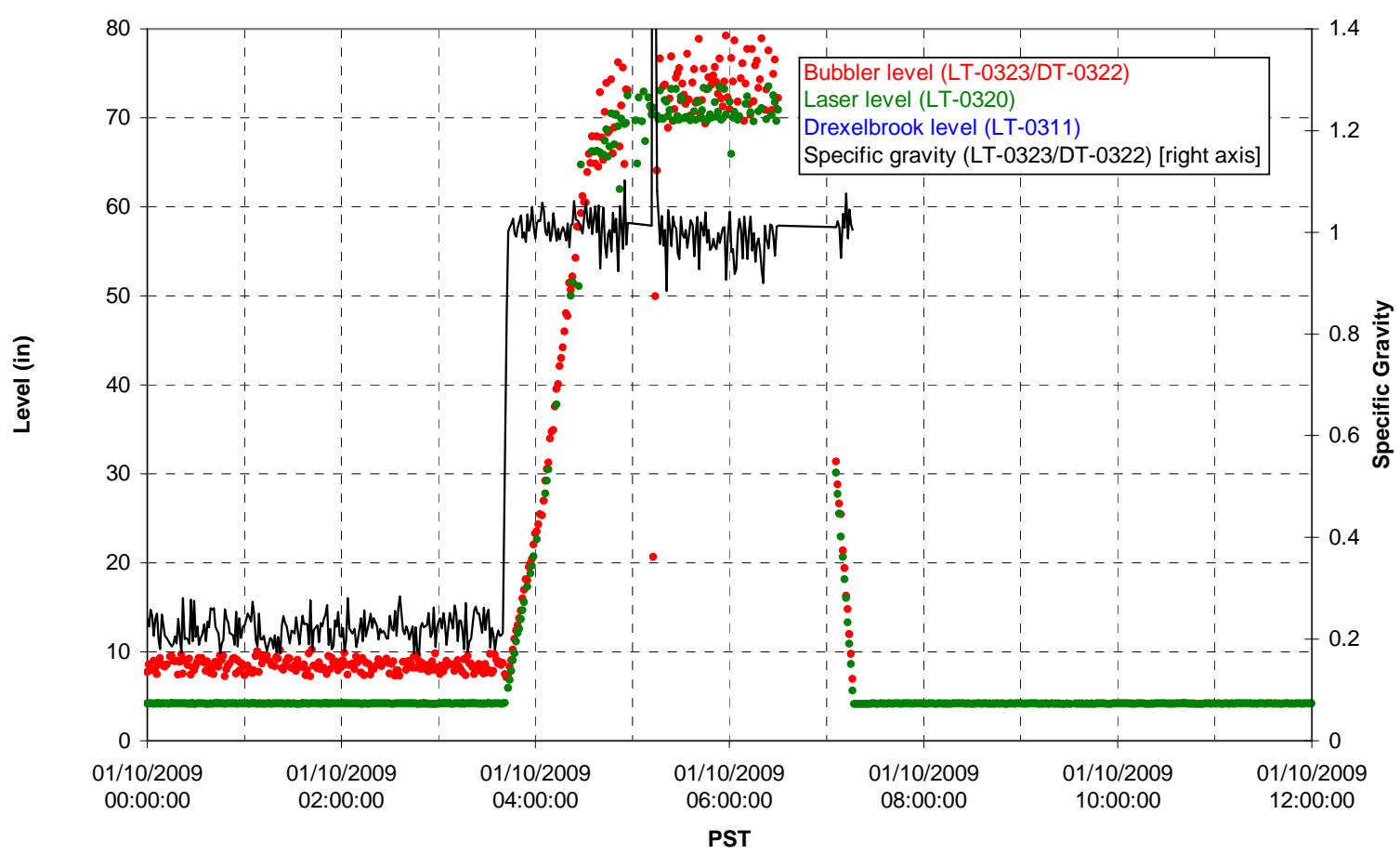

T01A temperatures

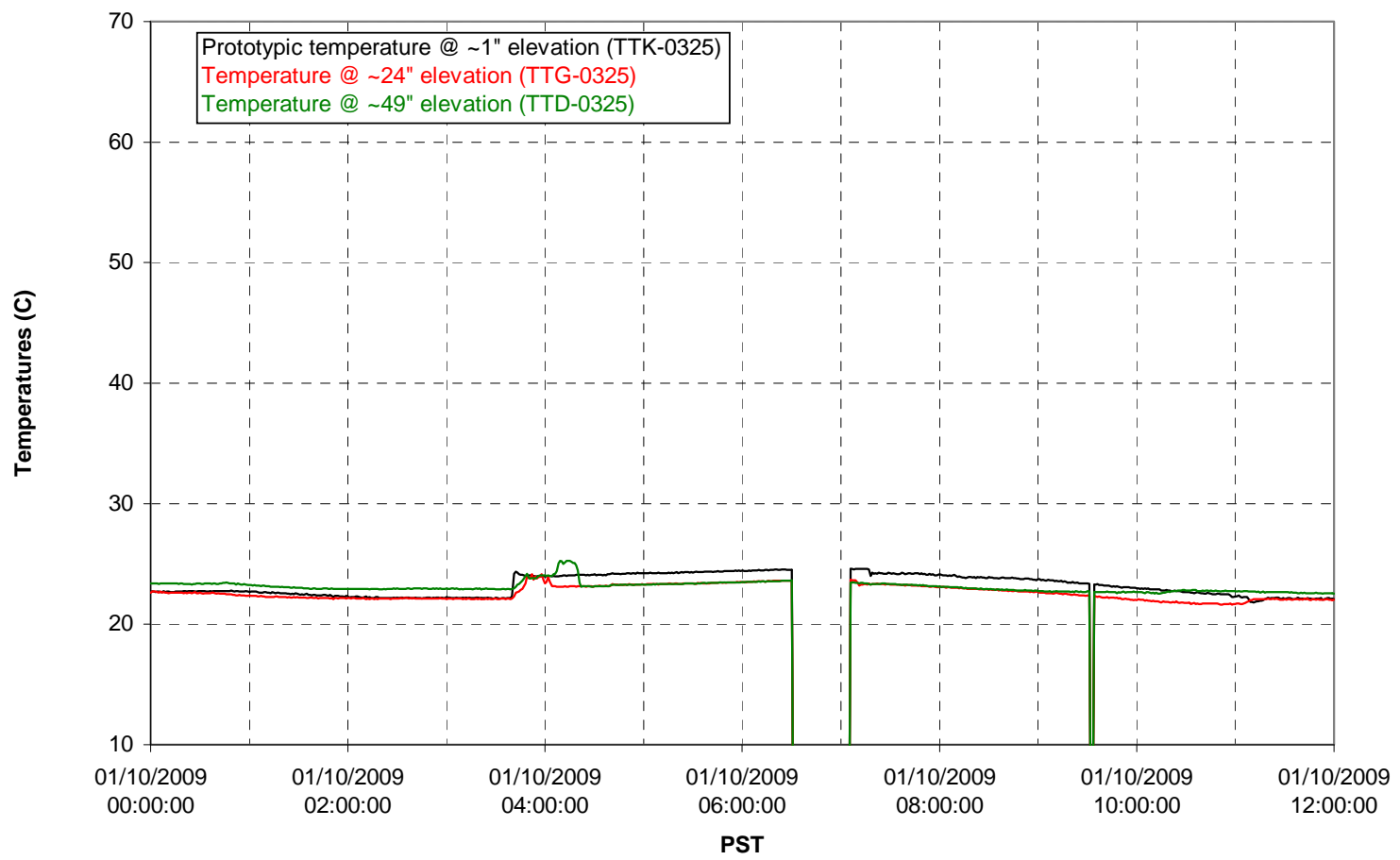


T01B level

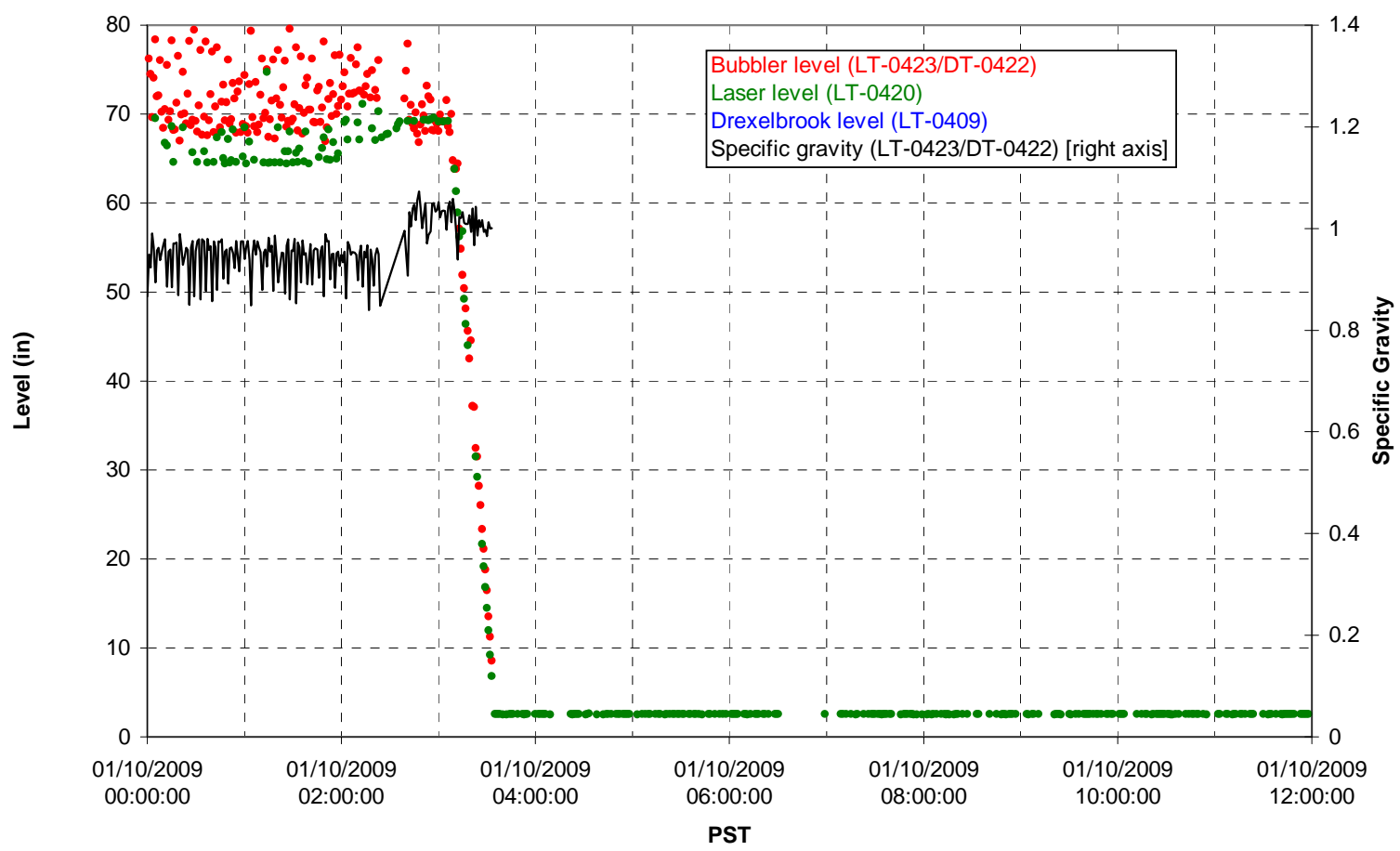

T01B temperatures

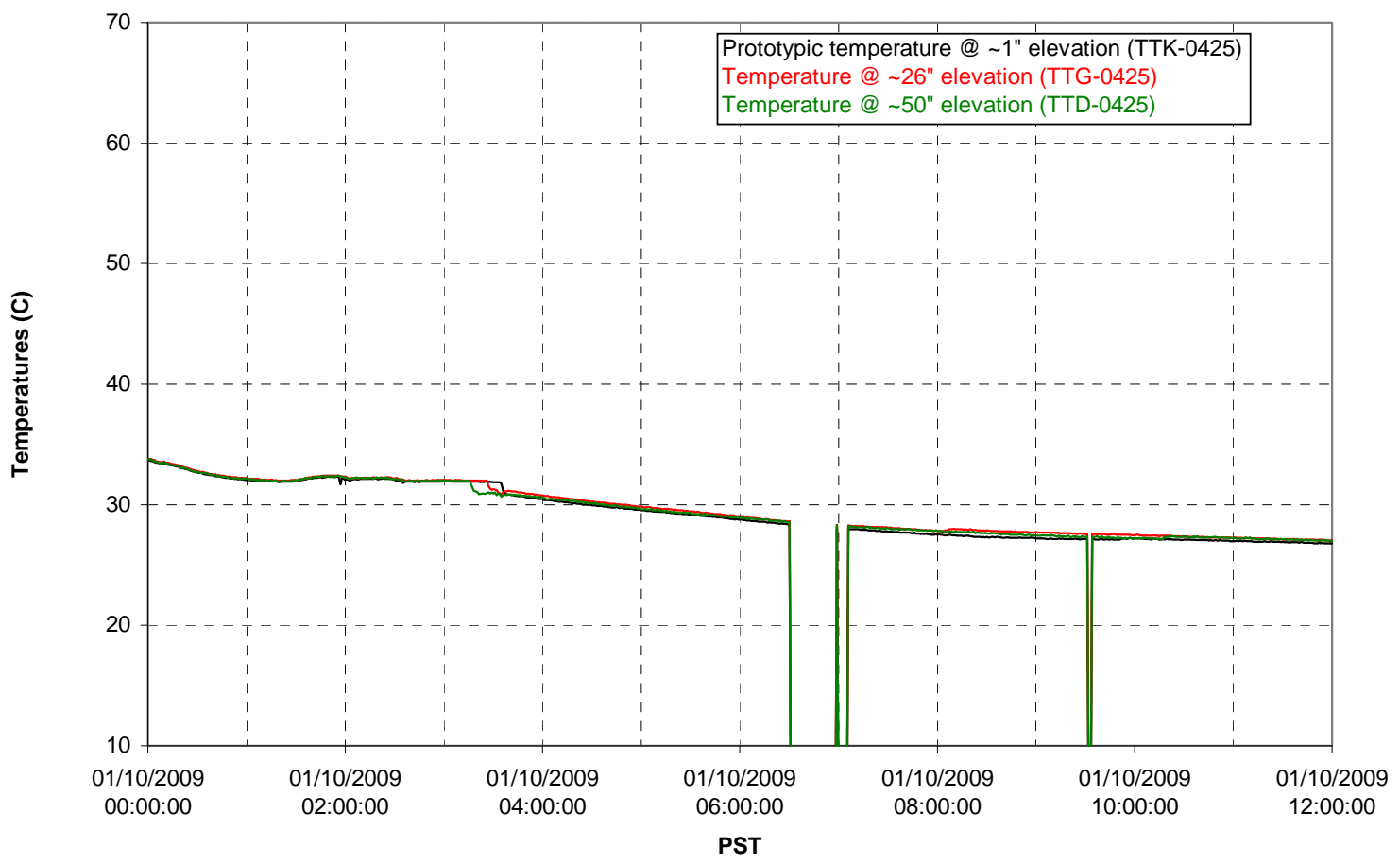


T02A level

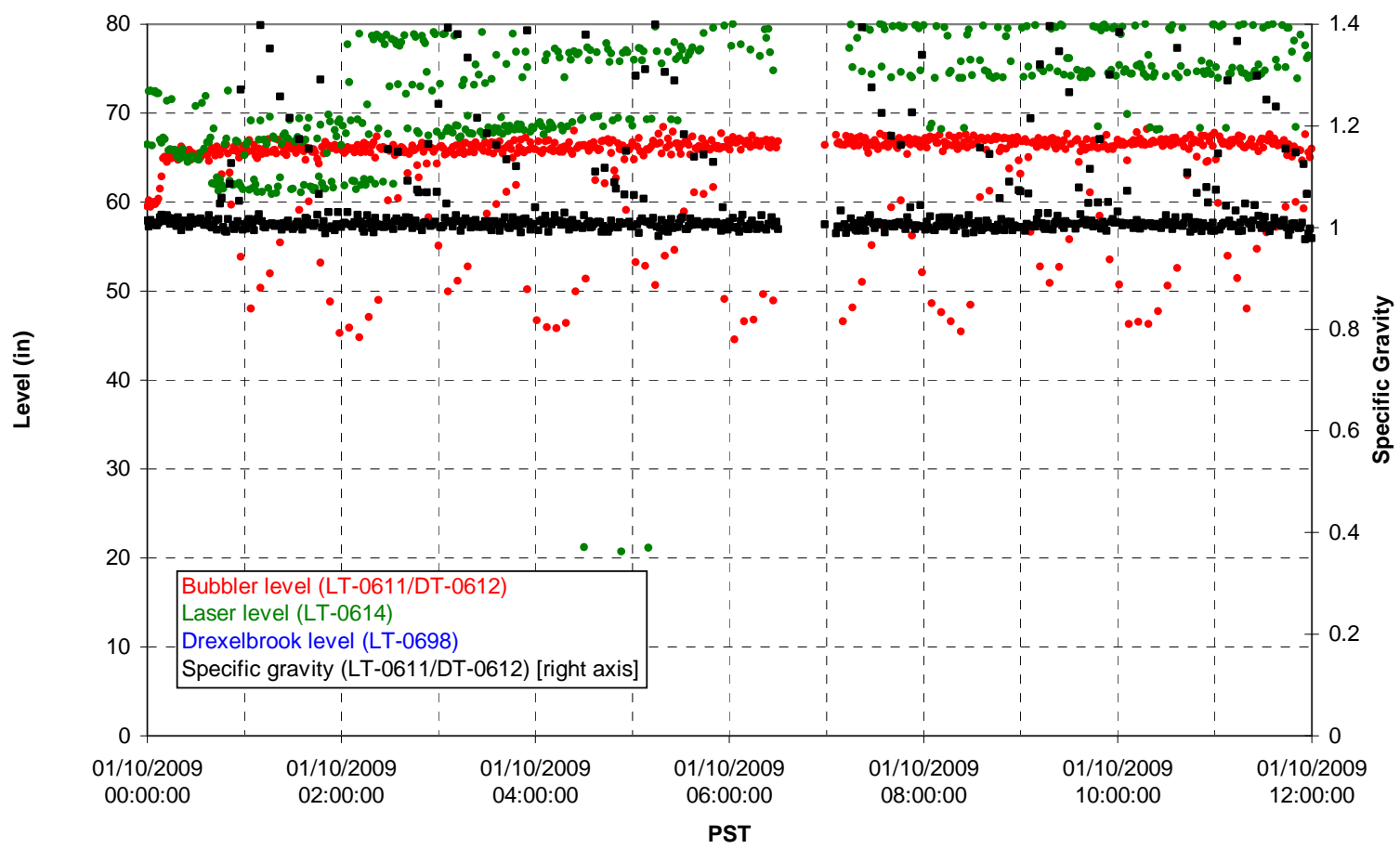

T02A temperatures

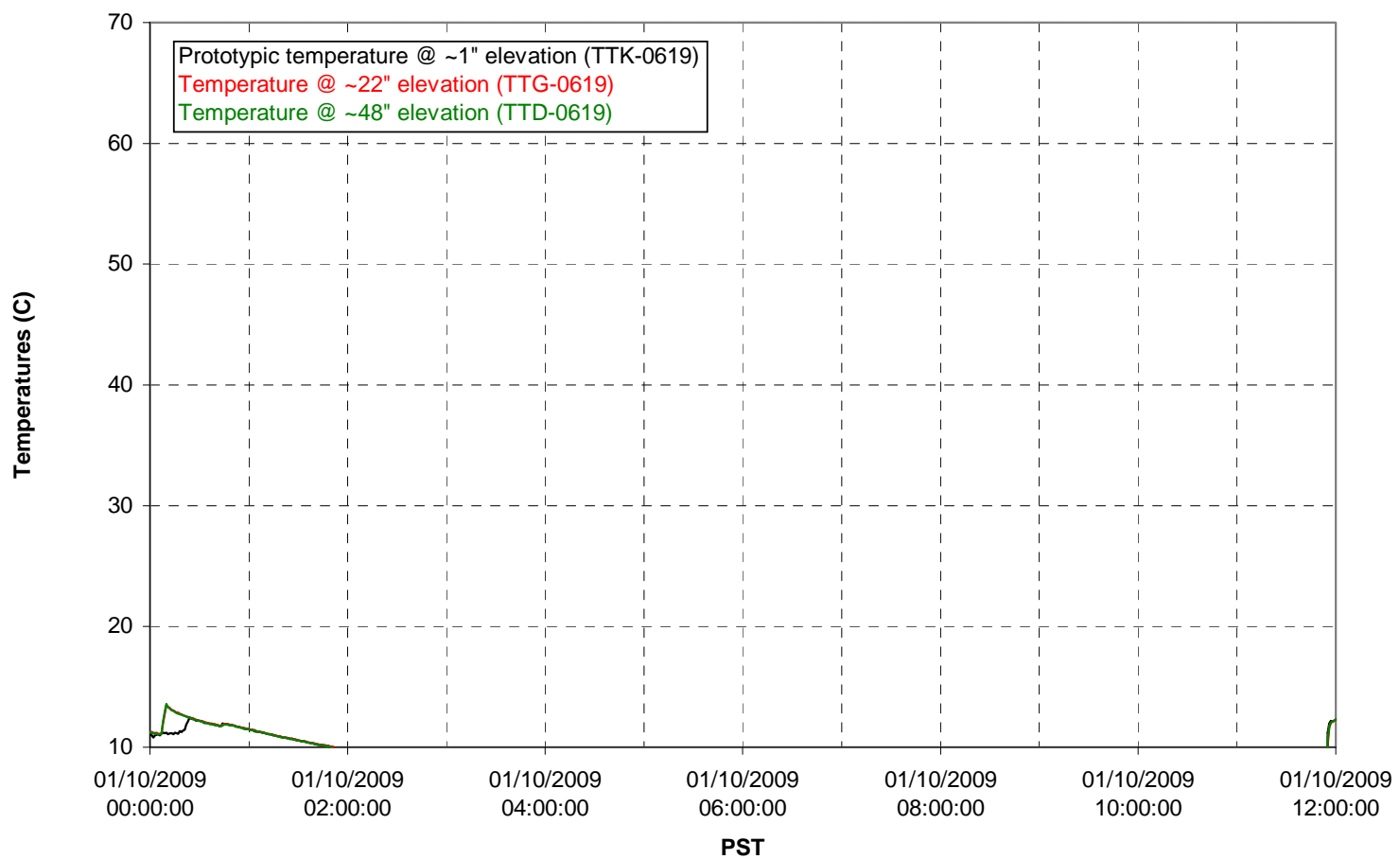


T02A and filter loop temperatures

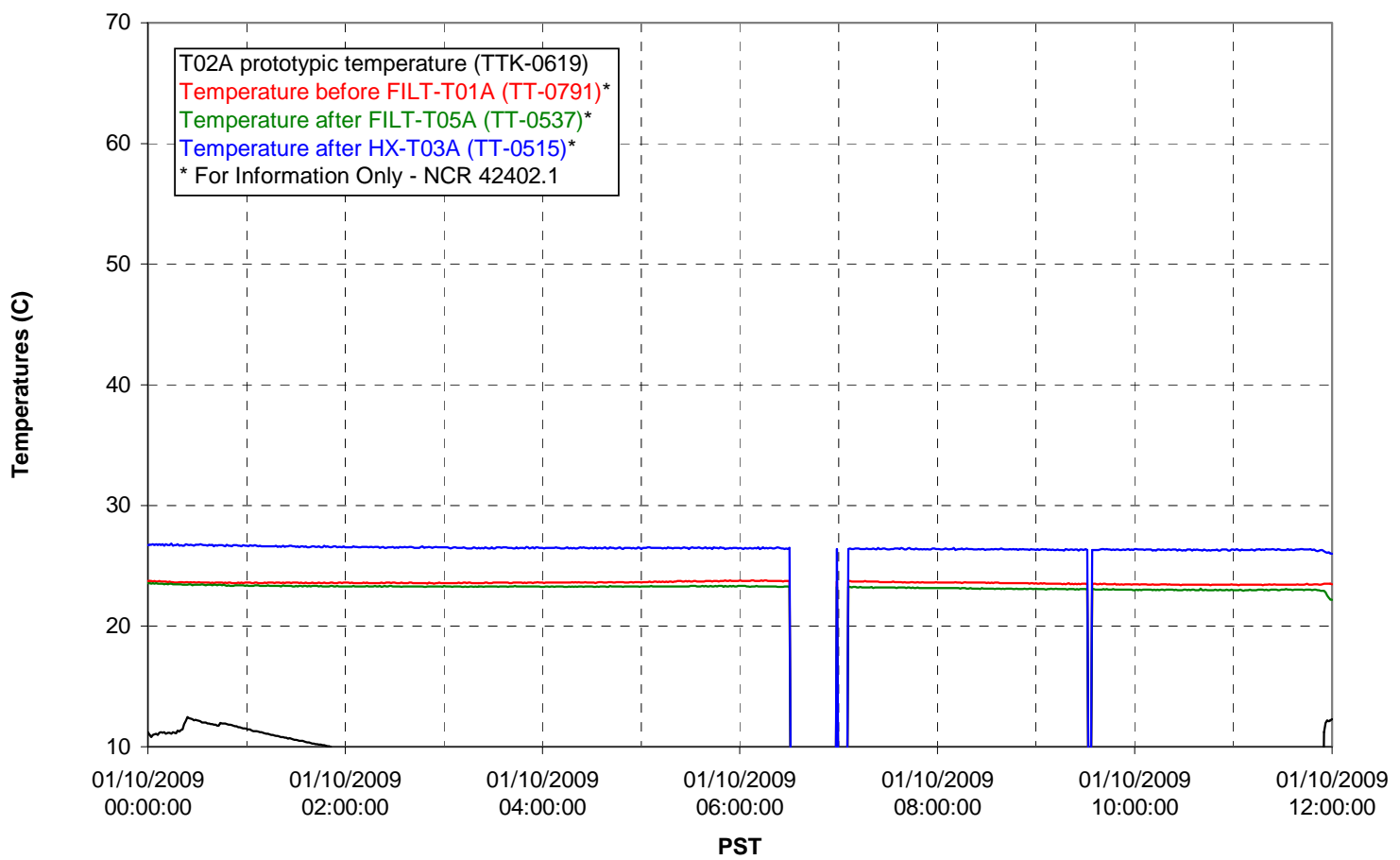

Pump Pressures and Flow

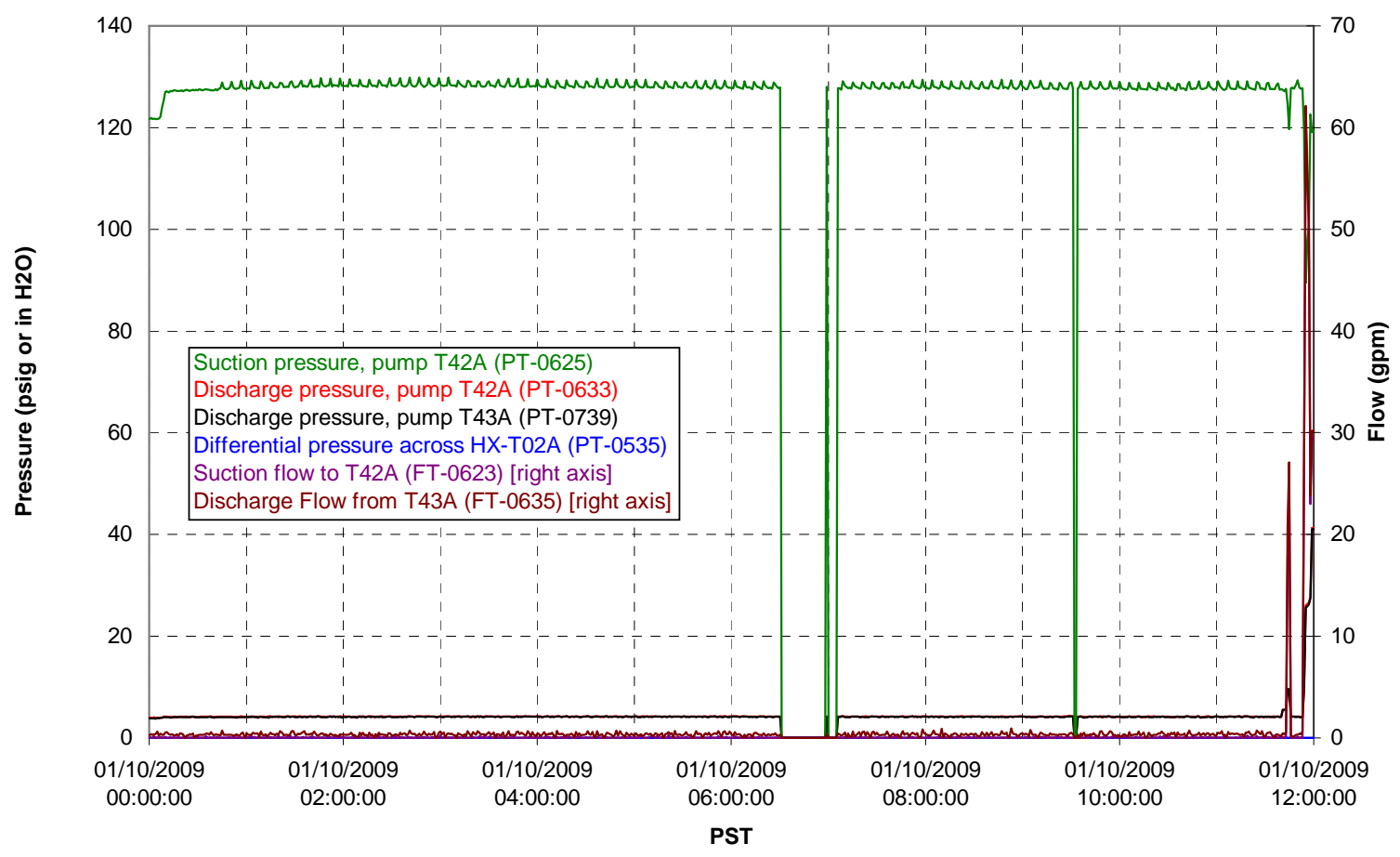




\section{Axial pressure drop}

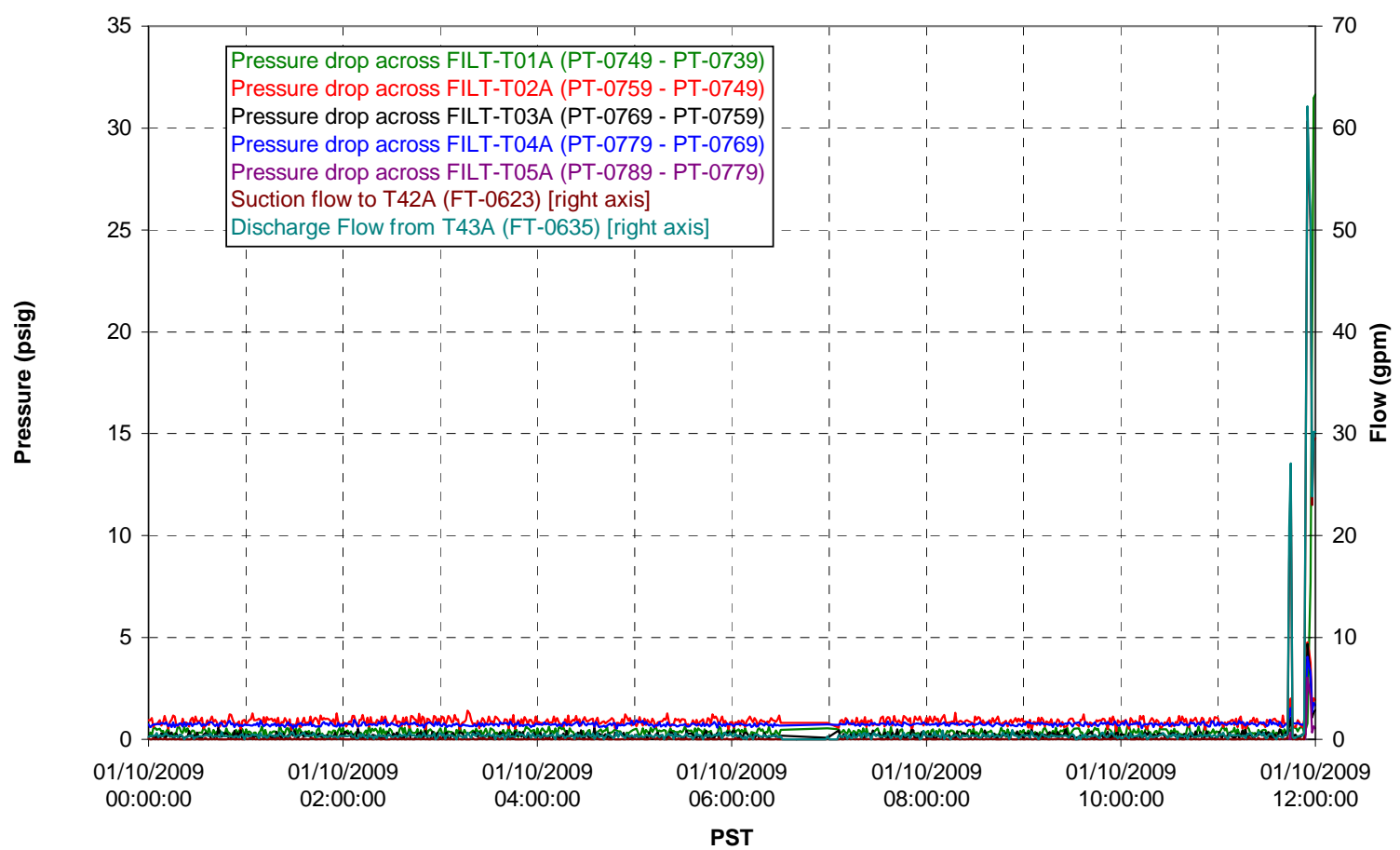

Permeate flow rates

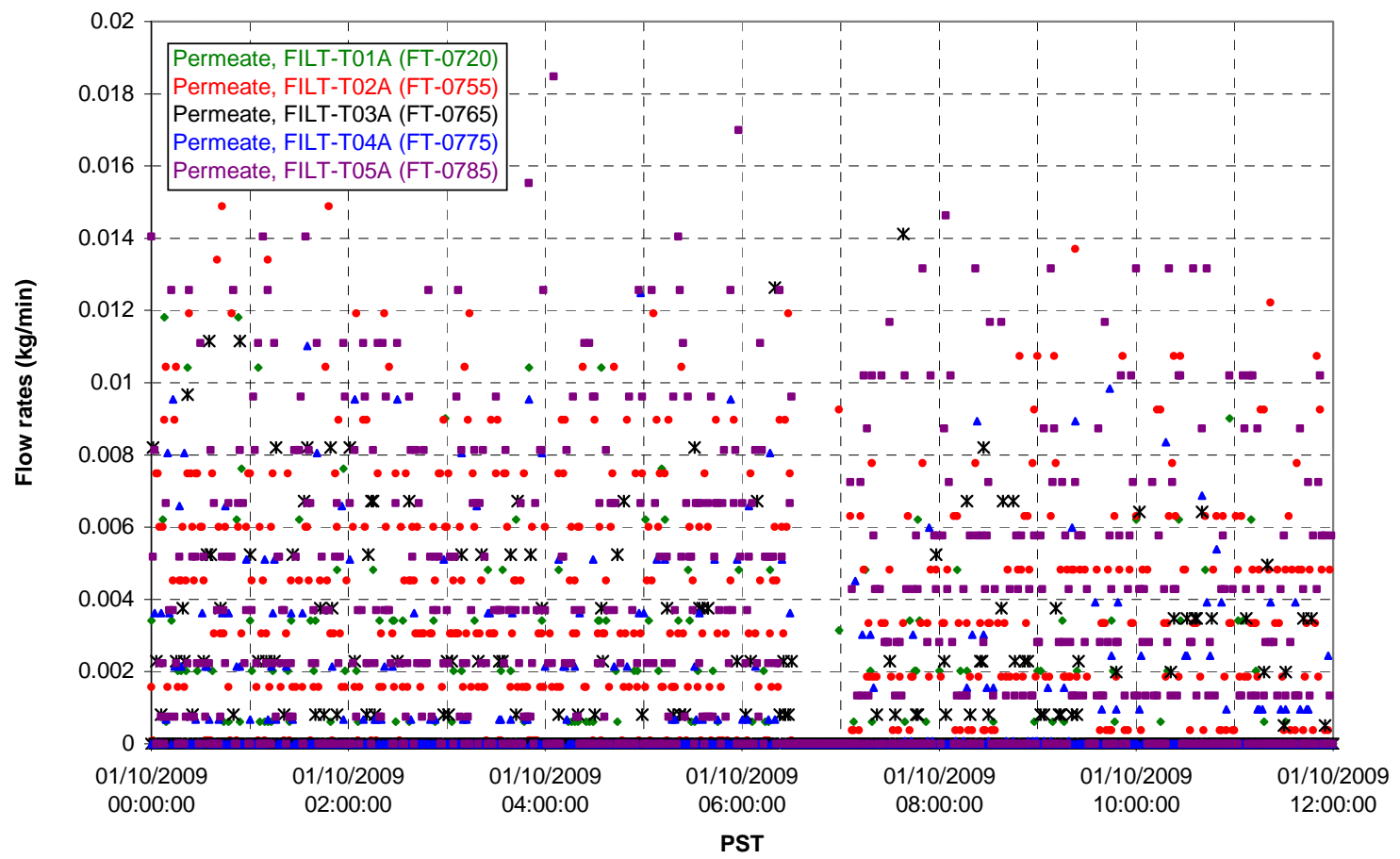


T02A Inner Temperature Tree

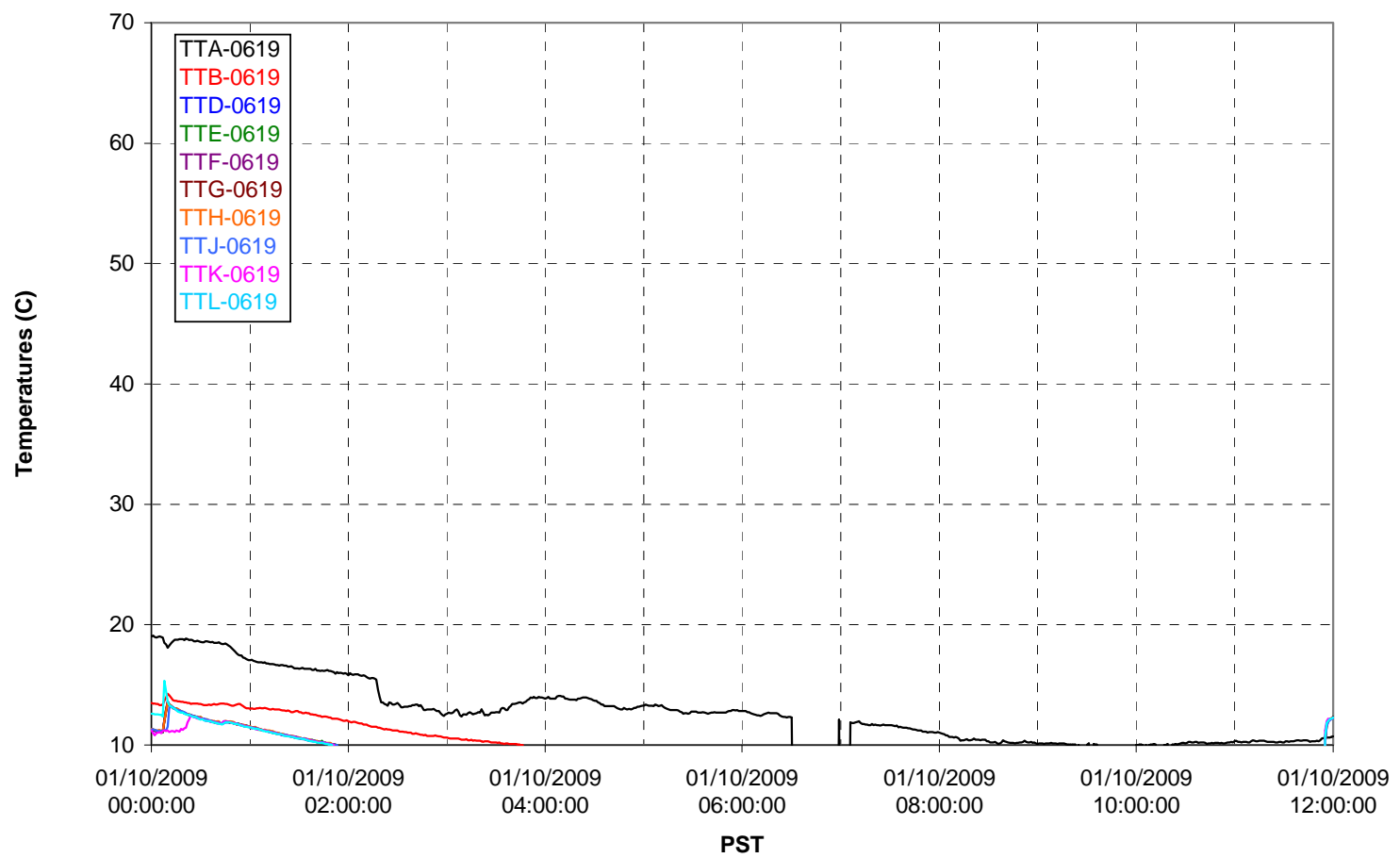

T02A Outer Temperature Tree

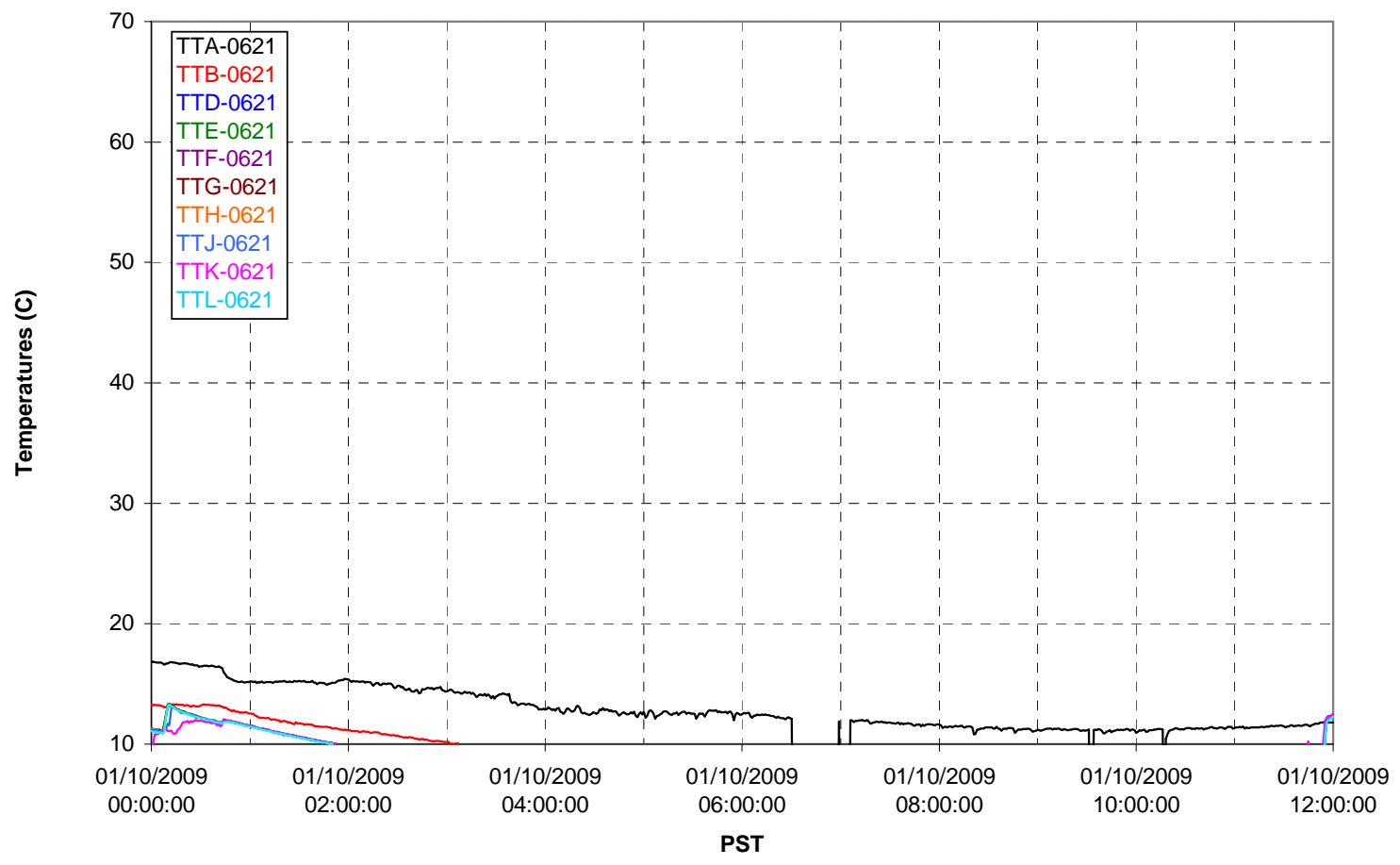


T02A temperatures

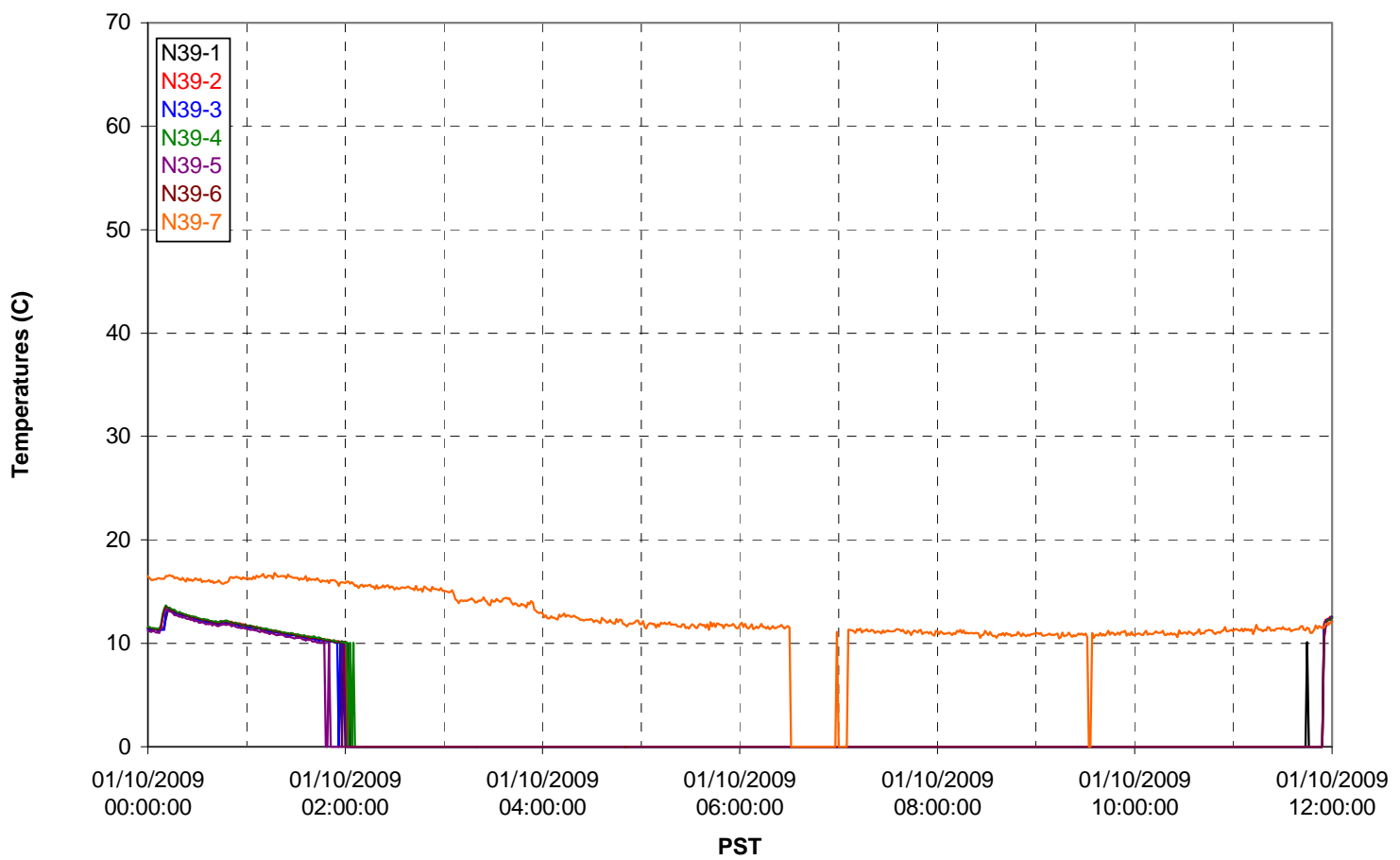

T02A temperatures

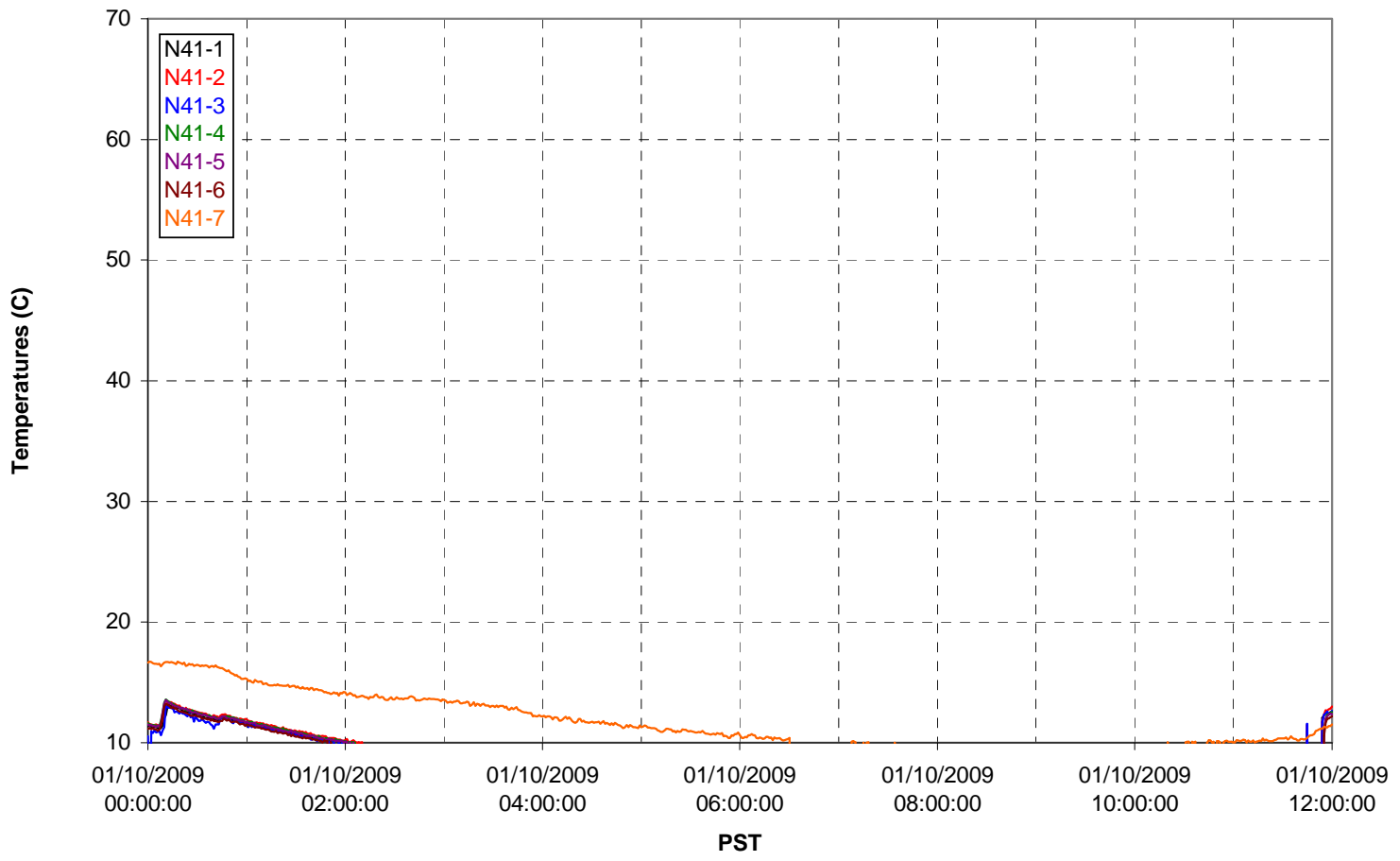


T02A temperatures

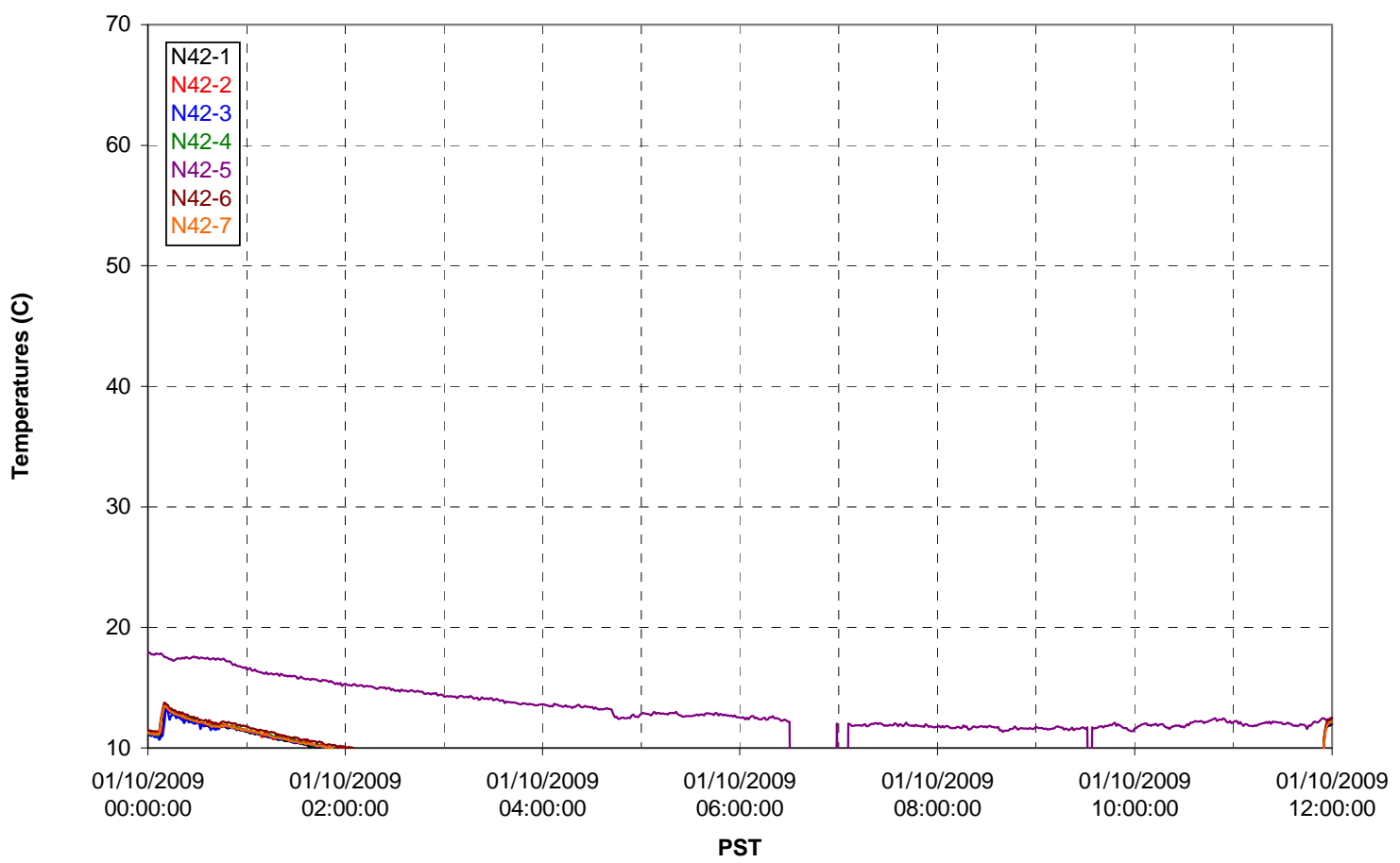

T02A temperatures

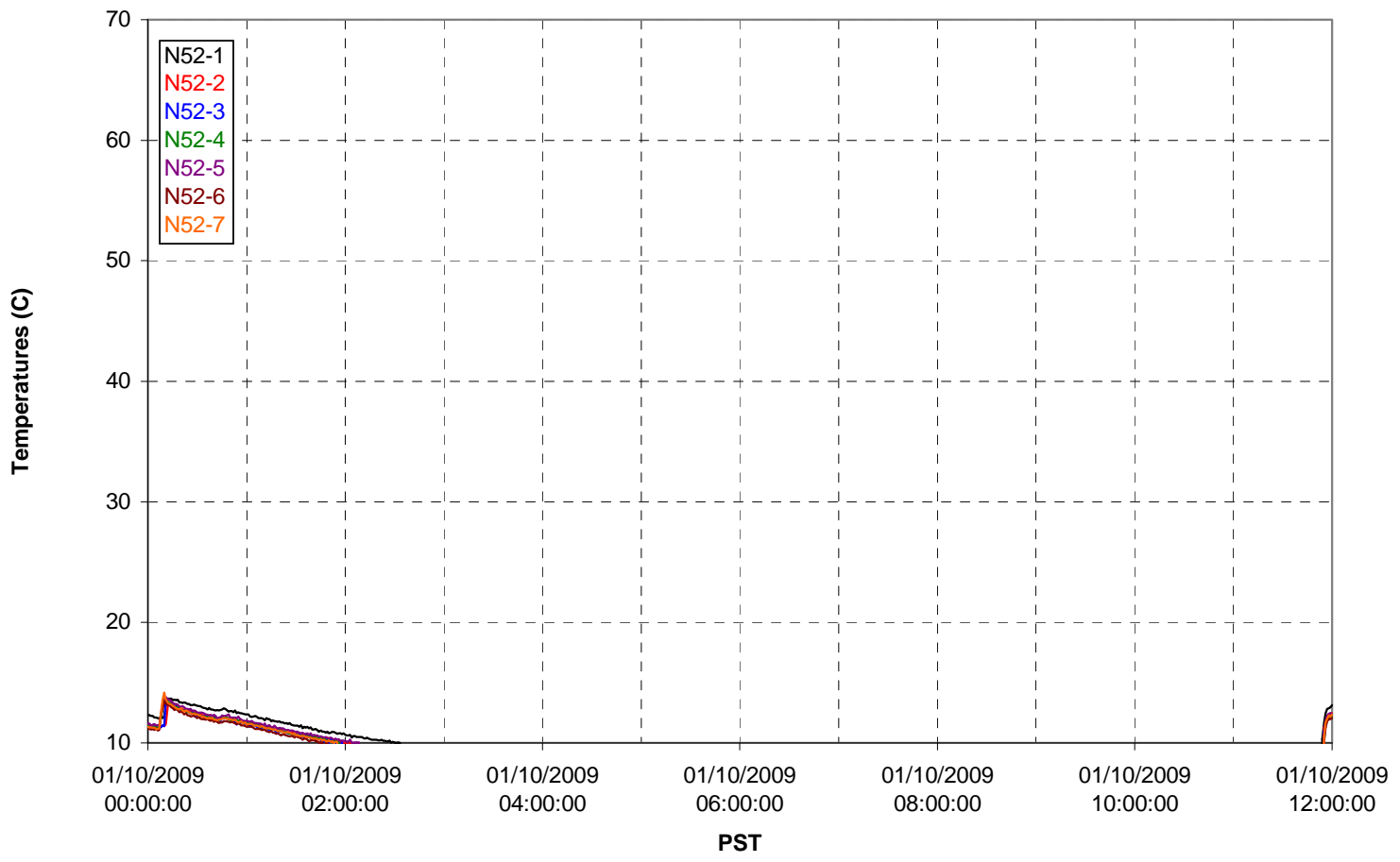


T02A Heating and Cooling

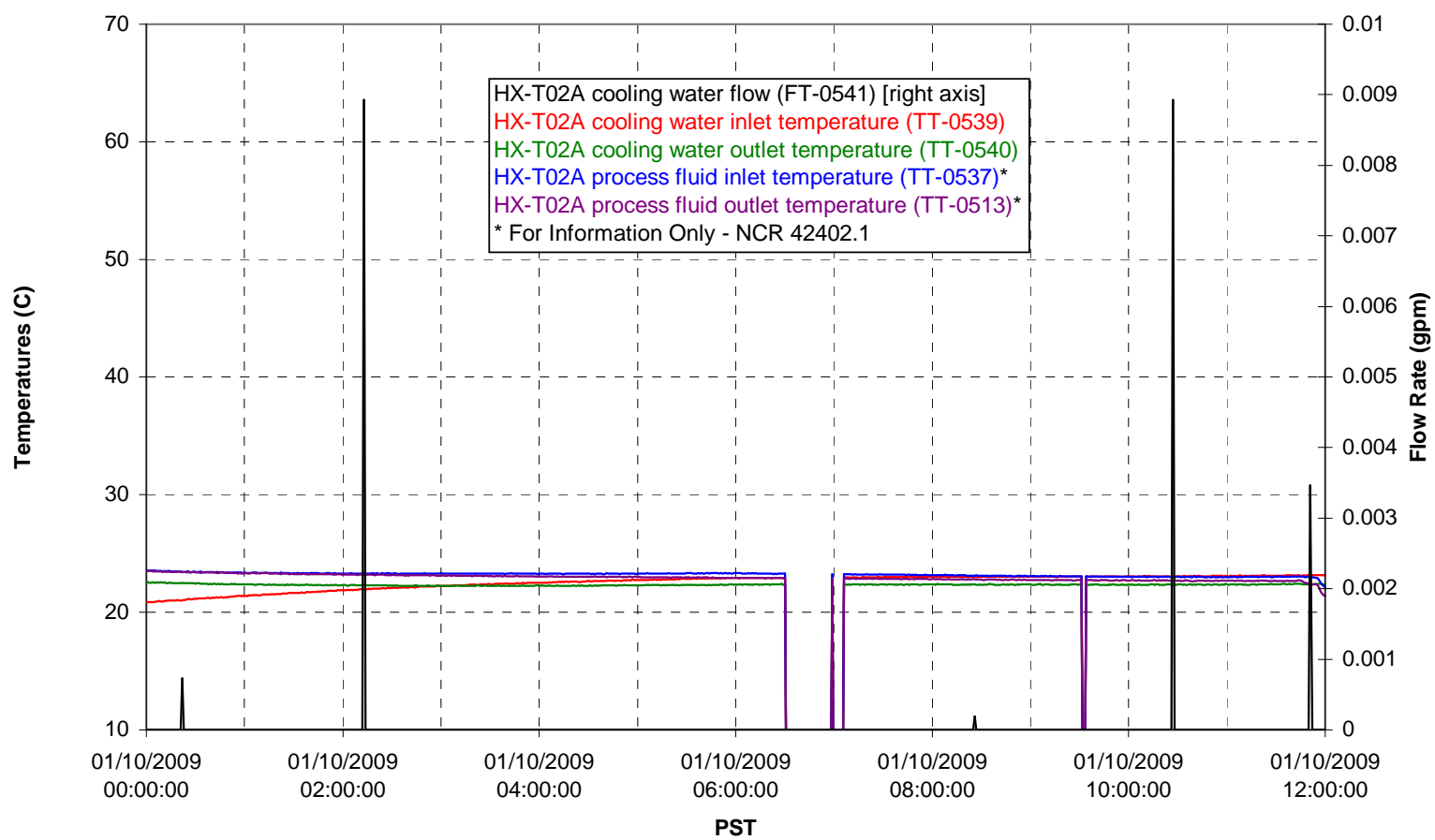

Pump Operation

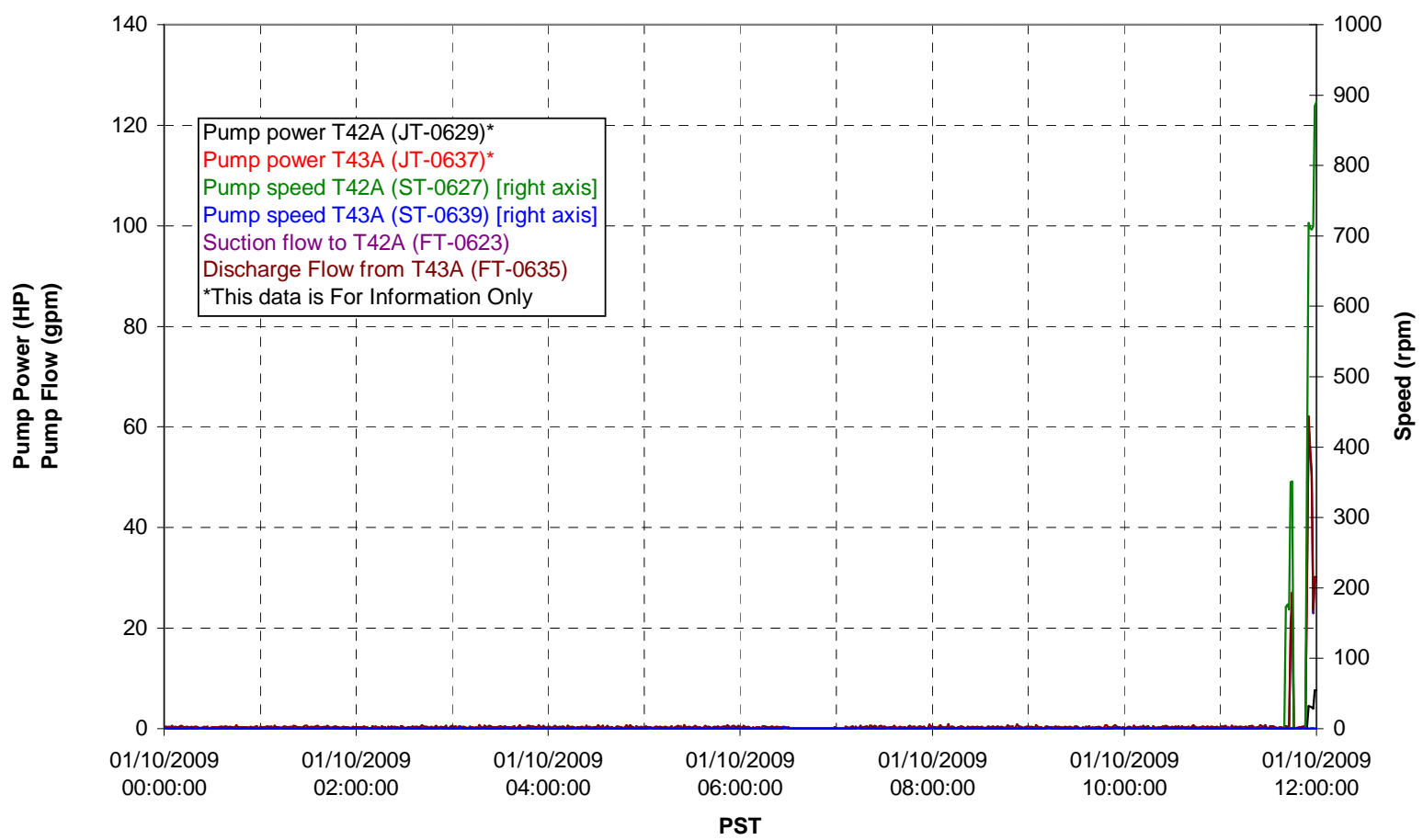


Pulsepot UFP-PP-T01A

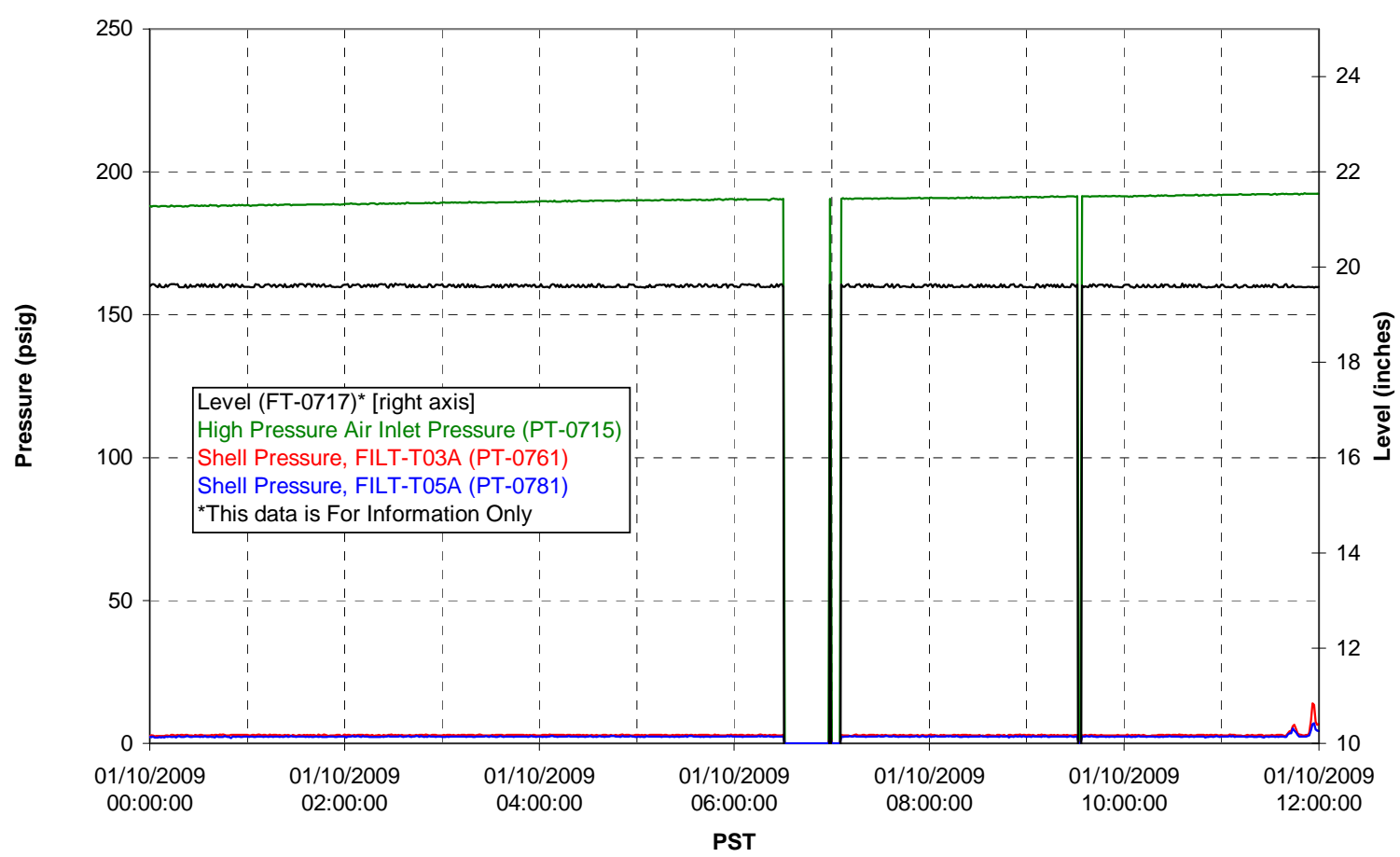

Pulsepot UFP-PP-T02A

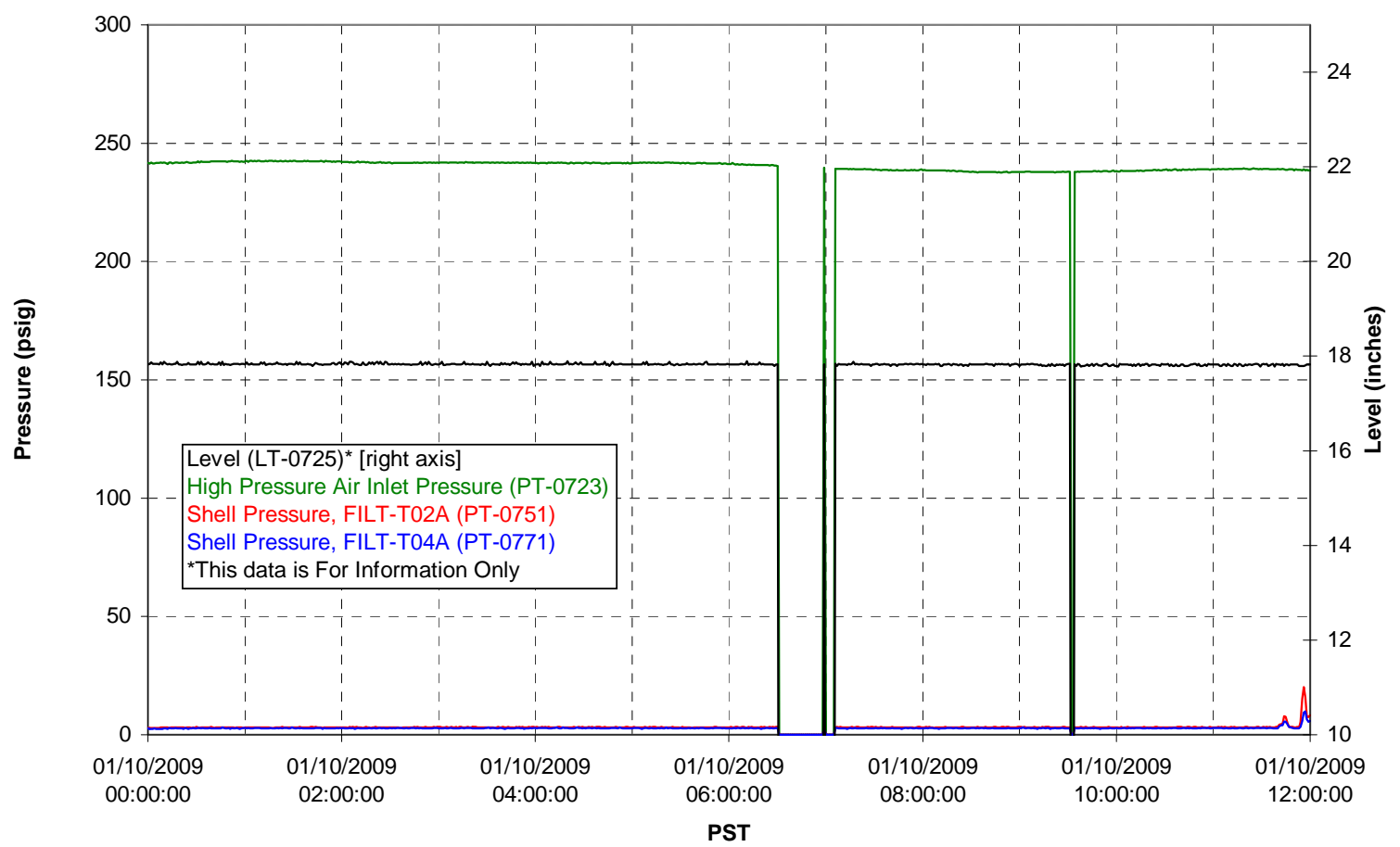


Pulsepot UFP-PP-T03A

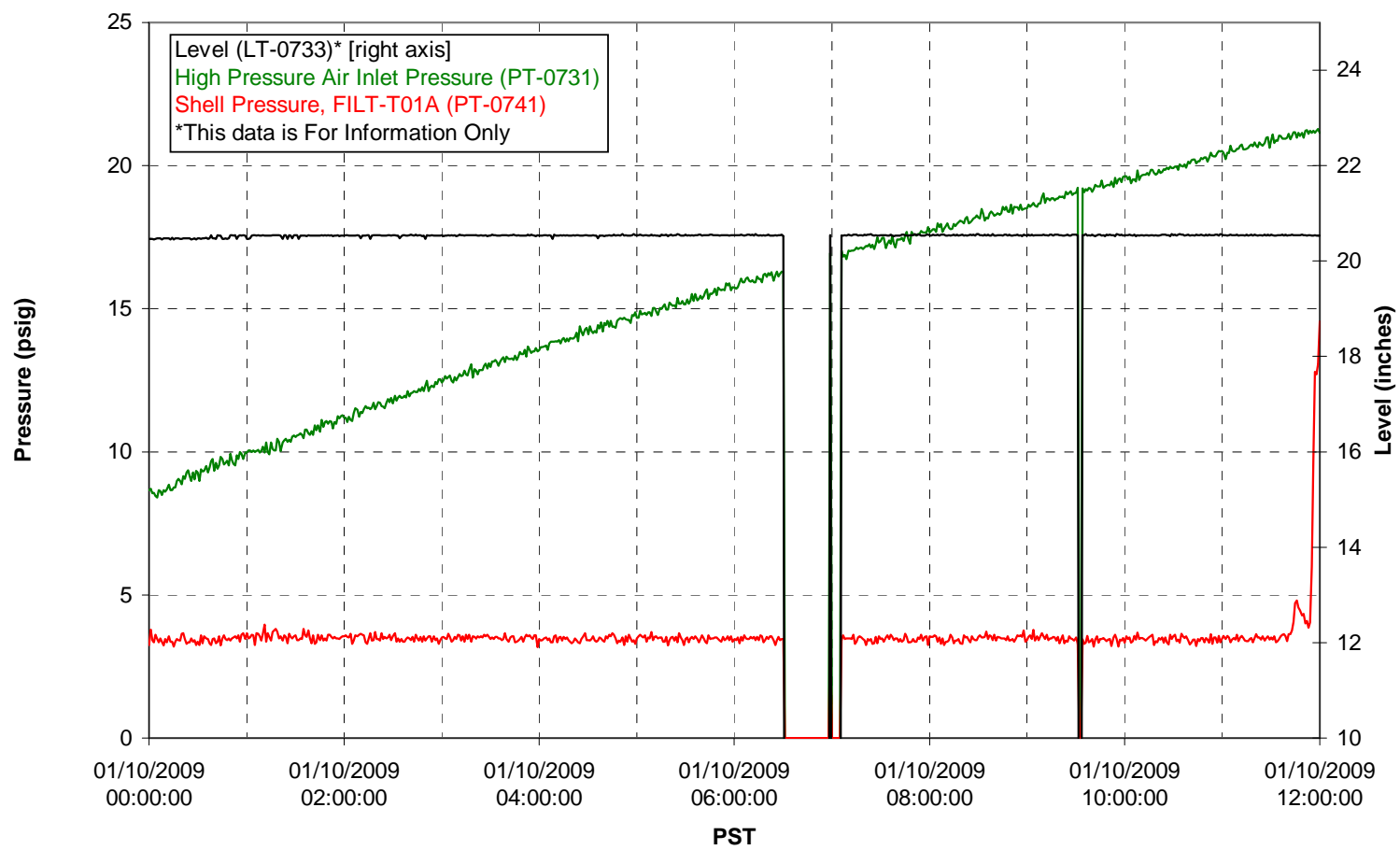

Pulsepot Levels

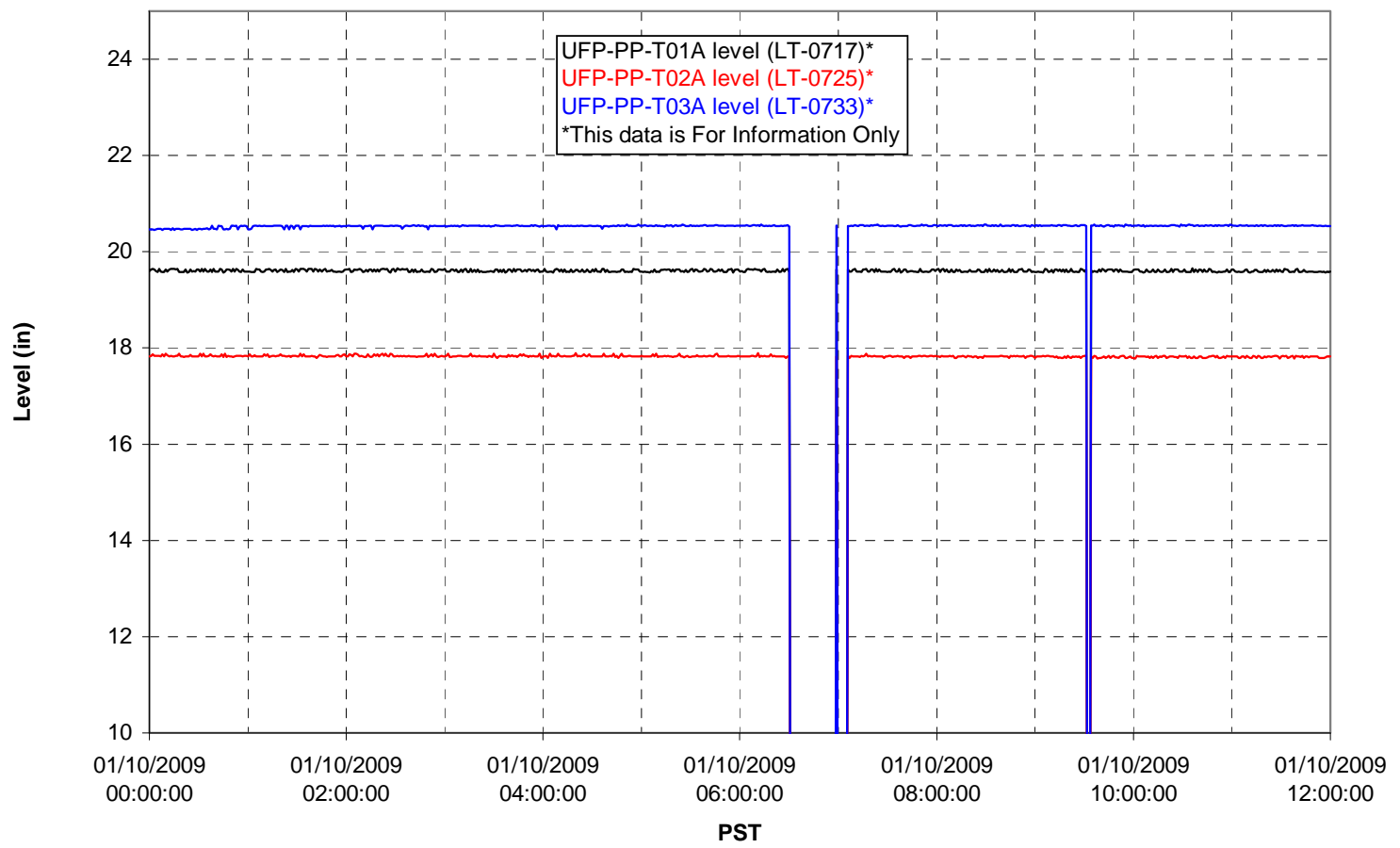


Filter UFP-FILT-T01A

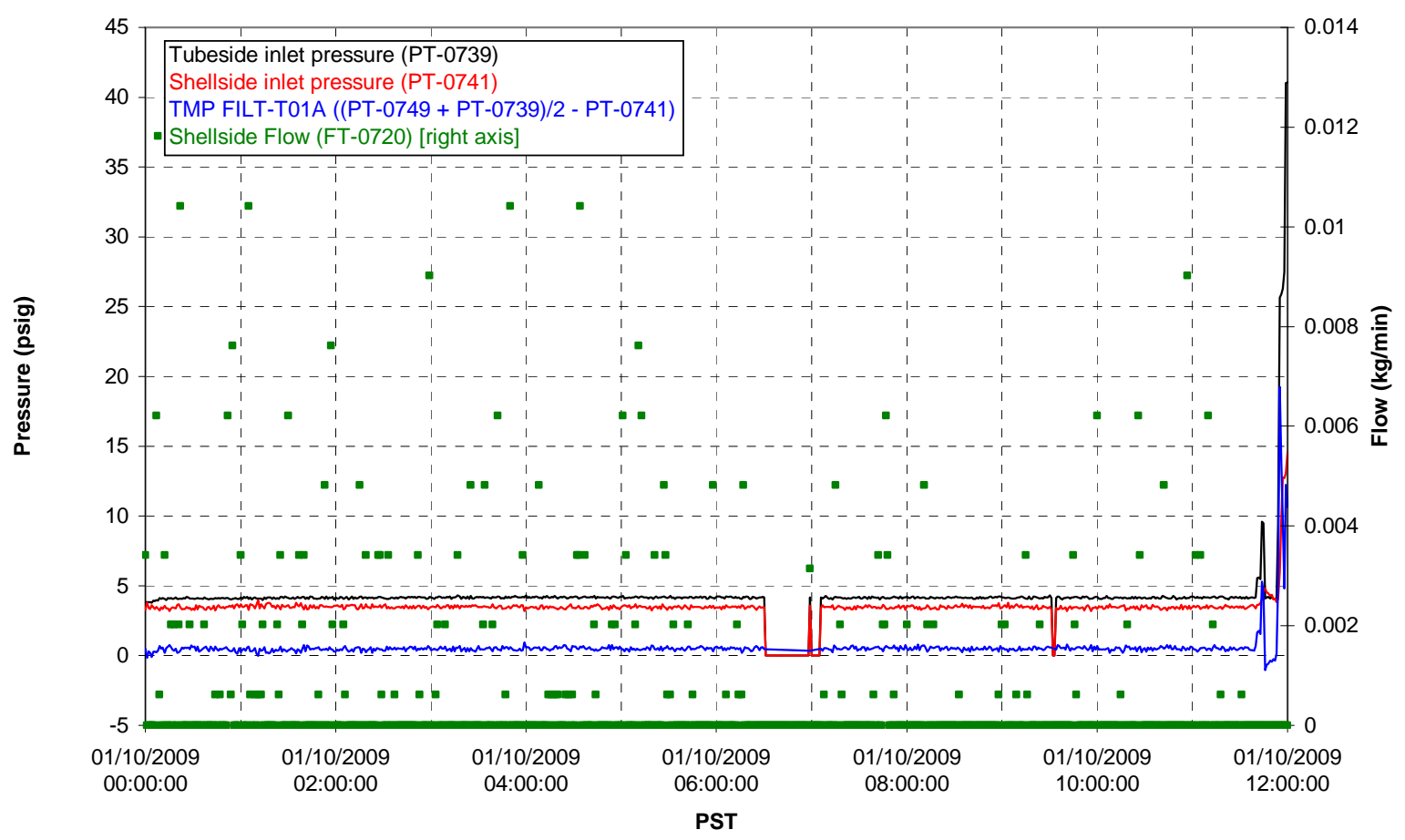

Filter UFP-FILT-T02A

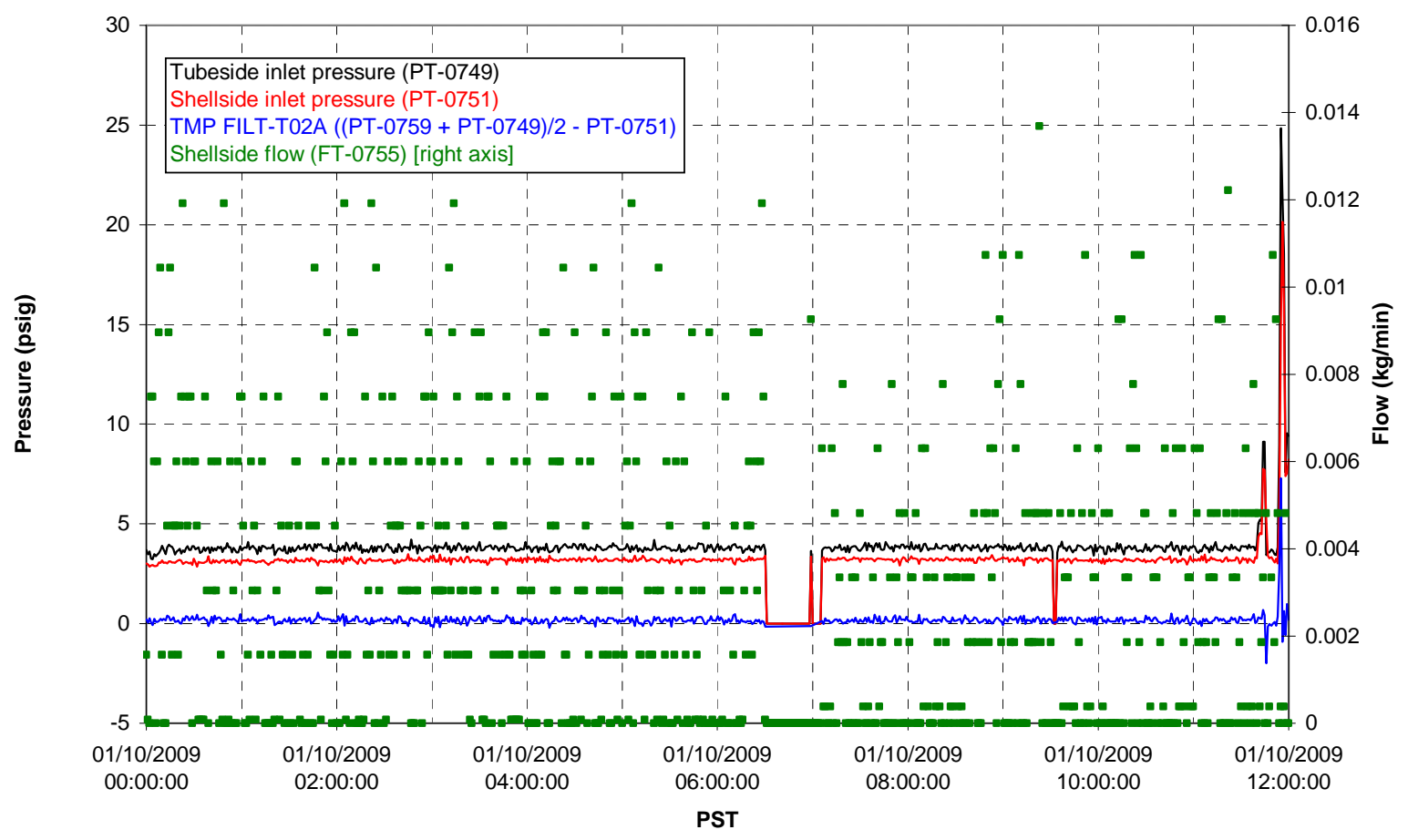


Filter UFP-FILT-T03A

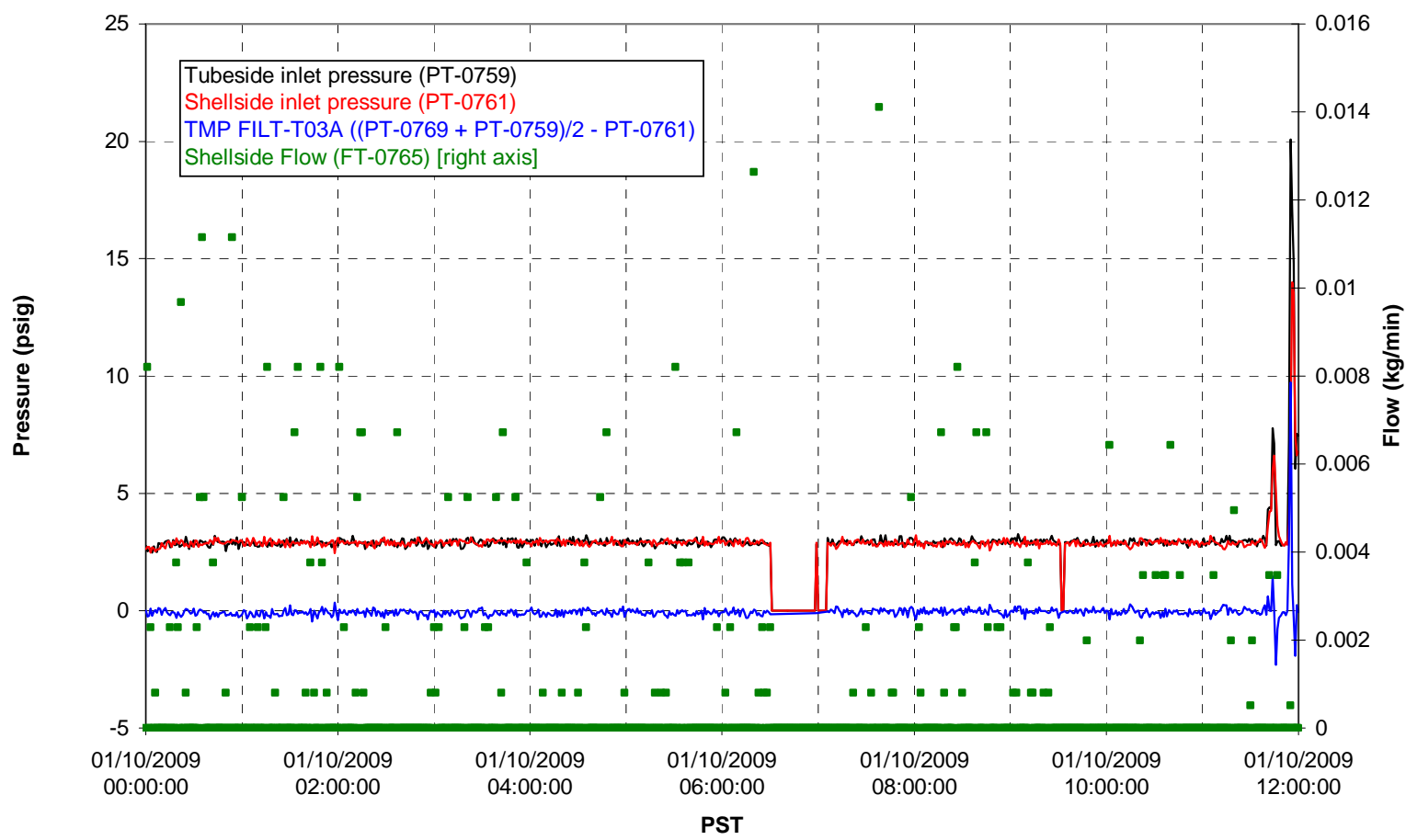

Filter UFP-FILT-T04A

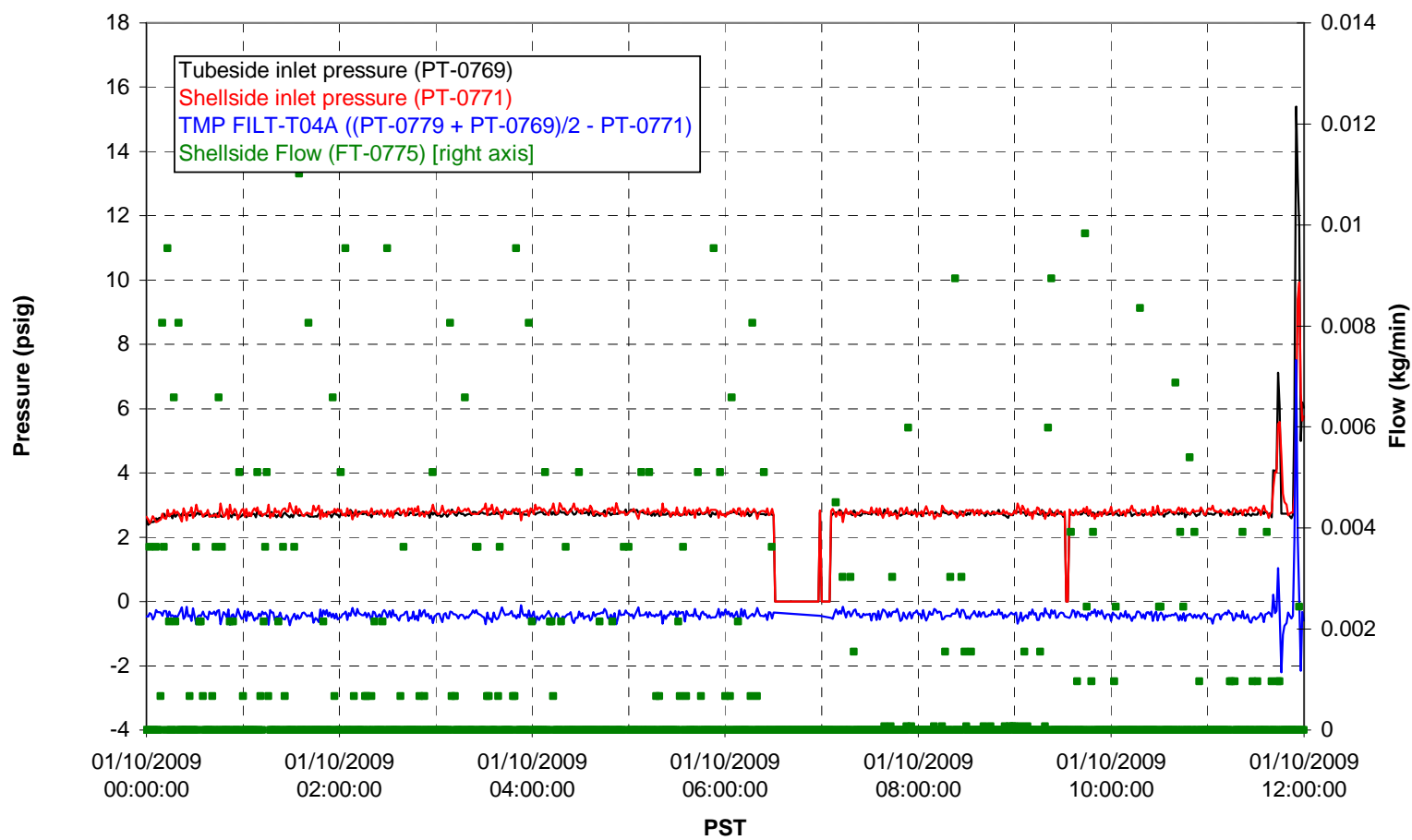


Filter UFP-FILT-T05A

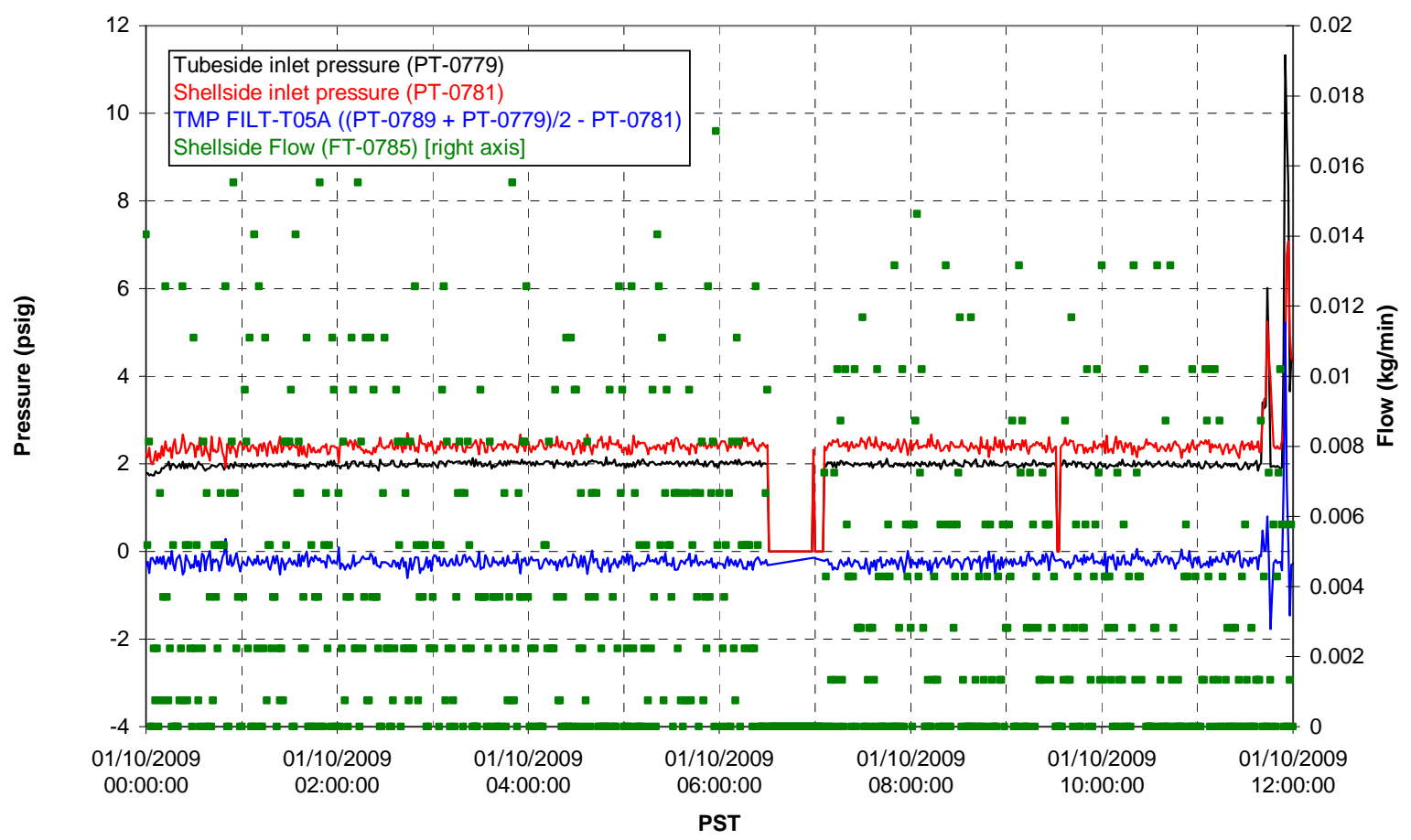

Chemical Flow

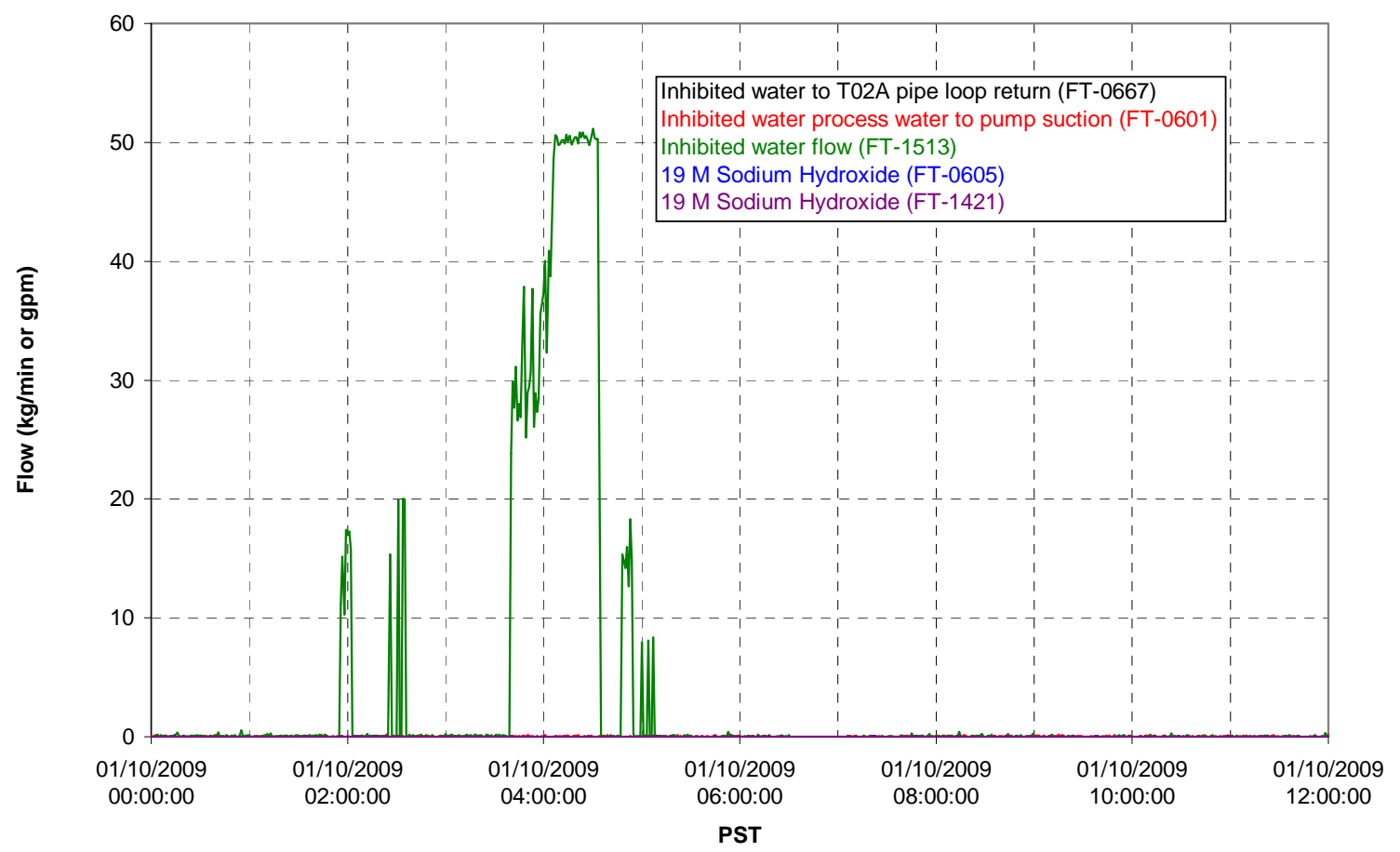




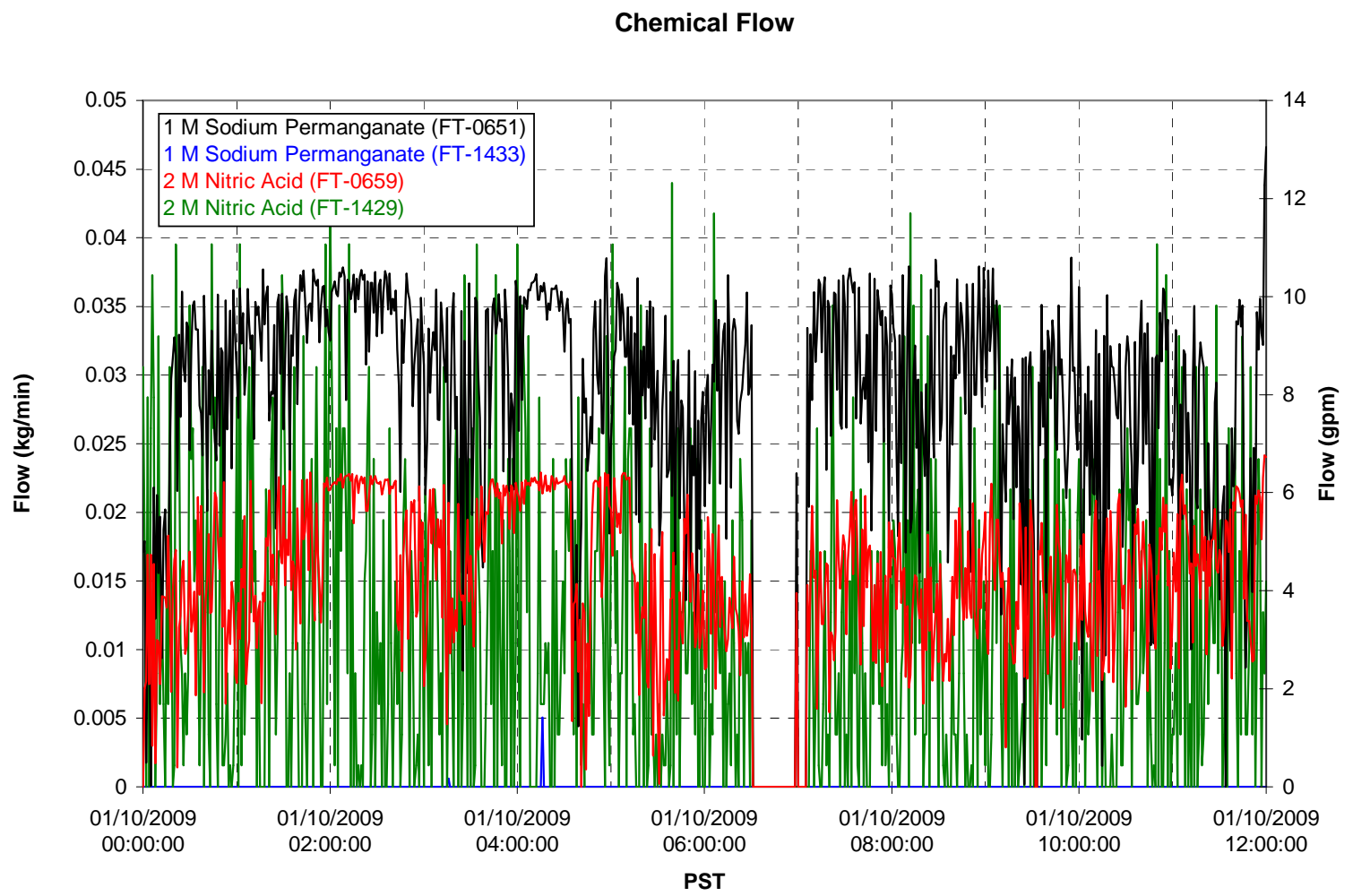

Air Flows

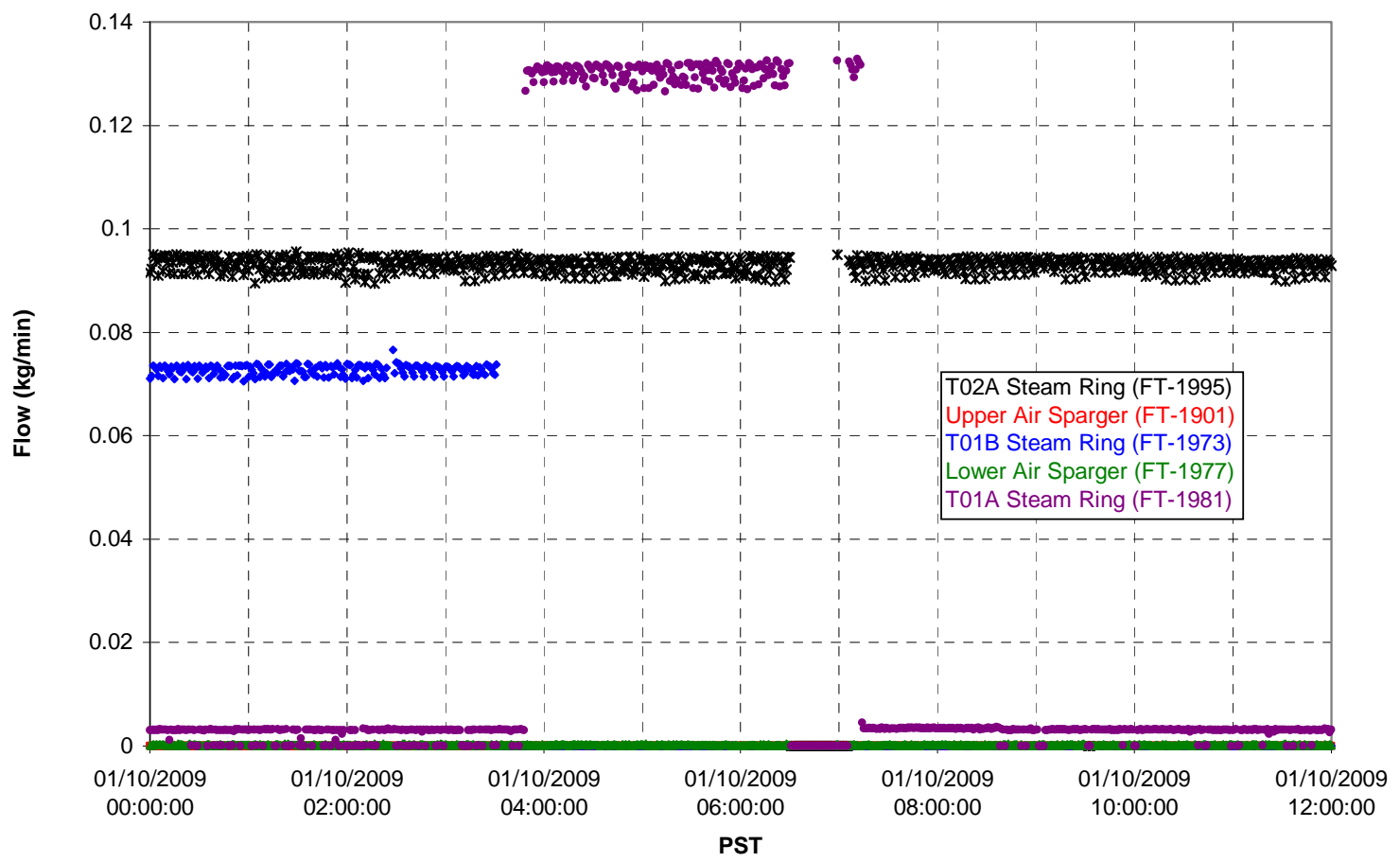


T02A Steam

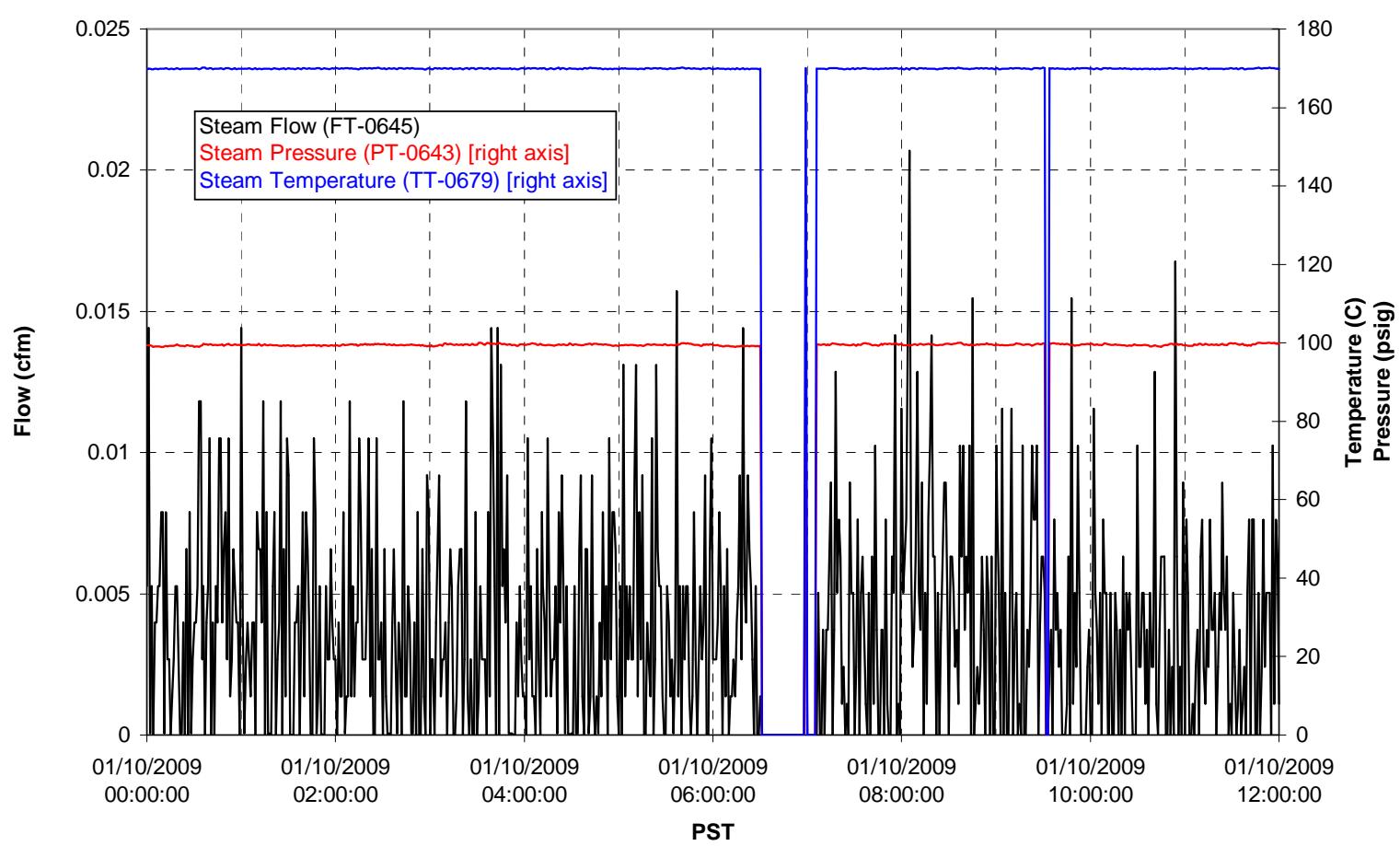

T01A Steam

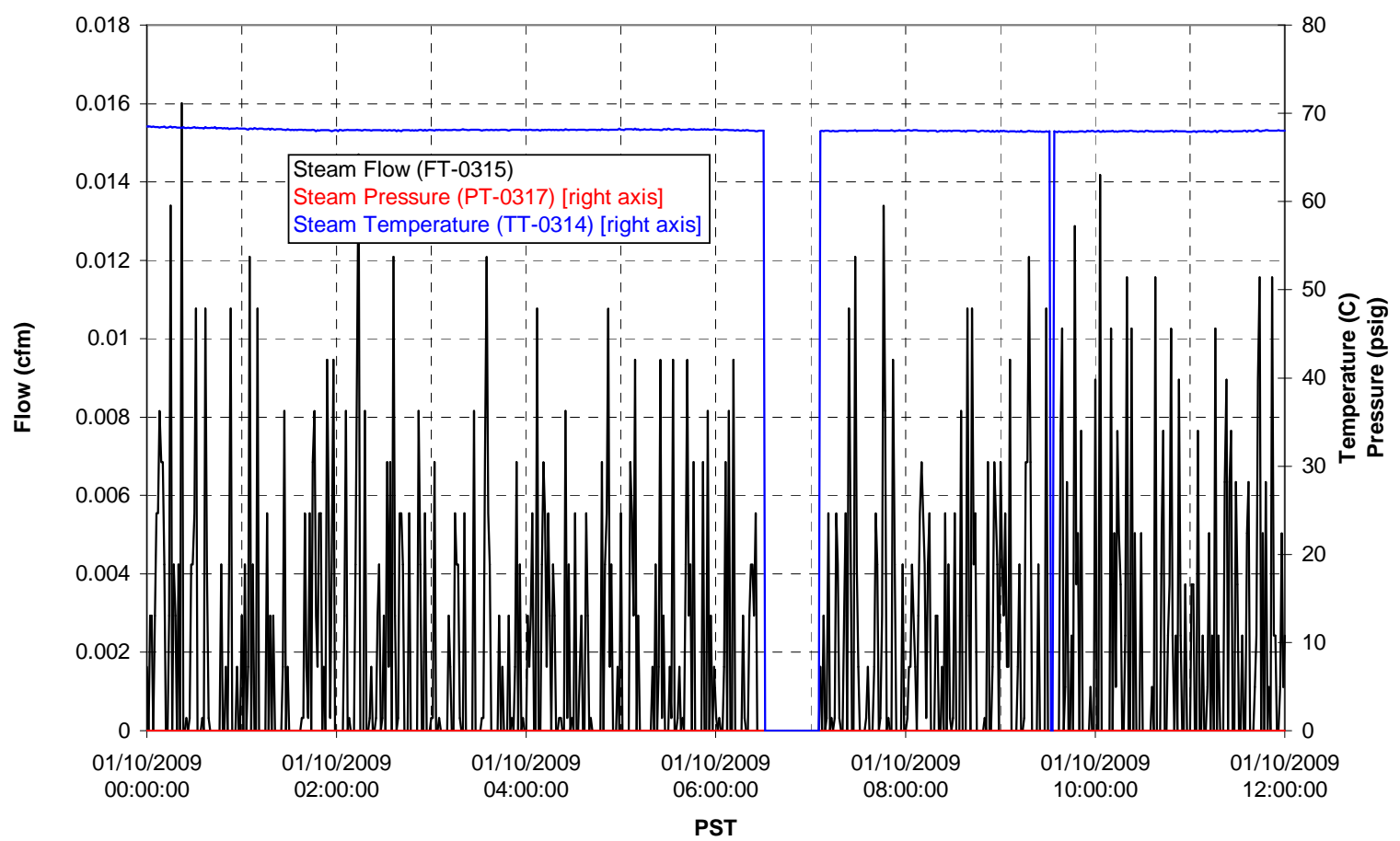


T01B Steam

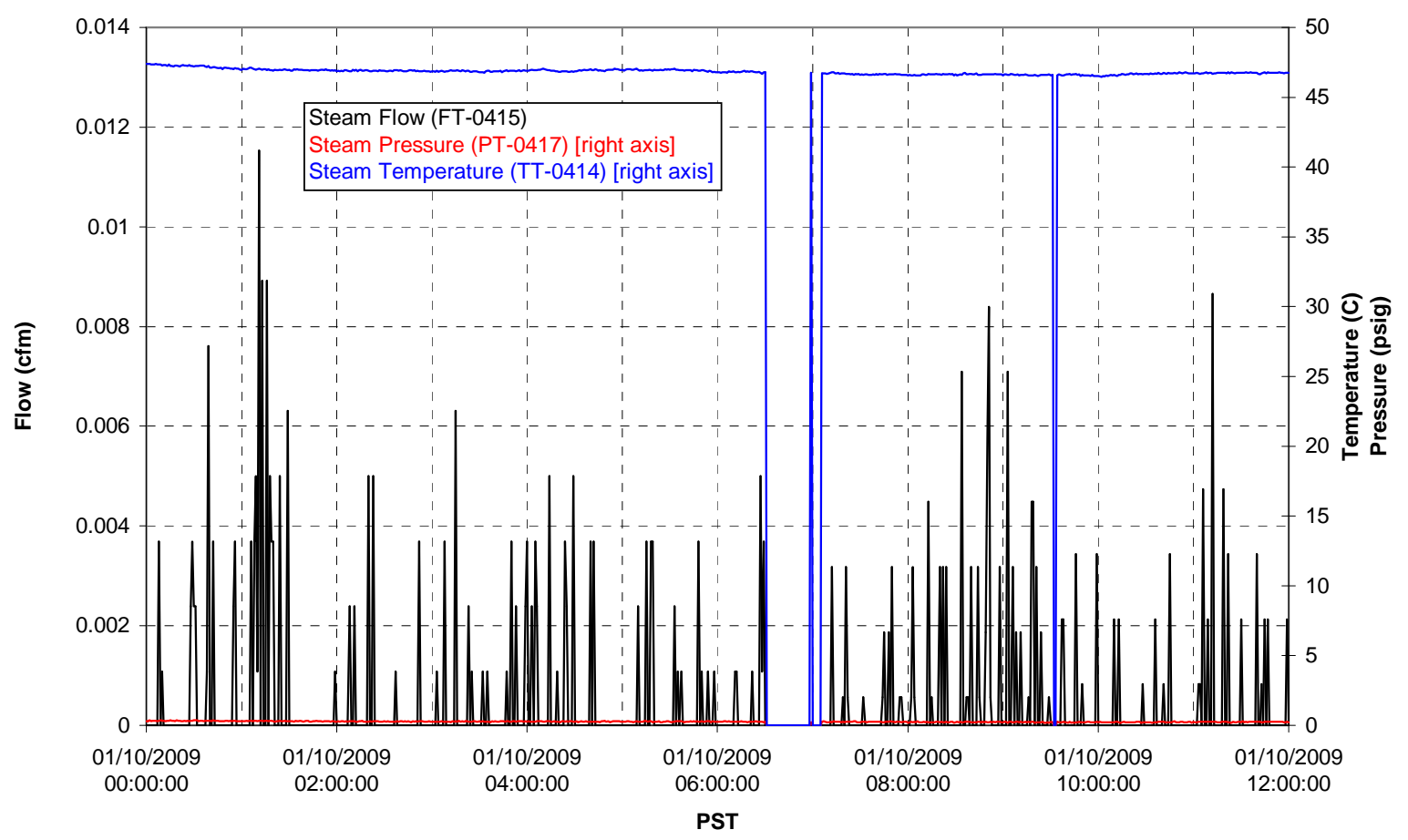


Functional Test Data Plots

01/10/2009 12:00 - 01/11/2009 00:00 
T01A level

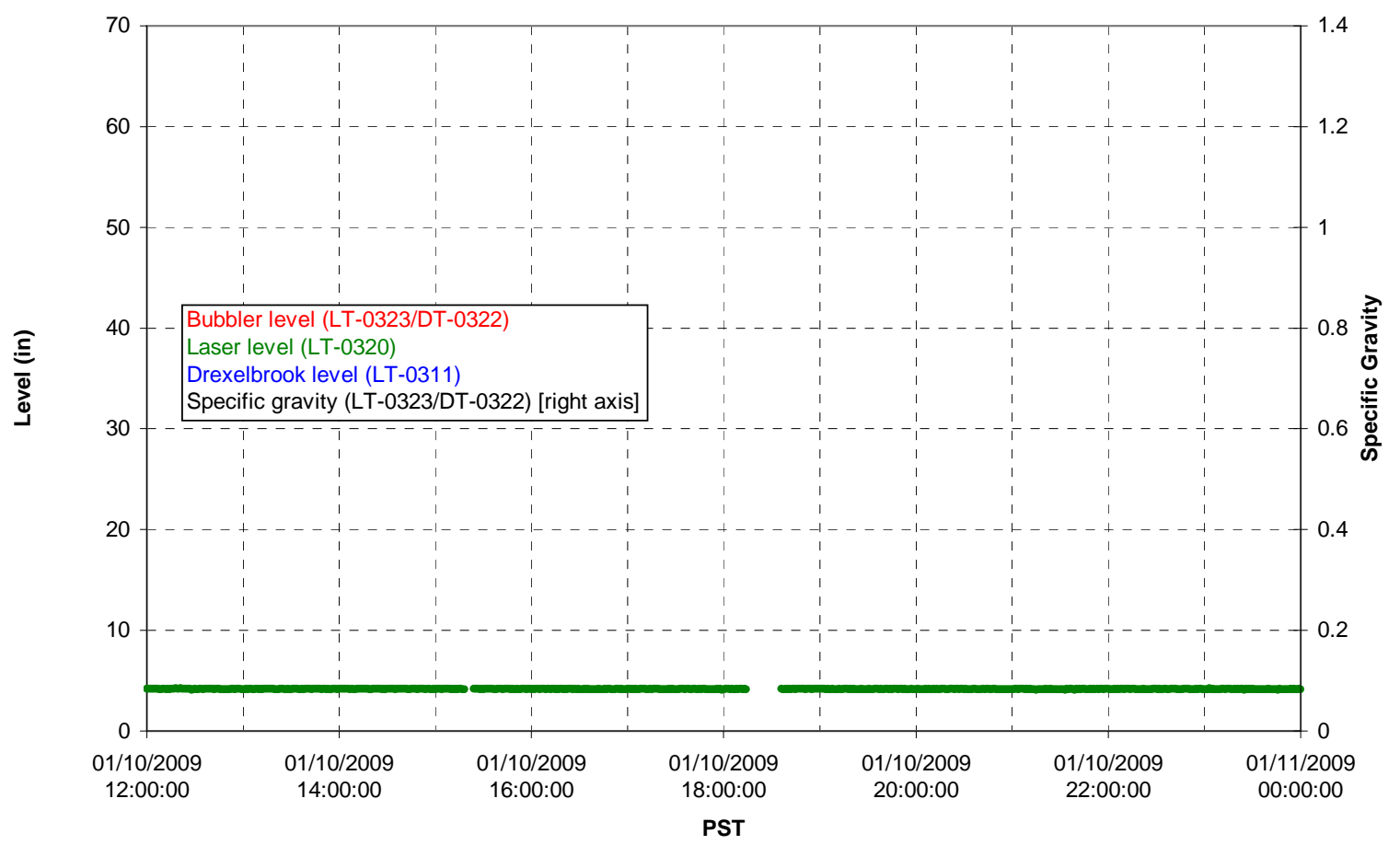

T01A temperatures

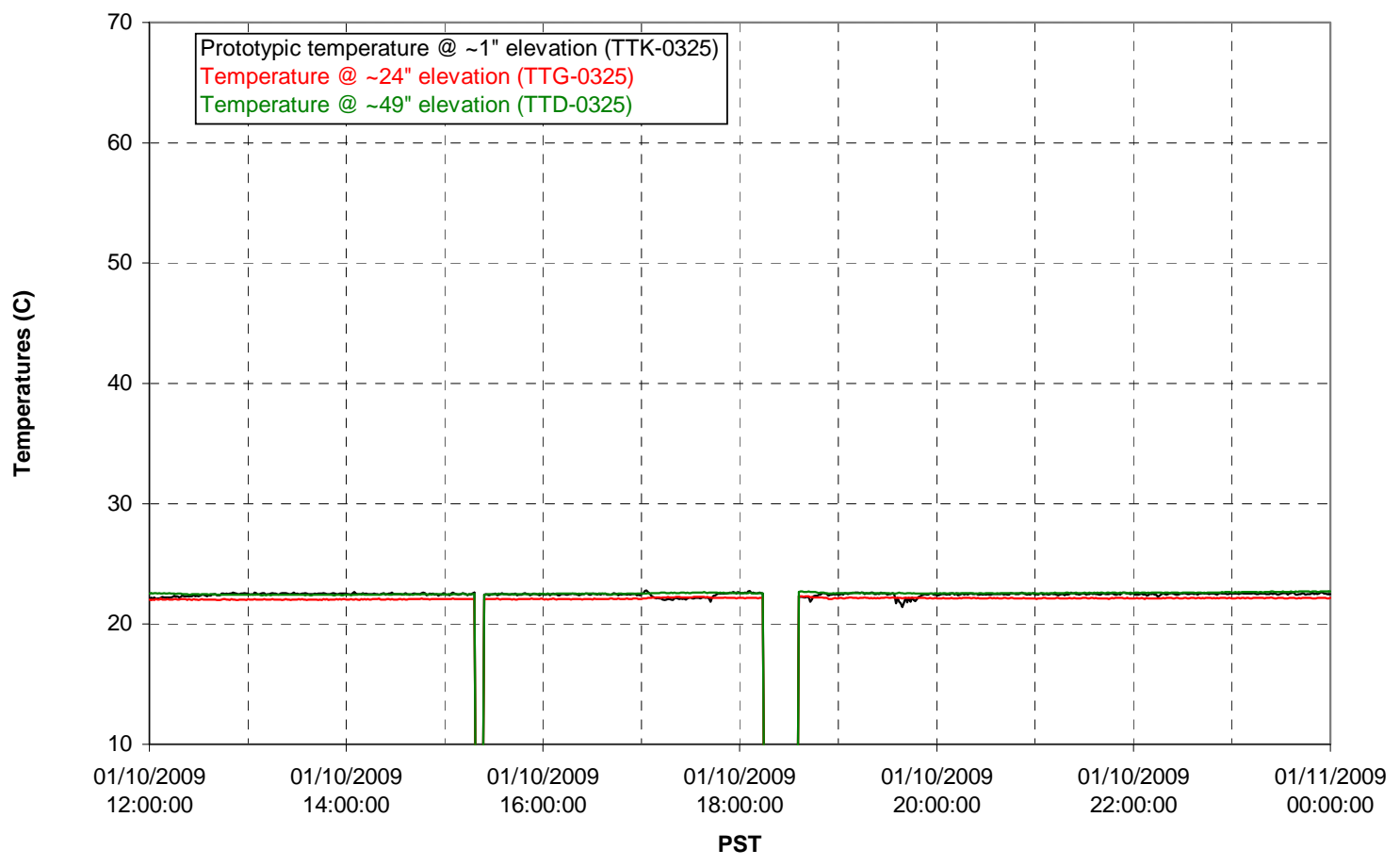


T01B level

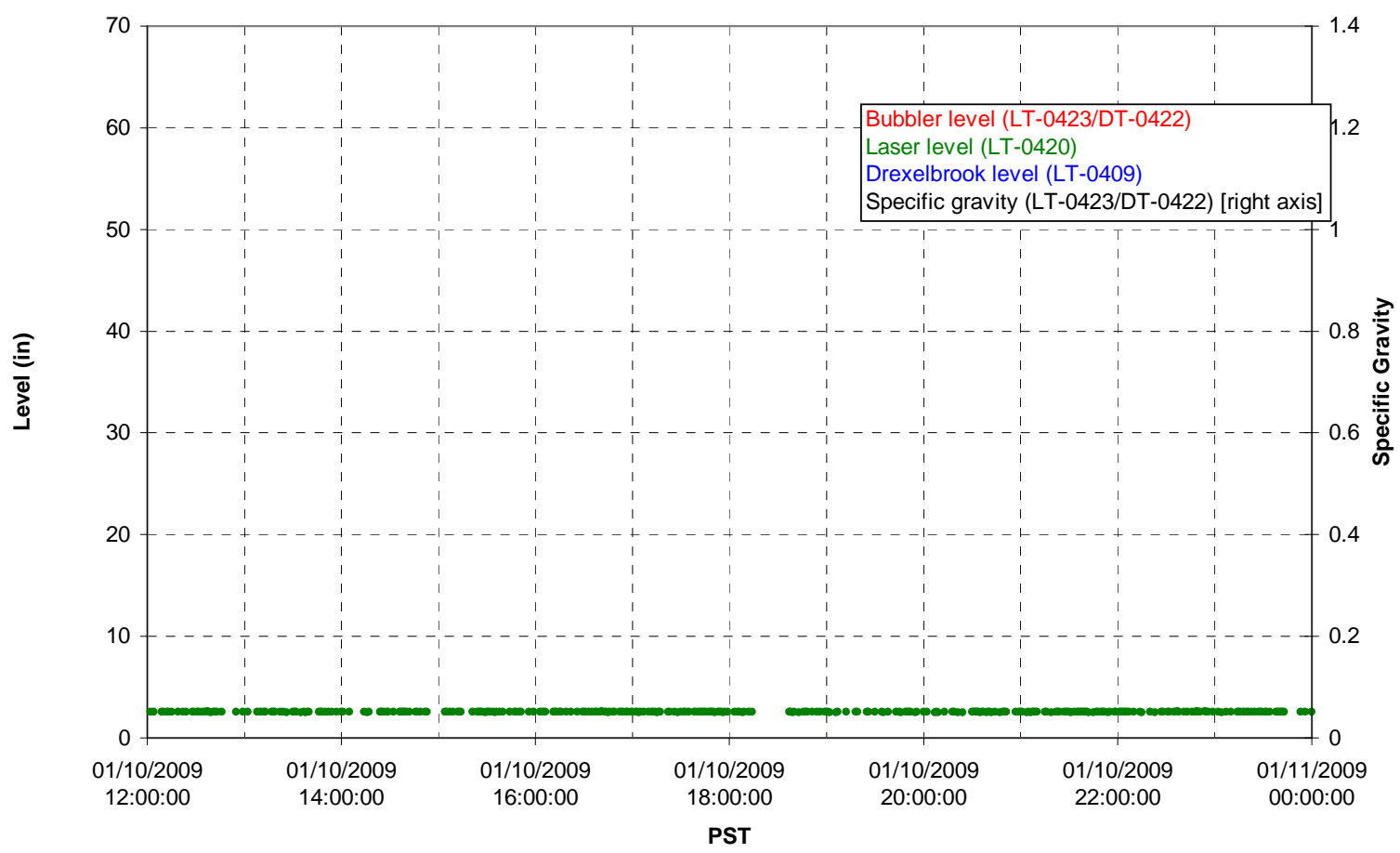

T01B temperatures

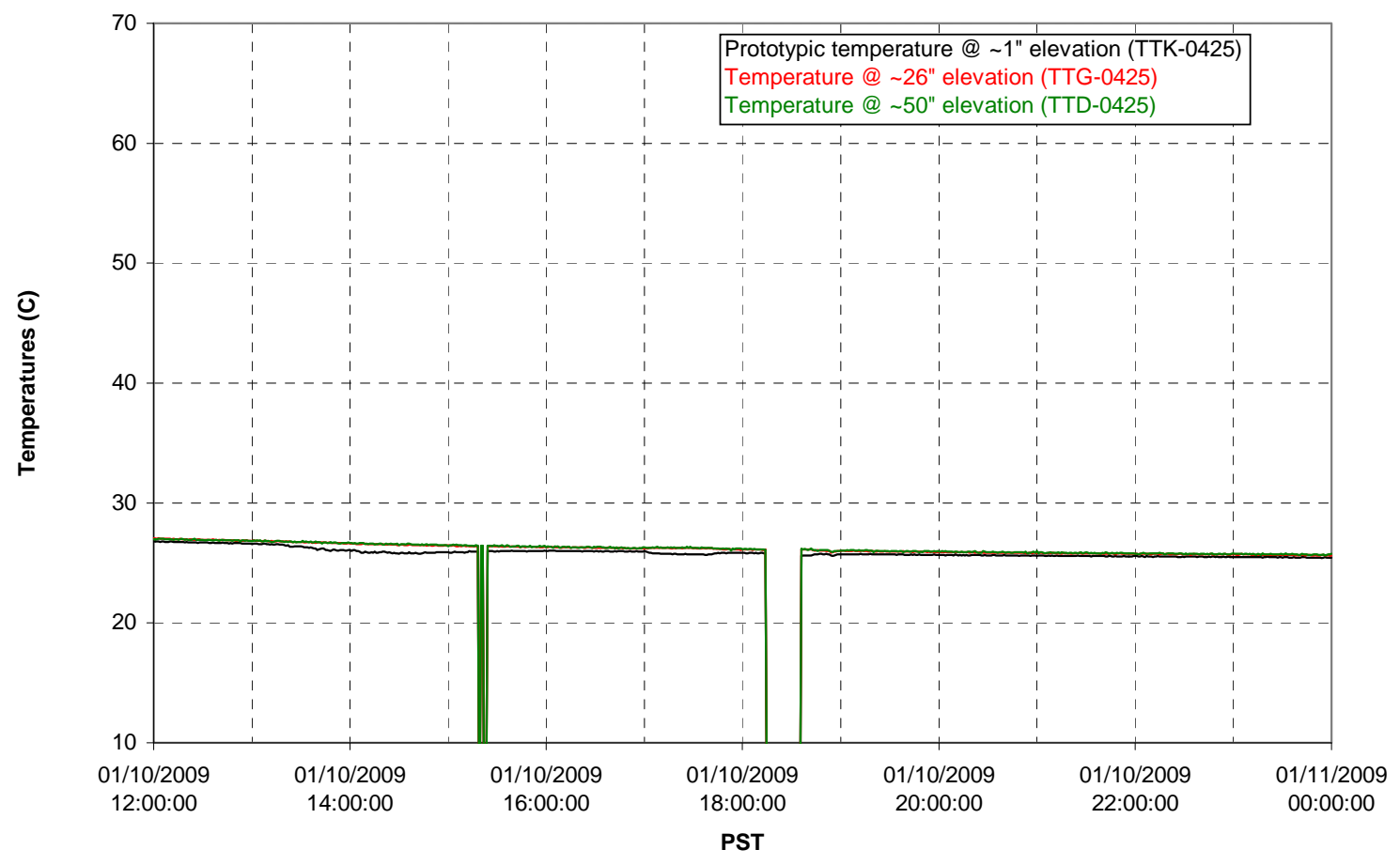


T02A level

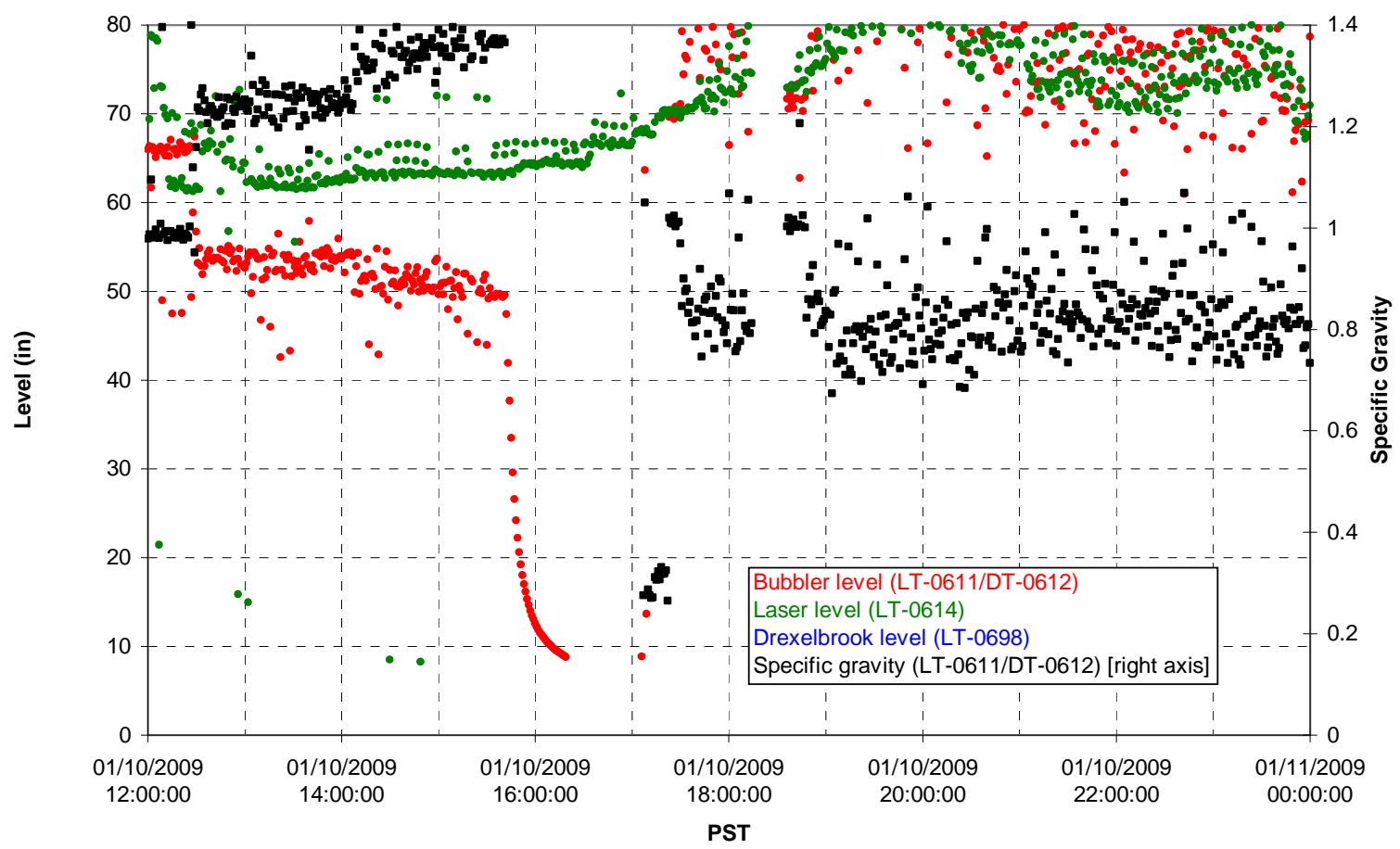

T02A temperatures

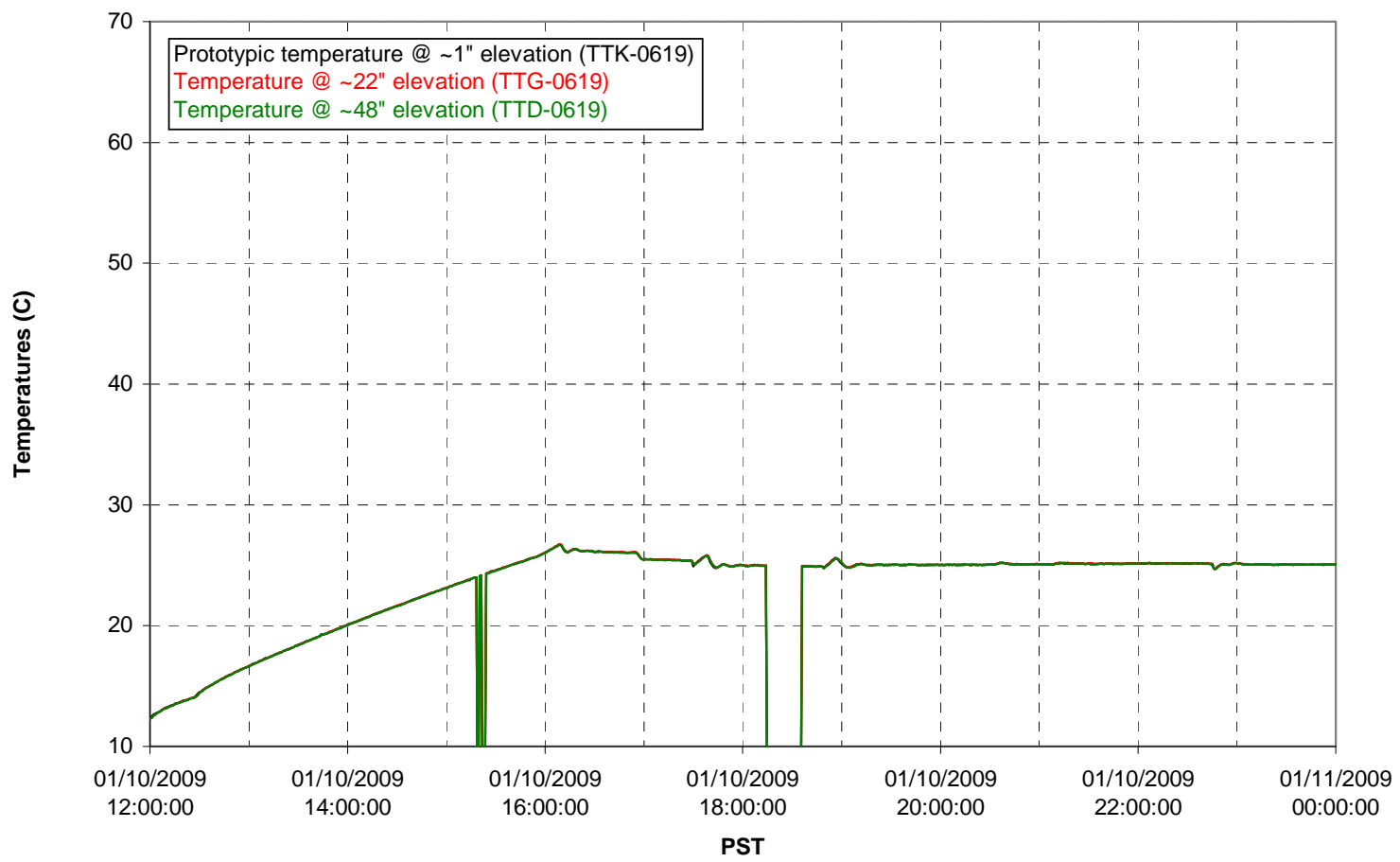


T02A and filter loop temperatures

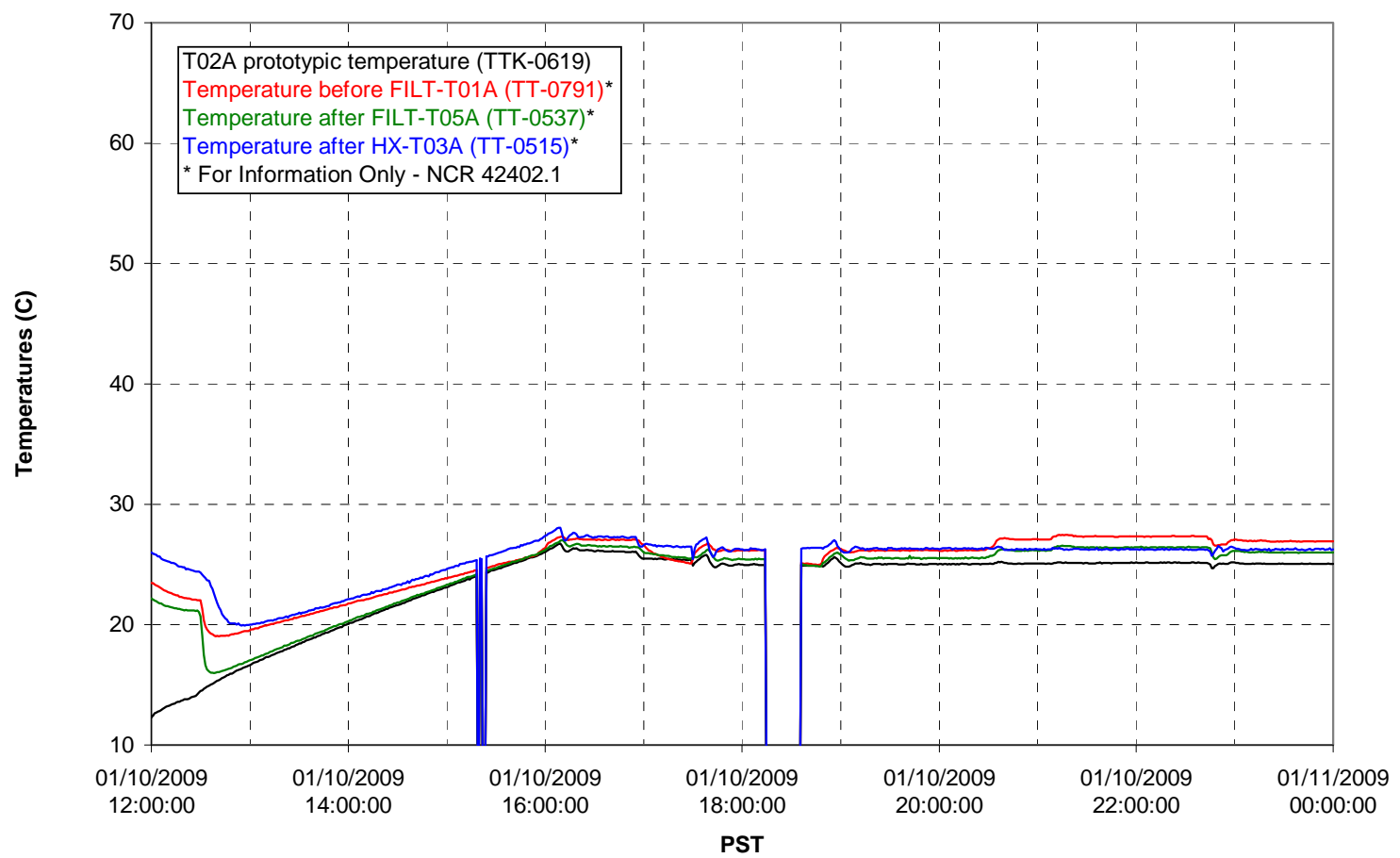

Pump Pressures and Flow

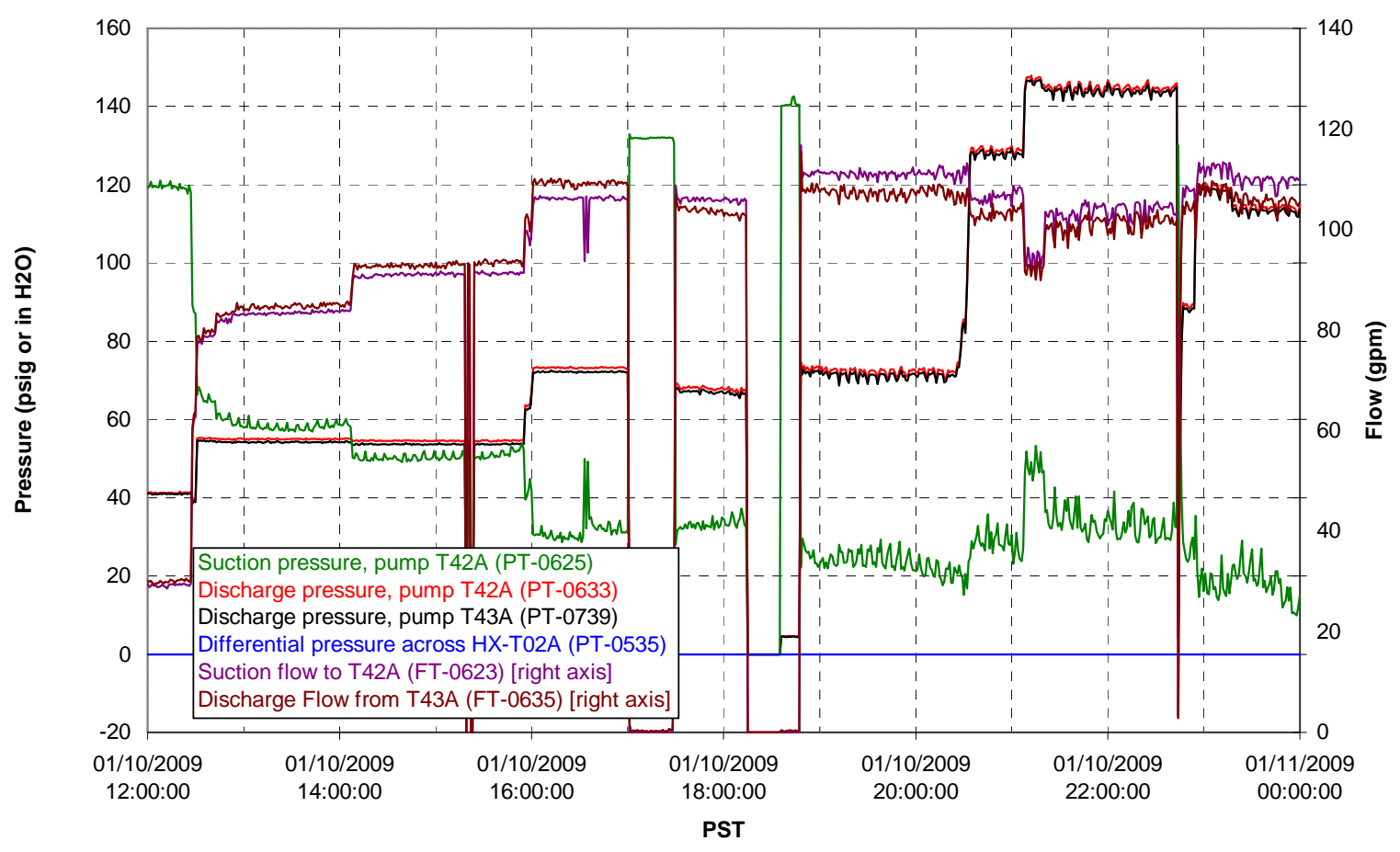




\section{Axial pressure drop}

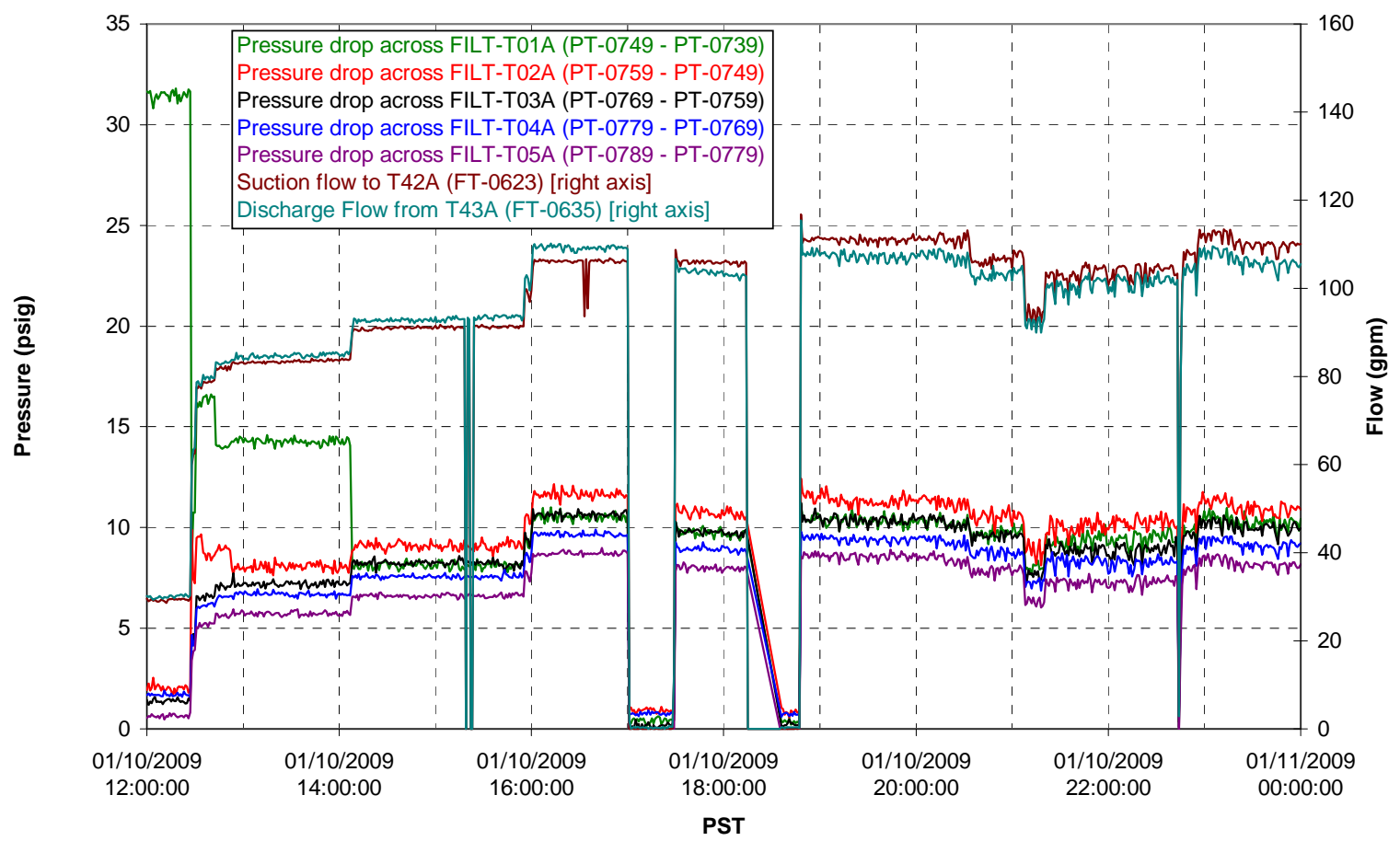

Permeate flow rates

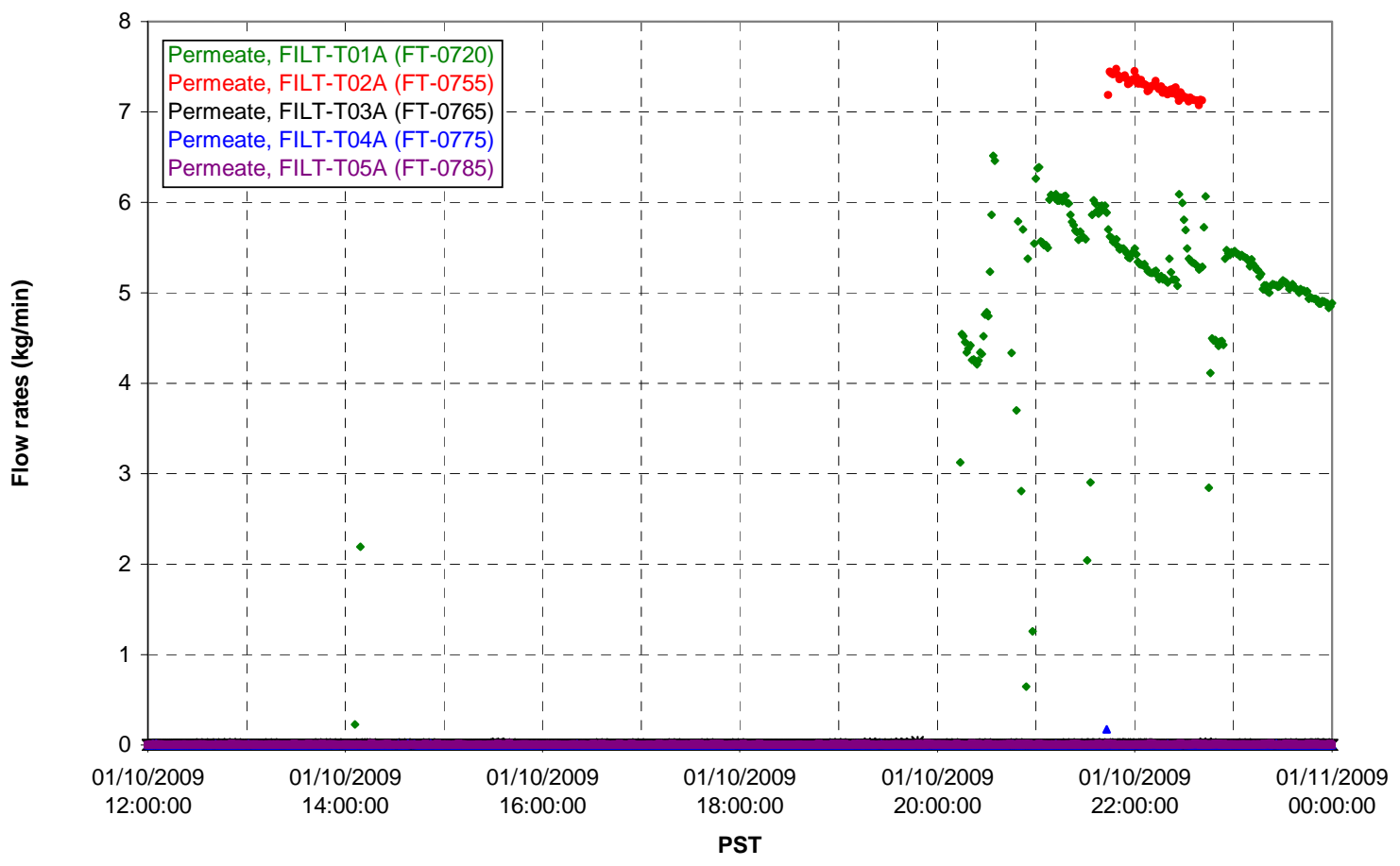


T02A Inner Temperature Tree

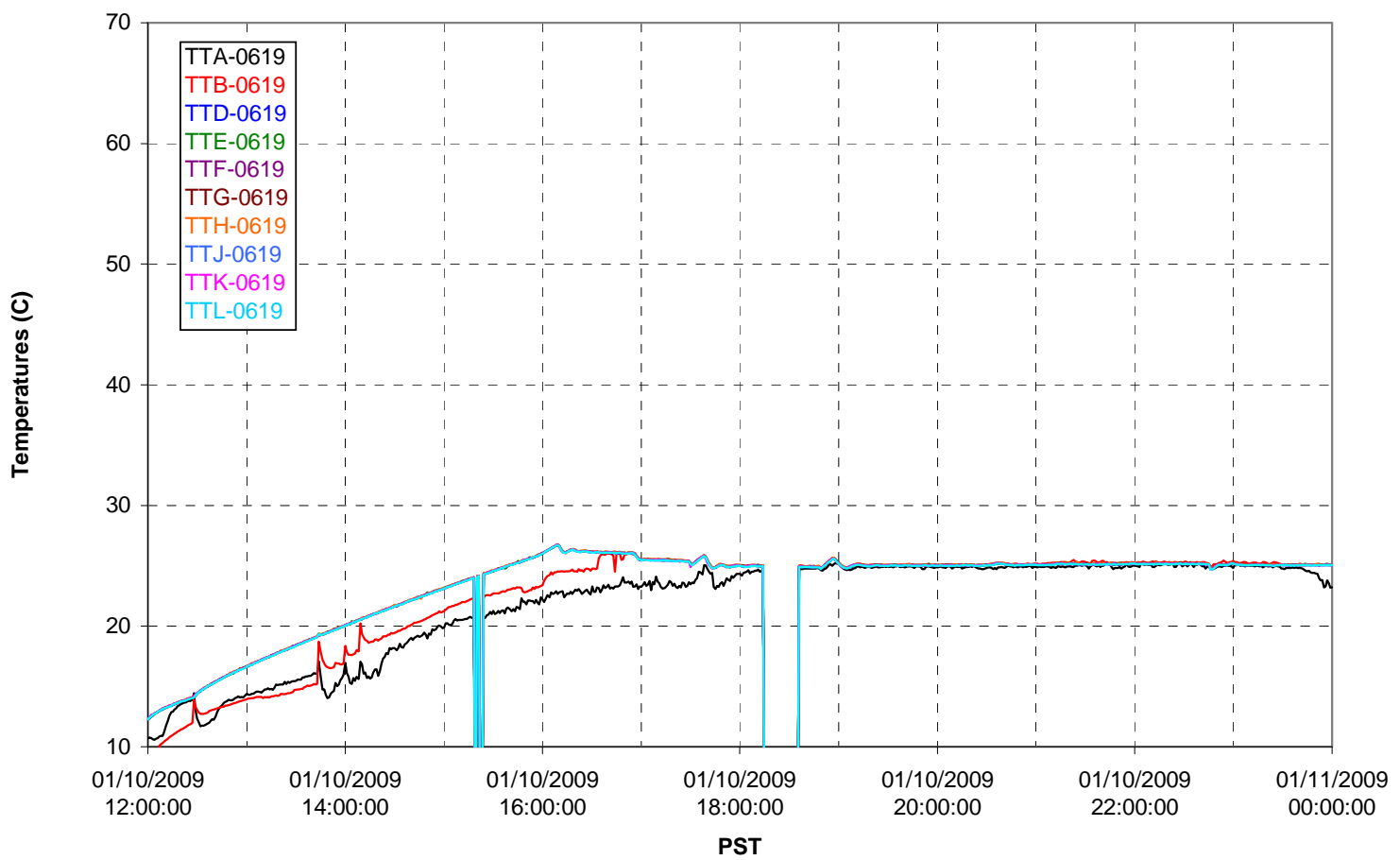

T02A Outer Temperature Tree

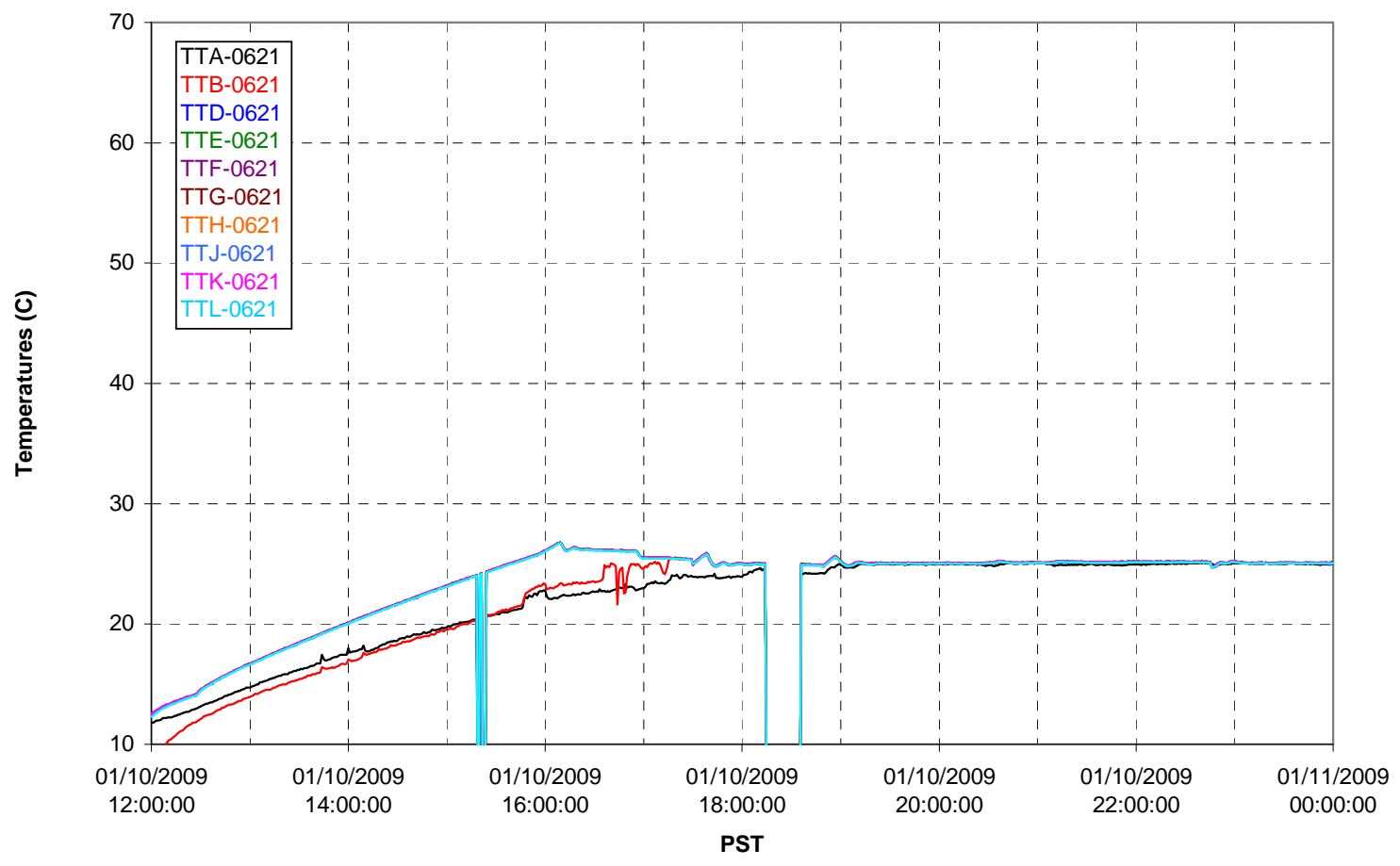


T02A temperatures

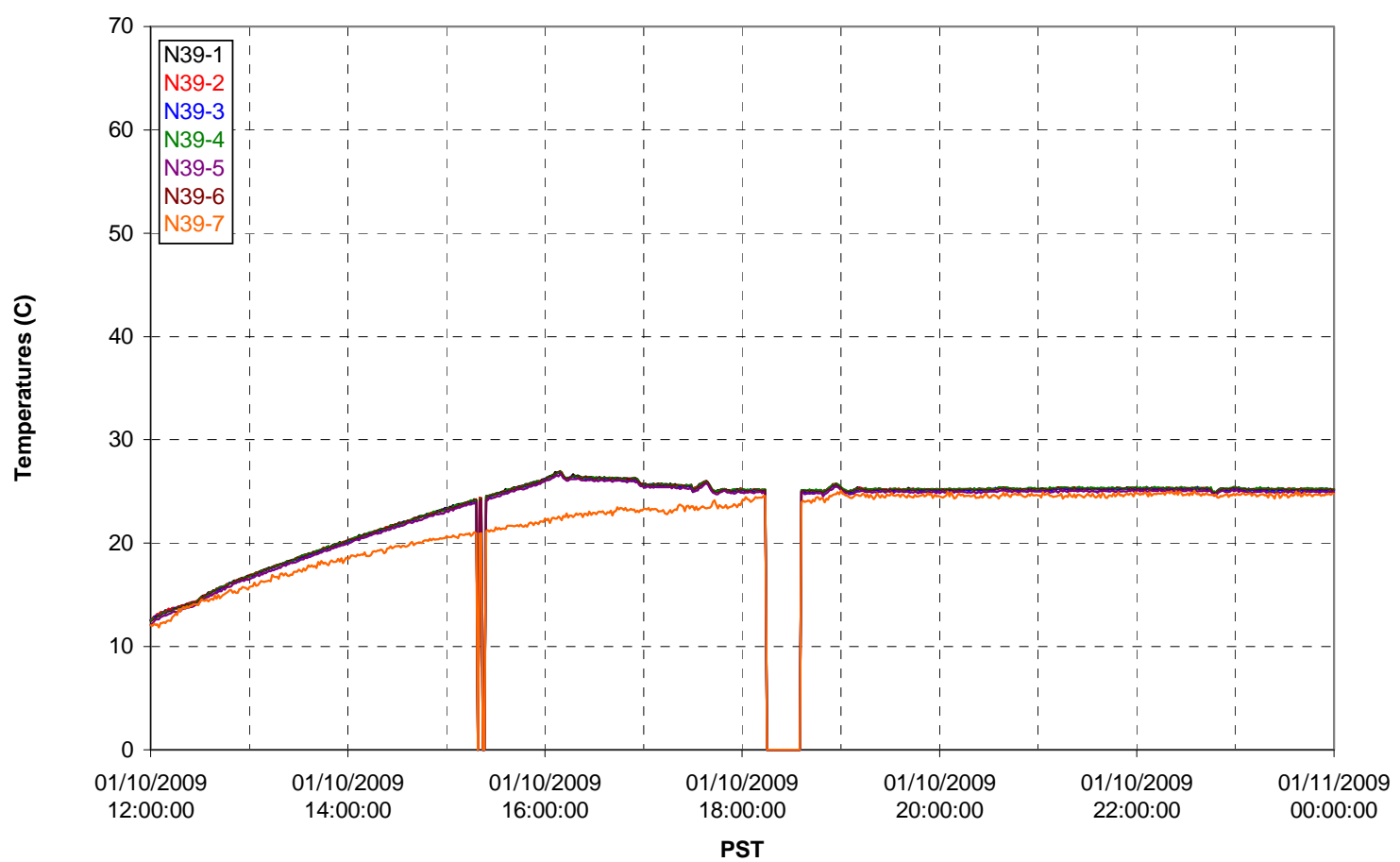

T02A temperatures

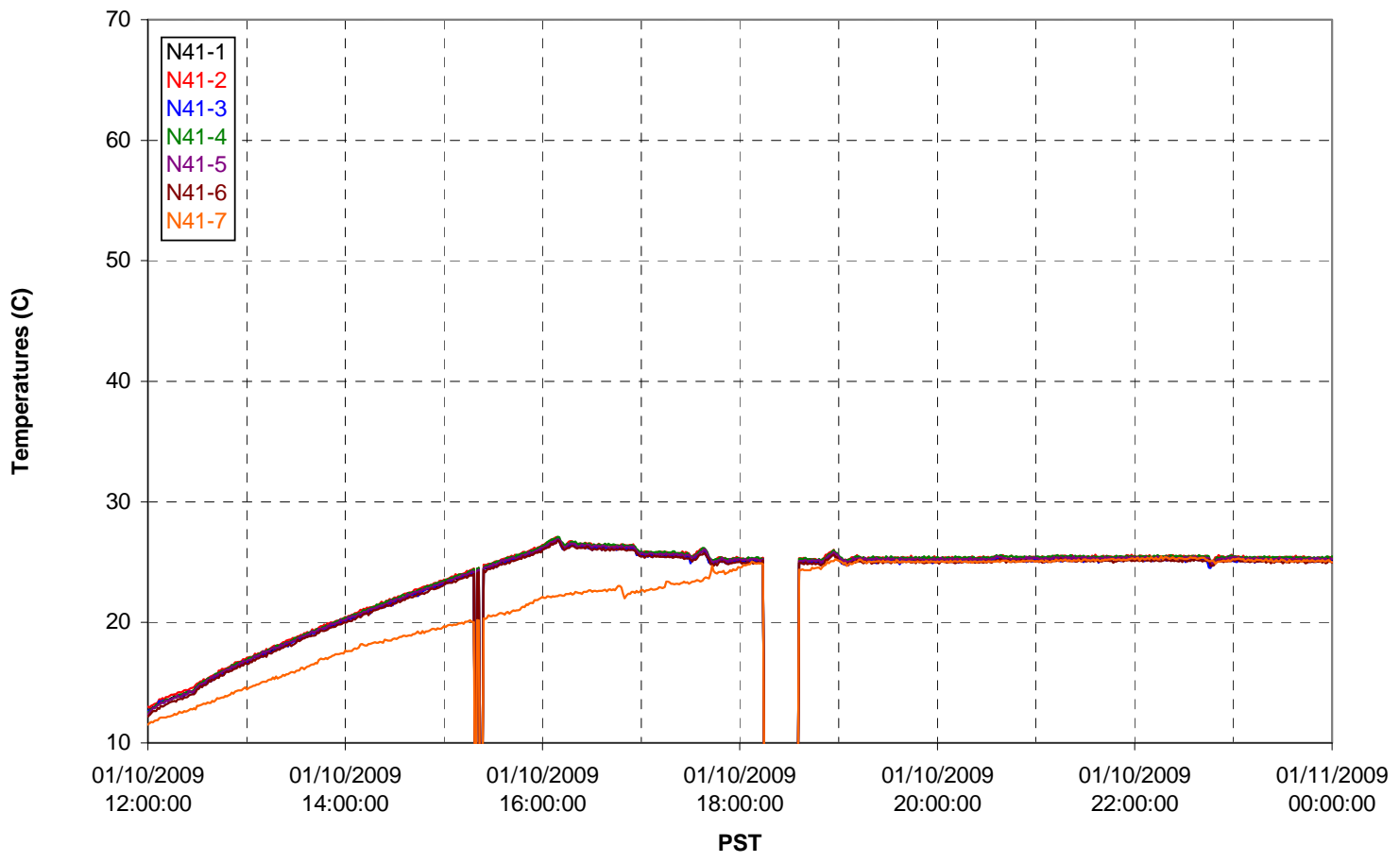


T02A temperatures

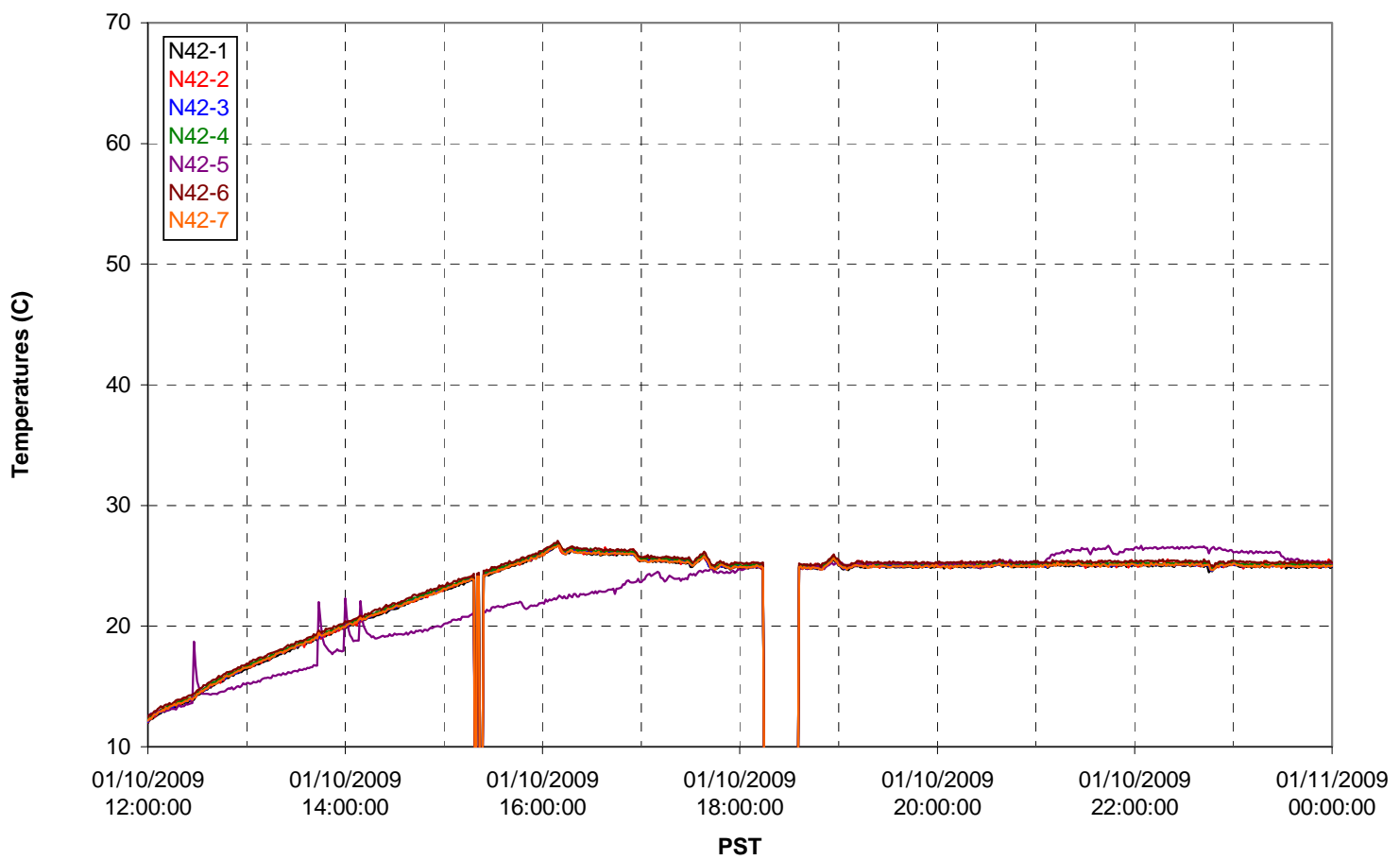

T02A temperatures

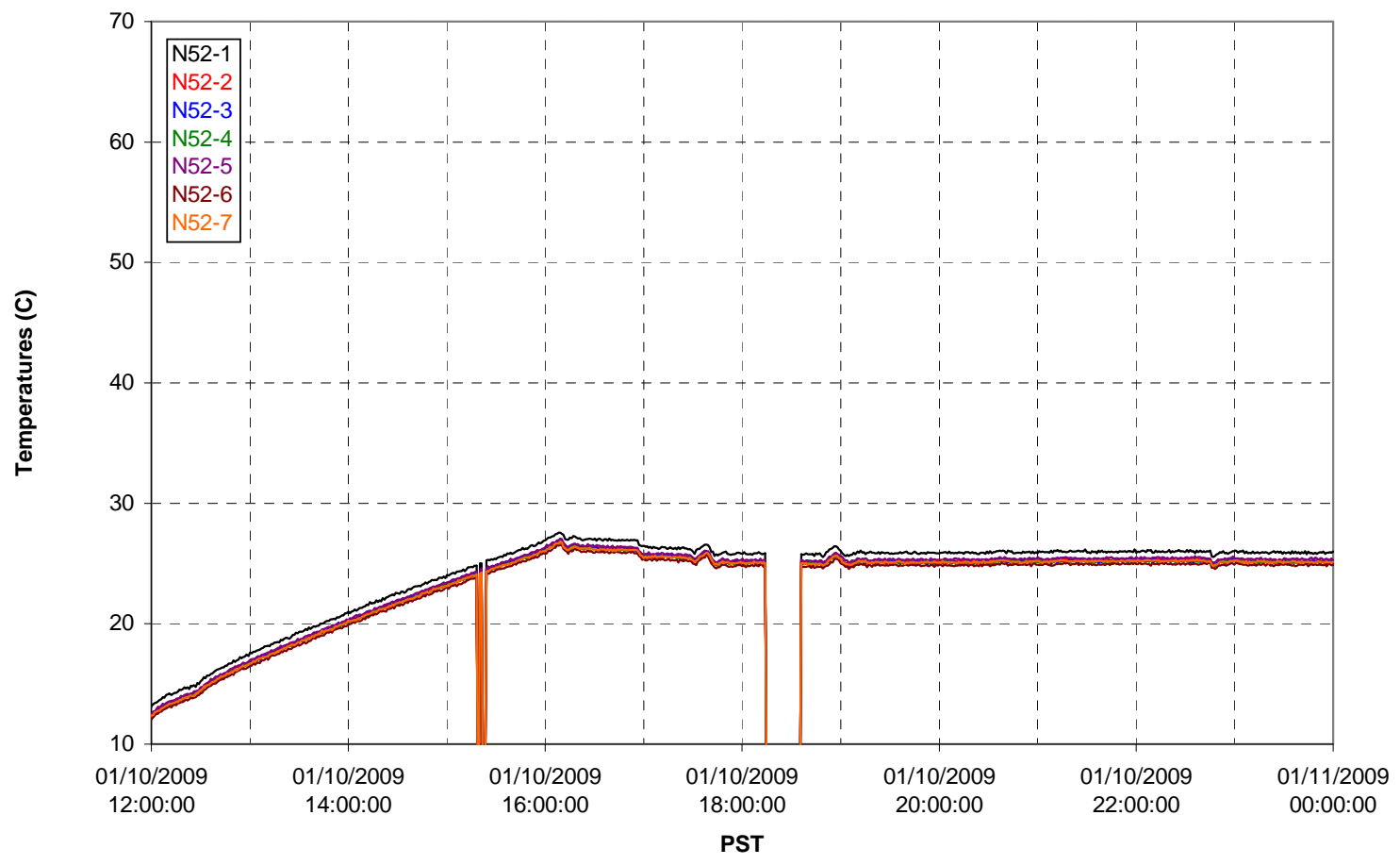


T02A Heating and Cooling

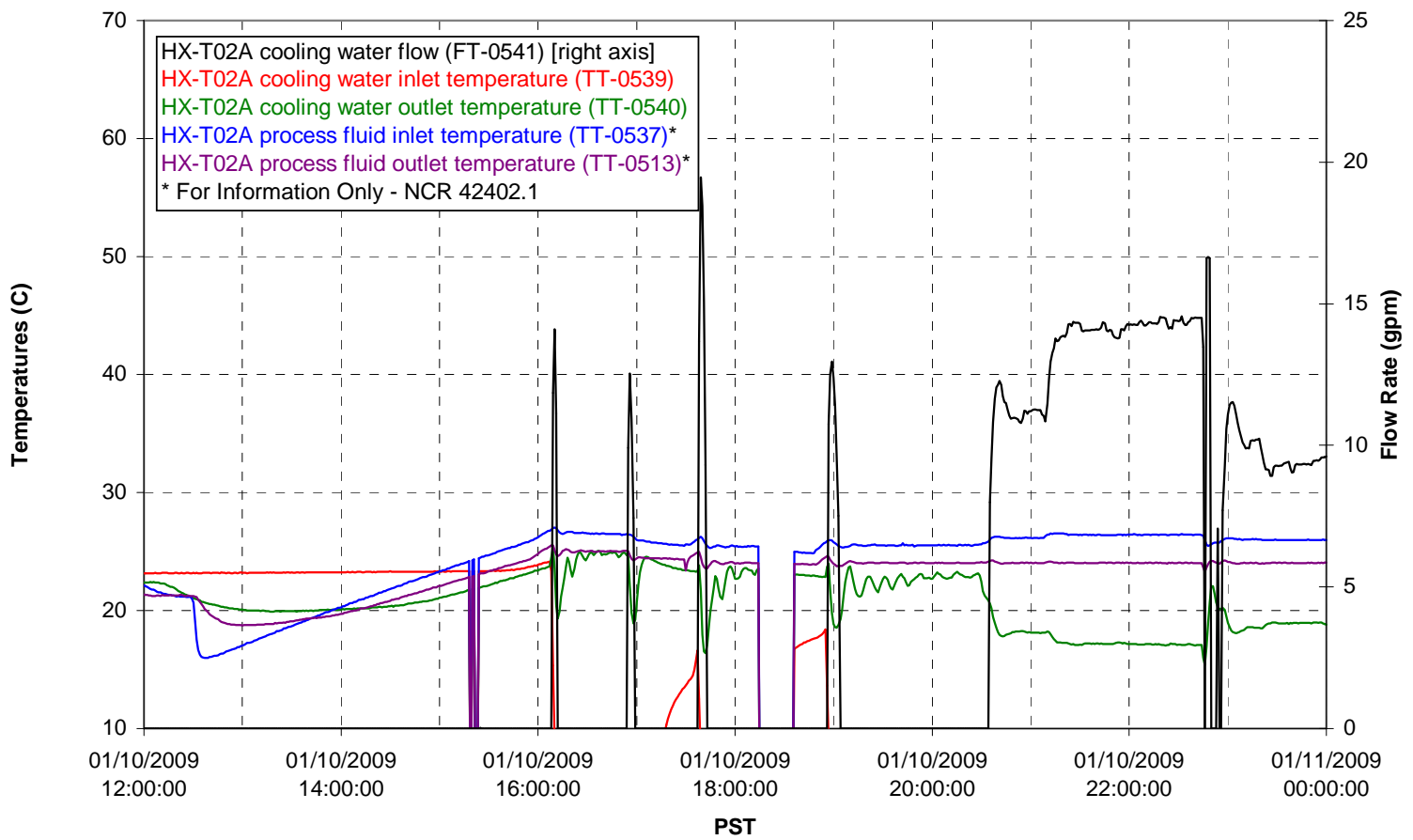

Pump Operation

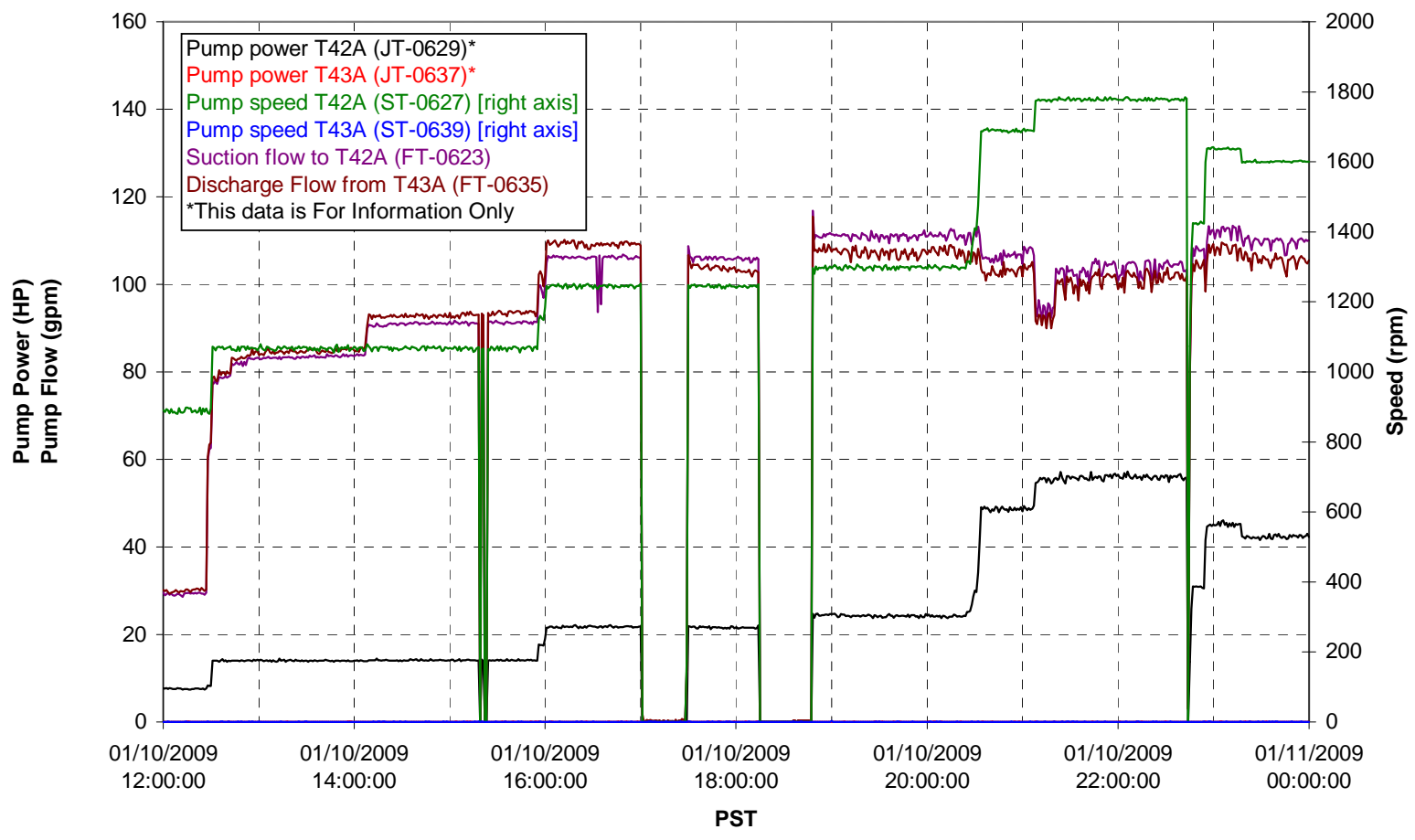


Pulsepot UFP-PP-T01A

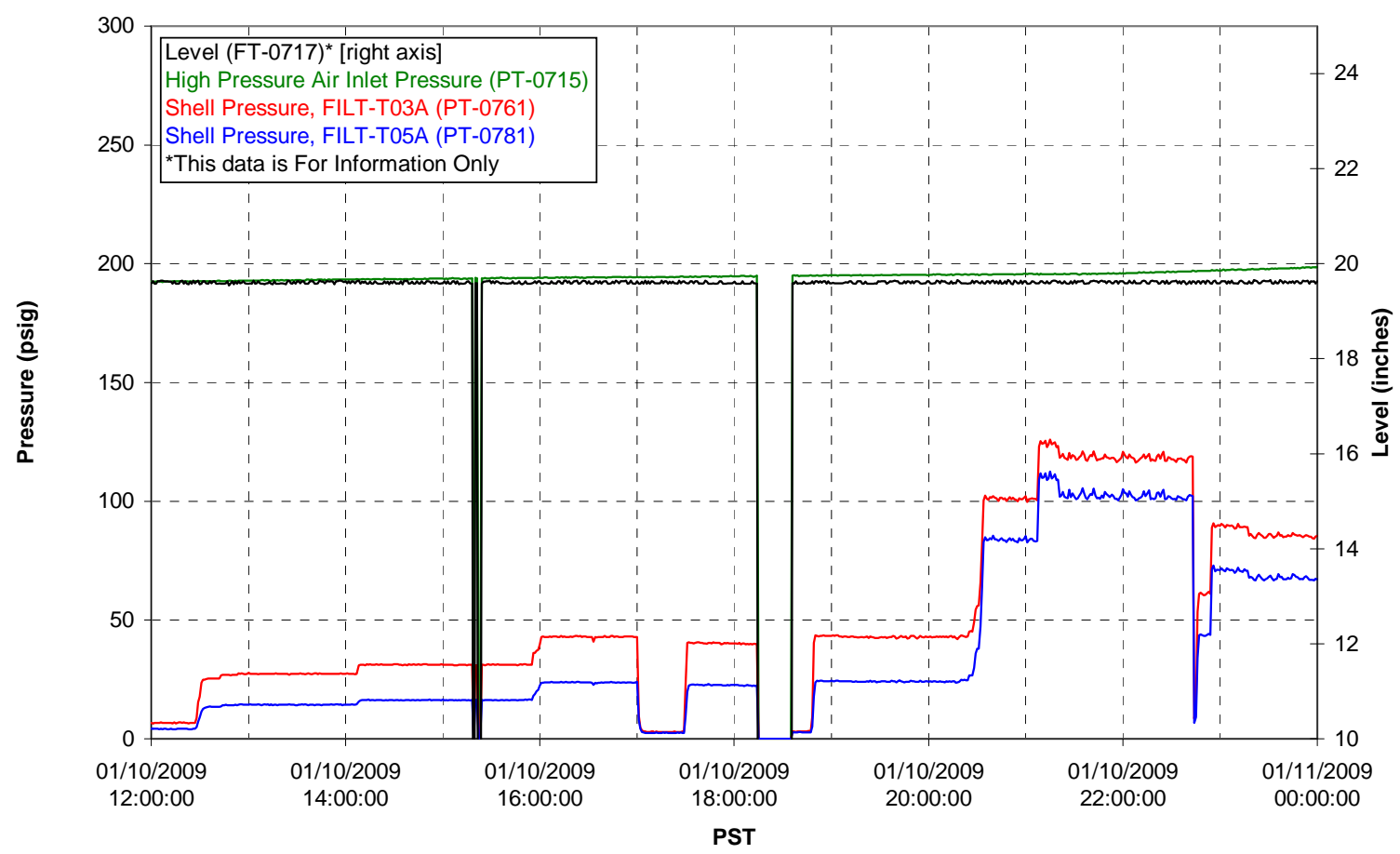

Pulsepot UFP-PP-T02A

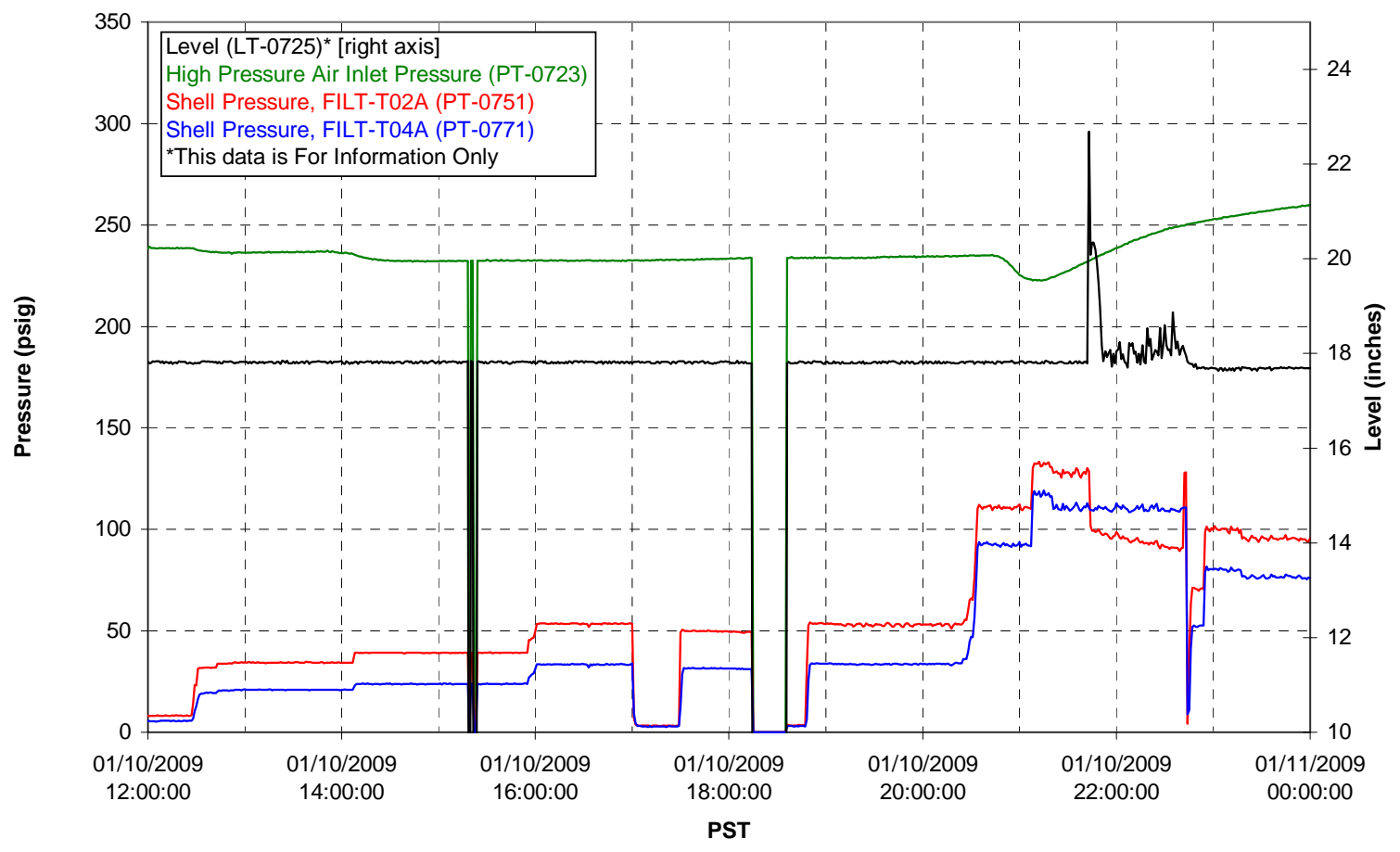


Pulsepot UFP-PP-T03A

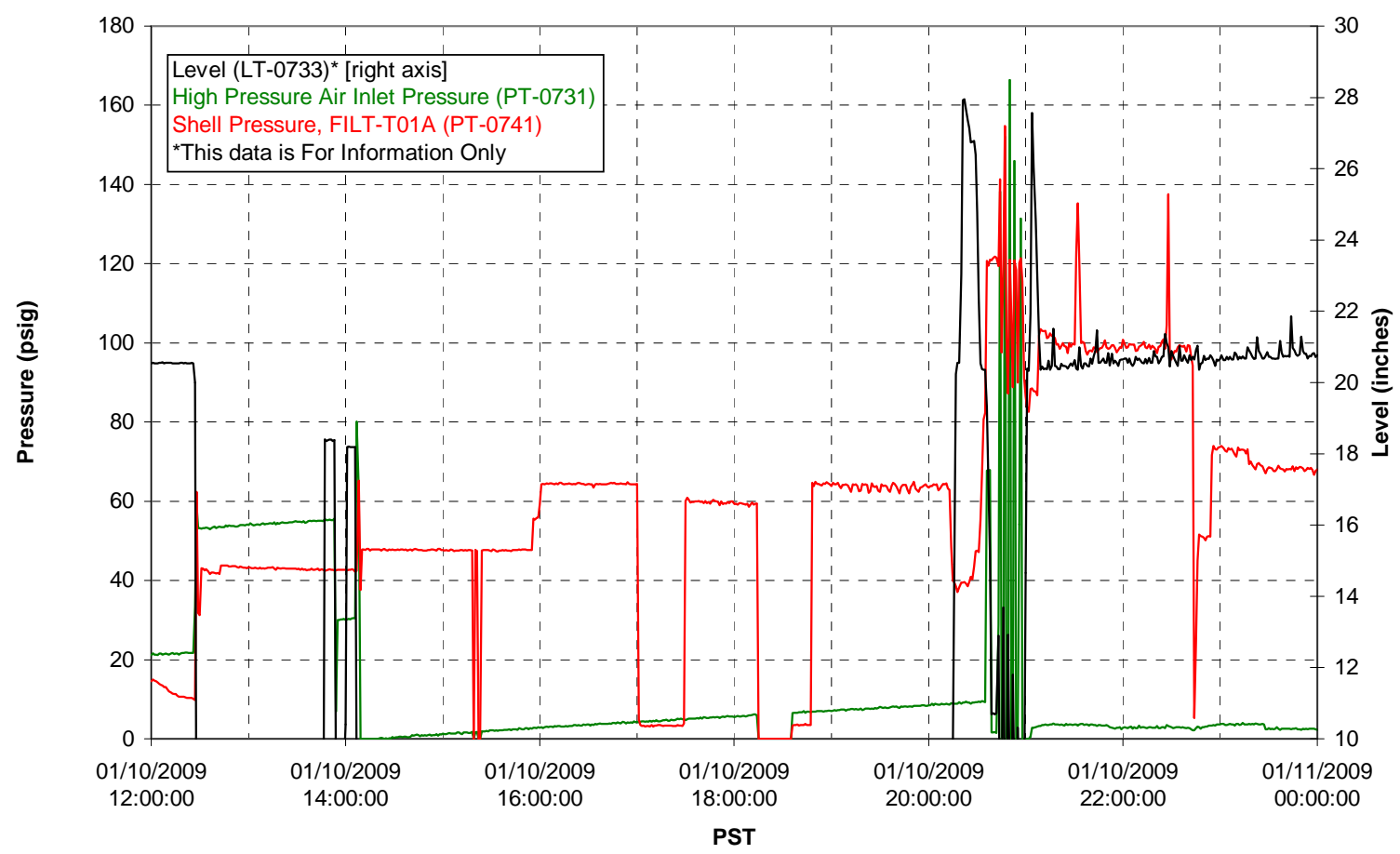

Pulsepot Levels

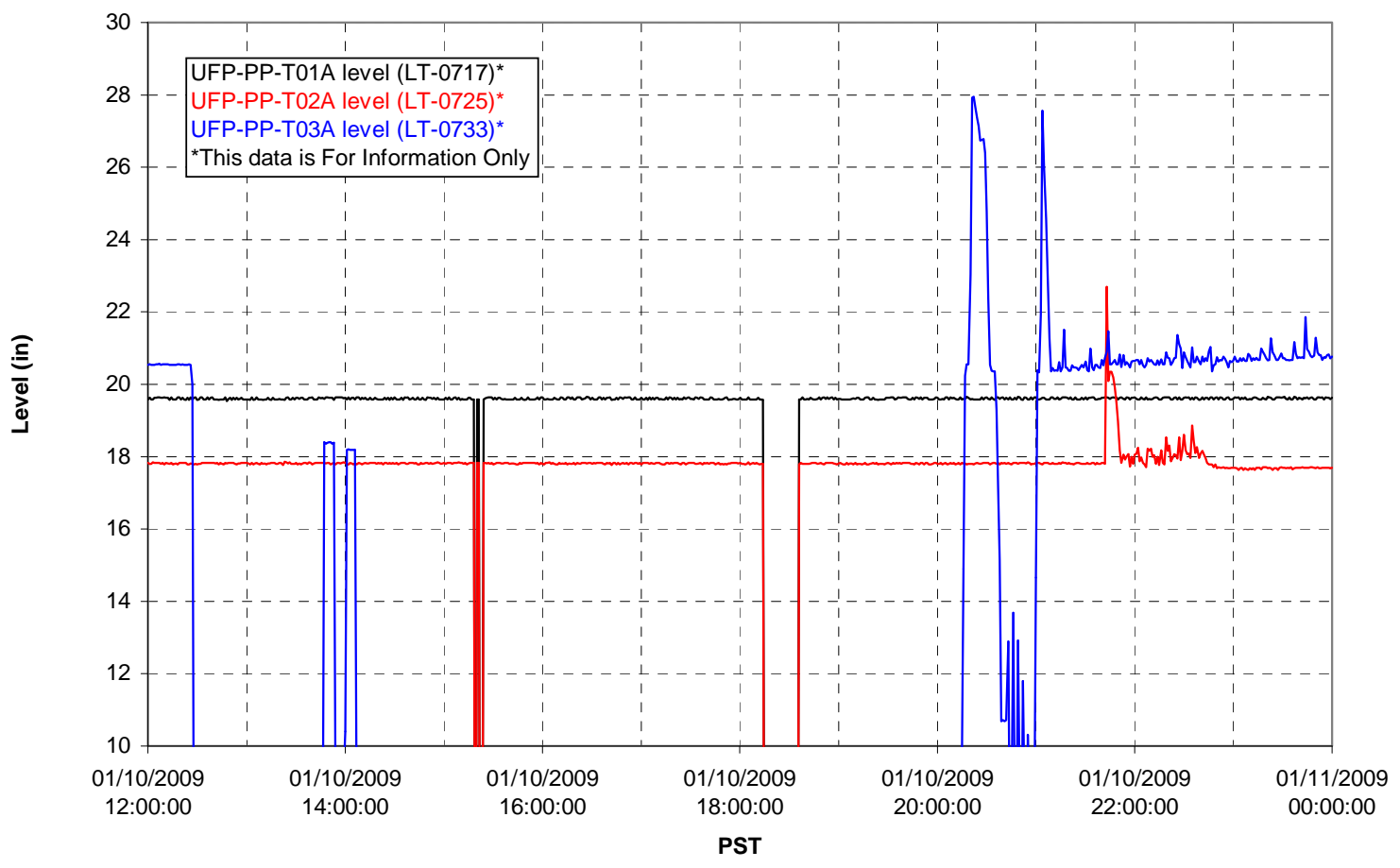


Filter UFP-FILT-T01A

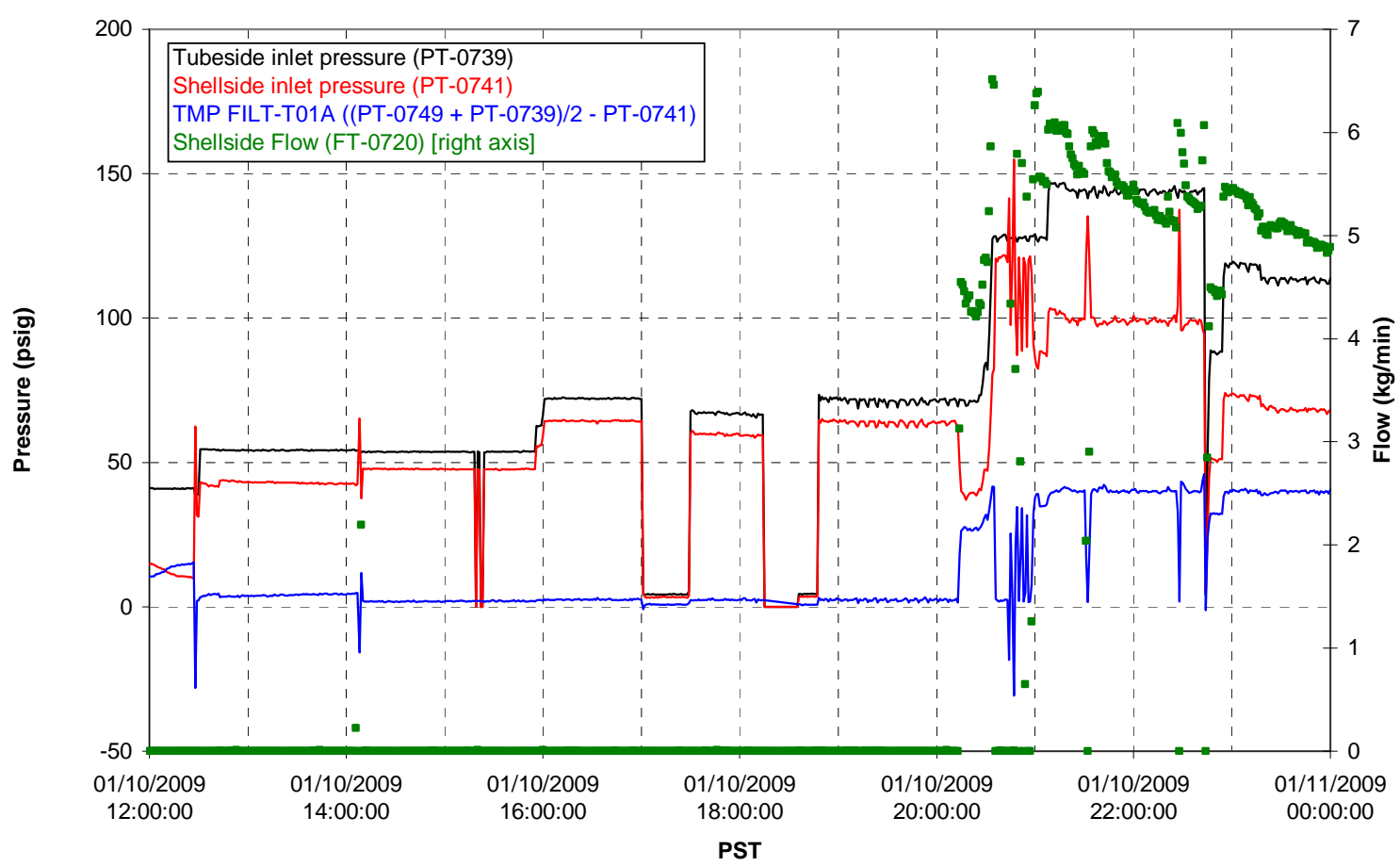

Filter UFP-FILT-T02A

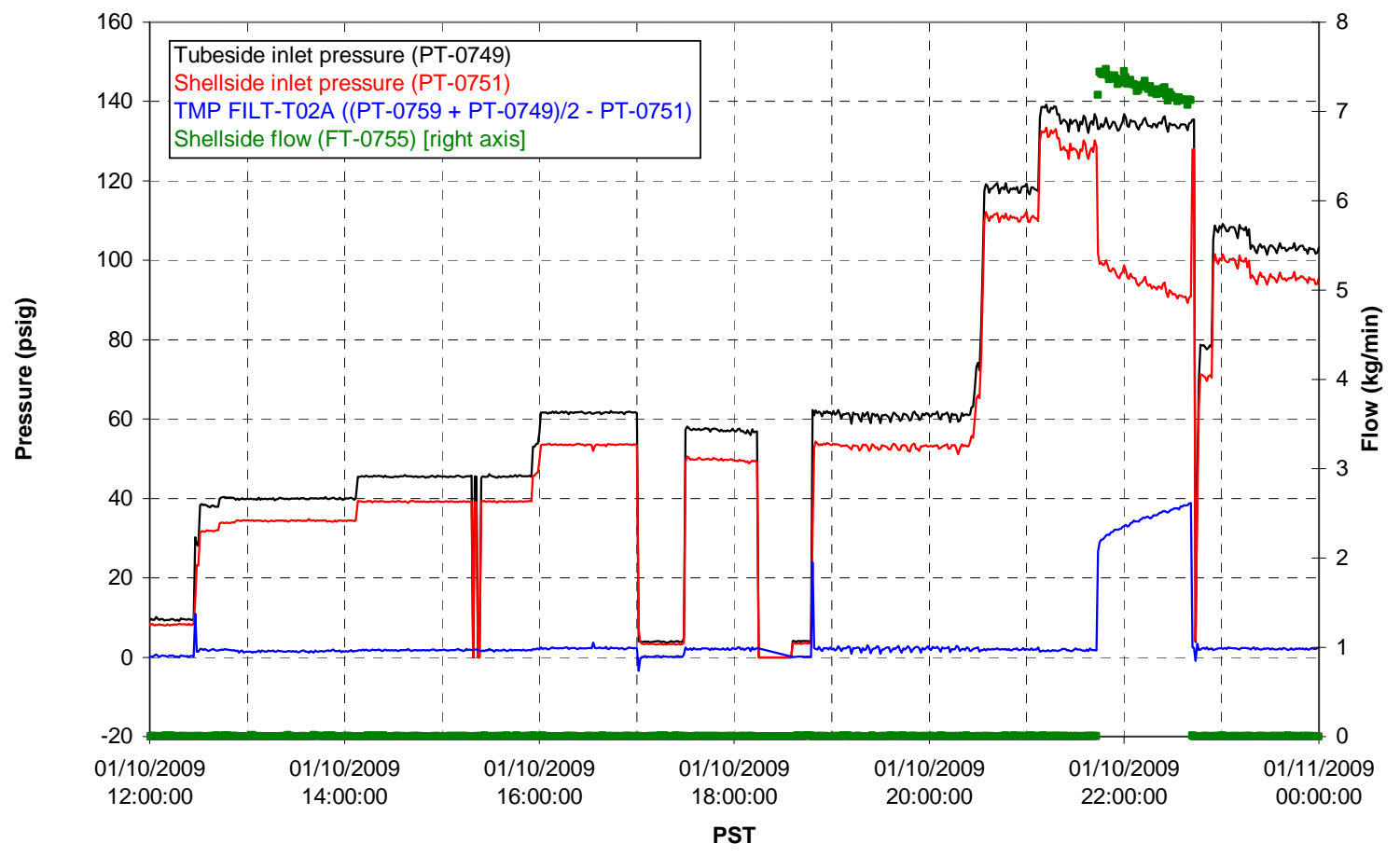


Filter UFP-FILT-T03A

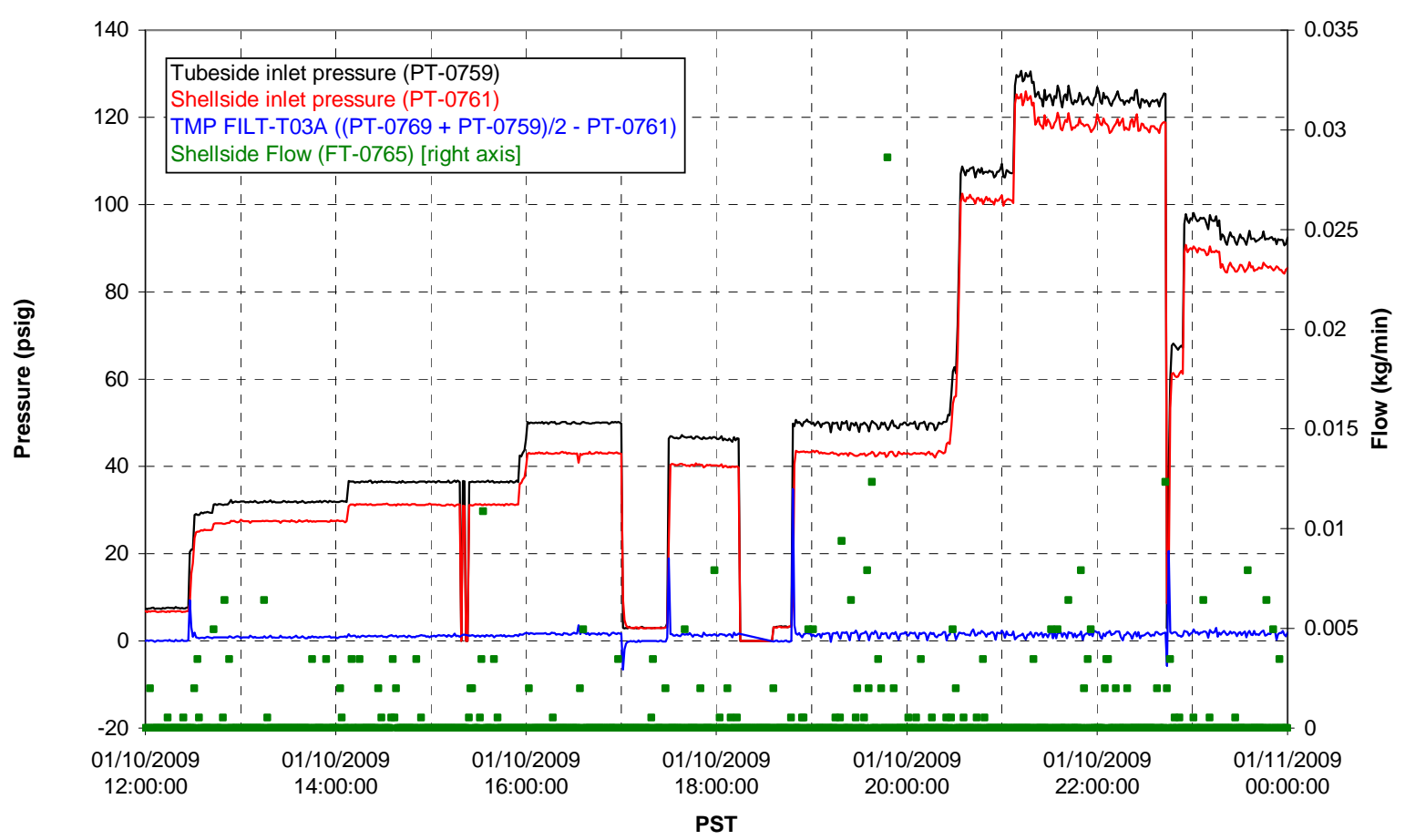

Filter UFP-FILT-T04A

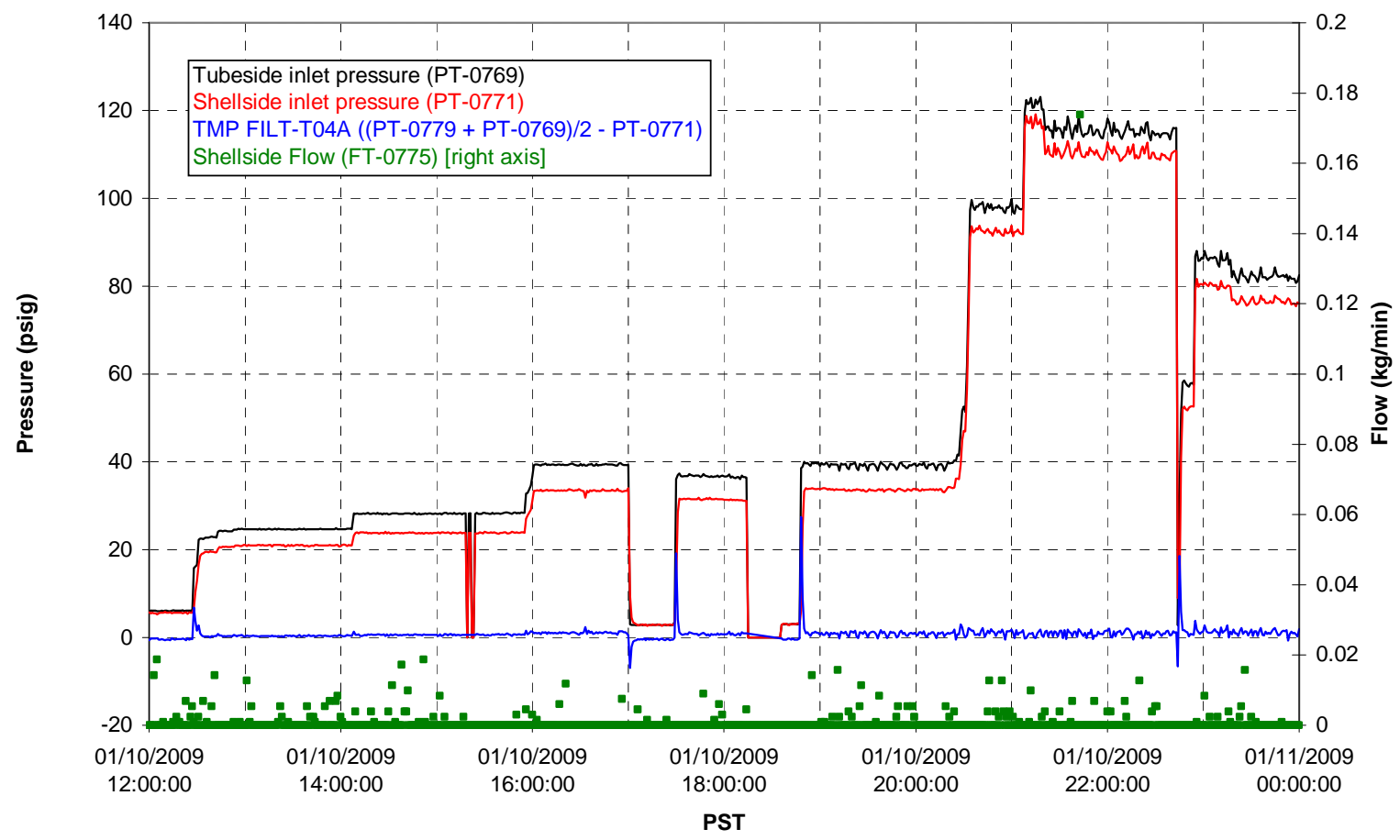


Filter UFP-FILT-T05A

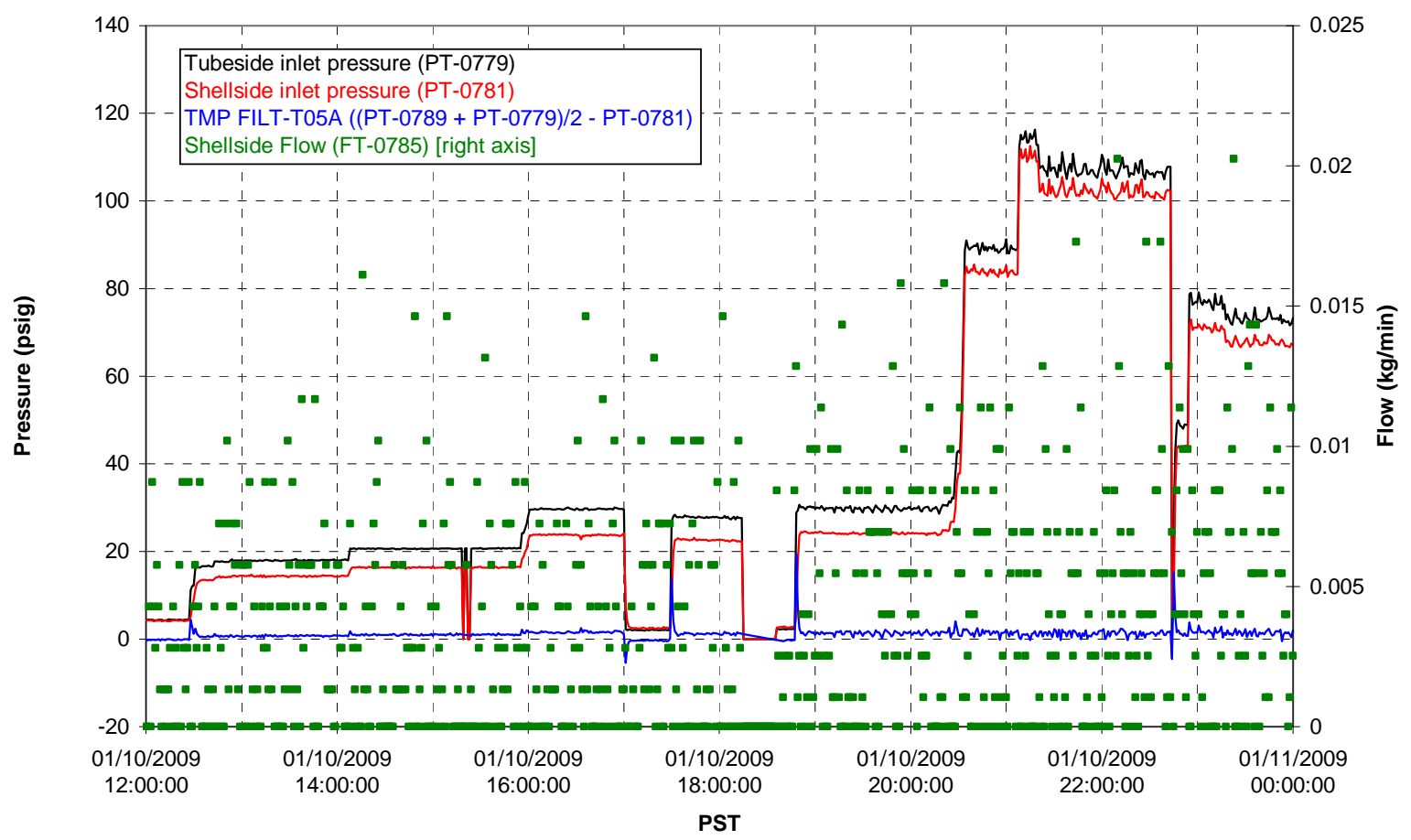

Chemical Flow

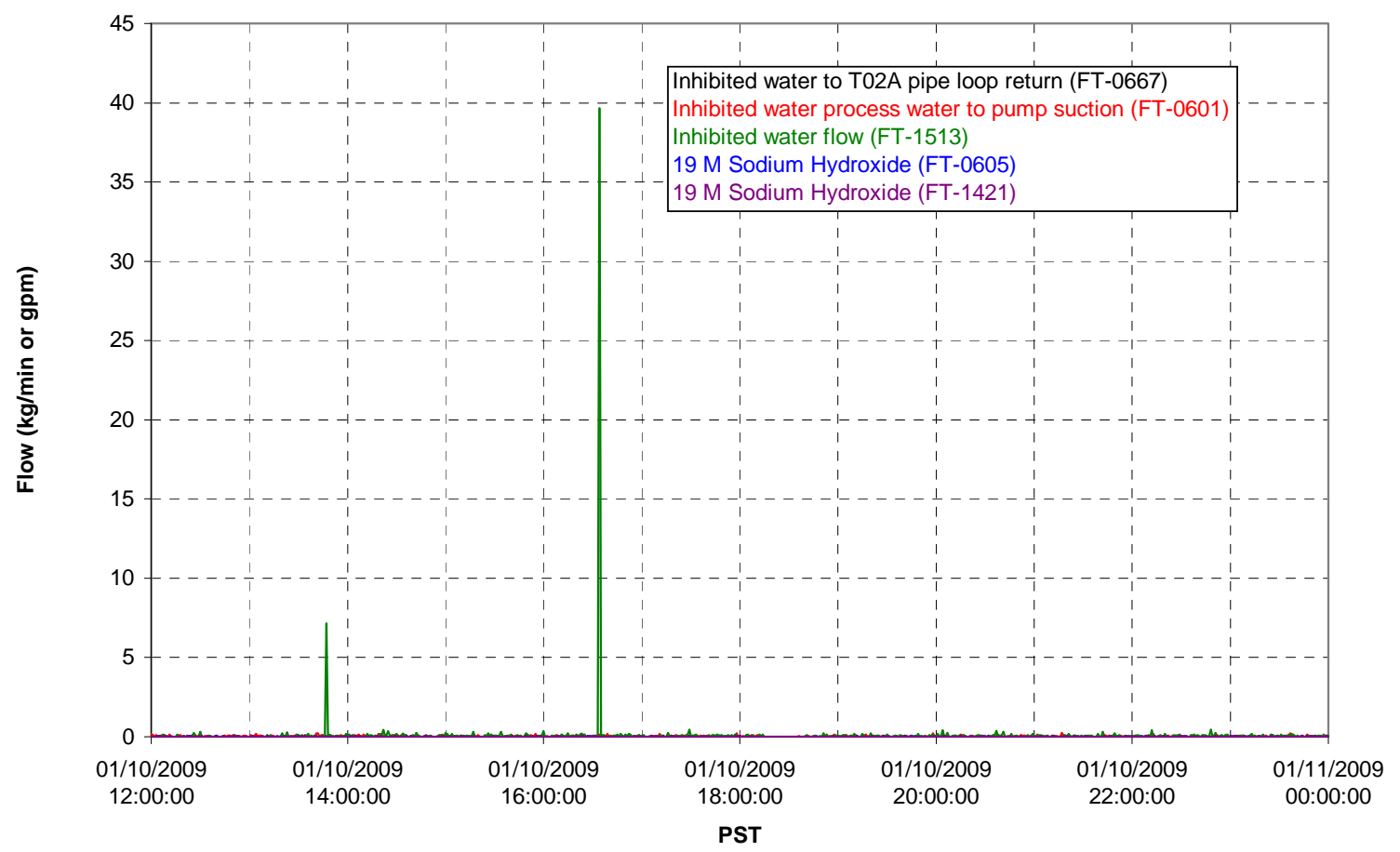




\section{Chemical Flow}

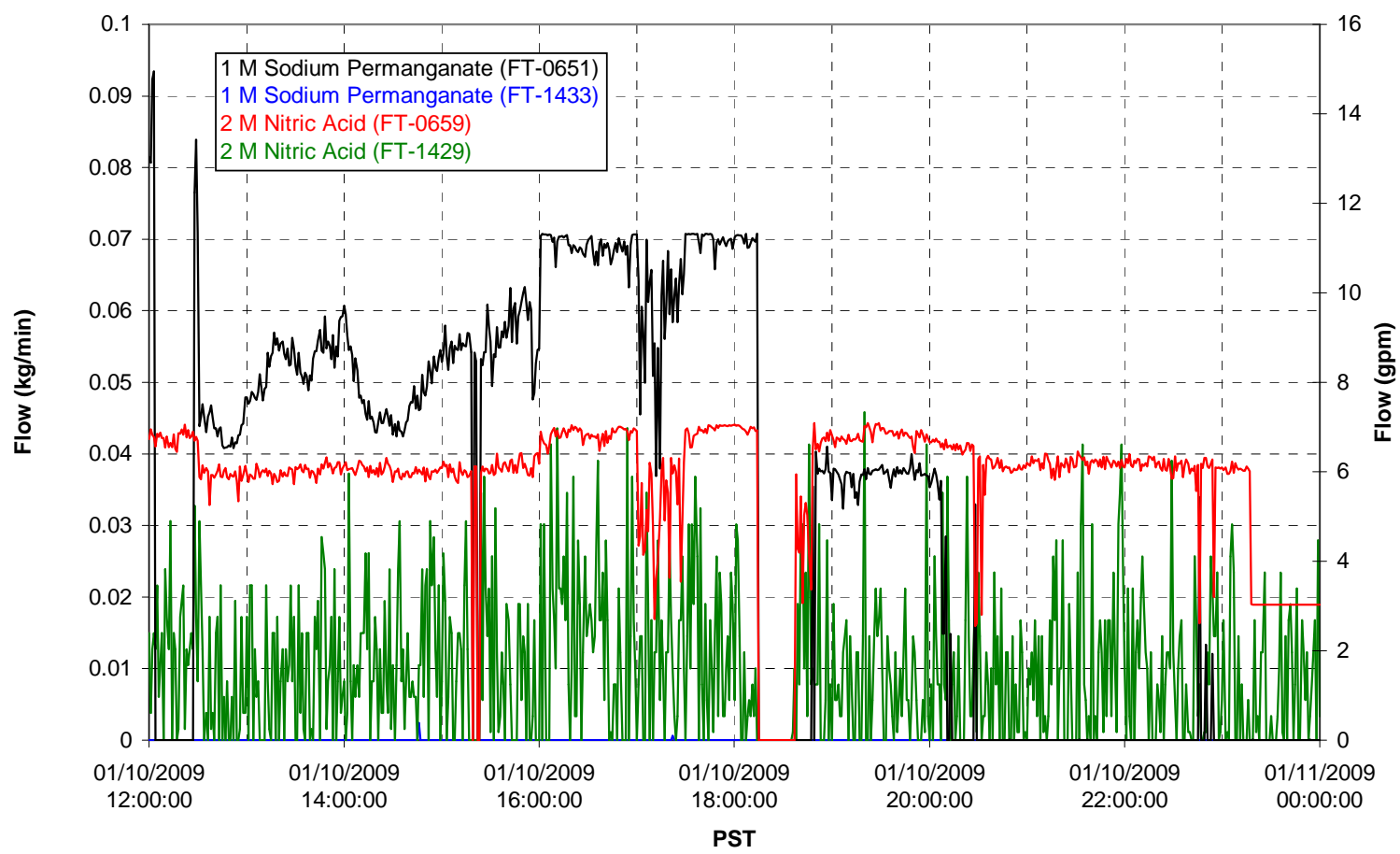

Air Flows

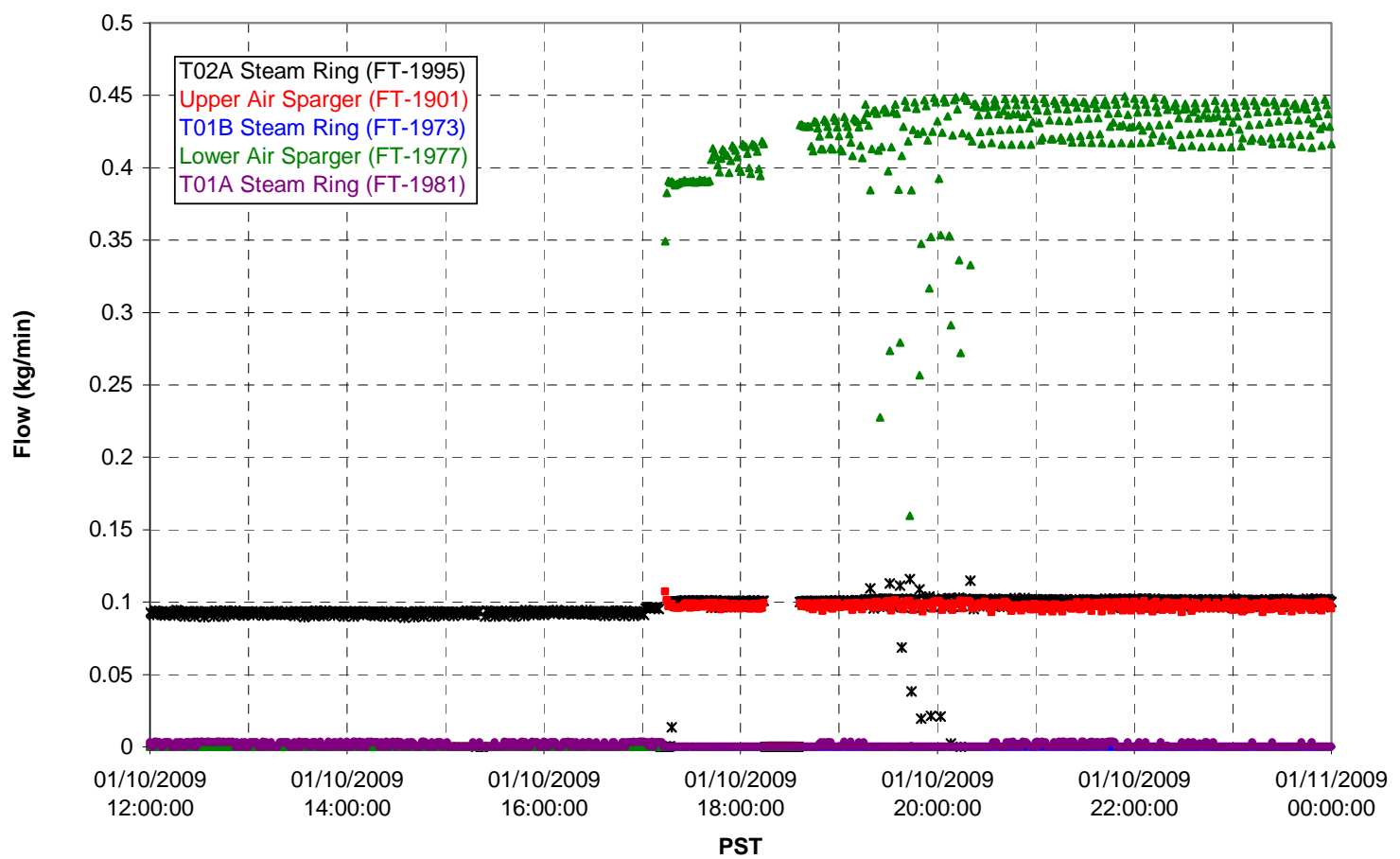


T02A Steam

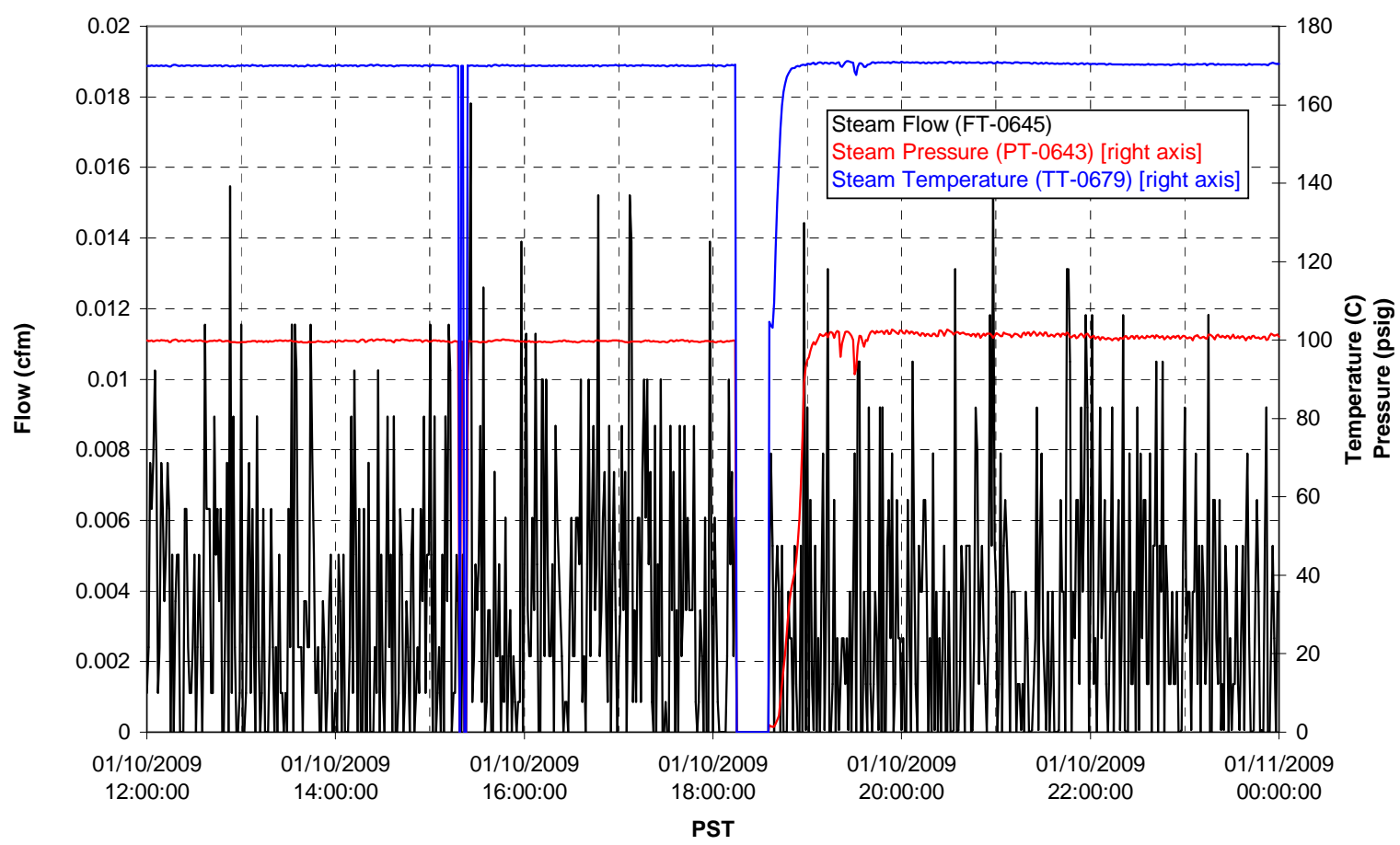

T01A Steam

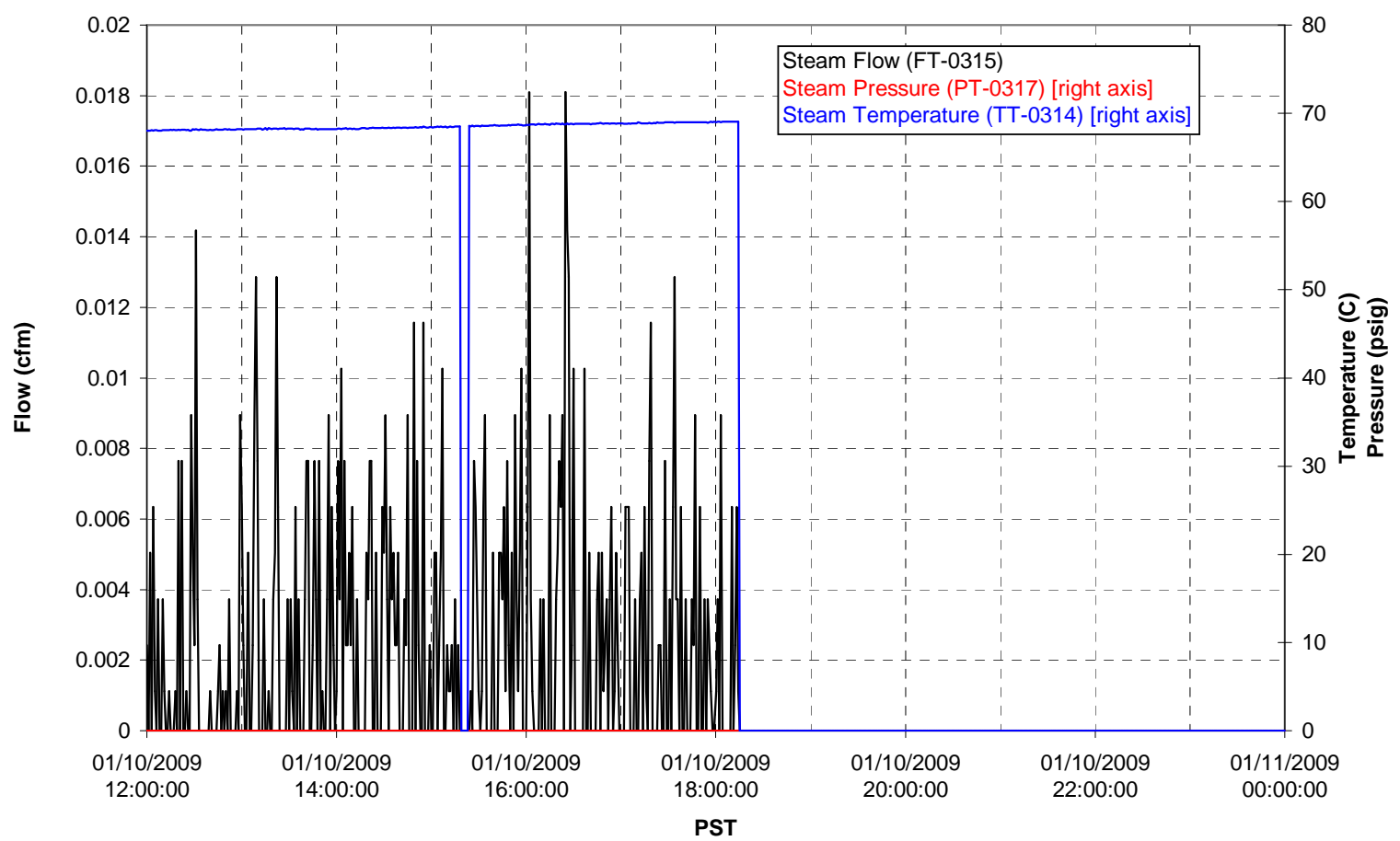


T01B Steam

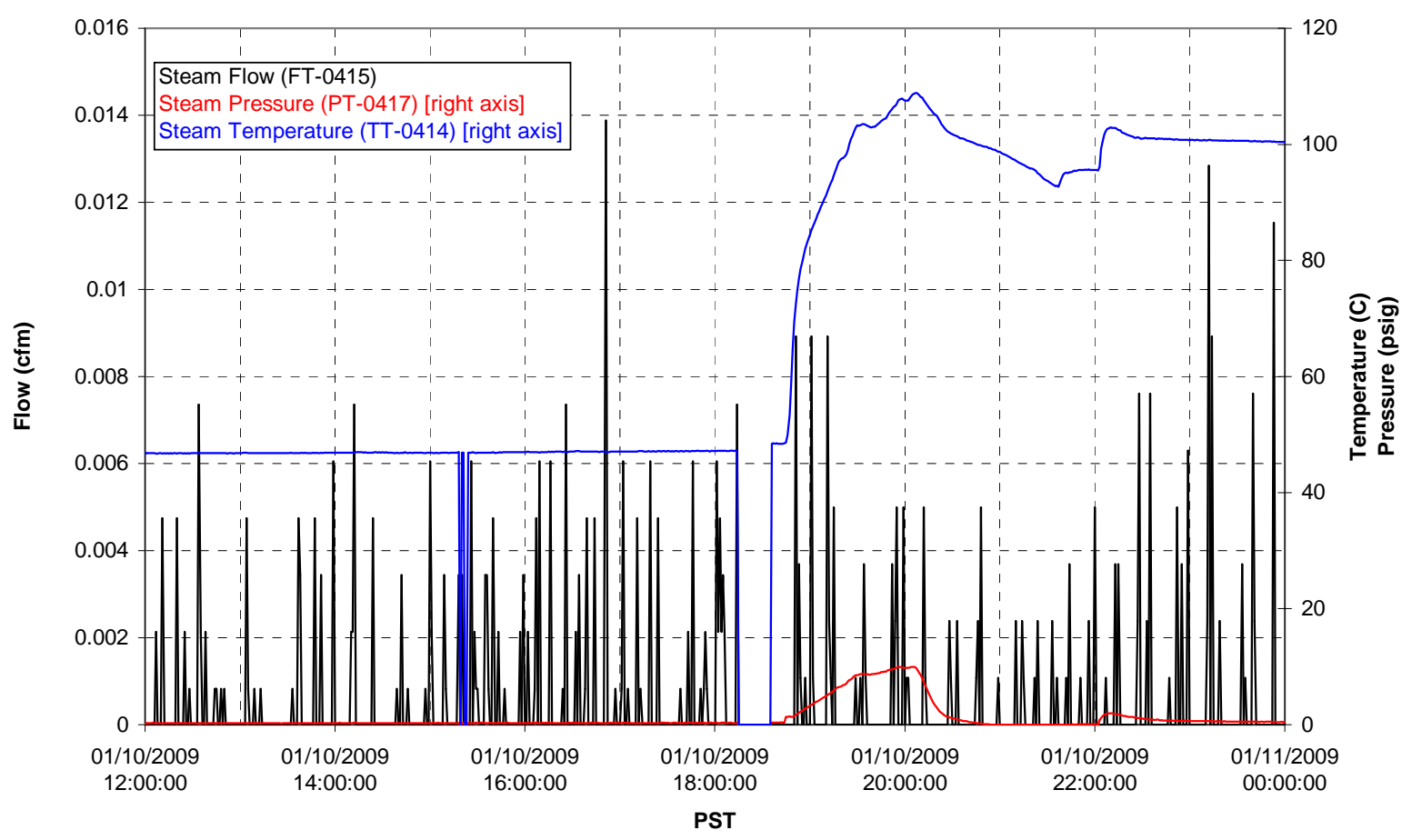


Functional Test Data Plots

01/11/2009 00:00 - 01/11/2009 12:00 
T01A level

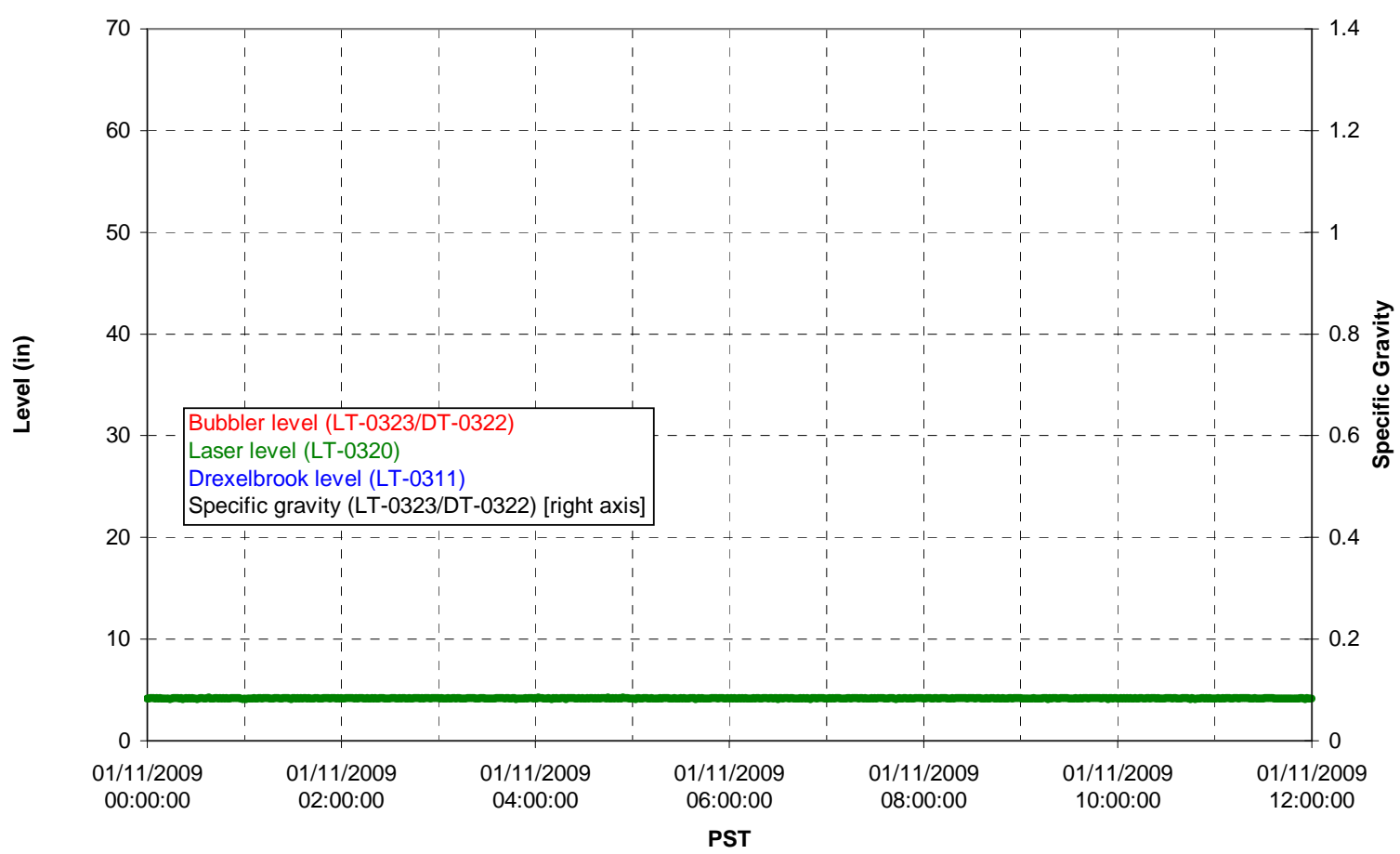

T01A temperatures

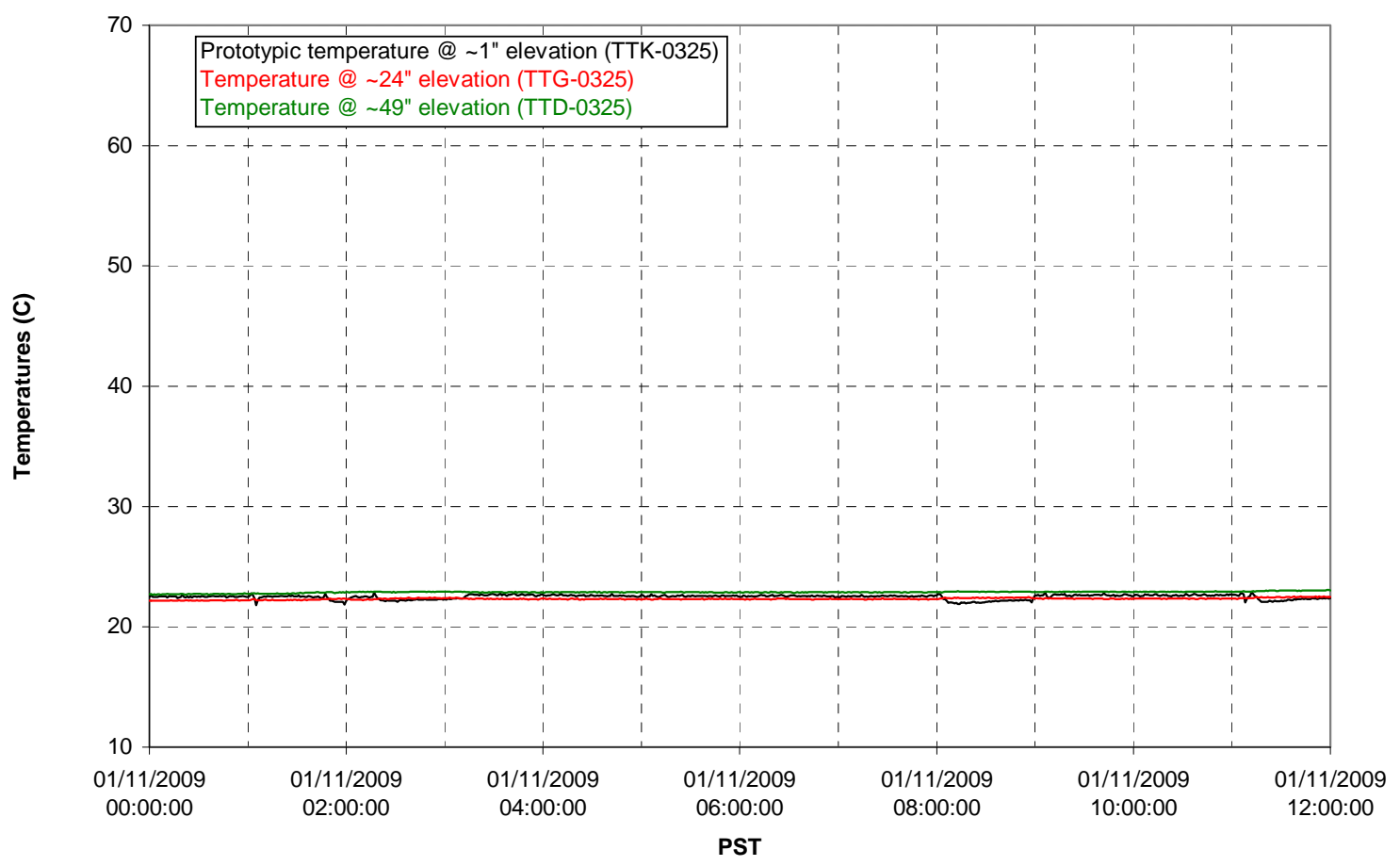


T01B level

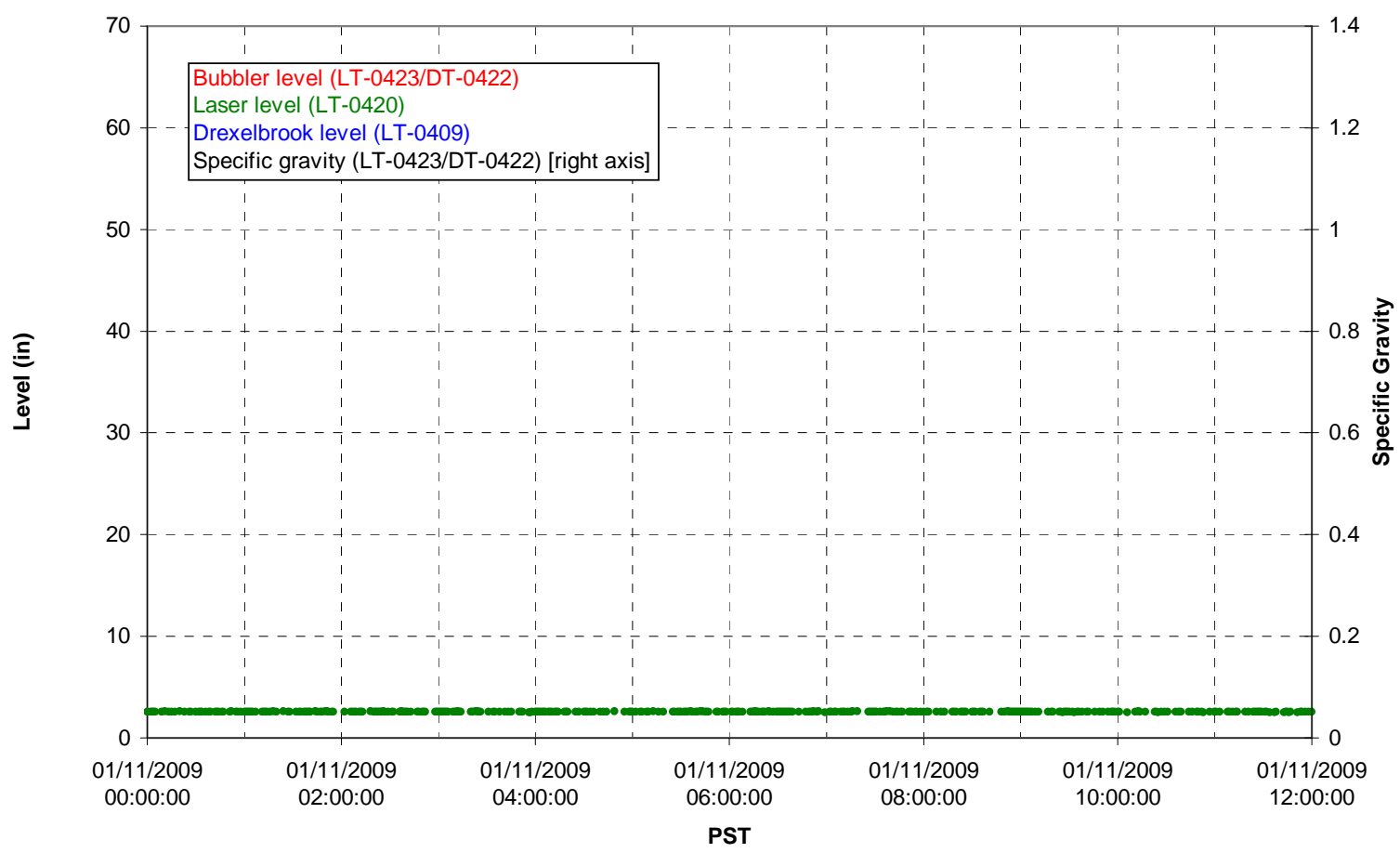

T01B temperatures

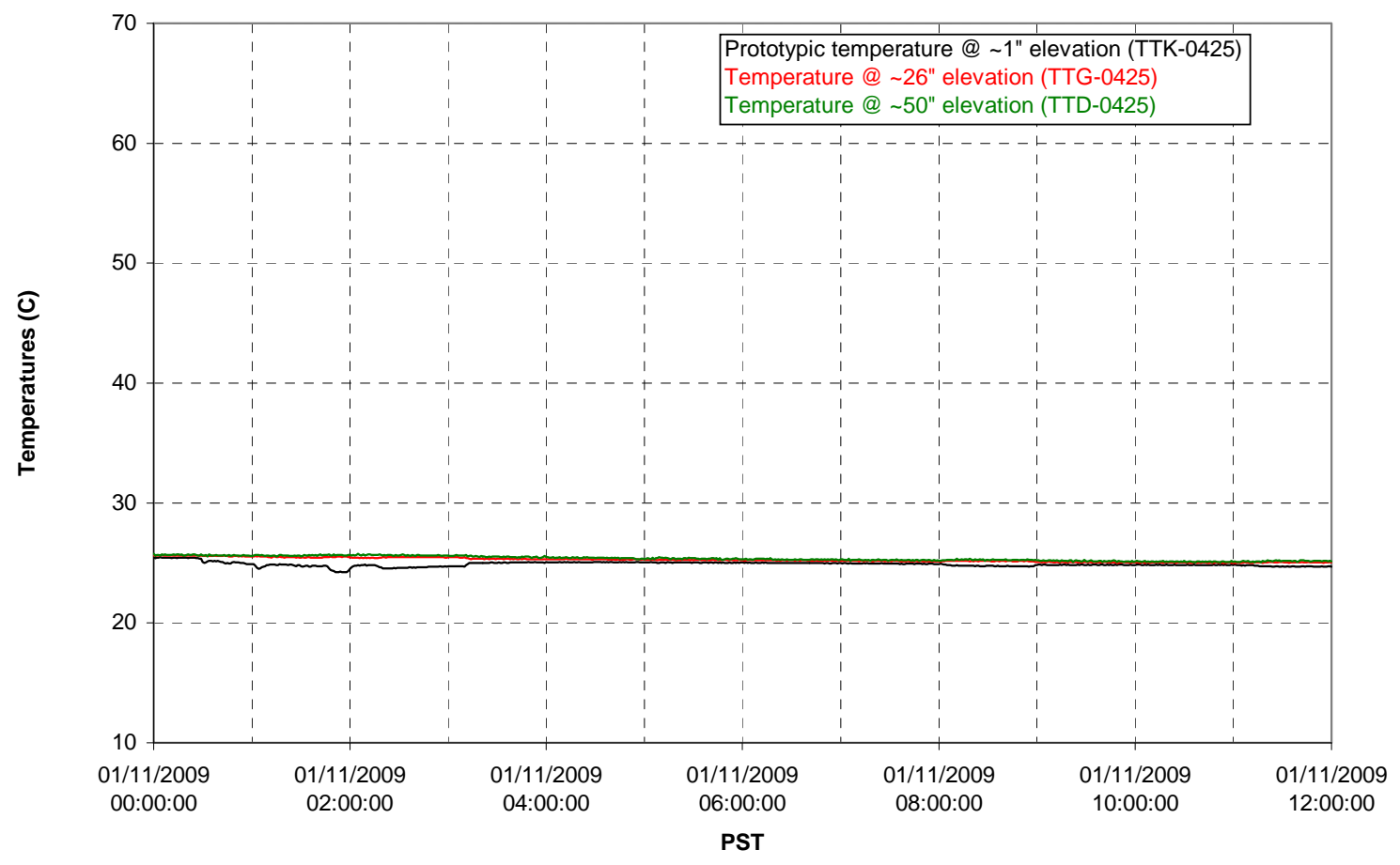


T02A level

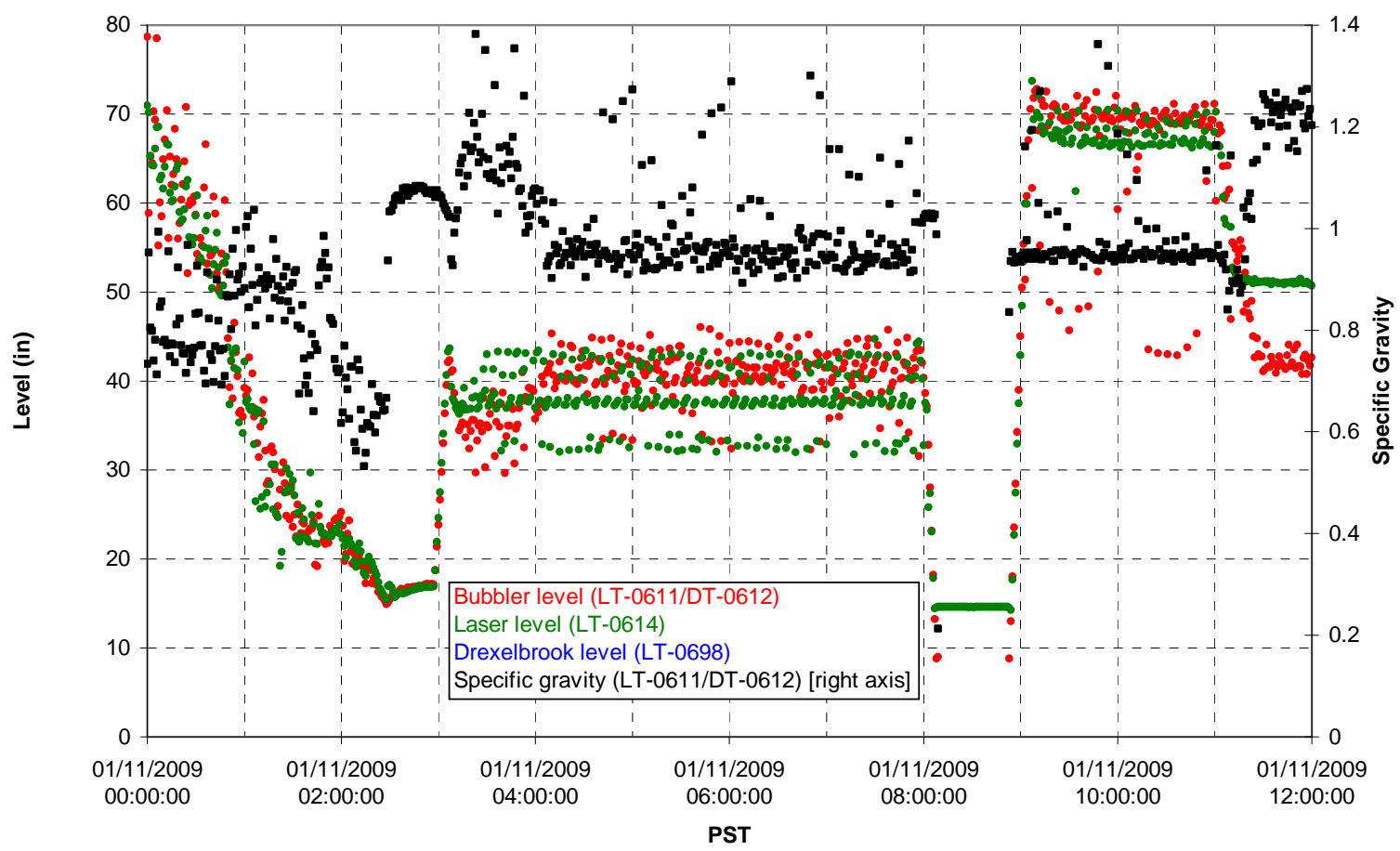

T02A temperatures

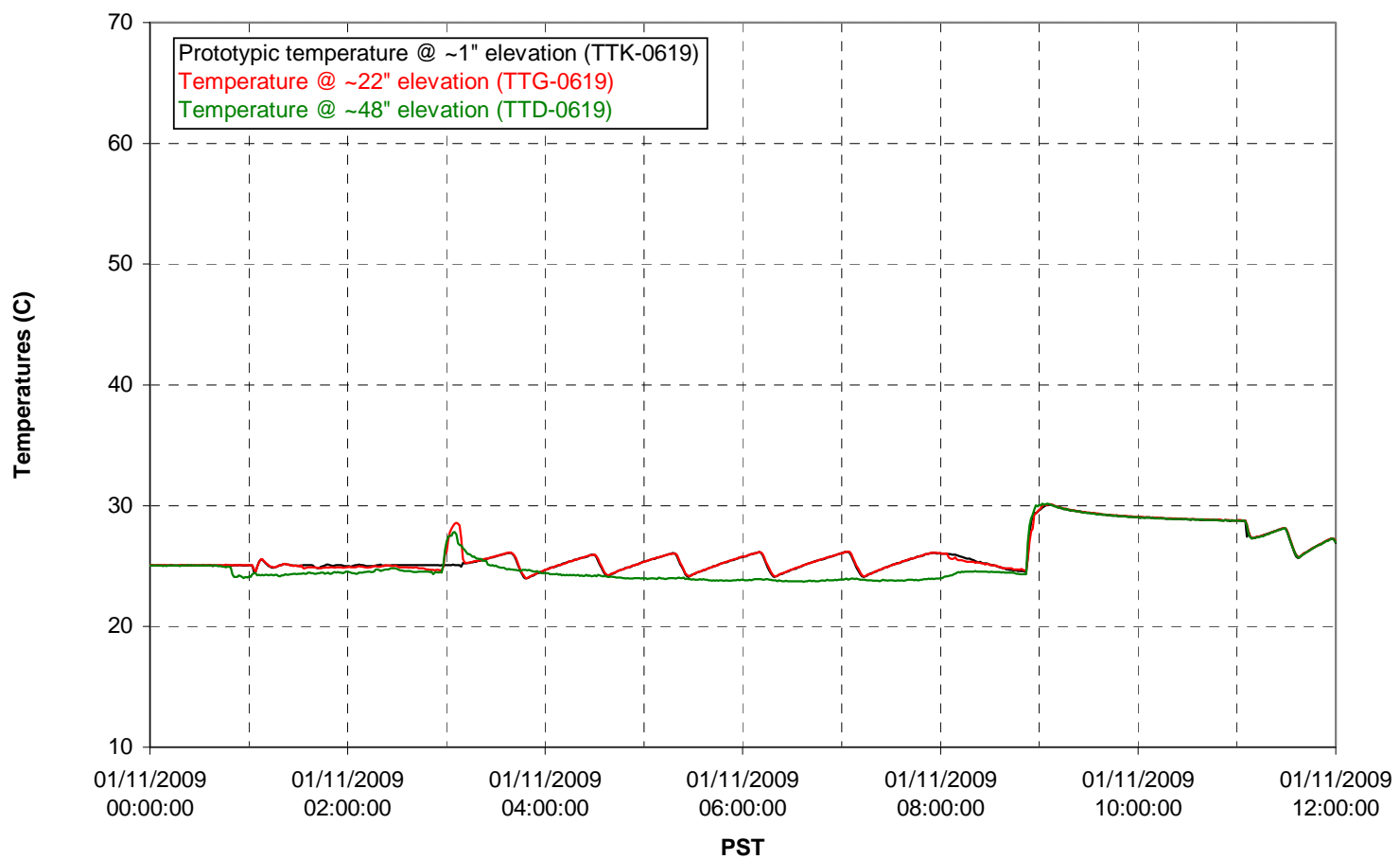


T02A and filter loop temperatures

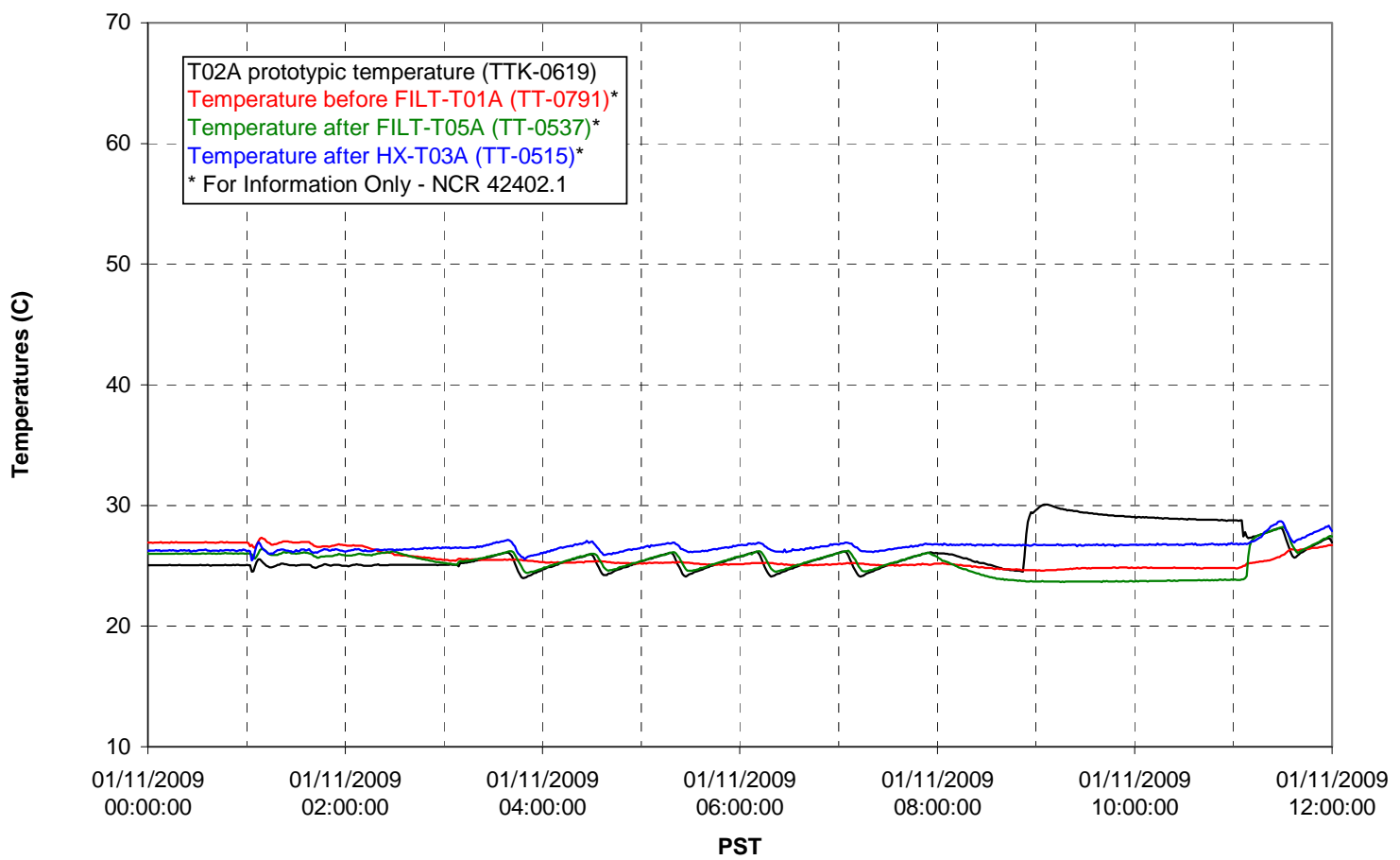

Pump Pressures and Flow

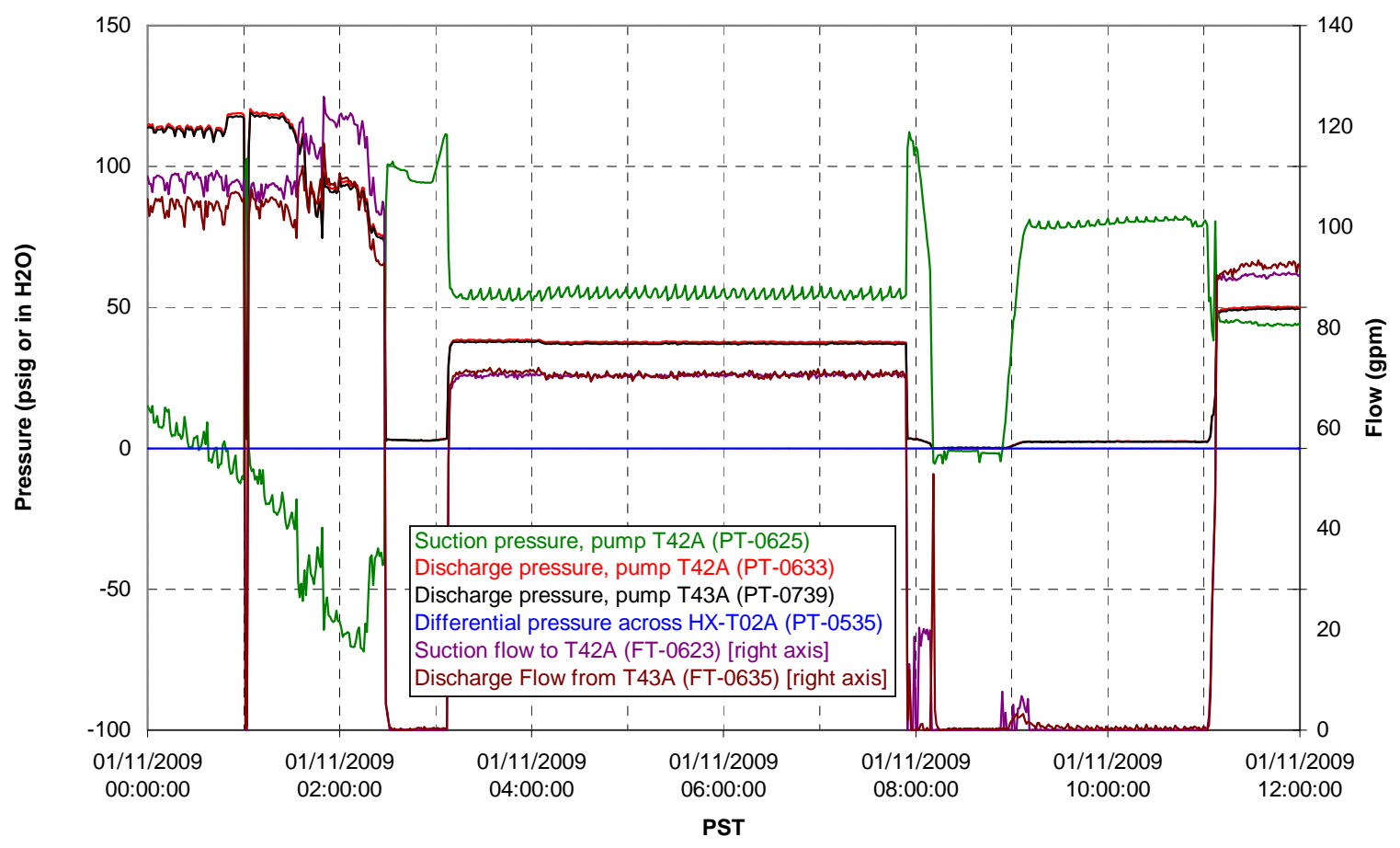


Axial pressure drop

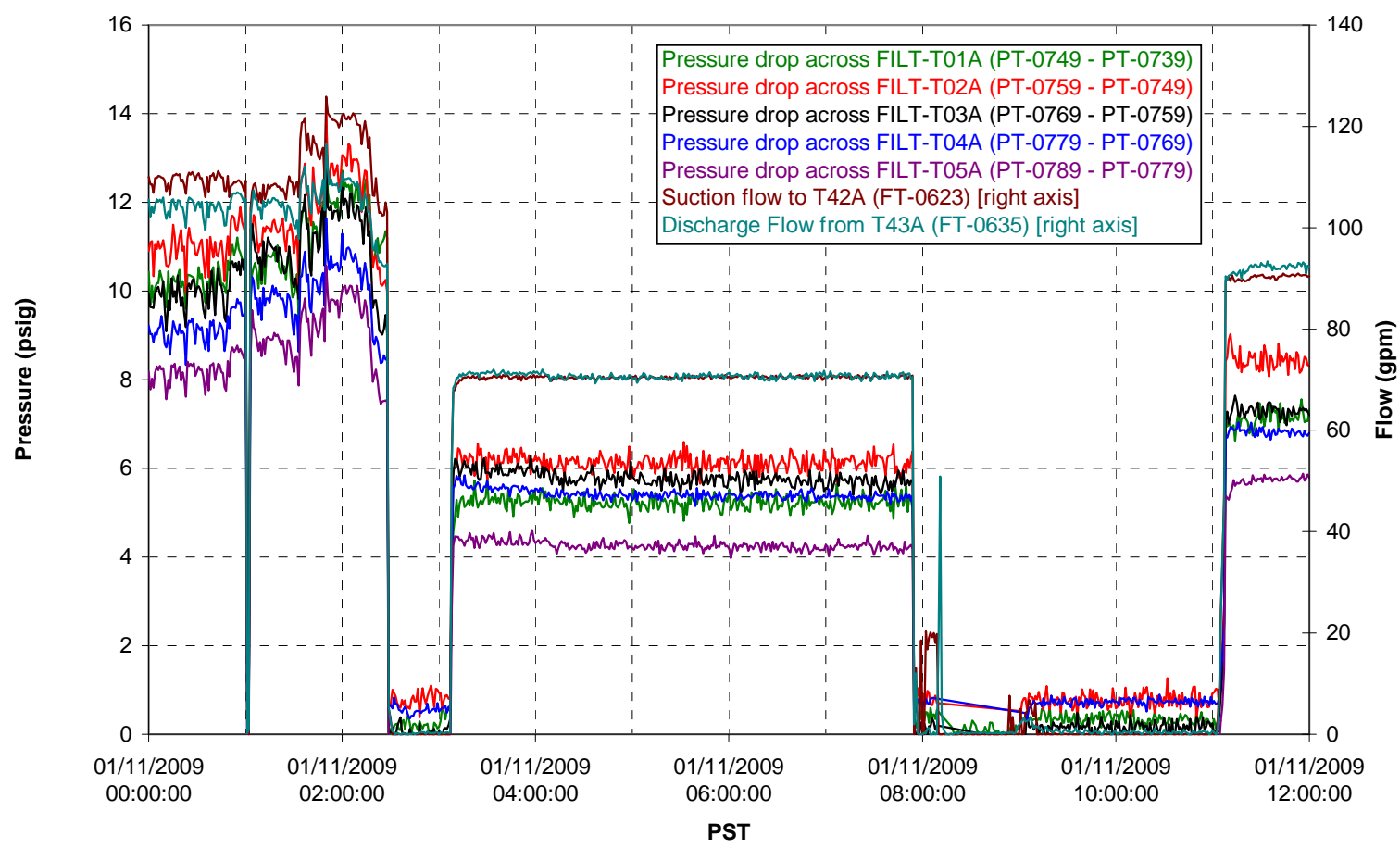

Permeate flow rates

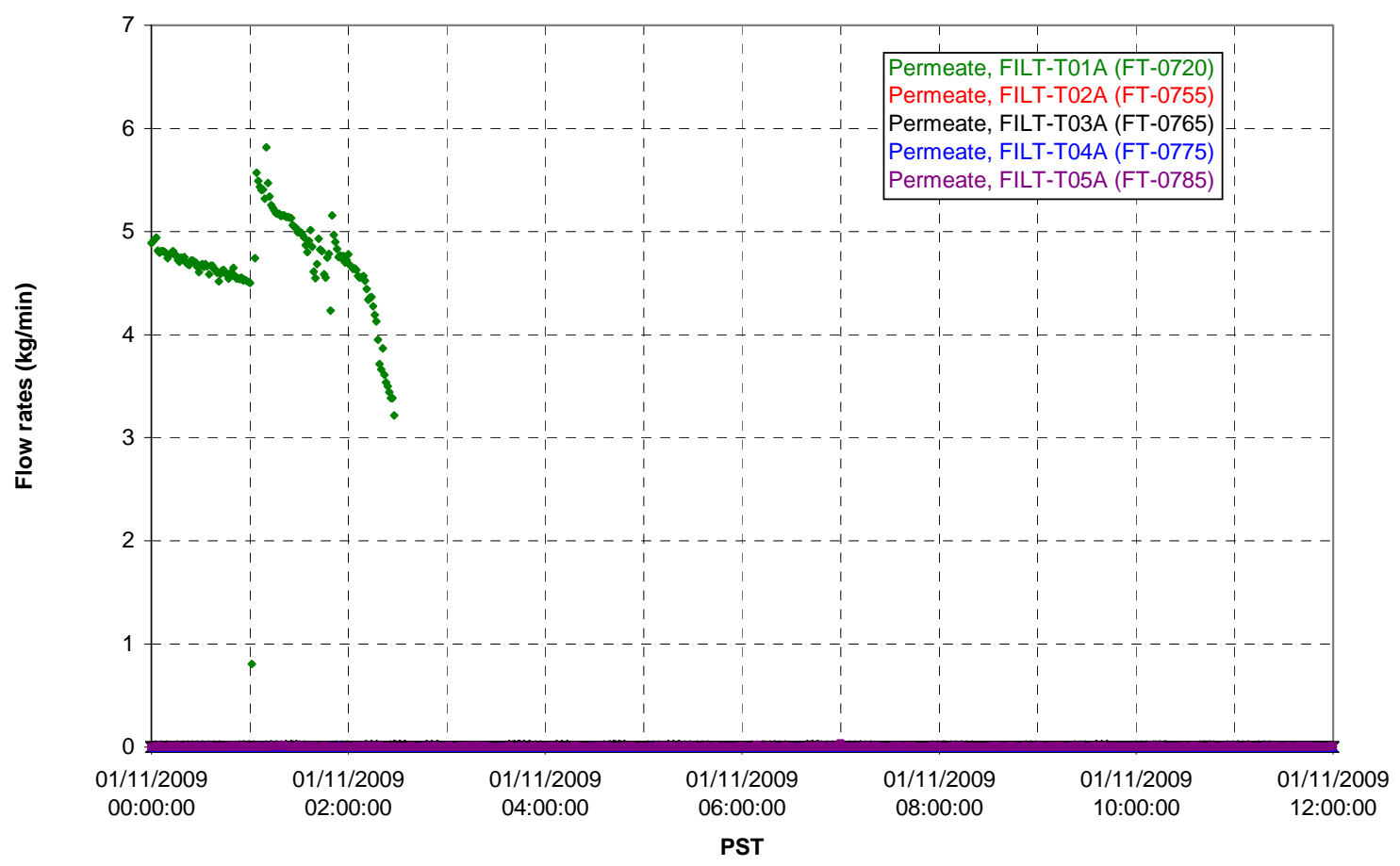


T02A Inner Temperature Tree

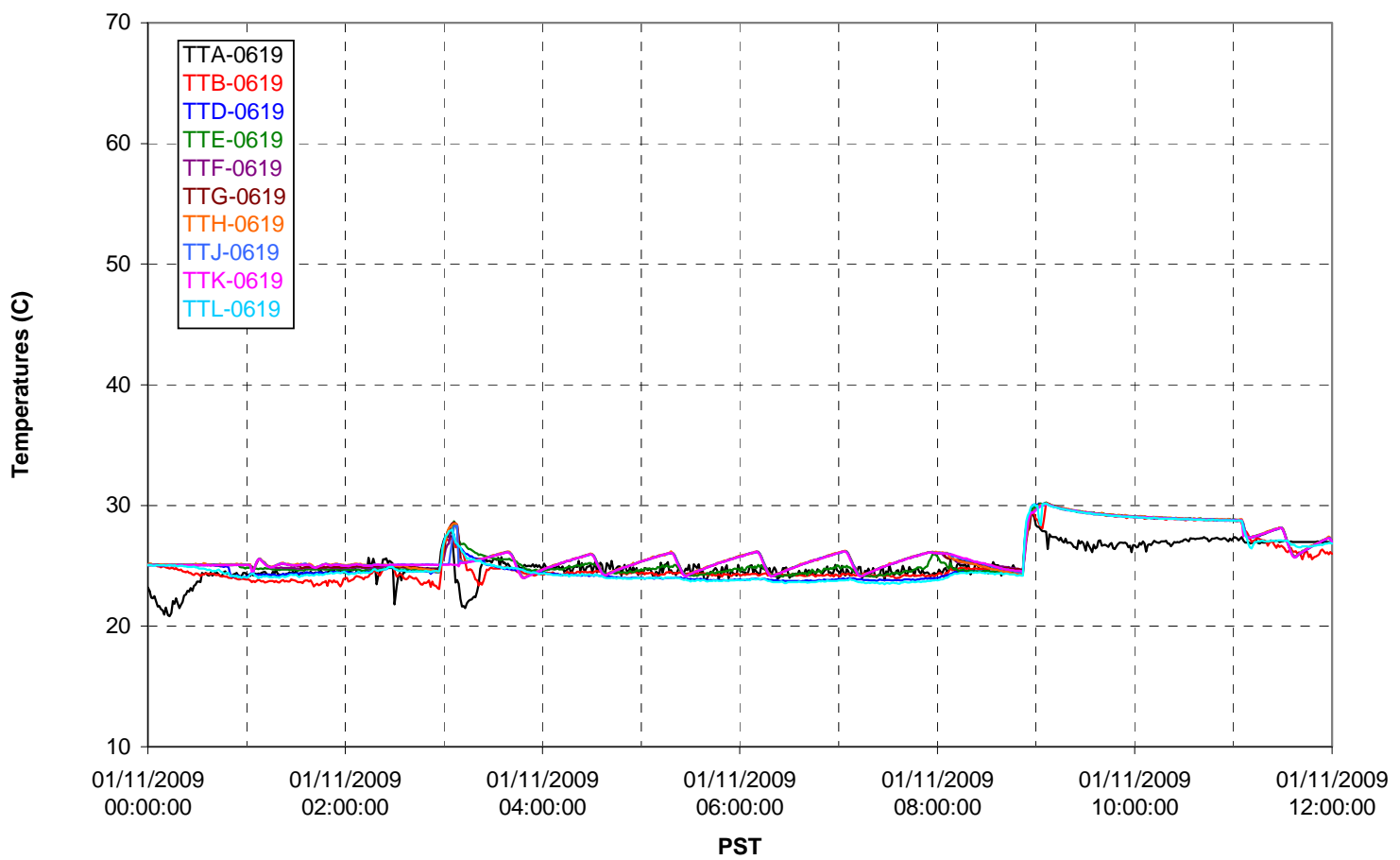

T02A Outer Temperature Tree

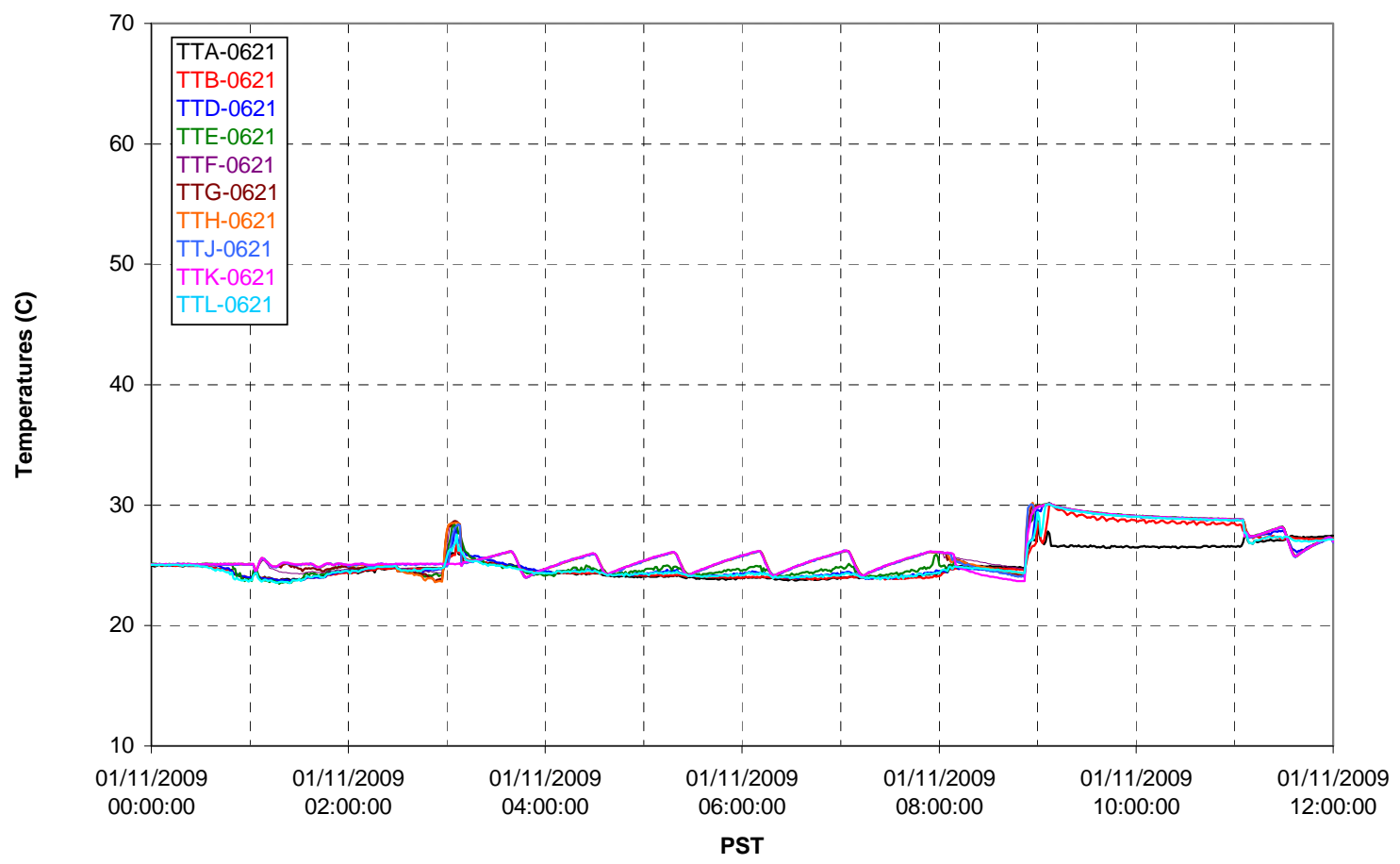


T02A temperatures

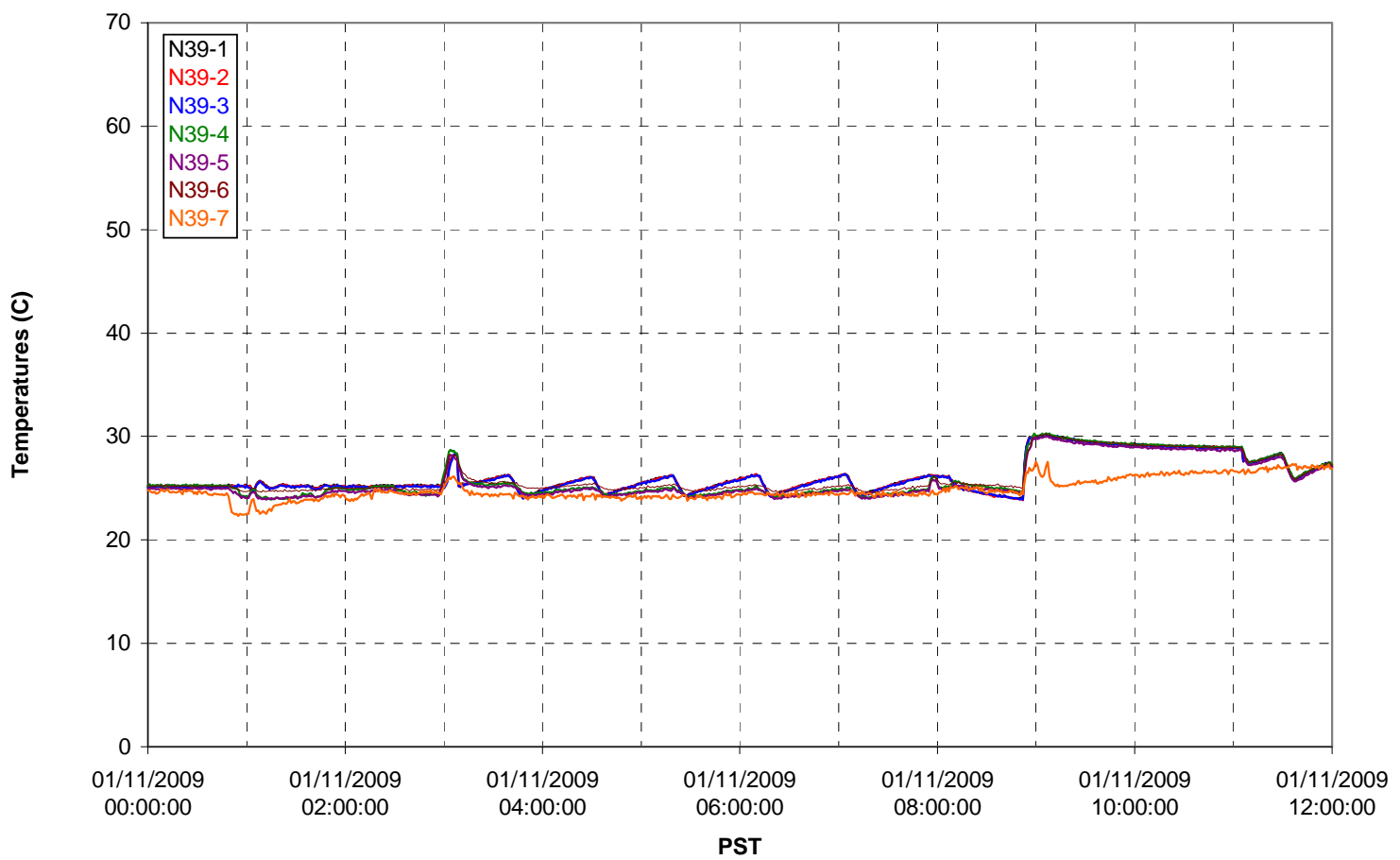

T02A temperatures

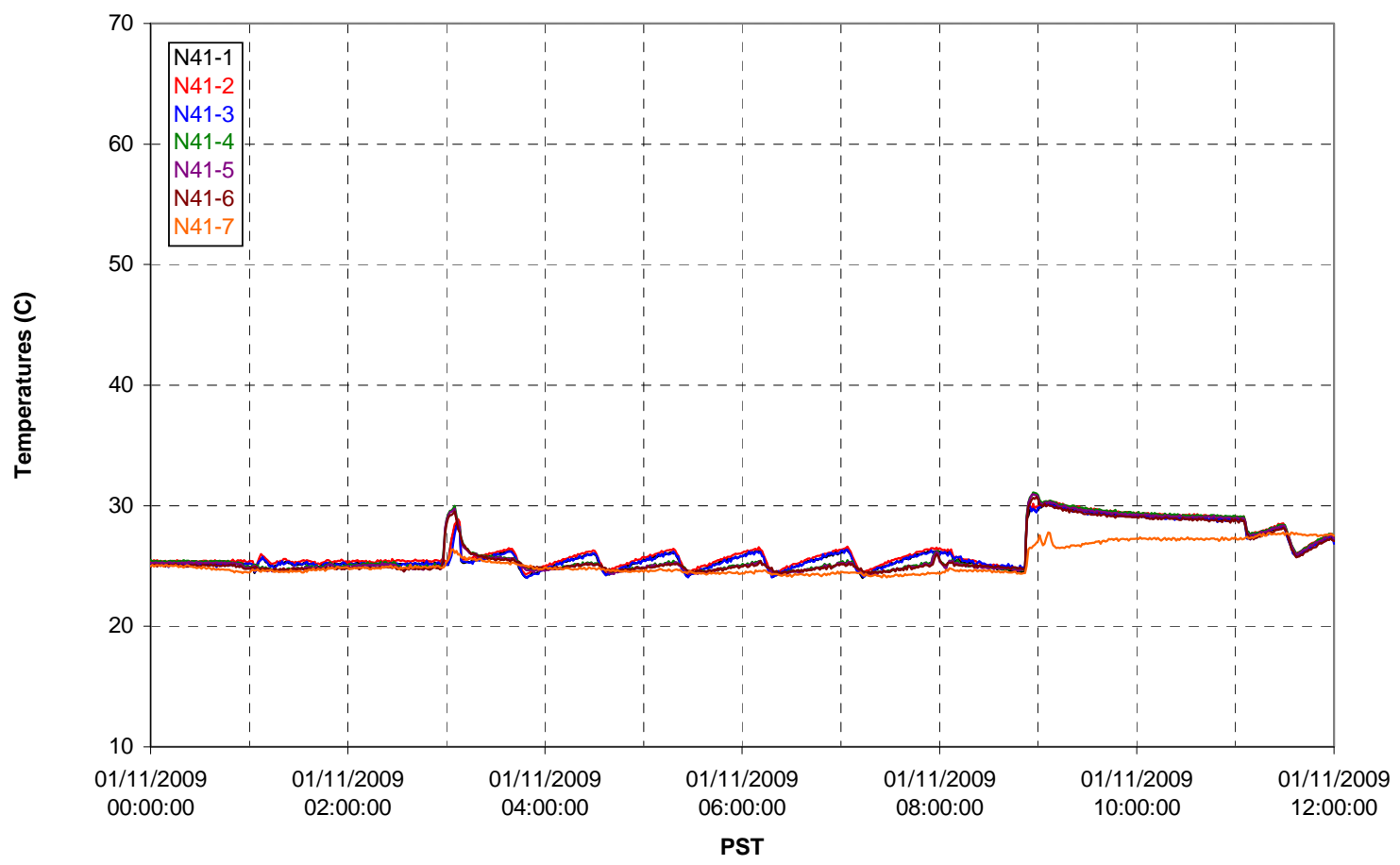


T02A temperatures

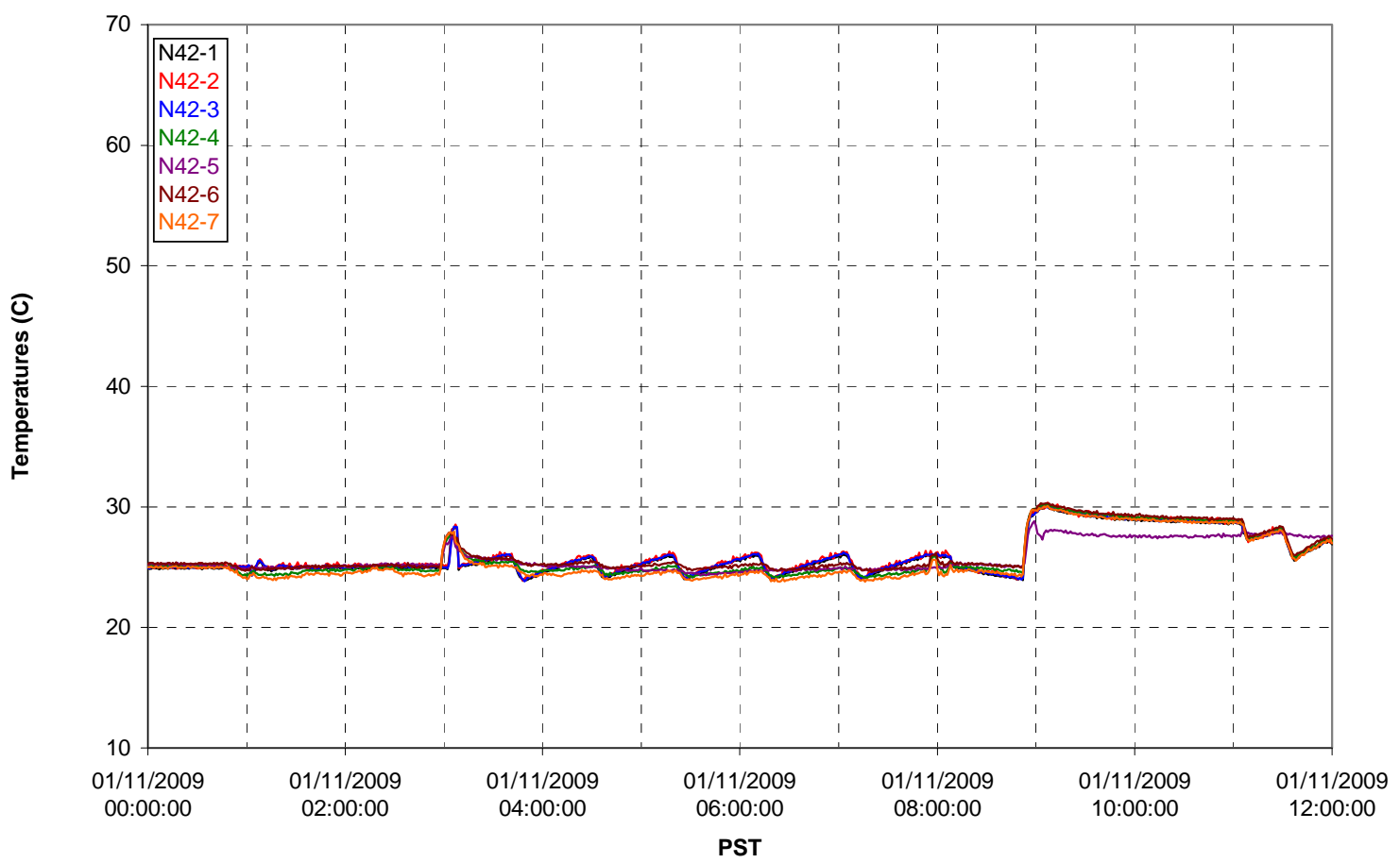

T02A temperatures

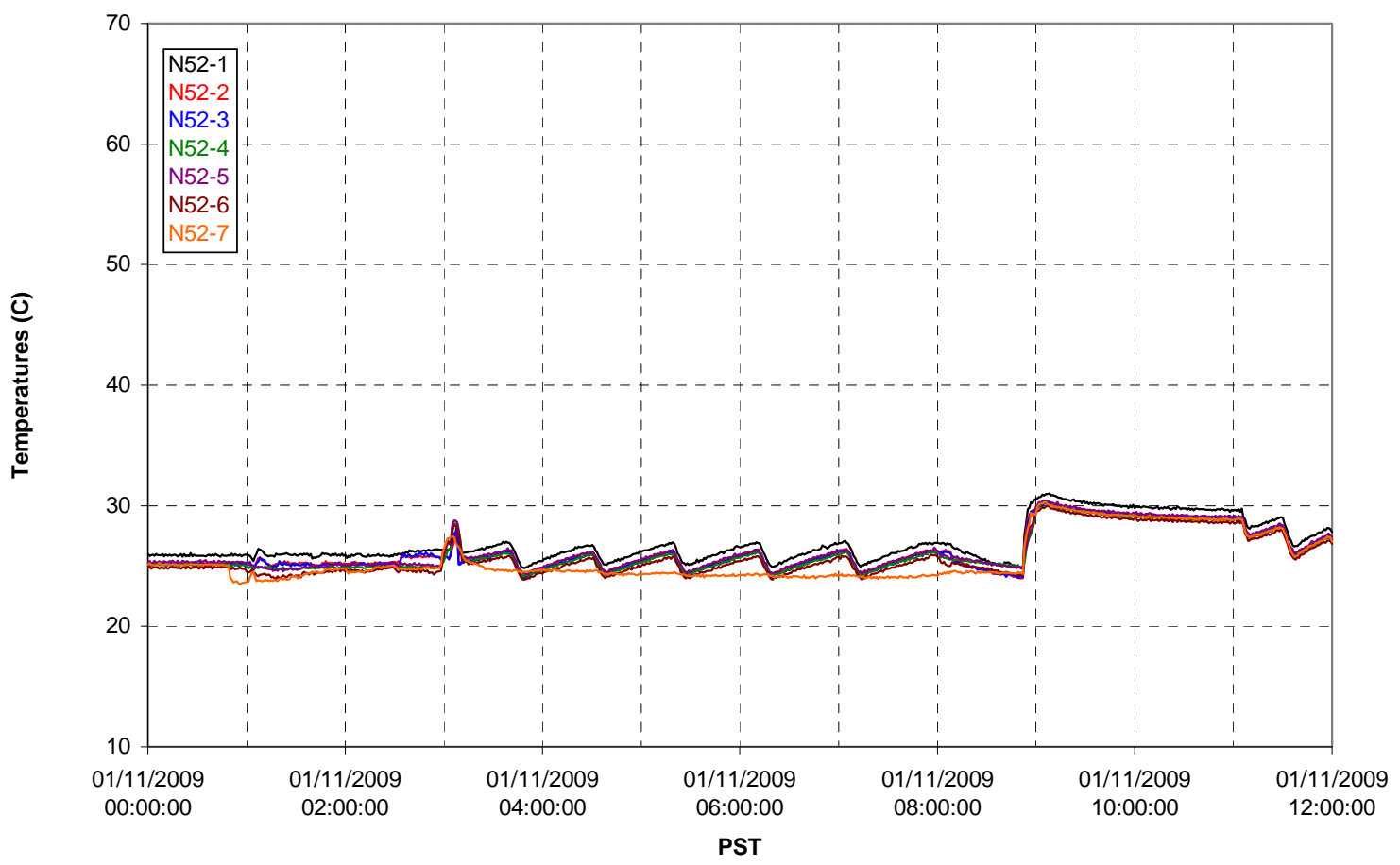


T02A Heating and Cooling

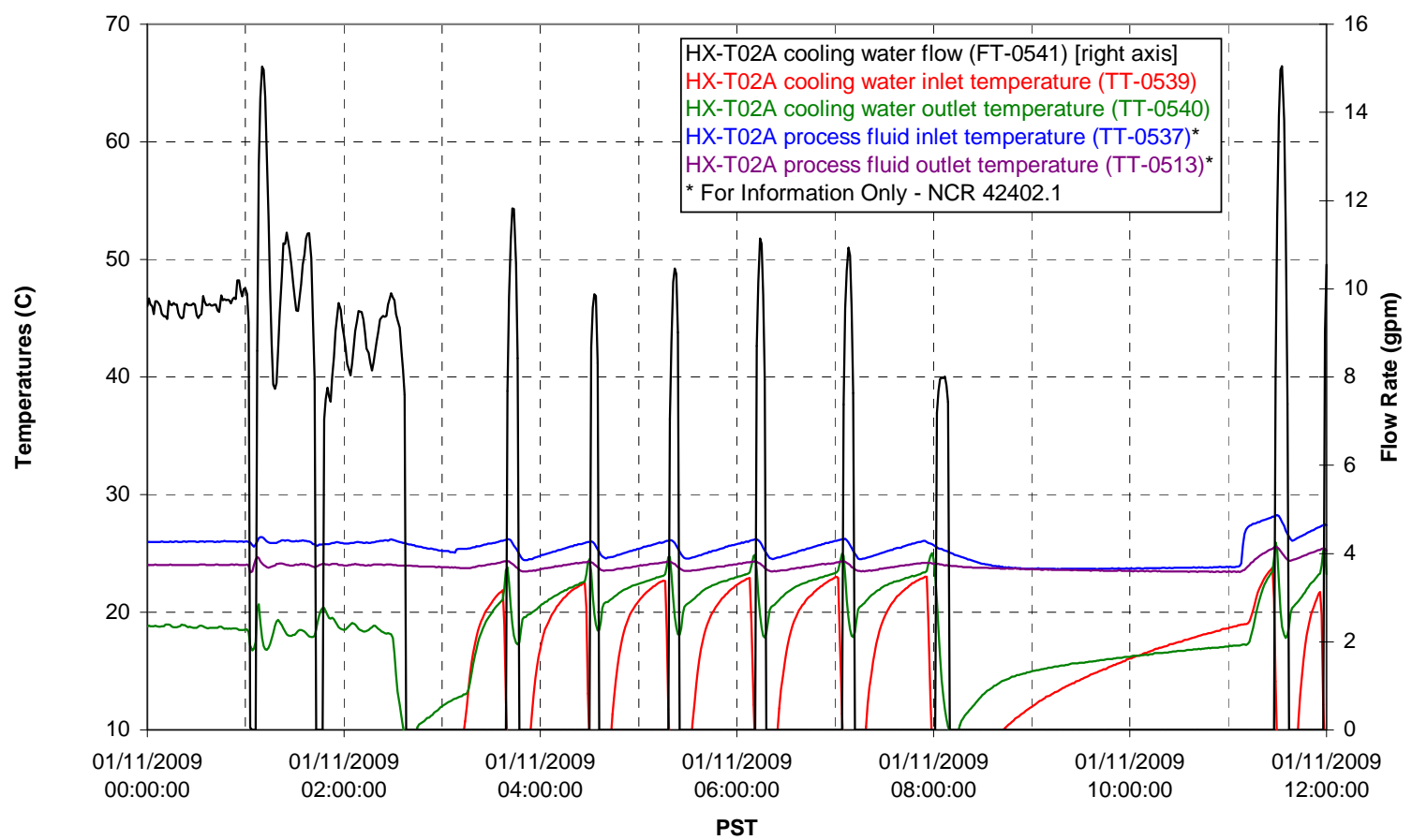

Pump Operation

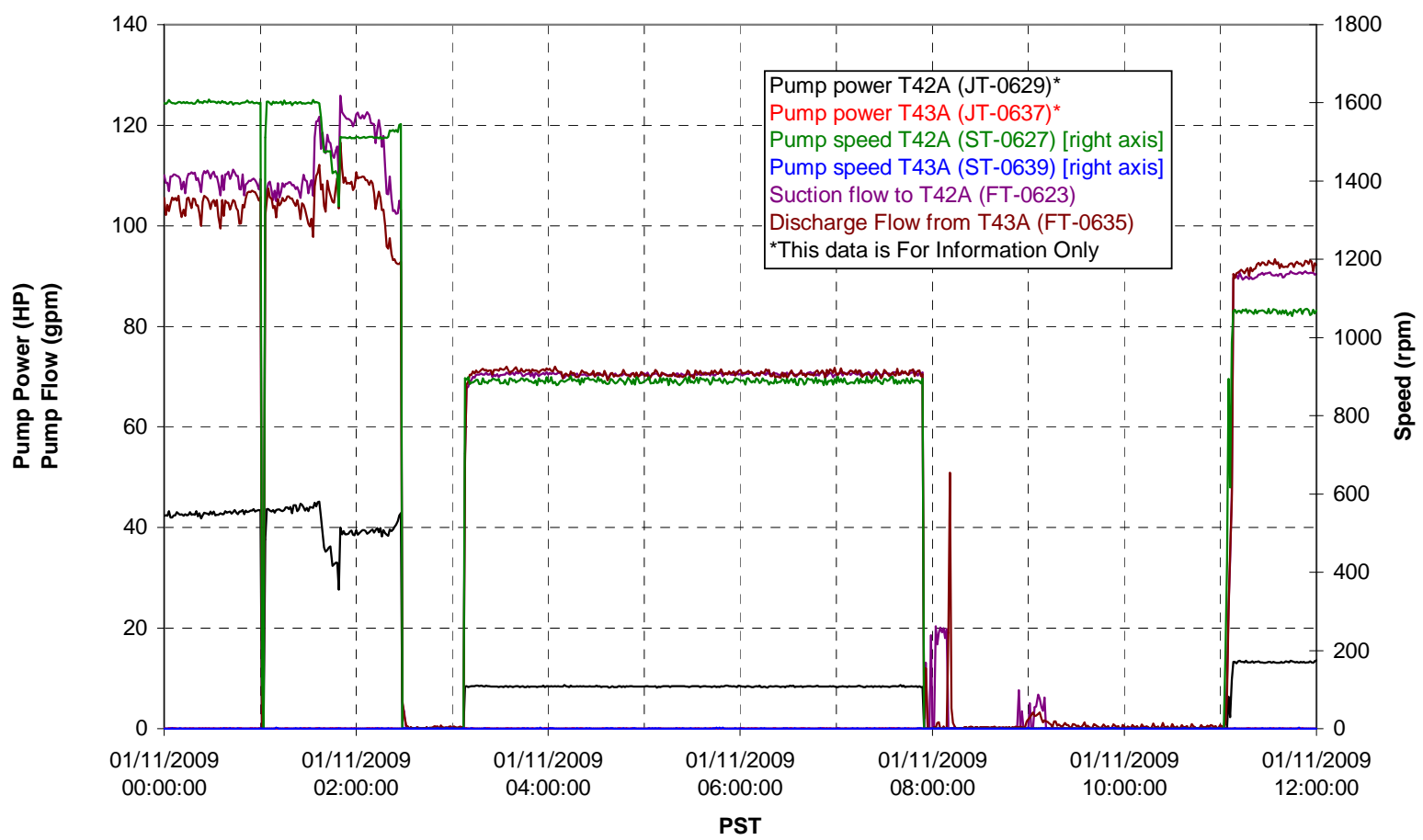


Pulsepot UFP-PP-T01A

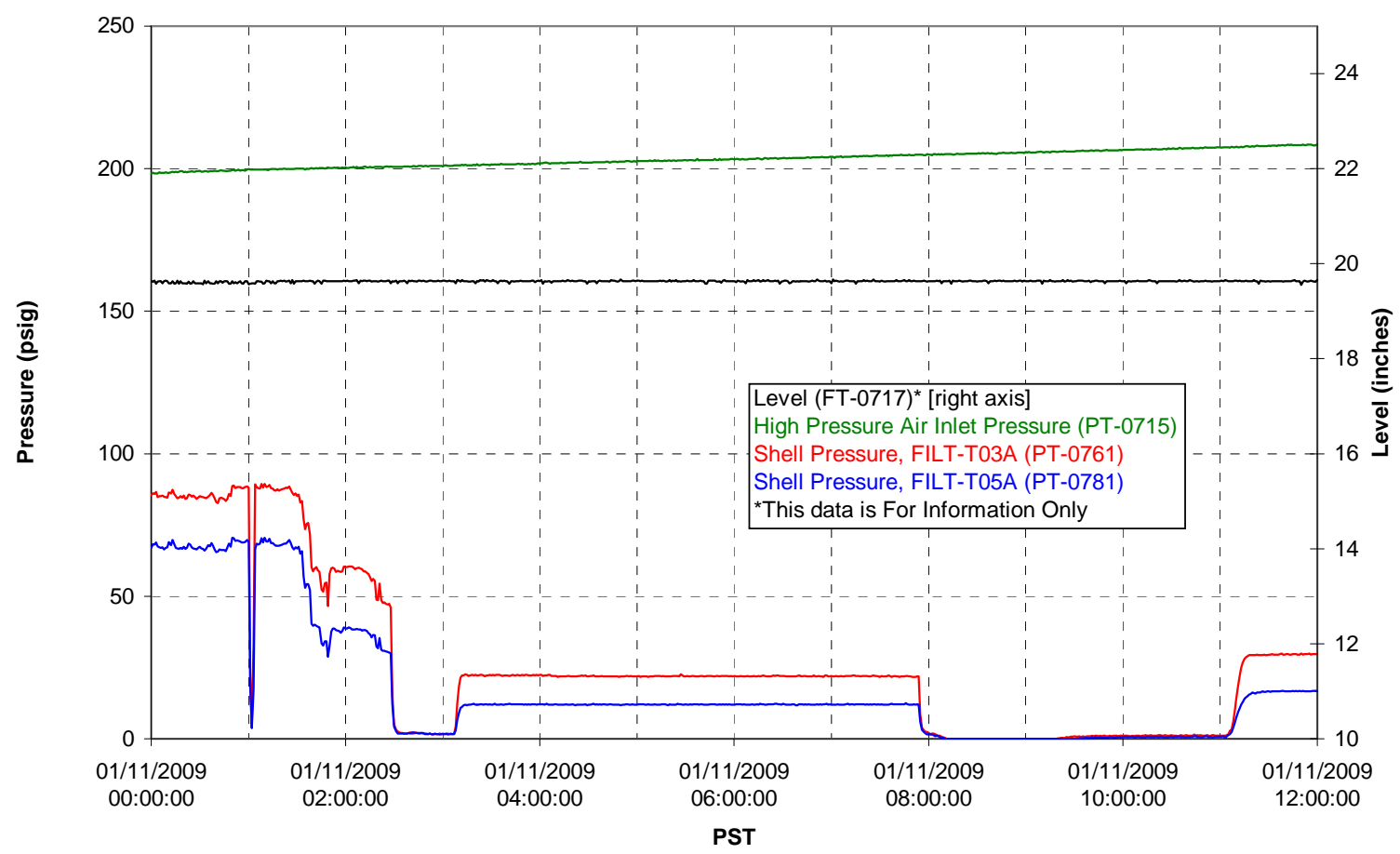

Pulsepot UFP-PP-T02A

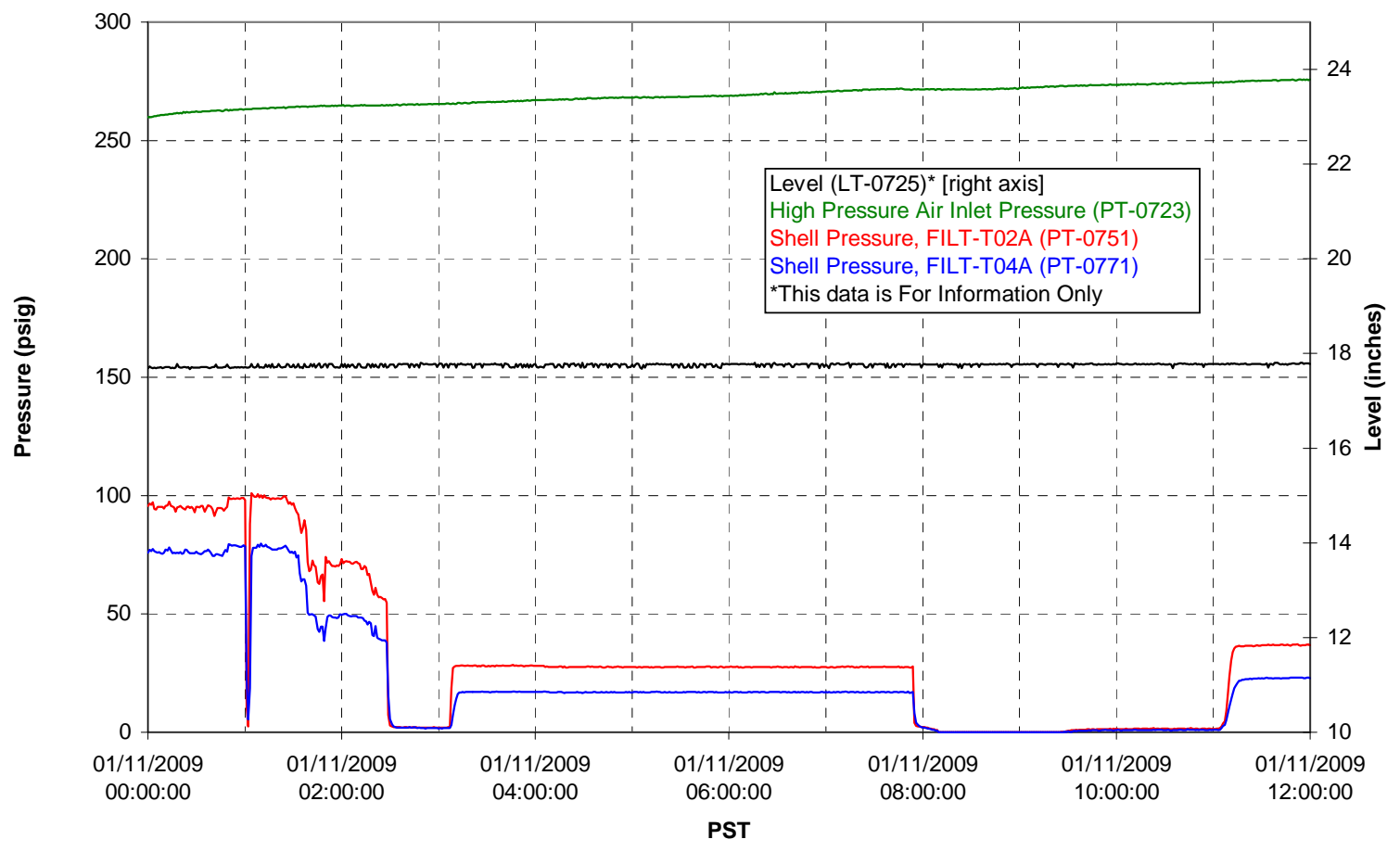


Pulsepot UFP-PP-T03A

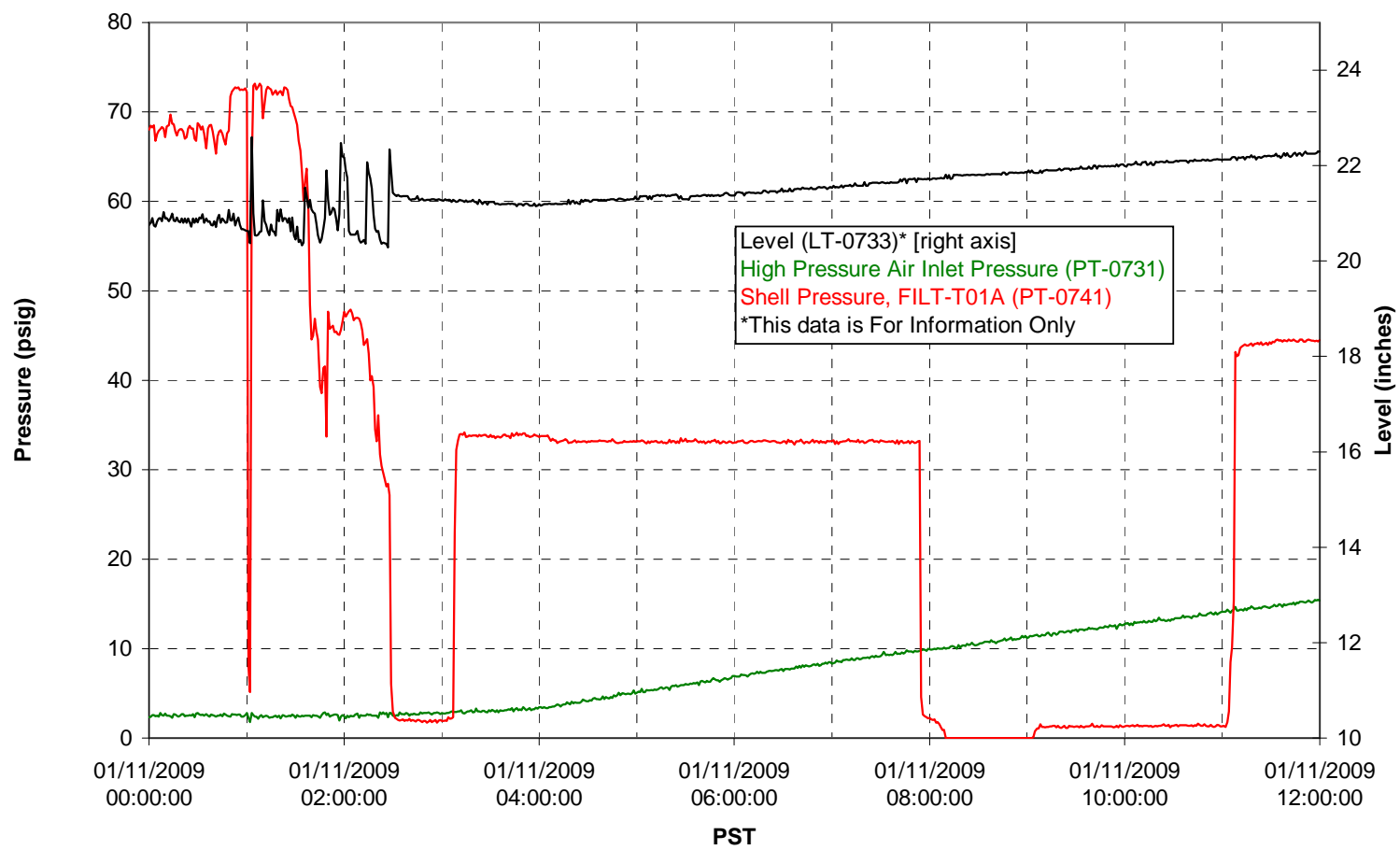

Pulsepot Levels

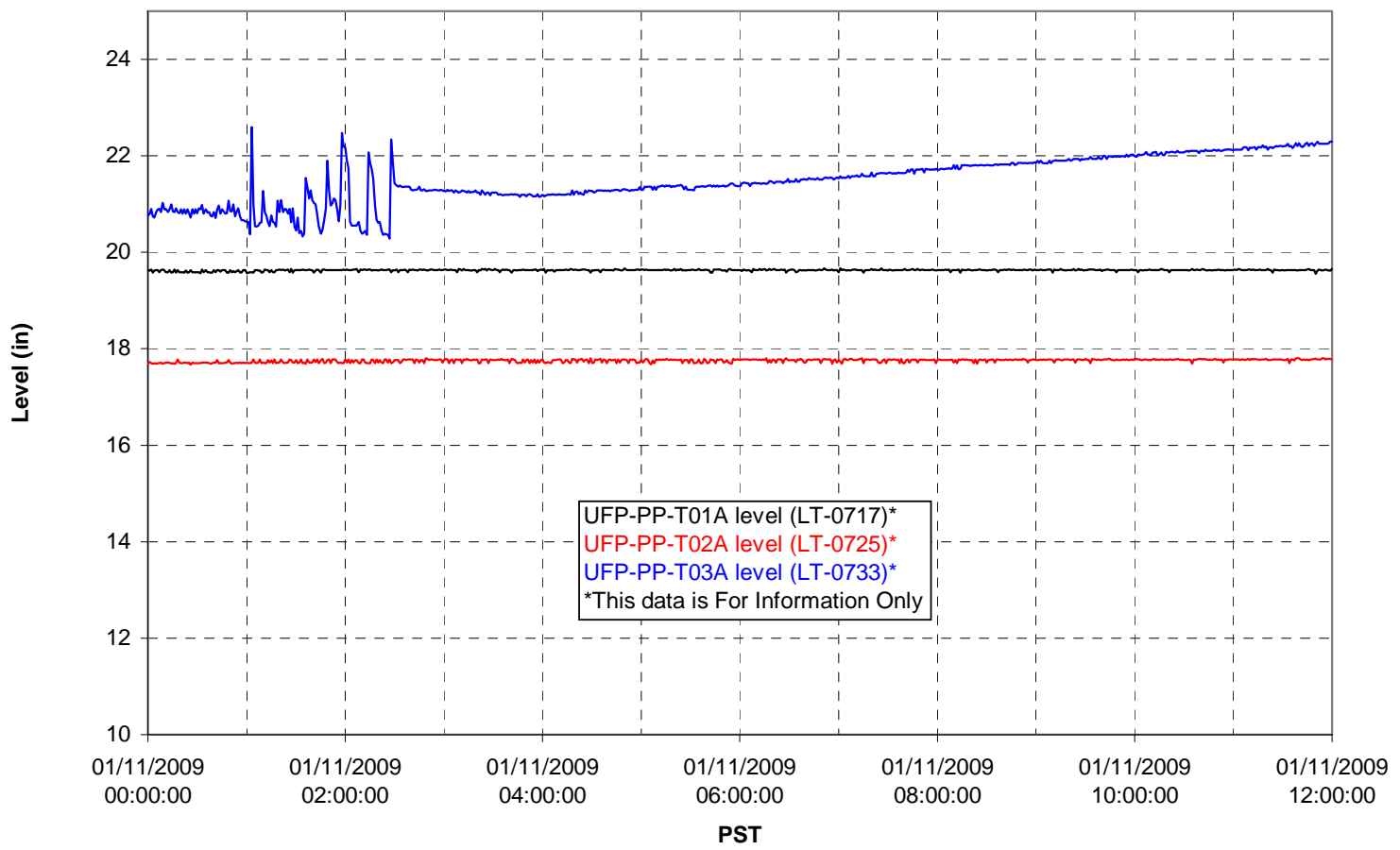


Filter UFP-FILT-T01A

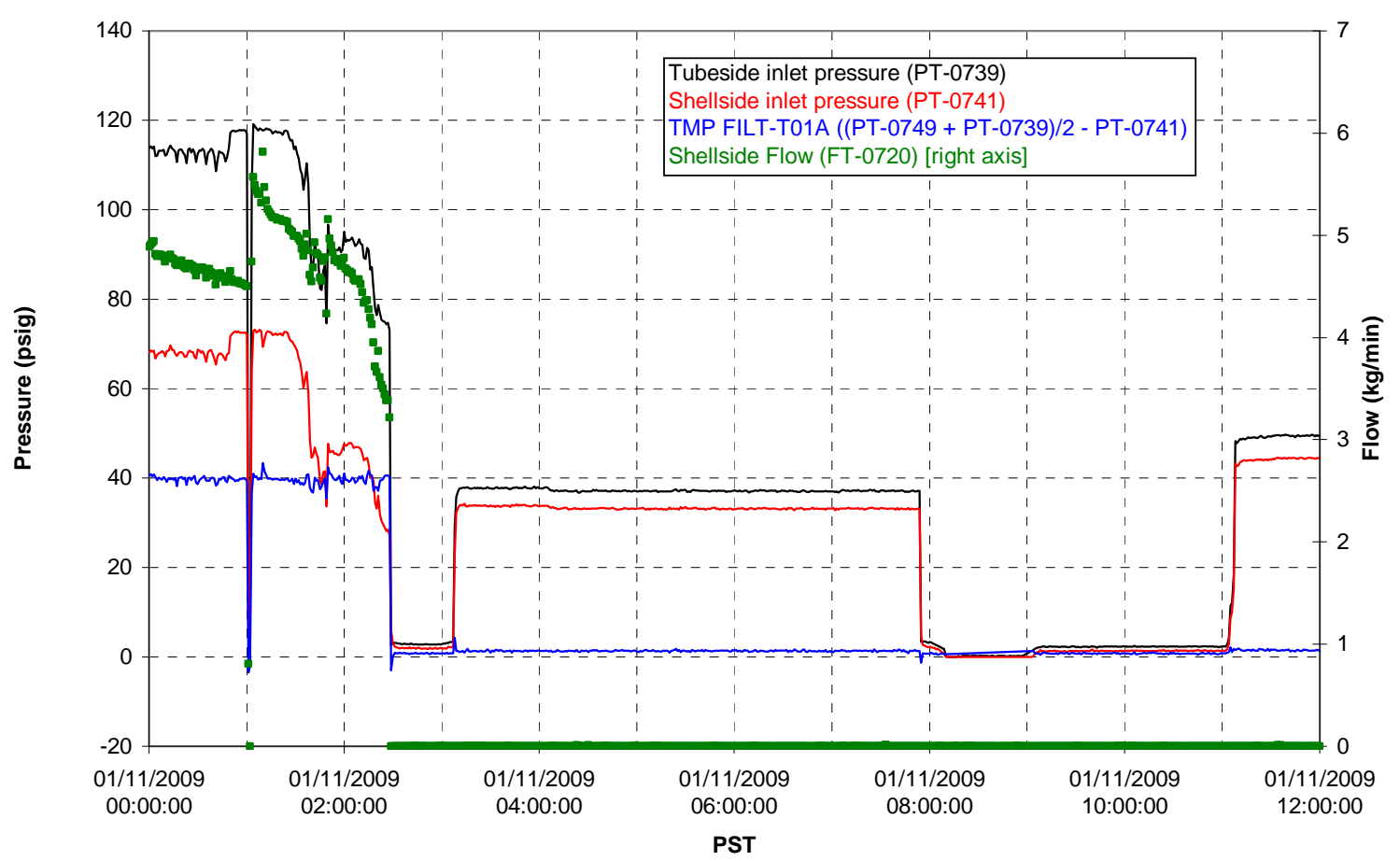

Filter UFP-FILT-T02A

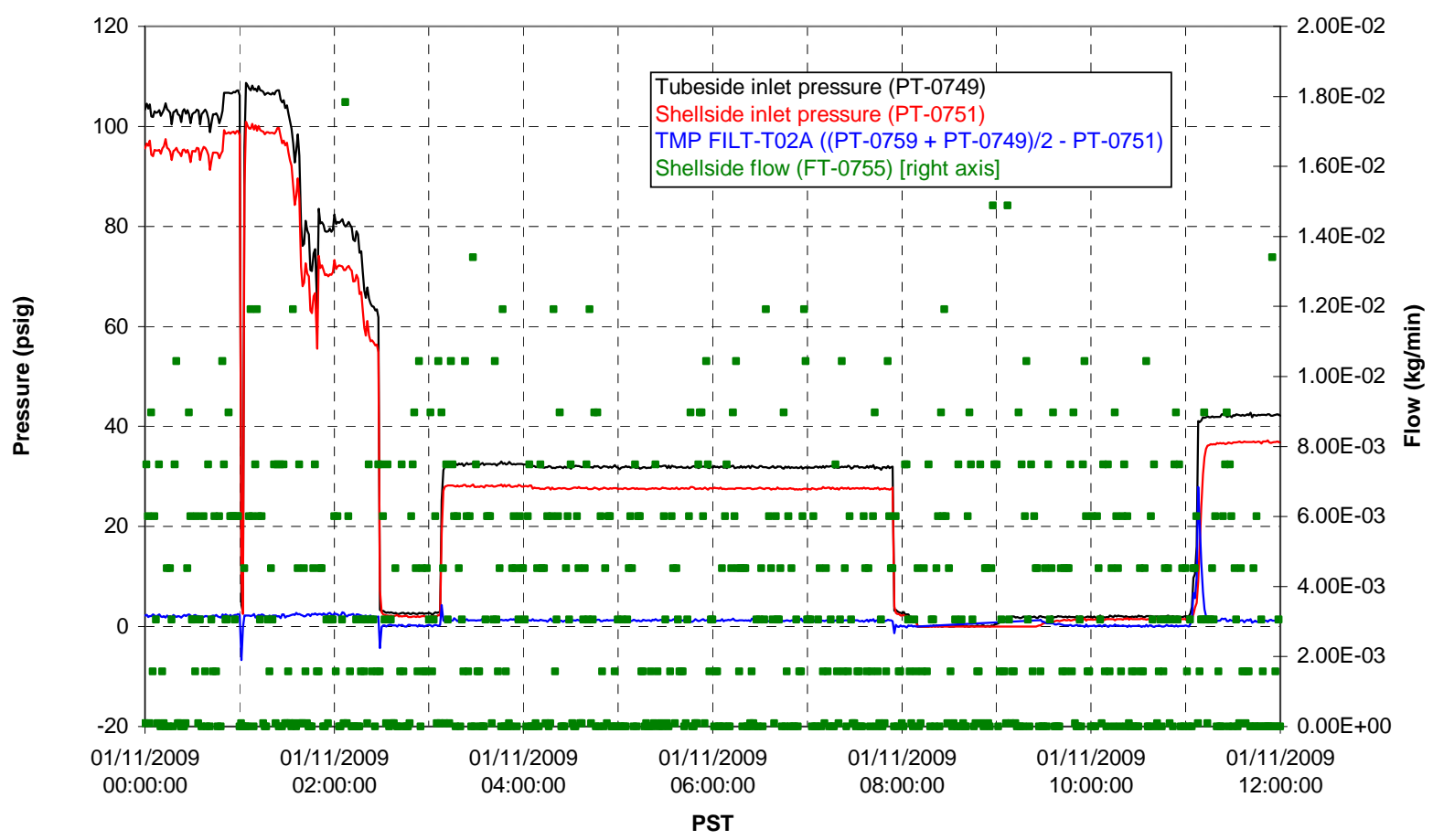


Filter UFP-FILT-T03A

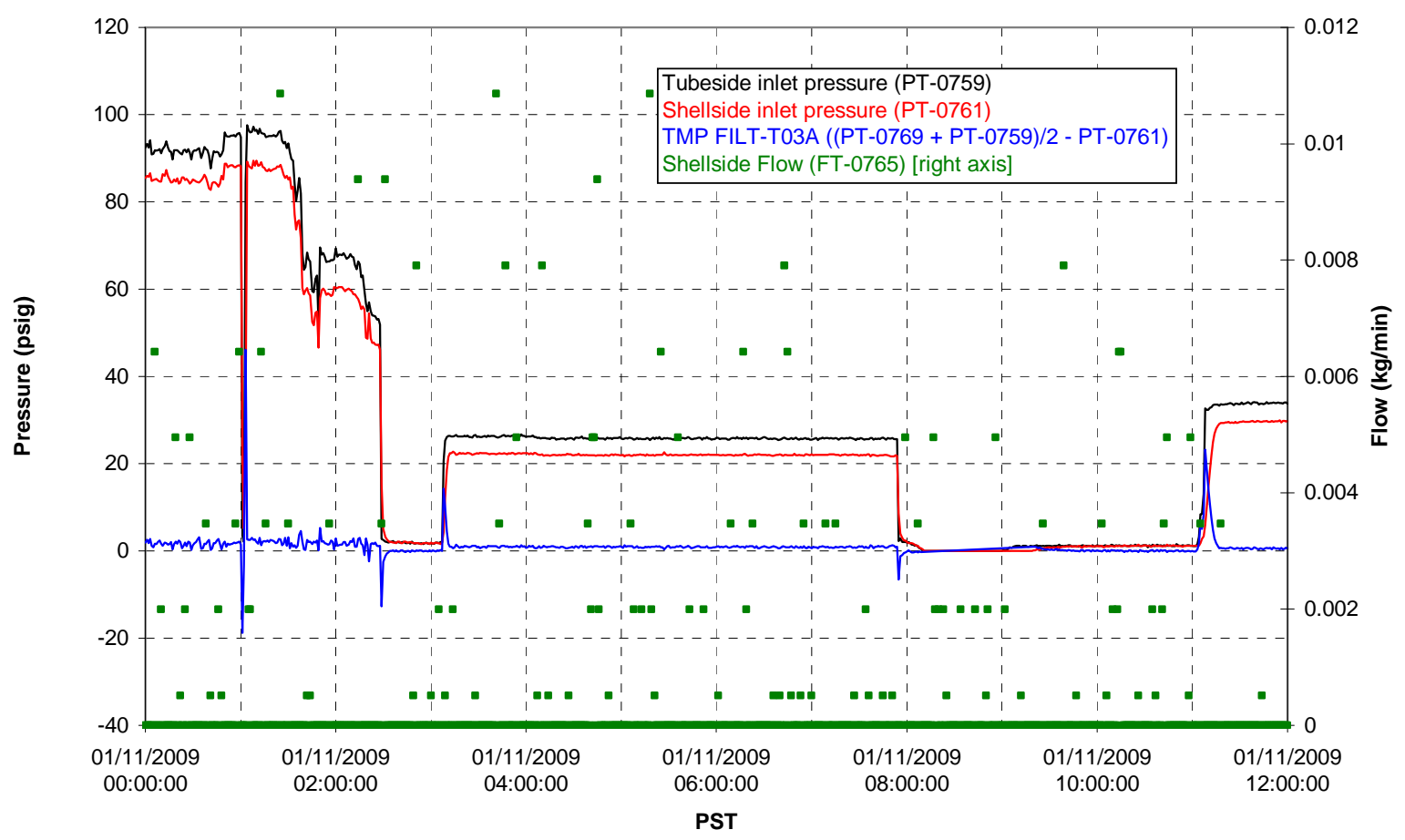

Filter UFP-FILT-T04A

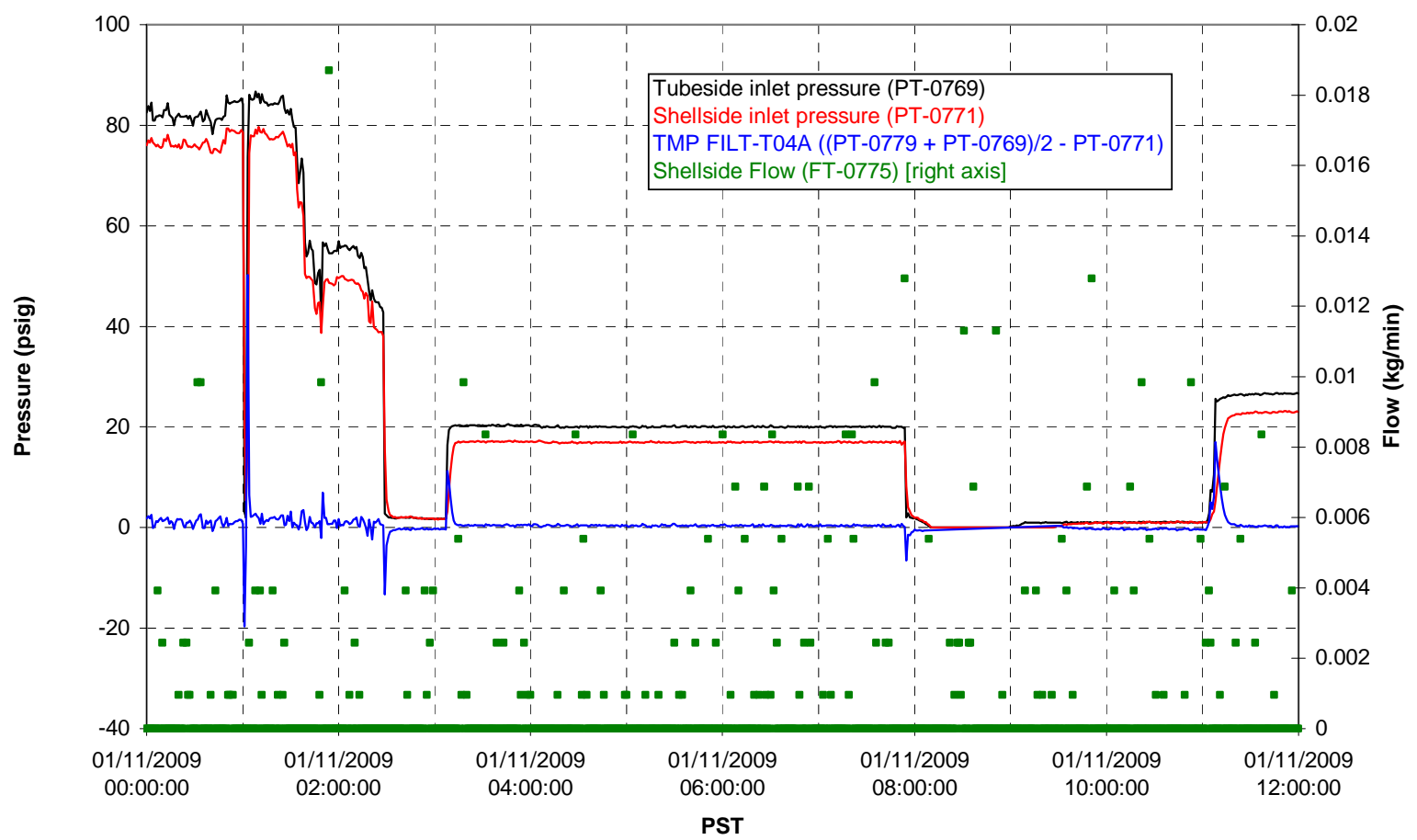


Filter UFP-FILT-T05A

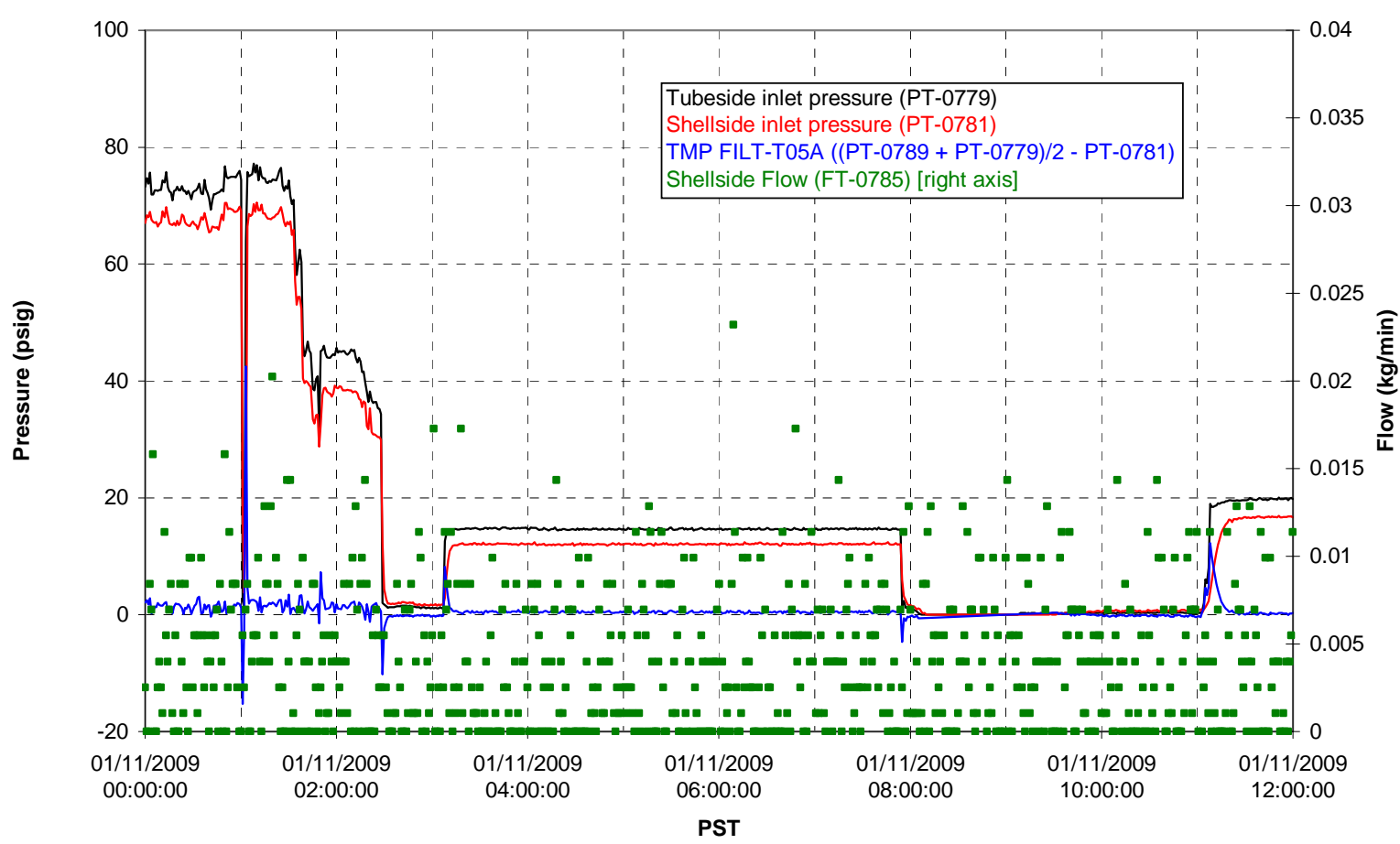

Chemical Flow

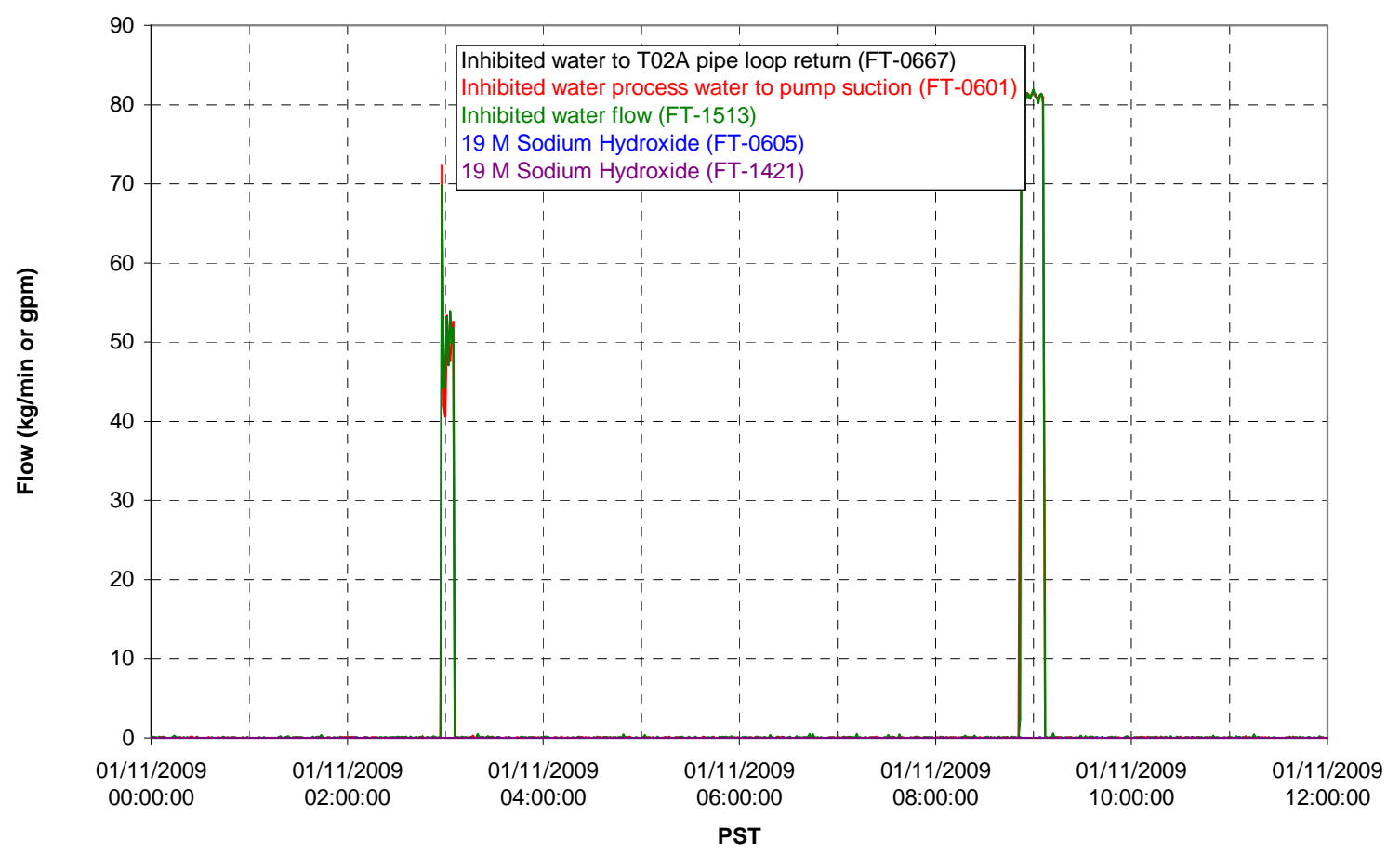




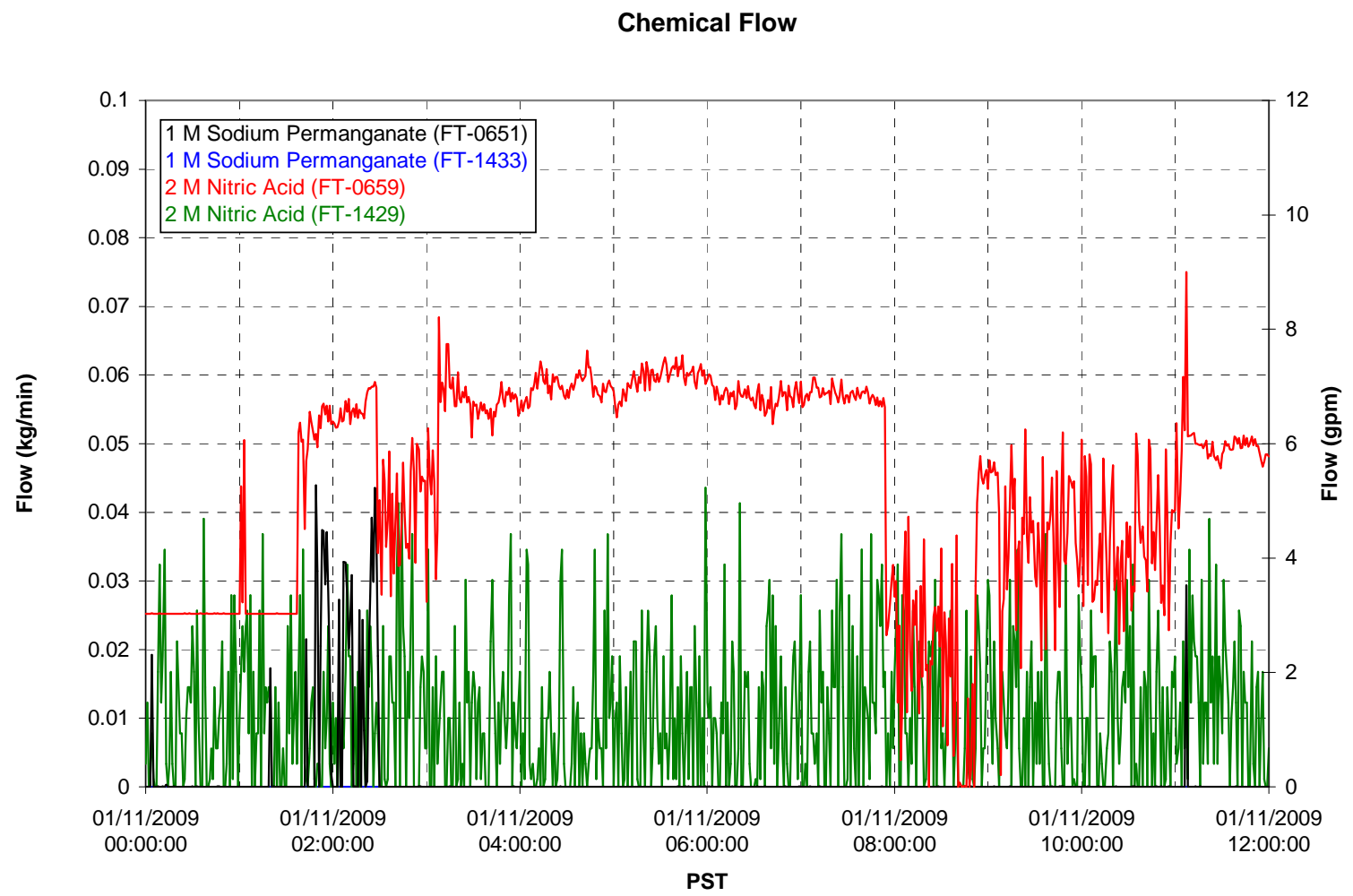

Air Flows

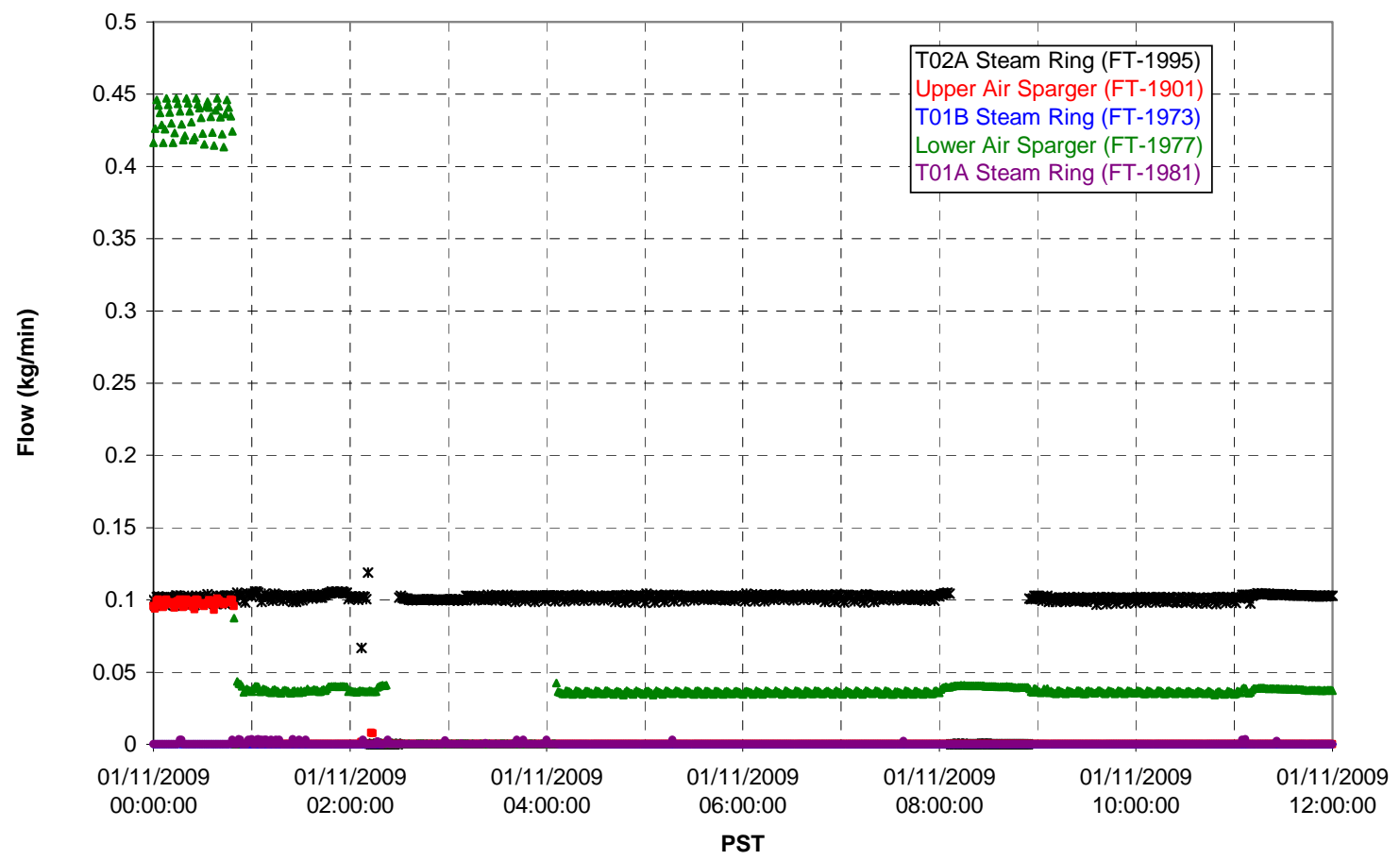


T02A Steam

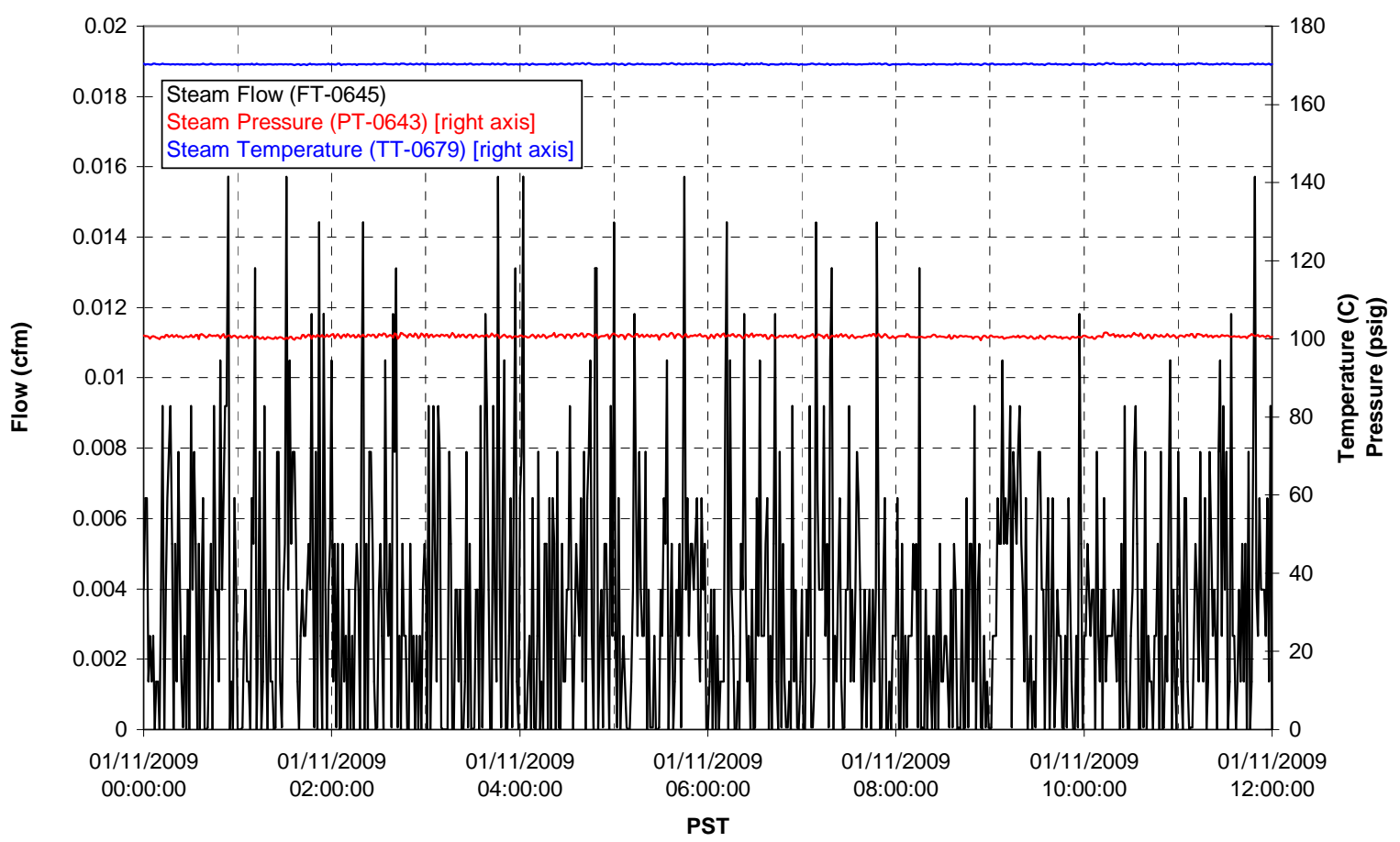

T01A Steam

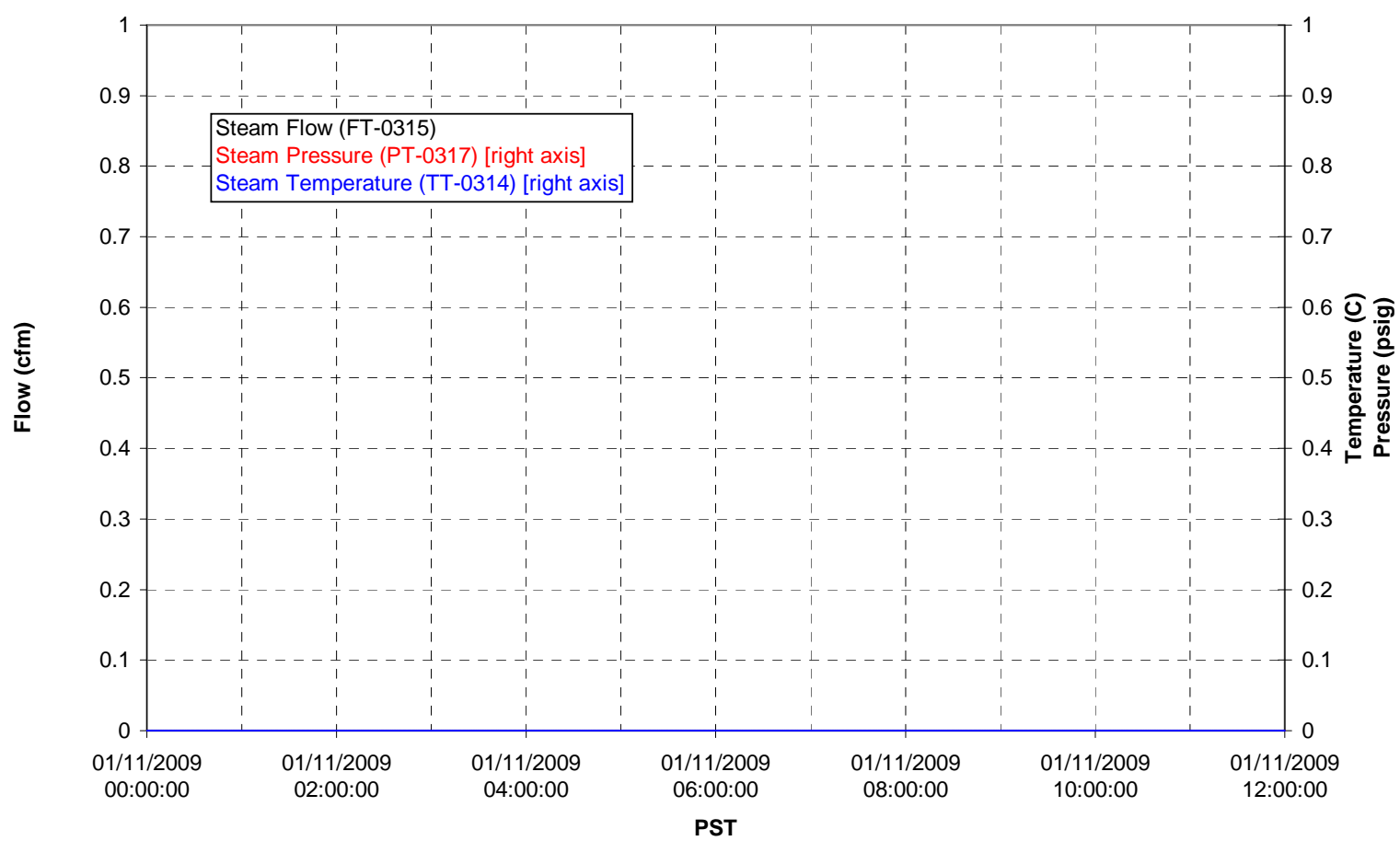


T01B Steam

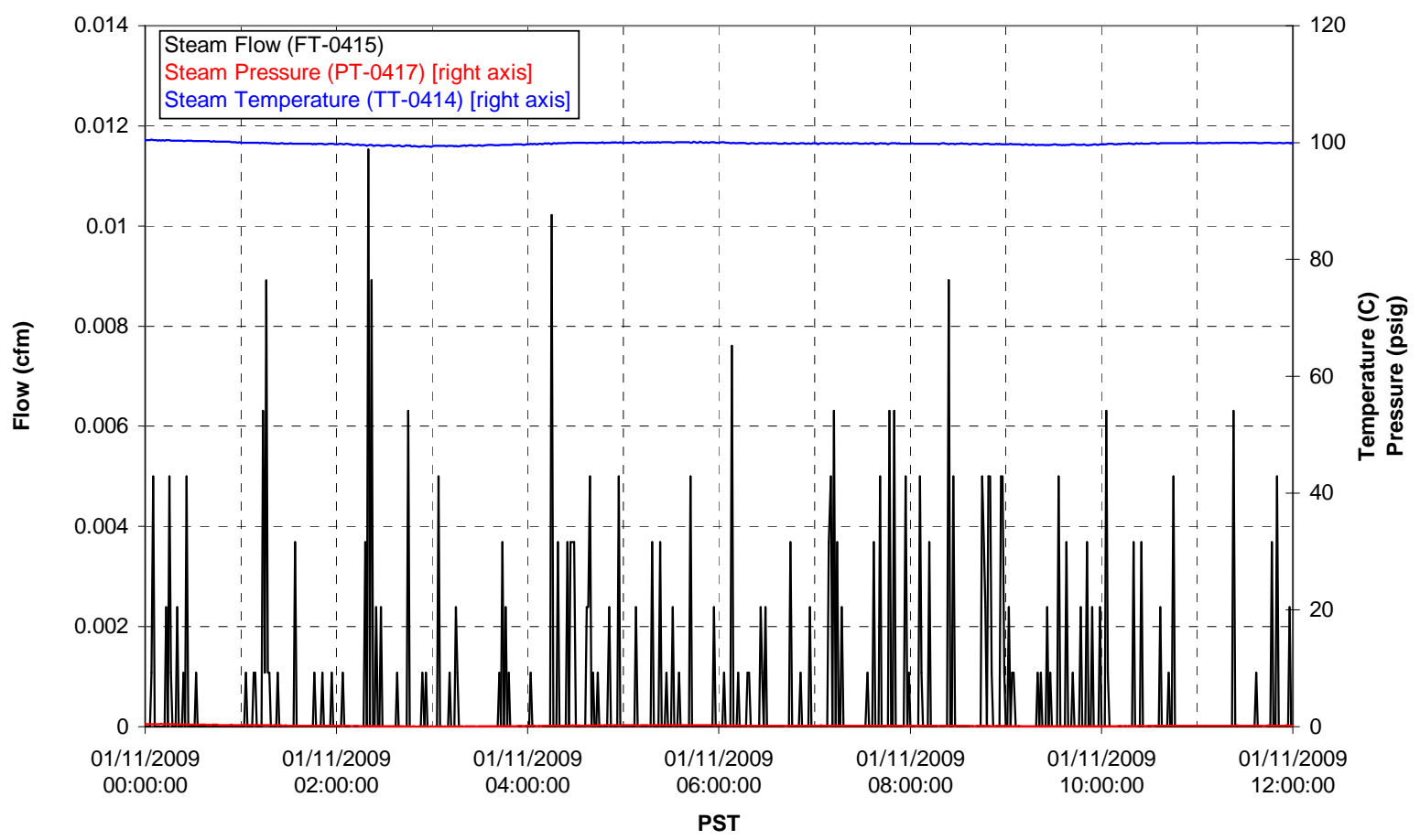



Appendix K

Shakedown/Functional Summary 



\section{Appendix K: Shakedown/Functional Summary}

A summary of the simulant Shakedown/Functional testing is presented as Table 4.3 in the main body of this report. 

Appendix $L$

Physical Properties 



\section{Appendix L: Physical Properties}

This appendix contains abstractions from Test Data Packages. Interested readers are referred to the listed Test Data Packages listed in the title of each section for more information.

\subsection{Rheology (TDP-WTP-355)}



Date: $\quad 01 / 30 / 2009$
Project No.:53569
To: Brad Johnson
Internal Distribution:
From: $\quad$ Lynette Jagoda
Subject: $\quad$ Shakedown Rheology Data Report on PEP
Slurry 11/24/2008 - 1/15/2009

Specimens collected for the Shakedown Test Plan were done under TI-062 and TI-032, Steps A.1.1, A.1.8, A.1.11, A.1.15, A.1.25, A.1.29, A.1.33

Multiple specimens of PEP slurry were delivered to APEL for rheological analysis between 11/24/2008 01/09/2009. Specimens were analyzed to determine their shear stress vs. shear rate properties. The analysis was done using an Anton Parr Rheometer MCR 301 SN80371304. Rheometer calibration verifications were done 4 times during this time period by Bryan Broocks in accordance with QA procedure TPR-WTP-PEP-049 using standard $9.4 \mathrm{cP}$ Brookfield Standard Lot \# 071008. All came in within the WTP defined specifications.

One of these calibration curves is shown here.

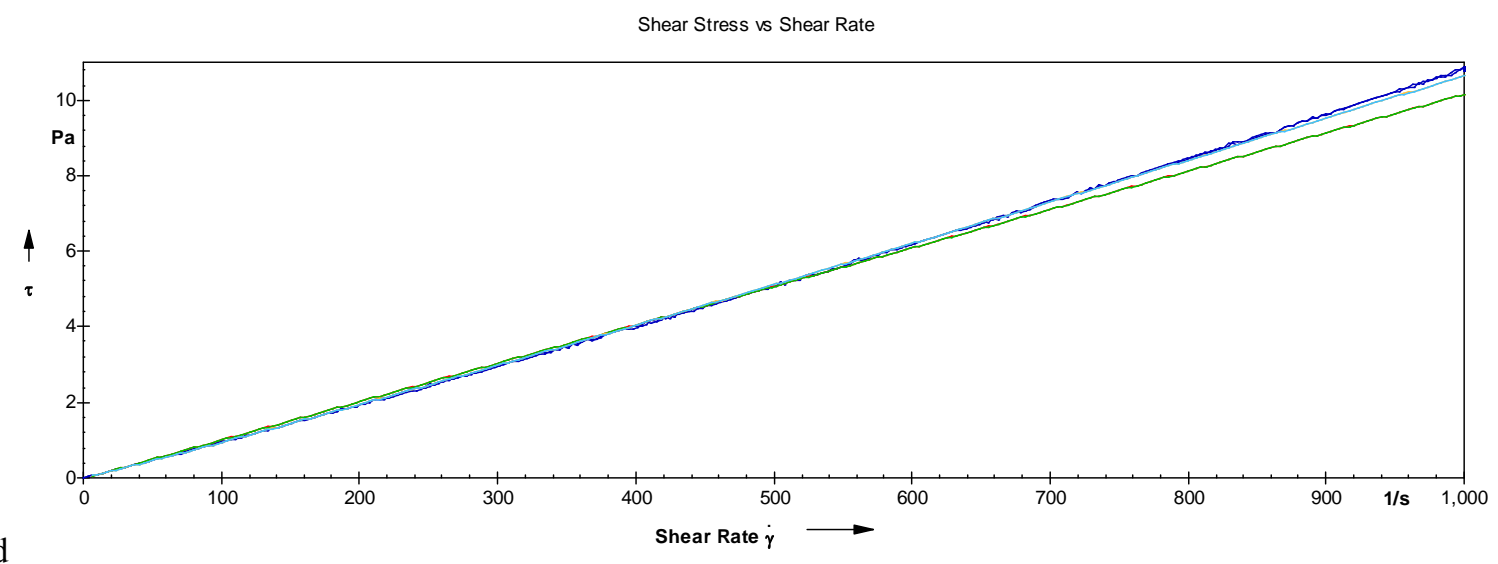

Standard

The analysis was performed in accordance with procedure TPR-WTP-PEP-049 and the data was collected digitally using the RHEOPLUS/32 V3.21 21003751-33024 software interface that is used to operate the rheometer. The specimens were prepared for analysis by shaking the bottle before extracting the sample. Experimental details were recorded in laboratory record book 60127. All measurements were done at $25^{\circ} \mathrm{C}$.

The testing was done with the cup and bob sensor system CC27-SN13634 at a ramp from 0-1,000 1/sec over 5-min., then a 1-min. hold at 1,000 1/sec followed by a ramp down over 5-min. Each specimen is generally measured three times: an initial measurement is made of a fresh sample taken from the specimen, that same sample is then re-run a second time. The rheometer is cleaned, and a third measurement (repeat) is made by taking a second aliquot from the specimen. 
The samples are placed in the cup up to a designated fill line, which take about 8-10-mL. Settling cannot be prevented if the particles are large and the viscosity low. However, no evidence of significant settling was seen in the data or the cup after testing.

The flow curves (plot of shear stress vs. shear rate) showed very little hysteresis and the data were fit with Newtonian and Bingham Plastic flow-curve descriptors via the Rheoplus curve fit software. However, almost all the samples displaced Taylor Vortices starting between the $350-6001 / \mathrm{sec}$, thus all the data curves had to be re-fit individually. The normal software fits the entire range of data collected. When Taylor Vortices are present a researcher has to go into the data and make a judgment call of the best range to fit for each specific data set. The independent technical review determined that many of these fits needed to be repeated a few times for some graphs to be considered optimal. Optimal fits have the calculated computer fit line that follows the data as closely as possible. Sometimes it is possible to get an excellent fit, sometimes a judgment must be made that a specific fit is not optimal for the data even when the fit is not perfect, since sometimes the fluids do not fit the model parameters exactly.

Rheological analysis was then performed on the supernatant in an identical manner as the slurry.

Two samples came in label Spare 91 and Spare 92; the official sample names were later added to the log book and data files.

\begin{tabular}{|c|c|c|c|}
\hline Sample Name & $\begin{array}{l}\text { Date } \\
\text { Received }\end{array}$ & $\begin{array}{l}\text { Date } \\
\text { Tested }\end{array}$ & Comments \\
\hline S-000FL-008-XX-0247-RHE-4 & $11 / 24 / 2008$ & $11 / 27 / 2008$ & $\begin{array}{l}\text { Very slight Bingham } \\
\text { or Newtonian }\end{array}$ \\
\hline S-000FL-008-XX-0248-RHE-4 & $11 / 24 / 2008$ & $11 / 27 / 2008$ & $\begin{array}{l}\text { Very slight Bingham } \\
\text { or Newtonian }\end{array}$ \\
\hline S-000FL-011-XX-0336-RHE-4 & $11 / 25 / 2008$ & $12 / 02 / 2008$ & Slight Bingham \\
\hline S-T22RL-001-XX-0162-RHE-4 & $12 / 01 / 2008$ & $12 / 09 / 2008$ & $\begin{array}{l}\text { Newtonian } \\
\text { (supernatant only) }\end{array}$ \\
\hline S-T22RL-001-XX-0161-RHE-4 & $12 / 01 / 2008$ & $12 / 09 / 2008$ & Newtonian \\
\hline S-T22RL-001-XX-0159-RHE-4 & $12 / 01 / 2008$ & $\begin{array}{l}12 / 15 / 2008 \\
- \\
12 / 19 / 2008\end{array}$ & $\begin{array}{l}\text { No Shear Strength } \\
\text { even after settling }\end{array}$ \\
\hline S-T22RL-001-XX-0160-RHE-4 & $12 / 01 / 2008$ & $\begin{array}{l}12 / 15 / 2008 \\
-12 / 19 / 08\end{array}$ & $\begin{array}{l}\text { No Shear Strength } \\
\text { even after settling }\end{array}$ \\
\hline S-T22GM-001-XX-0703-RHE-4 & $12 / 01 / 2008$ & $12 / 09 / 2008$ & $\begin{array}{l}\text { Newtonian } \\
\text { (supernatant only) }\end{array}$ \\
\hline S-000FL-015-XX-0350-RHE-4 & $12 / 01 / 2008$ & $12 / 09 / 2008$ & $\begin{array}{l}\text { Very slight Bingham } \\
\text { or Newtonian }\end{array}$ \\
\hline S-000FL-015-XX-0357-RHE-4 & $12 / 01 / 2008$ & $12 / 09 / 2008$ & Slight Bingham \\
\hline S-000FL-015-XX-0358-RHE-4 & $12 / 01 / 2008$ & $12 / 09 / 2008$ & $\begin{array}{l}\text { Newtonian } \\
\text { (supernatant only) }\end{array}$ \\
\hline $\begin{array}{l}\text { S-02AOL-016-XX-0703-RHE-4 } \\
\text { Spare } 91 \text { (rush) }\end{array}$ & $12 / 01 / 2008$ & $12 / 02 / 2008$ & $\begin{array}{l}\text { Very slight Bingham } \\
\text { or Newtonian }\end{array}$ \\
\hline
\end{tabular}




\begin{tabular}{|l|l|l|l|}
\hline $\begin{array}{l}\text { S-02AOL-016-XX-0704-RHE-4 } \\
\text { Spare 92 (rush) }\end{array}$ & $12 / 01 / 2008$ & $120 / 2 / 2008$ & $\begin{array}{l}\text { Very slight Bingham } \\
\text { or Newtonian }\end{array}$ \\
\hline S-02AML-008-XX-0865-RHE-4 & $12 / 31 / 2008$ & $01 / 02 / 2009$ & $\begin{array}{l}\text { Very slight Bingham } \\
\text { or Newtonian }\end{array}$ \\
\hline S-02AML-008-XX-0866-RHE-4 & $12 / 31 / 2008$ & $01 / 02 / 2009$ & $\begin{array}{l}\text { Very slight Bingham } \\
\text { or Newtonian }\end{array}$ \\
\hline S-02AML-011-XX-0895-RHE-4 & $01 / 02 / 2009$ & $01 / 07 / 2009$ & $\begin{array}{l}\text { Very slight Bingham } \\
\text { or Newtonian }\end{array}$ \\
\hline S-02AML-015-XX-0903-RHE-4 & $01 / 02 / 2009$ & $01 / 07 / 2009$ & Slight Bingham \\
\hline S-000FL-025-XX-0991-RHE-4 & $01 / 05 / 2009$ & $01 / 05 / 2009$ & $\begin{array}{l}\text { Very slight Bingham } \\
\text { or Newtonian }\end{array}$ \\
\hline S-02AML-029-XX-0928-RHE-4 & $01 / 09 / 2009$ & $01 / 09 / 2009$ & Slight Bingham \\
\hline S-02AML-029-XX-0998-RHE-4 & $01 / 12 / 2009$ & $01 / 15 / 2009$ & $\begin{array}{l}\text { Very slight Bingham } \\
\text { or Newtonian }\end{array}$ \\
\hline
\end{tabular}

\section{Summary of Results}

Samples S-T22RL-001-XX-0159-RHE-4 and S-T22RL-001-XX-0160-RHE-4 were tested with a Shear Vane and found to have no measureable shear strength.

Some samples were run only as a slurry, some only as a supernate, and others were tested both ways as was needed for the matrix support for that part of the Test Plan. Most slurry samples were slightly Bingham plastic in nature and the supernate samples were Newtonian.

The average results from rheological analysis are presented in Appendix A.

The data that was considered bad fits were removed from the summary table in Appendix A, and are highlighted in red in the more detailed table from an Excel spreadsheet in Appendix B.

The actual rheograms for each test and the curve fits are presented in Appendix C. 


\section{Appendix A}

Summary Table of Average Rheological Parameters 


\section{Appendix A}

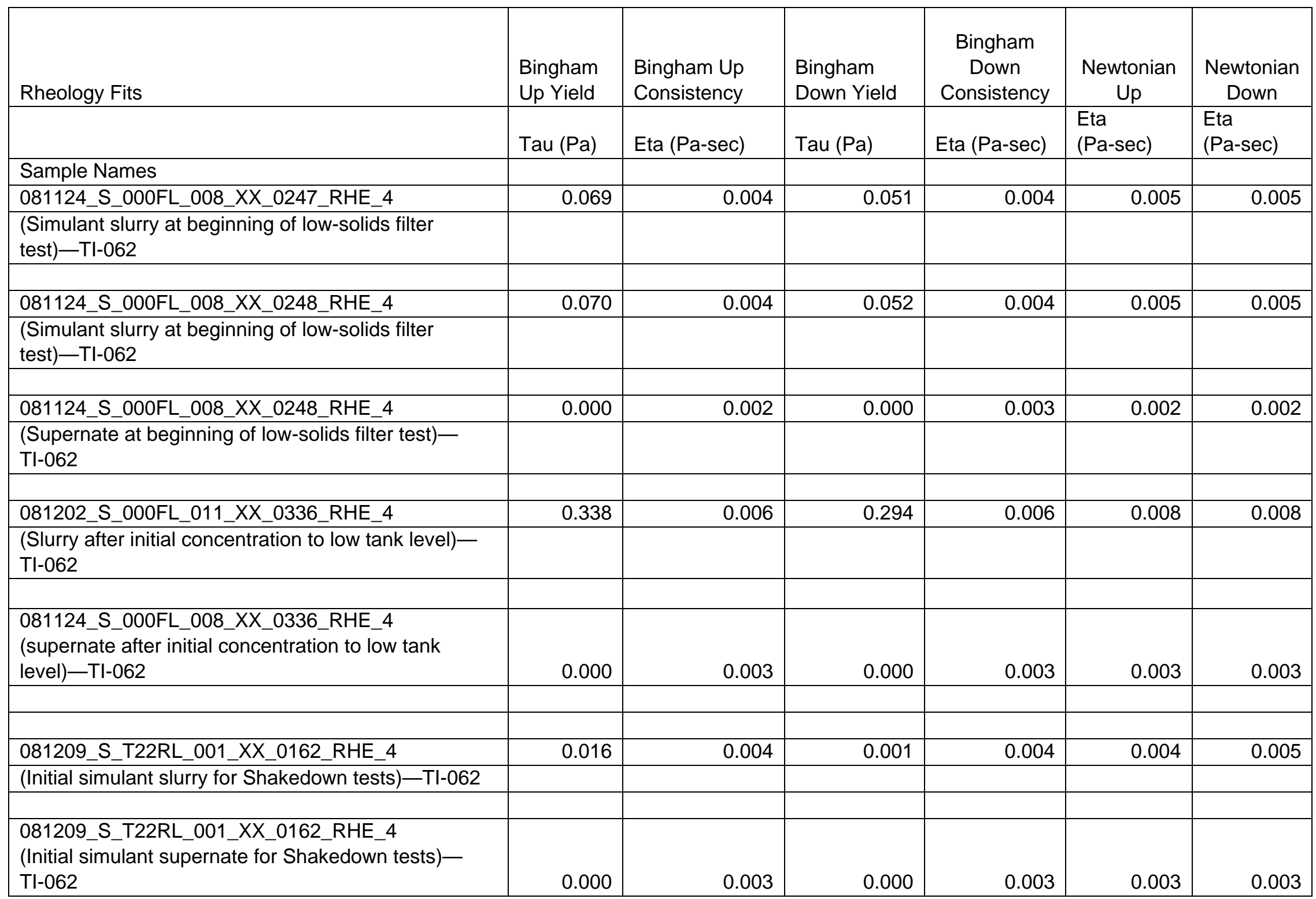




\section{Appendix A}

\begin{tabular}{|c|c|c|c|c|c|c|}
\hline Rheology Fits & $\begin{array}{l}\text { Bingham } \\
\text { Up Yield }\end{array}$ & $\begin{array}{l}\text { Bingham Up } \\
\text { Consistency }\end{array}$ & $\begin{array}{l}\text { Bingham } \\
\text { Down Yield }\end{array}$ & $\begin{array}{l}\text { Bingham } \\
\text { Down } \\
\text { Consistency }\end{array}$ & $\begin{array}{l}\text { Newtonian } \\
\text { Up }\end{array}$ & $\begin{array}{l}\text { Newtonian } \\
\text { Down }\end{array}$ \\
\hline & Tau (Pa) & Eta (Pa-sec) & Tau (Pa) & Eta (Pa-sec) & $\begin{array}{l}\text { Eta } \\
(P a-s e c)\end{array}$ & $\begin{array}{l}\text { Eta } \\
(\text { Pa-sec) }\end{array}$ \\
\hline \multicolumn{7}{|l|}{ Sample Names } \\
\hline 081209_S_T22RL_001_XX_0161_RHE_4 & 0.016 & 0.004 & 0.002 & 0.005 & 0.004 & 0.005 \\
\hline \multicolumn{7}{|c|}{ (Initial simulant slurry for Shakedown tests)—TI-062 } \\
\hline 081209_S_T22RL_001_XX_0703_RHE_4 & 0.013 & 0.004 & 0.009 & 0.004 & 0.004 & 0.004 \\
\hline \multicolumn{7}{|l|}{ (Simulant from HLP-T22, unknown time)-TI-062 } \\
\hline 081209_S_000FL_015_XX_0350_RHE_4 & 0.102 & 0.005 & 0.072 & 0.005 & 0.006 & 0.005 \\
\hline \multicolumn{7}{|l|}{$\begin{array}{l}\text { (Slurry at beginning of concentration with four filter } \\
\text { bundles) }-\mathrm{TI}-062\end{array}$} \\
\hline 081209_S_000FL_015_XX_0357_RHE_4 & 0.893 & 0.008 & 0.780 & 0.008 & 0.011 & 0.011 \\
\hline \multicolumn{7}{|l|}{$\begin{array}{l}\text { (Slurry after concentration to } 20 \% \text { with four filter } \\
\text { bundles) - TI-062 }\end{array}$} \\
\hline 081202_S_000FL_015_XX_0358_RHE_4 & 0.901 & 0.008 & 0.787 & 0.008 & 0.011 & 0.011 \\
\hline \multicolumn{7}{|l|}{$\begin{array}{l}\text { (Slurry after concentration to } 20 \% \text { with four filter } \\
\text { bundles) }-\mathrm{TI}-062\end{array}$} \\
\hline 081202_S_000FL_015_XX_0358_RHE_4 & 0.000 & 0.003 & 0.000 & 0.003 & 0.003 & 0.003 \\
\hline \multicolumn{7}{|l|}{$\begin{array}{l}\text { (Supernate after slurry concentration to } 20 \% \text { with } \\
\text { four filter bundles)_-TI-062 }\end{array}$} \\
\hline S_02AOL_016_XX_0703_RHE_4 (Spare 91) & 0.166 & 0.020 & 0.122 & 0.020 & 0.020 & 0.020 \\
\hline \multicolumn{7}{|l|}{ (Initial slurry simulant from HLP-T22)_-TI062 } \\
\hline S_02AOL_016_XX_0704_RHE_4 (Spare 92) & 0.170 & 0.020 & 0.124 & 0.020 & 0.020 & 0.020 \\
\hline Unknown & & & & & & \\
\hline
\end{tabular}


Appendix A

\begin{tabular}{|c|c|c|c|c|c|c|}
\hline Rheology Fits & $\begin{array}{l}\text { Bingham } \\
\text { Up Yield }\end{array}$ & $\begin{array}{l}\text { Bingham Up } \\
\text { Consistency }\end{array}$ & $\begin{array}{l}\text { Bingham } \\
\text { Down Yield }\end{array}$ & $\begin{array}{l}\text { Bingham } \\
\text { Down } \\
\text { Consistency }\end{array}$ & $\begin{array}{l}\text { Newtonian } \\
\text { Up }\end{array}$ & $\begin{array}{l}\text { Newtonian } \\
\text { Down }\end{array}$ \\
\hline & Tau (Pa) & Eta (Pa-sec) & Tau $(\mathrm{Pa})$ & Eta (Pa-sec) & $\begin{array}{l}\text { Eta } \\
(\mathrm{Pa}-\mathrm{sec})\end{array}$ & $\begin{array}{l}\text { Eta } \\
(\mathrm{Pa}-\mathrm{sec})\end{array}$ \\
\hline \multicolumn{7}{|l|}{ Sample Names } \\
\hline \multicolumn{7}{|l|}{ (Slurry at beginning of filter conditioning)-TI-032 } \\
\hline S_02AML_008_XX_0866_RHE_4 (slurry) & 0.077 & 0.005 & 0.057 & 0.005 & 0.005 & 0.005 \\
\hline \multicolumn{7}{|l|}{ (Slurry at beginning of filter conditioning)-TI-032 } \\
\hline S_02AML_008_XX_0866_RHE_4 (supernate) & 0.000 & 0.003 & 0.000 & 0.003 & 0.003 & 0.003 \\
\hline \multicolumn{7}{|l|}{$\begin{array}{l}\text { (Supernate at beginning of filter conditioning)- } \\
\text { TI-032 }\end{array}$} \\
\hline S_02AML_011_XX_0895_RHE_4 (slurry) & 0.663 & 0.008 & 0.572 & 0.008 & 0.010 & 0.010 \\
\hline \multicolumn{7}{|l|}{$\begin{array}{l}\text { (Slurry after initial concentration to low tank level)- } \\
\text { TI-032 }\end{array}$} \\
\hline S_02AML_015_XX_0903_RHE_4 (slurry) & 1.494 & 0.010 & 1.241 & 0.010 & 0.014 & 0.013 \\
\hline \multicolumn{7}{|l|}{ (Slurry after final concentration to 20\%)-TI-032 } \\
\hline S 000FI 025 XX 0991 RHF 4 (slurry) & 2367 & 0019 & 2027 & 0018 & Seo Plot & Seo Plot \\
\hline \multicolumn{7}{|l|}{ Unknown } \\
\hline & & & & & & \\
\hline S_02AML_029_XX_0928_RHE_4 (slurry) & 0.200 & 0.003 & 0.179 & 0.003 & 0.004 & 0.004 \\
\hline \multicolumn{7}{|l|}{$\begin{array}{l}\text { (Dilute slurry at start of high-solids filter test, first } \\
\text { attempt)—TI-032 }\end{array}$} \\
\hline S_02AML_029_XX_0998_RHE_4 (slurry) & 0.111 & 0.003 & 0.098 & 0.003 & 0.004 & 0.004 \\
\hline $\begin{array}{l}\text { (Dilute slurry at start of high-solids filter test, thirc } \\
\text { attempt)-TI-032 }\end{array}$ & & & & & & \\
\hline
\end{tabular}


1.2 Scanning Electron Microscopy (TDP-WTP-352) 
Date: $\quad 12 / 15 / 2009$

Project No.:

53596

To:

Ofelia Bredt

Internal

From: Brian Riley

Distribution:

Subject: PEP SEM Analyses for Shakedown

Test, TI-WTP-PEP-062

One specimen was received for scanning electron microscopy (SEM) examination in support of work being performed at the Pre-treatment Engineering Platform (PEP) during the course of the Shakedown Test. The morphologies of these specimens were analyzed using a JEOL 5900 SEM in accordance with procedure APEL102-SEM, Rev. 1.

\section{Sample Preparation}

Once received from PEP, the specimen was cleaned of nitrates. This cleaning process included 3 cycles of adding $10 \mathrm{~mL}$ of fresh $0.01 \mathrm{M} \mathrm{NaOH}$ to the centrifuge tube, vortexing for 3 sets of 15 seconds, centrifuging at $4500 \mathrm{rpm}$ for 10 minutes, decanting and then adding $10 \mathrm{~mL}$ of fresh $0.01 \mathrm{M} \mathrm{NaOH}$. These washing steps were recorded in laboratory record book BNW-60176.

After the last rinse, the $\mathrm{NaOH}$ and the pellet were vortexed, a small volume of the slurry was drawn up using a pipette, and a drop was placed on the top of an aluminum SEM sample stub. It was then placed in an oven at approximately $100^{\circ} \mathrm{C}$ to dry overnight. Once it was dry, it was coated with a thin layer of $\mathrm{Au} / \mathrm{Pd}$ using the Polaron Range plasma sputter coater in APEL/102. The Au/Pd layer was deposited using $800 \mathrm{~V}$ and $10 \mathrm{~mA}$ for $180 \mathrm{~s}$. The coated specimen was placed in an SEM sample holder and was placed in the SEM for analysis.

\section{Analysis}

Scanning electron micrographs were collected for informational purposes only using the Scan 4 option with $160 \mathrm{~s}$ collection times using the settings as seen below in Table 1. See Table 2 for information regarding the person that performed the analysis as well as the date of analysis. Images at the four magnifications listed in Table 1 were compiled in a $2 \times 2$ arrangement to show a macroscopic to microscopic view of the particles. See APPENDIX A: Micrograph Collages.

\section{Results}

Micrographs of the particles can be found in Figure 1. The specimen appeared to be a single phase consisting of particles that were someone tabular and faceted with sizes ranging from $\sim 1-10 \mu \mathrm{m}$ and appeared as agglomerates.

Table 1. Parameters used for capturing images.

\begin{tabular}{|l|l|l|l|l|l|}
\hline Sample ID & $\begin{array}{l}\text { Voltage } \\
(\mathrm{KV})\end{array}$ & $\begin{array}{l}\text { Spot } \\
\text { Size }\end{array}$ & Detector & $\begin{array}{l}\text { Working } \\
\text { Distance } \\
\text { (mm) }\end{array}$ & Magnification \\
\hline S_T22RL_001_XX_0166_XSP_4_B-1kx-SEI.tif & 20 & 31 & SEI & 12 & $1,000 \times$ \\
\hline S_T22RL_001_XX_0166_XSP_4_B-2500x-SEI(2).tif & 20 & 25 & SEI & 12 & $2,500 \times$ \\
\hline S_T22RL_001_XX_0166_XSP_4_B-5kx-SEI(2).tif & 20 & 25 & SEI & 12 & $5,000 \times$ \\
\hline S_T22RL_001_XX_0166_XSP_4_B-10kx-SEI(3).tif & 20 & 25 & SEI & 12 & $10,000 \times$ \\
\hline
\end{tabular}


TDP-WTP-352

Page 4 of 5

Table 2. Analysis information.

\begin{tabular}{|l|l|l|l|}
\hline Sample ID & Date of Analysis & Analysis Performed By & Signature \\
\hline S_T22RL_001_XX_0166_XSP_4_B & $12 / 15 / 2009$ & Brian Riley
\end{tabular}

L. 11 


\section{APPENDIX A: Micrograph Collages}

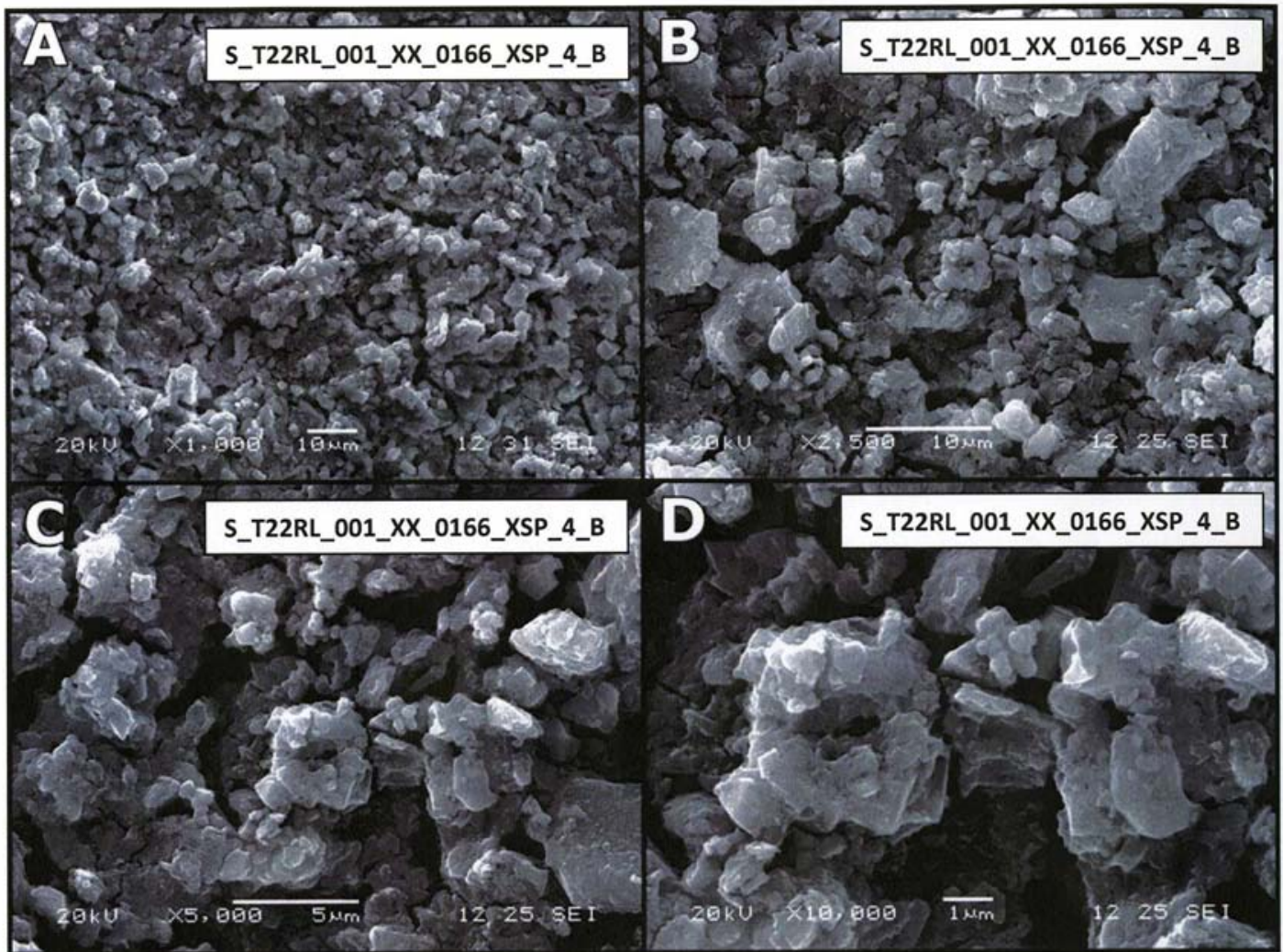

SEM micrographs from Pretreatment Engineering Platform (PEP) specimen S_T22RL_001_XX_0166_XSP_4_B. The specimen, while in the original $50 \mathrm{~mL}$ centrifuge tube, was rinsed with $0.01 \mathrm{M} \mathrm{NaOH}$, vortexed, centrifuged (4500 rpm for 10 minutes), and decanted $3 \times$ to clean off the nitrates. After the final rinse, the contents of the tube were vortexed in order to resuspend the pellet, and a drop of the suspended particles was placed on an Al SEM stub and placed in an oven at approximately $105^{\circ} \mathrm{C}$ to dry overnight. Once dry, the specimen was coated with $\mathrm{Au} / \mathrm{Pd}$ and analyzed using the APEL/102 SEM (JEOL 5900).

Figure 1. Scanning electron micrographs collected for informational purposes on S_T22RL_001_XX_0166_XSP_4_B at different magnifications.

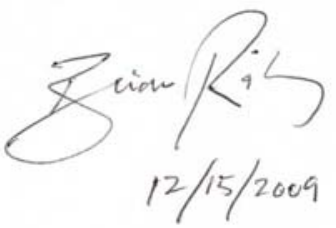




\subsection{X-Ray Diffraction (TDP-WTP-353)}

TDP-WTP-353

Page 3 of 11

Date: $\quad$ December 15,2008

$\begin{array}{ll}\text { To: } & \text { O. Bredt } \\ \text { From } & \text { J. V. Crum }\end{array}$

Subject: XRD Examination of: S_T22RL_001_XX_0166_XSP_4_B

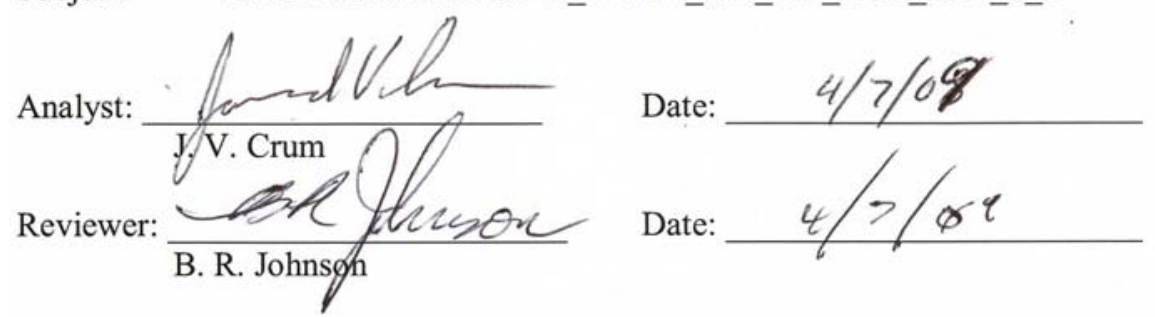

XRD data from the samples identified in Table I have been examined for identification of crystalline phases present.

Table I: Samples Examined in this Series

\begin{tabular}{|c|c|c|c|c|}
\hline Identification & XRD File ID $^{(\mathrm{a})}$ & $\begin{array}{c}\text { Count Time per } \\
\text { Step (Seconds) }\end{array}$ & $\begin{array}{c}\text { Number of } \\
\text { Replications }\end{array}$ & Internal Standard \\
\hline $\begin{array}{c}\text { S_T22RL_001_XX_0166_ } \\
\text { XSP_4_B }\end{array}$ & $121308 \mathrm{~A}$ & 2 & 9 & $\mathrm{TiO}_{2}$ \\
\hline
\end{tabular}

(a) The letter appended to the XRD File ID identifies the first sample in the replication series. The letter has been dropped in subsequent text to indicate that the referenced file is the summation of data from replicate runs.

Sample analysis was carried out by the Pacific Northwest National Laboratory Advanced Processing and Application Group. The XRD instrument used was the Scintag PAD V X-ray Diffractometer, Property Number WD33356, located in lab 102 in the APEL building. The data range for the sample was $5^{\circ}$ to $80^{\circ}$ 2-theta, with a step size of $0.02^{\circ} 2$-theta and count time 2.0 seconds per step. Copper $\mathrm{K} \alpha \mathrm{X}$-rays were used. The X-ray tube operating conditions were $45 \mathrm{kV}$ and $40 \mathrm{~mA}$.

Sample 120308 was prepared with $\mathrm{SRM} 674 \mathrm{~b}, \mathrm{TiO}_{2}$ internal standard. The dried powder was placed into a tungsten carbide milling chamber and milled for $1 \mathrm{~min}$ in the angstrom shaker mill. The TiO2 standard was then spiked in at $5.02 \mathrm{wt} \%$ with the dried powder and milled again for $2 \mathrm{~min}$ to insure a homogenous mixtur of the two powders. The sample was then mounted into an off-axis quartz sample holder (zero background holder). Replications were made and then summed for better counting statistics; the number of replications of each sample are shown in Table I.

Phase identification was done by use of the JADE search match routines (version 6.0, Materials Data Inc.) with comparison to the ICDD (International Centre for Diffraction Data) data base PDF-2 release 1999, which includes the ICSD (Inorganic Crystal Structure Database maintained by Fachinformationszentrum (FIZ), Karlsruhe, Germany). Searches were restricted to the PDF and ICSD Inorganic sections.

The phase identification plots are shown using "stick figures" to indicate the phases present; peak positions and line intensities. The phase showing the greatest peak intensity is shown at the top of the stick figures display (except the internal standard, if used, is always the topmost). Phases present at lower peak area/height are shown in decreasing order down the display. Sample identification is below the scan or 


\section{TDP-WTP-353}

Page 4 of 11

shown in the top most left of the plot figures. Scan running conditions are shown to the right on the identification.

The identified phases, ranked by relative peak intensities, are given below for each sample. Figure 1 shows the raw summed pattern. The background fitted pattern, along with the identified phases, are shown in figures 2,3 , and 4 .

S_T22RL_001_XX_0166_XSP_4_B, File: 121308

- Rutile, $\overline{\mathrm{TiO}} 2$, card\# $21-1 \overline{2} 7 \overline{6}$

- Gibbsite, $\mathrm{Al}(\mathrm{OH})_{3}$, card\# 60-2702, Good overall fit, Peak locations are an good match to the pattern. The $100 \%$ intensity peak is significantly more intense than the remaining peaks in the pattern, which indicates some preferred orientation.

- Bohmite, syn, $\mathrm{AlO}(\mathrm{OH})$, card\# 21-1307, Excellent overall fit. Peak locations and intensities are a good match to the pattern.

The pattern was also examined using RIQAS (release 4.0.0.26, 6/10/2002, Materials Data Inc.) rietveld analysis software. The phases identified above were input into the analysis along with a polynomial background and an amorphous hump at $\sim 35^{\circ} 2 \theta$, with fitted pattern shown in figure 5 .

The results of the analysis are:

- Gibbsite, $\mathrm{Al}(\mathrm{OH}) 3,69.3 \mathrm{wt} \%$, with a crystallite size of $130 \AA$

- Bohmite, $\mathrm{AlO}(\mathrm{OH}), 31.8 \mathrm{wt} \%$, with a crystallite size of $386 \AA$ 
TDP-WTP-353

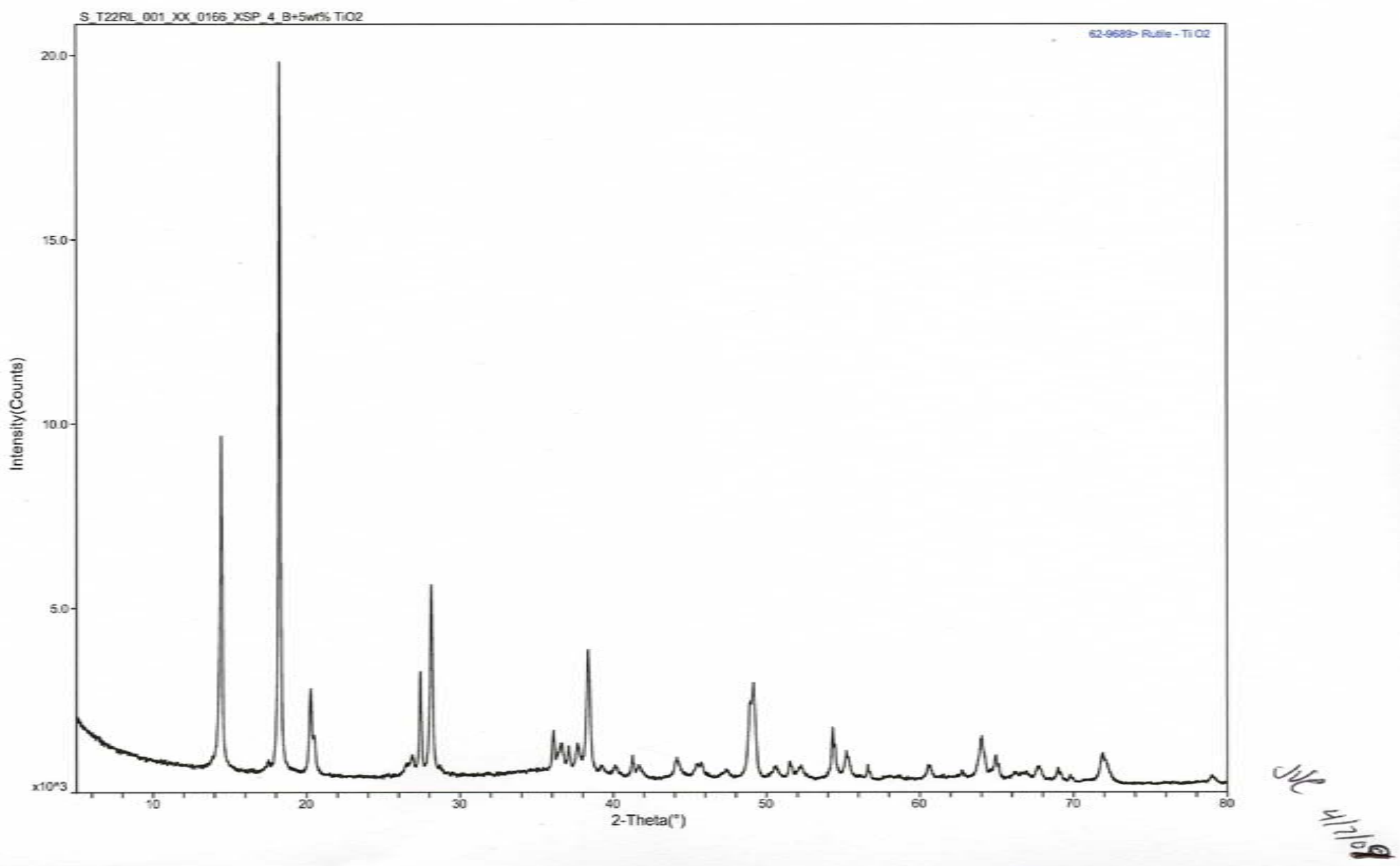


TDP-WTP-355
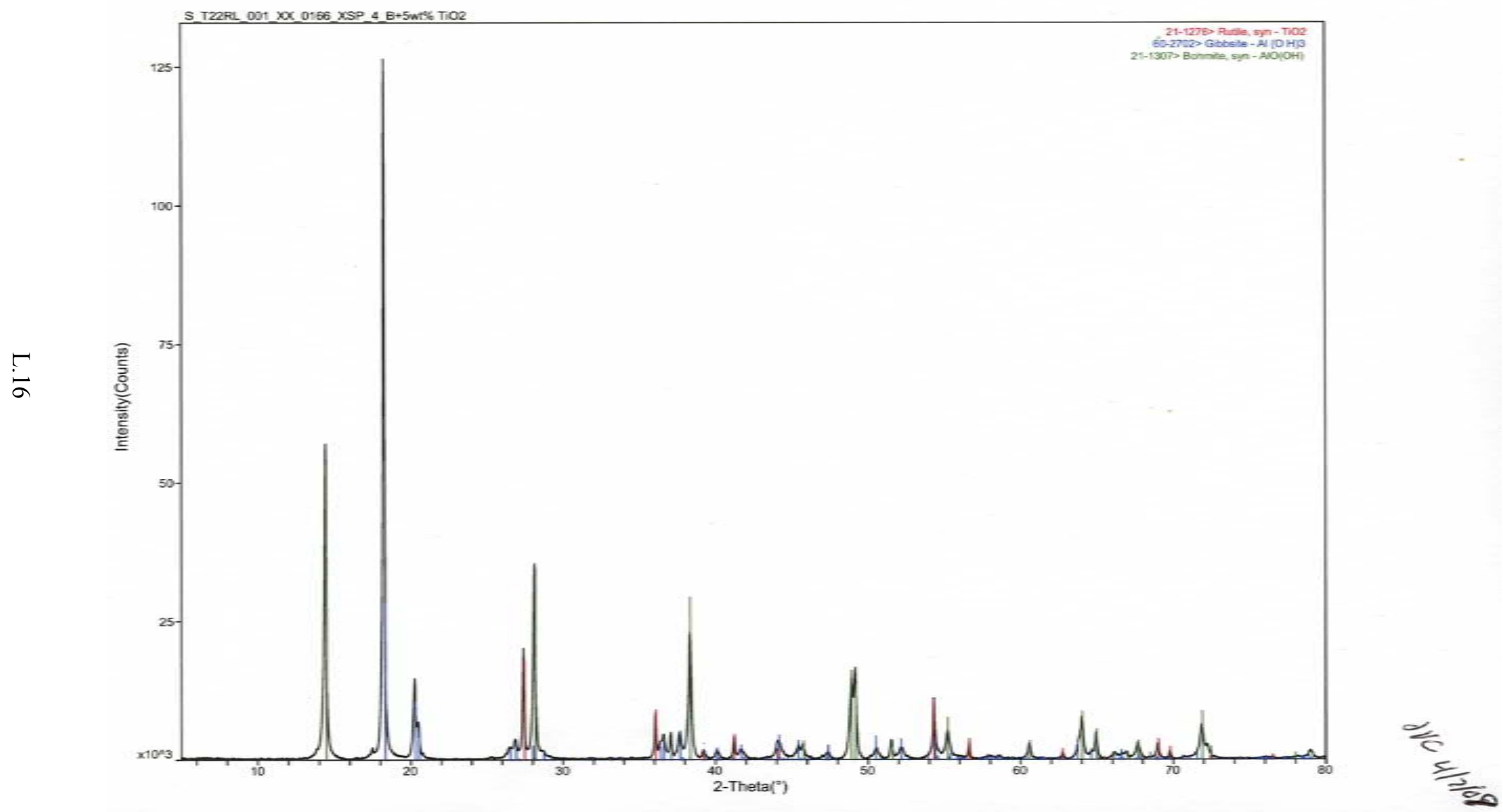


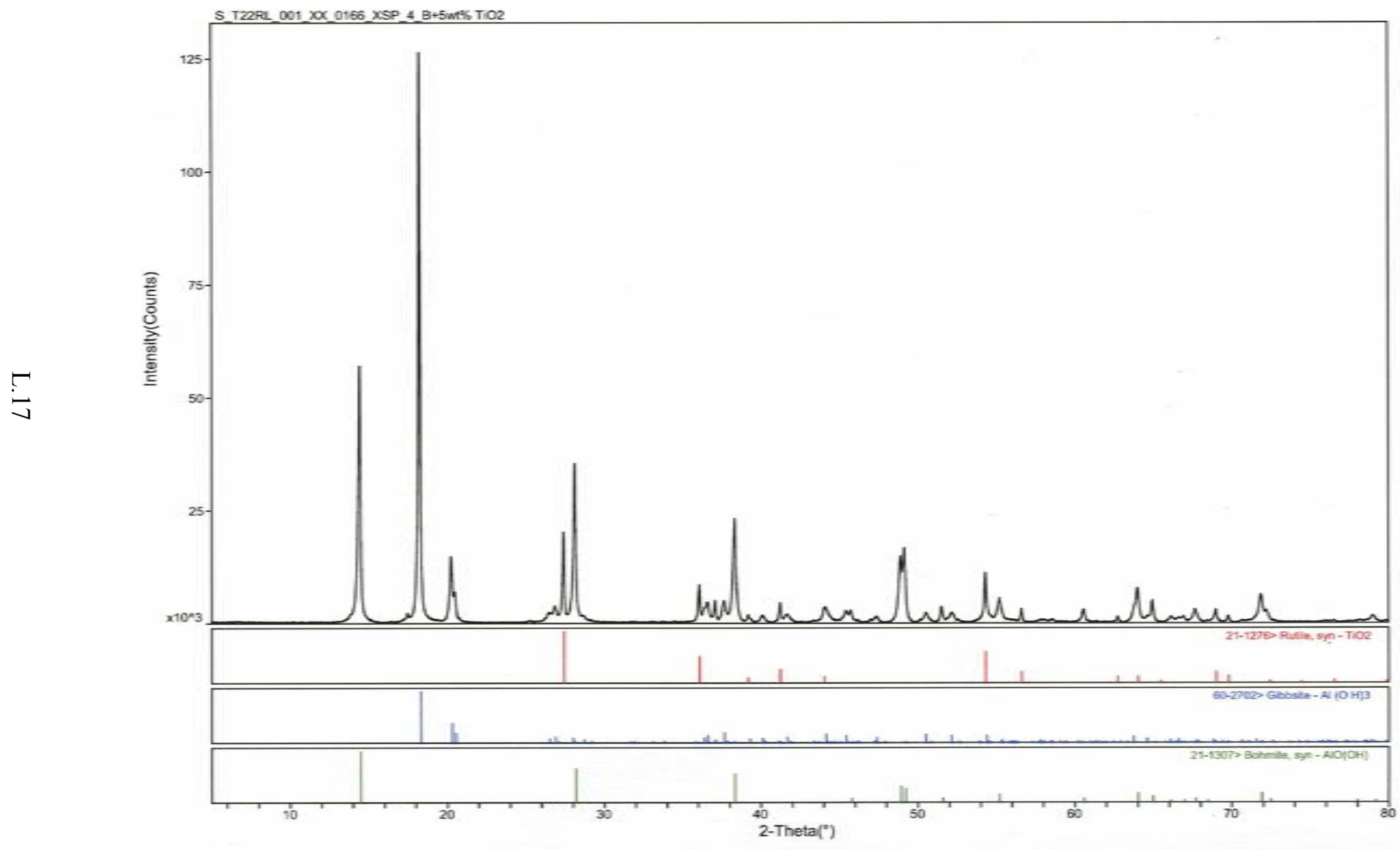


TDP-WTP-359
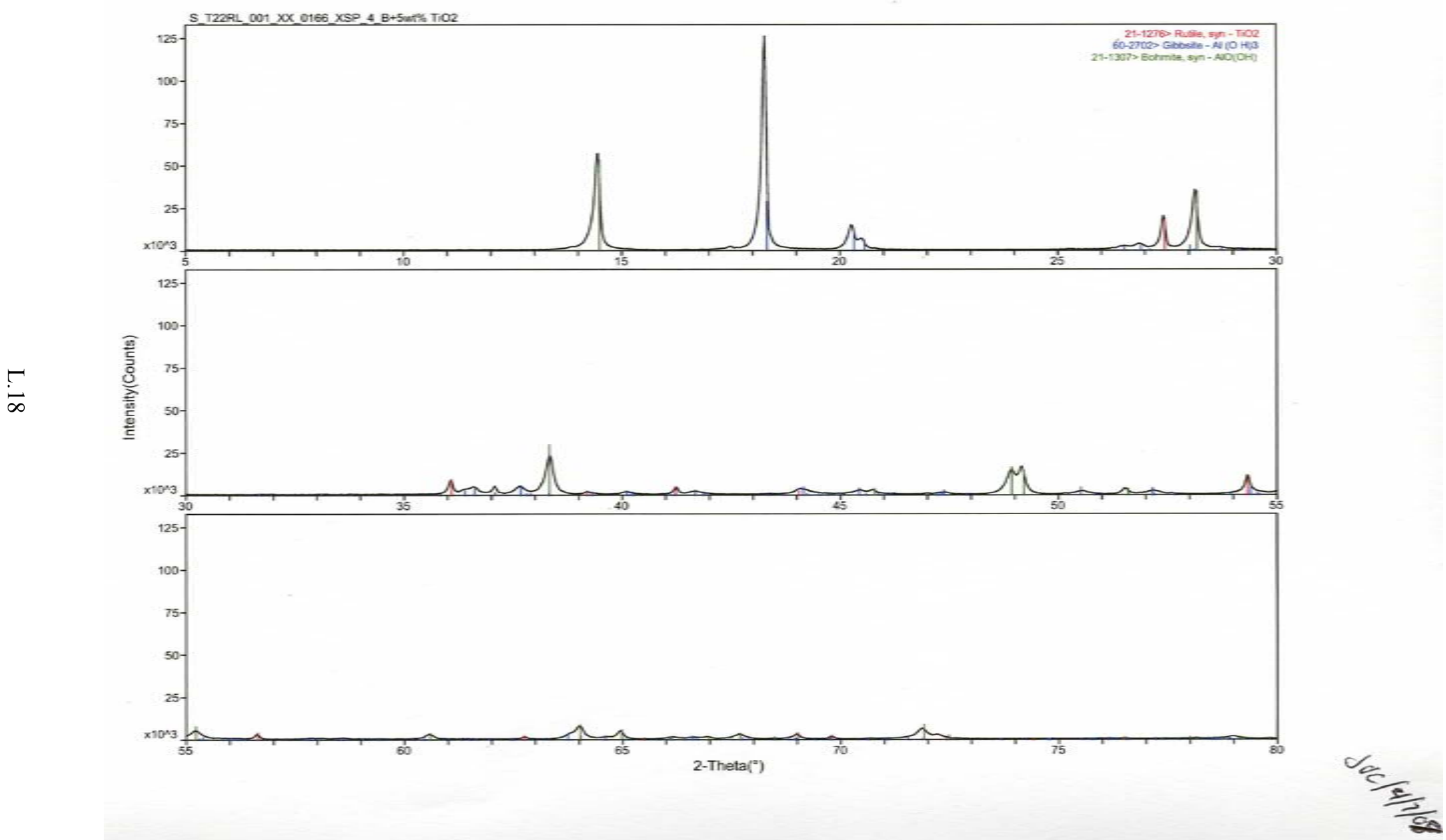

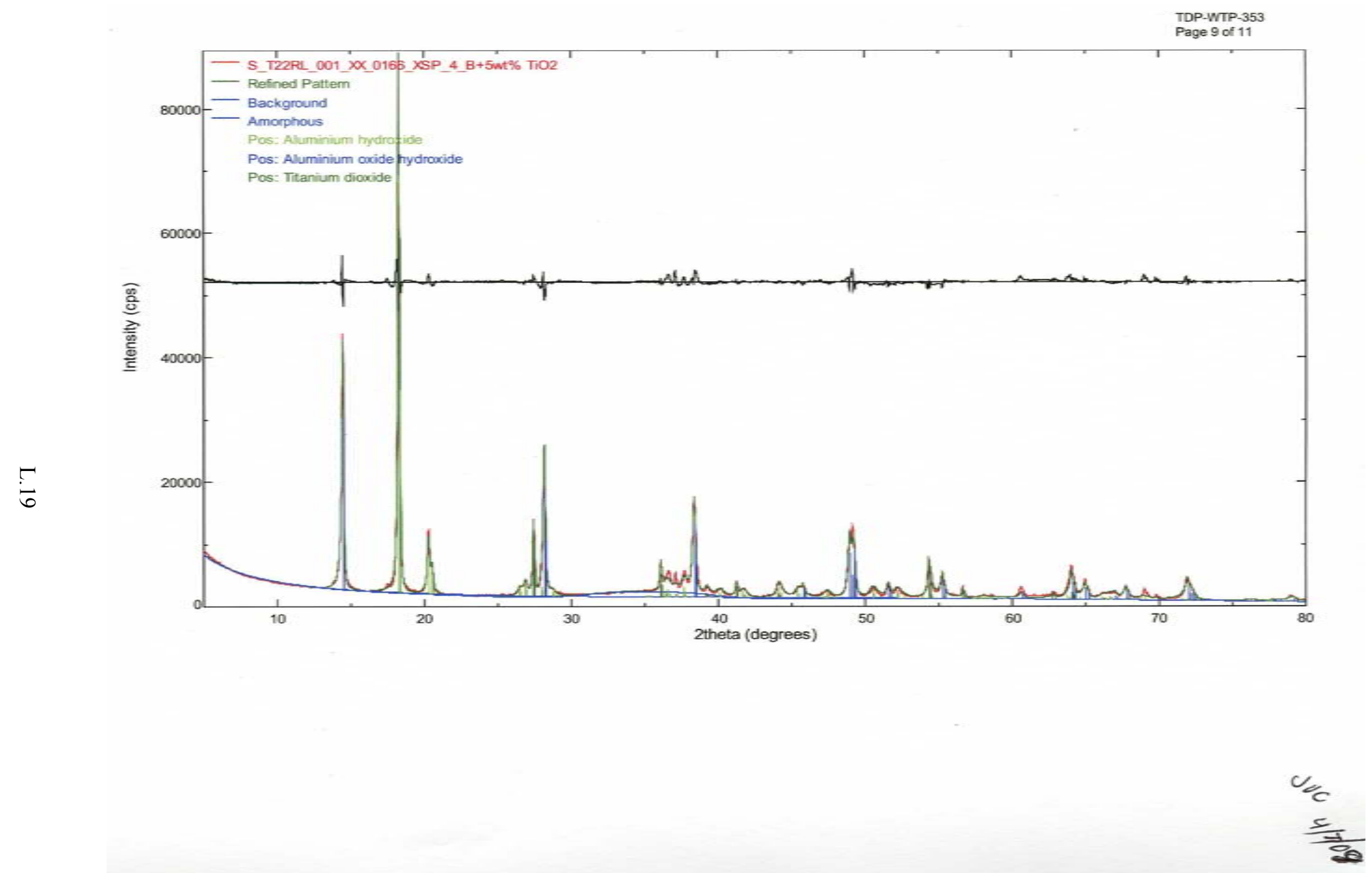
Appendix A

ICDD cards of interest for this sample 
- $\mathrm{PDF} \# 21-1276: \mathrm{QM}=$ Common $(+) ; \mathrm{d}=$ Other/Unknown; $\mathrm{I}=($ Unknown $)$

Rutile, syn

$\mathrm{TiO} 2$

Radiation $=\mathrm{CuKa} 1 \quad$ Lambda $=1.5406$ Filter $=$

Calibration $=\quad \mathrm{d}$-Cutoff $=\quad \mathrm{I} / \mathrm{Ic}(\mathrm{RIR})=3.4$

Ref: Level-1 PDF

Tetragonal, $\mathrm{P} 42 / \mathrm{mnm}(136) \quad \mathrm{Z}=2 \mathrm{mp}=$

CELL: $4.5933 \times 4.5933 \times 2.9592<90.0 \times 90.0 \times 90.0>\quad P . S=$

$\operatorname{Density}(\mathrm{c})=4.23$ Density $(\mathrm{m})=\quad \mathrm{Mwt}=\quad \mathrm{Vol}=62.4$

Ref: Ibid.

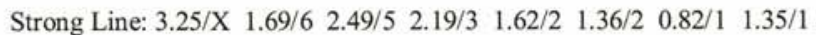

- $\mathrm{PDF} \# 60-2702: \mathrm{QM}=$ Calculated; $\mathrm{d}=\mathrm{O}$ ther/Unknown; $\mathrm{I}=($ Unknown $)$

Gibbsite

$\mathrm{Al}(\mathrm{O} \mathrm{H}) 3$

Radiation $=$ CuKal Lambda $=1.5406$ Filter $=$

Calibration $=\quad \mathrm{d}-$ Cutoff $=\quad \mathrm{I} / \mathrm{Ic}(\mathrm{RIR})=1.67$

Ref: Calculated from FIZ\#6162 (ICSD @08/01/01) by Jade 6.x

Monoclinic, $\mathrm{P} 21 / \mathrm{n}(14) \quad \mathrm{Z}=8 \quad \mathrm{mp}=$

CELL: $8.684 \times 5.078 \times 9.736<90.0 \times 94.54 \times 90.0>$ P.S $=$

Density $(\mathrm{c})=2.421$ Density $(\mathrm{m})=\quad \mathrm{Mwt}=\quad \mathrm{Vol}=428.0$

Ref: Ibid.

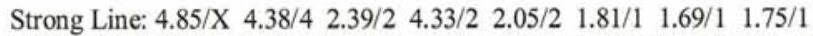

NOTE: Refinement of the crystal structure of gibbsite, $\mathrm{Al}(\mathrm{O} \mathrm{H}) 3$, Zeitschrift fuer

Kristallographie, Kristallgeometrie, Kristallphysik, Kristallchemie (-144,1977) [ZEKGA] 139

(1974) 129-135, Saalfeld H, Wedde M, Dx=2.4 .

M PDF 33-18.

$\mathrm{R}=0.032$

Calculated density is unusual, but tolerable.

At least one temperature factor is implausible or meaningless, but agrees with the value given in the paper.

- PDF\#21-1307: $\mathrm{QM}=$ Common $(+)$; $\mathrm{d}=$ Other/Unknown; $\mathrm{I}=($ Unknown)

Bohmite, syn

$\mathrm{AlO}(\mathrm{OH})$

Radiation $=\mathrm{CuKal} \quad$ Lambda $=1.5406$ Filter $=$

Calibration $=\quad \mathrm{d}$-Cutoff $=\quad \mathrm{I} / \mathrm{Ic}(\mathrm{RIR})=$

Ref: Level-1 PDF

Orthorhombic, Amam(63) $\mathrm{Z}=4 \quad \mathrm{mp}=$

CELL: $3.7 \times 12.227 \times 2.868<90.0 \times 90.0 \times 90.0>\quad P . S=$

$\operatorname{Density}(\mathrm{c})=3.07$ Density $(\mathrm{m})=\quad \mathrm{Mwt}=\quad \mathrm{Vol}=129.8$

Ref: Ibid.

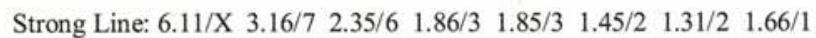




\section{L.5 Particle-Size Distribution (TDP-WTP-362)}

MEMORANDUM

\begin{tabular}{lll}
\hline & & \\
Date: & January 26, 2008 & Project No.: \\
To: & Brad Johnson & Internal Distribution: \\
From: & Bill Buchmiller & \\
Subject: & PEP PSD Analysis, Shakedown & \\
& Test Plan, TI-WTP-PEP-062 and & \\
& TI-WTP-PEP-032
\end{tabular}

Particle size distribution analysis was performed on slurries from the PEP (Pre-treatment Engineering Platform) project during shake-down testing. Under WP\# H14568, 10 samples were delivered per TI-WTP-PEP-062 and 9 samples delivered per TI-WTP-PEP-032. These samples were to be analyzed per instructions provided in an 11/25/2008 Letter to Analyst (LTA). Copies of the particle size distribution plots for the samples provided are attached in addition to a copy of the LTA. Equipment performance was verified using a vendor supplied transfer standard (QAS3001B, part No. CRM00016), and the particle distribution plot for that is attached as well.

\section{SAMPLE PREP}

All 19 samples were analyzed as provided using a Malvern MS2000 Analyzer with Hydro 2000S particle dispersion unit. Washed and centrifuged samples delivered in conical bottom centrifuge tubes, were initially agitated with a Scientific Industries, Vortex-T, Genie 2 vibrator after the addition of 5 to $10 \mathrm{mLs}$ nominal $0.01 \mathrm{M} \mathrm{NaOH}$ solution. Samples were agitated for a period of time determined at the discretion of the analyst. After the initial agitation, the samples were shaken by hand and the slurry transferred to the Malvern MS2000 using a poly transfer pipette. The transfer pipette was also used to keep the sample material suspended in the centrifuge tubes during transfer.

The requester of service indicated concern over potential agglomeration issues and requested that each sample, in addition to the as-received form, be analyzed after sonication. A transfer pipette was used to mix the slurry and transfer the required amount to the analyzer. Nominally 1 to 5 drops of each sample/sub-sample were placed in the Hydro 2000S particle dispersion unit using $0.01 \mathrm{M} \mathrm{NaOH}$ as the dispersion fluid. The dispersion unit has an internal sonication device with a variable speed pump/agitator. Unless noted differently, the pump/agitator was operated at a setpoint of 3000 and the sonicator operated at $0 \%$ (as-received) or $50 \%$ maximum setpoint. Sonicator setpoints of up to $100 \%$ maximum were an option at the analyst's discretion.

The amount of sub-sample required for analysis varied with the actual concentration of the sample. Neither the amount of sample nor the amount of $0.01 \mathrm{M} \mathrm{NaOH}$ dispersion solution was critical to the analytical process since the analytical software indicates graphically the amount of sample needed for analysis. The actual amounts of sample and water did not need to be, nor 
Brad Johnson

January 26, 2009

were they measured or recorded. Any additional sonication required to de-agglomerate sample(s) was noted on the respective particle size distribution data plot

\section{ANALYSIS}

Analysis was performed per procedure TPR-RPP-WTP-626, Rev. 0, Particle Size Analysis using Malvern MS2000 (current Revision) and per the LTA provided by the responsible scientist. The ID of the Malvern MS2000 Analyzer is property\# PT20096 (serial number MAL1019545 listed on the attached data plots). The pump/stirrer speed of the Hydro 2000S particle dispersion unit (ID property\# PT20083) integral to the analyzer was set at 3000 . Samples were allowed to circulate/sonicate in the Hydro $2000 \mathrm{~S}$ for at least 30 seconds prior to analysis.

The Malvern MS2000 Analyzer does not have the capability of calibration adjustment, and other than cleaning the lense or the sample chamber, or returning the equipment to the vendor, there are no adjustments, other than pump/stirrer speed and \% sonication, that will affect the operation of the equipment. Per the LTA, a QAS3001B, part No. CRM00016 standard was used to verify the analyzer performance on $11 / 25 / 2008$. Procedure TPR-RPP-WTP-626 requires that the performance analysis be repeated every 90 days. The following parameters were included in the standard analysis setup:

Setup name: Glass Beads (typical)

Dispersant Name: DI Water

Run number: 5

Adsorption: 0

Sensitivity: Enhanced

Analysis model: Single narrow mode

Particle refractive index: 1.520

Dispersant refractive index: 1.33

Standard analysis results were required to fall within $+/-10 \%$ of the manufacture's $D_{50}$ specifications. The glass standard microspheres were analyzed as non-agglomerating particles and the analysis results fell within $+/-10 \%$ of the manufacture specified volume weighted $\mathrm{D}_{50}$ value of 60.59 microns. See Figure 1 . 
Brad Johnson

January 26, 2009

11/25/2008 Standard Analysis Results

(microns)

\begin{tabular}{|c|c|c|c|c|c|c|c|}
\hline $\begin{array}{c}\text { Record } \\
\text { number }\end{array}$ & $\begin{array}{c}\text { Sample } \\
\text { Identification }\end{array}$ & $\begin{array}{c}\text { Measured } \\
\mathrm{D}_{50}\end{array}$ & $\begin{array}{c}\text { Vendor } \\
\text { specified } \mathrm{D}_{50}\end{array}$ & $\begin{array}{c}\text { Measured } \\
\mathrm{D}_{50} \\
+10 \%\end{array}$ & $\begin{array}{c}\text { Measured } \\
\mathrm{D}_{50} \\
-10 \%\end{array}$ & $\begin{array}{c}\text { Volume } \\
\text { weighted } \\
\text { mean (MV) }\end{array}$ & Comments \\
\hline 10 & QASstd - Average & 62.395 & 60.590 & 68.6345 & 56.156 & 63.224 & $\begin{array}{c}\text { Passed } \\
\text { performance } \\
\text { acceptance } \\
\text { criteria }\end{array}$ \\
\hline
\end{tabular}

Figure 1

Each sample was sub-sampled a minimum of 2 times to allow duplicate analysis of both the asreceived and sonicated portions of each sample. Per instructions on the LTA, 3 additional subsamples were analyzed if the initial duplicate MV(volume weighted mean diameter) values of the as-received or the sonicated sub-samples differed by more than $15 \%$. All duplicate analysis results varying by more than $100 \%$ were reviewed by the cognizant scientist. Any additional analysis requirements could be waived if the cognizant scientist agrees that agglomeration issues may cause results to be suspect.

The following parameters were included in the sample analysis setup:

Setup name: Ferric Oxide Hydroxide

Dispersant Name: $0.01 \mathrm{M} \mathrm{NaOH}$

Run number: 5

Adsorption: 0

Sensitivity: Normal

Analysis model: General purpose

Particle refractive index: 2.94

Dispersant refractive index: 1.33

Volume weighted mean diameter distributions for TI-WTP-PEP-062 and TI-WTP-PEP-032 are reported in Figures $2 \& 3$.

\section{COMMENTS AND OBSERVATIONS}

In addition to $0 \%$ sonication and $50 \%$ sonication, most samples were analyzed at $100 \%$ sonication to observe the effects of sonication on the breakdown of potentially agglomerated particles. With the technical input of the equipment custodian, it was decided to report only the $0 \%$ and $50 \%$ sonicated results. PSD results of samples analyzed at $100 \%$ sonication may be reported upon request or if it appears that agglomeration may be an issue at the $50 \%$ sonication level. 
Brad Johnson

January 26, 2009

\begin{tabular}{|c|c|c|c|c|c|c|}
\hline \multicolumn{7}{|c|}{$\begin{array}{c}\text { TI-WTP-PEP-062 Sample Analysis Results } \\
\text { (Microns) }\end{array}$} \\
\hline $\begin{array}{l}\text { Record } \\
\text { number }\end{array}$ & Sample Identification & D50 & $\begin{array}{l}\text { Volume } \\
\text { weighted } \\
\text { mean } \\
\text { diameter } \\
(\mathrm{MV})\end{array}$ & $\begin{array}{c}\mathrm{MV} \\
+15 \% \\
\end{array}$ & $\begin{array}{l}\text { MV - } \\
15 \%\end{array}$ & Comments \\
\hline & & & & & & \\
\hline 46 . & $\begin{array}{c}\text { S_000FL_008_XX_0249_XSP_4_B } \\
(0 \% \text { Son })-\text { Average }\end{array}$ & 5.89 & 8.305 & 9.551 & 7.059 & $\begin{array}{l}\text { Duplicate analysis } \\
\text { not performed }\end{array}$ \\
\hline 82 & $\begin{array}{l}\text { S_000FL_008_XX_0249_XSP_4_B } \\
\text { (50\% Son) - Average }\end{array}$ & 5.79 & 7.457 & 8.576 & 6.338 & \\
\hline 58 & $\begin{array}{l}\text { S_000FL_008_XX_0249_XSP_4_B } \\
(50 \% \text { Son })-\text { Average }\end{array}$ & 5.68 & 7.462 & 8.581 & 6.343 & \\
\hline & & & & & & \\
\hline 412 & $\begin{array}{c}\text { S_01AIL_006_12_0212_XSP_4_B } \\
\text { (0\% Son) - Average }\end{array}$ & 5.97 & 9.038 & 10.394 & 7.682 & \\
\hline 436 & $\begin{array}{c}\text { S_01AIL_006_12_0212_XSP_4_B } \\
\text { (0\% Son) - Average }\end{array}$ & 5.81 & 8.187 & 9.415 & 6.959 & \\
\hline 418 & $\begin{array}{l}\text { S_01AIL_006_12_0212_XSP_4_B } \\
(50 \% \text { Son) - Average }\end{array}$ & 5.93 & 8.061 & 9.270 & 6.852 & \\
\hline 442 & $\begin{array}{l}\text { S_01AIL_006_12_0212_XSP_4_B } \\
(50 \% \text { Son) - Average }\end{array}$ & 5.83 & 7.941 & 9.132 & 6.750 & \\
\hline & & & & & & \\
\hline 460 & $\begin{array}{c}\text { S_01AlH_006_12_0210_XSP_4_B } \\
(0 \% \text { Son }) \text { - Average }\end{array}$ & 6.13 & 20.844 & 23.971 & 17.717 & $\begin{array}{l}\text { Agglomeration } \\
\text { Issues }\end{array}$ \\
\hline 484 & $\begin{array}{c}\text { S_01AlH_006_12_0210_XSP_4_B } \\
\text { (0\%Son) - Average }\end{array}$ & 6.05 & 8.596 & 9.885 & 7.307 & 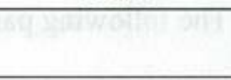 \\
\hline 466 & $\begin{array}{c}\text { S_01AlH_006_12_0210_XSP_4_B } \\
(50 \% \text { Son) }- \text { Average }\end{array}$ & 5.8 & 8.029 & 9.233 & 6.825 & \\
\hline 490 & $\begin{array}{c}\text { S_01AIH_006_12_0210_XSP_4_B } \\
(50 \% \text { Son })-\text { Average }\end{array}$ & 5.97 & 8.084 & 9.297 & 6.871 & 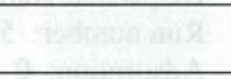 \\
\hline & & & & & & \\
\hline 118 & $\begin{array}{c}\text { S_000FL_009_XX_0327_XSP_4_B } \\
(0 \% \text { Son })-\text { Average }\end{array}$ & 4.94 & 10.337 & 11.888 & 8.786 & \\
\hline 142 & $\begin{array}{c}\text { S_000FL_009_XX_0327_XSP_4_B } \\
(0 \% \text { Son })-\text { Average }\end{array}$ & 5.04 & 9.697 & 11.152 & 8.242 & \\
\hline 100 & $\begin{array}{c}\text { S_000FL_009_XX_0327_XSP_4_B } \\
(50 \% \text { Son }) \text { - Average }\end{array}$ & 4.64 & 5.954 & 6.847 & 5.061 & \\
\hline 124 & $\begin{array}{l}\text { S_000FL_009_XX_0327_XSP_4_B } \\
(50 \% \text { Son })-\text { Average }\end{array}$ & 4.57 & 6.321 & 7.269 & 5.373 & \\
\hline & & & & & & \\
\hline 182 & $\begin{array}{c}\text { S_000FL_010_XX_0332_XSP_4_B } \\
\text { (0\% Son) - Average }\end{array}$ & 4.66 & 6.977 & 8.024 & 5.930 & \\
\hline 209 & 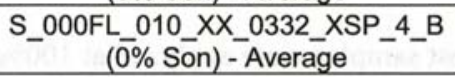 & 4.56 & 7.147 & 8.219 & 6.075 & \\
\hline 188 & $\begin{array}{l}\text { S_000FL_010_XX_0332_XSP_4_B } \\
(50 \% \text { Son) - Average }\end{array}$ & 4.62 & 6.551 & 7.534 & 5.568 & \\
\hline 215 & $\begin{array}{l}\text { S_000FL_010_XX_0332_XSP_4_B } \\
(50 \% \text { Son) }- \text { Average }\end{array}$ & 4.53 & 6.519 & 7.497 & 5.541 & \\
\hline
\end{tabular}

Figure 2 
Brad Johnson

January 26, 2009

\begin{tabular}{|c|c|c|c|c|c|c|}
\hline \multicolumn{7}{|c|}{$\begin{array}{l}\text { TI-WTP-PEP-062 Sample Analysis Results } \\
\text { (Microns) }\end{array}$} \\
\hline $\begin{array}{l}\text { Record } \\
\text { number }\end{array}$ & Sample Identification & D50 & $\begin{array}{l}\text { Volume } \\
\text { weighted } \\
\text { mean } \\
\text { diameter } \\
(\mathrm{MV})\end{array}$ & $\begin{array}{c}\text { MV } \\
+15 \% \\
\end{array}$ & $\begin{array}{l}\text { MV - } \\
15 \%\end{array}$ & Comments \\
\hline & & & & & & \\
\hline 364 & $\begin{array}{c}\text { S_01AIL_006_12_0700_XSP_4_B } \\
\text { (0\% Son) - Average }\end{array}$ & 6.22 & 8.781 & 10.098 & 7.464 & \\
\hline 388 & $\begin{array}{c}\text { S_01AlL_006_12_0700_XSP_4_B } \\
(0 \% \text { Son })-\text { Average }\end{array}$ & 6.05 & 9.715 & 11.172 & 8.258 & \\
\hline 370 & $\begin{array}{c}\text { S_01AlL_006_12_0700_XSP_4_B } \\
(50 \% \text { Son }) \text { - Average }\end{array}$ & 6.09 & 8.283 & 9.525 & 7.041 & \\
\hline 330 & 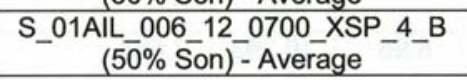 & 5.91 & 8.151 & 9.374 & 6.928 & \\
\hline 251 & 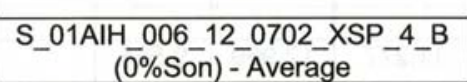 & 5.85 & 8.193 & 9.422 & 6.964 & \\
\hline 276 & $\begin{array}{c}\text { S_01AlH_006_12_0702_XSP_4_B } \\
(0 \% \text { Son }) \text { - Average }\end{array}$ & 5.89 & 10.204 & 11.735 & 8.673 & $\begin{array}{l}\text { Agglomeration } \\
\text { Issues }\end{array}$ \\
\hline 257 & $\begin{array}{c}\text { S_01AIH_006_12_0702_XSP_4_B } \\
\text { (50\%Son) - Average }\end{array}$ & 5.85 & 7.844 & 9.021 & 6.667 & \\
\hline 282 & $\begin{array}{c}\text { S_01AlH_006_12_0702_XSP_4_B } \\
(50 \% \text { Son) }- \text { Average }\end{array}$ & 5.84 & 8.040 & 9.246 & 6.834 & \\
\hline 508 & $\begin{array}{c}\text { S_T22RL_001_XX_0166_XSP_4_B } \\
\text { (0\% Son) - Average }\end{array}$ & 7.01 & 10.150 & 11.673 & 8.628 & \\
\hline 532 & $\begin{array}{c}\text { S_T22RL_001_XX_0166_XSP_4_B } \\
(0 \% \text { Son }) \text { - Average }\end{array}$ & 7.05 & 10.436 & 12.001 & 8.871 & \\
\hline 514 & $\begin{array}{l}\text { S_T22RL_001_XX_0166_XSP_4_B } \\
(50 \% \text { Son }) \text { - Average }\end{array}$ & 6.72 & 8.850 & 10.178 & 7.523 & \\
\hline 538 & $\begin{array}{c}\text { S_T22RL_001_XX_0166_XSP_4_B } \\
(50 \% \text { Son }) \text { - Average }\end{array}$ & 6.72 & 8.950 & 10.293 & 7.608 & \\
\hline & & & & & & \\
\hline 604 & $\begin{array}{c}\text { S_01AlL_006_36_0240_XSP_4_B } \\
(\overline{0 \%} \text { Son })-\text { Average }\end{array}$ & 6.47 & 9.152 & 10.525 & 7.779 & \\
\hline 628 & $\begin{array}{c}\text { S_01AIL_006_36_0240_XSP_4_B } \\
\text { (0\% Son })- \text { Average }\end{array}$ & 6.49 & 9.159 & 10.533 & 7.785 & \\
\hline 610 & $\begin{array}{c}\text { S_01AlL_006_36_0240_XSP_4_B } \\
(50 \% \text { Son })-\text { Average }\end{array}$ & 6.35 & 8.636 & 9.931 & 7.341 & \\
\hline 634 & $\begin{array}{c}\text { S_01AIL_006_36_0240_XSP_4_B } \\
(50 \% \text { Son) - Average }\end{array}$ & 6.42 & 8.739 & 10.050 & 7.428 & \\
\hline 556 & $\begin{array}{c}\text { S_01AlH_006_36_0238_XSP_4_B } \\
\text { (0\% Son) - Average }\end{array}$ & 6.36 & 8.888 & 10.221 & 7.555 & \\
\hline 580 & $\begin{array}{c}\text { S_01AlH_006_36_0238_XSP_4_B } \\
(0 \% \text { Son }) \text { - Average }\end{array}$ & 6.51 & 9.162 & 10.536 & 7.788 & \\
\hline 562 & $\begin{array}{c}\text { S_01AlH_006_36_0238_XSP_4_B } \\
(50 \% \text { Son }) \text { - Average }\end{array}$ & 6.24 & 8.416 & 9.678 & 7.154 & \\
\hline 586 & $\begin{array}{l}\text { S_01AlH_006_36_0238_XSP_4_B } \\
(50 \% \text { Son }) \text { - Average }\end{array}$ & 6.39 & 8.628 & 9.922 & 7.334 & \\
\hline
\end{tabular}

Figure 2 (continued) 
Brad Johnson

January 26, 2009

\begin{tabular}{|c|c|c|c|c|c|c|}
\hline \multicolumn{7}{|c|}{$\begin{array}{l}\text { TI-WTP-PEP-032 Sample Analysis Results } \\
\text { (Microns) }\end{array}$} \\
\hline $\begin{array}{l}\text { Record } \\
\text { number }\end{array}$ & Sample Identification & D50 & $\begin{array}{c}\text { Volume } \\
\text { weighted } \\
\text { mean } \\
\text { diameter } \\
\text { (MV) }\end{array}$ & $\begin{array}{c}\text { MV } \\
+15 \% \\
\end{array}$ & $\begin{array}{l}\text { MV - } \\
15 \%\end{array}$ & Comments \\
\hline & & & & & & \\
\hline 730 & $\begin{array}{c}\text { S_01BIH_006_12_0827_XSP_4_B } \\
(0 \% \text { Son })-\text { Average }\end{array}$ & 7.05 & 9.841 & 11.317 & 8.365 & \\
\hline 754 & $\begin{array}{c}\text { S_01BIH_006_12_0827_XSP_4_B } \\
0 \% \text { Son) - Average }\end{array}$ & 7.09 & 10.771 & 12.387 & 9.155 & \\
\hline 736 & $\begin{array}{c}\text { S_01BIH_006_12_0827_XSP_4_B } \\
(50 \% \text { Son })-\text { Average }\end{array}$ & 6.74 & 8.91 & 10.247 & 7.574 & \\
\hline 760 & $\begin{array}{c}\text { S_01BIH_006_12_0827_XSP_4_B } \\
(50 \% \text { Son })-\text { Average }\end{array}$ & 6.72 & 8.95 & 10.293 & 7.608 & \\
\hline 778 & $\begin{array}{c}\text { S_01BIL_006_12_0829_XSP_4_B } \\
\text { (0\% Son) - Average }\end{array}$ & 7.36 & 11.277 & 12.969 & 9.585 & \\
\hline 802 & $\begin{array}{c}\text { S_01BIL_006_12_0829_XSP_4_B } \\
(0 \% \text { Son })-\text { Average }\end{array}$ & 7.26 & 11.39 & 13.099 & 9.682 & \\
\hline 784 & $\begin{array}{c}\text { S_01BIL_006_12_0829_XSP_4_B } \\
\text { (50\% Son) - Average }\end{array}$ & 6.79 & 9.008 & 10.359 & 7.657 & \\
\hline 808 & $\begin{array}{c}\text { S_01BIL_006_12_0829_XSP_4_B } \\
\text { (50\% Son) - Average }\end{array}$ & 6.85 & 9.175 & 10.551 & 7.799 & \\
\hline 983 & S 02AML 008 XX 0867 XSP 4 B & 5.94 & 8.14 & 9.36 & 6.92 & \\
\hline & (0\% son) - Average & & 0.14 & 9.00 & 0.92 & \\
\hline 1007 & $\begin{array}{c}\text { S_02AML_008_XX_0867_XSP_4_B } \\
(0 \% \text { son) - Average }\end{array}$ & 5.93 & 8.191 & 9.42 & 6.96 & \\
\hline 989 & $\begin{array}{c}\text { S_02AML_008_XX_0867_XSP_4_B } \\
\text { (50\% son) - Average }\end{array}$ & 5.95 & 8.007 & 9.21 & 6.81 & \\
\hline 1013 & $\begin{array}{c}\text { S_02AML_008_XX_0867_XSP_4_B } \\
(50 \% \text { son })-\text { Average }\end{array}$ & 6.01 & 8.125 & 9.34 & 6.91 & \\
\hline 887 & $\begin{array}{c}\text { S_02AML_010_XX_0890_XSP_4_B } \\
(0 \% \text { Son }) \text { - Average }\end{array}$ & 4.28 & 6.361 & 7.315 & 5.407 & \\
\hline 911 & $\begin{array}{c}\text { S_02AML_010_XX_0890_XSP_4_B } \\
(0 \% \text { Son })-\text { Average }\end{array}$ & 4.29 & 6.379 & 7.336 & 5.422 & \\
\hline 893 & $\begin{array}{l}\text { S_02AML_010_XX_0890_XSP_4_B } \\
\text { (50\% Son) - Average }\end{array}$ & 4.36 & 6.319 & 7.267 & 5.371 & \\
\hline 917 & $\begin{array}{c}\text { S_02AML_010_XX_0890_XSP_4_B } \\
(50 \% \text { Son }) \text { - Average }\end{array}$ & 4.38 & 6.411 & 7.373 & 5.449 & \\
\hline & & & & & & \\
\hline 839 & $\begin{array}{c}\text { S_02AML_009_XX_0885_XSP_4_B } \\
(0 \% \text { Son })-\text { Average }\end{array}$ & 4.62 & 6.725 & 7.734 & 5.716 & \\
\hline 863 & $\begin{array}{c}\text { S_02AML_009_XX_0885_XSP_4_B } \\
(0 \% \text { Son }) \text { - Average }\end{array}$ & 4.62 & 6.763 & 7.777 & 5.749 & \\
\hline 845 & $\begin{array}{c}\text { S_02AML_009_XX_0885_XSP_4_B } \\
(50 \% \text { Son) - Average }\end{array}$ & 4.68 & 6.648 & 7.645 & 5.651 & \\
\hline 869 & $\begin{array}{c}\text { S_02AML_009_XX_0885_XSP_4_B } \\
(50 \% \text { Son) - Average }\end{array}$ & 4.72 & 6.769 & 7.784 & 5.754 & \\
\hline
\end{tabular}

Figure 3 
Brad Johnson

January 26, 2009

\begin{tabular}{|c|c|c|c|c|c|c|}
\hline \multicolumn{7}{|c|}{$\begin{array}{l}\text { TI-WTP-PEP-032 Sample Analysis Results } \\
\text { (Microns) }\end{array}$} \\
\hline $\begin{array}{l}\text { Record } \\
\text { number }\end{array}$ & Sample Identification & D50 & $\begin{array}{c}\text { Volume } \\
\text { weighted } \\
\text { mean } \\
\text { diameter } \\
\text { (MV) }\end{array}$ & $\begin{array}{c}\text { MV } \\
+15 \% \\
\end{array}$ & $\begin{array}{l}\text { MV - } \\
15 \%\end{array}$ & Comments \\
\hline & & & & & & \\
\hline 1031 & $\begin{array}{c}\text { S_01BIH_006_36_0855_XSP_4_B } \\
\text { (0\% son) - Average }\end{array}$ & 6.93 & 9.999 & 11.5 & 8.5 & \\
\hline 1055 & $\begin{array}{c}\text { S_01BIH_006_36_0855_XSP_4_B } \\
(0 \% \text { son }) \text { - Average }\end{array}$ & 6.8 & 9.927 & 11.42 & 8.44 & \\
\hline 1037 & $\begin{array}{c}\text { S_01BIH_006_36_0855_XSP_4_B } \\
(50 \% \text { son })-\text { Average }\end{array}$ & 6.66 & 8.944 & 10.29 & 7.6 & \\
\hline 1061 & $\begin{array}{l}\text { S_01BIH_006_36_0855_XSP_4_B } \\
(50 \% \text { son) }) \text { Average }\end{array}$ & 6.62 & 8.858 & 10.19 & 7.53 & \\
\hline 1103 & S 01BIL $006360857 \times$ XP 4 B & 6.39 & 9.305 & 10.7 & 7.91 & \\
\hline 1700 & 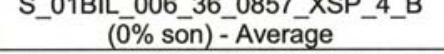 & 0.09 & 9.300 & & & \\
\hline 1079 & $\begin{array}{c}\text { S_01BIL_006_36_0857_XSP_4_B } \\
\text { (0\% son) - Average }\end{array}$ & 6.5 & 9.013 & 10.36 & 7.66 & \\
\hline 1085 & $\begin{array}{c}\text { S_01BIL_006_36_0857_XSP_4_B } \\
\text { (50\% son) - Average }\end{array}$ & 6.38 & 8.421 & 9.68 & 7.16 & \\
\hline 1109 & $\begin{array}{c}\text { S_01BIL_006_36_0857_XSP_4_B } \\
(50 \% \text { son) }) \text {-Average }\end{array}$ & 6.42 & 8.46 & 9.73 & 7.19 & \\
\hline 1127 & $\begin{array}{c}\text { S_02AML_029_XX_0929_XSP_4_B } \\
\text { (0\% son) - Average }\end{array}$ & 1.12 & 1.931 & 2.22 & 1.64 & \\
\hline 1151 & $\begin{array}{c}\text { S_02AML_029_XX_0929_XSP_4_B } \\
(0 \% \text { son) - Average }\end{array}$ & 1.16 & 2.966 & 3.41 & 2.52 & \\
\hline 1133 & $\begin{array}{c}\text { S_02AML_029_XX_0929_XSP_4_B } \\
(50 \% \text { son) }- \text {-Average }\end{array}$ & 1.08 & 1.894 & 2.18 & 1.61 & \\
\hline 1157 & $\begin{array}{c}\text { S_02AML_029_XX_0929_XSP_4_B } \\
(50 \% \text { son })-\text { Average }\end{array}$ & 1.09 & 1.912 & 2.2 & 1.63 & \\
\hline 935 & $\begin{array}{c}\text { S_02AML_029_XX_0999_XSP_4_B } \\
\text { (0\% son) - Average }\end{array}$ & 1.08 & 1.786 & 2.05 & 1.52 & \\
\hline 959 & $\begin{array}{c}\text { S_02AML_029_XX_0999_XSP_4_B } \\
\text { (0\% son)-Average }\end{array}$ & 1.08 & 1.927 & 2.22 & 1.64 & \\
\hline 941 & $\begin{array}{c}\text { S_02AML_029_XX_0999_XSP_4_B } \\
(50 \% \text { son })-\text { Average }\end{array}$ & 1.08 & 2.545 & 2.93 & 2.16 & \\
\hline 965 & $\begin{array}{c}\text { S_02AML_029_XX_0999_XSP_4_B } \\
(50 \% \text { son) }) \text { Average }\end{array}$ & 1.08 & 1.969 & 2.26 & 1.67 & \\
\hline
\end{tabular}

Figure 3 (continued) 
Appendix M

Operational Process Sheets 



\section{Appendix M: Operational Process Sheets}

The Operational Process sheets section of the Test Instructions provided the LTE target or set point values for key process operations. Usually, the LTE filled in the actual value during testing from available data. Often these data were from the HMI, which is not NQA-1 qualified. Data recorded were a snapshot in time rather than an average. The following is a reproduction of the operational process sheet with the "actual value" column filled in from various data sources. Data provided in the "actual value" column are generally from the original Test Instruction as filled in by the LTE. In cases where certified data were available as a result of data evaluation efforts, these data were used instead. The data source is provided in the "comments" column, with the following definitions:

HMI - The LTE obtained the value from the HMI screen, which does not provide certified calibrated readings. Reading taken as a snapshot in time with no averaging applied.

Local flow meter-The steam ring air purge and the air sparge values were read locally from the instrument (Coriolis gas flow meter). These local readouts were calibrated.

TI-The value or state was recorded on the Test Instruction. The timing of events, number of events (e.g., wash batches), equipment line ups, and AFA additions were documented in this manner and provide the best available record.

CCP-Selected PJM parameters were evaluated and documented in CCP-WTPSP-901.

$\mathrm{NP}$ - Indicates that the step was not performed as part of the Test Instruction.

$\mathrm{n} / \mathrm{r}$-indicates that the step was not recorded by the LTE.

The following operational process sheets, (Tables M.1, M.2, and M.3) are provided for information only.

Table M.1 TI-062 Operational Process Sheet

\begin{tabular}{|c|c|c|c|c|c|c|}
\hline \multicolumn{2}{|c|}{$\begin{array}{l}\text { Parameter Data Sheet } \\
\text { TI-062 }\end{array}$} & \multirow[b]{2}{*}{$\begin{array}{c}\text { Target or } \\
\text { Setpoint } \\
\text { Value }\end{array}$} & \multirow[b]{2}{*}{$\begin{array}{c}\text { Range } \\
(-/+)\end{array}$} & \multirow[b]{2}{*}{ Units } & \multirow[b]{2}{*}{$\begin{array}{l}\text { Actual } \\
\text { Value }\end{array}$} & \multirow[b]{2}{*}{ Comments } \\
\hline & Operation & & & & & \\
\hline \multirow{2}{*}{$\begin{array}{c}\text { Simulant storage } \\
\text { in } \\
\text { HLP-VSL-T22 } \\
\text { (Test Instruction } \\
\text { step 7.1.1.2) }\end{array}$} & $\begin{array}{c}\text { HLP-VSL-T22 } \\
\text { Operation }\end{array}$ & - & - & - & - & - \\
\hline & Agitator speed & 68 & $-10 /+10$ & rpm & $\mathrm{NP}$ & $\begin{array}{l}\text { Not performed under Test } \\
\text { Instruction. }\end{array}$ \\
\hline \multirow{3}{*}{$\begin{array}{c}\text { Transfer from } \\
\text { HLP-VSL-T22 to } \\
\text { UFP-VSL-T01A } \\
\text { and PJM tuning } \\
\text { (Test Instruction }\end{array}$} & $\begin{array}{l}\text { Total transfer volume } \\
\text { (endpoint) }\end{array}$ & 593 & $-5 /+5$ & gal & 594 & $\begin{array}{l}\text { Source: TI } \\
\text { Level should be } \sim 65 \text { in. }\end{array}$ \\
\hline & Transfer rate & 15 & $-2 /+2$ & gpm & $\mathrm{n} / \mathrm{r}$ & \\
\hline & $\begin{array}{c}\text { UFP-VSL-T01A } \\
\text { Operation }\end{array}$ & - & - & - & - & - \\
\hline
\end{tabular}




\begin{tabular}{|c|c|c|c|c|c|c|}
\hline \multirow[t]{7}{*}{ step 7.2.1) } & $\begin{array}{l}\text { Volume of antifoam to } \\
\text { add to } \\
\text { UFP-VSL-T01A }\end{array}$ & 1,000 & $-20 /+20$ & $\mathrm{~mL}$ & 1,003 & $\begin{array}{l}\text { Source: TI } \\
\text { This value is concentrate. } \\
\text { Dilute with } 3 \text { parts IW to } 1 \text { part } \\
\text { concentrate. }\end{array}$ \\
\hline & $\begin{array}{l}\text { Level to initiate PJM } \\
\text { operation }\end{array}$ & 65 & $-2 /+10$ & in. & NP & \\
\hline & PJM jet velocity & 4.8 & $\begin{array}{c}-0.3 /+ \\
0.3\end{array}$ & $\mathrm{~m} / \mathrm{s}$ & $4.5-5.0$ & LTE tuned. Not NQA-1. \\
\hline & PJM stroke & $80 \%$ & $-5 /+5$ & $\%$ & $75 \%-79 \%$ & LTE tuned Not NQA-1. \\
\hline & PJM cycle time & 35 & $-1 /+1$ & $\mathrm{~s}$ & 35 & HMI \\
\hline & $\begin{array}{l}\text { Level to adjust steam } \\
\text { ring air purge rotameter }\end{array}$ & 10 & $-0 /+10$ & in. & NP & \\
\hline & $\begin{array}{l}\text { Steam ring air purge } \\
\text { flow rate }\end{array}$ & 0.13 & $\begin{array}{c}-0.02 /+ \\
0.02\end{array}$ & $\mathrm{~kg} / \mathrm{min}$ & 0 & $\begin{array}{l}\sim 3.9 \text { scfm; air would not turn } \\
\text { on. }\end{array}$ \\
\hline \multirow{9}{*}{$\begin{array}{c}\text { Transfer from } \\
\text { HLP-VSL-T22 to } \\
\text { UFP-VSL-T01B } \\
\text { and PJM tuning } \\
\text { (Test Instruction } \\
\text { step } 7.2 .2 \text { ) }\end{array}$} & $\begin{array}{l}\text { Total transfer volume } \\
\text { (endpoint) }\end{array}$ & 593 & $-5 /+5$ & gal & 592 & $\begin{array}{l}\text { Source: TI } \\
\text { Level should be } \sim 65 \text { in. }\end{array}$ \\
\hline & Transfer rate & 30 & $-2 /+2$ & gpm & 15 & $\begin{array}{l}\text { HMI; target value is incorrect. } \\
\text { Should be } 15 \text { like transfer to } \\
\text { T01A. }\end{array}$ \\
\hline & $\begin{array}{c}\text { UFP-VSL-T01A } \\
\text { Operation }\end{array}$ & - & - & - & - & ( \\
\hline & $\begin{array}{l}\text { Level to initiate PJM } \\
\text { operation }\end{array}$ & 54 & $-2 /+10$ & in. & NP & \\
\hline & PJM jet velocity & 4.8 & $\begin{array}{c}-0.3 /+ \\
0.3\end{array}$ & $\mathrm{~m} / \mathrm{s}$ & 4.2 & $\begin{array}{l}\text { Data Source: PJM Parameters } \\
\text { for Functional Test.doc. }\end{array}$ \\
\hline & PJM stroke & $80 \%$ & $-5 /+5$ & $\%$ & $65 \%$ & $\begin{array}{l}\text { Data Source: PJM Parameters } \\
\text { for Functional Test.doc. }\end{array}$ \\
\hline & PJM cycle time & 35 & $-1 /+1$ & $\mathrm{~s}$ & 35 & $\begin{array}{l}\text { Data Source: PJM Parameters } \\
\text { for Functional Test.doc. }\end{array}$ \\
\hline & $\begin{array}{l}\text { Level to adjust steam } \\
\text { ring air purge rotameter }\end{array}$ & 10 & $-0 /+10$ & in. & NP & \\
\hline & $\begin{array}{l}\text { Steam ring air purge } \\
\text { flow rate }\end{array}$ & 0.13 & $\begin{array}{c}-0.02 /+ \\
0.02\end{array}$ & $\mathrm{~kg} / \mathrm{min}$ & 0.13 & $\begin{array}{l}\text { Source: pg. J.69. } \\
\sim 3.9 \mathrm{scfm}\end{array}$ \\
\hline \multirow{3}{*}{$\begin{array}{c}\begin{array}{c}\text { Fill and flush } \\
\text { transfer line } \\
\text { from }\end{array} \\
\text { HLP-VSL-T22 to } \\
\text { UFP-VSL-T01B } \\
\text { (Test Instruction } \\
\text { step 7.2.3) }\end{array}$} & Line Flush & - & - & - & - & - \\
\hline & $\begin{array}{l}\text { Total mass of inhibited } \\
\text { water flush }\end{array}$ & 127 & $-5 /+20$ & $\mathrm{~kg}$ & 130 & $\begin{array}{l}\text { Data Source: CCP } \\
5 \mathrm{x} \text { line volume of } \sim 6.7 \mathrm{gal}\end{array}$ \\
\hline & $\begin{array}{l}\text { Inhibited water flush } \\
\text { flow rate }\end{array}$ & 106 & $-50 /+10$ & $\mathrm{~kg} / \mathrm{min}$ & 69 & $\begin{array}{l}\text { Max achievable averaged over } \\
\text { transfer: Data Source CCP } \\
\text { Target } 28 \text { gpm to match } \\
\text { Reynolds number of plant. } \\
\text { Use Coriolis meter }(\mathrm{kg} / \mathrm{min}) \text { for } \\
\text { high flow rate. Swirl meter is } \\
\text { not accurate for high flow rate. }\end{array}$ \\
\hline \multirow{5}{*}{$\begin{array}{c}\begin{array}{c}\text { Initial transfer } \\
\text { from }\end{array} \\
\text { UFP-VSL-T01A } \\
\text { to } \\
\text { UFP-VSL-T02A } \\
\text { (Test Instruction } \\
\text { step 7.2.4) }\end{array}$} & $\begin{array}{l}\text { Transfer volume } \\
\text { (endpoint) }\end{array}$ & 329 & $-3 /+3$ & gal & 324 & $\begin{array}{l}\text { Source: TI } \\
\text { Transfer terminated due to } \\
\text { High-Level alarm. }\end{array}$ \\
\hline & Transfer rate & 6.9 & NA & gpm & 6.9 & HMI \\
\hline & $\begin{array}{c}\text { UFP-VSL-T02A } \\
\text { Operation }\end{array}$ & - & - & - & - & - \\
\hline & $\begin{array}{l}\text { Level to initiate PJM } \\
\text { operation }\end{array}$ & 55 & $-2 /+10$ & in. & NP & \\
\hline & PJM jet velocity & 7.3 & $\begin{array}{c}-0.4 /+ \\
0.4\end{array}$ & $\mathrm{~m} / \mathrm{s}$ & 5.9 & $\begin{array}{l}\text { Data Source: PJM Parameters } \\
\text { for Functional Test.doc. }\end{array}$ \\
\hline
\end{tabular}




\begin{tabular}{|c|c|c|c|c|c|c|}
\hline & PJM stroke & $80 \%$ & $-5 /+5$ & $\%$ & 82 & $\begin{array}{l}\text { Data Source: PJM Parameters } \\
\text { for Functional Test.doc. }\end{array}$ \\
\hline & PJM cycle time & 33 & $-1 /+1$ & $\mathrm{~s}$ & 33.2 & $\begin{array}{l}\text { Data Source: PJM Parameters } \\
\text { for Functional Test.doc. }\end{array}$ \\
\hline & $\begin{array}{l}\text { Level to adjust steam } \\
\text { ring air purge rotameter }\end{array}$ & 5 & $-0 /+10$ & in. & NP & \\
\hline & $\begin{array}{l}\text { Steam ring air purge } \\
\text { flow rate }\end{array}$ & 0.10 & $\begin{array}{c}-0.02 /+ \\
0.02\end{array}$ & $\mathrm{~kg} / \mathrm{min}$ & 0.1 & $\begin{array}{l}\text { Source: pg. J.87 } \\
\sim 3.1 \text { scfm }\end{array}$ \\
\hline & $\begin{array}{l}\text { Adjust lower air } \\
\text { spargers to full flow at } \\
\text { end of transfer }\end{array}$ & 72 & $-10 /+0$ & in. & NP & \\
\hline & $\begin{array}{l}\text { Total lower air sparger } \\
\text { flow rate (for } 5 \text { sparge } \\
\text { tubes) }\end{array}$ & 0.40 & $\begin{array}{c}-0.05 /+ \\
0.05\end{array}$ & $\mathrm{~kg} / \mathrm{min}$ & $\mathrm{n} / \mathrm{r}$ & $\begin{array}{l}\text { NCR } 41090.1 \\
\sim 2.4 \text { scfm for each sparge tube. }\end{array}$ \\
\hline & $\begin{array}{l}\text { Adjust upper air } \\
\text { spargers to full flow at } \\
\text { the end of transfer }\end{array}$ & 72 & $-10 /+0$ & in. & NP & \\
\hline & $\begin{array}{l}\text { Upper air sparger flow } \\
\text { rate }\end{array}$ & 0.10 & $\begin{array}{c}-0.02 /+ \\
0.02\end{array}$ & $\mathrm{~kg} / \mathrm{min}$ & 0.1 & $\begin{array}{l}\text { Source: pg. J.87 } \\
\sim 3.0 \mathrm{scfm}\end{array}$ \\
\hline \multirow{4}{*}{$\begin{array}{c}\text { Transfer from } \\
\text { HLP-VSL-T22 to } \\
\text { UFP-VSL-T01A } \\
\text { (refill) } \\
\text { (Test Instruction } \\
\text { step 7.2.5) }\end{array}$} & $\begin{array}{l}\text { Total transfer volume } \\
\text { (endpoint) }\end{array}$ & 329 & $-3 /+3$ & gal & $\mathrm{n} / \mathrm{r}$ & Level should be $\sim 65$ in. \\
\hline & Transfer rate & 30 & $-2 /+2$ & gpm & $\mathrm{n} / \mathrm{r}$ & \\
\hline & $\begin{array}{c}\text { UFP-VSL-T01A } \\
\text { Operation }\end{array}$ & - & - & - & - & - \\
\hline & $\begin{array}{l}\text { Volume of antifoam to } \\
\text { add to } \\
\text { UFP-VSL-T01A }\end{array}$ & 550 & $-10 /+10$ & $\mathrm{~mL}$ & 550 & $\begin{array}{l}\text { TI (addition recorded, volume } \\
\text { not recorded). This value is } \\
\text { concentrate. Dilute with } 3 \\
\text { parts IW to } 1 \text { part concentrate. }\end{array}$ \\
\hline \multirow{5}{*}{$\begin{array}{c}\text { Test for } \\
\text { stratification in } \\
\text { UFP-VSL-T01A } \\
(7.2 .5)\end{array}$} & $\begin{array}{c}\text { UFP-VSL-T01A } \\
\text { Operation }\end{array}$ & - & - & - & - & 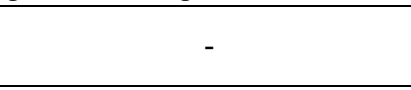 \\
\hline & PJM jet velocity & 4.8 & $\begin{array}{c}-0.3 /+ \\
0.3 \\
\end{array}$ & $\mathrm{~m} / \mathrm{s}$ & 4.9 & $\begin{array}{l}\text { Data Source: PJM Parameters } \\
\text { for Functional Test.doc. }\end{array}$ \\
\hline & PJM stroke & $80 \%$ & $-5 /+5$ & $\%$ & $78 \%$ & $\begin{array}{l}\text { Data Source: PJM Parameters } \\
\text { for Functional Test.doc. }\end{array}$ \\
\hline & PJM cycle time & 35 & $-1 /+1$ & s & 35 & $\begin{array}{l}\text { Data Source: PJM Parameters } \\
\text { for Functional Test.doc. }\end{array}$ \\
\hline & $\begin{array}{l}\text { Steam ring air purge } \\
\text { flow rate }\end{array}$ & 0.13 & $\begin{array}{c}-0.02 /+ \\
0.02 \\
\end{array}$ & $\mathrm{~kg} / \mathrm{min}$ & 0.13 & $\begin{array}{l}\text { Source: pg. J.105 } \\
\sim 3.9 \mathrm{scfm}\end{array}$ \\
\hline \multirow{8}{*}{$\begin{array}{c}\text { Low-solids filter } \\
\text { flux test } \\
\text { (includes filter } \\
\text { conditioning and } \\
\text { backpulsing) } \\
\text { (Test Instruction } \\
\text { steps } 7.2 .7 \text { and } \\
7.2 .8 \text { ) }\end{array}$} & $\begin{array}{c}\text { Ultrafiltration Loop } \\
\text { Operation }\end{array}$ & - & - & - & - & ( \\
\hline & $\begin{array}{l}\text { Number of filter } \\
\text { bundles }\end{array}$ & 5 & $-0 /+0$ & - & 5 & Data Source: TI Y (init.) \\
\hline & Filter-loop flow rate & 109 & $-10 /+10$ & gpm & $\sim 110$ & $\begin{array}{l}\text { Source: pgs. J.99, J.112, J.130, } \\
\text { J.148 }\end{array}$ \\
\hline & $\begin{array}{l}\text { Transmembrane } \\
\text { pressure }\end{array}$ & 40 & $-4 /+4$ & psi & 40 & $\begin{array}{l}\text { Source: pgs. J.102, J.103, } \\
\text { J.104, J.156, J.157, J.158 }\end{array}$ \\
\hline & Temperature & 25 & $-2 /+2$ & ${ }^{\circ} \mathrm{C}$ & $26-27$ & Source: pg. J.96 \\
\hline & $\begin{array}{l}\text { Axial } \Delta \mathrm{P} \text { for one filter } \\
\text { bundle }\end{array}$ & $<25$ & - & psi & $11-17$ & $\begin{array}{l}\text { Source: pgs. J.95, J.113, J.131, } \\
\text { J.149 }\end{array}$ \\
\hline & $\begin{array}{l}\text { Volume of first parallel } \\
\text { CUF sample }\end{array}$ & 4.2 & $\begin{array}{c}-0.1 /+ \\
0.5 \\
\end{array}$ & $\mathrm{~L}$ & 4.2 & Data Source: TI \\
\hline & $\begin{array}{l}\text { Volume of second } \\
\text { parallel CUF sample }\end{array}$ & 14.4 & $\begin{array}{c}-0.1 /+ \\
0.5\end{array}$ & $\mathrm{~L}$ & 4.4 & Data Source: TI \\
\hline
\end{tabular}




\begin{tabular}{|c|c|c|c|c|c|c|}
\hline \multirow{12}{*}{$\begin{array}{c}\text { Concentrate } \\
\text { solids to } 20 \% \text { in } \\
\text { UFP-VSL-T02A } \\
\text { (Test Instruction } \\
\text { step 7.2.10) }\end{array}$} & \multirow{2}{*}{$\begin{array}{l}\text { UFP-VSL-T02A level } \\
\text { (endpoint) by laser } \\
\text { measurement }\end{array}$} & \multirow{2}{*}{8} & \multirow{2}{*}{$-1 /+3$} & \multirow{2}{*}{ in. } & \multirow{2}{*}{22.5} & \multirow[t]{2}{*}{$\begin{array}{l}\text { Data Source: TI/HMI } \\
\text { Y (init.) }\end{array}$} \\
\hline & & & & & & \\
\hline & $\begin{array}{l}\text { UFP-VSL-T02A } \\
\text { Operation }\end{array}$ & - & - & - & - & - \\
\hline & $\begin{array}{l}\text { Level to reduce upper } \\
\text { air sparger flow rate to } \\
\text { idle }\end{array}$ & 52 & $-20 /+0$ & in. & NP & \\
\hline & $\begin{array}{l}\text { Upper air sparger flow } \\
\text { rate (idle) }\end{array}$ & 0.009 & $\begin{array}{l}-0.005 / \\
+0.005\end{array}$ & $\mathrm{~kg} / \mathrm{min}$ & 0.01 & Source: pg. J.159 \\
\hline & $\begin{array}{l}\text { Level to stop PJM } \\
\text { operation (measured } \\
\text { when PJMs are full) }\end{array}$ & 18 & $-0 /+10$ & in & NP & \\
\hline & $\begin{array}{l}\text { Ultrafiltration Loop } \\
\text { Operation }\end{array}$ & - & - & - & - & - \\
\hline & $\begin{array}{l}\text { Number of filter } \\
\text { bundles }\end{array}$ & 1 & $-0 /+0$ & - & 1 & $\begin{array}{l}\text { Data Source: TI } \\
\mathrm{Y} \text { (init.) }\end{array}$ \\
\hline & Filter-loop flow rate & 109 & $-10 /+10$ & gpm & $100-120$ & Source: pg. J.153 \\
\hline & $\begin{array}{l}\text { Transmembrane } \\
\text { pressure }\end{array}$ & 40 & $-4 /+4$ & psi & 40 & Source: pg. J.156 \\
\hline & Temperature & 25 & $-2 /+2$ & ${ }^{\circ} \mathrm{C}$ & $25-28$ & Source: pg. J.150 \\
\hline & $\begin{array}{l}\text { Axial } \Delta \mathrm{P} \text { for one filter } \\
\text { bundle }\end{array}$ & $<25$ & - & psi & - & Y (init.) \\
\hline $\begin{array}{c}\text { Flush filter-loop } \\
\text { (Test Instruction } \\
\text { step 7.1.11) }\end{array}$ & $\begin{array}{l}\text { Mass of water for } \\
\text { filter-loop flush to } \\
\text { UFP-VSL-T02A } \\
\text { (endpoint) }\end{array}$ & 163 & $-5 /+5$ & $\mathrm{~kg}$ & 201 & $\begin{array}{l}\text { Data Source: TI-HMI } \\
\sim 43 \text { gal. } \\
\text { Bypass filters } 2 \text { through } 5 .\end{array}$ \\
\hline & $\begin{array}{l}\text { Flush water addition } \\
\text { rate }\end{array}$ & $\begin{array}{l}\text { maximum } \\
\text { achievable } \\
\text { flow }\end{array}$ & - & $\mathrm{kg} / \mathrm{min}$ & 94 & $\begin{array}{l}\text { The pump capacity is } \sim 35 \mathrm{gpm} \\
\text { or } \sim 132-\mathrm{kg} / \mathrm{min} \text {. Higher levels } \\
\text { in SHR-VSL-T03 improve } \\
\text { pump suction head and increase } \\
\text { flow rate. }\end{array}$ \\
\hline & $\begin{array}{l}\text { Mass of water for } \\
\text { filter-loop flush to } \\
\text { UFP-VSL-T62A } \\
\text { (endpoint) } \\
\end{array}$ & 200 & $-5 /+5$ & $\mathrm{~kg}$ & 201 & Source: TI pg. 71 \\
\hline & $\begin{array}{c}\text { UFP-VSL-T02A } \\
\text { Operation }\end{array}$ & - & - & - & - & - \\
\hline & $\begin{array}{l}\text { Level to initiate PJM } \\
\text { operation }\end{array}$ & 13 & $-0 /+10$ & in. & NP & \\
\hline & PJM jet velocity & 7.3 & $\begin{array}{l}-0.4 / \\
+0.4 \\
\end{array}$ & $\mathrm{~m} / \mathrm{s}$ & 5.9 & $\begin{array}{l}\text { Data Source: PJM Parameters } \\
\text { for Functional Test.doc. }\end{array}$ \\
\hline & PJM stroke & $80 \%$ & $-5 /+5$ & $\%$ & 82 & $\begin{array}{l}\text { Data Source: PJM Parameters } \\
\text { for Functional Test.doc. }\end{array}$ \\
\hline & PJM cycle time & 33 & $-1 /+1$ & $\mathrm{~s}$ & 33.2 & $\begin{array}{l}\text { Data Source: PJM Parameters } \\
\text { for Functional Test.doc. }\end{array}$ \\
\hline $\begin{array}{l}\text { Transfer from } \\
\text { UFP-VSL-T01A }\end{array}$ & $\begin{array}{l}\text { UFP-VSL-T02A level } \\
\text { (endpoint) }\end{array}$ & 29 & $-1 /+1$ & in. & NP & \\
\hline & Transfer rate & 6.9 & NA & gpm & NP & 〜97-gal transfer \\
\hline $\begin{array}{l}\text { UFP-VSL-T02A } \\
\text { (Test Instruction }\end{array}$ & $\begin{array}{c}\text { Ultrafiltration Loop } \\
\text { Operation }\end{array}$ & - & - & - & - & - \\
\hline step 7.2.12) & Number of filter & 4 & $-0 /+0$ & - & 4 & Data Source: TI \\
\hline
\end{tabular}




\begin{tabular}{|c|c|c|c|c|c|c|}
\hline & $\begin{array}{l}\text { bundles (Filters } 2 \\
\text { through } 5 \text { should be } \\
\text { aligned at this point) }\end{array}$ & & & & & Y (init.) \\
\hline \multirow{12}{*}{$\begin{array}{c}\text { Concentrate } \\
\text { solids at } \\
\text { Prototypic Level } \\
\text { for UFP-VSL- } \\
\text { T02A Leaching } \\
\text { (Test Instruction } \\
\text { step 7.1.12) }\end{array}$} & $\begin{array}{l}\text { Make-up batch transfer } \\
\text { volume }\end{array}$ & 11 & $\begin{array}{l}-0.2 / \\
+0.2\end{array}$ & gal & 11 & $\begin{array}{l}\text { Data Source: TI } \\
\text { Total permeate removed after } \\
13 \text { batches is } \sim 143 \text { gal } \\
(\sim 660-\mathrm{kg})\end{array}$ \\
\hline & $\begin{array}{l}\text { Make-up batch } \\
\text { flowrate }\end{array}$ & 6.9 & NA & gpm & NP & \\
\hline & $\begin{array}{l}\text { Number of make-up } \\
\text { batches (endpoint) }\end{array}$ & 22 & $-0 /+0$ & - & 22 & $\begin{array}{l}\text { Data Source: TI } \\
\text { Y (init.) }\end{array}$ \\
\hline & $\begin{array}{c}\text { UFP-VSL-T02A } \\
\text { Operation }\end{array}$ & - & - & - & - & - \\
\hline & $\begin{array}{l}\text { Level to initiate each } \\
\text { make-up batch }\end{array}$ & 27 & $-1 /+1$ & in. & NP & \\
\hline & $\begin{array}{l}\text { Total lower air sparger } \\
\text { flowrate with } \\
\text { recirculation } \\
\text { (idle, for } 5 \text { sparge } \\
\text { tubes) }\end{array}$ & 0.037 & $\begin{array}{l}-0.005 / \\
+0.005\end{array}$ & $\mathrm{~kg} / \mathrm{min}$ & .037 & $\begin{array}{l}\text { Source: TI pg. } 75 \text { (field } \\
\text { transmitter) } \\
\sim 12.6 \text { scfh for each sparge tube } \\
\text { (record field transmitter value). }\end{array}$ \\
\hline & $\begin{array}{c}\text { Ultrafiltration Loop } \\
\text { Operation }\end{array}$ & - & - & - & - & 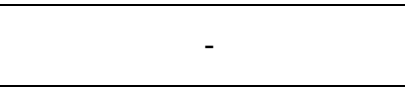 \\
\hline & $\begin{array}{l}\text { Number of filter } \\
\text { bundles }\end{array}$ & 4 & $-0 /+0$ & & & $\begin{array}{l}\text { Data source: TI } \\
\text { Y (init.) }\end{array}$ \\
\hline & Filter-loop flow rate & 109 & $-10 /+10$ & gpm & $110-118$ & Source: pg. J.189 \\
\hline & $\begin{array}{l}\text { Transmembrane } \\
\text { pressure }\end{array}$ & 40 & $-4 /+4$ & psi & 40 & Source: pg. J.192, J.193, J.194 \\
\hline & Temperature & 25 & $-2 /+2$ & ${ }^{\circ} \mathrm{C}$ & $23-25$ & Source: pg. J.185 \\
\hline & $\begin{array}{l}\text { Axial } \Delta \mathrm{P} \text { for one filter } \\
\text { bundle }\end{array}$ & $<25$ & - & psi & $12-19$ & Source: pg. J.185 \\
\hline \multirow{9}{*}{$\begin{array}{l}\text { Add caustic to } \\
\text { UFP-VSL-T02A } \\
\text { (Test Instruction } \\
\text { step 7.3.1.5) }\end{array}$} & $\begin{array}{l}\text { 19-M caustic to add } \\
\text { (endpoint) }\end{array}$ & 531 & $-5 /+5$ & $\mathrm{~kg}$ & 531 & Source: TI pg. 81 \\
\hline & Caustic addition rate & 10.3 & $\begin{array}{l}-0.5 / \\
+0.5\end{array}$ & $\mathrm{~kg} / \mathrm{min}$ & 10.3 & $\begin{array}{l}\text { Data Source: TI-HMI } \\
\sim 1.8 \text { gpm, over a } 49-\text { min } \\
\text { transfer. Range applies to } \\
\text { average transfer rate. }\end{array}$ \\
\hline & \multirow{2}{*}{$\begin{array}{l}\text { Tank level after caustic } \\
\text { addition }\end{array}$} & \multirow{2}{*}{34} & \multirow{2}{*}{$-2 /+2$} & \multirow{2}{*}{ in. } & \multirow{2}{*}{ NP } & Y (init.) \\
\hline & & & & & & $\sim 151$ gal in UFP-VSL-T02A \\
\hline & $\begin{array}{l}\text { Volume of antifoam to } \\
\text { add to } \\
\text { UFP-VSL-T02A }\end{array}$ & 270 & $-10 /+10$ & $\mathrm{~mL}$ & 270 & $\begin{array}{l}\text { Data Source: TI } \\
\text { Antifoam is added to account } \\
\text { for caustic and steam addition. } \\
\text { This value is concentrate. } \\
\text { Dilute with } 3 \text { parts IW to } 1 \text { part } \\
\text { concentrate. }\end{array}$ \\
\hline & $\begin{array}{c}\text { Ultrafiltration Loop } \\
\text { Operation }\end{array}$ & - & - & - & - & - \\
\hline & Filter-loop flow rate & 109 & $-10 /+10$ & gpm & $105-110$ & Source: pg. J.202 \\
\hline & Permeate valves & CLOSED & - & - & CLOSED & $\begin{array}{l}\text { Data Source: TI } \\
\text { Y (init.) }\end{array}$ \\
\hline & Temperature & $\leq 71$ & - & ${ }^{\circ} \mathrm{C}$ & $35-73$ & Source: pg. J.202 \\
\hline Initial heat-up of & Target temperature & 71 & $-2 /+2$ & ${ }^{\circ} \mathrm{C}$ & 72 & Data Source: TI-HMI \\
\hline
\end{tabular}




\begin{tabular}{|c|c|c|c|c|c|c|}
\hline \multirow{7}{*}{$\begin{array}{l}\text { UFP-VSL-T02A } \\
\text { (Non-prototypic } \\
\text { using external } \\
\text { exchanger) } \\
\text { (Test Instruction } \\
\text { step 7.2.1) }\end{array}$} & & & & & & Y (init.) \\
\hline & $\begin{array}{c}U F P-V S L-T 02 A \\
\text { Operation } \\
\end{array}$ & - & - & - & - & - \\
\hline & $\begin{array}{l}\text { Temperature to turn off } \\
\text { PJM suction refill }\end{array}$ & 60 & $-2 /+2$ & ${ }^{\circ} \mathrm{C}$ & 60 & Data Source: TI \\
\hline & $\begin{array}{l}\text { Ultrafiltration Loop } \\
\text { Operation }\end{array}$ & - & - & - & - & - \\
\hline & Filter-loop flow rate & 109 & $-10 /+10$ & gpm & $105-110$ & Source: pg. J.202 \\
\hline & Permeate valves & CLOSED & - & - & CLOSED & $\begin{array}{l}\text { Data Source: TI } \\
\text { Y (init.) }\end{array}$ \\
\hline & Temperature & $<60$ & - & ${ }^{\circ} \mathrm{C}$ & $35-73$ & $\begin{array}{l}\text { Source: pg. J.201 } \\
\text { Conflicts with target temp. } \\
\text { parameter above! }\end{array}$ \\
\hline \multirow{8}{*}{$\begin{array}{l}\text { Flush filter-loop } \\
\text { (Test Instruction } \\
\text { step 7.3.1.9.3) }\end{array}$} & $\begin{array}{l}\text { Filter-loop } \\
\text { recirculation rate }\end{array}$ & OFF & - & - & OFF & Data Source: TI \\
\hline & $\begin{array}{l}\text { Mass of water for } \\
\text { filter-loop flush to } \\
\text { UFP-VSL-T02A } \\
\text { (endpoint) }\end{array}$ & 201 & $-5 /+5$ & $\mathrm{~kg}$ & 200 & Data Source: TI \\
\hline & $\begin{array}{l}\text { Flush water addition } \\
\text { rate }\end{array}$ & $\begin{array}{l}\operatorname{maximum} \\
\text { achievable } \\
\text { flow }\end{array}$ & - & gpm & 22 & $\begin{array}{l}\text { Data Source: TI } \\
\text { The pump capacity is } \sim 35 \mathrm{gpm} \\
\text { or } \sim 132-\mathrm{kg} / \mathrm{min} \text {. Higher levels } \\
\text { in SHR-VSL-T03 improve } \\
\text { pump suction head and increase } \\
\text { flow rate. }\end{array}$ \\
\hline & $\begin{array}{l}\text { Mass of water for } \\
\text { filter-loop flush to } \\
\text { UFP-VSL-T62A } \\
\text { (endpoint) }\end{array}$ & 401 & $-10 /+10$ & $\mathrm{~kg}$ & NP & $\begin{array}{l}\text { UFP-VSL-T02A should be } \\
\text { isolated. }\end{array}$ \\
\hline & $\begin{array}{l}\text { Tank level after loop } \\
\text { flush }\end{array}$ & 46 & $-2 /+2$ & in. & 5 & $\begin{array}{l}\text { Data Source: TI } \sim 204 \text { gal in } \\
\text { UFP-VSL-T02A }\end{array}$ \\
\hline & $\begin{array}{c}\text { UFP-VSL-T02A } \\
\text { Operation }\end{array}$ & - & - & - & - & - \\
\hline & $\begin{array}{l}\text { Upper air sparger flow } \\
\text { rate }\end{array}$ & 0.10 & $\begin{array}{l}-0.02 / \\
+0.02\end{array}$ & $\mathrm{~kg} / \mathrm{min}$ & 0 & $\begin{array}{l}\text { Source: pg. J.213 } \\
\sim 3.0 \mathrm{scfm}\end{array}$ \\
\hline & $\begin{array}{l}\text { Total lower air sparger } \\
\text { flow rate (for } 5 \text { sparge } \\
\text { tubes) }\end{array}$ & 0.40 & $\begin{array}{l}-0.05 / \\
+0.05\end{array}$ & $\mathrm{~kg} / \mathrm{min}$ & $\mathrm{n} / \mathrm{r}$ & $\begin{array}{l}\text { NCR } 41090.1 \\
\sim 2.4 \text { scfm for each sparge tube. }\end{array}$ \\
\hline
\end{tabular}


Table M.2 TI-067 Operational Process Sheet

\begin{tabular}{|c|c|c|c|c|c|c|}
\hline \multicolumn{7}{|c|}{ Parameter Data Sheet TI-067 } \\
\hline & Operation & $\begin{array}{l}\text { Target } \\
\text { or } \\
\text { Setpoint } \\
\text { Value }\end{array}$ & $\begin{array}{c}\text { Range } \\
(-/+)\end{array}$ & Units & $\begin{array}{l}\text { Actual } \\
\text { Value }\end{array}$ & Comments \\
\hline \multirow{3}{*}{$\begin{array}{c}\text { Add caustic to } \\
\text { UFP-VSL- } \\
\text { T01A } \\
\text { (Test } \\
\text { Instruction step } \\
\text { 74.1.9) } \\
\end{array}$} & \multirow{2}{*}{$\begin{array}{l}\text { Volume of 19-M caustic to } \\
\text { add (endpoint) }\end{array}$} & \multirow{2}{*}{561} & \multirow{2}{*}{$-5 /+5$} & \multirow{2}{*}{$\mathrm{kg}$} & \multirow{2}{*}{561} & Source: TI pg. 105 \\
\hline & & & & & & $\sim 92$ gal \\
\hline & $\begin{array}{l}\text { Tank level after caustic } \\
\text { addition }\end{array}$ & 55 & $-1 /+1$ & in. & 58.6 & $\begin{array}{l}\text { Data source: TI-HMI } \\
\text { UFP-VSL-T01A should have } \\
\sim 481 \text { gal, including the heel. }\end{array}$ \\
\hline \multirow{2}{*}{$\begin{array}{c}\text { Initial heat-up } \\
\text { of } \\
\text { UFP-VSL- } \\
\text { T01A } \\
\text { (Nonprototypic } \\
\text { using external } \\
\text { exchanger) }\end{array}$} & $\begin{array}{l}\text { Target final temperature } \\
\text { (endpoint) }\end{array}$ & 63 & $-2 /+2$ & ${ }^{\circ} \mathrm{C}$ & 63 & TI \\
\hline & Heat-up time & NA & NA & $\mathrm{hr}$ & NA & $\begin{array}{l}\text { Heating loop is } \sim 9 \text { gal and will } \\
\text { be lost (unprototypic). Target } \\
\text { and range is not important. } \\
\text { Should be complete in under } \\
3 \mathrm{hr} \text {. }\end{array}$ \\
\hline \multirow{6}{*}{$\begin{array}{c}\text { Final heat-up } \\
\text { UFP-VSL- } \\
\text { T01A } \\
\text { (Test } \\
\text { Instruction step } \\
7.4 .2 .5 \text { ) }\end{array}$} & $\begin{array}{l}\text { Target final temperature } \\
\text { (endpoint) }\end{array}$ & 98 & $-2 /+2$ & ${ }^{\circ} \mathrm{C}$ & 98 & TI-HMI \\
\hline & Heat-up time & 3.0 & $\begin{array}{l}-0.5 / \\
+0.5\end{array}$ & $\mathrm{hr}$ & 3.1 & TI \\
\hline & UFP-VSL-T01A Operation & - & - & - & - & - \\
\hline & $\begin{array}{l}\text { Temperature to turn off } \\
\text { PJM suction refill }\end{array}$ & 60 & $-2 /+2$ & ${ }^{\circ} \mathrm{C}$ & $>60$ & $\begin{array}{l}\text { Temp not recorded. Time } \\
\text { recorded @ 13:44 12/09/2008 }\end{array}$ \\
\hline & \multirow[b]{2}{*}{ Level at end of heat-up } & \multirow[b]{2}{*}{62} & \multirow[b]{2}{*}{$-2 /+2$} & \multirow[b]{2}{*}{ in. } & \multirow[b]{2}{*}{67.0} & TI-HMI \\
\hline & & & & & & $\begin{array}{l}\text { UFP-VSL-T01A should have } \\
\sim 540 \text { gal, including the heel. }\end{array}$ \\
\hline \multirow{9}{*}{$\begin{array}{l}\text { Caustic leach in } \\
\text { UFP-VSL- } \\
\text { T01A } \\
\text { (Test } \\
\text { Instruction step } \\
7.4 .2 .7 \text { ) }\end{array}$} & $\begin{array}{l}\text { Caustic-leach duration } \\
\text { (endpoint) }\end{array}$ & 16 & $\begin{array}{c}-0.1 / \\
+1\end{array}$ & $\mathrm{hr}$ & 16.7 & $\begin{array}{l}\text { TI } \\
\text { May be extended to } \\
\text { accommodate sampling. }\end{array}$ \\
\hline & Temperature & 98 & $-2 /+2$ & ${ }^{\circ} \mathrm{C}$ & $95-98$ & Source: pgs. J.235, J.253 \\
\hline & UFP-VSL-T01A Operation & - & - & - & - & - \\
\hline & PJM jet velocity & 4.8 & $\begin{array}{l}-0.3 / \\
+0.3\end{array}$ & $\mathrm{~m} / \mathrm{s}$ & NP & \\
\hline & PJM stroke & $80 \%$ & $-5 /+5$ & $\%$ & NP & \\
\hline & PJM cycle time & 35 & $-1 /+1$ & $\mathrm{~s}$ & NP & \\
\hline & $\begin{array}{l}\text { Steam ring air purge flow } \\
\text { rate (automatic) }\end{array}$ & 0.13 & $\begin{array}{l}-0.02 / \\
+0.02\end{array}$ & $\mathrm{~kg} / \mathrm{min}$ & $\begin{array}{l}0.13 \\
\text { (when } \\
\text { on) }\end{array}$ & $\begin{array}{l}\sim 3.9 \text { scfm, purge will turn off } \\
\text { when steam exceeds } 6 \mathrm{cfm} \text { and } \\
\text { will turn back on when steam } \\
\text { flow drops below } 4 \mathrm{cfm} \text {. Source: } \\
\text { pg. J.267 }\end{array}$ \\
\hline & \multirow{2}{*}{ Level at end of leach } & \multirow{2}{*}{67} & \multirow{2}{*}{$-5 /+5$} & \multirow{2}{*}{ in. } & \multirow{2}{*}{64.2} & TI-bubbler HMI \\
\hline & & & & & & $\sim 593$ gal \\
\hline \multirow{4}{*}{$\begin{array}{c}\text { Cool-down of } \\
\text { UFP-VSL- } \\
\text { T01A } \\
\text { (Test } \\
\text { Instructions } \\
\text { step 7.4.2.9) }\end{array}$} & $\begin{array}{l}\text { Initial cool-down } \\
\text { temperature (endpoint) } \\
\text { using external chiller } \\
\text { UFP-HX-T05A }\end{array}$ & 60 & $-2 /+2$ & ${ }^{\circ} \mathrm{C}$ & 61 & TI \\
\hline & Initial cool-down duration & 4 & $\begin{array}{l}-0.5 / \\
+0.5 \\
\end{array}$ & $\mathrm{hr}$ & 3.8 & TI \\
\hline & UFP-VSL-T01A Operation & - & - & - & - & - \\
\hline & $\begin{array}{l}\text { Temperature to resume } \\
\text { PJM suction refill }\end{array}$ & 60 & $-2 /+2$ & ${ }^{\circ} \mathrm{C}$ & $<60$ & $\begin{array}{l}\text { Temp not recorded. Time } \\
\text { recorded @ 13:35 12/10/08 }\end{array}$ \\
\hline
\end{tabular}


Table M.3 TI-032 Operational Process Sheet

\begin{tabular}{|c|c|c|c|c|c|c|}
\hline Step & Operation & $\begin{array}{c}\text { Target or } \\
\text { Setpoint } \\
\text { Value }\end{array}$ & $\begin{array}{c}\text { Range } \\
(-/+)\end{array}$ & Units & $\begin{array}{l}\text { Actual } \\
\text { Value }\end{array}$ & Comments \\
\hline \multirow{2}{*}{$\begin{array}{c}\text { Simulant storage } \\
\text { in } \\
\text { HLP-VSL-T22 } \\
\text { (Test Instruction } \\
\text { step 7.1.1) }\end{array}$} & HLP-VSL-T22 Operation & - & - & - & - & - \\
\hline & Agitator speed & 68 & $\begin{array}{l}-10 / \\
+10\end{array}$ & Rpm & NP & $\begin{array}{l}\text { Not performed under Test } \\
\text { Instruction }\end{array}$ \\
\hline \multirow{9}{*}{$\begin{array}{c}\text { Transfer from } \\
\text { HLP-VSL-T22 } \\
\text { to } \\
\text { UFP-VSL-T01A } \\
\text { and PJM tuning } \\
\text { (Test Instruction } \\
\text { step 7.2.1) }\end{array}$} & $\begin{array}{l}\text { Total transfer volume } \\
\text { (endpoint) }\end{array}$ & 593 & $-5 /+5$ & Gal & NP & $\begin{array}{l}\text { Not performed under Test } \\
\text { Instruction } \\
\text { Assumes no previous heel in } \\
\text { UFP-VSL-T01A. Level } \\
\text { should be } \sim 66.5 \text { in. }\end{array}$ \\
\hline & Transfer rate & 15 & $-2 /+2$ & gpm & NP & $\begin{array}{l}\text { Not performed under Test } \\
\text { Instruction }\end{array}$ \\
\hline & UFP-VSL-T01A Operation & - & - & - & - & - \\
\hline & $\begin{array}{l}\text { Level to initiate PJM } \\
\text { operation }\end{array}$ & 66.5 & $-2 /+10$ & in. & NP & $\begin{array}{l}\text { Not performed under Test } \\
\text { Instruction }\end{array}$ \\
\hline & PJM jet velocity & 4.8 & $\begin{array}{l}-0.3 / \\
+0.3\end{array}$ & $\mathrm{~m} / \mathrm{s}$ & NP & Data Source: CCP \\
\hline & PJM stroke & $80 \%$ & $-5 /+5$ & $\%$ & NP & Data Source: CCP \\
\hline & PJM cycle time & 35 & $-1 /+1$ & $\mathrm{~s}$ & NP & Data Source: CCP \\
\hline & $\begin{array}{l}\text { Level to adjust steam ring } \\
\text { air purge rotameter }\end{array}$ & 10 & $-0 /+10$ & in. & NP & $\begin{array}{l}\text { Not performed under Test } \\
\text { Instruction }\end{array}$ \\
\hline & $\begin{array}{l}\text { Steam ring air purge flow } \\
\text { rate }\end{array}$ & 0.13 & $\begin{array}{l}-0.02 / \\
+0.02\end{array}$ & $\mathrm{~kg} / \mathrm{min}$ & NP & $\begin{array}{l}\text { Not performed under Test } \\
\text { Instruction } \sim 3.9 \mathrm{scfm}\end{array}$ \\
\hline \multirow[t]{2}{*}{$\begin{array}{c}\text { Transfer excess } \\
\text { simulant from } \\
\text { UFP-VSL-T01A } \\
\text { to } \\
\text { HLP-VSL-T22 } \\
\text { (Test Instruction } \\
\text { step 7.2.1.9) }\end{array}$} & $\begin{array}{l}\text { Target level in } \\
\text { UFP-VSL-T01A } \\
\text { (endpoint) }\end{array}$ & 45 & $\begin{array}{l}-0.5 / \\
+0.5\end{array}$ & in. & NP & $\begin{array}{l}\text { Not performed under Test } \\
\text { Instruction. } \\
\text { Volume in UFP-VSL-T01A } \\
\text { should be } ~ 391 \text { gal. } \\
\text { PJMs may be turned off as } \\
\text { target level is approach to } \\
\text { improve level measurement. }\end{array}$ \\
\hline & Transfer rate & $\begin{array}{c}\text { No } \\
\text { setpoint }\end{array}$ & - & - & NP & $\begin{array}{l}\text { Not performed under Test } \\
\text { Instruction }\end{array}$ \\
\hline \multirow{5}{*}{$\begin{array}{c}\text { Transfer from } \\
\text { HLP-VSL-T22 } \\
\text { to } \\
\text { UFP-VSL-T01B } \\
\text { and PJM tuning } \\
\text { (Test Instruction } \\
\text { step 7.2.2) }\end{array}$} & $\begin{array}{l}\text { Total transfer volume } \\
\text { (endpoint) }\end{array}$ & 593 & $-5 /+5$ & gal & 586 & $\begin{array}{l}\text { Data Source: HMI } \\
\text { Assumes no previous heel in } \\
\text { UFP-VSL-T01A. Level } \\
\text { should be } \sim 65 \text { in. }\end{array}$ \\
\hline & Transfer rate & 15 & $-2 /+2$ & g & 15.3 & Data Source: HMI \\
\hline & UFP-VSL-T01B Operation & - & - & - & - & - \\
\hline & $\begin{array}{l}\text { Volume of antifoam to add } \\
\text { to UFP-VSL-T01B }\end{array}$ & 1,000 & $\begin{array}{l}-20 / \\
+20\end{array}$ & $\mathrm{~mL}$ & 1000 & $\begin{array}{l}\text { Data Source: TI } \\
\text { This value is concentrate. } \\
\text { Dilute with } 3 \text { parts IW to } 1 \\
\text { part concentrate. }\end{array}$ \\
\hline & $\begin{array}{l}\text { Level to initiate PJM } \\
\text { operation }\end{array}$ & 65 & $-2 /+10$ & in. & 65 & Data Source: HMI \\
\hline
\end{tabular}




\begin{tabular}{|c|c|c|c|c|c|c|}
\hline Step & Operation & $\begin{array}{l}\text { Target or } \\
\text { Setpoint } \\
\text { Value }\end{array}$ & $\begin{array}{c}\text { Range } \\
(-/+)\end{array}$ & Units & $\begin{array}{l}\text { Actual } \\
\text { Value }\end{array}$ & Comments \\
\hline & PJM jet velocity & 4.8 & $\begin{array}{l}-0.3 / \\
+0.3\end{array}$ & $\mathrm{~m} / \mathrm{s}$ & 4.8 & Data Source: CCP \\
\hline & PJM stroke & $80 \%$ & $-5 /+5$ & $\%$ & 92 & Data Source: CCP \\
\hline & PJM cycle time & 35 & $-1 /+1$ & $\mathrm{~s}$ & 35.1 & Data Source: CCP \\
\hline & $\begin{array}{l}\text { Level to adjust steam ring } \\
\text { air purge rotameter }\end{array}$ & 10 & $-0 /+10$ & in. & 18 & Data Source: HMI \\
\hline & $\begin{array}{l}\text { Steam ring air purge flow } \\
\text { rate }\end{array}$ & 0.13 & $\begin{array}{l}-0.02 / \\
+0.02\end{array}$ & $\mathrm{~kg} / \mathrm{min}$ & 0.13 & $\begin{array}{l}\text { Data Source: Local flow } \\
\text { meter } \\
\sim 3.9 \mathrm{scfm}\end{array}$ \\
\hline \multirow{13}{*}{$\begin{array}{c}\begin{array}{c}\text { Initial transfer } \\
\text { from }\end{array} \\
\text { UFP-VSL-T01B } \\
\text { to } \\
\text { UFP-VSL-T02A } \\
\text { (Test Instruction } \\
\text { step 7.2.3) }\end{array}$} & $\begin{array}{l}\text { Transfer volume } \\
\text { (endpoint) }\end{array}$ & 283 & $-3 /+3$ & gal & 283 & $\begin{array}{l}\text { Data Source: HMI } \\
\text { UFP-VSL-T02A should be } \\
\sim 62.5 \text { in. }\end{array}$ \\
\hline & Transfer rate & 6.9 & NA & gpm & 7.1 & Data Source: HMI \\
\hline & UFP-VSL-T02A Operation & - & - & - & - & - \\
\hline & $\begin{array}{l}\text { Level to initiate PJM } \\
\text { operation }\end{array}$ & 72 & $-2 /+10$ & in. & 72 & Data Source: HMI \\
\hline & PJM jet velocity & 7.3 & $\begin{array}{l}-0.4 / \\
+0.4\end{array}$ & $\mathrm{~m} / \mathrm{s}$ & $\mathrm{n} / \mathrm{r}$ & \\
\hline & PJM stroke & $80 \%$ & $-5 /+5$ & $\%$ & $\mathrm{n} / \mathrm{r}$ & \\
\hline & PJM cycle time & 33 & $-1 /+1$ & $\mathrm{~s}$ & $\mathrm{n} / \mathrm{r}$ & \\
\hline & $\begin{array}{l}\text { Level to adjust steam ring } \\
\text { air purge rotameter }\end{array}$ & 5 & $-0 /+10$ & in. & 72 & Data Source: HMI \\
\hline & $\begin{array}{l}\text { Steam ring air purge flow } \\
\text { rate }\end{array}$ & 0.10 & $\begin{array}{l}-0.02 / \\
+0.02\end{array}$ & $\mathrm{~kg} / \mathrm{min}$ & $\mathrm{n} / \mathrm{r}$ & $\begin{array}{l}\text { Data Source: HMI } \\
\sim 3.1 \mathrm{scfm}\end{array}$ \\
\hline & $\begin{array}{l}\text { Adjust lower air spargers } \\
\text { to full flow at end of } \\
\text { transfer }\end{array}$ & 72 & $-10 /+0$ & in. & 72 & Data Source: HMI \\
\hline & $\begin{array}{l}\text { Total lower air sparger } \\
\text { flowrate (for } 5 \text { sparge } \\
\text { tubes) }\end{array}$ & 0.40 & $\begin{array}{l}-0.05 / \\
+0.05\end{array}$ & $\mathrm{~kg} / \mathrm{min}$ & 0.4 & $\begin{array}{l}\text { Data Source: Local flow } \\
\text { meter } \\
\sim 2.4 \text { scfm for each sparge } \\
\text { tube }\end{array}$ \\
\hline & $\begin{array}{l}\text { Adjust upper air spargers } \\
\text { to full flow at the end of } \\
\text { transfer }\end{array}$ & 72 & $-10 /+0$ & in. & 72 & Data Source: HMI \\
\hline & Upper air sparger flow rate & 0.10 & $\begin{array}{l}-0.02 / \\
+0.02\end{array}$ & $\mathrm{~kg} / \mathrm{min}$ & 0.1 & $\begin{array}{l}\text { Data Source: Local flow } \\
\text { meter } \sim 3.0 \mathrm{scfm}\end{array}$ \\
\hline \multirow{5}{*}{$\begin{array}{c}\text { Test for } \\
\text { stratification in } \\
\text { UFP-VSL-T01A } \\
\text { (Test Instruction } \\
\text { step 7.2.4) }\end{array}$} & UFP-VSL-T01A Operation & - & - & - & - & - \\
\hline & PJM jet velocity & 4.8 & $\begin{array}{l}-0.3 / \\
+0.3 \\
\end{array}$ & $\mathrm{~m} / \mathrm{s}$ & 4.8 & Data Source: TI (p. 46) \\
\hline & PJM stroke & $80 \%$ & $-5 /+5$ & $\%$ & 78 & Data Source: TI (p. 46) \\
\hline & PJM cycle time & 35 & $-1 /+1$ & $\mathrm{~s}$ & 35 & Data Source: TI (p. 46) \\
\hline & $\begin{array}{l}\text { Steam ring air purge flow } \\
\text { rate }\end{array}$ & 0.13 & $\begin{array}{l}-0.02 / \\
+0.02\end{array}$ & $\mathrm{~kg} / \mathrm{min}$ & 0.13 & $\begin{array}{l}\text { Data Source: Local flow } \\
\text { meter } \\
\sim 3.9 \mathrm{scfm}\end{array}$ \\
\hline \multirow{3}{*}{$\begin{array}{l}\text { PJM tuning in } \\
\text { UFP-VSL-T02A } \\
\text { (Test Instruction } \\
\text { step 7.2.5) }\end{array}$} & UFP-VSL-T02A Operation & - & - & - & - & - \\
\hline & $\begin{array}{l}\text { PJM supply pressure } \\
\text { (jet velocity }=7.3-\mathrm{m} / \mathrm{s} \text { ) }\end{array}$ & 5 & $-2 /+2$ & psig & 8 & $\begin{array}{l}\text { Data Source: TI } \\
\text { (nozzle velocity }=7.2-\mathrm{m} / \mathrm{s} \\
\text { and stroke length }=76 \% \text { per } \\
\text { CCP) }\end{array}$ \\
\hline & PJM stroke & 8 & & $\mathrm{~s}$ & 5.75 & Data Source: HMI \\
\hline
\end{tabular}




\begin{tabular}{|c|c|c|c|c|c|c|}
\hline Step & Operation & $\begin{array}{l}\text { Target or } \\
\text { Setpoint } \\
\text { Value }\end{array}$ & $\begin{array}{c}\text { Range } \\
(-/+)\end{array}$ & Units & $\begin{array}{l}\text { Actual } \\
\text { Value }\end{array}$ & Comments \\
\hline & PJM cycle time & 33 & $-1 /+1$ & $\mathrm{~s}$ & 33.2 & Data Source: CCP \\
\hline & $\begin{array}{l}\text { Steam ring air purge flow } \\
\text { rate }\end{array}$ & 0.10 & & $\mathrm{~kg} / \mathrm{min}$ & 0.1 & $\begin{array}{l}\text { Data Source: Local flow } \\
\text { meter } \\
\sim 3.1 \mathrm{scfm}\end{array}$ \\
\hline & Upper air sparger flow rate & Off & & $\mathrm{kg} / \mathrm{min}$ & Off & $\begin{array}{l}\text { Data Source: TI } \\
\sim 3.0 \mathrm{scfm}\end{array}$ \\
\hline & $\begin{array}{l}\text { Total lower air sparger } \\
\text { flow rate (for } 5 \text { sparge } \\
\text { tubes) }\end{array}$ & Off & & $\mathrm{kg} / \mathrm{min}$ & off & $\begin{array}{l}\text { Data Source: TI } \\
\sim 2.4 \text { scfm for each sparge } \\
\text { tube }\end{array}$ \\
\hline \multirow{9}{*}{$\begin{array}{c}\text { Low-solids filter } \\
\text { flux test } \\
\text { (Includes filter } \\
\text { conditioning and } \\
\text { backpulsing) } \\
\text { (Test Instruction } \\
\text { steps 7.2.6 } \\
\text { through 7.2.8) }\end{array}$} & $\begin{array}{l}\text { Target level in } \\
\text { UFP-VSL-T02A for refill } \\
\text { from UFP-VSL-T01A } \\
\text { (endpoint) }\end{array}$ & 62.5 & $-1 /+1$ & in. & 62.4 & $\begin{array}{l}\text { Data Source: HMI } \\
\text { Record volume transferred } \\
\text { for use in Test Instruction } \\
\text { step 7.2.6. }\end{array}$ \\
\hline & $\begin{array}{c}\text { Ultrafiltration Loop } \\
\text { Operation }\end{array}$ & - & - & - & - & s \\
\hline & Number of filter bundles & 5 & $-0 /+0$ & - & 5 & Data Source: TI \\
\hline & Filter-loop flow rate & 109 & $\begin{array}{l}-10 / \\
+10\end{array}$ & gpm & $105-115$ & Data Source: HMI \\
\hline & Transmembrane pressure & 40 & $-4 /+4$ & psi & 40 & $\begin{array}{l}\text { Data Source: HMI } \\
\text { Lower range not applicable } \\
\text { if back pressure valve is } \\
100 \% \text { open. }\end{array}$ \\
\hline & Temperature & 25 & $-2 /+2$ & ${ }^{\circ} \mathrm{C}$ & 28 & Data Source: HMI \\
\hline & $\begin{array}{l}\text { Axial } \Delta \mathrm{P} \text { for one filter } \\
\text { bundle }\end{array}$ & $<25$ & - & psi & $<25$ & Data Source: HMI \\
\hline & $\begin{array}{l}\text { Volume of first parallel } \\
\text { CUF sample }\end{array}$ & 4.7 & $\begin{array}{l}-0.1 / \\
+0.5\end{array}$ & $\mathrm{~L}$ & 4.7 & Data Source: TI (p.57) \\
\hline & $\begin{array}{l}\text { Volume of second parallel } \\
\text { CUF sample }\end{array}$ & 16.0 & $\begin{array}{l}-0.1 / \\
+0.5\end{array}$ & $\mathrm{~L}$ & 15 & Data Source: TI (p.60) \\
\hline \multirow{8}{*}{$\begin{array}{c}\text { Concentrate } \\
\text { solids to } 20-w t \% \\
\text { in } \\
\text { UFP-VSL-T02A } \\
\text { (Test Instruction } \\
\text { step 7.2.9) }\end{array}$} & $\begin{array}{l}\text { UFP-VSL-T02A level } \\
\text { (endpoint) by laser } \\
\text { measurement }\end{array}$ & 10 & $-1 /+3$ & in. & $\begin{array}{c}9.9 \\
\text { (bubbler) } \\
27.6 \\
\text { (laser) } \\
\end{array}$ & Data Source: TI (p.63) \\
\hline & UFP-VSL-T02A Operation & - & - & - & - & - \\
\hline & $\begin{array}{l}\text { Level to reduce upper air } \\
\text { sparger flow rate to idle }\end{array}$ & 52 & $-20 /+0$ & in. & 43 & Data Source: HMI \\
\hline & $\begin{array}{l}\text { Upper air sparger flow rate } \\
\text { (idle) }\end{array}$ & 0.01 & $\begin{array}{l}-0.005 / \\
+0.005\end{array}$ & $\mathrm{~kg} / \mathrm{min}$ & 0.01 & $\begin{array}{l}\text { Data Source: Local flow } \\
\text { meter } \sim 15.6 \mathrm{scfh} \text { (record } \\
\text { field transmitter value) }\end{array}$ \\
\hline & $\begin{array}{l}\text { Level to stop PJM } \\
\text { operation (measured when } \\
\text { PJMs are full) }\end{array}$ & 18 & $-0 /+10$ & in. & 27.4 & Data Source: HMI \\
\hline & $\begin{array}{c}\text { Ultrafiltration Loop } \\
\text { Operation }\end{array}$ & - & - & - & - & - \\
\hline & Number of filter bundles & 1 & $-0 /+0$ & - & 1 & Data Source: TI \\
\hline & Filter-loop flow rate & 109 & $-10 /+10$ & gpm & 109 & Data Source: HMI \\
\hline
\end{tabular}




\begin{tabular}{|c|c|c|c|c|c|c|}
\hline Step & Operation & $\begin{array}{l}\text { Target or } \\
\text { Setpoint } \\
\text { Value }\end{array}$ & $\begin{array}{c}\text { Range } \\
(-/+)\end{array}$ & Units & $\begin{array}{l}\text { Actual } \\
\text { Value }\end{array}$ & Comments \\
\hline & Transmembrane pressure & 40 & $-4 /+4$ & psi & 40 & $\begin{array}{l}\text { Data Source: HMI } \\
\text { Lower range not applicable } \\
\text { if back pressure valve is } \\
100 \% \text { open. }\end{array}$ \\
\hline & Temperature & 25 & $-2 /+2$ & ${ }^{\circ} \mathrm{C}$ & 25 & Data Source: HMI \\
\hline & $\begin{array}{l}\text { Axial } \Delta \mathrm{P} \text { for one filter } \\
\text { bundle }\end{array}$ & $<25$ & - & psi & 15.4 & Data Source: HMI \\
\hline \multirow[t]{8}{*}{$\begin{array}{l}\text { Flush filter-loop } \\
\text { (Test Instruction } \\
\text { step 7.2.10) }\end{array}$} & $\begin{array}{l}\text { Mass of water for } \\
\text { filter-loop flush to } \\
\text { UFP-VSL-T02A } \\
\text { (endpoint) }\end{array}$ & 163 & $-5 /+5$ & $\mathrm{~kg}$ & 163 & $\begin{array}{l}\text { Data Source: HMI } \\
\sim 43 \text { gal. } \\
\text { Bypass filters } 2 \text { through } 5 \text {. } \\
\text { Level in UPF-VSL-T02A } \\
\text { should rise } \sim 10 \mathrm{in.}\end{array}$ \\
\hline & Flush water addition rate & $\begin{array}{l}\operatorname{maximum} \\
\text { achievable } \\
\text { flow }\end{array}$ & - & - & $25-\mathrm{kg} / \mathrm{min}$ & $\begin{array}{l}\text { Data Source: HMI } \\
\text { The pump capacity is } \\
\sim 35 \text { gpm or } \sim 132-\mathrm{kg} / \mathrm{min} \text {. } \\
\text { Higher levels in } \\
\text { SHR-VSL-T03 improve } \\
\text { pump suction head and } \\
\text { increase flow rate. }\end{array}$ \\
\hline & $\begin{array}{l}\text { Mass of water for } \\
\text { filter-loop flush to } \\
\text { UFP-VSL-T62A } \\
\text { (endpoint) }\end{array}$ & 326 & $-5 /+5$ & $\mathrm{~kg}$ & 326 & $\begin{array}{l}\text { Data Source: HMI } \\
\sim 86 \text { gal. } \\
\text { UFP-VSL-T02A should be } \\
\text { isolated. }\end{array}$ \\
\hline & UFP-VSL-T02A Operation & - & - & - & - & - \\
\hline & $\begin{array}{l}\text { Level to initiate PJM } \\
\text { operation }\end{array}$ & 13 & $-0 /+10$ & in. & 34 & Data Source: HMI \\
\hline & PJM jet velocity & 7.3 & $\begin{array}{l}-0.4 / \\
+0.4\end{array}$ & $\mathrm{~m} / \mathrm{s}$ & $\mathrm{n} / \mathrm{r}$ & \\
\hline & PJM stroke & $80 \%$ & $-5 /+5$ & $\%$ & 80 & Data Source: HMI \\
\hline & PJM cycle time & 33 & $-1 /+1$ & $\mathrm{~s}$ & 3 & Data Source: HMI \\
\hline \multirow{5}{*}{ 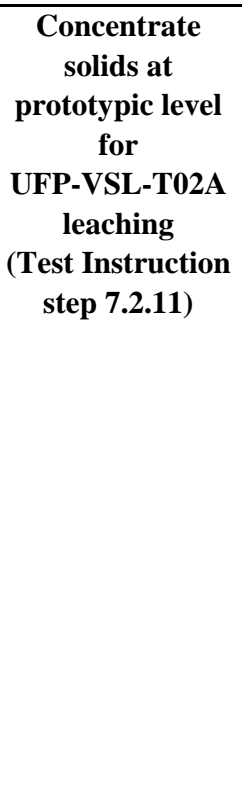 } & $\begin{array}{l}\text { Make-up batch transfer } \\
\text { volume }\end{array}$ & 11 & $\begin{array}{l}-0.2 / \\
+0.2 \\
\end{array}$ & gal & 11 & Data Source: HMI \\
\hline & Make-up batch flow rate & 6.9 & NA & gpm & 6.0 & Data Source: HMI \\
\hline & $\begin{array}{l}\text { Number of make-up } \\
\text { batches (endpoint) }\end{array}$ & Calculate & $-0 /+0$ & - & 18 & $\begin{array}{l}\text { Data Source: TI } \\
\text { Target volume to transfer = } \\
269 \text { gal - make-up volume at } \\
\text { start of filter conditioning. } \\
\text { Number of make-up batches } \\
=(\text { Target volume to transfer, } \\
\text { gal }) /(11 \text { gal }) .\end{array}$ \\
\hline & UFP-VSL-T02A Operation & - & - & - & - & - \\
\hline & $\begin{array}{l}\text { Level to initiate each } \\
\text { make-up batch }\end{array}$ & 18 & $-1 /+1$ & in. & 18 & $\begin{array}{l}\text { Data Source: HMI } \\
\text { Objective is to get loop } \\
\text { volume }(\sim 46 \text { gal })+\text { UFP- } \\
\text { VSL-T02A volume } \\
(\sim 78 \text { gal })=124 \text { gal of } \\
20-w t \% \text { slurry. Final level } \\
\text { in UFP-VSL-T02A should } \\
\text { be } 18 \text { in. after last make-up } \\
\text { batch. }\end{array}$ \\
\hline
\end{tabular}




\begin{tabular}{|c|c|c|c|c|c|c|}
\hline Step & Operation & $\begin{array}{l}\text { Target or } \\
\text { Setpoint } \\
\text { Value }\end{array}$ & $\begin{array}{c}\text { Range } \\
(-/+)\end{array}$ & Units & $\begin{array}{l}\text { Actual } \\
\text { Value }\end{array}$ & Comments \\
\hline & $\begin{array}{l}\text { Total lower air sparger } \\
\text { flow rate with recirculation } \\
\text { (idle, for } 5 \text { sparge tubes) }\end{array}$ & 0.037 & $\begin{array}{l}-0.005 / \\
+0.005\end{array}$ & $\mathrm{~kg} / \mathrm{min}$ & 0.04 & $\begin{array}{l}\text { Data Source: Local flow } \\
\text { meter } \\
\sim 12.6 \text { scfh for each sparge } \\
\text { tube (record field transmitter } \\
\text { value) }\end{array}$ \\
\hline & $\begin{array}{l}\text { Ultrafiltration Loop } \\
\text { Operation }\end{array}$ & - & - & - & - & - \\
\hline & Number of filter bundles & 4 & $-0 /+0$ & & 4 & Data Source: TI \\
\hline & Filter-loop flow rate & 109 & $-10 /+10$ & gpm & 109 & Data Source: HMI \\
\hline & Transmembrane pressure & 40 & $-4 /+4$ & psi & $41-43$ & $\begin{array}{l}\text { Data Source: HMI } \\
\text { Lower range not applicable } \\
\text { if back pressure valve is } \\
100 \% \text { open. }\end{array}$ \\
\hline & Temperature & 25 & $-2 /+2$ & ${ }^{\circ} \mathrm{C}$ & 25 & Data Source: HMI \\
\hline & $\begin{array}{l}\text { Axial } \Delta \mathrm{P} \text { for one filter } \\
\text { bundle }\end{array}$ & $<25$ & - & psi & 15 & Data Source: HMI \\
\hline \multirow{8}{*}{$\begin{array}{c}\text { Add caustic to } \\
\text { UFP-VSL-T02A } \\
\text { (Test Instruction } \\
\text { step 7.3.1) }\end{array}$} & $\begin{array}{l}\text { 19-M caustic to add } \\
\text { (endpoint) }\end{array}$ & 565 & $-5 /+5$ & $\mathrm{~kg}$ & 565 & Data Source: HMI $\sim 99$ gal \\
\hline & Caustic addition rate & 10.3 & $\begin{array}{l}-0.5 / \\
+0.5\end{array}$ & $\mathrm{~kg} / \mathrm{min}$ & 9.8 & $\begin{array}{l}\text { Data Source: HMI } \\
\sim 1.8 \text { gpm, over a } 49 \text {-min } \\
\text { transfer. Range applies to } \\
\text { average transfer rate. }\end{array}$ \\
\hline & $\begin{array}{l}\text { Tank level after caustic } \\
\text { addition }\end{array}$ & 40 & $-2 /+2$ & in. & 44 & $\begin{array}{l}\text { Data Source: HMI } \sim 177 \text { gal } \\
\text { in UFP-VSL-T02A }+ \\
\sim 46 \text { gal in filter-loop. }\end{array}$ \\
\hline & $\begin{array}{l}\text { Volume of antifoam to add } \\
\text { to UFP-VSL-T02A }\end{array}$ & 280 & $-10 /+10$ & $\mathrm{~mL}$ & 280 & $\begin{array}{l}\text { Data Source: TI } \\
\text { Antifoam is added to } \\
\text { account for caustic and } \\
\text { steam addition. This value } \\
\text { is concentrate. Dilute with } 3 \\
\text { parts IW to } 1 \text { part } \\
\text { concentrate. }\end{array}$ \\
\hline & $\begin{array}{c}\text { Ultrafiltration Loop } \\
\text { Operation } \\
\end{array}$ & - & - & - & - & - \\
\hline & Filter-loop flow rate & 109 & $-10 /+10$ & gpm & $\mathrm{n} / \mathrm{r}$ & \\
\hline & Permeate valves & CLOSED & - & - & Closed & Data Source: TI \\
\hline & Temperature & $\leq 71$ & $-2 /+2$ & ${ }^{\circ} \mathrm{C}$ & 71 & Data Source: HMI \\
\hline \multirow{6}{*}{$\begin{array}{l}\text { Initial heat-up of } \\
\text { UFP-VSL-T02A } \\
\text { (Test Instruction } \\
\text { step 7.3.1.9) }\end{array}$} & Target temperature & 71 & $-2 /+2$ & ${ }^{\circ} \mathrm{C}$ & 71 & Data Source: HMI \\
\hline & UFP-VSL-T02A Operation & - & - & - & - & - \\
\hline & $\begin{array}{l}\text { Temperature to turn off } \\
\text { PJM suction refill }\end{array}$ & 60 & $-2 /+2$ & ${ }^{\circ} \mathrm{C}$ & 60 & Data Source: HMI \\
\hline & $\begin{array}{c}\text { Ultrafiltration Loop } \\
\text { Operation } \\
\end{array}$ & - & - & - & - & - \\
\hline & Filter-loop flow rate & 109 & $-10 /+10$ & gpm & 105 & Data Source: HMI \\
\hline & Permeate valves & CLOSED & - & - & Closed & Data Source: TI \\
\hline Flush filter-loop & Filter-loop recirculation & OFF & - & - & Off & Data Source: TI \\
\hline
\end{tabular}




\begin{tabular}{|c|c|c|c|c|c|c|}
\hline Step & Operation & $\begin{array}{l}\text { Target or } \\
\text { Setpoint } \\
\text { Value }\end{array}$ & $\begin{array}{c}\text { Range } \\
(-/+)\end{array}$ & Units & $\begin{array}{l}\text { Actual } \\
\text { Value }\end{array}$ & Comments \\
\hline \multirow{8}{*}{$\begin{array}{l}\text { (Test Instruction } \\
\text { step 7.3.1.9) }\end{array}$} & rate & & & & & \\
\hline & $\begin{array}{l}\text { Mass of water for } \\
\text { filter-loop flush to } \\
\text { UFP-VSL-T02A } \\
\text { (endpoint) }\end{array}$ & 174 & $-5 /+5$ & $\mathrm{~kg}$ & 174 & $\begin{array}{l}\text { Data Source: HMI. } \\
\text { Assumes UFP-HX-T03A } \\
\text { has not been aligned. If } \\
\text { UFP-HX-T03A has been } \\
\text { used to assist heat-up, water } \\
\text { flush should be 201-kg. }\end{array}$ \\
\hline & Flush water addition rate & $\begin{array}{l}\text { maximum } \\
\text { achievable } \\
\text { flow }\end{array}$ & - & $\mathrm{kg} / \mathrm{min}$ & 83 & $\begin{array}{l}\text { Data Source: HMI. The } \\
\text { pump capacity is } \sim 35 \text { gpm } \\
\text { or } \sim 132-\mathrm{kg} / \mathrm{min} . \text { Higher } \\
\text { levels in SHR-VSL-T03 } \\
\text { improve pump suction head } \\
\text { and increase flow rate. }\end{array}$ \\
\hline & $\begin{array}{l}\text { Mass of water for } \\
\text { filter-loop flush to } \\
\text { UFP-VSL-T62A } \\
\text { (endpoint) }\end{array}$ & 348 & $-10 /+10$ & $\mathrm{~kg}$ & 348 & $\begin{array}{l}\text { Data Source: HMI. } \\
\text { Assumes UFP-HX-T03A } \\
\text { has not been aligned. If } \\
\text { UFP-HX-T03A has been } \\
\text { used to assist heat-up, water } \\
\text { flush should be 401-kg. }\end{array}$ \\
\hline & Tank level after loop flush & 50 & $-2 /+2$ & in. & 56 & $\begin{array}{l}\text { Data Source: HMI 223 gal } \\
\text { in UFP-VSL-T02A }\end{array}$ \\
\hline & UFP-VSL-T02A Operation & - & - & - & - & - \\
\hline & Upper air sparger flow rate & 0.10 & $\begin{array}{l}-0.02 / \\
+0.02\end{array}$ & $\mathrm{~kg} / \mathrm{min}$ & 0.1 & $\begin{array}{l}\text { Data Source: Local flow } \\
\text { meter } \\
\sim 3.0 \mathrm{scfm}\end{array}$ \\
\hline & $\begin{array}{l}\text { Total lower air sparger } \\
\text { flow rate (for } 5 \text { sparge } \\
\text { tubes) }\end{array}$ & 0.40 & $\begin{array}{l}-0.05 / \\
+0.05\end{array}$ & $\mathrm{~kg} / \mathrm{min}$ & 0.4 & $\begin{array}{l}\text { Data Source: Local flow } \\
\text { meter } \\
\sim 2.4 \text { scfm for each sparge } \\
\text { tube }\end{array}$ \\
\hline \multirow{7}{*}{$\begin{array}{l}\text { Final heat-up of } \\
\text { UFP-VSL-T02A } \\
\text { (Test Instruction } \\
\text { step 7.3.1.13) }\end{array}$} & $\begin{array}{l}\text { Target final temperature } \\
\text { (endpoint) }\end{array}$ & 98 & $-2 /+2$ & ${ }^{\circ} \mathrm{C}$ & 99 & Data Source: HMI \\
\hline & Heat-up time & 2.4 & $\begin{array}{l}-0.5 / \\
+0.5\end{array}$ & $\mathrm{hr}$ & 2.7 & Data Source: TI \\
\hline & UFP-VSL-T02A Operation & - & - & - & - & - \\
\hline & High-T for air spargers & 90 & $-2 /+2$ & ${ }^{\circ} \mathrm{C}$ & 90 & Data Source: HMI \\
\hline & $\begin{array}{l}\text { Upper air sparger flow rate } \\
\text { (above high } \mathrm{T} \text { ) }\end{array}$ & 0.02 & $\begin{array}{l}-0.005 / \\
+0.005\end{array}$ & $\mathrm{~kg} / \mathrm{min}$ & 0.02 & $\begin{array}{l}\text { Data Source: Local flow } \\
\text { meter } \\
\sim 0.5 \mathrm{scfm}\end{array}$ \\
\hline & $\begin{array}{l}\text { Total lower air sparger } \\
\text { flow rate } \\
\text { (above high } \mathrm{T} \text {, for } 5 \text { sparge } \\
\text { tubes) }\end{array}$ & 0.08 & $\begin{array}{l}-0.005 / \\
+0.005\end{array}$ & $\mathrm{~kg} / \mathrm{min}$ & 0.08 & $\begin{array}{l}\text { Data Source: Local flow } \\
\text { meter } \\
\sim 0.5 \text { scfm for each sparge } \\
\text { tube }\end{array}$ \\
\hline & Level at end of heat-up & 56 & $-5 /+7$ & in & 63 & $\begin{array}{l}\text { Data Source: HMI } \\
\text { Accumulate } \sim 27 \text { gal of } \\
\text { condensate during heat-up; } \\
\text { total } 250 \text { gal in } \\
\text { UFP-VSL-T02A. }\end{array}$ \\
\hline \multirow{2}{*}{$\begin{array}{l}\text { Caustic leach in } \\
\text { UFP-VSL-T02A } \\
\text { (Test Instruction } \\
\text { step 7.3.1.15) }\end{array}$} & $\begin{array}{l}\text { Caustic leach duration } \\
\text { (endpoint) }\end{array}$ & 16 & $-0.1 /+1$ & $\mathrm{hr}$ & 16 & $\begin{array}{l}\text { Data Source: TI } \\
\text { May be extended to } \\
\text { accommodate sampling. }\end{array}$ \\
\hline & Temperature & 98 & $-2 /+2$ & ${ }^{\circ} \mathrm{C}$ & 98 & Data Source: HMI \\
\hline
\end{tabular}




\begin{tabular}{|c|c|c|c|c|c|c|}
\hline Step & Operation & $\begin{array}{c}\text { Target or } \\
\text { Setpoint } \\
\text { Value }\end{array}$ & $\begin{array}{c}\text { Range } \\
(-/+)\end{array}$ & Units & $\begin{array}{l}\text { Actual } \\
\text { Value }\end{array}$ & Comments \\
\hline & UFP-VSL-T02A Operation & - & - & - & - & - \\
\hline & PJM jet velocity & 7.3 & $\begin{array}{l}-0.4 / \\
+0.4\end{array}$ & $\mathrm{~m} / \mathrm{s}$ & 7.1 & Data Source: CCP \\
\hline & PJM stroke & $80 \%$ & $-5 /+5$ & $\%$ & 70 & Data Source: CCP \\
\hline & PJM cycle time & 33 & $-1 /+1$ & $\mathrm{~s}$ & 33.1 & Data Source: CCP \\
\hline & $\begin{array}{l}\text { Steam ring air purge flow } \\
\text { rate (automatic) }\end{array}$ & 0.10 & $\begin{array}{l}-0.02 / \\
+0.02\end{array}$ & $\mathrm{~kg} / \mathrm{min}$ & 0.1 & $\begin{array}{l}\text { Data Source: Local flow } \\
\text { meter } \\
\sim 3.1 \text { scfm, purge will turn } \\
\text { off when steam exceeds } \\
6 \mathrm{cfm} \text { and will turn back on } \\
\text { when steam flow drops } \\
\text { below } 4 \mathrm{cfm}\end{array}$ \\
\hline & $\begin{array}{l}\text { Level at end of caustic } \\
\text { leach }\end{array}$ & 62.5 & $-5 /+9$ & in. & 6.9 & $\begin{array}{l}\text { Data Source: HMI } \\
\text { Accumulate } \sim 33 \text { gal of } \\
\text { condensate during leach; } \\
\text { total } \sim 283 \text { gal in } \\
\text { UFP-VSL-T02A }\end{array}$ \\
\hline \multirow{9}{*}{$\begin{array}{l}\text { Cool-down of } \\
\text { UFP-VSL-T02A } \\
\text { (Test Instruction } \\
\text { step 7.3.2) }\end{array}$} & Initial temperature setpoint & 60 & $-2 /+2$ & ${ }^{\circ} \mathrm{C}$ & 60 & Data Source: HMI \\
\hline & $\begin{array}{l}\text { Time to reach initial } \\
\text { temperature setpoint (from } \\
\text { start of cooling) }\end{array}$ & 4.3 & $\begin{array}{l}-0.5 / \\
+0.5\end{array}$ & $\mathrm{hr}$ & 3.5 & Data Source: TI \\
\hline & $\begin{array}{l}\text { Second temperature } \\
\text { setpoint }\end{array}$ & 25 & $-2 /+2$ & ${ }^{\circ} \mathrm{C}$ & 25 & Data Source: HMI \\
\hline & $\begin{array}{l}\text { Time to reach second } \\
\text { temperature setpoint (from } \\
\text { start of cooling) }\end{array}$ & 16.5 & $-1 /+1$ & $\mathrm{hr}$ & 15.75 & $\begin{array}{l}\text { Data Source: TI } \\
\sim 12 \mathrm{hr} \text { required to cool from } \\
60^{\circ} \mathrm{C} \text { to } 25^{\circ} \mathrm{C}\end{array}$ \\
\hline & UFP-VSL-T02A Operation & - & - & - & - & - \\
\hline & $\begin{array}{l}\text { Temperature to resume } \\
\text { PJM suction refill }\end{array}$ & 60 & $-2 /+2$ & ${ }^{\circ} \mathrm{C}$ & 60 & Data Source: HMI \\
\hline & High-T for air spargers & 90 & $-2 /+2$ & ${ }^{\circ} \mathrm{C}$ & 90 & Data Source: HMI \\
\hline & $\begin{array}{l}\text { Upper air sparger flow rate } \\
\text { (below high T) }\end{array}$ & 0.10 & $\begin{array}{l}-0.02 / \\
+0.02\end{array}$ & $\mathrm{~kg} / \mathrm{min}$ & 0.1 & $\begin{array}{l}\text { Data Source: Local flow } \\
\text { meter } \\
\sim 3.0 \mathrm{scfm}\end{array}$ \\
\hline & $\begin{array}{l}\text { Total lower air sparger } \\
\text { flowrate } \\
\text { (below high } \mathrm{T} \text {, for } 5 \text { sparge } \\
\text { tubes) }\end{array}$ & 0.40 & $\begin{array}{l}-0.05 / \\
+0.05\end{array}$ & $\mathrm{~kg} / \mathrm{min}$ & 0.4 & $\begin{array}{l}\text { Data Source: Local flow } \\
\text { meter } \\
\sim 2.4 \text { scfm for each sparge } \\
\text { tube }\end{array}$ \\
\hline \multirow{4}{*}{$\begin{array}{c}\text { Transfer from } \\
\text { HLP-VSL-T22 } \\
\text { to } \\
\text { UFP-VSL-T01A } \\
\text { (Test Instruction } \\
\text { step 7.4.1) }\end{array}$} & Vessel volume (endpoint) & 45 & $-1 /+1$ & in. & 45.1 & $\begin{array}{l}\text { Data Source: HMI } \\
\text { UFP-VSL-T01A should } \\
\text { have } \sim 391 \text { gal, including the } \\
\text { heel. }\end{array}$ \\
\hline & Transfer rate & 30 & $-2 /+2$ & gpm & NP & $\begin{array}{l}\text { (Simulant transfer did not } \\
\text { occur under Test } \\
\text { Instruction) } \\
\sim 34 \text {-gal transfer } \\
\end{array}$ \\
\hline & UFP-VSL-T01A Operation & - & - & - & - & - \\
\hline & $\begin{array}{l}\text { Volume of antifoam to add } \\
\text { to UFP-VSL-T01A }\end{array}$ & 990 & $-5 /+5$ & $\mathrm{~mL}$ & 990 & $\begin{array}{l}\text { Data Source: TI. Antifoam } \\
\text { is added to account for } \\
\text { simulant, caustic, and steam } \\
\text { addition. This value is } \\
\text { concentrate. Dilute with } 3\end{array}$ \\
\hline
\end{tabular}




\begin{tabular}{|c|c|c|c|c|c|c|}
\hline Step & Operation & $\begin{array}{c}\text { Target or } \\
\text { Setpoint } \\
\text { Value }\end{array}$ & $\begin{array}{c}\text { Range } \\
(-/+)\end{array}$ & Units & $\begin{array}{l}\text { Actual } \\
\text { Value }\end{array}$ & Comments \\
\hline & & & & & & $\begin{array}{l}\text { parts IW to } 1 \text { part } \\
\text { concentrate. }\end{array}$ \\
\hline \multirow{2}{*}{$\begin{array}{l}\text { Add caustic to } \\
\text { UFP-VSL-T01A } \\
\text { (Test Instruction } \\
\text { step 7.4.1.9) }\end{array}$} & $\begin{array}{l}\text { Volume of 19-M caustic to } \\
\text { add (endpoint) }\end{array}$ & 571 & $-5 /+5$ & $\mathrm{~kg}$ & 571 & Data Source: HMI 100 gal \\
\hline & $\begin{array}{l}\text { Tank level after caustic } \\
\text { addition }\end{array}$ & 55.3 & $-1 /+1$ & in. & 55.6 & $\begin{array}{l}\text { Data Source: HMI. } \\
\text { UFP-VSL-T01A should } \\
\text { have 491 gal, including the } \\
\text { heel. }\end{array}$ \\
\hline \multirow{4}{*}{$\begin{array}{l}\text { Initial heat-up of } \\
\text { UFP-VSL-T01A } \\
\text { (Non-prototypic } \\
\text { using external } \\
\text { exchanger) } \\
\text { (Test Instruction } \\
\text { step 7.4.2.1) }\end{array}$} & $\begin{array}{l}\text { Target final temperature } \\
\text { (endpoint) }\end{array}$ & 58 & $-2 /+2$ & ${ }^{\circ} \mathrm{C}$ & 58.2 & Data Source: HMI \\
\hline & Heat-up time & $<3$ & NA & $\mathrm{hr}$ & 0.9 & $\begin{array}{l}\text { Data Source: TI. Heating } \\
\text { loop is } ~ 9 \text { gal and will be } \\
\text { lost (nonprototypic). Target } \\
\text { and range is not important. } \\
\text { Should be complete in under } \\
3 \mathrm{hr} \text {. }\end{array}$ \\
\hline & UFP-VSL-T01A Operation & - & - & - & - & - \\
\hline & $\begin{array}{l}\text { Temperature to turn off } \\
\text { PJM suction refill }\end{array}$ & 60 & $-2 /+2$ & ${ }^{\circ} \mathrm{C}$ & 60 & Data Source: HMI \\
\hline \multirow{4}{*}{$\begin{array}{c}\text { Final heat-up } \\
\text { UFP-VSL-T01A } \\
\text { (Test Instruction } \\
\text { steps 7.4.2.3 to } \\
\text { 7.4.2.7) }\end{array}$} & $\begin{array}{l}\text { Target final temperature } \\
\text { (endpoint) }\end{array}$ & 98 & $-2 /+2$ & ${ }^{\circ} \mathrm{C}$ & 98 & Data Source: HMI \\
\hline & Heat-up time & 3.0 & $\begin{array}{l}-0.5 / \\
+0.5\end{array}$ & $\mathrm{hr}$ & 3 & Data Source: TI \\
\hline & UFP-VSL-T01A Operation & - & - & - & - & - \\
\hline & Level at end of heat-up & 62 & $-2 /+2$ & in. & 60.8 & $\begin{array}{l}\text { Data Source: HMI } \\
\text { Anticipate accumulation of } \\
\sim 65 \text { gal of condensate. }\end{array}$ \\
\hline \multirow{8}{*}{$\begin{array}{l}\text { Caustic leach in } \\
\text { UFP-VSL-T01A } \\
\text { (Test Instruction } \\
\text { step 7.4.2.8) }\end{array}$} & $\begin{array}{l}\text { Caustic-leach duration } \\
\text { (endpoint) }\end{array}$ & 16 & $-0.1 /+1$ & $\mathrm{hr}$ & 16.3 & $\begin{array}{l}\text { Data Source: TI. May be } \\
\text { extended to accommodate } \\
\text { sampling. }\end{array}$ \\
\hline & Temperature & 98 & $-2 /+2$ & ${ }^{\circ} \mathrm{C}$ & 98 & Data Source: HMI \\
\hline & UFP-VSL-T01A Operation & - & - & - & - & - \\
\hline & PJM jet velocity & 4.8 & $\begin{array}{l}-0.3 / \\
+0.3\end{array}$ & $\mathrm{~m} / \mathrm{s}$ & $\mathrm{n} / \mathrm{r}$ & \\
\hline & PJM stroke & $80 \%$ & $-5 /+5$ & $\%$ & $\mathrm{n} / \mathrm{r}$ & \\
\hline & PJM cycle time & 35 & $-1 /+1$ & $\mathrm{~s}$ & $\mathrm{n} / \mathrm{r}$ & \\
\hline & $\begin{array}{l}\text { Steam ring air purge flow } \\
\text { rate (automatic) }\end{array}$ & 0.13 & $\begin{array}{l}-0.02 / \\
+0.02\end{array}$ & $\mathrm{~kg} / \mathrm{min}$ & 0.13 & $\begin{array}{l}\text { Data Source: Local flow } \\
\text { meter } \\
\sim 3.9 \text { scfm, purge will turn } \\
\text { off when steam exceeds } \\
6 \mathrm{cfm} \text { and will turn back on } \\
\text { when steam flow drops } \\
\text { below } 4 \mathrm{cfm}\end{array}$ \\
\hline & Level at end of leach & 66 & $-5 /+5$ & in. & 63 & Data Source: HMI 593 gal \\
\hline \multirow[t]{2}{*}{$\begin{array}{l}\text { Cool-down of } \\
\text { UFP-VSL-T01A } \\
\text { (Test Instruction } \\
\text { step 7.4.2.9) }\end{array}$} & $\begin{array}{l}\text { Initial cool-down } \\
\text { temperature (endpoint) } \\
\text { using external chiller } \\
\text { UFP-HX-T05A }\end{array}$ & 60 & $-2 /+2$ & ${ }^{\circ} \mathrm{C}$ & 59 & Data Source: HMI \\
\hline & Initial cool-down duration & 4 & $-0.5 /$ & $\mathrm{hr}$ & 4.25 & Data Source: TI \\
\hline
\end{tabular}




\begin{tabular}{|c|c|c|c|c|c|c|}
\hline Step & Operation & $\begin{array}{l}\text { Target or } \\
\text { Setpoint } \\
\text { Value }\end{array}$ & $\begin{array}{c}\text { Range } \\
(-/+)\end{array}$ & Units & $\begin{array}{l}\text { Actual } \\
\text { Value }\end{array}$ & Comments \\
\hline & & & +0.5 & & & \\
\hline & UFP-VSL-T01A Operation & - & - & - & - & - \\
\hline & $\begin{array}{l}\text { Temperature to resume } \\
\text { PJM suction refill }\end{array}$ & 60 & $-2 /+2$ & ${ }^{\circ} \mathrm{C}$ & 60 & Data Source: HMI \\
\hline \multirow{14}{*}{$\begin{array}{c}\text { Concentrate } \\
\text { leached solids } \\
\text { (Test Instruction } \\
\text { step 7.5.2) }\end{array}$} & $\begin{array}{l}\text { Make-up batch transfer } \\
\text { volume }\end{array}$ & 11 & $\begin{array}{l}-0.2 / \\
+0.2\end{array}$ & gal & 11 & Data Source: HMI \\
\hline & Make-up batch flow rate & 6.9 & NA & gpm & 7.0 & Data Source: HMI \\
\hline & $\begin{array}{l}\text { UFP-VSL-T01A level } \\
\text { (endpoint to stop batch } \\
\text { transfers) }\end{array}$ & 10 & $-2 /+2$ & in. & 18 & $\begin{array}{l}\text { Data Source: HMI 47 } \\
\text { make-up batches }\end{array}$ \\
\hline & $\begin{array}{l}\text { UFP-VSL-T02A level to } \\
\text { initiate each make-up } \\
\text { batch }\end{array}$ & 37 & $-1 /+1$ & in. & 37 & $\begin{array}{l}\text { Data Source: HMI } \sim 163 \text { gal } \\
\text { in UFP-VSL-T02A }+43 \text { gal } \\
\text { in filter-loop. }\end{array}$ \\
\hline & UFP-VSL-T02A Operation & - & - & - & - & - \\
\hline & $\begin{array}{l}\text { Level to reduce air sparger } \\
\text { flow rates to idle }\end{array}$ & 52 & $-20 /+0$ & in & 47 & Data Source: HMI \\
\hline & $\begin{array}{l}\text { Upper air sparger flow rate } \\
\text { (idle) }\end{array}$ & 0.01 & $\begin{array}{l}-0.005 / \\
+0.005\end{array}$ & $\mathrm{~kg} / \mathrm{min}$ & 0.01 & $\begin{array}{l}\text { Data Source: Local flow } \\
\text { meter } \\
\sim 16 \mathrm{scfh} \text { (record field } \\
\text { transmitter value). }\end{array}$ \\
\hline & $\begin{array}{l}\text { Total lower air sparger } \\
\text { flow rate with recirculation } \\
\text { (idle) }\end{array}$ & 0.037 & & $\mathrm{~kg} / \mathrm{min}$ & 0.04 & $\begin{array}{l}\text { Data Source: Local flow } \\
\text { meter } \\
\sim 12.6 \text { scfh for each sparge } \\
\text { tube }\end{array}$ \\
\hline & $\begin{array}{l}\text { Ultrafiltration Loop } \\
\text { Operation }\end{array}$ & - & - & - & - & - \\
\hline & $\begin{array}{l}\text { Number of filter bundles } \\
\text { (Filters } 2 \text { through } 5 \text { should } \\
\text { be aligned) }\end{array}$ & 4 & $-0 /+0$ & & 4 & Data Source: TI \\
\hline & Filter-loop flow rate & 109 & $-10 /+10$ & gpm & 109 & Data Source: HMI \\
\hline & Transmembrane pressure & 40 & $-4 /+4$ & psi & 41 & $\begin{array}{l}\text { Data Source: HMI. Lower } \\
\text { range not applicable if back } \\
\text { pressure valve is } 100 \% \\
\text { open. }\end{array}$ \\
\hline & Temperature & 25 & $-2 /+2$ & ${ }^{\circ} \mathrm{C}$ & 24.8 & Data Source: HMI \\
\hline & $\begin{array}{l}\text { Axial } \Delta \mathrm{P} \text { for one filter } \\
\text { bundle }\end{array}$ & $<25$ & - & psi & $14-19$ & Data Source: HMI \\
\hline \multirow{3}{*}{$\begin{array}{c}\text { Tune PJMs for } \\
\text { Non-Newtonian } \\
\text { fluid } \\
\text { (Test Instruction } \\
\text { step 7.5.2.10) }\end{array}$} & PJM jet velocity & 12 & $\begin{array}{l}-0.6 / \\
+0.6\end{array}$ & $\mathrm{~m} / \mathrm{s}$ & 11.6 & Data Source: CCP \\
\hline & PJM stroke & $80 \%$ & $-5 /+5$ & $\%$ & 77 & Data Source: CCP \\
\hline & PJM cycle time & 20 & $-1 /+1$ & $\mathrm{~s}$ & 21.2 & Data Source: CCP \\
\hline \multirow{4}{*}{$\begin{array}{c}\text { Concentrate } \\
\text { solids for tracer } \\
\text { test } \\
\text { (Test } \\
\text { Instructions step } \\
7.5 .2 .12 \text { ) }\end{array}$} & $\begin{array}{l}\text { UFP-VSL-T02A level } \\
\text { (endpoint to stop } \\
\text { concentration) }\end{array}$ & $\begin{array}{c}13 \\
18 \text { per } \\
\text { Bechtel }\end{array}$ & $-1 /+1$ & in. & 18 & $\begin{array}{l}\text { Data Source: HMI. } \\
\text { UFP-VSL-T02A volume } \\
\sim 56 \text { gal }\end{array}$ \\
\hline & $\begin{array}{c}\text { Ultrafiltration Loop } \\
\text { Operation }\end{array}$ & - & - & - & - & - \\
\hline & $\begin{array}{l}\text { Number of filter bundles } \\
\text { (Filters } 2 \text { through } 5 \text { should } \\
\text { be aligned) }\end{array}$ & 4 & $-0 /+0$ & & 4 & Data Source: TI \\
\hline & Filter-loop flow rate & 109 & $-10 /+10$ & gpm & 102 & Data Source: HMI \\
\hline
\end{tabular}




\begin{tabular}{|c|c|c|c|c|c|c|}
\hline Step & Operation & $\begin{array}{l}\text { Target or } \\
\text { Setpoint } \\
\text { Value }\end{array}$ & $\begin{array}{c}\text { Range } \\
(-/+)\end{array}$ & Units & $\begin{array}{l}\text { Actual } \\
\text { Value }\end{array}$ & Comments \\
\hline & Transmembrane pressure & 40 & $-4 /+4$ & psi & 40 & $\begin{array}{l}\text { Data Source: HMI. Lower } \\
\text { range not applicable if back } \\
\text { pressure valve is } 100 \% \\
\text { open. }\end{array}$ \\
\hline & Temperature & 25 & $-2 /+2$ & ${ }^{\circ} \mathrm{C}$ & 26.8 & Data Source: HMI \\
\hline & $\begin{array}{l}\text { Axial } \Delta \mathrm{P} \text { for one filter } \\
\text { bundle }\end{array}$ & $<25$ & - & psi & 20 & Data Source: HMI \\
\hline \multirow[t]{5}{*}{$\begin{array}{l}\text { Inject tracer into } \\
\text { filter-loop } \\
\text { (Test Instruction } \\
\text { step 7.6) }\end{array}$} & $\begin{array}{l}\text { Volume of tracer solution } \\
\text { to inject }\end{array}$ & 500 & $-10 /+10$ & $\mathrm{~mL}$ & NP & $\begin{array}{l}\text { Tracer test deleted per } \\
\text { Bechtel. } \\
\text { Tracer is CsBr, target } \\
100 \text { ppm Cs when well } \\
\text { mixed }\end{array}$ \\
\hline & $\begin{array}{l}\text { Ultrafiltration Loop } \\
\text { Operation }\end{array}$ & - & - & - & - & - \\
\hline & $\begin{array}{l}\text { Number of filter bundles } \\
\text { (Filters } 2 \text { through } 5 \text { should } \\
\text { be aligned) }\end{array}$ & 4 & $-0 /+0$ & & NP & $\begin{array}{l}\text { Tracer test deleted per } \\
\text { Bechtel. }\end{array}$ \\
\hline & Filter-loop flow rate & 109 & $-10 /+10$ & gpm & NP & $\begin{array}{l}\text { Tracer test deleted per } \\
\text { Bechtel. }\end{array}$ \\
\hline & Permeate valves & CLOSED & & - & NP & $\begin{array}{l}\text { Tracer test deleted per } \\
\text { Bechtel. }\end{array}$ \\
\hline \multirow{9}{*}{$\begin{array}{c}\text { Wash post- } \\
\text { caustic-leach } \\
\text { slurry } \\
\text { (Test Instruction } \\
\text { step 7.7) }\end{array}$} & Total wash water & 1,416 & $-20 /+20$ & $\mathrm{~kg}$ & 1414 & $\begin{array}{l}\text { Data Source: HMI } \sim 374 \text { gal } \\
\text { total over } 34 \text { batches. }\end{array}$ \\
\hline & $\begin{array}{l}\text { Wash-water batch flow } \\
\text { rate }\end{array}$ & 16.3 & $\begin{array}{l}-0.1 / \\
+0.1\end{array}$ & $\mathrm{~kg} / \mathrm{min}$ & 19.3 & $\begin{array}{l}\text { Data Source: HMI } \\
\sim 4.3 \mathrm{gpm}\end{array}$ \\
\hline & $\begin{array}{l}\text { Volume of antifoam to add } \\
\text { initially and after every } 3 \text { rd } \\
11 \text {-gal batch to } \\
\text { UFP-VSL-T02A }\end{array}$ & 45 & $-2 /+2$ & $\mathrm{~mL}$ & 45 & $\begin{array}{l}\text { Data Source: TI. Antifoam } \\
(\sim 14-\mathrm{mL} \text { per } 11 \text { gallon wash } \\
\text { batch) is added to account } \\
\text { for wash-water dilution. } \\
\text { This value is concentrate. } \\
\text { Dilute with } 3 \text { parts IW to } 1 \\
\text { part concentrate. }\end{array}$ \\
\hline & $\begin{array}{c}\text { Ultrafiltration Loop } \\
\text { Operation } \\
\end{array}$ & - & - & - & - & 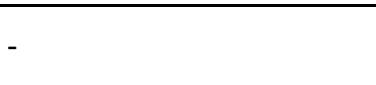 \\
\hline & $\begin{array}{l}\text { Number of filter bundles } \\
\text { (Filters } 2 \text { through } 5 \text { should } \\
\text { be aligned) }\end{array}$ & 4 & $-0 /+0$ & - & 4 & Data Source: TI \\
\hline & Filter-loop flow rate & 109 & $-10 /+10$ & gpm & 113 & Data Source: HMI \\
\hline & Transmembrane pressure & 40 & $-4 /+4$ & psi & 40 & $\begin{array}{l}\text { Data Source: HMI. Lower } \\
\text { range not applicable if back } \\
\text { pressure valve is } 100 \% \\
\text { open. }\end{array}$ \\
\hline & Temperature & 25 & $-2 /+2$ & ${ }^{\circ} \mathrm{C}$ & $\mathrm{n} / \mathrm{r}$ & \\
\hline & $\begin{array}{l}\text { Axial } \Delta \mathrm{P} \text { for one filter } \\
\text { bundle }\end{array}$ & $<25$ & - & psi & $\mathrm{n} / \mathrm{r}$ & Y (init.) \\
\hline \multirow[t]{2}{*}{$\begin{array}{l}\text { Add chromium } \\
\text { slurry } \\
\text { (Test Instruction } \\
\text { step } 7.8 .1 \text { ) }\end{array}$} & $\begin{array}{l}\text { Volume of chromium } \\
\text { solids slurry to add } \\
\text { (endpoint) }\end{array}$ & 53 & $-1 /+1$ & gal & $631 \mathrm{lbs}$ & $\begin{array}{l}\text { Data Source: TI. } \\
\text { Chromium slurry vessel may } \\
\text { be washed out with as much } \\
\text { as } 20 \text { gal of inhibited water. }\end{array}$ \\
\hline & $\begin{array}{l}\text { Chromium slurry addition } \\
\text { flow rate }\end{array}$ & $\begin{array}{c}\text { No } \\
\text { targeted }\end{array}$ & - & - & - & \\
\hline
\end{tabular}




\begin{tabular}{|c|c|c|c|c|c|c|}
\hline Step & Operation & $\begin{array}{l}\text { Target or } \\
\text { Setpoint } \\
\text { Value }\end{array}$ & $\begin{array}{c}\text { Range } \\
(-/+)\end{array}$ & Units & $\begin{array}{l}\text { Actual } \\
\text { Value }\end{array}$ & Comments \\
\hline & & rate & & & & \\
\hline & $\begin{array}{l}\text { Ultrafiltration Loop } \\
\text { Operation }\end{array}$ & - & - & - & - & - \\
\hline & $\begin{array}{l}\text { Number of filter bundles } \\
\text { (Filters } 2 \text { through } 5 \text { should } \\
\text { be aligned) }\end{array}$ & 4 & $-0 /+0$ & - & 4 & Data Source: TI \\
\hline & Filter-loop flow rate & 109 & $-10 /+10$ & gpm & 110 & Data Source: HMI \\
\hline & Permeate valves & CLOSED & - & - & Closed & Data Source: TI \\
\hline & Temperature & 25 & $-2 /+2$ & ${ }^{\circ} \mathrm{C}$ & 26 & Data Source: HMI \\
\hline & $\begin{array}{l}\text { Axial } \Delta \mathrm{P} \text { for one filter } \\
\text { bundle }\end{array}$ & $<25$ & - & psi & 13 & Data Source: HMI \\
\hline \multirow{7}{*}{$\begin{array}{c}\text { Reconcentrate } \\
\text { post-chromium } \\
\text { slurry } \\
\text { (Test Instruction } \\
\text { step 7.8.2.1) }\end{array}$} & $\begin{array}{l}\text { UFP-VSL-T02A level } \\
\text { (endpoint) }\end{array}$ & NA & NA & NA & & $\begin{array}{l}\text { To tank level prior to } \mathrm{Cr} \\
\text { addition. }\end{array}$ \\
\hline & $\begin{array}{c}\text { Ultrafiltration Loop } \\
\text { Operation }\end{array}$ & - & - & - & - & 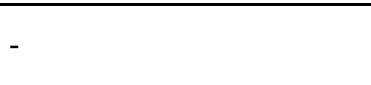 \\
\hline & $\begin{array}{l}\text { Number of filter bundles } \\
\text { (Filters } 2 \text { through } 5 \text { should } \\
\text { be aligned) }\end{array}$ & 4 & $-0 /+0$ & - & 4 & Data Source: TI \\
\hline & Filter-loop flow rate & 109 & $-10 /+10$ & gpm & 110 & Data Source: HMI \\
\hline & Transmembrane pressure & 40 & $-4 /+4$ & psi & 40 & $\begin{array}{l}\text { Data Source: HMI. Lower } \\
\text { range not applicable if back } \\
\text { pressure valve is } 100 \% \\
\text { open. }\end{array}$ \\
\hline & Temperature & 25 & $-2 /+2$ & ${ }^{\circ} \mathrm{C}$ & $\mathrm{n} / \mathrm{r}$ & \\
\hline & $\begin{array}{l}\text { Axial } \Delta \mathrm{P} \text { for one filter } \\
\text { bundle }\end{array}$ & $<25$ & - & psi & $\mathrm{n} / \mathrm{r}$ & \\
\hline \multirow{9}{*}{$\begin{array}{c}\text { Wash post- } \\
\text { chromium slurry } \\
\text { (Test Instruction } \\
\text { step 7.8.2.2) }\end{array}$} & Total wash-water & 833 & $-10 /+10$ & $\mathrm{~kg}$ & 833 & $\begin{array}{l}\text { Data Source: HMI } \sim 220 \text { gal } \\
\text { total over } 20 \text { batches }\end{array}$ \\
\hline & Wash-water batch size & 41.6 & $-1 /+1$ & $\mathrm{~kg}$ & 41.6 & Data Source: HMI \\
\hline & $\begin{array}{l}\text { Wash-water batch flow } \\
\text { rate }\end{array}$ & 16.3 & $\begin{array}{l}-0.1 / \\
+0.1 \\
\end{array}$ & $\mathrm{~kg} / \mathrm{min}$ & 21 & $\begin{array}{l}\text { Data Source: HMI } \\
\sim 4.3 \mathrm{gpm}\end{array}$ \\
\hline & $\begin{array}{c}\text { Ultrafiltration Loop } \\
\text { Operation }\end{array}$ & - & - & - & - & - \\
\hline & $\begin{array}{l}\text { Number of filter bundles } \\
\text { (Filters } 2 \text { through } 5 \text { should } \\
\text { be aligned) }\end{array}$ & 4 & $-0 /+0$ & - & 4 & Data Source: TI \\
\hline & Filter-loop flow rate & 109 & $-10 /+10$ & gpm & $\mathrm{n} / \mathrm{r}$ & Data Source: HMI \\
\hline & Transmembrane pressure & 40 & $-4 /+4$ & psi & 40 & $\begin{array}{l}\text { Data Source: HMI. Lower } \\
\text { range not applicable if back } \\
\text { pressure valve is } 100 \% \\
\text { open. }\end{array}$ \\
\hline & Temperature & 25 & $-2 /+2$ & ${ }^{\circ} \mathrm{C}$ & 25 & Data Source: HMI \\
\hline & $\begin{array}{l}\text { Axial } \Delta \mathrm{P} \text { for one filter } \\
\text { bundle }\end{array}$ & $<25$ & - & psi & 14.3 & Data Source: HMI \\
\hline $\begin{array}{l}\text { Partial transfer } \\
\text { from } \\
\text { UFP-VSL-T02A } \\
\text { to }\end{array}$ & $\begin{array}{l}\text { Target level in } \\
\text { UFP-VSL-T02A } \\
\text { (endpoint) }\end{array}$ & 10 & $-1 /+3$ & in. & NP & $\begin{array}{l}\text { Transfer deleted per Bechtel. } \\
\text { UFP-VSL-T02A volume } \\
\sim 44 \text { gal; loop volume } \\
\sim 46 \text { gal. }\end{array}$ \\
\hline
\end{tabular}




\begin{tabular}{|c|c|c|c|c|c|c|}
\hline Step & Operation & $\begin{array}{c}\text { Target or } \\
\text { Setpoint } \\
\text { Value } \\
\end{array}$ & $\begin{array}{c}\text { Range } \\
(-/+)\end{array}$ & Units & $\begin{array}{l}\text { Actual } \\
\text { Value }\end{array}$ & Comments \\
\hline \multirow{9}{*}{$\begin{array}{l}\text { HLP-VSL-T27 } \\
\text { (Test Instruction } \\
\text { step 7.9.1.1) }\end{array}$} & Transfer rate & $\begin{array}{c}\text { No } \\
\text { setpoint }\end{array}$ & - & - & NP & Transfer deleted per Bechtel. \\
\hline & UFP-VSL-T02A Operation & - & - & - & - & - \\
\hline & $\begin{array}{l}\text { Level to stop PJM } \\
\text { operation }\end{array}$ & 20 & $-5 /+5$ & in. & NP & Transfer deleted per Bechtel. \\
\hline & $\begin{array}{l}\text { Ultrafiltration Loop } \\
\text { Operation }\end{array}$ & - & - & - & - & - \\
\hline & Number of filter bundles & 4 & $-0 /+0$ & - & NP & Transfer deleted per Bechtel. \\
\hline & Permeate valves & CLOSED & - & - & NP & Transfer deleted per Bechtel. \\
\hline & Filter-loop flow rate & 109 & $-10 /+10$ & gpm & NP & Transfer deleted per Bechtel. \\
\hline & Temperature & 25 & $-2 /+2$ & ${ }^{\circ} \mathrm{C}$ & NP & Transfer deleted per Bechtel. \\
\hline & $\begin{array}{l}\text { Axial } \Delta \mathrm{P} \text { for one filter } \\
\text { bundle }\end{array}$ & $<25$ & - & psi & NP & Transfer deleted per Bechtel. \\
\hline \multirow{10}{*}{$\begin{array}{c}\text { Dilute } \\
\text { UFP-VSL-T02A } \\
\text { slurry with } \\
\text { inhibited water } \\
\text { (Test Instruction } \\
\text { step 7.9.1.3 and } \\
7.9 .1 .4 \text { ) }\end{array}$} & $\begin{array}{l}\text { Target UFP-VSL-T02A } \\
\text { level (endpoint) }\end{array}$ & 62.5 & $-5 /+5$ & in. & 64 & $\begin{array}{l}\text { Data Source: HMI. } \\
\text { UFP-VSL-T02A to have } \\
283 \text { gal inventory. }\end{array}$ \\
\hline & $\begin{array}{l}\text { Inhibited water addition } \\
\text { rate }\end{array}$ & $\begin{array}{c}\text { No } \\
\text { setpoint }\end{array}$ & - & - & $\begin{array}{r}25-34 \\
\mathrm{~kg} / \mathrm{min}\end{array}$ & Data Source: HMI \\
\hline & $\begin{array}{l}\text { Volume of antifoam to add } \\
\text { for water dilution }\end{array}$ & 330 & $-5 /+5$ & $\mathrm{~mL}$ & $\begin{array}{l}\text { Attempt } \\
\text { 1: } 280 \\
\text { Attempt } \\
\text { 2: } 0 \\
\text { Attempt } \\
\text { 3: } 400 \\
\end{array}$ & $\begin{array}{l}\text { Data Source: TI } \\
\text { Antifoam is added to } \\
\text { account for water dilution. } \\
\text { This value is concentrate. } \\
\text { Dilute with } 3 \text { parts IW to } 1 \\
\text { part concentrate. }\end{array}$ \\
\hline & UFP-VSL-T02A Operation & - & - & - & - & - \\
\hline & $\begin{array}{l}\text { Level to start PJM } \\
\text { operation }\end{array}$ & 20 & $-5 /+45$ & in & $\begin{array}{l}\text { Attempt } \\
\text { 1: } 45 \\
\text { Attempt } \\
\text { 2: } 20 \\
\text { Attempt } \\
\text { 3: }>20 \\
\end{array}$ & Data Source: HMI \\
\hline & PJM jet velocity & 12 & $\begin{array}{l}-0.6 / \\
+0.6\end{array}$ & $\mathrm{~m} / \mathrm{s}$ & $\mathrm{n} / \mathrm{r}$ & Data Source: HMI \\
\hline & PJM stroke & $80 \%$ & $-5 /+5$ & $\%$ & $\mathrm{n} / \mathrm{r}$ & \\
\hline & PJM cycle time & 20 & $-1 /+1$ & $\mathrm{~s}$ & $\begin{array}{l}\text { Attempt } \\
\text { 1: } 21 \\
\text { Attempt } \\
\text { 2: } \mathrm{n} / \mathrm{r} \\
\text { Attempt } \\
\text { 3: } 21 \\
\end{array}$ & Data Source: HMI \\
\hline & $\begin{array}{l}\text { Level to adjust upper air } \\
\text { sparger flowrate to full }\end{array}$ & 42 & $-0 /+10$ & in & $\begin{array}{l}\text { Attempt } \\
\text { 1: } 52 \\
\text { Attempt } \\
\text { 2: }>52 \\
\text { Attempt } \\
\text { 3: }>52 \\
\end{array}$ & Data Source: HMI \\
\hline & $\begin{array}{l}\text { Upper air sparger flowrate } \\
\text { (full) }\end{array}$ & 0.10 & $\begin{array}{l}-0.02 / \\
+0.02\end{array}$ & $\mathrm{~kg} / \mathrm{min}$ & $\begin{array}{l}\text { Attempt } \\
\text { 1: } 0.10 \\
\text { Attempt } \\
2: \mathrm{n} / \mathrm{r}\end{array}$ & $\begin{array}{l}\text { Data Source: Local flow } \\
\text { meter }\end{array}$ \\
\hline
\end{tabular}




\begin{tabular}{|c|c|c|c|c|c|c|}
\hline Step & Operation & $\begin{array}{c}\text { Target or } \\
\text { Setpoint } \\
\text { Value }\end{array}$ & $\begin{array}{c}\text { Range } \\
(-/+)\end{array}$ & Units & $\begin{array}{l}\text { Actual } \\
\text { Value }\end{array}$ & Comments \\
\hline & & & & & $\begin{array}{l}\text { Attempt } \\
\text { 3: n/r }\end{array}$ & \\
\hline & $\begin{array}{l}\text { Collect sample for parallel } \\
\text { testing }\end{array}$ & - & - & - & $\begin{array}{l}\text { Attempt } \\
\text { 1: yes } \\
\text { Attempt } \\
\text { 2: yes } \\
\text { Attempt } \\
\text { 3: yes } \\
\end{array}$ & Data Source: TI \\
\hline & $\begin{array}{l}\text { Volume of high-solids } \\
\text { filter flux CUF sample }\end{array}$ & 20.7 & $\begin{array}{l}-0.1 / \\
+0.5\end{array}$ & $\mathrm{~L}$ & $\begin{array}{l}\text { Attempt } \\
\text { 1: } 22.9 \\
\text { Attempt } \\
\text { 2: } 20.7 \\
\text { Attempt } \\
\text { 3: } 22.7\end{array}$ & Data Source: TI \\
\hline \multirow[t]{8}{*}{$\begin{array}{l}\text { High-solids filter } \\
\text { flux test } \\
\text { (Test Instruction } \\
\text { step 7.9.2) }\end{array}$} & $\begin{array}{l}\text { UFP-VSL-T02A level } \\
\text { (endpoint) }\end{array}$ & $\begin{array}{c}7 \\
10 \text { (per } \\
\text { Bechtel) }\end{array}$ & $-1 /+3$ & in. & $\begin{array}{l}\text { Attempt } \\
\text { 1: } 15.2 \\
\text { Attempt } \\
\text { 2: } 57.3 \\
\text { Attempt } \\
\text { 3: } 16.7\end{array}$ & $\begin{array}{l}\text { Data Source: HMI. } \\
\text { If low interlock is reached } \\
\text { (auto-shut down) stop } \\
\text { concentration step. }\end{array}$ \\
\hline & UFP-VSL-T02A Operation & - & - & - & - & - \\
\hline & $\begin{array}{l}\text { Level to adjust upper air } \\
\text { sparger flow rate to idle }\end{array}$ & 52 & $-10 /+0$ & in. & $\begin{array}{c}\text { Attempt } \\
\text { 1: } 45 \\
\text { Attempt } \\
\text { 2: n/r } \\
\text { Attempt } \\
\text { 3: }<52\end{array}$ & Data Source: HMI \\
\hline & $\begin{array}{l}\text { Upper air sparger flow rate } \\
\text { (idle) }\end{array}$ & 0.009 & $\begin{array}{l}-0.005 / \\
+0.005\end{array}$ & $\mathrm{~kg} / \mathrm{min}$ & $\begin{array}{c}\text { Attempt } \\
1: 0.009 \\
\text { Attempt } \\
2: 0.009 \\
\text { Attempt } \\
3: \mathrm{n} / \mathrm{r}\end{array}$ & $\begin{array}{l}\text { Data Source: Local flow } \\
\text { meter } \\
\sim 15.6 \text { scfh (record field } \\
\text { transmitter value) }\end{array}$ \\
\hline & $\begin{array}{l}\text { Level to stop PJM } \\
\text { operation }\end{array}$ & 20 & $-5 /+5$ & in. & $\begin{array}{c}\text { Attempt } \\
\text { 1: } 24 \\
\text { Attempt } \\
\text { 2: n/r } \\
\text { Attempt } \\
\text { 3: n/r } \\
\end{array}$ & Data Source: HMI \\
\hline & $\begin{array}{l}\text { Ultrafiltration Loop } \\
\text { Operation }\end{array}$ & - & - & - & - & - \\
\hline & Number of filter bundles & 1 & $-0 /+0$ & - & $\begin{array}{l}\text { Attempt } \\
\text { 1: } 1 \\
\text { Attempt } \\
\text { 2: } 1 \\
\text { Attempt } \\
\text { 3: } 1\end{array}$ & Data Source: TI \\
\hline & Filter-loop flow rate & 109 & $-10 /+10$ & gpm & $\begin{array}{l}\text { Attempt } \\
\text { 1: } 105 \\
\text { Attempt } \\
2: \mathrm{n} / \mathrm{r} \\
\text { Attempt }\end{array}$ & Data Source: HMI \\
\hline
\end{tabular}




\begin{tabular}{|c|c|c|c|c|c|c|}
\hline Step & Operation & $\begin{array}{l}\text { Target or } \\
\text { Setpoint } \\
\text { Value }\end{array}$ & $\begin{array}{c}\text { Range } \\
(-/+)\end{array}$ & Units & $\begin{array}{l}\text { Actual } \\
\text { Value }\end{array}$ & Comments \\
\hline & & & & & $3: \mathrm{n} / \mathrm{r}$ & \\
\hline & Transmembrane pressure & 40 & $-4 /+4$ & psi & $\begin{array}{c}\text { Attempt } \\
\text { 1: } 41 \\
\text { Attempt } \\
2: \mathrm{n} / \mathrm{r} \\
\text { Attempt } \\
3: \mathrm{n} / \mathrm{r}\end{array}$ & $\begin{array}{l}\text { Data Source: HMI. Lower } \\
\text { range not applicable if back } \\
\text { pressure valve is } 100 \% \\
\text { open. }\end{array}$ \\
\hline & Temperature & 25 & $-2 /+2$ & ${ }^{\circ} \mathrm{C}$ & $\begin{array}{c}\text { Attempt } \\
\text { 1: } 25.4 \\
\text { Attempt } \\
\text { 2: n/r } \\
\text { Attempt } \\
\text { 3: n/r }\end{array}$ & Data Source: HMI \\
\hline & $\begin{array}{l}\text { Axial } \Delta \mathrm{P} \text { for one filter } \\
\text { bundle }\end{array}$ & $<25$ & - & psi & $\begin{array}{c}\text { Attempt } \\
\text { 1: } 11 \\
\text { Attempt } \\
\text { 2: n/r } \\
\text { Attempt } \\
\text { 3: } \mathrm{n} / \mathrm{r}\end{array}$ & Data Source: HMI \\
\hline \multirow[t]{11}{*}{$\begin{array}{c}\text { Add sodium } \\
\text { permanganate } \\
\text { (Test Instruction } \\
\text { steps } 7.10 \text { ) }\end{array}$} & Inhibited water addition & 189 & $-5 /+5$ & $\mathrm{~kg}$ & 81.7 & $\begin{array}{l}\text { Data Source: HMI. } 50 \text { gal } \\
\text { of water added to prevent } \\
\text { cavitation of } \\
\text { UFP-PMP-T42A. }\end{array}$ \\
\hline & $\begin{array}{l}\text { Inhibited water addition } \\
\text { rate }\end{array}$ & $\begin{array}{c}\text { No } \\
\text { setpoint }\end{array}$ & - & - & $20-\mathrm{kg} / \mathrm{min}$ & Data Source: HMI \\
\hline & $\begin{array}{l}\text { Volume of sodium } \\
\text { permanganate to add } \\
\text { (endpoint) }\end{array}$ & 24.5 & $-1 /+1$ & $\mathrm{~kg}$ & 24.5 & Data Source: HMI \\
\hline & $\begin{array}{l}\text { Sodium permanganate } \\
\text { addition rate }\end{array}$ & 17 & $-2 /+2$ & $\mathrm{~kg} / \mathrm{min}$ & 15.5 & Data Source: HMI \\
\hline & $\begin{array}{l}\text { Foaming test duration } \\
\text { (endpoint) }\end{array}$ & 6 & $-0.1 /+1$ & $\mathrm{hr}$ & $\begin{array}{c}6 \mathrm{hr} \\
4-\mathrm{min} .\end{array}$ & Data Source: TI \\
\hline & $\begin{array}{l}\text { Ultrafiltration Loop } \\
\text { Operation }\end{array}$ & - & - & - & - & - \\
\hline & $\begin{array}{l}\text { Number of filter bundles } \\
\text { (Filters } 2 \text { through } 5 \text { should } \\
\text { be aligned) }\end{array}$ & 4 & $-0 /+0$ & - & 4 & Data Source: TI \\
\hline & Filter-loop flow rate & 109 & $-10 /+10$ & gpm & 106 & Data Source: HMI \\
\hline & Transmembrane pressure & 40 & $-4 /+4$ & psi & $\begin{array}{c}0 \text { (Not } \\
\text { filtering) }\end{array}$ & $\begin{array}{l}\text { Data Source: HMI } \\
\text { Lower range not applicable } \\
\text { if back pressure valve is } \\
100 \% \text { open. }\end{array}$ \\
\hline & Temperature & 25 & $-2 /+2$ & ${ }^{\circ} \mathrm{C}$ & 25.4 & Data Source: HMI \\
\hline & $\begin{array}{l}\text { Axial } \Delta \mathrm{P} \text { for one filter } \\
\text { bundle }\end{array}$ & $<25$ & - & psi & 13 & Data Source: HMI \\
\hline \multirow{2}{*}{$\begin{array}{c}\text { Filter-loop } \\
\text { backflush } \\
\text { (Test Instruction } \\
\text { step 7.10.1.6) }\end{array}$} & $\begin{array}{l}\text { Volume of flush water } \\
\text { (endpoint) }\end{array}$ & 174 & $-5 /+5$ & $\mathrm{~kg}$ & NP & $\begin{array}{l}\text { Not conducted as part of } \\
\text { testing. } \\
\sim 46 \text { gal flush }\end{array}$ \\
\hline & Flush water addition rate & $\begin{array}{l}\text { Maximum } \\
\text { achievable } \\
\text { flow }\end{array}$ & - & - & NP & $\begin{array}{l}\text { Not conducted as part of } \\
\text { testing. } \\
\text { The pump capacity is }\end{array}$ \\
\hline
\end{tabular}




\begin{tabular}{|c|c|c|c|c|c|c|}
\hline Step & Operation & $\begin{array}{c}\text { Target or } \\
\text { Setpoint } \\
\text { Value }\end{array}$ & $\begin{array}{c}\text { Range } \\
(-/+)\end{array}$ & Units & $\begin{array}{l}\text { Actual } \\
\text { Value }\end{array}$ & Comments \\
\hline & & & & & & 35 gpm or $132-\mathrm{kg} / \mathrm{min}$ \\
\hline & $\begin{array}{l}\text { Ultrafiltration Loop } \\
\text { Operation }\end{array}$ & - & - & - & - & - \\
\hline & Number of filter bundles & 5 & $-0 /+0$ & - & NP & $\begin{array}{l}\text { Not conducted as part of } \\
\text { testing. }\end{array}$ \\
\hline & Filter-loop flow rate & OFF & - & & NP & $\begin{array}{l}\text { Not conducted as part of } \\
\text { testing. }\end{array}$ \\
\hline & Permeate valves & CLOSED & - & - & NP & $\begin{array}{l}\text { Not conducted as part of } \\
\text { testing. }\end{array}$ \\
\hline \multirow[t]{8}{*}{$\begin{array}{c}\text { Transfer from } \\
\text { UFP-VSL-T02A } \\
\text { to } \\
\text { HLP-VSL-T27 } \\
\text { (Test Instruction } \\
\text { step 7.11.3) }\end{array}$} & Transfer rate & $\begin{array}{c}\text { No } \\
\text { setpoint }\end{array}$ & & gpm & NP & $\begin{array}{l}\text { Not conducted as part of } \\
\text { testing. } \\
\text { Transfer with diaphragm } \\
\text { pump. Record average } \\
\text { transfer rate. }\end{array}$ \\
\hline & $\begin{array}{l}\text { Level to stop PJM } \\
\text { operation }\end{array}$ & 15 & & in & NP & $\begin{array}{l}\text { Not conducted as part of } \\
\text { testing. }\end{array}$ \\
\hline & $\begin{array}{l}\text { Level to stop steam ring air } \\
\text { purge }\end{array}$ & 15 & & in & NP & $\begin{array}{l}\text { Not conducted as part of } \\
\text { testing. }\end{array}$ \\
\hline & Level to turn off air sparge & 13 & & in & NP & $\begin{array}{l}\text { Not conducted as part of } \\
\text { testing. }\end{array}$ \\
\hline & $\begin{array}{l}\text { Ultrafiltration Loop } \\
\text { Operation }\end{array}$ & - & - & - & - & 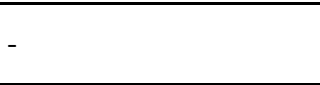 \\
\hline & Number of filter bundles & 5 & - & - & NP & $\begin{array}{l}\text { Not conducted as part of } \\
\text { testing. }\end{array}$ \\
\hline & Filter-loop flow rate & OFF & - & & NP & $\begin{array}{l}\text { Not conducted as part of } \\
\text { testing. }\end{array}$ \\
\hline & Permeate valves & CLOSED & - & - & NP & $\begin{array}{l}\text { Not conducted as part of } \\
\text { testing. }\end{array}$ \\
\hline
\end{tabular}


PNNL-18823

WTP-RPT-190, Rev 0

\section{Distribution}

No. of

Copies

OFFSITE
No. of

Copies

ONSITE

1 Pacific Northwest National Laboratory (authors will be notified electronically)

O.P. Bredt

K6-50

C.A. Burns

P7-25

J.G.H. Geeting

P7-28

C.E. Guzman-Leong

K5-22

G.B. Josephson

K9-69

G.J. Sevigny

P7-27

B.D. Hanson

P7-27

J.L. Huckaby

$\mathrm{H} 4-02$

D.E. Kurath

K3-52

Information Release (pdf)

Project File (1)

K3-52

2 Bechtel National Inc.

WTP R\&T Docs (1)

H4-02

S. Barnes

H4-02 


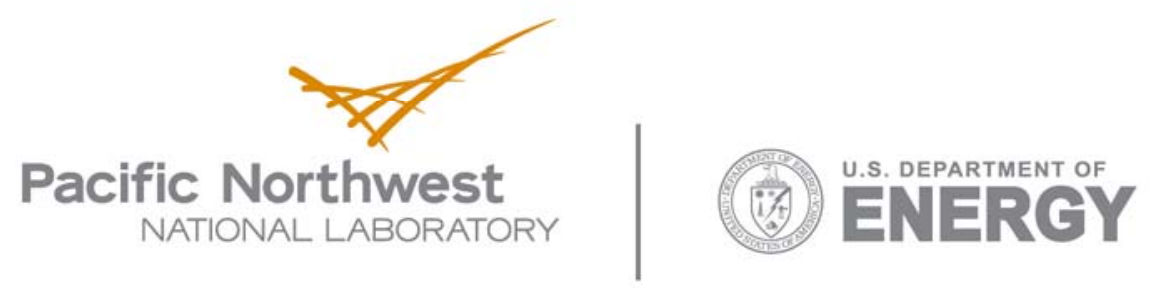

902 Battelle Boulevard

P.O. Box 999

Richland, WA 99352

1-888-375-PNNL (7665)

www.pnl.gov 